Argonne

\title{
Final Report: Site Investigation Results, 2009-2011, at Inman, Kansas
}

\author{
Environmental Science Division
}


About Argonne National Laboratory

Argonne is a U.S. Department of Energy laboratory managed by UChicago Argonne, LLC under contract DE-AC02-06CH11357. The Laboratory's main facility is outside Chicago, at 9700 South Cass Avenue, Argonne, Illinois 60439. For information about Argonne and its pioneering science and technology programs, see www.anl.gov.

\section{Availability of This Report}

This report is available, at no cost, at http://www.osti.gov/bridge. It is also available on paper to the U.S. Department of Energy and its contractors, for a processing fee, from:

U.S. Department of Energy

Office of Scientific and Technical Information

P.O. Box 62

Oak Ridge, TN 37831-0062

phone (865) 576-8401

fax (865) 576-5728

reports@adonis.osti.gov

\section{Disclaimer}

This report was prepared as an account of work sponsored by an agency of the United States Government. Reference herein to any specific commercial product, process, or service by trade name, trademark, manufacturer, or otherwise, does not necessarily constitute or imply its endorsement, recommendation, or favoring by the United States Government or any agency thereof. The views and opinions of document authors expressed herein do not necessarily state or reflect those of the United States Government or any agency thereof, Argonne National Laboratory, or UChicago Argonne, LLC. 


\section{Final Report: Site Investigation Results, 2009-2011, at Inman, Kansas}

by

Applied Geosciences and Environmental Management Section Environmental Science Division, Argonne National Laboratory

May 2015

USDA 


\section{Contents}

Notation.

viii

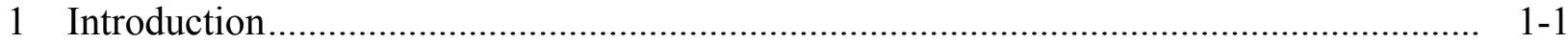

$1.1 \quad$ Background and Previous Investigations ......................................................... 1-1

1.2 Objectives of the Targeted Investigations........................................................ $1-5$

1.3 Field Investigation Activities .......................................................................... 1-5

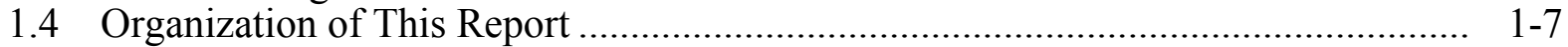

2 Investigative Methods .............................................................................................. 2-1

2.1 Methods to Identify Contaminant Sources and the Extent of Soil

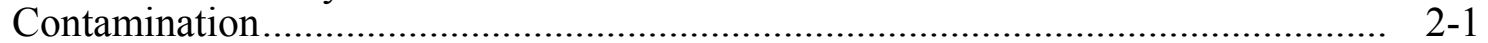

2.2 Methods to Characterize Groundwater Contamination ............................................ 2-2

2.2.1 Methods to Identify the Groundwater-Bearing System ................................ 2-2

2.2.2 Methods to Delineate the Extent of Groundwater Contamination................. 2-3

2.3 Methods to Determine and Monitor Groundwater Flow Patterns ............................ 2-5

2.4 Methods to Evaluate the Properties of the System of Multiple GroundwaterBearing Zones

3 Field and Laboratory Data ………….................................................................. 3-1

3.1 Electronic Cone Penetrometer Sensor Data ....................................................... 3-1

3.2 Geologic Coring Data ................................................................................ $3-1$

3.3 Soil Sampling Data ........................................................................................... 3-2

3.4 Groundwater Sampling Data.............................................................................. 3-2

3.5 Installation of Permanent Monitoring Wells............................................................. 3-3

3.6 Groundwater Level Data............................................................................ 3-3

3.7 Land Survey Data ................................................................................. 3-4

3.8 Results of Quality Control Activities..................................................................... 3-5

3.9 Waste Characterization, Handling, and Disposal .................................................. 3-6

4 Data Interpretation .............................................................................................

4.1 Extent of Soil Contamination and Potential Contaminant Sources ........................... 4-1

4.1.1 Contaminant Distribution in Soils at the Former CCC/USDA Facility.......... 4-2

4.1.2 Contaminant Distribution in Soils at the Former Private Grain Storage Facilities on the Sisson and Willems Properties............................................. 4-2

4.2 Hydrogeologic Setting and Groundwater-Bearing System...................................... 4-3

4.2.1 Local Lithostratigraphic Units ................................................................. 4-3

4.2.2 Groundwater-Bearing Zones.............................................................. 4-5

4.2.3 Spatial Distribution of the Groundwater-Bearing Zones ............................... 4-6

4.3 Groundwater Flow Patterns ............................................................................. 4. 4

4.3.1 Groundwater Levels and Flow Patterns in the Shallow GroundwaterBearing Zone. 
4.3.2 Groundwater Levels and Flow Patterns in the Middle GroundwaterBearing Zone....

4.3.3 Groundwater Levels and Flow Patterns in the Deep Groundwater-

Bearing Zone.

4.4 Characteristics of the Groundwater-Bearing System........................................... 4-15

4.4.1 Shallow Groundwater Zone ................................................................ 4-15

4.4.2 Middle Groundwater Zone.............................................................. 4-20

4.4.3 Deep Groundwater Zone..................................................................... 4-22

4.5 Delineation of Groundwater Contamination..................................................... 4-23

4.5.1 Contaminant Distribution in the Shallow Groundwater-Bearing Zone ......... 4-24

4.5.2 Contaminant Distribution in the Middle Groundwater-Bearing Zone........... 4-26

4.5.3 Contaminant Distribution in the Deep Groundwater-Bearing Zone............... 4-28

4.6 Conceptual Model of Contaminant Migration Pathways......................................... 4-29

5 Conclusions ............................................................................................ $5-1$

5.1 Physical Setting and Characteristics ....................................................... 5-1

5.2 Extent of Contamination and Migration Pathways ............................................. $5-4$

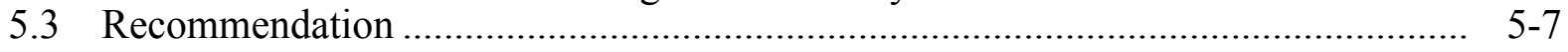

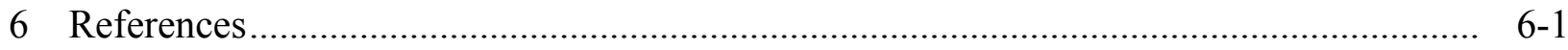

Appendix A: Summary of Investigation Results and Proposed Work Plan, Inman, Kansas, August 9, 2011

Appendix B: Lithology Logs and Electronic Cone Penetrometer Logs ............................ B-1

Appendix C: Soil and Groundwater Sampling Data ............................................. C-1

Appendix D: Well Registration Forms ............................................................... D-1

Appendix E: Hand-Measured Groundwater Level Data ............................................. E-1

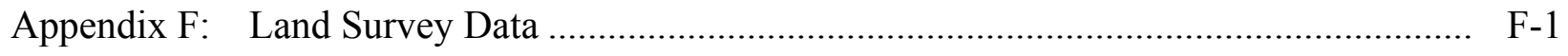

Appendix G: Evaluation of Drawdown and Recovery Data at Monitoring Wells ............... G-1

Supplement 1: Automatically Recorded Groundwater Level Data ..................................... CD

Supplement 2: Quality Control for Sample Collection, Handling, and Analysis ....................on CD

Supplement 3: Data from Pace Analytical Services for Investigation-Derived Waste ...........on CD

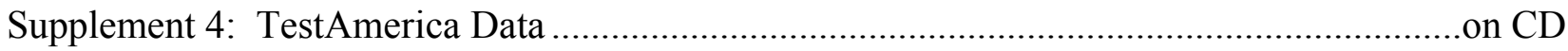




\section{Tables}

2.1 Information on private wells identified or sampled in the 2009-2011 site investigation

3.1 Analytical results from the AGEM Lababoratory for soil samples collected in 2009-2010

3.2 Analytical results for grab groundwater samples collected at temporary piezometers in 2009-2010.

3.3 Analytical results for groundwater samples collected from private wells and permanent monitoring wells in 2010-2011

3.4 Construction data for monitoring wells installed during the targeted investigation

4.1 Summary of hydraulic conductivity values estimated from the water level recovery event on November 9, 2010, as observed in monitoring wells in the shallow groundwater zone beneath the former CCC/USDA facility....

4.2 Hydraulic conductivity values estimated for the shallow groundwater zone beneath the former private grain storage facility on the Sisson property after the pumping event at the Sisson well on October 7, 2011, from observations made at well MW18S

4.3 Hydraulic conductivity values estimated for the middle groundwater zone beneath the former private grain storage facility on the Sisson property after the pumping event at the Sisson well on October 7, 2011, from observations made at well MW18M

5.1 Current contaminant levels in wells in the vicinity of the former CCC/USDA facility and private properties north of Buckskin Road

\section{Figures}

1.1 Locations of Inman, Kansas, former grain storage facilities, the Inman public water supply wells, and private wells

1.2 History of the former CCC/USDA and private grain storage facilities near the south edge of Inman, as interpreted from aerial photographs and property documents

1.3 History of the private grain storage facilities on the Klassen property, as interpreted from aerial photographs. 


\section{Figures (Cont.)}

1.4 Historical results for carbon tetrachloride in groundwater samples collected by the KDHE in 1997-2005 from private wells on the Sisson, Pankratz (now Newcombe), Willems, and Bengston properties

1.5 Field laboratory analytical results for soil samples collected by the KDHE in 1998 at the former CCC/USDA facility

2.1 Locations of soil sampling on the former CCC/USDA property and the Sisson and Willems properties in the targeted investigation.

2.2 Sampling locations and carbon tetrachloride concentrations in grab groundwater samples collected in 2009-2010

2.3 Locations of groundwater sampling from the monitoring wells installed during the targeted investigation

2.4 Locations of the private wells targeted for sampling during the investigation

2.5 Monitoring network for the shallow groundwater-bearing zone

2.6 Monitoring network for the middle groundwater-bearing zone

4.1 Maximum concentrations of carbon tetrachloride and chloroform in soil samples collected at and near the former CCC/USDA facility and the former private grain storage facilities on the Sisson and Willems properties.

4.2 Topographic surface at Inman

4.3 General sequence of local lithostratigraphic units near the former CCC/USDA facility

4.4 Locations of wells and hydrogeologic cross sections

4.5 Interpretive north-to-south hydrogeologic cross section A-A'

4.6 Interpretive west-to-east hydrogeologic cross section B-B'

4.7 Hydrographs in the shallow groundwater zone observed from December 2009 to March 2012 in monitoring wells installed in Phases 1-2 


\section{Figures (Cont.)}

4.8 Hydrographs in the shallow groundwater zone observed in September 2011 to March 2012 in monitoring wells installed in Phases 3-4

4.9 Detailed hydrographs for September and October 2011, showing the effects of pumping at private well(s) on the shallow water zone, as observed in monitoring wells installed in Phases 1-2

4.10 Detailed hydrographs for September and October 2011, showing the effects of pumping at private well(s) on the shallow water zone, as observed in monitoring wells installed in Phases 3-4

4.11a Potentiometric surface maps for the shallow groundwater zone north of Buckskin Road, for water levels measured on February 16 and August 20, 2011

4.11b Potentiometric surface maps for the shallow groundwater zone north of Buckskin Road, for water levels measured on November 11, 2011, and March 13, 2012.

4.12 Potentiometric surface and flow network in the shallow groundwater zone for water levels recorded at 11:00 a.m. on September 16, 2011, the time of maximum drawdown during the pumping event, in the area of the former CCC/USDA property and the private storage facilities to the west.

4.13 Potentiometric surface and flow network (ambient condition) in the shallow groundwater zone for water levels measured on March 13, 2012, in the area of the former CCC/USDA property and the private grain storage facilities to the west

4.14 Hydrographs observed from September 2011 to March 2012 in selected middle groundwater zone monitoring wells.

4.15 Detailed hydrographs for September and October 2011, showing the effects of pumping at private wells(s) on the middle water zone....

4.16 Hydrographs observed in the Klassen private wells south of Buckskin Road

4.17 Potentiometric surface map for the middle groundwater zone north of Buckskin Road on March 13, 2012.

4.18 Overall potentiometric surface maps in the middle water zone north of Buckskin Road under pumping at the Sisson well and nearby private wells on the Pankratz (Newcombe), Willems, and Bengston properties .... 


\section{Figures (Cont.)}

4.19 Potentiometric surface maps in the area affected by pumping at the Sisson well and nearby private wells on the Pankratz (Newcombe), Willems, and Bengston properties, showing a reversed flow pattern in the middle water zone.

4.20 Lateral distribution of carbon tetrachloride in groundwater samples collected in the shallow groundwater zone north of Buckskin Road

4.21 Lateral distribution of chloroform in groundwater samples collected in the shallow groundwater zone north of Buckskin Road....

4.22 Vertical distribution of carbon tetrachloride in groundwater along north-to-south hydrogeologic cross section A-A'

4.23 Vertical distribution of carbon tetrachloride in groundwater along west-to-east hydrogeologic cross section B-B'

4.24 Hydrogeologic cross section C-C', showing CPT electronic logs, lithologic logs, and carbon tetrachloride in groundwater at the monitoring well locations at and near the former CCC/USDA facility....

4.25 Potentiometric surface and flow network in the shallow groundwater zone for water levels measured on March 13, 2012, in the area bounded by the former CCC/USDA and private facilities on the north, Buckskin Road on the south, the MW17S location on the east, and the MW16 location on the west.

4.26 Lateral distribution of carbon tetrachloride in groundwater samples collected in the middle groundwater zone

4.27 Lateral distribution of chloroform in groundwater samples collected in the middle groundwater zone

4.28 Lateral distribution of carbon tetrachloride and chloroform in groundwater samples collected in the deep groundwater zone

4.29 Conceptual model of contaminant migration pathways north of Buckskin Road, east and west of Main Street 


\section{Notation}

AGEM Applied Geosciences and Environmental Management

AMSL above mean sea level

BGL below ground level

CCC Commodity Credit Corporation

CD compact disc

CLP Contract Laboratory Program

COC chain of custody

CPT cone penetrometer

EPA U.S. Environmental Protection Agency

$\mathrm{ft} \quad$ foot (feet)

gal gallon(s)

GC-MS gas chromatograph-mass spectrometer

gpm gallon(s) per minute

$\mathrm{hr} \quad$ hour(s)

in. inch(es)

KDHE Kansas Department of Health and Environment

$K_{h} \quad$ horizontal hydraulic conductivity

$K_{v} \quad$ vertical hydraulic conductivity

$\mu \mathrm{g} / \mathrm{kg} \quad$ microgram(s) per kilogram

$\mu \mathrm{g} / \mathrm{L} \quad$ microgram(s) per liter

MCL maximum contaminant level

$\mathrm{mg} / \mathrm{L} \quad$ milligram(s) per liter

mi mile(s)

min minute(s)

NAIP National Agricultural Imagery Program

QA/QC quality assurance/quality control

RPD relative percent difference

$S \quad$ storativity

SDG sample delivery group

SRE site reconnaissance and evaluation

$T$ transmissivity

TOC top of casing 
Inman Site Investigation Results

Version 02, 01/24/14

USDA U.S. Department of Agriculture

VOC volatile organic compound

yr

year(s) 


\section{Final Report: Site Investigation Results, 2009-2011, at Inman, Kansas}

\section{Introduction}

The Commodity Credit Corporation of the U.S. Department of Agriculture (CCC/USDA) operated a grain storage facility at the southern edge of the city of Inman, Kansas, from 1954 to 1965. During this time, commercial grain fumigants containing carbon tetrachloride were in common use by the grain storage industry to preserve grain in their facilities. In 1997, trace to low levels of carbon tetrachloride (below the maximum contamination level [MCL] of 5.0 $\mu \mathrm{g} / \mathrm{L}$ ) were detected in three private wells near the former grain storage facility at Inman, as part of a statewide USDA private well sampling program that was implemented by the Kansas Department of Health and Environment (KDHE) near former CCC/USDA facilities. No public water supply wells were identified in 1998 by the KDHE within $1 \mathrm{mi}$ of the town.

Carbon tetrachloride is the contaminant of primary concern at sites associated with grain storage operations. To determine whether the former CCC/USDA facility at Inman is a potential contaminant source and its possible relationship to the contamination in groundwater, the CCC/USDA agreed to conduct investigations at Inman. The investigations were performed by the Environmental Science Division of Argonne National Laboratory in accordance with the Intergovernmental Agreement between the KDHE and the Farm Service Agency of the USDA.

Argonne, on behalf of the CCC/USDA, developed a Work Plan (Argonne 2007) and subsequently a Summary of Investigation Results and Proposed Work Plan (Appendix A) for a phased site investigation. The proposed work was approved by the $\operatorname{KDHE}(2007,2011)$. The investigations were conducted from November 2009 to September 2011, as proposed in the two work plans. This report presents the findings of the 2009-2011 investigations at Inman.

\subsection{Background and Previous Investigations}

Inman, Kansas, is a city located in McPherson County, $10 \mathrm{mi}$ from McPherson and $13 \mathrm{mi}$ from Hutchinson (Figure 1.1). The town spans four sections (Sections 8, 9, 16, and 17) in Township 21 South, Range 4 West. Inman has numerous small businesses, a school district, a medical center, a library, churches, and a recreation facility. 
As of the census of 2010, the city of Inman was home to 1,377 people in 566 housing units. The city has been growing steadily, with a recent population increase of $20 \%(2000-2010)$. The residents of the city are served by a public water supply system that obtains water from wells located 2.5 mi east of Inman.

In response to the initial detection of trace levels of carbon tetrachloride in private wells at Inman in 1997, the KDHE and the CCC/USDA conducted the following investigations and studies:

- Private well sampling in 1997 and 1998, as a part of the statewide USDA private well sampling program.

- Pre-CERCLIS site reconnaissance and evaluation (SRE) by the KDHE in 1998.

- Private well sampling in 2005, as a part of the statewide USDA private well sampling program.

- Review of site historical data and the former grain storage facilities by Argonne in 2007.

- Review of historical information on private grain storage at the Klassen residence by Argonne in 2011.

The main findings of the previous investigations are summarized as follows:

- Former grain storage facilities as potential source areas:

- The CCC/USDA operated a grain storage facility with 30 metal circular bins from 1954 to 1965 on a strip of land ( $485 \mathrm{ft}$ by $128 \mathrm{ft}$ ) in the southern part of the city (Figure 1.2). The former facility is now part of the baseball field of the local school, USD 448. 
- Two small private grain storage facilities were identified on the Sisson and Willems properties. The northern facility on the Sisson property appears to have been active during the $1950 \mathrm{~s}$, consisting of nine circular storage structures and one Quonset located approximately 80-180 ft from the contaminated well on the Sisson property. The southern facility on the Willems property consisted of three circular bins, located 100-130 ft from the contaminated Willems well, in the 1950s-1960s (Figure 1.2).

- A commercial grain storage facility identified in the northwestern portion of Inman, along the railroad right-of-way and Front Street, included 3 elevators, 37 tall bins and silos, and 1 storage building (Figure 1.1). Carbon tetrachloride was recently identified in groundwater beneath this former facility (KDHE 2012).

- A private grain storage and operation facility at the Klassen residence was identified during the 2009-2011 investigations. This facility has grown gradually, from 3 circular bins and 1 storage structure in 1963, to 9 circular bins and 2 storage structures in 1979, and to 14 circular bins and 1 storage structure at present (Figure 1.3). The Klassen grain storage facility is about $60 \mathrm{ft}$ from the Klassen domestic well and $150 \mathrm{ft}$ from the Klassen test well, groundwater from which was found to be contaminated in the present investigations.

- Soil and groundwater contamination:

- In 1997-1998, four private wells were sampled as part of the USDA private well sampling program (in 1997) and the subsequent KDHE (1998) SRE investigation. Carbon tetrachloride was found in groundwater from two lawn and garden wells (Sisson and Pankratz [now owned by Newcombe]) and one domestic well (Bengston) at concentrations below the MCL. These wells are 80-180 ft from the closest former private grain storage facilities and 200-500 ft west and southwest of the former CCC/USDA facility (Figure 1.4). 
- In April 1998, the KDHE collected 10 soil samples above bedrock, at depths of $11.5 \mathrm{ft}$ and $21.5 \mathrm{ft}$ BGL (below ground level), at five locations across the former CCC/USDA facility. Carbon tetrachloride was detected by the field laboratory in 5 of the 10 samples collected, at concentrations of $\quad 0.3-2.5 \mu \mathrm{g} / \mathrm{kg}$ (Figure 1.5). The two samples with highest concentrations of carbon tetrachloride as analyzed by the field laboratory were submitted to an off-site laboratory for verification analysis. The offsite analyses detected no carbon tetrachloride or chloroform in either sample.

- In September 2005, the KDHE sampled two private wells (Pankratz [Newcombe] and Bengston), and carbon tetrachloride was detected at $7.4 \mu \mathrm{g} / \mathrm{L}$ and $6.4 \mu \mathrm{g} / \mathrm{L}$, respectively. After permission was obtained to sample the drinking water well at the Willems residence, the Pankratz (Newcombe), Bengston, and Willems wells were sampled in a second event in September 2005. On this second occasion, carbon tetrachloride was detected at concentrations below the MCL in all three wells where low-level contamination had been detected in the previous sampling event. (Figure 1.4).

- As a result of the September 2005 sampling, the CCC/USDA provided connections for the Bengston and Willems residences to the Inman public water supply. The connections to the two properties were completed in February-March 2006.

- Inman public water supply:

- In 1998, a KDHE survey indicated that no public wells were located within $1 \mathrm{mi}$ of the former CCC/USDA facility. The Inman public water supply wells are located $2.5 \mathrm{mi}$ from the former facility. 


\subsection{Objectives of the Targeted Investigations}

The 2009-2011 targeted investigations were conducted in a phased approach in accordance with the work plans (Argonne 2007 and Appendix A) approved by the KDHE. The primary goal of these investigations was to identify and characterize any subsurface contamination that might be associated with the past use of carbon tetrachloride-based grain fumigants at the former CCC/USDA facility and its relationship to the groundwater contamination found in the Sisson, Pankratz (Newcombe), Willems, and Bengston private wells to the west and southwest. The results of the targeted investigations provide the basis for determining whether response actions are warranted. To meet the investigational goal, the main technical objectives of the investigation at Inman were as follows:

- Evaluate the potential source of carbon tetrachloride at the former CCC/USDA facility.

- Determine the relationship of potential contamination at the former CCC/USDA facility to contamination identified in 1997 in groundwater samples from the Sisson, Pankratz (Newcombe), Willems, and Bengston private wells.

- Delineate the extent of any identified contamination potentially associated with the former CCC/USDA facility.

\subsection{Field Investigation Activities}

The targeted investigations were performed in five phases, as outlined in the Final Work Plan (Argonne 2007) and supplemental Work Plan (Appendix A), on the basis of results from Phase 1 and Phase 2. The five phases were as follows:

- Phase 1: Use of the cone penetrometer (CPT) unit to conduct vertical soil profiling through the vadose zone for analysis for volatile organic compounds (VOCs), in order to identify potential contaminant source areas on the former CCC/USDA property. 
- Phase 2: Characterization of the shallow water zone, as follows:

- Collection of groundwater samples (for VOCs analyses) from the shallow zone at selected locations beneath the former CCC/USDA facility; along Main Street between the former CCC/USDA facility and the Sisson, Pankratz (Newcombe), Willems, and Bengston private wells; upgradient from the former private grain storage facilities; and at the downgradient edge of the former CCC/USDA facility.

- Sampling of the Sisson, Pankratz (Newcombe), Willems, and Bengston private wells for VOCs analyses.

- Soil profiling at and near the former private grain bins and Quonset on the Sisson and Willems properties.

- Phase 3: Characterization of the deeper water zones to delineate the vertical extent of groundwater contamination and collection of water samples for VOCs analysis at the location showing the highest concentration of carbon tetrachloride in the shallow water zone on the former CCC/USDA property, as well as at locations immediately outside that property.

- Phase 4: Delineation of the lateral extent of carbon tetrachloride contamination downgradient from both the former CCC/USDA property and the former private grain storage facilities on the Sisson and Willems properties; identification of private wells south of the Sisson, Pankratz (Newcombe), Willems, and Bengston properties and collection of groundwater samples from those wells. This activity led to the discovery of contamination in the Klassen private well.

- Phase 5: Establishment of a groundwater monitoring network to record water level fluctuations and evaluate hydrogeologic properties by monitoring responses of various groundwater zones to pumping at the private wells on the Sisson, Pankratz (Newcombe), Willems, and Bengston properties. 
The Phase 1-Phase 2 investigations were performed in two main field mobilizations, on November 4-11, 2009, and November 8-14, 2010. The data collected in these two phases were evaluated, and a supplemental Work Plan (Appendix A) was developed as understanding of the site evolved. The supplement Work Plan for Phase 3 and Phase 4 was approved by the KDHE (2011). The main Phase 3-Phase 4 investigations were conducted on August 22-September 2, 2011, and September 13-16, 2011. For Phase 5, a complete groundwater monitoring network was established at the end of the field activities. Monitoring of the responses of water levels in various groundwater zones to pumping at the private Sisson, Pankratz (Newcombe), Willems, and Bengston wells continued until March 2012.

\subsection{Organization of This Report}

This report documents the data acquired during all five investigation phases. Section 1 presents background information, investigational objectives, and a summary of field activities. Section 2 describes the investigative methods used, and Section 3 presents the results of all investigative activities. Section 4 discusses and interprets the results in the context of the specific technical objectives outlined in Section 1.2. Section 5 summarizes the key findings of the investigations and provides recommendations for future consideration based on the investigation results. 


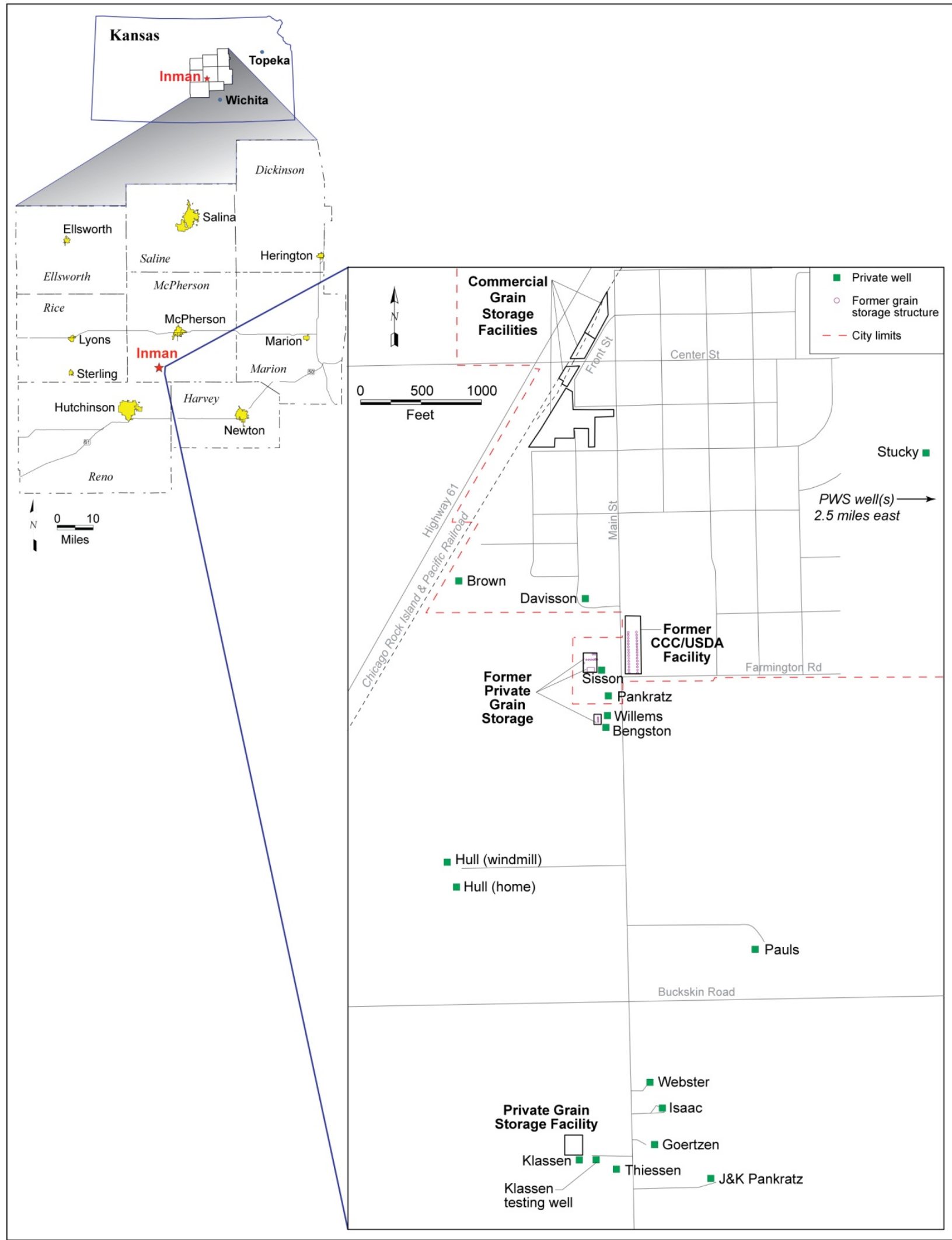

FIGURE 1.1 Locations of Inman, Kansas, former grain storage facilities, the Inman public water supply wells, and private wells. 

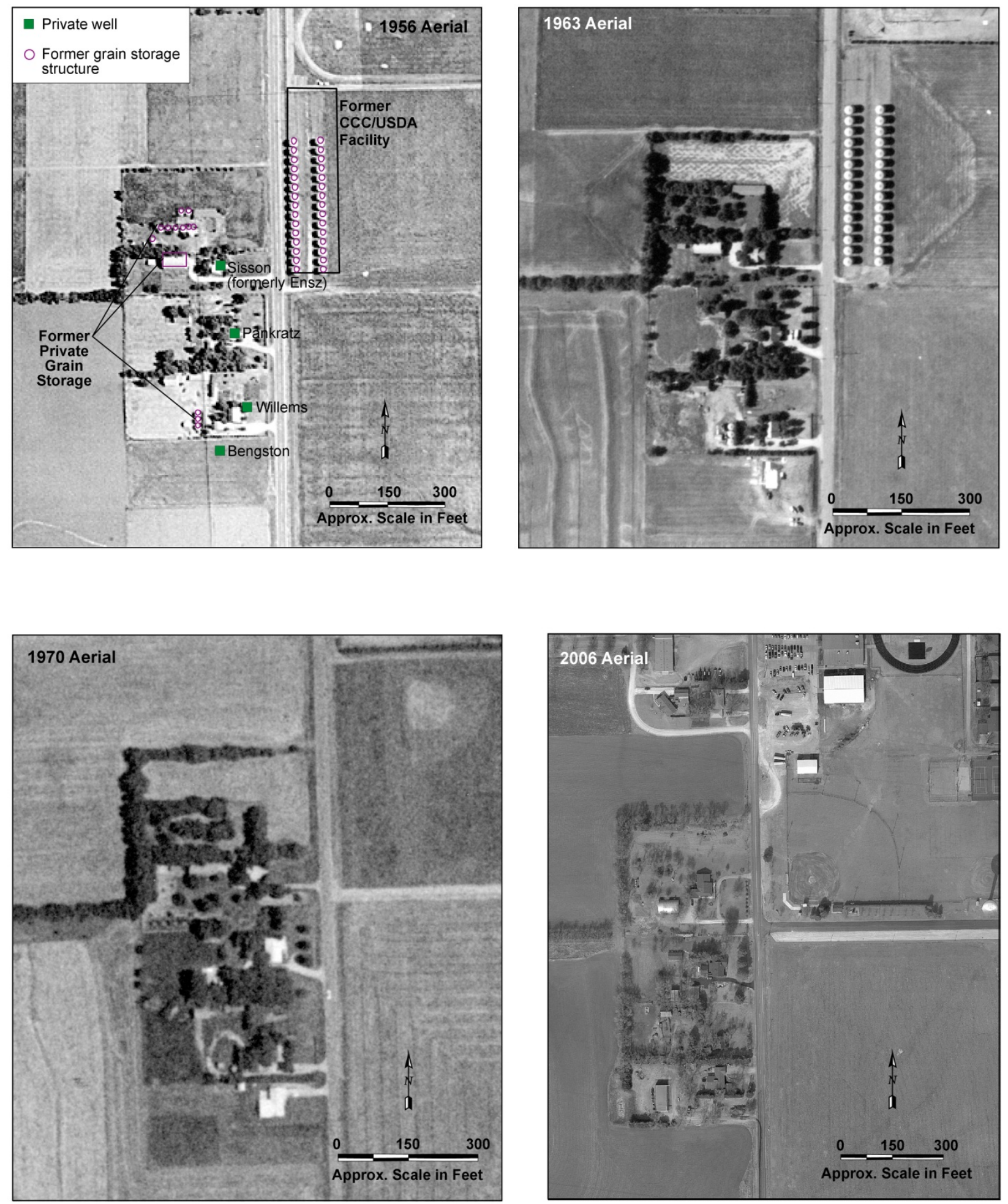

FIGURE 1.2 History of the former CCC/USDA and private grain storage facilities near the south edge of Inman, as interpreted from aerial photographs and property documents. Source of photographs: USDA (1956, 1963, 1970); McPherson Mapping Department (2006). 

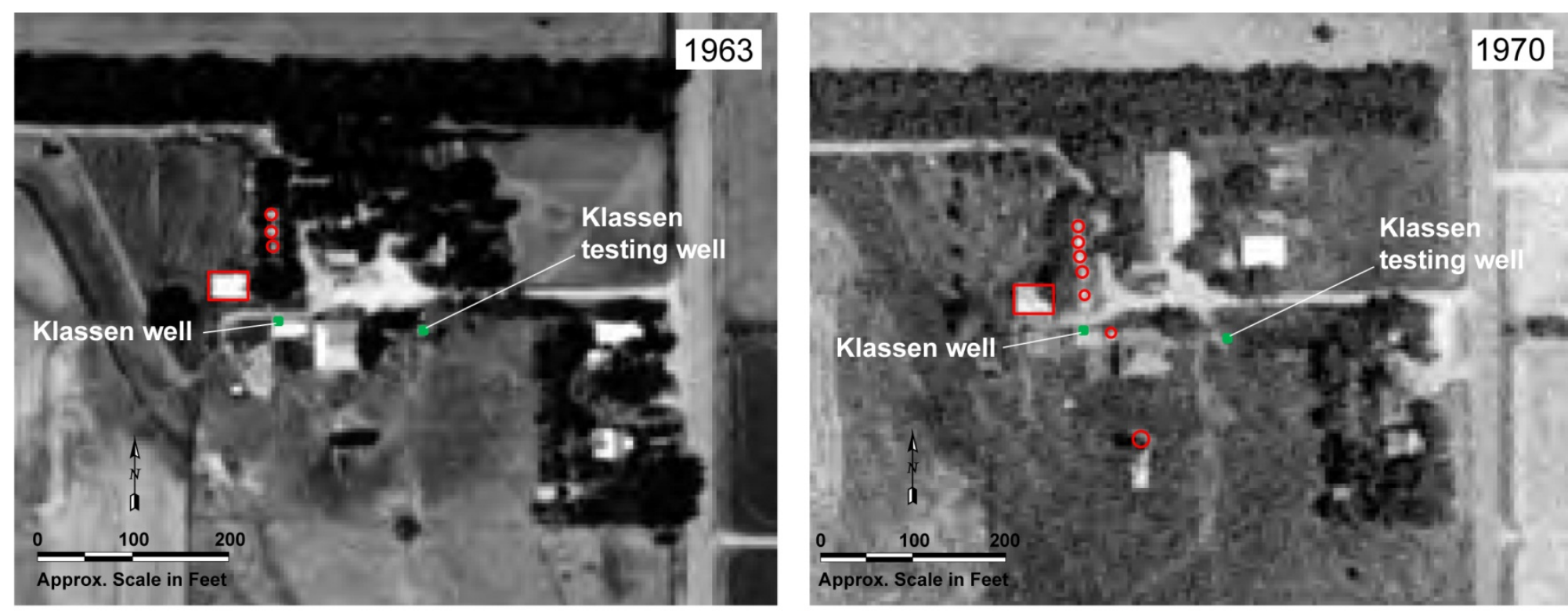

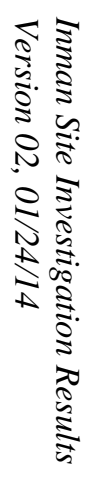
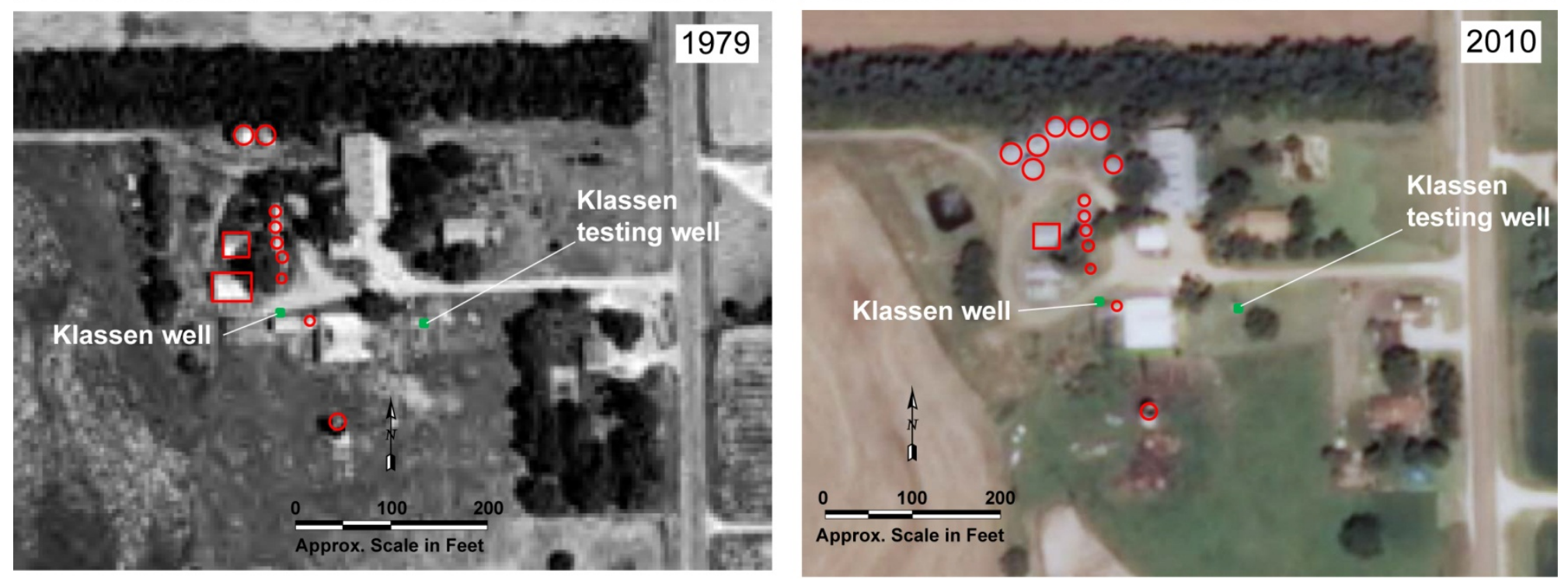

FIGURE 1.3 History of the private grain storage facilities on the Klassen property, as interpreted from aerial photographs. Source of photographs: USDA (1963, 1970, 1979); NAIP (2010). 


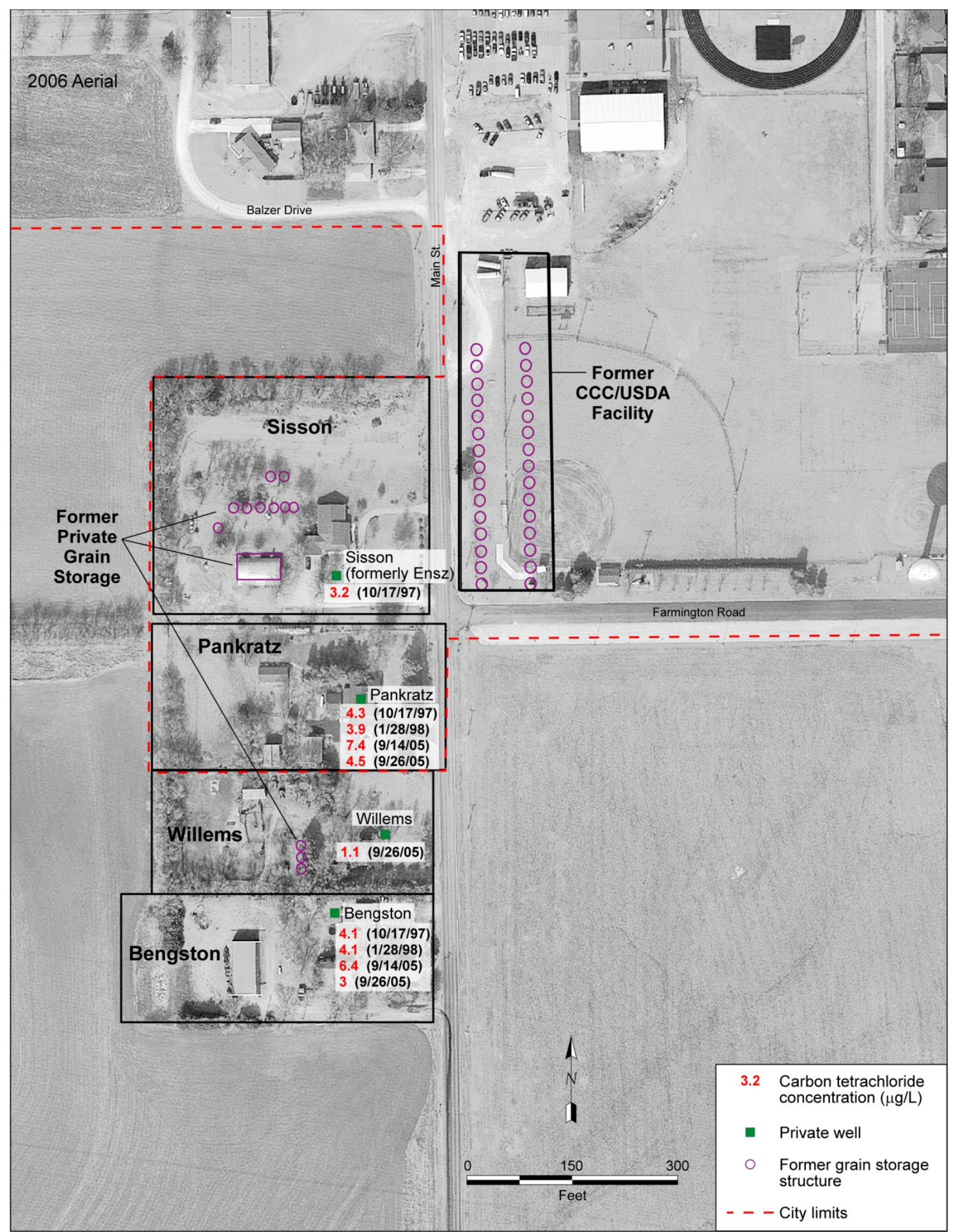

FIGURE 1.4 Historical results for carbon tetrachloride in groundwater samples collected by the KDHE in 1997-2005 from private wells on the Sisson, Pankratz (now Newcombe), Willems, and Bengston properties. Source of photograph: McPherson Mapping Department (2006). 


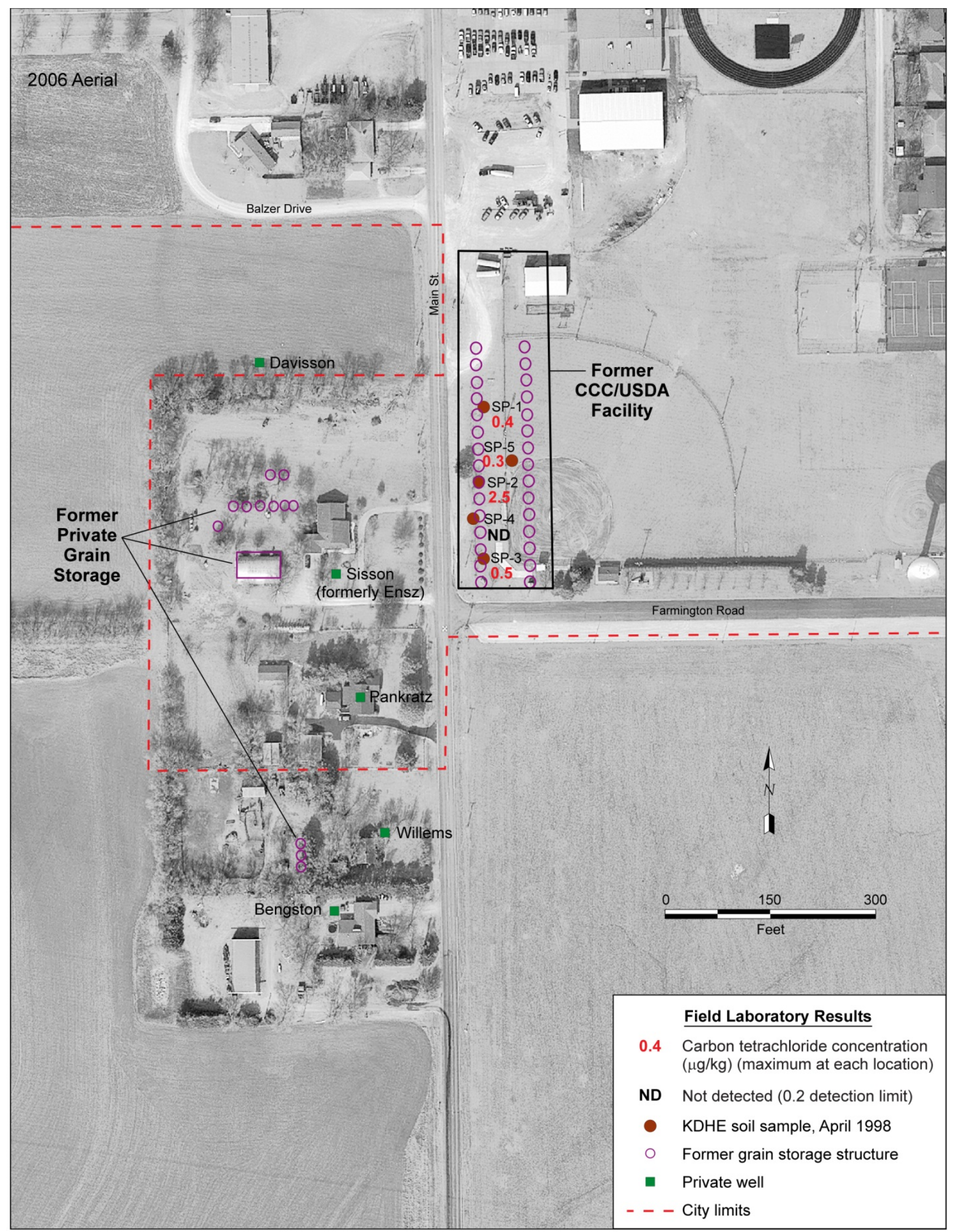

FIGURE 1.5 Field laboratory analytical results for soil samples collected by the KDHE in 1998 at the former CCC/USDA facility. Source of photograph: McPherson Mapping Department (2006). 


\section{Investigative Methods}

This section discusses the methods used to implement the targeted investigation and to achieve the specific technical objectives in Section 1.2. Individual activities were performed as specified in the Final Work Plan (Argonne 2007), the supplemental Work Plan (Appendix A), and the Master Work Plan (Argonne 2002). Detailed procedures are not repeated here.

Throughout the field investigations, a comprehensive quality assurance/quality control (QA/QC) program was implemented to confirm the reliability of all information as it was accumulated. The results of implementation of the QA/QC program are discussed in Section 3.8.

\subsection{Methods to Identify Contaminant Sources and the Extent of Soil Contamination}

The investigation activities described here focused on identification of soil contamination on and near the former CCC/USDA property, which is currently the location of the baseball field at the southwest corner of the USD 448 property. In addition, soil samples were also collected at selected locations near the former private grain storage facilities on the Sisson and Willems properties. To ensure that all soil sources and soil-to-groundwater pathways were identified, the following approaches were implemented:

- Continuous vertical soil profiling with sampling for VOCs analyses was conducted through the vadose zone and to the depth of the first water-bearing unit identified beneath the former CCC/USDA facility. The analytical results were evaluated to determine whether carbon tetrachloride was present in soil as a potential contaminant source.

- Vertical soil profiling was performed in two areas, as follows:

- Area 1. Subsurface soil samples were collected by using the CPT unit to the depth of refusal, at or near bedrock, at 11 locations (TI01-TI11) over the entire area of the former grains bins on the CCC/USDA facility (Figure 2.1). 
- Area 2. Subsurface soil samples were collected by using the CPT unit to the depth of the refusal, at or near bedrock, at 7 locations (TI19-TI25) selected on the basis of the estimated locations of the former grain storage structures on the Sisson and Willems properties (Figure 2.1).

\subsection{Methods to Characterize Groundwater Contamination}

\subsubsection{Methods to Identify the Groundwater-Bearing System}

Characterization of the groundwater-bearing system was conducted in two steps addressing (1) the shallow groundwater-bearing zone and (2) the middle and deep groundwaterbearing zones.

The shallow groundwater-bearing zone was initially evaluated at 7 locations (TI01, TI02, TI04-TI06, TI08, and TI11) on the former CCC/USDA property (Figure 2.2). Because of its lower permeability and limited water availability, the lithology and water contents of cores from the upper part of the bedrock, along with CPT electronic responses for tip and sleeve stresses and conductance, were evaluated carefully to identify the presence of this water-bearing zone. Correlations between the CPT electronic data and the lithology of the cores were also identified and used in subsequent investigations for the area immediately outside the former CCC/USDA facility. The contaminated portion of the shallow groundwater zone beneath the former CCC/USDA facility was the primary focus of this work.

The effort to identify the shallow groundwater-bearing zone was expanded beyond the former CCC/USDA facility to include 11 additional locations (TI12-TI18, TI22-TI24, and TI26; Figure 2.2). These additional locations included 3 near the former private grain storage facilities to the west and southwest, 2 along Main Street, 4 south of the former CCC/USDA facility, and 2 to the northwest.

Temporary piezometers were installed with the CPT unit at 18 locations on and near the former CCC/USDA property to collect grab groundwater samples for evaluation of the shallow groundwater zone and its contamination. Figure 2.2 shows the locations of the 18 temporary piezometers and the analytical results for the grab groundwater samples collected to assist in 
selection of monitoring well locations. On the basis of results from the temporary piezometers, monitoring wells were installed at 13 selected locations in the shallow zone.

The middle and deep groundwater zones were initially identified beneath the former CCC/USDA facility as the inferred interval(s) intersected by the contaminated Sisson, Pankratz (Newcombe), Willems, and Bengston private wells. The identification was later extended to the entire area on the basis of the characteristics of these zones.

At each boring location, multiple lines of evidence obtained from visual analyses of continuous cores, changes in lithology, observed relationships to previously identified waterbearing zones in nearby borings, and the elevation ranges of water-bearing zones in existing private wells were used to determine the locations, lithologic characteristics, and lateral continuity of the water-bearing intervals. Figure 2.3 shows all locations where groundwaterbearing intervals were characterized at monitoring wells (MW01-MW20).

\subsubsection{Methods to Delineate the Extent of Groundwater Contamination}

The shallow groundwater zone is an important interval that provides potential pathways for vertical and lateral migration of contaminants leached from the overlying soil. To fully delineate the contaminant distribution in the shallow water zone and understand the relationships of the contaminated Sisson, Pankratz (Newcombe), Willems, and Bengston private wells to the former CCC/USDA facility, the criteria for and approach to selecting investigation locations (Figures 2.2 and 2.3) were as follows:

- Define the contaminant distribution in shallow groundwater beneath the former CCC/USDA facility on the basis of the contaminant distribution in the overlying soil, as indicated by the analytical data for soils on the former CCC/USDA property and their spatial coverage. Five groundwater sampling points were selected (MW01-MW05; Figure 2.3) on this basis in Phase 1. The screen intervals of the monitoring wells were determined by considering the presence of saturated intervals, the elevation of the inferred groundwater zone, and decreasing carbon tetrachloride concentrations with depth at temporary piezometers TI01, TI06, and TI11 (Figure 2.2). 
- Determine any connection via the shallow water zone between the two potential source areas (the former CCC/USDA facility and the area encompassing the Sisson, Pankratz [Newcombe], Willems, and Bengston properties). Three investigation locations (MW09-MW11; Figure 2.3) along Main Street, between the two potential source areas, were selected in Phase 2.

- Identify any off-site migration in the shallow water zone from the former $\mathrm{CCC} / \mathrm{USDA}$ facility. Three sampling points were selected at downgradient locations near the southern edge of the former facility (MW06-MW08; Figure 2.3) in Phase 2.

- Rule out the presence of contaminant migration in the shallow groundwater zone from unknown upgradient source areas. Two monitoring points were selected upgradient from the contaminated Sisson, Pankratz (Newcombe), Willems, and Bengston private wells (MW12-MW13; Figure 2.3) in Phase 2.

- Delineate the lateral extent of groundwater contamination in the shallow water zone at distant downgradient locations on the basis of the groundwater flow pattern measured in Phase 2. Three investigation points were selected (MW15-MW17; Figure 2.3) in Phases 3-4.

For all locations where multiple water-bearing zones were identified, monitoring wells were installed separately to screen the shallow, middle, and deep groundwater zones. Groundwater samples collected from each well for VOCs analyses provided information on contaminant levels for the corresponding zones at that location.

The middle groundwater zone is the main source of local water supply to private wells. To assist in the delineation of contamination in the middle water-bearing zone, groundwater samples were also collected for VOCs analyses from accessible private wells, most of which penetrate both the shallow and middle groundwater-bearing zones. Figure 2.4 shows the locations of the 10 private wells sampled, in addition to the contaminated Sisson, Pankratz (Newcombe), Willems, and Bengston wells. The Davisson and Webster wells shown in Figure 2.4 were not sampled. The Davisson well had been abandoned, and access to the Webster well was denied. Information on private wells is in Table 2.1. In the Inman area, use of an open 
borehole without casing over the consolidated bedrock formation is a common well construction practice.

\subsection{Methods to Determine and Monitor Groundwater Flow Patterns}

Accurate determination of groundwater flow patterns was accomplished through a systematic process of (1) identification of all possible groundwater-bearing zones in the upper portion of the bedrock formation that potentially produce water to local private wells, (2) installation of monitoring wells to form a monitoring network for each water-bearing zone, (3) measurement of water levels for each contaminated groundwater-bearing zone, and (4) analysis of data to determine flow patterns in each zone.

The methods discussed in Section 2.2 were used to identify all groundwater-bearing zones. Monitoring wells were installed at most of the investigative locations to form monitoring networks adequate to configure complex flow patterns. Groundwater levels were periodically determined manually in the entire network of observation wells for each water-bearing zone. Water level fluctuations were also monitored continuously at selected locations through the use of automatic water level sensors and data loggers. Groundwater flow patterns were determined from stabilized water levels in wells that showed minimal, if any, interference from local pumping events. Determination of flow patterns was not attempted for the deep water-bearing zone because of the time required for the water levels to reach equilibrium.

To determine the groundwater flow pattern for the shallow water-bearing zone, water level data were obtained at 19 monitoring wells (at locations MW01-MW13, MW15-MW20; Figure 2.5). The network for the middle groundwater-bearing zone consists of 7 monitoring wells (at locations MW14-MW20; Figure 2.6) across the study area.

\subsection{Methods to Evaluate the Properties of the System of Multiple Groundwater- Bearing Zones}

In the system of multiple groundwater-bearing zones, understanding hydraulic communication among the different zones and/or different parts of the same zone can help to identify potential contaminant migration pathways. To facilitate this evaluation, long-term monitoring of water level fluctuations was implemented to capture aquifer responses to pumping 
events at the Sisson, Pankratz (Newcombe), Willems, and Bengston private wells. Automatic water level recorders were placed in selected wells installed in the shallow and middle groundwater-bearing zones. The following properties of the groundwater system were analyzed:

- The vertical hydraulic communication between the shallow and middle groundwater-bearing zones in response to pumping of private wells screened in the middle zone.

- The radius of influence of pumping in the middle groundwater zone and its heterogeneity. The radius of influence is the radial distance from the center of a pumping well to the point where there is no lowering of the water table or potentiometric surface. This point is the same as the edge of the cone of depression - the area affected by the pumping well, beyond which there is no measurable or observed effect.

- The estimated range of horizontal hydraulic conductivity $\left(K_{h}\right)$ values in the shallow and middle groundwater zone near the pumping well, based on a range of probable pumping rates.

In addition, for a water-bearing zone with extremely low permeability, water-level records showing slow recovery in response to a sampling event were evaluated. If recovery of the water level in a well took roughly a day or more after sampling, the relatively short sampling period (typically less than an hour) was treated as an instantaneous event, compared to the long recovery time. The data automatically recorded for the slow recovery were treated as the results of a slug test to estimate $K_{h}$. This estimation offers a quantitative value that is approximate to an order of magnitude. The accuracy of the estimate improves with increasing time required for full recovery. Thus, the lower the $K_{h}$ value, the more accurate the estimate. 
TABLE 2.1 Information on private wells identified or sampled in the 2009-2011 site investigation. ${ }^{\text {a }}$

\begin{tabular}{|c|c|c|c|c|c|}
\hline \multirow[b]{2}{*}{ Owner } & \multicolumn{3}{|c|}{ Depth (ft BGL) } & \multirow[b]{2}{*}{ Well Use ${ }^{b}$} & \multirow[b]{2}{*}{$\begin{array}{l}\text { Date of } \\
\text { Completion }\end{array}$} \\
\hline & Well Depth & $\begin{array}{l}\text { Screen/Open- } \\
\text { Borehole Interval }\end{array}$ & $\begin{array}{l}\text { Static } \\
\text { Water } \\
\text { Level }\end{array}$ & & \\
\hline \multicolumn{6}{|c|}{ Wells Identified during the 2009-2011 investigation } \\
\hline Jon and Amber Brown & 140 & Open borehole $40-140$ & 28 & Lawn-gardenc & 10/31/08 \\
\hline Judy Davisson & 114 & - & 26 & Abandoned & 05/01/93 \\
\hline \multirow{2}{*}{\multicolumn{6}{|c|}{ Gary and Jane Hull }} \\
\hline & & & & & \\
\hline House & 106 & Open borehole 54-106 & 31 & Domestic & $03 / 16 / 93$ \\
\hline Windmill & - & - & - & Lawn-garden & - \\
\hline $\begin{array}{l}\text { Terry and Audrey Isaac } \\
\text { Eldo and Janice Klassen }\end{array}$ & 97 & Open borehole 22-97 & 3 & Domestic & 07/08/99 \\
\hline Domestic & 174 & Open borehole $52-174$ & $32^{d}$ & Domestic & 09/08/08 \\
\hline Test well & 135 & - & $35^{d}$ & Not used & - \\
\hline Jerry and Kim Pankratz & - & - & - & Domestic & - \\
\hline Dennis and Janice Pauls & 101 & Open borehole 33-101 & 27 & Domestic & $01 / 24 / 06$ \\
\hline $\begin{array}{l}\text { Terry and Stephanie } \\
\text { Thiessen }\end{array}$ & 105 & Screen 75-95 & 25 & Lawn-garden & $10 / 19 / 77$ \\
\hline $\begin{array}{l}\text { Jeremy and Rachelle } \\
\text { Webster }\end{array}$ & - & - & - & - & - \\
\hline \multicolumn{6}{|c|}{ Four contaminated private wells identified previously } \\
\hline Jerry and Ruth Sisson & - & - & - & Lawn-garden & - \\
\hline $\begin{array}{l}\text { Emerson and Lilly Pankratz } \\
\text { (new owners: Willie and } \\
\text { Connie Newcombe) }\end{array}$ & - & - & - & Lawn-garden & - \\
\hline John Willems & - & - & - & Domestic ${ }^{\mathrm{e}}$ & - \\
\hline Kevin and Kim Bengston & - & - & - & Lawn-garden ${ }^{f}$ & - \\
\hline
\end{tabular}

a Source of data: KDHE water well database, except as specified otherwise.

b Information of water use is from KDHE water well database or as reported by the well owner.

c Lawn and garden well for a shop.

d Static water level measured during the site investigation.

e The property was connected to the Inman public water supply by the CCC/USDA in 2006. However, Mr. Willems has not implemented domestic use of the public water supply.

f The property was connected to the Inman public water supply by the CCC/USDA in 2006, and domestic use began at that time. 


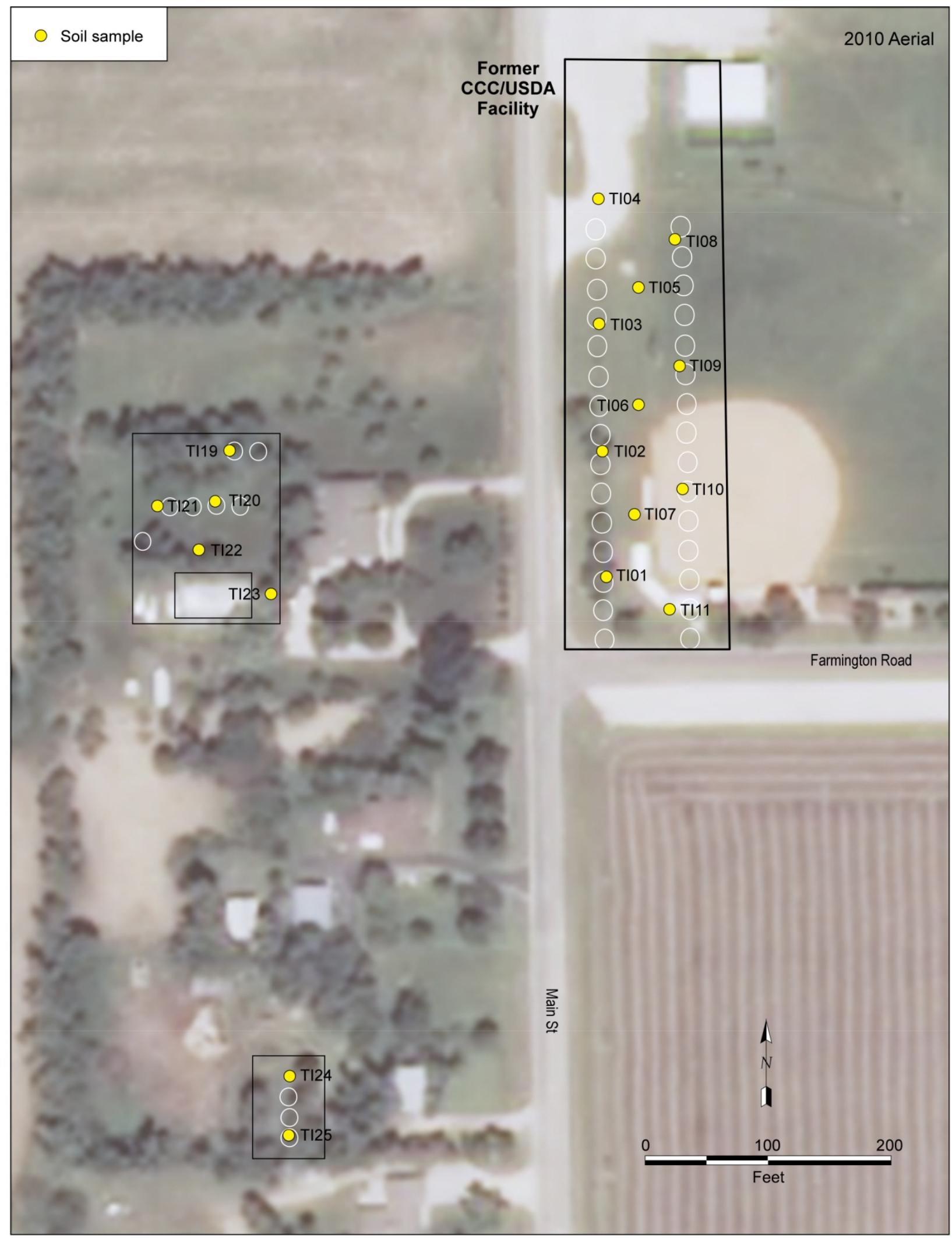

FIGURE 2.1 Locations of soil sampling on the former CCC/USDA property and the Sisson and Willems properties in the targeted investigation. Source of photograph: NAIP (2010). 


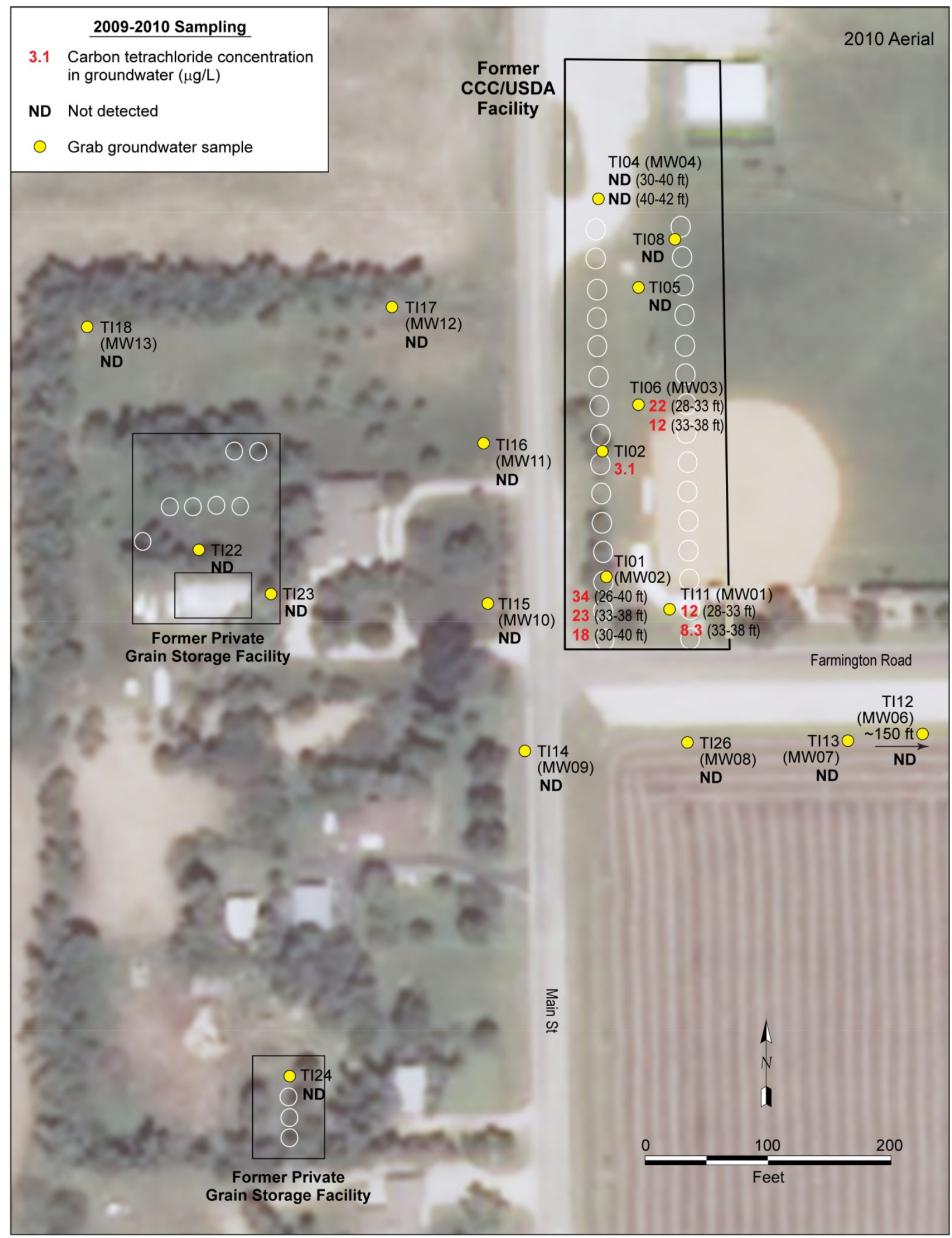

FIGURE 2.2 Sampling locations and carbon tetrachloride concentrations in grab groundwater samples collected in 2009-2010. Source of photograph: NAIP (2010). 


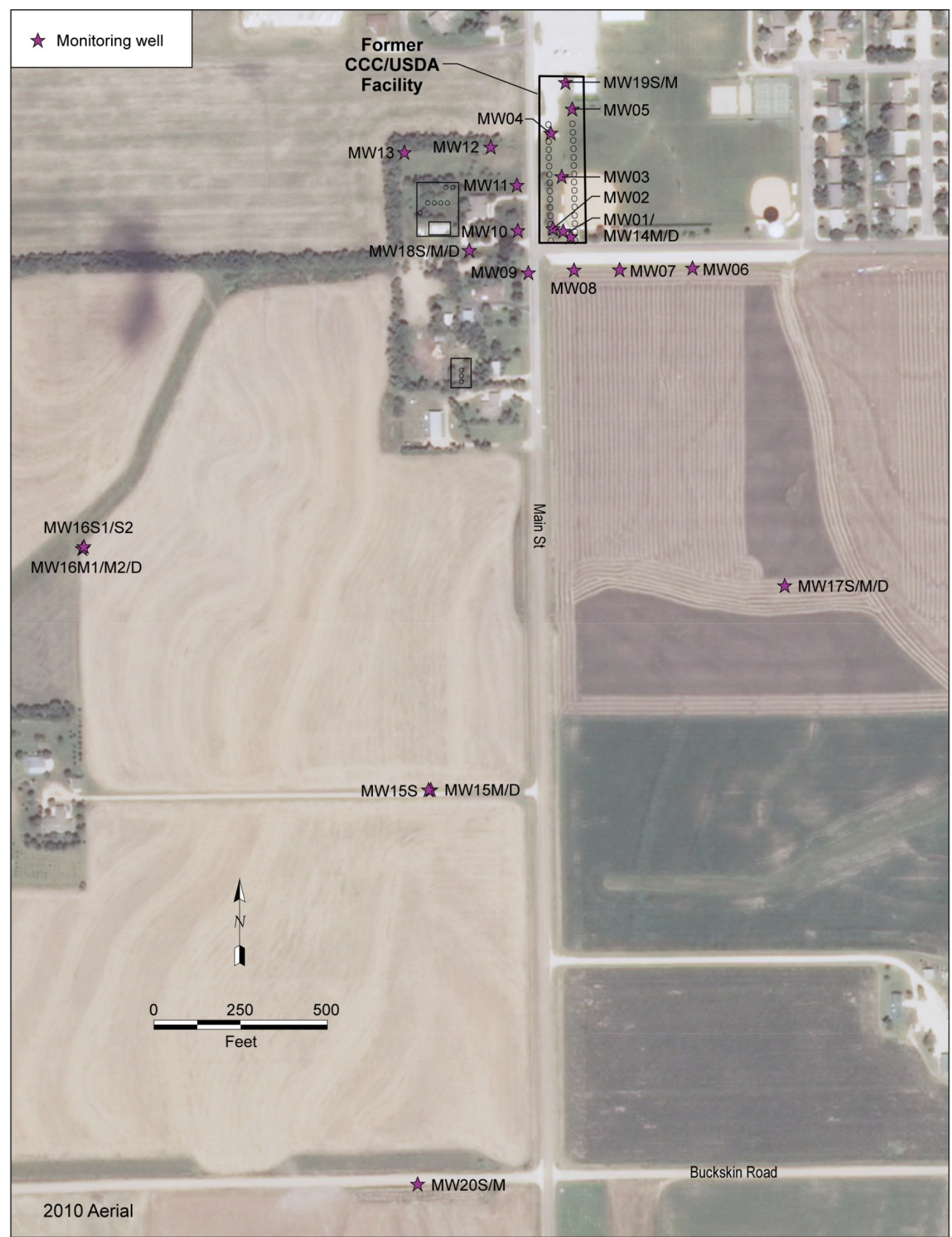

FIGURE 2.3 Locations of groundwater sampling from the monitoring wells installed during the targeted investigation. Source of photograph: NAIP (2010). 


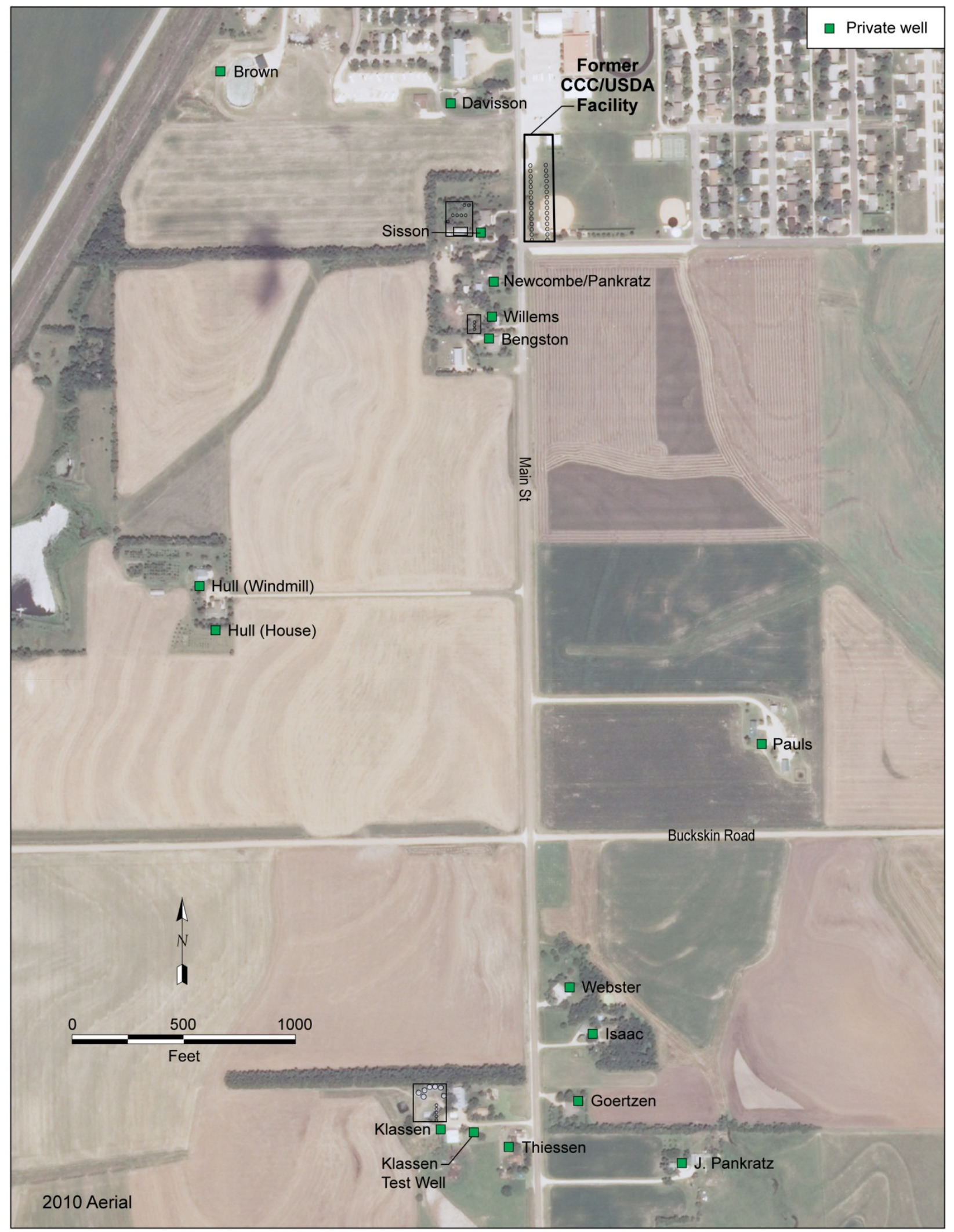

FIGURE 2.4 Locations of the private wells targeted for sampling during the investigation. Source of photograph: NAIP (2010). 


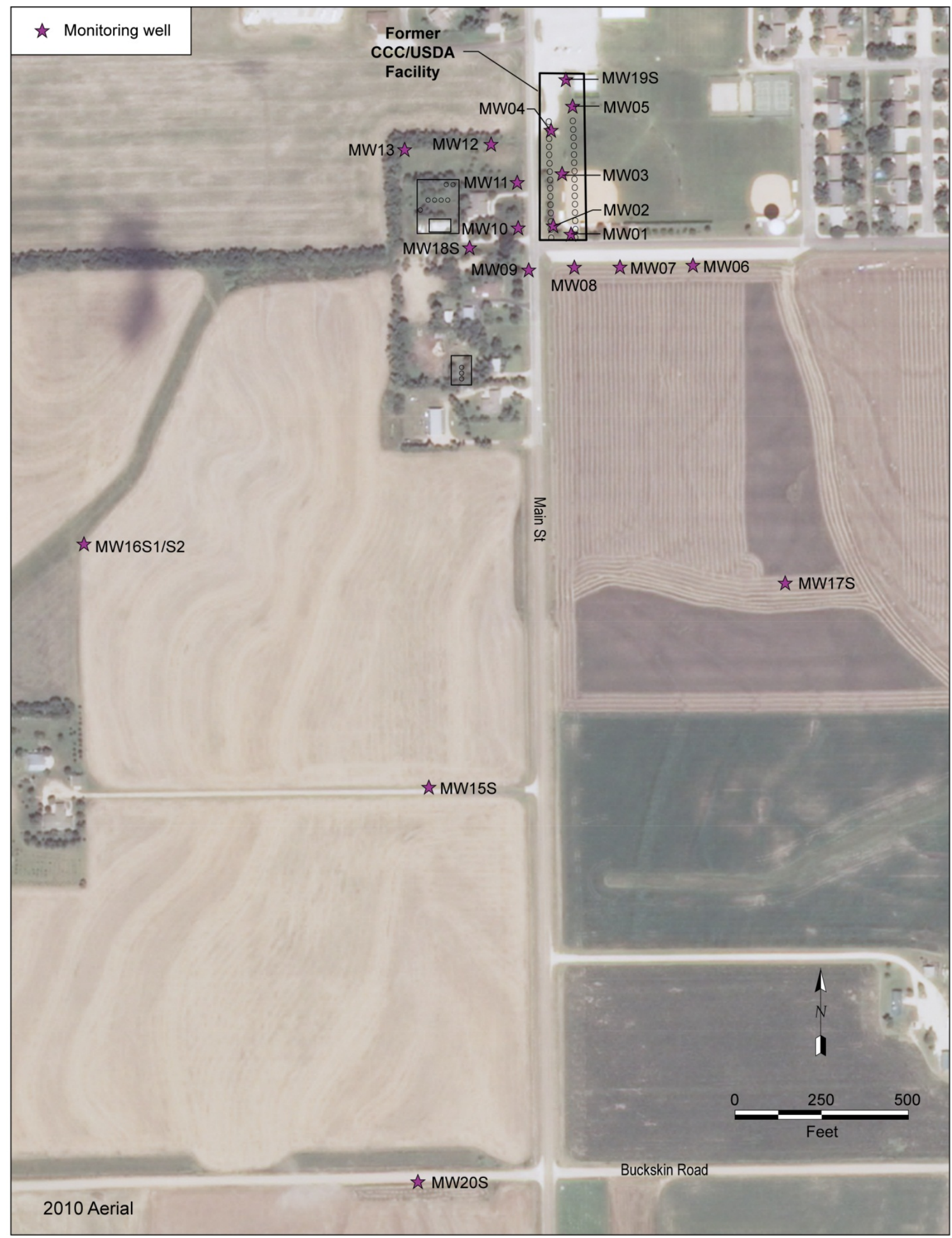

FIGURE 2.5 Monitoring network for the shallow groundwater-bearing zone. Source of photograph: NAIP (2010). 


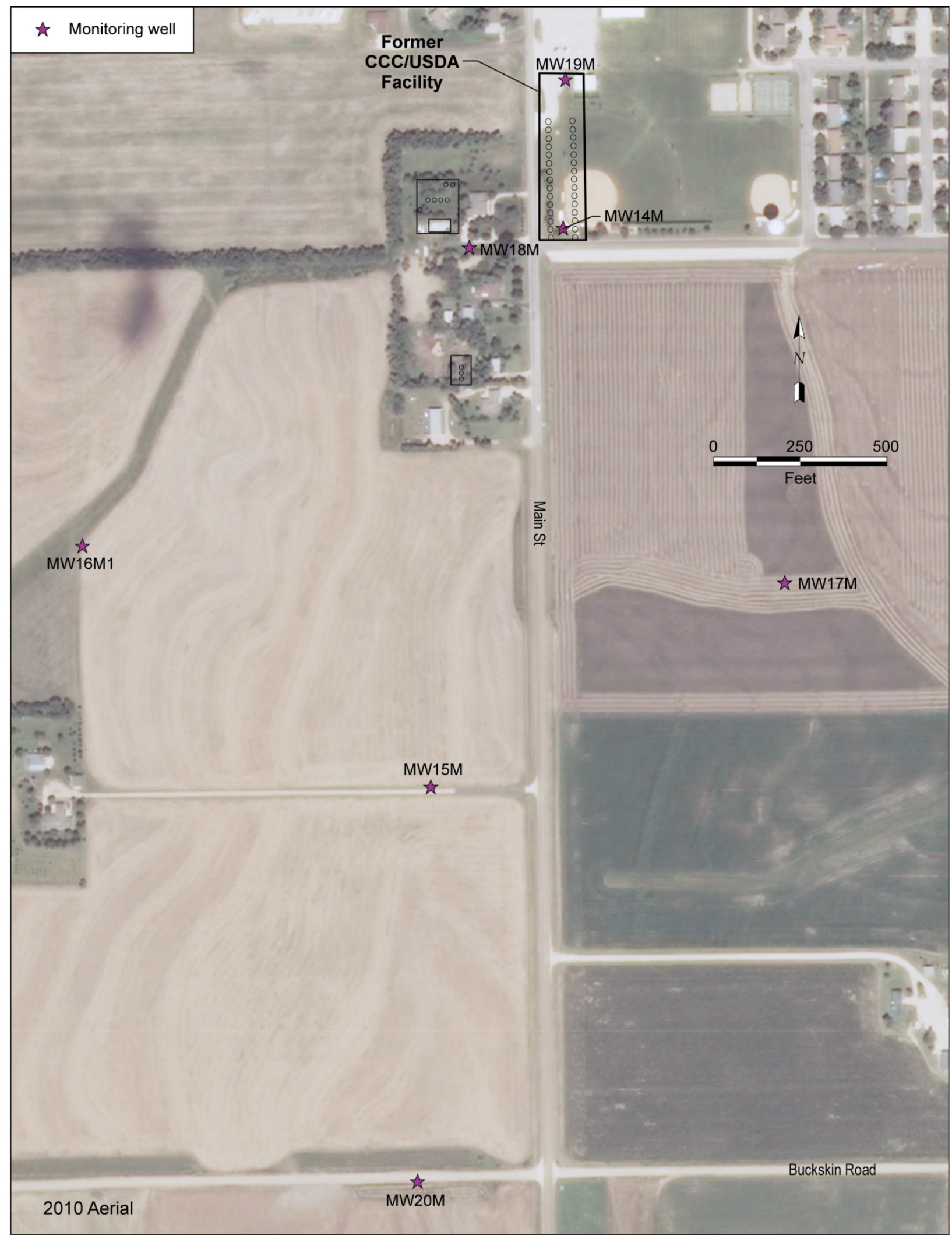

FIGURE 2.6 Monitoring network for the middle groundwater-bearing zone. Source of photograph: NAIP (2010). 


\section{Field and Laboratory Data}

The data generated by the 2009-2011 investigations are presented in this section according to the type of test performed or the medium analyzed. The methodologies for data collection are also discussed. Detailed procedures for the 2009-2011 investigations are described in the Final Work Plan (Argonne 2007), the supplemental Work Plan (Appendix A), and the Master Work Plan (Argonne 2002). A detailed interpretation of these data is in Section 4.

\subsection{Electronic Cone Penetrometer Sensor Data}

Electronic data for tip stress, sleeve stress, and conductance were collected by CPT sensors at investigation locations TI01 (MW02), TI02, TI03, TI04 (MW04), TI05, and TI12TI18, on or near the former CCC/USDA property and on the Sisson and Willems properties (Figures 2.1 and 2.2). The sensor logs were used as a guide for the general identification of lithology units, possible water-bearing zones, and optimal intervals for confirmatory soil and groundwater sampling. Correlations between sensor logs and core lithologies were identified at the former CCC/USDA facility. High tip and sleeve stresses with higher ratios of tip to sleeve and lower conductance were highly correlated with the shallow groundwater zone in the upper portion of the highly weathered bedrock at an equivalent range of elevations (TI01-TI05). These sensor logs were used as a guide for installing temporary wells and monitoring wells at locations MW06-MW07 (TI12-TI13) and MW09-MW13 (TI14-TI18). The electronic logs for locations TI01 (MW02), TI02, TI03, TI04 (MW4), and TI05 are in Appendix B.

\subsection{Geologic Coring Data}

At all locations drilled with the sonic rig, continuous cores were collected and evaluated to generate geologic logs. The lithology descriptions for these locations are in Appendix B. The lithology data were analyzed to identify the local lithostratigraphic and hydrostratigraphic units. 


\subsection{Soil Sampling Data}

Subsurface soil samples were collected for VOCs analyses to generate soil contamination data above the groundwater zones. Sampling was conducted primarily in vertical profiles through the vadose zone to the depth of the first water-bearing zone.

At borings TI01-TI11 on the former CCC/USDA property and borings TI19-TI25 on the Sisson and Willems properties (Figure 2.1), the CPT unit was used to collect subsurface soil samples at 4-ft intervals from the unconsolidated material, from the ground surface to the top of the bedrock and/or weathered bedrock material at the point of refusal. The uppermost groundwater-bearing zone (shallow water zone) is located in the upper portion of the bedrock. The lithology descriptions and VOCs results for the soil profiles are in Appendix B. Details are discussed in Section 4.

All soil samples were analyzed for VOCs at the Applied Geosciences and Environmental Management (AGEM) Laboratory at Argonne according to a modification of U.S. Environmental Protection Agency (EPA) Method 8260B (purge-and-trap method), as referenced in SW-846 (EPA 1998), to achieve a method detection limit of $10 \mu \mathrm{g} / \mathrm{kg}$. This method involves methanol extraction and analysis with a gas chromatograph-mass spectrometer (GC-MS) system. The results are in Table 3.1. Descriptions of all soil samples are in Appendix C, Table C.1. Replicate soil samples collected for verification analysis are discussed in Section 3.8.

\subsection{Groundwater Sampling Data}

Groundwater samples were collected for VOCs analyses from the three groundwaterbearing zones identified just above and within the bedrock formation. The shallow and deep water-bearing zones have low permeability and limited thicknesses and exhibit relatively poor production. The middle zone is the major production interval for the local private wells. Groundwater samples were collected from the shallow groundwater zone at 20 wells (MW01MW13, MW15S, MW16S1, MW16S2, MW17S-MW20S), from the middle groundwater zone at 8 wells (MW14M, MW15M, MW16M1, MW16M2, MW17M-MW20M), and from the deep groundwater zone at 5 wells (MW14D-MW18D) (Figure 2.3). Descriptions of the groundwater samples are in Appendix C, Table C.1. 
The selection of monitoring well locations for the shallow groundwater zone at and near the former grain storage facilities was based on analytical results for the initial grab groundwater samples collected during the drilling process or from the temporary monitoring wells (Section 2.2). Locations and results for grab water samples from the shallow groundwater zone are shown in Figure 2.2 and Table 3.2, respectively.

Groundwater samples were also collected from all accessible private wells around the former CCC/USDA facility and the former or existing private grain storage facilities at the Sisson, Willems, and Klassen residences. The locations of the private wells sampled are shown in Figure 2.4. Analytical results are in Table 3.3. Descriptions of water samples taken from the private wells are in Appendix C, Table C.1.

All groundwater samples were analyzed for VOCs at the AGEM Laboratory, according to a modification of EPA Method 524.2 (EPA 1995). Replicate groundwater samples collected for verification analysis with the EPA's Contract Laboratory Program (CLP) methodology are discussed in Section 3.8. The analytical results for groundwater samples from the private wells and the permanent monitoring wells are in Table 3.3.

\subsection{Installation of Permanent Monitoring Wells}

Thirty-three monitoring wells were installed at 19 locations to facilitate groundwater sampling and the monitoring of groundwater levels. At each location (Figure 2.3), the targeted groundwater-bearing zone was identified by evaluating continuous cores and analyzing their lithology, water contents, and stratigraphic relationships.

Monitoring wells were installed either by Argonne with the CPT or by Boart Longyear with a sonic rig. With the KDHE's approval, all of the wells were completed at the surface as flush mounts. Well construction data are in Table 3.4; well registration forms are in Appendix D.

\subsection{Groundwater Level Data}

Groundwater levels were measured both manually and automatically. The networks of observation points for water level measurements are shown in Figure 2.5 for the shallow waterbearing zone and Figure 2.6 for the middle water-bearing zone. Water levels were periodically 
measured manually in all wells. Continuous water level monitoring was conducted at selected wells in the shallow and middle groundwater-bearing zones to identify possible water level trends and to evaluate the responses to pumping at the Sisson, Pankratz (Newcombe), Willems, and Bengston private wells.

Manual measurements were taken by reading the depth to water in each well to the nearest $0.01 \mathrm{ft}$ with an electronic water level sensor, from a surveyed reference mark at the top of the well casing. The hand-measured water level data for the three groundwater-bearing zones are in Appendix E. Water levels were recorded automatically in selected wells by installing selfcontained water level sensors and data loggers (Instrumentation Northwest, Inc., Model PT2X ${ }^{\mathrm{TM}}$ ) programmed to collect measurements at intervals ranging from $30 \mathrm{~min}$ to $4 \mathrm{hr}$. The complete data logger records are in Supplement 1, on the compact disc (CD) inside the back cover of this report.

The gaps in the data for well MW16M1 (Supplement 1) were caused by an unknown electronic problem that resulted in depletion of the batteries. At the end of 2011, the recording of water levels in MW16M1 was terminated because of the electronic problem. The recorder was removed from the well during a site visit in March 2012.

For wells in which the observed water levels reached ambient static conditions, the water levels determined manually were used to construct estimates of the local potentiometric surfaces for the shallow and middle water-bearing zones. For the deep groundwater-bearing zone, monitoring data indicated that the groundwater levels in several wells were in recovery mode and had not reached equilibrium in the last data recovery in March 2012. To avoid generating potentially distorted flow patterns by using non-static water level data, no attempts were made to construct potentiometric surfaces for the deep water zone.

\subsection{Land Survey Data}

The exact locations and ground surface elevations of field activities are required to provide horizontal and vertical control for stratigraphic correlation, water level measurement, hydrogeologic mapping, and contaminant delineation in soil and groundwater. All investigative boring locations, all monitoring points, and most of the private wells were surveyed by licensed 
professional surveyors of Schwab-Eaton, P.A., Manhattan, Kansas. The results are in Appendix F, Table F.1.

\subsection{Results of Quality Control Activities}

The QA/QC procedures for sample collection, handling, and analysis during the 20092011 targeted investigation are described in detail in the Master Work Plan (Argonne 2002). A detailed QA/QC report addressing sample collection, handling, and analysis during the investigation is on CD, in Supplement 2.

The results of the QA/QC activities are summarized as follows:

- Sample integrity was maintained successfully during sample collection, shipping, and analysis through documentation of samples as they were collected and the use of custody seals and chain-of-custody records.

- All samples were received with custody seals intact and at the appropriate preservation conditions. Samples were analyzed within required holding times.

- Carbon tetrachloride and chloroform, the primary contaminants of concern, were not detected in laboratory method blanks.

- Quality control samples (field blanks, equipment rinsates, and trip blanks) were collected to monitor sample-handling activities. Carbon tetrachloride was not detected. Chloroform was detected in both field blanks collected (one of water used for drilling and the other of water used for equipment decontamination), both originating from the city water supply. The detections were both at low concentrations, consistent with the chlorination of the city water used during the investigation. Cross-contamination during sample collection and handling is not indicated.

- Soil and groundwater samples were analyzed for VOCs including carbon tetrachloride, chloroform, and methylene chloride at the AGEM Laboratory 
by using the purge-and-trap method (a GC-MS method) for quantitative determination of contaminant distribution. Dual analyses of samples indicated consistency in the sampling and analytical methodologies. Dual analyses were accomplished through either analysis of replicate samples submitted to the laboratory or duplicate analysis of samples selected by the laboratory. Consistency in both the sampling and analytical methodologies is indicated by the average relative percent difference values of $17 \%$ for carbon tetrachloride and $10 \%$ for chloroform in dual analyses with the contaminants present above the method detection limit.

- The analyses of soil and groundwater samples by the AGEM Laboratory were verified by a second laboratory (TestAmerica Laboratories, Inc., North Burlington, Vermont). Agreement in the results from the two laboratories is good over the range of contaminant concentrations detected. The concentrations detected in soil and groundwater in analyses at the AGEM Laboratory are supported by the verification analyses at TestAmerica.

- Groundwater samples collected for inorganic analyses to aid in geochemical characterization of the water-bearing zone were shipped to TestAmerica Laboratories, Inc., University Park, Illinois, for filtration and analysis. The analyses included total alkalinity by EPA Method SM 2320B, dissolved anion concentrations (chloride, sulfate, nitrate, and phosphate) by EPA Method 300, and dissolved metals (aluminum, calcium, iron, magnesium, manganese, phosphorus, potassium, silicon, sodium, and zinc) by EPA Method 6010B. The inorganic analyses are acceptable for geochemical characterization on the basis of the recovery of known concentrations of the analytes of concern in laboratory control samples analyzed with the groundwater samples.

\subsection{Waste Characterization, Handling, and Disposal}

Waste soil and water generated as potentially contaminated investigation-derived waste were containerized on-site. The accumulated waste soil and water were sampled on September 20, 2011, and analyzed by Pace Analytical Services, Inc., Lenexa, Kansas, on September 27-29, 2011. Waste soil was analyzed by EPA Method 8260 for VOCs and ASTM D2974-87 for percent moisture. Wastewater was analyzed by EPA Method 5030/8260 for VOCs, 
EPA Method 504.1 for ethylene dibromide, and EPA Method 353.2 for nitrate/nitrite nitrogen. No VOCs were detected in the soil or water. Nitrate/nitrite nitrogen was present in the wastewater at $4.8 \mathrm{mg} / \mathrm{L}$. The laboratory results are in Supplement 3, on CD. The soil cuttings were taken to the Reno County Landfill on November 14, 2011. The Inman wastewater (together with purge water from several other CCC/USDA investigation sites in Kansas) was delivered on December 19, 2011, for disposal at the Sabetha publicly owned wastewater treatment plant. 
TABLE 3.1 Analytical results from the AGEM Laboratory for soil samples collected in 2009-2010.

\begin{tabular}{|c|c|c|c|c|c|}
\hline \multirow[b]{2}{*}{ Location } & \multirow[b]{2}{*}{ Sample } & \multirow[b]{2}{*}{ Sample Date } & \multirow[b]{2}{*}{$\begin{array}{l}\text { Depth } \\
\text { (ft BGL) }\end{array}$} & \multicolumn{2}{|c|}{ Concentration $(\mu \mathrm{g} / \mathrm{kg})$} \\
\hline & & & & $\begin{array}{c}\text { Carbon } \\
\text { Tetrachloride }\end{array}$ & Chloroform \\
\hline \multicolumn{6}{|c|}{ Cone penetrometer sampling on the former CCC/USDA property } \\
\hline TI01 & INTI01-S-30620 & $11 / 4 / 09$ & 2 & $N^{a}$ & ND \\
\hline TI01 & INTI01-S-30621 & $11 / 4 / 09$ & 6 & ND & ND \\
\hline TI01 & INTI01-S-30622 & $11 / 4 / 09$ & 10 & ND & ND \\
\hline TIO1 & INTI01-S-30623 & $11 / 4 / 09$ & 14 & ND & ND \\
\hline TI01 & INTI01-S-30624 & $11 / 4 / 09$ & 18 & ND & ND \\
\hline TI01 & INTI01-S-30625 & $11 / 4 / 09$ & 22 & ND & ND \\
\hline TIO1 & INTI01-S-30630 & $11 / 4 / 09$ & 26 & ND & ND \\
\hline TI01 & INTI01-S-30626 & $11 / 4 / 09$ & 30 & $4.1 \mathrm{~J}^{\mathrm{b}}$ & ND \\
\hline TI01 & INTI01-S-30627 & 11/4/09 & 34 & ND & ND \\
\hline TIO1 & INTI01-S-30629 & $11 / 4 / 09$ & 38 & ND & ND \\
\hline TIO2 & INTI02-S-30631 & $11 / 4 / 09$ & 2 & ND & ND \\
\hline TI02 & INTI02-S-30632 & $11 / 4 / 09$ & 6 & ND & ND \\
\hline TIO2 & INTI02-S-30633 & $11 / 4 / 09$ & 10 & ND & ND \\
\hline TIO2 & INTI02-S-30634 & $11 / 4 / 09$ & 14 & ND & ND \\
\hline TI02 & INTI02-S-30635 & $11 / 4 / 09$ & 18 & ND & ND \\
\hline TIO2 & INTI02-S-30636 & $11 / 4 / 09$ & 22 & $4.2 \mathrm{~J}$ & ND \\
\hline TIO2 & INTI02-S-30637 & $11 / 4 / 09$ & 26 & $8.0 \mathrm{~J}$ & ND \\
\hline TIO2 & INTI02-S-30639 & $11 / 4 / 09$ & 30 & ND & ND \\
\hline TIO2 & INTI02-S-30640 & $11 / 4 / 09$ & 34 & ND & ND \\
\hline TIO2 & INTI02-S-30641 & $11 / 4 / 09$ & 38 & ND & ND \\
\hline TIO3 & INTI03-S-30643 & 11/5/09 & 2 & ND & ND \\
\hline TIO3 & INTI03-S-30644 & $11 / 5 / 09$ & 6 & ND & ND \\
\hline TI03 & INTI03-S-30645 & $11 / 5 / 09$ & 10 & ND & ND \\
\hline TIO3 & INTI03-S-30646 & $11 / 5 / 09$ & 14 & ND & ND \\
\hline TIO3 & INTI03-S-30647 & $11 / 5 / 09$ & 18 & ND & ND \\
\hline TI03 & INTI03-S-30648 & $11 / 5 / 09$ & 22 & ND & ND \\
\hline TIO3 & INTI03-S-30649 & $11 / 5 / 09$ & 26 & ND & ND \\
\hline TI03 & INTI03-S-30650 & $11 / 5 / 09$ & 30 & ND & ND \\
\hline TI03 & INTI03-S-30651 & 11/5/09 & 34 & ND & ND \\
\hline TI03 & INTI03-S-30652 & $11 / 5 / 09$ & 38 & ND & ND \\
\hline TI03 & INTI03-S-30653 & $11 / 5 / 09$ & 42 & ND & ND \\
\hline TIO4 & INTI04-S-30654 & $11 / 5 / 09$ & 2 & ND & ND \\
\hline TIO4 & INTI04-S-30655 & $11 / 5 / 09$ & 6 & ND & ND \\
\hline TIO4 & INTI04-S-30656 & 11/5/09 & 10 & ND & ND \\
\hline TI04 & INTI04-S-30657 & $11 / 5 / 09$ & 14 & ND & ND \\
\hline TIO4 & INTI04-S-30658 & $11 / 5 / 09$ & 18 & ND & ND \\
\hline TIO4 & INTI04-S-30659 & $11 / 5 / 09$ & 22 & ND & ND \\
\hline TIO4 & INTI04-S-30660 & $11 / 5 / 09$ & 26 & ND & ND \\
\hline TIO4 & INTI04-S-30661 & $11 / 5 / 09$ & 30 & ND & ND \\
\hline TI04 & INTI04-S-30662 & $11 / 5 / 09$ & 34 & ND & ND \\
\hline TIO4 & INTI04-S-30663 & $11 / 5 / 09$ & 38 & ND & ND \\
\hline TI05 & INTI05-S-30666 & 11/6/09 & 2 & ND & ND \\
\hline TI05 & INTI05-S-30667 & $11 / 6 / 09$ & 6 & ND & ND \\
\hline TI05 & INTI05-S-30668 & 11/6/09 & 10 & ND & ND \\
\hline TI05 & INTI05-S-30669 & $11 / 6 / 09$ & 14 & ND & ND \\
\hline
\end{tabular}


TABLE 3.1 (Cont.)

\begin{tabular}{|c|c|c|c|c|c|}
\hline \multirow[b]{2}{*}{ Location } & \multirow[b]{2}{*}{ Sample } & \multirow[b]{2}{*}{ Sample Date } & \multirow[b]{2}{*}{$\begin{array}{l}\text { Depth } \\
\text { (ft BGL) }\end{array}$} & \multicolumn{2}{|c|}{ Concentration $(\mu \mathrm{g} / \mathrm{kg})$} \\
\hline & & & & $\begin{array}{c}\text { Carbon } \\
\text { Tetrachloride }\end{array}$ & Chloroform \\
\hline \multicolumn{6}{|c|}{ Cone penetrometer sampling on the former CCC/USDA property (cont.) } \\
\hline TI05 & INTI05-S-30670 & 11/6/09 & 18 & ND & ND \\
\hline TI05 & INTI05-S-30671 & $11 / 6 / 09$ & 22 & ND & ND \\
\hline TI05 & INTI05-S-30672 & 11/6/09 & 26 & ND & ND \\
\hline TI05 & INTI05-S-30673 & 11/6/09 & 30 & ND & ND \\
\hline TI05 & INTI05-S-30674 & $11 / 6 / 09$ & 34 & ND & ND \\
\hline TI05 & INTI05-S-30675 & 11/6/09 & 38 & ND & ND \\
\hline TI06 & INTI06-S-30676 & 11/6/09 & 2 & ND & ND \\
\hline TI06 & INTI06-S-30677 & $11 / 6 / 09$ & 6 & ND & ND \\
\hline TI06 & INTI06-S-30678 & $11 / 6 / 09$ & 10 & ND & ND \\
\hline TI06 & INTI06-S-30679 & 11/6/09 & 14 & ND & ND \\
\hline TI06 & INTI06-S-30680 & $11 / 6 / 09$ & 18 & ND & ND \\
\hline TI06 & INTI06-S-30681 & 11/6/09 & 22 & ND & ND \\
\hline TI06 & INTI06-S-30682 & $11 / 6 / 09$ & 26 & ND & ND \\
\hline TIO6 & INTI06-S-30683 & $11 / 6 / 09$ & 30 & ND & ND \\
\hline TI06 & INTI06-S-30685 & 11/6/09 & 34 & ND & ND \\
\hline TI06 & INTI06-S-30686 & $11 / 6 / 09$ & 38 & ND & ND \\
\hline TI07 & INTI07-S-30687 & 11/6/09 & 2 & ND & ND \\
\hline TIO7 & INTI07-S-30688 & $11 / 6 / 09$ & 6 & ND & ND \\
\hline TI07 & INTI07-S-30689 & 11/6/09 & 10 & ND & ND \\
\hline TIO7 & INTI07-S-30690 & $11 / 6 / 09$ & 14 & ND & ND \\
\hline TI07 & INTI07-S-30691 & 11/6/09 & 18 & ND & ND \\
\hline TI07 & INTI07-S-30692 & $11 / 6 / 09$ & 22 & ND & ND \\
\hline TI07 & INTI07-S-30693 & $11 / 6 / 09$ & 26 & ND & ND \\
\hline TI07 & INTI07-S-30694 & $11 / 6 / 09$ & 30 & ND & ND \\
\hline TIO7 & INTI07-S-30695 & $11 / 6 / 09$ & 34 & ND & ND \\
\hline TIO7 & INTI07-S-30696 & 11/6/09 & 38 & ND & ND \\
\hline TI08 & INTI08-S-30698 & 11/7/09 & 2 & ND & ND \\
\hline TI08 & INTI08-S-30699 & $11 / 7 / 09$ & 6 & ND & ND \\
\hline TI08 & INTI08-S-30700 & $11 / 7 / 09$ & 10 & ND & ND \\
\hline TI08 & INTI08-S-30701 & 11/7/09 & 14 & ND & ND \\
\hline TI08 & INTI08-S-30702 & $11 / 7 / 09$ & 18 & ND & ND \\
\hline TI08 & INTI08-S-30703 & 11/7/09 & 22 & ND & ND \\
\hline TI08 & INTI08-S-30704 & 11/7/09 & 26 & ND & ND \\
\hline TI08 & INTI08-S-30706 & 11/7/09 & 30 & ND & ND \\
\hline TI08 & INTI08-S-30707 & 11/7/09 & 34 & ND & ND \\
\hline TI08 & INTI08-S-30708 & 11/7/09 & 38 & ND & ND \\
\hline TI09 & INTI09-S-30709 & 11/7/09 & 2 & ND & ND \\
\hline TI09 & INTI09-S-30710 & 11/7/09 & 6 & ND & ND \\
\hline TI09 & INTI09-S-30711 & 11/7/09 & 10 & ND & ND \\
\hline TI09 & INTI09-S-30712 & $11 / 7 / 09$ & 14 & ND & ND \\
\hline TI09 & INTI09-S-30713 & 11/7/09 & 18 & ND & ND \\
\hline TI09 & INTI09-S-30714 & $11 / 7 / 09$ & 22 & ND & ND \\
\hline TI09 & INTI09-S-30715 & 11/7/09 & 26 & ND & ND \\
\hline TI09 & INTI09-S-30716 & 11/7/09 & 30 & ND & ND \\
\hline TI09 & INTI09-S-30719 & $11 / 7 / 09$ & 34 & ND & ND \\
\hline TI09 & INTI09-S-30718 & 11/7/09 & 38 & ND & ND \\
\hline
\end{tabular}


TABLE 3.1 (Cont.)

\begin{tabular}{|c|c|c|c|c|c|}
\hline \multirow[b]{2}{*}{ Location } & \multirow[b]{2}{*}{ Sample } & \multirow[b]{2}{*}{ Sample Date } & \multirow[b]{2}{*}{$\begin{array}{c}\text { Depth } \\
\text { (ft BGL) }\end{array}$} & \multicolumn{2}{|c|}{ Concentration $(\mu \mathrm{g} / \mathrm{kg})$} \\
\hline & & & & $\begin{array}{c}\text { Carbon } \\
\text { Tetrachloride }\end{array}$ & Chlorof \\
\hline \multicolumn{6}{|c|}{ Cone penetrometer sampling on the former CCC/USDA property (cont.) } \\
\hline TI10 & INTI10-S-30720 & 11/7/09 & 2 & ND & ND \\
\hline TI10 & INTI10-S-30721 & $11 / 7 / 09$ & 6 & ND & ND \\
\hline TI10 & INTI10-S-30722 & 11/7/09 & 10 & ND & ND \\
\hline TI10 & INTI10-S-30723 & $11 / 7 / 09$ & 14 & ND & ND \\
\hline TI10 & INTI10-S-30724 & 11/7/09 & 18 & ND & ND \\
\hline TI10 & INTI10-S-30725 & 11/7/09 & 22 & ND & ND \\
\hline TI10 & INTI10-S-30726 & 11/7/09 & 26 & ND & ND \\
\hline TI10 & INTI10-S-30727 & 11/7/09 & 30 & ND & ND \\
\hline TI10 & INTI10-S-30728 & 11/7/09 & 34 & ND & ND \\
\hline TI10 & INTI10-S-30729 & 11/7/09 & 38 & $5.9 \mathrm{~J}$ & ND \\
\hline TI11 & INTI11-S-30734 & 11/8/09 & 2 & ND & ND \\
\hline TI11 & INTI11-S-30735 & $11 / 8 / 09$ & 6 & ND & ND \\
\hline TI11 & INTI11-S-30736 & 11/8/09 & 10 & ND & ND \\
\hline TI11 & INTI11-S-30737 & $11 / 8 / 09$ & 14 & ND & ND \\
\hline TI11 & INTI11-S-30738 & $11 / 8 / 09$ & 18 & ND & ND \\
\hline TI11 & INTI11-S-30739 & 11/8/09 & 22 & ND & ND \\
\hline TI11 & INTI11-S-30741 & 11/8/09 & 26 & ND & ND \\
\hline TI11 & INTI11-S-30742 & 11/8/09 & 30 & ND & ND \\
\hline TI11 & INTI11-S-30743 & 11/8/09 & 34 & ND & ND \\
\hline TI11 & INTI11-S-30744 & $11 / 8 / 09$ & 38 & ND & ND \\
\hline
\end{tabular}

Cone penetrometer sampling on private property west of the former CCC/USDA property

$\begin{array}{lllrrr}\text { TI19 } & \text { INTI19-S-30765 } & 11 / 10 / 10 & 4 & \text { ND } & \text { ND } \\ \text { TI19 } & \text { INTI19-S-30766 } & 11 / 10 / 10 & 8 & \text { ND } & \text { ND } \\ \text { TI19 } & \text { INTI19-S-30767 } & 11 / 10 / 10 & 12 & \text { ND } \\ \text { TI19 } & \text { INTI19-S-30768 } & 11 / 10 / 10 & 16 & \text { ND } & \text { ND } \\ \text { TI19 } & \text { INTI19-S-30769 } & 11 / 10 / 10 & 20 & \text { ND } \\ \text { TI19 } & \text { INTI19-S-30770 } & 11 / 10 / 10 & 24 & \text { ND } & \text { ND } \\ \text { TI19 } & \text { INTI19-S-30771 } & 11 / 10 / 10 & 28 & \text { ND } \\ \text { TI19 } & \text { INTI19-S-30772 } & 11 / 10 / 10 & 32 & \text { ND } & \text { ND } \\ \text { TI19 } & \text { INTI19-S-30774 } & 11 / 10 / 10 & 36 & \text { ND } \\ \text { TI19 } & \text { INTI19-S-30775 } & 11 / 10 / 10 & 39 & \text { ND } \\ & & & & \text { ND } & \text { ND } \\ \text { TI20 } & \text { INTI20-S-30790 } & 11 / 11 / 10 & 4 & \text { ND } \\ \text { TI20 } & \text { INTI20-S-30791 } & 11 / 11 / 10 & 8 & \text { ND } & \text { ND } \\ \text { TI20 } & \text { INTI20-S-30792 } & 11 / 11 / 10 & 12 & \text { ND } \\ \text { TI20 } & \text { INTI20-S-30793 } & 11 / 11 / 10 & 16 & \text { ND } & \text { ND } \\ \text { TI20 } & \text { INTI20-S-30794 } & 11 / 11 / 10 & 20 & \text { ND } \\ \text { TI20 } & \text { INTI22-S-30795 } & 11 / 11 / 10 & 24 & \text { ND } & \text { ND } \\ \text { TI20 } & \text { INTI20-S-30796 } & 11 / 11 / 10 & 28 & & \text { ND } \\ \text { TI20 } & \text { INTI20-S-30797 } & 11 / 11 / 10 & 32 & \text { ND } & \text { ND } \\ \text { TI20 } & \text { INTI20-S-30798 } & 11 / 11 / 10 & 36 & \text { ND } & \text { ND } \\ & & & & \text { ND } & \text { ND } \\ \text { TI21 } & \text { INTI21-S-30800 } & 11 / 11 / 10 & 4 & \text { ND } \\ \text { TI21 } & \text { INTI21-S-30801 } & 11 / 11 / 10 & 8 & \text { ND } \\ \text { TI21 } & \text { INTI21-S-30802 } & 11 / 11 / 10 & 12 & \text { ND } \\ \text { TI21 } & \text { INTI21-S-30803 } & 11 / 11 / 10 & 16 & 20 & \\ \text { TI21 } & \text { INTI21-S-30804 } & 11 / 11 / 10 & 20 & & \\ \text { TI21 } & \text { INTI21-S-30805 } & 11 / 11 / 10 & 24 & & \end{array}$


TABLE 3.1 (Cont.)

\begin{tabular}{|c|c|c|c|c|c|}
\hline \multirow[b]{2}{*}{ Location } & \multirow[b]{2}{*}{ Sample } & \multirow[b]{2}{*}{ Sample Date } & \multirow[b]{2}{*}{$\begin{array}{l}\text { Depth } \\
\text { (ft BGL) }\end{array}$} & \multicolumn{2}{|c|}{ Concentration $(\mu \mathrm{g} / \mathrm{kg})$} \\
\hline & & & & $\begin{array}{c}\text { Carbon } \\
\text { Tetrachloride }\end{array}$ & Chloroform \\
\hline \multicolumn{6}{|c|}{$\begin{array}{l}\text { Cone penetrometer sampling on private property west of the former CCC/USDA property } \\
\text { (cont.) }\end{array}$} \\
\hline TI21 & INTI21-S-30806 & $11 / 11 / 10$ & 28 & ND & ND \\
\hline $\mathrm{TI} 21$ & INTI21-S-30807 & $11 / 11 / 10$ & 32 & ND & ND \\
\hline TI21 & INTI21-S-30808 & $11 / 11 / 10$ & 36 & ND & ND \\
\hline TI22 & INTI22-S-30809 & $11 / 11 / 10$ & 4 & ND & ND \\
\hline TI22 & INTI22-S-30810 & $11 / 11 / 10$ & 8 & ND & ND \\
\hline TI22 & INTI22-S-30811 & $11 / 11 / 10$ & 12 & ND & ND \\
\hline TI22 & INTI22-S-30818 & $11 / 11 / 10$ & 16 & ND & ND \\
\hline TI22 & INTI22-S-30813 & $11 / 11 / 10$ & 20 & ND & ND \\
\hline $\mathrm{TI} 22$ & INTI22-S-30814 & $11 / 11 / 10$ & 24 & ND & ND \\
\hline TI22 & INTI22-S-30815 & $11 / 11 / 10$ & 28 & ND & ND \\
\hline $\mathrm{TI} 22$ & INTI22-S-30816 & $11 / 11 / 10$ & 32 & ND & ND \\
\hline TI22 & INTI22-S-30817 & $11 / 11 / 10$ & 36 & ND & ND \\
\hline TI23 & INTI23-S-30819 & $11 / 11 / 10$ & 4 & ND & ND \\
\hline TI23 & INTI23-S-30820 & $11 / 11 / 10$ & 8 & ND & ND \\
\hline TI23 & INTI23-S-30821 & $11 / 11 / 10$ & 12 & ND & ND \\
\hline TI23 & INTI23-S-30822 & $11 / 11 / 10$ & 16 & ND & ND \\
\hline $\mathrm{TI} 23$ & INTI23-S-30823 & $11 / 11 / 10$ & 20 & ND & ND \\
\hline TI23 & INTI23-S-30824 & $11 / 11 / 10$ & 24 & ND & ND \\
\hline TI23 & INTI23-S-30825 & $11 / 11 / 10$ & 28 & ND & ND \\
\hline TI23 & INTI23-S-30826 & $11 / 11 / 10$ & 32 & ND & ND \\
\hline $\mathrm{TI} 23$ & INTI23-S-30827 & $11 / 11 / 10$ & 36 & ND & ND \\
\hline TI23 & INTI23-S-30839 & $11 / 11 / 10$ & 38.2 & ND & ND \\
\hline TI24 & INTI24-S-30828 & $11 / 12 / 10$ & 4 & ND & ND \\
\hline TI24 & INTI24-S-30829 & $11 / 12 / 10$ & 8 & ND & ND \\
\hline TI24 & INTI24-S-30830 & $11 / 12 / 10$ & 12 & ND & ND \\
\hline TI24 & INTI24-S-30831 & $11 / 11 / 10$ & 16 & ND & ND \\
\hline TI24 & INTI24-S-30832 & $11 / 12 / 10$ & 20 & ND & ND \\
\hline TI24 & INTI24-S-30833 & $11 / 12 / 10$ & 24 & ND & ND \\
\hline TI24 & INTI24-S-30834 & $11 / 12 / 10$ & 28 & ND & ND \\
\hline TI24 & INTI24-S-30835 & $11 / 12 / 10$ & 32 & ND & ND \\
\hline TI24 & INTI24-S-30836 & $11 / 12 / 10$ & 36 & ND & ND \\
\hline TI24 & INTI24-S-30838 & $11 / 12 / 10$ & 39 & ND & ND \\
\hline TI25 & INTI25-S-30840 & $11 / 12 / 10$ & 4 & ND & ND \\
\hline TI25 & INTI25-S-30841 & $11 / 12 / 10$ & 8 & ND & ND \\
\hline TI25 & INTI25-S-30842 & $11 / 12 / 10$ & 12 & ND & ND \\
\hline TI25 & INTI25-S-30843 & $11 / 12 / 10$ & 16 & ND & ND \\
\hline TI25 & INTI25-S-30844 & $11 / 12 / 10$ & 20 & ND & ND \\
\hline TI25 & INTI25-S-30845 & $11 / 12 / 10$ & 24 & ND & ND \\
\hline TI25 & INTI25-S-30846 & $11 / 12 / 10$ & 28 & ND & ND \\
\hline TI25 & INTI25-S-30848 & $11 / 12 / 10$ & 36 & ND & ND \\
\hline
\end{tabular}

a ND, compound analyzed for but not detected at a level greater than or equal to the method detection limit $(<10 \mu \mathrm{g} / \mathrm{kg})$.

b $\mathrm{J}$, compound identified with an estimated concentration between the instrument detection limit and the method detection limit. 
TABLE 3.2 Analytical results for grab groundwater samples collected at temporary piezometers in 2009-2010.

\begin{tabular}{|c|c|c|c|c|c|}
\hline \multirow[b]{2}{*}{ Location } & \multirow[b]{2}{*}{ Sample } & \multirow[b]{2}{*}{$\begin{array}{l}\text { Sampling } \\
\text { Date }\end{array}$} & \multirow[b]{2}{*}{$\begin{array}{l}\text { Depth } \\
\text { (ft BGL) }\end{array}$} & \multicolumn{2}{|c|}{ Concentration $(\mu \mathrm{g} / \mathrm{L})$} \\
\hline & & & & $\begin{array}{c}\text { Carbon } \\
\text { Tetrachloride }\end{array}$ & Chloroform \\
\hline TI01 & INTI01-W-30733 & $11 / 7 / 09$ & $26-40$ & 34 & 3.4 \\
\hline TIO1 & INTI01-W-30750 & $11 / 9 / 09$ & $33-38$ & 23 & 2.4 \\
\hline TI01 & INTI01-W-30749 & $11 / 9 / 09$ & $30-40$ & 18 & 3.0 \\
\hline TIO2 & INTI02-W-30758 & 11/11/09 & $30-40$ & 3.1 & 1.0 \\
\hline TIO4 & INTI04B-W-30746 & $11 / 9 / 09$ & $40-42$ & $N D^{a}$ & $0.6 \mathrm{~J}^{\mathrm{b}}$ \\
\hline TIO4 & INTI04-W-30747 & $11 / 9 / 09$ & $30-40$ & ND & ND \\
\hline TI05 & INTI05-W-30748 & $11 / 9 / 09$ & $30-40$ & ND & ND \\
\hline TI06 & INTI06-W-30756 & 11/11/09 & $28-33$ & 22 & 3.1 \\
\hline TI06 & INTI06-W-30757 & 11/11/09 & $33-38$ & 12 & $0.9 \mathrm{~J}$ \\
\hline T108 & INTI08-W-30752 & $11 / 9 / 09$ & $30-40$ & ND & ND \\
\hline TI11 & INTI11-W-30751 & $11 / 9 / 09$ & $28-33$ & 12 & 4.5 \\
\hline TI11 & INTI11-W-30755 & 11/11/09 & $33-38$ & 8.3 & 3.2 \\
\hline TI12 & INTI12-W-32679 & $11 / 10 / 10$ & $32-42$ & ND & ND \\
\hline TI13 & INTI13-W-30783 & $11 / 11 / 10$ & $31.7-41.7$ & ND & ND \\
\hline TI14 & INTI14-W-30762 & 11/10/10 & 29.5-39.5 & ND & ND \\
\hline TI14 & INTI14-W-30763 & 11/10/10 & $29.5-39.5$ & ND & ND \\
\hline TI15 & INTI15-W-30784 & $11 / 11 / 10$ & $31.7-41.7$ & ND & ND \\
\hline TI16 & INTI16-W-30785 & $11 / 11 / 10$ & $31.2-41.2$ & ND & ND \\
\hline TI17 & INTI17-W-30853 & $11 / 12 / 10$ & $32.1-42.1$ & ND & ND \\
\hline TI18 & INTI18-W-30786 & $11 / 11 / 10$ & $30.6-40.6$ & ND & ND \\
\hline TI22 & INTI22-W-32680 & $11 / 14 / 10$ & $30-40$ & ND & ND \\
\hline TI22 & INTI22-W-32699c & $12 / 22 / 10$ & $30-40$ & $0.05 \mathrm{~J}$ & ND \\
\hline TI23 & INTI23-W-32681 & $11 / 14 / 10$ & $30-40$ & ND & ND \\
\hline TI23 & INTI23-W-32700' & $12 / 22 / 10$ & $30-40$ & $0.04 \mathrm{~J}$ & ND \\
\hline TI24 & INTI24-W-32682 & $11 / 14 / 10$ & 29.1-39.1 & ND & ND \\
\hline TI24 & INTI25-W-32701 c,d & $12 / 22 / 10$ & 29.1-39.1 & $0.3 \mathrm{~J}$ & ND \\
\hline TI26 & INTI26-W-32683 & 11/18/10 & $31.1-41.1$ & ND & ND \\
\hline
\end{tabular}




\section{TABLE 3.2 (Cont.)}

a ND, compound analyzed for but not detected at a level greater than or equal to the method detection limit $(<1 \mu \mathrm{g} / \mathrm{L}$ for analyses at the AGEM Laboratory or $<0.5 \mu \mathrm{g} / \mathrm{L}$ for analyses at TestAmerica).

b J, compound detected with an estimated concentration between the instrument detection limit and the method detection limit.

c Samples collected at TI22-TI24 in December 2010 were analyzed at TestAmerica, because the AGEM Laboratory was closed for the holiday season.

d Typographical error in the sample identifier; sample is actually from TI24, not TI25. 
TABLE 3.3 Analytical results for groundwater samples collected from private wells and permanent monitoring wells in 2010-2011. ${ }^{\text {a }}$

\begin{tabular}{|c|c|c|c|c|c|c|}
\hline \multirow[b]{2}{*}{ Location } & \multirow[b]{2}{*}{ Sample } & \multirow[b]{2}{*}{$\begin{array}{l}\text { Sample } \\
\text { Date }\end{array}$} & \multirow[b]{2}{*}{$\begin{array}{l}\text { Depth from } \\
\text { (ft BGL) }\end{array}$} & \multirow[b]{2}{*}{$\begin{array}{l}\text { Depth to } \\
\text { (ft BGL) }\end{array}$} & \multicolumn{2}{|c|}{ Concentration $(\mu \mathrm{g} / \mathrm{L})$} \\
\hline & & & & & $\begin{array}{c}\text { Carbon } \\
\text { Tetrachloride }\end{array}$ & Chloroform \\
\hline \multicolumn{7}{|l|}{ Private wells } \\
\hline Bengston & INBENGSTEN-W-32675 & $11 / 9 / 10$ & -- & -- & 5.0 & 1.4 \\
\hline $\begin{array}{l}\text { Newcombe } \\
\text { (formerly Pankratz) }\end{array}$ & INPANKRATZ-W-32674 & $11 / 9 / 10$ & -- & -- & 5.0 & 1.1 \\
\hline Sisson & INSISSON-W-30761 & $11 / 9 / 10$ & -- & -- & 4.9 & 1.2 \\
\hline Willems & INWILLEMS-W-32673 & $11 / 9 / 10$ & -- & -- & 4.0 & 1.0 \\
\hline Brown & INBROWN-W-32686 & $5 / 24 / 11$ & -- & -- & $N D^{b}$ & ND \\
\hline Goertzen & INGOERTZEN-W-32688 & $5 / 24 / 11$ & -- & -- & ND & ND \\
\hline Hull (Windmill) & INHULL-W-32691 & $5 / 24 / 11$ & -- & -- & ND & ND \\
\hline Hull (House) & INHULL-W-32694 & $6 / 23 / 11$ & -- & -- & ND & ND \\
\hline Isaac & INISAAC-W-32689 & $5 / 24 / 11$ & -- & -- & ND & $0.7 \mathrm{Jc}^{\mathrm{c}}$ \\
\hline Klassen & INKLASSEN-W-32687 & $5 / 24 / 11$ & -- & -- & 6.0 & $0.2 \mathrm{~J}$ \\
\hline Klassen & INKLASSEN-W-32693 & $6 / 23 / 11$ & -- & -- & 5.6 & ND \\
\hline J. Pankratz & INPANKRATZ-W-32690 & $5 / 24 / 11$ & -- & -- & ND & ND \\
\hline Pauls & INPAULS-W-32695 & $6 / 23 / 11$ & -- & -- & ND & ND \\
\hline Thiessen & INTHIESSEN-W-32685 & $5 / 24 / 11$ & -- & -- & ND & ND \\
\hline Klassen test well & INKTW-W-32743 & $8 / 25 / 11$ & -- & 135.2 & 14 & $0.5 \mathrm{~J}$ \\
\hline \multicolumn{7}{|l|}{ Monitoring wells } \\
\hline MW01 & INMW01-W-32670 & $11 / 9 / 10$ & 32 & 42 & 3.6 & 2.6 \\
\hline MW01 & INMW01-W-32685 & $12 / 22 / 10$ & 32 & 42 & 5.4 & 3.2 \\
\hline MW02 & INMW02-W-32671 & $11 / 9 / 10$ & 32 & 42 & 2.7 & 4.6 \\
\hline MW02 & INMW02-W-32686 & $12 / 22 / 10$ & 32 & 42 & 2.9 & 4.8 \\
\hline MW03 & INMW03-W-32672 & $11 / 9 / 10$ & 35 & 45 & $0.4 \mathrm{~J}$ & 1.4 \\
\hline MW03 & INMW03-W-32687 & $12 / 22 / 10$ & 35 & 45 & 2.2 & 1.5 \\
\hline MW04 & INMW04-W-32677 & $11 / 10 / 10$ & 30 & 40 & ND & ND \\
\hline MW04 & INMW04-W-32688 & $12 / 22 / 10$ & 30 & 40 & $0.22 \mathrm{~J}$ & $0.050 \mathrm{~J}$ \\
\hline MW05 & INMW05-W-32678 & $11 / 10 / 10$ & 35 & 45 & ND & ND \\
\hline MW05 & INMW05-W-32689 & $12 / 22 / 10$ & 35 & 45 & $0.091 \mathrm{~J}$ & ND \\
\hline MW06 & INMW06-W-32690 & $12 / 22 / 10$ & 32 & 42 & ND & ND \\
\hline MW07 & INMW07-W-32691 & $12 / 22 / 10$ & 31.7 & 41.7 & ND & ND \\
\hline MW08 & INMW08-W-32692 & $12 / 22 / 10$ & 31.1 & 41.1 & ND & ND \\
\hline MW09 & INMW09-W-32693 & $12 / 22 / 10$ & 29.5 & 39.5 & ND & ND \\
\hline MW10 & INMW10-W-32694 & $12 / 22 / 10$ & 31.7 & 41.7 & ND & ND \\
\hline MW11 & INMW11-W-32696 & $12 / 22 / 10$ & 31.2 & 41.2 & ND & ND \\
\hline MW12 & INMW12-W-32697 & $12 / 22 / 10$ & 32.1 & 42.1 & ND & ND \\
\hline MW13 & INMW13-W-32698 & $12 / 22 / 10$ & 30.6 & 40.6 & ND & ND \\
\hline MW14M & INMW14S-W-32736 & $8 / 24 / 11$ & 85 & 95 & 1.7 & 2.0 \\
\hline MW14D & INMW14D-W-32782 & $9 / 15 / 11$ & 115 & 125 & ND & $0.6 \mathrm{~J}$ \\
\hline MW15S & INMW15S-W-32757 & 8/29/11 & 35 & 45 & ND & ND \\
\hline MW15M & INMW15-W-32751 & $8 / 28 / 11$ & 86 & 96 & 2.9 & 1.5 \\
\hline MW15D & INMW15D-W-32759 & $8 / 29 / 11$ & 115 & 125 & ND & 2.3 \\
\hline MW16S1 & INMW16-W-32762 & $8 / 29 / 11$ & 25 & 35 & 1.3 & $0.5 \mathrm{~J}$ \\
\hline MW16S2 & INMW16-W-32765 & $8 / 29 / 11$ & 40 & 45 & 2.3 & $0.4 \mathrm{~J}$ \\
\hline MW16M1 & INMW16-W-32767 & 8/30/11 & 60 & 70 & ND & $0.4 \mathrm{~J}$ \\
\hline MW16M2 & INMW16-W-32764 & $8 / 29 / 11$ & 78 & 88 & ND & $0.4 \mathrm{~J}$ \\
\hline MW16D & INMW16D-W-32756 & $8 / 29 / 11$ & 100 & 110 & ND & 1.8 \\
\hline MW17S & INMW17S-W-32772 & 8/31/11 & 25 & 35 & ND & $0.8 \mathrm{~J}$ \\
\hline MW17M & INMW17M-W-32770 & $8 / 31 / 11$ & 72 & 82 & $0.9 \mathrm{~J}$ & ND \\
\hline MW17D & INMW17D-W-32773 & $8 / 31 / 11$ & 98 & 108 & ND & ND \\
\hline
\end{tabular}


TABLE 3.3 (Cont.)

\begin{tabular}{|c|c|c|c|c|c|c|}
\hline \multirow[b]{2}{*}{ Location } & \multirow[b]{2}{*}{ Sample } & \multirow[b]{2}{*}{$\begin{array}{l}\text { Sample } \\
\text { Date }\end{array}$} & \multirow[b]{2}{*}{$\begin{array}{l}\text { Depth from } \\
\text { (ft BGL) }\end{array}$} & \multirow[b]{2}{*}{$\begin{array}{l}\text { Depth to } \\
\text { (ft BGL) }\end{array}$} & \multicolumn{2}{|c|}{ Concentration $(\mu \mathrm{g} / \mathrm{L})$} \\
\hline & & & & & $\begin{array}{c}\text { Carbon } \\
\text { Tetrachloride }\end{array}$ & Chloroform \\
\hline \multicolumn{7}{|c|}{ Monitoring wells (cont.) } \\
\hline MW18S & INMW18S-W-32775 & $9 / 1 / 11$ & 40 & 50 & 4.5 & 1.0 \\
\hline MW18M & INMW18M-W-32776 & $9 / 1 / 11$ & 85 & 95 & 3.3 & 1.0 \\
\hline MW18D & INMW18D-W-32777 & $9 / 1 / 11$ & 115 & 125 & ND & 1.0 \\
\hline MW19S & INMW19S-W-32789 & $9 / 2 / 11$ & 38 & 48 & $0.8 \mathrm{~J}$ & $0.4 \mathrm{~J}$ \\
\hline MW19M & INMW19D-W-32790 & $9 / 2 / 11$ & 85 & 95 & ND & ND \\
\hline MW20S & INMW20S-W-32780 & $9 / 15 / 11$ & 30 & 40 & ND & $0.7 \mathrm{~J}$ \\
\hline MW20M & INMW20M-W-32781 & $9 / 15 / 11$ & 72 & 82 & ND & $0.4 \mathrm{~J}$ \\
\hline
\end{tabular}

a Samples were analyzed at the AGEM Laboratory by modified purge-and-trap Method E524.2, except for samples collected in December 2010, which were analyzed at TestAmerica by EPA Method SOM01 (trace volatiles).

b ND, compound analyzed for but not detected at a level greater than or equal to the method detection limit $(<1 \mu \mathrm{g} / \mathrm{L}$ for analyses at the AGEM Laboratory or $<0.5 \mu \mathrm{g} / \mathrm{L}$ for analyses at TestAmerica).

c J, compound identified with an estimated concentration between the instrument detection limit and the method detection limit. 
TABLE 3.4 Construction data for monitoring wells installed during the targeted investigation.

\begin{tabular}{|c|c|c|c|c|c|c|}
\hline \multirow[b]{2}{*}{ Well } & \multirow[b]{2}{*}{$\begin{array}{c}\text { KDHE } \\
\text { Registration } \\
\text { Number }\end{array}$} & \multicolumn{3}{|c|}{ Depth (ft BGL) } & \multirow[b]{2}{*}{$\begin{array}{l}\text { Casing } \\
\text { Diameter } \\
\text { (in.) }\end{array}$} & \multirow[b]{2}{*}{$\begin{array}{c}\text { Completion } \\
\text { Date }\end{array}$} \\
\hline & & $\begin{array}{l}\text { Well } \\
\text { Depth }\end{array}$ & $\begin{array}{l}\text { Screen } \\
\text { Interval }\end{array}$ & $\begin{array}{l}\text { Filter } \\
\text { Pack }\end{array}$ & & \\
\hline \multicolumn{7}{|c|}{ Shallow groundwater-bearing zone } \\
\hline MW01 & 460421 & 42 & $32-42$ & $30-42$ & 1 & $11 / 08 / 09$ \\
\hline MW02 & 460422 & 42 & $32-42$ & $30-42$ & 1 & $11 / 04 / 09$ \\
\hline MW03 & 460423 & 45 & $35-45$ & $33-45$ & 1 & $11 / 06 / 09$ \\
\hline MW04 & 460424 & 40 & $30-40$ & $28-40$ & 1 & $11 / 05 / 09$ \\
\hline MW05 & 460425 & 45 & $35-45$ & $33-45$ & 1 & $11 / 07 / 09$ \\
\hline MW06 & 460426 & 42 & $32-42$ & $30-42$ & 1 & $11 / 08 / 10$ \\
\hline MW07 & 460427 & 41.7 & $31.7-41.7$ & $30-41.7$ & 1 & $11 / 08 / 10$ \\
\hline MW08 & 460428 & 41.1 & 31.1-41.1 & $30-41.7$ & 1 & $11 / 13 / 10$ \\
\hline MW09 & 460429 & 39.5 & $29.5-39.5$ & 28-39.5 & 1 & $11 / 09 / 10$ \\
\hline MW10 & 460430 & 41.7 & $31.7-41.7$ & $30-41.7$ & 1 & $11 / 09 / 10$ \\
\hline MW11 & 460431 & 41.2 & $31.2-41.2$ & $29-41.2$ & 1 & $11 / 10 / 10$ \\
\hline MW12 & 460432 & 42.1 & $32.1-42.1$ & $30-42.1$ & 1 & $11 / 10 / 10$ \\
\hline MW13 & 460433 & 40.6 & $30.6-40.6$ & $28-40.6$ & 1 & $11 / 10 / 10$ \\
\hline MW15S & 449520 & 45 & 35-45 & $33-45$ & 2 & $8 / 27 / 11$ \\
\hline MW16S1 & 449516 & 35 & $25-35$ & $23-35$ & 2 & $8 / 26 / 11$ \\
\hline MW16S2 & 449517 & 45 & $40-45$ & $38-45$ & 2 & $8 / 26 / 11$ \\
\hline MW17S & 454724 & 35 & $25-35$ & $23-35$ & 1 & $8 / 28 / 11$ \\
\hline MW18S & 449512 & 25 & $40-50$ & $38-52$ & 1 & 8/30/11 \\
\hline MW19S & 449509 & 48 & $38-48$ & $36-50$ & 2 & $8 / 31 / 11$ \\
\hline MW20S & 449521 & 40 & $30-40$ & $28-40$ & 2 & $9 / 13 / 11$ \\
\hline \multicolumn{7}{|c|}{ Middle groundwater-bearing zone } \\
\hline MW14M & 449506 & 95 & $85-95$ & 83-95 & 2 & 8/23/11 \\
\hline MW15M & 449519 & 96 & $86-96$ & $84-96$ & 2 & $8 / 24 / 11$ \\
\hline MW16M1 & 449514 & 70 & $60-70$ & $58-70$ & 1 & $8 / 26 / 11$ \\
\hline MW16M2 & 429515 & 88 & $78-88$ & $76-88$ & 2 & $8 / 26 / 11$ \\
\hline MW17M & 454723 & 82 & $72-82$ & $70-84$ & 2 & $8 / 28 / 11$ \\
\hline MW18M & 449511 & 95 & $85-95$ & $83-97$ & 2 & $8 / 30 / 11$ \\
\hline MW19M & 449508 & 95 & $85-95$ & 83-100 & 2 & $8 / 31 / 11$ \\
\hline MW20M & 449522 & 82 & $72-82$ & $70-89.5$ & 2 & $9 / 13 / 11$ \\
\hline \multicolumn{7}{|c|}{ Deep groundwater-bearing zone } \\
\hline MW14D & 424087 & 125 & $115-125$ & $113-125$ & 2 & $8 / 23 / 11$ \\
\hline MW15D & 424088 & 125 & $115-125$ & 113-135 & 2 & $8 / 24 / 11$ \\
\hline MW16D & 424089 & 110 & $100-110$ & $98-110$ & 2 & $8 / 26 / 11$ \\
\hline MW17D & 424090 & 108 & $98-108$ & $96-111$ & 2 & $8 / 28 / 11$ \\
\hline MW18D & 424091 & 125 & $115-125$ & $113-125$ & 2 & 8/30/11 \\
\hline
\end{tabular}




\section{Data Interpretation}

This section presents the interpretation of data acquired in all five phases of the targeted investigation at Inman. As discussed in the previous sections, all investigation activities were designed and implemented to address the following specific technical objectives defined in Section 1.2:

- Evaluate the potential source of carbon tetrachloride at the former CCC/USDA facility.

- Determine the relationship of potential contamination at the former CCC/USDA facility to contamination identified in 1997 in groundwater samples from the Sisson, Pankratz (Newcombe), Willems, and Bengston private wells.

- Delineate the extent of any identified contamination potentially associated with the former CCC/USDA facility.

The results of the investigation are discussed and interpreted below in the context of these specific objectives.

\subsection{Extent of Soil Contamination and Potential Contaminant Sources}

An extensive program of near-surface and subsurface soil sampling was conducted, as proposed in the Final Work Plan (Argonne 2007), on the former CCC/USDA property in Phase 1. This program was extended in Phase 2 to selected locations near former private grain storage facilities on the Sisson and Willems properties. The purpose of the soil sampling was to identify any remaining carbon tetrachloride contamination in soil that might be associated with past operations at the former grain storage facilities and to determine the extent of contamination and its potential future contribution as a contaminant source to groundwater. The results of VOCs analyses on soil samples are in Table 3.1.

The vadose zone in the study area includes the unconsolidated intervals from the ground surface to depths of approximately 34.5-38 ft BGL and the underlying bedrock formation to the 
depth of the shallow groundwater zone $(<46 \mathrm{ft} \mathrm{BGL})$. The vadose zone usually represents an unsaturated zone of soils and/or rocks from the land surface to the uppermost water-bearing zone.

\subsubsection{Contaminant Distribution in Soils at the Former CCC/USDA Facility}

Soil samples were collected at 4 -ft intervals through the vadose zone at 11 boring locations (TI01-TI11) within the footprint of the former CCC/USDA facility (Figure 4.1). All soil samples were recovered by using the CPT unit and were analyzed for VOCs by the purgeand-trap method (Section 3.3).

Trace concentrations of carbon tetrachloride (below the purge-and-trap method detection limit of $10 \mu \mathrm{g} / \mathrm{kg}$ ) were identified in samples from three locations (TI01, TI02, and TI10; Table 3.1). No chloroform was detected in any soil sample from any location. The maximum concentrations of carbon tetrachloride at each location are illustrated in Figure 4.1. Soil contamination at trace levels was detected only in the southern portion of the former CCC/USDA property.

As Table 3.1 shows, the vertical distribution indicates that trace contamination in soil occurs primarily in two intervals: $20-30 \mathrm{ft}$ BGL at TI01-TI02 and $38 \mathrm{ft}$ BGL at TI10. The contamination in the upper interval might represent residual contamination adsorbed to soil. The contamination in the lower interval might be an effect of the underlying contaminated shallow groundwater zone in the upper part of the bedrock. However, the overall distribution and concentrations of carbon tetrachloride in the vadose zone are very limited and would not be a future source of contamination to groundwater.

\subsubsection{Contaminant Distribution in Soils at the Former Private Grain Storage Facilities on the Sisson and Willems Properties}

Soil profiling through the vadose zone was extended to the former private grain storage facilities on the Sisson and Willems properties, to identify likely locations of soil contamination as current and future contaminant sources to groundwater. The locations were selected on the basis of (1) the approximate locations of former grain bins and a Quonset and (2) accessibility for the CPT unit. 
Soil samples were collected at five locations (TI19-TI23) on the Sisson property and two locations (TI24-TI25) on the Willems property (Figure 4.1). No carbon tetrachloride or chloroform was detected. The results indicate that soils at these locations would not be a future source of contamination to groundwater.

\subsection{Hydrogeologic Setting and Groundwater-Bearing System}

This section describes the local geology, stratigraphy, and hydrostratigraphy of the groundwater-bearing system at Inman, determined on the basis of the site-specific investigation data. The following data were collected and integrated to develop this interpretation: (1) geologic and sensor logs generated from shallow CPT borings at 18 locations, (2) geologic logs from deep drilled borings at 7 locations, (3) supporting geologic data including drillers' logs for local private wells, and (4) topographic survey data for all of the borings and private wells. These data were analyzed to provide a detailed interpretation of the stratigraphic sequence and the spatial distribution of the lithologic units that host the groundwater-bearing system. The lithologic logs (for the investigative borings and monitoring wells) and the survey data are in Appendix B and Appendix F, respectively.

\subsubsection{Local Lithostratigraphic Units}

Inman lies in the northern part of the Wellington-McPherson Lowland physiographic province. The topography of the Inman area is generally flat to very gently rolling. The city of Inman and the former grain storage facilities to the south are located along the crest of a local surface drainage divide trending roughly north-south (Figure 4.2) at an elevation of approximately $1,520 \mathrm{ft}$ AMSL (above mean sea level). The headwaters of several intermittent creeks dissect the surface near the former CCC/USDA property to the southwest, south, and southeast, creating local topographic relief of 40-70 ft (Figure 4.2).

Regional geologic information indicates that the geologic sequence in the vicinity of Inman includes (1) surficial and near-surface Pleistocene eolian (wind-blown) deposits and (2) the underlying Permian Ninnescah Shale Formation, which is composed largely of brick red shale with some thin beds of gray and green shale, as well as argillaceous limestone (Argonne 2007). The site-specific geologic data obtained in the targeted investigation are consistent with this interpretation. 
Figure 4.3 summarizes the general sequence of the six lithostratigraphic units identified to a depth of approximately $125 \mathrm{ft}$ BGL in the study area. In order of increasing depth, these units are (1) surficial silt and clay with a lower section of caliche overlying gravelly clay to clayey gravel, (2) weathered red shale, (3) laminated shale, (4) shale with evaporitic minerals precipitated along bedding planes and fractures, (5) limy shale, and (6) gray dolomitic shale. The shales identified in Units 2-6 are inferred to be part of Permian Ninnescah Shale, on the basis of the regional geologic setting. The units are illustrated across the investigation area in two hydrogeologic cross sections constructed for the locations shown in Figure 4.4. The main characteristics of each unit are as follows:

- Unit 1 - Unconsolidated silt, clay, and caliche. This uppermost unit contains Pleistocene eolian (wind-blown) silts and clays and varies in thickness up to $44 \mathrm{ft}$. The upper section of the unit mainly contains clayey silt marked by scattered patches of sand, with iron oxide staining near the base. The lower section of the unit consistently includes a caliche horizon that contains red clay with abundant carbonate patches or gravel cemented by carbonate. Underlying the caliche horizon at locations to the south (MW15 and MW20) and southwest (MW16) of the former CCC/USDA facility (Figure 4.4), an interval of gravelly clay or clayey gravel, $3.5-11.5 \mathrm{ft}$ thick, overlies the eroded, unconformable surface of the Permian bedrock. Beneath the former CCC/USDA facility, however, the caliche horizon directly overlies the bedrock.

- Unit 2 - Weathered red shale. Unit 2 consists of a brick red to red, silty shale that frequently contains multiple thin lenses of calcareous, greenish gray to gray shale. The unit marks the top of the Permian age bedrock. Its thickness ranges from $26 \mathrm{ft}$ to $48 \mathrm{ft}$ (Figures 4.5-4.6). Near the erosion surface of the bedrock, Unit 2 is highly weathered and, together (locally) with the lowermost portion of Unit 1, hosts the uppermost water-bearing zone. See Section 4.2.2.

- Unit 3 - Laminated shale. Unit 3 consists predominately of calcareous, laminated shale varying in color from dark gray to red and light brown. The thickness of the unit ranges from $19 \mathrm{ft}$ to $32.5 \mathrm{ft}$. The presence of thin wet intervals was consistently identified along bedding planes in the laminated 
shale. Unit 3 hosts the most productive water-bearing zone in the section penetrated (to $125 \mathrm{ft} \mathrm{BGL}$ ) in the local area (Section 4.2.2).

- Unit 4-Shale with evaporitic deposit. This locally distinctive unit consists of dolomitic shale with secondary deposits of evaporitic minerals, such as white anhydrite and gypsum, along the bedding planes of the shale. Unit 4 is likely to be less permeable than the overlying (Unit 3) and underlying (Unit 5) shales because of the secondary precipitates filling the pore spaces along the bedding planes; hence, Unit 4 might serve as a barrier to vertical hydraulic communication. No moist-to-wet intervals were identified in Unit 4. The thickness of the unit ranges from $13 \mathrm{ft}$ to $25.5 \mathrm{ft}$.

- Unit 5 - Limy Shale. Unit 5 consists of highly calcareous gray shale to clayey limestone. Although the unit is thin (3-4.5 ft thick), it was consistently present at all borehole locations. Unit 5 hosts the deep water-bearing zone. See Section 4.2.2.

- Unit 6-Gray dolomitic shale. Unit 6 consists of dark gray, hard, dolomitic shale. The sediments are noncalcareous. The unit marks the confining base for the overlying water-bearing zone. No further attempt was made to penetrate the complete thickness of Unit 6 .

\subsubsection{Groundwater-Bearing Zones}

In the sequence of six lithostratigraphic units recognized near the former CCC/USDA facility, thin, saturated horizons can be identified and traced laterally as groundwater-bearing zones that form the local groundwater system. These saturated intervals are located primarily as follows (Figure 4.3):

1. In the upper portion of the weathered red shale of Unit 2 and the basal gravelly clay to clayey gravel in Unit 1 , near the erosional surface of the Permian bedrock (the shallow groundwater-bearing zone). 
2. In the laminated shale of Unit 3 (the middle groundwater-bearing zone, which is the most productive).

3. In the thin, limy shale of Unit 5 (the deep groundwater-bearing zone).

The intervals identified as the shallow, middle, and deep groundwater-bearing zones are illustrated in Figure 4.3. The groundwater present in these zones appears to occur primarily in secondary porosity developed along bedding planes and fractures in the bedrock formations, except for locations where the shallow groundwater zone is developed in the basal, unconsolidated, coarse interval at the erosional bedrock surface.

\subsubsection{Spatial Distribution of the Groundwater-Bearing Zones}

Determination of the distribution and characteristics of the water-bearing zones involved qualitative comparison and correlation of the sequence of lithologies, the occurrence of wet intervals, and water availability identified from cores obtained at 19 unique locations (Appendix B). The three-dimensional geometry and the structure of the main stratigraphic units and the significant water-bearing zones are illustrated in two interpretive hydrogeologic cross sections (A-A' and B-B') constructed for the locations shown in Figure 4.4.

Hydrogeologic cross section A-A' (Figure 4.5) extends from north to south and depicts the relationships among the six lithostratigraphic units (Section 4.2.1) and three groundwaterbearing zones (Section 4.2.2) identified beneath the former CCC/USDA facility, at the former private grain storage facilities on the Sisson and Willems properties, and at another private grain storage facility on the Klassen property farther south. The location of cross section A-A' (Figure 4.4) approximately coincides with the crest of the surface drainage divide described in Section 4.2.1, at elevations of approximately 1,520 ft AMSL (Figure 4.2). Data from five investigative borings and several local private wells are incorporated into the cross section.

Cross section A-A' (Figure 4.5) illustrates the following features associated with the groundwater-bearing zones:

- Shallow groundwater-bearing zone: 
- The elevation of the erosional surface at the top of the Permian bedrock gradually decreases to the south, resulting in the development of coarse deposits (gravelly clay and clayey gravel) beneath the caliche horizon at locations MW15 and MW20.

- At locations MW15 and MW20, the shallow groundwater-bearing zone is identified primarily in the coarse interval at the base of Unit 1.

- At the former CCC/USDA facility, where the caliche horizon directly overlies Unit 2 (MW01 and MW19 in Figure 4.5), the highly weathered and fractured upper portion of Unit 2, along with the basal portion of Unit 1, hosts the shallow groundwater-bearing zone. At the former CCC/USDA facility, the top of the bedrock surface (MW01) is 5-6 ft lower than elsewhere in the immediate vicinity of the facility, as indicated at locations MW18 and MW19 (Figure 4.5).

- The saturated intervals at locations MW19 and MW18 were found in the upper portion of Unit 2.

- Middle groundwater-bearing zone:

- The middle groundwater-bearing zone was consistently identified at elevations of 1,430-1,441 $\mathrm{ft}$ AMSL at all borehole locations along the line of section A-A' (Figure 4.5).

- Although the middle groundwater zone consists of only a few saturated layers, it provides a major source of groundwater to the local private wells.

- The saturated intervals in the middle groundwater-bearing zone are located primarily in the middle to lower portions of Unit 3 along cross section A-A' (Figure 4.5).

- Deep groundwater-bearing zone: 
- The deep groundwater-bearing zone occurs in Unit 5, a thin layer of limy shale to clayey limestone that is consistently present at elevations of 1,405-1,411 ft AMSL along the line of section A-A' (Figure 4.5).

- Groundwater recovery in the monitoring wells completed in this interval was very slow, requiring days to months to approach apparently stable levels.

Hydrogeologic cross section B-B' (Figure 4.6) extends from west to east across the local surface drainage divide with its north-south trend. The lithostratigraphic sequence is represented by boreholes MW16, MW15, and MW17. The following differences from cross section A-A' can be identified in cross section B-B':

- The topology of the bedrock surface does not mimic the topographic divide apparent at the ground surface along this section; the bedrock surface instead appears to rise continuously from west to east. No coarse material is present at the base of the unconsolidated section at MW17, as was observed at MW15 and MW16, with the result that the caliche horizon directly overlies Unit 2 at MW17. The shallow groundwater-bearing zone at MW17 is present only in the upper portion of Unit 2, as at locations on the former CCC/USDA property (Figure 4.5).

- At MW16, saturated intervals were found both in the basal gravelly clay to clayey gravel layers in Unit 1 and in the upper portion of Unit 2. Two monitoring wells with discrete screened intervals (MW16S1 and MW16S2) were installed to evaluate these saturated intervals. Information on water levels and contamination for these two wells confirms that these intervals are hydraulically well connected and together represent the shallow groundwater zone at this location.

- Multiple saturated intervals were also identified in the upper and lower portions of the laminated shale (Unit 3) at MW16. The elevation of the upper saturated interval is about 1,440 $\mathrm{ft}$ AMSL, similar to the elevation of the middle groundwater zone identified at locations near the former CCC/USDA facility (Figure 4.5). The elevation of the deeper saturated interval in Unit 3 at 
MW16 (1,428 ft AMSL) is about $12 \mathrm{ft}$ below the elevation of the upper saturated interval. Consequently, two wells (MW16M1 and MW16M2) with isolated screened intervals were completed in the upper and lower Unit 3 intervals. The hydraulic relationship between these two intervals is discussed in Section 4.3.2.

- Unit 5 and the deep groundwater-bearing zone were identified at slightly lower elevations to the west (1,400-1,403 ft AMSL at MW16) and to the east $(1,399-1,402 \mathrm{ft}$ AMSL at MW17) than at MW15 (1,406-1,411 ft AMSL) or at the borehole locations near the former CCC/USDA facility $(1,405-1,409 \mathrm{ft}$ AMSL).

\subsection{Groundwater Flow Patterns}

Groundwater levels were monitored for all three of the identified water-bearing zones to determine the pattern of groundwater flow in each zone. Manual measurements of the water levels were obtained periodically at 20 monitoring wells installed in the shallow groundwaterbearing zone; 8 wells in the middle groundwater-bearing zone; and 5 wells in the deep groundwater-bearing zone (Table 3.4). Groundwater levels were also recorded automatically at selected monitoring wells in the shallow and middle groundwater zones and in 2 private wells at the Klassen residence (Figure 2.4). To improve the understanding of potential groundwater fluctuations in response to local precipitation events, daily rainfall data collected at one of the Equus Beds Stations (Hutchinson), approximately $15 \mathrm{mi}$ southwest of Inman, were obtained for the period of observation from the Kansas Weather Data Library (http://wdl.agron.ksu.edu/).

The results of the groundwater level measurements are discussed below. The full sets of manually and automatically recorded water levels are in Appendix E and Supplement 1, respectively.

\subsubsection{Groundwater Levels and Flow Patterns in the Shallow Groundwater-Bearing Zone}

Automatic water level recorders were installed (1) at five monitoring wells (MW01MW05) in the shallow groundwater-bearing zone on the former CCC/USDA property in Phase 1 (2009); (2) at seven wells (MW06, MW07, and MW09-MW13) in the shallow groundwater- 
bearing zone around the former CCC/USDA property in Phase 2 (2010); and (3) at six wells (MW15S, MW16S2, MW17S-MW20S) upgradient and downgradient from the former CCC/USDA facility in Phase 3-Phase 4 (2011). Location MW18 is very near the Sisson lawn and garden well. The time series data for water levels are shown in Figure 4.7 for the monitoring wells installed in Phases 1-2 and in Figure 4.8 for the wells installed in Phases 3-4. The hydrographs illustrate the relationships of groundwater levels in the instrumented shallow groundwater wells since December 2009. Groundwater level fluctuations in response to local rainfall events were significant, with a total variation of up to $7 \mathrm{ft}$.

Figure 4.7 depicts one long-term period of decreasing groundwater levels between August 2010 and October 2011 and two shorter periods of increasing levels in June to July 2010 and in November 2011 to March 2012. Over the 24-month period of record, the observed groundwater fluctuations had little apparent effect on the relationships among water levels in most of the individual wells, indicating that the hydraulic gradients in the shallow groundwater zone across the site remained unchanged. This characteristic can be further verified at many locations by close examination of the groundwater fluctuations in two-month plots of water levels (Figures 4.9 and 4.10).

Figure 4.8 confirms the responses of water levels at MW16S2, MW18S, and MW19S to local rainfall events. Three limited water level increases in early November 2011, late December 2011, and early February 2012 are well correlated with rainfall events during these periods. However, unlike most of the shallow groundwater wells, water levels in MW15S, MW17S, and MW20S (south of the former CCC/USDA facility) showed no apparent responses to local rainfall events, continuing their decreasing trend from the previous drought period.

Figures 4.9 and 4.10 focus on a two-month period in the fall of 2011 to illustrate water level fluctuations observed in the shallow groundwater zone in response to pumping of the Sisson private well or another nearby well. Data for MW18M, which is near the Sisson private well and is screened in the middle groundwater zone, exhibit the pumping cycles and are used for comparison. The middle groundwater zone is the most productive, and the local private wells extract water primarily from this zone. As Figure 4.9 shows, the relationships among water level elevations in shallow wells (except for MW05) remained consistent over the pumping period, with little apparent fluctuation in response to the pumping cycles observed at MW18M. The consistent water levels observed at these locations indicate that the flow pattern undergoes little 
or no change under pumping versus ambient conditions in the area encompassing MW02MW04, MW06-MW07, and MW09-MW13.

However, at locations MW05, MW18S, and MW19S, water levels in the shallow groundwater zone fluctuated during September and October 2011 at a frequency that apparently coincided with pumping of the Sisson well or a nearby well (as reflected by the hydrograph for MW18M in Figures 4.9 and 4.10). The water level responses in the shallow groundwater zone at MW18S and MW19S (Figure 4.10) and MW05 (Figure 4.9) indicate hydraulic communication between the shallow and middle groundwater zones via the Sisson and nearby private wells. In addition, the quick recovery of water levels (within a few hours) in the shallow groundwater zone after each pumping episode suggests relatively high permeability $\left(K_{h}\right)$ at locations MW18S, MW19S, and MW05, compared to other locations at the former CCC/USDA facility, as discussed below. A more detailed analysis is in Section 4.4.

On November 9, 2010, significant drawdown (9-19ft) occurred in several shallow groundwater wells (e.g., MW01, MW02, MW03, MW05) as a result of groundwater sampling (Figure 4.7). After sampling, water level recovery was relatively rapid at MW05 (approximately $8 \mathrm{hr}$ ). In contrast, much slower recovery was observed at MW01 (9 days), MW02 (10 days), and MW03 (6 days), suggesting that the shallow groundwater zone has very low $K_{h}$ at MW01, MW02, and MW03. This interpretation is examined further in Section 4.4.

Several "spikes" in groundwater levels were observed at MW03 and occasionally at other wells, followed by a gradual decline (Figure 4.7). These spikes were caused by local flooding in the area surrounding these wells, as well as in the flush-mount well housings. The flooding, observed during several field visits, was most likely associated with snow melting and/or rainfall. The groundwater levels in MW03 have shown no apparent responses to local rainfall events, except for this interference.

Water levels measured manually on February 16, 2011, and August 20, 2011, at 13 shallow monitoring wells installed in Phases 1-2 and on November 11, 2011, and March 13, 2012, in all 20 shallow wells were used to generate four mechanically contoured representations of the potentiometric surface for the shallow groundwater zone. As Figure 4.7 shows, the groundwater levels in the shallow groundwater zone were relatively stable during periods of manual measurements, showing little short-term variation in response to local rainfall or pumping effects. 
The four potentiometric surface maps constructed for the shallow groundwater zone, representing February 2011, August 2011, November 2011, and March 2012, are illustrated in Figures $4.11 \mathrm{a}, \mathrm{b}$. The apparent flow patterns suggested by these four potentiometric surface maps are very similar. A local groundwater "high" is consistently located in the area around wells MW12 and MW13. For each of these four cases, an apparent semi-radial flow pattern from the groundwater high at MW12-MW13 to the southeast, south, and southwest is indicated across the northern area, including the former CCC/USDA and the private grain storage facility at the Sisson property. East of Main Street, at the former CCC/USDA facility, the southeastern component of the flow pattern is predominant. Thus, groundwater flows from the northern part of the Sisson property across Main Street to the former CCC/USDA facility. In the southern area (south of MW06-MW09), groundwater flows primarily to the south.

To assist further in visualizing groundwater flow patterns, flow principles were applied to the potentiometric surface maps to develop flow networks for the shallow groundwater zone in the vicinity of the former CCC USDA facility, under both pumping and ambient conditions. The potentiometric surface during pumping was generated by using water level data collected automatically by water level sensors and recorders. To ensure that the potentiometric surface represents maximum hydraulic stress during the pumping event, we used the shallow groundwater levels at the time of maximum drawdown in the middle groundwater zone under the influence of pumping at the private well.

Figure 4.12 shows the potentiometric surface observed when the pumping effect was greatest in one of daily pumping cycles. The flow lines were developed by using flow principles with the potentiometric surface data. As Figure 4.12 shows, the general flow pattern at the former CCC/USDA facility did not change during pumping at the private well. For comparison, Figure 4.13 shows both the potentiometric surface and the flow network in March 2012, reflecting one of ambient conditions shown in Figure 4.11b. The flow networks generated from the observed water level data show that the southeastern flow component predominates in the southern portion of the former CCC/USDA facility under both pumping (Figure 4.12) and ambient (Figure 4.13) conditions.

\subsubsection{Groundwater Levels and Flow Patterns in the Middle Groundwater-Bearing Zone}

Groundwater levels in the middle groundwater-bearing zone were monitored with automatic water level recorders in eight wells from September 3, 2011, to March 13, 2012. 
During this period, responses to local rainfall in the middle groundwater zone were minor (total variation less than $1 \mathrm{ft}$ ) because of low rainfall and few rainfall events (Figure 4.14). Rising groundwater levels in three episodes can be recognized at most of the wells, except for MW20M. These rising episodes were apparently associated with rainfall events in early November 2011, late December 2011, and early February 2012. Comparison with Figure 4.8 indicates that the rainfall responses were similar in the shallow groundwater zone.

Figure 4.14 shows several significant patterns of groundwater fluctuation in the middle groundwater zone that appear to represent responses to pumping at multiple frequencies. From September 3 to October 11, 2011, groundwater level fluctuations occurred every other day in all monitoring wells (MW14M-MW15M, MW16M1, MW17M-MW19M) except for MW20M and MW16M2 (Figure 4.15). The frequency of the fluctuations in this first period coincides with the pumping frequency of the Sisson well for lawn watering, as reported by the well owner. During a second period of water level fluctuations, from October 14 to November 1, 2011, more frequent fluctuations are recognizable, with three episodes of cycling each day (Figure 4.15). Before this second pumping series began, pumping of the Sisson lawn and garden well had ended for the year. The patterns of the water level responses at all impacted monitoring wells are very similar for the two periods, even though the fluctuations are at different frequencies. This similarity indicates that the pumping well operating in the second period is located very close to the Sisson well - probably at the Pankratz (Newcombe), Willems, or Bengston residence. After November 1, 2011, the monitoring record gives no evidence of pumping similar to the first and second pumping periods described here.

A third type of water level fluctuation with a much lower frequency (multiple days for each cycle) can be recognized only at MW16M2 (Figure 4.15). This unique response indicates that the (unknown) pumping well causing the fluctuations is hydraulically connected to a specific deeper section of the middle groundwater zone that is monitored by MW16M2 (Section 4.2.3). At the same location, no response to this unique pumping cycle was recorded in the shallower part of the middle water-bearing zone that is monitored by MW16M1. This distinction suggests that the water-bearing zone monitored by MW16M2 is hydraulically isolated from or poorly connected to the rest of the middle groundwater-bearing zone in the investigation area.

Groundwater levels in the Klassen wells were also recorded automatically to evaluate the effects of pumping. In this case, the Klassen domestic well is the pumping well; the nonpumping test well is located approximately $160 \mathrm{ft}$ east of the pumping domestic well. Figure 4.16 
illustrates the responses of the water level in the test well to pumping of the Klassen domestic well from September 15, 2011, to March 13, 2012. The pumping pattern and effects in this area are apparently not correlated with the response patterns recorded for the shallow and middle groundwater zones in the area north of MW20, including the former CCC/USDA facility and the former private grain storage facilities to the west and southwest (Figures 4.7, 4.8, and 4.14).

Groundwater levels were measured manually several times in monitoring wells screened in the middle groundwater zone after their completion in October 2011. Measurements made on March 13, 2012, reflecting a relatively stable period as indicated by the middle zone hydrographs (Figure 4.14), were used to estimate the ambient potentiometric surface for the middle groundwater zone. Because MW16M2 is monitoring a water-bearing zone that is isolated from the rest of the middle groundwater zone (as discussed above), only MW16M1 at this location was included for construction of the potentiometric surface.

Figure 4.17 shows a semi-radial flow pattern in the middle groundwater zone from the northern area (including the former CCC/USDA facility and the former private grain storage facilities on the Sisson and Willems properties) to the southeast, south, and southwest. The slope of the potentiometric surface (apparent hydraulic gradient) in the area that encompasses the former CCC/USDA facility and the Sisson, Pankratz (Newcombe), Willems, and Bengston properties is virtually flat, indicating relatively higher $K_{h}$ values in the northern area than in the southern area.

To evaluate the effects of pumping of the private wells on the flow pattern in the middle groundwater zone, Figure 4.18 illustrates two potentiometric surface maps constructed for periods of pumping stress, recorded on September 16, 2011 (first pumping cycle), and October 16, 2011 (second pumping cycle). The overall potentiometric surface shows significant changes in the northern area, including near the former CCC/USDA facility and the private grain storage facilities to the west and southwest. The groundwater high has shifted to the northwest from the area along the northern edge of the former CCC/USDA facility and Sisson property, whereas the groundwater level has dropped in the north-central and northeast areas. A locally reversed flow pattern due to pumping effects is observed at locations MW14M, MW15M, and MW17MW19M (Figure 4.19). The flow pattern in the southern area remains unchanged (Figure 4.18). 


\subsubsection{Groundwater Levels and Flow Patterns in the Deep Groundwater-Bearing Zone}

Groundwater levels in the deep groundwater-bearing zone have been measured manually several times since the deep wells were installed in September 2011. The water levels in most of the deep wells recovered very slowly. This slow water level recovery indicates that the deep groundwater zone has very low $K_{h}$. As of March 13, 2012, water levels measured for most of the deep wells ranged from $1,405 \mathrm{ft}$ to $1,410 \mathrm{ft}$ AMSL, except for a level of 1,487 ft AMSL measured at MW17D. Because the groundwater levels in the deep monitoring wells had not stabilized at that time, the flow patterns in the deep zone have not been determined.

\subsection{Characteristics of the Groundwater-Bearing System}

This section summarizes interpretations that integrate all available data and direct or indirect evidence pertaining to the site-specific groundwater-bearing zones that compose the hydrogeologic system at and near the former CCC/USDA facility. The characteristics of the local groundwater flow system provide a basis for analyzing contaminant migration pathways (Section 4.6).

\subsubsection{Shallow Groundwater Zone}

\subsubsection{Physical Distribution}

The shallow groundwater zone is the uppermost water-bearing zone near the unconformable bedrock surface, consisting of one or two saturated intervals at the base of the unconsolidated sediments of Unit 1 and/or in the uppermost portion of the bedrock (Unit 2). The shallow groundwater zone extends laterally to all of the locations investigated. 


\subsubsection{Hydrogeologic Condition}

The shallow groundwater zone is under confined to leaky-confined conditions. ${ }^{1}$ The water level (potentiometric surface) measured in each well is higher than the vertical position of the saturated interval that hosts groundwater at that location. The saturated intervals in the basal coarse section in Unit 1 and/or along the bedding planes in Unit 2 are confined mainly by the overlying clay and silt and the surrounding low-permeability bedrock matrix. This relationship is illustrated in cross sections A-A' and B-B' (Figures 4.5 and 4.6).

Vertical leakage in the overlying confining layer(s) occurs mainly through the unconsolidated materials in Unit 1 and also possibly through vertical fractures in the highly weathered upper portion of Unit 2. The vertical leakage is evident in (1) the responses of water levels to local rainfall in the shallow zone at all locations at and near the former CCC/USDA facility east of Main Street and the two private grain storage facilities west of Main Street and (2) contamination in the shallow groundwater zone beneath these facilities (Section 4.5).

\subsubsection{Lateral Flow and Hydraulic Gradient}

Groundwater in the shallow groundwater zone, in general, flows toward the southeast, south, and southwest from a local groundwater "high" at the northern edge of the Sisson property. The southeast flow component is predominant at the former CCC/USDA facility, where groundwater flows across Main Street from the Sisson property toward the former CCC/USDA property. During pumping of the private well on the Sisson property, the southeast flow remained unchanged over the southern portion of the former CCC/USDA facility.

The lateral hydraulic gradient (a dimensionless ratio) decreases from 0.006 in the area southeast of the groundwater high to 0.004 southwest of the high. To the south (in the vicinity of MW15 and MW20), the gradient is still lower at 0.0035. The higher hydraulic gradient to the southeast (near the Sisson and former CCC/USDA properties) might reflect lower permeability (or $K_{h}$ ) in this portion of the groundwater zone.

1 In a confined condition, the rock layers above and below the water-bearing zone (the confining layers) are impermeable to water. In a leaky-confined condition, the rock layers are somewhat less impermeable, and consequently some water is lost to or gained from the surrounding confining layers. 


\subsubsection{Vertical Flow and Hydraulic Gradient}

Vertical flow between the shallow groundwater zone and the underlying middle groundwater zone appears to occur via two mechanisms: (1) flow through the bedrock matrix, driven by a natural vertical hydraulic gradient, and (2) flow through possible conduits provided by either open boreholes or sand pack around the cased borehole in the Sisson, Pankratz (Newcombe), Willems, and Bengston private wells, driven by the vertical hydraulic gradient induced by periodic or seasonal pumping at the private wells.

The indicated directions of vertical groundwater flow driven by natural gradients are (1) downward in the northern area including the former CCC/USDA facility and the former private grain storage facilities on the Sisson and Willems properties (Figure 4.5), (2) upward in the vicinity of locations MW15-MW17 (Figure 4.6), and (3) downward in the southern portion of the study area (near MW20; Figure 4.5). On the basis of the water levels measured on November 11, 2011, and March 13, 2012, the natural downward gradient ranges from 0.02 to 0.05 at MW01, MW18, and MW19. The actual rates of downward flow at these locations depend, however, on the combined influence of the vertical gradient and the vertical hydraulic conductivity $\left(K_{v}\right)$ value of the bedrock matrix between the groundwater zones.

Vertical flow induced by pumping at private wells is also evident in water level responses recorded at locations MW05, MW18, and MW19. At these locations, the groundwater level in the shallow groundwater zone dropped by $0.1-0.4 \mathrm{ft}$ at MW05, 1-2 ft at MW18S, and 0.1-0.2 ft at MW19S (Figures 4.9 and 4.10). The drawdown at these locations appears to be induced by vertical flow via the open-borehole or sand pack conduit of the pumping well, resulting in groundwater extraction from the shallow zone to the pumping well. The data for the monitoring well near the pumping (Sisson) well that exhibits relatively greater water level responses (MW18S) can be used to estimate the $K_{h}$ of the shallow groundwater zone at this location. This analysis is presented in Section 4.4.1.5.

\subsubsection{Hydraulic Conductivity}

The hydraulic conductivity $\left(K_{h}\right)$ of the shallow groundwater zone was evaluated for two areas: (1) the southern portion of the former CCC/USDA facility and (2) the area surrounding the Sisson private well. The hydraulic conductivity in these areas plays a significant role in contaminant migration and its future fate. The $K_{h}$ value was estimated by interpretation of the 
available monitoring data. The estimates were derived from two sets of data: (1) water level recovery in wells MW01-MW03 and MW05 after a sampling event on November 9, 2010, and (2) water level recovery at MW18S following one pumping cycle on October 7, 2011, selected as an example for analysis. The water level data with time are summarized in Appendix G. The time after each drawdown event required for water level recovery to reach $90 \%$ of the initial static level varied significantly, from several hours (approximately $8 \mathrm{hr}$ at MW05) in the northern portion of the former CCC/USDA facility to several days (6 days at MW03, 9 days at MW01, and 10 days at MW02) in the southern portion of the former CCC/USDA facility. After the October 7 pumping event at the Sisson private well, the recovery period at MW18S was about $11 \mathrm{hr}$.

The water level recovery data recorded automatically in wells MW01-MW03 and MW05 after the sampling event on November 9, 2010, were interpreted by using the analysis methods of Bouwer and Rice (Bouwer and Rice 1976; Bouwer 1989) and Hvorslev (1951), as implemented in the commercial software package AqteSolv. Because of the slow recovery observed, the much shorter duration of the groundwater sampling event was considered to represent a relatively instantaneous water withdrawal of the type performed in conducting a conventional slug test. The recovery data are of reasonably good quality for the estimation of $K_{h}$ on this basis. The detailed data evaluation and analysis are presented in Appendix G. The resulting $K_{h}$ estimates are summarized in Table 4.1. For each data set, the estimated $K_{h}$ values calculated with the Bouwer and Rice method are consistently lower than the values calculated with the Hvorslev method. The average $K_{h}$ values for the two methods are $8.59 \times 10^{-5}$ to $1.03 \times 10^{-4} \mathrm{ft} / \mathrm{day}$ at MW01-MW03 and $>0.004 \mathrm{ft} / \mathrm{day}$ at MW05. Complete data (time versus residual drawdown) for the slug test analysis of the water level recovery data and representative interpretive curve fits for the data sets are in Appendix G.

During the water level monitoring period in September and October 2011, the effect of pumping at the Sisson lawn and garden well was observed at MW18S, screened in the shallow groundwater zone only and located $57 \mathrm{ft}$ south of the Sisson well. The pumping effect observed at MW18S confirmed that the open-borehole or sand pack construction of the Sisson well creates a hydraulic conduit for groundwater withdrawal from the shallow groundwater-bearing zone when the Sisson well is pumping. Water level data recorded at MW18S show good correlation with the fluctuation of water levels in the middle groundwater zone created by pumping of the Sisson well from September 3 to October 11, 2011 (Figure 4.10). 
The drawdown and recovery data for the shallow groundwater zone in response to the pumping event on October 7, 2011, were evaluated simultaneously by using the Theis (1935) method. Although no withdrawal rate was measured for the shallow groundwater zone, a range of rates can be estimated on the basis of the assumed pumping rate at the Sisson well and the fraction of total pumping rate exerted on the shallow zone, with the understanding that groundwater is drawn from both the shallow and middle groundwater zones when the Sisson well is pumped. The range of pumping rates at the Sisson well is plausibly 1-20 gpm for lawn watering, and the percentage of total pumped water from the shallow groundwater is likely in a range of $30 \%$ to $5 \%$ at best, as the shallow groundwater zone is demonstrably much less productive than the middle groundwater zone.

The resulting estimates of probable withdrawal rates for the shallow groundwater zone range from $0.05 \mathrm{gpm}$ to $6 \mathrm{gpm}$. Rough order-of-magnitude estimates obtained by this approach yielded reasonable fits to the theoretical Theis curves for the recorded data. The results of these analyses (Table 4.2) include transmissivity $(T)$ and storativity $(S)$ for the shallow groundwater zone near the Sisson lawn and garden well and MW18S. Dividing the resulting transmissivity values by the aquifer thickness ( $2 \mathrm{ft}$ for the shallow water-bearing interval) gives the estimated $K_{h}$ value near the Sisson well and MW18S. Given the range of withdrawal rates assumed (0.05-6 gpm) for the shallow groundwater zone, the estimates of $K_{h}$ are $10^{0}$ to $10^{2} \mathrm{ft} / \mathrm{day}$.

In summary, the estimated $K_{h}$ of the shallow groundwater zone appears to be highly variable, by as much as several orders of magnitude. The estimated values of $K_{h}$ are extremely low (approximately $10^{-4} \mathrm{ft} /$ day) in the southern portion of the former CCC/USDA facility, as suggested by the recovery data at MW01-MW03, and are slightly higher $\left(>4 \times 10^{-3} \mathrm{ft} / \mathrm{day}\right)$ in the northern portion of the former CCC/USDA facility, as indicated by the recovery data at MW05. In contrast, the estimated $K_{h}$ appears to be higher by at least three orders of magnitude $\left(10^{0}\right.$ to $10^{2} \mathrm{ft} /$ day) at the Sisson property than at the former CCC/USDA facility, on the basis of analysis of the data from MW18S for the October 7, 2011, pumping event at the Sisson well. 


\subsubsection{Middle Groundwater Zone}

\subsubsection{Physical Distribution}

The middle groundwater zone is well developed along the bedding planes and fractures in the laminated shale of Unit 3. This zone extends laterally to all the investigation locations and provides water to many local private wells.

\subsubsection{Hydrogeologic Condition}

The middle groundwater zone is under confined to leaky-confined conditions. Water levels (potentiometric surface) measured in each well completed in this zone are higher than the vertical position of the saturated interval, which is confined by the surrounding low-permeability bedrock matrix. This relationship is illustrated in cross sections A-A' and B-B' (Figures 4.5 and 4.6).

\subsubsection{Lateral Flow and Hydraulic Gradient}

Under ambient conditions, without pumping stress, the apparent flow in the middle groundwater zone is semi-radial toward the southeast, south, southwest, and west from the area north of the former CCC/USDA facility and the former grain storage facilities on the Sisson and Willems properties. During pumping at one of the four private wells (Sisson, Pankratz [Newcombe], Willems, or Bengston), the apparent groundwater "high" on the northern edge of the Sisson property and the former CCC/USDA facility shifted to the west (Figure 4.18) and was locally reversed flow toward the pumping well in the northeastern area near MW14M, MW15M, and MW17M-MW19M (Figure 4.19). The flow pattern to the south in the southern area of the study area (near MW20) remained unchanged.

The lateral hydraulic gradient is very low in the northern portion of the study area, ranging from 0.0001 in the northeast (between MW19M and MW17M) to 0.001 in the northwest (between MW18M and MW16M1). In the southern area (between MW15M and MW20M), the gradient increases by one or two orders of magnitude to 0.01 . This higher hydraulic gradient might imply lower permeability in this groundwater zone in the southern area. 


\subsubsection{Vertical Flow and Hydraulic Gradient}

To date, no evidence exists to indicate that vertical flow is present between the middle groundwater zone and the deep groundwater zone. As discussed in Section 4.2, the layer of shale filled with precipitated evaporitic minerals in pore spaces (Unit 4) appears to serve as a barrier inhibiting vertical hydraulic communication or flow between the middle and deep zones, even though a vertical hydraulic gradient could be present. The hydraulic gradient is predominantly downward between the two zones, as indicated by the water levels observed in monitoring wells installed exclusively in the middle and deep water-bearing zones. However, because the water levels in the deep groundwater zone had not reached equilibrium at the time of the last data retrieval in March 2012, the actual gradient could not be determined quantitatively.

The vertical flow and gradient between the middle and shallow groundwater zones are discussed in Section 4.4.1.4.

\subsubsection{Hydraulic Conductivity}

The $K_{h}$ value of the middle groundwater zone was estimated by analyzing water level response data recorded at MW18M, screened exclusively in the middle groundwater zone and located $57 \mathrm{ft}$ south of the Sisson lawn and garden well. The drawdown and recovery data for MW18M for the pumping event on October 7, 2011, were evaluated simultaneously by using the Theis (1935) method. To generate rough estimates, possible withdrawal rates from the middle groundwater zone were estimated for plausible pumping rates at the Sisson well and possible fractions of water withdrawn from the middle groundwater zone, under the assumption that groundwater is produced from both the shallow and middle groundwater zones by the Sisson well. As discussed in Section 4.4.1.5, pumping rates of 1-20 gpm are plausible for lawn watering, and the majority of the pumped water (70-95\%) is likely from the middle groundwater zone during pumping of the Sisson well. The estimated range of withdrawal rates from the middle groundwater zone (0.7-19 gpm) was considered in the analyses. The results of the analyses are in Table 4.3. The $K_{h}$ value was determined by dividing the resulting transmissivity values by the aquifer thickness ( $2 \mathrm{ft}$ for the middle water-bearing interval). Given the range of withdrawal rates of 0.7-19 gpm for the middle groundwater zone, the estimates of $K_{h}$ are $10^{1}$ to $10^{3} \mathrm{ft} /$ day. 
As shown in Tables 4.2 and 4.3, for a pumping rate of $10 \mathrm{gpm}$ at the Sisson well and an assumption that $95 \%$ of pumped water is from the middle groundwater zone and $5 \%$ from the shallow groundwater zone, the estimated $K_{h}$ values are $299.3 \mathrm{ft} /$ day for the middle zone and $28.37 \mathrm{ft} /$ day for the shallow zone. Under this scenario, the $K_{h}$ value is greater by approximately one order of magnitude in the middle groundwater zone than in the shallow groundwater zone at the MW18 location.

On the basis of the water level response patterns observed in monitoring wells screened in the middle groundwater zone during pumping events at the Sisson well, the $K_{h}$ values for this zone appear to be spatially heterogeneous (Figures 4.15, 4.18, and 4.19). The water level responses are similar in the area that includes MW14M, MW15M, and MW17M-MW19M. However, the responses appear to be different in the northwestern (near MW16M) and southern areas (between MW15M and MW20M).

\subsubsection{Deep Groundwater Zone}

\subsubsection{Physical Distribution}

The deep groundwater zone consists of a limited saturated interval in the thin, limy shale of Unit 5. The deep zone has very poor productivity, even though it is present at all investigative locations.

\subsubsection{Hydrogeologic Condition}

The deep groundwater zone might exist under confined conditions. The water levels (potentiometric surface) measured in the deep wells had not reached equilibrium at most locations at the time of the last data retrieval in March 2012. However, on the basis of measurements at MW16D-MW18D, the existing water levels are higher than the vertical positions of the corresponding saturated intervals. The deep groundwater zone is likely confined by (1) the overlying low-permeability shale that is filled with evaporitic minerals and (2) the underlying dolomitic shale. This relationship is illustrated in cross sections A-A' and B-B' (Figures 4.5 and 4.6). 


\subsubsection{Flow and Hydraulic Gradient}

Because groundwater levels in the deep wells (MW14D-MW18D) installed during Phase 3 and Phase 4 recovered so slowly to a static water level, no groundwater flow pattern or lateral or vertical gradient could be determined for the deep zone.

\subsubsection{Hydraulic Conductivity}

Water levels in most of the wells installed by drilling in the deep groundwater zone did not reach equilibrium for several months after installation. The extremely slow recovery of water levels indicates a much lower $K_{h}$ value in the deep zone than in the shallow groundwater zone, likely less than $10^{-4} \mathrm{ft} / \mathrm{day}$.

\subsection{Delineation of Groundwater Contamination}

The lithostratigraphic and hydrologic characteristics of the subsurface environment at Inman define the hydrogeologic framework controlling the potential distribution and movement of carbon tetrachloride contamination potentially associated with the former CCC/USDA facility. Groundwater sampling to delineate the groundwater contamination at Inman was therefore guided by the interpretation of multiple water-bearing zones and groundwater flow patterns, as presented in Sections 4.2-4.4, in conjunction with the contaminant source characteristics identified in Section 4.1.

Extensive characterization was performed to determine the distribution and concentrations of carbon tetrachloride and chloroform in the shallow, middle, and deep groundwater-bearing zones, to establish a conceptual model of contaminant migration pathways and mechanisms. Groundwater samples were collected from 20 wells for the shallow water zone, 8 wells for the middle zone, and 5 wells for the deep zone (Figure 2.3 and Table 3.4).

Groundwater samples were also collected for VOCs analyses from 14 private wells that produce water primarily from the middle zone (Figure 2.4).

The results of the analyses for VOCs in groundwater samples from the private wells and permanent monitoring wells are summarized in Table 3.3 and are discussed below. 


\subsubsection{Contaminant Distribution in the Shallow Groundwater-Bearing Zone}

The spatial distributions of carbon tetrachloride and chloroform in samples collected from the shallow groundwater zone are shown in Figures 4.20 and 4.21, respectively. The vertical distribution of carbon tetrachloride in the shallow zone is illustrated in hydrogeologic cross sections A-A' and B-B' spanning the wider study area (Figures 4.22 and 4.23) and a local hydrogeologic cross section $\mathrm{C}^{-} \mathrm{C}^{\prime}$ for the area at and near the former CCC/USDA facility (Figure 4.24).

As shown in Figure 4.20, carbon tetrachloride was identified at trace levels in shallow groundwater in three areas: on the former CCC/USDA property, near the former private grain storage facility on the Sisson property, and at location MW16 to the southwest.

At and near the former CCC/USDA facility, the shallow groundwater zone is hosted by the upper part of Unit 2 and the basal part of Unit 1, at the consistent range of elevations shown in cross section C-C' (Figure 4.24). Trace concentrations of carbon tetrachloride (2.2-5.4 $\mu \mathrm{g} / \mathrm{L}$ ) were detected at three locations (MW01-MW03) in the southern portion of the former facility, with the highest concentration at MW01. On the basis of the local shallow groundwater flow pattern discussed in Section 4.3.1, monitoring wells MW06-MW08 are located immediately downgradient of (and outside) the former CCC/USDA facility. The absence of contamination at these locations indicates that contaminants in the shallow groundwater zone beneath the former CCC/USDA facility have remained on the former property and have not migrated laterally off the property to the south and/or southeast. The lateral containment of carbon tetrachloride is consistent with the extremely low $K_{h}\left(10^{-4} \mathrm{ft} /\right.$ day $)$ of the shallow groundwater-bearing zone below the southern portion of the former CCC/USDA facility, as indicated by slow recovery of water levels in the shallow zone (Section 4.4.1.5).

On the Sisson property, west of Main Street, carbon tetrachloride was confirmed at a level $(4.5 \mu \mathrm{g} / \mathrm{L})$ near the MCL in shallow groundwater from well MW18S, located $80 \mathrm{ft}$ southeast of the former private grain storage facility on the Sisson property and $57 \mathrm{ft}$ south of the contaminated Sisson lawn and garden well. Contamination in the shallow groundwater-bearing zone appears to be a source for contamination in the middle groundwater-bearing zone, as the Sisson, Pankratz (Newcombe), Willems, and Bengston private wells intercept both groundwater zones. 
No contamination in shallow groundwater was detected at MW09-MW12, along the western edge of Main Street (Figure 4.20). The shallow groundwater zone monitored by these wells is consistent in elevation range with the contaminated portion of the shallow groundwater zone at the former CCC/USDA facility (Figure 4.24). The absence of contamination at locations MW09-MW12 confirms that contaminant contributions to the shallow groundwater come from two separate sources: (1) the former CCC/USDA facility east of Main Street and (2) the former private grain storage facilities on the Sisson and Willems properties west of Main Street. Although the flow pattern in the shallow groundwater-bearing zone includes a component to the southeast across Main Street (Figures 4.11a,b, 4.12, and 4.13), the complete absence of contamination in the shallow groundwater zone along Main Street indicates that contaminants from the two sources on each side of Main Street have remained separate and of limited lateral extent in the shallow groundwater-bearing zone.

A low level of carbon tetrachloride $(1.3-2.3 \mu \mathrm{g} / \mathrm{L})$ was found in the shallow groundwater zone at wells MW16S1 and MW16S2, 1,400 ft southwest of the former private grain storage facility on the Sisson property (Figure 4.20), where the contaminated Sisson lawn and garden well $(4.9 \mu \mathrm{g} / \mathrm{L})$ and MW18S $(4.5 \mu \mathrm{g} / \mathrm{L})$ are located. The source of contamination at MW16S1 and MW16S2 is unknown. On the basis of the shallow groundwater flow pattern under ambient conditions (Figure 4.25), groundwater at the northern edge of the Sisson property might be able to reach location MW16S2. The flow pattern also indicates that there is no hydraulic barrier between MW16S and the area north of the Sisson property - including the shallow groundwater contamination identified at the former Inman Elevator, as discussed in Section 1.1.

Cross sections A-A', B-B', and C-C' (Figures 4.22, 4.23, and 4.24) indicate that the contamination in the shallow groundwater-bearing zone is present vertically at depths of less than $46 \mathrm{ft}$ BGL and is limited laterally to a few locations, aside from the unknown migration pathways to the distant MW16 location to the southwest.

The spatial distribution of chloroform in the shallow groundwater-bearing zone is similar to that of carbon tetrachloride, as illustrated in Figure 4.21. Trace concentrations above the method detection limit, however, were found only in two separate former source areas: (1) the former CCC/USDA facility (1.5-4.8 $\mu \mathrm{g} / \mathrm{L}$ at MW01-MW03) and (2) the former private grain storage facility on the Sisson property $(1.0 \mu \mathrm{g} / \mathrm{L}$ at MW18S). The abundance of chloroform relative to carbon tetrachloride ranges from 59-165\% (MW01-MW03; southern portion of the former CCC/USDA property) to $22 \%$ (MW18S; near the former private storage facility on the 
Sisson property). The higher ratios of chloroform to carbon tetrachloride, in conjunction with evidence of low permeability, observed at the southern portion of the former CCC/USDA facility suggest that some degradation of carbon tetrachloride by reductive dechlorination is occurring in the shallow groundwater in this area, enhanced by the extended residence time of contaminants in the subsurface due to low permeability.

\subsubsection{Contaminant Distribution in the Middle Groundwater-Bearing Zone}

The spatial distribution of carbon tetrachloride and chloroform in the middle groundwater zone was determined on the basis of samples collected from 8 monitoring wells installed exclusively in the middle zone. In addition, because the middle groundwater-bearing zone is the most productive water zone over the upper $125 \mathrm{ft}$ of the soil profile in the area, many private lawn and garden wells and domestic wells have been installed into this zone to extract water. Groundwater from 14 private wells was analyzed to assist in delineation of contamination in the middle zone. The results of analyses of these samples for carbon tetrachloride and chloroform (Table 3.3) are summarized in Figures 4.26 and 4.27, respectively. The vertical distribution of carbon tetrachloride in the middle zone is illustrated in hydrogeologic cross sections A-A' and B-B' (Figures 4.22 and 4.23).

Figure 4.26 indicates that trace concentrations of carbon tetrachloride at or above the MCL $(5.0 \mu \mathrm{g} / \mathrm{L})$ in the middle groundwater-bearing zone are present mainly in two source areas associated with past grain storage activities: (1) the Sisson, Pankratz (Newcombe), Willems, and Bengston properties (encompassing two former private grain storage facilities and four lawn and garden wells) and (2) the Klassen property to the south.

On the Sisson, Pankratz (Newcombe), Willems, and Bengston properties, carbon tetrachloride was detected at all four private wells $(4.0-5.0 \mu \mathrm{g} / \mathrm{L})$, as well as at monitoring well MW18M $(3.3 \mu \mathrm{g} / \mathrm{L})$, near the Sisson well. In a common well construction practice in the area, most of private wells were installed with an open borehole, without casing over the consolidated bedrock formation (Argonne 2007). The open-borehole interval can directly intercept all waterbearing zones in the bedrock formation, including the shallow and middle groundwater-bearing zones in this local area. The other common construction practice for water wells in Kansas is use of a sand pack between casing and borehole in cased wells. The sand pack can also provide a conduit between multiple water-bearing zones. The concentration of carbon tetrachloride $(3.3 \mu \mathrm{g} / \mathrm{L})$ in groundwater from well MW18M apparently represents the actual contaminant level 
in the middle zone at this location, whereas the concentrations in the Sisson, Pankratz (Newcombe), Willems, and Bengston private wells likely result from the mixed contributions of carbon tetrachloride from both the shallow and middle groundwater zones.

At the Klassen residence, carbon tetrachloride was found in the domestic well at $6.0 \mu \mathrm{g} / \mathrm{L}$ and in the test well at $14 \mu \mathrm{g} / \mathrm{L}$. However, no contamination was detected at any of the other four private wells surrounding the Klassen property (Isaac, Goertzen, Thiessen, and J. Pankratz; Figure 4.26). The grain facility on the Klassen property has grown from 3 circular bins and 1 storage structure in 1963 to 14 circular bins and 1 storage structure at the present time (Figure 1.3). The grain facility is about $60 \mathrm{ft}$ from the Klassen domestic well and $150 \mathrm{ft}$ from the Klassen test well. A higher concentration of carbon tetrachloride in groundwater $(14 \mu \mathrm{g} / \mathrm{L})$ was found in the shallow Klassen test well (approximately $135 \mathrm{ft}$ BGL) than in the deep Klassen domestic well $(6.0 \mu \mathrm{g} / \mathrm{L}$ over a total depth of $172 \mathrm{ft} \mathrm{BGL})$, indicating a shallow source that results in decreasing concentration with depth. In addition, cross section A-A' (Figure 4.22) illustrates a discontinuity of contamination between the private grain storage facilities on the Sisson and Willems properties to the north and the facility on the Klassen property to the south. These multiple lines of evidence indicate that the contamination found in the Klassen wells is attributed to local shallow sources associated with past private grain storage on the Klassen property.

The extent of contamination in the middle zone can be defined laterally by the absence of contamination at MW16M and the Hull wells to the southwest, at MW20M to the south, and at the Pauls well to the southeast (Figure 4.26).

As shown in hydrologic cross section A-A' (Figure 4.22), the highest concentrations of carbon tetrachloride in the middle groundwater zone are limited, for the most part, to the Sisson, Pankratz (Newcombe), Willems, and Bengston properties, where the four private wells with open boreholes or sand packs intercept the shallow groundwater zone at or near the source.

At the former CCC/USDA facility, a very low concentration of carbon tetrachloride $(1.7 \mu \mathrm{g} / \mathrm{L})$ was found in the middle groundwater-bearing zone. The concentration is probably so low because of the absence of direct conduits, such as the open-borehole or sand pack in private wells to the west and southwest, that would provide a continuing source of contamination from the shallow groundwater zone. 
The identified distribution of chloroform in the middle groundwater zone is shown in Figure 4.27. All chloroform concentrations are extremely low $(\leq 2.0 \mu \mathrm{g} / \mathrm{L})$. The relative abundance of chloroform to carbon tetrachloride in the middle groundwater zone varies from $118 \%$ in the southern portion of the former CCC/USDA facility, to $22-30 \%$ at the Sisson, Pankratz (Newcombe), Willems, and Bengston properties, and to 3-4\% at the Klassen property. The ratios in the middle groundwater zone are consistent with those in the shallow groundwater zone below the former CCC/USDA facility (59-165\%) and the Sisson, Pankratz (Newcombe), Willems, and Bengston properties (22\%). The highest ratio observed at the southern portion of the former CCC/USDA facility implies the possibility of a limited amount of carbon tetrachloride degradation via reductive dechlorination inherited from the shallow groundwater.

\subsubsection{Contaminant Distribution in the Deep Groundwater-Bearing Zone}

Groundwater samples were collected from wells installed exclusively in the deep groundwater zone at five locations (MW14D-MW18D). No carbon tetrachloride was detected in any sample collected from these wells (Table 3.3; Figure 4.28). The finding of only trace concentrations of chloroform (maximum $2.3 \mu \mathrm{g} / \mathrm{L}$ ) at monitoring wells MW14D, MW15D, MW16D, and MW18D indicates that any migration from the middle groundwater zone to the deep groundwater zone is insignificant. The trace levels of chloroform observed could have been introduced with the chlorinated city water containing chloroform at $1.9-3.4 \mu \mathrm{g} / \mathrm{L}$ (see Section 3.8) that was used during drilling. Limited amounts of city water were used, as necessary, to ease the difficulty of penetrating the dolomitic shale (Unit 4) overlying the deep groundwater zone, as well as for well grouting and equipment decontamination. The extremely slow recovery of water in the deep groundwater zone (taking several months; Section 4.4.3.4) prevents adequate purging for groundwater sampling.

As discussed in Section 4.4.3.2, the deep groundwater zone is well confined by the overlying shale filled with evaporitic mineral. The absence of carbon tetrachloride contamination and the identification of only trace levels of chloroform in the deep water zone are consistent with the zone's hydrologic characteristics. 


\subsection{Conceptual Model of Contaminant Migration Pathways}

The data on geology, hydrogeology, groundwater-level monitoring, geochemistry, and contaminant distribution presented in Sections 4.1-4.5 provide the technical basis for development of an integrated conceptual model of groundwater flow and contaminant migration in the system of multiple groundwater-bearing zones north of Buckskin Road. The key elements of this working conceptual model are summarized as follows:

- Vertical infiltration in two separate shallow groundwater contamination areas that are associated with the past operation of former grain storage facilities (migration pathway I; $N E_{I}$ and $N W_{I}$ in Figure 4.29). The presence of carbon tetrachloride in the underlying shallow groundwater zone in two separate areas (the southern portion of the former CCC/USDA facility [MW01-MW03] and the Sisson property near the former private grain storage facility on that property [MW18S]; Figures 4.20 and 4.29) indicates the potential for vertical infiltration of carbon tetrachloride through the vadose zone (Unit 1). No carbon tetrachloride and chloroform were identified above the method detection limit of $10 \mu \mathrm{g} / \mathrm{kg}$ in vadose zone soils at the former CCC/USDA grain storage facility and the former private grain storage facilities on the Sisson and Willems properties, indicating the absence of soil sources of contamination to groundwater.

- Limited lateral migration in the shallow groundwater-bearing zone (migration pathway II).

- Shallow groundwater contamination on the former CCC/USDA facility east of Main Street: Contaminants in the shallow groundwater-bearing zone are confined to the southern portion of the former CCC/USDA facility (MW01-MW03) because of the apparently very low $K_{h}$ $\left(10^{-4} \mathrm{ft} /\right.$ day $)$ of the zone in this area. No evidence of lateral migration off the former facility was found in the shallow groundwater zone.

- Shallow groundwater contamination on the private properties west of Main Street: Lateral migration in the shallow groundwater zone might be limited to the private wells on the Sisson, Pankratz (Newcombe), Willems, 
and Bengston properties, near the former private grain storage facilities on the Sisson and Willems properties $\left(\mathrm{NW}_{\text {II }}\right.$ in Figure 4.29). Present data are inadequate to determine whether the trace level of carbon tetrachloride detected at MW16S emanated from the same location west of Main Street.

- Vertical migration from the shallow to the middle groundwater-bearing zone (migration pathway III). Contaminants have migrated from the shallow to the middle water zone primarily via two mechanisms:

- Hydraulic conduits (Open-borehole or sand pack conduits) (NW $W_{I I I}$ in Figure 4.29). Contaminants in the shallow groundwater zone have migrated directly via hydraulic conduits to the middle groundwater zone in the Sisson, Pankratz (Newcombe), Willems, and Bengston private wells west of Main Street, near the former private grain storage facilities on the Sisson and Willems properties. Concentrations of carbon tetrachloride in the four private wells are similar to those in the shallow groundwater zone at MW18S.

- Vertical leakage from the shallow to the middle water zone ( $N E_{\text {III }}$ and $N W_{\text {III }}$ in Figure 4.29). Vertical leakage is inferred primarily as an indirect pathway via the bedrock matrix beneath the southern portion of the former CCC/USDA facility, as well as beneath the Sisson, Pankratz (Newcombe), Willems, and Bengston properties. The conversion of carbon tetrachloride to chloroform has been observed at MW01/MW14M on the former CCC/USDA facility and MW18S/MW18M on the Sisson property. The ratios of chloroform to carbon tetrachloride remain the same in the shallow and middle groundwater zones in each shallow groundwater contamination area but differ between the two areas, east and west of Main Street.

- Lateral migration in the middle groundwater-bearing zone (migration pathway IV). Groundwater monitoring indicates that lateral migration of carbon tetrachloride from the inferred source area in the middle groundwater zone has been primarily toward the southwest, south, and southeast, in keeping with the identified natural semi-radial pattern of groundwater flow 
(Figure 4.17) in the middle zone. During the pumping event at any of the four private wells (Sisson, Pankratz [Newcombe], Willems, or Bengston), locally reversed flow back to the well occurs within the radius of influence, as observed primarily in the area around wells MW14M, MW15M, MW17M, and MW18M (Figure 4.19).

- Vertical migration from the middle to the deep water zone. The absence of carbon tetrachloride, along with the presence of only trace levels of chloroform possibly introduced with chlorinated city water used for drilling in a setting with poor purging potential, suggests that further vertical migration of contaminants from the middle to the deep groundwater zone either is not occurring or is insignificant. 
TABLE 4.1 Summary of hydraulic conductivity values estimated from the water level recovery event on November 9, 2010, as observed in monitoring wells in the shallow groundwater zone beneath the former CCC/USDA facility.

\begin{tabular}{cccc}
\hline & \multicolumn{3}{c}{ Calculated Hydraulic Conductivity ${ }^{\mathrm{a}}$ (ft/day) } \\
\cline { 2 - 4 } Well & $\begin{array}{c}\text { Bouwer and Rice Method } \\
\text { (Confined Aquifer) }\end{array}$ & $\begin{array}{c}\text { Hvorslev Method } \\
\text { (Confined Aquifer) }\end{array}$ & Average $^{\mathrm{b}}$ \\
\hline MW01 & $6.82 \times 10^{-5}$ & $1.18 \times 10^{-4}$ & $9.31 \times 10^{-5}$ \\
MW02 & $6.27 \times 10^{-5}$ & $1.09 \times 10^{-4}$ & $8.59 \times 10^{-5}$ \\
MW03 & $7.52 \times 10^{-5}$ & $1.30 \times 10^{-4}$ & $1.03 \times 10^{-4}$ \\
MW04 & NA $^{\mathrm{c}}$ & $\mathrm{NA}$ & $\mathrm{NA}$ \\
MW05 & $>0.003^{\mathrm{d}}$ & $>0.005^{\mathrm{d}}$ & $>0.004^{\mathrm{d}}$ \\
\hline
\end{tabular}

a Calculated with an assumed thickness of $2 \mathrm{ft}$ for the saturated interval.

b Averaged for the Bouwer and Rice and the Hvorslev calculation methods.

c Data are inadequate for analysis.

d Insufficient data points to capture the early portion of the recovery. The estimated values reflect minimum hydraulic conductivities. 
TABLE 4.2 Hydraulic conductivity values estimated for the shallow groundwater zone beneath the former private grain storage facility on the Sisson property after the pumping event at the Sisson well on October 7, 2011, from observations made at well MW18S.

\begin{tabular}{|c|c|c|c|c|c|}
\hline \multirow[b]{2}{*}{$\begin{array}{l}\text { Pumping } \\
\text { Rate at } \\
\text { Sisson Wella } \\
\quad(\mathrm{gpm})\end{array}$} & \multirow[b]{2}{*}{$\begin{array}{c}\text { Percent of Pumping } \\
\text { Rate Exerted on } \\
\text { Shallow Groundwater } \\
\text { Zone }\end{array}$} & \multirow[b]{2}{*}{$\begin{array}{c}\text { Withdrawal Rate } \\
\text { from Shallow } \\
\text { Groundwater Zone } \\
(\mathrm{gpm})^{\mathrm{c}}\end{array}$} & \multicolumn{3}{|c|}{$\begin{array}{l}\text { Values Estimated by Theis Method } \\
\text { (Confined Aquifer) }\end{array}$} \\
\hline & & & $\begin{array}{l}\text { Transmissivity } \\
\left(T, \mathrm{ft}^{2} / \text { day }\right)\end{array}$ & $\begin{array}{l}\text { Storativity } \\
(S)\end{array}$ & $\begin{array}{l}\text { Hydraulic } \\
\text { Conductivity } \\
\left(K_{h}, \mathrm{ft} / \text { day }\right)\end{array}$ \\
\hline 1 & $\begin{array}{c}5 \\
10 \\
30\end{array}$ & $\begin{array}{l}0.05 \\
0.1 \\
0.3\end{array}$ & $\begin{array}{l}5.673 \\
11.35 \\
34.04\end{array}$ & $\begin{array}{l}0.0001 \\
0.0002 \\
0.0007\end{array}$ & $\begin{array}{c}2.837 \\
5.675 \\
17.02\end{array}$ \\
\hline 10 & $\begin{array}{c}5 \\
10 \\
30\end{array}$ & $\begin{array}{l}0.5 \\
1.0 \\
3.0\end{array}$ & $\begin{array}{l}56.73 \\
113.5 \\
340.4\end{array}$ & $\begin{array}{l}0.0012 \\
0.002 \\
0.007\end{array}$ & $\begin{array}{l}28.37 \\
56.75 \\
170.2\end{array}$ \\
\hline 20 & $\begin{array}{c}5 \\
10 \\
30\end{array}$ & $\begin{array}{l}1.0 \\
2.0 \\
6.0\end{array}$ & $\begin{array}{l}113.5 \\
226.9 \\
680.8\end{array}$ & $\begin{array}{l}0.002 \\
0.005 \\
0.014\end{array}$ & $\begin{array}{l}56.75 \\
113.5 \\
340.4\end{array}$ \\
\hline \multicolumn{3}{|c|}{ Range of values (order of magnitude) ${ }^{d}$} & $10^{1}-10^{3}$ & $10^{-2}-10^{-4}$ & $10^{0}-10^{2}$ \\
\hline
\end{tabular}

a The range of pumping rates at the Sisson well is estimated on the basis of probable rates used for lawn watering.

b During pumping at the Sisson well, water is extracted from both the shallow groundwater zone and the middle groundwater zone via the well's uncased borehole. Because the shallow zone is less productive, water withdrawal from the shallow zone is assumed to range from $5 \%$ to $30 \%$ of the total pumping rate.

c Estimate based on the assumed probable pumping rate at the Sisson well (column 1) and the assumed percentage of total pumped water that is from the shallow zone (column 2).

d Range of hydraulic parameters, expressed in order of magnitude, for the assumed range of pumping rates (1-20 gpm) and fraction (percentage) of total pumped water from the shallow zone. 
TABLE 4.3 Hydraulic conductivity values estimated for the middle groundwater zone beneath the former private grain storage facility on the Sisson property after the pumping event at the Sisson well on October 7, 2011, from observations made at well MW18M.

\begin{tabular}{|c|c|c|c|c|c|}
\hline \multirow[b]{2}{*}{$\begin{array}{l}\text { Pumping } \\
\text { Rate at } \\
\text { Sisson Wella } \\
\text { (gpm) }\end{array}$} & \multirow{2}{*}{$\begin{array}{l}\text { Percent of } \\
\text { Pumping } \\
\text { Rate Exerted } \\
\text { on Middle } \\
\text { Groundwater } \\
\text { Zone }^{b}\end{array}$} & \multirow{2}{*}{$\begin{array}{l}\text { Withdrawal } \\
\text { Rate from } \\
\text { Middle } \\
\text { Groundwater } \\
\text { Zone } \\
\text { (gpm) }\end{array}$} & \multicolumn{3}{|c|}{$\begin{array}{l}\text { Value Estimated by Theis Method } \\
\text { (Confined Aquifer) }\end{array}$} \\
\hline & & & $\begin{array}{l}\text { Transmissivity } \\
\left(T, \mathrm{ft}^{2} / \mathrm{day}\right)\end{array}$ & Storativity $(S)$ & $\begin{array}{l}\text { Hydraulic } \\
\text { Conductivity } \\
\left(K_{h}, \mathrm{ft} / \text { day }\right)\end{array}$ \\
\hline 1 & $\begin{array}{l}95 \\
90 \\
70\end{array}$ & $\begin{array}{l}0.95 \\
0.90 \\
0.70\end{array}$ & $\begin{array}{l}59.85 \\
56.70 \\
44.10\end{array}$ & $\begin{array}{l}0.0006 \\
0.0006 \\
0.0004\end{array}$ & $\begin{array}{l}29.93 \\
28.35 \\
22.05\end{array}$ \\
\hline 10 & $\begin{array}{l}95 \\
90 \\
70\end{array}$ & $\begin{array}{l}9.5 \\
9.0 \\
7.0\end{array}$ & $\begin{array}{l}598.5 \\
567.0 \\
441.0\end{array}$ & $\begin{array}{l}0.006 \\
0.006 \\
0.004\end{array}$ & $\begin{array}{l}299.3 \\
283.5 \\
220.5\end{array}$ \\
\hline 20 & $\begin{array}{l}95 \\
90 \\
70\end{array}$ & $\begin{array}{l}19 \\
18 \\
14\end{array}$ & $\begin{array}{l}1157 \\
1134 \\
882.0\end{array}$ & $\begin{array}{l}0.012 \\
0.011 \\
0.009\end{array}$ & $\begin{array}{l}578.3 \\
567.0 \\
441.0\end{array}$ \\
\hline \multicolumn{3}{|c|}{ Range of values (order of magnitude) ${ }^{d}$} & $10^{1}-10^{3}$ & $10^{-2}-10^{-4}$ & $10^{1}-10^{3}$ \\
\hline
\end{tabular}

a The range of pumping rates at the Sisson well is estimated on the basis of probable rates used for lawn watering.

b During pumping at the Sisson well, water is extracted from both the shallow groundwater zone and the middle groundwater zone via the well's uncased borehole. Because the middle zone is more productive, water withdrawal from the middle zone is assumed to range from $95 \%$ to $70 \%$ of the total pumping rate.

c Estimate based on the assumed probable pumping rate at the Sisson well (column 1) and percentage of total pumped water that is from the middle zone (column 2).

d Range of hydraulic parameters, expressed in order of magnitude, for the assumed range of pumping rates (1-20 gpm) and fraction (percentage) of total pumped water from the middle zone. 


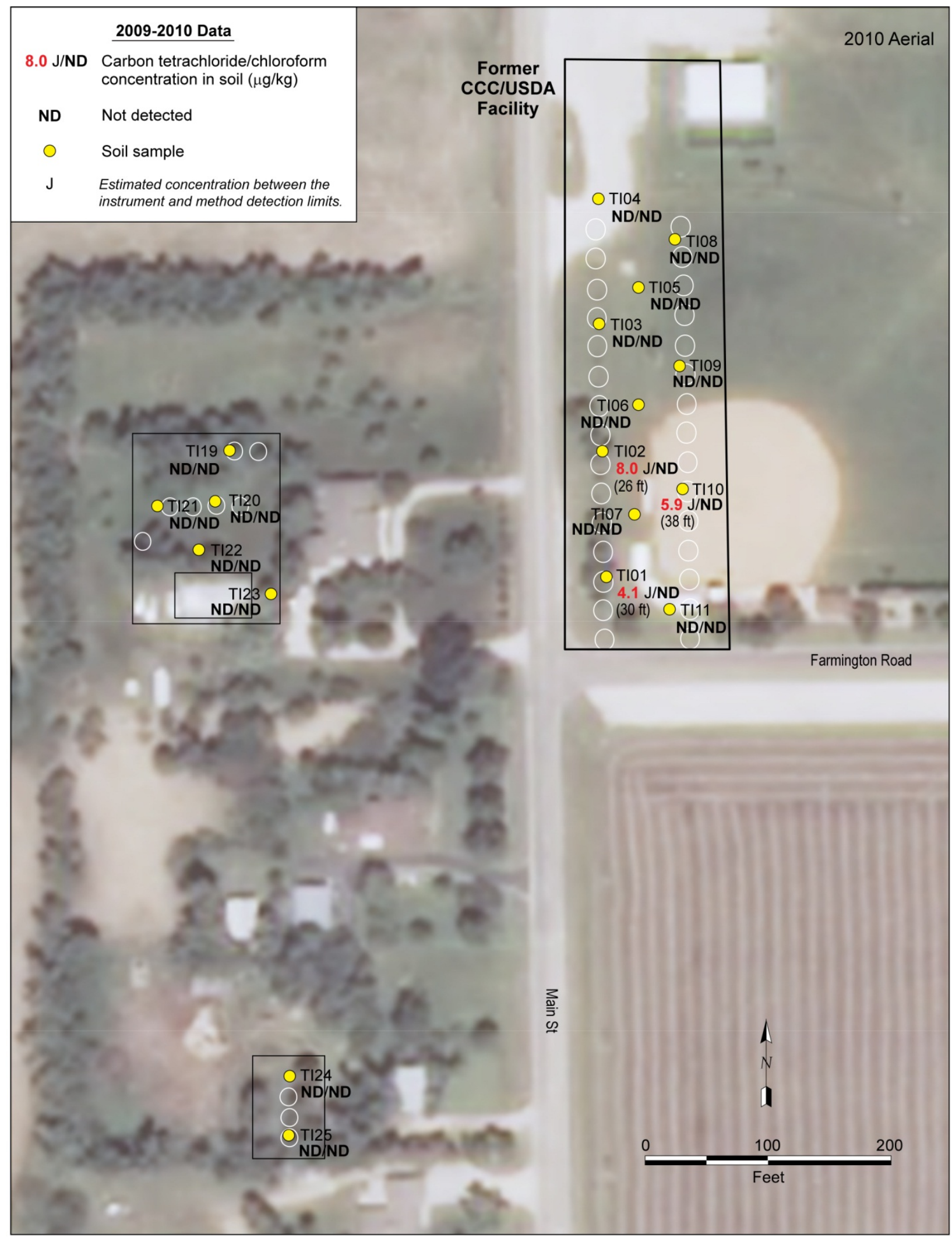

FIGURE 4.1 Maximum concentrations of carbon tetrachloride and chloroform in soil samples collected at and near the former CCC/USDA facility and the former private grain storage facilities on the Sisson and Willems properties. Source of photograph: NAIP (2010). 


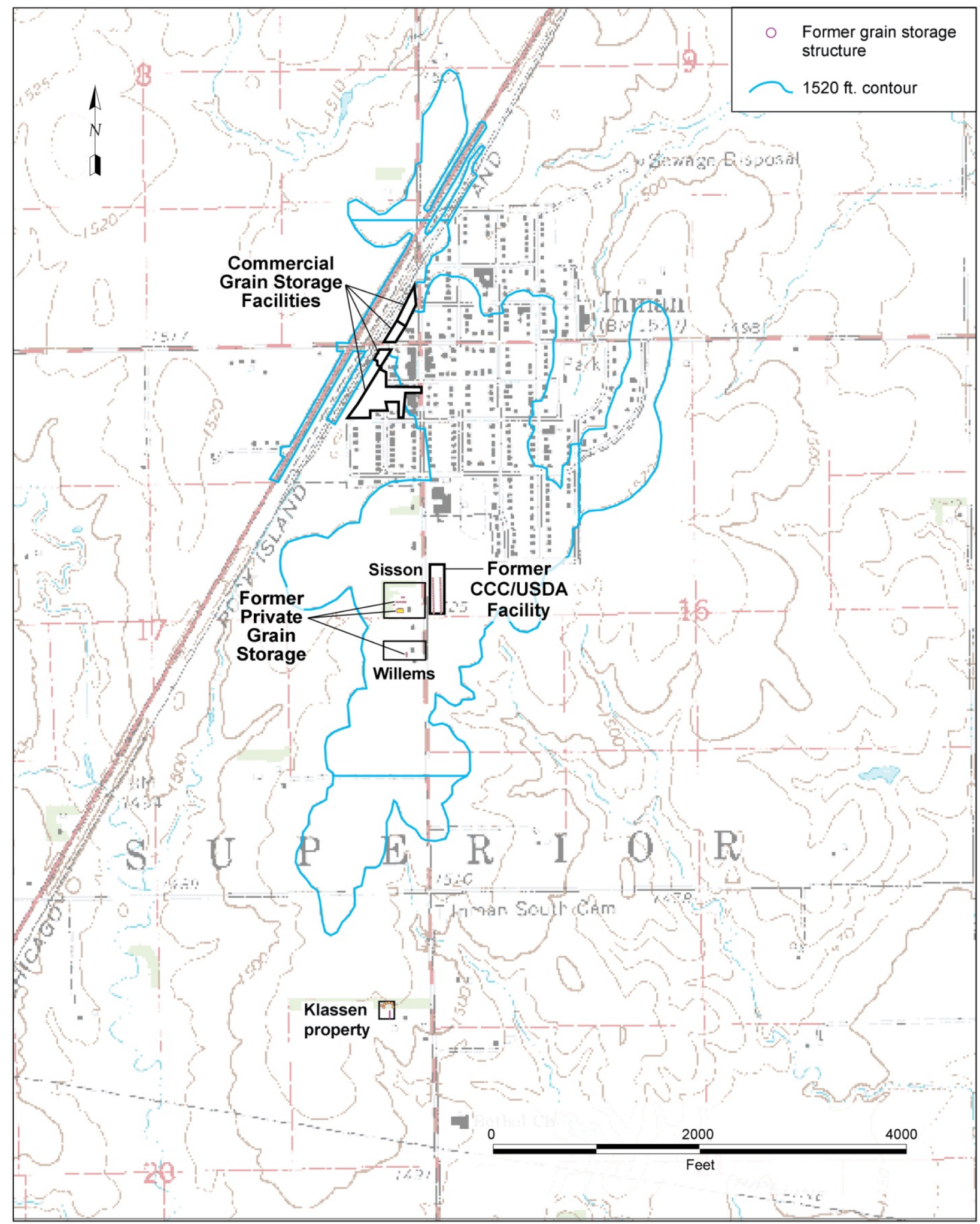

FIGURE 4.2 Topographic surface at Inman. Source of 1982 map: USGS (1997). 


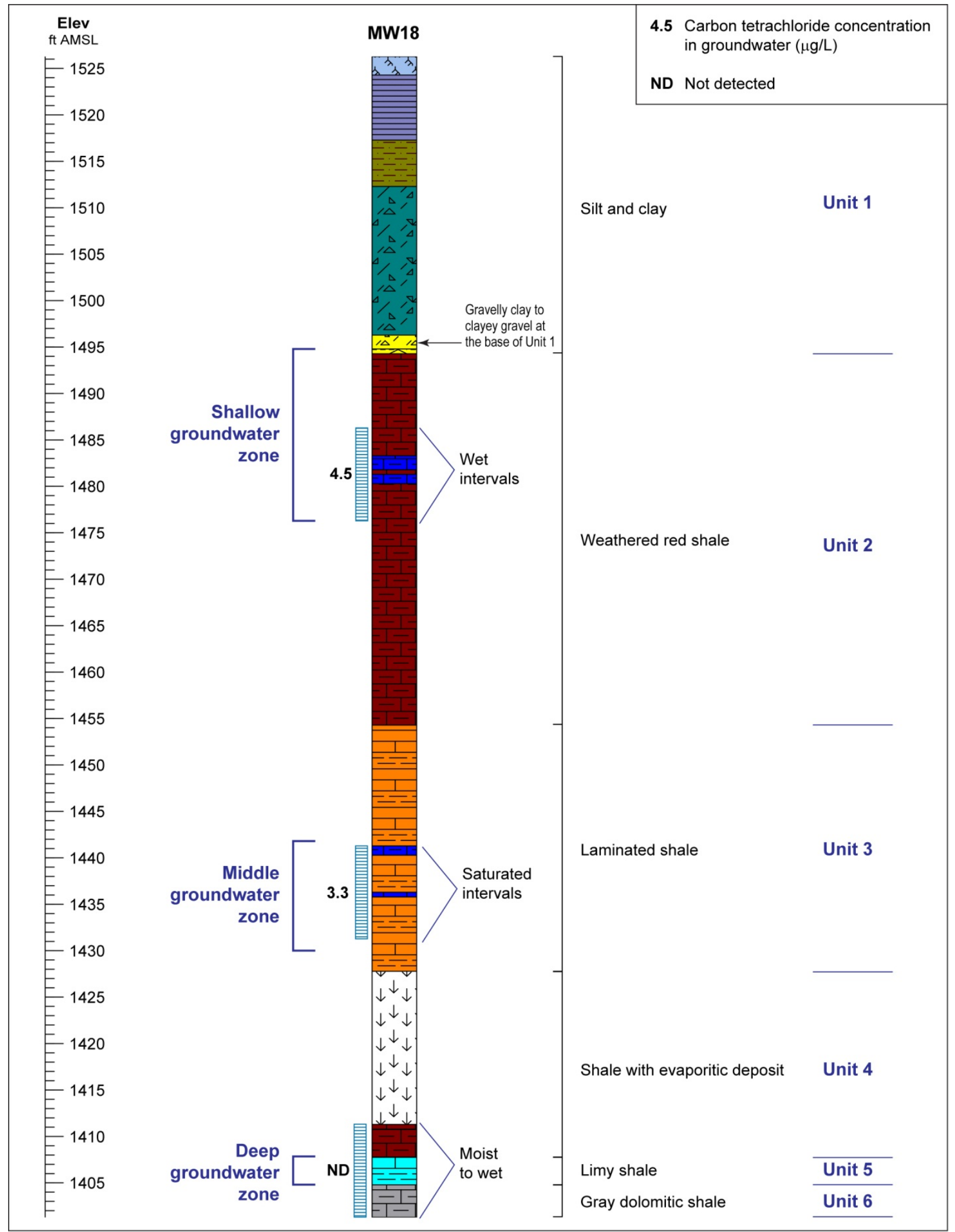

FIGURE 4.3 General sequence of local lithostratigraphic units near the former CCC/USDA facility. 


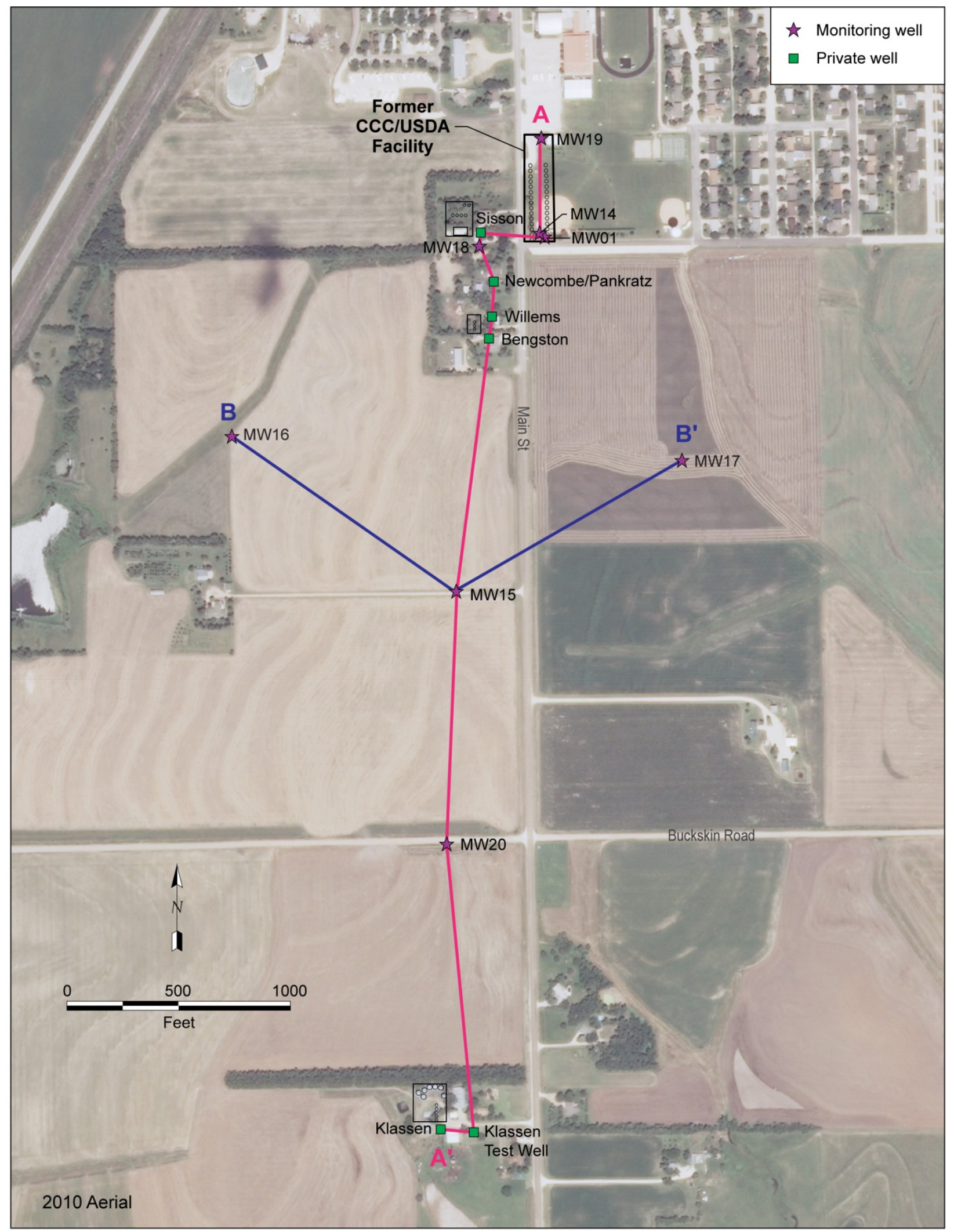

FIGURE 4.4 Locations of wells and hydrogeologic cross sections. Source of photograph: NAIP (2010). 


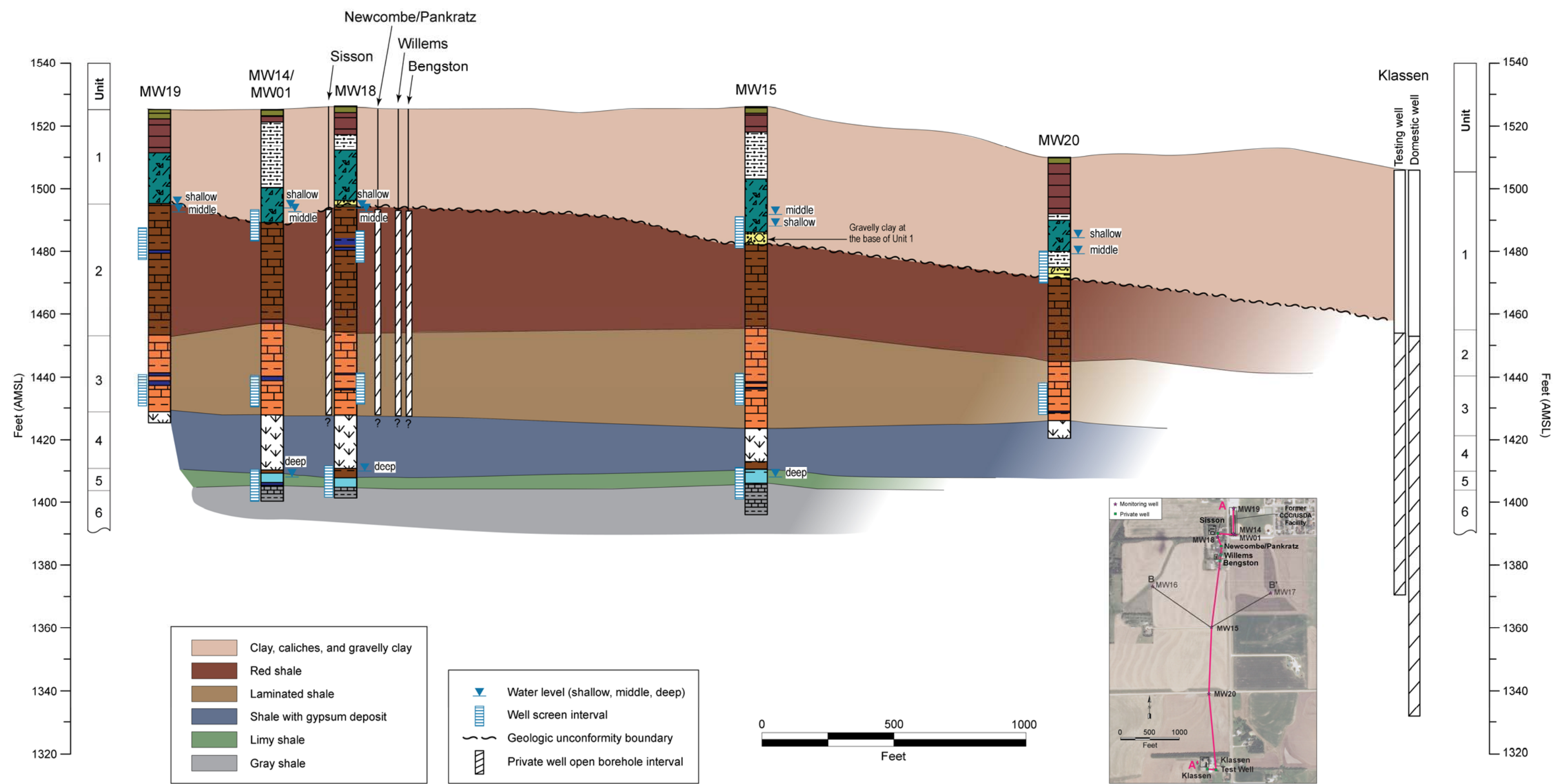

FIGURE 4.5 Interpretive north-to-south hydrogeologic cross section A-A'. The groundwater-bearing zones are hosted in the following units: shallow zone in Unit 1-Unit 2; middle zone in Unit 3; deep zone in Unit 5. 

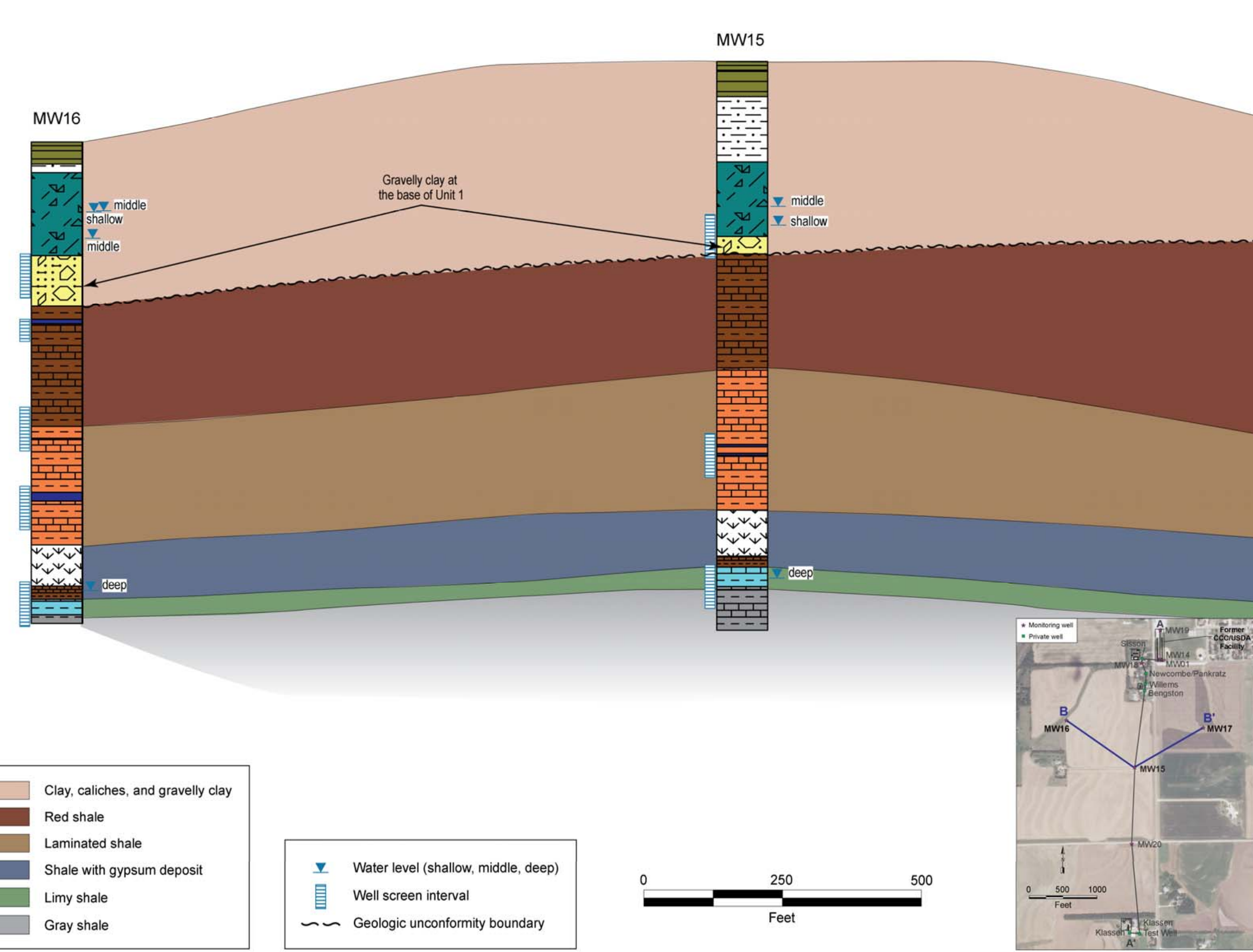

FIGURE 4.6 Interpretive west-to-east hydrogeologic cross section B-B'. The groundwater-bearing zones are hosted in the following units: shallow zone in Unit 1-Unit 2; middle zone in Unit 3; deep zone in Unit 5. 
Hydrographs in Shallow Groundwater Zone Recorded in Wells Installed in Phases 1-2

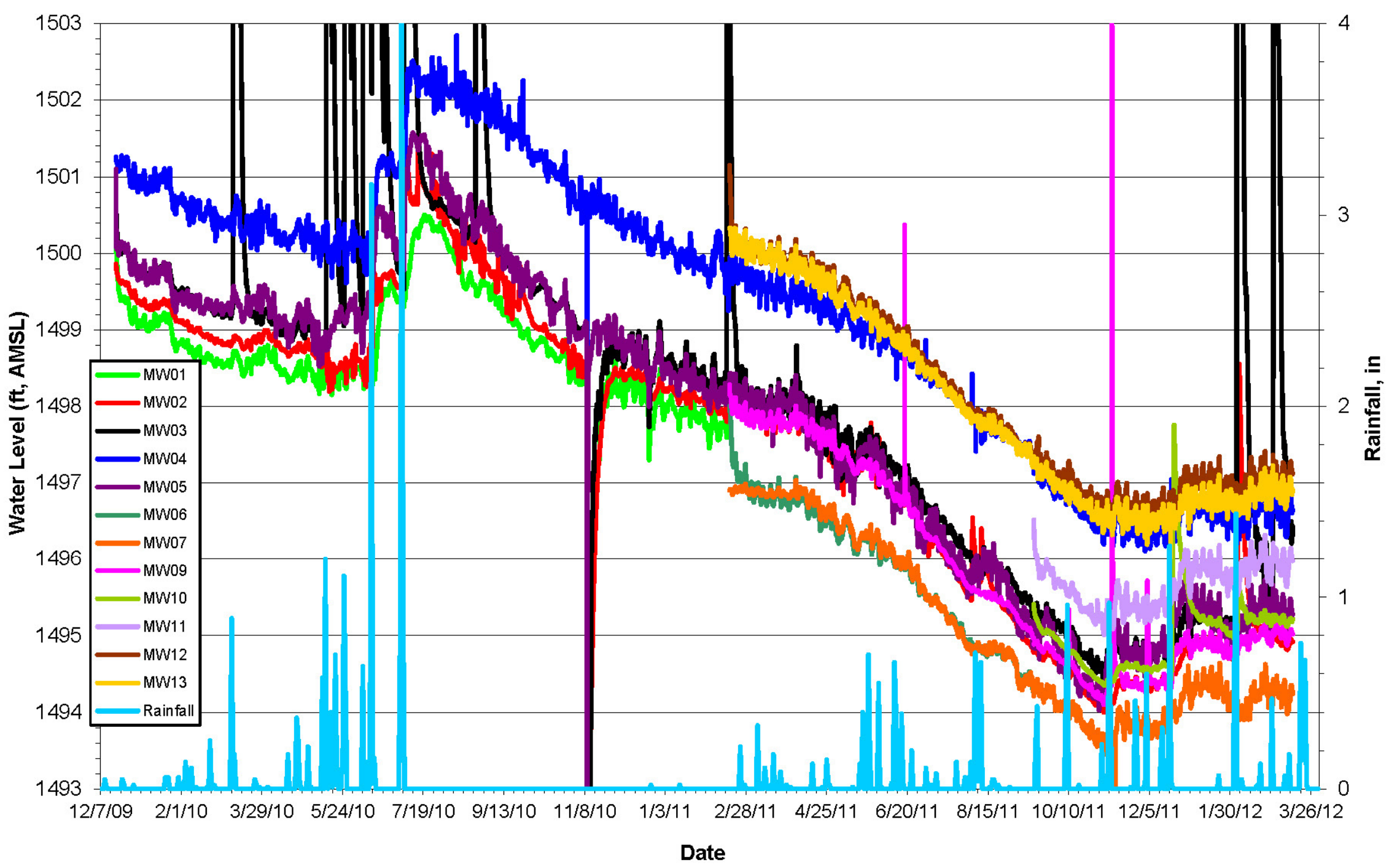

FIGURE 4.7 Hydrographs in the shallow groundwater zone observed from December 2009 to March 2012 in monitoring wells installed in Phases 1-2. 
Hydrographs in Shallow Groundwater Zone Recorded in Wells Installed in Phases 3-4

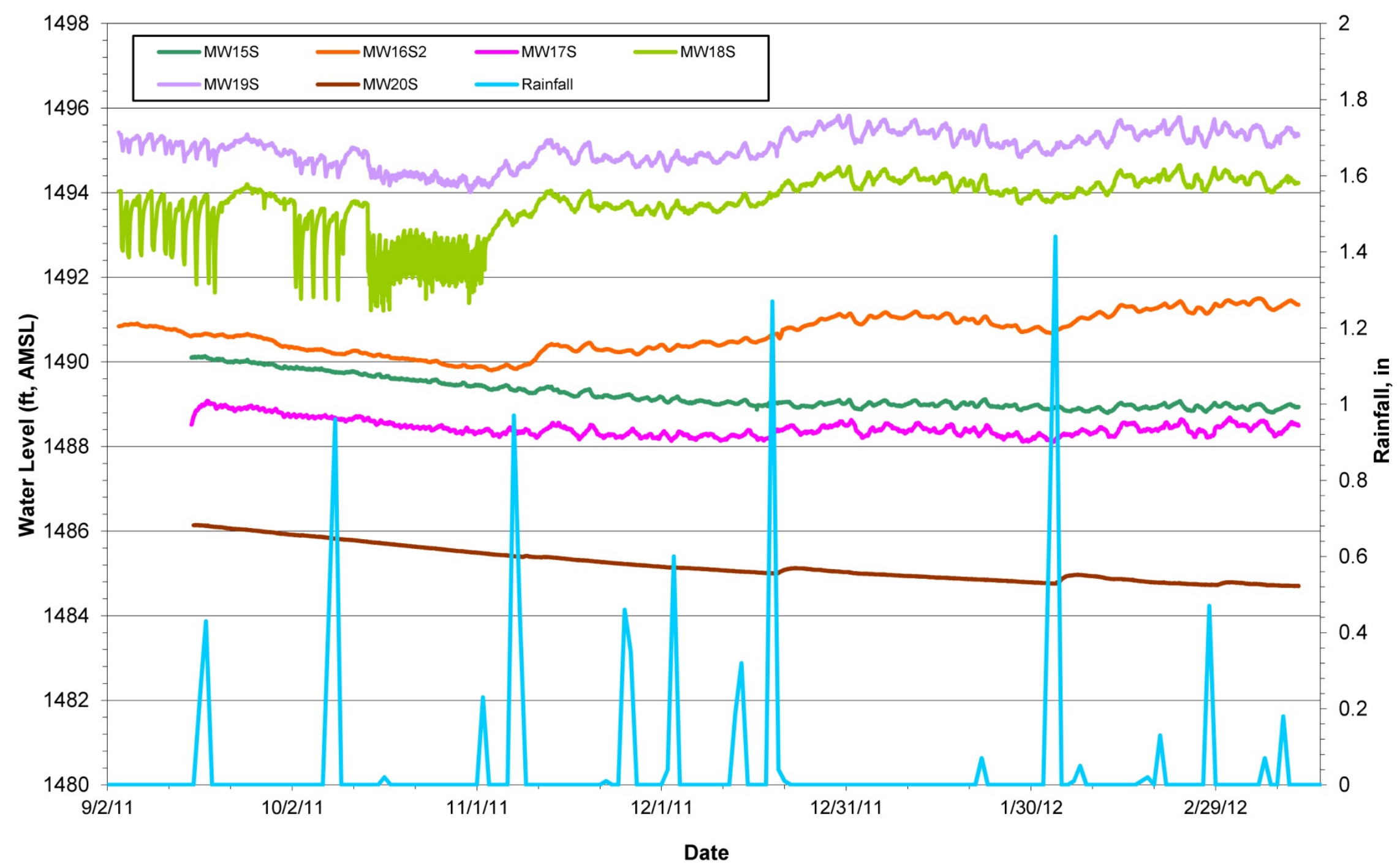


Hydrographs in Shallow Groundwater Zone Recorded in Wells Installed in Phases 1-2 and in Middle Groundwater Zone Recorded in Wells near Private Pumping Wells

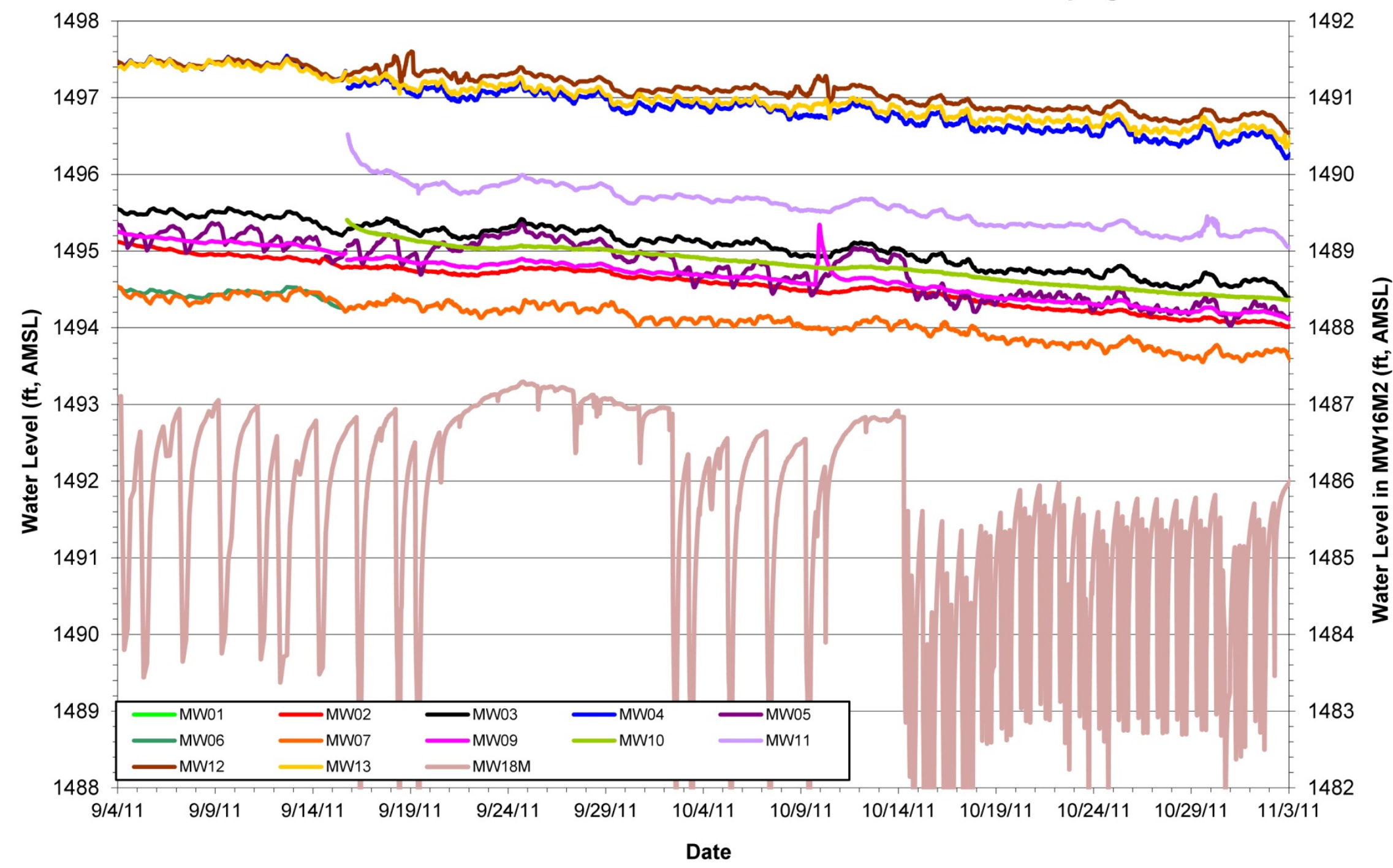

FIGURE 4.9 Detailed hydrographs for September and October 2011, showing the effects of pumping at private well(s) on the shallow water zone, as observed in monitoring wells installed in Phases 1-2. 
Hydrographs in Shallow Groundwater Zone Recorded in Wells Installed in Phases 3-4 and in Middle Groundwater Zone recorded in Wells near Private Pumping Wells

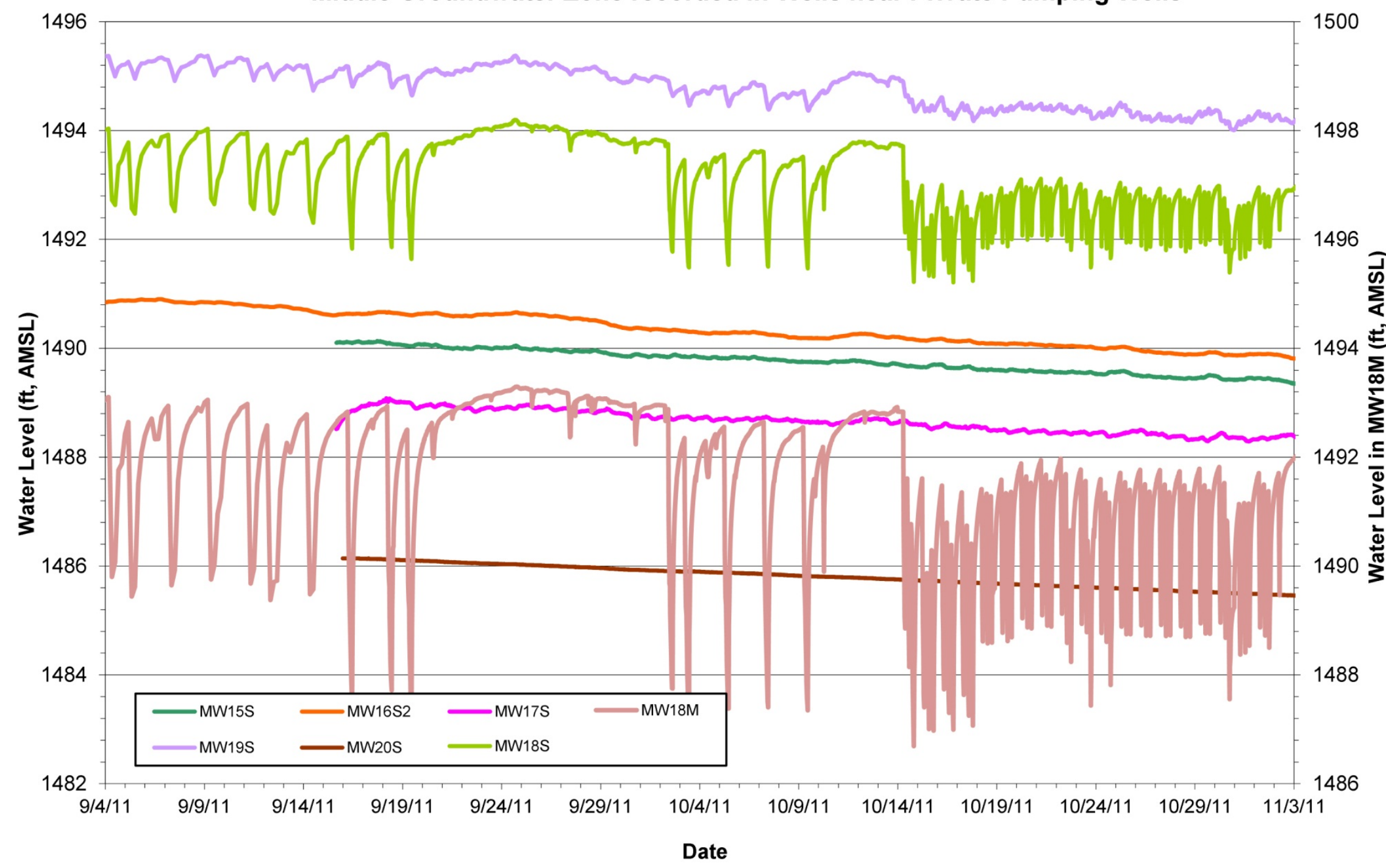

FIGURE 4.10 Detailed hydrographs for September and October 2011, showing the effects of pumping at private well(s) on the shallow water zone, as observed in monitoring wells installed in Phases 3-4. 

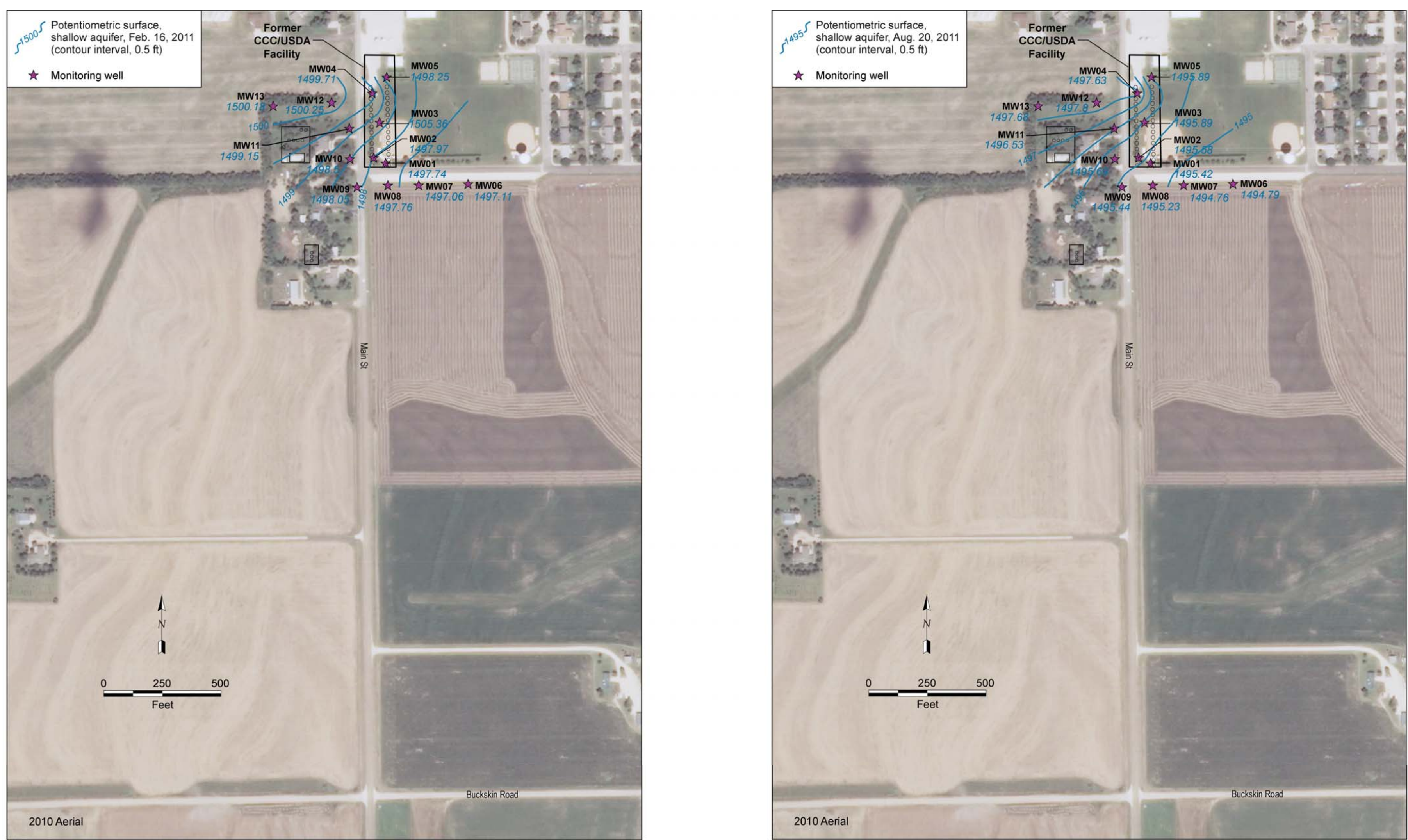

FIGURE 4.11a Potentiometric surface maps for the shallow groundwater zone north of Buckskin Road, for water levels measured on February 16 and August 20, 2011. Source of photograph: NAIP (2010). 

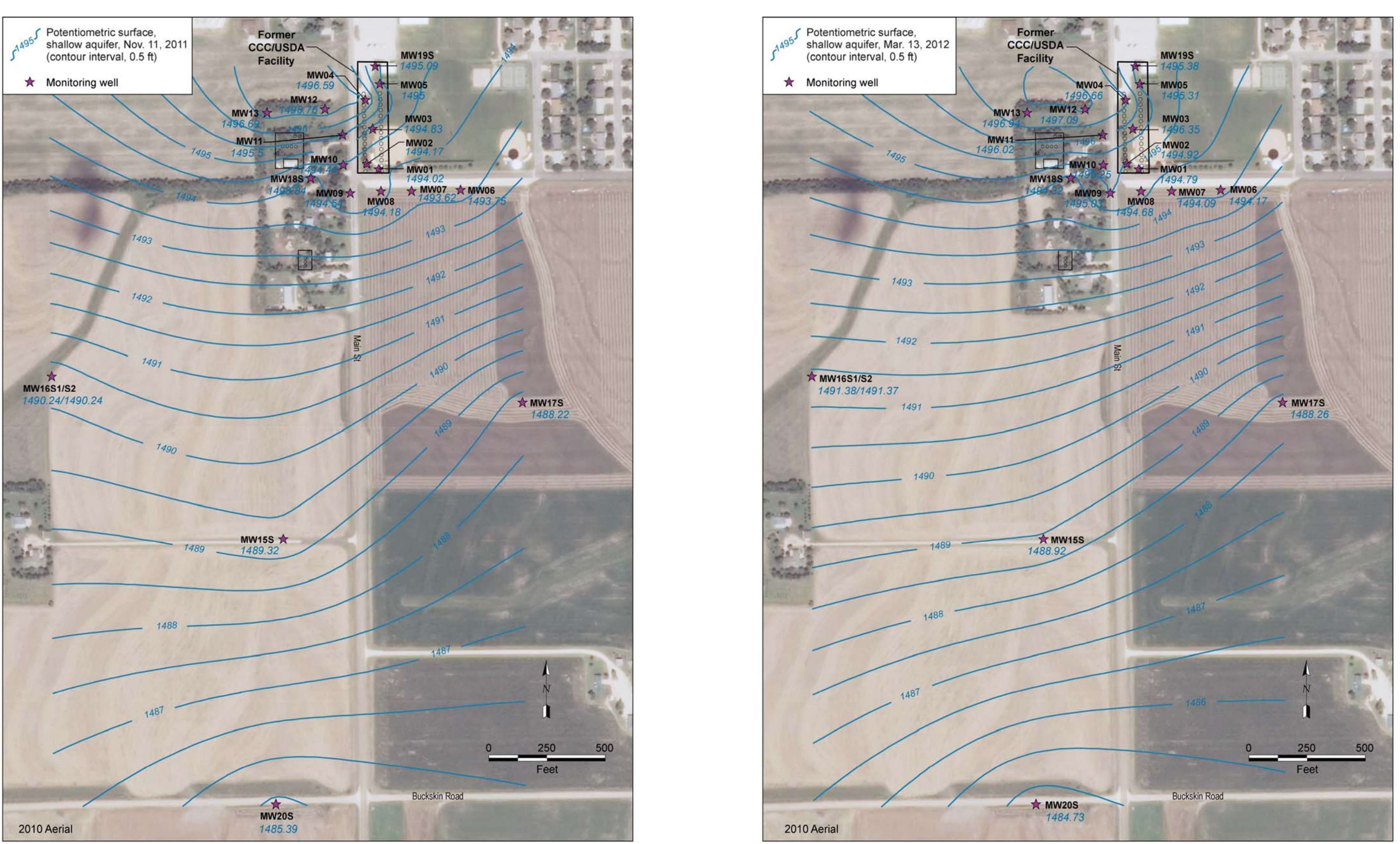

FIGURE 4.11b Potentiometric surface maps for the shallow groundwater zone north of Buckskin Road, for water levels measured on November 11, 2011, and March 13, 2012. Source of photograph: NAIP (2010). 


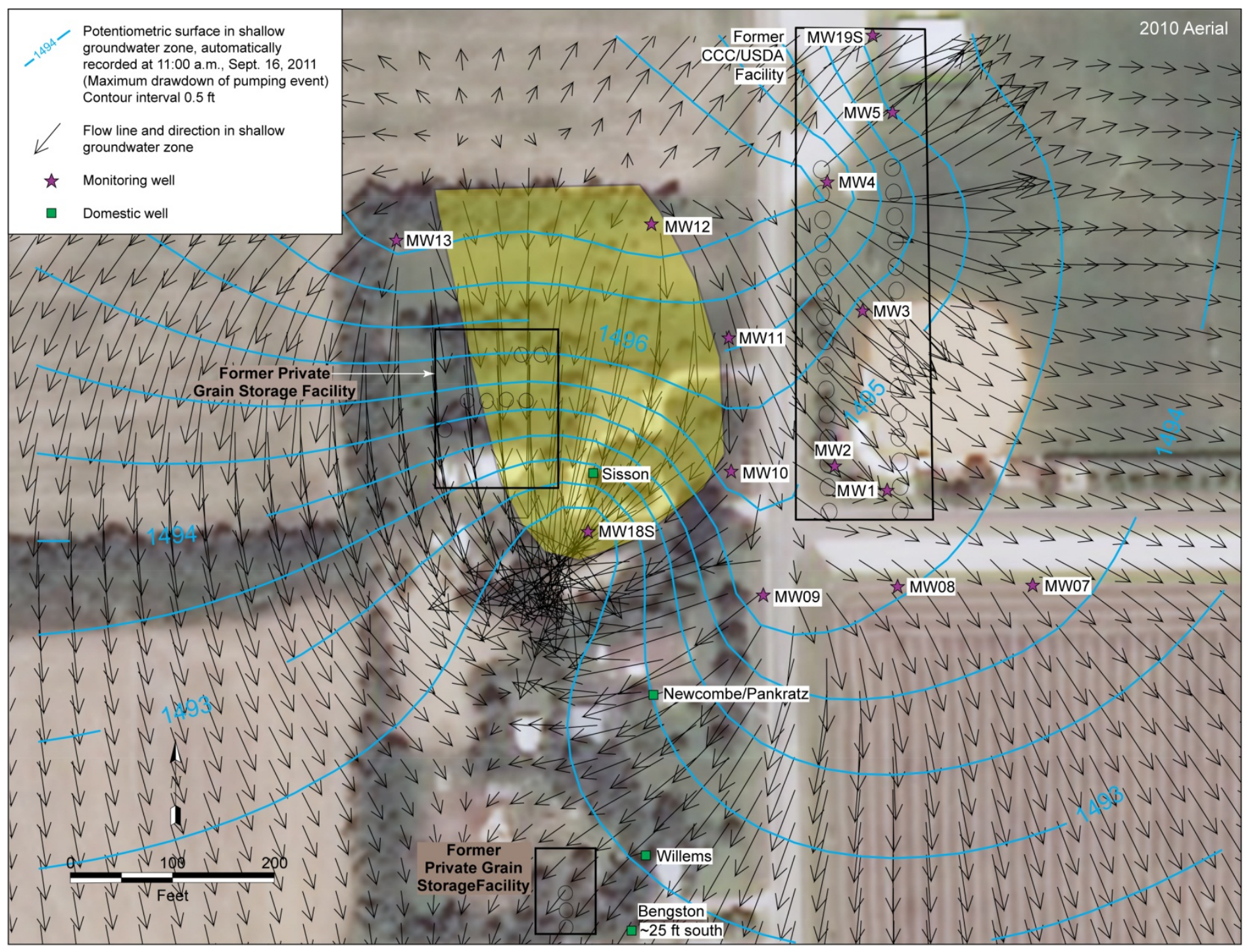

FIGURE 4.12 Potentiometric surface and flow network in the shallow groundwater zone for water levels recorded at 11:00 a.m. on September 16, 2011, the time of maximum drawdown during the pumping event, in the area of the former CCC/USDA property and the private storage facilities to the west. The shaded area indicates the approximate contribution area where shallow groundwater flowed toward the location of the pumping well. Source of photograph: NAIP (2010). 


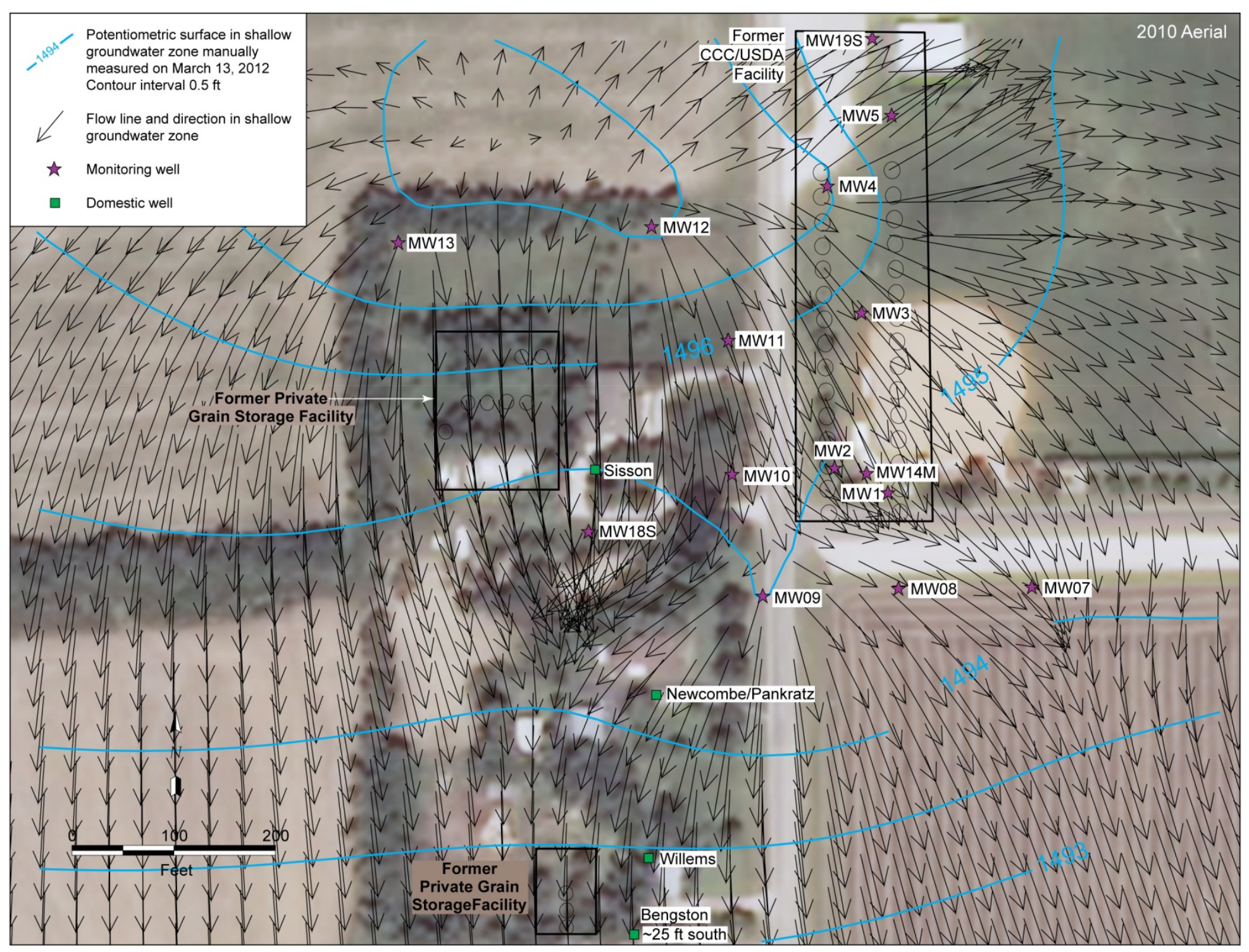

FIGURE 4.13 Potentiometric surface and flow network (ambient condition) in the shallow groundwater zone for water levels measured on March 13, 2012, in the area of the former CCC/USDA property and the private grain storage facilities to the west. Source of photograph: NAIP (2010). 
Hydrographs Observed in Middle Groundwater Zone

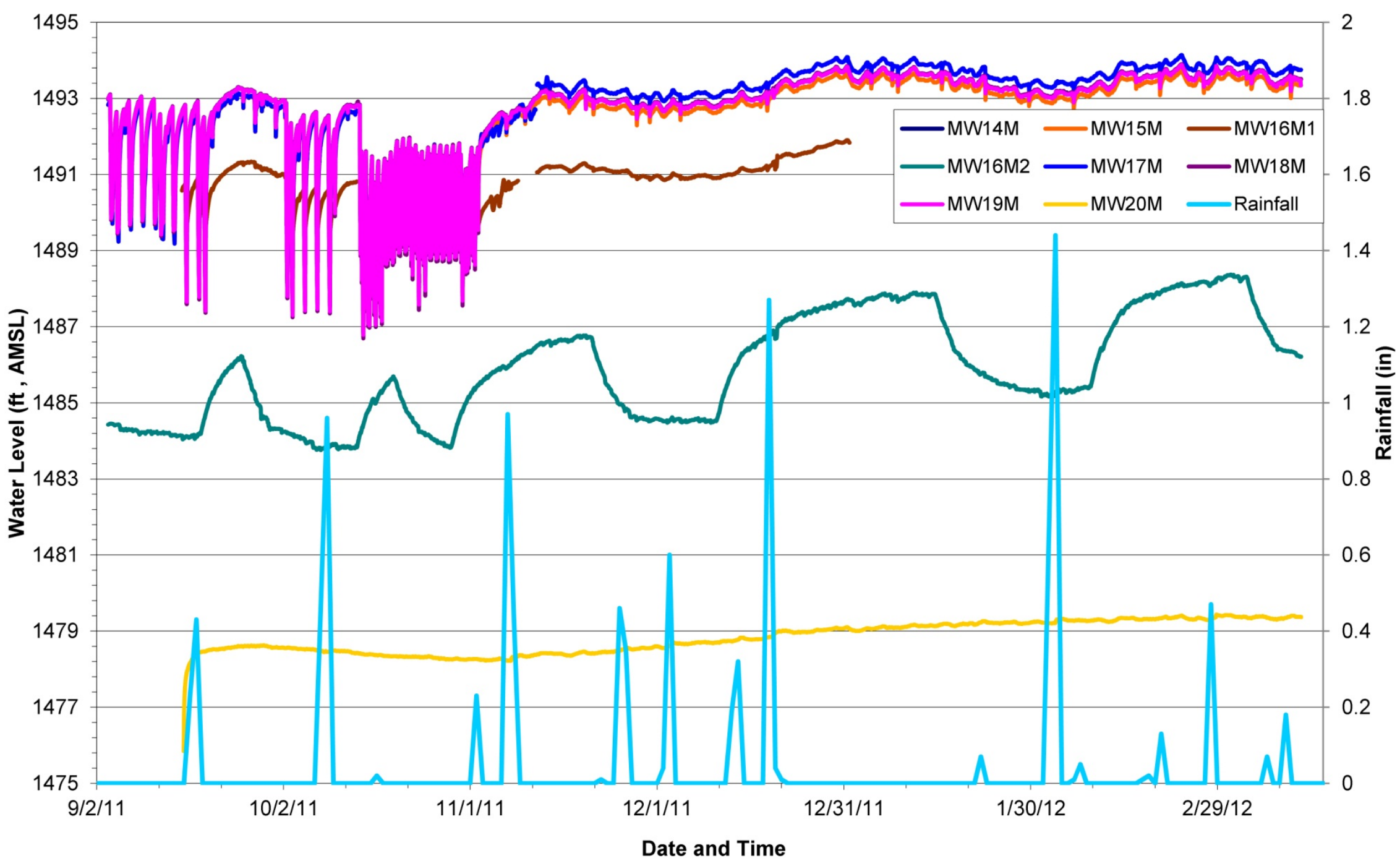

FIGURE 4.14 Hydrographs observed from September 2011 to March 2012 in selected middle groundwater zone monitoring wells. 
Hydrographs Observed in Middle Groundwater Zone During Pumping at Private Wells

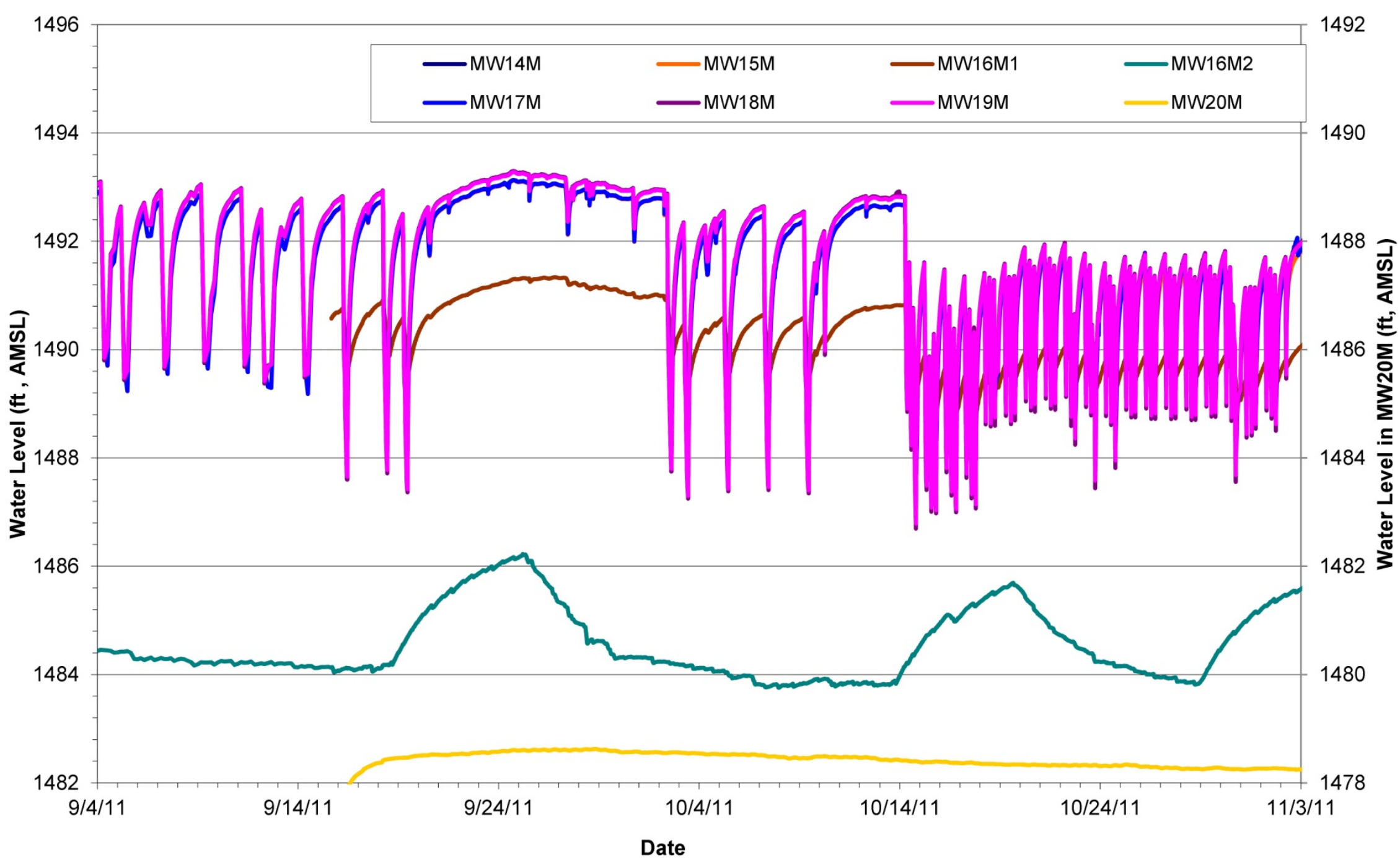

FIGURE 4.15 Detailed hydrographs for September and October 2011, showing the effects of pumping at private wells(s) on the middle water zone. 
Hydrographs Recorded in Wells in Klassen Residence

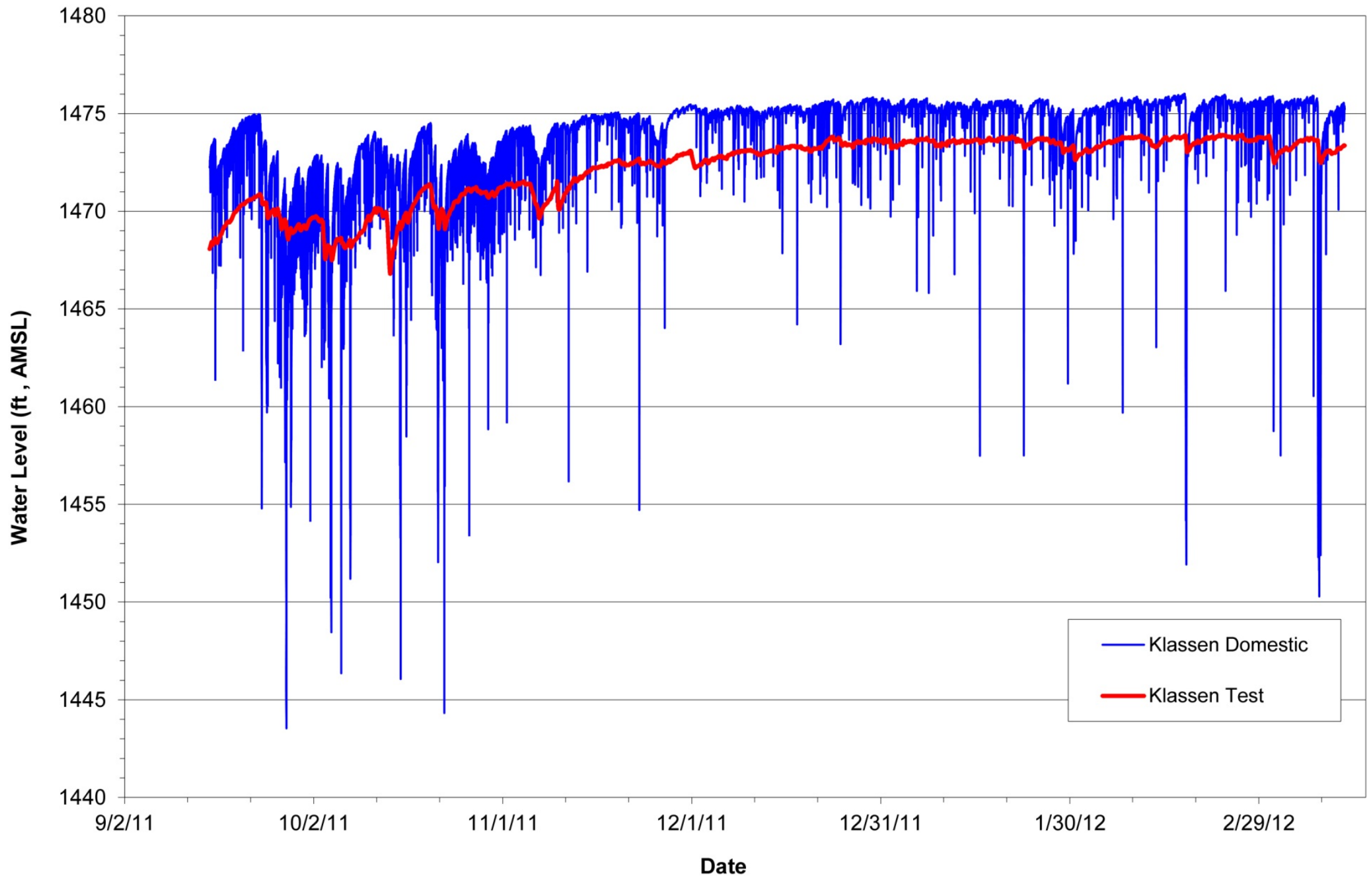

FIGURE 4.16 Hydrographs observed in the Klassen private wells south of Buckskin Road. 


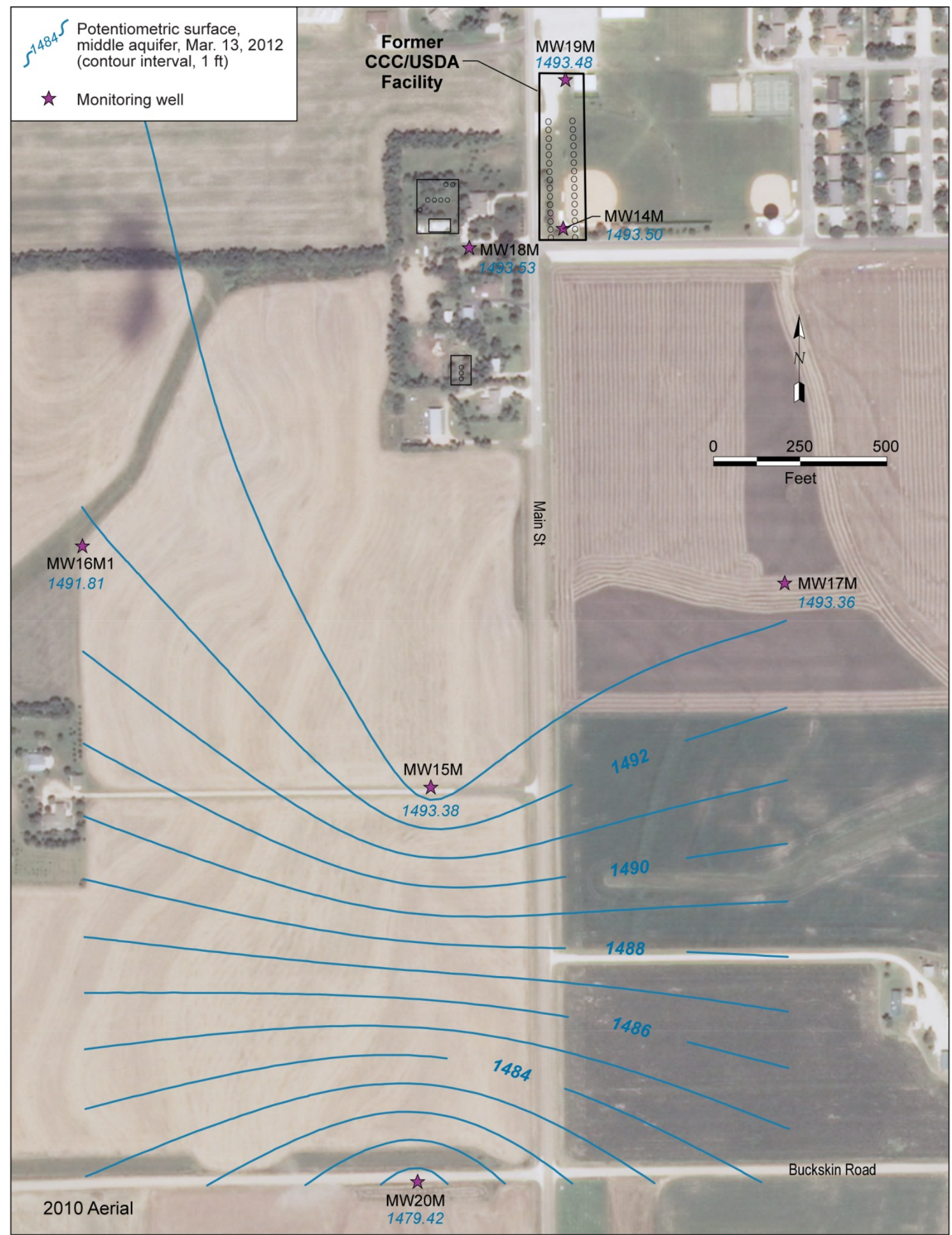

FIGURE 4.17 Potentiometric surface map for the middle groundwater zone north of Buckskin Road on March 13, 2012. Source of photograph: NAIP (2010). 

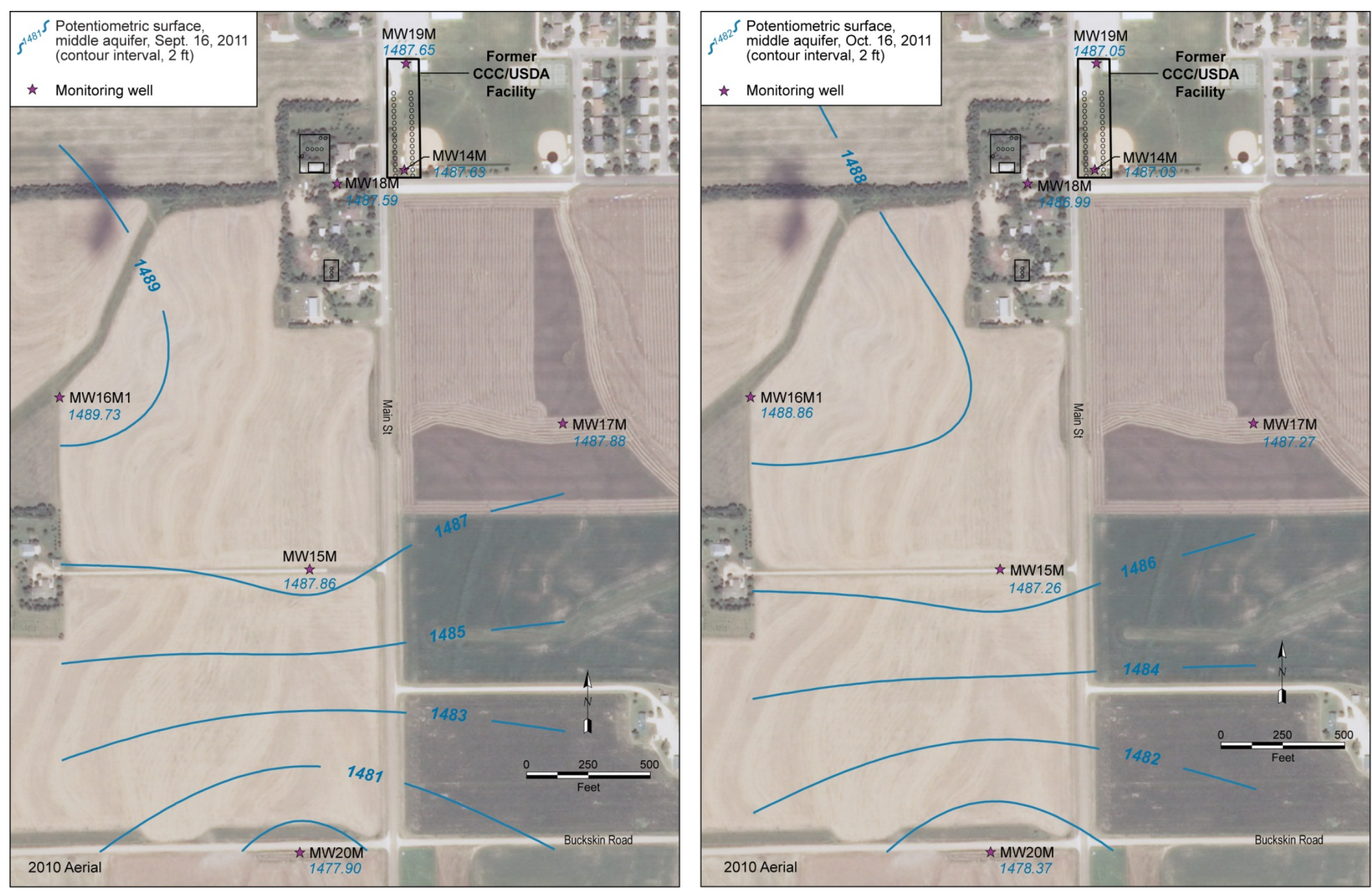

FIGURE 4.18 Overall potentiometric surface maps in the middle water zone north of Buckskin Road under pumping at the Sisson well and nearby private wells on the Pankratz (Newcombe), Willems, and Bengston properties. Water levels were recorded at 11:00:00 on September 16, 2011 (left), and at 17:30:00 on October 16, 2011 (right). Source of photograph: NAIP (2010). 


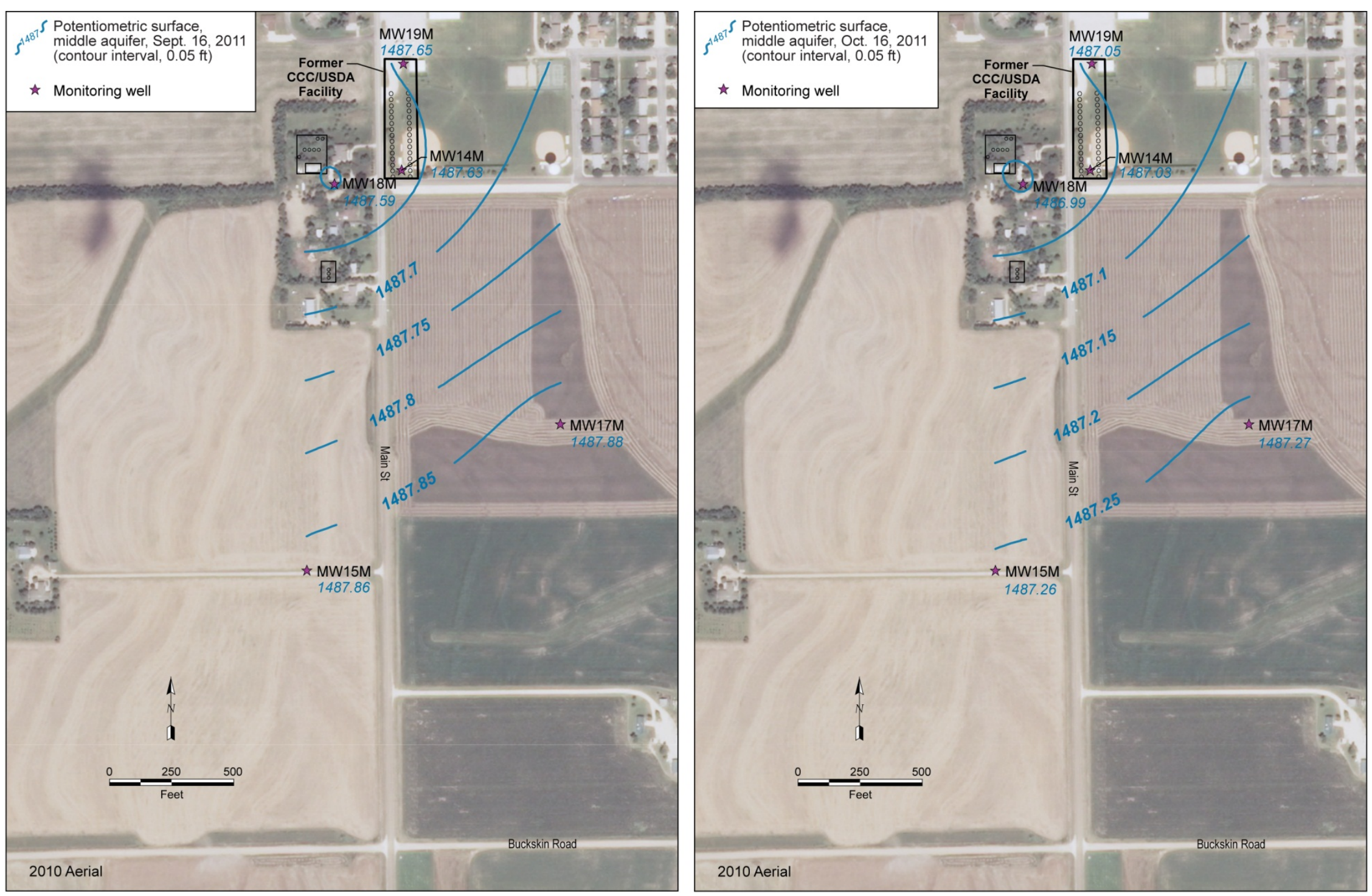

FIGURE 4.19 Potentiometric surface maps in the area affected by pumping at the Sisson well and nearby private wells on the Pankratz (Newcombe), Willems, and Bengston properties, showing a reversed flow pattern in the middle water zone. Water levels were recorded at 11:00:00 on September 16, 2011 (left), and at 17:30:00 on October 16, 2011 (right). Source of photograph: NAIP (2010). 


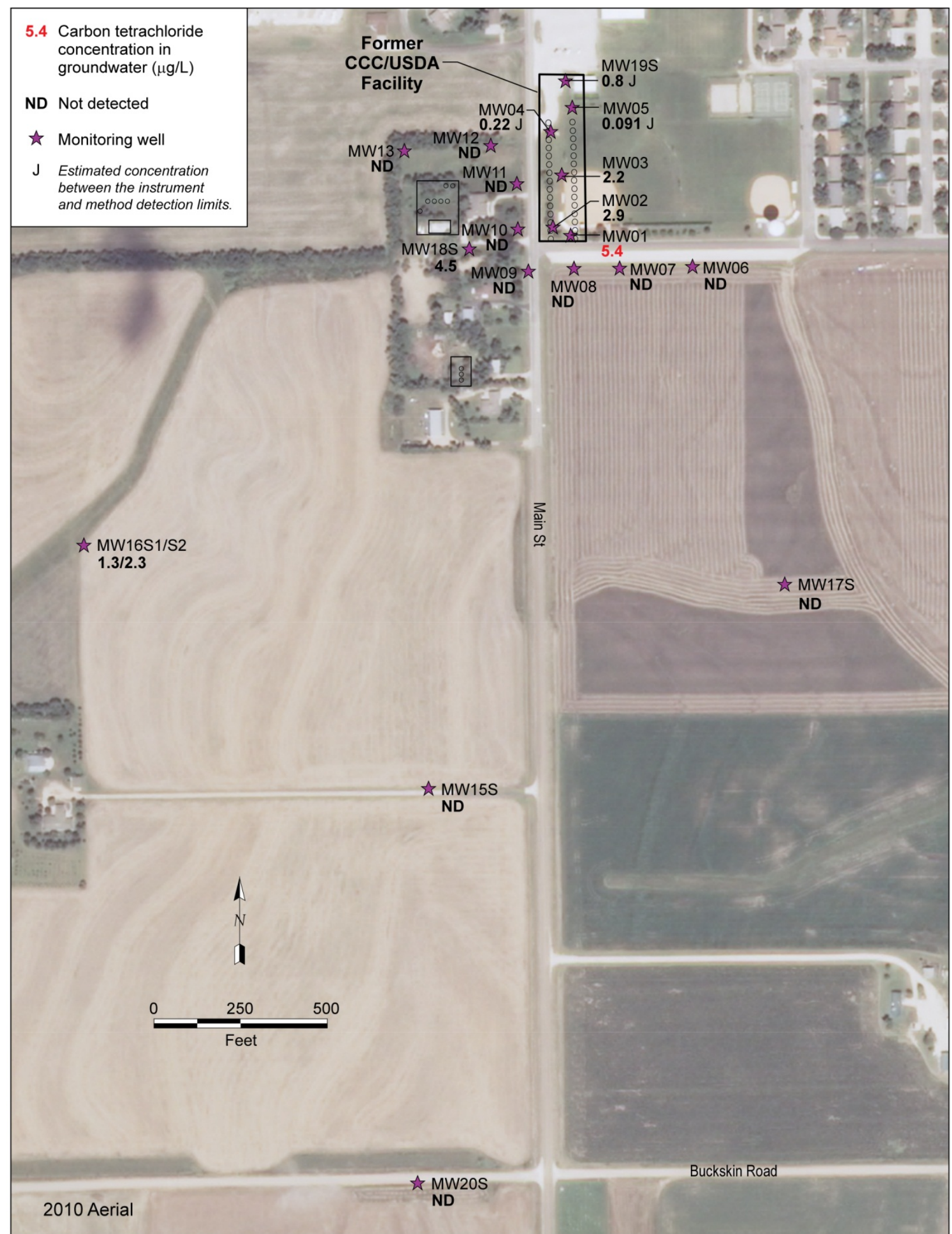

FIGURE 4.20 Lateral distribution of carbon tetrachloride in groundwater samples collected in the shallow groundwater zone north of Buckskin Road. Source of photograph: NAIP (2010). 


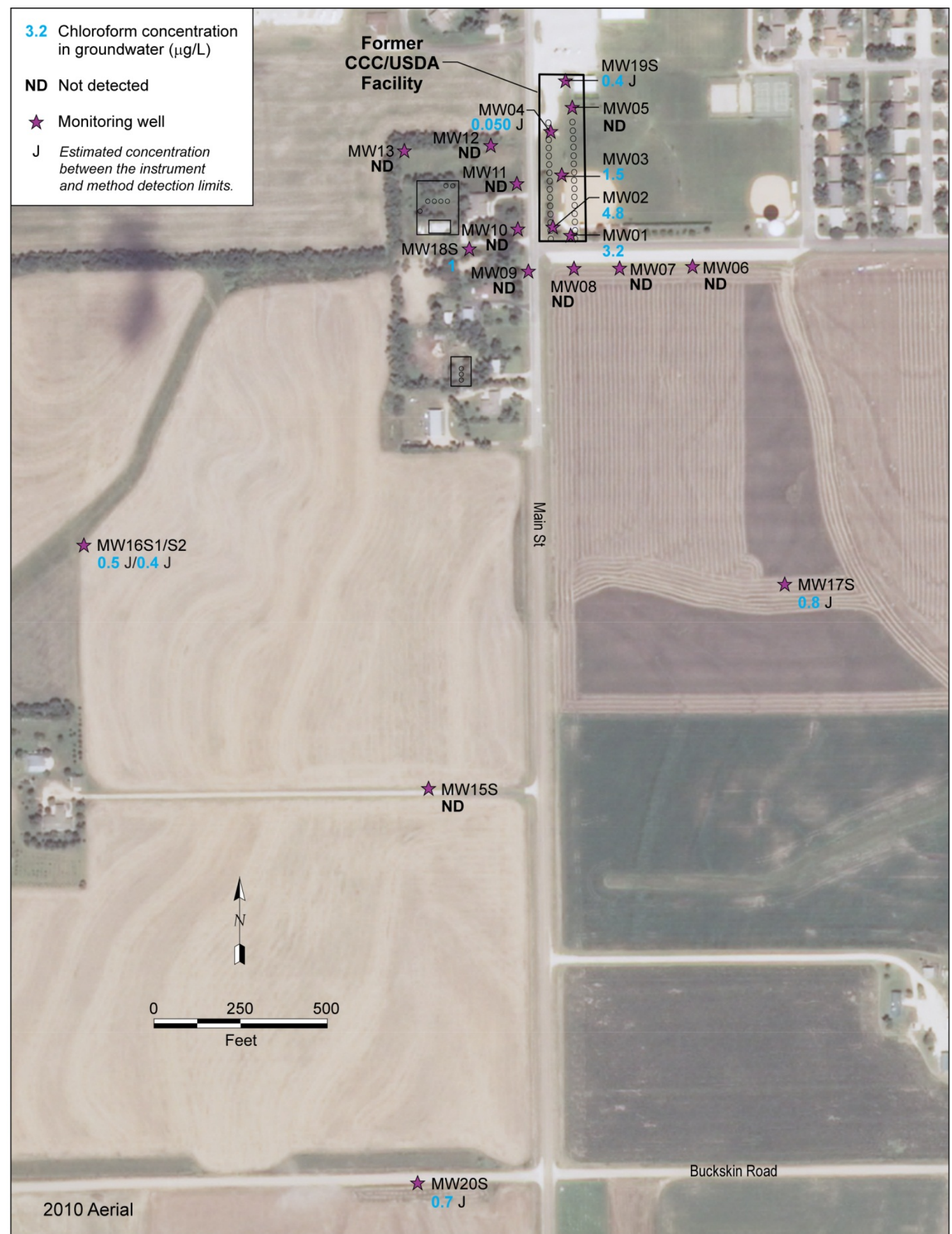

FIGURE 4.21 Lateral distribution of chloroform in groundwater samples collected in the shallow groundwater zone north of Buckskin Road. Source of photograph: NAIP (2010). 


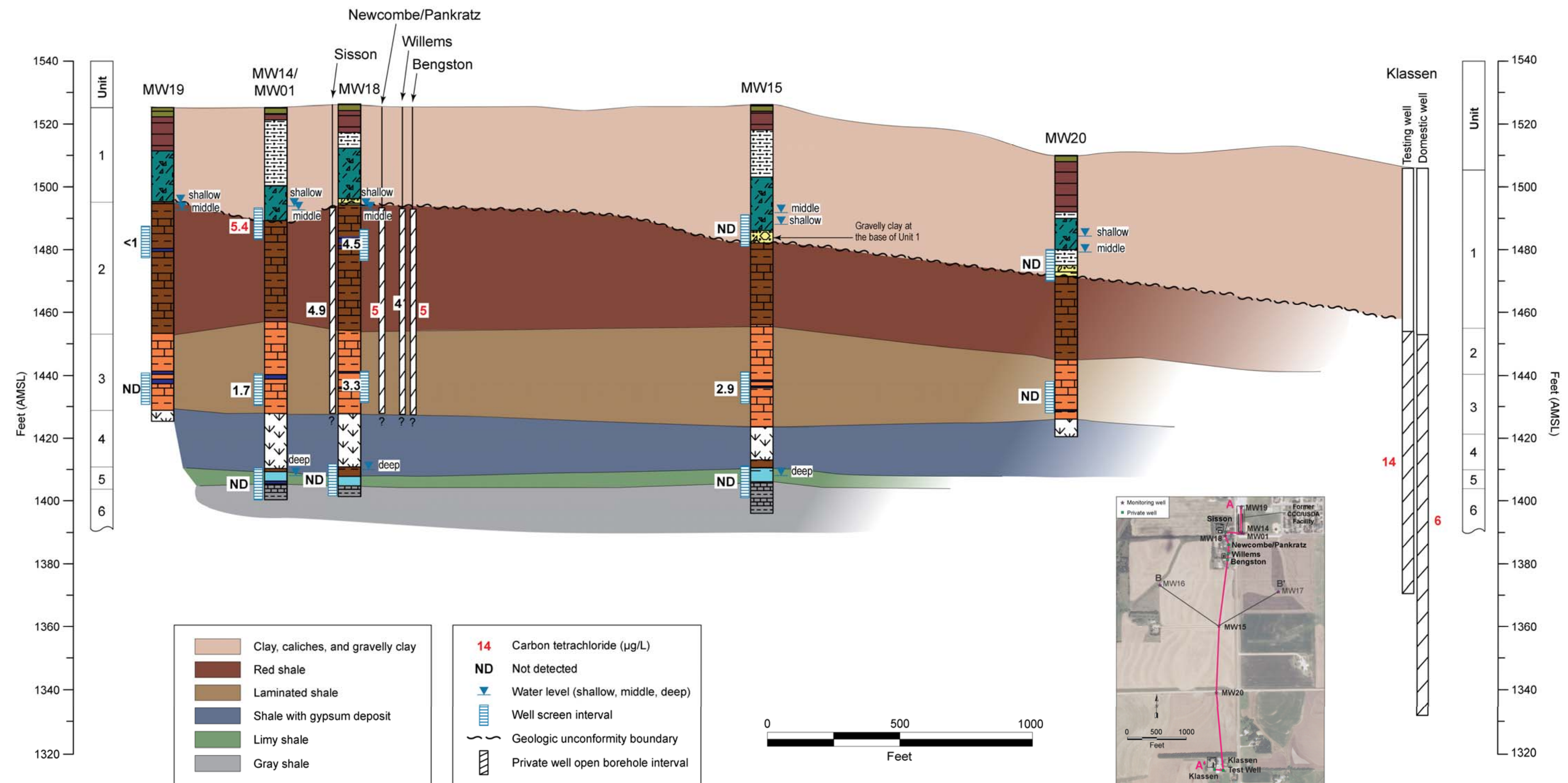

FIGURE 4.22 Vertical distribution of carbon tetrachloride in groundwater along north-to-south hydrogeologic cross section A-A'. 


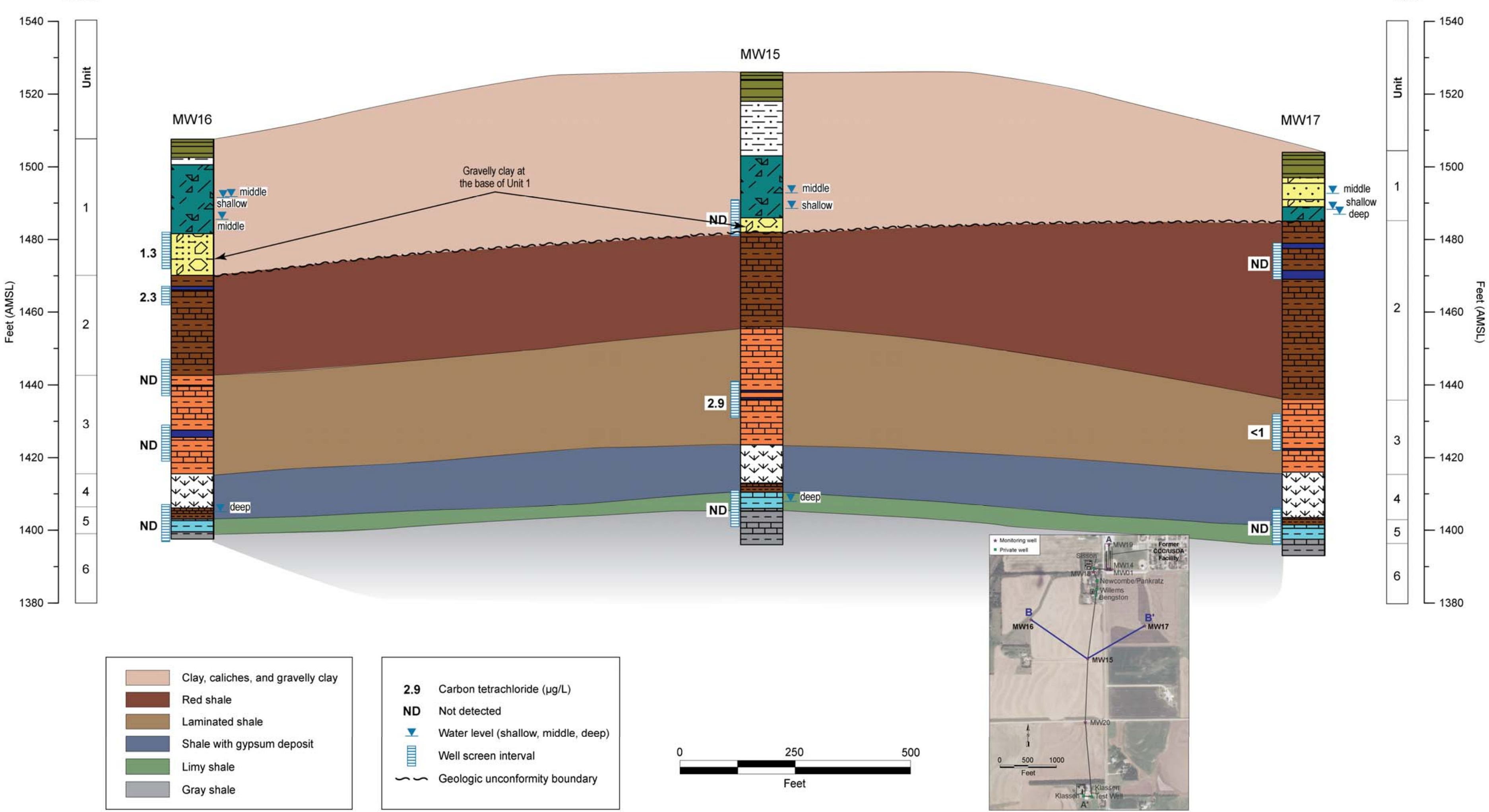




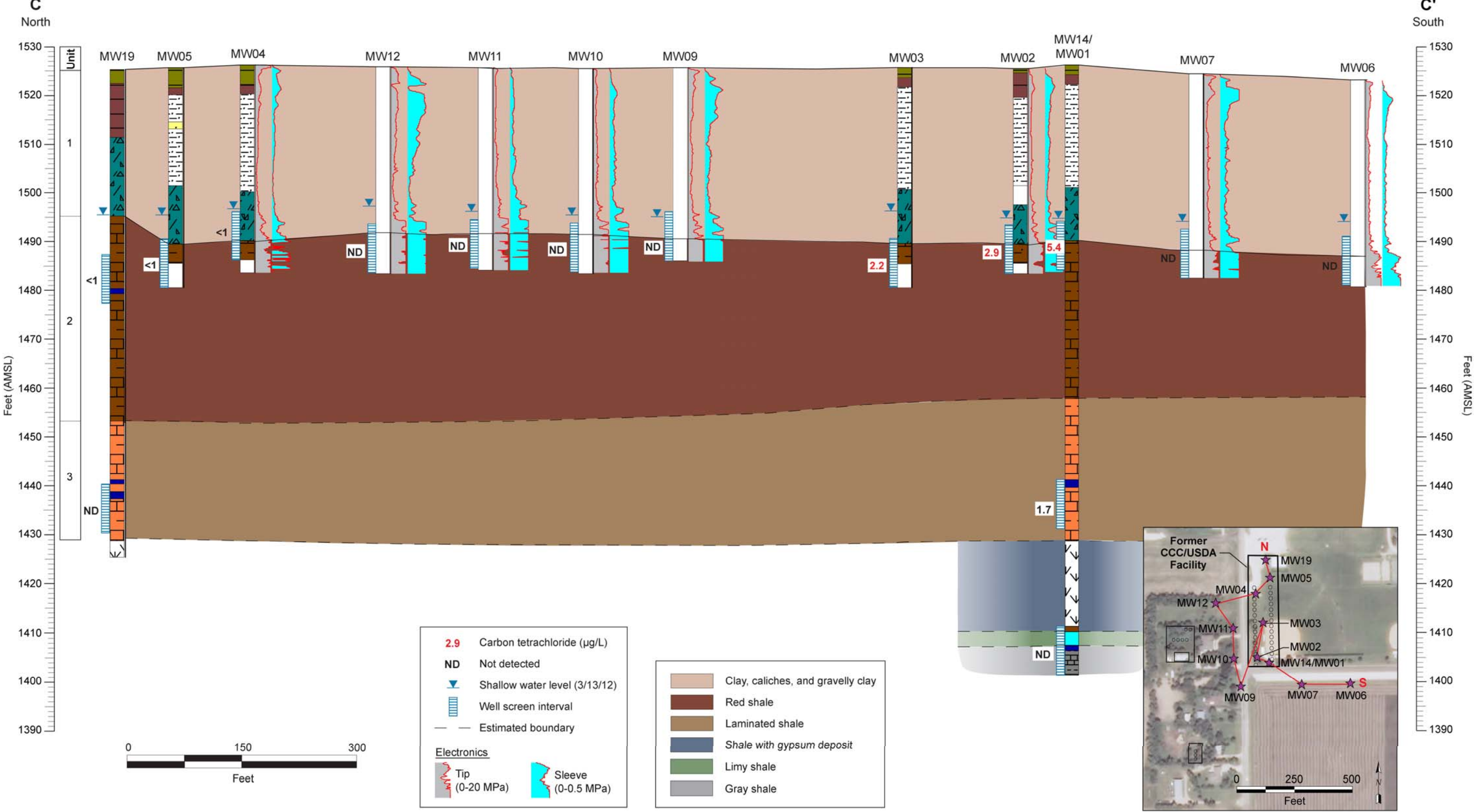

FIGURE 4.24 Hydrogeologic cross section C-C', showing CPT electronic logs, lithologic logs, and carbon tetrachloride in groundwater at the monitoring well locations at and near the former CCC/USDA facility. 


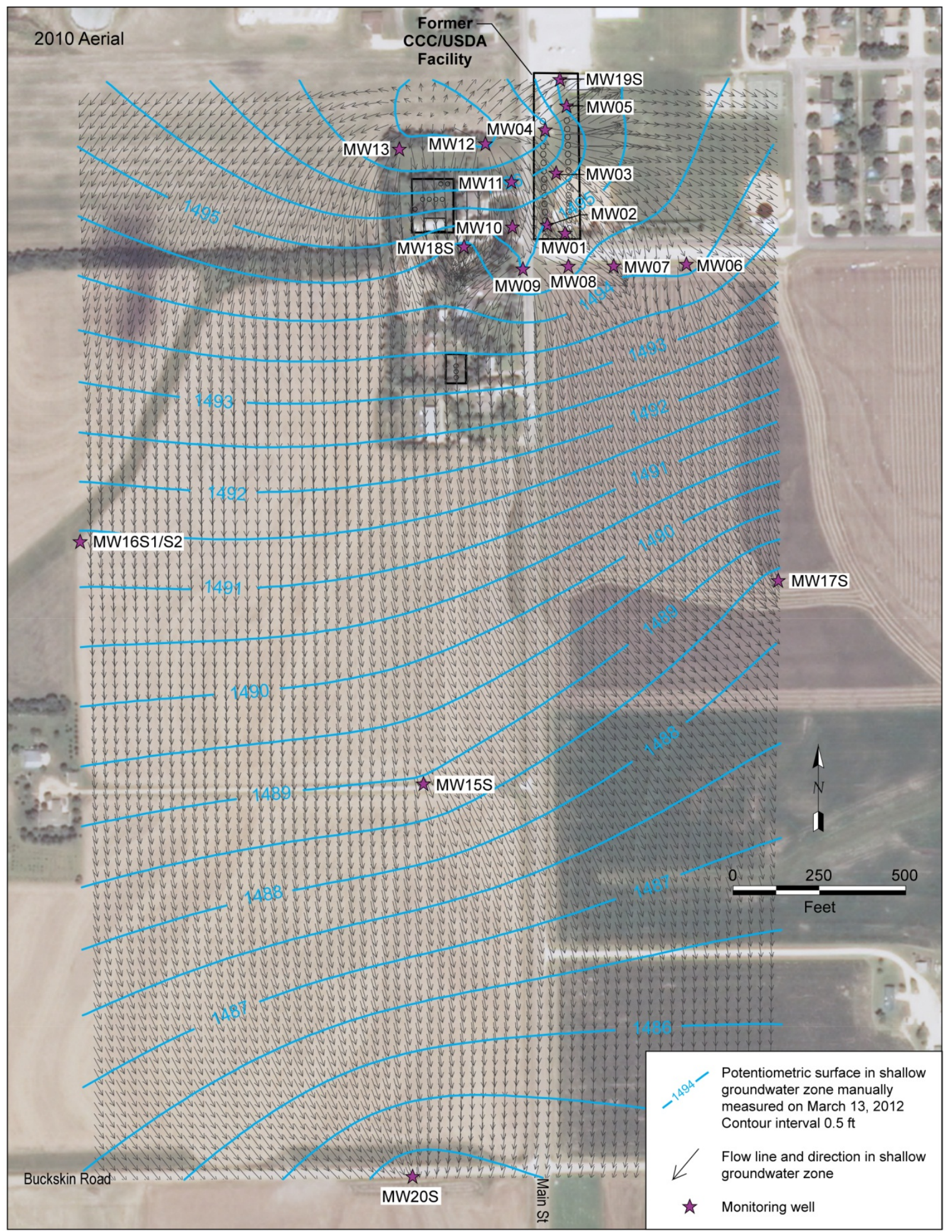

FIGURE 4.25 Potentiometric surface and flow network in the shallow groundwater zone for water levels measured on March 13, 2012, in the area bounded by the former CCC/USDA and private facilities on the north, Buckskin Road on the south, the MW17S location on the east, and the MW16 location on the west. Source of photograph: NAIP (2010). 


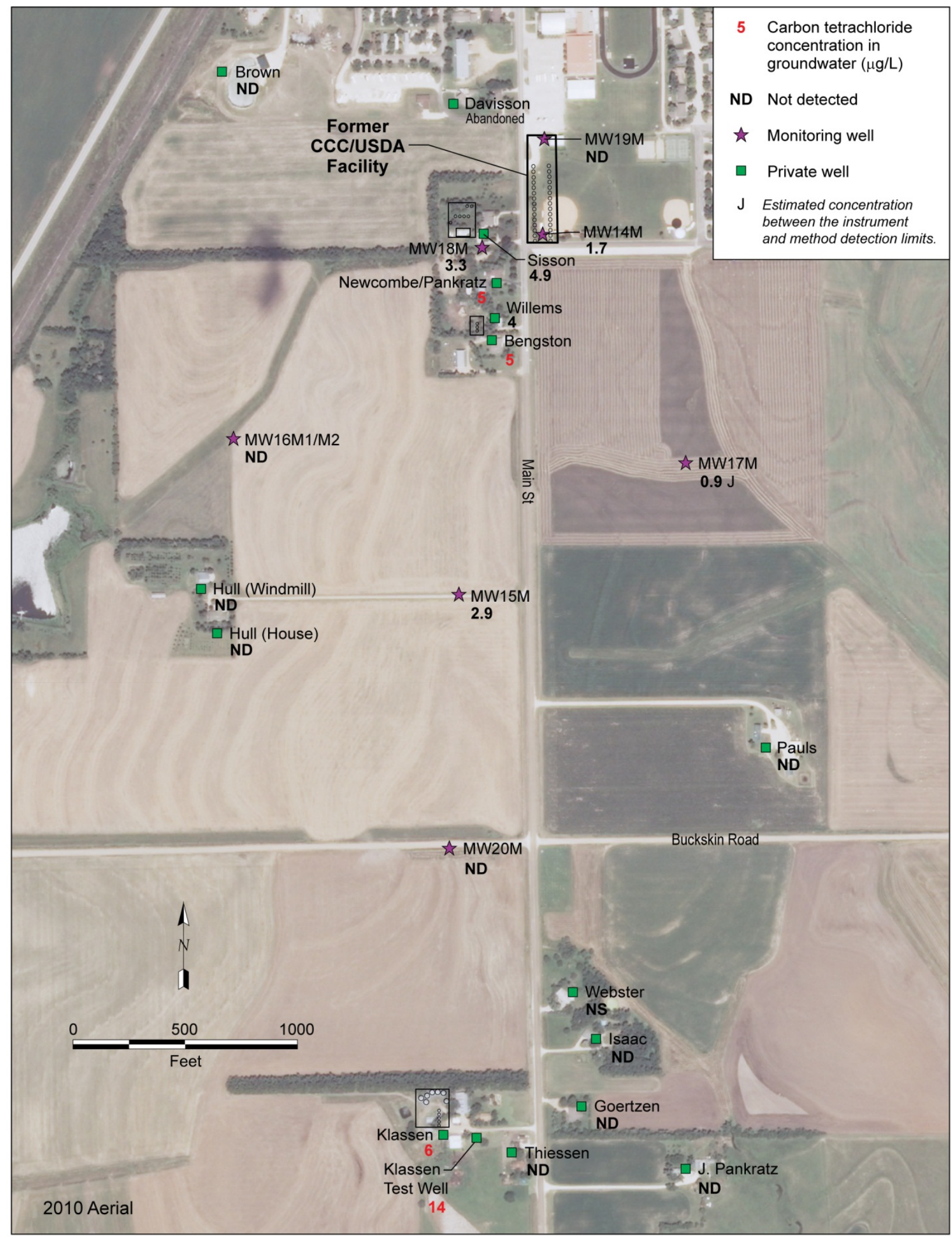

FIGURE 4.26 Lateral distribution of carbon tetrachloride in groundwater samples collected in the middle groundwater zone. Source of photograph: NAIP (2010). 


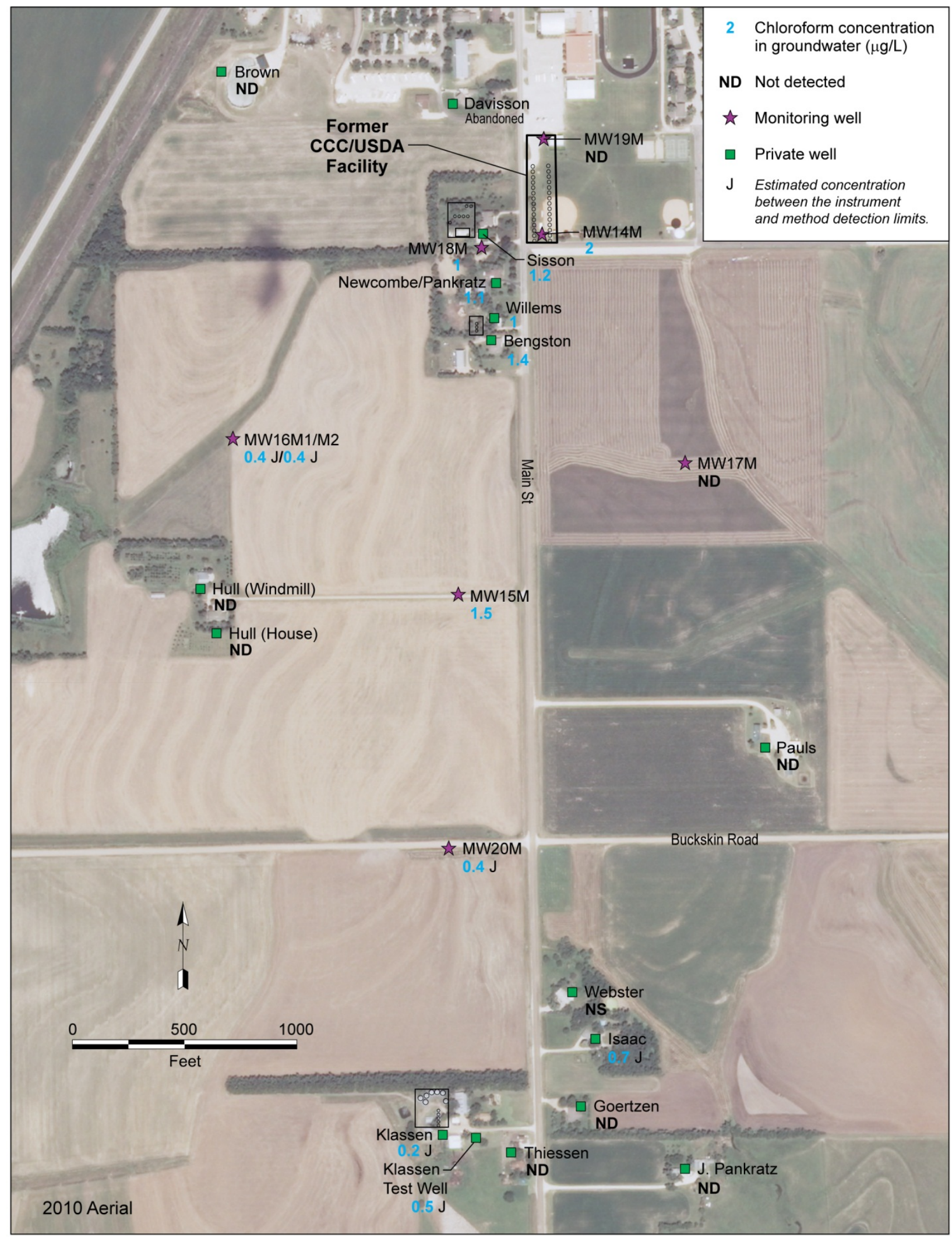

FIGURE 4.27 Lateral distribution of chloroform in groundwater samples collected in the middle groundwater zone. Source of photograph: NAIP (2010). 


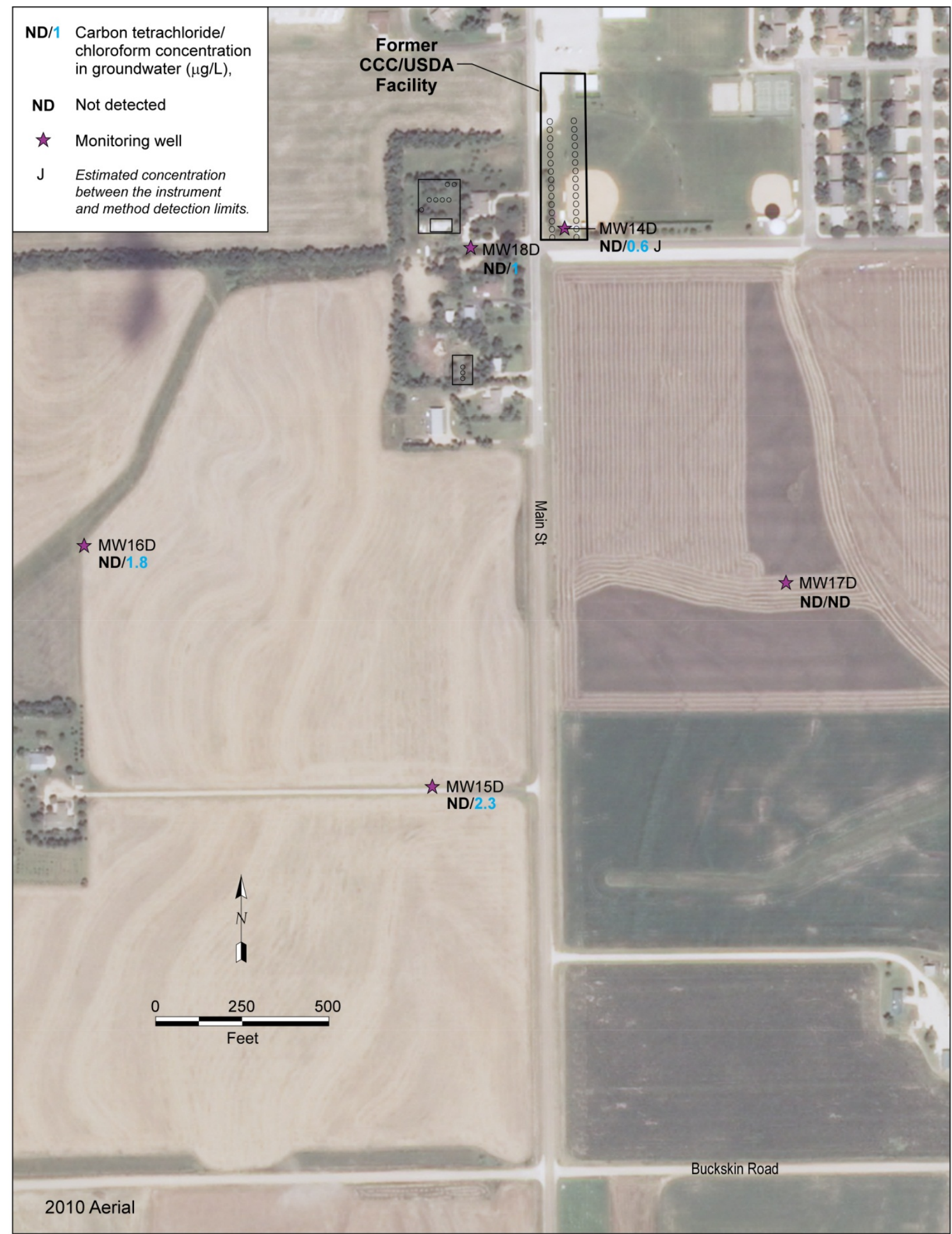

FIGURE 4.28 Lateral distribution of carbon tetrachloride and chloroform in groundwater samples collected in the deep groundwater zone. Source of photograph: NAIP (2010). 


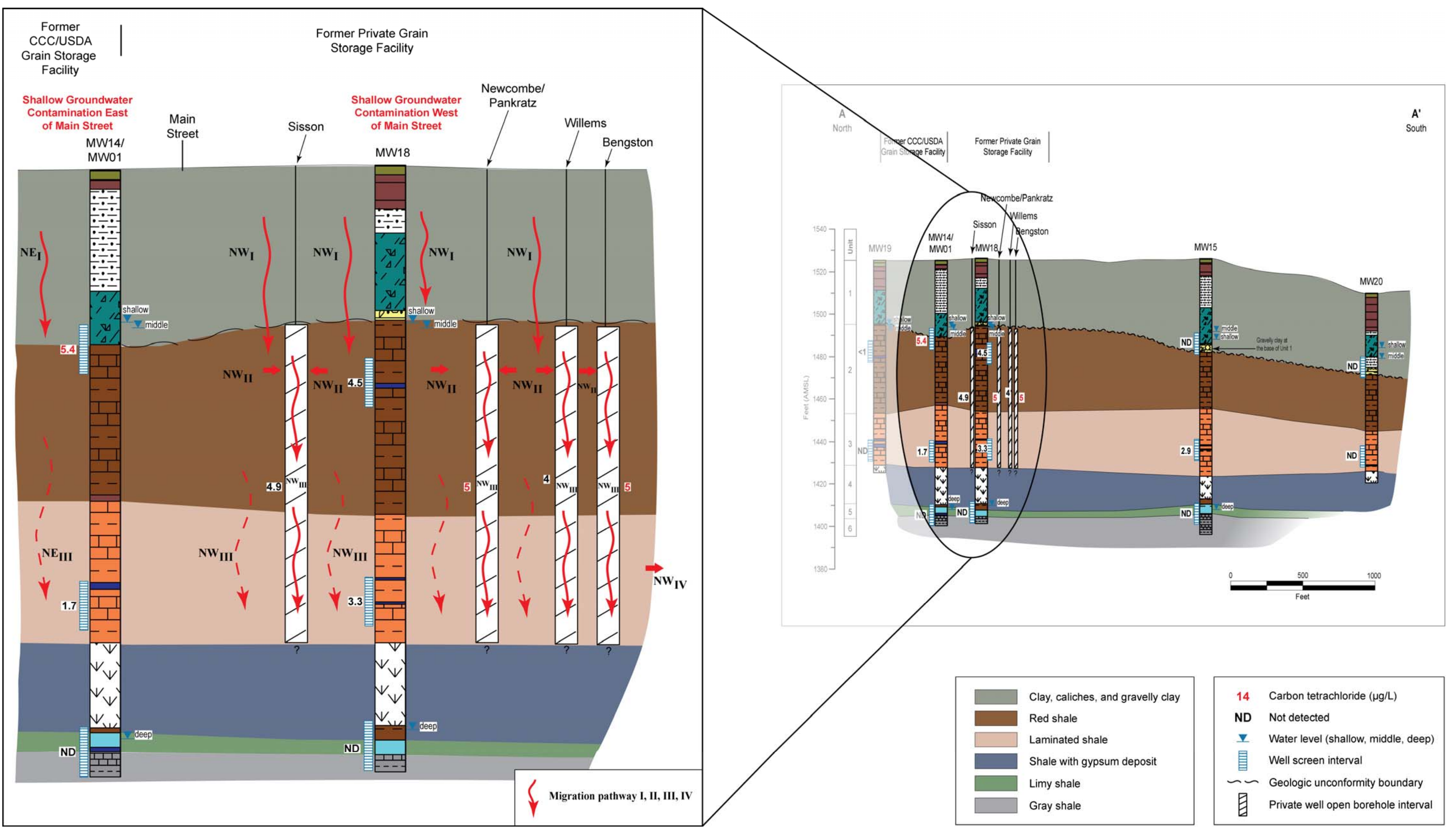

FIGURE 4.29 Conceptual model of contaminant migration pathways north of Buckskin Road, east and west of Main Street. 


\section{Conclusions and Recommendations}

The integrated analyses of geologic, hydrogeologic, groundwater monitoring, geochemical, and contaminant distribution data generated in all five phases of investigation provide a technical basis for understanding the groundwater flow and contaminant migration system at Inman. The key findings regarding the investigation area are summarized below.

\subsection{Physical Setting and Characteristics}

- The local geologic sequence in the vicinity of the former CCC/USDA facility generally consists of six primary lithostratigraphic units in the uppermost section to a depth of approximately $125 \mathrm{ft}$ BGL. From the surface downward, these units are (1) surficial silt and clay with a lower section of caliche overlying gravelly clay to clayey gravel, (2) weathered red shale, (3) laminated shale, (4) dolomitic shale filled with precipitated evaporitic minerals along the bedding planes and fractures, (5) limy shale, and (6) gray dolomitic shale. The shale formation identified in Units 2-6 is inferred to be part of Permian Ninnescah Shale.

- Three groundwater-bearing zones were identified in the local geologic sequence. The shallow groundwater zone is recognized in the upper portion of the weathered red shale of Unit 2 and the overlying basal gravelly clay to clayey gravel in Unit 1. The middle groundwater zone is the most productive and is consistently present in the laminated shale of Unit 3. The deep groundwater zone is the least productive and is hosted by the thin limy shale of Unit 5.

- The main characteristics of the shallow groundwater zone north of Buckskin Road are as follows:

- The shallow groundwater zone is the uppermost water-bearing zone near the unconformable bedrock surface; it exists under leaky-confined conditions. 
- The apparent flow pattern in the shallow zone is semi-radial from the groundwater high at MW12-MW13 to the southeast, south, and southwest across the northern area. At the former CCC/USDA facility, the southeastern component of the flow pattern is predominant for both scenarios under ambient condition and during pumping at private wells, resulting in groundwater flow from the Sisson property across Main Street to the former CCC/USDA facility.

- Vertical flow between the shallow groundwater zone and the underlying middle groundwater zone is recognized via two mechanisms: (1) infiltration through the bedrock matrix, driven by a natural vertical hydraulic gradient, and (2) direct flow through hydraulic conduits formed by open boreholes or sand packs in the Sisson, Pankratz (Newcombe), Willems, and Bengston private wells, which intercept both the shallow and middle groundwater zones. The apparent ambient vertical hydraulic gradient is (1) downward in the northern area including the former CCC/USDA facility and the private grain storage facilities on the Sisson and Willems properties, (2) upward at locations MW15-MW17, and (3) downward toward MW20 to the south.

- The estimated $K_{h}$ of the shallow groundwater zone is highly variable across several orders of magnitude - from an extremely low $K_{h}$ value (roughly $10^{-4} \mathrm{ft} /$ day) in the southern portion of the former CCC/USDA facility (MW01-MW03), to a slightly higher value ( $>4 \times 10^{-3} \mathrm{ft} /$ day) in the northern portion of the former CCC/USDA facility (MW05), and to a local maximum ( $10^{0}$ to $10^{2} \mathrm{ft} /$ day) at the Sisson property (MW18S).

- The main characteristics of the middle groundwater zone north of Buckskin Road are as follows:

- The middle groundwater zone is a well-developed water-bearing zone along the bedding planes and fractures in the laminated shale of Unit 3; it is confined by the surrounding low-permeability bedrock matrix. Most of local private wells produce from this water zone. 
- The apparent ambient flow pattern in the middle groundwater zone is semi-radial toward the southeast, south, southwest, and west from the area north of the former grain storage facilities on the Sisson and Willems properties and the former CCC/USDA facility. Under pumping at one of the four private wells (Sisson, Pankratz [Newcombe], Willems, or Bengston), locally reversed flow toward the pumping well was observed in the northeastern area around MW14M, MW15M, and MW17MMW19M. The flow pattern to the south (at MW20) remained unchanged.

- No evidence exists to indicate that vertical flow is present between the middle groundwater zone and the deep groundwater zone, even though the apparent hydraulic gradient is predominantly downward between the two zones.

- The estimated $K_{h}$ value of the middle groundwater zone is $10^{1}$ to $10^{3} \mathrm{ft} /$ day near the pumping well and the monitoring well (MW18) on the Sisson property for assumed pumping rates of 1-20 gpm and the assumption that $70-95 \%$ of the pumped water is from the middle groundwater zone.

- The main characteristics of the deep groundwater zone north of Buckskin Road are as follows:

- The deep groundwater zone is identified within a thin limy shale of Unit 5; it is confined by overlying low-permeability dolomitic shale filled with evaporitic minerals (Unit 4) and underlying gray dolomitic shale (Unit 6).

- The groundwater level in the deep groundwater zone was still under recovery at the time of the last data retrieval, and no flow pattern or lateral/vertical gradient could be determined.

- The $K_{h}$ value in the deep groundwater zone is likely less than $10^{-4} \mathrm{ft} /$ day, as suggested by extremely slow recovery of water levels (several months). 


\subsection{Extent of Contamination and Migration Pathways}

- No carbon tetrachloride and chloroform were identified above the method detection limit of $10 \mu \mathrm{g} / \mathrm{kg}$ in vadose zone soils at the former CCC/USDA grain storage facility and the former private grain storage facilities on the Sisson and Willems properties, indicating the absence of soil sources of contamination to groundwater.

- Carbon tetrachloride and chloroform detections in the shallow groundwater zone were limited primarily to two separate areas resulting from vertical infiltration from two separate overlying source areas. One area of shallow groundwater contamination represents the southern portion of the former CCC/USDA facility, whereas the other area involves the former private grain storage facilities on the Sisson and Willems properties.

- The lateral and vertical extents of contamination beneath the southern portion of the former CCC/USDA facility are as follows:

- Contamination (up to $5.4 \mu \mathrm{g} / \mathrm{L}$ for carbon tetrachloride) in the shallow groundwater-bearing zone has been confined to the southern portion of the former CCC/USDA facility (MW01-MW03) for the last $40 \mathrm{yr}$. This observation is consistent with the evidence of extremely low $K_{h}$ $\left(10^{-4} \mathrm{ft} /\right.$ day $)$ in the shallow zone in this area. No evidence of lateral migration off-site was found in the shallow groundwater-bearing zone. The local school baseball field is located over this portion of the former CCC/USDA facility.

- The highest abundance of chloroform relative to carbon tetrachloride (59-165\%) observed in this area suggests that degradation of carbon tetrachloride by reductive dechlorination has been enhanced by the extended residence time of contaminants in the subsurface in the southern portion of the former CCC/USDA facility due to extremely low permeability. 
- The trace level of carbon tetrachloride $(1.7 \mu \mathrm{g} / \mathrm{L})$ identified in the middle groundwater zone in this area reflects a reduction in concentration during vertical infiltration through the bedrock matrix from the shallow groundwater zone $(5.4 \mu \mathrm{g} / \mathrm{L})$ to the middle zone. The ratios of chloroform to carbon tetrachloride are similar in the shallow (59-165\%) and middle groundwater zones $(118 \%)$ on the southern portion of the former CCC/USDA facility but are distinct from the ratio (22\%) on the private properties to the west.

- Contaminants in the middle groundwater zone at the former CCC/USDA facility provide no significant contribution to contamination at the four private wells across Main Street. This interpretation is supported by three lines of evidence: (1) Higher contaminant levels have been found consistently in the private wells, which withdraw groundwater from both the more contaminated shallow zone and the less contaminated middle zone near the Sisson, Pankratz (Newcombe), Willems, and Bengston properties and the private grain storage facilities. (2) The carbon tetrachloride concentration in the middle zone at the former CCC/USDA facility is lower $(1.7 \mu \mathrm{g} / \mathrm{L})$ than the concentrations in the private wells $(4.0-5.0 \mu \mathrm{g} / \mathrm{L})$ and in the middle zone at MW18M (3.3 $\mu \mathrm{g} / \mathrm{L})$. (3) The concentration in the middle zone on the former CCC/USDA property east of Main Street is lower because no wells there provide a direct migration pathway between the shallow and middle groundwater zones.

- The lateral and vertical extents of contamination beneath the private properties west of Main Street and north of Buckskin Road are as follows:

- Carbon tetrachloride identified at a concentration $(4.5 \mu \mathrm{g} / \mathrm{L}$ at well MW18S) near the MCL in the shallow groundwater zone in this area has likely migrated within the area encompassing the four private wells on the Sisson, Pankratz (Newcombe), Willems, and Bengston properties because of relatively high $K_{h}\left(10^{0}\right.$ to $10^{2} \mathrm{ft} /$ day $)$. However, no evidence exists to indicate that contaminants have migrated off these four private properties, except for some uncertainty regarding potential migration pathways to location MW16. 
- Contaminants in the shallow groundwater zone have migrated directly via hydraulic conduits (well open-borehole or sand pack conduits) to the middle groundwater zone in the four private wells at the Sisson, Pankratz (Newcombe), Willems, and Bengston properties (Figure 4.29). The effect of vertical migration has been enhanced by pumping at these wells, as confirmed by water level responses recorded in MW18S (57 ft from the Sisson pumping well; Figure 4.10), which is screened exclusively in the shallow groundwater zone.

- The direct migration of contaminants in the shallow groundwater zone to the Sisson, Pankratz (Newcombe), Willems, and Bengston private wells is confirmed through multiple lines of evidence: (1) Concentrations of carbon tetrachloride in the four private wells $(4-5 \mu \mathrm{g} / \mathrm{L})$ are similar to that in the shallow groundwater zone $(4.5 \mu \mathrm{g} / \mathrm{L}$ at MW18S). (2) The abundance of chloroform relative to carbon tetrachloride (22-30\%) in the four private wells is also consistent with that in the shallow groundwater zone (22\% at MW18S). (3) Water withdrawal from the shallow groundwater was observed during pumping at a private well.

- Contaminants in the middle groundwater zone might have migrated to the south (MW15) and the southeast (MW17) from the Sisson, Pankratz (Newcombe), Willems, and Bengston properties, as indicated by the flow pattern in the middle zone. The contaminant levels are extremely low at these locations, ranging from $<1 \mu \mathrm{g} / \mathrm{L}$ at $\mathrm{MW} 17$ to $2.9 \mu \mathrm{g} / \mathrm{L}$ at $\mathrm{MW} 15$ (below the MCL of $5.0 \mu \mathrm{g} / \mathrm{L}$ ).

- North of Buckskin Road, the contamination areas in the shallow groundwater zone east and west of Main Street have remained separate, as confirmed by the complete absence of contamination in MW09-MW12 along Main Street, although the southeastern flow component is predominant from the Sisson property (west of Main Street) to the former CCC/USDA facility (east of Main Street), under both ambient and pumping conditions.

- The source of contamination at MW16S1 and MW16S2 is unknown. In addition to the former CCC/USDA facility and the private properties to the 
west, a low level of carbon tetrachloride $(1.3-2.3 \mu \mathrm{g} / \mathrm{L})$ was found in the shallow groundwater zone at locations MW16S1 and MW16S2, 1,400 ft southwest of the former private grain storage facility on the Sisson property. The shallow groundwater flow pattern indicates the absence of a hydraulic barrier between MW16S and the area at the northern edge and to the north of the Sisson property.

- The extent of contamination in the middle zone north of Buckskin Road can be defined laterally by the absence of contamination at MW16M and the Hull wells to the southwest, at MW20M to the south, and at the Pauls well to the southeast.

- Additional contamination was identified in groundwater from the Klassen domestic and test wells, south of Buckskin Road. Multiple lines of evidence suggest that this contamination is attributed to past grain storage operations at a private facility on the current Klassen property.

- Vertical migration of contaminants from the middle to the deep groundwater zone is either absent or insignificant at all investigative borehole locations.

\subsection{Recommendation}

On the basis of (1) the hydrologic setting (identified groundwater-bearing zones, flow pattern under both ambient and pumping conditions, and estimated hydraulic conductivities in the contaminated portions of shallow and middle groundwater zones), (2) the defined separate areas of groundwater contamination and their different migration pathways, (3) the laterally limited distribution of the contamination and the permeability constraints of the shallow groundwater in the southern portion of the former CCC/USDA facility, (4) the overall low-level contamination in both areas of groundwater contamination and in the four private wells north of Buckskin Road, and (5) the extremely low potential for risk associated with the former CCC/USDA facility, a monitoring evaluation for reclassification should be conducted at Inman.

The current concentrations of carbon tetrachloride in monitoring wells and private wells in the vicinity of the former CCC/USDA facility are summarized in Table 5.1. The ranges of 
carbon tetrachloride concentrations are $<1-5.4 \mu \mathrm{g} / \mathrm{L}$ in shallow groundwater on the southern portion of the former CCC/USDA property, $4.5 \mu \mathrm{g} / \mathrm{L}$ in shallow groundwater in monitoring well MW18S near the contaminated private wells west of Main Street, and $4-5 \mu \mathrm{g} / \mathrm{L}$ in the contaminated private wells, which intercept both the shallow and middle groundwater zones. The carbon tetrachloride concentration in the middle groundwater zone ranges from $<1$ to $3.3 \mu \mathrm{g} / \mathrm{L}$. The set of data in Table 5.1 can be used as a basis for developing a monitoring plan for reclassification. 
TABLE 5.1 Current contaminant levels in wells in the vicinity of the former CCC/USDA facility and private properties north of Buckskin Road.

\begin{tabular}{lcc}
\hline \multicolumn{1}{c}{ Contamination Area and Groundwater Zone } & $\begin{array}{c}\text { Carbon } \\
\text { Tetrachloride } \\
(\mu \mathrm{g} / \mathrm{L})\end{array}$ & Well \\
\hline $\begin{array}{l}\text { Shallow groundwater contamination in the southern portion of the } \\
\text { former CCC/USDA facility east of Main Street }\end{array}$ & $<1-5.4$ & MW01, MW02, MW03 \\
$\begin{array}{l}\text { Shallow groundwater contamination on private properties west of } \\
\text { Main Street }\end{array}$ & 4.5 & MW18S \\
$\begin{array}{l}\text { Private wells on private properties west of Main Street (intercepting } \\
\text { both the contaminated shallow and middle groundwater zones) }\end{array}$ & $4-5$ & $\begin{array}{c}\text { Sisson, } \\
\text { Pankratz (Newcombe), } \\
\text { Willems, Bengston }\end{array}$ \\
$\begin{array}{l}\text { Middle groundwater zone in the southern portion of the former } \\
\text { CCC/USDA facility east of Main Street }\end{array}$ & 1.7 & MW14M \\
$\begin{array}{l}\text { Middle groundwater zone on private properties west of Main Street } \\
\begin{array}{l}\text { Middle groundwater zone downgradient from the southern portion } \\
\text { of the former CCC/USDA facility east of Main Street and also } \\
\text { private properties west of Main Street }\end{array}\end{array}$ & 3.3 & MW18M \\
\hline
\end{tabular}




\section{References}

Argonne, 2002, Final Master Work Plan: Environmental Investigations at Former CCC/USDA Facilities in Kansas, 2002 Revision, ANL/ER/TR-02/004, prepared for the Commodity Credit Corporation, U.S. Department of Agriculture, Washington, D.C., by Argonne National Laboratory, Argonne, Illinois, December.

Argonne, 2007, Final Work Plan for Targeted Investigation at Inman, Kansas, ANL/EVS/AGEM/TR-07-02, prepared for the Commodity Credit Corporation, U.S. Department of Agriculture, Washington, D.C., by Argonne National Laboratory, Argonne, Illinois, October.

Bouwer, H., 1989, “The Bouwer and Rice Slug Test: An Update,” Ground Water 27(3):304-309.

Bouwer, H., and R. Rice, 1976, "A Slug Test for Determining Hydraulic Conductivity of Unconfined Aquifers with Completely or Partially Penetrating Wells," Water Resources Research 12(3):423-428.

EPA, 1995, Method 524.2: Measurement of Purgeable Organic Compounds in Water by Capillary Column Gas Chromatography/Mass Spectrometry, Revision 4.1, edited by J.W. Munch, National Exposure Research Laboratory, Office of Research and Development, U.S. Environmental Protection Agency, Cincinnati, Ohio.

EPA, 1997, "Definitions and Procedures for the Determination of the Method Detection Limit," pp. 317-319, Appendix B, Part 136, in Guidelines Establishing Test Procedures for the Analysis of Pollutants, U.S. Code of Federal Regulations, Title 40, Revised July 1 (http://www.epa.gov/region9/qa/pdfs/40cfr136_03.pdf).

EPA, 1998, Test Methods for Evaluating Solid Waste: Physical/Chemical Methods, EPA SW-846, 3rd edition, Draft Update IVA, U.S. Environmental Protection Agency, Washington, D.C., January.

EPA, 2008, National Functional Guidelines for Superfund Organic Methods Data Review, OSWER 9240.1-48, USEPA-540-R-08-01, Contract Laboratory Program, Office of Superfund Remediation and Technology Innovation, U.S. Environmental Protection Agency, Washington, D.C., June (http://www.epa.gov/superfund/programs/clp/download/somnfg.pdf). 
Hvorslev, M., 1951, Time Lag and Soil Permeability in Ground-Water Observations, Bulletin 36, U.S. Army Corps of Engineers, Waterways Experiment Station, Vicksburg, Mississippi.

KDHE, 1998, Site Reconnaissance and Evaluation — Inman USDA/CCC Grain Bin Site, Inman, Kansas, P5-059-7044, Bureau of Environmental Remediation, Kansas Department of Health and Environment, Topeka, Kansas, May.

KDHE, 2007, letter from C. Carey (Bureau of Environmental Remediation, Kansas Department of Health and Environment, Topeka, Kansas) to C. Roe (Commodity Credit Corporation, U.S. Department of Agriculture, Washington, D.C.), regarding the targeted investigation Work Plan, October 18.

KDHE, 2011, letter from M. Townsend (Bureau of Environmental Remediation, Kansas Department of Health and Environment, Topeka, Kansas) to C. Roe (Commodity Credit Corporation, U.S. Department of Agriculture, Washington, D.C.), regarding the revised Work Plan for Phase 3-Phase 4, August 10.

KDHE, 2012, memorandum from R. Weiser (Brownfields Program, Bureau of Environmental Remediation, Kansas Department of Health and Environment, Topeka, Kansas) to R. Brown (Site Assessment Program, Bureau of Environmental Remediation, Kansas Department of Health and Environment, Topeka, Kansas), regarding the Inman Elevator property at $201 \mathrm{~S}$. Spruce in Inman, Kansas, March 27.

McPherson Mapping Department, 2006, aerial photograph of McPherson County, Mapping Department, McPherson County, Kansas.

NAIP, 2010, aerial photograph ortho_1-1_1n_s_ks113_0210_1, National Agricultural Imagery Program, Farm Service Agency, U.S. Department of Agriculture, Salt Lake City, Utah, September 21.

Theis, C., 1935, "The Relation between the Lowering of the Piezometric Surface and the Rate and Duration of a Well Using Groundwater Storage," Transactions of the American Geophysical Union 16:519-524. 
USDA, 1956, aerial photograph AYL-3R-40D, U.S. Department of Agriculture, Washington, D.C., June 9.

USDA, 1963, aerial photograph AYL-2DD-179L, U.S. Department of Agriculture, Washington, D.C., July 15.

USDA, 1970, aerial photograph AYL-ILL-88EC, U.S. Department of Agriculture, Washington, D.C., June 27.

USDA, 1979, aerial photograph 20113-179-51EC, U.S. Department of Agriculture, Washington, D.C., August 4.

USGS, 1997, Digital Raster Graphics (DRG) UTM NAD 27 of Buhler Quadrangle, Kansas, 7.5 Minutes Series, U.S. Geological Survey, Reston, Virginia. 


\section{Appendix A:}

Summary of Investigation Results and Proposed Work Plan, Inman, Kansas, August 9, 2011 


\section{Appendix A:}

\section{Summary of Investigation Results and Proposed Work Plan, Inman, Kansas, August 9, 2011}

\section{A.1 Introduction}

Inman, Kansas, is located in southwest McPherson County, approximately $10 \mathrm{mi}$ southwest of the city of McPherson. In 1997, low levels of carbon tetrachloride were detected in groundwater samples from four private wells at Inman, by the Kansas Department of Health and Environment (KDHE).

The Commodity Credit Corporation of the U.S. Department of Agriculture (CCC/USDA) operated a grain storage facility at Inman from 1954 to 1965. Additional grain storage facilities also present in the area include a commercial facility located in the northwest part of Inman, along the railroad right-of-way, and small private facilities adjacent to the contaminated private wells.

Carbon tetrachloride is the contaminant of primary concern at sites associated with grain storage operations. To determine whether the former CCC/USDA facility at Inman is a potential contaminant source and its possible relationship to the contamination in groundwater, Argonne National Laboratory, on behalf of the CCC/USDA, developed a Work Plan ${ }^{1}$ (Argonne 2007) to conduct a phased targeted investigation. The Work Plan was approved by the KDHE in 2007.2

The major technical objectives of the investigation at Inman are as follows:

- Evaluate the potential source of carbon tetrachloride at the former CCC/USDA facility.

1 Argonne, 2007, Final Work Plan for Targeted Investigation at Inman, Kansas, ANL/EVS/AGEM/TR-07-02, prepared for the Commodity Credit Corporation, U.S. Department of Agriculture, Washington, D.C., by Argonne National Laboratory, Argonne, Illinois, October.

2 KDHE, 2007, letter from C. Carey (Bureau of Environmental Remediation, Kansas Department of Health and Environment, Topeka, Kansas) to C. Roe (Commodity Credit Corporation, U.S. Department of Agriculture, Washington, D.C.), regarding Final Work Plan for Targeted Investigation at Inman, Kansas, October 18. 
- Determine the relationship of potential contamination at the former CCC/USDA facility to contamination identified in 1997 in groundwater samples from four private wells to the west and southwest.

- Delineate the extent of potential contamination associated with the former CCC/USDA facility.

Argonne performed the initial part of investigation (Phases 1-2) in two main field mobilizations, on November 4-11, 2009, and November 8-14, 2010.

In the investigation in 2009 (Phase 1), Argonne collected data from unconsolidated soil ( $<45 \mathrm{ft}$ BGL [below ground level]) overlying the bedrock formation by using the cone penetrometer (CPT) unit. Argonne also collected groundwater samples from the shallow interval $(<45 \mathrm{ft}$ BGL) and installed monitoring wells to the top of the bedrock formation. The Phase 1 activity was confined to the former CCC/USDA property.

The detailed work during the Phase 2 investigation in 2010 included the following:

- Soil profiling for analysis for volatile organic compounds (VOCs) at the former private grain storage facilities.

- Shallow groundwater sampling for VOCs analysis and installation of temporary monitoring wells at the former private grain storage facilities and immediately outside the former CCC/USDA facility.

- Collection of groundwater samples for VOCs analysis from the existing private wells at and around the former grain storage facilities.

On the basis of the KDHE-approved general Work Plan developed in 2007 and results from the Phase 1 and Phase 2 investigations, the subsequent phases of targeted investigation are required to further determine the potential presence of contamination in the bedrock formation and to delineate the deep groundwater plume (if any) emanating from the former CCC/USDA facility. To implement the Phase 3 and Phase 4 investigations outlined in the KDHE-approved Work Plan, the detailed activities for these two phases are proposed below, along with a summary of results from the initial phases of the investigation. 


\section{A.2 Summary of Results from Phase 1 and Phase 2}

\section{A.2.1 Soil Sampling Results}

- Former CCC/USDA grain storage facility. Vertical soil profiling through unconsolidated soil was conducted with the CPT unit at 11 locations (TI01TI11) to a depth of $\sim 40 \mathrm{ft}$ BGL in November 2009 (Phase 1; Figure A.1). Carbon tetrachloride was detected at trace levels $(4.1-8.0 \mu \mathrm{g} / \mathrm{kg}$, below the method detection limit of $10 \mu \mathrm{g} / \mathrm{kg}$ ) in soil at only 3 locations in the southern portion of the former facility (Table A.1). The results indicate that no significant carbon tetrachloride source is currently present in the vadose zone soil on the former CCC/USDA property.

- Former private grain storage facilities. Vertical soil profiling through unconsolidated soil was conducted at 7 locations (TI19-TI25) to a depth of $\sim 40 \mathrm{ft}$ BGL in November 2010 (Phase 2; Figure A.1). Carbon tetrachloride was not detected by the purge-and-trap method (Table A.1). The results indicate that no carbon tetrachloride source is currently present in the vadose zone soil near the former private grain bins.

- Estimated bedrock depth is approximately $40 \mathrm{ft}$ BGL.

\section{A.2.2 Shallow Groundwater Sampling Results}

- Former CCC/USDA grain storage facility.

- Groundwater was recovered in temporary piezometers at various depths from $26 \mathrm{ft}$ to $42 \mathrm{ft}$ BGL. Grab samples for analysis for VOCs were collected without purging at 7 locations (TI01-TI02, TI04-TI06, TI08, and TI11). Carbon tetrachloride was identified (3.1-34 $\mu \mathrm{g} / \mathrm{L})$ in groundwater at 4 locations in the southern portion of the former facility (Table A.2).

- Five monitoring wells were installed with the CPT unit with 10-ft screen intervals at depths of $30-45 \mathrm{ft}$ BGL, near or at the top of the bedrock 
formation. Groundwater samples collected from purged monitoring wells were analyzed for VOCs. Carbon tetrachloride was detected at very low levels of $<1-5.4 \mu \mathrm{g} / \mathrm{L}$ (Figure A.2; Table A.2).

- Former private grain storage facilities. Groundwater was recovered in temporary piezometers at depths from $29.1 \mathrm{ft}$ to $41.1 \mathrm{ft}$ BGL. Grab samples for VOCs analysis were collected without purging at 3 locations, TI22-TI24 (Figure A.2). Carbon tetrachloride was found at trace levels $(<1 \mu \mathrm{g} / \mathrm{L})$ near the former grain bins and the Quonset.

- Area immediately outside of the former CCC/USDA property. Seven shallow monitoring wells ( $<45 \mathrm{ft}$ BGL), MW06-MW12, were installed with $10-\mathrm{ft}$ screen intervals in the areas immediately west, south, and southeast of the former CCC/USDA property (Figure A.2). Groundwater samples were collected from those wells for VOCs analysis. No carbon tetrachloride was detected. The results indicate that no contaminant migration from the former CCC/USDA facility to areas outside the facility has occurred in the shallow interval $(<45 \mathrm{ft}$ BGL).

\section{A.2.3 Private Well Sampling Results}

- Groundwater samples were collected from private wells at 4 residences (Sisson, Pankratz, Willems, and Bengston) across Main Street, west of the former CCC/USDA facility (Figure A.3). Carbon tetrachloride was found in all 4 private wells at consistently low levels (4.0-5.0 $\mu \mathrm{g} / \mathrm{L})$. Private grain bins and a Quonset were formerly located at 2 of the 4 residences.

- An extended private well sampling effort identified eight private wells in the vicinity of the former CCC/USDA grain storage facility and the private grain storage facilities. These wells were recently sampled (Figure A.3). Carbon tetrachloride was not identified, except in the Klassen residence well (carbon tetrachloride $=6 \mu \mathrm{g} / \mathrm{L}$ ), located approximately $4,000 \mathrm{ft}$ downgradient from the former CCC/USDA grain storage facility and the private grain storage 
facilities. In addition, 6-7 grain bins were found on the northern part of the Klassen residence property.

\section{A.3 Proposed Work Plan for the Phase 3-Phase 4 Investigation}

The Phase 3-Phase 4 investigations will involve deep well drilling. Initial deep drilling locations proposed for Phase 3 are illustrated in Figures A.4a-A.4c. The investigations will be implemented as follows:

- A phased approach will be taken to optimize the effectiveness of the investigation by drilling selectively into deeper horizons. This approach will minimize costs and limit disruption to the community - specifically to the landowners. The results from each step will be used to direct subsequent work as required. The CCC/USDA and KDHE project managers will be contacted during each step and kept apprised of the results. The implementation of each step will be discussed and mutually agreed upon by the CCC/USDA and KDHE project managers.

- The proposed initial drilling locations (Figures A.4a-A.4c) were selected on the basis of (1) the currently identified carbon tetrachloride distribution in shallow groundwater; (2) the potential groundwater flow pattern, as indicated by surface topography (Figure A.4b); and (3) the results of groundwater sampling at surrounding private wells.

- At the deep drilling location(s) in the potential source areas (i.e., the former CCC/USDA facility and/or former private grain storage facilities across Main Street from the former CCC/USDA facility), the water-bearing zone(s) within the bedrock formation will be identified and tested to determine the vertical distribution of contamination. The contaminated water-bearing zone(s) will be investigated further to delineate the lateral extent of contamination outside the source area(s) and to identify the groundwater flow pattern(s). 


\section{A.3.1 Phase 3 Investigation}

The Phase 3 investigation will be conducted at locations MW14-MW17 (Figure A.4aA.4c). MW14 is located at the point where the highest carbon tetrachloride concentration has currently been identified in shallow groundwater on the former CCC/USDA property (Figure A.4c). Locations MW15-MW17 lie to the southeast, south, and southwest of MW14 (Figure A.4b). These locations are downgradient from the former CCC/USDA property and the four contaminated private wells across Main Street and to the west of the former CCC/USDA property. One additional location (MW18) might be selected, as discussed below.

An analysis of regional and local hydrogeologic data was conducted and summarized in the 2007 Work Plan. The results indicate that the groundwater flow pattern in the Permian shale unit unconformably underlying the unconsolidated soil is expected to mimic the surface drainage pattern. This projection is consistent with the shallow water depths (3-44 ft BGL, mostly < $30 \mathrm{ft}$ ) reported in all private wells in the vicinity of the former CCC/USDA property. The former CCC/USDA facility and the former private grain storage facilities are located on the upland that extends from the north (Figure A.4b). Groundwater in the water-bearing zone(s) in the Permian shale unit appears likely to flow from the former facilities toward the southeast, south, or southwest. Locations MW14-MW17 were selected to capture and/or confirm all flow components in this anticipated general local and regional groundwater flow pattern in the bedrock formation.

Coring and groundwater sampling at location MW14 on the former CCC/USDA property will identify contaminated water-bearing zone(s) and their vertical interval(s) in the bedrock formation. Any identified water-bearing zone(s) in the bedrock at this location will be extrapolated as possible target zone(s) for further investigation at downgradient locations MW15-MW17.

If no contamination is found in the bedrock water-bearing zones at location MW14 on the former CCC/USDA property, an additional deep boring (MW18; Figures A.4a-A.4c) will be drilled into the bedrock formation next to the contaminated private well on the Sisson property west of Main Street, before work proceeds to the MW15-MW17 locations. The drilling at MW18 is contingent on the absence of contamination in the bedrock water-bearing zones at MW14 and on agreement by the landowner to allow access. Sampling and coring results at MW18 are anticipated to identify the exact vertical interval(s) of the contaminated groundwater-bearing 
zone(s). Any contaminated zone(s) identified at location MW18 will be extrapolated as possible target zone(s) for further investigation at downgradient locations MW15-MW17.

Groundwater sampling and groundwater level measurements at locations MW14-MW18 will (1) confirm whether contaminants are present in the deep bedrock groundwater interval(s) beneath the former CCC/USDA property or the former private grain storage facilities across Main Street; (2) identify groundwater flow pattern(s) in the bedrock water-bearing zone(s); and (3) determine downgradient contaminant migration pathways. Of particular interest is evidence for a possible southward contaminant migration pathway toward the Klassen residence from either the former CCC/USDA property or the former private grain storage facilities near the four contaminated private wells west of Main Street.

\section{A.3.2 Phase 4 Investigation}

The Phase 4 investigation, if warranted, will be guided by interpretation of the results from the Phase 3 investigation. The number, exact locations, and sampling intervals of deep drilling in Phase 4 will be selected to generate data further addressing or confirming the following issues:

- The lateral extent of any carbon tetrachloride contamination determined to have migrated from the former CCC/USDA property.

- The relationship of potential contamination at the former CCC/USDA facility to contamination identified in the four private wells to the west and southwest, across Main Street.

Prior to the Phase 4 investigation, the proposed drilling locations will be discussed in consultation with the CCC/USDA and KDHE project managers.

\section{A.3.3 Dynamic Drilling and Sampling Process}

The results of core examination and water sample analyses at each drilling location will be evaluated on-site to guide subsequent drilling activities. Throughout the investigation, the number, location, and sampling interval of wells will be evaluated prior to drilling at the next 
location. Plans will be modified as necessary on the basis of the integrated analysis of the previous field results and rapid-turnaround laboratory results for VOCs.

The available historical construction information for the private wells in the area indicates that each of these documented wells was installed as an open completion, without screened casing, through the entire section of the bedrock formation. No information is available regarding the depth(s) of the water-bearing zone(s) within the bedrock formation. The initial deep drilling will focus on identifying groundwater-bearing zone(s). Groundwater samples will be collected for VOCs analysis from all identified water-bearing zones to determine vertical distribution of contamination. The contaminated groundwater-bearing zone(s) will be the target zone(s) of further investigation to delineate their lateral extents.

If water-bearing zones are difficult to identify because of extremely low productivity, three or four nested wells with 10-ft screen intervals will be installed at one or two locations to cover the bedrock section from $50 \mathrm{ft}$ to120 ft BGL in the potential source areas at the former CCC/USDA property or the former private grain storage facilities across Main Street. Information on water recovery and carbon tetrachloride concentrations from the nested wells will be analyzed to determine the interval(s) of water-bearing zone(s) and the screen intervals for the remainder of the deep well drilling program. 


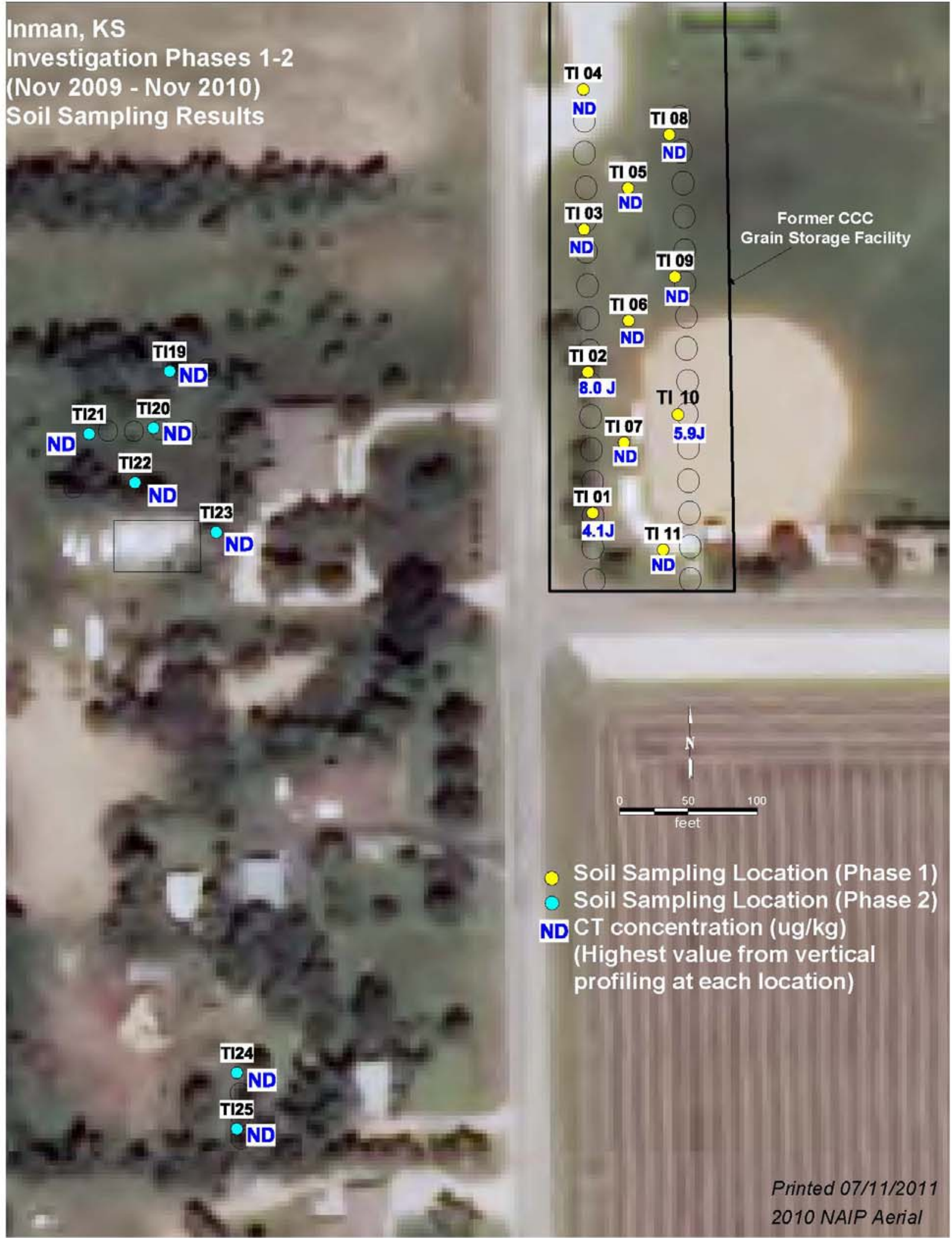

FIGURE A.1 Soil sampling results in November 2009-November 2010. Source of photograph: NAIP (2010). 


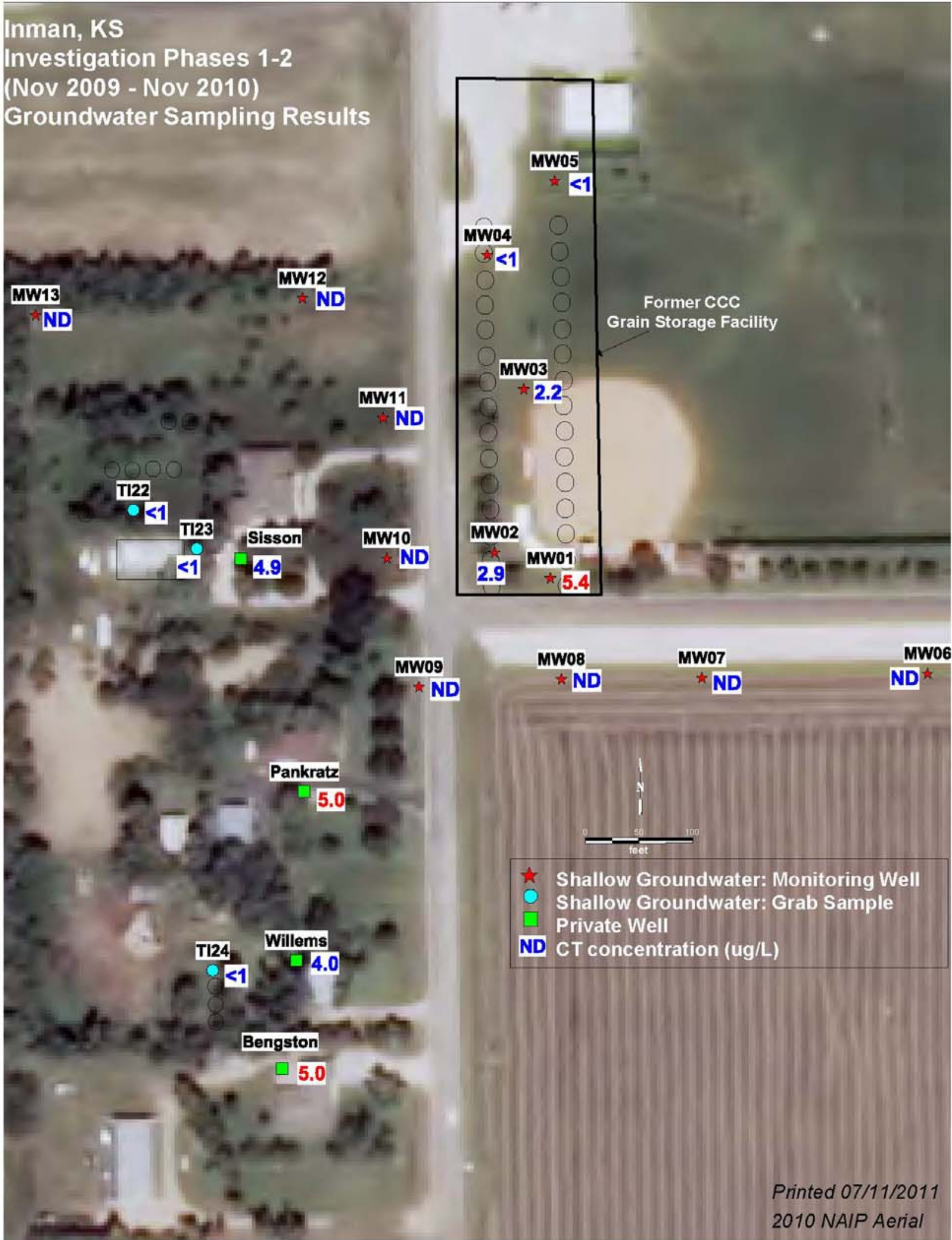

FIGURE A.2 Groundwater sampling results in November 2009-November 2010. Source of photograph: NAIP (2010). 


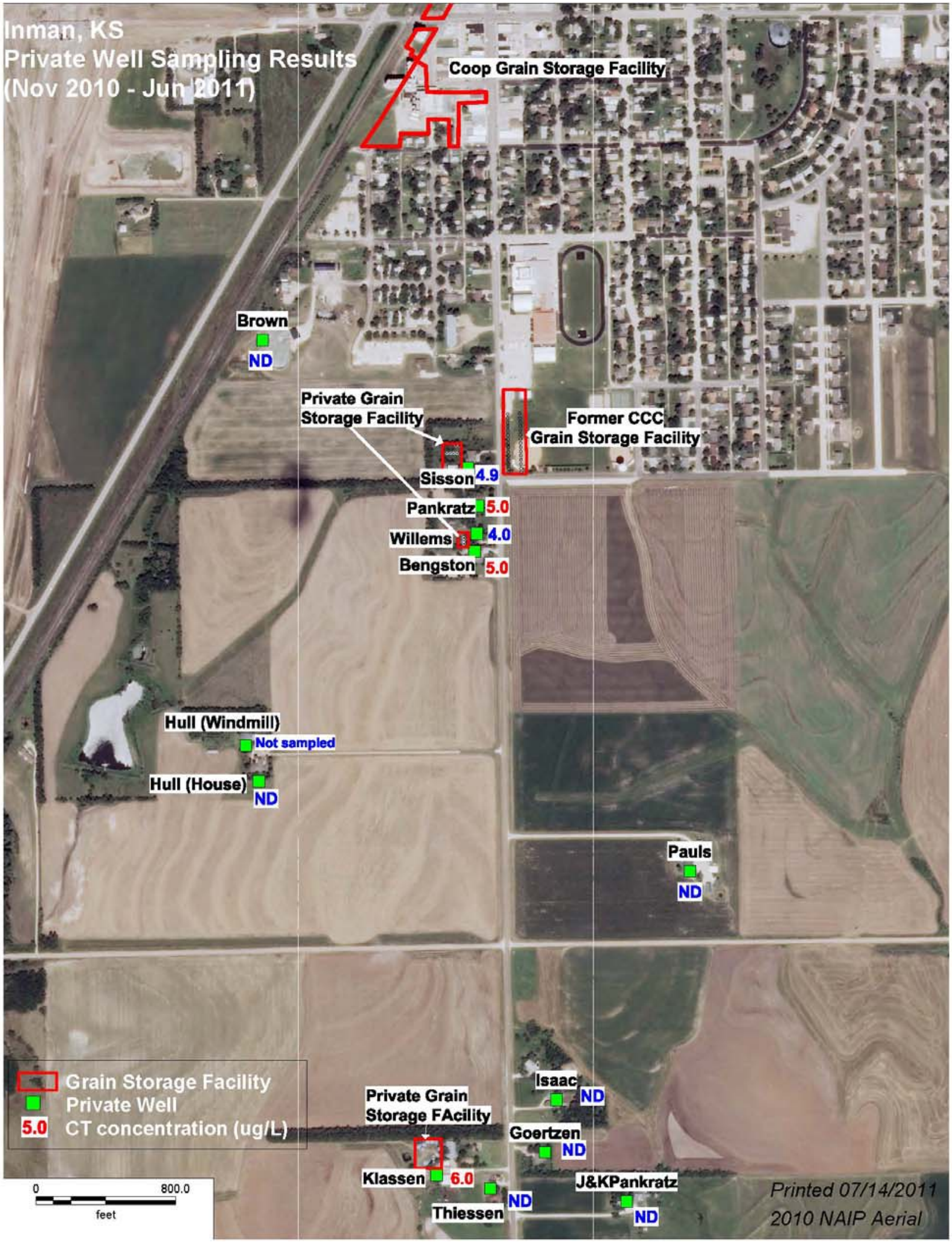

FIGURE A.3 Private well sampling results in November 2010-June 2011. Source of photograph: NAIP (2010). 


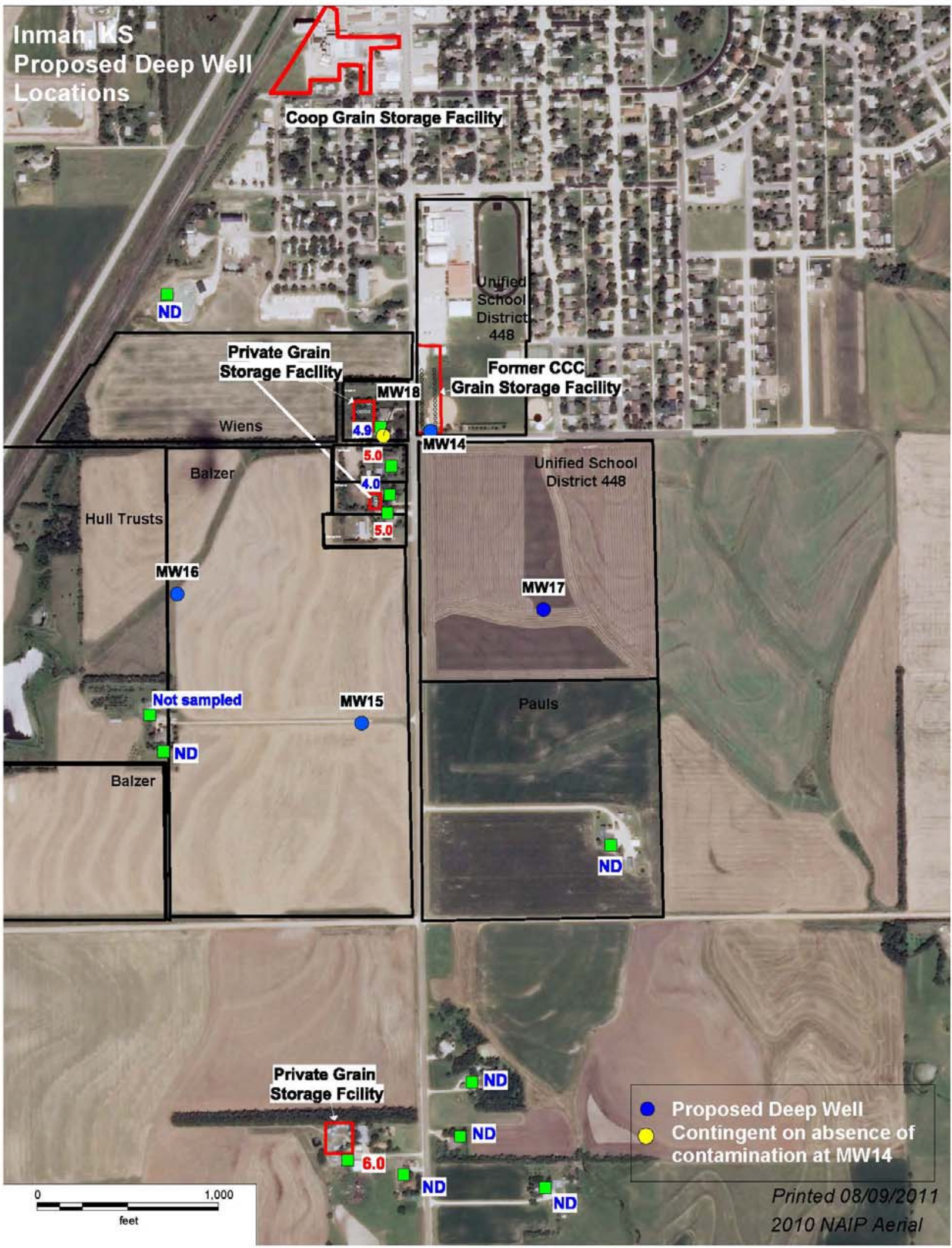

FIGURE A.4a Proposed initial deep well locations, with private well results in November 2010-June 2011. Source of photograph: NAIP (2010). 


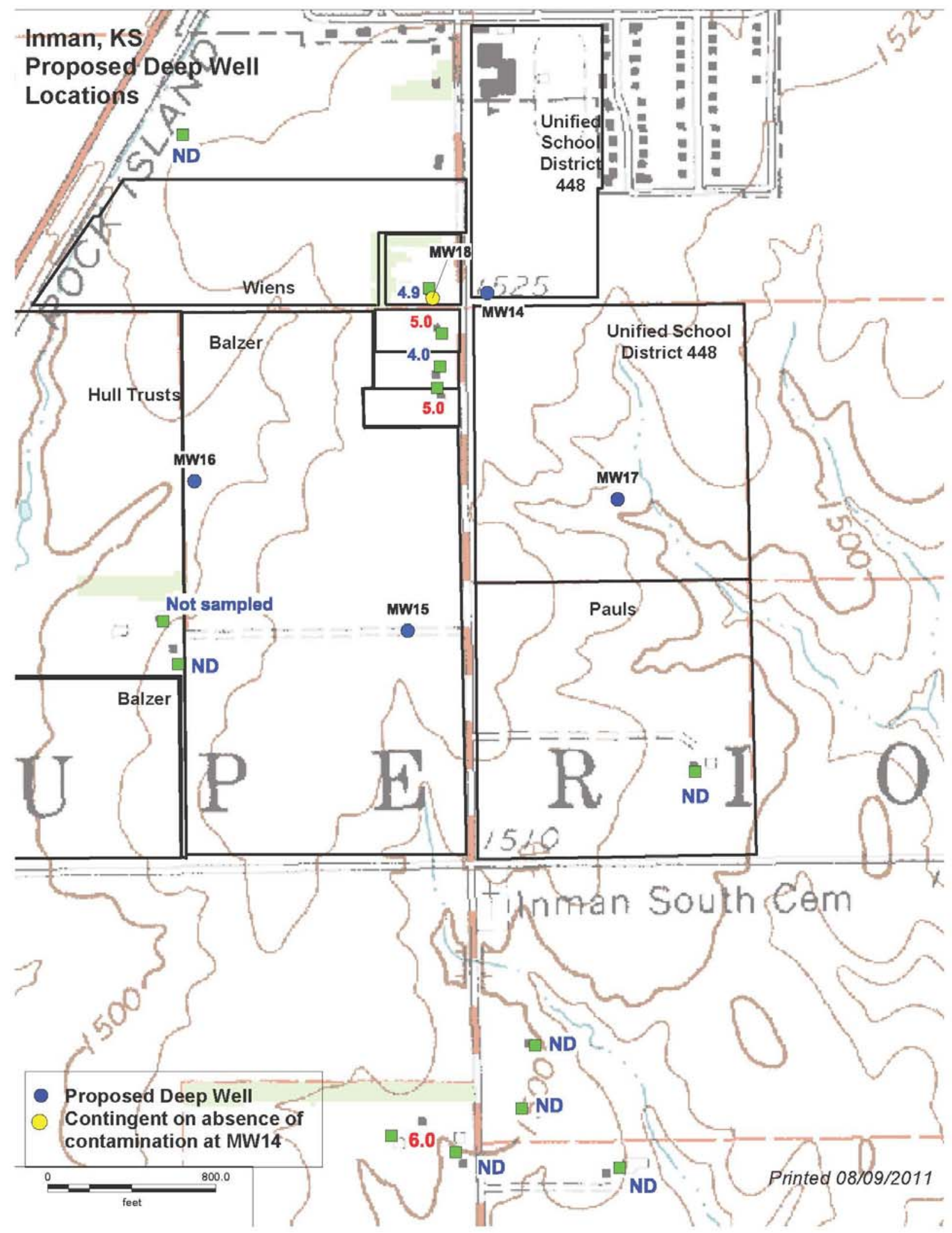

FIGURE A.4b Proposed initial deep well locations, with private well results in November 2010-June 2011 and surface topography. Source of 1982 map: USGS (1997). 


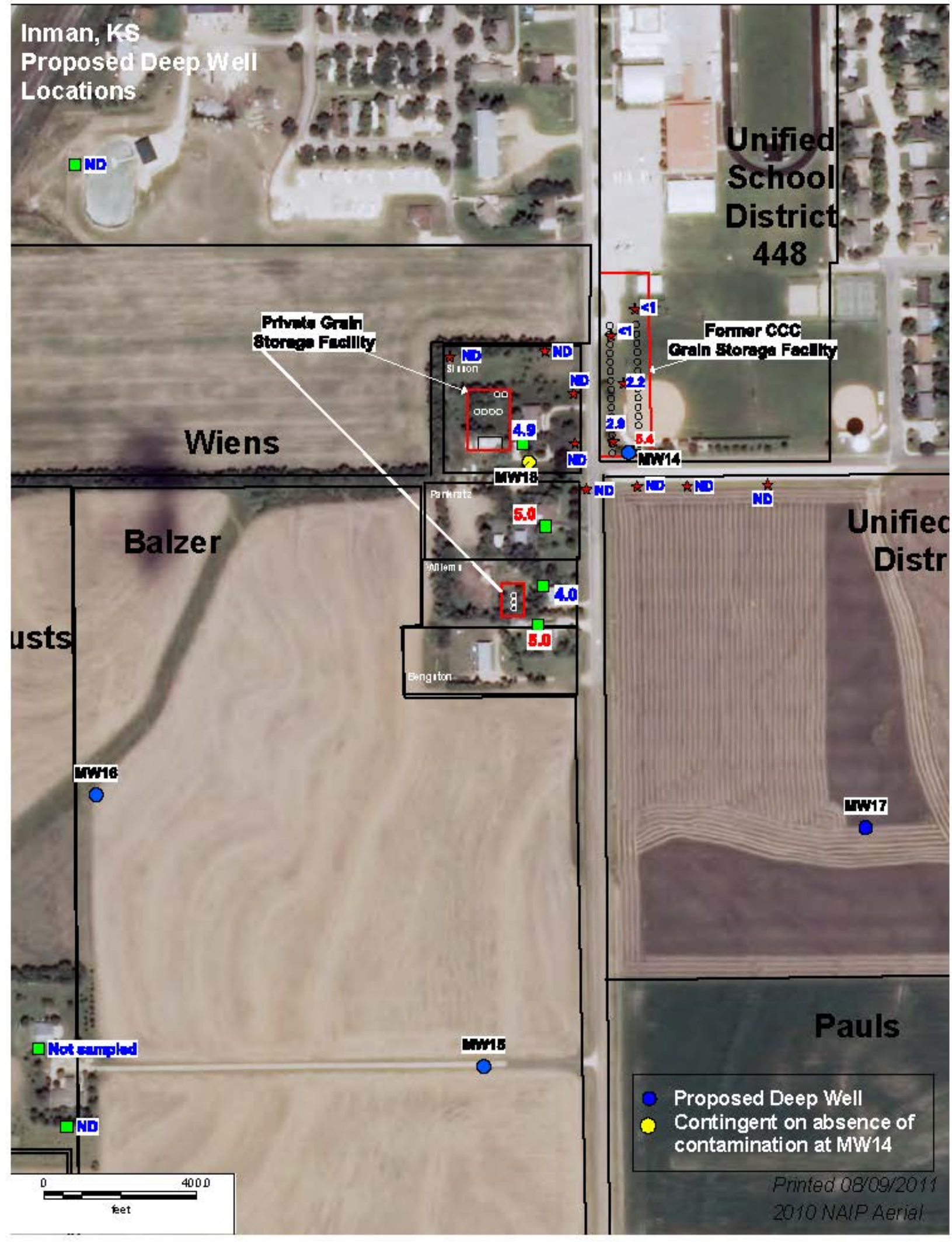

FIGURE A.4C Proposed initial deep well locations, with monitoring well and private well results in November 2009-June 2011. Source of photograph: NAIP (2010). 
TABLE A.1 Results from the AGEM Laboratory for soil samples collected at Inman, Kansas, in 2009-2010.

\begin{tabular}{|c|c|c|c|c|c|}
\hline Location & Sample & Sample Date & $\begin{array}{c}\text { Depth } \\
\text { (ft BGL) }\end{array}$ & $\begin{array}{c}\text { Carbon } \\
\text { Tetrachloride } \\
(\mu \mathrm{g} / \mathrm{kg})\end{array}$ & $\begin{array}{l}\text { Chloroform } \\
(\mu \mathrm{g} / \mathrm{kg})\end{array}$ \\
\hline
\end{tabular}

\section{CPT sampling on the former CCC/USDA facility property.}

\begin{tabular}{|c|c|c|c|c|c|c|}
\hline T101 & INTI01-S-30620 & $11 / 4 / 09$ & 2 & ND & ND & \\
\hline TI01 & INTI01-S-30621 & $11 / 4 / 09$ & 6 & ND & ND & \\
\hline TI01 & INTI01-S-30622 & $11 / 4 / 09$ & 10 & ND & ND & \\
\hline T101 & INTI01-S-30623 & $11 / 4 / 09$ & 14 & ND & ND & \\
\hline TI01 & INTI01-S-30624 & $11 / 4 / 09$ & 18 & ND & ND & \\
\hline TI01 & INTI01-S-30625 & $11 / 4 / 09$ & 22 & ND & ND & \\
\hline TI01 & INTI01-S-30630 & $11 / 4 / 09$ & 26 & ND & ND & \\
\hline TI01 & INTI01-S-30626 & $11 / 4 / 09$ & 30 & $4.1 \mathrm{~J}$ & ND & \\
\hline TI01 & INTI01-S-30627 & $11 / 4 / 09$ & 34 & ND & ND & QC Replicate $=3.5 \mathrm{ppb}$ \\
\hline TI01 & INTI01-S-30629 & $11 / 4 / 09$ & 38 & ND & ND & \\
\hline $\mathrm{TlO} 2$ & INTI02-S-30631 & $11 / 4 / 09$ & 2 & ND & ND & \\
\hline TI02 & INTI02-S-30632 & $11 / 4 / 09$ & 6 & ND & ND & \\
\hline $\mathrm{TIO} 2$ & INTI02-S-30633 & $11 / 4 / 09$ & 10 & ND & ND & \\
\hline TI02 & INTI02-S-30634 & $11 / 4 / 09$ & 14 & ND & ND & \\
\hline TI02 & INTI02-S-30635 & $11 / 4 / 09$ & 18 & ND & ND & \\
\hline $\mathrm{T} 102$ & INTI02-S-30636 & $11 / 4 / 09$ & 22 & $4.2 \mathrm{~J}$ & ND & \\
\hline $\mathrm{TIO} 2$ & INTI02-S-30637 & $11 / 4 / 09$ & 26 & $8 \mathrm{~J}$ & ND & QC Replicate = ND. \\
\hline TI02 & INTI02-S-30639 & $11 / 4 / 09$ & 30 & ND & ND & \\
\hline TI02 & INTI02-S-30640 & $11 / 4 / 09$ & 34 & ND & ND & \\
\hline TI02 & INTI02-S-30641 & $11 / 4 / 09$ & 38 & ND & ND & \\
\hline T103 & INTI03-S-30643 & $11 / 5 / 09$ & 2 & ND & ND & \\
\hline TI03 & INTI03-S-30644 & $11 / 5 / 09$ & 6 & ND & ND & \\
\hline TI03 & INTI03-S-30645 & $11 / 5 / 09$ & 10 & ND & ND & \\
\hline TI03 & INTI03-S-30646 & $11 / 5 / 09$ & 14 & ND & ND & \\
\hline TI03 & INTI03-S-30647 & $11 / 5 / 09$ & 18 & ND & ND & \\
\hline TI03 & INTI03-S-30648 & $11 / 5 / 09$ & 22 & ND & ND & \\
\hline TI03 & INTI03-S-30649 & $11 / 5 / 09$ & 26 & ND & ND & \\
\hline $\mathrm{TIO3}$ & INTI03-S-30650 & $11 / 5 / 09$ & 30 & ND & ND & \\
\hline TI03 & INTI03-S-30651 & $11 / 5 / 09$ & 34 & ND & ND & \\
\hline $\mathrm{TIO3}$ & INTI03-S-30652 & $11 / 5 / 09$ & 38 & ND & ND & \\
\hline TI03 & INTI03-S-30653 & $11 / 5 / 09$ & 42 & ND & ND & \\
\hline Tl04 & INTI04-S-30654 & $11 / 5 / 09$ & 2 & ND & ND & \\
\hline T104 & INTI04-S-30655 & $11 / 5 / 09$ & 6 & ND & ND & \\
\hline T104 & INTI04-S-30656 & $11 / 5 / 09$ & 10 & ND & ND & \\
\hline Tl04 & INTI04-S-30657 & $11 / 5 / 09$ & 14 & ND & ND & \\
\hline TI04 & INTI04-S-30658 & $11 / 5 / 09$ & 18 & ND & ND & \\
\hline TI04 & INTI04-S-30659 & $11 / 5 / 09$ & 22 & ND & ND & \\
\hline Tl04 & INTI04-S-30660 & $11 / 5 / 09$ & 26 & ND & ND & \\
\hline
\end{tabular}


TABLE A.1 Results from the AGEM Laboratory for soil samples collected at Inman, Kansas, in 2009-2010.

\begin{tabular}{|c|c|c|c|c|c|}
\hline Location & Sample & Sample Date & $\begin{array}{c}\text { Depth } \\
\text { (ft BGL) }\end{array}$ & $\begin{array}{c}\text { Carbon } \\
\text { Tetrachloride } \\
(\mu \mathrm{g} / \mathrm{kg})\end{array}$ & $\begin{array}{c}\text { Chloroform } \\
(\mu \mathrm{g} / \mathrm{kg})\end{array}$ \\
\hline T104 & INTI04-S-30661 & $11 / 5 / 09$ & 30 & ND & ND \\
\hline T104 & INTI04-S-30662 & $11 / 5 / 09$ & 34 & ND & ND \\
\hline T104 & INTI04-S-30663 & $11 / 5 / 09$ & 38 & ND & ND \\
\hline T105 & INTI05-S-30666 & $11 / 6 / 09$ & 2 & ND & ND \\
\hline T105 & INTI05-S-30667 & $11 / 6 / 09$ & 6 & ND & ND \\
\hline T105 & INTI05-S-30668 & $11 / 6 / 09$ & 10 & ND & ND \\
\hline T105 & INTI05-S-30669 & $11 / 6 / 09$ & 14 & ND & ND \\
\hline T105 & INTI05-S-30670 & $11 / 6 / 09$ & 18 & ND & ND \\
\hline T105 & INTI05-S-30671 & $11 / 6 / 09$ & 22 & ND & ND \\
\hline T105 & INTI05-S-30672 & $11 / 6 / 09$ & 26 & ND & ND \\
\hline TI05 & INTI05-S-30673 & $11 / 6 / 09$ & 30 & ND & ND \\
\hline TI05 & INTI05-S-30674 & $11 / 6 / 09$ & 34 & ND & ND \\
\hline T105 & INTI05-S-30675 & $11 / 6 / 09$ & 38 & ND & ND \\
\hline TI06 & INTI06-S-30676 & $11 / 6 / 09$ & 2 & ND & ND \\
\hline TI06 & INTI06-S-30677 & $11 / 6 / 09$ & 6 & ND & ND \\
\hline T106 & INTI06-S-30678 & $11 / 6 / 09$ & 10 & ND & ND \\
\hline TI06 & INTI06-S-30679 & $11 / 6 / 09$ & 14 & ND & ND \\
\hline TI06 & INTI06-S-30680 & $11 / 6 / 09$ & 18 & ND & ND \\
\hline TI06 & INTI06-S-30681 & $11 / 6 / 09$ & 22 & ND & ND \\
\hline TI06 & INTI06-S-30682 & $11 / 6 / 09$ & 26 & ND & ND \\
\hline TI06 & INTI06-S-30683 & $11 / 6 / 09$ & 30 & ND & ND \\
\hline TI06 & INTI06-S-30685 & $11 / 6 / 09$ & 34 & ND & ND \\
\hline TI06 & INTI06-S-30686 & $11 / 6 / 09$ & 38 & ND & ND \\
\hline T107 & INTI07-S-30687 & $11 / 6 / 09$ & 2 & ND & ND \\
\hline T107 & INT107-S-30688 & $11 / 6 / 09$ & 6 & ND & ND \\
\hline T107 & INTI07-S-30689 & $11 / 6 / 09$ & 10 & ND & ND \\
\hline Tl07 & INTI07-S-30690 & $11 / 6 / 09$ & 14 & ND & ND \\
\hline T107 & INTI07-S-30691 & $11 / 6 / 09$ & 18 & ND & ND \\
\hline T107 & INTI07-S-30692 & $11 / 6 / 09$ & 22 & ND & ND \\
\hline T107 & INTI07-S-30693 & $11 / 6 / 09$ & 26 & ND & ND \\
\hline T107 & INTI07-S-30694 & $11 / 6 / 09$ & 30 & ND & ND \\
\hline T107 & INT107-S-30695 & $11 / 6 / 09$ & 34 & ND & ND \\
\hline T107 & INTI07-S-30696 & $11 / 6 / 09$ & 38 & ND & ND \\
\hline T108 & INTI08-S-30698 & $11 / 7 / 09$ & 2 & ND & ND \\
\hline T108 & INTI08-S-30699 & $11 / 7 / 09$ & 6 & ND & ND \\
\hline T108 & INTI08-S-30700 & $11 / 7 / 09$ & 10 & ND & ND \\
\hline T108 & INTI08-S-30701 & $11 / 7 / 09$ & 14 & ND & ND \\
\hline T108 & INTI08-S-30702 & $11 / 7 / 09$ & 18 & ND & ND \\
\hline T108 & INTI08-S-30703 & $11 / 7 / 09$ & 22 & ND & ND \\
\hline
\end{tabular}


TABLE A.1 Results from the AGEM Laboratory for soil samples collected at Inman, Kansas, in 2009-2010.

\begin{tabular}{|c|c|c|c|c|c|c|}
\hline Location & Sample & Sample Date & $\begin{array}{c}\text { Depth } \\
\text { (ft BGL) }\end{array}$ & $\begin{array}{c}\text { Carbon } \\
\text { Tetrachloride } \\
(\mu \mathrm{g} / \mathrm{kg})\end{array}$ & $\begin{array}{l}\text { Chloroform } \\
(\mu \mathrm{g} / \mathrm{kg})\end{array}$ & \\
\hline T108 & INTI08-S-30704 & $11 / 7 / 09$ & 26 & ND & ND & \\
\hline T108 & INTI08-S-30706 & $11 / 7 / 09$ & 30 & ND & ND & \\
\hline T108 & INTI08-S-30707 & $11 / 7 / 09$ & 34 & ND & ND & \\
\hline T108 & INTI08-S-30708 & $11 / 7 / 09$ & 38 & ND & ND & \\
\hline T109 & INTI09-S-30709 & $11 / 7 / 09$ & 2 & ND & ND & \\
\hline T109 & INTI09-S-30710 & $11 / 7 / 09$ & 6 & ND & ND & \\
\hline T109 & INTI09-S-30711 & $11 / 7 / 09$ & 10 & ND & ND & \\
\hline T109 & INTI09-S-30712 & $11 / 7 / 09$ & 14 & ND & ND & \\
\hline T109 & INTI09-S-30713 & $11 / 7 / 09$ & 18 & ND & ND & \\
\hline TI09 & INTI09-S-30714 & $11 / 7 / 09$ & 22 & ND & ND & \\
\hline T109 & INTI09-S-30715 & $11 / 7 / 09$ & 26 & ND & ND & \\
\hline T109 & INTI09-S-30716 & $11 / 7 / 09$ & 30 & ND & ND & \\
\hline T109 & INTI09-S-30719 & $11 / 7 / 09$ & 34 & ND & ND & \\
\hline TI09 & INTI09-S-30718 & $11 / 7 / 09$ & 38 & ND & ND & \\
\hline TI10 & INTI10-S-30720 & $11 / 7 / 09$ & 2 & ND & ND & \\
\hline TI10 & INTI10-S-30721 & $11 / 7 / 09$ & 6 & ND & ND & \\
\hline TI10 & INTI10-S-30722 & $11 / 7 / 09$ & 10 & ND & ND & \\
\hline TI10 & INTI10-S-30723 & $11 / 7 / 09$ & 14 & ND & ND & \\
\hline TI10 & INTI10-S-30724 & $11 / 7 / 09$ & 18 & ND & ND & \\
\hline TI10 & INTI10-S-30725 & $11 / 7 / 09$ & 22 & ND & ND & \\
\hline TI10 & INTI10-S-30726 & $11 / 7 / 09$ & 26 & ND & ND & \\
\hline TI10 & INTI10-S-30727 & $11 / 7 / 09$ & 30 & ND & ND & \\
\hline TI10 & INTI10-S-30728 & $11 / 7 / 09$ & 34 & ND & ND & \\
\hline TI10 & INTI10-S-30729 & $11 / 7 / 09$ & 38 & $5.9 \mathrm{~J}$ & ND & QC Replicate $=$ ND. \\
\hline TI11 & INTI11-S-30734 & $11 / 8 / 09$ & 2 & ND & ND & \\
\hline TI11 & INTI11-S-30735 & $11 / 8 / 09$ & 6 & ND & ND & \\
\hline TI11 & INTI11-S-30736 & $11 / 8 / 09$ & 10 & ND & ND & \\
\hline TI11 & INTI11-S-30737 & $11 / 8 / 09$ & 14 & ND & ND & \\
\hline Tl11 & INTI11-S-30738 & $11 / 8 / 09$ & 18 & ND & ND & \\
\hline TI11 & INTI11-S-30739 & $11 / 8 / 09$ & 22 & ND & ND & \\
\hline TI11 & INTI11-S-30741 & $11 / 8 / 09$ & 26 & ND & ND & \\
\hline TI11 & INTI11-S-30742 & $11 / 8 / 09$ & 30 & ND & ND & \\
\hline TI11 & INTI11-S-30743 & $11 / 8 / 09$ & 34 & ND & ND & \\
\hline TI11 & INTI11-S-30744 & $11 / 8 / 09$ & 38 & ND & ND & \\
\hline
\end{tabular}

CPT sampling on private property west of the former CCC/USDA facility property.

$\begin{array}{lllccc}\text { TI19 } & \text { INTI19-S-30765 } & 11 / 10 / 10 & 4 & \text { ND } & \text { ND } \\ \text { TI19 } & \text { INTI19-S-30766 } & 11 / 10 / 10 & 8 & \text { ND } & \text { ND } \\ \text { TI19 } & \text { INTI19-S-30767 } & 11 / 10 / 10 & 12 & \text { ND } & \text { ND }\end{array}$


TABLE A.1 Results from the AGEM Laboratory for soil samples collected at Inman, Kansas, in 2009-2010.

\begin{tabular}{|c|c|c|c|c|c|}
\hline Location & Sample & Sample Date & $\begin{array}{c}\text { Depth } \\
\text { (ft BGL) }\end{array}$ & $\begin{array}{c}\text { Carbon } \\
\text { Tetrachloride } \\
(\mu \mathrm{g} / \mathrm{kg})\end{array}$ & $\begin{array}{c}\text { Chloroform } \\
(\mu \mathrm{g} / \mathrm{kg})\end{array}$ \\
\hline TI19 & INTI19-S-30768 & $11 / 10 / 10$ & 16 & ND & ND \\
\hline TI19 & INTI19-S-30769 & $11 / 10 / 10$ & 20 & ND & ND \\
\hline TI19 & INTI19-S-30770 & $11 / 10 / 10$ & 24 & ND & ND \\
\hline Tl19 & INTI19-S-30771 & $11 / 10 / 10$ & 28 & ND & ND \\
\hline TI19 & INTI19-S-30772 & $11 / 10 / 10$ & 32 & ND & ND \\
\hline TI19 & INTI19-S-30774 & $11 / 10 / 10$ & 36 & ND & ND \\
\hline TI19 & INTI19-S-30775 & $11 / 10 / 10$ & 39 & ND & ND \\
\hline T120 & INTI20-S-30790 & $11 / 11 / 10$ & 4 & ND & ND \\
\hline $\mathrm{TI} 20$ & INTI20-S-30791 & $11 / 11 / 10$ & 8 & ND & ND \\
\hline TI20 & INTI20-S-30792 & $11 / 11 / 10$ & 12 & ND & ND \\
\hline TI20 & INTI20-S-30793 & $11 / 11 / 10$ & 16 & ND & ND \\
\hline TI20 & INTI20-S-30794 & $11 / 11 / 10$ & 20 & ND & ND \\
\hline $\mathrm{T} 120$ & INTI20-S-30795 & $11 / 11 / 10$ & 24 & ND & ND \\
\hline $\mathrm{T} 120$ & INTI20-S-30796 & $11 / 11 / 10$ & 28 & ND & ND \\
\hline $\mathrm{T} 120$ & INTI20-S-30797 & $11 / 11 / 10$ & 32 & ND & ND \\
\hline $\mathrm{TI} 20$ & INTI20-S-30798 & $11 / 11 / 10$ & 36 & ND & ND \\
\hline TI21 & INTI21-S-30800 & $11 / 11 / 10$ & 4 & ND & ND \\
\hline TI21 & INTI21-S-30801 & $11 / 11 / 10$ & 8 & ND & ND \\
\hline $\mathrm{T} \mid 21$ & INTI21-S-30802 & $11 / 11 / 10$ & 12 & ND & ND \\
\hline TI21 & INTI21-S-30803 & $11 / 11 / 10$ & 16 & ND & ND \\
\hline TI21 & INTI21-S-30804 & $11 / 11 / 10$ & 20 & ND & ND \\
\hline TI21 & INTI21-S-30805 & $11 / 11 / 10$ & 24 & ND & ND \\
\hline TI21 & INTI21-S-30806 & $11 / 11 / 10$ & 28 & ND & ND \\
\hline $\mathrm{TI} 21$ & INTI21-S-30807 & $11 / 11 / 10$ & 32 & ND & ND \\
\hline $\mathrm{Tl} 21$ & INTI21-S-30808 & $11 / 11 / 10$ & 36 & ND & ND \\
\hline TI22 & INTI22-S-30809 & $11 / 11 / 10$ & 4 & ND & ND \\
\hline TI22 & INTI22-S-30810 & $11 / 11 / 10$ & 8 & ND & ND \\
\hline TI22 & INTI22-S-30811 & $11 / 11 / 10$ & 12 & ND & ND \\
\hline TI22 & INTI22-S-30818 & $11 / 11 / 10$ & 16 & ND & ND \\
\hline $\mathrm{T} 122$ & INTI22-S-30813 & $11 / 11 / 10$ & 20 & ND & ND \\
\hline TI22 & INTI22-S-30814 & $11 / 11 / 10$ & 24 & ND & ND \\
\hline TI22 & INTI22-S-30815 & $11 / 11 / 10$ & 28 & ND & ND \\
\hline TI22 & INTI22-S-30816 & $11 / 11 / 10$ & 32 & ND & ND \\
\hline TI22 & INTI22-S-30817 & $11 / 11 / 10$ & 36 & ND & ND \\
\hline TI23 & INTI23-S-30819 & $11 / 11 / 10$ & 4 & ND & ND \\
\hline $\mathrm{T} 123$ & INTI23-S-30820 & $11 / 11 / 10$ & 8 & ND & ND \\
\hline TI23 & INTI23-S-30821 & $11 / 11 / 10$ & 12 & ND & ND \\
\hline TI23 & INTI23-S-30822 & $11 / 11 / 10$ & 16 & ND & ND \\
\hline TI23 & INTI23-S-30823 & $11 / 11 / 10$ & 20 & ND & ND \\
\hline
\end{tabular}


TABLE A.1 Results from the AGEM Laboratory for soil samples collected at Inman, Kansas, in 2009-2010.

\begin{tabular}{|c|c|c|c|c|c|}
\hline Location & Sample & Sample Date & $\begin{array}{c}\text { Depth } \\
\text { (ft BGL) }\end{array}$ & $\begin{array}{c}\text { Carbon } \\
\text { Tetrachloride } \\
(\mu \mathrm{g} / \mathrm{kg})\end{array}$ & $\begin{array}{l}\text { Chloroform } \\
(\mu \mathrm{g} / \mathrm{kg})\end{array}$ \\
\hline TI23 & INTI23-S-30824 & $11 / 11 / 10$ & 24 & ND & ND \\
\hline TI23 & INTI23-S-30825 & $11 / 11 / 10$ & 28 & ND & ND \\
\hline TI23 & INTI23-S-30826 & $11 / 11 / 10$ & 32 & ND & ND \\
\hline TI23 & INTI23-S-30827 & $11 / 11 / 10$ & 36 & ND & ND \\
\hline TI23 & INTI23-S-30839 & $11 / 11 / 10$ & 38.2 & ND & ND \\
\hline TI24 & INTI24-S-30828 & $11 / 12 / 10$ & 4 & ND & ND \\
\hline TI24 & INTI24-S-30829 & $11 / 12 / 10$ & 8 & ND & ND \\
\hline T124 & INTI24-S-30830 & $11 / 12 / 10$ & 12 & ND & ND \\
\hline TI24 & INTI24-S-30831 & $11 / 11 / 10$ & 16 & ND & ND \\
\hline TI24 & INTI24-S-30832 & $11 / 12 / 10$ & 20 & ND & ND \\
\hline TI24 & INTI24-S-30833 & $11 / 12 / 10$ & 24 & ND & ND \\
\hline T124 & INTI24-S-30834 & $11 / 12 / 10$ & 28 & ND & ND \\
\hline TI24 & INTI24-S-30835 & $11 / 12 / 10$ & 32 & ND & ND \\
\hline TI24 & INTI24-S-30836 & $11 / 12 / 10$ & 36 & ND & ND \\
\hline TI24 & INTI24-S-30838 & $11 / 12 / 10$ & 39 & ND & ND \\
\hline TI25 & INTI25-S-30840 & $11 / 12 / 10$ & 4 & ND & ND \\
\hline TI25 & INTI25-S-30841 & $11 / 12 / 10$ & 8 & ND & ND \\
\hline TI25 & INTI25-S-30842 & $11 / 12 / 10$ & 12 & ND & ND \\
\hline TI25 & INTI25-S-30843 & $11 / 12 / 10$ & 16 & ND & ND \\
\hline TI25 & INTI25-S-30844 & $11 / 12 / 10$ & 20 & ND & ND \\
\hline TI25 & INTI25-S-30845 & $11 / 12 / 10$ & 24 & ND & ND \\
\hline TI25 & INTI25-S-30846 & $11 / 12 / 10$ & 28 & ND & ND \\
\hline TI25 & INTI25-S-30848 & $11 / 12 / 10$ & 36 & ND & ND \\
\hline
\end{tabular}

$\mathrm{ND}$, compound analyzed for but not detected at a level greater than or equal to the method detection limit $(<10 \mu \mathrm{g} / \mathrm{kg})$.

$\mathrm{J}$, compound identified with an estimated concentration between the instrument detection limit and the method detection limit. 
TABLE A.2 Results for groundwater sampling at Inman, Kansas, in 2009-2011.

\begin{tabular}{|c|c|c|c|c|c|}
\hline Location & Sample & $\begin{array}{c}\text { Sample } \\
\text { Date }\end{array}$ & $\begin{array}{l}\text { Depth from Depth to } \\
\begin{array}{ll}\text { (ft BGL) } & \text { (ft BGL) }\end{array}\end{array}$ & $\begin{array}{l}\text { Carbon } \\
\text { Tetrachloride } \\
\qquad(\mu \mathrm{g} / \mathrm{L}) \\
\quad(\mu \mathrm{g} / \mathrm{L})\end{array}$ & $\begin{array}{l}\text { Methylene } \\
\text { Chloride } \\
(\mu \mathrm{g} / \mathrm{L})\end{array}$ \\
\hline
\end{tabular}

CPT sampling on the former CCC/USDA facility property.

\begin{tabular}{|c|c|c|c|c|c|c|c|}
\hline TI01 & INTI01-W-30733 & $11 / 7 / 09$ & 26 & 40 & 34 & 3.4 & $N D^{a}$ \\
\hline TI01 & INTI01-W-30749 & $11 / 9 / 09$ & 30 & 40 & 18 & 3.0 & ND \\
\hline TI01 & INTI01-W-30750 & $11 / 9 / 09$ & 33 & 38 & 23 & 2.4 & ND \\
\hline TI02 & INTI02-W-30758 & $11 / 11 / 09$ & 30 & 40 & 3.1 & 1.0 & ND \\
\hline TI04 & INTI04-W-30747 & $11 / 9 / 09$ & 30 & 40 & ND & ND & ND \\
\hline TI04 & INTI04B-W-30746 & $11 / 9 / 09$ & 40 & 42 & ND & $0.6 \mathrm{~J}^{\mathrm{b}}$ & ND \\
\hline TI05 & INTI05-W-30748 & $11 / 9 / 09$ & 30 & 40 & ND & ND & ND \\
\hline T106 & INTI06-W-30756 & $11 / 11 / 09$ & 28 & 33 & 22 & 3.1 & ND \\
\hline TI06 & INTI06-W-30757 & $11 / 11 / 09$ & 33 & 38 & 12 & $0.9 \mathrm{~J}$ & ND \\
\hline TI08 & INTI08-W-30752 & $11 / 9 / 09$ & 30 & 40 & ND & ND & ND \\
\hline TI11 & INTI11-W-30751 & $11 / 9 / 09$ & 28 & 33 & 12 & 4.5 & ND \\
\hline TI11 & INTI11-W-30755 & $11 / 11 / 09$ & 33 & 38 & 8.3 & 3.2 & ND \\
\hline
\end{tabular}

Private well sampling west of the former CCC/USDA facility.

$\begin{array}{llllllll}\text { BENGSTEN } & \text { INBENGSTEN-W-32675 } & 11 / 9 / 10 & - & - & 5.0 & 1.4 & \text { ND } \\ \text { PANKRATZ } & \text { INPANKRATZ-W-32674 } & 11 / 9 / 10 & - & -- & 5.0 & 1.1 & \text { ND } \\ \text { SISSON } & \text { INSISSON-W-30761 } & 11 / 9 / 10 & - & -- & 4.9 & 1.2 & \text { ND } \\ \text { WILLEMS } & \text { INWILLEMS-W-32673 } & 11 / 9 / 10 & - & -- & 4.0 & 1.0 & \text { ND }\end{array}$

Monitoring wells installed on the former CCC/USDA facility property.

$\begin{array}{lllllllll}\text { MW01 } & \text { INMW01-W-32670 } & 11 / 9 / 10 & 32 & 42 & 3.6 & 2.6 & \text { ND } & \text { ND } \\ \text { MW02 } & \text { INMW02-W-32671 } & 11 / 9 / 10 & 32 & 42 & 2.7 & 4.6 & \text { ND } & \\ \text { MW03 } & \text { INMW03-W-32672 } & 11 / 9 / 10 & 35 & 45 & 0.4 \mathrm{~J} & 1.4 & \text { ND } & \text { Trace CT at TestAmerica=0.12 J } \\ \text { MW04 } & \text { INMW04-W-32677 } & 11 / 10 / 10 & 30 & 40 & \text { ND } & \text { ND } & \text { ND } \\ \text { MW05 } & \text { INMW05-W-32678 } & 11 / 10 / 10 & 35 & 45 & \text { ND } & \text { ND } & \text { ND } & \text { Trace CT at TestAmerica=0.08 J }\end{array}$


TABLE A.2 Results for groundwater sampling at Inman, Kansas, in 2009-2011.

CPT sampling off-site of the former CCC/USDA facility property.

\begin{tabular}{|c|c|c|c|c|c|c|c|c|}
\hline TI12 & INTI12-W-32679 & $11 / 10 / 10$ & 32 & 42 & ND & ND & ND & Completed as MW06. \\
\hline TI13 & INTI13-W-30783 & $11 / 11 / 10$ & 31.7 & 41.7 & ND & ND & ND & Completed as MW07. \\
\hline TI14 & INTI14-W-30762 & $11 / 10 / 10$ & 29.5 & 39.5 & ND & ND & ND & Completed as MW09. \\
\hline TI15 & INTI15-W-30784 & $11 / 11 / 10$ & 31.7 & 41.7 & ND & ND & ND & Completed as MW10. \\
\hline TI16 & INTI16-W-30785 & $11 / 11 / 10$ & 31.2 & 41.2 & ND & ND & ND & Completed as MW11. \\
\hline TI17 & INTI17-W-30853 & $11 / 12 / 10$ & 32.1 & 42.1 & ND & ND & ND & Completed as MW12. \\
\hline TI18 & INTI18-W-30786 & $11 / 11 / 10$ & 30.6 & 40.6 & ND & ND & ND & Completed as MW13. \\
\hline TI22 & INTI22-W-32680 & $11 / 14 / 10$ & 30 & 40 & ND & ND & ND & \\
\hline TI23 & INTI23-W-32681 & $11 / 14 / 10$ & 30 & 40 & ND & ND & ND & \\
\hline TI24 & INTI24-W-32682 & $11 / 14 / 10$ & 29.1 & 39.1 & ND & ND & ND & \\
\hline $\mathrm{TI} 26$ & INTI26-W-32683 & $11 / 18 / 10$ & 31.1 & 41.1 & ND & ND & ND & Completed as MW08. \\
\hline \multicolumn{9}{|c|}{ Comprehensive sampling of monitoring well network. } \\
\hline MW01 & INMW01-W-32685 & $12 / 22 / 10$ & 32 & 42 & 5.4 & 3.2 & ND & Analysis at TestAmerica. \\
\hline MW02 & INMW02-W-32686 & $12 / 22 / 10$ & 32 & 42 & 2.9 & 4.8 & 1.5 & Analysis at TestAmerica. \\
\hline MW03 & INMW03-W-32687 & $12 / 22 / 10$ & 35 & 45 & 2.2 & 1.5 & $0.078 \mathrm{~J}$ & Analysis at TestAmerica. \\
\hline MW04 & INMW04-W-32688 & $12 / 22 / 10$ & 30 & 40 & $0.22 \mathrm{~J}$ & $0.050 \mathrm{~J}$ & ND & Analysis at TestAmerica. \\
\hline MW05 & INMW05-W-32689 & $12 / 22 / 10$ & 35 & 45 & $0.091 \mathrm{~J}$ & ND & ND & Analysis at TestAmerica. \\
\hline MW06 & INMW06-W-32690 & $12 / 22 / 10$ & 32 & 42 & ND & ND & ND & Analysis at TestAmerica. \\
\hline MW07 & INMW07-W-32691 & $12 / 22 / 10$ & 31.7 & 41.7 & ND & ND & ND & Analysis at TestAmerica. \\
\hline MW08 & INMW08-W-32692 & $12 / 22 / 10$ & 31.1 & 41.1 & ND & ND & ND & Analysis at TestAmerica. \\
\hline MW09 & INMW09-W-32693 & $12 / 22 / 10$ & 29.5 & 39.5 & ND & ND & ND & Analysis at TestAmerica. \\
\hline MW10 & INMW10-W-32694 & $12 / 22 / 10$ & 31.7 & 41.7 & ND & ND & ND & Analysis at TestAmerica. \\
\hline MW11 & INMW11-W-32696 & $12 / 22 / 10$ & 31.2 & 41.2 & ND & ND & ND & Analysis at TestAmerica. \\
\hline MW12 & INMW12-W-32697 & $12 / 22 / 10$ & 32.1 & 42.1 & ND & ND & ND & Analysis at TestAmerica. \\
\hline MW13 & INMW13-W-32698 & $12 / 22 / 10$ & 30.6 & 40.6 & ND & ND & ND & Analysis at TestAmerica. \\
\hline TI22 (plugged) & INTI22-W-32699 & $12 / 22 / 10$ & 30 & 40 & $0.050 \mathrm{~J}$ & ND & ND & Analysis at TestAmerica. \\
\hline TI23 (plugged) & INTI23-W-32700 & $12 / 22 / 10$ & 30 & 40 & $0.041 \mathrm{~J}$ & ND & ND & Analysis at TestAmerica. \\
\hline TI24 (plugged) & INTI25-W-32701 ${ }^{c}$ & $12 / 22 / 10$ & 29.1 & 39.1 & $0.27 \mathrm{~J}$ & ND & ND & Analysis at TestAmerica. \\
\hline
\end{tabular}


TABLE A.2 Results for groundwater sampling at Inman, Kansas, in 2009-2011.

\begin{tabular}{|c|c|c|c|c|c|c|}
\hline Location & Sample & $\begin{array}{l}\text { Sample } \\
\text { Date }\end{array}$ & $\begin{array}{l}\text { Depth from } \\
\text { (ft BGL) }\end{array}$ & $\begin{array}{lc} & \text { Carbon } \\
\text { Depth to } & \text { Tetrachloride } \\
(\mathrm{ft} \mathrm{BGL}) & (\mu \mathrm{g} / \mathrm{L})\end{array}$ & $\underset{(\mu \mathrm{g} / L)}{\text { Chloroform }}$ & $\begin{array}{l}\text { Methylene } \\
\text { Chloride } \\
(\mu g / L)\end{array}$ \\
\hline
\end{tabular}

Private well sampling surrounding the former CCC/USDA facility and private grain storage.

$\begin{array}{llllllll}\text { Thiessen } & \text { INTHIESSEN-W-32685 } & 5 / 24 / 11 & - & - & \text { ND } & \text { ND } & \text { ND } \\ \text { Brown } & \text { INBROWN-W-32686 } & 5 / 24 / 11 & - & -- & \text { ND } & \text { ND } & \text { ND } \\ \text { Klassen } & \text { INKLASSEN-W-32687 } & 5 / 24 / 11 & - & -- & 6.0 & 0.2 \mathrm{~J} & \text { ND } \\ \text { Goertzen } & \text { INGOERTZEN-W-32688 } & 5 / 24 / 11 & - & -- & \text { ND } & \text { ND } & \text { ND } \\ \text { Isaac } & \text { INISAAC-W-32689 } & 5 / 24 / 11 & - & -- & \text { ND } & 0.7 \text { J } & \text { ND } \\ \text { Pankratz } & \text { INPANKRATZ-W-32690 } & 5 / 24 / 11 & -- & -- & \text { ND } & \text { ND } & \text { ND } \\ \text { Hull } & \text { INHULL-W-32691 } & 5 / 24 / 11 & - & -- & \text { ND } & \text { ND } & \text { ND } \\ \text { Klassen } & \text { INKLASSEN-W-32693 } & 6 / 23 / 11 & - & -- & 5.6 & \text { ND } & \text { ND } \\ \text { Hull } & \text { INHULL-W-32694 } & 6 / 23 / 11 & - & -- & \text { ND } & \text { ND } & \text { ND } \\ \text { Pauls } & \text { INPAULS-W-32695 } & 6 / 23 / 11 & - & -- & \text { ND } & \text { ND } & \text { ND }\end{array}$

${ }^{a} \mathrm{ND}$, compound analyzed for but not detected at a level greater than or equal to the method detection limit $(<1 \mu \mathrm{g} / \mathrm{L}$ for analyses at the AGEM Laboratory or $<0.5 \mu \mathrm{g} / \mathrm{L}$ for analyses at TestAmerica).

${ }^{D} \mathrm{~J}$, compound identified with an estimated concentration between the instrument detection limit and the method detection limit.

${ }^{c}$ Typographical error in sample identifier; sample is actually from TI24, not TI25. 


\section{Appendix B:}

Lithology Logs and Electronic Cone Penetrometer Logs 


\section{Argonne National Laboratory}

Project: Inman

Geologist: Eugene Yan
Elevation: $1525.081 \mathrm{ft}$

Depth: 42 ft BGL
Well ID: MW01

Log Date: November 08, 2009

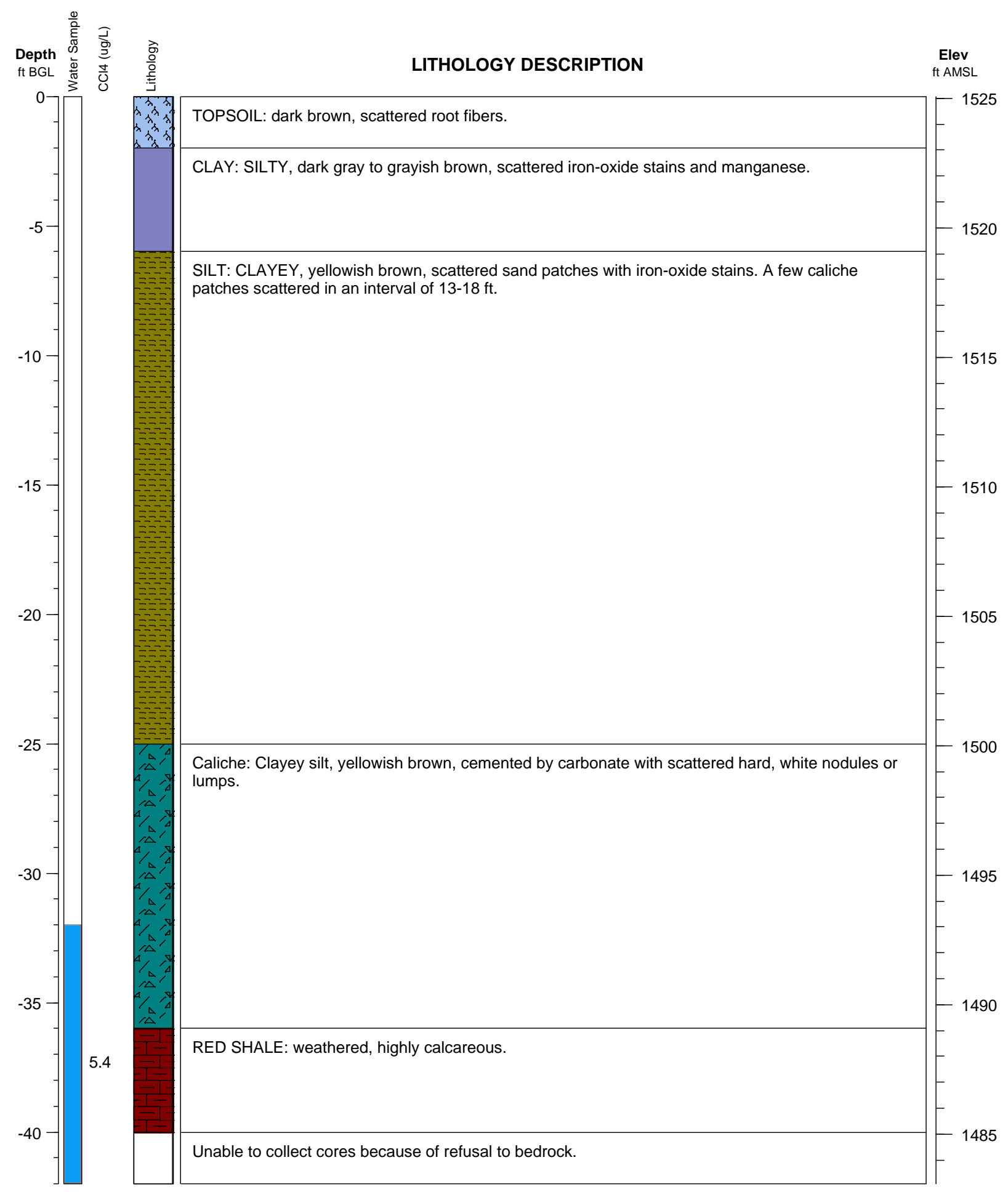




\section{Argonne National Laboratory}

Project: Inman

Geologist: Eugene Yan
Elevation: $1525.521 \mathrm{ft}$

Depth: $42 \mathrm{ft} \mathrm{BGL}$
Well ID: MW02

Log Date: November 04, 2009

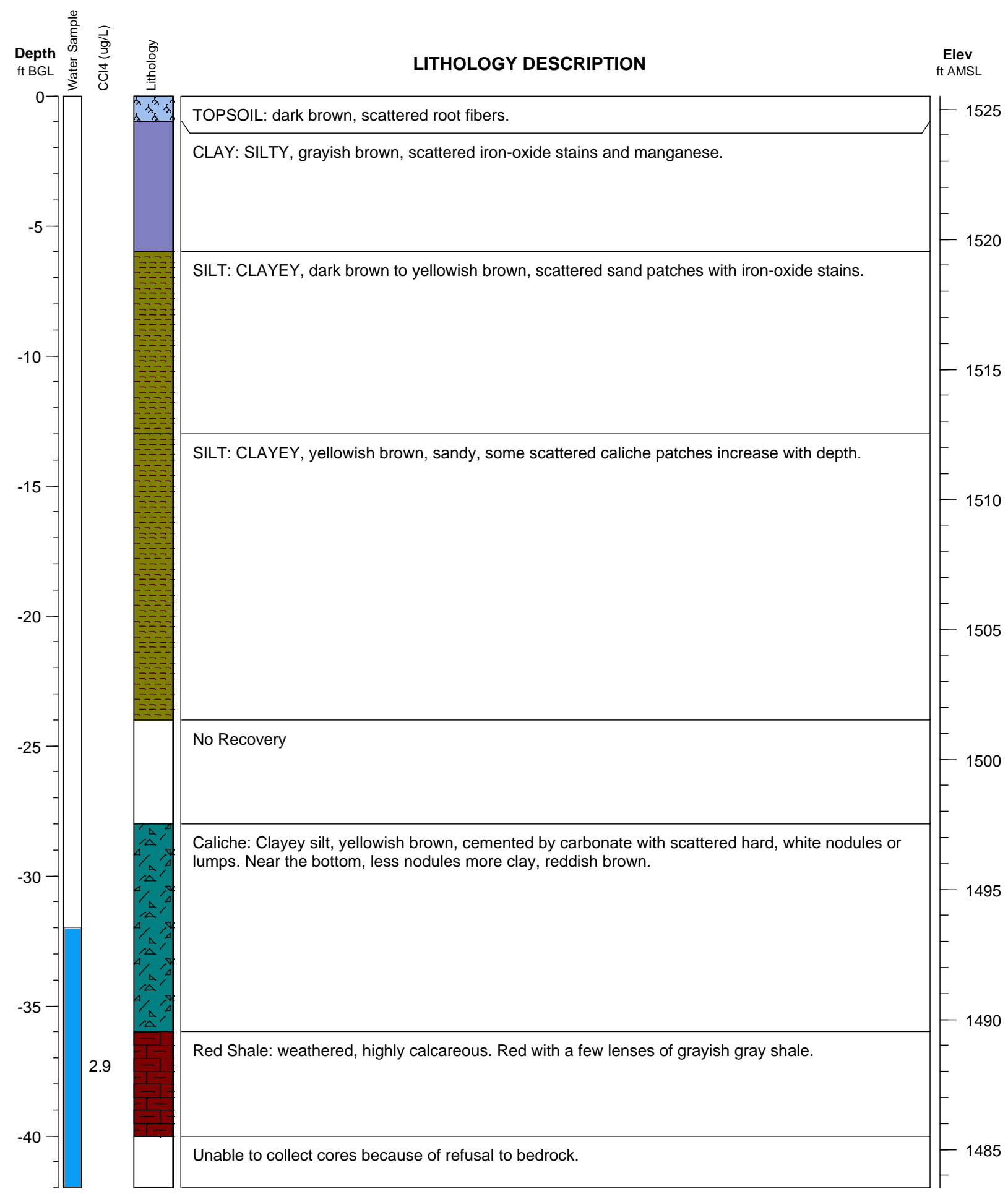




\section{Argonne National Laboratory}

Project: Inman

Geologist: Eugene Yan
Boring ID: MW02 (TI01)

Elevation: $1525.521 \mathrm{ft}$

Depth: $42 \mathrm{ft} \mathrm{BGL}$
Log Date: November 04, 2009

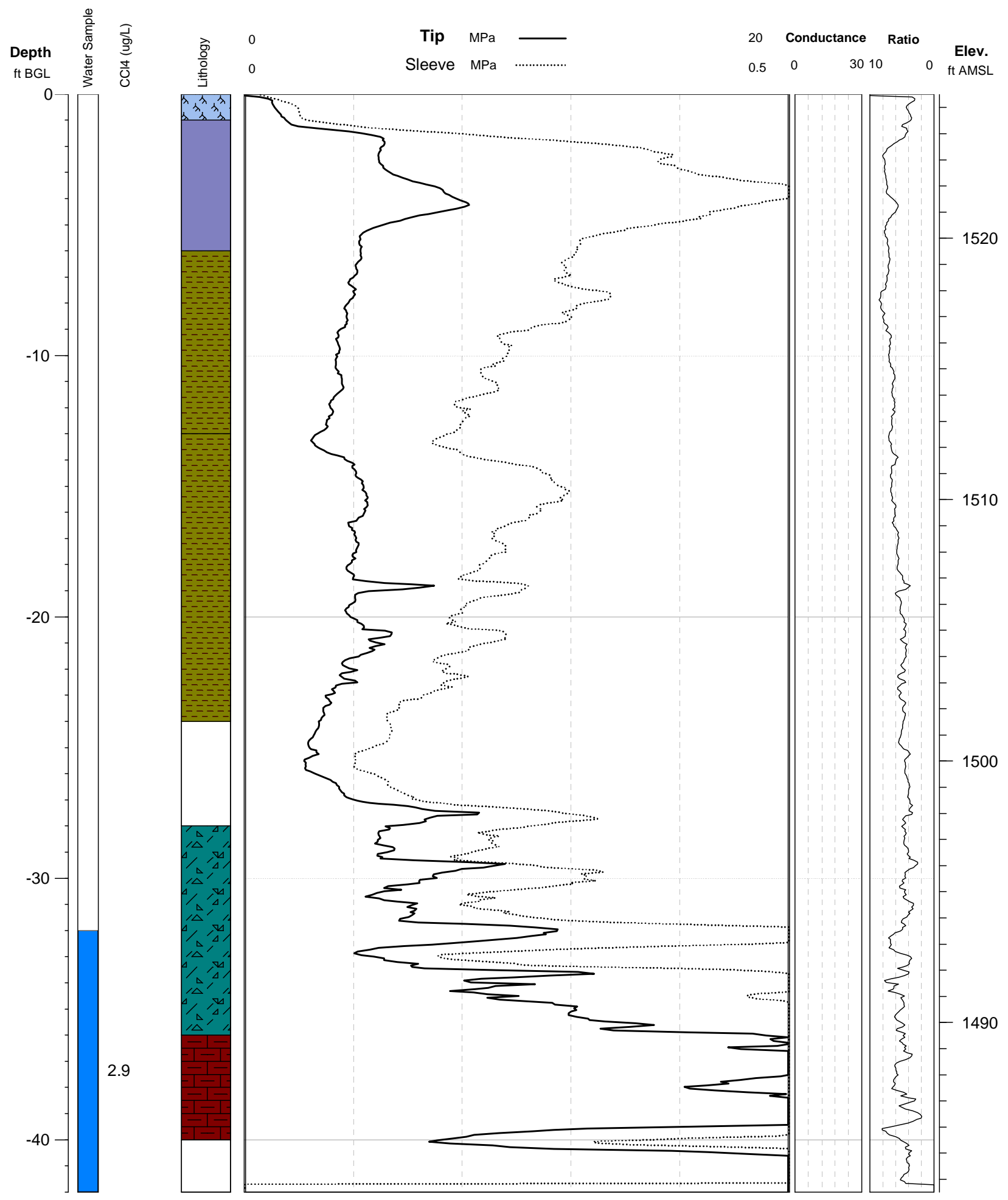




\section{Argonne National Laboratory}

Project: Inman

Geologist: Eugene Yan
Elevation: $1525.716 \mathrm{ft}$

Depth: $42 \mathrm{ft} \mathrm{BGL}$
Well ID: MW03

Log Date: November 06, 2009

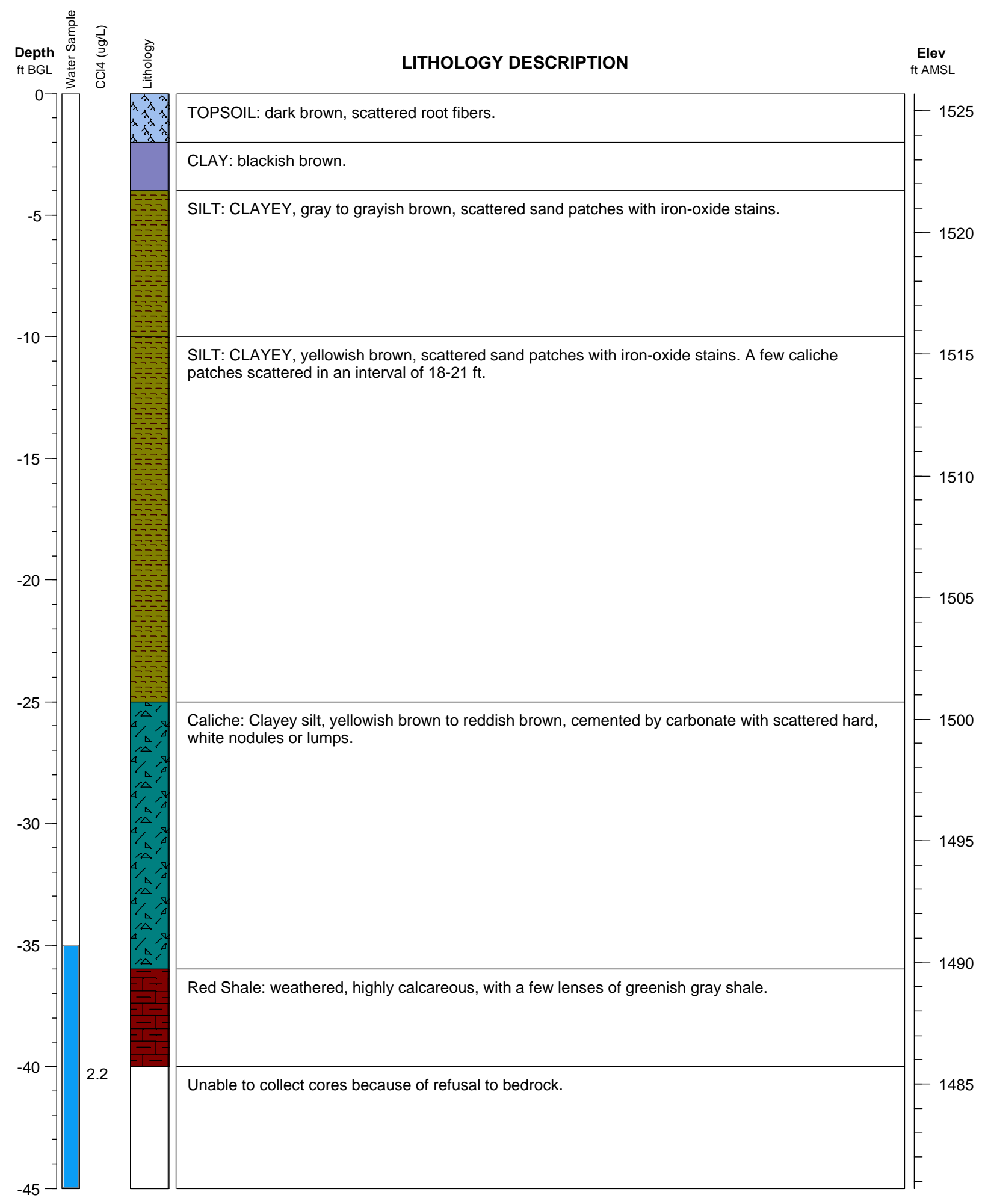




\section{Argonne National Laboratory}

Project: Inman

Geologist: Eugene Yan
Elevation: $1526.235 \mathrm{ft}$

Depth: $40 \mathrm{ft}$ BGL
Well ID: MW04

Log Date: November 05, 2009

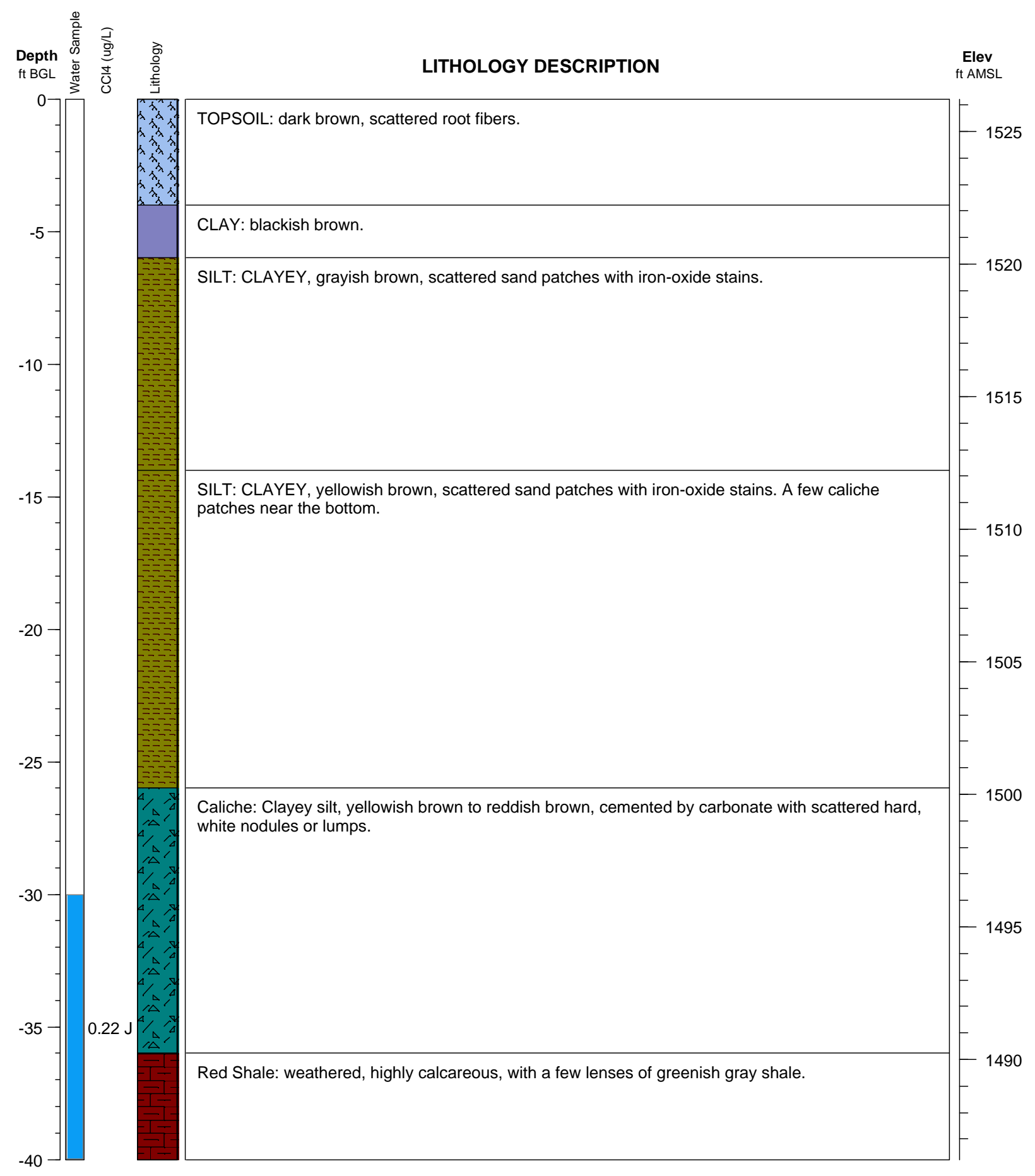




\section{Argonne National Laboratory}

Project: Inman

Geologist: Eugene Yan
Elevation: $1526.235 \mathrm{ft}$

Depth: $40 \mathrm{ft} B G L$
Boring ID: MW04 (TI04)

Log Date: November 05, 2009

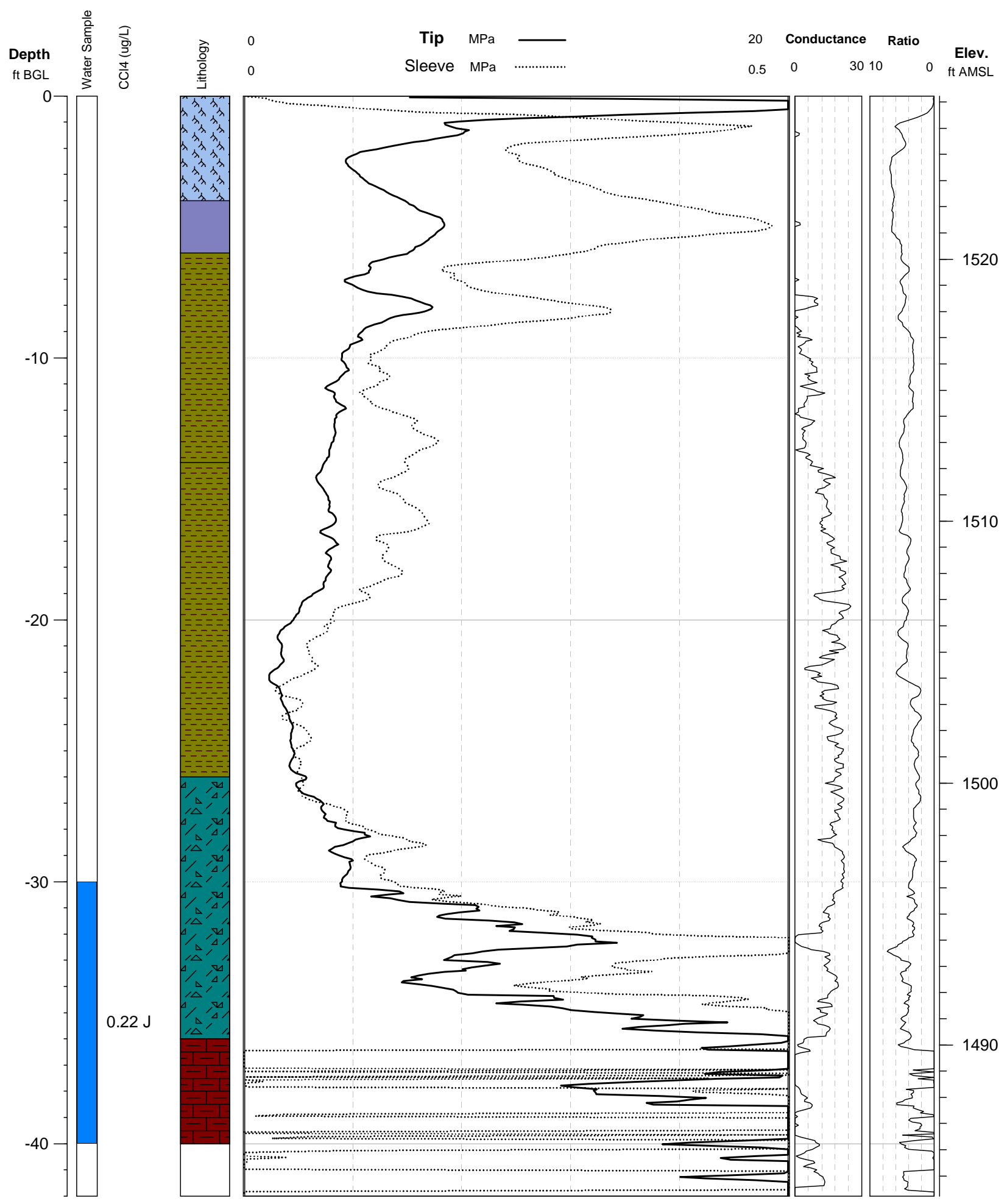




\section{Argonne National Laboratory}

Project: Inman

Geologist: Eugene Yan
Elevation: $1525.601 \mathrm{ft}$

Depth: $45 \mathrm{ft} B G \mathrm{~L}$
Well ID: MW05

Log Date: November 07, 2009

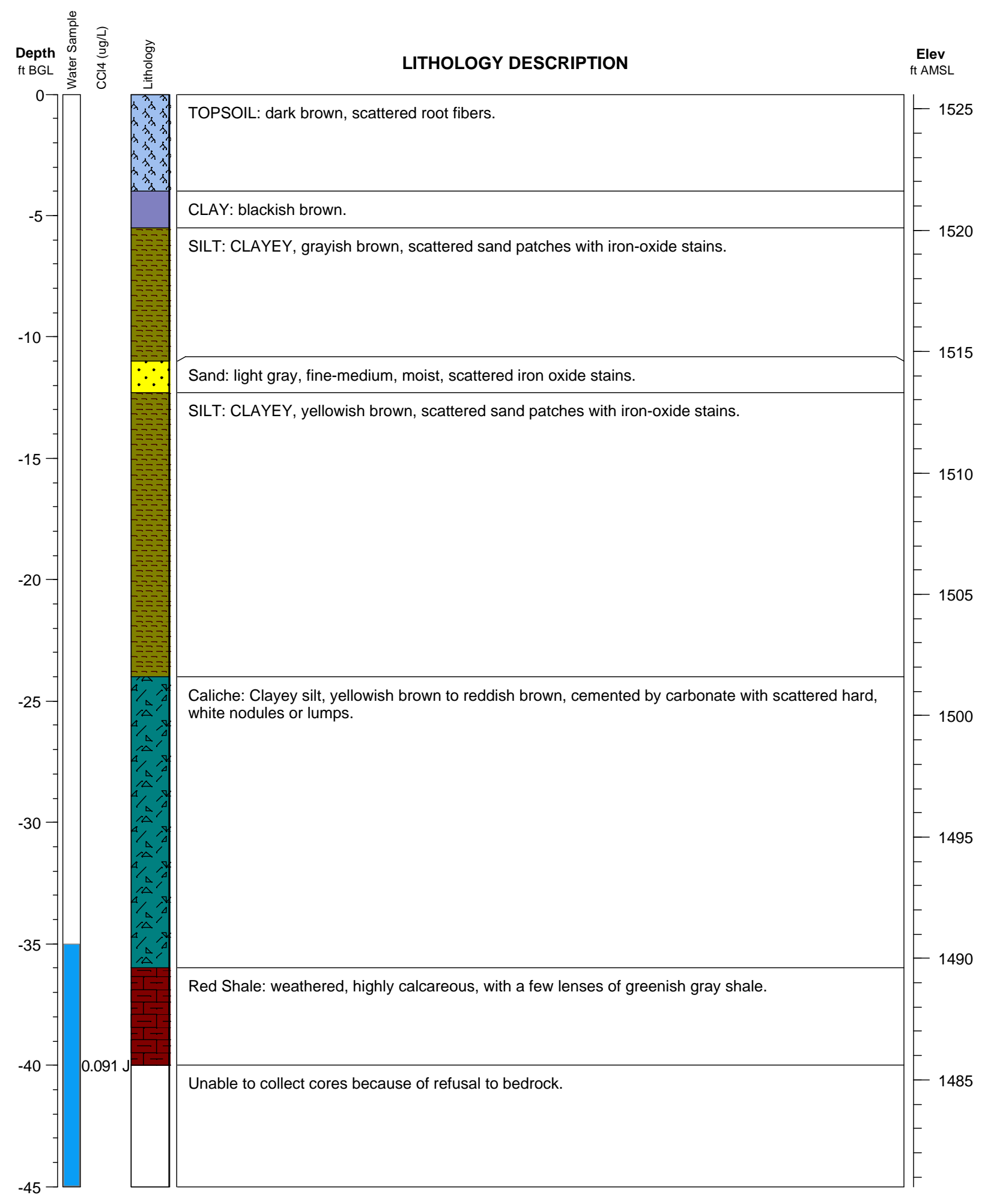




\section{Argonne National Laboratory}

Project: Inman

Geologist: Eugene Yan
Elevation: $1523.064 \mathrm{ft}$

Depth: $42.19 \mathrm{ft} B G L$

\section{Boring ID: MW06}

Log Date: November 8, 2010

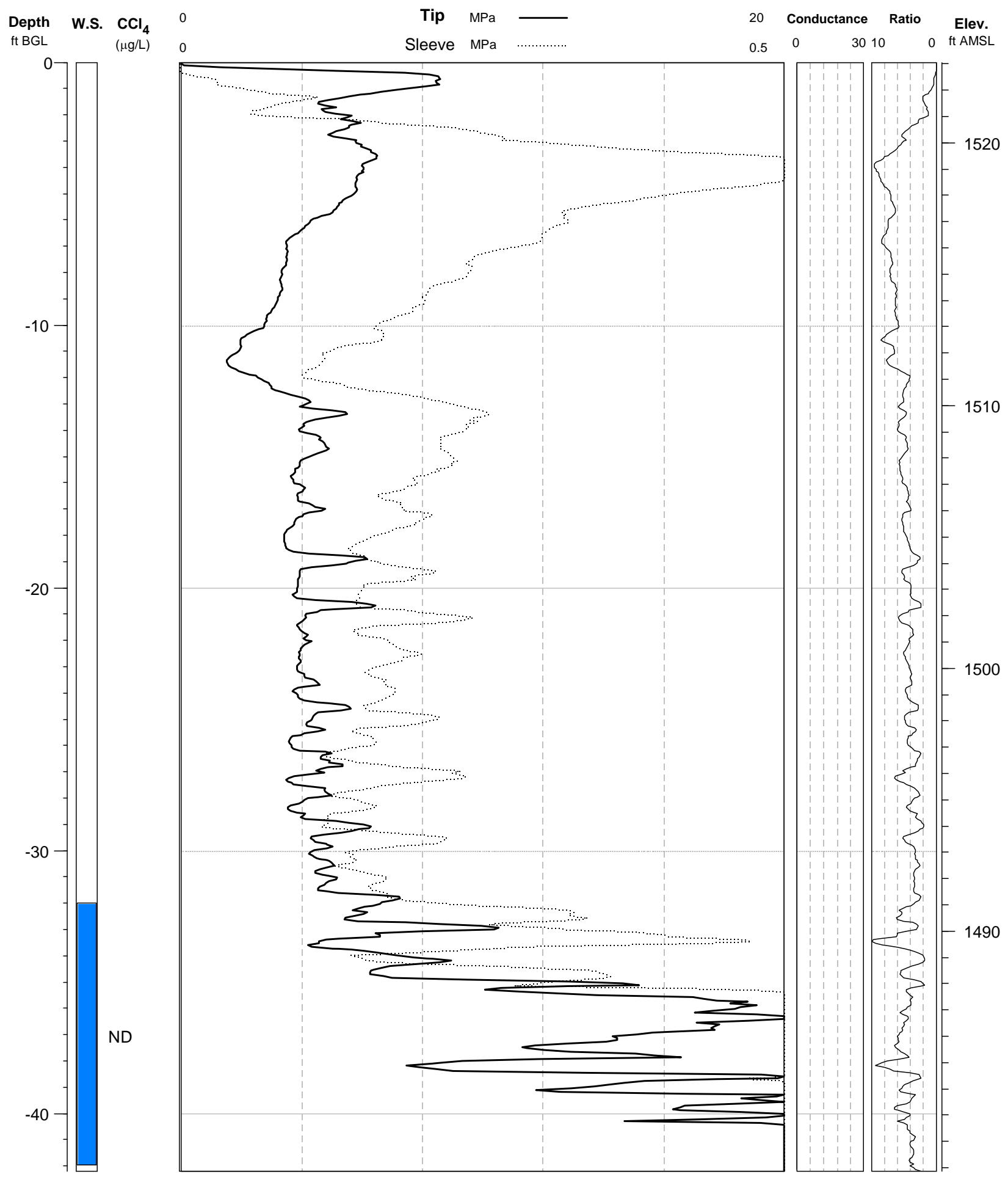




\section{Argonne National Laboratory}

Project: Inman

Geologist: Eugene Yan
Elevation: $1524.315 \mathrm{ft}$

Depth: $41.73 \mathrm{ft} \mathrm{BGL}$
Boring ID: MW07

Log Date: November 8, 2010

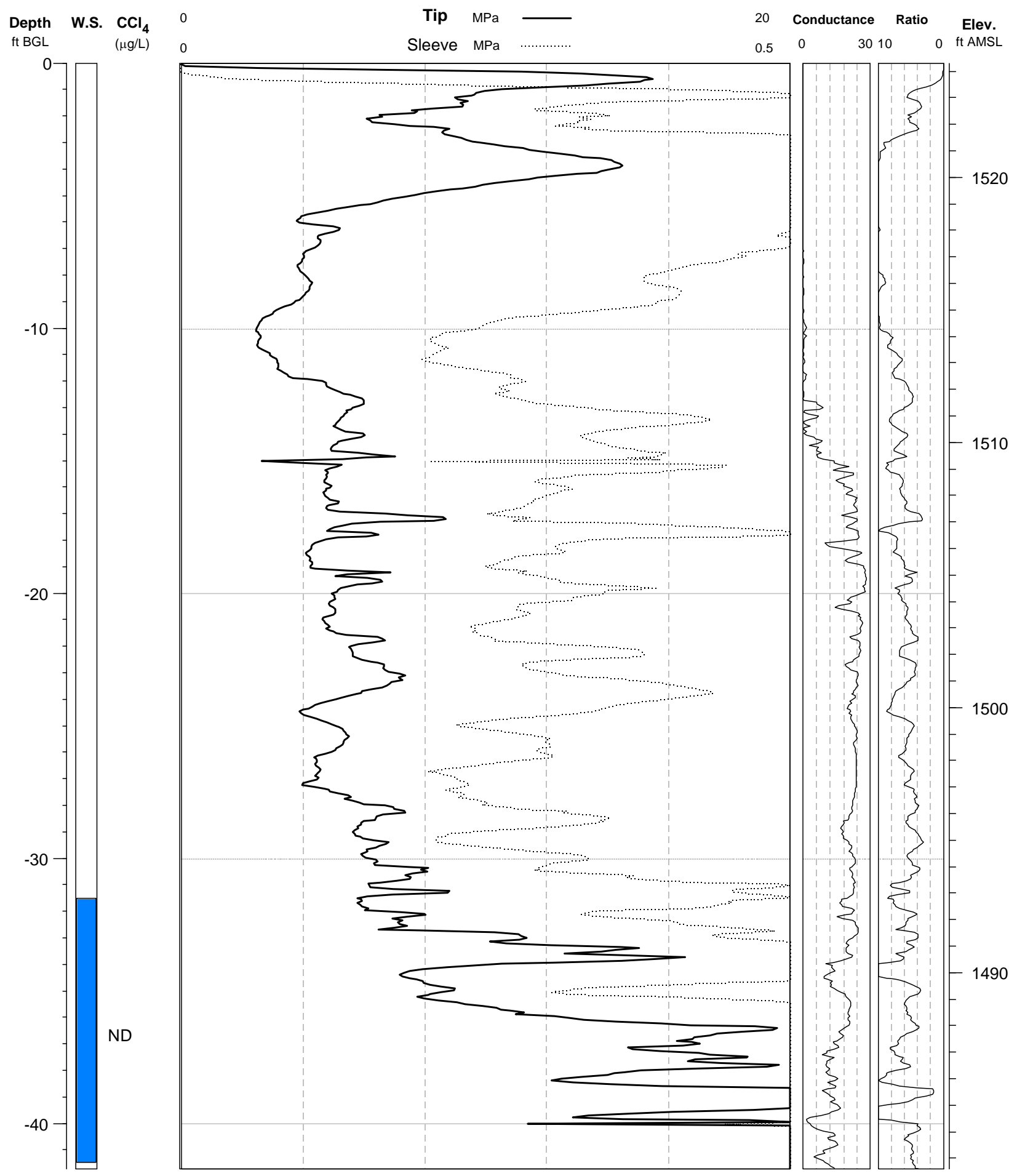




\section{Argonne National Laboratory}

Project: Inman

Geologist: Eugene Yan
Elevation: $1525.583 \mathrm{ft}$

Depth: $39.566 \mathrm{ft} \mathrm{BGL}$
Boring ID: MW09

Log Date: November 9, 2010

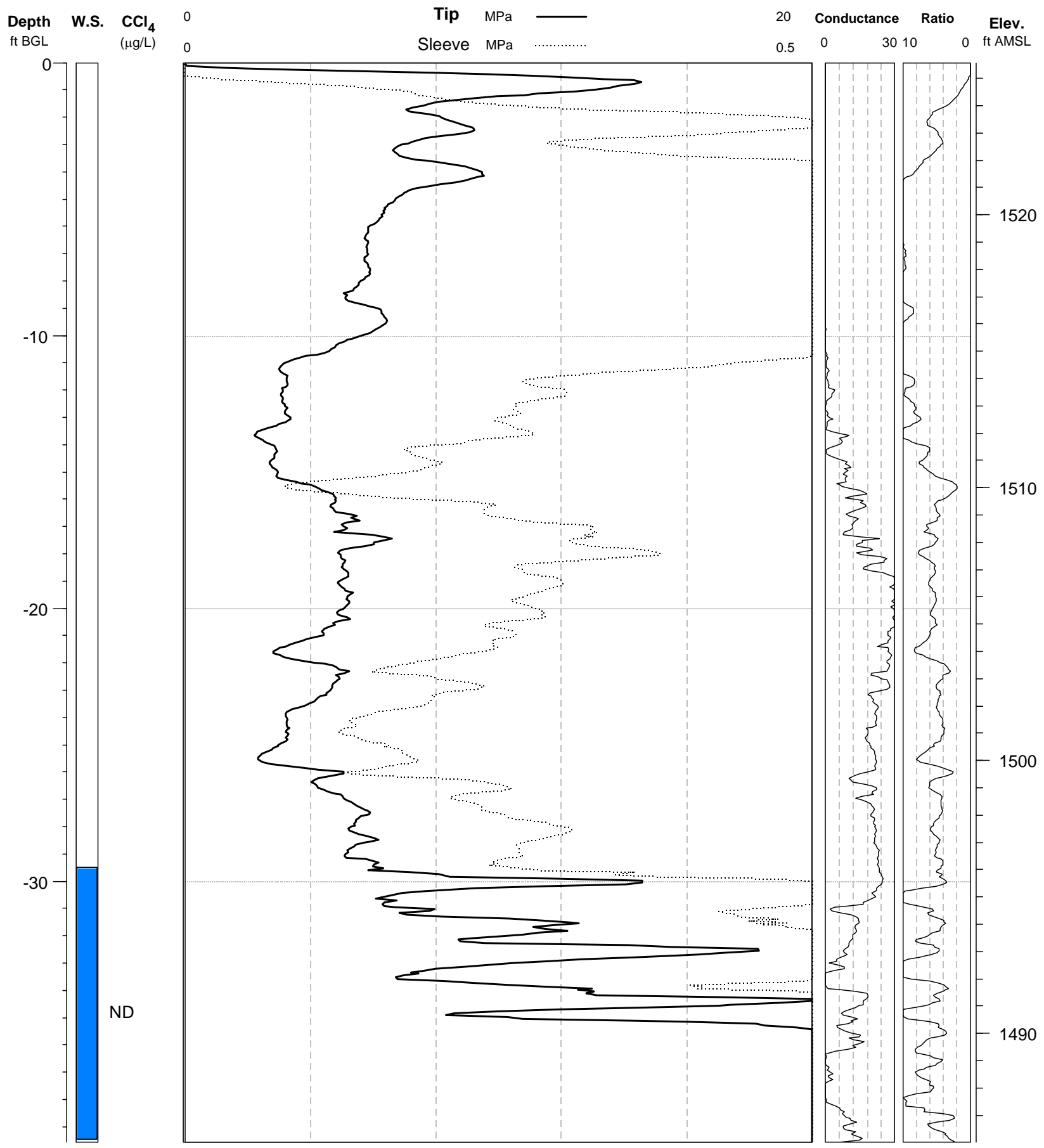




\section{Argonne National Laboratory}

\section{Project: Inman}

Geologist: Eugene Yan
Elevation: $1525.482 \mathrm{ft}$

Depth: $41.929 \mathrm{ft}$ BGL
Boring ID: MW10

Log Date: November 9, 2010

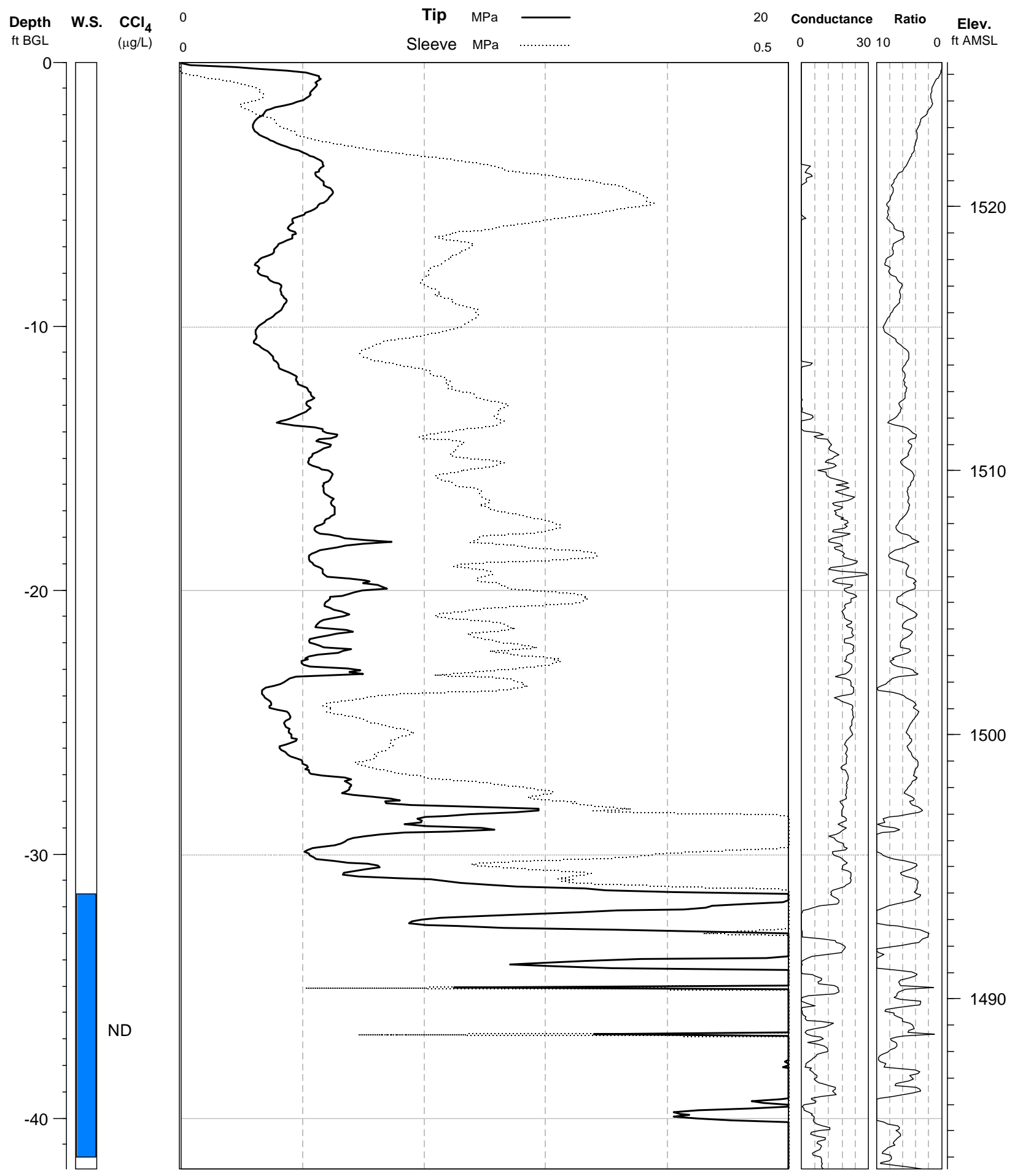


Inman Site Investigation Results

B-14

Version 02, 01/24/14

Argonne National Laboratory

Project: Unman

Geologist: Eugene Man
Elevation: $1525.716 \mathrm{ft}$

Depth: $41.601 \mathrm{ft} \mathrm{BGL}$
Boring ID: MW11

Log Date: November 10, 2010

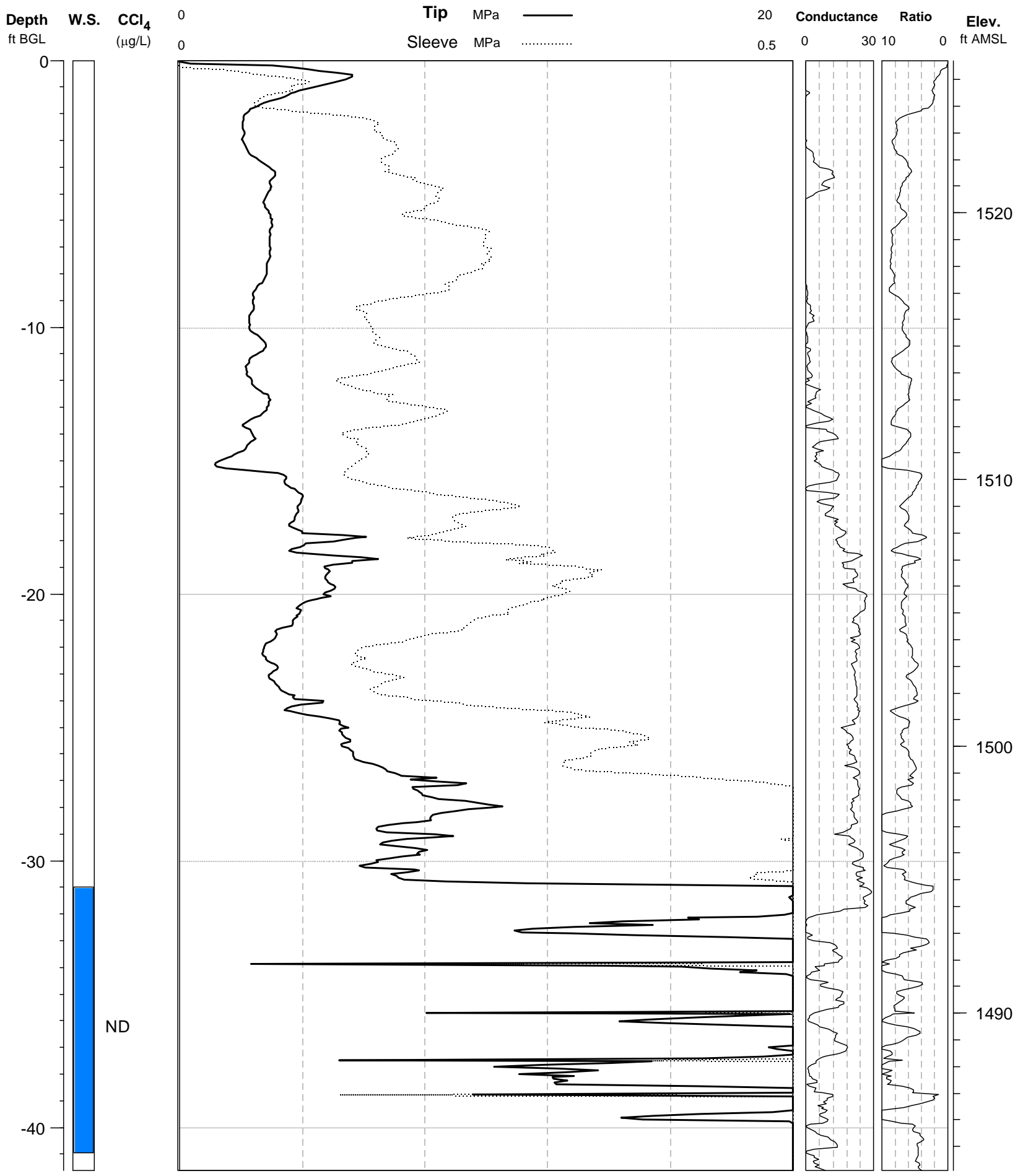




\section{Argonne National Laboratory}

Project: Inman

Geologist: Eugene Yan
Elevation: $1525.808 \mathrm{ft}$

Depth: $42.322 \mathrm{ft} \mathrm{BGL}$
Boring ID: MW12

Log Date: November 10, 2010

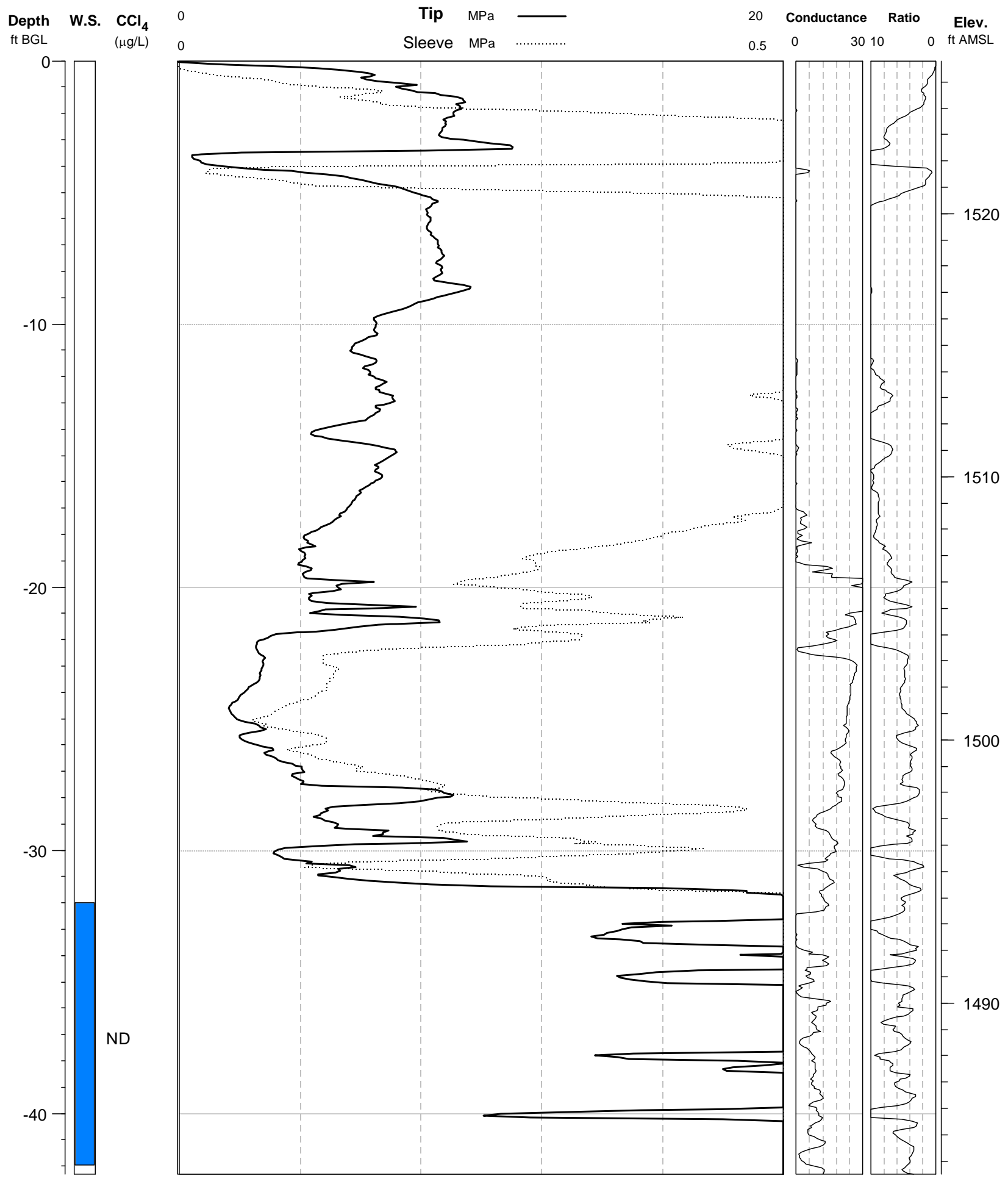




\section{Argonne National Laboratory}

Project: Inman

Geologist: Eugene Yan
Elevation: $1523.506 \mathrm{ft}$

Depth: $40.748 \mathrm{ft} \mathrm{BGL}$
Boring ID: MW13

Log Date: November 10, 2010

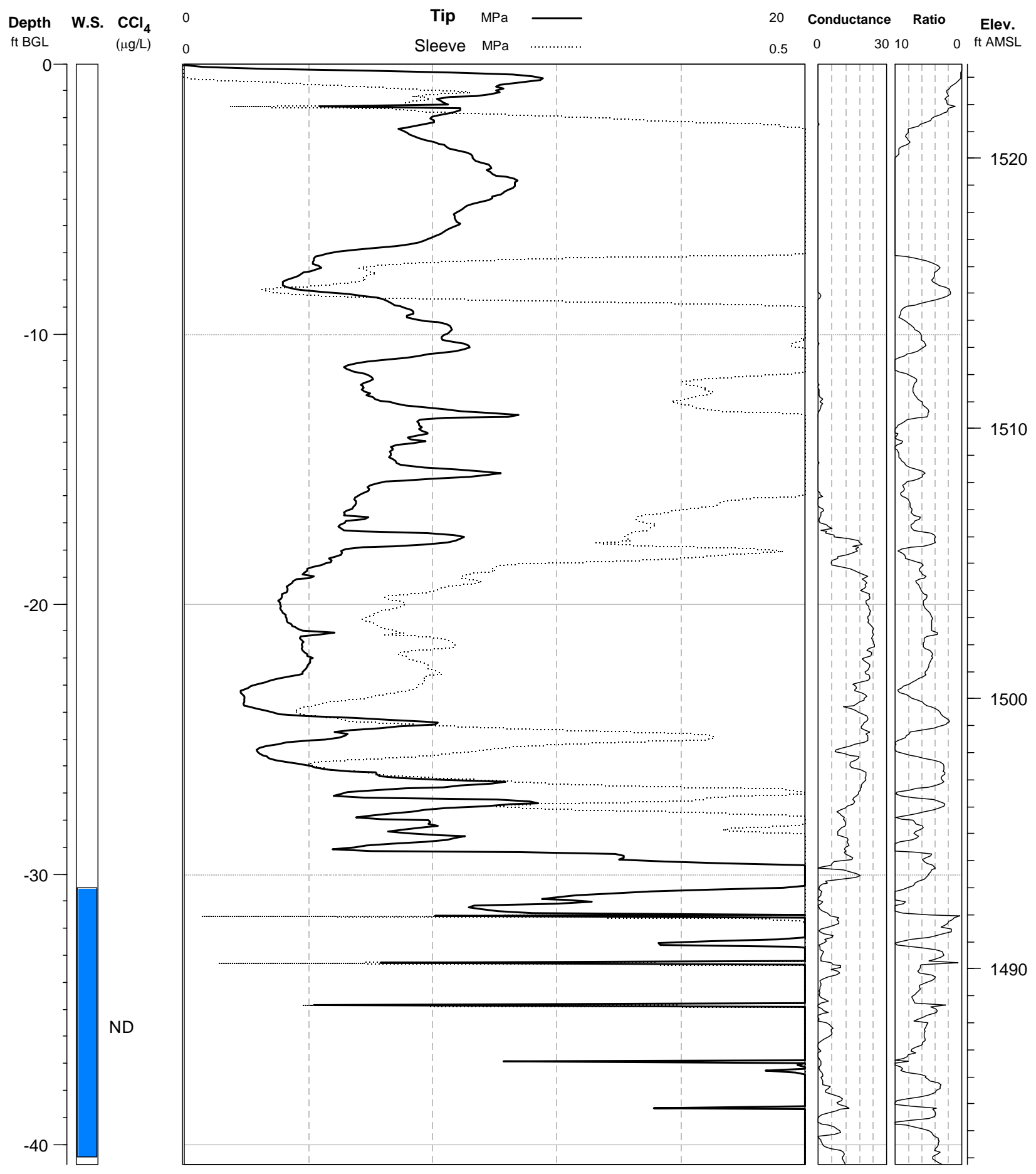




\section{Argonne National Laboratory}

Project: Inman

Geologist: Eugene Yan

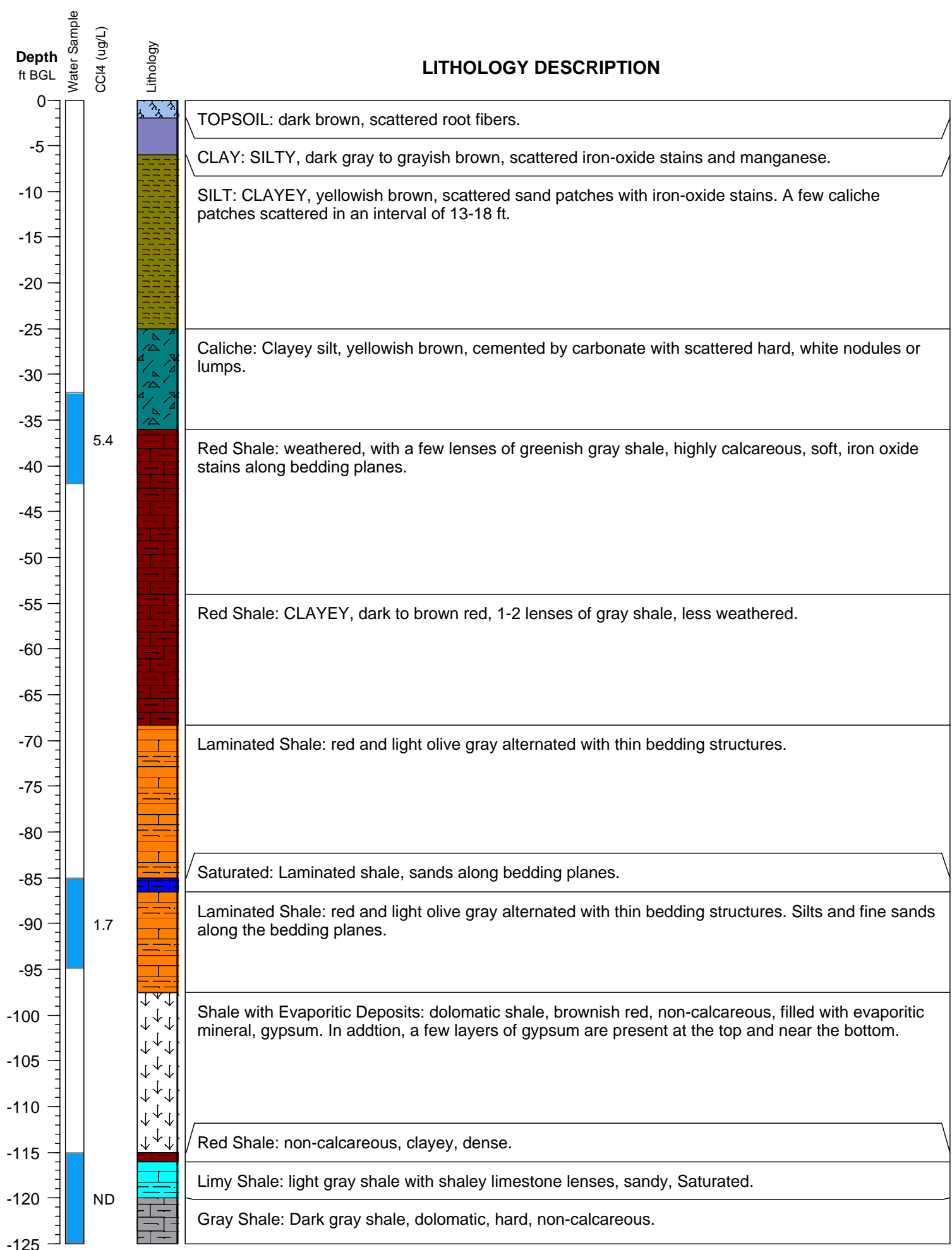

Well ID: MW14 (Wells: MW14M, MW14D)/MW01

Log Date: August 23, 2011
Depth: $125 \mathrm{ft} \mathrm{BGL}$
Elevation: $1525.267 \mathrm{ft}$ 


\section{Argonne National Laboratory}

Project: Inman

Geologist: Eugene Yan

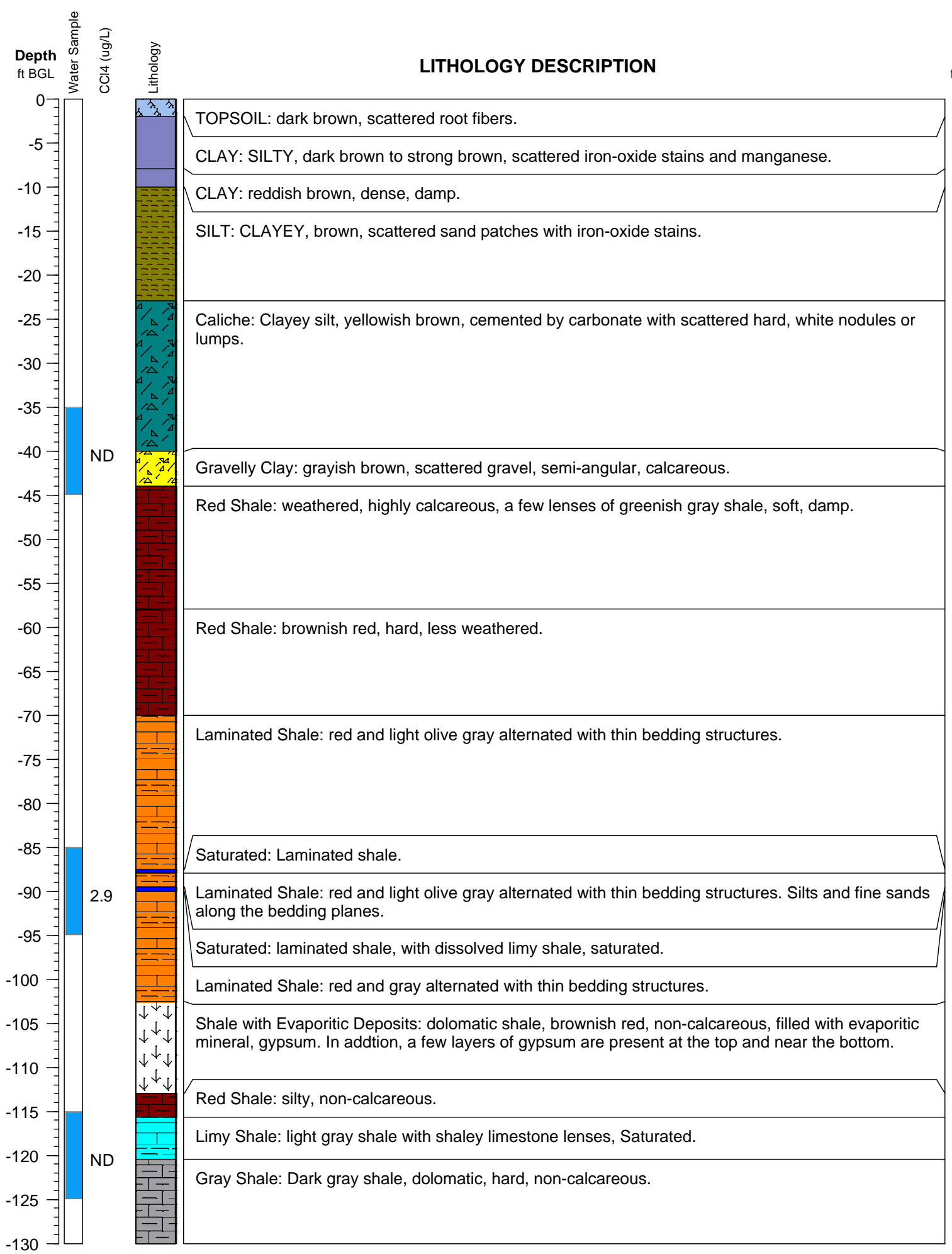

Well ID: MW15 (Wells: MW15S, MW15M, MW15D)

Log Date: August 24, 2011
Elevation: $1526.08 \mathrm{ft}$

Depth: $130 \mathrm{ft} \mathrm{BGL}$

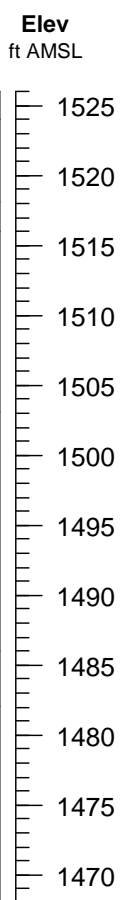

1465

1460

1455

1450

1445

1440

1435

1430

1425

1420

1415

1410

1405

1400 
Argonne National Laboratory

Project: Inman

Geologist: Eugene Yan

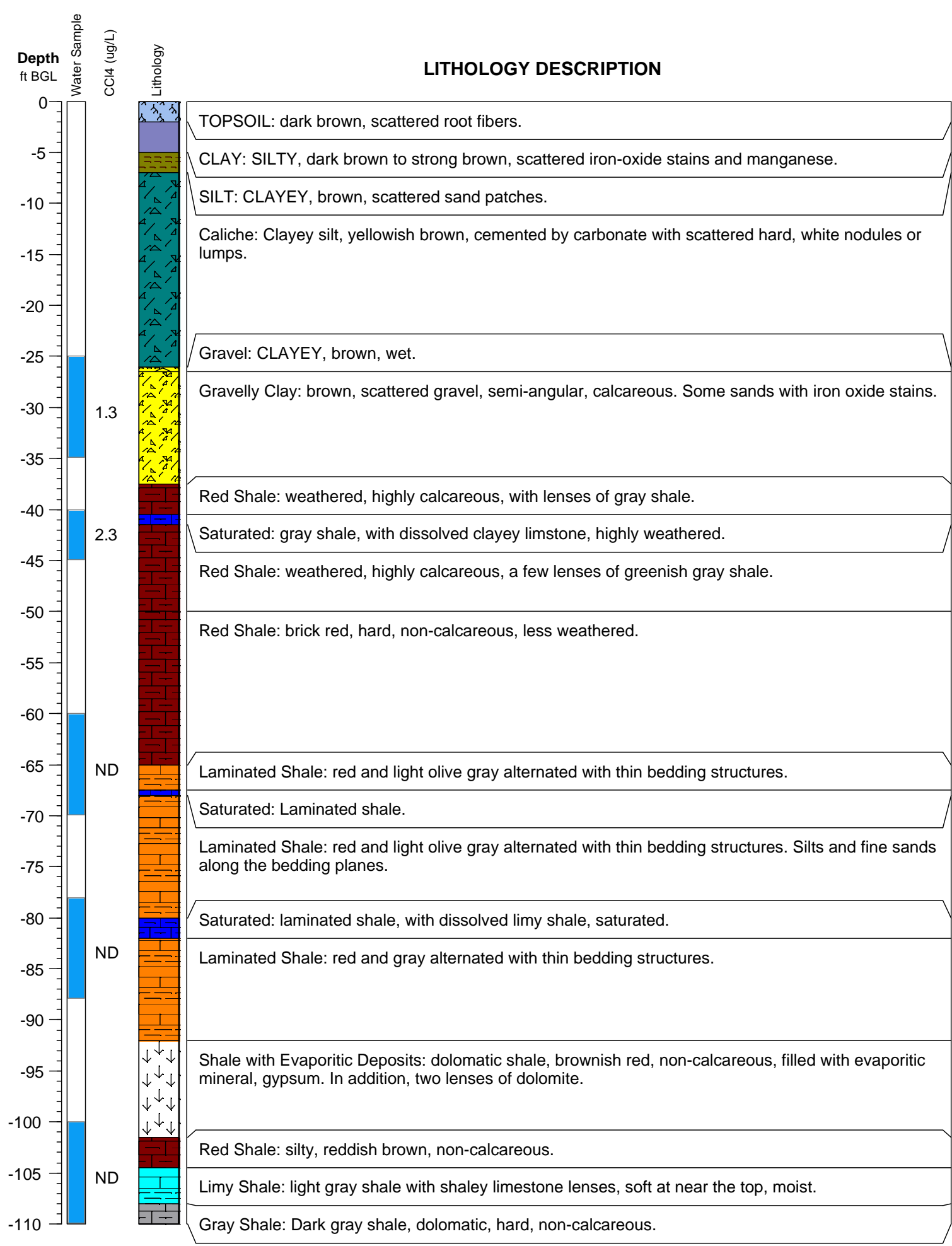

Well ID: MW16 (Wells: MW16S1, MW16S2, MW16M1, MW16M2, MW16D)

Elevation: $1507.678 \mathrm{ft}$

Depth: $110 \mathrm{ft} \mathrm{BGL}$
Log Date: August 25, 2011 
Argonne National Laboratory Project: Inman

Geologist: Eugene Yan

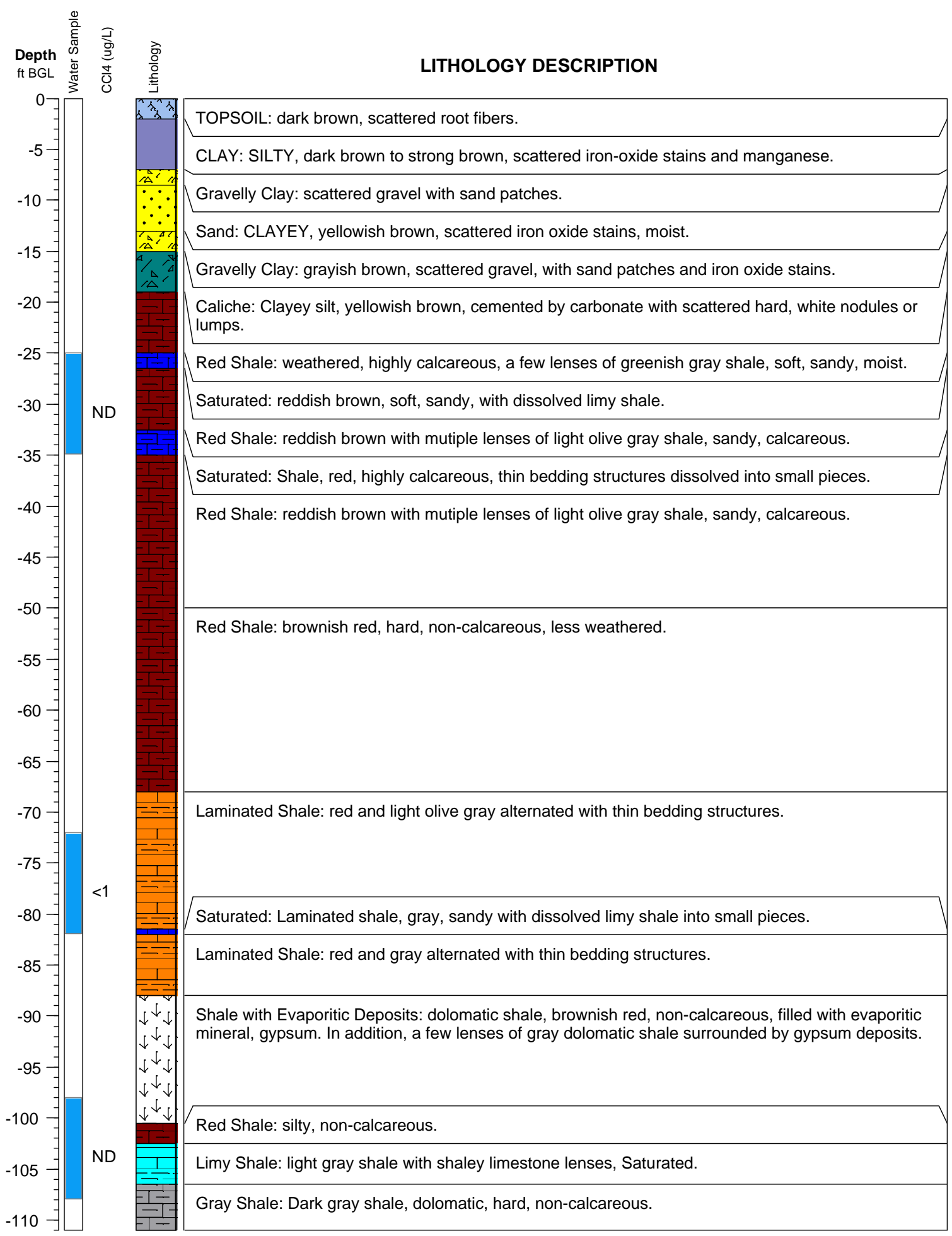

Well ID: MW17 (Wells: MW17S, MW17M, MW17D)

Elevation: $1504.672 \mathrm{ft}$

Depth: $111 \mathrm{ft} \mathrm{BGL}$
Log Date: August 27, 2011 


\section{Argonne National Laboratory}

Project: Inman

Geologist: Eugene Yan

SITHOLOGY DESCRIPTION

Elevation: $1526.29 \mathrm{ft}$

Depth: $125 \mathrm{ft} \mathrm{BGL}$
Well ID: MW18 (Wells MW18S, MW18M, MW18D)

Log Date: August 29, 2011

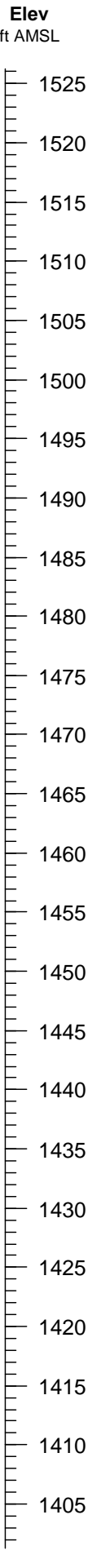




\section{Argonne National Laboratory}

Project: Inman

Geologist: Eugene Yan
Elevation: $1525.325 \mathrm{ft}$

Depth: $100 \mathrm{ft} \mathrm{BGL}$
Well ID: MW19 (Wells: MW19S, MW19M)

Log Date: August 30, 2011

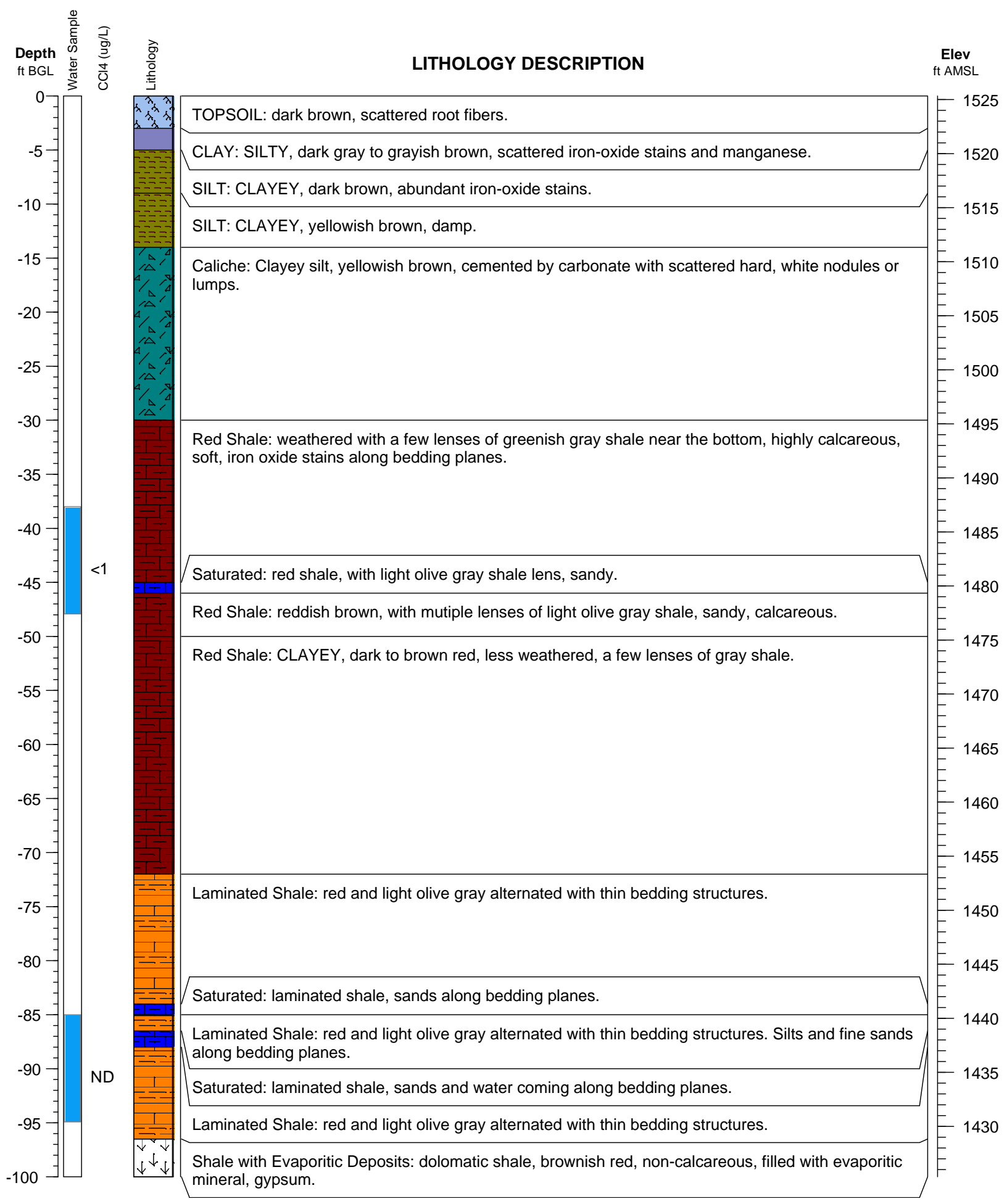




\section{Argonne National Laboratory}

Project: Inman

Geologist: Eugene Yan
Elevation: $1510.99 \mathrm{ft}$

Depth: $89.5 \mathrm{ft} \mathrm{BGL}$
Well ID: MW20 (Wells: MW20S, MW20M)

Log Date: September 13, 2011

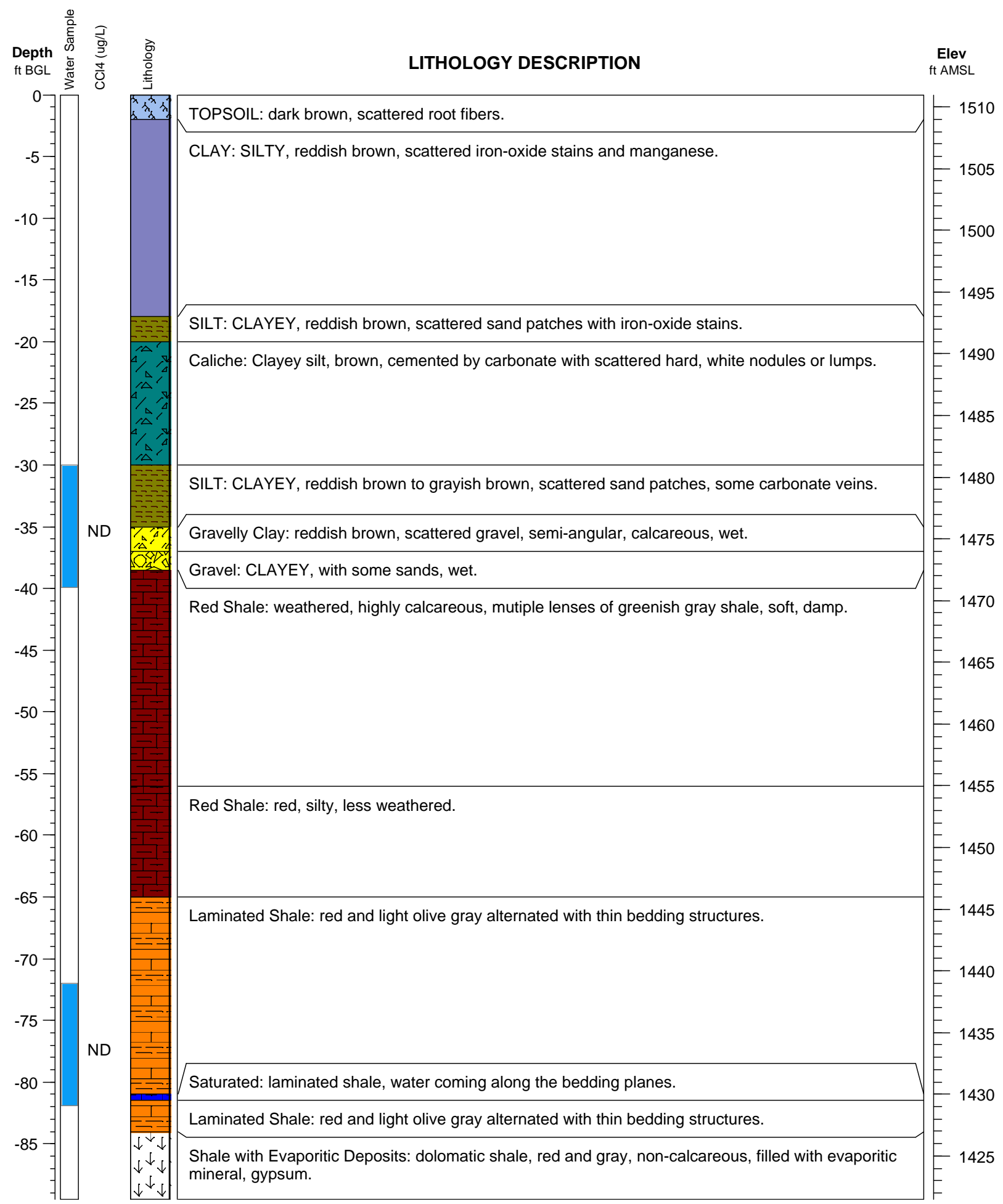




\section{Argonne National Laboratory}

Project: Inman

Geologist: Eugene Yan
Elevation: $1525.521 \mathrm{ft}$

Depth: $40 \mathrm{ft} \mathrm{BGL}$
Well ID: TI01 (MW02)

Log Date: November 04, 2009

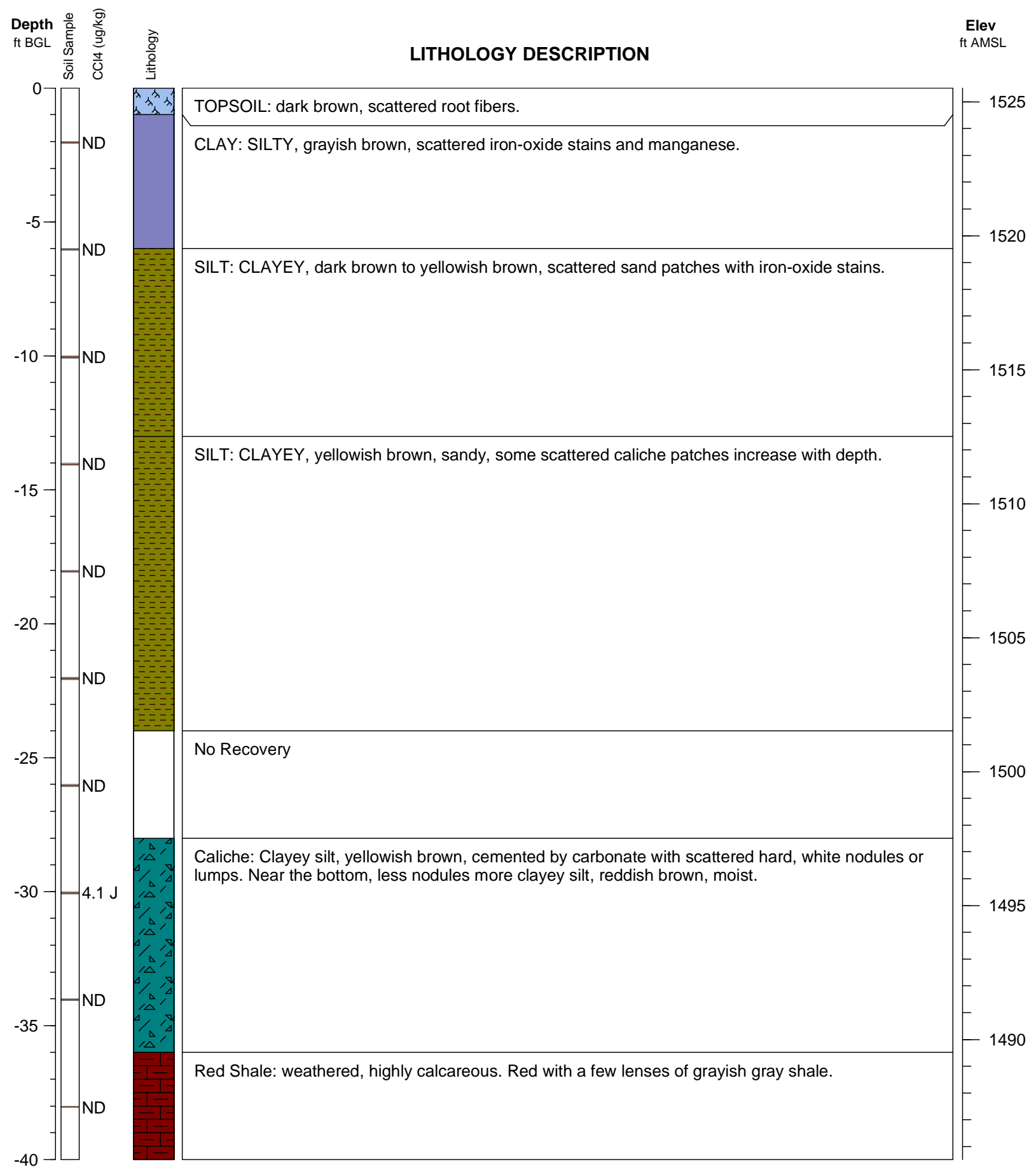


Argonne National Laboratory Project: Inman

Geologist: Eugene Yan
Well ID: TI02

Elevation: $1525.6 \mathrm{ft}$ (estimated)

Depth: $40 \mathrm{ft}$

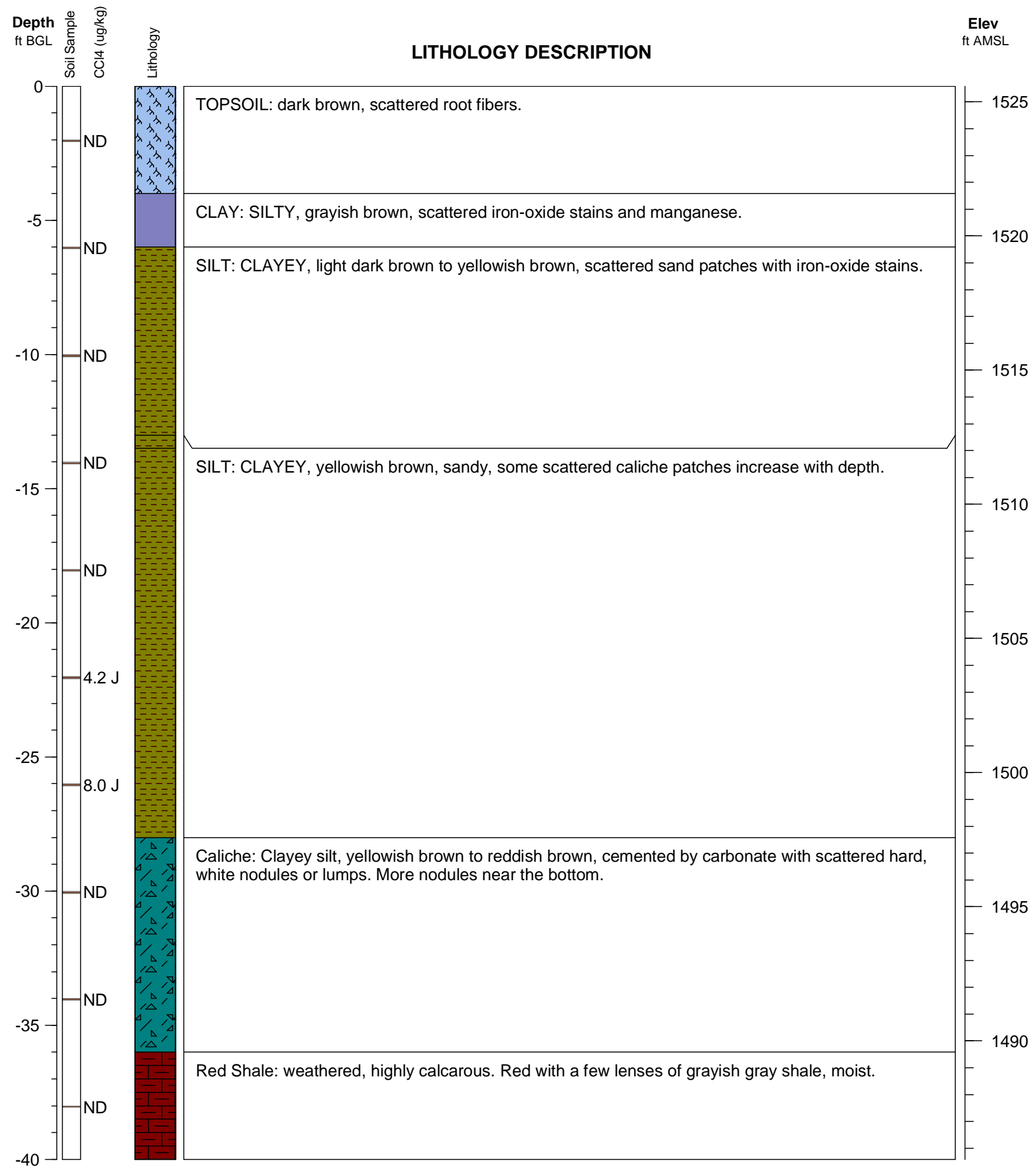




\section{Argonne National Laboratory}

Project: Inman

Geologist: Eugene Yan
Boring ID: TI02

Elevation: $1525.6 \mathrm{ft}$ (estimated)

Depth: $40 \mathrm{ft}$ BGL

Log Date: November 04, 2009

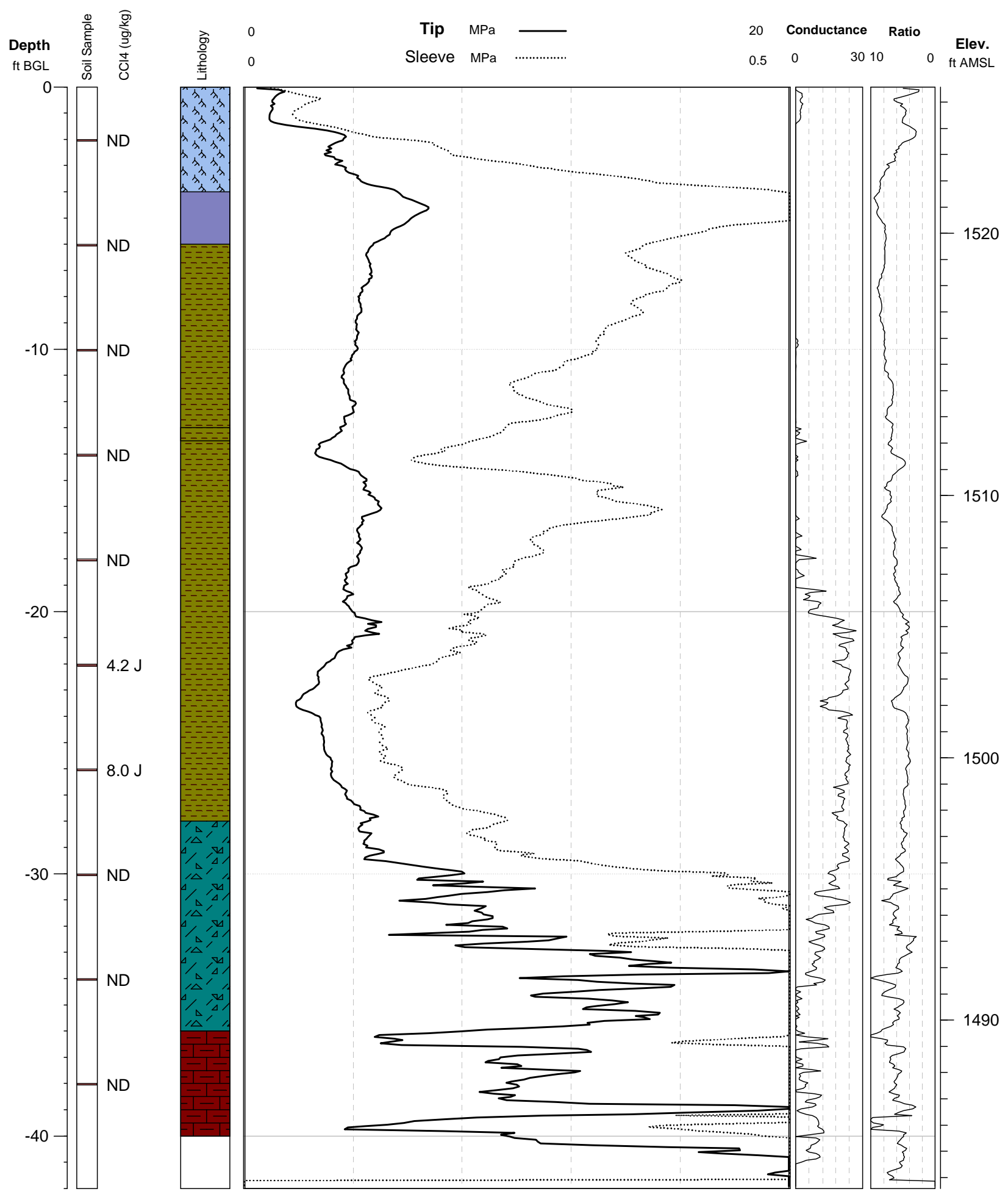


Argonne National Laboratory

Project: Inman

Geologist: Eugene Yan
Elevation: $1526 \mathrm{ft}$ (estimated)

Depth: $42 \mathrm{ft}$
Well ID: TI03

Log Date: November 05, 2009

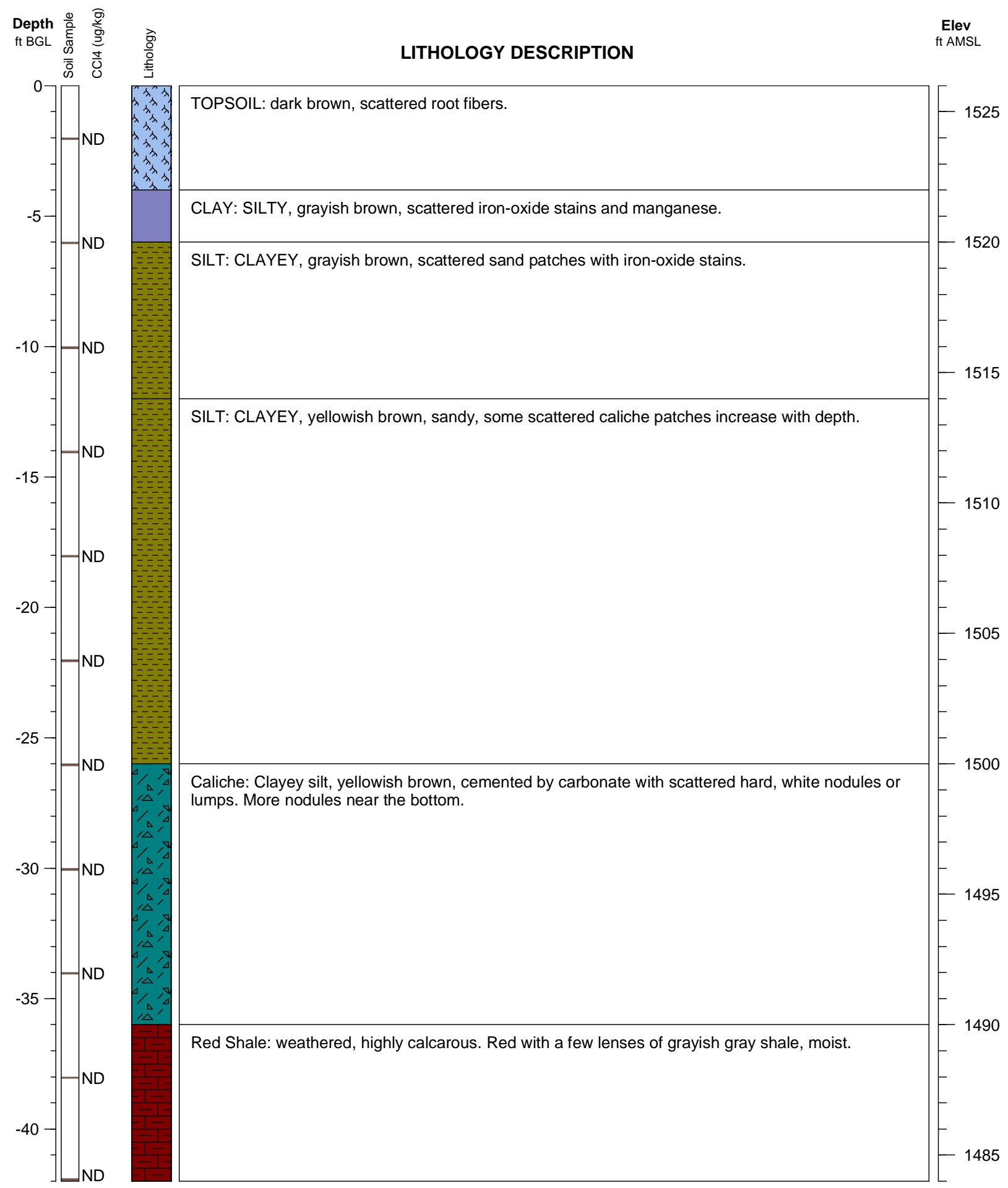




\section{Argonne National Laboratory}

Project: Inman

Geologist: Eugene Yan
Elevation: $1526 \mathrm{ft}$ (estimated)

Depth: $42 \mathrm{ft} B G L$
Boring ID: TI03

Log Date: November 05, 2009

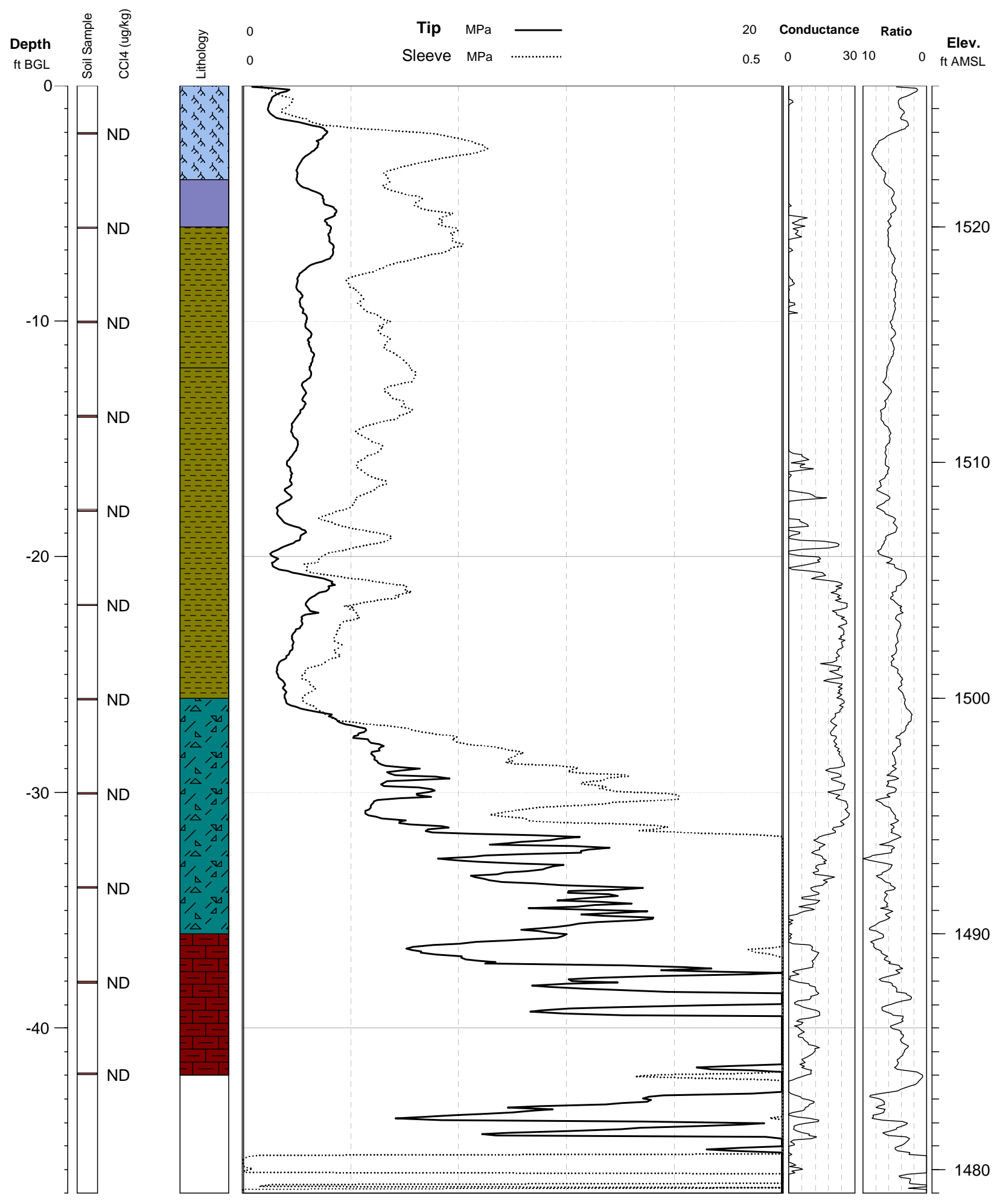




\section{Argonne National Laboratory}

Project: Inman

Geologist: Eugene Yan
Elevation: $1526.235 \mathrm{ft}$

Depth: $40 \mathrm{ft} B G L$

\section{Well ID: TI04/TI04b (MW04)}

Log Date: November 05, 2009

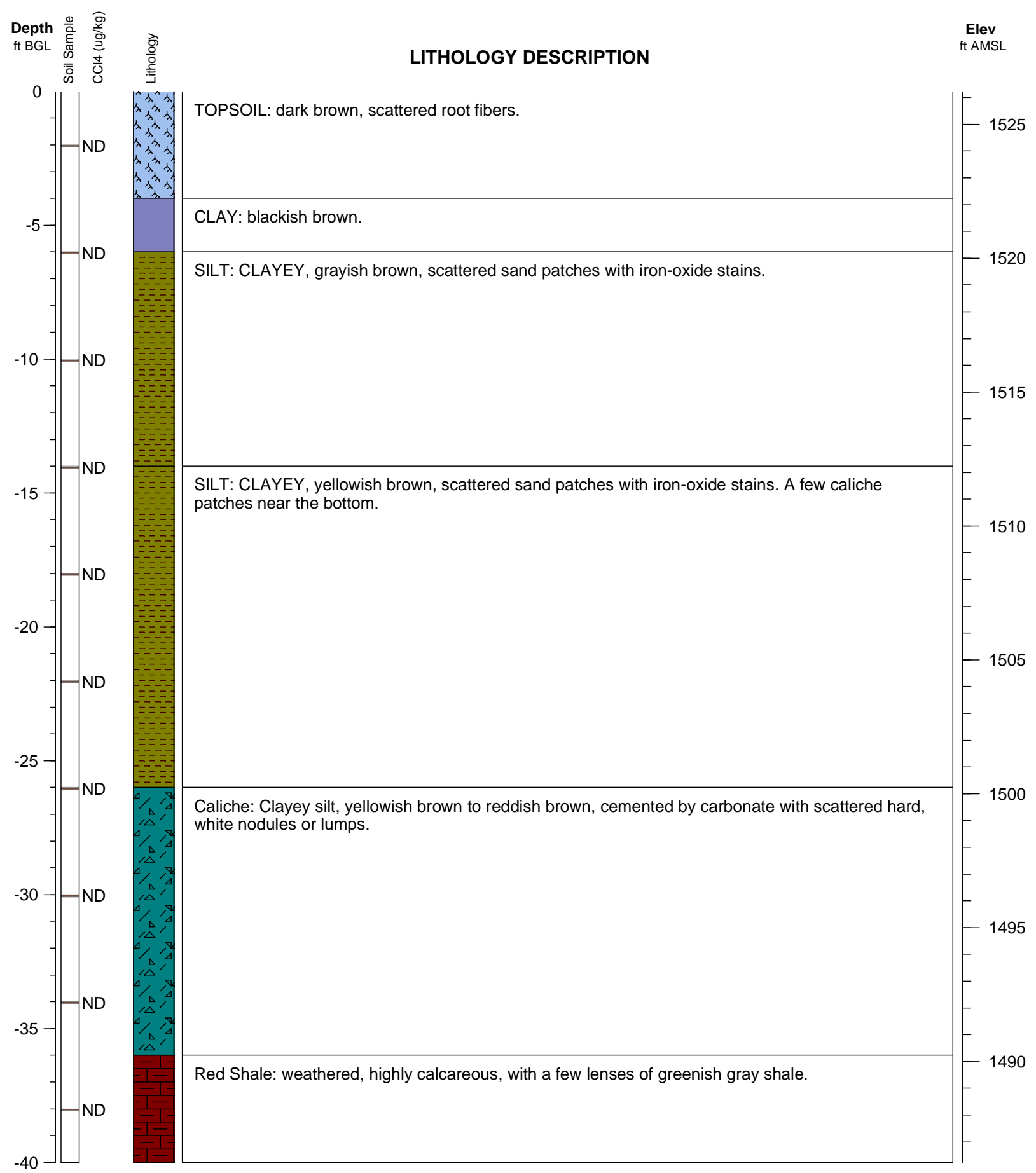


Argonne National Laboratory Project: Inman

Geologist: Eugene Yan
Well ID: TI05

Elevation: $1526.1 \mathrm{ft}$ (estimated)

Depth: $40 \mathrm{ft}$

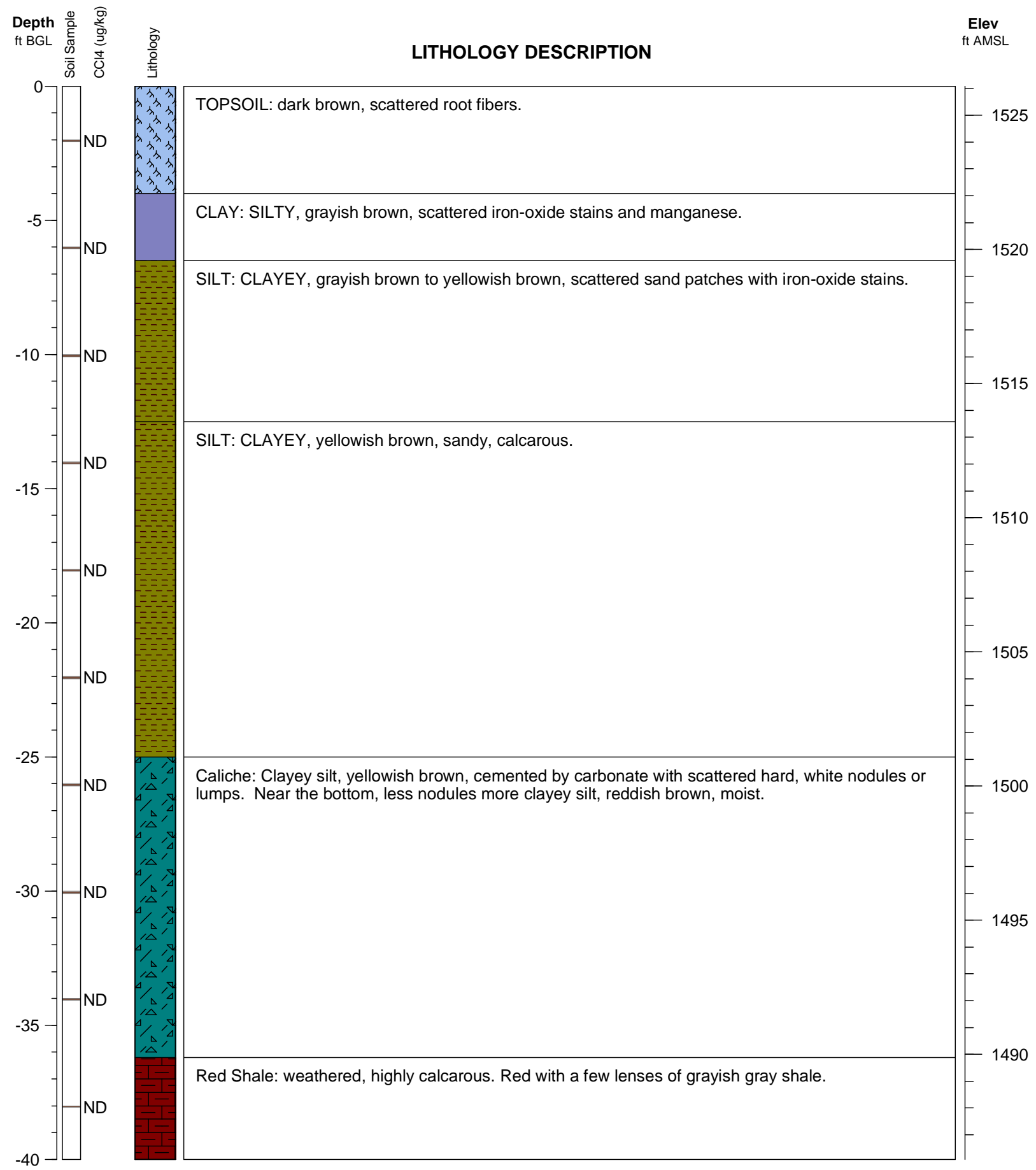




\section{Argonne National Laboratory}

Project: Inman

Geologist: Eugene Yan
Boring ID: TI05

Elevation: $1526.1 \mathrm{ft}$ (estimated)

Depth: $42 \mathrm{ft}$ BGL

Log Date: November 06, 2009

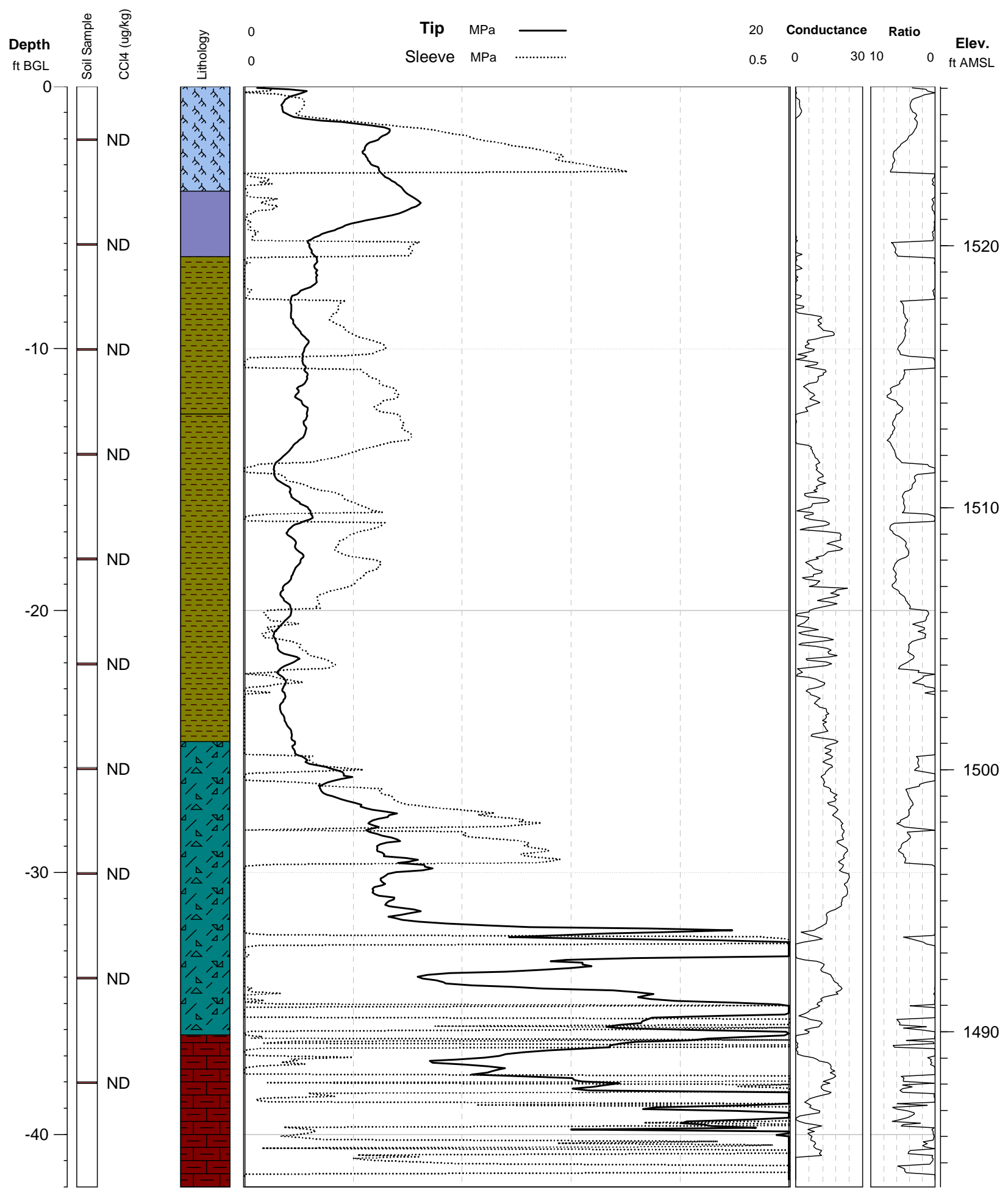




\section{Argonne National Laboratory}

Project: Inman

Geologist: Eugene Yan
Elevation: $1525.716 \mathrm{ft}$

Depth: $40 \mathrm{ft} B G L$
Well ID: TI06 (MW03)

Log Date: November 06, 2009

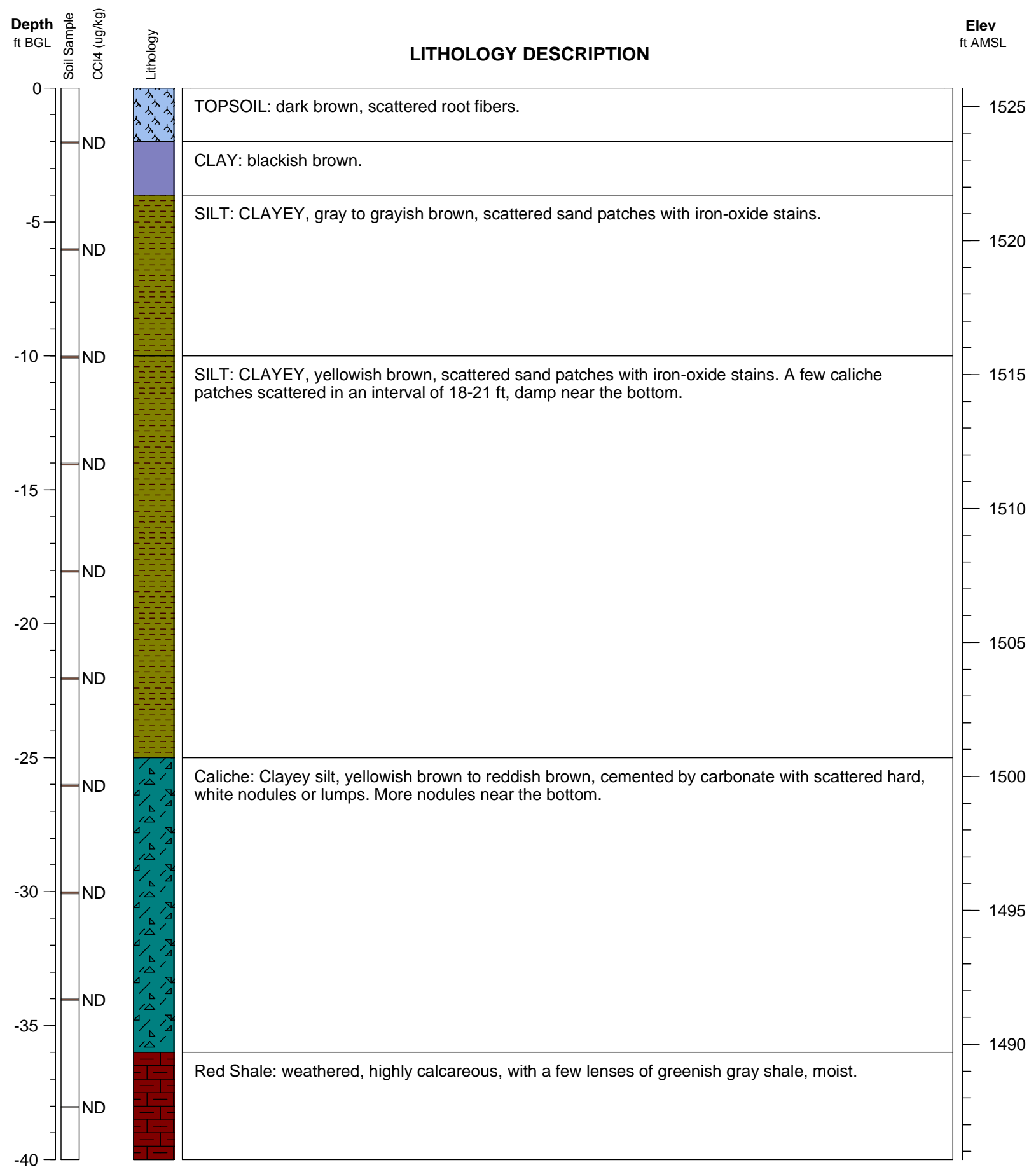


Argonne National Laboratory Project: Inman

Geologist: Eugene Yan
Well ID: TI07

Elevation: $1525.6 \mathrm{ft}$ (estimated)

Depth: $40 \mathrm{ft}$

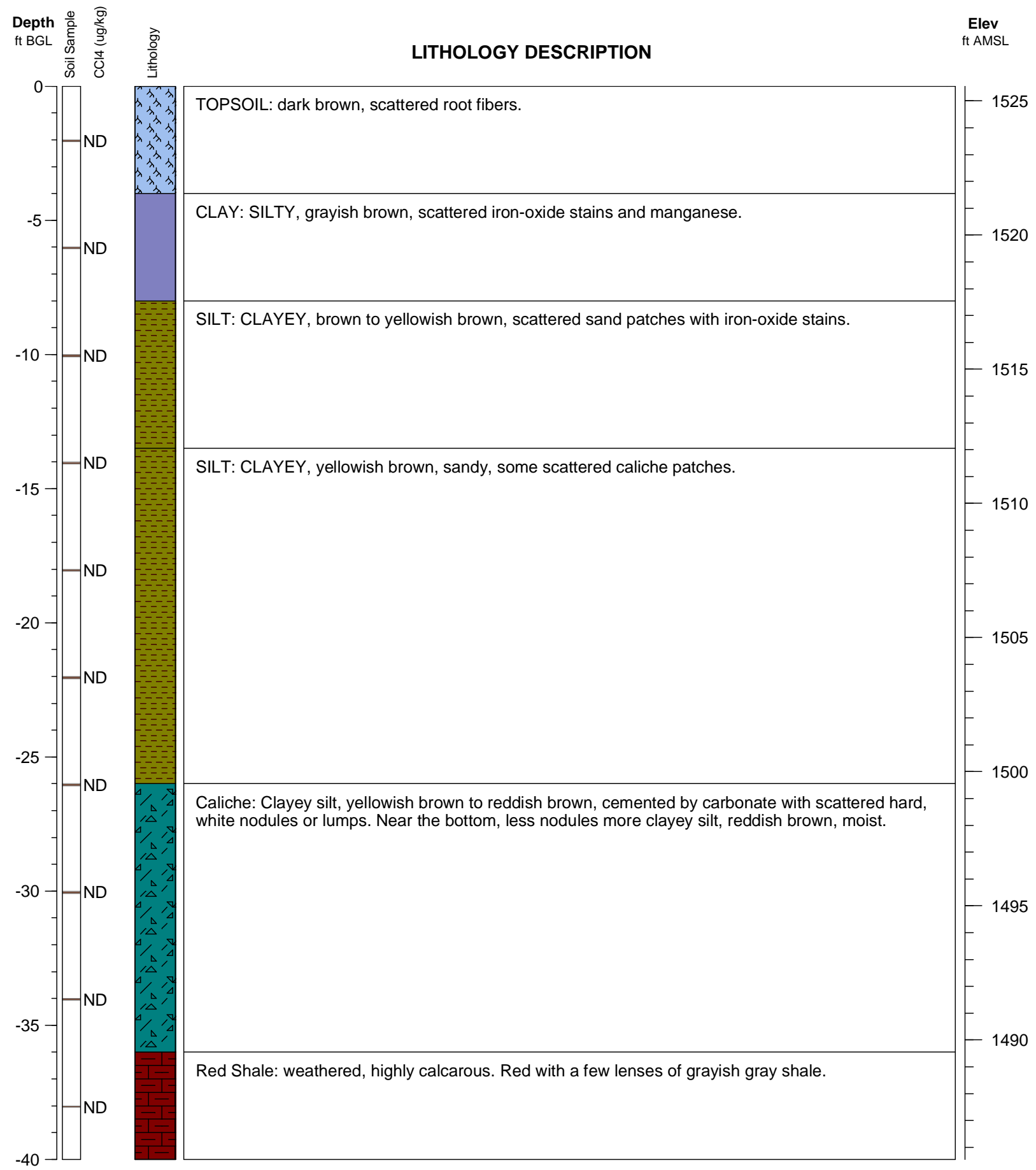




\section{Argonne National Laboratory}

Project: Inman

Geologist: Eugene Yan
Elevation: $1525.601 \mathrm{ft}$

Depth: $40 \mathrm{ft} \mathrm{BGL}$

\section{Well ID: T108 (MW05*)}

Log Date: November 07, 2009

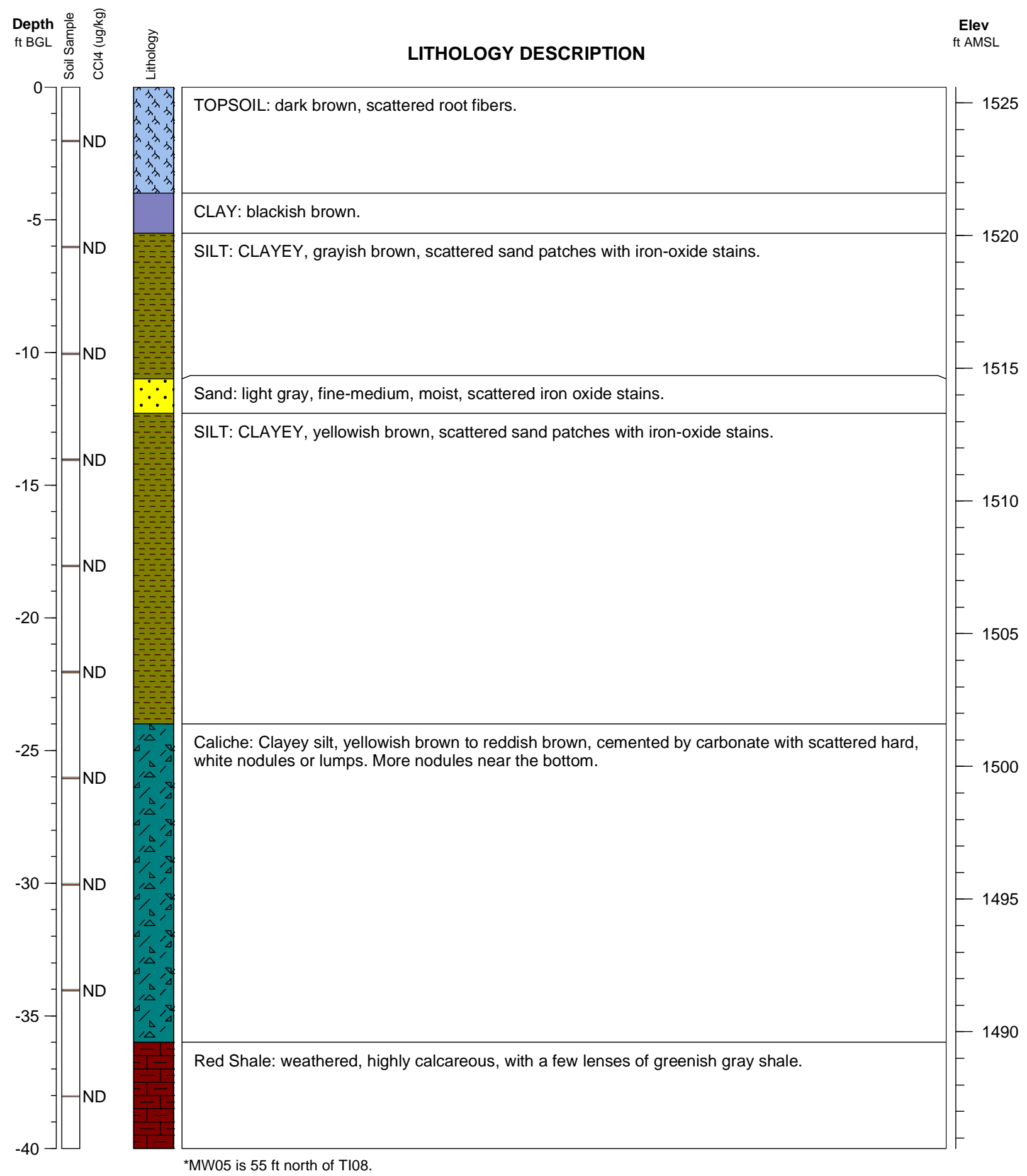


Argonne National Laboratory Project: Inman

Geologist: Eugene Yan
Well ID: TI09

Elevation: $1525.8 \mathrm{ft}$ (estimated) Depth: $40 \mathrm{ft}$

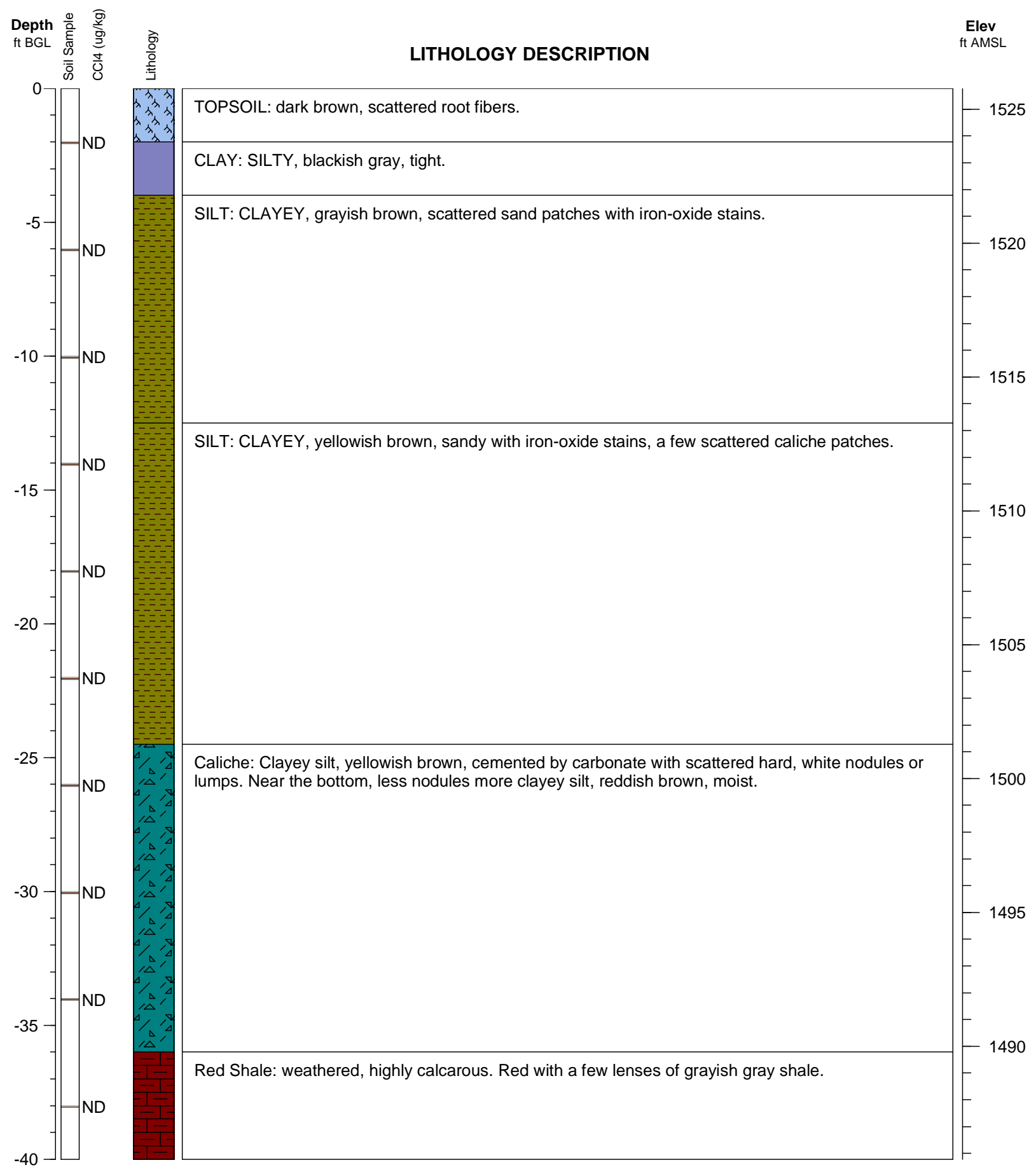


Argonne National Laboratory Project: Inman

Geologist: Eugene Yan
Well ID: TI10

Elevation: $1525.6 \mathrm{ft}$ (estimated)

Depth: $40 \mathrm{ft} \mathrm{BGL}$

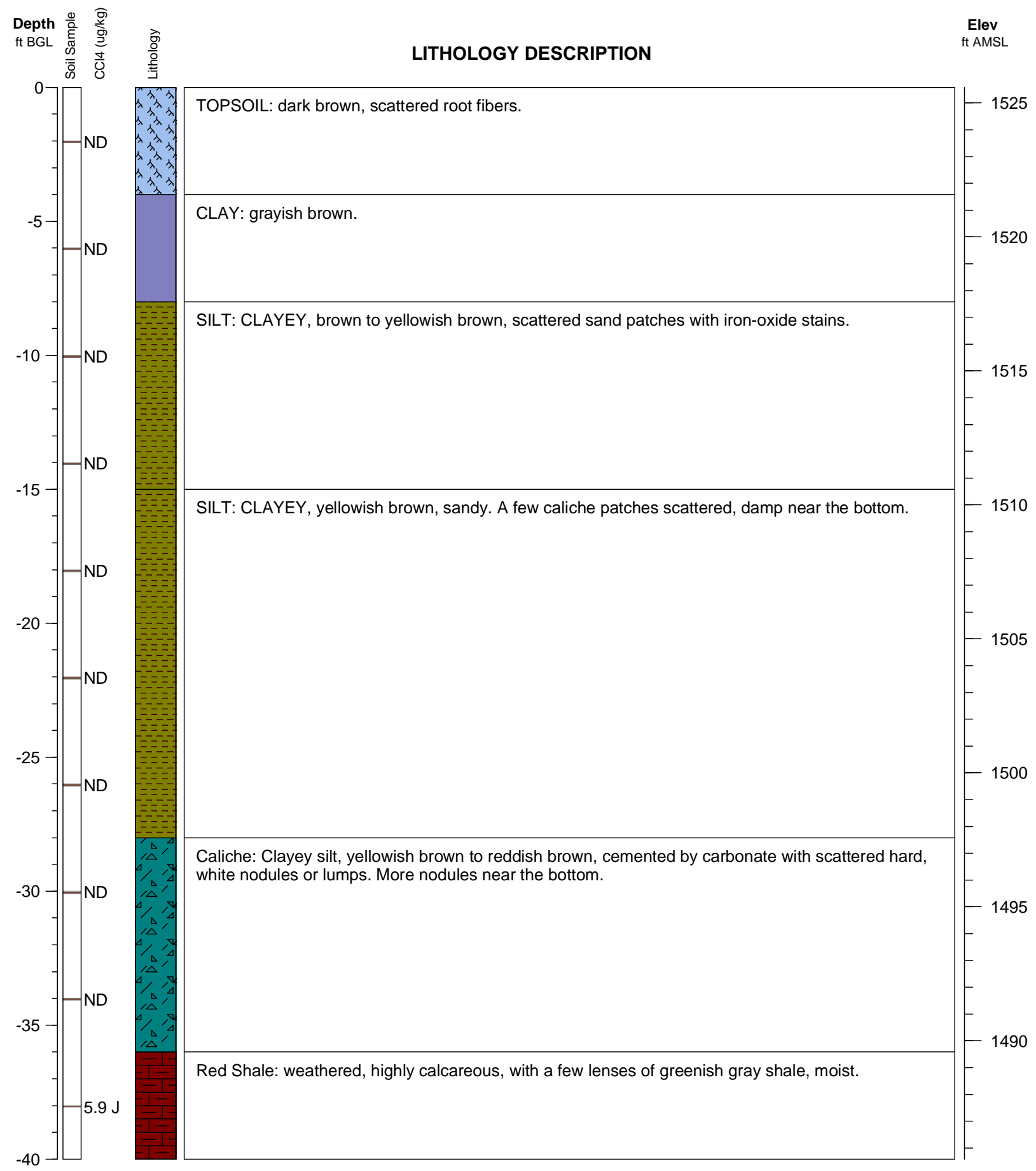




\section{Argonne National Laboratory}

Project: Inman

Geologist: Eugene Yan
Elevation: $1525.081 \mathrm{ft}$

Depth: $42 \mathrm{ft}$ BGL
Well ID: TI11 (MW01)

Log Date: November 08, 2009

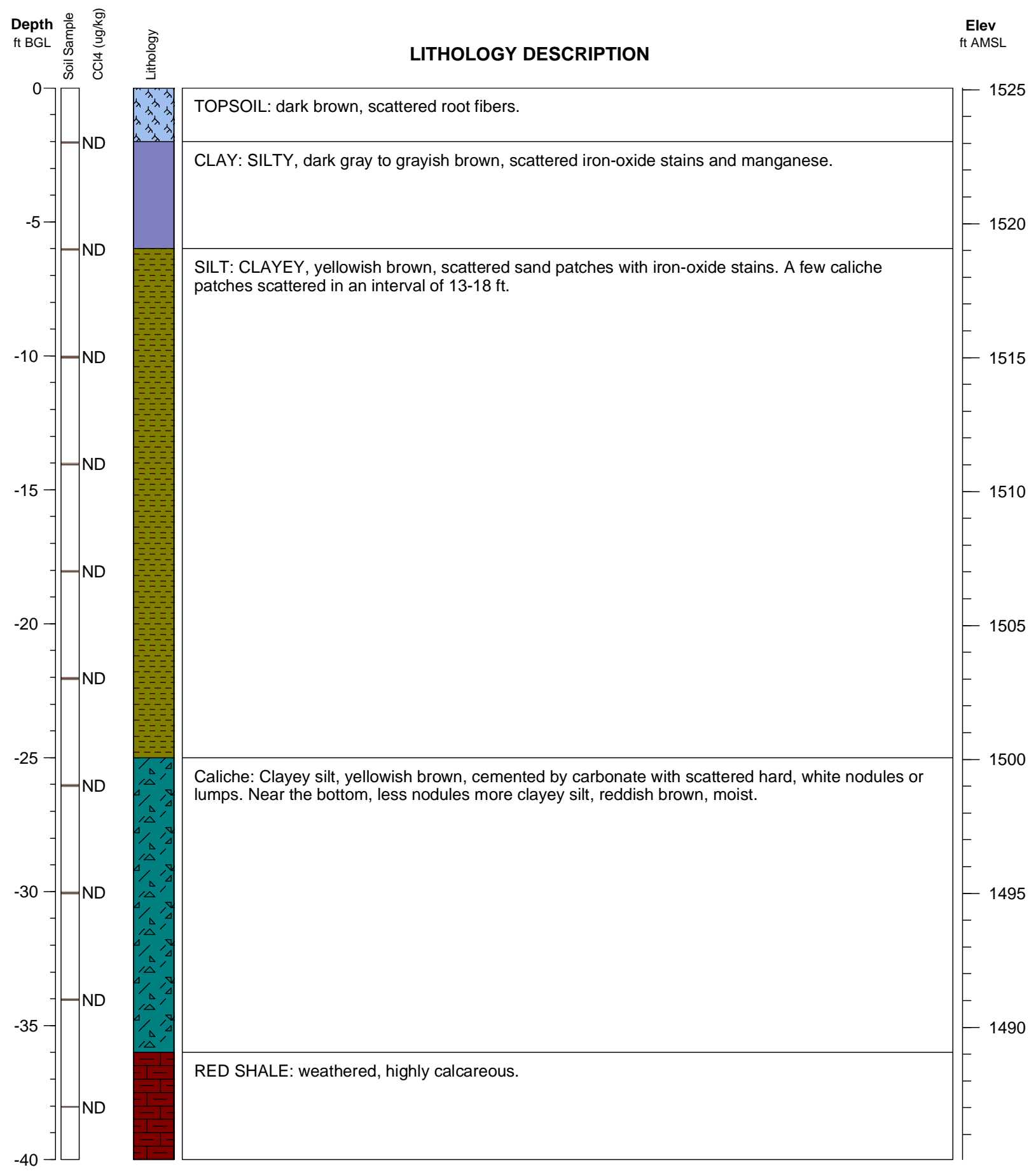




\section{Argonne National Laboratory}

Project: Inman

Geologist: Eugene Yan
Elevation: $1524.685 \mathrm{ft}$

Depth: $42 \mathrm{ft} \mathrm{BGL}$
Well ID: TI19

Log Date: November 10, 2010

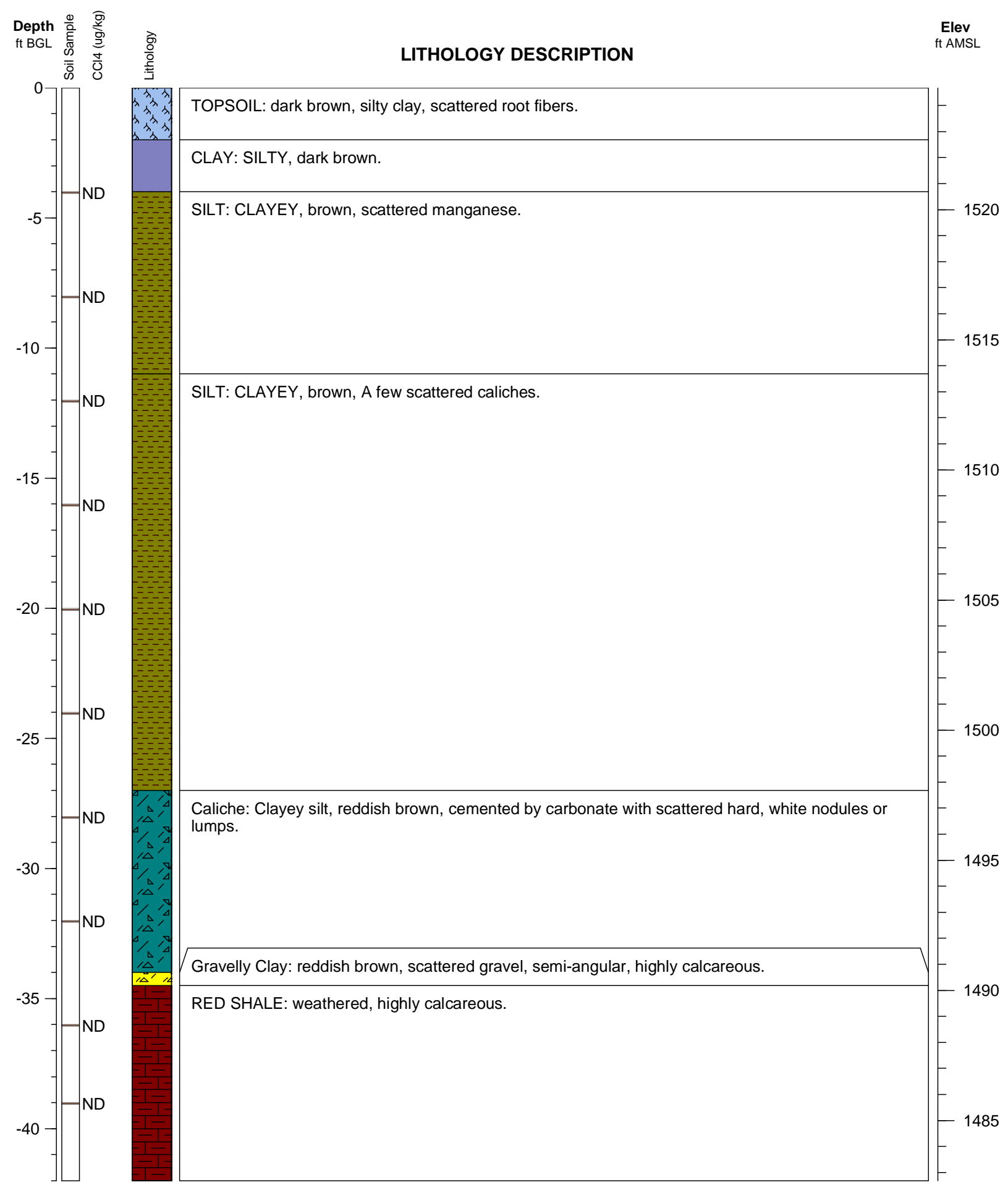




\section{Argonne National Laboratory}

Project: Inman

Geologist: Eugene Yan
Elevation: $1524.859 \mathrm{ft}$

Depth: $42 \mathrm{ft} \mathrm{BGL}$
Well ID: TI20

Log Date: November 11, 2010

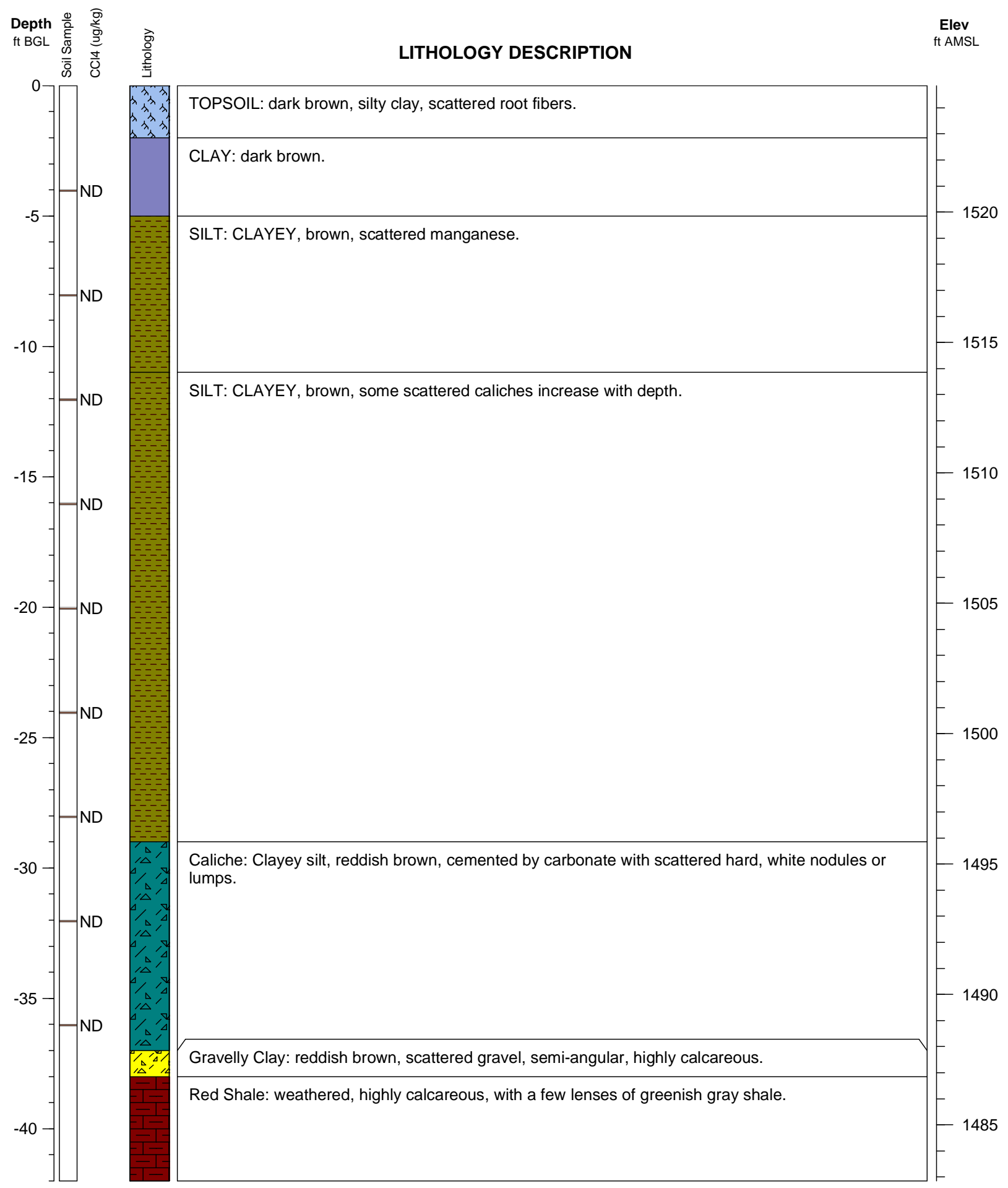




\section{Argonne National Laboratory}

Project: Inman

Geologist: Eugene Yan
Elevation: $1524.202 \mathrm{ft}$

Depth: $36 \mathrm{ft} \mathrm{BGL}$
Well ID: TI21

Log Date: November 11, 2010

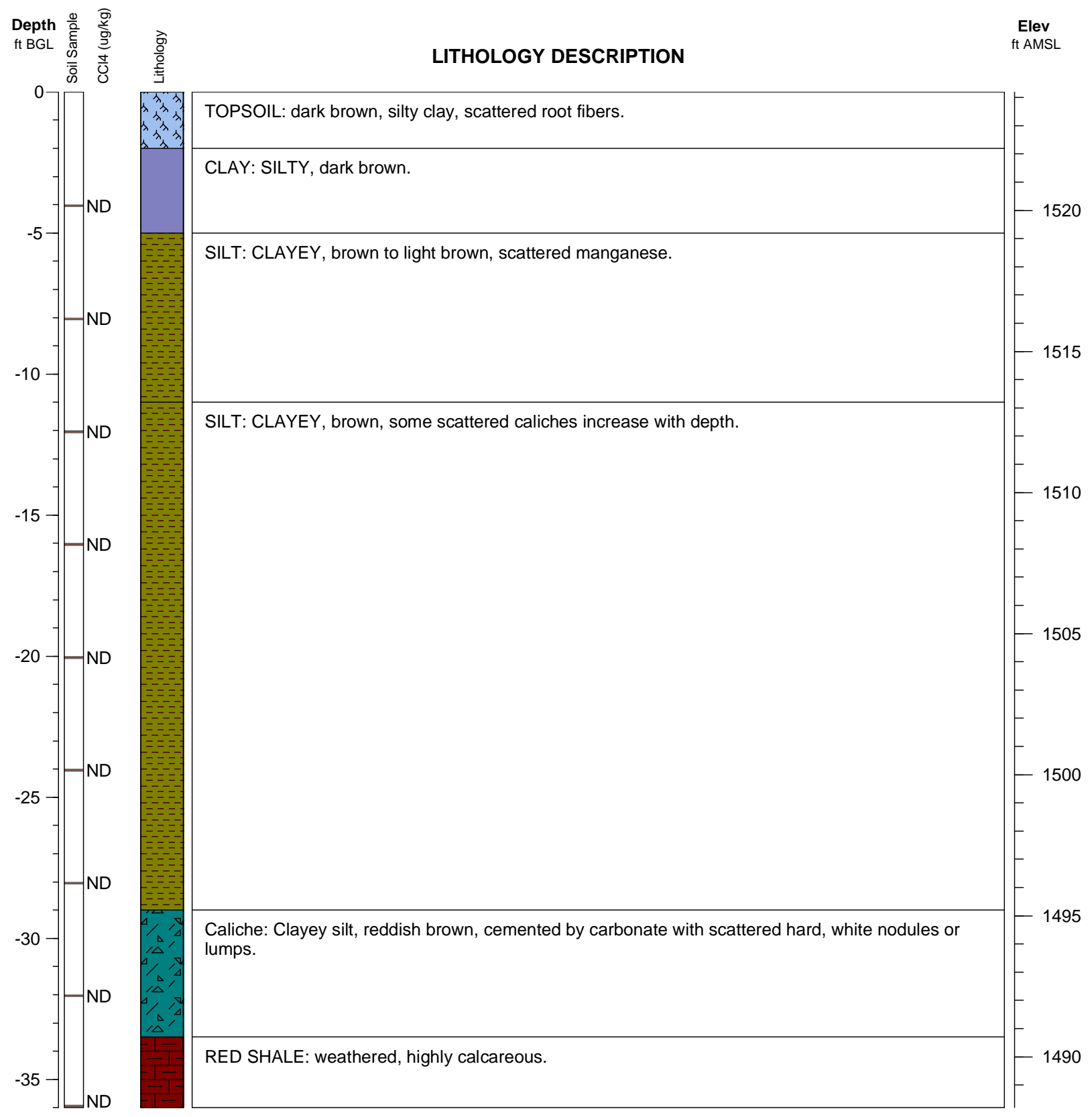




\section{Argonne National Laboratory}

Project: Inman

Geologist: Eugene Yan
Elevation: $1524.568 \mathrm{ft}$

Depth: $38 \mathrm{ft} \mathrm{BGL}$
Well ID: TI22

Log Date: November 11, 2010

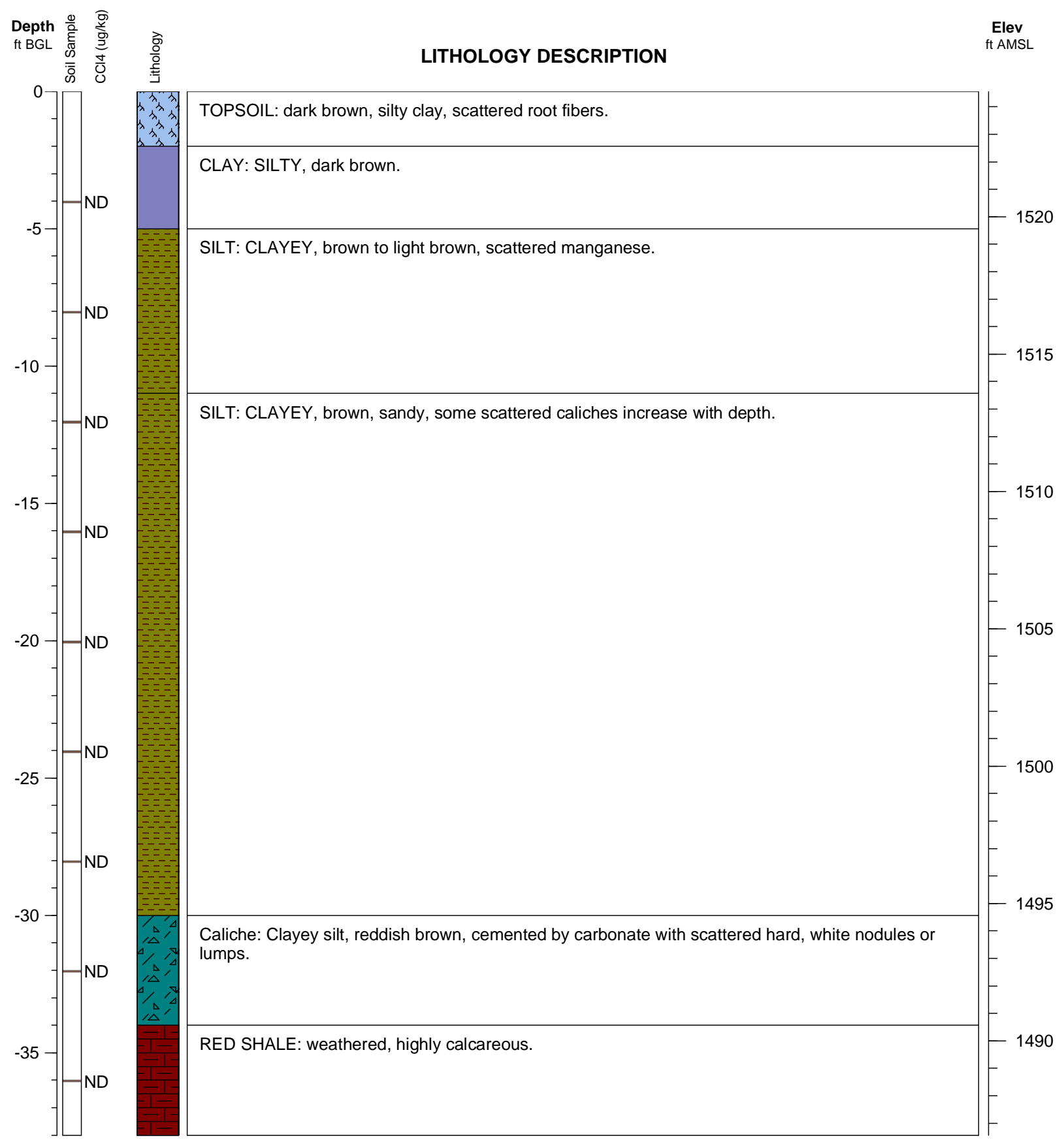




\section{Argonne National Laboratory}

Project: Inman

Geologist: Eugene Yan
Elevation: $1525.407 \mathrm{ft}$

Depth: $38.5 \mathrm{ft} \mathrm{BGL}$
Well ID: TI23

Log Date: November 11, 2010

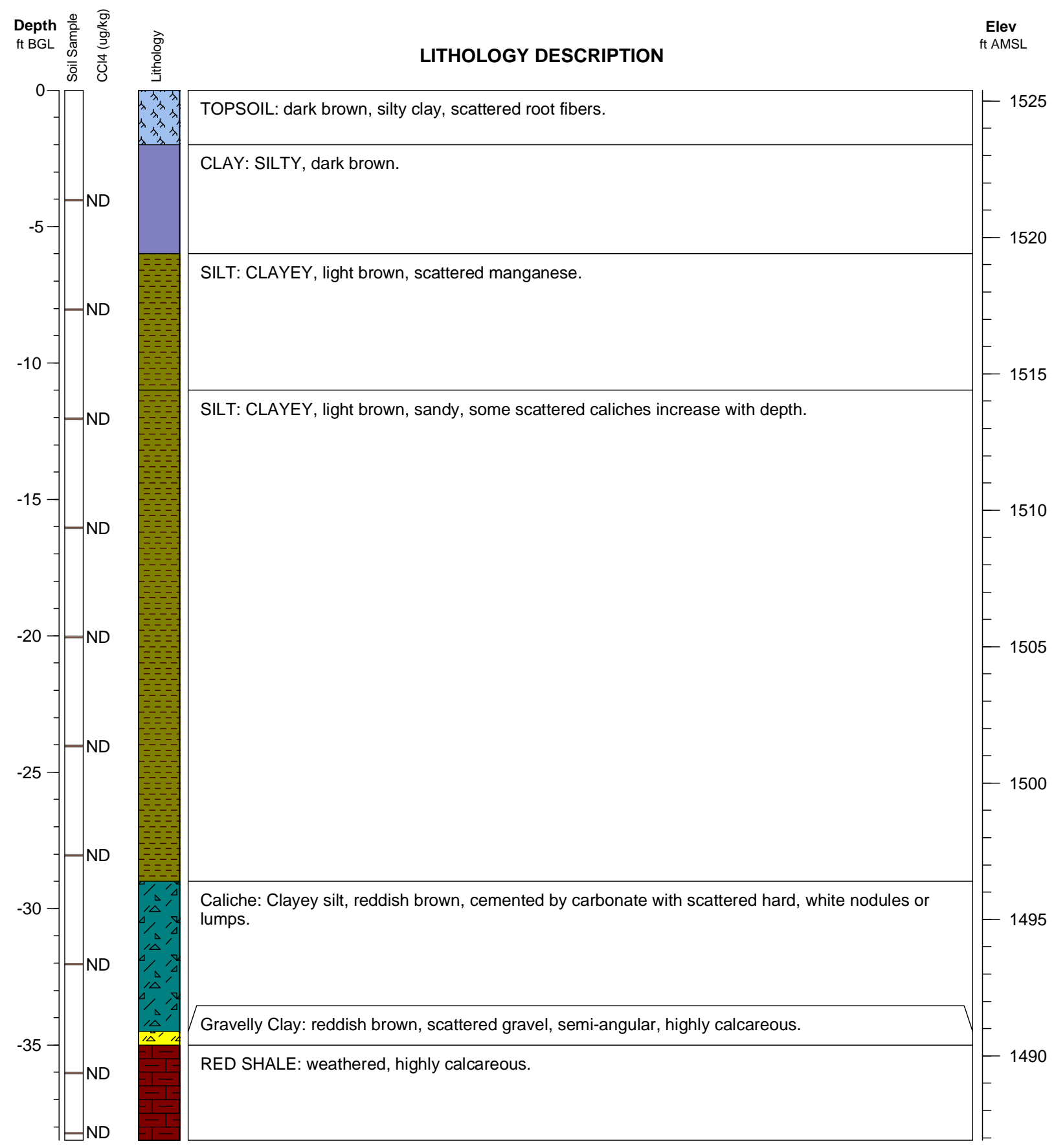




\section{Argonne National Laboratory}

Project: Inman

Geologist: Eugene Yan
Elevation: $1525.198 \mathrm{ft}$

Depth: $39 \mathrm{ft} \mathrm{BGL}$
Well ID: TI24

Log Date: November 12, 2010

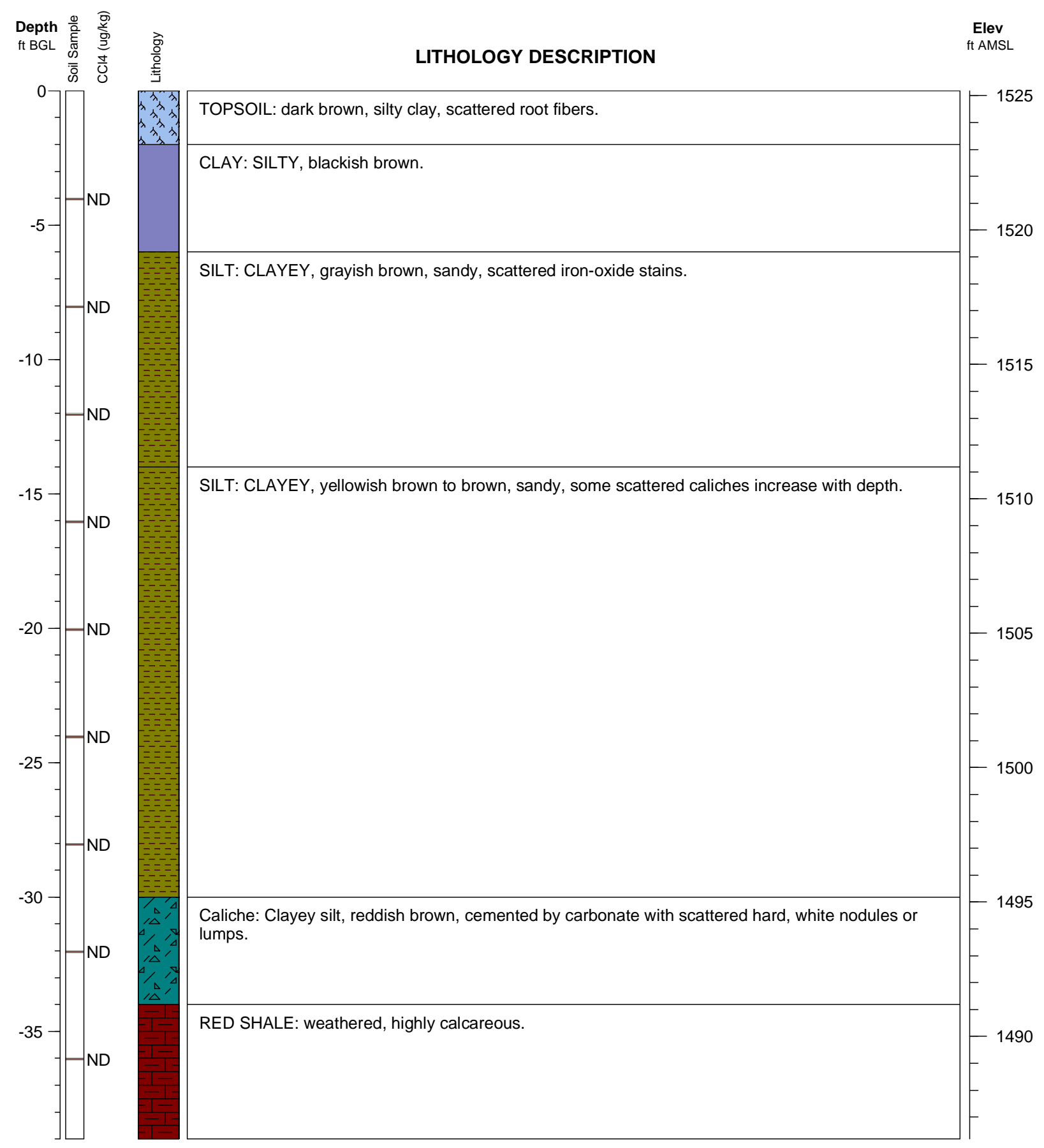




\section{Argonne National Laboratory}

Project: Inman

Geologist: Eugene Yan
Elevation: $1525.277 \mathrm{ft}$

Depth: $38 \mathrm{ft}$ BGL
Well ID: TI25

Log Date: November 12, 2010

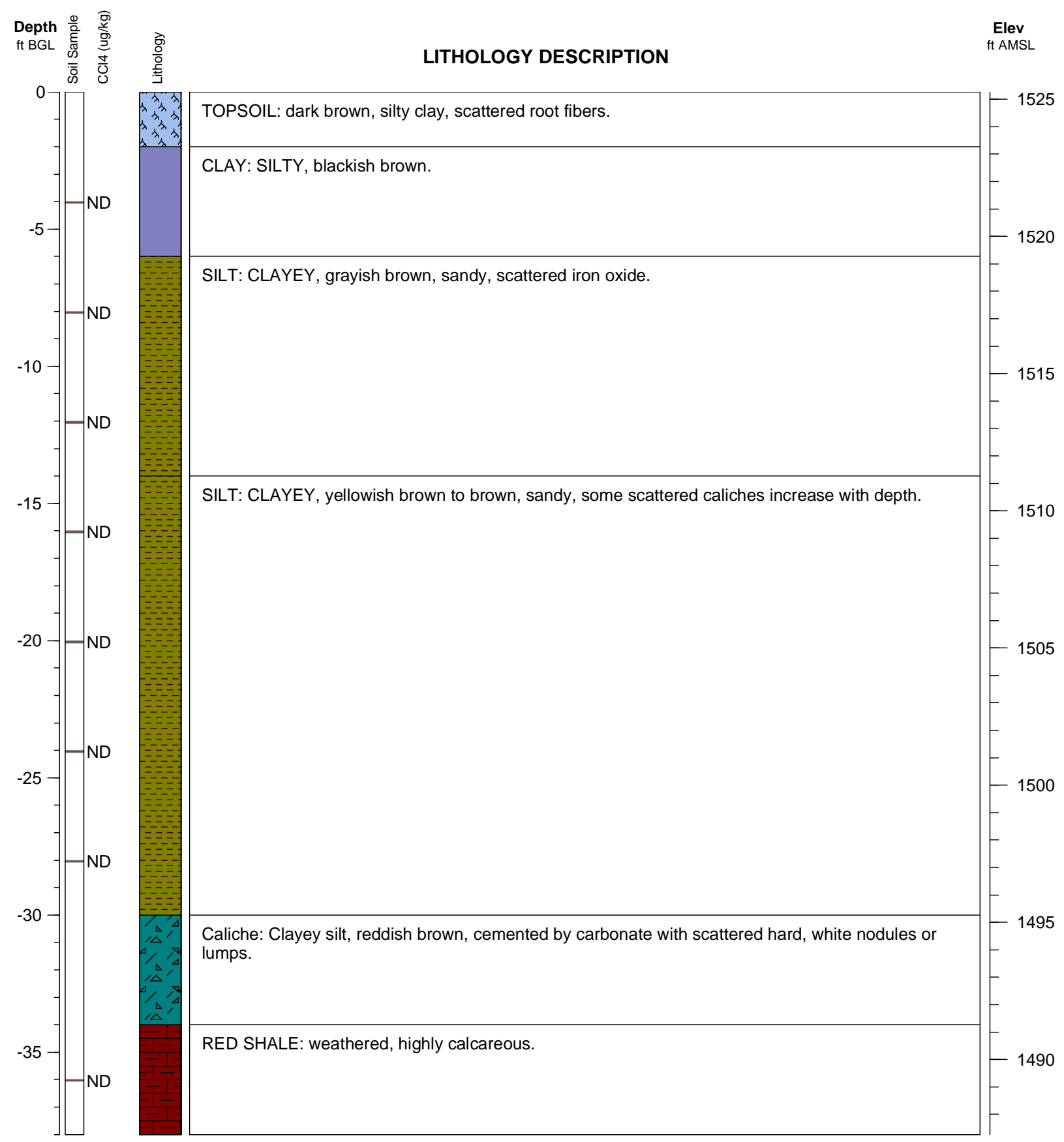




\section{Appendix C:}

Soil and Groundwater Sampling Data 
TABLE C.1 Sequence of sampling activities in 2009-2011.

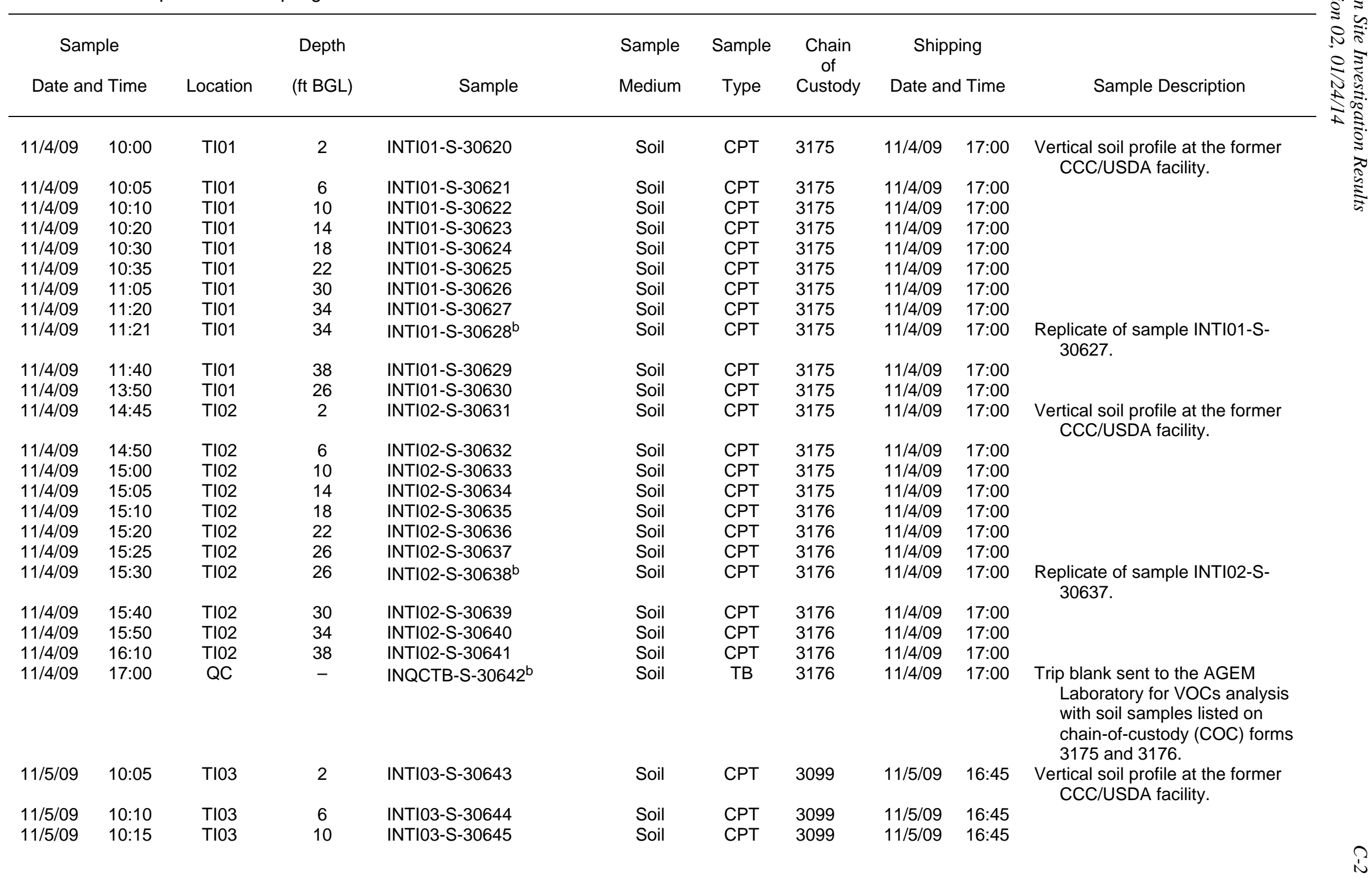


TABLE C.1 (Cont.)

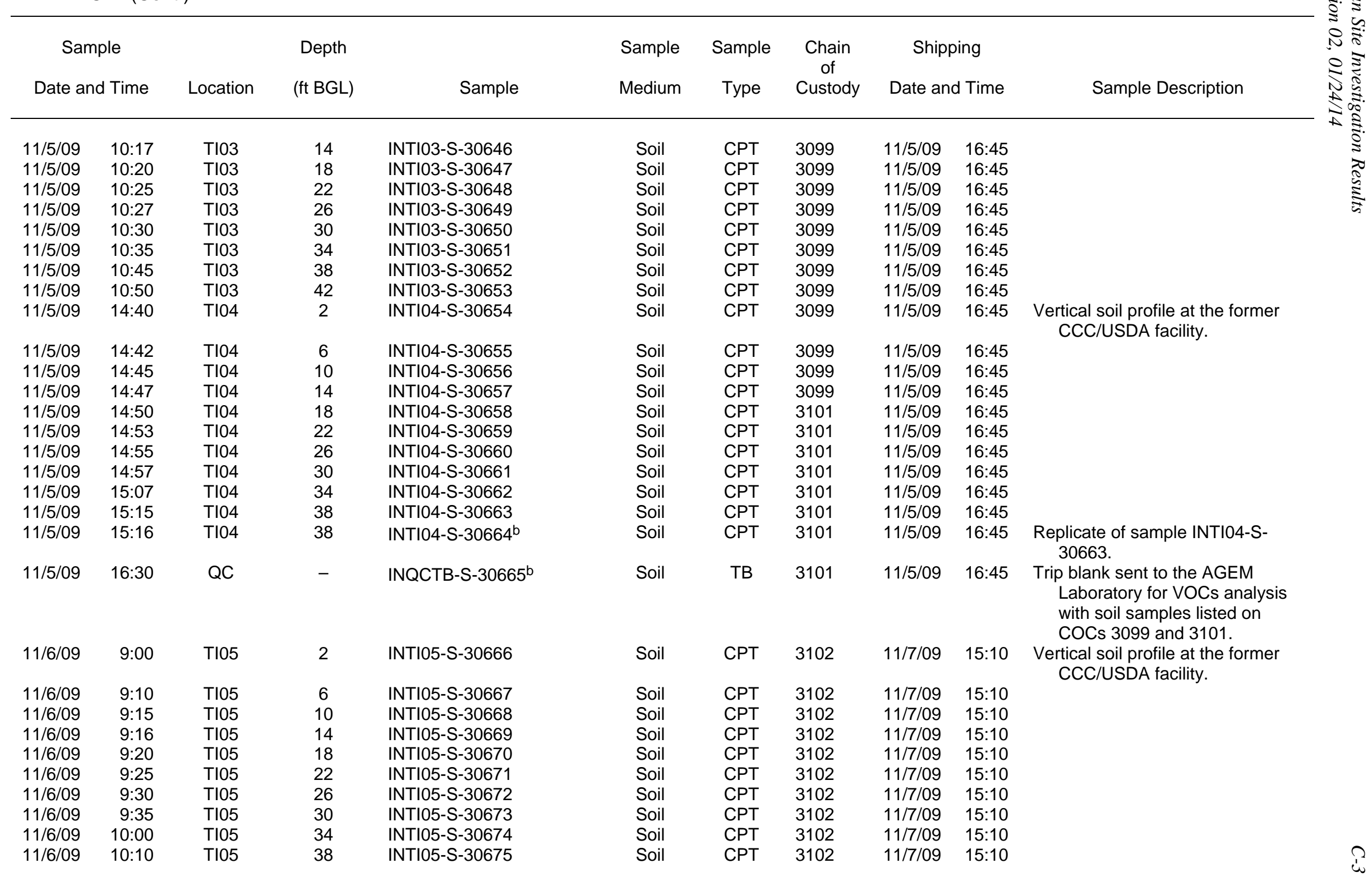


TABLE C.1 (Cont.)

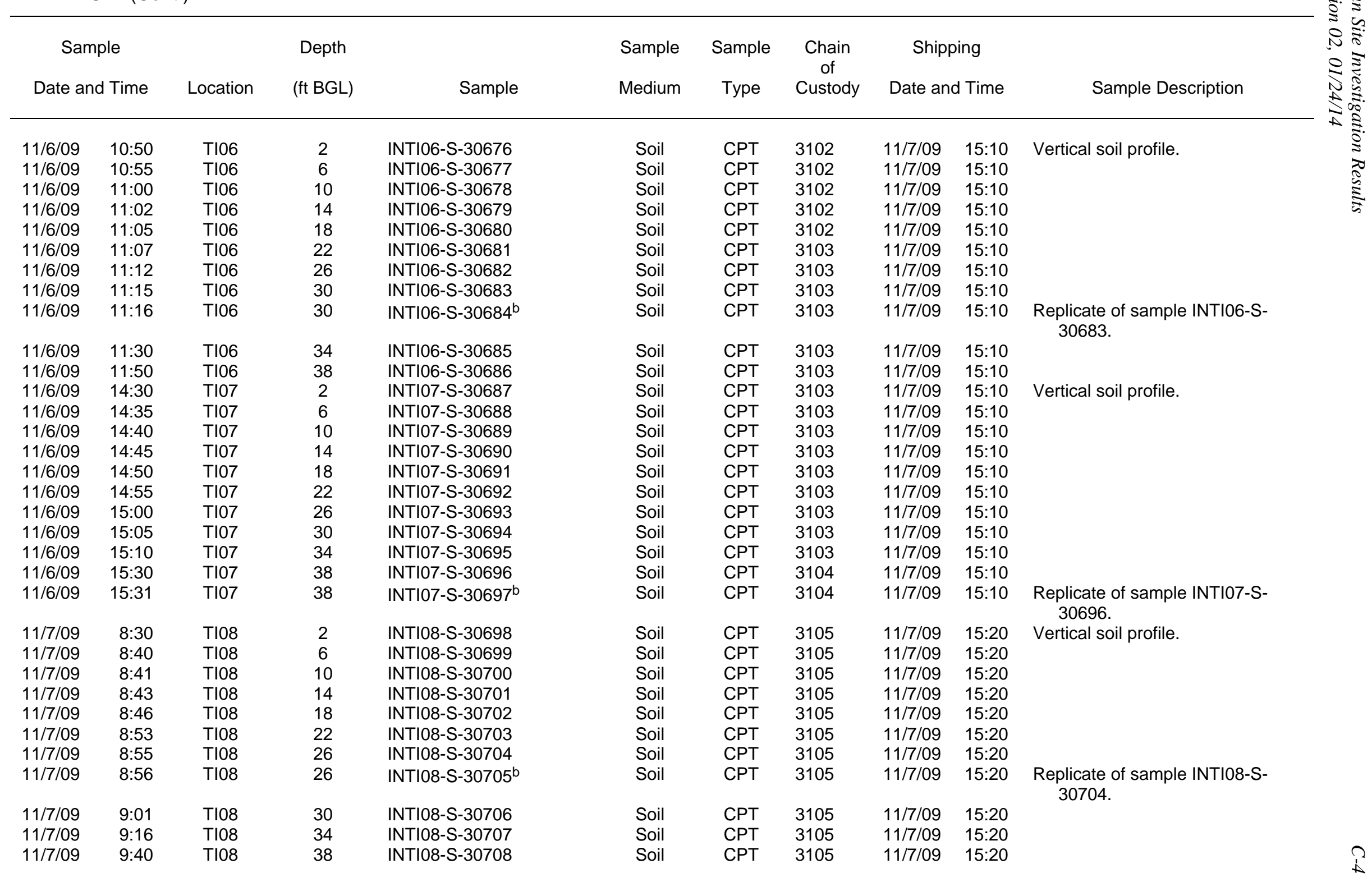


TABLE C.1 (Cont.)

\begin{tabular}{|c|c|c|c|c|c|c|c|c|c|c|}
\hline \multicolumn{2}{|c|}{ Sample } & \multirow{3}{*}{$\begin{array}{l}\text { Location } \\
\text { TI09 }\end{array}$} & \multirow{3}{*}{$\begin{array}{c}\text { Depth } \\
\text { (ft BGL) } \\
2\end{array}$} & \multirow{3}{*}{$\begin{array}{r}\text { Sample } \\
\text { INTI09-S-30709 }\end{array}$} & \multirow{3}{*}{$\begin{array}{c}\text { Sample } \\
\text { Medium } \\
\text { Soil }\end{array}$} & \multirow{3}{*}{$\begin{array}{c}\text { Sample } \\
\text { Type }\end{array}$} & \multirow{3}{*}{$\begin{array}{l}\begin{array}{c}\text { Chain } \\
\text { of } \\
\text { Custody }\end{array} \\
3105\end{array}$} & \multicolumn{2}{|c|}{ Shipping } & \multirow{3}{*}{$\begin{array}{l}\text { Sample Description } \\
\text { cal soil profile at the former } \\
\text { CC/USDA facility. }\end{array}$} \\
\hline Date ar & d Time & & & & & & & Date an & d Time & \\
\hline 11/7/09 & $10: 25$ & & & & & & & $11 / 7 / 09$ & $15: 20$ & \\
\hline 11/7/09 & $10: 27$ & TI09 & 6 & INTI09-S-30710 & Soil & CPT & 3105 & $11 / 7 / 09$ & $15: 20$ & \\
\hline $11 / 7 / 09$ & $10: 29$ & TI09 & 10 & INTI09-S-30711 & Soil & CPT & 3105 & $11 / 7 / 09$ & $15: 20$ & \\
\hline $11 / 7 / 09$ & $10: 32$ & TI09 & 14 & INTI09-S-30712 & Soil & CPT & 3105 & $11 / 7 / 09$ & $15: 20$ & \\
\hline $11 / 7 / 09$ & $10: 37$ & TI09 & 18 & INTI09-S-30713 & Soil & CPT & 3106 & $11 / 7 / 09$ & $15: 20$ & \\
\hline $11 / 7 / 09$ & $10: 38$ & TI09 & 38 & INTI09-S-30718 & Soil & CPT & 3106 & $11 / 7 / 09$ & $15: 20$ & \\
\hline 11/7/09 & $10: 39$ & TI09 & 22 & INTI09-S-30714 & Soil & CPT & 3106 & $11 / 7 / 09$ & $15: 20$ & \\
\hline $11 / 7 / 09$ & $10: 44$ & TI09 & 26 & INTI09-S-30715 & Soil & CPT & 3106 & $11 / 7 / 09$ & $15: 20$ & \\
\hline $11 / 7 / 09$ & $10: 48$ & TI09 & 30 & INTI09-S-30716 & Soil & CPT & 3106 & $11 / 7 / 09$ & $15: 20$ & \\
\hline $11 / 7 / 09$ & $10: 49$ & TI09 & 30 & INTI09-S-30717b & Soil & CPT & 3106 & $11 / 7 / 09$ & $15: 20$ & $\begin{array}{l}\text { Replicate of sample INTI09-S- } \\
30716 .\end{array}$ \\
\hline 11/7/09 & $12: 02$ & TI09 & 34 & INTI09-S-30719 & Soil & CPT & 3106 & $11 / 7 / 09$ & $15: 20$ & \\
\hline $11 / 7 / 09$ & $13: 50$ & TI10 & 2 & INTI10-S-30720 & Soil & CPT & 3106 & $11 / 7 / 09$ & $15: 20$ & Vertical soil profile. \\
\hline $11 / 7 / 09$ & $13: 52$ & TI10 & 6 & INTI10-S-30721 & Soil & CPT & 3106 & $11 / 7 / 09$ & $15: 20$ & \\
\hline $11 / 7 / 09$ & $13: 55$ & TI10 & 10 & INTI10-S-30722 & Soil & CPT & 3106 & $11 / 7 / 09$ & $15: 20$ & \\
\hline $11 / 7 / 09$ & $13: 58$ & TI10 & 14 & INTI10-S-30723 & Soil & CPT & 3106 & $11 / 7 / 09$ & $15: 20$ & \\
\hline $11 / 7 / 09$ & $14: 00$ & TI10 & 18 & INTI10-S-30724 & Soil & CPT & 3106 & $11 / 7 / 09$ & $15: 20$ & \\
\hline $11 / 7 / 09$ & $14: 04$ & TI10 & 22 & INTI10-S-30725 & Soil & CPT & 3106 & $11 / 7 / 09$ & $15: 20$ & \\
\hline $11 / 7 / 09$ & $14: 08$ & TI10 & 26 & INTI10-S-30726 & Soil & CPT & 3106 & $11 / 7 / 09$ & $15: 20$ & \\
\hline $11 / 7 / 09$ & $14: 13$ & TI10 & 30 & INTI10-S-30727 & Soil & CPT & 3106 & $11 / 7 / 09$ & $15: 20$ & \\
\hline $11 / 7 / 09$ & $14: 20$ & TI10 & 34 & INTI10-S-30728 & Soil & CPT & 3107 & $11 / 7 / 09$ & $15: 20$ & \\
\hline $11 / 7 / 09$ & $14: 30$ & TI10 & 38 & INTI10-S-30729 & Soil & CPT & 3107 & $11 / 7 / 09$ & $15: 20$ & \\
\hline $11 / 7 / 09$ & $14: 31$ & TI10 & 38 & INTI10-S-30730 & Soil & CPT & 3107 & $11 / 7 / 09$ & $15: 20$ & $\begin{array}{l}\text { Replicate of sample INTI10-S- } \\
30729 \text {. }\end{array}$ \\
\hline $11 / 7 / 09$ & $15: 10$ & $\mathrm{QC}$ & - & INTB-S-30731 b & Soil & TB & 3104 & $11 / 7 / 09$ & $15: 10$ & $\begin{array}{l}\text { Trip blank sent to the AGEM } \\
\text { Laboratory for VOCs analysis } \\
\text { with soil samples listed on } \\
\text { COCs } 3102,3103 \text {, and } 3104 .\end{array}$ \\
\hline 11/7/09 & $15: 15$ & $\mathrm{QC}$ & - & INQCTB-S-30732b & Soil & TB & 3107 & $11 / 7 / 09$ & $15: 20$ & $\begin{array}{l}\text { Trip blank sent to the AGEM } \\
\text { Laboratory for VOCs analysis } \\
\text { with soil samples listed on } \\
\text { COCs } 3105,3106 \text {, and } 3107 .\end{array}$ \\
\hline
\end{tabular}


TABLE C.1 (Cont.)

\begin{tabular}{|c|c|c|c|c|c|c|c|c|c|c|}
\hline \multicolumn{2}{|c|}{ Sample } & \multirow{2}{*}{$\begin{array}{l}\text { Location } \\
\text { TI01 }\end{array}$} & \multirow{2}{*}{$\begin{array}{c}\begin{array}{c}\text { Depth } \\
\text { (ft BGL) }\end{array} \\
26-40\end{array}$} & \multirow{2}{*}{$\begin{array}{r}\text { Sample } \\
\text { INTI01-W-30733 }\end{array}$} & \multirow{2}{*}{$\begin{array}{r}\text { Sample } \\
\text { Medium } \\
\text { Water }\end{array}$} & \multirow{2}{*}{$\begin{array}{c}\text { Sample } \\
\text { Type } \\
\text { CPT }\end{array}$} & \multirow{2}{*}{$\begin{array}{l}\begin{array}{c}\text { Chain } \\
\text { of } \\
\text { Custody }\end{array} \\
3109\end{array}$} & \multirow{2}{*}{\multicolumn{2}{|c|}{$\begin{array}{c}\text { Shipping } \\
\text { Date and Time }\end{array}$}} & \multirow{3}{*}{\begin{tabular}{l}
\multicolumn{1}{c}{ Sample Description } \\
Field evaluation sample. Grab \\
sample from open hole. Water \\
likely from $26-40 \mathrm{ft}$ interval \\
through $10-\mathrm{ft}$ screen at $30-40$ \\
without sand pack or grout. \\
Test hole to look for presence \\
of water.
\end{tabular}} \\
\hline Date ar & Time & & & & & & & & & \\
\hline $11 / 7 / 09$ & $17: 20$ & TI01 & $26-40$ & INTI01-W-30733 & Water & СРT & 3109 & $11 / 9 / 09$ & $16: 50$ & \\
\hline 11/8/09 & $11: 30$ & TI11 & 2 & INTI11-S-30734 & Soil & CPT & 3108 & 11/9/09 & $14: 30$ & $\begin{array}{l}\text { Vertical soil profile at the former } \\
\text { CCC/USDA facility. }\end{array}$ \\
\hline 11/8/09 & $11: 32$ & TI11 & 6 & INTI11-S-30735 & Soil & CPT & 3108 & 11/9/09 & $14: 30$ & \\
\hline $11 / 8 / 09$ & $11: 35$ & TI11 & 10 & INTI11-S-30736 & Soil & CPT & 3108 & $11 / 9 / 09$ & $14: 30$ & \\
\hline $11 / 8 / 09$ & $11: 37$ & TI11 & 14 & INTI11-S-30737 & Soil & CPT & 3108 & $11 / 9 / 09$ & $14: 30$ & \\
\hline $11 / 8 / 09$ & $11: 39$ & TI11 & 18 & INTI11-S-30738 & Soil & CPT & 3108 & $11 / 9 / 09$ & $14: 30$ & \\
\hline $11 / 8 / 09$ & $11: 43$ & TI11 & 22 & INTI11-S-30739 & Soil & CPT & 3108 & $11 / 9 / 09$ & $14: 30$ & \\
\hline 11/8/09 & 11:44 & TI11 & 22 & INTI11-S-30740 b & Soil & CPT & 3108 & 11/9/09 & $14: 30$ & $\begin{array}{l}\text { Replicate of sample INTI11-S- } \\
30739 \text {. }\end{array}$ \\
\hline 11/8/09 & $11: 45$ & TI11 & 26 & INTI11-S-30741 & Soil & CPT & 3108 & $11 / 9 / 09$ & $14: 30$ & \\
\hline $11 / 8 / 09$ & $11: 51$ & TI11 & 30 & INTI11-S-30742 & Soil & CPT & 3108 & $11 / 9 / 09$ & $14: 30$ & \\
\hline $11 / 8 / 09$ & $11: 55$ & TI11 & 34 & INTI11-S-30743 & Soil & CPT & 3108 & $11 / 9 / 09$ & $14: 30$ & \\
\hline $11 / 8 / 09$ & $12: 02$ & TI11 & 38 & INTI11-S-30744 & Soil & CPT & 3108 & $11 / 9 / 09$ & $14: 30$ & \\
\hline $11 / 9 / 09$ & $14: 30$ & $\mathrm{QC}$ & - & INQCTB-S-30745 & Soil & TB & 3108 & $11 / 9 / 09$ & $14: 30$ & $\begin{array}{l}\text { Trip blank sent to the AGEM } \\
\text { Laboratory for VOCs analysis } \\
\text { with soil samples listed on } \\
\text { COC } 3108 \text {. }\end{array}$ \\
\hline $11 / 9 / 09$ & $15: 00$ & TI04 & $40-42$ & INTI04B-W-30746 & Water & $\mathrm{CPT} / \mathrm{P}$ & 3109 & 11/9/09 & $16: 50$ & $\begin{array}{l}\text { Field evaluation sample. } \\
\text { Temporary piezometer. }\end{array}$ \\
\hline 11/9/09 & $15: 15$ & TI04 & $30-40$ & INTI04-W-30747 & Water & $\mathrm{CPT} / \mathrm{P}$ & 3109 & 11/9/09 & $16: 50$ & $\begin{array}{l}\text { Field evaluation sample. } \\
\text { Temporary piezometer. }\end{array}$ \\
\hline 11/9/09 & $15: 20$ & TI05 & $30-40$ & INTI05-W-30748 & Water & $\mathrm{CPT} / \mathrm{P}$ & 3109 & 11/9/09 & $16: 50$ & $\begin{array}{l}\text { Field evaluation sample. } \\
\text { Temporary piezometer. }\end{array}$ \\
\hline 11/9/09 & $15: 30$ & TI01 & $30-40$ & INTI01-W-30749 & Water & $\mathrm{CPT} / \mathrm{P}$ & 3109 & $11 / 9 / 09$ & $16: 50$ & Field evaluation sample. \\
\hline $11 / 9 / 09$ & $15: 35$ & TI01 & $33-38$ & INTI01-W-30750 & Water & $\mathrm{CPT} / \mathrm{P}$ & 3109 & $11 / 9 / 09$ & $16: 50$ & Field evaluation sample. \\
\hline $11 / 9 / 09$ & $15: 40$ & TI11 & $28-33$ & INTI11-W-30751 & Water & $\mathrm{CPT} / \mathrm{P}$ & 3109 & $11 / 9 / 09$ & $16: 50$ & $\begin{array}{l}\text { Field evaluation sample. } \\
\text { Temporary piezometer. Very } \\
\text { little water available. One } \\
\text { 20-mL vial collected. }\end{array}$ \\
\hline
\end{tabular}


TABLE C.1 (Cont.)

\begin{tabular}{|c|c|c|c|c|c|c|c|c|c|c|}
\hline \multicolumn{2}{|c|}{ Sample } & \multirow{3}{*}{$\begin{array}{l}\text { Location } \\
\text { TI08 }\end{array}$} & \multirow{3}{*}{$\begin{array}{c}\text { Depth } \\
\text { (ft BGL) } \\
30-40\end{array}$} & \multirow{3}{*}{$\begin{array}{r}\text { Sample } \\
\text { INTI08-W-30752 }\end{array}$} & \multirow{3}{*}{$\begin{array}{r}\text { Sample } \\
\text { Medium } \\
\text { Water }\end{array}$} & \multirow{3}{*}{$\begin{array}{c}\text { Sample } \\
\text { Type }\end{array}$} & \multirow{3}{*}{$\begin{array}{c}\begin{array}{c}\text { Chain } \\
\text { of } \\
\text { Custody }\end{array} \\
3109\end{array}$} & \multicolumn{2}{|c|}{ Shipping } & \multirow[b]{2}{*}{ Sample Description } \\
\hline Date anc & Time & & & & & & & Date and & Time & \\
\hline 11/9/09 & $15: 50$ & & & & & & & $11 / 9 / 09$ & 16:50 & $\begin{array}{l}\text { Field evaluation sample. } \\
\text { Temporary piezometer. }\end{array}$ \\
\hline $11 / 9 / 09$ & $15: 55$ & QC & - & INQCTR-W-30753 b & Water & $\mathrm{RI}$ & 3109 & $11 / 9 / 09$ & $16: 50$ & $\begin{array}{l}\text { Rinsate of decontaminated bailer } \\
\text { after collection of sample } \\
\text { INTI08-W-30752. }\end{array}$ \\
\hline $11 / 11 / 09$ & $10: 00$ & TI11 & $33-38$ & INTI11-W-30755 & Water & $\mathrm{CPT} / \mathrm{P}$ & 3179 & $11 / 11 / 09$ & $14: 20$ & $\begin{array}{l}\text { Field evaluation sample. } \\
\text { Temporary piezometer. }\end{array}$ \\
\hline 11/11/09 & $10: 30$ & TI06 & $28-33$ & INTI06-W-30756 & Water & CPT/P & 3179 & $11 / 11 / 09$ & $14: 20$ & $\begin{array}{l}\text { Field evaluation sample. } \\
\text { Temporary piezometer. }\end{array}$ \\
\hline 11/11/09 & $10: 35$ & TI06 & $33-38$ & INTI06-W-30757 & Water & CPT/P & 3179 & $11 / 11 / 09$ & $14: 20$ & $\begin{array}{l}\text { Field evaluation sample. } \\
\text { Temporary piezometer. }\end{array}$ \\
\hline 11/11/09 & 11:15 & QC & - & INQCTB-W-30760 & Water & TB & 3179 & $11 / 11 / 09$ & $14: 20$ & $\begin{array}{l}\text { Trip blank sent to the AGEM } \\
\text { Laboratory for VOCs analysis } \\
\text { with water samples listed on } \\
\text { COC } 3179 \text {. }\end{array}$ \\
\hline $11 / 12 / 09$ & $10: 00$ & $\mathrm{QC}$ & - & $\begin{array}{l}\text { IN-S-Meohblank- } \\
\text { 12Nov09b }\end{array}$ & Soil & TB & 3658 & $11 / 12 / 09$ & $10: 00$ & $\begin{array}{l}\text { Trip blank with soil samples for } \\
\text { verification organic analysis to } \\
\text { Test America Laboratory listed } \\
\text { on COC } 3658 \text {. }\end{array}$ \\
\hline $11 / 9 / 10$ & $10: 28$ & MW02 & $32-42$ & INMW02-W-32671 & Water & MW & 3020 & $11 / 9 / 10$ & $18: 00$ & Field evaluation sample. \\
\hline $11 / 9 / 10$ & $11: 10$ & MW01 & $32-42$ & INMW01-W-32670 & Water & MW & 3020 & $11 / 9 / 10$ & $18: 00$ & Field evaluation sample. \\
\hline $11 / 9 / 10$ & $15: 21$ & MW03 & $35-45$ & INMW03-W-32672 & Water & MW & 3020 & $11 / 9 / 10$ & $18: 00$ & Field evaluation sample. \\
\hline 11/9/10 & $16: 36$ & Willems & - & INWILLEMS-W-32673 & Water & DW & 3020 & $11 / 9 / 10$ & 18:00 & $\begin{array}{l}\text { Sample collected from outside } \\
\text { faucet at northeast corner of } \\
\text { house, after water was allowed } \\
\text { to run for } 5 \text { min. }\end{array}$ \\
\hline
\end{tabular}


TABLE C.1 (Cont.)

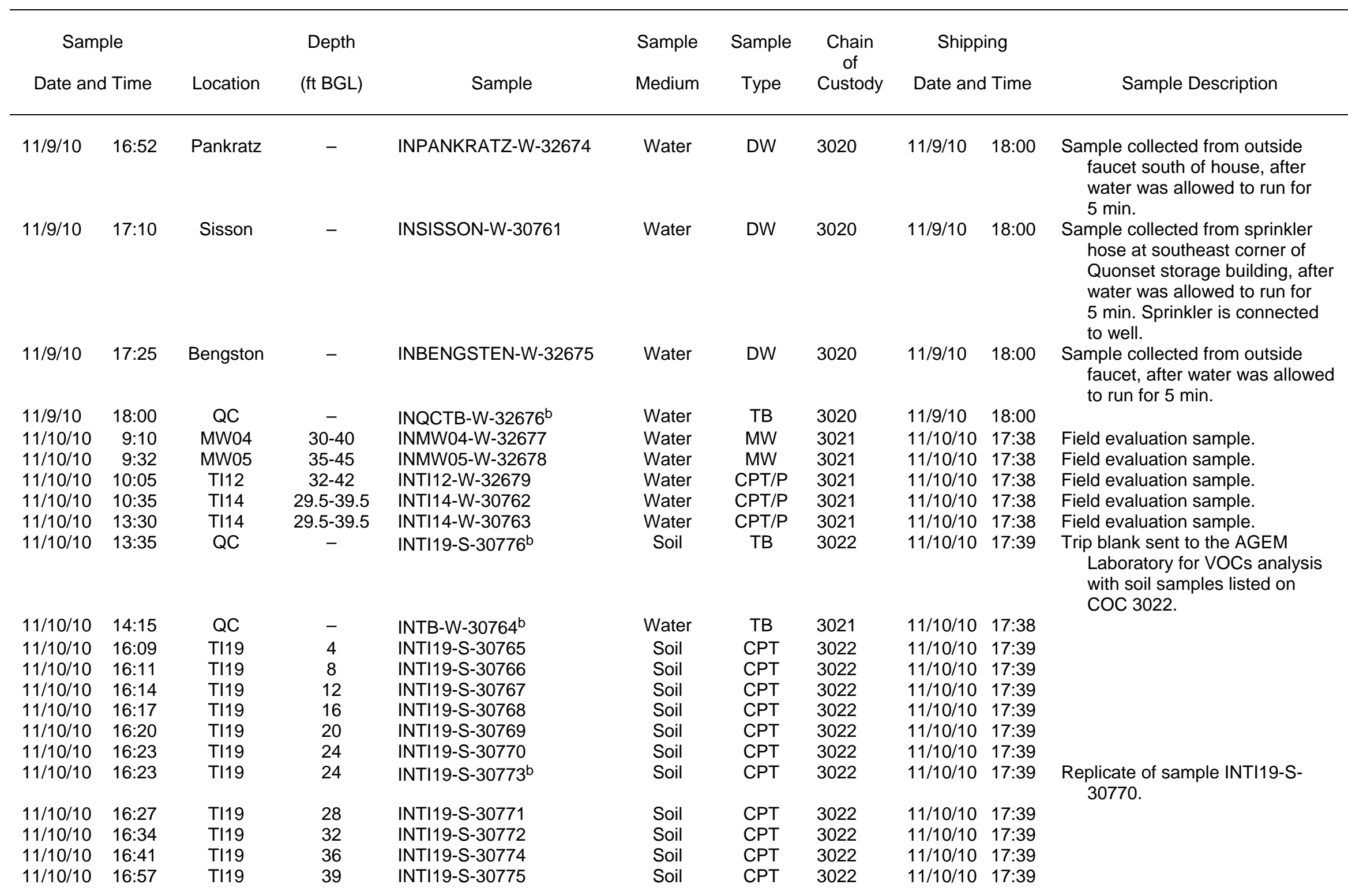


TABLE C.1 (Cont.)

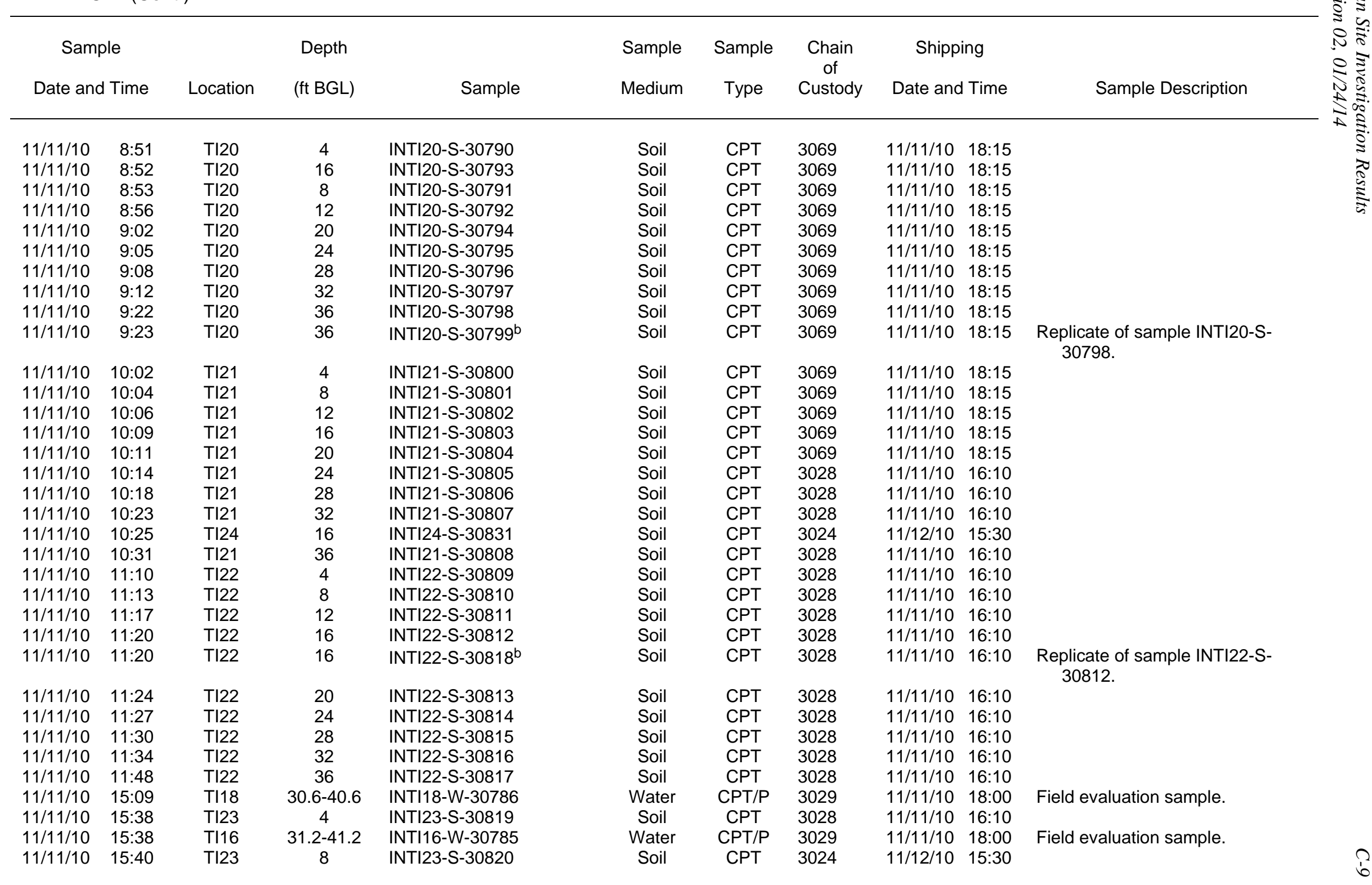


TABLE C.1 (Cont.)

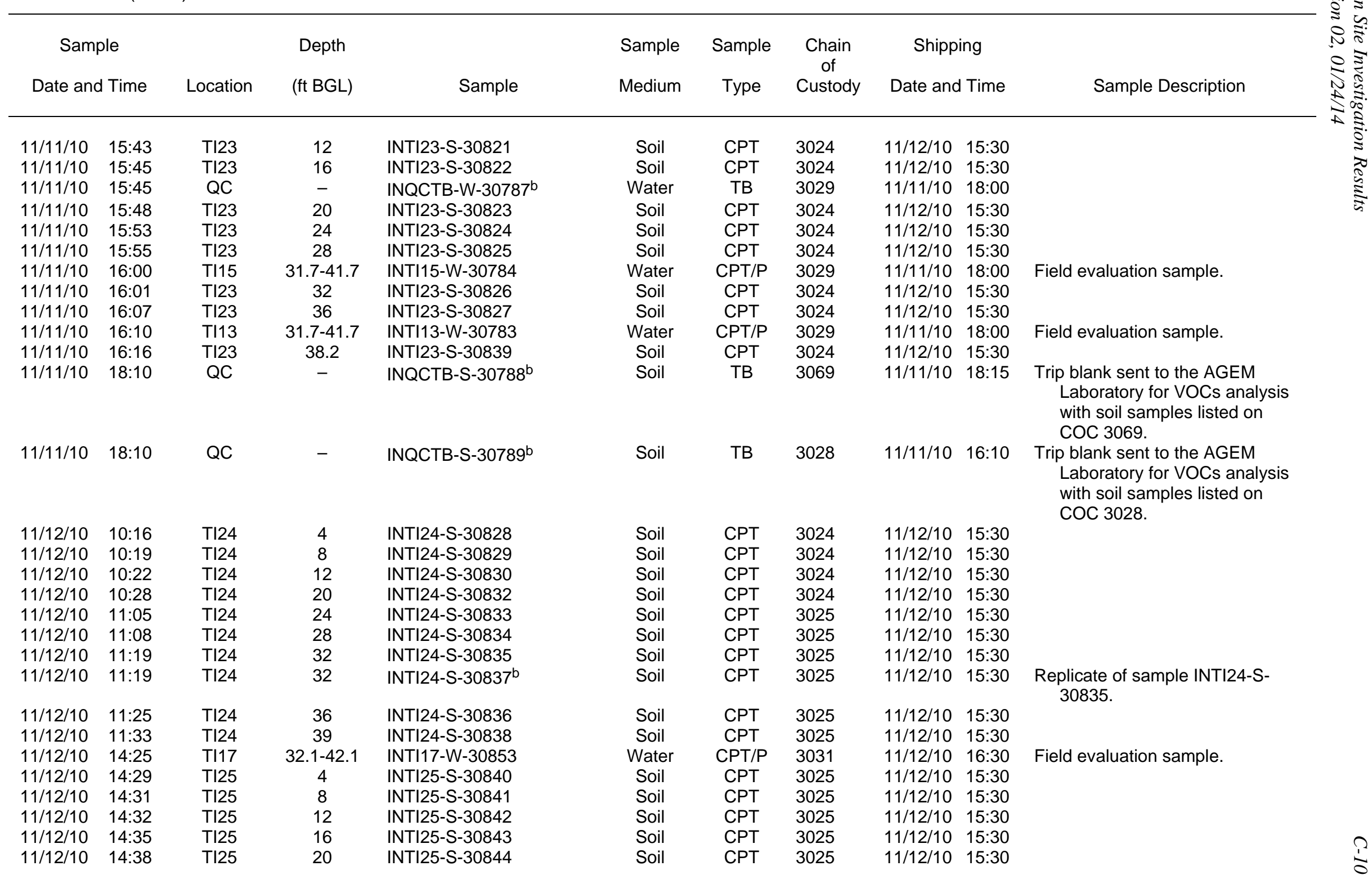


TABLE C.1 (Cont.)

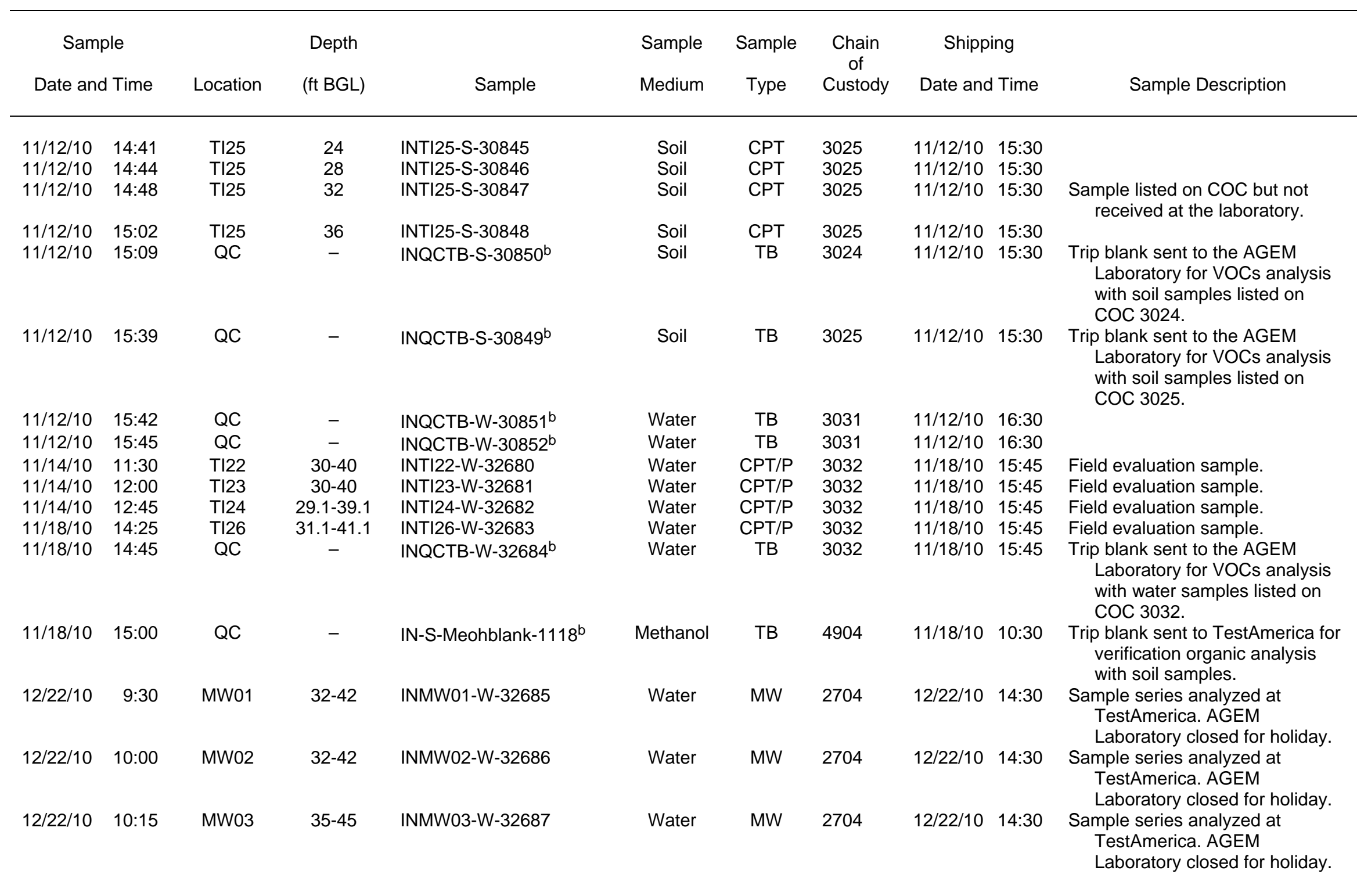


TABLE C.1 (Cont.)

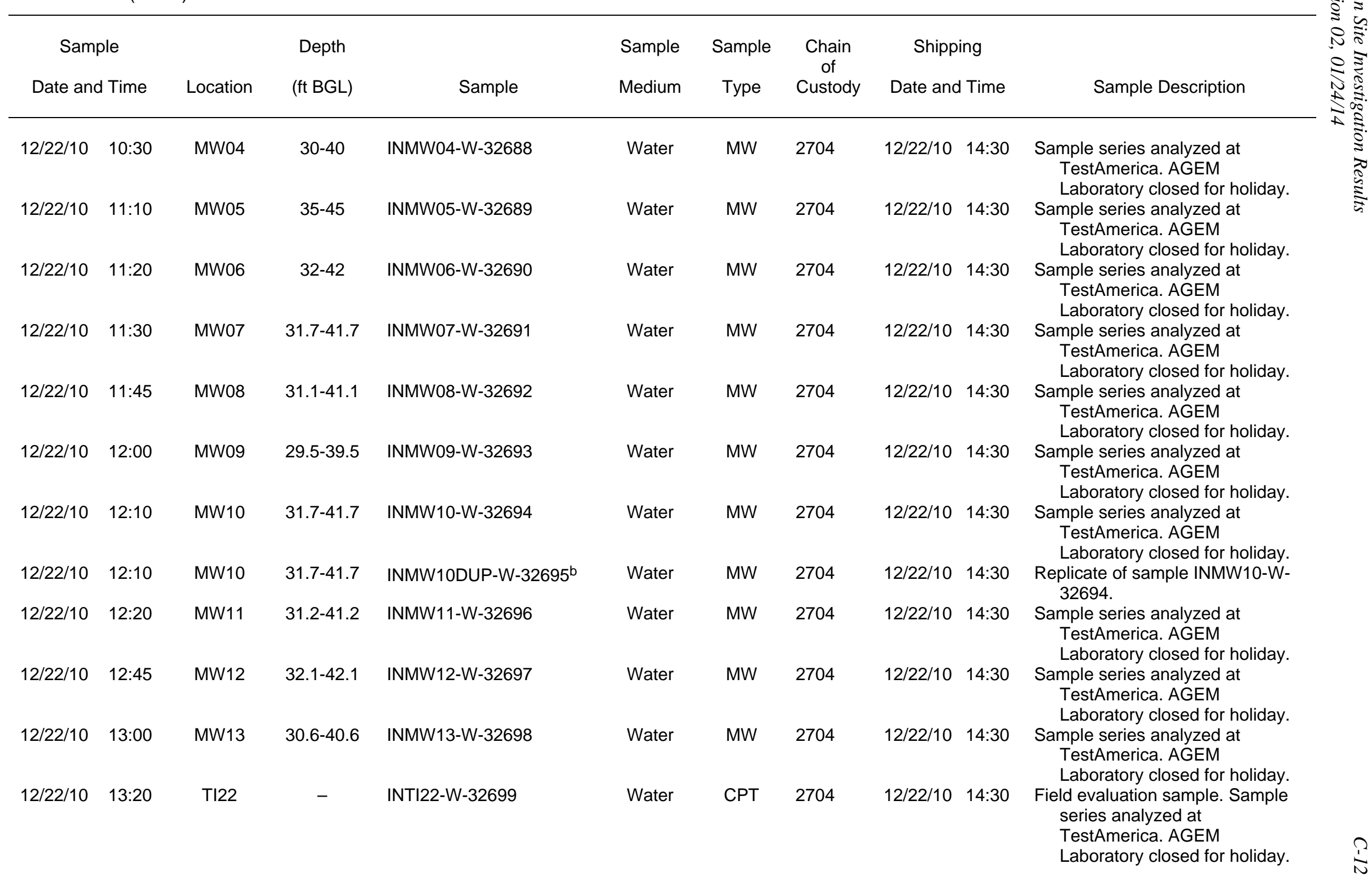


TABLE C.1 (Cont.)

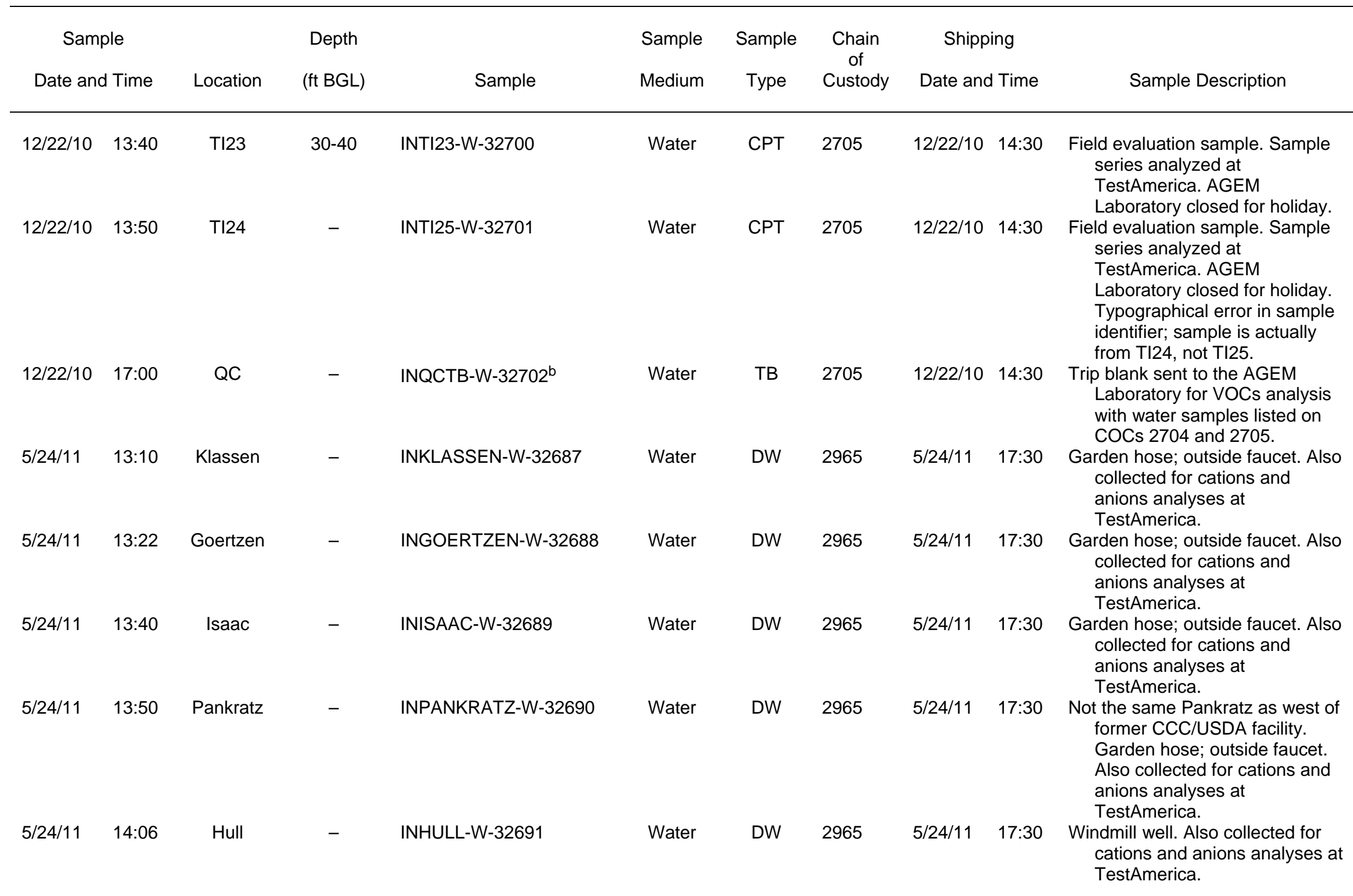


TABLE C.1 (Cont.)

\begin{tabular}{|c|c|c|c|c|c|c|c|c|c|c|}
\hline \multicolumn{2}{|c|}{$\begin{array}{c}\text { Sample } \\
\text { Date and Time }\end{array}$} & \multirow{2}{*}{$\begin{array}{l}\text { Location } \\
\text { QC }\end{array}$} & \multirow{2}{*}{$\begin{array}{c}\text { Depth } \\
\text { (ft BGL) } \\
-\end{array}$} & \multirow{2}{*}{$\begin{array}{l}\text { Sample } \\
3-W-32692^{b}\end{array}$} & \multirow{2}{*}{$\begin{array}{c}\text { Sample } \\
\text { Medium } \\
\text { Water }\end{array}$} & \multirow{2}{*}{$\begin{array}{c}\text { Sample } \\
\text { Type } \\
\text { TB }\end{array}$} & \multirow{2}{*}{$\begin{array}{c}\begin{array}{c}\text { Chain } \\
\text { of } \\
\text { Custody }\end{array} \\
2965\end{array}$} & \multicolumn{2}{|c|}{ Shipping } & Sample Description \\
\hline $5 / 24 / 11$ & $14: 32$ & & & & & & & $5 / 24 / 11$ & $17: 30$ & $\begin{array}{l}\text { Trip blank sent to the AGEM } \\
\text { Laboratory for VOCs analysis } \\
\text { with water samples listed on } \\
\text { COC } 2965 \text {. }\end{array}$ \\
\hline $5 / 24 / 11$ & $16: 05$ & Brown & - & INBROWN-W-32686 & Water & DW & 2965 & $5 / 24 / 11$ & $17: 30$ & $\begin{array}{l}\text { Hydrant south of pond. Water has } \\
\text { strong odor. Sulfur? Dead fish? }\end{array}$ \\
\hline $5 / 24 / 11$ & $16: 30$ & Thiessen & - & INTHIESSEN-W-32685 & Water & DW & 2965 & $5 / 24 / 11$ & $17: 30$ & $\begin{array}{l}\text { Faucet on south side of house. } \\
\text { Also collected for cations and } \\
\text { anions analyses at } \\
\text { TestAmerica. }\end{array}$ \\
\hline $6 / 23 / 11$ & $14: 26$ & Klassen & - & INKLASSEN-W-32693 & Water & DW & 2980 & $6 / 23 / 11$ & $17: 40$ & $\begin{array}{l}\text { Second set of samples collected } \\
\text { from the Klassen well. } \\
\text { Collected at the hydrant just } \\
\text { west of the house. }\end{array}$ \\
\hline $6 / 23 / 11$ & $16: 10$ & Hull & - & INHULL-W-32694 & Water & DW & 2980 & $6 / 23 / 11$ & $17: 40$ & $\begin{array}{l}\text { Samples collected from the well } \\
\text { supplying the Hull home. } \\
\text { Collected at faucet on south } \\
\text { side of house. }\end{array}$ \\
\hline $6 / 23 / 11$ & $16: 36$ & Pauls & - & INPAULS-W-32695 & Water & DW & 2980 & $6 / 23 / 11$ & $17: 40$ & $\begin{array}{l}\text { Samples collected from the } \\
\text { hydrant on the south end of the } \\
\text { place, near the garage. }\end{array}$ \\
\hline $6 / 23 / 11$ & $17: 45$ & QC & - & INQCTB-W-32696'b & Water & TB & 2980 & $6 / 23 / 11$ & $17: 40$ & $\begin{array}{l}\text { Trip blank sent to the AGEM } \\
\text { Laboratory for VOCs analysis } \\
\text { with water samples listed on } \\
\text { COC } 2980 \text {. }\end{array}$ \\
\hline $8 / 22 / 11$ & $13: 00$ & MW14 & $75-90$ & INMW14-W-32730 & Water & SB & 1519 & $8 / 22 / 11$ & 19:00 & Field evaluation sample. \\
\hline $8 / 22 / 11$ & $13: 25$ & QC & - & INTRUCK-W-32731 b & Water & FB & 1519 & $8 / 22 / 11$ & 19:00 & \\
\hline $8 / 22 / 11$ & $13: 30$ & QC & - & INCITY-W-32732 & Water & $\mathrm{FB}$ & 1519 & $8 / 22 / 11$ & 19:00 & \\
\hline $8 / 22 / 11$ & 13:35 & QC & - & INQCTB-W-32733 ${ }^{b}$ & Water & TB & 1519 & $8 / 22 / 11$ & 19:00 & $\begin{array}{l}\text { Trip blank sent to the AGEM } \\
\text { Laboratory for VOCs analysis } \\
\text { with water samples listed on } \\
\text { COC } 1519 .\end{array}$ \\
\hline $8 / 22 / 11$ & $14: 30$ & MW14 & $95-120$ & INMW14-W-32734 & Water & SB & 1519 & $8 / 22 / 11$ & 19:00 & Field evaluation sample. \\
\hline $8 / 24 / 11$ & $15: 00$ & MW15 & $55-75$ & INMW15-W-32735 & Water & SB & 2860 & $8 / 24 / 11$ & $16: 50$ & Field evaluation sample. \\
\hline $8 / 24 / 11$ & $15: 40$ & MW14M & $85-95$ & INMW14S-W-32736 & Water & MW & 2860 & $8 / 24 / 11$ & $16: 50$ & \\
\hline 8/24/11 & $15: 50$ & MW15 & $75-90$ & INMW15-W-32737 & Water & SB & 2860 & $8 / 24 / 11$ & $16: 50$ & Field evaluation sample. \\
\hline
\end{tabular}


TABLE C.1 (Cont.)

\begin{tabular}{|c|c|c|c|c|c|c|c|c|c|c|}
\hline \multirow{2}{*}{\multicolumn{2}{|c|}{$\begin{array}{c}\text { Sample } \\
\text { Date and Time }\end{array}$}} & \multirow{3}{*}{$\begin{array}{l}\text { Location } \\
\mathrm{QC}\end{array}$} & \multirow{3}{*}{$\begin{array}{c}\text { Depth } \\
\text { (ft BGL) } \\
-\end{array}$} & \multirow{3}{*}{$\begin{array}{l}\text { Sample } \\
\text { 3-W-32738b }\end{array}$} & \multirow{3}{*}{$\begin{array}{l}\text { Sample } \\
\text { Medium } \\
\text { Water }\end{array}$} & \multirow{3}{*}{$\begin{array}{c}\text { Sample } \\
\text { Type } \\
\text { TB }\end{array}$} & \multirow{3}{*}{$\begin{array}{c}\begin{array}{c}\text { Chain } \\
\text { of } \\
\text { Custody }\end{array} \\
2860\end{array}$} & \multirow{2}{*}{\multicolumn{2}{|c|}{$\begin{array}{l}\text { Shipping } \\
\text { Date and Time }\end{array}$}} & \multirow{3}{*}{ Sample Description } \\
\hline & & & & & & & & & & \\
\hline 8/24/11 & $16: 00$ & & & & & & & $8 / 24 / 11$ & $16: 50$ & \\
\hline $8 / 25 / 11$ & $13: 00$ & MW16 & $75-91$ & INMW16-W-32739 & Water & SB & 2850 & $8 / 25 / 11$ & $18: 12$ & Field evaluation sample. \\
\hline $8 / 25 / 11$ & $14: 00$ & MW16 & $35-45$ & INMW16-W-32740 & Water & SB & 2850 & $8 / 25 / 11$ & $18: 12$ & Field evaluation sample. \\
\hline $8 / 25 / 11$ & $14: 15$ & MW16 & $15-30$ & INMW16-W-32741 & Water & SB & 2850 & $8 / 25 / 11$ & $18: 12$ & Field evaluation sample. \\
\hline $8 / 25 / 11$ & $14: 25$ & MW16 & $55-70$ & INMW16-W-32742 & Water & SB & 2850 & $8 / 25 / 11$ & $18: 12$ & Field evaluation sample. \\
\hline $8 / 25 / 11$ & $14: 45$ & KTW & 135.2 & INKTW-W-32743 & Water & DW & 2850 & $8 / 25 / 11$ & $18: 12$ & \\
\hline $8 / 25 / 11$ & $15: 00$ & QC & - & INQCTB-W-32744 ${ }^{\mathrm{b}}$ & Water & TB & 2850 & $8 / 25 / 11$ & $18: 12$ & $\begin{array}{l}\text { Trip blank sent to the AGEM } \\
\text { Laboratory for VOCs analysis } \\
\text { with water samples listed on } \\
\text { COC } 2850 \text {. }\end{array}$ \\
\hline $8 / 27 / 11$ & $11: 00$ & MW17 & $15-31$ & INMW17-W-32745 & Water & SB & 2852 & $8 / 27 / 11$ & $15: 00$ & Field evaluation sample. \\
\hline $8 / 27 / 11$ & $11: 20$ & QC & - & INMW17QC-W-32746 ${ }^{\mathrm{b}}$ & Water & TB & 2852 & $8 / 27 / 11$ & $15: 00$ & $\begin{array}{l}\text { Trip blank sent to the AGEM } \\
\text { Laboratory for VOCs analysis } \\
\text { with water samples listed on } \\
\text { COC } 2852 \text {. }\end{array}$ \\
\hline $8 / 27 / 11$ & $11: 25$ & MW15M & $86-96$ & INMW15-W-32747 & Water & MW & 2852 & $8 / 27 / 11$ & $15: 00$ & Field evaluation sample. \\
\hline 8/27/11 & $11: 45$ & MW17 & $55-75$ & INMW17-W-32748 & Water & SB & 2852 & $8 / 27 / 11$ & $15: 00$ & Field evaluation sample. \\
\hline $8 / 27 / 11$ & $12: 00$ & MW17 & $55-75$ & INMW17-W-32749 & Water & SB & 2852 & $8 / 27 / 11$ & $15: 00$ & $\begin{array}{l}\text { Replicate of field evaluation } \\
\text { sample INMW17-W-32748. }\end{array}$ \\
\hline $8 / 28 / 11$ & $11: 45$ & MW15M & $86-96$ & INMW15-W-32750 & Water & MW & 3033 & $8 / 29 / 11$ & $17: 23$ & Field evaluation sample. \\
\hline $8 / 28 / 11$ & $12: 00$ & MW15M & $86-96$ & INMW15-W-32751 & Water & MW & 3033 & $8 / 29 / 11$ & $17: 23$ & \\
\hline 8/29/11 & $11: 45$ & MW18 & $85-90$ & INMW18-W-32752 & Water & $\mathrm{SB}$ & 3033 & 8/29/11 & $17: 23$ & Field evaluation sample. \\
\hline $8 / 29 / 11$ & $12: 50$ & MW18 & $38-52$ & INMW18-W-32753 & Water & SB & 3033 & $8 / 29 / 11$ & $17: 23$ & Field evaluation sample. \\
\hline $8 / 29 / 11$ & $13: 00$ & MW18 & $95-102.5$ & INMW18-W-32754 & Water & SB & 3033 & $8 / 29 / 11$ & $17: 23$ & Field evaluation sample. \\
\hline $8 / 29 / 11$ & $14: 20$ & MW18 & $85-90$ & INMW18-W-32755 & Water & SB & 3033 & $8 / 29 / 11$ & $17: 23$ & Field evaluation sample. \\
\hline $8 / 29 / 11$ & $14: 45$ & MW16D & $100-110$ & INMW16D-W-32756 & Water & MW & 3033 & $8 / 29 / 11$ & $17: 23$ & \\
\hline 8/29/11 & $16: 00$ & MW15S & $35-45$ & INMW15S-W-32757 & Water & MW & 3033 & $8 / 29 / 11$ & $17: 23$ & \\
\hline 8/29/11 & $16: 20$ & MW17D & $98-108$ & INMW17D-W-32758 & Water & MW & 3033 & 8/29/11 & $17: 23$ & Field evaluation sample. \\
\hline 8/29/11 & $16: 35$ & MW15D & $115-125$ & INMW15D-W-32759 & Water & MW & 3033 & $8 / 29 / 11$ & $17: 23$ & \\
\hline $8 / 29 / 11$ & $16: 40$ & MW16M2 & $78-88$ & INMW16-W-32764 & Water & MW & 2854 & $8 / 29 / 11$ & $15: 30$ & \\
\hline $8 / 29 / 11$ & $16: 45$ & MW18 & $65-80$ & INMW18-W-32760 & Water & $\mathrm{SB}$ & 3033 & $8 / 29 / 11$ & $17: 23$ & Field evaluation sample. \\
\hline $8 / 29 / 11$ & $16: 45$ & MW16S2 & $40-45$ & INMW16-W-32765 & Water & MW & 2854 & 8/29/11 & $15: 30$ & \\
\hline
\end{tabular}


TABLE C.1 (Cont.)

\begin{tabular}{|c|c|c|c|c|c|c|c|c|c|c|}
\hline \multicolumn{2}{|c|}{ Sample } & \multirow{2}{*}{$\begin{array}{l}\text { Location } \\
\text { MW18 }\end{array}$} & \multirow{2}{*}{$\begin{array}{c}\text { Depth } \\
\text { (ft BGL) } \\
65-80\end{array}$} & \multirow{2}{*}{$\begin{array}{c}\text { Sample } \\
8-W-32761^{b}\end{array}$} & \multirow{2}{*}{$\begin{array}{c}\text { Sample } \\
\text { Medium } \\
\text { Water }\end{array}$} & \multirow{2}{*}{$\begin{array}{c}\text { Sample } \\
\text { Type } \\
\text { SB }\end{array}$} & \multirow{2}{*}{$\begin{array}{c}\begin{array}{c}\text { Chain } \\
\text { of } \\
\text { Custody }\end{array} \\
3033\end{array}$} & \multicolumn{2}{|c|}{$\begin{array}{l}\text { Shipping } \\
\text { Date and Time }\end{array}$} & \multirow{2}{*}{$\begin{array}{l}\text { Sample Description } \\
\text { icate of field evaluation } \\
\text { ample INMW18-W-32760. }\end{array}$} \\
\hline $8 / 29 / 11$ & $16: 50$ & & & & & & & $8 / 29 / 11$ & $17: 23$ & \\
\hline 8/29/11 & $17: 00$ & MW16S1 & $25-35$ & INMW16-W-32762 & Water & MW & 3033 & $8 / 29 / 11$ & $17: 23$ & \\
\hline $8 / 29 / 11$ & $17: 00$ & QC & - & INTB-W-32766 ${ }^{\mathrm{b}}$ & Water & $\mathrm{TB}$ & 2854 & $8 / 29 / 11$ & $15: 30$ & $\begin{array}{l}\text { Trip blank sent to the AGEM } \\
\text { Laboratory for VOCs analysis } \\
\text { with water samples listed on } \\
\text { COC } 2854 \text {. }\end{array}$ \\
\hline $8 / 29 / 11$ & $17: 23$ & QC & - & INQC-W-32763 & Water & TB & 3033 & $8 / 29 / 11$ & $17: 23$ & $\begin{array}{l}\text { Trip blank sent to the AGEM } \\
\text { Laboratory for VOCs analysis } \\
\text { with water samples listed on } \\
\text { COC } 3033 \text {. }\end{array}$ \\
\hline 8/30/11 & $17: 45$ & MW16M1 & $60-70$ & INMW16-W-32767 & Water & MW & 2856 & $8 / 30 / 11$ & $18: 20$ & \\
\hline 8/30/11 & $18: 00$ & MW19 & $15-50$ & INMW19-W-32768 & Water & SB & 2856 & 8/30/11 & $18: 20$ & Field evaluation sample. \\
\hline 8/30/11 & $18: 20$ & QC & - & INQCTB-W-32769b & Water & TB & 2856 & $8 / 30 / 11$ & $18: 20$ & $\begin{array}{l}\text { Trip blank sent to the AGEM } \\
\text { Laboratory for VOCs analysis } \\
\text { with water samples listed on } \\
\text { COC } 2856 \text {. }\end{array}$ \\
\hline 8/31/11 & $17: 00$ & MW17M & $72-82$ & INMW17M-W-32770 & Water & MW & 3035 & $8 / 31 / 11$ & 19:00 & \\
\hline $8 / 31 / 11$ & $17: 30$ & MW19 & $75-90$ & INMW19-W-32771 & Water & SB & 3035 & $8 / 31 / 11$ & $19: 00$ & Field evaluation sample. \\
\hline $8 / 31 / 11$ & $17: 45$ & MW17S & $25-35$ & INMW17S-W-32772 & Water & MW & 3035 & $8 / 31 / 11$ & $19: 00$ & \\
\hline $8 / 31 / 11$ & $18: 00$ & MW17D & $98-108$ & INMW17D-W-32773 & Water & MW & 3035 & $8 / 31 / 11$ & $19: 00$ & \\
\hline $8 / 31 / 11$ & 19:00 & QC & - & INQCTB-W-32774 & Water & TB & 3035 & $8 / 31 / 11$ & 19:00 & $\begin{array}{l}\text { Trip blank sent to the AGEM } \\
\text { Laboratory for VOCs analysis } \\
\text { with water samples listed on } \\
\text { COC } 3035 \text {. }\end{array}$ \\
\hline $9 / 1 / 11$ & $18: 00$ & MW18S & $40-50$ & INMW18S-W-32775 & Water & MW & 3040 & $9 / 1 / 11$ & $19: 00$ & \\
\hline 9/1/11 & $18: 20$ & MW18M & $85-95$ & INMW18M-W-32776 & Water & MW & 3040 & $9 / 1 / 11$ & 19:00 & \\
\hline $9 / 1 / 11$ & $18: 40$ & MW18D & $115-125$ & INMW18D-W-32777 & Water & MW & 3040 & $9 / 1 / 11$ & 19:00 & \\
\hline 9/1/11 & 19:00 & QC & - & INQCTB-W-32778b & Water & TB & 3040 & $9 / 1 / 11$ & 19:00 & $\begin{array}{l}\text { Trip blank sent to the AGEM } \\
\text { Laboratory for VOCs analysis } \\
\text { with water samples listed on } \\
\text { COC } 3040 \text {. }\end{array}$ \\
\hline $9 / 2 / 11$ & $18: 00$ & MW19S & $38-48$ & INMW19S-W-32789 & Water & MW & 3037 & $9 / 2 / 11$ & $19: 00$ & \\
\hline $9 / 2 / 11$ & $18: 30$ & MW19M & $85-95$ & INMW19D-W-32790 & Water & MW & 3037 & $9 / 2 / 11$ & $19: 00$ & \\
\hline
\end{tabular}

Laboratory for VOCs analysis with water samples listed on

aboratory for VOCs analysis with water samples listed on

Field evaluation sample. blank sent to the AGEM ratory for VOCs analysis with water samples listed on with water samples listed on COC 3035 .

Laboratory for VOCs analysis with water samples listed on COC 3040 . 
TABLE C.1 (Cont.)

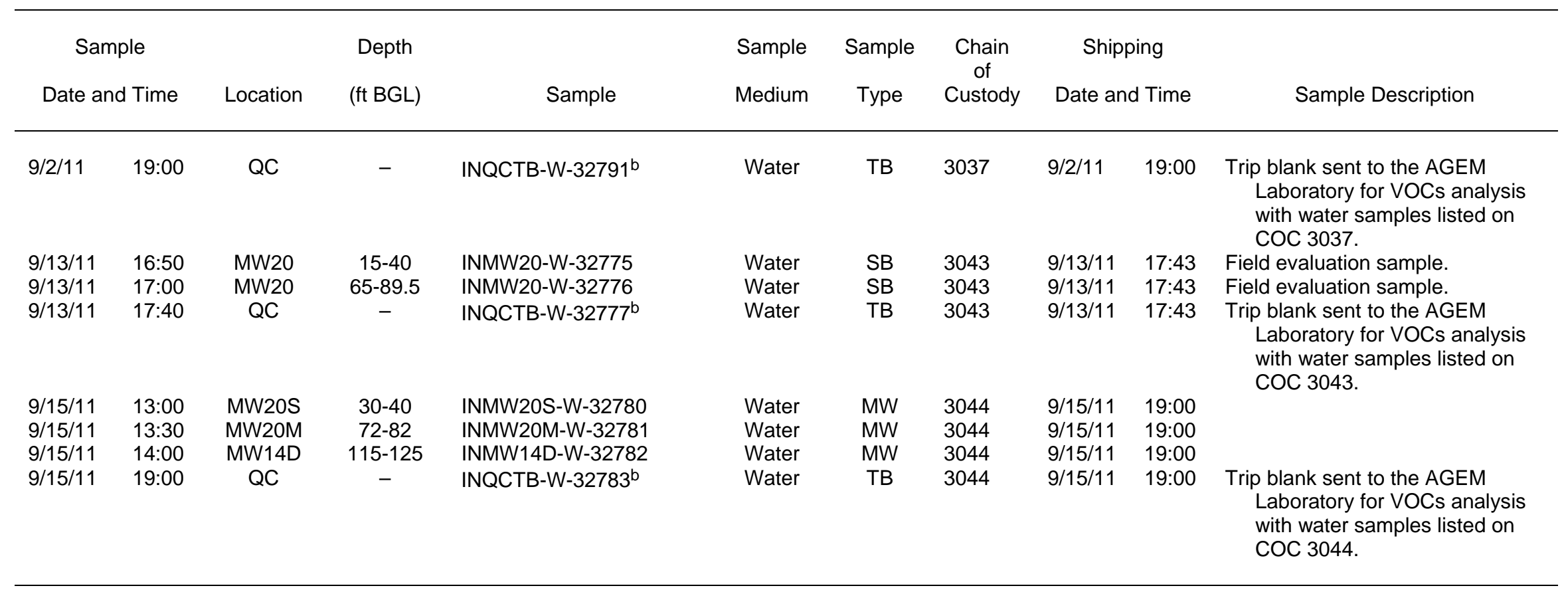

a Sample types: CPT, cone penetrometer; CPT/P, piezometer; DW, domestic well; FB, field blank; MW, monitoring well; RI, rinsate; SB, soil boring; TB, trip blank.

b Quality control sample collected to monitoring sample collection and handling procedures. 


\section{Appendix D:}

\section{Well Registration (WWC-5) Forms}




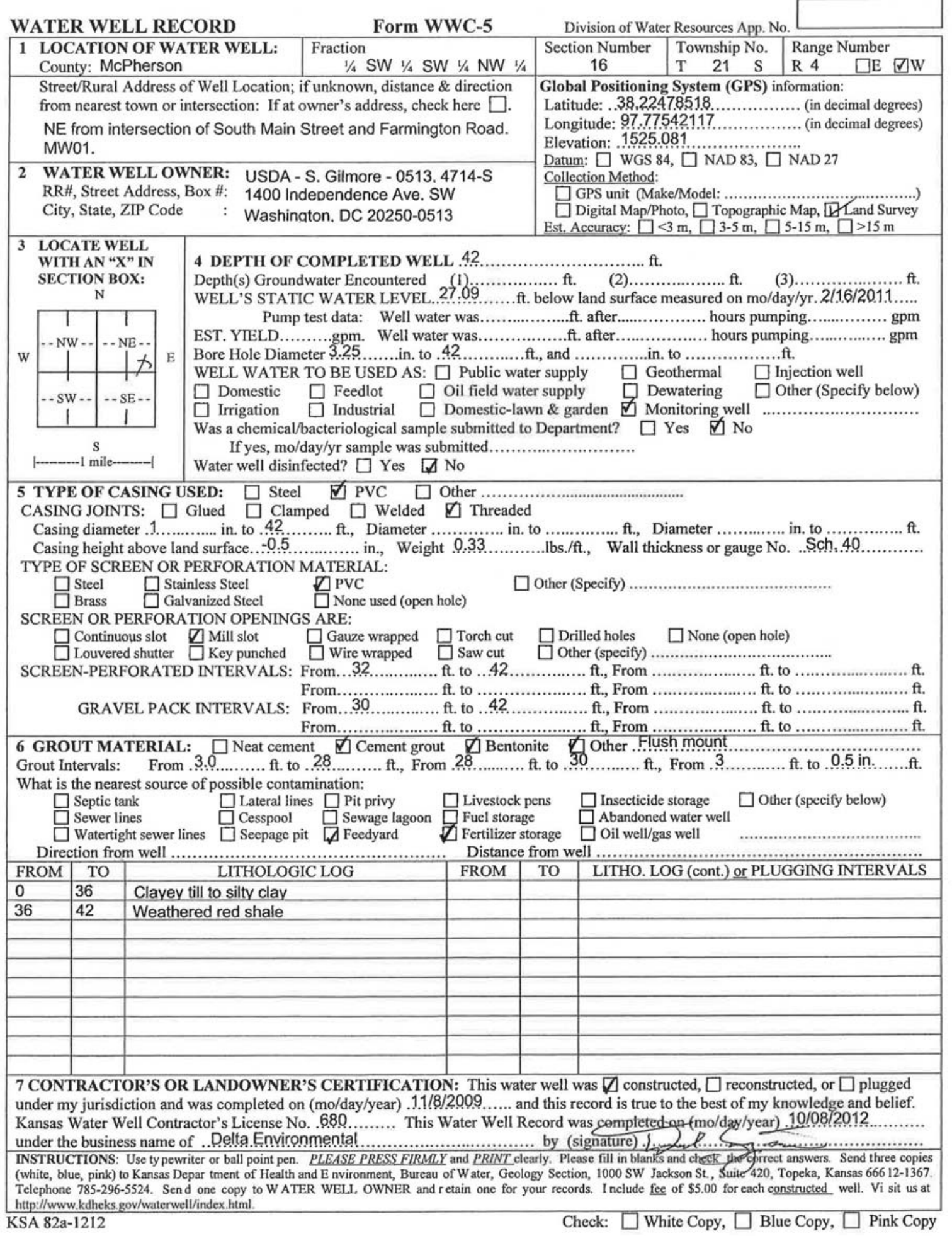


WATER WELL RECORD

\begin{tabular}{|c|c|c|c|}
\hline & \\
\hline & \multicolumn{3}{|c|}{$\begin{array}{l}\text { Street/Rural Address of } \\
\text { from nearest town or in }\end{array}$} \\
\hline & \multicolumn{3}{|c|}{$\begin{array}{l}\text { NE from intersectio } \\
\text { MW02. }\end{array}$} \\
\hline 2 & \multicolumn{3}{|c|}{$\begin{array}{l}\text { WATER WELL OW } \\
\text { RR\#, Street Address, } \\
\text { City, State, ZIP Code }\end{array}$} \\
\hline 3 & \multicolumn{3}{|c|}{$\begin{array}{l}\text { LOCATE WELL } \\
\text { WITH AN "X" IN } \\
\text { SECTION BOX: } \\
\text { N }\end{array}$} \\
\hline & & $\mid$ & \\
\hline & & $\begin{array}{r}\cdots \\
-N E\end{array}$ & \\
\hline & $-\begin{array}{c}-s w \\
1\end{array}$ & - SE - & \\
\hline
\end{tabular}

Form WWC-5

Fraction $1 / 4$ SW $1 / 4$ SW $1 / 4$ NW $1 / 4$

Well Location; if unknown, distance \& direction
.

South Main Street and Farmington Road.

USDA - S. Gilmore - 0513. 4714-S

1400 Independence Ave. SW

Washington. DC 20250-0513
Division of Water Resources App. No.

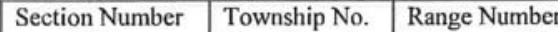
16 \begin{tabular}{lll|l}
$\mathrm{T}$ & $21 \mathrm{~S}$ & $\mathrm{R} 4$
\end{tabular} Global Positioning System (GPS) information:

Latitude: . 38,2248.525.

Longitude: 97.77560063

Elevation: 1525.521

Datum: $\square$ WGS 84, $\square$ NAD............ Collection Method:

$\square$ GPS unit (Make/Model: ........................................) Digital Map/Photo, $\square$ Topographic Map, $\square$ Land Survey Est. Accuracy: $\square<3 \mathrm{~m}, \square 3-5 \mathrm{~m}, \square 5-15 \mathrm{~m}, \square>15 \mathrm{~m}$ 4 DEPTH OF COMPLETED WELL 42

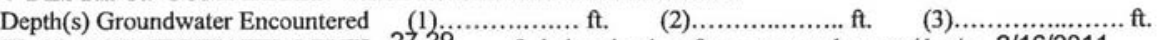
WELL'S STATIC WATER LEVEL..27.29 …...ft. below land surface measured on mo/day/yr. $2 / 16 / 201.1 \ldots .$. Pump test data: Well water was...................ft. after................... hours pumping................. gpm

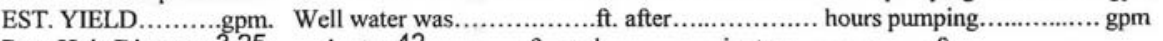

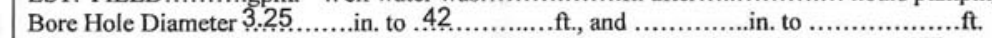
WELL WATER TO BE USED AS: $\square$ Public water supply $\quad \square$ Geothermal $\quad \square$ Injection well $\square$ Domestic $\square$ Feedlot $\square$ Oil field water supply $\square$ Dewatering $\square$ Other (Specify below) $\square$ Irrigation $\square$ Industrial $\square$ Domestic-lawn \& garden $\square$ Monitoring well Was a chemical/bacteriological sample submitted to Department? $\square$ Yes $\square$ No If yes, mo/day/yr sample was submitted.

Water well disinfected? $\square$ Yes $\square$ No

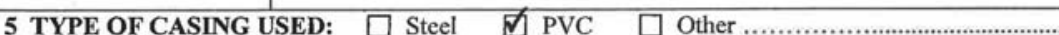

CASING JOINTS: $\square$ Glued $\square$ Clamped $\square$ Welded $\square$ Threaded

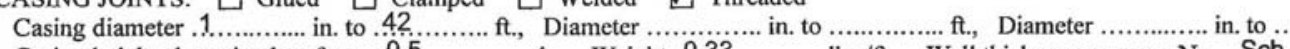

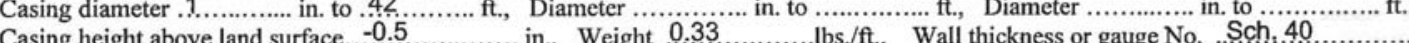
TYPE OF SCREEN OR PERFORATION MATERIAL:

$\square$ Steel $\square$ Stainless Steel $\square$ PVC

None used (open hole)

SCREEN OR PERFORATION OPENINGS ARE:

$\square$ Continuous slot $\square$ Mill slot $\square$ Gauze wrapped $\square$ Torch cut $\square$ Drilled holes $\square$ None (open hole)

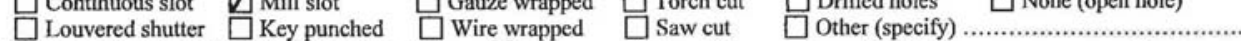

SCREEN-PERFORATED INTERVALS: From.... $32 \ldots \ldots \ldots \ldots \ldots . . . \mathrm{ft}$. to ...42 .

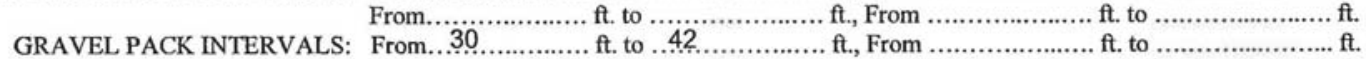

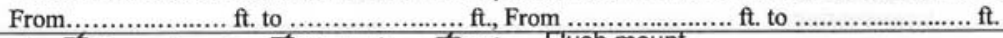

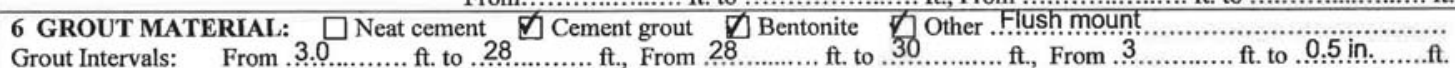
What is the nearest source of possible contamination:
$\square$ Septic tank
$\square$ Lateral lines $\square$ Pit privy $\square$ Livestock pens

Watertight sewer lines

Cesspool Sewage lagoon $\square$ Fuel storage

Fertilizer storage Abandoned water well

\begin{tabular}{|l|c|l|l|l|l|}
\hline FROM & TO & \multicolumn{1}{c|}{ LITHOLOGIC LOG } & FROM & TO & LITHO. LOG (cont.) or PLUGGING INTERVALS \\
\hline 0 & 36 & Clayey silt to silty clay & & & \\
\hline 36 & 42 & Weathered red and areen shale & & & \\
\hline & & & & & \\
\hline & & & & & \\
\hline & & & & & \\
\hline & & & & & \\
\hline & & & & & \\
\hline & & & & & \\
\hline
\end{tabular}

7 CONTRACTOR'S OR LANDOWNER'S CERTIFICATION: This water well was $\square$ constructed, $\square$ reconstructed, or $\square$ plugged under my jurisdiction and was completed on (mo/day/year) $1.1 / 4 / 2009 . \ldots . . .$. and this record is true to the best of my knowledge and belief. Kansas Water Well Contractor's License No. $680 . \ldots . . . .$. This Water Well Record was cempleted on (mo/day/year) .10/08/2012

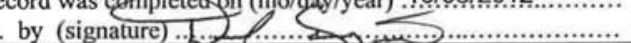

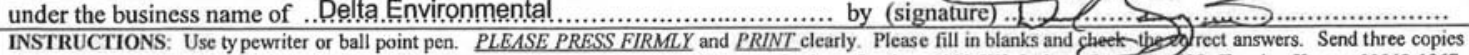
(white, blue, pink) to Kansas Depar tment of Health and E nvironment, Bureau of W ater, Geology Section, 1000 SW Jackson St, Swit 420, Topeka, Kansas 666 I2-1367. Telephone 785-296-5524. Send one copy to W ATER WELL OWNER and retain one for your records. Include fee of $\$ 5.00$ for each constructed well. Vi sit us at http://www.kdheks. gov/waterwell/index.html 
WATER WELL RECORD

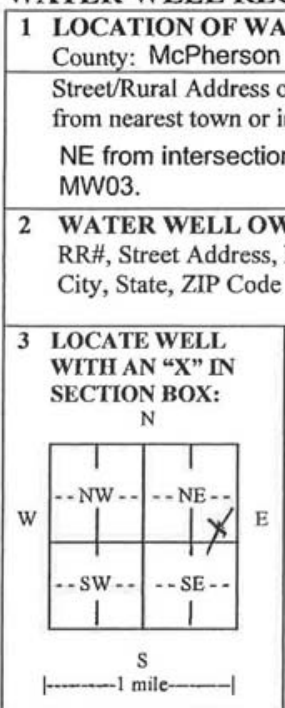

Form WWC-5 Fraction $1 / 4$ SW $1 / 4$ SW $1 / 4$ NW $1 / 4$ Well Location; if unknown, distance \& direction . If at owner's address, check here $\square$.

$$
\begin{aligned}
& \text { USDA - S. Gilmore - 0513. 4714-S } \\
& 1400 \text { Independence Ave. SW }
\end{aligned}
$$$$
\text { Washinaton. DC 20250-0513 }
$$

Division of Water Resources App. No. Section Number $\quad$ Township No. $\quad$ Range Number

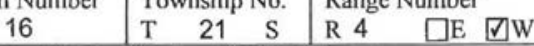
Global Positioning System (GPS) information:

Latitude: ..38,225526, $66.6 \ldots \ldots \ldots \ldots \ldots$ (in decimal degrees)

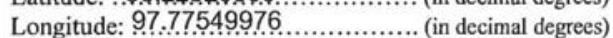
Elevation: 1525.716

Datum: $\square$ WGS 84, $\square$ NAD 83, $\square$ NAD 27 Collection Method:

$\square$ GPS unit (Make/Model: ..................................) Digital Map/Photo, $\square$ Topographic Map, [I Land Survey Est. Accuracy: $\square<3 \mathrm{~m}, \square 3-5 \mathrm{~m}, \square 5-15 \mathrm{~m}, \square>15 \mathrm{~m}$

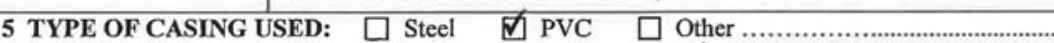

CASING JOINTS: $\square$ Glued $\square$ Clamped $\square$ Welded $\square$ Threaded

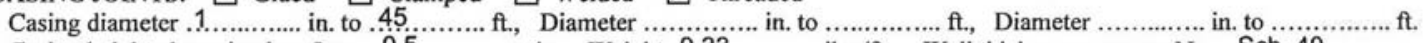

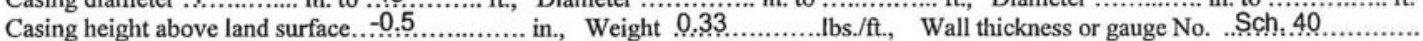

TYPE OF SCREEN OR PERFORATION MATERIAL:

$\square$ Steel $\square$ Stainless Steel $\square$ PVC

$\square$ Brass $\square$ Galvanized Steel $\square$ Non

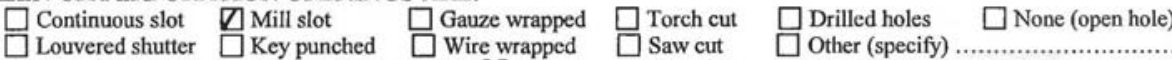

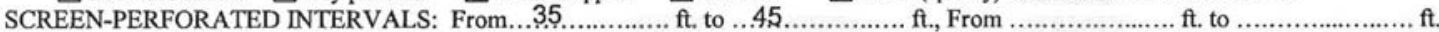

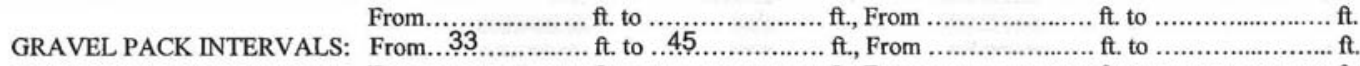

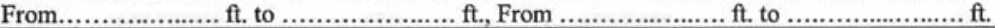

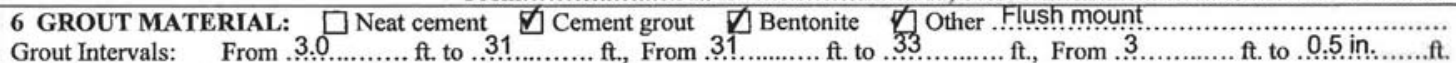

What is the nearest source of possible contamination:

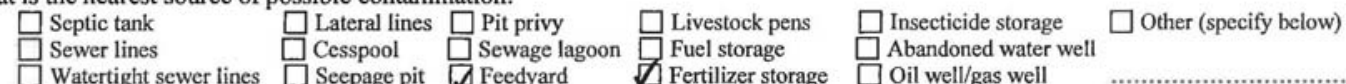

$\square$ Watertight sewer lines $\square$ Seepage pit $\square$ Feedyard $\square$ Fertilizer storage $\square$ Oil well/gas well

Direction from well ............................................... Distance from well

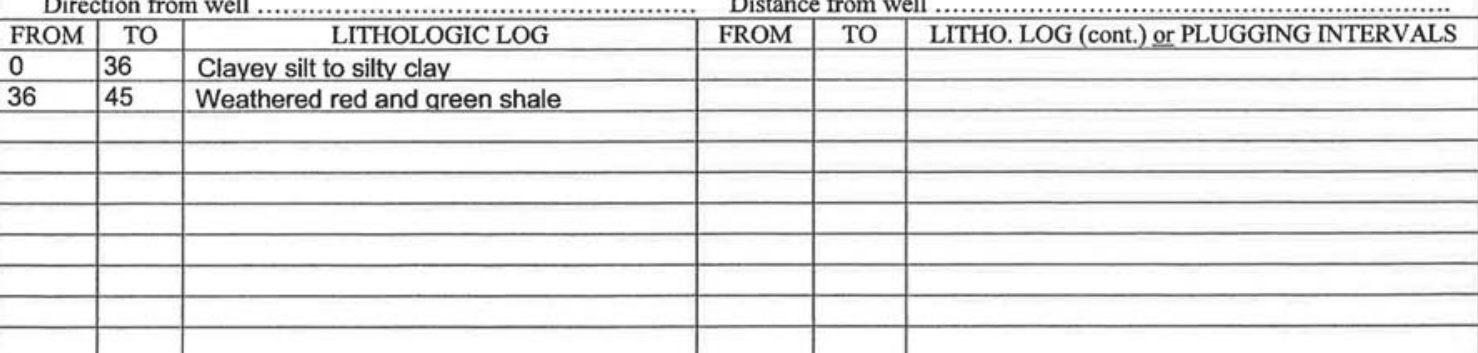

7 CONTRACTOR'S OR LANDOWNER'S CERTIFICATION: This water well was $\square$ constructed, $\square$ reconstructed, or $\square$ plugged under my jurisdiction and was completed on (mo/day/year) $.11 / 6 / 2009 . . . .$. and this record is true to the best of my knowledge and belief. Kansas Water Well Contractor's License No. 680......... This Water Well Record was completed on (ing/day/year) 10/08/2012

under the business name of Delta Environmental.

by (signature)

INSTRUCTIONS: Use ty pewriter or ball point pen. PLEASE PRESS FIRMLY and PRINT clearly. Please fill in blanks and ctieck the concrot answers. Send three copies (white, blue, pink) to Kansas Depar tment of Health and E nvironment, Bureau of W ater, Geology Section, 1000 SW Jackson St, Sujre 420, Topeka, Kansas $66612-1367$. Telephone 785-296-5524. Send one copy to W ATER WELL OWNER and r etain one for your records. I nclude fee of $\$ 5.00$ foreach constructed well. Vi sit us at http://www.kdheks. gov/waterwell/index.html.

KSA 82a-1212

Check: $\square$ White Copy, $\square$ Blue Copy, $\square$ Pink Copy 


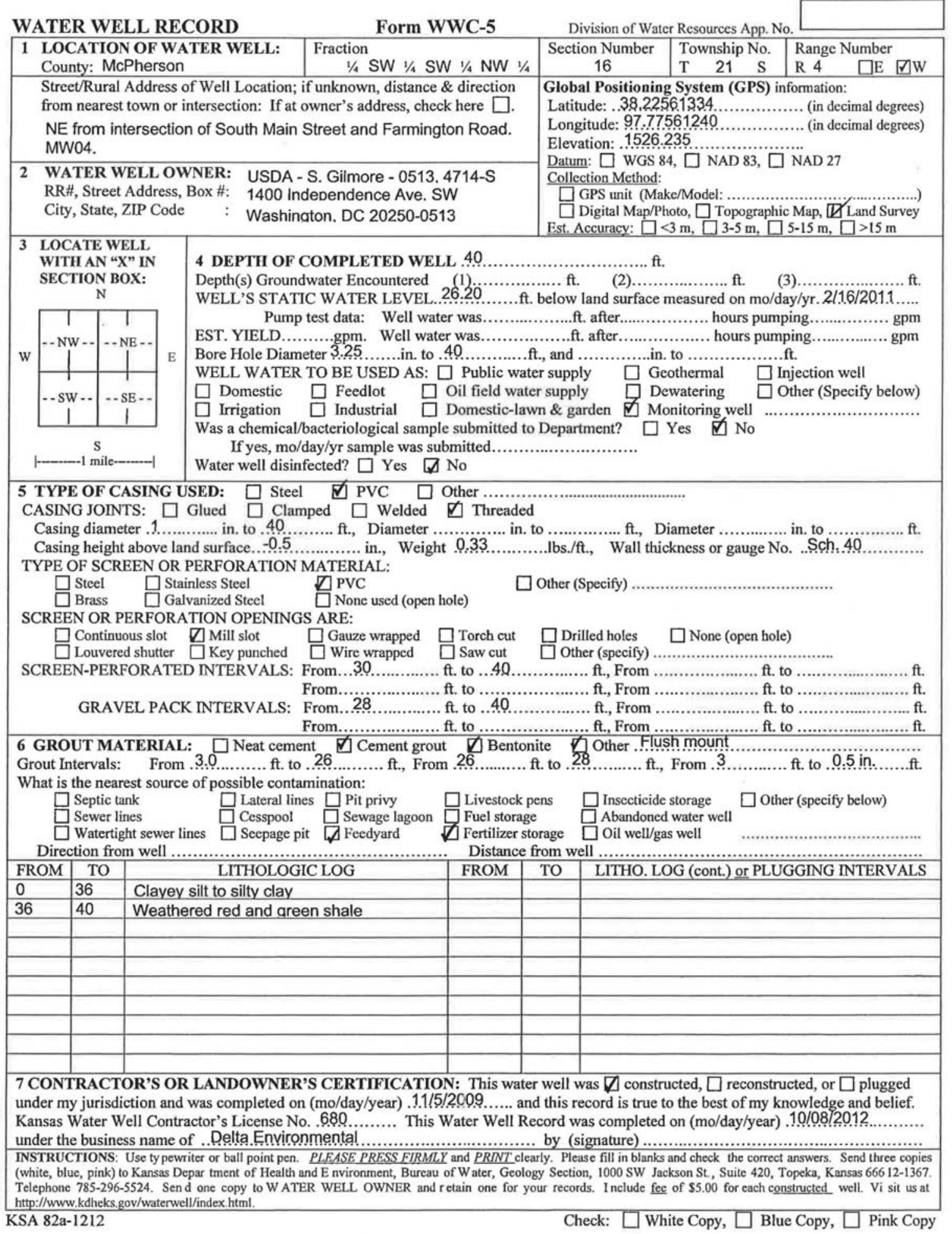




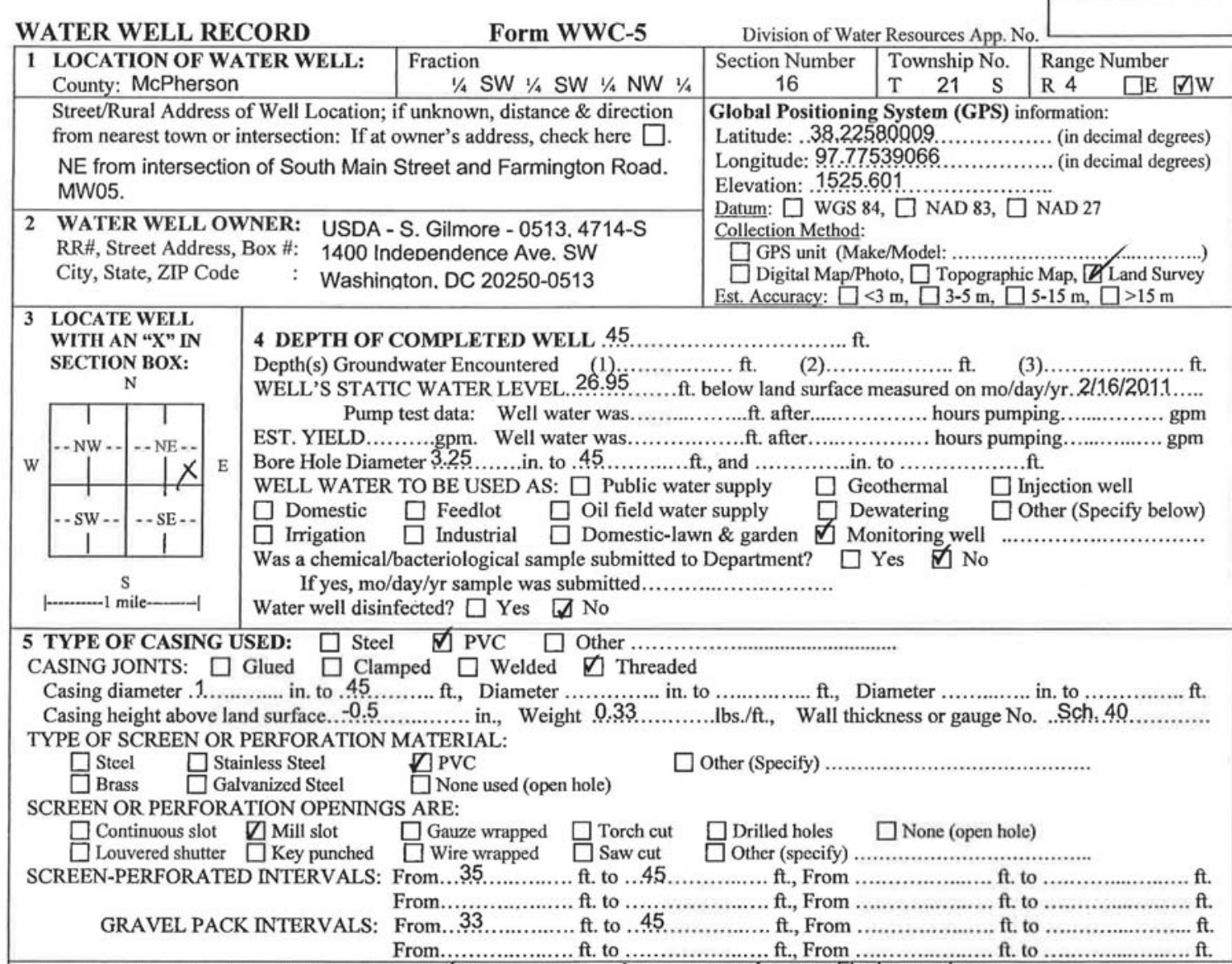

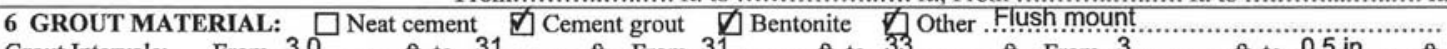

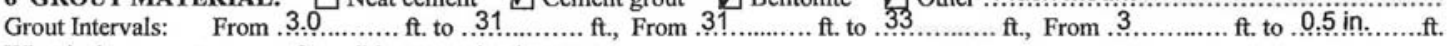
What is the nearest source of possible contamination:
$\square$ Septic tank
$\square$ Lateral lines $\square$ Pit privy
$\square$ Cesspool
$\square$ Secpage pit
$\square$ Insecticide storage
Abandoned water well
$\square$ Watertight sewer lines $\square$ Secpage pit $\square$ Feedyard $\square$ Fertilizer storage $\square$ Oil well/gas well

Distance from well .......

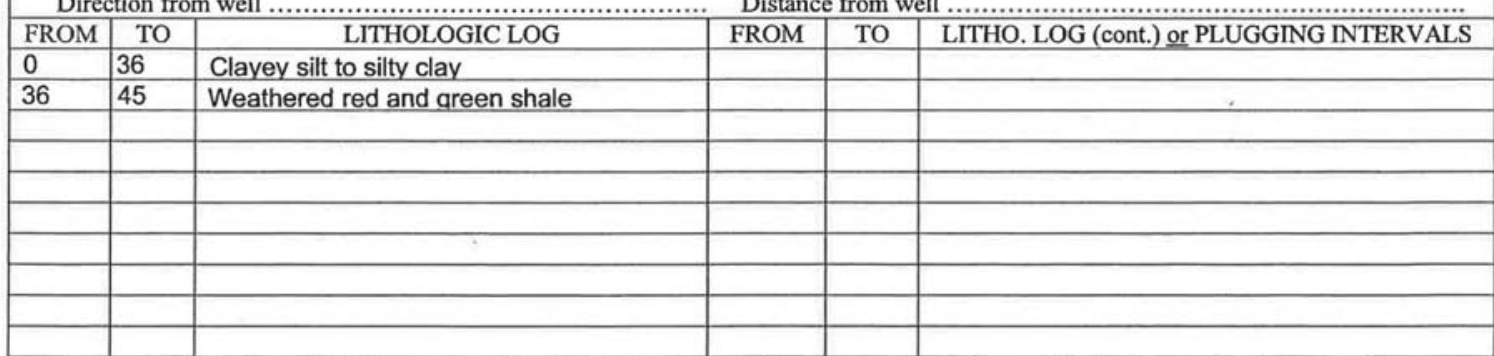

7 CONTRACTOR'S OR LANDOWNER'S CERTIFICATION: This water well was $\square$ constructed, $\square$ reconstructed, or $\square$ plugged under my jurisdiction and was completed on (mo/day/year) $.11 / 7 / 2009 . \ldots .$. and this recopt is true to the best of my knowledge and belief.

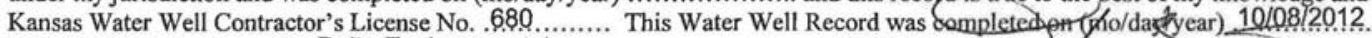

under the business name of ..Delta Environmenta! .............................. by (signature) $L$.

INSTRUCTIONS: Use ty pewriter or ball point pen. PLEASE PRESS FIRMIY and PRIVT el.......... Y Bureau of Water, Geology Section, 1000 SW Jackson St, Suite 20 X opeka, Kansas 666 12-1367 elephone 785-296-5524. Send one copy to

KSA $82 \mathrm{a}-1212$

Check: $\square$ White Copy, $\square$ Blue Copy, $\square$ Pink Copy 


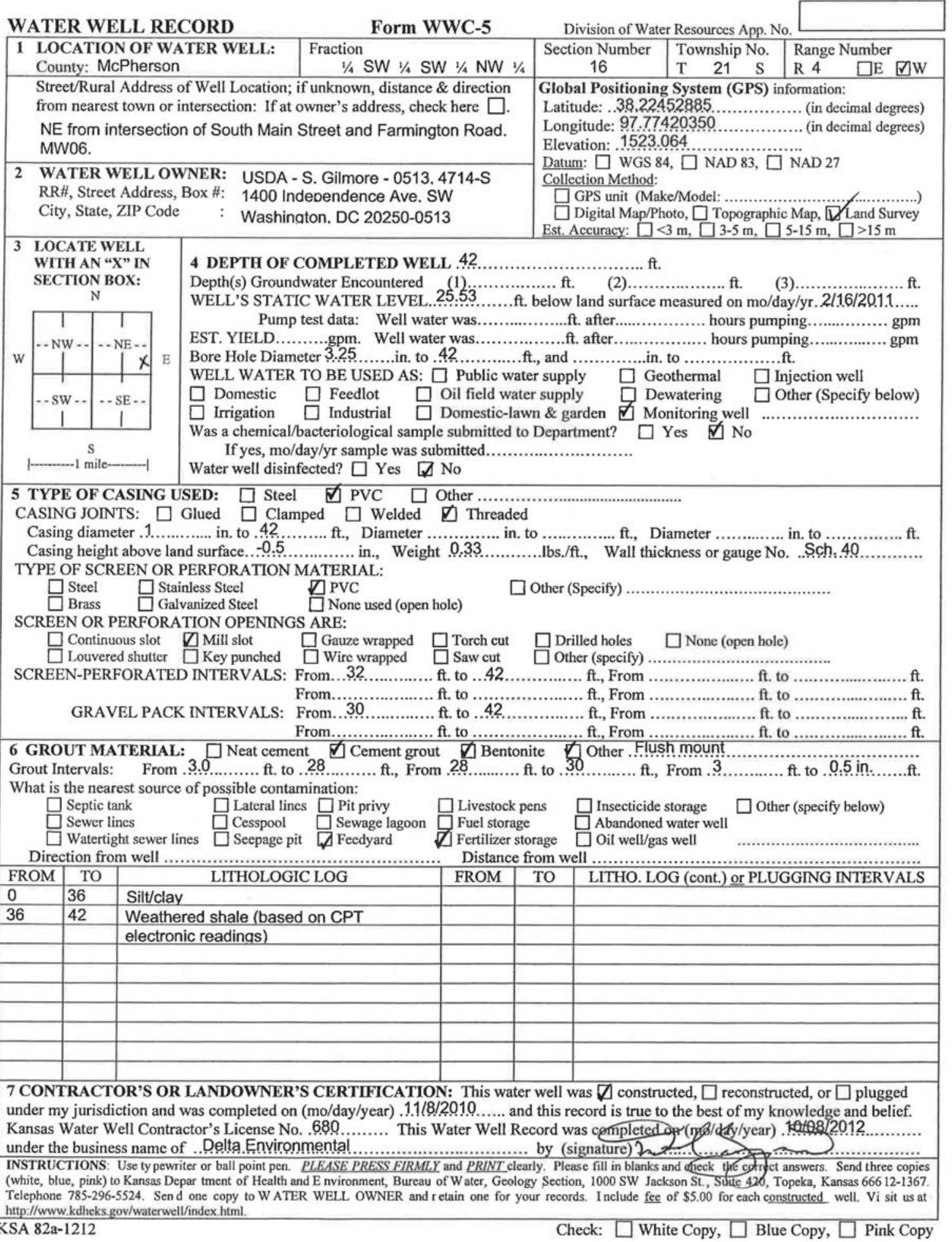




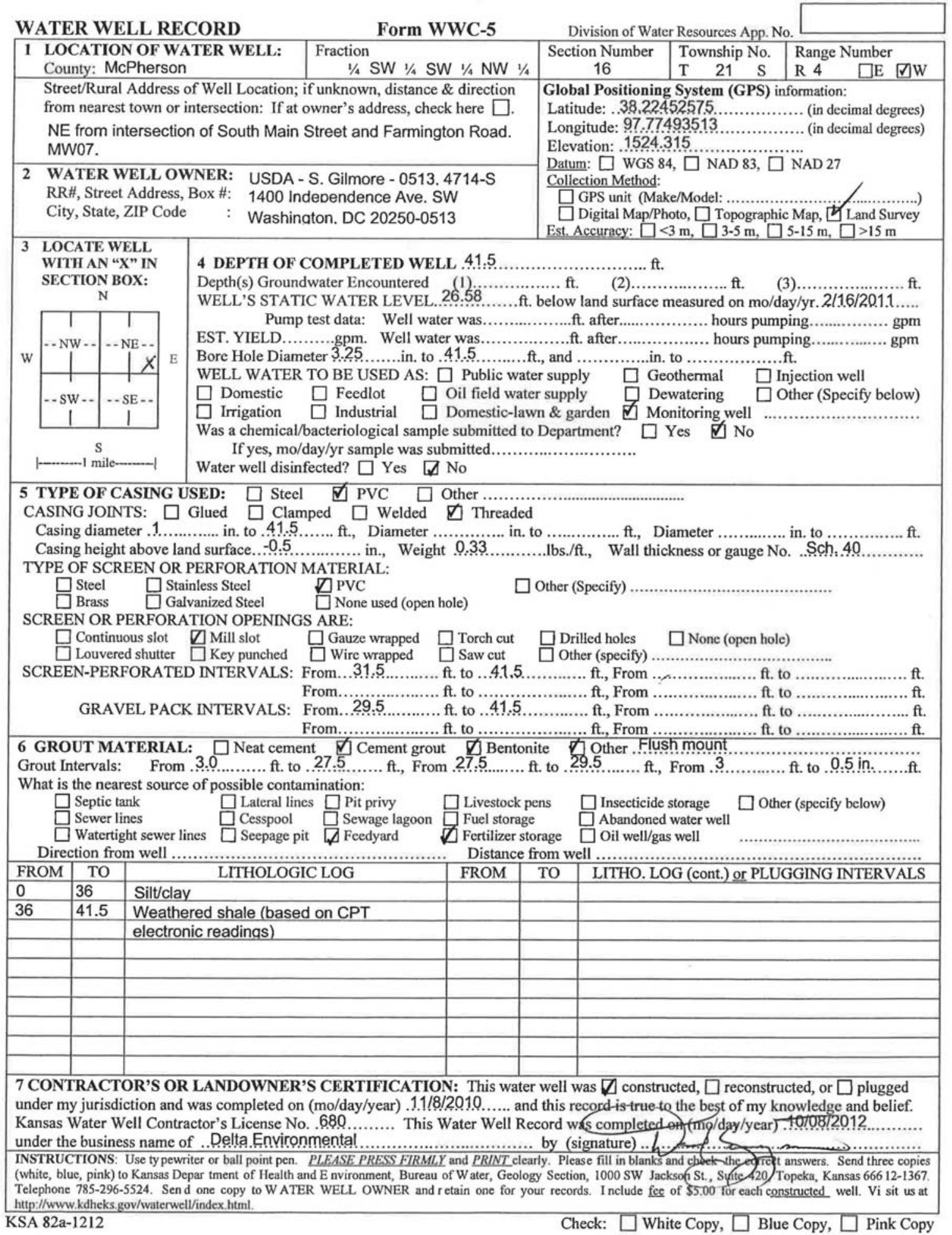


WATER WELL RECORD 1 LOCATION OF WATER WELL: County: McPherson NE from intersection of South Main Street and Farmington Road. MW08.

2 WATER WELL OWNER: USDA - S. Gilmore - 0513, 4714-S RR\#, Street Address, Box \#: 1400 Indedendence Ave. SW City, State, ZIP Code
Form WWC-5 Fraction $1 / 4$ SW $1 / 4$ SW $1 / 4$ NW $1 / 4$ Washinaton. DC 20250-0513

Division of Water Resources App. No. Section Number $\quad$ Township No. $\quad$ Range Number 16 $\begin{array}{lllll}\mathrm{T} & 21 & \mathrm{~S} & \mathrm{R} & 4\end{array} \mathrm{DE} \quad \mathrm{V} \mathrm{W}$ Global Positioning System (GPS) information:

Latitude: . 38, 22.452676. Longitude: 97.77539010 Elevation: 1524.848

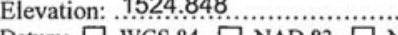
Datum: $\square$ WGS 84, $\square$ NAD 83, $\square$ NAD 27 Collection Method:

$\square$ GPS unit (Make/Model: .......................................) $\square$ Digital Map/Photo, $\square$ Topographic Map, $\square$ Land Survey Est. Accuracy: $\square<3 \mathrm{~m}, \square 3-5 \mathrm{~m}, \square 5-15 \mathrm{~m}, \square>15 \mathrm{~m}$

3 LOCATE WELL WITH AN "X" IN SECTION BOX:

W

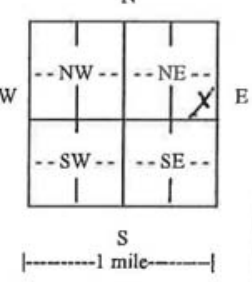
4 DEPTH OF COMPLETED WELL 41 ... ft.

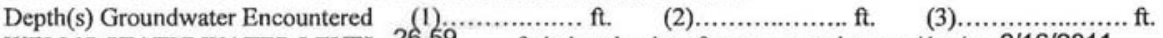
WELL'S STATIC WATER LEVEL..26.59 …....f. below land surface measured on mo/day/yr. 2l16/2011..... Pump test data: Well water was..................t. after................. hours pumping................. gpm EST. YIELD..........gpm. Well water was...............ft. after................ hours pumping ................. gpm Bore Hole Diameter $3.25 \ldots \ldots . .$. in. to .41 ............ft., and ................in. to ...................... WELL WATER TO BE USED AS: $\square$ Public water supply $\quad \square$ Geothermal $\quad \square$ Injection well $\square$ Domestic $\square$ Feedlot $\square$ Oil field water supply $\square$ Dewatering $\square$ Other (Specify below) $\square$ Irrigation $\square$ Industrial $\square$ Domestic-lawn \& garden Monitoring well Was a chemical/bacteriological sample submitted to Department? $\square$ Yes $\square$ No If yes, mo/day/yr sample was submitted.............................

Water well disinfected? $\square$ Yes $\square$ No

\section{TYPE OF CASING USED: $\square$ Steel $\square$ PVC $\square$ Other ...}

CASING JOINTS: $\square$ Glued $\square$ Clamped $\square$ welded $\square$ Threaded

Casing diameter .1............ in. to $41 \ldots \ldots \ldots \ldots$ ft., Diameter ............ in. to ............. ft., Diameter ............ in. to

Casing height above land surface... 0.5 ............... in., Weight $0.33 . . . . . . . .1$ lbs./ft., Wall thickness or gauge No. .Sch. 40

TYPE OF SCREEN OR PERFORATION MATERIAL:

$\square$ Steel $\square$ Stainless Steel $\quad \square$ PVC

$\square$ Brass $\square$ Galvanized Steel

SCREEN OR PERFORATION OPENINGS ARE:

$\square$ Continuous slot $\square$ Mill slot $\square$ Gauze wrapped $\square$ Torch cut $\square$ Drilled holes $\square$ None (open hole)

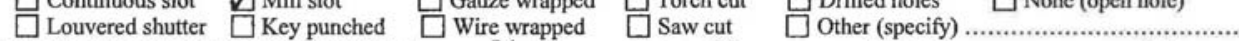

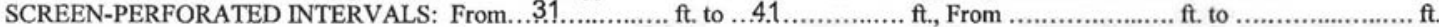

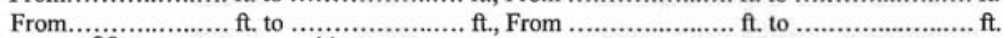

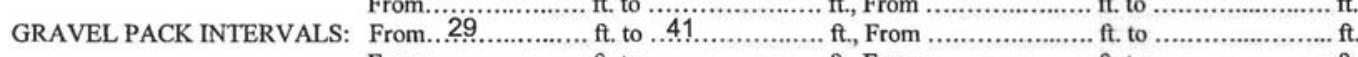

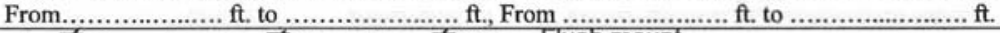

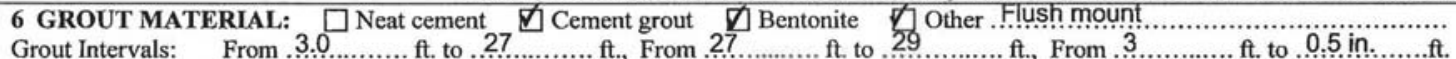
What is the nearest source of possible contamination:

$\square$ Septic tank $\square$ Lateral lines $\square$ Pit privy $\square$ Livestock pens $\square$ Insecticide storage $\square$ other (specify below)

$\square$ Sewer lines $\square$ Cesspool $\square$ Sewage lagoon $\square$ Fuel storage $\square$ Abandoned water well

$\square$ Watertight sewer lines $\square$ Seepage pit $\square$ Feedyard $\square$ Fertilizer storage $\square$ Oil well/gas well

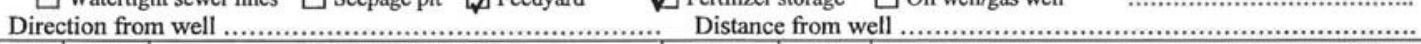
\begin{tabular}{|l|l|l|l|l|l|}
\hline FROM & TO & LITHOLOGIC LOG & FROM & TO & LITHO. LOG (cont.) or PLUGGING INTERVALS \\
\hline
\end{tabular}

\begin{tabular}{|l|l|l|l|l|l|}
\hline & No core, no CPT electronics & & & \\
\hline & & & & & \\
\hline & & & & & \\
\hline & & & & & \\
\hline & & & & & \\
\hline & & & & & \\
\hline & & & & & \\
\hline & & & & & \\
\hline & & & & \\
\hline
\end{tabular}

7 CONTRACTOR'S OR LANDOWNER'S CERTIFICATION: This water well was $\square$ constructed, $\square$ reconstructed, or $\square$ plugged under my jurisdiction and was completed on (mo/day/year) $.11 / 13 / 2010 \ldots$ and this record is true to the best of my knowledge and belief. Kansas Water Well Contractor's License No. 680 ......... This Water Well Record was completed on (fijo/day/year) 10/08/2012. under the business name of ..Delta Environmental ............................ by (signature) $h_{2}$. INSTRUCTIONS: Use ty pewriter or ball point pen. PLEASE PRESS FIRMLY.Y and PRINT clearly. Please fill in blanks and check the cofrect) nnswers. Send three copies (white, blue, pink) to Kansas Depar tment of Health and E nvironment, Bureau of Water, Geology Section, 1000 SW Jackson St., Suite (420, Thpeka, Kansas $66612-1367$ Telephone 785-296-5524. Send one copy to W ATER WELL. OWNER and r etain one for your records. I nclude fee of $\$ 5.00$ for each constructed well. Vi sit us at http://www.kdheks, gov/waterwell/index.htmL.

KSA 82a-1212

Check: $\square$ White Copy, $\square$ Blue Copy, $\square$ Pink Copy 


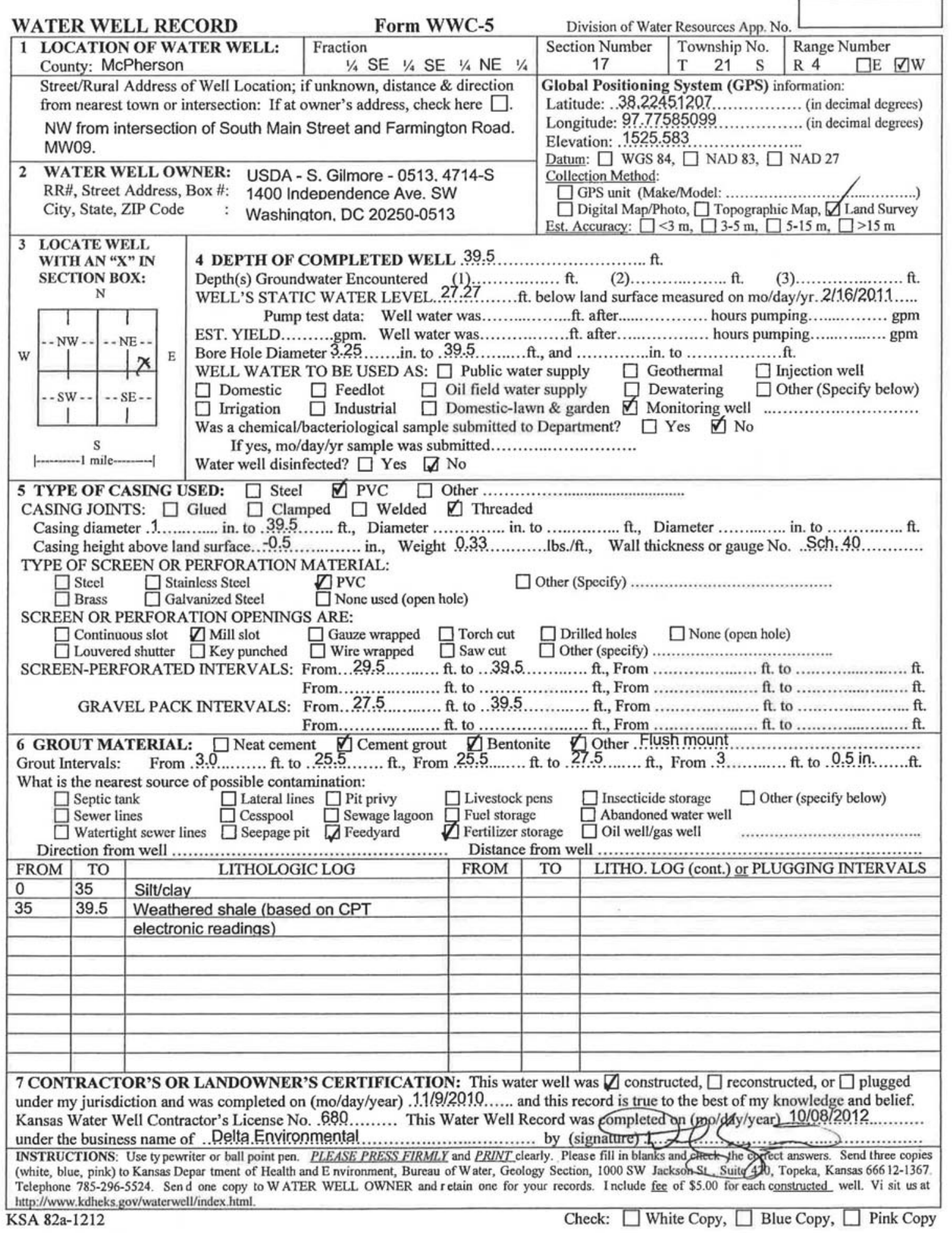


WATER WELL RECORD

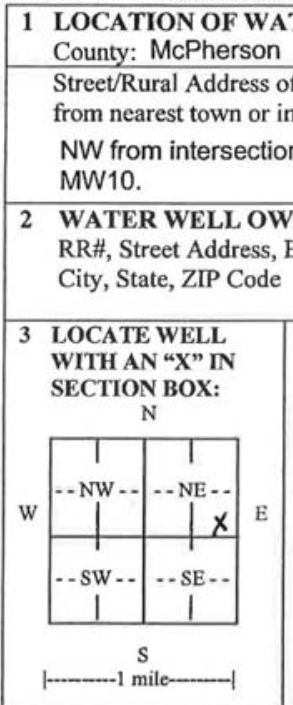

Form WWC-5

Fraction
$1 / 4$
$1 / 4$ SE $1 / 4$ SE $1 / 4$ NE $1 / 4$ Well Location; if unknown, distance \& direction foction: If at owner's address, check here $\square$

: USDA - S. Gilmore - 0513, 4714-S 1400 Indedendence Ave. SW Washinaton. DC 20250-0513
Division of Water Resources App. No.

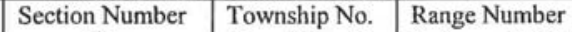
17 \begin{tabular}{lll|l}
$\mathrm{T}$ & 21 & $\mathrm{~S}$ & $\mathrm{R} 4$
\end{tabular}

Global Positioning System (GPS) information:

Latitude: . $38,2248,4.172 \ldots \ldots \ldots \ldots \ldots$ (in decimal degrees) Longitude: $97.77595005 . \ldots \ldots \ldots \ldots \ldots . . . .($ in decimal degrees)

Elevation: 1525.482

Datum: $\square$ WGS 84, $\square$ NAD 83, $\square$ NAD 27 Collection Method:

$\square$ GPS unit (Make/Model: ......................................) Digital Map/Photo, $\square$ Topographic Map, $\mathbb{D}$ Land Survey Est. Accuracy: $\square<3 \mathrm{~m}, \square 3-5 \mathrm{~m}, \square 5-15 \mathrm{~m}, \square>15 \mathrm{~m}$

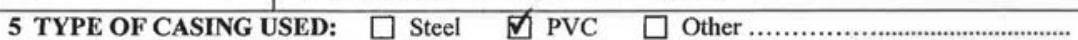

CASING JOINTS: $\square$ Glued $\square$ Clamped $\square$ Welded $\square$ Threaded

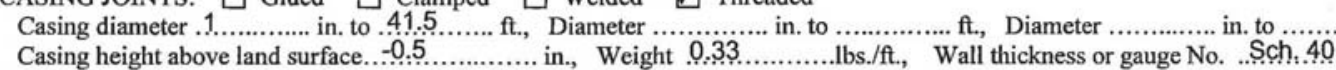
4 DEPTH OF COMPLETED WELL 41.5 ft.

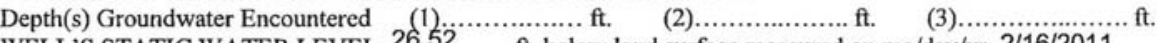
WELL'S STATIC WATER LEVEL. 26.52........f. below land surface measured on mo/day/yr. $2 / 16 / 20.1 .1 \ldots . .$. Pump test data: Well water was................ft. after............... hours pumping .............. gpm EST. YIELD ..........gpm. Well water was..............ft. after................. hours pumping ............... gpm

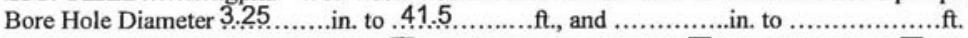
WELL WATER TO BE USED AS: $\square$ Public water supply $\square$ Geothermal $\square$ Injection well $\square$ Domestic $\square$ Feedlot $\square$ Oil field water supply $\square$ Dewatering $\square$ Other (Specify below) $\square$ Irrigation $\square$ Industrial $\square$ Domestic-lawn \& garden $\square$ Monitoring well Was a chemical/bacteriological sample submitted to Department? $\square$ Yes $\square$ No If yes, mo/day/yr sample was submitted.............................

If yes, mo/day/yr sample was submitted
Water well disinfected? $\square$ Yes $\square$ No

TYPE OF SCREEN OR PERFORATION MATERIAL:

$\square$ Steel $\square$ Stainless Steel $\square$ DVC

SCREEN OR PERFORATION OPENINGS ARE:

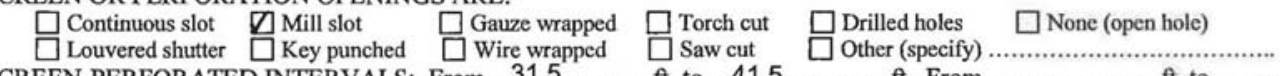

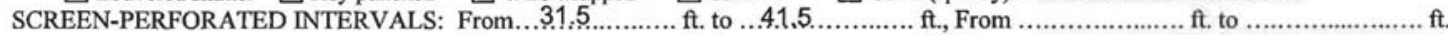

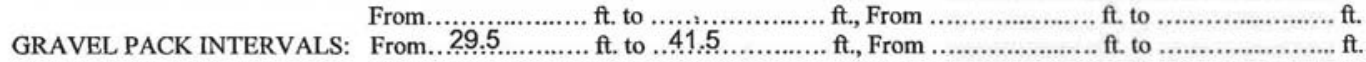

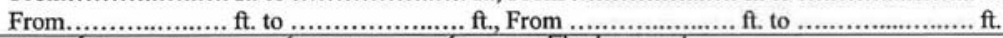

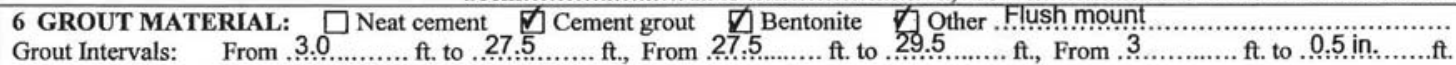
What is the nearest source of possible contamination:

$\square$ Septic tank $\square$ Lateral lines $\square$ Pit privy $\square$ Livestock pens $\square$ Insecticide storage $\square$ other (specify below)

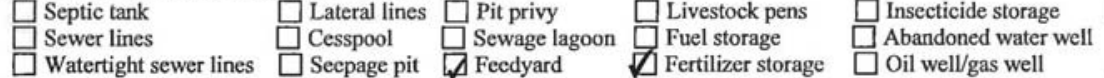

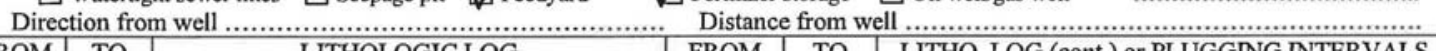
\begin{tabular}{|c|c|c|c|c|c|}
\hline FROM & TO & LITHOLOGIC LOG & FROM & TO & LITHO. LOG (cont.) or PLUGGING INTERVALS \\
\hline D
\end{tabular}

\begin{tabular}{|c|c|c|c|c|c|}
\hline KUM & & LMAULUGIC LUG & & & LITHU. LUU (cont.) or PLUUUINU INIEKVALS \\
\hline 0 & 34 & Silt/clay & & & \\
\hline 34 & 41.5 & Weathered shale (based on CPT & & & \\
\hline & & electronic readings) & & & \\
\hline & & & & & \\
\hline & & & & & \\
\hline & & & & & \\
\hline & & & & & \\
\hline & & & & & \\
\hline & & & & & \\
\hline 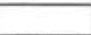 & - & ( & & & \\
\hline
\end{tabular}

7 CONTRACTOR'S OR LANDOWNER'S CERTIFICATION: This water well was $\nabla$ constructed, $\square$ reconstructed, or $\square$ plugged under my jurisdiction and was completed on (mo/day/year) $1.1 / 9 / 2010 \ldots . .$. and this record is true to the best of my knowledge and belief. Kansas Water Well Contractor's License No. $680 . . . . . .$. This Water Well Record was completed on (mo/dary/year) .10/0 $8 / 2012$.

under the business name of ... Delta Environmental ........................... by (signature) . Ho INSTRUCTIONS: Use ty pewriter or ball point pen. PLEASE PRESS FIRMLY and PRINT clearly. Please fill in blanks and eheck the - on ect answers. Send three copies (white, blue, pink) to Kansas Depar tment of Health and E nvironment, Bureau of W ater, Geology Section, 1000 SW Jackson St., Suite 420, Topeka, Kansas $66612-1367$. Telephone 785-296-5524. Send one copy to W ATER WELL OWNER and r etain one for your records. Include fee of $\$ 5.00$ for each constructed well. Vi sit us at http://www.kdheks.gov/waterwell/index.html.

KSA 82a-1212

Check: $\square$ White Copy, $\square$ Blue Copy, $\square$ Pink Copy 
WATER WELL RECORD

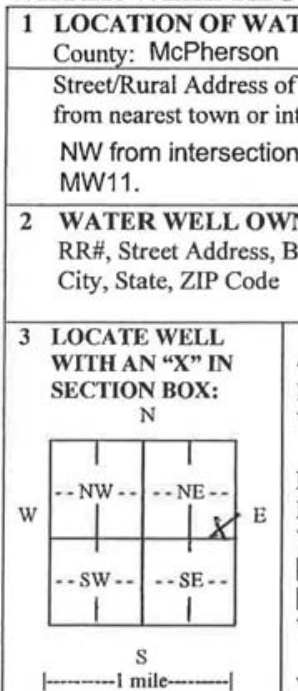

5 TYPE OF CASING USED:

CASING JOINTS:

Casing diameter .1. $\square$ Glued

\section{1.....} Glued $\square \mathrm{Cl}$ 4 DEPTH OF COMPLETED WELL 41

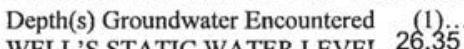

Form WWC-5

Fraction
$1 / 4$ SE $1 / 4$ SE $1 / 4$ NE $1 / 4$

Well Location; if unknown, distance \& direction

Casing height above land surface....
TYPE OF SCREEN OR PERFORATION MATERIAL: Pump test data: Well water was.....
EST. YIELD.............pm. Well water was..

USDA - S. Gilmore - 0513. 4714-S

1400 Independence Ave. SW

Washinaton. DC 20250-0513

Bore Hole Diameter 3.25 ........in. to .41

WELL WATER TO BE USED AS. $\square$ Public....t., and .....

$\square$ Domestic $\square$ Feedlot $\square$ Oil field water supply

Irrigation

as a chemical/bacteriological sample submitted to Department?

If yes, $\mathrm{mo} / \mathrm{day} / \mathrm{yr}$ sample was submitted.

ter well disinfected? $\square$ Yes $\square$ No

$\square$ Steel $\square$ Stainless Steel $\quad \square$ PVC

SCREEN OR PERFORATION OPENINGS ARE:

\begin{tabular}{lll}
$\square$ Continuous slot & $\nabla$ Mill slot \\
\hline Louvered shutter
\end{tabular} Key punched $\quad \square$ Wire wrapped $\quad \square$ Sorch cut $\quad \square$ Drilled holes $\quad \square$ None (open hole)

SCREEN-PERFORATED INTERVALS: From... 31

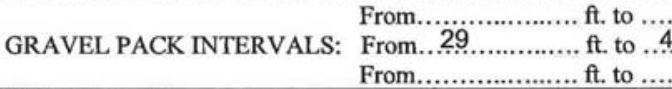

ft. From

ft. to .

ft., From ….................. ft. to

... in. to .

ft., Diameter

Sch, 40 ... $\mathrm{ft}$.

ft., From

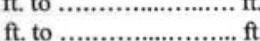

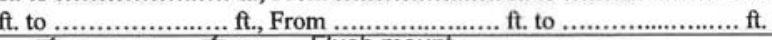

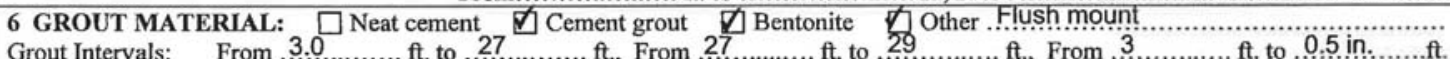

What is the nearest source of possible contamination:

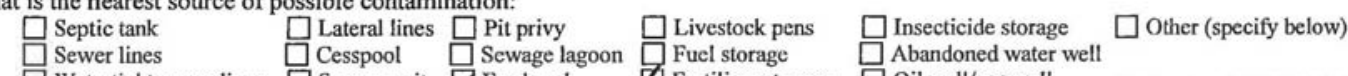

$\square$ Watertight sewer lines $\square$ Seepage pit $\square$ Feedyard $\quad \square$ Fertilizer storage $\quad \square$ Oil well/gas well

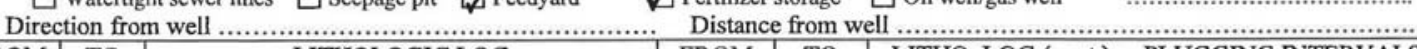

\begin{tabular}{|l|c|c|c|c|c|}
\hline FROM & TO & LITHOLOGIC LOG & FROM & TO & LITHO. LOG (cont.) or PLUGGING INTERVALS \\
\hline 0 & 34 & Silt/clay & & & \\
\hline 34 & 41 & W &
\end{tabular}

\begin{tabular}{l|lll}
\hline 0 & 34 & Siltclay \\
\hline 34 & 41 & Weathered shale (based on CPT
\end{tabular}

\begin{tabular}{|l|l|l|l|l|l|}
\hline 34 & 41 & Weathered shale (based on CPT & & & \\
\hline & & & & & \\
\hline & & & & & \\
\hline & & & & & \\
\hline & & & & & \\
\hline & & & & & \\
\hline & & & & & \\
\hline & & & & \\
\hline
\end{tabular}

7 CONTRACTOR'S OR LANDOWNER'S CERTIFICATION: This water well was $\square$ constructed, $\square$ reconstructed, or $\square$ plugged under my jurisdiction and was completed on (mo/day/year) $1.1 / 10 / 2010 \ldots .$. and this record is true to the best of my knowledge and belief.

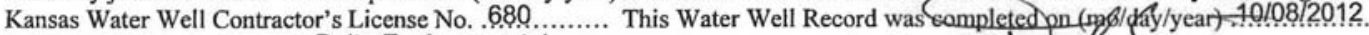

under the business name of ..Delta. Environmental.............................. by (signature) $h_{1 .}$

INSTRUCTIONS: Use ty pewriter or ball point pen. PLEASE PRESS FIRMLY and PRINT clearly. Please fill in blanks and check thereorect answers. Send three copies (white, blue, pink) to Kansas Depar tment of Health and E nvironment, Bureau of W ater, Geology Section, 1000 SW Jackson St, Suite 120, Topeka, Kansas 666 12-1367. Telephone 785-296-5524. Send one copy to W ATER WELL OWNER and retain one for your records. I nclude fee of $\$ 5.00$ for each constructed well. Vi sit us at http://www.kdheks. gov/waterwell//index.htmL 
WATER WELL RECORD 1 LOCATION OF WATER WELI County: McPherson

Form WWC-5 from nearest town or intersection: If at owner's address, check here $\square$ NW from intersection of South Main Street and Farmington Road. MW12.

2 WATER WELL OWNER: USDA - S. Gilmore - 0513, 4714-S RR\#, Street Address, Box \#: 1400 Indedendence Ave. SW City, State, ZIP Code $\quad$ : Washinaton, DC 20250-0513

Division of Water Resources App. No. \begin{tabular}{|l|l|l}
\hline Section Number & Township No. & Range Number
\end{tabular} Global Positioning System (GPS) information: Latitude: ..38.2255.090.1 ... (in decimal degrees) Longitude: $97.77621272 \ldots \ldots \ldots \ldots .$. (in decimal degrees) Elevation: 1525.808 Datum: $\square$ WGS 84, $\square$ NAD 83, $\square$ NAD 27 Collection Method:

$\square$ GPS unit (Make/Model: ......................................) $\square$ Digital Map/Photo, $\square$ Topographic Map, 1 Land Survey Est. Accuracy: $\square<3 \mathrm{~m}, \square 3-5 \mathrm{~m}, \square 5-15 \mathrm{~m}, \square>15 \mathrm{~m}$

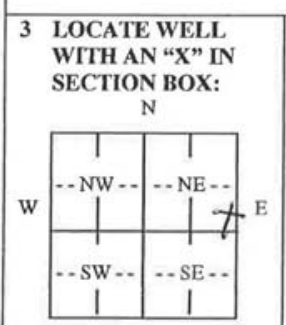
$\mathrm{S}$

\section{DEPTH OF COMPLETED WELL 42}

Depth(s) Groundwater Encountered 25.19 Pump test data: Well water was. EST. YIELD............. Wem. Well water was. Bore Hole Diameter $3.25 \ldots \ldots \ldots$ in. to $.42 \ldots \ldots \ldots \ldots$ ft., and $\square$ Domestic $\quad \square$ Feedlot $\square$ Oil field water supply Irrigation $\square$ Industrial

Domestic-lawn \& garden Was a chemical/bacteriological sample submitted to Department? If yes, mo/day/yr sample was submitted.

Water well disinfected? $\square$ Yes $\square$ No

... ft.

5 TYPE OF CASING USED: $\square$ Steel $\square$ PVC $\square$ Other

CASING JOINTS: $\square$ Glued $\square$ Clamped $\square$ Welded $\square$ Threaded Casing diameter 1 .............. in to $42 \ldots$

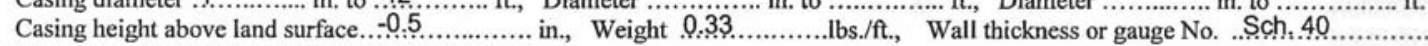
TYPE OF SCREEN OR PERFORATION MATERIAL:
$\square$ Steel
$\square$ Stainless Stcel
$\triangle \mathrm{PVC}$ $\square$ Brass $\quad \square$ Galvanized Steel $\quad \square$ None used (open hole)

(2).............

.... ft.

(3) ................ ft ..ft. below land surface measured on mo/day/yr. 2/16/201.1..... ft. after.................. hours pumping.................. gpm SCREEN OR PERFORATION OPENINGS ARE:
$\square$ Continuous slot $\square$ Mill slot
$\square$ Gauze wrapped $\square$ Torch cut

$\square$ Other (Specify)

...in. to

.

SCREEN-PERFORATED INTERVALS: From, 32 .

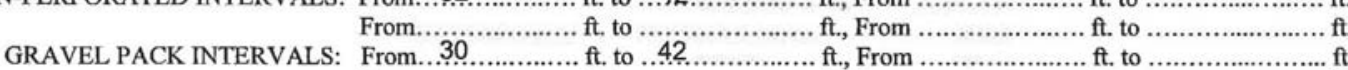

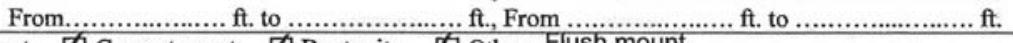
6 GROUT MATERIAL: $\square$ Neat cement $\square$ Cement grout $\nabla$ Bentonite

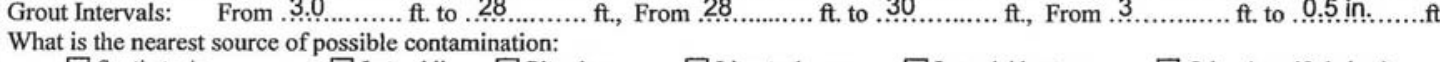
$\square$ Septic tank
$\square$ Lateral lines $\square$ Pit privy
$\square$ Livestock pen
$\square$ Insecticide storage
$\square$ Other (specify below)

ه Feedyard

$\square$ Abandoned water

$\square$ Watertight sewer lines

Direction from well

\begin{tabular}{|c|c|c|c|c|c|}
\hline FROM & TO & LITHOLOGIC LOG & FROM & TO & UTHO LOG (cont) or PLUGGING INTERVALS \\
\hline 0 & 34 & Silt/clay & & & \\
\hline 34 & 42 & Weathered shale (based on CPT & & & \\
\hline & & electronic readings) & & & \\
\hline & & & & & \\
\hline & & & & & \\
\hline & & & & & \\
\hline & & & & & \\
\hline & & & & & \\
\hline & & & & & \\
\hline & & & & & \\
\hline
\end{tabular}

7 CONTRACTOR'S OR LANDOWNER'S CERTIFICATION: This water well was $\square$ constructed, $\square$ reconstructed, or $\square$ plugged under my jurisdiction and was completed on (mo/day/year) $1.1 / 10 / 2010 \ldots$ and this record is true to the best of my knowledge and belief. Kansas Water Well Contractor's License No. .680. under the business name of ..Delta Environmental .. This Water Well Record was cometeted on (mo/day year) $10 / 0,0 / 2012$

INSTRUCTIONS: Use ty pewriter or ball point pen. PLEASE PRESS FIRMLY and PRINT clearly. Please fill in blanks and check the cet cect answers. Send three copies (white, blue, pink) to Kansas Depar tment of Health and E nvironment, Bureau of Water, Geology Section, 1000 SW Jackson St, Strito 420 , Topeka, Kansas 66612-1367 Telephone 785-296-5524. Send one copy to W ATER WELL OWNER and retain one for your records. Include fee of \$5.00 for each constructed well. Vi sit us at http://www.kdheks.gov/waterwell/index.html 
WATER WELL RECORD

\begin{tabular}{|ll|}
\hline 1 & LOCATION OF WA'T \\
County: McPherson \\
\hline \multicolumn{2}{l}{$\begin{array}{l}\text { Street/Rural Address of } \\
\text { from nearest town or in } \\
\text { NW from intersection } \\
\text { MW13. }\end{array}$} \\
\hline 2 & WATER WELL OWN \\
RR\#, Street Address, B \\
City, State, ZIP Code
\end{tabular}

Form WWC-5

\begin{tabular}{|lll} 
Fraction & $1 / 4$ SE $1 / 4$ SE $1 / 4$ NE $1 / 4$
\end{tabular}
Well Location; if unknown, distance \& direction of South Main Street and Farmington Road.

\section{: USD}

A - S. Gilmore - 0513, 4714-S

1400 Independence Ave. SW Washinaton. DC 20250-0513
Division of Water Resources App. No. \begin{tabular}{|l|l|l}
\hline Section Number & Township No. & Range Number
\end{tabular} Global Positioning System (GPS) information:

Latitude: . 38.2254.756.3 ................ (in decimal degrees) Longitude: 97.77707714

Elevation: 1523.506

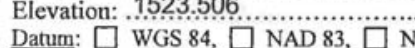
(in decimal degrees) Collection Method:

$\square$ GPS unit (Make/Model: ......................................) $\square$ Digital Map/Photo, $\square$ Topographic Map, $\square$ Land Survey Est. Accuracy: $\square<3 \mathrm{~m}, \square 3-5 \mathrm{~m}, \square 5-15 \mathrm{~m}, \square>15 \mathrm{~m}$

\section{TYPE OF CASING USED: $\square$ Steel 7 PVC $\square$ Other}

CASING JOINTS: $\square$ Glued $\square$ Clamped $\square$ welded $\square$ Threaded

Casing diameter .1............. in to 40.5 ...... ft., Diameter .............. in. to

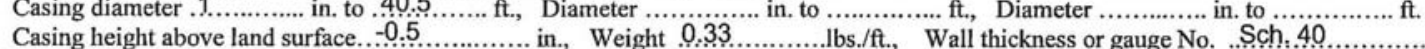
TYPE OF SCREEN OR PERFORATION MATERIAL:
$\square$ Steel
$\square$ Stainless Steel
$\triangle \mathrm{PVC}$
None used (open hole)
$\square$ Other (Specify)

SCREEN OR PERFORATION OPENINGS ARE:

$\begin{array}{lll}\square \text { Continuous slot } \quad \square \text { Mill slot } & \square \text { Gauze wrapped }\end{array} \quad \square$ Torch cut $\quad \square$ Drilled holes $\quad \square$ None (open hole)

SCREEN-PERFORATED INTERVALS: From... $30,5 \ldots \ldots \ldots \ldots \mathrm{ft}$, to $\ldots 40.5 \ldots \ldots \ldots \ldots . \mathrm{ft}$., From $\ldots \ldots \ldots \ldots \ldots \ldots \ldots \mathrm{ft}$. to $\ldots \ldots \ldots \ldots \ldots \ldots . \mathrm{ft}$

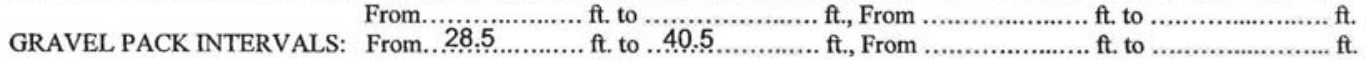

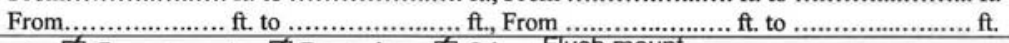

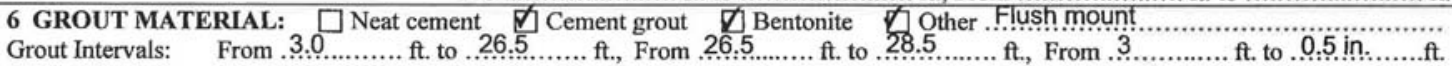
What is the nearest source of possible contamination:

$\square$ Septic tank $\square$ Lateral lines $\square$ Pit privy $\square$ Livestock pens $\square$ Insecticide storage $\square$ Other (specify below)

Sewer lines $\square$ Cesspool $\square$ Sewage lagoon

$\square$ Watertight sewer lines $\square$ seepage pit $\bar{\nabla}$ Feedyard $\quad \square$ Fertilizer storage $\square$ Oil well/gas well

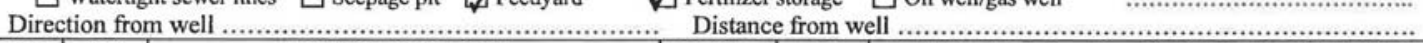

\begin{tabular}{|l|l|l|l|l|l|}
\hline FROM & TO & \multicolumn{1}{|c|}{ LITHOLOGIC LOG } & FROM & TO & LITHO. LOG (cont.) or PLUGGING INTERVALS \\
\hline 0 & 32 & Siltclay & & & \\
\hline 32 & 40.5 & Weathered shale (based on CPT & & & \\
\hline & & electronic readings) & & & \\
\hline & & & & & \\
\hline & & & & & \\
\hline & & & & & \\
\hline & & & & & \\
\hline & & & & & \\
\hline & & & & \\
\hline
\end{tabular}

7 CONTRACTOR'S OR LANDOWNER'S CERTIFICATION: This water well was $\square$ constructed, $\square$ reconstructed, or $\square$ plugged under my jurisdiction and was completed on (mo/day/year) $.11 / 10 / 2010 \ldots$ and this record is true to the best of my knowledge and belief. Kansas Water Well Contractor's License No. 680 ........ This Water Well Record was corppteted ong (mo/day/ygar) .10/08/2012.

under the business name of . . Delta Environmental by (signature) . Ln

INSTRUCTIONS: Use ty pewriter or ball point pen. PLEASE PRESS FIRMLY and PRINT clearly. Please fill in blanks and cheof tiecorrect answers. Send three..... copies (white, blue, pink) to Kansas Depar tment of Health and E nvironment, Bureau of W ater, Geology Section, 1000 SW Jackson St, Sutite 220 topeka, Kansas 666 12-1367. Telephone 785-296-5524. Send one copy to W ATER WELL OWNER and r etain one for your records. I nelude fee of $\$ 5.00$ for each constructed well. Vi sit us at http://www.kdheks.gov/waterwell//index.html. 


\section{$m \omega-14 m$}

WATER WELL RECORD

Form WWC-5

1 LOCATION OF W'ATER

County: M<Pherson $1 / 4$ SW $1 / 4 W_{1 / 4} N W_{1 / 4}$

ing

from nearest toyn or intersection: If at owner's address, check here $\square$

corner of Formington Road \& South Moin

street $100^{\circ}$ west $\alpha 50^{\circ}$ North

2 WATER WELL OWNER: USDA-SFeUE Gilmore

RR\#, Street Address, Box \#: Stop 0.513 Room $4714-5$

City, State, ZIP Code

1400 Ladependence Ave, sw

3 LOCATE WELL Weshingten, DC. $20250-05 / 3$

WITH AN "X" IN" 4 DEPTH OF COMPLETED WELL ..... 95

SECTION BOX: Depth(s) Groundwater Encountered (1)....

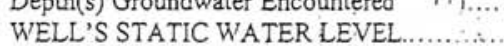

Pump test data: Well water was

$w$

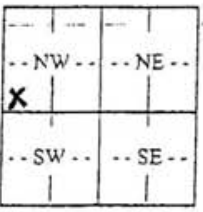

mile-...-.-.

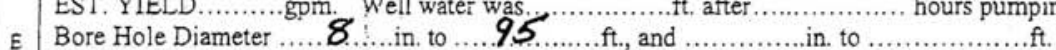

Division of Water Resources App. No.

\begin{tabular}{|l|l|l}
\hline Section Number & Township No. & Range Number \\
\hline
\end{tabular}

16

I 21

R $4 \quad \square E \&$

\section{Global Positioning System (GPS) information:}

Latitude: $38.22493 \ldots \ldots$ (in decimal degrees) Longitude: $9.7 .7 .755 \ldots \ldots \ldots$ (in decimal degrecs) Elevation:

Datum: $\square$ WGS $84, \square$ NAD 83, $\square$ NAD 27

Collection Method:

GPS unit (Make/Model: ...................................)
Digital Map/Photo, $\square$ Topographic Map, $\square$ Land Survey

Est. Accuracy: $\square<3 \mathrm{~m}, \square 3.5 \mathrm{~m}, \square 5.15 \mathrm{~m}, \square>15 \mathrm{~m}$

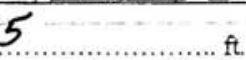

$\mathrm{ft}$ after.................. hours pumping

WELL WATER TO BE USED AS: $\square$ Public water supply $\square$ Geothermal $\square$ Injection well

$\square$ Domestic $\square$ Feedlot $\quad \square$ Oil field water supply $\square$ Dewatering $\square$ Other (Specify below)

$\square$ Irrigation $\square$ Industrial : $\square$ Domestic-lawn \& garden $\mathbb{Z}$ Monitoring well

Was a chemical/bacteriological sample submitted to Department? $\square$ Yes $\mathbb{X}$ No

If yes, mo/day/yr sample was submitted.

Water well disinfected? $\square$ Yes $\mathbb{X}$ No

\section{TYPE OF CASING USED: $\square$ Steel $\mathbb{X}$ PVC: $\square$ Other}

CASING JOINTS: $\square$ Glued $\square$ Clamped $\square$ Welded $\searrow$ Threaded

Casing diameter ........... in. to ....85 f... ft., Diameter ............. in. to ............. ft., Diameter ............. in to

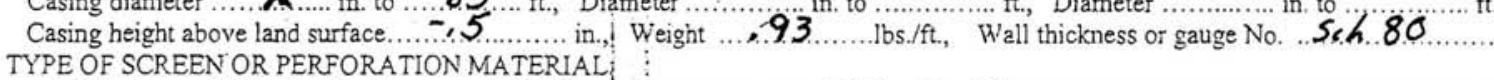
$\square$ Steel
$\square$ Stainless Steel
XPVC

$\square$ Brass $\square$ Galvanized Steel $\square$ Non
$\square$ Continuous slot
Xill siot
$\square$ Gauze wrepped
$\square$ Louvered shutter $\square$ Key punched $\square$ Wire wrapped
SCREEN-PERFORATED INTERVALS: From....8.5.
From...85
From ................ ft. to .........

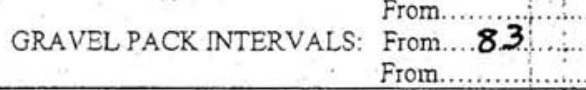
f. to 95
Drilled holes
Other (specify) $\square$ None (open hole)

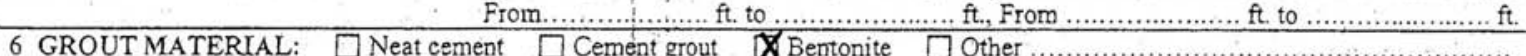

Other (Specify)

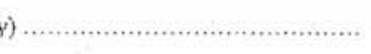

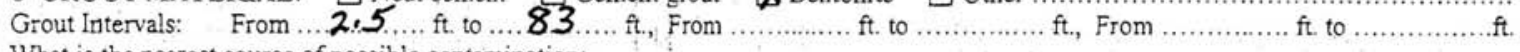

What is the nearest source of possible contamination:
$\square$ Septic tank
Sewer lines
Watertight sewer lines
$\square$ Lateral lines $\square$ Pit privu
$\square$ Cesspool
$\square$ Seepage pit
$\nabla$ Fewage iagoon
Livestock pens
Fuel storage
$\square$ Insecticide storage $\square$ Other (specify below)

Direction from well

\begin{tabular}{|c|c|c|c|c|c|c|}
\hline FROM & TO & LITHOLOGIC LOG & + & FROM & TO & LITHO. LOG (cont) or PLUGGING INTERVALS \\
\hline 0 & 40 & Clay & 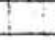 & & & \\
\hline 40 & 95 & Wedthered Shole & & & & \\
\hline & & & 3. & & 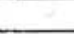 & \\
\hline & & & 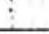 & & & 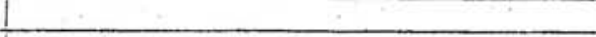 \\
\hline & & & + & & & \\
\hline & & & 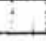 & & & $z$ \\
\hline & & & $\mathrm{i}_{\mathrm{i}}$ & & & \\
\hline & & & $\mathrm{i}$ & & & \\
\hline & & & ! & & & \\
\hline & & & & & & \\
\hline
\end{tabular}

7 CONTRACTOR'S OR LANDOWNER'S CERTIFICATION: This water well was $\varnothing$ constructed, $\square$ reconstructed, or $\square$ plugged under my jurisdiction and was completed on (mo/day/year) $8.1 .2 .3 / 1 / \ldots$ and this record is true to the best of my knowlegge and belief. Kansas Water Well Contractor's License No. 5.9.7...... This Water Well Record was completed on (mo/day/year) ...9/2/./.

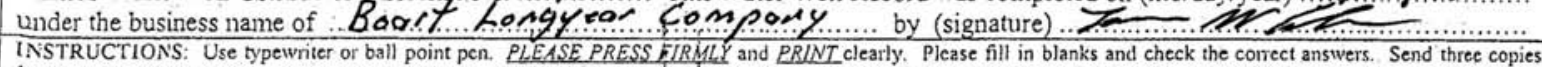
INSTRUCTIONS: Use typewriter or ball point pen. PLEASE PRESS FIRMLY and PRINT clearly. Please fill in blanks and check the correct answers. Send three copies
(white, blue, pink) to Kansas Department of Health and Enviromment, Burezu of Water, Geology Section, 1000 SW Jackson St, Suite 420, Topeka, Kansas 66612.1367 . Telephone 785-296-5522. Send one copy to WATER WELL OWNER and retain one for your records. Include fee of $\$ 5.00$ for each constructed well. Visit us at http: $/ /$ hunv. kdheks.gov/watenvell/index.html.

KSA $82 a-1212$

Check: $\square$ White Copy, $\square$ Blue Copy, $\square$ Pink Covv 
WATER WELL RECORD

Form WWC-5

Division of Water Resources App. No.

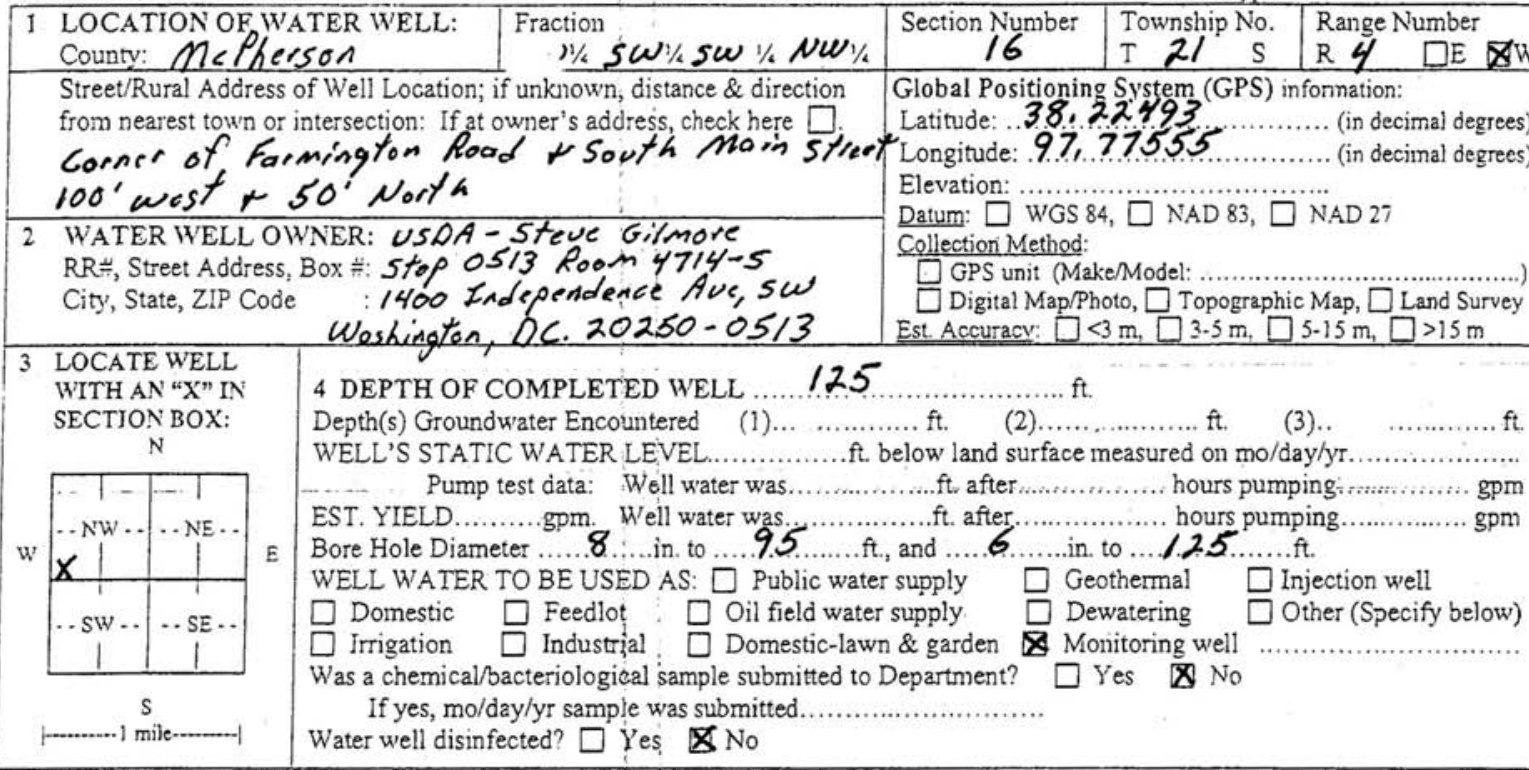

\section{TYPE OF CASING USED: $\square$ Steel $\triangle$ PVC $\square$ Other}

CASING JOINTS: $\square$ Glued $\square$ Clamped $\square$ Wyelded $\boldsymbol{\nabla}$ Threaded

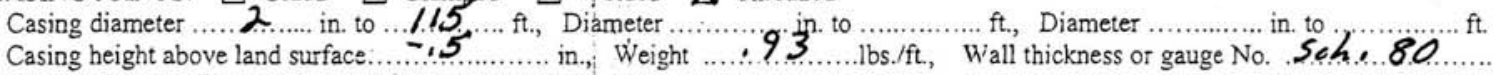
TYPE OF SCREEN OR PERFORATION MATERIAL:
$\square$ Steel
Stainless Steel
Q PVC
$\square$ None used (open hoie)

SCREEN OR PERFORATION OPENINGS ARE:

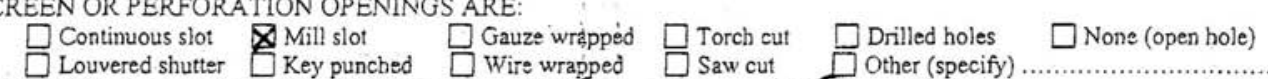

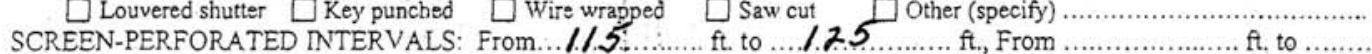

\begin{tabular}{|c|c|}
\hline GRAVEL PACK INTERVALS: & $\begin{array}{l}\text { ft. to } \\
\text { ft. to …/2 } 5 \ldots \ldots \text { ft., From }\end{array}$ \\
\hline & ft. to …… \\
\hline
\end{tabular}

6 GROUT MATERIAL: $\square$ Neat cement $\square$ Cement grout $\nabla$ Bentonite $\square$ Other ....................................................

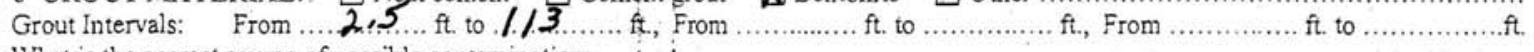
What is the nearest source of possible contamination: : :
$\square$ Septic tank
$\square$ Watertight sewer lines
$\square$ Lateral lines $\square$ Pit privy ! $\square$ Livestock pens
$\square$ Cesspool
$\square$ Insecticide storage $\square$ Other (specify below')
$\square$ Abandoned water well

Direction from well

\begin{tabular}{|c|c|c|c|c|c|c|}
\hline FROM & TO & LITHOLOGICLOG & i & FROM & TO & LITHO. LOG (cont) or PLUGGING INTERVALS \\
\hline 0 & 40 & Clay & & & & \\
\hline 40 & 125 & Weothered Shole & $\therefore i$ & & & \\
\hline & & & +1 & & & \\
\hline & & & $\therefore$ & & & \\
\hline & & & 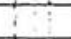 & & & \\
\hline & & & i & & & \\
\hline & & & 3 & & & \\
\hline
\end{tabular}

7 CONTRACTOR'S OR LANDOWNER'S CERTIFICATIIIN: This water well was $\mathbf{X}$ constructed, $\square$ reconstructed, or $\square$ plugged under my jurisdiction and was completed on (mo/jay/year) $8 / 23 / 1$.... and this record is true to the best of my knowgedge and belief.

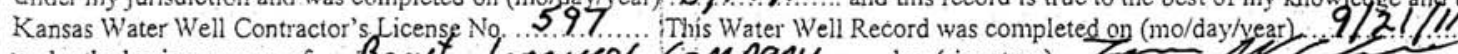

under the business name of .... Beait... Le ngy.e......... pany...... by (signature)

INSTRUCTIONS: Use typewriter or ball point pen. PLEASE PRESS FIRMLI and ERINT clearly. Please fill in blanks and check the correct answers. Send three copies (white, blue, pink) to Kansas Department of Heakh and Environment, Burcau of Water, Geology Section, 1000 SW Jackson St., Suitc 420, Topeka, Kansas 66612.1367. Telephone 785-296-5522. Send one copy to WATER WELL OWNER abd retain one for your records. Include fce of S5.00 for each constructed well. Visit us at http://www.kdheks.gov/waterwell/index.htmi.

KSA $82 \mathrm{a}-1212$

Check: $\square$ White Copy, $\square$ Blue Copy, $\square$ Pink Covy 
WATER WELL RECORD

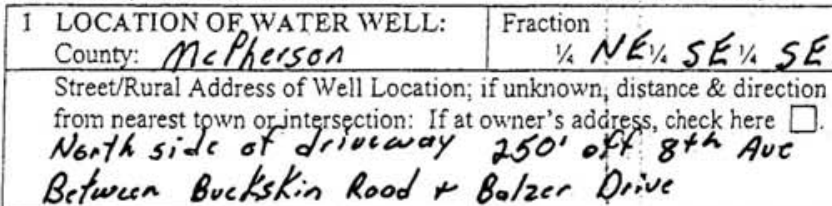

2 WATER WELL OWNER: USDA-Steve Gilmore $R R=$ Street Address, Box \#: Stop 0513 Roo in $4714-5$ City, State, ZIP Code : 1400 Independence Ave, sw Wesbingten, DC. $20250-05 / 3$

3 LOCATE WELL WITH AN "X" IN SECTION BOX:

4 DEPTH OF COMPLETED WELL …...44.

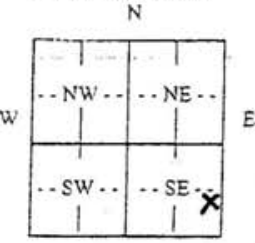
$\mathrm{s}$

Water well disinfected? $\square$ Yes $\square$ No

\section{$\operatorname{Depth}(s)$ Groundwater Encountered (1)}

Division of Water Resources App. No.

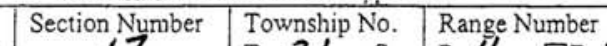

\section{Global Positioning System(GPS) information:}

Latitude: $38.22042 \ldots \ldots \ldots$ (in decimal degrees) Longitude: $97.77695 \ldots \ldots \ldots \ldots$ (in decimal degrees) Elevation:

Datum: $\square$ WGS $84, \square$ NAD 83, $\square$ NAD 27 Collection Method:

$\square$ GPS unit (Make/Model:

Digital Map/Photo, $\square$ Topo...........................)

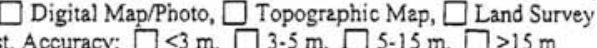

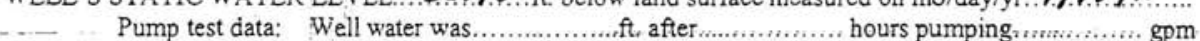

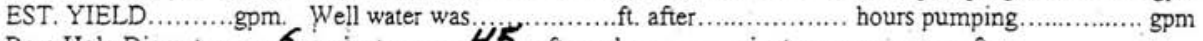

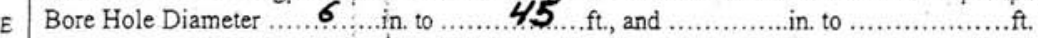
WELL WATER TO BE USED AS: $\square$ Public water supply $\square$ Geothermal $\square$ Injection well $\square$ Domestic $\square$ Feedlot : $\square$ Oil field water supply $\square$ Dewatering $\square$ Other (Specify below') $\square$ Irrigation $\square$ Industral : $\square$ Domestic-lawn \& garden $\mathbb{X}$ Monitoring well Was a chemical/bacteriologigal sample submitted to Department? $\square$ Yes $\bigotimes$ No If yes, mo/day/yr sample was submitted.

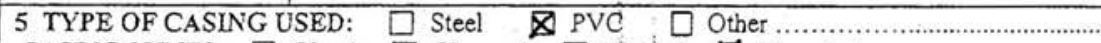

CASING JOINTS: $\square$ Glued $\square$ Clamped $\square$ welded $\otimes$ Threaded

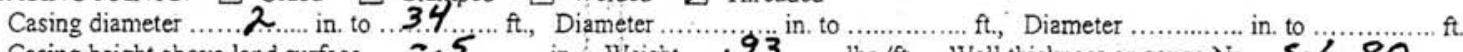

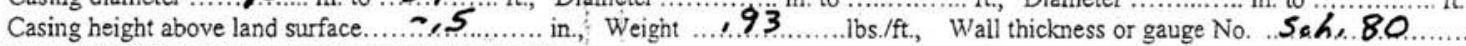
TYPE OF SCREEN OR PERFORATION MATERIAL
$\square$ Steel $\square$ Stainless Stee!
$\triangle \mathrm{PVC}$
$\triangle$ None used (open hole)
$\square$ Other (Specify')

SCREEN OR PERFORATION OPENINGS ARE:

$\square$ Continuous slot 叉Mill slot $\square$ Gauze wréppèd $\square$ Torch cut $\square$ Drilled holes $\square$ None (open hole)

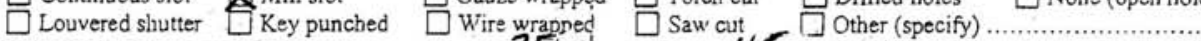

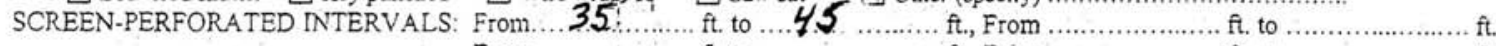

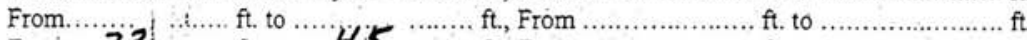
GRAVEL PACK INTERVALS: From....33 ….... ft. to … $45 \ldots \ldots \ldots$ ft., From ……........... ft. to …................. ft. From ............... ft. to ................... ft., From .................... ft. to ....................... f

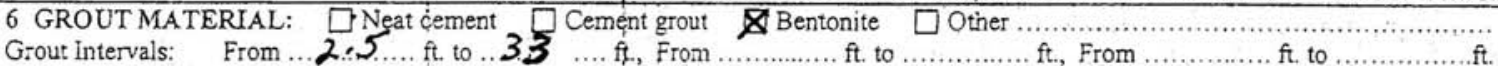
G:out intervals: From ...2.5.... ft. to ...3. 3 ... ff., From
What is the nearest source of possible contamination:
$\square$ Septic tank
$\square$ Lateral lines $\square$ Pit priv
$\square$ Insecticide storage $\square$ Other (specify below)

$\square$ Cesspool $\square$ Sewage jagoon $\square$ Fuel storage

$\square$ Watertight sewer lines

Direction from well

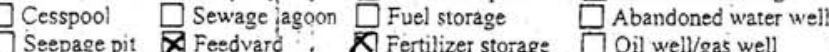

Distance from well

\begin{tabular}{|c|c|c|c|c|c|}
\hline FROM & TO & LITHOLOGIC LOG & FROM & TO & LITHO. LOG (cont.) Or PLUGGING INTERV \\
\hline 0 & 44 & ClOY & & & \\
\hline & & & & & \\
\hline & & & & & \\
\hline & & & & & \\
\hline & & & & & \\
\hline & & & & & \\
\hline & & & & & \\
\hline & & & & & \\
\hline
\end{tabular}

7 CONTRACTOR'S OR LANDOWNER'S CERTIFICA TION: This yater well was $\triangle$ constructed, $\square$ reconstructed, or $\square$ plugged under my jurisdiction and was completed on (mo/day/year),$. .8 / 2.7 .11 !$ and this record is true to the best of my knowledge and belief.

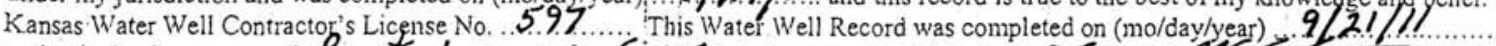

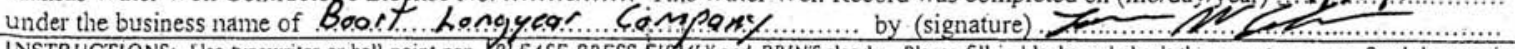

INSTRUCTIONS: Use typewriter or ball point pen. PLEASE PRESS FINALY and PRINT clearly. Please fill in blanks and check the correct answers. Send threc copies (white, bluc, pink) to Kansas Department of Health and Environment, Burehu of Water, Geology Section, 1000 SW Jackson St, Suite 420, Topeka, Kansas 66612-1367. Teiephone 785-296-5522. Send one copy to WATER WELL OWNER sind retsin one for your records. Include fee of $\$ 5.00$ for each constructed well. Visit us at hittp://Munw.kdheks.gov/waterweil/index.html. 


\section{$m \omega \cdot 15 m$}

WATER WELL RECORD

Form WWC-5

Division of Water Resources App. No.

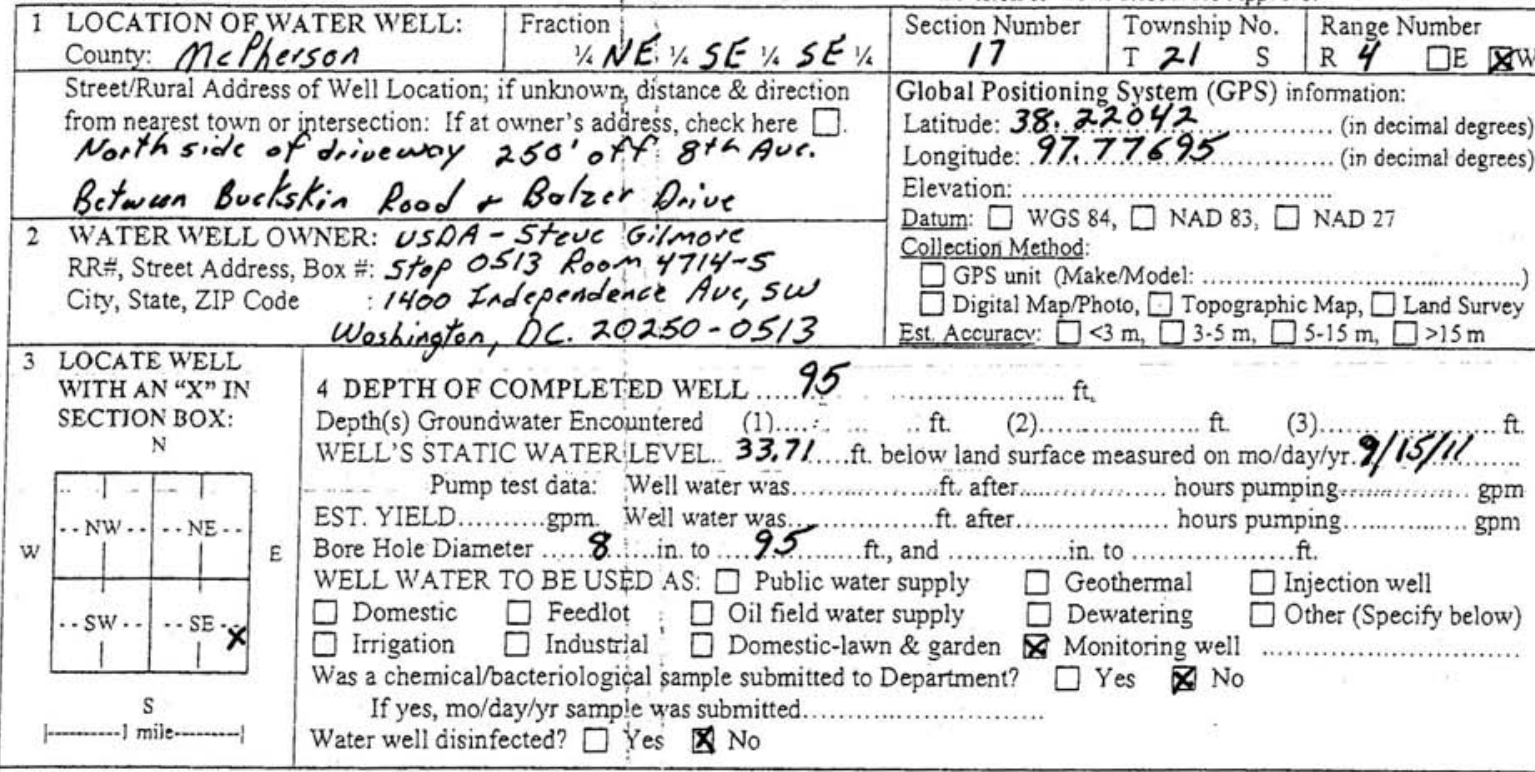

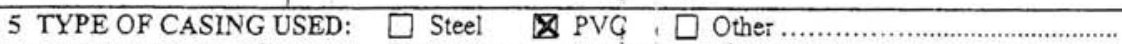

CASING JOINTS: $\square$ Glued $\square$ Clamped $\square$ Welded $\$ Threaded

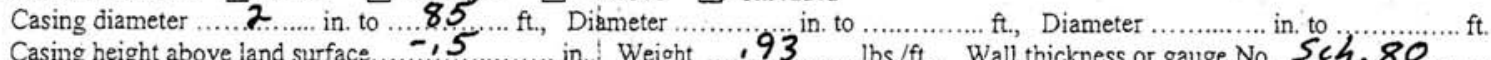
Casing hight TYPE OF SCREEN OR PERFORATION MATERIAL:
$\begin{array}{lll}\square \text { Steel } & \square \text { Stainless Steel } & \mathbb{X} \text { PVC } \\ \square \text { Brass } & \square \text { Galvanized Steel } & \square \text { None use }\end{array}$

CREEN OR PERFORATION OPENINGS ARE:

$\square$ Continuous siot $\bigotimes$ Mill slot : $\square$ Gauze wrapped $\square$ Torch cut $\square$ Drilled hoies $\square$ None (open hole)

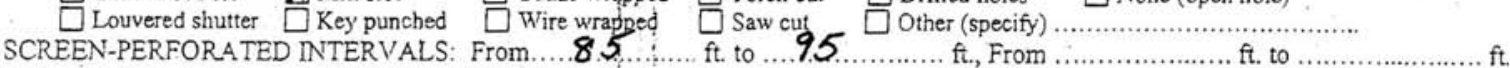

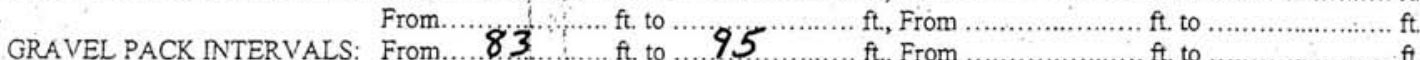

From …............ ft. to ................... ft., From ……............. ft. to ....................... ft.

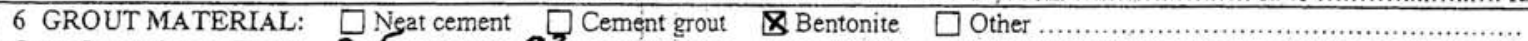

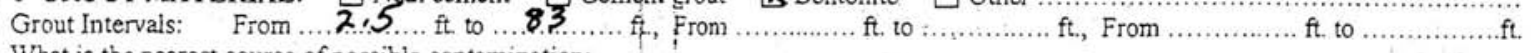

What is the nearest source of possible contamination:

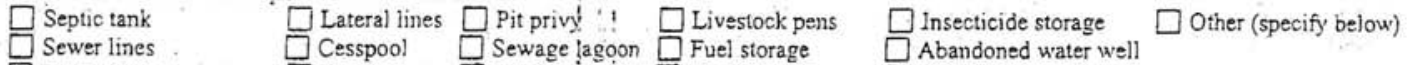

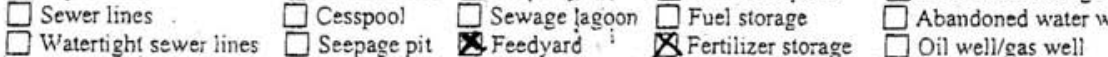

Direction from well .......................................... Distance from well .

\begin{tabular}{|c|c|c|c|c|c|}
\hline FROM & TO & LITHOLOGIC LOG & FROM & TO & LITHO. LOG (cont) OI PLUGGING INTERVALS \\
\hline 0 & 44 & Cloy & & & \\
\hline 44 & 95 & Wlothered Sha/t & & & \\
\hline & & & & & \\
\hline & & & & & \\
\hline & & $\vdots$ & & & \\
\hline & & & & & \\
\hline & & & & \\
\hline
\end{tabular}

7 CONTRACTOR'S OR LANDOWNER'S CERTIFICATION: This water well was $₫$ constructed, $\square$ reconstructed, or $\square$ plugged

under my jurisdiction and was completed on (mo/day/year) . 8/2.4/.1... and this record is true to the best of my knowledge and belief.

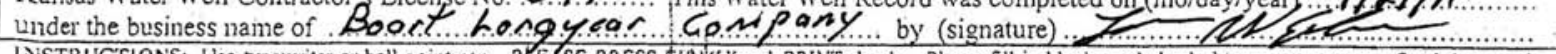

INSTRUCTIONS: Use typewriter or ball point pen. PLEASE PRESS BJ RMGLY and PRINT clearly. Plcase fill in blanks and check the correct answers. Send three copies

(white, blue, pink) to Kansas Department of Health and Environment, Bureav of Water, Geology Section, 1000 SW Jackson St., Suite 420, Topeka, Kansas 66612-1367.

Telephone 785-296-5522. Send one copy to WATER WELL OWNER and retain one for your records. Include fee of $\$ 5.00$ for cach constructed well. Visit us at hettp://munw.kdheks.gov/waterwell/index.htuml.

KSA $82 \mathrm{a}-1212$ 
WATER WELL RECORD

Form WWC-5

Division of Water Resources App. No.

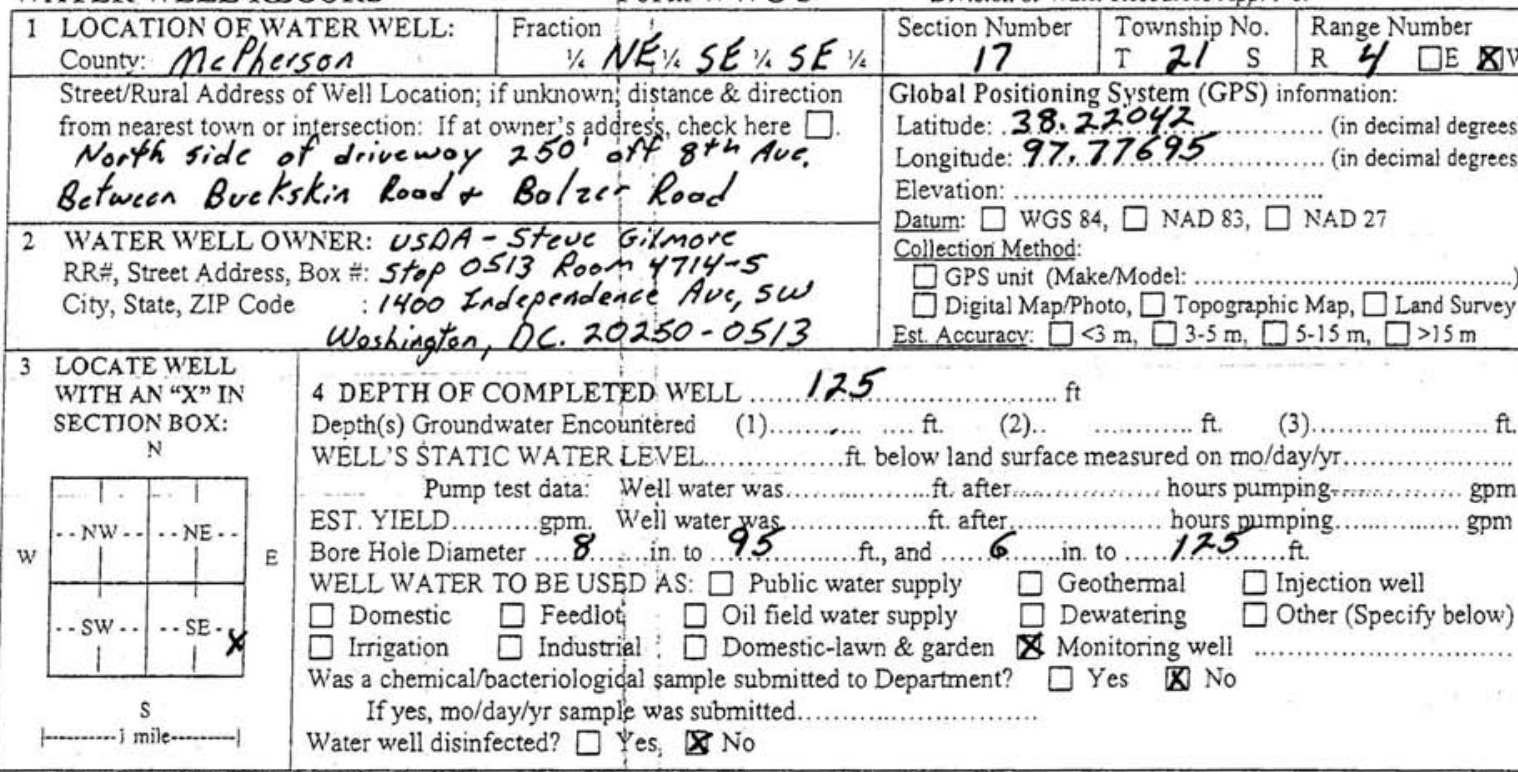

\section{TYPE OF CASING USED: $\square$ Steel $\bigotimes$ PVC $\square$ Other ...........
CASING JOINTS: $\square$ Glued $\square$ Clamped $\square$ Welded $\bigotimes$ Threaded}

Casing diameter ...2 $2 . . .$. in. to ...115 ... ft., Diameter .............. in to

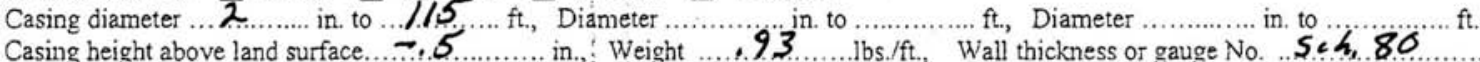
TYPE OF SCREEN OR PERFORATION MATERIAL
Steel
Brass
$\square$ Stainless Steel
$X$ PVC

SCREEN OR PERFORATION OPENINGS ARE:

$\square$ Continuous slot $\square$ Mill slot $\square$ Gauze wrapped $\square$ Torch cut $\square$ Drilled holes $\square$ None (open hole)

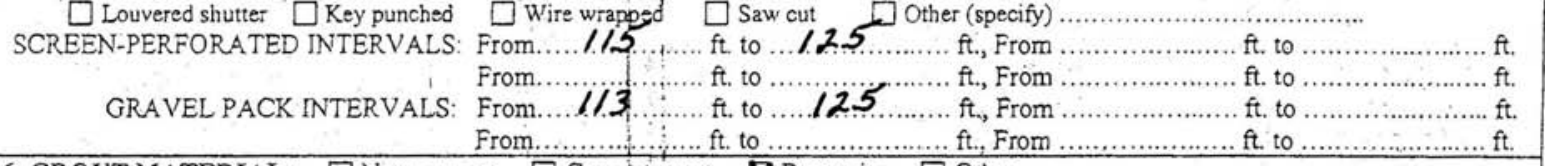

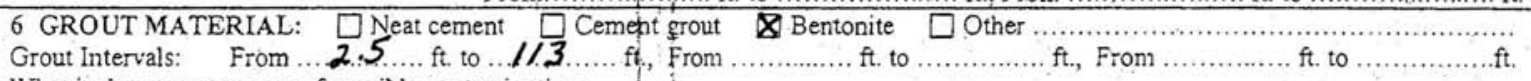
What is the nearest source of possible contamination:

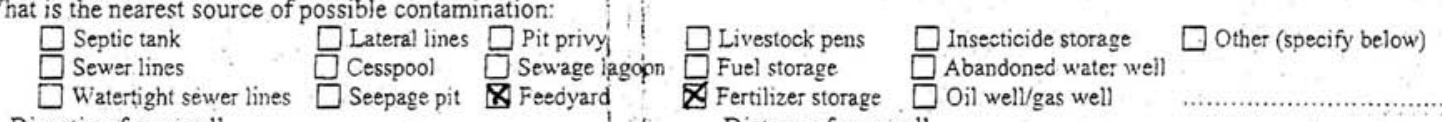

Direction from well ............................................. Distance from well .

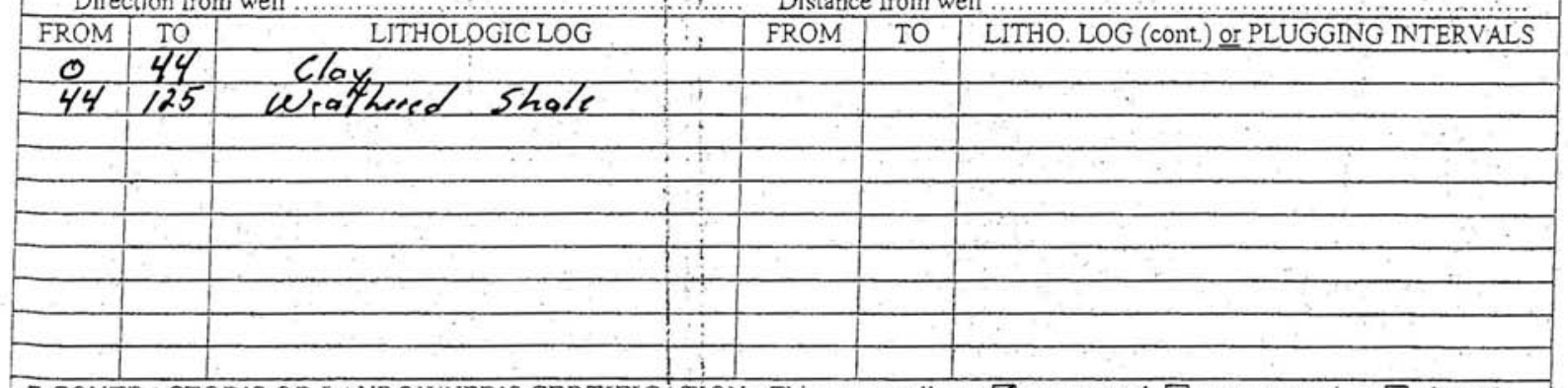

7 CONTRACTOR'S OR LANDOWNER'S CERTIFICATION: This water well was $\bigotimes$ constructed, $\square$ reconstructed, or $\square$ plugged under my jurisdiction and was completed on (mo/day/year) $8 / 24 / 1$..... and this record is true to the best of my knowledge and belief.

Kansas Water Well Contractor's License No. .5.9.7.... This Water Well Record was completed on (mo/day/year) . 9.1.21./.1........

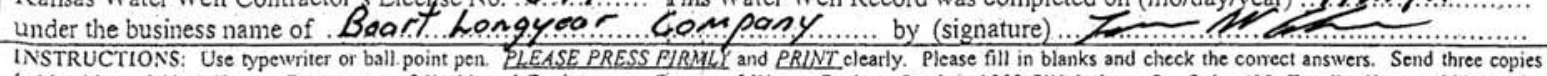

INSTRUCTIONS: Use typewriter or ball point pen. PLEASE PRESS FJRALLI and PRINT clearly. Please fill in blanks and check the correct answers. Send three copies
(white, bluc, pink) to Kansas Department of Health and Environment, Bureatu of W'ater, Geology Section, 1000 SW Jackson St., Suite 420 , Topeka, Kansas 66612.1367 . Telephone 785-296-5522. Send one copy to WATER WELL OWNER and retain one for your records. Include fee of S5.00 for each constructed well. Visit us at http://www.kdheks.gov/waterwell/index huml.

KSA 82a-1212

Check: $\square$ White Copv, $\Pi$ Blue Conv. $\Pi$ Pink Conv 
WATER WELL RECORD

Form WWC-5

Division of Water Resources App. No.

1 LOCATION OF WATER County: McPherson $\quad 1 / 4$ SW NE N SE $1 / 4$

Street/Rural Address of Well Location; if unknow'n; distance \& direction

from nearest town or intersection: If at owner's address, check here $\square$

Corner of fied
Bolzer Dive

2 WATER WELL OWNER: USDA - Steve Gilmore

RR ㅎ, Street Address, Box \#: Stop 0513 Roo in $4714-5$

City, State, ZIP Code

1400 Independeace Ave, sw

Weshingten, DC. $20250-05 / 3$

\begin{tabular}{l|l}
3 LOCATE WELL & 4 DEPTH OF COMPLETED WELL ...... 35 \\
WITH AN" "XN
\end{tabular}

Depth(s) Groundwater Encountered (1)...

WELL 1706 .

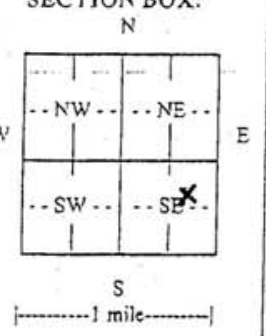

WELL'S STATIC WATER LEVEL.../7,
Pump test data: Well water was.

Pump test data: Well water was

EST. YIELD .........gpm. Well water was...............ft. after.

Bore Hole Diameter ....8. . . .....in. to ... 3.5.......ft., and Num

Township No.
I Z I S

Range Number

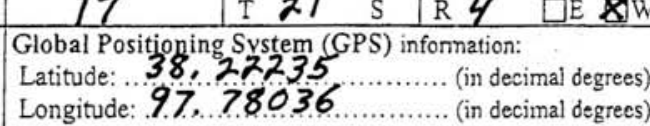
Elevation:

Datum: $\square$ WGS $84, \square$ NAD $83, \square$ NAD 27

Collection Method:

$\square$ GPS unit (Make/Model:

Digital Map/Photo, $\square$ Topo.............................)

Est. Accuracy: $\square<3 \mathrm{~m}, \square 3.5 \mathrm{~m}, \square 5.15 \mathrm{~m}, \square>15 \mathrm{~m}$

........in. to .................f.

WELL WATER TO BE USED AS: $\square$ Public water supply $\square$ Geothermal $\square$ Injection well

$\square$ Domestic $\square$ Feedlot $\square$ Oil field water supply $\square$ Dewatering . $\square$ Other (Specify below)

$\square$ Irrigation $\square$ Industrial $\square$ Domestic-lawn \& garden $\varangle$ Monitoring well ..........................

Was a chemical/bacteriologioal sample submitted to Department? $\square$ Yes $\otimes$ No

If yes, mo/day/yr sample was submitted.

Water well disinfected? $\square$ Yेes $\mathbb{X}$ No

\section{TYPE OF CASLNG USED: $\square$ Steel $\bigotimes$ PVC $\square$ Other \\ CASING JOINTS: $\square$ Glued $\square$ Clamped $\square$ welded $\otimes$ Threaded}

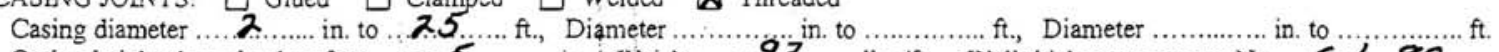

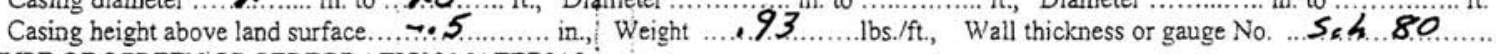
TYPE OF SCREEN OR PERFORATION MATERIAL:
$\square$ Steel
$\square$ Stainless Steel
XPVC
$\square$ Brass Galvanized Steel
$\square$ None uséd (open hoie)

SCREEN OR PERFORATION OPENINGS ARE

$\square$ Continuous slot $\quad$ Mill siot $\quad \square$ Gauze wrapped $\square$ Torch cut $\square$ Drilled holes $\square$ None (open hole)

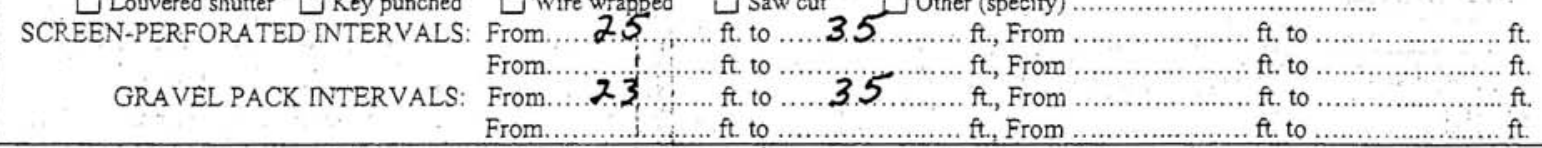

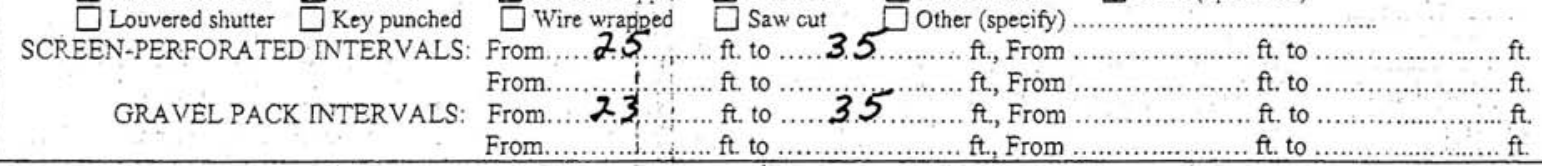

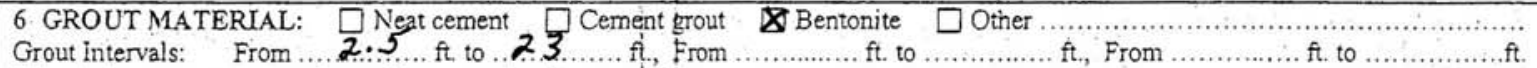

What is the nearest source of possible contamination:
$\square$ Septic tank
Sewer lines
$\square$ Lateral lines $\square$ Pit privy $\square$ Livestock pens
Cesspool
$\square$ Insecticide storage
Abandoned water wel

$\checkmark$ Watertight sewer lines

$\triangle$ Feedyard

Fertilizer storage 0 Oil well/gas well

Direction from well Distance from well

\begin{tabular}{|c|c|c|c|c|c|c|}
\hline FROM & TO & LITHOLOGIC LOG & FROM & TO & LITHO LOG (cont) Or PLUGGING INTERVALS \\
\hline 0 & 35 & ClaY & $\vdots$ & & & \\
\hline & & $\vdots$ & & & \\
\hline & & 1 & & & \\
\hline & & & & & \\
\hline & & & & & & \\
\hline & & & & & \\
\hline & & & & & \\
\hline
\end{tabular}

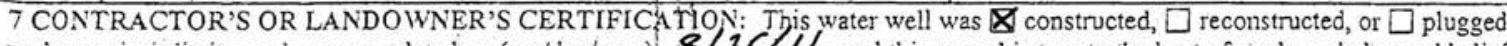
under my jurisdiction and was completed on (mo/day/year) $.8 / 2.6 / 11 \ldots$ and this record is true to the best of my knowlegisg and beljef

Kansas Water Well Contractor's License No. 5.7..... This Water Well Record was conipleted on (mo/day/year)

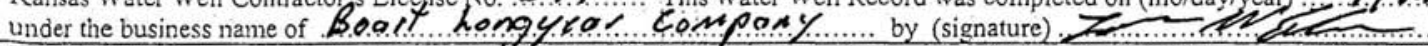

INSTRUCTIONS: Use typewriter or ball point pen. LLEASE PRESS FIRMLY and PRINT clearly. Please fill in blanks and check the coirect answers. Send three copies

(white, bluc, pink) to Kansas Department of Health and Environment, Bureau of Water, Geology Section, 1000 SW Jackson St., Suite 420, Topeka, Kansas 66612.1367.

Telephone 785-296-5522. Send one copy to WATER WELL OWNER anid retain one for your records. Include fee of $\$ S .00$ for cach canstructed well. Visit us at hitp: //Munw.kdheks.gov/waterwell/index.html. 
WATER WELL RECORD I LOCATION OF WATER

Form WWC-5

Division of Water Resources App. No.

County: McPherson Fraction $1 / \% \omega_{1 / 4 N E} N S E$

Street/Rural Address of Well Location; if unknown; distance \& direction

from nearest town or intersection: If at owner's address, check here $\square$. Corner of field offe $8^{\text {th }}$ Ave South $\alpha$

2 WATER WELL OWNER: USDA - SteUC Gilmore $R R \#$, Street Address, Box \#: Stop 0513 Roop $4714 /-5$ City, State, ZIP Code : 1400 Independence Ave, sW Weshingten, DC. 20250-05/3 WOCATE WELL $\quad 4$ DEPTH OF COMPLETED WELL ....... 45 SECTION BOX: Depth(s) Groundwater Encountered \begin{tabular}{l|l|l}
\hline & Township No. & Range Number \\
\hline
\end{tabular} $\begin{array}{llll}17 & \mathrm{~T} & 2 / \mathrm{S} & \mathrm{R}\end{array}$ Latitude: .. $38.22235 \ldots \ldots \ldots$ (in decimal degrees) Longitude: $97.78036 \ldots \ldots \ldots \ldots$ (in decimal degrees) Elevation: Datum: $\square$ WGS 84, $\square$ NAD 83, $\square$ NAD 27 Collection Method:

$\square$ GPS unit (Make/Model: ......................................) $\square$ Digital Map/Photo, $\square$ Topographic Map, $\square$ Land Survey Est. Accuracy: $\square<3 \mathrm{~m}, \square 3.5 \mathrm{~m}, \square 5.15 \mathrm{~m}, \square>15 \mathrm{~m}$

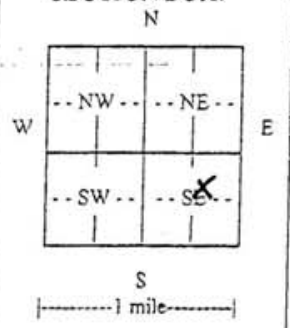
WELL'S STATIC WATER LEVEL...17.0.2ft. below land surface measured on mo/day/yr $9 \% 1.5 / 1 / 1 . \ldots$ Pump test data: Well water was..................ft after .............. hours pumping

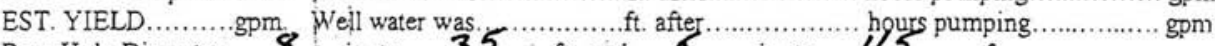

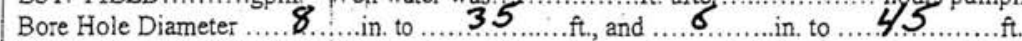

WELL WATER TO BE USED AS: $\square$ Public water supply $\square$ Geothermal $\square$ Injection well $\square$ Domestic $\square$ Feedlot,$\square$ Oil field water supply $\square$ Dewatering $\square$ Other (Specify below) $\square$ Irrigation $\square$ Industrial $\square$ Domestic-lawn \& garden $\otimes$ Monitoring well W/as a chemical/bacteriological sample submitted to Department? $\square$ Yes $\boldsymbol{Q}$ No If yes, mo/day/yr sample was submitted. Water well disinfected? $\square$ Yes $\mathrm{X}$ No

\section{TYPE OF CASING USED: $\square$ Steel $\mathbb{X}$ PVC $\square$ Other}

CASING JOINTS: $\square$ Glued $\square$ Clamped $\square$ Welded Threaded

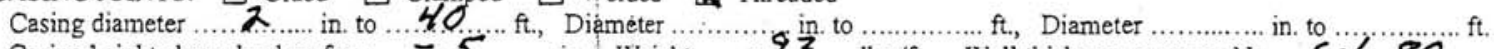

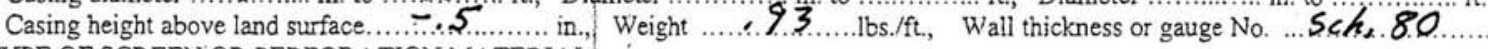
TYPE OF SCREEN OR PERFORATION MATERIAL:
$\square$ Steel
$\square$ Stainless Steel
XPVC
$\square$ None used (open hole)

SCREEN OR PERFORATION OPENINGS ARE:

$\square$ Continuous siot $\quad$ Mill slot $\quad \square$ Gauze wrapped $\square$ Torch cut $\square$ Drilled holes $\quad \square$ None (open hole)

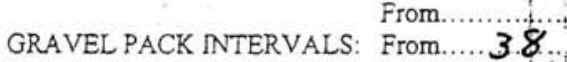

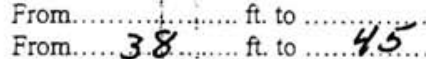
ft., From
ft. to From....................... to ft., From

f., From

ft. to

6 GROUT MATERIAL: $\square$ Neat cement $\square$ Cement grout $\nabla$ Bentonite $\square$ Other

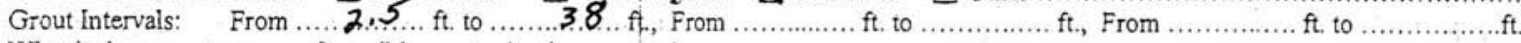
What is the nearest source of possible contamination:
$\square$ Septic tank
Sewer lines Cesspoo!
$\square$ Lateral lines $\square$ Pit privy $\square$ Livestock pens
Cesspool Sewage fagoon $\square$ Fuel storage
Fertilizer storage
$\square$ Insecticide storage
Abandoned water well
$\square$ Other (specify below)

irection from well

\begin{tabular}{|c|c|c|c|c|c|}
\hline FROM & TO & LITHOLOGIC LOG & FROM & TO & LITHO. LOG (cont) or PLUGGING INTERVALS \\
\hline 0 & 38 & Clay & & & \\
\hline 38 & 45 & Weothered Shale & & & \\
\hline & & & & & \\
\hline & & $\vdots$ & & & \\
\hline & & & & & \\
\hline & & & & & \\
\hline & & & & & \\
\hline & & & & \\
\hline
\end{tabular}

7 CONTRACTOR'S OR LANDOWNER'S CERTIFICATION: This water well was $\$ constructed, $\square$ reconstructed, or $\square$ plugged under my jurisdiction and was completed on (mg/day/year) $1.8 / 26 / 1 /$ and this record is true to the best of my knowledge and belief.

Kansas Water Well Contractor's License No. 5.9.7..... This Water Well Record was completed on (mo/day/year) .. 9.721. 1/

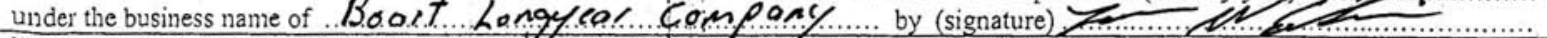

INSTRUCTIONS: Use typewriter or ball point pen. PLEASE PRESS AJRMLI and PRINT clearly. Plcase fill in blanks and check the conect answers. Send three copies (white, blue, pink) to Kansas Department of Heaith and Environment, Burcau of Water, Geology Section, 1000 SW Jackson St., Suite 420, Topeka, Kansas 66612.1367. Telephone 785-296-5522. Send onc copy to WATER WELL OWNER and retain one for your records. Include fee of $\$ 5.00$ for each constincted well. Visit us at hattp://munv.kulheks.gov/waterwell/index.html. 
WATER WELL RECORD

1 LOCATION OF W'ATER WELL: County: McPherson

SW $1 / 4 E^{1 / 4}$ SE $1 /$

Street/Rural Address of Well Location; if unknowri, distance \& direction from nearest town or intersection: If at owner's address, check here $\square$

Corner of firld off 8 th Aue south

\& Bolzer Deive

2 WATER WELL OWNER: USDA - Steve Gilmore RR\#, Street Address, Box \#: stop 0513 Rooin 4714-5 City, State, ZIP Code : 1400 Independence Ave, sw

3 LOCATE WELL WITH AN" "X" IN" SECTION BOX: $\mathrm{N}$

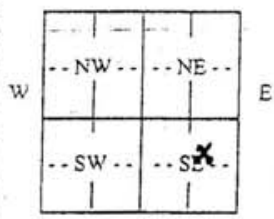

$S$

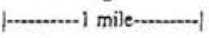

Woshingten, DC. $20250-0513$

\section{DEPTH OF COMPLETED WELL ...... 70}

Depth(s) Groundwater Encóuntered

(1)....

Pump test data: Well water was

EST. YIELD …......gpm. Well water was............. aft after.

Division of Water Resources App. No. Section Number $\quad$ Township No. $\quad$ Range Number Global Positioning System (GPS) infornation:

Latitude: 38.22235 .......... (in decimal degrees) Longitude: $97,78036 \ldots \ldots \ldots \ldots$ (in decinal degrees) Elevation: Datum: $\square$ WGS 84, $\square$ NAD 83, $\square$ NAD 27 Collection Method: Est. Accuracy: $\square<3 \mathrm{~m}, \square 3.5 \mathrm{~m}, \square 5-15 \mathrm{~m}, \square>15 \mathrm{~m}$

Bore Hole Diameter ...8. 8 in. to ....70 ....ft., and ............in. to ................f.
WELL WATER TO BE USED AS: $\square$ Public water supply $\square$ Geothermal $\square$ Injection well

$\square$ Domestic $\square$ Feedlot $\square$ Oil field water supply Dewatering Was a chemical/bacteriologícal sample submitted to Department? $\square$ Yes $\boldsymbol{X}$ No

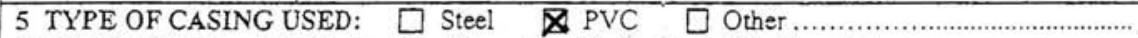

CASING JOINTS: $\square$ Glued $\square$ Clamped $\square$ Welded $\otimes$ Threaded

Casing diameter ............ in to ...6. 60 ... ft., Diameter ........... in. to ............. ft., Diameter ............. in. to ........... ft. Casing height above land surface........ TYPE OF SCREEN OR PERFORATION MATERIAL:
$\square$ Steel
$\square$ Stainless Steel
$\triangle P V C$
$\triangle$ None used (open hole)

$\square$ Other (Specify)

SCREEN OR PERFORATION OPENINGS ARE:

$\square$ Continuous slot $\mathbf{X}$ Mill slot $\square$ Gauze wrapped $\square$ Torch cut $\square$ Drilled holes $\square$ None (open hole)

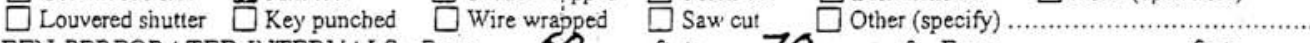

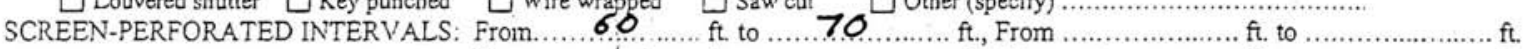

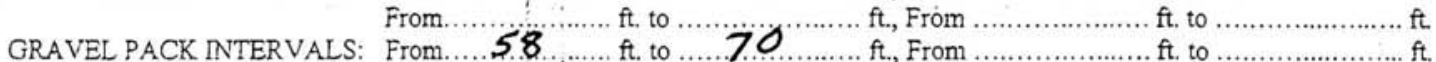

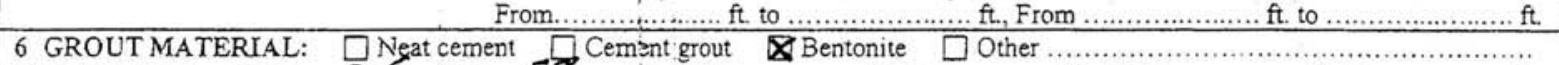

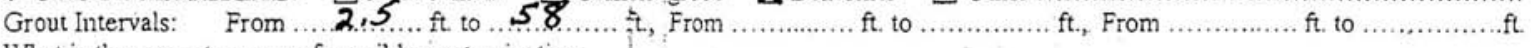

What is the nearest source of possible contamination:
$\begin{array}{ll}\square \text { Septic tank } & \square \text { Lateral lines } \square \text { Pit privy } \\ \square \text { Sewer lines } & \square \text { Cesspool } \square \text { Sewage lagoo }\end{array}$
Watertight sewer lines $\square$ Seepage pit $\square$ Feedyard

Direction from well

\begin{tabular}{|c|c|c|c|c|c|c|}
\hline FROM & TO & LITHOLOGIC LOG & $\therefore$ & FROM & TO & LITHO. LOG (cont.) or PLUGGING INTERVALS \\
\hline 0 & 38 & Clay & 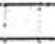 & +2 & & + \\
\hline 38 & 70 & Weothered shale & 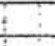 & & & - \\
\hline & & & 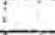 & - & 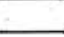 & 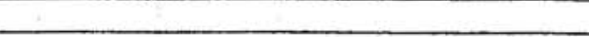 \\
\hline & & & 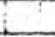 & 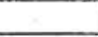 & & \\
\hline & & & $\therefore$ & & & \\
\hline & & & $\therefore$ & 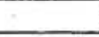 & & 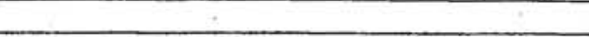 \\
\hline & & & ! $:$ & & & 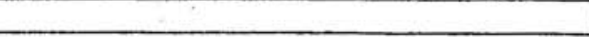 \\
\hline & & & 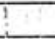 & & & \\
\hline & & & $\vdots$ & & & \\
\hline & & & 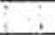 & 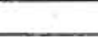 & & - \\
\hline
\end{tabular}

7 CONTRACTOR'S OR LANDOWNER'S CERTIFICATION: This water well was $\$$ constructed, $\square$ reconstructed, or $\square$ plugged under my jurisdiction and was completed on (mo/day/year) ..8/26/.1!... and this record is true to the best of my knowlegge and belief. Kansas Water Well Contractor's ficense No...5.9. .... This Water Well Record was completed on (mo/day/year) ..9. 2.1\% II!....

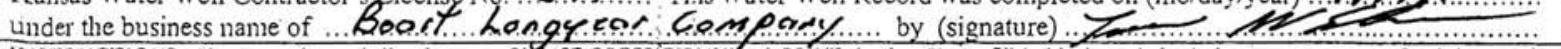

INSTRUCTIONS: Use typewriter or ball point pen. Pl.EASE PRESSFFIRMLY and PRINT clearly. Please fill in blanks and check the correct answers. Send three copies (white, blue, pink) to Kansas Department of Health and Environment, Bureau of Water, Geology Section, 1000 SW' Jackson St., Suite 420, 'Topeka, Kansas 66612.1367.

Telephone 785-296-5522. Send one copy to WATER WELL OWNER and retain one for your records. Include fee of S5.00 for cach constuvcted well. Visit us at hetp://nnww.kdheks.gov/watenvell/index.htmi. 


\section{$m \omega-16 m 2$}

WATER WELL RECORD

\begin{tabular}{l}
1 LOCATION OF WATER \\
County: McPherson \\
\hline Street/Rural Address of Well
\end{tabular}

Form WWC-5

Division of Water Resources App. No. Street/Rural Address of Well Location; if unknowr, distance \& direction from nearest town or intersection: If at owner's adjress, check here $\square$.

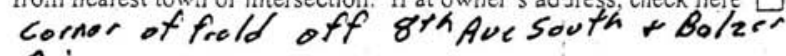
Drive

2 WATER WELL OWNER: USDA-Steve Gilmore RR \#, Street Address, Box \#: 5 top 0513 Room $4714-5$ City, State, ZIP Code : 1400 Independence Ave, sw

3. LOCATE WELL WITH AN "X" IN SECTION BOX:

w

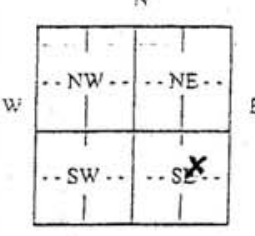

S Weshingten, DC. $20250-0513$ 4 DEPTH OF COMPLETED WELL …....88 Depth(s) Groundwater Encciuntered (1). (2) $34 \ldots \ldots \ldots \ldots \ldots \ldots \ldots$ ft. \begin{tabular}{|l|l|l|l}
17 & Township No. & Range Number \\
17 & R \& DE QW
\end{tabular} No. $R$

Range Number Global Positioning System (GPS) infornation:
Latitude: $38,2,235$ Longitude: $97,78036 \ldots \ldots \ldots \ldots$ (in decimal degrees) Elevation: Datum: $\square$ WGS 84, $\square$ NAD 83, $\square$ NAD 27 Collection Method:

7 GPS unit (Make/Model:

Digital Map/Photo $\square$ Topographic Map....................) Est. Accuracy: $\square<3 \mathrm{~m}, \square 3.5 \mathrm{~m}, \square 5.15 \mathrm{~m}, \square>15 \mathrm{~m}$

88

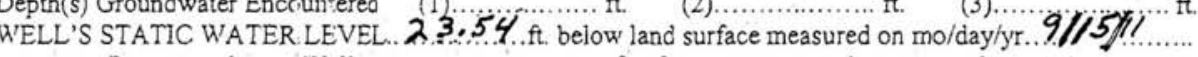

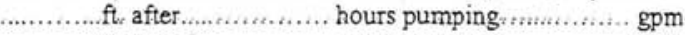
EST. YIELD …........ $\mathrm{gmm}_{\mathbf{8}}$ Well water was.

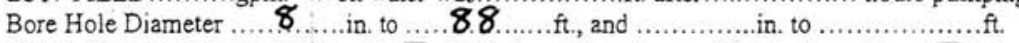
WELL WATER TO BE US DD AS: $\square$ Public water supply $\quad \square$ Geothermal $\quad \square$ Injection well $\square$ Domestic $\square$ Feedlot $\square$ Oil field water supply $\square$ Dewatering $\square$ Other (Specify below) $\square$ Irrigation $\square$ Industrial $\square$ Domestic-lawn \& garden $\mathbf{X}$ Monitoring well Was a chemical/bacteriological sample submitted to Department? $\square$ Yes $\otimes$ No If yes, mo/day/yr sample was submitted.

(-....... mile

Water well disinfected? $\square$ Yes $\boldsymbol{X}$ No

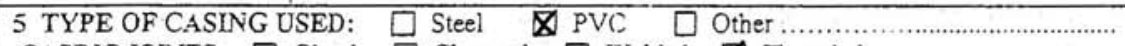

CASING JOINTS: $\square$ Glued $\square$ Clamped $\square$ Welded $\bigotimes$ Threaded

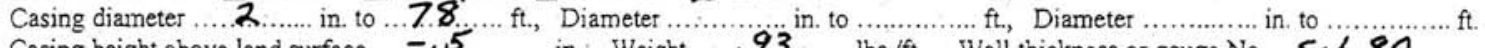

Casing height above land surface...... TYPE OF SCREEN OR PERFORATION MATERIAL:

$\square$ Steel $\square$ Stainiess Steel X PVC

None us:d (open hole)

SCREEN OR PERFORATION OPENINGS ARE:

$\square$ Continuous siot 叉Mill slot $\square$ Gauze wrapped $\square$ Torch cut $\square$ Drilled holes $\square$ None (open hole)

$\square$ Louvered shutter $\square$ Key punched $\square$ Wire wrapped

SCREEN-PERFORATED INTERVALS: From...7.8..........

$\square$ Saw cut 8 Other (specify) ....................................

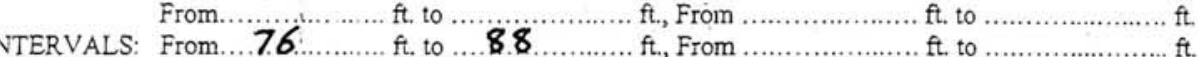
From

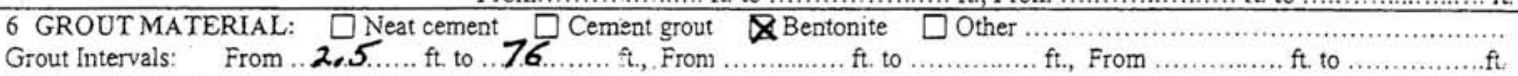
What is the nearest source of possible contamination:
$\square$ Septic tank
$\square$ Wewer lines
$\square$ Lateral lines $\square$ Pit privy
$\square$ Pivestock pens
Fuel storage
$\square$ Insecticide storage
Abandoned water well
$\square$ Other (specify below)

Direction from well

\begin{tabular}{|c|c|c|c|c|c|}
\hline FROM & TO & LITHOLOGIC LOG & FROM & TO & L \\
\hline 0 & 38 & Clav & & & \\
\hline 38 & 88 & Weathered Shote & & & \\
\hline
\end{tabular}

\begin{tabular}{|l|l|l|l|l|l|}
\hline 0 & 38 & & & \\
\hline 38 & 88 & Weathered Shote & & \\
\hline & & & & \\
\hline & & & & & \\
\hline & & & & \\
\hline & & & & \\
\hline & & & & \\
\hline
\end{tabular}

7 CONTRACTOR'S OR LANDOWNER'S CERTIFICATION: This water well was $\boldsymbol{\nabla}$ constructed, $\square$ reconstructed, or $\square$ plugged under my jurisdiction and was completed on (mo/day/year) . $8 / 26 / .6$ ! . and this record is true to the best of my knowledge and belief. Kansas Water Well Contractor's License No. 5.9.7..... This Water Well Record was completed on (mo/day/year) ...9/2 $1.1 / 1 . . . . .$. under the business name of Boart... ho.rgy.ea ..... Compony ...... by (signature)

INSTRUCTIONS: Use typewriter or ball point pen. PLEASE PRESS FIRMLY and PRINT clearly. Please fill in blanks and check the correct answers. Send three copies (white, bluc, pink) to Kansas Department of Heaith and Environment, Bureau of Water, Geology Section, 1000 SW Jackson St., Suite 420, Topeka, Kansas 66612-1367. Telephione 785-296-5522. Send one copy to WATER WVLL OWNER and retain one for your records. Include fee of S5.00 for each constnucted well. Visit us at http://www.kdheks,goviwatenwell/index.html, 


\section{$M w \cdot 160$}

WATER WELL RECORD

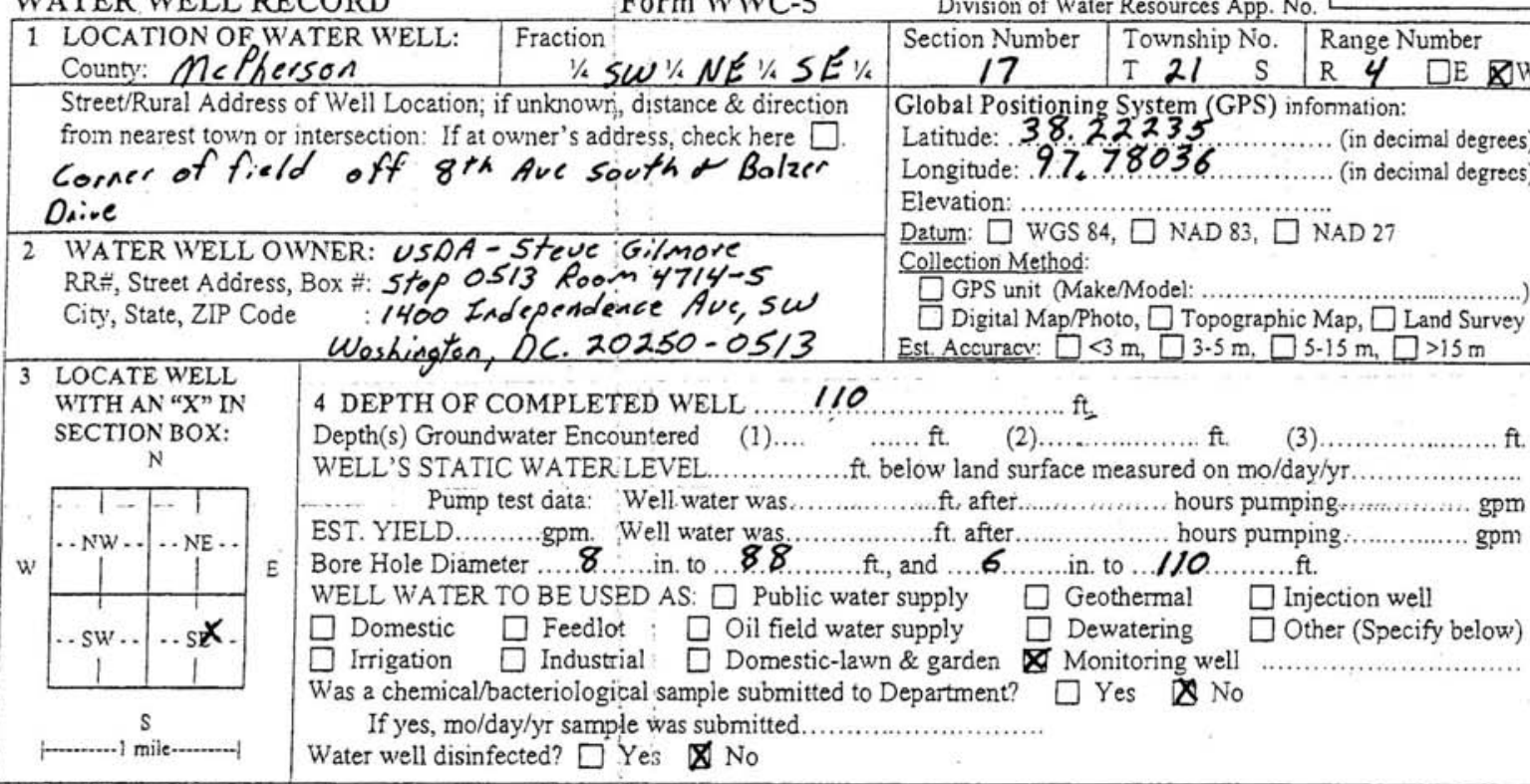

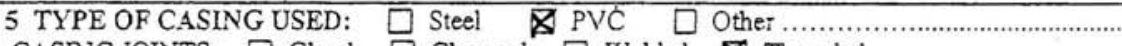

CASING JOINTS: $\square$ Glued $\square$ Clamped $\square$ Welded $\otimes$ Threaded

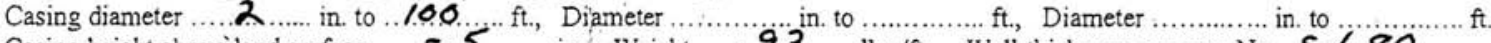

Casing height above land surface......, $5 \ldots \ldots . .$. in. Weight .... $9.3 \ldots \ldots$ lbs./ft., Wall thickness or gauge No. Sch.8.

TYPE OF SCREEN OR PERFORATION MATERIAL:

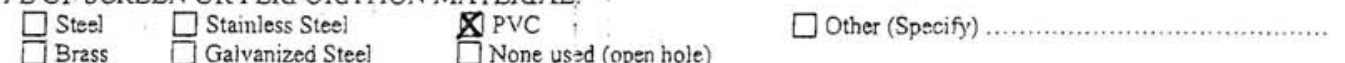

SCREEN OR PERFORATION OPENINGS ARE:

$\square$ Continuous slot $\square$ Mill slot $\square$ Gauze wrápped $\square$ Torch cut $\square$ Drilled holes $\square$ None (open hole)

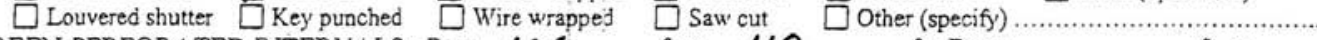

SCREEN-PERFORATED INTERVALS: From $10.0 \ldots \ldots \ldots \mathrm{ft}$ to $\ldots / 10 \ldots \ldots \ldots \ldots \mathrm{ft}$., From .................................................... ft.

GRAVEL PACK NNERVALS: Frot

From .................. ft. to

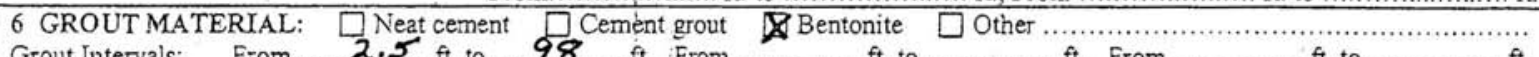

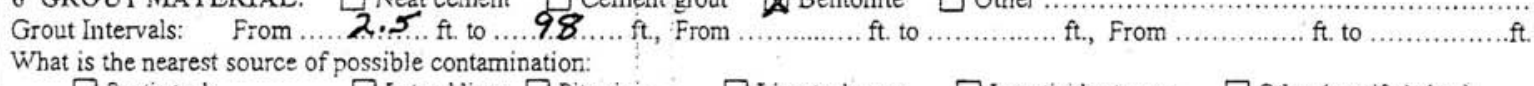
Septic tank
$\square$ Sewer lines
Watertight se
$\square$ Lateral lines $\square$ Pit privy $\quad \square$ Livestock pens

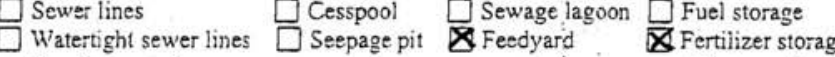
$\square$ Insecticide storage
Abandoned water well
Oil weli/gas well
Direction from well ...... Distance from well

\begin{tabular}{|c|c|c|c|c|c|}
\hline FROM & TO & LITHOLOGIC LOG & FROM & TO & LITHO LOG (cont.) Or PLUGGING INTERVALS \\
\hline 0 & 38 & Cloy & & & \\
\hline 38 & 110 & Weathered Shale & & & \\
\hline & & & & & \\
\hline & & & & & \\
\hline & & $\vdots$ & & & \\
\hline & & & & & \\
\hline & & & & & \\
\hline
\end{tabular}

7 CONTRACTOR'S OR LANDOWNER'S CERTIFICATION: This water well was $\triangle$ constructed, $\square$ reconstructed, or $\square$ plugged under my jurisdiction and was completed on (mo/day/year) . $8 / 26 / 11 \ldots$ and this record is true to the best of my knowledge and belief.

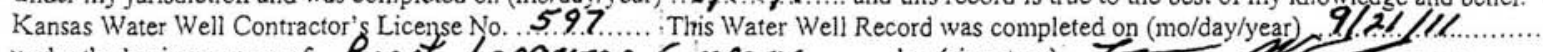

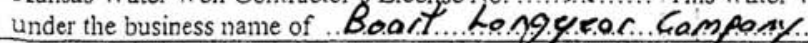

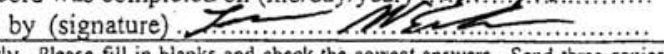

INSTRUCTIONS: Use typewriter or ball point pen. PLEASE PRESS FJRALY and PRINT ciearly. Please fill in blanks and check the correct answers. Send three copies

(white, bluc, pink) to Kansas Department of Health and Environment, Burcau of Water, Geology Section, 1000 SW Jackson St, Suite 420, Topeka, Kansas $66612-1367$.

Telephone 785.296-5522. Send one copy to WATER WELL OWNER aind retain one for your records. Include fee of $\$ 5.00$ for cach construcled well. Visit us at hettp://hunw.kdheks.gov/waterwell/index.html.

KSA $82 a-1212$

Check: П White Conv. $\prod$ Blue Conv $\prod$ Pink Conv 
WATER WELL RECORD Form WWC-5

Division of Water Resources App. No.

\begin{tabular}{|l|c|c|c|c|c|}
\hline 1 LOCATION OF WATER WELL: & Fraction \\
County: MCPherson & $1 / 4$ SE. $1 / 4 \omega_{1 / 4} S \omega_{1 / 4}$ & 16 & Section Number & Township No. & Range Number \\
\hline
\end{tabular}
County: McPherson

own, distance \& direction from nearest town or intersection: If at owner's address, check here $\square$ In field east of $8^{\text {th }}$ Aue. Between East Morgan street r Formington Road

2 WATER WELL OWNER: USDA-Steve Gilmore $R R \#$, Street Address, Box \#: Stop 0 513 Room $4714-5$ City, State, ZIP Code 1400 Independerce Ave, sw

3 LOCATE WELL Weshingten, DC. $20250-05 / 3$ WITH AN "X" IN SECTION BOX:

w

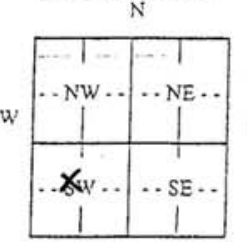

4 DEPTH OF COMPLETEE WELL ....... 35

Depth(s) Groundwater Encountered

\section{G}

Global Positioning System (GPS) information:

Latitude: $38,12205 \ldots . . . \ldots$ (in decimal degrees) Longitude: $97.7733 .5 \ldots \ldots \ldots \ldots$ (in decimal degrees) Elevation:

Datum: $\square$ WGS 84, $\square$ NAD 83, $\square$ NAD 27 Collection Method:

GPS unit (Make/Model: ...................................) Digital Map/Photo, $\square$ Topographic Map, $\square$ Land Survey Est Accuracv: $\square<3 \mathrm{~m}, \square 3.5 \mathrm{~m}, \square 5.15 \mathrm{~m}, \square>15 \mathrm{~m}$ STATIC WATER LEVEL......16.1. . ft. below land surface measured on mo/day/yr. $9 / 15 / 1 / \ldots \ldots$ ............ft after

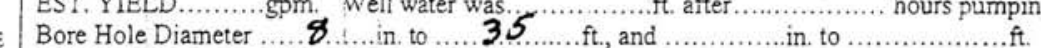
WELL WATER TO BE USED AS: $\square$ Public water supply $\square$ Geothermal $\square$ Injection well $\square$ Domestic $\square$ Feedlo $\square$ Oil field water supply $\square$ Dewatering $\square$ Other (Specify below) $\square$ Irrigation $\square$ Industrial $\square$ Domestic-lawn \& garden $\square$ Monitoring well Was a chemical/bacteriological sample submitted to Department? $\square$ Yes $\bigotimes$ No If yes, mo/day/yr sampie was submitted.

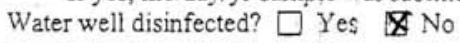

5 TYPE OF CASING USED: $\square$ Steel $\triangle$ PVC $\square$ Other.

CASING JOINTS: $\square$ Glued $\square$ Clamped $\square$ Welded $\boldsymbol{X}$ Threaded

Casing diameter ........... in. to ...25 ... ft., Diameter ............ in. to ............. ft., Diameter ............. in. to ........... ft. Casing height above land surface.......5...... in., Weight ....3.3....lbs./ft., Wall thickness or gauge No. Sch. 40...... TYPE OF SCREEN OR PERFORATION MATERIAL:
$\square$ Steel
$\square$ Stainless Steel
XPVC
Done used (open hole)
$\square$ Other (Specif̂́)

$\square$ Brass $\square$ Galvanized Steel SCREEN OR PERFORATION OPENINGS ARE:

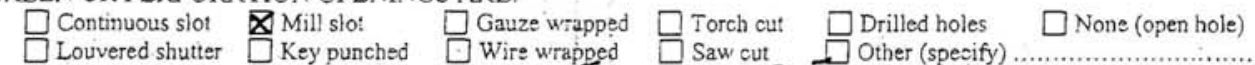

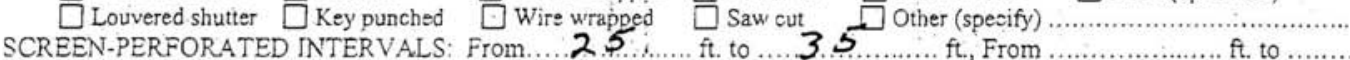
GRAVEEL PACK INTERVALS: From
From..........
ft. to ....
3.5 From.
f., From

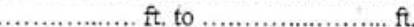

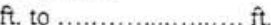

6 GROUT MATERIAL: $\square$ Neat cement $\square$ Cement grout $\$$ Bentonite $\square$ Other

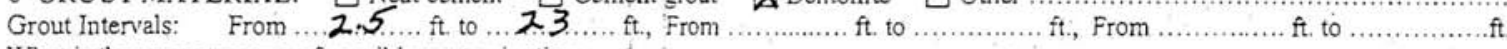

What is the nearest source of possible contamination:
$\square$ Septic tank
Sewer lines
$\square$ Lateral lines $\square$ Pit privy $\square$ Livestock pens
Cesspool
¿Fertilizer storage
$\square$ Insecticide storage
Abandoned water well
Oil well/gas well

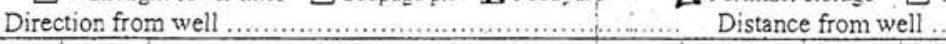

\begin{tabular}{|c|c|c|c|c|c|}
\hline FROM & TO & LITHOLOGIC LOG & FROM & TO & LITHO. LOG (cont.) OI PLUGGING INTERVALS \\
\hline 0 & 35 & Cloy & & & \\
\hline & & & & & \\
\hline & & & & & \\
\hline & & & & & \\
\hline & & & & & \\
\hline & & & & & \\
\hline & & & & \\
\hline
\end{tabular}

7 CONTRACTOR'S OR LANDOWNER'S CERTIFICATION: This water well was $\bigotimes$ constructed, $\square$ reconstructed, or $\square$ plugged under my jurisdiction and was completed on (mo/day/year) . $8 / 2.8 / 11 \ldots$ and this record is true to the best of my knowledge and belief.

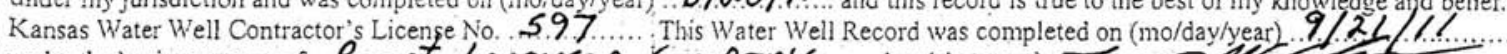

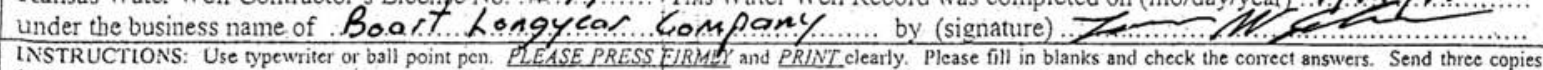

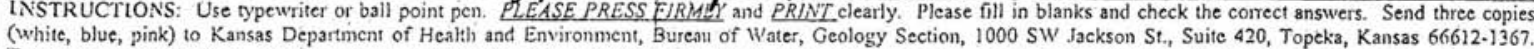
Telephone 785-296-5522. Send one copy to WATER WELL OWNER and retain one for your records. Include fee of $\$ 5.00$ for each censtincted well. Visit us a hetp://hunw.kdheks.gov/waterwell/index.html.

KSA $82 \mathrm{a}-1212$

Check: $\square$ White Conv, $\Pi$ Blue Conv. $\Pi$ Pink Conv 


\section{CORRECTION(S) TO WATER WELL RECORD (WWC-5)}

(to rectify lacking or incorrect information)

\section{Location listed as:}

Section-Township-Range: $\quad 16-26 \leq-4 W$

Fraction $(1 / 41 / 4 \quad 1 / 4)$ :
County: Location changed to:

McPherson

$$
16-21 s-4 w
$$

NW SE NW SW

Other changes: Initial statements:

Changed to:

Comments: well is just south of Inman, 15 .

verification method: Latitude \& longitude, KGS" "LEO" conversion tool, and mapping tool on KGS website initials: $Q R A$ date: $3 / 9 / 20 / 2$

submitted by: Kansas Geological Survey, Data Resources Library, 1930 Constant Ave., Lawrence, KS 66047-3726 to: Kansas Dept of Health \& Environment, Bureau of Water, 1000 SW Jackson, Suite 420, Topeka, KS 66612-1367. 
WATER WELL RECORD Form WWC-5 Division of Water Resources App. No.

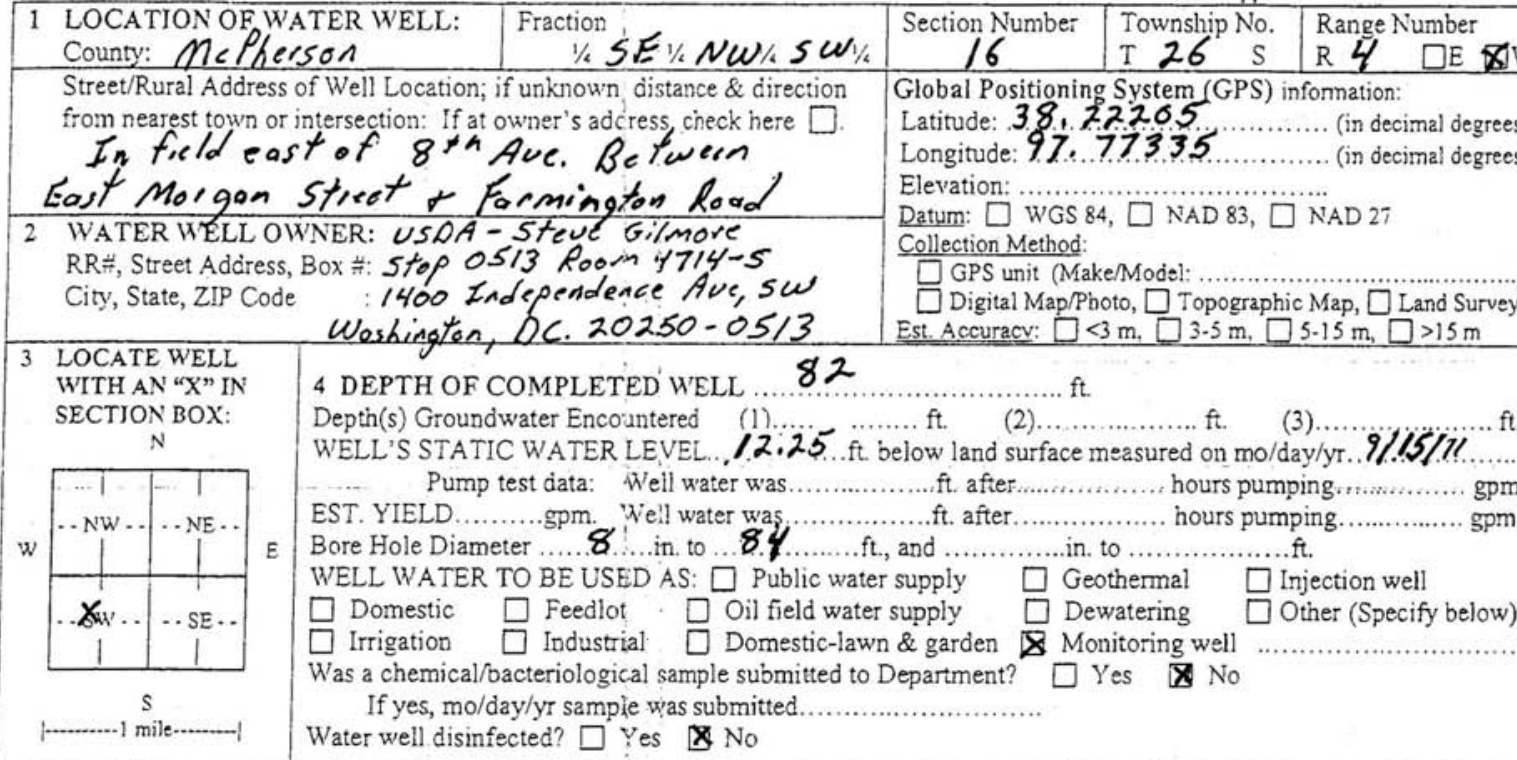

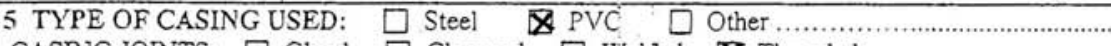

CASING JOINTS: $\square$ Glued $\square$ Clamped $\square$ welded $\boldsymbol{X}$ Threaded

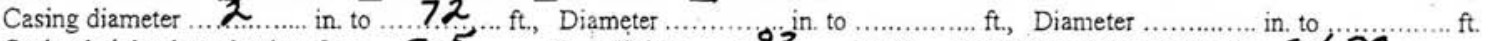

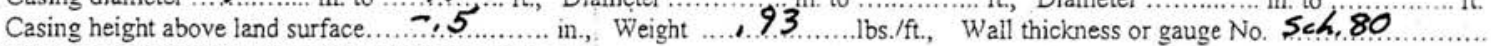

TYPE OF SCREEN OR PERFORATION MATERIAL:
$\square$ Steel
$\square$ Stainless Steel
XPVC
None used (open hole)
$\square$ Other (Specify)

SCREEN OR PERFORATION OPENINGS ARE:

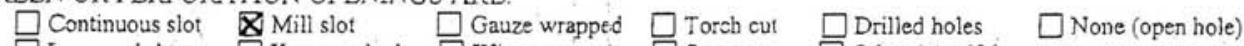

$\square$ Louvered shutter $\square$ Keypunched $\square$ Wire wrapped $\square$ Saw cut

SCREEN-PERFORATED NERVALS: From.....7. 7....... ft. to 87

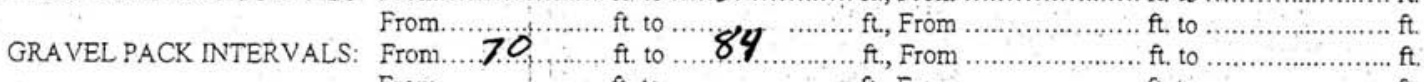

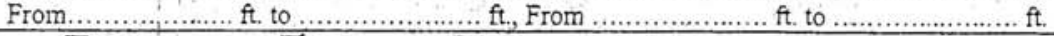

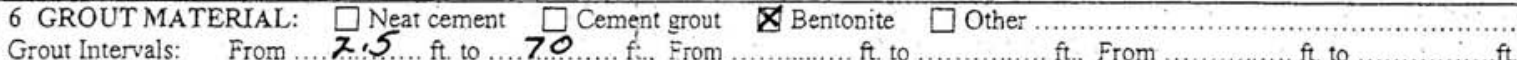

What is the nearest source of possible contamination: ,

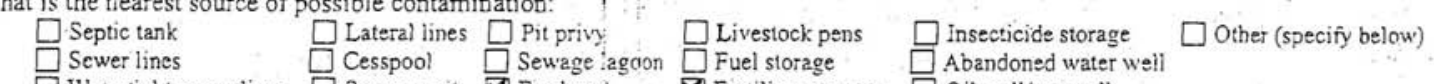

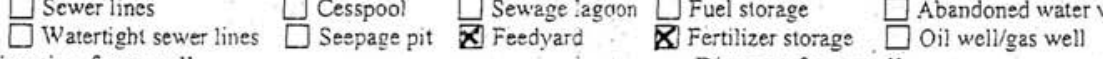

Direction from well ............................................ Distance from well

\begin{tabular}{|c|c|c|c|c|c|}
\hline FROM & TO & LITHOLOGIC LOG & FROM & TO & LITHO LOG (cont.) OI PLUGGING INTERV ALS \\
\hline 0 & 20 & Clay & & & \\
\hline 20 & 82 & Weathered Shale & & & \\
\hline & & & & & \\
\hline & & & & & \\
\hline & & & & & \\
\hline & & & & \\
\hline & & & & \\
\hline & & &
\end{tabular}

7 CONTRACTOR'S OR LANDOWNER'S CERTIFICATION: This water well was $\$ constructed, $\square$ reconstructed, or $\square$ plugged under ny jurisdiction and was completed on (mo/day/year) $8 / 28 / 1] \ldots$ and this record is true to the best of my knowledge and belief.

Kansas Water Well Contractor's License No. 5.9.7..... This Water Well Record was completed on (mo/day/year) .97. 2! I. I.l.........

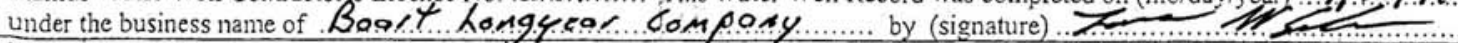

INSTRUCTIONS: Use typewriter or ball point pen. PLEASE PRESS FIRILY and PRINT clearly. Please fill in blanks and check the correct answers. Send three copies

(white, bluc, pink) to Kansas Department of Health and Environment, Burchu of W'ater, Geology Section, 1000 SW Jackson St, Suite 420, Topeka, Kansas 66612-1367.

Telephone 785-296-5522. Send one copy to WATER WELL OWNER and retain one for your records. Include fee of $\$ 5.00$ for each constructed well. Visit us at hittp: $: /$ hunw.kdheks.gov/waterwell/index.htmi.

KSA $\$ 2 a-1212$

Check: $\square$ White Conv. $\Pi$ Blue Conv. $\Pi$ Pink Conv 


\section{CORRECTION(S) TO WATER WELL RECORD (WWC-5)}

(to rectify lacking or incorrect information)

\section{Location listed as:}

Section-Township-Range:

Fraction $\left(\begin{array}{lll}1 / 4 & 1 / 4 & 1 / 4\end{array}\right)$ :
$16-26 s-4 \omega$

SE NW $5 \omega$
Location changed to:

McPherson

$16-215-4 \omega$

NW SE NW SW

Other changes: Initial statements:

Changed to:

Comments: well is just south of Inman, $\pi s$

verification method: Latitude \& longitude, KGS" "LEO" conversion too/, and mapping tool on KGS website 
WATER WELL RECORD

Form WWC-5 Division of Water Resources App. No.

1 LOCATION OF WATER WELL:

County: MePherson

\begin{tabular}{l|l|l} 
Township No. & Range Number \\
\hline
\end{tabular}

Street/Rural Address of Well Location; if unknown, distance \& direction

from nearest town or intersection: If at owner's address, check here $\square$

In firld east of $8^{\text {th }}$ Ave. Betwien East Morgen streot + Farmingtor Rood

2 WATER WELL OWNER: USDA-Steve Gilmore

$R R=$, Street Address, Box $\#$ : stop 0513 Room $4714-5$

City, State, ZIP Code : 1400 Independence Ave, sw

Weshingten, DC. $20250-0513$

\begin{tabular}{ll|l}
3 LOCATE WELL & 4 DEPTH OF COMPLETED WELL .... 108 \\
WITH AN" "XN & 4 DN
\end{tabular}

SECTION BOX:

Depth(s) Groundwater Enccuntered (1)...

(2).......... ft.

I 26

$R \quad 4 \quad \square E X W$

\section{Global Positioning System (GPS) infornation:}

Latitude: 38.22205 ........... (in decimal degrees)

Longitude $97,77335 \ldots \ldots \ldots \ldots$ (in decimal degrees)

Elevation:

Datum: $\square$ WGS 84, $\square$ NAD 83, $\square$ NAD 27

Coliection Method:

GPS unit (Make/Model: .........................................)

$\square$ Digital Map/Photo, $\square$ Topographic Map, $\square$ Land Survey Est. Accuracv: $\square<3 \mathrm{~m}, \square 3.5 \mathrm{~m}, \square 5.15 \mathrm{~m}, \square>15 \mathrm{~m}$

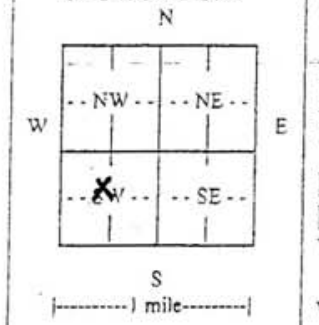

WELL'S STATIC WATER LEVEL. 20,7.3

Pump test data: Well water was.

..........ft after............... hours pumping

EST. YIELD ......... gpm. Well water was ...............ft. after................ hom

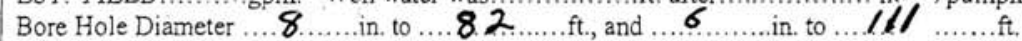

WELL WATER TO BE USED AS: $\square$ Public water supply $\square$ Geothermal $\square$ Injection well

$\square$ Domestic $\quad \square$ Feedlot $\square$ Oil field water supply $\square$ Dewatering $\square$ Other (Specify below)

$\square$ Irrigation $\square$ Industial $\square$ Domestic-lawn \& garden $\square$ Monitoring well

Was a chemical/bacteriologijal sample submitted to Department? $\square$ Yes $\boldsymbol{X}$ No

If yes, mo/day/yr sample was submitted.

Water well disinfected? $\square$ Yes $\square$ No

\section{TYPE OF CASING USED: $\square$ Steel $\triangle$ PVC $\square$ Other ...............
CASING JOINTS: $\square$ Glued $\square$ Clamped $\square$ Velded $\triangle$ Threaded
Casing diameter 2 in to 98 f., Diameter}

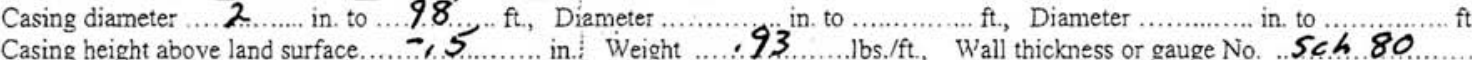

TYPE OF SCREEN OR PERFORATION MATERIAL:

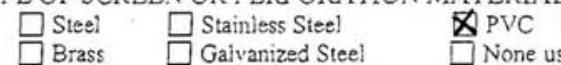

SCREEN OR PERFORATION OPENINGS ARE:

$\square$ Continuous siot $\bigotimes$ Mill siot $\square$ Gauze wrapped $\square$ Torch cut $\square$ Drilled holes $\square$ None (open hole)

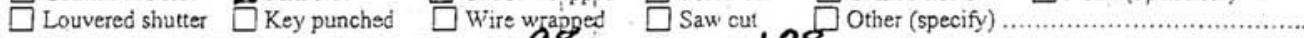

SCREEN-PERFORATED INTERVALS: From.....98 $98 \ldots \ldots$ ft. to .... 108 ........ ft., From ….................. ft. to ...................... ft.

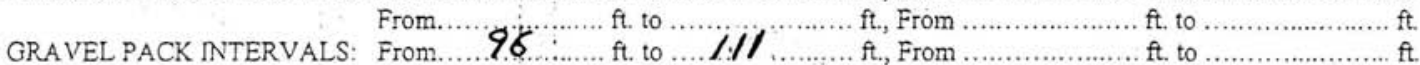

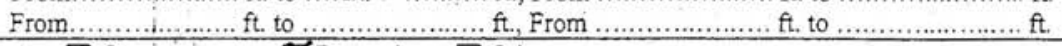

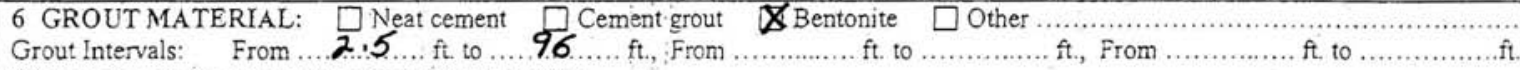

What is the nearest source of possible contamination
$\square$ Septic tank
1.
Other (specify below)

Watertight se

Direction from well

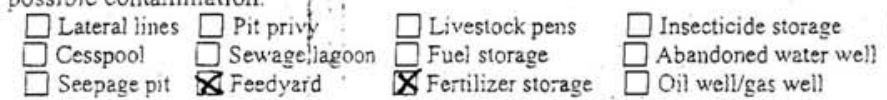

Fertilizer storage $\square$ Oil well/gas well

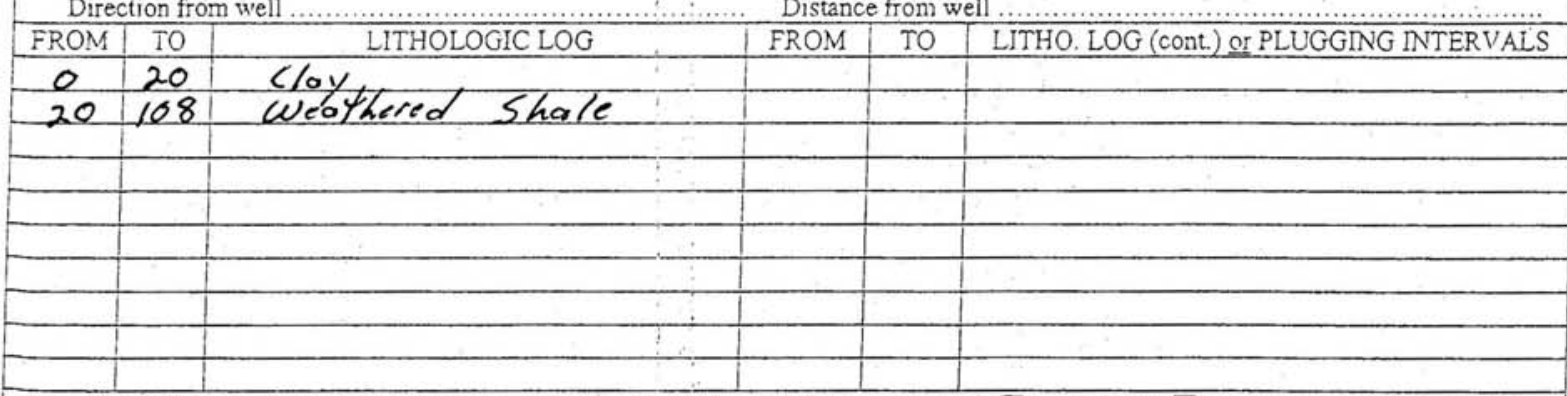

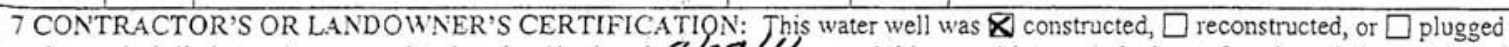
under my jurisdiction and was completed on (mo/day/year) $8 / 2.8 / / 1 \ldots \ldots$ and this record is true to the best of my knowledge ayd belief. Kansas Water Well Contractor's License No .5.9...... This Water Well Record was completed on (mo/day/year) .97.2/. $/ 1 . . . . .$.

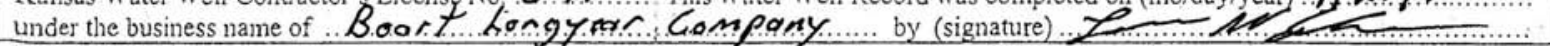

INSTRUCTIONS: Use typewriter or ball point pen. PLEASE PRESSFIRMLP and PRINT clearly. Please fill in blanks and check the correct answers. Send three copies (white, blue, pink) to Kansas Depaitment of Health and Enviromment, Bureau of Water, Geology Section, 1000 SW Jackson St., Suite 420, Topeka, Kansas 66612.1367. Telephone 785-296-5522. Send one copy to WATER WELL OWNTR and retain one for your records. Include fee of S5.00 for cach constructed well. Visit us at http:/hwnw.kdheks,gov/waterweil/index.html.

KSA $\$ 2 a-1212$

Check: $\square$ White Copy, $\square$ Blue Copy, $\square$ Pink Copy 


\section{CORRECTION(S) TO WATER WELL RECORD (WWC-5)}

(to rectify lacking or incorrect information)

Location listed as:

Section-Township-Range:

Fraction $\left(1 / 4{ }^{1 / 4} \quad 1 / 4\right)$
County: Location changed to:

$16-215-4 u$ NW SE NW SW

Other changes: Initial statements:

Changed to:

Comments: well is just south of Inman, $\mathrm{TS}_{\mathrm{s}}$

verification method: Latitude \& longitude, KGS" "LEO" conversion tool, and mapping tool on KGS website initials: $\operatorname{RR} \&$ date: $3 / 9 / 20 / 2$

submitted by: Kansas Geological Survey, Data Resources Library, 1930 Constant Ave., Lawrence, KS 66047-3726 to: Kansas Dept of Health \& Environment, Bureau of Water, 1000 SW Jackson, Suite 420, Topeka, KS 66612-1367. 


\section{Mw-185}

WATER WELL RECORD

Form WWW-5

Division of Water Resources App. No.

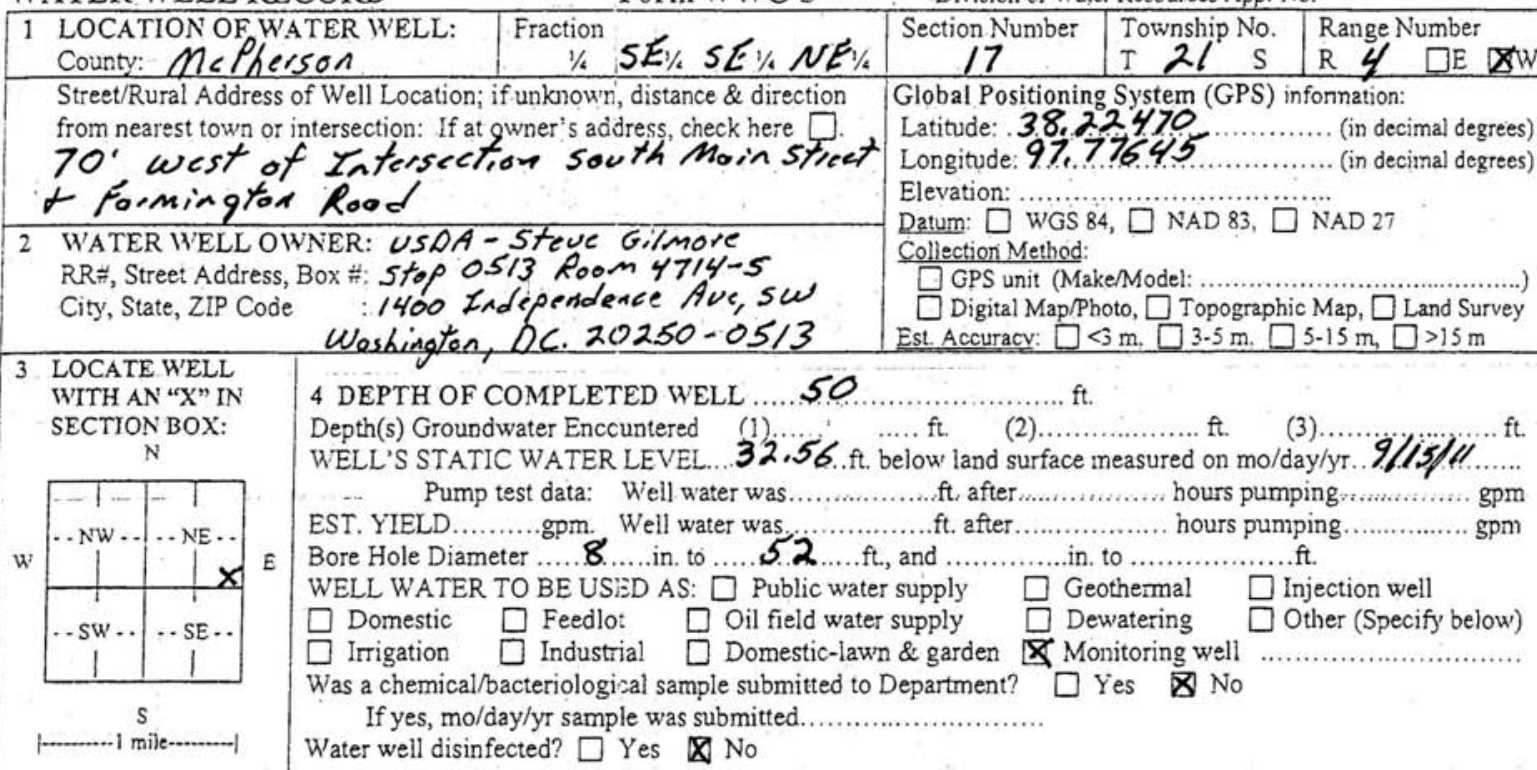

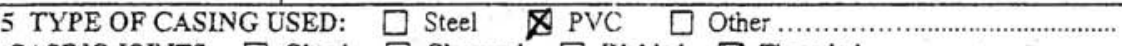

CASING JOINTS: $\square$ Glued $\square$ Clamped $\square$ Welded $\otimes$ Threaded

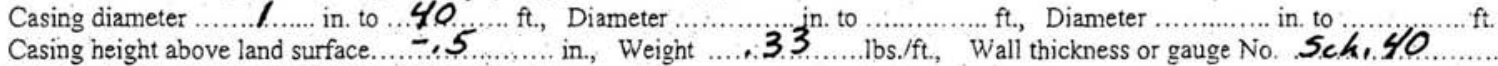

TYPE OF SCREEN OR PERFORATION MATERIAL:

$\square$ Steel $\square$ Stainiess Steel Х PVC

None used (open hole)

SCREEN OR PERFORATION OPENINGS ARE:

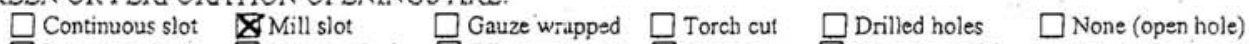

$\square$ Louvered shutter Key punched $\square$ Wire wrapped

SCREEN-PERFORATED INTERVALS: From...... 40 .........ft. to ....50

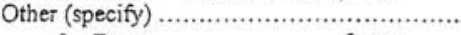

ft., From …............... ft. to .................... ft.

GRAVEL PACK NTERVALS: From......38 ........ ft. to .....

ft., From ................. ft, to …................ ft.

From................ ft. to

th to

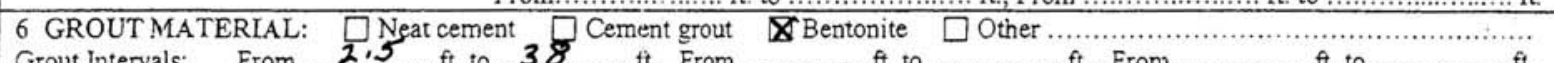

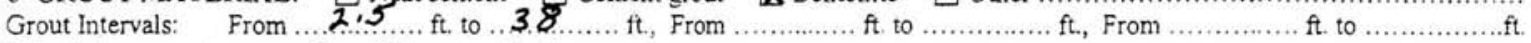
What is the nearest source of possible contamination:
$\square$ Septic tank
Sewer lines
$\square$ Watertight sewer lines $\square$ Seepage pit $\bar{\nabla}$ Feedyard
$\square$ Livestock pens
Fuel storage
insecticide storage
Abandoned water well
$\boldsymbol{X}$ Fertilizer storage $\square$ Oil well/gas well

Distance from well

\begin{tabular}{|c|c|c|c|c|c|}
\hline FROM & TO & LITHOLOGIC LOG & FROM & TO & LITHO. LOG (cont) OI PLUGGING INTERVALS \\
\hline 6 & 32 & Cloy & & & \\
\hline 32 & 50 & Weathere Shale & & & \\
\hline & & & & & \\
\hline & & & & & \\
\hline & & & & \\
\hline
\end{tabular}

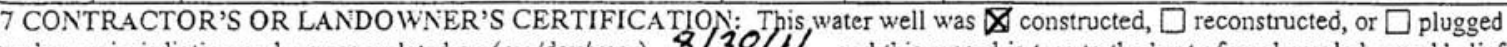
under my jurisdiction and was completed on (mo/day/year) . $8 / 30 / 1 / \ldots$ and this record is true to the best of my knowledge ayd belief

Kansas Water Well Contractor's Licepse No. 5.9.7.... This Water Well Record was completed on (mo/day/year) 9.7. Z1./11.......

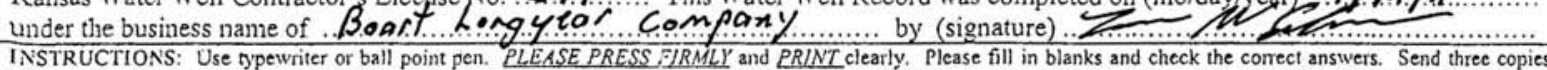

INSTRUCTIONS: Use typewriter or ball point pen. PLEASE PRESS FIRMLY and PRINT clearly. Please fill in blanks and check the correct answers. Send three copies
(white, blue, pink) to Kansas Departument of Health and Environment, Bureau of Water, Geology Section, 1000 SW Jackson St., Suite 420, Topeka, Kansas $66612-1367$. Telephone 785-296-5522. Send onc copy to WATER WVELL OWNER and retain one for your records. Include fee of S5.00 for each constructed well. Visit us at hutp://wwww.kdheks.gov/waterwell/index.htmi.

KSA $82 a-1212$ 
WATER WELL RECORD

Form WWC-5

Division of Water Resources App. No.

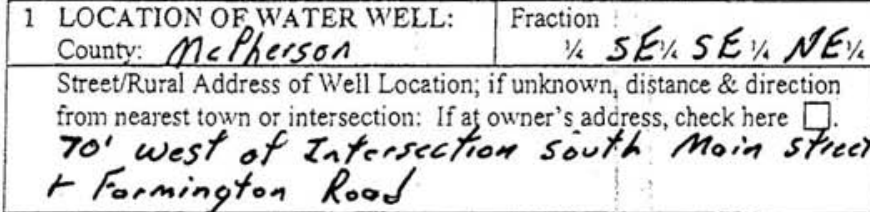

2 WATER WELL OWNER: USDA - Steve Gilmore RR\#, Street Address, Box \#: Stop 0.513 Room 4714-5

City, State, ZIP Code 1400 Ladependence Ave, SW
Weshingten, DC. 20250-0513

3 LOCATE WELL WITH AN "X" IN" SECTION BOX: $\mathrm{N}$

$\mathrm{w}$

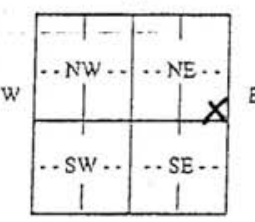

$S$

$1+\ldots \mid$

4 DEPTH OF COMPLETED WELL 95

Depth(s) Groundwater Encointered (1)... ection Number

Township No. Range Number 17 $\mathrm{T} 2 l^{\mathrm{S}} \mathrm{R} 4$

\section{Global Positioning System (GPS) infonnation:}

Latitude: 38,22470 ......... (in decimal degrees) Longitude: $97,77645 \ldots \ldots \ldots . . .$. (in decimal degrees) Elevation: Datum: $\square$ wGS 84, $\square$ NAD 83, $\square$ NAD 27 Collection Method:

G GS unit (Make/Model: Land Survey Est. Accuracy: $\square<3 \mathrm{~m}, \square 3.5 \mathrm{~m}, \square 5.15 \mathrm{~m}, \square>15 \mathrm{~m}$

CASING JOINTS: $\square$ Glued $\square$ Clamped $\square$ Welded $\bigotimes$ Threaded

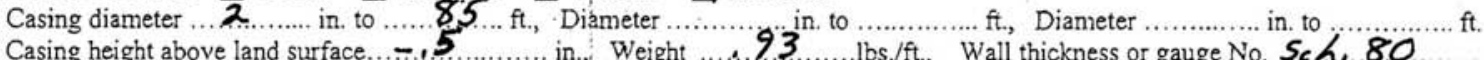
TYPE OF SCREEN OR PERFORATION MATERIAL:
$\square$ Steel
$\square$ Stainless Steel
X PVC
BEEN OR PERFORATION OPENINGS ARE:

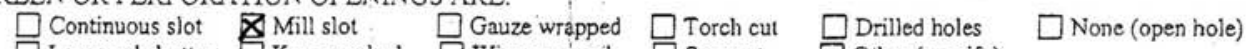

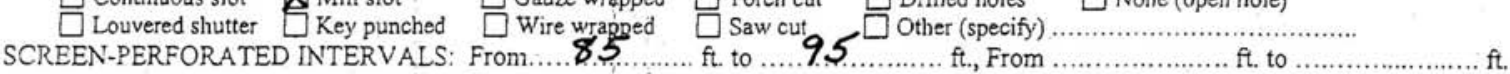

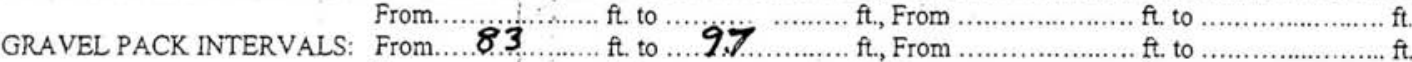

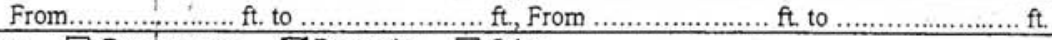

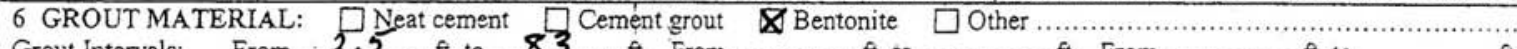

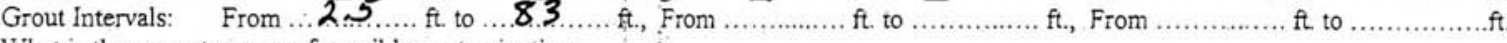
What is the nearest source of possible contamination:
$\square$ Septic tank
Watertight sewer lines
$\square$ Lateral lines $\square$ Pit privy $\square$ Livestock pens
Cesspool
Sewagetagoon Livestock pens $\square$ Insecticide storage
Abandoned water well
$\square$ Other (specify below)

Direction from well

\begin{tabular}{|c|c|c|c|c|c|}
\hline FROM & TO & LITHOLOGIC LOG & FROM & TO & LITHO LOG (cont.) OI PLUGGING INTERVALS \\
\hline 0 & 32 & Cloy & & & \\
\hline 32 & 95 & Weathered Shale & & & \\
\hline & & & & & \\
\hline & & & & & \\
\hline & & & & \\
\hline & & & & \\
\hline & & & & \\
\hline & & & & \\
\hline
\end{tabular}

7 CONTRACTOR'S OR LANDOWNER'S CERTIFICATION: This water well was $\bigotimes$ constructed, $\square$ reconstructed, or $\square$ plugged under my jurisdiction and was completed on (mo/day/year) . 8/.3.0/1! ... and this record is true to the best of my knowledge and belief.

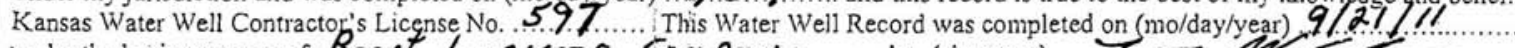

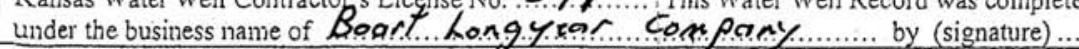

INSTRUCTIONS: Use typewriter or ball point pen. PLEASE PRESS F/RMLLY and PRINT clearly. Please fill in blanks and check the correct answers. Send three copies (white, blue, pink) to Kansas Department of Bealth and Environment, Burcau of Water, Geology Section, 1000 SW Jackson St., Suite 420, Topeka, Kansas 66612-1367. Telephone 785.296-5\$22. Send one copy to WATER WELL OWNER aid retain one for your records. Include fee of $\$ 5.00$ for each constructed well. Visit us at htto://whw.kdheks.gov/waterwell/index.htmil.

KSA $82 a-1212$

Check: $\prod$ White Couv. $\prod$ Blue Conv. $\prod$ Pink C.nnv 


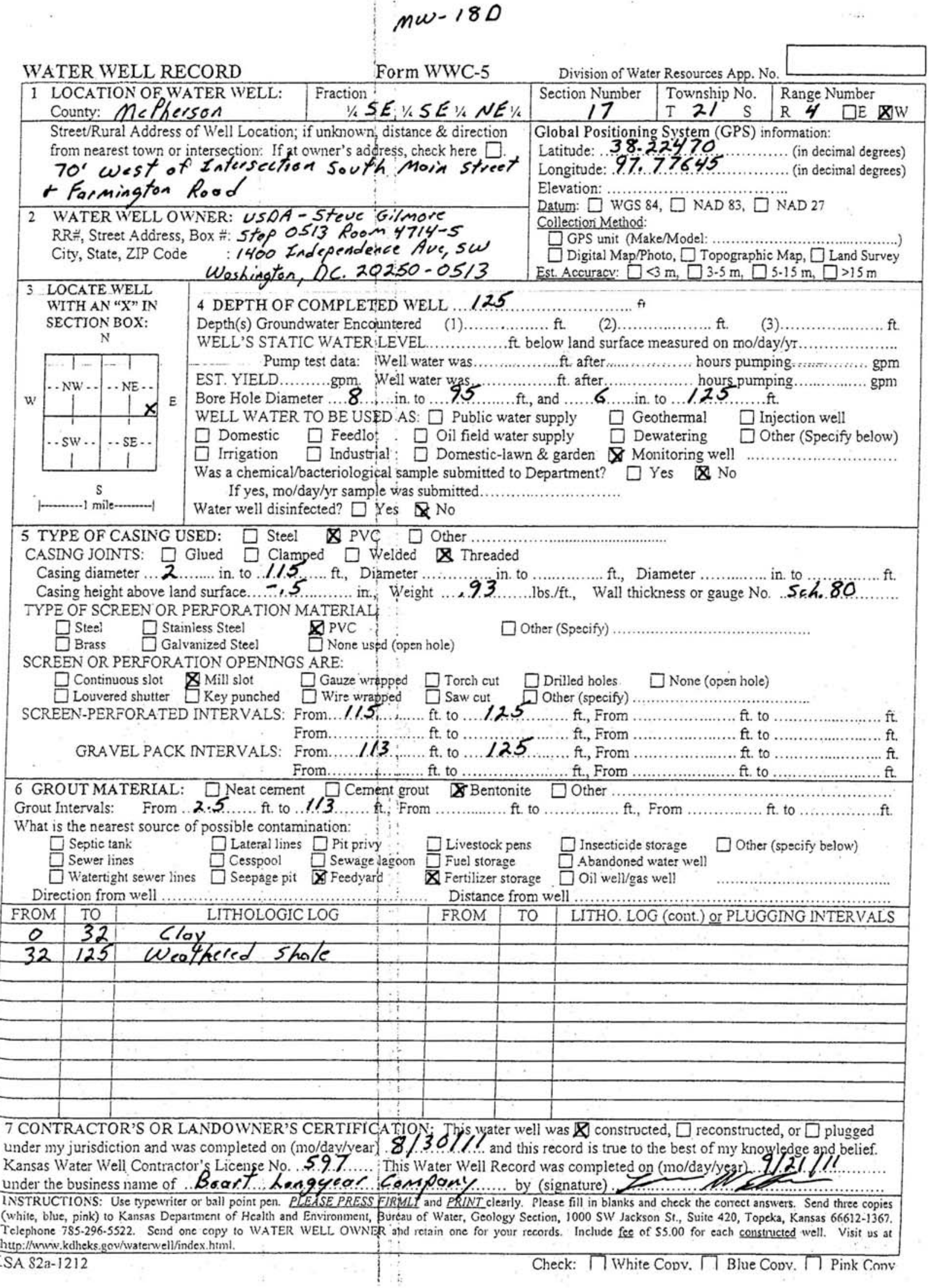


WATER WELL RECORD Form WWC-5 Division of Water Resources App. No.

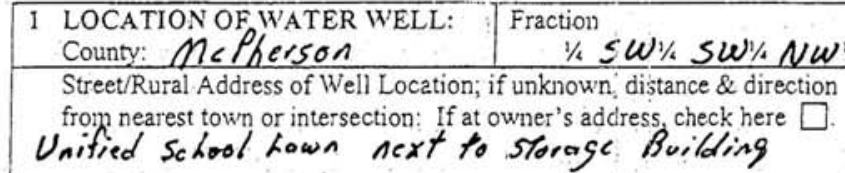

2 WATER WELL OWNER: USDA-Steve Gilmore RRH, Street Address, Box $\#$ : Stop 0513 Room 4714-5 City, State, ZIP Code : 1400 Independeace Ave, sW 3 LOCATE WELL
WITH AN" $X$ " IN SECTION BOX: 4 DEPTH OF COMPLETED WELL .... $4 / 8$

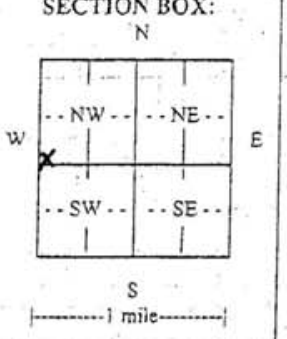
EST. YIELD ...........gpm. Well water was..............

Bore Hole Diameter ..... 8. ......... to ...5.0 If yes, mo/day/yr sample was submitted.

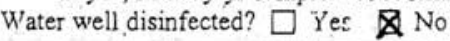
Weshingten, DC. $20250-05 / 3$
Depth(s) Groundwater Encountered: $(1) \ldots \ldots$.....ft
WELL'S STATIC WATER LEVEL... $30,2 / . \mathrm{ft}$ below Pump test data: Well water was... ..............t. after.

ion Number.

Township No.

Range Number Global Positioning System (GPS) information:

Latitude: $3.8,226.02 \ldots \ldots \ldots \ldots$ (in decimal degrees) Longitude: 9.7.7.75.4I............ (in decimal degrees) Elevation: Datum: $\square$ WGS 84, $\square$ NAD 83, $\square$ NAD 27 Collection Method: GPS unit (Make/Model: .....................................) $\square$ Digital Map/Photo, $\square$ Topographic Map, $\square$ Land Survey Est. Accuracv: $\square<3 \mathrm{~m}, \square 3-5 \mathrm{~m}, \square 5-15 \mathrm{~m}, \square>15 \mathrm{~m}$

and ................ in to ....................

WELL WATER TO BE USED AS: $\square$ Public water supply $\square$ Geothermal $\square$ injection well

$\square$ Domestic $\square$ Feedlo $\quad \square$ Oil field water supply $\quad \square$ Dewatering $\square$ Other (Specify below)

$\square$ Irrigation $\square$ Industr al $\square$ Domestic-lawn \& garden $\mathbb{X}$ Monitoring well ..............................

Was a chemical/bacteriological sample submitted to Department? $\square$ Yes $\mathbb{X}$ No

5 TYPE OF CASING USED: $\square$ Steel $\triangle$ PVC $\square$ Other

CASING JOINTS: $\square$ Glued $\square$ Clamped $\square$ Velded $\bigotimes$ Threaded

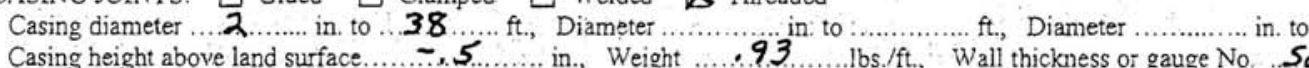

Casing height above land surface $\quad-.5 \ldots$ in., Weight ....93 ...lbs./ft., Wall thickness or gauge No $5 \mathrm{ch} 80 . . . \mathrm{ft}$. TYPE OF SCREEN OR PERFORATION MATERIAL:
$\square$ Siteel
$\square$ Stainless Stee!
$\triangle P \vee C$
$\square$ None us:d (open hole)
$\square$ Other (Specif')

SCREEN OR PERFORATION OPENINGS ARE:

$\square$ Continuous slo: $\nexists$ Mill slot $\square$ Gauze wrapped $\square$ Torch cut $\square$ Drilled holes $\square$ None (open hole)

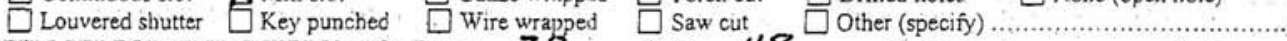

SCREEN-PERFORATED INTERVALS: From..... 38 ...... ft to 48

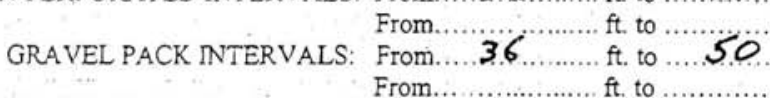

ft., From

f. to

ft., From

ft. to

ft., From ….................. ft. to

From .................. ft. to ................. ft., From

.

6 GROUT MATERIAL: $\square$ Neat cement $\square$ Cement grout $\mathbf{X}$ Bentonite $\square$ Othe

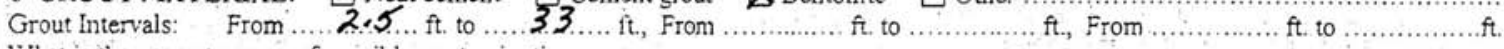

What is the nearest source of possible contamination:

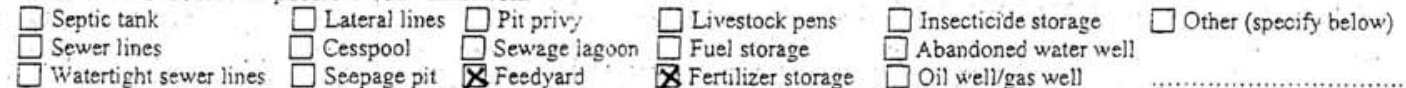

Direction from well ........................................... Distance from well

\begin{tabular}{|c|c|c|c|c|c|}
\hline FROM & TO & LITHOLOGIC LOG & FROM & TO & LITHO LOG (cont) Or PLUGGING INTERVALS \\
\hline 0 & 30 & Cloy & & & \\
\hline 30 & 50 & Weathered Shote & & & \\
\hline & & & & & \\
\hline & & & & \\
\hline & & & & \\
\hline & & & & \\
\hline & & & & \\
\hline & & & & \\
\hline
\end{tabular}

7 CONTRACTOR'S OR LANDOWNER'S CERTIFICATION: This water well was $\boldsymbol{Q}$ constructed, $\square$ reconstructed, or $\square$ plugged under my jurisdiction and was completed on (mo/day/year) $.8 / .31 / 1 / \ldots .$. and this record is true to the best of my knowledge and belief. Kansas Water Well Contractor's License No. 5.9 .7 .... This Water Well Record was completed on (mo/day/year). $9 / 21 / .11 .$.

under the business name of Boart hangyar. Co.mpany

by (signature)

INSTRUCTIONS: Use typewriter or ball point pen. LLEASE PRESS FIRILIY and PRINT clearly. Please fill in blanks and check the correct answers. Send threc copies (White, blue, pink) to Kansas Departument of Health and Environment, Bureau of Water, Geology Section, 1000 SW Jackson St, Suite 420, Topeka, Kansas 66612-1367. Tclephone 785-296-5S22. Send one copy to W/ATER WELL OWNER and sciain one for your records. Include face of S5.00 for each constructed well. Visit us at hitp://hunw, kdheks. gov/waterwell/index.html. 
WATER WELL RECORD Form WWC-5

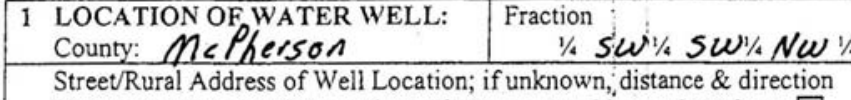
from nearest town or intersection: If at owner's address, check here $\square$. Unified school hawn, next to storoge building

2 WATER WELL OWNER: USDA - Steve Gilmore RR\#, Street Address, Box \#: stop 0.513 Room $4714-5$ City, State, ZIP Code

Division of Water Resources App. No. Section Number Township No. Range Number $T 2 / \mathrm{S}$

R 4 DE XW

\section{Global Positioning System (GPS) information:}

Latitude: $38.22602 \ldots \ldots \ldots \ldots$ (in decimal degrees) Longitude: $9.7 .7 .7,5.41 \ldots \ldots \ldots \ldots$ (in decimal degrees) Elevation:

Datum: $\square$ WGS 84, $\square$ NAD 83, $\square$ NAD 27

Collection Method:

$\square$ GPS unit (Make/Model: ....................................) $\square$ Digital Map/Photo, $\square$ Topographic Map, $\square$ Land Survey Est. Accuracy: $\square<3 \mathrm{~m}, \square 3.5 \mathrm{~m}, \square 5.15 \mathrm{~m}, \square>15 \mathrm{~m}$

3 LOCATE WELL WITH AN "X" IN SECTION BOX: 4 DEPTH OF COMPLETED WELL .......75........

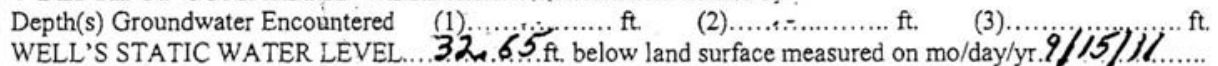
Pump test data: Well water was................ft. after............... hours pumping ............... gpm EST. YIELD .........gpm. Well water was...............ft. after............... hours pumping ............... gpm

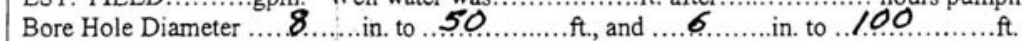

w

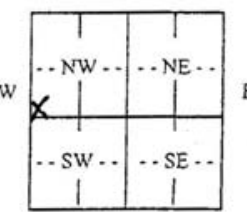
$s$ WELL WATER TO BE USED AS: $\square$ Public water supply $\square$ Geothermal $\square$ Injection well $\square$ Irrigation $\square$ Industrial $\square$ Domestic-lawn \& garden $\mathbb{X}$ Dewatering $\square$ Other (Specify below) Was a chemical/bacteriological sample submitted to Department? $\square$ Yes $\otimes$ No

If yes, $\mathrm{mo} / \mathrm{day} / \mathrm{yr}$ sample was submitted.. Water well disinfected? $\square$ Yेes $\mathbb{X}$ No

\section{TYPE OF CASING USED: $\square$ Steel $\triangle$ PVC $\square$ Other...............
CASING JOINTS: $\square$ Glued $\square$ Clamped $\square$ Welded $\otimes$ Threaded}

Casing height above land surface................ in., Weight .... 9.3 ........lbs./ft., Wall thickness or gauge No. .5.ch.8. TYPE OF SCREEN OR PERFORATION MATERIAL;
$\square$ Steel
Stainless Steel
XPVC
Non:

\section{$\square$ Continuous siot $\bigotimes$ Mill slot $\square$ Gauze wrapped $\square$ Torch cut $\square$ Drilled holes $\square$ None (open hole) \\ $\square$ Louvered shutter $\square$ Key punched $\square$ Wire wrapped $\square$ Saw cut $\square$ Other (specify) ..........................................}

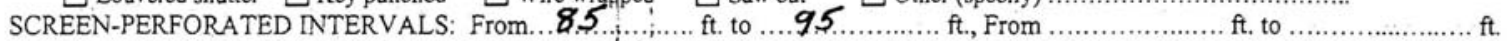

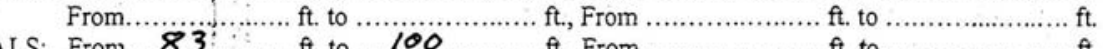

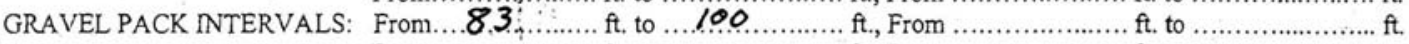

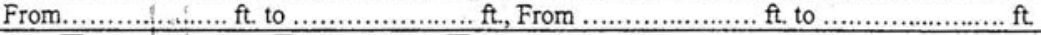

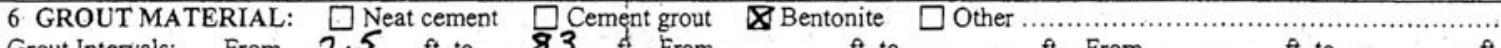

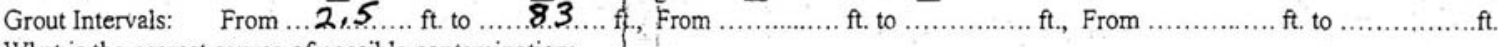

What is the nearest source of possible contamination:

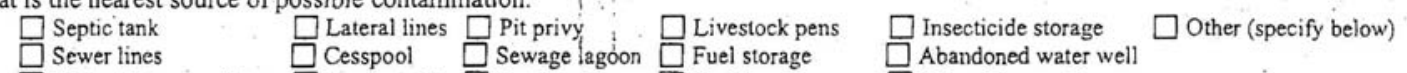

$\square$ Watertight sewer lines $\square$ Seepage pit $\bigotimes$ Feedyard $\square$ Fertilizer storage. $\square$ Oil well/gas well

Direction from well .............................................. Distance from well

\begin{tabular}{|c|c|c|c|c|c|}
\hline FROM & TO & LITHOLOGIC LOG & FROM & TO & LITHO. LOG (cont.) Or PLUGGING INTERVALS \\
\hline 0 & 30 & Cloy & & & \\
\hline 30 & 100 & Weothered Shale & & & \\
\hline & & & & & \\
\hline & & & & & \\
\hline & & & & & \\
\hline & & & & \\
\hline & & & & \\
\hline & & & & \\
\hline
\end{tabular}

7 CONTRACTOR'S OR LANDOWNER'S CERTIFICATION: This water well was $\bigotimes$ constructed, $\square$ reconstructed, or $\square$ plugged under my jurisdiction and was completed on (mo/day/year) . $8 /, 3 / 1 ! ! \ldots$ and this record is true to the best of my knowledge and belief.

Kansas Water Well Contractor's License No. S.9.7..... This Water Well Record was completed on (mo/day/vear) 9.21 .111 .

under the busiress name of . Bodtt... han gyraf.. Compory ….......... by (signature)

INSTRUCTIONS: Use typewriter or ball point pen. PLEASE PRESS FIRMLY and PRINT clearly. Please fill in blanks and check the correct answers. Send three copies (white, blue, pink) to Kansas Department of Health and Environment, Bureau of Water, Geology Section, 1000 SW Jackson St, Suite 420, Topeka, Kansas 66612-1367.

Telephone 785-296-5522. Send one copy to WATER WELL OWNER ahd retain one for your records. Include fee of $\$ 5.00$ for each constructed well. "Visit us at hittp://wnww.kdheks.gov/waterwell/index.html. 


$$
\text { Mw }-205
$$

WATER WELL RECORD

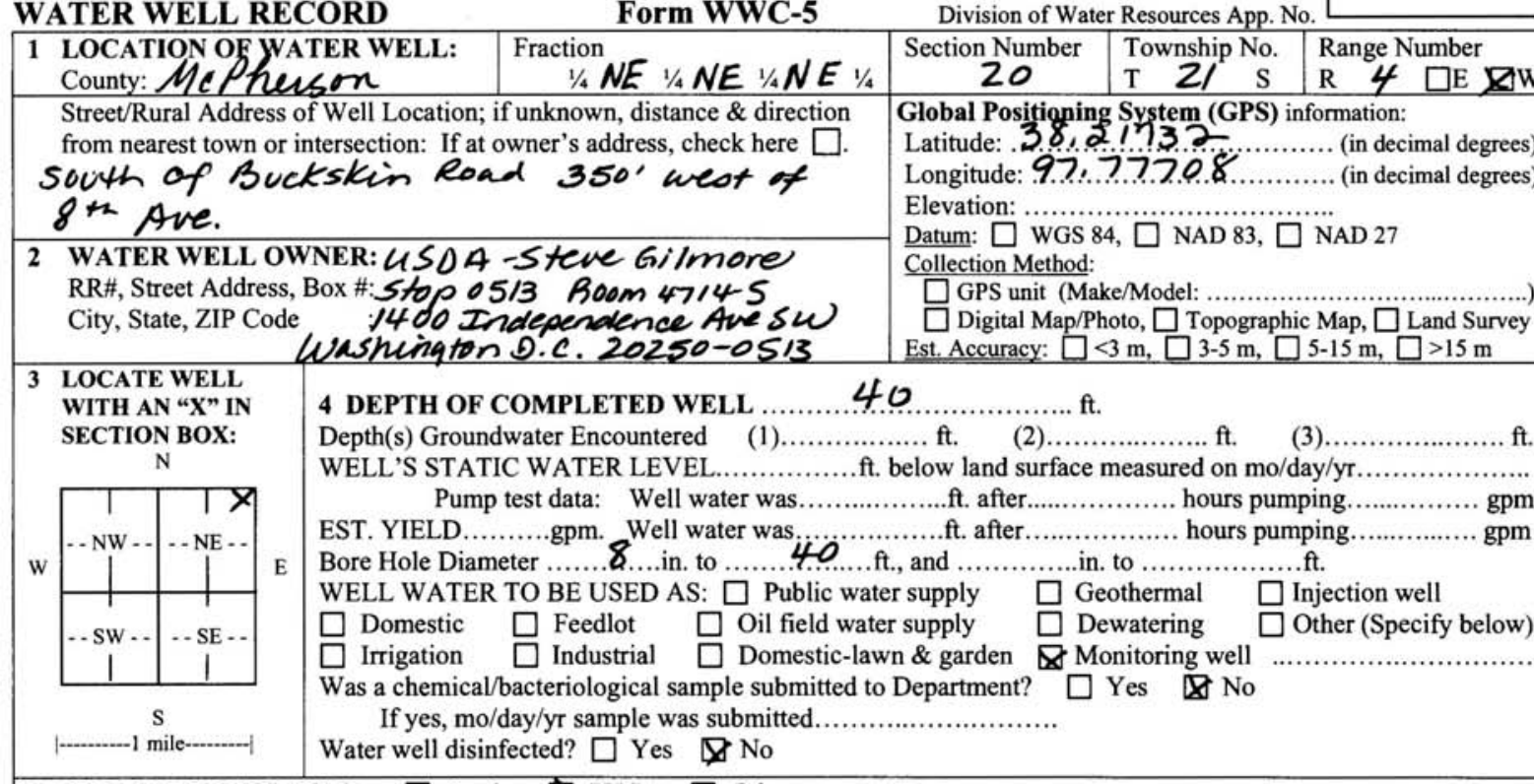

\section{TYPE OF CASING USED: $\square$ Steel $\square$ PVC $\square$ Other}

CASING JOINTS: $\square_{2}$ Glued $\square$ Clamped $\square$ welded $\square$ Threaded

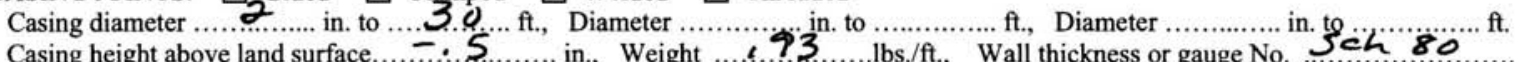
TYPE OF SCREEN OR PERFORATION MATERIAL:
$\square$ Steel
$\square$ Stainless Steel
QPVC
$\square$ None used (open hole)

SCREEN OR PERFORATION OPENINGS ARE:

$\square$ Continuous slot $\mathbf{X}^{\text {Mill slot }} \square$ Gauze wrapped $\square$ Torch cut $\square$ Drilled holes $\square$ None (open hole)

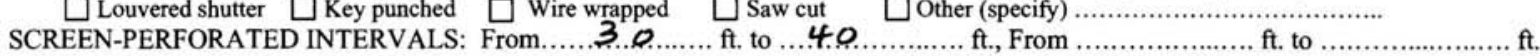
GRAVEL PACK INTERVALS: F
From.....
ft. to ....................... ft.

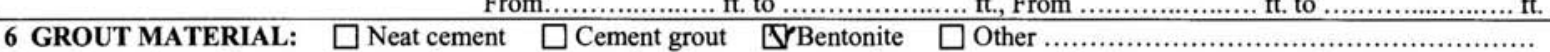

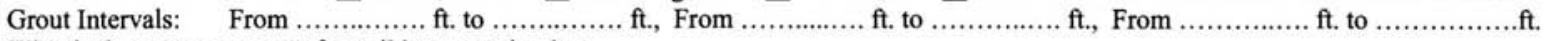

What is the nearest source of possible contamination:
$\square$ Septic tank
$\square$ Sewer lines
$\square$ Lateral lines $\square$ Pit privy
$\square$ Cesspool
$\square$ Livestock pens
Fuel storage
Insecticide storage
$\square$ Watertight sewer lines $\square$ Seepage pit $\quad$ Feedyard $\quad$ Fertilizer storage $\quad$ Oil well/gas well

Direction from well ........................ Distance from well ....

\begin{tabular}{|c|c|c|c|c|c|}
\hline FROM & TO & LITHOLOGIC LOG & FROM & TO & LITHO. LOG (cont.) or PLUGGING INTERVALS \\
\hline 0 & 38 & Cay & & & \\
\hline 38 & 40 & weathered Shale & & & \\
\hline & & & & & \\
\hline & & & & & \\
\hline & & & & & \\
\hline & & & & & \\
\hline & & & & & \\
\hline & & & & & \\
\hline & & & & & \\
\hline
\end{tabular}

7 CONTRACTOR'S OR LANDOWNER'S CERTIFICATION: This water well was $\bar{\nabla}$ constructed, $\square$ reconstructed, or $\square$ plugged under my jurisdiction and was completed on (mo/day/year) ...9.1/3.1.1.... and this record is true to the best of my knowledge and belief.

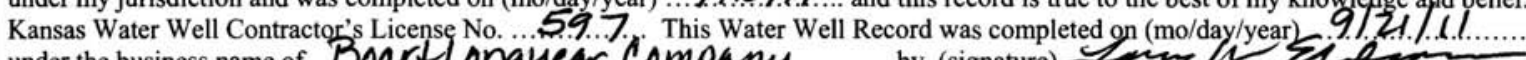
under the business name of Boar. Longyear. Company ......... by (signature

INSTRUCTIONS: Use typewriter or ball point pen. PLEASE PRESS FIRMLY and PRINT clearly. Please fill in blanks and check the correct answers. Send three copies (white, blue, pink) to Kansas Department of Health and Environment, Bureau of Water, Geology Section, 1000 SW Jackson St., Suite 420, Topeka, Kansas 66612-1367. Telephone 785-296-5524. Send one copy to WATER WELL OWNER and retain one for your records. Include fee of $\$ 5.00$ for each constructed well. Visit us at http://www.kdheks.gov/waterwell/index.html.

KSA $82 \mathrm{a}-1212$ 
$M W-20 M$

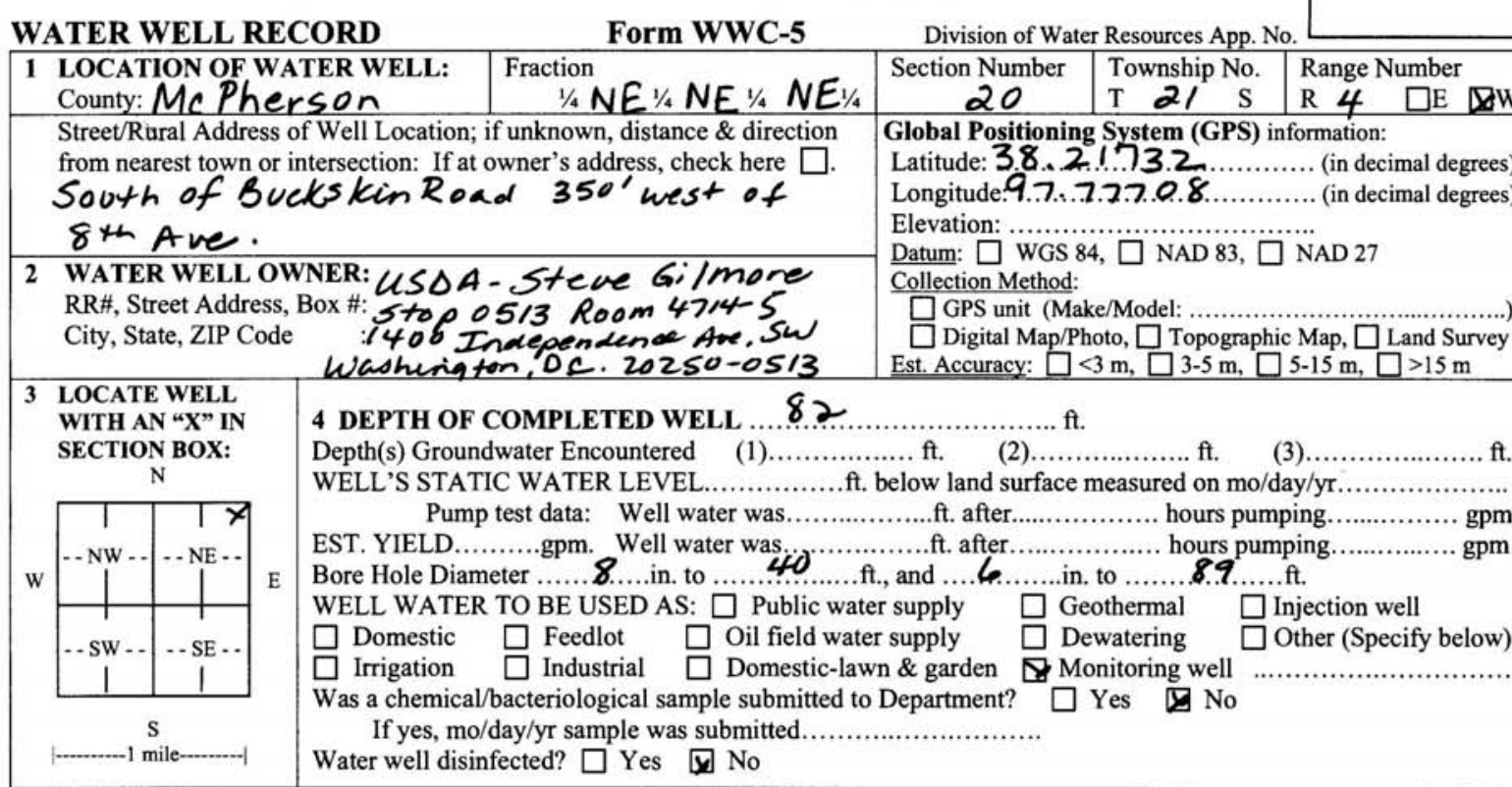

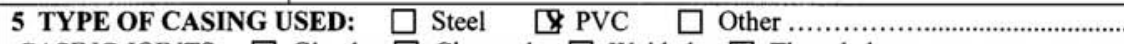

CASING JOINTS: $\square$ Glued $\square$ Clamped $\square$ welded $\square$ Threaded

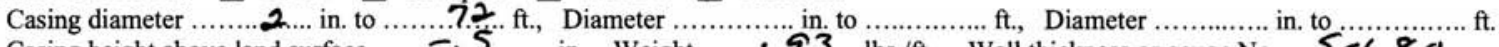
Casing height above land surface......... 5 ...... in., Weight .......9.3...lbs./ft., Wall thickness or gauge No. ... $5.4 .8 .0 . \ldots .$. TYPE OF SCREEN OR PERFORATION MATERIAL:
몬el
$\square$ Stainless Steel
XPVC
$\square$ None used (open hole)
$\square$ Other (Specify)

SCREEN OR PERFORATION OPENINGS ARE:

$\square$ Continuous slot $\mathbb{B}$ Mill slot $\square$ Gauze wrapped $\square$ Torch cut $\square$ Drilled holes $\square$ None (open hole)

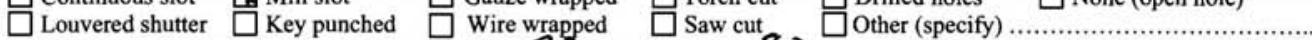

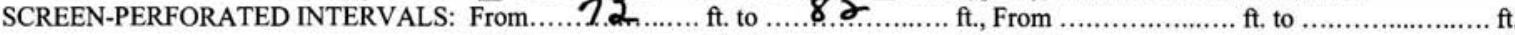

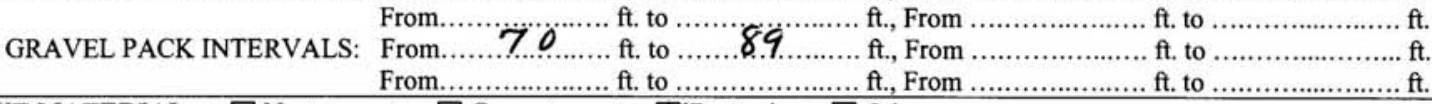

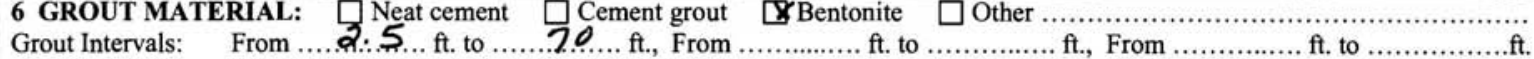
What is the nearest source of possible contamination:
$\square$ Septic tank
$\square$ Lateral lines $\square$ Pit privy
$\square$ Lateral lines $\square$ Pit privy
$\square$ Cesspool
Seepage pit
$\square$ Insecticide storage

XFertilizer storage

Abandoned water

Direction from well.

Distance from well

\begin{tabular}{|c|c|c|c|c|c|}
\hline FROM & TO & LITHOLOGIC LOG & FROM & TO & LITHO. LOG (cont.) or PLUGGING INTERVALS \\
\hline 0 & 38 & Clay & & & \\
\hline 38 & 82 & wearhered Shale & & & \\
\hline & & & & & \\
\hline & & & & & \\
\hline & & & & & \\
\hline & & & & & \\
\hline & & & & & \\
\hline & & & & \\
\hline
\end{tabular}

7 CONTRACTOR'S OR LANDOWNER'S CERTIFICATION: This water well was $\boldsymbol{X}$ constructed, $\square$ reconstructed, or $\square$ plugged under my jurisdiction and was completed on (mo/day/year) . .9.13.11.... and this record is true to the best of my knowledge and belief. Kansas Water Well Contractor's License No. 5.9.7..... This Water Well Record was completed gn (mo/day/year) $9.91 .2 \mathrm{~J} .1 .1 \ldots \ldots \ldots$ under the business name of B.OART.LOngyear. CO.

by (signature)

INSTRUCTIONS: Use typewriter or ball point pen. PLEASE PRESS FIRMLY and PRINT clearly. Please fill in blanks and check the correct answers. Send three copies
(white, blue, pink) to Kansas Department of Health and Environment, Bureau of Water, Geology Section, $1000 \mathrm{SW}$ Jackson St., Suite 420, Topeka, Kansas 66612-1367, (white, blue, pink) to Kansas Department of Health and Environment, Bureau of Water, Geology Section, 1000 SW Jackson St., Suite 420, Topeka, Kansas 66612-1367.
Telephone 785-296-5524. Send one copy to WATER WELL OWNER and retain one for your records. Include fee of $\$ 5.00$ for cach constructed well. Visit us at http://www.kdheks.gov/waterwell/index.html.

KSA 82a-1212 


\section{Appendix E:}

Hand-Measured Groundwater Level Data 
TABLE E.1 Hand-measured groundwater levels, February 16, 2011, to March 13, 2012.

\begin{tabular}{|c|c|c|c|c|c|c|c|c|c|c|c|c|c|c|c|c|c|c|c|c|c|c|}
\hline \multirow[b]{2}{*}{ Well } & \multirow[b]{2}{*}{$\begin{array}{l}\text { Reference } \\
\text { Elevation } \\
\text { (t AMSLL) }\end{array}$} & \multicolumn{3}{|c|}{ February 16, 2011} & \multicolumn{3}{|c|}{ August 20, 2011} & \multicolumn{3}{|c|}{ September 3, 2011} & \multicolumn{3}{|c|}{ September 8, 2011} & \multicolumn{3}{|c|}{ September 15, 2011} & \multicolumn{3}{|c|}{ November 11, 2011} & \multicolumn{3}{|c|}{ March 13, 2012} \\
\hline & & Time & $\begin{array}{l}\text { Depth to } \\
\text { water } \\
\text { (tt TOC) }\end{array}$ & $\begin{array}{l}\text { Waver Level } \\
\text { Elevataion } \\
\text { (t AMSLL) }\end{array}$ & Time & $\begin{array}{l}\text { Depth to } \\
\text { WWater } \\
\text { (t TOC) }\end{array}$ & $\begin{array}{l}\text { Waver Level } \\
\text { Elevation } \\
\text { (tt AMSLL) }\end{array}$ & Time & $\begin{array}{l}\text { Depth to } \\
\text { water } \\
\text { (tt TOC) }\end{array}$ & $\begin{array}{l}\text { Waver Level } \\
\text { Elevation } \\
\text { (tt AMSLL) }\end{array}$ & Time & $\begin{array}{l}\text { Depth to } \\
\text { water } \\
\text { (tt TOC) }\end{array}$ & $\begin{array}{l}\text { Waver Level } \\
\text { Elevataion } \\
\text { (tA AMSLL) }\end{array}$ & Time & $\begin{array}{l}\text { Depth to } \\
\text { water } \\
\text { (tt TOC) }\end{array}$ & $\begin{array}{l}\text { Waver Level } \\
\text { Elevation } \\
\text { (tt AMSLL) }\end{array}$ & Time & $\begin{array}{l}\text { Depth to } \\
\text { Water } \\
\text { (tt TOC) }\end{array}$ & $\begin{array}{l}\text { Waver Level } \\
\text { Elevation } \\
\text { (t AMSLL) }\end{array}$ & Time & $\begin{array}{l}\text { Depth to } \\
\text { water } \\
\text { (tt TOC) }\end{array}$ & $\begin{array}{l}\text { Waver Leve } \\
\text { Elevation } \\
\text { (tt AMSLL) }\end{array}$ \\
\hline MW01 & 1524.831 & 10:53 & 27.09 & 1497.74 & 18:00 & 29.41 & 1495.42 & 17:13 & 29.47 & 1495.36 & 11:48 & 29.92 & 1494.91 & $15: 28$ & 30.21 & 1494.62 & 16:40 & 30.68 & $1494.03^{\mathrm{a}}$ & $12: 20$ & 29.91 & $1494.80^{\mathrm{a}}$ \\
\hline MW02 & $\begin{array}{l}15552.264 \\
{ }_{1525}\end{array}$ & $\begin{array}{l}11: 100 \\
11: 22\end{array}$ & $\begin{array}{l}27.29 \\
27\end{array}$ & & & & & $\begin{array}{l}11: 717 \\
17: 71\end{array}$ & $\begin{array}{l}30.09 \\
30.05\end{array}$ & & & $\begin{array}{l}30.30 \\
30.77 \\
30\end{array}$ & & & & $\begin{array}{l}1499.45 \\
149.57\end{array}$ & $\begin{array}{l}16: 42 \\
15: 45\end{array}$ & $\begin{array}{l}31.09 \\
321\end{array}$ & $\begin{array}{l}1494.17 \\
149.17\end{array}$ & $\begin{array}{l}12: 25 \\
12: 25\end{array}$ & $\begin{array}{l}30.34 \\
20\end{array}$ & $\begin{array}{l}{ }^{14949.92} \\
140.92\end{array}$ \\
\hline $\begin{array}{l}\text { MWO3 } \\
\text { MW04 }\end{array}$ & $\begin{array}{l}1525.24 ! \\
1525.918\end{array}$ & $\begin{array}{l}11: 23 \\
11: 35\end{array}$ & $\begin{array}{l}19.88 \\
26.2\end{array}$ & $\begin{array}{l}1509.37 \\
1499.72\end{array}$ & $\begin{array}{l}16: 54 \\
16: 21\end{array}$ & $\begin{array}{l}28.35 \\
28.28\end{array}$ & $\begin{array}{l}14995.90 \\
1497.64\end{array}$ & $\begin{array}{l}17: 21 \\
17: 59\end{array}$ & $\begin{array}{l}29.55 \\
28.45\end{array}$ & $\begin{array}{l}1995.10 \\
1497.47\end{array}$ & $\begin{array}{l}12: 40 \\
12: 31\end{array}$ & $\begin{array}{l}29.17 \\
28.65\end{array}$ & $\begin{array}{l}14955.48 \\
14972.7\end{array}$ & $\begin{array}{l}118: 65 \\
1835\end{array}$ & $\begin{array}{l}29.88 \\
28.78\end{array}$ & $\begin{array}{l}1495.72 \\
1497.14\end{array}$ & 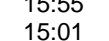 & $\begin{array}{l}30.41 \\
29.32\end{array}$ & $\begin{array}{l}1194.48 \\
199660\end{array}$ & $\begin{array}{l}1: 129 \\
\text { 1:48 }\end{array}$ & $\begin{array}{l}28.989 \\
29\end{array}$ & $\begin{array}{l}1499.36 \\
14967\end{array}$ \\
\hline $\begin{array}{l}\text { MW05 } \\
\text { MWO5 }\end{array}$ & 1525.201 & 11:48 & $\begin{array}{l}26.95 \\
26.52\end{array}$ & $\begin{array}{l}1498.25 \\
140725\end{array}$ & $\begin{array}{l}15: 48 \\
10: 42\end{array}$ & $\begin{array}{l}22.31 \\
0.71\end{array}$ & 1495.89 & $18: 17$ & 29.69 & 1495.51 & $12: 25$ & 29.93 & 1495.27 & $18: 28$ & 30.19 & 1495.01 & $14: 45$ & 30.2 & 1495.00 & $10: 56$ & 29.89 & 1495.31 \\
\hline $\begin{array}{l}\text { MW06 } \\
\text { MW07 }\end{array}$ & $\begin{array}{l}1522.646 \\
1523.643\end{array}$ & $\begin{array}{l}12: 26 \\
13: 02\end{array}$ & $\begin{array}{l}25.53 \\
2658\end{array}$ & $\begin{array}{l}1497.12 \\
14906\end{array}$ & $\begin{array}{l}18: 23 \\
18: 41\end{array}$ & $\begin{array}{l}27.85 \\
2288\end{array}$ & $\begin{array}{l}\begin{array}{l}1494.80 \\
149776\end{array} \\
\end{array}$ & $\begin{array}{l}17: 41 \\
17: 36\end{array}$ & $\begin{array}{l}28.03 \\
29.06\end{array}$ & $\begin{array}{l}1494.62 \\
19945\end{array}$ & $\begin{array}{l}11: 25 \\
11: 13\end{array}$ & $\begin{array}{l}28.29 \\
2932\end{array}$ & $\begin{array}{l}1494.36 \\
149432\end{array}$ & 17:06 & $\begin{array}{l}28.42 \\
2017\end{array}$ & $\begin{array}{l}1494.23 \\
11917\end{array}$ & $\begin{array}{l}17: 09 \\
16: 57\end{array}$ & $\begin{array}{l}28.89 \\
20{ }^{2}\end{array}$ & $\begin{array}{l}1493.76 \\
1103\end{array}$ & $\begin{array}{l}13: 07 \\
12.57\end{array}$ & $\begin{array}{l}28.47 \\
20.55\end{array}$ & $\begin{array}{l}1494.18 \\
119010\end{array}$ \\
\hline 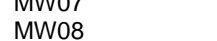 & $\begin{array}{l}1553.643 \\
1524.356\end{array}$ & $\begin{array}{l}13: 02 \\
13: 32\end{array}$ & $\begin{array}{l}2.658 \\
2659\end{array}$ & $\begin{array}{l}{ }_{1497.06} \\
149777\end{array}$ & $\begin{array}{l}18: 41 \\
18: 55\end{array}$ & $\begin{array}{l}28.88 \\
2212\end{array}$ & $\begin{array}{l}1494.76 \\
149524\end{array}$ & $\begin{array}{l}17: 36 \\
17: 32\end{array}$ & $\begin{array}{l}29.06 \\
29.27\end{array}$ & $\begin{array}{l}1494.58 \\
149509\end{array}$ & $\begin{array}{l}11: 31 \\
11: 38\end{array}$ & $\begin{array}{l}29.32 \\
2949\end{array}$ & $\begin{array}{l}14944.32 \\
149487\end{array}$ & $\begin{array}{l}17: 14 \\
11: 23\end{array}$ & $\begin{array}{l}29.47 \\
2967\end{array}$ & $\begin{array}{l}1494.17 \\
14969\end{array}$ & $\begin{array}{l}16: 57 \\
16: 36\end{array}$ & $\begin{array}{l}30.02 \\
30.17\end{array}$ & $\begin{array}{l}1493.62 \\
1494.19\end{array}$ & $\begin{array}{l}12: 57 \\
12.15\end{array}$ & $\begin{array}{l}29.55 \\
2967\end{array}$ & $\begin{array}{l}1494.09 \\
149696\end{array}$ \\
\hline MW09 & $\begin{array}{l}1525.324 \\
15252\end{array}$ & $\begin{array}{l}13: 42 \\
13: 42\end{array}$ & $\begin{array}{l}27.27 \\
2.27\end{array}$ & $\begin{array}{l}1498.05 \\
1495\end{array}$ & $\begin{array}{l}\text { 10.50 } \\
19: 02\end{array}$ & 29.88 & $\begin{array}{l}1495.44 \\
1495.44\end{array}$ & $\begin{array}{l}17: 29 \\
17: 29\end{array}$ & $\begin{array}{l}30.08 \\
30.08\end{array}$ & $\begin{array}{l}14955.24 \\
1495\end{array}$ & $\begin{array}{l}11: 42 \\
11: 42\end{array}$ & 30.25 & $\begin{array}{l}14945.07 \\
149.07\end{array}$ & 17:39 & 30.45 & $\begin{array}{l}1494.89 \\
1494.87\end{array}$ & $\begin{array}{l}10.30 \\
16: 25\end{array}$ & 30.78 & $\begin{array}{l}1494.19 \\
1494.54\end{array}$ & $12: 36$ & 30.29 & $\begin{array}{l}1495.03 \\
1495\end{array}$ \\
\hline MW10 & $\begin{array}{l}1525.025 \\
152500\end{array}$ & 14:10 & $\begin{array}{l}26.52 \\
2.52\end{array}$ & 1498.51 & $17: 22$ & 29.33 & $\begin{array}{l}1495.70 \\
1406\end{array}$ & $17: 47$ & 29.53 & & 11:59 & 29.69 & $\begin{array}{l}1495.34 \\
10651\end{array}$ & $\begin{array}{l}17: 34 \\
17750\end{array}$ & 29.91 & 1495.12 & $16: 11$ & 30.58 & $\begin{array}{l}1494.45 \\
105.50\end{array}$ & $12: 46$ & 29.77 & 1495.26 \\
\hline $\begin{array}{l}\text { MW11 } \\
\text { MW12 }\end{array}$ & $\begin{array}{l}1555.500 \\
1525.447\end{array}$ & $\begin{array}{l}\text { 14:18 } \\
15: 18\end{array}$ & $\begin{array}{l}26.35 \\
25.19\end{array}$ & $\begin{array}{l}\begin{array}{l}44999.15 \\
1500.26\end{array} \\
\text {. }\end{array}$ & $\begin{array}{l}17: 17 \\
16: 45\end{array}$ & $\begin{array}{l}28.97 \\
27.64\end{array}$ & $\begin{array}{l}\begin{array}{l}14966.53 \\
1497.81\end{array} \\
\end{array}$ & $\begin{array}{l}17: 51 \\
18: 03\end{array}$ & $\begin{array}{l}29.23 \\
27.86\end{array}$ & $\begin{array}{l}1446.27 \\
1497.59\end{array}$ & $\begin{array}{l}12: 04 \\
12: 16\end{array}$ & $\begin{array}{l}29.35 \\
28.03\end{array}$ & $\begin{array}{l}11966.15 \\
1497.42\end{array}$ & $\begin{array}{l}17: 58 \\
18: 21\end{array}$ & $\begin{array}{l}29.51 \\
2822\end{array}$ & $\begin{array}{l}1495.99 \\
1497.23\end{array}$ & $\begin{array}{l}15: 44 \\
15: 34\end{array}$ & $\begin{array}{l}30 \\
28.68\end{array}$ & $\begin{array}{l}1495.50 \\
1496.77\end{array}$ & $\begin{array}{l}11: 41 \\
12: 04\end{array}$ & $\begin{array}{l}29.48 \\
28.85\end{array}$ & $\begin{array}{l}1496.02 \\
1497.10\end{array}$ \\
\hline & 1523.028 & $14: 46$ & $22.84^{\mathrm{b}}$ & 1500.19 & $16: 34$ & 25.34 & $\begin{array}{l}1497.69 \\
149\end{array}$ & 18:09 & & 1497.48 & 12:09 & 25.69 & 1497.34 & $18: 13$ & 25.79 & 1497.24 & $15: 21$ & 26.33 & 70 & 11:52 & 26.08 & .95 \\
\hline MW14M & 1524.973 & & & & & & & 17:00 & 31.89 & 1493.08 & $12: 58$ & 32.17 & 1492.80 & $15: 23$ & 32.36 & 1492.61 & $10: 10$ & 31.96 & $\begin{array}{l}1493.01 \\
1493\end{array}$ & 14:12 & 31.47 & 1493.50 \\
\hline MW14D & $\begin{array}{l}1524.957 \\
1525659\end{array}$ & & & & & & & $\begin{array}{l}17: 02 \\
12: 22\end{array}$ & $\begin{array}{l}124.18 \\
25220\end{array}$ & $\begin{array}{l}1400.78 \\
1002\end{array}$ & $\begin{array}{l}13: 01 \\
12.57\end{array}$ & $\begin{array}{l}123.92 \\
25.9\end{array}$ & $\begin{array}{l}1401.04 \\
1002\end{array}$ & $\begin{array}{l}15: 21 \\
10: 10\end{array}$ & $\begin{array}{r}123.64 \\
2564\end{array}$ & $\begin{array}{r}1401.32 \\
\end{array}$ & $\begin{array}{l}10: 08 \\
11: 52\end{array}$ & $\begin{array}{l}121.67 \\
2627\end{array}$ & $\begin{array}{l}1403.29 \\
100222\end{array}$ & 14:10 & $\begin{array}{r}116.85 \\
26.7\end{array}$ & $\begin{array}{l}1408.11 \\
0\end{array}$ \\
\hline $\begin{array}{l}\text { MWW15s } \\
\text { MW }\end{array}$ & $\begin{array}{l}15525.699 \\
15283\end{array}$ & & & & & & & $\begin{array}{l}13: 33 \\
13: 30\end{array}$ & $\begin{array}{l}353.38 \\
33.02\end{array}$ & $\begin{array}{l}1940.32 \\
19282\end{array}$ & $\begin{array}{l}13: 57 \\
13: 59\end{array}$ & $\begin{array}{l}355.48 \\
33.16\end{array}$ & $\begin{array}{l}11940.222 \\
14928\end{array}$ & $\begin{array}{l}12: 19 \\
12: 21\end{array}$ & $\begin{array}{l}35.64 \\
3.47\end{array}$ & $\begin{array}{l}1490.06 \\
149.37\end{array}$ & $\begin{array}{l}11: 53 \\
1151\end{array}$ & $\begin{array}{l}36.37 \\
3298\end{array}$ & $\begin{array}{l}1489.33 \\
190288\end{array}$ & $\begin{array}{l}14: 55 \\
11.54\end{array}$ & $\begin{array}{l}36.77 \\
3245\end{array}$ & $\begin{array}{l}1488.93 \\
1493939\end{array}$ \\
\hline MW15D & 1525.815 & & & & & & & $\begin{array}{l}13: 29 \\
13.00\end{array}$ & $\begin{array}{r}33.02 \\
123.95\end{array}$ & $\begin{array}{l}11442.82 \\
1401.87\end{array}$ & 14:01 & 123.69 & $\begin{array}{l}14942.08 \\
1402.13\end{array}$ & $\begin{array}{l}11.21 \\
12: 23\end{array}$ & $\begin{array}{l}3.4 .4 I \\
123.45\end{array}$ & $\begin{array}{l}1492.37 \\
1402.37\end{array}$ & $\begin{array}{l}11.511 \\
11: 49\end{array}$ & $\begin{array}{l}32.98 \\
121.59\end{array}$ & $\begin{array}{l}1442.80 \\
1404.23\end{array}$ & $\begin{array}{l}14.54 \\
14: 52\end{array}$ & $\begin{array}{l}32.45 \\
117.4\end{array}$ & $\begin{array}{l}1493.399 \\
1408.42\end{array}$ \\
\hline MW16S1 & $\begin{array}{l}1507.257 \\
1507\end{array}$ & & & & & & & 13:47 & 16.66 & $\begin{array}{l}1490.60 \\
\end{array}$ & $14: 19$ & 16.39 & 1490.87 & 13:10 & 16.63 & 1490.63 & $\begin{array}{l}12: 20 \\
12: 21\end{array}$ & 17.01 & 1490.25 & $15: 18$ & 15.87 & 1491.39 \\
\hline MW1652 & $\begin{array}{l}1557.264 \\
{ }_{1507}^{267}\end{array}$ & & & & & & & 13:48 & 16.67 & $\begin{array}{l}1490.59 \\
140.59\end{array}$ & 14:20 & 16.43 & 14 & 13:11 & 16.66 & 60 & 12:21 & 17.02 & 1490.24 & 15:19 & 15.89 & 1491.37 \\
\hline 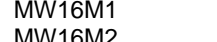 & $\begin{array}{l}1507.1 / 6 \\
1507\end{array}$ & & & & & & & $\begin{array}{l}13: 5 \\
13: 50\end{array}$ & $\begin{array}{l}16.23 \\
162\end{array}$ & 1490.95 & 14:22 & $\begin{array}{l}16.40 \\
16.40\end{array}$ & 1490.78 & 13:12 & 16.65 & 1490.53 & 12:28 & 16.11 & 1491.07 & 15:29 & 15.36 & 1491.82 \\
\hline W16D & 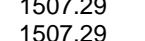 & & & & & & & $\begin{array}{l}13: 59 \\
11: 01\end{array}$ & $\begin{array}{l}22.83 \\
1064\end{array}$ & $\begin{array}{l}14844.46 \\
139965\end{array}$ & $\begin{array}{l}11.23 \\
1025\end{array}$ & $\begin{array}{l}23.05 \\
106.51\end{array}$ & $\begin{array}{l}1844.24 \\
10078\end{array}$ & $\begin{array}{l}13: 33 \\
13: 5\end{array}$ & $\begin{array}{l}23.15 \\
10582\end{array}$ & $\begin{array}{l}1444.14 \\
140147\end{array}$ & $\begin{array}{l}212: 26 \\
12: 24\end{array}$ & $\begin{array}{l}20.82 \\
1006\end{array}$ & $\begin{array}{l}18460.47 \\
10043\end{array}$ & $\begin{array}{l}115242 \\
1523\end{array}$ & & $\begin{array}{l}1486.22 \\
140579\end{array}$ \\
\hline MW17S & $\begin{array}{l}1504.007 \\
504\end{array}$ & & & & & & & $\begin{array}{l}13: 05 \\
13: 05\end{array}$ & 15.14 & $\begin{array}{l}1988.87 \\
1488.8\end{array}$ & $\begin{array}{l}11: 37 \\
13: 37\end{array}$ & 15.26 & 1488.75 & $\begin{array}{l}14: 23 \\
14: 23\end{array}$ & $\begin{array}{l}15.44 \\
154\end{array}$ & $\begin{array}{l}1488.57 \\
\end{array}$ & 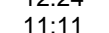 & 1578 & 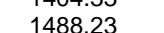 & $\begin{array}{l}1.3 .36 \\
1136\end{array}$ & 1574 & $\begin{array}{l}148087 \\
14887\end{array}$ \\
\hline MW17M & $\begin{array}{l}1504.08 \\
1504\end{array}$ & & & & & & & 13:07 & 11.28 & 1492.80 & $13: 38$ & 11.42 & $\begin{array}{l}1490.166 \\
14926\end{array}$ & $\begin{array}{l}14: 5 \\
11: 4\end{array}$ & $\begin{array}{l}1.146 \\
11.66\end{array}$ & $\begin{array}{l}1492.42 \\
1429\end{array}$ & 盀1:11 & $\begin{array}{l}151.22 \\
11.22\end{array}$ & $\begin{array}{l}1402.86 \\
142.86\end{array}$ & $\begin{array}{l}103: 35 \\
13: 3\end{array}$ & 10.72 & $\begin{array}{l}1490.3 .6 \\
1493\end{array}$ \\
\hline MW17D & 1504.085 & & & & & & & 13:15 & 25.88 & 1478.21 & 13:40 & 20.79 & 1483.30 & 14:25 & 20.14 & 1483.95 & 11:14 & 19.56 & 1484.53 & 13:33 & 17.09 & 1487.00 \\
\hline MW18S & 1525.88 & & & & & & & 16:38 & 31.70 & 1494.18 & 13:13 & 31.98 & 1493.90 & 15:41 & 32.15 & 1493.73 & 10:35 & 32.04 & 1493.84 & 14:29 & 31.56 & 1494.32 \\
\hline $\begin{array}{l}\text { MW } \\
\text { MW }\end{array}$ & $\begin{array}{l}1525.875 \\
152589\end{array}$ & & & & & & & $\begin{array}{l}16: 45 \\
11: 51\end{array}$ & $\begin{array}{r}32.78 \\
12020\end{array}$ & $\begin{array}{l}1493.10 \\
1001610\end{array}$ & 13:14 & $\begin{array}{r}33.04 \\
12384\end{array}$ & $\begin{array}{l}1492.84 \\
100205\end{array}$ & $\begin{array}{l}15: 42 \\
11.54\end{array}$ & $\begin{array}{r}33.25 \\
12341\end{array}$ & $\begin{array}{l}1492.63 \\
100248\end{array}$ & $\begin{array}{l}10: 37 \\
10: 30\end{array}$ & $\begin{array}{l}32.86 \\
105\end{array}$ & $\begin{array}{l}1493.02 \\
105020\end{array}$ & $\begin{array}{l}14: 28 \\
11: 25\end{array}$ & $\begin{array}{r}32.34 \\
11515\end{array}$ & 1493.54 \\
\hline & $\begin{array}{l}1552.89 \\
152905\end{array}$ & & & & & & & $\begin{array}{l}10.51 \\
11.25\end{array}$ & $\begin{array}{l}114.20 \\
29.43\end{array}$ & $\begin{array}{l}14015589 \\
19958\end{array}$ & $13: 17$ & $\begin{array}{l}123.84 \\
29.58\end{array}$ & 149 & 15: & $\begin{array}{l}123.41 \\
2978\end{array}$ & $\begin{array}{l}1402.48 \\
119512\end{array}$ & & & $\begin{array}{l}14455.39 \\
1059\end{array}$ & 25 & $\begin{array}{l}115015 \\
2052\end{array}$ & $\begin{array}{l}14110.074 \\
119059\end{array}$ \\
\hline MW19M & 1524.919 & & & & & & & $\begin{array}{l}18.23 \\
18: 23\end{array}$ & $\begin{array}{l}32.02 \\
32.02\end{array}$ & $\begin{array}{l}14949.40 \\
1492.90\end{array}$ & $\begin{array}{l}14: 45 \\
14.45\end{array}$ & 32.06 & $\begin{array}{l}14992.836 \\
1492.56\end{array}$ & 19:11 & 32.23 & $\begin{array}{l}1492.69 \\
149.10\end{array}$ & $\begin{array}{l}14: 26 \\
14: 26\end{array}$ & $\begin{array}{l}2.1 .91 \\
3.9\end{array}$ & $\begin{array}{l}1493.02 \\
1933.02\end{array}$ & $\begin{array}{l}11: 06 \\
11: 06\end{array}$ & 31.43 & $\begin{array}{l}14993.39 \\
149.49\end{array}$ \\
\hline $\begin{array}{l}\text { MW20S } \\
\text { Mw20M }\end{array}$ & $\begin{array}{l}1512.84 \\
152.82\end{array}$ & & & & & & & & & & & & & & & & $13: 10$ & 27.45 & $\begin{array}{l}1485.39 \\
\end{array}$ & $16: 06$ & 28.11 & $\begin{array}{l}1484.73 \\
143\end{array}$ \\
\hline $\begin{array}{l}\text { MW2 } \\
\text { Klass }\end{array}$ & $\begin{array}{l}1512.82 \\
1508.87\end{array}$ & & & & & & & & & & & & & & & & 13 & 34 & $\begin{array}{l}1478.39 \\
1773652\end{array}$ & $\begin{array}{l}16: 05 \\
116.56\end{array}$ & $\begin{array}{l}33.4 \\
323\end{array}$ & $\begin{array}{l}1479.42 \\
147537\end{array}$ \\
\hline Klassen Test & $\begin{array}{l}1509.12 \\
1509.12\end{array}$ & & & & & & & & & & & & & & & & $\begin{array}{l}13340 \\
1341\end{array}$ & $\begin{array}{l}35.94 \\
37.94\end{array}$ & 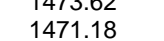 & $\begin{array}{l}105060 \\
10: 4\end{array}$ & $\begin{array}{l}35.46 \\
35.76\end{array}$ & $\begin{array}{l}14753.36 \\
14736\end{array}$ \\
\hline
\end{tabular}

a The top of the casing (TOC) at MW01 was cut lower by $1.5 \mathrm{in}$. (0.125 tt) on November 11, 2011. The new reference elevation is $1524.706 \mathrm{ft}$ AMSL.

${ }^{b}$ The water level at MW13 was rising very slowly at time of measurement on February 16, 2011. 


\section{Appendix F:}

\section{Land Survey Data}


TABLE F.1 Survey data for 2009-2011 investigations at Inman. ${ }^{a}$

\begin{tabular}{|c|c|c|c|c|c|}
\hline Location & $\begin{array}{l}\text { Easting } \\
\quad(\mathrm{ft})\end{array}$ & $\begin{array}{l}\text { Northing } \\
\quad(\mathrm{ft})\end{array}$ & $\begin{array}{l}\text { Ground } \\
\text { Elevation } \\
\text { (ft AMSL) }\end{array}$ & $\begin{array}{c}\text { Top of } \\
\text { Casing } \\
\text { Elevation } \\
\text { (ft AMSL) }\end{array}$ & $\begin{array}{l}\text { Type } \\
\text { of } \\
\text { Data }\end{array}$ \\
\hline \multicolumn{6}{|c|}{ Monitoring well locations } \\
\hline MW01 & 1520479.230 & 1880494.719 & 1525.081 & 1524.831 & Surveyed \\
\hline MW02 & 1520427.488 & 1880518.867 & 1525.521 & 1525.264 & Surveyed \\
\hline MW03 & 1520455.285 & 1880670.602 & 1525.716 & 1525.247 & Surveyed \\
\hline MW04 & 1520421.956 & 1880795.862 & 1526.235 & 1525.918 & Surveyed \\
\hline MW05 & 1520485.121 & 1880864.361 & 1525.601 & 1525.201 & Surveyed \\
\hline MW06 & 1520829.742 & 1880404.100 & 1523.064 & 1522.646 & Surveyed \\
\hline MW07 & 1520619.584 & 1880401.335 & 1524.315 & 1523.643 & Surveyed \\
\hline MW08 & 1520488.888 & 1880400.685 & 1524.848 & 1524.356 & Surveyed \\
\hline MW09 & 1520356.534 & 1880394.307 & 1525.583 & 1525.324 & Surveyed \\
\hline MW10 & 1520327.144 & 1880514.127 & 1525.482 & 1525.025 & Surveyed \\
\hline MW11 & 1520324.427 & 1880644.886 & 1525.716 & 1525.500 & Surveyed \\
\hline MW12 & 1520249.805 & 1880756.532 & 1525.808 & 1525.447 & Surveyed \\
\hline MW13 & 1520001.588 & 1880742.450 & 1523.506 & 1523.028 & Surveyed \\
\hline MW14M & 1520459.130 & 1880512.599 & 1525.267 & 1524.973 & Surveyed \\
\hline MW14D & 1520458.957 & 1880512.475 & 1525.238 & 1524.957 & Surveyed \\
\hline MW15S & 1520061.509 & 1878905.161 & 1526.039 & 1525.699 & Surveyed \\
\hline MW15M & 1520068.065 & 1878904.972 & 1526.080 & 1525.836 & Surveyed \\
\hline MW15D & 1520067.866 & 1878905.124 & 1526.080 & 1525.815 & Surveyed \\
\hline MW16S1 & 1519071.534 & 1879612.174 & 1507.687 & 1507.257 & Surveyed \\
\hline MW16S2 & 1519071.583 & 1879612.141 & 1507.687 & 1507.264 & Surveyed \\
\hline MW16M1 & 1519068.693 & 1879607.145 & 1507.678 & 1507.176 & Surveyed \\
\hline MW16M2 & 1519068.501 & 1879606.877 & 1507.678 & 1507.290 & Surveyed \\
\hline MW16D & 1519068.343 & 1879607.083 & 1507.678 & 1507.290 & Surveyed \\
\hline MW17S & 1521091.428 & 1879487.520 & 1504.672 & 1504.007 & Surveyed \\
\hline MW17M & 1521091.708 & 1879487.512 & 1504.672 & 1504.080 & Surveyed \\
\hline MW17D & 1521091.626 & 1879487.900 & 1504.672 & 1504.085 & Surveyed \\
\hline MW18S & 1520186.549 & 1880456.959 & 1526.288 & 1525.880 & Surveyed \\
\hline MW18M & 1520186.464 & 1880457.066 & 1526.288 & 1525.875 & Surveyed \\
\hline MW18D & 1520186.454 & 1880456.877 & 1526.288 & 1525.890 & Surveyed \\
\hline MW19S & 1520466.270 & 1880939.688 & 1525.335 & 1524.905 & Surveyed \\
\hline MW19M & 1520466.562 & 1880939.691 & 1525.335 & 1524.919 & Surveyed \\
\hline MW20S & 1520023.992 & 1877768.765 & 1510.992 & 1512.841 & Surveyed \\
\hline MW20M & 1520024.191 & 1877768.420 & 1510.992 & 1512.822 & Surveyed \\
\hline \multicolumn{6}{|c|}{ Cone penetrometer soil vertical profiling locations } \\
\hline TI01 & 1520423.750 & 1880535.970 & - & - & GPS \\
\hline TI02 & 1520420.850 & 1880638.380 & - & - & GPS \\
\hline TIO3 & 1520418.520 & 1880741.970 & - & - & GPS \\
\hline TI04 & 1520418.570 & 1880843.510 & - & - & GPS \\
\hline TI05 & 1520450.630 & 1880771.740 & - & - & GPS \\
\hline TI06 & 1520450.550 & 1880675.540 & - & - & GPS \\
\hline TIO7 & 1520446.870 & 1880587.010 & - & - & GPS \\
\hline
\end{tabular}


TABLE F.1 (Cont.)

\begin{tabular}{|c|c|c|c|c|c|}
\hline Location & $\begin{array}{c}\text { Easting } \\
(\mathrm{ft})\end{array}$ & $\begin{array}{l}\text { Northing } \\
(\mathrm{ft})\end{array}$ & $\begin{array}{l}\text { Ground } \\
\text { Elevation } \\
\text { (ft AMSL) }\end{array}$ & $\begin{array}{c}\text { Top of } \\
\text { Casing } \\
\text { Elevation } \\
\text { (ft AMSL) }\end{array}$ & $\begin{array}{l}\text { Type } \\
\text { of } \\
\text { Data }\end{array}$ \\
\hline \multicolumn{6}{|c|}{ Cone penetrometer soil vertical profiling locations (cont.) } \\
\hline TI08 & 1520480.920 & 1880810.390 & - & - & GPS \\
\hline TI09 & 1520484.420 & 1880707.090 & - & - & GPS \\
\hline TI10 & 1520486.140 & 1880607.050 & - & - & GPS \\
\hline TI11 & 1520474.810 & 1880509.000 & - & - & GPS \\
\hline TI19 & 1520117.057 & 1880640.634 & 1524.685 & - & Surveyed \\
\hline TI20 & 1520105.298 & 1880599.382 & 1524.859 & - & Surveyed \\
\hline $\mathrm{TI} 21$ & 1520058.200 & 1880595.486 & 1524.202 & - & Surveyed \\
\hline TI22 & 1520091.482 & 1880559.842 & 1524.568 & - & Surveyed \\
\hline TI23 & 1520150.476 & 1880523.591 & 1525.407 & - & Surveyed \\
\hline TI24 & 1520163.255 & 1880130.931 & 1525.198 & - & Surveyed \\
\hline TI25 & 1520162.930 & 1880128.313 & 1525.277 & - & Surveyed \\
\hline \multicolumn{6}{|l|}{ Private well locations } \\
\hline Bengston & 1520227.095 & 1880039.624 & 1525.723 & - & Surveyed \\
\hline Newcombe/Pankratz & 1520249.300 & 1880297.300 & - & - & Estimated \\
\hline Sisson & 1520191.490 & 1880513.863 & 1526.165 & - & Surveyed \\
\hline Willems & 1520241.000 & 1880140.100 & - & - & Estimated \\
\hline Brown & 1519025.200 & 1881249.100 & - & - & GPS \\
\hline Goertzen & 1520609.100 & 1876610.300 & - & - & GPS \\
\hline Hull Windmill & 1518917.800 & 1878937.400 & - & - & GPS \\
\hline Hull House & 1518990.500 & 1878734.200 & - & - & GPS \\
\hline Isaac & 1520675.000 & 1876909.900 & - & - & GPS \\
\hline Klassen & 1519989.158 & 1876487.303 & 1507.712 & 1508.769 & Surveyed \\
\hline J. Pankratz & 1521073.200 & 1876327.500 & - & - & GPS \\
\hline Pauls Well & 1521442.600 & 1878209.000 & - & - & GPS \\
\hline Klassen Test Well & 1520145.625 & 1876470.095 & 1508.024 & 1509.122 & Surveyed \\
\hline Davisson & 1520060.300 & 1881098.500 & - & - & Estimated \\
\hline
\end{tabular}

a Coordinates are State Plane Kansas northern zone; horizontal datum = NAD83; vertical datum = NAVD29. 


\section{Appendix G:}

\section{Evaluation of Drawdown and Recovery Data at Monitoring Wells}




\section{Appendix G:}

\section{Evaluation of Drawdown and Recovery Data at Monitoring Wells}

The hydraulic properties of the shallow and middle groundwater zones and the potential variations in these properties are the primary factors that govern groundwater flow and the fate of contaminants at the Inman site. To provide quantitative estimates for these properties, the following two types of drawdown and/or recovery data were identified and analyzed:

- Water level recovery data observed in monitoring wells MW01, MW02, MW03, and MW05 after a sampling event on November 9, 2010.

- Water level drawdown and recovery data observed at monitoring wells MW18S and MW18M in response to one pumping cycle at the Sisson well on October 7, 2011.

\section{G.1 Analysis of Water Level Recovery Data for MW01-MW03 and MW05}

The water level recovery data after a sampling event on November 9, 2010, were automatically recorded (Table G.1). The recovery process was very slow (8 $\mathrm{hr}$ to a few days), from an initial drawdown of 8.7-18.4 ft to a negligible level of $<1 \mathrm{ft}$. Because of the slow recovery observed, the much shorter duration of the groundwater sampling event was considered to represent a relatively instantaneous water withdrawal of the type performed in conducting a conventional slug test. On this basis, the recovery data are of reasonably good quality for the estimation of lateral hydraulic conductivity $\left(K_{h}\right)$. The methods of Bouwer and Rice (Bouwer and Rice 1976, Bouwer 1989) and Hvorslev (1951), which are commonly applied to interpret slug test data, were used as implemented in the commercial software package AqteSolv.

On the basis of the analysis of lithologic logs for the shallow groundwater-bearing zone, an effective thickness of $2 \mathrm{ft}$ was assumed as having remained fully saturated and hence "confined." For this groundwater-bearing zone developed along bedding planes or in the basal gravelly clay to clayey gravel near the erosional surface, an anisotropy ratio $K_{v} / K_{h}=0.1$ was assumed. The porosity of the gravel pack in the well is approximately 30\%. The input data and representative interpretive curves fitted to the recovery data are shown in Figures G.1-G.9. 
The data recorded indicate a more rapid recovery at MW05 (approximately $8 \mathrm{hr}$ ) than at MW01-MW03 (Table G.1). A small number of data points (four) recorded during the entire recovery period for MW05 might not capture the early portion of the recovery, which is usually faster than the later portion. The insufficiency of data points for the faster recovery at MW05 would result in underestimation of $K_{h}$ at this location. However, the estimate will reflect a minimum value for $K_{h}$.

For each data set, the $K$ values calculated with the Bouwer and Rice method are consistently lower than the values calculated with the Hvorslev method (Table G.2). The average $K_{h}$ values for the two methods are $8.59 \times 10^{-5}$ to $1.03 \times 10^{-4} \mathrm{ft} /$ day at MW01-MW03 and $>0.004 \mathrm{ft} /$ day at MW05.

\section{G.2 Analysis of Water Level Drawdown and Recovery Data for MW18S and MW18M}

During the water level monitoring period in September-October 2011, a significant effect of pumping at the Sisson well was identified at MW18S (in the shallow groundwater-bearing zone) and MW18M (in the middle groundwater-bearing zone). Wells MW18S and MW18M are located $57 \mathrm{ft}$ south of the Sisson well. As an example of data analysis, the drawdown and recovery data automatically recorded on October 7, 2011, were evaluated for estimation of hydraulic conductivity in the shallow and middle groundwater-bearing zones at location MW18. The data were recorded every $30 \mathrm{~min}$ for the entire event, including the pumping period of approximately $6 \mathrm{hr}$ (360 min) and the recovery period of roughly $41 \mathrm{hr}$ (2,460 min) (Table G.3).

The pumping effects observed at MW18S and MW18M indicate that groundwater was drawn from both the shallow and middle groundwater zones when the Sisson well was pumped. The range of pumping rates at the Sisson well is plausibly 1-20 gpm for lawn watering. The percentage of total pumped water is likely in the range of $30 \%$ to $5 \%$ from the shallow groundwater zone and $70 \%$ to $95 \%$ from the middle groundwater zone, as the shallow groundwater zone is demonstrably much less productive than the middle groundwater zone. On this basis, we derived a range of withdrawal rates of $0.05-6 \mathrm{gpm}$ for the shallow zone and 0.7-19 gpm for the middle zone (Tables G.4 and G.5, respectively). Use of theoretical Theis curves at various selected pumping rates in the range of withdrawal rates for each zone to fit the drawdown and recovery data resulted in a range of estimates for transmissivity $(T)$ and storativity $(S)$ in each zone at MW18. The representative interpretive curve fits for time versus residual 
drawdown data are shown in Figures G.9-G.17 for the shallow zone and in Figures G.18-G.26 for the middle zone. The $K_{h}$ values were determined by dividing the resulting transmissivity values by the aquifer thickness (average $2 \mathrm{ft}$ for both the shallow and middle zones). Use of this approach yielded rough order-of-magnitude estimates of $K_{h}$ of $10^{0}$ to $10^{2} \mathrm{ft}$ /day for the shallow groundwater zone and $10^{1}$ to $10^{3} \mathrm{ft} /$ day for the middle groundwater zone. 
TABLE G.1 Residual drawdown data recorded in wells MW01-MW03 and MW05 during the recovery period after a sampling event on November 9, 2010.

\begin{tabular}{|c|c|c|c|c|c|c|c|c|c|}
\hline \multirow{2}{*}{\multicolumn{2}{|c|}{ Date and Time }} & \multicolumn{2}{|c|}{ MW01 } & \multicolumn{2}{|c|}{ MW02 } & \multicolumn{2}{|c|}{ MW03 } & \multicolumn{2}{|c|}{ MW05 } \\
\hline & & $\begin{array}{l}\text { Recovery } \\
\text { Time } \\
\text { (min) }\end{array}$ & $\begin{array}{l}\text { Residual } \\
\text { Drawdown } \\
\text { (ft) }\end{array}$ & $\begin{array}{l}\text { Recovery } \\
\text { Time } \\
\text { (min) }\end{array}$ & $\begin{array}{l}\text { Residual } \\
\text { Drawdown } \\
\text { (ft) }\end{array}$ & $\begin{array}{c}\text { Recovery } \\
\text { Time } \\
\text { (min) }\end{array}$ & $\begin{array}{c}\text { Residual } \\
\text { Drawdown } \\
\text { (ft) }\end{array}$ & $\begin{array}{l}\text { Recovery } \\
\text { Time } \\
\text { (min) }\end{array}$ & $\begin{array}{c}\text { Residual } \\
\text { Drawdown } \\
\text { (ft) }\end{array}$ \\
\hline $11 / 9 / 10$ & $12: 00$ & 0 & 8.789 & 0 & 8.716 & & & & \\
\hline $11 / 9 / 10$ & $16: 00$ & 240 & 8.01 & 240 & 8.22 & 0 & 11.169 & 0 & 18.440 \\
\hline $11 / 9 / 10$ & $20: 00$ & 480 & 7.67 & 480 & 7.90 & 240 & 10.21 & 240 & 2.87 \\
\hline $11 / 10 / 10$ & $0: 00$ & 720 & 7.39 & 720 & 7.62 & 480 & 9.78 & 480 & 0.77 \\
\hline 11/10/10 & $4: 00$ & 960 & 7.13 & 960 & 7.35 & 720 & 9.39 & 720 & 0.33 \\
\hline $11 / 10 / 10$ & $8: 00$ & 1200 & 6.85 & 1200 & 7.09 & 960 & 9.05 & & \\
\hline $11 / 10 / 10$ & $12: 00$ & 1440 & 6.58 & 1440 & 6.88 & 1200 & 8.73 & & \\
\hline $11 / 10 / 10$ & $16: 00$ & 1680 & 6.33 & 1680 & 6.67 & 1440 & 8.37 & & \\
\hline $11 / 10 / 10$ & $20: 00$ & 1920 & 6.14 & 1920 & 6.45 & 1680 & 7.89 & & \\
\hline $11 / 11 / 10$ & $0: 00$ & 2160 & 5.95 & 2160 & 6.24 & 1920 & 7.50 & & \\
\hline $11 / 11 / 10$ & $4: 00$ & 2400 & 5.77 & 2400 & 6.03 & 2160 & 7.09 & & \\
\hline $11 / 11 / 10$ & $8: 00$ & 2640 & 5.61 & 2640 & 5.79 & 2400 & 6.72 & & \\
\hline $11 / 11 / 10$ & $12: 00$ & 2880 & 5.43 & 2880 & 5.57 & 2640 & 6.60 & & \\
\hline $11 / 11 / 10$ & $16: 00$ & 3120 & 5.24 & 3120 & 5.38 & 2880 & 6.26 & & \\
\hline $11 / 11 / 10$ & $20: 00$ & 3360 & 5.06 & 3360 & 5.21 & 3120 & 6.03 & & \\
\hline $11 / 12 / 10$ & $0: 00$ & 3600 & 4.87 & 3600 & 5.06 & 3360 & 5.79 & & \\
\hline $11 / 12 / 10$ & $4: 00$ & 3840 & 4.62 & 3840 & 4.88 & 3600 & 5.57 & & \\
\hline $11 / 12 / 10$ & $8: 00$ & 4080 & 4.44 & 4080 & 4.76 & 3840 & 5.30 & & \\
\hline $11 / 12 / 10$ & $12: 00$ & 4320 & 4.31 & 4320 & 4.59 & 4080 & 5.01 & & \\
\hline $11 / 12 / 10$ & $16: 00$ & 4560 & 4.17 & 4560 & 4.43 & 4320 & 3.43 & & \\
\hline $11 / 12 / 10$ & $20: 00$ & 4800 & 4.00 & 4800 & 4.22 & 4560 & 2.72 & & \\
\hline $11 / 13 / 10$ & $0: 00$ & 5040 & 3.86 & 5040 & 4.07 & 4800 & 2.56 & & \\
\hline $11 / 13 / 10$ & $4: 00$ & 5280 & 3.72 & 5280 & 3.90 & 5040 & 2.47 & & \\
\hline $11 / 13 / 10$ & $8: 00$ & 5520 & 3.59 & 5520 & 3.70 & 5280 & 2.38 & & \\
\hline $11 / 13 / 10$ & $12: 00$ & 5760 & 3.54 & 5760 & 3.54 & 5520 & 2.46 & & \\
\hline $11 / 13 / 10$ & $16: 00$ & 6000 & 3.10 & 6000 & 3.37 & 5760 & 2.12 & & \\
\hline $11 / 13 / 10$ & $20: 00$ & 6240 & 2.91 & 6240 & 3.23 & 6000 & 1.99 & & \\
\hline $11 / 14 / 10$ & $0: 00$ & 6480 & 2.79 & 6480 & 3.11 & 6240 & 1.86 & & \\
\hline $11 / 14 / 10$ & $4: 00$ & 6720 & 2.64 & 6720 & 2.99 & 6480 & 1.78 & & \\
\hline $11 / 14 / 10$ & $8: 00$ & 6960 & 2.54 & 6960 & 2.87 & 6720 & 1.73 & & \\
\hline $11 / 14 / 10$ & $12: 00$ & 7200 & 2.38 & 7200 & 2.75 & 6960 & 1.87 & & \\
\hline $11 / 14 / 10$ & $16: 00$ & 7440 & 2.21 & 7440 & 2.63 & 7200 & 1.60 & & \\
\hline $11 / 14 / 10$ & $20: 00$ & 7680 & 2.11 & 7680 & 2.53 & 7440 & 1.54 & & \\
\hline $11 / 15 / 10$ & 0:00 & 7920 & 1.98 & 7920 & 2.43 & 7680 & 1.45 & & \\
\hline $11 / 15 / 10$ & $4: 00$ & 8160 & 1.85 & 8160 & 2.34 & 7920 & 1.38 & & \\
\hline $11 / 15 / 10$ & $8: 00$ & 8400 & 1.76 & 8400 & 2.25 & 8160 & 1.31 & & \\
\hline $11 / 15 / 10$ & $12: 00$ & 8640 & 1.68 & 8640 & 2.15 & 8400 & 1.45 & & \\
\hline $11 / 15 / 10$ & $16: 00$ & 8880 & 1.60 & 8880 & 2.07 & 8640 & 1.29 & & \\
\hline $11 / 15 / 10$ & $20: 00$ & 9120 & 1.56 & 9120 & 1.98 & 8880 & 1.15 & & \\
\hline $11 / 16 / 10$ & $0: 00$ & 9360 & 1.48 & 9360 & 1.89 & 9120 & 1.09 & & \\
\hline $11 / 16 / 10$ & $4: 00$ & 9600 & 1.40 & 9600 & 1.81 & 9360 & 1.04 & & \\
\hline $11 / 16 / 10$ & $8: 00$ & 9840 & 1.35 & 9840 & 1.74 & 9600 & 0.99 & & \\
\hline $11 / 16 / 10$ & $12: 00$ & 10080 & 1.26 & 10080 & 1.66 & & & & \\
\hline $11 / 16 / 10$ & $16: 00$ & 10320 & 1.14 & 10320 & 1.59 & & & & \\
\hline $11 / 16 / 10$ & $20: 00$ & 10560 & 1.12 & 10560 & 1.53 & & & & \\
\hline $11 / 17 / 10$ & $0: 00$ & 10800 & 1.07 & 10800 & 1.47 & & & & \\
\hline $11 / 17 / 10$ & $4: 00$ & 11040 & 1.04 & 11040 & 1.42 & & & & \\
\hline $11 / 17 / 10$ & $8: 00$ & 11280 & 1.12 & 11280 & 1.39 & & & & \\
\hline $11 / 17 / 10$ & $12: 00$ & 11520 & 1.15 & 11520 & 1.31 & & & & \\
\hline $11 / 17 / 10$ & $16: 00$ & 11760 & 1.11 & 11760 & 1.19 & & & & \\
\hline $11 / 17 / 10$ & $20: 00$ & 12000 & 1.06 & 12000 & 1.13 & & & & \\
\hline $11 / 18 / 10$ & $0: 00$ & 12240 & 1.19 & 12240 & 1.22 & & & & \\
\hline $11 / 18 / 10$ & $4: 00$ & 12480 & 1.15 & 12480 & 1.20 & & & & \\
\hline $11 / 18 / 10$ & $8: 00$ & 12720 & 1.11 & 12720 & 1.17 & & & & \\
\hline $11 / 18 / 10$ & $12: 00$ & 12960 & 1.04 & 12960 & 1.13 & & & & \\
\hline $11 / 18 / 10$ & $16: 00$ & 13200 & 0.90 & 13200 & 1.07 & & & & \\
\hline $11 / 18 / 10$ & $20: 00$ & 13440 & 0.80 & 13440 & 1.03 & & & & \\
\hline $11 / 19 / 10$ & $0: 00$ & 13680 & 0.71 & 13680 & 0.99 & & & & \\
\hline $11 / 19 / 10$ & $4: 00$ & 13920 & 0.61 & 13920 & 0.94 & & & & \\
\hline $11 / 19 / 10$ & $8: 00$ & 14160 & 0.58 & 14160 & 0.91 & & & & \\
\hline $11 / 19 / 10$ & $12: 00$ & 14400 & 0.55 & 14400 & 0.87 & & & & \\
\hline $11 / 19 / 10$ & $16: 00$ & 14640 & 0.51 & 14640 & 0.84 & & & & \\
\hline $11 / 19 / 10$ & $20: 00$ & 14880 & 0.53 & 14880 & 0.81 & & & & \\
\hline
\end{tabular}


TABLE G.2 Summary of hydraulic conductivity values estimated from the water level recovery event on November 9,2010, as observed in monitoring wells in the shallow groundwater zone beneath the former CCC/USDA facility.

\begin{tabular}{ccccc}
\hline & \multicolumn{3}{c}{ Calculated Hydraulic Conductivity ${ }^{\mathrm{a}}$ (ft/day) } & \\
\cline { 2 - 4 } Well & $\begin{array}{c}\text { Bouwer and Rice Method } \\
\text { (Confined Aquifer) }\end{array}$ & $\begin{array}{c}\text { Hvorslev Method } \\
\text { (Confined Aquifer) }\end{array}$ & Average $^{\mathrm{b}}$ & Figures \\
\hline MW01 & $6.82 \times 10^{-5}$ & $1.18 \times 10^{-4}$ & $9.31 \times 10^{-5}$ & G.1, G.2 \\
MW02 & $6.27 \times 10^{-5}$ & $1.09 \times 10^{-4}$ & $8.59 \times 10^{-5}$ & G.3, G.4 \\
MW03 & $7.52 \times 10^{-5}$ & $1.30 \times 10^{-4}$ & $1.03 \times 10^{-4}$ & G.5, G.6 \\
MW04 & NA $^{\mathrm{c}}$ & $\mathrm{NA}$ & $\mathrm{NA}$ & - \\
MW05 & $>0.003^{\mathrm{d}}$ & $>0.005^{\mathrm{d}}$ & $>0.004^{\mathrm{d}}$ & G.7, G.8 \\
\hline
\end{tabular}

a Calculated with an assumed thickness of $2 \mathrm{ft}$ for the saturated interval.

b Averaged for the Bouwer and Rice and the Hvorslev calculation methods.

c Data are inadequate for analysis.

d Insufficient data points to capture the early portion of the recovery. The estimated values reflect minimum hydraulic conductivities. 
TABLE G.3 Drawdown data recorded for one pumping cycle at the Sisson well on October 7 , 2011.

\begin{tabular}{|c|c|c|c|c|c|}
\hline \multicolumn{2}{|c|}{ Time and Date } & \multirow{2}{*}{$\begin{array}{c}\text { Time since Pumping } \\
\text { Started at the } \\
\text { Sisson Well (min) } \\
0\end{array}$} & \multirow{2}{*}{$\begin{array}{c}\text { MW18S } \\
\begin{array}{c}\text { Drawdown } \\
(\mathrm{ft})\end{array} \\
0.000\end{array}$} & \multirow{2}{*}{$\begin{array}{c}\text { MW18M } \\
\begin{array}{c}\text { Drawdown } \\
(\mathrm{ft})\end{array} \\
0.000\end{array}$} & \multirow{2}{*}{$\begin{array}{c}\text { Note } \\
\text { Pumping started }\end{array}$} \\
\hline 10/7/11 & $5: 30$ & & & & \\
\hline $10 / 7 / 11$ & $6: 00$ & 30 & 0.221 & 1.076 & \\
\hline $10 / 7 / 11$ & $6: 30$ & 60 & 0.821 & 2.306 & \\
\hline $10 / 7 / 11$ & 7:00 & 90 & 1.079 & 2.870 & \\
\hline $10 / 7 / 11$ & $7: 30$ & 120 & 1.245 & 3.161 & \\
\hline $10 / 7 / 11$ & 8:00 & 150 & 1.308 & 3.329 & \\
\hline 10/7/11 & $8: 30$ & 180 & 1.511 & 3.891 & \\
\hline $10 / 7 / 11$ & 9:00 & 210 & 1.688 & 4.336 & \\
\hline $10 / 7 / 11$ & $9: 30$ & 240 & 1.847 & 4.706 & \\
\hline $10 / 7 / 11$ & $10: 00$ & 270 & 1.977 & 4.998 & \\
\hline $10 / 7 / 11$ & $10: 30$ & 300 & 2.077 & 5.142 & \\
\hline $10 / 7 / 11$ & $11: 00$ & 330 & 2.110 & 5.239 & \\
\hline $10 / 7 / 11$ & $11: 30$ & 360 & 1.728 & 3.680 & Pumping stopped \\
\hline $10 / 7 / 11$ & $12: 00$ & 390 & 1.453 & 3.026 & \\
\hline $10 / 7 / 11$ & $12: 30$ & 420 & 1.278 & 2.619 & \\
\hline $10 / 7 / 11$ & $13: 00$ & 450 & 1.159 & 2.318 & \\
\hline $10 / 7 / 11$ & $13: 30$ & 480 & 1.063 & 2.088 & \\
\hline $10 / 7 / 11$ & $14: 00$ & 510 & 0.974 & 1.898 & \\
\hline $10 / 7 / 11$ & $14: 30$ & 540 & 0.902 & 1.740 & \\
\hline $10 / 7 / 11$ & $15: 00$ & 570 & 0.842 & 1.607 & \\
\hline $10 / 7 / 11$ & $15: 30$ & 600 & 0.783 & 1.488 & \\
\hline $10 / 7 / 11$ & $16: 00$ & 630 & 0.741 & 1.384 & \\
\hline $10 / 7 / 11$ & $16: 30$ & 660 & 0.695 & 1.296 & \\
\hline $10 / 7 / 11$ & $17: 00$ & 690 & 0.662 & 1.216 & \\
\hline $10 / 7 / 11$ & $17: 30$ & 720 & 0.627 & 1.142 & \\
\hline $10 / 7 / 11$ & $18: 00$ & 750 & 0.592 & 1.078 & \\
\hline $10 / 7 / 11$ & $18: 30$ & 780 & 0.578 & 1.093 & \\
\hline 10/7/11 & $19: 00$ & 810 & 0.550 & 0.991 & \\
\hline 10/7/11 & $19: 30$ & 840 & 0.524 & 0.936 & \\
\hline 10/7/11 & $20: 00$ & 870 & 0.503 & 0.889 & \\
\hline $10 / 7 / 11$ & $20: 30$ & 900 & 0.485 & 0.851 & \\
\hline $10 / 7 / 11$ & $21: 00$ & 930 & 0.466 & 0.808 & \\
\hline 10/7/11 & $21: 30$ & 960 & 0.447 & 0.770 & \\
\hline $10 / 7 / 11$ & $22: 00$ & 990 & 0.429 & 0.737 & \\
\hline 10/7/11 & $22: 30$ & 1020 & 0.408 & 0.706 & \\
\hline $10 / 7 / 11$ & $23: 00$ & 1050 & 0.394 & 0.678 & \\
\hline 10/7/11 & $23: 30$ & 1080 & 0.380 & 0.647 & \\
\hline 10/8/11 & $0: 00$ & 1110 & 0.366 & 0.621 & \\
\hline $10 / 8 / 11$ & $0: 30$ & 1140 & 0.340 & 0.595 & \\
\hline $10 / 8 / 11$ & $1: 00$ & 1170 & 0.338 & 0.571 & \\
\hline $10 / 8 / 11$ & $1: 30$ & 1200 & 0.322 & 0.545 & \\
\hline $10 / 8 / 11$ & $2: 00$ & 1230 & 0.317 & 0.529 & \\
\hline $10 / 8 / 11$ & $2: 30$ & 1260 & 0.310 & 0.510 & \\
\hline 10/8/11 & $3: 00$ & 1290 & 0.296 & 0.491 & \\
\hline 10/8/11 & $3: 30$ & 1320 & 0.291 & 0.470 & \\
\hline 10/8/11 & $4: 00$ & 1350 & 0.280 & 0.453 & \\
\hline
\end{tabular}


TABLE G.3 (Cont.)

\begin{tabular}{|c|c|c|c|c|c|}
\hline \multicolumn{2}{|c|}{ Time and Date } & \multirow{2}{*}{$\begin{array}{c}\text { Time since Pumping } \\
\text { Started at the } \\
\text { Sisson Well (min) } \\
1380\end{array}$} & \multirow{2}{*}{$\begin{array}{c}\text { MW18S } \\
\text { Drawdown } \\
(\mathrm{ft})\end{array}$} & \multirow{2}{*}{$\begin{array}{c}\text { MW18M } \\
\begin{array}{c}\text { Drawdown } \\
(\mathrm{ft})\end{array} \\
0.434\end{array}$} & \multirow{2}{*}{ Note } \\
\hline 10/8/11 & $4: 30$ & & & & \\
\hline $10 / 8 / 11$ & $5: 00$ & 1410 & 0.256 & 0.420 & \\
\hline $10 / 8 / 11$ & $5: 30$ & 1440 & 0.259 & 0.406 & \\
\hline $10 / 8 / 11$ & $6: 00$ & 1470 & 0.259 & 0.396 & \\
\hline $10 / 8 / 11$ & $6: 30$ & 1500 & 0.261 & 0.384 & \\
\hline $10 / 8 / 11$ & $7: 00$ & 1530 & 0.249 & 0.377 & \\
\hline $10 / 8 / 11$ & $7: 30$ & 1560 & 0.245 & 0.363 & \\
\hline 10/8/11 & $8: 00$ & 1590 & 0.245 & 0.351 & \\
\hline $10 / 8 / 11$ & $8: 30$ & 1620 & 0.245 & 0.344 & \\
\hline $10 / 8 / 11$ & 9:00 & 1650 & 0.254 & 0.344 & \\
\hline $10 / 8 / 11$ & 9:30 & 1680 & 0.249 & 0.334 & \\
\hline 10/8/11 & $10: 00$ & 1710 & 0.247 & 0.330 & \\
\hline $10 / 8 / 11$ & $10: 30$ & 1740 & 0.277 & 0.327 & \\
\hline $10 / 8 / 11$ & $11: 00$ & 1770 & 0.263 & 0.320 & \\
\hline $10 / 8 / 11$ & $11: 30$ & 1800 & 0.263 & 0.316 & \\
\hline 10/8/11 & $12: 00$ & 1830 & 0.256 & 0.308 & \\
\hline 10/8/11 & $12: 30$ & 1860 & 0.249 & 0.299 & \\
\hline $10 / 8 / 11$ & $13: 00$ & 1890 & 0.242 & 0.287 & \\
\hline 10/8/11 & $13: 30$ & 1920 & 0.228 & 0.278 & \\
\hline $10 / 8 / 11$ & $14: 00$ & 1950 & 0.210 & 0.266 & \\
\hline $10 / 8 / 11$ & $14: 30$ & 1980 & 0.207 & 0.256 & \\
\hline $10 / 8 / 11$ & $15: 00$ & 2010 & 0.203 & 0.252 & \\
\hline $10 / 8 / 11$ & $15: 30$ & 2040 & 0.184 & 0.237 & \\
\hline $10 / 8 / 11$ & $16: 00$ & 2070 & 0.172 & 0.223 & \\
\hline $10 / 8 / 11$ & $16: 30$ & 2100 & 0.177 & 0.216 & \\
\hline $10 / 8 / 11$ & $17: 00$ & 2130 & 0.163 & 0.209 & \\
\hline 10/8/11 & $17: 30$ & 2160 & 0.151 & 0.195 & \\
\hline $10 / 8 / 11$ & $18: 00$ & 2190 & 0.147 & 0.190 & \\
\hline $10 / 8 / 11$ & $18: 30$ & 2220 & 0.151 & 0.183 & \\
\hline $10 / 8 / 11$ & $19: 00$ & 2250 & 0.151 & 0.178 & \\
\hline 10/8/11 & $19: 30$ & 2280 & 0.158 & 0.178 & \\
\hline 10/8/11 & $20: 00$ & 2310 & 0.161 & 0.176 & \\
\hline $10 / 8 / 11$ & $20: 30$ & 2340 & 0.158 & 0.171 & \\
\hline 10/8/11 & $21: 00$ & 2370 & 0.156 & 0.169 & \\
\hline $10 / 8 / 11$ & $21: 30$ & 2400 & 0.151 & 0.169 & \\
\hline $10 / 8 / 11$ & $22: 00$ & 2430 & 0.158 & 0.166 & \\
\hline $10 / 8 / 11$ & $22: 30$ & 2460 & 0.163 & 0.164 & \\
\hline $10 / 8 / 11$ & $23: 00$ & 2490 & 0.154 & 0.159 & \\
\hline 10/8/11 & $23: 30$ & 2520 & 0.151 & 0.157 & \\
\hline $10 / 9 / 11$ & $0: 00$ & 2550 & 0.144 & 0.152 & \\
\hline $10 / 9 / 11$ & $0: 30$ & 2580 & 0.140 & 0.145 & \\
\hline $10 / 9 / 11$ & $1: 00$ & 2610 & 0.133 & 0.143 & \\
\hline $10 / 9 / 11$ & $1: 30$ & 2640 & 0.137 & 0.138 & \\
\hline $10 / 9 / 11$ & $2: 00$ & 2670 & 0.133 & 0.133 & \\
\hline $10 / 9 / 11$ & $2: 30$ & 2700 & 0.126 & 0.126 & \\
\hline $10 / 9 / 11$ & $3: 00$ & 2730 & 0.116 & 0.124 & \\
\hline 10/9/11 & $3: 30$ & 2760 & 0.105 & 0.116 & \\
\hline
\end{tabular}


TABLE G.3 (Cont.)

\begin{tabular}{lccccc}
\hline Time and Date & $\begin{array}{c}\text { Time since Pumping } \\
\text { Started at the } \\
\text { Sisson Well (min) }\end{array}$ & $\begin{array}{c}\text { MW18S } \\
\text { Drawdown } \\
(\mathrm{ft})\end{array}$ & $\begin{array}{c}\text { MW18M } \\
\text { Drawdown } \\
(\mathrm{ft})\end{array}$ & Note \\
\hline 10/9/11 & $4: 00$ & 2790 & 0.102 & 0.109 \\
$10 / 9 / 11$ & $4: 30$ & 2820 & 0.100 & 0.102 \\
$10 / 9 / 11$ & $5: 00$ & 2850 & 0.088 & 0.098 \\
\hline
\end{tabular}


TABLE G.4 Hydraulic conductivity values estimated for the shallow groundwater zone beneath the former private grain storage facility on the Sisson property after the pumping event at the Sisson well on October 7, 2011, from observations made at well MW18S.

\begin{tabular}{|c|c|c|c|c|c|c|}
\hline \multirow{2}{*}{$\begin{array}{c}\text { Pumping } \\
\text { Rate at } \\
\text { Sisson } \\
\text { Wella }^{a} \\
\text { (gpm) }\end{array}$} & \multirow{2}{*}{$\begin{array}{l}\text { Percent of } \\
\text { Pumping Rate } \\
\text { Exerted to } \\
\text { Shallow } \\
\text { Groundwater } \\
\text { Zone }^{b}\end{array}$} & \multirow[b]{2}{*}{$\begin{array}{l}\text { Withdrawal Rate } \\
\text { from Shallow } \\
\text { Groundwater } \\
\text { Zone } \\
(\mathrm{gpm})^{\mathrm{c}}\end{array}$} & \multicolumn{3}{|c|}{$\begin{array}{l}\text { Values Estimated by Theis Method } \\
\text { (Confined Aquifer) }\end{array}$} & \multirow[b]{2}{*}{ Figure } \\
\hline & & & $\begin{array}{c}\text { Transmisivity } \\
\left(T, \mathrm{ft}^{2} / \text { day }\right)\end{array}$ & Storativity (S) & $\begin{array}{l}\text { Hydraulic } \\
\text { Conductivity } \\
\left(K_{h}, \mathrm{ft} / \text { day }\right)\end{array}$ & \\
\hline 1 & $\begin{array}{c}5 \\
10 \\
30\end{array}$ & $\begin{array}{l}0.05 \\
0.1 \\
0.3\end{array}$ & $\begin{array}{l}5.673 \\
11.35 \\
34.04\end{array}$ & $\begin{array}{l}0.0001 \\
0.0002 \\
0.0007\end{array}$ & $\begin{array}{l}2.837 \\
5.675 \\
17.02\end{array}$ & $\begin{array}{l}\text { G.9 } \\
\text { G.10 } \\
\text { G.11 }\end{array}$ \\
\hline 10 & $\begin{array}{c}5 \\
10 \\
30\end{array}$ & $\begin{array}{l}0.5 \\
1.0 \\
3.0\end{array}$ & $\begin{array}{l}56.73 \\
113.5 \\
340.4\end{array}$ & $\begin{array}{l}0.0012 \\
0.002 \\
0.007\end{array}$ & $\begin{array}{c}28.37 \\
56.75 \\
170.2\end{array}$ & $\begin{array}{l}\text { G.12 } \\
\text { G.13 } \\
\text { G.14 }\end{array}$ \\
\hline 20 & $\begin{array}{c}5 \\
10 \\
30\end{array}$ & $\begin{array}{l}1.0 \\
2.0 \\
6.0\end{array}$ & $\begin{array}{l}113.5 \\
226.9 \\
680.8\end{array}$ & $\begin{array}{l}0.002 \\
0.005 \\
0.014\end{array}$ & $\begin{array}{l}56.75 \\
113.5 \\
340.4\end{array}$ & $\begin{array}{l}\text { G.15 } \\
\text { G.16 } \\
\text { G.17 }\end{array}$ \\
\hline Range of & ues (order of ma & ude $)^{d}$ & $10^{1}-10^{3}$ & $10^{-2}-10^{-4}$ & $10^{0}-10^{2}$ & - \\
\hline
\end{tabular}

a The range of pumping rates at the Sisson well is estimated on the basis of probable rates used for lawn watering.

b During pumping at the Sisson well, water is extracted from both the shallow groundwater zone and middle groundwater zone via the well's uncased borehole. Because the shallow zone is less productive, water withdrawal from the shallow zone is assumed to range from $5 \%$ to $30 \%$ of the total pumping rate.

c Estimate based on the assumed probable pumping rate at the Sisson well (column 1) and the assumed percentage of total pumped water that is from the shallow zone (column 2).

d Range of hydraulic parameters, expressed in order of magnitude, for the assumed range of pumping rates (1-20 gpm) and fraction (percentage) of total pumped water from the shallow zone. 
TABLE G.5 Hydraulic conductivity values estimated for the middle groundwater zone beneath the former private grain storage facility on the Sisson property after the pumping event at the Sisson well on October 7, 2011, from observations made at well MW18M.

\begin{tabular}{|c|c|c|c|c|c|c|}
\hline \multirow[b]{2}{*}{$\begin{array}{c}\text { Pumping } \\
\text { Rate at } \\
\text { Sisson Wella } \\
\text { (gpm) }\end{array}$} & \multirow{2}{*}{$\begin{array}{l}\text { Percent of } \\
\text { Pumping } \\
\text { Rate Exerted } \\
\text { to Middle } \\
\text { Groundwater } \\
\text { Zone }^{\mathrm{b}}\end{array}$} & \multirow{2}{*}{$\begin{array}{l}\text { Withdrawal } \\
\text { Rate from } \\
\text { Middle } \\
\text { Groundwater } \\
\text { Zone } \\
(\mathrm{gpm})^{\mathrm{C}}\end{array}$} & \multicolumn{3}{|c|}{$\begin{array}{l}\text { Value Estimated by Theis Method } \\
\text { (Confined Aquifer) }\end{array}$} & \multirow[b]{2}{*}{ Figure } \\
\hline & & & $\begin{array}{l}\text { Transmisivity } \\
\left(T, \mathrm{ft}^{2} / \text { day }\right)\end{array}$ & \multicolumn{2}{|r|}{$\begin{array}{l}\text { Hydraulic } \\
\text { Conductivity } \\
\left(K_{h}, \text { ft/day }\right)\end{array}$} & \\
\hline 1 & $\begin{array}{l}95 \\
90 \\
70\end{array}$ & $\begin{array}{l}0.95 \\
0.90 \\
0.70\end{array}$ & $\begin{array}{l}59.85 \\
56.70 \\
44.10\end{array}$ & $\begin{array}{l}0.0006 \\
0.0006 \\
0.0004\end{array}$ & $\begin{array}{l}29.93 \\
28.35 \\
22.05\end{array}$ & $\begin{array}{l}\text { G.18 } \\
\text { G.19 } \\
\text { G.20 }\end{array}$ \\
\hline 10 & $\begin{array}{l}95 \\
90 \\
70\end{array}$ & $\begin{array}{l}9.5 \\
9.0 \\
7.0\end{array}$ & $\begin{array}{l}598.5 \\
567.0 \\
441.0\end{array}$ & $\begin{array}{l}0.006 \\
0.006 \\
0.004\end{array}$ & $\begin{array}{l}299.3 \\
283.5 \\
220.5\end{array}$ & $\begin{array}{l}\text { G.21 } \\
\text { G.22 } \\
\text { G.23 }\end{array}$ \\
\hline 20 & $\begin{array}{l}95 \\
90 \\
70\end{array}$ & $\begin{array}{l}19 \\
18 \\
14\end{array}$ & $\begin{array}{c}1157 \\
1134 \\
882.0\end{array}$ & $\begin{array}{l}0.012 \\
0.011 \\
0.009\end{array}$ & $\begin{array}{l}578.3 \\
567.0 \\
441.0\end{array}$ & $\begin{array}{l}\text { G.24 } \\
\text { G.25 } \\
\text { G.26 }\end{array}$ \\
\hline \multicolumn{3}{|c|}{ Range of values (order of magnitude) ${ }^{d}$} & $10^{1}-10^{3}$ & $10^{-2}-10^{-4}$ & $10^{1}-10^{3}$ & - \\
\hline
\end{tabular}

a The range of pumping rates at the Sisson well is estimated on the basis of probable rates used for lawn watering.

b During pumping at the Sisson well, water is extracted from both the shallow groundwater zone and middle groundwater zone via the well's uncased borehole. Because the middle zone is more productive, water withdrawal from the middle zone is assumed to range from $95 \%$ to $70 \%$ of the total pumping rate.

c Estimate based on the assumed probable pumping rate at the Sisson well (column 1) and percentage of total pumped water that is from the middle zone (column 2).

d Range of hydraulic parameters, expressed in order of magnitude, for the assumed range of pumping rates (1-20 gpm) and fraction (percentage) of total pumped water from the middle zone. 


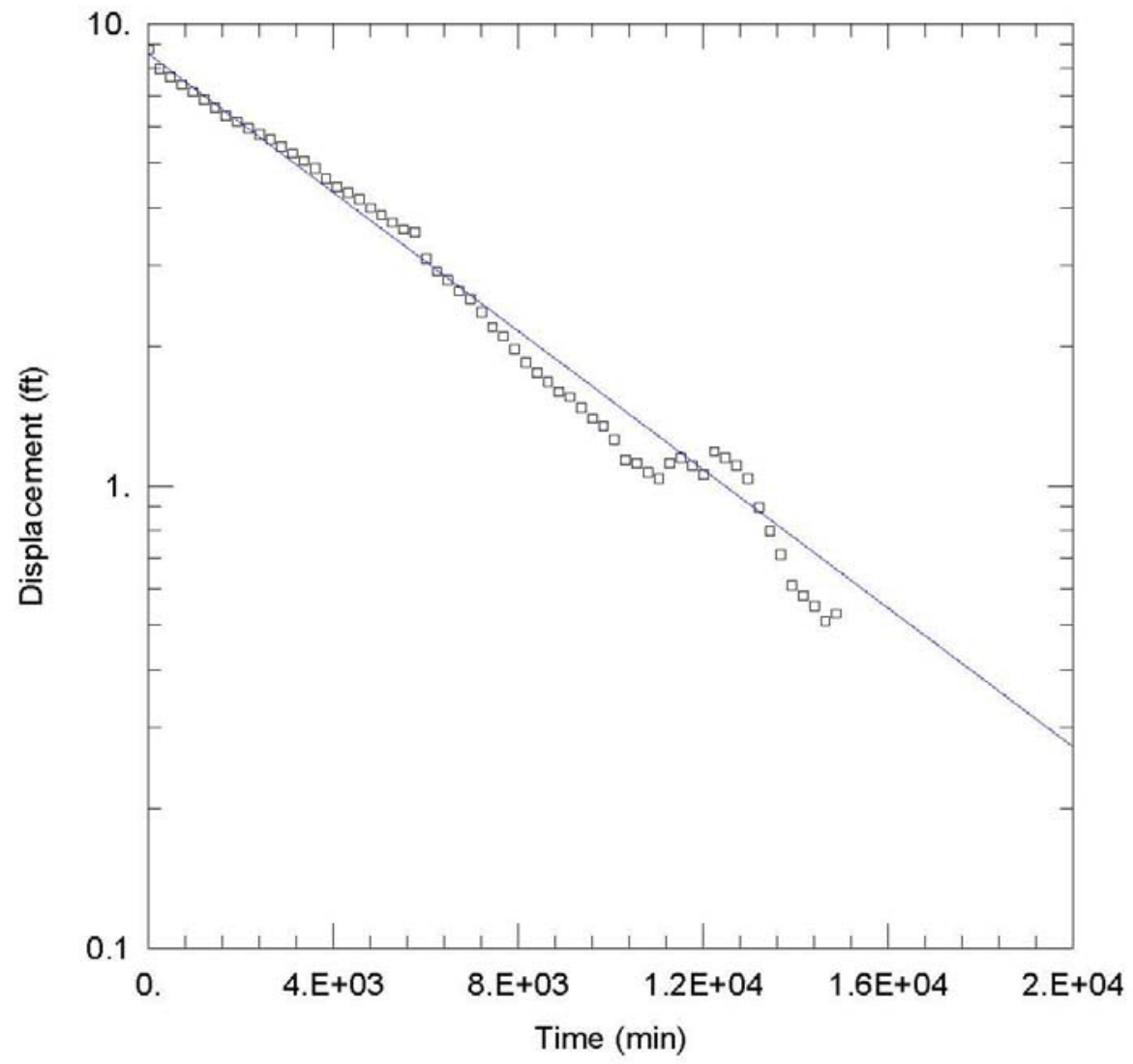

\begin{tabular}{|c|c|}
\hline \multicolumn{2}{|c|}{ WELL TEST ANALYSIS } \\
\hline \multicolumn{2}{|c|}{ PROJECT INFORMATION } \\
\hline \multicolumn{2}{|l|}{ 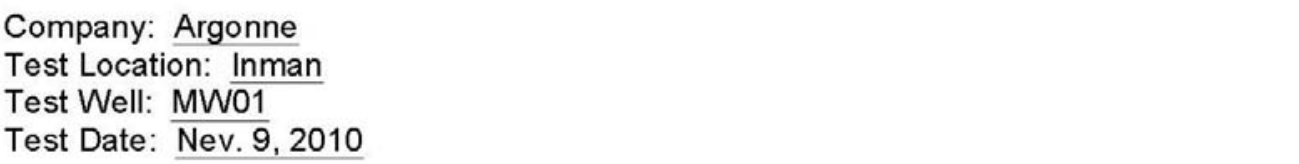 } \\
\hline \multicolumn{2}{|c|}{ AQUIFER DATA } \\
\hline Saturated Thickness: $\underline{2} . \mathrm{ft}$ & Anisotropy Ratio $(\mathrm{Kz} / \mathrm{Kr}): \underline{0.1}$ \\
\hline \multicolumn{2}{|c|}{ WELL DATA (MW01) } \\
\hline $\begin{array}{l}\text { Initial Displacement: } \underline{8.789} \mathrm{ft} \\
\text { Wellbore Radius: } 0.04167 \mathrm{ft} \\
\text { Screen Length: } 10 . \mathrm{ft} \\
\text { Gravel Pack Porosity: } 0.3\end{array}$ & $\begin{array}{l}\text { Casing Radius: } \underline{0.04167 \mathrm{ft}} \\
\text { Well Skin Radius: } 0.08333 \mathrm{ft} \\
\text { Total Well Penetration Depth: } 2 . \mathrm{ft}\end{array}$ \\
\hline \multicolumn{2}{|c|}{ SOLUTION } \\
\hline Aquifer Model: Confined & Solution Method: Bouwer-Rice \\
\hline$K=6.823 \mathrm{E}-05 \mathrm{ft} / \mathrm{day}$ & $y 0=8.607 \mathrm{ft}$ \\
\hline
\end{tabular}

FIGURE G.1 Bouwer-Rice analysis of recovery data for well MW01 after sampling on November 9, 2010. 


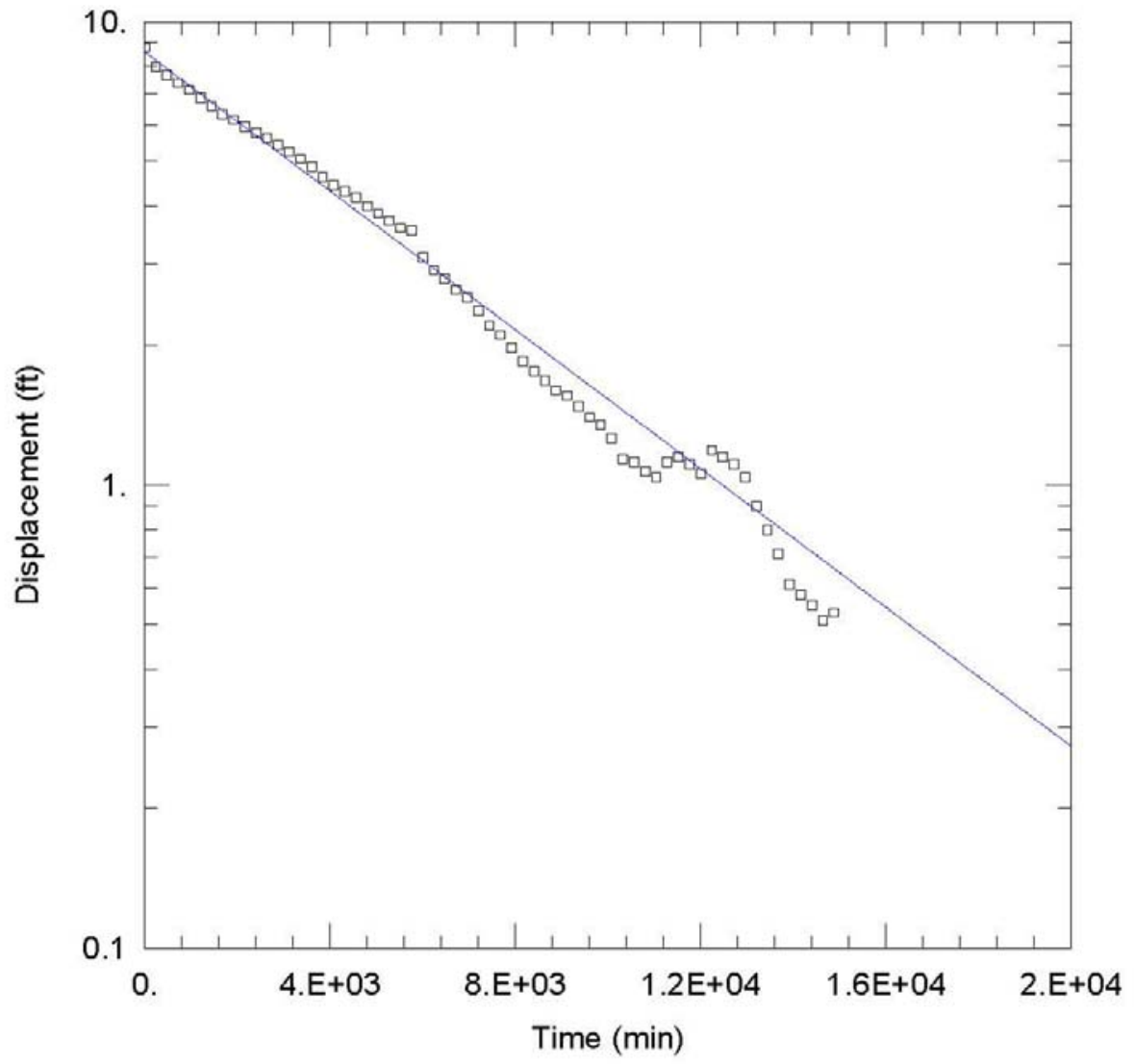

\begin{tabular}{|c|c|}
\hline \multicolumn{2}{|c|}{ WELL TEST ANALYSIS } \\
\hline \multicolumn{2}{|c|}{ PROJECT INFORMATION } \\
\hline \multicolumn{2}{|l|}{ 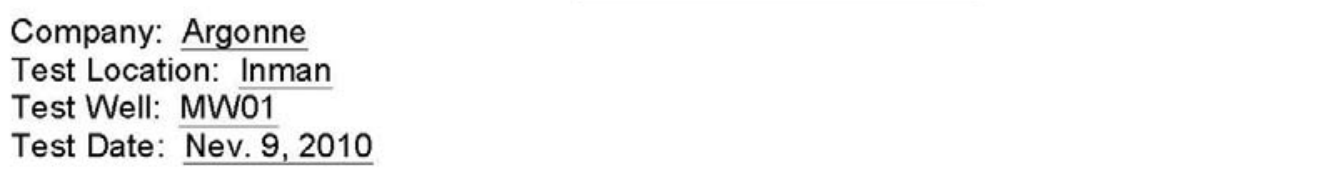 } \\
\hline \multicolumn{2}{|c|}{ AQUIFER DATA } \\
\hline Saturated Thickness: $\underline{2 .} \mathrm{ft}$ & Anisotropy Ratio $(\mathrm{Kz} / \mathrm{Kr})$ : $\underline{0.1}$ \\
\hline \multicolumn{2}{|c|}{ WELL DATA (MW01) } \\
\hline $\begin{array}{l}\text { Initial Displacement: } \underline{8.789} \mathrm{ft} \\
\text { Wellbore Radius: } 0.04167 \mathrm{ft} \\
\text { Screen Length: } 10 . \mathrm{ft} \\
\text { Gravel Pack Porosity: } \underline{0.3}\end{array}$ & $\begin{array}{l}\text { Casing Radius: } \underline{0.04167 \mathrm{ft}} \\
\text { Well Skin Radius: } 0.08333 \mathrm{ft} \\
\text { Total Well Penetration Depth: } 2 . \mathrm{ft}\end{array}$ \\
\hline \multicolumn{2}{|c|}{ SOLUTION } \\
\hline Aquifer Model: Confined & Solution Method: Hvorslev \\
\hline 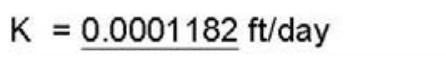 & $\mathrm{y} 0=\underline{8.608} \mathrm{ft}$ \\
\hline
\end{tabular}

FIGURE G.2 Hvorslev analysis of recovery data for well MW01 after sampling on November 9, 2010. 


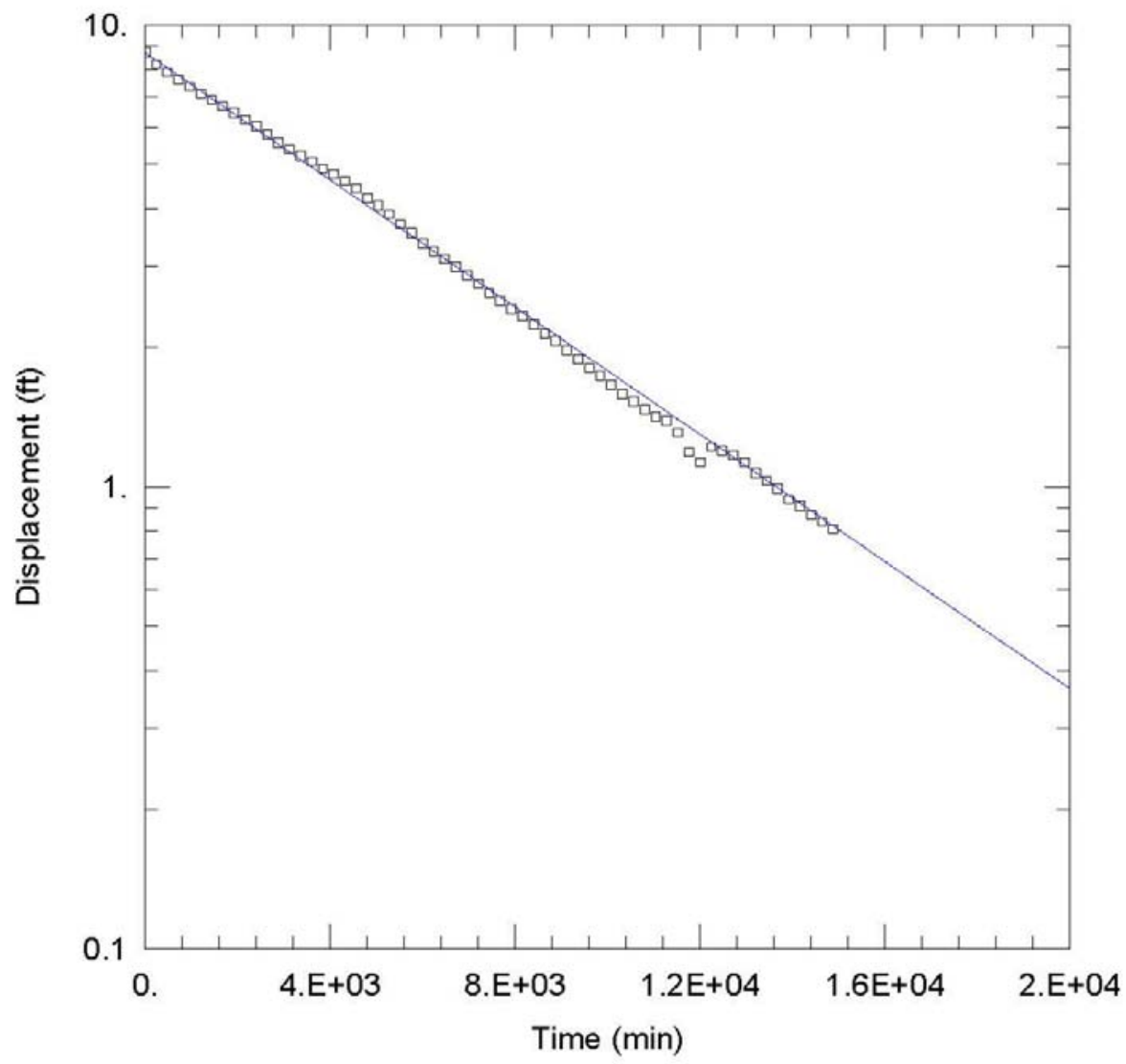

\begin{tabular}{|c|c|}
\hline \multicolumn{2}{|c|}{ WELL TEST ANALYSIS } \\
\hline \multicolumn{2}{|c|}{ PROJECT INFORMATION } \\
\hline \multicolumn{2}{|l|}{$\begin{array}{l}\text { Company: Argonne } \\
\text { Test Location: Inman } \\
\text { Test Well: MW02 } \\
\text { Test Date: } \underline{\text { Nev. 9, } 2010}\end{array}$} \\
\hline \multicolumn{2}{|c|}{ AQUIFER DATA } \\
\hline Saturated Thickness: $\underline{2} . \mathrm{ft}$ & Anisotropy Ratio $(\mathrm{Kz} / \mathrm{Kr}): \underline{0.1}$ \\
\hline \multicolumn{2}{|c|}{ WELL DATA (MWO2) } \\
\hline $\begin{array}{l}\text { Initial Displacement: } 8.716 \mathrm{ft} \\
\text { Wellbore Radius: } 0.04167 \mathrm{ft} \\
\text { Screen Length: } 10 . \mathrm{ft} \\
\text { Gravel Pack Porosity: } \underline{0.3}\end{array}$ & $\begin{array}{l}\text { Casing Radius: } 0.04167 \mathrm{ft} \\
\text { Well Skin Radius: } 0.08333 \mathrm{ft} \\
\text { Total Well Penetration Depth: } \underline{2 .} \mathrm{ft}\end{array}$ \\
\hline \multicolumn{2}{|c|}{ SOLUTION } \\
\hline Aquifer Model: Confined & Solution Method: Bouwer-Rice \\
\hline $\mathrm{K}=6.267 \mathrm{E}-05 \mathrm{ft} / \mathrm{day}$ & $y 0=8.702 \mathrm{ft}$ \\
\hline
\end{tabular}

FIGURE G.3 Bouwer-Rice analysis of recovery data for well MW02 after sampling on November 9, 2010. 


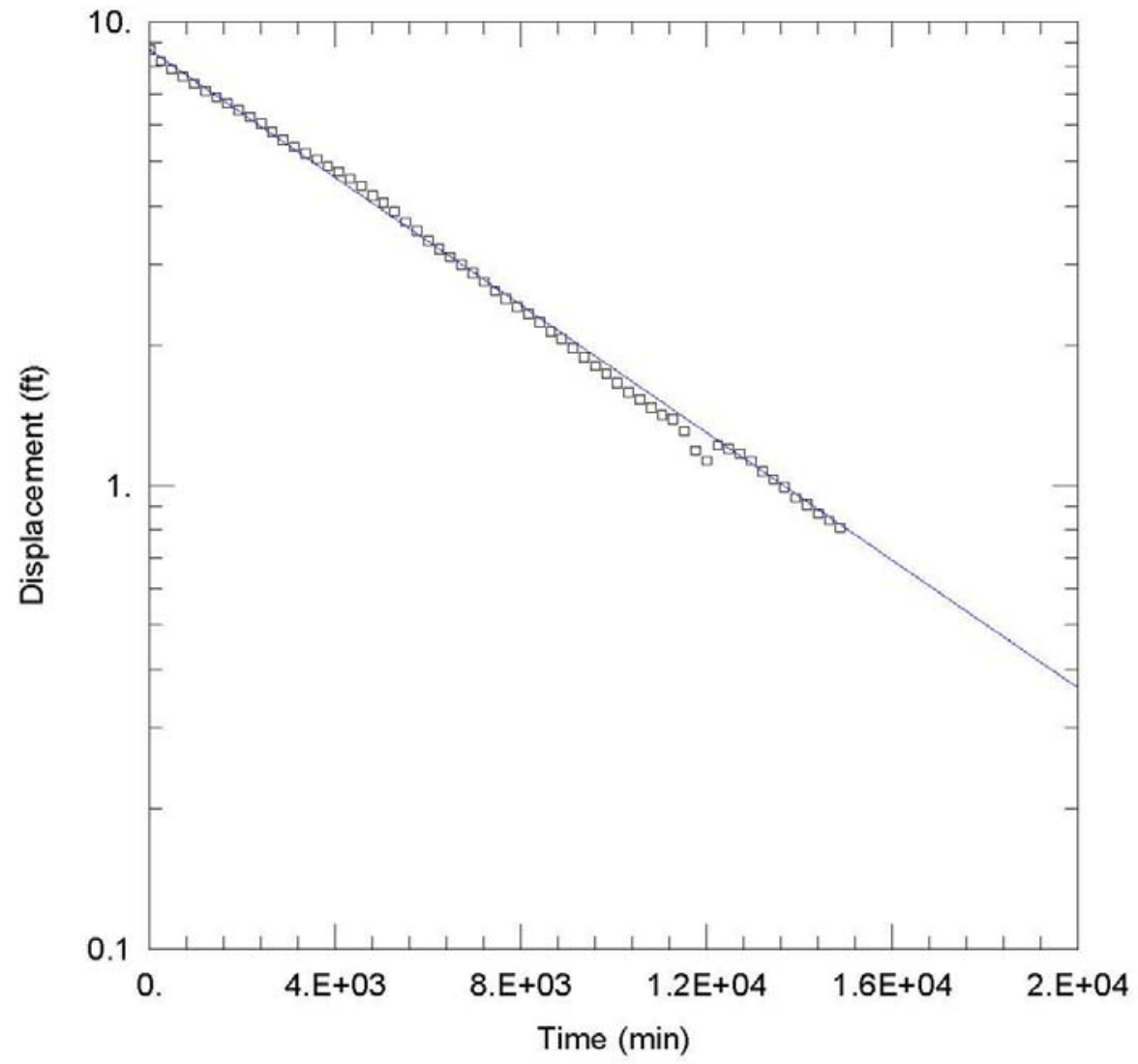

\begin{tabular}{|c|c|}
\hline \multicolumn{2}{|c|}{ WELL TEST ANALYSIS } \\
\hline $\begin{array}{l}\text { Company: Argonne } \\
\text { Test Location: Inman } \\
\text { Test Well: } \frac{\text { MW02 }}{\text { Nev. } 9,2010} \\
\text { Test Date: }\end{array}$ & ORMATION \\
\hline \multicolumn{2}{|c|}{ AQUIFER DATA } \\
\hline Saturated Thickness: $\underline{2} . \mathrm{ft}$ & Anisotropy Ratio $(\mathrm{Kz} / \mathrm{Kr})$ : $\underline{0.1}$ \\
\hline \multicolumn{2}{|c|}{ WELL DATA (MWO2) } \\
\hline $\begin{array}{l}\text { Initial Displacement: } \underline{8.716} \mathrm{ft} \\
\text { Wellbore Radius: } \underline{0.04167} \mathrm{ft} \\
\text { Screen Length: } 10 . \mathrm{ft} \\
\text { Gravel Pack Porosity: } \underline{0.3}\end{array}$ & $\begin{array}{l}\text { Casing Radius: } \underline{0.04167 \mathrm{ft}} \\
\text { Well Skin Radius: } \underline{0.08333} \mathrm{ft} \\
\text { Total Well Penetration Depth: } \underline{2 .} \mathrm{ft}\end{array}$ \\
\hline \multicolumn{2}{|c|}{ SOLUTION } \\
\hline Aquifer Model: Confined & Solution Method: Hvorslev \\
\hline$K=0.0001086 \mathrm{ft} / \mathrm{day}$ & $\mathrm{y} 0=8.703 \mathrm{ft}$ \\
\hline
\end{tabular}

FIGURE G.4 Hvorslev analysis of recovery data for well MW02 after sampling on November 9, 2010. 


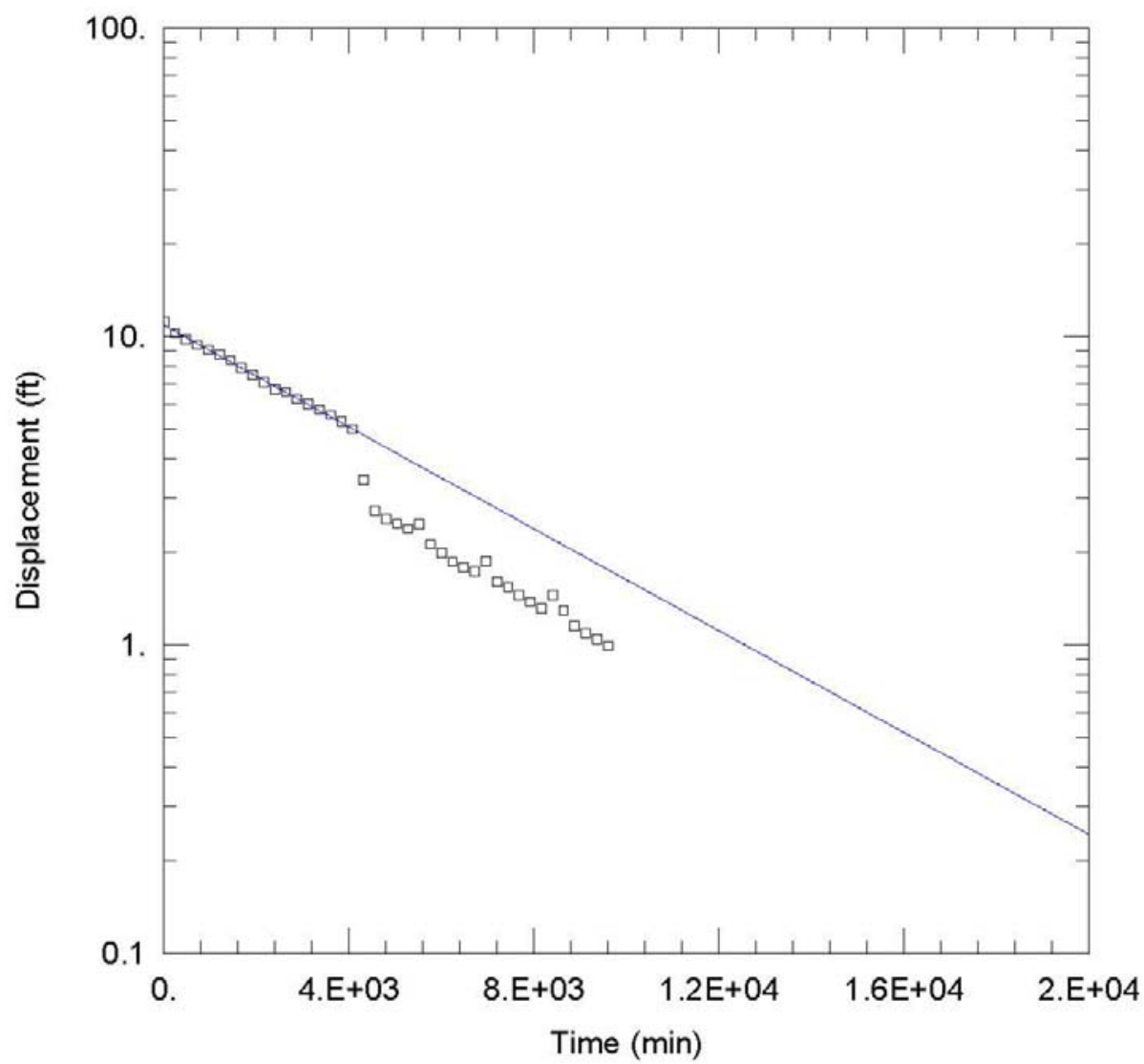

WELL TEST ANALYSIS

PROJECT INFORMATION

Company: Argonne

Test Location: Inman

Test Well: MW03

Test Date: Nev. 9, 2010

\begin{tabular}{|c|c|}
\hline \multicolumn{2}{|c|}{ AQUIFER DATA } \\
\hline Saturated Thickness: $\underline{2} . \mathrm{ft}$ & Anisotropy Ratio $(\mathrm{Kz} / \mathrm{Kr}): \underline{0.1}$ \\
\hline \multicolumn{2}{|c|}{ WELL DATA (MWO3) } \\
\hline $\begin{array}{l}\text { Initial Displacement: } 11.17 \mathrm{ft} \\
\text { Wellbore Radius: } 0.04167 \mathrm{ft} \\
\text { Screen Length: } 10 . \mathrm{ft} \\
\text { Gravel Pack Porosity: } \underline{0.3}\end{array}$ & $\begin{array}{l}\text { Casing Radius: } \underline{0.04167} \mathrm{ft} \\
\text { Well Skin Radius: } \underline{0.08333} \mathrm{ft} \\
\text { Total Well Penetration Depth: } \underline{2 .} \mathrm{ft}\end{array}$ \\
\hline \multicolumn{2}{|c|}{ SOLUTION } \\
\hline Aquifer Model: Confined & Solution Method: Bouwer-Rice \\
\hline 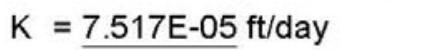 & $y 0=10.86 \mathrm{ft}$ \\
\hline
\end{tabular}

FIGURE G.5 Bouwer-Rice analysis of recovery data for well MW03 after sampling on November 9, 2010. 


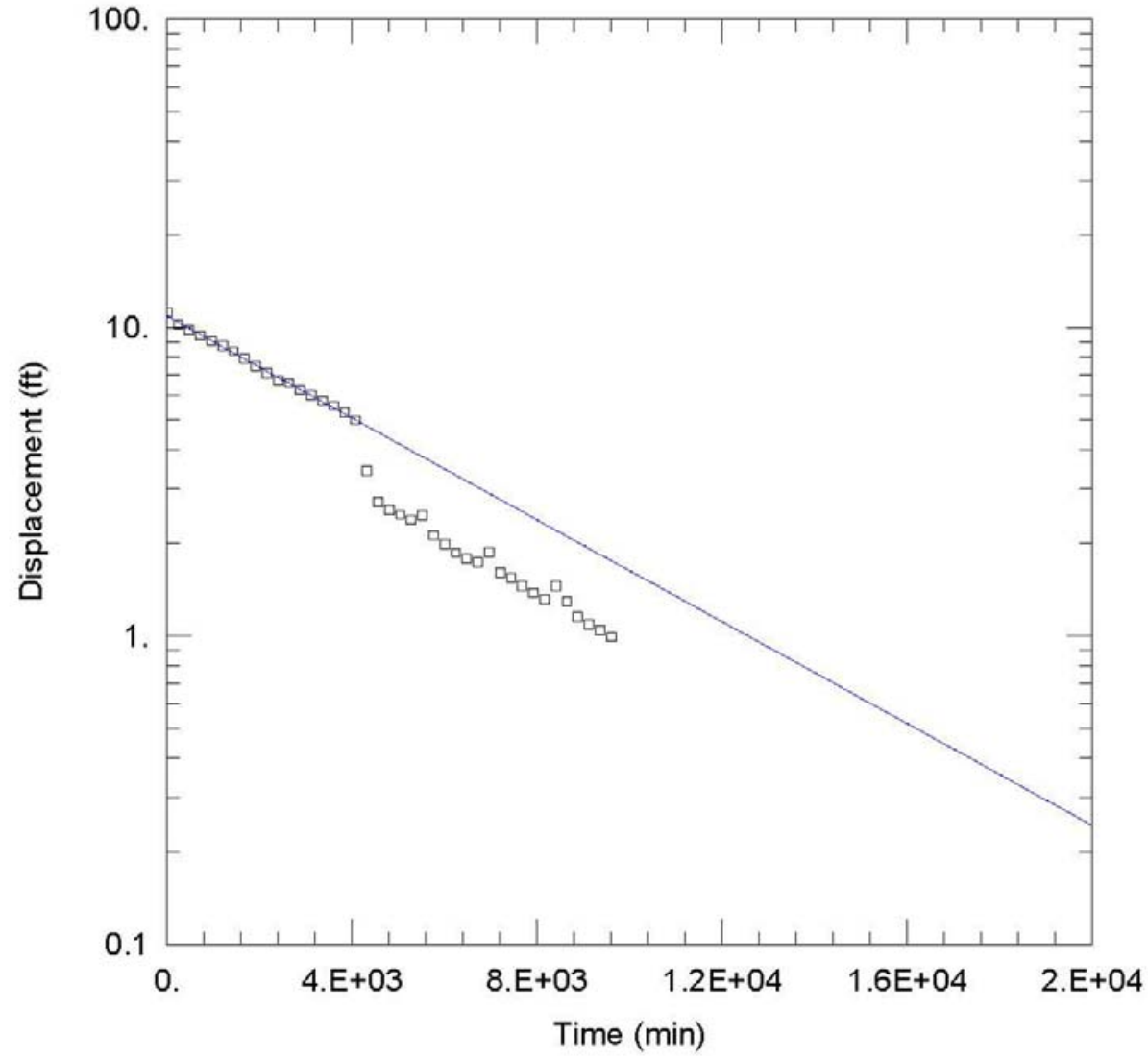

\begin{tabular}{|c|c|}
\hline \multicolumn{2}{|c|}{ WELL TEST ANALYSIS } \\
\hline \multicolumn{2}{|c|}{ PROJECT INFORMATION } \\
\hline $\begin{array}{l}\text { Company: Argonne } \\
\text { Test Location: Inman } \\
\text { Test Well: MW03 } \\
\text { Test Date: } \underline{\text { Nev. } 9,2010}\end{array}$ & \\
\hline \multicolumn{2}{|c|}{ AQUIFER DATA } \\
\hline Saturated Thickness: $\underline{2} . \mathrm{ft}$ & Anisotropy Ratio $(\mathrm{Kz} / \mathrm{Kr}): \underline{0.1}$ \\
\hline \multicolumn{2}{|c|}{ WELL DATA (MW03) } \\
\hline $\begin{array}{l}\text { Initial Displacement: } 11.17 \mathrm{ft} \\
\text { Wellbore Radius: } 0.04167 \mathrm{ft} \\
\text { Screen Length: } 10 . \mathrm{ft} \\
\text { Gravel Pack Porosity: } 0.3\end{array}$ & $\begin{array}{l}\text { Casing Radius: } \underline{0.04167} \mathrm{ft} \\
\text { Well Skin Radius: } \underline{0.08333} \mathrm{ft} \\
\text { Total Well Penetration Depth: } \underline{2 .} \mathrm{ft}\end{array}$ \\
\hline \multicolumn{2}{|c|}{ SOLUTION } \\
\hline Aquifer Model: Confined & Solution Method: Hvorslev \\
\hline$K=\underline{0.0001302} \mathrm{ft} / \mathrm{day}$ & $y 0=\underline{10.86} \mathrm{ft}$ \\
\hline
\end{tabular}

FIGURE G.6 Hvorslev analysis of recovery data for well MW03 after sampling on November 9, 2010. 


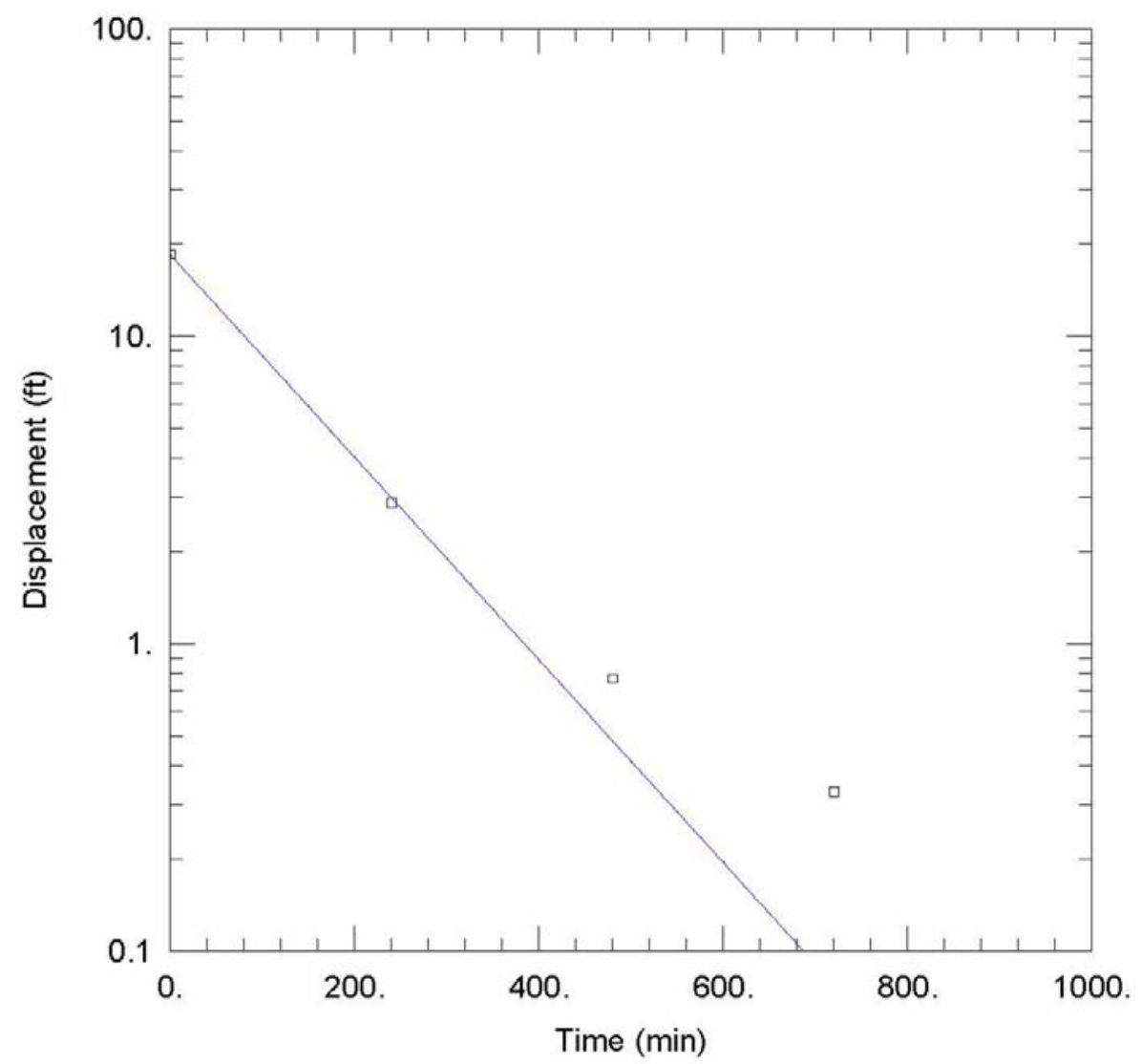

\begin{tabular}{|c|c|}
\hline \multicolumn{2}{|c|}{ WELL TEST ANALYSIS } \\
\hline \multicolumn{2}{|c|}{ PROJECT INFORMATION } \\
\hline \multicolumn{2}{|l|}{$\begin{array}{l}\text { Company: Argonne } \\
\text { Test Location: Inman } \\
\text { Test Well: MW05 } \\
\text { Test Date: } \frac{\text { Nev. } 9,2010}{}\end{array}$} \\
\hline \multicolumn{2}{|c|}{ AQUIFER DATA } \\
\hline Saturated Thickness: $\underline{2} . \mathrm{ft}$ & Anisotropy Ratio $(\mathrm{Kz} / \mathrm{Kr})$ : 1 . \\
\hline \multicolumn{2}{|c|}{ WELL DATA (MW05) } \\
\hline $\begin{array}{l}\text { Initial Displacement: } 18.44 \mathrm{ft} \\
\text { Wellbore Radius: } \underline{0.04167} \mathrm{ft} \\
\text { Screen Length: } 10 . \mathrm{ft} \\
\text { Gravel Pack Porosity: } \underline{0.3}\end{array}$ & $\begin{array}{l}\text { Casing Radius: } 0.04167 \mathrm{ft} \\
\text { Well Skin Radius: } 0.08333 \mathrm{ft} \\
\text { Total Well Penetration Depth: } \underline{2 .} \mathrm{ft}\end{array}$ \\
\hline \multicolumn{2}{|c|}{ SOLUTION } \\
\hline Aquifer Model: $\underline{\text { Confined }}$ & Solution Method: Bouwer-Rice \\
\hline$K=0.003001 \mathrm{ft} / \mathrm{day}$ & $y 0=18.43 \mathrm{ft}$ \\
\hline
\end{tabular}

FIGURE G.7 Bouwer-Rice analysis of recovery data for well MW05 after sampling on November 9, 2010. 


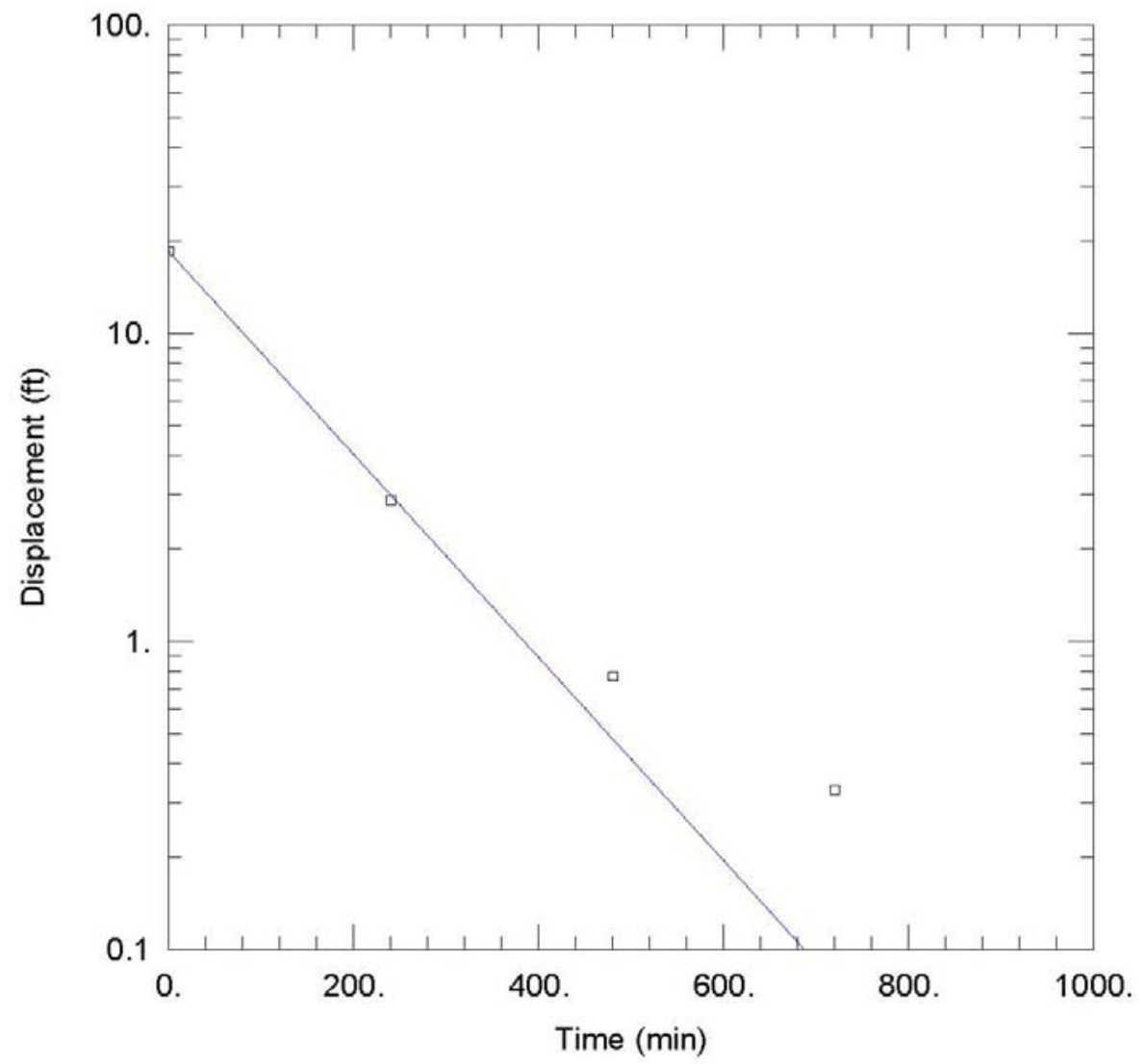

\begin{tabular}{|c|c|}
\hline \multicolumn{2}{|c|}{ WELL TEST ANALYSIS } \\
\hline \multicolumn{2}{|c|}{ PROJECT INFORMATION } \\
\hline \multicolumn{2}{|l|}{ 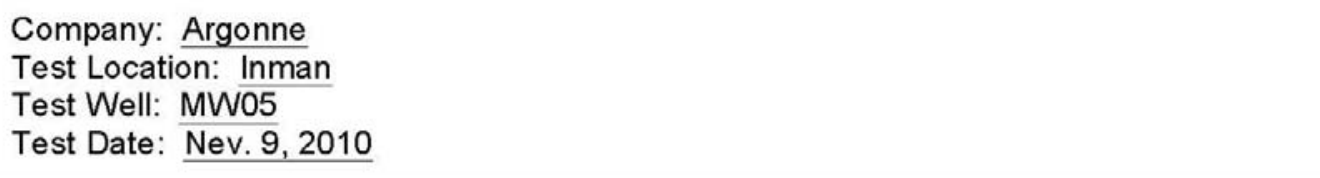 } \\
\hline \multicolumn{2}{|c|}{ AQUIFER DATA } \\
\hline Saturated Thickness: $\underline{2} . \mathrm{ft}$ & Anisotropy Ratio $(\mathrm{Kz} / \mathrm{Kr}): \underline{1}$ \\
\hline \multicolumn{2}{|c|}{ WELL DATA (MW05) } \\
\hline $\begin{array}{l}\text { Initial Displacement: } 18.44 \mathrm{ft} \\
\text { Wellbore Radius: } \underline{0.04167} \mathrm{ft} \\
\text { Screen Length: } \underline{10 . \mathrm{ft}} \\
\text { Gravel Pack Porosity: } \underline{0.3}\end{array}$ & $\begin{array}{l}\text { Casing Radius: } 0.04167 \mathrm{ft} \\
\text { Well Skin Radius: } \underline{0.08333} \mathrm{ft} \\
\text { Total Well Penetration Depth: } \underline{2 .} \mathrm{ft}\end{array}$ \\
\hline \multicolumn{2}{|c|}{ SOLUTION } \\
\hline Aquifer Model: Confined & Solution Method: Hvorslev \\
\hline$K=\underline{0.005199} \mathrm{ft} / \mathrm{day}$ & $y 0=18.43 \mathrm{ft}$ \\
\hline
\end{tabular}

FIGURE G.8 Hvorslev analysis of recovery data for well MW05 after sampling on November 9, 2010. 


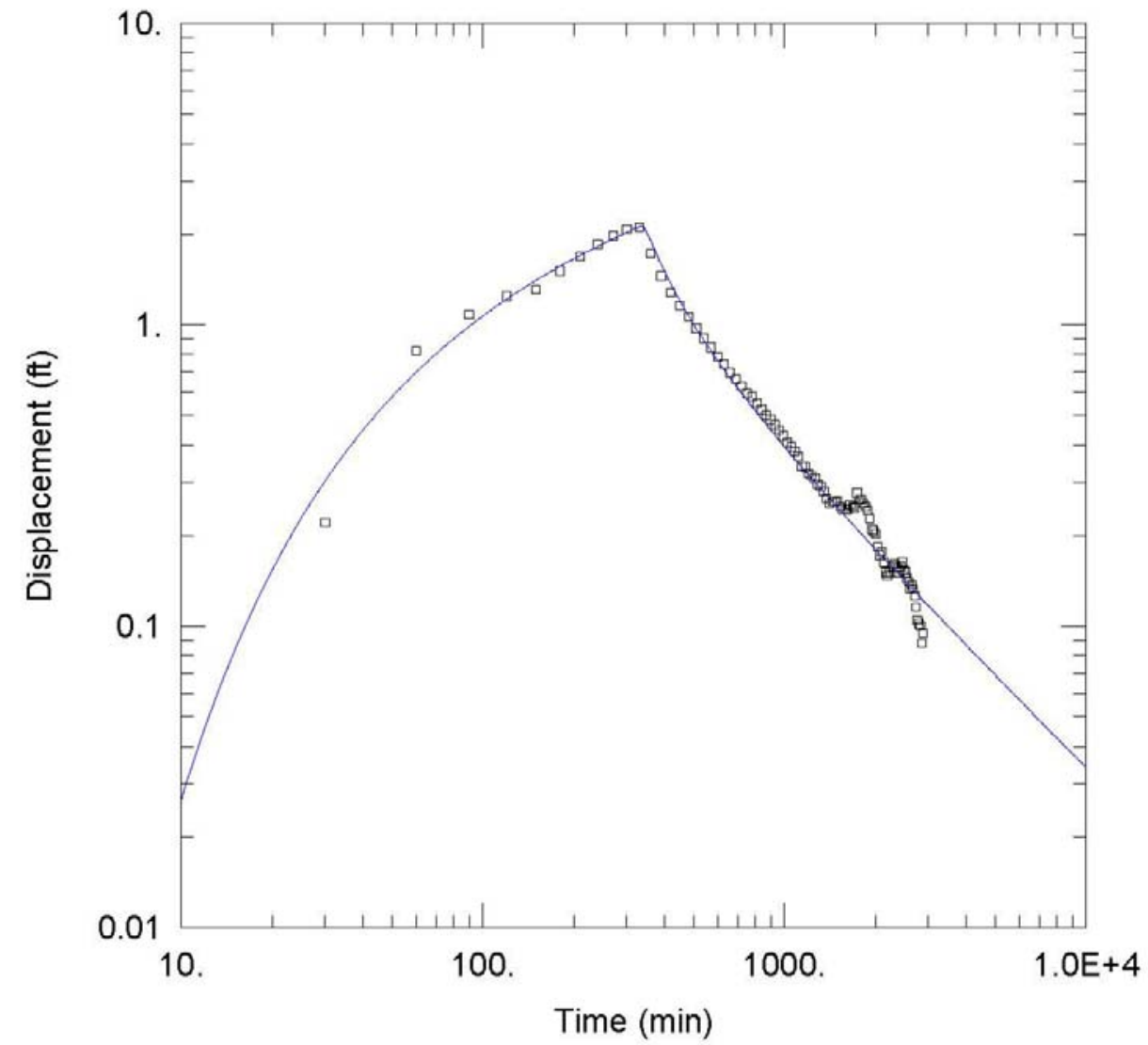

\begin{tabular}{|c|c|c|c|c|c|}
\hline \multicolumn{6}{|c|}{ PRIVATE WELL PUMPING DATA ANALYSIS } \\
\hline \multicolumn{6}{|c|}{ PROJECT INFORMATION } \\
\hline \multicolumn{6}{|c|}{$\begin{array}{l}\text { Company: Argonne } \\
\text { Location: Inman } \\
\text { Test Well: Sisson } \\
\text { Test Date: Oct. 7, } 2011\end{array}$} \\
\hline \multicolumn{6}{|c|}{ WELL DATA } \\
\hline \multicolumn{3}{|c|}{ Pumping Wells } & \multicolumn{3}{|c|}{ Observation Wells } \\
\hline Well Name & $X(\mathrm{ft})$ & $\mathrm{Y}(\mathrm{ft})$ & Well Name & $X(\mathrm{ft})$ & $\mathrm{Y}(\mathrm{ft})$ \\
\hline Sisson $0.05 \mathrm{gpm}$ & 0 & 0 & MW18S & 4.94 & 56.9 \\
\hline \multicolumn{6}{|c|}{ SOLUTION } \\
\hline \multicolumn{3}{|c|}{ Aquifer Model: Confined } & \multicolumn{3}{|c|}{ Solution Method: Theis } \\
\hline \multicolumn{2}{|c|}{$\begin{array}{l}T=5.673 \mathrm{ft}^{2} / \text { day } \\
\mathrm{Kz} / \mathrm{Kr}=0.1\end{array}$} & & \multicolumn{2}{|c|}{$\begin{array}{l}=0.0001186 \\
=2 \mathrm{ft}\end{array}$} & \\
\hline
\end{tabular}

FIGURE G.9 Theis interpretive curve fit for shallow zone well MW18S during drawdown and recovery after the start of pumping at the Sisson well on October 7, 2011, with assumed pumping at the Sisson well at $1 \mathrm{gpm}, 5 \%$ of the pumping rate exerted on the shallow zone, and withdrawal from the shallow groundwater zone at $0.05 \mathrm{gpm}$. 


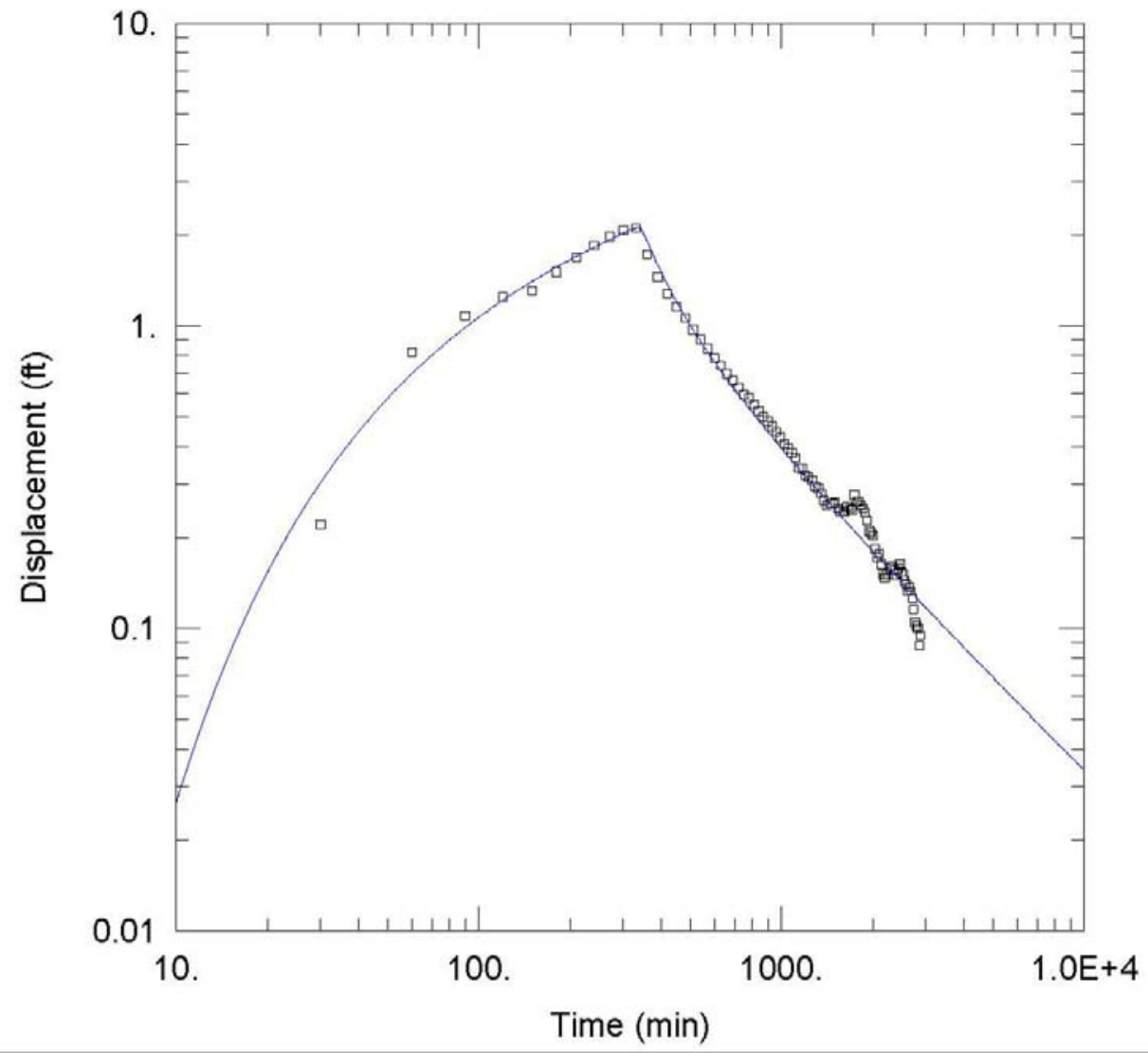

\begin{tabular}{|c|c|c|c|c|c|}
\hline \multicolumn{6}{|c|}{ PRIVATE WELL PUMPING DATA ANALYSIS } \\
\hline \multicolumn{6}{|c|}{ PROJECT INFORMATION } \\
\hline \multicolumn{6}{|c|}{$\begin{array}{l}\text { Company: Argonne } \\
\text { Location: Inman } \\
\text { Test Well: Sisson } \\
\text { Test Date: Oct. 7, } 2011\end{array}$} \\
\hline \multicolumn{6}{|c|}{ WELL DATA } \\
\hline \multicolumn{3}{|c|}{ Pumping Wells } & \multicolumn{3}{|c|}{ Observation Wells } \\
\hline Well Name & $X(\mathrm{ft})$ & $\mathrm{Y}(\mathrm{ft})$ & Well Name & $X(\mathrm{ft})$ & $\mathrm{Y}(\mathrm{ft})$ \\
\hline Sisson $0.1 \mathrm{gpm}$ & 0 & 0 & MW18S & 4.94 & 56.9 \\
\hline \multicolumn{6}{|c|}{ SOLUTION } \\
\hline \multicolumn{3}{|c|}{ Aquifer Model: Confined } & \multicolumn{3}{|c|}{ Solution Method: Theis } \\
\hline \multicolumn{2}{|c|}{$\begin{array}{l}T=11.35 \mathrm{ft}^{2} / \mathrm{day} \\
\mathrm{Kz} / \mathrm{Kr}=\underline{0.1}\end{array}$} & & \multicolumn{2}{|c|}{$\begin{array}{ll}\mathrm{S} & =0.0002373 \\
\mathrm{~b} & =\underline{2 . \mathrm{ft}}\end{array}$} & \\
\hline
\end{tabular}

FIGURE G.10 Theis interpretive curve fit for shallow zone well MW18S during drawdown and recovery after the start of pumping at the Sisson well on October 7, 2011, with assumed pumping at the Sisson well at $1 \mathrm{gpm}, 10 \%$ of the pumping rate exerted on the shallow zone, and withdrawal from the shallow groundwater zone at $0.1 \mathrm{gpm}$. 


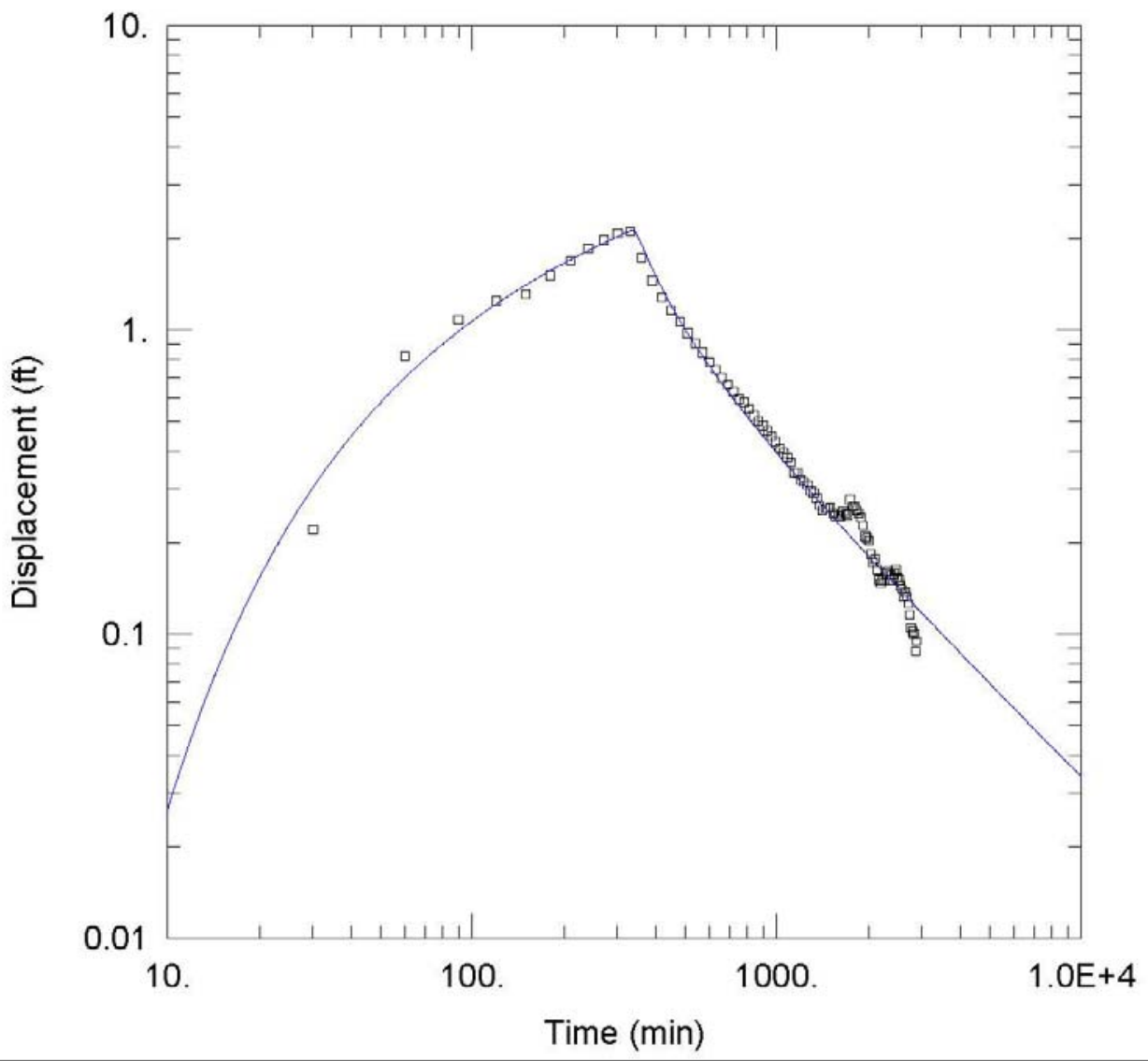

\begin{tabular}{|c|c|c|c|c|c|}
\hline \multicolumn{6}{|c|}{ PRIVATE WELL PUMPING DATA ANALYSIS } \\
\hline \multicolumn{6}{|c|}{ PROJECT INFORMATION } \\
\hline \multicolumn{6}{|c|}{$\begin{array}{l}\text { Company: Argonne } \\
\text { Location: Inman } \\
\text { Test Well: Sisson } \\
\text { Test Date: Oct. } 7,2011\end{array}$} \\
\hline \multicolumn{6}{|c|}{ WELL DATA } \\
\hline \multicolumn{3}{|c|}{ Pumping Wells } & \multicolumn{3}{|c|}{ Observation Wells } \\
\hline Well Name & $X(\mathrm{ft})$ & $Y(\mathrm{ft})$ & Well Name & $X(\mathrm{ft})$ & $\mathrm{Y}(\mathrm{ft})$ \\
\hline Sisson $0.3 \mathrm{gpm}$ & 0 & 0 & - MW18S & 4.94 & 56.9 \\
\hline \multicolumn{6}{|c|}{ SOLUTION } \\
\hline \multicolumn{3}{|c|}{ Aquifer Model: Confined } & \multicolumn{3}{|c|}{ Solution Method: Theis } \\
\hline \multicolumn{2}{|c|}{$\begin{array}{l}\mathrm{T}=34.04 \mathrm{ft}^{2} / \text { day } \\
\mathrm{Kz} / \mathrm{Kr}=0.1\end{array}$} & & \multicolumn{2}{|c|}{$\begin{aligned} \mathrm{S} & =0.0007119 \\
\mathrm{~b} & =2 \mathrm{ft}\end{aligned}$} & \\
\hline
\end{tabular}

FIGURE G.11 Theis interpretive curve fit for shallow zone well MW18S during drawdown and recovery after the start of pumping at the Sisson well on October 7, 2011, with assumed pumping at the Sisson well at $1 \mathrm{gpm}, 30 \%$ of the pumping rate exerted on the shallow zone, and withdrawal from the shallow groundwater zone at $0.3 \mathrm{gpm}$. 


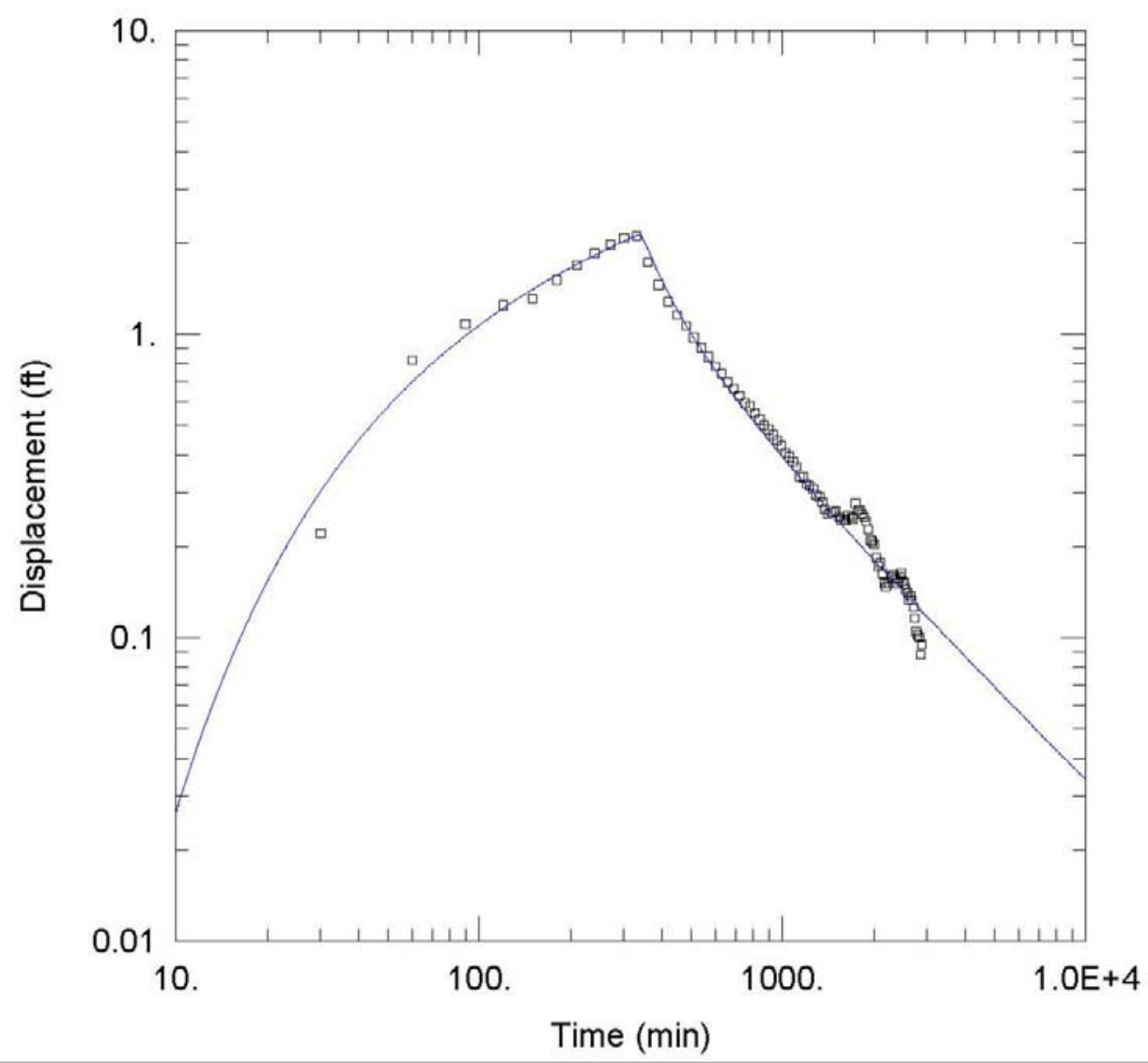

\begin{tabular}{|c|c|c|c|c|c|}
\hline \multicolumn{6}{|c|}{ PRIVATE WELL PUMPING DATA ANALYSIS } \\
\hline \multicolumn{6}{|c|}{ PROJECT INFORMATION } \\
\hline \multicolumn{6}{|c|}{$\begin{array}{l}\text { Company: Argonne } \\
\text { Location: Inman } \\
\text { Test Well: } \frac{\text { Sisson }}{\text { Test Date: }} \text { Oct. 7, } 2011\end{array}$} \\
\hline \multicolumn{6}{|c|}{ WELL DATA } \\
\hline \multicolumn{3}{|c|}{ Pumping Wells } & \multicolumn{3}{|c|}{ Observation Wells } \\
\hline Well Name & $X(\mathrm{ft})$ & $\mathrm{Y}(\mathrm{ft})$ & Well Name & $X(\mathrm{ft})$ & $Y(\mathrm{ft})$ \\
\hline Sisson $0.5 \mathrm{gpm}$ & 0 & 0 & MW18S & 4.94 & 56.9 \\
\hline \multicolumn{6}{|c|}{ SOLUTION } \\
\hline \multicolumn{3}{|c|}{ Aquifer Model: Confined } & \multicolumn{3}{|c|}{ Solution Method: Theis } \\
\hline \multicolumn{2}{|c|}{$\begin{aligned} T & =56.73 \mathrm{ft}^{2} / \text { day } \\
\mathrm{Kz} / \mathrm{Kr} & =\underline{0.1}\end{aligned}$} & & \multicolumn{2}{|c|}{$\begin{array}{ll}\mathrm{S} & =0.001186 \\
\mathrm{~b} & =2 \mathrm{ft}\end{array}$} & \\
\hline
\end{tabular}

FIGURE G.12 Theis interpretive curve fit for shallow zone well MW18S during drawdown and recovery after the start of pumping at the Sisson well on October 7, 2011, with assumed pumping at the Sisson well at $10 \mathrm{gpm}, 5 \%$ of the pumping rate exerted on the shallow zone, and withdrawal from the shallow groundwater zone at $0.5 \mathrm{gpm}$. 


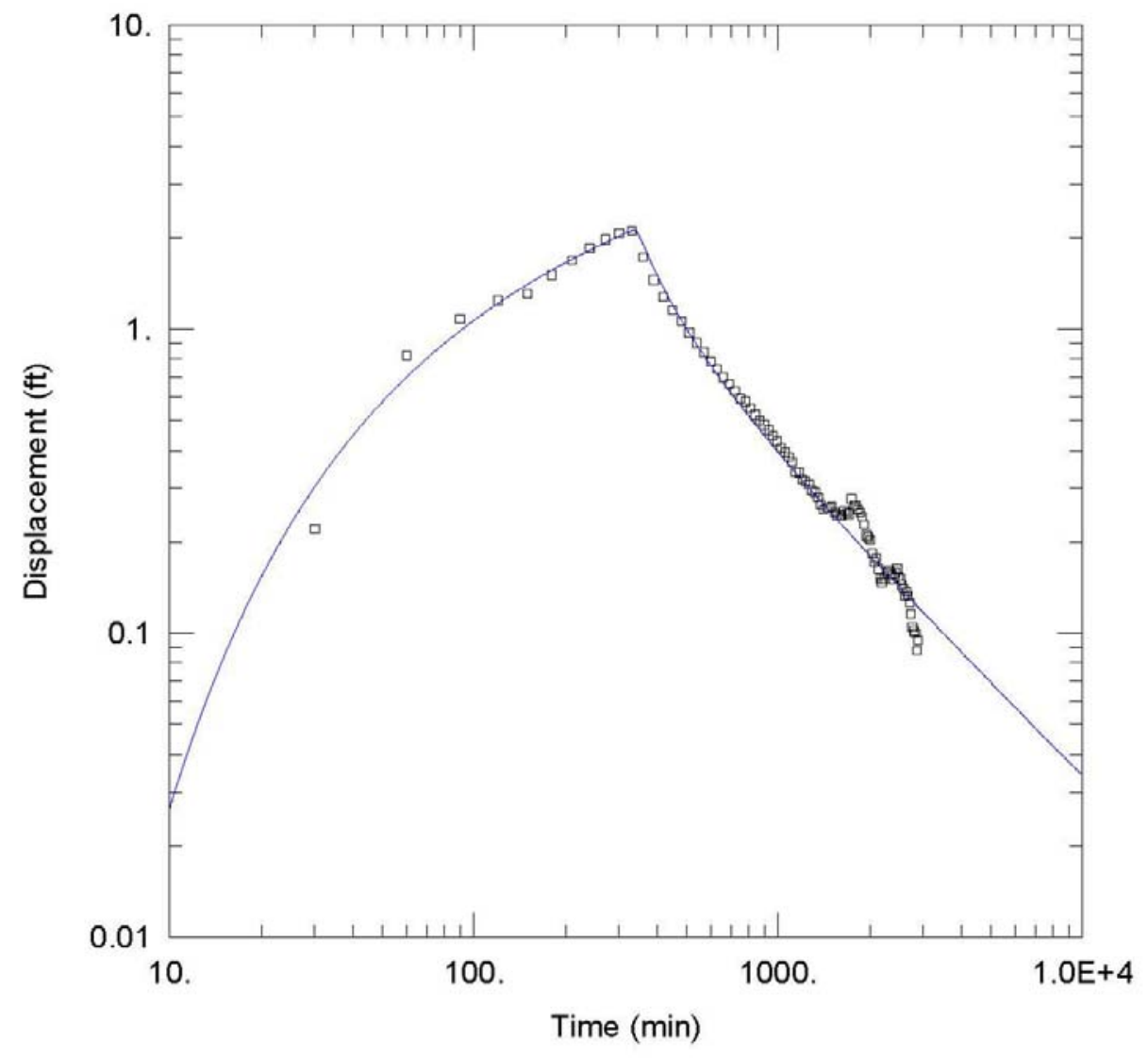

PRIVATE WELL PUMPING DATA ANALYSIS

PROJECT INFORMATION

Company: Argonne

Location: Inman

Test Well: Sisson

Test Date: Oct. 7, 2011

WELL DATA

Pumping Wells

\begin{tabular}{|l|c|c|}
\hline Well Name & $\mathrm{X}(\mathrm{ft})$ & $\mathrm{Y}(\mathrm{ft})$ \\
\hline Sisson $1.0 \mathrm{gpm}$ & 0 & 0 \\
\hline
\end{tabular}

\begin{tabular}{|l}
\hline Well \\
$\square$ MV \\
\hline TION \\
\hline
\end{tabular}

\section{SOLUTION}

Aquifer Model: Confined

Solution Method: Theis

$T=113.5 \mathrm{ft}^{2} /$ day

$\mathrm{Kz} / \mathrm{Kr}=0.1$

$\begin{aligned} \mathrm{S} & =0.002373 \\ \mathrm{~b} & =\underline{2 . \mathrm{ft}}\end{aligned}$

FIGURE G.13 Theis interpretive curve fit for shallow zone well MW18S during drawdown and recovery after the start of pumping at the Sisson well on October 7, 2011, with assumed pumping at the Sisson well at $10 \mathrm{gpm}, 10 \%$ of the pumping rate exerted on the shallow zone, and withdrawal from the shallow groundwater zone at $1.0 \mathrm{gpm}$. 


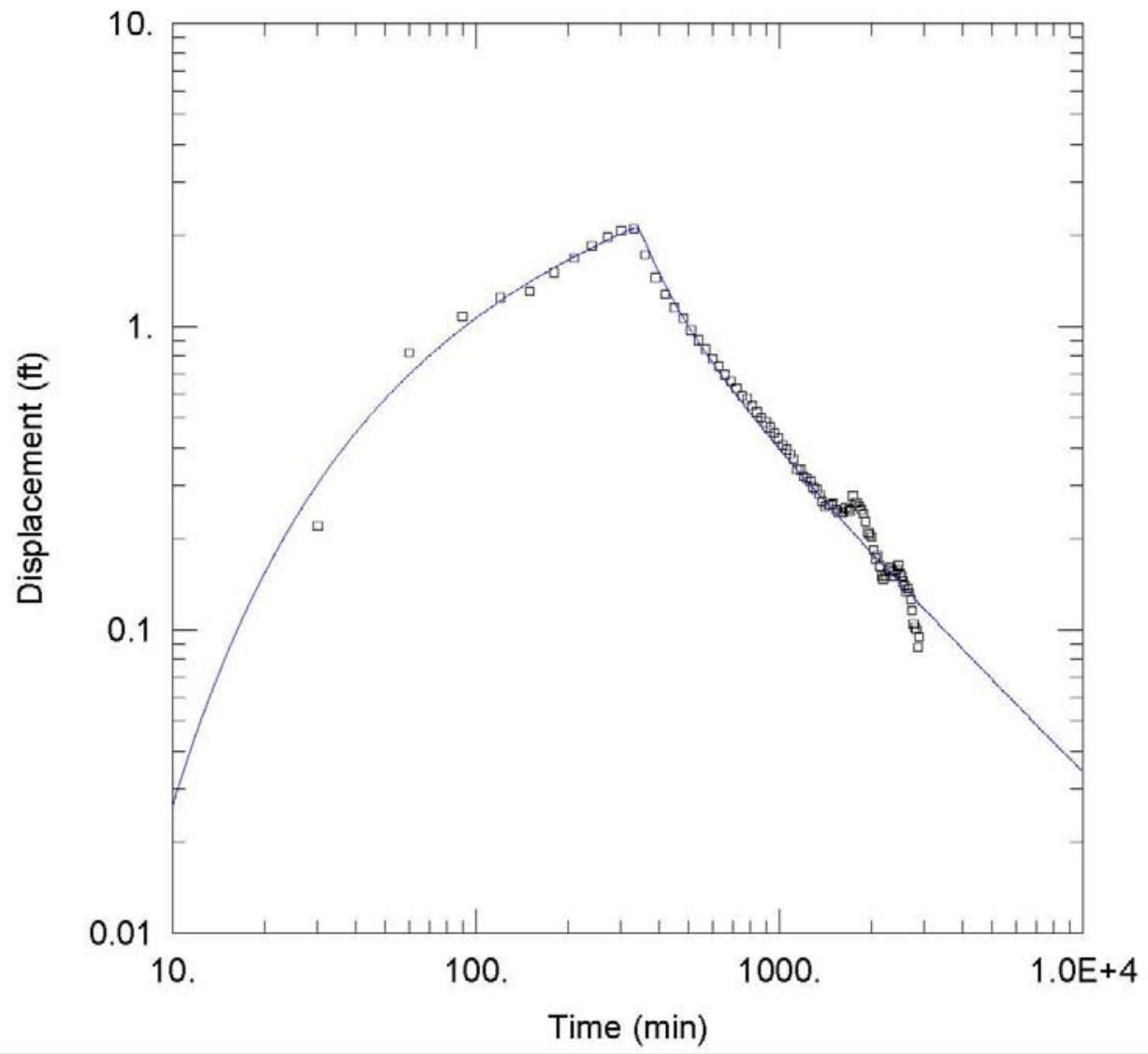

PRIVATE WELL PUMPING DATA ANALYSIS

PROJECT INFORMATION

Company: Argonne

Location: Inman

Test Well: Sisson

Test Date: $\underline{\text { Oct. 7, } 2011}$

WELL DATA

Pumping Wells

\begin{tabular}{|l|c|c|}
\hline Well Name & $X(\mathrm{ft})$ & $\mathrm{Y}(\mathrm{ft})$ \\
\hline Sisson $3.0 \mathrm{gpm}$ & 0 & 0 \\
\hline
\end{tabular}

Observation Wells

\begin{tabular}{|l|c|c|}
\hline Well Name & $\mathrm{X}(\mathrm{ft})$ & $\mathrm{Y}(\mathrm{ft})$ \\
\hline 口 MW18S & 4.94 & 56.9 \\
\hline
\end{tabular}

SOLUTION

Aquifer Model: Confined

Solution Method: Theis

$T \quad=340.4 \mathrm{ft}^{2} /$ day

$\mathrm{Kz} / \mathrm{Kr}=\underline{0.1}$

$S \quad=0.007119$

$\mathrm{b} \quad \underline{2 . \mathrm{ft}}$

FIGURE G.14 Theis interpretive curve fit for shallow zone well MW18S during drawdown and recovery after the start of pumping at the Sisson well on October 7, 2011, with assumed pumping at the Sisson well at $10 \mathrm{gpm}, 30 \%$ of the pumping rate exerted on the shallow zone, and withdrawal from the shallow groundwater zone at $3.0 \mathrm{gpm}$. 


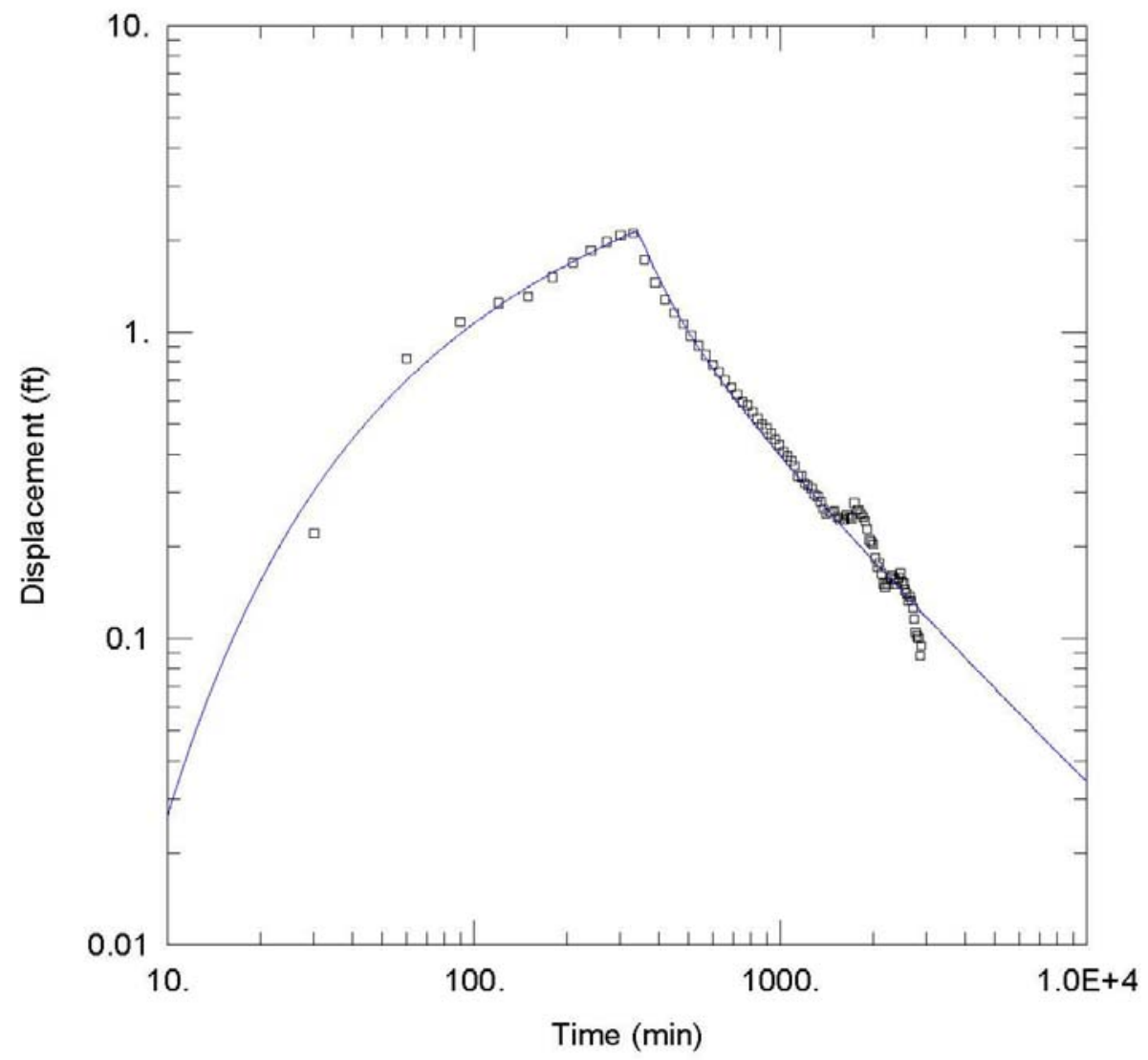

\begin{tabular}{|c|c|c|c|c|c|}
\hline \multicolumn{6}{|c|}{ PRIVATE WELL PUMPING DATA ANALYSIS } \\
\hline \multicolumn{6}{|c|}{ PROJECT INFORMATION } \\
\hline \multicolumn{6}{|c|}{$\begin{array}{l}\text { Company: Argonne } \\
\text { Location: Inman } \\
\text { Test Well: Sisson } \\
\text { Test Date: } \text { Oct. } 7,2011\end{array}$} \\
\hline \multicolumn{6}{|c|}{ WELL DATA } \\
\hline \multicolumn{3}{|c|}{ Pumping Wells } & \multicolumn{3}{|c|}{ Observation Wells } \\
\hline Well Name & $X(\mathrm{ft})$ & $Y(\mathrm{ft})$ & Well Name & $X(\mathrm{ft})$ & $Y(\mathrm{ft})$ \\
\hline Sisson $1.0 \mathrm{gpm}$ & 0 & 0 & - MW18S & 4.94 & 56.9 \\
\hline \multicolumn{6}{|c|}{ SOLUTION } \\
\hline \multicolumn{3}{|c|}{ Aquifer Model: Confined } & \multicolumn{3}{|c|}{ Solution Method: Theis } \\
\hline \multicolumn{3}{|c|}{$\begin{aligned} T & =113.5 \mathrm{ft}^{2} / \text { day } \\
\mathrm{Kz} / \mathrm{Kr} & =0.1\end{aligned}$} & \multicolumn{2}{|c|}{$S \quad=0.002373$} & \\
\hline
\end{tabular}

FIGURE G.15 Theis interpretive curve fit for shallow zone well MW18S during drawdown and recovery after the start of pumping at the Sisson well on October 7, 2011, with assumed pumping at the Sisson well at $20 \mathrm{gpm}, 5 \%$ of the pumping rate exerted on the shallow zone, and withdrawal from the shallow groundwater zone at $1.0 \mathrm{gpm}$. 


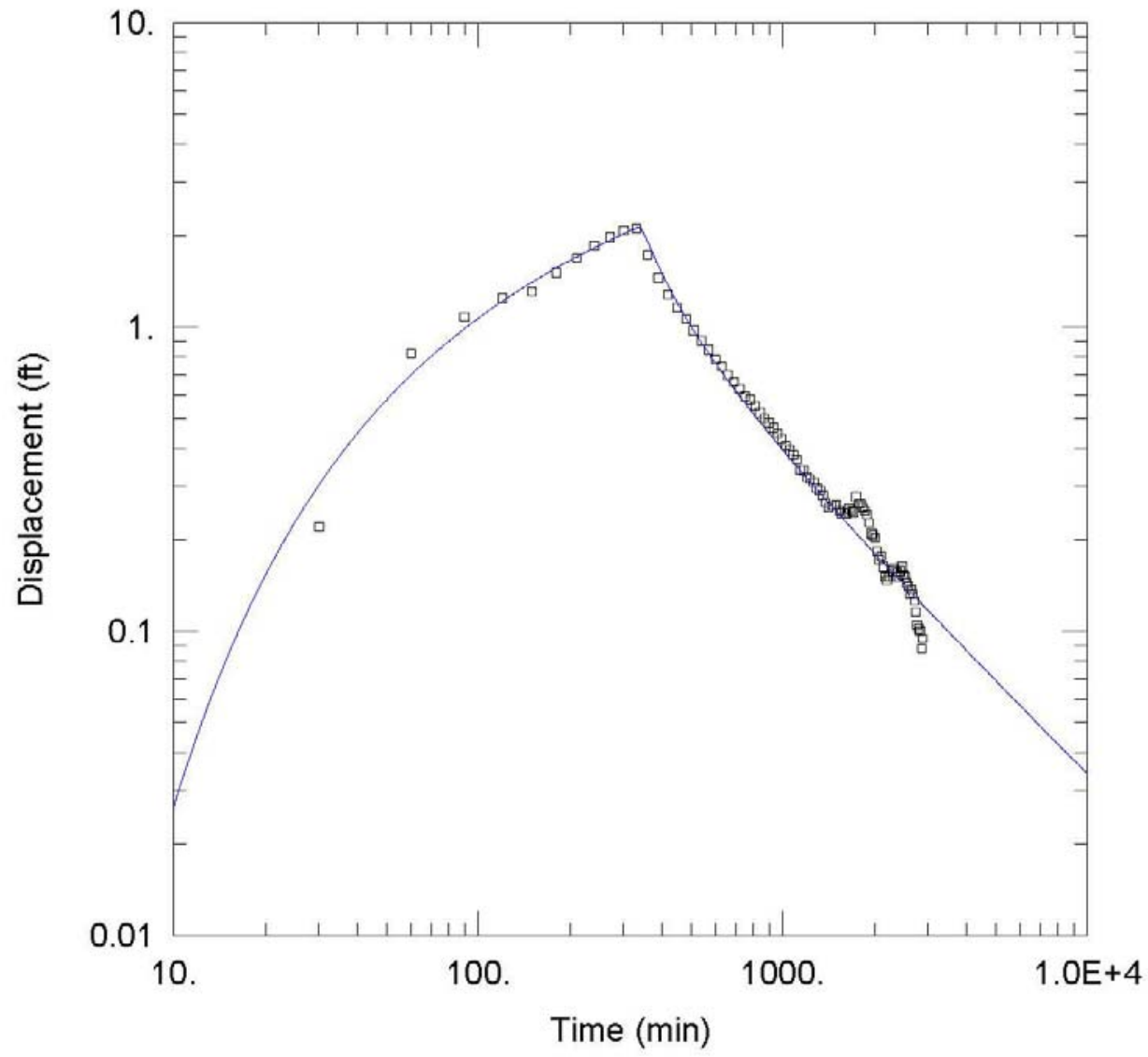

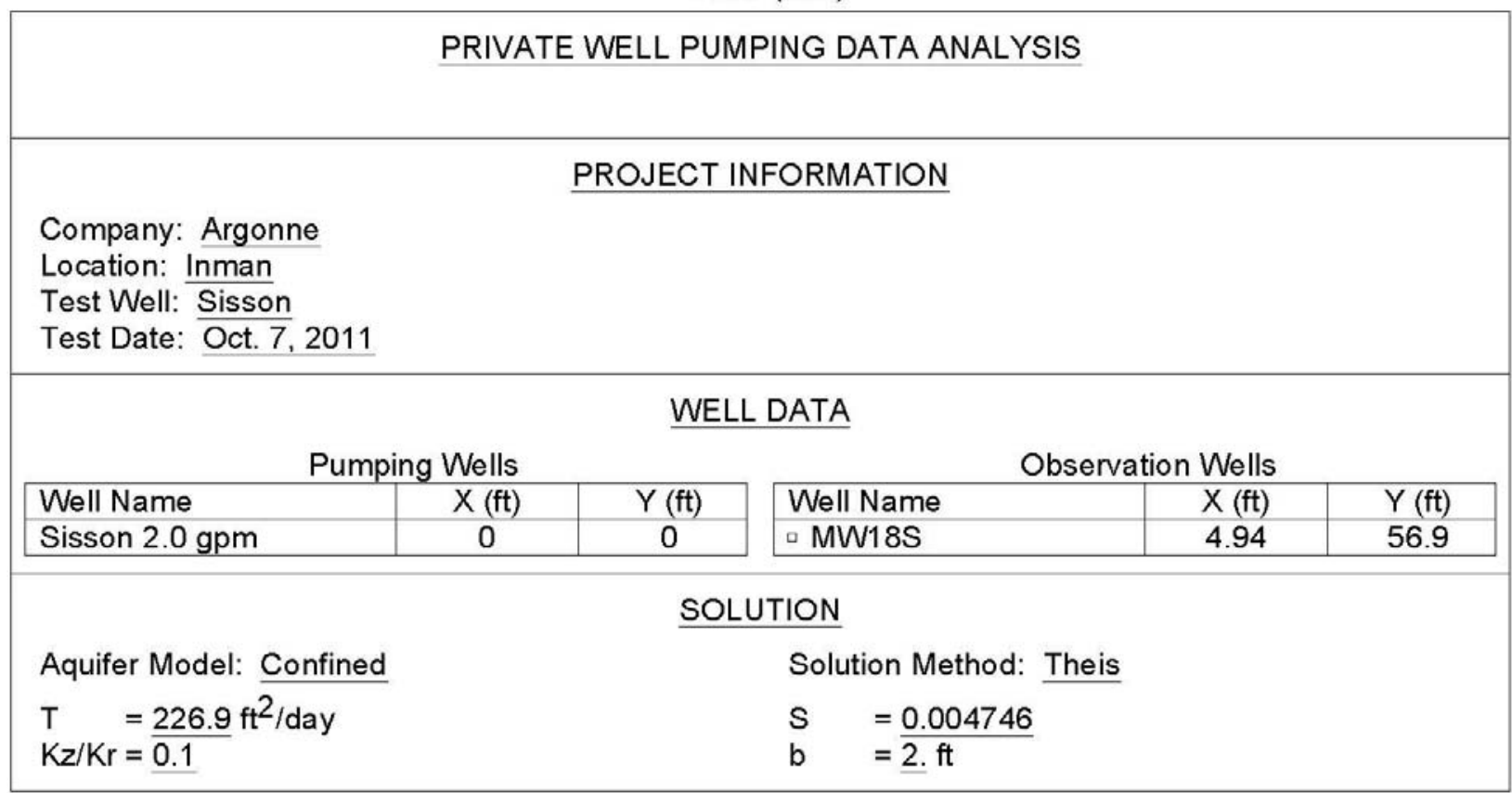

FIGURE G.16 Theis interpretive curve fit for shallow zone well MW18S during drawdown and recovery after the start of pumping at the Sisson well on October 7, 2011, with assumed pumping at the Sisson well at $20 \mathrm{gpm}, 10 \%$ of the pumping rate exerted on the shallow zone, and withdrawal from the shallow groundwater zone at $2.0 \mathrm{gpm}$. 


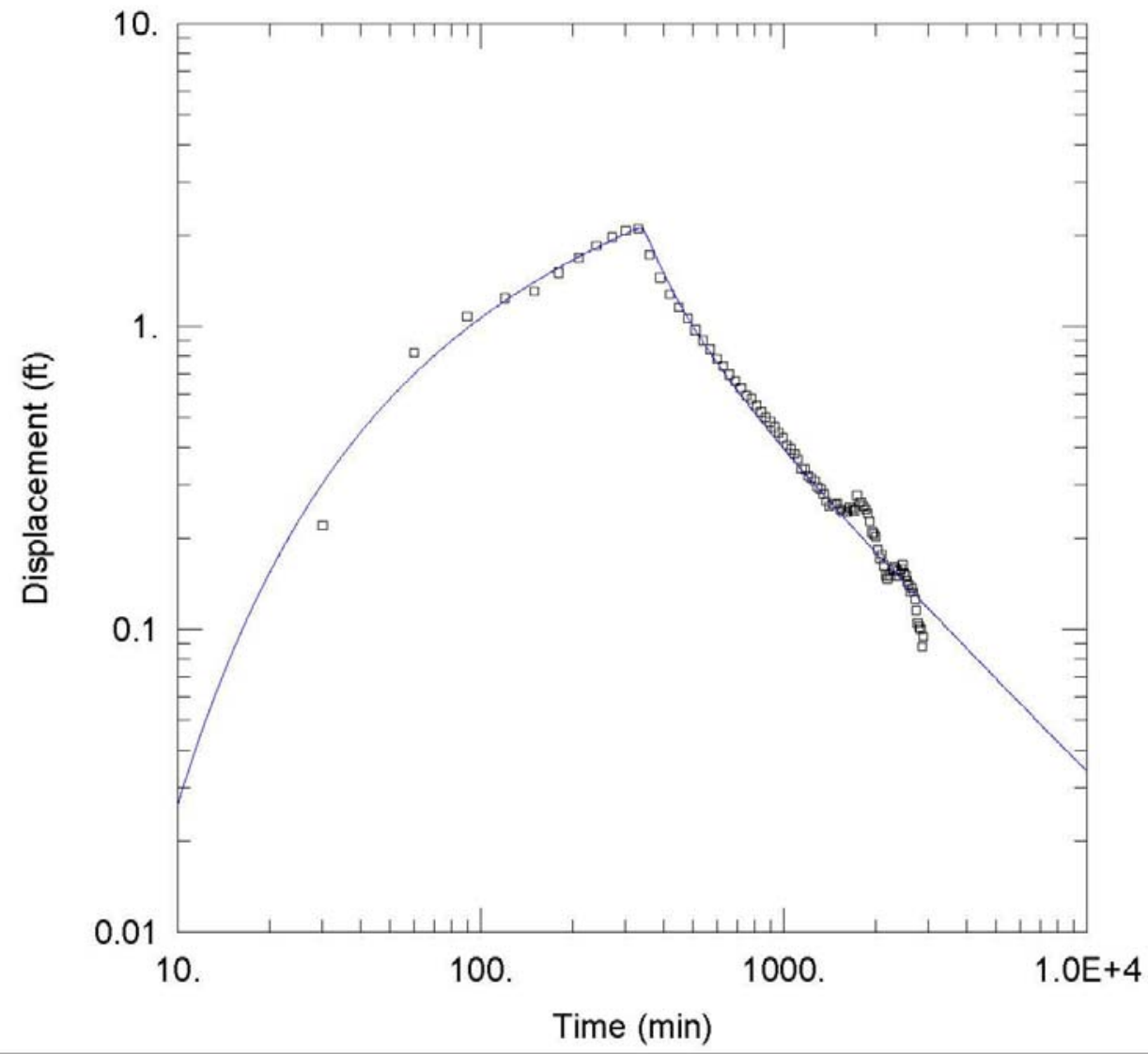

\begin{tabular}{|c|c|c|c|c|c|}
\hline \multicolumn{6}{|c|}{ PRIVATE WELL PUMPING DATA ANALYSIS } \\
\hline \multicolumn{6}{|c|}{ PROJECT INFORMATION } \\
\hline \multicolumn{6}{|c|}{$\begin{array}{l}\text { Company: Argonne } \\
\text { Location: Inman } \\
\text { Test Well: Sisson } \\
\text { Test Date: } \text { Oct. 7, } 2011\end{array}$} \\
\hline \multicolumn{6}{|c|}{ WELL DATA } \\
\hline \multicolumn{3}{|c|}{ Pumping Wells } & \multicolumn{3}{|c|}{ Observation Wells } \\
\hline Well Name & $X(\mathrm{ft})$ & $Y(\mathrm{ft})$ & Well Name & $X(\mathrm{ft})$ & $Y(\mathrm{ft})$ \\
\hline Sisson $6.0 \mathrm{gpm}$ & 0 & 0 & MW18S & 4.94 & 56.9 \\
\hline \multicolumn{6}{|c|}{ SOLUTION } \\
\hline \multicolumn{2}{|c|}{ Aquifer Model: Confined } & & \multicolumn{3}{|c|}{ Solution Method: Theis } \\
\hline \multicolumn{2}{|c|}{$\begin{array}{l}\mathrm{T}=680.8 \mathrm{ft}^{2} / \text { day } \\
\mathrm{Kz} / \mathrm{Kr}=\underline{0.1}\end{array}$} & & \multicolumn{2}{|c|}{$\begin{array}{ll}\mathrm{S} & =0.01424 \\
\mathrm{~b} & =\underline{\mathrm{2}} \mathrm{ft}\end{array}$} & \\
\hline
\end{tabular}

FIGURE G.17 Theis interpretive curve fit for shallow zone well MW18S during drawdown and recovery after the start of pumping at the Sisson well on October 7, 2011, with assumed pumping at the Sisson well at $20 \mathrm{gpm}, 30 \%$ of the pumping rate exerted on the shallow zone, and withdrawal from the shallow groundwater zone at $6.0 \mathrm{gpm}$. 


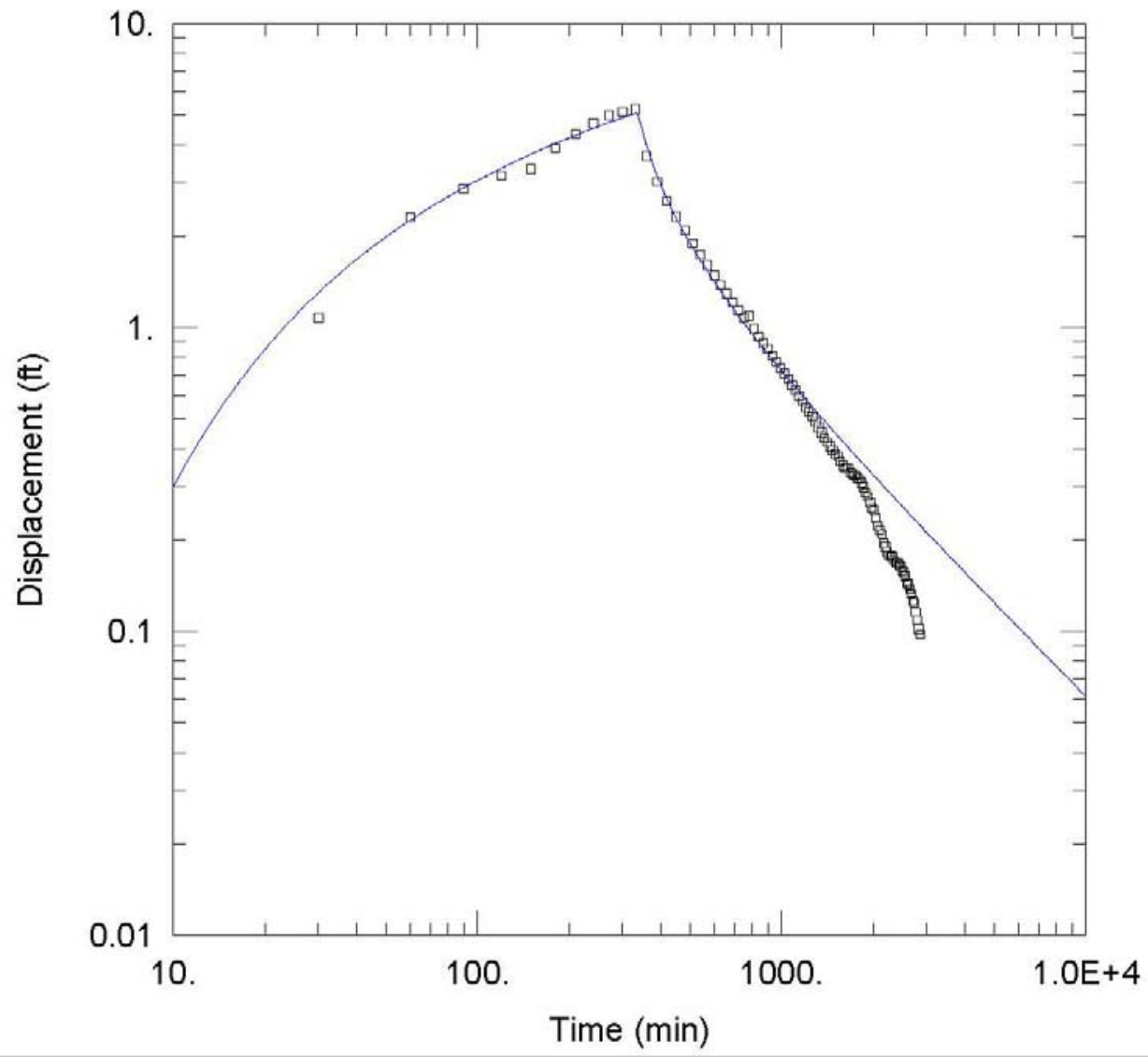

\begin{tabular}{|c|c|c|c|c|c|}
\hline \multicolumn{6}{|c|}{ PRIVATE WELL PUMPING DATA ANALYSIS } \\
\hline \multicolumn{6}{|c|}{ PROJECT INFORMATION } \\
\hline \multicolumn{6}{|c|}{$\begin{array}{l}\text { Company: Argonne } \\
\text { Location: Inman } \\
\text { Test Well: Sisson } \\
\text { Test Date: Oct. 7, } 2011\end{array}$} \\
\hline \multicolumn{6}{|c|}{ WELL DATA } \\
\hline \multicolumn{3}{|c|}{ Pumping Wells } & \multicolumn{3}{|c|}{ Observation Wells } \\
\hline Well Name & $X(\mathrm{ft})$ & $Y(\mathrm{ft})$ & Well Name & $X(\mathrm{ft})$ & $Y(\mathrm{ft})$ \\
\hline Sisson $0.95 \mathrm{gpm}$ & 0 & 0 & - MW18M & 5.03 & 56.8 \\
\hline \multicolumn{6}{|c|}{ SOLUTION } \\
\hline \multicolumn{3}{|c|}{ Aquifer Model: Confined } & \multicolumn{3}{|c|}{ Solution Method: Theis } \\
\hline $\begin{aligned} T & =59.85 \mathrm{ft}^{2} / \\
\mathrm{Kz} / \mathrm{Kr} & =\underline{0.1}\end{aligned}$ & & & $\begin{array}{ll}\mathrm{S} & =0.00 \\
\mathrm{~b} & =2 . \mathrm{ft}\end{array}$ & & \\
\hline
\end{tabular}

FIGURE G.18 Theis interpretive curve fit for shallow zone well MW18M during drawdown and recovery after the start of pumping at the Sisson well on October 7, 2011, with assumed pumping at the Sisson well at $1 \mathrm{gpm}, 95 \%$ of the pumping rate exerted on the shallow zone, and withdrawal from the shallow groundwater zone at $0.95 \mathrm{gpm}$. 


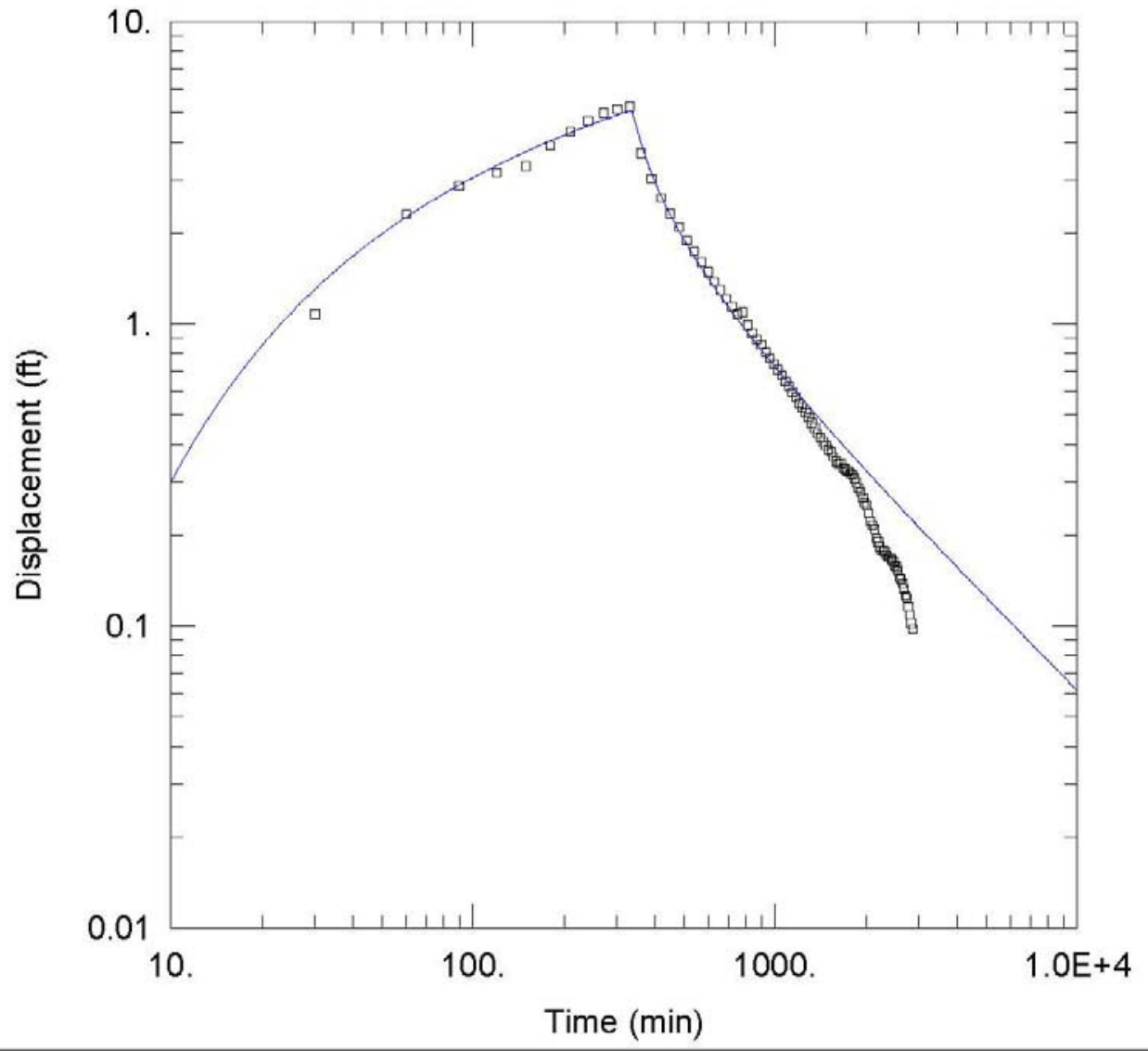

PRIVATE WELL PUMPING DATA ANALYSIS

PROJECT INFORMATION

Company: Argonne

Location: Inman

Test Well: Sisson

Test Date: Oct. 7, 2011

WELL DATA

Pumping Wells

\begin{tabular}{|l|c|c|}
\hline Well Name & $X(\mathrm{ft})$ & $\mathrm{Y}(\mathrm{ft})$ \\
\hline Sisson $0.9 \mathrm{gpm}$ & 0 & 0 \\
\hline
\end{tabular}

Observation Wells

\begin{tabular}{|l|c|c|}
\hline Well Name & $X(f t)$ & $Y(f t)$ \\
\hline$\square$ MW18M & 5.03 & 56.8 \\
\hline
\end{tabular}

\section{SOLUTION}

Aquifer Model: Confined

Solution Method: Theis

$T \quad=\underline{56.7} \mathrm{ft}^{2} /$ day

$\mathrm{Kz} / \mathrm{Kr}=\underline{0.1}$

$S \quad=0.0005699$

b $\quad=\underline{\text {. }} \mathrm{ft}$

FIGURE G.19 Theis interpretive curve fit for shallow zone well MW18M during drawdown and recovery after the start of pumping at the Sisson well on October 7, 2011, with assumed pumping at the Sisson well at $1 \mathrm{gpm}, 90 \%$ of the pumping rate exerted on the shallow zone, and withdrawal from the shallow groundwater zone at $0.90 \mathrm{gpm}$. 


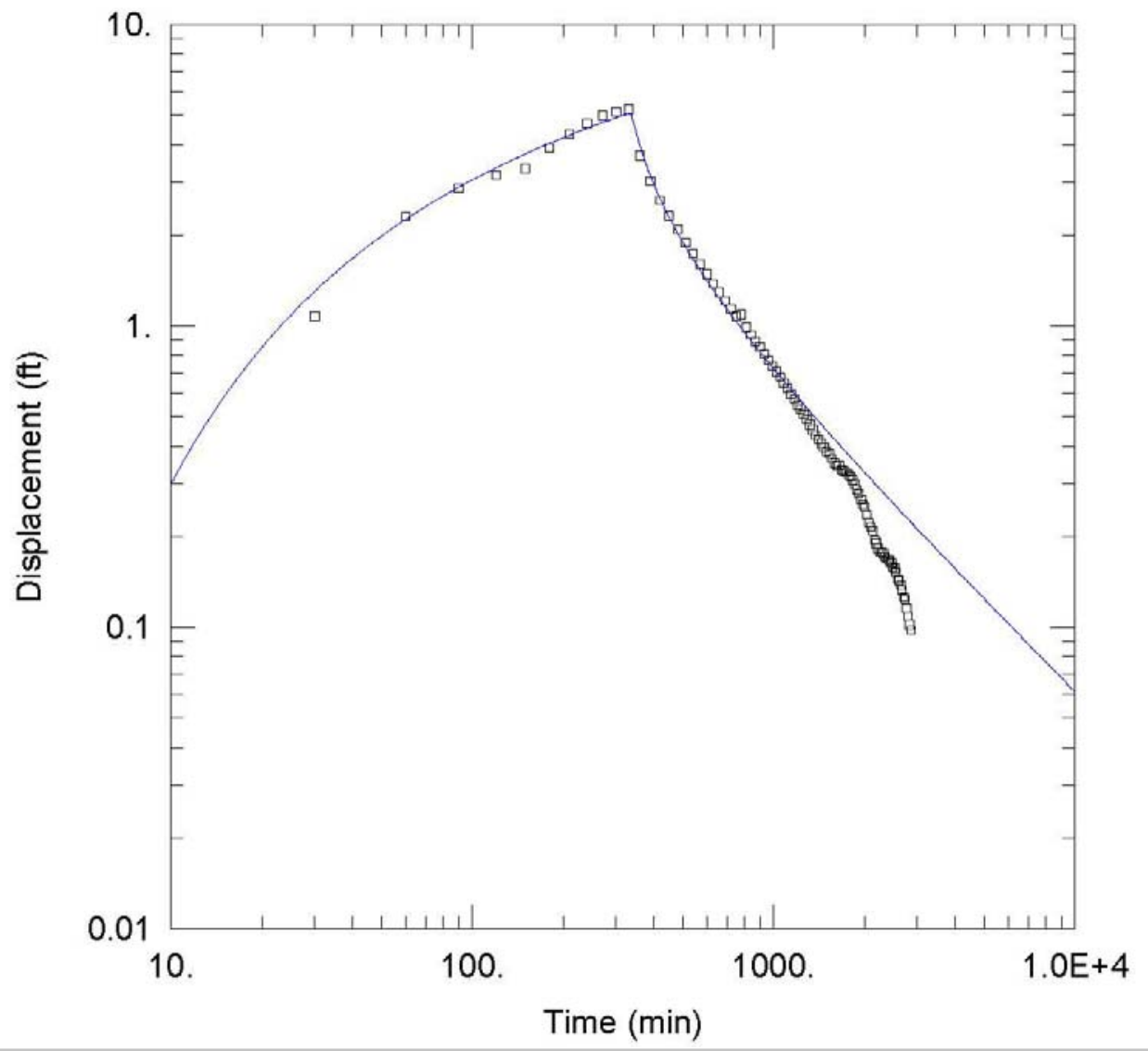

PRIVATE WELL PUMPING DATA ANALYSIS

\section{PROJECT INFORMATION}

Company: Argonne

Location: Inman

Test Well: Sisson

Test Date: Oct. 7, 2011

WELL DATA

Pumping Wells
\begin{tabular}{|l|c|c|c|c|c|c|}
\hline Well Name & $\mathrm{X}(\mathrm{ft})$ & $\mathrm{Y}(\mathrm{ft})$ & Well Name & $\mathrm{X}(\mathrm{ft})$ & $\mathrm{Y}(\mathrm{ft})$ \\
\hline Sisson 0.7 gpm & 0 & 0 & 口 MW18M & 5.03 & 56.8 \\
\hline
\end{tabular}

\section{SOLUTION}

Aquifer Model: Confined

$$
\mathrm{T}=\underline{44.1} \mathrm{ft}^{2} / \mathrm{day}
$$$$
\mathrm{Kz} / \mathrm{Kr}=\underline{0.1}
$$

Solution Method: Theis

$\mathrm{S}=0.0004432$

$\mathrm{b} \quad \underline{2} . \mathrm{ft}$

FIGURE G.20 Theis interpretive curve fit for shallow zone well MW18M during drawdown and recovery after the start of pumping at the Sisson well on October 7, 2011, with assumed pumping at the Sisson well at $1 \mathrm{gpm}, 70 \%$ of the pumping rate exerted on the shallow zone, and withdrawal from the shallow groundwater zone at $0.70 \mathrm{gpm}$. 


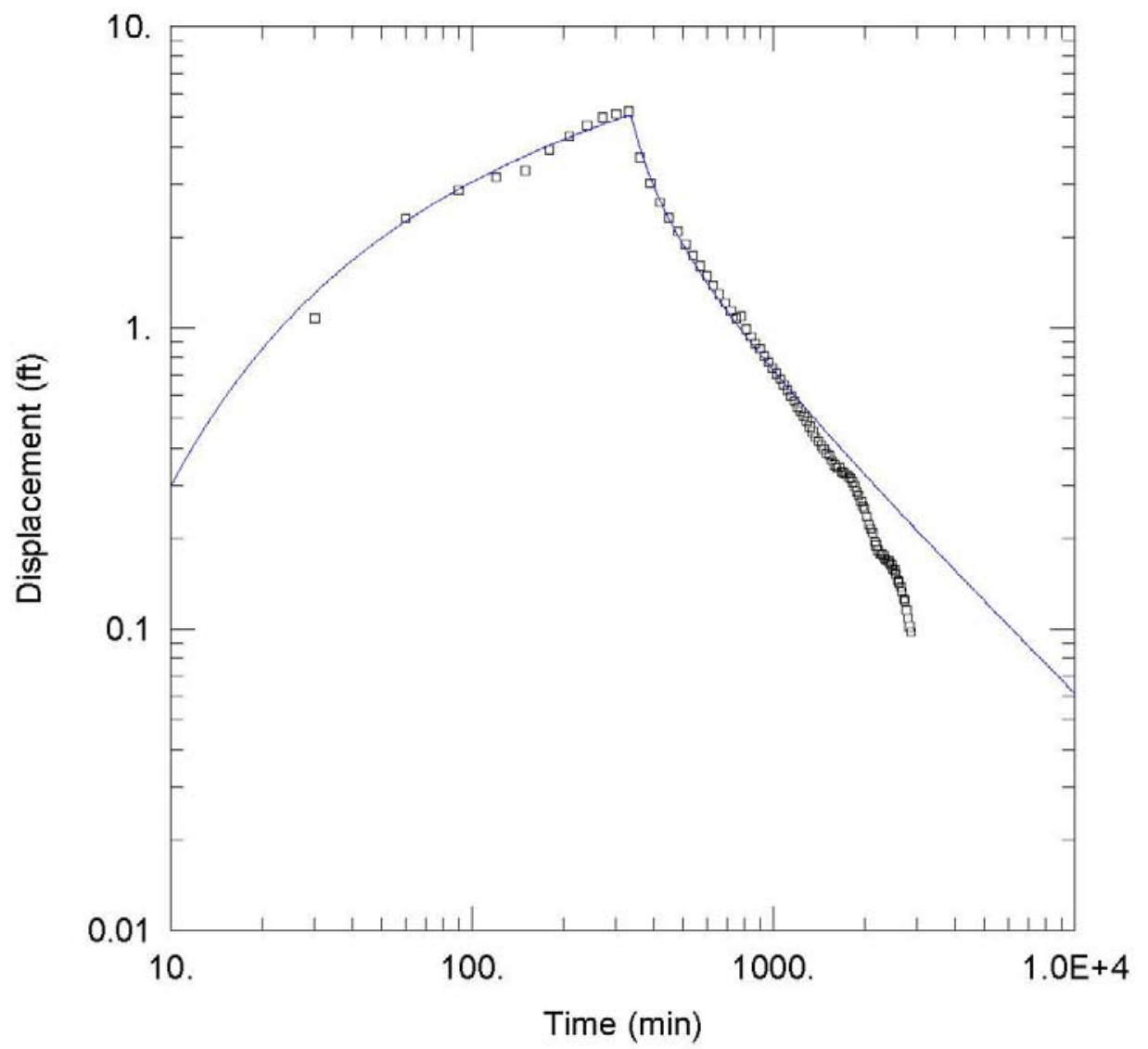

\begin{tabular}{|c|c|c|c|c|c|}
\hline \multicolumn{6}{|c|}{ PRIVATE WELL PUMPING DATA ANALYSIS } \\
\hline \multicolumn{6}{|c|}{ PROJECT INFORMATION } \\
\hline \multicolumn{6}{|c|}{$\begin{array}{l}\text { Company: Argonne } \\
\text { Location: Inman } \\
\text { Test Well: Sisson } \\
\text { Test Date: } \text { Oct. } 7,2011\end{array}$} \\
\hline \multicolumn{6}{|c|}{ WELL DATA } \\
\hline \multicolumn{3}{|c|}{ Pumping Wells } & \multicolumn{3}{|c|}{ Observation Wells } \\
\hline Well Name & $X(\mathrm{ft})$ & $Y(\mathrm{ft})$ & Well Name & $X(\mathrm{ft})$ & $Y(\mathrm{ft})$ \\
\hline Sisson $9.5 \mathrm{gpm}$ & 0 & 0 & MW18M & 5.03 & 56.8 \\
\hline \multicolumn{6}{|c|}{ SOLUTION } \\
\hline \multicolumn{3}{|c|}{ Aquifer Model: Confined } & \multicolumn{3}{|c|}{ Solution Method: Theis } \\
\hline \multicolumn{3}{|c|}{$\begin{array}{l}T=598.5 \mathrm{ft}^{2} / \text { day } \\
\mathrm{Kz} / \mathrm{Kr}=\frac{0.1}{}\end{array}$} & $\begin{array}{ll}\mathrm{s} & =0.00 \\
\mathrm{~b} & =2 . \mathrm{ft}\end{array}$ & \multicolumn{2}{|c|}{$=0.006015$} \\
\hline
\end{tabular}

FIGURE G.21 Theis interpretive curve fit for shallow zone well MW18M during drawdown and recovery after the start of pumping at the Sisson well on October 7, 2011, with assumed pumping at the Sisson well at $10 \mathrm{gpm}, 95 \%$ of the pumping rate exerted on the shallow zone, and withdrawal from the shallow groundwater zone at $9.5 \mathrm{gpm}$. 


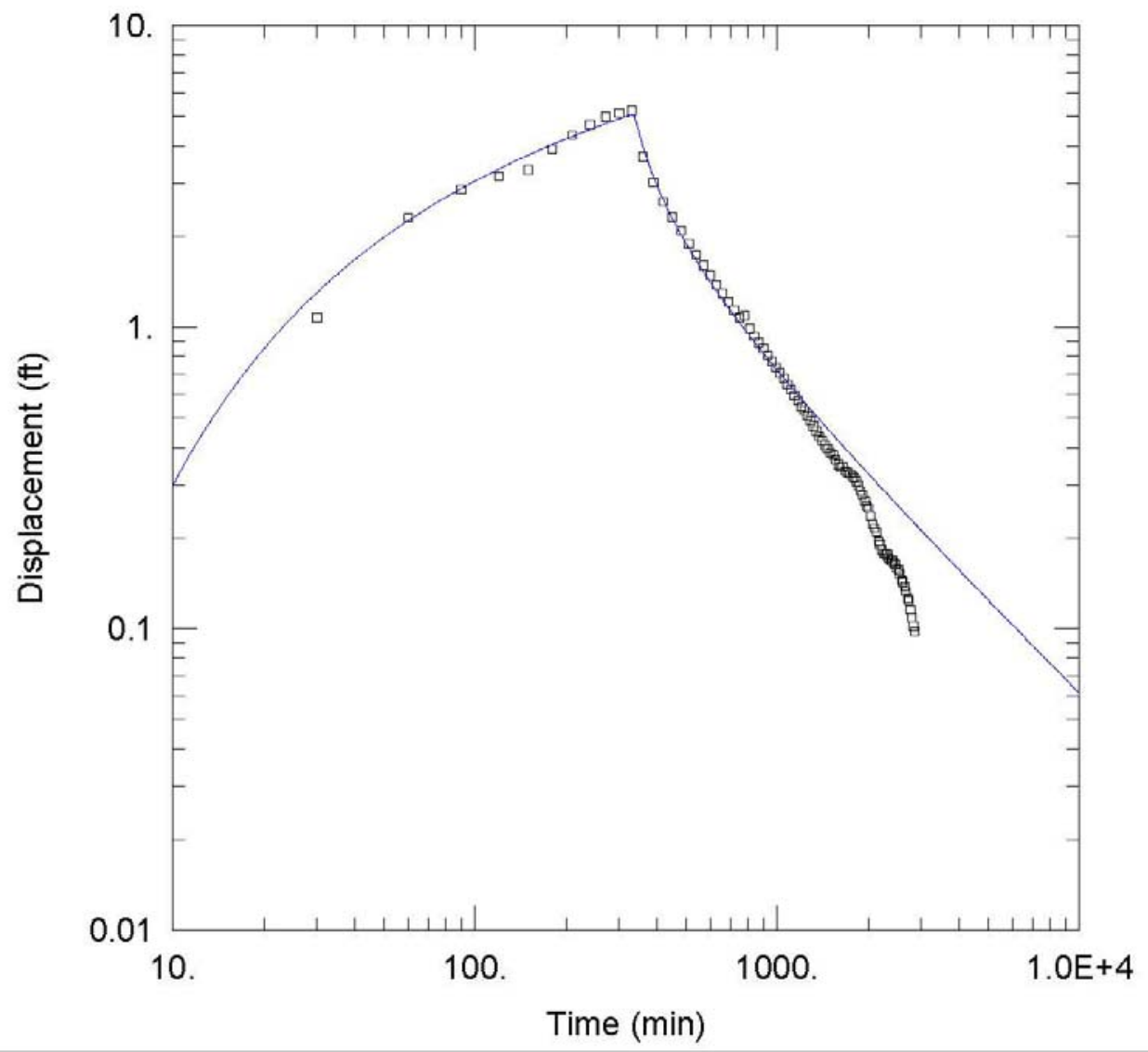

\begin{tabular}{|c|c|c|c|c|c|}
\hline \multicolumn{6}{|c|}{ PRIVATE WELL PUMPING DATA ANALYSIS } \\
\hline \multicolumn{6}{|c|}{ PROJECT INFORMATION } \\
\hline \multicolumn{6}{|c|}{$\begin{array}{l}\text { Company: Argonne } \\
\text { Location: Inman } \\
\text { Test Well: Sisson } \\
\text { Test Date: Oct. } 7,2011\end{array}$} \\
\hline \multicolumn{6}{|c|}{ WELL DATA } \\
\hline \multicolumn{3}{|c|}{ Pumping Wells } & \multicolumn{3}{|c|}{ Observation Wells } \\
\hline Well Name & $X(\mathrm{ft})$ & $Y(\mathrm{ft})$ & Well Name & $X(\mathrm{ft})$ & $Y(\mathrm{ft})$ \\
\hline Sisson $9 \mathrm{gpm}$ & 0 & 0 & $\square$ MW18M & 5.03 & 56.8 \\
\hline \multicolumn{6}{|c|}{ SOLUTION } \\
\hline \multicolumn{3}{|c|}{ Aquifer Model: Confined } & \multicolumn{3}{|c|}{ Solution Method: Theis } \\
\hline $\begin{aligned} T & =567 . \mathrm{ft} \\
\mathrm{Kz} / \mathrm{Kr} & =\underline{0.1}\end{aligned}$ & & & $\begin{array}{l}\mathrm{S}=0.00 \\
\mathrm{~b}=2 . \mathrm{ft}\end{array}$ & & \\
\hline
\end{tabular}

FIGURE G.22 Theis interpretive curve fit for shallow zone well MW18M during drawdown and recovery after the start of pumping at the Sisson well on October 7, 2011, with assumed pumping at the Sisson well at $10 \mathrm{gpm}, 90 \%$ of the pumping rate exerted on the shallow zone, and withdrawal from the shallow groundwater zone at $9.0 \mathrm{gpm}$. 


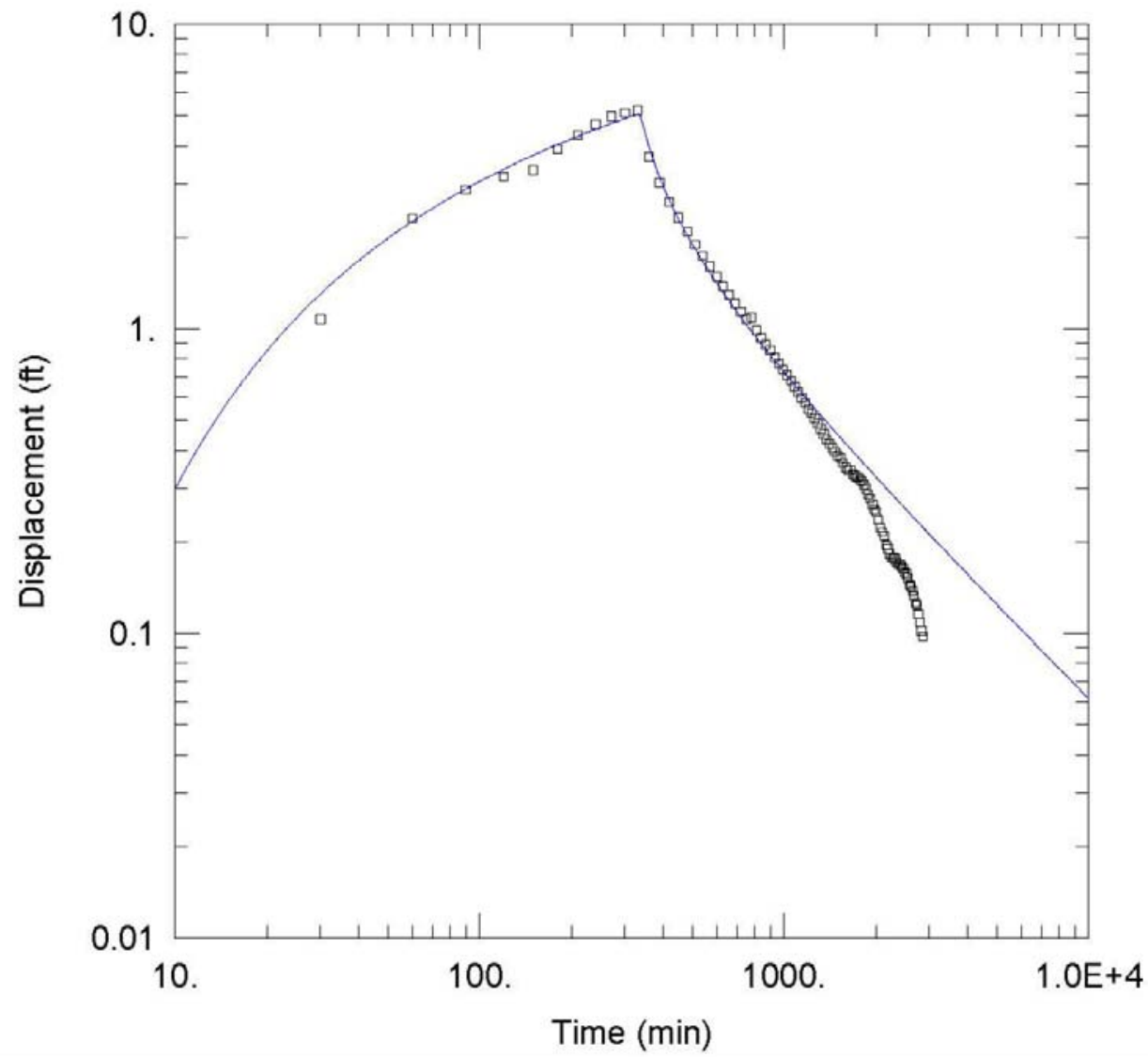

\begin{tabular}{|c|c|c|c|c|c|}
\hline \multicolumn{6}{|c|}{ PRIVATE WELL PUMPING DATA ANALYSIS } \\
\hline \multicolumn{6}{|c|}{ PROJECT INFORMATION } \\
\hline \multicolumn{6}{|c|}{$\begin{array}{l}\text { Company: Argonne } \\
\text { Location: Inman } \\
\text { Test Well: Sisson } \\
\text { Test Date: Oct. 7, } 2011\end{array}$} \\
\hline \multicolumn{6}{|c|}{ WELL DATA } \\
\hline \multicolumn{3}{|c|}{ Pumping Wells } & \multicolumn{3}{|c|}{ Observation Wells } \\
\hline Well Name & $X(\mathrm{ft})$ & $\mathrm{Y}(\mathrm{ft})$ & Well Name & $X(\mathrm{ft})$ & $\mathrm{Y}(\mathrm{ft})$ \\
\hline Sisson $7.0 \mathrm{gpm}$ & 0 & 0 & $\square$ MW18M & 5.03 & 56.8 \\
\hline \multicolumn{6}{|c|}{ SOLUTION } \\
\hline \multicolumn{3}{|c|}{ Aquifer Model: Confined } & \multicolumn{3}{|c|}{ Solution Method: Theis } \\
\hline \multicolumn{3}{|c|}{$\begin{aligned} \mathrm{T} & =441 . \mathrm{ft}^{2} / \text { day } \\
\mathrm{Kz} / \mathrm{Kr} & =0.1\end{aligned}$} & \multicolumn{3}{|c|}{$S \quad=0.004432$} \\
\hline
\end{tabular}

FIGURE G.23 Theis interpretive curve fit for shallow zone well MW18M during drawdown and recovery after the start of pumping at the Sisson well on October 7, 2011, with assumed pumping at the Sisson well at $10 \mathrm{gpm}, 70 \%$ of the pumping rate exerted on the shallow zone, and withdrawal from the shallow groundwater zone at $7.0 \mathrm{gpm}$. 


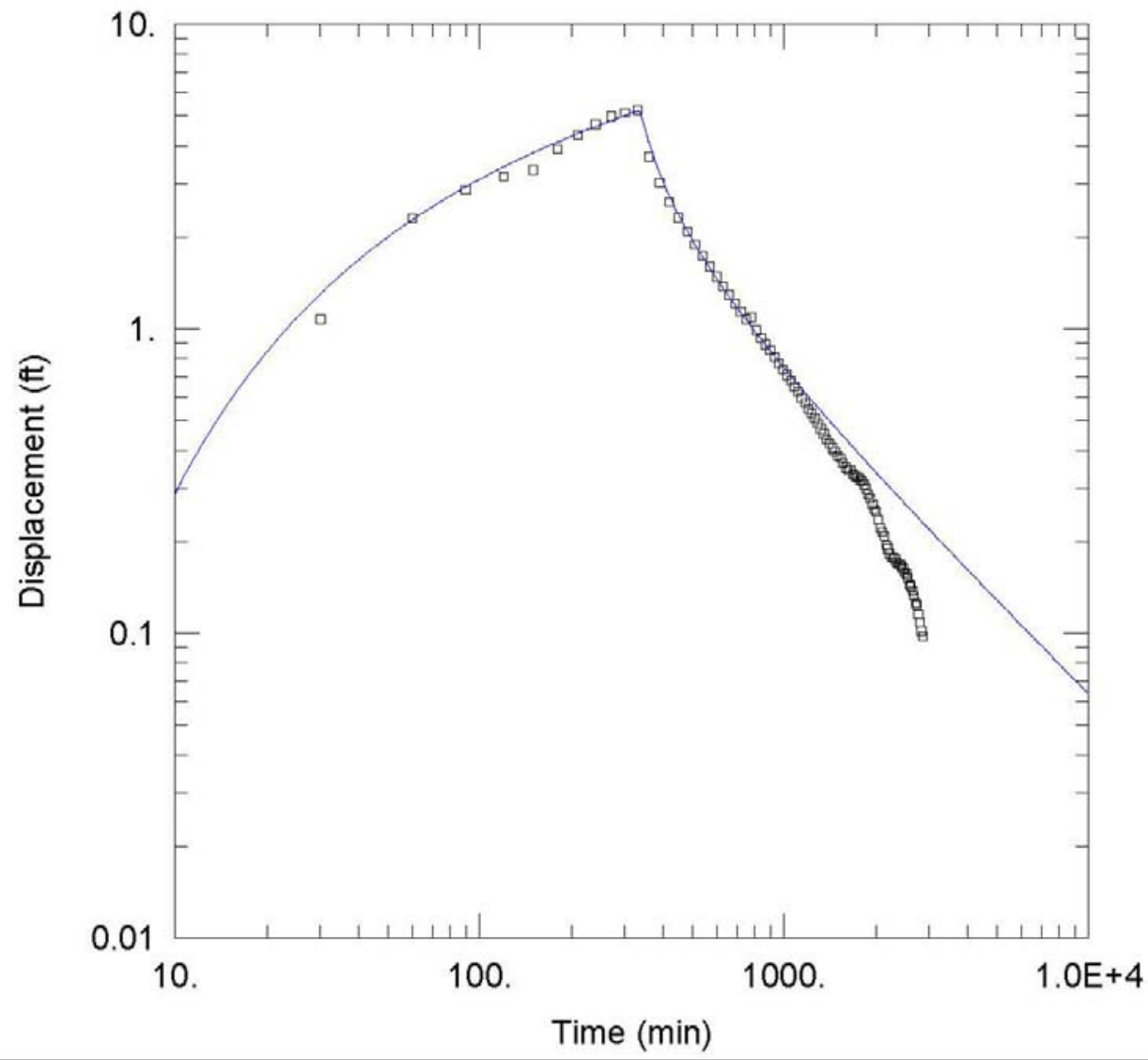

\begin{tabular}{|c|c|c|c|c|c|}
\hline \multicolumn{6}{|c|}{ PRIVATE WELL PUMPING DATA ANALYSIS } \\
\hline \multicolumn{6}{|c|}{ PROJECT INFORMATION } \\
\hline \multicolumn{6}{|c|}{$\begin{array}{l}\text { Company: Argonne } \\
\text { Location: Inman } \\
\text { Test Well: Sisson } \\
\text { Test Date: } \text { Oct. 7, } 2011\end{array}$} \\
\hline \multicolumn{6}{|c|}{ WELL DATA } \\
\hline \multicolumn{3}{|c|}{ Pumping Wells } & \multicolumn{3}{|c|}{ Observation Wells } \\
\hline Well Name & $\mathrm{X}(\mathrm{ft})$ & $\mathrm{Y}(\mathrm{ft})$ & Well Name & $X(\mathrm{ft})$ & $\mathrm{Y}(\mathrm{ft})$ \\
\hline Sisson $19 \mathrm{gpm}$ & 0 & 0 & - MW18M & 5.03 & 56.8 \\
\hline \multicolumn{6}{|c|}{ SOLUTION } \\
\hline \multicolumn{3}{|c|}{ Aquifer Model: Confined } & \multicolumn{3}{|c|}{ Solution Method: Theis } \\
\hline \multicolumn{2}{|c|}{$\begin{array}{l}\mathrm{T}=1156.6 \mathrm{ft}^{2} / \text { day } \\
\mathrm{Kz} / \mathrm{Kr}=0.1\end{array}$} & & \multicolumn{2}{|c|}{$\begin{array}{ll}\mathrm{S} & =0.01203 \\
\mathrm{~b} & =2 \mathrm{ft}\end{array}$} & \\
\hline
\end{tabular}

FIGURE G.24 Theis interpretive curve fit for shallow zone well MW18M during drawdown and recovery after the start of pumping at the Sisson well on October 7, 2011, with assumed pumping at the Sisson well at $20 \mathrm{gpm}, 95 \%$ of the pumping rate exerted on the shallow zone, and withdrawal from the shallow groundwater zone at $19 \mathrm{gpm}$. 


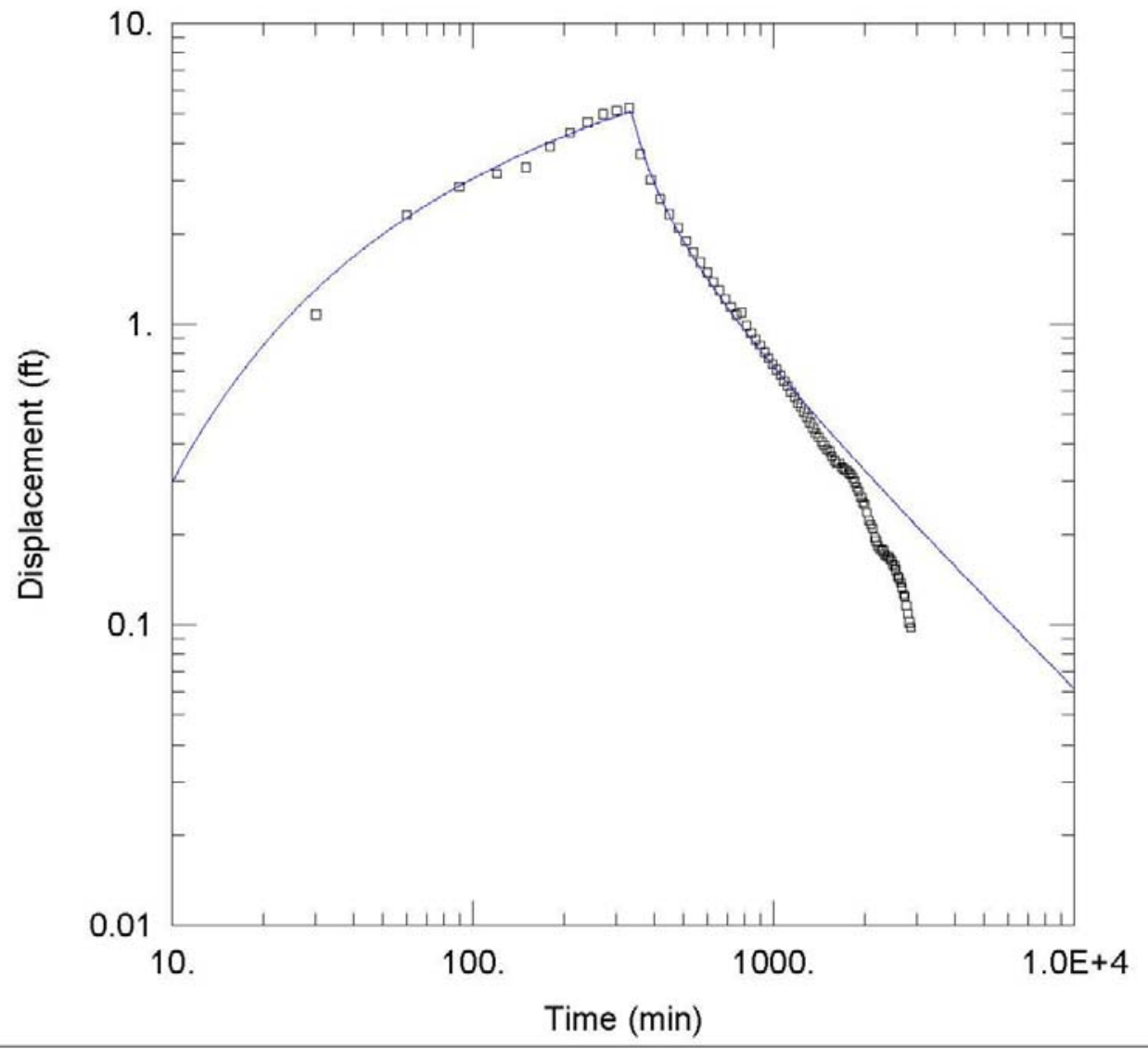

\begin{tabular}{|c|c|c|c|c|c|}
\hline \multicolumn{6}{|c|}{ PRIVATE WELL PUMPING DATA ANALYSIS } \\
\hline \multicolumn{6}{|c|}{ PROJECT INFORMATION } \\
\hline \multicolumn{6}{|c|}{$\begin{array}{l}\text { Company: Argonne } \\
\text { Location: Inman } \\
\text { Test Well: Sisson } \\
\text { Test Date: Oct. 7, } 2011\end{array}$} \\
\hline \multicolumn{6}{|c|}{ WELL DATA } \\
\hline \multicolumn{3}{|c|}{ Pumping Wells } & \multicolumn{3}{|c|}{ Observation Wells } \\
\hline Well Name & $X(\mathrm{ft})$ & $\mathrm{Y}(\mathrm{ft})$ & Well Name & $X(\mathrm{ft})$ & $Y(\mathrm{ft})$ \\
\hline Sisson $18 \mathrm{gpm}$ & 0 & 0 & $\circ \mathrm{MW} 18 \mathrm{M}$ & 5.03 & 56.8 \\
\hline \multicolumn{6}{|c|}{ SOLUTION } \\
\hline \multicolumn{3}{|c|}{ Aquifer Model: Confined } & \multicolumn{3}{|c|}{ Solution Method: Theis } \\
\hline \multicolumn{2}{|c|}{$\begin{array}{l}T=1134 . \mathrm{ft}^{2} / \mathrm{day} \\
\mathrm{Kz} / \mathrm{Kr}=\underline{0.1}\end{array}$} & & \multicolumn{2}{|c|}{$\begin{array}{ll}\mathrm{S} & =0.0114 \\
\mathrm{~b} & =2 \mathrm{ft}\end{array}$} & \\
\hline
\end{tabular}

FIGURE G.25 Theis interpretive curve fit for shallow zone well MW18M during drawdown and recovery after the start of pumping at the Sisson well on October 7, 2011, with assumed pumping at the Sisson well at $20 \mathrm{gpm}, 90 \%$ of the pumping rate exerted on the shallow zone, and withdrawal from the shallow groundwater zone at $18 \mathrm{gpm}$. 


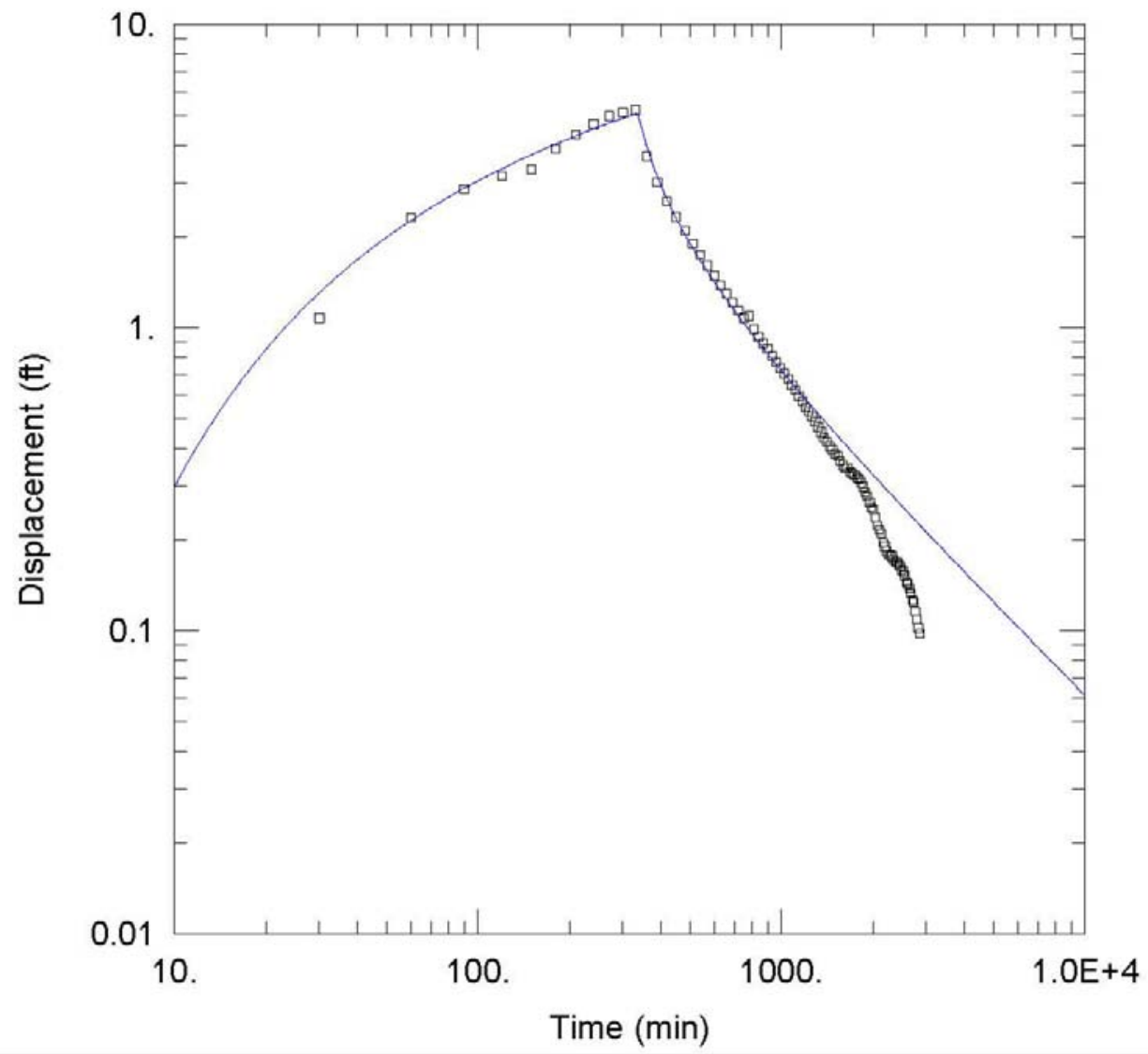

\begin{tabular}{|c|c|c|c|c|c|}
\hline \multicolumn{6}{|c|}{ PRIVATE WELL PUMPING DATA ANALYSIS } \\
\hline \multicolumn{6}{|c|}{ PROJECT INFORMATION } \\
\hline \multicolumn{6}{|c|}{$\begin{array}{l}\text { Company: Argonne } \\
\text { Location: Inman } \\
\text { Test Well: Sisson } \\
\text { Test Date: Oct. } 7,2011\end{array}$} \\
\hline \multicolumn{6}{|c|}{ WELL DATA } \\
\hline \multicolumn{3}{|c|}{ Pumping Wells } & \multicolumn{3}{|c|}{ Observation Wells } \\
\hline Well Name & $X(\mathrm{ft})$ & $\mathrm{Y}(\mathrm{ft})$ & Well Name & $X(\mathrm{ft})$ & $\mathrm{Y}(\mathrm{ft})$ \\
\hline Sisson $14 \mathrm{gpm}$ & 0 & 0 & - MW18M & 5.03 & 56.8 \\
\hline \multicolumn{6}{|c|}{ SOLUTION } \\
\hline \multicolumn{3}{|c|}{ Aquifer Model: Confined } & \multicolumn{3}{|c|}{ Solution Method: Theis } \\
\hline \multicolumn{2}{|c|}{$\begin{array}{l}T=882 . \mathrm{ft}^{2} / \text { day } \\
\mathrm{Kz} / \mathrm{Kr}=\underline{0.1}\end{array}$} & & \multicolumn{2}{|c|}{$\begin{array}{l}=0.008865 \\
=2 . \mathrm{ft}\end{array}$} & \\
\hline
\end{tabular}

FIGURE G.26 Theis interpretive curve fit for shallow zone well MW18M during drawdown and recovery after the start of pumping at the Sisson well on October 7, 2011, with assumed pumping at the Sisson well at $20 \mathrm{gpm}, 70 \%$ of the pumping rate exerted on the shallow zone, and withdrawal from the shallow groundwater zone at $14 \mathrm{gpm}$. 


\section{Supplement 1:}

Automatically Recorded Groundwater Level Data 


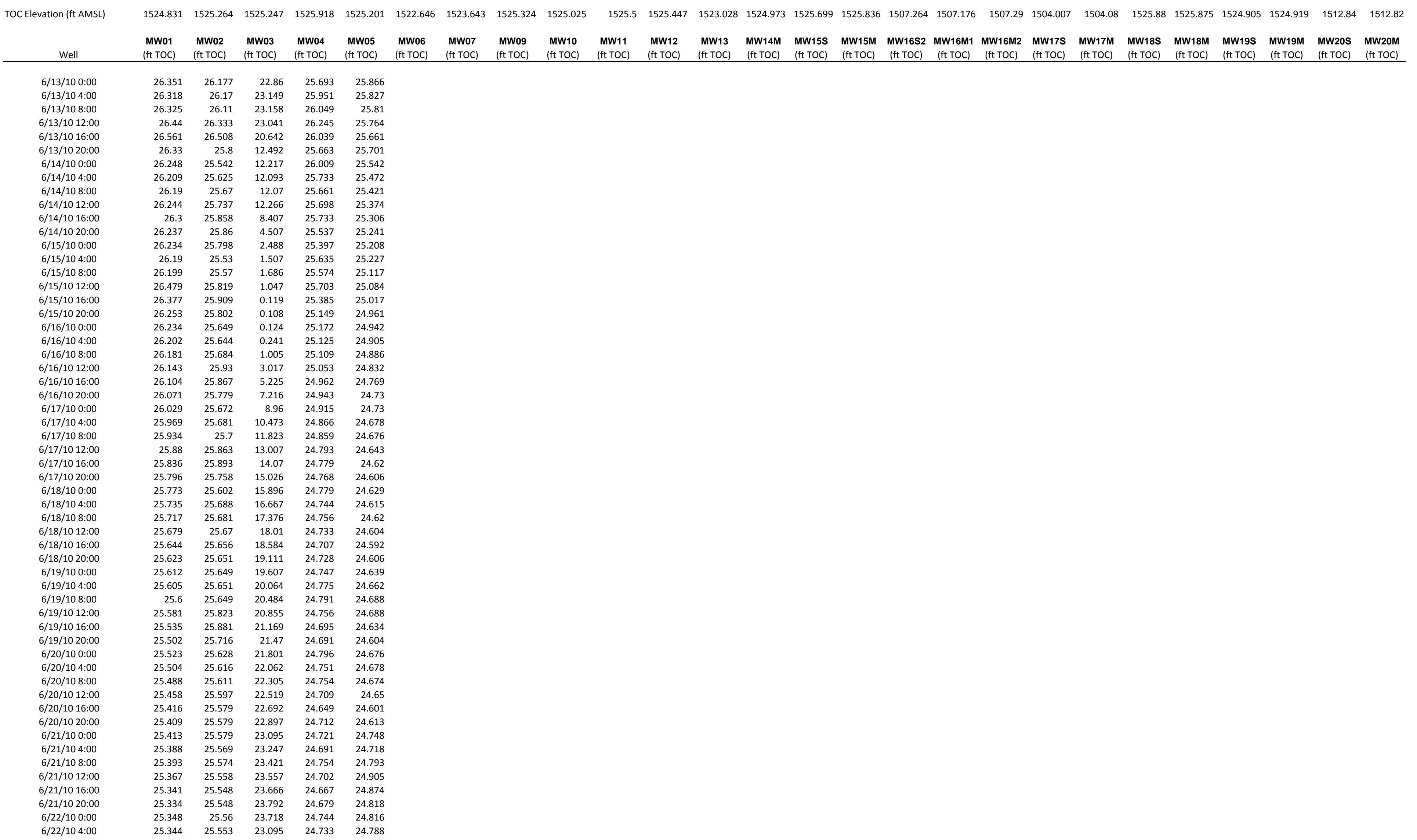




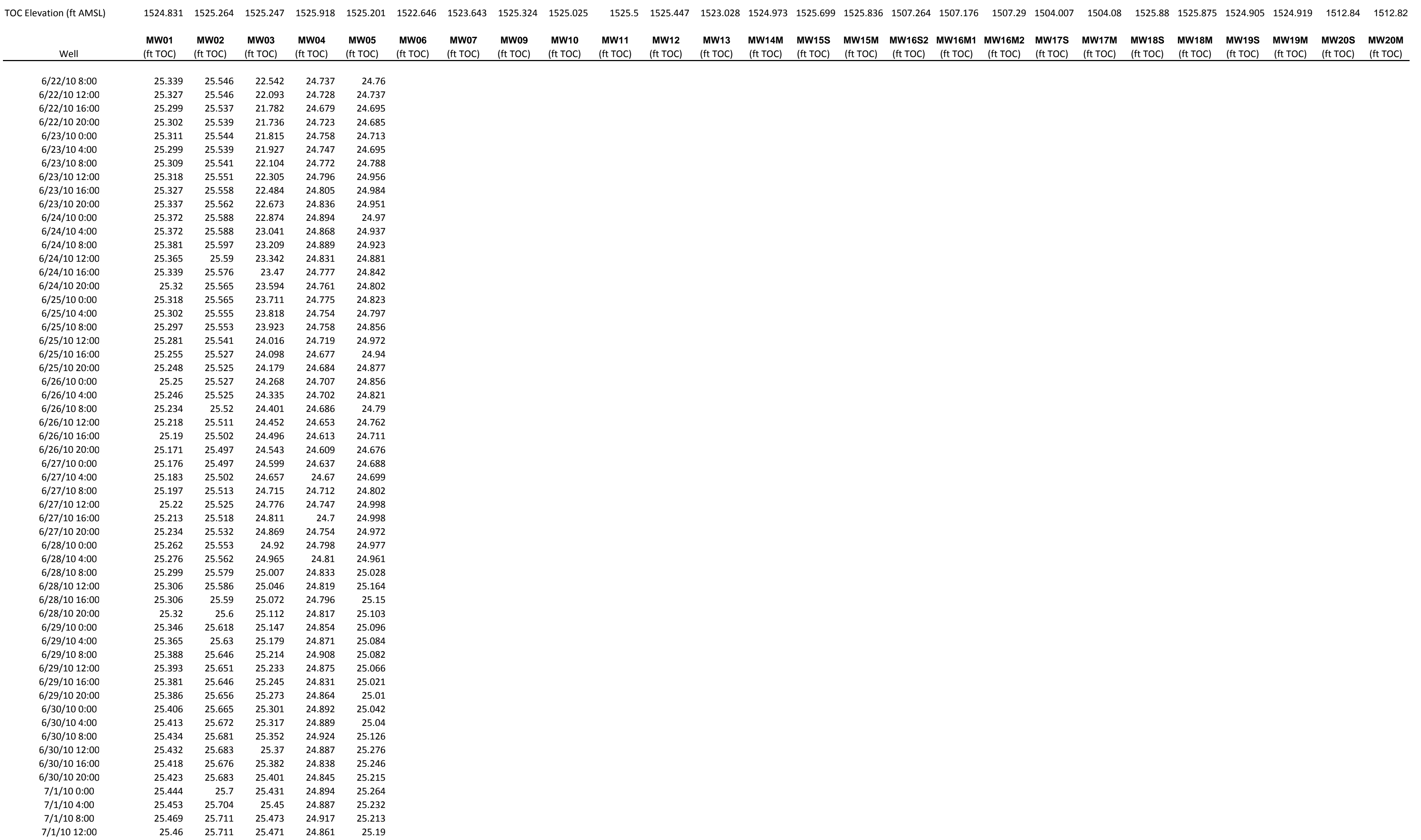




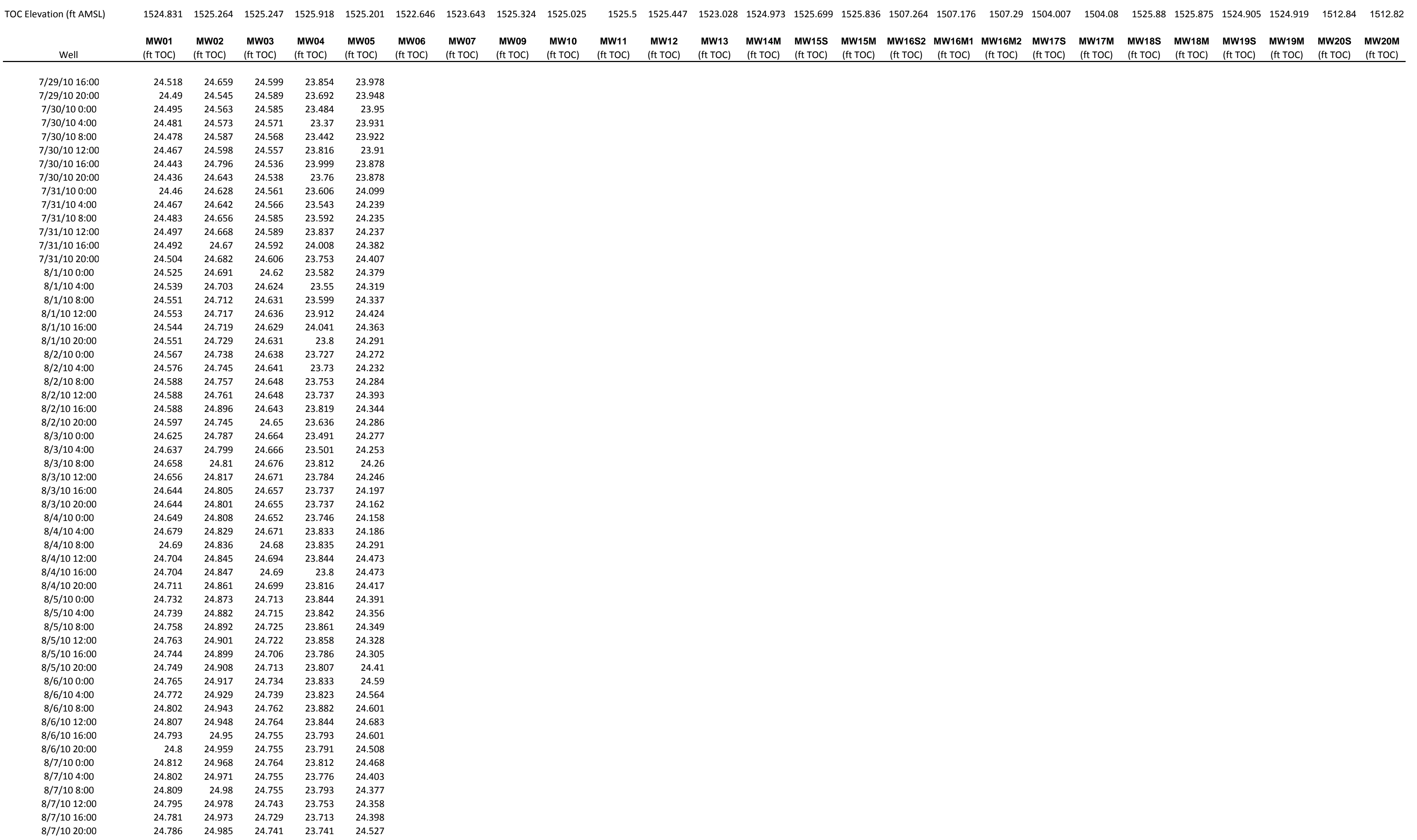




\begin{tabular}{|c|c|c|c|c|c|}
\hline Well & (ft TOC) & $\begin{array}{l}\text { (ft TOC) } \\
\text { (a) }\end{array}$ & (ft TOC) & (ft TOC) & (ft TOC) \\
\hline $8 / 8 / 100: 00$ & 24.8 & 24.994 & 24.753 & 23.779 & 24.548 \\
\hline 8/8/10 4:00 & 24.8 & 25.001 & 24.753 & 23.762 & 24.492 \\
\hline 8/8/10 8:00 & 24.823 & 25.013 & 24.755 & 23.755 & 24.519 \\
\hline $8 / 8 / 1012: 00$ & 24.823 & 25.013 & 24.762 & 23.781 & 24.629 \\
\hline $8 / 8 / 1016: 00$ & 24.812 & 25.015 & 24.757 & 23.725 & 24.578 \\
\hline $8 / 8 / 1020: 00$ & 24.819 & 25.022 & 24.76 & 23.748 & 24.515 \\
\hline $8 / 9 / 100: 00$ & 24.844 & 25.036 & 24.774 & 23.805 & 24.501 \\
\hline $\begin{array}{l}8 / 9 / 104: 00 \\
8 / 100\end{array}$ & 24.856 & 25.043 & 24.778 & 23.802 & 24.475 \\
\hline 8/9/10 8:00 & 24.882 & 25.055 & 24.795 & 23.851 & 24.557 \\
\hline 8/9/10 12:00 & 24.891 & 25.064 & 24.804 & 23.844 & 24.702 \\
\hline $8 / 9 / 1016: 00$ & 24.891 & 25.169 & 24.799 & 23.809 & 24.667 \\
\hline $8 / 9 / 1020: 00$ & 24.903 & 25.003 & 24.809 & 23.83 & 24.606 \\
\hline $8 / 10 / 100: 00$ & 24.924 & 25.09 & 24.823 & 23.868 & 24.589 \\
\hline 8/10/10 4:00 & 24.919 & 25.094 & 24.818 & 23.828 & 24.536 \\
\hline 8/10/10 8:00 & 24.94 & 25.108 & 24.83 & 23.882 & 24.541 \\
\hline 8/10/10 12:00 & 24.945 & 25.113 & 24.83 & 23.861 & 24.529 \\
\hline 8/10/10 16:00 & 24.931 & 25.111 & 24.813 & 23.798 & 24.475 \\
\hline $8 / 10 / 1020: 00$ & 24.921 & 24.98 & 24.827 & 23.41 & 24.482 \\
\hline $8 / 11 / 100: 00$ & 24.947 & 24.971 & 24.83 & 23.173 & 24.489 \\
\hline 8/11/10 4:00 & 24.949 & 24.987 & 24.83 & 23.073 & $\begin{array}{r}24.409 \\
24.48\end{array}$ \\
\hline 8/11/10 8:00 & $\begin{array}{r}24.949 \\
24.97\end{array}$ & $\begin{array}{l}24.981 \\
25.015\end{array}$ & $\begin{array}{r}24.843 \\
24.84\end{array}$ & 23.169 & $\begin{array}{r}24.473 \\
24.573\end{array}$ \\
\hline 8/11/10 12:00 & $\begin{array}{r}24.973 \\
24.973\end{array}$ & 25.034 & $\begin{array}{l}24.844 \\
24.846\end{array}$ & $\begin{array}{l}23.169 \\
24.003\end{array}$ & $\begin{array}{l}24.7 / 322 \\
24.722\end{array}$ \\
\hline $8 / 11 / 1016: 00$ & 24.956 & 25.276 & 24.839 & 23.821 & 24.713 \\
\hline $8 / 11 / 1020: 00$ & 24.963 & 25.113 & 24.844 & 23.835 & 4.666 \\
\hline $8 / 12 / 100: 00$ & 24.975 & 25.083 & 24.853 & 23.849 & 24.641 \\
\hline 8/12/10 4:00 & 24.977 & 25.048 & 24.851 & 23.837 & 24.599 \\
\hline 8/12/10 8:00 & 24.987 & 25.02 & 24.858 & 23.865 & . \\
\hline $8 / 12 / 1012: 00$ & $\begin{array}{l}24.901 \\
24.982\end{array}$ & 25.36 & 24.848 & 23.835 & .557 \\
\hline 8/12/10 16:00 & 24.956 & $\begin{array}{l}25.50 \\
25.518\end{array}$ & 24.825 & $\begin{array}{l}23.053 \\
23.952 \\
\end{array}$ & 24.492 \\
\hline $8 / 12 / 1020: 00$ & 24.952 & 25.243 & 24.823 & 23.744 & 24.466 \\
\hline $8 / 13 / 100: 00$ & 24.966 & 25.034 & 24.827 & 23.575 & 24.494 \\
\hline 8/13/10 4:00 & 24.959 & 25.131 & 24.816 & 23.557 & 24.475 \\
\hline 8/13/10 8:00 & 24.959 & 25.138 & 24.816 & 23.615 & 24.541 \\
\hline $8 / 13 / 1012: 00$ & 24.954 & 25.141 & 24.816 & 23.964 & 24.674 \\
\hline $8 / 13 / 1016: 00$ & 24.938 & 25.141 & 24.806 & 23.751 & 24.641 \\
\hline $8 / 13 / 1020: 00$ & 24.973 & 25.162 & 24.834 & 23.627 & 24.643 \\
\hline $8 / 14 / 100: 00$ & 25.015 & 25.18 & 24.862 & 23.907 & 24.685 \\
\hline $8 / 14 / 104: 00$ & 25.038 & 25.192 & 24.874 & 23.926 & 24.688 \\
\hline 8/14/10 8:00 & 25.059 & 25.206 & 24.888 & $\begin{array}{l}23.940 \\
23.949\end{array}$ & 24.697 \\
\hline 8/14/10 12:00 & 25.073 & 25.213 & 24.89 & 23.942 & 24.695 \\
\hline $8 / 14 / 1016: 00$ & 25.078 & 25.222 & 24.89 & 23.924 & 24.676 \\
\hline 8/14/10 20:00 & $\begin{array}{l}25.018 \\
25.113\end{array}$ & $\begin{array}{l}25.242 \\
25.248\end{array}$ & $\begin{array}{l}24.99 \\
24.92\end{array}$ & $\begin{array}{l}23.924 \\
24.017\end{array}$ & 4.727 \\
\hline $8 / 15 / 100: 00$ & 25.138 & 25.257 & 24.932 & 24.02 & 4.783 \\
\hline $8 / 15 / 104: 00$ & 25.171 & 25.274 & 24.96 & 24.071 & 4.814 \\
\hline & 25.206 & 25.294 & 24.976 & 24.108 & 24.907 \\
\hline & 25.225 & 25.311 & 25 & 24.101 & 25.073 \\
\hline & 25.232 & 2 & 24.995 & 23.956 & 68 \\
\hline $8 / 15$ & 25.22 & 25.208 & & 23.791 & 96 \\
\hline $8 / 16 / 100: 00$ & 25.243 & 25.236 & & 23.643 & 24.944 \\
\hline 8/16/10 4:00 & 25.264 & 25.271 & & 23.552 & 24.916 \\
\hline 8/16/10 8:00 & 25.276 & 25.299 & 25.046 & 23.58 & 24.961 \\
\hline $8 / 16 / 1012: 00$ & 25.28 & 25.32 & 25.053 & 23.872 & 25.08 \\
\hline $8 / 16 / 1016: 00$ & 25.259 & 25.32 & 25.039 & 24.087 & 25.003 \\
\hline $8 / 16 / 1020: 00$ & 25.248 & 25.327 & 25.037 & 23.994 & 24.916 \\
\hline $8 / 17 / 100: 00$ & 25.264 & 25.339 & 25.049 & 23.886 & 24.879 \\
\hline & & & & & \\
\hline
\end{tabular}




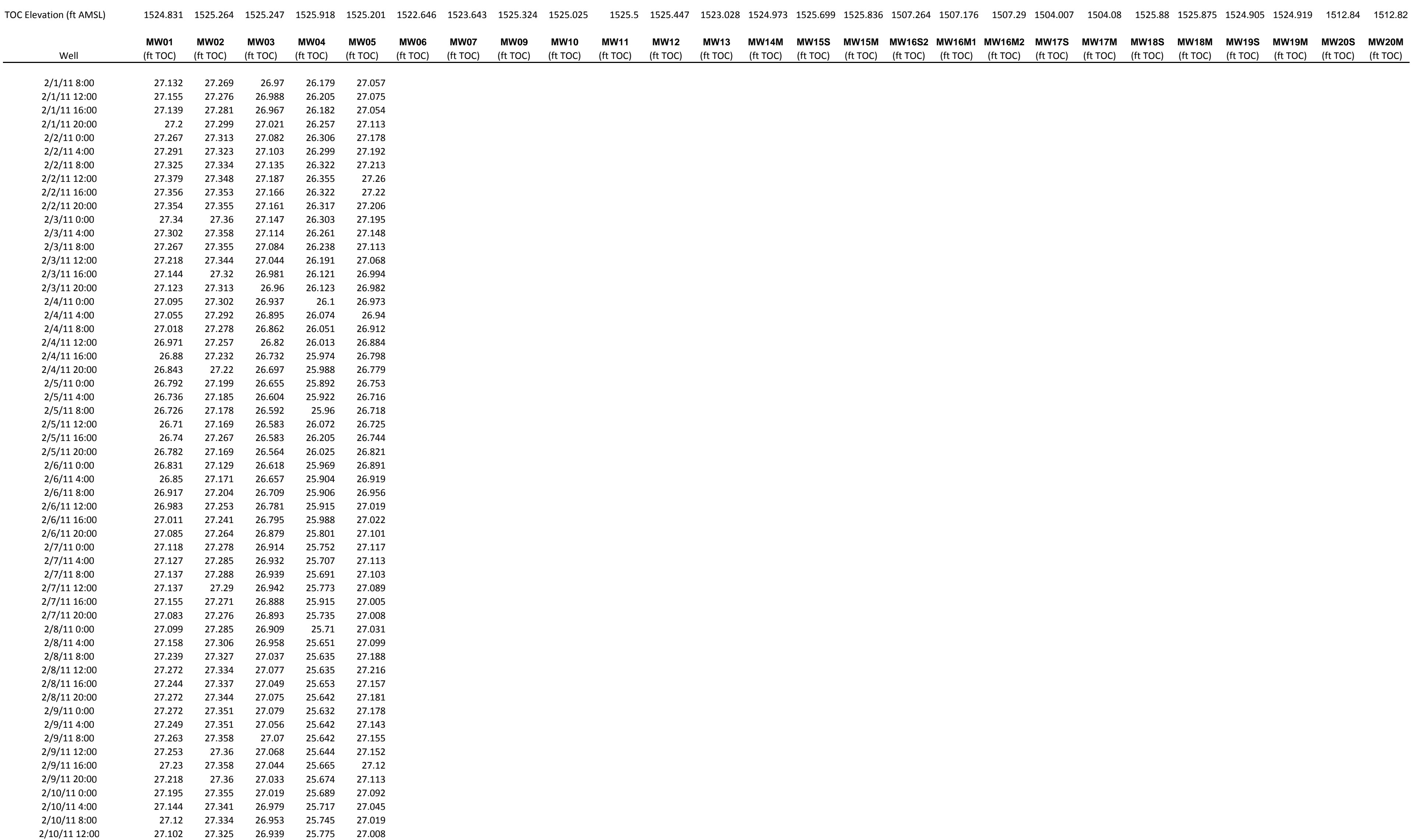




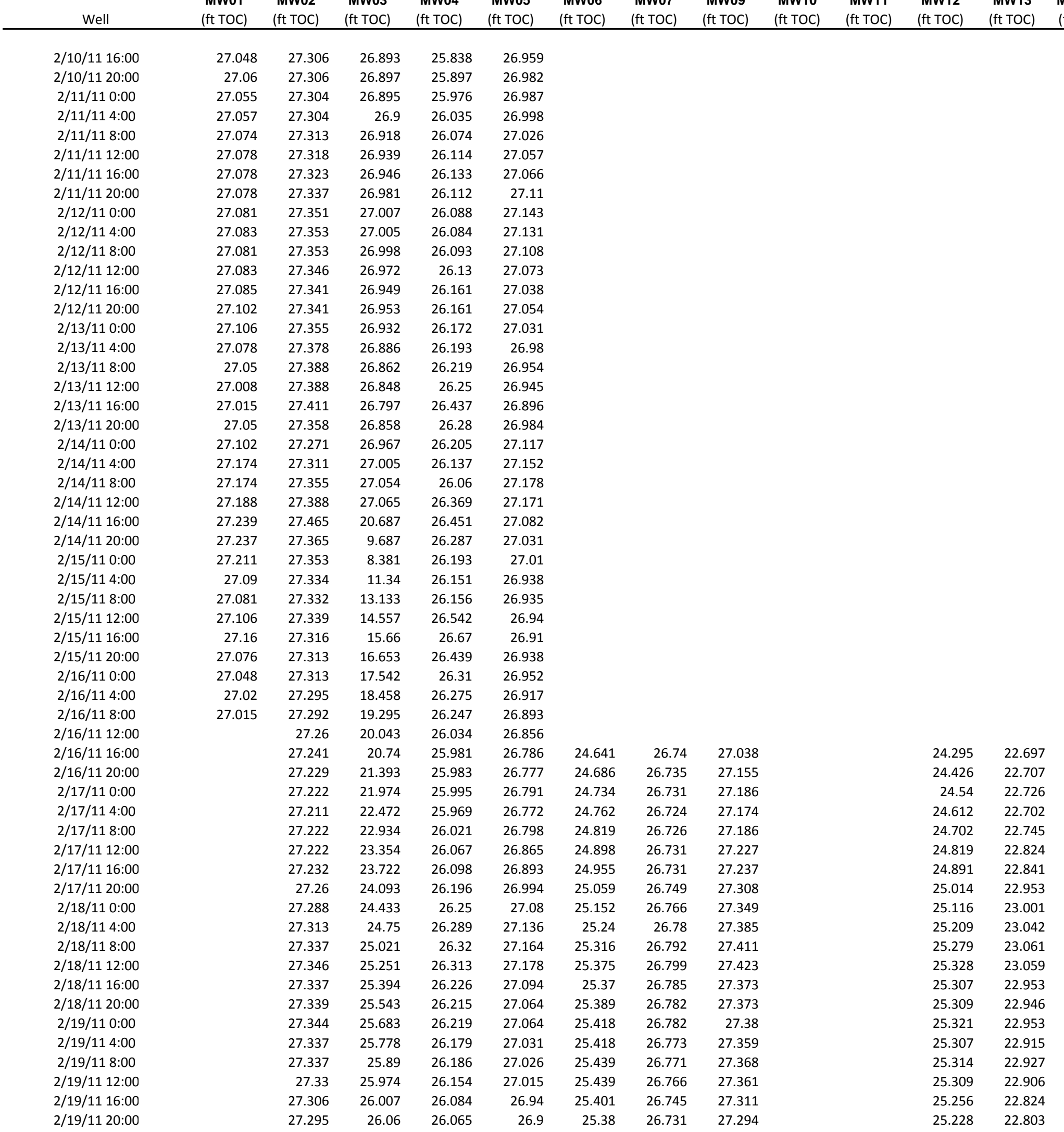


TABLE S1.1 Automatically recorded groundwater depths in monitoring wells, December 2009 to March 2013, measured from the top of the casing (ft TOC).

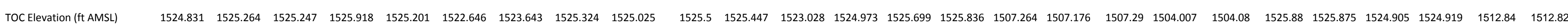

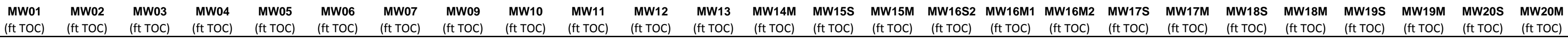

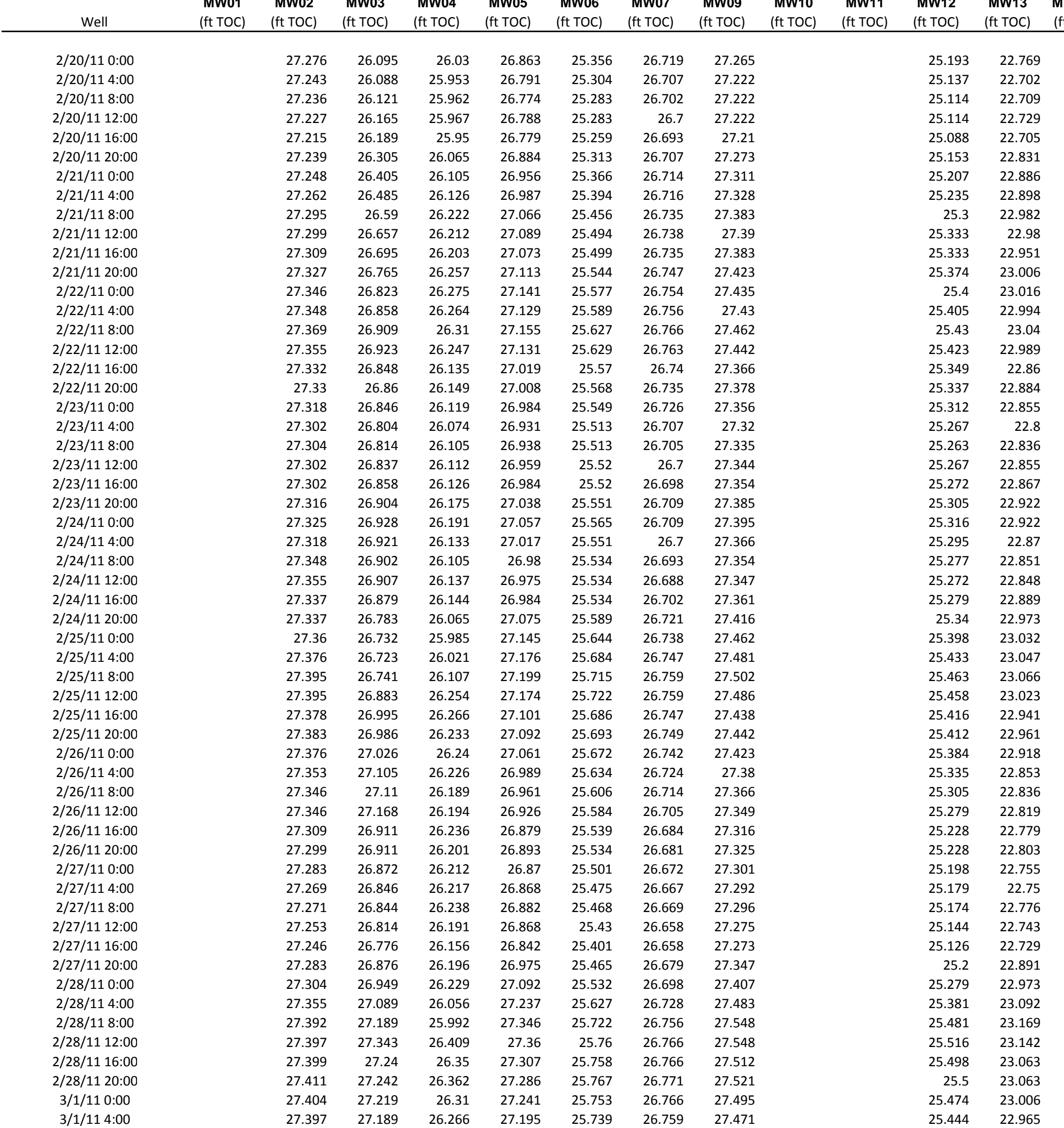


TABLE S1.1 Automatically recorded groundwater depths in monitoring wells, December 2009 to March 2013, measured from the top of the casing (ft TOC).

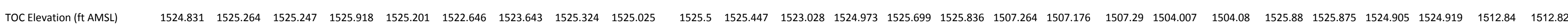

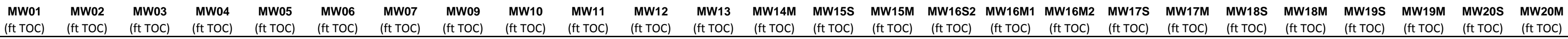

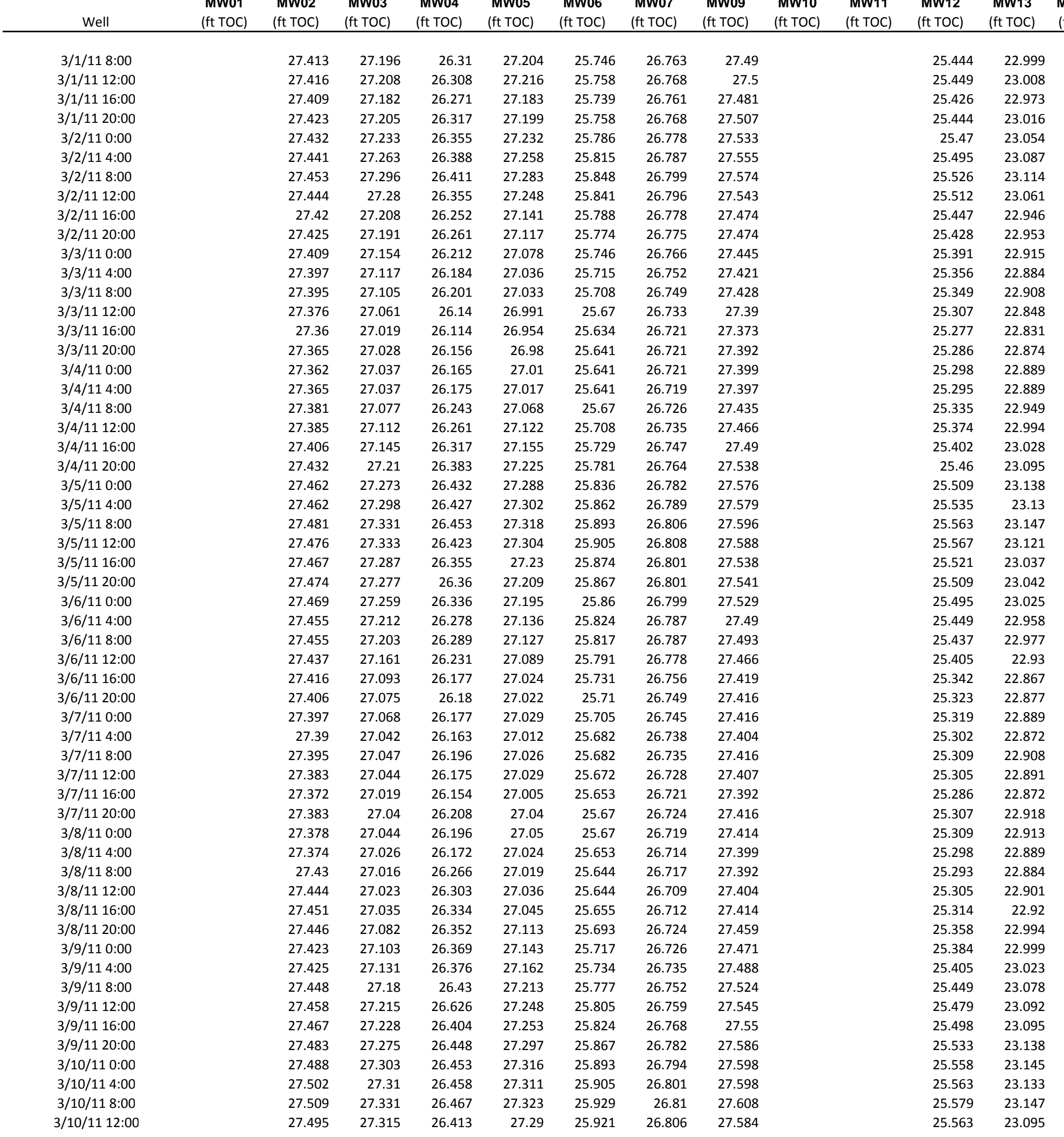


TABLE S1.1 Automatically recorded groundwater depths in monitoring wells, December 2009 to March 2013, measured from the top of the casing (ft TOC).

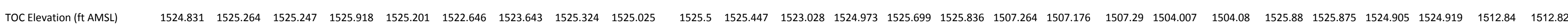

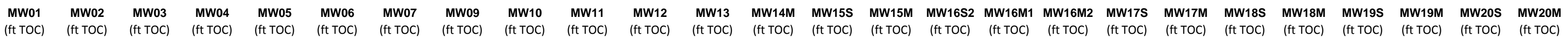

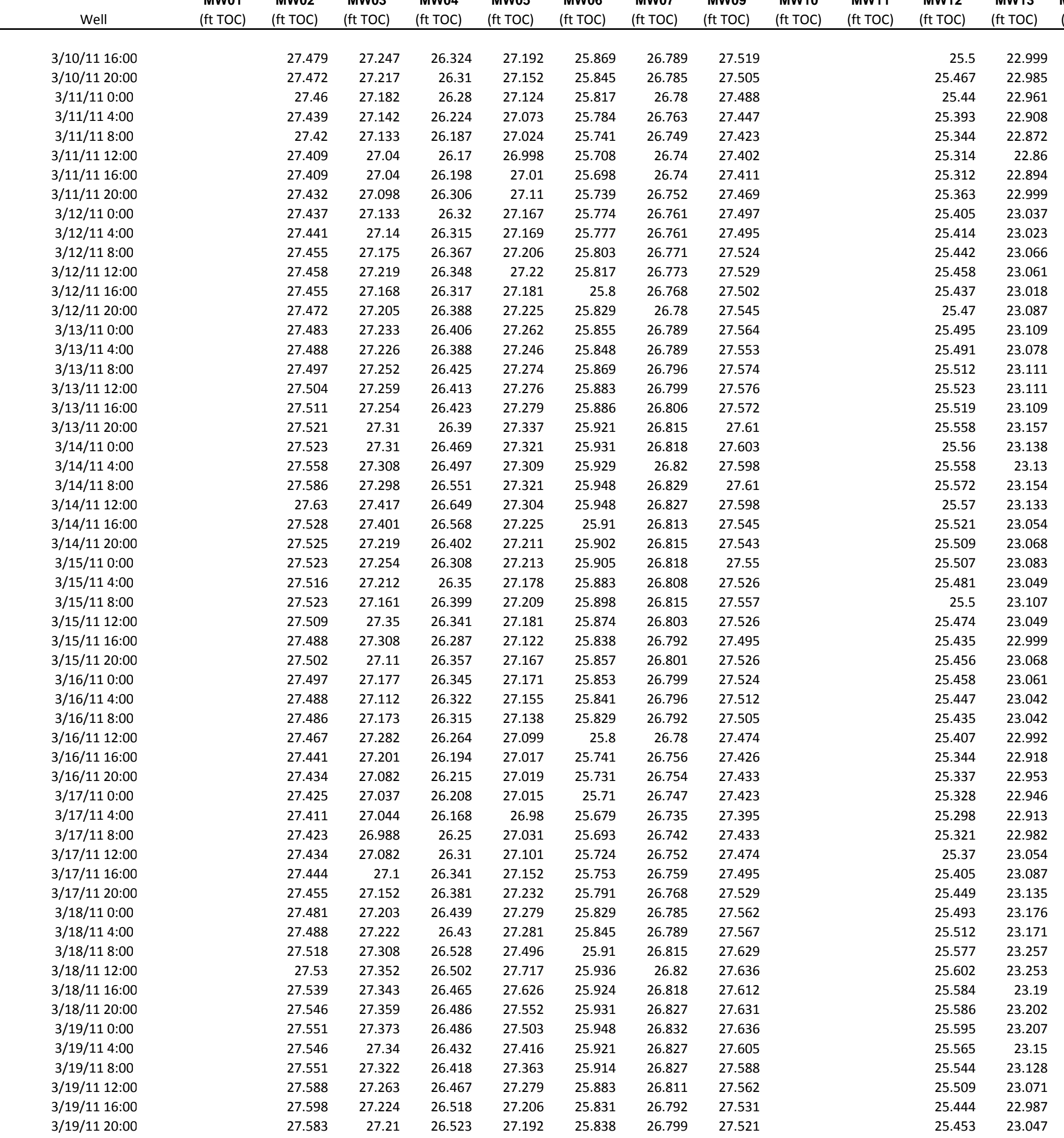


TABLE S1.1 Automatically recorded groundwater depths in monitoring wells, December 2009 to March 2013, measured from the top of the casing (ft TOC).

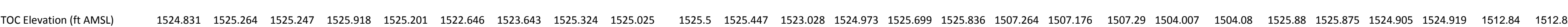

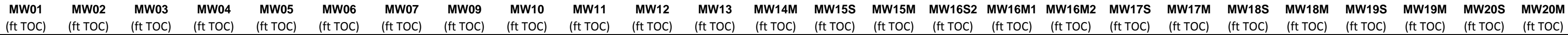

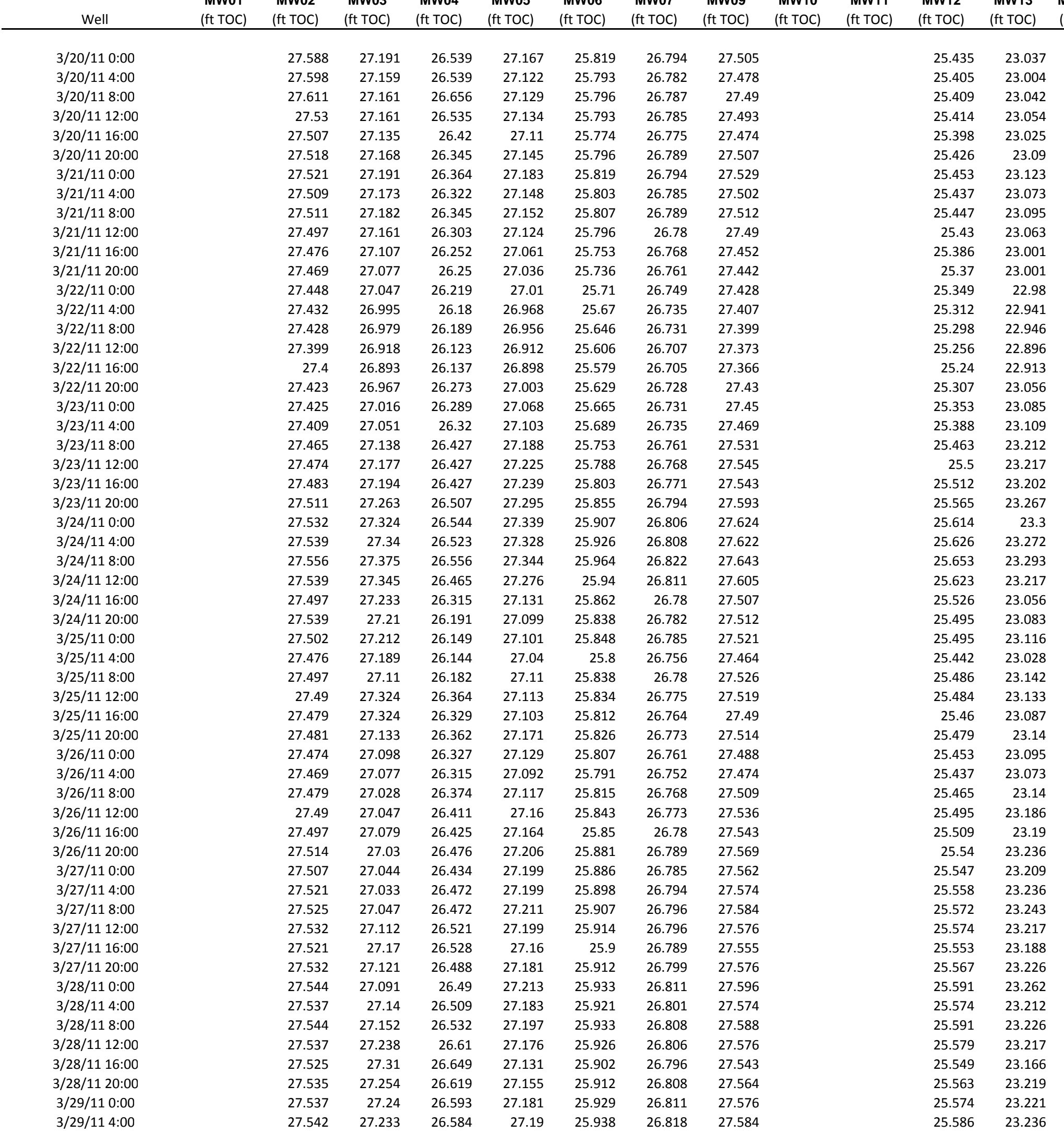


TABLE S1.1 Automatically recorded groundwater depths in monitoring wells, December 2009 to March 2013, measured from the top of the casing (ft TOC).

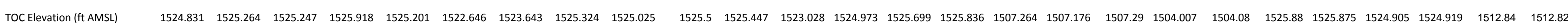

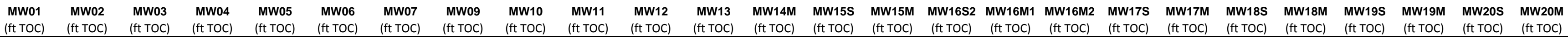

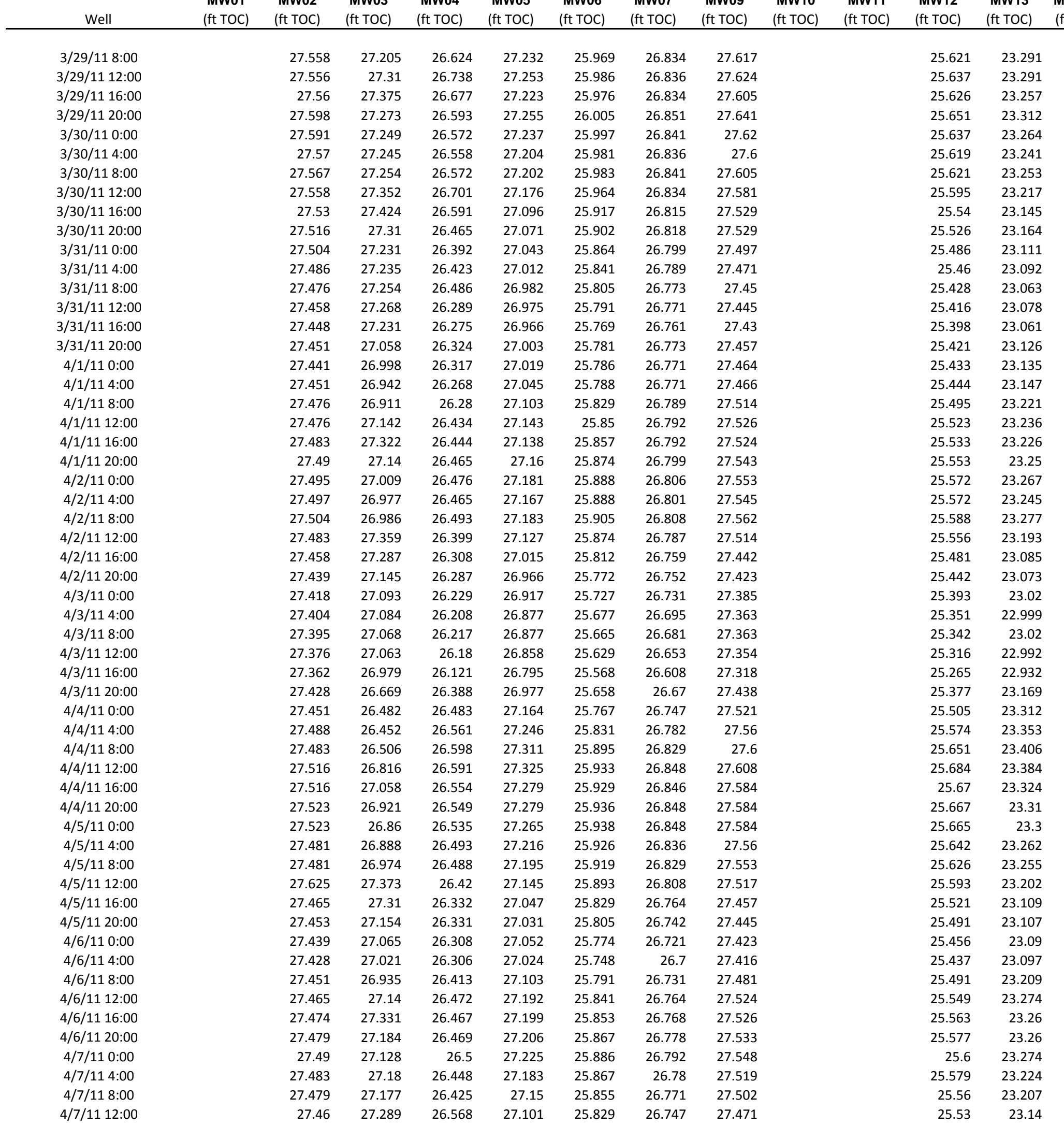


TABLE S1.1 Automatically recorded groundwater depths in monitoring wells, December 2009 to March 2013, measured from the top of the casing (ft TOC).

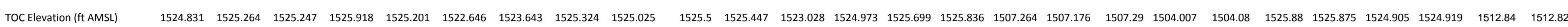

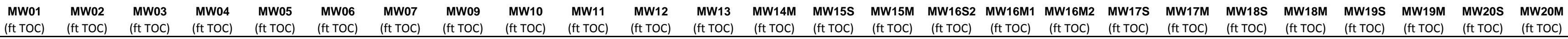

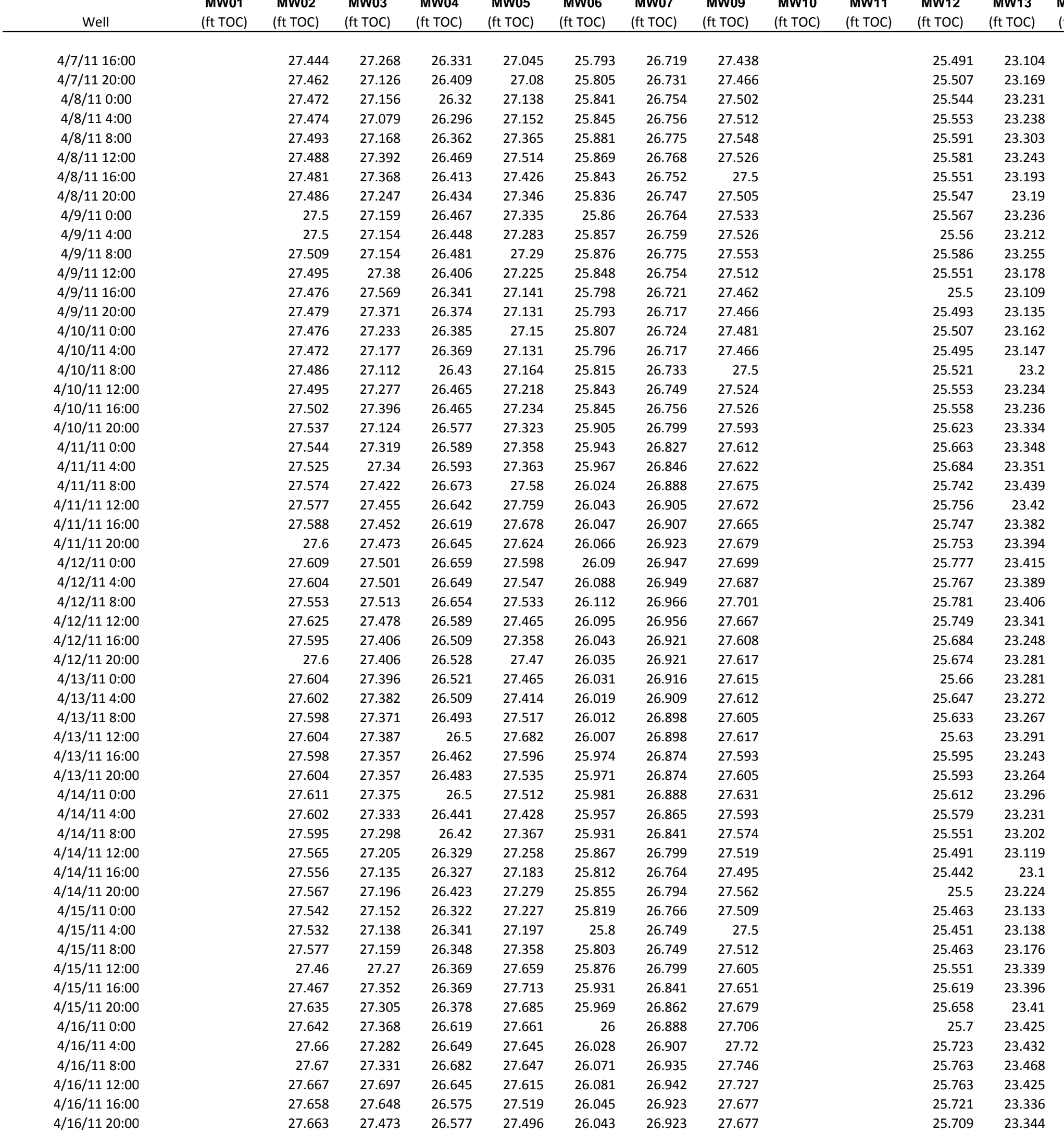


TABLE S1.1 Automatically recorded groundwater depths in monitoring wells, December 2009 to March 2013, measured from the top of the casing (ft TOC).

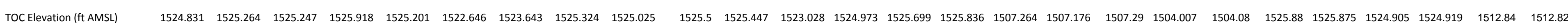

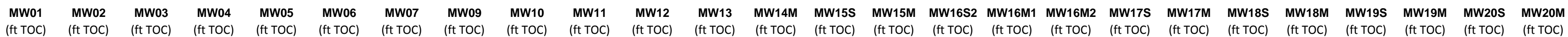

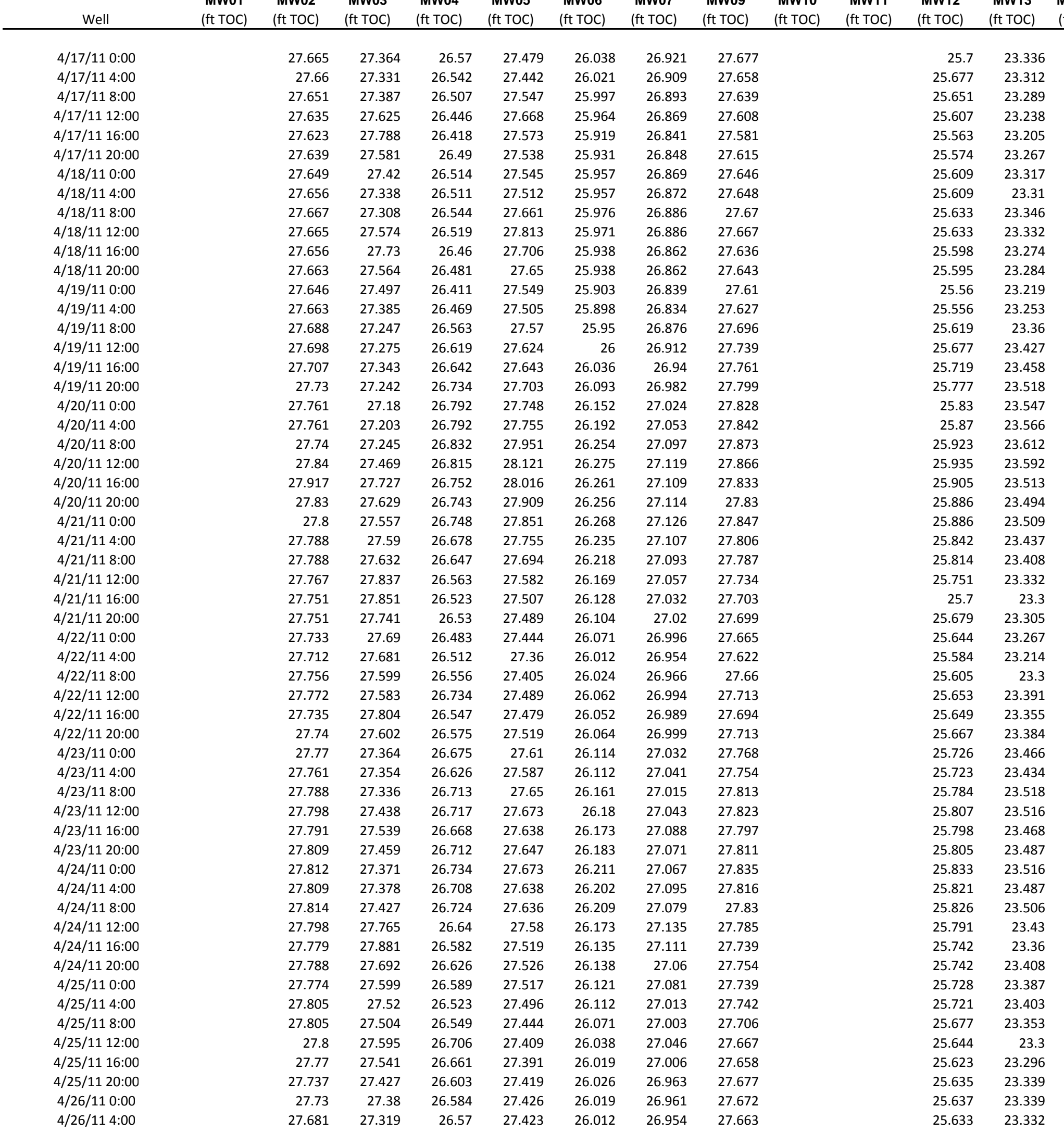


TABLE S1.1 Automatically recorded groundwater depths in monitoring wells, December 2009 to March 2013, measured from the top of the casing (ft TOC).

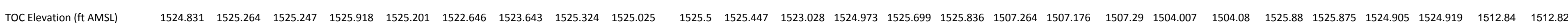

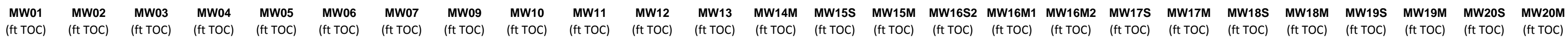

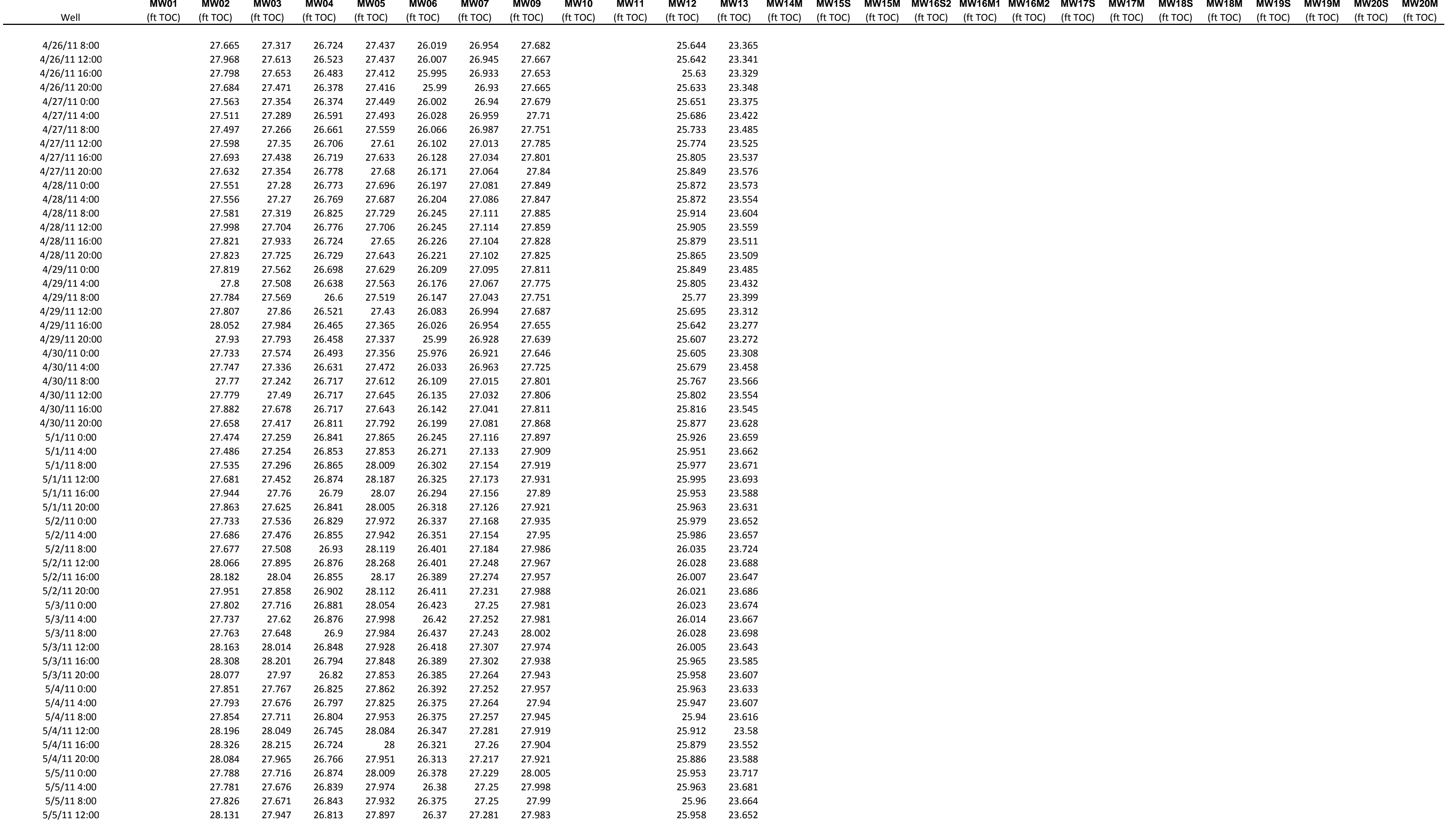


TABLE S1.1 Automatically recorded groundwater depths in monitoring wells, December 2009 to March 2013, measured from the top of the casing (ft TOC).

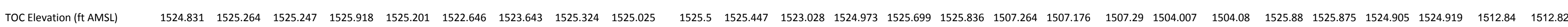

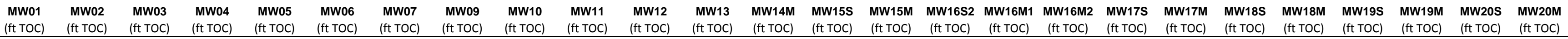

\begin{tabular}{|c|c|c|c|c|c|c|c|c|c|c|c|}
\hline Well & $\begin{array}{l}\text { MW01 } \\
\text { (ft TOC) }\end{array}$ & $\begin{array}{l}\text { MW02 } \\
\text { (ft TOC) }\end{array}$ & $\begin{array}{r}\text { MW03 } \\
\text { (ft TOC) }\end{array}$ & $\begin{array}{l}\text { MW04 } \\
\text { (ft TOC) }\end{array}$ & $\begin{array}{l}\text { MW05 } \\
\text { (ft TOC) }\end{array}$ & $\begin{array}{l}\text { MW06 } \\
\text { (ft TOC) }\end{array}$ & $\begin{array}{l}\text { MW07 } \\
\text { (ft Toc) }\end{array}$ & $\begin{array}{l}\text { MW09 } \\
\text { (ft TOC) }\end{array}$ & $\begin{array}{cc}\text { MW10 } & \text { MW11 } \\
(\mathrm{ft} \text { TOC) } & \text { (ft TOC) }\end{array}$ & $\begin{array}{r}\text { MW12 } \\
\text { (ft TOC) }\end{array}$ & $\begin{array}{l}\begin{array}{l}\text { WW13 } \\
\text { (ft Toc) }\end{array} \\
\end{array}$ \\
\hline 5/5/11 16:00 & & 28.238 & 28.096 & 26.771 & 27.832 & 26.34 & 27.288 & 27.95 & & 25.926 & 23.599 \\
\hline 5/5/11 20:00 & & 28.049 & 27.87 & 26.78 & 27.874 & 26.335 & 27.257 & 27.95 & & 25.923 & 23.612 \\
\hline 5/6/11 0:00 & & 27.854 & 27.681 & 26.785 & 27.883 & 26.332 & 27.243 & 27.959 & & 25.926 & 23.624 \\
\hline 5/6/111 4:00 & & 27.823 & 27.611 & 26.734 & 27.82 & 26.309 & 27.255 & 27.931 & & 25.9 & 23.58 \\
\hline 5/6/11 8:00 & & 27.905 & 27.676 & 26.694 & 27.921 & 26.28 & 27.255 & 27.904 & & 25.87 & 23.554 \\
\hline $5 / 6 / 1112: 00$ & & 28.263 & 28.014 & 26.642 & 28.04 & 26.233 & 27.22 & 27.861 & & 25.821 & 23.499 \\
\hline $5 / 6 / 1116: 00$ & & 28.42 & 28.175 & 26.589 & 27.928 & 26.176 & 27.182 & 27.833 & & 25.77 & 23.451 \\
\hline 5/6/11 20:00 & & 28.182 & 27.975 & 26.612 & 27.879 & 26.164 & 27.156 & 27.837 & & 25.76 & 23.473 \\
\hline 5/7/11 0:00 & & 27.944 & 27.762 & 26.633 & 27.851 & 26.166 & 27.121 & 27.859 & & 25.772 & 23.513 \\
\hline 5/7/11 4:00 & & 27.83 & 27.632 & $\begin{array}{l}20.033 \\
26.649\end{array}$ & 27.82 & $\begin{array}{l}26.160 \\
26.166\end{array}$ & 27.111 & 27.866 & & 25.781 & 23.523 \\
\hline 5/7/11 8:00 & & 27.816 & 27.609 & 26.687 & 27.815 & 26.176 & 27.097 & 27.892 & & 25.807 & 23.568 \\
\hline 5/7/11 12:00 & & 28.121 & 27.874 & 26.703 & 27.825 & 26.195 & 27.13 & 27.914 & & 25.83 & 23.592 \\
\hline 5/7/11 16:00 & & 28.252 & 28.079 & 26.68 & 27.79 & 26.183 & 27.149 & 27.9 & & 25.826 & 23.559 \\
\hline 5/7/11 20:00 & & 28.017 & 27.823 & 26.724 & 27.804 & 26.197 & 27.135 & 27.921 & & 25.849 & 23.602 \\
\hline 5/8/11 0:00 & & 27.823 & 27.62 & 26.71 & 27.794 & 26.204 & 27.133 & 27.928 & & 25.858 & 23.6 \\
\hline 5/8/11 4:00 & & 27.942 & 27.56 & 26.67 & 27.745 & 26.178 & 27.147 & 27.902 & & 25.842 & 23.554 \\
\hline 5/8/11 8:00 & & 27.942 & 27.581 & 26.675 & 27.895 & 26.178 & 27.137 & 27.902 & & 25.84 & 23.566 \\
\hline 5/8/11 12:00 & & 27.928 & 27.907 & 26.617 & 28.044 & 26.135 & 27.154 & 27.866 & & 25.802 & 23.516 \\
\hline 5/8/11 16:00 & & 27.919 & 28.098 & 26.582 & 27.984 & 26.1 & 27.133 & 27.859 & & 25.77 & 23.485 \\
\hline 5/8/11 20:00 & & 27.93 & 27.851 & 26.635 & 27.958 & 26.104 & 27.076 & 27.878 & & 25.784 & 23.533 \\
\hline 5/9/11 0:00 & & 27.933 & 27.655 & 26.647 & 27.949 & 26.114 & 27.067 & 27.902 & & 25.802 & 23.564 \\
\hline 5/9/11 4:00 & & 27.935 & 27.553 & 26.635 & 27.888 & 26.109 & 27.079 & 27.902 & & 25.8 & 23.547 \\
\hline 5/9/11 8:00 & & 27.94 & 27.55 & 26.656 & 28.014 & 26.116 & 27.076 & 27.916 & & 25.814 & 23.573 \\
\hline 5/9/11 12:00 & & 27.947 & 27.818 & 26.659 & 28.175 & 26.116 & 27.097 & 27.926 & & 25.821 & 23.58 \\
\hline 5/9/11 16:00 & & 27.942 & 28.012 & 26.624 & 28.093 & 26.1 & 27.133 & 27.926 & & 25.805 & 23.547 \\
\hline $5 / 9 / 1120: 00$ & & 27.958 & 27.79 & 26.687 & 28.075 & 26.107 & 27.081 & 27.952 & & 25.83 & 23.602 \\
\hline $5 / 10 / 110: 00$ & & 27.968 & 27.62 & 26.708 & 28.091 & 26.138 & 27.1 & 27.978 & & 25.863 & 23.638 \\
\hline 5/10/11 4:00 & & 27.979 & 27.553 & 26.717 & 28.04 & 26.142 & 27.107 & 27.986 & & 25.879 & 23.643 \\
\hline 5/10/11 8:00 & & 27.998 & 27.518 & 26.783 & $\begin{array}{l}28.044 \\
28.042\end{array}$ & $\begin{array}{l}26.142 \\
26.185\end{array}$ & 27.142 & 28.022 & & $\begin{array}{l}25.823 \\
25.923\end{array}$ & $\begin{array}{l}23.695 \\
23.695\end{array}$ \\
\hline $5 / 10 / 1112: 00$ & & $\begin{array}{l}27.9983 \\
27.993\end{array}$ & $\begin{array}{l}27.5818 \\
27.835\end{array}$ & $\begin{array}{l}26.1755 \\
26.755\end{array}$ & $\begin{array}{l}28.042 \\
27.995\end{array}$ & $\begin{array}{l}26.185 \\
26.188\end{array}$ & $\begin{array}{l}27.142 \\
27.142\end{array}$ & $\begin{array}{l}28.022 \\
28.007\end{array}$ & & $\begin{array}{l}25.923 \\
25.928\end{array}$ & $\begin{array}{l}23.659 \\
23.662\end{array}$ \\
\hline 5/10/11 16:00 & & 28.007 & 27.837 & 26.792 & 27.977 & 26.209 & 27.161 & 28.026 & & 25.951 & 23.693 \\
\hline 5/10/11 20:00 & & 28.019 & 27.678 & 26.822 & 27.981 & 26.23 & 27.18 & $\begin{array}{l}28.020 \\
28.043\end{array}$ & & 25.977 & 23.719 \\
\hline 5/11/11 0:00 & & 28.019 & 27.569 & 26.822 & 27.967 & 26.247 & 27.194 & 28.041 & & 25.991 & 23.71 \\
\hline 5/11/11 4:00 & & 28.024 & 27.539 & 26.822 & 27.944 & 26.256 & 27.198 & $\begin{array}{l}28.041 \\
28.038\end{array}$ & & $\begin{array}{l}25.995 \\
25.995\end{array}$ & 23.702 \\
\hline 5/11/11 8:00 & & 28.031 & 27.588 & 26.839 & 28.093 & 26.273 & 27.213 & 28.048 & & 26.009 & 23.717 \\
\hline $5 / 11 / 1112: 00$ & & 28.021 & 27.746 & 26.834 & 28.303 & 26.292 & 27.276 & 28.055 & & 26.023 & 23.731 \\
\hline $5 / 11 / 1116: 00$ & & 28.026 & 27.853 & 26.799 & 28.191 & 26.271 & 27.22 & 28.026 & & 25.988 & 23.688 \\
\hline $5 / 11 / 1120: 00$ & & 28.049 & 27.697 & 26.867 & 28.152 & 26.292 & 27.198 & 28.067 & & 26.014 & 23.741 \\
\hline $5 / 12 / 110: 00$ & & 28.052 & 27.616 & 26.867 & 28.124 & 26.316 & 27.234 & 28.081 & & 26.037 & 23.753 \\
\hline $5 / 12 / 114: 00$ & & 28.056 & 27.643 & 26.85 & 28.058 & 26.309 & 27.255 & 28.067 & & 26.028 & 23.724 \\
\hline 5/12/11 8:00 & & 28.073 & 27.881 & 26.914 & 28.072 & 26.349 & 27.236 & 28.105 & & 26.063 & 23.779 \\
\hline 5/12/11 12:00 & & 28.068 & 27.872 & 26.872 & 28.028 & 26.351 & 27.292 & 28.084 & & 26.058 & 23.743 \\
\hline $5 / 12 / 1116: 00$ & & 28.077 & 27.867 & 26.876 & 28.012 & 26.349 & 27.29 & 28.079 & & 26.053 & 23.736 \\
\hline 5/12/11 20:00 & & 28.087 & 27.891 & 26.909 & 28.128 & 26.375 & 27.267 & 28.105 & & 26.072 & 23.774 \\
\hline 5/13/11 0:00 & & 28.094 & 27.907 & 26.916 & 28.152 & 26.389 & 27.274 & 28.11 & & 26.079 & 23.777 \\
\hline 5/13/11 4:00 & & 28.126 & 27.954 & 26.86 & 28.156 & 26.435 & 27.29 & 28.165 & & 26.123 & 23.834 \\
\hline 5/13/11 8:00 & & $\begin{array}{l}28.120 \\
27.921\end{array}$ & $\begin{array}{l}27.934 \\
28\end{array}$ & $\begin{array}{l}20.80 \\
26.89\end{array}$ & 28.315 & $\begin{array}{r}\begin{array}{r}2.453 \\
26.47\end{array} \\
\end{array}$ & 27.318 & $\begin{array}{r}28.103 \\
28.16\end{array}$ & & 26.156 & $\begin{array}{l}23.845 \\
23.865 \\
\end{array}$ \\
\hline $\begin{array}{l}5 / 13 / 111 \\
5 / 02: 00\end{array}$ & & $\begin{array}{l}21.921 \\
28.005\end{array}$ & 28.037 & $\begin{array}{l}27.049 \\
272\end{array}$ & $\begin{array}{l}28.462 \\
28.452\end{array}$ & $\begin{array}{l}26.47 \\
26.496\end{array}$ & 27.347 & $\begin{array}{l}28.170 \\
28.172\end{array}$ & & 26.177 & $\begin{array}{l}23.8302 \\
23.882\end{array}$ \\
\hline 5/13/11 16:00 & & 27.863 & 28.051 & 26.986 & 28.376 & 26.494 & 27.379 & 28.17 & & 26.174 & 23.851 \\
\hline 5/13/11 20:00 & & 27.891 & 28.082 & 27.033 & 28.327 & $\begin{array}{l}20.4534 \\
26.534\end{array}$ & 27.375 & 28.196 & & 26.2 & 23.884 \\
\hline 5/14/11 0:00 & & 27.924 & 28.089 & 27.009 & 28.268 & 26.551 & 27.415 & 28.191 & & 26.205 & 23.868 \\
\hline 5/14/11 4:00 & & 27.956 & 28.079 & 26.995 & 28.198 & 26.551 & 27.436 & 28.184 & & 26.195 & 23.841 \\
\hline 5/14/11 8:00 & & 27.991 & 28.091 & 27.019 & 28.177 & 26.57 & 27.422 & 28.206 & & 26.205 & 23.863 \\
\hline 5/14/11 12:00 & & 28 & 28.061 & 26.956 & 28.11 & 26.558 & 27.481 & 28.175 & & 26.179 & 23.81 \\
\hline 5/14/111 16:00 & & 28.014 & 28.019 & 26.925 & 28.037 & 26.532 & 27.478 & 28.146 & & 26.146 & 23.767 \\
\hline 5/14/11 20:00 & & 28.045 & 28.037 & 26.991 & 28.054 & 26.556 & 27.436 & 28.182 & & 26.163 & 23.829 \\
\hline
\end{tabular}


TABLE S1.1 Automatically recorded groundwater depths in monitoring wells, December 2009 to March 2013, measured from the top of the casing (ft TOC).

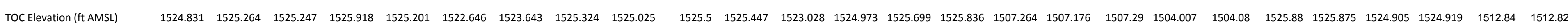

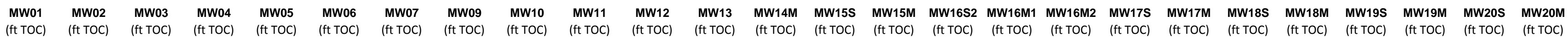

Well

$5 / 15 / 110: 00$

5/15/114:00

5/15/111 12:00

5/15/1120:00

$5 / 16 / 110: 00$

$5 / 16 / 118.00$

$5 / 16 / 1112: 00$

$5 / 16 / 1116: 00$

$5 / 16 / 1120: 00$

$5 / 17 / 114: 00$

$5 / 17 / 118: 00$

5/17/11 16:00

5/17/11 20:00

$5 / 18 / 114: 00$

$5 / 18 / 118: 00$

$5 / 18 / 111600$

5/18/11 20:00

5/19/11 0:00

5/19/11 4:00

5/19/11 12:00

5/19/11 16:00

$5 / 20 / 110: 00$

5/20/11 4:00

5/20/11 8:00

5/20/11 16:00

5/20/11 20:00

5/21/11 0:00

$5 / 21 / 114: 00$

5/21/11 12:00

5/21/11 20:00

5/22/11 0:00

5/22/11 8:00

5/22/11 12:00

5/22/11 16:00

5/23/11 0:00

5/23/11 12:00

5/23/11 16:00

5/23/11 20:00

\begin{tabular}{rrrrrrr}
28.045 & 28.033 & 26.97 & 28.047 & 26.563 & 27.459 & 28.184 \\
\hline & 28.059 & 26963 & 28.016 & 2656 & 27.469 & 28.172
\end{tabular}

$\begin{array}{rrrrrrr}28.059 & 28 & 26.963 & 28.016 & 26.556 & 27.469 & 28.172 \\ 28.073 & 28.003 & 26.991 & 28.026 & 26.568 & 27.452 & 28.187\end{array}$

$\begin{array}{rrrrrrr}28.07 & 28.154 & 26.958 & 28 & 26.56 & 27.481 & 28.175\end{array}$

$\begin{array}{rrrrrrrr}28.082 & 28.119 & 26.963 & 27.988 & 26.56 & 27.483 & 28.172 \\ 28.091 & 27.963 & 26.984 & 27.995 & 26.555 & 27.462 & 28.187\end{array}$

$\begin{array}{lllllll}28.091 & 27.963 & 26.984 & 27.995 & 26.565 & 27.462 & 28.187 \\ 28.094 & 27.886 & 26.995 & 28.007 & 26.577 & 27.457 & 28.198\end{array}$

$\begin{array}{lllllllll}28.103 & 27.879 & 26.981 & 27.988 & 26.565 & 27.473 & 28.184 \\ 28.107 & 27.902 & 26.993 & 27.93 & 26577 & 27.776 & 28.94\end{array}$

$\begin{array}{llllllll}28.1 & 28.126 & 26.946 & 27.963 & 26.565 & 27.518 & 28.172 \\ & 22.58 & 26.872 & 27.383 & 26.522 & 27.999 & 28.117\end{array}$

$\begin{array}{lllllll}28.089 & 28.058 & 26.872 & 27.883 & 26.522 & 27.499 & 28.117\end{array}$

$\begin{array}{rrrrrrr}28.096 & 27.886 & 26.89 & 27.872 & 26.515 & 27.457 & 28.117 \\ 28.103 & 27.704 & 26.902 & 27.921 & 26.52 & 27.45 & 28.127\end{array}$

$\begin{array}{rrrrrrr}28.103 & 27.704 & 26.902 & 27.921 & 26.52 & 27.45 & 28.127 \\ 28.105 & 27.758 & 26.89 & 27.9 & 26.501 & 27.441 & 28.12\end{array}$

\begin{tabular}{llllllll}
28.112 & 27.816 & 26.909 & 27.907 & 26.511 & 27.431 & 28.134 \\
\hline & 27.996 & 26.95 & 27.85 & 26.482 & 27.471 & 28.098
\end{tabular}

$28.077 \quad 27.93$

$\begin{array}{lllllll}28.084 & 27.795 & 26.822 & 27.773 & 26.427 & 27.386 & 28.067 \\ 2877 & 27.71 & 26.78 & 27.73 & 2623 & 2739 & 28065\end{array}$

$\begin{array}{llllllll}28.077 & 27.671 & 26.818 & 27.783 & 26.423 & 27.379 & 28.065 \\ 28.107 & 27.609 & 26.776 & 27.757 & 26.401 & 27.379 & 28.048 \\ 28.758 & 27.799 & 2682 & 27.77 & 26.401 & 27.350 & \end{array}$

$\begin{array}{lllllll}28.107 & 27.609 & 26.776 & 27.757 & 26.401 & 27.379 & 28.048 \\ 28.035 & 27.599 & 26.822 & 27.776 & 26.401 & 27.358 & 28.06\end{array}$

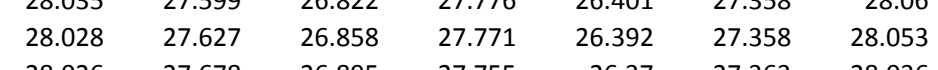

\begin{tabular}{rrrrrr}
28.026 & 27.678 & 26.895 & 27.755 & 26.37 & 27.363 \\
\hline & 27.671 & 269 & 27.76 & 26.378 & 27.332
\end{tabular}

$\begin{array}{rrrrrrrr}28.045 & 27.671 & 26.99 & 26.995 & 27.762 & 26.378 & 27.335 & 28036 \\ & 27.69 & 26.368 & 27.342 & 28.038\end{array}$

$\begin{array}{rrrrrrrr}28.049 & 27.713 & 26.935 & 27.734 & 26.349 & 27.332 & 28.026 \\ 28.035 & 27.69 & 26.946 & 27.78 & 26.373 & 27.3 & 28.06 \\ 28098 & 27.788 & 27.143 & 27.76 & 26361 & 27.328 & 2800\end{array}$

$\begin{array}{rrrrrrr}28.035 & 27.69 & 26.946 & 27.78 & 26.373 & 27.3 & 28.06 \\ 28.098 & 27.788 & 27.143 & 27.769 & 26.361 & 27.328 & 28.048\end{array}$

$\begin{array}{rrrrrrrr}28 & 27.811 & 27.977 & 27.769 & 26.361 & 27.328 & 28.048 \\ 27.339 & 28.01 \\ 27.993 & 27.72 & 26825 & 27.59 & 2639 & 27.309 & 28.52\end{array}$

\begin{tabular}{lll}
2637 & 27.295 & 28.052 \\
\hline & 28.076
\end{tabular}

\begin{tabular}{llllllll}
27.998 & 27.636 & 26.881 & 27.808 & 26.375 & 27.328 & 28.139 \\
\hline
\end{tabular}

$\begin{array}{rrrrrrr}27.877 & 27.625 & 26.879 & 27.808 & 26.392 & 27.321 & 28.124 \\ 27.84 & 27.744 & 27.122 & 27.797 & 26.397 & 27.332 & 28.06 \\ 27.82 & 27.81 & 27005 & 27.78 & 2737 & 27.35 & 28.08\end{array}$

$\begin{array}{rrrrrrr}27.84 & 27.744 & 27.122 & 27.797 & 26.397 & 27.332 & 28.06 \\ 27.802 & 27.881 & 27.075 & 27.748 & 26.37 & 27.365 & 28.028 \\ 27.81 & 27.751 & 26.35 & 27.75 & 26385 & 27.38 & 28.05\end{array}$

$\begin{array}{llllllll}27.819 & 27.751 & 26.825 & 27.755 & 26.385 & 27.318 & 28.05 \\ 27.85 & 27.734 & 26.89 & 27.74 & 2635 & 27.34 & 2802\end{array}$

$\begin{array}{rrrrrrrr}27.835 & 27.634 & 26.9 & 27.764 & 26.385 & 27.314 & 28.062 \\ 27.849 & 27.634 & 27.375 & 27.73 & 26.375 & 27.33 & 28.057\end{array}$

\begin{tabular}{lllllllll}
27.872 & 27.643 & 26.914 & 27.769 & 26.399 & 27.314 & 28.083 \\
\hline
\end{tabular}

$\begin{array}{llllllll}27.877 & 27.881 & 26.862 & 27.731 & 26.378 & 27.368 & 28.055 \\ 27.886 & 27.975 & 26.55 & 27.77 & 26368 & 27.321 & 28.055\end{array}$

$\begin{array}{rrrrrrr}27.886 & 27.975 & 26.855 & 27.701 & 26.363 & 27.356 & 28.04 \\ 27.9 & 27.802 & 26.874 & 27.71 & 26.368 & 27.321 & 28.055\end{array}$

\begin{tabular}{rlllllll}
27.91 & 27.823 & 26.874 & 27.872 & 27.711 & 26.368 & 27.321 & 28.055 \\
27.307 & 28.06 \\
\hline 27.921 & 27.571 & 27.36 & 27.65 & 2635 & 27.307 & 28053
\end{tabular}

\begin{tabular}{llllllll}
27.921 & 27.571 & 26.869 & 27.696 & 26.356 & 27.307 & 28.053 \\
27.942 & 27.555 & 26.935 & 27.748 & 26385 & 27.271 & 28.095 \\
\hline
\end{tabular}

$\begin{array}{llllllll}27.949 & 27.9 & 26.928 & 27.764 & 26.394 & 27.314 & 28.103\end{array}$

$\begin{array}{llllllll}27.954 & 27.886 & 26.909 & 27.75 & 26.385 & 27.344 & 28.091 \\ 27.956 & 27.725 & 27.693 & 27.72 & 26.375 & 27.85 & 28.127\end{array}$

$\begin{array}{llllllll}27.956 & 27.725 & 26.893 & 27.727 & 26.375 & 27.335 & 28.081 \\ 27.979 & 27.769 & 26.97 & 27.778 & 26.408 & 27.281 & 28.127\end{array}$

\begin{tabular}{llllllll}
27.979 & 27.769 & 26.89 & 27.727 & 26.375 & 27.335 & 28.081 \\
27.979 & 27.699 & 26944 & 27.765 & 26.408 & 27.281 & 28.127 \\
\hline & 27.999 & 27.311 & 28115
\end{tabular}

\begin{tabular}{lllllllll}
27.986 & 27.695 & 26.958 & 27.778 & 26.413 & 27.307 & 28.131 \\
\hline
\end{tabular}

$\begin{array}{llllllll}27.989 & 27.886 & 26.96 & 27.788 & 26.413 & 27.337 & 28.134\end{array}$

$\begin{array}{llllllll}27.982 & 27.907 & 26.914 & 27.762 & 26.406 & 27.372 & 28.112 \\ 28.003 & 27.765 & 26.949 & 27.769 & 26.408 & 27.335 & 28.127 \\ 28007 & 27.781 & 26.96 & 27.792 & 26.42 & 27.328 & 28.139\end{array}$

\begin{tabular}{rrrrrrrr}
28.003 & 27.765 & 26.949 & 27.769 & 26.408 & 27.335 & 28.127 \\
28.007 & 27.781 & 2696 & 27.792 & 26.42 & 27.328 & 28.139 \\
\hline
\end{tabular}

$\begin{array}{rrrrrrr}28.007 & 27.781 & 26.96 & 27.792 & 26.42 & 27.328 & 28.139 \\ 28.005 & 27.779 & 26.93 & 27.766 & 26.411 & 27.347 & 28.122\end{array}$

$\begin{array}{rr}26.167 & 23.832 \\ 26.153 & 23.81 \\ 26.163 & 23.839 \\ 26.151 & 23.815 \\ 26.144 & 23.815 \\ 26.156 & 23.841 \\ 26.17 & 23.853 \\ 26.163 & 23.836 \\ 26.17 & 23.848 \\ 26.153 & 23.81 \\ 26.102 & 23.734 \\ 26.091 & 23.755 \\ 26.098 & 23.779 \\ 26.088 & 23.772 \\ 26.098 & 23.801 \\ 26.07 & 23.746 \\ 26.014 & 23.674 \\ 26.016 & 23.722 \\ 26.016 & 23.726 \\ 26.002 & 23.705 \\ 26.014 & 23.731 \\ 26.012 & 23.729 \\ 25.998 & 23.712 \\ 26.007 & 23.736 \\ 26.005 & 23.722 \\ 25.9911 & 23.714 \\ 26.021 & 23.772 \\ 26.016 & 23.746 \\ 25.988 & 23.681 \\ 26.016 & 23.757 \\ 26.046 & 23.803 \\ 26.06 & 23.798 \\ 26.084 & 23.805 \\ 26.084 & 23.813 \\ 26.065 & 23.769 \\ 26.079 & 23.796 \\ 26.091 & 23.813 \\ 26.088 & 23.793 \\ 26.109 & 23.844 \\ 26.086 & 23.779 \\ 26.07 & 23.762 \\ 26.081 & 23.791 \\ 26.084 & 23.796 \\ 26.074 & 23.779 \\ 26.116 & 23.853 \\ 26.128 & 23.848 \\ 26.121 & 23.82 \\ 26.112 & 23.803 \\ 26.149 & 23.882 \\ 26.144 & 23.836 \\ 26.158 & 23.868 \\ 26.165 & 23.868 \\ 26.146 & 23.827 \\ 26.153 & 23.853 \\ 26.167 & 23.86 \\ 26.153 & 23.834 \\ & \\ & \end{array}$


TABLE S1.1 Automatically recorded groundwater depths in monitoring wells, December 2009 to March 2013, measured from the top of the casing (ft TOC).

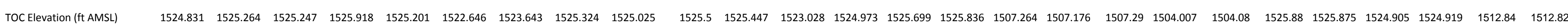

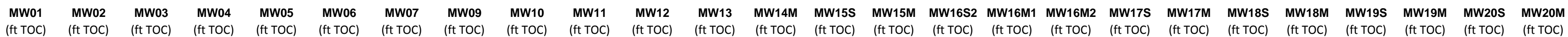

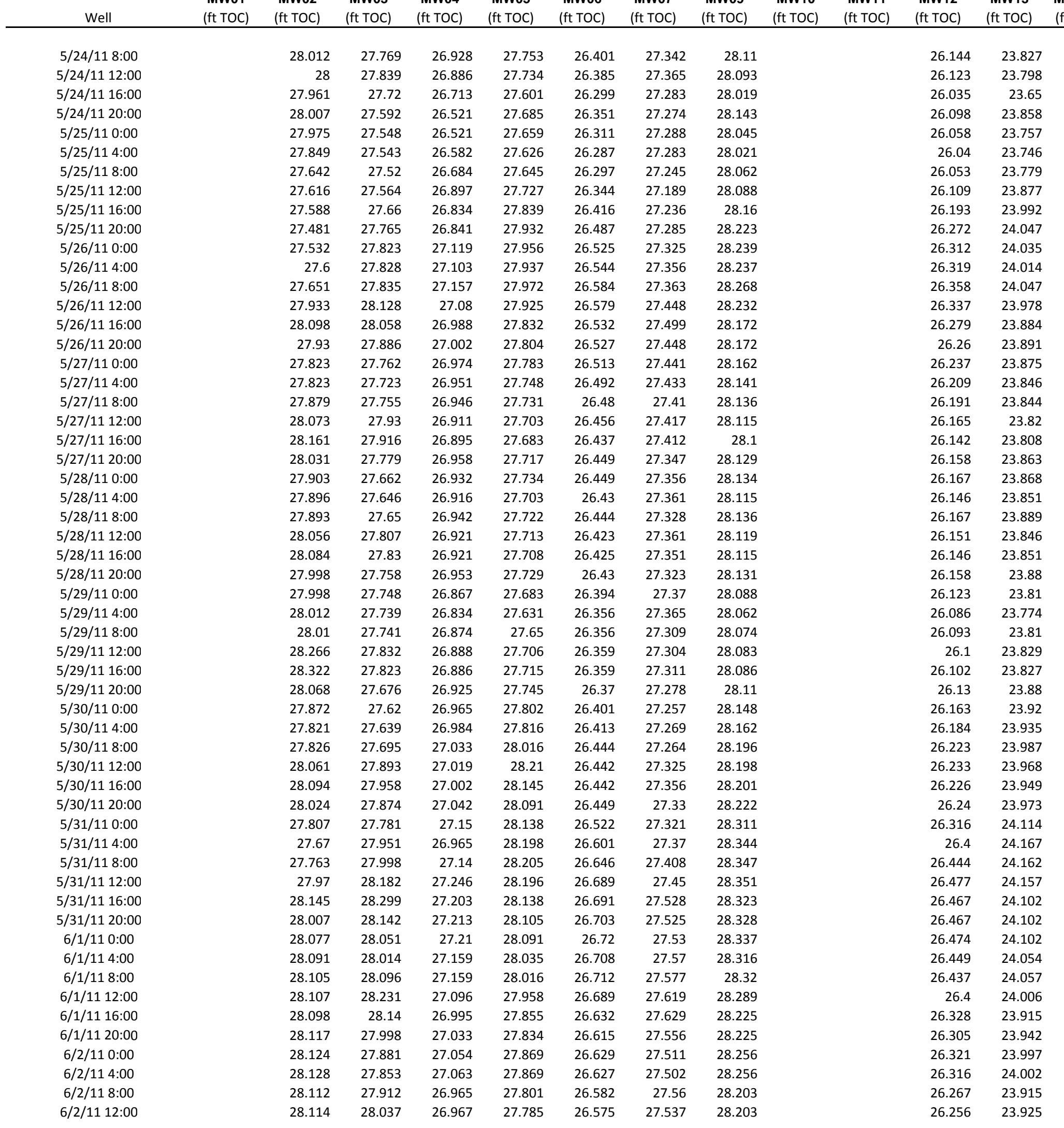


TABLE S1.1 Automatically recorded groundwater depths in monitoring wells, December 2009 to March 2013, measured from the top of the casing (ft TOC).

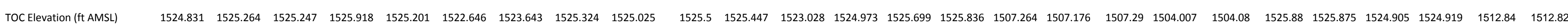

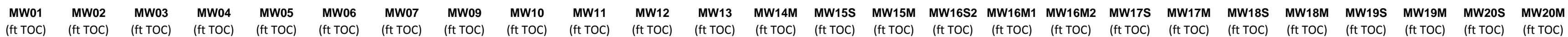

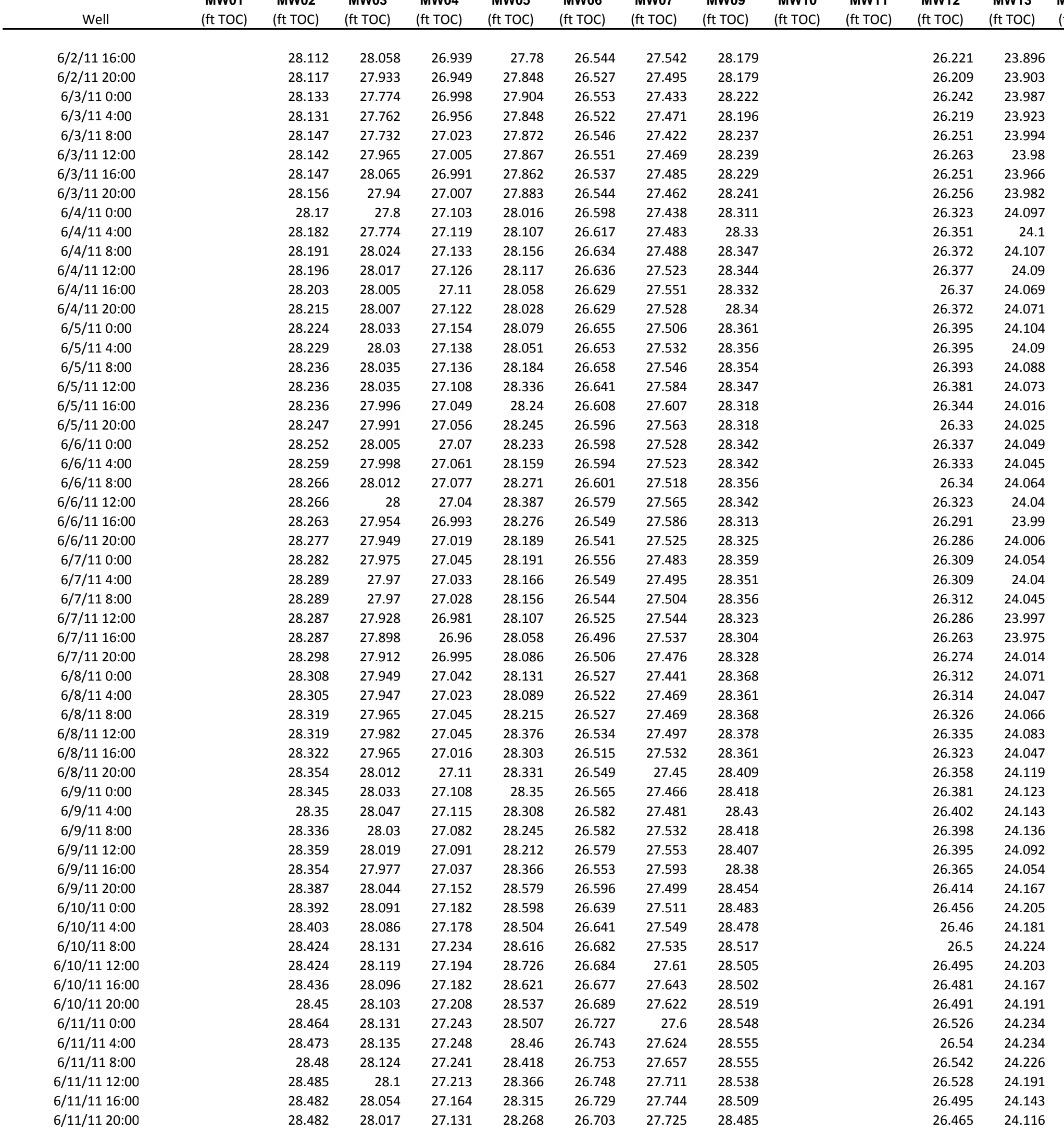


TABLE S1.1 Automatically recorded groundwater depths in monitoring wells, December 2009 to March 2013, measured from the top of the casing (ft TOC).

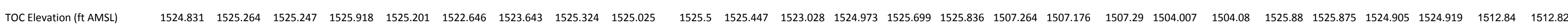

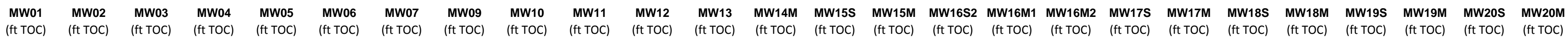

\begin{tabular}{|c|c|c|c|c|c|c|c|c|c|c|c|}
\hline Well & $\begin{array}{l}\text { MW01 } \\
\text { (ft TOC) }\end{array}$ & $\begin{array}{l}\text { MW02 } \\
(\mathrm{ft} \text { ToC) }\end{array}$ & $\begin{array}{l}\text { MW03 } \\
\text { (ft ToC) }\end{array}$ & $\begin{array}{l}\text { MW04 } \\
\text { (ft TOC) }\end{array}$ & $\begin{array}{l}\text { MW05 } \\
\text { (ft TOC) }\end{array}$ & $\begin{array}{l}\text { MW06 } \\
\text { (ft TOC) }\end{array}$ & $\begin{array}{l}\text { MW07 } \\
\text { (ft TOC) }\end{array}$ & $\begin{array}{l}\text { MW09 } \\
\text { (ft TOC) }\end{array}$ & $\begin{array}{cc}\text { MW10 } & \text { MW11 } \\
(\mathrm{ft} \text { TOC) } & \text { (ft TOC) }\end{array}$ & $\begin{array}{r}\text { MW12 } \\
\text { (ft TOC) }\end{array}$ & $\begin{array}{l}\begin{array}{l}\text { WW13 } \\
\text { (ft Toc) }\end{array} \\
\end{array}$ \\
\hline 6/12/11 0:00 & & 28.55 & 28.007 & 27.194 & 28.254 & 26.705 & 27.744 & 28.488 & & 26.472 & 24.136 \\
\hline $6 / 12 / 114: 00$ & & 28.487 & 28.007 & 27.15 & 28.21 & 26.696 & 27.706 & 28.469 & & 26.451 & 24.104 \\
\hline 6/12/11 8:00 & & 28.317 & 28.089 & 27.187 & 28.439 & 26.76 & 27.615 & 28.557 & & 26.523 & 24.253 \\
\hline $6 / 12 / 1112: 00$ & & 28.324 & 28.061 & 27.561 & 28.556 & 26.732 & 27.711 & 28.505 & & 26.486 & 24.167 \\
\hline 6/12/11 16:00 & & 28.326 & 28.049 & 27.145 & 28.467 & 26.717 & 27.723 & 28.5 & & 26.47 & 24.145 \\
\hline 6/12/11 20:00 & & 28.35 & 28.056 & 27.159 & 28.392 & 26.717 & 27.685 & 28.509 & & 26.465 & 24.157 \\
\hline $6 / 13 / 110: 00$ & & 28.366 & 28.056 & 27.145 & 28.341 & 26.712 & 27.676 & 28.519 & & 26.463 & 24.164 \\
\hline 6/13/11 4:00 & & 28.38 & 28.033 & 27.122 & 28.261 & 26.691 & 27.687 & 28.497 & & 26.44 & 24.126 \\
\hline $6 / 13 / 118: 00$ & & 28.389 & 28.047 & 27.129 & 28.355 & 26.689 & 27.666 & 28.504 & & 26.44 & 24.143 \\
\hline 6/13/11 12:00 & & 28.399 & 28.051 & 27.115 & 28.479 & 26.682 & 27.68 & 28.505 & & 26.433 & 24.145 \\
\hline $6 / 13 / 1116: 00$ & & 28.401 & 28.021 & 27.073 & 28.385 & 26.646 & 27.709 & 28.488 & & 26.398 & 24.095 \\
\hline 6/13/11 20:00 & & 28.42 & 28.061 & 27.126 & 28.343 & 26.663 & 27.64 & 28.521 & & 26.423 & 24.152 \\
\hline 6/14/11 0:00 & & 28.422 & 28.065 & 27.117 & 28.292 & 26.663 & 27.638 & 28.526 & & 26.428 & 24.155 \\
\hline 6/14/11 4:00 & & 28.44 & 28.072 & 27.143 & 28.257 & 26.67 & 27.619 & 28.533 & & 26.437 & 24.169 \\
\hline 6/14/11 8:00 & & 28.454 & 28.098 & 27.173 & 28.259 & 26.686 & 27.615 & 28.555 & & 26.46 & 24.198 \\
\hline $6 / 14 / 1112: 00$ & & 28.457 & 28.112 & 27.18 & 28.25 & 26.693 & 27.64 & 28.557 & & 26.474 & 24.2 \\
\hline $6 / 14 / 1116: 00$ & & 28.461 & 28.091 & 27.15 & 28.205 & 26.686 & 27.683 & 28.54 & & 26.467 & 24.174 \\
\hline $6 / 14 / 1120: 00$ & & 28.471 & 28.117 & 27.192 & 28.245 & 26.705 & 27.65 & 28.567 & & 26.493 & 24.222 \\
\hline 6/15/11 0:00 & & 28.492 & 28.145 & 27.241 & 28.268 & 26.734 & 27.626 & 28.591 & & 26.521 & 24.253 \\
\hline 6/15/11 4:00 & & 28.503 & 28.156 & 27.257 & 28.268 & 26.746 & 27.64 & 28.598 & & 26.542 & 24.258 \\
\hline 6/15/11 8:00 & & 28.515 & 28.191 & 27.285 & 28.434 & 26.784 & 27.659 & 28.615 & & 26.577 & 24.293 \\
\hline 6/15/11 12:00 & & 28.513 & 28.184 & 27.253 & 28.581 & 26.784 & 27.727 & 28.608 & & 26.577 & 24.277 \\
\hline 6/15/11 16:00 & & 28.515 & 28.145 & 27.196 & 28.479 & 26.758 & 27.777 & 28.574 & & 26.542 & 24.21 \\
\hline 6/15/11 20:00 & & 28.522 & 28.128 & 27.182 & 28.38 & 26.743 & 27.748 & 28.567 & & 26.521 & 24.195 \\
\hline 6/16/11 0:00 & & 28.52 & 28.107 & 27.154 & 28.331 & 26.727 & 27.753 & 28.55 & & 26.505 & 24.162 \\
\hline 6/16/11 4:00 & & 28.524 & 28.096 & 27.147 & 28.271 & 26.712 & 27.734 & 28.541 & & 26.486 & 24.159 \\
\hline 6/16/11 8:00 & & $\begin{array}{l}28.555 \\
28.524\end{array}$ & 28.135 & 27.236 & $\begin{array}{l}28.289 \\
28.28\end{array}$ & 26.739 & 27.652 & $\begin{array}{l}28.541 \\
28.593\end{array}$ & & $\begin{array}{l}20.400 \\
26.519\end{array}$ & 24.236 \\
\hline 6/16/11 12:00 & & $\begin{array}{l}28.524 \\
28.524\end{array}$ & $\begin{array}{l}28.1305 \\
28.105\end{array}$ & $\begin{array}{l}27.230 \\
27.15\end{array}$ & $\begin{array}{l}28.289 \\
28.245\end{array}$ & 26.717 & 27.723 & 28.564 & & $\begin{array}{l}26.495 \\
26.495\end{array}$ & $\begin{array}{l}24.200 \\
24.186\end{array}$ \\
\hline 6/16/11 16:00 & & $\begin{array}{r}28.524 \\
28.52\end{array}$ & 28.075 & $\begin{array}{l}27.13 \\
27.11\end{array}$ & $\begin{array}{l}28.245 \\
28.175\end{array}$ & 26.693 & 27.739 & $\begin{array}{r}28.304 \\
28.54\end{array}$ & & $\begin{array}{l}26.495 \\
26.463\end{array}$ & $\begin{array}{l}24.128 \\
24.128\end{array}$ \\
\hline 6/16/11 20:00 & & 28.559 & 28.117 & 27.236 & 28.196 & 26.712 & 27.621 & 28.588 & & 26.498 & 24.258 \\
\hline 6/17/11 0:00 & & 28.548 & 28.051 & 27.208 & 28.149 & 26.655 & 27.753 & $\begin{array}{r}28.5 \\
280\end{array}$ & & $\begin{array}{l}26.450 \\
26.437\end{array}$ & 24.061 \\
\hline 6/17/11 4:00 & & 28.541 & 28.086 & 27.185 & $\begin{array}{l}28.147 \\
28.147\end{array}$ & 26.684 & 27.664 & $\begin{array}{r}28.5 \\
28.54\end{array}$ & & $\begin{array}{l}26.451 \\
26.472\end{array}$ & 24.195 \\
\hline 6/17/11 8:00 & & 28.543 & 28.11 & 27.299 & 28.308 & 26.696 & 27.676 & 28.56 & & 26.493 & 24.212 \\
\hline 6/17/11 12:00 & & 28.55 & 28.126 & 27.175 & 28.467 & 26.701 & 27.692 & 28.567 & & 26.495 & 24.222 \\
\hline 6/17/11 16:00 & & 28.555 & 28.133 & 27.168 & 28.427 & 26.696 & 27.704 & 28.576 & & 26.5 & 24.224 \\
\hline $6 / 17 / 1120: 00$ & & 28.457 & 28.142 & 27.201 & 28.39 & 26.717 & 27.657 & 28.605 & & 26.523 & 24.231 \\
\hline 6/18/11 0:00 & & 28.245 & 28.166 & 26.925 & 28.35 & 26.724 & 27.687 & 24.954 & & 26.537 & 24.253 \\
\hline $6 / 18 / 114: 00$ & & 28.308 & 28.196 & 26.942 & 28.338 & 26.753 & 27.711 & 27.332 & & 26.565 & 24.26 \\
\hline 6/18/11 8:00 & & 28.359 & 28.25 & 27.285 & 28.352 & 26.793 & 27.654 & 28.117 & & 26.616 & 24.351 \\
\hline 6/18/11 12:00 & & 28.378 & 28.224 & 27.253 & 28.283 & 26.779 & 27.741 & 28.363 & & 26.593 & 24.277 \\
\hline 6/18/11 16:00 & & 28.482 & 28.21 & 27.224 & 28.226 & 26.769 & 27.767 & 28.466 & & 26.581 & 24.25 \\
\hline $6 / 18 / 1120: 00$ & & 28.389 & 28.191 & 27.22 & 28.182 & 26.753 & 27.751 & 28.507 & & 26.563 & 24.231 \\
\hline $6 / 19 / 110: 00$ & & 28.366 & 28.191 & 27.161 & 28.18 & 26.784 & 27.718 & 28.579 & & 26.591 & 24.348 \\
\hline 6/19/11 4:00 & & 28.42 & 28.168 & 27.21 & 28.138 & 26.755 & 27.701 & 28.55 & & 26.556 & 24.226 \\
\hline 6/19/11 8:00 & & 28.429 & 28.152 & 27.175 & 28.238 & 26.736 & 27.723 & 28.538 & & 26.537 & 24.2 \\
\hline 6/19/11 12:00 & & 28.431 & 28.119 & 27.119 & 28.341 & 26.701 & 27.746 & $\begin{array}{l}28.505 \\
28.505\end{array}$ & & 26.495 & 24.152 \\
\hline 6/19/11 16:00 & & $\begin{array}{l}28.451 \\
28.426\end{array}$ & 28.061 & 27.061 & $\begin{array}{l}28.341 \\
28.245\end{array}$ & 26.651 & 27.744 & $\begin{array}{l}28.403 \\
28.478\end{array}$ & & $\begin{array}{r}20.459 \\
26.44\end{array}$ & 24.102 \\
\hline 6/19/11 20:00 & & 28.431 & 28.037 & 27.063 & 28.217 & 26.627 & 27.694 & 28.471 & & 26.416 & 24.104 \\
\hline 6/20/11 0:00 & & 28.438 & 28.044 & 27.068 & 28.21 & 26.627 & 27.65 & 28.48 & & 26.419 & 24.136 \\
\hline 6/20/11 4:00 & & 28.443 & 28.019 & 27.056 & 28.135 & 26.603 & 27.643 & 28.476 & & 26.402 & 24.114 \\
\hline 6/20/11 8:00 & & 28.457 & 28.051 & 27.098 & 28.252 & 26.625 & 27.607 & 28.502 & & 26.423 & 24.176 \\
\hline 6/20/11 12:00 & & 28.461 & 28.072 & 27.101 & 28.39 & 26.625 & 27.615 & 28.512 & & 26.437 & 24.191 \\
\hline 6/20/11 16:00 & & 28.473 & 28.077 & 27.105 & 28.339 & 26.622 & 27.633 & 28.507 & & 26.428 & 24.167 \\
\hline 6/20/11 20:00 & & 28.494 & 28.131 & 27.203 & 28.338 & 26.655 & 27.544 & 28.586 & & 26.486 & 24.281 \\
\hline $6 / 21 / 110: 00$ & & 28.499 & 28.149 & 27.192 & 28.325 & 26.663 & 27.593 & 28.593 & & 26.502 & 24.265 \\
\hline $6 / 21 / 114: 00$ & & 28.522 & 28.21 & 27.269 & 28.336 & 26.708 & 27.582 & 28.646 & & 26.558 & 24.341 \\
\hline
\end{tabular}


TABLE S1.1 Automatically recorded groundwater depths in monitoring wells, December 2009 to March 2013, measured from the top of the casing (ft TOC).

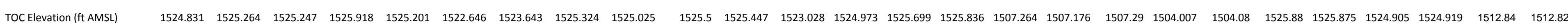

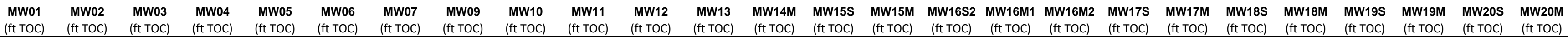

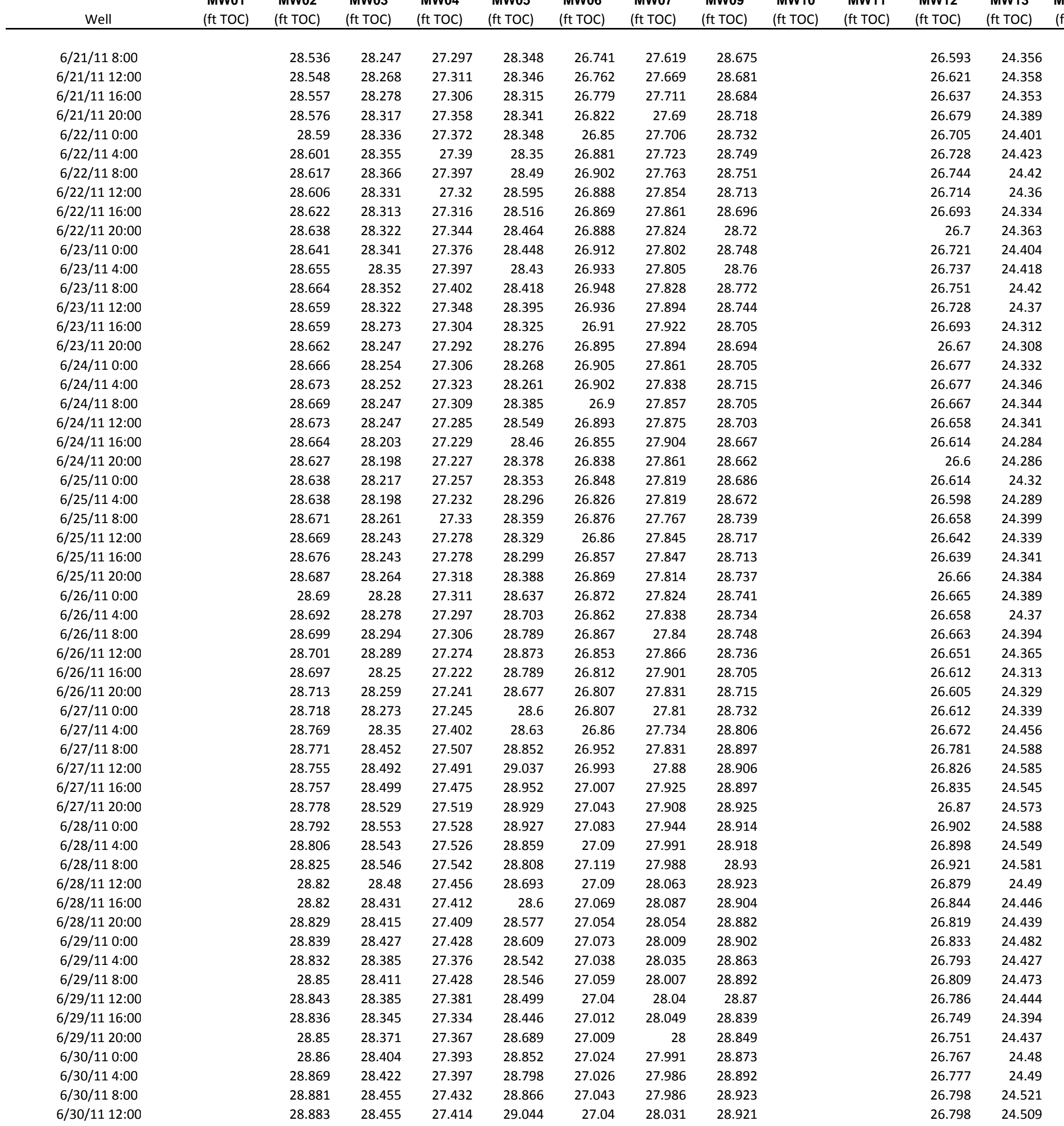


TABLE S1.1 Automatically recorded groundwater depths in monitoring wells, December 2009 to March 2013, measured from the top of the casing (ft TOC).

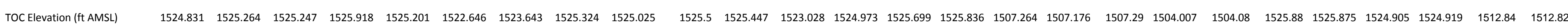

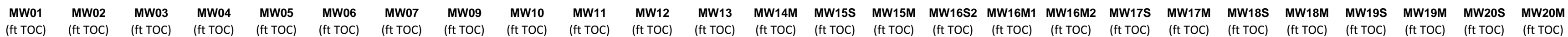

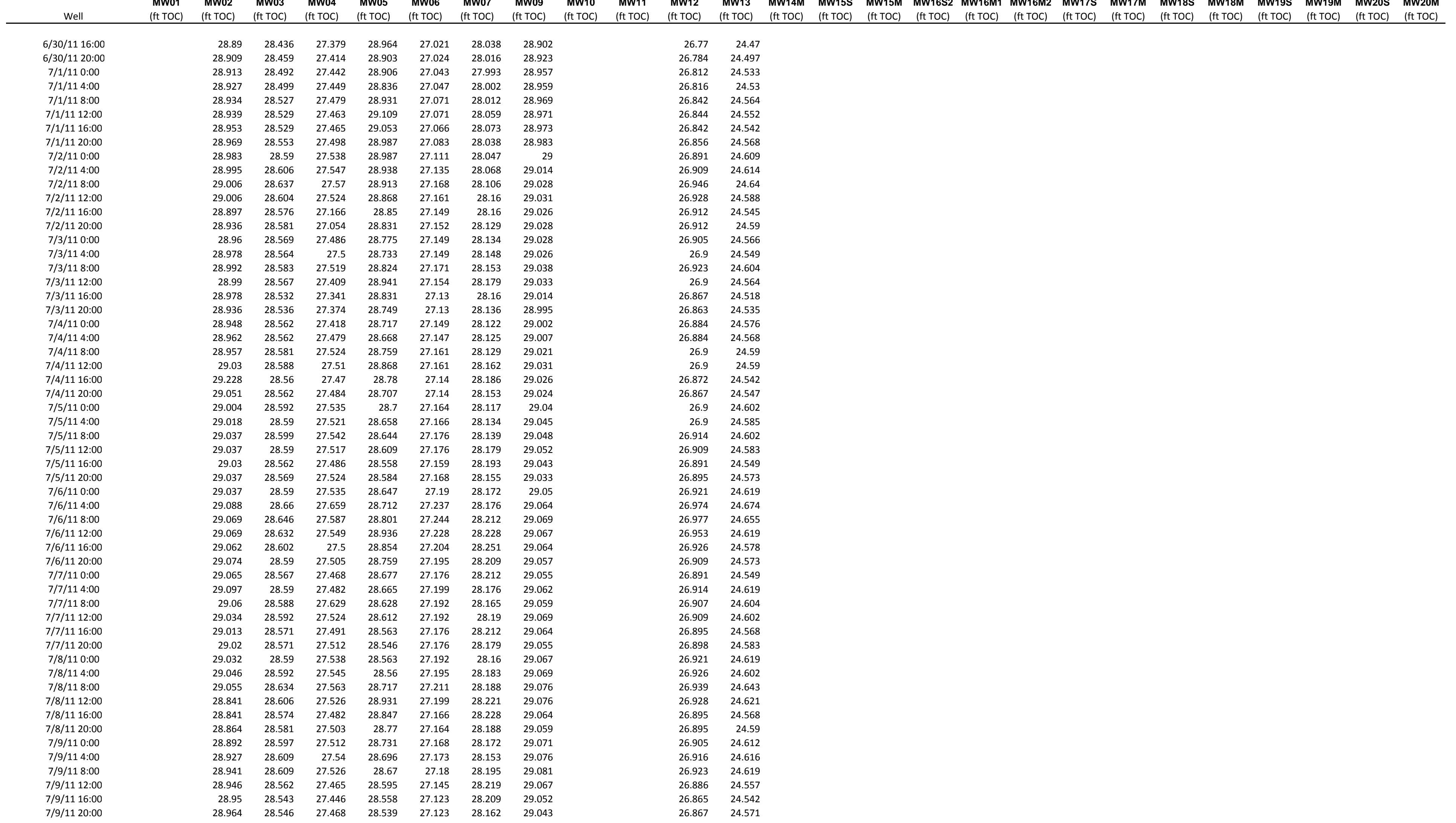


TABLE S1.1 Automatically recorded groundwater depths in monitoring wells, December 2009 to March 2013, measured from the top of the casing (ft TOC).

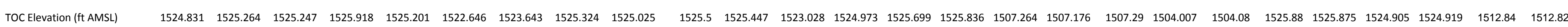

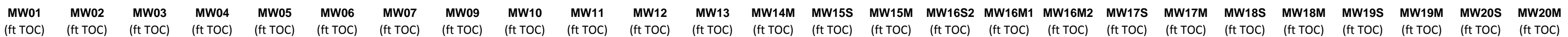

\begin{tabular}{|c|c|c|c|c|c|c|c|c|c|c|c|}
\hline well & $\begin{array}{l}\text { (ft TOC) } \\
\end{array}$ & $\begin{array}{l}\text { MwOz } \\
\text { (ft TOC) }\end{array}$ & $\begin{array}{r}\text { Wvos } \\
\text { (ft TOC) }\end{array}$ & $\begin{array}{l}\text { MWO4 } \\
\text { (ft TOC) }\end{array}$ & $\begin{array}{l}\text { Mit Toc) } \\
\text { (T) }\end{array}$ & $\begin{array}{l}\text { (ft TOC) } \\
\text { (f) }\end{array}$ & $\begin{array}{l}\text { Mwor } \\
\text { (ft TOC) }\end{array}$ & $\begin{array}{l}\text { Mvo } \\
\text { (ft TOC) }\end{array}$ & $\begin{array}{l}\text { Now10 } \\
\text { (ft TOC) }\end{array}$ & $\begin{array}{l}\text { mW12 } \\
\text { (ft TOC) }\end{array}$ & $\begin{array}{l}\text { MW13 } \\
\text { (ft TOC) }\end{array}$ \\
\hline $7 / 10 / 110: 00$ & & 28.985 & 28.578 & 27.521 & 28.577 & 27.145 & 28.136 & 29.055 & & 26.9 & 24.631 \\
\hline 7/10/11 4:00 & & 28.999 & 28.59 & 27.538 & 28.586 & 27.157 & 28.139 & 29.074 & & 26.919 & 24.638 \\
\hline 7/10/11 8:00 & & 29.016 & 28.62 & 27.573 & 28.752 & 27.176 & 28.16 & 29.086 & & 26.946 & 24.669 \\
\hline $7 / 10 / 1112: 00$ & & 29.016 & 28.632 & 27.552 & 28.938 & 27.18 & 28.19 & 29.091 & & 26.949 & 24.659 \\
\hline $7 / 10 / 1116: 00$ & & 29.023 & 28.623 & 27.528 & 28.88 & 27.161 & 28.212 & 29.091 & & 26.933 & 24.633 \\
\hline $7 / 10 / 1120: 00$ & & 29.041 & 28.641 & 27.559 & 28.878 & 27.176 & 28.162 & 29.093 & & 26.944 & 24.657 \\
\hline 7/11/11 0:00 & & 29.06 & 28.693 & 27.633 & 28.952 & 27.214 & 28.193 & 29.107 & & 26.991 & 24.734 \\
\hline 7/11/11 4:00 & & 29.074 & 28.725 & 27.659 & 28.945 & 27.247 & 28.209 & 29.117 & & 27.028 & 24.758 \\
\hline $7 / 11 / 118: 00$ & & 29.083 & 28.742 & 27.647 & 29.004 & 27.256 & 28.226 & 29.129 & & 27.042 & 24.743 \\
\hline 7/11/11 12:00 & & 29.086 & 28.723 & 27.598 & 29.088 & 27.242 & 28.266 & 29.134 & & 27.026 & 24.702 \\
\hline $7 / 11 / 1116: 00$ & & 29.088 & 28.69 & 27.563 & 28.992 & 27.228 & 28.277 & 29.134 & & 27 & 24.662 \\
\hline $7 / 11 / 1120: 00$ & & 29.106 & 28.688 & 27.58 & 28.952 & 27.223 & 28.242 & 29.138 & & 26.993 & 24.667 \\
\hline $7 / 12 / 110: 00$ & & 29.116 & 28.723 & 27.624 & 28.978 & 27.249 & 28.226 & 29.148 & & 27.023 & 24.726 \\
\hline $7 / 12 / 114: 00$ & & 29.123 & 28.723 & 27.619 & 28.922 & 27.252 & 28.242 & 29.155 & & 27.023 & 24.714 \\
\hline $7 / 12 / 118: 00$ & & 29.139 & 28.746 & 27.659 & 28.899 & 27.28 & 28.259 & 29.165 & & 27.051 & 24.748 \\
\hline $7 / 12 / 1112: 00$ & & 29.153 & 28.73 & 27.622 & 28.845 & 27.275 & 28.287 & 29.169 & & 27.042 & 24.71 \\
\hline $7 / 12 / 1116: 00$ & & 29.127 & 28.697 & 27.587 & 28.77 & 27.259 & 28.296 & 29.169 & & 27.021 & 24.674 \\
\hline 7/12/11 20:00 & & 29.141 & 28.707 & 27.624 & 28.819 & 27.266 & 28.247 & 29.172 & & 27.033 & 24.712 \\
\hline 7/13/11 0:00 & & 29.148 & 28.732 & 27.64 & 28.922 & 27.287 & 28.273 & 29.182 & & 27.051 & 24.741 \\
\hline 7/13/11 4:00 & & 29.158 & 28.728 & 27.629 & 28.873 & 27.287 & 28.27 & 29.184 & & 27.042 & 24.717 \\
\hline 7/13/11 8:00 & & 29.162 & 28.744 & 27.652 & 28.957 & 27.301 & 28.282 & 29.191 & & 27.063 & 24.755 \\
\hline $7 / 13 / 1112: 00$ & & 29.167 & 28.735 & 27.624 & 29.078 & 27.294 & 28.313 & 29.196 & & 27.051 & 24.724 \\
\hline $7 / 13 / 1116: 00$ & & 29.165 & 28.702 & 27.58 & 28.992 & 27.271 & 28.327 & 29.191 & & 27.026 & 24.686 \\
\hline $7 / 13 / 1120: 00$ & & 29.183 & 28.718 & 27.615 & 28.943 & 27.278 & 28.266 & 29.198 & & 27.03 & 24.717 \\
\hline 7/14/11 0:00 & & 29.188 & 28.742 & 27.647 & 28.948 & 27.294 & 28.284 & 29.203 & & 27.053 & 24.76 \\
\hline 7/14/11 4:00 & & 29.193 & 28.739 & 27.636 & 28.892 & 27.294 & 28.284 & 29.208 & & 27.051 & 24.743 \\
\hline $7 / 14 / 118: 00$ & & 29.195 & 28.725 & 27.617 & 28.836 & 27.287 & 28.294 & 29.208 & & 27.042 & 24.719 \\
\hline $7 / 14 / 1112: 00$ & & 29.197 & 28.723 & 27.615 & 28.822 & 27.287 & 28.301 & 29.213 & & 27.042 & 24.719 \\
\hline 7/14/11 16:00 & & 29.188 & 28.695 & 27.575 & 28.815 & 27.263 & 28.317 & 29.203 & & 27.016 & 24.681 \\
\hline 7/14/11 20:00 & & 29.197 & 28.695 & 27.591 & 28.791 & 27.266 & 28.273 & 29.203 & & 27.016 & 24.702 \\
\hline 7/15/11 0:00 & & 29.204 & 28.714 & 27.617 & 28.798 & 27.278 & 28.268 & 29.213 & & 27.035 & 24.738 \\
\hline 7/15/11 4:00 & & 29.209 & $\begin{array}{l}28.714 \\
2814\end{array}$ & 27.622 & 28.775 & 27.275 & 28.266 & 29.213 & & 27.035 & 24.729 \\
\hline 7/15/11 8:00 & & 29.214 & $\begin{array}{l}28.737 \\
28.74\end{array}$ & 27.645 & 28.892 & 27.29 & 28.284 & 29.219 & & 27.056 & 24.76 \\
\hline 7/15/11 12:00 & & 29.211 & 28.737 & 27.622 & 29.036 & 27.285 & 28.31 & 29.222 & & 27.049 & 24.748 \\
\hline 7/15/11 16:00 & & 29.214 & 28.716 & 27.591 & 28.987 & 27.268 & 28.324 & 29.217 & & 27.028 & 24.712 \\
\hline $7 / 15 / 1120: 00$ & & 29.228 & 28.735 & 27.629 & 28.973 & 27.273 & 28.273 & 29.222 & & 27.042 & 24.743 \\
\hline $7 / 16 / 110: 00$ & & 29.242 & 28.793 & 27.701 & 29.018 & 27.318 & 28.298 & 29.236 & & 27.095 & 24.824 \\
\hline 7/16/11 4:00 & & 29.246 & 28.795 & 27.687 & 28.971 & 27.325 & 28.306 & 29.244 & & 27.1 & 24.805 \\
\hline 7/16/11 8:00 & & 29.251 & 28.816 & 27.708 & 28.957 & 27.347 & 28.327 & 29.253 & & 27.126 & 24.827 \\
\hline $7 / 16 / 1112: 00$ & & 29.253 & 28.816 & 27.687 & 28.99 & 27.347 & 28.35 & 29.258 & & 27.128 & 24.805 \\
\hline $7 / 16 / 1116: 00$ & & 29.262 & 28.809 & 27.683 & 29.027 & 27.349 & 28.357 & 29.265 & & 27.123 & 24.796 \\
\hline $7 / 16 / 1120: 00$ & & 29.274 & 28.83 & 27.72 & 29.039 & 27.363 & 28.343 & 29.27 & & 27.142 & 24.827 \\
\hline 7/17/11 0:00 & & 29.281 & 28.858 & 27.741 & 29.074 & 27.392 & 28.367 & 29.282 & & 27.17 & 24.86 \\
\hline 7/17/11 4:00 & & 29.293 & 28.865 & 27.743 & 29.039 & 27.406 & 28.374 & 29.292 & & 27.177 & 24.851 \\
\hline 7/17/11 8:00 & & 29.302 & 28.895 & 27.776 & 29.137 & 27.434 & 28.4 & 29.301 & & 27.205 & 24.884 \\
\hline $7 / 17 / 1112: 00$ & & 29.304 & 28.898 & 27.757 & 29.277 & 27.439 & 28.423 & 29.313 & & 27.207 & 24.877 \\
\hline 7/17/11 16:00 & & 29.309 & 28.874 & 27.727 & 29.198 & 27.43 & 28.449 & 29.315 & & 27.191 & 24.836 \\
\hline 7/17/11 20:00 & & 29.316 & 28.872 & 27.741 & 29.123 & 27.432 & 28.407 & 29.325 & & 27.191 & 24.848 \\
\hline 7/18/11 0:00 & & 29.325 & 28.888 & 27.76 & 29.088 & 27.449 & 28.425 & 29.33 & & 27.205 & 24.865 \\
\hline 7/18/11 4:00 & & 29.337 & 28.891 & 27.771 & 29.051 & 27.461 & 28.428 & 29.337 & & 27.212 & 24.87 \\
\hline 7/18/11 8:00 & & 29.339 & $\begin{array}{l}2.091 \\
28.905\end{array}$ & 27.781 & 29.142 & 27.472 & $\begin{array}{l}20.420 \\
28.451\end{array}$ & 29.344 & & 27.226 & 24.889 \\
\hline $7 / 18 / 1112: 00$ & & 29.335 & 28.877 & 27.727 & 29.27 & 27.458 & 28.487 & 29.346 & & 27.2 & 24.846 \\
\hline 7/18/11 16:00 & & 29.337 & 28.849 & 27.699 & 29.195 & 27.439 & 28.487 & 29.346 & & 27.177 & 24.817 \\
\hline $7 / 18 / 1120: 00$ & & 29.346 & 28.846 & 27.706 & 29.111 & 27.434 & 28.442 & 29.346 & & 27.165 & 24.82 \\
\hline 7/19/11 0:00 & & 29.353 & 28.872 & 27.743 & 29.085 & 27.449 & 28.435 & 29.351 & & 27.186 & 24.865 \\
\hline 7/19/11 4:00 & & 29.353 & 28.865 & 27.729 & 29.034 & 27.451 & 28.44 & 29.358 & & 27.181 & 24.853 \\
\hline
\end{tabular}


TABLE S1.1 Automatically recorded groundwater depths in monitoring wells, December 2009 to March 2013, measured from the top of the casing (ft TOC).

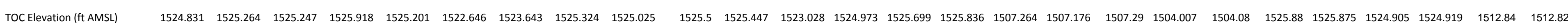

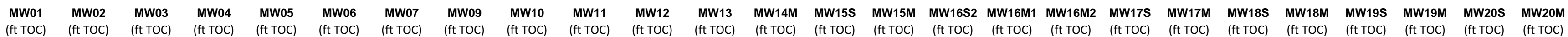

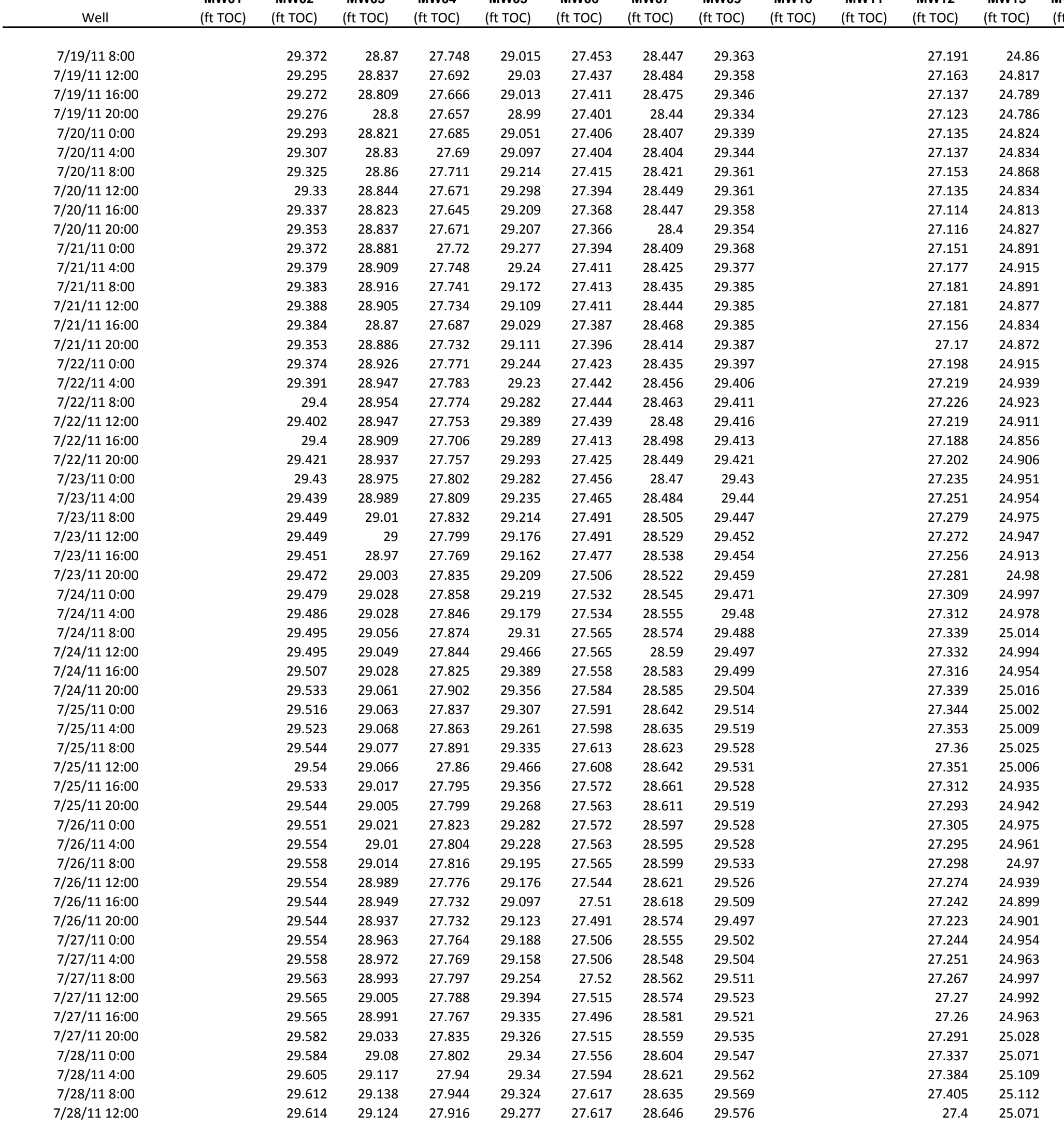


TABLE S1.1 Automatically recorded groundwater depths in monitoring wells, December 2009 to March 2013, measured from the top of the casing (ft TOC).

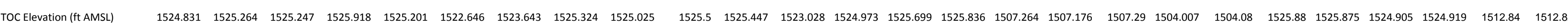

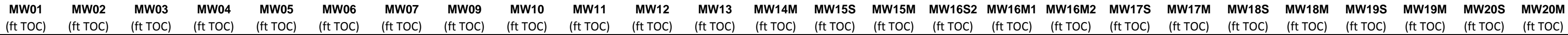

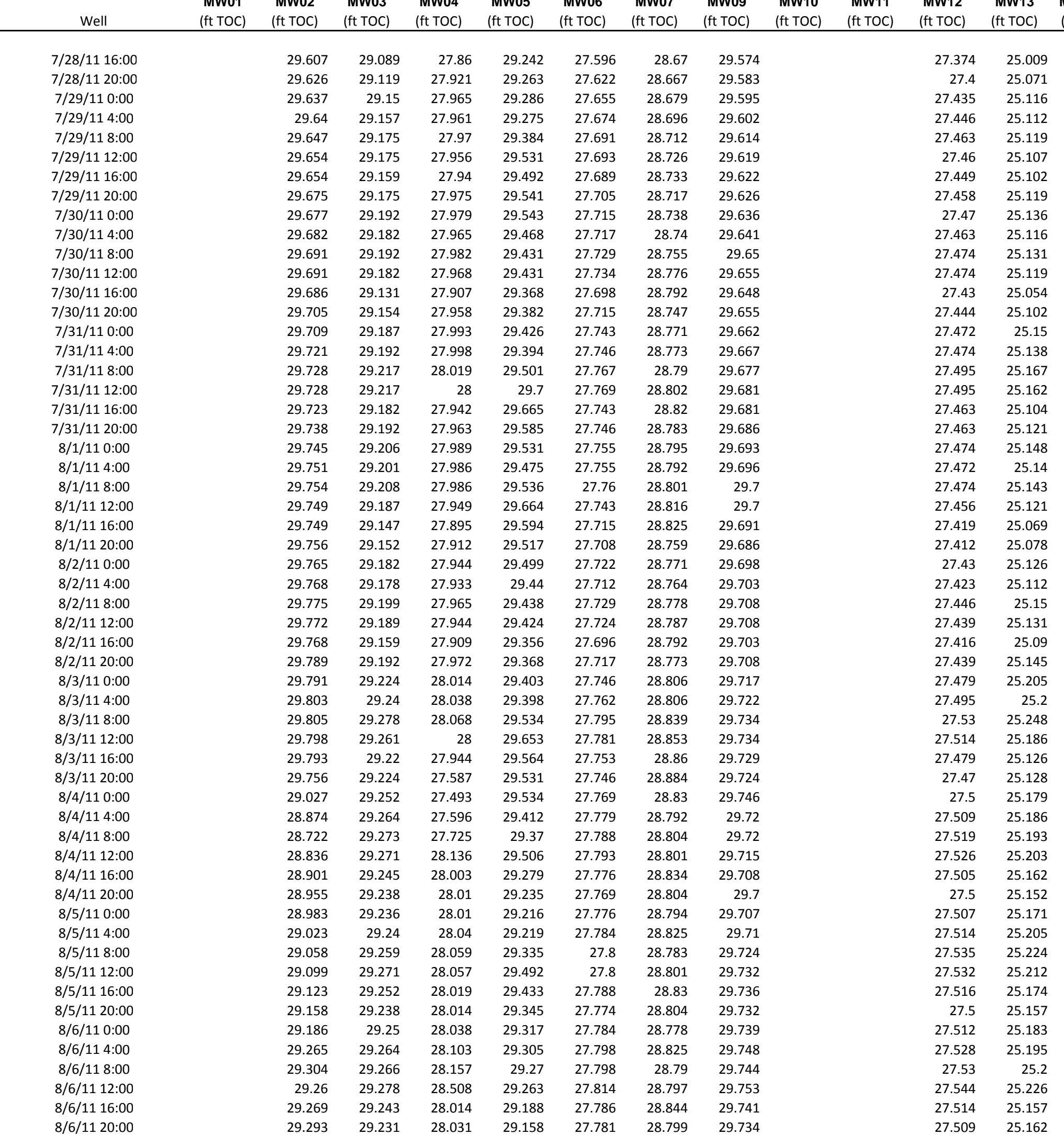


TABLE S1.1 Automatically recorded groundwater depths in monitoring wells, December 2009 to March 2013, measured from the top of the casing (ft TOC).

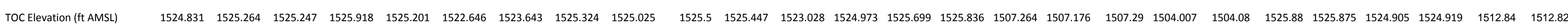

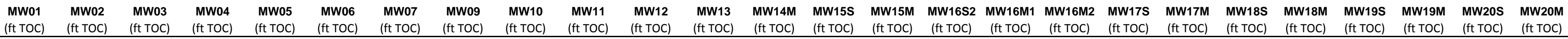

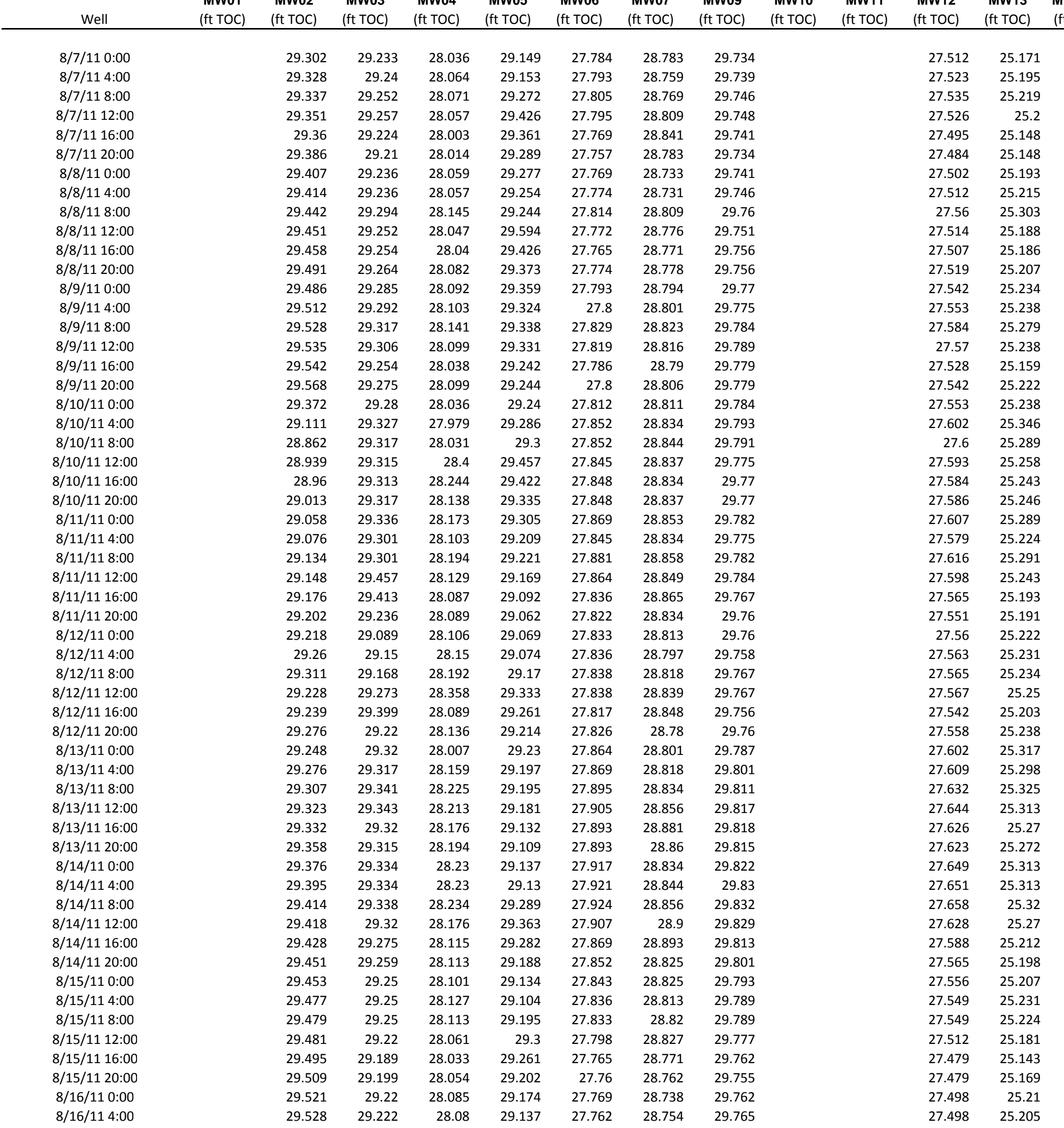


TABLE S1.1 Automatically recorded groundwater depths in monitoring wells, December 2009 to March 2013, measured from the top of the casing (ft TOC).

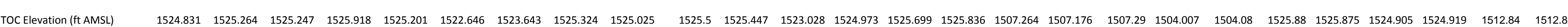

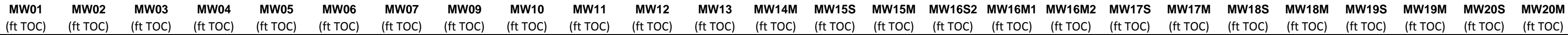

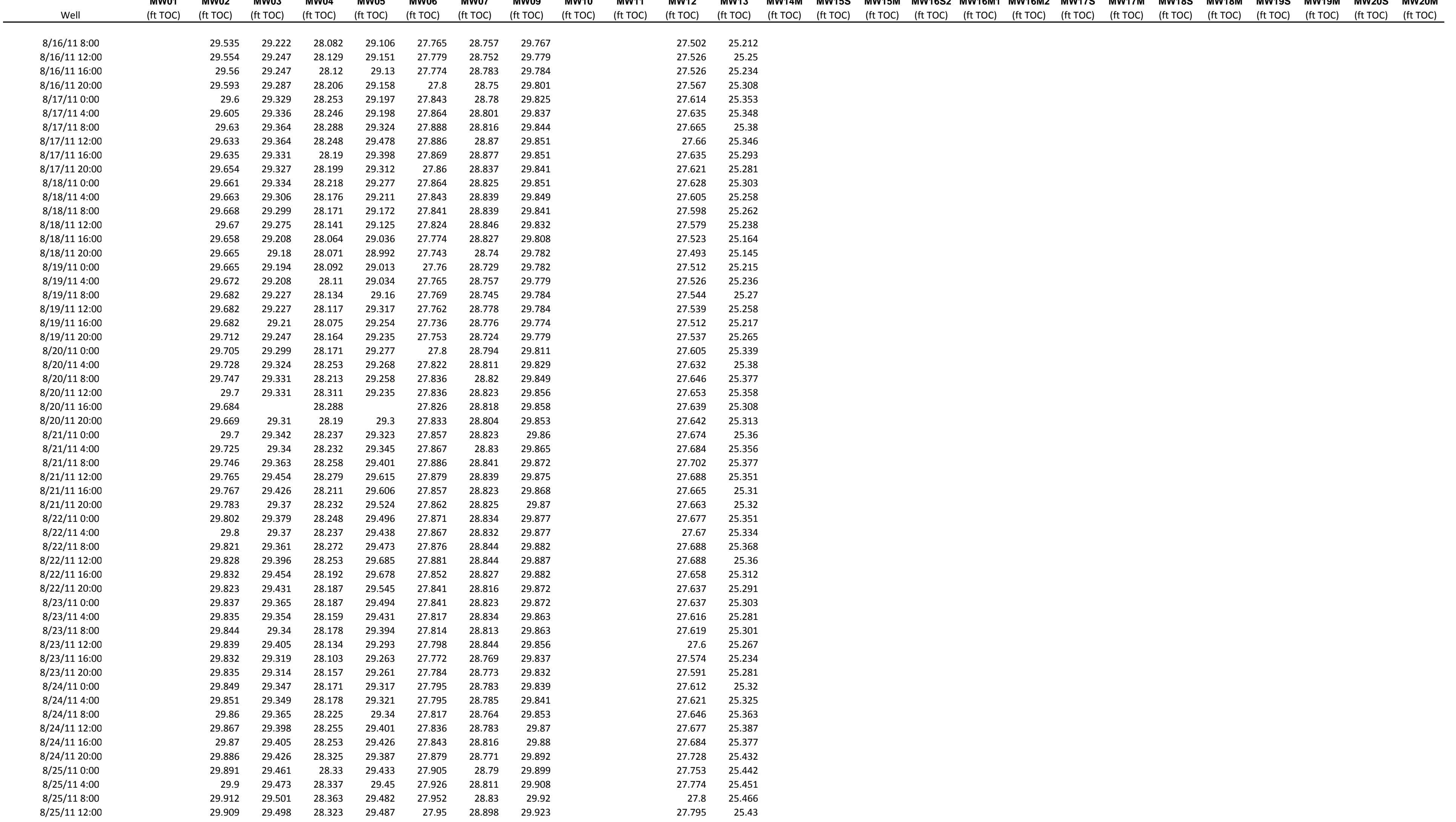


TABLE S1.1 Automatically recorded groundwater depths in monitoring wells, December 2009 to March 2013, measured from the top of the casing (ft TOC).

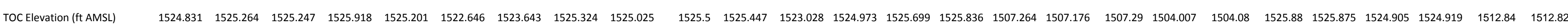

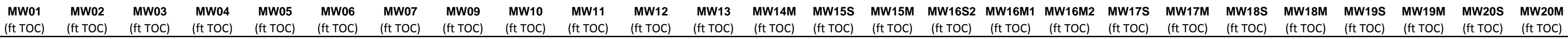

\begin{tabular}{|c|c|c|c|c|c|c|c|c|c|}
\hline & & & & & & & & & \\
\hline 8/25/11 16:00 & & 29.9 & 29.456 & 28.251 & 29.429 & 27.921 & 28.942 & 29.91: & \\
\hline $8 / 25 / 1120: 00$ & & 29.9 & 29.428 & 28.272 & 29.384 & 27.917 & 28.896 & 29.906 & \\
\hline 8/26/11 0:00 & & 29.914 & 29.445 & 28.304 & 29.408 & 27.938 & 28.863 & 29.916 & \\
\hline 8/26/11 4:00 & & 29.914 & 29.442 & 28.281 & 29.412 & 27.931 & 28.881 & 29.916 & \\
\hline 8/26/11 8:00 & & 29.921 & 29.447 & 28.325 & 29.419 & 27.95 & 28.863 & 29.923 & \\
\hline $8 / 26 / 1112: 00$ & & 29.923 & 29.459 & 28.281 & 29.443 & 27.938 & 28.919 & & \\
\hline $8 / 26 / 1116: 00$ & & 29.907 & 29.407 & 28.22 & 29.387 & 27.902 & 28.957 & & \\
\hline $8 / 26 / 1120: 00$ & & 29.912 & 29.403 & 28.295 & 29.373 & 27.921 & 28.848 & & \\
\hline $8 / 27 / 110: 00$ & & 29.923 & 29.424 & 28.288 & 29.41 & 27.924 & 28.865 & 29.913 & \\
\hline 8/27/11 4:00 & & 29.93 & 29.428 & 28.297 & 29.417 & 27.931 & 28.867 & 29.918 & \\
\hline $8 / 27 / 118: 00$ & & 29.937 & 29.442 & 28.314 & 29.438 & 27.943 & 28.872 & 29.927 & \\
\hline $8 / 27 / 1112: 00$ & & 29.932 & 29.442 & 28.274 & 29.443 & 27.931 & 28.914 & 29.927 & \\
\hline 8/27/11 16:00 & & 29.925 & 29.414 & 28.241 & 29.408 & 27.912 & 28.931 & 29.916 & \\
\hline $8 / 27 / 11$ 20:00 & & 29.923 & 29.398 & 28.253 & 29.394 & 27.912 & 28.891 & 29.906 & \\
\hline $8 / 28 / 110: 00$ & & 29.93 & 29.407 & 28.26 & 29.408 & 27.907 & 28.872 & 29.906 & \\
\hline $8 / 28 / 114: 00$ & & 29.932 & 29.407 & 28.253 & 29.41 & 27.907 & 28.874 & 29.906 & \\
\hline $8 / 28 / 118: 00$ & & 29.935 & 29.407 & 28.234 & 29.408 & 27.893 & $\begin{array}{l}20.084 \\
28.886\end{array}$ & $29.90<$ & \\
\hline 8/28/11 12:00 & & 29.932 & 29.4 & 28.213 & 29.536 & 27.881 & 28.896 & 29.896 & \\
\hline 8/28/11 16:00 & & 29.921 & 29.372 & 28.185 & 29.671 & 27.857 & 28.891 & 29.887 & \\
\hline $8 / 28 / 1120: 00$ & & 29.928 & 29.382 & 28.218 & 29.646 & 27.86 & 28.848 & & \\
\hline $8 / 29 / 110: 00$ & & 29.946 & 29.414 & 28.274 & 29.646 & 27.883 & 28.804 & & \\
\hline 8/29/11 4:00 & & 29.951 & 29.44 & 28.265 & 29.634 & 27.883 & $\begin{array}{l}28.8044 \\
28.837\end{array}$ & $\begin{array}{l}29.906 \\
29.92\end{array}$ & \\
\hline 8/29/11 8:00 & & 29.974 & 29.48 & 28.321 & 29.69 & 27.917 & $\begin{array}{l}28.83 \\
28.891\end{array}$ & $\begin{array}{l}29.992 \\
29.932\end{array}$ & \\
\hline 8/29/11 12:00 & & 29.958 & 29.498 & 28.29 & 29.891 & 27.909 & $\begin{array}{l}28.917 \\
28.917\end{array}$ & $\begin{array}{l}29.933 \\
29.935\end{array}$ & \\
\hline $8 / 29 / 1116: 00$ & & 29.953 & 29.468 & 28.234 & 29.87 & 27.879 & 28.928 & $\begin{array}{l}29.935 \\
29.93\end{array}$ & \\
\hline $8 / 29 / 1120: 00$ & & 29.96 & 29.449 & 28.26 & 29.723 & 27.879 & 28.872 & $\begin{array}{l}29.933 \\
29.937\end{array}$ & \\
\hline $8 / 30 / 110: 00$ & & 29.965 & 29.463 & 28.244 & 29.671 & 27.874 & 28.872 & $\begin{array}{l}29.93 \\
29.935 \\
\end{array}$ & \\
\hline $8 / 30 / 114: 00$ & & 29.967 & 29.445 & 28.367 & 29.611 & 27.893 & 28.853 & $\begin{array}{l}29.934 \\
29.944\end{array}$ & \\
\hline $8 / 30 / 118: 00$ & & 29.968 & 29.468 & 28.356 & 29.608 & 27.883 & 28.87 & $\begin{array}{l}29.944 \\
29.947\end{array}$ & \\
\hline $8 / 30 / 1112 \cdot 00$ & & 29986 & 29.473 & 28.25 & 29.587 & 27881 & 28.893 & $\begin{array}{l}29.947 \\
29.944\end{array}$ & \\
\hline $8 / 30 / 1116.00$ & & 29.974 & 29.449 & 28.22 & 29.585 & 2786 & & 29.944 & \\
\hline $\begin{array}{l}\text { 8/30/11 16:00 } \\
8 / 30 / 11200\end{array}$ & & 29.986 & 29456 & $28267 \mathrm{r}$ & 29.587 & 27876 & $\begin{array}{l}28.881 \\
28.839\end{array}$ & 29.942 & \\
\hline 8/30/11 20:00 & & & & & & & 28.839 & 29.944 & \\
\hline $8 / 31 / 110: 00$ & & 29.993 & 29.484 & 28.281 & 29.608 & 27.893 & 28.837 & 29.956 & \\
\hline 8/31/11 4:00 & & 29.995 & 29.494 & 28.288 & 29.597 & 27.905 & 28.846 & 29.961 & \\
\hline 8/31/11 8:00 & & 30.007 & 29.512 & 28.316 & 29.657 & 27.919 & 28.874 & 29.966 & \\
\hline 8/31/11 12:00 & & 30.007 & 29.536 & 28.295 & 29.884 & 27.919 & 28.912 & 29.971 & \\
\hline 8/31/11 16:00 & & 30 & 29.515 & 28.255 & 29.884 & 27.902 & 28.919 & 29.971 & \\
\hline 8/31/11 20:00 & & 30.012 & 29.512 & 28.295 & 29.795 & 27.912 & 28.867 & 29.975 & \\
\hline 9/1/11 0:00 & & 30.026 & 29.554 & 28.335 & 29.786 & 27.938 & 28.91 & $29.9 \varepsilon$ & \\
\hline 9/1/11 4:00 & & 30.033 & 29.571 & 28.351 & 29.762 & 27.952 & 28.893 & 29.992 & \\
\hline 9/1/11 8:00 & & 30.04 & 29.587 & 28.349 & 29.744 & 27.962 & 28.914 & 29.995 & \\
\hline 9/1/11 12:00 & & 30.04 & 29.587 & 28.321 & 29.718 & 27.964 & 28.959 & 30.002 & \\
\hline $9 / 1 / 1116: 00$ & & 30.033 & 29.547 & 28.281 & 29.685 & 27.943 & 28.95 & 29.997 & \\
\hline 9/1/11 20:00 & & 30.044 & 29.55 & 28.33 & 29.671 & 27.962 & 28.914 & 30.006 & \\
\hline 9/2/11 0:00 & & 30.056 & 29.578 & 28.349 & 29.692 & 27.978 & 28.954 & 30.011 & \\
\hline 9/2/11 4:00 & & 30.061 & 29.582 & 28.356 & 29.685 & 27.988 & 28.947 & $30.01 \varepsilon$ & \\
\hline 9/2/11 8:00 & & 30.07 & 29.603 & 28.356 & 29.744 & 28 & 28.957 & 30.023 & \\
\hline 9/2/11 12:00 & & 29.981 & 29.61 & 28.325 & 29.998 & 27.995 & 28.999 & 30.028 & \\
\hline $9 / 2 / 1116: 00$ & & 30.009 & 29.571 & 28.29 & 29.965 & 27.981 & 29.015 & 30.026 & \\
\hline $9 / 2 / 1120: 00$ & & 30.03 & 29.568 & 28.33 & 29.865 & 27.983 & 28.961 & 30.0 & \\
\hline $9 / 3 / 110: 00$ & & 30.051 & 29.596 & 28.349 & 29.833 & 27.995 & 28.95 & 30.039 & \\
\hline 9/3/11 4:00 & & 30.065 & 29.606 & 28.356 & 29.797 & 28 & 28.957 & 30.0 & \\
\hline 9/3/11 8:00 & & 30.079 & 29.617 & 28.349 & 29.779 & 28.004 & 28.978 & 30.045 & \\
\hline 9/3/11 12:00 & & 30.093 & 29.624 & 28.353 & 29.76 & 3.016 & 28.987 & 30.052 & \\
\hline $9 / 3 / 1116: 00$ & & 30.091 & 29.599 & 28.323 & 29.72 & & 29.027 & 30.052 & \\
\hline 9/3/11 20:00 & & 30.128 & 29.641 & 28.47 & 29.774 & 28.069 & 29.055 & 30.05 & \\
\hline
\end{tabular}

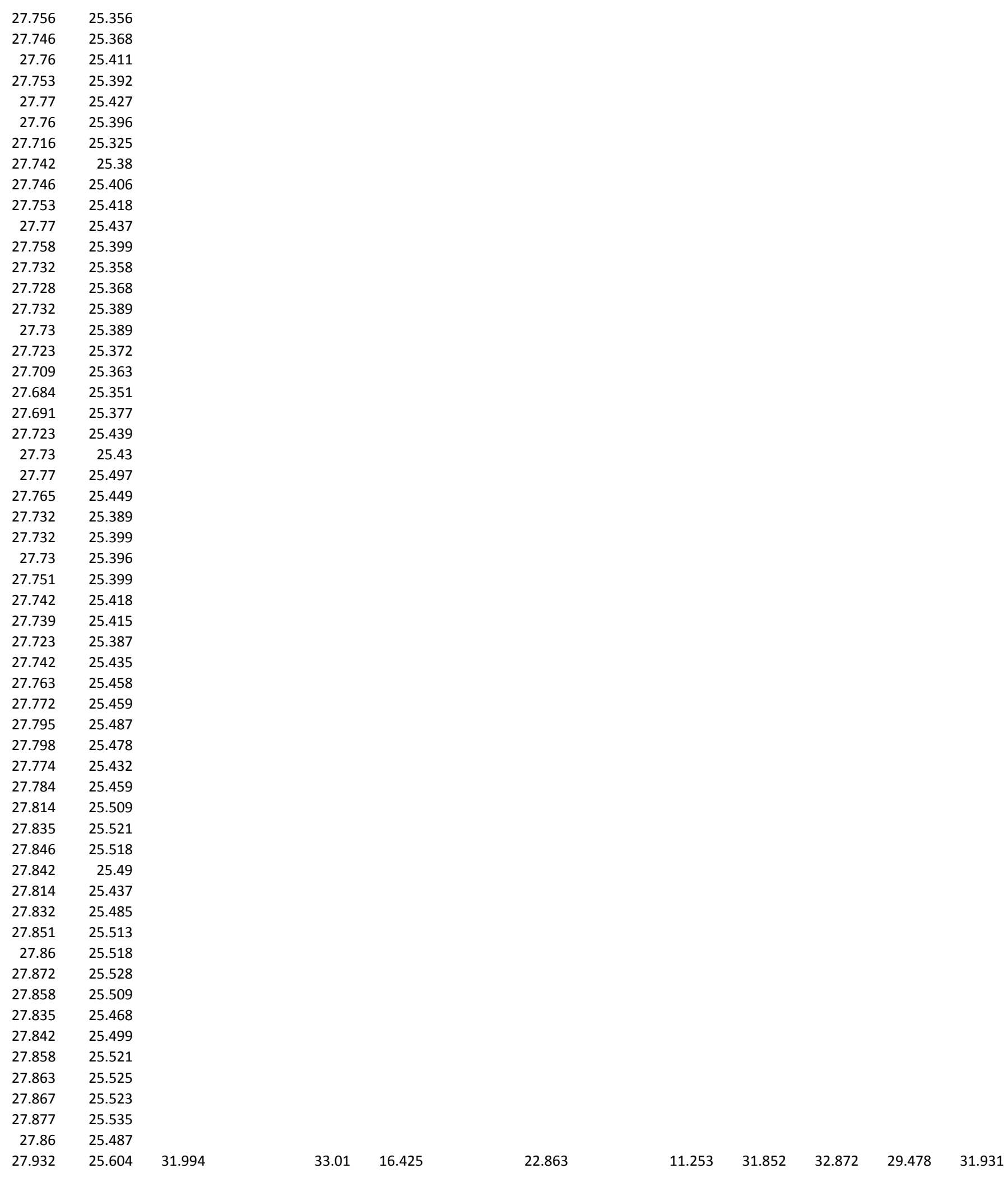




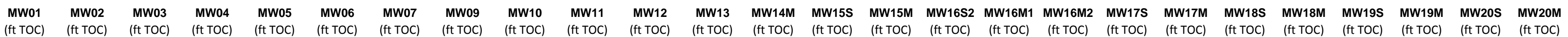

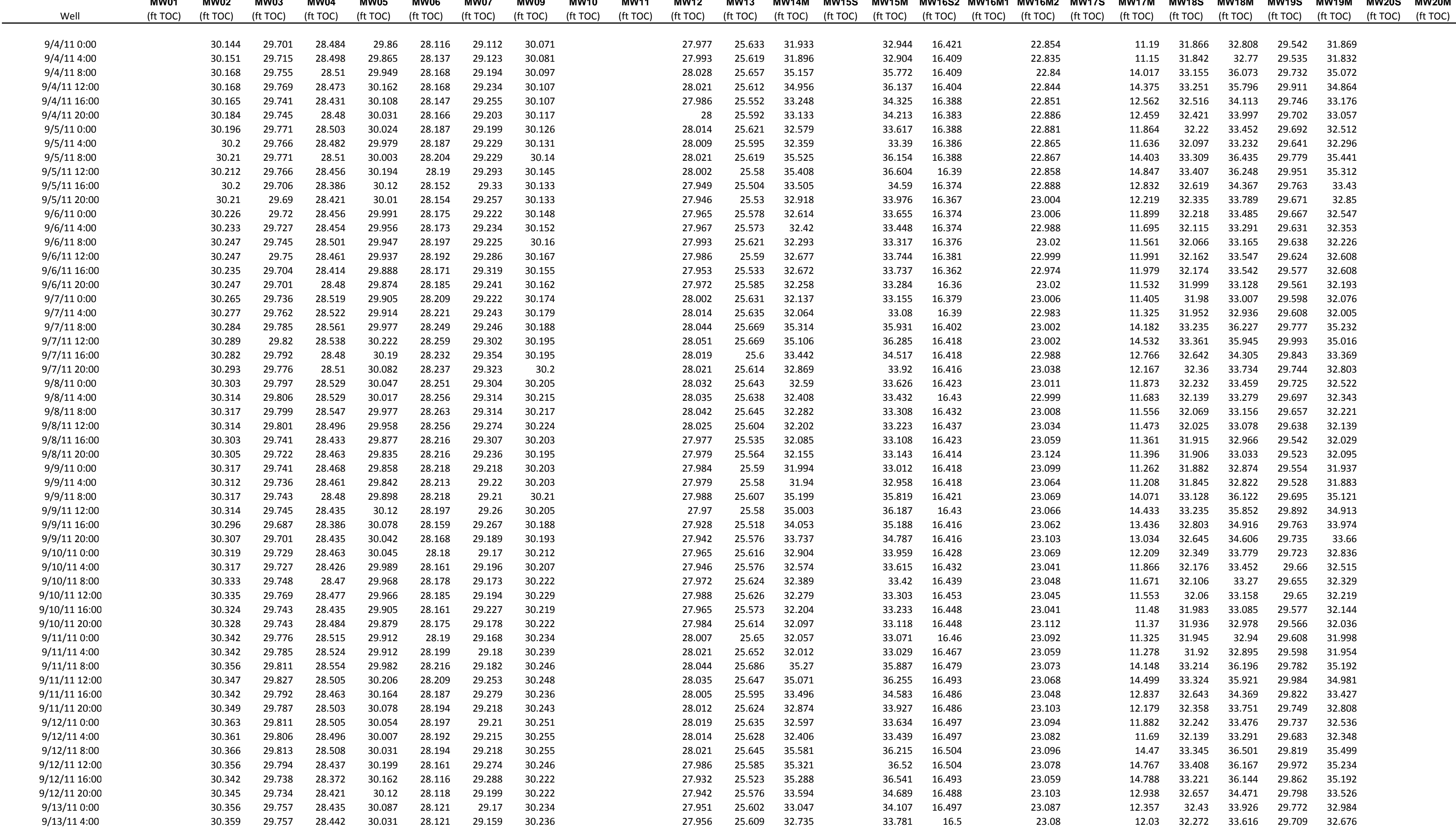




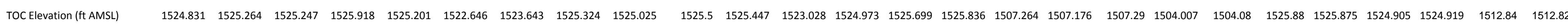

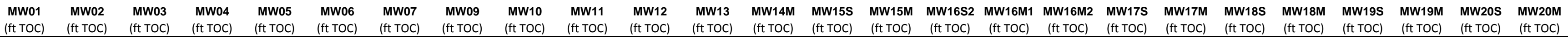

\begin{tabular}{|c|c|c|c|c|c|c|c|c|c|c|c|c|c|c|c|c|c|c|c|c|c|c|c|c|c|c|}
\hline Well & $\begin{array}{l}\text { (ft TOC) } \\
\text { (ft }\end{array}$ & $\begin{array}{l}\text { (ft TOC) } \\
\text { (f) }\end{array}$ & $\begin{array}{l}\text { (ft TOC) } \\
\text { (f) }\end{array}$ & $\begin{array}{l}\text { (ft TOC) } \\
\text { (ft) }\end{array}$ & $\begin{array}{l}\text { (ft TOC) } \\
\text { (f) }\end{array}$ & $\begin{array}{l}(\mathrm{ft} \text { TOC) } \\
\text { (n) }\end{array}$ & $\begin{array}{l}\text { (ft TOC) } \\
\text { (ft }\end{array}$ & $\begin{array}{l}\text { (ft TOC) } \\
\text { (f) }\end{array}$ & $\begin{array}{l}\text { (ft TOC) } \\
\text { (f) }\end{array}$ & $\begin{array}{l}\text { (ft TOC) } \\
\text { (n) }\end{array}$ & (ft TOC) & $\begin{array}{l}\text { (ft TOC) } \\
\text { (f) }\end{array}$ & (ft TOC) & $\begin{array}{l}\text { (ft TOC) } \\
\end{array}$ & (ft TOC) & (ft TOC) & (ft TOC) & (ft TOC) & $\begin{array}{l}\text { (ft TOC) } \\
\text { (1) }\end{array}$ & (ft TOC) & $\begin{array}{l}\text { (ft TOC) } \\
\text { (1) }\end{array}$ & $\begin{array}{l}\text { (ft TOC) } \\
\text { (f) }\end{array}$ & $\begin{array}{l}\text { (ft TOC) } \\
\text { (n) }\end{array}$ & (ft TOC) & $\begin{array}{l}\text { (ft TOC) } \\
\end{array}$ & (ft TOC) \\
\hline $18: 00$ & & 30.38 & 29.799 & 28.517 & 30.038 & 28.156 & 29.126 & 30.257 & & & 28.002 & 25.679 & 32.902 & & 33.981 & 16.516 & & 23.089 & & 12.232 & 32.305 & 33 & 29.744 & 32.838 & & \\
\hline 9/13/11 12:00 & & 30.387 & 29.839 & 28.522 & 30.096 & 28.173 & 29.18 & 30.27 & & & 28.025 & 25.688 & 32.679 & & 33.72 & 16.535 & & 23.082 & & 11.974 & 32.298 & 33.561 & 29.789 & 32.618 & & \\
\hline 9/13/11 16:00 & & 30.38 & 29.827 & 28.498 & 30.045 & 28.164 & 29.22 & 30.267 & & & 28.016 & 25.647 & 32.462 & & 33.495 & 16.532 & & 23.08 & & 11.744 & 32.167 & 33.345 & 29.723 & 32.402 & & \\
\hline 9/13/11 20:00 & & 30.387 & 29.832 & 28.55 & 30.017 & 28.183 & 29.178 & 30.274 & & & 28.037 & 25.676 & 32.331 & & 33.352 & $2 \quad 16.537$ & & 23.154 & & 11.6 & 32.099 & 33.21 & 29.704 & 32.268 & & \\
\hline 9/14/11 0:00 & & 30.403 & 29.871 & 28.557 & 30.052 & 28.204 & 29.182 & 30.282 & & & 28.063 & 25.71 & 32.27 & & 33.291 & $1 \quad 16.553$ & & 23.156 & & 11.541 & 32.106 & 33.153 & 29.749 & 32.212 & & \\
\hline 9/14/11 4:00 & & 30.391 & 29.864 & 28.599 & 30.019 & 28.218 & 29.182 & 30.289 & & & 28.084 & 25.719 & 32.204 & & 33.223 & $3 \quad 16.556$ & & 23.133 & & 11.473 & 32.043 & 33.087 & 29.704 & 32.146 & & \\
\hline 9/14/11 8:00 & & 30.422 & 29.916 & 28.636 & 30.12 & 28.256 & 29.185 & 30.301 & & & 28.121 & 25.772 & 35.469 & & 36.095 & $5 \quad 16.574$ & & 23.147 & & 14.343 & 33.368 & 36.392 & 29.927 & 35.391 & & \\
\hline 9/14/11 12:00 & & 30.363 & 29.955 & 28.634 & & 28.285 & 29.229 & 30.31 & & & 28.149 & 25.789 & 35.461 & & 36.656 & $6 \quad 16.595$ & & 23.133 & & 14.898 & 33.575 & 36.305 & 30.17 & 35.368 & & \\
\hline 9/14/11 16:00 & & 30.366 & 29.944 & & 30.271 & 28.28 & 29.295 & 30.318 & & & 28.137 & 25.746 & 33.667 & & 34.754 & $4 \quad 16.602$ & & 23.131 & & 13.001 & 32.841 & 34.542 & 30.043 & 33.599 & & \\
\hline 9/14/11 20:00 & & 30.398 & 29.967 & 28.662 & 30.276 & 28.318 & 29.25 & 30.33 & & & 28.174 & 25.796 & 33.098 & & 34.147 & $7 \quad 16.616$ & & 23.17 & & 12.395 & 32.596 & 33.973 & 29.996 & 33.031 & & \\
\hline 9/15/11 0:00 & & 30.428 & 30.011 & 28.669 & 30.358 & 28.349 & 29.272 & 30.344 & & & 28.2 & 25.822 & 32.829 & & 33.868 & $8 \quad 16.635$ & & 23.168 & & 12.12 & 32.491 & 33.71 & 29.989 & 32.766 & & \\
\hline 9/15/11 4:00 & & 30.442 & 30.011 & 28.683 & 30.313 & 28.368 & 29.293 & 30.353 & & & 28.214 & 25.808 & 32.647 & & 33.681 & $1 \quad 16.639$ & & 23.152 & & 11.929 & 32.382 & 33.53 & 29.944 & 32.587 & & \\
\hline 9/15/11 8:00 & & 30.461 & 30.03 & 28.676 & 30.299 & 28.382 & 29.328 & 30.363 & & & 28.219 & 25.803 & 32.523 & & 33.549 & $\begin{array}{l}9 \\
9\end{array}$ & & 23.154 & & 11.797 & 32.323 & 33.405 & 29.923 & 32.463 & & \\
\hline 9/15/11 12:00 & & 30.484 & 30.044 & 28.66 & 30.281 & 28.387 & 29.375 & 30.368 & & & 28.219 & 25.774 & 32.441 & & 33.465 & 16.66 & & 23.145 & & 11.713 & 32.281 & 33.322 & 29.909 & 32.385 & & \\
\hline 9/15/11 16:00 & & 30.473 & 29.995 & 28.568 & 30.19 & & 29.434 & 30.356 & & & 28.165 & 25.688 & 32.346 & 35.599 & 33.397 & 16.655 & 16.601 & 23.161 & 15.492 & 11.629 & 32.145 & 33.246 & 29.808 & 32.287 & & \\
\hline 9/15/11 16:30 & & & & & & & & & & & & & 32.334 & 35.596 & 33.383 & 16.652 & 16.587 & 23.158 & 15.48 & 11.617 & 32.128 & 33.237 & & & & \\
\hline 9/15/11 17:00 & & & & & & & & & & & & & 32.32 & 35.589 & 33.369 & 16.65 & 16.578 & 23.154 & 15.48 & 11.601 & 32.114 & 33.22 & & & & \\
\hline 9/15/11 17:30 & & & & & & & & & & & & & 32.309 & 35.592 & 33.355 & 16.648 & 16.566 & 23.172 & 15.461 & 11.587 & 32.105 & 33.206 & & & & \\
\hline 9/15/11 18:00 & & 30.468 & & & & & 29.417 & 30.441 & 29.619 & & & & 32.297 & 35.592 & 33.345 & 16.648 & 16.554 & 23.193 & 15.447 & 11.577 & 32.098 & 33.199 & & & & \\
\hline 9/15/11 18:30 & & 30.468 & & & & & 29.413 & 30.439 & 29.622 & & 28.149 & 25.799 & 32.285 & 35.587 & 33.334 & 16.645 & 16.545 & 23.223 & 15.435 & 11.566 & 32.086 & 33.187 & & & & \\
\hline 9/15/11 19:00 & & 30.473 & 29.962 & 28.783 & 30.134 & & 29.41 & 30.436 & 29.631 & 98 & 28.149 & 25.787 & 32.276 & 35.589 & 33.324 & 16.643 & 16.536 & 23.251 & 12 & 11.559 & 32.086 & 33.175 & & & & \\
\hline 9/15/11 19:30 & & 30.47 & 29.96 & 28.788 & 30.129 & & 29.399 & 30.439 & 29.638 & 29.008 & 28.151 & 25.801 & 32.266 & 35.587 & 33.313 & 16.643 & 16.526 & 23.249 & 07 & 11.547 & 32.075 & 33.166 & 9.774 & 2.227 & & \\
\hline 9/15/11 20:00 & & 30.468 & 29.955 & 28.797 & 30.122 & & 29.394 & 30.436 & 29.643 & 29.031 & 28.151 & 25.808 & 32.259 & 35.582 & 33.306 & 16.641 & 16.517 & 23.237 & 5.398 & 11.535 & 32.065 & 33.158 & 29.767 & 32.215 & & \\
\hline 9/15/11 20:30 & & 30.475 & 29.96 & 28.797 & 30.124 & & 29.38 & 30.439 & 29.653 & 29.059 & 28.153 & 25.82 & 32.255 & 35.592 & 33.299 & 16.641 & 16.51 & 23.235 & 15.369 & 11.528 & 32.072 & 33.151 & 29.778 & 32.211 & & \\
\hline 9/15/11 21:00 & & 30.473 & 29.958 & 28.797 & 30.124 & & 29.387 & 30.439 & 29.655 & 29.08 & 28.149 & 25.815 & 32.243 & 35.589 & 33.292 & 16.641 & 16.503 & 23.228 & 15.365 & 11.523 & 32.07 & 33.144 & 29.776 & 32.204 & & \\
\hline 9/15/11 21:30 & & 30.48 & 29.965 & 28.795 & 30.127 & & 29.38 & 30.439 & 29.66 & 29.106 & 28.149 & 25.815 & 32.241 & 35.587 & 33.282 & 16.643 & 16.498 & 23.235 & 15.344 & 11.519 & 32.075 & 33.139 & 29.783 & 32.197 & & \\
\hline 9/15/11 22:00 & & 30.473 & 29.96 & 28.79 & 30.122 & & 29.377 & 30.439 & 29.662 & 29.12 & 28.149 & 25.815 & 32.234 & 35.594 & 33.277 & 16.641 & 16.493 & 23.232 & 15.346 & 11.509 & 32.065 & 33.132 & 29.776 & 32.19 & & \\
\hline 9/15/11 22:30 & & 30.477 & 29.962 & 28.797 & 30.122 & & 29.38 & 30.436 & 29.672 & 29.14 & 28.146 & 25.811 & 32.229 & 35.594 & 33.273 & 16.641 & 16.494 & 23.228 & 15.327 & 11.505 & 32.063 & 33.128 & 29.778 & 32.19 & & \\
\hline 9/15/11 23:00 & & 30.477 & 29.96 & 28.792 & 30.12 & & 29.373 & 30.441 & 29.674 & 29.154 & 28.146 & 25.818 & 32.222 & 35.594 & 33.268 & $3 \quad 16.641$ & 16.493 & 23.223 & 15.318 & 11.5 & 32.056 & 33.123 & 29.778 & 32.185 & & \\
\hline 9/15/11 23:30 & & 30.475 & 29.955 & 28.788 & 30.115 & & 29.375 & 30.439 & 29.681 & 29.164 & 28.144 & 25.815 & 32.215 & 35.596 & 33.259 & 16.641 & 16.494 & 23.219 & 15.316 & 11.493 & 32.056 & 33.116 & 29.769 & 32.175 & 26.701 & 36.974 \\
\hline 9/16/11 0:00 & & 30.48 & 29.958 & 28.776 & 30.117 & & 29.387 & 30.436 & 29.679 & 29.182 & 28.135 & 25.811 & 32.213 & 35.596 & 33.256 & 16.638 & 16.489 & 23.216 & 15.297 & 11.491 & 32.058 & 33.113 & 29.783 & 32.173 & 26.701 & 36.444 \\
\hline $9 / 16 / 110: 30$ & & 30.477 & 29.955 & 28.774 & 30.115 & & 29.387 & 30.436 & 29.683 & 29.194 & 28.132 & 25.799 & 32.203 & 35.596 & 33.249 & 16.638 & 16.484 & 23.214 & 15.292 & 11.486 & 32.047 & 33.106 & 29.771 & 32.166 & 26.701 & 36.114 \\
\hline 9/16/11 1:00 & & 30.477 & 29.951 & 28.764 & 30.11 & & 29.391 & 30.434 & 29.683 & 29.203 & 28.128 & 25.799 & 32.199 & 35.599 & 33.245 & 16.636 & 16.482 & 23.225 & 15.287 & 11.477 & 32.04 & 33.102 & 29.76 & 32.161 & 26.701 & 35.897 \\
\hline $9 / 16 / 111: 30$ & & 30.475 & 29.946 & 28.755 & 30.103 & & 29.396 & 30.431 & 29.686 & 29.21 & 28.123 & 25.791 & 32.194 & 35.587 & 33.24 & 16.636 & 16.477 & 23.221 & 15.278 & 11.472 & 32.033 & 33.094 & 29.752 & 32.154 & 26.701 & 35.743 \\
\hline 9/16/11 2:00 & & 30.473 & 29.939 & 28.75 & 30.096 & & 29.391 & 30.431 & 29.693 & 29.219 & 28.119 & 25.779 & 32.187 & 35.594 & 33.231 & 16.636 & 16.47 & 23.219 & 15.273 & 11.462 & 32.023 & 33.087 & 29.748 & 32.145 & 26.701 & 35.626 \\
\hline 9/16/11 2:30 & & 30.47 & 29.934 & 28.746 & 30.087 & & 29.396 & 30.427 & 29.693 & 29.224 & 28.114 & 25.777 & 32.18 & 35.589 & 33.223 & 16.634 & 16.461 & 23.214 & 15.271 & 11.458 & 32.019 & 33.083 & 29.741 & 32.138 & 26.701 & 35.535 \\
\hline 9/16/11 3:00 & & $\begin{array}{l}30.468 \\
30.47\end{array}$ & 29.927 & 28.748 & 30.08 & & 29.394 & $\begin{array}{l}30.424 \\
30.424\end{array}$ & 29.698 & 29.229 & 28.112 & 25.772 & 32.171 & 35.587 & 33.214 & 16.634 & $\begin{array}{l}10.401 \\
16.456\end{array}$ & 23.209 & 15.259 & $\begin{array}{l}11.450 \\
11.446\end{array}$ & 32.007 & $\begin{array}{l}33.073 \\
3.073\end{array}$ & 29.731 & 32.131 & 26.701 & 35.458 \\
\hline 9/16/11 3:30 & & $\begin{array}{l}30.468 \\
30.468\end{array}$ & 29.923 & 28.762 & $\begin{array}{l}30.0073 \\
30.073\end{array}$ & & 29.382 & $\begin{array}{l}30.427 \\
30.427\end{array}$ & 29.702 & 29.236 & 28.114 & 25.775 & 32.163 & 35.585 & 33.205 & 16.631 & $\begin{array}{l}16.449 \\
16.499\end{array}$ & 23.202 & 15.259 & 11.439 & 32.002 & 33.061 & 29.724 & 32.121 & 26.701 & $\begin{array}{l}35.393 \\
35.35\end{array}$ \\
\hline 9/16/11 4:00 & & 30.468 & 29.918 & 28.76 & 30. & & 29.377 & 30.427 & 29.707 & 29.243 & 28 & 25.789 & 32.156 & 35.589 & 33.2 & 16.629 & 16.442 & 23.2 & 5 & 11.432 & 31.991 & 33.057 & 29.72 & 32.117 & 26.701 & 35.334 \\
\hline & & 30. & 29.918 & 28.757 & 30 & & 29.373 & 30.427 & 29.707 & 29 & 28 & 25.787 & 32.149 & 35.58 & 33.195 & 16 & 33 & 23.195 & 8 & 11.427 & 31.993 & 52 & 22 & 32.11 & 1 & 35.285 \\
\hline & & 30.475 & 29.92 & 28.757 & & & 29.373 & 30. & 25 & 29 & 28 & 25 & 32.145 & 35. & 33.188 & 16.631 & 16.428 & 23 & 9 & 11.418 & 31.998 & 47 & 29 & 07 & 1 & 35.241 \\
\hline $9 / 16$ & & 30.475 & 29. & 28.76 & & & 29.37 & 30.42 & 29 & & 28.109 & 25. & 32 & 35.585 & 33.179 & 16.631 & 16.423 & 23.188 & 15.215 & 11.415 & 31.993 & .042 & 29.729 & 32.098 & 26.701 & 35.201 \\
\hline 9/16/11 6:00 & & 30.47 & 29.918 & 28.764 & 30.0 & & 29.366 & 30.4 & & 29. & 28.112 & 25 & 32.95 & 35.587 & 33.388 & 16.629 & 16.423 & 23.184 & 15.212 & 11.622 & 32.161 & 33.992 & 29.724 & 32.917 & 26.698 & 35.164 \\
\hline 9/16/11 6:30 & & 30.47 & 29.918 & 28.771 & 30.06 & & 29.359 & 30.424 & 29.7 & 29. & 28.112 & 25.796 & 34.298 & 35.589 & 34.673 & 16.631 & 16.484 & 23.184 & 15.203 & 12.908 & 32.784 & 35.305 & 29.76 & 4.254 & 6.701 & 35.131 \\
\hline 9/16/11 7:00 & & 30.473 & 29. & 28.767 & 30. & & & 30.424 & 29.7 & & 28.112 & 25. & 34.906 & 35.587 & 35.367 & 16.629 & 16.589 & 23.182 & 15.194 & 13.604 & 33.04 & 35.886 & 29.804 & 34.853 & 26.703 & 35.099 \\
\hline 9/16/11 7:30 & & 30.473 & 29.92 & 28.769 & 30.103 & & & 30.424 & 29.731 & & 28. & 25.803 & 35.255 & 35.589 & 35.852 & 16.629 & 16.706 & 23.182 & 15.187 & 14.086 & 33.217 & 36.198 & 29.856 & 35.195 & 26.701 & 35.071 \\
\hline 9/16/11 8:00 & & 30.477 & 29.927 & 28.767 & 30. & & 29. & 30.424 & 29.733 & & 28.109 & 25.806 & 35.411 & 35.592 & 36.06 & 16.629 & 16.811 & 23.179 & 15.172 & 14.295 & 33.269 & 36.355 & 29.891 & 35.35 & 26.703 & 35.045 \\
\hline 9/16/11 8:30 & & 30.475 & 29.92 & 28.771 & 30.159 & & 29. & 30.426 & 29.7 & 29.324 & 28.112 & 25.803 & 35.9 & 35.592 & 36.459 & 16.631 & 16.912 & 23.175 & 15.172 & 14.694 & 33.458 & 36.916 & 29.922 & 35.883 & 26.701 & 35.022 \\
\hline 9/16/11 9:00 & & 30.475 & 29.925 & 28.774 & 30.187 & & 29.351 & 30.427 & 29.74 & 29.331 & 28.112 & 25.813 & 36.36 & 35.594 & 36.855 & 16.631 & 17.019 & 23.17 & 15.168 & 15.087 & 33.63 & 37.34 & 29.952 & 36.303 & 26.7 & 34.998 \\
\hline $9 / 16 / 119: 30$ & & 30.475 & 29.923 & 28.771 & 30.218 & & 29.356 & 30.426 & 29.74 & 29.34 & 28.109 & 25.815 & 36.722 & 35.594 & 37.226 & 16.631 & 17.129 & 23.17 & 15.163 & 15.458 & 33.777 & 37.686 & 29.985 & 36.65 & 26.703 & 34.977 \\
\hline 9/16/11 10:00 & & 30.48 & 29.927 & 28.767 & 30.25 & & 29.354 & 30.427 & 29.743 & 29.347 & 28.107 & 25.818 & 37.035 & 35.596 & 37.552 & 16.631 & 17.237 & 23.168 & 15.158 & 15.785 & 33.91 & 37.999 & 30.03 & 36.965 & 26.703 & 34.959 \\
\hline 9/16/11 10:30 & & 30.477 & 29.927 & 28.764 & 30.281 & & 29.359 & 30.426 & 29.745 & 29.354 & 28.105 & 25.813 & 37.325 & 35.596 & 37.854 & 16.634 & 17.339 & 23.175 & 15.156 & 16.091 & 34.017 & 38.269 & 30.056 & 37.248 & 26.703 & 34.94 \\
\hline 9/16/11 11:00 & & 30.48 & 29.93 & 28.757 & 30.309 & & 29.366 & 30.427 & 29.75 & 29.363 & 28.102 & 25.813 & 37.339 & 35.601 & 37.967 & 16.634 & 17.438 & 23.179 & 15.149 & 16.199 & 34.05 & 38.279 & 30.091 & 37.263 & 26.703 & 34.921 \\
\hline 9/16/11 11:30 & & 30.477 & 29.927 & 28.748 & 30.334 & & 29.37 & 30.427 & 29.75 & 29.366 & 28.098 & 25.806 & 35.9 & 35.594 & 37.144 & 16.636 & 17.505 & 23.179 & 15.151 & 15.372 & 33.67 & 36.755 & 30.096 & 35.82 & 26.703 & 34.907 \\
\hline 9/16/11 12:00 & & 30.477 & 29.927 & 28.739 & 30.355 & & 29.37 & 30.427 & 29.748 & 29.368 & 28.095 & 25.801 & 35.205 & 35.596 & 36.417 & 16.636 & 17.501 & 23.175 & 15.151 & 14.645 & 33.388 & 36.075 & 30.093 & 35.134 & 26.703 & 34.891 \\
\hline 9/16/11 12:30 & & 30.473 & 29.92 & 28.732 & 30.362 & & 29.38 & 30.424 & 29.75 & 29.37 & 28.088 & 25.791 & 34.777 & 35.594 & 35.962 & 16.634 & 17.47 & 23.172 & 15.156 & 14.187 & 33.21 & 35.651 & 30.07 & 34.71 & 26.703 & 34.875 \\
\hline
\end{tabular}




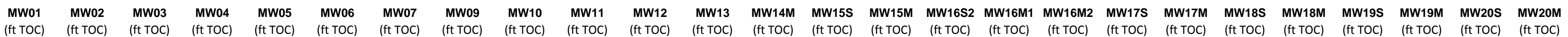

\begin{tabular}{|c|c|c|c|c|c|c|c|c|c|c|c|c|c|c|c|c|c|c|c|c|c|c|c|c|c|c|}
\hline Well & $\begin{array}{l}\text { (ft TOC) } \\
\text { (is }\end{array}$ & $\begin{array}{l}\text { (ft TOC) } \\
\text { (f) }\end{array}$ & $\begin{array}{l}\text { (ft TOC) } \\
\text { (f) }\end{array}$ & (ft TOC) & $\begin{array}{l}\text { (ft Toc) } \\
\text { (f) }\end{array}$ & $\begin{array}{l}\text { (ft TOC) } \\
\text { (f) }\end{array}$ & (ft toc) & $\begin{array}{l}\text { (ft TOC) } \\
\text { (f) }\end{array}$ & $\begin{array}{l}\text { (ft TOC) } \\
\text { (ft) }\end{array}$ & $\begin{array}{l}\text { (ft TOC) } \\
\text { (t) }\end{array}$ & $\begin{array}{l}\text { (ft TOC) } \\
\text { (f) }\end{array}$ & $\begin{array}{l}\text { (ft Toc) } \\
\text { (f) }\end{array}$ & $\begin{array}{l}\text { (ft TOC) } \\
\text { (t) }\end{array}$ & $\begin{array}{l}\text { (ft TOC) } \\
\end{array}$ & (ft TOC) & (ft TOC) & (ft TOC) & (ft TOC) & $\begin{array}{l}\text { (ft TOC) } \\
\text { (ft) }\end{array}$ & (ft TOC) & $\begin{array}{l}\text { (ft TOC) } \\
\end{array}$ & $\begin{array}{l}\text { (ft TOC) } \\
\text { (1) }\end{array}$ & $\begin{array}{l}\text { (ft TOC) } \\
\text { (n) }\end{array}$ & (ft TOC) & $\begin{array}{l}\text { (ft TOC) } \\
\end{array}$ & (ft TOC) \\
\hline 9/16/11 13:00 & & 30.475 & 29.92 & 28.722 & 30.369 & & 29.382 & 30.422 & 29.752 & 29.375 & 28.084 & 25.782 & 34.468 & 35.592 & 35.629 & $\begin{array}{l}9 \\
9\end{array}$ & 17.433 & 23.172 & 15.144 & 13.862 & 33.091 & 35.348 & 30.056 & 34.405 & 26.698 & 34.861 \\
\hline 9/16/11 13:30 & & 30.473 & 29.913 & 28.713 & 30.362 & & 29.384 & 30.419 & 29.755 & 29.375 & 28.079 & 25.775 & 34.225 & 35.589 & 35.371 & $1 \quad 16.634$ & 17.396 & 23.175 & 15.158 & 13.599 & 32.982 & 35.108 & 30.032 & 34.165 & 26.7 & 34.851 \\
\hline 9/16/11 14:00 & & 30.473 & 29.906 & 28.713 & 30.358 & & 29.384 & 30.417 & 29.759 & 29.375 & 28.074 & 25.77 & 34.028 & 35.585 & 35.163 & $\begin{array}{l}3 \\
16.634\end{array}$ & 17.358 & 23.177 & 15.154 & 13.395 & 32.898 & 34.912 & 30.013 & 33071 & 26.7 & 34.84 \\
\hline $1114: 30$ & & 30.47 & 29.902 & 28.711 & 30.346 & & 29.387 & 30.415 & 29.757 & 29.375 & 28.072 & 25.765 & 33.865 & 35.587 & 34.987 & 16.631 & 17.318 & 23.179 & 15.149 & 13.221 & 32.828 & & 95 & 09 & 26.7 & 34.825 \\
\hline 9/16/11 15:00 & & 30.47 & 29.897 & 28.711 & 30.334 & & 29.382 & 30.417 & 29.759 & 29.377 & 28.067 & 25.763 & 33.724 & 35.582 & 34.841 & 16.631 & 17.283 & 23.184 & 15.147 & 13. & 32.763 & 34.611 & 976 & 68 & 26.7 & 34.811 \\
\hline 9/16/11 15:30 & & 30.468 & 29.892 & 28.706 & 30.32 & & 29.382 & 30.422 & 29.762 & 29.38 & 28.063 & 25.763 & 33.603 & 35.578 & 34.712 & 16.631 & 17.248 & 23.184 & 15.146 & 12.943 & 32.711 & 34.49 & 29.964 & 3.548 & 26.7 & 34.8 \\
\hline $9 / 16 / 1116: 00$ & & 30.468 & 29.888 & 28.711 & 30.306 & & 29.377 & 30.417 & 29.764 & 29.38 & 28.063 & 25.76 & 33.495 & 35.578 & 34.602 & $2 \quad 16.629$ & 17.216 & 23.188 & 15.142 & 12.83 & 32.658 & 34.388 & 29.945 & 33.445 & 26.7 & 34.788 \\
\hline 9/16/11 16:30 & & 30.468 & 29.888 & 28.711 & 30.295 & & 29.37 & 30.415 & 29.767 & 29.382 & 28.063 & 25.765 & 33.399 & 35.578 & 34.504 & $4 \quad 16.627$ & 17.183 & 23.191 & 15.13 & 12.734 & 32.618 & 34.291 & 29.929 & 33.349 & 26.7 & 34.776 \\
\hline 9/16/11 17:00 & & 30.468 & 29.885 & 28.715 & 30.281 & & 29.363 & 30.417 & 29.771 & 29.384 & 28.063 & 25.767 & 33.317 & 35.575 & 34.415 & $5 \quad 16.627$ & 17.152 & 23.191 & 15.13 & 12.642 & 32.578 & 34.21 & 29.917 & 33.264 & 26.7 & 34.767 \\
\hline 9/16/11 17:30 & & 30.47 & 29.885 & 28.72 & 30.271 & & 29.359 & 30.417 & 29.771 & 29.391 & 28.063 & 25.772 & 33.242 & 35.575 & 34.335 & $5 \quad 16.629$ & 17.125 & 23.193 & 15.121 & 12.572 & 32.546 & 34.137 & 29.912 & 33.194 & 26.7 & 34.755 \\
\hline 9/16/11 18:00 & & 30.47 & 29.888 & 28.722 & 30.262 & & 29.351 & 30.412 & 29.771 & 29.396 & 28.06 & 25.777 & 33.2 & 35.578 & 34.29 & $\begin{array}{l}9 \\
9\end{array}$ & 17.092 & 23.205 & 15.118 & 12.52 & 32.52 & 34.094 & 29.908 & 33.149 & 26.703 & 34.746 \\
\hline 9/16/11 18:30 & & 30.47 & 29.885 & 28.722 & 30.255 & & 29.347 & 30.415 & 29.774 & 29.4 & 28.063 & 25.779 & 33.123 & 35.571 & 34.208 & $8 \quad 16.629$ & 17.068 & 23.202 & 15.118 & 12.44 & 32.487 & 34.019 & 29.896 & 33.074 & 26.7 & 34.737 \\
\hline 9/16/11 19:00 & & 30.475 & 29.89 & 28.727 & 30.248 & & 29.342 & 30.417 & 29.776 & 29.407 & 28.065 & 25.779 & 33.062 & 35.571 & 34.147 & $7 \quad 16.629$ & 17.043 & 23.214 & 15.109 & 12.377 & 32.469 & 33.959 & 29.893 & 33.013 & 26.703 & 34.727 \\
\hline 9/16/11 19:30 & & 30.475 & 29.892 & 28.743 & 30.239 & & 29.328 & 30.419 & 29.776 & 29.41 & 28.067 & 25.787 & 33.011 & 35.58 & 34.089 & 16.629 & 17.015 & 23.214 & 15.107 & 12.323 & 32.443 & 33.907 & 29.886 & 32.962 & 26.703 & 34.718 \\
\hline $9 / 16 / 1120: 00$ & & 30.473 & 29.89 & 28.753 & 30.229 & & 29.321 & 30.422 & 29.781 & 29.412 & 28.072 & 25.801 & 32.961 & 35.578 & 34.037 & 16.629 & 16.991 & 23.202 & 15.107 & 12.271 & 32.413 & 33.857 & 29.879 & 32.912 & 26.705 & 34.709 \\
\hline 9/16/11 20:30 & & 30.48 & 29.895 & 28.757 & 30.229 & & 29.312 & 30.424 & 29.783 & 29.421 & 28.077 & 25.811 & 32.917 & 35.582 & 33.993 & 16.631 & 16.968 & 23.198 & 15.095 & 12.226 & 32.408 & 33.815 & 29.886 & 32.872 & 26.705 & 34.702 \\
\hline 9/16/11 21:00 & & 30.482 & 29.899 & 28.762 & 30.227 & & 29.314 & 30.427 & 29.783 & 29.431 & 28.077 & 25.818 & 32.877 & 35.582 & 33.953 & 16.631 & $\begin{array}{l}16.947 \\
\text {. }\end{array}$ & 23.202 & 15.088 & 12.182 & 32.39 & 33.774 & 29.884 & 32.832 & 26.705 & 34.695 \\
\hline 9/16/11 21:30 & & 30.482 & 29.904 & 28.753 & 30.227 & & 29.314 & 30.427 & 29.783 & 29.438 & 28.074 & 25.82 & 32.842 & 35.585 & 33.913 & 16.634 & 16.928 & 23.2 & $\begin{array}{l}15.081 \\
15.08\end{array}$ & 12.144 & 32.371 & 33.741 & 29.886 & 32.795 & 26.705 & 34.688 \\
\hline 9/16/11 22:00 & & $\begin{array}{l}30.482 \\
30.482\end{array}$ & 29.906 & 28.76 & 30.225 & & 29.321 & 30.427 & 29.788 & 29.445 & 28.077 & $\begin{array}{l}25.82 \\
25.811\end{array}$ & 32.807 & 35.592 & 33.878 & 16.636 & $\begin{array}{l}10.560 \\
16.907\end{array}$ & 23.198 & $\begin{array}{l}15.081 \\
15.081\end{array}$ & $\begin{array}{l}12.109 \\
12.109\end{array}$ & 32.362 & $\begin{array}{l}33.706 \\
33140\end{array}$ & 29.886 & 32.764 & 26.705 & 34.683 \\
\hline 9/16/11 22:30 & & $\begin{array}{l}30.402 \\
30.487\end{array}$ & 29.913 & $\begin{array}{l}28.757 \\
287\end{array}$ & 30.225 & & 29.314 & 30.429 & 29.786 & 29.454 & 28.079 & $\begin{array}{l}2.011 \\
25.82\end{array}$ & 32.777 & 35.592 & $\begin{array}{l}33.845 \\
33.81\end{array}$ & 16.636 & 16.888 & 23.195 & $\begin{array}{l}15.067 \\
15.067\end{array}$ & 12.08 & $\begin{array}{l}32.348 \\
32.348\end{array}$ & $\begin{array}{l}33.673 \\
337\end{array}$ & $\begin{array}{l}29.000 \\
29.884\end{array}$ & $\begin{array}{l}32.732 \\
32.732\end{array}$ & 26.707 & 34.676 \\
\hline 9/16/11 23:00 & & 30.482 & 29.909 & 28.741 & 30.213 & & 29.328 & 30.431 & 29.783 & 29.452 & 28.072 & 25.815 & 32.744 & 35.596 & 33.812 & 16.636 & 16.87 & 23.193 & 15.076 & 12.043 & 32.327 & 33.642 & 29.875 & 32.699 & 26.705 & 34.669 \\
\hline 9/16/11 23:30 & & 30.487 & 29.911 & 28.736 & & & 29.33 & 30.429 & 29.786 & 29.458 & 28.07 & 25.801 & & .596 & 33.784 & 16.636 & 16.853 & 23.191 & .069 & 12.015 & 32.32 & 33.616 & 875 & .673 & 26.707 & 34.664 \\
\hline 9/17/11 0:00 & & 30.484 & 29.911 & 28.743 & 30.206 & & 29.328 & 30.429 & 29.79 & 29.461 & 28.067 & 25.794 & 32.69 & .596 & 33.756 & 16.636 & 16.837 & 23.186 & .074 & 11.986 & 32.299 & 33.59 & .863 & .647 & 26.705 & 34.66 \\
\hline $9 / 17 / 1$ & & 30.48 & 29.906 & 28.743 & 30.197 & & 29.328 & 30.431 & 29.793 & 29.456 & 28.072 & 25.806 & 32.662 & 35.596 & .725 & 16.634 & $\begin{array}{l}16.8316 \\
16.816\end{array}$ & & 5.074 & $\begin{array}{l}11.500 \\
11.958\end{array}$ & 32.278 & 33.559 & 8.851 & 619 & 26.705 & 34.653 \\
\hline 9/17/11 1:00 & & 30.48 & 29.904 & 28.734 & 30.187 & & 29.33 & 30.429 & 29.793 & 29.458 & 28.067 & 25.806 & 32.639 & 35.594 & 33.699 & 16.634 & 16.802 & 23.182 & 15.074 & 11.932 & 32.266 & 33.535 & 9.844 & 2.595 & 26.707 & 34.646 \\
\hline 9/17/11 1:30 & & 30.482 & 29.906 & 28.725 & 30.183 & & 29.342 & 30.429 & 29.788 & 29.465 & 28.06 & 25.794 & 32.615 & 35.594 & 33.676 & 16.634 & 16.788 & 23.177 & 15.067 & 11.907 & 32.254 & 33.514 & 29.842 & .574 & 26.707 & 34.643 \\
\hline 9/17/11 2:00 & & 30.484 & 29.906 & 28.725 & 30.178 & & 29.34 & 30.429 & 29.79 & 29.468 & 28.058 & 25.789 & 32.594 & 35.596 & 33.655 & 16.636 & 16.772 & 23.177 & 15.064 & 11.888 & 32.245 & 33.495 & 29.839 & 32.548 & 26.707 & 34.639 \\
\hline 9/17/11 2:30 & & 30.48 & 29.902 & 28.722 & 30.169 & & 29.34 & 30.426 & 29.793 & 29.463 & 28.058 & 25.787 & 32.571 & 35.592 & 33.632 & 16.631 & 16.758 & 23.175 & 15.074 & 11.864 & 32.219 & 33.466 & 29.821 & 32.527 & 26.705 & 34.632 \\
\hline 9/17/11 3:00 & & 30.482 & 29.897 & 28.722 & 30.159 & & 29.347 & 30.427 & 29.788 & 29.465 & 28.051 & 25.784 & 32.55 & 35.592 & 33.608 & 316.634 & 16.741 & 23.175 & 15.064 & 11.841 & 32.217 & 33.45 & 29.818 & 32.506 & 26.707 & 34.627 \\
\hline 9/17/11 3:30 & & 30.482 & 29.897 & 28.732 & 30.152 & & 29.333 & 30.427 & 29.797 & 29.465 & 28.06 & 25.784 & 32.531 & 35.592 & 33.585 & 16.631 & 16.727 & 23.184 & 15.06 & 11.817 & 32.203 & 33.431 & 29.814 & 32.488 & 26.707 & 34.622 \\
\hline 9/17/11 4:00 & & 30.477 & 29.892 & 28.722 & 30.143 & & 29.33 & 30.426 & 29.793 & 29.463 & 28.058 & 25.796 & 32.51 & 35.592 & 33.566 & 16.631 & 16.711 & 23.184 & 15.069 & 11.801 & 32.184 & 33.41 & 29.804 & 32.469 & 26.705 & 34.618 \\
\hline 9/17/11 4:30 & & 30.484 & 29.892 & 28.727 & 30.141 & & 29.333 & 30.424 & 29.797 & 29.468 & 28.039 & 25.784 & 32.491 & 35.589 & 33.547 & 16.631 & 16.699 & 23.182 & 15.055 & 11.782 & 32.187 & 33.391 & 29.802 & 32.45 & 26.707 & 34.613 \\
\hline 9/17/11 5:00 & & 30.484 & 29.895 & 28.706 & 30.138 & & 29.349 & 30.422 & 29.79 & 29.472 & 28.021 & 25.787 & 32.475 & 35.587 & 33.528 & 16.631 & 16.685 & 23.179 & 15.055 & 11.766 & 32.18 & 33.374 & 29.804 & 32.434 & 26.71 & 34.606 \\
\hline 9/17/11 5:30 & & 30.48 & 29.89 & 28.685 & 30.131 & & 29.347 & 30.424 & 29.79 & 29.468 & 28.023 & 25.76 & 32.454 & 35.594 & 33.507 & 16.631 & 16.666 & 23.168 & 15.05 & 11.74 & 32.156 & 33.353 & 29.79 & 32.412 & 26.707 & 34.599 \\
\hline 9/17/11 6:00 & & 30.48 & 29.89 & 28.683 & 30.124 & & 29.359 & 30.414 & 29.793 & 29.472 & 28.023 & 25.763 & 32.43 & 35.594 & 33.488 & $\begin{array}{l}36.629 \\
\end{array}$ & 16.65 & 23.161 & 15.048 & 11.723 & 32.147 & 33.334 & 29.783 & 32.387 & 26.707 & 34.59 \\
\hline 9/17/11 6:30 & & & 29.874 & 28.732 & 30. & & 29.33 & 30.422 & 29.802 & 29.461 & 28.019 & 25.765 & 32.405 & 35.585 & $\begin{array}{r}3.400 \\
33.46\end{array}$ & 16.627 & 16.627 & 23.147 & 15.055 & 11.695 & 32.119 & 33.305 & 29.757 & 32.363 & 26.705 & 34.576 \\
\hline 9/17/11 7:00 & & 30.466 & 29.864 & 28.739 & 30.0 & & 29.309 & 30.427 & 29.807 & 29.452 & 28.028 & 25.796 & 32.383 & $\begin{array}{l}35.58 \\
35\end{array}$ & $\begin{array}{l}33.440 \\
33.444\end{array}$ & 16.62 & $\begin{array}{l}10.071 \\
16.61\end{array}$ & 23.147 & 15.057 & 11.676 & 32.096 & 33.286 & 29.734 & 32.344 & 26.703 & 34.566 \\
\hline 9/17/11 7:30 & & $\begin{array}{l}30.400 \\
30.475\end{array}$ & 29.864 & 28.734 & 30.0 & & 29.307 & 30.424 & 29.805 & 29.456 & $\begin{array}{l}28.037 \\
28.037\end{array}$ & 25.811 & 32.372 & 35.578 & $\begin{array}{l}33.423 \\
33.423\end{array}$ & $\begin{array}{l}16.62 \\
16.62\end{array}$ & $\begin{array}{l}16.059 \\
16.59\end{array}$ & 23.145 & 15.046 & $\begin{array}{l}11.657 \\
11.657\end{array}$ & $\begin{array}{r}32.1 \\
32.1\end{array}$ & 33.27 & 29.743 & 32.328 & 26.705 & 34.562 \\
\hline 9/17/11 8:00 & & $\begin{array}{l}30.489 \\
30.470\end{array}$ & $\begin{array}{l}29.004 \\
29.885\end{array}$ & 28.722 & 30.1 & & 29.319 & $\begin{array}{l}30.424 \\
30.422\end{array}$ & 29.797 & 29.472 & 28.065 & 25.822 & 32.365 & 35.582 & $\begin{array}{l}33.413 \\
33.413\end{array}$ & $\begin{array}{r}10.02 \\
16.622\end{array}$ & $\begin{array}{l}10.599 \\
16.592\end{array}$ & 23.145 & $\begin{array}{l}15.018 \\
15.018\end{array}$ & $\begin{array}{l}11.078 \\
11.648\end{array}$ & 32.117 & $\begin{array}{l}33.27 \\
3.265\end{array}$ & .783 & 26 & 26.712 & 34.559 \\
\hline $9 / 17$ & & 30 & 29.888 & 28.727 & 30 & & & 30 & 29 & 29 & 28 & 25 & 32 & 35 & 33.404 & 16 & 1 & 23 & 15.025 & 11.636 & 32.114 & 3 & 35 & 12 & 71 & 34.555 \\
\hline & & 30.482 & 29.88 & 28.727 & & & & 30 & 25 & & & & & 35.587 & 33.39 & 16.627 & 16.566 & & 7 & 11.622 & 32.103 & 33.239 & 57 & 77 & 26.71 & 34.55 \\
\hline 9/17/11 9:30 & & 30.48 & 29.8 & 28 & & & & & & & 28.084 & & & - & 33.376 & 16.622 & & & & 11.6 & (2) & & & 36 & 26.712 & 34.545 \\
\hline $9 / 17 /$ & & 30.482 & 29.883 & & & & & 30 & & & 28 & & & 35.582 & 33.364 & 16.622 & & & 15. & 11. & 86 & & & 32.274 & & 34.541 \\
\hline 9/17 & & 30.48 & 29.883 & 28 & & & & 30 & & & & 25 & 32. & 35.592 & 33.355 & 16.624 & 16.538 & & 15. & 11.587 & 32.079 & & & 32.26 & 71 & 34.539 \\
\hline $9 / 1$ & & & 29.8 & 28 & & & & 30 & & & 28 & 25. & 32 & 35.592 & 33.343 & 16.6 & 16. & & 15. & 11.575 & 32.07 & & 29.745 & & 26.71 & 34.531 \\
\hline 111:30 & & & 29.8 & 28. & & & & 30 & & & 28. & & 32. & 35.589 & 33.47 & 16 & 16. & 23. & 15. & 11.704 & 32.084 & & & & 26.71 & 34.529 \\
\hline 9/17/11 12:00 & & 30. & 29.8 & 28.697 & 30. & & & 30.4 & 29.8 & 29. & 28.1 & 25.765 & 32 & 35.594 & 33.503 & 16.62 & 16.547 & 23.145 & 15.034 & 11.735 & 32.117 & 33.343 & 29.738 & 32.398 & 26.71 & 34.527 \\
\hline 9/17/11 12:30 & & 30.475 & 29.8 & 28.692 & 30. & & & 30.4 & 29.8 & 29.4 & 28.1 & 25. & 32.3 & 35.589 & 33.43 & 16.617 & 16.545 & 23.14 & 15.0 & 11.6 & 32.091 & 33.277 & 29.736 & 32.333 & 26.71 & 34.522 \\
\hline 9/17/11 13:00 & & 30.475 & 29.867 & 28.687 & 30.061 & & 29.337 & 30.415 & 29.809 & 29.477 & 28.151 & 25.755 & 32.339 & 35.585 & 33.397 & 16.615 & 16.531 & 23.138 & 15.036 & 11.627 & 32.079 & 33.241 & 29.727 & 32.297 & 26.71 & 34.52 \\
\hline 9/17/11 13:30 & & 30.473 & 29.869 & 28.68 & 30.057 & & 29.335 & 30.415 & 29.809 & 29.477 & 28.149 & 25.753 & 32.313 & 35.587 & 33.369 & 16.615 & 16.524 & 23.138 & 15.034 & 11.601 & 32.061 & 33.213 & 29.727 & 32.274 & 26.71 & 34.515 \\
\hline 9/17/11 14:00 & & 30.47 & 29.864 & 28.678 & 30.05 & & 29.337 & 30.41 & 29.809 & 29.472 & 28.146 & 25.746 & 32.295 & 35.582 & 33.345 & 16.615 & 16.512 & 23.131 & 15.034 & 11.58 & 32.047 & 33.196 & 29.72 & 32.253 & 26.712 & 34.513 \\
\hline 9/17/11 14:30 & & 30.47 & 29.862 & 28.676 & 30.043 & & 29.337 & 30.41 & 29.809 & 29.472 & 28.137 & 25.746 & 32.276 & 35.582 & 33.327 & 16.613 & 16.503 & 23.133 & 15.034 & 11.559 & 32.04 & 33.177 & 29.717 & 32.234 & 26.71 & 34.511 \\
\hline 9/17/11 15:00 & & 30.47 & 29.857 & 28.669 & 30.038 & & 29.333 & 30.408 & 29.809 & 29.468 & 28.135 & 25.739 & 32.262 & 35.582 & 33.313 & 16.61 & 16.491 & 23.135 & 15.036 & 11.544 & 32.03 & 33.161 & 29.705 & 32.22 & 26.71 & 34.506 \\
\hline 9/17/11 15:30 & & 30.47 & 29.853 & 28.657 & 30.033 & & 29.34 & 30.405 & 29.812 & 29.465 & 28.132 & 25.736 & 32.259 & 35.582 & 33.31 & 16.61 & 16.479 & 23.142 & 15.034 & 11.54 & 32.026 & 33.161 & 29.703 & 32.218 & 26.712 & 34.503 \\
\hline 9/17/11 16:00 & & 30.468 & 29.85 & 28.657 & 30.028 & & 29.337 & 30.403 & 29.812 & 29.463 & 28.125 & 25.724 & 32.238 & 35.578 & 33.289 & 16.608 & 16.472 & 23.145 & 15.036 & 11.521 & 32.014 & 33.142 & 29.696 & 32.201 & 26.71 & 34.499 \\
\hline 9/17/11 16:30 & & 30.466 & 29.841 & 28.662 & 30.021 & & 29.333 & 30.403 & 29.812 & 29.461 & 28.116 & 25.722 & 32.224 & 35.58 & 33.273 & 16.606 & 16.461 & 23.198 & 15.034 & 11.507 & 32.002 & 33.125 & 29.689 & 32.182 & 26.71 & 34.494 \\
\hline
\end{tabular}




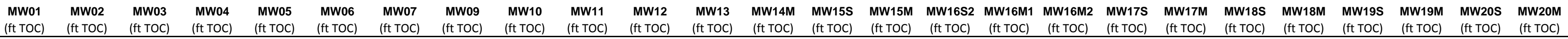

\begin{tabular}{|c|c|c|c|c|c|c|c|c|c|c|c|c|c|c|c|c|c|c|c|c|c|c|c|c|c|}
\hline well & $\begin{array}{l}\text { (ft TOC) } \\
\text { (f) }\end{array}$ & $\begin{array}{l}\text { (ft TOC) } \\
\text { (ft) }\end{array}$ & $\begin{array}{l}\text { (ft TOC) } \\
\text { (f) }\end{array}$ & $\begin{array}{l}\text { (ft TOC) } \\
\text { (ft) }\end{array}$ & $\begin{array}{ll}(\mathrm{ft} \text { TOC) } & \text { (ft TOC) }\end{array}$ & (ft toc) & $\begin{array}{l}\text { (ift TOC) } \\
\text { (n) }\end{array}$ & $\begin{array}{l}\text { (ft TOC) } \\
\text { (f) }\end{array}$ & $\begin{array}{l}\text { (ft TOC) } \\
\text { (f) }\end{array}$ & $\begin{array}{l}\text { (ft TOC) } \\
\text { (n) }\end{array}$ & $\begin{array}{l}\text { (ft TOC) } \\
\text { (ft }\end{array}$ & (ft TOC) & $\begin{array}{l}\text { (ft TOC) } \\
\end{array}$ & (ft TOC) & (ft TOC) & $\begin{array}{l}\text { (ft TOC) } \\
\text { (f) }\end{array}$ & (ft TOC) & $\begin{array}{l}\text { (ft TOC) } \\
\text { (ft) }\end{array}$ & (ft TOC) & $\begin{array}{l}\text { (ft TOC) } \\
\text { (f) }\end{array}$ & $\begin{array}{l}\text { (ft TOC) } \\
\text { (f) }\end{array}$ & $\begin{array}{l}\text { (ft TOC) } \\
\text { (n) }\end{array}$ & (ft TOC) & $\begin{array}{l}\text { (ft TOC) } \\
\text { (1) }\end{array}$ & (ft TOC) \\
\hline 7:00 & & 30.461 & 29.83 & 28.64 & 30.0 & 29.33 & 30.4 & 29 & 29.4 & 28.116 & 25.7 & 32.208 & 35.5 & 33.2 & 16.604 & 16.452 & 23.237 & 15.039 & 11.493 & 31.986 & 33.111 & 29.675 & .168 & & 34.489 \\
\hline 11 17:30 & & 30.461 & 29.832 & 28.64 & 30.003 & 29.337 & 30.396 & 29.809 & 29.452 & 28.091 & 25.71 & 32.196 & 35.56 & 33.245 & 16.601 & 16.44 & 23.237 & 15.029 & 11.479 & 31.981 & .097 & 67 & 2.154 & & \\
\hline 9/17/11 18:00 & & 30.463 & 29.832 & 28.664 & 30.001 & 29.326 & 30.393 & 29.814 & 29.454 & 28.067 & 25.712 & 32.187 & 35.573 & 33.233 & 16.601 & 16.433 & 23.232 & 15.027 & 11.465 & & 33.087 & .668 & .145 & 12 & 34.482 \\
\hline 9/17/11 18:30 & & 30.456 & 29.822 & 28.683 & 29.989 & 29.312 & 30.398 & 29.819 & 29.445 & 28.039 & 25.732 & 32.173 & 35.568 & 33.219 & 16.599 & 16.423 & 23.225 & 15.029 & 11.455 & 31.963 & 33.073 & 29.656 & 32.133 & 12 & 34.48 \\
\hline 9/17/11 19:00 & & 30.459 & 29.822 & 28.687 & 29.984 & 29.29 & 30.396 & 29.814 & 29.445 & 28.025 & 25.753 & 32.163 & 35.568 & 33.207 & 16.597 & 16.414 & 23.219 & 15.027 & 11.444 & 31.958 & 33.061 & 29.651 & 32.121 & 26.712 & 34.478 \\
\hline 9/17/11 19:30 & & 30.463 & 29.827 & 28.676 & 29.986 & 29.293 & 30.398 & 29.816 & 29.449 & 28.028 & 25.758 & 32.154 & 35.568 & 33.198 & 16.597 & 16.407 & 23.214 & 15.015 & 11.432 & 31.96 & 33.056 & 29.663 & 32.114 & 26.712 & 34.475 \\
\hline 9/17/11 20:00 & & 30.473 & 29.836 & 28.676 & 29.998 & 29.297 & 30.396 & 29.816 & 29.456 & 28.028 & 25.746 & 32.152 & 35.573 & 33.195 & 16.599 & 16.4 & 23.209 & 14.996 & 11.429 & 31.972 & 33.052 & 29.689 & 32.11 & 26.717 & 34.473 \\
\hline 9/17/11 20:30 & & 30.468 & 29.836 & 28.671 & 29.998 & 29.295 & 30.395 & 29.821 & 29.458 & 28.028 & 25.746 & 32.142 & 35.578 & 33.186 & 16.599 & 16.395 & 23.205 & 15.006 & 11.418 & 31.967 & 33.045 & 29.682 & 32.105 & 26.714 & 34.471 \\
\hline 9/17/11 21:00 & & 30.461 & 29.832 & 28.685 & 29.989 & 29.297 & 30.393 & 29.816 & 29.456 & 28.025 & 25.746 & 32.133 & 35.568 & 33.179 & 16.597 & 16.384 & 23.195 & 15.015 & 11.411 & 31.951 & 33.033 & 29.666 & 32.093 & 26.712 & 34.464 \\
\hline 9/17/11 21:30 & & 30.461 & 29.834 & 28.685 & 29.991 & 29.288 & 30.395 & 29.819 & 29.456 & 28.028 & 25.758 & 32.124 & 35.575 & 33.172 & 16.597 & 16.377 & 23.191 & 15.006 & 11.401 & 31.947 & 33.026 & 29.666 & 32.084 & 26.712 & 34.461 \\
\hline 9/17/11 22:00 & & 30.461 & 29.832 & 28.678 & 29.986 & 29.286 & 30.393 & 29.819 & 29.456 & 28.025 & 25.76 & 32.117 & 35.575 & 33.163 & 16.597 & 16.37 & 23.186 & 15.003 & 11.394 & 31.947 & 33.019 & 29.668 & 32.077 & 26.712 & 34.459 \\
\hline 9/17/11 22:30 & & 30.466 & 29.834 & 28.692 & 29.993 & 29.281 & 30.396 & 29.819 & 29.461 & 28.032 & 25.751 & 32.114 & 35.578 & 33.158 & 16.599 & 16.367 & 23.179 & 14.994 & 11.387 & 31.949 & 33.016 & 29.675 & 32.072 & 26.714 & 34.459 \\
\hline 9/17/11 23:00 & & 30.466 & 29.839 & 28.697 & 29.993 & 29.272 & 30.398 & 29.821 & 29.463 & 28.023 & 25.77 & 32.11 & 35.582 & 33.153 & 16.597 & 16.36 & 23.179 & 14.994 & 11.383 & 31.951 & 33.011 & 29.677 & 32.068 & 26.714 & 34.457 \\
\hline 9/17/11 23:30 & & 30.466 & 29.839 & 28.704 & 29.991 & 29.265 & 30.398 & 29.826 & 29.461 & 28.019 & 25.775 & 32.1 & 35.58 & 33.146 & 16.597 & 16.353 & 23.175 & 14.989 & 11.382 & 31.947 & 33.004 & 29.673 & 32.06 & 26.717 & 34.454 \\
\hline 9/18/11 0:00 & & 30.466 & 29.839 & 28.722 & 29.996 & 29.257 & 30.4 & 29.821 & 29.465 & 28.012 & 25.782 & 32.098 & 35.58 & 33.139 & 16.597 & 16.349 & 23.172 & 14.994 & 11.371 & 31.944 & 33.002 & 29.677 & 32.06 & 26.714 & 34.454 \\
\hline 9/18/11 0:30 & & 30.466 & 29.843 & 28.704 & & 29.257 & 30.402 & 29.816 & 29.47 & 28.021 & 25.808 & 32.096 & 35.585 & 33.137 & 16.597 & 16.344 & 23.184 & 14.982 & 11.371 & 31.949 & 32.997 & 29.684 & 32.056 & 26.717 & 34.454 \\
\hline 9/18/11 1:00 & & 30.475 & 29.853 & 28.704 & 30.007 & 29.274 & 30.4 & 29.821 & 29.477 & 28.019 & 25.782 & 32.096 & 35.589 & 33.132 & 16.601 & 16.339 & 23.186 & 14.971 & 11.368 & 31.963 & 32.995 & 29.701 & 32.053 & 26.719 & 34.454 \\
\hline 9/18/11 1:30 & & 30.477 & 29.862 & 28.711 & 30.017 & 29.265 & 30.403 & 29.826 & 29.484 & 28.009 & 25.779 & 32.093 & 35.592 & 33.132 & 16.601 & 16.337 & 23.186 & 14.959 & 11.368 & 31.967 & 32.995 & 29.715 & 32.053 & 26.719 & 34.452 \\
\hline 9/18/11 2:00 & & 30.463 & 29.848 & 28.711 & 30.005 & 29.26 & 30.4 & 29.828 & 29.477 & 28.005 & 25.791 & 32.086 & 35.589 & 33.125 & 16.601 & 16.332 & 23.182 & 14.989 & 11.359 & 31.944 & 32.985 & 29.684 & 32.044 & 26.717 & 34.45 \\
\hline 9/18/11 2:30 & & 30.466 & 29.85 & 28.715 & 30.005 & 29.257 & 30.405 & 29.833 & 29.479 & 27.998 & 25.791 & 32.082 & 35.592 & 33.123 & 16.599 & 16.328 & 23.177 & 14.982 & 11.354 & 31.944 & 32.981 & 29.691 & 32.042 & 26.717 & 34.45 \\
\hline 9/18/11 3:00 & & 30.47 & 29.855 & 28.767 & 30.012 & 29.255 & 30.402 & 29.833 & 29.482 & 27.995 & 25.796 & 32.077 & 35.596 & 33.116 & 16.601 & 16.323 & 23.175 & 14.978 & 11.352 & 31.947 & 981 & 29.701 & 32.039 & 26.719 & 34.447 \\
\hline 9/18/11 3:30 & & 30.466 & 29.855 & 28.79 & 30.01 & 29.21 & 30.415 & 29.845 & 29.484 & 27.939 & 25.854 & 32.072 & 35.592 & 33.118 & 16.601 & 16.316 & 23.17 & 4.982 & 11.347 & 31.937 & 971 & 29.689 & 32.032 & 26.719 & .445 \\
\hline 9/18/11 4:00 & & 30.47 & 29.857 & 28.767 & 30.017 & 29.236 & 30.415 & 29.828 & 29.489 & 27.909 & 25.851 & 32.07 & 35.599 & 33.109 & 16.604 & 16.313 & 23.168 & 14.975 & 11.342 & 31.947 & .974 & 9.698 & .03 & 26.717 & 34.445 \\
\hline 9/18/11 4:30 & & 30.496 & 29.89 & 28.769 & 30.047 & 29.239 & 30.417 & 29.833 & 29.51 & 27.905 & 25.858 & 32.07 & 35.61 & 33.104 & 16.61 & 16.304 & 23.156 & 14.928 & 11.34 & 31.988 & 32.969 & 29.752 & 32.03 & 26.724 & 34.445 \\
\hline 9/18/11 5:00 & & 30.487 & 29.892 & 28.722 & 30.054 & 29.257 & 30.415 & 29.821 & 29.516 & 27.923 & 25.854 & 32.046 & 35.615 & 33.085 & 16.61 & 16.276 & 23.128 & 14.935 & 11.319 & 31.972 & 32.95 & 29.741 & 32.009 & 26.719 & 34.424 \\
\hline 9/18/11 5:30 & & 30.48 & 29.885 & 28.718 & 30.047 & 29.29 & 30.41 & 29.821 & 29.512 & 27.93 & 25.846 & 32.037 & 35.613 & 33.073 & 16.61 & 16.267 & 23.121 & 14.949 & 11.309 & 31.956 & 32.938 & 29.734 & 31.999 & 26.721 & 34.412 \\
\hline 9/18/11 6:00 & & 30.477 & 29.888 & 28.72 & 30.047 & 29.269 & 30.412 & 29.831 & 29.514 & 27.919 & 25.834 & 32.863 & 35.61 & 33.296 & 16.608 & 16.26 & 23.121 & 14.964 & 11.533 & 32.117 & 33.9 & 29.724 & 32.832 & 26.719 & 34.408 \\
\hline 9/18/11 6:30 & & 30.456 & 29.86 & 28.713 & 30.026 & 29.288 & 30.41 & 29.824 & 29.498 & 27.928 & 25.825 & 34.195 & 35.601 & 34.562 & 16.601 & 16.314 & 23.112 & 14.992 & 12.8 & 32.709 & 35.201 & 29.715 & 34.151 & 26.712 & 34.401 \\
\hline 9/18/11 7:00 & & 30.473 & 29.869 & 28.781 & 30.04 & 29.265 & 30.41 & 29.843 & 29.505 & 27.919 & 25.834 & 34.801 & 35.603 & 35.259 & 16.601 & 16.421 & 23.112 & 14.961 & 13.496 & 32.987 & 35.781 & 29.783 & 34.747 & 26.719 & 34.398 \\
\hline 9/18/11 7:30 & & 30.466 & 29.869 & 28.788 & 30.052 & 29.234 & 30.415 & 29.843 & 29.503 & 27.935 & 25.849 & 35.147 & 35.601 & 35.739 & 16.601 & 16.531 & 23.112 & 14.973 & 13.975 & 33.15 & 36.089 & 29.809 & 35.087 & 26.719 & 34.396 \\
\hline 9/18/11 8:00 & & 30.47 & 29.867 & 28.79 & 30.073 & 29.246 & 30.417 & 29.838 & 29.503 & 27.953 & 25.861 & 35.308 & 35.599 & 35.95 & 16.601 & 16.631 & 23.11 & 14.964 & 14.189 & 33.199 & 36.25 & 29.846 & 35.247 & 26.719 & 34.391 \\
\hline $9 / 18 / 118: 30$ & & 30.491 & 29.897 & 28.788 & 30.129 & 29.243 & 30.419 & 29.843 & 29.526 & 27.977 & 25.878 & 35.847 & 35.61 & 36.356 & 16.606 & 16.736 & 23.115 & 14.933 & 14.589 & 33.439 & 36.814 & 29.929 & 35.787 & 26.724 & 34.394 \\
\hline 9/18/11 9:00 & & 30.487 & 29.902 & 28.792 & 30.162 & 29.246 & 30.422 & 29.847 & 29.53 & 27.981 & 25.885 & 36.268 & 35.613 & 36.752 & 16.608 & 16.842 & 23.115 & 14.945 & 14.986 & 33.602 & 37.241 & 29.966 & 36.202 & 26.724 & 34.394 \\
\hline 9/18/11 9:30 & & 30.484 & 29.909 & 28.795 & 30.197 & 29.248 & 30.422 & 29.847 & 29.535 & 28.019 & 25.901 & 36.621 & 35.617 & 37.12 & 16.608 & 16.951 & 23.112 & 14.947 & 15.357 & 33.756 & 37.584 & 30.004 & 36.552 & 26.724 & 34.391 \\
\hline 9/18/11 10:00 & & 30.484 & 29.909 & 28.793 & 30. & 29.253 & 30.424 & 29.845 & 29.537 & 28.025 & 25.937 & 36.939 & 35.618 & 37.448 & 16.61 & 17.054 & 23.11 & 14.954 & 15.682 & 33.889 & .902 & 30.034 & 36.868 & 26.724 & 34.389 \\
\hline 9/18/11 10:30 & & 30.489 & 29.916 & 28.792 & 30. & 29.26 & & 29.847 & 29.54 & 28.086 & 25.973 & 37.131 & 35.617 & 37.72 & 16.61 & 17.16 & & 14.954 & 15.957 & 33.989 & .075 & 30.072 & 37.056 & 26.726 & 34.389 \\
\hline 9/18/11 11:00 & & 30.489 & 29.918 & 28.788 & 30. & 29.267 & 30.427 & $\begin{array}{l}29.047 \\
29.85\end{array}$ & $\begin{array}{r}29.544 \\
\end{array}$ & 28.179 & 25.875 & 37.215 & 35.617 & 37.838 & $\begin{array}{l}16.615 \\
16.615\end{array}$ & 17.258 & 23.108 & $\begin{array}{l}14.959 \\
14.959\end{array}$ & $\begin{array}{l}16.072 \\
\end{array}$ & $\begin{array}{l}34.02 \\
34.02\end{array}$ & .158 & 30.103 & 37.138 & 26.724 & 34.384 \\
\hline $\begin{array}{l}9 / 18 / 1 \\
9 / 10\end{array}$ & & 30.487 & 29.92 & $\begin{array}{l}28.790 \\
28.795\end{array}$ & 30. & 29.272 & 30.426 & 29.85 & 29.549 & 28.191 & 25.901 & 35.783 & $\begin{array}{r}35.62 \\
35\end{array}$ & 37.022 & 16.615 & 17.325 & 23.105 & 14.964 & 15.252 & $\begin{array}{l}33.056 \\
33.656\end{array}$ & 641 & 14 & 35.705 & 26.726 & 34.384 \\
\hline 9/1 & & $\begin{array}{l}30.489 \\
30.489\end{array}$ & 29.5 & 28.797 & 30. & 29.272 & $\begin{array}{l}30.431 \\
30.41\end{array}$ & 29.847 & 29.549 & 28.239 & 25.878 & $\begin{array}{r}35.150 \\
35.1\end{array}$ & $\begin{array}{r}35.624 \\
35.624\end{array}$ & $\begin{array}{l}36.304 \\
36.304\end{array}$ & 16.613 & 17.325 & 7 & $\begin{array}{l}14.974 \\
14.971\end{array}$ & 14.532 & $\begin{array}{l}33.364 \\
33.04\end{array}$ & 71 & $\frac{4}{7}$ & 35.026 & 26.726 & .382 \\
\hline $9 / 18 /$ & & 30. & 29 . & 28.792 & 30 & 29.279 & 30 & 29 & 29. & 28.232 & 25.878 & 34.677 & 35. & 35.854 & 16.615 & 17.297 & 5 & 14.98 & 14.083 & 96 & 2 & 1 & 34 & 26 & .382 \\
\hline & & & & 28.792 & 30. & 29.286 & & 29 & & 28. & 25.87 & 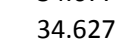 & 35 & & 16.617 & 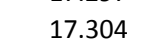 & 1 & 14.975 & 14.032 & & 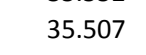 & 1 & 34.559 & & 34.382 \\
\hline & & & & 28 & & & & 29 & & & & 34 & & & & & 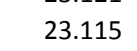 & 14.9 & 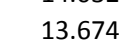 & 33.038 & & & 34.229 & & \\
\hline $9 / 1$ & & 30 & 29.932 & 28.788 & & 29 & & 29.852 & & 28. & & 34.0 & 35. & 35.1 & & & 23.103 & 14. & 13.432 & 32.5 & 34.947 & & 34.006 & & 34.38 \\
\hline 14:30 & & 30 & 29.93 & 28.79 & & 29.297 & & 29.8 & & 28.1 & & 33. & 35.624 & & 16. & 17.2 & 23.096 & 14. & 13.239 & & 34.77 & 6 & 33.827 & 26.726 & 34.377 \\
\hline 15:00 & & & 29. & 28.79 & & 29.3 & & 29. & & 28 & & 33.734 & 35.624 & 34.848 & 16. & 17 & 23 & 14.994 & 13.082 & 32.814 & & $y_{1}$ & & & 34.377 \\
\hline $9 / 1$ & & & 29. & 28.788 & & 29.302 & & 29. & 29.5 & 28. & & 33.605 & 35. & 34.717 & 16. & 17.141 & 23.068 & 14.996 & 12.948 & 32.758 & & & & & 34.375 \\
\hline 9/18/11 16:00 & & & 29.5 & 28.792 & & 29.307 & & $29 . \varepsilon$ & & 28. & 25. & 33.497 & 35.6 & 34.6 & 16. & 17.108 & 23.064 & 14.994 & 12.833 & 32.711 & 34.386 & 30.013 & 33.445 & 26.728 & 34.375 \\
\hline 9/18/11 16:30 & & 30. & 29.5 & 28.792 & 30. & 29.302 & 30.6 & 29.8 & 29 & 28.6 & 25.863 & 33.401 & 35.627 & 34.499 & 16.6 & 17.0 & 23.094 & 15.003 & 12.727 & 32.665 & 34.293 & 29.995 & 33.349 & 26.728 & 34.373 \\
\hline 9/18/11 17:00 & & 30.496 & 29.93 & 28.799 & 30.3 & 29.3 & 30.4 & 29.859 & 29.572 & 28.0 & 25.86 & 33.315 & 35.624 & 34.41 & 16.617 & 17.0 & 23.108 & 14.999 & 12.64 & 32.625 & 34.208 & 29.99 & 33.262 & 26.728 & 34.373 \\
\hline 9/18/11 17:30 & & 30.498 & 29.944 & 28.802 & 30.32 & 29.3 & 30.4 & 29.862 & 29.575 & 28.044 & 25.868 & 33.238 & 35.632 & 34.328 & 16.617 & 17.017 & 23.096 & 15.001 & 12.558 & 32.595 & 34.132 & 29.98 & 33.187 & 26.728 & 34.373 \\
\hline 9/18/11 18:00 & & 30.501 & 29.941 & 28.806 & 30.318 & 29.297 & 30.446 & 29.862 & 29.579 & 28.025 & 25.868 & 33.167 & 35.627 & 34.255 & 16.62 & 16.989 & 23.078 & 15.001 & 12.489 & 32.564 & 34.064 & 29.971 & 33.121 & 26.728 & 34.371 \\
\hline 9/18/11 18:30 & & 30.503 & 29.948 & 28.813 & 30.311 & 29.293 & 30.448 & 29.864 & 29.581 & 28 & 25.873 & 33.106 & 35.624 & 34.19 & 16.622 & 16.963 & 23.061 & 15.001 & 12.421 & 32.536 & 34.002 & 29.966 & 33.058 & 26.731 & 34.373 \\
\hline 9/18/11 19:00 & & 30.501 & 29.948 & 28.823 & 30.304 & 29.288 & 30.448 & 29.864 & 29.584 & 27.967 & 25.878 & 33.05 & 35.629 & 34.131 & 16.622 & 16.937 & 23.045 & 14.999 & 12.36 & 32.508 & 33.947 & 29.957 & 33.001 & 26.731 & 34.37 \\
\hline 9/18/11 19:30 & & 30.503 & 29.948 & 28.832 & 30.295 & 29.283 & 30.451 & 29.869 & 29.586 & 27.939 & 25.885 & 32.999 & 35.627 & 34.077 & 16.622 & 16.914 & 23.027 & 14.994 & 12.308 & 32.485 & 895 & 29.955 & 32.95 & 26.731 & 34.368 \\
\hline $9 / 1$ & & 30.508 & 29.955 & 28.846 & 30.29 & 29.276 & 30.455 & 29.871 & 29.591 & 27.916 & 25.894 & 32.95 & 35.631 & 34.025 & 16.624 & 16.891 & 23.015 & 14.999 & 12.259 & 32.464 & 33.846 & 29.95 & 32.903 & 26.731 & 34.366 \\
\hline 9/18/11 20:30 & & 30.508 & 29.96 & 28.851 & 30.288 & 29.272 & 30.458 & 29.871 & 29.595 & 27.9 & 25.909 & 32.908 & 35.631 & 33.981 & 16.624 & 16.867 & 22.997 & 14.996 & 12.212 & 32.45 & 33.805 & 29.952 & 32.863 & 26.731 & .363 \\
\hline
\end{tabular}




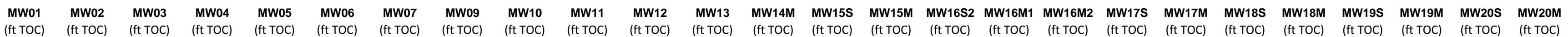

\begin{tabular}{|c|c|c|c|c|c|c|c|c|c|c|c|c|c|c|c|c|c|c|c|c|c|c|c|c|c|c|}
\hline Well & $\begin{array}{l}\text { MW01 } \\
\text { (ft TOC) }\end{array}$ & $\begin{array}{l}\text { MW02 } \\
\text { (ft TOC) }\end{array}$ & $\begin{array}{l}\text { MW03 } \\
\text { (ft ToC) }\end{array}$ & $\begin{array}{r}\text { MW04 } \\
\text { (ft ToC) }\end{array}$ & $\begin{array}{r}\text { MW05 } \\
\text { (ft TOC) }\end{array}$ & $\begin{array}{r}\text { MW06 } \\
\text { (ft TOC) }\end{array}$ & $\begin{array}{l}\text { MW07 } \\
\text { (ft TOC) }\end{array}$ & $\begin{array}{l}\text { MW09 } \\
\text { (ft TOC) }\end{array}$ & $\begin{array}{l}\text { MW10 } \\
\text { (ft TOC) }\end{array}$ & $\begin{array}{l}\text { MW11 } \\
\text { (ft TOC) }\end{array}$ & $\begin{array}{r}\mathrm{MW12} \\
\text { (ft TOC) }\end{array}$ & $\begin{array}{l}\text { MW13 } \\
\text { (ft TOC) }\end{array}$ & $\begin{array}{l}\text { MW14M } \\
\text { (ft TOC) }\end{array}$ & $\begin{array}{l}\text { MWW15S } \\
\text { (ft TOC) }\end{array}$ & $\begin{array}{l}\text { MW15M } \\
\text { (ft TOC) }\end{array}$ & $\begin{array}{l}\text { MW16S2 } \\
\text { (ft TOC) }\end{array}$ & $\begin{array}{c}\text { MW16M1 } \\
\text { (ft TOC) }\end{array}$ & $\begin{array}{l}\text { MW16M2 } \\
\text { (ft TOC) }\end{array}$ & $\begin{array}{l}\text { MW175 } \\
\text { (ft TOC) }\end{array}$ & $\begin{array}{l}\text { MW17M } \\
\text { (ft TOC) }\end{array}$ & $\begin{array}{l}\text { MW18S } \\
\text { (ft TOC) }\end{array}$ & $\begin{array}{l}\text { MWw18M } \\
\text { (ft TOC) }\end{array}$ & $\begin{array}{l}\text { MWW19S } \\
\text { (ft TOC) }\end{array}$ & $\begin{array}{l}\begin{array}{l}\text { WW19M } \\
\text { (ft TOC) }\end{array} \\
\text { (a) }\end{array}$ & $\begin{array}{l}\mathrm{MW} 20 \mathrm{~S} \\
(\mathrm{ft} \text { TOC) }\end{array}$ & $\begin{array}{l}\text { MW20M } \\
\text { (ft toc) }\end{array}$ \\
\hline 9/18/11 21:00 & & 30.512 & 29.965 & 28.846 & 30.288 & & 29.272 & 30.46 & 29.874 & 29.602 & 27.886 & 25.909 & 32.87 & 35.631 & 33.941 & 16.627 & 16.849 & 22.978 & 14.992 & 12.172 & 32.434 & 33.77 & 29.95 & 32.825 & 26.733 & 34.366 \\
\hline 9/18/11 21:30 & & 30.517 & 29.972 & 28.851 & 30.285 & & 29.279 & 30.46 & 29.869 & 29.609 & 27.877 & 25.906 & 32.835 & 35.632 & 33.903 & 16.631 & 16.83 & 22.964 & 14.989 & 12.132 & 32.427 & 33.729 & 29.952 & 32.79 & 26.733 & 34.366 \\
\hline 9/18/11 22:00 & & 30.515 & 29.976 & 28.849 & 30.285 & & 29.281 & 30.462 & 29.874 & 29.612 & 27.872 & 25.904 & 32.8 & 35.636 & 33.866 & $5 \quad 16.629$ & 16.809 & 22.946 & 14.992 & 12.097 & 32.406 & $5 \quad 33.699$ & 29.95 & 32.753 & 26.733 & 34.364 \\
\hline 9/18/11 22:30 & & 30.515 & 29.979 & 28.846 & 30.278 & & 29.283 & 30.462 & 29.874 & 29.614 & 27.87 & 25.901 & 32.767 & 35.636 & 33.835 & ; 16.631 & 16.793 & 22.932 & 14.996 & 12.064 & 32.39 & 33.665 & 29.94 & 32.724 & 26.733 & 34.363 \\
\hline 9/18/11 23:00 & & 30.512 & 29.979 & 28.849 & 30.274 & & 29.286 & 30.465 & 29.874 & 29.619 & 27.872 & 25.899 & 32.739 & 35.641 & 33.8 & 16.634 & 16.776 & 22.916 & 14.996 & 12.033 & 32.373 & 33.635 & 29.94 & 32.694 & 26.733 & 34.363 \\
\hline 9/18/11 23:30 & & 30.515 & 29.981 & 28.856 & 30.271 & & 29.286 & 30.465 & 29.874 & 29.621 & 27.872 & 25.901 & 32.709 & 35.641 & 33.77 & 16.636 & 16.76 & 22.902 & 14.996 & 12.005 & 32.357 & 33.606 & 29.936 & 32.666 & 26.733 & 34.361 \\
\hline 9/19/11 0:00 & & 30.515 & 29.981 & 28.865 & 30.264 & & 29.288 & 30.467 & 29.876 & 29.623 & 27.865 & 25.906 & 32.681 & 35.639 & 33.744 & 16.634 & 16.744 & 22.886 & 14.996 & 11.975 & 32.343 & 33.58 & 29.931 & 32.638 & 26.733 & 34.361 \\
\hline 9/19/11 0:30 & & 30.517 & 29.983 & 28.865 & 30.26 & & 29.286 & 30.467 & 29.878 & 29.626 & 27.853 & 25.911 & 32.657 & 35.639 & 33.718 & $3 \quad 16.636$ & 16.727 & 22.886 & 14.996 & 11.951 & 32.334 & 33.554 & 29.922 & 32.614 & 26.733 & 34.361 \\
\hline 9/19/11 1:00 & & 30.519 & 29.988 & 28.863 & 30.257 & & 29.288 & 30.47 & 29.881 & 29.628 & 27.844 & 25.911 & 32.634 & 35.641 & 33.692 & 16.636 & 16.713 & 22.872 & 14.999 & 11.925 & 32.324 & 33.533 & 29.917 & 32.591 & 26.733 & 34.359 \\
\hline 9/19/11 1:30 & & 30.519 & 29.99 & 28.867 & 30.255 & & 29.288 & 30.469 & 29.881 & 29.633 & 27.849 & 25.909 & 32.613 & 35.643 & 33.671 & 16.636 & 16.699 & 22.858 & 14.996 & 11.904 & 32.313 & 33.511 & 29.922 & 32.57 & 26.735 & 34.361 \\
\hline 9/19/11 2:00 & & 30.519 & 29.993 & 28.872 & 30.253 & & 29.29 & 30.47 & 29.885 & 29.635 & 27.851 & 25.909 & 32.592 & 35.641 & 33.65 & 16.638 & 16.685 & 22.842 & 14.999 & 11.881 & 32.301 & 33.49 & 29.915 & 32.549 & 26.735 & 34.361 \\
\hline $9 / 19 / 112: 30$ & & 30.522 & 29.993 & 28.877 & 30.248 & & 29.29 & 30.472 & 29.883 & 29.64 & 27.851 & 25.913 & 32.571 & 35.643 & 33.629 & 16.641 & 16.673 & 22.828 & 14.996 & 11.857 & 32.292 & 33.471 & 9.915 & 32.53 & 26.735 & 34.361 \\
\hline 9/19/11 3:00 & & 30.522 & 29.995 & 28.879 & 30.246 & & 29.295 & 30.475 & 29.881 & 29.642 & 27.856 & 25.918 & 32.554 & 35.643 & 33.608 & $\begin{array}{ll}3 & 16.641\end{array}$ & 16.662 & 22.814 & 15.001 & 11.843 & 32.282 & 33.452 & 29.91 & 32.511 & 26.735 & 34.359 \\
\hline 9/19/11 3:30 & & 30.524 & 30.002 & 28.879 & 30.246 & & 29.293 & 30.477 & 29.883 & 29.646 & 28.07 & 25.921 & 32.536 & 35.65 & 33.589 & 16.643 & 16.648 & 22.798 & 4.999 & 11.824 & 32.278 & 33.433 & 29.912 & 32.495 & 26.735 & 34.359 \\
\hline 9/19/11 4:00 & & 30.526 & 30.004 & 28.879 & 30.241 & & 29.3 & 30.475 & 29.885 & 29.649 & 28.121 & 25.918 & 32.517 & 35.646 & 33.573 & 16.645 & 16.636 & 22.784 & 15.001 & 11.805 & 32.273 & 33.419 & 29.912 & 32.478 & 26.738 & 34.361 \\
\hline 9/19/11 4:30 & & 30.529 & 30.007 & 28.879 & 30.243 & & 29.302 & 30.477 & 29.885 & 29.651 & 28.125 & 25.916 & 32.503 & 35.653 & 33.556 & 16.645 & 16.627 & 22.77 & 15.001 & 11.787 & 32.261 & 33.402 & 29.912 & 32.462 & 26.738 & 34.359 \\
\hline 9/19/11 5:00 & & 30.526 & 30.009 & 28.886 & 30.239 & & 29.302 & 30.477 & 29.885 & 29.656 & 28.13 & 25.916 & 32.486 & 35.648 & 33.54 & 16.645 & 16.613 & 22.754 & 15.006 & 11.773 & 32.252 & 33.388 & 29.903 & 32.445 & 26.738 & 34.359 \\
\hline 9/19/11 5:30 & & 30.526 & 30.007 & 28.886 & 30.234 & & 29.304 & 30.477 & 29.89 & 29.656 & 28.132 & 25.918 & 32.472 & 35.65 & 33.524 & 16.645 & 16.603 & 22.738 & 15.003 & 11.756 & 32.24 & 33.374 & 29.903 & 32.431 & 26.738 & 34.359 \\
\hline 9/19/11 6:00 & & 30.529 & 30.009 & 28.895 & 30.232 & & 29.304 & 30.479 & 29.893 & 29.66 & 28.137 & 25.923 & 33.298 & 35.648 & 33.744 & 16.645 & 16.594 & 22.738 & 15.006 & 11.977 & 32.406 & 34.331 & 29.901 & 33.266 & 26.74 & 34.357 \\
\hline 9/19/11 6:30 & & 30.531 & 30.014 & 28.902 & 30.234 & & 29.304 & 30.481 & 29.895 & 29.653 & 28.144 & 25.93 & 34.63 & 35.653 & 35.012 & $\quad 16.65$ & 16.648 & 22.728 & 15.001 & 13.249 & 33.022 & 35.63 & 29.933 & 34.583 & 26.74 & 34.357 \\
\hline 9/19/11 7:00 & & 30.536 & 30.018 & 28.905 & 30.243 & & 29.307 & 30.481 & 29.893 & 29.642 & 28.149 & 25.937 & 35.222 & 35.653 & 35.695 & 16.65 & 16.751 & 22.71 & 14.999 & 13.926 & 33.278 & 36.198 & 29.983 & 35.165 & 26.74 & 34.357 \\
\hline 9/19/11 7:30 & & 30.536 & 30.023 & 28.905 & 30.267 & & 29.309 & 30.484 & 29.895 & 29.644 & 28.151 & 25.937 & 35.559 & 35.66 & 36.166 & $\quad 16.652$ & 16.858 & 22.703 & 14.999 & 14.401 & 33.446 & 36.504 & 30.027 & 35.498 & 26.74 & 34.359 \\
\hline 9/19/11 8:00 & & 30.538 & 30.028 & 28.905 & 30.29 & & 29.316 & 30.486 & 29.895 & 29.64 & 28.151 & 25.937 & 35.713 & 35.657 & 36.37 & 16.652 & 16.954 & 22.689 & 15.001 & 14.603 & 33.497 & 36.653 & 30.06 & 35.648 & 26.74 & 34.357 \\
\hline 9/19/11 8:30 & & 30.536 & 30.028 & 28.909 & 30.316 & & 29.319 & 30.486 & 29.9 & 29.642 & 28.158 & 25.937 & 36.247 & 35.657 & 36.769 & 16.655 & 17.05 & 22.673 & 15.006 & 15 & 33.686 & 537.217 & 30.091 & 36.183 & 26.74 & 34.357 \\
\hline 9/19/11 9:00 & & 30.538 & 30.032 & 28.909 & 30.341 & & 29.323 & 30.486 & 29.9 & 29.667 & 28.163 & 25.944 & 36.661 & 35.657 & 37.158 & 16.655 & 17.148 & 22.657 & 15.008 & 15.39 & 33.852 & & 30.128 & 36.594 & 26.74 & 34.357 \\
\hline 9/19/11 9:30 & & 30.538 & 30.037 & 28.909 & 30.367 & & 29.326 & 30.486 & 29.9 & 29.709 & 28.165 & 25.944 & 37.031 & 35.66 & 37.54 & 16.657 & $\begin{array}{l}17.251 \\
\end{array}$ & 22.641 & $\begin{array}{l}15.013 \\
\end{array}$ & 15.776 & $\begin{array}{l}33.999 \\
33.996\end{array}$ & 37.994 & 30.161 & 36.958 & 26.742 & 34.357 \\
\hline 9/19/11 10:00 & & 30.54 & 30.042 & 28.902 & $\begin{array}{r}30.4 \\
30.51\end{array}$ & & 29.337 & 30.489 & 29.902 & 29.751 & 28.165 & 25.944 & 37.325 & 35.657 & $\begin{array}{l}37.849 \\
-1.849\end{array}$ & 16.657 & $\begin{array}{l}17.351 \\
17.351\end{array}$ & 22.627 & $\begin{array}{l}15.015 \\
\end{array}$ & 16.084 & 34.124 & 38.286 & 192 & 37.253 & 26.742 & 34.357 \\
\hline 9/19/11 10:30 & & $\begin{array}{l}30.544 \\
30.543\end{array}$ & 30.044 & 28.898 & 30.43 & & 29.344 & $\begin{array}{l}30.489 \\
30.489\end{array}$ & 29.897 & 29.691 & 28.165 & $\begin{array}{l}25.944 \\
25.94\end{array}$ & 37.496 & $\begin{aligned} \\
353.071 \\
35.66\end{aligned}$ & $\begin{array}{r}3.049 \\
38.11\end{array}$ & 16.657 & $\begin{array}{l}17.452 \\
17.42\end{array}$ & 22.615 & $\begin{array}{r}15.02 \\
15.02\end{array}$ & $\begin{array}{l}10.004 \\
16.34\end{array}$ & $\begin{array}{l}34.222 \\
34.22\end{array}$ & $\begin{array}{l}38.438 \\
38.20\end{array}$ & .227 & 37.422 & 26.742 & 34.354 \\
\hline 9/19/11 11:00 & & $\begin{array}{l}30.345 \\
30.54\end{array}$ & $\begin{array}{l}30.044 \\
30.046\end{array}$ & $\begin{array}{l}28.898 \\
28.893\end{array}$ & $\begin{array}{r}30.456 \\
30.456\end{array}$ & & 29.354 & $\begin{array}{l}30.499 \\
30.491\end{array}$ & 29.902 & $\begin{array}{l}29.6993 \\
29.693\end{array}$ & $\begin{array}{l}28.165 \\
28.165\end{array}$ & $\begin{array}{l}25.944 \\
25.935\end{array}$ & $\begin{array}{l}37.450 \\
37.573\end{array}$ & $\begin{array}{l}35.00 \\
35.66\end{array}$ & $\begin{array}{l}38.11 \\
38.215\end{array}$ & $\begin{array}{l}10.037 \\
16.659\end{array}$ & $\begin{array}{l}17.452 \\
17.543\end{array}$ & $\begin{array}{l}22.5913 \\
22.59\end{array}$ & $\begin{array}{l}15.022 \\
15.027\end{array}$ & $\begin{array}{l}16.44 \\
16.443\end{array}$ & $\begin{array}{l}34.241 \\
34.242\end{array}$ & $\begin{array}{l}38.450 \\
38.511\end{array}$ & $\begin{array}{l}30.247 \\
30.246\end{array}$ & $\begin{array}{l}37.422 \\
37.497\end{array}$ & $\begin{array}{l}26.742 \\
26.742\end{array}$ & 34.354 \\
\hline $9 / 19 / 1$ & & & 30.049 & 28.877 & 30.4 & & 29.366 & 30.486 & 29.9 & 29.693 & 28 & 25.928 & 36.127 & 35.662 & 37.376 & 16.659 & 17.606 & 22.585 & 15.036 & 15.607 & 33.875 & 36.98 & 26 & 36.045 & 26.742 & 34.354 \\
\hline 9/ & & & 30.049 & 28 & 30 & & & 30.491 & 29.902 & 29.693 & 28.153 & 25.913 & 35.432 & 35.662 & 36.647 & 16.657 & 17.601 & 22.571 & 15.046 & 14.878 & 33.574 & 36.303 & 46 & 35.36 & 42 & 34.352 \\
\hline 9/19/11 & & & & & 30.509 & & & 30.489 & 29.902 & & 28.149 & 25.901 & 002 & 35.66 & 89 & & 68 & & 15.05 & 19 & 33.406 & 376 & 25 & 34.932 & 742 & 352 \\
\hline 9/19/11 13:00 & & 30.531 & 30.044 & 28.839 & 30.507 & & 29.401 & 30.486 & 29.9 & 29.688 & 28.142 & 25.889 & 34.684 & 35.655 & 35.854 & 16.657 & 17.529 & 22.548 & $\begin{array}{l}15.069 \\
15.069\end{array}$ & $\begin{array}{l}14.419 \\
14.083\end{array}$ & $\begin{array}{l}33.400 \\
33.269\end{array}$ & $\begin{array}{l}35.070 \\
35.561\end{array}$ & 197 & $\begin{array}{l}54.532 \\
34.62\end{array}$ & 6.742 & $\begin{array}{l}34.35 \\
34.53\end{array}$ \\
\hline 9/19/11 13:30 & & 30.533 & 30.037 & 28.825 & 30.5 & & 29.415 & 30.486 & 29.897 & 29.686 & 28.137 & 25.873 & 34.44 & 35.653 & 35.594 & 16.655 & 17.484 & 22.536 & 15.074 & 13.822 & 33.166 & 535.322 & 30.175 & 34.379 & 26.745 & 34.347 \\
\hline 9/19/11 14:00 & & 30.531 & 30.035 & 28.811 & 30.4 & & & 30.484 & 29.897 & & 28.128 & 25.856 & 34.237 & 35.655 & 35.378 & 16.652 & 17.445 & 22.523 & 15.081 & 13.608 & 33.073 & 35.12 & .147 & 34.179 & 26.742 & 34.347 \\
\hline 9/19/11 14:30 & & 30.529 & 30.025 & 28.802 & 30. & & 29.429 & 30.479 & 29.902 & & 28.123 & 25.844 & 34.066 & 35.648 & 35.198 & 16.652 & 17.403 & 22.509 & 15.093 & 13.43 & 32.994 & 34.952 & 114 & 34.01 & 26.742 & 34.345 \\
\hline 9/19/11 15:00 & & 30.524 & 30.016 & 28.795 & 30. & & 29.431 & 30.477 & 29.902 & 29.674 & 28.116 & 25.832 & 33.918 & 35.646 & 35.043 & 16.648 & 17.36 & 22.495 & 15.097 & 13.272 & 32.919 & 34.807 & 30.096 & 33.865 & 26.742 & 34.34 \\
\hline 9/19/11 15:30 & & 30.524 & 30.007 & 28.788 & 30. & & 29.431 & 30.477 & 29.9 & 29.667 & 28.112 & 25.825 & 33.792 & 35.636 & 34.909 & 16.645 & 17.323 & 22.481 & 15.104 & 13.138 & 32.856 & 34.682 & 30.07 & 33.736 & 26.742 & 34.34 \\
\hline 9/19/11 16:00 & & 30.524 & & 28.79 & 30. & & 29.429 & 30.472 & 29.9 & 29.663 & 28.109 & 25.82 & 33.68 & 35.636 & 34.792 & 16.645 & 17.288 & 22.469 & 15.111 & 13.021 & 32.802 & 34.568 & 30.044 & 33.626 & 26.742 & 34.338 \\
\hline 9/19/11 16:30 & & 30.522 & 29.995 & 28.785 & 30.393 & & 29.427 & 30.472 & 29.907 & 29.658 & 28.105 & 25.82 & 33.579 & 35.634 & 34.684 & 16.643 & 17.253 & 22.469 & 15.109 & 12.917 & 32.749 & 34.471 & 30.027 & 33.527 & 26.74 & 34.335 \\
\hline 9/19/11 17:00 & & 30.522 & 29.988 & 28.783 & 30.374 & & 29.422 & 30.469 & 29.902 & 29.653 & 28.105 & 25.815 & 33.486 & 35.629 & 34.59 & 16.641 & 17.218 & 22.46 & 15.109 & 12.821 & 32.704 & 34.383 & 30.011 & 33.438 & 26.742 & 34.335 \\
\hline 9/19/11 17:30 & & 30.524 & 29.988 & 28.783 & 30.358 & & 29.417 & 30.47 & 29.904 & 29.651 & 28.102 & 25.815 & 33.408 & 35.629 & 34.506 & 16.641 & 17.188 & 22.444 & 15.111 & 12.732 & 32.665 & 34.303 & 29.997 & 33.358 & 26.742 & 34.333 \\
\hline 9/19/11 18:00 & & 30.524 & 29.981 & 28.785 & 30.341 & & 29.41 & 30.467 & 29.904 & 29.646 & 28.098 & 25.815 & 33.336 & 35.627 & 34.429 & 16.638 & 17.157 & 22.43 & 15.111 & 12.661 & 32.627 & 34.227 & 29.978 & 33.283 & 26.742 & 34.331 \\
\hline 9/19/11 18:30 & & 30.52 & 29.981 & 28.783 & 30.325 & & 29.406 & 30.467 & 29.907 & 29.644 & 28.1 & 25.815 & 33.266 & 35.624 & 34.361 & 16.636 & 17.129 & 22.421 & 15.1 & 12.586 & 32.595 & 34.161 & 69 & 33.217 & 26.745 & 34.331 \\
\hline $9 / 19 / 11$ & & 30.526 & 29.979 & 28.785 & 30. & & 29.396 & 30.465 & 29.904 & 29.644 & 28.098 & 25.815 & 33.207 & 35.624 & 34.295 & 16.63 & 17.096 & 22.409 & 15.104 & 12.522 & 32.567 & 11 & .957 & 33.154 & 26.745 & 34.328 \\
\hline $9 / 19 / 1$ & & 30.526 & 29.979 & 28.795 & 30.2 & & 29.389 & 30.467 & 29.907 & 29.642 & 28.1 & 25.82 & 33.149 & 35.622 & 34.234 & 16.636 & $\begin{array}{l}17.071 \\
\end{array}$ & 22.398 & $\begin{array}{r}15.1 \\
15.1\end{array}$ & 12.466 & 32.537 & 34.042 & 9.948 & 33.1 & 26.745 & 34.328 \\
\hline $9 / 1$ & & 30.526 & 29.979 & 28.804 & 30. & & 29.375 & 30.47 & 29.912 & $\begin{array}{l}29.642 \\
29.642\end{array}$ & $\begin{array}{r}28.1 \\
28.105\end{array}$ & $\begin{array}{l}25.02 \\
25.83\end{array}$ & $\begin{array}{l}33.149 \\
33.095\end{array}$ & $\begin{array}{l}53.022 \\
35.622\end{array}$ & $\begin{array}{l}34.178 \\
34.178\end{array}$ & $\begin{array}{l}10.030 \\
16.631\end{array}$ & 17.045 & $\begin{array}{l}22.384 \\
22.384\end{array}$ & $\begin{array}{l}15.1 \\
15.1\end{array}$ & $\begin{array}{l}12.400 \\
12.414\end{array}$ & 32.508 & $\begin{array}{l}5.4042 \\
33.99\end{array}$ & 36 & 33.048 & 26.745 & 34.326 \\
\hline & & 30 & 29.9 & 28 & & & 2 & 30.474 & 29. & 29.64 & 28.107 & 25.839 & 33 & 35 & 34 & 16 & 17 & 22 & 15.093 & 12.358 & 32.487 & 33.943 & 8 & 11 & 26.745 & 34.324 \\
\hline & & 30. & 29. & 28. & & & & 30. & 29 & 29. & 28. & & & 35 & 34 & & 16 & & 15 & 12. & 32 & & & 57 & 26.745 & 34.324 \\
\hline & & 30. & 29. & 28. & & & & & & & 28.1 & & & 35. & 34. & & & & & 12. & 32. & & & 15 & 45 & \\
\hline & & & & & & & & & & & 28.1 & & & & 33. & & & & & 20 & & & & & & \\
\hline & & & & & & & & & & & 2 & & & & & & & & & & & & & & & \\
\hline 9/ & & & & & & & & & & & 2 & & & & & & & & & & & & & & & 34. \\
\hline & & 30 & 29 & 28 & & & & & 29 & & 28 & 25. & & 35 & & & 16. & & & 12 & & & & 1 & & 34. \\
\hline 9/20/11 0:00 & & 30.531 & 29.981 & 28.795 & & & 29.361 & 30.474 & 29.912 & 29.64 & (20.1 & $\begin{array}{r}25.83 \\
\end{array}$ & 32.781 & 35.629 & 33.852 & 16.631 & 16.87 & 22.298 & 15.076 & 12.083 & 32.359 & 33.682 & 29.889 & 32.739 & 26.745 & 34.321 \\
\hline 9/20/11 0:30 & & 30.531 & 29.974 & 28.79 & 30.218 & & 29.361 & 30.472 & 29.912 & 29.637 & 28.098 & 25.827 & 32.753 & 35.625 & 33.821 & 16.631 & 16.849 & 22.289 & 15.074 & 12.057 & 32.338 & 33.651 & 29.877 & 32.708 & 26.745 & 34.319 \\
\hline
\end{tabular}




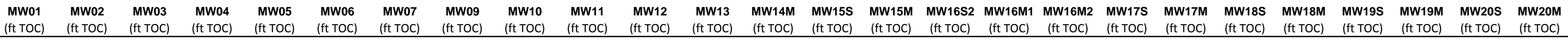

\begin{tabular}{|c|c|c|c|c|c|c|c|c|c|c|c|c|c|c|c|c|c|c|c|c|c|c|c|c|c|}
\hline well & $\begin{array}{l}\text { (ft TOC) } \\
\text { (f) }\end{array}$ & $\begin{array}{l}\text { (ft TOC) } \\
\text { (ft) }\end{array}$ & $\begin{array}{l}\text { (ft TOC) } \\
\end{array}$ & $\begin{array}{l}\text { (ft TOC) } \\
\text { (f) }\end{array}$ & $\begin{array}{l}\text { (ift TOC) } \\
\text { (ft TOC) }\end{array}$ & $\begin{array}{l}\text { (ft TOC) } \\
\end{array}$ & $\begin{array}{l}\text { (ift TOC) } \\
\end{array}$ & $\begin{array}{l}\text { (ft TOC) } \\
\text { (f) }\end{array}$ & $\begin{array}{l}\text { (ft TOC) } \\
\text { (f) }\end{array}$ & $\begin{array}{l}\text { (ft TOC) } \\
\text { (n) }\end{array}$ & $\begin{array}{l}\text { (ft TOC) } \\
\text { (ft) }\end{array}$ & (ft TOC) & $\begin{array}{l}\text { (ft TOC) } \\
\end{array}$ & (ft TOC) & (ft TOC) & (ft TOC) & (ft TOC) & $\begin{array}{l}\text { (nt TOC) } \\
\text { (ft }\end{array}$ & (ft TOC) & $\begin{array}{l}\text { (ft TOC) } \\
\text { (f) }\end{array}$ & (ft TOC) & $\begin{array}{l}\text { (ft TOC) } \\
\text { (n) }\end{array}$ & (ft TOC) & $\begin{array}{l}\text { (ft TOC) } \\
\text { (1) }\end{array}$ & (ft TOC) \\
\hline 1:00 & & 30.531 & 29.976 & 28.7 & 30.2 & 29.361 & 30.4 & 29 & 29.6 & 28.095 & 25 & 32.727 & 35.631 & 33. & 16.629 & 16.832 & 22.278 & 15.067 & 12.029 & & 33.623 & 29.882 & 2.682 & 47 & \\
\hline 11 1:30 & & 30.531 & 29.974 & 28.785 & 30.204 & 29.363 & 30.475 & 29.914 & 29.635 & 28.093 & 25.825 & 32.699 & 35.634 & 33.765 & 16.629 & 16.814 & 22.268 & 15.067 & 11.996 & 32.308 & 33.599 & 9.87 & 2.654 & & 34.319 \\
\hline 9/20/11 2:00 & & 30.531 & 29.972 & 28.788 & 30.197 & 29.359 & 30.472 & 29.921 & 29.633 & 28.095 & 25.822 & 32.674 & 35.632 & 33.737 & 16.629 & 16.797 & 22.257 & 15.067 & 11.97 & 32.294 & 33.571 & & 2.628 & & 317 \\
\hline 9/20/11 2:30 & & 30.531 & 29.969 & 28.785 & 30.187 & 29.356 & 30.472 & 29.916 & 29.63 & 28.093 & 25.825 & 32.648 & 35.622 & 33.711 & 16.627 & 16.779 & 22.245 & 15.064 & 11.944 & 32.278 & 33.545 & 29.854 & 32.605 & 26.747 & 34.317 \\
\hline 9/20/11 3:00 & & 30.533 & 29.967 & 28.783 & 30.18 & 29.356 & 30.47 & 29.919 & 29.63 & 28.093 & 25.822 & 32.624 & 35.631 & 33.69 & 16.627 & 16.765 & 22.236 & 15.06 & 11.918 & 32.266 & 33.523 & 29.844 & 32.581 & 26.747 & 34.317 \\
\hline 9/20/11 3:30 & & 30.533 & 29.969 & 28.785 & 30.173 & 29.354 & 30.472 & 29.919 & 29.63 & 28.091 & 25.82 & 32.603 & 35.631 & 33.664 & 16.627 & 16.751 & 22.222 & 15.057 & 11.897 & 32.254 & 33.5 & 29.844 & 32.558 & 26.747 & 34.317 \\
\hline 9/20/11 4:00 & & 30.533 & 29.967 & 28.79 & 30.166 & 29.351 & 30.472 & 29.919 & 29.626 & 28.093 & 25.822 & 32.582 & 35.627 & 33.646 & 16.624 & 16.734 & 22.215 & 15.055 & 11.874 & 32.243 & 33.481 & 29.835 & 32.534 & 26.749 & 34.317 \\
\hline 9/20/11 4:30 & & 30.533 & 29.965 & 28.783 & 30.162 & 29.351 & 30.475 & 29.919 & 29.626 & 28.091 & 25.825 & 32.559 & 35.631 & 33.62 & 16.624 & 16.72 & 22.204 & 15.055 & 11.852 & 32.229 & 33.459 & 29.832 & 32.516 & 26.749 & 34.314 \\
\hline 9/20/11 5:00 & & 30.533 & 29.965 & 28.788 & 30.157 & 29.354 & 30.472 & 29.916 & 29.626 & 28.088 & 25.82 & 32.54 & 35.636 & 33.599 & 16.624 & 16.706 & 22.192 & 15.05 & 11.836 & 32.219 & 33.44 & 29.823 & 32.497 & 26.747 & 34.317 \\
\hline 9/20/11 5:30 & & 30.533 & 29.965 & 28.79 & 30.155 & 29.342 & 30.472 & 29.923 & 29.626 & 28.095 & 25.827 & 32.522 & 35.629 & 33.58 & 16.624 & 16.694 & 22.18 & 15.05 & 11.812 & 32.208 & 33.421 & 29.823 & 32.478 & 26.749 & 34.314 \\
\hline 9/20/11 6:00 & & 30.533 & 29.962 & 28.783 & 30.145 & 29.347 & 30.474 & 29.919 & 29.621 & 28.095 & 25.825 & 32.503 & 35.632 & 33.561 & 16.624 & 16.68 & 22.185 & 15.055 & 11.794 & 32.191 & 33.402 & 29.814 & 32.457 & 26.749 & 34.314 \\
\hline 9/20/11 6:30 & & 30.536 & 29.965 & 28.774 & 30.143 & 29.349 & 30.472 & 29.919 & 29.621 & 28.088 & 25.822 & 32.486 & 35.631 & 33.545 & 16.627 & 16.666 & 22.18 & 15.046 & 11.775 & 32.194 & 33.386 & 29.821 & 32.443 & 26.749 & 34.314 \\
\hline 9/20/11 7:00 & & 30.533 & 29.965 & 28.797 & 30.138 & 29.342 & 30.472 & 29.926 & 29.621 & 28.093 & 25.818 & 32.468 & 35.634 & 33.526 & 16.624 & 16.652 & 22.171 & 15.05 & 11.758 & 32.18 & 33.367 & 29.811 & 32.426 & 26.747 & 34.314 \\
\hline 9/20/11 7:30 & & 30.533 & 29.962 & 28.804 & 30.134 & 29.335 & 30.475 & 29.926 & 29.619 & 28.095 & 25.837 & 32.451 & 35.634 & 33.51 & 16.622 & 16.641 & 22.169 & 15.053 & 11.744 & 32.17 & 33.353 & 29.799 & 32.408 & 26.749 & 34.312 \\
\hline 9/20/11 8:00 & & 30.536 & 29.96 & 28.813 & 30.129 & 29.333 & 30.477 & 29.928 & 29.616 & 28.1 & 25.844 & 32.433 & 35.634 & 33.493 & 16.622 & 16.629 & 22.164 & 15.048 & 11.723 & 32.156 & 33.334 & 29.799 & 32.391 & 26.749 & 34.312 \\
\hline 9/20/11 8:30 & & 30.54 & 29.965 & 28.832 & 30.131 & 29.328 & 30.477 & 29.928 & 29.623 & 28.105 & 25.854 & 32.421 & 35.639 & 33.479 & 16.624 & 16.62 & 22.153 & 15.043 & 11.711 & 32.161 & 33.322 & 29.816 & 32.377 & 26.749 & 34.315 \\
\hline 9/20/11 9:00 & & 30.54 & 29.969 & 28.83 & 30.134 & 29.323 & 30.482 & 29.926 & 29.623 & 28.109 & 25.87 & 32.407 & 35.641 & 33.465 & 16.624 & 16.606 & 22.141 & 15.043 & 11.697 & 32.159 & 33.308 & 29.811 & 32.365 & 26.752 & 34.312 \\
\hline 9/20/11 9:30 & & 30.54 & 29.972 & 28.82 & 30.136 & 29.328 & 30.481 & 29.926 & 29.628 & 28.109 & 25.87 & 32.395 & 35.646 & 33.451 & 16.627 & 16.594 & 22.132 & 15.039 & 11.683 & 32.149 & 33.296 & 29.816 & 32.354 & 26.752 & 34.312 \\
\hline 9/20/11 10:00 & & 30.547 & 29.981 & 28.804 & 30.141 & 29.342 & 30.482 & 29.926 & 29.633 & 28.107 & 25.858 & 32.383 & 35.643 & 33.439 & 16.627 & 16.587 & 22.123 & 15.032 & 11.674 & 32.156 & 33.284 & 29.828 & 32.342 & 26.754 & 34.314 \\
\hline 30 & & 30.545 & 29.981 & 28.806 & 30.1 & 29.349 & 30.481 & 29.928 & 29.635 & 28.105 & 25.842 & 32.372 & 35.65 & 33.428 & 16.629 & 16.575 & 22.116 & 15.041 & 11.66 & 32.142 & 33.274 & 29.818 & 32.333 & 26.752 & 34.312 \\
\hline 30 & & 30.54 & 29.976 & 28.806 & 30.1 & 29.349 & 30.481 & 29.926 & 29.633 & 28.102 & 25.846 & 32.355 & 35.655 & 33.413 & 16.627 & 16.564 & 22.10 & 15.0 & 11.648 & $32.1 .>2>$ & 255 & 29.811 & 32.316 & 26.752 & .312 \\
\hline $9 / 2$ & & 30.536 & 29.972 & 28.802 & 30.1 & 29.359 & 30.482 & 29.931 & 29.628 & 28.102 & 25.842 & 32.346 & 35.648 & 33.397 & 16.624 & 16.552 & .093 & 15.0 & 11.629 & 32.119 & 246 & 9.795 & 2.302 & 26.752 & .312 \\
\hline $9 / 20 /$ & & 30.538 & 29.972 & 28.788 & 30.122 & 29.366 & 30.479 & 29.926 & 29.628 & 28.1 & 25.839 & 32.685 & 35.65 & 33.779 & 16.627 & 16.55 & 22.083 & 15.055 & 12.012 & 212 & 585 & 9.802 & 38 & 26.752 & 4.31 \\
\hline 9/20/11 12:30 & & 30.536 & 29.972 & 28.776 & 30.117 & 29.375 & & 29.923 & 29.626 & 28.091 & 25.827 & 32.835 & 35.648 & 33.943 & 16.624 & 16.557 & 22.069 & 15.057 & 12.174 & 32.268 & 33.732 & 29.799 & 32.785 & 26.754 & 34.31 \\
\hline 9/20/11 13:00 & & 30.536 & 29.967 & 28.769 & 30.113 & 29.382 & 30.477 & 29.926 & 29.626 & 28.088 & 25.818 & 32.968 & 35.646 & 34.086 & 16.624 & 16.566 & 22.06 & 15.067 & 12.313 & 32.313 & 33.86 & 29.802 & 32.915 & 26.754 & 34.307 \\
\hline 9/20/11 13:30 & & 30.531 & 29.965 & 28.76 & 30.108 & 29.389 & 30.472 & 29.926 & 29.621 & 28.084 & 25.806 & 32.997 & 35.646 & 34.112 & 16.622 & 16.58 & 22.056 & 15.074 & 12.341 & 32.331 & 33.891 & 29.797 & 32.945 & 26.752 & 34.305 \\
\hline 9/20/11 14:00 & & 30.529 & 29.958 & 28.755 & 30.103 & 29.394 & 30.472 & 29.926 & 29.614 & 28.077 & 25.796 & 32.917 & 35.643 & 34.021 & 16.62 & 16.585 & 22.044 & 15.076 & 12.247 & 32.32 & 33.812 & 29.795 & 32.87 & 26.754 & 34.303 \\
\hline 9/20/111 14:30 & & 30.526 & 29.946 & 28.76 & 30.099 & 29.387 & 30.469 & 29.928 & 29.609 & 28.077 & 25.796 & 32.73 & 35.639 & 33.81 & 16.62 & 16.585 & 22.037 & 15.083 & 12.043 & 32.259 & 33.625 & 29.788 & 32.682 & 26.752 & 34.3 \\
\hline 9/20/11 15:00 & & 30.524 & 29.944 & 28.767 & 30.094 & 29.377 & 30.472 & 29.931 & 29.602 & 28.079 & 25.801 & 32.629 & 35.634 & 33.702 & 16.617 & 16.575 & 22.026 & 15.086 & 11.932 & 32.215 & 33.526 & 29.781 & 32.584 & 26.752 & 34.3 \\
\hline 9/20/11 15:30 & & 30.526 & 29.939 & 28.774 & 30.087 & 29.373 & 30.469 & 29.933 & 29.6 & 28.081 & 25.808 & 32.559 & 35.636 & 33.629 & 16.615 & 16.568 & 22.014 & 15.083 & 11.862 & 32.182 & 33.457 & 29.771 & 32.516 & 26.754 & 34.298 \\
\hline 9/20/11 16:00 & & 30.529 & 29.941 & 28.788 & 30.087 & 29.363 & 30.472 & 29.935 & 29.6 & 28.086 & 25.818 & 32.51 & 35.632 & 33.575 & 16.615 & 16.559 & 22.014 & 15.083 & 11.81 & 32.166 & 33.407 & 29.769 & 32.466 & 26.754 & 34.298 \\
\hline $9 / 20 / 1116: 30$ & & 30.531 & 29.941 & 28.795 & 30.089 & 29.354 & 30.472 & 29.935 & 29.6 & 28.088 & 25.83 & 32.468 & 35.629 & 33.531 & 16.615 & 16.55 & 22.046 & 15.081 & 11.763 & 32.149 & 33.367 & 29.771 & 32.424 & 26.754 & 34.296 \\
\hline 9/20/11 17:00 & & 30.533 & 29.946 & 28.813 & 30.089 & 29.34 & 30.475 & 29.94 & 29.602 & 28.098 & 25.839 & 32.437 & 35.636 & 33.498 & 16.617 & 16.54 & 22.06 & 15.074 & 11.73 & 32.14 & 33.336 & 29.776 & 32.394 & 26.754 & 34.296 \\
\hline 9/20/11 17:30 & & 30.533 & 29.951 & 28.83 & 30.092 & 29.323 & 30.479 & 29.942 & 29.605 & 28.105 & 25.858 & 32.409 & 35.636 & 33.467 & 16.615 & 16.528 & 22.053 & 15.072 & 11.702 & 32.133 & 33.31 & 29.776 & 32.365 & 26.754 & 34.296 \\
\hline 9/20/11 18:00 & & 30. & 29.953 & 28.842 & 30. & 29.316 & 30.482 & 29.94 & 29.609 & 28.109 & 25.875 & 32.386 & 35.641 & $\begin{array}{l}33.444 \\
33.4\end{array}$ & 16.617 & 16.519 & 22.042 & 15.06 & 11.676 & 32.133 & 33.286 & 29.788 & 32.344 & 26.756 & 34.296 \\
\hline $9 / 2$ & & 30.543 & 29.965 & 28.846 & & 29.3 & & 29.938 & 29.616 & 28. & 25. & 32.367 & 35.648 & 33.42 & 16.62 & 16.512 & 22.046 & 15.055 & 11.655 & 32.131 & 33.27 & 29.802 & 32.326 & 26.759 & 34.298 \\
\hline $\begin{array}{l}9 / 20 / 1 \\
9\end{array}$ & & 30.545 & 29.972 & $\begin{array}{l}2.084046 \\
28.846\end{array}$ & 30. & 29.316 & $\begin{array}{l}30.484 \\
30.484\end{array}$ & 29.938 & 29.621 & 28 & 25.889 & 32.353 & $\begin{array}{l}35.040 \\
35.653\end{array}$ & $\begin{array}{l}33.42 \\
33.402\end{array}$ & $\begin{array}{l}16.622 \\
16.622\end{array}$ & $\begin{array}{l}16.503 \\
1603\end{array}$ & 22.039 & 15.053 & 11.636 & 32.128 & 33.251 & 29.809 & 32.307 & 26.759 & 34.298 \\
\hline $\begin{array}{l}9 / 20 / 1 \\
9\end{array}$ & & 30.545 & 29.974 & $\begin{array}{l}2.0 .040 \\
28.858\end{array}$ & 30. & 29.304 & $\begin{array}{l}30.489 \\
30.489\end{array}$ & 29.942 & 29.626 & 28.123 & 25.889 & 32.334 & $\begin{array}{r}35.65 \\
35\end{array}$ & $\begin{array}{l}33.402 \\
33.388\end{array}$ & $\begin{array}{l}10.622 \\
16.622\end{array}$ & 16.496 & 22.028 & 15.048 & $\begin{array}{l}11.622 \\
11.62\end{array}$ & 32.126 & $\begin{array}{l}33.234 \\
334\end{array}$ & 29.811 & 32.295 & 26.759 & 34.298 \\
\hline & & 30. & 29. & 28.872 & 30 & 29. & 30. & 29.945 & 29.628 & 28. & 25.904 & 32.32 & 35.657 & 33.374 & 16.624 & 16.484 & 22.016 & 15 & 11.608 & 21 & 33.22 & 29.809 & 32.276 & 26.759 & 34.298 \\
\hline & & 30 & 29. & 28.884 & 30 & 25 & & 29. & 29 & 28. & 25. & 32.306 & 35.662 & 33 & 16.627 & 75 & 7 & 15 & 11.592 & 7 & & 6 & 32.267 & 59 & 34.3 \\
\hline & & & 29. & 28.893 & & & & & & 28. & & ( > & 35.6 & 33. & & 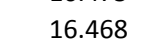 & 21.9 & 15. & 11. & 32.119 & & 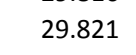 & 3 & 26.759 & 34.301 \\
\hline & & & & 200 & & & & & & 28. & 25 & 32 & & & & & 5 & 15. & 11. & 32.121 & & 29.8 & 43 & & \\
\hline & & 30 & 30.00 & 28.909 & & & & 29. & & 28. & & 32.2 & 35. & & & & 21. & 15. & 11.5 & 32.119 & 33.1 & 7 & 32.236 & & .301 \\
\hline & & 30 & $30.0 \mathrm{c}$ & 28.914 & & 29 & & 29 & & 28.1 & 25 & 32.269 & 35.669 & 33. & 16.6 & & 21. & 15.039 & 11.554 & 32.121 & & 29.842 & 32.227 & 26.761 & 34.301 \\
\hline & & & $30 . c$ & 28. & & 29.2 & & 29.954 & & 28 & & 32.259 & & & 16. & 16.442 & 21.954 & 15.034 & 11.54 & 32.121 & & 29.846 & 32.22 & & 34.301 \\
\hline $9 / 20$ & & & $30 . c$ & 28. & & 29. & & 29.952 & 29. & 28. & & 32.252 & 35.674 & 33.301 & 16.641 & & 21.945 & 15.034 & 11.535 & 32.117 & & 29.858 & & & 34.303 \\
\hline $9 / 21$ & & & $30 . c$ & 28.923 & & 29 & & 29. & 29.67 & 28. & 25. & 32.243 & 35.681 & 33.292 & 16. & 16.428 & 21.935 & 15.034 & 11.526 & 32.121 & 33.147 & 29.858 & 32.206 & 26.763 & 34.303 \\
\hline 9/21/11 0:30 & & 30. & 30 & 28.921 & 30.1 & $29.33>>>3$ & & 29.954 & $29.6 \mathrm{C}>\mathrm{C}$ & 28.1 & 25.9 & 32.238 & 35.681 & $33.2>>3$ & 16.6 & 16.423 & 21.926 & 15.032 & 11.519 & 32.119 & 33.142 & 29.858 & 32.199 & 26.763 & 34.301 \\
\hline 9/21/11 1:00 & & 30.564 & 30.03 & 28.928 & 30.18 & 29.304 & 30.5 & 29.954 & 29.681 & 28.1 & 25.95 & 32.234 & 35.683 & 33.282 & 16.648 & 16.416 & 21.919 & 15.029 & 11.512 & 32.121 & 33.137 & 29.865 & 32.192 & 26.763 & 34.303 \\
\hline $9 / 21 / 111: 30$ & & 30.559 & 30.035 & 28.921 & 30.1 & 29.316 & 30.506 & 29.95 & 29.684 & 28.186 & 25.959 & 32.227 & 35.681 & 33.273 & 16.648 & 16.412 & 21.912 & 15.036 & 11.505 & 32.112 & 33.128 & 29.856 & 32.185 & 26.763 & 34.303 \\
\hline $9 / 21 / 112: 00$ & & 30.559 & 30.037 & 28.947 & 30.1 & 29.309 & 30.508 & 29.959 & 29.686 & 28.193 & 25.952 & 32.22 & 35.683 & 33.266 & 16.648 & 16.405 & 21.903 & 15.036 & 11.497 & 32.11 & 33.12 & 29.861 & 32.178 & 26.766 & 34.303 \\
\hline $9 / 21 / 112: 30$ & & 30.564 & 30.039 & 28.951 & 30.183 & 29.297 & 30.51 & 29.957 & 29.691 & 28.205 & 25.983 & 32.215 & 35.685 & 33.261 & 16.65 & 16.398 & 21.894 & 15.036 & 11.495 & 32.112 & 33.116 & 29.865 & 32.173 & 26.766 & 34.303 \\
\hline 9/21/11 3:00 & & 30.561 & 30.039 & 28.944 & 30.183 & 29.302 & 30.513 & 29.964 & 29.693 & 28.167 & 25.976 & 32.206 & 35.683 & 33.252 & 16.652 & 16.395 & 21.884 & 15.041 & 11.486 & 32.103 & 33.109 & 29.861 & 32.166 & 26.766 & 34.303 \\
\hline 9/21/11 3:30 & & 30.575 & 30.063 & 28.949 & 30.201 & 29.323 & 30.51 & 29.957 & 29.709 & 28.153 & 25.973 & 32.206 & 35.69 & 33.249 & 16.657 & 16.391 & 21.88 & 15.022 & 11.483 & 32.126 & 33.109 & 29.889 & 32.166 & 26.77 & 34.305 \\
\hline 9/21/11 4:00 & & 30.568 & 30.056 & 28.926 & 30.199 & 29.333 & 30.513 & 29.954 & 29.709 & 28.153 & 25.973 & 32.201 & 35.69 & 33.247 & 16.657 & 16.384 & 21.868 & 15.036 & 11.479 & 32.114 & 33.099 & 29.879 & 32.161 & 26.768 & 34.305 \\
\hline $9 / 21 / 114: 30$ & & 30.566 & 30.058 & 28.926 & 30.199 & 29.342 & 30.513 & 29.959 & 29.709 & 28.139 & 25.952 & 32.194 & 35.69 & 33.238 & 16.657 & 16.379 & 21.861 & 15.041 & 11.472 & 32.105 & 33.097 & 29.875 & 32.154 & 26.768 & 13 \\
\hline
\end{tabular}




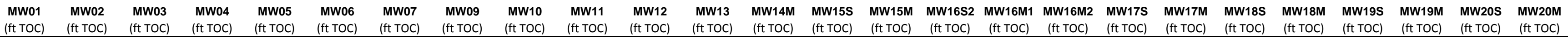

\begin{tabular}{|c|c|c|c|c|c|c|c|c|c|c|c|c|c|c|c|c|c|c|c|c|c|c|c|c|c|}
\hline well & $\begin{array}{l}\text { (ft TOC) } \\
\text { (f) }\end{array}$ & $\begin{array}{l}\text { (ft TOC) } \\
\text { (ft) }\end{array}$ & $\begin{array}{l}\text { (ft TOC) } \\
\text { (f) }\end{array}$ & $\begin{array}{l}\text { (ft TOC) } \\
\text { (ft) }\end{array}$ & $\begin{array}{ll}(\mathrm{ft} \text { TOC) } & \text { (ft TOC) }\end{array}$ & (ft toc) & $\begin{array}{l}\text { (ift TOC) } \\
\text { (n) }\end{array}$ & $\begin{array}{l}\text { (ft TOC) } \\
\text { (f) }\end{array}$ & $\begin{array}{l}\text { (ft TOC) } \\
\text { (f) }\end{array}$ & $\begin{array}{l}\text { (ft TOC) } \\
\text { (n) }\end{array}$ & $\begin{array}{l}\text { (ft TOC) } \\
\text { (ft }\end{array}$ & (ft TOC) & $\begin{array}{l}\text { (ft TOC) } \\
\end{array}$ & (ft TOC) & (ft TOC) & $\begin{array}{l}\text { (ft TOC) } \\
\text { (f) }\end{array}$ & (ft TOC) & $\begin{array}{l}\text { (ft TOC) } \\
\text { (ft) }\end{array}$ & (ft TOC) & $\begin{array}{l}\text { (ft TOC) } \\
\text { (f) }\end{array}$ & $\begin{array}{l}\text { (ft TOC) } \\
\text { (f) }\end{array}$ & $\begin{array}{l}\text { (ft TOC) } \\
\text { (n) }\end{array}$ & (ft TOC) & $\begin{array}{l}\text { (ft TOC) } \\
\text { (1) }\end{array}$ & (ft TOC) \\
\hline 0 & & 30.564 & 30.06 & 28. & $30.1 \mathrm{~s}$ & 29.3 & 30.5 & 29.964 & 29. & 28.13 & 25 & 32.189 & 35.693 & 33.2 & 16.659 & 16.374 & 21.852 & 15.046 & 11.467 & 32.107 & 33.092 & 29.875 & 32.15 & 26.768 & 3 \\
\hline $1115: 30$ & & 30.554 & 30.051 & 28.923 & 30.187 & 29.344 & 30.518 & 29.957 & 29.707 & 28.139 & 25.971 & 32.182 & 35.688 & 33.224 & 16.659 & 16.367 & 21.843 & 15.057 & 11.458 & 32.089 & 33.083 & .851 & 32.138 & & 34.303 \\
\hline 9/21/11 6:00 & & 30.564 & 30.056 & 28.93 & 30.187 & 29.354 & 30.515 & 29.959 & 29.711 & 28.128 & 25.942 & 32.178 & 35.69 & 33.219 & 16.657 & 16.36 & 21.848 & 15.046 & 11.453 & 32.093 & 33.08 & .863 & 2.135 & 68 & 34.303 \\
\hline 9/21/11 6:30 & & 30.568 & 30.063 & 28.94 & 30.194 & 29.351 & 30.515 & 29.966 & 29.718 & 28.123 & 25.949 & 32.173 & 35.693 & 33.217 & 16.662 & 16.358 & 21.843 & 15.046 & 11.45 & 32.098 & 33.075 & 87 & 32.133 & 77 & 34.305 \\
\hline 9/21/11 7:00 & & 30.559 & 30.056 & 28.954 & 30.185 & 29.347 & 30.52 & 29.966 & 29.714 & 28.125 & 25.961 & 32.166 & 35.69 & 33.207 & 16.662 & 16.353 & 21.848 & 15.057 & 11.443 & 32.082 & 33.066 & 29.849 & 32.124 & 26.77 & 34.305 \\
\hline 9/21/11 7:30 & & 30.559 & 30.058 & 28.961 & 30.18 & 29.34 & 30.522 & 29.969 & 29.714 & 28.142 & 25.973 & 32.161 & 35.688 & 33.2 & 16.659 & 16.346 & 21.848 & 15.06 & 11.439 & 32.079 & 33.061 & 29.849 & 32.121 & 26.768 & 34.303 \\
\hline 9/21/11 8:00 & & 30.566 & 30.065 & 28.956 & 30.185 & 29.349 & 30.52 & 29.964 & 29.721 & 28.17 & 25.978 & 32.159 & 35.69 & 33.2 & 16.662 & 16.344 & 21.838 & 15.053 & 11.434 & 32.084 & 33.061 & 29.858 & 32.119 & 26.77 & 34.305 \\
\hline 9/21/11 8:30 & & 30.573 & 30.072 & 28.954 & 30.194 & 29.359 & 30.522 & 29.969 & 29.73 & 28.198 & 25.976 & 32.159 & 35.697 & 33.198 & 16.666 & 16.342 & 21.836 & 15.046 & 11.434 & 32.093 & 33.059 & 29.87 & 32.117 & 26.773 & 34.308 \\
\hline 9/21/11 9:00 & & 30.575 & 30.074 & 28.961 & 30.197 & 29.359 & 30.522 & 29.971 & 29.735 & 28.219 & 25.971 & 32.154 & 35.695 & 33.193 & 16.666 & 16.337 & 21.824 & 15.053 & 11.429 & 32.086 & 33.054 & 29.875 & 32.114 & 26.77 & 34.308 \\
\hline 9/21/11 9:30 & & 30.571 & 30.074 & 28.968 & 30.197 & 29.359 & 30.525 & 29.973 & 29.732 & 28.235 & 25.978 & 32.152 & 35.699 & 33.191 & 16.669 & 16.332 & 21.815 & 15.062 & 11.425 & 32.089 & 33.054 & 29.872 & 32.11 & 26.77 & 34.305 \\
\hline 9/21/11 10:00 & & 30.571 & 30.077 & 28.965 & 30.194 & 29.366 & 30.527 & 29.971 & 29.735 & 28.228 & 25.983 & 32.145 & 35.695 & 33.188 & 16.669 & 16.328 & 21.806 & 15.067 & 11.418 & 32.082 & 33.049 & 29.863 & 32.105 & 26.77 & 34.308 \\
\hline 9/21/11 10:30 & & 30.578 & 30.081 & 28.956 & 30.199 & 29.375 & 30.524 & 29.969 & 29.742 & 28.253 & 25.98 & 32.142 & 35.702 & 33.184 & 16.671 & 16.323 & 21.797 & 15.057 & 11.418 & 32.093 & 33.045 & 29.879 & 32.103 & 26.773 & 34.308 \\
\hline 9/21/11 11:00 & & 30.578 & 30.086 & 28.958 & 30.201 & 29.377 & 30.527 & 29.973 & 29.746 & 28.23 & 25.971 & 32.14 & 35.697 & 33.179 & 16.671 & 16.321 & 21.79 & 15.067 & 11.413 & 32.089 & 33.04 & 29.875 & 32.1 & 26.773 & 34.307 \\
\hline 9/21/11 11:30 & & 30.575 & 30.084 & 28.97 & 30.199 & 29.377 & 30.529 & 29.976 & 29.746 & 28.228 & 25.973 & 32.135 & 35.702 & 33.179 & 16.671 & 16.316 & 21.78 & 15.074 & 11.411 & 32.086 & 33.038 & 29.875 & 32.096 & 26.773 & 34.308 \\
\hline 9/21/11 12:00 & & 30.573 & 30.081 & 28.97 & 30.194 & 29.377 & 30.529 & 29.976 & 29.746 & 28.246 & 25.983 & 32.131 & 35.707 & 33.172 & 16.671 & 16.309 & 21.767 & 15.076 & 11.406 & 32.077 & 33.03 & 29.868 & 32.089 & 26.773 & 34.305 \\
\hline 9/21/11 12:30 & & 30.578 & 30.086 & 28.961 & 30.197 & 29.382 & 30.532 & 29.978 & 29.749 & 28.246 & 25.98 & 32.262 & 35.702 & 33.313 & 16.673 & 16.318 & 21.764 & 15.076 & 11.547 & 32.117 & 33.165 & 29.872 & 32.218 & 26.775 & 34.305 \\
\hline 9/21/11 13:00 & & 30.58 & 30.091 & 28.949 & 30.201 & 29.394 & 30.532 & 29.969 & 29.753 & 28.239 & 25.973 & 32.201 & 35.711 & 33.245 & 16.673 & 16.323 & 21.755 & 15.072 & 11.476 & 32.112 & 33.101 & 29.872 & 32.159 & 26.775 & 34.305 \\
\hline 9/21/11 13:30 & & 30.58 & 30.091 & 28.93 & 30.201 & 29.415 & 30.527 & 29.969 & 29.756 & 28.216 & 25.961 & 32.178 & 35.702 & 33.221 & 16.678 & 16.321 & 21.746 & 15.076 & 11.455 & 32.105 & 33.075 & 29.879 & 32.135 & 26.775 & 34.305 \\
\hline 9/21/11 14:00 & & 30.575 & 30.088 & 28.923 & 30.199 & 29.422 & 30.529 & 29.973 & 29.753 & 28.212 & 25.94 & 32.159 & 35.704 & 33.202 & 16.676 & 16.313 & 21.741 & 15.079 & 11.436 & 32.089 & 33.061 & 29.868 & 32.119 & 26.773 & 34.303 \\
\hline & & 30.573 & 30.086 & 28.912 & 30.1 & 29.429 & 30.529 & 29.971 & 29.753 & 28.242 & 25.93 & 32.147 & 35.704 & 33.191 & 16.676 & 16.306 & 21.732 & 15.083 & 11.422 & 32.084 & 33.049 & 29.858 & 32.107 & 26.773 & 34.303 \\
\hline $9 / 2$ & & 30.564 & 30.077 & 28.907 & 30.17 & 29.436 & 30.525 & 29.973 & 29.746 & 28.246 & 25.918 & 32.131 & 35.7 & 33.172 & 16.676 & 16.297 & 21.72 & 15.095 & 11.406 & 32.058 & .035 & 29.837 & 32.091 & 26.773 & 298 \\
\hline $9 / 21 / 1$ & & 30.566 & 30.072 & 28.909 & 30.1 & 29.436 & 30.527 & 29.976 & 29.742 & 28.228 & 25.916 & 32.121 & 35.695 & 33.163 & 16.673 & .288 & 21.711 & 15.095 & 11.399 & .049 & 021 & 29.835 & 32.079 & 26.775 & 294 \\
\hline $9 / 21 / 1$ & & 30.564 & 30.065 & 28.912 & 30.159 & 29.436 & 30.524 & 29.973 & 29.739 & 28.219 & 25.916 & 32.112 & 35.697 & 33.151 & 16.671 & 16.285 & 21.713 & 15.107 & 11.387 & 32.038 & .011 & 9.818 & 32.07 & 26.773 & 34.294 \\
\hline 9/21/11 16:30 & & 30.568 & 30.067 & 28.907 & 30.152 & 29.438 & 30.525 & 29.973 & 29.737 & 28.221 & 25.918 & 32.105 & 35.695 & 33.146 & 16.671 & 16.278 & 21.743 & 15.104 & 11.38 & 32.033 & 33.004 & 29.811 & 32.063 & 26.775 & 34.291 \\
\hline 9/21/11 17:00 & & 30.568 & 30.065 & 28.909 & 30.15 & 29.441 & & 29.976 & 29.735 & 28.219 & 25.913 & 32.096 & 35.695 & 33.139 & 16.671 & 16.276 & 21.771 & 15.102 & 11.368 & & 32.997 & 29.814 & 32.056 & 26.775 & 34.291 \\
\hline 9/21/11 17:30 & & 30.566 & 30.063 & 28.919 & 30.143 & 29.429 & 30.53 & 29.98 & 29.735 & 28.209 & 25.913 & 32.089 & 35.697 & 33.132 & 16.669 & 16.271 & 21.767 & 15.102 & 11.363 & 32.026 & 32.99 & 29.814 & 32.049 & 26.775 & 34.291 \\
\hline 9/21/11 18:00 & & 30.568 & 30.063 & 28.921 & 30.141 & 29.427 & 30.53 & 29.978 & 29.732 & 28.179 & 25.925 & 32.084 & 35.692 & 33.125 & 16.669 & 16.264 & 21.757 & 15.107 & 11.359 & 32.019 & 32.985 & 29.809 & 32.044 & 26.775 & 34.289 \\
\hline 9/21/11 18:30 & & 30.568 & 30.06 & 28.921 & 30.136 & 29.429 & 30.527 & 29.978 & 29.732 & 28.188 & 25.923 & 32.077 & 35.688 & 33.118 & 16.669 & 16.26 & 21.746 & 15.1 & 11.352 & 32.021 & 32.978 & 29.804 & 32.037 & 26.775 & 34.289 \\
\hline 9/21/11 19:00 & & 30.573 & 30.065 & 28.923 & 30.141 & 29.427 & 30.527 & 29.976 & 29.735 & 28.181 & 25.925 & 32.075 & 35.695 & 33.113 & 16.669 & 16.257 & 21.737 & 15.095 & 11.347 & 32.024 & 32.978 & 29.809 & 32.035 & 26.777 & 34.291 \\
\hline 9/21/11 19:30 & & 30.573 & 30.065 & 28.93 & 30.136 & 29.424 & 30.53 & 29.978 & 29.732 & 28.179 & 25.928 & 32.07 & 35.695 & 33.109 & 16.669 & 16.253 & 21.725 & 15.107 & 11.342 & 32.019 & 32.971 & 29.809 & 32.028 & 26.777 & 34.289 \\
\hline 9/21/11 20:00 & & 30.573 & 30.065 & 28.937 & 30.134 & 29.42 & 30.532 & 29.983 & 29.735 & 28.163 & 25.933 & 32.065 & 35.7 & 33.104 & 16.669 & 16.248 & 21.716 & 15.102 & 11.338 & 32.014 & 32.966 & 29.809 & 32.028 & 26.777 & 34.291 \\
\hline $9 / 21 / 1120: 30$ & & 30.573 & 30.067 & 28.942 & 30.136 & 29.417 & 30.532 & 29.983 & 29.737 & 28.144 & 25.94 & 32.063 & 35.695 & 33.102 & 16.671 & 16.243 & 21.706 & 15.102 & 11.335 & 32.014 & 32.962 & 29.811 & 32.023 & 26.777 & 34.291 \\
\hline $9 / 21 / 1121: 00$ & & 30.575 & 30.07 & 28.947 & 30.138 & 29.413 & 30.532 & 29.983 & 29.737 & 28.135 & 25.944 & 32.058 & 35.7 & 33.099 & 16.671 & 16.241 & 21.697 & 15.095 & 11.333 & 32.019 & 32.962 & 29.816 & 32.018 & 26.78 & 34.291 \\
\hline 9/21/11 21:30 & & 30.58 & 30.072 & 28.951 & 30.138 & 29.408 & 30.534 & 29.985 & 29.739 & 28.13 & 25.949 & 32.058 & 35.704 & 33.095 & 16.671 & 16.239 & 21.69 & 15.097 & 11.333 & 32.019 & 32.959 & 29.818 & 32.016 & 26.78 & 34.291 \\
\hline $9 / 21 / 1122: 00$ & & 30.58 & 30.072 & 28.944 & 30. & 29.415 & 30.534 & 29.985 & 29.742 & 28.153 & 25.952 & 32.054 & 35.7 & 33.095 & 16.676 & 16.236 & 21.681 & 15.097 & 11.326 & 32.01 & .957 & 29.818 & 32.013 & 26.777 & 34.291 \\
\hline $9 / 21 / 1$ & & 30.582 & 30.077 & 28.944 & & 29.417 & & 29.985 & 29.744 & 28.142 & 25.944 & 32.051 & 35.707 & 33.09 & 16.673 & 16.232 & 21.674 & 15.093 & 11.326 & 32.024 & .955 & 29.828 & 32.011 & 26.78 & 34.291 \\
\hline 9/21/1 & & 30.582 & 30.079 & $\begin{array}{r}2.0344 \\
28.94\end{array}$ & 30. & 29.424 & 30. & 29.983 & 29.746 & 28.158 & $\begin{array}{l}25.942 \\
2.942\end{array}$ & 32.049 & 35.707 & $\begin{array}{l}33.099 \\
33.092\end{array}$ & 16.673 & $\begin{array}{l}10.229 \\
16.229\end{array}$ & 21.665 & 15.095 & 11.321 & 32.017 & . 952 & 29.821 & 32.011 & 26.78 & 34.291 \\
\hline 9/21/1 & & 30.578 & 30.074 & $\begin{array}{l}28.928 \\
28.928\end{array}$ & 30. & 29.431 & 30. & 29.983 & 29.744 & 28.179 & 25.942 & 32.044 & 35.707 & 33.083 & 16.676 & 16.225 & 21.658 & 15.102 & 11.316 & 32.012 & 947 & 29.816 & 32.006 & 26.78 & 34.291 \\
\hline $9 / 2$ & & 30.578 & 30 & 28.914 & 30 & 29 & 30 & 2 & 29.744 & 28.195 & 25 & 32 & 35.709 & 33. & 16 & 1 & 21.649 & 15. & 11 & 32.01 & 3 & 29.811 & 32.002 & 26.78 & .289 \\
\hline $9 / 221$ & & & 30 & 28. & & 29 & 30 & 29. & & 28. & 25 & 32. & 35. & 33 & 16 & 16 & 21.642 & 15 & 11 & 32 & 8 & 29. & 31 & 26.78 & .289 \\
\hline & & 30. & 30. & 28.909 & 30. & & & 29 & & 28. & & 32 & 35. & 33.071 & 16.673 & & 21.632 & 15.114 & 11.307 & 93 & 3 & 29.797 & & 26.78 & 34.287 \\
\hline & & & & 28.905 & & & & 29.985 & & 28. & 25 & 32.025 & & & 16.671 & 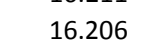 & 21.626 & 15.114 & 11.298 & 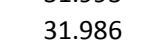 & 4 & 29.788 & 31.985 & & 34.287 \\
\hline & & 30 & 30.0 & 28.895 & & & 30. & 29.978 & & & & 32.018 & & & & & 21.6 & 15.1 & 11.293 & & & & & & 287 \\
\hline & & 30 & 30.0 & 28.912 & 30. & & & 29. & & 28 & & 32.014 & 35.6 & 33. & 16. & & 21.6 & 15.118 & 11.291 & & & 29.778 & 31.974 & & 34.284 \\
\hline & & & 30. & 28.912 & & & & 29. & & 28 & & 32.009 & & & & 16. & 21. & 15.123 & 11.281 & & & 29.767 & 31.969 & & 34.284 \\
\hline $9 / 2$ & & & & 28.912 & & 29. & & & & & & 32.002 & & & & 16.192 & 21. & 15. & 11.279 & & & 29. & 31.964 & & 34.282 \\
\hline 14:00 & & 30. & 30. & 28.909 & & 29.45 & & 29. & & 28.2 & 25. & 32.007 & 35.697 & 33. & 16. & 16.19 & 21.5 & 15. & 11.277 & 31.975 & 32.905 & 29.781 & 31.964 & 26.785 & 34.284 \\
\hline 9/22/11 4:30 & & 30. & 30. & 28.921 & 30. & 29.443 & & 29.9 & 29. & 28.2. & 25.9 & 32 & 35.695 & 33.0 & 16.6 & 16.185 & 21.5. & 15.1 & 11.277 & 31.965 & 32.902 & 29.776 & 31.962 & 26.782 & 34.282 \\
\hline 9/22/11 5:00 & & 30.573 & 30.0 & 28.916 & 30.0 & 29.438 & 30.5 & 29.992 & 29.725 & 28.219 & 25.91 & 31.997 & 35.7 & 33.034 & 16.666 & 16.185 & 21.5 & 15.109 & 11.269 & 31.965 & 32.898 & 29.771 & 31.957 & 26.782 & 34.282 \\
\hline $9 / 22 / 115: 30$ & & 30.575 & 30.051 & 28.933 & 30.0 & 29.436 & 30.534 & 29.99 & 29.723 & 28.221 & 25.916 & 31.995 & 35.7 & 33.034 & 16.669 & 16.183 & 21.565 & 15.107 & 11.267 & 31.968 & 32.898 & 29.776 & 31.955 & 26.782 & 34.284 \\
\hline 9/22/11 6:00 & & 30.578 & 30.056 & 28.937 & 30.099 & 29.424 & 30.537 & 29.99 & 29.725 & 28.228 & 25.93 & 31.995 & 35.704 & 33.031 & 16.669 & 16.18 & 21.561 & 15.1 & 11.265 & 31.97 & 32.895 & 29.778 & 31.952 & 26.785 & 34.284 \\
\hline $9 / 22 / 116: 30$ & & 30.575 & 30.056 & 28.949 & 30.099 & 29.417 & 30.539 & 29.995 & 29.725 & 28.232 & 25.94 & 31.99 & 35.702 & 33.029 & 16.669 & 16.176 & 21.558 & 15.097 & 11.265 & 31.965 & 32.895 & 29.776 & 31.95 & 26.785 & 34.284 \\
\hline 9/22/11 7:00 & & 30.582 & 30.06 & 28.954 & 30.108 & 29.417 & 30.537 & 29.997 & 29.73 & 28.237 & 25.944 & 31.99 & 35.699 & 33.029 & 16.671 & 16.176 & 21.568 & 15.088 & 11.262 & 31.977 & 32.893 & 29.792 & 31.95 & 26.787 & 34.287 \\
\hline 9/22/11 7:30 & & 30.585 & 30.063 & 28.951 & 30.11 & 29.42 & 30.537 & 29.988 & 29.732 & 28.237 & 25.954 & 31.993 & 35.709 & 33.029 & 16.673 & 16.176 & 21.579 & 15.093 & 11.265 & 31.979 & 32.895 & 29.795 & 31.95 & 26.787 & 34.287 \\
\hline 9/22/11 8:00 & & 30.585 & 30.065 & 28.944 & 30.115 & 29.424 & 30.539 & 29.988 & 29.737 & 28.237 & 25.949 & 31.99 & 35.709 & 33.029 & 16.673 & 16.176 & 21.577 & 15.088 & 11.262 & 31.977 & 32.893 & 29.799 & 31.95 & 26.787 & 34.289 \\
\hline $9 / 22 / 118: 30$ & & 30.585 & 30.07 & 28.947 & 30.12 & 29.427 & 30.541 & 29.995 & 29.739 & 28.235 & 25.942 & 31.99 & 35.711 & 33.029 & 16.673 & 16.173 & 21.568 & 15.088 & 11.265 & 31.982 & 32.893 & 29.799 & 31.952 & 26.787 & 9 \\
\hline
\end{tabular}




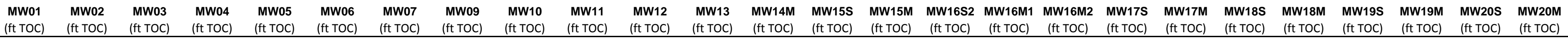

\begin{tabular}{|c|c|c|c|c|c|c|c|c|c|c|c|c|c|c|c|c|c|c|c|c|c|c|c|c|c|c|}
\hline Well & $\begin{array}{l}\text { (ft TOC) } \\
\text { (ft }\end{array}$ & $\begin{array}{l}\text { (ft TOC) } \\
\text { (f) }\end{array}$ & $\begin{array}{l}\text { (ft TOC) } \\
\text { (f) }\end{array}$ & $\begin{array}{l}\text { (ft TOC) } \\
\text { (f }\end{array}$ & $\begin{array}{l}\text { (ft TOC) } \\
\text { (f) }\end{array}$ & $\begin{array}{l}\text { (ft TOC) } \\
\text { (f) }\end{array}$ & $\begin{array}{l}\text { (ft TOC) } \\
\text { (ft }\end{array}$ & $\begin{array}{l}\text { (ft TOC) } \\
\text { (f) }\end{array}$ & $\begin{array}{l}\text { (ft TOC) } \\
\text { (ft) }\end{array}$ & $\begin{array}{l}\text { (ft TOC) } \\
\text { (f) }\end{array}$ & $\begin{array}{l}\text { (ft TOC) } \\
\text { (f) }\end{array}$ & $\begin{array}{l}\text { (ft TOC) } \\
\text { (f) }\end{array}$ & (ft TOC) & $\begin{array}{l}\text { (ft TOC) } \\
\text { (f) }\end{array}$ & (ft TOC) & (ft TOC) & $\begin{array}{l}\text { (ft TOC) } \\
\text { (f) }\end{array}$ & (ft TOC) & $\begin{array}{l}\text { (ft TOC) } \\
\text { (1) }\end{array}$ & (ft TOC) & $\begin{array}{l}\text { (ft TOC) } \\
\end{array}$ & $\begin{array}{l}\text { (ft TOC) } \\
\text { (f) }\end{array}$ & $\begin{array}{l}\text { (ft TOC) } \\
\end{array}$ & (ft TOC) & $\begin{array}{l}\text { (ft TOC) } \\
\text { (1) }\end{array}$ & (ft TOC) \\
\hline 9:00 & & 30.582 & 30.067 & 28.944 & 30. & & 29.4 & 30.542 & 29.9 & 29. & 28.235 & 25 & 31.988 & 35711 & $33 . c$ & 16 & 16 & 21.558 & 15. & 11.26 & 31.982 & 32.891 & & 31.95 & 5.787 & 34.291 \\
\hline 11 9:30 & & 30.582 & 30.065 & 28.947 & 30.117 & & 29.438 & 30.541 & 29.992 & 29.739 & 28.235 & 25.942 & 31.986 & 35.709 & 33.027 & 16.676 & 16.169 & 21.554 & 15.1 & 11 & & 32.891 & 29.799 & 1.948 & 5.787 & 34.291 \\
\hline 9/22/11 10:00 & & 30.582 & 30.067 & 28.94 & 30.117 & & 29.443 & 30.544 & 29.992 & 29.739 & 28.237 & 25.94 & 31.983 & 35.711 & 33.022 & 16.676 & 16.166 & 21.547 & 15.111 & 11.258 & 31.975 & 32.886 & 29.792 & 1.943 & 5.787 & 34.291 \\
\hline 9/22/11 10:30 & & 30.582 & 30.067 & 28.933 & 30.117 & & 29.448 & 30.542 & 29.995 & 29.737 & 28.235 & 25.937 & 31.981 & 35.714 & 33.022 & $\begin{array}{l}2 \\
16.673\end{array}$ & 16.162 & 21.542 & 15.107 & 11.253 & 31.97 & 32.886 & 29.79 & 31.943 & 87 & 34.291 \\
\hline 9/22/11 11:00 & & 30.585 & 30.067 & 28.921 & 30.117 & & 29.462 & 30.542 & 29.99 & 29.739 & 28.228 & 25.928 & 31.979 & 35.711 & 33.02 & $2 \quad 16.678$ & 16.162 & 21.533 & 15.114 & 11.248 & 31.975 & 32.883 & 29.797 & 31.943 & 26.787 & 34.291 \\
\hline 9/22/11 11:30 & & 30.58 & 30.065 & 28.907 & 30.113 & & 29.474 & 30.539 & 29.99 & 29.737 & 28.223 & 25.916 & 31.979 & 35.711 & 33.017 & $7 \quad 16.676$ & 16.155 & 21.528 & 15.118 & 11.246 & 31.968 & 32.879 & 29.788 & 31.938 & 26.789 & 34.291 \\
\hline 9/22/11 12:00 & & 30.578 & 30.06 & 28.898 & 30.11 & & 29.483 & 30.534 & 29.99 & 29.735 & 28.219 & 25.901 & 31.974 & 35.709 & 33.01 & $1 \quad 16.676$ & 16.152 & 21.519 & 15.128 & 11.246 & 31.961 & 32.876 & 29.783 & 31.934 & 26.787 & 34.289 \\
\hline 9/22/11 12:30 & & 30.573 & 30.053 & 28.886 & 30.099 & & 29.493 & 30.534 & 29.99 & 29.73 & 28.212 & 25.892 & 31.967 & 35.707 & 33.008 & $8 \quad 16.673$ & 16.148 & 21.512 & 15.142 & 11.241 & 31.954 & 32.869 & 29.769 & 31.927 & 26.787 & 34.289 \\
\hline 9/22/11 13:00 & & 30.568 & 30.049 & 28.872 & 30.089 & & 29.502 & 30.529 & 29.99 & 29.723 & 28.205 & 25.882 & 31.962 & 35.709 & 33.001 & $1 \quad 16.671$ & 16.143 & 21.503 & 15.144 & 11.234 & 31.944 & 32.865 & 29.76 & 31.922 & 26.789 & 34.287 \\
\hline 9/22/11 13:30 & & 30.568 & 30.044 & 28.858 & 30.082 & & 29.509 & 30.527 & 29.983 & 29.718 & 28.195 & 25.866 & 31.955 & 35.7 & 32.996 & $6 \quad 16.669$ & 16.136 & 21.496 & 15.151 & 11.232 & 31.933 & 32.857 & 29.748 & 31.917 & 26.787 & 34.284 \\
\hline 9/22/11 14:00 & & 30.568 & 30.032 & 28.849 & 30.073 & & 29.516 & 30.525 & 29.983 & 29.714 & 28.191 & 25.854 & 31.951 & 35.697 & 32.987 & $7 \quad 16.666$ & 16.134 & 21.489 & 15.151 & 11.225 & 31.923 & 32.85 & 29.738 & 31.91 & 26.787 & 34.282 \\
\hline 9/22/11 14:30 & & 30.561 & 30.023 & 28.839 & 30.059 & & 29.516 & 30.52 & 29.985 & 29.705 & 28.184 & 25.846 & 31.941 & 35.693 & 32.98 & $8 \quad 16.666$ & 16.131 & 21.485 & 15.165 & 11.215 & 31.909 & 32.843 & 29.72 & 31.906 & 26.787 & 34.277 \\
\hline 9/22/11 15:00 & & 30.561 & 30.014 & 28.832 & 30.047 & & 29.514 & 30.515 & 29.983 & 29.7 & 28.177 & 25.834 & 31.937 & 35.69 & 32.97 & $7 \quad 16.664$ & 16.124 & 21.478 & 15.165 & 11.208 & 31.898 & 32.834 & 29.715 & 31.896 & 26.787 & 34.275 \\
\hline 9/22/11 15:30 & & 30.559 & 30.002 & 28.825 & 30.04 & & 29.511 & 30.51 & 29.983 & 29.691 & 28.174 & 25.832 & 31.927 & 35.688 & 32.968 & 316.659 & 16.12 & 21.471 & 15.17 & 11.199 & 31.891 & 32.831 & 29.703 & 31.889 & 26.787 & 34.275 \\
\hline 9/22/11 16:00 & & 30.557 & 29.997 & 28.823 & 30.026 & & 29.509 & 30.508 & 29.985 & 29.688 & 28.167 & 25.825 & 31.92 & 35.683 & 32.961 & 16.655 & 16.115 & 21.461 & 15.172 & 11.194 & 31.881 & 32.82 & 29.694 & 31.884 & 26.787 & 34.273 \\
\hline 9/22/11 16:30 & & 30.557 & 29.993 & 28.83 & 30.021 & & 29.504 & 30.51 & 29.983 & 29.681 & 28.167 & 25.827 & 31.915 & 35.681 & 32.956 & 16.655 & 16.11 & 21.466 & 15.168 & 11.19 & 31.879 & 32.817 & 29.694 & 31.875 & 26.787 & 34.273 \\
\hline 9/22/11 17:00 & & 30.552 & 29.986 & 28.83 & 30.01 & & 29.495 & 30.506 & 29.983 & 29.677 & 28.167 & 25.832 & 31.908 & 35.678 & 32.949 & 16.652 & 16.108 & 21.466 & 15.172 & 11.18 & 31.87 & 32.812 & 29.684 & 31.87 & 26.787 & 34.27 \\
\hline 9/22/11 17:30 & & 30.554 & 29.983 & 28.83 & 30.005 & & 29.488 & 30.506 & 29.98 & 29.672 & 28.165 & 25.834 & 31.904 & 35.676 & 32.945 & 16.65 & 16.103 & 21.487 & 15.17 & 11.182 & 31.865 & 32.808 & 29.677 & 31.866 & 26.789 & 34.268 \\
\hline 9/22/11 18:00 & & 30.554 & 29.983 & 28.832 & 30.005 & & 29.481 & 30.506 & 29.985 & 29.672 & 28.165 & 25.834 & 31.901 & 35.674 & 32.942 & 16.652 & 16.098 & 21.496 & 15.163 & 11.173 & 31.865 & 32.803 & 29.682 & 31.861 & 26.789 & 34.268 \\
\hline $9 / 22 /$ & & 30.557 & 29.981 & 28.832 & 30 & & 29.476 & 30.501 & 29.983 & 29.67 & 28.163 & 25.837 & 31.899 & 35.681 & 32.935 & 16.648 & 16.096 & 21.491 & 15.161 & 11.171 & 31.87 & 32.798 & 9.677 & 1.861 & 26.789 & 34.268 \\
\hline bo & & 30.557 & 29.979 & 28.83 & 29.998 & & 29.469 & 30.503 & 29.985 & 29.667 & 28.163 & 25.842 & 31.894 & 35.674 & 32.933 & 16.648 & 16.094 & 21.485 & 88 & 11.166 & 31.865 & 798 & .677 & 54 & 26.789 & 34.268 \\
\hline $9 / 22 / 1$ & & 30.554 & 29.981 & 28.835 & 29.998 & & 29.462 & 30.503 & 29.983 & 29.665 & 28.163 & 25.842 & 31.89 & 35.671 & 32.931 & 16.648 & 16.091 & 21.473 & 154 & 11.164 & 31.86 & 793 & .682 & .852 & 5.789 & 266 \\
\hline $9 / 22 / 11$ & & 30.554 & 29.979 & 28.842 & 29.996 & & 29.455 & 30.501 & 29.98 & 29.663 & 28.163 & 25.846 & 31.887 & 35.674 & 32.926 & 16.648 & 16.089 & 21.464 & 15.151 & 11.161 & 31.863 & .789 & 677 & 1.849 & 89 & 34.266 \\
\hline 9/22/11 20:30 & & 30.554 & 29.979 & 28.846 & 29.996 & & 29.446 & 30.501 & 29.985 & 29.663 & 28.165 & 25.854 & 31.885 & 35.671 & 32.926 & 16.645 & 16.087 & 21.461 & 15.147 & 11.159 & 31.858 & 32.789 & 29.68 & 31.847 & 26.789 & 34.266 \\
\hline 9/22/11 21:00 & & 30.554 & 29.979 & 28.846 & 29.996 & & 29.441 & 30.503 & 29.985 & & 28.167 & 25.861 & 31.883 & 35.676 & 32.921 & & 16.084 & 21.461 & 15.149 & 11.157 & 31.856 & & 29.68 & 31.847 & 26.792 & 34.266 \\
\hline 9/22/11 21:30 & & 30.554 & 29.979 & 28.853 & 29.998 & & 29.436 & 30.501 & 29.988 & 29.66 & 28.167 & 25.861 & 31.883 & 35.676 & 32.923 & 16.645 & 16.084 & 21.461 & 15.14 & 11.157 & 31.86 & 32.786 & 29.689 & 31.845 & 26.792 & 34.266 \\
\hline 9/22/11 22:00 & & 30.554 & 29.981 & 28.858 & 30 & & 29.431 & 30.501 & 29.985 & 29.66 & 28.17 & 25.87 & 31.883 & 35.676 & 32.919 & 16.648 & 16.082 & 21.454 & 15.135 & 11.154 & 31.865 & 32.786 & 29.689 & 31.842 & 26.792 & 34.266 \\
\hline 9/22/11 22:30 & & 30.557 & 29.981 & 28.858 & 30 & & 29.429 & 30.501 & 29.988 & 29.66 & 28.17 & 25.873 & 31.88 & 35.676 & 32.916 & 16.645 & 16.08 & 21.445 & 15.13 & 11.152 & 31.865 & 32.782 & 29.691 & 31.842 & 26.792 & 34.266 \\
\hline 9/22/11 23:00 & & 30.557 & 29.983 & 28.853 & 30.005 & & 29.431 & 30.501 & 29.99 & 29.66 & 28.167 & 25.873 & 31.876 & 35.685 & 32.916 & 16.648 & 16.075 & 21.441 & 15.128 & 11.15 & 31.867 & 32.779 & 29.696 & 31.84 & 26.792 & 34.268 \\
\hline 9/22/11 23:30 & & 30.557 & 29.983 & 28.851 & 30.01 & & 29.431 & 30.498 & 29.988 & 29.66 & 28.167 & 25.87 & 31.876 & 35.683 & 32.914 & 16.648 & 16.073 & 21.431 & 15.123 & 11.147 & 31.865 & 32.777 & 29.701 & 31.837 & 26.792 & 34.268 \\
\hline 9/23/11 0:00 & & 30.552 & 29.986 & 28.844 & 30.007 & & 29.434 & 30.498 & 29.985 & 29.658 & 28.165 & 25.868 & 31.876 & 35.688 & 32.912 & 16.648 & 16.07 & 21.427 & 15.128 & 11.145 & 31.865 & 32.777 & 29.696 & 31.837 & 26.792 & 34.266 \\
\hline 9/23/11 0:30 & & 30.554 & 29.983 & 28.844 & 30.007 & & 29.431 & 30.498 & 29.988 & 29.656 & 28.163 & 25.863 & 31.871 & 35.685 & 32.909 & 16.645 & 16.066 & 21.422 & 15.121 & 11.143 & 31.863 & 32.772 & 29.694 & 31.833 & 26.792 & 34.263 \\
\hline 9/23/11 1:00 & & 30.552 & 29.979 & 28.844 & 30.005 & & 29.429 & 30.496 & 29.99 & 29.653 & 28.163 & 25.861 & 31.869 & 35.683 & 32.905 & 16.645 & 16.063 & 21.415 & 15.121 & 11.14 & 31.86 & 32.772 & 29.691 & 31.83 & 26.792 & 34.263 \\
\hline 9/23/11 1:30 & & 30.55 & 29.979 & 28.846 & 30.003 & & 29.427 & 30.496 & 29.988 & 29.651 & 28.163 & 25.863 & 31.864 & 35.683 & 32.902 & 16.645 & 16.059 & 21.413 & 15.121 & 11.138 & 31.856 & 32.765 & 29.684 & 31.826 & 26.792 & 34.261 \\
\hline 9/23/11 2:00 & & 30.55 & 29.976 & 28.844 & 30.003 & & 29.424 & 30.496 & 29.99 & 29.651 & 28.16 & 25.866 & 31.862 & 35.683 & 32.898 & 16. & 16 & 21.406 & 15.116 & 11.133 & 31.853 & 32.763 & 9.682 & 31.823 & 26.794 & 34.261 \\
\hline 9/23/11 2:30 & & & 29.976 & 28.846 & 30 & & 29.422 & 30.493 & 29.99 & 29.649 & 28.163 & 25.866 & 31.859 & 35.685 & 32.898 & 16.643 & $\begin{array}{l}16.054 \\
\end{array}$ & 21.401 & 15.1 & 11.131 & 31.853 & 32.76 & 29.687 & 31.819 & 26.792 & 34.261 \\
\hline 9/23/11 3:00 & & 30.55 & 29.976 & $\begin{array}{l}28.040 \\
28.846\end{array}$ & 30 & & 29.427 & $\begin{array}{l}30.495 \\
30.496\end{array}$ & 29.988 & 29.649 & 28.16 & 25.866 & 31.857 & 35.685 & 32.895 & 16.643 & $\begin{array}{l}10.044 \\
16.049\end{array}$ & 21.394 & 15.111 & $\begin{array}{l}11.111 \\
11.128\end{array}$ & $\begin{array}{l}31.851 \\
31.851\end{array}$ & 32.758 & 29.687 & 31.819 & 26.794 & 34.259 \\
\hline $9 / 23 / 11$ & & 30.547 & 29.976 & $\begin{array}{l}28.840 \\
28.846\end{array}$ & 30 & & 29.42 & 30.496 & 29.988 & 29.646 & 28.163 & 25.868 & 31.852 & 35.688 & 32.891 & 16.641 & $\begin{array}{l}10.047 \\
16.047\end{array}$ & 21.387 & $\begin{array}{l}15.109 \\
1509\end{array}$ & $\begin{array}{l}11.124 \\
11.124\end{array}$ & $\begin{array}{l}31.849 \\
31.849\end{array}$ & 32.753 & .687 & 16 & 26.794 & 34.259 \\
\hline $9 / 23 / 1$ & & 30.545 & 29.976 & 28. & 30 & & 29 & 30.494 & 29.988 & 16 & 2 & 25.866 & 3 & 35.6 & 32.891 & 16.643 & 16.045 & 21.38 & 15.111 & 11.124 & 31.844 & 53 & 32 & 12 & 26.794 & 34.259 \\
\hline $9 / 2$ & & 30 & 29.97 & 28.8 & 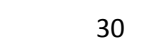 & & 29. & 30 & 29 & 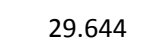 & 28 & 25.87 & 3 & 35 & 32.886 & 16 & 16.042 & 21 & 15.102 & 11.124 & 31 & 51 & 2 & 9 & 26.794 & .256 \\
\hline & & & & 28 & 29 & & & 36 & 29 & 29.644 & 28 & 25.875 & 31 & 35.688 & 84 & 16 & 1 & 99 & 15.104 & 11.121 & 31 & 32.751 & & 9 & 94 & 34.256 \\
\hline $9 / 23 /$ & & & & & & & & 30 & & & 28.165 & 25 & & & 32.884 & & 16.038 & 4 & 15.1 & 11.119 & 31.849 & 51 & 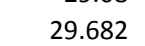 & 07 & 26.796 & 34.259 \\
\hline 9/23/11 6:00 & & 30.547 & & 28.856 & & & & & & & 28.165 & & & 35.688 & 32.881 & 16.641 & & 21.374 & 15.095 & 11.117 & & & & 7 & 26.796 & 34.259 \\
\hline $116: 30$ & & 30.55 & 29 & 28. & & & & 30. & & & 28.167 & 25. & & 35.6 & 32.881 & 16.641 & 16.033 & & 15.1 & 11.114 & 31.846 & 32.748 & & 07 & 26.796 & 34.259 \\
\hline $9 / 2$ & & 30 & & 28 & & & & & 29. & & 28 & & & & 32.881 & 16.641 & & & & 11.117 & 31.846 & & & 77 & 26.796 & 34.259 \\
\hline 117:30 & & 30.547 & & 28. & & & & & & & 28. & & & 35.6 & 32.881 & 16.643 & & 21. & & 11.119 & 31.856 & & & & & 34.259 \\
\hline 9/23/11 8:00 & & 30 & 29. & 28. & 30 & & & 30. & 29.995 & & 28.172 & 25. & 31. & 35.6 & 32.879 & 16.643 & 16.035 & 21.374 & 15.093 & 11.114 & 31.856 & 32.748 & 29.698 & 31.807 & 26.796 & 34.261 \\
\hline 9/23/11 8:30 & & 30. & 29. & $28.8 \mathrm{Y}-\mathrm{Y}$ & 30. & & & 30. & 29. & & 28.172 & $25.8->2$ & $31.8 \mathrm{x}$ & 35.7 & 32.881 & 16.645 & 16.035 & 21.38 & 15.093 & 11.117 & 31.858 & 32.748 & 29.703 & 31.807 & 26.796 & 34.263 \\
\hline 9/23/11 9:00 & & 30.54 & 29.9 & 28.879 & 30.0 & & 29. & 30.5 & 29.995 & 29.6 & 28.177 & 25.899 & 31.84 & 35.702 & 32.881 & 16.645 & 16.033 & 21.376 & 15.095 & 11.117 & 31.858 & 32.746 & 29.698 & 31.807 & 26.799 & 34.263 \\
\hline 9/23/11 9:30 & & 30.55 & 29.983 & 28.886 & 30.0 & & 29.403 & 30.501 & 29.995 & 29.646 & 28.179 & 25.906 & 31.845 & 35.7 & 32.884 & 16.645 & 16.031 & 21.371 & 15.093 & 11.117 & 31.858 & 32.748 & 29.696 & 31.809 & 26.796 & 34.263 \\
\hline 9/23/11 10:00 & & 30.55 & 29.983 & 28.884 & 30.017 & & 29.403 & 30.503 & 29.997 & 29.649 & 28.181 & 25.913 & 31.845 & 35.702 & 32.879 & 16.645 & 16.031 & 21.364 & 15.09 & 11.114 & 31.858 & 32.748 & 29.705 & 31.807 & 26.799 & 34.266 \\
\hline 9/23/11 10:30 & & 30.55 & 29.988 & 28.881 & 30.021 & & 29.41 & 30.501 & 29.995 & 29.649 & 28.179 & 25.909 & 31.848 & 35.707 & 32.879 & 16.645 & 16.031 & 21.36 & 15.088 & 11.117 & 31.865 & 32.748 & 29.71 & 31.807 & 26.799 & 34.266 \\
\hline 9/23/11 11:00 & & 30.552 & 29.988 & 28.881 & 30.026 & & 29.413 & 30.503 & 29.992 & 29.653 & 28.181 & 25.909 & 31.848 & 35.707 & 32.879 & 16.648 & 16.028 & 21.353 & 15.093 & 11.117 & 31.865 & 32.748 & 29.717 & 31.807 & 26.799 & 34.268 \\
\hline 9/23/11 11:30 & & 30.55 & 29.986 & 28.872 & 30.026 & & 29.422 & 30.503 & 29.995 & 29.651 & 28.179 & 25.909 & 31.927 & 35.709 & 32.968 & 16.65 & 16.026 & 21.346 & 15.095 & 11.204 & 31.872 & 32.829 & 29.71 & 31.884 & 26.799 & 34.266 \\
\hline 9/23/11 12:00 & & 30.552 & 29.988 & 28.867 & 30.028 & & 29.429 & 30.501 & 29.992 & 29.653 & 28.177 & 25.899 & 31.866 & 35.709 & 32.902 & 16.648 & 16.026 & 21.339 & 15.095 & 11.138 & 31.877 & 32.767 & 29.715 & 31.828 & 26.801 & 34.268 \\
\hline 9/23/11 12:30 & & 30.55 & 29.988 & 28.851 & 30.026 & & 29.438 & 30.501 & 29.99 & 29.651 & 28.17 & 25.894 & 31.857 & 35.711 & 32.893 & 16.648 & 16.024 & 21.332 & 15.1 & 11.128 & 31.867 & 32.76 & 29.713 & 31.816 & 26.801 & 34.266 \\
\hline
\end{tabular}




\begin{tabular}{|c|c|c|c|c|c|c|c|c|c|c|c|c|c|c|c|c|c|c|c|c|c|c|c|c|c|c|}
\hline Well & $\begin{array}{l}\text { (ft TOC) } \\
\text { (f) }\end{array}$ & $\begin{array}{l}\text { (ft TOC) } \\
\text { (f) }\end{array}$ & $\begin{array}{l}\text { (ft TOC) } \\
\text { (f) }\end{array}$ & $\begin{array}{l}\text { (ft TOC) } \\
\text { (f) }\end{array}$ & $\begin{array}{l}\text { (ft TOC) } \\
\text { (n) }\end{array}$ & $\begin{array}{l}\text { (ft TOC) } \\
\text { (f) }\end{array}$ & (ft TOC) & $\begin{array}{l}\text { (ft TOC) } \\
\text { (f) }\end{array}$ & $\begin{array}{l}\text { (ft TOC) } \\
\text { (f) }\end{array}$ & $\begin{array}{l}\text { (ft TOC) } \\
\text { (n) }\end{array}$ & $\begin{array}{l}\text { (ift TOC) } \\
\end{array}$ & $\begin{array}{l}\text { (ft TOC) } \\
\text { (f) }\end{array}$ & (ft TOC) & $\begin{array}{l}\text { (ft TOC) } \\
\text { (f) }\end{array}$ & (ft TOC) & $\begin{array}{l}\text { (ft TOC) } \\
\text { (n) }\end{array}$ & (ft TOC) & (ft TOC) & (ft TOC) & (ft TOC) & $\begin{array}{l}\text { (ft TOC) } \\
\end{array}$ & $\begin{array}{l}\text { (ft TOC) } \\
\end{array}$ & $\begin{array}{l}\text { (ft TOC) } \\
\end{array}$ & (ft TOC) & $\begin{array}{l}\text { (nt TOC) } \\
\text { (nt) }\end{array}$ & (ft TOC) \\
\hline 9/23/11 13:00 & & 30.545 & 29.983 & 28.837 & 30.024 & & 29.45 & 30.496 & 29.988 & 29.649 & 28.163 & 25.878 & 31.85 & 35.771 & 32.886 & 16.648 & 16.021 & 21.33 & 15.111 & 11.119 & 31.858 & 32.748 & 29,705 & 31.809 & 26.801 & 34.263 \\
\hline 9/23/11 13:30 & & 30.543 & 29.981 & 28.827 & 30.019 & & 29.455 & 30.493 & 29.985 & 29.646 & 28.158 & 25.863 & 31.841 & 35.707 & 32.879 & 16.648 & 16.019 & 21.32 & 15.114 & 11.114 & 31.853 & 32.744 & 29.698 & 31.805 & 26.801 & 34.261 \\
\hline 9/23/11 14:00 & & 30.538 & 29.976 & 28.823 & 30.012 & & 29.46 & 30.491 & 29.985 & 29.64 & 28.153 & 25.854 & 31.834 & 35.702 & 32.87 & 16.645 & 16.014 & $\begin{array}{r}41.316 \\
4\end{array}$ & 15.126 & $\begin{array}{l}11.105 \\
11.14\end{array}$ & 31.844 & 32.734 & 29.682 & 31.793 & 26.801 & 34.261 \\
\hline 4:30 & & 30.533 & 29.967 & 28.818 & 30 & & 29.46 & 30.491 & 29.985 & 29.635 & 28.151 & 25.851 & 31.827 & 35.7 & 32.863 & 16.643 & 16.01 & $1 \quad 21.309$ & 15.128 & 11.098 & 31.83 & 32.727 & 29.67 & 31.786 & 26.801 & 34.259 \\
\hline 11 15:00 & & 30.533 & 29.96 & 28.816 & 29.991 & & 29.462 & 30.489 & 29.983 & 29.628 & 28.149 & 25.846 & 31.822 & 35.695 & 32.855 & 16.643 & 16.005 & 21.304 & 15.13 & 11.093 & רכ) & & 29.663 & 31.781 & & 34.254 \\
\hline 9/23/11 15:30 & & 30.533 & 29.958 & 28.823 & 29.989 & & 29.457 & 30.486 & 29.985 & 29.626 & 28.146 & 25.844 & 31.815 & 35.693 & 32.855 & 16.641 & 16 & 21.295 & 15. & 11.091 & 1.818 & 2.718 & 9.661 & & 5.801 & 4.254 \\
\hline $9 / 23 / 1116: 00$ & & 30.533 & 29.953 & 28.825 & 29.982 & & 29.448 & 30.486 & 29.983 & 29.623 & 28.149 & 25.854 & 31.813 & 35.688 & 32.848 & 16.638 & 15.996 & $6 \quad 21.293$ & 15.135 & 11.084 & 31.814 & 32.713 & 29.654 & 31.774 & 6.801 & 34.252 \\
\hline 9/23/11 16:30 & & 30.533 & 29.951 & 28.827 & 29.977 & & 29.448 & 30.484 & 29.985 & 29.621 & 28.146 & 25.854 & 31.81 & 35.688 & 32.844 & 16.638 & 15.993 & $\begin{array}{ll}3 & 21.325\end{array}$ & 15.128 & 11.077 & 31.814 & 32.711 & 29.654 & 31.769 & 26.801 & 34.249 \\
\hline 9/23/11 17:00 & & 30.533 & 29.951 & 28.827 & 29.977 & & 29.443 & 30.484 & 29.985 & 29.621 & 28.146 & 25.856 & 31.808 & 35.688 & 32.841 & 16.638 & 15.991 & 21.35 & 15.128 & 11.079 & 31.811 & 32.708 & 29.654 & 31.767 & 26.801 & 34.249 \\
\hline 9/23/11 17:30 & & 30.533 & 29.951 & 28.832 & 29.975 & & 29.438 & 30.484 & 29.988 & 29.619 & 28.151 & 25.861 & 31.803 & 35.69 & 32.839 & 16.638 & 15.989 & 21.35 & 15.126 & 11.074 & 31.814 & 32.706 & 29.658 & 31.765 & 26.801 & 34.247 \\
\hline 9/23/11 18:00 & & 30.533 & 29.948 & 28.827 & 29.972 & & 29.431 & 30.484 & 29.985 & 29.619 & 28.151 & 25.861 & 31.801 & 35.683 & 32.837 & 16.636 & 15.989 & $9 \quad 21.341$ & 15.128 & 11.072 & 31.811 & 32.703 & 29.658 & 31.762 & 26.801 & 34.247 \\
\hline 9/23/11 18:30 & & 30.533 & 29.951 & 28.825 & 29.975 & & 29.429 & 30.482 & 29.988 & 29.619 & 28.149 & 25.861 & 31.801 & 35.69 & 32.834 & 16.638 & 15.989 & $\begin{array}{ll}9 & 21.334\end{array}$ & 15.123 & 11.07 & 31.811 & 32.701 & 29.654 & 31.762 & 26.803 & 34.247 \\
\hline 9/23/11 19:00 & & 30.533 & 29.951 & 28.823 & 29.975 & & 29.429 & 30.481 & 29.983 & 29.619 & 28.146 & 25.858 & 31.798 & 35.69 & 32.834 & 16.638 & 15.986 & $6 \quad 21.325$ & 15.118 & 11.07 & 31.814 & 32.699 & 29.663 & 31.762 & 26.803 & 34.247 \\
\hline 9/23/11 19:30 & & 30.531 & 29.951 & 28.82 & 29.972 & & 29.424 & 30.481 & 29.98 & 29.616 & 28.146 & 25.858 & 31.798 & 35.69 & 32.83 & 16.638 & 15.982 & 21.32 & 15.126 & 11.067 & 31.809 & 32.701 & 29.654 & 31.76 & 26.801 & 34.247 \\
\hline 9/23/11 20:00 & & 30.531 & 29.946 & 28.832 & 29.97 & & 29.417 & 30.481 & 29.985 & 29.614 & 28.149 & 25.858 & 31.796 & 35.688 & 32.832 & 16.638 & 15.982 & 21.313 & 15.126 & 11.067 & 31.807 & 32.696 & 29.654 & 31.758 & 26.803 & 34.247 \\
\hline $9 / 23 / 1120: 30$ & & 30.529 & 29.946 & 28.837 & 29.97 & & 29.41 & 30.481 & 29.985 & 29.614 & 28.149 & 25.87 & 31.794 & 35.688 & 32.83 & 16.638 & 15.979 & 21.306 & 15.126 & 11.063 & 31.807 & 32.696 & 29.651 & 31.753 & 26.803 & 34.247 \\
\hline 9/23/11 21:00 & & 30.529 & 29.944 & 28.835 & 29.97 & & 29.403 & 30.481 & 29.983 & 29.612 & 28.153 & 25.873 & 31.794 & 35.688 & 32.83 & 16.636 & 15.979 & 21.3 & 15.121 & 11.06 & 31.804 & 32.694 & 29.654 & 31.753 & 26.803 & 34.247 \\
\hline 9/23/11 21:30 & & 30.531 & 29.951 & 28.832 & 29.975 & & 29.403 & 30.481 & 29.983 & 29.614 & 28.149 & 25.87 & 31.794 & 35.695 & 32.827 & 16.636 & 15.979 & 21.29 & 15.114 & 11.065 & 31.809 & 32.696 & 29.661 & 31.753 & 26.803 & 34.247 \\
\hline 9/23/11 22:00 & & 30.533 & 29.951 & 28.832 & 29.977 & & 29.406 & 30.481 & 29.985 & 29.616 & 28.149 & 25.868 & 31.794 & 35.695 & 32.827 & 16.638 & 15.977 & 21.288 & 15.109 & 11.065 & 31.811 & 32.694 & 29.668 & $3 \quad 31.755$ & 26.806 & 34.247 \\
\hline 9/23/11 22:30 & & 30.531 & 29.951 & $\begin{array}{l}20.02 \\
28.83\end{array}$ & 29.975 & & 29.403 & $\begin{array}{l}30.401 \\
30.479\end{array}$ & $\begin{array}{l}29.985 \\
29.985\end{array}$ & 29.612 & 28.146 & $\begin{array}{l}25.000 \\
25.87\end{array}$ & $\begin{array}{l}31.791 \\
31.791\end{array}$ & $\begin{array}{l}530.093 \\
35.697\end{array}$ & $\begin{array}{l}32.827 \\
32.827\end{array}$ & $\begin{array}{l}10.030 \\
16.636\end{array}$ & 15.977 & $\begin{array}{l}21.280 \\
21.281\end{array}$ & $\begin{array}{l}15.111 \\
15.199\end{array}$ & 11.06 & $\begin{array}{l}31.814 \\
31.814\end{array}$ & $\begin{array}{l}32.044 \\
32.696\end{array}$ & 29.666 & 31.755 & $\begin{array}{l}20.000 \\
26.803\end{array}$ & $\begin{array}{l}34.249 \\
34.249\end{array}$ \\
\hline 9/23/11 23:00 & & 30.526 & 29.946 & $\begin{array}{l}28.83 \\
28.827\end{array}$ & 29.972 & & 29.406 & $\begin{array}{l}30.482 \\
30.48\end{array}$ & 29.983 & $\begin{array}{l}29.612 \\
29.609\end{array}$ & $\begin{array}{l}28.140 \\
28.146\end{array}$ & $\begin{array}{r}25.87 \\
25.866\end{array}$ & $\begin{array}{l}31.781 \\
31.789\end{array}$ & $\begin{array}{r}53.097 \\
35.69\end{array}$ & $\begin{array}{l}32.825 \\
32.825\end{array}$ & $\begin{array}{l}10.030 \\
16.638\end{array}$ & 15.972 & $\begin{array}{l}21.281 \\
21.274\end{array}$ & $\begin{array}{l}15.114 \\
15.114\end{array}$ & $\begin{array}{l}11.00 \\
11.06\end{array}$ & $\begin{array}{l}31.797 \\
31.797\end{array}$ & $\begin{array}{l}32.096 \\
32.692\end{array}$ & 29.661 & 31.751 & $\begin{array}{l}2.0035 \\
26.803\end{array}$ & $\begin{array}{l}34.247 \\
34.247\end{array}$ \\
\hline 9/23/11 23:30 & & 30.526 & 29.944 & 28.823 & 29.972 & & 29.408 & 30.477 & 29.98 & 29.609 & 28.144 & 25.866 & 31.789 & 35.695 & 32.82 & 16.638 & 15.972 & 21.269 & 15.111 & 11.058 & 31.811 & 32.692 & 29.663 & 31.751 & 26.806 & 34.247 \\
\hline $9 / 24 / 11$ & & 30.526 & 29.944 & 28.818 & & & 29 & 30.477 & & 29.607 & 28.142 & 25.861 & 31.787 & 35.697 & 32.82 & .638 & 15.968 & & 15.107 & 11.053 & .804 & 32.689 & & 48 & .803 & \\
\hline $9 / 24 / 110: 30$ & & 30.526 & 29.944 & & & & & 30.477 & & $\begin{array}{l}29.007 \\
29.607\end{array}$ & $\begin{array}{l}20.142 \\
28.139\end{array}$ & 25.858 & 31.782 & $\begin{array}{l}53.097 \\
35.693\end{array}$ & $\begin{array}{l}32.82 \\
32.818\end{array}$ & 638 & 15.968 & $\begin{array}{l}31.258 \\
3\end{array}$ & $\begin{array}{l}15.105 \\
15.105\end{array}$ & 1.056 & .804 & $\begin{array}{l}32.687 \\
32.687\end{array}$ & 29.654 & 746 & .803 & $\begin{array}{l}4.245 \\
4.245\end{array}$ \\
\hline 9/24/11 1:00 & & 30.524 & 29.941 & & & & & 30.474 & 29.98 & & 28.139 & 25.858 & 31.78 & 35.697 & 32.816 & 636 & 15.963 & 21.251 & 15.105 & 11.051 & .802 & 32.682 & 29.651 & 744 & 806 & 4.245 \\
\hline 9/24/11 1:30 & & 30.522 & 29.934 & & 29.965 & & 29.413 & 30.474 & 29.978 & 29.602 & 28.132 & 25.851 & 31.777 & 35.69 & 32.813 & 16.636 & 15.958 & 3. 21.244 & 15.102 & 11.046 & 1.793 & 32.68 & 29.649 & 31.739 & 806 & 4.245 \\
\hline 9/24/11 2:00 & & 30.522 & 29.937 & 28.804 & 29.965 & & 29.415 & 30.472 & 29.978 & 29.6 & 28.13 & 25.846 & 31.775 & 35.69 & 32.811 & 16.636 & 15.958 & $\begin{array}{l}31.237 \\
3\end{array}$ & $\begin{array}{l}15.102 \\
15.102\end{array}$ & 11.048 & 1.793 & 32.677 & 29.654 & 31.737 & 5.806 & 34.242 \\
\hline 9/24/11 2:30 & & 30.517 & 29.93 & 28.802 & 29.961 & & 29.408 & 30.469 & 29.976 & 29.598 & 28.13 & 25.846 & 31.773 & 35.693 & 32.806 & 16.634 & 15.954 & 21.232 & 15.102 & 11.041 & 31.786 & 32.673 & 29.64 & 31.732 & 26.806 & 34.24 \\
\hline 9/24/11 3:00 & & 30.517 & 29.925 & 28.802 & 29.956 & & 29.408 & 30.472 & 29.976 & 29.593 & 28.13 & 25.846 & 31.766 & 35.688 & 32.802 & 16.634 & 15.949 & 21.228 & 15.104 & 11.037 & 31.781 & 32.67 & 29.63 & 31.727 & 26.803 & 34.238 \\
\hline 9/24/11 3:30 & & 30.517 & 29.925 & 28.802 & 29.954 & & 29.403 & 30.467 & 29.983 & 29.593 & 28.128 & 25.846 & 31.763 & 35.681 & 32.799 & 16.631 & 15.947 & 21.221 & 15.098 & 11.034 & 31.779 & 32.668 & 29.637 & 31.727 & 26.806 & 34.238 \\
\hline 9/24/11 4:00 & & 30.517 & 29.923 & 28.802 & 29.951 & & 29.399 & 30.467 & 29.978 & 29.591 & 28.128 & 25.846 & 31.761 & 35.685 & 32.797 & 16.631 & 15.942 & 21.214 & 15.1 & 11.03 & 31.776 & 32.663 & 29.628 & 31.725 & 26.806 & 34.235 \\
\hline 9/24/11 4:30 & & 30.515 & 29.923 & 28.802 & 29.947 & & 29.401 & 30.467 & 29.978 & 29.591 & 28.123 & 25.849 & 31.756 & 35.688 & 32.795 & 16.631 & 15.94 & 21.209 & 15.098 & 11.03 & 31.779 & 32.663 & 29.63 & 31.72 & 26.806 & 34.235 \\
\hline 9/24/11 5:00 & & 30.515 & 29.923 & 28.811 & 29.947 & & 29.394 & 30.465 & 29.978 & 29.588 & 28.13 & 25.849 & 31.756 & 35.685 & 32.792 & 16.629 & 15.94 & 21.202 & 15.09 & 11.025 & 31.776 & 32.661 & 29.63 & 31.718 & 26.806 & 34.233 \\
\hline 9/24/11 5:30 & & 30.515 & 29.92 & 28.811 & 29.947 & & 29.382 & 30.467 & 29.983 & 29.586 & 28.132 & 25.856 & 31.754 & 35.688 & 32.79 & 16.629 & 15.935 & 21.205 & 15.093 & 11.03 & 31.772 & 32.658 & 29.63 & 31.715 & 26.806 & 34.233 \\
\hline $9 / 24 / 116: 00$ & & 30.515 & 29.92 & 28.811 & 29.947 & & 29.387 & 30.467 & 29.978 & 29.586 & 28.13 & 25.858 & 31.754 & $\begin{array}{l}35.69 \\
\end{array}$ & 32.79 & 16.631 & 15.935 & 21.223 & 15.086 & 11.018 & 31.774 & 32.658 & 29.635 & 31.715 & 26.808 & 34.233 \\
\hline $9 / 24 / 116: 30$ & & 30.517 & 29.925 & 28.806 & 29.951 & & 29.389 & 30.465 & 29.976 & 29.586 & 28.128 & 25.861 & 31.754 & 35.685 & 32.788 & 16.631 & 15.933 & 21.221 & $\begin{array}{l}15.081 \\
15.08\end{array}$ & 11.006 & 31.783 & 32.658 & 29.64 & 31.718 & 26.808 & 34.233 \\
\hline 9/24/117 & & 30.517 & 29.927 & 28.8 & 29. & & 29.391 & $\begin{array}{l}30.465 \\
30.465\end{array}$ & 29.973 & 29.586 & 28.128 & 25.856 & 31.754 & $\begin{array}{r}35.69 \\
35.6\end{array}$ & $\begin{array}{r}32.79 \\
32.00\end{array}$ & 16.631 & 15.932 & 21. & $\begin{array}{l}15.001 \\
15.081\end{array}$ & 11.001 & 31.783 & 32.656 & $\begin{array}{l}29.637 \\
\end{array}$ & 31.718 & 6.808 & 34.235 \\
\hline 9/24/11 7:30 & & 30.512 & $\begin{array}{l}29.92 \\
29.92\end{array}$ & 28. & 29.949 & & 29.389 & $\begin{array}{l}30.465 \\
30.465\end{array}$ & 29.976 & 29.586 & 28.125 & 25.8 & 31.752 & 35.69 & 32.788 & $\begin{array}{l}10.031 \\
16.631\end{array}$ & 15.935 & 21.212 & $\begin{array}{l}15.081 \\
15.083\end{array}$ & 11.004 & 31.779 & 32.6 & 29.637 & 31.718 & 5.808 & 34.233 \\
\hline 9/24/11 8:00 & & $\begin{array}{l}30.51 \\
30.51\end{array}$ & 29.918 & 28.788 & 29.947 & & 29.396 & $\begin{array}{l}30.400 \\
30.462\end{array}$ & 29.973 & 29.584 & 28. & 25.8 & $\begin{array}{l}31.749 \\
31.749\end{array}$ & $\begin{array}{l}35.693 \\
35.693\end{array}$ & 32.788 & $\begin{array}{l}10.051 \\
16.629\end{array}$ & 15.933 & 21.207 & $\begin{array}{l}13.008 \\
15.086\end{array}$ & 11.011 & 74 & $\begin{array}{l}32.056 \\
32.656\end{array}$ & 29.633 & $\begin{array}{l}31.715 \\
3\end{array}$ & 5.808 & 34.235 \\
\hline $9 / 24 / 11$ & & $\begin{array}{l}30.51 \\
30.51\end{array}$ & 29.918 & $\begin{array}{l}28.780 \\
28.781\end{array}$ & $\begin{array}{l}29.947 \\
29.947\end{array}$ & & 29.403 & $\begin{array}{r}30.402 \\
30.46\end{array}$ & 29.971 & $\begin{array}{l}29.084 \\
29.581\end{array}$ & 28. & 25.8 & $\begin{array}{l}11.149 \\
31.749\end{array}$ & $\begin{array}{r}53.093 \\
35.69\end{array}$ & 32.785 & $\begin{array}{l}10.029 \\
16.629\end{array}$ & $\begin{array}{r}13.953 \\
15.93\end{array}$ & $\begin{array}{r}21.201 \\
3\end{array}$ & $\begin{array}{l}13.080 \\
15.083\end{array}$ & 11 & 74 & $\begin{array}{l}32.050 \\
32.651\end{array}$ & $\begin{array}{l}29.635 \\
29.635\end{array}$ & 31.713 & .808 & $\begin{array}{l}34.235 \\
34.235\end{array}$ \\
\hline $\begin{array}{l}9 / 24 / 11 \\
9 / 241\end{array}$ & & $\begin{array}{l}30.51 \\
30.508\end{array}$ & 29.918 & $\begin{array}{l}28.781 \\
28.785\end{array}$ & 29 & & 29 & 30 & 29.976 & 25 & 28. & 25.8 & $\begin{array}{l}31 . \\
31 .\end{array}$ & $\begin{array}{l}35.69 \\
35.69\end{array}$ & $\begin{array}{l}32.185 \\
32.783\end{array}$ & 29 & $\begin{array}{l}13.93 \\
15.93 \\
\end{array}$ & 21. & 15 & $\begin{array}{l}0 \\
5\end{array}$ & 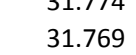 & $\begin{array}{l}32.051 \\
32.654\end{array}$ & $\begin{array}{l}29.628 \\
29.628\end{array}$ & 713 & $\begin{array}{l}20.808 \\
26.808\end{array}$ & $\begin{array}{l}34.235 \\
34.235\end{array}$ \\
\hline $9 /$ & & 30 & 29 & 28 & 2 & & 29 & 30 & 29 & 25 & 28 & 25 & 31 & $35.6 \varepsilon$ & 32 & 9 & 1 & 21 & 1 & 7 & 5 & 32. & 29 & 8 & 26.81 & 34.238 \\
\hline $9 / 241$ & & 30. & 29 & 28 & & & 29 & & & 29 & & 25 & 31 & 35 & 32 & 7 & 15 & 21 & & 11 & & 32 & 29 & 6 & 26.808 & 34.238 \\
\hline $9 / 24$ & & 30.505 & & 28. & & & 29. & 30 & & & & 25 & 31. & 35 & & & 15 & 21 & 15 & 11. & & 32 & 29 & & 26.81 & 34.238 \\
\hline 11:00 & & 30.503 & 29.902 & 28.7 & 29.93 & & 29.415 & 30. & 29. & 29.5 & & 25.8 & 31 & 35. & 32.776 & 7 & 15.926 & 21 & & 11.065 & 31 & 32.644 & 29.611 & 704 & 26.81 & 34.238 \\
\hline 11:30 & & & 29.899 & 28. & & & 29.422 & & & 29.565 & & 25.813 & 31.738 & & & 16. & 15.921 & 21. & 15.093 & 11.07 & 31.753 & 32.642 & 29.609 & 31.701 & 26.808 & 34.238 \\
\hline $9 / 2$ & & & & & & & & & & & & & 31. & & 32 & 16. & & 21. & & 11. & 31.751 & 32.637 & 25.6 & 31.697 & $\begin{array}{l}2.0000 \\
26.81\end{array}$ & 8 \\
\hline $9 / 2$ & & & & & & & & & & & & & & & 32. & 16.622 & & 21. & & & 31. & 32. & & & & \\
\hline 9/24/11 13:00 & & 30.489 & 29.8 & & & & & 30. & & & 28. & & 31. & 35. & 32.7 & 16.62 & 15.912 & 21. & 15.107 & 10.992 & 31.727 & 32.625 & 29.576 & 31.685 & 26.808 & 34.233 \\
\hline 9/24/11 13:30 & & 30.484 & 29.8 & 28. & 29. & & 29.446 & 30. & 29. & 29.8 & 28. & 25. & 31.7 & 35.6 & 32.755 & 16.62 & 15.909 & 21.161 & 15.111 & 10.99 & 31.718 & 32.62 & 29.567 & 31.68 & 26.808 & 34.233 \\
\hline 9/24/11 14:00 & & 30.482 & 29.857 & 28.6 & 29.8 & & 29.436 & 30.436 & 29.9 & 29.535 & $28 . C$ & 25.758 & 31.71 & 35.674 & 32.75 & 16.615 & 15.905 & 21.154 & 15.114 & 10.983 & 31.711 & 32.611 & 29.56 & 31.673 & 26.808 & 34.228 \\
\hline 9/24/11 14:30 & & 30.477 & 29.846 & 28.7 & 29.874 & & 29.436 & 30.434 & 29.957 & 29.526 & 28.051 & 25.76 & 31.705 & 35.667 & 32.743 & 16.613 & 15.898 & 21.152 & 15.118 & 10.978 & 31.697 & 32.606 & 29.546 & 31.666 & 26.808 & 34.226 \\
\hline 9/24/11 15:00 & & 30.477 & 29.841 & 28.701 & 29.863 & & 29.424 & 30.431 & 29.954 & 29.519 & 28.053 & 25.758 & 31.698 & 35.66 & 32.734 & 16.608 & 15.893 & 21.147 & 15.119 & 10.969 & 31.692 & 32.602 & 29.539 & 31.662 & 26.808 & 34.224 \\
\hline 9/24/11 15:30 & & 30.477 & 29.836 & 28.704 & 29.858 & & 29.42 & 30.429 & 29.957 & 29.516 & 28.053 & 25.763 & 31.693 & 35.66 & 32.727 & 16.608 & 15.888 & 21.142 & 15.119 & 10.966 & 31.688 & 32.597 & 29.532 & 31.657 & 26.808 & 34.221 \\
\hline 9/24/11 16:00 & & 30.48 & 29.832 & 28.711 & 29.851 & & 29.408 & 30.429 & 29.952 & 29.512 & 28.051 & 25.767 & 31.684 & 35.66 & 32.722 & 16.608 & 15.886 & 21.138 & 15.114 & 10.959 & 31.685 & 32.592 & 29.532 & 31.65 & 26.81 & 4.219 \\
\hline & & & & & & & & & & & & & & & & & & & & & & & & & & \\
\hline
\end{tabular}




\begin{tabular}{|c|c|c|c|c|c|c|c|c|c|c|c|c|c|c|c|c|c|c|c|c|c|c|c|c|c|c|}
\hline Well & $\begin{array}{l}\text { (ft TOC) } \\
\text { (f) }\end{array}$ & $\begin{array}{l}\text { (ft TOC) } \\
\text { (f }\end{array}$ & $\begin{array}{l}\text { (ft TOC) } \\
\text { (ft }\end{array}$ & $\begin{array}{l}\text { (ft TOC) } \\
\text { (f) }\end{array}$ & $\begin{array}{l}\text { (ft TOC) } \\
\text { (n) }\end{array}$ & $\begin{array}{l}\text { (ft TOC) } \\
\text { (f) }\end{array}$ & $\begin{array}{l}\text { (ft TOC) } \\
\text { (f) }\end{array}$ & $\begin{array}{l}\text { (ft TOC) } \\
\text { (f) }\end{array}$ & $\begin{array}{l}\text { (ft TOC) } \\
\text { (ft }\end{array}$ & $\begin{array}{l}\text { (ft TOC) } \\
\text { (f) }\end{array}$ & $\begin{array}{l}\text { (ft TOC) } \\
\text { (f) }\end{array}$ & $\begin{array}{l}\text { (ft TOC) } \\
\text { (f) }\end{array}$ & $\begin{array}{l}\text { (ft TOC) } \\
\end{array}$ & $\begin{array}{l}\text { (ft TOC) } \\
\text { (f) }\end{array}$ & $\begin{array}{l}\text { (ft TOC) } \\
\text { (f) }\end{array}$ & $\begin{array}{l}\text { (ft TOC) } \\
\text { (n) }\end{array}$ & $\begin{array}{l}\text { (ft TOC) } \\
\end{array}$ & (ft TOC) & $\begin{array}{l}\text { (ft TOC) } \\
\text { (1) }\end{array}$ & $\begin{array}{l}\text { (ft TOC) } \\
\text { (f) }\end{array}$ & $\begin{array}{l}\text { (ft TOC) } \\
\end{array}$ & $\begin{array}{l}\text { (ft TOC) } \\
\text { (f) }\end{array}$ & $\begin{array}{l}\text { (ft TOC) } \\
\text { (ft }\end{array}$ & $\begin{array}{l}\text { (ft TOC) } \\
\text { (f) }\end{array}$ & $\begin{array}{l}\text { (ft TOC) } \\
\text { (f) }\end{array}$ & $\begin{array}{l}\text { (ft TOC) } \\
\text { (1) }\end{array}$ \\
\hline 17:00 & & 30.48 & 29.832 & 28.725 & 29.84 & & 29.389 & 30.4 & 29.9 & 25 & 28. & 25.784 & 31.6 & 35. & 32 & 16.606 & 15.879 & 21.128 & 15 & 10 & & 5 & 29 & 31.643 & .81 & \\
\hline 9/24/11 17:30 & & 30.477 & 29.834 & 28.739 & 29.851 & & 29.37 & 30.427 & 29.952 & 29.507 & 28.058 & 25.789 & 31.679 & 35.65 & 32.715 & 16.604 & 15.876 & 21.124 & 15.112 & 10.95 & 31.685 & 2.583 & 9.539 & 31.643 & 6.81 & 1.214 \\
\hline 9/24/11 18:00 & & 30.477 & 29.834 & 28.753 & 29.851 & & 29.356 & 30.426 & 29.961 & 29.507 & 28.065 & 25.811 & 31.679 & 35.65 & 32.715 & 16.604 & 15.874 & 21.119 & 15.112 & 10.947 & 31.688 & 32.585 & 29.543 & 31.64 & .81 & .214 \\
\hline 9/24/11 18:30 & & 30.48 & 29.836 & 28.764 & 29.856 & & 29.344 & 30.429 & 29.957 & 29.51 & 28.072 & 25.825 & 31.677 & 35.657 & 32.712 & 16.604 & 15.874 & 21.142 & 15.102 & 10.947 & 31.697 & 32.58 & 29.553 & 31.638 & 26.813 & 34.214 \\
\hline 9/24/11 19:00 & & 30.484 & 29.841 & 28.79 & 29.865 & & 29.321 & 30.429 & 29.969 & 29.512 & 28.086 & 25.837 & 31.681 & 35.66 & 32.715 & 16.606 & 15.874 & 21.149 & 15.09 & 10.952 & 31.706 & 32.583 & 29.564 & 31.643 & 26.813 & 34.214 \\
\hline 9/24/11 19:30 & & 30.487 & 29.848 & 28.809 & 29.874 & & 29.3 & 30.434 & 29.961 & 29.516 & 28.095 & 25.868 & 31.684 & 35.664 & 32.717 & 16.608 & 15.872 & 21.161 & 15.088 & 10.954 & 31.711 & 32.585 & 29.574 & 31.647 & 26.813 & 34.214 \\
\hline 9/24/11 20:00 & & 30.489 & 29.853 & 28.82 & 29.884 & & 29.29 & 30.436 & 29.961 & 29.519 & 28.102 & 25.887 & 31.686 & 35.671 & 32.72 & 16.61 & 15.872 & 21.159 & 15.081 & 10.957 & 31.723 & 32.587 & 29.59 & 31.647 & 26.813 & 34.217 \\
\hline 9/24/11 20:30 & & 30.496 & 29.869 & 28.83 & 29.902 & & 29.286 & 30.441 & 29.966 & 29.528 & 28.109 & 25.899 & 31.696 & 35.671 & 32.727 & 16.615 & 15.876 & 21.154 & 15.069 & 10.959 & 31.741 & 32.592 & 29.611 & 31.654 & 26.815 & 34.219 \\
\hline 9/24/11 21:00 & & 30.498 & 29.878 & 28.832 & 29.919 & & 29.283 & 30.443 & 29.966 & 29.537 & 28.116 & 25.909 & 31.698 & 35.681 & 32.729 & 16.617 & 15.879 & 21.152 & 15.067 & 10.964 & 31.753 & 32.599 & 29.628 & 31.662 & 26.815 & 34.221 \\
\hline 9/24/11 21:30 & & 30.496 & 29.883 & 28.83 & 29.93 & & 29.288 & 30.446 & 29.961 & 29.542 & 28.116 & 25.911 & 31.703 & 35.685 & 32.736 & 16.62 & 15.876 & 21.147 & 15.06 & 10.971 & 31.753 & 32.609 & 29.644 & 31.666 & 26.815 & 34.224 \\
\hline 9/24/11 22:00 & & 30.496 & 29.89 & 28.827 & 29.942 & & 29.288 & 30.446 & 29.969 & 29.547 & 28.119 & 25.909 & 31.707 & 35.693 & 32.738 & 16.622 & 15.879 & 21.142 & 15.062 & 10.973 & 31.767 & 32.609 & 29.649 & 31.671 & 26.817 & 34.224 \\
\hline 9/24/11 22:30 & & 30.496 & 29.895 & 28.825 & 29.949 & & 29.297 & 30.446 & 29.964 & 29.551 & 28.119 & 25.906 & 31.712 & 35.695 & 32.743 & 16.622 & 15.881 & 21.14 & 15.06 & 10.978 & 31.772 & 32.613 & 29.654 & 31.671 & 26.815 & 34.226 \\
\hline 9/24/11 23:00 & & 30.494 & 29.897 & 28.818 & 29.954 & & 29.302 & 30.448 & 29.961 & 29.551 & 28.119 & 25.904 & 31.712 & 35.697 & 32.743 & 16.624 & 15.879 & 21.138 & 15.06 & 10.98 & 31.772 & 32.616 & 29.656 & 31.676 & 26.817 & 34.226 \\
\hline 9/24/11 23:30 & & 30.491 & 29.895 & 28.818 & 29.958 & & 29.304 & 30.448 & 29.961 & 29.554 & 28.119 & 25.899 & 31.712 & 35.702 & 32.745 & 16.624 & 15.879 & 21.133 & 15.065 & 10.983 & 31.767 & 32.616 & 29.654 & 31.676 & 26.817 & 34.226 \\
\hline 9/25/11 0:00 & & 30.491 & 29.895 & 28.809 & 29.958 & & 29.312 & 30.448 & 29.961 & 29.554 & 28.119 & 25.897 & 31.714 & 35.702 & 32.743 & 16.627 & 15.876 & 21.128 & 15.065 & 10.98 & 31.772 & 32.616 & 29.651 & 31.676 & 26.817 & 34.226 \\
\hline 9/25/11 0:30 & & 30.489 & 29.895 & 28.811 & 29.958 & & 29.314 & 30.448 & 29.961 & 29.554 & 28.119 & 25.889 & 31.712 & 35.702 & 32.745 & 16.627 & 15.876 & 21.122 & 15.069 & 10.98 & 31.767 & 32.613 & 29.654 & 31.673 & 26.815 & 34.226 \\
\hline 9/25/11 1:00 & & 30.491 & 29.892 & 28.816 & 29.958 & & 29.316 & 30.446 & 29.961 & 29.554 & 28.119 & 25.889 & 31.712 & 35.709 & 32.743 & 16.624 & 15.874 & 21.117 & 15.069 & 10.98 & 31.767 & 32.613 & 29.649 & 31.676 & 26.817 & 34.226 \\
\hline 9/25/11 1:30 & & 30.487 & 29.89 & 28.82 & 29.958 & & 29.312 & 30.45 & 29.961 & 29.554 & 28.123 & 25.894 & 31.707 & 35.704 & 32.741 & 16.627 & 15.874 & 21.115 & 15.067 & 10.978 & 31.765 & 32.609 & 29.64 & 31.673 & 26.817 & 34.224 \\
\hline 9/25/11 2:00 & & 30.487 & 29.89 & 28.82 & 29.956 & & 29.316 & 30.448 & 29.961 & 29.554 & 28.125 & 25.897 & 31.71 & 35.704 & 32.743 & 16.624 & 15.872 & 21.11 & 15.064 & 10.978 & 31.765 & 32.609 & 29.644 & 31.673 & 26.815 & 34.224 \\
\hline 9/25/11 2:30 & & 30.491 & 29.895 & 28.82 & 29.961 & & 29.316 & 30.448 & 29.964 & 29.556 & 28.125 & 25.897 & 31.707 & 35.704 & 32.741 & 16.627 & 15.867 & 21.105 & 15.057 & 10.973 & 31.767 & 32.611 & 29.647 & 31.671 & 26.817 & 34.221 \\
\hline 00 & & 30.487 & 29.895 & 28.818 & 29.96 & & 29.319 & 30.45 & 29.961 & 29.558 & 28.128 & 25.897 & 31.705 & 35.711 & 32.738 & 16.627 & 15.865 & 21.101 & 15.057 & 10.978 & 31.769 & 32.609 & 29.647 & 31.669 & 26.817 & 4.221 \\
\hline 9/25/11 3:30 & & 30.489 & 29.897 & 28.82 & 29.963 & & 29.321 & 30.448 & 29.961 & 29.558 & 28.128 & 25.897 & 31.707 & 35.707 & 32.736 & 16.629 & 15.862 & 21.094 & 15.06 & .976 & 1.767 & 2.609 & 29.649 & 31.669 & 6.817 & 4.221 \\
\hline 9/25/11 4:00 & & 30.487 & 29.895 & 28.825 & 29.963 & & 29.319 & 30.45 & 29.969 & 29.561 & 28.132 & 25.899 & 31.705 & 35.704 & 32.738 & 16.627 & 15.862 & 21.089 & 15.057 & 10.973 & 1.765 & 2.606 & 29.651 & 31.666 & 6.817 & 4.219 \\
\hline 9/25/11 4:30 & & 30.489 & 29.895 & 28.835 & 29.963 & & 29.314 & 30.45 & 29.969 & 29.561 & 28.135 & 25.904 & 31.705 & 35.707 & 32.734 & 16.629 & 15.86 & 21.085 & 15.06 & 10.976 & 31.765 & 2.606 & 29.647 & 31.664 & 26.817 & 4.217 \\
\hline 9/25/11 5:00 & & 30.489 & 29.897 & 28.846 & 29.965 & & 29.309 & 30.453 & 29.969 & 29.563 & 28.142 & 25.911 & 31.703 & 35.707 & 32.736 & 16.631 & 15.858 & 21.064 & 15.053 & 10.971 & 31.767 & 32.604 & 29.651 & 31.666 & 26.817 & 34.214 \\
\hline 9/25/11 5:30 & & 30.491 & 29.902 & 28.851 & 29.968 & & 29.304 & 30.455 & 29.969 & 29.565 & 28.144 & 25.925 & 31.705 & 35.709 & 32.736 & 16.631 & 15.858 & 21.078 & 15.051 & 10.969 & 31.774 & 2.606 & 29.654 & 31.664 & 26.82 & 4.214 \\
\hline 9/25/11 6:00 & & 30.491 & 29.904 & 28.858 & 29.972 & & 29.304 & 30.458 & 29.973 & 29.57 & 28.151 & 25.928 & 31.707 & 35.711 & 32.736 & 16.634 & 15.858 & 21.089 & 15.046 & 10.973 & 31.776 & 32.609 & 29.666 & 31.669 & 26.82 & 34.214 \\
\hline 9/25/11 6:30 & & 30.496 & 29.911 & 28.863 & 29.982 & & 29.304 & 30.458 & 29.969 & 29.575 & 28.156 & 25.935 & 31.712 & 35.711 & 32.741 & 16.634 & 15.858 & 21.085 & 15.043 & 10.976 & 31.786 & 32.613 & 29.673 & 31.673 & 26.82 & 34.217 \\
\hline 9/25/11 7:00 & & 30.496 & 29.918 & 28.867 & 29.986 & & 29.302 & 30.46 & 29.971 & 29.577 & 28.158 & 25.942 & 31.712 & 35.716 & 32.741 & 16.638 & 15.858 & 21.082 & 15.046 & 10.978 & 31.79 & 32.616 & 29.68 & 31.673 & 26.822 & 34.217 \\
\hline 9/25/11 7:30 & & 30.496 & 29.916 & 28.877 & 29.989 & & 29.302 & 30.462 & 29.973 & 29.579 & 28.165 & 25.94 & 31.714 & 35.714 & 32.745 & 16.638 & 15.858 & 21.078 & 15.048 & 10.983 & 31.793 & 32.613 & 29.682 & 31.676 & 26.822 & 34.217 \\
\hline 9/25/11 8:00 & & 30.496 & 29.923 & 28.877 & 29.996 & & 29.302 & 30.465 & 29.973 & 29.584 & 28.167 & 25.949 & 31.717 & 35.716 & 32.748 & 16.641 & 15.86 & 21.075 & 15.048 & 10.983 & 31.797 & 32.623 & 29.691 & 31.678 & 26.822 & 34.219 \\
\hline $9 / 25 / 118: 30$ & & 30.498 & 29.927 & 28.881 & 30 & & 29.304 & 30.467 & 29.976 & 29.588 & 28.17 & 25.949 & 31.719 & 35.716 & 32.752 & 16.641 & 15.86 & 21.078 & 15.046 & 10.987 & 31.797 & 32.623 & 29.691 & 31.685 & 26.822 & 34.221 \\
\hline 9/25/11 9:00 & & 30.498 & 29.932 & 28.886 & 30.007 & & 29.309 & 30.467 & 29.973 & 29.593 & 28.174 & 25.954 & 31.724 & 35.718 & 32.755 & 16.643 & 15.862 & 21.098 & 15.044 & 10.992 & 31.804 & 32.628 & 29.701 & 31.687 & 26.824 & 34.221 \\
\hline 9/25/11 9:30 & & 30.498 & 29.932 & 28.884 & 30.01 & & 29.319 & 30.469 & 29.973 & 29.595 & 28.174 & 25.952 & 31.726 & 35.721 & 32.762 & 16.645 & 15.865 & 21.117 & 15.048 & 10.992 & 31.809 & 32.63 & 29.703 & 31.69 & 26.824 & 34.226 \\
\hline 9/25/11 10:00 & & 30.501 & 29.937 & 28.879 & 30.017 & & 29.3 & 30.472 & 29.973 & 29.6 & 28.177 & 25.949 & 31.731 & 35.721 & 32.762 & 16.648 & 15.867 & 21.147 & 15.053 & 10.994 & 1.814 & 32.632 & 29.705 & 31.692 & 26.824 & 34.226 \\
\hline $9 / 25 / 1110: 30$ & & 30.496 & 29.939 & 28.87 & 30.017 & & 29 & 30.475 & 29.976 & 29.6 & 28.177 & 25.944 & 31.733 & 35.728 & 32.764 & 16.648 & 15.867 & 21.177 & $\begin{array}{l}15.062 \\
15.06\end{array}$ & 10.997 & 1.814 & 32.635 & 29.71 & $\begin{array}{l}31.694 \\
\end{array}$ & 26.824 & 4.228 \\
\hline 9/25/11 11:00 & & $\begin{array}{l}30.4708 \\
30.498\end{array}$ & 29.939 & 28.863 & 30.021 & & 29.347 & 30.472 & 29.973 & 29.602 & 28.172 & 25.935 & 31.735 & 35.723 & 32.766 & $\begin{array}{r}16.65 \\
16.65\end{array}$ & $\begin{array}{l}15.867 \\
15.867\end{array}$ & 21.198 & $\begin{array}{l}15.06 \\
15.06\end{array}$ & 11.001 & .816 & 32.639 & 29.71 & 31.697 & 26.824 & 4.231 \\
\hline 9/25/11 11:30 & & $\begin{array}{l}30.470 \\
30.496\end{array}$ & 29.939 & 28.856 & 30.021 & & 29.356 & 30.47 & 29.971 & 29.605 & 28.17 & 25.925 & 31.735 & 35.725 & 32.764 & 16.65 & $\begin{array}{l}15.869 \\
15.869\end{array}$ & 21.212 & $\begin{array}{l}15.067 \\
1506\end{array}$ & $\begin{array}{l}11.001 \\
11.001\end{array}$ & .809 & 2.637 & 29.705 & 31.697 & 6.824 & 4.231 \\
\hline 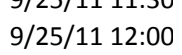 & & $\begin{array}{l}30.490 \\
30.494\end{array}$ & 29.932 & $\begin{array}{l}28.0500 \\
28.844\end{array}$ & 30.017 & & 29.363 & $\begin{array}{l}30.469 \\
30.469\end{array}$ & 29.973 & $\begin{array}{r}29.6 \\
29.6\end{array}$ & $\begin{array}{l}28.167 \\
28.167\end{array}$ & $\begin{array}{l}25.923 \\
25.918\end{array}$ & 31.733 & 35.721 & $\begin{array}{l}32.764 \\
32.764\end{array}$ & 16.65 & 5.865 & 21.219 & $\begin{array}{l}13.076 \\
15.076\end{array}$ & $\begin{array}{l}11.001 \\
11.001\end{array}$ & 02 & 35 & 29.694 & 31.694 & 6.824 & 4.231 \\
\hline & & 30.494 & 29 & 28. & 30 & & & 30 & & 29.602 & $2 \varepsilon$ & 25. & 31. & 35. & 32.7 & 16.648 & 62 & 21.209 & 15 & 11.027 & 77 & 53 & 29.691 & 31.72 & 827 & 4.231 \\
\hline & & 30 & & 28. & & & & & & 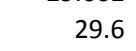 & 28 & 25 & 32 & 35 & 33 & & 05 & 21.198 & 15 & 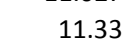 & 88 & 32 & 29 & 31.995 & .827 & 4.231 \\
\hline & & & & 28 & & & & & & 2 & 28 & 25 & 31 & & 32 & & 26 & 21. & 15.09 & 11 & 0 & & 29.687 & 31.877 & 827 & 4.228 \\
\hline $9 / 2$ & & 30.487 & 29 & & & & & 30 & & & & 25. & 31. & & & & $\sigma$ & 21. & 15.095 & 11.143 & & & 29.677 & 31.826 & 26.824 & 4.226 \\
\hline $9 / 2$ & & 30.482 & 29 & & & & 2 & & & & & 25.873 & 31.8 & & & 16 & 15. & 21. & 15.1 & & & 39 & 29.67 & 31.795 & 26.824 & 4.224 \\
\hline .15:00 & & 30.482 & & 28 & & & & & & & & 25.87 & 31. & & & 1 & 15. & 21 & 5.1 & & & 18 & 29.666 & 31.774 & & 4.221 \\
\hline $115: 30$ & & 30.484 & & & 29 & & & & & & & 25 & 31.801 & & & 16.643 & 15.902 & 21.193 & 15.105 & & & & 29.658 & 31.762 & 26.827 & 4.219 \\
\hline $116: 00$ & & 30.482 & 29 & 28. & 29. & & & & 29 & 29 & 28 & 25. & 31.787 & 35 & 32 & 16.643 & 15.895 & 21.202 & 15.1 & 11.058 & 31.802 & 32.689 & 29.656 & 31.748 & 26.827 & 34.217 \\
\hline 9/25/11 16:30 & & 30.48 & 29. & 28. & 29 & & 29. & 30. & 29 . $>>$ & 29 . & 28. & 25.868 & 31. & 35. & $32.8 \mathrm{~S}-2$ & 16.643 & 15.891 & 21.244 & 15.104 & 11.048 & 31.797 & 32.677 & 29.654 & 31.737 & 26.827 & 34.214 \\
\hline 9/25/11 17:00 & & 30.482 & 29.8 & 28.8 & 29.97 & & 29 & 30. & 29.5 & 29.577 & 28.144 & 25 & 31.7 & 35. & 32.802 & 16.641 & 15.8 & 21.283 & 15.107 & 11.041 & 31.793 & 32.668 & 29.649 & 31.732 & 26.827 & 34.214 \\
\hline 9/25/11 17:30 & & 30.48 & 29.897 & 28.823 & 29.968 & & 29.37 & 30.455 & 29.964 & 29.577 & 28.146 & 25.878 & 31.761 & 35.702 & 32.799 & 16.641 & 15.881 & 21.302 & 15.104 & 11.032 & 31.79 & 32.663 & 29.654 & 31.723 & 26.827 & 34.212 \\
\hline 9/25/11 18:00 & & 30.484 & 29.899 & 28.827 & 29.968 & & 29.366 & 30.455 & 29.966 & 29.579 & 28.146 & 25.887 & 31.756 & 35.704 & 32.79 & 16.641 & 15.879 & 21.309 & 15.102 & 11.03 & 31.793 & 32.658 & 29.651 & 31.72 & 26.827 & 34.212 \\
\hline $9 / 25 / 1118: 30$ & & 30.482 & 29.902 & 28.832 & 29.968 & & 29.356 & 30.455 & 29.966 & 29.579 & 28.151 & 25.889 & 31.754 & 35.704 & 32.79 & 16.643 & 15.876 & 21.325 & 15.1 & 11.023 & 31.795 & 32.654 & 29.658 & 31.713 & 26.827 & 34.212 \\
\hline 9/25/11 19:00 & & 30.484 & 29.902 & 28.839 & 29.972 & & 29.351 & 30.455 & 29.969 & 29.581 & 28.156 & 25.897 & 31.752 & 35.707 & 32.788 & 16.643 & 15.876 & 21.332 & 15.098 & 11.02 & 31.79 & 32.654 & 29.663 & 31.711 & 26.827 & 34.21 \\
\hline 9/25/11 19:30 & & 30.484 & 29.906 & 28.858 & 29.975 & & 29.337 & 30.455 & 29.971 & 29.581 & 28.16 & 25.901 & 31.747 & 35.702 & 32.785 & 16.645 & 15.874 & 21.357 & 15.097 & 11.018 & 31.8 & 32.651 & 29.666 & 31.711 & 26.827 & 34.212 \\
\hline 9/25/11 20:00 & & 30.484 & 29.909 & 28.863 & 29.979 & & 29.326 & 30.46 & 29.971 & 29.584 & 28.167 & 25.921 & 31.749 & 35.704 & 32.783 & 16.645 & 15.874 & 21.369 & 15.098 & 11.018 & 31.8 & 32.651 & 29.67 & 31.711 & 26.829 & 34.212 \\
\hline 9/25/11 20:30 & & 30.489 & 29.913 & 28.858 & 29.984 & & 29.326 & 30.463 & 29.971 & 29.586 & 28.167 & 25.925 & 31.752 & 35.714 & 32.783 & 16.645 & 15.874 & 21.378 & 15.09 & 11.023 & 31.804 & 32.649 & 29.682 & 31.713 & 26.829 & 34.212 \\
\hline
\end{tabular}




\begin{tabular}{|c|c|c|c|c|c|c|c|c|c|c|c|c|c|c|c|c|c|c|c|c|c|c|c|c|c|c|}
\hline Well & $\begin{array}{l}\text { (ft TOC) } \\
\text { (f) }\end{array}$ & $\begin{array}{l}\text { (ft TOC) } \\
\text { (f) }\end{array}$ & $\begin{array}{l}\text { (ft TOC) } \\
\text { (f) }\end{array}$ & $\begin{array}{l}\text { (ft TOC) } \\
\text { (ft }\end{array}$ & $\begin{array}{l}\text { (ft TOC) } \\
\text { (n) }\end{array}$ & $\begin{array}{l}\text { (ft TOC) } \\
\text { (ft }\end{array}$ & (ft TOC) & $\begin{array}{l}\text { (ft TOC) } \\
\text { (f) }\end{array}$ & $\begin{array}{l}\text { (ft TOC) } \\
\text { (f) }\end{array}$ & $\begin{array}{l}\text { (ft TOC) } \\
\text { (f) }\end{array}$ & $\begin{array}{l}\text { (ft TOC) } \\
\text { (f) }\end{array}$ & $\begin{array}{l}\text { (ft TOC) } \\
\text { (f) }\end{array}$ & (ft TOC) & $\begin{array}{l}\text { (nt TOC) } \\
\text { (ft }\end{array}$ & (ft TOC) & $\begin{array}{l}\text { (ft TOC) } \\
\end{array}$ & (ft TOC) & (ft TOC) & (ft TOC) & (ft TOC) & $\begin{array}{l}\text { (ft TOC) } \\
\end{array}$ & $\begin{array}{l}\text { (ft TOC) } \\
\end{array}$ & $\begin{array}{l}\text { (ft TOC) } \\
\end{array}$ & $\begin{array}{l}\text { (ft TOC) } \\
\text { (f) }\end{array}$ & $\begin{array}{l}\text { (ft TOC) } \\
\end{array}$ & (ft TOC) \\
\hline 9/25/11 21:00 & & 30.491 & 29.92 & 28.856 & 29.993 & & 29.333 & 30.46 & 29.971 & 29.593 & 28.167 & 25.923 & 31.752 & 35.718 & 32.785 & 16.65 & 15.874 & $\begin{array}{ll}4 & 21.385\end{array}$ & 15.083 & 11.02 & 31.818 & 32.654 & 29.696 & 31,713 & 26.829 & 34.217 \\
\hline 9/25/11 21:30 & & 30.491 & 29.923 & 28.858 & 29.998 & & 29.333 & 30.46 & 29.971 & 29.595 & 28.17 & 25.918 & 31.754 & 35.718 & 32.788 & 16.65 & 15.876 & $\begin{array}{ll}6 & 21.387\end{array}$ & 15.088 & 11.023 & 31.816 & 32.658 & 29.696 & 31.715 & 26.831 & 34.217 \\
\hline 9/25/11 22:00 & & 30.487 & 29.92 & 28.856 & 29.998 & & 29.335 & 30.465 & 29.969 & 29.595 & 28.167 & 25.918 & 31.756 & 35.718 & 32.79 & 16.65 & 15.874 & $\begin{array}{l}41.397 \\
4\end{array}$ & $\begin{array}{l}15.000 \\
15.095\end{array}$ & 11.02 & 31.811 & 32.656 & 29.689 & 31.715 & 26.829 & 34.217 \\
\hline $2: 30$ & & 30.489 & 29.92 & 28.851 & 30 & & 29.335 & 30.465 & 29.969 & 29.595 & 28.167 & 25.918 & 31.752 & 35.721 & 32.785 & 16.652 & 15.874 & $4 \quad 21.401$ & 15.09 & 11.023 & . & 32.656 & 29.691 & 31.715 & .829 & 34.217 \\
\hline 23:00 & & 30.489 & 29.92 & 28.851 & 30 & & 29.337 & 30.465 & 29.973 & 29.595 & 28.167 & 25.913 & 31.754 & 35.723 & 32.785 & 16.652 & 15.872 & 21.411 & 15.093 & 11.023 & 18 & & 29.691 & 31.713 & .831 & 34.219 \\
\hline 9/25/11 23:30 & & 30.487 & 29.92 & 28.849 & 30.003 & & 29.34 & 30.463 & 29.973 & 29.598 & 28.167 & 25.913 & 31.754 & 35.728 & 32.785 & 16.655 & 15.872 & 21.417 & 15.093 & 11.023 & 1.814 & 2.656 & 9.696 & 31.718 & 5.829 & 4.221 \\
\hline 9/26/11 0:00 & & 30.484 & 29.92 & 28.846 & 30.003 & & 29.34 & 30.463 & 29.971 & 29.598 & 28.167 & 25.911 & 31.752 & 35.721 & 32.785 & 16.655 & 15.872 & 21.422 & 15.095 & 11.023 & 31.811 & 32.656 & 29.691 & 31.711 & 6.831 & 34.219 \\
\hline 9/26/11 0:30 & & 30.487 & 29.918 & 28.844 & 30.003 & & 29.342 & 30.465 & 29.971 & 29.598 & 28.165 & 25.909 & 31.752 & 35.721 & 32.783 & $\begin{array}{l}16.652 \\
-652\end{array}$ & 15.872 & 21.431 & $\begin{array}{l}15.095 \\
\end{array}$ & 11.02 & 31.811 & 32.656 & 29.691 & 31.713 & 26.831 & 34.221 \\
\hline 9/26/11 1:00 & & 30.484 & 29.916 & 28.849 & 30.003 & & 29.342 & 30.463 & 29.971 & 29.595 & 28.165 & 25.906 & 31.752 & 35.723 & 32.78 & 16.652 & 15.869 & $\begin{array}{l}9 \\
9\end{array}$ & 15.093 & 11.02 & 31.809 & 32.654 & 29.687 & 31.713 & 26.831 & 34.219 \\
\hline 9/26/11 1:30 & & 30.484 & 29.916 & 28.851 & 30 & & 29.342 & 30.465 & 29.973 & 29.595 & 28.167 & 25.911 & 31.747 & 35.723 & 32.78 & 16.652 & 15.865 & $5 \quad 21.445$ & 15.093 & 11.018 & 31.807 & 32.651 & 29.687 & 31.708 & 26.831 & 34.219 \\
\hline 9/26/11 2:00 & & 30.484 & 29.916 & 28.851 & 30 & & 29.34 & 30.465 & 29.969 & 29.595 & 28.17 & 25.909 & 31.747 & 35.723 & 32.778 & 16.655 & 15.862 & $2 \quad 21.45$ & 15.09 & 11.015 & 31.804 & 32.649 & 29.684 & 31.708 & 26.831 & 34.217 \\
\hline $9 / 26 / 112: 30$ & & 30.487 & 29.92 & 28.849 & 30.003 & & 29.342 & 30.465 & 29.969 & 29.598 & 28.167 & 25.911 & 31.745 & 35.721 & 32.778 & 16.655 & 15.862 & 2121.457 & 15.083 & 11.015 & 31.809 & 32.647 & 29.689 & 31.708 & 26.831 & 34.217 \\
\hline 9/26/11 3:00 & & 30.484 & 29.918 & 28.863 & 30 & & 29.333 & 30.462 & 29.973 & 29.598 & 28.172 & 25.911 & 31.74 & 35.721 & 32.776 & 16.657 & 15.86 & $6 \quad 21.464$ & 15.086 & 11.011 & 31.804 & 32.644 & 29.684 & 31.704 & 26.831 & 34.214 \\
\hline 9/26/11 3:30 & & 30.484 & 29.918 & 28.867 & 30.003 & & 29.33 & 30.467 & 29.973 & 29.598 & 28.177 & 25.923 & 31.74 & 35.725 & 32.773 & 16.657 & 15.858 & $3 \quad 21.468$ & 15.083 & 11.011 & 31.804 & 32.644 & 29.689 & 31.704 & 26.831 & 34.214 \\
\hline 9/26/11 4:00 & & 30.487 & 29.918 & 28.874 & 30.003 & & 29.328 & 30.467 & 29.973 & 29.598 & 28.179 & 25.928 & 31.74 & 35.725 & 32.771 & 16.655 & 15.855 & 21.475 & 15.081 & 11.009 & 31.804 & 32.642 & 29.691 & 31.704 & 26.834 & 34.212 \\
\hline 9/26/11 4:30 & & 30.489 & 29.923 & 28.879 & 30.007 & & 29.326 & 30.467 & 29.976 & 29.602 & 28.181 & 25.935 & 31.742 & 35.725 & 32.773 & 16.659 & 15.855 & 21.485 & 15.076 & 11.011 & 31.811 & 32.644 & 29.701 & 31.704 & 26.834 & 34.21 \\
\hline 9/26/11 5:00 & & 30.489 & 29.927 & 28.886 & 30.01 & & 29.319 & 30.472 & 29.98 & 29.605 & 28.188 & 25.937 & 31.742 & 35.725 & 32.771 & 16.659 & 15.853 & 21.491 & 15.076 & 11.009 & 31.814 & 32.644 & 29.705 & 31.704 & 26.834 & 34.21 \\
\hline 9/26/11 5:30 & & 30.491 & 29.927 & 28.891 & 30.017 & & 29.321 & $\begin{array}{l}30.474 \\
\end{array}$ & 29.976 & 29.607 & 28.188 & 25.944 & 31.742 & 35.725 & 32.773 & 16.662 & 15.851 & 21.505 & $\begin{array}{l}15.074 \\
\end{array}$ & 11.011 & 31.818 & 32.647 & 29.708 & 31.706 & 26.834 & 34.21 \\
\hline 9/26/11 6:00 & & 30.491 & 29.93 & $\begin{array}{l}28.051 \\
28.909\end{array}$ & 30.019 & & 29.316 & 30.475 & 29.978 & 29.609 & $\begin{array}{l}28.195 \\
28.195\end{array}$ & 25.952 & 31.745 & 35.728 & 32.773 & $\begin{array}{l}10.062 \\
16.644\end{array}$ & 15.848 & 21.524 & $\begin{array}{l}15.074 \\
\end{array}$ & 11.013 & $\begin{array}{l}31.010 \\
31.821\end{array}$ & 32.647 & 29.715 & 31.706 & 26.836 & 34.207 \\
\hline 9/26/11 6:30 & & $\begin{array}{l}30.494 \\
30.494\end{array}$ & 29.937 & 28.919 & 30.026 & & $\begin{array}{l}29.307 \\
29.307\end{array}$ & $\begin{array}{l}30.479 \\
30.479\end{array}$ & 29.983 & 29.614 & 28.205 & 25.966 & 31.745 & $\begin{array}{l}5.170 \\
35.73\end{array}$ & 32.776 & $\begin{array}{l}10.004 \\
16.664\end{array}$ & $\begin{array}{l}13.040 \\
15.848\end{array}$ & $\begin{array}{l}21.524 \\
3\end{array}$ & $\begin{array}{l}13.074 \\
15.074\end{array}$ & 11.013 & $\begin{array}{l}31.021 \\
31.823\end{array}$ & $\begin{array}{l}32.041 \\
32.649\end{array}$ & 29.715 & 31.706 & $\begin{array}{l}20.000 \\
26.836\end{array}$ & 34.207 \\
\hline 9/26/11 7:00 & & $\begin{array}{l}30.494 \\
30.496\end{array}$ & 29.941 & $\begin{array}{l}28.928 \\
28.928\end{array}$ & $\begin{array}{l}50.020 \\
30.033\end{array}$ & & 29.302 & $\begin{array}{l}30.481 \\
30.41\end{array}$ & 29.983 & $\begin{array}{l}29.619 \\
29.619\end{array}$ & 28.212 & $\begin{array}{l}25.976 \\
25.906\end{array}$ & $\begin{array}{l}1.1 .73 \\
31.749\end{array}$ & $\begin{array}{l}35.735 \\
35.73\end{array}$ & 32.778 & $\begin{array}{l}10.04 \\
16.666\end{array}$ & $\begin{array}{l}13.848 \\
15.848\end{array}$ & $\begin{array}{l}21.533 \\
3\end{array}$ & $\begin{array}{l}13.069 \\
15.069\end{array}$ & $\begin{array}{l}11.016 \\
11.016\end{array}$ & $\begin{array}{l}31.823 \\
31.832\end{array}$ & $\begin{array}{l}32.651 \\
32.651\end{array}$ & 29.729 & $\begin{array}{l}31.708 \\
31.708\end{array}$ & $\begin{array}{l}20.830 \\
26.836\end{array}$ & $\begin{array}{r}34.201 \\
34.21\end{array}$ \\
\hline 9/26/11 7:30 & & 30.501 & 29.948 & 28.93 & 30.04 & & 29.307 & 30.486 & 29.978 & 29.623 & 28.214 & 25.985 & 31.752 & 35.732 & 32.783 & 16.671 & 15.851 & 21.572 & 15.069 & 11.02 & 31.842 & 32.654 & 29.738 & 31.715 & 26.838 & 34.21 \\
\hline $9 / 26 / 118: 00$ & & 30.501 & 29.958 & 28.94 & 30.05 & & & 30.489 & 29.983 & & 28.221 & 25.985 & 31.756 & 35.732 & 32.785 & 671 & 15.853 & 21.579 & 15.069 & 11.025 & .849 & 32.658 & 29.752 & 718 & 838 & 34.212 \\
\hline $9 / 26 / 1$ & & 30.503 & 29.962 & 28.947 & 30.059 & & & 30.491 & 29.985 & 29.635 & 28.225 & 92 & 31.761 & 35.737 & 792 & 673 & 15.855 & 21.589 & 15.069 & 11.025 & .856 & 32.666 & 29.757 & .725 & .838 & 34.212 \\
\hline 9/26/11 9:00 & & 30.505 & 29.967 & 28.942 & 30.064 & & & 30.491 & 29.985 & 29.64 & 28.23 & 26 & 31.763 & 35.737 & 795 & 676 & 15.855 & 21.598 & 15.072 & 11.032 & 31.86 & 32.668 & 29.762 & .727 & 838 & 34.214 \\
\hline 9/26/11 9:30 & & 30.505 & 29.969 & & 30.071 & & & 30.494 & 29.983 & 29.644 & 28.225 & 25.995 & 31.77 & 35.737 & 32.802 & 16.678 & 15.858 & 21.605 & $\begin{array}{l}15.074 \\
\end{array}$ & 11.034 & 31.87 & 32.673 & 29.769 & 31.732 & 838 & 4.217 \\
\hline 9/26/11 10:00 & & 30.508 & 29.976 & 28.919 & 30.08 & & 29.342 & 30.493 & 29.983 & 29.649 & 28.223 & 25.978 & 31.796 & 35.737 & 32.827 & 16.68 & 15.86 & 21.614 & 15.079 & 11.065 & 31.881 & 32.699 & 29.776 & $\begin{array}{l}51.758 \\
\end{array}$ & 5.841 & 34.221 \\
\hline 9/26/11 10:30 & & 30.505 & 29.976 & 28.909 & 30.085 & & 29.351 & 30.491 & 29.978 & 29.649 & 28.221 & 25.966 & 31.787 & 35.739 & 32.818 & 16.68 & 15.862 & 21.623 & 15.088 & 11.053 & 31.874 & 32.689 & 29.771 & 31.744 & 6.841 & 34.221 \\
\hline 9/26/11 11:00 & & 30.501 & 29.967 & 28.905 & 30.078 & & 29.363 & 30.493 & 29.985 & 29.646 & 28.219 & 25.954 & 31.782 & 35.742 & 32.816 & 16.68 & 15.862 & 21.63 & 15.1 & 11.048 & 31.867 & 32.687 & 29.762 & 31.746 & 841 & 34.221 \\
\hline 9/26/11 11:30 & & 30.501 & 29.967 & 28.898 & 30.075 & & 29.373 & 30.494 & 29.98 & 29.646 & 28.214 & 25.947 & 31.782 & 35.746 & 32.813 & 16.68 & 15.865 & 21.639 & 15.105 & 11.051 & 31.865 & 32.684 & 29.757 & 31.744 & 26.838 & 34.224 \\
\hline 9/26/11 12:00 & & 30.498 & 29.965 & 28.891 & 30.071 & & 29.38 & 30.494 & 29.98 & 29.644 & 28.212 & 25.942 & 31.78 & 35.732 & 32.813 & 16.68 & 15.865 & 21.649 & 15.112 & 11.051 & 31.856 & 32.684 & 29.748 & $\begin{array}{l}31.741 \\
\end{array}$ & 26.841 & 34.221 \\
\hline 9/26/11 12:30 & & 30.498 & 29.96 & 28.879 & 30.066 & & 29.389 & 30.491 & 29.98 & 29.642 & 28.207 & 25.933 & 31.777 & 35.744 & 32.809 & 16.68 & 15.862 & 21.66 & 15.112 & 11.044 & 31.853 & 32.68 & 29.745 & 31.739 & 26.841 & 34.224 \\
\hline 9/26/11 13:00 & & 30.498 & 29.958 & 28.87 & 30.064 & & 29.396 & 30.489 & 29.98 & 29.64 & 28.202 & 25.923 & 31.777 & 35.735 & 32.811 & 16.68 & 15.865 & 21.667 & 15.116 & 11.046 & 31.851 & 32.684 & 29.741 & 31.741 & 26.841 & 34.221 \\
\hline 9/26/11 13:30 & & 30.494 & 29.951 & 28.86 & 30.057 & & 29.408 & 30.489 & 29.976 & 29.637 & 28.198 & 25.911 & 31.777 & 35.735 & 32.809 & 16.68 & 15.862 & 21.674 & 15.121 & 11.041 & 31.839 & 32.677 & 29.729 & 31.739 & 26.841 & 34.221 \\
\hline 9/26/11 14:00 & & 30.494 & 29.946 & 28.856 & 30.047 & & 29.413 & 30.484 & 29.976 & 29.635 & 28.193 & 25.901 & 31.773 & 35.728 & 32.804 & 16.678 & 15.862 & 21.683 & 15.13 & 11.044 & 31.835 & 32.675 & 29.722 & 31.734 & 26.838 & 34.221 \\
\hline 9/26/11 14:30 & & 30.489 & 29.941 & 28.851 & 30.038 & & 29.415 & $\begin{array}{l}30.481 \\
\end{array}$ & 29.973 & 29.63 & 28.191 & 25.897 & 31.768 & 35.73 & 32.804 & 16.68 & 15.86 & 21.688 & 15.133 & 11.039 & 31.828 & 32.67 & 29.71 & 31.732 & 26.841 & 34.219 \\
\hline 11 15:00 & & 30.487 & 29.937 & 28. & 30 & & 29 & 30.481 & 29.973 & 29.628 & 28.184 & 25.892 & 31.766 & 35.723 & 32.797 & $\begin{array}{l}16.060 \\
16.678\end{array}$ & 15.858 & 21.693 & 15.14 & 11.032 & 31.821 & 32.67 & 29.705 & 31.727 & $\begin{array}{l}2.0 .841 \\
26.841\end{array}$ & 34.217 \\
\hline 9/26/11 15:30 & & $\begin{array}{l}30.487 \\
30.40\end{array}$ & 29.932 & 28. & 30 & & 29.415 & $\begin{array}{l}30.479 \\
30.479\end{array}$ & 29.973 & 29.626 & 28. & 25.8 & 31. & 35.725 & 32.795 & $\begin{array}{l}10.676 \\
16.676\end{array}$ & 15.853 & 21.7 & 15.14 & $\begin{array}{l}11.032 \\
\end{array}$ & 31. & 32.663 & 29.698 & 31.725 & $\begin{array}{l}2.0 .841 \\
26.841\end{array}$ & 34.214 \\
\hline 9/26/11 16:00 & & $\begin{array}{l}30.487 \\
30.487\end{array}$ & 29.93 & $\begin{array}{l}20.044 \\
28.842\end{array}$ & $\begin{array}{l}30.019 \\
30.019\end{array}$ & & 29.415 & 30.477 & 29.976 & 29.621 & 28. & 25.8 & 31. & 35. & 32.79 & $\begin{array}{l}10.076 \\
16.676\end{array}$ & 15.851 & 21.706 & 15.14 & $\begin{array}{l}11.025 \\
11.025\end{array}$ & 6 & $\begin{array}{l}32.061 \\
32.661\end{array}$ & 29.691 & 31.72 & $\begin{array}{l}20.041 \\
26.841\end{array}$ & 34.212 \\
\hline 9/26/11 16:30 & & $\begin{array}{l}30.481 \\
30.487\end{array}$ & 29.925 & $\begin{array}{l}28.842 \\
28.846\end{array}$ & 30.012 & & $\begin{array}{r}29.41 \\
29.41\end{array}$ & $\begin{array}{l}30.479 \\
30.479\end{array}$ & 29.976 & 29.621 & $\begin{array}{l}28.181 \\
28.181\end{array}$ & $\begin{array}{l}25.003 \\
25.885\end{array}$ & 31. & 35.721 & $\begin{array}{l}\begin{array}{l}22.179 \\
32.79\end{array} \\
\end{array}$ & $\begin{array}{l}10.078 \\
16.673\end{array}$ & $\begin{array}{l}13.831 \\
15.846\end{array}$ & 21.753 & $\begin{array}{l}15.144 \\
15.144\end{array}$ & $\begin{array}{r}11.023 \\
11.02\end{array}$ & 67 & $\begin{array}{l}32.001 \\
32.658\end{array}$ & $\begin{array}{l}29.689 \\
29.689\end{array}$ & $\begin{array}{l}31.72 \\
31.715\end{array}$ & .841 & $\begin{array}{r}34.212 \\
34.21\end{array}$ \\
\hline 9/ & & 30 & 29.923 & 28 & 30 & & 29 & 30 & 29 & 25 & 28 & 25 & 31 & 35 & 32.785 & 1 & 15.848 & 21 & 15 & 1 & 7 & 32. & 29.68 & 713 & 41 & 34.21 \\
\hline 9/ & & 30 & 29 & 28 & 30 & & 29 & 30 & 29 & 25 & 28 & 25 & 31 & 35 & 32. & 16.673 & 15 & 21 & 1 & 11 & 7 & 32. & 29.689 & 5 & 41 & 34.207 \\
\hline & & 30. & 29 & & & & & & & & 28 & 25 & & 35 & & 16.6 & 15 & 21 & 15 & & 7 & 32 & 29 & 88 & 3 & 34.205 \\
\hline 9/26/11 18:30 & & 30.489 & 29. & & & & 29 & & & 29 & & 25 & & & & & 15 & 21 & 15 & & 77 & 32 & 29 & 11 & 41 & 34.205 \\
\hline 9/26/11 19:00 & & 30.489 & 29.923 & 28.867 & & & & & 29. & 29.6 & & 25. & & & 32 & 16.6 & 15.841 & 21. & & 11. & 31.804 & 32.651 & 29.689 & 31.711 & 843 & 34.205 \\
\hline 19: & & & 29.925 & & & & & & & & & 25.913 & & & 32. & 16.673 & 15.844 & 21.8 & 15.137 & & 31.811 & 32.654 & 29.694 & & & 34.203 \\
\hline $9 / 2$ & & & & & & & & & & & & & & & & & 15 & & & & & 32. & & & & 34.205 \\
\hline $120: 30$ & & & & & & & & & & & & 25. & & & 32. & & 15. & 21 & 15 & & 31 & 32. & 29. & & & 34.205 \\
\hline 9/26/11 21:00 & & 30.494 & 29. & 28. & & & & 30. & & & 28. & 25 & & 35. & 32.7 & 16.6 & 15. & 21. & 15. & 11.022 & 31.823 & 32.658 & 29.71 & 31.715 & 26.843 & 34.207 \\
\hline 9/26/11 21:30 & & 30.496 & 29.9 & 28.898 & 30. & & 29. & 30.4 & 29.4 & 29.6 & 28. & 25. & 31.7 & 35.7 & 32. & 16.678 & 15.848 & 21.9 & 15.126 & 11.025 & 31.835 & 32.6 & 29.72 & 31.72 & 26.845 & 34.21 \\
\hline 9/26/11 22:00 & & 30.494 & 29.937 & 28.9 & 30.0 & & 29.34 & 30.482 & 29.9 & 29.6 & 28.2 & 25.9 & 31.76 & 35.7 & 32.792 & 16. & 15.848 & 21.926 & 15.126 & 11.029 & 31.83 & 32.666 & 29.722 & 31.72 & 26.845 & 34.212 \\
\hline $9 / 26 / 1122: 30$ & & 30.496 & 29.941 & 28.902 & 30.031 & & 29.337 & 30.486 & 29.983 & 29.626 & 28.207 & 25.947 & 31.766 & 35.732 & 32.795 & 16.683 & 15.848 & 21.931 & 15.128 & 11.029 & 31.837 & 32.663 & 29.722 & 31.727 & 26.845 & 34.21 \\
\hline 9/26/11 23:00 & & 30.496 & 29.946 & 28.905 & 30.038 & & 29.337 & 30.484 & 29.98 & 29.63 & 28.209 & 25.952 & 31.77 & 35.739 & 32.795 & 16.683 & 15.853 & 21.947 & 15.123 & 11.034 & 31.842 & 32.67 & 29.734 & 31.732 & 26.845 & 34.212 \\
\hline 9/26/11 23:30 & & 30.498 & 29.946 & 28.909 & 30.042 & & 29.337 & 30.489 & 29.983 & 29.63 & 28.212 & 25.952 & 31.773 & 35.737 & 32.802 & 16.687 & 15.853 & 21.949 & 15.121 & 11.036 & 31.844 & 32.675 & 29.736 & 31.734 & 26.845 & 34.214 \\
\hline 9/27/11 0:00 & & 30.496 & 29.951 & 28.909 & 30.045 & & 29.337 & 30.489 & 29.988 & 29.635 & 28.212 & 25.954 & 31.773 & 35.742 & 32.806 & 16.687 & 15.855 & 21.954 & 15.118 & 11.039 & 31.849 & 32.677 & 29.743 & 31.737 & 26.848 & 34.217 \\
\hline & & & & & & & & & & & & & & & & 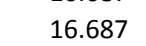 & & & & & & & & & & \\
\hline
\end{tabular}




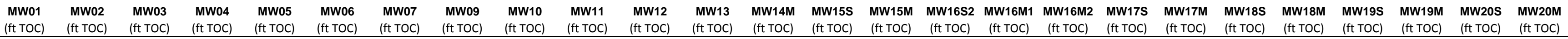

\begin{tabular}{|c|c|c|c|c|c|c|c|c|c|c|c|c|c|c|c|c|c|c|c|c|c|c|c|c|c|c|}
\hline Well & $\begin{array}{l}\text { (ft TOC) } \\
\text { (is }\end{array}$ & $\begin{array}{l}\text { (ft TOC) } \\
\text { (f) }\end{array}$ & $\begin{array}{l}\text { (ft TOC) } \\
\text { (f) }\end{array}$ & $\begin{array}{l}\text { (ft TOC) } \\
\text { (f }\end{array}$ & $\begin{array}{l}\text { (ft TOC) } \\
\text { (f) }\end{array}$ & $\begin{array}{l}\text { (ft TOC) } \\
\text { (f) }\end{array}$ & $\begin{array}{l}\text { (ft TOC) } \\
\text { (ft }\end{array}$ & $\begin{array}{l}\text { (ft TOC) } \\
\text { (f) }\end{array}$ & $\begin{array}{l}\text { (ft TOC) } \\
\text { (ft) }\end{array}$ & $\begin{array}{l}\text { (ft TOC) } \\
\text { (f) }\end{array}$ & $\begin{array}{l}\text { (ft TOC) } \\
\text { (f) }\end{array}$ & $\begin{array}{l}\text { (ft TOC) } \\
\text { (f) }\end{array}$ & (ft TOC) & $\begin{array}{l}\text { (ft TOC) } \\
\text { (f) }\end{array}$ & (ft TOC) & $\begin{array}{l}\text { (ft TOC) } \\
\text { (a) }\end{array}$ & $\begin{array}{l}\text { (ft TOC) } \\
\text { (f) }\end{array}$ & (ft TOC) & $\begin{array}{l}\text { (ft TOC) } \\
\text { (1) }\end{array}$ & (ft TOC) & $\begin{array}{l}\text { (ft TOC) } \\
\end{array}$ & $\begin{array}{l}\text { (ft TOC) } \\
\text { (f) }\end{array}$ & $\begin{array}{l}\text { (ft TOC) } \\
\end{array}$ & (ft TOC) & $\begin{array}{l}\text { (ft TOC) } \\
\text { (1) }\end{array}$ & (ft TOC) \\
\hline 1:00 & & 30.498 & 29.953 & 28.921 & 30.054 & & 29.33 & 30.49 & 29.98 & 29.6 & 28.216 & 25 & 31 & 35746 & 32.809 & 16.69 & 15.855 & 21.958 & 15.121 & 11.044 & 31.853 & 32.682 & 29.745 & 31.739 & 48 & 3.219 \\
\hline 9/27/11 1:30 & & 30.498 & 29.955 & 28.93 & 30.057 & & 29.33 & 30.496 & 29.988 & 29.64 & 28.223 & 25.966 & 31.78 & 35.744 & 32.811 & 16.692 & 15.855 & 21.961 & 5.123 & 1.044 & 31.851 & 32.682 & 29.745 & .741 & 26.848 & 34.219 \\
\hline 9/27/11 2:00 & & 30.501 & 29.955 & 28.928 & 30.061 & & 29.333 & 30.496 & 29.988 & 29.64 & 28.223 & 25.976 & 31.78 & 35.744 & 32.811 & 16.692 & 15.855 & 21.963 & 15.116 & 11.046 & 31.856 & 32.682 & 9.75 & .741 & 2685 & 3.219 \\
\hline 9/27/11 2:30 & & 30.505 & 29.962 & 28.933 & 30.066 & & 29.333 & 30.498 & 29.985 & 29.646 & 28.225 & 25.973 & 31.782 & 35.744 & 32.811 & 16.694 & 15.855 & 21.968 & 15.109 & 11.048 & 31.865 & 32.684 & 29.757 & 31.746 & 26.848 & 34.217 \\
\hline 9/27/11 3:00 & & 30.505 & 29.967 & 28.944 & 30.073 & & 29.33 & 30.498 & 29.988 & 29.649 & 28.23 & 25.978 & 31.784 & 35.749 & 32.813 & 3 16.694 & 15.855 & 21.972 & 15.111 & 11.051 & 31.87 & 32.687 & 29.764 & 31.746 & 26.85 & 34.217 \\
\hline 9/27/11 3:30 & & 30.505 & 29.967 & 28.947 & 30.073 & & 29.328 & 30.503 & 29.992 & 29.651 & 28.237 & 25.985 & 31.787 & 35.749 & 32.816 & $5 \quad 16.697$ & 15.855 & 21.975 & 15.112 & 11.053 & 31.87 & 32.687 & 29.767 & 31.746 & 26.85 & 34.217 \\
\hline 9/27/11 4:00 & & 30.508 & 29.969 & 28.944 & 30.078 & & 29.33 & 30.505 & 29.992 & 29.653 & 28.237 & 25.988 & 31.787 & 35.751 & 32.816 & $5 \quad 16.697$ & 15.853 & 21.977 & 15.109 & 11.051 & . 31.872 & 32.687 & 29.769 & 31.746 & 26.85 & 34.214 \\
\hline 9/27/11 4:30 & & 30.51 & 29.974 & 28.944 & 30.087 & & 29.335 & 30.505 & 29.99 & 29.658 & 28.239 & 25.983 & 31.789 & 35.751 & 32.816 & $5 \quad 16.699$ & 15.853 & 21.982 & 15.107 & 11.053 & 31.874 & 32.692 & 29.776 & 31.748 & 26.85 & 34.214 \\
\hline 9/27/11 5:00 & & 30.51 & 29.979 & 28.947 & 30.092 & & 29.34 & 30.508 & 29.992 & 29.663 & 28.239 & 25.985 & 31.789 & 35.749 & 32.818 & $3 \quad 16.704$ & 15.851 & 21.979 & 15.109 & 11.055 & 31.879 & 32.692 & 29.781 & 31.751 & 26.85 & 34.212 \\
\hline 9/27/11 5:30 & & 30.51 & 29.979 & 28.954 & 30.092 & & 29.337 & 30.508 & 29.992 & 29.663 & 28.242 & 25.985 & 31.787 & 35.756 & 32.816 & $\quad 16.701$ & 15.851 & 21.986 & 15.109 & 11.056 & 31.877 & 32.692 & 29.778 & 31.751 & 26.85 & 34.212 \\
\hline 9/27/11 6:00 & & 30.51 & 29.981 & 28.961 & 30.094 & & 29.337 & 30.51 & 29.992 & 29.665 & 28.246 & 25.992 & 31.789 & 35.758 & 32.818 & $3 \quad 16.704$ & 15.848 & 21.991 & 15.112 & 11.058 & 31.881 & 32.692 & 29.781 & 31.751 & 26.85 & 34.21 \\
\hline $9 / 27 / 116: 30$ & & 30.51 & 29.981 & 28.97 & 30.094 & & 29.33 & 30.513 & 29.997 & 29.667 & 28.253 & 26 & 31.789 & 35.758 & 32.82 & 16.704 & 15.851 & 21.993 & 15.109 & 11.053 & 31.881 & 32.692 & 29.781 & 31.751 & 26.852 & 34.21 \\
\hline 9/27/11 7:00 & & 30.517 & 29.986 & 28.979 & 30.101 & & 29.326 & 30.517 & 29.999 & 29.672 & 28.26 & 26.009 & 31.791 & 35.753 & 32.82 & 16.706 & 15.853 & 22.007 & 15.107 & 11.058 & 31.891 & 32.696 & 29.79 & 31.755 & 26.852 & 34.207 \\
\hline 9/27/11 7:30 & & 30.519 & 29.99 & 28.982 & 30.106 & & 29.33 & 30.518 & 29.997 & 29.677 & 28.263 & 26.016 & 31.794 & 35.756 & 32.825 & 16.708 & 15.851 & 22.018 & 15.105 & 11.058 & 31.895 & 32.701 & 29.797 & 31.758 & 26.852 & 34.21 \\
\hline 9/27/11 8:00 & & 30.522 & 29.997 & 28.991 & 30.113 & & 29.33 & 30.522 & 29.999 & 29.681 & 28.267 & 26.019 & 31.798 & 35.758 & 32.825 & $5 \quad 16.711$ & 15.853 & 22.03 & 15.102 & 11.056 & 31.905 & 32.703 & 29.811 & 31.76 & 26.855 & 34.21 \\
\hline 9/27/11 8:30 & & 30.522 & 30.002 & 28.998 & 30.12 & & 29.33 & 30.522 & 30.007 & 29.686 & 28.274 & 26.026 & 31.845 & 35.763 & 32.881 & 16.711 & 15.855 & 22.037 & 15.107 & 11.1 & 31.916 & 32.748 & 29.811 & 31.805 & 26.855 & 34.212 \\
\hline 9/27/11 9:00 & & 30.526 & 30.004 & 28.998 & 30.124 & & 29.333 & 30.529 & 30.002 & 29.691 & 28.281 & 26.033 & 31.972 & 35.763 & 33.017 & 16.715 & 15.86 & 22.039 & 15.107 & 11.237 & 31.958 & 32.872 & 29.828 & 31.934 & 26.855 & 34.212 \\
\hline 9/27/11 9:30 & & 30.529 & 30.014 & 28.996 & 30.136 & & 29.342 & 30.532 & 30.002 & 29.698 & 28.281 & 26.033 & 32.058 & 35.765 & 33.109 & 16.718 & 15.872 & 22.046 & 15.109 & 11.328 & 32.003 & 32.959 & 29.832 & 32.013 & 26.857 & 34.217 \\
\hline 9/27/11 10:00 & & 30.529 & 30.018 & 28.986 & 30.143 & & 29.354 & 30.532 & 29.999 & 29.702 & 28.279 & 26.031 & 32.14 & 35.768 & 33.205 & 16.72 & 15.886 & 22.051 & 15.112 & 11.417 & 32.035 & 33.04 & 29.844 & 32.098 & 26.855 & 34.217 \\
\hline 30 & & 30.529 & 30.021 & 28.977 & 30.15 & & 29.368 & 30.532 & 29.999 & 29.705 & 28.277 & 26.019 & 32.449 & 35.768 & 33.542 & 16.72 & 15.907 & 22.06 & 15.119 & 11.765 & 32.142 & 33.348 & 8.851 & 32.401 & 26.855 & 34.219 \\
\hline Do & & 30.529 & 30.021 & 28.968 & 30.155 & & 29.38 & 30.532 & 29.999 & 29.707 & 28.274 & 26.011 & 32.61 & 35.77 & 33.716 & 16.722 & 15.935 & 22.088 & 15.123 & 11.949 & 32.215 & 33.509 & .863 & 32.56 & 26.857 & 34.221 \\
\hline $9 / 27 / 1$ & & 30.524 & 30.018 & 28.956 & 30.162 & & 29.394 & 30.529 & 29.999 & 29.707 & 28.27 & 26.002 & 32.592 & 35.772 & 33.683 & 16.725 & 15.965 & 22.143 & 133 & 11.932 & 32.247 & 33.49 & .872 & .541 & 26.857 & 34.221 \\
\hline $9 / 27 / 11$ & & 30.524 & 30.016 & 28.944 & 30.164 & & 29.406 & 30.529 & 29.999 & 29.705 & 28.267 & 25.99 & 32.358 & 35.768 & 33.423 & 3 $\quad 16.722$ & 15.979 & 22.18 & 15.14 & 11.688 & 32.17 & 33.258 & 29.87 & 2.311 & 6.857 & 34.224 \\
\hline 9/27/11 12:30 & & 30.522 & 30.011 & 28.93 & 30.166 & & 29.42 & 30.527 & 30.002 & 29.705 & 28.26 & 25.978 & 32.252 & 35.77 & 33.308 & $3 \quad 16.722$ & 15.981 & 22.206 & 15.147 & 11.582 & 32.124 & 33.149 & 29.868 & 32.208 & 26.857 & 34.224 \\
\hline 9/27/11 13:00 & & 30.519 & 30.007 & 28.921 & 30.164 & & 29.427 & 30.525 & 29.997 & 29.702 & 28.258 & 25.966 & 32.185 & 35.763 & 33.231 & 16.722 & 15.984 & 22.208 & 15.154 & 11.523 & 32.089 & 33.08 & 29.856 & 32.14 & 26.857 & 34.221 \\
\hline 9/27/11 13:30 & & 30.519 & 30.004 & 28.905 & 30.162 & & 29.441 & 30.524 & 29.995 & 29.7 & 28.246 & 25.952 & 32.133 & 35.763 & 33.177 & 16.722 & 15.979 & 22.206 & 15.159 & 11.415 & 32.061 & 33.033 & 29.847 & 32.091 & 26.857 & 34.224 \\
\hline 9/27/11 14:00 & & 30.519 & 29.997 & 28.9 & 30.155 & & 29.446 & 30.522 & 29.995 & 29.695 & 28.244 & 25.937 & 32.096 & 35.761 & 33.139 & $\quad 16.722$ & 15.979 & 22.208 & 15.168 & 11.375 & 32.038 & 32.995 & 29.835 & 32.053 & 26.857 & 34.221 \\
\hline 9/27/11 14:30 & & 30.517 & 29.993 & 28.9 & 30.145 & & 29.448 & 30.517 & 29.995 & 29.691 & 28.242 & 25.933 & 32.058 & 35.753 & 33.104 & 16.72 & 15.975 & 22.204 & 15.172 & 11.338 & 32.019 & 32.959 & 29.821 & 32.018 & 26.857 & 34.219 \\
\hline 9/27/11 15:00 & & 30.512 & 29.983 & 28.9 & 30.136 & & 29.446 & 30.518 & 29.995 & 29.686 & 28.242 & 25.933 & 32.032 & 35.753 & 33.074 & $\quad 16.718$ & 15.967 & 22.206 & 15.18 & 11.312 & 31.998 & 32.933 & 29.807 & 31.992 & 26.857 & 34.219 \\
\hline 9/27/11 15:30 & & 30.515 & 29.981 & 28.895 & 30.129 & & 29.446 & 30.515 & 29.997 & 29.684 & 28.237 & 25.933 & 32.014 & 35.751 & 33.05 & 16.715 & 15.961 & 22.241 & 15.177 & 11.288 & 31.989 & 32.912 & 29.802 & 31.971 & 26.857 & 34.217 \\
\hline 9/27/11 16:00 & & 30.517 & 29.981 & 28.893 & 30.124 & & 29.443 & 30.515 & 29.99 & 29.684 & 28.235 & 25.93 & 31.993 & 35.751 & 33.034 & 16.715 & 15.958 & 22.264 & 15.177 & 11.269 & 31.979 & 32.893 & 29.8 & 31.952 & 26.857 & 34.214 \\
\hline 9/27/11 16:30 & & 30.515 & 29.979 & 28.898 & 30.117 & & 29.441 & 30.513 & 29.995 & 29.681 & 28.235 & 25.928 & 31.974 & 35.749 & 33.015 & 16.715 & 15.954 & 22.264 & 15.182 & 11.253 & 31.968 & 32.876 & 29.797 & 31.938 & 26.859 & 34.212 \\
\hline 9/27/11 17:00 & & 30.515 & 29.976 & 28.902 & 30.113 & & 29.436 & 30.513 & 29.997 & 29.679 & 28.237 & 25.93 & 31.96 & 35.744 & 33.001 & 16.715 & 15.951 & 22.264 & 15.184 & 11.234 & 31.961 & 32.862 & 29.788 & 31.922 & 26.859 & 34.212 \\
\hline 9/27/11 17:30 & & 30.517 & 29.974 & 28.902 & 30.108 & & 29.429 & 30.513 & 29.995 & 29.677 & 28.237 & 25.937 & 32.192 & 35.746 & 33.057 & 16.715 & 15.946 & 22.271 & 15.184 & 11.288 & 31.982 & 33.116 & 29.785 & 32.15 & 26.857 & 34.21 \\
\hline 9/27/11 18:00 & & 30 & 29.976 & 28.907 & & & 29.424 & 30 & 29.997 & 29.677 & 28.237 & 25.937 & 31.967 & 35.746 & 33.008 & $3 \quad 16.715$ & 15.946 & 22.282 & 15.184 & 11.241 & 31.963 & 32.867 & 29.785 & 31.927 & 26.862 & 34.21 \\
\hline $9 / 27 / 11$ & & 30 & 29. & 28.905 & & & 29.42 & 30. & 29.997 & 29.677 & 28.235 & 25.94 & & 35.749 & 32.987 & 16.715 & $\begin{array}{l}15.944 \\
\end{array}$ & 22.291 & 15.182 & 11.222 & 31.951 & 32.848 & 29.785 & 31.908 & 26.859 & 34.207 \\
\hline $\begin{array}{l}9 \\
9 / 27 / 11\end{array}$ & & 30 & 29.976 & 28.907 & 30. & & 29.415 & 30.513 & 29.997 & 29.677 & 28.235 & 25.94 & 31.937 & 35.739 & 32.973 & 16.715 & $\begin{array}{l}15.94 \\
1544\end{array}$ & 22.305 & 15.18 & $\begin{array}{l}11.222 \\
11.208\end{array}$ & 31.949 & $\begin{array}{l}32.836 \\
32.046\end{array}$ & 29.79 & 31.899 & 26.859 & 34.207 \\
\hline $9 / 27 / 1119: 30$ & & 30 & 29.979 & 28.907 & 30. & & 29.413 & 30.513 & 29.997 & 29.677 & 28.235 & 25.94 & 31.948 & 35.749 & 32.984 & 16.718 & $\begin{array}{l}15.944 \\
15.942\end{array}$ & 22.317 & $\begin{array}{l}15.117 \\
157\end{array}$ & 11.22 & 31.947 & .853 & 29.785 & 08 & 26.859 & 34.207 \\
\hline $20: 00$ & & 30 & 29.976 & 28 & 30 & & 2 & 30.5 & 29 & 29. & 28 & 25.942 & 31 & 35.7 & 32.966 & 16 & 15.944 & 22.324 & 1 & 11 & 31.947 & 834 & 38 & 91 & 26.859 & 34.205 \\
\hline & & 30 & 29. & 28. & & & & 3 & & 29. & 28 & 25.942 & & 35.7 & 32.959 & 16 & 47 & 22 & 15. & 11 & 31.942 & 24 & 38 & 32 & 59 & 34.207 \\
\hline & & & & & & & & 30. & & & 28. & & 31 & 357 & 32.9 & 16 & 15.954 & 22 & 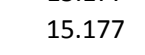 & 11.187 & 31.942 & 32.82 & & 77 & 52 & 34.207 \\
\hline & & & & & & & & & & & 28.2 & & & 35.7 & 32. & 16.7 & 15.953 & & 15 & 11.18 & 31 & 32. & & 31.873 & 99 & 34.21 \\
\hline $9 / 2$ & & & 29.97 & 28. & & & & & & & 28.235 & & & 35.7 & & 16.718 & 15.963 & & 2 & 11.178 & & & & & & 34.21 \\
\hline 22:30 & & 30 & 29.9 & 28. & & & & 30 & & & 28 & & & 35.756 & 32.94 & 16.72 & 15. & & 15.173 & 11.175 & 31.935 & & & & 52 & 34.21 \\
\hline $9 / 2$ & & & & 28 & & & & & & & 28 & & & 35.753 & 32.935 & 16.718 & & & 15.173 & 11.17 & 31.935 & & & 33 & 02 & 34.212 \\
\hline $9 / 27 /$ & & & & 28. & & & & 30 & & & 28. & & & 35.756 & 32.931 & & 15. & 22. & 15.173 & 11.166 & 31.93 & 32.803 & & 31.861 & 26.862 & 34.214 \\
\hline 9/28/11 0:00 & & 30. & 29. & 28. & 30. & & & 30. & 30 & & 28. & 25. & 31.899 & 35.756 & 32.933 & 3 16.722 & 15.989 & 22.345 & 15.166 & 11.166 & 31.937 & 32.801 & 29.792 & 31.859 & 26.862 & 34.217 \\
\hline 9/28/11 0:30 & & 30. & 29.5 & 28. & 30. & & & 30. & 30 & & 28.2 & 25. & 31.8 & 35.7 & 32. & 16.725 & 15. & 22.347 & 15.1 & 11.166 & 31.928 & 32.798 & 29.792 & 31.859 & 26.862 & 34.217 \\
\hline 9/28/11 1:00 & & 30.5 & 29.9 & 28.914 & 30. & & 29. & 30.5 & 30. & 29. & 28.237 & 25.954 & 31.894 & 35.7 & 32.926 & 16.725 & 15.991 & 22.354 & 15.166 & 11.164 & 31.928 & 32.796 & 29.79 & 31.856 & 26.864 & 34.217 \\
\hline $9 / 28 / 111: 30$ & & 30.524 & 29.983 & 28.909 & 30.1 & & 29.394 & 30.515 & 30.002 & 29.677 & 28.237 & 25.954 & 31.892 & 35.753 & 32.926 & 16.725 & 15.991 & 22.356 & 15.161 & 11.161 & 31.933 & 32.796 & 29.79 & 31.852 & 26.864 & 34.217 \\
\hline 9/28/11 2:00 & & 30.522 & 29.983 & 28.907 & 30.101 & & 29.396 & 30.515 & 29.999 & 29.677 & 28.235 & 25.949 & 31.89 & 35.761 & 32.919 & 16.727 & 15.991 & 22.358 & 15.163 & 11.159 & 31.93 & 32.791 & 29.788 & 31.849 & 26.864 & 34.219 \\
\hline 9/28/11 2:30 & & 30.522 & 29.979 & 28.902 & 30.101 & & 29.399 & 30.515 & 29.999 & 29.677 & 28.232 & 25.947 & 31.885 & 35.758 & 32.919 & 16.732 & 15.986 & 22.363 & 15.161 & 11.156 & 31.926 & 32.789 & 29.795 & 31.847 & 26.864 & 34.217 \\
\hline 9/28/11 3:00 & & 30.522 & 29.976 & 28.9 & 30.101 & & 29.403 & 30.513 & 29.999 & 29.674 & 28.23 & 25.94 & 31.883 & 35.753 & 32.916 & $\begin{array}{l}16.729 \\
5\end{array}$ & 15.984 & 22.365 & 15.163 & 11.152 & 31.923 & 32.786 & 29.788 & 31.845 & 26.862 & 34.217 \\
\hline 9/28/11 3:30 & & 30.519 & 29.976 & 28.895 & 30.096 & & 29.399 & 30.513 & 30.004 & 29.674 & 28.23 & 25.94 & 31.878 & 35.761 & 32.909 & 16.732 & 15.981 & 22.365 & 15.163 & 11.147 & 31.919 & 32.779 & 29.783 & 31.84 & 26.864 & 34.217 \\
\hline 9/28/11 4:00 & & 30.519 & 29.974 & 28.891 & 30.094 & & 29.401 & 30.515 & 30.002 & 29.672 & 28.228 & 25.937 & 31.876 & 35.753 & 32.905 & 16.734 & 15.979 & 22.365 & 15.163 & 11.142 & 31.919 & 32.779 & 29.778 & 31.835 & 26.864 & 34.214 \\
\hline 9/28/11 4:30 & & 30.522 & 29.974 & 28.886 & 30.094 & & 29.406 & 30.508 & 30.002 & 29.67 & 28.223 & 25.933 & 31.871 & 35.756 & 32.907 & 16.734 & 15.972 & 22.365 & 15.159 & 11.142 & 31.914 & 32.775 & 29.776 & 31.833 & 26.864 & 3.212 \\
\hline
\end{tabular}




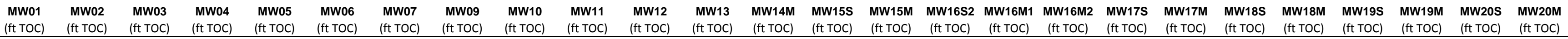

\begin{tabular}{|c|c|c|c|c|c|c|c|c|c|c|c|c|c|c|c|c|c|c|c|c|c|c|c|c|c|}
\hline well & $\begin{array}{l}\text { MW01 } \\
\text { (ft TOC) }\end{array}$ & $\begin{array}{l}\text { MW02 } \\
\text { (ft TOC) }\end{array}$ & $\begin{array}{l}\text { MW03 } \\
\text { (ft Toc) }\end{array}$ & $\begin{array}{l}\text { MWO04 } \\
\text { (ft TOC) }\end{array}$ & $\begin{array}{cc}\text { MW05 } & \text { MW06 } \\
\text { (ft TOC) } & \text { (ft TOC) } \\
\end{array}$ & $\begin{array}{l}\text { MWW7 } \\
\text { (ft TOC) }\end{array}$ & $\begin{array}{l}\text { MW09 } \\
\text { (ft ToC) }\end{array}$ & $\begin{array}{l}\text { MW10 } \\
\text { (ft TOC) }\end{array}$ & $\begin{array}{l}\text { MW11 } \\
\text { (ft TOC) }\end{array}$ & $\begin{array}{l}\text { MW12 } \\
\text { (ft TOC) }\end{array}$ & $\begin{array}{l}M W 13 \\
(\mathrm{ft} \text { Toc) } \\
\end{array}$ & $\begin{array}{l}\text { MW14M } \\
\text { (ft TOC) }\end{array}$ & $\begin{array}{l}\text { MW15S } \\
\text { (ft ToC) }\end{array}$ & $\begin{array}{l}\text { MW W } 15 \mathrm{M} \\
\text { (ft TOC) }\end{array}$ & $\begin{array}{l}\text { MW16S2 } \\
\text { (ft TOC) }\end{array}$ & $\begin{array}{c}\text { MW16M1 } \\
\text { (ft TOC) }\end{array}$ & $\begin{array}{l}\text { MW16M2 } \\
\text { (ft TOC) }\end{array}$ & $\begin{array}{l}\text { MWW7S } \\
\text { (ft TOC) }\end{array}$ & $\begin{array}{l}\text { MW17M } \\
\text { (ft Toc) }\end{array}$ & $\begin{array}{l}\text { MWW } 18 \mathrm{~S} \\
\text { (ft TOC) }\end{array}$ & $\begin{array}{l}\text { MW18M } \\
\text { (ft TOC) }\end{array}$ & $\begin{array}{l}\text { MW19S } \\
\text { (ft TOC) }\end{array}$ & $\begin{array}{l}\text { MWW19M } \\
\text { (ft TOC) }\end{array}$ & $\begin{array}{l}\begin{array}{l}\text { MW20S } \\
\text { (ft Toc) }\end{array} \\
\text { (t) }\end{array}$ & $\begin{array}{l}\mathrm{MW} 20 \mathrm{M} \\
\text { (ft Toc) }\end{array}$ \\
\hline 11 5:00 & & 30.519 & 29.969 & 28.886 & .089 & 29.408 & 51 & 29.999 & 29.667 & 28.221 & 25.93 & 31 & 6 & 9 & 16.734 & 15.972 & 22 & 9 & 11.135 & 07 & & 29.774 & 26 & 64 & \\
\hline $9 / 28 / 115: 30$ & & 30.517 & 29.967 & 28.886 & 30.085 & 29.406 & 30.508 & 30.002 & 29.665 & 28.219 & 25.925 & 31.862 & 35.751 & 32.895 & 16.734 & $\quad 15.968$ & 22.368 & 15.159 & 11.133 & 31.905 & 32.763 & 29.774 & 31.823 & 26.864 & 34.207 \\
\hline 9/28/11 6:00 & & 30.517 & 29.965 & 28.888 & 30.08 & 29.403 & 30.508 & 29.999 & 29.665 & 28.221 & 25.928 & 31.857 & 35.751 & 32.893 & 16.734 & 15.97 & 22.393 & 15.163 & 11.126 & 31.898 & 32.76 & 29.762 & 31.819 & 26.864 & 34.205 \\
\hline 9/28/11 6:30 & & 30.517 & 29.967 & 28.895 & 30.078 & 29.399 & 30.51 & 29.999 & 29.663 & 28.221 & 25.928 & 31.857 & 35.753 & 32.886 & $5 \quad 16.734$ & $4 \quad 15.972$ & 22.405 & 15.159 & 11.124 & 31.9 & 32.758 & $\begin{array}{l}89.762 \\
\end{array}$ & 31.816 & $5 \quad 26.866$ & 34.203 \\
\hline 9/28/11 7:00 & & 30.517 & 29.962 & 28.898 & 30.078 & 29.391 & 30.51 & 30.002 & 29.66 & 28.225 & 25.937 & 31.855 & 35.753 & 32.886 & $5 \quad 16.736$ & $5 \quad 15.975$ & 22.407 & 15.151 & 11.123 & 31.898 & 32.753 & $\begin{array}{l}3 \quad 29.764 \\
\end{array}$ & 31.814 & 26.866 & 34.203 \\
\hline 9/28/11 7:30 & & 30.519 & 29.965 & 28.9 & 30.075 & 29.394 & 30.51 & 30.002 & 29.66 & 28.223 & 25.944 & 31.85 & 35.753 & 32.884 & 16.736 & $\begin{array}{ll}5 & 15.981\end{array}$ & 22.409 & 15.154 & 11.119 & 31.893 & 32.753 & $\begin{array}{l}3 \quad 29.764 \\
\end{array}$ & 31.814 & 26.866 & 34.203 \\
\hline 9/28/11 8:00 & & 30.522 & 29.967 & 28.898 & 30.078 & 29.394 & 30.51 & 30.002 & 29.663 & 28.225 & 25.944 & 31.85 & 35.758 & 32.884 & 16.736 & $\begin{array}{l}5 \quad 15.989 \\
\end{array}$ & 22.412 & 15.149 & 11.119 & 31.9 & 32.753 & $\begin{array}{l}3 \quad 29.769 \\
\end{array}$ & 31.812 & 26.866 & 34.2 \\
\hline $9 / 28 / 118: 30$ & & 30.522 & 29.969 & 28.895 & 30.08 & 29.399 & 30.51 & 30.004 & 29.663 & 28.223 & 25.942 & 31.852 & 35.753 & 32.881 & 16.741 & $1 \quad 15.993$ & 22.472 & 15.147 & 11.119 & 31.9 & 32.753 & $\begin{array}{l}3 \quad 29.769 \\
\end{array}$ & 31.812 & 26.866 & 34.2 \\
\hline 9/28/11 9:00 & & 30.522 & 29.969 & 28.898 & 30.082 & 29.401 & 30.51 & 30.007 & 29.665 & 28.221 & 25.94 & 31.85 & 35.758 & 32.884 & 16.739 & $\quad 15.998$ & 22.534 & 15.151 & 11.119 & 31.902 & 32.751 & $1 \quad 29.771$ & 31.814 & 26.866 & 34.2 \\
\hline 9/28/11 9:30 & & 30.519 & 29.969 & 28.881 & 30.08 & 29.403 & 30.51 & 29.999 & 29.663 & 28.221 & 25.942 & 32.007 & 35.753 & 33.048 & 16.743 & 36.026 & 22.638 & 15.154 & 11.286 & 31.958 & 32.907 & 729.774 & 31.964 & 26.869 & 34.2 \\
\hline 9/28/11 10:00 & & 30.519 & 29.967 & 28.884 & 30.08 & 29.41 & 30.508 & 30.002 & 29.66 & 28.216 & 25.928 & 31.948 & 35.756 & 32.987 & 16.746 & $5 \quad 16.033$ & 22.71 & 15.158 & 11.222 & 31.94 & 32.85 & 59.774 & 31.908 & 26.866 & 34.2 \\
\hline 9/28/11 10:30 & & $\begin{array}{l}30.522 \\
30.52\end{array}$ & $\begin{array}{l}29.901 \\
29.967\end{array}$ & $\begin{array}{l}28.884 \\
28.877\end{array}$ & $\begin{array}{r}30.080 \\
30.082\end{array}$ & $\begin{array}{l}29.41 \\
29.413\end{array}$ & $\begin{array}{l}30.508 \\
30.508\end{array}$ & $\begin{array}{l}29.999 \\
29.969\end{array}$ & $\begin{array}{l}29.00 \\
29.66\end{array}$ & $\begin{array}{l}28.214 \\
28.214\end{array}$ & $\begin{array}{l}25.986 \\
25.93\end{array}$ & $\begin{array}{l}31.948 \\
31.927\end{array}$ & 35.765 & $\begin{array}{l}32.901 \\
32.963 \\
\end{array}$ & $\begin{array}{l}10.740 \\
16.746\end{array}$ & $\begin{array}{ll}0 & 16.0335 \\
5 & 16.035\end{array}$ & 22.717 & $\begin{array}{l}15.150 \\
15.156\end{array}$ & $\begin{array}{l}11.226 \\
11.199\end{array}$ & $\begin{array}{l}31.934 \\
31.933\end{array}$ & $\begin{array}{r}32.03 \\
32.829 \\
\end{array}$ & $\begin{array}{l}0.778 \\
9\end{array}$ & $\begin{array}{l}31.887 \\
31.807\end{array}$ & $\begin{array}{l}26.0000 \\
26.869\end{array}$ & $\begin{array}{r}34.203 \\
34.20\end{array}$ \\
\hline 9/28/11 11:00 & & 30.515 & 29.96 & 28.872 & 30.075 & 29.42 & 30.505 & 29.999 & 29.656 & 28.212 & 25.923 & 31.908 & 35.758 & 32.945 & 16.746 & $5 \quad 16.033$ & 22.708 & 15.166 & 11.182 & 31.919 & 32.812 & 29.767 & 31.868 & $\begin{array}{l}3 \\
3\end{array}$ & 34.203 \\
\hline 9/28/11 11:30 & & 30.515 & 29.96 & 28.863 & 30.075 & 29.427 & 30.503 & 29.995 & 29.656 & 28.205 & 25.918 & 31.901 & 35.753 & 32.938 & 16.748 & $3 \quad 16.033$ & 22.698 & 15.166 & 11.173 & 31.916 & 32.803 & 29.767 & 31.861 & 26.869 & 34.205 \\
\hline 9/28/11 12:00 & & 30.515 & 29.958 & 28.858 & 30.075 & 29.429 & 30.503 & 29.999 & 29.653 & 28.202 & 25.913 & 31.892 & 35.749 & 32.931 & 16.748 & 16.031 & 22.687 & 15.173 & 11.163 & 31.912 & 32.796 & 29.762 & 1.852 & 26.869 & 34.205 \\
\hline $9 / 28 / 1112: 30$ & & 30.515 & 29.953 & 28.849 & 30.068 & 29.436 & 30.501 & 29.997 & 29.649 & 28.198 & 25.906 & 31.887 & 35.758 & 32.924 & 16.748 & 16.028 & 22.68 & 15.17 & 11.159 & 31.9 & 32.789 & 29.757 & 1.847 & 6.869 & 34.205 \\
\hline 9/28/11 13:00 & & 30.51 & 29.951 & 28.844 & 30.064 & 29.436 & 30.498 & 29.999 & 29.646 & 28.193 & 25.899 & 32.126 & 35.746 & 33.179 & 16.748 & 16.049 & 22.671 & 15.177 & 11.415 & 31.97 & 33.03 & 29.755 & 2.084 & 6.869 & 34.205 \\
\hline $9 / 28 / 1$ & & 30.51 & 29.944 & 28.839 & 30.059 & 29.441 & 30.496 & 29.995 & 29.642 & 28.186 & 25.894 & 32.023 & 35.744 & 33.067 & 16.748 & 16.068 & 22.664 & 15.18 & 11.302 & 31.958 & 32.926 & 29.75 & 1.983 & 6.869 & 34.207 \\
\hline 4:00 & & 30.508 & 29.941 & 28.839 & 30.057 & 29.441 & 30.493 & 29.997 & 29.64 & 28.186 & 25.889 & 32.014 & 35.746 & 33.059 & 16.748 & 16.061 & 22.654 & 15.182 & 95 & 31.949 & 32.917 & 29.748 & 1.974 & 6.871 & 34.207 \\
\hline 30 & & 30.505 & 29.937 & 28.839 & 30.052 & 29.436 & 30.491 & 29.995 & 29.635 & 28.186 & 25.892 & 31.972 & 35.744 & 33.013 & 16.748 & 16.061 & 22.65 & 15.187 & 46 & 31.9 & 32.872 & 29.741 & 929 & 5.869 & $\begin{array}{l}34.205 \\
34.205\end{array}$ \\
\hline 9/28/11 15:00 & & 30.505 & 29.932 & 28.837 & $\begin{array}{l}30.052 \\
30.05\end{array}$ & $\begin{array}{l}29.436 \\
29.436\end{array}$ & $\begin{array}{l}30.491 \\
30.491\end{array}$ & 29.995 & $\begin{array}{l}29.635 \\
29.63\end{array}$ & $\begin{array}{l}28.100 \\
28.181\end{array}$ & 25.892 & 31.946 & 35.744 & 32.987 & $\begin{array}{l}10.740 \\
16.748\end{array}$ & $\begin{array}{l}16.054 \\
3\end{array}$ & $\begin{array}{r}22.643 \\
22.643\end{array}$ & $\begin{array}{l}15.191 \\
15.191\end{array}$ & $\begin{array}{l}11.440 \\
11.222\end{array}$ & 31.916 & 32.85 & 29.741 & 1.906 & 6.869 & 34.205 \\
\hline 9/28/11 15:30 & & $\begin{array}{l}30.005 \\
30.505\end{array}$ & $\begin{array}{l}29.932 \\
29.932 \\
\end{array}$ & 28.835 & $\begin{array}{l}50.03 \\
30.05\end{array}$ & $\begin{array}{l}29.430 \\
29.434\end{array}$ & $\begin{array}{l}30.491 \\
30.491\end{array}$ & 29.995 & $\begin{array}{l}29.63 \\
29.628\end{array}$ & $\begin{array}{l}28.1717 \\
28.177\end{array}$ & 25.889 & $\begin{array}{l}31.940 \\
31.929\end{array}$ & 35.742 & $\begin{array}{l}32.901 \\
32.968 \\
\end{array}$ & $\begin{array}{r}10.740 \\
36.75\end{array}$ & $\begin{array}{ll}0 & 16.034 \\
5 & 16.049\end{array}$ & 22.641 & $\begin{array}{l}15.191 \\
15.191\end{array}$ & $\begin{array}{l}11.222 \\
11.201\end{array}$ & $\begin{array}{l}31.910 \\
31.914\end{array}$ & $\begin{array}{r}32.03 \\
32.831 \\
\end{array}$ & $\begin{array}{ll}1 & 29.741 \\
1\end{array}$ & 31.891 & 6.0871 & $\begin{array}{l}34.205 \\
34.203\end{array}$ \\
\hline 9/28/11 16:00 & & 30.508 & 29.932 & 28.837 & 30.045 & 29.429 & 30.489 & 29.995 & 29.626 & 28.177 & 25.889 & 31.918 & 35.737 & 32.954 & 16.748 & $3 \quad 16.042$ & 22.645 & 15.196 & 11.192 & 31.907 & 32.822 & 29.734 & 31.877 & 26.869 & 34.203 \\
\hline 9/28/11 16:30 & & 30.505 & 29.93 & 28.837 & 30.042 & 29.422 & 30.489 & 29.995 & 29.623 & 28.179 & 25.894 & 32.082 & 35.739 & 33.024 & 16.748 & $3 \quad 16.035$ & 22.678 & 15.196 & 11.257 & 31.935 & 32.992 & $2 \quad 29.731$ & 32.065 & $\quad 26.869$ & 34.2 \\
\hline 9/28/11 17:00 & & 30.505 & 29.925 & 28.846 & 30.042 & 29.415 & 30.486 & 29.992 & 29.619 & 28.181 & 25.897 & 32.018 & 35.739 & 33.057 & 16.75 & 16.04 & 22.705 & 15.196 & 11.295 & 31.944 & 32.919 & $\begin{array}{l}9 \\
9\end{array} 29.741$ & 31.978 & $\begin{array}{l}3 \\
26.871\end{array}$ & 34.2 \\
\hline 9/28/11 17:30 & & 30.505 & 29.927 & 28.856 & 30.045 & 29.403 & 30.486 & 29.997 & 29.619 & 28.184 & 25.906 & 31.967 & 35.732 & 33.006 & 16.753 & $\begin{array}{l}3 \\
16.035\end{array}$ & 22.703 & 15.196 & 11.243 & 31.933 & 32.869 & $\begin{array}{l}9 \\
9\end{array} 29.748$ & 31.927 & 26.869 & 34.198 \\
\hline 9/28/11 18:00 & & 30.505 & 29.927 & 28.86 & 30.047 & 29.394 & 30.489 & 29.997 & 29.619 & 28.186 & 25.918 & 31.944 & 35.739 & 32.98 & $\begin{array}{l}36.75 \\
3\end{array}$ & $\quad 16.031$ & 22.696 & 15.196 & 11.215 & 31.921 & 32.843 & $\begin{array}{l}3 \quad 29.736 \\
\end{array}$ & 31.903 & $\begin{array}{l}36.871 \\
\end{array}$ & 34.198 \\
\hline 9/28/11 18:30 & & 30.508 & 29.93 & $\begin{array}{l}28.80 \\
28.87\end{array}$ & 30.047 & 29.387 & 30.489 & 29.997 & 29.619 & 28.191 & 25.923 & 31.927 & 35.737 & 32.963 & 16.753 & $3 \quad 16.028$ & 22.694 & 15.189 & 11.201 & 31.916 & 32.829 & $\begin{array}{ll}9 & 29.752\end{array}$ & 31.887 & 26.871 & 34.196 \\
\hline 9/28/11 19:00 & & 30.512 & 29.937 & 28.877 & 30.057 & 29.375 & $\begin{array}{l}30.407 \\
30.489\end{array}$ & 29.999 & 29.621 & 28.195 & 25.933 & 31.918 & 35.744 & $\begin{array}{l}32.952 \\
32.952\end{array}$ & 16.755 & $\begin{array}{l}16.024 \\
5\end{array}$ & 22.687 & 15.177 & $\begin{array}{l}11.212 \\
11.189\end{array}$ & 31.919 & 32.82 & $\begin{array}{l}29.757 \\
2 \quad 25\end{array}$ & $\begin{array}{l}31.8017 \\
31.87\end{array}$ & 26.871 & 34.196 \\
\hline 9/28/11 19:30 & & 30.51 & 29.937 & 28.891 & 30.059 & 29.363 & $\begin{array}{l}30.409 \\
30.491\end{array}$ & 29.999 & 29.623 & 28.2 & 25.942 & 31.908 & 35.746 & $\begin{array}{l}32.942 \\
32.942\end{array}$ & 16.757 & $\begin{array}{l}16.024 \\
7\end{array}$ & 22.68 & 15.182 & $\begin{array}{l}11.199 \\
11.182\end{array}$ & 31.921 & 32.81 & $\begin{array}{l}1 \\
1 \quad 29.762\end{array}$ & $\begin{array}{l}31.87 \\
31.87\end{array}$ & 26.871 & 34.196 \\
\hline 9/28/11 20:00 & & $\begin{array}{r}30.515 \\
30.515\end{array}$ & 29.941 & $\begin{array}{l}20.091 \\
28.895\end{array}$ & 30.066 & 29.356 & $\begin{array}{l}30.491 \\
30.493\end{array}$ & 29.999 & 29.626 & $\begin{array}{r}28.2 \\
28.205\end{array}$ & 25.956 & 31.904 & $\begin{array}{l}35.749 \\
35.740\end{array}$ & $\begin{array}{r}32.94 \\
32.94\end{array}$ & $\begin{array}{l}16.757 \\
+\end{array}$ & 16.019 & $\begin{array}{l}22.600 \\
22.678\end{array}$ & $\begin{array}{l}15.175 \\
15.102\end{array}$ & $\begin{array}{l}11.102 \\
11.173\end{array}$ & 31.919 & $\begin{array}{r}32.01 \\
32.805\end{array}$ & $\begin{array}{l}29.762 \\
5\end{array}$ & 31.863 & $\begin{array}{l}26.873 \\
3\end{array}$ & 34.196 \\
\hline 9/28/11 20:30 & & $\begin{array}{l}30.517 \\
30.517\end{array}$ & $\begin{array}{l}29.941 \\
29.948\end{array}$ & $\begin{array}{r}28.859 \\
28.9\end{array}$ & $\begin{array}{l}30.000 \\
30.075\end{array}$ & 29.354 & $\begin{array}{l}30.493 \\
30.496\end{array}$ & 30.002 & $\begin{array}{r}29.060 \\
29.63\end{array}$ & $\begin{array}{l}28.207 \\
28.207\end{array}$ & 25.961 & $\begin{array}{l}31.904 \\
31.899\end{array}$ & $\begin{array}{l}33.749 \\
35.749\end{array}$ & $\begin{array}{r}32.94 \\
32.935 \\
\end{array}$ & $\begin{array}{r}16.157 \\
\quad 16.76\end{array}$ & $5 \quad 16.014$ & 22.675 & $\begin{array}{l}15.168 \\
15.178\end{array}$ & $\begin{array}{l}11.1 / 16 \\
11.168\end{array}$ & $\begin{array}{r}31.919 \\
31.93\end{array}$ & $\begin{array}{l}32.005 \\
32.803 \\
\end{array}$ & $\begin{array}{l}3.1782 \\
3 \quad 29.778\end{array}$ & $\begin{array}{l}31.803 \\
31.861\end{array}$ & 26.873 & $\begin{array}{l}34.190 \\
34.196\end{array}$ \\
\hline 9/28/11 21:00 & & & 29.953 & 28.905 & 30.082 & 29.351 & 30.498 & 30.002 & 29.635 & 28.212 & 25.966 & 31.899 & 35.756 & 32.931 & 16.764 & $4 \quad 16.014$ & 22.673 & 66 & 71 & 31.933 & 32.801 & .783 & 1.859 & 26.873 & 34.198 \\
\hline 9/28/11 21:30 & & & 29.953 & 28.912 & 30.087 & 29.347 & 30.501 & 30.002 & 29.637 & 28.214 & 25.971 & 31.897 & 35.753 & 32.931 & 16.766 & $\begin{array}{ll}5 & 16.017\end{array}$ & 22.671 & 15.163 & 11.166 & 31.935 & 32.801 & 29.792 & 31.856 & 26.873 & 34.198 \\
\hline 9/28/11 22:00 & & 30.517 & 29.958 & 28.914 & 30.092 & 29.344 & 30.501 & 29.999 & 29.637 & 28.216 & 25.976 & 31.897 & 35.761 & 32.928 & 16.766 & $5 \quad 16.019$ & 22.668 & 15.168 & 11.166 & 31.933 & 32.801 & $1 \quad 29.792$ & 31.856 & 26.873 & 34.2 \\
\hline $22: 30$ & & & 29.962 & 28.919 & 30. & 29.342 & 30.503 & 30.007 & 29.642 & 28.223 & 25.98 & 31.897 & 35.761 & 32.928 & 16.766 & 16.026 & 22.671 & 15.156 & 1.1 .50 & & 32.798 & $\quad 29.802$ & 1.856 & 26.873 & 34.2 \\
\hline & & & 29.9 & 28. & 30. & & 36 & 30 & 29. & 28 & 25. & & 35.7 & 32.931 & 16. & 16 & 22.6 & & 11. & & 32.796 & 04 & 6 & 76 & 34.205 \\
\hline & & & 29.969 & 28 & & & 30 & & 29 & 28.2 & 25.992 & & & 32.931 & 16. & 16 & 24 & & $I_{1}$ & & 32.801 & 16 & & 76 & 34.205 \\
\hline 9/29/11 0:00 & & & 29.974 & & 30. & & & & 29.6 & & 25.997 & & .768 & 32.931 & 16.7 & 16.04 & $\begin{array}{l}22.671 \\
22.671\end{array}$ & 9 & 11. & 47 & 32.803 & 814 & 59 & 5.876 & 34.207 \\
\hline $9 / 29 / 110: 30$ & & 30 & 29.974 & 28 & 30.122 & 7 & 30.513 & & 29.653 & 28 & 26 & 31 & 72 & 32.931 & 16.776 & $\begin{array}{r}5 \\
5 \\
5\end{array}$ & 22.6 & & $\begin{array}{l}11.100 \\
11.171\end{array}$ & 354 & $\begin{array}{l}32.005 \\
32.803\end{array}$ & .823 & 61 & 76 & $\begin{array}{l}34.21 \\
34.21\end{array}$ \\
\hline 9/29/11 1:00 & & & 29.979 & $2 \varepsilon$ & 30.127 & & 30.518 & & 29.656 & 28 & 26.002 & & $3 !$ & 32.935 & 16.778 & $3 \quad 16.045$ & 22.673 & 1 & 11.171 & 31.954 & 32.803 & 025 & 31.866 & 76 & 34.212 \\
\hline 9/29/11 1:30 & & & 29.983 & 28.94 & 30.131 & & 30 & & 29.66 & 28 & 26 & 31. & & 32.935 & 16.778 & & 22. & & 11.17 & 31.961 & 32.805 & & 31.866 & 78 & 34.214 \\
\hline 9/29/11 2:00 & & & 29.986 & 28.937 & 30.1 & & 30.518 & & 29.663 & & 26.002 & 31. & & 32.935 & 16.78 & $\begin{array}{l}3 \quad 16.042 \\
\end{array}$ & 22.673 & 15 & 11.173 & 31.954 & 32.808 & & 31.863 & $3 \quad 26.878$ & 34.214 \\
\hline 9/29/11 2:30 & & 30 & 29.986 & 28.942 & 30.1 & 29 & 30.52 & 30 & 29.665 & 28 & 25.997 & 31. & 35 & 32.935 & 16.785 & $5 \quad 16.042$ & 22.673 & 15.149 & 11.17 & 31.963 & 32.805 & $5 \quad 29.835$ & 31.866 & 5 26.878 & 34.214 \\
\hline 9/29/11 3:00 & & 30. & 29.988 & 28.937 & 30.1 & 29.356 & 30.52 & 30. & 29.667 & 28.2 & 25.995 & 31.904 & 35.777 & 32.933 & 16.785 & 16.04 & 22.675 & 15.151 & 11.168 & 31.961 & 32.808 & $\begin{array}{l}3 \quad 29.835 \\
\end{array}$ & 31.866 & $5 \quad 26.878$ & 34.214 \\
\hline 9/29/11 3:30 & & 30. & 29.99 & 28.942 & 30.1 & 29.359 & 30.522 & 30. & 29.667 & 28. & 25.992 & 31.899 & 35.777 & 32.933 & 16.787 & $\begin{array}{l}76.035 \\
7\end{array}$ & 22.673 & 15.151 & 11.168 & 31.954 & 32.803 & 29.83 & 31.863 & $\begin{array}{l}36.878 \\
\end{array}$ & 34.214 \\
\hline 9/29/11 4:00 & & 30.529 & 29.99 & 28.949 & 30.141 & 29.351 & 30.527 & 30.014 & 29.667 & 28.249 & 25.997 & 31.899 & 35.784 & 32.931 & 16.787 & 16.033 & 22.673 & 15.147 & 11.168 & 31.961 & 32.805 & 29.83 & 31.861 & 26.878 & 34.214 \\
\hline 9/29/11 4:30 & & 30.529 & 29.988 & $\begin{array}{l}20.949 \\
28.951\end{array}$ & 30.143 & 29.351 & 30.527 & 30.014 & 29.672 & $\begin{array}{l}28.251 \\
28.251\end{array}$ & 26.002 & 31.901 & 35.779 & 32.928 & $\begin{array}{r}16.79 \\
3\end{array}$ & $\begin{array}{ll}16.031 \\
\end{array}$ & 22.671 & $\begin{array}{l}15.149 \\
\end{array}$ & $\begin{array}{l}11.1 .160 \\
1.166\end{array}$ & 31.958 & 32.801 & $\begin{array}{l}1 \quad 29.832 \\
1.832\end{array}$ & 31.859 & 26.878 & 34.214 \\
\hline 9/29/11 5:00 & & 30.533 & 29.993 & $\begin{array}{l}20.951 \\
28.968\end{array}$ & 30.143 & 29.349 & 30.53 & 30.011 & 29.674 & 28.256 & $\begin{array}{l}20.0004 \\
26.004\end{array}$ & 31.899 & 35.775 & $\begin{array}{l}32.920 \\
32.928 \\
\end{array}$ & 16.79 & $\begin{array}{l}16.026 \\
\end{array}$ & 22.67 & $\begin{array}{l}15.142 \\
15.142\end{array}$ & $\begin{array}{l}11.100 \\
11.168\end{array}$ & 31.963 & 32.801 & $\begin{array}{l}1 \\
1 \quad 29.839\end{array}$ & $\begin{array}{l}31.859 \\
31.859\end{array}$ & $\begin{array}{l}26.881 \\
\quad 261\end{array}$ & 34.212 \\
\hline 9/29/115:30 & & 30.536 & 29.995 & $\begin{array}{r}20.908 \\
28.97\end{array}$ & $\begin{array}{l}30.143 \\
30.148\end{array}$ & $\begin{array}{l}29.349 \\
29.349\end{array}$ & $\begin{array}{r}30.532 \\
30.532\end{array}$ & 30.014 & $\begin{array}{l}29.674 \\
29.677\end{array}$ & $\begin{array}{r}28.250 \\
28.26\end{array}$ & $\begin{array}{l}20.004 \\
26.016\end{array}$ & $\begin{array}{l}31.899 \\
31.899\end{array}$ & 35.777 & $\begin{array}{l}32.928 \\
32.928 \\
\end{array}$ & $\begin{array}{r}10.79 \\
3 \\
3\end{array}$ & $\begin{array}{l}116.026 \\
4 \quad 160\end{array}$ & $\begin{array}{l}22.677 \\
22.677\end{array}$ & $\begin{array}{l}15.142 \\
15.142\end{array}$ & $\begin{array}{l}11.100 \\
11.166\end{array}$ & $\begin{array}{l}31.903 \\
31.963\end{array}$ & $\begin{array}{l}32.801 \\
32.801\end{array}$ & $\begin{array}{l}29.844 \\
1 \quad 29\end{array}$ & 1.859 & 6.0881 & $\begin{array}{l}34.212 \\
34.212 \\
\end{array}$ \\
\hline $9 / 29 / 116: 00$ & & 30.536 & 30 & 29.026 & 30.15 & 29.342 & 30.534 & 30.016 & 29.679 & 28.265 & 26.023 & 31.897 & 35.777 & 32.926 & 16.794 & $\begin{array}{l}46.024 \\
4\end{array}$ & 22.698 & 15.14 & 11.163 & 31.972 & 32.798 & 29.842 & 1.859 & 6.883 & 34.21 \\
\hline $9 / 29 / 116: 30$ & & 30.538 & 30.004 & 29.024 & 30.159 & 29.309 & 30.541 & 30.016 & 29.684 & 28.288 & 26.078 & 31.899 & 35.782 & 32.928 & $3 \quad 16.794$ & $\quad 16.019$ & 22.703 & 15.137 & 11.166 & 31.968 & 32.801 & .851 & 1.859 & 26.881 & 34.21 \\
\hline 9/29/11 7:00 & & 30.543 & 30.009 & 29.04 & 30.164 & 29.312 & 30.541 & 30.021 & 29.688 & 28.298 & 26.074 & 31.899 & 35.784 & 32.926 & 16.797 & 16.019 & 22.71 & 15.135 & 11.163 & 31.975 & 32.803 & $3 \quad 29.863$ & 31.859 & 26.883 & 34.207 \\
\hline $9 / 29 / 117: 30$ & & 30.564 & 30.039 & 29.054 & 30.192 & 29.316 & 30.546 & 30.021 & 29.711 & 28.302 & 26.088 & 31.911 & 35.789 & 32.938 & 36.804 & $\quad 16.028$ & 22.724 & 15.112 & 11.178 & 32.01 & 32.812 & 29.905 & 31.87 & 6.883 & 34.212 \\
\hline & & . & & & & & & & 2.1 & & & & & & & 0.020 & & & 11. & דיט. & 5.012 & & J & & \\
\hline $18: 30$ & & 30.559 & 30.049 & 9.066 & 30.213 & 29.319 & 30.549 & 30.028 & 29.723 & 28.323 & 26.107 & 31.915 & 35.793 & 32.947 & 16.811 & 16.04 & 22.719 & 15.123 & $\begin{array}{l}11.185 \\
11.185\end{array}$ & 32.014 & 32.82 & 29.917 & 31.875 & 6.883 & 12 \\
\hline
\end{tabular}




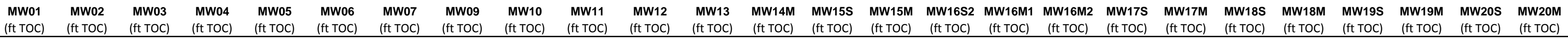

\begin{tabular}{|c|c|c|c|c|c|c|c|c|c|c|c|c|c|c|c|c|c|c|c|c|c|c|c|c|c|c|}
\hline Well & $\begin{array}{l}\text { (ft TOC) } \\
\text { (is }\end{array}$ & $\begin{array}{l}\text { (ft TOC) } \\
\text { (f) }\end{array}$ & $\begin{array}{l}\text { (ft TOC) } \\
\text { (f) }\end{array}$ & (ft TOC) & $\begin{array}{l}\text { (ift TOC) } \\
\text { (ft }\end{array}$ & $\begin{array}{l}\text { (ft TOC) } \\
\text { (f) }\end{array}$ & $\begin{array}{l}\text { (ft TOC) } \\
\text { (ft }\end{array}$ & $\begin{array}{l}\text { (ft TOC) } \\
\text { (f) }\end{array}$ & $\begin{array}{l}\text { (ft TOC) } \\
\text { (ft) }\end{array}$ & $\begin{array}{l}\text { (ft TOC) } \\
\text { (f) }\end{array}$ & $\begin{array}{l}\text { (ft TOC) } \\
\text { (f) }\end{array}$ & $\begin{array}{l}\text { (ft Toc) } \\
\text { (f) }\end{array}$ & (ft TOC) & $\begin{array}{l}\text { (ft TOC) } \\
\end{array}$ & (ft TOC) & (ft TOC) & (ft TOC) & (ft TOC) & $\begin{array}{l}\text { (ft TOC) } \\
\text { (ft) }\end{array}$ & (ft TOC) & $\begin{array}{l}\text { (ft TOC) } \\
\end{array}$ & $\begin{array}{l}\text { (ft TOC) } \\
\text { (n) }\end{array}$ & $\begin{array}{l}\text { (ft TOC) } \\
\text { (n) }\end{array}$ & (ft TOC) & $\begin{array}{l}\text { (ft TOC) } \\
\text { (1) }\end{array}$ & (ft TOC) \\
\hline 9/29/11 9:00 & & 30.564 & 30.058 & 29.073 & 30.227 & & 29.316 & 30.553 & 30.028 & 29.735 & 28.332 & 26.11 & 31.925 & 35.796 & 32.949 & 16.815 & 16.045 & 22.717 & 15.123 & 11.189 & 32.031 & 32.824 & 29.931 & 31.884 & 26.885 & 34.214 \\
\hline $9 / 29 / 119: 30$ & & 30.566 & 30.067 & 29.073 & 30.241 & & 29.328 & 30.556 & 30.021 & 29.742 & 28.337 & 26.119 & 31.927 & 35.8 & 32.956 & 16.818 & 16.049 & 22.719 & 15.13 & 11.194 & 32.035 & 32.834 & 29.938 & 31.889 & 26.885 & 34.217 \\
\hline $9 / 29 / 1110: 00$ & & 30.568 & 30.077 & 29.075 & 30.25 & & 29.335 & 30.558 & 30.023 & 29.749 & 28.344 & 26.114 & 31.934 & 35.8 & 32.959 & 16.822 & 16.052 & 22.731 & 15.126 & 11.197 & 32.04 & 32.836 & 29.95 & 31.894 & 26.888 & 34.219 \\
\hline 10:30 & & 30.571 & 30.081 & 29.073 & 30.262 & & 29.347 & 30.56 & 30.028 & 29.758 & 28.346 & 26.117 & 31.974 & 35.805 & 33.008 & 16.829 & 16.056 & 22.756 & 15.13 & 11.246 & 32.056 & 32.874 & & 31 & 200 & 34.221 \\
\hline 9/29/11 11:00 & & 30.573 & 30.091 & 29.078 & 30.267 & & 29.354 & 30.558 & 30.028 & 29.763 & 28.351 & 26.119 & 31.953 & 35.805 & 32.98 & 16.829 & 16.059 & 22.791 & 15.14 & 11.218 & 32.054 & 32.86 & 29.957 & & 26.888 & 34.224 \\
\hline 9/29/11 11:30 & & 30.571 & 30.093 & 29.075 & 30.269 & & 29.363 & 30.56 & 30.028 & 29.769 & 28.356 & 26.119 & 31.953 & 35.814 & 32.982 & 16.832 & 16.059 & 22.812 & 15.147 & 11.225 & 32.054 & 32.855 & 29.959 & 31.913 & 26.89 & 34.224 \\
\hline 9/29/11 12:00 & & 30.575 & 30.095 & 29.071 & 30.278 & & 29.373 & 30.56 & 30.026 & 29.772 & 28.356 & 26.117 & 31.958 & 35.812 & 32.984 & 16.836 & 16.066 & 22.821 & 15.151 & 11.22 & 32.061 & 32.86 & 29.971 & 31.915 & 6.89 & 34.226 \\
\hline 9/29/11 12:30 & & 30.578 & 30.102 & 29.064 & 30.281 & & 29.389 & 30.563 & 30.023 & 29.776 & 28.356 & 26.11 & 31.962 & 35.812 & 32.989 & 16.836 & 16.068 & 22.823 & 15.159 & 11.225 & 32.063 & 32.857 & 29.966 & 31.917 & 26.89 & 34.226 \\
\hline 9/29/11 13:00 & & 30.578 & 30.105 & 29.052 & 30.285 & & 29.406 & 30.561 & 30.026 & 29.781 & 28.351 & 26.1 & 31.965 & 35.814 & 32.991 & 16.841 & 16.068 & 22.825 & 15.159 & 11.229 & 32.063 & 32.867 & 29.969 & 31.922 & 26.89 & 34.231 \\
\hline 9/29/11 13:30 & & 30.575 & 30.102 & 29.052 & 30.283 & & 29.415 & 30.563 & 30.026 & 29.781 & 28.351 & 26.088 & 31.965 & 35.81 & 32.991 & 16.841 & 16.07 & 22.828 & 15.173 & 11.234 & 32.061 & 32.865 & 29.964 & 31.922 & 26.892 & 34.233 \\
\hline 9/29/11 14:00 & & 30.575 & 30.1 & 29.05 & 30.283 & & 29.417 & 30.565 & 30.026 & 29.781 & 28.353 & 26.088 & 31.967 & 35.819 & 32.994 & 16.841 & 16.07 & 22.879 & 15.175 & 11.232 & 32.059 & 32.865 & 29.957 & 31.92 & 26.892 & 34.233 \\
\hline 9/29/11 14:30 & & 30.573 & 30.098 & 29.05 & 30.278 & & 29.42 & 30.565 & 30.03 & 29.781 & 28.358 & 26.081 & 31.965 & 35.812 & 32.989 & 16.841 & 16.073 & 22.909 & 15.187 & 11.229 & 32.056 & 32.864 & 29.955 & 31.922 & 26.888 & 34.231 \\
\hline 9/29/11 15:00 & & 30.575 & 30.1 & 29.052 & 30.278 & & 29.429 & 30.568 & 30.03 & 29.783 & 28.358 & 26.083 & 31.965 & 35.817 & 32.994 & 16.843 & 16.07 & 22.916 & 15.184 & 11.229 & 32.056 & 32.867 & 29.952 & 31.922 & 26.892 & 34.233 \\
\hline 9/29/11 15:30 & & 30.578 & 30.1 & 29.054 & 30.278 & & 29.427 & 30.565 & 30.033 & 29.786 & 28.358 & 26.083 & 31.965 & 35.814 & 32.991 & 16.846 & 16.068 & 22.916 & 15.189 & 11.234 & 32.047 & 32.867 & 29.95 & 31.922 & 26.892 & 34.233 \\
\hline 9/29/11 16:00 & & 30.58 & 30.1 & 29.052 & 30.274 & & 29.436 & 30.565 & 30.033 & 29.788 & 28.358 & 26.083 & 31.965 & 35.817 & 32.994 & 16.843 & 16.07 & 22.916 & 15.191 & 11.234 & 32.054 & 32.867 & 29.952 & 31.924 & 26.892 & 34.233 \\
\hline 9/29/11 16:30 & & 30.58 & 30.1 & 29.054 & 30.276 & & 29.434 & 30.57 & 30.033 & 29.788 & 28.365 & 26.083 & 31.967 & 35.814 & 32.994 & 16.848 & 16.066 & 22.916 & 15.187 & 11.229 & 32.056 & 32.865 & 29.95 & 31.924 & 26.892 & 34.231 \\
\hline 9/29/11 17:00 & & 30.582 & 30.102 & 29.061 & 30.276 & & 29.434 & 30.573 & 30.035 & 29.79 & 28.367 & 26.083 & 31.965 & 35.817 & 32.994 & 16.85 & 16.066 & 22.92 & 15.198 & 11.234 & 32.054 & 32.867 & 29.952 & 31.927 & 26.892 & 34.233 \\
\hline 9/29/11 17:30 & & 30.582 & 30.105 & 29.061 & 30.274 & & 29.434 & 30.573 & 30.033 & 29.795 & 28.367 & 26.088 & 31.969 & 35.817 & 32.992 & 16.848 & $\begin{array}{l}16.068 \\
\end{array}$ & 22.927 & 15.198 & 11.232 & 32.056 & 32.867 & 29.948 & 31.924 & 26.895 & 34.231 \\
\hline 9/29/11 18:00 & & 30.587 & 30.107 & 29.071 & 30.274 & & 29.429 & 30.573 & 30.035 & 29.795 & 28.372 & $\begin{array}{l}26.000 \\
26.088\end{array}$ & 31.967 & 35.819 & 32.996 & $\begin{array}{l}10.060 \\
16.85\end{array}$ & $\begin{array}{l}10.000 \\
16.063\end{array}$ & 22.934 & 15.201 & 11.232 & 32.058 & 32.867 & 29.952 & 31.924 & 26.895 & 34.231 \\
\hline 9/29/11 18:30 & & $\begin{array}{l}30.501 \\
30.591\end{array}$ & 30.114 & 29.075 & 30.278 & & 29.427 & 30.575 & 30.033 & $\begin{array}{r}29.8 \\
29.8\end{array}$ & 28.377 & $\begin{array}{l}20.000 \\
26.095\end{array}$ & 31.967 & $\begin{array}{l}35.019 \\
35.821\end{array}$ & 32.996 & $\begin{array}{l}1.05 \\
16.853\end{array}$ & $\begin{array}{l}10.003 \\
16.063\end{array}$ & $\begin{array}{l}22.954 \\
22.971\end{array}$ & $\begin{array}{l}15.198 \\
15.198\end{array}$ & $\begin{array}{l}11.236 \\
11.236\end{array}$ & $\begin{array}{l}32.063 \\
32.03\end{array}$ & $\begin{array}{l}32.869 \\
32.069\end{array}$ & 29.952 & 31.927 & $\begin{array}{l}20.059 \\
26.895\end{array}$ & 34.231 \\
\hline 9/29/11 19:00 & & $\begin{array}{l}30.591 \\
30.591\end{array}$ & $\begin{array}{l}30.114 \\
30.114\end{array}$ & 29.082 & $\begin{array}{l}30.278 \\
30.278\end{array}$ & & 29.424 & 30.575 & $\begin{array}{l}30.037 \\
30.037\end{array}$ & 29.802 & 28.381 & $\begin{array}{r}26.095 \\
26.1\end{array}$ & 31.967 & $\begin{array}{l}53.821 \\
35.821\end{array}$ & $\begin{array}{l}32.900 \\
33.001\end{array}$ & $\begin{array}{l}10.033 \\
16.853\end{array}$ & $\begin{array}{l}10.003 \\
16.066\end{array}$ & $\begin{array}{r}22.99 \\
22.99\end{array}$ & $\begin{array}{l}13.1203 \\
15.203\end{array}$ & $\begin{array}{l}11.230 \\
11.236\end{array}$ & $\begin{array}{l}32.063 \\
32.065\end{array}$ & $\begin{array}{l}32.899 \\
32.872\end{array}$ & 29.962 & $\begin{array}{l}31.929 \\
31.929\end{array}$ & $\begin{array}{l}26.8995 \\
26.895\end{array}$ & $\begin{array}{l}34.231 \\
34.231\end{array}$ \\
\hline 9/29/11 19:30 & & & 30.119 & 29.092 & 30.283 & & 29.42 & 30.577 & 30.04 & 29.809 & 28.388 & 26.107 & 31.969 & 35.826 & 33.001 & 16.855 & 16.063 & 22.999 & 5.203 & 11.234 & 32.068 & 32.874 & 969 & 1.929 & 26.897 & 34.231 \\
\hline $9 / 29 / 1$ & & 30.596 & 30.123 & 29.096 & 30.28 & & 29.422 & 30.58 & 30.037 & 29.811 & 28.391 & 26.117 & 31.974 & .826 & 33.003 & & 16.063 & 22.997 & 203 & 11.239 & 32.072 & 874 & .973 & 1.931 & 5.897 & .231 \\
\hline 9/29/1 & & 30.598 & 30.13 & 29.106 & 30.292 & & 29.42 & 30.58 & 30.042 & 29.816 & 28.395 & 26.122 & 31.979 & 35.824 & 33.006 & 16.86 & 16.063 & 22.992 & 203 & 11.243 & 32.08 & 879 & 29.98 & 1.938 & 6.897 & 34.233 \\
\hline 9/29/11 21:00 & & 30.601 & 30.135 & 29.106 & 30.299 & & & 30.582 & 30.042 & 29.823 & 28.4 & 26.126 & 31.983 & 35.824 & 33.01 & 16.864 & 16.063 & 22.99 & 203 & 11.243 & 32.082 & 32.883 & 29.988 & 1.941 & 26.897 & 34.233 \\
\hline 9/29/11 21:30 & & 30.601 & 30.139 & 29.11 & 30.304 & & 29.422 & 30.584 & 30.04 & 29.83 & 28.405 & 26.126 & 31.986 & 35.833 & 33.013 & 16.864 & 16.068 & 22.987 & 196 & 11.25 & 32.091 & 32.888 & 29.995 & 1.945 & 26.897 & 34.235 \\
\hline 9/29/11 22:00 & & 30.603 & 30.144 & 29.11 & 30.309 & & 29.427 & 30.584 & 30.042 & 29.834 & 28.405 & 26.126 & 31.988 & 35.831 & 33.017 & 16.869 & 16.075 & 22.987 & 15.198 & 11.255 & 32.096 & 32.895 & 29.999 & 31.95 & 26.899 & 34.238 \\
\hline 9/29/11 22:30 & & 30.603 & 30.146 & 29.11 & 30.311 & & 29.431 & 30.589 & 30.042 & 29.837 & 28.409 & 26.129 & 31.995 & 35.831 & 33.022 & 16.869 & 16.082 & 22.987 & 15.201 & 11.26 & 32.098 & 32.895 & 30.002 & 31.955 & 26.899 & 34.24 \\
\hline 9/29/11 23:00 & & 30.601 & 30.149 & 29.11 & 30.318 & & 29.438 & 30.589 & 30.045 & 29.844 & 28.412 & 26.129 & 32 & 35.831 & 33.027 & 16.871 & 16.091 & 22.983 & 15.203 & 11.262 & 32.105 & 32.902 & 30.004 & 31.957 & 26.899 & 34.24 \\
\hline 9/29/11 23:30 & & 30.603 & 30.153 & 29.11 & 30.323 & & 29.443 & 30.589 & 30.047 & 29.846 & 28.414 & 26.129 & 32.002 & 35.831 & 33.034 & 16.874 & 16.096 & 22.98 & 15.198 & 11.269 & 32.105 & 32.905 & 30.011 & 31.962 & 26.899 & 34.242 \\
\hline 9/30/11 0:00 & & 30.603 & 30.153 & 29.117 & 30.325 & & 29.446 & 30.592 & 30.047 & 29.848 & 28.419 & 26.126 & 32.002 & 35.838 & 33.031 & 16.876 & 16.103 & 22.978 & 15.205 & 11.272 & 32.11 & 32.907 & 30.011 & 31.964 & 26.899 & 34.247 \\
\hline 9/30/11 0:30 & & 30.603 & 30.156 & 29.12 & 30.325 & & 29.446 & 30.592 & 30.047 & 29.851 & 28.421 & 26.134 & 32.009 & 35.84 & 33.038 & 16.876 & 16.108 & 22.976 & 15.208 & 11.276 & 32.105 & 32.91 & 30.011 & 31.969 & 26.899 & 34.247 \\
\hline 9/30/11 1:00 & & 30.603 & 30.16 & 29.129 & 30.327 & & 29.443 & 30.594 & 30.049 & 29.853 & 28.425 & 26.136 & 32.014 & 35.838 & 33.038 & 16.878 & 16.11 & 22.971 & 15.203 & 11.276 & 32.112 & 32.912 & 30.011 & 31.971 & 26.899 & 34.252 \\
\hline 9/30/11 1:30 & & 30.608 & 30.163 & 29.131 & 30.332 & & 29.443 & 30.594 & 30.047 & 29.858 & 28.43 & 26.145 & 32.016 & 35.838 & 33.045 & 16.881 & 16.115 & 22.971 & 15.21 & 11.283 & 32.114 & 32.919 & 30.018 & 31.976 & 26.899 & 34.252 \\
\hline 9/30/11 2:00 & & 30.61 & 30.167 & 29.127 & 30.337 & & 29.45 & 30.594 & 30.047 & 29.862 & 28.432 & 26.145 & 32.018 & 35.843 & 33.045 & 16.883 & 16.115 & 22.971 & 15.205 & 11.281 & 32.124 & 32.921 & .025 & 31.981 & 26.902 & 34.254 \\
\hline $9 / 30 / 112: 30$ & & 30.612 & 30.174 & 29.12 & 30. & & 29.46 & 30.596 & 30.049 & 29.869 & 28.43 & 26.141 & 32.021 & 35.845 & 33.052 & 16.888 & 16.117 & 22.973 & 15.2 & 11.288 & 32.128 & 32.926 & 0.027 & 31.983 & 26.902 & 34.256 \\
\hline 9/30/11 3:00 & & $\begin{array}{r}30.61 \\
30\end{array}$ & 30.174 & 29.117 & 30.346 & & 29.469 & 30.599 & 30.047 & 29.872 & $\begin{array}{l}20.45 \\
28.43\end{array}$ & 26.131 & 32.028 & 35.847 & $\begin{array}{l}33.055 \\
33.055\end{array}$ & 16.888 & 16.12 & 22.973 & 15.205 & $\begin{array}{l}11.200 \\
11.288\end{array}$ & 32.124 & 32.928 & 30.032 & 31.988 & 26.902 & 34.259 \\
\hline 9/30/11 3:30 & & 30.61 & 30.177 & 29.122 & 30.348 & & 29.471 & 30.599 & 30.049 & 29.874 & $\begin{array}{l}28.435 \\
28.435\end{array}$ & 26.129 & 32.028 & 35.845 & 33.057 & $\begin{array}{l}\begin{array}{l}0.000 \\
16.89\end{array} \\
\end{array}$ & $\begin{array}{l}16.119 \\
16.19\end{array}$ & 22.971 & 15.208 & $\begin{array}{l}11.200 \\
11.293\end{array}$ & 32.131 & 32.931 & 30.03 & 85 & 26.902 & 34.259 \\
\hline 9/30/11 4:00 & & $\begin{array}{l}30.608 \\
30.608\end{array}$ & 30.17 & 29.124 & 30. & & 29.474 & 30.599 & $\begin{array}{l}30.052 \\
30.052\end{array}$ & 29.872 & $\begin{array}{l}28.435 \\
28.432\end{array}$ & 26.131 & 32.028 & $\begin{array}{l}35.847 \\
35.847\end{array}$ & $\begin{array}{l}33.055 \\
33.055\end{array}$ & 10 & $\begin{array}{l}10.117 \\
16.117\end{array}$ & 22.969 & 10.200 & $\begin{array}{l}11.295 \\
11.295\end{array}$ & 32.121 & 31 & 30.023 & 85 & 26.904 & 34.259 \\
\hline $9 / 30 / 1$ & & 30 & 30 & 29 & & & 29. & 30 & 30 & 29 & 28 & 26 & 32 & $3 !$ & 33.057 & 16.89 & 16 & 22 & 15.215 & 11. & 32.131 & 8 & 22 & 38 & 26.902 & 34.256 \\
\hline $9 / 30 / 1$ & & & 30 & 29 & & & & & & & 28 & & 32 & 35.847 & 33.0 & 16.892 & 16.117 & 22 & 15. & 11.293 & 32.126 & 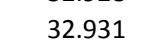 & 3 & 38 & 34 & 34.259 \\
\hline $9 / 3$ & & & 30 & 29 & & & & & & 29.8 & 28.435 & & & 35.85 & 3 & 16.895 & 16.117 & 22.967 & 15 & 11.295 & 32.128 & 32.933 & & 1.988 & $\begin{array}{l}3 \\
3\end{array} 26.902$ & 34.256 \\
\hline 11 6:00 & & 30.608 & 30 & 29.106 & 30 & & & & & 29.8 & 28.432 & 26.122 & 32. & 35.847 & 33. & 16.892 & & & & 11.293 & 32.119 & & & 31.988 & $\begin{array}{l}3 \\
3\end{array}$ & 34.259 \\
\hline $16: 30$ & & 30.61 & 30 & 29.108 & & & & & & & 28 & & & 35.847 & & 16. & 16. & & 15.222 & 11.295 & 32.122 & & 16 & & 34 & 34.256 \\
\hline $17: 00$ & & 30.612 & & 29.11 & & & & & & & 28 & & 32. & 35.85 & & & 16. & 22 & 15 & 11.293 & 32.119 & & & & & 34.254 \\
\hline 9/30/11 7:30 & & 30.61 & & 29.12 & & & & 30 & & & 28. & & 32 & 35.847 & 33.055 & 16. & 16.138 & 22.987 & 15.2 & 11.293 & 32.117 & 32.928 & & & $3 \quad 26.904$ & 34.254 \\
\hline 9/30/11 8:00 & & 30.612 & 30.1 & 29.113 & 30. & & & 30. & 30. & & 28. & 26.119 & 32.0 & 35.852 & 33.057 & 16.897 & 16.143 & 22.983 & 15. & 11.295 & 32.117 & 32.929 & 30.013 & 31.985 & $5 \quad 26.906$ & 34.252 \\
\hline 9/30/11 8:30 & & 30.615 & 30.1 & 29.115 & & & 29. & 30. & 30. & 29. & 28.4 & 26.114 & 32.03 & 35.847 & 33.055 & 16.899 & 16.15 & 22.98 & 15.217 & 11.297 & 32.117 & 32.929 & 30.013 & 31.988 & $\begin{array}{l}3 \\
3\end{array}$ & 34.252 \\
\hline 9/30/11 9:00 & & 30.617 & 30.181 & 29.11 & 30.3 & & 29.521 & 30.611 & 30.059 & 29.881 & 28.437 & 26.117 & 32.03 & 35.85 & 33.057 & 16.902 & 16.152 & 22.978 & 15.224 & 11.297 & 32.121 & 32.933 & 30.02 & 31.99 & 26.906 & 34.252 \\
\hline 9/30/11 9:30 & & 30.615 & 30.179 & 29.108 & 30.337 & & 29.53 & 30.608 & 30.056 & 29.881 & 28.435 & 26.11 & 32.028 & 35.85 & 33.057 & 16.902 & 16.157 & 22.976 & 15.229 & 11.295 & 32.114 & 32.931 & 30.009 & 31.988 & $\begin{array}{l}3 \\
3\end{array}$ & 34.254 \\
\hline 9/30/11 10:00 & & 30.615 & 30.181 & 29.103 & 30.334 & & 29.537 & 30.611 & 30.054 & 29.881 & 28.432 & 26.107 & 32.03 & 35.85 & 33.059 & 16.902 & 16.157 & 22.973 & 15.236 & 11.297 & 32.117 & 32.936 & 30.009 & 31.99 & 26.906 & 34.254 \\
\hline 9/30/11 10:30 & & 30.612 & 30.177 & 29.094 & 30.33 & & 29.547 & 30.611 & 30.054 & 29.881 & 28.428 & 26.1 & 32.03 & 35.85 & 33.06 & 16.904 & 16.157 & 22.971 & 15.243 & 11.297 & 32.115 & 32.929 & 30.011 & 31.988 & $\begin{array}{l}3 \quad 26.909 \\
\end{array}$ & 34.254 \\
\hline 9/30/11 11:00 & & 30.617 & 30.174 & 29.085 & 30.33 & & 29.558 & 30.609 & 30.054 & 29.881 & 28.425 & 26.09 & 32.03 & 35.847 & 33.06 & 16.906 & 16.157 & 22.969 & 15.245 & 11.297 & 32.112 & 32.931 & 30.006 & 31.988 & $\begin{array}{l}3 \quad 26.909 \\
\end{array}$ & 34.254 \\
\hline 9/30/11 11:30 & & 30.615 & 30.177 & 29.075 & 30.325 & & 29.565 & 30.611 & 30.054 & 29.879 & 28.421 & 26.078 & 32.028 & 35.845 & 33.062 & 16.906 & 16.157 & 22.967 & 15.25 & 11.297 & 32.11 & 32.931 & 29.999 & 31.988 & $\begin{array}{l}36.909 \\
3\end{array}$ & 34.254 \\
\hline 9/30/11 12:00 & & 30.61 & 30.172 & 29.068 & 30.318 & & 29.572 & 30.611 & 30.054 & 29.876 & 28.416 & 26.071 & 32.03 & 35.847 & 33.057 & 16.904 & 16.157 & 22.964 & 15.259 & 11.295 & 32.105 & 32.931 & 29.992 & 31.988 & $\begin{array}{l}3 \\
26.909\end{array}$ & 34.254 \\
\hline 9/30/11 12:30 & & 30.61 & 30.165 & 29.05 & 30.311 & & 29.587 & 30.608 & 30.054 & 29.872 & 28.409 & 26.062 & 32.028 & 35.852 & 33.057 & 16.906 & 16.157 & 22.964 & 15.266 & 11.293 & 32.098 & 32.928 & 29.983 & 31.985 & $5 \quad 26.909$ & 34.254 \\
\hline
\end{tabular}




\begin{tabular}{|c|c|c|c|c|c|c|c|c|c|c|c|c|c|c|c|c|c|c|c|c|c|c|c|c|c|c|}
\hline Well & $\begin{array}{l}\text { (ft TOC) } \\
\text { (f) }\end{array}$ & $\begin{array}{l}\text { (ft TOC) } \\
\text { (f }\end{array}$ & $\begin{array}{l}\text { (ft TOC) } \\
\text { (ft }\end{array}$ & $\begin{array}{l}\text { (ft TOC) } \\
\text { (f) }\end{array}$ & $\begin{array}{l}\text { (ft TOC) } \\
\text { (n) }\end{array}$ & $\begin{array}{l}\text { (ft TOC) } \\
\text { (f) }\end{array}$ & $\begin{array}{l}\text { (ift TOC) } \\
\text { (ft }\end{array}$ & $\begin{array}{l}\text { (ft TOC) } \\
\text { (f) }\end{array}$ & $\begin{array}{l}\text { (ft TOC) } \\
\text { (f) }\end{array}$ & $\begin{array}{l}\text { (ft TOC) } \\
\text { (f) }\end{array}$ & $\begin{array}{l}\text { (ft TOC) } \\
\text { (f) }\end{array}$ & $\begin{array}{l}\text { (ft TOC) } \\
\text { (f) }\end{array}$ & $\begin{array}{l}\text { (ft TOC) } \\
\end{array}$ & $\begin{array}{l}\text { (ft TOC) } \\
\text { (1) Ton }\end{array}$ & $\begin{array}{l}\text { (ft TOC) } \\
\text { (f) }\end{array}$ & $\begin{array}{l}\text { (ft TOC) } \\
\text { (n) }\end{array}$ & $\begin{array}{l}\text { (ft TOC) } \\
\end{array}$ & (ft TOC) & $\begin{array}{l}\text { (ft TOC) } \\
\text { (1) }\end{array}$ & $\begin{array}{l}\text { (ft TOC) } \\
\text { (f) }\end{array}$ & $\begin{array}{l}\text { (ft TOC) } \\
\end{array}$ & $\begin{array}{l}\text { (ft TOC) } \\
\text { (f) }\end{array}$ & $\begin{array}{l}\text { (ft TOC) } \\
\text { (ft }\end{array}$ & $\begin{array}{l}\text { (ft TOC) } \\
\text { (f) }\end{array}$ & $\begin{array}{l}\text { (ft TOC) } \\
\text { (f) }\end{array}$ & $\begin{array}{l}\text { (ft TOC) } \\
\text { (1) }\end{array}$ \\
\hline 3:00 & & 30.61 & 30.163 & 29.036 & 30.306 & & 29.596 & 30.604 & 30 & 25 & 28.4 & 26.0 & 32. & 35 & 33 & 16.906 & 16.157 & 22.967 & 15 & 11.293 & 4 & 26 & 98 & 31.983 & 26.909 & 34.254 \\
\hline 11 13:30 & & 30.608 & 30.156 & 29.026 & 30.297 & & 29.605 & 30.603 & 30.049 & 29.862 & 28.393 & 26.031 & 32.023 & 35.84 & 33.052 & 16.906 & 16.157 & 22.969 & 15.281 & 11.29 & 32.084 & .926 & 29.971 & 31.985 & 26.909 & 34.254 \\
\hline $1114: 00$ & & 30.605 & 30.149 & 29.015 & 30.283 & & 29.612 & 30.599 & 30.049 & 29.858 & 28.384 & 26.016 & 32.018 & 35.838 & 33.048 & 16.904 & 16.155 & 22.962 & 15.288 & 11.288 & 32.077 & 2.921 & 9.952 & 31.978 & 26.909 & 34.254 \\
\hline o/11 14:30 & & 30.601 & 30.139 & 29.005 & 30.271 & & 29.615 & 30.599 & 30.045 & 29.848 & 28.379 & 26.011 & 32.016 & 35.836 & 33.045 & 16.902 & 16.154 & 22.962 & 15.297 & 11.286 & 32.066 & 8.919 & 29.945 & 31.976 & 26.909 & 34.254 \\
\hline 9/30/11 15:00 & & 30.598 & 30.132 & 28.996 & 30.262 & & 29.619 & 30.594 & 30.047 & 29.841 & 28.372 & 26.002 & 32.009 & 35.824 & 33.043 & 16.902 & 16.15 & 22.967 & 15.299 & 11.279 & 32.059 & 32.914 & 29.931 & 31.971 & 26.909 & 34.252 \\
\hline 9/30/11 15:30 & & 30.598 & 30.128 & 28.989 & 30.253 & & 29.622 & 30.589 & 30.047 & 29.837 & 28.365 & 25.992 & 32.007 & 35.831 & 33.041 & 16.897 & 16.145 & 22.969 & 15.297 & 11.274 & 32.049 & 32.907 & 29.922 & 31.967 & 26.909 & $\begin{array}{l}34.25 \\
34.252\end{array}$ \\
\hline 9/30/11 16:00 & & 30.598 & 30.119 & 28.979 & 30.241 & & 29.619 & 30.589 & 30.047 & 29.83 & 28.358 & 25.983 & 32 & 35.819 & 33.034 & 16.897 & 16.145 & 22.971 & 15.304 & 11.272 & 32.045 & 32.902 & 29.912 & 31.962 & 26.909 & $\begin{array}{l}34.25 \\
34.252\end{array}$ \\
\hline 9/30/11 16:30 & & 30.596 & 30.112 & 28.977 & 30.232 & & 29.619 & 30.587 & 30.045 & 29.823 & 28.353 & 25.978 & 32 & 35.821 & 33.031 & 16.895 & 16.14 & 22.971 & 15.309 & 11.269 & 32.038 & 32.902 & 29.898 & 31.96 & 26.909 & 34.249 \\
\hline 9/30/11 17:00 & & 30.596 & 30.105 & 28.977 & 30.22 & & 29.612 & 30.584 & 30.047 & 29.818 & 28.349 & 25.976 & 31.993 & 35.817 & 33.027 & 16.895 & 16.136 & 22.973 & 15.313 & 11.262 & 32.028 & 32.898 & 29.893 & 31.952 & 26.909 & 34.247 \\
\hline 9/30/11 17:30 & & 30.594 & 30.102 & 28.979 & 30.213 & & 29.605 & 30.582 & 30.047 & 29.811 & 28.349 & 25.976 & 32.316 & 35.814 & 33.388 & 16.892 & 16.138 & 22.978 & 15.309 & 11.624 & 32.103 & 33.22 & 29.889 & 32.272 & 26.909 & 34.249 \\
\hline 9/30/11 18:00 & & 30.596 & 30.098 & 28.979 & 30.208 & & 29.601 & 30.58 & 30.047 & 29.807 & 28.344 & 25.98 & 32.571 & 35.807 & 33.667 & 16.892 & 16.154 & 22.976 & 15.302 & 11.902 & 32.203 & 33.469 & 29.896 & 32.525 & 26.909 & 34.247 \\
\hline 9/30/11 18:30 & & 30.594 & 30.093 & 28.984 & 30.208 & & 29.589 & 30.58 & 30.052 & 29.804 & 28.344 & 25.98 & 32.737 & 35.812 & 33.847 & 16.89 & 16.178 & 22.976 & 15.304 & 12.083 & 32.278 & 33.639 & 29.91 & 32.689 & 26.911 & 34.245 \\
\hline 9/30/11 19:00 & & 30.596 & 30.091 & 28.993 & 30.208 & & 29.577 & 30.58 & 30.054 & 29.8 & 28.346 & 25.988 & 32.596 & 35.807 & 33.674 & 16.89 & 16.201 & 22.973 & 155.302 & 11.911 & 32.28 & 33.492 & 29.915 & 32.548 & 26.909 & 34.245 \\
\hline 9/30/11 19:30 & & 30.596 & 30.088 & 28.996 & 30.211 & & 29.568 & 30.577 & 30.052 & 29.797 & 28.344 & 25.997 & 32.423 & 35.807 & 33.486 & 16.89 & 16.208 & 22.973 & 15.306 & 11.721 & 32.212 & 33.319 & 29.919 & 32.377 & 26.909 & 34.245 \\
\hline 9/30/11 20:00 & & 30.598 & 30.091 & 29.003 & 30.218 & & 29.558 & 30.58 & 30.052 & 29.797 & 28.349 & 26.002 & 32.334 & 35.81 & 33.39 & 16.89 & 16.208 & 22.976 & 15.295 & 11.624 & 32.184 & 33.234 & 29.922 & 32.293 & 26.911 & 34.245 \\
\hline 9/30/11 20:30 & & 30.598 & 30.091 & 29.007 & 30.225 & & 29.551 & 30.582 & 30.056 & 29.8 & 28.346 & 26.009 & 32.278 & 35.814 & 33.327 & 16.89 & 16.208 & 22.971 & 15.29 & 11.563 & 32.166 & 33.177 & 29.931 & 32.236 & 26.911 & 34.242 \\
\hline 9/30/11 21:00 & & 30.601 & 30.093 & 29.007 & 30.227 & & 29.544 & 30.584 & 30.054 & 29.797 & 28.349 & 26.019 & 32.236 & 35.812 & 33.285 & 16.888 & 16.204 & 22.973 & 15.285 & 11.521 & 32.15 & 33.137 & 29.931 & 32.194 & 26.911 & 34.245 \\
\hline 9/30/11 21:30 & & 30.603 & 30.098 & 29.007 & 30.232 & & 29.544 & 30.582 & 30.054 & 29.8 & 28.346 & 26.016 & 32.208 & 35.81 & 33.249 & 16.89 & 16.204 & 22.973 & 15.283 & 11.488 & 32.145 & 33.106 & 29.933 & 32.166 & 26.911 & 34.242 \\
\hline 9/30/11 22:00 & & 30.603 & 30.098 & 29.019 & 30.239 & & 29.537 & 30.582 & 30.056 & 29.8 & 28.349 & 26.019 & 32.182 & 35.817 & 33.226 & 16.89 & 16.206 & 22.973 & 15.278 & 11.46 & 32.135 & 33.085 & 29.938 & 32.143 & 26.911 & 34.247 \\
\hline 9/30/11 22:30 & & 30.603 & 30.098 & 29.022 & 30.239 & & 29.53 & 30.584 & 30.059 & 29.797 & 28.351 & 26.031 & 32.163 & 35.812 & 33.205 & 16.892 & 16.211 & 22.971 & 15.276 & 11.443 & 32.126 & 3.064 & 29.936 & 32.124 & 26.911 & 34.245 \\
\hline 9/30/11 23:00 & & 30.605 & 30.098 & 29.024 & 30.24 & & 29.525 & 30.587 & 30.059 & 29.797 & 28.353 & 26.033 & 32.149 & 35.812 & 33.191 & 16.892 & 6.213 & 22.971 & 15.269 & 11.427 & 2.121 & 52 & 29.938 & 32.11 & 5.913 & 34.247 \\
\hline 9/30/11 23:30 & & 30.608 & 30.102 & 29.029 & 30.246 & & 29.523 & 30.591 & 30.059 & 29.797 & 28.353 & 26.038 & 32.14 & 35.821 & 33.177 & 16.895 & 6.218 & 22.971 & 15.266 & 11.415 & 1.121 & 3.038 & 29.938 & 32.098 & 9911 & 4.249 \\
\hline $10 / 1 / 110: 00$ & & 30.61 & 30.105 & 29.029 & 30.25 & & 29.521 & 30.592 & 30.059 & 29.8 & 28.353 & 26.043 & 32.128 & 35.824 & 33.167 & 16.895 & 16.218 & 22.971 & 15.262 & 11.406 & 2.119 & 33.03 & 29.95 & 32.089 & .913 & 4.249 \\
\hline 10/1/11 0:30 & & 30.608 & 30.105 & 29.029 & 30.253 & & 29.523 & 30.594 & 30.059 & 29.8 & 28.353 & 26.043 & 32.121 & 35.826 & 33.158 & 16.895 & 16.218 & 22.971 & 15.264 & 11.394 & 32.122 & 33.023 & 29.948 & 32.079 & 26.913 & 34.252 \\
\hline 10/1/11 1:00 & & 30.61 & 30.107 & 29.033 & 30.255 & & 29.518 & 30.594 & 30.061 & 29.8 & 28.356 & 26.043 & 32.117 & 35.826 & 33.151 & 16.895 & 16.22 & 22.973 & 15.257 & 11.391 & 32.114 & 33.014 & 29.95 & 32.074 & 26.913 & 34.254 \\
\hline 10/1/11 1:30 & & 30.61 & 30.107 & 29.043 & 30.255 & & 29.518 & 30.594 & 30.061 & 29.8 & 28.358 & 26.047 & 32.107 & 35.826 & 33.144 & 16.897 & 16.218 & 22.973 & 15.257 & 11.384 & 32.107 & 33.009 & 29.952 & 32.07 & 26.913 & 4.256 \\
\hline 10/1/11 2:00 & & 30.61 & 30.107 & 29.038 & 30.26 & & 29.516 & 30.596 & 30.059 & 29.8 & 28.36 & 26.057 & 32.103 & 35.831 & 33.137 & 16.897 & 16.218 & 22.973 & 15.257 & 11.377 & 32.11 & 33.002 & 29.955 & 32.06 & 26.913 & 34.256 \\
\hline 10/1/11 2:30 & & 30.612 & 30.112 & 29.033 & 30.262 & & 29.521 & 30.599 & 30.059 & 29.802 & 28.358 & 26.052 & 32.098 & 35.833 & 33.132 & 16.899 & 16.213 & 22.971 & 15.252 & 11.368 & 32.105 & 33 & 29.952 & 32.056 & 26.913 & 34.259 \\
\hline 10/1/11 3:00 & & 30.615 & 30.112 & 29.036 & 30.267 & & 29.523 & 30.599 & 30.061 & 29.804 & 28.356 & 26.05 & 32.098 & 35.831 & 33.13 & 16.902 & 16.215 & 22.976 & 15.252 & 11.368 & 32.112 & 32.997 & 29.959 & 32.056 & 26.916 & 34.261 \\
\hline 10/1/11 3:30 & & 30.612 & 30.114 & 29.04 & 30.262 & & 29.518 & 30.603 & 30.064 & 29.802 & 28.358 & 26.05 & 32.086 & 35.833 & 33.123 & 16.902 & 16.211 & 22.976 & 15.255 & 11.359 & 32.105 & 32.99 & 29.955 & 32.049 & 26.913 & 34.261 \\
\hline $10 / 1 / 114: 00$ & & 30.612 & 30.112 & 29.043 & 30.262 & & 29.516 & 30.601 & 30.061 & 29.802 & 28.36 & 26.055 & 32.086 & 35.836 & 33.118 & 16.904 & 16.208 & 22.978 & 15.255 & 11.359 & 32.103 & 32.985 & 29.952 & 32.046 & 26.916 & 34.261 \\
\hline $10 / 1 / 114: 30$ & & 30.615 & 30.112 & 29.045 & 30.264 & & 29.518 & 30.601 & 30.064 & 29.802 & 28.363 & 26.057 & 32.082 & 35.836 & 33.113 & 16.902 & 16.204 & 22.976 & 15.257 & 11.351 & 32.101 & 32.983 & 29.957 & 32.039 & 26.916 & 34.261 \\
\hline $10 / 1 / 11$ 5:00 & & 30.617 & 30.112 & 29.05 & 30.264 & & 29.518 & 30.606 & 30.068 & 29.804 & 28.36 & 26.059 & 32.077 & 35.838 & 33.111 & 16.904 & 16.201 & 22.976 & 15.252 & 11.349 & 32.103 & 32.978 & 29.955 & 32.037 & 26.916 & 34.261 \\
\hline 10/1/11 5:30 & & 30.617 & 30.114 & 29.054 & 30.267 & & 29.514 & 30.606 & 30.066 & 29.804 & 28.363 & 26.064 & 32.075 & 35.838 & 33.106 & 16.904 & 16.196 & 22.973 & 15.25 & 11.347 & 32.1 & 32.976 & 29.962 & 32.035 & 26.916 & 34.261 \\
\hline 10/1/11 6:00 & & 30.617 & 30.114 & 29.064 & & & & & 30.068 & 29.804 & 28.367 & 26.069 & & 35.836 & 33.104 & 16.906 & 16.196 & 22.971 & 15.252 & 11.342 & 32.105 & 73 & 29.957 & 32.03 & 6.916 & 34.261 \\
\hline $10 / 1 / 1$ & & & 30.119 & & & & & & & & 28.372 & 26.076 & 32.07 & 35.84 & 33.099 & 16.906 & 16.192 & 22.973 & 15.243 & 11.34 & 32.103 & & 29.966 & 32.028 & 6.918 & 4.261 \\
\hline 10/1/11 7:00 & & 30.622 & 30.121 & 29.078 & 30.274 & & 29.504 & 30.611 & $\begin{array}{l}30.000 \\
30.068\end{array}$ & 29.809 & 28.374 & 26.088 & 32.068 & $\begin{array}{l}35.84 \\
35.845\end{array}$ & 33.097 & $\begin{array}{l}16.909 \\
\end{array}$ & $\begin{array}{l}10.192 \\
16.192\end{array}$ & 22.973 & $\begin{array}{l}15.243 \\
15.243\end{array}$ & $\begin{array}{l}11.344 \\
1.337\end{array}$ & 32.105 & 32.966 & 29.969 & 32.028 & 6.918 & 4.261 \\
\hline 10/1/11 7:30 & & 30.626 & 30.125 & 29.085 & 30.278 & & 29.507 & 30.611 & 30.068 & 29.814 & 28.379 & 26.09 & 32.068 & 35.845 & 33.099 & 16.909 & 16.192 & 22.971 & 15.234 & 11.335 & 32.11 & 32.966 & 29.971 & 32.025 & 26.918 & 4.259 \\
\hline & & 30.629 & 2012 & 29. & 30 & & 2 & 30 & & 29.816 & 28.381 & 201 & 32.065 & 35.847 & 33.097 & 16.911 & 16.199 & 22.971 & 15.236 & 11.335 & 32.115 & 2.966 & 29.983 & 32.025 & 26.92 & 4.261 \\
\hline & & 30 & 30 & 29. & & & & & & & 28 & 26. & 32 & & 33 & & 1 & 22 & 15 & 11. & & 6 & 29.983 & 32 & 26.92 & 4.261 \\
\hline & & & & 29. & & & & 30 & & & & 26. & & & & & & 22 & & & & & 29.992 & 2 & 26.92 & 1.261 \\
\hline & & & 30 & 29. & & & & & & & & 26 & & & & 16. & & 22 & 15 & 11 & & & 29.995 & 32.023 & 26.92 & 4.261 \\
\hline $10 /$ & & & & 29 & & & & & & 29 & & & 32 & & & 1 & 04 & 22. & 15 & 11 & & & 29.999 & 32.023 & 26.92 & 4.261 \\
\hline 10:30 & & 30.626 & 30 & 29. & & & & & & 2 & 28 & 26 & 32.063 & 35. & 33 & 16.918 & 34 & 22. & & 11. & & 2 & 29.995 & 32.023 & 26.923 & 4.261 \\
\hline 10 & & 30.624 & 30 & 29. & & & & & 30 & & 28 & 26 & 32.063 & & 33 & 16.92 & 16.201 & 22.985 & 15.255 & 11.333 & 32.117 & 52 & 29.987 & 32.023 & 26.923 & 4.261 \\
\hline $111: 30$ & & 30 & 30 & 29. & & & & & & & & 26 & 32.061 & & & & 1 & 22.987 & 15. & 11.33 & & & & 32.021 & & 4.259 \\
\hline 10/1/11 12:00 & & 30.622 & 30. & 29.6 & & & & 30. & 30 & 29 & & 26 & 32.056 & 35.852 & 33.088 & 16.918 & 16. & 22.985 & 15. & 11.328 & 32.103 & 32.957 & 29.976 & 32.016 & 26.923 & 34.259 \\
\hline $10 / 1 / 1112: 30$ & & 30.619 & 30. & 29 & & & & 30. & 30. & 29. & 28. & 26. & 32. & 35 & 33. & 16.932 & 16.1 & 22. & 15. & 11.326 & 32.101 & 32.957 & 29.978 & 32.016 & 26.923 & 34.261 \\
\hline 10/1/11 13:00 & & 30.619 & 30.128 & 29.0 & 30. & & 29.589 & 30. & 30. & 29.821 & 28.363 & 26.047 & 32.051 & 35.852 & 33.085 & 16.918 & 16.194 & 22.985 & 15.281 & 11.319 & 32.091 & 32.952 & 29.966 & 32.011 & 26.923 & 34.261 \\
\hline 10/1/11 13:30 & & 30.619 & 30.123 & 29.015 & 30.281 & & 29.598 & 30.608 & 30.064 & 29.816 & 28.353 & 26.033 & 32.049 & 35.85 & 33.083 & 16.92 & 16.192 & 22.987 & 15.285 & 11.316 & 32.091 & 32.95 & 29.959 & 32.006 & 26.923 & 34.259 \\
\hline 10/1/11 14:00 & & 30.617 & 30.114 & 29.012 & 30.271 & & 29.603 & 30.606 & 30.064 & 29.814 & 28.349 & 26.021 & 32.046 & 35.84 & 33.078 & 16.918 & 16.189 & 22.983 & 15.292 & 11.316 & 32.084 & 32.945 & 29.945 & 32.006 & 26.923 & 34.259 \\
\hline 10/1/11 14:30 & & 30.612 & 30.105 & 29.007 & 30.262 & & 29.601 & 30.603 & 30.066 & 29.807 & 28.346 & 26.019 & 32.039 & 35.84 & 33.074 & 16.916 & 16.185 & 22.983 & 15.299 & 11.312 & 32.07 & 32.943 & 29.938 & 32.002 & 26.923 & 34.259 \\
\hline 10/1/11 15:00 & & 30.612 & 30.098 & 29.007 & 30.253 & & 29.601 & 30.603 & 30.066 & 29.802 & 28.344 & 26.016 & 32.035 & 35.84 & 33.067 & 16.913 & 16.185 & 22.985 & 15.302 & 11.307 & 32.066 & 32.938 & 29.926 & 31.997 & 26.923 & 34.259 \\
\hline 10/1/11 15:30 & & 30.615 & 30.095 & 29.005 & 30.248 & & 29.598 & 30.603 & 30.068 & 29.8 & 28.342 & 26.016 & 32.032 & 35.833 & 33.067 & 16.916 & 16.182 & 22.985 & 15.304 & 11.304 & 32.058 & 32.936 & 29.926 & 31.992 & 26.923 & 4.256 \\
\hline $10 / 1 / 1116: 00$ & & 30.612 & 30.091 & 29.005 & 30.241 & & 29.594 & 30.601 & 30.064 & 29.795 & 28.339 & 26.016 & 32.03 & 35.833 & 33.064 & 16.911 & 16.18 & 23.001 & 15.306 & 11.3 & 32.056 & 32.931 & 29.919 & 31.988 & 26.925 & 4.259 \\
\hline 10/1/11 16:30 & & 30.615 & 30.088 & 29 & 30.239 & & 29.596 & 30.599 & 30.059 & 29.793 & 28.337 & 26.014 & 32.028 & 35.833 & 33.06 & 16.916 & 16.175 & 23.052 & 15.306 & 11.295 & 32.056 & 32.928 & 29.915 & 31.988 & 26.923 & 34.256 \\
\hline
\end{tabular}




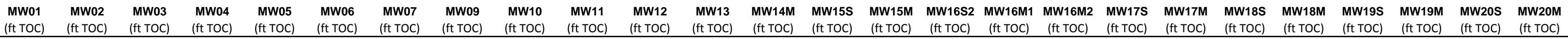

\begin{tabular}{|c|c|c|c|c|c|c|c|c|c|c|c|c|c|c|c|c|c|c|c|c|c|c|c|c|c|c|c|}
\hline Well & $\begin{array}{l}\text { (ft TOC) } \\
\text { (is }\end{array}$ & $\begin{array}{l}\text { (ft TOC) } \\
\text { (f) }\end{array}$ & $\begin{array}{l}\text { (ft TOC) } \\
(\text { tect }\end{array}$ & (ft TOC) & $\begin{array}{l}\text { (ft TOC) } \\
\text { (f) }\end{array}$ & $\begin{array}{l}\text { (ft ToC) } \\
\text { (f) }\end{array}$ & (ft TOC) & $\begin{array}{l}\text { (ft TOC) } \\
\text { (f) }\end{array}$ & $\begin{array}{l}\text { (ft TOC) } \\
\text { (ft) }\end{array}$ & $\begin{array}{l}\text { (ft TOC) } \\
\text { (l) }\end{array}$ & $\begin{array}{l}\text { (ft TOC) } \\
\text { (f) }\end{array}$ & $\begin{array}{l}\text { (ft Toc) } \\
\text { (f) }\end{array}$ & (ft TOC) & $\begin{array}{l}\text { (ft TOC) } \\
\end{array}$ & (ft TOC) & (ft TOC) & $\begin{array}{l}\text { (ft TOC) } \\
\text { (f) }\end{array}$ & (ft TOC) & $\begin{array}{l}\text { (ft TOC) } \\
\end{array}$ & (ft TOC) & $\begin{array}{l}\text { (ft TOC) } \\
\end{array}$ & $\begin{array}{l}\text { (ft TOC) } \\
\text { (f) }\end{array}$ & $\begin{array}{l}\text { (ft TOC) } \\
\end{array}$ & $\begin{array}{l}\text { (ft TOC) } \\
\text { (f) }\end{array}$ & fft TC & b) & (ft TOC) \\
\hline $10 / 1 / 1117: 00$ & & 30.615 & 30.086 & 29.005 & 30.232 & & 29.591 & 30.599 & 30.066 & 29.79 & 28.335 & 26.009 & 32.023 & 35.831 & 33.06 & $5 \quad 16.913$ & 16.175 & 23.078 & 15.306 & 11.295 & 32.049 & 32.926 & 29.915 & 31985 & & 925 & 34.256 \\
\hline $10 / 1 / 1117: 30$ & & 30.612 & 30.084 & 29.005 & 30.229 & & 29.584 & 30.599 & 30.066 & 29.79 & 28.337 & 26.014 & 32.021 & 35.831 & 33.057 & $7 \quad 16.913$ & 16.171 & 23.075 & 15.309 & 11.29 & 32.052 & 32.921 & 29.912 & 31.981 & & .925 & 34.256 \\
\hline $10 / 1 / 1118: 00$ & & 30.612 & 30.081 & 29.005 & 30.227 & & 29.58 & 30.599 & 30.068 & 29.786 & 28.335 & 26.019 & 32.018 & 35.829 & 33.05 & $5 \quad 16.913$ & 16.168 & 23.075 & 15.306 & 11.288 & 32.045 & & & & & .925 & 34.256 \\
\hline $10 / 1 / 1118: 30$ & & 30.615 & 30.081 & 29.007 & 30.225 & & 29.575 & 30.599 & 30.068 & 29.786 & 28.335 & 26.019 & 32.016 & 35.829 & 33.048 & 16.911 & 16.166 & 23.068 & 15.302 & 11.288 & 32.049 & 32.919 & 29.912 & 78 & & .925 & 34.254 \\
\hline 10/1/11 19:00 & & 30.617 & 30.081 & 29.012 & 30.22 & & 29.565 & 30.599 & 30.073 & 29.786 & 28.337 & 26.023 & 32.014 & 35.831 & 33.05 & 16.911 & 16.164 & 23.064 & 15.304 & 11.2 & 32.049 & 32.917 & 29.912 & & & 25 & 34.254 \\
\hline $10 / 1 / 1119: 30$ & & 30.615 & 30.081 & 29.015 & 30.225 & & 29.558 & 30.599 & 30.073 & 29.783 & 28.337 & 26.028 & 32.025 & 35.829 & 33.055 & 16.911 & 16.161 & 23.064 & 15.302 & 11.295 & 32.047 & 32.926 & .912 & .985 & & .925 & 34.254 \\
\hline $10 / 1 / 1120: 00$ & & 30.617 & 30.081 & 29.024 & 30.225 & & 29.551 & 30.601 & 30.073 & 29.783 & 28.339 & 26.033 & 32.016 & 35.831 & 33.05 & 16.911 & 16.161 & 23.059 & $\begin{array}{l}15.297 \\
\end{array}$ & 11.288 & 32.052 & 32.921 & 29.917 & 1.978 & & .925 & 34.254 \\
\hline $10 / 1 / 1120: 30$ & & 30.619 & 30.084 & 29.026 & 30.225 & & 29.544 & 30.603 & 30.075 & 29.786 & 28.342 & 26.038 & 32.014 & 35.833 & 33.048 & $8 \quad 16.913$ & 16.159 & 23.059 & 15.295 & 11.286 & 32.052 & 32.917 & 29.917 & 31.978 & & .925 & 34.254 \\
\hline 10/1/11 21:00 & & 30.619 & 30.086 & 29.026 & 30.229 & & 29.542 & 30.601 & 30.075 & 29.786 & 28.342 & 26.045 & 32.016 & 35.836 & 33.048 & $8 \quad 16.911$ & 16.159 & 23.057 & 15.29 & 11.284 & 32.056 & $5 \quad 32.917$ & 29.926 & 31.976 & & .927 & 34.254 \\
\hline $10 / 1 / 1121: 30$ & & 30.622 & 30.088 & 29.031 & 30.232 & & 29.54 & 30.603 & 30.075 & 29.786 & 28.344 & 26.045 & 32.014 & 35.836 & 33.045 & $5 \quad 16.913$ & 16.159 & 23.054 & 15.288 & 11.283 & 32.059 & 32.917 & 29.929 & 31.976 & & .925 & 34.256 \\
\hline 10/1/11 22:00 & & 30.622 & 30.091 & 29.033 & 30.236 & & 29.535 & 30.603 & 30.073 & 29.788 & 28.342 & 26.047 & 32.018 & 35.838 & 33.045 & $5 \quad 16.913$ & 16.164 & 23.054 & 15.278 & 11.286 & 32.059 & 32.917 & 29.931 & 31.976 & & .927 & 34.254 \\
\hline 10/1/11 22:30 & & 30.622 & 30.091 & 29.033 & 30.236 & & 29.535 & 30.603 & 30.075 & 29.788 & 28.344 & 26.052 & 32.014 & 35.84 & 33.048 & $8 \quad 16.916$ & 16.166 & 23.052 & 15.283 & 11.283 & 32.058 & $\begin{array}{l}32.917 \\
\end{array}$ & 29.931 & 31.976 & & .927 & 34.256 \\
\hline 10/1/11 23:00 & & 30.622 & 30.095 & 29.031 & 30.239 & & 29.537 & 30.606 & 30.071 & 29.788 & 28.342 & 26.052 & 32.014 & 35.838 & 33.048 & $8 \quad 16.916$ & 16.173 & 23.05 & 15.281 & 11.286 & 32.063 & 32.917 & 29.936 & 31.976 & & .927 & 34.256 \\
\hline 10/1/11 23:30 & & 30.624 & 30.093 & 29.033 & 30.239 & & 29.537 & 30.608 & 30.071 & 29.788 & 28.342 & 26.05 & 32.018 & 35.845 & 33.048 & $3 \quad 16.916$ & 16.175 & 23.05 & 15.278 & 11.286 & 32.066 & 52.919 & 29.936 & 31.978 & & .927 & 34.256 \\
\hline $10 / 2 / 110: 00$ & & 30.624 & 30.095 & 29.031 & 30.243 & & 29.535 & 30.608 & 30.075 & 29.788 & 28.344 & 26.052 & 32.018 & 35.843 & 33.05 & 16.916 & 16.178 & 23.054 & 15.274 & 11.288 & 32.065 & 32.919 & 29.941 & 31.978 & & .927 & 34.259 \\
\hline $10 / 2 / 110: 30$ & & 30.624 & 30.093 & 29.04 & 30.241 & & 29.533 & 30.609 & 30.078 & 29.788 & 28.344 & 26.055 & 32.018 & 35.85 & 33.05 & 16.916 & 16.182 & 23.052 & 15.273 & 11.286 & 32.063 & 32.919 & 29.936 & 31.978 & & .927 & 34.259 \\
\hline 10/2/11 1:00 & & 30.624 & 30.093 & 29.045 & 30.243 & & 29.525 & 30.611 & 30.078 & 29.788 & 28.349 & 26.059 & 32.018 & 35.843 & 33.048 & 16.918 & 16.185 & 23.05 & 15.269 & 11.286 & 32.066 & 32.921 & 29.936 & 31.981 & & 5.927 & 34.261 \\
\hline 10/2/11 1:30 & & 30.624 & 30.095 & 29.047 & 30.246 & & 29.523 & 30.611 & 30.078 & 29.788 & 28.351 & 26.069 & 32.021 & 35.843 & 33.048 & 16.918 & 16.185 & 23.052 & 15.271 & 11.29 & 32.065 & $\begin{array}{r}32.921 \\
5\end{array}$ & 29.941 & 31.981 & & 5.927 & 34.261 \\
\hline 10/2/11 2:00 & & 30.629 & 30.098 & 29.045 & 30.248 & & 29.528 & 30.613 & 30.073 & 29.79 & $\begin{array}{l}28.349 \\
28.51\end{array}$ & 26.067 & 32.021 & 35.847 & $\begin{array}{l}33.052 \\
33.052\end{array}$ & 16.92 & $\begin{array}{l}16.187 \\
16.187\end{array}$ & 23.05 & 15.266 & $\begin{array}{l}11.29 \\
11.29\end{array}$ & 32.07 & 32.921 & 29.948 & 31.983 & & 6.93 & 34.263 \\
\hline $10 / 2 / 112: 30$ & & 30.629 & $\begin{array}{r}30.090 \\
30.1\end{array}$ & 29.04 & 30.253 & & 29.53 & 30.613 & 30.075 & 29.793 & $\begin{array}{l}28.349 \\
28.349\end{array}$ & 26.067 & 32.025 & $\begin{array}{l}35.845 \\
35.845\end{array}$ & $\begin{array}{l}33.055 \\
33.052\end{array}$ & $\begin{array}{r}10.922 \\
16.922\end{array}$ & $\begin{array}{l}10.181 \\
16.185\end{array}$ & 23.054 & $\begin{array}{l}15.260 \\
15.264\end{array}$ & $\begin{array}{l}11.29 \\
11.29\end{array}$ & $\begin{array}{l}32.075 \\
32.075\end{array}$ & 32.921 & $\begin{array}{l}29.948 \\
29.948\end{array}$ & 1.985 & & 66.93 & 34.263 \\
\hline 10/2/11 3:00 & & $\begin{array}{l}30.629 \\
30.629\end{array}$ & $\begin{array}{r}30.1 \\
30.102\end{array}$ & $\begin{array}{l}29.045 \\
29.045\end{array}$ & $\begin{array}{l}30.255 \\
30.255\end{array}$ & & $\begin{array}{r}29.533 \\
29.53\end{array}$ & 30.615 & $\begin{array}{l}30.075 \\
30.075\end{array}$ & 29.795 & $\begin{array}{l}28.349 \\
28.349\end{array}$ & $\begin{array}{l}20.001 \\
26.062\end{array}$ & 32.025 & $\begin{array}{r}3.045 \\
35.85\end{array}$ & $\begin{array}{l}33.052 \\
33.052\end{array}$ & $\begin{array}{l}10.922 \\
16.922\end{array}$ & $\begin{array}{l}10.183 \\
16.185\end{array}$ & $\begin{array}{l}23.034 \\
23.054\end{array}$ & $\begin{array}{l}15.264 \\
15.264\end{array}$ & $\begin{array}{l}11.299 \\
11.293\end{array}$ & $\begin{array}{l}32.075 \\
32.075\end{array}$ & $\begin{array}{l}32.924 \\
32.924\end{array}$ & 29.952 & 31.985 & & 66.93 & $\begin{array}{l}34.263 \\
34.266\end{array}$ \\
\hline 10/2/11 3:30 & & 30.629 & 30.102 & 29.047 & 30.257 & & 29.53 & 30.616 & 30.08 & 29.795 & 28.351 & 26.064 & 32.023 & 35.85 & 33.055 & 16.922 & 16.185 & 23.052 & 15.271 & 11.293 & 32.077 & 32.926 & 29.945 & 1.983 & & 6.93 & 34.266 \\
\hline $10 / 2 / 11$ & & & 30.1 & 29.052 & 30.2 & & 29.525 & 30.618 & 30.083 & 29.793 & 28.353 & $\begin{array}{l}20.0069 \\
26.069\end{array}$ & 32.023 & 35.854 & 33.055 & 16.922 & $\begin{array}{l}16.180 \\
16.187\end{array}$ & .052 & 15.264 & $\begin{array}{l}11.295 \\
11.295\end{array}$ & 32.075 & .926 & 948 & 1.985 & & 6.93 & 34.268 \\
\hline $10 / 2 / 1$ & & 30.629 & 30.102 & 29.054 & 30.2 & & 29.53 & 30.615 & 30.075 & 29.793 & 28.353 & 26.071 & & 847 & 33.055 & 16.925 & 16.185 & 23.05 & 266 & 11.295 & 32.077 & 32.928 & 948 & 1.985 & & 6.93 & 268 \\
\hline 10/2/11 5:00 & & 30.631 & 30.105 & 29.061 & 30.26 & & 29.525 & 618 & 30.08 & 29.797 & & & & 35.847 & 33.055 & 16.925 & 16.185 & 23.047 & .255 & $\begin{array}{l}11.295 \\
11.295\end{array}$ & 32.08 & $\begin{array}{r}25.258 \\
32.926\end{array}$ & 9.952 & 1.988 & & 5.932 & $\begin{array}{l}34.270 \\
34.27\end{array}$ \\
\hline 10/2/11 5:30 & & & 30.109 & & 30.262 & & & 30.618 & 30.078 & & 28.358 & 26.081 & 32.028 & 35.854 & 33.057 & 16.925 & 16.185 & 23.052 & 15.259 & 11.297 & 32.077 & 32.928 & 1.957 & 1.985 & & .932 & 34.268 \\
\hline 10/2/11 6:00 & & 30.633 & 30.107 & 29.066 & 30.264 & & 29.523 & 30.62 & 30.078 & 29.797 & 28.363 & 26.081 & 32.028 & 35.854 & 33.055 & 16.927 & 16.192 & 23.047 & 15.255 & 11.288 & 32.082 & 32.929 & .962 & 1.988 & & .932 & 34.27 \\
\hline 10/2/11 6:30 & & 30.636 & 30.112 & 29.068 & 30.269 & & 29.523 & 30.621 & 30.083 & 29.802 & 28.363 & 26.086 & 32.306 & 35.854 & 33.357 & 16.927 & 16.229 & 23.05 & 15.255 & 11.577 & 32.166 & $\begin{array}{l}53.208 \\
\end{array}$ & 29.969 & 2.262 & & .932 & 34.27 \\
\hline 10/2/11 7:00 & & 30.633 & 30.112 & 29.071 & 30.271 & & 29.523 & 30.62 & 30.08 & & 28.365 & 26.088 & 32.196 & 35.854 & 33.24 & 16.929 & 16.253 & 23.052 & 15.259 & 11.455 & 32.161 & 33.101 & 29.971 & 2.157 & & .932 & 34.268 \\
\hline 10/2/11 7:30 & & 30.636 & 30.116 & 29.073 & 30.278 & & 29.523 & 30.623 & 30.085 & 29.804 & 28.367 & 26.09 & 32.156 & 35.859 & 33.196 & 16.929 & 16.26 & 23.052 & 15.245 & 11.406 & 32.147 & 33.061 & 29.983 & 32.117 & & .932 & 34.27 \\
\hline $10 / 2 / 118: 00$ & & 30.636 & 30.116 & 29.075 & 30.281 & & 29.53 & 30.623 & 30.08 & 29.807 & 28.367 & 26.09 & 32.135 & 35.861 & 33.17 & 16.932 & 16.262 & 23.054 & 15.252 & 11.384 & 32.143 & 33.035 & 29.98 & 32.096 & & .934 & 34.27 \\
\hline 10/2/11 8:30 & & 30.636 & 30.119 & 29.078 & 30.285 & & 29.533 & 30.623 & 30.085 & 29.807 & 28.37 & 26.093 & 32.121 & 35.864 & 33.156 & 16.932 & 16.267 & 23.057 & 15.25 & 11.37 & 32.133 & 33.023 & 29.99 & 32.082 & & .932 & 34.27 \\
\hline 10/2/11 9:00 & & 30.636 & 30.119 & 29.073 & 30.288 & & 29.537 & 30.623 & 30.085 & 29.809 & 28.372 & 26.09 & 32.107 & 35.854 & 33.146 & 16.934 & 16.269 & 23.057 & 15.255 & 11.361 & 32.126 & 33.014 & 29.985 & 32.067 & & .932 & 34.27 \\
\hline 10/2/11 9:30 & & 30.636 & 30.125 & 29.066 & 30.292 & & 29.549 & 30.625 & 30.083 & 29.811 & 28.365 & 26.086 & 32.1 & 35.861 & 33.137 & 16.936 & 16.267 & 23.057 & 15.255 & 11.361 & 32.131 & 33.004 & 29.985 & 32.065 & & 8934 & 34.27 \\
\hline 10/2/11 10:00 & & 30.636 & 30.123 & 29.057 & 30.295 & & 29.556 & 30.625 & 30.08 & 29.811 & 28.365 & 26.078 & 32.096 & 35.861 & 33.132 & 16.936 & 16.264 & 23.059 & 15.259 & 11.366 & 32.126 & 32.997 & 29.987 & 32.058 & & 934 & 34.27 \\
\hline 10/2/11 10:30 & & 30.633 & 30.123 & 29.045 & 30.295 & & 29.568 & 30.625 & 30.08 & 29.811 & 28.36 & 26.071 & 34.047 & 35.857 & 34.41 & 16.939 & 16.306 & 23.061 & 15.266 & 12.663 & 32.847 & 35.051 & 30.009 & 34.013 & & .934 & 34.268 \\
\hline 10/2/11 11:00 & & 30.631 & 30.114 & 29.031 & 30. & & 29.577 & 30.62 & $\begin{array}{l}30.083 \\
30.083\end{array}$ & 29.809 & 28.353 & 26.062 & 34.726 & 35.859 & 35.177 & 16. & 16.4 & 23.061 & 15.271 & $\begin{array}{l}13.044 \\
13.44\end{array}$ & 33.138 & 35.713 & 30.053 & 34.684 & & 9.934 & 34.268 \\
\hline 10/2/11 11:30 & & 30.629 & 30.112 & 29.017 & 30. & & 29.594 & 30.618 & 30.078 & 29.807 & 28.344 & 26.047 & 35.219 & 35.857 & 35.697 & 16.93 & $\begin{array}{l}16.4517 \\
16.517\end{array}$ & 23.059 & 15.281 & $\begin{array}{l}13.4989 \\
13.989\end{array}$ & $\begin{array}{l}33.332 \\
33.32\end{array}$ & 191 & 30.089 & 35.169 & & 5.934 & 34.266 \\
\hline $10 / 2 / 1112: 00$ & & 30.624 & 30.107 & 29.007 & 30.3 & & 29.598 & 30.616 & $\begin{array}{l}30.078 \\
30.078\end{array}$ & 29.804 & $\begin{array}{l}20.344 \\
28.342\end{array}$ & $\begin{array}{l}20.041 \\
26.035\end{array}$ & 35.294 & $\begin{array}{l}35.854 \\
35.851\end{array}$ & 35.932 & $\begin{array}{l}10.939 \\
16.939\end{array}$ & $\begin{array}{l}10.511 \\
16.619\end{array}$ & $\begin{array}{l}23.059 \\
23.059\end{array}$ & $\begin{array}{l}15.281 \\
15.283\end{array}$ & $\begin{array}{l}13.539 \\
14.238\end{array}$ & $\begin{array}{l}33.382 \\
33.381\end{array}$ & 236 & 0.119 & 35.24 & & 8934 & 34.263 \\
\hline $10 / 2 / 11$ & & $\begin{array}{l}30.024 \\
30.624\end{array}$ & 30.1 & 28.989 & $\begin{array}{l}30.3 \\
30.3\end{array}$ & & 29.608 & 30.611 & $\begin{array}{l}30.075 \\
30.075\end{array}$ & $\begin{array}{l}29.8749 \\
29.797\end{array}$ & $\begin{array}{l}28.342 \\
28.32\end{array}$ & $\begin{array}{l}20.035 \\
26.026\end{array}$ & $\begin{array}{l}33.244 \\
35.788\end{array}$ & $\begin{array}{l}53.854 \\
35.847\end{array}$ & $\begin{array}{l}36.293 \\
36.293 \\
5\end{array}$ & 16.936 & $\begin{array}{l}10.019 \\
16.715\end{array}$ & 23.073 & $\begin{array}{l}15.29 \\
15.29\end{array}$ & $\begin{array}{l}14.28 \\
14.621\end{array}$ & $\begin{array}{l}33.381 \\
33.544\end{array}$ & 762 & 1.138 & $\begin{array}{l}35.24 \\
35.737\end{array}$ & & 5.932 & $\begin{array}{l}34.263 \\
34.263\end{array}$ \\
\hline 10 & & 30 & $\begin{array}{r}30.1 \\
30.091\end{array}$ & $\begin{array}{l}28.989 \\
28.975\end{array}$ & 30. & & 2 & $\begin{array}{l}30.611 \\
30.611\end{array}$ & 30 & $\begin{array}{r}29.179 \\
29.79\end{array}$ & 28 & $\begin{array}{l}20.026 \\
26.011\end{array}$ & $\begin{array}{r}35.178 \\
36.16\end{array}$ & $\begin{array}{r}35.84 t \\
35.84\end{array}$ & $\begin{array}{l}36.687 \\
36.687\end{array}$ & $\begin{array}{l}10.930 \\
16.934\end{array}$ & $\begin{array}{r}1 \\
1\end{array}$ & $\begin{array}{l}23.087 \\
23.087\end{array}$ & $\begin{array}{l}15.299 \\
15\end{array}$ & $\begin{array}{l}14.021 \\
14.922\end{array}$ & $\begin{array}{l}33.544 \\
33.696\end{array}$ & 125 & 66 & 3 & & 8.934 & $\begin{array}{l}34.263 \\
34.261\end{array}$ \\
\hline $10 /$ & & & 30.086 & 28 & $3 c$ & & & 30 & & 29. & 28 & 25.997 & 36 & 35 & 37.081 & & 16 & 23 & 15 & 15 & - & 8 & 2 & 36 & & 4 & 34.261 \\
\hline $10 /$ & & & 30 & 28 & 30 & & & 30 & & & & & & 35 & 37 & & 17 & & 15 & 15. & 33 & & 1 & & & & 34.261 \\
\hline & & & & & & & & & & & & 25.99 & & & 37. & & & & & 15.9 & & & & & & & 34.259 \\
\hline $10 /$ & & & & & & & 29. & & & & 28 & 25.992 & & 35.826 & 37. & & & & 15. & 16.0 & 34.108 & & 30. & 7 & & & 34.259 \\
\hline & & & & & & & & & & & 28 & & & & 37 & & 17. & & 15.311 & 15. & 33.84 & & & 35. & & & 34.259 \\
\hline $10 /$ & & & & & & & & & & & & & & & 36. & & 17. & & 15. & 14 & 33 & & & & & & 34.256 \\
\hline $10 /$ & & & & 28. & & & & & & & & & & 35. & 35 & & & & 15.318 & 14. & 33.32 & & 30.255 & & & & 34.256 \\
\hline 10/2/11 17:00 & & 30. & $30 . \mathrm{c}$ & $2 \varepsilon$ & & & & 30. & 30. & & 28. & & 34.4 & 35.821 & 35.575 & 16. & 17.2 & 23.082 & 15.313 & 13 & 33.19 & 3 & 30.241 & 34.358 & & .937 & 34.256 \\
\hline 10/2/11 17:30 & & 30.6 & 30.058 & 28.5 & & & & 30. & $30 . \bar{c}$ & 29 & 28. & 26. & 34.1 & 35.821 & 35. & 16. & 17.22 & 23.08 & 15.313 & 13.5 & 33.099 & 35.054 & 30.23 & 34.111 & & 5.937 & 34.256 \\
\hline $10 / 2 / 1118: 00$ & & 30.619 & 30.06 & 28.979 & 30.5 & & 29.5 & 30.5 & 30. & 29 . & 28.3 & 26.016 & 33.9 & 35.821 & 35.102 & 16. & 17.19 & 23.0 & 15.313 & 13.336 & 33.012 & 34.857 & 30.223 & 33.916 & & .937 & 34.259 \\
\hline 10/2/11 18:30 & & 30.619 & 30.058 & 28.984 & 30.5 & & 29.565 & 30.599 & 30.085 & 29.76 & 28.3 & 26.021 & 33.808 & 35.814 & 34.923 & 16.927 & 17.157 & 23.094 & 15.309 & 13.159 & 32.945 & 34.694 & 30.213 & 33.755 & & .937 & 34.256 \\
\hline 10/2/11 19:00 & & 30.622 & 30.06 & 28.989 & 30.516 & & 29.556 & 30.599 & 30.085 & 29.76 & 28.305 & 26.028 & 33.668 & 35.824 & 34.776 & 16.929 & 17.122 & 23.096 & 15.304 & 13.013 & 32.886 & 34.559 & 30.201 & 33.618 & & .937 & 34.256 \\
\hline 10/2/11 19:30 & & 30.624 & 30.065 & 28.993 & 30.514 & & 29.551 & 30.601 & 30.09 & 29.765 & 28.309 & 26.033 & 33.551 & 35.824 & 34.654 & 16.929 & 17.092 & 23.101 & 15.302 & 12.889 & 32.844 & 34.44 & 30.194 & 33.501 & & .939 & 34.256 \\
\hline 10/2/11 20:00 & & 30.626 & 30.067 & 28.998 & 30.509 & & 29.542 & 30.603 & 30.09 & 29.765 & 28.312 & 26.038 & 33.448 & 35.821 & 34.544 & 16.929 & 17.061 & 23.098 & 15.297 & 12.778 & 32.8 & & 30.187 & 33.398 & & & 34.259 \\
\hline & & & & & & & & & & & & & & & & & & & & & & & & & & & \\
\hline
\end{tabular}




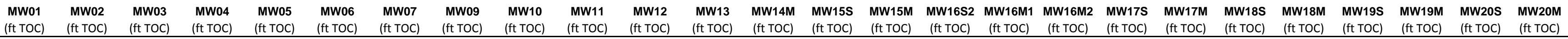

\begin{tabular}{|c|c|c|c|c|c|c|c|c|c|c|c|c|c|c|c|c|c|c|c|c|c|c|c|c|c|c|}
\hline Well & $\begin{array}{l}\text { MW01 } \\
\text { (ft TOC) }\end{array}$ & $\begin{array}{l}\text { MW02 } \\
\text { (ft TOC) }\end{array}$ & $\begin{array}{l}\text { MW03 } \\
\text { (ft ToC) }\end{array}$ & $\begin{array}{r}\text { MW04 } \\
\text { (ft ToC) }\end{array}$ & $\begin{array}{r}\text { MW05 } \\
\text { (ft TOC) }\end{array}$ & $\begin{array}{r}\text { MW06 } \\
\text { (ft TOC) }\end{array}$ & $\begin{array}{l}\text { MW07 } \\
\text { (ft ToC) }\end{array}$ & $\begin{array}{l}\text { MW09 } \\
\text { (ft TOC) }\end{array}$ & $\begin{array}{l}\text { MW10 } \\
\text { (ft TOC) }\end{array}$ & $\begin{array}{l}\text { MW11 } \\
\text { (ft TOC) }\end{array}$ & $\begin{array}{r}\mathrm{MW12} \\
\text { (ft TOC) }\end{array}$ & $\begin{array}{l}\text { MW13 } \\
\text { (ft TOC) }\end{array}$ & $\begin{array}{l}\text { MW144M } \\
\text { (ft TOC) }\end{array}$ & $\begin{array}{l}\text { MW15S } \\
\text { (ft TOC) } \\
\end{array}$ & $\begin{array}{l}\text { MW15M } \\
\text { (ft TOC) }\end{array}$ & $\begin{array}{l}\text { MW16S2 } \\
\text { (ft TOC) }\end{array}$ & $\begin{array}{c}\text { MW16M1 } \\
\text { (ft TOC) }\end{array}$ & $\begin{array}{l}\text { MW16M2 } \\
\text { (ft TOC) }\end{array}$ & $\begin{array}{l}\text { MWW17S } \\
(\mathrm{ft} \text { TOC) }\end{array}$ & $\begin{array}{l}\text { MW17M } \\
\text { (ft TOC) }\end{array}$ & $\begin{array}{l}\text { MW18S } \\
\text { (ft TOC) }\end{array}$ & $\begin{array}{l}\mathrm{MW18M} \\
\text { (ft TOC) }\end{array}$ & $\begin{array}{l}\text { MWW19S } \\
\text { (ft TOC) }\end{array}$ & $\begin{array}{l}\begin{array}{l}\text { WW19M } \\
\text { (ft TOC) }\end{array} \\
\text { (a) }\end{array}$ & $\begin{array}{l}\mathrm{MW} 20 \mathrm{~S} \\
(\mathrm{ft} \text { TOC) }\end{array}$ & $\begin{array}{l}\text { MW20M } \\
\text { (ft toc) }\end{array}$ \\
\hline 10/2/11 21:00 & & 30.629 & 30.074 & 29.005 & 30.5 & & 29.533 & 30.606 & 30.092 & 29.769 & 28.314 & 26.045 & 33.28 & 35.831 & 34.363 & 16.932 & 17.005 & 23.101 & 15.29 & 12.602 & 32.728 & 34.172 & 30.178 & 33.231 & 26.939 & 34.259 \\
\hline 10/2/11 21:30 & & 30.629 & 30.077 & 29.015 & 30.498 & & 29.525 & 30.611 & 30.092 & 29.769 & 28.316 & 26.05 & 33.207 & 35.831 & 34.288 & $3 \quad 16.932$ & 16.979 & 23.096 & 15.285 & 12.525 & 32.695 & 34.099 & 30.173 & 33.159 & 26.939 & 34.259 \\
\hline 10/2/111 22:00 & & 30.629 & 30.079 & 29.017 & 30.491 & & 29.523 & 30.613 & 30.094 & 29.772 & 28.319 & 26.059 & 33.142 & 35.833 & 34.225 & 16.934 & 16.961 & 23.098 & 15.285 & 12.459 & 32.665 & 34.04 & 30.166 & 33.095 & 26.939 & 34.259 \\
\hline 10/2/111 22:30 & & 30.633 & 30.084 & 29.019 & 30.486 & & 29.521 & 30.615 & 30.09 & 29.774 & 28.321 & 26.064 & 33.085 & 35.838 & 34.161 & 16.936 & 16.944 & 23.101 & 15.281 & 12.398 & 32.646 & 33.983 & 30.164 & 33.041 & 26.939 & 34.259 \\
\hline 10/2/11 23:00 & & 30.636 & 30.086 & 29.017 & 30.486 & & 29.518 & 30.613 & 30.094 & 29.776 & 28.321 & 26.064 & 33.036 & 35.838 & 34.11 & 16.936 & 16.928 & 23.101 & 15.276 & 12.344 & 32.621 & 33.931 & 30.161 & 32.99 & 26.941 & 34.261 \\
\hline 10/2/11 23:30 & & 30.638 & 30.093 & 29.022 & 30.481 & & 29.521 & 30.62 & 30.092 & 29.781 & 28.323 & 26.064 & 32.989 & 35.84 & 34.061 & 16.936 & 16.912 & 23.101 & 15.278 & 12.296 & 32.602 & 33.883 & 30.157 & 32.943 & 26.939 & 34.259 \\
\hline 10/3/11 0:00 & & 30.636 & 30.093 & 29.026 & 30.474 & & 29.518 & 30.62 & 30.09 & 29.781 & 28.325 & 26.069 & 32.943 & 35.838 & 34.014 & 16.936 & 16.895 & 23.103 & 15.281 & 12.249 & 32.579 & 33.841 & 30.147 & 32.898 & 26.941 & 34.261 \\
\hline 10/3/11 0:30 & & 30.638 & 30.093 & 29.026 & 30.467 & & 29.518 & 30.623 & 30.097 & 29.781 & 28.325 & 26.069 & 32.905 & 35.845 & 33.972 & 16.939 & 16.877 & 23.103 & 15.276 & 12.21 & 32.558 & 33.803 & 30.147 & 32.863 & 26.939 & 34.261 \\
\hline 10/3/11 1:00 & & 30.64 & 30.098 & 29.031 & 30.465 & & 29.516 & 30.623 & 30.099 & 29.783 & 28.328 & 26.074 & 32.865 & 35.845 & 33.934 & 16.939 & 16.858 & 23.11 & 15.274 & 12.17 & 32.546 & 33.765 & 30.145 & 32.823 & 26.941 & 34.261 \\
\hline $10 / 3 / 111: 30$ & & 30.64 & 30.1 & 29.026 & 30.46 & & 29.521 & 30.623 & 30.097 & 29.786 & 28.325 & 26.076 & 32.833 & 35.85 & 33.899 & 16.943 & 16.844 & 23.107 & 15.269 & 12.134 & 32.527 & 33.732 & 30.136 & 32.79 & 26.941 & 34.263 \\
\hline $10 / 3 / 112: 00$ & & 30.643 & 30.102 & 29.024 & 30.458 & & 29.521 & 30.625 & 30.097 & 29.788 & 28.328 & 26.074 & 32.802 & 35.852 & 33.864 & 16.943 & 16.827 & 23.108 & 15.269 & 12.101 & 32.518 & 33.701 & 30.136 & 32.76 & 26.941 & 34.266 \\
\hline $10 / 3 / 112: 30$ & & 30.64 & 30.105 & 29.022 & 30.453 & & 29.525 & 30.625 & 30.097 & 29.788 & 28.323 & 26.071 & 32.774 & 35.85 & 33.833 & 316.943 & 16.811 & 23.107 & 15.269 & 12.071 & 32.499 & 33.673 & 30.133 & 32.732 & 26.941 & 34.266 \\
\hline 10/3/11 3:00 & & 30.64 & 30.105 & 29.024 & 30.449 & & 29.528 & 30.625 & 30.097 & 29.79 & 28.325 & 26.064 & 32.746 & 35.854 & 33.803 & $\begin{array}{l}36.943 \\
3\end{array}$ & 16.795 & 23.105 & 15.271 & 12.043 & 32.485 & 33.644 & .131 & 32.703 & 26.944 & 34.266 \\
\hline 10/3/11 3:30 & & 30.64 & 30.105 & 29.022 & 30.442 & & 29.525 & 30.625 & 30.097 & 29.79 & 28.325 & 26.069 & 32.718 & 35.85 & 33.777 & & 16.781 & 23.105 & 15.269 & 12.017 & 32.467 & 33.616 & 30.119 & 32.675 & 26.941 & 34.266 \\
\hline 10/3/11 4:00 & & 30.638 & 30.1 & 29.019 & 30.435 & & 29.528 & 30.625 & 30.099 & 29.79 & 28.325 & 26.067 & 32.695 & 35.852 & 33.749 & 16.946 & 16.764 & 23.108 & 15.274 & 11.986 & 32.448 & 33.59 & 30.11 & 32.652 & 26.941 & 34.266 \\
\hline 10/3/11 4:30 & & 30.64 & 30.102 & 29.017 & 30.432 & & 29.533 & 30.625 & 30.094 & 29.79 & 28.325 & 26.067 & 32.669 & 35.852 & 33.725 & 16.948 & 16.75 & 23.107 & 15.264 & 11.963 & 32.441 & 33.568 & 30.112 & 32.626 & 26.944 & 34.268 \\
\hline 10/3/11 5:00 & & 30.64 & 30.105 & 29.017 & 30.425 & & 29.535 & 30.627 & 30.094 & 29.79 & 28.323 & 26.062 & 32.648 & 35.859 & 33.702 & 16.948 & 16.736 & 23.105 & 15.269 & 11.939 & 32.429 & 33.547 & 30.103 & 32.605 & 26.944 & 34.268 \\
\hline 10/3/11 5:30 & & 30.643 & 30.105 & 29.019 & 30.418 & & 29.533 & 30.628 & 30.102 & 29.793 & 28.323 & 26.062 & 32.627 & 35.854 & 33.681 & 16.948 & 16.725 & 23.107 & 15.266 & 11.918 & 32.42 & 33.526 & 30.1 & 32.584 & 26.944 & 34.268 \\
\hline 10/3/11 6:00 & & 30.64 & 30.102 & 29.024 & 30.414 & & 29.533 & 30.63 & 30.099 & 29.79 & 28.325 & 26.067 & 33.537 & 35.847 & 33.969 & 16.948 & 16.718 & 23.124 & 15.269 & 12.205 & 32.609 & 34.563 & 30.093 & 33.522 & 26.944 & 34.268 \\
\hline 10/3/11 6:30 & & 30.643 & 30.102 & 29.026 & 30.414 & & 29.528 & 30.627 & 30.099 & 29.79 & 28.328 & 26.071 & 34.796 & 35.85 & 35.188 & 16.946 & 16.783 & 23.124 & 15.266 & 13.432 & 33.201 & 35.796 & 30.131 & 34.756 & 26.944 & 34.268 \\
\hline 10/3/11 7:00 & & 30.643 & 30.102 & 29.036 & 30.421 & & 29.525 & 30.628 & 30.099 & 29.79 & 28.33 & 26.074 & 35.372 & 35.85 & 35.854 & 16.95 & 16.886 & 23.128 & 15.266 & 14.095 & 33.441 & 36.348 & 30.168 & 35.324 & 26.944 & 34.268 \\
\hline 10/3/11 7:30 & & 30.645 & 30.107 & 29.031 & 30.437 & & 29.525 & 30.627 & 30.102 & 29.793 & 28.33 & 26.081 & 35.687 & 35.859 & 36.307 & 16.953 & 17 & 23.135 & 15.262 & 14.546 & 33.605 & 36.63 & 30.213 & 35.632 & 26.946 & 34.27 \\
\hline 10/3/11 8:00 & & 30.647 & 30.109 & 29.038 & 30.463 & & 29.53 & 30.63 & 30.102 & 29.795 & 28.335 & 26.083 & 35.847 & 35.854 & 36.508 & $3 \quad 16.95$ & 17.101 & 23.138 & 15.257 & 14.744 & 33.658 & 36.784 & 30.246 & 35.789 & 26.946 & 34.27 \\
\hline $10 / 3 / 118: 30$ & & 30.647 & 30.114 & 29.038 & 30.488 & & 29.528 & 30.63 & 30.104 & 29.8 & 28.335 & 26.088 & 36.389 & 35.857 & 36.912 & 16.953 & 17.194 & 23.138 & 15.257 & 15.15 & 33.852 & 37.355 & 30.279 & 36.336 & 26.946 & 34.27 \\
\hline 10/3/11 9:00 & & 30.647 & 30.116 & 29.047 & 30.509 & & 29.53 & 30.63 & 30.106 & 29.8 & 28.337 & 26.09 & 36.806 & 35.857 & 37.301 & 16.955 & 17.297 & 23.133 & 15.259 & 15.536 & 34.017 & & 30.312 & 36.751 & 26.946 & 34.27 \\
\hline 10/3/11 9:30 & & 30.65 & 30.119 & 29.038 & 30.542 & & 29.533 & 30.632 & 30.104 & 29.8 & 28.337 & 26.09 & 37.157 & 35.859 & 37.669 & 16.957 & 17.4 & 23.135 & 15.259 & 15.903 & 34.164 & 38.125 & 30.349 & 37.098 & 26.946 & 34.273 \\
\hline 10/3/11 10:00 & & 30.65 & 30.121 & $\begin{array}{l}29.038 \\
29.038\end{array}$ & 30.568 & & 29.54 & 30.63 & 30.104 & $\begin{array}{r}29.80 \\
\end{array}$ & $\begin{array}{l}28.337 \\
28.37\end{array}$ & 26.088 & 37.461 & 35.859 & 37.995 & 16.957 & $\begin{array}{l}17.501 \\
\end{array}$ & 23.133 & 15.264 & 16.229 & 34.288 & 38.419 & 30.38 & 37.396 & 26.937 & 34.277 \\
\hline 10/3/11 10:30 & & $\begin{array}{l}30.05 \\
30.65\end{array}$ & 30.125 & $\begin{array}{l}29.031 \\
29.031\end{array}$ & $\begin{array}{l}30.500 \\
30.593 \\
\end{array}$ & & 29.547 & $\begin{array}{l}30.632 \\
30.632\end{array}$ & $\begin{array}{l}30.104 \\
30.104\end{array}$ & 29.804 & $\begin{array}{l}28.337 \\
28.337\end{array}$ & $\begin{array}{l}20.000 \\
26.093\end{array}$ & 37.606 & $\begin{array}{l}35.051 \\
35.861\end{array}$ & 38.222 & 16.962 & $\begin{array}{l}17.599 \\
17.599\end{array}$ & 23.131 & $\begin{array}{l}15.264 \\
15.264\end{array}$ & 16.46 & $\begin{array}{l}34.200 \\
34.379\end{array}$ & $\begin{array}{l}38.546 \\
38.546\end{array}$ & $\begin{array}{l}30.400 \\
30.411\end{array}$ & 37.539 & 26.937 & 34.277 \\
\hline 10/3/11 11:00 & & $\begin{array}{l}30.603 \\
30.647\end{array}$ & 30.125 & 29.024 & $\begin{array}{l}30.393 \\
30.619\end{array}$ & & $\begin{array}{l}29.547 \\
29.554\end{array}$ & $\begin{array}{l}30.032 \\
30.632\end{array}$ & $\begin{array}{l}30.102 \\
30.102\end{array}$ & $\begin{array}{l}29.804 \\
29.804\end{array}$ & 28.332 & $\begin{array}{l}20.083 \\
26.083\end{array}$ & 37.686 & $\begin{array}{l}35.801 \\
35.859\end{array}$ & $\begin{array}{l}38.222 \\
38.325\end{array}$ & $\begin{array}{l}\begin{array}{r}10.962 \\
16.96\end{array} \\
\end{array}$ & $\begin{array}{l}17.687 \\
17.597\end{array}$ & 23.128 & $\begin{array}{l}15.264 \\
15.266\end{array}$ & $\begin{array}{l}16.40 \\
16.563\end{array}$ & $\begin{array}{l}34.395 \\
34.395\end{array}$ & $\begin{array}{l}30.360 \\
38.627\end{array}$ & $\begin{array}{l}30.411 \\
30.432\end{array}$ & $\begin{array}{l}37.619 \\
37.619\end{array}$ & 26.937 & 34.277 \\
\hline 10 & & & 30 & 29.017 & & & 61 & 30.632 & 30.104 & 29.807 & 28 & 26.078 & 36.209 & 35.857 & 37.451 & 16.962 & 17.751 & 23.131 & 15.274 & 15.686 & 34.017 & 066 & .448 & 6.127 & 26.937 & 34.28 \\
\hline 10 & & 30 & 21 & 29.01 & & & & 30.63 & 30.102 & 29.804 & 28.328 & 26.074 & 35.526 & 35.859 & 36.736 & 16.96 & 17.737 & 28 & 78 & 14.972 & 33.726 & 93 & 27 & 56 & 37 & 277 \\
\hline $10 / 3 / 11$ & & & & 29.003 & 30. & & & 30.632 & 30.104 & 29.804 & $\begin{array}{l}28.323 \\
28.323\end{array}$ & $\begin{array}{l}26.064 \\
\end{array}$ & 35.098 & 35.864 & 36.281 & 16.964 & $\begin{array}{l}17.704 \\
\end{array}$ & 128 & $\begin{array}{l}15.278 \\
15.283\end{array}$ & $\begin{array}{r}14.9 / 2 \\
14.52\end{array}$ & $\begin{array}{l}33.126 \\
33.556\end{array}$ & 971 & 422 & 5.031 & 6.939 & 275 \\
\hline 10/3/11 13:00 & & 30.645 & 30.119 & 28.989 & 30.675 & & 29.582 & 30.63 & 30.104 & 29.802 & 28.316 & 26.057 & 34.784 & 35.854 & 35.948 & 16.962 & 17.662 & 23.126 & 15.29 & $\begin{array}{l}14.187 \\
14.27\end{array}$ & $\begin{array}{l}33.427 \\
33.400\end{array}$ & $\begin{array}{l}35.66 \\
35.17\end{array}$ & 394 & 4.721 & 26.939 & 34.275 \\
\hline 10/3/11 13:30 & & 30.645 & 30.112 & 28.984 & 30.675 & & 29.589 & 30.627 & 30.104 & 29.8 & 28.309 & 26.043 & 34.536 & 35.854 & 35.685 & 16.964 & 17.62 & 23.126 & 15.297 & 13.921 & 33.322 & 35.416 & 30.373 & 34.477 & 26.939 & 34.275 \\
\hline 10/3/11 14:00 & & 30.643 & 30.109 & 28.982 & & & & 30.627 & 30.104 & 29.795 & 28.307 & 26.04 & 34.333 & 35.85 & 35.472 & 16.962 & 17.575 & 23.121 & 15.304 & 13.709 & 33.229 & 35.22 & 354 & 4.283 & 26.939 & 34.273 \\
\hline 10/3/11 14:30 & & 30.64 & 30.102 & 28.984 & 30. & & & 30.627 & 30.104 & 29.793 & 28.309 & 26.038 & 34.164 & 35.843 & 35.292 & 16.962 & 17.536 & 23.121 & 15.309 & 13.531 & 33.15 & & 30.328 & 34.114 & 26.937 & 34.273 \\
\hline $10 / 3 / 1115: 00$ & & 30.64 & 30.098 & 28.982 & 30.642 & & 29.587 & 30.625 & 30.104 & 29.79 & 28.305 & 26.038 & 34.019 & 35.847 & 35.142 & 16.96 & 17.494 & 23.121 & 15.309 & 13.375 & 33.089 & 34.909 & 30.309 & 33.968 & 26.937 & 34.27 \\
\hline $10 / 3 / 1115: 30$ & & 30.643 & 30.095 & 28.984 & 30.631 & & 29.582 & 30.627 & 30.111 & 29.788 & 28.307 & 26.038 & 33.895 & 35.843 & 35.01 & 16.96 & 17.454 & 23.126 & 15.311 & 13.249 & 33.029 & 34.784 & 30.293 & 33.844 & 26.941 & 34.27 \\
\hline $10 / 3 / 1116: 00$ & & 30.643 & 30.098 & 28.982 & 30.621 & & 29.58 & 30.625 & 30.104 & 29.788 & 28.305 & 26.04 & 33.785 & 35.845 & 34.895 & 16.96 & 17.419 & 23.128 & 15.313 & 13.131 & 32.982 & 34.677 & 30.279 & 33.736 & 26.939 & 34.273 \\
\hline 10/3/11 16:30 & & 30.645 & 30.095 & 28.984 & 30.61 & & 29.577 & 30.625 & 30.106 & 29.786 & 28.305 & 26.038 & 33.691 & 35.843 & 34.79 & 16.96 & 17.381 & 23.163 & 15.313 & 13.028 & 32.94 & 34.58 & 30.272 & 33.642 & 26.939 & 34.273 \\
\hline 10/3/11 17:00 & & 30.645 & 30.098 & 28.986 & 30.598 & & 29.57 & 30.628 & 30.109 & 29.788 & 28.307 & 26.043 & 33.603 & 35.84 & 34.701 & 16.962 & 17.351 & 23.186 & 15.318 & 12.934 & 32.9 & 34.495 & 30.26 & 33.555 & 26.941 & 34.273 \\
\hline 10/3/11 17:30 & & 30.645 & 30.098 & 28.991 & 30.589 & & 29.568 & 30.63 & 30.106 & 29.788 & 28.305 & 26.047 & 33.525 & 35.838 & 34.619 & 16.962 & 17.318 & 23.186 & 15.313 & 12.854 & 32.861 & 34.419 & 30.246 & 33.478 & 26.939 & 34.273 \\
\hline 10/3/11 18:00 & & 30.647 & 30.098 & 28.991 & 30.577 & & 29.561 & 30.63 & 30.111 & 29.788 & 28.307 & 26.047 & 33.453 & 35.845 & 34.544 & 16.962 & 17.288 & 23.184 & 15.313 & 12.778 & 32.83 & 34.345 & 30.244 & 33.407 & 26.941 & 34.273 \\
\hline 10/3/11 18:30 & & 30.65 & 30.1 & 28.996 & 30.568 & & 29.556 & 30.63 & 30.111 & 29.788 & 28.309 & 26.05 & 33.387 & 35.843 & 34.476 & 16.962 & 17.26 & 23.184 & 15.316 & 12.713 & 32.805 & 34.284 & .234 & 33.342 & 26.941 & 34.273 \\
\hline 10/3/11 19:00 & & 30.652 & 30.102 & 29.007 & 30.55 & & 29.544 & 30.632 & 30.111 & 29.79 & 28.314 & 26.057 & 33.331 & 35.845 & 34.417 & 16.9 & 17.234 & 23.186 & 15.3 & 12.649 & 32.781 & 34.225 & 0.227 & 85 & 26.941 & 34.273 \\
\hline 10/3/11 19:30 & & 30.654 & 30.102 & 29.017 & 30.549 & & 29.537 & 30.632 & 30.113 & 29.79 & 28.316 & 26.069 & 33.422 & 35.845 & 34.511 & 16.964 & 17.218 & 23.184 & 15.306 & 12.745 & 32.788 & 34.315 & 30.218 & 74 & 26.941 & 34.273 \\
\hline $10 / 3 / 1$ & & $\begin{array}{l}30.044 \\
30.657\end{array}$ & 30.107 & 29.026 & 30.547 & & 29.525 & 30.635 & 30.118 & 29.793 & $\begin{array}{l}28.321 \\
28.310\end{array}$ & 26.076 & $\begin{array}{l}3.42 \\
33.31\end{array}$ & $\begin{array}{l}53.845 \\
35.845\end{array}$ & $\begin{array}{l}34.389 \\
34.311\end{array}$ & $\begin{array}{l}10.904 \\
16.967\end{array}$ & $\begin{array}{l}17.204 \\
17.204\end{array}$ & $\begin{array}{l}23.1749 \\
23.179\end{array}$ & $\begin{array}{l}15.304 \\
\end{array}$ & $\begin{array}{l}12.626 \\
12.626\end{array}$ & 32.76 & 34 & $\begin{array}{l}r .210 \\
30.22\end{array}$ & $\begin{array}{l}3.262 \\
3.262\end{array}$ & 26.941 & 34.275 \\
\hline $10 / 3$ & & & 30. & 29. & 30 & & & 30. & & 29.795 & 28. & 26. & 33 & 35. & 34 & & 17 & 23. & 15. & 12. & 32.737 & & & 88 & 26.941 & 34.275 \\
\hline & & & 30. & 29. & & & & & & 2 & 28 & & & 35 & 34 & & 17. & 2 & 15. & 12. & 32 & 34.0 & & 17 & 26.941 & 34.275 \\
\hline & & & 30. & 29 & 30. & & & & & & 28 & & & 35. & 34 & & 17 & & 15 & 12. & 32. & 34 & & 8 & 44 & . \\
\hline & & & & 29.0 & & & & & & & & & & & & & & & & 12. & $22-12-1$ & & & & & \\
\hline & & & & & & & & & & & & & & & & & & & 15 & 12 & & & & & & 34. \\
\hline & & & & & & & & & & & & 26.0 & & & & & & & & & & & & & & 34.2 \\
\hline $10 /$ & & & & 29. & & & & & 30 & & 28 & 26 & & 35 & 34. & & 17 & 23 & 15. & 12 & 32 & & & 32 & & 34.2 \\
\hline 10/4/11 0:00 & & 30.664 & 30.135 & 29.045 & 30.526 & & 29.516 & 30.642 & 30.116 & 29.816 & $\begin{array}{l}28.337 \\
20.027\end{array}$ & 20.1 & 32.964 & 35.861 & 34.03 & 16.976 & 17.026 & 23.172 & 15.288 & 12.268 & 32.616 & 33.862 & 30.211 & 32.922 & 26.944 & 34.277 \\
\hline 10/4/11 0:30 & & 30.666 & 30.139 & 29.045 & 30.523 & & 29.521 & 30.644 & 30.121 & 29.818 & 28.337 & 26.098 & 32.936 & 35.864 & 34 & 16.978 & 17.007 & 23.17 & 15.29 & 12.235 & 32.6 & 33.831 & 30.204 & 32.893 & 26.944 & 34.28 \\
\hline
\end{tabular}




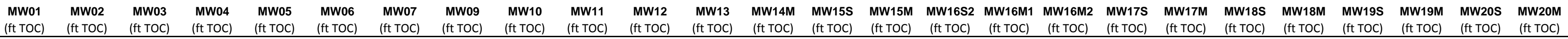

\begin{tabular}{|c|c|c|c|c|c|c|c|c|c|c|c|c|c|c|c|c|c|c|c|c|c|c|c|c|c|c|}
\hline Well & $\begin{array}{l}\text { (ft TOC) } \\
\text { (f) }\end{array}$ & $\begin{array}{l}\text { (ft TOC) } \\
\text { (f) }\end{array}$ & $\begin{array}{l}\text { (ft TOC) } \\
\text { (f) }\end{array}$ & (ft TOC) & (ft Toc) & $\begin{array}{l}\text { (ft ToC) } \\
\text { (f) }\end{array}$ & 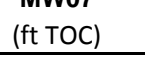 & (ft TOC) & $\begin{array}{l}\text { (ft TOC) } \\
\text { (f) }\end{array}$ & $\begin{array}{l}\text { (ft TOC) } \\
\text { (l) }\end{array}$ & $\begin{array}{l}\text { (ft TOC) } \\
\text { (n) }\end{array}$ & $\begin{array}{l}\text { ft TOC) } \\
\text { flats }\end{array}$ & (ft TOC) & $\begin{array}{l}\text { (ft Toc) } \\
\text { (f Tor }\end{array}$ & (ft TOC) & (ft TOC) & (ft TOC) & (ft TOC) & $\begin{array}{l}\text { (ft TOC) } \\
\text { (ft) }\end{array}$ & (ft TOC) & $\begin{array}{l}\text { (ft TOC) } \\
\text { (1) }\end{array}$ & (ft TOC) & $\begin{array}{l}\text { (ft TOC) } \\
\text { (n) }\end{array}$ & (ft TOC) & $\begin{array}{l}\text { (nt TOC) } \\
\text { (nt }\end{array}$ & (ft TOC) \\
\hline 1/11 1:00 & & 30.664 & 30.139 & 29.052 & 30.521 & & 29.514 & 30.644 & 30.121 & 29.821 & 28.339 & 26.098 & 32.908 & 35.866 & 33.969 & 16.978 & 16.989 & 23.168 & 15.288 & 12.21 & 32.588 & 33.805 & 30.194 & 32.865 & 26.946 & 34.28 \\
\hline $10 / 4 / 111: 30$ & & 30.664 & 30.139 & 29.05 & 30.514 & & 29.516 & 30.644 & 30.123 & 29.821 & 28.342 & 26.105 & 32.884 & 35.868 & 33.946 & 16.978 & 16.972 & 23.17 & 15.288 & 12.181 & 32.574 & 33.781 & 30.197 & 32.839 & 26.946 & 34.28 \\
\hline 10/4/11 2:00 & & 30.668 & 30.144 & 29.045 & 30.509 & & 29.523 & 30.647 & 30.118 & 29.823 & 28.342 & 26.102 & 32.858 & 35.864 & 33.92 & 16.978 & 16.956 & 23.168 & 15.283 & 12.155 & 32.565 & 33.758 & 30.199 & 32.816 & 26.946 & 34.282 \\
\hline $\begin{array}{l}10 / 4 / 112: 30 \\
\text { lat }\end{array}$ & & 30.668 & 30.146 & 29.045 & 30.509 & & 29.525 & 30.644 & 30.121 & 29.825 & 28.342 & 26.095 & 32.84 & 35.868 & 33.894 & 16.981 & 16.942 & 23.168 & 15.283 & 12.132 & 32.553 & 33.736 & 30.19 & & & 34.282 \\
\hline $\begin{array}{l}\text { 10/4/11 3:00 }\end{array}$ & & 30.666 & 30.149 & 29.05 & 30.502 & & 29.525 & 30.647 & 30.123 & 29.825 & 28.342 & 26.095 & 32.814 & 35.866 & 33.871 & 16.981 & 16.926 & 23.168 & 15.288 & 12.111 & 32.541 & 33.713 & 85 & 74 & & 34.282 \\
\hline 10/4/11 3:30 & & 30.666 & 30.146 & 29.057 & 30.495 & & 29.521 & 30.649 & 30.123 & 29.825 & 28.346 & 26.1 & 32.795 & 35.868 & 33.85 & 16.981 & 16.909 & 23.168 & 15.285 & 12.085 & 32.525 & 33.691 & 0.173 & 2.753 & 26.946 & 34.282 \\
\hline $10 / 4 / 114: 00$ & & 30.668 & 30.149 & 29.064 & 30.493 & & 29.518 & 30.647 & 30.123 & 29.828 & 28.349 & 26.105 & 32.774 & 35.871 & 33.831 & 16.983 & 16.895 & 23.168 & 15.288 & 12.068 & 32.518 & 33.67 & 30.171 & 32.732 & 26.946 & 34.282 \\
\hline $10 / 4 / 114: 30$ & & 30.668 & 30.151 & 29.071 & 30.488 & & 29.514 & 30.651 & 30.13 & 29.83 & 28.353 & 26.112 & 32.755 & 35.864 & 33.812 & 16.981 & 16.884 & 23.165 & 15.281 & 12.05 & 32.511 & 33.656 & 30.173 & 32.715 & 26.946 & 34.284 \\
\hline 10/4/11 5:00 & & 30.675 & 30.153 & 29.078 & 30.491 & & 29.514 & 30.654 & 30.125 & 29.832 & 28.356 & 26.117 & 32.741 & 35.868 & 33.793 & 16.981 & 16.87 & 23.165 & 15.276 & 12.033 & 32.506 & 33.639 & 30.173 & 32.699 & 26.948 & 34.287 \\
\hline 10/4/11 5:30 & & 30.675 & 30.158 & 29.082 & 30.491 & & 29.509 & 30.651 & 30.13 & 29.834 & 28.36 & 26.122 & 32.723 & 35.866 & 33.777 & 16.983 & 16.858 & 23.165 & 15.274 & 12.014 & 32.499 & 33.623 & 30.176 & 32.682 & 26.949 & 34.287 \\
\hline $10 / 4 / 116: 00$ & & 30.678 & 30.16 & 29.089 & 30.491 & & 29.509 & 30.654 & 30.13 & 29.837 & 28.365 & 26.126 & 32.709 & 35.871 & 33.763 & 16.985 & 16.844 & 23.177 & 15.269 & 11.998 & 32.495 & 33.608 & 30.18 & 32.666 & 26.948 & 34.287 \\
\hline 10/4/11 6:30 & & 30.682 & 30.167 & 29.096 & 30.493 & & 29.509 & 30.654 & 30.128 & 29.841 & 28.37 & 26.134 & 32.697 & 35.871 & 33.746 & 16.985 & 16.834 & 23.181 & 15.271 & 11.986 & 32.492 & 33.594 & 30.183 & 32.654 & 26.951 & 34.289 \\
\hline $10 / 4 / 117: 00$ & & 30.682 & 30.17 & 29.096 & 30.493 & & 29.509 & 30.656 & 30.13 & 29.846 & 28.372 & 26.136 & 32.683 & 35.873 & 33.732 & 16.988 & 16.823 & 23.186 & 15.269 & 11.972 & 32.485 & 33.582 & 30.183 & 32.64 & 26.951 & 34.289 \\
\hline $10 / 4 / 117: 30$ & & 30.68 & 30.174 & 29.106 & 30.495 & & 29.507 & 30.656 & 30.132 & 29.848 & 28.377 & 26.138 & 32.795 & 35.875 & 33.864 & 16.99 & 16.813 & 23.193 & 15.271 & 12.099 & 32.499 & 33.694 & 30.183 & 32.755 & 26.951 & 34.291 \\
\hline $10 / 4 / 118: 00$ & & 30.682 & 30.179 & 29.11 & 30.498 & & 29.509 & 30.661 & 30.135 & 29.853 & 28.384 & 26.145 & 33.048 & 35.878 & 34.143 & 16.988 & 16.813 & 23.193 & 15.269 & 12.376 & 32.59 & 33.947 & 30.194 & 33.001 & 26.951 & 34.294 \\
\hline $10 / 4 / 118: 30$ & & 30.685 & 30.184 & 29.113 & 30.5 & & 29.514 & 30.661 & 30.137 & 29.855 & 28.386 & 26.148 & 33.177 & 35.878 & 34.265 & $\begin{array}{l}16.992 \\
\end{array}$ & 16.82 & 23.191 & 15.264 & 12.508 & 32.649 & 34.075 & 30.199 & 33.123 & 26.951 & 34.294 \\
\hline 10/4/11 9:00 & & 30.687 & 30.188 & 29.11 & 30.505 & & 29.523 & 30.661 & 30.132 & 29.86 & 28.386 & 26.15 & 33.247 & 35.882 & 34.354 & 16.992 & 16.832 & 23.191 & 15.266 & 12.588 & 32.686 & 34.142 & 30.213 & 33.198 & 26.951 & 34.294 \\
\hline 10/4/11 9:30 & & 30.687 & 30.193 & 29.108 & 30.512 & & 29.528 & 30.663 & 30.132 & 29.865 & 28.388 & 26.145 & 33.305 & 35.878 & 34.412 & $\begin{array}{l}16.992 \\
1692\end{array}$ & $\begin{array}{l}16.841 \\
\end{array}$ & 23.191 & 15.269 & 12.649 & 32.707 & 34.198 & 30.223 & 33.255 & 26.951 & 34.296 \\
\hline 10/4/11 10:00 & & 30.687 & 30.195 & 29.103 & 30.514 & & 29.535 & 30.663 & 30.135 & 29.867 & $\begin{array}{l}28.380 \\
28.386\end{array}$ & 26.143 & $\begin{array}{l}33.347 \\
3.34\end{array}$ & 35.882 & $\begin{array}{l}34.459 \\
34.42\end{array}$ & $\begin{array}{l}10.992 \\
16.992\end{array}$ & $\begin{array}{l}10.041 \\
16.851\end{array}$ & $\begin{array}{l}23.193 \\
23.193\end{array}$ & 15.274 & $\begin{array}{l}12.694 \\
12.694\end{array}$ & 32.735 & 34.239 & 30.23 & 33.295 & 26.953 & 34.296 \\
\hline $10 / 4 / 1110: 30$ & & 30.685 & 30.195 & 29.094 & $\begin{array}{l}30.519 \\
30.519\end{array}$ & & 29.547 & 30.663 & 30.13 & 29.867 & $\begin{array}{l}28.380 \\
28.386\end{array}$ & 26.138 & $\begin{array}{l}33.345 \\
33.345\end{array}$ & $\begin{array}{ll} & \\
35.002 & 358\end{array}$ & $\begin{array}{l}34.4552 \\
34.452\end{array}$ & $\begin{array}{l}10.995 \\
16.995\end{array}$ & 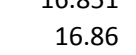 & 23.191 201 & $\begin{array}{l}15.278 \\
15.278\end{array}$ & 12.687 & 32.739 & 34.239 & $\begin{array}{l}30.225 \\
30.25\end{array}$ & $\begin{array}{l}33.292 \\
33.292\end{array}$ & 26.953 & 34.294 \\
\hline 10/4/11 11:00 & & $\begin{array}{l}30.0032 \\
30.682\end{array}$ & 30.198 & 29.085 & 30.523 & & 29.558 & $\begin{array}{l}30.663 \\
30.663\end{array}$ & $\begin{array}{r}30.132 \\
30.132\end{array}$ & 29.867 & $\begin{array}{l}28.300 \\
28.381\end{array}$ & $\begin{array}{l}20.130 \\
26.129\end{array}$ & $\begin{array}{l}33.345 \\
33.275\end{array}$ & $\begin{array}{l}35.86 \\
35.88\end{array}$ & $\begin{array}{l}54.432 \\
34.375\end{array}$ & $\begin{array}{l}10.995 \\
16.995\end{array}$ & $\begin{array}{l}10.80 \\
16.865\end{array}$ & 23.191 & $\begin{array}{l}13.2818 \\
15.288\end{array}$ & 12.609 & 32.725 & $\begin{array}{l}34.169 \\
34.168\end{array}$ & $\begin{array}{l}30.227 \\
30.227\end{array}$ & $\begin{array}{l}33.222 \\
33.224\end{array}$ & $\begin{array}{l}26.953 \\
26.953\end{array}$ & 34.296 \\
\hline 10/4/11 11:30 & & 30.68 & 30.195 & 29.075 & 30.523 & & 29.57 & 30.666 & 30.13 & 29.867 & 28.377 & 26.119 & 33.099 & 35.882 & 34.18 & 16.997 & 16.86 & .188 & 15.29 & 12.419 & 32.67 & .995 & 30.227 & 33.053 & 26.953 & 34.296 \\
\hline $10 / 4 / 1$ & & & & 29.068 & 30.52 & & 29.577 & 30.663 & 30.132 & 29.867 & 28.377 & 26.11 & .999 & 35.882 & 34.072 & 16.995 & $\begin{array}{l}16.800 \\
16.853\end{array}$ & 186 & 15.29 & 12.308 & 32.63 & .895 & .218 & 32.952 & 26.956 & .296 \\
\hline $10 / 4 / 1$ & & 30.68 & 30.191 & 29.054 & 30.516 & & 29.589 & 30.664 & 30.132 & 29.865 & 28.37 & 26.102 & 32.926 & 35.878 & 33.995 & 16.995 & 16.839 & 23.186 & 15.302 & 12.231 & 32.59 & .822 & .204 & 2884 & 26.956 & 34.294 \\
\hline 10/4/11 13:00 & & 30.678 & 30.186 & 29.038 & 30.509 & & 29.601 & 30.659 & 30.13 & 29.862 & 28.363 & 26.088 & 32.87 & 35.88 & 33.939 & 16.992 & 16.825 & 23.184 & 15.304 & 12.172 & 32.562 & 33.77 & 30.192 & 32.828 & 26.956 & 34.294 \\
\hline 10/4/11 13:30 & & 30.675 & 30.179 & 29.031 & 30.502 & & 29.612 & 30.658 & 30.13 & 29.86 & 28.356 & 26.074 & 32.826 & 35.88 & 33.89 & 16.99 & 16.816 & 23.184 & 15.316 & 12.125 & 32.539 & 33.725 & 30.18 & 32.781 & 26.956 & 34.291 \\
\hline 10/4/11 14:00 & & 30.673 & 30.17 & 29.024 & 30.491 & & 29.615 & & 30.13 & 29.855 & 28.351 & 26.062 & 32.786 & 35.875 & 33.845 & 16.99 & 16.799 & 23.181 & 15.32 & 12.083 & 32.506 & 33.682 & 30.164 & 32.741 & 26.953 & 34.289 \\
\hline 10/4/11 14:30 & & 30.671 & 30.158 & 29.024 & 30.474 & & 29.615 & 30.656 & 30.13 & 29.848 & 28.349 & 26.059 & 32.748 & 35.871 & 33.81 & 16.988 & 16.785 & 23.179 & 15.33 & 12.043 & 32.481 & 33.646 & 30.145 & 32.706 & 26.953 & 34.287 \\
\hline $10 / 4 / 1115: 00$ & & 30.671 & 30.156 & 29.017 & 30.463 & & 29.617 & 30.654 & 30.128 & 29.846 & 28.346 & 26.057 & 32.716 & 35.864 & 33.772 & 16.985 & 16.776 & 23.182 & 15.332 & 12.01 & 32.467 & 33.616 & 30.138 & 32.675 & 26.956 & 34.284 \\
\hline $10 / 4 / 1115: 30$ & & 30.673 & 30.151 & 29.012 & 30.456 & & 29.619 & 30.654 & 30.13 & 29.844 & 28.342 & 26.052 & 32.69 & 35.866 & 33.746 & 16.983 & 16.764 & 23.179 & 15.33 & 11.979 & 32.448 & 33.587 & 30.129 & 32.647 & 26.956 & 34.287 \\
\hline $10 / 4 / 1116: 00$ & & 30.671 & 30.149 & 29.012 & 30.446 & & 29.615 & 30.651 & 30.132 & 29.839 & 28.339 & 26.047 & 32.664 & 35.864 & 33.721 & 16.983 & 16.753 & 23.184 & 15.335 & 11.958 & 32.432 & 33.561 & 30.121 & 32.621 & 26.953 & 34.284 \\
\hline 10/4/11 16:30 & & 30.671 & 30.142 & 29.012 & 30.435 & & 29.608 & 30.649 & 30.13 & 29.834 & 28.339 & 26.05 & 32.641 & 35.864 & 33.695 & 16.983 & 16.741 & 23.218 & 15.337 & 11.93 & 32.415 & 33.542 & 30.107 & 32.6 & 26.956 & 34.284 \\
\hline 10/4/11 17:00 & & 30.671 & 30.139 & 29.015 & 30.425 & & 29.605 & 30.649 & 30.13 & 29.832 & 28.339 & 26.05 & 32.62 & 35.857 & 33.676 & 16.978 & 16.732 & 23.237 & 15.339 & 11.909 & 32.404 & 33.521 & 30.103 & 32.579 & 26.956 & 34.287 \\
\hline 10/4/11 17:30 & & 30.673 & 30.139 & 29.024 & 30.421 & & 29.598 & 30.649 & 30.132 & 29.83 & 28.339 & 26.055 & 32.601 & 35.857 & 33.657 & 16.976 & 16.722 & 23.237 & 15.337 & 11.892 & 32.399 & 33.502 & 30.1 & 32.56 & 26.956 & 34.284 \\
\hline 10/4/11 18:00 & & 30.675 & 30.139 & 29.026 & 30. & & 29.587 & 30.651 & 30.132 & 29.83 & 28.342 & 26.059 & 32.585 & 35.859 & 33.636 & 16.978 & 16.711 & 23.235 & 15.335 & 11.873 & 32.387 & 33.485 & 30.096 & 32.546 & 26.956 & 34.284 \\
\hline $10 / 4 / 11$ & & 30.675 & 30.139 & 29.038 & 30. & & 29.577 & 30.649 & 30.142 & 29. & 28.346 & 26.064 & 32.573 & 35.859 & 33.62 & 16.978 & 16.701 & 23.23 & 15.332 & 11.857 & 32.376 & 469 & 30.096 & 32.53 & 26.956 & 34.287 \\
\hline $10 / 4 / 11$ & & 30.678 & 30.142 & 29.045 & 30.4 & & 29.568 & 30.651 & 30.142 & 29.83 & 28.351 & 26.076 & 32.554 & 35.859 & $\begin{array}{l}33.06 \\
33.606\end{array}$ & $\begin{array}{l}16.981 \\
16.981\end{array}$ & $\begin{array}{l}16.694 \\
16.14\end{array}$ & 23.232 & 15.328 & 11.84 & 32.378 & 457 & 30.098 & 32.516 & 26.956 & 34.287 \\
\hline $10 /$ & & 30.68 & 30.146 & 29.052 & 30.4 & & 29.561 & 30.656 & 30.142 & 29.832 & 28.351 & 26.083 & 32.603 & 35.866 & $\begin{array}{lll}33.66 & \\
33.66\end{array}$ & $\begin{array}{l}10.51 \\
16.978\end{array}$ & $\begin{array}{l}10.684 \\
16.685\end{array}$ & 23.23 & 15.323 & $\begin{array}{l}11.044 \\
11.895\end{array}$ & 32.376 & .504 & 0.103 & 32.567 & 26.958 & 34.287 \\
\hline 10 & & 30.685 & 30.149 & 29.057 & $\begin{array}{l}30.4 \\
30.4\end{array}$ & & 29.551 & $\begin{array}{l}30.658 \\
30.058\end{array}$ & 30.142 & 29.834 & $\begin{array}{l}28.356 \\
28.356\end{array}$ & $\begin{array}{l}26.003 \\
26.093\end{array}$ & $\begin{array}{l}32.807 \\
32.807\end{array}$ & $\begin{array}{l}35.8001 \\
35.861\end{array}$ & $\begin{array}{l}33.071 \\
33.871\end{array}$ & $\begin{array}{l}16.978 \\
10.978\end{array}$ & $\begin{array}{l}10.003 \\
16.725\end{array}$ & $\begin{array}{r}23.232 \\
23.232\end{array}$ & 15.316 & 12.108 & 32.457 & 6 & 14 & 32.76 & 26.958 & 289 \\
\hline 10 & & 30 & 30 & 29 & 30 & & & 30 & 30 & 29 & 28. & 26 & 32 & 35. & 33 & 16 & 16 & 23. & 1 & 11 & 1 & 2 & 9 & 32. & 8 & 289 \\
\hline & & 30. & 30. & & & & & & & & & 26.1 & 32 & 35. & 33. & 16 & 16 & 23 & 15. & 11 & 5 & & 6 & 32 & 88 & 34.291 \\
\hline 10/4/11 21:30 & & 30. & 30. & 29.064 & & & & & & & 28.358 & 20 & 32.613 & 35.873 & 33.662 & & & & 15.316 & 11.902 & - & & 6 & 2 & 96 & 34.291 \\
\hline 122:00 & & 30. & 30. & & & & & & 30 & & 28.363 & 26.102 & 32.589 & 35.88 & 33.639 & & & & 15.318 & 11.878 & & & & 2 & 96 & 34.291 \\
\hline 2::30 & & 30. & 30.163 & 29 & & & & & 30 & & & & 32.571 & 35.875 & 33.6 & & & 23.2 & 15.311 & $11 . \varepsilon$ & & & 6 & 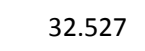 & .96 & 34.291 \\
\hline $10 / 4$ & & & 30.16 & & 30. & & & & 30. & & & & 32.552 & 35.873 & 33. & & & 23.225 & 15.311 & 11.838 & & & 6 & 32.511 & 6.96 & 34.291 \\
\hline $10 / 4$ & & 30. & 30. & & 30.4 & & & & 30.144 & & 28. & 26 & 32.538 & 35.88 & 33. & & 16.673 & 23.2 & 15.306 & 11.819 & 32.383 & & 30.129 & 32.497 & 26.96 & 34.294 \\
\hline $10 / 5 / 110: 00$ & & 30. & 30.1 & 29.061 & & & 29. & & 30.144 & 29. & 28.363 & 26.102 & 32.526 & 35.882 & 33. & 16. & 16.666 & 23.232 & 15.309 & 11.805 & 32.378 & 33.421 & 30.124 & 32.485 & 26.96 & 34.294 \\
\hline 10/5/11 0:30 & & 30. & 30.16 & 29.061 & 30 & & 29.5 & & 30.142 & 29.8 & 28. & 26.102 & 32.512 & 35.88 & 33.5 & 16.985 & 16.655 & 23.232 & 15.311 & 11.793 & 32.371 & 33.412 & 30.124 & 32.469 & 26.96 & 34.294 \\
\hline 10/5/11 1:00 & & 30.689 & 30.165 & 29.059 & 30.428 & & 29.554 & 30.6 & 30.147 & 29.848 & 28.365 & 26.1 & 32.498 & 35.882 & 33.543 & 16.983 & 16.648 & 23.23 & 15.313 & 11.782 & 32.364 & 33.398 & 30.117 & 32.457 & 26.96 & 34.294 \\
\hline 10/5/11 1:30 & & 30.689 & 30.167 & 29.057 & 30.423 & & 29.556 & 30.668 & 30.142 & 29.846 & 28.363 & 26.1 & 32.489 & 35.882 & 33.533 & 16.983 & 16.641 & 23.225 & 15.309 & 11.768 & 32.352 & 33.388 & 30.114 & 32.448 & 26.96 & 34.294 \\
\hline 10/5/11 2:00 & & 30.689 & 30.167 & 29.057 & 30.421 & & 29.558 & 30.668 & 30.147 & 29.846 & 28.36 & 26.098 & 32.477 & 35.882 & 33.521 & 16.983 & 16.631 & 23.228 & 15.309 & 11.761 & 32.352 & 33.379 & 30.11 & 32.438 & 26.96 & 34.294 \\
\hline $\begin{array}{l}10 / 5 / 112: 30 \\
\text { l }\end{array}$ & & 30.689 & 30.165 & 29.054 & 30.418 & & 29.558 & 30.668 & 30.144 & 29.846 & 28.36 & 26.095 & 32.47 & 35.885 & 33.512 & 16.985 & 16.624 & 23.225 & 15.309 & 11.746 & 32.343 & 33.367 & 30.107 & 32.429 & 26.96 & 34.294 \\
\hline $\begin{array}{l}\text { 10/5/11 3:00 }\end{array}$ & & 30.692 & 30.165 & 29.054 & 30.416 & & 29.558 & 30.668 & 30.147 & 29.846 & 28.358 & 26.093 & 32.458 & 35.878 & 33.5 & 16.983 & 16.617 & 23.223 & 15.306 & 11.735 & 32.336 & 357 & 30.112 & 32.417 & 26.96 & 34.294 \\
\hline 10/5/11 3:30 & & 30.692 & 30.165 & 29.057 & 30.414 & & 29.558 & 30.668 & 30.147 & 29.846 & 28.36 & 26.093 & 32.449 & 35.88 & 33.493 & 16.985 & 16.61 & 23.221 & 15.304 & 11.732 & 32.334 & 348 & 30.103 & 32.408 & 26.963 & 34.294 \\
\hline 10/5/11 4:00 & & 30.689 & 30.167 & 29.061 & 30.411 & & 29.556 & 30.668 & 30.147 & 29.846 & 28.36 & 26.095 & 32.442 & 35.878 & 33.484 & 16.983 & 16.606 & 23.221 & 15.306 & 11.718 & 32.331 & 33.343 & 30.105 & 32.403 & 26.963 & 34.294 \\
\hline $10 / 5 / 114: 30$ & & 30.692 & 30.165 & 29.059 & 30.407 & & 29.556 & 30.668 & 30.149 & 29.846 & 28.363 & 26.098 & 32.433 & 35.882 & 33.475 & 16.983 & 16.598 & 23.218 & 15.304 & 11.711 & 32.324 & 33.336 & 30.096 & 32.394 & 26.963 & 34.294 \\
\hline
\end{tabular}




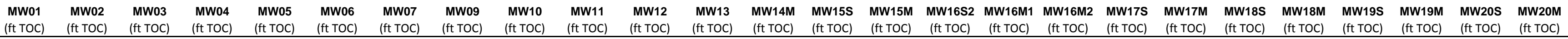

\begin{tabular}{|c|c|c|c|c|c|c|c|c|c|c|c|c|c|c|c|c|c|c|c|c|c|c|c|c|c|}
\hline Well & $\begin{array}{l}\text { (ft TOC) } \\
\text { (is }\end{array}$ & $\begin{array}{l}\text { (ft TOC) } \\
\text { (ft) }\end{array}$ & $\begin{array}{l}\text { (ft TOC) } \\
\text { (f) }\end{array}$ & (ft TOC) & $\begin{array}{ll}(\mathrm{ft} \text { TOC) } & \text { (ft TOC) }\end{array}$ & $\begin{array}{l}\text { (ft TOC) } \\
\text { (ft }\end{array}$ & $\begin{array}{l}\text { (ft TOC) } \\
\text { (f) }\end{array}$ & $\begin{array}{l}\text { (ft TOC) } \\
\text { (ft) }\end{array}$ & $\begin{array}{l}\text { (ft TOC) } \\
\text { (f) }\end{array}$ & (ft TOC) & $\begin{array}{l}\text { (ft TOC) } \\
\text { (f) }\end{array}$ & (ft TOC) & $\begin{array}{l}\text { (ft TOC) } \\
\end{array}$ & (ft TOC) & (ft TOC) & (ft TOC) & (ft TOC) & $\begin{array}{l}\text { (ft TOC) } \\
\end{array}$ & (ft TOC) & $\begin{array}{l}\text { (ft TOC) } \\
\end{array}$ & $\begin{array}{l}\text { (ft TOC) } \\
\text { (1) }\end{array}$ & $\begin{array}{l}\text { (ft TOC) } \\
\text { (n) }\end{array}$ & (ft TOC) & $\begin{array}{l}\text { (ft TOC) } \\
\end{array}$ & (ft TOC) \\
\hline ;/11 5:00 & & 30.692 & 30.165 & 29.059 & 30.407 & 29.558 & 30.668 & 30.151 & 29.846 & 28.363 & 26.098 & 32.425 & 35.882 & 17 & 16.983 & 16.591 & 23.218 & 15.304 & 11.704 & 32.32 & 33.327 & 30.098 & 32.384 & 26.963 & 34.296 \\
\hline $10 / 5 / 115: 30$ & & 30.692 & 30.167 & 29.061 & 30.407 & 29.556 & 30.67 & 30.151 & 29.846 & 28.365 & 26.098 & 32.418 & 35.88 & 33.463 & 16.985 & 16.587 & 23.218 & 15.299 & 11.697 & 32.317 & 33.317 & 30.096 & 32.38 & 26.963 & 34.296 \\
\hline 10/5/11 6:00 & & 30.694 & 30.167 & 29.064 & 30.404 & 29.551 & 30.67 & 30.151 & 29.848 & 28.365 & 26.1 & 33.357 & 35.88 & 33.77 & 16.983 & 16.58 & 23.235 & 15.299 & 12.003 & 32.532 & 34.383 & & 33.344 & 26.963 & 34.298 \\
\hline 10/5/11 6:30 & & 30.694 & 30.167 & 29.064 & 30.404 & 29.554 & 30.671 & 30.151 & 29.846 & 28.367 & 26.102 & 34.616 & 35.882 & 34.996 & 16.985 & 16.648 & 23.237 & 15.297 & 13.234 & 33.124 & 35.615 & 33 & & 26.963 & 34.296 \\
\hline 10/5/11 7:00 & & 30.696 & 30.17 & 29.066 & 30.421 & 29.554 & 30.67 & 30.151 & 29.851 & 28.367 & 26.105 & 35.201 & 35.878 & 35.669 & 16.983 & 16.753 & 23.244 & 15.292 & 13.907 & 33.381 & 36.179 & 30.185 & 58 & 65 & 34.301 \\
\hline 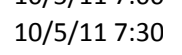 & & 30.696 & 30.17 & 29.068 & 30.439 & 29.554 & 30.67 & 30.149 & 29.851 & 28.367 & 26.11 & 35.517 & 35.887 & 36.126 & 16.983 & 16.865 & 23.248 & 15.295 & 14.365 & 33.539 & 36.459 & 30.225 & 5.463 & 6.963 & 34.301 \\
\hline 10/5/11 8:00 & & 30.696 & 30.17 & 29.068 & 30.463 & 29.556 & 30.67 & 30.154 & 29.851 & 28.37 & 26.11 & 35.641 & 35.882 & 36.295 & 16.985 & 16.965 & 23.249 & 15.295 & 14.534 & 33.577 & 36.58 & 30.26 & 35.587 & 26.965 & 34.301 \\
\hline 10/5/11 8:30 & & 30.699 & 30.174 & 29.066 & 30.491 & 29.565 & 30.673 & 30.151 & 29.851 & 28.37 & 26.11 & 36.214 & 35.882 & 36.729 & 16.985 & 17.061 & 23.249 & 15.292 & 14.962 & 33.786 & 37.177 & 30.295 & 36.157 & 26.965 & 34.301 \\
\hline 10/5/11 9:00 & & 30.699 & 30.177 & 29.061 & 30.516 & 29.57 & 30.673 & 30.154 & 29.853 & 28.367 & 26.107 & 36.647 & 35.885 & 37.125 & 16.985 & 17.166 & 23.246 & 15.295 & 15.362 & 33.959 & 37.618 & 30.331 & 36.589 & 26.965 & 34.303 \\
\hline 10/5/11 9:30 & & 30.696 & 30.174 & 29.052 & 30.544 & 29.575 & 30.673 & 30.151 & 29.853 & 28.363 & 26.107 & 37.014 & 35.885 & 37.51 & 16.985 & 17.276 & 23.249 & 15.295 & 15.745 & 34.111 & 37.983 & 30.359 & 36.955 & 26.965 & 34.305 \\
\hline 10/5/11 10:00 & & 30.696 & 30.174 & 29.054 & 30.572 & 29.582 & 30.67 & 30.154 & 29.853 & 28.363 & 26.105 & 37.307 & 35.887 & 37.826 & 16.985 & 17.381 & 23.253 & 15.299 & 16.062 & 34.23 & 38.267 & 30.389 & 37.246 & 26.965 & 34.303 \\
\hline 10/5/11 10:30 & & 30.696 & 30.172 & 29.047 & 30.6 & 29.582 & 30.67 & 30.154 & 29.853 & 28.363 & 26.102 & 37.468 & 35.882 & 38.077 & 16.985 & 17.484 & 23.272 & 15.304 & 16.312 & 34.32 & 38.409 & 30.422 & 37.399 & 26.967 & 34.303 \\
\hline 10/5/11 11:00 & & 30.692 & 30.172 & 29.036 & 30.626 & 29.594 & 30.671 & 30.149 & 29.851 & 28.358 & 26.095 & 37.555 & 35.882 & 38.187 & 16.985 & 17.573 & 23.293 & 15.306 & 16.424 & 34.348 & 38.492 & 30.446 & 37.49 & 26.965 & 34.303 \\
\hline 10/5/11 11:30 & & 30.696 & 30.172 & 29.029 & 30.654 & 29.598 & 30.673 & 30.154 & 29.851 & 28.353 & 26.086 & 36.08 & 35.88 & 37.313 & 16.985 & 17.643 & 23.304 & 15.311 & 15.552 & 33.966 & 36.938 & 30.455 & 36.002 & 26.967 & 34.305 \\
\hline $10 / 5 / 1112: 00$ & & 30.692 & 30.167 & 29.012 & 30.668 & 29.608 & 30.668 & 30.151 & 29.846 & 28.346 & 26.078 & 35.409 & 35.88 & 36.609 & 16.983 & 17.638 & 23.309 & 15.313 & 14.849 & 33.679 & 36.274 & 30.439 & 35.336 & 26.967 & 34.303 \\
\hline 10/5/11 12:30 & & 30.692 & 30.163 & 28.993 & 30.677 & 29.622 & 30.668 & 30.147 & 29.841 & 28.335 & 26.064 & 34.988 & 35.873 & 36.168 & 16.983 & 17.606 & 23.313 & 15.325 & 14.4 & 33.504 & 35.867 & 30.422 & 34.921 & 26.967 & 34.303 \\
\hline 10/5/11 13:00 & & 30.689 & 30.158 & 28.989 & 30.677 & 29.627 & 30.666 & 30.149 & 29.839 & 28.328 & 26.047 & 34.684 & 35.875 & 35.836 & 16.981 & 17.568 & 23.316 & 15.332 & 14.076 & 33.376 & 35.559 & 30.399 & 34.618 & 26.965 & 34.298 \\
\hline 10/5/11 13:30 & & 30.682 & 30.149 & 28.989 & 30.673 & 29.622 & 30.661 & 30.149 & 29.83 & 28.325 & 26.043 & $\begin{array}{r}34.44 \\
\end{array}$ & 35.871 & 35.587 & $\begin{array}{l}16.978 \\
16.978\end{array}$ & 17.529 & 23.316 & 15.335 & 13.817 & 33.271 & 35.317 & 30.366 & 34.379 & 26.965 & 34.296 \\
\hline 10/5/11 14:00 & & $\begin{array}{l}30.082 \\
30.682\end{array}$ & 30.142 & 28.991 & 30.661 & 29.619 & 30.661 & 30.149 & 29.823 & 28.325 & 26.045 & $\begin{array}{l}34.26 \\
\end{array}$ & $\begin{array}{l}35.864 \\
35.04\end{array}$ & 35.397 & $\begin{array}{l}16.976 \\
16.976\end{array}$ & $\begin{array}{l}17.491 \\
\text { 1. }\end{array}$ & 23.311 & 15.339 & $\begin{array}{l}13.034 \\
13.634\end{array}$ & $\begin{array}{r}33.19 \\
33.19\end{array}$ & 35.146 & 30.347 & 34.207 & 26.965 & 34.294 \\
\hline 10/5/11 14:30 & & $\begin{array}{l}30.002 \\
30.682\end{array}$ & $\begin{array}{l}30.142 \\
30.135\end{array}$ & 28.989 & $\begin{array}{l}30.011 \\
30.649\end{array}$ & 29.617 & 30.658 & $\begin{array}{l}30.151 \\
30.151\end{array}$ & 29.818 & $\begin{array}{l}20.320 \\
28.323\end{array}$ & 26.045 & $\begin{array}{l}34.089 \\
34.089\end{array}$ & $\begin{array}{l}35.0061 \\
35.861\end{array}$ & 35.212 & 16.976 & $\begin{array}{l}17.454 \\
17.454\end{array}$ & $\begin{array}{l}23.311 \\
23.311\end{array}$ & 15.335 & $\begin{array}{l}13.044 \\
13.446\end{array}$ & 33.117 & 34.971 & 30.328 & 34.031 & 26.963 & 34.294 \\
\hline $\begin{array}{l}10 / 5 / 11 \\
15: 00\end{array}$ & & $\begin{array}{l}30.082 \\
30.687\end{array}$ & 30.135 & $\begin{array}{l}28.979 \\
28.977\end{array}$ & $\begin{array}{l}30.049 \\
30.64\end{array}$ & 29.617 & $\begin{array}{l}50.058 \\
30.658\end{array}$ & 30.151 & $\begin{array}{l}29.818 \\
29.816\end{array}$ & $\begin{array}{l}28.323 \\
28.319\end{array}$ & $\begin{array}{l}20.045 \\
26.045\end{array}$ & $\begin{array}{l}34.089 \\
33.949\end{array}$ & $\begin{array}{l}35.001 \\
35.861\end{array}$ & $\begin{array}{l}35.064 \\
35.064\end{array}$ & $\begin{array}{l}10.970 \\
16.976\end{array}$ & $\begin{array}{l}1 . .454 \\
17.416\end{array}$ & $\begin{array}{l}23.341 \\
23.341\end{array}$ & $\begin{array}{l}15.337 \\
15.33\end{array}$ & $\begin{array}{l}13.440 \\
13.298\end{array}$ & $\begin{array}{l}33.054 \\
33.054\end{array}$ & 34.831 & $\begin{array}{l}30.328 \\
30.321\end{array}$ & $\begin{array}{l}54.011 \\
33.893\end{array}$ & $\begin{array}{l}26.965 \\
26.965\end{array}$ & $\begin{array}{l}34.294 \\
34.294\end{array}$ \\
\hline $5: 30$ & & 30.685 & 30.132 & 28.972 & 30.633 & 29.622 & 30.654 & 30.149 & 29.816 & 28.312 & 26.035 & 33.823 & 35.859 & 34.933 & 16.974 & 17.381 & 23.36 & 5.339 & 13.171 & 32.998 & .715 & 30.3 & 33.769 & 26.965 & 34.294 \\
\hline $10 /$ & & 30.685 & 30.13 & 28.972 & 30.621 & 29.617 & 30.656 & 30.151 & 29.814 & 28.312 & 26.031 & $\begin{array}{l}33.020 \\
33.722\end{array}$ & .866 & $\begin{array}{r}34.82 \\
34.82\end{array}$ & 16.974 & $\begin{array}{l}17.349 \\
17.349\end{array}$ & $\begin{array}{r}23.359 \\
2359\end{array}$ & 342 & $\begin{array}{l}3.117 \\
13.06\end{array}$ & 32.947 & 34.608 & .286 & 33.67 & 26.965 & 34.291 \\
\hline $10 / 5 / 1$ & & 30.68 & 30.123 & 28.984 & 30.603 & 29.605 & 30.654 & 30.144 & 29.809 & 28.312 & 26.031 & 33.626 & 35.859 & 34.722 & 16.971 & $\begin{array}{l}17.349 \\
17.316\end{array}$ & $\begin{array}{l}23.359 \\
23.359\end{array}$ & 5.346 & $\begin{array}{l}12.900 \\
12.955\end{array}$ & 32.903 & $\begin{array}{l}34.016 \\
34.516\end{array}$ & .265 & $\begin{array}{l}33.574 \\
3.574\end{array}$ & $\begin{array}{l}26.9065 \\
26.965\end{array}$ & 34.291 \\
\hline 10/5/11 17:00 & & 30.685 & 30.123 & 28.986 & 30.589 & 29.598 & 30.656 & 30.154 & 29.804 & 28.312 & 26.04 & 33.539 & 35.857 & 34.635 & 16.971 & 17.286 & 23.355 & 15.342 & 12.87 & 32.861 & 34.431 & .255 & 3.489 & 26.967 & 34.289 \\
\hline 10/5/11 17:30 & & 30.685 & 30.121 & 28.984 & 30.579 & 29.589 & 30.654 & 30.151 & 29.802 & 28.312 & 26.043 & 33.467 & 35.852 & 34.553 & 16.969 & 17.257 & 23.353 & 15.337 & 12.79 & 32.83 & 34.357 & 30.246 & 33.417 & 26.965 & 34.289 \\
\hline $10 / 5 / 1118: 00$ & & 30.689 & 30.121 & 28.993 & 30.572 & 29.584 & 30.656 & 30.154 & 29.804 & 28.314 & 26.047 & 33.399 & 35.857 & 34.485 & 16.969 & 17.232 & 23.35 & 15.335 & 12.722 & 32.8 & 34.291 & 30.241 & 33.351 & 26.967 & 34.291 \\
\hline $10 / 5 / 1118: 30$ & & 30.689 & 30.123 & 28.996 & 30.563 & 29.577 & 30.658 & 30.154 & 29.804 & 28.314 & 26.055 & 33.336 & 35.854 & 34.419 & 16.971 & 17.208 & 23.346 & 15.335 & 12.659 & 32.77 & 34.232 & 30.232 & 33.29 & 26.97 & 34.291 \\
\hline 10/5/11 19:00 & & 30.692 & 30.128 & 29.005 & 30.554 & 29.568 & 30.658 & 30.161 & 29.804 & 28.316 & 26.057 & 33.305 & 35.859 & 34.389 & 16.969 & 17.183 & 23.353 & 15.332 & 12.621 & 32.749 & 34.198 & 30.23 & 33.262 & 26.97 & 34.294 \\
\hline $10 / 5 / 1119: 30$ & & 30.692 & 30.128 & 29.007 & 30.549 & 29.561 & 30.661 & 30.161 & 29.807 & 28.319 & 26.069 & 33.362 & 35.859 & 34.445 & 16.971 & 17.187 & 23.353 & 15.328 & 12.684 & 32.772 & 34.253 & 30.227 & 33.313 & 26.97 & 34.294 \\
\hline $10 / 5 / 1120: 00$ & & 30.694 & 30.132 & 29.017 & 30.544 & 29.556 & 30.663 & 30.159 & 29.807 & 28.321 & 26.069 & 33.27 & 35.859 & 34.351 & 16.969 & 17.166 & 23.348 & 15.325 & 12.586 & 32.742 & 34.165 & 30.23 & 33.222 & 26.97 & 34.296 \\
\hline 10/5/11 20:30 & & 30.699 & 30.135 & 29.026 & 30.542 & 29.542 & 30.663 & 30.163 & 29.811 & 28.328 & 26.081 & 33.212 & 35.866 & 34.286 & 16.974 & 17.145 & 23.346 & 15.32 & 12.52 & 32.718 & 34.104 & 30.23 & 33.163 & 26.97 & 34.296 \\
\hline 10/5/11 $21: 00$ & & 30.696 & 30.137 & 29.024 & 30.537 & 29.54 & 30.668 & 30.161 & 29.811 & 28.328 & 26.093 & 33.16 & 35.871 & 34.232 & 16.971 & 17.122 & 23.348 & 15.32 & 12.468 & 32.695 & 34.054 & 30.227 & 33.114 & 26.97 & 34.296 \\
\hline 10/5/11 21:30 & & 30.701 & 30.144 & 29.022 & 30.54 & 29.544 & 30.668 & 30.156 & 29.814 & 28.328 & 26.09 & 33.116 & 35.868 & 34.187 & 16.974 & 17.101 & 23.343 & 15.313 & 12.426 & 32.684 & 34.014 & 30.227 & 33.072 & 26.97 & 34.298 \\
\hline $10 / 5 / 1122: 00$ & & 30.703 & 30.149 & 29.029 & 30.537 & 29.54 & 30.668 & 30.156 & 29.818 & 28.328 & 26.088 & 33.081 & 35.868 & 34.147 & 16.976 & 17.085 & 23.343 & 15.311 & 12.383 & 32.667 & 33.971 & 30.227 & 33.034 & 26.972 & 34.301 \\
\hline $10 / 5 / 1122: 30$ & & 30.701 & 30.149 & 29.012 & 30.5 & 29.544 & 30.673 & 30.159 & 29.821 & 28.328 & 26.09 & 33.041 & 35.866 & 34.108 & 16.976 & 17.064 & 23.336 & 15.318 & 12.346 & 32.644 & 33.938 & .218 & 32.997 & 26.972 & 34.301 \\
\hline $10 / 5 / 1123: 00$ & & 30.703 & 30.149 & 29.017 & 30.5 & 29.549 & 30.668 & 30.161 & 29.821 & $\begin{array}{l}20.320 \\
28.325\end{array}$ & 26.078 & $\begin{array}{l}33.006 \\
33.006\end{array}$ & 35.868 & 34.072 & 16.976 & $\begin{array}{l}17.045 \\
17.045\end{array}$ & 23.334 & 15.313 & $\begin{array}{l}12.308 \\
12.308\end{array}$ & 32.63 & $\begin{array}{r}33.9 \\
33.9\end{array}$ & .215 & 32.961 & 26.972 & 34.301 \\
\hline 10/5/11 23:30 & & 30.703 & 30.151 & 29.015 & 30.5 & 29.549 & $\begin{array}{r}30.67 \\
30.67\end{array}$ & 30.161 & 29.821 & $\begin{array}{l}28.325 \\
28.325\end{array}$ & 26.086 & 32.975 & 35.875 & $\begin{array}{r}34.04 \\
\end{array}$ & 16.976 & $\begin{array}{l}17.024 \\
\end{array}$ & $\begin{array}{l}23.334 \\
23.34\end{array}$ & 15.316 & $\begin{array}{l}12.275 \\
12.50\end{array}$ & 32.618 & 33.874 & 211 & 32.931 & 26.972 & 34.301 \\
\hline $\begin{array}{l}10 / 6 / 11 \\
10 / 11\end{array}$ & & $\begin{array}{l}30.699 \\
30\end{array}$ & 30.14 & 29.007 & 30. & 29.5 & 30 & $\begin{array}{l}30.159 \\
30.159\end{array}$ & 29.821 & $\begin{array}{l}20.023 \\
28.323\end{array}$ & $\begin{array}{l}20.000 \\
26.081\end{array}$ & 32.943 & 35.873 & $\begin{array}{r}34.007 \\
34.007\end{array}$ & $\begin{array}{l}16.978 \\
16.978\end{array}$ & $\begin{array}{l}17.044 \\
17.007\end{array}$ & $\begin{array}{l}23.334 \\
23.34\end{array}$ & 15.318 & $\begin{array}{l}12.242 \\
12.242\end{array}$ & $\begin{array}{l}32.010 \\
32.593\end{array}$ & 841 & 30.197 & 2.898 & 26.972 & 34.298 \\
\hline 10 & & 30 & 30 & 2 & 30 & 2 & & & 29 & 28 & 26 & 32 & $3 !$ & 33.976 & 16 & 16 & 23 & 6 & 12 & 32.579 & 315 & 9 & 5 & 26.972 & 34.301 \\
\hline $10 / 6 / 1$ & & 30 & 30 & 29 & & 25 & 36 & & & 28 & & & 3 & 33.95 & & 16.97 & 23 & 3 & 12.186 & 32.565 & 33.786 & 35 & 16 & 72 & 34.301 \\
\hline $\begin{array}{l}\text { 10/6/11 1:30 } \\
\text { l }\end{array}$ & & & 30.149 & 29.003 & & & 30.673 & & 29 & 28.321 & & 32.861 & 35.878 & 33.925 & 16.976 & 16.954 & & 15.311 & 12.158 & 32.546 & 33. & .183 & 2.818 & 26.974 & 34.298 \\
\hline 10/6/11 2:00 & & 30 & 30.149 & 28.993 & 30 & & 30.67 & & & 28.316 & & & 35.871 & 33.899 & 16.976 & & & 11 & 12.134 & 32.537 & & & 2.795 & 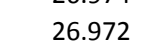 & 34.298 \\
\hline $10 /$ & & 30 & 30 & & & & & & & 28 & & & 35.871 & 33.873 & 16. & 16. & & 1 & 12.108 & 32.523 & & 30.166 & 32.774 & 74 & 34.298 \\
\hline $10 /$ & & 30. & & & & & 30. & & & 28. & & 32.793 & 35.873 & & & & & 15.311 & 12.087 & 32.509 & & 30.157 & 32.75 & 72 & 34.296 \\
\hline 10/6/11 3:30 & & 30. & & & & & 30 & & & 28. & & 32.77 & 35.871 & 33.826 & 16. & 16. & 23. & 15.311 & 12.064 & 32.492 & 33.668 & 30.15 & 32.727 & 26.972 & 34.296 \\
\hline 10/6/11 4:00 & & 30. & 30.1 & 28. & 30. & & 30. & 30.161 & & 28. & 26. & 32.748 & 35.871 & 33.807 & 16.974 & 16.879 & 23.313 & 15.311 & 12.043 & 32.474 & 33.649 & 30.133 & 32.71 & 26.974 & 34.296 \\
\hline 10/6/11 4:30 & & 30.6 & 30.128 & 28.5 & 30. & & 30. & 30.161 & 29. & 28.309 & 26.059 & 32.73 & 35.868 & 33.784 & 16.971 & 16.865 & 23.309 & 15.311 & 12.024 & 32.467 & 33.63 & 30.126 & 32.687 & 26.972 & 34.296 \\
\hline 10/6/11 5:00 & & 30.65 & 30.13 & 28.993 & 30.4 & 29.554 & 30.66 & 30.161 & 29.8 & 28.312 & 26.059 & 32.713 & 35.873 & 33.768 & 16.974 & 16.853 & 23.309 & 15.306 & 12.01 & 32.455 & 33.611 & 30.131 & 32.67 & 26.974 & 34.296 \\
\hline $10 / 6 / 115: 30$ & & 30.699 & 30.13 & 28.996 & $30.4=$ & 29.554 & 30.666 & 30.163 & 29.804 & 28.314 & 26.064 & 32.695 & 35.866 & 33.749 & 16.974 & 16.841 & 23.306 & 15.304 & 11.986 & 32.45 & 33.594 & 30.124 & 32.656 & 26.972 & 34.296 \\
\hline 10/6/11 6:00 & & 30.701 & 30.13 & 28.993 & 30.437 & 29.547 & 30.668 & 30.161 & 29.802 & 28.312 & 26.069 & 32.681 & 35.866 & 33.737 & 16.971 & 16.83 & 23.304 & 15.304 & 11.974 & 32.439 & 33.578 & 30.126 & 32.64 & 26.974 & 34.298 \\
\hline 10/6/11 6:30 & & 30.701 & 30.13 & 28.998 & 30.435 & 29.544 & 30.668 & 30.163 & 29.804 & 28.314 & 26.069 & 32.664 & 35.871 & 33.718 & 16.974 & 16.818 & 23.311 & 15.302 & 11.956 & 32.432 & 33.566 & 30.124 & 32.626 & 26.974 & 34.298 \\
\hline $\begin{array}{l}\text { 10/6/11 7:00 }\end{array}$ & & 30.703 & 30.135 & 29.005 & 30.432 & 29.54 & 30.668 & 30.168 & 29.807 & 28.319 & 26.074 & 32.653 & 35.873 & 33.702 & 16.974 & 16.809 & 23.313 & 15.299 & 11.942 & 32.422 & 33.554 & 30.129 & 32.612 & 26.974 & 34.301 \\
\hline $\begin{array}{l}\text { 10/6/11 7:30 } \\
\text { l }\end{array}$ & & 30.706 & 30.135 & 29.01 & 30.428 & 29.54 & 30.67 & 30.166 & 29.804 & 28.321 & 26.078 & 32.639 & 35.871 & 33.693 & 16.974 & 16.799 & 23.316 & 15.297 & 11.927 & 32.42 & 33.537 & 30.124 & 32.6 & 26.977 & 34.301 \\
\hline 10/6/11 8:00 & & 30.706 & 30.137 & 29.012 & 30.43 & 29.535 & 30.67 & 30.163 & 29.807 & 28.323 & 26.083 & 32.627 & 35.871 & 33.678 & 16.976 & 16.79 & 23.311 & 15.292 & 11.916 & 32.415 & 33.526 & 30.126 & 32.586 & 26.977 & 34.301 \\
\hline $10 / 6 / 118: 30$ & & 30.706 & 30.139 & 29.017 & 30.43 & 29.535 & 30.673 & 30.166 & 29.807 & 28.323 & 26.088 & 32.617 & 35.873 & 33.667 & 16.976 & 16.781 & 23.316 & 15.292 & 11.904 & 32.411 & 33.511 & 30.126 & 32.574 & 26.974 & 34.303 \\
\hline
\end{tabular}




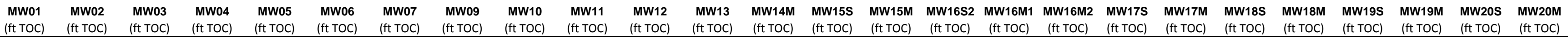

\begin{tabular}{|c|c|c|c|c|c|c|c|c|c|c|c|c|c|c|c|c|c|c|c|c|c|c|c|c|c|c|}
\hline Well & $\begin{array}{l}\text { MW01 } \\
\text { (ft TOC) }\end{array}$ & $\begin{array}{l}\text { MW02 } \\
\text { (ft TOC) }\end{array}$ & $\begin{array}{l}\text { MW03 } \\
\text { (ft TOC) }\end{array}$ & $\begin{array}{l}\text { MW04 } \\
\text { (ft TOC) }\end{array}$ & $\begin{array}{r}\text { MW05 } \\
\text { (ft TOC) }\end{array}$ & $\begin{array}{r}\text { MW06 } \\
\text { (ft TOC) }\end{array}$ & $\begin{array}{l}\text { MW07 } \\
\text { (ft ToC) }\end{array}$ & $\begin{array}{l}\text { MW09 } \\
\text { (ft TOC) }\end{array}$ & $\begin{array}{l}\text { MW10 } \\
\text { (ft TOC) }\end{array}$ & $\begin{array}{l}\text { MW11 } \\
\text { (ft TOC) }\end{array}$ & $\begin{array}{r}\mathrm{MW12} \\
\text { (ft TOC) }\end{array}$ & $\begin{array}{l}\text { MW13 } \\
\text { (ft TOC) }\end{array}$ & $\begin{array}{l}\text { MW14M } \\
\text { (ft TOC) }\end{array}$ & $\begin{array}{l}\text { MWW15S } \\
\text { (ft TOC) }\end{array}$ & $\begin{array}{l}\text { MW15M } \\
\text { (ft TOC) }\end{array}$ & $\begin{array}{l}\text { MW16S2 } \\
\text { (ft TOC) }\end{array}$ & $\begin{array}{c}\text { MW16M1 } \\
\text { (ft TOC) }\end{array}$ & $\begin{array}{l}\text { MW16M2 } \\
\text { (ft TOC) }\end{array}$ & $\begin{array}{l}\text { MW175 } \\
\text { (ft TOC) }\end{array}$ & $\begin{array}{l}\text { MW17M } \\
\text { (ft TOC) }\end{array}$ & $\begin{array}{l}\text { MW18S } \\
\text { (ft TOC) }\end{array}$ & $\begin{array}{l}\mathrm{MW18M} \\
\text { (ft TOC) }\end{array}$ & $\begin{array}{l}\text { MWW19S } \\
\text { (ft TOC) }\end{array}$ & $\begin{array}{l}\text { MW19M } \\
\text { (ft TOC) }\end{array}$ & $\begin{array}{l}\mathrm{MW} 20 \mathrm{~S} \\
(\mathrm{ft} \text { TOC) }\end{array}$ & $\begin{array}{l}\text { MW20M } \\
\text { (ft toc) }\end{array}$ \\
\hline 10/6/11 9:00 & & 30.706 & 30.144 & 29.022 & 30.43 & & 29.533 & 30.673 & 30.168 & 29.809 & 28.328 & 26.09 & 32.601 & 35.871 & 33.655 & 16.978 & 16.774 & 23.318 & 15.29 & 11.89 & 32.411 & 33.499 & 30.124 & 32.56 & 26.974 & 34.305 \\
\hline 10/6/11 9:30 & & 30.708 & 30.146 & 29.019 & 30.432 & & 29.533 & 30.678 & 30.168 & 29.814 & 28.328 & 26.098 & 32.594 & 35.878 & 33.641 & 16.978 & 16.767 & 23.313 & 15.29 & 11.88 & 32.406 & 33.49 & 30.131 & 32.553 & 26.981 & 34.308 \\
\hline 10/6/11 10:00 & & 30.71 & 30.146 & 29.007 & 30.428 & & 29.54 & 30.676 & 30.166 & 29.814 & 28.33 & 26.098 & 32.582 & 35.885 & 33.634 & 16.978 & 16.755 & 23.311 & 15.295 & 11.871 & 32.399 & 33.483 & 30.126 & 32.541 & 26.977 & 34.308 \\
\hline $10 / 6 / 1110: 30$ & & 30.713 & 30.149 & 29.003 & 30.432 & & 29.547 & 30.675 & 30.166 & 29.816 & 28.321 & 26.071 & 32.575 & 35.882 & 33.625 & 16.978 & 16.748 & 23.309 & 15.292 & 11.859 & 32.399 & 33.471 & 30.124 & 32.532 & 26.979 & 34.305 \\
\hline 10/6/11 11:00 & & 30.71 & 30.149 & 29.005 & 30.432 & & 29.554 & 30.673 & 30.166 & 29.816 & 28.325 & 26.081 & 32.564 & 35.882 & 33.613 & 16.978 & 16.741 & 23.309 & 15.292 & 11.852 & 32.39 & 33.464 & 30.124 & 32.52 & 26.979 & 34.308 \\
\hline 10/6/11 11:30 & & 30.703 & 30.144 & 28.989 & 30.423 & & 29.556 & 30.673 & 30.166 & 29.816 & 28.321 & 26.078 & 32.552 & 35.887 & 33.601 & 16.981 & 16.734 & 23.309 & $\begin{array}{l}15.299 \\
\end{array}$ & 11.836 & 32.376 & 33.452 & 30.11 & 32.509 & 26.977 & 34.308 \\
\hline 10/6/11 12:00 & & 30.706 & 30.142 & 28.972 & 30.421 & & 29.568 & 30.67 & 30.161 & 29.811 & 28.312 & 26.067 & 32.54 & 35.885 & 33.589 & 16.978 & 16.727 & 23.309 & 15.299 & 11.829 & 32.373 & 33.438 & 30.103 & 32.492 & 26.979 & 34.31 \\
\hline 10/6/11 12:30 & & 30.703 & 30.142 & 28.954 & 30.418 & & 29.584 & 30.668 & 30.161 & 29.818 & 28.3 & 26.055 & 32.529 & 35.882 & 33.578 & 3 16.978 & 16.72 & 23.311 & 15.302 & 11.815 & 32.364 & 33.428 & 30.105 & 32.492 & 26.974 & 34.303 \\
\hline 10/6/11 13:00 & & 30.701 & 30.135 & $\begin{array}{l}20.944 \\
28.942\end{array}$ & $\begin{array}{l}30.410 \\
30.404\end{array}$ & & $\begin{array}{l}29.589 \\
29.59\end{array}$ & $\begin{array}{l}30.060 \\
30.664\end{array}$ & 30.159 & 29.816 & $\begin{array}{r}28.295 \\
28.295\end{array}$ & 26.031 & 32.519 & $\begin{array}{l}35.002 \\
35.878\end{array}$ & $\begin{array}{l}33.566 \\
33.560\end{array}$ & $\begin{array}{l}16.976 \\
5\end{array}$ & $\begin{array}{l}16.712 \\
16.713\end{array}$ & 23.313 & $\begin{array}{l}15.311 \\
15.311\end{array}$ & 11.803 & $\begin{aligned} 32.504 \\
32.35\end{aligned}$ & $\begin{array}{l}0.480 \\
33.419\end{array}$ & 30.089 & 32.473 & $\begin{array}{l}26.979 \\
26.979\end{array}$ & 34.305 \\
\hline $10 / 6 / 1113: 30$ & & 30.692 & 30.123 & 28.942 & 30.393 & & 29.591 & 30.658 & 30.159 & 29.818 & 28.286 & 26.021 & 32.503 & 35.871 & 33.55 & 16.976 & 16.704 & 23.316 & 15.32 & 11.791 & 32.329 & 33.405 & 30.067 & 32.459 & 26.977 & 34.303 \\
\hline $10 / 6 / 1114: 00$ & & 30.689 & 30.114 & 28.94 & 30.376 & & 29.589 & 30.658 & 30.159 & 29.816 & 28.291 & 26.028 & 32.489 & 35.871 & 33.538 & 16.974 & 16.697 & 23.318 & 15.325 & 11.775 & 32.308 & 33.383 & 30.056 & 32.443 & 26.974 & 34.298 \\
\hline 10/6/11 14:30 & & 30.689 & 30.107 & 28.935 & 30.369 & & 29.589 & 30.656 & 30.156 & 29.814 & 28.284 & 26.021 & 32.475 & 35.871 & 33.524 & 16.971 & 16.687 & 23.318 & 15.323 & 11.768 & 32.301 & 374 & 30.042 & 32.433 & 26.974 & 34.296 \\
\hline 10/6/11 15:00 & & 30.692 & 30.102 & 28.93 & 30.36 & & 29.594 & 30.654 & 30.156 & 29.814 & 28.272 & 26.016 & 32.465 & 35.866 & 33.512 & 16.969 & 16.678 & 23.322 & 15.323 & 11.753 & 32.299 & 33.367 & 0.044 & 32.424 & 26.977 & 34.296 \\
\hline 10/6/11 15:30 & & 30.687 & 30.1 & 28.928 & 30.348 & & 29.591 & 30.649 & 30.156 & 29.811 & 28.277 & 26.011 & 32.454 & 35.861 & 33.505 & 16.967 & 16.671 & 23.325 & 15.318 & 11.737 & 32.285 & 33.353 & 30.03 & 32.41 & 26.979 & 34.296 \\
\hline 10/6/11 16:00 & & 30.689 & 30.093 & 28.937 & 30.344 & & 29.582 & 30.654 & 30.156 & 29.809 & 28.279 & 26.011 & 32.444 & 35.861 & 33.491 & 16.967 & $\begin{array}{l}16.659 \\
-65\end{array}$ & 23.341 & 15.33 & 11.73 & 32.278 & 33.343 & 30.027 & 32.403 & 26.974 & 34.294 \\
\hline 10/6/11 16:30 & & 30.689 & 30.091 & 28.942 & 30.337 & & 29.575 & 30.649 & 30.159 & 29.807 & 28.279 & 26.016 & 32.433 & 35.857 & 33.479 & 16.969 & 16.652 & 23.376 & 15.332 & 11.718 & 32.271 & 33.334 & 30.018 & 32.394 & 26.974 & 34.294 \\
\hline 10/6/11 17:00 & & 30.689 & 30.091 & 28.944 & 30.334 & & 29.565 & 30.649 & 30.151 & 29.809 & 28.279 & 26.023 & 32.425 & 35.859 & 33.475 & 16.964 & 16.645 & 23.401 & 15.323 & 11.709 & 32.266 & 33.324 & 30.02 & 32.382 & 26.974 & 34.296 \\
\hline 10/6/11 17:30 & & 30.692 & 30.088 & 28.949 & 30.33 & & 29.558 & 30.649 & 30.156 & 29.807 & 28.281 & 26.026 & 32.416 & 35.854 & 33.465 & 16.967 & 16.638 & 23.41 & 15.323 & 11.704 & 32.264 & 33.319 & 30.018 & 32.377 & 26.979 & 34.296 \\
\hline $10 / 6 / 1118: 00$ & & 30.694 & 30.091 & 28.961 & 30.327 & & 29.547 & 30.649 & 30.159 & 29.804 & 28.286 & 26.033 & 32.409 & 35.854 & 33.458 & 3 16.967 & 16.631 & 23.42 & 15.32 & 11.697 & 32.261 & 33.31 & 30.025 & 32.368 & 26.977 & 34.296 \\
\hline $10 / 6 / 1118: 30$ & & 30.694 & 30.095 & 28.972 & 30.33 & & 29.533 & 30.649 & 30.163 & 29.804 & 28.288 & 26.047 & 32.407 & 35.857 & 33.451 & 16.967 & 16.626 & 23.454 & 15.32 & 11.69 & 32.266 & 33.298 & 30.02 & 32.363 & 26.979 & 34.296 \\
\hline 10/6/11 19:00 & & 30.696 & 30.093 & 28.982 & 30.33 & & 29.521 & 30.651 & 30.166 & 29.804 & 28.298 & 26.059 & 32.4 & 35.857 & 33.444 & 16.967 & 16.622 & 23.473 & 15.318 & 11.681 & 32.266 & 33.3 & 30.027 & 32.358 & 26.981 & 34.298 \\
\hline 10/6/11 19:30 & & 30.696 & 30.1 & 28.996 & 30.334 & & 29.514 & 30.656 & 30.17 & 29.807 & 28.302 & 26.069 & 32.395 & 35.861 & 33.439 & 16.969 & 16.617 & 23.473 & 15.309 & 11.676 & 32.266 & 33.296 & 30.042 & 32.356 & 26.979 & 34.301 \\
\hline 10/6/11 20:00 & & 30.701 & 30.107 & 29.003 & 30.339 & & 29.502 & 30.656 & 30.173 & 29.807 & 28.305 & 26.081 & 32.393 & 35.864 & 33.435 & 16.971 & 16.615 & 23.471 & 15.302 & 11.674 & 32.271 & 33.291 & 30.051 & 32.349 & 26.981 & 34.303 \\
\hline 10/6/11 20:30 & & 30.703 & 30.112 & 29.005 & 30.344 & & 29.5 & 30.658 & 30.166 & 29.809 & 28.309 & 26.093 & 32.39 & 35.868 & 33.432 & 16.971 & 16.608 & 23.473 & 15.302 & 11.669 & 32.273 & 33.286 & 30.058 & 32.349 & 26.979 & 34.303 \\
\hline 10/6/11 21:00 & & 30.703 & 30.114 & 29.01 & 30.351 & & 29.502 & 30.659 & 30.17 & 29.814 & 28.312 & 26.095 & 32.39 & 35.868 & 33.432 & 16.976 & 16.605 & 23.468 & 15.295 & 11.664 & 32.275 & 33.289 & 30.06 & 32.347 & 26.981 & 34.305 \\
\hline 10/6/11 21:30 & & 30.708 & 30.121 & 29.019 & 30.36 & & 29.495 & 30.661 & 30.168 & 29.816 & 28.316 & 26.095 & 32.386 & 35.878 & 33.428 & 16.978 & 16.601 & 23.468 & 15.292 & 11.664 & 32.282 & 33.284 & 30.072 & 32.347 & 26.984 & 34.308 \\
\hline $10 / 6 / 1122: 00$ & & 30.703 & 30.123 & 29.022 & 30.365 & & 29.49 & 30.664 & $\begin{array}{l}30.173 \\
30.173\end{array}$ & 29.818 & 28.321 & 26.107 & 32.381 & $\begin{array}{l}35.082 \\
35.82\end{array}$ & $\begin{array}{l}53.420 \\
33.423\end{array}$ & 16.978 & $\begin{array}{l}10.01 \\
16.598\end{array}$ & $\begin{array}{l}23.468 \\
23.468\end{array}$ & $\begin{array}{l}15.297 \\
15.297\end{array}$ & $\begin{array}{l}11.004 \\
11.659\end{array}$ & 32.28 & $\begin{array}{l}33.279 \\
3.249\end{array}$ & 30.067 & 32.342 & $\begin{array}{l}20.504 \\
26.981\end{array}$ & 34.308 \\
\hline $10 / 6 / 1122: 30$ & & 30.708 & 30.125 & 29.015 & 30.367 & & 29.495 & $\begin{array}{l}30.064 \\
30.666\end{array}$ & 30.168 & 29.818 & $\begin{array}{l}28.323 \\
28.323\end{array}$ & 26.11 & $\begin{array}{l}32.31 \\
32.376\end{array}$ & $\begin{array}{l}5.002 \\
35.88\end{array}$ & $\begin{array}{l}33.421 \\
33.421\end{array}$ & 16.978 & $\begin{array}{l}10.590 \\
16.591\end{array}$ & $\begin{array}{r}23.400 \\
23.47\end{array}$ & $\begin{array}{l}15.291 \\
15.292\end{array}$ & $\begin{array}{l}11.039 \\
11.659\end{array}$ & $\begin{array}{r}32.28 \\
32.282\end{array}$ & $\begin{array}{l}33.279 \\
33.279\end{array}$ & 0.074 & 32.337 & $\begin{array}{l}26.981 \\
26.986\end{array}$ & 34.308 \\
\hline $10 / 6 / 1123: 00$ & & 30.708 & 30.13 & 29.005 & $\begin{array}{l}50.301 \\
30.374\end{array}$ & & $\begin{array}{l}29.495 \\
29.504\end{array}$ & $\begin{array}{l}30.006 \\
30.666\end{array}$ & $\begin{array}{l}30.100 \\
30.17\end{array}$ & $\begin{array}{l}29.828 \\
29.823\end{array}$ & $\begin{array}{l}28.325 \\
28.319\end{array}$ & $\begin{array}{l}26.11 \\
26.105\end{array}$ & $\begin{array}{l}32.376 \\
32.376\end{array}$ & $\begin{array}{l}35.88 \\
35.887\end{array}$ & $\begin{array}{l}53.421 \\
33.418\end{array}$ & $\begin{array}{l}10.978 \\
16.983\end{array}$ & $\begin{array}{l}10.591 \\
16.587\end{array}$ & $\begin{array}{r}23.41 \\
23.468\end{array}$ & $\begin{array}{l}13.292 \\
15.29\end{array}$ & $\begin{array}{l}11.039 \\
11.657\end{array}$ & $\begin{array}{l}32.285 \\
32.282\end{array}$ & $\begin{array}{l}33.274 \\
3.274\end{array}$ & .084 & $\begin{array}{l}32.331 \\
32.337\end{array}$ & 26.981 & $\begin{array}{l}34.312 \\
34.312\end{array}$ \\
\hline $10 / 6 / 1$ & & 3 & 30 & 29.007 & & & 29.509 & 30.668 & 30.168 & 29.825 & 28 & 26.095 & 32.372 & 35.882 & 33.414 & & 16.584 & 23.47 & 15.292 & 11.652 & 32.285 & 72 & .084 & 32.335 & 26.984 & 34.312 \\
\hline $10 /$ & & 30 & 30 & & & & & 30.668 & 30.168 & 3 & 28.319 & 26.098 & 32.369 & 35.887 & 33.407 & 16.985 & $\begin{array}{l}16.577 \\
\end{array}$ & 23.47 & 15.297 & 11.648 & 32.278 & 27 & 882 & 28 & 984 & 34.312 \\
\hline $\begin{array}{l}10 / 7 / 11 \\
10\end{array}$ & & 8 & & 29.005 & 30.374 & & & 30.668 & 30.17 & & 28.321 & 26.09 & 362 & 35.882 & 3.404 & 16.985 & 16.573 & 23.47 & $\begin{array}{l}15.295 \\
15.295\end{array}$ & 11.641 & 32.271 & 33.263 & .067 & 2.321 & 6.984 & $\begin{array}{l}34.315 \\
315\end{array}$ \\
\hline 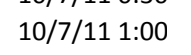 & & 30.708 & 30.128 & 29.003 & 30.372 & & 29.516 & 30.668 & 30.173 & 29.821 & 28.323 & 26.095 & 32.36 & 35.885 & 33.399 & 16.983 & 16.568 & 23.473 & 15.295 & 11.636 & 32.268 & 33.255 & 30.072 & 32.314 & 26.986 & 34.312 \\
\hline 10/7/11 1:30 & & 30.706 & 30.13 & 29.005 & 30.369 & & 29.518 & 30.67 & 30.175 & 29.818 & 28.319 & 26.093 & 32.353 & 35.887 & 33.395 & 16.985 & 16.561 & 23.475 & 15.295 & 11.631 & 32.268 & 33.253 & 30.07 & 32.311 & 26.986 & 34.31 \\
\hline 10/7/11 2:00 & & 30.708 & 30.132 & 29.015 & & & & 30.67 & 30.173 & & 28.328 & 26.09 & 32.348 & 35.887 & 33.39 & & 16.559 & 23.477 & 15.297 & 11.629 & 32.257 & 33.248 & 30.063 & 32.307 & 26.984 & 34.31 \\
\hline 10/7/11 2:30 & & 30.708 & 30.132 & 29.022 & & & p4 & 30.67 & 30.17 & & 28.325 & 26.105 & 32.344 & 35.889 & 33.383 & 16.985 & 16.552 & 23.477 & 15.295 & 11.624 & 32.259 & 33.244 & .067 & 32.302 & 26.984 & 34.31 \\
\hline 10/7/11 3:00 & & 30.708 & 30.128 & 29.026 & 30.367 & & 29.504 & 30.67 & 30.173 & 29.821 & 28.337 & 26.107 & 32.339 & 35.889 & 33.383 & 16.988 & 16.549 & 23.482 & 15.292 & 11.615 & 32.259 & 33.239 & 30.063 & 32.3 & 26.986 & 34.31 \\
\hline 10/7/11 3:30 & & 30.713 & 30.135 & 29.029 & 30.372 & & 29.504 & 30.675 & 30.17 & 29.821 & 28.335 & 26.117 & 32.337 & 35.892 & 33.376 & 16.992 & 16.545 & 23.482 & 15.29 & 11.615 & 32.261 & 33.236 & 30.072 & 32.295 & 26.988 & 34.312 \\
\hline 10/7/11 4:00 & & 30.715 & 30.139 & 29.036 & 30. & & 29.502 & 30.675 & 30.173 & 29.825 & 28.339 & 26.117 & 32.334 & 35.892 & 33.374 & 16.99 & 16.54 & 23.48 & 15.285 & 11.612 & 32.264 & 33.234 & 30.079 & 32.293 & 26.984 & 34.312 \\
\hline 10/7/11 4:30 & & 30.713 & 30.144 & 29.04 & 30.374 & & 29.502 & 30.675 & 30.168 & 29.828 & 28.339 & 26.119 & 32.332 & 35.887 & 33.374 & 16.99 & 16.538 & 23.48 & 15.283 & 11.61 & 32.264 & 33.232 & 30.072 & 32.293 & 26.988 & 34.315 \\
\hline 10/7/11 5:00 & & 30.717 & 30.146 & 29.045 & 30.379 & & 29.502 & 30.675 & 30.173 & 29.83 & 28.346 & 26.124 & 32.33 & 35.892 & 33.371 & 16.99 & 16.533 & 23.487 & 15.278 & 11.608 & 32.268 & 33.229 & 30.084 & 32.288 & 26.988 & 34.315 \\
\hline 10/7/11 5:30 & & 30.717 & 30.146 & 29.05 & 30.386 & & 29.504 & 30.678 & 30.178 & 29.828 & 28.351 & 26.126 & 32.332 & 35.892 & 33.367 & 16.992 & 16.533 & 23.489 & 15.283 & 11.608 & 32.271 & 33.229 & 30.086 & 32.293 & 26.986 & 34.315 \\
\hline 10/7/11 6:00 & & 30.72 & 30.151 & 29.052 & 30.388 & & 29.497 & 30.678 & 30.178 & 29.832 & 28.353 & 26.136 & 33.277 & 35.901 & 33.69 & 16.995 & 16.528 & 23.498 & 15.281 & 11.925 & 32.492 & 34.305 & 30.096 & 33.269 & 26.988 & 34.317 \\
\hline 10/7/11 6:30 & & 30.72 & 30.153 & 29.064 & 30.395 & & 29.497 & 30.68 & 30.178 & 29.834 & 28.36 & 26.136 & 34.534 & 35.894 & 34.912 & 16.995 & 16.59 & 23.512 & 15.278 & 13.155 & 33.092 & 5 & .136 & 34.498 & 26.986 & 34.317 \\
\hline $10 / 7 / 117$ & & 30.722 & 30.16 & 29.078 & 30.409 & & 29.495 & 30.682 & 30.18 & 29.8 & 28.363 & 26.148 & 35.121 & 35.894 & 35.587 & 16.9 & 16.706 & 23.521 & 15.2 & 13. & $\begin{array}{r}33.35 \\
\end{array}$ & 36.099 & 33 & 35.078 & 26.988 & 34.317 \\
\hline 10/7/11 7 & & 30.722 & 30.165 & 29.089 & 30.432 & & 29.488 & 30.682 & 30.182 & 29.839 & 28.37 & 26.157 & 35.444 & 35.899 & 36.047 & 16.997 & 16.82 & 23.524 & 15.274 & 14.288 & 33.516 & 36.39 & .232 & 35.385 & 26.988 & 34.319 \\
\hline $10 / 7 / 1$ & & 30.724 & 30.167 & 29.094 & $\begin{array}{l}30.452 \\
30.465\end{array}$ & & $\begin{array}{r}29.400 \\
29.49\end{array}$ & 30.683 & $\begin{array}{l}30.182 \\
30.182\end{array}$ & 29.846 & 28.377 & 26.172 & $\begin{array}{l}5.444 \\
35.62\end{array}$ & $\begin{array}{l}35.894 \\
35.894\end{array}$ & $\begin{array}{l}36.265 \\
36.24 !\end{array}$ & 17.002 & $\begin{array}{l}16.02 \\
16.923 \\
\end{array}$ & $\begin{array}{l}23.526 \\
23.526\end{array}$ & $\begin{array}{l}15.274 \\
15.274\end{array}$ & $\begin{array}{l}14.200 \\
14.504\end{array}$ & $\begin{array}{l}33.510 \\
33.579\end{array}$ & $\begin{array}{l}36.559 \\
36.558\end{array}$ & 2 & 35.564 & $\begin{array}{l}26.900 \\
26.993\end{array}$ & 34.324 \\
\hline $10 /$ & & 30 & 30. & 29. & 30 & & 29. & 30.6 & & 29 & 28. & 26 & 36 & 35 & 36 & 17 & 17 & 23.524 & 15. & 14. & 33 & 37.12 & 7 & 1 & 26.991 & 34.327 \\
\hline $10 / 7 /$ & & 30. & 30. & 29. & 30. & & 29 & 30. & & & 28 & & & 35. & 37 & & & 23 & 15. & 15 & 33 & 37 & & 3 & 26.991 & 34.329 \\
\hline Nor & & & & 29.1 & & & 29.4 & & & & & & & & 37. & & & & 15. & 15. & 34 & & & 04 & 38 & \\
\hline & & & & 29 & & & & 30. & & & & & & & & & & & & (1) & & & & & & \\
\hline & & & & & & & & & & & & & & & & & & & & & & & & & & \\
\hline & & & & & & & & & & & & & & & & & & & 15 & & & & & & & 34. \\
\hline 10 & & 30 & & 29. & & & 29 . & & & & 28 & & 36 & & & & 17 & 23. & 15. & 15 & & & & 35 & & 34. \\
\hline 10/7/11 12:00 & & 30.731 & 30.214 & 29.094 & 30.731 & & 29.53 & 30.692 & 30.187 & 29.881 & 28.391 & 26.177 & 35.39 & 35.911 & 36.588 & 17.016 & 17.634 & 23.512 & 15.29 & 14.819 & 33.724 & 36.255 & 30.516 & 35.315 & 26.993 & 34.336 \\
\hline 10/7/11 12:30 & & 30.729 & 30.214 & 29.087 & 30.745 & & 29.54 & 30.692 & 30.182 & 29.881 & 28.391 & 26.172 & 34.971 & 35.906 & 36.145 & 17.018 & 17.608 & 23.51 & 15.295 & 14.379 & 33.549 & 35.848 & 30.505 & 34.904 & 26.993 & 34.336 \\
\hline
\end{tabular}




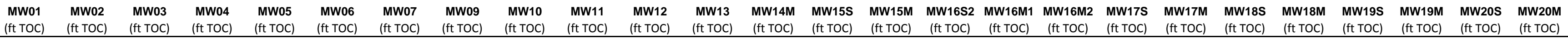

\begin{tabular}{|c|c|c|c|c|c|c|c|c|c|c|c|c|c|c|c|c|c|c|c|c|c|c|c|c|c|c|}
\hline Well & $\begin{array}{l}\text { (ft TOC) } \\
\text { (ft }\end{array}$ & $\begin{array}{l}\text { (ft TOC) } \\
\text { (f) }\end{array}$ & $\begin{array}{l}\text { (ft TOC) } \\
\text { (f) }\end{array}$ & $\begin{array}{l}\text { (ft TOC) } \\
\text { (f }\end{array}$ & $\begin{array}{l}\text { (ft TOC) } \\
\text { (f) }\end{array}$ & $\begin{array}{l}\text { (ft TOC) } \\
\text { (f) }\end{array}$ & $\begin{array}{l}\text { (ft TOC) } \\
\text { (ft }\end{array}$ & $\begin{array}{l}\text { (ft TOC) } \\
\text { (f) }\end{array}$ & $\begin{array}{l}\text { (ft TOC) } \\
\text { (f) }\end{array}$ & $\begin{array}{l}\text { (ft TOC) } \\
\text { (f) }\end{array}$ & $\begin{array}{l}\text { (ft TOC) } \\
\text { (f) }\end{array}$ & $\begin{array}{l}\text { (ft TOC) } \\
\text { (f) }\end{array}$ & (ft TOC) & $\begin{array}{l}\text { (ft TOC) } \\
\end{array}$ & (ft TOC) & (ft TOC) & $\begin{array}{l}\text { (ft TOC) } \\
\text { (f) }\end{array}$ & (ft TOC) & $\begin{array}{l}\text { (ft TOC) } \\
\text { (1) }\end{array}$ & (ft TOC) & $\begin{array}{l}\text { (ft TOC) } \\
\end{array}$ & (ft TOC) & $\begin{array}{l}\text { (ft TOC) } \\
\end{array}$ & (ft TOC) & $\begin{array}{l}\text { (ft TOC) } \\
\text { (1) }\end{array}$ & (ft TOC) \\
\hline 13:00 & & 30.727 & 30.214 & 29.075 & 30.7 & & 29.549 & 30.65 & 30 & $29 . \varepsilon$ & 28.386 & 26. & 34.67 & 35.913 & $35 . \varepsilon$ & 17. & 17 & 23.507 & 15.299 & 14.062 & 33.43 & 35.547 & 30.483 & 06 & 26.995 & 34.338 \\
\hline 111 13:30 & & 30.727 & 30.216 & 29.075 & 30.759 & & 29.556 & 30.695 & 30.187 & 29.883 & 28.384 & 26.15 & 34.433 & 35.908 & 35.571 & 17.018 & 17.538 & 23.503 & 15.304 & 13.808 & 33.334 & 35.317 & 172 & & 6.993 & 34.333 \\
\hline 10/7/11 14:00 & & 30.724 & 30.216 & 29.064 & 30.75 & & 29.561 & 30.694 & 30.182 & 29.879 & 28.386 & 26.15 & 34.244 & 35.911 & 35.371 & 17.018 & 17.503 & 23.501 & 15.311 & 13.606 & 33.245 & 35.127 & 30.448 & 1.184 & 5.995 & 34.336 \\
\hline 10/7/11 14:30 & & 30.724 & 30.212 & 29.064 & 30.745 & & 29.572 & 30.694 & 30.185 & 29.876 & 28.381 & 26.138 & 34.08 & 35.906 & 35.2 & $2 \quad 17.018$ & 17.463 & 23.496 & 15.311 & 13.437 & 33.173 & 34.969 & 30.427 & 34.029 & 26.993 & 34.336 \\
\hline 10/7/11 15:00 & & 30.727 & 30.212 & 29.061 & 30.736 & & 29.572 & 30.694 & 30.187 & 29.876 & 28.379 & 26.136 & 33.949 & 35.908 & 35.055 & $\begin{array}{ll}5 & 17.018\end{array}$ & 17.43 & 23.494 & 15.313 & 13.296 & 33.113 & 34.836 & 30.415 & 33.893 & 26.995 & 34.333 \\
\hline 10/7/11 15:30 & & 30.722 & 30.209 & 29.061 & 30.724 & & 29.572 & 30.694 & 30.185 & 29.874 & 28.381 & 26.134 & 33.832 & 35.904 & 34.931 & $1 \quad 17.016$ & 17.395 & 23.487 & 15.325 & 13.166 & 33.054 & 34.717 & 30.385 & 33.773 & 26.995 & 34.333 \\
\hline 10/7/11 16:00 & & 30.727 & 30.209 & 29.061 & 30.71 & & 29.575 & 30.694 & 30.187 & 29.874 & 28.381 & 26.134 & 33.729 & 35.906 & 34.823 & $\begin{array}{ll}3 & 17.016\end{array}$ & 17.363 & 23.484 & 15.323 & 13.06 & 33.012 & 34.613 & 30.382 & 33.672 & 26.995 & 34.331 \\
\hline 10/7/11 16:30 & & 30.727 & 30.212 & 29.061 & 30.701 & & 29.572 & 30.694 & 30.189 & 29.879 & 28.379 & 26.131 & 33.635 & 35.901 & 34.729 & $\begin{array}{l}9 \\
9\end{array}$ & 17.335 & 23.482 & 15.32 & 12.966 & 32.966 & 534.525 & 30.364 & 33.581 & 26.998 & 34.333 \\
\hline 10/7/11 17:00 & & 30.729 & 30.214 & 29.064 & 30.694 & & 29.57 & 30.697 & 30.192 & 29.874 & 28.381 & 26.131 & 33.556 & 35.901 & 34.644 & $4 \quad 17.016$ & 17.307 & 23.477 & 15.323 & 12.882 & 32.933 & 34.445 & 30.359 & 33.508 & 26.998 & 34.333 \\
\hline 10/7/11 17:30 & & 30.729 & 30.214 & 29.064 & 30.68 & & 29.57 & 30.697 & 30.185 & 29.876 & 28.381 & 26.131 & 33.481 & 35.901 & 34.567 & $7 \quad 17.018$ & 17.279 & 23.475 & 15.323 & 12.804 & 32.898 & $3 \quad 34.371$ & 30.345 & 33.435 & 26.995 & 34.331 \\
\hline $10 / 7 / 1118: 00$ & & 30.731 & 30.214 & 29.068 & 30.67 & & 29.565 & 30.697 & 30.189 & 29.876 & 28.379 & 26.131 & 33.418 & 35.904 & 34.497 & $7 \quad 17.018$ & 17.25 & 23.477 & 15.323 & 12.736 & 32.863 & 34.307 & 30.335 & 33.367 & 26.998 & 34.331 \\
\hline $10 / 7 / 1118: 30$ & & 30.731 & 30.214 & 29.078 & 30.659 & & 29.563 & 30.697 & 30.192 & 29.876 & 28.388 & 26.138 & 33.429 & 35.904 & 34.513 & $\begin{array}{l}317.02 \\
\end{array}$ & 17.229 & 23.473 & 15.328 & 12.753 & 32.849 & 34.322 & 30.326 & 33.377 & 26.995 & 34.333 \\
\hline 10/7/11 19:00 & & 30.731 & 30.214 & 29.08 & 30.649 & & 29.558 & 30.699 & 30.192 & 29.876 & 28.386 & 26.145 & 33.329 & 35.908 & 34.41 & 17.02 & 17.208 & 23.473 & 15.328 & 12.647 & 32.821 & $\quad 34.22$ & 30.316 & 33.281 & 26.995 & 34.331 \\
\hline 10/7/11 19:30 & & 30.736 & 30.219 & 29.078 & 30.642 & & 29.561 & 30.702 & 30.192 & 29.879 & 28.388 & 26.145 & 33.273 & 35.901 & 34.347 & 17.02 & 17.185 & 23.475 & 15.323 & 12.586 & 32.795 & 34.165 & 30.314 & 33.227 & 26.998 & 34.336 \\
\hline 10/7/11 20:00 & & 30.738 & 30.221 & 29.08 & 30.638 & & 29.556 & 30.702 & 30.194 & 29.883 & 28.388 & 26.145 & 33.226 & 35.906 & 34.3 & 17.023 & 17.164 & 23.473 & 15.325 & 12.534 & 32.774 & 34.118 & 30.314 & 33.182 & 27 & 34.336 \\
\hline 10/7/11 20:30 & & 30.738 & 30.223 & 29.08 & 30.633 & & 29.556 & 30.699 & 30.194 & 29.883 & 28.391 & 26.145 & 33.181 & 35.908 & 34.251 & 17.023 & 17.145 & 23.47 & 15.325 & 12.487 & 32.756 & 34.08 & 30.309 & 33.135 & 27 & 34.338 \\
\hline 10/7/11 21:00 & & 30.738 & 30.226 & 29.078 & 30.624 & & 29.561 & 30.704 & 30.192 & 29.886 & 28.388 & 26.145 & 33.142 & 35.911 & 34.211 & 17.025 & 17.127 & 23.473 & 15.325 & 12.447 & 32.737 & 34.037 & 30.305 & 33.098 & 27 & 34.34 \\
\hline 10/7/11 21:30 & & 30.738 & 30.226 & 29.08 & 30.619 & & 29.563 & 30.702 & 30.197 & 29.888 & 28.388 & 26.143 & 33.106 & 35.911 & 34.173 & 17.025 & 17.108 & 23.489 & 15.33 & 12.412 & 32.718 & 33.999 & 30.3 & 33.062 & 27 & 34.34 \\
\hline 10/7/11 22:00 & & 30.736 & 30.226 & 29.078 & 30.614 & & 29.561 & 30.699 & 30.194 & 29.888 & 28.388 & 26.143 & 33.074 & 35.908 & 34.138 & $3 \quad 17.025$ & 17.089 & 23.498 & 15.33 & 12.374 & 32.7 & 33.966 & 30.293 & 33.029 & 27 & 34.343 \\
\hline $10 / 7 / 1122: 30$ & & 30.736 & 30.223 & 29.078 & 30.607 & & 29.561 & 30.702 & 30.194 & 29.886 & 28.391 & 26.141 & 33.039 & 35.911 & 34.103 & 17.025 & 17.071 & 23.514 & 15.33 & 12.336 & 32.679 & 33.935 & 30.281 & 32.994 & 26.998 & 34.343 \\
\hline 00 & & 30.736 & 30.226 & 29.071 & 30. & & 29.565 & 30.704 & 30.194 & 29.888 & 28.388 & 26.141 & 33.01 & 35.908 & 34.072 & 17.027 & 17.057 & 23.531 & 15.33 & 12.306 & 32.665 & 33.907 & .279 & 2.964 & 26.998 & 34.345 \\
\hline $10 / 7 / 11$ & & 30.738 & 30.228 & 29.066 & 30.591 & & 29.57 & 30.704 & 30.194 & 29.89 & 28.386 & 26.134 & 32.982 & .908 & 34.042 & 17.027 & 17.04 & 23.533 & 15.33 & 12.278 & 32.651 & 876 & .272 & 2.938 & 27 & 34.343 \\
\hline $10 / 8 / 1$ & & 38 & 30.228 & 29.075 & 30.586 & & 29.565 & 30.699 & 30.197 & 29.888 & 28.388 & 26.131 & 32.954 & 35.913 & 34.014 & 17.027 & 17.021 & 23.528 & 15.335 & 12.247 & 32.637 & 3.85 & 267 & 2.912 & .002 & 34.345 \\
\hline 10/8/11 0:30 & & 30.734 & 30.221 & 29.08 & 30.577 & & 29.565 & 30.702 & 30.199 & 29.888 & 28.391 & 26.136 & 32.929 & 35.911 & 33.988 & 317.027 & 17.005 & 23.524 & 15.337 & 12.226 & 32.611 & 33.824 & .251 & 2.886 & 27 & 34.343 \\
\hline 10/8/11 1:00 & & 30.738 & 30.223 & 29.089 & 30.572 & & 29.561 & 30.706 & 30.199 & 29.888 & 28.393 & 26.138 & 32.903 & 35.913 & 33.965 & 17.03 & 16.991 & 23.514 & 15.335 & 12.202 & 32.609 & 33.8 & 30.248 & 2.861 & 7.002 & 34.345 \\
\hline 10/8/11 1:30 & & 30.741 & 30.223 & 29.089 & 30.568 & & 29.554 & 30.706 & 30.196 & 29.888 & 28.395 & 26.148 & 32.882 & 35.913 & 33.939 & 17.03 & 16.972 & 23.507 & 15.33 & 12.177 & 32.593 & 33.774 & 246 & 2.837 & 27 & 34.345 \\
\hline 10/8/11 2:00 & & 30.741 & 30.226 & 29.087 & 30.563 & & 29.558 & 30.709 & 30.201 & 29.89 & 28.393 & 26.148 & 32.861 & 35.913 & 33.915 & 17.027 & 16.961 & 23.503 & 15.33 & 12.151 & 32.588 & 33.758 & 30.244 & 32.818 & 27.002 & 34.347 \\
\hline 10/8/11 2:30 & & 30.745 & 30.23 & 29.089 & 30.563 & & 29.558 & 30.709 & 30.201 & 29.893 & 28.395 & 26.143 & 32.84 & 35.918 & 33.894 & 17.032 & 16.947 & 23.501 & 15.328 & 12.132 & 32.581 & 33.739 & 30.253 & 32.8 & 27.002 & 34.345 \\
\hline 10/8/11 3:00 & & 30.745 & 30.23 & 29.085 & 30.561 & & 29.558 & 30.707 & 30.199 & 29.897 & 28.398 & 26.145 & 32.821 & 35.915 & 33.878 & $\begin{array}{ll}3 & 17.032\end{array}$ & 16.933 & 23.496 & 15.33 & 12.111 & 32.567 & 33.72 & 30.241 & 32.778 & 27 & 34.345 \\
\hline 10/8/11 3:30 & & 30.743 & 30.233 & 29.082 & 30.556 & & 29.563 & 30.706 & 30.201 & 29.895 & 28.395 & 26.143 & 32.802 & 35.913 & 33.854 & 17.032 & 16.919 & 23.489 & 15.33 & 12.097 & 32.562 & 33.699 & 30.237 & 32.76 & 27.002 & 34.347 \\
\hline 10/8/11 4:00 & & 30.745 & 30.235 & 29.094 & 30.551 & & 29.561 & 30.709 & 30.201 & 29.895 & 28.4 & 26.138 & 32.786 & 35.915 & 33.836 & 17.034 & 16.907 & 23.489 & 15.328 & 12.076 & 32.551 & 33.682 & 30.237 & 32.746 & 27.005 & 34.345 \\
\hline $10 / 8 / 114: 30$ & & 30.743 & 30.233 & 29.11 & 30.547 & & 29.551 & 30.709 & 30.206 & 29.895 & 28.405 & 26.15 & 32.767 & 35.918 & 33.817 & 17.034 & 16.895 & 23.484 & 15.332 & 12.054 & 32.537 & 33.663 & 30.23 & 32.727 & 27.002 & 34.345 \\
\hline 10/8/11 5:00 & & 30.743 & 30.233 & 29.122 & 30.542 & & 29.54 & 30.711 & 30.208 & 29.895 & 28.412 & 26.169 & 32.751 & 35.915 & 33.803 & 17.034 & 16.884 & 23.482 & 15.33 & 12.04 & 32.527 & 33.649 & 30.23 & 32.713 & 27.005 & 34.343 \\
\hline 10/8/11 5:30 & & 30.748 & 30.233 & 29.12 & 30.544 & & 29.54 & 30.714 & 30.208 & 29.897 & 28.416 & 26.177 & 32.739 & 35.913 & 33.789 & 17.034 & 16.874 & 23.482 & 15.32 & 12.024 & 32.53 & 33.635 & 30.234 & 32.696 & 27.005 & 34.345 \\
\hline 10/8/11 6:00 & & 30.754 & 30.244 & 29.117 & 30.5 & & 29.544 & 30.716 & 30.204 & 29.904 & 28.414 & 26.174 & 32.73 & 35.92 & 33.779 & 17.037 & 16.863 & 23.494 & 15.316 & 12.014 & 32.53 & 33.625 & 30.246 & 32.689 & 27.005 & 34.347 \\
\hline 10/8/11 6:30 & & 30.755 & 30.247 & 29.115 & 30. & & 29.551 & 30.716 & 30.201 & 29.909 & 28.414 & 26.174 & 32.716 & & 33.765 & 17.039 & $\begin{array}{l}16.0856 \\
\end{array}$ & 23.494 & 15.3 & $\begin{array}{l}12.005 \\
12.005\end{array}$ & 32.532 & 33.613 & 30.248 & 32.675 & 27.007 & $\begin{array}{r}34.35 \\
345\end{array}$ \\
\hline 10/8/11 7:00 & & 30.752 & $\begin{array}{l}30.249 \\
30.249\end{array}$ & 29.131 & 30. & & 29.544 & 30.716 & 30.206 & 29.911 & $\begin{array}{l}28.423 \\
28.423\end{array}$ & 26.169 & 32.706 & $\begin{array}{l}35.918 \\
35.918\end{array}$ & 33.754 & 17.041 & $\begin{array}{l}10.086 \\
16.846\end{array}$ & $\begin{array}{l}23.489 \\
23.489\end{array}$ & 15.32 & 11.991 & 32.52 & 33.606 & 30.244 & 32.666 & 27.005 & 34.35 \\
\hline 10/8/11 7:30 & & 30 & 30.249 & 29.159 & 30. & & 29 & 30.719 & 30.216 & 29.911 & 28.435 & 26.186 & 32.695 & 35.918 & 33.742 & 17.041 & 16.834 & 23.484 & 15.325 & 11.979 & 32.516 & 33.592 & 34 & 52 & 27.007 & 34.352 \\
\hline & & 30 & 30.2 & 29.171 & 30 & & 29. & 30 & 30 & 29 & 28 & 26 & 32 & 35.9 & 33.732 & 17.044 & 1 & 23.484 & 15.318 & 1 & 32.516 & 33.58 & 18 & 45 & 27.007 & 34.352 \\
\hline & & 30. & 30.26 & 29.164 & 30. & & & 30 & $3 c$ & 29 & 28 & 26 & 32 & 35.922 & 33.723 & 17.044 & 16.82 & 23 & 15.32 & 11 & 32.516 & 53 & 5 & 38 & 27.009 & 34.354 \\
\hline & & & 30.2 & 29.199 & & & & 30 & 30 & 26 & 28 & 26 & 32 & 35.927 & 33.718 & 17 & 16.816 & 23 & 15.306 & 11.956 & 32.525 & 72 & 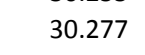 & 33 & 27.009 & 34.362 \\
\hline & & & 30. & & & & & 30. & & 29. & 28. & 26 & 32.6 & . & 33.7 & 17 & 16 & 23.4 & & 11.949 & 32.52 & 33.563 & 30.272 & 4 & 27.009 & 34.364 \\
\hline $10 /$ & & 30.76 & 30.282 & 29.199 & & & & 30. & & & 28. & & & 35. & 33.7 & & & & & 11.944 & 32.518 & .559 & & 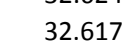 & 27.009 & 34.366 \\
\hline 10:30 & & 30.78 & 30.3 & 29.194 & 30.5 & & & 30 & & & 28. & 26 & 32.6 & 35. & 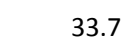 & 17.058 & 16.802 & & 15.297 & 11.939 & 32.548 & 33.556 & 09 & 19 & 27.014 & 34.371 \\
\hline 10 & & 30. & $30.3 c$ & 29. & $2 x+2$ & & & 30. & & & & & 32 & 35. & & & & & 15.311 & 11.927 & 32.534 & & & 12 & 27.012 & 34.371 \\
\hline 11:30 & & & 30.3 & 29.187 & 30. & & & & & & & 26. & 32. & 35. & 33.688 & 17. & 16. & & 15.311 & 11.923 & 32.534 & & & 32.605 & 27.012 & 34.371 \\
\hline $10 / 8 / 1112: 00$ & & 30. & 30. & 29.171 & 30. & & & 30. & 30.2 & & 28.421 & 26.236 & 32.639 & 35.9 & 33.681 & 17. & 16.7 & 23.494 & 15.316 & 11.918 & 32.527 & 33.537 & 30.302 & 32.6 & 27.014 & 34.371 \\
\hline 10/8/11 12:30 & & 30. & 30 & 29.159 & 30.66 & & & 30.7 & $30.2 \mathrm{x}-\mathrm{s}$ & 29. & 28.421 & 26.22 & 32.629 & 35.9 > & 33.671 & 17.0 & 16.77 & 23.489 & 15.318 & 11.906 & 32.52 & 33.528 & 30.295 & 32.591 & 27.012 & 34.371 \\
\hline 10/8/11 13:00 & & 30.7 & 30.307 & 29.15 & 36 & & 29. & 30.7 & $30.2>-2$ & 29.5 & 28.407 & 26.21 & $32.6 \mathrm{~s}>\mathrm{s}$ & 35.9 & 33.66 & 17.067 & 16.767 & 23.487 & 15.325 & 11.899 & 32.513 & $\begin{array}{l}3 \\
3\end{array} 33.516$ & 30.286 & 32.581 & 27.014 & 34.371 \\
\hline 10/8/11 13:30 & & 30.759 & 30.305 & 29.152 & 30.593 & & 29.587 & 30.733 & 30.213 & 29.962 & 28.4 & 26.198 & 32.61 & 35.939 & 33.655 & 17.065 & 16.76 & 23.482 & 15.33 & 11.887 & 32.499 & 33.507 & 30.279 & 32.567 & 27.014 & 34.371 \\
\hline 10/8/11 14:00 & & 30.759 & 30.3 & 29.15 & 30.582 & & 29.594 & 30.735 & 30.218 & 29.96 & 28.384 & 26.196 & 32.596 & 35.936 & 33.639 & 17.065 & 16.75 & 23.48 & 15.335 & 11.88 & 32.481 & 33.495 & 30.26 & 32.56 & 27.014 & 34.366 \\
\hline 10/8/11 14:30 & & 30.759 & 30.298 & 29.127 & 30.575 & & 29.605 & 30.733 & 30.211 & 29.955 & 28.388 & 26.193 & 32.587 & 35.941 & 33.625 & 17.065 & 16.743 & 23.475 & 15.335 & 11.866 & 32.478 & 33.485 & 30.251 & 32.548 & 27.014 & 34.366 \\
\hline 10/8/11 15:00 & & 30.764 & 30.3 & 29.131 & 30.572 & & 29.617 & 30.733 & 30.211 & 29.958 & 28.379 & 26.186 & 32.578 & 35.941 & 33.622 & 17.065 & 16.734 & 23.473 & 15.328 & 11.859 & 32.474 & 33.481 & 30.255 & 32.539 & 27.014 & 34.364 \\
\hline 10/8/11 15:30 & & 30.759 & 30.298 & 29.129 & 30.561 & & 29.605 & 30.733 & 30.211 & 29.955 & 28.377 & 26.174 & 32.568 & 35.939 & 33.606 & 17.065 & 16.722 & 23.468 & 15.346 & 11.845 & 32.455 & 33.466 & 30.232 & 32.527 & 27.012 & 34.362 \\
\hline $10 /$ & & 30.757 & 30.289 & 29.11 & 30.549 & & 29.622 & 30.73 & 30.211 & 29.948 & 28.356 & 26.167 & 32.554 & 35.932 & 33.596 & 17.062 & 16.715 & 23.461 & 15.351 & 11.831 & 32.443 & 33.452 & 30.223 & 32.516 & 27.012 & 34.359 \\
\hline 10/8/11 16:30 & & 30.766 & 30.293 & 29.134 & 30.551 & & 29.615 & 30.733 & 30.213 & 29.951 & 28.344 & 26.145 & 32.55 & 35.934 & 33.589 & 17.062 & 16.706 & 23.503 & 15.332 & 11.826 & 32.448 & 33.445 & 30.23 & 32.509 & 27.014 & 4.359 \\
\hline
\end{tabular}




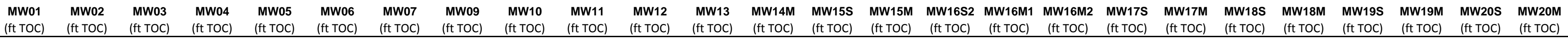

\begin{tabular}{|c|c|c|c|c|c|c|c|c|c|c|c|c|c|c|c|c|c|c|c|c|c|c|c|c|c|c|}
\hline well & $\begin{array}{l}\text { (ft TOC) } \\
\text { (f) }\end{array}$ & $\begin{array}{l}\text { (ft TOC) } \\
\text { (ft) }\end{array}$ & $\begin{array}{l}\text { (ft TOC) } \\
\text { (f) }\end{array}$ & $\begin{array}{l}\text { (ft TOC) } \\
\text { (ft) }\end{array}$ & $\begin{array}{l}\text { (ft TOC) } \\
\text { (f) }\end{array}$ & $\begin{array}{l}\text { (ft toc) } \\
\text { (ftocts }\end{array}$ & (ft toc) & $\begin{array}{l}\text { (ift TOC) } \\
\text { (n) }\end{array}$ & $\begin{array}{l}\text { (ft TOC) } \\
\text { (ft) }\end{array}$ & $\begin{array}{l}\text { (ft TOC) } \\
\text { (f) }\end{array}$ & $\begin{array}{l}\text { (ft TOC) } \\
\text { (n) }\end{array}$ & $\begin{array}{l}\text { (ft TOC) } \\
\text { (f) }\end{array}$ & (ft TOC) & $\begin{array}{l}\text { (ft TOC) } \\
\end{array}$ & (ft TOC) & (ft TOC) & $\begin{array}{l}\text { (ft TOC) } \\
\text { (f) }\end{array}$ & (ft TOC) & $\begin{array}{l}\text { (ft TOC) } \\
\text { (ft) }\end{array}$ & (ft TOC) & $\begin{array}{l}\text { (ft ToC) } \\
\text { (ft }\end{array}$ & $\begin{array}{l}\text { (ft TOC) } \\
\text { (f) }\end{array}$ & $\begin{array}{l}\text { (ft TOC) } \\
\text { (n) }\end{array}$ & (ft TOC) & $\begin{array}{l}\text { (ft TOC) } \\
\text { (1) }\end{array}$ & (ft TOC) \\
\hline 7:00 & & 30.761 & 30.291 & 29.141 & 30.5 & & 29.61 & 30.735 & 30.216 & 29.9 & 28.328 & 26 & 32.538 & 35.9 & 33.5 & 17. & 16.699 & 23.514 & 15.344 & 11.819 & 32.434 & 33.438 & 30.218 & 99 & 14 & 4 \\
\hline 11 17:30 & & 30.759 & 30.284 & 29.131 & 30.528 & & 29.61 & 30.735 & 30.213 & 29.944 & 28.325 & 26.153 & 32.529 & 35.932 & 33.566 & 17.06 & 16.69 & 23.51 & 15.344 & 11.805 & 32.422 & 24 & .206 & 2.485 & 14 & 34.352 \\
\hline 11 18:00 & & 30.768 & 30.291 & 29.164 & 30.533 & & 29.594 & 30.737 & 30.22 & 29.951 & 28.3 & 26.153 & 32.522 & 35.936 & 33.561 & 17.065 & 16.687 & 23.512 & 15.337 & 11.8 & 32.418 & 33.419 & .225 & 2.483 & 7.016 & 354 \\
\hline 10/8/11 18:30 & & 30.768 & 30.296 & 29.169 & 30.528 & & 29.594 & 30.737 & 30.218 & 29.953 & 28.309 & 26.145 & 32.512 & 35.936 & 533.552 & 17.062 & 16.678 & 23.503 & 15.337 & 11.791 & 32.422 & 33.412 & 30.213 & .473 & 14 & 34.352 \\
\hline 10/8/11 19:00 & & 30.771 & 30.293 & 29.169 & 30.528 & & 29.587 & 30.738 & 30.22 & 29.951 & 28.302 & 26.141 & 32.51 & 35.936 & $5 \quad 33.547$ & 17.062 & 16.676 & 23.496 & 15.335 & 11.786 & 32.422 & 33.407 & 30.215 & 32.471 & 27.019 & 34.35 \\
\hline 10/8/11 19:30 & & 30.775 & 30.303 & 29.169 & 30.533 & & 29.589 & 30.74 & 30.223 & 29.96 & 28.302 & 26.145 & 32.507 & 35.939 & $\begin{array}{l}7 \\
73.547\end{array}$ & 17.067 & 16.671 & 23.496 & 15.328 & 11.784 & 32.429 & 33.407 & 30.225 & 32.469 & 27.016 & 34.354 \\
\hline 10/8/11 20:00 & & 30.778 & 30.31 & 29.169 & 30.54 & & 29.591 & 30.74 & 30.22 & 29.964 & 28.312 & 26.138 & 32.503 & 35.941 & 133.545 & 17.067 & 16.666 & 23.494 & 15.32 & 11.782 & 32.432 & 33.405 & 30.23 & 32.469 & 27.016 & 34.354 \\
\hline 10/8/11 20:30 & & 30.778 & 30.307 & 29.173 & 30.537 & & 29.601 & 30.742 & 30.218 & 29.964 & 28.307 & 26.124 & 32.5 & 35.948 & $3 \quad 33.54$ & 17.069 & 16.662 & 23.491 & 15.33 & 11.779 & 32.429 & 33.4 & 30.234 & 32.464 & 27.016 & 34.357 \\
\hline 10/8/11 21:00 & & 30.775 & 30.307 & 29.187 & 30.537 & & 29.587 & 30.743 & 30.225 & 29.964 & 28.302 & 26.117 & 32.498 & 35.943 & 333.538 & 17.067 & 16.662 & 23.489 & 15.325 & 11.777 & 32.427 & 33.398 & 30.225 & 32.462 & 27.019 & 34.359 \\
\hline 10/8/11 21:30 & & 30.775 & 30.31 & 29.187 & 30.537 & & 29.589 & 30.742 & 30.223 & 29.967 & 28.307 & 26.124 & 32.498 & 35.948 & $3 \quad 33.533$ & 17.069 & 16.657 & 23.487 & 15.335 & 11.775 & 32.422 & 33.398 & 30.23 & 32.455 & 27.019 & 34.359 \\
\hline 10/8/111 22:00 & & 30.782 & 30.314 & 29.178 & 30.542 & & 29.598 & 30.745 & 30.223 & 29.971 & 28.33 & 26.126 & 32.496 & 35.946 & $5 \quad 33.533$ & 17.071 & 16.655 & 23.482 & 15.325 & 11.77 & 32.429 & 33.395 & 30.237 & 32.457 & 27.019 & 34.364 \\
\hline 10/8/11 22:30 & & 30.782 & 30.321 & 29.18 & 30.544 & & 29.603 & 30.745 & 30.225 & 29.976 & 28.332 & 26.122 & 32.493 & 35.95 & 533.531 & 17.074 & 16.652 & 23.477 & 15.33 & 11.768 & 32.434 & 33.393 & 30.241 & 32.455 & 27.019 & 34.364 \\
\hline 10/8/11 23:00 & & 30.78 & 30.319 & 29.166 & 30.544 & & 29.615 & 30.747 & 30.22 & 29.976 & 28.353 & 26.126 & 32.493 & 35.953 & 33.528 & 17.074 & 16.648 & 23.477 & 15.337 & 11.765 & 32.425 & 33.388 & 30.234 & 32.452 & 27.019 & 34.366 \\
\hline 10/8/11 23:30 & & 30.778 & 30.319 & 29.155 & 30.542 & & 29.624 & 30.745 & 30.22 & 29.976 & 28.374 & 26.129 & 32.486 & 35.948 & 33.521 & 17.074 & 16.643 & 23.477 & 15.342 & 11.761 & 32.422 & 33.386 & 30.232 & 32.45 & 27.021 & 34.369 \\
\hline 10/9/11 0:00 & & 30.778 & 30.319 & 29.169 & 30.537 & & 29.617 & 30.747 & 30.225 & 29.976 & 28.372 & 26.131 & 32.484 & 35.953 & 33.521 & 17.076 & 16.641 & 23.473 & 15.342 & 11.756 & 32.415 & 33.381 & 30.225 & 32.445 & 27.021 & 34.369 \\
\hline 10/9/11 0:30 & & 30.778 & 30.317 & 29.178 & 30.53 & & 29.612 & 30.747 & 30.225 & 29.974 & 28.367 & 26.129 & 32.477 & 35.953 & 33.512 & 17.076 & 16.636 & 23.468 & 15.342 & 11.751 & 32.411 & 33.374 & 30.218 & 32.438 & 27.019 & 34.369 \\
\hline 10/9/11 1:00 & & 30.775 & 30.314 & 29.171 & 30.526 & & 29.619 & 30.749 & 30.227 & 29.971 & 28.374 & 26.134 & 32.47 & 35.948 & 33.505 & 17.074 & 16.629 & 23.461 & 15.342 & 11.744 & 32.404 & 33.372 & 30.213 & 32.431 & 27.021 & 34.369 \\
\hline 10/9/11 1:30 & & 30.782 & 30.319 & 29.164 & 30.526 & & 29.624 & 30.752 & 30.223 & 29.974 & 28.379 & 26.129 & 32.468 & 35.95 & 33.505 & 17.076 & 16.629 & 23.466 & 15.339 & 11.737 & 32.408 & 33.367 & 30.22 & 32.429 & 27.021 & 34.369 \\
\hline 10/9/11 2:00 & & 30.78 & 30.317 & 29.155 & 30.526 & & 29.634 & 30.747 & 30.223 & 29.976 & 28.391 & 26.131 & 32.463 & 35.95 & 33.498 & 17.076 & 16.619 & 23.468 & 15.342 & 11.735 & 32.404 & 33.362 & 30.213 & 32.424 & 27.021 & 34.369 \\
\hline $10 / 9 / 112: 30$ & & 30.78 & 30.317 & 29.15 & 30.523 & & 29.641 & 30.749 & 30.223 & 29.976 & 28.398 & 26.131 & 32.458 & 35.953 & 33.493 & 17.078 & 16.617 & 23.466 & 15.349 & 11.73 & 32.397 & 33.355 & 30.208 & 32.419 & 27.021 & 34.366 \\
\hline 10/9/11 3:00 & & 30.778 & 30.314 & 29.15 & 30.5 & & 29.645 & 30.747 & 30.22 & 29.974 & 28.4 & 26.129 & 32.451 & 35.95 & 33.489 & 17.076 & 16.61 & 23.464 & 15.351 & 11.725 & 32.387 & 353 & 0.201 & 32.412 & 27.021 & .366 \\
\hline 10/9/11 3:30 & & 30.775 & 30.31 & 29.145 & 30. & & 29.643 & 30.747 & 30.22 & 29.971 & 28.402 & 26.134 & 32.447 & 35.95 & 33.477 & 17.076 & 16.605 & 23.464 & 15.356 & 11.718 & 376 & 345 & 87 & 2408 & 27.021 & .364 \\
\hline 10/9/11 4:00 & & 30.778 & 30.305 & 29.152 & 30.502 & & 29.643 & 30.747 & 30.227 & 29.971 & 28.4 & 26.131 & 32.44 & 35.939 & 33.475 & 17.076 & 16.601 & 23.459 & 15.36 & 11.714 & 32.373 & 338 & 187 & 01 & 27.021 & 34.364 \\
\hline 10/9/11 4:30 & & 30.778 & 30.307 & 29.148 & 30.498 & & 29.641 & 30.749 & 30.227 & 29.969 & 28.398 & 26.131 & 32.433 & 35.948 & 33.47 & 17.076 & 16.596 & 23.452 & 15.351 & 11.706 & 32.371 & 33.331 & 30.18 & 32.394 & 27.021 & 34.362 \\
\hline 10/9/11 5:00 & & 30.778 & 30.3 & 29.15 & 30.493 & & 29.645 & 30.749 & 30.227 & 29.967 & 28.409 & 26.134 & 32.428 & 35.946 & 33.463 & 17.074 & 16.591 & 23.45 & 15.356 & 11.702 & 32.359 & 33.327 & 30.18 & 32.391 & 27.023 & 34.362 \\
\hline 10/9/11 5:30 & & 30.782 & 30.305 & 29.145 & 30.493 & & 29.643 & 30.749 & 30.223 & 29.969 & 28.414 & 26.136 & 32.425 & 35.95 & 33.458 & 17.076 & 16.589 & 23.447 & 15.353 & 11.697 & 32.366 & 33.327 & 30.18 & 32.389 & 27.023 & 34.364 \\
\hline 10/9/11 6:00 & & 30.78 & 30.305 & 29.145 & 30.488 & & 29.643 & 30.747 & 30.223 & 29.969 & 28.419 & 26.138 & 33.383 & 35.948 & 33.793 & 17.076 & 16.587 & 23.452 & 15.356 & 12.024 & 32.586 & 34.407 & 30.178 & 33.37 & 27.026 & 34.364 \\
\hline 10/9/11 6:30 & & 30.782 & 30.303 & 29.141 & 30.491 & & 29.655 & 30.749 & 30.227 & 29.969 & 28.421 & 26.138 & 34.632 & 35.948 & 35.006 & 17.076 & 16.652 & 23.454 & 15.356 & 13.241 & 33.178 & 35.63 & 30.215 & 34.592 & 27.023 & 34.364 \\
\hline 10/9/11 7:00 & & 30.78 & 30.303 & 29.148 & 30.5 & & 29.645 & 30.75 & 30.225 & 29.967 & 28.419 & 26.141 & 35.215 & 35.946 & 35.676 & 17.076 & 16.76 & 23.457 & 15.358 & 13.914 & 33.427 & 36.191 & 30.258 & 35.169 & 27.026 & 34.364 \\
\hline 10/9/11 7:30 & & 30.778 & 30.296 & 29.138 & 30.514 & & 29.65 & 30.749 & 30.225 & 29.964 & 28.43 & 26.143 & 35.533 & 35.948 & 36.133 & 17.076 & 16.877 & 23.454 & 15.365 & 14.372 & 33.579 & 36.476 & 30.293 & 35.472 & 27.023 & 34.364 \\
\hline 10/9/11 8:00 & & 30.78 & 30.296 & 29.141 & 30.533 & & 29.657 & 30.747 & 30.227 & 29.962 & 28.432 & 26.15 & 35.704 & 35.948 & 36.351 & 17.074 & 16.977 & 23.45 & 15.36 & 14.588 & 33.64 & 36.644 & 30.331 & 35.648 & 27.021 & 34.366 \\
\hline 10/9/11 8:30 & & 30.782 & 30.298 & 29.152 & 30.561 & & 29.645 & 30.749 & 30.23 & 29.964 & 28.416 & 26.15 & 36.242 & 35.95 & 36.759 & 17.076 & 17.08 & 23.454 & 15.36 & 14.995 & 33.835 & 37.201 & 30.359 & 36.183 & 27.026 & 34.366 \\
\hline 10/9/11 9:00 & & 30.778 & 30.293 & 29.157 & 30.582 & & 29.641 & 30.752 & 30.23 & 29.96 & 28.402 & 26.155 & 36.67 & 35.946 & 37.146 & 17.074 & 17.187 & 23.452 & 15.363 & 15.383 & 33.992 & 37.641 & 30.387 & 36.61 & 27.023 & 34.366 \\
\hline $\begin{array}{l}\text { 10/9/11 9:30 }\end{array}$ & & 30.785 & 30.298 & 29.152 & 30.612 & & 29.641 & 30.752 & 30.227 & 29.962 & 28.386 & 26.153 & 37.035 & 35.948 & 37.531 & 17.074 & 17.295 & 23.45 & 15.358 & 15.764 & 34.157 & 38.001 & 30.429 & 36.969 & 27.026 & 34.366 \\
\hline 10/9/11 10:00 & & 30.787 & 30.303 & 29.15 & 30. & & 29.645 & 30.752 & 30.227 & 29.964 & 28.391 & 26.162 & 37.332 & 35.95 & $37 . \varepsilon$ & 17.076 & 17.402 & 23.445 & 15.353 & 16.081 & 34.29 & 293 & 30.472 & 37.265 & 27.026 & 34.369 \\
\hline $10 / 5$ & & 30.789 & 30.305 & & & & 29.65 & & 30.235 & 29.967 & 28.386 & 26.169 & 37.494 & 35.9 & 38.093 & 17.076 & 17.505 & 23.445 & 15.353 & 16.335 & 34.383 & .433 & 30.502 & 37.42 & 27.026 & 34.364 \\
\hline $10 / 9 / 1$ & & 30.787 & 30.305 & 29.159 & 30.7 & & 29.641 & 30.754 & 30.227 & 29.967 & 28.4 & 26.177 & 37.587 & $\begin{array}{l}55.95 \\
35.95\end{array}$ & 38.208 & 17.076 & 17.596 & 23.44 & 15.358 & 16.446 & 34.414 & .527 & 30.523 & 37.516 & 27.028 & 34.366 \\
\hline $10 / 9 / 1$ & & 30.785 & 30.305 & 29.152 & 30.7 & & 29.641 & 30.754 & 30.232 & 29.964 & $\begin{aligned} 28.4 & \\
28.395 & \end{aligned}$ & 26.189 & 36.104 & 35.95 & 37.327 & 17.078 & $\begin{array}{l}17.664 \\
\end{array}$ & 23.433 & 15.36 & 15.566 & 34 & 961 & 30.542 & 36.021 & 27.026 & 34.364 \\
\hline & & 30.792 & 36 & 29 & 30. & & 29.65 & 30 & 30 & 29 & 6 & 26 & 35 & 35.946 & 36 & 17 & 2 & 3 & 15.356 & 14.871 & 33 & 5 & 4 & 35.367 & 27.028 & .364 \\
\hline & & 30. & 30 & 29 & & & 29. & & 30 & 29 & 28.332 & 26 & 35 & 35.953 & 36 & 17 & 4 & 23.431 & 15. & 14 & 1 & 7 & 3 & 66 & 27.028 & .364 \\
\hline & & & & & & & & & & & 28 & & 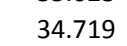 & 35. & & & 6 & & 15.372 & 14.109 & 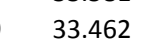 & 6 & & 34.656 & 27.028 & .364 \\
\hline & & & & 29 & & & & & & & & & 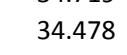 & & & & & 234 & 15.3 & 13.848 & 33.357 & 7 & 1 & 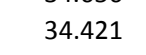 & 27.026 & .359 \\
\hline $10 /$ & & 30 & 30.303 & & 30 & & & & & & & & 34.2 & & & & & 23.4 & 15. & 13. & & & & & & 357 \\
\hline 14:30 & & 30 & 30.305 & 29 & & & & & 30 & & 28 & & 34. & 35.943 & 35.2 & & & 23.4 & 15.3 & 13. & 33. & & & & & 34.357 \\
\hline $15: 00$ & & & 30. & & & & & & 30 & & & & 33.989 & 35.95 & 35.0 & 17. & 17 & 23.4 & 15.36 & 13.333 & 33.155 & & 3 & 33.935 & 27.028 & 34.354 \\
\hline $10 / 4$ & & & 30.3 & & & & & & & & & & & & 34 & & & 23.408 & 15.363 & 13.206 & & & 2 & 33.82 & & 34.352 \\
\hline 10/9/11 16:00 & & 30. & 30.3 & 29. & & & 29. & & 30.2 & & 28. & 26. & 33.766 & 35.953 & 34 & 17. & 17.374 & 23.403 & 15.363 & 13.1 & 33.059 & 34.656 & 30.418 & 33.719 & 27.031 & 34.347 \\
\hline 10/9/11 16:30 & & 30. & 30.3 & 29.1 & & & $29.6 \mathrm{C}>\mathrm{C}$ & & 30.237 & 29. & 28.2 & 26.1 & 33.675 & 35.953 & 34.766 & 17.0 & 17.344 & 23.4. & 15.3 & 13.002 & 33.026 & 34.566 & 30.415 & 33.63 & 27.031 & 34.347 \\
\hline $10 / 9 / 1117: 00$ & & 30.792 & 30.3 & 29.159 & 30.7 & & 29.645 & 30.7 & 30.242 & 29.971 & 28.235 & 26.119 & 33.591 & 35.957 & 34.677 & 17.078 & 17.31 & 23.3 & 15.367 & 12.915 & 32.975 & 34.48 & 30.392 & 33.543 & 27.03 & 34.345 \\
\hline 10/9/11 17:30 & & 30.794 & 30.312 & 29.173 & 30.7 & & 29.643 & 30.761 & 30.239 & 29.971 & 28.216 & 26.112 & 33.516 & 35.95 & 34.6 & 17.078 & 17.281 & 23.387 & 15.363 & 12.837 & 32.94 & 34.407 & 30.375 & 33.468 & 27.031 & 34.34 \\
\hline 10/9/11 18:00 & & 30.796 & 30.317 & 29.171 & 30.71 & & 29.641 & 30.749 & 30.242 & 29.974 & 28.212 & 26.112 & 33.448 & 35.953 & 34.527 & 17.078 & 17.255 & 23.385 & 15.36 & 12.767 & 32.91 & 34.341 & 30.368 & 33.403 & 27.031 & 34.338 \\
\hline 10/9/11 18:30 & & 30.796 & 30.317 & 29.155 & 30.701 & & 29.65 & 30.623 & 30.235 & 29.974 & 28.212 & 26.112 & 33.387 & 35.951 & 34.462 & 17.078 & 17.227 & 23.383 & 15.363 & 12.701 & 32.879 & 34.279 & 30.359 & 33.342 & 27.031 & 34.336 \\
\hline 10/9/11 19:00 & & 30.803 & 30.321 & 29.159 & 30.696 & & 29.65 & 30.534 & 30.244 & 29.981 & 28.205 & 26.114 & 33.415 & 35.953 & 34.497 & 17.078 & 17.204 & 23.383 & 15.358 & 12.731 & 32.868 & 34.31 & 30.356 & 33.372 & 27.031 & 34.336 \\
\hline 10/9/11 19:30 & & 30.799 & 30.321 & 29.162 & 30.682 & & 29.65 & 30.501 & 30.242 & 29.981 & 28.202 & 26.112 & 33.645 & 35.948 & 34.745 & 17.078 & 17.234 & 23.378 & 15.36 & 12.978 & 32.928 & 34.537 & 30.347 & 33.595 & 27.033 & 34.336 \\
\hline $10 /$ & & 30.794 & 30.317 & 29.152 & 30.67 & & 29.655 & 30.489 & 30.242 & 29.976 & 28.186 & 26.11 & 33.703 & 35.955 & 34.804 & 17.078 & 17.276 & 23.383 & 15.367 & 13.042 & 32.956 & 34.594 & 30.333 & 33.654 & 27.03 & 34.336 \\
\hline 10/9/11 20:30 & & 30.796 & 30.317 & 29.15 & 30.661 & & 29.655 & 30.489 & 30.244 & 29.978 & 28.179 & 26.105 & 33.511 & 35.95 & 34.598 & 17.076 & 17.267 & 23.378 & 15.37 & 12.835 & 32.905 & 34.405 & 30.328 & 33.464 & 27.033 & 6 \\
\hline
\end{tabular}




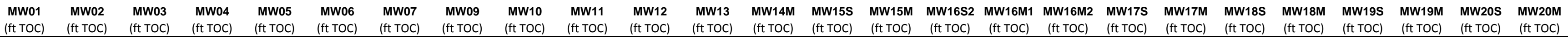

\begin{tabular}{|c|c|c|c|c|c|c|c|c|c|c|c|c|c|c|c|c|c|c|c|c|c|c|c|c|c|c|}
\hline well & $\begin{array}{l}\text { (ft TOC) } \\
\text { (ft }\end{array}$ & $\begin{array}{l}\text { (ft TOC) } \\
\text { (f) }\end{array}$ & $\begin{array}{l}\text { (ft TOC) } \\
\text { (f) }\end{array}$ & (ft TOC) & $\begin{array}{l}\text { (ift TOC) } \\
\text { (ft }\end{array}$ & $\begin{array}{l}\text { (ft TOC) } \\
\text { (f) }\end{array}$ & $\begin{array}{l}\text { (ft TOC) } \\
\text { (n) }\end{array}$ & $\begin{array}{l}\text { (ft TOC) } \\
\text { (f) }\end{array}$ & $\begin{array}{l}\text { (ft TOC) } \\
\text { (ft) }\end{array}$ & $\begin{array}{l}\text { (ft TOC) } \\
\text { (f) }\end{array}$ & $\begin{array}{l}\text { (ft TOC) } \\
\text { (f) }\end{array}$ & $\begin{array}{l}\text { (ft Toc) } \\
\text { (f) }\end{array}$ & (ft TOC) & $\begin{array}{l}\text { (ft TOC) } \\
\end{array}$ & (ft TOC) & (ft TOC) & (ft TOC) & (ft TOC) & $\begin{array}{l}\text { (ft TOC) } \\
\text { (ft) }\end{array}$ & (ft TOC) & $\begin{array}{l}\text { (ft TOC) } \\
\end{array}$ & $\begin{array}{l}\text { (ft TOC) } \\
\text { (n) }\end{array}$ & $\begin{array}{l}\text { (ft TOC) } \\
\text { (n) }\end{array}$ & (ft TOC) & $\begin{array}{l}\text { (ft TOC) } \\
\end{array}$ & (ft TOC) \\
\hline 10/9/11 21:00 & & 30.799 & 30.319 & 29.159 & 30.654 & & 29.657 & 30.469 & 30.244 & 29.978 & 28.165 & 26.102 & 33.404 & 35.948 & 34.483 & $\begin{array}{l}3 \quad 17.078 \\
\end{array}$ & 17.239 & 23.376 & 15.367 & 12.724 & 32.863 & 34.296 & 30.324 & 33.36 & 27.033 & 34.336 \\
\hline 10/9/11 21:30 & & 30.794 & 30.312 & 29.166 & 30.64 & & 29.641 & 30.28 & 30.246 & 29.974 & 28.165 & 26.107 & 33.319 & 35.955 & 34.398 & $8 \quad 17.078$ & 17.211 & 23.373 & 15.374 & 12.633 & 32.823 & 34.215 & 30.302 & 33.276 & 27.033 & 34.333 \\
\hline 10/9/11 22:00 & & 30.796 & 30.312 & 29.178 & 30.633 & & 29.634 & 30.113 & 30.244 & 29.974 & 28.167 & 26.11 & 33.259 & 35.957 & 34.33 & $\begin{array}{l}317.078 \\
\end{array}$ & 17.187 & 23.373 & 15.365 & 12.569 & 32.793 & 34.153 & 30.3 & & 27.033 & 34.333 \\
\hline 10/9/11 22:30 & & 30.796 & 30.31 & 29.176 & 30.624 & & 29.643 & 29.998 & 30.244 & 29.976 & 28.177 & 26.105 & 33.202 & 35.946 & 34.272 & 17.076 & 17.164 & 23.385 & 15.365 & 12.508 & 32.765 & & & & 27.033 & 34.331 \\
\hline 10/9/11 23:00 & & 30.808 & 30.321 & 29.164 & 30.626 & & 29.652 & 29.981 & 30.244 & 29.978 & 28.186 & 26.112 & 33.158 & 35.955 & 34.227 & 17.081 & 17.143 & 23.403 & 15.349 & 12.461 & 32.76 & & 30.298 & .119 & 35 & 34.333 \\
\hline 10/9/11 23:30 & & 30.803 & 30.319 & 29.162 & 30.617 & & 29.659 & 30.012 & 30.244 & 29.983 & 28.209 & 26.107 & 33.121 & 35.95 & 34.183 & 17.078 & 17.122 & 23.404 & 15.367 & 12.421 & 32.732 & 34.014 & 30.277 & 3.076 & 7.033 & 34.336 \\
\hline 10/10/11 0:00 & & 30.806 & 30.324 & 29.152 & 30.612 & & 29.659 & 30.063 & 30.244 & 29.988 & 28.214 & 26.11 & 33.085 & 35.95 & 34.147 & $\begin{array}{l}7 \\
7\end{array}$ & 17.101 & 23.401 & 15.363 & 12.386 & 32.718 & 33.98 & 30.274 & 33.044 & 27.035 & 34.336 \\
\hline 10/10/11 0:30 & & 30.794 & 30.312 & 29.15 & 30.591 & & 29.666 & 30.115 & 30.242 & 29.981 & 28.214 & 26.11 & 33.048 & 35.955 & 34.108 & $8 \quad 17.078$ & 17.08 & 23.396 & 15.379 & 12.348 & 32.684 & 33.943 & 30.246 & 33.004 & 27.033 & 34.336 \\
\hline 10/10/11 1:00 & & 30.801 & 30.317 & 29.15 & 30.586 & & 29.666 & 30.158 & 30.244 & 29.981 & 28.216 & 26.107 & 33.018 & 35.953 & 34.077 & $7 \quad 17.081$ & 17.064 & 23.394 & 15.37 & 12.315 & 32.674 & 33.912 & 30.239 & 32.976 & 27.035 & 34.336 \\
\hline 10/10/11 1:30 & & 30.799 & 30.314 & 29.159 & 30.575 & & 29.657 & 30.192 & 30.249 & 29.978 & 28.221 & 26.112 & 32.985 & 35.953 & 34.044 & $4 \quad 17.078$ & 17.045 & 23.39 & 15.37 & 12.28 & 32.651 & 33.881 & 30.225 & 32.943 & 27.038 & 34.336 \\
\hline 10/10/11 2:00 & & 30.801 & 30.31 & 29.162 & 30.561 & & 29.659 & 30.218 & 30.246 & 29.978 & 28.228 & 26.117 & 32.954 & 35.953 & 34.014 & $\begin{array}{l}4 \quad 17.078 \\
\end{array}$ & 17.026 & 23.387 & 15.372 & 12.252 & 32.637 & 33.85 & 30.218 & 32.915 & 27.035 & 34.333 \\
\hline $10 / 10 / 112: 30$ & & 30.803 & 30.31 & 29.164 & 30.554 & & 29.657 & 30.245 & 30.249 & 29.978 & 28.228 & 26.119 & 32.929 & 35.953 & 33.986 & $\begin{array}{ll}6 & 17.078\end{array}$ & 17.01 & 23.385 & 15.37 & 12.221 & 32.623 & 33.826 & 30.213 & 32.889 & 27.035 & 34.333 \\
\hline 10/10/11 3:00 & & 30.803 & 30.314 & 29.166 & 30.549 & & 29.655 & 30.266 & 30.249 & 29.978 & 28.23 & 26.117 & 32.903 & 35.95 & 33.962 & $2 \quad 17.081$ & 16.993 & 23.383 & 15.367 & 12.2 & 32.614 & 33.8 & 30.199 & 32.863 & 27.035 & 34.333 \\
\hline 10/10/11 3:30 & & 30.806 & 30.314 & 29.166 & 30.537 & & 29.659 & 30.29 & 30.246 & 29.978 & 28.235 & 26.117 & 32.879 & 35.953 & 33.934 & 17.078 & 16.975 & 23.38 & 15.365 & 12.172 & 32.595 & 33.777 & 30.199 & 32.839 & 27.038 & 34.333 \\
\hline 10/10/11 4:00 & & 30.808 & 30.314 & 29.169 & 30.533 & & 29.657 & 30.312 & 30.249 & 29.981 & 28.235 & 26.114 & 32.858 & 35.957 & 33.913 & 17.081 & 16.961 & 23.378 & 15.363 & 12.148 & 32.583 & 33.755 & 30.19 & 32.818 & 27.038 & 34.333 \\
\hline 10/10/11 4:30 & & 30.808 & 30.317 & 29.171 & 30.523 & & 29.657 & 30.331 & 30.251 & 29.981 & 28.223 & 26.107 & 32.837 & 35.957 & 33.89 & 17.078 & 16.947 & 23.376 & 15.372 & 12.127 & 32.569 & 33.732 & 30.183 & 32.795 & 27.038 & 34.331 \\
\hline $10 / 10 / 115: 00$ & & 30.806 & 30.317 & 29.176 & 30.519 & & 29.65 & 30.352 & 30.254 & 29.981 & 28.198 & 26.093 & 32.814 & 35.957 & 33.866 & 17.081 & 16.93 & 23.373 & 15.367 & 12.106 & 32.558 & 33.71 & 30.171 & 32.776 & 27.038 & 34.331 \\
\hline $10 / 10 / 115: 30$ & & 30.81 & 30.317 & 29.171 & 30.514 & & 29.652 & 30.374 & 30.251 & 29.983 & 28.181 & 26.081 & 32.795 & 35.962 & 33.847 & 17.081 & 16.916 & 23.371 & 15.365 & 12.085 & 32.551 & 33.691 & 30.173 & 32.757 & 27.038 & 34.331 \\
\hline $10 / 10 / 116: 00$ & & 30.81 & 30.317 & 29.169 & 30.507 & & 29.655 & 30.393 & 30.246 & 29.983 & $\begin{array}{l}28.177 \\
28.177\end{array}$ & 26.071 & 33.75 & 35.962 & 34.176 & 17.083 & $\begin{array}{l}10.900 \\
16.907\end{array}$ & 23.369 & 15.363 & $\begin{array}{l}12.405 \\
12.05\end{array}$ & 32.756 & $\begin{array}{l}34.77 \\
34.77\end{array}$ & 30.166 & 33.733 & 27.038 & 34.329 \\
\hline $10 / 10 / 116: 30$ & & $\begin{array}{l}30.01 \\
30.81\end{array}$ & $\begin{array}{l}30.311 \\
30.319\end{array}$ & 29.171 & 30.502 & & 29.655 & 30.41 & $\begin{array}{l}30.250 \\
30.251\end{array}$ & 29.985 & 28.165 & $\begin{array}{l}20.064 \\
26.064\end{array}$ & 34.981 & $\begin{array}{l}35.955 \\
35.952\end{array}$ & 35.374 & 17.081 & $\begin{array}{l}10.968 \\
16.968\end{array}$ & $\begin{array}{l}23.309 \\
23.369\end{array}$ & $\begin{array}{l}13.03 \\
15.365\end{array}$ & $\begin{array}{l}13.4613 \\
13.613\end{array}$ & 33.327 & 35.976 & 30.199 & 34.939 & $\begin{array}{l}27.038 \\
278\end{array}$ & $\begin{array}{l}34.331 \\
34.331\end{array}$ \\
\hline $10 / 10 / 117: 00$ & & $\begin{array}{l}30.81 \\
30.81\end{array}$ & $\begin{array}{l}30.319 \\
30.321\end{array}$ & 29.178 & 30.507 & & $\begin{array}{l}29.653 \\
29.652\end{array}$ & $\begin{array}{r}30.41 \\
30.429\end{array}$ & 30.256 & 29.983 & $\begin{array}{l}28.167 \\
28.167\end{array}$ & $\begin{array}{l}20.004 \\
26.055\end{array}$ & $\begin{array}{l}3.991 \\
33.918\end{array}$ & $\begin{aligned} 35.950 \\
35.96\end{aligned}$ & $\begin{array}{l}53.514 \\
35.022\end{array}$ & 17.081 & $\begin{array}{l}17.0300 \\
17.031\end{array}$ & 23.371 & $\begin{array}{l}13.03 \\
15.363\end{array}$ & $\begin{array}{l}13.013 \\
13.262\end{array}$ & $\begin{array}{l}33.321 \\
33.066\end{array}$ & $\begin{array}{l}3.9160 \\
34.805\end{array}$ & 30.22 & $\begin{array}{l}34.939 \\
33.862\end{array}$ & $\begin{array}{l}27.038 \\
278\end{array}$ & $\begin{array}{l}34.351 \\
34.331\end{array}$ \\
\hline 10/10/11 7:30 & & & 30.319 & 29.18 & 30.512 & & 29.65 & 30.443 & 30.254 & 29.983 & 28.179 & 26.057 & 33.556 & 35.962 & 34.647 & 17.081 & 17.031 & 23.371 & 15.36 & 12.887 & 32.886 & 34.445 & 30.22 & 33.508 & 27.038 & 34.331 \\
\hline $10 / 10 / 118: 00$ & & & 19 & 29.187 & 30.51 & & 29.648 & 30.46 & 30.254 & 29.985 & 28.212 & 26.071 & 33.371 & .962 & 34.452 & 17.081 & $\begin{array}{l}17.021 \\
17.021\end{array}$ & 23.371 & 15.36 & $\begin{array}{l}12.009 \\
12.689\end{array}$ & 32.812 & 34.262 & $\begin{array}{r}30.218 \\
30.218\end{array}$ & $\begin{array}{r}3.000 \\
33.32\end{array}$ & 7.04 & 34.331 \\
\hline $10 / 10 / 118: 30$ & & 30.813 & 30.321 & 29.187 & 30.521 & & 29.65 & 30.474 & 30.256 & 29.988 & 28.265 & 26.095 & & .96 & 34.321 & 17.083 & 7.01 & & 15.363 & 12.56 & 32.756 & 34.137 & .215 & 201 & .04 & 34.333 \\
\hline 10/10/11 9:00 & & 30.817 & 30.324 & 29.187 & 30.523 & & 29.655 & 30.489 & 30.254 & 29.99 & 28.3 & 26.124 & 33.158 & 35.96 & 34.227 & 17.083 & 17 & 23.378 & .363 & 12.463 & 32.718 & 34.054 & .204 & 8.112 & 7.04 & 34.336 \\
\hline 10/10/11 9:30 & & 30.817 & 30.328 & 29.185 & 30.526 & & 29.655 & 30.503 & 30.256 & 29.992 & 28.351 & 26.134 & 33.088 & 35.964 & 34.154 & 17.083 & 16.986 & 23.371 & 365 & 12.393 & 32.693 & 33.98 & .201 & 33.044 & 7.04 & 34.338 \\
\hline 10/10/11 10:00 & & 30.815 & 30.328 & 29.183 & 30.521 & & 29.657 & 30.517 & 30.258 & 29.992 & 28.432 & 26.201 & 33.032 & 35.967 & 34.096 & 17.083 & 16.972 & 23.378 & 15.367 & 12.336 & 32.665 & 33.928 & 30.197 & 32.99 & 27.04 & 34.338 \\
\hline 10/10/11 10:30 & & 30.817 & 30.328 & 29.176 & 30.519 & & 29.666 & 30.529 & 30.254 & 29.992 & 28.502 & 26.224 & 32.985 & 35.962 & 34.047 & 17.085 & 16.961 & 23.387 & 15.367 & 12.285 & 32.644 & 33.879 & 30.194 & 32.945 & 27.042 & 34.343 \\
\hline 10/10/11 11:00 & & 30.815 & 30.326 & 29.169 & 30.514 & & 29.676 & 30.539 & 30.251 & 29.992 & 28.563 & 26.282 & 32.947 & 35.969 & 34.004 & 17.083 & 16.951 & 23.404 & 15.374 & 12.245 & 32.621 & 33.841 & 30.185 & 32.903 & 27.04 & 34.343 \\
\hline 10/10/11 11:30 & & 30.813 & 30.328 & 29.159 & 30.509 & & 29.683 & 30.551 & 30.256 & 29.992 & 28.642 & 26.301 & 32.908 & 35.964 & 33.967 & 17.083 & 16.937 & 23.42 & 15.377 & 12.205 & 32.6 & 33.803 & 30.173 & 32.865 & 27.042 & 34.345 \\
\hline $10 / 10 / 1112: 00$ & & 30.815 & 30.326 & 29.148 & 30.502 & & 29.69 & 30.563 & 30.249 & 29.99 & 28.5 & 26.177 & 32.877 & 35.964 & 33.932 & 17.085 & 16.926 & 23.42 & 15.382 & 12.17 & 32.583 & 33.772 & 30.159 & 32.835 & 27.042 & 34.345 \\
\hline $10 / 10 / 1112: 30$ & & 30.813 & 30.317 & 29.129 & 30.488 & & 29.704 & 30.568 & 30.251 & 29.985 & 28.488 & 26.165 & 32.847 & 35.962 & 33.901 & 17.083 & 16.914 & 23.417 & 15.389 & 12.139 & 32.56 & 33.741 & 30.147 & 32.807 & 27.042 & 34.348 \\
\hline 10/10/11 13:00 & & 30.808 & 30.312 & 29.115 & 30.479 & & 29.714 & 30.58 & 30.251 & 29.985 & 28.477 & 26.148 & 32.816 & 35.962 & 33.873 & 17.083 & 16.902 & 23.415 & 15.391 & 12.111 & 32.539 & 33.713 & 30.133 & 32.776 & 27.042 & 34.347 \\
\hline $10 / 10 / 1113: 30$ & & 30.806 & 30.303 & 29.108 & 30.465 & & 29.718 & 30.587 & 30.251 & 29.978 & 28.474 & 26.136 & 32.788 & 35.962 & 33.845 & 17.081 & 16.886 & 23.42 & 15.4 & 12.08 & 32.513 & 33.684 & 30.117 & 32.748 & 27.042 & 34.347 \\
\hline 10/10/11 14:00 & & 30.803 & 30.293 & 29.099 & 30. & & 29. & 30.594 & 30.246 & 29.974 & 28.467 & 26.126 & 32.763 & 35.95 & 33.817 & 17.078 & $\begin{array}{l}16.874 \\
\text { S }\end{array}$ & 23.438 & 15.403 & 12.057 & 32.495 & 33.658 & 0.103 & 32.724 & 27.04 & 34.345 \\
\hline 10 & & 30. & 30.284 & 29.101 & & & 29. & 30. & 30.251 & 29. & 28.451 & 26.114 & 32.739 & 35.948 & 33.791 & 17.076 & 16.86 & 23.473 & 15 & 12.029 & 32.471 & 33.635 & 30.089 & $\begin{array}{l}32.699 \\
\end{array}$ & 27.042 & 34.343 \\
\hline $10 / 10 / 1$ & & 30.799 & $\begin{array}{l}30.274 \\
30.275\end{array}$ & 29.099 & 30. & & 29.716 & 30.611 & $\begin{array}{l}30.249 \\
30\end{array}$ & 29.962 & $\begin{array}{r}20.471 \\
28.446\end{array}$ & 26.119 & 32.716 & 35.946 & $\begin{array}{l}33.765 \\
3\end{array}$ & 17.074 & $\begin{array}{l}16.080 \\
16.849\end{array}$ & 23.487 & $\begin{array}{r}15.412 \\
15.412\end{array}$ & $\begin{array}{l}12.005 \\
12.05\end{array}$ & $\begin{array}{l}32.457 \\
32\end{array}$ & $\begin{array}{l}33.608 \\
3.008\end{array}$ & 30.07 & 32.675 & 27.042 & 34.345 \\
\hline $10 / 10 / 1$ & & 30.799 & 30.268 & 29.092 & 30. & & 29.716 & 30.615 & $\begin{array}{l}30.246 \\
30.45\end{array}$ & 29.955 & $\begin{array}{l}20.440 \\
28.437\end{array}$ & 26.119 & 32.69 & 35.948 & 33.742 & 17.071 & $\begin{array}{l}1.0 .837 \\
16.837\end{array}$ & 23.482 & 15.412 & $\begin{array}{l}11.981 \\
11.981\end{array}$ & 32.436 & $\begin{array}{l}33.585 \\
33.585\end{array}$ & 30.056 & 32.652 & 27.042 & 34.343 \\
\hline 0 & & 30. & $\begin{array}{l}30.20 \\
30.26\end{array}$ & 29.087 & 30. & & 29.718 & 30.62 & $\begin{array}{l}30.240 \\
30.249\end{array}$ & 29.955 & $\begin{array}{r}20.451 \\
28.4\end{array}$ & 26.11 & $\begin{array}{l}32.6971 \\
32.671\end{array}$ & $\begin{array}{l}35.940 \\
35.943\end{array}$ & $\begin{array}{l}33.725 \\
3.725\end{array}$ & 17.069 & $\begin{array}{l}10.07 \\
16.825\end{array}$ & $\begin{array}{l}23.402 \\
23.477\end{array}$ & $\begin{array}{l}15.407 \\
15.40\end{array}$ & $\begin{array}{l}11.501 \\
11.958\end{array}$ & $\begin{array}{l}32.400 \\
32.432\end{array}$ & 33.568 & 58 & 31 & 27.042 & $\begin{array}{l}34.343 \\
34.343\end{array}$ \\
\hline 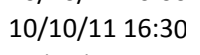 & & 30 & 30 & 29 & & & 2 & 30 & $3 c$ & 25 & 28. & 26.105 & . & 3 & 33.702 & 17 & 16 & 23 & 1 & 11.939 & 32.418 & 9 & 9 & 4 & 27.042 & 34.34 \\
\hline & & & & 29 & & & & & & & & 2 & & 3 & 33. & 17 & 16 & 23 & 15 & 11.92 & 32.401 & 33.528 & 2 & 35 & 42 & \\
\hline $117: 30$ & & & & & & & & & & & 28 & 1 & & 35.939 & 33.6 & & 16.788 & 23 & & 11.904 & 32.39 & & 27 & 77 & 27.042 & 34.338 \\
\hline 18:00 & & 30 & 30.249 & 29.087 & & & & & 30 & & 28.314 & 1 & 32.601 & 35.939 & 33.648 & $\begin{array}{ll}3 & 17.062\end{array}$ & 16.778 & & 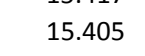 & 11.887 & 32.38 & & .018 & 2.562 & 27.042 & 34.34 \\
\hline 10 & & 30 & 30.2 & 29.089 & & & & & & & 28.316 & 26.107 & & 35.936 & 33.634 & 17. & 16.769 & 23.4 & 15.407 & 11.871 & 32.364 & & 13 & 2.546 & 27.042 & 34.34 \\
\hline 10 & & & & & & & & & & & 28. & 26.11 & & & 33.62 & & 16 & & 15. & 11.855 & 32.359 & & & 32.532 & 27.042 & 34.338 \\
\hline 19:30 & & 30 & & 29. & & & & & & & 28 & 26. & & 35.934 & 33.606 & & 16. & 23. & 15. & 11.843 & 32.355 & & & & 27.042 & 34.341 \\
\hline 11 20:00 & & 30 & 30.2 & 29.106 & & & & 30. & 30.2 & & 28.325 & 26.122 & 32. & 35.934 & 33.594 & 17.0 & 16.746 & 23.501 & 15.398 & 11.831 & 32.348 & 33.445 & 30.009 & 32.509 & 27.045 & 34.34 \\
\hline /11 20:30 & & 30. & 30.2 & 29.11 & & & & 30. & 30.8 & 29.5 & 28.325 & 26.126 & 32.5 & 35.936 & 33.585 & 17.0 & 16.73 & 23.494 & 15.396 & 11.822 & 32.345 & 33.433 & 30.013 & 32.502 & 27.045 & 34.343 \\
\hline 10/10/11 21:00 & & 30.801 & 30.249 & 29.11 & 30.3 & & 29.652 & 30.661 & 30.254 & 29.932 & 28.33 & 26.131 & 32.529 & 35.939 & 33.575 & 17.055 & 16.729 & 23.489 & 15.391 & 11.81 & 32.343 & 33.424 & 30.018 & 32.49 & 27.045 & 34.345 \\
\hline $10 / 10 / 1121: 30$ & & 30.801 & 30.251 & 29.11 & $30.3=$ & & 29.648 & 30.666 & 30.254 & 29.932 & 28.335 & 26.131 & 32.517 & 35.941 & 33.566 & 17.055 & 16.725 & 23.489 & 15.389 & 11.803 & 32.336 & 33.417 & 30.016 & 32.483 & 27.045 & 34.347 \\
\hline 10/10/11 22:00 & & 30.801 & 30.251 & 29.108 & 30.334 & & 29.65 & 30.67 & 30.251 & 29.934 & 28.33 & 26.134 & 32.51 & 35.941 & 33.552 & 17.055 & 16.718 & 23.484 & 15.384 & 11.793 & 32.336 & 33.407 & 30.016 & 32.471 & 27.047 & 34.347 \\
\hline $10 / 10 / 1122: 30$ & & 30.801 & 30.251 & 29.106 & 30.337 & & 29.65 & 30.673 & 30.254 & 29.932 & 28.332 & 26.131 & 32.503 & 35.939 & 33.545 & 17.055 & 16.713 & 23.477 & 15.384 & 11.784 & 32.331 & 33.4 & 30.016 & 32.464 & 27.045 & 34.35 \\
\hline $10 / 10 / 1123: 00$ & & 30.803 & 30.251 & 29.099 & 30.334 & & 29.655 & 30.675 & 30.249 & 29.932 & 28.33 & 26.129 & 32.493 & 35.95 & 33.538 & 17.055 & 16.706 & 23.473 & 15.384 & 11.779 & 32.331 & 33.391 & 30.013 & 32.452 & 27.047 & 34.35 \\
\hline 10/10/11 23:30 & & 30.801 & 30.251 & 29.092 & 30.33 & & 29.659 & 30.678 & 30.251 & 29.932 & 28.328 & 26.122 & 32.486 & 35.943 & 33.528 & 17.055 & 16.699 & 23.47 & 15.382 & 11.768 & 32.322 & 33.383 & 30.013 & 32.448 & 27.047 & 34.352 \\
\hline 10/11/11 0:00 & & 30.799 & 30.249 & 29.085 & 30.325 & & 29.659 & 30.68 & 30.256 & 29.927 & 28.328 & 26.114 & 32.477 & 35.948 & 33.519 & 17.053 & 16.692 & 23.466 & 15.382 & 11.758 & 32.313 & 33.374 & 30.002 & 32.441 & 27.047 & 34.352 \\
\hline 10/11/11 0:30 & & 30.796 & 30.244 & 29.085 & 30.32 & & 29.659 & 30.682 & 30.254 & 29.925 & 28.328 & 26.11 & 32.465 & 35.948 & 33.512 & 17.051 & 16.687 & 23.461 & 15.384 & 11.751 & 32.301 & 33.362 & 29.992 & 32.429 & 27.045 & 34.352 \\
\hline
\end{tabular}




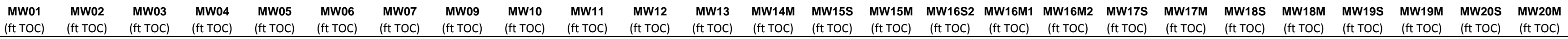

\begin{tabular}{|c|c|c|c|c|c|c|c|c|c|c|c|c|c|c|c|c|c|c|c|c|c|c|c|c|c|}
\hline well & (ft TOC) & (ft TOC) & (ft TOC) & (ft TOC) & $\begin{array}{ll}(\mathrm{ft} \text { TOC) } & \text { (ft TOC) } \\
\end{array}$ & (ft TOC) & (ft TOC) & (ft TOC) & (ft TOC) & (ft TOC) & (ft TOC) & (ft TOC) & (ft TOC) & (ft TOC) & (ft TOC) & (ft TOC) & (ft TOC) & (ft TOC) & (ft TOC) & (ft TOC) & (ft TOC) & (ft TOC) & (ft TOC) & (ft TOC) & (ft TOC) \\
\hline .1:00 & & 30.794 & 30. & 29.087 & 30.316 & 29.6 & 30.685 & 30 & 29. & 28.33 & & 32.456 & 35.948 & & 17. & 16 & 23.457 & 15.379 & 11.739 & 32.294 & 33 & 29.988 & 32. & 27.047 & 34.352 \\
\hline 1/111 1:30 & & 30.794 & 30.24 & 29.087 & 30.309 & 29.659 & 30.687 & 30.254 & 29.918 & 28.328 & 26.112 & 32.444 & 35.941 & 33.489 & 17.048 & 16.671 & 23.452 & 15.379 & 1.725 & 32.289 & 33.345 & .976 & & 7047 & 34.352 \\
\hline 10/11/11 2:00 & & 30.796 & 30.237 & 29.078 & 30.306 & 29.655 & 30.69 & 30.251 & 29.918 & 28.328 & 26.112 & 32.437 & 35.948 & 33.482 & 17.046 & 16.664 & 23.452 & 15.372 & 11.718 & 32.287 & 33.334 & 29.98 & 98 & 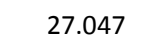 & 34.352 \\
\hline 10/11/11 2:30 & & 30.794 & 30.235 & 29.078 & 30.299 & 29.657 & 30.69 & 30.249 & 29.916 & 28.325 & 26.105 & 32.428 & 35.943 & 33.47 & 17.048 & 16.657 & 23.452 & 15.372 & 11.709 & 32.275 & 33.327 & 29.976 & 32.389 & 27.047 & 34.352 \\
\hline 10/11/11 3:00 & & 30.796 & 30.235 & 29.073 & 30.297 & 29.655 & 30.69 & 30.251 & 29.913 & 28.328 & 26.102 & 32.419 & 35.943 & 33.463 & $\begin{array}{l}3 \quad 17.046 \\
\end{array}$ & 16.65 & 23.464 & 15.374 & 11.699 & 32.275 & 33.317 & 29.976 & 32.382 & 27.047 & 34.35 \\
\hline 10/11/11 3:30 & & 30.792 & 30.228 & 29.075 & 30.29 & 29.652 & 30.692 & 30.249 & 29.911 & 28.325 & 26.1 & 32.409 & 35.943 & 33.451 & $1 \quad 17.041$ & 16.641 & 23.477 & 15.372 & 11.69 & 32.261 & 33.308 & 29.962 & 32.372 & 27.049 & 34.35 \\
\hline 10/11/11 4:00 & & 30.792 & 30.228 & 29.078 & 30.288 & 29.65 & 30.692 & 30.251 & 29.909 & 28.325 & 26.102 & 32.397 & 35.941 & 33.442 & $2 \quad 17.044$ & 16.631 & 23.475 & 15.367 & 11.678 & 32.257 & 33.296 & 29.964 & 32.361 & 27.047 & 34.347 \\
\hline 10/11/11 4:30 & & 30.789 & 30.226 & 29.078 & 30.283 & 29.648 & 30.694 & 30.249 & 29.906 & 28.328 & 26.105 & 32.39 & 35.946 & 33.435 & $5 \quad 17.041$ & 16.626 & 23.468 & 15.367 & 11.671 & 32.252 & 33.289 & 29.959 & 32.354 & 27.049 & 34.345 \\
\hline 10/11/11 5:00 & & 30.792 & 30.226 & 29.08 & 30.281 & 29.643 & 30.697 & 30.254 & 29.904 & 28.33 & 26.105 & 32.381 & 35.946 & 33.428 & $8 \quad 17.041$ & 16.619 & 23.464 & 15.36 & 11.659 & 32.247 & 33.279 & 29.959 & 32.347 & 27.047 & 34.345 \\
\hline 10/11/11 5:30 & & 30.789 & 30.226 & 29.078 & 30.276 & 29.648 & 30.699 & 30.249 & 29.904 & 28.328 & 26.107 & 32.374 & 35.941 & 33.414 & $4 \quad 17.041$ & 16.612 & 23.457 & 15.363 & 11.657 & 32.245 & 33.272 & 29.952 & 32.337 & 27.049 & 34.343 \\
\hline 10/11/11 6:00 & & 30.792 & 30.226 & 29.075 & 30.276 & 29.643 & 30.699 & 30.251 & 29.902 & 28.33 & 26.105 & 32.369 & 35.939 & 33.407 & $7 \quad 17.039$ & 16.605 & 23.466 & 15.353 & 11.647 & 32.238 & 33.265 & 29.957 & 32.33 & 27.049 & 34.343 \\
\hline 10/11/11 6:30 & & 30.789 & 30.226 & 29.078 & 30.274 & 29.641 & 30.702 & 30.251 & 29.902 & 28.328 & 26.102 & 32.36 & 35.943 & 33.402 & $2 \quad 17.039$ & 16.598 & 23.464 & 15.358 & 11.643 & 32.233 & 33.26 & 29.952 & 32.323 & 27.049 & 34.343 \\
\hline 10/11/11 7:00 & & 30.789 & 30.221 & 29.078 & 30.271 & 29.641 & 30.702 & 30.249 & 29.899 & 28.33 & 26.105 & 32.353 & 35.948 & 33.39 & $\begin{array}{ll}9 & 17.037\end{array}$ & 16.591 & 23.459 & 15.356 & 11.634 & 32.231 & 33.251 & 29.95 & 32.314 & 27.049 & 34.343 \\
\hline 10/11/11 7:30 & & 30.789 & 30.223 & 29.075 & 30.269 & 29.638 & 30.702 & 30.249 & 29.897 & 28.328 & 26.105 & 32.348 & 35.943 & 33.388 & $\begin{array}{l}317.037 \\
\end{array}$ & 16.589 & 23.454 & 15.353 & 11.629 & 32.222 & 33.246 & 29.948 & 32.309 & 27.049 & 34.34 \\
\hline 10/11/11 8:00 & & 30.789 & 30.221 & 29.071 & 30.264 & 29.641 & 30.702 & 30.251 & 29.897 & 28.328 & 26.102 & 32.341 & 35.943 & 33.383 & 17.037 & 16.582 & 23.45 & 15.353 & 11.619 & 32.224 & 33.239 & 29.945 & 32.304 & 27.049 & 34.343 \\
\hline 10/11/11 8:30 & & 30.789 & 30.221 & 29.066 & 30.264 & 29.643 & 30.702 & 30.249 & 29.895 & 28.325 & 26.1 & 32.337 & 35.941 & 33.376 & 17.034 & 16.58 & 23.447 & 15.351 & 11.615 & 32.219 & 33.234 & 29.945 & 32.3 & 27.049 & 34.343 \\
\hline 10/11/11 9:00 & & 30.787 & 30.219 & 29.059 & 30.262 & 29.648 & 30.702 & 30.246 & 29.893 & 28.325 & 26.095 & 32.33 & 35.946 & 33.369 & 17.034 & 16.575 & 23.443 & 15.356 & 11.61 & 32.212 & 33.229 & 29.941 & 32.293 & 27.049 & 34.343 \\
\hline 10/11/11 9:30 & & 30.785 & 30.216 & 29.057 & 30.257 & 29.65 & 30.702 & 30.246 & 29.893 & 28.321 & 26.088 & 32.327 & 35.934 & 33.367 & 17.032 & 16.57 & 23.438 & 15.353 & 11.603 & 32.21 & 33.225 & 29.936 & 32.286 & 27.049 & 34.345 \\
\hline 10/11/11 10:00 & & 30.782 & 30.212 & 29.057 & 30.255 & 29.65 & 30.702 & 30.249 & 29.888 & 28.325 & 26.086 & 32.318 & 35.943 & 33.36 & 17.032 & 16.566 & 23.433 & 15.358 & 11.601 & 32.203 & 33.218 & 29.931 & 32.283 & 27.049 & 34.345 \\
\hline 30 & & 30.782 & 30.207 & 29.052 & 30.2 & 29. & 30.704 & 30.249 & 29.886 & 28.321 & 26.088 & 32.313 & 35.943 & 33.355 & 17.032 & 16.561 & 23.436 & 15.36 & 11.591 & 32.198 & 33.21 & .926 & 32.276 & 27.049 & 34.345 \\
\hline bo & & 30.782 & 30.202 & 29.05 & 30. & 29.652 & 30.702 & 30.244 & 29.881 & 28.319 & 26.083 & 32.309 & 35.943 & 33.348 & 17.0 & 16.559 & 23.44 & 15.356 & 11.587 & 32.194 & 33.208 & 24 & 32.272 & 27.052 & 34.347 \\
\hline 10 & & 30.78 & 30.202 & 29.038 & 30.241 & 29.655 & 30.704 & 30.242 & 29.879 & 28.319 & 26.078 & 32.301 & 5.943 & 33.343 & 17.02 & 16.554 & 23.438 & .358 & 11.582 & 32.189 & 201 & 15 & 32.267 & 27.052 & 34.347 \\
\hline $10 / 11 / 1$ & & 30.78 & 30.2 & 29.024 & 30.236 & 66 & 30.699 & 30.242 & 29.879 & 28.309 & 26.071 & 32.297 & 35.939 & 33.339 & 17.027 & 16.552 & 23.438 & 15.358 & 11.575 & 32.187 & 33.194 & .912 & 32.26 & 27.052 & 34.35 \\
\hline 10/11/11 12:30 & & 30.775 & 30.195 & 29.015 & 30.234 & 29.669 & 30.699 & 30.239 & 29.874 & 28.305 & 26.059 & 32.29 & 35.943 & 33.331 & 17.025 & 16.547 & 23.438 & 15.36 & 11.57 & 32.18 & 33.189 & 29.912 & 32.255 & 27.052 & 34.352 \\
\hline 10/11/11 13:00 & & 30.773 & 30.186 & 29.003 & 30.225 & 29.674 & 30.697 & 30.239 & 29.869 & 28.3 & 26.05 & 32.285 & 35.939 & 33.327 & 17.025 & 16.542 & 23.436 & 15.367 & 11.568 & 32.166 & 33.184 & 29.894 & 32.243 & 27.049 & 34.352 \\
\hline 10/11/11 13:30 & & 30.766 & 30.177 & & 30.215 & 29.676 & 30.692 & 30.235 & 29.867 & 28.293 & 26.04 & 32.276 & 35.939 & 33.317 & 17.023 & 16.538 & 23.436 & 15.372 & 11.558 & 32.157 & 33.175 & 884 & 2.239 & 27.052 & 34.352 \\
\hline 10/11/11 14:00 & & 30.766 & 30.17 & 28.998 & 30.206 & 29.671 & 30.692 & 30.239 & 29.862 & 28.288 & 26.035 & 32.269 & 35.932 & 33.31 & 17.02 & 16.531 & 23.431 & 15.374 & 11.549 & 32.147 & 33.17 & 29.875 & 32.232 & 27.052 & 34.352 \\
\hline 10/11/11 14:30 & & 30.761 & 30.16 & 28.996 & 30.194 & 29.669 & 30.69 & 30.239 & 29.86 & 28.288 & 26.035 & 32.262 & 35.925 & 33.303 & $3 \quad 17.016$ & 16.524 & 23.431 & 15.379 & 11.544 & 32.136 & 33.158 & 29.868 & 32.225 & 27.052 & 34.35 \\
\hline 10/11/11 15:00 & & 30.764 & 30.156 & 28.993 & 30.19 & 29.664 & 30.687 & 30.232 & 29.858 & 28.284 & 26.033 & 32.255 & 35.927 & 33.296 & 17.016 & 16.519 & 23.429 & 15.374 & 11.535 & 32.129 & 33.151 & 29.861 & 32.215 & 27.052 & 34.347 \\
\hline $10 / 11 / 1115: 30$ & & 30.761 & 30.153 & 28.991 & 30.185 & 29.662 & 30.685 & 30.235 & 29.855 & 28.281 & 26.031 & 32.245 & 35.927 & 33.289 & 17.016 & 16.512 & 23.424 & 15.377 & 11.523 & 32.129 & 33.146 & 29.856 & 32.211 & 27.054 & 34.347 \\
\hline 10/11/11 16:00 & & 30.761 & 30.151 & 28.984 & 30.178 & 29.657 & 30.69 & 30.235 & 29.851 & 28.279 & 26.028 & 32.241 & 35.925 & 33.282 & 17.011 & 16.51 & 23.424 & 15.379 & 11.521 & 32.122 & 33.137 & 29.854 & 32.201 & 27.052 & 34.348 \\
\hline $10 / 11 / 1116: 30$ & & 30.759 & 30.149 & 28.991 & 30.173 & 29.652 & 30.682 & 30.237 & 29.848 & 28.279 & 26.026 & 32.234 & 35.927 & 33.275 & 17.011 & 16.505 & 23.459 & 15.374 & 11.514 & 32.119 & 33.13 & 29.849 & 32.194 & 27.052 & 34.345 \\
\hline 10/11/11 17:00 & & 30.757 & 30.144 & 28.993 & 30.169 & 29.641 & 30.682 & 30.237 & 29.846 & 28.281 & 26.033 & 32.227 & 35.92 & 33.268 & $3 \quad 17.009$ & 16.496 & 23.475 & 15.37 & 11.509 & 32.11 & 33.125 & 29.847 & 32.187 & 27.054 & 34.343 \\
\hline $10 / 11 / 1117: 30$ & & 30.755 & 30.137 & 29 & 30.162 & 29.634 & 30.68 & 30.237 & 29.841 & 28.281 & 26.035 & 32.22 & 35.92 & 33.259 & 17.006 & 16.491 & 23.47 & 15.377 & 11.5 & 32.101 & 33.12 & 29.842 & 32.182 & 27.052 & 34.343 \\
\hline 10/11/11 18:00 & & 30 & 30.144 & 29.007 & 30 & 29 & 30.682 & 30.237 & 29.841 & 28.284 & 26.045 & 32.217 & 35 & 33.256 & 17.004 & 16.489 & 23.466 & 15.367 & 11.493 & 32.105 & 33.116 & 29.849 & 32.178 & 27.054 & 34.343 \\
\hline & & & 30 & 29.015 & & 29 & 30.682 & 30.239 & 29.841 & 28.288 & 26.05 & 32.21 & 35.918 & 33.252 & 17.004 & $\begin{array}{l}16.484 \\
\end{array}$ & 23.461 & 15. & 11.49 & 32.101 & 33.111 & 29.842 & 32.173 & 27.054 & 34.343 \\
\hline 10 & & 30.759 & 30.144 & 29.015 & 30. & 29.608 & 30.678 & 30.235 & 29.839 & $\begin{array}{l}20.200 \\
28.288\end{array}$ & 26.059 & 32.208 & $\begin{array}{l}35.92 \\
35.92\end{array}$ & 33.245 & 17.004 & $\begin{array}{l}10.404 \\
16.482\end{array}$ & 23.457 & 15.356 & $\begin{array}{l}11.485 \\
1.485\end{array}$ & 32.108 & $\begin{array}{l}33.106 \\
3.114\end{array}$ & 29.849 & 32.173 & 27.054 & 34.343 \\
\hline 10 & & 30.757 & 30.144 & 29.021 & 30. & 29.594 & 30.68 & 30.239 & 29.839 & $\begin{array}{l}20.200 \\
28.293\end{array}$ & 26.064 & 32.206 & $\begin{array}{l}35.925 \\
35.925\end{array}$ & 33.242 & 17.004 & $\begin{array}{l}16.402 \\
16.477\end{array}$ & 23.457 & 15.358 & $\begin{array}{l}11.403 \\
11.483\end{array}$ & 32.108 & 33.104 & .856 & 32.168 & 27.054 & 34.345 \\
\hline & & 30. & 30 & 29 & 30 & 2 & 36 & 30 & 29 & 28.295 & 26.071 & 32 & 35.927 & 33. & 17 & 16 & 23 & 15.356 & 11.478 & 32.112 & 01 & 88 & 66 & 27.054 & 34.345 \\
\hline & & & 30. & 29 & 30 & 29 & 30. & & 29 & 28.3 & & 32 & $3 !$ & 33.238 & & 16.47 & 23 & 15.349 & 11.478 & 32.108 & 33.099 & 33 & 54 & 27.056 & 34.347 \\
\hline & & & & & & & & & & 28.298 & & & - & 33 & & 16.472 & 23. & 15.3 & 11.478 & 32 & 3 & 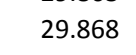 & 54 & 27.056 & 34.347 \\
\hline & & & & & & & 30. & & & 28.2 & & & 35.9 & & & 16 & 23.4 & 15.3 & 11. & 32. & 33.099 & & 64 & 27.056 & 34.352 \\
\hline 10 & & & 30 & 29.017 & 30. & & 30 & & & 28. & & & & 33. & & & & & 11. & 32. & & & 4 & 2705 & 34.352 \\
\hline 10 & & 30 & 30 & 29. & & & 30.6 & & & 28.2 & & & 35 & 33.233 & & 16.465 & 23. & 15. & 11.471 & 32.112 & & & 9 & 56 & 34.354 \\
\hline 10/ & & 30. & & 29 & & & 30 & 30.2 & & 28 & & & & 33.231 & & 16. & 23.475 & & 11.469 & 32.108 & & & 57 & 27.056 & 34.354 \\
\hline $10 / 1$ & & & & & & & 30. & 30.23 & & 28 & & & 35. & 33.228 & & & 23.47 & 15. & 11.464 & & & & 32.154 & & 34.354 \\
\hline 10/12/11 0:00 & & 30 & 30. & 28. & 30. & & 30. & 30.23 & & 28. & 26. & 32. & 35. & 33.226 & 17.002 & 16.458 & 23.47 & 15.346 & 11.462 & 32.103 & 33.085 & 29.856 & 32.15 & 27.056 & 34.355 \\
\hline 10/12/11 0:30 & & 30.7 & 30. & 28.5 & 30. & & 30. & 30.232 & 29. & 28.8 & 26. & 32.1 & 35.9 & 33.221 & 17.0 & 16.454 & 23.468 & 15.344 & 11.457 & 32.096 & 33.082 & 29.851 & 32.15 & 27.059 & 34.354 \\
\hline 10/12/11 1:00 & & 30. & 30.1 & 29.003 & 30. & 29.5 & 30. & 30.2 & 29. & 28.288 & 26.052 & 32.1 & 35.9 & 33.217 & 16.999 & 16.451 & 23.468 & 15.3 & 11.455 & 32.084 & 33.075 & 29.844 & 32.143 & 27.059 & 34.357 \\
\hline 10/12/11 1:30 & & 30.745 & 30.135 & 29.005 & 30.1 & 29.58 & 30.673 & 30.227 & 29.8 & 28.288 & 26.059 & 32.17 & 35.932 & 33.21 & 16.999 & 16.449 & 23.464 & 15.339 & 11.448 & 32.089 & 33.075 & 29.844 & 32.138 & 27.056 & 34.354 \\
\hline 10/12/11 2:00 & & 30.745 & 30.135 & 29.015 & 30.162 & 29.572 & 30.673 & 30.232 & 29.818 & 28.293 & 26.062 & 32.173 & 35.929 & 33.207 & 16.997 & 16.444 & 23.461 & 15.337 & 11.448 & 32.089 & 33.068 & 29.849 & 32.133 & 27.059 & 34.357 \\
\hline 10/12/11 2:30 & & 30.745 & 30.137 & 29.019 & 30.164 & 29.565 & 30.673 & 30.232 & 29.821 & 28.293 & 26.071 & 32.166 & 35.932 & 33.205 & 16.997 & 16.442 & 23.457 & 15.328 & 11.443 & 32.087 & 33.068 & 29.849 & 32.131 & 27.059 & 34.354 \\
\hline 10/12/11 3:00 & & 30.748 & 30.137 & 29.024 & 30.166 & 29.565 & 30.673 & 30.23 & 29.818 & 28.298 & 26.076 & 32.163 & 35.929 & 33.203 & 16.999 & 16.437 & 23.45 & 15.323 & 11.443 & 32.089 & 33.064 & 29.856 & 32.131 & 27.059 & 34.354 \\
\hline 10/12/11 3:30 & & 30.748 & 30.142 & 29.031 & 30.169 & 29.556 & 30.676 & 30.232 & 29.821 & 28.302 & 26.081 & 32.163 & 35.936 & 33.2 & 16.999 & 16.435 & 23.45 & 15.318 & 11.436 & 32.091 & 33.064 & 29.861 & 32.128 & 27.059 & 34.354 \\
\hline 10/12/11 4:00 & & 30.748 & 30.142 & 29.033 & 30.171 & 29.556 & 30.675 & 30.23 & 29.821 & 28.305 & 26.088 & 32.161 & 35.936 & 33.196 & 16.997 & 16.43 & 23.443 & 15.313 & 11.431 & 32.089 & 33.056 & 29.858 & 32.124 & 27.061 & 34.354 \\
\hline $10 / 12 / 114: 30$ & & 30.75 & 30.146 & 29.026 & 30.176 & 29.556 & 30.676 & 30.23 & 29.823 & 28.302 & 26.09 & 32.159 & 35.939 & 33.196 & 16.999 & 16.428 & 23.44 & 15.309 & 11.434 & 32.094 & 33.059 & 29.872 & 32.124 & 27.061 & 34.352 \\
\hline
\end{tabular}




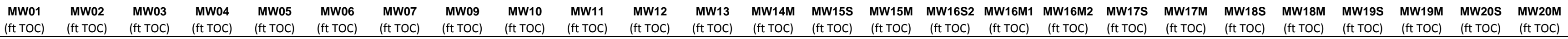

\begin{tabular}{|c|c|c|c|c|c|c|c|c|c|c|c|c|c|c|c|c|c|c|c|c|c|c|c|c|c|c|}
\hline Well & $\begin{array}{l}\text { MW01 } \\
\text { (ft TOC) }\end{array}$ & $\begin{array}{l}\text { MW02 } \\
\text { (ft TOC) }\end{array}$ & $\begin{array}{l}\text { Mw03 } \\
\text { (ft ToC) }\end{array}$ & $\begin{array}{r}\text { MWW4 } \\
\text { (ft TOC) }\end{array}$ & $\begin{array}{l}\text { MW05 } \\
\text { (ft TOC) }\end{array}$ & $\begin{array}{l}\text { MWvo6 } \\
\text { (ft TOC) }\end{array}$ & $\begin{array}{l}\text { MW07 } \\
\text { (ft TOC) }\end{array}$ & $\begin{array}{r}\text { Mw09 } \\
\text { (ft TOC) }\end{array}$ & $\begin{array}{r}\text { MW10 } \\
\text { (ft TOC) }\end{array}$ & $\begin{array}{r}\text { MWi1 } \\
\text { (ft TOC) }\end{array}$ & $\begin{array}{r}\text { MW12 } \\
\text { (ft TOC) }\end{array}$ & $\begin{array}{l}\text { MW13 } \\
(\mathrm{ft} \text { Toc) }\end{array}$ & $\begin{array}{l}\text { MW14M } \\
(\mathrm{ft} \text { TOC) }\end{array}$ & $\begin{array}{l}\text { MW15S } \\
\text { (ft TOC) }\end{array}$ & $\begin{array}{l}\text { MW15M } \\
\text { (ft TOC) }\end{array}$ & $\begin{array}{l}\text { MW1652 } \\
\text { (ft TOC) }\end{array}$ & $\begin{array}{l}\text { (ft TOC) } \\
\text { (ft }\end{array}$ & $\begin{array}{c}\text { MW16162 } \\
\text { (ft TOC) }\end{array}$ & $\begin{array}{l}\text { MW17s } \\
(\mathrm{ft} \text { TOC) }\end{array}$ & (ft TOC) & $\begin{array}{l}\text { MW18S } \\
\text { (ft TOC) } \\
\end{array}$ & $\begin{array}{l}\text { MW18M } \\
\text { (ft TOC) }\end{array}$ & $\begin{array}{l}\text { MW19S } \\
\text { (ft TOC) }\end{array}$ & $\begin{array}{l}\text { MW19M } \\
\text { (ft TOC) }\end{array}$ & $\begin{array}{l}\text { MW20S } \\
\text { (ft TOC) }\end{array}$ & $\begin{array}{l}\text { MWwaM } \\
\text { (ft TOC) }\end{array}$ \\
\hline $10 / 12 / 11$ 5:00 & & 30.745 & 30.149 & 29.026 & 30.176 & & 29.549 & 30.675 & 30.23 & 29.823 & 28.307 & 26.086 & 32.154 & 35.941 & 33.191 & $1 \quad 16.999$ & 16.423 & 23.436 & 15.309 & 11.429 & 32.096 & 33.056 & 29.868 & 32.119 & 27.061 & 34.352 \\
\hline 10/12/11 5:30 & & 30.745 & 30.146 & 29.026 & 30.18 & & 29.556 & 30.673 & 30.232 & 29.823 & 28.307 & 26.083 & 32.154 & 35.946 & 33.188 & $\begin{array}{ll}8 & 17.002\end{array}$ & 16.419 & 23.431 & 15.309 & 11.429 & 32.098 & 33.052 & 29.872 & 32.119 & 27.061 & 34.352 \\
\hline 10/12/11 6:00 & & 30.745 & 30.149 & 29.024 & 30.183 & & 29.554 & 30.675 & 30.232 & 29.823 & 28.305 & 26.083 & 32.149 & 35.946 & 33.186 & $\begin{array}{ll}6 & 17.002\end{array}$ & 16.414 & 23.44 & 15.304 & 11.424 & 32.091 & 33.052 & 29.87 & 32.114 & 27.061 & 34.35 \\
\hline 10/12/11 6:30 & & 30.743 & 30.146 & 29.026 & 30.18 & & 29.556 & 30.673 & 30.234 & 29.821 & 28.305 & 26.081 & 32.147 & 35.939 & 33.181 & $1 \quad 16.999$ & 16.409 & 23.44 & 15.309 & 11.42 & 32.089 & 33.045 & 29.863 & 32.112 & 27.061 & 34.35 \\
\hline 10/12/11 7:00 & & 30.743 & 30.149 & 29.033 & 30.18 & & 29.551 & 30.673 & 30.232 & 29.821 & 28.307 & 26.086 & 32.145 & 35.946 & 33.179 & $\begin{array}{l}9 \\
9\end{array}$ & 16.409 & 23.436 & 15.302 & 11.42 & 32.087 & 33.042 & 29.865 & 32.11 & 27.061 & 34.347 \\
\hline 10/12/11 7:30 & & 30.741 & 30.146 & 29.04 & 30.178 & & 29.547 & 30.675 & 30.23 & 29.818 & 28.312 & 26.09 & 32.14 & 35.943 & 33.179 & $\begin{array}{l}9 \\
9\end{array}$ & 16.407 & 23.433 & 15.304 & 11.415 & 32.087 & 33.042 & 29.863 & 32.107 & 27.063 & 34.347 \\
\hline $10 / 12 / 118: 00$ & & 30.741 & 30.149 & 29.04 & 30.178 & & 29.549 & 30.675 & 30.232 & 29.818 & 28.314 & 26.098 & 32.334 & 35.941 & 33.385 & $5 \quad 16.997$ & 16.426 & 23.429 & 15.299 & 11.622 & 32.145 & 33.236 & 29.868 & 32.295 & 27.063 & 34.347 \\
\hline $10 / 12 / 118: 30$ & & 30.745 & 30.151 & 29.047 & 30.183 & & 29.547 & 30.675 & 30.235 & 29.821 & 28.316 & 26.1 & 32.255 & 35.946 & 33.299 & $\begin{array}{ll}9 & 16.997\end{array}$ & 16.44 & 23.436 & 15.29 & 11.535 & 32.136 & 33.154 & 29.879 & 32.218 & 27.063 & 34.35 \\
\hline 10/12/11 9:00 & & 30.743 & 30.151 & 29.052 & 30.187 & & 29.544 & 30.678 & 30.235 & 29.821 & 28.321 & 26.105 & 32.224 & 35.953 & 33.266 & $6 \quad 16.999$ & 16.437 & 23.45 & 15.292 & 11.504 & 32.131 & 33.125 & 29.877 & 32.189 & 27.061 & 34.35 \\
\hline 10/12/11 9:30 & & 30.743 & 30.151 & 29.05 & 30.19 & & 29.544 & 30.678 & 30.232 & 29.823 & 28.321 & 26.11 & 32.208 & 35.95 & 33.249 & $\begin{array}{l}9 \\
9\end{array}$ & 16.433 & 23.454 & 15.295 & 11.485 & 32.122 & 33.109 & 29.882 & 32.173 & 27.061 & 34.352 \\
\hline 10/12/11 10:00 & & 30.745 & 30.156 & 29.047 & 30.194 & & 29.551 & 30.678 & 30.23 & 29.823 & 28.323 & 26.107 & 32.199 & 35.95 & 33.235 & $5 \quad 17.002$ & 16.433 & 23.459 & 15.297 & 11.478 & 32.122 & 33.097 & 29.884 & 32.164 & 27.063 & 34.352 \\
\hline $10 / 12 / 1110: 30$ & & 30.743 & 30.16 & 29.045 & 30.199 & & 29.558 & 30.678 & 30.23 & 29.828 & 28.321 & 26.107 & 32.201 & 35.953 & 33.238 & $8 \quad 17.002$ & 16.433 & 23.459 & 15.292 & 11.476 & 32.122 & 33.097 & 29.896 & 2.166 & 27.063 & 34.355 \\
\hline $12 / 1111: 00$ & & 30.741 & 30.158 & 29.036 & 30.199 & & 29.568 & 30.676 & 30.23 & 29.825 & 28.319 & 26.102 & 32.191 & 35.96 & 33.231 & 17.004 & 16.428 & 23.457 & 15.299 & 11.467 & 32.122 & 33.09 & 29.894 & 2.157 & 7.066 & 34.354 \\
\hline $12 / 1111: 30$ & & 30.738 & 30.156 & 29.045 & 30.199 & & 29.563 & 30.675 & 30.232 & 29.825 & 28.321 & 26.095 & 32.185 & 35.953 & 33.221 & 17.002 & 16.43 & 23.452 & 15.306 & 11.46 & 32.117 & 33.082 & 29.886 & 2.147 & 7.063 & 34.357 \\
\hline $12: 00$ & & 30.736 & 30.156 & 29.047 & 30.19 & & 29.561 & 30.676 & 30.23 & 29.823 & 28.325 & 26.102 & 32.178 & 35.955 & 33.214 & 17.004 & 16.423 & 23.454 & 15.311 & 11.455 & 32.105 & 33.078 & 29.884 & 2.143 & 27.063 & 34.359 \\
\hline 30 & & 30.738 & 30.153 & 29.04 & 30.1 & & 29.565 & 30.678 & 30.232 & 29.821 & 28.323 & 26.107 & 32.173 & 35.955 & 33.212 & 17.004 & 16.423 & 23.452 & 15.309 & 11.448 & 32.11 & 33.071 & 9.884 & 38 & 27.063 & 34.359 \\
\hline 00 & & 30.741 & 30.158 & 29.038 & 30.2 & & 29.57 & 30.676 & 30.227 & 29.823 & 28.319 & 26.095 & 32.17 & 35.955 & 33.205 & 17.006 & 16.421 & 23.454 & 5.309 & 11.446 & 32.115 & 068 & 9.889 & 35 & 7.063 & 34.359 \\
\hline $13: 30$ & & 30.741 & 30.156 & 29.036 & 30.201 & & 29.572 & 30.675 & 30.232 & 29.828 & 28.321 & 26.095 & 32.168 & 35.957 & 33.205 & 17.006 & 16.419 & 23.45 & 509 & 11.443 & 32.11 & 066 & .889 & 31 & 7.063 & 34.362 \\
\hline $10 / 12 / 1$ & & 30.736 & 30.153 & 29.036 & 30.197 & & 29.572 & 30.675 & 30.225 & 29.821 & 28.323 & 26.095 & 32.161 & 35.958 & 33.2 & 17.004 & 16.416 & 23.447 & 15.313 & 11.436 & 32.103 & 33.061 & 29.879 & 2.128 & 27.063 & 34.364 \\
\hline 10/12/11 14:30 & & 30.734 & 30.151 & 29.05 & 30.197 & & 29.568 & 30.678 & 30.23 & 29.821 & 28.323 & 26.095 & 32.161 & 35.95 & 33.196 & 17.006 & 16.414 & 23.445 & 15.318 & 11.434 & 32.101 & 33.056 & 29.882 & 32.124 & 27.063 & 34.364 \\
\hline 10/12/11 15:00 & & 30.734 & 30.151 & 29.057 & 30.192 & & 29.558 & 30.676 & 30.232 & 29.818 & 28.33 & 26.107 & 32.154 & 35.953 & 33.191 & 17.004 & 16.411 & 23.443 & 15.323 & 11.429 & 32.098 & 33.054 & 29.877 & 32.117 & 27.063 & 34.364 \\
\hline 10/12/11 15:30 & & 30.736 & 30.153 & 29.071 & 30.194 & & 29.556 & 30.675 & 30.232 & 29.818 & 28.332 & 26.114 & 32.149 & 35.955 & 33.186 & 17.006 & 16.407 & 23.438 & 15.313 & 11.429 & 32.101 & 33.047 & 29.884 & 32.114 & 27.066 & 34.362 \\
\hline 10/12/11 16:00 & & 30.741 & 30.16 & 29.075 & 30.201 & & 29.547 & 30.68 & 30.235 & 29.825 & 28.339 & 26.129 & 32.152 & 35.962 & 33.189 & 17.009 & 16.407 & 23.44 & 15.309 & 11.427 & 32.11 & 33.047 & 29.894 & 32.117 & 27.066 & 34.364 \\
\hline 10/12/11 16:30 & & 30.738 & 30.163 & 29.082 & 30.204 & & 29.54 & 30.68 & 30.23 & 29.823 & 28.346 & 26.134 & 32.147 & 35.96 & 33.186 & 17.009 & 16.404 & 23.464 & 15.311 & 11.42 & 32.11 & 33.047 & 29.891 & 32.112 & 27.066 & 34.362 \\
\hline $10 / 12 / 1117: 00$ & & 30.743 & 30.167 & 29.092 & 30.211 & & 29.537 & 30.683 & 30.235 & 29.832 & 28.351 & 26.136 & 32.147 & 35.964 & 33.184 & 17.011 & 16.402 & 23.487 & 15.306 & 11.422 & 32.119 & 33.047 & 29.905 & 32.112 & 27.068 & 34.362 \\
\hline $10 / 12 / 1117: 30$ & & 30.743 & 30.17 & 29.106 & 30.218 & & 29.528 & 30.687 & 30.237 & 29.834 & 28.358 & 26.148 & 32.149 & 35.964 & 33.184 & 17.009 & 16.4 & 23.494 & 15.306 & 11.422 & 32.122 & 33.047 & 29.908 & 32.112 & 27.066 & 34.362 \\
\hline 10/12/11 18:00 & & 30.741 & 30.174 & 29.12 & 30.222 & & 29.518 & 30.687 & 30.239 & 29.837 & 28.365 & 26.16 & 32.149 & 35.967 & 33.181 & 17.013 & 16.397 & 23.484 & 15.309 & 11.42 & 32.124 & 33.047 & 29.915 & 32.112 & 27.068 & 34.364 \\
\hline $10 / 12 / 1118: 30$ & & 30.748 & 30.179 & 29.129 & 30.232 & & 29.511 & 30.692 & 30.242 & 29.841 & 28.374 & 26.174 & 32.152 & 35.969 & 33.181 & 17.013 & 16.395 & 23.489 & 15.304 & $\begin{array}{r}11.422 \\
\end{array}$ & 32.131 & 33.045 & 29.924 & 32.114 & 27.07 & 34.364 \\
\hline 10/12/11 19:00 & & 30.75 & 30.188 & 29.136 & 30.241 & & 29.509 & 30.695 & 30.239 & 29.848 & 28.379 & 26.181 & 32.152 & 35.974 & 33.186 & 17.018 & 16.395 & 23.484 & 15.297 & 11.422 & 32.14 & $\begin{array}{l}33.049 \\
3.049\end{array}$ & 29.943 & 32.117 & 27.07 & 34.366 \\
\hline 10/12/11 19:30 & & 30.75 & 30.195 & 29.141 & 30.248 & & 29.509 & 30.697 & 30.244 & $\begin{array}{l}29.080 \\
29.853\end{array}$ & 28.384 & 26.186 & 32.152 & 35.972 & 33.188 & $\begin{array}{r}17.02 \\
3\end{array}$ & $\begin{array}{l}16.395 \\
1695\end{array}$ & $\begin{array}{r}23.404 \\
23.48\end{array}$ & 15.299 & 11.427 & 32.143 & $\begin{array}{l}33.052 \\
3.052\end{array}$ & 29.948 & 32.117 & 27.07 & 34.369 \\
\hline / $/ 11120: 00$ & & 30.752 & 30.2 & 29.15 & & & 29.509 & 30.699 & 30.246 & 29.86 & 28.388 & 26.191 & 32.154 & 35.979 & 33.186 & 17.023 & $\begin{array}{l}16.395 \\
1695\end{array}$ & 23.487 & 15.295 & 11.427 & 32.152 & 056 & 29.952 & 32.121 & 27.07 & 34.371 \\
\hline / $/ 1120.30$ & & 30.752 & 30.207 & 29.164 & & & 29.504 & 30.704 & $\begin{array}{l}30.240 \\
30.249\end{array}$ & $\begin{array}{r}29.800 \\
29.82\end{array}$ & $\begin{array}{l}28.300 \\
28.398\end{array}$ & 26.198 & 32.156 & $\begin{array}{l}35.976 \\
35.976\end{array}$ & 33.191 & 17.025 & $\begin{array}{l}16.395 \\
16.395\end{array}$ & 23.484 & $\begin{array}{l}15.302 \\
15.302\end{array}$ & 11.431 & 32.154 & 059 & 29.962 & 32.121 & 27.07 & $\begin{array}{l}34.373 \\
34.37\end{array}$ \\
\hline / $/ 112100$ & & $\begin{array}{l}30.12 \\
30.75\end{array}$ & 30.212 & 29.171 & $\begin{array}{l}30.20 \\
30.27\end{array}$ & & $\begin{array}{r}2.504 \\
29.5\end{array}$ & 30.704 & $\begin{array}{l}50.249 \\
30.244\end{array}$ & $\begin{array}{l}29.802 \\
29.865\end{array}$ & $\begin{array}{l}28.3902 \\
28.402\end{array}$ & $\begin{array}{l}26.198 \\
26.215\end{array}$ & $\begin{array}{l}32.150 \\
32.159\end{array}$ & $\begin{array}{l}35.970 \\
35.979\end{array}$ & 33.191 & 17.027 & $\begin{array}{l}10.395 \\
16.393\end{array}$ & $\begin{array}{l}23.464 \\
23.489\end{array}$ & $\begin{array}{l}13.02 \\
15.299\end{array}$ & $\begin{array}{l}11.41 \\
11.434\end{array}$ & 32.161 & $\begin{array}{l}33.059 \\
33.059\end{array}$ & $\begin{array}{l}29.902 \\
29.962\end{array}$ & 32.126 & 27.07 & $\begin{array}{l}34.378 \\
34.378\end{array}$ \\
\hline /111 21:30 & & 30.757 & 30.216 & 29.173 & 30.28 & & 29.504 & 30.706 & 30.244 & 29.874 & 28.409 & 26.217 & 32.161 & 35.983 & 33.196 & 17.03 & 16.395 & 23.491 & 15.297 & 11.434 & 32.166 & .064 & 29.98 & 32.128 & 27.073 & 34.38 \\
\hline & & 30. & 30.22 & 29.176 & . & & & 30. & & & 28.4 & & 32.16 & 35.9 & 33.203 & 17.032 & 16 & 32 & 15205 & 11.436 & 32.173 & 56 & 29.985 & 33 & 27.073 & 383 \\
\hline $22: 30$ & & & 30.228 & & & & & & & & 28.4 & & 32 & 8 & 33.2 & 17.0 & 16.4 & 3.4 & 15.2 & 11.441 & 32.18 & 1 & & 32.135 & 27.073 & 34.385 \\
\hline $10 / 12 / 1123: 00$ & & 30 & 30.23 & 29.183 & & & 29. & 30.711 & 46 & 29.888 & 28.421 & & 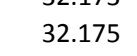 & 33 & 33.205 & 17.0 & 16.4 & 23.482 & 15.299 & 11.443 & 32.177 & 073 & 29.992 & $\quad 32.14$ & 27.075 & 34.39 \\
\hline 10/12/11 23:30 & & 30.759 & 30.235 & 29.183 & 30 & & 29.518 & 30.714 & 30.246 & 29.895 & 28.423 & 26.222 & 32.177 & 35.988 & 33.21 & 17.039 & 16.402 & 23.48 & 15.295 & 11.448 & 32.182 & 33.075 & 29.999 & 32.14 & 27.075 & 34.39 \\
\hline $10 / 13 /$ & & 30.761 & 30.237 & 29.18 & 30 & & & 30.716 & 30.246 & 29. & 28.425 & 26.222 & 32.178 & 35.993 & 33.212 & 17.041 & 16.402 & 23.482 & 15.295 & 11.448 & 32.187 & & 29.997 & 32.143 & 27.075 & 34.392 \\
\hline 10/13/11 0:30 & & 30 & 30.237 & 29.173 & & & & 30.716 & & 29.899 & 28.423 & 26.215 & 32.18 & 35.988 & 33.212 & 17.041 & 16.402 & 23.482 & 15.299 & 11.45 & 32.189 & & 30.004 & 32.145 & 27.075 & 34.394 \\
\hline 10/13/11 1:00 & & 30 & 30.24 & 29.169 & & & 29 & 30.719 & 30 & 29.902 & 28.423 & 26.21 & 32.182 & 35.995 & 33.212 & 17.044 & 16.402 & 23.477 & 15.306 & 11.453 & 32.184 & 33.082 & 29.997 & 32.145 & 27.075 & 34.394 \\
\hline 10/13/11 1:30 & & 30. & 30.242 & 29.173 & & & 29. & 30.718 & 30.249 & 29.902 & 28.425 & 26.203 & 32.18 & 35.995 & 33.212 & 17.044 & 16.402 & 23.48 & 15.313 & 11.453 & 32.184 & 33.078 & 29.992 & 32.145 & 27.073 & 34.397 \\
\hline $10 / 13 / 112: 00$ & & 30. & 30.24 & 29.176 & & & 29.544 & 30.7 & 30.2 & 29.904 & 28.43 & 26.205 & 32.178 & 35.993 & 33.212 & 17.044 & 16.4 & 23.477 & 15.3 & 11.45 & 32.18 & 33.08 & 29.995 & 32.145 & 27.075 & 34.397 \\
\hline 10/13/11 2:30 & & 30.759 & 30.235 & 29.178 & & & 29.554 & 30.721 & 30.251 & 29.904 & 28.43 & 26.205 & 32.178 & 35.993 & 33.21 & 17.046 & 16.4 & 23.475 & 15.318 & 11.45 & 32.18 & 33.078 & 29.99 & 32.145 & 27.077 & 34.397 \\
\hline 10/13/11 3:00 & & 30.761 & 30.24 & 29.185 & 30.316 & & 29.551 & 30.721 & 30.249 & 29.909 & 28.435 & 26.205 & 32.175 & 35.993 & 33.21 & 17.046 & 16.395 & 23.473 & 15.309 & 11.448 & 32.184 & 33.08 & 29.997 & 32.145 & 27.077 & 34.397 \\
\hline $10 / 13 / 113: 30$ & & 30.757 & 30.24 & 29.185 & 30.313 & & 29.556 & 30.723 & 30.249 & 29.906 & 28.437 & 26.212 & 32.177 & 35.997 & 33.21 & 17.046 & 16.395 & 23.473 & 15.318 & 11.448 & 32.175 & 33.075 & 29.99 & 32.14 & 27.075 & 34.397 \\
\hline $10 / 13 / 11$ 4:00 & & 30.761 & 30.242 & 29.19 & 30.316 & & 29.554 & 30.723 & 30.249 & 29.911 & 28.439 & 26.21 & 32.177 & 35.995 & 33.21 & 17.048 & 16.395 & 23.468 & 15.313 & 11.45 & 32.182 & 33.075 & 29.997 & 32.14 & 27.077 & 34.397 \\
\hline $10 / 13 / 114: 30$ & & 30.761 & 30.244 & 29.192 & 30.3 & & 29.556 & 30.728 & 30.251 & 29.913 & 28.442 & 26.215 & 32.175 & 35.995 & 33.207 & 17.051 & $\begin{array}{l}16.393 \\
\end{array}$ & 23.466 & 15.313 & 11.45 & 32.18 & .073 & 29.997 & 32.14 & 27.077 & 34.397 \\
\hline $10 / 13 / 11$ 5:00 & & 30.764 & 30.249 & 29.194 & 30.32 & & 29.556 & 30.728 & 30.249 & 29.916 & 28.446 & 26.212 & 32.173 & 36 & 33.207 & 17.053 & $\begin{array}{l}16.388 \\
1688\end{array}$ & $\quad 23.47$ & 15.311 & $\begin{array}{l}11.446 \\
1.446\end{array}$ & 32.182 & 1 & 30.002 & 32.14 & 27.077 & 34.397 \\
\hline $10 / 13 / 115: 30$ & & 30.7 & 30.249 & 29.194 & 30.323 & & 29.556 & 30.73 & 30.254 & 29.918 & 28.449 & 26.215 & 32.173 & 35.997 & 33.207 & 17.053 & 16.388 & 23.466 & 15.313 & 11.443 & 32.182 & 3 & 30.002 & 32.14 & 27.077 & 34.394 \\
\hline $10 / 13 / 116: 00$ & & 30.764 & 30.251 & 29.197 & 30.323 & & 29.563 & 30.73 & 30.251 & 29.92 & 28.449 & 26.215 & 32.173 & 35.997 & 33.203 & 17.053 & 16.386 & 23.477 & 15.309 & 11.443 & 32.184 & 33.073 & 30.006 & 32.138 & 27.08 & 34.394 \\
\hline $10 / 13$ & & 30.7 & 30.25 & 29.202 & 30.3 & & 29. & 30.7 & 30. & 29.923 & 28.453 & 26.215 & 32.171 & 36.002 & 33.205 & 17.053 & 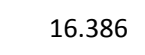 & 23. & 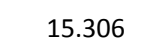 & 11.443 & 84 & 71 & 6 & 32.135 & 08 & 34.394 \\
\hline 10 & & & 30.2 & 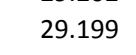 & & & & & 30.254 & & 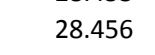 & & . & & & & & & & & & & & & 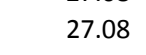 & 4 \\
\hline & & & 30. & & & & & & & & & & & 50 & & & & & & & & & & & & \\
\hline & & & & & & & & & & & 28.46 & & & & & & & & & & & & & & & \\
\hline 111 8:30 & & 30.768 & 30.258 & 29.223 & 30.33 & & 29.565 & 30.74 & 30.254 & 29.932 & 28.47 & 26.236 & 32.175 & .007 & 33.205 & 17.06 & 16.383 & 8.475 & 5.316 & 1.443 & 1.189 & 33.073 & .011 & 2.138 & 87.08 & 394 \\
\hline
\end{tabular}




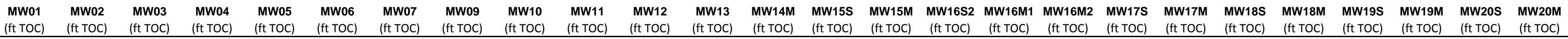

\begin{tabular}{|c|c|c|c|c|c|c|c|c|c|c|c|c|c|c|c|c|c|c|c|c|c|c|c|c|c|c|}
\hline Well & $\begin{array}{l}\text { (ft TOC) } \\
\text { (ft }\end{array}$ & $\begin{array}{l}\text { (ft TOC) } \\
\text { (f) }\end{array}$ & $\begin{array}{l}\text { (ft TOC) } \\
\text { (f) }\end{array}$ & $\begin{array}{l}\text { (ft TOC) } \\
\text { (f) }\end{array}$ & $\begin{array}{l}\text { (ft TOC) } \\
\text { (f) }\end{array}$ & $\begin{array}{l}\text { (ft TOC) } \\
\text { (f) }\end{array}$ & $\begin{array}{l}\text { (ft TOC) } \\
\text { (ft }\end{array}$ & $\begin{array}{l}\text { (ft TOC) } \\
\text { (f) }\end{array}$ & $\begin{array}{l}\text { (ft TOC) } \\
\text { (ft) }\end{array}$ & $\begin{array}{l}\text { (ft TOC) } \\
\text { (f) }\end{array}$ & $\begin{array}{l}\text { (ft TOC) } \\
\text { (f) }\end{array}$ & $\begin{array}{l}\text { (ft TOC) } \\
\text { (f) }\end{array}$ & (ft TOC) & $\begin{array}{l}\text { (ft TOC) } \\
\text { (f) }\end{array}$ & (ft TOC) & (ft TOC) & $\begin{array}{l}\text { (ft TOC) } \\
\text { (f) }\end{array}$ & (ft TOC) & $\begin{array}{l}\text { (ft TOC) } \\
\text { (1) }\end{array}$ & (ft TOC) & $\begin{array}{l}\text { (ft TOC) } \\
\end{array}$ & $\begin{array}{l}\text { (ft TOC) } \\
\text { (1) }\end{array}$ & $\begin{array}{l}\text { (ft TOC) } \\
\text { (n) }\end{array}$ & (ft TOC) & $\begin{array}{l}\text { (ft TOC) } \\
\text { (1) }\end{array}$ & (ft TOC) \\
\hline 19:00 & & 30.773 & 30.26 & 29.227 & 30.339 & & 29.568 & 30.745 & 30 & 29.9 & 28.474 & 26 & 32 & 36.004 & 33.2 & 17.06 & 16.386 & 23.475 & 15.313 & 11.443 & 32. & 33. & 30.023 & & 82 & 34.397 \\
\hline 3/11 9:30 & & 30.773 & 30.268 & 29.227 & 30.344 & & 29.568 & 30.745 & 30.258 & 29.944 & 28.479 & 26.241 & 32.18 & 36.009 & 33.21 & 17 & 16.386 & 23.47 & 15.311 & 11.455 & 32.203 & 33.075 & 30.027 & .143 & 7.082 & 34.397 \\
\hline 10/13/11 10:00 & & 30.775 & 30.272 & 29.22 & 30.346 & & 29.58 & 30.745 & 30.256 & 29.946 & 28.479 & 26.241 & 32.177 & 36.004 & 33.214 & 17.065 & 16.386 & 23.47 & 15.318 & 11.457 & 32.205 & 33.078 & 30.027 & .145 & 7.082 & 34.399 \\
\hline 10/13/11 10:30 & & 30.775 & 30.275 & 29.211 & 30.351 & & 29.589 & 30.747 & 30.254 & 29.951 & 28.477 & 26.234 & 32.196 & 36.009 & 33.226 & 17.067 & 16.386 & 23.47 & 15.32 & 11.469 & 32.21 & 33.094 & 30.035 & 32.161 & 27.082 & 34.401 \\
\hline 10/13/11 11:00 & & 30.775 & 30.277 & 29.199 & 30.353 & & 29.603 & 30.747 & 30.254 & 29.955 & 28.474 & 26.222 & 32.189 & 36.009 & 33.224 & $4 \quad 17.067$ & 16.388 & 23.477 & 15.328 & 11.464 & 32.21 & 33.09 & 30.032 & 32.154 & 27.082 & 34.404 \\
\hline 10/13/11 11:30 & & 30.773 & 30.277 & 29.192 & 30.348 & & 29.612 & 30.749 & 30.254 & 29.953 & 28.47 & 26.212 & 32.189 & 36.007 & 33.219 & $\begin{array}{l}9 \\
9\end{array}$ & 16.388 & 23.484 & 15.337 & 11.483 & 32.208 & 33.087 & 30.051 & 32.152 & 27.082 & 34.404 \\
\hline 10/13/11 12:00 & & 30.771 & 30.272 & 29.171 & 30.348 & & 29.627 & 30.747 & 30.254 & 29.951 & 28.465 & 26.203 & 32.189 & 36.009 & 33.221 & $1 \quad 17.067$ & 16.386 & 23.48 & 15.349 & 11.493 & 32.194 & 33.085 & 30.082 & 32.152 & 27.084 & 34.406 \\
\hline 10/13/11 12:30 & & 30.768 & 30.265 & 29.155 & 30.341 & & 29.641 & 30.747 & 30.246 & 29.951 & 28.456 & 26.186 & 32.184 & 36.007 & 33.219 & $\begin{array}{l}9 \\
9\end{array}$ & 16.388 & 23.464 & 15.353 & 11.507 & 32.191 & 33.085 & 30.011 & 32.15 & 27.082 & 34.406 \\
\hline 10/13/11 13:00 & & 30.768 & 30.261 & 29.138 & 30.334 & & 29.652 & 30.745 & 30.249 & 29.948 & 28.449 & 26.167 & 32.182 & 36.009 & 33.217 & $\begin{array}{l}7 \quad 17.067 \\
\end{array}$ & 16.386 & 23.473 & 15.365 & 11.455 & 32.187 & 33.08 & 30.006 & 32.147 & 27.082 & 34.406 \\
\hline 10/13/11 13:30 & & 30.761 & 30.256 & 29.122 & 30.323 & & 29.662 & 30.745 & 30.246 & 29.941 & 28.442 & 26.15 & 32.178 & 36.002 & 33.212 & $2 \quad 17.067$ & 16.386 & 23.47 & 15.374 & 11.453 & 32.175 & 33.078 & 29.99 & 32.145 & 27.082 & 34.406 \\
\hline 10/13/11 14:00 & & 30.759 & 30.244 & 29.108 & 30.311 & & 29.674 & 30.737 & 30.246 & 29.934 & 28.432 & 26.134 & 32.175 & 35.995 & 33.207 & $7 \quad 17.062$ & 16.381 & 23.461 & 15.379 & 11.446 & 32.163 & 33.075 & 29.976 & 32.138 & 27.082 & 34.406 \\
\hline $10 / 13 / 1114: 30$ & & 30.755 & 30.235 & 29.103 & 30.299 & & 29.676 & 30.735 & 30.242 & 29.93 & 28.428 & 26.122 & 32.166 & 35.995 & 33.203 & $\begin{array}{l}317.062 \\
\end{array}$ & 16.383 & 23.45 & 15.382 & 11.438 & 32.15 & 33.071 & 29.962 & 32.131 & 27.082 & 34.404 \\
\hline $10 / 13 / 1115: 00$ & & 30.752 & 30.228 & 29.108 & 30.285 & & 29.674 & 30.733 & 30.246 & 29.923 & 28.428 & 26.114 & 32.161 & 35.988 & 33.196 & $\begin{array}{ll}6 & 17.06\end{array}$ & 16.379 & 23.447 & 15.391 & 11.434 & 32.14 & 33.061 & 29.948 & 32.126 & 27.082 & 34.401 \\
\hline $10 / 13 / 1115: 30$ & & 30.75 & 30.219 & 29.106 & 30.276 & & 29.671 & 30.73 & 30.244 & 29.918 & 28.423 & 26.119 & 32.156 & 35.983 & 33.191 & 17.055 & 16.374 & 23.436 & 15.393 & 11.427 & 32.131 & 33.056 & 29.941 & 32.121 & 27.084 & 34.401 \\
\hline 10/13/11 16:00 & & 30.75 & 30.214 & 29.106 & 30.264 & & 29.664 & 30.73 & 30.244 & 29.913 & 28.421 & 26.117 & 32.152 & 35.983 & 33.181 & 17.055 & 16.372 & 23.424 & 15.393 & 11.422 & 32.126 & 33.054 & 29.931 & 32.117 & 27.084 & 34.399 \\
\hline 10/13/11 16:30 & & 30.75 & 30.212 & 29.106 & 30.257 & & 29.657 & 30.73 & 30.244 & 29.911 & 28.423 & 26.117 & 32.147 & 35.979 & 33.181 & 17.055 & 16.369 & 23.417 & 15.393 & 11.417 & 32.122 & 33.049 & 29.926 & 32.112 & 27.082 & 34.401 \\
\hline 10/13/11 17:00 & & 30.752 & 30.209 & 29.103 & 30.255 & & 29.657 & 30.728 & 30.244 & 29.909 & 28.419 & 26.117 & 32.145 & 35.983 & 33.177 & 17.053 & 16.365 & 23.422 & 15.391 & 11.415 & 32.126 & 33.042 & 29.929 & 32.11 & 27.084 & 34.399 \\
\hline 10/13/11 17:30 & & 30.75 & 30.212 & 29.106 & 30.25 & & 29.65 & 30.725 & 30.246 & 29.906 & 28.419 & 26.117 & 32.142 & 35.981 & 33.179 & 17.053 & 16.367 & 23.415 & 15.391 & 11.41 & 32.122 & 33.042 & 29.931 & 32.105 & 27.084 & 34.397 \\
\hline 10/13/11 18:00 & & 30.75 & 30.207 & 29.115 & 30.248 & & 29.638 & 30.725 & 30.246 & 29.904 & 28.421 & 26.119 & 32.138 & 35.972 & 33.172 & 17.053 & 16.362 & 23.406 & 15.393 & 11.41 & 32.119 & 33.037 & 29.922 & 32.1 & 27.084 & 34.394 \\
\hline $3 / 11$ 18:30 & & 30.75 & 30.207 & 29.122 & 30.24 & & 29.624 & 30.728 & 30.244 & 29.904 & 28.425 & 26.134 & 32.133 & 35.974 & 33.17 & 17.051 & 16.362 & 23.429 & 15.391 & 11.406 & 32.119 & 33.035 & .919 & 32.1 & 27.084 & 34.394 \\
\hline$: 00$ & & 30.75 & 30.207 & 29.129 & 30. & & 29.617 & 30.725 & 30.249 & 29.902 & 28.425 & 26.138 & 32.133 & 35.976 & 33.167 & 17.053 & 16.36 & 23.447 & 15.389 & 11.403 & 32.119 & 33.026 & .926 & 32.1 & 27.084 & 34.392 \\
\hline /111 19:30 & & 30.752 & 30.212 & 29.127 & 30.248 & & 29.61 & 30.726 & 30.246 & 29.904 & 28.428 & 26.141 & 32.133 & 35.976 & 33.17 & 17.053 & 16.36 & 23.459 & 379 & 11.403 & 32.129 & .007 & .931 & 32.098 & 27.084 & 34.394 \\
\hline 10/13/11 20:00 & & 30.755 & 30.214 & 29.127 & 30.25 & & 29.608 & 30.728 & 30.246 & 306 & 28.428 & 26.143 & 32.131 & 35.981 & 33.165 & 17.055 & 16.358 & 23.445 & 377 & 11.408 & 32.129 & 32.995 & 9.941 & 32.1 & 27.084 & 34.394 \\
\hline 10/13/11 20:30 & & 30.754 & 30.216 & 29.129 & 30.255 & & 29.605 & 30.728 & 30.246 & 29.906 & 28.428 & 26.141 & 32.135 & 35.976 & 33.167 & 17.055 & 16.358 & 23.429 & 15.379 & 11.403 & 32.124 & 32.983 & 29.943 & 32.098 & 27.087 & 34.394 \\
\hline 10/13/11 21:00 & & 30.752 & 30.214 & 29.131 & 30.253 & & 29.601 & 30.728 & 30.249 & & 28.428 & 26.145 & 32.135 & 35.986 & 33.165 & 17.055 & 16.358 & 23.41 & 15.382 & 11.406 & 32.129 & 32.978 & 29.943 & 32.098 & 27.084 & 34.394 \\
\hline 10/13/11 21:30 & & 30.752 & 30.212 & 29.131 & 30.255 & & 29.601 & 30.725 & 30.249 & 29.904 & 28.43 & 26.148 & 32.133 & 35.981 & 33.165 & 17.055 & 16.358 & 23.394 & 15.379 & 11.403 & 32.122 & 32.969 & 29.943 & .096 & 27.087 & 34.394 \\
\hline 10/13/11 22:00 & & 30.755 & 30.216 & 29.131 & 30.257 & & 29.601 & 30.728 & 30.246 & 29.906 & 28.425 & 26.148 & 32.135 & 35.981 & 33.165 & 17.055 & 16.358 & 23.378 & 15.377 & 11.403 & 32.129 & 32.964 & 29.948 & 2.098 & 27.087 & 34.397 \\
\hline 10/13/11 22:30 & & 30.75 & 30.214 & 29.138 & 30.26 & & 29.596 & 30.728 & 30.246 & 29.904 & 28.43 & 26.148 & 32.133 & 35.988 & 33.167 & 17.058 & 16.358 & 23.359 & 15.374 & 11.403 & 32.126 & 32.966 & 29.945 & 32.098 & 27.087 & 34.397 \\
\hline 10/13/11 23:00 & & 30.752 & 30.214 & 29.136 & 30.26 & & 29.594 & 30.725 & 30.249 & 29.904 & 28.432 & 26.155 & 32.133 & 35.986 & 33.165 & 17.058 & 16.36 & 23.346 & 15.374 & 11.406 & 32.131 & 32.962 & 29.95 & 32.1 & 27.087 & 34.399 \\
\hline 10/13/11 23:30 & & 30.752 & 30.216 & 29.134 & 30.262 & & 29.591 & 30.728 & 30.249 & 29.906 & 28.43 & 26.153 & 32.133 & 35.986 & 33.167 & 17.058 & 16.36 & 23.329 & 15.372 & 11.403 & 32.129 & 32.959 & 29.948 & 32.098 & 27.089 & 34.401 \\
\hline $10 / 14 / 110: 00$ & & 30.752 & 30.216 & 29.138 & 30.262 & & 29.591 & 30.728 & 30.249 & 29.906 & 28.43 & 26.153 & 32.135 & 35.99 & 33.167 & 17.058 & 16.36 & 23.313 & 15.367 & 11.408 & 32.131 & 33.035 & 29.95 & 32.1 & 27.087 & 34.404 \\
\hline 10/14/11 0:30 & & 30.75 & 30.214 & 29.15 & 30.264 & & 29.587 & 30.73 & 30.251 & 29.904 & 28.432 & 26.155 & 32.135 & 35.99 & 33.167 & 17.058 & 16.358 & 23.297 & 15.37 & 11.403 & 32.129 & 33.035 & 29.95 & 32.098 & 27.089 & 34.404 \\
\hline 10/14/11 1:00 & & 30.75 & 30.214 & 29.159 & 30.262 & & 29.575 & 30.73 & 30.251 & 29.904 & 28.442 & 26.167 & 32.135 & 35.99 & 33.167 & 17.06 & 16.36 & 23.281 & 15.367 & 11.406 & 32.133 & 33.033 & 29.945 & 32.098 & 27.087 & 34.406 \\
\hline 10/14/11 1:30 & & 30.752 & 30.216 & 29.18 & 30.267 & & 29.558 & 30.735 & 30.258 & 29.906 & 28.453 & 26.179 & 32.133 & 35.993 & 33.167 & 17.058 & 16.36 & 23.267 & 15.363 & 11.405 & 32.133 & 33.033 & 29.955 & 32.098 & 27.089 & 34.406 \\
\hline $10 / 14 / 112: 00$ & & 30.757 & 30.223 & 29.185 & 30. & & 29. & 30.74 & 30.256 & 29.909 & 28.456 & 26.198 & 32.138 & 35.995 & 33.167 & 17.06 & 16 & 23.253 & 15.356 & 11.408 & 32.143 & 33.04 & 29.966 & 32.1 & 27.089 & 34.408 \\
\hline $10 / 14 / 112: 30$ & & 30 & 30.235 & 29.18 & & & 29.563 & 30.74 & 30.254 & 29.916 & 28.453 & 26.203 & 32.14 & 36.002 & 33.17 & 17.065 & 16.36 & 23.237 & 15.339 & 11.413 & 32.159 & 33.037 & 29.985 & 32.105 & 27.091 & 34.408 \\
\hline $10 / 14 / 113: 00$ & & 30.761 & 30.235 & 29.178 & 30. & & 29.565 & 30.742 & 30.256 & 29.92 & 28.456 & 26.198 & 32.14 & 36.002 & 33.172 & 17.067 & 16.36 & 23.223 & 15.349 & 11.41 & 32.161 & $\begin{array}{l}33.042 \\
3.042\end{array}$ & 29.99 & 32.107 & 27.091 & 34.411 \\
\hline $10 / 14 / 113: 30$ & & 30.761 & 30.237 & 29.183 & 30. & & 29.57 & 30.74 & 30.246 & 29.923 & $\begin{array}{l}28.456 \\
28.456\end{array}$ & 26.198 & 32.142 & 36 & 33.172 & 17.067 & $\begin{array}{l}16.30 \\
16.362\end{array}$ & 23.209 & 15.351 & $\begin{array}{l}11.413 \\
11.413\end{array}$ & 32.161 & $\begin{array}{l}5.042 \\
33.04\end{array}$ & 29.995 & 32.105 & 27.089 & 34.411 \\
\hline $10 / 14 / 114: 00$ & & 30 & $\begin{array}{l}30.237 \\
30.237\end{array}$ & 29.185 & 30 & & 29.568 & $\begin{array}{l}30.745 \\
30.745\end{array}$ & $\begin{array}{l}30.256 \\
30.256\end{array}$ & 29.923 & $\begin{array}{r}20.450 \\
28.46\end{array}$ & 26.198 & 32. & $\begin{array}{l}36.009 \\
\end{array}$ & 33.172 & 17.069 & $\begin{array}{l}\begin{array}{c}10.020 \\
16.36\end{array} \\
\end{array}$ & 23.191 & 1 & $\begin{array}{l}11.413 \\
11.413\end{array}$ & 32.161 & $\begin{array}{l}33.04 \\
33.04\end{array}$ & 29.99 & 2.105 & 27.091 & 34.411 \\
\hline 4:30 & & 30 & 30. & 29 & & & & 30 & 30 & 25 & 28 & 26 & 32 & 36 & 33. & 17 & 16 & 5 & 15.353 & 11.408 & 32 & 33.04 & 8 & 5 & 27.091 & 34.408 \\
\hline 5:00 & & & 30 & 29.199 & & & & 30.7 & & & 28. & & & 36 & $3=$ & 17.069 & 16 & 23 & 15 & 11.413 & 57 & 33.037 & 38 & 3 & 27.091 & 34.408 \\
\hline /111 5:30 & & & 30.2 & 29.209 & & & & 30.7 & & & $2 \varepsilon$ & & 32.13 & 36.009 & & & & & & 11.408 & 32. & & 5 & 05 & 91 & 34.408 \\
\hline $10 / 14 / 116: 00$ & & & 30. & 29.22 & & & & & & & 28. & & & 36.014 & 33.167 & 17.071 & & & 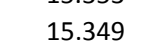 & 11.408 & - & & & & & 34.406 \\
\hline 111 6:30 & & & 30. & 29.23 & & & & 30. & & & 28. & & 32.1 & 36. & 33 & 17.074 & 16. & 23 & 15. & 11.408 & 32.166 & 33.04 & & 5 & 27.094 & 34.408 \\
\hline /11 7:00 & & & 30. & 29.237 & & & & & & & 28 & & 34 & & & & 16. & & 15. & 12.793 & 32.924 & 35.146 & 30.049 & & 27.094 & 34.406 \\
\hline 10/14/11 7:30 & & & 30.2 & 29.251 & & & & & & & 28. & & & & 35. & & 16. & & 15. & 13. & 33.134 & & 30.1 & 11 & 27.096 & 34.408 \\
\hline $10 / 14 / 118: 00$ & & & 30.265 & 29.258 & 30.3 & & & 30. & 30. & & 28.9 & 26. & 35. & 36.0 & 35.723 & 17.078 & 16. & 23.089 & 15.3 & 13. & 33.383 & 36.258 & 30.145 & 35.282 & 27.096 & 34.408 \\
\hline 10/14/11 8:30 & & 30. & 30 & 29.262 & 30 & & & 30. & $30.2 \mathrm{x}-\mathrm{s}$ & 29.5 & 28.507 & 26 & $35.8 \mathrm{~s}$ & 36.0 & 36.281 & 17.081 & 16. & 23.073 & 15. & 14. & 33.637 & 36.821 & 30.197 & 35.791 & 27.096 & 34.411 \\
\hline 10/14/11 9:00 & & 30.778 & 30.279 & 29.265 & 30.4 & & 29. & 30.7 & 30.2 & 29 & 28.512 & 26.277 & 36.0 & 36.0 & 36.6 & 17.083 & 16.856 & 23.075 & 15. & 14.8 & 33.751 & 37.023 & 30.248 & 36.007 & 27.096 & 34.411 \\
\hline 10/14/11 9:30 & & 30.78 & 30.289 & 29.262 & 30.4 & & 29.561 & 30.766 & 30.265 & 29.9 & 28.516 & 26.279 & 34.48 & 36.025 & 35.629 & 17.085 & 16.916 & 23.0 & 15.337 & 13.848 & 33.278 & 35.355 & 30.277 & 34.414 & 27.096 & 34.413 \\
\hline 10/14/11 10:00 & & 30.782 & 30.293 & 29.262 & 30.507 & & 29.568 & 30.769 & 30.265 & 29.974 & 28.516 & 26.277 & 34.012 & 36.025 & 35.139 & 17.088 & 16.916 & 23.082 & 15.339 & 13.354 & 33.089 & 34.897 & 30.279 & 33.956 & 27.099 & 34.413 \\
\hline $10 / 14 / 1110: 30$ & & 30.78 & 30.298 & 29.258 & 30.53 & & 29.577 & 30.771 & 30.265 & 29.978 & 28.521 & 26.275 & 33.727 & 36.028 & 34.839 & 17.09 & 16.905 & 23.075 & 15.342 & 13.053 & 32.97 & 34.613 & 30.274 & 33.675 & 27.099 & 34.415 \\
\hline 10/14/11 11:00 & & 30.782 & 30.303 & 29.246 & 30.547 & & 29.591 & 30.771 & 30.265 & 29.981 & 28.516 & 26.272 & 33.525 & 36.03 & 34.621 & 17.092 & 16.891 & 23.061 & 15.349 & 12.847 & 32.886 & 34.414 & 30.267 & 33.475 & 27.098 & 34.418 \\
\hline 10/14/11 11:30 & & 30.785 & 30.303 & 29.241 & 30.556 & & 29.601 & 30.771 & 30.268 & 29.983 & 28.516 & 26.26 & 33.376 & 36.028 & 34.462 & 17.092 & 16.877 & 23.045 & 15.356 & 12.691 & 32.823 & 34.265 & 30.265 & 33.325 & 27.098 & 34.42 \\
\hline $10 / 14 / 1112: 00$ & & 30.78 & 30.303 & 29.239 & 30.558 & & 29.61 & 30.776 & 30.265 & 29.983 & 28.514 & 26.256 & 34.248 & 36.033 & 34.708 & 17.092 & 16.858 & 23.031 & 15.37 & 12.941 & 32.98 & 35.253 & 30.255 & 34.226 & 27.098 & 34.42 \\
\hline 10/14/11 12:30 & & 30.78 & 30.3 & 29.234 & 30.561 & & 29.619 & 30.773 & 30.265 & 29.983 & 28.512 & 26.251 & 35.304 & 36.03 & 35.779 & 17.095 & 16.928 & 23.013 & 15.367 & 14.036 & 33.479 & 36.277 & 30.272 & 35.254 & 27.098 & 2 \\
\hline
\end{tabular}




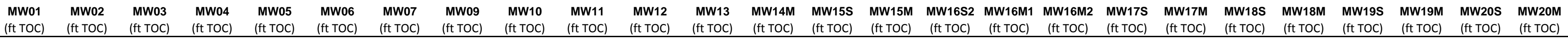

\begin{tabular}{|c|c|c|c|c|c|c|c|c|c|c|c|c|c|c|c|c|c|c|c|c|c|c|c|c|c|}
\hline Well & $\begin{array}{l}\text { (ft TOC) } \\
\text { (ft }\end{array}$ & $\begin{array}{l}\text { (ft TOC) } \\
\text { (f) }\end{array}$ & $\begin{array}{l}\text { (ft TOC) } \\
\text { (f) }\end{array}$ & (ft TOC) & $\begin{array}{ll}(\mathrm{ft} \text { TOC) } & \text { (ft TOC) }\end{array}$ & $\begin{array}{l}\text { (ft TOC) } \\
\text { (ft }\end{array}$ & $\begin{array}{l}\text { (ft TOC) } \\
\text { (f) }\end{array}$ & $\begin{array}{l}\text { (ft TOC) } \\
\text { (ft) }\end{array}$ & $\begin{array}{l}\text { (ft TOC) } \\
\text { (t) }\end{array}$ & (ft TOC) & $\begin{array}{l}\text { (ft Toc) } \\
\text { (f) }\end{array}$ & $\begin{array}{l}\text { (ft TOC) } \\
\text { (n) }\end{array}$ & $\begin{array}{l}\text { (ft TOC) } \\
\end{array}$ & (ft toc) & (ft TOC) & (ft TOC) & (ft TOC) & $\begin{array}{l}\text { (ft TOC) } \\
\text { (ft) }\end{array}$ & (ft TOC) & $\begin{array}{l}\text { (ft TOC) } \\
\end{array}$ & $\begin{array}{l}\text { (ft TOC) } \\
\text { (1) }\end{array}$ & $\begin{array}{l}\text { (ft TOC) } \\
\text { (n) }\end{array}$ & (ft TOC) & $\begin{array}{l}\text { (ft TOC) } \\
\end{array}$ & (ft TOC) \\
\hline 10/14/11 13:00 & & 30.78 & 30.303 & 29.225 & 30.568 & 29.629 & 30.773 & 30.265 & 29.985 & 28.512 & 26.246 & 35.708 & 36.032 & 36.22 & 17.095 & 17.017 & 22.999 & 15.377 & 14.487 & 33.626 & 36.682 & 30.302 & 35.66 & 27.101 & 34.422 \\
\hline 10/14/11 13:30 & & 30.78 & 30.298 & 29.211 & 30.579 & 29.641 & 30.771 & 30.263 & 29.985 & 28.505 & 26.236 & 36.34 & 36.028 & 36.799 & 17.095 & 17.117 & 22.983 & 15.382 & 15.075 & 33.88 & 37.314 & 30.331 & 36.279 & 27.101 & 34.425 \\
\hline 10/14/11 14:00 & & 30.78 & 30.3 & 29.209 & 30.596 & 29.648 & 30.774 & 30.263 & 29.985 & 28.505 & 26.224 & 36.759 & 36.032 & 37.242 & 17.095 & 17.225 & 22.969 & 15.382 & 15.526 & 34.055 & 37.727 & 30.361 & 36.692 & 27.098 & 34.425 \\
\hline $14 / 11$ 14:30 & & 30.78 & 30.298 & 29.211 & 30.61 & 29.65 & 30.773 & 30.263 & 29.983 & 28.502 & 26.224 & 35.762 & 36.028 & 36.94 & 17.092 & 17.323 & 22.955 & 15.393 & 15.249 & 33.875 & 36.625 & 30.38 & & 27.101 & 34.425 \\
\hline 10/14/11 15:00 & & 30.778 & 30.298 & 29.213 & 30.624 & 29.648 & 30.773 & 30.265 & 29.981 & 28.505 & 26.224 & 34.939 & 36.021 & 36.107 & 17.092 & 17.339 & 22.939 & 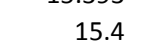 & & & & & 867 & 01 & 34.425 \\
\hline 10/14/11 15:30 & & 30.778 & 30.293 & 29.209 & 30.635 & 29.65 & 30.776 & 30.265 & 29.978 & 28.5 & 26.227 & 34.515 & 36.018 & 35.662 & 17.092 & 17.316 & 22.922 & 15.4 & 13.975 & 33.322 & 35.39 & 30.361 & 4.449 & 7.101 & 34.422 \\
\hline $10 / 14 / 1116: 00$ & & 30.782 & 30.298 & 29.216 & 30.645 & 29.65 & 30.776 & 30.268 & 29.981 & 28.502 & 26.222 & 34.227 & 36.021 & 35.357 & 17.092 & 17.29 & 22.916 & 15.403 & 13.665 & 33.211 & 35.108 & 30.354 & 34.168 & 27.101 & 34.425 \\
\hline 10/14/11 16:30 & & 30.785 & 30.298 & 29.22 & 30.649 & 29.645 & 30.776 & 30.275 & 29.981 & 28.505 & 26.227 & 34.358 & 36.032 & 35.292 & 17.095 & 17.26 & 22.939 & 15.403 & 13.596 & 33.187 & 35.26 & 30.345 & 34.313 & 27.103 & 34.425 \\
\hline 10/14/11 17:00 & & 30.785 & 30.298 & 29.23 & 30.647 & 29.636 & 30.778 & 30.27 & 29.983 & 28.512 & 26.234 & 34.7 & 36.021 & 35.634 & 17.095 & 17.264 & 22.941 & 15.403 & 13.94 & 33.353 & 35.604 & 30.335 & 34.644 & 27.103 & 34.422 \\
\hline 10/14/11 17:30 & & 30.787 & 30.3 & 29.232 & 30.652 & 29.636 & 30.778 & 30.27 & 29.985 & 28.512 & 26.241 & 36.279 & 36.026 & 36.574 & 17.095 & 17.311 & 22.929 & 15.405 & 14.873 & 33.854 & 37.295 & 30.364 & 36.23 & 27.103 & 34.422 \\
\hline 10/14/11 18:00 & & 30.789 & 30.305 & 29.241 & 30.663 & 29.629 & 30.778 & 30.273 & 29.988 & 28.514 & 26.244 & 36.801 & 36.026 & 37.275 & 17.095 & 17.416 & 22.913 & 15.396 & 15.557 & 34.12 & 37.769 & 30.403 & 36.735 & 27.103 & 34.422 \\
\hline 10/14/11 18:30 & & 30.789 & 30.31 & 29.251 & 30.677 & 29.624 & 30.781 & 30.275 & 29.992 & 28.521 & 26.256 & 37.365 & 36.028 & 37.786 & 17.097 & 17.519 & 22.897 & 15.405 & 16.062 & 34.323 & 38.345 & 30.436 & 37.263 & 27.103 & 34.422 \\
\hline 10/14/11 19:00 & & 30.794 & 30.312 & 29.255 & 30.696 & 29.617 & 30.783 & 30.273 & 29.992 & 28.521 & 26.265 & 37.95 & 36.032 & 38.311 & 17.099 & 17.629 & 22.883 & 15.403 & 16.575 & 34.537 & 38.942 & 30.476 & 37.882 & 27.103 & 34.422 \\
\hline 10/14/11 19:30 & & 30.796 & 30.321 & 29.255 & 30.724 & 29.615 & 30.785 & 30.277 & 29.999 & 28.525 & 26.268 & 38.21 & 36.032 & 38.668 & $3 \quad 17.099$ & 17.739 & 22.865 & 15.403 & 16.911 & 34.659 & 39.181 & 30.516 & 38.133 & 27.106 & 34.425 \\
\hline $10 / 14 / 1120: 00$ & & 30.799 & 30.326 & 29.262 & 30.752 & 29.617 & 30.785 & 30.275 & 30.002 & 28.528 & 26.27 & 36.96 & 36.032 & 37.964 & 17.102 & 17.821 & 22.86 & 15.4 & 16.196 & 34.337 & 37.85 & 30.542 & 36.873 & 27.106 & 34.425 \\
\hline $10 / 14 / 1120: 30$ & & 30.799 & 30.328 & 29.265 & 30.776 & 29.615 & 30.788 & 30.277 & 30.006 & 28.528 & 26.275 & 36.546 & 36.032 & 37.54 & 17.102 & 17.856 & 22.849 & 15.405 & 15.754 & 34.16 & 37.44 & 30.556 & 36.469 & 27.106 & 34.425 \\
\hline 10/14/11 21:00 & & 30.801 & 30.333 & 29.274 & 30.794 & 29.61 & 30.79 & 30.282 & 30.009 & 28.535 & 26.279 & 36.02 & 36.035 & 37.198 & 17.104 & 17.877 & 22.837 & 15.405 & 15.409 & 34.034 & 36.881 & 30.556 & 35.932 & 27.106 & 34.427 \\
\hline 10/14/11 21:30 & & 30.803 & 30.34 & 29.279 & 30.811 & 29.608 & 30.792 & 30.282 & 30.013 & 28.537 & 26.289 & 35.404 & 36.042 & 36.593 & 17.106 & 17.853 & 22.825 & 15.403 & 14.786 & 33.775 & 36.277 & 30.554 & 35.334 & 27.106 & 34.427 \\
\hline $10 / 14 / 1122: 00$ & & 30.806 & 30.345 & 29.283 & 30.825 & 29.608 & 30.792 & 30.282 & 30.016 & $\begin{array}{l}28.544 \\
28.541\end{array}$ & 26.291 & 35.051 & 36.037 & 36.218 & 17.106 & 17.816 & 22.802 & 15.398 & $\begin{array}{l}14.1398 \\
14.308\end{array}$ & 33.635 & 35.923 & 30.54 & 34.984 & 27.106 & 34.429 \\
\hline $10 / 14 / 1122: 30$ & & $\begin{array}{r}30.000 \\
30.81\end{array}$ & $\begin{array}{l}50.345 \\
30.352\end{array}$ & 29.276 & 30.834 & 29.612 & 30.795 & 30.277 & $\begin{array}{l}30.022 \\
30.022\end{array}$ & $\begin{array}{l}20.344 \\
28.544\end{array}$ & $\begin{array}{l}20.291 \\
26.296\end{array}$ & 34.789 & 36.037 & $\begin{array}{l}35.210 \\
35.936\end{array}$ & 17.111 & $\begin{array}{l}17.010 \\
17.776\end{array}$ & $\begin{array}{l}22.798 \\
22.798\end{array}$ & $\begin{array}{r}1.350 \\
15.4\end{array}$ & $\begin{array}{l}14.50 \\
14.102\end{array}$ & $\begin{array}{l}33.035 \\
33.532\end{array}$ & $\begin{array}{l}35.665 \\
3.53\end{array}$ & $\begin{array}{r}30.534 \\
30.533\end{array}$ & $\begin{array}{l}34.504 \\
34.724\end{array}$ & 27.108 & $\begin{array}{l}34.432 \\
34.42\end{array}$ \\
\hline $10 / 14 / 1123: 00$ & & $\begin{array}{r}30.813 \\
30.813\end{array}$ & $\begin{array}{l}30.352 \\
30.359\end{array}$ & 29.274 & $\begin{array}{l}30.844 \\
30.836\end{array}$ & 29.617 & 30.795 & 30.28 & $\begin{array}{l}30.022 \\
30.025\end{array}$ & $\begin{array}{l}28.344 \\
28.544\end{array}$ & $\begin{array}{l}20.250 \\
26.289\end{array}$ & $\begin{array}{l}34.597 \\
34.574\end{array}$ & $\begin{array}{l}30.031 \\
36.037\end{array}$ & $\begin{array}{l}35.714 \\
35.714\end{array}$ & 17.111 & 17.734 & $\begin{array}{l}22.798 \\
22.788\end{array}$ & $\begin{array}{l}13.4 \\
15.4\end{array}$ & $\begin{array}{l}14.162 \\
13.872\end{array}$ & $\begin{array}{l}33.032 \\
33.444\end{array}$ & $\begin{array}{l}35.457 \\
35.037\end{array}$ & $\begin{array}{l}30.528 \\
30.528\end{array}$ & $\begin{array}{l}34.144 \\
34.517\end{array}$ & 27.108 & $\begin{array}{l}34.452 \\
34.434\end{array}$ \\
\hline $10 / 14 / 1$ & & & 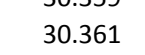 & 29.267 & 30.836 & 29.624 & 30.797 & 30.282 & 30.029 & 28.542 & 26.284 & 34.4 & 36.037 & 35.528 & 17.113 & 17.692 & 22.775 & 5.403 & $\begin{array}{l}13.679 \\
13.679\end{array}$ & 33.376 & 284 & .512 & 4.346 & 27.108 & 34.436 \\
\hline $10 / 15$ & & & & 29.265 & 30.829 & 29.634 & 30.795 & 30.28 & 30.032 & $\begin{array}{l}28.542 \\
28.539\end{array}$ & 26.277 & $\begin{array}{r}34.251 \\
34.45\end{array}$ & 36.044 & $\begin{array}{l}35.369 \\
35.369\end{array}$ & 17.113 & $\begin{array}{l}17.052 \\
17.652\end{array}$ & 22.768 & 405 & 13.526 & 33.311 & 137 & .495 & 1.198 & 27.108 & 34.436 \\
\hline $10 / 15$ & & 30 & 61 & 29.272 & 30.822 & 29.631 & 30.797 & 30.284 & 30.032 & 28.544 & 26.275 & 34.122 & 36.04 & 35.235 & & 17.615 & 22.756 & 405 & 13.472 & 33.248 & 35.006 & .476 & .071 & 27.108 & 34.439 \\
\hline 10/15/11 1:00 & & 30.81 & 30.359 & 29.274 & 30.806 & & 30.8 & 30.284 & & 28.546 & 26.282 & 34.007 & 36.042 & 35.113 & 17.113 & 17.578 & 22.742 & 15.414 & 13.352 & 33.19 & 34.893 & 30.45 & 3.956 & 27.108 & 34.439 \\
\hline 10/15/11 1:30 & & 30.813 & 30.359 & 29.279 & 30.797 & 29.629 & 30.802 & 30.284 & & 28.549 & 26.284 & 33.907 & 36.042 & 35.008 & $3 \quad 17.116$ & 17.543 & 22.731 & 15.41 & 13.241 & 33.15 & 34.793 & 30.446 & 3.858 & 27.108 & 34.441 \\
\hline 10/15/11 2:00 & & 30.82 & 30.366 & 29.286 & 30.79 & 29.631 & 30.802 & 30.287 & & 28.549 & 26.289 & 33.818 & 36.044 & 34.914 & 17.116 & 17.512 & 22.717 & 15.412 & 13.15 & 33.113 & 34.706 & 30.439 & 33.769 & 27.108 & 34.441 \\
\hline 10/15/11 2:30 & & 30.822 & 30.373 & 29.283 & 30.783 & 29.631 & 30.802 & 30.284 & 30.039 & 28.553 & 26.291 & 33.738 & 36.042 & 34.83 & 17.118 & 17.479 & 22.705 & 15.407 & 13.068 & 33.073 & 34.627 & 30.429 & 33.689 & 27.108 & 34.443 \\
\hline 10/15/11 3:00 & & 30.822 & 30.375 & 29.276 & 30.771 & 29.636 & 30.804 & 30.284 & 30.041 & 28.551 & 26.289 & 33.663 & 36.047 & 34.752 & 17.12 & 17.449 & 22.694 & 15.407 & 12.992 & 33.045 & 34.556 & 30.425 & 33.618 & 27.108 & 34.443 \\
\hline $10 / 15 / 113: 30$ & & 30.827 & 30.375 & 29.281 & 30.769 & 29.645 & 30.804 & 30.282 & 30.048 & 28.553 & 26.282 & 33.598 & 36.049 & 34.684 & 17.12 & 17.421 & 22.682 & 15.407 & 12.919 & 33.01 & 34.488 & 30.418 & 33.553 & 27.11 & 34.443 \\
\hline 10/15/11 4:00 & & 30.824 & 30.377 & 29.276 & 30.757 & 29.645 & 30.804 & 30.289 & 30.048 & 28.553 & 26.287 & 33.535 & 36.051 & 34.616 & 17.123 & 17.391 & 22.67 & 15.41 & 12.854 & 32.973 & 34.426 & 30.406 & 33.489 & 27.11 & 34.443 \\
\hline 10/15/11 4:30 & & 30.824 & 30.38 & 29.267 & 30.745 & 29.652 & 30.809 & 30.284 & 30.048 & 28.551 & 26.282 & 33.479 & 36.047 & 34.558 & $3 \quad 17.123$ & 17.363 & 22.657 & 15.403 & 12.795 & 32.954 & 34.369 & 30.399 & 33.433 & 27.11 & 34.443 \\
\hline 10/15/11 5:00 & & 30.824 & 30.38 & 29.255 & 30.736 & 29.662 & 30.807 & 30.284 & 30.05 & 28.544 & 26.268 & 33.425 & 36.047 & 34.499 & 17.12 & 17.337 & 22.643 & 15.407 & 12.731 & 32.921 & 34.317 & 30.387 & 33.379 & 27.11 & 34.443 \\
\hline 10/15/11 5:30 & & 30.822 & 30.377 & 29.255 & 30.726 & 29.664 & 30.805 & 30.289 & 30.05 & 28.546 & 26.256 & 33.373 & 36.049 & 34.45 & 17.123 & 17.309 & 22.633 & 15.412 & 12.68 & 32.891 & 34.267 & 30.371 & 33.33 & 27.113 & 34.441 \\
\hline 10/15/11 6:00 & & 30.82 & 30.377 & 29.248 & 30. & 29.666 & 30.807 & 30.289 & 30.048 & 28.544 & 26.256 & 34.531 & 36.049 & 34.834 & 17.123 & 17.286 & 22.622 & 15.414 & 13.06 & 33.136 & 35.587 & .364 & 34.52 & 27.11 & 34.441 \\
\hline $10 / 15 / 116: 30$ & & & 30.373 & 29.248 & 30.6 & 29.674 & 30.809 & 30.289 & 30.046 & 28.539 & 26.251 & 35.917 & 36.044 & 36.192 & 17.123 & 17.355 & 22.608 & 15.4 & $\begin{array}{l}14.431 \\
\end{array}$ & 33.772 & 36.94 & 0.387 & 35.871 & 27.11 & 34.439 \\
\hline 10/15/11 7:00 & & 30.822 & 30.373 & 29.26 & 30.7 & 29.669 & 30.807 & 30.294 & $\begin{array}{l}30.046 \\
30.046\end{array}$ & 28.544 & 26.248 & 36.509 & $\begin{array}{r}36.04 \\
36.04\end{array}$ & 36.855 & 17.123 & 17.461 & 22.596 & 15.412 & $\begin{array}{l}14.411 \\
15.089\end{array}$ & $\begin{array}{l}33.994 \\
33.94\end{array}$ & $\begin{array}{l}30.54 \\
37.52\end{array}$ & .425 & 36.458 & 27.11 & 34.439 \\
\hline 10/15/11 7:30 & & $\begin{array}{r}30.82 \\
30.82\end{array}$ & 30.37 & 29.274 & 30. & 29.662 & 30.809 & 30.289 & $\begin{array}{l}30.043 \\
30.043\end{array}$ & 28.549 & 26.26 & 37.22 & 36.047 & 37.538 & $\quad 17.12$ & 17.575 & 22.583 & 15.417 & $\begin{array}{l}15.78 \\
15\end{array}$ & 34.274 & 38.227 & .455 & 37.159 & 27.113 & 34.436 \\
\hline $10 / 15 /$ & & $\begin{array}{l}30.024 \\
30.824\end{array}$ & 30.3 & 29.283 & 30.7 & 29.652 & 30.812 & $\begin{array}{l}30.299 \\
30.294\end{array}$ & $\begin{array}{l}30.043 \\
30.043\end{array}$ & $\begin{array}{l}20.349 \\
28.553\end{array}$ & $\begin{array}{l}26.270 \\
26.275\end{array}$ & 37.508 & 36.042 & $\begin{array}{l}38.007 \\
38.007\end{array}$ & 17.12 & $\begin{array}{l}17.692 \\
17.692\end{array}$ & 22.576 & 2 & $\begin{array}{l}16.10 \\
16.243\end{array}$ & $\begin{array}{l}34.432 \\
34.42\end{array}$ & $\begin{array}{l}38.468 \\
38.27\end{array}$ & .497 & 34 & 27.113 & 34.436 \\
\hline 10 & & 30 & 30 & 29 & 30 & 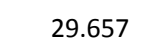 & 30 & & 30 & 28 & 26 & 36 & 36 & 37.655 & 1 & 17 & 22 & 15.405 & 15.881 & 34.223 & 1 & 5 & 77 & 27.113 & 34.436 \\
\hline & & & & 29 & & & 30 & & 30 & 28 & 26. & 35 & 36 & 36.748 & $\begin{array}{ll}3 & 17 .\end{array}$ & 17 & 22 & 15.403 & 14.962 & 38 & 3 & 2 & 99 & 27.115 & 34.439 \\
\hline 10/15/11 9:30 & & 30 & 30 & 29 & & & & & & & & & 36.044 & 36.605 & 17.127 & 17.802 & & & 14.816 & 33.807 & 36.537 & 1.547 & 5.573 & 27.115 & 34.439 \\
\hline 10/15/11 10:00 & & 30 & 30.389 & 29.244 & & & & & & 28.542 & & & 36.051 & 36.59 & 17.125 & & & & 14.821 & & & 1.537 & 5.568 & 27.113 & 34.439 \\
\hline 10:30 & & 30 & 30.3 & & & & 30 & & & 28.5 & 26. & & 36.051 & 36.558 & 17. & 17. & 22 & 15.4 & 14.809 & 33.789 & & & 35.538 & 27.115 & 34.439 \\
\hline 10 & & 30. & 30.3 & & & & & & & 28 & & 35.5 & & 36.532 & 17. & 17. & & 15.431 & 14.788 & 33.779 & & & & 27.115 & 34.439 \\
\hline 10 & & 30.824 & 30 & 29 & & & 30 & & & 28. & & 35 & 36.044 & 36.29 & 17. & 17.818 & 22. & 15.44 & 14.572 & 33.668 & 36.004 & 30.509 & 35.059 & 27.113 & 34.439 \\
\hline 10/15/11 12:00 & & 30.82 & 30 & 29.202 & & & 30. & 30.2 & & 28.518 & 26. & 35.7 & 36.042 & 36.281 & 17.123 & 17. & 22.5 & 15.452 & 14.508 & 33.675 & 36.731 & 30.495 & 35.712 & 27.115 & 34.436 \\
\hline 10/15/11 12:30 & & 30 & 30.3 & 29.18 & 30. & & 30. & 30.8 & 30. & 28.509 & 26.208 & 36.7 & 36.04 & 37.238 & 17.12 & 17.8 & 22.518 & 15.45 & 15.474 & 34.113 & 37.691 & 30.5 & 36.662 & 27.115 & 34.439 \\
\hline 10/15/11 13:00 & & 30.82 & 30.363 & 29.166 & 30.7 & 29.746 & 30.804 & 30.287 & 30.034 & 28.5 & 26.186 & 37.019 & 36.037 & 37.585 & 17.118 & 17.919 & 22.506 & 15.457 & 15.82 & 34.211 & 37.98 & 30.514 & 36.96 & 27.115 & 34.439 \\
\hline $10 / 15 / 1113: 30$ & & 30.817 & 30.356 & 29.164 & 30.7 & 29.751 & 30.802 & 30.287 & 30.032 & 28.493 & 26.174 & 37.559 & 36.025 & 38.079 & 17.118 & 17.996 & 22.497 & 15.466 & 16.314 & 34.416 & 38.523 & 30.533 & 37.49 & 27.115 & 34.441 \\
\hline 10/15/11 14:00 & & 30.815 & 30.342 & 29.157 & $30.7 \mathrm{c}$ & 29.746 & 30.8 & 30.292 & 30.022 & 28.488 & 26.172 & 37.913 & 36.028 & 38.457 & 17.116 & 18.08 & 22.49 & 15.471 & 16.692 & 34.549 & 38.871 & 30.54 & 37.84 & 27.115 & 34.439 \\
\hline $10 / 15 / 1114: 30$ & & 30.813 & 30.335 & 29.145 & 30.79 & 29.749 & 30.797 & 30.287 & 30.018 & 28.481 & 26.165 & 36.86 & 36.021 & 38.084 & 17.113 & 18.155 & 22.49 & 15.478 & 16.33 & 34.365 & 37.71 & 30.552 & 36.76 & 27.115 & 34.436 \\
\hline 10/15/11 15:00 & & 30.815 & 30.331 & 29.131 & 30.80 & 29.753 & 30.798 & 30.287 & 30.016 & 28.474 & 26.155 & 35.98 & 36.018 & 37.195 & 17.111 & 18.155 & 22.486 & 15.48 & 15.432 & 33.978 & 36.845 & 30.542 & 35.902 & 27.115 & 34.436 \\
\hline 10/15/11 15:30 & & 30.815 & 30.326 & 29.124 & 30.8 & 29.758 & 30.79 & 30.284 & 30.011 & 28.467 & 26.143 & 35.51 & 36.016 & 36.698 & $3 \quad 17.111$ & 18.117 & 22.476 & 15.478 & 14.936 & 33.784 & 36.376 & 30.523 & 35.439 & 27.117 & 34.436 \\
\hline 10/15/11 16:00 & & 30.81 & 30.317 & 29.122 & 30.799 & 29.753 & 30.788 & 30.287 & 30.002 & 28.463 & 26.136 & 35.175 & 36.014 & 36.347 & 17.109 & 18.071 & 22.465 & 15.489 & 14.584 & 33.637 & 36.047 & 30.49 & 35.111 & 27.115 & 34.436 \\
\hline 10/15/11 16:30 & & 30.81 & 30.307 & 29.124 & 30.79 & 29.742 & 30.788 & 30.292 & 29.999 & 28.458 & 26.136 & 34.918 & 36.007 & 36.075 & 17.104 & 18.019 & 22.486 & 15.492 & 14.309 & 33.535 & 35.791 & 30.462 & 34.855 & 27.115 & 34.434 \\
\hline
\end{tabular}




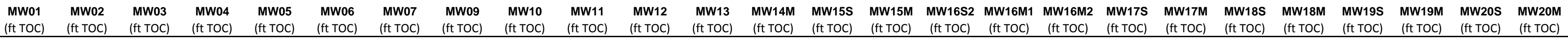

\begin{tabular}{|c|c|c|c|c|c|c|c|c|c|c|c|c|c|c|c|c|c|c|c|c|c|c|c|c|c|c|}
\hline Well & $\begin{array}{l}\text { (ft TOC) } \\
\text { (ft }\end{array}$ & $\begin{array}{l}\text { (ft TOC) } \\
\text { (f) }\end{array}$ & $\begin{array}{l}\text { (ft TOC) } \\
\text { (f) }\end{array}$ & (ft TOC) & $\begin{array}{l}\text { (ft TOC) } \\
\text { (f) }\end{array}$ & $\begin{array}{l}\text { (ft TOC) } \\
\text { (f) }\end{array}$ & (ft toc) & $\begin{array}{l}\text { (ft TOC) } \\
\text { (f) }\end{array}$ & $\begin{array}{l}\text { (ft TOC) } \\
\text { (ft) }\end{array}$ & $\begin{array}{l}\text { (ft TOC) } \\
\text { (f) }\end{array}$ & $\begin{array}{l}\text { (ft TOC) } \\
\text { (f) }\end{array}$ & $\begin{array}{l}\text { (ft Toc) } \\
\text { (f) }\end{array}$ & $\begin{array}{l}\text { (ft TOC) } \\
\text { (t) }\end{array}$ & $\begin{array}{l}\text { (ft TOC) } \\
\end{array}$ & (ft TOC) & (ft TOC) & (ft TOC) & (ft TOC) & $\begin{array}{l}\text { (ft TOC) } \\
\text { (ft) }\end{array}$ & (ft TOC) & $\begin{array}{l}\text { (ft TOC) } \\
\end{array}$ & $\begin{array}{l}\text { (ft TOC) } \\
\text { (1) }\end{array}$ & $\begin{array}{l}\text { (ft TOC) } \\
\text { (n) }\end{array}$ & (ft TOC) & $\begin{array}{l}\text { (ft TOC) } \\
\end{array}$ & (ft TOC) \\
\hline 10/15/11 17:00 & & 30.81 & 30.3 & 29.124 & 30.778 & & 29.737 & 30.788 & 30.294 & 29.992 & 28.46 & 26.141 & 34.705 & 36.009 & 35.852 & 17.102 & 17.97 & 22.488 & 15.494 & 14.09 & 33.437 & 35.587 & 30.443 & 34.648 & 27.117 & 34.432 \\
\hline 10/15/11 17:30 & & 30.813 & 30.298 & 29.12 & 30.766 & & 29.735 & 30.785 & 30.291 & 29.99 & 28.453 & 26.141 & 36.303 & 36.004 & 36.79 & 17.102 & 17.966 & 22.481 & 15.487 & 15.023 & 33.898 & 37.281 & 30.443 & 36.256 & 27.117 & 34.432 \\
\hline $15 / 11$ 18:00 & & 30.813 & 30.3 & 29.12 & 30.762 & & 29.725 & 30.788 & 30.289 & 29.99 & 28.451 & 26.136 & 36.745 & 36.007 & 37.423 & 17.102 & 18.029 & 22.462 & 15.482 & 15.66 & 34.122 & 37.674 & 30.462 & 36.678 & 27.117 & 34.432 \\
\hline $5 / 1118: 30$ & & 30.81 & 30.293 & 29.122 & 30.759 & & 29.718 & 30.785 & 30.292 & 29.985 & 28.449 & 26.141 & 37.311 & 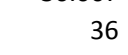 & 37.861 & 17.099 & 18.096 & 22.451 & 15.487 & 16.1 & 34.295 & 38.269 & 30.479 & .241 & 27.117 & 34.429 \\
\hline 10/15/11 19:00 & & 30.81 & 30.293 & 29.134 & 30.762 & & 29.704 & 30.786 & 30.296 & 29.981 & 28.451 & 26.145 & 37.803 & 36 & 38.335 & 17.099 & 18.176 & 22.437 & 15.482 & 16.57 & 34.479 & 38.76 & 30.502 & & 17 & 34.427 \\
\hline 10/15/11 19:30 & & 30.813 & 30.293 & 29.127 & 30.771 & & 29.695 & 30.788 & 30.294 & 29.981 & 28.451 & 26.157 & 37.962 & 35.997 & 38.6 & 17.097 & 18.258 & 22.43 & 15.48 & 16.838 & 34.565 & 38.9 & 30.519 & 7.884 & 7.117 & 34.427 \\
\hline 10/15/11 20:00 & & 30.817 & 30.298 & 29.134 & 30.783 & & 29.685 & 30.788 & 30.296 & 29.981 & 28.449 & 26.153 & 36.401 & 35.995 & 37.632 & 17.097 & 18.297 & 22.425 & 15.466 & 15.872 & 34.148 & 37.257 & 30.537 & 36.314 & 27.117 & 34.427 \\
\hline 10/15/11 20:30 & & 30.82 & 30.3 & 29.134 & 30.794 & & 29.685 & 30.79 & 30.294 & 29.983 & 28.449 & 26.16 & 35.804 & 35.995 & 37.01 & 17.097 & 18.269 & 22.414 & 15.468 & 15.249 & 33.903 & 36.672 & 30.535 & 35.73 & 27.117 & 34.427 \\
\hline 10/15/11 21:00 & & 30.817 & 30.298 & 29.134 & 30.799 & & 29.678 & 30.79 & 30.296 & 29.981 & 28.449 & 26.165 & 35.421 & 35.997 & 36.602 & 17.097 & 18.223 & 22.407 & 15.468 & 14.842 & 33.751 & 36.293 & 30.516 & 35.355 & 27.117 & 34.427 \\
\hline 10/15/11 21:30 & & 30.82 & 30.3 & 29.131 & 30.801 & & 29.676 & 30.788 & 30.296 & 29.981 & 28.446 & 26.162 & 35.137 & 35.997 & 36.304 & 17.097 & 18.171 & 22.395 & 15.466 & 14.541 & 33.633 & 36.013 & 30.5 & 35.073 & 27.117 & 34.427 \\
\hline 10/15/11 22:00 & & 30.822 & 30.303 & 29.134 & 30.799 & & 29.674 & 30.792 & 30.299 & 29.981 & 28.446 & 26.162 & 34.911 & 36.002 & 36.063 & 17.097 & 18.122 & 22.386 & 15.464 & 14.302 & 33.542 & 35.788 & 30.486 & 34.85 & 27.117 & 34.429 \\
\hline 10/15/11 22:30 & & 30.82 & 30.3 & 29.129 & 30.794 & & 29.671 & 30.792 & 30.294 & 29.978 & 28.442 & 26.165 & 34.723 & 36.002 & 35.866 & 517.097 & 18.075 & 22.379 & 15.459 & 14.102 & 33.462 & 35.604 & 30.469 & 34.667 & 27.117 & 34.429 \\
\hline 10/15/11 23:00 & & 30.82 & 30.298 & 29.124 & 30.787 & & 29.669 & 30.792 & 30.296 & 29.978 & 28.437 & 26.157 & 34.564 & 36 & 35.7 & 17.097 & 18.026 & 22.368 & 15.459 & 13.937 & 33.392 & 35.445 & 30.455 & 34.508 & 27.117 & 34.429 \\
\hline 10/15/11 23:30 & & 30.822 & 30.3 & 29.117 & 30.776 & & 29.674 & 30.792 & 30.296 & 29.978 & 28.437 & 26.155 & 34.428 & 36 & 35.554 & 17.097 & 17.984 & 22.356 & 15.457 & 13.794 & 33.334 & 35.31 & 30.441 & 34.374 & 27.12 & 34.432 \\
\hline $10 / 16 / 110: 00$ & & 30.822 & 30.298 & 29.11 & 30.764 & & 29.676 & 30.792 & 30.296 & 29.976 & 28.43 & 26.15 & 34.307 & 35.997 & 35.428 & $3 \quad 17.095$ & 17.94 & 22.347 & 15.454 & 13.664 & 33.283 & 35.191 & 30.432 & 34.254 & 27.12 & 34.432 \\
\hline 10/16/11 0:30 & & 30.82 & 30.293 & 29.113 & 30.75 & & 29.674 & 30.792 & 30.299 & 29.974 & 28.428 & 26.145 & 34.197 & 36 & 35.313 & 17.095 & 17.898 & 22.338 & 15.464 & 13.552 & 33.227 & 35.085 & 30.406 & 34.146 & 27.117 & 34.432 \\
\hline 10/16/11 1:00 & & 30.817 & 30.289 & 29.113 & 30.736 & & 29.669 & 30.792 & 30.296 & 29.971 & 28.428 & 26.145 & 34.101 & 36 & 35.212 & 17.092 & 17.858 & 22.328 & 15.464 & 13.448 & 33.178 & 34.988 & 30.389 & 34.052 & 27.117 & 34.434 \\
\hline 10/16/11 1:30 & & 30.82 & 30.286 & 29.11 & 30.719 & & 29.666 & 30.792 & 30.299 & 29.967 & 28.425 & 26.145 & 34.012 & 36.002 & 35.12 & 17.092 & 17.821 & 22.314 & 15.454 & $\begin{array}{l}13.435 \\
13.357\end{array}$ & 33.138 & 34.897 & 30.375 & 33.966 & 27.12 & 34.432 \\
\hline $10 / 16 / 112: 00$ & & 30.822 & 30.289 & 29.113 & 30.708 & & 29.666 & 30.792 & 30.299 & 29.967 & 28.423 & 26.145 & 33.932 & 35.995 & $\begin{array}{r}35.034 \\
35.12\end{array}$ & 17.092 & $\begin{array}{l}17.783 \\
\end{array}$ & 22.31 & $\begin{array}{l}15.452 \\
152\end{array}$ & 13.272 & $\begin{array}{l}33.1096 \\
33.096\end{array}$ & 34.819 & 30.373 & 33.884 & 27.12 & 34.434 \\
\hline $10 / 16 / 112: 30$ & & $\begin{array}{r}r .022 \\
30.82\end{array}$ & $\begin{array}{l}30.209 \\
30.286\end{array}$ & 29.108 & 30.696 & & 29.664 & 30.792 & 30.301 & 29.967 & $\begin{array}{l}28.423 \\
28.423\end{array}$ & 26.148 & 33.855 & $\begin{array}{l}35.997 \\
35.997\end{array}$ & 34.956 & $\begin{array}{l}17.092 \\
5\end{array}$ & $\begin{array}{l}17.748 \\
\end{array}$ & 22.305 & $\begin{array}{l}13.452 \\
15.452\end{array}$ & $\begin{array}{l}13.27 \\
13.194\end{array}$ & $\begin{array}{l}33.057 \\
33.057\end{array}$ & 34.748 & 30.354 & $\begin{array}{l}53.004 \\
33.811\end{array}$ & 27.12 & 34.434 \\
\hline $10 / 16 / 113: 00$ & & $\begin{array}{r}30.82 \\
30.822 \\
\end{array}$ & $\begin{array}{l}30.280 \\
30.284\end{array}$ & 29.106 & $\begin{array}{l}50.000 \\
30.68\end{array}$ & & 29.664 & $\begin{array}{l}30.192 \\
30.792\end{array}$ & $\begin{array}{l}30.501 \\
30.299\end{array}$ & 29.964 & $\begin{array}{l}28.419 \\
28.419\end{array}$ & $\begin{array}{l}20.148 \\
26.141\end{array}$ & $\begin{array}{l}33.787 \\
33.787\end{array}$ & $\begin{aligned} 36 \\
36\end{aligned}$ & $\begin{array}{l}34.980 \\
34.884\end{array}$ & $\begin{array}{r}17.092 \\
17.09\end{array}$ & $\begin{array}{l}17.713 \\
17.713\end{array}$ & $\begin{array}{l}22.305 \\
22.296\end{array}$ & $\begin{array}{l}13.422 \\
15.45\end{array}$ & $\begin{array}{l}13.124 \\
13.124\end{array}$ & $\begin{array}{l}33.026 \\
33.026\end{array}$ & $\begin{array}{l}34.677 \\
34.677\end{array}$ & $\begin{array}{l}30.544 \\
30.338\end{array}$ & $\begin{array}{l}53.011 \\
33.743\end{array}$ & 27.12 & $\begin{array}{l}34.454 \\
34.436\end{array}$ \\
\hline 10/16/11 3:30 & & 30.822 & 30.284 & 29.101 & 30.673 & & 29.662 & 30.79 & 30.299 & 29.962 & 28.416 & 26.141 & 33.722 & .997 & 34.816 & 17.09 & 17.68 & 22.287 & 15.447 & 13.058 & 32.991 & 34.613 & 328 & 3.679 & 27.122 & 34.436 \\
\hline 10/16/11 4:00 & & & 82 & 29.108 & 30.65 & & 29.662 & 30.795 & 30.299 & & 28.414 & 26.138 & 33.661 & 995 & 34.755 & 17.09 & 17.645 & 22.275 & 15.445 & 12.997 & 32.961 & 34.552 & .314 & 621 & 7.12 & 434 \\
\hline $10 / 16 /$ & & 30.824 & 30.282 & 29.124 & 30.645 & & 29.65 & 30.795 & 30.301 & 29.957 & 28.419 & 26.145 & 33.607 & 36.002 & 34.694 & 17.09 & 17.617 & 22.266 & 15.443 & 12.933 & 32.931 & 34.497 & .307 & .564 & 122 & 34.434 \\
\hline $10 / 16 / 115: 00$ & & 30.822 & 30.277 & 29.183 & 30.633 & & 29.601 & 30.802 & 30.306 & 29.957 & 28.432 & 26.16 & 33.556 & 35.993 & 34.64 & 17.09 & 17.587 & 22.25 & 15.44 & 12.875 & 32.9 & 34.443 & .293 & 3.511 & 27.12 & 34.432 \\
\hline 10/16/11 5:30 & & 30.824 & 30.282 & 29.169 & 30.624 & & 29.619 & 30.802 & 30.299 & 29.957 & 28.444 & 26.22 & 33.507 & 35.993 & 34.591 & 17.09 & 17.557 & 22.245 & 15.438 & 12.825 & 32.882 & 34.398 & 30.291 & 3.464 & 27.122 & 34.432 \\
\hline 10/16/11 6:00 & & 30.836 & 30.289 & 29.152 & 30.626 & & 29.624 & 30.807 & 30.301 & & 28.439 & 26.208 & 34.478 & 35.997 & 34.931 & 17.092 & 17.531 & 22.234 & 15.414 & 13.161 & 33.094 & 35.492 & 30.307 & .465 & 27.124 & 34.434 \\
\hline 10/16/11 6:30 & & 30.834 & 30.298 & 29.173 & 30.633 & & 29.612 & 30.807 & 30.303 & 29.976 & 28.442 & 26.193 & 35.652 & 36.004 & 36.098 & $3 \quad 17.092$ & 17.58 & 22.217 & 15.428 & 14.334 & 33.633 & 36.637 & 30.349 & 5.606 & 27.117 & 34.432 \\
\hline $10 / 16 / 117: 00$ & & 30.841 & 30.312 & 29.194 & 30.652 & & 29.601 & 30.812 & 30.308 & 29.976 & 28.456 & 26.212 & 36.083 & 36.016 & 36.598 & 17.099 & 17.664 & 22.217 & 15.405 & 14.83 & 33.803 & 37.061 & 30.387 & 36.033 & 27.124 & 34.434 \\
\hline $10 / 16 / 117: 30$ & & 30.836 & 30.307 & 29.22 & 30.659 & & 29.582 & 30.816 & 30.318 & 29.976 & 28.465 & 26.236 & 36.736 & 36.011 & 37.205 & 17.097 & 17.751 & 22.21 & 15.41 & 15.439 & 34.066 & 37.705 & 30.418 & 36.671 & 27.124 & 34.432 \\
\hline 10/16/11 8:00 & & 30.841 & 30.317 & 29.237 & 30.68 & & 29.572 & 30.819 & 30.313 & 29.983 & 28.479 & 26.263 & 37.173 & 36.011 & 37.671 & 17.099 & 17.853 & 22.199 & 15.4 & 15.909 & 34.251 & 38.141 & 30.46 & 37.108 & 27.124 & 34.434 \\
\hline $10 / 16 / 118: 30$ & & 30.85 & 30.328 & 29.253 & 30.715 & & 29.57 & 30.821 & 30.318 & 29.99 & 28.486 & 26.279 & 36.198 & 36.026 & 37.385 & 17.102 & 17.945 & 22.194 & 15.391 & 15.628 & 34.108 & 37.058 & 30.512 & 36.106 & 27.124 & 34.434 \\
\hline 10/16/11 9:00 & & 30.857 & 30.342 & 29.265 & 30.75 & & 29.568 & 30.824 & 30.315 & 30.002 & 28.495 & 26.294 & 35.395 & 36.03 & 36.576 & 17.104 & 17.949 & 22.187 & 15.389 & 14.816 & 33.763 & 36.265 & 30.53 & 35.322 & 27.127 & 34.439 \\
\hline 10/16/11 9:30 & & 30.859 & 30.354 & 29.276 & 30.78 & & 29.57 & 30.828 & 30.322 & 30.011 & 28.502 & 26.301 & 34.983 & 36.037 & 36.143 & 17.109 & 17.924 & 22.183 & 15.386 & 14.381 & 33.605 & 35.857 & 30.542 & 34.918 & 27.127 & 34.439 \\
\hline 10/16/11 10:00 & & 30.862 & 30.366 & 29.286 & 30.8 & & 29.57 & 30.831 & 30.32 & 30.02 & 28.512 & 26.315 & 34.705 & 36.044 & 35.847 & 17.111 & 17.888 & 22.183 & 15.389 & 14.088 & 33.502 & 35.585 & 30.537 & 34.646 & 27.127 & 34.439 \\
\hline $10 / 16 / 11$ & & 30.862 & 30.3 & 29.279 & 30.8 & & 29.582 & 30.831 & 30.318 & 30.029 & 28.512 & 26.32 & 34.496 & 36.049 & 35.629 & 17.116 & $\begin{array}{l}17.853 \\
\end{array}$ & 22.192 & 15.3 & $\begin{array}{l}13.064 \\
13.864\end{array}$ & 33.423 & 35.378 & 30.54 & 34.437 & 27.129 & 34.441 \\
\hline $\begin{array}{l}10 / 16 / 11 \\
\end{array}$ & & 30.864 & 30.382 & 29.29 & $\begin{array}{l}30.8 \\
30.8\end{array}$ & & 29.589 & 30.831 & $\begin{array}{r}30.510 \\
30.32\end{array}$ & 30.034 & 28.519 & 26.313 & 34.33 & 36.051 & 35.451 & 17.118 & 17.821 & 22.194 & $\begin{array}{l}15.300 \\
15.391\end{array}$ & $\begin{array}{l}13.004 \\
13.688\end{array}$ & 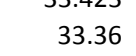 & $\begin{array}{l}35.211 \\
35\end{array}$ & 30.533 & 34.275 & 27.127 & 34.443 \\
\hline $10 / 16 / 1$ & & $\begin{array}{l}30.004 \\
30.859\end{array}$ & $\begin{array}{l}30.302 \\
30.389\end{array}$ & 29.281 & $\begin{array}{l}30.8 \\
30.8\end{array}$ & & 29.591 & 30.836 & $\begin{array}{l}30.32 \\
30.32\end{array}$ & $\begin{array}{l}30.043 \\
30.043\end{array}$ & 28.523 & 26.323 & 34.1999 & 36.054 & 35.308 & $\begin{array}{r}17.12 \\
3\end{array}$ & $\begin{array}{l}17.788 \\
17.788\end{array}$ & 22.197 & 15.398 & $\begin{array}{l}13.000 \\
13.547\end{array}$ & $\begin{array}{l}33.3004 \\
3.304\end{array}$ & 35.078 & 0.523 & 34.144 & 27.129 & 34.448 \\
\hline 10 & & 30. & $\begin{array}{l}30.3 \mathrm{c} \\
30.3 \varepsilon\end{array}$ & 29.269 & 30. & & 29.617 & $\begin{array}{l}30.050 \\
30.836\end{array}$ & $\begin{array}{l}30.311 \\
30.311\end{array}$ & $\begin{array}{l}30.043 \\
30.043\end{array}$ & $\begin{array}{l}28.523 \\
28.516\end{array}$ & 26.311 & 35.091 & $\begin{array}{l}30.054 \\
36.058\end{array}$ & $\begin{array}{l}35.500 \\
35.578\end{array}$ & $\begin{array}{l}17.123 \\
3\end{array}$ & $\begin{array}{l}11.100 \\
17.753\end{array}$ & 22.201 & 8 & $\begin{array}{l}13.54 \\
13.808\end{array}$ & $\begin{array}{l}33.044 \\
33.455\end{array}$ & $\begin{array}{l}3.0089 \\
36.089\end{array}$ & 23 & $\begin{array}{l}35.144 \\
35.066\end{array}$ & 27.129 & $\begin{array}{l}34.448 \\
34.448\end{array}$ \\
\hline 10 & & 30 & 30 & 29 & & & & 30 & & 30 & 28 & 26 & 3 & 36 & 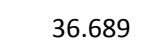 & 17 & 17 & 22 & 15.405 & 14.924 & 33.968 & 91 & 19 & 6 & 27.129 & 34.448 \\
\hline & & & 30 & 29. & & & & 36 & & 30 & 28 & & 36 & 36 & 37.1 & 17 & 17 & 22.215 & 4 & 15.362 & 34.09 & $a_{1}$ & 6 & 35 & 27.131 & 34.448 \\
\hline 111 13:30 & & & 30 & 29.251 & & & & 30.8 & 30.318 & & 28.512 & & 37.206 & 36 & 3 & 17.125 & & 22.229 & 1 & 15.938 & 34.332 & 38.174 & & 38 & 27.129 & 34.448 \\
\hline 11 14:00 & & 30.852 & 30.396 & 29.258 & & & & & ר? & 30.0 & 28.5 & & 37.613 & 36 & 38.133 & 17.127 & 18.066 & & $2+$ & 16.368 & 34.491 & 38.573 & 30.608 & 39 & 27.131 & 34.448 \\
\hline 10/ & & 30 & 30.3 & 29.26 & & & & 30.8 & & & 28. & & 36.6 & & 37.805 & 5 17.1 & 18.15 & 22.2 & 3 & 16.046 & 34.318 & & 30.62 & 02 & 27.129 & 34.448 \\
\hline 10/ & & 30. & 30. & & & & & 30 & & & 28. & & 35.7 & & & 17. & 18.155 & 22.2 & 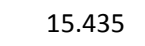 & 15.202 & 33.952 & & & & 27.131 & 34.45 \\
\hline 10/ & & 30. & 30.3 & 25 & & & & $30 . \varepsilon$ & 30. & & 28. & & & & & 17. & 18. & & 15.431 & 14.739 & 33.779 & 36.201 & & & 27.131 & 34.45 \\
\hline 10/16/11 16:00 & & 30. & 30.4 & 29.267 & & & & 30.8 & 30.327 & 30.0 & 28.5 & 26. & 35. & 36.054 & 36.183 & 17.1 & 18.08 & 22.254 & 15.438 & 14.419 & 33.656 & 35.9 & 30.599 & 34.958 & 27.131 & 34.453 \\
\hline 10/16/11 16:30 & & 30.862 & 30.405 & 29.26 & 30. & & 29. & 30. & 30. & 30 & 28.5 & 26.287 & 34.79 & 36.063 & 35.936 & 17.13 & 18.036 & 22.284 & 15.44 & 14.172 & 33.558 & 35.672 & 30.584 & 34.735 & 27.134 & 34.453 \\
\hline 10/16/11 17:00 & & 30.864 & 30.405 & 29.258 & 30.8 & & 29.671 & 30.845 & 30.32 & 30.06 & 28.516 & 26.282 & 34.606 & 36.056 & 35.742 & 17.127 & 17.996 & 22.305 & 15.443 & 13.977 & 33.481 & 35.487 & 30.573 & 34.55 & 27.131 & 34.45 \\
\hline $10 / 16 / 1117: 30$ & & 30.864 & 30.41 & 29.262 & 30. & & 29.671 & 30.848 & 30.325 & 30.062 & 28.519 & 26.277 & 36.223 & 36.058 & 36.696 & 17.13 & 17.991 & 22.31 & 15.445 & 14.929 & 33.947 & 37.203 & 30.575 & 36.174 & 27.131 & 34.453 \\
\hline $10 / 16 / 1118: 00$ & & 30.864 & 30.41 & 29.274 & 30.885 & & 29.666 & 30.845 & 30.33 & 30.062 & 28.523 & 26.277 & 36.682 & 36.058 & 37.348 & 17.13 & 18.064 & 22.307 & 15.447 & 15.583 & 34.19 & 37.615 & 30.594 & 36.615 & 27.134 & 34.45 \\
\hline $10 / 16 / 1118: 30$ & & 30.862 & 30.41 & 29.279 & 30.888 & & 29.657 & 30.845 & 30.33 & 30.064 & 28.528 & 26.294 & 37.286 & 36.058 & 37.817 & 17.132 & 18.138 & 22.31 & 15.447 & 16.05 & 34.374 & 38.248 & 30.61 & 37.218 & 27.131 & 34.45 \\
\hline 10/16/11 19:00 & & 30.864 & 30.412 & 29.279 & 30.899 & & 29.657 & 30.85 & 30.325 & 30.064 & 28.53 & 26.296 & 37.77 & 36.061 & 38.295 & $\quad 17.13$ & 18.223 & 22.305 & 15.445 & 16.53 & 34.565 & 38.731 & 30.638 & 37.697 & 27.134 & 34.45 \\
\hline 10/16/11 19:30 & & 30.871 & 30.419 & 29.281 & 30.918 & & 29.659 & 30 & 30.332 & 30.071 & 28.532 & 26.296 & 37.941 & 36.063 & 38.574 & 17.132 & 18.309 & 22.298 & 15.44 & 16.81 & 34.668 & 38.881 & 30.681 & 37.863 & 27.134 & 34.45 \\
\hline $10 / 16 / 1120: 00$ & & 30.873 & 30.426 & 29.274 & 30.934 & & 29.666 & 30.85 & 30.33 & 30.074 & 28.53 & 26.299 & 36.389 & 36.061 & 37.615 & 17.132 & 18.354 & 22.291 & 15.443 & 15.853 & 34.244 & 37.248 & 30.688 & 36.303 & 27.134 & 34.453 \\
\hline 10/16/11 20:30 & & 30.871 & 30.426 & 29.269 & 30.946 & & 29.669 & 30.852 & 30.327 & 30.076 & 28.528 & 26.294 & 35.804 & 36.058 & 37.001 & 17.134 & 18.325 & 22.282 & 15.445 & 15.24 & 33.994 & 36.672 & 30.678 & 35.73 & 27.134 & 34.453 \\
\hline
\end{tabular}




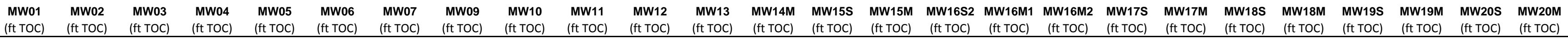

\begin{tabular}{|c|c|c|c|c|c|c|c|c|c|c|c|c|c|c|c|c|c|c|c|c|c|c|c|c|c|}
\hline Well & $\begin{array}{l}\text { (ft TOC) } \\
\text { (ft }\end{array}$ & $\begin{array}{l}\text { (ft TOC) } \\
\text { (f) }\end{array}$ & $\begin{array}{l}\text { (ft TOC) } \\
\text { (f) }\end{array}$ & (ft TOC) & $\begin{array}{ll}(\mathrm{ft} \text { TOC) } & \text { (ft TOC) }\end{array}$ & $\begin{array}{l}\text { (ft TOC) } \\
\text { (ft }\end{array}$ & $\begin{array}{l}\text { (ft TOC) } \\
\text { (f) }\end{array}$ & $\begin{array}{l}\text { (ft TOC) } \\
\text { (ft) }\end{array}$ & $\begin{array}{l}\text { (ft TOC) } \\
\text { (f) }\end{array}$ & $\begin{array}{l}\text { (ft TOC) } \\
\text { (f) }\end{array}$ & $\begin{array}{l}\text { (ft Toc) } \\
\text { (f) }\end{array}$ & $\begin{array}{l}\text { (ft TOC) } \\
\text { (n) }\end{array}$ & $\begin{array}{l}\text { (ft TOC) } \\
\end{array}$ & (ft TOC) & (ft TOC) & $\begin{array}{l}\text { (ft TOC) } \\
\text { (f) }\end{array}$ & (ft TOC) & $\begin{array}{l}\text { (ft TOC) } \\
\text { (ft) }\end{array}$ & (ft TOC) & $\begin{array}{l}\text { (ft TOC) } \\
\end{array}$ & $\begin{array}{l}\text { (ft TOC) } \\
\text { (1) }\end{array}$ & $\begin{array}{l}\text { (ft TOC) } \\
\text { (n) }\end{array}$ & (ft TOC) & $\begin{array}{l}\text { (ft TOC) } \\
\end{array}$ & (ft TOC) \\
\hline 10/16/11 21:00 & & 30.871 & 30.426 & 29.267 & 30.953 & 29.678 & 30.852 & 30.325 & 30.078 & 28.53 & 26.287 & 35.432 & 36.063 & 36.612 & $2 \quad 17.134$ & 18.286 & 22.273 & 15.45 & 14.845 & 33.849 & 36.303 & 30.664 & 35.364 & 27.134 & 34.45 \\
\hline 10/16/11 21:30 & & 30.871 & 30.429 & 29.262 & 30.951 & 29.678 & 30.855 & 30.327 & 30.078 & 28.528 & 26.284 & 35.35 & 36.058 & 36.525 & $5 \quad 17.137$ & 18.251 & 22.261 & 15.452 & 14.758 & 33.758 & 36.227 & 30.643 & 35.284 & 27.134 & 34.45 \\
\hline $10 / 16 / 1122: 00$ & & 30.869 & 30.429 & 29.262 & 30.948 & 29.683 & 30.855 & 30.329 & 30.078 & 28.523 & 26.277 & 35.182 & 36.063 & 36.347 & 17.134 & 18.253 & 22.245 & 15.454 & 14.584 & 33.719 & 36.061 & & 35.118 & 27.136 & 34.453 \\
\hline $10 / 16 / 1$ & & 30.871 & 30.431 & 29.265 & 30.941 & 29.681 & 30.855 & 30.332 & 30.078 & 28.528 & 26.275 & 34.922 & 36.063 & 36.068 & 17.137 & 18.211 & 22.245 & 15.452 & 14.306 & 33.626 & & & & 27.136 & 34.453 \\
\hline $10 / 16 / 1123: 00$ & & 30.866 & 30.429 & 29.267 & 30.934 & 29.683 & 30.855 & 30.33 & 30.078 & 28.528 & 26.277 & 34.733 & 36.065 & 35.871 & 17.137 & 18.162 & 22.24 & 15.459 & 14.107 & 33.539 & 5.611 & 30.596 & & 36 & 34.453 \\
\hline 10/16/11 23:30 & & 30.873 & 30.431 & 29.265 & 30.925 & 29.681 & 30.857 & 30.329 & 30.078 & 28.528 & 26.279 & 34.576 & 36.058 & 35.707 & 17.137 & 18.115 & 22.234 & 15.452 & 13.944 & 33.483 & 35.461 & 30.587 & 4.524 & 7.136 & 34.453 \\
\hline 10/17/11 0:00 & & 30.873 & 30.431 & 29.255 & 30.913 & 29.685 & 30.857 & 30.329 & 30.081 & 28.525 & 26.277 & 34.471 & 36.061 & 35.592 & $2 \quad 17.137$ & 18.075 & 22.224 & 15.457 & 13.831 & 33.432 & 35.355 & 30.575 & 34.419 & 27.136 & 34.455 \\
\hline 10/17/11 0:30 & & 30.875 & 30.433 & 29.255 & 30.904 & 29.692 & 30.857 & 30.33 & 30.081 & 28.525 & 26.268 & 34.349 & 36.061 & 35.465 & $\begin{array}{ll}5 & 17.137\end{array}$ & 18.038 & 22.215 & 15.452 & 13.704 & 33.378 & 35.236 & 30.563 & 34.297 & 27.136 & 34.455 \\
\hline 10/17/11 1:00 & & 30.876 & 30.433 & 29.258 & 30.892 & 29.69 & 30.862 & 30.332 & 30.083 & 28.525 & 26.265 & 34.246 & 36.063 & 35.355 & $\begin{array}{l}5 \\
5\end{array}$ & 17.996 & 22.208 & 15.452 & 13.594 & 33.334 & 35.132 & 30.549 & 34.196 & 27.136 & 34.457 \\
\hline 10/17/11 1:30 & & 30.869 & 30.424 & 29.26 & 30.874 & 29.688 & 30.859 & 30.337 & 30.078 & 28.528 & 26.268 & 34.148 & 36.058 & 35.259 & $\begin{array}{l}9 \\
9\end{array}$ & 17.956 & 22.199 & 15.464 & 13.495 & 33.28 & 35.037 & 30.523 & 34.102 & 27.134 & 34.457 \\
\hline 10/17/11 2:00 & & 30.873 & 30.426 & 29.255 & 30.864 & 29.692 & 30.859 & 30.334 & 30.081 & 28.525 & 26.27 & 34.068 & 36.063 & 35.17 & $7 \quad 17.137$ & 17.919 & 22.185 & 15.457 & 13.406 & 33.243 & 34.954 & 30.516 & 34.02 & 27.136 & 34.457 \\
\hline 10/17/11 2:30 & & 30.878 & 30.429 & 29.253 & 30.853 & 29.699 & 30.859 & 30.334 & 30.083 & 28.523 & 26.263 & 33.991 & 36.061 & 35.09 & $\begin{array}{l}9 \\
9\end{array}$ & 17.884 & 22.178 & 15.452 & 13.328 & 33.213 & 34.881 & 30.512 & 33.945 & 27.138 & 34.46 \\
\hline 10/17/11 3:00 & & 30.876 & 30.429 & 29.239 & 30.843 & 29.706 & 30.859 & 30.334 & 30.083 & 28.516 & 26.26 & 33.921 & 36.061 & 35.015 & $5 \quad 17.139$ & 17.849 & 22.169 & 15.459 & 13.265 & 33.171 & 34.812 & 30.493 & 33.877 & 27.136 & 34.46 \\
\hline 10/17/11 3:30 & & 30.876 & 30.426 & 29.234 & 30.829 & 29.711 & 30.857 & 30.332 & 30.081 & 28.514 & 26.248 & 33.86 & 36.063 & 34.949 & 17.137 & 17.816 & 22.16 & 15.454 & 13.192 & 33.141 & 34.748 & 30.483 & 33.811 & 27.138 & 34.46 \\
\hline $10 / 17 / 114: 00$ & & 30.873 & 30.424 & 29.218 & 30.815 & 29.716 & 30.859 & 30.334 & 30.081 & 28.509 & 26.241 & 33.797 & 36.061 & 34.884 & 17.137 & 17.783 & 22.15 & 15.461 & 13.133 & 33.11 & 34.687 & 30.462 & 33.747 & 27.138 & 34.46 \\
\hline 10/17/11 4:30 & & 30.871 & 30.417 & 29.202 & 30.799 & 29.728 & 30.855 & 30.33 & 30.078 & 28.5 & 26.227 & 33.736 & 36.058 & 34.825 & 17.137 & 17.751 & 22.139 & 15.464 & $\begin{array}{l}13.079 \\
\end{array}$ & 33.071 & 34.63 & 30.448 & 33.693 & 27.136 & 34.457 \\
\hline $10 / 17 / 11$ 5:00 & & 30.869 & 30.41 & 29.206 & 30.783 & 29.725 & 30.857 & 30.327 & 30.074 & 28.495 & 26.208 & 33.682 & 36.054 & 34.771 & 17.134 & 17.72 & 22.129 & 15.468 & 13.025 & 33.038 & 34.57 & 30.432 & 33.64 & 27.136 & 34.457 \\
\hline $10 / 17 / 115: 30$ & & 30.869 & 30.401 & 29.199 & 30.764 & 29.725 & 30.855 & 30.334 & 30.069 & 28.495 & 26.215 & 33.631 & 36.058 & 34.713 & 17.134 & 17.687 & 22.118 & 15.466 & $\begin{array}{l}12.978 \\
\end{array}$ & 33.008 & 34.523 & 30.413 & 33.588 & 27.138 & 34.457 \\
\hline $10 / 17 / 116: 00$ & & 30.864 & 30.394 & 29.197 & 30.745 & 29.725 & 30.852 & $\begin{array}{l}30.344 \\
30.332\end{array}$ & 30.064 & 28.493 & 26.21 & 34.595 & 36.056 & 35.055 & 17.132 & $\begin{array}{l}17.657 \\
17.657\end{array}$ & 22.106 & 15.466 & $\begin{array}{l}13.316 \\
13.316\end{array}$ & $\begin{array}{l}33.187 \\
3.067\end{array}$ & 35.604 & 30.399 & 34.578 & 27.138 & 34.455 \\
\hline $10 / 17 / 116: 30$ & & $\begin{array}{l}30.004 \\
30.869\end{array}$ & $\begin{array}{l}30.594 \\
30.389\end{array}$ & 29.19 & 30.733 & 29.725 & 30.852 & $\begin{array}{l}30.334 \\
30.334\end{array}$ & $\begin{array}{l}30.04 \\
30.062\end{array}$ & $\begin{array}{l}28.491 \\
28.491\end{array}$ & $\begin{array}{l}26.211 \\
26.205\end{array}$ & 35.76 & 36.047 & 36.211 & 17.132 & $\begin{array}{l}17.704 \\
17.04\end{array}$ & 22.099 & $\begin{array}{l}13.400 \\
15.464\end{array}$ & $\begin{array}{l}13.010 \\
14.48\end{array}$ & $\begin{array}{l}33.1717 \\
33.717\end{array}$ & $\begin{array}{l}3.0 .74 \\
36.748\end{array}$ & 30.415 & $\begin{array}{l}35.714 \\
35.714\end{array}$ & 27.136 & $\begin{array}{l}34.455 \\
34.455\end{array}$ \\
\hline $10 / 17 / 117: 00$ & & 30.869 & 30.387 & 29.194 & 30.731 & 29.725 & 30.85 & 30.334 & 30.06 & 28.488 & 26.201 & 36.197 & 36.044 & 36.703 & 17.132 & 17.783 & 22.088 & 15.461 & 14.969 & 33.866 & 37.177 & 30.441 & 36.15 & 27.138 & 34.453 \\
\hline 10/17/11 7:30 & & 30.869 & 30.384 & 29.202 & 30.73 & 29.714 & 30.855 & 30.339 & 30.057 & 28.493 & 26.205 & 36.846 & 36.04 & 37.32 & 17.13 & 17.87 & 22.083 & 15.461 & 15.592 & 34.129 & 37.819 & 30.474 & 36.784 & 27.136 & 34.453 \\
\hline $10 / 17 /$ & & 30.866 & 30.382 & 29.211 & 30. & 29.706 & 30.855 & 30.337 & 30.053 & 28.495 & 26.212 & 37.279 & 36.035 & 37.782 & 17.1 & 17.968 & .076 & 5.468 & 16.06 & 34.302 & .243 & .497 & 11 & 27.138 & 34.45 \\
\hline $10 / 17 / 1$ & & 30.871 & 30.382 & 29.22 & 30.762 & 29.704 & 30.855 & 30.334 & 30.055 & 28.498 & 26.224 & 36.291 & .044 & 37.479 & 17.1 & 18.061 & .062 & .454 & 15.757 & 34.146 & 148 & .528 & 97 & 27.138 & 34.45 \\
\hline 10/17/11 9:00 & & 30.873 & 30.384 & 29.199 & 30.778 & 29.702 & 30.857 & 30.337 & 30.055 & 28.493 & 26.236 & 35.481 & 36.04 & 36.666 & 17.13 & 18.061 & 22.06 & 15.457 & 14.931 & 33.791 & 36.35 & 30.53 & 5.409 & 27.141 & 34.45 \\
\hline 10/17/11 9:30 & & 30.876 & 30.389 & 29.202 & 30.794 & 29.711 & 30.855 & 30.337 & 30.057 & 28.493 & 26.212 & 35.065 & 36.042 & 36.229 & 17.13 & 18.031 & 22.058 & 15.454 & 14.49 & 33.621 & 35.942 & 30.526 & 5.001 & 27.138 & 34.45 \\
\hline 10/17/11 10:00 & & 30.88 & 30.396 & 29.199 & & 29.714 & 30.855 & 30.339 & & 28.488 & 26.217 & 34.782 & 36.044 & 35.927 & 17.132 & 17.996 & 22.055 & 15.445 & 14.179 & 33.516 & 35.66 & 30.53 & 1.724 & 27.141 & 34.45 \\
\hline 10/17/11 10:30 & & 30.876 & 30.389 & 29.173 & 30.804 & 29.737 & 30.855 & 30.33 & 30.055 & 28.479 & 26.215 & 34.567 & 36.047 & 35.7 & 17.13 & 17.954 & 22.046 & 15.459 & 13.97 & 33.42 & 35.447 & 30.507 & 34.508 & 27.141 & 34.448 \\
\hline 10/17/11 11:00 & & 30.873 & 30.384 & 29.166 & 30.801 & 29.732 & 30.852 & 30.33 & 30.055 & 28.472 & 26.191 & 34.391 & 36.042 & 35.517 & 17.13 & 17.917 & 22.037 & 15.461 & 13.796 & 33.343 & 35.274 & 30.481 & 34.339 & 27.141 & 34.448 \\
\hline $10 / 17 / 1111: 30$ & & 30.873 & 30.38 & 29.159 & 30.792 & 29.735 & 30.852 & 30.334 & 30.05 & 28.47 & 26.184 & 34.251 & 36.042 & 35.367 & 17.132 & 17.879 & 22.03 & 15.468 & 13.66 & 33.278 & 35.134 & 30.472 & 34.2 & 27.138 & 34.448 \\
\hline $10 / 17 / 1112: 00$ & & 30.866 & 30.368 & 29.152 & 30.776 & 29.739 & 30.85 & 30.334 & 30.041 & 28.465 & 26.177 & 35.145 & 36.037 & 35.636 & $5 \quad 17.127$ & 17.842 & 22.016 & 15.48 & 13.867 & 33.413 & 36.141 & 30.441 & 35.118 & 27.141 & 34.448 \\
\hline $10 / 17 / 1112: 30$ & & 30.866 & 30.361 & 29.159 & 30.766 & 29.735 & 30.85 & 30.337 & 30.036 & 28.465 & 26.172 & 36.263 & 36.035 & 36.738 & $3 \quad 17.125$ & 17.893 & 22.016 & 15.478 & 14.978 & 33.905 & 37.239 & 30.453 & 36.207 & 27.143 & 34.446 \\
\hline 10/17/11 13:00 & & 30.866 & 30.359 & 29.162 & 30.759 & 29.735 & 30.848 & 30.337 & 30.039 & 28.46 & 26.179 & 36.649 & 36.032 & 37.181 & 17.125 & 17.966 & 22.007 & 15.478 & 15.418 & 34.038 & 37.625 & 30.469 & 36.596 & 27.141 & 34.446 \\
\hline $10 / 17 / 1113: 30$ & & 30.869 & 30.354 & 29.173 & 30.764 & 29.725 & 30.85 & 30.344 & 30.041 & 28.463 & 26.181 & 37.257 & 36.032 & 37.758 & $\quad 17.123$ & 18.057 & 22.005 & 15.475 & 15.996 & 34.285 & 38.222 & 30.495 & 37.19 & 27.141 & 34.446 \\
\hline 10/17/11 14:00 & & 30.871 & 30.352 & 29.19 & 30. & 29.714 & 30.85 & 30.344 & 30.039 & 28.47 & 26.193 & 37.658 & 36.035 & 38.18 & $\begin{array}{l}37.123 \\
3\end{array}$ & 18.148 & 21.995 & 15.475 & 16.42 & 34.442 & 38.622 & 30.521 & 37.589 & 27.141 & 34.446 \\
\hline 10 & & 30.871 & 30.359 & 29.216 & & 29.688 & 30.855 & $\begin{array}{l}30.349 \\
3\end{array}$ & 30.041 & 28.479 & 26.217 & 36.644 & 36.03 & 37.85 & 17.123 & 18.23 & 21.995 & 15.471 & $\begin{array}{l}16.094 \\
16.093\end{array}$ & 34.281 & 37.504 & 54 & 36.542 & 27.143 & 34.448 \\
\hline $10 /$ & & 30.873 & 30.361 & 29.237 & $\begin{array}{l}30.8 \\
30.8\end{array}$ & 29.674 & $\begin{array}{l}30.857 \\
30.857\end{array}$ & 30.351 & $\begin{array}{l}30.041 \\
30.043\end{array}$ & 28.491 & 26.241 & 35.807 & 36.03 & 37.006 & 17.123 & 18.23 & 21.988 & $\begin{array}{l}15.464 \\
1564\end{array}$ & $\begin{array}{l}15.247 \\
\end{array}$ & $\begin{array}{l}33.922 \\
33.922\end{array}$ & 36.679 & .563 & 35.726 & 27.143 & 34.448 \\
\hline $10 /$ & & 30.883 & 30.373 & 29.251 & $\begin{array}{l}30.8 \\
30.8\end{array}$ & 29.659 & 30.864 & 30.353 & $\begin{array}{l}30.048 \\
30.048\end{array}$ & $\begin{array}{r}0.471 \\
28.5\end{array}$ & $\begin{array}{l}20.2641 \\
26.263\end{array}$ & 35.364 & 36.04 & 36.546 & 17.125 & $\begin{array}{l}18.197 \\
187\end{array}$ & 21.981 & $\begin{array}{l}15.4404 \\
15.452\end{array}$ & $\begin{array}{l}14.786 \\
14.786\end{array}$ & 33.761 & 36.224 & .573 & 35.296 & 27.145 & $\begin{array}{r}r 4.440 \\
34.45\end{array}$ \\
\hline 10 & & 30. & 30.38 & 29.262 & 30. & 29.652 & $\begin{array}{l}30.004 \\
30.864\end{array}$ & 30.351 & $\begin{array}{l}30.005 \\
30.057\end{array}$ & $\begin{array}{r}28.5 \\
28.505\end{array}$ & 26.279 & $\begin{array}{l}35.04 \\
35.065\end{array}$ & $\begin{array}{l}36.044 \\
36.044\end{array}$ & $\begin{array}{l}30.540 \\
36.222\end{array}$ & 17.127 & $\begin{array}{l}18.157 \\
1.157\end{array}$ & 21.979 & $\begin{array}{l}13.42 \\
15.45\end{array}$ & $\begin{array}{l}14.160 \\
14.464\end{array}$ & 33.654 & $\begin{array}{l}35.907 \\
35.94\end{array}$ & 3 & 35.003 & 27.145 & $\begin{array}{l}34.453 \\
3.453\end{array}$ \\
\hline 10 & & 30 & 30 & 29.276 & & & 30 & & 30 & 28 & 26 & - & 36 & 35. & 17 & 18 & 22 & 15.445 & 14.219 & 33 & 6 & 2 & 8 & 27.145 & 34.455 \\
\hline & & & 30.4 & 2 & & & & & & 28. & 26 & 34 & 36 & 35.786 & 17 & 18 & 22 & 15.443 & 14.029 & 33.504 & 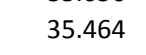 & 7 & 77 & 27.145 & 34.455 \\
\hline 11117:30 & & 30.901 & 30.41 & 29.311 & 30. & 2 & 30. & & & 28.532 & & & 36.058 & 36 & & 18.068 & 22.021 & 33 & 14.986 & 33.98 & 163 & 37 & 28 & 27.145 & 34.457 \\
\hline $10 /$ & & 30.906 & 30.424 & 29.335 & & & & & & 28.542 & & & 36.061 & 37.432 & 17.139 & & & 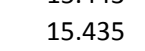 & 15.667 & 34.239 & & & & 27.145 & 34.457 \\
\hline & & 30 & 30.43 & & & & & & & 28. & & & 36 & 37.901 & 17. & & & 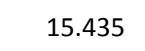 & 16.142 & 34.446 & & & 77 & 27.148 & 34.46 \\
\hline 10/ & & 30. & & & & & & & & 28 & & & 36 & & 17. & & & 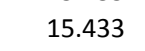 & 16.615 & 34.642 & & & & 27.148 & 34.462 \\
\hline $10 / 3$ & & & 30. & 29. & & & 30. & & 30. & 28. & & & & & 17. & 18. & 22. & 15.431 & 15.587 & 34.19 & & & 36.073 & 27.148 & 34.462 \\
\hline 10/17/11 20: & & 30 & 30.4 & 29.314 & 30.5 & & 30.8 & 30. & 30 & 28. & 26. & 35. & 36. & 36.811 & 17.153 & 18.318 & 22.0 & 15.438 & 15.021 & 33.973 & 36.497 & 30.728 & 35.554 & 27.148 & 34.464 \\
\hline 10/17/11 20:30 & & 30.9 & 30.4 & 29.325 & 30 & 29. & 30.8 & 30. & 30. & 28.5 & 26. & 35.2 & 36.079 & 36.448 & 17.153 & 18.276 & 21.998 & 15. & 14.661 & 33.824 & 36.158 & 30.711 & 35.219 & 27.148 & 34.464 \\
\hline 10/17/11 21:00 & & 30.904 & 30.466 & 29.346 & 30.95 & 29.643 & $30.8 \varepsilon$ & 30.365 & 30.12 & 28.567 & 26.349 & 35.032 & 36.079 & 36.18 & 17.155 & 18.23 & 21.991 & 15.4 & 14.382 & 33.714 & 35.907 & 30.692 & 34.968 & 27.148 & 34.46 \\
\hline $10 / 17 / 1121: 30$ & & 30.901 & 30.461 & 29.36 & 30.98 & 29.636 & 30.888 & 30.372 & 30.118 & 28.579 & 26.363 & 34.826 & 36.079 & 35.967 & 17.155 & 18.185 & 21.986 & 15.457 & 14.163 & 33.628 & 35.708 & 30.667 & 34.768 & 27.15 & 34.46 \\
\hline 10/17/11 22:00 & & 30.913 & 30.473 & 29.36 & 30.99 & 29.641 & 30.891 & 30.363 & 30.125 & 28.579 & 26.378 & 34.667 & 36.082 & 35.798 & 17.158 & 18.141 & 21.979 & 15.445 & 13.966 & 33.57 & 35.547 & 30.671 & 34.611 & 27.148 & 34.462 \\
\hline $10 / 17 / 1122: 30$ & & 30.92 & 30.485 & 29.356 & 30.993 & 29.643 & 30.893 & 30.365 & 30.134 & 28.584 & 26.38 & 34.529 & 36.082 & 35.65 & 17.16 & 18.103 & 21.972 & 15.438 & 13.794 & 33.525 & 35.414 & 30.674 & 34.475 & 27.15 & 34.464 \\
\hline 10/17/11 23:00 & & 30.922 & 30.492 & 29.36 & 30.995 & 29.652 & 30.893 & 30.365 & 30.141 & 28.588 & 26.373 & 34.412 & 36.089 & 35.528 & 17.162 & 18.061 & 21.965 & 15.438 & 13.65 & 33.474 & 35.298 & 30.662 & 34.36 & 27.152 & 34.464 \\
\hline 10/17/11 23:30 & & 30.924 & 30.499 & 29.36 & 30.5 & 29.657 & 30.895 & 30.37 & 30.146 & 28.593 & 26.373 & 34.311 & 36.089 & 35.421 & 17.165 & 18.026 & 21.956 & 15.438 & 13.528 & 33.434 & 35.194 & 30.657 & 34.257 & 27.152 & 34.467 \\
\hline 10/18/11 0:00 & & 30.92 & 30.501 & 29.351 & 30.986 & 29.664 & 30.898 & 30.365 & 30.148 & 28.593 & 26.373 & 34.218 & 36.089 & 35.32 & 17.167 & 17.987 & 21.949 & 15.445 & 13.43 & 33.385 & 35.101 & 30.648 & 34.165 & 27.15 & 34.467 \\
\hline 10/18/11 0:30 & & 30.922 & 30.506 & 29.353 & 30.979 & 29.678 & 30.898 & 30.365 & 30.152 & 28.591 & 26.363 & 34.131 & 36.096 & 35.231 & 17.167 & 17.952 & 21.942 & 15.445 & 13.469 & 33.35 & 35.021 & 30.636 & 34.083 & 27.15 & 34.467 \\
\hline
\end{tabular}




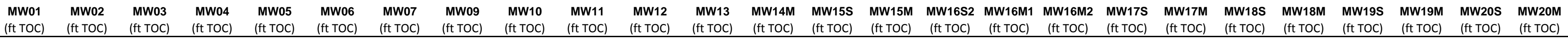

\begin{tabular}{|c|c|c|c|c|c|c|c|c|c|c|c|c|c|c|c|c|c|c|c|c|c|c|c|c|c|c|}
\hline well & $\begin{array}{l}\text { (ft TOC) } \\
\text { (is }\end{array}$ & $\begin{array}{l}\text { (ft TOC) } \\
\text { (ft) }\end{array}$ & $\begin{array}{l}\text { (ft TOC) } \\
\text { (f) }\end{array}$ & (ft TOC) & $\begin{array}{l}\text { (ft toc) } \\
\text { (foc) }\end{array}$ & $\begin{array}{l}\text { (ft TOC) } \\
\text { (f) }\end{array}$ & (ft toc) & $\begin{array}{l}\text { (ft TOC) } \\
\text { (f) }\end{array}$ & $\begin{array}{l}\text { (ft TOC) } \\
\text { (ft) }\end{array}$ & $\begin{array}{l}\text { (ft TOC) } \\
\text { (f) }\end{array}$ & $\begin{array}{l}\text { (ft TOC) } \\
\text { (f) }\end{array}$ & $\begin{array}{l}\text { (ft Toc) } \\
\text { (f) }\end{array}$ & (ft TOC) & $\begin{array}{l}\text { (ft TOC) } \\
\end{array}$ & (ft TOC) & (ft TOC) & $\begin{array}{l}\text { (ft TOC) } \\
\text { (f) }\end{array}$ & (ft TOC) & $\begin{array}{l}\text { (ft TOC) } \\
\text { (ft) }\end{array}$ & (ft TOC) & $\begin{array}{l}\text { (ft TOC) } \\
\end{array}$ & $\begin{array}{l}\text { (ft TOC) } \\
\text { (n) }\end{array}$ & $\begin{array}{l}\text { (ft TOC) } \\
\text { (n) }\end{array}$ & (ft TOC) & $\begin{array}{l}\text { (ft TOC) } \\
\end{array}$ & (ft TOC) \\
\hline 10/18/11 1:00 & & 30.922 & 30.508 & 29.356 & 30.969 & & 29.678 & 30.903 & 30.368 & 30.155 & 28.595 & 26.363 & 34.054 & 36.091 & 35.153 & $\begin{array}{l}3 \quad 17.169 \\
3\end{array}$ & 17.917 & 21.938 & 15.447 & 13.389 & 33.313 & 34.945 & 30.622 & 34.006 & 27.152 & 34.469 \\
\hline 10/18/11 1:30 & & 30.92 & 30.506 & 29.358 & 30.958 & & 29.681 & 30.903 & 30.37 & 30.155 & 28.598 & 26.366 & 33.984 & 36.091 & 35.081 & $1 \quad 17.167$ & 17.886 & 21.933 & 15.452 & 13.319 & 33.273 & 34.874 & 30.61 & 33.938 & 27.15 & 34.471 \\
\hline $10 / 18 / 112: 00$ & & 30.924 & 30.51 & 29.344 & 30.948 & & 29.692 & 30.903 & 30.37 & 30.157 & 28.595 & 26.366 & 33.923 & 36.094 & 35.015 & 17.172 & 17.853 & 21.928 & 15.447 & 13.251 & 33.245 & 34.812 & & 874 & 27.152 & 34.471 \\
\hline $10 / 18 / 112: 30$ & & 30.924 & 30.515 & 29.332 & 30.939 & & 29.706 & 30.905 & 30.368 & 30.159 & 28.593 & 26.351 & 33.865 & 36.09 & 34.952 & 17.172 & 17.823 & 21.924 & 15.45 & 13 & 33.222 & & & 82 & 55 & 34.471 \\
\hline $10 / 18 / 113: 00$ & & 30.924 & 30.517 & 29.325 & 30.93 & & 29.721 & 30.905 & 30.368 & 30.162 & 28.588 & 26.339 & 33.808 & 36.093 & 34.893 & 17.172 & 17.795 & 21.919 & 15.45 & 13.1 & 33.194 & 34.698 & .584 & 64 & 152 & 34.474 \\
\hline 10/18/11 3:30 & & 30.92 & 30.513 & 29.318 & 30.913 & & 29.728 & 30.905 & 30.363 & 30.162 & 28.586 & 26.33 & 33.755 & 36.096 & 34.841 & 17.172 & 17.765 & 21.91 & 15.464 & 13.079 & 33.157 & 34.649 & 30.566 & 33.71 & 7.152 & 34.474 \\
\hline 10/18/11 4:00 & & 30.918 & 30.508 & 29.307 & 30.895 & & 29.732 & 30.905 & 30.37 & 30.157 & 28.584 & 26.32 & 33.705 & 36.091 & 34.788 & $\begin{array}{ll}8 & 17.172\end{array}$ & 17.734 & 21.903 & 15.471 & 13.025 & 33.124 & 34.597 & 30.54 & 33.663 & 27.152 & 34.471 \\
\hline 10/18/11 4:30 & & 30.92 & 30.508 & 29.314 & 30.883 & & 29.739 & 30.905 & 30.368 & 30.157 & 28.581 & 26.311 & 33.659 & 36.091 & 34.738 & $\begin{array}{ll}8 & 17.172\end{array}$ & 17.708 & 21.898 & 15.471 & 12.976 & 33.096 & 34.549 & 30.535 & 33.614 & 27.152 & 34.474 \\
\hline 10/18/11 5:00 & & 30.92 & 30.503 & 29.314 & 30.867 & & 29.737 & 30.903 & 30.372 & 30.155 & 28.584 & 26.315 & 33.614 & 36.086 & 34.694 & $\begin{array}{l}4 \\
17.169\end{array}$ & 17.683 & 21.889 & 15.471 & 12.933 & 33.068 & 34.507 & 30.519 & 33.572 & 27.152 & 34.474 \\
\hline 10/18/11 5:30 & & 30.918 & 30.499 & 29.314 & 30.85 & & 29.739 & 30.905 & 30.37 & 30.15 & 28.584 & 26.311 & 33.572 & 36.086 & 34.647 & $7 \quad 17.169$ & 17.657 & 21.88 & 15.475 & 12.889 & 33.038 & 34.462 & 30.495 & 33.529 & 27.152 & 34.474 \\
\hline 10/18/11 6:00 & & 30.924 & 30.503 & 29.314 & 30.843 & & 29.737 & 30.907 & 30.372 & 30.152 & 28.586 & 26.315 & 34.555 & 36.084 & 35.006 & $\begin{array}{ll}6 & 17.169\end{array}$ & 17.631 & 21.875 & 15.466 & 13.232 & 33.231 & 35.563 & 30.497 & 34.538 & 27.155 & 34.474 \\
\hline 10/18/11 6:30 & & 30.927 & 30.503 & 29.318 & 30.834 & & 29.739 & 30.907 & 30.372 & 30.152 & 28.584 & 26.313 & 35.652 & 36.091 & 36.089 & $\begin{array}{ll}9 & 17.169\end{array}$ & 17.68 & 21.868 & 15.461 & 14.318 & 33.735 & 36.641 & 30.523 & 35.608 & 27.155 & 34.474 \\
\hline 10/18/11 7:00 & & 30.927 & 30.503 & 29.321 & 30.834 & & 29.737 & 30.907 & 30.377 & 30.155 & 28.588 & 26.313 & 36.3 & 36.096 & 36.827 & $7 \quad 17.169$ & 17.774 & 21.864 & 15.464 & 15.058 & 34.027 & 37.26 & 30.556 & 36.244 & 27.155 & 34.474 \\
\hline 10/18/11 7:30 & & 30.929 & 30.506 & 29.332 & 30.843 & & 29.735 & 30.91 & 30.379 & 30.155 & 28.591 & 26.318 & 34.897 & 36.086 & 36.037 & 17.169 & 17.811 & 21.866 & 15.466 & 14.266 & 33.621 & 35.774 & 30.575 & 34.827 & 27.155 & 34.471 \\
\hline 10/18/11 8:00 & & 30.931 & 30.508 & 29.342 & 30.85 & & 29.735 & 30.915 & 30.379 & 30.155 & 28.595 & 26.327 & 34.499 & 36.093 & 35.629 & 17.169 & 17.797 & 21.857 & 15.464 & 13.843 & 33.446 & 35.381 & 30.573 & 34.444 & 27.155 & 34.471 \\
\hline $10 / 18 / 118: 30$ & & 30.934 & 30.508 & 29.351 & 30.857 & & 29.728 & 30.915 & 30.377 & 30.157 & 28.6 & 26.337 & 34.267 & 36.093 & 35.381 & 17.172 & 17.769 & 21.85 & 15.464 & 13.589 & 33.353 & 35.151 & 30.563 & 34.212 & 27.157 & 34.471 \\
\hline $10 / 18 / 119: 00$ & & 30.936 & 30.513 & 29.356 & 30.864 & & 29.728 & 30.915 & 30.377 & 30.162 & 28.605 & 26.344 & 34.101 & 36.094 & 35.207 & 17.172 & 17.746 & 21.847 & 15.464 & 13.425 & 33.285 & 34.99 & 30.561 & 34.05 & 27.155 & 34.471 \\
\hline $10 / 18 / 119: 30$ & & 30.941 & 30.522 & 29.36 & 30.871 & & 29.728 & 30.917 & 30.377 & 30.166 & 28.605 & 26.351 & 33.979 & 36.093 & 35.078 & $3 \quad 17.174$ & $\begin{array}{l}17.72 \\
\end{array}$ & 21.84 & 15.457 & $\begin{array}{l}13.4279 \\
\end{array}$ & 33.238 & 34.867 & 30.556 & 33.93 & 27.157 & 34.471 \\
\hline 10/18/11 10:00 & & $\begin{array}{l}30.945 \\
30.945\end{array}$ & 30.524 & 29.36 & 30.869 & & 29.732 & 30.917 & 30.382 & 30.171 & 28.607 & 26.351 & 33.876 & 36.098 & 34.968 & 17.179 & $\begin{array}{l}17.692 \\
\end{array}$ & 21.836 & 15.459 & 13.168 & $\begin{array}{l}33.201 \\
33.201\end{array}$ & 34.765 & 30.556 & 33.832 & 27.157 & 34.471 \\
\hline $10 / 18 / 1110: 30$ & & $\begin{array}{l}30.943 \\
30.943\end{array}$ & $\begin{array}{l}30.524 \\
30.529\end{array}$ & 29.351 & 30.871 & & 29.742 & 30.919 & $\begin{array}{l}30.302 \\
30.379\end{array}$ & 30.173 & 28.607 & $\begin{array}{l}20.371 \\
26.351\end{array}$ & $\begin{array}{l}33.0102 \\
33.792\end{array}$ & 36.098 & $\begin{array}{l}34.900 \\
34.881\end{array}$ & 17.176 & $\begin{array}{l}17.069 \\
17.669\end{array}$ & $\begin{array}{r}21.030 \\
21.82\end{array}$ & $\begin{array}{l}15.461 \\
15.49\end{array}$ & $\begin{array}{l}13.100 \\
13.079\end{array}$ & $\begin{array}{l}33.162 \\
33.162\end{array}$ & 34.684 & $\begin{array}{l}30.549 \\
30.549\end{array}$ & $\begin{array}{l}33.024 \\
33.745\end{array}$ & 27.157 & $\begin{array}{l}34.474 \\
34.47\end{array}$ \\
\hline $10 / 18 / 1111: 00$ & & $\begin{array}{l}30.943 \\
30.943\end{array}$ & $\begin{array}{l}30.529 \\
30.531\end{array}$ & 29.339 & 30.871 & & 29.749 & $\begin{array}{l}30.919 \\
30.922\end{array}$ & $\begin{array}{l}30.379 \\
30.379\end{array}$ & $\begin{array}{l}30.176 \\
30.176\end{array}$ & 28.607 & $\begin{array}{l}20.351 \\
26.342\end{array}$ & 33.719 & $\begin{array}{r}30.050 \\
36.1\end{array}$ & $\begin{array}{l}54.801 \\
34.806\end{array}$ & 17.179 & $\begin{array}{l}17.0643 \\
17.643\end{array}$ & $\begin{array}{r}21.02 \\
21.822\end{array}$ & $\begin{array}{l}13.412 \\
15.464\end{array}$ & $\begin{array}{l}13.009 \\
13.09\end{array}$ & $\begin{array}{l}33.127 \\
33.127\end{array}$ & $\begin{array}{l}34.064 \\
34.611\end{array}$ & $\begin{array}{l}30.549 \\
30.549\end{array}$ & $\begin{array}{l}33.677 \\
33.67\end{array}$ & 27.157 & $\begin{array}{l}34.474 \\
34.474\end{array}$ \\
\hline $1: 30$ & & & 30.533 & 29.33 & 30.864 & & 29.761 & 30.919 & 30.379 & 30.178 & 28.6 & & 33.659 & 36.101 & 34.738 & $3 \quad 17.179$ & & 21.817 & .475 & 12.95 & 33.099 & 34.547 & .533 & .614 & 27.157 & 34.474 \\
\hline 10 & & & 529 & 29.321 & 30.857 & & 29.775 & 30.917 & 30.377 & 30.178 & 28.593 & & 34.62 & 36.101 & 35.076 & 17.179 & 17.594 & .81 & 15.48 & 13.295 & 33.285 & 627 & .526 & 34.602 & 27.157 & 476 \\
\hline $10 / 18 /$ & & & 30.529 & 29.311 & 30.848 & & 29.784 & 30.917 & 30.377 & 30.176 & 28.591 & 26.308 & 35.701 & 36.105 & 36.143 & 17.179 & 17.648 & 21.801 & 15.487 & 388 & 33.754 & 689 & .542 & 35.66 & 27.157 & 34.474 \\
\hline 10/18/11 13:00 & & 30.934 & 30.522 & 29.304 & 30.846 & & 29.786 & 30.924 & 30.382 & 30.169 & 28.588 & 26.301 & 36.34 & 36.103 & 36.867 & 17.179 & 17.737 & 21.797 & 15.499 & 5.141 & 34.043 & 37.298 & 1.561 & 36.279 & 27.159 & 34.474 \\
\hline 10/18/11 13:30 & & 30.931 & 30.517 & 29.3 & 30.848 & & 29.796 & 30.919 & 30.377 & & 28.586 & 26.294 & 34.922 & 36.089 & 36.068 & $3 \quad 17.176$ & 17.779 & 21.79 & 15.506 & 14.363 & 33.623 & 35.798 & .566 & 34.855 & 27.157 & 34.474 \\
\hline 10/18/11 14:00 & & 30.934 & 30.513 & 29.302 & & & 29.798 & 30.922 & 30.384 & & 28.584 & 26.289 & 34.52 & 36.093 & 35.648 & $3 \quad 17.179$ & 17.765 & 21.785 & 15.513 & 13.949 & 33.441 & 35.4 & 30.556 & .461 & 27.159 & 34.471 \\
\hline 10/18/11 14:30 & & 30.936 & 30.508 & 29.3 & 30.848 & & 29.793 & 30.92 & 30.384 & 30.162 & 28.584 & 26.294 & 34.276 & 36.091 & 35.395 & 17.176 & 17.739 & 21.778 & 15.515 & 13.709 & 33.341 & 35.163 & 30.544 & 4.224 & 27.159 & 34.474 \\
\hline 10/18/11 15:00 & & 30.934 & 30.506 & 29.302 & 30.846 & & 29.796 & 30.919 & 30.379 & 30.162 & 28.579 & 26.289 & 34.103 & 36.094 & 35.212 & 17.176 & 17.716 & 21.771 & 15.515 & 13.533 & 33.259 & 34.992 & 30.533 & 34.057 & 27.155 & 34.474 \\
\hline $10 / 18 / 1115: 30$ & & 30.936 & 30.506 & 29.3 & 30.841 & & 29.796 & 30.919 & 30.387 & 30.162 & 28.581 & 26.291 & 33.975 & 36.089 & 35.076 & 17.174 & 17.687 & 21.766 & 15.518 & 13.397 & 33.206 & 34.857 & 30.526 & 33.926 & 27.159 & 34.474 \\
\hline 10/18/11 16:00 & & 30.938 & 30.503 & 29.304 & 30.836 & & 29.791 & 30.922 & 30.387 & 30.159 & 28.581 & 26.289 & 33.867 & 36.089 & 34.963 & 17.172 & 17.662 & 21.769 & 15.518 & 13.291 & 33.159 & 34.755 & 30.507 & 33.82 & 27.159 & 34.474 \\
\hline $10 / 18 / 1116: 30$ & & 30.941 & 30.501 & 29.307 & 30.832 & & 29.786 & 30.92 & 30.389 & 30.157 & 28.584 & 26.291 & 33.778 & 36.093 & 34.87 & 17.176 & 17.638 & 21.799 & 15.522 & 13.197 & 33.115 & 34.665 & 30.5 & 33.736 & 27.159 & 34.474 \\
\hline 10/18/11 17:00 & & 30.943 & 30.501 & 29.311 & 30.822 & & 29.784 & 30.922 & 30.384 & 30.159 & 28.581 & 26.291 & 33.701 & 36.086 & 34.788 & 317.174 & 17.61 & 21.81 & 15.52 & 13.119 & 33.078 & 34.592 & 30.493 & 33.656 & 27.162 & 34.474 \\
\hline $10 / 18 / 1117: 30$ & & 30.943 & 30.501 & 29.321 & 30.818 & & 29.777 & 30.924 & 30.389 & 30.157 & 28.584 & 26.296 & 35.397 & 36.082 & 35.833 & 17.174 & 17.615 & 21.81 & 15.515 & 14.149 & 33.616 & 36.409 & 30.502 & 35.31 & 27.162 & 34.474 \\
\hline $10 / 18 / 1118: 00$ & & 30.943 & 30.503 & 29.328 & 30 & & 29.77 & 30.924 & 30.394 & 30.159 & 28.591 & 26.308 & 36.247 & 36.093 & 36.63 & 17.172 & $\begin{array}{l}17.692 \\
\text { S }\end{array}$ & 21.808 & 15.52 & 14.943 & 33.943 & 37.281 & 1.533 & 36.171 & 27.162 & 34.476 \\
\hline 10 & & 30.95 & 30.506 & 29.337 & 30. & & 29.765 & 30.926 & $\begin{array}{l}30.394 \\
3\end{array}$ & 30. & 28.591 & 26.315 & 35.479 & 36.091 & 36.621 & 17.1 & 17.79 & 21.797 & 15.5 & $\begin{array}{l}14.934 \\
\end{array}$ & 33.894 & 36.35 & .573 & 35.399 & 27.162 & 34.474 \\
\hline 10 & & 30.952 & 30.51 & 29.342 & 30.841 & & 29.758 & $\begin{array}{l}30.926 \\
30.920\end{array}$ & 30.391 & 30.162 & 28.598 & 26.325 & 35.481 & 36.096 & 36.426 & 17.174 & 17.816 & 21.792 & $\begin{array}{l}15.513 \\
15.513\end{array}$ & $\begin{array}{r}14.547 \\
14.71\end{array}$ & $\begin{array}{l}53.074 \\
33.784\end{array}$ & 36.378 & .584 & 35.416 & 27.164 & 34.478 \\
\hline 10 & & $\begin{array}{l}30.955 \\
30.95\end{array}$ & 30.515 & 29.342 & $\begin{array}{l}30.855 \\
30.855\end{array}$ & & 29.758 & $\begin{array}{l}30.929 \\
30.920\end{array}$ & $\begin{array}{l}30.394 \\
304\end{array}$ & $\begin{array}{l}30.164 \\
30.164\end{array}$ & 28.598 & 26.33 & 35.432 & 36.098 & 36.372 & 17.176 & $\begin{array}{l}17.0832 \\
17.832\end{array}$ & 21.783 & 15.511 & 14.64 & $\begin{array}{l}33.756 \\
33.756\end{array}$ & 36.331 & .591 & $\begin{array}{l}35.467 \\
35.367\end{array}$ & 27.164 & $\begin{array}{l}34.478 \\
34.48\end{array}$ \\
\hline 10 & & $\begin{array}{l}30.957 \\
30\end{array}$ & 30.52 & 29.339 & 30. & & 29.758 & 30.929 & $\begin{array}{l}30.394 \\
30.394\end{array}$ & $\begin{array}{l}30.104 \\
30.169\end{array}$ & $\begin{array}{l}28.5500 \\
28.598\end{array}$ & $\begin{array}{l}26.33 \\
26.33\end{array}$ & $\begin{array}{l}35.421 \\
35.421\end{array}$ & $\begin{array}{l}30.090 \\
36.098\end{array}$ & 36.363 & 17. & $\begin{array}{l}11.028 \\
17.849\end{array}$ & 21.776 & 15.511 & $\begin{array}{l}14.644 \\
14.609\end{array}$ & 33.763 & 36.319 & .608 & 35.362 & 27.164 & $\begin{array}{l}34.478 \\
34.478\end{array}$ \\
\hline 0 & & & 30 & 29 & 30. & & 2 & 30 & & 30 & 28 & 26 & 2 & 36 & 36. & 17 & 17.858 & 21 & 15.508 & 14.544 & 33.742 & 62 & 5 & 3 & 27.162 & 34.478 \\
\hline & & & 30.5 & 29.3 & & & 29. & 30 & & 30 & & & & 36 & 36 & 17 & & 21 & 15.508 & 14.546 & 33.754 & - & 30.615 & 5 & 27.164 & 34.478 \\
\hline $1121: 30$ & & & 30. & 29.335 & 30. & & & & & & & 26. & & 36 & 36.3 & 17.1 & 17.879 & & $1+$ & 14.553 & 33.756 & 36.291 & 52 & & 27.164 & 34.478 \\
\hline .22:00 & & & 30.52 & 29.33 & & & 29 & & & & & & 34.784 & 36 & 35. & 17.181 & & 21.757 & 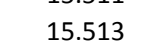 & 14.142 & 33.579 & & & 26 & 27.164 & 34.476 \\
\hline 10 & & 30 & 30.5 & 29. & & & & 30 & & & 28 & 26 & & 36 & 35.641 & 17.181 & 17. & 21. & 15.5 & 13.848 & 33.458 & & 66 & 56 & 27.164 & 34.478 \\
\hline 10/ & & & & 29. & & & & & & & & & & 36 & 35. & 17.181 & 17. & 21. & & 13.643 & 33.381 & & & 1 & 27.166 & 34.478 \\
\hline $10 / 1$ & & & 30. & 29 & & & & & & & & & & 36.101 & 35.287 & 17.181 & 17. & 21.7 & 15. & 13.491 & 33.318 & & & 34.128 & 27.166 & 34.478 \\
\hline 10/19/11 0:00 & & 30 & 30.5 & 29.309 & & & & 30. & 30. & & 28. & 26. & 34. & 36.103 & 35.163 & 17.1 & 17.7 & 21.732 & 15.518 & 13.366 & 33.262 & 34.947 & 30.559 & 34.01 & 27.166 & 34.478 \\
\hline 10/19/11 0:30 & & 30. & 30.5 & 29.318 & & & & $30.5>>>$ & 30.3 & & 28.9 & 26.296 & 33.94 & 36.101 & 35.055 & 17.179 & 17.7 & 21.7. & 15.522 & 13.26 & 33.206 & 34.848 & 30.547 & 33.912 & 27.166 & 34.478 \\
\hline 10/19/11 1:00 & & 30.9 & 30.513 & 29.323 & 30.8 & & 29.779 & 30.93 & 30.396 & 30.1 & 28.58 & 26.303 & 33.872 & 36.1 & 34.963 & 17.179 & 17.702 & 21.718 & 15.52 & 13.166 & 33.169 & 34.76 & 30.533 & 33.825 & 27.164 & 34.478 \\
\hline 10/19/11 1:30 & & 30.955 & 30.508 & 29.325 & 30.857 & & 29.779 & 30.936 & 30.398 & 30.164 & 28.591 & 26.308 & 33.794 & 36.098 & 34.884 & 17.176 & 17.673 & 21.713 & 15.52 & 13.084 & 33.131 & 34.684 & 30.519 & 33.75 & 27.166 & 34.478 \\
\hline 10/19/11 2:00 & & 30.959 & 30.513 & 29.323 & 30.85 & & 29.779 & 30.936 & 30.401 & 30.164 & 28.591 & 26.311 & 33.729 & 36.098 & 34.813 & 17.176 & 17.648 & 21.709 & 15.511 & 13.002 & 33.103 & 34.618 & 30.516 & 33.684 & 27.166 & 34.478 \\
\hline 10/19/11 2:30 & & 30.962 & 30.513 & 29.321 & 30.843 & & 29.784 & 30.936 & 30.398 & 30.166 & 28.588 & 26.306 & 33.668 & 36.098 & 34.752 & 17.179 & 17.622 & 21.704 & 15.508 & 12.929 & 33.078 & 34.559 & 30.507 & 33.623 & 27.166 & 34.481 \\
\hline 10/19/11 3:00 & & 30.964 & 30.515 & 29.318 & 30.836 & & 29.786 & 30.936 & 30.398 & 30.166 & 28.586 & 26.303 & 33.612 & 36.1 & 34.694 & 17.179 & 17.596 & 21.699 & 15.506 & 12.87 & 33.047 & 34.504 & .495 & 33.571 & 27.169 & 34.481 \\
\hline 10/19/11 3:30 & & 30.959 & 30.512 & 29.321 & 30.82 & & 29.786 & 30.938 & 30.401 & 30.166 & 28.586 & 26.303 & 33.563 & 36.103 & 34.64 & 17.179 & 17.573 & 21.692 & 15.511 & 12.821 & 33.024 & 34.454 & .488 & 33.517 & 27.169 & 34.481 \\
\hline 10/19/11 4:00 & & 30.962 & 30.51 & 29.325 & 30.818 & & 29.786 & 30.936 & 30.396 & 30.166 & 28.588 & 26.303 & 33.516 & 36.105 & 34.593 & 17.181 & 17.55 & 21.686 & 15.511 & 12.767 & 32.994 & 34.407 & 30.481 & 33.471 & 27.169 & 34.483 \\
\hline 10/19/11 4:30 & & 30.962 & 30.508 & 29.328 & 30.808 & & 29.782 & 30.939 & 30.401 & 30.164 & 28.588 & 26.308 & 33.469 & 36.098 & 34.548 & 17.179 & 17.529 & 21.683 & 15.515 & 12.724 & 32.963 & 34.364 & 30.472 & 33.431 & 27.169 & 34.483 \\
\hline
\end{tabular}




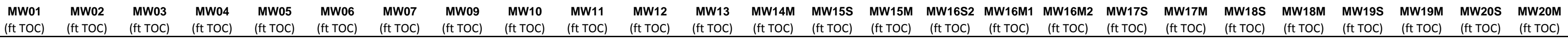

\begin{tabular}{|c|c|c|c|c|c|c|c|c|c|c|c|c|c|c|c|c|c|c|c|c|c|c|c|c|c|c|}
\hline well & $\begin{array}{l}\text { (ft TOC) } \\
\text { (f) }\end{array}$ & $\begin{array}{l}\text { (ft TOC) } \\
\text { (ft) }\end{array}$ & $\begin{array}{l}\text { (ft TOC) } \\
\text { (f) }\end{array}$ & $\begin{array}{l}\text { (ft TOC) } \\
\text { (ft) }\end{array}$ & $\begin{array}{l}\text { (ft TOC) } \\
\text { (f) }\end{array}$ & $\begin{array}{l}\text { (ft toc) } \\
\text { (ftocts }\end{array}$ & (ft toc) & $\begin{array}{l}\text { (ift TOC) } \\
\text { (n) }\end{array}$ & $\begin{array}{l}\text { (ft TOC) } \\
\text { (f) }\end{array}$ & $\begin{array}{l}\text { (ft TOC) } \\
\text { (f) }\end{array}$ & $\begin{array}{l}\text { (ft TOC) } \\
\text { (n) }\end{array}$ & $\begin{array}{l}\text { (ft TOC) } \\
\text { (f) }\end{array}$ & (ft TOC) & $\begin{array}{l}\text { (ft TOC) } \\
\end{array}$ & (ft TOC) & (ft TOC) & $\begin{array}{l}\text { (ft TOC) } \\
\text { (f) }\end{array}$ & (ft TOC) & $\begin{array}{l}\text { (ft TOC) } \\
\text { (ft) }\end{array}$ & (ft TOC) & $\begin{array}{l}\text { (ft TOC) } \\
\text { (f) }\end{array}$ & $\begin{array}{l}\text { (ft TOC) } \\
\text { (f) }\end{array}$ & $\begin{array}{l}\text { (ft TOC) } \\
\text { (n) }\end{array}$ & (ft TOC) & $\begin{array}{l}\text { (ft TOC) } \\
\text { (1) }\end{array}$ & (ft TOC) \\
\hline 5:00 & & 30.962 & 30.51 & 29.332 & 30.801 & & 29 & 30.941 & 30 & 30.1 & 28.59 & 26 & 33.434 & 36.0 & 34.5 & 17 & 17.505 & 21.676 & 15.511 & 12.6 & 32.954 & 34.326 & 30.462 & 3.391 & 69 & 34.483 \\
\hline 8/11 5:30 & & 30.966 & 30.508 & 29.33 & 30.794 & & 29.782 & 30.938 & 30.398 & 30.166 & 28.593 & 26.315 & 3.39 & 36.105 & 34.469 & 17.181 & 17.484 & 21.674 & 13 & 12.642 & 32.938 & 289 & 458 & 3.356 & 7.169 & 34.485 \\
\hline 10/19/11 6:00 & & 30.969 & 30.513 & 29.328 & 30.79 & & 29.789 & 30.938 & 30.401 & 30.169 & 28.588 & 26.311 & 34.389 & 36.105 & 34.83 & 17.181 & 17.465 & 21.683 & 15.511 & 12.987 & 33.15 & 35.402 & 30.46 & 4.374 & 7.169 & 34.485 \\
\hline 10/19/11 6:30 & & 30.969 & 30.515 & 29.339 & 30.785 & & 29.782 & 30.943 & 30.403 & 30.169 & 28.593 & 26.306 & 35.491 & 36.105 & 35.915 & 17.183 & 17.517 & 21.683 & 15.508 & 14.078 & 33.644 & 36.485 & 30.488 & 35.449 & 27.169 & 34.485 \\
\hline 10/19/11 7:00 & & 30.964 & 30.513 & 29.351 & 30.787 & & 29.775 & 30.941 & 30.408 & 30.169 & 28.598 & 26.32 & 36.139 & 36.103 & $3 \quad 36.659$ & 17.181 & 17.613 & 21.683 & 15.511 & 14.833 & 33.94 & 37.099 & 30.523 & 36.08 & 27.169 & 34.485 \\
\hline 10/19/11 7:30 & & 30.969 & 30.513 & 29.365 & 30.799 & & 29.765 & 30.946 & 30.408 & 30.169 & 28.605 & 26.332 & 34.733 & 36.105 & $5 \quad 35.871$ & 17.183 & 17.657 & 21.681 & 15.504 & 14.038 & 33.532 & 35.608 & 30.542 & 34.663 & 27.169 & 34.485 \\
\hline 10/19/11 8:00 & & 30.971 & 30.52 & 29.367 & 30.815 & & 29.763 & 30.948 & 30.41 & 30.171 & 28.609 & 26.346 & 34.344 & 36.1 & 35.465 & 17.183 & 17.645 & 21.683 & 15.504 & 13.617 & 33.367 & 35.227 & 30.544 & 34.289 & 27.171 & 34.485 \\
\hline 10/19/11 8:30 & & 30.978 & 30.524 & 29.374 & 30.829 & & 29.765 & 30.948 & 30.41 & 30.178 & 28.612 & 26.346 & 34.279 & 36.105 & $5 \quad 35.399$ & 17.186 & 17.636 & 21.679 & 15.497 & 13.535 & 33.306 & 35.165 & 30.549 & 34.224 & 27.171 & 34.488 \\
\hline 10/19/11 9:00 & & 30.98 & 30.531 & 29.37 & 30.836 & & 29.768 & 30.948 & 30.408 & 30.183 & 28.614 & 26.351 & 34.103 & 36.11 & 35.212 & 17.186 & 17.645 & 21.676 & 15.499 & 13.345 & 33.269 & 34.99 & 30.552 & 34.048 & 27.171 & 34.488 \\
\hline 10/19/11 9:30 & & 30.978 & 30.531 & 29.37 & 30.846 & & 29.777 & 30.948 & 30.408 & 30.185 & 28.614 & 26.354 & 33.937 & 36.11 & 35.036 & 17.188 & 17.624 & 21.669 & 15.499 & 13.175 & 33.206 & 34.826 & 30.547 & 33.888 & 27.171 & 34.488 \\
\hline 10/19/11 10:00 & & 30.98 & 30.54 & 29.37 & 30.85 & & 29.784 & 30.948 & 30.406 & 30.187 & 28.614 & 26.346 & 33.818 & 36.11 & 34.912 & 17.188 & 17.599 & 21.667 & 15.499 & 13.056 & 33.162 & 34.706 & 30.547 & 33.771 & 27.174 & 34.488 \\
\hline $10 / 19 / 1110: 30$ & & 30.976 & 30.538 & 29.353 & 30.848 & & 29.793 & 30.95 & 30.41 & 30.19 & 28.612 & 26.344 & 33.722 & 36.115 & 34.813 & 17.19 & 17.571 & 21.658 & 15.511 & 12.971 & 33.117 & 34.613 & 30.528 & 33.677 & 27.174 & 34.488 \\
\hline 10/19/11 11:00 & & 30.976 & 30.54 & 29.346 & 30.848 & & 29.803 & 30.946 & 30.41 & 30.187 & 28.609 & 26.334 & 33.642 & 36.115 & $5 \quad 34.727$ & 17.19 & 17.545 & 21.651 & 15.518 & 12.898 & 33.08 & 34.535 & 30.523 & 33.597 & 27.174 & 34.488 \\
\hline $10 / 19 / 1111: 30$ & & 30.976 & 30.538 & 29.337 & 30.841 & & 29.81 & 30.95 & 30.41 & 30.187 & 28.605 & 26.325 & 33.67 & 36.117 & 34.757 & 17.19 & 17.543 & 21.646 & 15.518 & 12.947 & 33.08 & 34.563 & 30.516 & 33.626 & 27.174 & 34.485 \\
\hline 10/19/11 12:00 & & 30.971 & 30.533 & 29.323 & 30.829 & & 29.822 & 30.948 & 30.408 & 30.187 & 28.6 & 26.318 & 34.604 & 36.115 & 35.06 & 17.19 & 17.519 & 21.637 & 15.529 & 13.263 & 33.255 & 35.613 & 30.505 & 34.59 & 27.171 & 34.488 \\
\hline 10/19/11 12:30 & & 30.973 & 30.531 & 29.309 & 30.825 & & 29.836 & 30.946 & 30.408 & 30.183 & 28.591 & 26.301 & 35.669 & 36.112 & 36.11 & 17.19 & 17.571 & 21.63 & 15.534 & 14.332 & 33.728 & 36.658 & 30.521 & 35.622 & 27.174 & 34.488 \\
\hline 10/19/11 13:00 & & 30.971 & 30.526 & 29.293 & 30.825 & & 29.848 & 30.943 & 30.406 & 30.18 & 28.584 & 26.289 & 36.291 & 36.112 & 36.825 & 17.188 & 17.666 & 21.63 & 15.536 & 15.068 & 34.008 & 37.253 & 30.544 & 36.232 & 27.171 & 34.483 \\
\hline 10/19/11 13:30 & & 30.966 & 30.513 & 29.288 & 30.82 & & 29.85 & 30.943 & 30.408 & 30.176 & 28.579 & 26.275 & 34.864 & 36.11 & 36.009 & 17.186 & 17.704 & 21.621 & 15.55 & 14.29 & 33.581 & 35.741 & 30.549 & 34.794 & 27.171 & 34.483 \\
\hline $9 / 11$ 14:00 & & 30.966 & 30.506 & 29.283 & 30.822 & & 29.855 & 30.938 & 30.408 & 30.171 & 28.574 & 26.27 & 34.459 & 36.103 & 35.585 & 17.183 & 17.687 & 21.612 & 15.553 & 13.881 & 33.402 & 35.338 & 30.535 & 34.397 & 27.174 & 34.483 \\
\hline & & 30.964 & 30.499 & 29.276 & 30.81 & & 29.855 & 30.941 & 30.408 & 30.164 & 28.57 & 26.268 & 34.211 & 36.096 & 35.327 & 17.181 & 17.662 & 21.607 & 15.56 & 13.639 & 33.287 & .097 & 30.514 & 34.156 & 27.171 & 34.481 \\
\hline 0 & & 30.966 & 30.494 & 29.276 & 30. & & 29.852 & 30.936 & 30.41 & 30.162 & 28.57 & 26.26 & 34.035 & 36.098 & 35.142 & 17.181 & 17.636 & 21.6 & 15.558 & 13.469 & 33.213 & .919 & 30.495 & 33.985 & 27.174 & .481 \\
\hline 10 & & 30.964 & 30.487 & 29.279 & 30. & & 29.848 & 30.938 & 30.41 & 30.157 & 28.567 & 26.26 & 33.9 & 36.091 & 35.001 & 17.179 & .608 & 21.598 & 15.56 & 13.338 & 145 & .791 & .483 & 33.853 & 27.174 & .481 \\
\hline $10 /$ & & 30.964 & 30.485 & 29.279 & 30.799 & & 29.845 & 30.936 & 30.415 & 30.152 & 28.567 & 26.263 & 33.79 & 36.089 & 34.881 & 17.179 & 17.58 & 21.598 & 15.558 & 13.232 & 33.096 & 679 & 469 & 3.745 & 27.174 & 34.481 \\
\hline 10/19/11 16:30 & & 30.969 & 30.485 & 29.281 & 30.792 & & 29.843 & 30.939 & 30.413 & 30.152 & 28.565 & 26.263 & 33.703 & 36.091 & 34.792 & 17.176 & 17.557 & 21.625 & 15.553 & 13.133 & 33.068 & 34.592 & 30.465 & 33.654 & 27.174 & 34.478 \\
\hline 10/19/11 17:00 & & 30.969 & 30.482 & 29.281 & 30.783 & & & & 30.415 & 30.15 & 28.565 & 26.265 & 33.624 & 36.093 & & 17.176 & 17.531 & 21.637 & 15.555 & 13.056 & 33.024 & 34.516 & 30.45 & 33.579 & 27.174 & 34.481 \\
\hline 10/19/11 17:30 & & 30.969 & 30.482 & 29.283 & 30.776 & & 29.831 & & 30.415 & 30.15 & 28.567 & 26.265 & 35.304 & 36.091 & 35.749 & 17.176 & 17.538 & 21.639 & 15.553 & 14.097 & 33.563 & 307 & 30.462 & 35.219 & 27.176 & 34.481 \\
\hline 10/19/11 18:00 & & 30.969 & 30.48 & 29.288 & 30.773 & & 29.824 & 30.938 & 30.415 & 30.148 & 28.567 & 26.27 & 36.158 & 36.091 & 36.546 & 17.176 & 17.615 & 21.642 & 15.55 & 14.892 & 33.88 & 37.186 & 30.483 & 36.087 & 27.176 & 34.481 \\
\hline 10/19/11 18:30 & & 30.973 & 30.482 & 29.293 & 30.78 & & 29.817 & 30.938 & 30.417 & 30.146 & 28.567 & 26.275 & 35.269 & 36.089 & 36.41 & 17.176 & 17.706 & 21.642 & 15.55 & 14.755 & 33.763 & 36.141 & 30.521 & 35.188 & 27.176 & 34.481 \\
\hline 10/19/11 19:00 & & 30.973 & 30.482 & 29.295 & 30.79 & & 29.81 & 30.939 & 30.42 & 30.148 & 28.57 & 26.282 & 34.651 & 36.093 & 35.791 & 17.176 & 17.702 & 21.644 & 15.546 & 14.113 & 33.472 & 35.53 & 30.526 & 34.59 & 27.178 & 34.481 \\
\hline 10/19/11 19:30 & & 30.976 & 30.482 & 29.297 & 30.801 & & 29.808 & 30.944 & 30.42 & 30.148 & 28.57 & 26.284 & 34.347 & 36.089 & 35.467 & 17.176 & 17.678 & 21.649 & 15.546 & 13.78 & 33.35 & 35.232 & 30.521 & 34.29 & 27.176 & 34.481 \\
\hline $10 / 19 / 1120: 00$ & & 30.978 & 30.487 & 29.3 & 30.808 & & 29.803 & 30.943 & 30.42 & 30.15 & 28.572 & 26.287 & 34.145 & 36.093 & 35.254 & 17.179 & 17.655 & 21.655 & 15.539 & 13.545 & 33.267 & 35.03 & 30.521 & 34.092 & 27.178 & 34.483 \\
\hline $10 / 19 / 1120: 30$ & & 30.978 & 30.487 & 29.297 & 30.808 & & 29.805 & 30.946 & 30.415 & 30.148 & 28.57 & 26.289 & 33.991 & 36.096 & 35.097 & 17.176 & 17.627 & 21.66 & 15.543 & 13.373 & 33.204 & 34.881 & 30.502 & 33.942 & 27.178 & 34.481 \\
\hline 10/19/11 21:00 & & 30.98 & 30.489 & 29.302 & 30.808 & & 29.803 & 30.943 & 30.42 & 30.148 & 28.572 & 26.284 & 33.874 & 36.096 & 34.968 & 17.174 & 17.603 & 21.665 & 15.539 & 13.234 & 33.15 & 34.762 & 30.497 & 33.825 & 27.178 & 34.481 \\
\hline $10 / 19 / 1121: 30$ & & 30.98 & 30.489 & 29.307 & 30.806 & & 29.798 & 30.948 & 30.42 & 30.148 & 28.572 & 26.294 & 33.776 & 36.096 & 34.863 & 17.176 & 17.575 & 21.672 & 15.536 & 13.115 & 33.106 & 34.665 & 30.49 & 33.726 & 27.178 & 34.481 \\
\hline 10/19/11 22:00 & & & 30.489 & 29.309 & 30 & & 29.796 & 30. & 30.42 & 30.148 & 28.5 & 26.296 & 33.689 & 36.101 & 34.776 & 17.179 & 17.552 & 21.681 & 15.539 & 13.02 & 33.068 & 34.58 & 30.476 & 33.642 & 27.181 & 34.481 \\
\hline 22:30 & & 30.983 & $\begin{array}{l}30.492 \\
30.45\end{array}$ & 29.314 & & & 29.796 & 30.948 & 30.42 & $\begin{array}{l}30.148 \\
30\end{array}$ & 28.5 & 26.299 & $\begin{array}{l}33.619 \\
3.619\end{array}$ & 36.098 & 34.701 & 17.179 & 17.526 & 21.688 & 15.529 & 12.933 & 33.043 & 34.509 & 30.472 & 33.574 & 27.178 & 34.483 \\
\hline 23:00 & & $\begin{array}{l}30.985 \\
30.985\end{array}$ & $\begin{array}{l}30.492 \\
30.496\end{array}$ & 29.307 & 30. & & 29.793 & $\begin{array}{l}30.951 \\
301\end{array}$ & $\begin{array}{l}30.422 \\
30.422\end{array}$ & $\begin{array}{l}30.140 \\
30.148\end{array}$ & 28. & 26.303 & 33.556 & $\begin{array}{l}36.098 \\
36.098\end{array}$ & 34.635 & 17.179 & 17.501 & 21.697 & 15.527 & 12.854 & 33.012 & 34.447 & $\begin{array}{l}30.472 \\
30.472\end{array}$ & $\begin{array}{r}33.51 \\
331\end{array}$ & 27.178 & 34.483 \\
\hline 23:30 & & 30.985 & $\begin{array}{l}30.494 \\
30.494\end{array}$ & 29.309 & 30. & & 29.798 & 30.951 & $\begin{array}{r}r .422 \\
30.42\end{array}$ & 30.15 & 28.572 & 26.296 & $\begin{array}{l}33.495 \\
33.05\end{array}$ & 36.105 & 34.574 & 17.179 & 17.479 & 21.702 & 15.529 & $\begin{array}{r}1.044 \\
12.79\end{array}$ & 32.982 & $\begin{array}{r}r .447 \\
34.39 \\
\end{array}$ & $\begin{array}{l}30.465 \\
30.46\end{array}$ & $\begin{array}{l}33.454 \\
3.454\end{array}$ & 27.178 & $\begin{array}{l}34.483 \\
3.483\end{array}$ \\
\hline $10 /$ & & 30.987 & 30.4 & 29.3 & 30 & & 29.796 & 30. & 30 & 30. & 28.572 & 26.299 & 33 & 36.108 & 34.523 & 17.179 & 17.456 & 21. & 15.525 & 12.726 & 63 & 8 & 88 & 33.407 & 27.181 & .483 \\
\hline & & 30. & 30.4 & 29. & & & 29. & 30. & 30 & 30.148 & 28. & 26 & 33.401 & 36.108 & 34.473 & 17.179 & 17.435 & 2 & 15.5 & 12.677 & 32.935 & & 6 & 33.358 & 27.178 & .483 \\
\hline & & & 30.4 & 29 & & & & & 30 & & 28. & & & 36.1 & & 17 & & 21.7 & 15. & 12.628 & 32.919 & 3 & 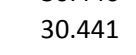 & 33.316 & 27.181 & .481 \\
\hline & & 30. & & 29 & & & & & & & 28. & & & 36.1 & & 17 & & & 15.5 & 12.574 & 32.898 & 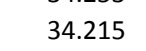 & & 33.278 & 27.181 & \\
\hline $10 /$ & & . & 30.499 & 29. & & & & & & & & & & 36. & & & & 21.7 & 15.5 & 12.529 & & & & 3 & 27.181 & .483 \\
\hline 2:30 & & 30. & 30.4 & 29. & & & & & 30 & 30 & 28 & & 33 & 36.112 & 34. & 17. & 17. & 21. & 15.522 & 12.494 & 32.861 & & 5 & 33.212 & 27.181 & 34.483 \\
\hline & & & 30. & 29. & & & & & & & 28 & & 33 & 36.112 & 34. & 17. & & 21.7 & 15.522 & 12.454 & & & 7 & 33.182 & 27.181 & 34.485 \\
\hline 10/ & & & 30. & 29.3 & & & & & & & & & 33. & 36.112 & 34.2 & 17.183 & & 21.7 & 15.518 & 12.426 & & & 3 & 33.154 & 27.183 & 34.483 \\
\hline 10/20/11 4:00 & & 30.9 & 30. & 29.3 & & & 29.8 & & 30. & 30 & 28. & & 33.167 & 36.112 & 34 & 17.181 & 17.304 & 21.7 & 15.52 & 12.402 & 32.814 & 34.061 & 30.401 & 33.126 & 27.181 & 34.485 \\
\hline 10/20/11 4:30 & & 30. & 30.4 & 29.318 & & & 29.7 & & 30.429 & 30.148 & 28.9 & 26.3 & 33.139 & 36.11 & 34.201 & 17.1 & 17.286 & 21.797 & 15.518 & 12.376 & 32.798 & 34.033 & 30.394 & 33.1 & 27.183 & 34.483 \\
\hline $10 / 20 / 115: 00$ & & 30.987 & 30.4 & 29.323 & & & 29.798 & 30.9 & 30.427 & 30.148 & 28.5 & 26.303 & 33.116 & 36.112 & 34.173 & 17.181 & 17.274 & 21.808 & 15.515 & 12.346 & 32.786 & 34.011 & 30.392 & 33.076 & 27.183 & 34.485 \\
\hline 10/20/11 5:30 & & 30.99 & 30.492 & 29.33 & 30.7 & & 29.791 & 30.953 & 30.429 & 30.15 & 28.581 & 26.311 & 33.092 & 36.112 & 34.152 & 17.181 & 17.255 & 21.822 & 15.511 & 12.313 & 32.779 & 33.992 & 30.392 & 33.055 & 27.183 & 34.485 \\
\hline 10/20/11 6:00 & & 30.992 & 30.496 & 29.325 & 30.7 & & 29.793 & 30.953 & 30.427 & 30.15 & 28.579 & 26.315 & 34.096 & 36.112 & 34.53 & 17.183 & 17.243 & 21.847 & 15.511 & 12.675 & 33.003 & 35.113 & 30.392 & 34.085 & 27.183 & 34.488 \\
\hline 10/20/11 6:30 & & 30.994 & 30.496 & 29.337 & 30.717 & & 29.796 & 30.955 & 30.432 & 30.152 & 28.581 & 26.313 & 35.208 & 36.119 & 35.622 & 17.183 & 17.3 & 21.866 & 15.504 & 13.765 & 33.509 & 36.203 & 30.432 & 35.167 & 27.185 & 34.49 \\
\hline 10/20/11 7:00 & & 30.994 & 30.501 & 29.344 & 30.724 & & 29.786 & 30.958 & 30.429 & 30.155 & 28.586 & 26.325 & 35.868 & 36.112 & 36.375 & 17.186 & 17.402 & 21.882 & 15.506 & 14.52 & 33.81 & 36.836 & 30.472 & 35.81 & 27.185 & 34.49 \\
\hline 10/20/11 7:30 & & 30.994 & 30.501 & 29.358 & 30.738 & & 29.784 & 30.96 & 30.434 & 30.155 & 28.593 & 26.332 & 34.471 & 36.115 & 35.596 & 17.186 & 17.449 & 21.894 & 15.508 & 13.745 & 33.399 & 35.355 & 30.493 & 34.409 & 27.185 & 34.49 \\
\hline $10 / 20 /$ & & 30.999 & 30.506 & 29.367 & 30.755 & & 29.777 & 30.962 & 30.436 & 30.159 & 28.6 & 26.344 & 34.096 & 36.117 & 35.205 & 17.186 & 17.444 & 21.901 & 15.497 & 13.335 & 33.238 & 34.983 & 30.495 & 34.038 & 27.185 & 34.49 \\
\hline $10 / 20 / 118: 30$ & & 30.999 & 30.51 & 29.372 & 30.769 & & 29.779 & 30.965 & 30.436 & 30.159 & 28.605 & 26.351 & 33.879 & 36.112 & 34.977 & 17.186 & 17.43 & 21.91 & 15.497 & 13.103 & 33.152 & 34.765 & 30.497 & 33.83 & 27.185 & 12 \\
\hline
\end{tabular}




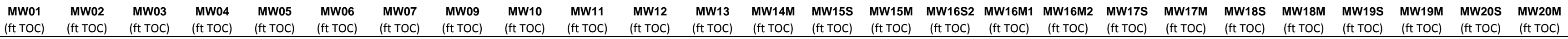

\begin{tabular}{|c|c|c|c|c|c|c|c|c|c|c|c|c|c|c|c|c|c|c|c|c|c|c|c|c|c|c|}
\hline Well & $\begin{array}{l}\text { MW01 } \\
\text { (ft TOC) }\end{array}$ & $\begin{array}{l}\text { MW02 } \\
\text { (ft TOC) }\end{array}$ & $\begin{array}{l}\text { Mw03 } \\
\text { (ft ToC) }\end{array}$ & $\begin{array}{r}\text { MWW4 } \\
\text { (ft TOC) }\end{array}$ & $\begin{array}{l}\text { MW05 } \\
\text { (ft TOC) }\end{array}$ & $\begin{array}{l}\text { MWvo6 } \\
\text { (ft TOC) }\end{array}$ & $\begin{array}{l}\text { MW07 } \\
\text { (ft TOC) }\end{array}$ & $\begin{array}{r}\text { Mw09 } \\
\text { (ft TOC) }\end{array}$ & $\begin{array}{r}\text { MW10 } \\
\text { (ft TOC) }\end{array}$ & $\begin{array}{r}\text { MWi1 } \\
\text { (ft TOC) }\end{array}$ & $\begin{array}{r}\text { MW12 } \\
\text { (ft TOC) }\end{array}$ & $\begin{array}{l}\text { MW13 } \\
(\mathrm{ft} \text { Toc) }\end{array}$ & $\begin{array}{l}\text { MW14M } \\
(\mathrm{ft} \text { TOC) }\end{array}$ & $\begin{array}{l}\text { MW15S } \\
\text { (ft TOC) }\end{array}$ & $\begin{array}{l}\text { MW15M } \\
\text { (ft TOC) }\end{array}$ & $\begin{array}{l}\text { MW16s2 } \\
(\mathrm{ft} \text { TOC) }\end{array}$ & $\begin{array}{l}\text { (ft TOC) } \\
\text { (ft }\end{array}$ & $\begin{array}{l}\text { MW16M2 } \\
\text { (ft TOC) }\end{array}$ & $\begin{array}{l}\text { MW17s } \\
(\mathrm{ft} \text { TOC) }\end{array}$ & (ft TOC) & $\begin{array}{l}\text { MW18S } \\
\text { (ft TOC) } \\
\end{array}$ & $\begin{array}{l}\text { MW18M } \\
\text { (ft TOC) }\end{array}$ & $\begin{array}{l}\text { MW19S } \\
\text { (ft TOC) }\end{array}$ & $\begin{array}{l}\text { MW19M } \\
\text { (ft TOC) }\end{array}$ & $\begin{array}{l}\text { MWW20S } \\
\text { (ft TOC) }\end{array}$ & $\begin{array}{l}\text { MWwaM } \\
\text { (ft TOC) }\end{array}$ \\
\hline 10/20/11 9:00 & & 31.004 & 30.517 & 29.37 & 30.78 & & 29.784 & 30.96 & 30.434 & 30.166 & 28.605 & 26.356 & 33.731 & 36.122 & 34.82 & 17.19 & 17.412 & 21.919 & 15.494 & 12.938 & 33.099 & 34.62 & 30.5 & 33.682 & 27.185 & 34.492 \\
\hline 10/20/11 9:30 & & 31.006 & 30.522 & 29.365 & 30.792 & & 29.793 & 30.962 & 30.434 & 30.171 & 28.605 & 26.354 & 33.617 & 36.122 & 34.701 & 17.193 & 17.395 & 21.931 & 15.494 & 12.82 & 33.054 & 34.509 & 30.5 & 33.574 & 27.185 & 34.492 \\
\hline 10/20/11 10:00 & & 31.004 & 30.526 & 29.358 & 30.794 & & 29.803 & 30.965 & 30.434 & 30.173 & 28.607 & 26.346 & 33.528 & 36.124 & 34.607 & 17.195 & 17.374 & 21.935 & 15.501 & 12.741 & 33.012 & 34.424 & 30.495 & 33.485 & 27.185 & 34.495 \\
\hline 10/20/11 10:30 & & 31.004 & 30.526 & 29.349 & 30.797 & & 29.812 & 30.965 & 30.432 & 30.173 & 28.607 & 26.339 & 33.455 & 36.124 & 34.53 & 17.195 & 17.356 & 21.944 & 15.511 & 12.675 & 32.98 & 34.348 & 30.483 & 33.41 & 27.188 & 34.495 \\
\hline 10/20/11 11:00 & & 31.001 & 30.524 & 29.339 & 30.792 & & 29.819 & 30.965 & 30.432 & 30.176 & 28.6 & 26.33 & 33.394 & 36.122 & 34.464 & 17.195 & 17.337 & 21.951 & 15.515 & 12.628 & 32.952 & 34.286 & 30.479 & 33.349 & 27.185 & 34.495 \\
\hline 10/20/11 11:30 & & 30.997 & 30.522 & 29.33 & 30.785 & & 29.829 & 30.962 & 30.434 & 30.173 & 28.6 & 26.32 & 33.336 & 36.124 & 34.408 & 17.193 & 17.316 & 21.958 & 15.522 & 12.595 & 32.917 & 34.232 & 30.46 & 33.295 & 27.185 & 34.495 \\
\hline 10/20/11 12:00 & & 30.999 & 30.522 & 29.309 & 30.778 & & 29.843 & 30.962 & 30.432 & 30.171 & 28.593 & 26.313 & 34.314 & 36.122 & 34.757 & 17.193 & 17.3 & 21.965 & 15.527 & 12.952 & 33.117 & 35.326 & 30.458 & 34.299 & 27.188 & 34.492 \\
\hline 10/20/11 12:30 & & 30.994 & 30.52 & 29.297 & 30.771 & & 29.857 & 30.96 & 30.427 & 30.169 & 28.584 & 26.294 & 35.402 & 36.119 & 35.824 & 17.193 & 17.353 & 21.972 & 15.532 & 14.05 & 33.6 & 36.395 & 30.474 & 35.357 & 27.188 & 34.492 \\
\hline $10 / 20 / 11$ 13:00 & & 30.992 & 30.51 & 29.286 & 30.773 & & 29.866 & 30.958 & 30.432 & 30.164 & 28.577 & 26.279 & 36.041 & 36.122 & 36.555 & 17.193 & 17.454 & 21.981 & 15.543 & 14.807 & 33.887 & 37.002 & 30.502 & 35.979 & 27.188 & 34.49 \\
\hline 10/20/11 13:30 & & 30.987 & 30.496 & 29.274 & 30.771 & & 29.873 & 30.955 & 30.429 & 30.159 & 28.57 & 26.27 & 34.623 & 36.117 & 35.758 & 17.19 & 17.496 & 21.986 & 15.543 & 14.045 & 33.462 & 35.502 & 30.5 & 34.555 & 27.188 & 34.488 \\
\hline 10/20/11 14:00 & & 30.985 & 30.492 & 29.274 & 30.773 & & 29.876 & 30.95 & 30.432 & 30.155 & 28.565 & 26.263 & 34.232 & 36.112 & 35.348 & 17.19 & 17.484 & 21.991 & 15.55 & 13.657 & 33.278 & 35.113 & & 34.172 & 27.188 & 34.488 \\
\hline $10 / 20 / 1114: 30$ & & 30.983 & 30.482 & 29.276 & 30.769 & & 29.873 & 30.95 & 30.434 & 30.148 & 28.565 & 26.263 & 33.993 & 36.11 & 35.099 & 17.186 & 17.465 & 21.998 & 15.558 & 13.432 & 33.171 & 34.876 & 30.469 & 33.94 & 27.188 & 34.485 \\
\hline $10 / 20 / 1115: 00$ & & 30.985 & 30.475 & 29.274 & 30.764 & & 29.869 & 30.95 & 30.432 & 30.143 & 28.565 & 26.26 & 33.829 & 36.105 & 34.926 & 17.183 & 17.442 & 22.005 & 15.56 & 13.272 & 33.101 & 34.717 & 30.453 & 33.78 & 27.188 & 34.485 \\
\hline $10 / 20 / 1115: 30$ & & 30.987 & 30.475 & 29.281 & 30.762 & & 29.864 & 30.948 & 30.432 & 30.143 & 28.563 & 26.263 & 33.708 & 36.105 & 34.795 & 17.183 & 17.421 & 22.012 & 15.555 & 13.15 & 33.045 & 34.597 & 30.446 & 33.654 & 27.19 & 34.483 \\
\hline 10/20/11 16:00 & & 30.987 & 30.473 & 29.288 & 30.757 & & 29.857 & 30.951 & 30.436 & 30.141 & 28.563 & 26.268 & 33.607 & 36.098 & 34.691 & 17.183 & 17.4 & 22.023 & 15.558 & 13.051 & 33.001 & 34.497 & .436 & 33.56 & 27.19 & 34.483 \\
\hline 30 & & 30.987 & 30.473 & 29.295 & 30.75 & & 29.848 & 30.948 & 30.439 & 30.139 & 28.565 & 26.277 & 33.521 & 36.103 & 34.602 & 17.181 & 17.381 & 22.065 & 15.555 & 12.969 & 32.968 & .412 & .427 & 33.478 & 27.19 & 34.483 \\
\hline 00 & & 30.99 & 30.475 & 29.302 & 30.743 & & 29.84 & 30.953 & 30.441 & 30.139 & 28.57 & 26.282 & 33.455 & 36.103 & 34.532 & 17.181 & 17.36 & 22.09 & 5.555 & 12.894 & 32.94 & .345 & .425 & 7 & 7.19 & 34.485 \\
\hline $10 / 20 /$ & & 30.994 & 30.478 & 29.314 & 30.743 & & 29.831 & 30.953 & 30.441 & 30.141 & 28.572 & 26.289 & 35.137 & 36.105 & 35.58 & 17.183 & 17.372 & 22.099 & 15.55 & 13.935 & 33.481 & .132 & 443 & 5.057 & 27.19 & 34.483 \\
\hline $10 / 20 /$ & & 30.997 & 30.48 & 29.318 & 30.75 & & 29.822 & 30.953 & 30.441 & 30.141 & 28.577 & 26.301 & 35.994 & 36.105 & 36.38 & 17.183 & 17.456 & 22.106 & 15.548 & 14.725 & 33.81 & .023 & 479 & 5.925 & 27.192 & 34.485 \\
\hline 10/20/11 18:30 & & 30.997 & 30.485 & 29.33 & 30.762 & & 29.812 & 30.958 & 30.441 & 30.143 & 28.581 & 26.311 & 35.114 & 36.107 & 36.248 & 17.186 & 17.547 & 22.116 & 15.546 & 14.595 & 33.698 & 35.987 & 30.512 & 35.033 & 27.192 & 34.485 \\
\hline 10/20/11 19:00 & & 30.999 & 30.489 & 29.335 & 30.776 & & 29.808 & 30.96 & 30.444 & 30.146 & 28.584 & 26.32 & 34.508 & 36.112 & 35.639 & 17.186 & 17.55 & 22.123 & 15.543 & 13.961 & 33.42 & 35.388 & 30.521 & 34.447 & 27.192 & 34.488 \\
\hline 10/20/11 19:30 & & 31.004 & 30.494 & 29.332 & 30.792 & & 29.805 & 30.962 & 30.441 & 30.148 & 28.586 & 26.327 & 34.211 & 36.108 & 35.324 & 17.188 & 17.533 & 22.129 & 15.541 & 13.627 & 33.294 & 35.094 & 30.521 & 34.153 & 27.192 & 34.488 \\
\hline $10 / 20 / 1120: 00$ & & 31.006 & 30.499 & 29.335 & 30.804 & & 29.81 & 30.962 & 30.441 & 30.152 & 28.584 & 26.325 & 34.017 & 36.115 & 35.118 & 17.19 & 17.512 & 22.139 & 15.539 & 13.399 & 33.22 & 34.905 & 30.523 & 33.963 & 27.192 & 34.49 \\
\hline 10/20/11 20:30 & & 31.008 & 30.503 & 29.339 & 30.811 & & 29.808 & 30.965 & 30.444 & 30.155 & 28.586 & 26.323 & 33.872 & 36.119 & 34.966 & 17.19 & 17.491 & 22.148 & 15.536 & 13.227 & 33.162 & 34.76 & 30.519 & 33.82 & 27.195 & 34.49 \\
\hline 10/20/11 21:00 & & 31.006 & 30.503 & 29.342 & 30.811 & & 29.805 & 30.965 & 30.441 & 30.155 & 28.591 & 26.327 & 33.757 & 36.124 & 34.848 & 17.193 & 17.468 & 22.16 & 15.543 & 13.1 & 33.11 & 34.646 & 30.509 & 33.708 & 27.192 & 34.49 \\
\hline $10 / 20 / 1121: 30$ & & 31.006 & 30.503 & 29.344 & 30.811 & & 29.805 & 30.967 & 30.441 & 30.157 & 28.588 & 26.33 & 33.663 & 36.122 & 34.745 & 17.193 & 17.447 & 22.169 & 15.541 & 12.992 & 33.071 & 34.552 & 30.5 & 33.616 & 27.192 & 34.49 \\
\hline 10/20/11 22:00 & & 31.008 & 30.508 & 29.342 & 30.811 & & 29.808 & 30.97 & 30.443 & 30.159 & 28.591 & 26.334 & 33.586 & 36.119 & 34.666 & 17.195 & 17.423 & 22.176 & 15.532 & 12.896 & 33.038 & 34.476 & 30.495 & 33.539 & 27.192 & 34.49 \\
\hline 10/20/11 22:30 & & 31.011 & 30.51 & 29.339 & 30.806 & & 29.81 & 30.97 & 30.444 & 30.159 & 28.591 & 26.33 & 33.518 & 36.124 & 34.595 & 17.195 & 17.402 & 22.185 & 15.539 & 12.811 & 33.01 & 34.409 & 30.493 & 33.475 & 27.192 & 34.49 \\
\hline 10/20/11 23:00 & & 31.008 & 30.508 & 29.342 & 30.801 & & 29.808 & 30.967 & 30.444 & 30.162 & 28.593 & 26.327 & 33.457 & 36.124 & 34.53 & 17.195 & 17.381 & 22.192 & 15.536 & $\begin{array}{l}12.748 \\
12.748\end{array}$ & 32.98 & 34.35 & 30.483 & 33.412 & 27.195 & 34.49 \\
\hline 10/20/11 23:30 & & 31.008 & 30.512 & 29.346 & 30.799 & & 29.808 & 30.97 & 30.446 & 30.159 & 28.595 & 26.332 & $\begin{array}{l}33.406 \\
3.406\end{array}$ & 36.124 & 34.476 & 17.195 & $\begin{array}{r}17.36 \\
\end{array}$ & 22.197 & 15.534 & $\begin{array}{l}12.686 \\
12.686\end{array}$ & 32.956 & $\begin{array}{r}34.3 \\
34.0\end{array}$ & 30.474 & $\begin{array}{r}\quad 3.412 \\
\quad 33.36 \\
\end{array}$ & 27.195 & 34.49 \\
\hline $10 / 21 / 110: 00$ & & 31.011 & 30.51 & 29.351 & 30.7 & & 29.808 & 30.972 & 30.446 & 30.162 & 28.595 & 26.332 & $\begin{array}{l}33.357 \\
33.357\end{array}$ & 36.124 & $\begin{array}{l}34.429 \\
\end{array}$ & 17.197 & $\begin{array}{l}17.300 \\
\end{array}$ & 22.201 & 15.532 & $\begin{array}{l}12.621 \\
12.60\end{array}$ & 32.938 & 34.251 & 30.469 & 33.313 & 27.195 & 34.49 \\
\hline $10 / 21 / 110: 30$ & & 31.011 & 30.515 & 29.353 & 30. & & 29.808 & 30.972 & $\begin{array}{l}30.440 \\
30.448\end{array}$ & $\begin{array}{l}30.164 \\
30.164\end{array}$ & $\begin{array}{l}28.598 \\
28.598\end{array}$ & $\begin{array}{l}20.532 \\
26.337\end{array}$ & $\begin{array}{l}33.315 \\
3315\end{array}$ & $\begin{array}{l}36.126 \\
36.126\end{array}$ & 34.384 & 17.197 & $\begin{array}{l}17.323 \\
17.323\end{array}$ & 22.208 & 15.532 & $\begin{array}{l}12.574 \\
12.574\end{array}$ & 32.912 & 34.208 & 30.465 & 33.274 & 27.197 & $\begin{array}{l}34.49 \\
34.49\end{array}$ \\
\hline 10/21/11 1:00 & & 31.011 & 30.517 & 29.358 & 30.783 & & 29.808 & 30.97 & $\begin{array}{l}\begin{array}{r}50.446 \\
30.446\end{array} \\
\end{array}$ & $\begin{array}{l}30.164 \\
30.164\end{array}$ & 28.6 & $\begin{array}{l}20.351 \\
26.337\end{array}$ & $\begin{array}{l}33.271 \\
33.277\end{array}$ & $\begin{array}{l}30.126 \\
36.129\end{array}$ & $\begin{array}{l}34.384 \\
34.344\end{array}$ & 17.2 & 17.304 & 22.213 & $\begin{array}{l}13.52 \\
15.529\end{array}$ & $\begin{array}{l}12.527 \\
12.52\end{array}$ & $\begin{array}{l}32.912 \\
32.891 \\
\end{array}$ & 168 & $\begin{array}{r}30.403 \\
30.46\end{array}$ & $\begin{array}{l}33.274 \\
33.234\end{array}$ & 27.195 & $\begin{array}{l}34.49 \\
34.49\end{array}$ \\
\hline 10/21/11 1:30 & & 31.015 & 30.517 & 29.358 & 30.7 & & 29.808 & 30.972 & 30.448 & 30.166 & 28.602 & 26.342 & 33.242 & 36.131 & 34.309 & 17.2 & 17.288 & 22.222 & 15.527 & 12.484 & 32.875 & .137 & 30.458 & 33.198 & 27.195 & 34.492 \\
\hline & & & 30. & & 30.7 & & & & & & 28 & 26.339 & 33.2 & 36.126 & 34.2 & 17.2 & 269 & 22.231 & ריר & 107 & 32.861 & & .453 & 3.168 & 27.197 & 34.49 \\
\hline $10 /$ & & & & & & & & & & & 28 & & 33.1 & 36.1 & 34.2 & & & 6 & 2 & 12.404 & 32.849 & & 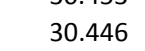 & & 97 & 34.492 \\
\hline $10 / 21 / 113: 00$ & & 31 & 30.52 & 29.353 & 30 & & & 30.972 & 18 & 30.169 & 28.6 & 26.337 & 33.151 & 36.131 & 34.211 & 17.202 & 239 & 13 & 25 & 12.374 & 32.833 & 047 & 46 & 12 & 77.197 & 34.49 \\
\hline $10 / 21 /$ & & 31. & 30.52 & 29.351 & 30. & & & 30.974 & 30.448 & 30.169 & 28.602 & 26.334 & 33.125 & 36.126 & 34.185 & 17.202 & 17.222 & 22.247 & 5.529 & 12.35 & 32.812 & 34.021 & 27 & 33.086 & 27.197 & 34.49 \\
\hline 10/21/11 4:00 & & 31. & 30.524 & 29.349 & 30. & & & 30.972 & 30.453 & 30.171 & 28.598 & 26.332 & 33.102 & 36.129 & 34.159 & 17.202 & 17.208 & 22.254 & 15.529 & 12.322 & 32.805 & & 29 & 33.062 & 27.197 & 34.492 \\
\hline 10/21/11 4:30 & & 31. & 30.522 & 29.344 & 30 & & & 30. & & 30.171 & 28.598 & 26.327 & 33.081 & 36.129 & 34.133 & 17.204 & 17.194 & 22.259 & 15.527 & 12.294 & 32.795 & & 7 & 33.039 & 27.199 & 34.492 \\
\hline $10 / 21 / 11$ 5:00 & & 31. & 30.522 & 29.346 & 30. & & & 30. & & 30.173 & 28.598 & 26.32 & 33.057 & 36.129 & 34.112 & 17.202 & 17.18 & 22.268 & 15.532 & 12.275 & 32.779 & 33.952 & 30.418 & 33.018 & 27.197 & 34.492 \\
\hline $10 / 21 / 115: 30$ & & 31. & 30.52 & 29 & & & & 30. & $30.4 \div$ & 30.169 & 28.6 & 26.323 & 33.036 & 36.129 & 34.091 & 17.204 & 17.166 & 22.275 & 15.534 & 12.259 & 32.763 & 33.933 & 30.408 & 32.997 & 27.199 & 34.492 \\
\hline 10/21/11 6:00 & & 31.013 & 30.517 & 29. & & & 29.8 & 30. & 30.4 & 30.169 & 28.602 & 26.332 & 34.047 & 36.124 & 34.473 & 17.204 & 17.155 & 22.282 & 15.529 & 12.628 & 32.989 & 35.063 & 30.408 & 34.038 & 27.197 & 34.492 \\
\hline 10/21/11 6:30 & & 31.015 & 30.52 & 29.363 & & & 29.819 & 30.977 & 30.45 & 30.169 & 28.607 & 26.337 & 35.159 & 36.136 & 35.566 & 17.204 & 17.215 & 22.291 & 15.522 & 13.723 & 33.493 & 36.153 & 30.441 & 35.118 & 27.199 & 34.492 \\
\hline 10/21/11 7:00 & & 31.018 & 30.524 & 29.374 & 30.7 & & 29.815 & 30.979 & 30.455 & 30.171 & 28.609 & 26.342 & 35.814 & 36.129 & 36.314 & 17.204 & 17.321 & 22.303 & 15.52 & 14.466 & 33.805 & 36.781 & 30.493 & 35.756 & 27.199 & 34.495 \\
\hline 10/21/11 7:30 & & 31.02 & 30.524 & 29.379 & 30.757 & & 29.81 & 30.982 & 30.458 & 30.173 & 28.614 & 26.356 & 34.419 & 36.133 & 35.54 & 17.204 & 17.37 & 22.321 & 15.515 & 13.69 & 33.395 & 35.3 & 30.512 & 34.355 & 27.199 & 34.497 \\
\hline $10 / 21 / 118: 00$ & & 31.022 & 30.529 & 29.386 & 30.773 & & 29.812 & 30.982 & 30.451 & 30.176 & 28.618 & 26.358 & 34.047 & 36.131 & 35.153 & 17.207 & 17.367 & 22.328 & 15.515 & 13.298 & 33.231 & 34.931 & 30.512 & 33.991 & 27.199 & 34.497 \\
\hline $10 / 21 / 118: 30$ & & 31.027 & 30.536 & 29.384 & 30.79 & & 29.815 & 30.982 & 30.458 & 30.18 & 28.621 & 26.366 & 33.832 & 36.133 & 34.926 & 17.209 & 17.353 & 22.335 & 15.508 & $\begin{array}{l}13.056 \\
\end{array}$ & 33.143 & 34.722 & 0.519 & 33.783 & 27.202 & 34.499 \\
\hline $10 / 21 / 1$ & & 31.027 & 30.538 & 29.384 & 30.79 & & 29.819 & 30.982 & $\begin{array}{l}30.453 \\
30.45\end{array}$ & $\begin{array}{l}30.180 \\
30.183\end{array}$ & 28.621 & 26.366 & $\begin{array}{l}33.082 \\
33.687\end{array}$ & 36.136 & 34.773 & 17.209 & $\begin{array}{l}17.339 \\
\end{array}$ & 22.344 & 15.515 & 12.903 & 33.087 & $\begin{array}{r}34.58 \\
34.78\end{array}$ & 0.521 & 33.64 & 27.202 & 34.502 \\
\hline 10 & & 31. & 30. & 29.377 & 30.8 & & 29.826 & 30.979 & 30.455 & 30.187 & 28.623 & 26.361 & 33.577 & 36.136 & 34.656 & 17.211 & 17.323 & 22.351 & 15.518 & 12.797 & 33.043 & 34.466 & 30.514 & 33.529 & 27.202 & 34.502 \\
\hline $10 / 21 /$ & & 31.025 & 30.54 & 29.374 & 30.8 & & 29.836 & 30.982 & 30.455 & 30.187 & 28.618 & 26.356 & 33.49 & 36.138 & 34.562 & 17.214 & 17.307 & 22.356 & 15.52 & 12.72 & 33.005 & 34.381 & 30.509 & 33.442 & 27.202 & 34.502 \\
\hline $10 / 21 /$ & & 31.025 & 30.54 & 29.367 & 30. & & 29 & 30.9 & 30.453 & 30.19 & 28.618 & 26.351 & 33.415 & 36.138 & 34.487 & 17.214 & 17.288 & 22.391 & 15.525 & 12.656 & 32.97 & 34.305 & 30.497 & 33.372 & 27.202 & 34.504 \\
\hline 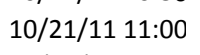 & & 31.0 & 30.5 & 29. & & & 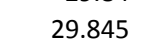 & & & 30 & & & 33.3 & 36. & 34. & & 1702 & 22.414 & 15. & 12.614 & 200 & & & 9 & 27.202 & 504 \\
\hline & & 31. & 30. & & & & & & & & & . & 20 & & & & & 2 & te & 12. & & & & 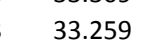 & 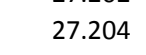 & 22 \\
\hline & & & & & & & & & & & & & & & & & & & & & & & & & & \\
\hline $112: 30$ & & 31.02 & 30.538 & 29.325 & 30.79 & & 29.88 & 30.982 & 455 & 30.185 & 28.6 & 26.318 & 35.374 & 36.138 & 35.793 & 17.214 & 17.293 & 22.423 & 5.543 & 1.033 & 33.602 & 6.369 & 497 & 5.331 & 7.204 & 02 \\
\hline
\end{tabular}




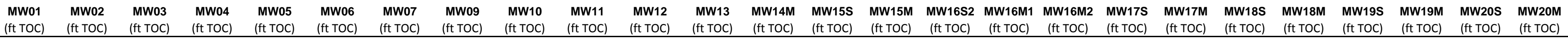

\begin{tabular}{|c|c|c|c|c|c|c|c|c|c|c|c|c|c|c|c|c|c|c|c|c|c|c|c|c|c|c|}
\hline Well & $\begin{array}{l}\text { MW01 } \\
\text { (ft TOC) }\end{array}$ & $\begin{array}{l}\text { MW02 } \\
\text { (ft TOC) }\end{array}$ & $\begin{array}{l}\text { MW03 } \\
\text { (ft TOC) }\end{array}$ & $\begin{array}{l}\begin{array}{l}\text { MW04 } \\
\text { (ft TOC) }\end{array}\end{array}$ & $\begin{array}{l}\text { MW05 } \\
\text { (ft Toc) }\end{array}$ & $\begin{array}{l}\text { MW06 } \\
\text { (ft TOC) }\end{array}$ & 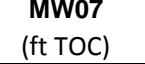 & $\begin{array}{l}\text { MW09 } \\
\text { (ft TOC) }\end{array}$ & $\begin{array}{l}\text { MW10 } \\
\text { (ft Toc) }\end{array}$ & $\begin{array}{l}\text { MW11 } \\
\text { (ft Toc) }\end{array}$ & $\begin{array}{l}\text { MW12 } \\
\text { (ft TOC) }\end{array}$ & $\begin{array}{l}\text { MW13 } \\
\text { (ft TOC) }\end{array}$ & $\begin{array}{l}\text { MW14M } \\
\text { (ft TOC) }\end{array}$ & $\begin{array}{l}\begin{array}{l}\text { MWW15S } \\
\text { (ft Toc) }\end{array}\end{array}$ & $\begin{array}{l}\text { MWW15M } \\
\text { (ft ToC) }\end{array}$ & $\begin{array}{l}\text { MW1652 } \\
\text { (ft TOC) }\end{array}$ & $\begin{array}{l}\text { MW16161 } \\
\text { (ft TOC) }\end{array}$ & $\begin{array}{c}\text { MW16162 } \\
\text { (ft TOC) }\end{array}$ & $\begin{array}{l}\text { MW17S } \\
\text { (ft TOC) }\end{array}$ & $\begin{array}{l}\text { MW17M } \\
\text { (ft TOC) }\end{array}$ & $\begin{array}{l}\text { MWW18S } \\
\text { (ft TOC) }\end{array}$ & $\begin{array}{l}\text { MW18M } \\
\text { (ft Toc) }\end{array}$ & $\begin{array}{l}\text { MWWigS } \\
\text { (ft TOC) }\end{array}$ & $\begin{array}{l}\text { MW19M } \\
\text { (ft TOC) }\end{array}$ & $\begin{array}{l}\text { MW20S } \\
\text { (ft TOC) }\end{array}$ & 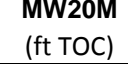 \\
\hline 10/21/11 13:00 & & 31.018 & 30.531 & 29.321 & 30.787 & & 29.887 & 30.977 & 30.451 & 30.18 & 28.598 & 26.306 & 36.008 & 36.133 & 36.52 & 17.214 & 17.393 & 22.425 & 15.548 & 14.79 & 33.894 & 36.971 & 30.521 & 35.946 & 27.202 & 34.499 \\
\hline 10/21/11 13:30 & & 31.013 & 30.522 & 29.309 & 30.792 & & 29.89 & 30.977 & 30.455 & 30.178 & 28.593 & 26.301 & 34.59 & 36.131 & 35.723 & 17.211 & 17.44 & 22.425 & 15.56 & 14.024 & 33.462 & 35.473 & 30.526 & 34.526 & 27.204 & 34.499 \\
\hline 10/21/11 14:00 & & 31.013 & 30.517 & 29.309 & 30.794 & & 29.895 & 30.972 & 30.455 & 30.171 & 28.591 & 26.289 & 34.202 & 36.131 & 35.315 & 17.209 & 17.428 & 22.43 & 15.565 & 13.639 & 33.285 & 35.087 & 30.512 & 34.144 & 27.204 & 34.495 \\
\hline $10 / 21 / 1114: 30$ & & 31.013 & 30.51 & 29.311 & 30.794 & & 29.89 & 30.974 & 30.455 & 30.171 & 28.586 & 26.291 & 33.968 & 36.124 & 35.071 & 17.209 & 17.412 & 22.432 & 15.569 & 13.408 & 33.183 & 34.855 & 30.502 & 33.914 & 27.204 & 34.492 \\
\hline $10 / 21 / 1115: 00$ & & 31.011 & 30.503 & 29.309 & 30.79 & & 29.892 & 30.972 & 30.458 & 30.164 & 28.584 & 26.289 & 33.804 & 36.124 & 34.9 & 17.207 & 17.388 & 22.435 & 15.576 & 13.258 & 33.115 & 34.694 & 30.486 & 33.754 & 27.204 & 34.492 \\
\hline $10 / 21 / 11$ 15:30 & & 31.013 & 30.503 & 29.307 & 30.787 & & 29.885 & 30.972 & 30.458 & 30.162 & 28.586 & 26.291 & 33.682 & 36.124 & 34.769 & 17.207 & 17.37 & 22.439 & 15.576 & 13.135 & 33.059 & 34.573 & 30.481 & 33.635 & 27.204 & 34.492 \\
\hline $10 / 21 / 1116: 00$ & & 31.013 & 30.503 & 29.307 & $\begin{array}{l}30.783 \\
303\end{array}$ & & 29.887 & $\begin{array}{l}30.97 \\
30.97\end{array}$ & $\begin{array}{l}30.455 \\
30.455\end{array}$ & 30.162 & 28.581 & 26.291 & $\begin{array}{l}33.584 \\
3.584\end{array}$ & 36.119 & 34.668 & 17.207 & 17.351 & 22.446 & 15.576 & 13.039 & 33.01 & 34.473 & $\begin{array}{l}30.467 \\
30.467\end{array}$ & $\begin{array}{l}33.056 \\
33.536\end{array}$ & 27.206 & $\begin{aligned} 54.472 \\
34.49\end{aligned}$ \\
\hline $10 / 21 / 1116: 30$ & & 31.015 & 30.501 & 29.307 & $\begin{array}{l}30.776 \\
30.776\end{array}$ & & $\begin{array}{l}29.881 \\
29.883\end{array}$ & 30.97 & $\begin{array}{r}\begin{array}{r}5.453 \\
30.46\end{array} \\
\end{array}$ & $\begin{array}{l}30.162 \\
30.162\end{array}$ & 28.581 & $\begin{array}{l}26.289 \\
26.289\end{array}$ & $\begin{array}{l}33.504 \\
33.504\end{array}$ & 36.122 & $\begin{array}{l}34.000 \\
34.579\end{array}$ & 17.207 & $\begin{array}{r}17.33 \\
17.33\end{array}$ & 22.481 & $\begin{array}{l}15.574 \\
15.57\end{array}$ & $\begin{array}{l}13.952 \\
12.952\end{array}$ & 32.977 & $\begin{array}{l}34.395 \\
34.395\end{array}$ & 30.458 & $\begin{array}{l}33.456 \\
33.456\end{array}$ & 27.206 & $\begin{array}{l}34.49 \\
34.49 \\
\end{array}$ \\
\hline 10/21/11 17:00 & & 31.015 & $\begin{array}{l}30.491 \\
30.09\end{array}$ & 29.316 & 30.771 & & 29.876 & 30.97 & $\begin{array}{l}30.456 \\
30.458\end{array}$ & $\begin{array}{l}30.159 \\
30.159\end{array}$ & $\begin{array}{l}28.581 \\
28.581\end{array}$ & $\begin{array}{l}26.299 \\
26.291\end{array}$ & $\begin{array}{l}33.044 \\
33.436\end{array}$ & $\begin{array}{l}30.117 \\
36.117\end{array}$ & 34.506 & 17.204 & $\begin{array}{l}17.33 \\
17.311\end{array}$ & 22.504 & $\begin{array}{l}13.5746 \\
15.576\end{array}$ & 12.889 & 32.94 & 34.326 & $\begin{array}{l}3.4 .48 \\
30.446\end{array}$ & $\begin{array}{l}33.430 \\
33.391\end{array}$ & 27.206 & $\begin{array}{l}54.49 \\
34.49 \\
\end{array}$ \\
\hline 10/21/11 17:30 & & 31.015 & 30.499 & 29.325 & 30.764 & & 29.866 & 30.97 & 30.462 & 30.157 & 28.586 & 26.299 & 35.109 & 36.124 & 35.559 & 17.204 & 17.325 & 22.506 & 15.581 & 13.942 & 33.49 & 36.099 & 30.458 & 35.031 & 27.206 & 34.49 \\
\hline 00 & & 31.018 & 30.501 & 29.335 & 30.766 & & 29.857 & 30.974 & 30.463 & 30.157 & 28.591 & 26.311 & 35.971 & 36.119 & 36.358 & 17.204 & 17.409 & 22.509 & 15.574 & 14.734 & 33.814 & 36.99 & 30.488 & 35.904 & 27.206 & 34.49 \\
\hline $18: 30$ & & 31.02 & 30.503 & 29.339 & 30.778 & & 29.85 & 30.974 & 30.465 & 30.159 & 28.595 & 26.32 & 35.093 & 36.124 & 36.225 & 17.207 & 17.503 & 22.516 & 15.572 & 14.602 & 33.7 & 35.966 & 30.53 & 5.015 & 27.206 & 34.492 \\
\hline $10 / 21 /$ & & 31.022 & 30.508 & 29.342 & 30.794 & & 29.845 & 30.977 & 30.463 & 30.162 & 28.595 & 26.325 & 34.489 & 36.126 & 35.615 & 17.209 & 17.51 & 22.52 & 5.569 & 13.97 & 33.418 & 35.371 & 30.54 & 4.428 & 27.209 & 34.495 \\
\hline $10 / 21$ & & 31.025 & 30.512 & 29.344 & 30.808 & & 29.845 & 30.979 & 30.46 & 30.162 & 28.595 & 26.33 & 34.195 & 36.124 & 35.303 & 17.211 & 17.494 & 22.527 & 5.567 & 13.646 & 33.297 & .075 & 30.54 & 4.137 & 27.206 & 34.495 \\
\hline $10 / 21 /$ & & 31.025 & 30.515 & 29.344 & 30.815 & & 29.843 & 30.979 & 30.462 & 30.164 & 28.598 & 26.33 & 33.998 & 36.131 & 35.102 & 17.211 & 17.472 & 22.532 & 15.565 & 13.422 & 33.215 & 34.886 & 30.535 & 33.945 & 27.206 & 34.495 \\
\hline $10 / 21 /$ & & 31. & 30.515 & 29.349 & 30.82 & & 29.843 & 30.984 & 30.46 & 30.166 & 28.598 & 26.332 & 33.855 & 36.129 & 34.947 & 17.211 & 17.451 & 22.534 & 15.565 & 13.258 & 33.157 & 34.743 & 30.526 & 33.806 & 27.209 & 34.497 \\
\hline 10/21/11 21:00 & & 31.027 & 30.517 & 29.353 & 30.822 & & 29.84 & 30.984 & 30.462 & 30.166 & 28.598 & 26.334 & 33.743 & 36.133 & 34.827 & 17.214 & 17.433 & 22.539 & 15.565 & 13.129 & 33.108 & 34.632 & .519 & 33.694 & 27.206 & 34.497 \\
\hline $10 / 21 / 1121: 30$ & & 31.029 & 30.52 & 29.358 & 30.822 & & 29.833 & 30.984 & 30.465 & 30.166 & 28.605 & 26.339 & 33.647 & 36.133 & 34.731 & 17.214 & 17.409 & 22.543 & 15.565 & 13.02 & 33.071 & 34.537 & .512 & 33.6 & 27.209 & 34.497 \\
\hline $10 / 21 / 1122: 00$ & & 31.027 & 30.522 & 29.363 & 30.82 & & 29.831 & 30.984 & 30.463 & 30.169 & 28.607 & 26.344 & 33.572 & 36.143 & 34.649 & 17.214 & 17.386 & 22.546 & 15.565 & 12.931 & 33.033 & 34.462 & 30.507 & 33.525 & 27.209 & 34.497 \\
\hline $10 / 21 / 1122: 30$ & & 31.034 & 30.526 & 29.365 & 30.82 & & 29.833 & 30.984 & 30.467 & 30.173 & 28.607 & 26.349 & 33.504 & 36.138 & 34.579 & 17.216 & 17.37 & 22.55 & 15.555 & 12.839 & 33.015 & 34.395 & 30.509 & 33.459 & 27.209 & 34.499 \\
\hline $10 / 21 / 1123: 00$ & & 31.034 & 30.529 & 29.36 & 30.818 & & 29.833 & 30.987 & 30.462 & 30.173 & 28.607 & 26.349 & 33.448 & 36.136 & 34.52 & 17.216 & 17.349 & 22.557 & 15.558 & 12.769 & 32.984 & 34.338 & 30.502 & 33.403 & 27.211 & 34.499 \\
\hline 10/21/11 23:30 & & 31.032 & 30.529 & 29.358 & 30.815 & & 29.838 & 30.989 & 30.465 & 30.176 & 28.607 & 26.344 & 33.397 & 36.138 & 34.464 & 17.218 & 17.328 & 22.56 & 15.558 & 12.708 & 32.961 & 34.286 & 30.493 & 33.353 & 27.209 & 34.499 \\
\hline 10/22/11 0:00 & & 31.034 & 30.533 & 29.353 & 30.813 & & 29.843 & 30.987 & 30.467 & 30.178 & 28.605 & 26.342 & 33.35 & 36.138 & 34.415 & 17.221 & 17.309 & 22.562 & 15.555 & 12.647 & 32.94 & 34.246 & 30.493 & 33.306 & 27.211 & 34.499 \\
\hline 10/22/11 0:30 & & 31.029 & 30.529 & 29.353 & 30.804 & & 29.845 & 30.989 & 30.465 & 30.178 & 28.605 & 26.337 & 33.305 & 36.145 & 34.37 & 17.221 & 17.293 & 22.566 & 15.555 & 12.602 & 32.912 & 34.201 & 30.483 & 33.264 & 27.209 & 34.497 \\
\hline 10/22/11 1:00 & & 31.032 & 30.531 & 29.353 & 30.797 & & 29.845 & 30.989 & 30.465 & 30.176 & 28.605 & 26.334 & 33.27 & 36.14 & 34.333 & 17.221 & 17.274 & 22.569 & 15.555 & 12.562 & 32.898 & & 30.474 & 33.227 & 27.211 & 34.497 \\
\hline 10/22/11 1:30 & & 31.029 & 30.529 & 29.351 & 30.79 & & 29.848 & 30.989 & 30.465 & 30.176 & 28.602 & $\begin{array}{l}20.534 \\
26.337\end{array}$ & 33.23 & 36.143 & 34.293 & 17.218 & 17.255 & 22.569 & 15.553 & 12.517 & 32.877 & 34.127 & 30.465 & 33.191 & 27.211 & 34.497 \\
\hline $10 / 22 / 112: 00$ & & 31.032 & 30.531 & 29.346 & 30.783 & & $\begin{array}{r}2.0406 \\
29.85\end{array}$ & 30.989 & $\begin{array}{l}30.403 \\
30.463\end{array}$ & 30.176 & $\begin{array}{l}20.002 \\
28.602\end{array}$ & $\begin{array}{l}2.531 \\
26.33\end{array}$ & $\begin{array}{r}r 3.25 \\
33.2\end{array}$ & $\begin{array}{l}30.135 \\
36.138\end{array}$ & 34.26 & $\begin{array}{l}17.210 \\
17.218\end{array}$ & 17.241 & 22.573 & $\begin{array}{l}15.555 \\
15.555\end{array}$ & 12.478 & 32.863 & 1.094 & $\begin{array}{l}\begin{array}{r}5.4403 \\
30.46\end{array} \\
\end{array}$ & 33.158 & 27.211 & 34.495 \\
\hline $10 / 22 / 112: 30$ & & 31.032 & 30.529 & $\begin{array}{l}29.340 \\
29.342\end{array}$ & 30.776 & & $\begin{array}{l}29.857 \\
2957\end{array}$ & 30.989 & $\begin{array}{l}30.405 \\
30.465\end{array}$ & $\begin{array}{l}30.176 \\
30.176\end{array}$ & $\begin{array}{l}28.0068 \\
28.598\end{array}$ & $\begin{array}{l}26.33 \\
2637\end{array}$ & 33.167 & $\begin{array}{l}50.130 \\
36.14\end{array}$ & $\begin{array}{l}34.227 \\
34.22\end{array}$ & $\begin{array}{l}17.218 \\
17.221\end{array}$ & $\begin{array}{l}17.222 \\
17.222\end{array}$ & 22.576 & $\begin{array}{l}1.553 \\
15.53\end{array}$ & $\begin{array}{l}12.474 \\
12.44\end{array}$ & 32.84 & 1.063 & $\begin{array}{l}30.460 \\
30.488\end{array}$ & $\begin{array}{l}33.128 \\
33.128\end{array}$ & 27.211 & $\begin{array}{l}34.495 \\
34.495\end{array}$ \\
\hline $10 / 22 / 113: 00$ & & $\begin{array}{l}31.022 \\
31.029\end{array}$ & $\begin{array}{l}30.529 \\
30.526\end{array}$ & 29.342 & $\begin{array}{l}30.7 \\
30.7\end{array}$ & & $\begin{array}{l}29.851 \\
29.855\end{array}$ & 30.986 & $\begin{array}{l}30.465 \\
30.465\end{array}$ & $\begin{array}{l}30.176 \\
30.176\end{array}$ & $\begin{array}{l}28.598 \\
28.598\end{array}$ & $\begin{array}{l}20.327 \\
26.323\end{array}$ & $\begin{array}{l}33.137 \\
33.139\end{array}$ & $\begin{array}{l}30.14 \\
36.14\end{array}$ & $\begin{array}{l}34.227 \\
34.199\end{array}$ & $\begin{array}{l}17.221 \\
17.221\end{array}$ & 17.206 & $\begin{array}{l}22.586 \\
22.583\end{array}$ & $\begin{array}{l}13.533 \\
15.558\end{array}$ & $\begin{array}{l}12.444 \\
12.409\end{array}$ & $\begin{array}{r}32.84 \\
32.826\end{array}$ & .035 & $\begin{array}{l}30.48 \\
30.441\end{array}$ & $\begin{array}{l}33.188 \\
33.097\end{array}$ & 27.211 & $\begin{array}{l}34.495 \\
34.495\end{array}$ \\
\hline $10 / 22 /$ & & 31.029 & 30.522 & 29.349 & 30. & & 29.852 & 30.989 & 30.465 & 30.173 & 28.598 & 26.323 & 33.113 & 36.143 & 34.171 & 17.218 & 17.187 & 22.58 & 15.555 & 12.383 & 32.807 & 009 & .432 & 72 & 27.211 & 34.492 \\
\hline $10 / 22 /$ & & & 30.522 & 29.353 & & & 29.848 & 30.989 & 30.467 & 30.171 & 28.602 & 26.33 & 33.085 & 36.138 & $\begin{array}{r}34.14 \\
34.14\end{array}$ & 17.221 & 17.173 & 22.592 & 855 & 12.358 & 32.793 & 83 & 25 & 48 & 27.211 & $\begin{array}{l}34.492 \\
34.42\end{array}$ \\
\hline $10 / 221$ & & & 30.522 & 29.358 & & & 29.843 & $\begin{array}{l}30.989 \\
3099\end{array}$ & $\begin{array}{l}3.4017 \\
30.47\end{array}$ & 30.171 & $\begin{array}{l}28.007 \\
28.607\end{array}$ & $\begin{array}{l}26.033 \\
2634\end{array}$ & $\begin{array}{l}33.064 \\
33.064\end{array}$ & $\begin{array}{l}30.130 \\
36.136\end{array}$ & $\begin{array}{l}34.119 \\
34.119\end{array}$ & 17.221 & 17.162 & 22.596 & $\begin{array}{l}1.555 \\
15.55\end{array}$ & $\begin{array}{l}12.358 \\
12.327\end{array}$ & $\begin{array}{l}32.93 \\
32.784\end{array}$ & 954 & 4.425 & 3.025 & $\begin{array}{l}27.211 \\
27.213\end{array}$ & $\begin{array}{l}34.442 \\
34.492\end{array}$ \\
\hline $10 / 22 / 115: 00$ & & & 30.522 & 29.356 & 30.743 & & 29.843 & 30.991 & 30.47 & 30.171 & 28.607 & 26.339 & 33.046 & 36.138 & 34.094 & 17.218 & 17.148 & 22.601 & 15.55 & 12.294 & 32.777 & 928 & 422 & 3.004 & 27.211 & 34.492 \\
\hline 10/22/11 5:30 & & 31.034 & 30.526 & 29.349 & 30.738 & & 29.85 & 30.991 & 30.465 & 30.173 & 28.605 & 26.334 & 33.025 & 36.147 & 34.079 & 17.223 & 17.138 & 22.603 & 15.548 & 12.268 & 32.765 & 33.902 & 30.422 & 32.987 & 27.213 & 34.495 \\
\hline 10/22/11 6:00 & & 31. & 30.526 & 29.353 & 30. & & 29.855 & 30.991 & 30.47 & 30.176 & 28.602 & 26.327 & 34.04 & 36.14 & 34.466 & 17.223 & 17.129 & 22.624 & 15.548 & 12.639 & 32.994 & 35.03 & 22 & 34 & 27.213 & 34.495 \\
\hline $10 / 22 / 116: 30$ & & 31. & 30.524 & 29.351 & & & 29.852 & 30.993 & 30.472 & 30.176 & 28.602 & 26.33 & 35.152 & 36.143 & 35.559 & 17.225 & 17.187 & 22.629 & 15.546 & 13.744 & 33.497 & 36.125 & & 35.115 & 27.213 & 34.495 \\
\hline $10 / 22 / 117: 00$ & & 31.032 & 30.524 & 29.358 & 30. & & 29.85 & 30.991 & 30.47 & 30.173 & 28.605 & 26.332 & 35.807 & 36.14 & 36.307 & 17.223 & 17.293 & 22.631 & 15.548 & 14.494 & 33.798 & 36.75 & 30.493 & 35.751 & 27.213 & 34.497 \\
\hline $10 / 22 / 117: 30$ & & 31.032 & 30.526 & 29.363 & 30.7 & & 29.848 & 30.993 & 30.47 & 30.173 & 28.607 & 26.337 & 34.41 & 36.143 & 35.528 & 17.225 & 17.344 & 22.633 & 15.546 & 13.726 & 33.381 & 35.267 & 30.507 & 34.346 & 27.213 & 34.497 \\
\hline $10 / 22 / 118: 00$ & & 31.034 & 30.524 & 29.365 & 30.7 & & 29.843 & 30.991 & 30.477 & 30.173 & 28.609 & 26.342 & 34.038 & 36.145 & 35.144 & 17.225 & 17.339 & 22.645 & 15.546 & 13.331 & 33.217 & 34.895 & 30.509 & 33.982 & 27.216 & 34.499 \\
\hline $10 / 22 / 118: 30$ & & 31.039 & 30.529 & 29.365 & 30. & & 29.845 & 30.993 & 30.472 & 30.176 & 28.612 & 26.349 & 33.82 & 36.143 & 34.914 & 17.225 & 17.328 & 22.652 & 15.539 & 13.096 & 33.134 & 34.675 & 30.509 & 33.771 & 27.216 & 34.499 \\
\hline 10/22/11 9:00 & & 31.041 & 30.531 & 29.365 & 30.7 & & 29.855 & 30.994 & 30.472 & 30.176 & 28.609 & 26.346 & 33.675 & 36.147 & 34.762 & 17.228 & 17.309 & 22.657 & 15.539 & 12.936 & 33.073 & 34.525 & 30.509 & 33.626 & 27.216 & 34.499 \\
\hline 10/22/11 9:30 & & 31.041 & 30.533 & 29.363 & 30.794 & & 29.852 & 30.996 & 30.47 & 30.178 & 28.612 & 26.344 & 33.565 & 36.147 & 34.645 & 17.228 & 17.297 & 22.659 & 15.543 & 12.828 & 33.024 & 34.421 & 30.505 & 33.518 & 27.216 & 34.502 \\
\hline 10/22/11 10:00 & & 31.036 & 30.533 & 29.363 & 30.792 & & 29.859 & 30.993 & 30.47 & 30.178 & 28.612 & 26.344 & 33.478 & 36.145 & 34.551 & 17.228 & 17.279 & 22.664 & 15.55 & 12.75 & 32.984 & 34.345 & 30.488 & 33.428 & 27.216 & 34.504 \\
\hline 10/22/11 10:30 & & 31.036 & 30.536 & 29.36 & 30.7 & & 29.862 & 30.993 & 30.472 & 30.178 & 28.609 & 26.342 & 33.406 & 36.143 & 34.473 & 17.228 & 17.264 & 22.666 & 15.539 & 12.682 & 32.952 & .284 & 30.483 & 33.358 & 27.216 & 34.504 \\
\hline 10/22/11 11:00 & & 31.039 & 30.533 & 29.351 & 30.79 & & 29.866 & 30.993 & 30.47 & 30.178 & 28.609 & 26.339 & 33.343 & 36.15 & 34.412 & 17.228 & $\begin{array}{l}17.246 \\
\end{array}$ & 22.668 & 15.55 & 12.628 & 32. & .234 & 6 & 33.302 & 27.218 & 34.504 \\
\hline $10 / 22 /$ & & 31.039 & 30.536 & 29.346 & 30.787 & & 29.876 & 30.996 & 30.472 & 30.18 & 28.605 & 26.33 & $\begin{array}{l}33.289 \\
3.289\end{array}$ & 36.147 & $\begin{array}{l}34.358 \\
34.358\end{array}$ & $\begin{array}{l}17.228 \\
17.228\end{array}$ & $\begin{array}{l}17.232 \\
\end{array}$ & 22.673 & 15.55 & 12.585 & 32.905 & .189 & 4.469 & 33.248 & 27.218 & 34.504 \\
\hline $10 /$ & & 31.039 & 30.533 & $\begin{array}{l}29.340 \\
2935\end{array}$ & $\begin{array}{l}30.78 \\
30.78\end{array}$ & & $\begin{array}{l}29.88 \\
29.88\end{array}$ & 30.993 & $\begin{array}{l}30.472 \\
30.472\end{array}$ & $\begin{array}{r}30.178 \\
30.178\end{array}$ & $\begin{array}{r}28.009 \\
28.6\end{array}$ & $\begin{array}{l}26.33 \\
2637\end{array}$ & $\begin{array}{l}33.289 \\
34.281\end{array}$ & $\begin{array}{l}30.147 \\
36.147\end{array}$ & $\begin{array}{l}54.350 \\
34.717\end{array}$ & $\begin{aligned} 17.28 \\
17.23\end{aligned}$ & $\begin{array}{l}17.222 \\
17.215\end{array}$ & 22.675 & $\begin{array}{l}15.53 \\
15.53\end{array}$ & $\begin{array}{l}12.380 \\
12.952\end{array}$ & 33.106 & 17 & 52 & $\begin{array}{l}33.248 \\
34.266\end{array}$ & 27.218 & $\begin{array}{l}34.502 \\
34.502\end{array}$ \\
\hline & & 31. & 30. & 29 & 30. & & 29.8 & 30. & 30 & 30. & 28.5 & 26 & 35. & 36. & 35 & 17. & 17 & 22.682 & 15.558 & 14 & 1 & & 6 & 35. & 27.218 & 34.502 \\
\hline & & 31. & 30. & 29.3 & & & & & 30 & & 28 & & 36. & 36. & 36 & 17 & & 22 & 15 & 14. & & & & & 27.218 & 34.502 \\
\hline & & 31. & & 29. & & & & & & & 28 & & 35. & 36. & & & & & & & & & & & 27.218 & . \\
\hline & & & 30.5 & 29.3 & & & & & & & & & & & & & & & & & & & & & 8 & \\
\hline & & & & & & & & & & & & & & & & & & 22 & & & 33 & & & & 27. & \\
\hline & & & & & & & & & & & & & & & & & & & & & & & & & 27.218 & \\
\hline 10 & & & 30. & & & & & & & & & 26. & 35.0 & & & 17 & 17. & 22 & 15 & & & & & & 27.22 & 34. \\
\hline 10/22/11 16:00 & & 31.034 & 30.508 & 29.304 & 30.832 & & 29.899 & 30.984 & 30.474 & 30.162 & 28.577 & 26.296 & 35.1 & 36.138 & 36.035 & 17.225 & 17.557 & 22.684 & 15.574 & 14.37 & 33.598 & 35.999 & 30.561 & 35.04 & 27.22 & 34.495 \\
\hline 10/22/11 16:30 & & 31.032 & 30.506 & 29.274 & 30.834 & & 29.904 & 30.984 & 30.472 & 30.159 & 28.57 & 26.291 & 34.7 & 36.133 & 35.826 & 17.223 & 17.57 & 22.689 & 15.579 & 14.177 & 33.509 & 35.577 & 30.556 & 34.632 & 27.22 & 34.492 \\
\hline
\end{tabular}




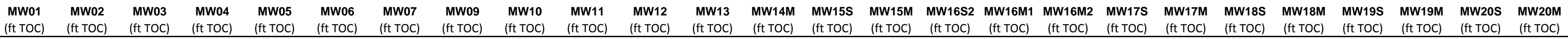

\begin{tabular}{|c|c|c|c|c|c|c|c|c|c|c|c|c|c|c|c|c|c|c|c|c|c|c|c|c|c|c|}
\hline Well & $\begin{array}{l}\text { (ft TOC) } \\
\text { (ft }\end{array}$ & $\begin{array}{l}\text { (ft TOC) } \\
\text { (f) }\end{array}$ & $\begin{array}{l}\text { (ft TOC) } \\
\text { (f) }\end{array}$ & $\begin{array}{l}\text { (ft TOC) } \\
\text { (f }\end{array}$ & $\begin{array}{l}\text { (ft TOC) } \\
\text { (f) }\end{array}$ & $\begin{array}{l}\text { (ft TOC) } \\
\text { (f) }\end{array}$ & $\begin{array}{l}\text { (ft TOC) } \\
\text { (ft }\end{array}$ & $\begin{array}{l}\text { (ft TOC) } \\
\text { (f) }\end{array}$ & $\begin{array}{l}\text { (ft TOC) } \\
\text { (ft) }\end{array}$ & $\begin{array}{l}\text { (ft TOC) } \\
\text { (f) }\end{array}$ & $\begin{array}{l}\text { (ft TOC) } \\
\text { (f) }\end{array}$ & $\begin{array}{l}\text { (ft TOC) } \\
\text { (f) }\end{array}$ & (ft TOC) & $\begin{array}{l}\text { (ft TOC) } \\
\text { (f) }\end{array}$ & (ft TOC) & (ft TOC) & $\begin{array}{l}\text { (ft TOC) } \\
\text { (f) }\end{array}$ & (ft TOC) & $\begin{array}{l}\text { (ft TOC) } \\
\text { (1) }\end{array}$ & (ft TOC) & $\begin{array}{l}\text { (ft TOC) } \\
\end{array}$ & $\begin{array}{l}\text { (ft TOC) } \\
\text { (1) }\end{array}$ & $\begin{array}{l}\text { (ft TOC) } \\
\text { (n) }\end{array}$ & (ft TOC) & $\begin{array}{l}\text { (ft TOC) } \\
\text { (1) }\end{array}$ & (ft TOC) \\
\hline 00 & & 31.029 & 30.499 & 29.269 & 30.834 & & 29.9 & 30.979 & 30 & 30.1 & 28.56 & 26 & 34.337 & 36.133 & 35.4 & 17. & 17. & 22.707 & 15.583 & 13.817 & 33.343 & & 30.542 & 34.282 & 18 & 92 \\
\hline 10/22/11 17:30 & & 31.025 & 30.494 & 29.279 & 30.829 & & 29.909 & 30.977 & 30.472 & 30.152 & 28.558 & 26.26 & 35.868 & 36.133 & 36.37 & 17.223 & & 22.719 & 15.586 & & 33.801 & 36.838 & 30.542 & .782 & 18 & 34.492 \\
\hline 10/22/11 18:00 & & 31.022 & 30.48 & 29.281 & 30.822 & & 29.902 & 30.974 & 30.472 & 30.141 & 28.558 & 26.27 & 36.628 & 36.129 & 37.059 & 17.218 & 17.652 & 22.744 & 15.593 & 15.446 & 34.073 & 37.634 & 30.547 & .559 & ר? & 34.49 \\
\hline 10/22/11 18:30 & & 31.022 & 30.475 & 29.295 & 30.825 & & 29.887 & 30.974 & 30.477 & 30.139 & 28.563 & 26.275 & 35.673 & 36.129 & 36.841 & 17.218 & 17.741 & 22.758 & 15.59 & 15.239 & 33.929 & 36.54 & 30.577 & 35.592 & 22 & 34.49 \\
\hline 10/22/11 19:00 & & 31.025 & 30.478 & 29.318 & 30.836 & & 29.866 & 30.977 & 30.482 & 30.139 & 28.57 & 26.291 & 35.327 & 36.124 & 36.497 & $\begin{array}{l}7 \quad 17.218 \\
\end{array}$ & 17.783 & 22.77 & 15.583 & 14.884 & 33.707 & 36.203 & 30.582 & 35.252 & 27.22 & 34.49 \\
\hline 10/22/11 19:30 & & 31.027 & 30.48 & 29.332 & 30.846 & & 29.85 & 30.982 & 30.481 & 30.136 & 28.579 & 26.313 & 34.852 & 36.124 & 36 & $\begin{array}{ll}5 & 17.218\end{array}$ & 17.781 & 22.779 & 15.586 & 14.377 & 33.553 & 35.729 & 30.575 & 34.787 & 27.22 & 34.492 \\
\hline 10/22/11 20:00 & & 31.034 & 30.487 & 29.339 & 30.857 & & 29.843 & 30.984 & 30.479 & 30.143 & 28.581 & 26.33 & 34.571 & 36.124 & 35.702 & $2 \quad 17.221$ & 17.753 & 22.786 & 15.569 & 14.05 & 33.458 & 35.452 & 30.582 & 34.512 & 27.223 & 34.492 \\
\hline 10/22/11 20:30 & & 31.036 & 30.496 & 29.335 & 30.869 & & 29.845 & 30.987 & 30.479 & 30.148 & 28.581 & 26.334 & 34.368 & 36.136 & 35.486 & $\begin{array}{ll}6 & 17.225\end{array}$ & 17.723 & 22.791 & 15.56 & 13.803 & 33.388 & 35.253 & 30.589 & 34.313 & 27.223 & 34.495 \\
\hline 10/22/11 21:00 & & 31.039 & 30.501 & 29.332 & 30.876 & & 29.845 & 30.986 & 30.477 & 30.15 & 28.581 & 26.332 & 34.211 & 36.136 & 35.32 & $2 \quad 17.225$ & 17.692 & 22.795 & 15.56 & 13.622 & 33.325 & 35.097 & 30.589 & 34.158 & 27.223 & 34.499 \\
\hline 10/22/11 21:30 & & 31.039 & 30.506 & 29.337 & 30.881 & & 29.843 & 30.989 & 30.477 & 30.155 & 28.586 & 26.332 & 34.085 & 36.133 & 35.186 & $\begin{array}{ll}6 & 17.225\end{array}$ & 17.659 & 22.802 & 15.56 & 13.472 & 33.276 & 34.969 & 30.582 & 34.031 & 27.223 & 34.499 \\
\hline $10 / 22 / 1122: 00$ & & 31.039 & 30.508 & 29.337 & 30.878 & & 29.845 & 30.991 & 30.477 & 30.155 & 28.584 & 26.337 & 33.975 & 36.138 & 35.069 & $\begin{array}{l}9 \\
9\end{array} 17.225$ & 17.631 & 22.802 & 15.562 & 13.354 & 33.225 & 34.862 & 30.568 & 33.923 & 27.223 & 34.499 \\
\hline $10 / 22 / 1122: 30$ & & 31.039 & 30.508 & 29.33 & 30.874 & & 29.845 & 30.993 & 30.479 & 30.155 & 28.581 & 26.334 & 33.879 & 36.138 & 34.97 & $7 \quad 17.228$ & 17.601 & 22.805 & 15.562 & 13.244 & 33.183 & 34.77 & 30.561 & 33.832 & 27.223 & 34.502 \\
\hline 10/22/11 23:00 & & 31.041 & 30.51 & 29.33 & 30.869 & & 29.85 & 30.993 & 30.479 & 30.157 & 28.581 & 26.33 & 33.797 & 36.143 & 34.881 & $1 \quad 17.228$ & 17.575 & 22.809 & 15.56 & 13.152 & 33.152 & 34.687 & 30.554 & 33.75 & 27.223 & 34.499 \\
\hline $10 / 22 / 1123: 30$ & & 31.041 & 30.51 & 29.328 & 30.864 & & 29.848 & 30.993 & 30.481 & 30.157 & 28.584 & 26.327 & 33.724 & 36.14 & 34.806 & $\quad 17.23$ & 17.547 & 22.809 & 15.562 & 13.07 & 33.12 & 34.618 & 30.542 & 33.677 & 27.223 & 34.502 \\
\hline 10/23/11 0:00 & & 31.039 & 30.508 & 29.325 & 30.855 & & 29.85 & 30.993 & 30.482 & 30.157 & 28.581 & 26.327 & 33.659 & 36.143 & 34.736 & 17.23 & 17.517 & 22.812 & 15.567 & 12.997 & 33.08 & 34.552 & 30.533 & 33.611 & 27.223 & 34.499 \\
\hline 10/23/11 0:30 & & 31.041 & 30.51 & 29.33 & 30.85 & & 29.852 & 30.993 & 30.482 & 30.157 & 28.581 & 26.325 & 33.598 & 36.143 & 34.675 & 17.23 & 17.493 & 22.816 & 15.562 & 12.933 & 33.059 & 34.49 & & 33.555 & 27.225 & 34.497 \\
\hline 10/23/11 1:00 & & 31.041 & 30.508 & 29.328 & 30.839 & & 29.85 & 30.996 & 30.482 & 30.155 & 28.581 & 26.327 & 33.542 & 36.147 & 34.616 & 17.23 & 17.468 & 22.816 & 15.567 & 12.875 & 33.024 & 34.435 & 30.512 & 33.501 & 27.225 & 34.499 \\
\hline 10/23/11 1:30 & & 31.041 & 30.51 & 29.325 & 30.829 & & 29.852 & 30.996 & 30.481 & 30.155 & 28.579 & 26.325 & 33.493 & 36.145 & 34.567 & 17.232 & 17.444 & 22.818 & 15.562 & 12.816 & 33.003 & 34.388 & 30.502 & 33.452 & 27.225 & 34.497 \\
\hline 10/23/11 2:00 & & 31.041 & 30.51 & 29.323 & 30.822 & & 29.855 & 30.996 & 30.479 & 30.157 & 28.579 & 26.323 & 33.448 & 36.147 & 34.516 & 17.23 & 17.421 & 22.821 & 15.56 & 12.759 & 32.98 & 34.341 & 30.497 & 33.407 & 27.225 & 34.497 \\
\hline $2: 30$ & & 31.043 & 30.513 & 29.323 & 30. & & 29.855 & 30.996 & 30.482 & 30. & 28.579 & 26.323 & 33.406 & 36.143 & 34.473 & 17.23 & 17.4 & 22.823 & 15.558 & 12.712 & 32.959 & 34.3 & .488 & 33.365 & 27.225 & 34.495 \\
\hline 3:00 & & 31.041 & 30.51 & 29.318 & 30. & & 29.855 & 30.993 & 30.479 & 30. & 28.577 & 26.32 & 33.364 & 36.145 & 34.434 & 17.2 & 17.379 & 22.825 & 15.558 & 12.67 & 32.938 & 4.262 & .474 & 33.323 & 27.225 & 34.495 \\
\hline 10/23/11 3:30 & & 31.041 & 30.51 & 29.323 & 30.799 & & 29.855 & 30.996 & 30.486 & 30.155 & 28.579 & 26.318 & 33.331 & 36.143 & 34.396 & $\quad 17.232$ & 17.358 & 22.825 & 33 & 12.6 & 32.919 & .222 & .474 & 33.29 & 7.227 & 34.492 \\
\hline $10 / 23 / 1$ & & 31.041 & 30.506 & 29.335 & 30.79 & & 29.845 & 30.996 & 30.482 & 30.155 & 28.584 & 26.323 & 33.294 & 36.14 & 34.356 & 17.232 & 17.337 & 22.828 & 15.558 & 12.592 & 32.898 & 34.189 & .462 & 33.252 & 7.225 & 34.492 \\
\hline 10/23/11 4:30 & & 31.041 & 30.508 & 29.335 & 30.78 & & 29.843 & 30.996 & 30.484 & 30.152 & 28.586 & 26.332 & 33.263 & 36.143 & 34.326 & 17.23 & 17.318 & 22.83 & 15.553 & 12.56 & 32.882 & 34.156 & .455 & 33.222 & 27.227 & 34.49 \\
\hline 10/23/11 5:00 & & 31.045 & 30.51 & 29.335 & 30.778 & & 29.845 & 30.996 & 30.484 & 30.155 & 28.584 & 26.334 & 33.235 & 36.145 & 34.293 & 17.23 & 17.302 & 22.83 & 15.548 & 12.515 & 32.87 & 34.13 & 30.458 & 3.194 & 27.227 & 34.49 \\
\hline 10/23/11 5:30 & & 31.048 & 30.517 & 29.328 & 30.776 & & 29.848 & 30.996 & 30.482 & 30.157 & 28.584 & 26.332 & 33.209 & 36.143 & 34.267 & 17.232 & 17.283 & 22.837 & 15.543 & 12.48 & 32.861 & 34.104 & 30.462 & 3.168 & 27.227 & 34.492 \\
\hline 10/23/11 6:00 & & 31.048 & 30.515 & 29.332 & 30.771 & & 29.85 & 30.998 & 30.484 & 30.157 & 28.584 & 26.325 & 34.223 & 36.15 & 34.654 & 17.232 & 17.269 & 22.851 & 15.548 & 12.853 & 33.08 & 35.239 & 30.455 & 34.214 & 27.227 & 34.49 \\
\hline 10/23/11 6:30 & & 31.043 & 30.513 & 29.337 & 30.769 & & 29.845 & 30.999 & 30.484 & 30.157 & 28.586 & 26.33 & 35.325 & 36.15 & 35.742 & 17.234 & 17.328 & 22.853 & 15.55 & 13.951 & 33.572 & 36.322 & 30.483 & 35.289 & 27.227 & 34.492 \\
\hline 10/23/11 7:00 & & 31.046 & 30.513 & 29.337 & 30.773 & & 29.845 & 30.998 & 30.489 & 30.155 & 28.588 & 26.337 & 35.973 & 36.147 & 36.483 & 17.234 & 17.43 & 22.858 & 15.548 & 14.694 & 33.875 & 36.938 & 30.521 & 35.916 & 27.227 & 34.492 \\
\hline 10/23/11 7:30 & & 31.046 & 30.515 & 29.346 & 30.787 & & 29.845 & 30.998 & 30.486 & 30.157 & 28.586 & 26.339 & 34.571 & 36.15 & 35.695 & 17.234 & 17.479 & 22.86 & 15.546 & 13.904 & 33.469 & 35.447 & 30.537 & 34.503 & 27.227 & 34.492 \\
\hline $10 / 23 / 118: 00$ & & 31.045 & 30.517 & 29.356 & 30.799 & & 29.838 & 31.001 & 30.489 & 30.159 & 28.595 & 26.346 & 34.192 & 36.147 & 35.301 & 17.234 & 17.47 & 22.862 & 15.55 & 13.502 & 33.302 & 35.075 & 30.54 & 34.135 & 27.23 & 34.495 \\
\hline $10 / 23 / 118: 30$ & & 31.048 & 30.52 & 29.36 & 30.811 & & 29.833 & 30.998 & 30.491 & 30.159 & 28.598 & 26.354 & 33.972 & 36.147 & 35.069 & 17.237 & 17.454 & 22.874 & 15.546 & 13.272 & 33.208 & 34.857 & 30.537 & 33.919 & 27.227 & 34.497 \\
\hline 10/23/11 9:00 & & 31.053 & 30.524 & 29.36 & 30.818 & & 29.84 & 31.001 & 30.491 & 30.162 & 28.598 & 26.356 & 33.82 & 36.152 & 34.912 & 17.237 & 17.437 & 22.886 & 15.543 & 13.112 & 33.148 & 34.708 & 30.54 & 33.773 & 27.23 & 34.499 \\
\hline 10/23/11 9:30 & & 31.055 & 30.529 & 29.358 & 30.827 & & 29.845 & 31.003 & 30.486 & 30.166 & 28.6 & 26.356 & 33.708 & 36.152 & 34.79 & 17.239 & 17.421 & 22.886 & 15.539 & 12.987 & 33.108 & 34.597 & 30.542 & 33.661 & 27.23 & 34.502 \\
\hline 10/23/11 10:00 & & & 30 & 29.353 & & & 29.848 & 31.001 & 30.486 & & 28.6 & 26.354 & 33 & 36.154 & 34.696 & $\quad 17.23$ & 17.4 & 22.89 & 15.5 & 12.898 & 33.066 & 34.507 & 37 & 33.572 & 27.232 & 34.502 \\
\hline & & & & 29.358 & & & & 31.003 & & & 28.6 & 26.351 & & 36.152 & 34.614 & 17.239 & 17.384 & 22.895 & 15.5 & 12.83 & 33.033 & & & 33.499 & 27.23 & 34.502 \\
\hline $10 / 23 /$ & & & 30.533 & 29.349 & 30. & & 29.857 & 31.005 & 30.486 & 30.169 & 28.6 & 26.351 & 33.476 & 36.152 & 34.548 & 17.239 & $\begin{array}{l}17.04 \\
17.365\end{array}$ & 22.897 & 15.55 & $\begin{array}{l}12.03 \\
12.776\end{array}$ & $\begin{array}{l}33.003 \\
3.003\end{array}$ & .369 & 0.516 & $\begin{array}{l}33.473 \\
33.433\end{array}$ & 27.232 & 34.504 \\
\hline 11:30 & & 31.05 & 30.533 & 29.346 & 30 & & 29.864 & 31.003 & 30.491 & 30.171 & 28.598 & 26.346 & 33.422 & 54 & 34.492 & 17.244 & 17 & 22.899 & 15.553 & 12.729 & 32.973 & 34.315 & 12 & 79 & 27.232 & 34.504 \\
\hline & & 31.053 & 30 & 29.33 & 30 & & 29.8 & 31 & 30 & 30 & 28. & 26.339 & 34 & 36 & 34.853 & 17.241 & 17 & 22.899 & 15.558 & 13.096 & 33.183 & 35.428 & 77 & 34.404 & 27.232 & 34.504 \\
\hline & & & 30 & 29.318 & & & & 31 & & 30 & 28.586 & 26 & 35 & 36.154 & 35.9 & & 17.386 & 22.902 & 15.567 & 14 & 33.665 & ( & 28 & 58 & 27.232 & 34.504 \\
\hline & & & & 29 & & & & & & & 28.581 & & & 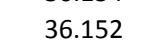 & 36. & & & & & 14 & 33 & & & 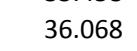 & 32 & 34.502 \\
\hline & & & & & & & & & & & & & & & & & & & & 14.158 & 33.528 & 5.582 & 3 & 34 & 27.234 & \\
\hline $10 /$ & & & 30.515 & 29.302 & 30. & & & & & & & & & 36.1 & & & & & 15. & 13.808 & 33.357 & & & & 27.232 & \\
\hline 10 & & & 30 & 29.297 & & & & 30 & & & 28 & & & 36.1 & 35.723 & 17. & 17.519 & 22 & 15. & 14.078 & 33.474 & & & & 27.234 & 34.499 \\
\hline 10 & & & & 29.29 & & & & & & & & & & 36.1 & & 17.237 & 17. & & & 14.189 & & & & & 27.232 & 34.495 \\
\hline $15: 30$ & & & & 29.293 & & & & & & & 28 & & & 36.1 & & 17. & & & 15. & 14.243 & 33.523 & & & & 27.234 & 34.495 \\
\hline 11 16:00 & & & 30 & 29.293 & & & 29. & 30. & 30.489 & 30. & 28. & & 34. & 36.1 & 35.911 & 17. & 17.566 & 22.943 & 15.5 & 14.311 & 33.544 & 35.881 & 30.549 & 34.925 & 27.234 & 34.492 \\
\hline 10/23/11 16:30 & & 31. & 30. & 29.3 & & & & 30. & 30. & & 28.9 & 26. & 35.0 & 36.1 & 35.96 & 17.2 & 17.5 & 22.99 & 15.5 & 14.367 & 33.563 & 35.928 & 30.547 & 34.972 & 27.234 & 34.495 \\
\hline 10/23/11 17:00 & & 31.0 & 30.4 & 29.314 & 30. & & 29.8 & 30.5 & 30.493 & 30.1 & 28.567 & 26.301 & 35.0 & 36.138 & 6 & 17.234 & 17.601 & 23.024 & 15.59 & 14.407 & 33.581 & 35.968 & 30.554 & 35.01 & 27.234 & 34.492 \\
\hline $10 / 23 / 1117: 30$ & & 31.041 & 30.49 & 29.328 & 30.8 & & 29.866 & 30.991 & 30.496 & 30.146 & 28.574 & 26.315 & 36.605 & 36.133 & 36.949 & 17.234 & 17.657 & 23.047 & 15.593 & 15.355 & 34.085 & 37.613 & 30.584 & 36.528 & 27.234 & 34.492 \\
\hline 10/23/11 18:00 & & 31.043 & 30.499 & 29.342 & 30.8 & & 29.852 & 30.994 & 30.501 & 30.143 & 28.581 & 26.332 & 37.41 & 36.136 & 37.702 & 17.237 & 17.769 & 23.059 & 15.588 & 16.1 & 34.395 & 38.437 & 30.617 & 37.34 & 27.234 & 34.492 \\
\hline $10 / 23 / 1118: 30$ & & 31.048 & 30.506 & 29.356 & 30.869 & & 29.84 & 30.998 & 30.498 & 30.148 & 28.591 & 26.346 & 36.708 & 36.14 & 37.669 & 17.237 & 17.879 & 23.054 & 15.583 & 16.055 & 34.328 & 37.601 & 30.662 & 36.617 & 27.237 & 34.492 \\
\hline 10/23/11 19:00 & & 31.05 & 30.513 & 29.37 & 30.895 & & 29.833 & 31.003 & 30.498 & 30.152 & 28.595 & 26.361 & 35.662 & 36.143 & 36.841 & 17.239 & 17.917 & 23.052 & 15.576 & 15.204 & 33.952 & 36.53 & 30.681 & 35.582 & 27.237 & 34.495 \\
\hline 10/23/11 19:30 & & 31.055 & 30.52 & 29.377 & 30.918 & & 29.824 & 31.005 & 30.498 & 30.157 & 28.602 & 26.375 & 35.173 & 36.15 & 36.335 & 17.239 & 17.898 & 23.052 & 15.574 & 14.671 & 33.756 & 36.051 & 30.69 & 35.106 & 27.237 & 34.497 \\
\hline $10 / 23 / 1120: 00$ & & 31.057 & 30.526 & 29.389 & 30.939 & & 29.819 & 31.005 & 30.501 & 30.164 & 28.607 & 26.385 & 34.859 & 36.147 & 36.004 & 17.246 & 17.87 & 23.052 & 15.572 & 14.318 & 33.64 & 35.741 & 30.688 & 1.801 & 27.237 & 34.499 \\
\hline 10/23/11 20:30 & & 31.062 & 30.531 & 29.403 & 30.953 & & 29.812 & 31.008 & 30.501 & 30.169 & 28.616 & 26.392 & 34.634 & 36.157 & 35.761 & 17.244 & 17.839 & 23.05 & 15.567 & 14.048 & 33.546 & 35.518 & 30.685 & 4.576 & 27.237 & 4.502 \\
\hline
\end{tabular}




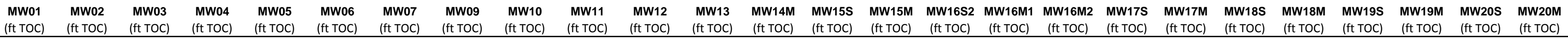

\begin{tabular}{|c|c|c|c|c|c|c|c|c|c|c|c|c|c|c|c|c|c|c|c|c|c|c|c|c|c|}
\hline Well & $\begin{array}{l}\text { (ft TOC) } \\
\text { (is }\end{array}$ & $\begin{array}{l}\text { (ft TOC) } \\
\text { (ft) }\end{array}$ & $\begin{array}{l}\text { (ft TOC) } \\
\text { (f) }\end{array}$ & (ft TOC) & $\begin{array}{ll}(\mathrm{ft} \text { TOC) } & \text { (ft TOC) }\end{array}$ & (ft toc) & $\begin{array}{l}\text { (ft TOC) } \\
\text { (f) }\end{array}$ & $\begin{array}{l}\text { (ft TOC) } \\
\text { (ft) }\end{array}$ & $\begin{array}{l}\text { (ft TOC) } \\
\text { (f) }\end{array}$ & $\begin{array}{l}\text { (ft TOC) } \\
\text { (f) }\end{array}$ & $\begin{array}{l}\text { (ft Toc) } \\
\text { (f) }\end{array}$ & (ft TOC) & $\begin{array}{l}\text { (ft TOC) } \\
\end{array}$ & (ft TOC) & (ft TOC) & (ft TOC) & (ft TOC) & $\begin{array}{l}\text { (ft TOC) } \\
\text { (ft) }\end{array}$ & (ft TOC) & $\begin{array}{l}\text { (ft TOC) } \\
\end{array}$ & $\begin{array}{l}\text { (ft TOC) } \\
\text { (n) }\end{array}$ & $\begin{array}{l}\text { (ft TOC) } \\
\text { (n) }\end{array}$ & (ft TOC) & $\begin{array}{l}\text { (ft TOC) } \\
\text { (1) }\end{array}$ & (ft TOC) \\
\hline $10 / 23 / 1121: 00$ & & 31.064 & 30.536 & 29.412 & 30.96 & 29.81 & 31.01 & 30.5 & 30.173 & 28.621 & 26.404 & 34.454 & 36.159 & 35.573 & 17.248 & 17.809 & 23.052 & 15.567 & 13.846 & 33.481 & 35.338 & 30.683 & 34.4 & 27.237 & 34.504 \\
\hline $10 / 23 / 1121: 30$ & & 31.067 & 30.547 & 29.412 & 30.972 & 29.808 & 31.013 & 30.498 & 30.18 & 28.625 & 26.411 & 34.311 & 36.159 & 35.418 & 17.251 & 17.778 & 23.052 & 15.56 & 13.669 & 33.43 & 35.198 & 30.681 & 34.259 & 27.237 & 34.506 \\
\hline $10 / 23 / 1122: 00$ & & 31.071 & 30.554 & 29.412 & 30.976 & 29.815 & 31.013 & 30.498 & 30.185 & 28.628 & 26.413 & 34.464 & 36.161 & 35.582 & 17.253 & 17.783 & 23.047 & 15.565 & 13.81 & 33.446 & 35.35 & 30.681 & 34.404 & 27.237 & 34.511 \\
\hline $\begin{array}{l}10 / 23 / 1122: 30 \\
\text { L }\end{array}$ & & 31.069 & 30.561 & 29.417 & 30.981 & 29.815 & 31.015 & 30.5 & 30.192 & 28.63 & 26.411 & 34.248 & 36.164 & 35.355 & 17.255 & 17.774 & 23.052 & 15.558 & 13.566 & 33.404 & 35.139 & 0.681 & .196 & 7.237 & \\
\hline $10 / 23 / 1123: 00$ & & 31.069 & 30.564 & 29.414 & 30.979 & 29.819 & 31.015 & 30.501 & 30.194 & 28.632 & 26.413 & 34.117 & 36.162 & 35.214 & 17.258 & 17.746 & 23.054 & 15.562 & 13.411 & דרט & 35.006 & & & 39 & 513 \\
\hline 10/23/11 23:30 & & 31.069 & 30.566 & 29.412 & 30.976 & 29.822 & 31.017 & 30.503 & 30.197 & 28.635 & 26.413 & 34.014 & 36.169 & 35.106 & 17.26 & 17.713 & 23.054 & 15.558 & 13.293 & 33.306 & 34.905 & 30.667 & 3.966 & 7.239 & 34.511 \\
\hline 10/24/11 0:00 & & 31.069 & 30.571 & 29.414 & 30.974 & 29.826 & 31.02 & 30.503 & 30.201 & 28.639 & 26.411 & 33.928 & 36.164 & 35.015 & 17.262 & 17.683 & 23.057 & 15.56 & 13.197 & 33.267 & 34.819 & 30.66 & 33.881 & 27.239 & 34.513 \\
\hline 10/24/11 0:30 & & 31.069 & 30.571 & 29.412 & 30.967 & 29.833 & 31.02 & 30.503 & 30.201 & 28.637 & 26.411 & 33.853 & 36.176 & 34.938 & 17.262 & 17.657 & 23.057 & 15.558 & 13.105 & 33.238 & 34.741 & 30.65 & 33.808 & 27.242 & 34.513 \\
\hline 10/24/11 1:00 & & 31.069 & 30.575 & 29.41 & 30.965 & 29.836 & 31.017 & 30.505 & 30.204 & 28.637 & 26.406 & 33.785 & 36.169 & 34.865 & 17.262 & 17.627 & 23.057 & 15.562 & 13.032 & 33.208 & 34.677 & 30.641 & 33.738 & 27.239 & 34.513 \\
\hline 10/24/11 1:30 & & 31.069 & 30.575 & 29.405 & 30.958 & 29.848 & 31.022 & 30.498 & 30.206 & 28.635 & 26.404 & 33.722 & 36.171 & 34.802 & 17.262 & 17.599 & 23.057 & 15.558 & 12.964 & 33.176 & 34.618 & 30.634 & 33.679 & 27.242 & 34.513 \\
\hline 10/24/11 2:00 & & 31.069 & 30.578 & 29.403 & 30.951 & 29.845 & 31.02 & 30.505 & 30.206 & 28.637 & 26.397 & 33.668 & 36.176 & 34.743 & 17.265 & 17.575 & 23.057 & 15.558 & 12.9 & 33.15 & 34.563 & 30.624 & 33.625 & 27.242 & 34.509 \\
\hline 10/24/11 2:30 & & 31.067 & 30.575 & 29.4 & 30.939 & 29.85 & 31.022 & 30.505 & 30.208 & 28.637 & 26.397 & 33.619 & 36.171 & 34.691 & 17.265 & 17.549 & 23.052 & 15.56 & 12.851 & 33.117 & 34.509 & 30.608 & 33.574 & 27.239 & 34.509 \\
\hline 10/24/11 3:00 & & 31.069 & 30.58 & 29.405 & 30.932 & 29.855 & 31.022 & 30.501 & 30.208 & 28.639 & 26.39 & 33.57 & 36.173 & 34.64 & 17.267 & 17.526 & 23.052 & 15.555 & 12.795 & 33.099 & 34.464 & 30.603 & 33.527 & 27.242 & 34.509 \\
\hline 10/24/11 3:30 & & 31.069 & 30.578 & 29.407 & 30.923 & 29.852 & 31.025 & 30.503 & 30.208 & 28.642 & 26.394 & 33.528 & 36.169 & 34.598 & 17.267 & 17.501 & 23.05 & 15.553 & 12.748 & 33.073 & 34.421 & 30.591 & 33.485 & 27.242 & 34.506 \\
\hline $10 / 24 / 114: 00$ & & 31.067 & 30.578 & 29.41 & 30.911 & 29.85 & 31.025 & 30.51 & 30.208 & 28.644 & 26.397 & 33.485 & 36.171 & 34.555 & 17.265 & 17.479 & 23.05 & 15.555 & 12.708 & 33.05 & 34.381 & 30.582 & 33.445 & 27.242 & 34.506 \\
\hline $10 / 24 / 114: 30$ & & 31.071 & 30.582 & 29.412 & 30.909 & 29.857 & 31.025 & 30.505 & 30.21 & 28.644 & 26.401 & 33.45 & 36.173 & 34.516 & 17.267 & 17.456 & 23.05 & 15.548 & 12.654 & 33.033 & 34.345 & 30.577 & 33.41 & 27.242 & 34.504 \\
\hline $10 / 24 / 11$ 5:00 & & 31.073 & 30.587 & 29.414 & 30.904 & 29.855 & 31.025 & 30.505 & 30.213 & 28.649 & 26.399 & 33.415 & 36.176 & 34.48 & 17.267 & 17.437 & 23.052 & 15.546 & 12.611 & 33.017 & 34.312 & 30.577 & 33.374 & 27.244 & 34.504 \\
\hline $10 / 24 / 115: 30$ & & 31.074 & 30.587 & 29.407 & 30.897 & 29.862 & 31.025 & 30.503 & 30.215 & 28.646 & 26.399 & 33.385 & 36.169 & 34.448 & 17.269 & 17.416 & 23.052 & 15.546 & 12.574 & 33.003 & 34.279 & 30.57 & 33.344 & 27.242 & 34.504 \\
\hline $10 / 24 / 116: 00$ & & 31.074 & 30.587 & 29.421 & 30.892 & 29.864 & 31.025 & 30.505 & 30.217 & $\begin{array}{l}28.649 \\
28.649\end{array}$ & 26.394 & 34.41 & 36.176 & 34.844 & 17.269 & $\begin{array}{r}17.40 \\
17.4\end{array}$ & 23.061 & 15.546 & 12.959 & $\begin{array}{l}33.222 \\
3.222\end{array}$ & 35.424 & 30.566 & $\begin{array}{r}53.544 \\
34.4\end{array}$ & 27.244 & 34.504 \\
\hline $10 / 24 / 116: 30$ & & 31.074 & $\begin{array}{l}30.301 \\
30.589\end{array}$ & 29.426 & 30.888 & $\begin{array}{l}29.004 \\
29.855\end{array}$ & 31.029 & 30.51 & 30.217 & $\begin{array}{l}20.049 \\
28.653\end{array}$ & 26.406 & $\begin{array}{r}35.44 \\
35.496\end{array}$ & 36.173 & $\begin{array}{r}54.044 \\
35.92 \\
\end{array}$ & 17.269 & $\begin{array}{l}11.44 \\
17.458\end{array}$ & 23.066 & $\begin{array}{l}15.340 \\
15.548\end{array}$ & $\begin{array}{l}12.959 \\
14.036\end{array}$ & $\begin{array}{l}33.719 \\
33.719\end{array}$ & $\begin{array}{l}\begin{array}{r}0.3444 \\
36.49\end{array} \\
3\end{array}$ & 30.596 & $\begin{array}{r}35.44 \\
35.456\end{array}$ & 27.244 & 34.504 \\
\hline $10 / 24 / 117: 00$ & & 31.074 & $\begin{array}{l}30.389 \\
30.592\end{array}$ & $\begin{array}{l}29.420 \\
29.424\end{array}$ & $\begin{array}{l}30.800 \\
30.892\end{array}$ & 29.857 & 31.027 & $\begin{array}{l}30.511 \\
30.505\end{array}$ & 30.217 & $\begin{array}{l}28.035 \\
28.658\end{array}$ & $\begin{array}{l}20.400 \\
26.411\end{array}$ & $\begin{array}{l}3.406 \\
36.137\end{array}$ & 36.171 & $\begin{array}{r}36.92 \\
36.651\end{array}$ & $\begin{array}{l}17.209 \\
17.269\end{array}$ & $\begin{array}{l}11.480 \\
17.559\end{array}$ & 23.066 & $\begin{array}{l}13.348 \\
15.543\end{array}$ & $\begin{array}{l}14.050 \\
14.769\end{array}$ & 34.015 & $\begin{array}{l}30.49 \\
37.103\end{array}$ & $\begin{array}{l}30.634 \\
30.634\end{array}$ & $\begin{aligned} 50.450 \\
36.08\end{aligned}$ & 27.244 & $\begin{array}{l}34.504 \\
34.504\end{array}$ \\
\hline 10/24/11 7:30 & & 31.081 & 30.599 & 29.417 & 30.909 & 29.866 & 31.03 & 30.505 & 30.224 & 28.653 & 26.409 & 34.733 & 36.178 & 35.859 & & 17.608 & 23.077 & 15.541 & 13.97 & 33.605 & 35.611 & 30.66 & 34.665 & 27.244 & 34.506 \\
\hline 10/24/11 8:00 & & 31.081 & 30.599 & 29.419 & 30. & 29.864 & 31.03 & 30.51 & 30.227 & 28.656 & 26.404 & 34.625 & 36.18 & 35.763 & 17.274 & 17.624 & 23.08 & 14 & 13.867 & 33.5 & 511 & 30.66 & 34.566 & 27.244 & .506 \\
\hline $10 / 24 / 118: 30$ & & 31.078 & 30.596 & 29.417 & 30.93 & 29.871 & 31.032 & 30.512 & 30.227 & 28.658 & 26.406 & 34.361 & 36.176 & 35.479 & 17.274 & 17.65 & 23.07 & .543 & 13.584 & 33.43 & 251 & 30.662 & 34.308 & 27.246 & 34.509 \\
\hline 10/24/11 9:00 & & 31.076 & 30.601 & 29.412 & 30.934 & 29.878 & 31.032 & 30.508 & & 28.656 & 26.401 & 34.141 & 36.18 & 35.245 & 17.276 & 17.631 & 23.077 & 15.546 & 13.364 & 33.348 & 35.028 & 30.65 & 1.088 & 27.244 & 34.509 \\
\hline 10/24/11 9:30 & & 31.076 & 30.601 & 29.405 & 30.937 & 29.887 & 31.032 & 30.508 & 30.227 & 28.653 & 26.397 & 33.993 & 36.18 & 35.085 & 17.274 & 17.608 & 23.082 & 15.553 & 13.218 & 33.283 & 34.879 & 30.643 & 3.942 & 27.244 & 34.511 \\
\hline 10/24/11 10:00 & & 31.076 & 30.601 & 29.4 & 30.934 & 29.897 & 31.032 & 30.508 & & 28.653 & 26.387 & 33.876 & 36.18 & 34.963 & 17.274 & 17.585 & 23.082 & 15.56 & 13.11 & 33.231 & 34.765 & 30.631 & 3.827 & 27.244 & 34.513 \\
\hline 10/24/11 10:30 & & 31.076 & 30.599 & 29.386 & 30.932 & 29.902 & 31.029 & 30.51 & 30.224 & 28.649 & 26.382 & 33.785 & 36.18 & 34.87 & 17.276 & 17.561 & 23.082 & 15.562 & 13.034 & 33.19 & 34.675 & 30.622 & 33.738 & 27.246 & 34.513 \\
\hline 10/24/11 11:00 & & 31.074 & 30.601 & 29.374 & 30.925 & 29.916 & 31.027 & 30.508 & 30.224 & 28.642 & 26.373 & 33.705 & 36.178 & 34.783 & 17.276 & 17.538 & 23.082 & 15.567 & 12.971 & 33.15 & 34.597 & 30.608 & 33.658 & 27.246 & 34.516 \\
\hline $10 / 24 / 1111: 30$ & & 31.071 & 30.596 & 29.358 & 30.918 & 29.93 & 31.027 & 30.508 & 30.222 & 28.635 & 26.356 & 33.638 & 36.175 & 34.713 & 17.276 & 17.512 & 23.084 & 15.576 & 12.919 & 33.12 & 34.528 & 30.596 & 33.593 & 27.246 & 34.516 \\
\hline $10 / 24 / 1112: 00$ & & 31.066 & 30.589 & 29.337 & 30.904 & 29.939 & 31.025 & 30.508 & 30.22 & 28.625 & 26.344 & 34.625 & 36.178 & 35.076 & 17.276 & 17.493 & 23.082 & 15.583 & 13.302 & 33.309 & 35.637 & 30.577 & 34.613 & 27.246 & 34.516 \\
\hline $10 / 24 / 1112: 30$ & & 31.067 & 30.582 & 29.321 & 30.892 & 29.953 & 31.022 & 30.508 & 30.215 & 28.616 & 26.325 & 35.69 & 36.173 & 36.126 & 17.274 & 17.547 & 23.084 & 15.59 & 14.386 & 33.775 & 36.682 & 30.594 & 35.646 & 27.246 & 34.516 \\
\hline 10/24/11 13:00 & & 31.064 & 30.571 & 29.297 & 30.885 & 29.97 & 31.015 & 30.5 & 30.206 & 28.602 & 26.306 & 36.312 & 36.171 & 36.837 & 17.272 & 17.643 & 23.08 & 15.602 & 15.134 & 34.052 & 37.269 & 30.615 & 36.251 & 27.246 & 34.516 \\
\hline $10 / 24 / 1113: 30$ & & 31.057 & 30.557 & 29.286 & 30.883 & 29.974 & 31.008 & 30.505 & 30.201 & 28.591 & 26.287 & 34.882 & 36.166 & 36.018 & 17.269 & 17.683 & 23.08 & 15.607 & 14.353 & 33.623 & 35.758 & 30.613 & 34.813 & 27.246 & 34.511 \\
\hline 10/24/11 14:00 & & 31.055 & 30.547 & 29.279 & 30. & 29.979 & 31.008 & 30.503 & 30.194 & 28.586 & 26.272 & 34.478 & 36.166 & 35.601 & 17.267 & 17.666 & 23.08 & 15.619 & 13.965 & 33.434 & 35.362 & .589 & 34.421 & 27.244 & 34.509 \\
\hline $10 / 24 / 1$ & & 31.05 & 30.531 & 29.279 & & & 31.005 & 30.508 & 30. & 28.581 & 26.268 & 34.234 & 36.152 & 35.346 & 17.262 & 17.643 & 23.08 & 15.626 & 13.742 & 33.322 & 35.12 & 0.568 & 34.179 & 27.244 & 34.506 \\
\hline 10 & & & 30.524 & 29.272 & 30. & 29.972 & 30.999 & 30.501 & 30.178 & $\begin{array}{l}20.001 \\
28.577\end{array}$ & $\begin{array}{l}20.260 \\
26.265\end{array}$ & 34.061 & $\begin{array}{r}36.15 \\
36\end{array}$ & 35.163 & 17.26 & $\begin{array}{l}17.0613 \\
17.6\end{array}$ & 23.077 & 15.63 & 13.582 & $\begin{array}{l}33.241 \\
33.52\end{array}$ & 34.95 & 0.542 & 34.01 & 27.244 & 34.504 \\
\hline 10 & & 31.05 & 30.515 & 29.265 & 30. & 29.967 & 31.001 & 30.501 & $\begin{array}{l}30.176 \\
30.176\end{array}$ & 28.57 & 26.26 & 33.93 & 36.14 & 35.027 & $\begin{array}{l}17.258 \\
17.258\end{array}$ & $\begin{array}{l}17.589 \\
17.59\end{array}$ & 23.077 & $\begin{array}{l}15.628 \\
15.628\end{array}$ & $\begin{array}{l}13.46 \\
13.42\end{array}$ & $\begin{array}{l}33.184 \\
33.183\end{array}$ & 34.819 & .526 & 33.879 & 27.246 & 34.502 \\
\hline 10 & & 31.05 & 30.51 & 29.26 & 30. & 29.965 & 30.996 & 30.501 & 30.171 & 28.567 & $\begin{array}{l}26.250 \\
26.258\end{array}$ & $\begin{array}{l}33.822 \\
3.822\end{array}$ & $\begin{array}{l}30.14 \\
36.138\end{array}$ & $\begin{array}{l}34.912 \\
34.912\end{array}$ & 17.258 & $\begin{array}{l}17.559 \\
17.559\end{array}$ & 23.077 & $\begin{array}{l}13.060 \\
15.628\end{array}$ & $\begin{array}{r}13.40 \\
13.359\end{array}$ & 33.131 & $\begin{array}{r}5.0079 \\
34.71\end{array}$ & 16 & $\begin{array}{l}33.0773 \\
33.773\end{array}$ & 27.244 & 34.502 \\
\hline $10 / 24 /$ & & 31. & 30.503 & 29 & & ? & 30 & 30 & 30 & 28. & 26 & 34 & 36 & 35 & 17 & 17 & 23 & 1 & 13 & 33 & 3 & 55 & 34.186 & 27.246 & 34.497 \\
\hline 10 & & & 30.499 & 29 & & & & & & 28 & 26. & & 36 & 35.399 & 17 & 17 & 23. & & 13. & 33 & 35.388 & 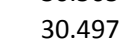 & 3 & 27.246 & 34.497 \\
\hline & & & & 29.274 & & & 30. & & & 28.563 & & & 36.136 & 36.464 & & & & 15.628 & 14.934 & 33.896 & .156 & .514 & 36.078 & $\begin{array}{ll}3 & 27.246\end{array}$ & 34.495 \\
\hline $10 /$ & & & 30 & 29.281 & & & & & & 28.567 & & & 36.133 & 37.296 & 17.251 & 17.706 & 23 & 15.626 & 15.764 & & & & 6.964 & $\quad 27.246$ & 34.495 \\
\hline & & & 30 & 29.288 & & & & & & 28 & & & 36.136 & 37.32 & 17. & 17. & & 15. & 15.785 & 34 & & & 36.279 & 49 & 34.497 \\
\hline 10/ & & 31. & & 29 & & & & & & 28. & & & 36.131 & 36. & & $17 . \varepsilon$ & 23. & & 15.369 & & & & & $\quad 27.246$ & 34.497 \\
\hline $10 /$ & & 31 & & 29. & & & 30 & 30.512 & & 28. & & & 36.131 & 36. & & 17.884 & 23. & 15. & 14.863 & 33.761 & & & & $\begin{array}{l}3 \\
\quad 27.249\end{array}$ & 34.497 \\
\hline 10/24/11 20: & & 31.6 & 30.5 & 29.293 & & & 30. & 30.51 & & 28.57 & 26. & $34 . \varepsilon$ & 36.136 & 36.009 & 17. & 17 & 23.1 & 15.6 & 14.424 & 33.593 & 35.743 & 30.622 & 34.803 & $\begin{array}{l}3 \\
3\end{array}$ & 34.499 \\
\hline 10/24/11 20:30 & & 31.0 & 30.5 & 29.29 & & 29.8 & 30.5 & 30.515 & 30. & 28.565 & 26. & 34.6 & 36.136 & 35.732 & 17.253 & 17.832 & 23.135 & 15.609 & 14.142 & 33.493 & 35.487 & 30.613 & 34.55 & $\begin{array}{l}57.249 \\
5\end{array}$ & 34.504 \\
\hline 10/24/11 21:00 & & 31.053 & 30.51 & 29.288 & 30.8 & 29.887 & 30.95 & 30.51 & 30.1 & 28.565 & 26.301 & 34.412 & 36.14 & 35.533 & 17.253 & 17.802 & 23.138 & 15.6 & 13.93 & 33.413 & 35.298 & 30.603 & 34.36 & $\quad 27.249$ & 34.502 \\
\hline $10 / 24 / 1121: 30$ & & 31.053 & 30.508 & 29.283 & 30.8 & 29.887 & 30.989 & 30.508 & 30.157 & 28.563 & 26.299 & 34.26 & 36.14 & 35.369 & 17.255 & 17.771 & 23.135 & 15.607 & 13.761 & 33.348 & 35.146 & 30.591 & 34.21 & 27.251 & 34.504 \\
\hline $10 / 24 / 1122: 00$ & & 31.05 & 30.508 & 29.279 & 30.883 & 29.89 & 30.991 & 30.508 & 30.155 & 28.563 & 26.296 & 34.136 & 36.143 & 35.238 & 17.255 & 17.743 & 23.135 & 15.609 & 13.622 & 33.29 & 35.025 & 30.58 & 34.085 & $\quad 27.249$ & 34.506 \\
\hline $10 / 24 / 1122: 30$ & & 31.053 & 30.508 & 29.272 & 30.878 & 29.89 & 30.993 & 30.508 & 30.152 & 28.553 & 26.296 & 34.031 & 36.136 & 35.125 & 17.255 & 17.713 & 23.138 & 15.609 & 13.507 & 33.243 & 34.916 & 30.573 & 33.982 & 27.251 & 34.504 \\
\hline $10 / 24 / 1123: 00$ & & 31.05 & 30.506 & 29.262 & 30.871 & 29.887 & 30.989 & 30.505 & 30.152 & 28.549 & 26.287 & 33.935 & 36.138 & 35.027 & 17.253 & 17.685 & 23.135 & 15.607 & 13.408 & 33.201 & 34.826 & 30.556 & 33.888 & $\begin{array}{r}3 \\
3\end{array}$ & 34.509 \\
\hline $10 / 24 / 1123: 30$ & & 31.048 & 30.503 & 29.253 & 30. & 29.892 & 30.991 & 30.508 & 30.148 & 28.551 & 26.275 & 33.855 & 36.143 & 34.945 & 17.251 & 17.659 & 23.135 & 15.614 & 13.324 & 33.162 & 34.748 & 30.542 & 33.808 & $\begin{array}{l}37.251 \\
\end{array}$ & 34.506 \\
\hline 10/25/11 0:00 & & 31.046 & 30.499 & 29.248 & 30.848 & 29.897 & 30.987 & 30.51 & 30.146 & 28.542 & 26.27 & 33.783 & 36.143 & 34.867 & 17.255 & 17.631 & 23.133 & 15.609 & 13.251 & 33.113 & 34.668 & 30.526 & 33.733 & $\begin{array}{l}3 \\
27.246\end{array}$ & 34.504 \\
\hline 10/25/11 0:30 & & 31.046 & 30.494 & 29.244 & 30.836 & 29.892 & 30.986 & 30.508 & 30.146 & 28.539 & 26.265 & 33.715 & 36.138 & 34.795 & 17.253 & 17.606 & 23.138 & 15.607 & 13.18 & 33.092 & 34.604 & 30.512 & 33.67 & 27.249 & 34.506 \\
\hline
\end{tabular}




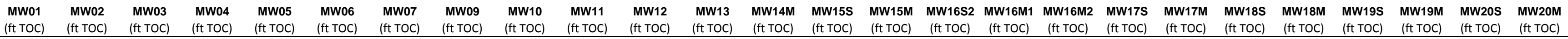

\begin{tabular}{|c|c|c|c|c|c|c|c|c|c|c|c|c|c|c|c|c|c|c|c|c|c|c|c|c|c|}
\hline well & (ft TOC) & (ft TOC) & (ft TOC) & (ft TOC) & $\begin{array}{ll}(\mathrm{ft} \text { TOC) } & \text { (ft TOC) } \\
\end{array}$ & (ft TOC) & (ft TOC) & (ft TOC) & (ft TOC) & (ft TOC) & (ft TOC) & (ft TOC) & (ft TOC) & (ft TOC) & (ft TOC) & (ft TOC) & (ft TOC) & (ft TOC) & (ft TOC) & (ft TOC) & (ft TOC) & (ft TOC) & (ft TOC) & (ft TOC) & (ft TOC) \\
\hline 1:00 & & 31.043 & 30.487 & 29.237 & 30.825 & 29.8 & 30.986 & 30 & 30. & 28 & 26 & 33.652 & 36.131 & 34.731 & 17. & 17 & 2314 & 15612 & 13.121 & 33.054 & 34.544 & 30.495 & og & 51 & 04 \\
\hline 5/11 1:30 & & 31.043 & 30.482 & 29.237 & 30.811 & 29.892 & 30.984 & 30.508 & 30.139 & 28.53 & 26.258 & 33.595 & 36.13 & 34.6 & 17.248 & 17.554 & 23.135 & 15.607 & 3.063 & 33.022 & 4.49 & 30.481 & & 7.251 & 34.502 \\
\hline 10/25/11 2:00 & & 31.041 & 30.478 & 29.234 & 30.799 & 29.887 & 30.982 & 30.505 & 30.139 & 28.525 & 26.26 & 33.544 & 36.133 & 34.621 & 17.248 & 17.528 & 23.133 & 15.607 & 13.009 & 32.989 & 34.435 & 30.472 & 3.501 & & 34.499 \\
\hline 10/25/11 2:30 & & 31.043 & 30.473 & 29.232 & 30.785 & 29.89 & 30.982 & 30.51 & 30.136 & 28.519 & 26.26 & 33.497 & 36.129 & 34.572 & 17.246 & 17.503 & 23.131 & 15.602 & 12.962 & 32.968 & 34.388 & 30.46 & 33.459 & .251 & 34.499 \\
\hline 10/25/11 3:00 & & 31.041 & 30.471 & 29.23 & 30.778 & 29.887 & 30.977 & 30.505 & 30.134 & 28.521 & 26.256 & 33.457 & 36.133 & 34.525 & $5 \quad 17.246$ & 17.482 & 23.133 & 15.595 & 12.905 & 32.947 & 34.353 & 30.458 & 33.41 & 27.249 & 34.495 \\
\hline 10/25/11 3:30 & & 31.039 & 30.471 & 29.22 & 30.762 & 29.883 & 30.977 & 30.505 & 30.132 & 28.519 & 26.248 & 33.411 & 36.129 & 34.483 & $\begin{array}{l}37.246 \\
\end{array}$ & 17.456 & 23.133 & 15.6 & 12.863 & 32.919 & 34.305 & 30.436 & 33.372 & 27.251 & 34.495 \\
\hline 10/25/11 4:00 & & 31.041 & 30.466 & 29.213 & 30.752 & 29.885 & 30.974 & 30.508 & 30.129 & 28.512 & 26.246 & 33.376 & 36.126 & 34.443 & $\begin{array}{ll}3 & 17.246\end{array}$ & 17.433 & 23.133 & 15.593 & 12.823 & 32.905 & 34.265 & 30.427 & 33.332 & 27.251 & 34.492 \\
\hline 10/25/11 4:30 & & 31.036 & 30.459 & 29.211 & 30.741 & 29.89 & 30.972 & 30.505 & 30.127 & 28.509 & 26.244 & 33.336 & 36.124 & 34.403 & $\begin{array}{l}3 \\
3\end{array} 17.244$ & 17.412 & 23.128 & 15.593 & 12.781 & 32.877 & 34.232 & 30.42 & 33.295 & 27.251 & 34.49 \\
\hline 10/25/11 5:00 & & 31.036 & 30.459 & 29.204 & 30.731 & 29.883 & 30.97 & 30.505 & 30.125 & 28.505 & 26.239 & 33.303 & 36.133 & 34.368 & $\begin{array}{ll}8 & 17.244\end{array}$ & 17.391 & 23.131 & 15.595 & 12.748 & 32.858 & 34.198 & 30.413 & 33.264 & 27.249 & 34.488 \\
\hline 10/25/11 5:30 & & 31.032 & 30.452 & 29.204 & 30.717 & 29.88 & 30.97 & 30.508 & 30.122 & 28.498 & 26.236 & 33.27 & 36.124 & 34.335 & $5 \quad 17.241$ & 17.372 & 23.131 & 15.593 & 12.72 & 32.837 & 34.163 & 30.392 & 33.229 & 27.249 & 34.485 \\
\hline 10/25/11 6:00 & & 31.034 & 30.45 & 29.211 & 30.705 & 29.873 & 30.967 & 30.508 & 30.12 & 28.502 & 26.234 & 34.293 & 36.119 & 34.727 & $7 \quad 17.241$ & 17.353 & 23.128 & 15.583 & 13.096 & 33.057 & 35.303 & 30.394 & 34.287 & 27.251 & 34.485 \\
\hline $10 / 25 / 116: 30$ & & 31.032 & 30.443 & 29.209 & 30.701 & 29.866 & 30.967 & 30.505 & 30.115 & 28.502 & 26.241 & 35.383 & 36.122 & 35.805 & $5 \quad 17.239$ & 17.409 & 23.131 & 15.586 & 14.182 & 33.544 & 36.378 & 30.413 & 35.348 & 27.253 & 34.483 \\
\hline 10/25/11 7:00 & & 31.032 & 30.443 & 29.199 & 30.703 & 29.869 & 30.965 & 30.508 & 30.115 & 28.495 & 26.244 & 36.029 & 36.119 & 36.541 & $1 \quad 17.239$ & 17.508 & 23.133 & 15.581 & 14.917 & 33.838 & 36.99 & 30.45 & 35.97 & 27.253 & 34.483 \\
\hline 10/25/11 7:30 & & 31.034 & 30.445 & 29.206 & 30.715 & 29.862 & 30.965 & 30.51 & 30.108 & 28.495 & 26.236 & 34.613 & 36.122 & 35.749 & 17.237 & 17.552 & 23.135 & 15.579 & 14.116 & 33.439 & 35.497 & 30.474 & 34.552 & 27.253 & 34.483 \\
\hline 10/25/11 8:00 & & 31.032 & 30.443 & 29.209 & 30.722 & 29.857 & 30.965 & 30.501 & 30.108 & 28.5 & 26.246 & 34.234 & 36.119 & 35.35 & 17.239 & 17.545 & 23.135 & 15.583 & 13.714 & 33.262 & 35.12 & 30.467 & 34.182 & 27.251 & 34.483 \\
\hline $10 / 25 / 118: 30$ & & 31.029 & 30.44 & 29.211 & 30.726 & 29.852 & 30.965 & 30.51 & 30.106 & 28.498 & 26.251 & 34.01 & 36.119 & 35.113 & 17.239 & 17.526 & 23.142 & 15.574 & 13.479 & 33.169 & 34.895 & 30.462 & 33.963 & 27.253 & 34.481 \\
\hline 10/25/11 9:00 & & 31.032 & 30.445 & 29.225 & 30.731 & 29.838 & 30.963 & 30.508 & 30.106 & 28.502 & 26.253 & 33.858 & 36.119 & 34.952 & 17.237 & 17.508 & 23.144 & 15.569 & 13.312 & 33.11 & 34.746 & 30.458 & 33.808 & 27.256 & 34.485 \\
\hline 10/25/11 9:30 & & 31.032 & 30.44 & 29.218 & 30.733 & 29.843 & 30.965 & 30.51 & 30.101 & 28.5 & 26.268 & 33.738 & 36.117 & 34.825 & 17.237 & 17.486 & 23.147 & 15.576 & 13.187 & 33.052 & 34.627 & 30.443 & 33.693 & 27.253 & 34.485 \\
\hline 10/25/11 10:00 & & 31.034 & 30.447 & 29.22 & 30.741 & 29.843 & 30.963 & 30.512 & 30.104 & 28.5 & 26.263 & 33.647 & 36.122 & 34.727 & 17.237 & 17.468 & 23.156 & 15.562 & 13.079 & 33.024 & 34.537 & 30.45 & 33.6 & 27.258 & 34.49 \\
\hline 30 & & 31.034 & 30.45 & 29.23 & 30. & 29.836 & 30.965 & 30.512 & 30.1 & 28.505 & 26.265 & 33.574 & 36.119 & 34.647 & 17.239 & 17.449 & 23.158 & 15.567 & 12.994 & 32.991 & 34.459 & 30.441 & 33.527 & 27.258 & 34.492 \\
\hline 10 & & 31.029 & 30.447 & 29.246 & 30. & 29.826 & 30.967 & 30.517 & 30. & 28.512 & 26.279 & 33.504 & 36.119 & 34.581 & 17.241 & 17.428 & 23.161 & 15577 & 12.936 & 32.954 & 34.395 & 30.441 & 33.464 & 27.256 & 34.492 \\
\hline $10 / 25$ & & 31.032 & 30.445 & 29.258 & 30 & 29.815 & 30.97 & 30.515 & 30.101 & 28.516 & 26.296 & 33.448 & 36.124 & 34.52 & 17.239 & 17.414 & 23.163 & .567 & 12.872 & 32.935 & 338 & .439 & 3.412 & 27.258 & 34.495 \\
\hline 10/25/11 12:00 & & 31 & 30.454 & 29.255 & 30 & 29.812 & 30.97 & 30.515 & 30.101 & 28.519 & 26.311 & 34.45 & 36.126 & 34.898 & $3 \quad 17.241$ & 17.395 & 23.161 & 5.565 & 13.23 & 33.15 & 35.457 & .446 & 1.444 & 27.258 & 34.495 \\
\hline 10/25/11 12:30 & & 31.041 & 30.461 & 29.265 & 30.741 & 29.817 & 30.97 & 30.517 & 30.106 & 28.519 & 26.308 & 35.533 & 36.136 & 35.962 & 17.244 & 17.456 & 23.175 & 15.553 & 14.292 & 33.64 & 36.525 & 30.483 & 35.496 & 27.258 & 34.497 \\
\hline 10/25/11 13:00 & & 31.043 & 30.468 & 29.274 & 30.757 & 29.81 & 30.974 & 30.517 & & 28.528 & 26.315 & 36.165 & 36.136 & 36.684 & 17.248 & 17.556 & 23.179 & 15.555 & 15.014 & 33.938 & 37.127 & 30.526 & 36.108 & 27.258 & 34.497 \\
\hline 10/25/11 13:30 & & 31.039 & 30.466 & 29.276 & & 29.81 & 30.977 & 30.512 & 30.108 & 28.528 & 26.327 & 34.74 & 36.138 & 35.875 & 17.246 & 17.601 & 23.179 & 15.558 & 14.215 & 33.523 & 35.618 & 30.54 & 34.677 & 27.258 & 34.497 \\
\hline 10/25/11 14:00 & & 31.041 & 30.473 & 29.276 & 30.785 & 29.808 & 30.977 & 30.517 & 30.111 & 28.53 & 26.327 & 34.354 & 36.136 & 35.467 & 17.248 & 17.589 & 23.181 & 15.555 & 13.803 & 33.355 & 35.234 & 30.544 & 34.297 & 27.26 & 34.497 \\
\hline 10/25/11 14:30 & & 31.043 & 30.475 & 29.281 & 30.801 & 29.815 & 30.977 & 30.515 & 30.115 & 28.537 & 26.327 & 34.124 & 36.143 & 35.231 & 17.253 & 17.57 & 23.181 & 15.558 & 13.554 & 33.267 & 35.006 & 30.547 & 34.074 & 27.258 & 34.499 \\
\hline 10/25/11 15:00 & & 31.046 & 30.482 & 29.295 & 30.811 & 29.805 & 30.982 & 30.52 & 30.115 & 28.542 & 26.334 & 33.961 & 36.15 & 35.057 & 17.251 & 17.547 & 23.181 & 15.558 & 13.38 & 33.201 & 34.845 & 30.542 & 33.912 & 27.258 & 34.497 \\
\hline $10 / 25 / 1115: 30$ & & 31.041 & 30.482 & 29.304 & 30.811 & 29.803 & 30.982 & 30.517 & 30.115 & 28.546 & 26.349 & 33.836 & 36.145 & 34.926 & 17.251 & 17.524 & 23.181 & 15.56 & 13.251 & 33.148 & 34.725 & 30.528 & 33.792 & 27.26 & 34.497 \\
\hline 10/25/11 16:00 & & 31.048 & 30.487 & 29.314 & 30.82 & 29.798 & 30.989 & 30.52 & 30.12 & 28.551 & 26.356 & 33.736 & 36.154 & 34.82 & 17.255 & 17.505 & 23.186 & 15.55 & 13.138 & 33.11 & 34.63 & 30.535 & 33.693 & 27.26 & 34.497 \\
\hline $10 / 25 / 1116: 30$ & & 31.05 & 30.494 & 29.321 & 30.825 & 29.796 & 30.991 & 30.52 & 30.125 & 28.556 & 26.366 & 33.656 & 36.15 & 34.736 & 17.255 & 17.482 & 23.191 & 15.553 & 13.042 & 33.08 & 34.547 & 30.537 & 33.611 & 27.26 & 34.497 \\
\hline $10 / 25 / 1117: 00$ & & 31.055 & 30.501 & 29.332 & 30.829 & 29.789 & 30.993 & 30.522 & 30.129 & 28.563 & 26.373 & 33.588 & 36.157 & 34.663 & 17.258 & 17.463 & 23.205 & 15.546 & 12.954 & 33.057 & 34.478 & 30.537 & 33.546 & 27.263 & 34.497 \\
\hline $10 / 25 / 1117: 30$ & & 31.055 & 30.506 & 29.342 & 30.829 & 29.779 & 31.001 & 30.524 & 30.132 & 28.57 & 26.385 & 35.254 & 36.159 & 35.728 & $3 \quad 17.26$ & 17.477 & 23.225 & 15.548 & 14.008 & 33.607 & 36.231 & 30.547 & 35.183 & 27.26 & 34.497 \\
\hline 10/25/11 18:00 & & 31 . & 30.51 & 29.358 & 30. & 29.77 & 31.003 & 30.524 & 30. & 28.581 & 26.397 & 36.125 & 36.164 & 36.527 & 17.2 & 17.561 & 23.225 & 15.543 & 14.788 & 33.933 & 37.127 & .594 & 36.066 & 27.263 & 34.497 \\
\hline & & & 30.52 & 29.367 & & 29. & 31.01 & 30.527 & & 28.588 & 26.411 & 35.243 & 36.169 & 36.375 & 17.267 & 17.655 & 23.228 & . & 14.628 & 33.819 & 36.118 & .641 & 35.162 & 27.263 & 34.497 \\
\hline 10 & & $\begin{array}{l}31.064 \\
31.064\end{array}$ & 30.526 & 29.382 & 30.8 & 29.765 & 31.013 & 30.524 & 30.148 & $\begin{array}{l}20.000 \\
28.595\end{array}$ & 26.418 & 34.644 & 36.173 & $\begin{aligned} 35.77 \\
\end{aligned}$ & 17.267 & $\begin{array}{l}17.059 \\
\end{array}$ & 23.225 & 15.536 & $\begin{array}{l}14.060 \\
13.998\end{array}$ & $\begin{array}{l}33.544 \\
3.544\end{array}$ & 35.523 & 30.643 & 34.58 & 27.263 & 34.499 \\
\hline :30 & & 31 & 30 & 29.396 & 30 & 29.758 & 7 & 30 & 30. & 28 & 26.433 & 34.51 & 36.178 & 35.632 & 17. & 17.673 & 23.23 & 15.534 & 13.845 & 33.476 & .395 & 30.653 & 34.451 & 27.263 & 34.502 \\
\hline & & 31. & 30 & 29 & 30 & 2 & 31 & 30 & 30 & 28 & 26.442 & 34 & 36 & 35.362 & 17 & 17.659 & 23.23 & 17 & 13. & 33.392 & 35.142 & 30.66 & 34.2 & 27.265 & 34.504 \\
\hline & & 31 & 30. & 29. & & & 31 & & 30 & 28 & 26.442 & 34 & 36.187 & 35.1 & 17.279 & 17.638 & 23.232 & 15.522 & 13.347 & 33.339 & 34 & 30.662 & 34.041 & 27.265 & 34.509 \\
\hline & & 31. & & & & & & & & 28 & 26 & 33 & & & 17.281 & & & 15 & 13.208 & 33.278 & 34.8 & & 33.921 & 27.263 & 34.511 \\
\hline & & 31 & & 29 & & & & & & 28 & & & 36.1 & 34. & & & & & 13. & & & & & 27.265 & 13 \\
\hline 1 1 22:00 & & 31.6 & & 29.393 & & & & & & 28 & & & 36. & & & & & 15. & 12.999 & & & & & 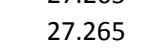 & 34.516 \\
\hline 22:30 & & & 30 & 29.4 & & 29 & & & & 28. & & & 36.1 & 34. & 17. & & & 15. & 12.924 & 64 & 34.604 & & & 27.265 & 34.518 \\
\hline 10/ & & & & 29.389 & & 29 & & & & 28.621 & 26 & & 36.1 & 34. & & & & & 12.856 & 33.141 & & & 33 & 27.265 & 34.52 \\
\hline & & & & 29.403 & & 25 & & & & 28.63 & & & 36.19 & 34.673 & 17.2 & & 23. & 15. & 12.788 & 33.122 & & 30.631 & 33.553 & 27.267 & 34.52 \\
\hline (11 0:00 & & 31. & & 29.396 & 30. & & 31. & 30. & & 28.623 & 26.433 & 33.5 & 36.19 & 34.621 & 17.2 & 17.491 & 23.244 & 15. & 12.741 & 33.092 & 34.443 & 30.617 & 33.508 & 27.267 & 34.523 \\
\hline 10/26/11 0:30 & & 31.6 & 30. & 29.407 & 30. & & 31. & 30. & & 28.63 & 26.421 & 33. & 36.1 & 34.5 & 17. & 17. & 23.244 & 15.529 & 12.686 & 33.068 & 34.398 & 30.66 & 33.464 & 27.267 & 34.523 \\
\hline 10/26/11 1:00 & & 31.0 & 30.578 & 29.389 & 30 & & 31.6 & 30.5 & 30. & 28.628 & 26.43 & 33.4 & 36.1 & 34.5 & 17.2 & 17.456 & 23.251 & 15.5 & 12.639 & 33.052 & 34.357 & 30.606 & 33.426 & 27.265 & 34.525 \\
\hline 10/26/11 1:30 & & 31.078 & 30.573 & 29.398 & 30.9 & 29.833 & 31.037 & 30.531 & 30.1 & 28.632 & 26.411 & 33.427 & 36.197 & 34.487 & 17.29 & 17.437 & 23.251 & 15.541 & 12.611 & 33.022 & 34.317 & 30.587 & 33.381 & 27.267 & 34.523 \\
\hline 10/26/11 2:00 & & 31.085 & 30.58 & 29.403 & 30.909 & 29.822 & 31.037 & 30.529 & 30.204 & 28.635 & 26.418 & 33.392 & 36.194 & 34.457 & 17.293 & 17.421 & 23.251 & 15.527 & 12.557 & 33.015 & 34.291 & 30.599 & 33.351 & 27.267 & 34.525 \\
\hline $10 / 26 / 112: 30$ & & 31.076 & 30.575 & 29.417 & 30.895 & 29.819 & 31.041 & 30.529 & 30.199 & 28.639 & 26.421 & 33.357 & 36.192 & 34.417 & 17.293 & 17.4 & 23.251 & 15.541 & 12.538 & 32.989 & 34.251 & 30.57 & 33.316 & 27.27 & 34.523 \\
\hline $10 / 26 / 113: 00$ & & 31.087 & 30.587 & 29.4 & 30.897 & 29.829 & 31.044 & 30.524 & 30.206 & 28.639 & 26.43 & 33.331 & 36.197 & 34.389 & 17.295 & 17.384 & 23.251 & 15.522 & 12.484 & 32.984 & 34.22 & 30.582 & 33.288 & 27.267 & 34.525 \\
\hline 10/26/11 3:30 & & 31.09 & 30.587 & 29.496 & 30.897 & 29.815 & 31.041 & 30.543 & 30.21 & 28.667 & 26.413 & 33.301 & 36.197 & 34.358 & 17.295 & 17.365 & 23.251 & 15.522 & 12.447 & 32.973 & 34.196 & 30.58 & 33.259 & 27.27 & 34.523 \\
\hline 10/26/11 4:00 & & 31.09 & 30.592 & 29.449 & 30.89 & 29.815 & 31.044 & 30.524 & 30.21 & 28.663 & 26.512 & 33.277 & 36.199 & 34.33 & 17.297 & 17.348 & 23.251 & 15.525 & 12.414 & 32.959 & 34.168 & 30.577 & 33.236 & 27.27 & 34.52 \\
\hline $10 / 26 / 114: 30$ & & 31.09 & 30.592 & 29.459 & 30.885 & 29.808 & 31.049 & 30.529 & 30.213 & 28.67 & 26.459 & 33.249 & 36.197 & 34.305 & 17.297 & 17.33 & 23.246 & 15.518 & 12.383 & 32.947 & 34.142 & 30.575 & 33.21 & 27.272 & 8 \\
\hline
\end{tabular}




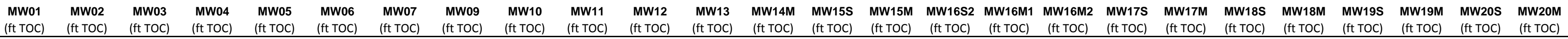

\begin{tabular}{|c|c|c|c|c|c|c|c|c|c|c|c|c|c|c|c|c|c|c|c|c|c|c|c|c|c|}
\hline well & $\begin{array}{l}\text { (ft TOC) } \\
\text { (is }\end{array}$ & $\begin{array}{l}\text { (ft TOC) } \\
\text { (f) }\end{array}$ & $\begin{array}{l}\text { (ft TOC) } \\
\text { (f) }\end{array}$ & (ft TOC) & $\begin{array}{ll}(\mathrm{ft} \text { TOC) } & \text { (ft TOC) }\end{array}$ & $\begin{array}{l}\text { (ft TOC) } \\
\text { (ft }\end{array}$ & $\begin{array}{l}\text { (ft TOC) } \\
\text { (f) }\end{array}$ & $\begin{array}{l}\text { (ft TOC) } \\
\text { (ft) }\end{array}$ & $\begin{array}{l}\text { (ft TOC) } \\
\text { (f) }\end{array}$ & $\begin{array}{l}\text { (ft TOC) } \\
\text { (f) }\end{array}$ & $\begin{array}{l}\text { (ft TOC) } \\
\text { (f) }\end{array}$ & (ft TOC) & $\begin{array}{l}\text { (ft TOC) } \\
\end{array}$ & (ft TOC) & (ft TOC) & (ft TOC) & (ft TOC) & $\begin{array}{l}\text { (ft TOC) } \\
\text { (ft) }\end{array}$ & (ft TOC) & $\begin{array}{l}\text { (ft TOC) } \\
\end{array}$ & $\begin{array}{l}\text { (ft TOC) } \\
\text { (n) }\end{array}$ & $\begin{array}{l}\text { (ft TOC) } \\
\text { (n) }\end{array}$ & (ft TOC) & $\begin{array}{l}\text { (ft TOC) } \\
\end{array}$ & (ft TOC) \\
\hline $10 / 26 / 11$ 5:00 & & 31.097 & 30.613 & 29.473 & 30.906 & 29.817 & 31.049 & 30.529 & 30.248 & 28.674 & 26.468 & 33.235 & 36.199 & 34.288 & $8 \quad 17.304$ & 17.32 & 23.251 & 15.52 & 12.334 & 32.952 & 34.13 & 30.594 & 33.194 & 27.265 & 34.523 \\
\hline $10 / 26 / 11$ 5:30 & & 31.099 & 30.617 & 29.463 & 30.909 & 29.81 & 31.053 & 30.534 & 30.241 & 28.681 & 26.48 & 33.214 & 36.204 & 34.265 & $5 \quad 17.307$ & 17.307 & 23.248 & 15.506 & 12.292 & 32.949 & 34.108 & 30.599 & 33.175 & 27.272 & 34.518 \\
\hline $10 / 26 / 116: 00$ & & 31.099 & 30.622 & 29.475 & 30.904 & 29.819 & 31.051 & 30.539 & 30.243 & 28.684 & 26.471 & 34.251 & 36.204 & 34.67 & 17.309 & 17.295 & 23.262 & 15.513 & 12.689 & 33.18 & 35.265 & 30.594 & 34.243 & 27.27 & 34.518 \\
\hline $10 / 26 / 116: 30$ & & 31.106 & 30.629 & 29.477 & 30.913 & 29.824 & 31.056 & 30.539 & 30.252 & 28.686 & 26.48 & 35.386 & 36.211 & 35.77 & 17.309 & 17.355 & 23.269 & 15.504 & 13.77 & 33.705 & & .648 & 35.35 & 27.272 & 34.518 \\
\hline $10 / 26 / 11$ 7:00 & & 31.097 & 30.629 & 29.484 & 30.92 & 29.819 & 31.056 & 30.539 & 30.25 & 28.695 & 26.483 & 36.001 & 36.204 & 36.506 & 17.311 & 17.458 & 23.276 & 15.515 & 14.5 & 33.992 & 36.964 & .676 & 44 & 72 & 34.518 \\
\hline 10/26/11 7:30 & & 31.101 & 30.634 & 29.48 & 30.939 & 29.831 & 31.058 & 30.534 & 30.257 & 28.691 & 26.49 & 34.597 & 36.204 & 35.714 & 17.311 & 17.51 & 23.285 & 15.515 & 13.728 & 33.579 & 35.478 & 30.697 & 4.529 & 7.272 & 34.518 \\
\hline $10 / 26 / 118: 00$ & & 31.106 & 30.638 & 29.48 & 30.955 & 29.836 & 31.056 & 30.541 & 30.259 & 28.698 & 26.483 & 34.225 & 36.211 & 35.331 & 17.316 & 17.508 & 23.283 & 15.508 & 13.333 & 33.418 & 35.111 & 30.707 & 34.172 & 27.272 & 34.52 \\
\hline $10 / 26 / 118: 30$ & & 31.106 & 30.643 & 29.494 & 30.969 & 29.838 & 31.058 & 30.539 & 30.264 & 28.705 & 26.48 & 34.01 & 36.213 & 35.102 & $2 \quad 17.316$ & 17.493 & 23.285 & 15.515 & 13.107 & 33.329 & 34.9 & 30.702 & 33.959 & 27.272 & 34.52 \\
\hline 10/26/11 9:00 & & 31.104 & 30.643 & 29.477 & 30.974 & 29.848 & 31.063 & 30.536 & 30.266 & 28.702 & 26.492 & 33.862 & 36.218 & 34.947 & $7 \quad 17.316$ & 17.475 & 23.283 & 15.522 & 12.959 & 33.259 & 34.753 & 30.692 & 33.811 & 27.272 & 34.52 \\
\hline 10/26/11 9:30 & & 31.106 & 30.648 & 29.466 & 30.981 & 29.862 & 31.06 & 30.536 & 30.269 & 28.7 & 26.478 & 33.752 & 36.211 & 34.832 & $2 \quad 17.318$ & 17.458 & 23.285 & 15.518 & 12.844 & 33.215 & 34.644 & 30.69 & 33.708 & 27.274 & 34.523 \\
\hline 10/26/11 10:00 & & 31.106 & 30.652 & 29.463 & 30.983 & 29.878 & 31.058 & 30.539 & 30.275 & 28.7 & 26.464 & 33.666 & 36.215 & 34.741 & $1 \quad 17.321$ & 17.447 & 23.285 & 15.527 & 12.762 & 33.178 & 34.556 & 30.683 & 33.623 & 27.274 & 34.525 \\
\hline 10/26/11 10:30 & & 31.099 & 30.648 & 29.47 & 30.979 & 29.871 & 31.063 & 30.543 & 30.273 & 28.705 & 26.457 & 33.593 & 36.213 & 34.663 & $\begin{array}{ll}3 & 17.321\end{array}$ & 17.43 & 23.283 & 15.536 & 12.708 & 33.141 & 34.488 & 30.66 & 33.548 & 27.274 & 34.525 \\
\hline 10/26/11 11:00 & & 31.101 & 30.648 & 29.463 & 30.972 & 29.88 & 31.063 & 30.541 & 30.269 & 28.705 & 26.468 & 33.532 & 36.215 & 34.6 & $5 \quad 17.321$ & 17.414 & 23.285 & 15.543 & 12.656 & 33.106 & 34.426 & 30.648 & 33.489 & 27.274 & 34.53 \\
\hline 10/26/11 11:30 & & 31.101 & 30.645 & 29.454 & 30.965 & 29.895 & 31.066 & 30.539 & 30.271 & 28.698 & 26.459 & 33.481 & 36.211 & 34.546 & 17.321 & 17.398 & 23.285 & 15.546 & 12.616 & 33.085 & 34.374 & 30.645 & 33.438 & 27.277 & 34.53 \\
\hline $10 / 26 / 1112: 00$ & & 31.104 & 30.65 & 29.445 & 30.962 & 29.899 & 31.065 & 30.539 & 30.273 & 28.698 & 26.447 & 34.496 & 36.211 & 34.928 & $3 \quad 17.325$ & 17.388 & 23.285 & 15.553 & 12.997 & 33.299 & 35.506 & 30.641 & 34.484 & 27.277 & 34.532 \\
\hline $10 / 26 / 1112: 30$ & & 31.104 & 30.65 & 29.421 & 30.958 & 29.92 & 31.065 & 30.536 & 30.273 & 28.688 & 26.437 & 35.575 & 36.213 & 35.99 & 17.323 & 17.449 & 23.285 & 15.558 & 14.088 & 33.775 & 36.568 & 30.664 & 35.533 & 27.274 & 34.532 \\
\hline 10/26/11 13:00 & & 31.101 & 30.648 & 29.407 & 30.958 & 29.937 & 31.06 & 30.536 & 30.271 & 28.679 & 26.416 & 36.193 & 36.213 & 36.71 & 17.325 & 17.549 & 23.288 & 15.562 & 14.833 & 34.064 & 37.158 & 30.692 & 36.134 & 27.277 & 34.532 \\
\hline 10/26/11 13:30 & & 31.094 & 30.638 & 29.407 & $\begin{array}{l}30.962 \\
302\end{array}$ & 29.937 & 31.058 & 30.539 & 30.269 & 28.677 & 26.401 & 34.77 & 36.22 & 35.901 & 17.321 & 17.596 & 23.285 & 15.579 & 14.055 & 33.64 & 35.651 & 30.7 & 34.705 & 27.274 & 34.532 \\
\hline $10 / 26 / 1114: 00$ & & 31.092 & $\begin{array}{l}30.634 \\
30.634\end{array}$ & 29.412 & 30.958 & 29.937 & 31.056 & 30.536 & 30.259 & 28.679 & 26.401 & 34.379 & 36.204 & 35.493 & 17.321 & $\begin{array}{l}17.587 \\
\end{array}$ & 23.281 & 15.586 & $\begin{array}{l}13.674 \\
13.674\end{array}$ & $\begin{array}{l}33.404 \\
3.453\end{array}$ & $\begin{array}{l}35.265 \\
3.265\end{array}$ & 30.681 & 34.32 & 27.277 & 34.53 \\
\hline $10 / 26 / 1114: 30$ & & $\begin{array}{r}31.09 \\
31.09\end{array}$ & $\begin{array}{l}30.034 \\
30.624\end{array}$ & $\begin{array}{l}29.417 \\
29.417\end{array}$ & $\begin{array}{l}30.955 \\
30.955\end{array}$ & 29.93 & 31.058 & $\begin{array}{l}30.541 \\
30.541\end{array}$ & 30.257 & 28.681 & $\begin{array}{l}20.401 \\
26.404\end{array}$ & 34.15 & 36.211 & $\begin{array}{l}35.439 \\
35.249\end{array}$ & 17.318 & 17.568 & 23.283 & $\begin{array}{l}13.000 \\
15.588\end{array}$ & $\begin{array}{l}13.044 \\
13.453\end{array}$ & $\begin{array}{l}33.450 \\
33.346\end{array}$ & $\begin{array}{ll}35.035 \\
353\end{array}$ & $\begin{array}{l}30.061 \\
30.662\end{array}$ & $\begin{array}{l}34.095 \\
34.095\end{array}$ & 27.277 & 34.53 \\
\hline $10 / 26 / 1115: 00$ & & $\begin{array}{r}31.092 \\
31.092\end{array}$ & $\begin{array}{l}30.024 \\
30.624\end{array}$ & $\begin{array}{l}29.426 \\
29.426\end{array}$ & $\begin{array}{l}30.951 \\
30.951\end{array}$ & 29.925 & 31.06 & $\begin{array}{l}30.541 \\
30.541\end{array}$ & $\begin{array}{l}30.255 \\
30.25\end{array}$ & $\begin{array}{l}28.684 \\
28.684\end{array}$ & $\begin{array}{l}20.404 \\
26.409\end{array}$ & $\begin{array}{l}33.989 \\
33.989\end{array}$ & $\begin{array}{l}30.211 \\
36.206\end{array}$ & $\begin{array}{l}53.249 \\
35.081\end{array}$ & 17.316 & $\begin{array}{l}17.500 \\
17.547\end{array}$ & 23.281 & $\begin{array}{l}13.58 \\
15.588\end{array}$ & $\begin{array}{l}13.435 \\
13.288\end{array}$ & $\begin{array}{l}53.340 \\
33.281\end{array}$ & $\begin{array}{l}34.874 \\
34.074\end{array}$ & $\begin{array}{l}30.02 \\
30.653\end{array}$ & $\begin{array}{l}34.093 \\
33.938\end{array}$ & 27.274 & $\begin{array}{l}54.53 \\
34.53\end{array}$ \\
\hline //11 15:30 & & 31.099 & 30.629 & 29.445 & 30.955 & 29.916 & 31.063 & 30.548 & 30.257 & 28.691 & 26.416 & 33.865 & 36.204 & 34.949 & & 17.528 & 23.279 & 15.581 & 13.159 & 33.234 & 753 & .653 & .818 & 27.277 & 34.527 \\
\hline $10 /$ & & 31.101 & & 29.461 & 30 & 29.902 & 31.065 & 30.548 & 30.257 & 28.698 & 26.437 & 33.766 & 201 & 34.848 & $3 \quad 17.318$ & 17.505 & 23.281 & 15.579 & 13.051 & 33.192 & 658 & .648 & .724 & 27.277 & .527 \\
\hline $10 / 2$ & & 31 & 30.636 & 29.459 & 30.95 & 9.895 & 31.065 & 30.546 & 30.259 & 28.7 & 26.452 & 33.684 & 36.206 & 34.764 & 17.318 & 17.487 & .32 & .579 & 12.964 & 33.157 & 578 & .641 & 642 & 27.277 & 34.527 \\
\hline 10/26/11 17:00 & & 31 & 30.645 & 29.449 & 30.958 & 29.904 & 31.068 & 30.543 & 30.266 & 28.698 & 26.449 & 33.624 & 36.204 & 34.691 & 17.321 & 17.468 & 23.339 & 15.572 & 12.872 & 33.143 & 34.511 & .653 & 3.576 & 27.279 & 34.527 \\
\hline 10/26/11 17:30 & & 31.115 & 30.65 & 29.449 & 30.96 & 29.909 & 31.065 & 30.543 & 30.271 & 28.695 & 26.437 & 35.29 & 36.215 & 35.761 & 17.325 & 17.484 & 23.339 & 15.572 & 13.921 & 33.703 & 36.26 & 30.671 & 35.216 & 27.279 & 34.527 \\
\hline 10/26/11 18:00 & & 31.111 & 30.652 & 29.466 & 30.962 & 29.909 & 31.068 & 30.546 & 30.273 & 28.698 & 26.435 & 36.158 & 36.215 & 36.558 & $3 \quad 17.328$ & 17.57 & 23.343 & 15.576 & 14.715 & 34.017 & 37.156 & 30.7 & 6.096 & 27.279 & 34.527 \\
\hline 10/26/11 18:30 & & 31.108 & 30.65 & 29.477 & 30.969 & 29.885 & 31.072 & 30.55 & 30.271 & 28.712 & 26.452 & 35.269 & 36.215 & 36.401 & 17.323 & 17.659 & 23.336 & 15.583 & 14.569 & 33.887 & 36.144 & 30.723 & 5.188 & 27.277 & 34.527 \\
\hline 10/26/11 19:00 & & 31.106 & 30.648 & 29.48 & 30.976 & 29.892 & 31.065 & 30.548 & 30.271 & 28.712 & 26.464 & 34.667 & 36.215 & 35.793 & 17.325 & 17.662 & 23.339 & 15.583 & 13.958 & 33.598 & 35.547 & 30.723 & 34.606 & 27.277 & 34.527 \\
\hline 10/26/11 19:30 & & 31.12 & 30.662 & 29.484 & 31 & 29.89 & 31.072 & 30.548 & 30.28 & 28.714 & 26.466 & 34.377 & 36.22 & 35.486 & 17.328 & 17.65 & 23.336 & 15.562 & 13.613 & 33.493 & 35.262 & 30.742 & 34.322 & 27.281 & 34.53 \\
\hline 10/26/11 20:00 & & 31.115 & 30.662 & 29.484 & 31.004 & 29.89 & 31.072 & 30.548 & 30.282 & 28.716 & 26.468 & 34.18 & 36.22 & 35.282 & 17.33 & 17.629 & 23.334 & 15.579 & 13.411 & 33.404 & 35.073 & 30.725 & 34.13 & 27.279 & 34.53 \\
\hline $10 / 26 / 1120: 30$ & & 31.12 & 30.666 & 29.496 & 31.014 & 29.883 & 31.077 & 30.553 & 30.285 & 28.723 & 26.471 & 34.042 & 36.22 & 35.135 & 17.33 & 17.61 & 23.332 & 15.574 & 13.248 & 33.35 & 34.928 & 30.721 & 33.991 & 27.281 & 34.532 \\
\hline 10/26/11 21:00 & & 31.12 & 30.669 & 29.498 & 31.016 & 29.885 & 31.077 & 30.553 & 30.289 & 28.725 & 26.48 & 33.93 & 36.222 & 35.017 & 17.332 & 17.589 & 23.339 & 15.574 & 13.126 & 33.304 & 34.822 & 30.718 & 33.884 & 27.281 & 34.534 \\
\hline $10 / 26 / 1121: 30$ & & 31.125 & 30.676 & 29.501 & 31.021 & 29.89 & 31.077 & 30.55 & 30.292 & 28.728 & 26.483 & 33.839 & 36.229 & 34.921 & 17.335 & 17.575 & 23.339 & 15.567 & 13.016 & 33.269 & 34.732 & 30.718 & 33.797 & 27.284 & 34.537 \\
\hline $10 / 26 / 1$ & & 31.122 & 30.68 & 29.491 & 31. & 29.895 & 31.08 & 30.55 & 30.296 & 28.728 & 26.485 & 33.762 & 36.225 & 34.841 & 17.337 & 17.552 & 23.339 & 15.574 & 12.936 & 33.241 & 34.658 & 11 & 33.722 & 27.281 & 34.539 \\
\hline 10 & & 31.125 & 30.683 & 29.496 & & 29.902 & 31.077 & 30.55 & 30. & 28.728 & 26.473 & 33.703 & 36.225 & 34.778 & 17. & 17.535 & 23.341 & 15.576 & 12.86 & $\begin{array}{l}33.211 \\
33.211\end{array}$ & 34.592 & 0.707 & 33.656 & 27.284 & 34.544 \\
\hline $10 / 26 /$ & & 31.125 & $\begin{array}{l}30.685 \\
30.085\end{array}$ & 29.496 & 31. & 29.904 & 31.08 & 30.555 & 30.301 & 28.73 & 26.478 & 33.645 & 36.227 & 34.715 & 17.339 & 17.517 & 23.341 & 15.574 & $\begin{array}{l}12.700 \\
12.799\end{array}$ & $\begin{array}{l}33.183 \\
33.183\end{array}$ & $\begin{array}{r}34.54 \\
34.54\end{array}$ & .702 & $\begin{array}{l}33.004 \\
33.604\end{array}$ & 27.284 & 34.544 \\
\hline $10 / 26 /$ & & 31.12 & 30.683 & 29.503 & 31. & 29.892 & 31.082 & 30.555 & 30.301 & 28.737 & 26.476 & $\begin{array}{l}33.593 \\
33.593\end{array}$ & 36.222 & 34.663 & 17.339 & $\begin{array}{l}17.498 \\
\end{array}$ & 23.343 & 15.581 & 12.757 & $\begin{array}{l}33.157 \\
33.157\end{array}$ & $\begin{array}{l}34.484 \\
3488\end{array}$ & .692 & $\begin{array}{l}33.053 \\
33.553\end{array}$ & 27.281 & 34.546 \\
\hline $10 / 27$ & & $\begin{array}{l}31.122 \\
31.122\end{array}$ & $\begin{array}{l}30.003 \\
30.687\end{array}$ & 29.503 & 31. & $\begin{array}{l}29.0989 \\
29.909\end{array}$ & 31.082 & 30.55 & 30.301 & 28.732 & $\begin{array}{l}20.410 \\
26.483\end{array}$ & 33.551 & 36.225 & $\begin{array}{l}34.0016 \\
34.616\end{array}$ & $\begin{array}{l}17.339 \\
5\end{array}$ & $\begin{array}{l}11.490 \\
17.482\end{array}$ & $\begin{array}{l}23.345 \\
23.343\end{array}$ & $\begin{array}{l}15.510 \\
15.579\end{array}$ & $\begin{array}{l}12.703 \\
12.71\end{array}$ & $\begin{array}{l}33.143 \\
33.143\end{array}$ & $\begin{array}{l}34.400 \\
34.443\end{array}$ & $\begin{array}{l}3.0 .682 \\
30.685\end{array}$ & $\begin{array}{r}3.530 \\
33.51\end{array}$ & 27.284 & $\begin{array}{l}34.540 \\
34.549\end{array}$ \\
\hline $10 /$ & & 31 & 30 & 29 & & 2 & 31 & 30 & 30 & 28 & 26 & 33 & 36 & 34. & 17 & 17 & 23 & 15.567 & 12.649 & 3 & 55 & 9 & 1 & 27.284 & 34.548 \\
\hline $10 / 27 / 1$ & & & 30 & 29 & & & 31 & 3 & 30 & & & 33 & 36 & 34 & 17 & 17.449 & 23.343 & 3 & 12.616 & 33 & 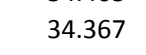 & 4 & 3 & 27.284 & 34.551 \\
\hline $10 / 27 / 1$ & & 31 & 30.692 & 29.473 & & & 31.082 & & & & & & 36.232 & 34.499 & & & & 99 & 12.578 & 33.082 & 34.336 & 30.676 & 3.398 & 27.284 & 34.553 \\
\hline 2:00 & & 31 & 30.69 & 29.475 & & & 31. & & & 28 & & & 36.227 & 34.466 & 17.344 & 17.419 & & 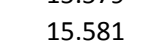 & 12.548 & 33.064 & 34.3 & & & רา & 34.551 \\
\hline 1 : :30 & & 31. & 30 & 29. & & & 31 & & & & 26. & & 36.227 & 34.431 & 17.344 & 17.4 & & 15. & 12.522 & 43 & 34.267 & & & 27.284 & 34.551 \\
\hline 11 3:00 & & 31. & 30 & 29. & & & & & & & 26 & & 36.229 & 34. & 17. & 17.384 & & & 12.517 & 33 & & & & 27.284 & 34.549 \\
\hline 10/27/11 3:30 & & 31.12 & 30 & 29.468 & & & 31. & & & & 26. & & 36.227 & 34.373 & 17. & 17.37 & 23. & 15. & 12.489 & 33.001 & & 30.624 & 33.276 & 27.284 & 34.549 \\
\hline 10/27/11 4:00 & & 31.118 & 30.6 & 29.47 & & & 31. & 30.4 & & 28. & 26. & 33.2 & 36.225 & 34.342 & 17.342 & 17.351 & 23.334 & 15.5 & 12. & 32.98 & 34.184 & 30.613 & 33.248 & 27.284 & 34.546 \\
\hline 10/27/11 4:30 & & 31.12 & 30.6 & 29.47 & 30. & & 31. & 30.9 & & 28.728 & 26.442 & 33.26 & 36.227 & 34.316 & 17.342 & 17.334 & 23.3 & 15.597 & 12.447 & 32.968 & 34.158 & 30.601 & 33.224 & 27.284 & 34.544 \\
\hline $10 / 27 / 11$ 5:00 & & 31.122 & 30.678 & 29.461 & 30.9 & 29.946 & 31.08 & 30.553 & 30.2 & 28.723 & 26.442 & 33.23 & 36.227 & 34.29 & 17.342 & 17.321 & 23.334 & 15.593 & 12.414 & 32.956 & 34.137 & 30.601 & 33.201 & 27.284 & 34.544 \\
\hline 10/27/11 5:30 & & 31.127 & 30.68 & 29.466 & 30.5 & 29.953 & 31.082 & 30.555 & 30.301 & 28.716 & 26.435 & 33.216 & 36.227 & 34.269 & 17.344 & 17.306 & 23.336 & 15.59 & 12.388 & 32.947 & 34.113 & 30.601 & 33.177 & 27.286 & 34.541 \\
\hline 10/27/11 6:00 & & 31.127 & 30.683 & 29.477 & 30.918 & 29.937 & 31.087 & 30.558 & 30.301 & 28.725 & 26.433 & 34.258 & 36.229 & 34.68 & 17.344 & 17.295 & 23.346 & 15.588 & 12.781 & 33.178 & 35.272 & 30.594 & 34.254 & 27.286 & 34.539 \\
\hline $10 / 27 / 116: 30$ & & 31.122 & 30.676 & 29.503 & 30.906 & 29.925 & 31.087 & 30.565 & 30.299 & 28.737 & 26.447 & 35.35 & 36.227 & 35.756 & 17.342 & 17.351 & 23.343 & 15.593 & 13.876 & 33.67 & 36.35 & 30.617 & 35.315 & 27.286 & 34.539 \\
\hline 10/27/11 7:00 & & 31.127 & 30.68 & 29.512 & 30.91 & 29.916 & 31.089 & 30.567 & 30.299 & 28.744 & 26.473 & 35.989 & 36.222 & 36.494 & 17.344 & 17.456 & 23.35 & 15.579 & 14.602 & 33.973 & 36.954 & 30.669 & 35.934 & 27.288 & 34.537 \\
\hline 10/27/11 7:30 & & 31.132 & 30.685 & 29.519 & 30.9 & 29.911 & 31.092 & 30.562 & 30.303 & 28.746 & 26.483 & 34.585 & 36.229 & 35.702 & 17.346 & 17.508 & 23.353 & 15.581 & 13.801 & 33.565 & 35.469 & 30.697 & 34.522 & 27.288 & 34.537 \\
\hline 10/27/11 8:00 & & 31.139 & 30.694 & 29.522 & 30.958 & 29.916 & 31.094 & 30.562 & 30.313 & 28.751 & 26.49 & 34.22 & 36.237 & 35.32 & 17.349 & 17.503 & 23.355 & 15.574 & 13.392 & 33.416 & 35.104 & 30.711 & 34.165 & 27.288 & 34.539 \\
\hline 10/27/11 8:30 & & 31.139 & 30.699 & 29.508 & 30.972 & 29.92 & 31.092 & 30.56 & 30.317 & 28.751 & 26.492 & 34.007 & 36.232 & 35.097 & 17.349 & 17.491 & 23.357 & 15.574 & 13.161 & 33.327 & 34.895 & 30.714 & 33.956 & 27.288 & 34.539 \\
\hline
\end{tabular}




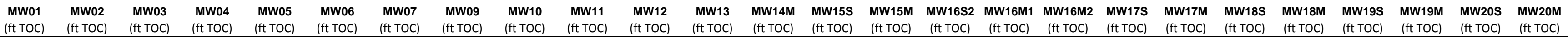

\begin{tabular}{|c|c|c|c|c|c|c|c|c|c|c|c|c|c|c|c|c|c|c|c|c|c|c|c|c|c|}
\hline well & $\begin{array}{l}\text { (ft TOC) } \\
\text { (f) }\end{array}$ & $\begin{array}{l}\text { (ft TOC) } \\
\text { (ft) }\end{array}$ & $\begin{array}{l}\text { (ft TOC) } \\
\text { (f) }\end{array}$ & $\begin{array}{l}\text { (ft TOC) } \\
\text { (ft) }\end{array}$ & $\begin{array}{ll}(\mathrm{ft} \text { TOC) } & \text { (ft TOC) }\end{array}$ & (ft TOC) & $\begin{array}{l}\text { (ift TOC) } \\
\text { (n) }\end{array}$ & $\begin{array}{l}\text { (ft TOC) } \\
\text { (ft) }\end{array}$ & $\begin{array}{l}\text { (ft TOC) } \\
\text { (f) }\end{array}$ & $\begin{array}{l}\text { (ft TOC) } \\
\text { (n) }\end{array}$ & $\begin{array}{l}\text { (ft TOC) } \\
\text { (f) }\end{array}$ & (ft TOC) & $\begin{array}{l}\text { (ft TOC) } \\
\end{array}$ & (ft TOC) & (ft TOC) & $\begin{array}{l}\text { (ft TOC) } \\
\text { (f) }\end{array}$ & (ft TOC) & $\begin{array}{l}\text { (ft TOC) } \\
\text { (ft) }\end{array}$ & (ft TOC) & $\begin{array}{l}\text { (ft TOC) } \\
\text { (f) }\end{array}$ & $\begin{array}{l}\text { (ft TOC) } \\
\text { (f) }\end{array}$ & $\begin{array}{l}\text { (ft TOC) } \\
\text { (n) }\end{array}$ & (ft TOC) & $\begin{array}{l}\text { (ft TOC) } \\
\text { (1) }\end{array}$ & (ft TOC) \\
\hline 00 & & 31.139 & 30.704 & 29. & 30.986 & 29.939 & 31.0 & 30 & 30. & 28.746 & 26.48 & 33.862 & 36.2 & 34. & 17.351 & 17.477 & 23.359 & 15.579 & 13.006 & 33.269 & 34.753 & 30.709 & 3.816 & 88 & 1 \\
\hline $19: 30$ & & 31.139 & 30.706 & 29.496 & 30.993 & 29.939 & 31.094 & 30.56 & 30.327 & 28.746 & 26.476 & 33.75 & 36.236 & 34.83 & 17.353 & 17.461 & 23.362 & 15.586 & 12.893 & 33.224 & 644 & 30.7 & & & \\
\hline 10/27/11 10:00 & & 31.129 & 30.704 & 29.494 & 30.986 & 29.958 & 31.094 & 30.558 & 30.322 & 28.739 & 26.471 & 33.663 & 36.234 & 34.738 & 17.353 & 17.444 & 23.362 & 15.597 & 12.835 & 33.173 & 34.554 & & 33.621 & 88 & .544 \\
\hline 10/27/11 10:30 & & 31.134 & 30.704 & 29.498 & 30.986 & 29.951 & 31.097 & 30.562 & 30.322 & 28.746 & 26.464 & 33.593 & 36.239 & 34.663 & 17.353 & 17.428 & 23.359 & 15.597 & 12.769 & 33.148 & 34.488 & 30.676 & {$[-$} & 88 & 34.544 \\
\hline 10/27/11 11:00 & & 31.132 & 30.699 & 29.498 & 30.979 & 29.956 & 31.094 & 30.562 & 30.32 & 28.746 & 26.466 & 33.53 & 36.236 & 34.6 & 17.353 & 17.414 & 23.359 & 15.612 & 12.734 & 33.113 & 34.426 & 30.662 & 33.492 & 27.288 & 34.546 \\
\hline 10/27/11 11:30 & & 31.134 & 30.701 & 29.491 & 30.976 & 29.96 & 31.096 & 30.562 & 30.32 & 28.744 & 26.464 & 33.483 & 36.237 & 34.548 & 17.356 & 17.398 & 23.359 & 15.604 & 12.687 & 33.089 & 34.379 & 30.662 & 33.442 & 27.291 & 34.546 \\
\hline 10/27/11 12:00 & & 31.134 & 30.701 & 29.477 & 30.969 & 29.972 & 31.096 & 30.562 & 30.322 & 28.739 & 26.461 & 34.503 & 36.241 & $1 \quad 34.938$ & 17.356 & 17.388 & 23.357 & 15.612 & 13.074 & 33.311 & 35.516 & 30.655 & 34.494 & 27.291 & 34.546 \\
\hline 10/27/11 12:30 & & 31.134 & 30.701 & 29.477 & 30.969 & 29.979 & 31.094 & 30.56 & 30.322 & 28.739 & 26.445 & 35.575 & 36.237 & 35.993 & 17.353 & 17.449 & 23.357 & 15.607 & 14.151 & 33.794 & 36.566 & 30.683 & 35.533 & 27.291 & 34.548 \\
\hline 10/27/11 13:00 & & 31.134 & 30.701 & 29.466 & 30.969 & 29.984 & 31.094 & 30.562 & 30.32 & 28.732 & 26.442 & 36.197 & 36.239 & 36.71 & 17.353 & 17.549 & 23.357 & 15.621 & 14.892 & 34.078 & 37.16 & 30.716 & 36.141 & 27.291 & 34.549 \\
\hline 10/27/11 13:30 & & 31.129 & 30.697 & 29.449 & 30.976 & 29.998 & 31.094 & 30.558 & 30.315 & 28.723 & 26.435 & 34.775 & 36.232 & 35.904 & 17.351 & 17.601 & 23.357 & 15.628 & 14.102 & 33.651 & 35.656 & 30.723 & 34.71 & 27.291 & 34.549 \\
\hline $10 / 27 / 1114: 00$ & & 31.132 & 30.694 & 29.447 & 30.983 & 30.003 & 31.092 & 30.56 & 30.313 & 28.723 & 26.418 & 34.386 & 36.234 & 35.503 & 17.353 & 17.592 & 23.357 & 15.633 & 13.709 & 33.479 & 35.269 & 30.718 & 34.332 & 27.293 & 34.549 \\
\hline 10/27/11 14:30 & & 31.127 & 30.687 & 29.447 & 30.981 & 30 & 31.092 & 30.56 & 30.31 & 28.723 & 26.416 & 34.159 & 36.237 & 35.256 & 17.351 & 17.573 & 23.355 & 15.644 & 13.493 & 33.369 & 35.047 & 30.695 & 34.109 & 27.291 & 34.549 \\
\hline $10 / 27 / 1115: 00$ & & 31.125 & 30.678 & 29.449 & 30.979 & 30 & 31.092 & 30.565 & 30.303 & 28.721 & 26.416 & 33.996 & 36.227 & 35.085 & 17.349 & 17.552 & 23.353 & 15.644 & 13.338 & 33.297 & 34.886 & 30.681 & 33.949 & 27.291 & 34.549 \\
\hline $10 / 27 / 1115: 30$ & & 31.127 & 30.676 & 29.452 & 30.976 & 29.993 & 31.092 & 30.562 & 30.301 & 28.721 & 26.418 & 33.872 & 36.229 & 34.956 & 17.349 & 17.533 & 23.348 & 15.649 & 13.218 & 33.245 & 34.762 & 30.669 & 33.827 & 27.291 & 34.546 \\
\hline 10/27/11 16:00 & & 31.129 & 30.673 & 29.456 & 30.972 & 29.991 & 31.092 & 30.565 & 30.299 & 28.721 & 26.423 & 33.776 & 36.227 & 34.856 & 17.349 & 17.514 & 23.346 & 15.647 & 13.121 & 33.204 & 34.665 & 30.657 & 33.729 & 27.293 & 34.544 \\
\hline 10/27/11 16:30 & & 31.132 & 30.676 & 29.459 & 30.962 & 29.989 & 31.094 & 30.562 & 30.299 & 28.718 & 26.425 & 33.694 & 36.225 & 34.769 & 17.349 & 17.491 & 23.348 & 15.644 & 13.034 & 33.164 & & 30.65 & 33.649 & 27.293 & 34.546 \\
\hline 10/27/11 17:00 & & 31.134 & 30.678 & 29.468 & 30.962 & 29.981 & 31.094 & 30.567 & 30.299 & 28.723 & 26.428 & 33.626 & 36.225 & 34.698 & 17.351 & 17.472 & 23.346 & 15.642 & 12.959 & 33.141 & 34.518 & 30.65 & 33.586 & 27.293 & 34.544 \\
\hline 10/27/11 17:30 & & 31.134 & 30.673 & 29.477 & 30.958 & 29.972 & 31.096 & 30.565 & 30.299 & 28.728 & 26.437 & 35.285 & 36.229 & 35.763 & 17.349 & 17.489 & 23.343 & 15.642 & 14.026 & 33.689 & 36.26 & 30.66 & 35.214 & 27.293 & 34.544 \\
\hline $10 /$ & & 31.136 & 30.676 & 29.484 & 30.96 & 29.965 & 31.096 & 30.569 & 30.301 & 28.73 & 26.445 & 36.151 & 36.229 & 36.558 & 17.351 & 17.573 & 23.343 & 15.647 & 14.816 & 34.01 & 37.146 & 30.69 & 36.092 & 27.295 & 34.544 \\
\hline & & 31.141 & 30.683 & 29.496 & 30.97 & 29.956 & 31.099 & 30.569 & 30.303 & 28.735 & 26.457 & 35.269 & 36.232 & 36.398 & 17.351 & 17.666 & 23.35 & 15.6 & 14.652 & 33.884 & 4 & 73 & 35.186 & 27.295 & 34.544 \\
\hline bo & & 31.143 & 30.687 & 29.498 & 30.98 & 29.953 & 31.099 & 30.574 & 30.306 & 28.737 & 26.466 & 34.67 & 36.232 & 35.796 & 17.353 & 17.669 & 23.373 & 15.635 & 14.033 & 33607 & 4 & 30.732 & 34.611 & 27.295 & 34.544 \\
\hline $10 / 27 / 1$ & & 31.146 & 30.692 & 29.503 & 31. & 29.953 & 31.099 & 30.572 & 30.308 & 28.739 & 26.468 & 34.379 & 36.234 & 35.489 & 17.353 & 17.655 & 23.401 & 15.63 & 13.69 & 33.486 & 5 & 35 & 25 & 27295 & 34.544 \\
\hline $10 / 27 / 1$ & & & 30.694 & 29.501 & & 29.953 & 31.101 & 30.574 & 30.313 & 28.742 & 26.473 & 34.185 & 36.241 & 35.285 & 17.358 & 17.636 & 23.422 & 15.628 & 13.474 & 411 & 073 & .737 & 35 & 27.295 & 34.546 \\
\hline 10/27/11 20:30 & & 31.146 & 30.697 & 29.505 & 31.016 & 29.953 & 31.104 & 30.572 & 30.315 & 28.744 & 26.471 & 34.045 & 36.241 & 35.135 & 17.356 & 17.615 & 23.424 & 15.63 & 13.316 & 33.353 & .931 & 1732 & 33.996 & 27.295 & 34.546 \\
\hline 10/27/11 21:00 & & 31.148 & 30.699 & 29.51 & 31.018 & 29.949 & 31.101 & 30.574 & 30.315 & 28.746 & 26.473 & 33.932 & 36.241 & 35.017 & 17.358 & 17.594 & 23.424 & 15.626 & 13.185 & 33.304 & 34.822 & 30.725 & 33.886 & 27.295 & 34.549 \\
\hline 10/27/11 21:30 & & 31.146 & 30.704 & 29.515 & 31.018 & 29.946 & 31.106 & 30.572 & 30.317 & 28.751 & 26.476 & 33.841 & 36.241 & 34.924 & 17.358 & 17.575 & 23.422 & 15.628 & 13.079 & 33.262 & 332 & .716 & 33.797 & 27.295 & 34.551 \\
\hline 10/27/11 22:00 & & 31.148 & 30.704 & 29.519 & 31.018 & 29.949 & 31.106 & 30.574 & 30.32 & 28.751 & 26.483 & 33.769 & 36.244 & 34.841 & 17.36 & 17.559 & 23.417 & 15.628 & 12.99 & 33.234 & .656 & 30.711 & 33.724 & 27.295 & 34.551 \\
\hline 10/27/11 22:30 & & 31.153 & 30.708 & 29.517 & 31.018 & 29.949 & 31.106 & 30.574 & 30.322 & 28.753 & 26.485 & 33.701 & 36.243 & 34.776 & 17.363 & 17.538 & 23.417 & 15.623 & 12.908 & 33.215 & 34.594 & 30.711 & 33.661 & 27.295 & 34.553 \\
\hline 10/27/11 23:00 & & 31.15 & 30.711 & 29.51 & 31.016 & 29.956 & 31.108 & 30.572 & 30.324 & 28.749 & 26.483 & 33.647 & 36.243 & 34.717 & 17.363 & 17.524 & 23.417 & 15.623 & 12.842 & 33.18 & 34.54 & 30.707 & 33.607 & 27.295 & 34.558 \\
\hline 10/27/11 23:30 & & 31.153 & 30.713 & 29.505 & 31.016 & 29.965 & 31.109 & 30.574 & 30.327 & 28.746 & 26.473 & 33.598 & 36.246 & 34.668 & 17.365 & 17.507 & 23.415 & 15.621 & 12.781 & 33.164 & 34.49 & 30.697 & 33.557 & 27.298 & 34.558 \\
\hline $10 / 28 / 110: 00$ & & 31.153 & 30.715 & 29.51 & 31.009 & 29.965 & 31.106 & 30.577 & 30.327 & 28.749 & 26.471 & 33.553 & 36.244 & 34.621 & 17.365 & 17.489 & 23.415 & 15.626 & 12.734 & 33.138 & 34.447 & 30.692 & 33.515 & 27.298 & 34.56 \\
\hline 10/28/11 0:30 & & 31.148 & 30.711 & 29.512 & 31 & 29.965 & 31.111 & 30.574 & 30.324 & 28.751 & 26.473 & 33.514 & 36.243 & 34.577 & 17.365 & 17.472 & 23.417 & 15.63 & 12.696 & 33.11 & 34.407 & 30.683 & 33.473 & 27.298 & 34.563 \\
\hline 10/28/11 1:00 & & 31.15 & 30.711 & 29.517 & 30.993 & 29.96 & 31.108 & 30.577 & 30.324 & 28.753 & 26.478 & 33.476 & 36.241 & 34.539 & 17.367 & 17.456 & 23.417 & 15.628 & 12.656 & 33.094 & 34.369 & 30.674 & 33.435 & 27.298 & 34.562 \\
\hline 10/28/11 1:30 & & 31.15 & 30.711 & 29.519 & 30.986 & 29.963 & 31.111 & 30.577 & 30.324 & 28.756 & 26.483 & 33.441 & 36.251 & 34.504 & 17.365 & 17.442 & 23.42 & 15.626 & 12.616 & 33.078 & 34.336 & 30.669 & 33.403 & 27.298 & 34.565 \\
\hline $10 / 28 / 112: 00$ & & 31.155 & 30.713 & 29.519 & 30 & 29.963 & 31. & 30.574 & 30.327 & 28.758 & 26.48 & 33.411 & 36.248 & 34.469 & 17.365 & 17.426 & 23.42 & 15.623 & 12.578 & 33.064 & .305 & 30.664 & 33.37 & 27.3 & 34.565 \\
\hline $2: 30$ & & 31. & 30.718 & 29.517 & & 29.967 & & 30 & 30.329 & 28.756 & 26.483 & 33.383 & 36.248 & 34.438 & 17.367 & 17.412 & 23. & 15.626 & 12.543 & 33.047 & 77 & 30.669 & 33.346 & 27.3 & 34.565 \\
\hline $10 / 28 / 1$ & & 31. & 30.718 & 29.517 & 30. & 29.974 & 31.111 & 30.577 & 30.331 & 28.756 & 26.478 & $\begin{array}{l}33.354 \\
3.354\end{array}$ & $\begin{array}{l}36.248 \\
36.48\end{array}$ & $\begin{array}{r}34.450 \\
34.41\end{array}$ & 17.367 & 17.398 & 23.42 & 15.621 & 12.506 & $\begin{array}{l}33.038 \\
33.047\end{array}$ & 251 & 30.667 & 33.316 & 27.3 & 34.565 \\
\hline $10 / 28 / 113: 30$ & & 31. & 30.718 & 29.517 & 30. & 29.97 & 31.113 & 30.577 & 30.334 & 28.758 & 26.473 & $\begin{array}{l}33.329 \\
33.34\end{array}$ & $\begin{array}{l}36.251 \\
36.25\end{array}$ & 34.384 & 17.37 & 17.381 & $\begin{array}{r}23.413 \\
23.413\end{array}$ & 15.623 & 12.482 & 33.026 & 225 & .655 & 33.29 & 7.3 & 34.567 \\
\hline & & 31 & 30 & 29.5 & 30 & 29.972 & 31 & 30 & 30 & 28.758 & 26.4 & 33.303 & 36.251 & 34 & 17 & 17.365 & 7 & 15.621 & 12.458 & 33.008 & 1 & 5 & 33. & 27.3 & 34.565 \\
\hline & & 31. & 30. & 29. & & & 31. & $3 c$ & 30 & 28.758 & 26 & 33.277 & 36. & 34 & 17.37 & 17 & 3 & 15.623 & 12.426 & 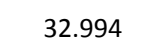 & 7 & 3 & & 27.3 & 34.563 \\
\hline & & & 30.7 & & & & & & & & & 33.259 & 36. & & 17.372 & & 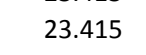 & 15.6 & 12.405 & & 6 & 5 & & .3 & 34.563 \\
\hline & & & & & & & & & & & & 202 & & & & & 23.4 & 15.6 & 12. & & & & 33. & 3 & 34.563 \\
\hline $16: 00$ & & 31.157 & 30.718 & & & & & & & & & 34.2 & & & & & 23.4 & 15. & 12.7 & & & & & & \\
\hline 6:30 & & 31 & 30.718 & 29 & & & 31 & & & 28.763 & 26 & 35 & 36.251 & 35. & & & 23.4 & 15. & 13.8 & & & 4 & 35. & 27.302 & 34.558 \\
\hline 7:00 & & & 30 & & & & & & & 28.7 & & & 36.253 & 36. & & & 23.4 & 15.619 & 14.605 & & & 30.709 & & & 34.558 \\
\hline 17:30 & & 31. & 30.7 & & & & & & & 28 & 26. & & 36. & & & & & 15.614 & 13.808 & & & & & & 34.556 \\
\hline 10/28/11 8:00 & & & 30.7 & 29.543 & & & & & & 28.7 & 26.492 & 34.234 & 36. & 35. & 17. & 17.519 & 23.443 & 15.614 & 13.415 & 33.427 & 35.123 & 30.728 & 34.184 & 27.302 & 34.556 \\
\hline 10/28/11 8:30 & & & 30.7 & 29.55 & & 29. & 31. & $30.5 \mathrm{~S}$ & $30.3 \mathrm{~K}$ & 28.7 & 26.5 & 34.021 & 36.251 & 35.113 & 17. & 17.505 & 23.438 & 15.6 & 13.18 & 33.339 & 34.914 & 30.73 & 33.975 & 27.305 & 34.556 \\
\hline 10/28/11 9:00 & & 31.167 & 30.7 & 29.557 & 31.0 & 29.9 & 31.123 & 30.584 & 30.345 & 28.779 & 26.50 & 33.874 & 36.255 & 34.954 & 17.3 & 17.491 & 23.4 & 15.619 & 13.034 & 33.283 & 34.765 & 30.725 & 33.827 & 27.302 & 34.555 \\
\hline 10/28/11 9:30 & & 31.169 & 30.736 & 29.552 & 31.00 & 29.977 & 31.123 & 30.581 & 30.35 & 28.779 & 26.512 & 33.764 & 36.255 & 34.846 & 17.377 & 17.475 & 23.436 & 15.619 & 12.924 & 33.238 & 34.656 & 30.725 & 33.722 & 27.305 & 34.558 \\
\hline 10/28/11 10:00 & & 31.171 & 30.739 & 29.547 & 31.016 & 29.984 & 31.123 & 30.588 & 30.354 & 28.781 & 26.509 & 33.68 & 36.258 & 34.755 & 17.377 & 17.458 & 23.436 & 15.621 & 12.851 & 33.204 & 34.573 & 30.723 & 33.64 & 27.305 & 34.558 \\
\hline $10 / 28 / 1110: 30$ & & 31.171 & 30.743 & 29.536 & 31.018 & 30 & 31.123 & 30.586 & 30.357 & 28.779 & 26.502 & 33.612 & 36.26 & 34.682 & 17.379 & 17.447 & 23.436 & 15.623 & 12.79 & 33.173 & 34.509 & 30.721 & 33.571 & 27.305 & 34.56 \\
\hline $10 / 28 / 1111: 00$ & & 31.171 & 30.743 & 29.531 & 31.018 & 30.01 & 31.125 & 30.581 & 30.357 & 28.774 & 26.49 & 33.553 & 36.26 & 34.619 & 17.381 & 17.43 & 23.436 & 15.63 & 12.745 & 33.145 & 34.447 & 30.716 & 33.513 & 27.307 & 34.56 \\
\hline 10/28/11 11:30 & & 31.167 & 30.741 & 29.524 & 31.011 & 30.014 & 31.125 & 30.581 & 30.357 & 28.774 & 26.488 & 33.502 & 36.258 & 34.565 & 17.381 & 17.414 & 23.436 & 15.637 & 12.715 & 33.12 & 34.393 & 30.692 & 33.464 & 27.307 & 34.563 \\
\hline 11 12:00 & & 31.164 & 30.736 & 29.508 & 31.004 & 30.026 & 31.123 & 30.581 & 30.352 & 28.767 & 26.478 & 34.524 & 36.262 & 34.959 & 17.381 & 17.402 & 23.433 & 15.649 & 13.124 & 33.336 & 35.537 & 30.688 & 34.519 & 27.307 & 34.563 \\
\hline 10/28/11 12:30 & & 31.162 & 30.734 & 29.498 & 30.997 & 30.04 & 31.123 & 30.581 & 30.352 & 28.763 & 26.464 & 35.594 & 36.258 & 36.016 & 17.381 & 17.463 & 23.433 & 15.651 & 14.205 & 33.815 & 36.589 & 30.711 & 35.557 & 27.307 & 3 \\
\hline
\end{tabular}




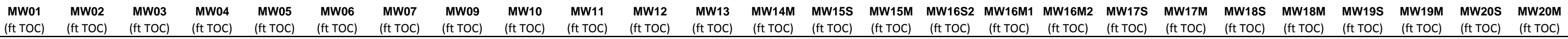

\begin{tabular}{|c|c|c|c|c|c|c|c|c|c|c|c|c|c|c|c|c|c|c|c|c|c|c|c|c|c|c|}
\hline Well & $\begin{array}{l}\text { MW01 } \\
\text { (ft TOC) }\end{array}$ & $\begin{array}{l}\text { MW02 } \\
\text { (ft TOC) }\end{array}$ & $\begin{array}{l}\text { MW03 } \\
\text { (ft TOC) }\end{array}$ & $\begin{array}{r}\text { MW04 } \\
\text { (ft TOC) }\end{array}$ & $\begin{array}{l}\text { MW05 } \\
\text { (ft TOC) }\end{array}$ & $\begin{array}{r}\text { MW06 } \\
\text { (ft TOC) }\end{array}$ & $\begin{array}{l}\begin{array}{l}\text { WW07 } \\
\text { (ft TOC) }\end{array} \\
\text { (f) }\end{array}$ & $\begin{array}{l}\text { MW09 } \\
\text { (ft TOC) }\end{array}$ & $\begin{array}{l}\text { MW10 } \\
\text { (ft TOC) }\end{array}$ & $\begin{array}{l}\text { MW11 } \\
\text { (ft TOC) }\end{array}$ & $\begin{array}{r}\text { MW12 } \\
\text { (ft TOC) }\end{array}$ & $\begin{array}{l}\text { MW13 } \\
\text { (ft Toc) }\end{array}$ & $\begin{array}{l}\text { MW14M } \\
\text { (ft TOC) }\end{array}$ & $\begin{array}{l}\text { MWW15S } \\
\text { (ft TOC) }\end{array}$ & $\begin{array}{l}\text { MW15M } \\
(\mathrm{ft} \text { TOC) }\end{array}$ & $\begin{array}{l}\text { MW16S2 } \\
\text { (ft TOC) }\end{array}$ & $\begin{array}{c}\text { MW16M1 } \\
\text { (ft TOC) }\end{array}$ & $\begin{array}{c}\text { MW16M2 } \\
\text { (ft TOC) }\end{array}$ & $\begin{array}{l}\text { MW17s } \\
\text { (ft TOC) }\end{array}$ & $\begin{array}{l}\text { MW17M } \\
\text { (ft TOC) }\end{array}$ & $\begin{array}{l}\mathrm{MW} 18 \mathrm{~S} \\
(\mathrm{ft} \text { TOC) }\end{array}$ & $\begin{array}{r}\text { MW18M } \\
\text { (ft TOC) }\end{array}$ & $\begin{array}{l}\text { MW19S } \\
(\mathrm{ft} \text { TOC) }\end{array}$ & $\begin{array}{l}\text { MW19M } \\
\text { (ft TOC) }\end{array}$ & $\begin{array}{l}\mathrm{MW} 20 \mathrm{~S} \\
\text { (ft TOC) }\end{array}$ & $\begin{array}{l}\text { MW20M } \\
\text { (ft TOC) }\end{array}$ \\
\hline 10/28/11 13:00 & & 31.162 & 30.729 & 29.487 & 31.002 & & 30.047 & 31.121 & 30.581 & 30.35 & 28.758 & 26.457 & 36.216 & 36.258 & 36.734 & 17.381 & 17.568 & 23.431 & 15.663 & 14.948 & 34.101 & 37.179 & 30.742 & 36.16 & 27.307 & 34.565 \\
\hline 10/28/11 13:30 & & 31.157 & 30.718 & 29.477 & 31.002 & & 30.057 & 31.118 & 30.581 & 30.343 & 28.751 & 26.445 & 34.791 & 36.251 & 35.918 & 17.379 & 17.615 & 23.431 & 15.668 & 14.167 & 33.672 & 35.672 & 30.747 & 34.726 & 27.307 & 34.565 \\
\hline 10/28/11 14:00 & & 31.157 & 30.713 & 29.477 & 31.007 & & 30.054 & 31.118 & 30.584 & 30.34 & 28.749 & 26.435 & 34.403 & 36.253 & 35.517 & 17.377 & 17.608 & 23.427 & 15.675 & 13.784 & 33.495 & 35.291 & 30.732 & 34.35 & 27.307 & 34.563 \\
\hline $10 / 28 / 1114: 30$ & & 31.155 & 30.706 & 29.475 & 31.004 & & 30.057 & 31.118 & 30.586 & 30.336 & 28.744 & 26.435 & 34.176 & 36.255 & 35.278 & 17.377 & 17.589 & 23.427 & 15.687 & 13.57 & 33.39 & 35.063 & 30.714 & 34.125 & 27.307 & 34.563 \\
\hline $10 / 28 / 1115: 00$ & & 31.155 & 30.701 & 29.475 & 31.002 & & 30.054 & 31.118 & 30.584 & 30.331 & 28.746 & 26.43 & 34.017 & 36.246 & 35.106 & 17.377 & 17.57 & 23.427 & 15.684 & 13.411 & 33.316 & 34.905 & 30.704 & 33.966 & 27.307 & 34.563 \\
\hline $10 / 28 / 1115: 30$ & & 31.157 & 30.697 & 29.477 & 31 & & 30.054 & 31.116 & 30.584 & 30.329 & 28.742 & 26.435 & 33.89 & 36.246 & 34.977 & 17.374 & 17.549 & 23.424 & 15.689 & 13.298 & 33.269 & 34.784 & 30.695 & 33.846 & 27.307 & 34.562 \\
\hline $10 / 28 / 1116: 00$ & & 31.16 & 30.694 & 29.477 & 30.995 & & $\begin{array}{l}30.047 \\
304\end{array}$ & 31.116 & $\begin{array}{l}30.584 \\
30.584\end{array}$ & 30.327 & 28.742 & 26.437 & $\begin{array}{l}33.793 \\
3.794\end{array}$ & 36.239 & 34.877 & 17.374 & 17.531 & $\begin{array}{l}23.422 \\
23.422\end{array}$ & $\begin{array}{l}15.687 \\
15.687\end{array}$ & 13.197 & 33.225 & 34.687 & 30.683 & $\begin{array}{r}5.040 \\
33.75\end{array}$ & 27.31 & 34.563 \\
\hline $10 / 28 / 1116: 30$ & & $\begin{array}{l}31.10 \\
31.16\end{array}$ & $\begin{array}{l}30.697 \\
30.697\end{array}$ & 29.482 & 30.988 & & $\begin{array}{l}30.045 \\
30.045\end{array}$ & 31.118 & $\begin{array}{l}30.594 \\
30.591\end{array}$ & 30.324 & 28.742 & $\begin{array}{l}26.431 \\
26.437\end{array}$ & $\begin{array}{l}33.712 \\
3.712\end{array}$ & $\begin{array}{l}30.246 \\
36.246\end{array}$ & 34.785 & 17.374 & $\begin{array}{r}1.371 \\
17.51\end{array}$ & $\begin{array}{l}23.422 \\
23.457\end{array}$ & $\begin{array}{l}13.694 \\
15.694\end{array}$ & 13.121 & $\begin{array}{l}33.187 \\
3.257\end{array}$ & $\begin{array}{l}34.601 \\
34.601\end{array}$ & $\begin{array}{l}30.669 \\
30.669\end{array}$ & 33.672 & 27.31 & $\begin{array}{l}5.0303 \\
34.56\end{array}$ \\
\hline $10 / 28 / 1117: 00$ & & $\begin{array}{l}31.160 \\
31.162\end{array}$ & $\begin{array}{l}30.694 \\
30.694\end{array}$ & $\begin{array}{l}29.482 \\
29.487\end{array}$ & 30.981 & & $\begin{array}{l}30.038 \\
30.038\end{array}$ & 31.118 & $\begin{array}{l}30.586 \\
30.586\end{array}$ & $\begin{array}{l}30.324 \\
30.324\end{array}$ & 28.744 & $\begin{array}{r}20.431 \\
26.44\end{array}$ & $\begin{array}{l}33.112 \\
33.642\end{array}$ & $\begin{array}{l}30.240 \\
36.243\end{array}$ & 34.717 & $\begin{array}{l}17.374 \\
17.374\end{array}$ & $\begin{array}{l}17.411 \\
17.491\end{array}$ & 23.475 & $\begin{array}{l}13.0944 \\
15.687\end{array}$ & $\begin{array}{l}13.049 \\
13.049\end{array}$ & $\begin{array}{l}33.157 \\
33.157\end{array}$ & $\begin{array}{l}34.031 \\
34.537\end{array}$ & $\begin{array}{l}30.099 \\
30.669\end{array}$ & $\begin{array}{l}33.072 \\
33.604\end{array}$ & 27.31 & $\begin{array}{l}54.30 \\
34.56\end{array}$ \\
\hline 10/28/11 17:30 & & 31.162 & 30.697 & 29.496 & 30.976 & & 30.033 & 31.118 & 30.588 & 30.322 & 28.749 & 26.445 & 35.299 & 36.241 & 35.784 & 17.374 & 17.507 & 23.473 & 15.691 & 14.113 & 33.71 & 36.274 & 30.678 & 35.235 & 27.31 & 34.56 \\
\hline bo & & 31.164 & 30.694 & 29.505 & 30.97 & & 30.019 & 31.123 & 30.588 & 30.322 & 28.751 & 26.454 & 36.183 & 36.244 & 36.593 & 17.374 & 17.592 & 23.475 & 15.687 & 14.917 & 34.036 & 37.177 & 30.714 & 36.127 & 27.312 & 34.56 \\
\hline$: 30$ & & 31.164 & 30.699 & 29.508 & 30.988 & & 30.012 & 31.123 & 30.586 & 30.324 & 28.756 & 26.466 & 35.294 & 36.246 & 36.422 & 17.374 & 17.687 & 23.473 & 15.687 & 14.75 & 33.903 & 36.168 & 30.747 & 35.214 & 27.31 & 34.558 \\
\hline 10/28/11 19:00 & & 31.169 & 30.701 & 29.508 & 31.007 & & 30.012 & 31.123 & 30.588 & 30.327 & 28.753 & 26.471 & 34.695 & 36.246 & 35.822 & 17.374 & 17.687 & 23.47 & 15.682 & 14.123 & 33.626 & 575 & 0.751 & 34.634 & 31 & 34.56 \\
\hline $10 / 28 /$ & & 31.174 & 30.706 & 29.508 & 31.018 & & 30.017 & 31.125 & 30.586 & 30.329 & 28.753 & 26.468 & 34.4 & 36.246 & 35.51 & 17.377 & 17.673 & 23.466 & 5.677 & $\begin{array}{l}13.794 \\
13.72\end{array}$ & $\begin{array}{l}33.060 \\
33.502\end{array}$ & $\begin{array}{l}35.269 \\
35.71\end{array}$ & .761 & $\begin{array}{l}4.0344 \\
4.348\end{array}$ & 27.312 & $\begin{array}{r}34.50 \\
34.558\end{array}$ \\
\hline $10 / 28 / 1$ & & 31.171 & 30.708 & 29.501 & 31.025 & & 30.019 & 31.125 & 30.588 & 30.331 & 28.749 & 26.466 & 34.206 & 36.253 & 35.306 & 17.377 & 17.655 & 23.466 & 15.677 & 13.573 & 33.427 & 35.068 & 0.751 & 34.158 & 27.312 & 34.56 \\
\hline $10 / 28 / 112$ & & 31.171 & 30.711 & 29.498 & 31.03 & & 30.017 & 31.125 & 30.588 & 30.331 & 28.746 & 26.461 & 34.061 & 36.255 & 35.153 & 17.379 & 17.631 & 23.466 & 15.684 & 13.413 & 33.362 & 34.921 & 30.737 & 34.013 & 27.312 & 34.56 \\
\hline 10/28/11 21:00 & & 31.169 & 30.706 & 29.494 & 31.025 & & 30.021 & 31.127 & 30.591 & 30.329 & 28.746 & 26.459 & 33.949 & 36.253 & 35.036 & 17.379 & 17.613 & 23.464 & 15.68 & 13.291 & 33.311 & 34.803 & 0.73 & 3.902 & 27.312 & 34.56 \\
\hline $10 / 28 / 1121: 30$ & & 31.169 & 30.704 & 29.489 & 31.023 & & 30.021 & 31.125 & 30.588 & 30.329 & 28.744 & 26.452 & 33.86 & 36.255 & 34.94 & 17.379 & 17.589 & 23.459 & 15.68 & 13.185 & 33.269 & 34.713 & 30.718 & 33.813 & 27.312 & 34.56 \\
\hline $10 / 28 / 1122: 00$ & & 31.167 & 30.701 & 29.487 & 31.016 & & 30.024 & 31.127 & 30.593 & 30.324 & 28.744 & 26.452 & 33.778 & 36.255 & 34.856 & 17.379 & 17.57 & 23.454 & 15.684 & 13.103 & 33.229 & 34.637 & 30.7 & 33.736 & 27.312 & 34.562 \\
\hline $10 / 28 / 1122: 30$ & & 31.171 & 30.699 & 29.489 & 31.009 & & 30.021 & 31.125 & 30.593 & 30.324 & 28.742 & 26.449 & 33.71 & 36.251 & 34.785 & 17.377 & 17.552 & 23.445 & 15.675 & 13.023 & 33.197 & 34.563 & 30.692 & 33.668 & 27.312 & 34.563 \\
\hline $10 / 28 / 1123: 00$ & & 31.169 & 30.697 & 29.48 & 31.002 & & 30.026 & 31.128 & 30.588 & 30.324 & 28.739 & 26.449 & 33.652 & 36.251 & 34.722 & 17.379 & 17.533 & 23.438 & 15.682 & 12.962 & 33.169 & 34.504 & 30.678 & 33.611 & 27.312 & 34.565 \\
\hline $10 / 28 / 1123: 30$ & & 31.171 & 30.694 & 29.477 & 30.995 & & 30.031 & 31.125 & 30.591 & 30.322 & 28.735 & 26.44 & 33.602 & 36.253 & 34.67 & 17.379 & 17.515 & 23.431 & 15.675 & 12.898 & 33.141 & 34.447 & 30.676 & 33.56 & 27.314 & 34.565 \\
\hline 10/29/11 0:00 & & 31.169 & 30.694 & 29.473 & 30.983 & & 30.031 & 31.125 & 30.591 & 30.322 & 28.732 & 26.437 & 33.556 & 36.251 & 34.621 & 17.379 & 17.498 & 23.422 & 15.677 & 12.849 & 33.12 & 34.402 & 30.667 & 33.515 & 27.312 & 34.567 \\
\hline $10 / 29 / 110: 30$ & & 31.167 & 30.687 & 29.468 & 30.974 & & 30.031 & 31.125 & 30.588 & 30.317 & 28.732 & 26.435 & 33.511 & 36.253 & 34.574 & 17.377 & 17.482 & 23.41 & 15.677 & 12.802 & 33.089 & 34.364 & 30.655 & 33.473 & 27.312 & 34.567 \\
\hline 10/29/11 1:00 & & 31.167 & 30.683 & 29.463 & 30.965 & & 30.031 & & $\begin{array}{l}30.501 \\
30.591\end{array}$ & 30.315 & 28.728 & 26.428 & 33.471 & 36.248 & 34.534 & 17.377 & 17.465 & 23.399 & 15.677 & 12.762 & 33.068 & & 30.643 & 33.433 & 27.312 & 34.567 \\
\hline 10/29/11 1:30 & & 31.167 & 30.68 & 29.463 & 30.5 & & 30.033 & 31.123 & 30.591 & 30.313 & 28.725 & 26.425 & $\begin{array}{l}33.436 \\
33.436\end{array}$ & 36.253 & 34.499 & 17.377 & $\begin{array}{l}17.449 \\
\end{array}$ & 23.392 & 15.673 & 12.72 & $\begin{array}{l}33.047 \\
3.047\end{array}$ & 34.291 & 30.634 & $\begin{array}{l}33.398 \\
33.398\end{array}$ & 27.314 & 34.569 \\
\hline $10 / 29 / 112: 00$ & & 31.167 & $\begin{array}{l}30.678 \\
30.678\end{array}$ & 29.454 & 30.946 & & $\begin{array}{l}30.036 \\
30.036\end{array}$ & 31.123 & 30.591 & 30.31 & 28.721 & 26.425 & $\begin{array}{l}33.400 \\
33.401\end{array}$ & $\begin{array}{l}36.248 \\
36.258\end{array}$ & $\begin{array}{l}34.464 \\
34.464\end{array}$ & 17.377 & $\begin{array}{l}17.433 \\
17.433\end{array}$ & 23.38 & $\begin{array}{l}15.675 \\
15.675\end{array}$ & 12.686 & 33.026 & 1.262 & $\begin{array}{l}30.024 \\
30.622\end{array}$ & $\begin{array}{l}33.367 \\
33.367\end{array}$ & 27.314 & $\begin{array}{l}5.009 \\
34.57\end{array}$ \\
\hline $10 / 29 / 112: 30$ & & 31.167 & 30.673 & $\begin{array}{l}29.444 \\
29.445\end{array}$ & $\begin{array}{l}30.934 \\
30.934\end{array}$ & & $\begin{array}{l}30.043 \\
30.043\end{array}$ & 31.118 & 30.591 & $\begin{array}{r}30.31 \\
30.306\end{array}$ & 28.716 & $\begin{array}{l}26.425 \\
26.416\end{array}$ & $\begin{array}{l}33.411 \\
33.373\end{array}$ & $\begin{array}{l}30.240 \\
36.251\end{array}$ & $\begin{array}{l}54.404 \\
34.431\end{array}$ & 17.377 & $\begin{array}{l}17.435 \\
17.416\end{array}$ & $\begin{array}{r}23.38 \\
23.369\end{array}$ & $\begin{array}{l}13.675 \\
15.675\end{array}$ & $\begin{array}{l}12.080 \\
12.656\end{array}$ & $\begin{array}{r}53.020 \\
33.01\end{array}$ & 1.234 & $\begin{array}{l}30.022 \\
30.615\end{array}$ & $\begin{array}{l}33.501 \\
33.334\end{array}$ & 27.314 & $\begin{array}{l}34.57 \\
34.57\end{array}$ \\
\hline $10 / 29 / 113: 00$ & & & $\begin{array}{l}30.6 / 3 \\
30.671\end{array}$ & $\begin{array}{l}29.435 \\
29.435\end{array}$ & $\begin{array}{l}30.927 \\
30.927\end{array}$ & & $\begin{array}{l}30.045 \\
30.045\end{array}$ & 31.118 & 30.588 & $\begin{array}{l}50.506 \\
30.303\end{array}$ & $\begin{array}{l}28.709 \\
28.709\end{array}$ & $\begin{array}{l}26.410 \\
26.411\end{array}$ & $\begin{array}{l}33.375 \\
33.343\end{array}$ & $\begin{array}{l}30.251 \\
36.248\end{array}$ & $\begin{array}{l}54.431 \\
34.401\end{array}$ & 17.377 & $\begin{array}{r}17.410 \\
17.4\end{array}$ & $\begin{array}{l}23.369 \\
23.355\end{array}$ & $\begin{array}{l}13.6 / 7 \\
15.675\end{array}$ & $\begin{array}{l}12.650 \\
12.623\end{array}$ & $\begin{array}{r}33.91 \\
32.994\end{array}$ & .203 & $\begin{array}{l}30.613 \\
30.613\end{array}$ & $\begin{array}{l}33.344 \\
33.304\end{array}$ & 27.314 & $\begin{array}{l}34.51 \\
34.57\end{array}$ \\
\hline 10 & & & 30 & 29.431 & & & 30.047 & & 30.588 & 30.301 & 28.707 & 26.397 & 33.315 & 11 & & 17.374 & 17.384 & 23.343 & 15.675 & 12.604 & 32.97 & 184 & .591 & 76 & 27.314 & 34.569 \\
\hline 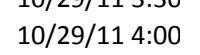 & & & 30 & $\begin{array}{l}29.435 \\
29.435\end{array}$ & 30. & & & & $\begin{array}{l}30.500 \\
30.58\end{array}$ & 30.296 & 28.705 & 26.394 & $\begin{array}{l}33.289 \\
3.289\end{array}$ & 44 & 34.344 & 17.374 & $\begin{array}{l}17.374 \\
17.37\end{array}$ & 29 & 75 & 12.578 & 32.956 & $\begin{array}{l}84 \\
58\end{array}$ & 84 & 48 & 27.314 & 1.567 \\
\hline $10 / 29 / 1$ & & & & & & & & & 30.591 & 30.289 & 28.705 & & 259 & 39 & 14 & & & & 15.677 & 12.562 & 32.935 & 44 & .568 & 22 & 27.314 & 565 \\
\hline $10 / 29 / 115: 00$ & & & 30.648 & 29.438 & 30.881 & & & 31.113 & 30.593 & 30.287 & 28.702 & 26.399 & 33.233 & 36.239 & 34.288 & 17.372 & 17.337 & 23.297 & 15.67 & 12.531 & 32.921 & 118 & .561 & 3.198 & 27.314 & 34.565 \\
\hline 10/29/11 5:30 & & 31.162 & 30.645 & 29.435 & 30.874 & & 30.029 & 31.111 & 30.596 & 30.285 & 28.705 & 26.404 & 33.212 & 36.239 & 34.265 & 17.37 & 17.323 & 23.285 & 15.668 & 12.503 & 32.91 & 34.092 & 30.559 & 33.175 & 27.314 & 34.563 \\
\hline 10/29/11 6:00 & & 31.164 & 30.645 & 29.433 & 30. & & & 31.111 & 30.593 & 30.285 & 28.702 & 26.401 & 34.26 & 36.239 & 34.68 & 17.372 & 17.309 & 23.269 & 15.658 & 12.903 & 33.148 & 35.255 & & 34.257 & 27.314 & 34.563 \\
\hline 10/29/11 6:30 & & 31.164 & 30.643 & 29.438 & 30. & & & 31.113 & 30.593 & 30.282 & 28.7 & 26.404 & 35.346 & 36.237 & 35.758 & 17.37 & 17.365 & 23.258 & 15.658 & 13.982 & 33.642 & & & 35.315 & 27.314 & 34.56 \\
\hline 10/29/11 7:00 & & 31.164 & 30.643 & 29.442 & 30. & & 30.021 & 31.111 & 30.598 & 30.287 & 28.702 & 26.406 & 35.987 & 36.234 & 36.492 & 17.37 & 17.472 & 23.237 & 15.658 & 14.715 & 33.936 & 36.928 & 30.624 & 35.93 & 27.317 & 34.558 \\
\hline $10 / 29 / 117: 30$ & & 31.164 & 30.645 & 29.447 & 30. & & 30.014 & 31.111 & 30.593 & 30.282 & 28.705 & 26.411 & 34.574 & 36.239 & 35.693 & 17.367 & 17.522 & 23.223 & 15.651 & 13.918 & 33.525 & 35.433 & 30.645 & 34.512 & 27.317 & 34.558 \\
\hline $10 / 29 / 118: 00$ & & 31.164 & 30.645 & 29.442 & 30. & & 30.012 & 31.111 & 30.591 & 30.275 & 28.702 & 26.421 & 34.206 & 36.229 & 35.308 & 17.37 & 17.512 & 23.205 & 15.649 & 13.526 & 33.36 & 35.061 & 30.648 & 34.151 & 27.314 & 34.556 \\
\hline 10/29/11 8:30 & & 31.167 & 30.645 & 29.44 & 30.5 & & 30.012 & 31.111 & 30.593 & 30.266 & 28.702 & 26.418 & 33.989 & 36.239 & 35.078 & 17.37 & 17.498 & 23.195 & 15.649 & 13.293 & 33.269 & 34.841 & 30.645 & 33.938 & 27.317 & 34.556 \\
\hline 10/29/11 9:00 & & 31.169 & 30.65 & 29.431 & 30.9 & & 30.019 & 31.111 & 30.591 & 30.264 & 28.698 & 26.416 & 33.839 & 36.239 & 34.924 & 17.37 & 17.482 & 23.179 & 15.644 & 13.135 & 33.211 & 34.694 & 30.643 & 33.792 & 27.317 & 34.555 \\
\hline 10/29/11 9:30 & & 31.167 & 30.648 & 29.417 & 30.916 & & 30.029 & 31.111 & 30.591 & 30.278 & 28.691 & 26.406 & 33.727 & 36.241 & 34.804 & 17.372 & 17.463 & 23.172 & 15.644 & 13.027 & 33.157 & 34.589 & 30.631 & 33.684 & 27.317 & 34.556 \\
\hline 10/29/11 10:00 & & 31.164 & 30.645 & 29.407 & 30.913 & & 30.04 & 31.106 & 30.591 & 30.308 & 28.684 & 26.394 & 33.638 & 36.237 & 34.708 & 17.367 & 17.447 & 23.165 & 15.654 & 12.957 & 33.117 & 34.511 & 30.62 & 33.595 & 27.319 & 34.553 \\
\hline 10/29/11 10:30 & & 31.162 & 30.643 & 29.393 & 30. & & 30.045 & 31.106 & 30.596 & 30.271 & 28.679 & 26.382 & 33.56 & 36.241 & 34.63 & 17.367 & 17.428 & 23.149 & 15.663 & 12.905 & 33. & 1.459 & 30.606 & 33.522 & 27.319 & 34.556 \\
\hline 10/29/11 11:00 & & 31.157 & 30.629 & 29.386 & 30. & & 30.047 & 31.104 & 30.593 & 30.269 & 28.674 & 26.373 & 33.5 & 36.234 & 34.565 & 17.367 & $\begin{array}{l}17.407 \\
\end{array}$ & 23. & 15.67 & 12.865 & 33. & .419 & 39 & 33.456 & 27.317 & 34.553 \\
\hline $10 / 29 /$ & & 31.157 & 30.622 & 29.377 & 30. & & 30.054 & 31.099 & 30.591 & 30.259 & 28.667 & 26.366 & 33.443 & 36.237 & 34.511 & 17.363 & 17.395 & 23.114 & 15.677 & 12.832 & $\begin{array}{l}33.042 \\
33.012\end{array}$ & 1.393 & 58 & $\begin{array}{l}33.405 \\
33.405\end{array}$ & 27.319 & 34.553 \\
\hline $10 /$ & & 31.155 & 30.617 & 29.36 & 30. & & 30.064 & 31.097 & 30.584 & 30.255 & 28.653 & 26.354 & 34.464 & 36.232 & 34.902 & 17.36 & 17.377 & 23.101 & 15.68 & 13.234 & 33.22 & 6 & 1 & 34.458 & 27.319 & 34.553 \\
\hline & & 31.1 & 30. & 29.3 & 30 & & & & 30 & 30 & 28 & 26. & 35 & 36. & 35 & 17. & 17. & 2 & 15. & 14 & 33. & 4 & 2 & 35.5 & 27.319 & 34.553 \\
\hline & & 31.1 & 30. & 29. & 30. & & & & & & 28. & 26 & 36. & 36.2 & & & 17 & 23 & 15. & 15 & & 5 & 3 & 36.094 & 27.319 & 34.553 \\
\hline & & 31. & & & & & & & & & 28. & & 34. & 36 & & & & & 13. & & & 1 & & & 27.319 & . \\
\hline & & & & & & & & & & & & & & 我 & & & & & & & & & & & & \\
\hline & & & & & & & & & & & & & & & & & & & 15. & & 33. & & & & & \\
\hline & & & & & & & & & & & 28.6 & & & & & & & & & & & & & & & \\
\hline 10 & & 31. & 30. & & & & & & & & & 26 & 33. & & & & 17 & 23 & 15. & & & & & & 27 & 34. \\
\hline 10/29/11 16:00 & & 31.132 & 30.533 & 29.295 & & & 30.054 & 31.065 & 30.586 & 30.187 & 28.602 & 26.282 & 33.78 & 36.201 & 34.872 & 17.339 & 17.535 & 22.996 & 15.71 & 13.394 & 33.099 & 34.672 & 30.519 & 33.733 & 27.317 & 34.544 \\
\hline 10/29/11 16:30 & & 31.132 & 30.529 & 29.297 & 30.813 & & 30.043 & 31.063 & 30.586 & 30.185 & 28.6 & 26.294 & 33.687 & 36.197 & 34.769 & 17.339 & 17.51 & 22.978 & 15.703 & 13.295 & 33.061 & 34.578 & 30.507 & 33.642 & 27.319 & 34.544 \\
\hline
\end{tabular}




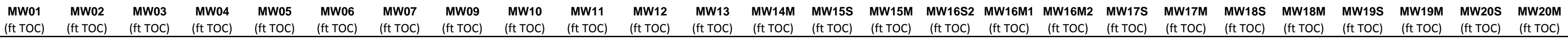

\begin{tabular}{|c|c|c|c|c|c|c|c|c|c|c|c|c|c|c|c|c|c|c|c|c|c|c|c|c|c|}
\hline Well & $\begin{array}{l}\text { (ft TOC) } \\
\text { (is }\end{array}$ & $\begin{array}{l}\text { (ft TOC) } \\
\text { (ft) }\end{array}$ & $\begin{array}{l}\text { (ft TOC) } \\
\text { (f) }\end{array}$ & (ft TOC) & $\begin{array}{ll}(\mathrm{ft} \text { TOC) } & \text { (ft TOC) }\end{array}$ & (ft toc) & $\begin{array}{l}\text { (ft TOC) } \\
\text { (f) }\end{array}$ & $\begin{array}{l}\text { (ft TOC) } \\
\text { (ft) }\end{array}$ & $\begin{array}{l}\text { (ft TOC) } \\
\text { (f) }\end{array}$ & $\begin{array}{l}\text { (ft TOC) } \\
\text { (f) }\end{array}$ & $\begin{array}{l}\text { (ft Toc) } \\
\text { (f) }\end{array}$ & $\begin{array}{l}\text { (ft TOC) } \\
\text { (t) }\end{array}$ & $\begin{array}{l}\text { (ft TOC) } \\
\end{array}$ & (ft TOC) & (ft TOC) & (ft TOC) & (ft TOC) & $\begin{array}{l}\text { (ft TOC) } \\
\end{array}$ & (ft TOC) & $\begin{array}{l}\text { (ft TOC) } \\
\end{array}$ & $\begin{array}{l}\text { (ft TOC) } \\
\text { (n) }\end{array}$ & $\begin{array}{l}\text { (ft TOC) } \\
\text { (n) }\end{array}$ & (ft TOC) & $\begin{array}{l}\text { (ft TOC) } \\
\end{array}$ & (ft TOC) \\
\hline 10/29/11 17:00 & & 31.129 & 30.529 & 29.307 & 30.806 & 30.036 & 31.06 & 30.586 & 30.183 & 28.595 & 26.299 & 33.607 & 36.19 & 34.684 & 17.337 & 17.489 & 22.973 & 15.701 & 13.211 & 33.022 & 34.499 & 30.5 & 33.564 & 27.319 & 34.542 \\
\hline $10 / 29 / 1117: 30$ & & 31.134 & $\begin{array}{l}30.529 \\
30.529\end{array}$ & 29.323 & $\begin{array}{l}30.806 \\
30.806\end{array}$ & $\begin{array}{l}50.016 \\
30.017\end{array}$ & $\begin{array}{r}31.06 \\
31.061\end{array}$ & $\begin{array}{l}30.580 \\
30.586\end{array}$ & $\begin{array}{l}30.183 \\
30.164\end{array}$ & $\begin{array}{l}28.505 \\
28.605\end{array}$ & $\begin{array}{l}26.299 \\
26.308\end{array}$ & 35.254 & $\begin{array}{r}36.194 \\
36.194\end{array}$ & $\begin{array}{l}34.084 \\
35.749\end{array}$ & $\begin{array}{l}11.331 \\
17.337\end{array}$ & $\begin{array}{r}17.49 \\
17.5\end{array}$ & $\begin{array}{l}22.973 \\
22.959\end{array}$ & 15.691 & $\begin{array}{l}13.211 \\
14.259\end{array}$ & $\begin{array}{l}33.022 \\
33.574\end{array}$ & $\begin{array}{l}3.449 \\
36.222\end{array}$ & $\begin{array}{r}30.5 \\
30.514\end{array}$ & $\begin{array}{l}35.504 \\
35.191\end{array}$ & 27.321 & $\begin{array}{l}34.542 \\
34.542\end{array}$ \\
\hline $29 / 1118: 00$ & & 31.132 & 30.526 & 29.332 & 30.806 & 29.996 & 31.063 & 30.588 & 30.143 & 28.607 & 26.327 & 36.127 & 36.192 & 36.544 & 17.337 & 17.582 & 22.943 & 15.687 & 15.049 & 33.894 & 37.118 & & 6.073 & 27.321 & 34.542 \\
\hline :9/11 18:30 & & 31.134 & 30.533 & 29.337 & 30.818 & 29.986 & 31.06 & 30.588 & 30.12 & 28.612 & 26.339 & 35.231 & 36.194 & 36.363 & 17.337 & 17.673 & 22.932 & 15.682 & 14.861 & 7 & & 30.589 & & 27.321 & 34.539 \\
\hline 10/29/11 19:00 & & 31.141 & 30.543 & 29.339 & 30.839 & 29.981 & 31.063 & 30.584 & 30.078 & 28.612 & 26.346 & 34.632 & 36.194 & 35.765 & 17.339 & 17.673 & 22.918 & 15.668 & 14.219 & 33.5 & 35.516 & 30.603 & .571 & 21 & \\
\hline 10/29/11 19:30 & & 31.139 & 30.545 & 29.337 & 30.85 & 29.974 & 31.063 & 30.588 & 30.048 & 28.614 & 26.349 & 34.333 & 36.197 & 35.449 & 17.339 & 17.655 & 22.906 & 15.666 & 13.897 & 33.376 & 5.22 & 30.603 & 4.278 & 7.321 & 34.539 \\
\hline 10/29/11 20:00 & & 31.139 & 30.547 & 29.332 & 30.862 & 29.974 & 31.06 & 30.588 & 30.162 & 28.609 & 26.349 & 34.134 & 36.201 & 35.238 & $\begin{array}{l}37.339 \\
3\end{array}$ & 17.631 & 22.892 & 15.663 & 13.671 & 33.295 & 35.023 & 30.596 & 34.083 & 27.321 & 34.539 \\
\hline 10/29/11 20:30 & & 31.136 & 30.547 & 29.335 & 30.867 & 29.981 & 31.061 & 30.586 & 30.139 & 28.607 & 26.346 & 33.989 & 36.201 & 35.083 & 17.339 & 17.608 & 22.879 & 15.661 & 13.502 & 33.234 & 34.879 & 30.587 & 33.942 & 27.321 & 34.539 \\
\hline 10/29/11 21:00 & & 31.134 & 30.545 & 29.344 & 30.867 & 29.963 & 31.06 & 30.596 & 30.125 & 28.614 & 26.349 & 33.872 & 36.199 & 34.961 & 17.339 & 17.585 & 22.865 & 15.663 & 13.375 & 33.183 & 34.76 & 30.582 & 33.825 & 27.321 & 34.542 \\
\hline 10/29/11 21:30 & & 31.129 & 30.543 & 29.344 & 30.857 & 29.96 & 31.06 & 30.588 & 30.118 & 28.612 & 26.363 & 33.776 & 36.197 & 34.86 & 17.339 & 17.561 & 22.842 & 15.663 & 13.274 & 33.138 & 34.663 & 30.559 & 33.729 & 27.321 & 34.542 \\
\hline 10/29/11 22:00 & & 31.136 & 30.547 & 29.346 & 30.864 & 29.951 & 31.063 & 30.591 & 30.108 & 28.616 & 26.361 & 33.694 & 36.197 & 34.778 & $3 \quad 17.339$ & 17.542 & 22.842 & 15.651 & 13.171 & 33.11 & 34.587 & 30.566 & 33.654 & 27.326 & 34.542 \\
\hline 10/29/11 22:30 & & 31.136 & 30.552 & 29.342 & 30.862 & 29.96 & 31.063 & 30.588 & 30.097 & 28.612 & 26.363 & 33.626 & 36.199 & 34.705 & 17.339 & 17.522 & 22.849 & 15.647 & 13.084 & 33.082 & 34.518 & 30.566 & 33.586 & 27.321 & 34.542 \\
\hline 10/29/11 23:00 & & 31.139 & 30.557 & 29.337 & 30.86 & 29.953 & 31.06 & 30.591 & 30.087 & 28.612 & 26.363 & 33.57 & 36.208 & 34.642 & 17.339 & 17.5 & 22.851 & 15.642 & 13.004 & 33.054 & 34.462 & 30.566 & 33.529 & 27.324 & 34.544 \\
\hline 10/29/11 23:30 & & 31.134 & 30.554 & 29.344 & 30.855 & 29.958 & 31.063 & 30.588 & 30.094 & 28.609 & 26.358 & 33.514 & 36.204 & 34.586 & 17.339 & 17.482 & 22.839 & 15.647 & 12.954 & 33.024 & 34.409 & 30.552 & 33.473 & 27.324 & 34.544 \\
\hline $10 / 30 / 110: 00$ & & 31.136 & 30.554 & 29.346 & 30.85 & 29.951 & 31.063 & 30.591 & 30.094 & 28.612 & 26.366 & 33.467 & 36.206 & 34.537 & 17.342 & 17.461 & 22.828 & 15.642 & 12.891 & 33.003 & 34.36 & 30.547 & 33.428 & 27.324 & 34.546 \\
\hline 10/30/11 0:30 & & 31.134 & 30.554 & 29.356 & 30.841 & 29.942 & 31.063 & 30.593 & 30.101 & 28.616 & 26.37 & 33.427 & 36.204 & 34.487 & 17.337 & 17.444 & 22.812 & 15.644 & 12.849 & 32.975 & 34.317 & 30.535 & 33.386 & 27.324 & 34.549 \\
\hline 10/30/11 1:00 & & 31.136 & 30.557 & 29.363 & 30.836 & 29.937 & 31.06 & 30.591 & 30.09 & 28.618 & 26.375 & 33.387 & 36.206 & 34.452 & 17.342 & 17.428 & 22.8 & 15.63 & 12.792 & 32.963 & 34.281 & 30.533 & 33.351 & 27.326 & 34.549 \\
\hline 10/30/11 1:30 & & 31.139 & 30.559 & 29.365 & 30.839 & 29.934 & 31.066 & 30.593 & 30.081 & 28.621 & 26.387 & 33.35 & 36.211 & 34.412 & 17.342 & 17.412 & 22.786 & 15.63 & 12.743 & 32.952 & 34.248 & 30.537 & 33.313 & 27.326 & 34.551 \\
\hline $10 / 30 / 112: 00$ & & 31.139 & 30.559 & 29.374 & 30.834 & 29.927 & 31.066 & 30.596 & $\begin{array}{l}30.074 \\
30.074\end{array}$ & 28.625 & 26.39 & 33.319 & 36.211 & 34.382 & 17.344 & $\begin{array}{l}17.438 \\
17.398\end{array}$ & 22.775 & $\begin{array}{l}15.628 \\
\end{array}$ & 12.701 & 32.938 & 34.215 & 30.53 & 33.285 & 27.326 & 34.551 \\
\hline $10 / 30 / 112: 30$ & & 31.141 & 30.566 & $\begin{array}{l}29.391 \\
29.391\end{array}$ & $\begin{array}{l}30.044 \\
30.834\end{array}$ & 29.918 & 31.07 & 30.6 & 30.136 & $\begin{array}{l}20.028 \\
28.635\end{array}$ & $\begin{array}{l}26.039 \\
26.401\end{array}$ & 33.291 & $\begin{array}{l}30.211 \\
36.213\end{array}$ & $\begin{array}{l}34.351 \\
34.351\end{array}$ & 17.342 & $\begin{array}{l}17.380 \\
17.381\end{array}$ & 22.763 & $\begin{array}{l}13.020 \\
15.621\end{array}$ & $\begin{array}{l}12.654 \\
12.654\end{array}$ & 32.926 & 34.187 & 30.535 & $\begin{array}{l}33.252 \\
33.252\end{array}$ & 27.326 & 34.553 \\
\hline $10 / 30 / 113: 00$ & & 31.141 & $\begin{array}{l}30.300 \\
30.568\end{array}$ & $\begin{array}{r}29.4 \\
29.4\end{array}$ & $\begin{array}{l}30.834 \\
30.834\end{array}$ & 29.913 & 31.07 & $\begin{array}{l}30.6 \\
30.6\end{array}$ & $\begin{array}{l}30.120 \\
30.122\end{array}$ & $\begin{array}{l}28.053 \\
28.639\end{array}$ & $\begin{array}{l}20.401 \\
26.413\end{array}$ & $\begin{array}{l}33.263 \\
33.263\end{array}$ & $\begin{array}{l}30.213 \\
36.218\end{array}$ & $\begin{array}{l}34.531 \\
34.323\end{array}$ & 17.344 & $\begin{array}{l}17.381 \\
17.367\end{array}$ & $\begin{array}{l}22.703 \\
22.754\end{array}$ & $\begin{array}{l}13.021 \\
15.619\end{array}$ & $\begin{array}{l}12.044 \\
12.611\end{array}$ & $\begin{array}{l}32.926 \\
32.914\end{array}$ & 34.161 & $\begin{array}{l}30.535 \\
30.535\end{array}$ & $\begin{array}{l}33.222 \\
33.229\end{array}$ & 27.326 & $\begin{array}{l}34.535 \\
34.553\end{array}$ \\
\hline 10/30/11 3:30 & & 31.146 & 30.575 & 29.407 & 30.839 & 29.911 & 31.075 & 30.598 & 30.104 & 28.642 & 26.428 & 33.24 & 36.22 & 34.298 & 317.346 & 17.353 & 22.742 & 15.604 & 12.56 & 32.914 & 137 & .537 & 33.203 & 27.326 & 34.556 \\
\hline $10 / 30 / 114: 00$ & & 31.148 & 30.582 & 29.421 & 30.839 & 29.904 & 31.078 & $\begin{array}{r}30.60 \\
30.6\end{array}$ & 30.085 & $\begin{array}{l}20.042 \\
28.649\end{array}$ & 26.43 & $\begin{array}{r}33.24 \\
33.221\end{array}$ & 36.22 & 34.274 & 17.349 & $\begin{array}{l}17.339 \\
\end{array}$ & 22.733 & $\begin{array}{l}15.0597 \\
\end{array}$ & 12.52 & 32.905 & 113 & .544 & 3.182 & 27.328 & 34.558 \\
\hline $10 / 30 /$ & & 31.148 & 30.587 & 29.438 & 30.841 & 29.892 & 31.082 & 30.605 & 30.169 & 28.66 & 26.445 & 33.195 & 36.234 & 34.253 & 17.349 & & 22.724 & 5.602 & 12.487 & .893 & .094 & 1.549 & 161 & 27.326 & 34.558 \\
\hline 10/30/11 5:00 & & 31.153 & 30.592 & 29.456 & 30.846 & 29.887 & 31.087 & 30.6 & 30.146 & 28.67 & 26.461 & 33.177 & 36.232 & 34.234 & 17.351 & 17.311 & 22.712 & 15.59 & 12.437 & 32.893 & 34.075 & 30.556 & 3.142 & 27.326 & 34.558 \\
\hline 10/30/11 5:30 & & 31.157 & 30.601 & 29.47 & 30.855 & 29.887 & 31.089 & 30.605 & 30.125 & 28.677 & 26.478 & 33.16 & 36.234 & 34.215 & 17.356 & 17.299 & 22.703 & 15.581 & 12.395 & 32.898 & 34.056 & 30.57 & 33.126 & 27.331 & 34.558 \\
\hline 10/30/11 6:00 & & 31.162 & 30.61 & 29.491 & & 29.873 & 31.097 & 30.61 & & 28.691 & 26.49 & 34.216 & 36.244 & 34.64 & 17.358 & 17.29 & 22.689 & 15.579 & 12.792 & 33.15 & 35.232 & 30.582 & 4.217 & 27.328 & 34.56 \\
\hline 10/30/11 6:30 & & 31.162 & 30.617 & 29.501 & 30.874 & 29.873 & 31.101 & 30.603 & 30.104 & 28.698 & 26.512 & 35.311 & 36.244 & 35.718 & $3 \quad 17.363$ & 17.351 & 22.68 & 15.579 & 13.869 & 33.654 & 36.312 & 30.617 & 35.277 & 27.328 & 34.56 \\
\hline 10/30/11 7:00 & & 31.169 & 30.629 & 29.512 & 30.897 & 29.871 & 31.106 & 30.605 & 30.192 & 28.707 & 26.521 & 35.952 & 36.253 & 36.459 & 17.365 & 17.456 & 22.67 & 15.567 & 14.581 & 33.961 & 36.916 & 30.678 & 35.894 & 27.331 & 34.56 \\
\hline $10 / 30 / 117: 30$ & & 31.169 & 30.641 & 29.526 & 30.923 & 29.869 & 31.113 & 30.61 & 30.171 & 28.716 & 26.533 & 34.541 & 36.258 & 35.662 & 17.367 & 17.503 & 22.659 & 15.569 & 13.777 & 33.558 & 35.428 & 30.711 & 34.482 & 27.328 & 34.563 \\
\hline 10/30/11 8:00 & & 31.174 & 30.65 & 29.538 & 30.951 & 29.871 & 31.118 & 30.607 & 30.155 & 28.728 & 26.545 & 34.178 & 36.262 & 35.278 & 17.37 & 17.498 & 22.647 & 15.562 & 13.378 & 33.404 & 35.066 & 30.718 & 34.123 & 27.331 & 34.563 \\
\hline $10 / 30 / 118: 30$ & & 31.178 & 30.659 & 29.545 & 30.972 & 29.88 & 31.12 & 30.603 & 30.141 & 28.73 & 26.555 & 33.968 & 36.265 & 35.057 & 17.374 & 17.486 & 22.636 & 15.56 & 13.135 & 33.318 & 34.857 & 30.728 & 33.919 & 27.331 & 34.563 \\
\hline 10/30/11 9:00 & & 31.183 & 30.669 & 29.55 & 30.99 & 29.887 & 31.125 & 30.607 & 30.264 & 28.737 & 26.559 & 33.825 & 36.269 & 34.905 & 17.377 & 17.472 & 22.624 & 15.565 & 12.973 & 33.269 & 34.717 & 30.732 & 33.778 & 27.331 & 34.563 \\
\hline 10/30/11 9:30 & & 31.183 & 30.676 & 29.552 & 31.002 & 29.895 & 31.13 & 30.61 & 30.271 & 28.742 & 26.559 & 33.717 & 36.267 & 34.792 & 17.379 & 17.456 & 22.615 & 15.567 & 12.851 & 33.222 & 34.608 & 30.732 & 33.672 & 27.333 & 34.565 \\
\hline 10/30/11 10:00 & & 31.185 & 30.683 & 29.557 & 31.0 & 29.897 & 31.127 & 30.61 & 30.28 & 28.749 & 26.559 & 33.631 & 36.272 & 34.703 & 17.381 & $\begin{array}{r}17.44 \\
\end{array}$ & 22.606 & 15.574 & 12.769 & 33.19 & 34.525 & 30.728 & 33.588 & 27.333 & 34.565 \\
\hline 10/30/11 10:30 & & 31.188 & $\begin{array}{l}30.69 \\
\end{array}$ & 29.561 & 31. & 29.906 & 31.133 & 30.61 & 30.282 & 28.751 & 26.564 & 33.565 & 36.274 & 34.63 & 17.384 & 17.423 & 22.596 & 15.576 & 12.703 & 33.162 & 34.457 & 0.725 & 33.522 & 27.331 & 34.565 \\
\hline 10/30/11 11:00 & & $\begin{array}{l}31.180 \\
31.188\end{array}$ & 30.692 & 29.552 & 31.0 & 29.92 & 31.132 & 30.612 & 30.289 & 28.753 & $\begin{array}{l}20.504 \\
26.562\end{array}$ & 33.507 & 36.272 & 34.572 & 17.386 & 17.407 & 22.585 & 15.579 & 12.644 & $\begin{array}{l}33.136 \\
3.136\end{array}$ & 34.402 & .732 & $\begin{array}{l}33.466 \\
33.466\end{array}$ & 27.333 & 34.567 \\
\hline 10/30/11 11:30 & & $\begin{array}{l}31.180 \\
31.188\end{array}$ & $\begin{array}{l}30.692 \\
30.699\end{array}$ & 29.54 & 31.0 & 29.937 & 31.135 & 30.61 & 30.294 & 28.753 & 26.552 & 33.457 & 36.274 & 34.52 & 17.386 & 17.393 & 22.576 & 15.586 & $\begin{array}{l}12.004 \\
12.604\end{array}$ & 33.122 & 34.353 & 1.725 & $\begin{array}{l}33.414 \\
33.400\end{array}$ & 27.333 & 34.567 \\
\hline 10 & & $\begin{array}{r}31.100 \\
31.19\end{array}$ & $\begin{array}{l}30.099 \\
30.704\end{array}$ & $\begin{array}{l}29.529 \\
29.52\end{array}$ & 31.0 & 29.953 & 31.135 & $\begin{array}{l}30.607 \\
30.607\end{array}$ & $\begin{array}{l}30.301 \\
30.301\end{array}$ & 28.751 & 26.538 & $\begin{array}{l}34.489 \\
34.489\end{array}$ & 36.276 & $\begin{array}{l}34.924 \\
34.924\end{array}$ & $\begin{array}{r}17.300 \\
17.39\end{array}$ & $\begin{array}{l}17.381 \\
17.31\end{array}$ & 22.571 & 3 & $\begin{array}{l}12.044 \\
12.999\end{array}$ & $\begin{array}{l}33.346 \\
33.346\end{array}$ & 35.502 & 21 & 82 & 27.333 & $\begin{array}{l}34.507 \\
34.57\end{array}$ \\
\hline 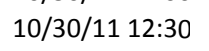 & & 31 & 30 & 29 & & 2 & 3 & & 30 & 28 & 26 & 35 & 36 & 35.974 & 17.388 & 1 & 22 & 4 & 14. & 33.826 & 4 & 30.744 & 1 & 27.335 & 34.567 \\
\hline & & & 30.65 & & 31 & & 3. & & & & 26 & 36 & 36 & 36 & 17.388 & 17.554 & 22 & 4 & 14 & 13 & 37 & & 57 & 27.335 & 34.567 \\
\hline $13: 30$ & & 31 & 30.692 & 29.508 & & 29.989 & 31 & & & 28 & 26.507 & & 36.279 & 35.901 & 17 & & & 15.621 & 14.059 & 33.684 & 35.651 & 34 & 05 & 27.333 & 34.567 \\
\hline 14:00 & & 31 & 30. & 29.501 & & & 32 & & & 28 & 26.5 & & 36.274 & 35.493 & 17.388 & 17.594 & & 15.628 & 13.676 & 33.507 & & & & 27232 & 34.57 \\
\hline $10 /$ & & 31 & 30.68 & & & $30 . c$ & 31 & & & 28.735 & & & 36.279 & 35.249 & 17. & 17.578 & & 15.63 & 13. & 33.406 & & 1 & & 35 & 34.57 \\
\hline 10/ & & & & & & & 31. & & & 28.732 & 26. & & 36.274 & 35.132 & & 17. & & & 13.335 & 33.339 & & & & 27.333 & 34.57 \\
\hline $10 /$ & & & 30. & 29 & & & 31.132 & & & 28.73 & 26 & 34. & 36.276 & 35.479 & 17. & 17. & & 15.644 & 13.707 & 33.497 & & & 34.522 & 27.335 & 34.567 \\
\hline 10/30/11 16:00 & & 31.176 & 30.6 & 29.491 & 31 & & 31 & 30.6 & & 28.73 & 26.473 & 34.7 & 36.272 & 35.636 & 17.3 & 17.9 & 22.504 & 15.651 & 13.876 & 33.56 & 35.623 & 30.735 & 34.67 & 27.335 & 34.57 \\
\hline 10/30/11 16:30 & & 31.17 & 30.68 & 29.496 & 31. & & 31.13 & 30 & 30. & 28.732 & 26.48 & 34.829 & 36.269 & 35.751 & 17.384 & 17.589 & 22.5 & 15.649 & 13.996 & 33.598 & 35.732 & 30.742 & 34.778 & 27.335 & 34.567 \\
\hline 10/30/11 17:00 & & 31.178 & 30.68 & 29.498 & 31.025 & 30.012 & 31.135 & 30.615 & 30.2 & 28.735 & 26.48 & 34.911 & 36.269 & 35.831 & 17.384 & 17.608 & 22.536 & 15.654 & 14.092 & 33.635 & 35.812 & 30.744 & 34.855 & 27.335 & 34.567 \\
\hline $10 / 30 / 1117: 30$ & & 31.181 & 30.68 & 29.508 & 31.03 & 30.007 & 31.135 & 30.612 & 30.292 & 28.739 & 26.483 & 36.471 & 36.269 & 36.818 & 317.386 & 17.671 & 22.529 & 15.656 & 15.073 & 34.169 & 37.468 & 30.765 & 36.399 & 27.338 & 34.567 \\
\hline $10 / 30 / 1118: 00$ & & 31.183 & 30.685 & 29.508 & 31.042 & 30.003 & 31.135 & 30.615 & 30.294 & 28.739 & 26.49 & 37.302 & 36.269 & 37.592 & 17.386 & 17.783 & 22.518 & 15.649 & 15.846 & 34.486 & 38.321 & 30.805 & 37.239 & 27.338 & 34.567 \\
\hline $10 / 30 / 1118: 30$ & & 31.185 & 30.687 & 29.512 & 31.058 & 30 & 31.139 & 30.617 & 30.296 & 28.742 & 26.492 & 36.82 & 36.274 & 37.782 & 17.386 & 17.902 & 22.506 & 15.651 & 16.034 & 34.444 & 37.717 & 30.841 & 36.73 & 27.338 & 34.567 \\
\hline 10/30/11 19:00 & & 31.185 & 30.692 & 29.51 & 31.079 & 29.998 & 31.139 & 30.615 & 30.299 & 28.744 & 26.497 & 36.586 & 36.272 & 37.573 & 17.386 & 18.001 & 22.492 & 15.647 & 15.806 & 34.311 & 37.487 & 30.862 & 36.509 & 27.338 & 34.567 \\
\hline 10/30/11 19:30 & & 31.18 & 30.694 & 29.512 & 31.05 & 30 & 31.14 & 30.615 & 30.299 & 28.742 & 26.495 & 36.254 & 36.274 & 37.226 & 17.388 & 18.057 & 22.483 & 15.644 & 15.451 & 34.225 & 37.151 & 30.876 & 36.178 & 27.338 & 34.567 \\
\hline $10 / 30 / 112$ & & 31.19 & 30.697 & 29.512 & 31.114 & 29.998 & 31.139 & 30.615 & 30.303 & 28.744 & 26.495 & 36.069 & 36.274 & 37.038 & $3 \quad 17.388$ & 18.075 & 22.476 & 15.649 & 15.249 & 34.164 & 36.968 & 30.871 & 35.998 & 27.338 & 34.567 \\
\hline 10/30/11 20:30 & & 31.188 & 30.697 & 29.519 & 31.123 & 29.998 & 31.139 & 30.619 & 30.303 & 28.746 & 26.497 & 35.959 & 36.276 & 36.923 & 17.39 & 18.085 & 22.465 & 15.649 & 15.127 & 34.122 & 36.857 & 30.883 & 35.892 & 27.338 & 34.567 \\
\hline
\end{tabular}




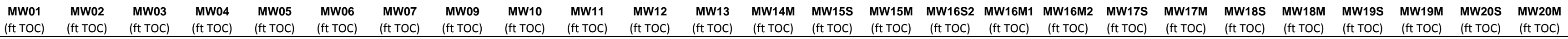

\begin{tabular}{|c|c|c|c|c|c|c|c|c|c|c|c|c|c|c|c|c|c|c|c|c|c|c|c|c|c|c|}
\hline Well & $\begin{array}{l}\text { (ft TOC) } \\
\text { (ft }\end{array}$ & $\begin{array}{l}\text { (ft TOC) } \\
\text { (f) }\end{array}$ & $\begin{array}{l}\text { (ft TOC) } \\
\text { (flocts }\end{array}$ & $\begin{array}{l}\text { (ft TOC) } \\
\text { (f }\end{array}$ & $\begin{array}{l}\text { (ft TOC) } \\
\text { (f) }\end{array}$ & $\begin{array}{l}\text { (ft TOC) } \\
\text { (f) }\end{array}$ & $\begin{array}{l}\text { (ft TOC) } \\
\text { (ft }\end{array}$ & $\begin{array}{l}\text { (ft TOC) } \\
\text { (f) }\end{array}$ & $\begin{array}{l}\text { (ft TOC) } \\
\text { (f) }\end{array}$ & $\begin{array}{l}\text { (ft TOC) } \\
\text { (f) }\end{array}$ & $\begin{array}{l}\text { (ft TOC) } \\
\text { (f) }\end{array}$ & $\begin{array}{l}\text { (ft TOC) } \\
\text { (f) }\end{array}$ & (ft TOC) & $\begin{array}{l}\text { (ft TOC) } \\
\text { (f) }\end{array}$ & (ft TOC) & (ft TOC) & $\begin{array}{l}\text { (ft TOC) } \\
\text { (f) }\end{array}$ & (ft TOC) & $\begin{array}{l}\text { (ft TOC) } \\
\text { (1) }\end{array}$ & (ft TOC) & $\begin{array}{l}\text { (ft TOC) } \\
\end{array}$ & $\begin{array}{l}\text { (ft TOC) } \\
\text { (1) }\end{array}$ & $\begin{array}{l}\text { (ft TOC) } \\
\text { (n) }\end{array}$ & (ft TOC) & $\begin{array}{l}\text { (ft TOC) } \\
\text { (1) }\end{array}$ & (ft TOC) \\
\hline 21:00 & & 31.188 & 30.699 & 29.524 & 31.135 & & 29.993 & 31.14 & 30 & 30.306 & 28.749 & 26 & 35.886 & 36274 & 36.848 & 17.386 & 18.089 & 22.455 & 15.649 & 15.044 & 34097 & 36.786 & 30.885 & 35.817 & 27.338 & 34.567 \\
\hline 10/30/11 21:30 & & 31.19 & 30.704 & 29.529 & 31.147 & & 29.991 & 31.142 & 30.619 & 30.308 & 28.753 & 26.504 & 35.837 & 36.276 & 36.792 & 17.39 & 18.096 & 22.437 & 15.64 & 1.978 & 34.078 & 36.736 & 0.89 & & 27.34 & 34.567 \\
\hline 10/30/11 22:00 & & 31.192 & 30.708 & 29.524 & 31.156 & & 29.996 & 31.144 & 30.615 & 30.31 & 28.751 & 26.509 & 35.8 & 36.276 & 36.757 & 17.39 & 18.096 & 22.435 & 15.64 & 14.931 & 34.066 & 36.701 & 0.89 & & & 34.567 \\
\hline 10/30/11 22:30 & & 31.195 & 30.711 & 29.524 & 31.163 & & 30 & 31.144 & 30.612 & 30.313 & 28.751 & 26.507 & 35.774 & 36.279 & 36.731 & $1 \quad 17.393$ & 18.103 & 22.423 & 15.642 & 14.899 & 34.064 & 36.675 & 30.895 & 35.709 & 27.34 & 34.57 \\
\hline 10/30/11 23:00 & & 31.192 & 30.713 & 29.519 & 31.168 & & 30.003 & 31.144 & 30.617 & 30.315 & 28.751 & 26.504 & 35.755 & 36.283 & 36.708 & $\begin{array}{ll}8 & 17.393\end{array}$ & 18.108 & 22.412 & 15.642 & 14.87 & 34.055 & 36.656 & 30.897 & 35.69 & 27.338 & 34.57 \\
\hline 10/30/11 23:30 & & 31.192 & 30.711 & 29.519 & 31.172 & & 30.007 & 31.145 & 30.617 & 30.315 & 28.749 & 26.502 & 35.737 & 36.279 & 36.696 & $\begin{array}{ll}6 & 17.393\end{array}$ & 18.11 & 22.407 & 15.644 & 14.856 & 34.05 & 36.639 & 30.892 & 35.676 & 27.34 & 34.57 \\
\hline 10/31/11 0:00 & & 31.192 & 30.715 & 29.517 & 31.175 & & 30.007 & 31.147 & 30.622 & 30.317 & 28.751 & 26.497 & 35.208 & 36.276 & 36.351 & $1 \quad 17.393$ & 18.108 & 22.395 & 15.644 & 14.511 & 33.905 & 36.087 & 30.895 & 35.141 & 27.34 & 34.572 \\
\hline 10/31/11 0:30 & & 31.192 & 30.713 & 29.515 & 31.172 & & 30.007 & 31.149 & 30.617 & 30.317 & 28.749 & 26.5 & 34.899 & 36.281 & 36.037 & $\begin{array}{l}7 \quad 17.393 \\
\end{array}$ & 18.078 & 22.386 & 15.647 & 14.193 & 33.765 & 35.781 & 30.878 & 34.841 & 27.34 & 34.572 \\
\hline 10/31/11 1:00 & & 31.192 & 30.713 & 29.515 & 31.17 & & 30.012 & 31.147 & 30.619 & 30.317 & 28.751 & 26.497 & 34.695 & 36.281 & 35.817 & $7 \quad 17.393$ & 18.043 & 22.379 & 15.644 & 13.97 & 33.686 & 35.58 & 30.869 & 34.642 & 27.34 & 34.572 \\
\hline 10/31/11 1:30 & & 31.192 & 30.713 & 29.508 & 31.168 & & 30.014 & 31.147 & 30.619 & 30.317 & 28.746 & 26.492 & 34.539 & 36.279 & 35.648 & $\begin{array}{ll}8 & 17.393\end{array}$ & 18.008 & 22.37 & 15.647 & 13.801 & 33.609 & 35.426 & 30.857 & 34.484 & 27.34 & 34.574 \\
\hline 10/31/11 2:00 & & 31.192 & 30.713 & 29.503 & 31.161 & & 30.019 & 31.149 & 30.619 & 30.317 & 28.744 & 26.485 & 34.407 & 36.279 & 35.512 & $2 \quad 17.395$ & 17.975 & 22.361 & 15.649 & 13.664 & 33.553 & 35.296 & 30.841 & 34.36 & 27.34 & 34.574 \\
\hline 10/31/11 2:30 & & 31.192 & 30.715 & 29.494 & 31.149 & & 30.026 & 31.147 & 30.617 & 30.317 & 28.742 & 26.483 & 34.297 & 36.276 & 35.395 & $5 \quad 17.395$ & 17.942 & 22.351 & 15.647 & 13.549 & 33.502 & 35.187 & 30.826 & 34.25 & 27.342 & 34.577 \\
\hline 10/31/11 3:00 & & 31.19 & 30.713 & 29.489 & 31.137 & & 30.031 & 31.144 & 30.617 & 30.317 & 28.737 & 26.471 & 34.202 & 36.279 & 35.294 & $\begin{array}{l}4 \\
47.393\end{array}$ & 17.909 & 22.335 & 15.651 & 13.45 & 33.462 & 35.092 & 30.81 & 34.156 & 27.34 & 34.574 \\
\hline 10/31/11 3:30 & & 31.19 & 30.711 & 29.484 & 31.126 & & 30.033 & 31.147 & 30.619 & 30.315 & 28.735 & 26.466 & 34.115 & 36.281 & 35.207 & 17.395 & 17.879 & 22.333 & 15.651 & 13.368 & 33.413 & 35.004 & 30.798 & 34.069 & 27.342 & 34.576 \\
\hline 10/31/11 4:00 & & 31.19 & 30.706 & 29.48 & 31.109 & & 30.033 & 31.144 & 30.617 & 30.313 & 28.73 & 26.464 & 34.038 & 36.274 & 35.123 & 17.393 & 17.849 & 22.326 & 15.658 & 13.295 & 33.367 & 34.928 & 30.777 & 33.991 & 27.342 & 34.577 \\
\hline 10/31/11 4:30 & & 31.188 & 30.704 & 29.48 & 31.095 & & 30.036 & 31.147 & 30.622 & 30.31 & 28.732 & 26.459 & 33.968 & 36.274 & 35.052 & 17.393 & 17.821 & 22.317 & 15.658 & 13.227 & 33.334 & 34.862 & 30.761 & 33.923 & 27.342 & 34.574 \\
\hline 10/31/11 5:00 & & 31.19 & 30.704 & 29.48 & 31.081 & & 30.036 & 31.147 & 30.624 & 30.308 & 28.732 & 26.457 & 33.904 & 36.272 & 34.987 & 17.393 & 17.79 & 22.305 & 15.651 & 13.159 & 33.297 & 34.798 & 30.749 & 33.865 & 27.342 & 34.574 \\
\hline 10/31/11 5:30 & & 31.19 & 30.701 & 29.477 & 31.07 & & 30.033 & 31.147 & 30.619 & 30.308 & 28.728 & 26.457 & 33.846 & 36.272 & 34.926 & 17.393 & 17.762 & 22.303 & 15.651 & 13.103 & 33.269 & 34.739 & 30.737 & 33.804 & 27.342 & 34.574 \\
\hline 10/31/11 6:00 & & 31.19 & 30.699 & 29.477 & 31.056 & & 30.031 & 31.145 & 30.622 & 30.308 & 28.728 & 26.454 & 34.875 & 36.269 & 35.331 & 17.393 & 17.737 & 22.305 & 15.654 & 13.493 & 33.483 & 35.883 & 30.73 & 34.867 & 27.342 & 34.574 \\
\hline $10 / 31 / 116: 30$ & & 31.19 & 30.699 & 29.477 & 31.049 & & 30.029 & 31.144 & 30.624 & 30.306 & 28.728 & 26.457 & 35.938 & 36.267 & 36.372 & 17.39 & 17.79 & 22.314 & 15.656 & 14.548 & 33.957 & 36.928 & 30.751 & 35.894 & 27.345 & 34.574 \\
\hline$: 00$ & & 31.192 & 30.697 & 29.482 & 31.04 & & 30.021 & 31.149 & 30.626 & 30.30 & 28.73 & 26.459 & 36.541 & 36.269 & 37.078 & 17.39 & 17.884 & 22.307 & 15.649 & 15.258 & 34.237 & .501 & .782 & 36.481 & 27.342 & 34.572 \\
\hline $10 / 31 / 1$ & & 31.192 & 30.697 & 29.482 & 31.05 & & 30.024 & 31.147 & 30.624 & 30.306 & 28.73 & 26.464 & 35.102 & 36.269 & 36.241 & 17.388 & 17.921 & 2.298 & 5.651 & 14.426 & 33.824 & 35.98 & .798 & 5.031 & 27.342 & 34.572 \\
\hline 10/31/11 8:00 & & & 30.699 & 29.489 & 31.063 & & 30.019 & 31.147 & 30.624 & 30.306 & 28.732 & 26.464 & 34.709 & 36.269 & 35.831 & 17.393 & 17.902 & 22.289 & 15.647 & 14.008 & 33.647 & 35.592 & 1796 & 1.648 & 27.342 & 34.572 \\
\hline 10/31/11 8:30 & & 31.195 & 30.699 & 29.487 & 31.067 & & & 31.147 & 30.624 & 30.306 & 28.732 & 26.468 & 34.468 & 36.274 & 35.58 & $3 \quad 17.393$ & 17.877 & 22.28 & 15.647 & 13.754 & 33.551 & 35.357 & .786 & 34.416 & 27.342 & 34.57 \\
\hline 10/31/11 9:00 & & 31.197 & 30.699 & 29.482 & 31.07 & & 30.024 & 31.149 & 30.619 & 30.308 & 28.728 & 26.468 & 34.3 & 36.267 & 35.402 & 17.393 & 17.849 & 22.268 & 15.647 & 13.584 & 33.481 & 35.187 & 30.779 & 34.25 & 27.345 & 34.57 \\
\hline 10/31/11 9:30 & & 31.199 & 30.706 & 29.477 & 31.072 & & 30.029 & 31.149 & 30.626 & 30.308 & 28.728 & 26.464 & 34.169 & 36.272 & 35.263 & $\quad 17.39$ & 17.821 & 22.259 & .647 & 13.455 & 33.423 & 35.056 & 30.77 & 34.123 & 27.345 & 34.57 \\
\hline 10/31/11 10:00 & & 31.195 & 30.701 & 29.48 & 31.065 & & 30.031 & 31.149 & 30.626 & 30.306 & 28.725 & 26.461 & 34.061 & 36.274 & 35.151 & 17.393 & 17.793 & 22.25 & 15.658 & 13.361 & 33.374 & 34.95 & 30.754 & 34.015 & 27.345 & 34.57 \\
\hline 10/31/11 10:30 & & 31.195 & 30.701 & 29.475 & 31.058 & & 30.033 & 31.147 & 30.629 & 30.306 & 28.725 & 26.459 & 33.972 & 36.272 & 35.06 & $\quad 17.39$ & 17.765 & 22.24 & 15.658 & 13.281 & 33.329 & 34.862 & 30.747 & 33.928 & 27.347 & 34.57 \\
\hline 10/31/11 11:00 & & 31.192 & 30.697 & 29.468 & 31.049 & & 30.036 & 31.149 & 30.624 & 30.306 & 28.723 & 26.459 & 33.895 & 36.274 & 34.977 & 17.39 & 17.739 & 22.231 & 15.665 & 13.22 & 33.292 & 34.786 & 30.732 & 33.851 & 27.345 & 34.57 \\
\hline 10/31/11 11:30 & & 31.195 & 30.694 & 29.456 & 31.044 & & 30.045 & 31.149 & 30.624 & 30.303 & 28.718 & 26.452 & 33.827 & 36.267 & 34.905 & 17.388 & 17.711 & 22.222 & 15.668 & 13.161 & 33.257 & 34.72 & 30.725 & 33.78 & 27.345 & 34.567 \\
\hline $10 / 31 / 1112: 00$ & & 31.195 & 30.697 & 29.442 & 31.035 & & 30.057 & 31.147 & 30.622 & 30.306 & 28.709 & 26.44 & 34.852 & 36.269 & 35.306 & 17.39 & 17.692 & 22.215 & 15.668 & 13.554 & 33.469 & 35.859 & 30.716 & 34.841 & 27.347 & 34.567 \\
\hline $10 / 31 / 1112: 30$ & & 31.192 & 30.69 & 29.424 & 31.025 & & 30.066 & 31.142 & 30.619 & 30.301 & 28.705 & 26.428 & 35.907 & 36.274 & 36.344 & 17.39 & 17.746 & 22.206 & 15.677 & 14.631 & 33.931 & 36.897 & 30.735 & 35.866 & 27.347 & 34.567 \\
\hline 10/31/11 13:00 & & 31.188 & 30.68 & 29.412 & 31.021 & & 30.073 & 31.139 & 30.622 & 30.294 & 28.695 & 26.411 & 36.506 & 36.267 & 37.043 & 17.388 & 17.839 & 22.194 & 15.687 & 15.357 & 34.206 & 37.466 & 30.756 & 36.444 & 27.347 & 34.565 \\
\hline $10 / 31 / 1113: 30$ & & 31.185 & 30.673 & 29.403 & 31.021 & & 30.08 & 31.137 & 30.622 & 30.289 & 28.688 & 26.397 & 35.063 & 36.258 & 36.204 & 17.388 & 17.879 & 22.19 & 15.696 & 14.551 & 33.78 & 35.94 & 30.756 & 34.993 & 27.349 & 34.565 \\
\hline 10/31/11 14:00 & & & 30.662 & 29.393 & 31 & & & 31.1 & 30 & 30. & 28.681 & 26.392 & 34.662 & 36.2 & 35.789 & 17.3 & 17.86 & 22.183 & 15. & 14.165 & 33.591 & 35.549 & 1737 & 34.606 & 27.347 & 34.563 \\
\hline 10 & & & 30.652 & 29.389 & & & & 31.133 & & & 28.677 & 26.382 & 34.421 & 36.255 & 35.535 & 17.379 & 17.835 & 22.171 & 15 & $\begin{array}{l}13.932 \\
13.932\end{array}$ & 33.483 & 35.307 & 30.716 & 34.369 & 27.347 & 34.563 \\
\hline $10 / 31 /$ & & 31 & 30.648 & 29.386 & 31. & & 30.078 & 31.127 & 30.622 & 30.269 & 28.674 & 26.382 & 34.251 & 36.248 & 35.353 & 17.379 & $\begin{array}{l}17.087 \\
\end{array}$ & 22.166 & $\begin{array}{l}15.715 \\
\end{array}$ & $\begin{array}{l}13.77 \\
13.77\end{array}$ & $\begin{array}{l}33.406 \\
33.406\end{array}$ & 35.134 & 30.7 & $\begin{array}{r}34.539 \\
34.2\end{array}$ & 27.349 & 34.56 \\
\hline $10 / 31 / 1$ & & 31 & $\begin{array}{l}30.040 \\
30.641\end{array}$ & 29.386 & 30. & & 30.073 & 31.1 & 30.622 & 30.264 & 28.67 & 26.382 & 34.11 & $\begin{array}{l}30.250 \\
36.251\end{array}$ & 35.212 & 17.377 & $\begin{array}{l}17.071 \\
17.71\end{array}$ & 22.155 & 15.71 & 13.643 & $\begin{array}{l}33.343 \\
33.343\end{array}$ & 35.004 & 30.685 & 34.067 & 27.347 & 34.56 \\
\hline $10 / 31 / 1$ & & 31 & 30. & 29.384 & 30 & & 30 & 31. & 30 & 30 & 28.665 & 26.378 & 34 & 36 & 35.099 & 17 & 17.753 & 2 & 15 & 1 & 95 & 34.897 & 74 & 1 & 27.347 & 34.56 \\
\hline & & & 30.6 & 29.384 & & & & & & & 28.665 & 26.378 & .914 & 36.2 & $35 . c$ & 17 & 17.727 & 22.141 & 15.715 & 13 & 33 & 34.805 & 2 & 99 & 47 & 34.56 \\
\hline & & & & 29.382 & & & & 31. & & 30. & 28.663 & & & 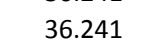 & 34.9. & 17 & & & 15.7 & 13.378 & 33 & & 3 & 2 & 49 & 34.558 \\
\hline & & & 30 & 29 & & & & & & & & & & & & & 17 & & 15. & 14. & 33 & 447 & 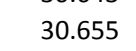 & 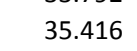 & (7) & 34.558 \\
\hline 10 & & 31 & 30 & 29.398 & & & & . & & 30 & 28. & & & 36.2 & & 17.374 & & & & 15.2 & 34.066 & & & 3 & 49 & \\
\hline & & & 30.6 & 29.4 & & & & 31 & & & 28. & & 35. & 36.2 & 36.591 & 17 & 17. & 22 & 15 & 15.061 & 33.926 & & & 67 & 49 & 34.558 \\
\hline 10/ & & & & 29.396 & & & & & & & 28.665 & & & 36.2 & & 17.372 & 17. & 22.141 & & 14.426 & & & & & 99 & 34.556 \\
\hline $10 /$ & & & 30. & 29.41 & & & 30.012 & 31. & & & & & 34. & 36.2 & 35. & 17. & 17. & & & 14.092 & & & & 4 & 99 & 34.558 \\
\hline 10/31/11 20:00 & & & 30.6 & 29.421 & 30. & & & 31.1 & 30. & & 28. & 26. & 34. & 36.2 & 35.456 & 17.372 & 17.828 & 22.153 & 15.6 & 13.871 & 33.444 & 35.232 & 30.704 & 34.292 & 27.349 & 34.558 \\
\hline 10/31/11 20: & & 31 & 30.6 & 29.417 & 30.5 & & 29.5 & 31.1 & 30.6 & 30. & 28.6 & 26.428 & 34.1 & 36.2 & 35.294 & 17.372 & 17.8 & 22.15 & 15.6 & 13.7 & 33.378 & 35.08 & 30.695 & 34.144 & 27.349 & 34.558 \\
\hline 10/31/11 21:00 & & 31.1 & 30.638 & 29.424 & 30 & & 29. & 31.12 & 30.6 & 30.2 & 28.677 & 26.425 & 34.0 & 36.241 & 35.17 & 17.372 & 17.772 & 22.15 & 15.6 & 13.554 & 33.339 & 34.964 & 30.702 & 34.029 & 27.352 & 34.558 \\
\hline $10 / 31 / 1121: 30$ & & 31.185 & 30.643 & 29.424 & 30.9 & & 29.988 & 31.125 & 30.629 & 30.25 & 28.674 & 26.43 & 33.977 & 36.251 & 35.067 & 17.374 & 17.746 & 22.139 & 15.684 & 13.432 & 33.299 & 34.869 & 30.692 & 33.933 & 27.352 & 34.558 \\
\hline $10 / 31 / 1122: 00$ & & 31.188 & 30.641 & 29.421 & 30.988 & & 29.989 & 31.125 & 30.631 & 30.25 & 28.677 & 26.43 & 33.895 & 36.253 & 34.98 & 17.374 & 17.723 & 22.129 & 15.68 & 13.34 & 33.26 & 34.784 & 30.685 & 33.851 & 27.352 & 34.558 \\
\hline $10 / 31 / 1122: 30$ & & 31.188 & 30.645 & 29.424 & 30.986 & & 29.989 & 31.125 & 30.629 & 30.25 & 28.677 & 26.43 & 33.822 & 36.251 & 34.905 & 17.377 & 17.697 & 22.123 & 15.677 & 13.253 & 33.234 & 34.715 & 30.685 & 33.778 & 27.352 & 34.558 \\
\hline $10 / 31 / 1123: 00$ & & 31.188 & 30.645 & 29.412 & 30.981 & & 29.991 & 31.125 & 30.624 & 30.25 & 28.672 & 26.43 & 33.757 & 36.255 & 34.834 & 17.377 & 17.671 & 22.111 & 15.68 & 13.18 & 33.201 & 34.651 & 30.671 & 33.715 & 27.352 & 34.558 \\
\hline $10 / 31 / 1123: 30$ & & 31.188 & 30.645 & 29.407 & 30.976 & & 30 & 31.125 & 30.624 & 30.248 & 28.67 & 26.421 & 33.701 & 36.255 & 34.776 & 17.377 & 17.65 & 22.104 & 15.68 & 13.112 & 33.171 & 34.594 & 30.664 & 33.658 & 27.352 & 34.558 \\
\hline 11/1/11 0:C & & 31.188 & 30.648 & 29.403 & 30.967 & & 30 & 31.123 & 30.626 & 30.25 & 28.67 & 26.418 & 33.647 & 36.26 & 34.724 & 17.374 & 17.627 & 22.095 & 15.682 & 13.056 & 33.145 & 34.542 & 30.655 & 33.607 & 27.352 & 34.558 \\
\hline $11 / 1 / 110: 30$ & & 31.185 & 30.641 & 29.396 & 30.955 & & 30.005 & 31.123 & 30.624 & 30.245 & 28.663 & 26.411 & 33.6 & 36.258 & 34.673 & 17.374 & 17.606 & 22.086 & 15.682 & 13.011 & 33.117 & 34.495 & 30.641 & 33.562 & 27.352 & 4.558 \\
\hline
\end{tabular}




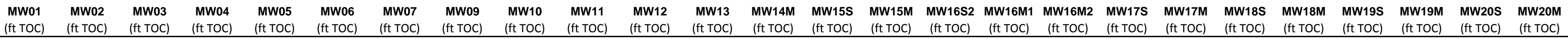

\begin{tabular}{|c|c|c|c|c|c|c|c|c|c|c|c|c|c|c|c|c|c|c|c|c|c|c|c|c|c|}
\hline Well & $\begin{array}{l}\text { (ft TOC) } \\
\text { (ft }\end{array}$ & $\begin{array}{l}\text { (ft TOC) } \\
\text { (f) }\end{array}$ & $\begin{array}{l}\text { (ft TOC) } \\
\text { (f) }\end{array}$ & $\begin{array}{l}\text { (ft TOC) } \\
\text { (f }\end{array}$ & $\begin{array}{ll}(\mathrm{ft} \text { TOC) } & \text { (ft TOC) }\end{array}$ & $\begin{array}{l}\text { (ft TOC) } \\
\text { (ft) }\end{array}$ & $\begin{array}{l}\text { (ft TOC) } \\
\text { (f) }\end{array}$ & $\begin{array}{l}\text { (ft TOC) } \\
\text { (ft) }\end{array}$ & $\begin{array}{l}\text { (ft TOC) } \\
\text { (f) }\end{array}$ & $\begin{array}{l}\text { (ft TOC) } \\
\text { (f) }\end{array}$ & $\begin{array}{l}\text { (ft TOC) } \\
\text { (f) }\end{array}$ & (ft TOC) & $\begin{array}{l}\text { (ft TOC) } \\
\end{array}$ & (ft TOC) & $\begin{array}{l}\text { (ft TOC) } \\
\end{array}$ & $\begin{array}{l}\text { (ft TOC) } \\
\text { (f) }\end{array}$ & (ft TOC) & $\begin{array}{l}\text { (ft TOC) } \\
\text { (1) }\end{array}$ & (ft TOC) & $\begin{array}{l}\text { (ft TOC) } \\
\end{array}$ & $\begin{array}{l}\text { (ft TOC) } \\
\text { (f) }\end{array}$ & $\begin{array}{l}\text { (ft TOC) } \\
\end{array}$ & (ft TOC) & $\begin{array}{l}\text { (ft TOC) } \\
\text { (1) }\end{array}$ & (ft TOC) \\
\hline 1:00 & & 31.185 & 30.641 & 29.3 & 30.946 & 30.0 & 31.123 & 30 & 30.2 & 28.66 & 26. & 33.558 & 36.253 & 34. & 17. & 17. & 22.079 & 15 & 12.966 & 33. & 34.452 & 30.627 & 33.5 & 7.352 & 34.558 \\
\hline $11 / 1 / 111: 30$ & & 31.183 & 30.636 & 29.386 & 30.937 & 30.003 & 31.121 & 30.626 & 30.243 & 28.658 & 26.406 & 33.518 & 36.251 & 34.584 & 17.374 & 17.564 & 22.069 & 15. & 2.924 & 33.073 & 412 & 30.617 & & 7354 & .56 \\
\hline $11 / 1 / 112: 00$ & & 31.183 & 30.634 & 29.384 & 30.925 & 30.005 & 31.12 & 30.624 & 30.238 & 28.656 & 26.399 & 33.478 & 36.253 & 34.546 & 17.372 & 17.543 & 22.065 & 15.68 & 12.886 & 33 & 34.374 & 30.608 & .442 & .352 & 3.558 \\
\hline 11/1/11 2:30 & & 31.181 & 30.631 & 29.377 & 30.916 & 30.005 & 31.121 & 30.626 & 30.236 & 28.656 & 26.399 & 33.443 & 36.251 & 34.511 & 17.372 & 17.524 & 22.055 & 15.682 & 12.851 & 33.022 & 34.343 & 30.599 & 33.405 & 27.352 & 34.558 \\
\hline 11/1/11 3:00 & & 31.183 & 30.627 & 29.372 & 30.906 & 30.007 & 31.118 & 30.626 & 30.234 & 28.651 & 26.392 & 33.411 & 36.251 & 34.478 & $3 \quad 17.372$ & 17.508 & 22.049 & 15.68 & 12.818 & 33.008 & 34.305 & 30.587 & 33.374 & 27.352 & 34.558 \\
\hline 11/1/11 3:30 & & 31.181 & 30.624 & 29.374 & 30.897 & 30.007 & 31.116 & 30.624 & 30.231 & 28.649 & 26.39 & 33.383 & 36.246 & 34.443 & 17.372 & 17.487 & 22.039 & 15.677 & 12.785 & 32.982 & 34.274 & 30.58 & 33.342 & 27.354 & 34.558 \\
\hline 11/1/11 4:00 & & 31.178 & 30.62 & 29.374 & 30.885 & 30.003 & 31.116 & 30.626 & 30.227 & 28.649 & 26.39 & 33.35 & 36.248 & 34.412 & 17.37 & 17.47 & 22.032 & 15.68 & 12.757 & 32.966 & 34.246 & 30.568 & 33.313 & 27.352 & 34.558 \\
\hline 11/1/11 4:30 & & 31.176 & 30.617 & 29.382 & 30.876 & 30 & 31.113 & 30.626 & 30.222 & 28.649 & 26.394 & 33.319 & 36.246 & 34.382 & 17.367 & 17.451 & 22.025 & 15.68 & 12.729 & 32.947 & 34.217 & 30.556 & 33.285 & 27.354 & 34.558 \\
\hline 11/1/11 5:00 & & 31.178 & 30.617 & 29.382 & 30.869 & 29.991 & 31.113 & 30.629 & 30.222 & 28.651 & 26.397 & 33.296 & 36.248 & 34.354 & 17.367 & 17.435 & 22.021 & 15.673 & 12.696 & 32.938 & 34.194 & 30.552 & 33.262 & 27.354 & 34.558 \\
\hline 11/1/11 5:30 & & 31.178 & 30.613 & 29.379 & 30.86 & 29.989 & 31.113 & 30.624 & 30.222 & 28.649 & 26.401 & 33.273 & 36.246 & 34.33 & 17.367 & 17.421 & 22.016 & 15.668 & 12.663 & 32.921 & 34.17 & 30.552 & 33.236 & 27.354 & 34.558 \\
\hline $11 / 1 / 116: 00$ & & 31.181 & 30.617 & 29.382 & 30.857 & 29.991 & 31.116 & 30.626 & 30.222 & 28.646 & 26.397 & 34.333 & 36.244 & 34.762 & 17.367 & 17.407 & 22.023 & 15.665 & 13.067 & 33.166 & 35.345 & 30.554 & 34.329 & 27.354 & 34.558 \\
\hline $11 / 1 / 116: 30$ & & 31.181 & 30.62 & 29.386 & 30.857 & 29.986 & 31.113 & 30.629 & 30.22 & 28.649 & 26.401 & 35.407 & 36.244 & 35.822 & 17.367 & 17.463 & 22.028 & 15.661 & 14.128 & 33.649 & 36.407 & 30.587 & 35.369 & 27.354 & 34.56 \\
\hline $11 / 1 / 117: 00$ & & 31.178 & 30.615 & 29.396 & 30.86 & 29.974 & 31.113 & 30.631 & 30.217 & 28.651 & 26.406 & 36.036 & 36.246 & 36.551 & 17.367 & 17.564 & 22.028 & 15.665 & 14.866 & 33.945 & 37.002 & 30.617 & 35.979 & 27.354 & 34.558 \\
\hline 11/1/11 7:30 & & 31.178 & 30.617 & 29.403 & 30.874 & 29.97 & 31.113 & 30.634 & 30.217 & 28.658 & 26.418 & 34.625 & 36.246 & 35.746 & 17.37 & 17.608 & 22.018 & 15.654 & 14.057 & 33.532 & 35.506 & 30.636 & 34.555 & 27.356 & 34.558 \\
\hline $11 / 1 / 118: 00$ & & 31.181 & 30.62 & 29.405 & 30.89 & 29.965 & 31.116 & 30.631 & 30.217 & 28.66 & 26.425 & 34.251 & 36.251 & 35.36 & 17.37 & 17.599 & 22.012 & 15.651 & 13.651 & 33.374 & 35.137 & 30.645 & 34.196 & 27.356 & 34.558 \\
\hline $11 / 1 / 118: 30$ & & 31.183 & 30.624 & 29.405 & 30.902 & 29.965 & 31.116 & 30.629 & 30.222 & 28.66 & 26.43 & 34.035 & 36.251 & 35.13 & 17.372 & 17.585 & 22.005 & 15.649 & 13.413 & 33.288 & 34.923 & 30.643 & 33.984 & 27.356 & 34.558 \\
\hline $11 / 1 / 119: 00$ & & 31.185 & 30.629 & 29.41 & 30.911 & 29.963 & 31.116 & 30.634 & 30.222 & 28.667 & 26.43 & 33.881 & 36.253 & 34.973 & 17.372 & 17.564 & 21.995 & 15.647 & 13.251 & 33.227 & & 30.643 & 33.839 & 27.356 & 34.558 \\
\hline $11 / 1 / 119: 30$ & & 31.185 & 30.631 & 29.407 & 30.916 & 29.97 & 31.116 & 30.626 & 30.222 & 28.66 & 26.433 & 33.773 & 36.258 & 34.853 & 17.372 & 17.545 & 21.988 & 15.642 & 13.128 & 33.18 & 34.663 & 30.641 & 33.724 & 27.356 & 34.558 \\
\hline $11 / 1 / 1110: 00$ & & 31.185 & 30.631 & 29.405 & 30.92 & 29.974 & 31.118 & 30.634 & 30.222 & 28.663 & 26.43 & 33.68 & 36.258 & 34.757 & 17.372 & 17.524 & 21.981 & 15.644 & 13.034 & 33.143 & 34.573 & 30.634 & 33.635 & 27.356 & 34.556 \\
\hline $11 / 1$ & & 31.185 & 30.634 & 29.4 & 30.92 & 29.979 & 31.118 & 30.631 & 30.224 & 28.66 & 26.428 & 33.605 & 36.26 & 34.682 & 17.374 & 17.505 & 21.975 & 15.647 & 12.957 & 33.108 & 34.497 & 30.629 & 33.564 & 27.359 & 34.558 \\
\hline Do & & 31.183 & 30.631 & 29.398 & 30.91 & 29.979 & 31.118 & 30.631 & 30.22 & 28.66 & 26.428 & 33.539 & 36.265 & 34.612 & 17.374 & 17.484 & 21.963 & 15.654 & 12.91 & 33.071 & 34.433 & 30.615 & 33.499 & 27.359 & 34.556 \\
\hline $11 /$ & & 31.185 & 30.631 & 29.391 & 30.91 & 29.984 & 31.118 & 30.631 & 30.222 & 28.658 & 26.423 & 33.488 & 36.262 & 34.555 & 17.372 & 17.465 & 21.947 & 15.651 & 12.853 & 33.054 & 34.381 & 30.61 & 33.447 & 27.359 & 34.556 \\
\hline $11 / 1 / 1$ & & 31.183 & 30.629 & 29.384 & 30.906 & 29.993 & 31.118 & 30.629 & 30.222 & 28.653 & 26.421 & 34.524 & 36.262 & 34.963 & 17.372 & 17.449 & 21.951 & 5.656 & 13.26 & 33.274 & 35.535 & 30.596 & 4.517 & 27.359 & 34.558 \\
\hline 11/1/11 12:30 & & 31.181 & 30.629 & 29.372 & 30.904 & 29.996 & 31.113 & 30.629 & 30.22 & 28.649 & 26.413 & 35.577 & 36.26 & 36 & 17.372 & 17.508 & 21.942 & 15.656 & 14.318 & 33.733 & 36.573 & 30.627 & 5.538 & 27.359 & 34.556 \\
\hline 11/1/11 13:00 & & 31.181 & 30.629 & 29.374 & 30.906 & 30 & 31.113 & 30.626 & 30.22 & 28.649 & 26.404 & 36.188 & 36.265 & 36.715 & 17.374 & 17.608 & 21.938 & 15.658 & 15.044 & 34.024 & 37.151 & 30.66 & 36.127 & 27.359 & 34.556 \\
\hline 11/1/11 13:30 & & 31.181 & 30.624 & 29.37 & 30.913 & 30 & 31.113 & 30.629 & 30.22 & 28.646 & 26.406 & 34.758 & 36.26 & 35.885 & 17.372 & 17.652 & 21.933 & 15.663 & 14.24 & . 33.602 & 35.637 & 30.667 & 34.691 & 27.361 & 34.553 \\
\hline $11 / 1 / 1114: 00$ & & 31.176 & 30.62 & 29.367 & 30.916 & 30 & 31.113 & 30.631 & 30.22 & 28.642 & 26.401 & 34.372 & 36.258 & 35.489 & 17.372 & 17.638 & 21.924 & 15.668 & 13.855 & 33.425 & 35.253 & 30.66 & 34.315 & 27.359 & 34.551 \\
\hline $11 / 1 / 1114: 30$ & & 31.178 & 30.617 & 29.36 & 30.92 & 30.005 & 31.111 & 30.631 & 30.222 & 28.639 & 26.401 & 34.143 & 36.258 & 35.245 & 17.372 & 17.62 & 21.917 & 15.67 & 13.622 & 33.332 & 35.03 & 30.648 & 34.095 & 27.361 & 34.553 \\
\hline $11 / 1 / 1115: 00$ & & 31.176 & 30.617 & 29.358 & 30.92 & 30.005 & 31.108 & 30.631 & 30.22 & 28.637 & 26.394 & 33.982 & 36.255 & 35.074 & 17.367 & 17.601 & 21.912 & 15.67 & 13.46 & 33.26 & 34.869 & 30.636 & 33.933 & 27.361 & 34.553 \\
\hline $11 / 1 / 1115: 30$ & & 31.174 & 30.615 & 29.363 & 30.916 & 30 & 31.109 & 30.629 & 30.217 & 28.635 & 26.392 & 33.858 & 36.253 & 34.945 & 17.367 & 17.575 & 21.903 & 15.68 & 13.342 & 33.201 & 34.748 & 30.62 & 33.811 & 27.361 & 34.551 \\
\hline $11 / 1 / 1116: 00$ & & 31.174 & 30.61 & 29.367 & 30.911 & 29.996 & 31.109 & 30.631 & 30.217 & 28.637 & 26.399 & 33.757 & 36.251 & 34.839 & 17.367 & 17.554 & 21.898 & 15.675 & 13.248 & 33.162 & 34.649 & 30.613 & 33.712 & 27.361 & 34.553 \\
\hline $11 / 1 / 1116: 30$ & & 31.176 & 30.608 & 29.37 & 30.906 & 29.989 & 31.106 & 30.631 & 30.217 & 28.639 & 26.404 & 33.675 & 36.248 & 34.755 & 17.365 & 17.531 & 21.933 & 15.677 & 13.159 & 33.117 & 34.566 & 30.606 & 33.632 & 27.361 & 34.551 \\
\hline 11/1/11 17:00 & & 31.176 & 30.61 & 29.377 & 30.904 & 29.986 & 31.109 & 30.631 & 30.217 & 28.642 & 26.406 & 33.607 & 36.253 & 34.68 & 17.367 & 17.512 & 21.951 & 15.677 & 13.084 & 33.092 & 34.499 & 30.601 & 33.564 & 27.361 & 34.551 \\
\hline $11 / 1 / 1117: 30$ & & 31.176 & 30.61 & 29.379 & 30.899 & 29.977 & 31.108 & 30.634 & 30.215 & 28.642 & 26.413 & 35.236 & 36.251 & 35.756 & 17.367 & 17.526 & 21.949 & 15.673 & 14.149 & 33.64 & 36.198 & 30.615 & 35.169 & 27.363 & 34.551 \\
\hline $11 / 1 / 1118: 00$ & & 31 . & 30.613 & 29.393 & 30. & 29.97 & 31.108 & 30.634 & 30.217 & 28.649 & 26.421 & 36.345 & 36.251 & 36.614 & 17.367 & 17.61 & 21.944 & 15.673 & 15.002 & 34.015 & 37.374 & 30.648 & 36.293 & 27.363 & 34.551 \\
\hline $\begin{array}{l}11 / 1 / 11 \\
11 / 1\end{array}$ & & 31 . & 30.615 & 29.403 & 30. & & 31.1 & 30.636 & 30. & 28.651 & 26.433 & 35.804 & 36.253 & 36.743 & 17.367 & 17.72 & 21.942 & 15.668 & $\begin{array}{l}15.129 \\
1529\end{array}$ & 34.008 & 36.705 & 30.685 & 35.726 & 27.363 & 34.553 \\
\hline $11 / 1 / 11$ 19:00 & & 31 & 30.617 & 29.41 & 30. & 29.949 & 31. & 30.636 & 30.217 & $\begin{array}{l}20.051 \\
28.658\end{array}$ & 26.445 & 35.514 & 36.253 & 36.457 & 17.367 & 17.758 & 21.933 & $\begin{array}{l}15.600 \\
15.665\end{array}$ & 14.83 & $\begin{array}{l}33.859 \\
33.859\end{array}$ & 36.412 & .704 & 35.444 & 27.363 & 34.553 \\
\hline $11 / 1 / 1119: 30$ & & 31 & 30.622 & $\begin{array}{r}29.424 \\
29.424\end{array}$ & 30. & 29.939 & 31. & 30.638 & 30.222 & $\begin{array}{l}20.050 \\
28.663\end{array}$ & 26.452 & 35.194 & 36.26 & 36.295 & 17.37 & $\begin{array}{l}17.783 \\
1.783\end{array}$ & 21.928 & 15.658 & $\begin{array}{l}14.6039 \\
14.649\end{array}$ & $\begin{array}{l}33.037 \\
33.803\end{array}$ & 36.075 & 0.721 & 35.104 & 27.363 & 34.553 \\
\hline & & 31 & 30 & 29.424 & 30 & 2 & 3 & 30 & 30 & 28. & 26.461 & 34 & 36 & 35.796 & 17.372 & 17.774 & 21 & 15.651 & 1 & 33.586 & 35.554 & 28 & 09 & 27.363 & 34.553 \\
\hline & & 31 & 30 & 29 & 30 & & 3. & $3 c$ & 30 & 2 & 26 & 34 & 36.267 & 35.526 & 17 & 51 & 2 & 15.651 & 13. & 33.483 & .3 & 25 & 88 & 27.366 & 34.556 \\
\hline & & & 30 & 29.4 & & & 3 & & & 28. & 26 & 34 & 36.2 & 35. & 17 & 17 & 21 & 15 & 13 & 33.411 & 23 & 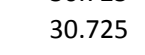 & 32 & 27.363 & 34.556 \\
\hline & & 31. & 30.6 & 29.4 & & & & & & 28. & & & & & 17 & 17 & 21 & 9 & 13.479 & 33 & 183 & 30.71 & 46 & 27.363 & 34.555 \\
\hline 2:00 & & 31 & 30.648 & 29.435 & & & 31.12 & & & 28. & & 33.92 & 36.2 & & & & & 15.647 & 13.342 & 33.306 & & & & 27.366 & 34.556 \\
\hline 2::30 & & 31. & 30.65 & 29. & & & 31.1 & & & 28. & 26.478 & & 36.274 & 34.975 & 17. & 17. & 21 & 15.642 & 13.2 & 33.274 & & & 44 & 27.366 & 34.556 \\
\hline $11 /$ & & 31 & 30.6 & 29. & & & 31.1 & & & 28. & & & 36.2 & & 17. & 17. & 21.882 & 15. & 13.157 & 33.229 & 34.698 & & 52 & 27.366 & 34.556 \\
\hline $11 / 1$ & & 31 & 30. & 29.419 & & & 31 & & & 28. & & & 36.2 & 34 & 17. & 17. & & 15.647 & 13.079 & 33. & 34.63 & & 33.693 & 27.366 & 34.555 \\
\hline $11 / 2 / 110: 00$ & & 31.1 & 30 & 29.421 & 30 & & & 30. & & 28. & 26. & 33. & 36.2 & 34.748 & 17.381 & 17. & 21.873 & 15.647 & 13.009 & 33.171 & 34.57 & 30.676 & 33.632 & 27.366 & 34.556 \\
\hline $11 / 2 / 110: 30$ & & 31 & 30. & 29.421 & & & 31. & 30. & & 28. & 26.461 & 33.6 & 36.2 & 34.689 & 17.381 & 17. & 21.866 & 15.649 & 12.952 & 33.138 & 34.511 & 30.669 & 33.576 & 27.366 & 34.556 \\
\hline $11 / 2 / 111: 00$ & & 31. & 30.652 & 29.431 & 30. & 29.951 & 31.133 & 30.6 & 30. & 28.679 & 26.461 & 33.4 & 36.276 & 34.64 & 17.381 & 17.53 & 21.864 & 15.651 & 12.903 & 33.115 & 34.462 & 30.657 & 33.527 & 27.368 & 34.556 \\
\hline $11 / 2 / 111: 30$ & & 31.188 & 30.648 & 29.424 & 30.9 & 29.956 & 31.132 & 30.636 & 30.2 & 28.681 & 26.471 & 33.523 & 36.274 & 34.591 & 17.381 & 17.515 & 21.854 & 15.654 & 12.853 & 33.087 & 34.416 & 30.643 & 33.482 & 27.366 & 34.556 \\
\hline $11 / 2 / 112: 00$ & & 31.192 & 30.652 & 29.419 & 30.95 & 29.96 & 31.132 & 30.634 & 30.241 & 28.677 & 26.464 & 33.483 & 36.276 & 34.546 & 17.381 & 17.496 & 21.85 & 15.637 & 12.797 & 33.075 & 34.376 & 30.645 & 33.44 & 27.368 & 34.558 \\
\hline $11 / 2 / 112: 30$ & & 31.192 & 30.65 & 29.433 & 30.951 & 29.965 & 31.135 & 30.643 & 30.243 & 28.679 & 26.457 & 33.443 & 36.276 & 34.509 & 17.381 & 17.479 & 21.845 & 15.647 & 12.752 & 33.05 & 34.341 & 30.641 & 33.405 & 27.366 & 34.556 \\
\hline 11/2/11 3:00 & & 31.188 & 30.648 & 29.435 & 30.941 & 29.951 & 31.135 & 30.638 & 30.241 & 28.686 & 26.468 & 33.408 & 36.281 & 34.469 & 17.379 & 17.458 & 21.836 & 15.647 & 12.719 & 33.029 & 34.305 & 30.627 & 33.367 & 27.366 & 34.558 \\
\hline $11 / 2 / 113: 30$ & & 31.19 & 30.652 & 29.438 & 30.937 & 29.953 & 31.137 & 30.638 & 30.241 & 28.684 & 26.471 & 33.376 & 36.279 & 34.441 & 17.381 & 17.442 & 21.834 & 15.644 & 12.682 & 33.012 & 34.27 & 30.624 & 33.339 & 27.368 & 34.558 \\
\hline $11 / 2 / 114: 00$ & & 31.199 & 30.657 & 29.445 & 30.934 & 29.951 & 31.135 & 30.641 & 30.245 & 28.691 & 26.471 & 33.35 & 36.281 & 34.405 & 17.384 & 17.426 & 21.829 & 15.63 & 12.635 & 33.005 & 34.243 & 30.629 & 33.309 & 27.368 & 34.558 \\
\hline $11 / 2 / 114: 30$ & & 31.195 & 30.655 & 29.454 & 30.927 & 29.951 & 31.139 & 30.643 & 30.245 & 28.693 & 26.478 & 33.319 & 36.281 & 34.377 & 17.384 & 17.407 & 21.822 & 15.642 & 12.604 & 32.987 & 34.215 & 30.617 & 33.281 & 27.368 & 34.56 \\
\hline
\end{tabular}




\begin{tabular}{|c|c|c|c|c|c|c|c|c|c|c|c|c|c|c|c|c|c|c|c|c|c|c|c|c|c|c|}
\hline Well & $\begin{array}{l}\text { (ft TOC) } \\
\text { (flocts }\end{array}$ & $\begin{array}{l}\text { (ft TOC) } \\
\text { (f) }\end{array}$ & $\begin{array}{l}\text { (ft TOC) } \\
\text { (ft) }\end{array}$ & $\begin{array}{l}\text { (ft TOC) } \\
\text { (f) }\end{array}$ & $\begin{array}{l}\text { (ft TOC) } \\
\text { (n) }\end{array}$ & (ft TOC) & $\begin{array}{l}\text { (ift TOC) } \\
\text { (ft }\end{array}$ & $\begin{array}{l}\text { (ft TOC) } \\
\text { (f) }\end{array}$ & $\begin{array}{l}\text { (ft TOC) } \\
\text { (f) }\end{array}$ & $\begin{array}{l}\text { (ft TOC) } \\
\text { (n) }\end{array}$ & $\begin{array}{l}\text { (ft TOC) } \\
\text { (f) }\end{array}$ & $\begin{array}{l}\text { (ft TOC) } \\
\text { (f) }\end{array}$ & (ft TOC) & $\begin{array}{l}\text { (ft TOC) } \\
\text { (f) }\end{array}$ & (ft TOC) & $\begin{array}{l}\text { (ft TOC) } \\
\end{array}$ & $\begin{array}{l}\text { (ft TOC) } \\
\end{array}$ & (ft TOC) & $\begin{array}{l}\text { (ft TOC) } \\
\end{array}$ & (ft TOC) & $\begin{array}{l}\text { (ft TOC) } \\
\end{array}$ & $\begin{array}{l}\text { (ft TOC) } \\
\text { (1) }\end{array}$ & $\begin{array}{l}\text { (ft TOC) } \\
\end{array}$ & $\begin{array}{l}\text { (ft TOC) } \\
\text { (f) }\end{array}$ & $\begin{array}{l}\text { (ft TOC) } \\
\text { (1) }\end{array}$ & (ft TOC) \\
\hline$: 00$ & & 31.197 & 30.659 & 29.4 & 30.925 & & 29.946 & 31 & 30.6 & 30.2. & $2 \varepsilon$ & 26.4 & 33. & 36 & 34.349 & 17.386 & 17. & 21 & 15 & 12.567 & 32.977 & 91 & 30. & 33 & 68 & \\
\hline 11/2/11 5:30 & & 31.197 & 30.664 & 29.461 & 30.923 & & 29.944 & 31.145 & 30.641 & 30.25 & 28.698 & 26.49 & 33.273 & 36.274 & 34.326 & 17.386 & 17.379 & 21.815 & 15.628 & 12.534 & 2.97 & 68 & 17 & 33.234 & & 34.56 \\
\hline 11/2/11 6:00 & & 31.202 & 30.669 & 29.477 & 30.925 & & 29.939 & 31.147 & 30.641 & 30.252 & 28.702 & 26.492 & 34.342 & 36.281 & 34.764 & 17.386 & 17.367 & 21.824 & 15.628 & 12.943 & 33.218 & $=255$ & 30.624 & & & 3.563 \\
\hline 11/2/11 6:30 & & 31.202 & 30.669 & 29.482 & 30.927 & & 29.942 & 31.147 & 30.641 & 30.257 & 28.712 & 26.504 & 35.414 & 36.283 & 35.824 & 17.386 & 17.428 & $8 \quad 21.834$ & 15.626 & 14.005 & 33.705 & 36.412 & 30.662 & 35.376 & 27.37 & 34.563 \\
\hline 11/2/11 7:00 & & 31.204 & 30.671 & 29.501 & 30.937 & & 29.932 & 31.152 & 30.645 & 30.259 & 28.718 & 26.512 & 34.379 & 36.286 & 35.477 & 17.388 & 17.491 & $1 \quad 21.831$ & 15.626 & 13.667 & 33.486 & 35.262 & 30.683 & $3 \quad 34.311$ & 27.37 & 34.563 \\
\hline 11/2/11 7:30 & & 31.206 & 30.673 & 29.515 & 30.948 & & 29.92 & 31.151 & 30.645 & 30.264 & 28.725 & 26.524 & 34.014 & 36.283 & 35.104 & 17.39 & 17.494 & $\begin{array}{l}41.824 \\
\end{array}$ & 15.626 & 13.279 & 33.323 & 34.9 & 30.69 & 33.954 & 27.373 & 34.565 \\
\hline 11/2/11 8:00 & & 31.209 & 30.683 & 29.519 & 30.965 & & 29.92 & 31.151 & 30.645 & 30.266 & 28.732 & 26.538 & 33.825 & 36.288 & 34.905 & 17.393 & 17.475 & 21.82 & 15.616 & 13.065 & 33.243 & 34.715 & 30.697 & 33.776 & 27.373 & 34.567 \\
\hline 11/2/11 8:30 & & 31.216 & 30.694 & 29.526 & 30.979 & & 29.923 & 31.154 & 30.648 & 30.275 & 28.739 & 26.54 & 33.703 & 36.288 & 34.776 & 17.395 & 17.463 & 21.82 & 15.607 & 12.905 & 33.204 & 34.592 & 30.716 & 533.656 & 27.373 & 34.567 \\
\hline 11/2/11 9:00 & & 31.216 & 30.701 & 29.543 & 30.99 & & 29.923 & 31.156 & 30.645 & 30.282 & 28.746 & 26.547 & 33.612 & 36.295 & 34.68 & 17.397 & 17.449 & $\begin{array}{l}9 \\
9\end{array}$ & 15.612 & 12.799 & 33.169 & 34.504 & 30.711 & $\quad 33.567$ & 27.37 & 34.57 \\
\hline 11/2/11 9:30 & & 31.213 & 30.704 & 29.545 & 31 & & 29.923 & 31.156 & 30.643 & 30.287 & 28.749 & 26.557 & 33.544 & 36.297 & 34.607 & 17.4 & 17.433 & $\begin{array}{l}31.808 \\
\end{array}$ & 15.612 & 12.719 & 33.136 & 34.433 & 30.711 & 33.494 & 27.373 & 34.57 \\
\hline 11/2/11 10:00 & & 31.216 & 30.706 & 29.564 & 31.002 & & 29.92 & 31.159 & 30.648 & 30.294 & 28.76 & 26.562 & 33.483 & 36.293 & 34.546 & 17.4 & 17.419 & $\begin{array}{l}9 \\
91.801\end{array}$ & 15.612 & 12.644 & 33.11 & 34.376 & 30.711 & 33.438 & 27.37 & 34.57 \\
\hline 11/2/11 10:30 & & 31.22 & 30.715 & 29.559 & 31.007 & & 29.92 & 31.164 & 30.645 & 30.299 & 28.765 & 26.576 & 33.434 & 36.297 & 34.492 & 17.404 & 17.405 & $5 \quad 21.797$ & 15.607 & 12.576 & 33.106 & 34.326 & 30.714 & 33.391 & 27.375 & 34.57 \\
\hline 11/2/11 11:00 & & 31.218 & 30.718 & 29.569 & 31.011 & & 29.93 & 31.163 & 30.648 & 30.306 & 28.765 & 26.567 & 33.392 & 36.297 & 34.448 & 17.404 & 17.386 & $6 \quad 21.79$ & 15.612 & 12.522 & 33.08 & 34.284 & 30.714 & 33.346 & 27.373 & 34.567 \\
\hline 11/2/11 11:30 & & 31.223 & 30.729 & 29.573 & 31.018 & & 29.927 & 31.161 & 30.65 & 30.313 & 28.772 & 26.574 & 33.354 & 36.302 & 34.412 & 17.409 & 17.37 & 21.783 & 15.604 & 12.466 & 33.075 & 34.248 & 30.718 & 333.311 & 27.375 & 34.572 \\
\hline 11/2/11 12:00 & & 31.218 & 30.732 & 29.569 & 31.016 & & 29.932 & 31.164 & 30.645 & 30.313 & 28.772 & 26.579 & 33.322 & 36.302 & 34.377 & 17.409 & 17.353 & 21.78 & 15.609 & 12.428 & 33.05 & 34.215 & 30.711 & 33.283 & 27.375 & 34.57 \\
\hline 11/2/11 12:30 & & 31.223 & 30.736 & 29.561 & 31.018 & & 29.942 & 31.168 & 30.645 & 30.32 & 28.774 & 26.567 & 33.294 & 36.305 & 34.349 & 17.414 & 17.342 & 21.773 & 15.6 & 12.386 & 33.04 & 34.189 & 30.714 & $\quad 33.252$ & 27.373 & 34.57 \\
\hline 11/2/11 13:00 & & 31.222 & 30.743 & 29.571 & 31.023 & & 29.949 & 31.166 & 30.648 & 30.324 & 28.779 & 26.564 & 33.268 & 36.307 & 34.319 & 17.414 & 17.328 & $3 \quad 21.771$ & 15.607 & 12.357 & 33.033 & 34.163 & 30.709 & 33.227 & 27.375 & 34.57 \\
\hline 11/2/11 13:30 & & 31.218 & 30.739 & 29.576 & 31.009 & & 29.949 & 31.171 & 30.653 & 30.327 & 28.786 & 26.569 & 33.24 & 36.307 & 34.295 & 17.411 & 17.316 & 21.762 & 15.614 & 12.336 & 33.015 & 34.139 & 30.697 & 33.203 & 27.375 & 34.57 \\
\hline$/ 1114: 00$ & & 31.213 & 30.739 & 29.589 & 31.009 & & 29.951 & 31.168 & 30.65 & 30.327 & 28.791 & 26.569 & 33.214 & 36.307 & 34.269 & 17.414 & 17.297 & 21.757 & 15.619 & 12.315 & 33.001 & 34.113 & 30.685 & 33.18 & 27.375 & 34.57 \\
\hline 114:30 & & 31.223 & 30.746 & 29.601 & 31.011 & & 29.953 & 31.171 & 30.653 & 30.331 & 28.798 & 26.583 & 33.2 & 36.312 & 34.251 & 17.418 & 17.29 & 21.755 & 15.607 & 12.273 & 32.998 & 34.097 & 30.697 & 33.156 & 27.375 & 34.572 \\
\hline 5:00 & & 31.22 & 30.746 & 29.618 & 31.009 & & 29.934 & 31.176 & 30.655 & 30.336 & 28.807 & 26.593 & 33.179 & 36.307 & 34.23 & 17.418 & 17.279 & 21.748 & 15.616 & 12.259 & 32.982 & 34.075 & 30.692 & 233.135 & 7.377 & 34.57 \\
\hline $11 / 2 / 1115: 30$ & & 31.23 & 30.757 & 29.62 & 31.016 & & 29.937 & 31.178 & 30.657 & 30.343 & 28.816 & 26.61 & 33.163 & 36.314 & 34.213 & 17.418 & 17.272 & 21.743 & 15.597 & .209 & .994 & 34.063 & 30.714 & 126 & 7.375 & 34.572 \\
\hline 11/2/11 16:00 & & 31.234 & 30.771 & 29.629 & 31.023 & & 29.939 & 31.173 & 30.655 & 30.352 & 28.821 & 26.607 & 33.151 & 36.316 & 34.199 & 17.423 & 17.262 & 21.748 & 15.595 & .183 & 2.994 & 34.047 & 30.723 & 33.114 & 27.38 & 4.572 \\
\hline 11/2/11 16:30 & & 31.23 & 30.774 & 29.646 & 31.025 & & 29.927 & 31.18 & 30.662 & 30.359 & 28.832 & 26.595 & 33.139 & 36.316 & 34.183 & 17.421 & 17.25 & 21.743 & 15.604 & 12.169 & 32.982 & 34.033 & 30.707 & 33.095 & 27.377 & 34.572 \\
\hline 11/2/11 17:00 & & 31.232 & 30.778 & 29.646 & 31.03 & & 29.939 & 31.183 & 30.655 & 30.366 & 28.837 & 26.583 & 33.125 & 36.321 & 34.171 & 17.425 & 17.236 & $5 \quad 21.743$ & 15.602 & 12.143 & 32.98 & 34.021 & 30.721 & 33.086 & 27.38 & 34.577 \\
\hline 11/2/11 17:30 & & 31.232 & 30.783 & 29.66 & 31.032 & & 29.932 & 31.185 & 30.662 & 30.371 & 28.849 & 26.567 & 33.111 & 36.319 & 34.157 & 17.425 & 17.227 & 21.757 & 15.604 & 12.127 & 32.973 & 34.004 & 30.723 & 33.074 & 27.377 & 34.572 \\
\hline 11/2/11 18:00 & & 31.241 & 30.797 & 29.674 & 31.042 & & 29.934 & 31.185 & 30.66 & 30.38 & 28.856 & 26.552 & 33.102 & 36.321 & 34.147 & 17.43 & 17.218 & $\begin{array}{l}3 \quad 21.776 \\
\end{array}$ & 15.597 & 12.096 & 32.984 & 33.999 & 30.73 & 333.067 & 27.377 & 34.579 \\
\hline 11/2/11 18:30 & & 31.241 & 30.802 & 29.674 & 31.044 & & 29.939 & 31.187 & 30.655 & 30.389 & 28.863 & 26.531 & 33.088 & 36.323 & 34.133 & 17.432 & 17.213 & 31.776 & 15.597 & 12.08 & 32.98 & 33.99 & 30.735 & 533.053 & 27.377 & 34.574 \\
\hline 11/2/11 19:00 & & 31.243 & 30.809 & 29.685 & 31.056 & & 29.942 & 31.19 & 30.662 & 30.396 & 28.87 & 26.516 & 33.081 & 36.328 & 34.122 & 17.435 & 17.204 & 21.769 & 15.597 & 12.056 & 32.984 & 33.978 & 30.747 & 33.044 & 27.377 & 34.574 \\
\hline 11/2/11 19:30 & & 31.251 & 30.823 & 29.697 & 31.06 & & 29.946 & 31.194 & 30.662 & 30.405 & 28.879 & 26.667 & 33.074 & 36.328 & 34.119 & 17.437 & 17.194 & 21.766 & 15.593 & 12.028 & 32.982 & 33.971 & 30.754 & $\begin{array}{l}73.039 \\
7\end{array}$ & 27.38 & 34.574 \\
\hline $11 / 2 / 1120: 00$ & & 31.244 & 30.827 & 29.709 & 31.06 & & 29.944 & 31.195 & 30.664 & 30.41 & 28.888 & 26.674 & 33.064 & 36.33 & 34.108 & 17.442 & 17.185 & $\quad 21.76$ & 15.595 & 12.019 & 32.98 & 33.959 & 30.758 & $3 \quad 33.029$ & 27.375 & 34.574 \\
\hline $11 / 2 / 1120: 30$ & & 31.251 & 30.837 & 29.704 & 31.07 & & 29.949 & 31.195 & 30.66 & 30.422 & 28.891 & 26.681 & 33.057 & 36.328 & 34.101 & 17.446 & 17.176 & $\quad 21.755$ & 15.597 & 12.341 & 32.984 & 33.957 & 30.758 & 33.025 & 27.38 & 34.574 \\
\hline $11 / 2 / 1121: 00$ & & 31.253 & 30.841 & 29.709 & 31.077 & & 29.96 & 31.2 & 30.664 & 30.429 & 28.898 & 26.653 & 33.048 & 36.342 & 34.091 & 17.446 & 17.169 & 21.746 & 15.593 & 12.334 & 32.982 & 33.945 & 30.768 & $3 \quad 33.013$ & 27.38 & 34.577 \\
\hline $11 / 2 / 1121: 30$ & & 31.255 & 30.853 & 29.706 & 31.081 & & 29.965 & 31.202 & 30.664 & 30.438 & 28.905 & 26.65 & 33.039 & 36.342 & 34.082 & 17.446 & 17.157 & 21.736 & 15.593 & 12.301 & 32.98 & 33.935 & 30.77 & 33.001 & 27.38 & 34.572 \\
\hline $11 / 2 / 1122: 00$ & & 31.248 & 30.851 & 29.695 & 31 . & & 29.984 & 31.204 & 30.662 & 30.44 & 28.907 & 26.662 & 33.032 & 36.333 & 34.072 & 17.446 & 17.145 & 21.732 & 15.602 & 12.287 & 32.968 & 33.926 & 30.761 & $\begin{array}{r}32.994 \\
\end{array}$ & 27.377 & 34.574 \\
\hline $22: 30$ & & 31.255 & 30.86 & & & & 29.996 & 31.202 & 30.66 & 30.447 & 28.905 & 26.662 & 33.027 & 36.349 & 34.068 & 17.451 & 17.1 & 21.729 & 15.6 & 12.277 & 32.977 & 33.926 & 30.772 & $\begin{array}{l}32.992 \\
2\end{array}$ & 27.382 & 34.574 \\
\hline $11 / 2 / 1123: 00$ & & 31.248 & 30.86 & 29.69 & 31.081 & & 30.01 & 31.204 & 30.662 & $\begin{array}{r}r 0.441 \\
30.45\end{array}$ & 28.905 & 26.658 & $\begin{array}{r}3.071 .02 \\
33.02\end{array}$ & 36.347 & 34.061 & $\begin{array}{l}17.451 \\
17.451\end{array}$ & 17.134 & 21.725 & $\begin{array}{l}15.609 \\
15.609\end{array}$ & 12.266 & 32.966 & 33.917 & 30.758 & 32.982 & 27.382 & 34.577 \\
\hline 11/2/11 23:30 & & 31.246 & 30.858 & 29.683 & 31 & & 30.017 & 31.204 & 30.66 & 30.447 & 28.907 & 26.641 & 33.01 & 36.347 & 34.051 & 17.453 & 17.127 & 21.718 & 15.616 & 99 & 59 & 33.909 & 30.751 & .975 & 7.382 & 34.574 \\
\hline $11 / 3 / 110$ & & 3 & 30.86 & 29 & 31 & & 30. & 31 & 30. & 30 & 28.902 & 9 & 33.003 & 36.344 & 4 & 53 & 17.12 & 21.715 & 23 & 9 & 2 & 33.9 & 30.744 & .968 & 7.385 & 4.577 \\
\hline $11 / 3 / 1$ & & 31 & 30.86 & 29 & 31 & & 30 & 31 & 30 & 30.4 & 2 & 2 & 32.996 & 36 & 7 & 3 & 17.113 & 21.709 & 15 & 12 & 5 & 33.895 & 30.739 & .959 & 82 & 4.574 \\
\hline $11 / 3 / 111: 00$ & & 3 & 30.855 & 29 & 31 & & 30 & 31 & & 30 & 28. & 2 & & 36 & 6 & 1 & 17.108 & 21 & 37 & & 1 & 33.886 & 30 & 95 & 82 & 4.574 \\
\hline $11 / 3 / 111: 30$ & & 31.244 & 30.855 & 29.662 & & & & & & 30.4 & 28.9 & 26. & & & 34 & 3 & 17.099 & 21.697 & 15.637 & & 4 & 33.874 & 30.718 & $\begin{array}{l}32.943 \\
\end{array}$ & .382 & 4.577 \\
\hline $11 / 3 / 112: 00$ & & 31.246 & 30.855 & 29.66 & & & & 31.212 & & 30.45 & 28.9 & 26.571 & & & & 17.453 & 17.094 & 21.697 & 15.635 & & & 33.869 & 30.718 & 32.936 & 27.385 & 34.574 \\
\hline $11 / 3 / 112: 30$ & & 31.246 & 30.853 & & & & & & & & 28.898 & 26.564 & & & & 17.453 & 17.087 & 21.692 & 15.644 & 12.198 & 32.903 & 33.86 & 30.709 & 32.926 & 7.384 & 34.574 \\
\hline 11/3/11 3:00 & & 31.244 & 30 & & & & & & & 30.447 & & 26 & 32. & & & 17.453 & 17.08 & 21. & 15 & 12.183 & 3 & 33.852 & & & 27.385 & 77 \\
\hline 11/3/11 3:30 & & 31.248 & 30. & & & & & & & 30.447 & & 26.538 & 32.5 & 36. & & 17.453 & 17.073 & 21. & & 12.172 & 32.889 & 33.843 & & & 27.387 & 79 \\
\hline $11 / 3 / 114: 0$ & & 31.2 & 30.846 & & & & & 31.211 & & 30 & 28. & 26.531 & 32 & 36. & 33. & 17.453 & 17.068 & 21. & 15.654 & 12.16 & 32.882 & 33.836 & 30.683 & 32.905 & 27.385 & 34.577 \\
\hline $11 / 3 / 114: 30$ & & 31.2 & 30.844 & & 31. & & 30. & 31.211 & & 30.4 & 28. & 26.5 & 32. & 36.3 & 33.972 & 17.453 & 17.066 & 21.667 & 15.654 & 12.148 & 32.875 & 33.829 & 30.676 & 32.898 & 27.385 & 34.577 \\
\hline $11 / 3 / 11$ 5:00 & & 31.251 & 30.848 & 29. & 31.0 & & 30.064 & 31.214 & 30.6 & 30.447 & 28.9 & 26.519 & 32.5 & 36.3 & 33.967 & 17.451 & 17.057 & 21.672 & 15.651 & 12.132 & 32.875 & 33.826 & 30.681 & 32.891 & 27.387 & 34.579 \\
\hline $11 / 3 / 11$ 5:30 & & 31.257 & 30.853 & 29.678 & 31.007 & & 30.073 & 31.214 & 30.667 & 30.454 & 28.912 & 26.502 & 32.922 & 36.356 & 33.962 & 17.451 & 17.054 & 21.667 & 15.647 & 12.099 & 32.877 & 33.822 & 30.688 & 32.886 & 27.387 & 34.579 \\
\hline $11 / 3 / 116: 00$ & & 31.255 & 30.855 & 29.683 & 31.007 & & 30.073 & 31.216 & 30.669 & 30.454 & 28.914 & 26.495 & 32.919 & 36.349 & 33.955 & 17.456 & 17.049 & 21.674 & 15.647 & 12.087 & 32.877 & 33.815 & 30.688 & 32.884 & 27.387 & 34.581 \\
\hline $11 / 3 / 116: 30$ & & 31.255 & 30.858 & 29.681 & 31.002 & & 30.073 & 31.216 & 30.669 & 30.459 & 28.914 & 26.488 & 32.912 & 36.349 & 33.953 & 17.453 & 17.047 & 21.683 & 15.654 & 12.075 & 32.875 & 33.81 & 30.681 & 32.879 & 27.387 & 34.581 \\
\hline $11 / 3 / 11$ 7:00 & & 31.257 & 30.86 & 29.697 & 31.004 & & 30.073 & 31.219 & 30.669 & 30.459 & 28.921 & 26.485 & 32.91 & 36.354 & 33.946 & 17.456 & 17.04 & 21.683 & 15.654 & 12.064 & 32.872 & 33.808 & 30.685 & 32.875 & 27.387 & 34.583 \\
\hline $11 / 3 / 117: 30$ & & 31.257 & 30.86 & 29.711 & 31.004 & & 30.064 & 31.221 & 30.669 & 30.461 & 28.93 & 26.48 & 32.905 & 36.354 & 33.941 & 17.456 & 17.035 & 21.676 & 15.654 & 12.054 & 32.872 & 33.803 & 30.692 & 32.87 & 27.387 & 34.586 \\
\hline $11 / 3 / 118: 00$ & & 31.257 & 30.86 & 29.723 & 31.004 & & 30.064 & 31.221 & 30.672 & 30.463 & 28.935 & 26.646 & 32.9 & 36.356 & 33.936 & 17.458 & 17.031 & 21.674 & 15.651 & 12.04 & 32.868 & 33.796 & 30.688 & $3 \quad 32.863$ & 27.387 & 34.584 \\
\hline $11 / 3 / 118: 30$ & & 31.267 & 30.869 & 29.718 & 31.011 & & 30.068 & 31.223 & 30.669 & 30.473 & 28.939 & 26.658 & 32.898 & 36.356 & 33.934 & 17.46 & 17.028 & 21.665 & 15.651 & 12.042 & 32.875 & 33.774 & 30.702 & 32.865 & 27.389 & 34.588 \\
\hline
\end{tabular}




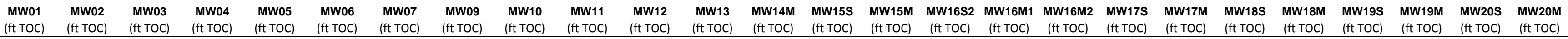

\begin{tabular}{|c|c|c|c|c|c|c|c|c|c|c|c|c|c|c|c|c|c|c|c|c|c|c|c|c|c|c|}
\hline Well & $\begin{array}{l}\text { (ft TOC) } \\
\text { (ft }\end{array}$ & $\begin{array}{l}\text { (ft TOC) } \\
\text { (f) }\end{array}$ & $\begin{array}{l}\text { (ft TOC) } \\
\text { (flocts }\end{array}$ & $\begin{array}{l}\text { (ft TOC) } \\
\text { (f }\end{array}$ & $\begin{array}{l}\text { (ft TOC) } \\
\text { (f) }\end{array}$ & $\begin{array}{l}\text { (ft TOC) } \\
\text { (f) }\end{array}$ & $\begin{array}{l}\text { (ft TOC) } \\
\text { (ft }\end{array}$ & $\begin{array}{l}\text { (ft TOC) } \\
\text { (f) }\end{array}$ & $\begin{array}{l}\text { (ft TOC) } \\
\text { (f) }\end{array}$ & $\begin{array}{l}\text { (ft TOC) } \\
\text { (f) }\end{array}$ & $\begin{array}{l}\text { (ft TOC) } \\
\text { (f) }\end{array}$ & $\begin{array}{l}\text { (ft TOC) } \\
\text { (f) }\end{array}$ & (ft TOC) & $\begin{array}{l}\text { (ft TOC) } \\
\text { (f) }\end{array}$ & (ft TOC) & $\begin{array}{l}\text { (ft TOC) } \\
\end{array}$ & $\begin{array}{l}\text { (ft TOC) } \\
\text { (f) }\end{array}$ & (ft TOC) & $\begin{array}{l}\text { (ft TOC) } \\
\text { (1) }\end{array}$ & (ft TOC) & $\begin{array}{l}\text { (ft TOC) } \\
\end{array}$ & $\begin{array}{l}\text { (ft TOC) } \\
\text { (f) }\end{array}$ & $\begin{array}{l}\text { (ft TOC) } \\
\end{array}$ & (ft TOC) & $\begin{array}{l}\text { (ft TOC) } \\
\text { (1) }\end{array}$ & (ft TOC) \\
\hline 9:00 & & 31.267 & 30.874 & 29.711 & 31.016 & & $30.07 \varepsilon$ & .22 & 30 & 30.48 & 28.9 & 26.65 & 32.896 & 36.361 & 33.5 & 17. & 17. & 21.665 & 15.6 & 12.033 & 32. & & 30.707 & 32.86 & 89 & 34.588 \\
\hline /11 9:30 & & 31.269 & 30.881 & 29.697 & 31.023 & & 30.092 & 31.226 & 30.667 & 30.489 & 28.935 & 26.641 & 32.893 & 36.363 & 33.929 & 17.463 & 17.021 & 21.662 & 15.654 & 2.026 & 32.879 & 33.741 & 30.714 & 32.86 & 7.389 & \\
\hline $11 / 3 / 11$ 10:00 & & 31.267 & 30.881 & 29.697 & 31.021 & & 30.101 & 31.228 & 30.667 & 30.487 & 28.935 & 26.629 & 32.889 & 36.358 & 33.925 & 17.465 & 17.019 & 21.655 & 15.661 & 12.033 & 32.875 & 33.736 & 30.704 & 32.853 & 7.389 & 34.593 \\
\hline 11/3/11 10:30 & & 31.262 & 30.879 & 29.683 & 31.018 & & 30.108 & 31.226 & 30.669 & 30.489 & 28.932 & 26.626 & 32.884 & 36.361 & 33.92 & 17.467 & 17.01 & 21.653 & 15.67 & 12.054 & 32.87 & 33.736 & 30.702 & 32.851 & .392 & 34.591 \\
\hline 11/3/11 11:00 & & 31.257 & 30.874 & 29.669 & 31.009 & & 30.125 & 31.223 & 30.669 & 30.482 & 28.923 & 26.614 & 32.875 & 36.363 & 33.915 & 17.465 & 17.003 & 21.644 & 15.68 & 12.075 & 32.858 & 33.746 & 30.685 & 32.844 & 27.389 & 34.593 \\
\hline 11/3/11 11:30 & & 31.262 & 30.874 & 29.66 & 31.004 & & 30.137 & 31.223 & 30.669 & 30.482 & 28.918 & 26.6 & 32.872 & 36.363 & 33.911 & 17.465 & 17.003 & 21.644 & 15.684 & 12.082 & 32.856 & 33.755 & 30.685 & 32.835 & 27.389 & 34.59 \\
\hline 11/3/11 12:00 & & 31.255 & 30.869 & 29.648 & 30.997 & & 30.151 & 31.224 & 30.669 & 30.48 & 28.914 & 26.588 & 32.868 & 36.358 & 33.901 & 17.465 & 16.993 & 21.637 & 15.698 & 12.096 & 32.844 & 33.784 & 30.669 & 32.83 & 27.392 & 34.591 \\
\hline 11/3/11 12:30 & & 31.253 & 30.862 & 29.636 & 30.986 & & 30.158 & 31.223 & 30.667 & 30.475 & 28.907 & 26.574 & 32.856 & 36.358 & 33.894 & 17.463 & 16.989 & 21.632 & 15.71 & 12.118 & 32.835 & 33.808 & 30.645 & 32.825 & 27.392 & 34.591 \\
\hline 11/3/11 13:00 & & 31.253 & 30.855 & 29.62 & 30.976 & & 30.17 & 31.218 & 30.664 & 30.47 & 28.9 & 26.562 & 32.851 & 36.356 & 33.885 & $\quad 17.46$ & 16.982 & 21.621 & 15.717 & 12.132 & 32.821 & 33.831 & 30.643 & 32.814 & 27.394 & 34.588 \\
\hline 11/3/11 13:30 & & 31.251 & 30.851 & 29.606 & 30.965 & & 30.181 & 31.216 & 30.664 & 30.463 & 28.891 & 26.545 & 32.842 & 36.354 & 33.88 & 17.46 & 16.975 & 21.623 & 15.724 & 12.146 & 32.809 & 33.85 & 30.631 & 32.807 & 27.392 & 34.588 \\
\hline $11 / 3 / 11$ 14:00 & & 31.25 & 30.844 & 29.597 & 30.953 & & 30.186 & 31.214 & 30.667 & 30.459 & 28.886 & 26.531 & 32.835 & 36.351 & 33.871 & 17.456 & 16.97 & 21.618 & 15.731 & 12.16 & 32.798 & 33.857 & 30.62 & 32.799 & 27.392 & 34.586 \\
\hline $11 / 3 / 11$ 14:30 & & 31.246 & 30.834 & 29.592 & 30.939 & & 30.188 & 31.209 & 30.664 & 30.452 & 28.881 & 26.521 & 32.826 & 36.347 & 33.861 & 17.456 & 16.963 & 21.614 & 15.738 & 12.174 & 32.784 & 33.725 & 30.603 & 32.788 & 27.394 & 34.586 \\
\hline $11 / 3 / 1115: 00$ & & 31.244 & 30.825 & 29.589 & 30.923 & & 30.191 & 31.206 & 30.667 & 30.445 & 28.877 & 26.514 & 32.814 & 36.34 & 33.852 & 17.451 & 16.956 & 21.607 & 15.743 & 12.186 & 32.768 & 33.715 & 30.589 & 32.778 & 27.394 & 34.584 \\
\hline $11 / 3 / 1115: 30$ & & 31.243 & 30.818 & 29.585 & 30.911 & & 30.191 & 31.207 & 30.667 & 30.438 & 28.872 & 26.514 & 32.807 & 36.342 & 33.845 & 17.449 & 16.954 & 21.6 & 15.748 & 12.193 & 32.763 & 33.703 & 30.582 & 32.774 & 27.394 & 34.584 \\
\hline 11/3/11 16:00 & & 31.244 & 30.816 & 29.58 & 30.899 & & 30.188 & 31.204 & 30.667 & 30.433 & 28.87 & 26.509 & 32.798 & 36.337 & 33.836 & 17.449 & 16.944 & 21.598 & 15.752 & 12.195 & 32.756 & 33.699 & 30.57 & 32.762 & 27.392 & 34.584 \\
\hline $11 / 3 / 1116: 30$ & & 31.246 & 30.811 & 29.592 & 30.89 & & 30.179 & 31.204 & 30.669 & 30.429 & 28.87 & 26.507 & 32.793 & 36.335 & 33.831 & 17.446 & 16.94 & 21.632 & 15.752 & 12.191 & 32.747 & 33.691 & 30.568 & 32.76 & 27.394 & 34.584 \\
\hline 11/3/11 17:00 & & 31.244 & 30.809 & 29.594 & 30.883 & & 30.167 & 31.204 & 30.669 & 30.426 & 28.87 & 26.516 & 32.786 & 36.335 & 33.824 & 17.444 & 16.937 & 21.644 & 15.755 & 12.193 & 32.74 & 33.684 & 30.556 & 32.753 & 27.394 & 34.584 \\
\hline $11 / 3 / 1117: 30$ & & 31.244 & 30.802 & 29.599 & 30.876 & & 30.163 & 31.202 & 30.672 & 30.422 & 28.87 & 26.521 & 32.781 & 36.333 & 33.817 & 17.444 & 16.933 & 21.639 & 15.755 & 12.186 & 32.737 & 33.68 & 30.554 & 32.746 & 27.394 & 34.584 \\
\hline $11 / 3 / 1118: 00$ & & 31.248 & 30.806 & 29.601 & 30.878 & & 30.155 & 31.202 & 30.667 & 30.419 & 28.87 & 26.524 & 32.779 & 36.335 & 33.815 & 17.444 & 16.928 & 21.635 & 15.748 & 12.167 & 32.74 & 33.675 & 30.561 & 32.741 & 27.396 & 34.584 \\
\hline $11 / 3$ & & 31.248 & 30.806 & 29.608 & 30. & & 30.146 & 31.204 & 30.667 & 30.422 & 28.87 & 26.526 & 32.774 & 36.335 & 33.81 & 17.444 & 16.926 & 21.628 & 15.75 & 12.153 & 32.74 & 33.672 & .561 & 32.738 & 27.394 & 34.586 \\
\hline $11 /$ & & 31.248 & 30.806 & 29.601 & 30. & & 30.146 & 31.204 & 30.667 & 30.417 & 28.867 & 26.535 & 32.769 & 36.335 & 33.803 & 17.444 & 16.921 & 21.635 & 15.75 & 12.132 & 32.737 & 33.668 & .563 & 32.734 & 27.396 & 34.586 \\
\hline $11 / 3 / 1$ & & 31.248 & 30.806 & 29.597 & 30.874 & & 30.148 & 31.202 & 30.664 & 30.417 & 28.865 & 26.528 & 32.762 & 36.335 & 33.8 & 17.444 & 16.916 & 21.635 & 15.745 & 12.108 & 32.735 & .663 & .561 & 2.727 & 27.396 & 34.586 \\
\hline $11 / 3 / 11$ & & 31.25 & 30.806 & 29.599 & 30.874 & & 30.139 & 31.202 & 30.669 & 30.419 & 28.865 & 26.526 & 32.76 & 36.342 & 33.796 & 17.444 & 16.914 & 21.63 & 15.745 & 12.089 & 32.732 & 33.661 & .563 & 32.727 & 27.396 & 34.588 \\
\hline 11/3/11 20:30 & & 31.248 & 30.804 & 29.59 & 30.871 & & 48 & 31.202 & 30.669 & 30.412 & 28.86 & 26.528 & 32.755 & 36.335 & 33.791 & 17.444 & 16.909 & 21.628 & 15.743 & 12.078 & 32.728 & 33.658 & .559 & 32.72 & 27.396 & 34.586 \\
\hline 11/3/11 21:00 & & 31.25 & 30.802 & 29.59 & 30.869 & & 30.148 & 31.199 & 30.667 & 30.41 & 28.856 & 26.514 & 32.753 & 36.337 & 33.786 & 17.444 & 16.907 & 21.621 & 15.741 & 12.064 & 32.723 & 33.651 & 30.556 & 32.717 & 27.396 & 34.588 \\
\hline 11/3/11 21:30 & & 31.246 & 30.799 & 29.599 & 30.862 & & 30.141 & 31.202 & 30.672 & 30.41 & 28.858 & 26.514 & 32.748 & 36.335 & 33.782 & 17.442 & 16.902 & 21.616 & .745 & 12.056 & 32.714 & 33.646 & 549 & 32.713 & 27.396 & 34.588 \\
\hline 11/3/11 22:00 & & 31.248 & 30.797 & 29.608 & 30.86 & & 30.13 & 31.202 & 30.674 & 30.405 & 28.86 & 26.526 & 32.741 & 36.337 & 33.777 & 17.442 & 16.897 & 21.609 & 15.741 & 12.047 & 32.716 & 33.642 & 30.547 & 32.706 & 27.396 & 34.588 \\
\hline 11/3/11 22:30 & & 31.248 & 30.792 & 29.608 & 30.855 & & 30.127 & 31.202 & 30.674 & 30.403 & 28.863 & 26.535 & 32.737 & 36.337 & 33.77 & 17.439 & 16.893 & 21.6 & 15.738 & 12.033 & 32.709 & 33.637 & 30.547 & 32.703 & 27.399 & 34.586 \\
\hline 11/3/11 23:00 & & 31.253 & 30.795 & 29.604 & 30.857 & & 30.13 & 31.202 & 30.669 & 30.403 & 28.86 & 26.535 & 32.734 & 36.344 & 33.77 & 17.439 & 16.891 & 21.6 & 15.727 & 12.005 & 32.714 & 33.632 & 30.552 & 32.699 & 27.399 & 34.588 \\
\hline $11 / 3 / 1123: 30$ & & 31.253 & 30.797 & 29.599 & 30.859 & & 30.13 & 31.204 & 30.669 & 30.405 & 28.858 & 26.533 & 32.727 & 36.337 & 33.763 & 17.442 & 16.886 & 21.6 & 15.727 & 11.991 & 32.709 & 33.632 & 30.556 & 32.696 & 27.399 & 34.588 \\
\hline $11 / 4 / 110: 00$ & & 31.248 & 30.795 & 29.592 & 30.855 & & 30.132 & 31.202 & 30.672 & 30.403 & 28.856 & 26.526 & 32.727 & 36.337 & 33.758 & 17.442 & 16.881 & 21.593 & 15.734 & 11.979 & 32.709 & 33.625 & 30.547 & 32.694 & 27.399 & 34.588 \\
\hline $11 / 4 / 110: 30$ & & 31.248 & 30.795 & 29.585 & 30.857 & & 30.141 & 31.2 & 30.667 & 30.401 & 28.849 & 26.521 & 32.72 & 36.342 & 33.756 & 17.442 & 16.877 & 21.591 & 15.729 & 11.977 & 32.707 & 33.623 & 30.549 & 32.689 & 27.399 & 34.588 \\
\hline 11/4/11 1:00 & & 31.248 & 30.79 & 29.578 & 30.85 & & 30.144 & 31.2 & 30.669 & 30.399 & 28.849 & 26.516 & 32.716 & 36.342 & 33.751 & 17.439 & 16.872 & 21.586 & 15.731 & 11.972 & 32.698 & 33.613 & 30.542 & 32.682 & 27.399 & 34.586 \\
\hline $11 / 4 / 111: 30$ & & 31.244 & 30.785 & 29.575 & 30.843 & & 30.146 & 31.199 & 30.672 & 30.394 & 28.842 & 26.512 & 32.711 & 36.344 & 33.747 & 17.437 & 16.87 & 21.582 & 15.738 & 11.97 & 32.695 & 33.611 & 30.535 & 32.677 & 27.399 & 34.586 \\
\hline $11 / 4 / 112: 00$ & & 31 & 30.781 & 29.564 & $\begin{array}{l}30.8 \\
30.8\end{array}$ & & 30 & 31.197 & 30.669 & 30.389 & 28.839 & 26.504 & 32.706 & 36.337 & 33.74 & 17.4 & 16.863 & 21.575 & 15.734 & 11.962 & 32.688 & 33.604 & 1.528 & 32.67 & 27.399 & 34.586 \\
\hline $11 / 4 / 112: 30$ & & & 30.778 & 29.566 & 30. & & 30.146 & 31.195 & 30.672 & 30.387 & 28.837 & 26.495 & 32.699 & 36.34 & 33.737 & 17.435 & $\begin{array}{l}16.058 \\
\end{array}$ & 21.568 & 15.736 & $\begin{array}{l}11.965 \\
11.965\end{array}$ & 32.677 & $\begin{array}{l}33.599 \\
3399\end{array}$ & 0.521 & 32.666 & 27.399 & 34.584 \\
\hline $11 / 4 / 113: 00$ & & 31.239 & 30.771 & 29.569 & 30. & & 30.141 & 31.195 & 30.667 & $\begin{array}{l}30.382 \\
30.01\end{array}$ & $\begin{array}{l}28.831 \\
28.835\end{array}$ & 26.502 & 32.695 & 36.337 & 33.73 & 17.435 & $\begin{array}{l}10.000 \\
16.865\end{array}$ & 21.563 & 15.736 & 11.96 & 32.672 & $\begin{array}{l}33.592 \\
3.592\end{array}$ & 0.512 & 32.663 & 27.399 & 34.584 \\
\hline $11 / 4 / 113: 30$ & & 31 . & 30.769 & 29.561 & 30. & & 30.144 & 31.192 & 30.669 & $\begin{array}{l}30.378 \\
30.378\end{array}$ & 28.83 & 26.5 & 32.69 & 36.335 & 33.723 & 17.435 & $\begin{array}{l}10.0056 \\
16.856\end{array}$ & 21.556 & 15.731 & $\begin{array}{l}11.953 \\
1.953\end{array}$ & 32.665 & 33.587 & .514 & 2.656 & 27.399 & 34.584 \\
\hline & & 31 & 30 & 29.55 & 30 & & 30 & 3. & 30 & 30 & 28 & 26.495 & 32 & 36 & 33.718 & 2 & 46 & 21 & 15.731 & 11.944 & 32.663 & 33.582 & 77 & 2 & 27.399 & 34.581 \\
\hline & & & 30. & 29.554 & 30 & & $3 c$ & & & 30 & 2 & 26.488 & 32.683 & 36 & 33.714 & 17 & 16 & 21 & 15.731 & 11.937 & 32.663 & 33.58 & 22 & 17 & 27.399 & 34.581 \\
\hline & & & & 29 & & & & 31 & & & 28 & & 32 & 36.3 & 22700 & 17. & 16 & 21 & 15.7 & 11.934 & 32.656 & 75 & 7 & 12 & 1 & 34.581 \\
\hline $11 /$ & & & & & & & & 31. & & & & & & s & & & & 21 & & 11.934 & 32. & 771 & 30.493 & 5 & 01 & 34.581 \\
\hline $11 / 4 / 116: 00$ & & 31 & 30. & 29.564 & & & 30 & 31.1 & & & & 26.495 & & 36.3 & 33. & 17 & & & & 11.92 & 32. & & & 3 & (7) & 34.584 \\
\hline $11 / 4 / 116: 30$ & & & 30.7 & 29. & & & & 31 & & & & 26.5 & & 36.3 & 33. & 17.43 & 16.799 & 21. & 15.7 & 11.906 & 32.646 & 33.563 & & 8 & 27.401 & 34.584 \\
\hline $11 / 4 / 117$ & & & & 29. & & & & 31.1 & & & & 26. & & 36.3 & & 17 & 16.79 & 21.542 & 15. & 11.897 & 32.649 & & & 26 & 27.401 & 34.584 \\
\hline 11/4/11 7:30 & & & & 29. & & & & & & & & & & 36.3 & 33 & 17 & 16.781 & & 15. & 11.885 & 32.649 & & & 32.626 & 27.401 & 34.584 \\
\hline $11 / 4 / 118: 00$ & & 31. & 30. & 29.578 & & & 30. & 31 & 30. & & 28. & & 32.655 & 36.3 & 33.683 & 17.428 & 16.771 & 21.535 & 15.7 & 11.883 & 32.644 & 33.554 & 30.493 & 32.621 & 27.401 & 34.584 \\
\hline 11/4/11 8: & & 31.2. & 30.7 & 29.571 & & & 30 & 31.1. & $30.6 \mathrm{r}-\mathrm{r}$ & & $28.8 \mathrm{Y}-\mathrm{Y}$ & 26. & 32.652 & 36.335 & 33.686 & 17.43 & 16.76 & 21.533 & $15.7 \mathrm{r}-\mathrm{c}$ & 11.866 & 32.649 & 33.554 & 30.495 & 32.619 & 27.403 & 34.586 \\
\hline 11/4/11 9:00 & & 31.241 & 30.755 & 29.561 & 30. & & 30.127 & 31.19 & 30.6 & 30. & 28.823 & 26.509 & 32.65 & 36.333 & 33.683 & 17.428 & 16.764 & 21.5 & 15.7 & 11.873 & 32.646 & 33.552 & 30.502 & 32.616 & 27.403 & 34.586 \\
\hline $11 / 4 / 119: 30$ & & 31.241 & 30.757 & 29.554 & 30.8 & & 30.137 & 31.188 & 30.669 & 30.359 & 28.818 & 26.502 & 32.65 & 36.34 & 33.683 & 17.432 & 16.771 & 21.533 & 15.712 & 11.873 & 32.649 & 33.547 & 30.505 & 32.614 & 27.403 & 34.588 \\
\hline 11/4/11 10:00 & & 31.236 & 30.75 & 29.545 & 30.801 & & 30.144 & 31.185 & 30.667 & 30.354 & 28.814 & 26.495 & 32.645 & 36.34 & 33.676 & 17.428 & 16.802 & 21.526 & 15.722 & 11.894 & 32.646 & 33.542 & 30.493 & 32.609 & 27.403 & 34.586 \\
\hline 11/4/11 10:30 & & 31.234 & 30.746 & 29.538 & 30.794 & & 30.146 & 31.183 & 30.672 & 30.35 & 28.812 & 26.485 & 32.638 & 36.335 & 33.672 & 17.428 & 16.846 & 21.526 & 15.729 & 11.92 & 32.632 & 33.54 & 30.488 & 32.602 & 27.403 & 34.586 \\
\hline 11/4/11 11:00 & & 31.232 & 30.739 & 29.526 & 30.787 & & 30.151 & 31.18 & 30.667 & 30.345 & 28.802 & 26.483 & 32.634 & 36.335 & 33.667 & 17.425 & 16.879 & 21.521 & 15.738 & 11.941 & 32.625 & 33.533 & 30.481 & 32.6 & 27.403 & 34.586 \\
\hline 11/4/11 11:30 & & 31.23 & 30.734 & 29.512 & 30.783 & & 30.16 & 31.178 & 30.664 & 30.34 & 28.795 & 26.468 & 32.627 & 36.337 & 33.662 & 17.428 & 16.904 & 21.514 & 15.738 & 11.953 & 32.618 & 33.526 & 30.472 & 32.593 & 27.403 & 34.586 \\
\hline $11 / 4 / 1112: 00$ & & 31.227 & 30.732 & 29.501 & 30.778 & & 30.172 & 31.175 & 30.664 & 30.336 & 28.786 & 26.457 & 32.622 & 36.333 & 33.657 & 17.425 & 16.935 & 21.512 & 15.748 & 11.97 & 32.614 & 33.521 & 30.465 & 32.588 & 27.403 & 34.586 \\
\hline $11 / 4 / 1112: 30$ & & 31.225 & 30.722 & 29.484 & 30.768 & & 30.174 & 31.171 & 30.66 & 30.329 & 28.781 & 26.445 & 32.615 & 36.33 & 33.65 & 17.423 & 16.963 & 21.501 & 15.752 & 11.988 & 32.604 & 33.516 & 30.453 & 32.579 & 27.403 & 3.584 \\
\hline
\end{tabular}




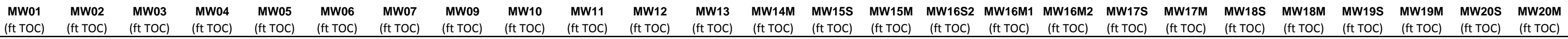

\begin{tabular}{|c|c|c|c|c|c|c|c|c|c|c|c|c|c|c|c|c|c|c|c|c|c|c|c|c|c|c|}
\hline Well & $\begin{array}{l}\text { MW01 } \\
\text { (ft TOC) }\end{array}$ & $\begin{array}{l}\text { MW02 } \\
\text { (ft TOC) }\end{array}$ & $\begin{array}{l}\text { MW03 } \\
\text { (ft TOC) }\end{array}$ & $\begin{array}{r}\text { MW04 } \\
\text { (ft TOC) }\end{array}$ & $\begin{array}{l}\text { MW05 } \\
\text { (ft TOC) }\end{array}$ & $\begin{array}{r}\text { MW06 } \\
\text { (ft TOC) }\end{array}$ & $\begin{array}{l}\text { MW07 } \\
\text { (ft TOC) }\end{array}$ & $\begin{array}{l}\text { MW09 } \\
\text { (ft TOC) }\end{array}$ & $\begin{array}{l}\text { MW10 } \\
\text { (ft TOC) }\end{array}$ & $\begin{array}{l}\text { MW11 } \\
\text { (ft TOC) }\end{array}$ & $\begin{array}{l}\mathrm{MW12} \\
(\mathrm{ft} \text { TOC) }\end{array}$ & $\begin{array}{l}\text { MW13 } \\
\text { (ft TOC) }\end{array}$ & $\begin{array}{l}\text { MW14M } \\
(\mathrm{ft} \text { TOC) }\end{array}$ & $\begin{array}{l}\text { MWW15S } \\
\text { (ft TOC) }\end{array}$ & $\begin{array}{l}\text { MWW15M } \\
(\mathrm{ft} \text { TOC) }\end{array}$ & 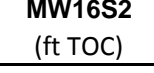 & $\begin{array}{c}\text { MW16M1 } \\
\text { (ft TOC) }\end{array}$ & $\begin{array}{l}\begin{array}{l}\text { MW16M2 } \\
\text { (ft TOC) }\end{array} \\
\end{array}$ & $\begin{array}{l}\text { MW17S } \\
\text { (ft TOC) }\end{array}$ & $\begin{array}{l}\text { MW17M } \\
\text { (ft TOC) }\end{array}$ & $\begin{array}{l}\text { MWW18S } \\
\text { (ft TOC) }\end{array}$ & $\begin{array}{l}\text { MW18M } \\
\text { (ft TOC) }\end{array}$ & $\begin{array}{l}\text { MWW19S } \\
\text { (ft TOC) }\end{array}$ & $\begin{array}{l}\text { MW19M } \\
\text { (ft TOC) }\end{array}$ & $\begin{array}{l}\mathrm{MW} 20 \mathrm{~S} \\
(\mathrm{ft} \text { TOC) }\end{array}$ & $\begin{array}{l}\text { MW20M } \\
\text { (ft TOC) }\end{array}$ \\
\hline 11/4/11 13:00 & & 31.22 & 30.711 & 29.473 & 30.757 & & 30.181 & 31.168 & 30.662 & 30.322 & 28.77 & 26.433 & 32.608 & 36.33 & 33.643 & 17.421 & 16.998 & 21.501 & 15.757 & 12.009 & 32.59 & 33.509 & 30.436 & 32.574 & 27.403 & 34.581 \\
\hline 11/4/11 13:30 & & 31.218 & 30.701 & 29.461 & 30.745 & & 30.186 & 31.164 & 30.66 & 30.315 & 28.758 & 26.418 & 32.601 & 36.321 & 33.639 & $\begin{array}{l}9 \\
9\end{array} 17.418$ & 17.019 & 21.496 & 15.759 & 12.026 & 32.576 & 33.504 & 30.427 & 32.567 & 27.403 & 34.581 \\
\hline 11/4/11 14:00 & & 31.213 & 30.69 & 29.456 & 30.733 & & 30.186 & 31.161 & 30.657 & 30.308 & 28.756 & 26.411 & 32.594 & 36.314 & 33.629 & $\begin{array}{l}9 \\
9\end{array}$ & 17.045 & 21.489 & 15.766 & 12.042 & 32.572 & 33.497 & 30.411 & 32.558 & 27.403 & 34.577 \\
\hline 11/4/11 14:30 & & 31.209 & 30.678 & 29.44 & 30.722 & & 30.188 & 31.154 & 30.655 & 30.299 & 28.746 & 26.406 & 32.587 & 36.319 & 33.622 & $2 \quad 17.411$ & 17.068 & 21.484 & 15.776 & 12.054 & 32.558 & 33.485 & 30.401 & 32.553 & 27.403 & 34.579 \\
\hline $11 / 4 / 11$ 15:00 & & 31.208 & 30.671 & 29.431 & 30.71 & & 30.188 & 31.152 & 30.653 & 30.289 & 28.739 & 26.39 & 32.578 & 36.311 & 33.613 & $\begin{array}{ll}3 & 17.411\end{array}$ & 17.082 & 21.482 & 15.773 & 12.064 & 32.548 & 33.476 & 30.392 & 32.544 & 27.403 & 34.574 \\
\hline $11 / 4 / 11$ 15:30 & & 31.206 & 30.662 & 29.424 & 30.701 & & 30.186 & 31.149 & 30.655 & 30.285 & 28.73 & 26.382 & 32.571 & 36.312 & 33.608 & $\begin{array}{l}8 \quad 17.409 \\
8\end{array}$ & $\begin{array}{l}17.094 \\
\end{array}$ & 21.475 & 15.778 & 12.071 & 32.541 & $\begin{array}{l}33.471 \\
331\end{array}$ & 30.382 & 32.539 & 27.406 & 34.574 \\
\hline $11 / 4 / 11$ 16:00 & & 31.202 & 30.645 & 29.424 & 30.687 & & 30.181 & 31.147 & 30.65 & 30.275 & 28.725 & 26.38 & 32.564 & 36.305 & 33.599 & $\begin{array}{l}9 \\
9\end{array} 17.404$ & 17.113 & 21.477 & 15.783 & 12.082 & 32.527 & 33.464 & 30.368 & 32.53 & 27.406 & 34.572 \\
\hline $11 / 4 / 1116: 30$ & & 31.199 & 30.641 & 29.421 & 30.677 & & 30.172 & 31.145 & 30.655 & 30.269 & 28.721 & 26.378 & 32.557 & 36.297 & 33.594 & 17.4 & 17.115 & 21.475 & 15.778 & 12.082 & 32.52 & 33.457 & 30.364 & 32.523 & 27.406 & 34.57 \\
\hline $11 / 4 / 11$ 17:00 & & 31.197 & $\begin{array}{l}30.631 \\
30.636\end{array}$ & $\begin{array}{l}29.426 \\
29.426\end{array}$ & $\begin{array}{l}30.668 \\
30.17\end{array}$ & & $\begin{array}{l}30.172 \\
30.16\end{array}$ & 31.142 & $\begin{array}{l}30.035 \\
30.655\end{array}$ & $\begin{array}{l}30.269 \\
30.262\end{array}$ & 28.723 & $\begin{array}{l}20.378 \\
26.375\end{array}$ & $\begin{array}{l}32.55 \\
32.55\end{array}$ & $\begin{array}{l}30.291 \\
36.302\end{array}$ & $\begin{array}{l}33.587 \\
33.58\end{array}$ & $\begin{array}{l}17.4 \\
17.4\end{array}$ & 17.115 & 21.475 & $\begin{array}{l}15.776 \\
15.776\end{array}$ & $\begin{array}{l}12.082 \\
12.082\end{array}$ & $\begin{array}{l}32.52 \\
32.516\end{array}$ & 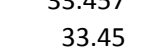 & $\begin{array}{l}30.54 \\
30.356\end{array}$ & $\begin{array}{l}32.516 \\
32.516\end{array}$ & 27.403 & $\begin{array}{l}34.51 \\
34.57\end{array}$ \\
\hline $11 / 4 / 11$ 17:30 & & 31.199 & 30.636 & 29.424 & 30.663 & & 30.155 & 31.137 & 30.653 & 30.259 & 28.716 & 26.385 & 32.545 & 36.295 & 33.582 & $2 \quad 17.397$ & 17.108 & 21.482 & 15.771 & 12.073 & 32.511 & 33.445 & 30.352 & 32.509 & 27.406 & 34.569 \\
\hline $11 / 4 / 1118: 00$ & & 31.197 & 30.627 & 29.435 & 30.656 & & 30.137 & 31.135 & 30.657 & 30.252 & 28.723 & 26.382 & 32.54 & 36.297 & 33.575 & $5 \quad 17.395$ & 17.092 & 21.487 & 15.769 & 12.066 & 32.506 & 33.44 & 30.349 & 32.506 & 27.406 & 34.57 \\
\hline $11 / 4 / 1118: 30$ & & 31.195 & 30.627 & 29.438 & 30.654 & & 30.125 & 31.137 & 30.655 & 30.252 & 28.721 & 26.397 & 32.538 & 36.295 & 33.571 & $1 \quad 17.395$ & 17.078 & 21.487 & 15.769 & 12.061 & 32.502 & 33.438 & 30.345 & 32.502 & 27.406 & 34.57 \\
\hline 11/4/11 19:00 & & 31.192 & 30.62 & 29.438 & 30.649 & & 30.115 & 31.133 & 30.65 & 30.248 & 28.721 & 26.401 & 32.531 & 36.293 & 33.566 & $\begin{array}{ll}6 & 17.393\end{array}$ & 17.049 & 21.489 & 15.762 & 12.045 & 32.502 & 33.431 & 30.349 & 32.497 & 27.406 & 34.57 \\
\hline $11 / 4 / 11$ 19:30 & & 31.197 & 30.622 & 29.435 & 30.654 & & 30.113 & 31.132 & 30.65 & 30.245 & 28.716 & 26.404 & 32.531 & 36.293 & 33.564 & 17.393 & 17.014 & 21.487 & 15.755 & 12.019 & 32.506 & 33.433 & 30.354 & .494 & 27.406 & 34.572 \\
\hline $11 / 4 / 1120: 00$ & & 31.197 & 30.627 & 29.442 & 30.656 & & 30.106 & 31.13 & 30.648 & 30.245 & 28.718 & 26.404 & 32.528 & 36.295 & 33.564 & 17.393 & 16.984 & 21.484 & 15.748 & 11.998 & 32.509 & 33.428 & 30.364 & 32.497 & 27.408 & 34.574 \\
\hline 11/4/11 20:30 & & 31. & 30.624 & 29.438 & 30.654 & & 30.099 & 31.13 & 30.648 & 30.243 & 28.716 & 26.409 & 32.526 & 36.293 & 33.559 & 17.39 & 16.968 & 21.482 & 15.748 & 11.989 & 32.509 & 33.426 & 30.359 & 32.492 & 27.408 & 34.572 \\
\hline 11/4/11 21:00 & & 31.192 & 30.622 & 29.442 & 30.654 & & 30.092 & 31.13 & 30.65 & 30.241 & 28.716 & 26.411 & 32.524 & 36.295 & 33.559 & 17.393 & 16.947 & 21.48 & 15.741 & 11.974 & 32.506 & 33.424 & 0.356 & 32.49 & 27.408 & 34.572 \\
\hline 11/4/11 21:30 & & 31.192 & 30.622 & 29.44 & 30.656 & & 30.087 & 31.127 & 30.65 & 30.238 & 28.716 & 26.413 & 32.521 & 36.293 & 33.554 & 17.39 & 16.921 & 21.48 & 15.736 & 11.955 & 32.504 & 33.419 & .361 & 32.487 & 27.408 & 34.572 \\
\hline $11 / 4 / 1122: 00$ & & 31.192 & 30.62 & 29.44 & 30.659 & & 30.083 & 31.127 & 30.65 & 30.236 & 28.714 & 26.413 & 32.521 & 36.295 & 33.552 & 17.39 & 16.898 & 21.48 & 15.731 & 11.941 & 32.509 & 33.417 & 30.361 & 32.485 & 27.408 & 34.574 \\
\hline $11 / 4 / 1122: 30$ & & 31.19 & 30.62 & 29.428 & 30.659 & & 30.085 & 31.125 & 30.648 & 30.234 & 28.709 & 26.413 & 32.517 & 36.295 & 33.547 & 17.39 & 16.879 & 21.475 & 15.729 & 11.927 & 32.506 & 33.414 & 30.359 & 32.483 & 27.408 & 34.574 \\
\hline $11 / 4 / 1123: 00$ & & 31.188 & 30.617 & 29.424 & 30.67 & & 30.092 & 31.123 & 30.638 & 30.231 & 28.705 & 26.406 & 32.512 & 36.297 & 33.545 & 17.39 & 16.863 & 21.471 & 15.727 & 11.915 & 32.499 & 33.412 & 30.364 & 32.478 & 27.408 & 34.574 \\
\hline $11 / 4 / 1123: 30$ & & 31.185 & 30.617 & 29.417 & 30.666 & & 30.085 & 31.118 & 30.643 & 30.231 & 28.702 & 26.399 & 32.51 & 36.293 & 33.543 & $\quad 17.388$ & 16.849 & 21.471 & 15.727 & 11.908 & 32.499 & 33.407 & 30.356 & 32.476 & 27.408 & 34.574 \\
\hline $11 / 5 / 110: 00$ & & 31.181 & 30.61 & 29.416 & 30.656 & & 30.083 & 31.118 & 30.648 & 30.224 & 28.7 & 26.397 & 32.505 & 36.295 & 33.538 & $\begin{array}{l}37.388 \\
3\end{array}$ & 16.846 & 21.466 & 15.724 & 11.906 & 32.488 & 33.405 & 30.345 & 32.471 & 27.408 & 34.574 \\
\hline $11 / 5 / 110: 30$ & & 31.181 & 30.608 & 29.414 & 30.654 & & 30.083 & 31.116 & 30.645 & 30.222 & 28.695 & 26.399 & 32.498 & 36.295 & 33.531 & 17.386 & 16.832 & 21.461 & 15.719 & 11.894 & 32.492 & 33.398 & 30.345 & 32.464 & 27.408 & 34.572 \\
\hline 11/5/11 1:00 & & 31.181 & 30.608 & 29.412 & 30.647 & & 30.08 & 31.116 & 30.645 & 30.217 & 28.693 & 26.394 & 32.493 & 36.293 & 33.528 & 17.386 & 16.82 & 21.457 & 15.712 & $\begin{array}{l}11.89 \\
11.89\end{array}$ & 32.485 & & 30.342 & 32.462 & 27.41 & 34.57 \\
\hline 11/5/11 1:30 & & 31.178 & 30.601 & 29.405 & $\begin{array}{l}30.642 \\
30.62\end{array}$ & & 30.08 & 31.113 & 30.643 & 30.215 & 28.691 & 26.397 & 32.491 & 36.29 & $\begin{array}{l}33.524 \\
33.524\end{array}$ & 17.384 & $\begin{array}{r}16.02 \\
16.809\end{array}$ & 21.45 & 15.712 & 11.878 & 32.481 & $\begin{array}{ll}33.39 \\
33.59\end{array}$ & 30.338 & 32.459 & 27.408 & 34.567 \\
\hline $11 / 5 / 112: 00$ & & $\begin{array}{l}31.178 \\
31.178\end{array}$ & 30.599 & 29.407 & $\begin{array}{l}3.046 \\
30.64\end{array}$ & & $\begin{array}{r}30.000 \\
30.078\end{array}$ & 31.111 & $\begin{array}{l}30.645 \\
30.645\end{array}$ & $\begin{array}{l}30.21 \\
30.21\end{array}$ & $\begin{array}{l}28.688 \\
28.08\end{array}$ & 26.39 & $\begin{array}{l}32.481 \\
32.486\end{array}$ & $\begin{array}{l}36.289 \\
36.28\end{array}$ & $\begin{array}{l}33.524 \\
33.519\end{array}$ & 17.384 & 16.799 & $\begin{array}{l}21.43 \\
21.45\end{array}$ & 15.71 & $\begin{array}{l}11.010 \\
11.868\end{array}$ & 32.476 & $\begin{array}{l}33.396 \\
33.386\end{array}$ & $\begin{array}{l}30.300 \\
30.335\end{array}$ & $\begin{array}{l}32.452 \\
32.452\end{array}$ & $\begin{array}{r}27.400 \\
27.41\end{array}$ & 34.567 \\
\hline $11 / 5 / 112: 30$ & & 31.176 & 30.596 & 29.403 & $\begin{array}{r}30.64 \\
30.635 \\
-\end{array}$ & & $\begin{array}{l}30.073 \\
30.073\end{array}$ & 31.111 & $\begin{array}{l}30.045 \\
30.641\end{array}$ & $\begin{array}{r}30.21 \\
30.208\end{array}$ & 28.686 & $\begin{array}{l}26.39 \\
26.392\end{array}$ & $\begin{array}{l}32.480 \\
32.479\end{array}$ & $\begin{array}{r}50.280 \\
36.29\end{array}$ & $\begin{array}{l}33.519 \\
33.517\end{array}$ & 17.381 & 16.792 & $\begin{array}{l}21.447 \\
21.447\end{array}$ & $\begin{array}{l}15.711 \\
15.708\end{array}$ & $\begin{array}{l}11.800 \\
11.861\end{array}$ & 32.471 & $\begin{array}{l}33.300 \\
33.381\end{array}$ & $\begin{array}{l}30.33 \\
30.331\end{array}$ & $\begin{array}{l}32.452 \\
32.448\end{array}$ & 27.41 & 34.567 \\
\hline $11 / 5 / 11$ 3:00 & & $\begin{array}{l}31.176 \\
31.174\end{array}$ & $\begin{array}{l}30.596 \\
30.594\end{array}$ & $\begin{array}{l}29.4353 \\
29.393\end{array}$ & $\begin{array}{l}30.633 \\
30.633\end{array}$ & & $\begin{array}{l}30.076 \\
30.076\end{array}$ & 31.109 & $\begin{array}{l}30.641 \\
30.641\end{array}$ & $\begin{array}{l}30.208 \\
30.201\end{array}$ & $\begin{array}{l}28.680 \\
28.681\end{array}$ & $\begin{array}{l}\begin{array}{l}20.392 \\
26.39\end{array} \\
2\end{array}$ & $\begin{array}{l}32.479 \\
32.475\end{array}$ & $\begin{array}{r}36.289 \\
36.286\end{array}$ & $\begin{array}{l}33.511 \\
33.512\end{array}$ & $\begin{array}{l}11.381 \\
17.379\end{array}$ & $\begin{array}{l}16 / 722 \\
16.785\end{array}$ & $\begin{array}{l}21.447 \\
21.447\end{array}$ & $\begin{array}{l}13.08 \\
15.705\end{array}$ & $\begin{array}{l}11.801 \\
11.854\end{array}$ & $\begin{array}{l}32.464 \\
32.464\end{array}$ & .376 & $\begin{array}{l}30.311 \\
30.326\end{array}$ & $\begin{array}{l}32.448 \\
32.443\end{array}$ & 27.41 & $\begin{array}{l}34.561 \\
34.565\end{array}$ \\
\hline 3:30 & & & 30.594 & 29.386 & & & & & 30.636 & 30.201 & 28.674 & 26.382 & 32.472 & 3 & 33.507 & 17.379 & 16.774 & 21.445 & 15.701 & 11.843 & 32.464 & 374 & .326 & 32.44 & 7.41 & 34.565 \\
\hline 4:00 & & 31 & 30 & 29.393 & 30 & & 30.076 & 4 & 30.641 & 30.197 & 4 & 26.375 & 468 & 36 & 33.503 & 17.379 & 16.771 & 21.44 & $\begin{array}{l}15.701 \\
\end{array}$ & $\begin{array}{l}11.045 \\
11.843\end{array}$ & 32.457 & 69 & 24 & 33 & 84 & 34.563 \\
\hline $11 / 5 / 114: 30$ & & & 30.582 & & & & & & & & & & 65 & & 198 & & & 21.434 & 15.703 & $\begin{array}{l}11.045 \\
11.847\end{array}$ & 32.455 & 364 & 312 & 29 & 84 & .563 \\
\hline 11/5/11 5:00 & & 31.164 & 30.578 & 29.393 & 30.614 & & & 31.101 & 30.641 & 30.185 & 28.672 & 26.38 & 32.461 & 36.279 & 33.493 & 17.374 & 16.774 & 21.431 & 15.694 & 11.845 & 32.448 & 33.36 & 0.309 & 2.426 & 27.41 & 34.563 \\
\hline 11/5/11 5:30 & & 31.167 & 30.58 & 29.382 & 30. & & 30.061 & 31.101 & 30.636 & 30.185 & 28.667 & 26.385 & 32.456 & 36.283 & 33.486 & 17.374 & 16.767 & 21.427 & 15.698 & 11.833 & 32.45 & 33.355 & 30.312 & 32.424 & 27.41 & 34.563 \\
\hline 11/5/11 6:00 & & 31.164 & 30.575 & 29.379 & 30. & & & 31.096 & 30.631 & 30.18 & 28.66 & 26.378 & 32.454 & 36.283 & 33.486 & $5 \quad 17.372$ & 16.762 & 21.436 & 15.691 & 11.833 & 32.443 & 33.353 & & 32.417 & 27.41 & 34.56 \\
\hline 11/5/11 6:30 & & 31.162 & 30.575 & 29.379 & & & & 31.096 & 30.636 & 30.178 & 28.665 & 26.375 & 32.451 & 36.281 & 33.486 & 17.372 & 16.757 & 21.431 & 15.689 & 11.831 & 32.446 & 33.353 & & 32.417 & 27.41 & 34.56 \\
\hline 11/5/11 7:00 & & 31.157 & 30.571 & 29.377 & 30. & & 30.059 & 31.094 & 30.636 & 30.173 & 28.658 & 26.375 & 32.449 & 36.288 & 33.482 & 17.37 & 16.757 & 21.431 & 15.689 & 11.828 & 32.441 & 33.348 & 30.305 & 32.415 & 27.41 & 34.563 \\
\hline $11 / 5 / 117: 30$ & & 31.16 & 30.568 & 29.374 & 30.6 & & 30.054 & 31.092 & 30.634 & 30.171 & 28.658 & 26.375 & 32.444 & 36.279 & 33.479 & 17.37 & 16.748 & 21.427 & 15.684 & 11.824 & 32.441 & 33.345 & 30.302 & 32.41 & 27.41 & 34.563 \\
\hline $11 / 5 / 118: 00$ & & 31.157 & 30.566 & 29.367 & 30.603 & & 30.054 & 31.092 & 30.631 & 30.169 & 28.656 & 26.378 & 32.454 & 36.279 & 33.491 & 17.37 & 16.748 & 21.427 & 15.687 & 11.836 & 32.441 & 33.355 & 30.298 & 32.422 & 27.413 & 34.563 \\
\hline 11/5/11 8:30 & & 31.157 & 30.564 & 29.365 & 30.6 & & 30.054 & 31.09 & 30.631 & 30.166 & 28.651 & 26.368 & 32.447 & 36.281 & 33.479 & 17.367 & 16.743 & 21.429 & 15.677 & 11.824 & 32.443 & 33.348 & 30.3 & 32.412 & 27.413 & 34.563 \\
\hline 11/5/11 9:00 & & 31.153 & 30.564 & 29.358 & 30.596 & & 30.054 & 31.09 & 30.626 & 30.164 & 28.644 & 26.368 & 32.442 & 36.279 & 33.477 & 17.365 & 16.75 & 21.427 & 15.682 & 11.826 & 32.434 & 33.341 & 30.293 & 32.41 & 27.41 & 34.563 \\
\hline 11/5/11 9:30 & & 31.148 & 30.559 & 29.36 & 30.591 & & 30.054 & 31.085 & 30.626 & 30.157 & 28.644 & 26.361 & 32.435 & 36.279 & 33.47 & 17.367 & 16.769 & 21.424 & 15.687 & 11.84 & 32.429 & 33.334 & 30.286 & 32.401 & 27.41 & 34.56 \\
\hline 11/5/11 10:00 & & 31.15 & 30.557 & 29.351 & 30.589 & & 30.059 & 31.085 & 30.629 & 30.155 & 28.639 & 26.366 & 32.43 & 36.281 & 33.468 & $3 \quad 17.365$ & 16.781 & 21.422 & 15.68 & 11.84 & 32.429 & 33.331 & 30.286 & 32.396 & 27.413 & 34.565 \\
\hline $11 / 5 / 11$ 10:30 & & 31.146 & 30.55 & 29.337 & 30.5 & & 30.059 & 31.078 & 30.626 & 30.148 & 28.63 & 26.354 & 32.428 & 36.279 & 33.465 & 17.363 & $\begin{array}{l}16.799 \\
\end{array}$ & 21.422 & 15.68 & 11.847 & 32.42 & 33.326 & 30.284 & 32.391 & 27.41 & 34.56 \\
\hline 11/5/11 11:00 & & 31.143 & 30.547 & 29.332 & 30.5 & & 30.054 & 31.08 & 30.629 & 30.146 & 28.625 & 26.346 & 32.421 & 36.279 & 33.456 & 17.365 & 16.816 & 21.42 & $\begin{array}{l}15.680 \\
\end{array}$ & 11.854 & 32.415 & .324 & .274 & 32.384 & 27.41 & 34.563 \\
\hline $11 / 5 / 1$ & & 31.141 & 30.54 & 29.323 & 30.5 & & 30.071 & 31.078 & 30.619 & $\begin{array}{l}30.139 \\
30.139\end{array}$ & 28.623 & 26.342 & 32.416 & 36.279 & $\begin{array}{l}33.450 \\
33.451\end{array}$ & 17.363 & $\begin{array}{l}10.010 \\
16.839\end{array}$ & $\begin{array}{l}21.415 \\
21.415\end{array}$ & $\begin{array}{l}13.004 \\
15.689\end{array}$ & $\begin{array}{l}11.034 \\
11.866\end{array}$ & 32.408 & .319 & .267 & $\begin{array}{l}32.379 \\
32.379\end{array}$ & 27.413 & 34.563 \\
\hline $11 / 5 / 1$ & & 31.136 & 30.533 & 29.318 & 30. & & 30.064 & 31.075 & 30.624 & 30.132 & 28.609 & 26.332 & 32.409 & 36.274 & 33.446 & 17.36 & 16.851 & 21.408 & 15.689 & 11.871 & 32.387 & 2 & 8 & 32.372 & 27.41 & 34.558 \\
\hline & & 31 & 30.5 & 29.3 & 30 & & 30 & 31. & 30 & 30. & 28. & 26 & 32.407 & 36.274 & 33.439 & 17. & 16 & 21 & 15.689 & 11. & 2 & & 1 & 32. & 27.41 & 34.56 \\
\hline & & 31. & 30. & 29 & & & 30. & 31 & & 30 & 28. & 26. & 32 & 36. & 33. & 17 & 16.8 & 21 & 15 & 11 & & & & 8 & 27.415 & 34.558 \\
\hline $11 / 5$ & & 31. & 30. & 29 & & & 30. & 31. & & 30. & 28. & & 32.3 & 36 & & & & & 15. & 11. & & & & 1 & 27.413 & 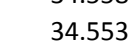 \\
\hline & & & & & & & & & & & & & 32.3 & & 33. & & & 21.3 & & & & & & & 3 & \\
\hline & & & & & & & & & & & & & & & & & & & & & & & & & 27. & \\
\hline & & & & & 30.5 & & & & & & & & & & & & & 21 & & & & & & & 27. & 34. \\
\hline $11 / 5$ & & & 30. & 29 & & & & & & & & 26 & 32 & & & 17 & & 21. & 15 & 12 & & & & 2 & 27.415 & 34. \\
\hline 11/5/11 16:00 & & 31.108 & 30.464 & 29.237 & 30.491 & & 30.071 & 31.051 & 30.617 & 30.064 & 28.549 & 26.251 & 32.402 & 36.248 & 33.437 & 17.339 & 16.993 & 21.378 & 15.708 & 11.988 & 32.345 & 33.298 & 30.183 & 32.365 & 27.41 & 34.544 \\
\hline 11/5/11 16:30 & & 31.101 & 30.447 & 29.246 & 30.474 & & 30.05 & 31.046 & 30.612 & 30.05 & 28.551 & 26.265 & 32.386 & 36.255 & 33.425 & 17.335 & 17.012 & 21.408 & 15.717 & & 32.322 & 33.281 & 30.157 & 32.349 & 27.413 & 34.539 \\
\hline
\end{tabular}




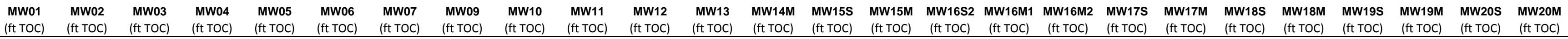

\begin{tabular}{|c|c|c|c|c|c|c|c|c|c|c|c|c|c|c|c|c|c|c|c|c|c|c|c|c|c|}
\hline well & $\begin{array}{l}\text { (ft TOC) } \\
\text { (f) }\end{array}$ & $\begin{array}{l}\text { (ft TOC) } \\
\text { (ft) }\end{array}$ & $\begin{array}{l}\text { (ft TOC) } \\
\text { (f) }\end{array}$ & $\begin{array}{l}\text { (ft TOC) } \\
\text { (ft) }\end{array}$ & $\begin{array}{ll}(\mathrm{ft} \text { TOC) } & \text { (ft TOC) }\end{array}$ & (ft toc) & $\begin{array}{l}\text { (ift TOC) } \\
\text { (n) }\end{array}$ & $\begin{array}{l}\text { (ft TOC) } \\
\text { (ft) }\end{array}$ & $\begin{array}{l}\text { (ft TOC) } \\
\text { (f) }\end{array}$ & $\begin{array}{l}\text { (ft TOC) } \\
\text { (n) }\end{array}$ & $\begin{array}{l}\text { (ft TOC) } \\
\text { (f) }\end{array}$ & (ft TOC) & $\begin{array}{l}\text { (ft TOC) } \\
\end{array}$ & (ft TOC) & (ft TOC) & $\begin{array}{l}\text { (ft TOC) } \\
\text { (f) }\end{array}$ & (ft TOC) & $\begin{array}{l}\text { (ft TOC) } \\
\text { (ft) }\end{array}$ & $\begin{array}{l}\text { (ft toc) } \\
\text { (fol) }\end{array}$ & $\begin{array}{l}\text { (ft TOC) } \\
\text { (f) }\end{array}$ & $\begin{array}{l}\text { (ft TOC) } \\
\text { (f) }\end{array}$ & $\begin{array}{l}\text { (ft TOC) } \\
\text { (n) }\end{array}$ & (ft TOC) & $\begin{array}{l}\text { (ft TOC) } \\
\text { (1) }\end{array}$ & (ft TOC) \\
\hline 7:00 & & 31.099 & 30.44 & 29.26 & 30.4 & 30.026 & $31 . c$ & 30.615 & 30. & 28.5 & 26 & 32.372 & 36.248 & 33. & & 17.007 & 21.42 & 15.717 & 11.991 & 32.313 & 33.272 & 30.152 & 32.335 & 41 & \\
\hline $11 / 5 / 1117: 30$ & & 31.111 & 30.443 & 29.26 & 30.467 & 30.021 & 31.046 & 30.612 & 30.043 & 28.551 & 26.294 & 32.367 & 36.248 & 33.404 & 17.33 & 16.982 & 21.42 & 5.698 & 11.962 & 32. & 33.267 & 166 & 32.33 & & 34.542 \\
\hline $11 / 5 / 1118: 00$ & & 31.118 & 30.447 & 29.279 & 30.474 & 30.003 & 31.042 & 30.617 & 30.043 & 28.558 & 26.294 & 32.365 & 36.251 & 33.402 & 17.332 & 16.944 & 21.424 & 15.687 & 11.932 & 32.336 & 33.262 & 185 & 32.33 & 27.413 & 34.542 \\
\hline 11/5/11 18:30 & & 31.113 & 30.445 & 29.3 & 30.472 & 29.981 & 31.046 & 30.61 & 30.043 & 28.567 & 26.315 & 32.36 & 36.248 & 33.397 & 17.328 & 16.935 & 21.422 & 15.691 & 11.925 & 32.331 & 33.258 & 30.178 & 32.323 & 27.413 & 34.542 \\
\hline 11/5/11 19:00 & & 31.115 & 30.45 & 29.309 & 30.477 & 29.965 & 31.044 & 30.615 & 30.041 & 28.572 & 26.337 & 32.355 & 36.246 & 33.393 & 17.33 & 16.9 & 21.413 & 15.682 & 11.895 & 32.334 & 33.258 & 30.197 & 32.325 & 27.415 & 34.544 \\
\hline 11/5/11 19:30 & & 31.122 & 30.454 & 29.314 & 30.488 & 29.953 & 31.042 & 30.612 & 30.048 & 28.574 & 26.349 & 32.358 & 36.248 & 33.393 & 17.335 & 16.853 & 21.408 & 15.665 & 11.857 & 32.35 & 33.255 & 30.213 & 32.323 & 27.417 & 34.546 \\
\hline 11/5/11 20:00 & & 31.12 & 30.461 & 29.311 & 30.498 & 29.949 & 31.044 & 30.612 & 30.053 & 28.577 & 26.356 & 32.358 & 36.253 & 33.39 & 17.332 & 16.827 & 21.408 & 15.665 & 11.843 & 32.35 & 33.26 & 30.22 & 32.323 & 27.413 & 34.549 \\
\hline 11/5/11 20:30 & & 31.122 & 30.468 & 29.304 & 30.509 & 29.956 & 31.041 & 30.605 & 30.055 & 28.572 & 26.354 & 32.358 & 36.255 & 33.393 & 17.335 & 16.802 & 21.406 & 15.658 & 11.821 & 32.366 & 33.258 & 30.234 & 32.323 & 27.415 & 34.549 \\
\hline 11/5/11 21:00 & & 31.12 & 30.471 & 29.302 & 30.514 & 29.949 & 31.041 & 30.605 & 30.055 & 28.572 & 26.351 & 32.358 & 36.258 & 33.39 & 17.337 & 16.776 & 21.399 & 15.654 & 11.807 & 32.364 & 33.26 & 30.239 & 32.325 & 27.415 & 34.551 \\
\hline 11/5/11 21:30 & & 31.113 & 30.466 & 29.304 & 30.519 & 29.953 & 31.039 & 30.607 & 30.055 & 28.57 & 26.346 & 32.355 & 36.26 & 33.39 & 17.337 & 16.774 & 21.397 & 15.665 & 11.805 & 32.362 & 33.255 & 30.23 & 32.321 & 27.417 & 34.551 \\
\hline $11 / 5 / 1122: 00$ & & 31.111 & 30.466 & 29.311 & 30.516 & 29.942 & 31.037 & 30.607 & 30.053 & 28.579 & 26.356 & 32.353 & 36.26 & 33.388 & 17.335 & 16.755 & 21.39 & 15.651 & 11.791 & 32.359 & 33.251 & 30.237 & 32.321 & 27.417 & 34.551 \\
\hline $11 / 5 / 1122: 30$ & & 31.108 & 30.461 & 29.307 & 30.516 & 29.942 & 31.037 & 30.605 & 30.053 & 28.579 & 26.358 & 32.351 & 36.258 & 33.385 & 17.337 & 16.748 & 21.383 & 15.656 & 11.788 & 32.355 & 33.251 & 30.23 & 32.318 & 27.417 & 34.553 \\
\hline $11 / 5 / 1123: 00$ & & 31.113 & 30.468 & 29.309 & 30.523 & 29.937 & 31.034 & 30.605 & 30.055 & 28.579 & 26.351 & 32.348 & 36.262 & 33.383 & 17.335 & 16.727 & 21.378 & 15.642 & 11.774 & 32.359 & 33.248 & 30.237 & 32.316 & 27.415 & 34.553 \\
\hline 11/5/11 23:30 & & 31.108 & 30.471 & 29.314 & 30.528 & 29.934 & 31.034 & 30.603 & 30.055 & 28.579 & 26.358 & 32.348 & 36.267 & 33.383 & 17.337 & 16.718 & 21.373 & 15.644 & 11.763 & 32.359 & 33.248 & 30.237 & 32.314 & 27.415 & 34.553 \\
\hline $11 / 6 / 110: 00$ & & 31.106 & 30.468 & 29.325 & 30.523 & 29.927 & 31.034 & 30.6 & 30.055 & 28.584 & 26.368 & 32.346 & 36.267 & 33.381 & 17.335 & 16.706 & 21.366 & 15.642 & 11.753 & 32.364 & 33.246 & 30.237 & 32.316 & 27.415 & 34.551 \\
\hline 11/6/11 0:30 & & 31.104 & 30.468 & 29.33 & 30.53 & 29.923 & 31.032 & 30.605 & 30.053 & 28.586 & 26.378 & 32.344 & 36.267 & 33.376 & 17.337 & 16.694 & 21.364 & 15.637 & 11.746 & 32.357 & 33.251 & 30.237 & 32.314 & 27.42 & 34.556 \\
\hline $11 / 6 / 111: 00$ & & 31.108 & 30.468 & 29.344 & 30.533 & 29.913 & 31.034 & 30.605 & 30.055 & 28.593 & 26.382 & 32.344 & 36.265 & 33.374 & 17.337 & 16.673 & 21.36 & 15.628 & 11.734 & 32.369 & 33.244 & 30.244 & 32.307 & 27.417 & 34.551 \\
\hline $11 / 6 / 111: 30$ & & 31.108 & 30.475 & 29.358 & 30.54 & 29.906 & 31.034 & 30.607 & 30.06 & 28.598 & 26.397 & 32.346 & 36.267 & 33.374 & 17.337 & 16.652 & 21.355 & 15.623 & 11.716 & 32.364 & 33.239 & 30.253 & 32.309 & 27.42 & 34.553 \\
\hline $11 / 6 / 112: 00$ & & 31.108 & 30.478 & 29.37 & 30.544 & 29.897 & 31.037 & 30.605 & 30.06 & 28.607 & 26.409 & 32.346 & 36.269 & 33.374 & 17.337 & 16.631 & 21.355 & 15.619 & 11.699 & 32.371 & 33.244 & 30.255 & 32.309 & 27.417 & 34.551 \\
\hline $11 / 6 / 112: 30$ & & 31.113 & 30.487 & 29.393 & 30.556 & 29.885 & 31.037 & 30.612 & 30.067 & 28.616 & 26.425 & 32.346 & 36.276 & 33.374 & 17.339 & 16.605 & 21.346 & 15.612 & 11.68 & 32.376 & 4 & 30.272 & 32.309 & 27.42 & 34.553 \\
\hline $11 / 6 / 11$ & & 31.115 & 30.492 & 29.403 & 30.5 & 29.876 & 31.042 & 30.605 & 30.071 & 28.625 & 26.445 & 32.346 & 36.274 & 33.376 & 17.344 & 16.58 & 21.343 & 15.6 & 11.662 & 32.392 & 6 & 0.281 & 32.311 & 27.422 & .553 \\
\hline $11 / 6 / 113: 30$ & & 31.118 & 30.503 & 29.407 & 30. & 29.876 & 31.044 & 30.605 & 30.076 & 28.632 & 26.457 & 32.348 & 36.281 & 33.376 & 17.346 & 16.556 & 21.343 & 15.595 & 11.643 & 397 & 8 & .298 & 314 & 27.42 & 553 \\
\hline $11 / 6 / 11$ & & 31.125 & 30.52 & 29.414 & 30.591 & 29.878 & 31.046 & 30.605 & 30.085 & 28.637 & 26.459 & 32.353 & 36.286 & 33.381 & 17.349 & 16.521 & 21.341 & 15.588 & 11.612 & 415 & 253 & .317 & 2.318 & 27.422 & .556 \\
\hline 11/6/11 4:30 & & 31.122 & 30.524 & 29.426 & 30.603 & 29.873 & 31.046 & 30.607 & 30.092 & 28.644 & 26.468 & 32.355 & 36.29 & 33.383 & 17.351 & 16.498 & 21.336 & 15.586 & 11.598 & 32.42 & 33.253 & 30.326 & 32.323 & 27.422 & 34.556 \\
\hline 11/6/11 5:00 & & 31.118 & 30.526 & 29.431 & 30.607 & 29.873 & 31.046 & 30.605 & & 28.649 & 26.478 & 32.355 & 36.293 & & 17.351 & 16.486 & 21.332 & 15.586 & 11.598 & 32.42 & 33.255 & 30.328 & 32.323 & 27.422 & 34.556 \\
\hline 11/6/11 5:30 & & 31.12 & 30.531 & 29.449 & 30.619 & 29.866 & 31.049 & 30.61 & 30.104 & 28.66 & 26.483 & 32.36 & 36.295 & 33.386 & 17.353 & 16.463 & 21.329 & 15.586 & 11.582 & 32.429 & 33.26 & 30.335 & 32.323 & 27.424 & 34.558 \\
\hline 11/6/11 6:00 & & 31.122 & 30.543 & 29.461 & 30.631 & 29.864 & 31.053 & 30.607 & 30.108 & 28.67 & 26.5 & 32.362 & 36.3 & 33.388 & 17.356 & 16.44 & 21.325 & 15.579 & 11.565 & 32.439 & 33.263 & 30.345 & 32.33 & 27.424 & 34.558 \\
\hline 11/6/11 6:30 & & 31.122 & 30.545 & 29.47 & 30.638 & 29.862 & 31.056 & 30.61 & 30.113 & 28.677 & 26.512 & 32.365 & 36.305 & 33.393 & 17.36 & 16.419 & 21.329 & 15.574 & 11.553 & 32.439 & 33.265 & 30.352 & 32.333 & 27.424 & 34.56 \\
\hline 11/6/11 7:00 & & 31.125 & 30.559 & 29.484 & 30.649 & 29.859 & 31.058 & 30.612 & 30.122 & 28.686 & 26.521 & 32.372 & 36.314 & 33.397 & 17.363 & 16.393 & 21.339 & 15.569 & 11.535 & 32.45 & 33.27 & 30.368 & 32.335 & 27.424 & 34.563 \\
\hline $11 / 6 / 117: 30$ & & 31.127 & 30.564 & 29.498 & 30.661 & 29.855 & 31.063 & 30.615 & 30.129 & 28.695 & 26.533 & 32.374 & 36.314 & 33.402 & 17.365 & 16.372 & 21.343 & 15.567 & 11.525 & 32.462 & 33.274 & 30.378 & 32.342 & 27.424 & 34.563 \\
\hline $11 / 6 / 118: 00$ & & 31.129 & 30.575 & 29.517 & 30.67 & 29.855 & 31.068 & 30.615 & 30.136 & 28.705 & 26.547 & 32.379 & 36.319 & 33.407 & 17.367 & 16.353 & 21.341 & 15.569 & 11.506 & 32.469 & 33.279 & 30.389 & 32.347 & 27.424 & 34.565 \\
\hline $11 / 6 / 118: 30$ & & 31.134 & 30.589 & 29.515 & 30.687 & 29.859 & 31.072 & 30.612 & 30.146 & 28.712 & 26.562 & 32.386 & 36.326 & 33.414 & 17.372 & 16.323 & 21.339 & 15.558 & 11.488 & 32.485 & 33.284 & 30.403 & 32.349 & 27.424 & 34.567 \\
\hline 11/6/11 9:00 & & 31.134 & 30.596 & 29.531 & 30.696 & 29.866 & 31.073 & 30.615 & 30.155 & 28.718 & 26.559 & 32.39 & 36.33 & 33.416 & 17.372 & 16.318 & 21.336 & 15.56 & 11.485 & 32.49 & 33.291 & 30.415 & 32.356 & 27.427 & 34.57 \\
\hline $\begin{array}{l}11 / 6 / 119: 30 \\
\text { 11/ }\end{array}$ & & 31.136 & 30.608 & 29.54 & 30.71 & 29.864 & 31.08 & 30.617 & 30.166 & 28.728 & 26.571 & 32.397 & 36.337 & 33.421 & 17.377 & 16.32 & 21.339 & 15.562 & 11.483 & 32.502 & 33.298 & 30.429 & 32.365 & 27.424 & 34.574 \\
\hline 11/6/11 10:00 & & 31. & 30.61 & 29.536 & 30.7 & 29.88 & 31.082 & 30.607 & 30.169 & 28.732 & 26.581 & 32.4 & 36.342 & 33.4 & 17.381 & 16.346 & 21.339 & 15.565 & 11.497 & 32.499 & .298 & 30.429 & 32.368 & 27.427 & 34.577 \\
\hline $11 / 6 / 11$ & & 31. & 30.624 & 29.54 & 30.7 & 29.89 & 31.087 & 30.617 & 30.18 & 28.739 & 26.574 & 32.407 & 36.342 & 33.43 & 17.384 & $\begin{array}{l}16.348 \\
1640\end{array}$ & 21.346 & 15.558 & $\begin{array}{c}11.49 \\
11.49\end{array}$ & 32.506 & 8 & 30.446 & 32.372 & 27.427 & 34.579 \\
\hline 11/6/11 11:00 & & 31. & 30.631 & 29.538 & 30.7 & 29.892 & $\begin{array}{l}31.09 \\
31.09\end{array}$ & 30.615 & 30.187 & 28.744 & 26.576 & 32.409 & 36.347 & $\begin{array}{l}33.435 \\
33.435\end{array}$ & $\begin{array}{l}17.384 \\
17.386\end{array}$ & $\begin{array}{l}10.360 \\
16.367\end{array}$ & 21.346 & $\begin{array}{l}15.558 \\
15.558\end{array}$ & $\begin{array}{l}11.49 \\
11.497\end{array}$ & 32.52 & 308 & 30.453 & 32.375 & 27.427 & 34.581 \\
\hline 11/6/11 11:30 & & 31. & 30.634 & 29.519 & 30.7 & 29.916 & 31.092 & 30.612 & 30.19 & 28.737 & 26.574 & 32.411 & 36.349 & $\begin{array}{l}33.4535 \\
33.435\end{array}$ & $\begin{array}{l}17.388 \\
17.38\end{array}$ & 16.388 & 21.346 & 15.574 & 11.513 & 32.52 & 312 & $\begin{array}{l}30.448 \\
30.45\end{array}$ & 32.377 & 27.429 & 34.581 \\
\hline & & 31 & 30 & 29.512 & & 29 & 31.092 & 30. & 30. & 28.737 & 26 & 32 & 36.351 & 33 & 17.39 & 16.407 & 6 & 15.574 & 11.523 & 32.527 & 7 & 5 & 32.379 & 27.427 & .584 \\
\hline & & & 30 & 29.501 & & & 31.0 & & 30 & 28.7 & 26 & 32 & 36.354 & 33 & 17.393 & 137 & 3 & 15.593 & 11.5 & 6 & 5 & 8 & 32.384 & 27.427 & .584 \\
\hline & & 31. & 30. & 29.498 & & 29 & & & & 28.7 & 26 & 32.4 & 36. & 33 & 17.393 & $x_{1}$ & 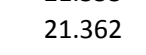 & 15.597 & 11 & 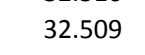 & 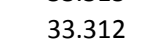 & 36 & 32.379 & 29 & .581 \\
\hline & & & & & & & & 30.612 & & & & & & & 17.393 & $x_{1}$ & 21.3 & 15.609 & 11. & & & 30.427 & 32.375 & 27.429 & .581 \\
\hline $11 / 6$ & & 31 & 30.6 & & & 29. & & 30.61 & & 28 & & 32.4 & 36. & & & & 21.3 & 15. & 11. & & & & 32.375 & 27.429 & 34.581 \\
\hline $114: 30$ & & & 30. & 29 & & & & 30 & & & & 32.407 & 36 & 33.4 & 17.3 & & 21.3 & 15.619 & 11.624 & & & & 32.372 & 27.429 & 34.579 \\
\hline $115: 00$ & & & 30 & 29. & & 29. & & 30 & & & & 32.404 & 36. & 33.4 & 17. & & 21.3 & 15.619 & 11.631 & & & 3 & 32.372 & 27.431 & 34.579 \\
\hline $11 / 6 / 1115: 30$ & & & 30.6 & 29.505 & & 29. & & & & & & 32.404 & & & & & 21. & 15.6 & 11.638 & 32.492 & & 5 & 32.37 & 27.431 & 34.579 \\
\hline $11 / 6 / 1116: 00$ & & & 30.6 & 29.51 & & 29 & & 30.612 & & 28. & 26. & 32.404 & 36.349 & 33.4 & 17. & 16. & 21 & 15.623 & 11.643 & 32.49 & & 30.411 & 32.37 & 27.431 & 34.579 \\
\hline $11 / 6 / 1116: 30$ & & 31. & 30.6 & 29.515 & & 29 & 31.0 & 30.612 & 30. & 28.7 & 26.5 & 32.404 & 36.347 & 33.432 & 17. & 16.591 & 21.3 & 15. & 11.652 & 32.492 & 33. & 30.408 & 32.37 & 27.431 & 34.579 \\
\hline $11 / 6 / 1117: 00$ & & 31.132 & 30.6 & 29.522 & 30.7 & 29.965 & 31.0 & 30.612 & 30.201 & 28.749 & 26.535 & 32.407 & 36.354 & 33.432 & 17.395 & 16.584 & 21.399 & 15.623 & 11.647 & 32.497 & 33.305 & 30.413 & 32.37 & 27.431 & 34.581 \\
\hline $11 / 6 / 1117: 30$ & & 31.132 & 30.627 & 29.533 & 30.72 & 29.963 & 31.099 & 30.612 & 30.204 & 28.753 & 26.54 & 32.409 & 36.351 & 33.435 & 17.395 & 16.58 & 21.397 & 15.63 & 11.65 & 32.497 & 33.308 & 30.42 & 32.372 & 27.431 & 34.581 \\
\hline $11 / 6 / 11118: 00$ & & 31.132 & 30.629 & 29.547 & 30.731 & 29.953 & 31.101 & 30.615 & 30.208 & 28.76 & 26.55 & 32.599 & 36.354 & 33.62 & 17.397 & 16.573 & 21.392 & 15.628 & 11.836 & 32.595 & 33.497 & 30.427 & 32.558 & 27.431 & 34.584 \\
\hline $11 / 6 / 1118: 30$ & & 31.134 & 30.634 & 29.554 & 30.736 & 29.949 & 31.104 & 30.615 & 30.213 & 28.77 & 26.562 & 32.51 & 36.356 & 33.538 & 17.4 & 16.556 & 21.39 & 15.623 & 11.739 & 32.553 & 33.407 & 30.436 & 32.471 & 27.431 & 34.586 \\
\hline 11/6/11 19:00 & & 31.136 & 30.638 & 29.559 & 30.743 & 29.949 & 31.108 & 30.617 & 30.217 & 28.77 & 26.569 & 32.746 & 36.356 & 33.798 & 17.402 & 16.554 & 21.392 & 15.621 & 11.979 & 32.614 & 33.644 & 30.45 & 32.708 & 27.434 & 34.586 \\
\hline 11/6/11 19:30 & & 31.139 & 30.645 & 29.561 & 30.752 & 29.949 & 31.111 & 30.615 & 30.224 & 28.774 & 26.571 & 32.718 & 36.358 & 33.761 & 17.404 & 16.57 & 21.387 & 15.619 & 11.927 & 32.646 & 33.618 & 30.469 & 32.675 & 27.431 & 34.588 \\
\hline $11 / 6 / 1120: 00$ & & 31.139 & 30.652 & 29.571 & 30.762 & 29.949 & 31.109 & 30.617 & 30.229 & 28.781 & 26.574 & 32.641 & 36.365 & 33.681 & 17.407 & 16.547 & 21.38 & 15.621 & 11.831 & 32.628 & 33.542 & 30.472 & 32.602 & 27.434 & 34.591 \\
\hline /6/11 20:30 & & 31.141 & 30.655 & 29.58 & 30.769 & 29.946 & 31.116 & 30.617 & 30.231 & 28.788 & 26.581 & 32.603 & 36.363 & 33.636 & 17.409 & 16.528 & 21.378 & 15.621 & 11.779 & 32.614 & 33.502 & 30.479 & 32.567 & 27.434 & 13 \\
\hline
\end{tabular}




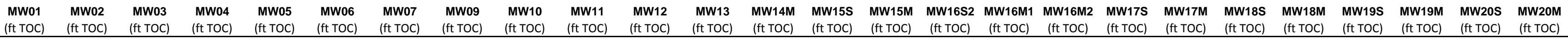

\begin{tabular}{|c|c|c|c|c|c|c|c|c|c|c|c|c|c|c|c|c|c|c|c|c|c|c|c|c|c|}
\hline Well & $\begin{array}{l}\text { (ft TOC) } \\
\text { (is }\end{array}$ & $\begin{array}{l}\text { (ft TOC) } \\
\text { (f) }\end{array}$ & $\begin{array}{l}\text { (ft TOC) } \\
\text { (f) }\end{array}$ & (ft TOC) & $\begin{array}{ll}(\mathrm{ft} \text { TOC) } & \text { (ft TOC) }\end{array}$ & $\begin{array}{l}\text { (ft TOC) } \\
\text { (ft }\end{array}$ & $\begin{array}{l}\text { (ft TOC) } \\
\text { (f) }\end{array}$ & $\begin{array}{l}\text { (ft TOC) } \\
\text { (ft) }\end{array}$ & $\begin{array}{l}\text { (ft TOC) } \\
\text { (f) }\end{array}$ & $\begin{array}{l}\text { (ft TOC) } \\
\text { (f) }\end{array}$ & $\begin{array}{l}\text { (ft Toc) } \\
\text { (f) }\end{array}$ & $\begin{array}{l}\text { (ft TOC) } \\
\text { (t) }\end{array}$ & $\begin{array}{l}\text { (ft TOC) } \\
\end{array}$ & (ft TOC) & (ft TOC) & (ft TOC) & (ft TOC) & $\begin{array}{l}\text { (ft TOC) } \\
\text { (ft) }\end{array}$ & (ft TOC) & $\begin{array}{l}\text { (ft TOC) } \\
\end{array}$ & $\begin{array}{l}\text { (ft TOC) } \\
\text { (1) }\end{array}$ & $\begin{array}{l}\text { (ft TOC) } \\
\text { (n) }\end{array}$ & (ft TOC) & $\begin{array}{l}\text { (ft TOC) } \\
\end{array}$ & (ft TOC) \\
\hline $11 / 6 / 1121: 00$ & & 31.139 & 30.657 & 29.585 & 30.773 & 29.944 & 31.116 & 30.617 & 30.236 & 28.791 & 26.588 & 32.58 & 36.365 & 33.613 & $\begin{array}{l}3 \quad 17.407 \\
\end{array}$ & 16.51 & 21.373 & 15.623 & 11.749 & 32.609 & 33.481 & 30.481 & 32.544 & 27.434 & 34.593 \\
\hline $11 / 6 / 11$ 21:30 & & 31.143 & 30.664 & 29.592 & 30.785 & 29.944 & 31.118 & 30.622 & 30.243 & 28.798 & 26.593 & 32.564 & 36.365 & 33.594 & $4 \quad 17.411$ & 16.482 & 21.371 & 15.621 & 11.716 & 32.602 & 33.464 & 30.488 & 32.525 & 27.434 & 34.595 \\
\hline $11 / 6 / 1122: 00$ & & 31.146 & 30.673 & 29.59 & 30.792 & 29.949 & 31.123 & 30.619 & 30.248 & 28.798 & 26.598 & 32.554 & 36.365 & 33.582 & $2 \quad 17.414$ & 16.46 & 21.371 & 15.619 & 11.687 & 32.604 & 33.452 & 30.5 & 32.518 & 27.434 & 34.598 \\
\hline $11 / 6 / 1122: 30$ & & 31.148 & 30.673 & 29.592 & 30.797 & 29.953 & 31.123 & 30.619 & 30.252 & 28.8 & 26.595 & 32.542 & 36.373 & 33.573 & 17.416 & 16.444 & 21.369 & 15.619 & 11.673 & 32.614 & & & 32.511 & 27.434 & 34.6 \\
\hline 11/6/11 23:00 & & 31.146 & 30.678 & 29.583 & 30.804 & 29.96 & 31.125 & 30.619 & 30.259 & 28.802 & 26.595 & 32.538 & 36.373 & 33.566 & 17.418 & 16.433 & 21.364 & 15.619 & 11.652 & 32.602 & 33.435 & 30.502 & & 7.434 & 34.6 \\
\hline $11 / 6 / 1123: 30$ & & 31.146 & 30.678 & 29.573 & 30.804 & 29.972 & 31.125 & 30.617 & 30.262 & 28.8 & 26.586 & 32.531 & 36.375 & 33.559 & 17.421 & 16.426 & 21.364 & 15.623 & 11.652 & 32.604 & 33.433 & 30.5 & 2.494 & 7.436 & 34.6 \\
\hline 11/7/11 0:00 & & 31.148 & 30.68 & 29.576 & 30.808 & 29.977 & 31.128 & 30.617 & 30.264 & 28.8 & 26.574 & 32.528 & 36.37 & 33.554 & $4 \quad 17.421$ & 16.414 & 21.362 & 15.621 & 11.636 & 32.602 & 33.426 & 30.502 & 32.492 & 27.436 & 34.6 \\
\hline 11/7/11 0:30 & & 31.143 & 30.676 & 29.576 & 30.804 & 29.981 & 31.13 & 30.619 & 30.262 & 28.8 & 26.574 & 32.517 & 36.373 & 33.547 & $7 \quad 17.421$ & 16.418 & 21.357 & 15.635 & 11.64 & 32.59 & 33.417 & 30.49 & 32.483 & 27.436 & 34.6 \\
\hline 11/7/11 1:00 & & 31.143 & 30.673 & 29.573 & 30.801 & 29.984 & 31.13 & 30.619 & 30.262 & 28.802 & 26.571 & 32.51 & 36.368 & 33.54 & $4 \quad 17.421$ & 16.414 & 21.353 & 15.633 & 11.638 & 32.581 & 33.412 & 30.49 & 32.476 & 27.436 & 34.6 \\
\hline 11/7/11 1:30 & & 31.143 & 30.673 & 29.58 & 30.801 & 29.986 & 31.13 & 30.619 & 30.266 & 28.802 & 26.569 & 32.505 & 36.373 & 33.533 & $3 \quad 17.421$ & 16.404 & 21.348 & 15.63 & 11.629 & 32.586 & 33.407 & 30.486 & 32.471 & 27.436 & 34.598 \\
\hline 11/7/11 2:00 & & 31.143 & 30.673 & 29.585 & 30.799 & 29.986 & 31.13 & 30.622 & 30.266 & 28.807 & 26.571 & 32.5 & 36.373 & 33.529 & $\begin{array}{l}9 \\
9\end{array} 17.423$ & 16.4 & 21.341 & 15.635 & 11.629 & 32.576 & 33.4 & 30.483 & 32.466 & 27.436 & 34.598 \\
\hline 11/7/11 2:30 & & 31.143 & 30.673 & 29.587 & 30.799 & 29.984 & 31.133 & 30.622 & 30.264 & 28.809 & 26.576 & 32.498 & 36.377 & 33.524 & $\begin{array}{l}4 \quad 17.423 \\
\end{array}$ & 16.393 & 21.339 & 15.635 & 11.622 & 32.576 & 33.395 & 30.481 & 32.464 & 27.436 & 34.595 \\
\hline 11/7/11 3:00 & & 31.146 & 30.673 & 29.585 & 30.799 & 29.986 & 31.135 & 30.622 & 30.269 & 28.809 & 26.579 & 32.493 & 36.373 & 33.521 & $1 \quad 17.421$ & 16.386 & 21.334 & 15.637 & 11.619 & 32.572 & 33.393 & 30.483 & 32.459 & 27.436 & 34.595 \\
\hline 11/7/11 3:30 & & 31.148 & 30.68 & 29.58 & 30.801 & 29.991 & 31.135 & 30.622 & 30.273 & 28.809 & 26.574 & 32.491 & 36.373 & 33.519 & 17.425 & 16.376 & 21.332 & 15.633 & 11.603 & 32.579 & 33.393 & 30.493 & 32.455 & 27.438 & 34.595 \\
\hline 11/7/11 4:00 & & 31.148 & 30.678 & 29.583 & 30.801 & 30 & 31.137 & 30.622 & 30.275 & 28.809 & 26.569 & 32.489 & 36.377 & 33.514 & 17.425 & 16.369 & 21.327 & 15.633 & 11.6 & 32.574 & 33.388 & 30.479 & 32.452 & 27.436 & 34.595 \\
\hline $11 / 7 / 114: 30$ & & 31.146 & 30.678 & 29.592 & 30.799 & 29.996 & 31.137 & 30.624 & 30.275 & 28.814 & 26.567 & 32.486 & 36.373 & 33.512 & 17.425 & 16.566 & 21.32 & 15.635 & 11.6 & 32.574 & 33.383 & 30.49 & 32.448 & 27.438 & 34.593 \\
\hline 11/7/11 5:00 & & 31.146 & 30.676 & 29.594 & 30.797 & 29.991 & 31.137 & 30.624 & 30.273 & 28.818 & 26.574 & 32.479 & 36.373 & 33.51 & 17.425 & 16.563 & 21.313 & 15.64 & 11.605 & 32.567 & 33.381 & 30.481 & 32.445 & 27.438 & 34.593 \\
\hline $11 / 7 / 115: 30$ & & 31.146 & 30.676 & 29.594 & 30.794 & 29.996 & 31.139 & 30.622 & 30.278 & 28.816 & 26.576 & 32.482 & 36.377 & 33.505 & 17.428 & 16.561 & 21.311 & 15.637 & 11.598 & 32.567 & $\begin{array}{l}33.379 \\
3379\end{array}$ & 30.481 & 32.441 & 27.438 & 34.593 \\
\hline 11/7/11 6:00 & & 31.153 & 30.683 & 29.594 & 30.801 & 30.003 & 31.139 & 30.622 & 30.28 & 28.816 & 26.574 & 32.479 & 36.377 & 33.505 & 17.425 & $\begin{array}{l}10.559 \\
16.599\end{array}$ & 21.311 & 15.63 & $\begin{array}{l}11.586 \\
11.586\end{array}$ & 32.574 & $\begin{array}{l}33.379 \\
379\end{array}$ & $\begin{array}{l}30.493 \\
30.493\end{array}$ & 32.445 & 27.441 & 34.593 \\
\hline $11 / 7 / 116: 30$ & & 31.146 & 30.68 & 29.594 & $\begin{array}{l}30.011 \\
30.797\end{array}$ & 30.007 & 31.14 & $\begin{array}{l}30.024 \\
30.624\end{array}$ & $\begin{array}{l}30.28 \\
30.282\end{array}$ & $\begin{array}{l}20.010 \\
28.818\end{array}$ & 26.574 & 32.477 & 36.375 & $\begin{array}{l}33.503 \\
3.503\end{array}$ & 17.428 & $\begin{array}{l}10.539 \\
16.559\end{array}$ & 21.306 & $\begin{array}{l}15.03 \\
15.64\end{array}$ & $\begin{array}{l}11.500 \\
11.591\end{array}$ & 32.569 & $\begin{array}{l}33.376 \\
3776\end{array}$ & 30.483 & 32.44 & 27.438 & 34.593 \\
\hline $11 / 7 / 11$ 7:00 & & 31.15 & 30.685 & 29.573 & 30.801 & 30.017 & 31.142 & 30.619 & 30.285 & 28.814 & 26.571 & 32.475 & 36.379 & 33.503 & 17.43 & 16.559 & 21.306 & 15.64 & 11.589 & 32.574 & 33.376 & 30.488 & 32.44 & 27.441 & 34.593 \\
\hline 11/7/11 7:30 & & 31.148 & 30.683 & 29.58 & 30.799 & 30.024 & 31.14 & 30.619 & 30.285 & 28.812 & 26.552 & 32.477 & 36.377 & 33.503 & 17.43 & 16.559 & 21.311 & 15.647 & 11.596 & 32.562 & 374 & 0.481 & 32.44 & 27.441 & 34.595 \\
\hline $11 / 7 / 118: 00$ & & & & & & 30.01 & 31.142 & & 30.285 & $\begin{array}{l}28.821 \\
28.821\end{array}$ & 26.555 & 32.475 & 36.375 & 33.503 & 17.43 & $\begin{array}{l}10.556 \\
16.556\end{array}$ & 21.318 & 15.651 & 11.603 & 32.565 & .374 & .481 & 32.438 & 27.438 & 34.595 \\
\hline 11/7/11 8:30 & & 31.141 & 30.669 & 29.587 & 30 & 30.019 & 31.14 & 30.626 & 30.28 & 28.821 & 26.567 & 32.47 & 36.372 & 33.496 & $\quad 17.428$ & 16.552 & 21.316 & 5.661 & 11.619 & 32.546 & 33.367 & .467 & 32.433 & 27.441 & 34.595 \\
\hline 11/7/11 9:00 & & & 30.678 & 29.576 & 30.792 & 30.021 & 31.142 & 30.624 & 30.282 & 28.821 & 26.564 & 32.47 & 36.377 & 33.498 & 17.43 & 16.554 & 21.313 & 15.647 & 11.605 & 32.56 & 33.369 & .481 & 2.436 & 27.443 & 34.598 \\
\hline 11/7/11 9:30 & & 31.15 & 30.678 & 29.576 & 30.794 & 30.024 & 31.142 & 30.622 & & 28.816 & 26.55 & 32.47 & 36.38 & 33.498 & 17.43 & 16.556 & 21.316 & 15.651 & 11.617 & 32.565 & 33.371 & 30.483 & 32.436 & 27.441 & 34.597 \\
\hline 11/7/11 10:00 & & 31.148 & 30.676 & 29.559 & 30.792 & 30.038 & 31.142 & 30.619 & 30.287 & 28.809 & 26.55 & 32.47 & 36.38 & 33.496 & 17.43 & 16.554 & 21.311 & 15.654 & 11.624 & 32.562 & 33.369 & 479 & .433 & 27.441 & 34.6 \\
\hline 11/7/11 10:30 & & 31.146 & 30.673 & 29.554 & 30.787 & 30.045 & 31.14 & 30.622 & 30.282 & 28.802 & 26.531 & 32.465 & 36.373 & 33.493 & 17.43 & 16.552 & 21.309 & 15.665 & 11.643 & 32.553 & 33.364 & 30.469 & 32.429 & 27.441 & 34.6 \\
\hline 11/7/11 11:00 & & 31.143 & 30.671 & 29.552 & 30.785 & 30.045 & 31.14 & 30.622 & 30.285 & 28.805 & 26.531 & 32.465 & 36.375 & 33.491 & 17.43 & 16.549 & 21.304 & 15.673 & 11.657 & 32.546 & 33.364 & 30.465 & 32.429 & 27.443 & 34.602 \\
\hline 11/7/11 11:30 & & 31.136 & 30.659 & 29.531 & 30.771 & 30.05 & 31.14 & 30.622 & 30.278 & 28.8 & 26.524 & 32.461 & 36.373 & 33.484 & 17.43 & 16.545 & 21.302 & 15.684 & 11.687 & 32.53 & 33.36 & 30.446 & 32.424 & 27.443 & 34.598 \\
\hline $11 / 7 / 1112: 00$ & & 31.143 & 30.662 & 29.515 & 30.771 & 30.076 & 31.135 & 30.612 & 30.278 & 28.784 & 26.504 & 32.458 & 36.375 & 33.486 & 17.428 & 16.545 & 21.302 & 15.673 & 11.685 & 32.541 & 33.36 & 30.45 & 32.422 & 27.443 & 34.6 \\
\hline $11 / 7 / 1112: 30$ & & 31.141 & 30.657 & 29.508 & 30.764 & 30.076 & 31.13 & 30.624 & 30.275 & 28.786 & 26.48 & 32.451 & 36.37 & 33.479 & 17.428 & 16.542 & 21.295 & 15.684 & 11.694 & 32.53 & 33.355 & 30.443 & 32.417 & 27.443 & 34.598 \\
\hline 11/7/11 13:00 & & 31.125 & 30.641 & 29.515 & 30.745 & 30.059 & 31.127 & 30.622 & 30.266 & 28.788 & 26.478 & 32.44 & 36.365 & 33.468 & $3 \quad 17.423$ & 16.533 & 21.297 & 15.705 & 11.725 & 32.499 & 33.341 & 30.411 & 32.405 & 27.438 & 34.591 \\
\hline 11/7/11 13:30 & & 31.127 & 30.634 & 29.463 & 30.736 & 30.078 & 31.125 & 30.605 & 30.262 & 28.767 & 26.492 & 32.414 & 36.361 & 33.442 & 17.421 & 16.503 & 21.283 & 15.696 & 11.72 & 32.485 & 33.315 & 30.394 & 32.379 & 27.441 & 34.572 \\
\hline 11/7/11 14:00 & & 31.136 & 30.636 & 29.459 & 30.7 & 30.094 & 31.118 & 30.612 & 30.257 & 28.758 & 26.435 & 32.395 & 36.358 & 33.418 & $\begin{array}{l}37.421 \\
3\end{array}$ & 16.486 & 21.26 & 15.673 & 11.678 & 32.485 & 33.296 & 30.403 & 32.358 & 27.441 & 34.556 \\
\hline $11 / 7 / 1114: 30$ & & 31.122 & 30.627 & 29.505 & 30. & 30.031 & 31.118 & 30.638 & 30.255 & 28.786 & 26.425 & 32.367 & 36.351 & $\begin{array}{l}33.495 \\
33.395\end{array}$ & 17.416 & 16.47 & 21.251 & 15.698 & 11.683 & 32.455 & 33.27 & 30.375 & 32.333 & 27.436 & 34.544 \\
\hline $11 / 7 / 1115: 00$ & & 31.12 & 30.613 & 29.463 & 30.65 & 30.066 & 31.116 & $\begin{array}{l}30.007 \\
30.607\end{array}$ & 30.241 & 28.763 & 26.485 & 32.358 & 36.344 & 33.383 & 17.411 & $\begin{array}{l}16.458 \\
16.48\end{array}$ & 21.244 & $\begin{array}{l}15.696 \\
15.696\end{array}$ & $\begin{array}{l}11.003 \\
11.692\end{array}$ & 32.441 & 33.258 & 30.352 & 32.323 & 27.443 & 34.539 \\
\hline $11 / 7 / 1115: 30$ & & 31.122 & 30.606 & 29.484 & 30.68 & 30.08 & 31.106 & 30.612 & 30.234 & 28.753 & 26.437 & 32.339 & $\begin{array}{r}36.34 \\
36.34\end{array}$ & $\begin{array}{l}33.364 \\
33.364\end{array}$ & 17.404 & $\begin{array}{l}10.400 \\
16.499\end{array}$ & 21.228 & 15.684 & $\begin{array}{l}11.671 \\
11.671\end{array}$ & 32.427 & $\begin{array}{l}33.239 \\
3.239\end{array}$ & 0.349 & 32.307 & 27.441 & 34.527 \\
\hline $\begin{array}{l}11 / 7 / 1 \\
11\end{array}$ & & 31. & 30.62 & $\begin{array}{l}29.404 \\
29.463\end{array}$ & $\begin{array}{l}30.6 \\
30.6\end{array}$ & $\begin{array}{l}30.050 \\
30.054\end{array}$ & 31.101 & $\begin{array}{l}30.012 \\
30.615\end{array}$ & $\begin{array}{l}30.245 \\
30.245\end{array}$ & 28.758 & $\begin{array}{l}20.451 \\
26.459\end{array}$ & 32.33 & $\begin{array}{l}36.344 \\
36.347\end{array}$ & $\begin{array}{l}33.354 \\
33.353\end{array}$ & 17.407 & $\begin{array}{l}10.449 \\
16.437\end{array}$ & 21.216 & $\begin{array}{l}13.004 \\
15.68\end{array}$ & $\begin{array}{l}11.031 \\
11.631\end{array}$ & 32.429 & 33.229 & .364 & 95 & 27.438 & 34.523 \\
\hline 11 & & 31 & 30.6 & 29 & 30 & 3 & 31 & 30 & 30 & 28 & 26 & 32 & 36 & 3 & 17 & 16.433 & 21 & 4 & $\begin{array}{l}11.662 \\
11.62\end{array}$ & 32.408 & 33.225 & 31 & 32.288 & 27.441 & 34.518 \\
\hline $11 / 7 / 1$ & & & 30 & 29 & & & 31 & & 30 & & 26 & 32 & 36.337 & 33.353 & & 16.43 & 21 & 15.682 & 11.645 & 32.418 & 33.225 & 7 & 9 & 27.438 & 34.516 \\
\hline $11 / 7 / 1$ & & 31 & & 29.468 & 30 & & 30.774 & & & & & & 36.33 & 33.35 & & 16.426 & 21. & 39 & 11.666 & 32.406 & 33.225 & 24 & 88 & 27.441 & 34.513 \\
\hline $11 / 7$ & & 31 & & 29.477 & & & & & & & & 32.316 & 36.328 & 33.339 & 17.395 & 16.419 & 21.242 & 15.689 & 11.666 & 32.394 & 33.21 & 0.309 & 2.276 & 27.443 & 34.506 \\
\hline & & & & & & & 30.4 & & & & & 32. & 36.328 & 33.339 & & 16.414 & 21.23 & 15.687 & 11.659 & 32.39 & 33.208 & .307 & .274 & 27.443 & 34.504 \\
\hline & & & & & & & 30.4 & & & & & 32.3 & 36.33 & 33.339 & 17. & 16.416 & 21. & 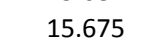 & 11.64 & 32.406 & 33.213 & 26 & 32.279 & 27.445 & 34.506 \\
\hline 11/7/11 19:30 & & 31.127 & & 29. & & & 30 & 30.6 & & & & 32 & 36.328 & 33.346 & 17.3 & 16.416 & 21 & 15.663 & 11.626 & 32.418 & 33.22 & 30.338 & 32.286 & 27.443 & 34.511 \\
\hline $11 / 7 / 1120: 00$ & & 31.132 & 30. & 29.473 & 30. & & 30.5 & 30.61 & & 28. & 26.457 & 32.325 & 36.33 & 33.348 & 17. & 16.416 & 21.228 & 15.651 & 11.6 & 32.429 & 33.222 & 30.354 & 32.288 & 27.445 & 34.511 \\
\hline 11/7/11 20:30 & & 31.12 & 30.5 & 29.503 & 30.6 & & 30.5 & 30.617 & 30.2 & 28.753 & 26.457 & 32.3 & 36.33 & 33.35 & 17.3 & 16.416 & 21.228 & 15.6 & 11.624 & 32.408 & 33.225 & 30.328 & 32.286 & 27.441 & 34.511 \\
\hline 11/7/11 21:00 & & 31.115 & 30.592 & 29.505 & 30. & 29.998 & 30.58 & 30.612 & 30.217 & 28.758 & 26.485 & 32.318 & 36.328 & 33.346 & 17.388 & 16.414 & 21.221 & 15.677 & 11.633 & 32.404 & 33.217 & 30.319 & 32.286 & 27.443 & 34.511 \\
\hline $11 / 7 / 1121: 30$ & & 31.115 & 30.592 & 29.489 & 30.638 & 30.003 & 30.623 & 30.612 & 30.217 & 28.753 & 26.49 & 32.323 & 36.328 & 33.348 & 17.388 & 16.419 & 21.221 & 15.677 & 11.631 & 32.408 & 33.222 & 30.314 & 32.283 & 27.443 & 34.513 \\
\hline $\begin{array}{l}11 / 7 / 1122: 00 \\
\text { 11/ }\end{array}$ & & 31.129 & 30.608 & 29.496 & 30.647 & 30 & 30.644 & 30.617 & 30.224 & 28.756 & 26.478 & 32.327 & 36.335 & 33.35 & 17.39 & 16.419 & 21.219 & 15.654 & 11.596 & 32.427 & 33.225 & 30.342 & 32.29 & 27.445 & 34.516 \\
\hline $11 / 7 / 1122: 30$ & & 31.122 & 30.603 & 29.496 & 30.647 & 30 & 30.668 & 30.607 & 30.224 & 28.756 & 26.483 & 32.327 & 36.337 & 33.35 & 17.39 & 16.416 & 21.221 & 15.665 & 11.607 & 32.418 & 33.227 & 30.326 & 32.293 & 27.443 & 34.518 \\
\hline 11/7/11 23:00 & & 31.115 & 30.596 & 29.48 & 30.642 & 30 & 30.688 & 30.603 & 30.22 & 28.746 & 26.483 & 32.325 & 36.335 & 33.35 & 17.39 & 16.416 & 21.216 & 15.675 & 11.612 & 32.413 & 33.222 & 30.319 & 32.288 & 27.443 & 34.516 \\
\hline 11/7/11 23:30 & & 31.115 & 30.596 & 29.494 & 30.64 & 30.003 & 30.702 & 30.615 & 30.222 & 28.756 & 26.468 & 32.323 & 36.335 & 33.35 & 17.388 & 16.416 & 21.212 & 15.67 & 11.607 & 32.408 & 33.222 & 30.317 & 32.288 & 27.443 & 34.516 \\
\hline 11/8/11 0:00 & & 31.113 & 30.596 & 29.51 & 30.635 & 29.993 & 30.723 & 30.61 & 30.22 & 28.753 & 26.478 & 32.323 & 36.34 & 33.348 & 17.386 & 16.416 & 21.207 & 15.673 & 11.607 & 32.406 & 33.222 & 30.312 & 32.288 & 27.445 & 34.516 \\
\hline 11/8/11 0:30 & & 31.108 & 30.589 & 29.508 & 30.626 & 29.981 & 30.74 & 30.61 & 30.213 & 28.758 & 26.497 & 32.318 & 36.335 & 33.343 & 17.386 & 16.411 & 21.2 & 15.68 & 11.617 & 32.397 & 33.217 & 30.293 & 32.279 & 27.443 & 34.516 \\
\hline
\end{tabular}




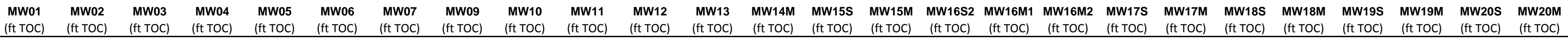

\begin{tabular}{|c|c|c|c|c|c|c|c|c|c|c|c|c|c|c|c|c|c|c|c|c|c|c|c|c|c|c|}
\hline Well & $\begin{array}{l}\text { MW01 } \\
\text { (ft TOC) }\end{array}$ & $\begin{array}{r}\text { MW02 } \\
\text { (ft TOC) }\end{array}$ & $\begin{array}{l}\text { MW03 } \\
\text { (ft ToC) }\end{array}$ & $\begin{array}{l}\text { MW04 } \\
\text { (ft ToC) }\end{array}$ & $\begin{array}{r}\text { MW05 } \\
\text { (ft TOC) }\end{array}$ & $\begin{array}{r}\text { MW06 } \\
\text { (ft TOC) }\end{array}$ & $\begin{array}{l}\text { MW07 } \\
\text { (ft TOC) }\end{array}$ & $\begin{array}{l}\text { MW09 } \\
\text { (ft TOC) }\end{array}$ & $\begin{array}{l}\text { MW10 } \\
\text { (ft TOC) }\end{array}$ & $\begin{array}{l}\text { MW11 } \\
\text { (ft TOC) }\end{array}$ & $\begin{array}{l}\text { MW12 } \\
\text { (ft TOC) }\end{array}$ & $\begin{array}{l}\mathrm{MW13} \\
\text { (ft TOC) }\end{array}$ & $\begin{array}{l}\text { MW14M } \\
\text { (ft TOC) }\end{array}$ & $\begin{array}{l}\text { MWW15S } \\
\text { (ft TOC) }\end{array}$ & $\begin{array}{l}\text { MWW15M } \\
(\mathrm{ft} \text { TOC) }\end{array}$ & $\begin{array}{l}\text { MW16S2 } \\
\text { (ft TOC) }\end{array}$ & $\begin{array}{c}\text { MW16M1 } \\
\text { (ft TOC) }\end{array}$ & $\begin{array}{l}\text { MW16M2 } \\
\text { (ft TOC) }\end{array}$ & $\begin{array}{l}\text { MW17S } \\
(\mathrm{ft} \text { TOC) }\end{array}$ & $\begin{array}{l}\text { MW17M } \\
\text { (ft TOC) }\end{array}$ & $\begin{array}{l}\mathrm{MW18S} \\
\text { (ft TOC) }\end{array}$ & $\begin{array}{r}\text { MW18M } \\
\text { (ft TOC) } \\
\end{array}$ & $\begin{array}{l}\text { MWW19S } \\
\text { (ft TOC) }\end{array}$ & $\begin{array}{l}\text { MW19M } \\
\text { (ft TOC) }\end{array}$ & $\begin{array}{l}\text { MWO20S } \\
\text { (ft TOC) }\end{array}$ & $\begin{array}{l}\text { MW2OM } \\
\text { (ft TOC) }\end{array}$ \\
\hline 11/8/11 1:00 & & 31.118 & 30.601 & 29.477 & 30.633 & & 30.005 & 30.757 & 30.607 & 30.217 & 28.751 & 26.495 & 32.32 & 36.344 & 33.343 & 17.386 & 16.414 & 21.198 & 15.661 & 11.591 & 32.415 & 33.22 & 30.312 & 32.283 & 27.448 & 34.516 \\
\hline $11 / 8 / 111: 30$ & & 31.125 & 30.608 & 29.435 & 30.638 & & 30.029 & 30.769 & 30.603 & 30.222 & 28.737 & 26.468 & 32.32 & 36.342 & 33.348 & $\begin{array}{l}37.388 \\
\end{array}$ & 16.411 & 21.193 & 15.654 & 11.575 & 32.418 & 33.22 & 30.324 & 32.286 & 27.445 & 34.518 \\
\hline $11 / 8 / 112: 00$ & & 31.111 & 30.596 & 29.482 & 30.631 & & 30.019 & 30.783 & 30.607 & 30.222 & 28.739 & 26.428 & 32.318 & 36.344 & 33.343 & 3 17.384 & 16.407 & 21.191 & 15.67 & 11.593 & 32.406 & 533.215 & 30.302 & 32.279 & 27.445 & 34.516 \\
\hline $11 / 8 / 112: 30$ & & 31.099 & 30.582 & 29.505 & 30.612 & & 29.991 & 30.8 & 30.615 & 30.21 & 28.753 & 26.471 & 32.308 & 36.34 & 33.334 & 17.381 & 16.402 & 21.182 & 15.687 & 11.619 & 32.38 & 33.208 & 30.26 & 32.272 & 27.443 & 34.511 \\
\hline $11 / 8 / 113: 00$ & & 31.094 & 30.573 & 29.498 & 30.596 & & 29.989 & 30.814 & 30.6 & 30.197 & 28.753 & 26.497 & 32.299 & 36.333 & 33.327 & 17.377 & 16.395 & 21.175 & 15.687 & 11.624 & 32.366 & 33.201 & 30.253 & 32.262 & 27.445 & 34.504 \\
\hline $11 / 8 / 113: 30$ & & 31.118 & 30.592 & 29.491 & 30.607 & & 29.986 & 30.826 & 30.603 & 30.21 & 28.751 & 26.49 & 32.301 & 36.337 & 33.332 & 17.379 & 16.4 & 21.172 & 15.654 & 11.58 & 32.394 & 33.201 & 30.291 & 32.269 & 27.448 & 34.509 \\
\hline $11 / 8 / 114: 00$ & & 31.12 & 30.599 & 29.496 & 30.619 & & 29.981 & 30.838 & 30.612 & 30.217 & 28.753 & 26.48 & 32.304 & 36.342 & 33.329 & 17.381 & 16.397 & 21.17 & 15.651 & 11.568 & 32.404 & 33.208 & 30.298 & 32.269 & 27.448 & 34.509 \\
\hline $11 / 8 / 114: 30$ & & 31.12 & 30.599 & 29.498 & 30.617 & & 29.996 & 30.85 & 30.603 & 30.213 & 28.751 & 26.485 & 32.301 & 36.347 & 33.329 & 17.379 & 16.393 & 21.163 & 15.651 & 11.558 & 32.404 & 33.201 & 30.3 & 32.267 & 27.448 & 34.509 \\
\hline $11 / 8 / 11$ 5:00 & & 31.111 & 30.594 & 29.501 & 30.61 & & 29.981 & 30.86 & 30.605 & 30.213 & 28.756 & 26.485 & 32.299 & 36.342 & 33.329 & 17.379 & 16.39 & 21.161 & 15.656 & 11.565 & 32.392 & 33.196 & 30.291 & 32.264 & 27.448 & 34.504 \\
\hline $11 / 8 / 115: 30$ & & 31.111 & $\begin{array}{l}30.594 \\
30.594\end{array}$ & 29.505 & $\begin{array}{l}30.01 \\
30.61\end{array}$ & & 29.981 & $\begin{array}{l}30.80 \\
30.867\end{array}$ & 30.607 & $\begin{array}{l}30.213 \\
30.213\end{array}$ & 28.753 & $\begin{array}{l}26.489 \\
26.492\end{array}$ & $\begin{array}{l}32.299 \\
32.297\end{array}$ & $\begin{array}{l}30.342 \\
36.347\end{array}$ & $\begin{array}{l}33.329 \\
33.325\end{array}$ & $\begin{array}{l}17.377 \\
5\end{array}$ & $\begin{array}{l}10.39 \\
16.39\end{array}$ & $\begin{array}{l}21.156 \\
21.156\end{array}$ & $\begin{array}{l}13.030 \\
15.658\end{array}$ & $\begin{array}{l}11.030 \\
11.565\end{array}$ & $\begin{array}{l}32.392 \\
32.392\end{array}$ & $\begin{array}{l}33.199 \\
3.199\end{array}$ & $\begin{array}{l}30.281 \\
30.281\end{array}$ & $\begin{array}{l}32.204 \\
32.262\end{array}$ & $\begin{array}{r}27.48 \\
27.45\end{array}$ & $\begin{array}{l}34.504 \\
34.504\end{array}$ \\
\hline $11 / 8 / 116: 00$ & & 31.113 & 30.592 & 29.501 & 30.607 & & 29.984 & 30.874 & 30.598 & 30.213 & 28.751 & 26.492 & 32.294 & 36.351 & 33.325 & 17.374 & 16.388 & 21.154 & 15.656 & 11.553 & 32.399 & 33.196 & 30.284 & 32.257 & 27.45 & 34.504 \\
\hline $11 / 8 / 116: 30$ & & 31.108 & 30.594 & 29.505 & 30.607 & & 29.979 & 30.884 & 30.61 & 30.213 & 28.758 & 26.488 & 32.294 & 36.342 & 33.322 & 17.377 & 16.388 & 21.161 & 15.661 & 11.556 & 32.385 & 33.194 & 30.284 & 32.257 & 27.448 & 34.504 \\
\hline $11 / 8 / 11$ 7:00 & & 31.113 & 30.599 & 29.501 & 30.607 & & 29.972 & 30.893 & 30.607 & 30.215 & 28.76 & 26.492 & 32.294 & 36.347 & 33.32 & 17.374 & 16.386 & 21.168 & 15.654 & 11.546 & 32.394 & 33.194 & 30.286 & 32.26 & 27.45 & 34.506 \\
\hline $11 / 8 / 117: 30$ & & 31.108 & 30.594 & 29.515 & 30.605 & & 29.972 & 30.903 & 30.607 & 30.213 & 28.763 & 26.495 & 32.292 & 36.351 & 33.317 & 17.374 & 16.383 & 21.168 & 15.656 & 11.549 & 32.387 & 33.194 & 30.284 & 32.257 & 27.45 & 34.504 \\
\hline $11 / 8 / 11$ 8:00 & & 31.111 & 30.596 & 29.533 & 30.605 & & 29.963 & 30.91 & 30.607 & 30.213 & 28.772 & 26.504 & 32.292 & 36.356 & $\begin{array}{r}33.32 \\
33\end{array}$ & 17.372 & 16.386 & 21.168 & 15.656 & 11.542 & 32.387 & $\begin{array}{l}33.194 \\
\end{array}$ & 30.284 & 32.257 & 27.452 & 34.504 \\
\hline $11 / 8 / 118: 30$ & & 31.111 & 30.596 & 29.531 & 30.607 & & 29.97 & 30.919 & 30.607 & 30.21 & 28.77 & 26.524 & 32.292 & 36.354 & $\begin{array}{l}35.32 \\
33.32\end{array}$ & 17.372 & $\begin{array}{l}10.500 \\
16.383\end{array}$ & 21.165 & $\begin{array}{l}15.050 \\
15.654\end{array}$ & 11.537 & 32.392 & 33.191 & 30.286 & 32.257 & 27.45 & 34.504 \\
\hline $11 / 8 / 119: 00$ & & 31 & 30.601 & 29.526 & 30.61 & & 29.977 & 30.924 & 30.603 & 30.217 & 28.767 & 26.519 & 32.294 & 36.356 & $\begin{array}{l}33.32 \\
33.32\end{array}$ & $\begin{array}{r}17.37 \\
2\end{array}$ & $\begin{array}{l}10.503 \\
16.386\end{array}$ & 21.163 & $\begin{array}{l}15.044 \\
15.649\end{array}$ & 11.53 & 32.394 & 33.194 & 30.293 & 32.26 & 7.45 & 34.506 \\
\hline 11/8/11 9:30 & & 31 & 30.606 & 29.524 & 30.617 & & 29.972 & 30.934 & 30.598 & 30.222 & 28.765 & 26.512 & 32.297 & 36.358 & 33.325 & 17.37 & 16.386 & 21.158 & 15.654 & 11.528 & 32.399 & 33.194 & 30.293 & 32.262 & 7.45 & 34.509 \\
\hline $11 / 8 / 1110: 00$ & & 31.111 & 30.606 & 29.515 & 30.617 & & 29.996 & 30.939 & 30.588 & 30.222 & 28.756 & 26.514 & 32.294 & 36.358 & 33.322 & 17.37 & 16.383 & 21.154 & 15.651 & 11.528 & 32.397 & 33.196 & 30.295 & 32.26 & 27.45 & 34.509 \\
\hline $11 / 8 / 1110: 30$ & & 31.108 & 30.606 & 29.519 & 30.614 & & 29.984 & 30.941 & 30.6 & 30.222 & 28.765 & 26.502 & 32.294 & 36.365 & 33.32 & 17.367 & 16.383 & 21.151 & 15.649 & 11.521 & 32.399 & $\begin{array}{ll}9 & 33.196\end{array}$ & 30.295 & 32.257 & 27.452 & 34.506 \\
\hline 11/8/11 11:00 & & 31.106 & 30.601 & 29.503 & 30.612 & & 30 & 30.946 & 30.605 & 30.22 & 28.765 & 26.507 & 32.283 & 36.363 & 33.306 & 17.365 & 16.374 & 21.14 & 15.651 & 11.521 & 32.385 & 33.182 & 30.281 & 32.248 & 27.45 & 34.499 \\
\hline 11/8/11 11:30 & & 31.106 & 30.601 & 29.496 & 30.605 & & 29.991 & 30.948 & 30.6 & 30.217 & 28.758 & 26.492 & 32.273 & 36.363 & 33.303 & 17.365 & 16.367 & 21.133 & 15.649 & 11.518 & 32.38 & $\begin{array}{l}33.172 \\
\end{array}$ & 30.277 & 32.239 & 27.45 & 34.497 \\
\hline $11 / 8 / 1112: 00$ & & 31.108 & 30.601 & 29.468 & 30.605 & & 30.019 & 30.951 & 30.588 & 30.217 & 28.739 & 26.485 & 32.276 & 36.365 & 33.299 & 17.363 & 16.367 & 21.128 & 15.651 & 11.523 & 32.38 & $\begin{array}{l}33.175 \\
3\end{array}$ & 30.274 & 32.241 & 27.453 & 34.497 \\
\hline $11 / 8 / 1112: 30$ & & 31.099 & 30.594 & 29.459 & 30.596 & & 30.024 & 30.953 & 30.596 & 30.213 & 28.739 & 26.457 & 32.271 & 36.358 & 33.299 & 17.358 & $\begin{array}{l}16.362 \\
\text {. }\end{array}$ & 21.121 & 15.663 & 11.53 & 32.369 & 33.17 & 30.262 & 32.232 & 27.45 & 34.497 \\
\hline $11 / 8 / 1113: 00$ & & 31.09 & 30.582 & 29.491 & 30.579 & & 30.021 & 30.953 & 30.596 & 30.208 & 28.735 & 26.447 & 32.264 & 36.354 & 33.289 & 17.353 & $\begin{array}{l}16.358 \\
\end{array}$ & 21.117 & 15.677 & 11.56 & 32.348 & $\begin{array}{l}33.163 \\
3\end{array}$ & 30.234 & 32.227 & 27.448 & 34.495 \\
\hline 11/8/11 13:30 & & 31.083 & 30.568 & 29.468 & 30.563 & & 30.019 & 30.958 & 30.588 & $\begin{array}{l}30.199 \\
309\end{array}$ & 28.732 & 26.476 & 32.248 & 36.351 & 33.271 & 17.349 & $\begin{array}{l}10.30 \\
16.36\end{array}$ & 21.108 & 15.68 & $\begin{array}{l}11.563 \\
1.563\end{array}$ & 32.329 & 33.144 & $\begin{array}{l}30.211 \\
30.211\end{array}$ & 32.213 & 27.448 & 34.485 \\
\hline $11 / 8 / 1114: 00$ & & $\begin{array}{l}31.087 \\
31.087\end{array}$ & $\begin{array}{l}30.500 \\
30.571\end{array}$ & $\begin{array}{l}29.400 \\
29.482\end{array}$ & 30.556 & & 30.01 & 30.958 & $\begin{array}{l}30.500 \\
30.596\end{array}$ & 30.194 & 28.739 & $\begin{array}{l}20.410 \\
26.457\end{array}$ & $\begin{array}{l}32.240 \\
32.245\end{array}$ & $\begin{array}{l}36.347 \\
36.37\end{array}$ & $\begin{array}{l}33.275 \\
33.271\end{array}$ & $\begin{array}{l}17.346 \\
5\end{array}$ & $\begin{array}{l}10.340 \\
16.348\end{array}$ & $\begin{array}{l}21.105 \\
21.105\end{array}$ & $\begin{array}{l}15.600 \\
15.668\end{array}$ & $\begin{array}{r}11.003 \\
11.56\end{array}$ & 32.331 & $\begin{array}{l}33.144 \\
33.146\end{array}$ & 30.215 & 32.213 & 27.445 & 34.485 \\
\hline $11 / 8 / 11$ 14:30 & & 31.106 & $\begin{array}{l}30.5 / 1 \\
30.582 \\
\end{array}$ & $\begin{array}{l}29.482 \\
29.45\end{array}$ & $\begin{array}{l}30.560 \\
30.568\end{array}$ & & $\begin{array}{r}30.998 \\
29.998\end{array}$ & $\begin{array}{l}30.950 \\
30.963\end{array}$ & 30.598 & $\begin{array}{l}30.1244 \\
30.204\end{array}$ & 28.744 & $\begin{array}{l}20.471 \\
26.471\end{array}$ & $\begin{array}{l}32.25 \\
32.25\end{array}$ & $\begin{array}{l}30.341 \\
36.351\end{array}$ & $\begin{array}{l}33.275 \\
33.275\end{array}$ & $\begin{array}{l}17.340 \\
17.346\end{array}$ & $\begin{array}{l}10.348 \\
16.346\end{array}$ & 21.105 & $\begin{array}{l}13.060 \\
15.651\end{array}$ & $\begin{array}{l}11.530 \\
11.537\end{array}$ & $\begin{array}{l}32.331 \\
32.345\end{array}$ & $\begin{array}{l}33.140 \\
33.151\end{array}$ & $\begin{array}{l}30.237 \\
30.237\end{array}$ & $\begin{array}{l}32.213 \\
32.215\end{array}$ & $\begin{array}{r}27.443 \\
27.45\end{array}$ & $\begin{array}{l}34.483 \\
34.488\end{array}$ \\
\hline $11 / 8 / 1115: 00$ & & 31.094 & $\begin{array}{l}30.582 \\
30.566\end{array}$ & $\begin{array}{l}29.478 \\
29.47\end{array}$ & $\begin{array}{l}30.568 \\
30.549\end{array}$ & & $\begin{array}{l}\begin{array}{l}29.998 \\
30.003\end{array}\end{array}$ & $\begin{array}{l}30.903 \\
30.963\end{array}$ & $\begin{array}{l}30.598 \\
30.598\end{array}$ & $\begin{array}{l}50.244 \\
30.192\end{array}$ & $\begin{array}{l}28.744 \\
28.739\end{array}$ & $\begin{array}{l}20.4 / 1 \\
26.466\end{array}$ & $\begin{array}{r}32.243 \\
32.241\end{array}$ & $\begin{array}{l}30.351 \\
36.347\end{array}$ & $\begin{array}{l}33.273 \\
33.268\end{array}$ & $\begin{array}{l}11.740 \\
3 \\
3\end{array}$ & $\begin{array}{l}10.340 \\
16.339\end{array}$ & $\begin{array}{l}21.094 \\
21.094\end{array}$ & $\begin{array}{l}13.051 \\
15.665\end{array}$ & $\begin{array}{l}11.531 \\
11.551\end{array}$ & $\begin{array}{l}32.345 \\
32.324\end{array}$ & $\begin{array}{l}33.131 \\
33.139\end{array}$ & .208 & $\begin{array}{l}32.215 \\
32.206\end{array}$ & $\begin{array}{r}27.45 \\
27.445\end{array}$ & $\begin{array}{l}34.480 \\
34.481\end{array}$ \\
\hline $11 / 8 / 1115: 30$ & & $\begin{array}{l}31.044 \\
31.097\end{array}$ & $\begin{array}{l}30.566 \\
30.571\end{array}$ & $\begin{array}{l}29.484 \\
29.484\end{array}$ & $\begin{array}{l}30.549 \\
30.551\end{array}$ & & $\begin{array}{l}\begin{array}{l}30.003 \\
29.993\end{array}\end{array}$ & $\begin{array}{l}30.963 \\
30.965\end{array}$ & $\begin{array}{l}30.598 \\
30.596\end{array}$ & $\begin{array}{l}30.192 \\
30.197\end{array}$ & $\begin{array}{l}28.749 \\
28.74\end{array}$ & $\begin{array}{l}26.466 \\
26.459\end{array}$ & $\begin{array}{l}32.241 \\
32.236\end{array}$ & $\begin{array}{l}30.34 ! \\
36.344\end{array}$ & $\begin{array}{l}33.268 \\
33.261\end{array}$ & $\begin{array}{l}11.339 \\
17.342\end{array}$ & $\begin{array}{l}10.399 \\
16.337\end{array}$ & $\begin{array}{l}21.084 \\
21.087\end{array}$ & $\begin{array}{l}13.665 \\
15.658\end{array}$ & $\begin{array}{l}11.511 \\
11.532\end{array}$ & $\begin{array}{l}32.324 \\
32.331\end{array}$ & $\begin{array}{l}33.139 \\
33.132\end{array}$ & $\begin{array}{l}30.288 \\
30.215\end{array}$ & $\begin{array}{l}32.206 \\
32.196\end{array}$ & $\begin{array}{l}27.445 \\
27.443\end{array}$ & $\begin{array}{l}\begin{array}{l}34.441 \\
34.478\end{array} \\
\end{array}$ \\
\hline $11 / 8 / 1116: 00$ & & $\begin{array}{l}31.097 \\
31.092\end{array}$ & $\begin{array}{l}30.5 / 1 \\
30.568\end{array}$ & $\begin{array}{l}29.484 \\
29.515\end{array}$ & $\begin{array}{l}30.551 \\
30.542\end{array}$ & & $\begin{array}{l}29.993 \\
29.974\end{array}$ & $\begin{array}{l}30.965 \\
30.967\end{array}$ & $\begin{array}{l}30.596 \\
30.598\end{array}$ & $\begin{array}{l}30.197 \\
30.192\end{array}$ & $\begin{array}{l}28.744 \\
28.751\end{array}$ & $\begin{array}{l}26.459 \\
26.471\end{array}$ & $\begin{array}{l}32.266 \\
32.227\end{array}$ & 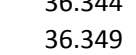 & $\begin{array}{l}33.261 \\
33.254\end{array}$ & $\begin{array}{l}17.342 \\
17.337\end{array}$ & $\begin{array}{l}16.331 \\
16.341\end{array}$ & $\begin{array}{l}21.081 \\
21.084\end{array}$ & $\begin{array}{l}13.658 \\
15.663\end{array}$ & $\begin{array}{r}11.532 \\
11.53\end{array}$ & $\begin{array}{l}32.331 \\
32.324\end{array}$ & $\begin{array}{l}33.132 \\
33.127\end{array}$ & 0.206 & $\begin{array}{l}32.196 \\
32.192\end{array}$ & $\begin{array}{l}27.443 \\
27.441\end{array}$ & $\begin{array}{l}34.48 \\
34.474\end{array}$ \\
\hline $11 / 8 / 1116: 30$ & & 31 & 30 & 29 & 30. & & 29.958 & 30.967 & 30.596 & 30.19 & 28 & 26.507 & 32.224 & 36.347 & 33.254 & 17.335 & & 21.119 & 15.654 & 11.523 & 32.32 & 33.125 & .204 & 32.192 & 27.441 & $\begin{array}{l}34.474 \\
34.474\end{array}$ \\
\hline $17: 00$ & & & & & & & & & & 30. & & $\begin{array}{l}20.501 \\
26.507\end{array}$ & $\begin{array}{l}32.24 \\
32.227\end{array}$ & $\begin{array}{l}30.341 \\
36.349\end{array}$ & $\begin{array}{l}33.234 \\
33.252\end{array}$ & $\begin{array}{l}17.3332 \\
2.332\end{array}$ & & 21.131 & $\begin{array}{l}13.034 \\
15.654\end{array}$ & $\begin{array}{l}11.525 \\
11.509\end{array}$ & $\begin{array}{l}32.32 \\
32.324\end{array}$ & $\begin{array}{l}33.125 \\
3.125\end{array}$ & 11 & $\begin{array}{l}32.192 \\
32.192\end{array}$ & 27.438 & $\begin{array}{l}34.474 \\
34.474\end{array}$ \\
\hline $11 / 8 / 11$ 17:30 & & 31.111 & $\begin{array}{l}30.5 / 1 \\
30.587\end{array}$ & $\begin{array}{l}29.534 \\
29.538\end{array}$ & $\begin{array}{l}30.544 \\
30.558\end{array}$ & & 29.946 & $\begin{array}{l}30.972 \\
30.972\end{array}$ & 30.598 & 30.204 & 28.772 & 26.533 & $\begin{array}{l}32.227 \\
32.229\end{array}$ & $\begin{array}{l}30.349 \\
36.356\end{array}$ & $\begin{array}{l}33.252 \\
33.257\end{array}$ & $\begin{array}{l}17.332 \\
17.335\end{array}$ & & 21.128 & $\begin{array}{l}13.034 \\
15.633\end{array}$ & $\begin{array}{l}11.099 \\
11.473\end{array}$ & $\begin{array}{l}32.324 \\
32.345\end{array}$ & $\begin{aligned} 33.1250 \\
33.13\end{aligned}$ & 34 & 94 & $\begin{array}{l}27.440 \\
\end{array}$ & $\begin{array}{l}34.444 \\
34.474\end{array}$ \\
\hline $11 / 8 / 1118: 00$ & & 31.108 & 30.592 & 29.569 & 30.558 & & 29.939 & 30.161 & 30.605 & 30.204 & 28.779 & 26.526 & 32.222 & 36.356 & $\begin{array}{r}33.25 \\
33.25\end{array}$ & $\begin{array}{l}17.332 \\
5\end{array}$ & & 21.121 & 15.633 & $\begin{array}{l}11.455 \\
11.45\end{array}$ & 32.338 & 33.125 & 30.239 & 32.187 & 27.438 & 34.471 \\
\hline $11 / 8 / 1118: 30$ & & 31.111 & 30.599 & 29.587 & 30 & & 18 & 29.505 & 30.603 & 30.213 & 28.793 & 26.557 & 32.217 & 36.363 & 33.242 & 17.332 & & 21.117 & 15.628 & 11.434 & 32.348 & 33.118 & 30.244 & 32.185 & 27.436 & 34.467 \\
\hline $11 / 8 / 1119: 00$ & & 31.108 & 30.601 & 29.604 & 30 & & & 29.242 & 30.603 & 30.21 & 28.8 & 26.571 & 32.213 & 36.363 & 33.24 & $\quad 17.33$ & & 21.11 & 15.628 & 11.417 & 32.352 & 33.113 & 30.244 & 32.18 & 27.434 & 34.464 \\
\hline $11 / 8 / 1119: 30$ & & 31.118 & 30.617 & 29.62 & 30. & & & 28.78 & 30.607 & & 28.809 & 26.583 & 32.22 & 36.37 & 33.245 & 17.332 & & 21.108 & 15.614 & 11.389 & 32.364 & 33.123 & 30.267 & 32.185 & 27.434 & 34.467 \\
\hline $11 / 8 / 1120: 00$ & & 31.118 & 30.622 & 29.629 & 30.596 & & 29 & 27.01 & 30.603 & 30.234 & 28.818 & 26.6 & 32.227 & 36.377 & 33.25 & 17.335 & & 21.103 & 15.614 & 11.377 & 32.371 & 33.125 & 30.27 & 32.192 & 27.436 & 34.464 \\
\hline $11 / 8 / 1120: 30$ & & 31.12 & 30.636 & 29.641 & 30. & & 29.904 & 26.6 & 30.605 & 30.241 & 28.825 & 26.61 & 32.227 & 36.38 & 33.252 & 17.335 & & 21.096 & 15.609 & 11.351 & 32.38 & 33.127 & 30.279 & 32.192 & 27.436 & 34.464 \\
\hline $11 / 8 / 1121: 00$ & & 31.125 & 30.645 & 29.648 & & & 29.904 & 26.512 & 30.607 & 30.248 & 28.832 & 26.619 & 32.227 & 36.38 & 33.254 & 177.335 & & 21.089 & 15.6 & 11.325 & 32.387 & 33.127 & 30.288 & 32.196 & 27.434 & 34.464 \\
\hline 11/8/11 21:30 & & 31.122 & 30.655 & 29.662 & 30. & & 29.899 & 26.751 & 30.61 & 30.257 & 28.844 & 26.626 & 32.229 & 36.38 & 33.254 & 17.337 & & 21.08 & 15.6 & 11.307 & 32.392 & 33.13 & 30.288 & 32.194 & 27.431 & 34.457 \\
\hline $11 / 8 / 1122: 00$ & & 31.125 & 30.664 & 29.669 & 30.631 & & 29.904 & 26.799 & 30.605 & 30.266 & 28.849 & 26.638 & 32.229 & 36.387 & 33.254 & $\begin{array}{l}17.335 \\
7\end{array}$ & & 21.071 & 15.597 & 11.285 & 32.39 & 33.127 & 30.291 & 32.194 & 27.434 & 34.453 \\
\hline $11 / 8 / 1122: 30$ & & 31.127 & 30.671 & 29.671 & 30.638 & & 29.909 & 24.933 & 30.61 & 30.273 & 28.853 & 26.643 & 32.236 & 36.391 & 33.259 & 17.335 & & 21.066 & 15.595 & 11.276 & 32.399 & $\begin{array}{l}33.137 \\
\end{array}$ & 30.298 & 32.201 & 27.431 & 34.455 \\
\hline $11 / 8 / 1123: 00$ & & 31.127 & 30.683 & 29.669 & $\begin{array}{r}30.64 \\
\end{array}$ & & 29.916 & 23.38 & 30.603 & 30.28 & 28.858 & 26.641 & 32.238 & 36.382 & 33.264 & 17.337 & & 21.068 & 15.597 & 11.269 & 32.404 & $\begin{array}{r}33.142 \\
7\end{array}$ & 30.302 & 32.206 & 27.431 & 34.455 \\
\hline $11 / 8 / 11$ 23:30 & & 31.127 & 30.685 & 29.671 & 30.645 & & 29.927 & 22.119 & 30.605 & $\begin{array}{l}30.280 \\
30.287\end{array}$ & $\begin{array}{l}20.030 \\
28.86\end{array}$ & 26.636 & 32.241 & 36.389 & $\begin{array}{l}53.204 \\
33.266\end{array}$ & $\begin{array}{l}17.335 \\
\end{array}$ & & 21.066 & 15.6 & $\begin{array}{l}11.262 \\
11.262\end{array}$ & 32.408 & $\begin{array}{r}33.139 \\
3\end{array}$ & 30.298 & 32.206 & 27.431 & 34.455 \\
\hline $11 / 9 / 110$ & & 31.125 & 30.687 & 29.676 & 30.64 & & 29.932 & $\begin{array}{l}21.609 \\
21.609\end{array}$ & 30.607 & $\begin{array}{l}50.291 \\
30.292\end{array}$ & $\begin{array}{l}28.80 \\
28.865\end{array}$ & $\begin{array}{l}20.030 \\
26.636\end{array}$ & $\begin{array}{l}32.241 \\
32.241\end{array}$ & $\begin{array}{l}30.389 \\
36.396\end{array}$ & $\begin{array}{l}33.200 \\
33.266\end{array}$ & $\begin{array}{l}11.3 \\
5.3\end{array}$ & & $\begin{array}{l}21.000 \\
21.061\end{array}$ & $\begin{array}{r}15.607 \\
15.607\end{array}$ & $\begin{array}{l}11.202 \\
11.267\end{array}$ & $\begin{array}{l}32.408 \\
32.408\end{array}$ & $\begin{array}{l}33.159 \\
3\end{array}$ & $\begin{array}{r}30.290 \\
30.3\end{array}$ & $\begin{array}{l}32.200 \\
32.208\end{array}$ & 27.429 & $\begin{array}{l}34.457 \\
34.457\end{array}$ \\
\hline $11 / 9 / 110: 30$ & & 31.125 & $\begin{array}{r}50.681 \\
30.69\end{array}$ & $\begin{array}{l}29.674 \\
29.674\end{array}$ & $\begin{array}{l}30.641 \\
30.645\end{array}$ & & $\begin{array}{l}29.932 \\
29.939\end{array}$ & $\begin{array}{l}21.609 \\
21.599\end{array}$ & $\begin{array}{l}30.607 \\
30.607\end{array}$ & $\begin{array}{l}30.292 \\
30.294\end{array}$ & $\begin{array}{l}28.805 \\
28.867\end{array}$ & $\begin{array}{l}20.030 \\
26.641\end{array}$ & $\begin{array}{l}32.241 \\
32.245\end{array}$ & $\begin{array}{l}30.396 \\
36.398\end{array}$ & $\begin{array}{l}33.260 \\
33.268\end{array}$ & $\begin{array}{l}17.332 \\
17.332\end{array}$ & & $\begin{array}{l}21.001 \\
21.059\end{array}$ & $\begin{array}{l}13.0012 \\
15.612\end{array}$ & $\begin{array}{l}11.26 \\
11.271\end{array}$ & $\begin{array}{l}32.408 \\
32.406\end{array}$ & $\begin{array}{l}33.144 \\
5 \\
5\end{array}$ & $\begin{array}{r}30.35 \\
30.295\end{array}$ & 32.211 & 27.429 & $\begin{array}{l}34.457 \\
34.457\end{array}$ \\
\hline $11 / 9 / 111$ & & 31.127 & 30.694 & 29.674 & 30.647 & & 29.949 & 21.781 & 30.61 & 30.299 & 28.87 & 26.636 & 32.245 & 36.396 & 33.268 & $\begin{array}{l}17.332 \\
3\end{array}$ & & 21.054 & $\begin{array}{l}15.609 \\
\end{array}$ & 11.274 & 32.408 & $\begin{array}{l}33.146 \\
3\end{array}$ & 30.291 & 32.211 & 27.429 & 34.46 \\
\hline & & 31.1 & 30.65 & 29. & 30. & & and & 22.3 & 30.61 & 30. & 28. & 26 & 32 & 36. & 33.271 & 17 & & & 15. & 11.274 & 32.404 & & 1 & 32.213 & 27.429 & 34.46 \\
\hline & & & 30. & 29. & & & & & 30.61 & & 28. & & 32 & 36. & 220 & 17 & & & 15. & 11. & 32.404 & & 30 & 32.215 & 27.429 & 34.46 \\
\hline & & & & & & & & & & & 28. & & 32. & 36.401 & & 17. & & 21 & 15.619 & 11.278 & 32.401 & & & 13 & 27. & $3+1.70$ \\
\hline 11 & & & & & & & & & & & 28 & & & 36. & & & & & 10. & 11.2 & & & & 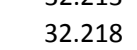 & & 34. \\
\hline $11 /$ & & & & 29 & & & & & & & 28. & & 32 & 36. & & & & & & & & & & 32. $2>>$ & & \\
\hline 11/9/11 4:00 & & 31.134 & 30.715 & 29.716 & & & 29.967 & 26.203 & 30.61 & & 20 & 26.653 & 32.2 & 36.4 & 33.278 & 37. & & 21 & 15. & 11 & 32. & & 30 & 32.22 & 27.429 & 34 \\
\hline 11/9/11 4:30 & & 31.134 & 30.722 & 29.718 & 30.645 & & 29.963 & 26.672 & 30.612 & 30.331 & 28.905 & 26.658 & 32.252 & 36.412 & 33.28 & $\begin{array}{l}37.328 \\
\end{array}$ & & 21.034 & 15.616 & 11.255 & 32.418 & 33.154 & 30.279 & 32.218 & 27.429 & 34.457 \\
\hline
\end{tabular}




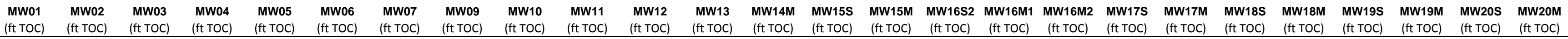

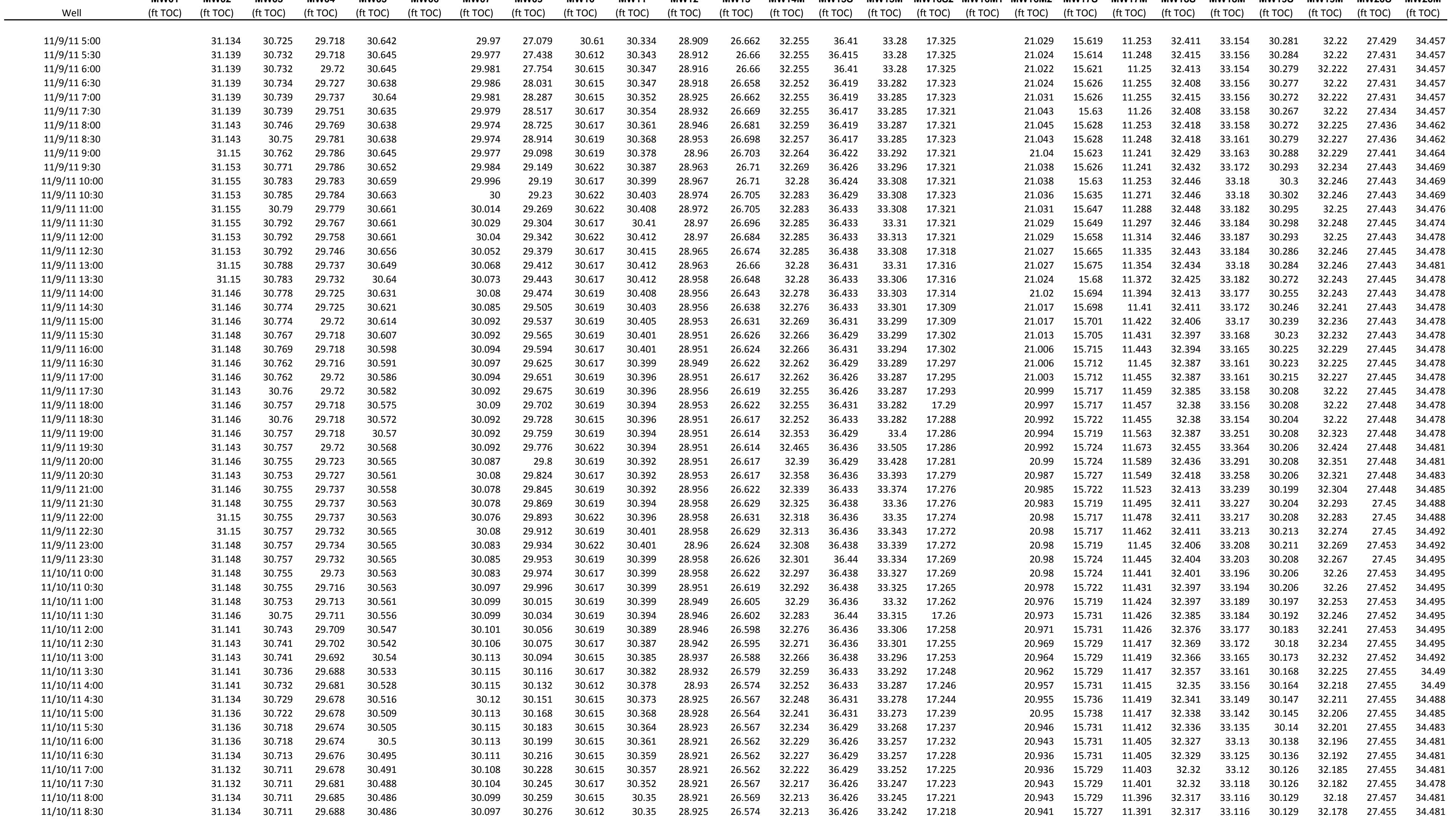




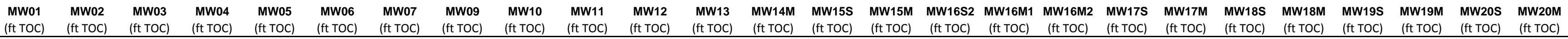

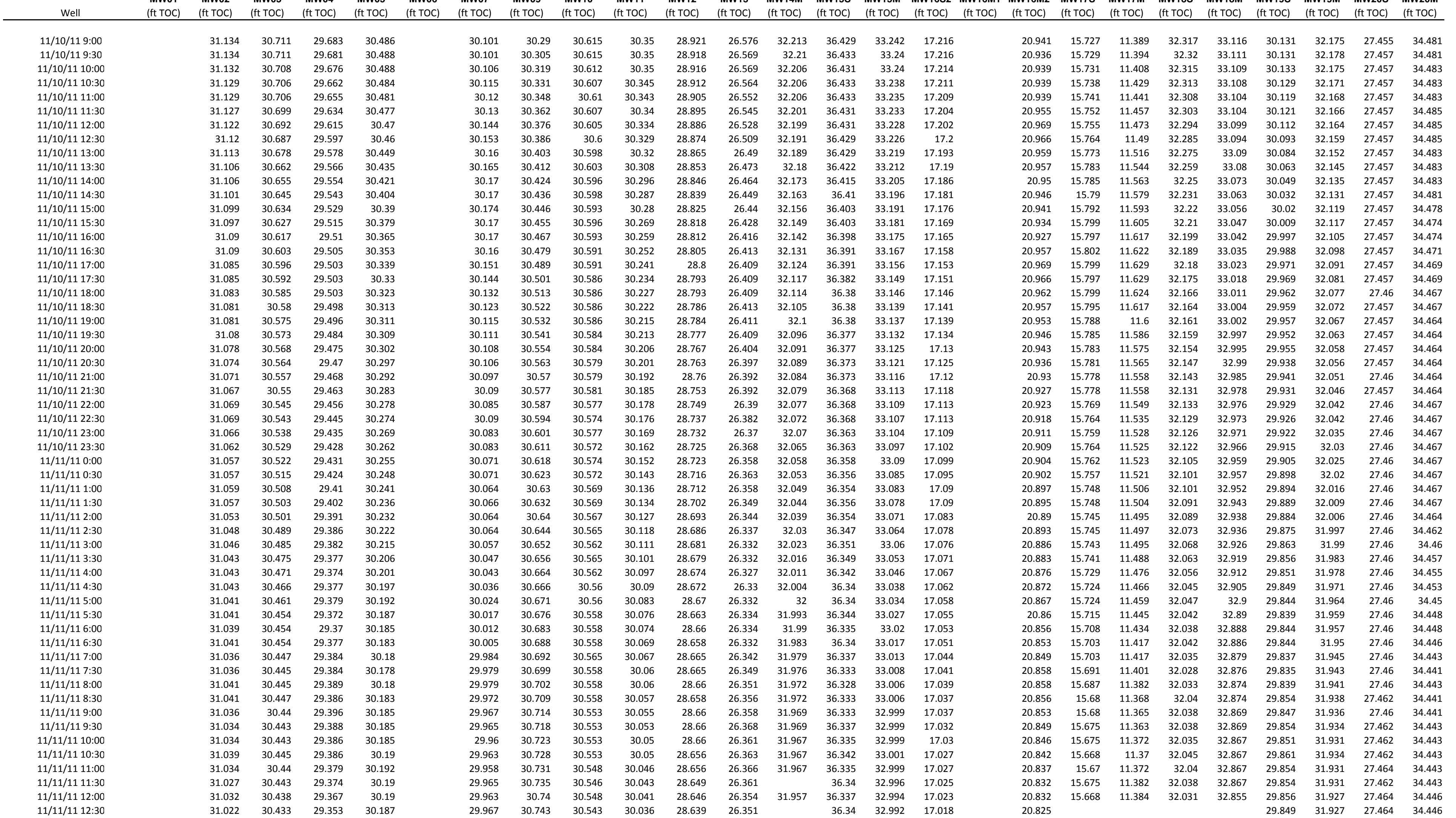




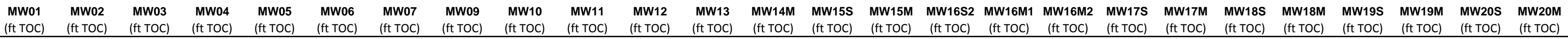

\begin{tabular}{|c|c|c|c|c|c|c|c|c|c|c|c|c|c|c|c|c|c|c|c|c|c|c|c|c|c|c|}
\hline Well & $\begin{array}{l}\text { Mwo1 } \\
\text { (ft TOC) }\end{array}$ & $\begin{array}{l}\text { MW02 } \\
\text { (ft TOC) }\end{array}$ & $\begin{array}{l}\text { Mwo3 } \\
\text { (ft TOC) }\end{array}$ & $\begin{array}{r}\text { MWva } \\
\text { (ft TOC) } \\
\end{array}$ & $\begin{array}{l}\text { Mwos } \\
\text { (ft TOC) }\end{array}$ & $\begin{array}{l}\text { MWvor } \\
\text { (ft TOC) }\end{array}$ & $\begin{array}{l}\text { (ft TOC) } \\
\text { (f ToC }\end{array}$ & $\begin{array}{l}\text { Mwo9 } \\
\text { (ft ToC) }\end{array}$ & $\begin{array}{l}\text { MW10 } \\
\text { (ft ToC) }\end{array}$ & $\begin{array}{l}\text { MWII } \\
\text { (ft TOC) }\end{array}$ & $\begin{array}{l}\text { MWI2 } \\
\text { (ft TOC) }\end{array}$ & $\begin{array}{l}\text { MW13 } \\
\text { (ft ToC) }\end{array}$ & $\begin{array}{l}\text { (ft TOC) } \\
\end{array}$ & $\begin{array}{l}\text { MWvitss } \\
\text { (ft TOC) }\end{array}$ & (ft TOC) & $\begin{array}{l}\text { (ft TOC) } \\
\end{array}$ & $\begin{array}{l}\text { (ft TOC) } \\
\text { (ft }\end{array}$ & $\begin{array}{l}\text { MWW10ML2 } \\
\text { (ft TOC) }\end{array}$ & (ft TOC) & $\begin{array}{l}\text { (ft TOC) } \\
\end{array}$ & $\begin{array}{l}\text { MW18s } \\
\text { (ft TOC) }\end{array}$ & $\begin{array}{l}\text { MWI8M } \\
\text { (ft TOC) }\end{array}$ & $\begin{array}{l}\text { (ft TOC) } \\
\end{array}$ & $\begin{array}{l}\text { (ft TOC) } \\
\end{array}$ & $\begin{array}{l}\text { MWLos } \\
\text { (ft TOC) }\end{array}$ & $\begin{array}{l}\text { (ft TOC) } \\
\end{array}$ \\
\hline 11/11/11 13:00 & & 31.022 & 30.431 & 29.346 & 30.185 & & 29.972 & 30.745 & 30.543 & 30.032 & 28.635 & 26.337 & 31.948 & 36.34 & 32.989 & 17.016 & 16.148 & 20.825 & 15.682 & 10.618 & 32.017 & 32.845 & 29.844 & 31.924 & 27.464 & 34.446 \\
\hline 11/11/11 13:30 & & 31.02 & 30.424 & 29.344 & 30.18 & & 29.97 & 30.747 & 30.541 & 30.029 & 28.63 & 26.33 & & & & 17.016 & & 20.821 & & & & & 29.839 & 31.922 & 27.464 & 34.446 \\
\hline 11/11/11 14:00 & & 31.015 & 30.419 & 29.337 & 30.173 & & 29.97 & 30.752 & 30.543 & 30.02 & 28.625 & 26.332 & 31.941 & 36.333 & 32.975 & 17.006 & 16.143 & 20.807 & 15.68 & 10.668 & 32.007 & 32.838 & 29.83 & 31.915 & 27.464 & 34.446 \\
\hline 11/11/11 14:30 & & 31.013 & 30.412 & 29.335 & 30.166 & & 29.963 & 30.757 & 30.541 & 30.013 & 28.623 & 26.325 & & & & & & & & & & & 29.825 & 31.91 & & \\
\hline $11 / 11 / 1115: 00$ & & 31.013 & 30.41 & & 30.164 & & 29.958 & 30.759 & 30.539 & 30.011 & 28.621 & 26.325 & 31.932 & 36.328 & 32.966 & 17.002 & 16.138 & 20.8 & 15.675 & 10.708 & 32.003 & 32.831 & 29.821 & 31.905 & 27.453 & 34.434 \\
\hline $11 / 11 / 1115: 30$ & & 31.011 & 30.405 & & 30.159 & & 29.949 & 30.762 & 30.539 & 30.006 & & 26.339 & & & & & & & & & & & & 31.906 & & \\
\hline $11 / 11 / 1116: 00$ & & 31.013 & 30.403 & 29.295 & 30.166 & & 29.946 & 30.766 & 30.555 & 30.002 & 28.621 & 26.334 & 31.927 & 36.328 & 32.959 & 16.995 & 16.129 & 20.793 & 15.658 & 10.731 & 32.003 & 32.829 & 29.818 & 31.894 & 27.451 & 34.431 \\
\hline $11 / 11 / 1116: 30$ & & 31.013 & 30.403 & & & & 29.942 & & & 30.002 & & & & & & & & & & & & & & & & \\
\hline 11/11/11 17:00 & & 31.011 & 30.401 & 29.295 & 30.166 & & & 30.769 & 30.548 & 29.99 & 28.618 & 26.337 & 31.92 & 36.328 & 32.954 & 16.99 & 16.122 & 20.784 & 15.658 & 10.745 & 32.003 & 32.822 & 29.818 & 31.889 & 27.451 & 34.429 \\
\hline $\begin{array}{l}11 / 11 / 1117: 30 \\
11 / 11 / 1118: 00\end{array}$ & & $\begin{array}{l}31.008 \\
31.015\end{array}$ & 30.403 & 29.309 & 30.173 & & 29.911 & 30.778 & 30.548 & 29.992 & 28.623 & & & & & & 122 & & 15.644 & 10.726 & & & & & & \\
\hline $\begin{array}{l}111 / 111 / 1118: 00 \\
11 / 11 / 119: 00\end{array}$ & & $\begin{array}{l}31.015 \\
31.008\end{array}$ & $\begin{array}{l}30.403 \\
30.401\end{array}$ & $\begin{array}{l}29.399 \\
29311\end{array}$ & $\begin{array}{l}30.178 \\
30.178\end{array}$ & & $\begin{array}{l}29.911 \\
29.902\end{array}$ & 30.783 & $\begin{array}{l}30.548 \\
30.543\end{array}$ & $\begin{array}{r}29.992 \\
29.99\end{array}$ & $\begin{array}{l}28.623 \\
28.621\end{array}$ & $\begin{array}{l}26.354 \\
26.356\end{array}$ & 31.918 & $\begin{array}{l}36.328 \\
36.328\end{array}$ & $\begin{array}{l}32.952 \\
32.947 \\
3\end{array}$ & $\begin{array}{l}16.995 \\
16.983\end{array}$ & 16.115 & 20.782 & $\begin{array}{r}15.664 \\
15.64\end{array}$ & $\begin{array}{l}10 / 266 \\
10.717\end{array}$ & $\begin{array}{r}32.01 \\
32.007\end{array}$ & $\begin{array}{l}32.819 \\
32.819\end{array}$ & $\begin{array}{l}29.888 \\
29.832\end{array}$ & $\begin{array}{l}31.887 \\
31.884\end{array}$ & $\begin{array}{l}27.453 \\
27.43\end{array}$ & $\begin{array}{l}34.429 \\
34.429\end{array}$ \\
\hline $11 / 11 / 1120: 00$ & & 31.004 & 30.401 & 29.309 & 30.178 & & 29.897 & 30.79 & 30.536 & 29.985 & 28.618 & 26.356 & 31.918 & 36.33 & 32.947 & 16.981 & 16.115 & 20.777 & 15.637 & 10.703 & 32.005 & 32.817 & 29.828 & $\begin{array}{l}51.004 \\
31.882\end{array}$ & $\begin{array}{l}27.453 \\
27.453\end{array}$ & $\begin{array}{l}34.429 \\
34.429\end{array}$ \\
\hline $11 / 11 / 1121: 00$ & & 31.001 & 30.398 & 29.306 & 30.18 & & 29.887 & 30.795 & 30.539 & 29.983 & 28.616 & 26.358 & 31.918 & 36.337 & 32.945 & 16.976 & 16.113 & 20.775 & 15.623 & 10.694 & 32.005 & 32.817 & 29.835 & 31.884 & 27.453 & $\begin{array}{l}34.431 \\
34.431\end{array}$ \\
\hline 11 & & 30.997 & 30.396 & 29.297 & 30.178 & & 29.887 & 30.798 & 30.531 & 29.978 & 28.609 & 26.351 & 31.915 & 36.33 & 32.945 & 16.969 & 16.113 & .775 & 15.623 & 10.691 & 32.005 & .815 & 29.825 & 31.882 & 27.453 & .434 \\
\hline $11 / 11 / 1123: 00$ & & 30.983 & 30.377 & 29.269 & 30.162 & & 29.902 & 30.8 & 30.527 & 29.964 & 28.595 & 26.325 & 31.908 & 36.333 & 32.94 & 16.962 & 16.11 & .768 & 15.623 & 10.71 & 31.982 & 32.808 & 29.8 & 31.873 & 27.453 & 34.434 \\
\hline 11/12/11 0:00 & & 30.98 & 30.366 & 29.255 & 30.15 & & 29.902 & 30.804 & 30.527 & 29.955 & 28.586 & 26.313 & 31.901 & 36.326 & 32.935 & 16.957 & 16.103 & 20.77 & 15.616 & 10.712 & 31.975 & 32.8 & 29.792 & 31.866 & 27.453 & 34.434 \\
\hline 11/12/11 1:00 & & 30.978 & 30.361 & 29.243 & 30.134 & & 29.902 & 30.807 & 30.52 & 29.944 & 28.577 & 26.301 & 31.892 & 36.321 & 32.926 & 16.953 & 16.096 & 20.765 & 15.614 & 10.722 & 31.961 & 32.791 & 29.781 & 31.859 & 27.453 & 34.431 \\
\hline 11/12/11 2:00 & & 30.973 & 30.345 & 29.225 & 30.117 & & 29.899 & 30.807 & 30.522 & 29.932 & 28.56 & 26.287 & 31.883 & 36.319 & 32.914 & 16.948 & 16.092 & 20.761 & 15.609 & 10.729 & 31.949 & 32.779 & 29.764 & 31.849 & 27.456 & 34.429 \\
\hline 11/12/11 3:00 & & 30.964 & 30.331 & 29.199 & 30.096 & & 29.909 & 30.807 & 30.515 & 29.913 & 28.544 & 26.263 & 31.869 & 36.314 & 32.902 & 16.939 & 16.08 & 20.751 & 15.609 & 10.743 & 31.928 & 32.767 & 29.743 & 31.835 & 27.453 & 34.424 \\
\hline 11/12/11 4:00 & & 30.957 & 30.312 & 29.176 & 30.075 & & 29.909 & 30.807 & 30.512 & 29.895 & 28.525 & 26.241 & 31.852 & 36.312 & 32.886 & 16.932 & 16.071 & 20.745 & 15.607 & 10.759 & 31.907 & 32.753 & 29.72 & 31.821 & 27.453 & 34.417 \\
\hline 11/12/11 5:00 & & 30.959 & 30.303 & 29.171 & 30.059 & & 29.895 & 30.81 & 30.517 & 29.883 & 28.516 & 26.239 & 31.84 & 36.302 & 32.874 & 16.927 & 16.061 & 20.738 & 15.602 & 10.762 & 31.895 & 32.741 & 29.71 & 31.807 & 27.453 & 34.41 \\
\hline 11/12/11 6:00 & & 30.957 & 30.296 & 29.164 & 30.05 & & 29.883 & 30.807 & 30.512 & 29.872 & 28.505 & 26.236 & 31.831 & 36.302 & 32.863 & 16.918 & 16.052 & 20.731 & 15.59 & 10.759 & 31.888 & 32.729 & 29.703 & 31.795 & 27.456 & 34.408 \\
\hline 11/12/11 7:00 & & 30.952 & 30.289 & 29.159 & 30.04 & & 29.869 & 30.81 & 30.508 & 29.865 & 28.5 & 26.236 & 31.822 & 36.297 & 32.856 & 16.913 & 16.042 & 20.717 & 15.59 & 10.764 & 31.881 & 32.72 & 29.694 & 31.786 & 27.456 & 34.406 \\
\hline $11 / 12 / 118: 00$ & & 30.943 & 30.272 & 29.14 & 30.028 & & 29.869 & 30.807 & 30.505 & 29.846 & 28.484 & 26.217 & 31.81 & 36.293 & 32.842 & 16.904 & 16.035 & 20.714 & 15.595 & 10.785 & 31.865 & 32.708 & 29.677 & 31.776 & 27.453 & 34.399 \\
\hline 11/12/11 9:00 & & 30.95 & 30.275 & 29.157 & 30.033 & & 29.84 & 30.81 & 30.505 & 29.848 & 28.488 & 26.241 & 31.805 & 36.295 & 32.839 & 16.899 & 16.033 & 20.712 & 15.581 & 10.783 & 31.874 & 32.708 & 29.687 & 31.774 & 27.456 & 34.401 \\
\hline $11 / 12 / 1110: 00$ & & 30.943 & 30.27 & 29.152 & 30.028 & & 29.831 & 30.81 & 30.503 & 29.841 & 28.484 & 26.241 & 31.803 & 36.293 & 32.837 & 16.897 & 16.028 & 20.708 & 15.581 & 10.797 & 31.867 & 32.703 & 29.687 & 31.767 & 27.456 & 34.401 \\
\hline 11/12/11 11:00 & & 30.938 & 30.261 & 29.145 & 30. & & 29.831 & 30.81 & 30.5 & 29.834 & 28.477 & 26.236 & 31.801 & 36.29 & 32.832 & 16.892 & 16.024 & 20.708 & 15.583 & 10.83 & 31.863 & 32.699 & 29.684 & 31.765 & 27.453 & 34.401 \\
\hline 12:00 & & 30.927 & 30.251 & 29.129 & & & 29.829 & 30.805 & 30.493 & 29.818 & 28.465 & 26.22 & 31.791 & 36.286 & 32.823 & 16.885 & 16.019 & 20.724 & 15.59 & 10.865 & 31.851 & 689 & 29.668 & 31.758 & 27.458 & 34.403 \\
\hline 13:00 & & 30.931 & 30.251 & 29.133 & 30.01 & & 29.81 & 30.805 & 30.496 & 29.818 & 28.465 & 26.234 & 31.787 & 36.288 & 32.82 & 16.883 & 16.017 & 20.724 & $\begin{array}{l}15.581 \\
\end{array}$ & 10.875 & 31.853 & 687 & 29.675 & 31.755 & 27.458 & 34.403 \\
\hline 00 & & 30.927 & 30.247 & 29.133 & 30.0 & & 29.8 & 30.805 & 30.491 & 29.814 & 28.46 & 26.234 & 31.784 & 36.286 & 32.816 & 16.876 & 16.01 & 20.714 & 15.581 & 10.891 & 31.856 & .684 & 29.675 & 31.751 & 27.458 & 34.403 \\
\hline & & 30.934 & 30.251 & 29.1 & 30. & & 29.779 & 30. & 30.49 & 29.816 & 28.465 & 26.258 & 31.782 & 36.288 & 32.816 & 16.876 & 16.005 & 714 & 15.567 & 10.884 & 31.863 & 682 & 29.691 & 31.748 & 27.458 & 34.403 \\
\hline & & & 30.2 & 29.1 & & & 29.7 & & & & 28.4 & 26.279 & 3178 & 36.293 & 32.813 & 16.876 & 16.003 & 769 & 15.562 & 10.86 & 31.879 & 2 & 29.708 & 31.751 & 27.46 & 34.403 \\
\hline $11 /$ & & 30.931 & 30.265 & 29.183 & & & 29.739 & 30.807 & 30.491 & & 28.484 & 26.294 & 31.784 & 36.3 & 32.8 & 16.874 & 1 & & 150 & 10.837 & 31.879 & 684 & 29.72 & 31.751 & 27.458 & 34.403 \\
\hline 11/12/11 18:00 & & 4 & 30.277 & 29.204 & & & 29.721 & 30.807 & 30.491 & 29 & 28.491 & 26.315 & 31.787 & 36.305 & 32.816 & 16.874 & 15.998 & 20.763 & 15.543 & 10.795 & 31.9 & 689 & 29.743 & 31.755 & 27.46 & 34.403 \\
\hline $11 / 12 /$ & & 30. & 30.279 & 29.199 & & & 29.718 & & 30.493 & 29 & 28.495 & 26.313 & 31.791 & 36.309 & 32.818 & 16.871 & 15.996 & 756 & 15.539 & 10.766 & 31.9 & 32.689 & 29.745 & 31.755 & 27.46 & 34.403 \\
\hline 20:00 & & & 30.275 & 29.19 & & & 29.725 & $30 . \varepsilon$ & 30 & & 28.491 & 26.303 & 31.789 & 36.307 & 32.82 & 16.869 & 15.993 & 11 & 15.543 & 10.762 & & 32.689 & 29.736 & 31.753 & 7.46 & 34.403 \\
\hline 21:00 & & & 30.265 & 29.169 & & & 29.735 & 30.805 & 30.481 & & 28.481 & 26.284 & 31.787 & 36.309 & 32.816 & 16.867 & & 2 & 15.546 & 10.766 & 31.879 & 32.684 & 29.717 & 31.753 & 27.46 & 34.401 \\
\hline $11 / 12 / 1122: 00$ & & 30.901 & 30.254 & 29.143 & & & 29.746 & 30.8 & 30.477 & 29.818 & 28.47 & 26.263 & 31.78 & 36.295 & 32.811 & 16.864 & 15.989 & 20.742 & 15.548 & 10.776 & 31.865 & 32.68 & 29.698 & 31.741 & 27.46 & 34.403 \\
\hline $11 / 12 / 1123: 00$ & & 30.889 & 30.23 & 29.105 & & & 29.77 & 30.7 & 30.474 & 29.793 & 28.446 & 26.222 & 31.77 & 36.302 & 32.799 & 16.857 & 15.984 & 20.731 & 15.548 & 10.797 & 31.839 & 32.67 & 29.668 & 31.734 & 27.458 & 34.401 \\
\hline 11/13/11 0:00 & & 30.897 & 30.223 & 29.101 & 30.007 & & 29.753 & 30.7 & 30.479 & 29.786 & 28.439 & 26.222 & 31.763 & 36.29 & 32.795 & 16.855 & 15.982 & 20.726 & 15.536 & 10.785 & 31.837 & 32.663 & 29.663 & 31.729 & 27.463 & 34.401 \\
\hline 11/13/11 1:00 & & 30.89 & 30.216 & 29.096 & 0 & & 29.746 & 30.788 & 30.47 & 29.781 & 28.435 & 26.22 & 31.756 & 36.29 & 32.79 & 16.853 & 15.977 & 20.719 & 15.534 & 10.78 & 31.832 & 32.656 & 29.659 & 31.722 & 27.463 & 34.401 \\
\hline $11 / 13 / 112: 00$ & & 30.896 & 30.219 & 29.112 & 30.0 & & 29.721 & 30.786 & 30.474 & 29.783 & 28.439 & 26.239 & 31.756 & 36.29 & 32.788 & 16.848 & 15.973 & 20.712 & 15.515 & 10.755 & 31.842 & 32.656 & 29.673 & 31.722 & 27.465 & 34.401 \\
\hline $11 / 13 / 11$ 3:00 & & 30.894 & 30.219 & 29.117 & 30.007 & & 29.709 & 30.783 & 30.472 & 29.781 & 28.439 & 26.244 & 31.749 & 36.286 & 32.783 & 16.843 & 15.968 & 20.705 & 15.513 & 10.738 & 31.839 & 32.649 & 29.675 & 31.718 & 27.465 & 34.401 \\
\hline $11 / 13 / 11$ 4:00 & & 30.91 & 30.249 & 29.176 & 30.04 & & 29.659 & 30.788 & 30.477 & 29.802 & 28.465 & 26.306 & 31.761 & 36.302 & 32.79 & 16.848 & 15.97 & 20.703 & 15.482 & 10.668 & 31.877 & 32.658 & 29.72 & 31.725 & 27.463 & 34.401 \\
\hline $11 / 13 / 11$ 5:00 & & 30.91 & 30.27 & 29.206 & 30.068 & & 29.641 & 30.792 & 30.474 & 29.816 & 28.481 & 26.334 & 31.766 & 36.312 & 32.795 & 16.848 & 15.968 & 20.698 & 15.473 & 10.625 & 31.893 & .668 & 29.75 & 31.732 & 27.465 & 34.401 \\
\hline $11 / 13 / 116: 00$ & & 30.911 & 30.296 & 29.234 & 30.0 & & 29.622 & 30.795 & 30.472 & 29.83 & 28.5 & 26.361 & 31.773 & 36.321 & 32.802 & 16.85 & 15. & 20.6 & 15.459 & 10.574 & 4 & 2 & 29.776 & 31.739 & 27.467 & 34.401 \\
\hline $11 / 13 / 11$ 7:00 & & 30.908 & 30.312 & 29.253 & 30.1 & & 29.617 & 30.8 & 30.472 & 29.841 & 28.514 & 26.38 & 31.782 & 36.335 & 32.806 & 16.85 & 15.961 & 20.687 & 15.454 & 10.543 & 31.928 & 682 & 29.797 & 31.746 & 27.467 & 34.401 \\
\hline $11 / 13 / 118: 00$ & & 30.91 & 30.328 & 29.274 & 30.1 & & 29.61 & 30.807 & 30.472 & 29.858 & 28.528 & 26.399 & 31.789 & 36.335 & 32.816 & 16.855 & 15.963 & 20.684 & $\begin{array}{l}15.454 \\
\end{array}$ & $\begin{array}{l}10.52 \\
1.52\end{array}$ & 31.944 & 9 & 29.814 & 31.753 & 27.467 & 34.401 \\
\hline & & 30.9 & 30.3 & בר & & & & & & & & & & 36.3 & 32.8 & & & & & 10.5 & & & & & 27.467 & .406 \\
\hline & & & & & & & & & & & & & 31. & & & & & & & & & & & & & \\
\hline & & & & 29.2 & & & & & & & & & & & & & & & & & & & & & & \\
\hline & & & & & & & & & & & & & 31. & & & & & & & & & & & & & \\
\hline & & & & & & & & & & 29. & & & & & & & & & & & & & & & & \\
\hline 1114.00 & & 30.887 & 30.349 & 29.264 & & & 29. & 30. & & & & & 31.8 & & & & & 20.675 & 15.4 & 10.7 & 31. & & & & & 1 \\
\hline 11/13/11 15:00 & & 30.885 & 30.345 & 29.264 & 30.169 & & 29.692 & 30.812 & 30.458 & 29.893 & 28.551 & 26.373 & 31.81 & 36.361 & 32.839 & 16.869 & 15.963 & 20.668 & 15.504 & 10.729 & 31.954 & 32.708 & 29.821 & 31.774 & 27.47 & 34.41 \\
\hline
\end{tabular}




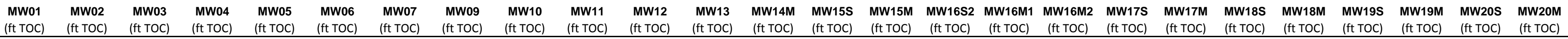

\begin{tabular}{|c|c|c|c|c|c|c|c|c|c|c|c|c|c|c|c|c|c|c|c|c|c|c|c|c|c|c|}
\hline Well & $\begin{array}{l}\text { MW01 } \\
\text { (ft TOC) }\end{array}$ & $\begin{array}{l}\text { MW02 } \\
\text { (ft TOC) }\end{array}$ & $\begin{array}{l}\text { MW03 } \\
\text { (ft TOC) }\end{array}$ & $\begin{array}{l}\text { MW04 } \\
\text { (ft TOC) }\end{array}$ & $\begin{array}{l}\text { MW05 } \\
\text { (ft ToC) }\end{array}$ & $\begin{array}{l}\text { MW06 } \\
\text { (ft TOC) }\end{array}$ & $\begin{array}{l}\text { MW07 } \\
\text { (ft TOC) }\end{array}$ & $\begin{array}{l}\text { MW09 } \\
\text { (ft TOC) }\end{array}$ & $\begin{array}{l}\text { MW10 } \\
\text { (ft ToC) }\end{array}$ & $\begin{array}{l}\text { MW11 } \\
\text { (ft TOC) }\end{array}$ & $\begin{array}{l}\text { MW12 } \\
\text { (ft TOC) }\end{array}$ & $\begin{array}{l}\text { MW13 } \\
(\mathrm{ft} \text { TOC) }\end{array}$ & $\begin{array}{l}\text { MW14M } \\
\text { (ft Toc) }\end{array}$ & $\begin{array}{l}\text { MWW5s } \\
\text { (ft TOC) }\end{array}$ & $\begin{array}{l}\text { MW15M } \\
\text { (ft TOC) }\end{array}$ & $\begin{array}{l}\text { MW16S2 } \\
\text { (ft TOC) }\end{array}$ & $\begin{array}{l}\text { MW16161 } \\
\text { (ft TOC) }\end{array}$ & $\begin{array}{l}\text { MW16M2 } \\
\text { (ft TOC) }\end{array}$ & $\begin{array}{l}\text { MW175 } \\
\text { (ft Toc) }\end{array}$ & $\begin{array}{l}\text { MWw7M } \\
\text { (ft TOC) }\end{array}$ & $\begin{array}{l}\text { MW18S } \\
\text { (ft TOC) }\end{array}$ & $\begin{array}{l}\text { MWWigM } \\
\text { (ft TOC) }\end{array}$ & $\begin{array}{l}\text { MW19S } \\
\text { (ft TOC) }\end{array}$ & $\begin{array}{l}\text { MW19M } \\
\text { (ft TOC) }\end{array}$ & $\begin{array}{l}\text { MW20S } \\
\text { (ft TOC) }\end{array}$ & $\begin{array}{l}\mathrm{MW} \text { M.OM } \\
\text { (ft TOC) }\end{array}$ \\
\hline $11 / 13 / 1116: 00$ & & 30.878 & 30.342 & 29.257 & 30.164 & & 29.699 & 30.81 & 30.451 & 29.89 & 28.551 & 26.363 & 31.81 & 36.363 & 32.839 & 16.869 & 15.961 & 20.664 & 15.511 & 10.743 & 31.947 & 32.708 & 29.814 & 31.774 & 27.472 & 34.408 \\
\hline $11 / 13 / 1117: 00$ & & 30.883 & 30.349 & 29.271 & 30.166 & & 29.688 & 30.812 & 30.453 & 29.897 & 28.558 & 26.375 & 31.81 & 36.365 & 32.837 & 16.869 & 15.961 & 20.659 & 15.501 & 10.722 & 31.954 & 32.708 & 29.823 & 31.772 & 27.472 & 34.408 \\
\hline $11 / 13 / 1118: 00$ & & 30.873 & 30.338 & 29.253 & 30.157 & & 29.702 & 30.81 & 30.448 & 29.893 & 28.549 & 26.356 & 31.957 & 36.365 & 32.989 & 16.869 & 15.963 & 20.68 & 15.511 & 10.879 & 31.968 & $\begin{array}{l}3 \quad 32.857 \\
\end{array}$ & 29.804 & 31.915 & 27.472 & 34.403 \\
\hline 11/13/11 19:00 & & 30.871 & 30.335 & 29.246 & 30.152 & & 29.699 & 30.81 & 30.448 & 29.893 & 28.549 & 26.346 & 31.929 & 36.365 & 32.966 & 16.867 & 16.003 & 20.708 & 15.508 & 10.842 & 31.996 & 32.829 & 29.807 & 31.889 & 27.472 & 34.403 \\
\hline $11 / 13 / 1120: 00$ & & 30.869 & 30.331 & 29.239 & 30.148 & & 29.702 & 30.805 & 30.446 & 29.89 & 28.544 & 26.342 & 31.885 & 36.361 & 32.919 & 16.864 & 15.996 & 20.708 & 15.513 & 10.79 & 31.97 & 32.781 & 29.802 & 31.849 & 27.472 & 34.403 \\
\hline 11/13/11 21:00 & & 30.859 & 30.317 & 29.222 & 30.136 & & 29.714 & 30.802 & 30.443 & 29.883 & 28.535 & 26.32 & 31.862 & 36.361 & 32.893 & $\begin{array}{l}36.862 \\
3\end{array}$ & 15.991 & 20.712 & 15.518 & 10.769 & 31.947 & 32.76 & 29.783 & 31.823 & 27.472 & 34.401 \\
\hline $11 / 13 / 1122: 00$ & & 30.864 & 30.312 & 29.215 & 30.127 & & 29.706 & 30.8 & 30.444 & 29.881 & 28.53 & 26.315 & 31.848 & 36.356 & 32.877 & 16.86 & 15.989 & 20.721 & 15.513 & 10.748 & 31.942 & 32.744 & 29.778 & 31.809 & 27.474 & 34.403 \\
\hline $11 / 13 / 1123: 00$ & & 30.857 & 30.303 & 29.204 & 30.12 & & 29.711 & 30.798 & 30.439 & 29.876 & 28.523 & 26.303 & 31.836 & 36.354 & 32.867 & 16.86 & 15.982 & 20.788 & 15.52 & 10.743 & 31.928 & 32.734 & 29.769 & 31.802 & 27.472 & 34.403 \\
\hline $11 / 14 / 110: 00$ & & $\begin{array}{l}30.851 \\
30.857\end{array}$ & $\begin{array}{l}30.305 \\
30.298\end{array}$ & 29.197 & $\begin{array}{r}30.113 \\
30.113\end{array}$ & & 29.704 & $\begin{array}{l}30.793 \\
30.793\end{array}$ & $\begin{array}{l}30.499 \\
30.446\end{array}$ & $\begin{array}{l}29.810 \\
29.872\end{array}$ & $\begin{array}{l}28.523 \\
28.521\end{array}$ & 26.301 & $\begin{array}{l}31.830 \\
31.826\end{array}$ & $\begin{array}{l}30.344 \\
36.349\end{array}$ & $\begin{array}{l}32.858 \\
32.858\end{array}$ & $\begin{array}{r}10.00 \\
3 \\
3\end{array}$ & $\begin{array}{l}13.982 \\
15.98\end{array}$ & 20.782 & $\begin{array}{l}15.52 \\
15.506\end{array}$ & 10.726 & $\begin{array}{l}31.926 \\
31.926\end{array}$ & $\begin{array}{l}32.725 \\
32.725\end{array}$ & 29.767 & $\begin{array}{l}31.802 \\
31.788\end{array}$ & 27.477 & $\begin{array}{l}34.453 \\
34.406\end{array}$ \\
\hline $11 / 14 / 11$ 1:00 & & 30.859 & 30.298 & 29.204 & 30.11 & & 29.69 & 30.795 & 30.443 & 29.872 & 28.521 & 26.306 & 31.822 & 36.351 & 32.851 & 16.855 & 15.98 & 20.768 & 15.499 & 10.71 & 31.926 & 32.72 & 29.771 & 31.783 & 27.477 & 34.406 \\
\hline $11 / 14 / 112: 00$ & & 30.857 & 30.298 & 29.208 & 30.11 & & 29.681 & 30.795 & 30.444 & 29.872 & 28.523 & 26.313 & 31.817 & 36.351 & 32.846 & $5 \quad 16.853$ & 15.975 & 20.756 & 15.499 & 10.696 & 31.93 & 32.715 & .771 & 31.783 & 27.477 & 34.406 \\
\hline $11 / 14 / 113: 00$ & & 30.862 & 30.31 & 29.227 & 30.122 & & 29.659 & 30.79 & 30.441 & 29.876 & 28.53 & 26.33 & 31.815 & 36.356 & 32.846 & $5 \quad 16.853$ & 15.972 & 20.745 & 15.485 & 10.661 & 31.935 & 32.715 & 1785 & 31.779 & 27.477 & 34.408 \\
\hline $11 / 14 / 11$ 4:00 & & 30.875 & 30.345 & 29.285 & 30.155 & & 29.619 & 30.798 & 30.443 & 29.897 & 28.556 & 26.387 & 31.822 & 36.365 & 32.853 & $\begin{array}{ll}3 & 16.855\end{array}$ & 15.975 & 20.735 & 15.471 & 10.691 & 31.968 & 725 & 837 & 31.79 & 27.477 & 34.41 \\
\hline $11 / 14 / 11$ 5:00 & & 30.885 & 30.375 & 29.33 & 30.192 & & 29.589 & 30.805 & 30.451 & 29.918 & 28.581 & 26.43 & 31.836 & 36.377 & 32.86 & 16.86 & 15.973 & 20.726 & 5.449 & 10.698 & 31.998 & 32.734 & 9.872 & 31.793 & 27.479 & 34.413 \\
\hline $11 / 14 / 116: 00$ & & 30.885 & 30.405 & 29.367 & 30.229 & & 29.577 & 30.812 & 30.444 & 29.941 & 28.605 & 26.466 & 31.845 & 36.387 & 32.872 & 16.864 & 15.977 & 20.712 & $\begin{array}{l}15.452 \\
\end{array}$ & 10.708 & 32.026 & 32.746 & 29.908 & 31.807 & 27.479 & 34.415 \\
\hline 11/14/11 7:00 & & 30.88 & 30.41 & 29.37 & 30.243 & & 29.594 & 30.812 & 30.446 & 29.951 & 28.612 & 26.464 & 31.85 & 36.396 & 32.877 & 16.867 & 15.973 & 20.719 & 15.466 & 10.715 & 32.021 & 32.751 & 29.91 & 31.812 & 27.479 & 34.413 \\
\hline $11 / 14 / 11$ 8:00 & & 30.887 & 30.433 & 29.398 & 30.267 & & 29.587 & 30.817 & 30.446 & 29.967 & 28.628 & 26.488 & 31.859 & 36.401 & 32.884 & 16.871 & 15.972 & 20.721 & 15.454 & 10.719 & 32.047 & 32.76 & 29.933 & 31.821 & 27.481 & 34.415 \\
\hline 11/14/11 9:00 & & 30.892 & 30.454 & 29.428 & 30.292 & & 29.584 & 30.822 & 30.446 & 29.988 & 28.651 & 26.514 & 31.869 & 36.419 & 32.895 & 16.878 & 15.975 & 20.721 & 15.454 & 10.726 & 32.068 & $3 \quad 32.767$ & 29.959 & 31.833 & 27.481 & 34.417 \\
\hline $11 / 14 / 1110: 00$ & & 30.892 & 30.464 & 29.433 & 30.306 & & 29.601 & 30.824 & 30.441 & 29.999 & 28.66 & 26.516 & 31.878 & 36.415 & 32.903 & 316.885 & 15.98 & 20.717 & 15.468 & 10.741 & 32.066 & 32.777 & 29.971 & 31.842 & 27.481 & 34.42 \\
\hline $11 / 14 / 1111: 00$ & & 30.892 & 30.473 & 29.44 & 30.32 & & 29.612 & 30.829 & 30.441 & 30.011 & 28.67 & 26.519 & 31.887 & 36.429 & 32.912 & 16.888 & 15.982 & 20.71 & 15.478 & 10.745 & 32.084 & 32.786 & 29.976 & 31.849 & 27.484 & 34.422 \\
\hline $11 / 14 / 1112: 00$ & & 30.88 & 30.464 & 29.414 & 30.311 & & 29.655 & 30.829 & 30.432 & 30.009 & 28.66 & 26.488 & 31.885 & 36.426 & 32.91 & 16.89 & 15.982 & 20.701 & 15.506 & 10.748 & 32.063 & 32.784 & 29.955 & 31.854 & 27.481 & 34.42 \\
\hline $11 / 14 / 1113: 00$ & & 30.875 & 30.454 & 29.395 & 30.297 & & 29.681 & 30.829 & 30.434 & 30.006 & 28.656 & 26.464 & 31.885 & 36.422 & 32.91 & 16.892 & 15.98 & 20.696 & 15.52 & 10.745 & 32.049 & 32.784 & 29.941 & 31.851 & 27.481 & 34.42 \\
\hline $11 / 14 / 1114: 00$ & & 30.876 & 30.447 & 29.386 & 30.29 & & 29.692 & 30.829 & 30.432 & 30.006 & 28.656 & 26.454 & 31.88 & 36.429 & 32.91 & 16.895 & 15.979 & 20.689 & 15.525 & 10.745 & 32.052 & 32.781 & 29.933 & 31.847 & 27.484 & 34.422 \\
\hline $11 / 14 / 1115: 00$ & & 30.869 & 30.436 & 29.363 & 30.274 & & 29.716 & 30.831 & $\begin{array}{l}30.432 \\
\end{array}$ & 29.999 & 28.644 & 26.425 & 31.878 & 36.419 & 32.907 & 16.895 & 15.975 & 20.677 & 15.541 & 10.741 & 32.031 & 32.774 & 29.91 & 31.842 & 27.481 & 34.42 \\
\hline $11 / 14 / 1116: 00$ & & 30.866 & 30.426 & 29.356 & 30.26 & & 29.721 & 30.828 & $\begin{array}{l}30.434 \\
\end{array}$ & 29.997 & 28.642 & 26.418 & 31.871 & 36.412 & 32.9 & $\begin{array}{l}36.895 \\
9\end{array}$ & 15.975 & 20.726 & 15.546 & 10.736 & 32.026 & 32.772 & 29.901 & 31.835 & 27.484 & 34.417 \\
\hline $11 / 14 / 1117: 00$ & & $\begin{array}{l}30.0064 \\
30.864\end{array}$ & $\begin{array}{l}30.426 \\
30.426\end{array}$ & 29.36 & 30.2 & & 29.716 & 30.831 & $\begin{array}{l}30.432 \\
30.432\end{array}$ & 30.002 & $\begin{array}{l}20.042 \\
28.644\end{array}$ & 26.418 & 31.871 & 36.419 & 32.9 & 16.895 & 15.975 & 20.717 & $\begin{array}{l}15.546 \\
\end{array}$ & 10.733 & 32.026 & 32.77 & 29.905 & 31.833 & 27.486 & 34.417 \\
\hline $11 / 14 / 1118: 00$ & & $\begin{array}{l}30.004 \\
30.871\end{array}$ & $\begin{array}{l}30.420 \\
30.433 \\
\end{array}$ & 29.379 & 30.26 & & 29.697 & 30.833 & $\begin{array}{l}30.434 \\
30.434\end{array}$ & $\begin{array}{l}30.009 \\
30.009\end{array}$ & $\begin{array}{l}20.044 \\
28.649\end{array}$ & $\begin{array}{l}20.410 \\
26.435\end{array}$ & $\begin{array}{l}31.871 \\
31.871\end{array}$ & $\begin{array}{l}36.415 \\
36.415\end{array}$ & $\begin{array}{r}32.898 \\
32.9\end{array}$ & $\begin{array}{l}16.895 \\
3\end{array}$ & 15.972 & 20.712 & $\begin{array}{l}15.536 \\
15.536\end{array}$ & 10.736 & $\begin{array}{l}32.020 \\
32.033\end{array}$ & 32.772 & 29.912 & $\begin{array}{l}31.833 \\
31.833\end{array}$ & 27.486 & 34.415 \\
\hline $11 / 14 / 1119: 00$ & & 30.864 & $\begin{array}{l}30.453 \\
30.429 \\
\end{array}$ & 29.377 & $\begin{array}{l}30.2 \\
30.2\end{array}$ & & 29.702 & 30.831 & $\begin{array}{l}30.434 \\
30.432\end{array}$ & 30.006 & $\begin{array}{l}28.049 \\
28.649\end{array}$ & $\begin{array}{r}20.430 \\
26.43\end{array}$ & $\begin{array}{l}31.869 \\
31.869\end{array}$ & $\begin{array}{l}30.410 \\
36.417\end{array}$ & $\begin{array}{l}32.898 \\
32.898\end{array}$ & $\begin{array}{l}30.0597 \\
36.897\end{array}$ & $\begin{array}{l}15.973 \\
15.973\end{array}$ & 20.703 & $\begin{array}{l}13.530 \\
15.541\end{array}$ & $\begin{array}{l}10.730 \\
10.733\end{array}$ & $\begin{array}{l}32.033 \\
32.033\end{array}$ & 32.77 & 29.912 & $\begin{array}{l}31.833 \\
31.833\end{array}$ & 27.486 & $\begin{array}{l}34.415 \\
34.415\end{array}$ \\
\hline $\begin{array}{l}11 / 14 / \\
11 / 4\end{array}$ & & $\begin{array}{l}30.804 \\
30.862\end{array}$ & $\begin{array}{l}30.429 \\
30.424 \\
\end{array}$ & 29.37 & 30. & & 29.704 & $\begin{array}{l}30.831 \\
30.833\end{array}$ & $\begin{array}{l}30.452 \\
30.429\end{array}$ & $\begin{array}{l}30.006 \\
30.006\end{array}$ & $\begin{array}{l}28.049 \\
28.646\end{array}$ & $\begin{array}{l}26.433 \\
26.423\end{array}$ & $\begin{array}{l}31.869 \\
31.869\end{array}$ & $\begin{array}{l}30.41 \prime \\
36.424\end{array}$ & $\begin{array}{l}32.898 \\
32.895\end{array}$ & $\begin{array}{l}10.897 \\
16.895\end{array}$ & $\begin{array}{l}13.973 \\
15.97\end{array}$ & $\begin{array}{l}0.003 \\
20.696\end{array}$ & $\begin{array}{l}13.541 \\
15.546\end{array}$ & $\begin{array}{l}10.733 \\
10.733\end{array}$ & $\begin{array}{l}32.033 \\
32.028\end{array}$ & $\begin{array}{r}32.17 \\
32.765\end{array}$ & .908 & 31.833 & $\begin{array}{l}27.480 \\
27.486\end{array}$ & $\begin{array}{l}34.415 \\
34.415\end{array}$ \\
\hline & & & 30.424 & 29.377 & & & & 30.836 & 30.432 & & 28 & 26.428 & 31.871 & 36.422 & 32.9 & 16.895 & 15.97 & 20.691 & 15.548 & 10.736 & 32.028 & 32.772 & & 1.835 & 27.488 & 34.415 \\
\hline 11/14/11 22:00 & & & 30.422 & 29.379 & & & & 30.836 & 30.427 & $36>2>3$ & 28.651 & 26.428 & 31.873 & 36.419 & 32.9 & 16.897 & 15.975 & 20.684 & 53 & 10.736 & 32.021 & 32.772 & 03 & 35 & 27.488 & 34.417 \\
\hline 11/14/11 23: & & & 30.419 & 29.374 & 30. & & 29.706 & 30.838 & 30.425 & 30.009 & 28.649 & & 31.873 & 36.424 & 2.902 & 16.895 & 15.975 & 20.687 & 3.555 & 10.738 & 32.026 & .772 & 908 & .84 & 27.488 & 34.42 \\
\hline $11 / 15 / 110: 00$ & & & 30.412 & 29.363 & 30. & & 29.711 & 30.838 & 30.424 & 30.006 & 28.644 & 26.409 & 31.871 & 36.417 & 32.898 & 16.895 & 15.973 & 20.68 & 15.555 & 10.733 & 32.021 & 32.772 & .896 & .833 & 27.488 & 4.42 \\
\hline 11/15/11 1:00 & & 30.845 & 30.398 & 29.339 & 30. & & 29.725 & 30.836 & 30.42 & 29.997 & 28.63 & 26.385 & 31.864 & 36.415 & 32.893 & 16.892 & 15.972 & 20.673 & 15.567 & 10.729 & 32 & 32.763 & 29.879 & 31.828 & 27.488 & 34.419 \\
\hline $11 / 15 / 112: 00$ & & 30.845 & 30.389 & 29.334 & & & & 30.836 & 30.422 & & 28.628 & 26.382 & 31.864 & 36.412 & 32.891 & 16.89 & 15.972 & & 15.562 & 10.729 & 32 & 32.763 & & 26 & 27.491 & 34.42 \\
\hline 11/15/11 3:00 & & 30.843 & 30.387 & 29.33 & & & 23 & 30.833 & 30.42 & & 28.623 & 26.378 & 31.859 & 36.41 & 32.891 & 16.89 & 15.968 & 20.659 & 15.565 & 10.726 & 31.996 & 52.758 & 29.863 & 31.823 & 27.491 & 34.417 \\
\hline $11 / 15 / 11$ 4:00 & & 30.841 & 30.377 & 29.32 & 30. & & 29.723 & 30.833 & 30.42 & 29.985 & 28.618 & 26.368 & 31.855 & 36.41 & 32.884 & 16.888 & 15.968 & 20.65 & 15.567 & 10.722 & 31.986 & 532.755 & 29.858 & 31.816 & 27.491 & 34.417 \\
\hline $11 / 15 / 11$ 5:00 & & 30.866 & 30.398 & 29.367 & 30. & & 29.676 & 30.836 & 30.432 & 30.002 & 28.639 & 26.416 & 31.862 & 36.412 & 32.884 & 16.89 & 15.968 & 20.643 & 15.529 & 10.724 & 32.021 & 32.763 & 29.901 & 31.816 & 27.491 & 34.417 \\
\hline $11 / 15 / 116: 00$ & & 30.855 & 30.401 & 29.37 & 30.2 & & 29.683 & 30.838 & 30.429 & 30.002 & 28.639 & 26.416 & 31.859 & 36.412 & 32.886 & 16.888 & 15.963 & 20.64 & 15.543 & 10.724 & 32.014 & 32.758 & 29.896 & 31.821 & 27.493 & 34.417 \\
\hline 11/15/11 7:00 & & 30.859 & 30.403 & 29.381 & 30.241 & & 29.676 & 30.838 & 30.427 & 30.006 & 28.642 & 26.423 & 31.859 & 36.422 & 32.886 & 16.89 & 15.963 & 20.647 & 15.541 & 10.724 & 32.024 & 32.758 & 29.901 & 31.821 & 27.493 & 34.415 \\
\hline $11 / 15 / 118: 00$ & & 30.855 & 30.408 & 29.388 & 30.25 & & 29.674 & 30.841 & 30.422 & 30.011 & 28.646 & 26.43 & 31.857 & 36.426 & 32.888 & 16.888 & 15.961 & 20.647 & 15.543 & 10.722 & 32.021 & 32.763 & 29.908 & 31.821 & 27.493 & 34.415 \\
\hline $11 / 15 / 119: 00$ & & 30.859 & 30.417 & 29.402 & 30.262 & & 29.666 & 30.843 & 30.42 & 30.018 & 28.656 & 26.445 & 31.862 & 36.429 & 32.891 & 16.888 & 15.961 & 20.647 & 15.546 & 10.726 & 32.033 & 32.765 & 29.922 & 31.826 & 27.493 & 34.413 \\
\hline 11/15/11 10:00 & & 30.859 & 30.426 & 29.421 & 30.276 & & 29.664 & 30.845 & 30.422 & 30.027 & 28.665 & 26.461 & 31.869 & 36.436 & 32.895 & 16.892 & 15.961 & 20.645 & 15.548 & 10.731 & 32.042 & 32.767 & 9.936 & 31.833 & 27.495 & 34.415 \\
\hline $11 / 15 / 1111: 00$ & & 30.8 & 30.433 & 29.423 & 30. & & 29.671 & 30.848 & 30.417 & 30.032 & 28.672 & 26.464 & 31.873 & 36.436 & 32.9 & 16.895 & 15.963 & 20.64 & 15.558 & 10.733 & 32.049 & $\begin{array}{l}62 \\
92\end{array}$ & 43 & 31.84 & 27.495 & 34.417 \\
\hline $11 / 15$ & & 30.855 & 30.429 & 29.419 & 30. & & 29.685 & 30.848 & 30.42 & 30. & 28.67 & 26.457 & 31.876 & $\begin{array}{r}30.44 \\
36.44\end{array}$ & 32.903 & 16.8 & 15.963 & 20.636 & $\begin{array}{l}15.574 \\
\end{array}$ & 10.738 & 32.047 & 32.774 & 936 & 31.842 & 27.498 & 34.415 \\
\hline $\begin{array}{l}11 / 15 \\
11 / 1\end{array}$ & & 30.848 & $\begin{array}{l}30.426 \\
30.426\end{array}$ & 29.405 & 30. & & 29.702 & 30.848 & $\begin{array}{l}30.415 \\
30.415\end{array}$ & $\begin{array}{l}30.032 \\
30.032\end{array}$ & 28.665 & 26.442 & 31.878 & 36.433 & 32.905 & 16.895 & 15.966 & 20.629 & $\begin{array}{l}15.588 \\
\end{array}$ & 10.736 & 32.038 & 32.772 & 1.924 & $\begin{array}{l}31.84 \\
31.84\end{array}$ & 27.498 & $\begin{array}{l}34.42 \\
34.42\end{array}$ \\
\hline $11 / 1$ & & 30. & $\begin{array}{l}30.420 \\
30.419\end{array}$ & 29.402 & 30. & & $\begin{array}{l}29.709 \\
2909\end{array}$ & $\begin{array}{r}30.848 \\
30.85\end{array}$ & $\begin{array}{r}30.413 \\
30.42\end{array}$ & $\begin{array}{l}30.029 \\
30.029\end{array}$ & $\begin{array}{l}28.003 \\
28.663\end{array}$ & $\begin{array}{l}20.442 \\
26.435\end{array}$ & 31.876 & $\begin{array}{l}30.433 \\
36.433\end{array}$ & $\begin{array}{l}32.903 \\
32.903\end{array}$ & $\begin{array}{l}10.0595 \\
16.895\end{array}$ & 15.9 & 20.624 & $\begin{array}{l}13.580 \\
15.593\end{array}$ & $\begin{array}{l}10.730 \\
10.736\end{array}$ & $\begin{array}{l}32.030 \\
32.035\end{array}$ & 52 & 26 & $\begin{array}{l}31.84 \\
31.842\end{array}$ & $\begin{array}{l}27.498 \\
27.498\end{array}$ & $\begin{array}{r}34.44 \\
34.417\end{array}$ \\
\hline & & & 30. & 29.4 & & & 25 & 30. & 30. & & 28 & 26 & & 36 & 32 & & 15 & $2 c$ & 15 & 10 & 32 & & & 7 & 27.498 & 34.42 \\
\hline & & & 30. & 29 & & & & 30 & 30 & 30 & 28. & 26 & & 36. & 32 & & 15 & & 15 & 10 & 32 & & & 2 & 27.498 & 34.42 \\
\hline $11 / 1$ & & & 30 & 29.4 & & & & & & & 28.684 & 26.471 & & 36.438 & & & 15.968 & & & 10 & & & & & & 政 \\
\hline & & & & 29. & & & & & & & 20.0 & & & & & & & & & & & & & & & \\
\hline & & & & & & & & & & & 28. & & & & & & & & & 10 & & & & & & 34 \\
\hline 11 & & & & & & & & & & & & & 31 & & & & & 20.6 & & & & 32.812 & & & 27 & 34 \\
\hline 11 & & 30 & & 29 & & & & & & & 28 & & & & & & & & & 10 & 32 & 32 & & & 27 & 34. \\
\hline 11/15/11 22:00 & & 30.882 & 30.522 & 29.557 & & & 29.652 & 30.872 & 30.424 & 30.127 & 28.758 & 26.574 & 31.934 & 36.459 & 32.959 & 16.92 & 15.987 & 20.65 & 15.581 & 10.792 & 32.147 & 32.831 & & 31.896 & 27.505 & 34.431 \\
\hline 11/15/11 23:00 & & 30.883 & 30.526 & 29.557 & 30.409 & & 29.664 & 30.874 & 30.427 & 30.136 & 28.765 & 26.569 & 31.941 & 36.466 & 32.963 & 16.925 & 15.989 & 20.65 & 15.588 & 10.799 & 32.15 & 32.841 & 30.063 & 31.901 & 27.505 & 34.436 \\
\hline
\end{tabular}




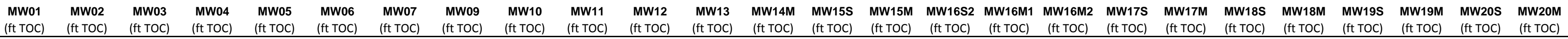

\begin{tabular}{|c|c|c|c|c|c|c|c|c|c|c|c|c|c|c|c|c|c|c|c|c|c|c|c|c|c|c|}
\hline Well & $\begin{array}{l}\text { MW01 } \\
\text { (ft TOC) }\end{array}$ & $\begin{array}{l}\text { MW02 } \\
\text { (ft TOC) }\end{array}$ & $\begin{array}{l}\text { MW03 } \\
\text { (ft ToC) }\end{array}$ & $\begin{array}{l}\text { MW04 } \\
\text { (ft TOC) }\end{array}$ & $\begin{array}{l}\text { MW05 } \\
\text { (ft TOC) }\end{array}$ & $\begin{array}{l}\text { MW06 } \\
\text { (ft Toc) }\end{array}$ & $\begin{array}{l}\text { MW07 } \\
\text { (ft TOC) }\end{array}$ & $\begin{array}{l}\text { MW09 } \\
\text { (ft TOC) }\end{array}$ & $\begin{array}{l}\text { MW10 } \\
\text { (ft Toc) }\end{array}$ & $\begin{array}{l}\text { MW11 } \\
\text { (ft Toc) }\end{array}$ & $\begin{array}{l}\text { MW12 } \\
\text { (ft TOC) }\end{array}$ & $\begin{array}{l}\text { MW13 } \\
\text { (ft TOC) }\end{array}$ & $\begin{array}{l}\text { MW14M } \\
\text { (ft TOC) }\end{array}$ & $\begin{array}{l}\begin{array}{l}\text { MWW15S } \\
\text { (ft Toc) }\end{array}\end{array}$ & $\begin{array}{l}\text { MWW15M } \\
\text { (ft ToC) }\end{array}$ & $\begin{array}{l}\text { MW16S2 } \\
\text { (ft TOC) }\end{array}$ & $\begin{array}{l}\text { MW16161 } \\
\text { (ft TOC) }\end{array}$ & $\begin{array}{l}\text { MW16M2 } \\
\text { (ft TOC) }\end{array}$ & $\begin{array}{l}\text { MW17S } \\
\text { (ft TOC) }\end{array}$ & $\begin{array}{l}\text { MWw7M } \\
\text { (ft TOC) }\end{array}$ & $\begin{array}{l}\text { MW18S } \\
\text { (ft ToC) }\end{array}$ & $\begin{array}{l}\text { MWWidM } \\
\text { (ft TOC) }\end{array}$ & $\begin{array}{l}\text { MWWigS } \\
\text { (ft TOC) }\end{array}$ & $\begin{array}{l}\text { MW19M } \\
\text { (ft TOC) }\end{array}$ & $\begin{array}{l}\text { MW20S } \\
\text { (ft Toc) }\end{array}$ & 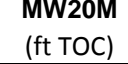 \\
\hline $11 / 16 / 110: 00$ & & 30.887 & 30.531 & 29.561 & 30.418 & & 29.674 & 30.877 & 30.427 & 30.143 & 28.772 & 26.574 & 31.948 & 36.469 & 32.971 & 16.929 & 15.993 & 20.647 & 15.593 & 10.809 & 32.157 & 32.848 & 30.07 & 31.91 & 27.507 & 34.436 \\
\hline 11/16/11 1:00 & & 30.889 & 30.538 & 29.566 & 30.425 & & 29.685 & 30.879 & 30.429 & 30.15 & 28.777 & 26.571 & 31.955 & 36.471 & 32.978 & $3 \quad 16.934$ & 15.998 & 20.643 & 15.59 & 10.816 & 32.164 & 32.855 & 30.077 & 31.915 & 27.507 & 34.441 \\
\hline 11/16/11 2:00 & & 30.887 & 30.538 & 29.564 & 30.425 & & 29.699 & 30.884 & 30.429 & 30.155 & 28.781 & 26.569 & 31.96 & 36.471 & 32.982 & 16.939 & 16 & 20.64 & 15.6 & 10.823 & 32.166 & 32.857 & 30.074 & 31.922 & 27.507 & 34.443 \\
\hline $11 / 16 / 113: 00$ & & 30.897 & 30.55 & 29.58 & 30.432 & & 29.697 & 30.886 & 30.432 & 30.166 & 28.791 & 26.576 & 31.965 & 36.476 & 32.989 & 16.941 & 16.007 & 20.636 & 15.593 & 10.825 & 32.178 & 32.867 & 30.089 & 31.927 & 27.507 & 34.448 \\
\hline $11 / 16 / 11$ 4:00 & & 30.894 & 30.552 & 29.585 & 30.442 & & 29.709 & 30.893 & 30.427 & 30.173 & 28.795 & 26.581 & 31.972 & 36.476 & 32.996 & 16.941 & 16.01 & 20.636 & 15.602 & 10.835 & 32.18 & 32.872 & 30.086 & 31.934 & 27.507 & 34.445 \\
\hline $11 / 16 / 11$ 5:00 & & 30.892 & 30.547 & 29.571 & 30.435 & & 29.732 & 30.893 & 30.427 & 30.173 & 28.793 & 26.564 & 31.972 & 36.478 & 32.994 & 16.946 & 16.008 & 20.627 & 15.616 & 10.835 & 32.171 & 32.869 & 30.079 & 31.934 & 27.507 & 34.445 \\
\hline $11 / 16 / 116: 00$ & & 30.906 & 30.566 & 29.606 & 30.456 & & 29.711 & 30.898 & 30.432 & 30.19 & 28.812 & 26.595 & 31.981 & $\begin{array}{l}50.410 \\
36.48\end{array}$ & 33.001 & $\begin{array}{l}16.940 \\
16.95\end{array}$ & $\begin{array}{l}10.0012 \\
16.012\end{array}$ & 20.636 & $\begin{array}{r}15.60 \\
15.6\end{array}$ & $\begin{array}{l}10.039 \\
\end{array}$ & 32.199 & $\begin{array}{l}32.879 \\
32.079\end{array}$ & 30.107 & 31.938 & 27.509 & 34.448 \\
\hline $11 / 16 / 117: 00$ & & $\begin{array}{l}30.900 \\
30.91\end{array}$ & $\begin{array}{l}30.587 \\
30.58\end{array}$ & 29.638 & $\begin{array}{l}30.450 \\
30.479\end{array}$ & & 29.704 & $\begin{array}{l}30.0903 \\
30.903\end{array}$ & $\begin{array}{l}30.426 \\
30.436\end{array}$ & 30.21 & $\begin{array}{l}20.016 \\
28.832\end{array}$ & 26.624 & 31.988 & $\begin{array}{r}36.480 \\
36.485\end{array}$ & $\begin{aligned} 33.01 \\
33.01\end{aligned}$ & 16.955 & $\begin{array}{l}10.012 \\
16.017\end{array}$ & $\begin{array}{l}2.000 \\
20.65\end{array}$ & $\begin{array}{r}15.00 \\
15.602\end{array}$ & $\begin{array}{l}10.039 \\
10.849\end{array}$ & 32.215 & 32.888 & 30.129 & $\begin{array}{l}31.948 \\
31.948\end{array}$ & 27.512 & 34.448 \\
\hline $11 / 16 / 118: 00$ & & $\begin{array}{r}30.922 \\
30.922\end{array}$ & $\begin{array}{l}30.581 \\
30.61\end{array}$ & $\begin{array}{l}29.680 \\
29.671\end{array}$ & $\begin{array}{l}30.505 \\
30.57\end{array}$ & & $\begin{array}{l}29.695 \\
29.695\end{array}$ & $\begin{array}{l}30.903 \\
30.91\end{array}$ & $\begin{array}{l}30.430 \\
30.439\end{array}$ & $\begin{array}{r}30.21 \\
30.234\end{array}$ & 28.851 & $\begin{array}{l}20.024 \\
26.653\end{array}$ & $\begin{array}{c}580 \\
32\end{array}$ & $\begin{array}{l}30.480 \\
36.49\end{array}$ & $\begin{array}{r}33.024 \\
33.024\end{array}$ & $\begin{array}{l}10.9302 \\
16.962\end{array}$ & $\begin{array}{l}10.011 \\
16.019\end{array}$ & $\begin{array}{l}20.659 \\
20.659\end{array}$ & $\begin{array}{l}13.002 \\
15.597\end{array}$ & $\begin{array}{l}1.849 \\
10.86\end{array}$ & 32.234 & $\begin{array}{l}32.8000 \\
32.902\end{array}$ & 30.154 & $\begin{array}{l}31.960 \\
31.962\end{array}$ & 27.512 & $\begin{array}{l}34.448 \\
34.452\end{array}$ \\
\hline $11 / 16 / 119: 00$ & & 30.924 & 30.629 & 29.694 & 30.528 & & 29.699 & 30.915 & 30.441 & 30.252 & 28.87 & 26.669 & 32.014 & 36.497 & 33.034 & 16.967 & 16.026 & 20.659 & 15.607 & 10.872 & 32.252 & 32.912 & 30.183 & 31.974 & 27.512 & 34.452 \\
\hline $11 / 16 / 1110: 00$ & & 30.931 & 30.643 & 29.708 & 30.544 & & 29.706 & 30.917 & 30.443 & 30.269 & 28.884 & 26.681 & 32.023 & 36.497 & 33.043 & 16.971 & 16.028 & 20.654 & 15.612 & 10.882 & 32.269 & 32.921 & 30.199 & 31.981 & 27.514 & 34.455 \\
\hline $16 / 1$ & & 30.941 & 30.659 & 29.73 & 30.565 & & 29.716 & 30.927 & 30.451 & 30.287 & 28.9 & 26.693 & 32.032 & 36.501 & 33.055 & 16.981 & 16.033 & 20.654 & 15.614 & 10.893 & 32.28 & 32.933 & .211 & 1.995 & 27.514 & 34.459 \\
\hline $11 / 16 / 1112: 00$ & & 30.931 & 30.652 & 29.708 & 30.563 & & 29.753 & 30.929 & 30.441 & 30.287 & 28.9 & 26.674 & 32.042 & 36.506 & 33.064 & 16.983 & 16.04 & 20.65 & 15.642 & 10.9 & 32.271 & 32.938 & 199 & 2.002 & 27.514 & 34.457 \\
\hline $11 / 16 / 1113: 00$ & & 30.934 & 30.645 & 29.69 & 30.554 & & 29.782 & 30.934 & 30.446 & 30.282 & 28.893 & 26.648 & 32.039 & 36.506 & 33.064 & 16.985 & 16.038 & 20.643 & 654 & 10.9 & 32.266 & 32.938 & 187 & 31.999 & 27.517 & 34.457 \\
\hline $11 / 16 / 1114: 00$ & & 30.938 & 30.652 & 29.699 & 30.556 & & 29.789 & 30.936 & 30.451 & 30.289 & 28.902 & 26.655 & 32.046 & 36.501 & 33.069 & 16.99 & $\begin{array}{l}16.045 \\
\end{array}$ & 20.64 & 15.663 & 10.905 & 32.271 & 32.945 & 30.194 & 32.006 & 27.516 & 34.459 \\
\hline 11/16/11 15:00 & & 30.938 & 30.65 & 29.701 & 30.556 & & 29.805 & 30.941 & 30.446 & 30.294 & 28.902 & 26.65 & 32.049 & 36.508 & 33.071 & 16.992 & 16.047 & 20.634 & 15.675 & 10.91 & 32.271 & 32.95 & 30.194 & 32.013 & 27.517 & 34.462 \\
\hline 11/16/11 16:00 & & 30.945 & 30.652 & 29.706 & 30.558 & & 29.808 & 30.946 & 30.451 & 30.299 & 28.907 & 26.65 & 32.051 & 36.511 & 33.078 & 3 $\quad 16.997$ & 16.052 & 20.677 & 15.677 & 10.912 & 32.275 & 32.954 & 194 & 32.013 & 27.517 & 34.462 \\
\hline 11/16/11 17:00 & & 30.952 & 30.659 & 29.723 & 30.568 & & 29.805 & 30.951 & 30.455 & 30.31 & 28.916 & 26.662 & 32.058 & 36.513 & 33.083 & 17.002 & 16.054 & 20.687 & 15.675 & 10.919 & 32.285 & 32.959 & .208 & 32.018 & 27.519 & 34.462 \\
\hline $11 / 16 / 1118: 00$ & & 30.957 & 30.666 & 29.734 & 30.577 & & 29.808 & 30.956 & 30.46 & 30.322 & 28.925 & 26.672 & 32.063 & 36.516 & 33.09 & 17.004 & 16.059 & 20.68 & 15.673 & 10.926 & 32.294 & 32.964 & 30.215 & 32.025 & 27.519 & 34.464 \\
\hline $11 / 16 / 1119: 00$ & & 30.957 & 30.664 & 29.73 & 30.575 & & 29.822 & 30.96 & 30.455 & 30.324 & 28.925 & 26.662 & 32.067 & 36.513 & 33.092 & 17.006 & 16.059 & 20.675 & 15.691 & 10.931 & 32.294 & 32.969 & 30.22 & 32.03 & 27.519 & 34.464 \\
\hline $11 / 16 / 1120: 00$ & & 30.959 & 30.669 & 29.732 & 30.577 & & 29.829 & 30.963 & 30.458 & 30.329 & 28.93 & 26.662 & 32.072 & 36.523 & 33.099 & 17.009 & 16.061 & 20.668 & 15.694 & 10.933 & 32.294 & 32.969 & 30.215 & 32.032 & 27.519 & 34.464 \\
\hline $11 / 16 / 1121: 00$ & & 30.962 & 30.669 & 29.737 & 30.582 & & 29.833 & 30.965 & 30.46 & 30.336 & 28.935 & 26.662 & 32.074 & 36.518 & 33.099 & 17.011 & 16.066 & 20.664 & 15.694 & 10.933 & 32.299 & 32.976 & 30.223 & 32.037 & 27.519 & 34.464 \\
\hline $11 / 16 / 1122: 00$ & & 30.959 & 30.666 & 29.725 & 30.577 & & 29.85 & 30.972 & 30.463 & 30.334 & 28.93 & 26.65 & 32.184 & 36.52 & 33.217 & 17.011 & $\begin{array}{l}16.099 \\
\end{array}$ & 20.661 & 15.705 & 11.053 & 32.343 & 33.082 & 30.218 & 32.145 & 27.521 & 34.464 \\
\hline $11 / 16 / 1123: 00$ & & 30.959 & 30.659 & 29.711 & 30.57 & & 29.869 & 30.975 & 30.462 & 30.329 & 28.925 & 26.631 & 32.142 & 36.518 & 33.172 & 17.013 & 16.101 & 20.661 & 15.712 & 11.009 & 32.315 & $\begin{array}{l}33.04 \\
5\end{array}$ & 30.204 & 32.105 & 27.521 & 34.464 \\
\hline $11 / 17 / 110: 00$ & & 30.964 & 30.657 & 29.711 & 30.565 & & 29.871 & 30.975 & 30.46 & 30.329 & 28.925 & 26.631 & 32.126 & 36.516 & 33.156 & 17.016 & $\begin{array}{l}16.099 \\
1.099\end{array}$ & 20.664 & 15.71 & $\begin{array}{l}10.992 \\
\end{array}$ & 32.313 & 33.028 & 30.206 & 32.086 & 27.521 & 34.464 \\
\hline 11/17/11 1:00 & & 30.966 & 30.655 & 29.716 & 30.568 & & 29.871 & 30.982 & $\begin{array}{l}30.465 \\
30.465\end{array}$ & 30.331 & 28.928 & 26.631 & 32.119 & 36.518 & 33.149 & 17.016 & $\begin{array}{l}16.0101 \\
16.0\end{array}$ & 20.661 & $\begin{array}{l}15.712 \\
\end{array}$ & $\begin{array}{l}10.985 \\
\end{array}$ & 32.315 & 33.021 & .208 & 32.081 & 27.524 & 34.466 \\
\hline $11 / 17 / 112: 00$ & & $\begin{array}{l}30.906 \\
30.966\end{array}$ & 30.652 & 29.708 & 30.563 & & 29.883 & $\begin{array}{l}30.987 \\
30.987\end{array}$ & $\begin{array}{l}30.400 \\
30.462\end{array}$ & 30.331 & $\begin{array}{l}28.920 \\
28.923\end{array}$ & $\begin{array}{l}20.021 \\
26.624\end{array}$ & 32.114 & $\begin{array}{l}30.510 \\
36.516\end{array}$ & $\begin{array}{l}53.149 \\
33.142\end{array}$ & 17.018 & $\begin{array}{l}10.101 \\
16.101\end{array}$ & 20.654 & $\begin{array}{l}15.722 \\
1322\end{array}$ & $\begin{array}{l}10.9076 \\
10.976\end{array}$ & 32.306 & $\begin{array}{l}33.014 \\
33.014\end{array}$ & .201 & 32.077 & 27.524 & $\begin{array}{l}34.469 \\
34.469\end{array}$ \\
\hline $11 / 17 / 113: 00$ & & $\begin{array}{l}30.966 \\
30.966\end{array}$ & $\begin{array}{l}30.052 \\
30.648\end{array}$ & 29.701 & $\begin{array}{l}30.558 \\
30.558\end{array}$ & & 29.892 & $\begin{array}{l}30.989 \\
30.989\end{array}$ & $\begin{array}{l}30.402 \\
30.465\end{array}$ & $\begin{array}{l}30.327 \\
30.327\end{array}$ & $\begin{array}{l}28.923 \\
28.921\end{array}$ & $\begin{array}{l}20.024 \\
26.614\end{array}$ & $\begin{array}{l}32.14 \\
32.11\end{array}$ & $\begin{array}{l}30.510 \\
36.52\end{array}$ & $\begin{array}{l}33.142 \\
33.137\end{array}$ & 17.018 & $\begin{array}{l}10.101 \\
16.101\end{array}$ & $\begin{array}{l}20.034 \\
20.652\end{array}$ & $\begin{array}{l}13.722 \\
15.729\end{array}$ & $\begin{array}{l}10.970 \\
10.973\end{array}$ & $\begin{array}{l}32.300 \\
32.294\end{array}$ & $\begin{array}{l}33.014 \\
33.011\end{array}$ & 0.197 & 32.072 & 27.524 & $\begin{array}{l}34.499 \\
34.469\end{array}$ \\
\hline $11 / 17 / 114: 00$ & & $\begin{array}{l}30.960 \\
30.962\end{array}$ & $\begin{array}{l}30.648 \\
30.636\end{array}$ & 29.683 & $\begin{array}{l}30.538 \\
30.544\end{array}$ & & $\begin{array}{l}29.892 \\
29.909\end{array}$ & $\begin{array}{l}30.999 \\
30.991\end{array}$ & $\begin{array}{l}30.465 \\
30.462\end{array}$ & $\begin{array}{l}30.371 \\
30.32\end{array}$ & $\begin{array}{l}28.921 \\
28.909\end{array}$ & $\begin{array}{l}20.014 \\
26.593\end{array}$ & $\begin{array}{l}32.111 \\
32.103\end{array}$ & $\begin{array}{l}\begin{array}{l}0.522 \\
36.52\end{array} \\
\end{array}$ & $\begin{array}{l}33.131 \\
33.132\end{array}$ & $\begin{array}{l}17.016 \\
17.016\end{array}$ & $\begin{array}{l}10.101 \\
16.099\end{array}$ & $\begin{array}{l}20.052 \\
20.647\end{array}$ & $\begin{array}{l}13 . / 749 \\
15.741\end{array}$ & $\begin{array}{l}10.973 \\
10.964\end{array}$ & $\begin{array}{l}32.294 \\
32.282\end{array}$ & $\begin{array}{l}33.001 \\
33.002\end{array}$ & 1.178 & $\begin{array}{l}32.012 \\
32.063\end{array}$ & 27.524 & $\begin{array}{l}34.4499 \\
34.469\end{array}$ \\
\hline 11 & & & 30 & 29.664 & 30 & & & 30 & 30.462 & 30.313 & 28.9 & 26.576 & 32.093 & 36.513 & 33.123 & 17.013 & 16.094 & 20.64 & 15.748 & 10.959 & 32.266 & 32.995 & 159 & 32.058 & 27.524 & 34.466 \\
\hline 11 & & & 3 & 29.657 & 30 & & & 30 & $\begin{array}{l}30.465 \\
30.465\end{array}$ & 30.306 & $\begin{array}{r}28.895 \\
28.895\end{array}$ & 26.567 & 32.084 & 36.506 & 33.114 & 17.016 & $\begin{array}{l}10.094 \\
16.094\end{array}$ & 20.647 & $\begin{array}{r}15.75 \\
15\end{array}$ & 10.95 & 32.264 & 85 & 54 & 32.046 & 27.526 & $\begin{array}{l}34.464 \\
34.404\end{array}$ \\
\hline $11 / 17 / 11$ 7:00 & & & & 29.657 & & & & & & & & & & 506 & 109 & & 89 & 20.652 & 15.752 & 10.943 & 32.255 & 78 & 30.15 & 42 & 27.526 & 34.462 \\
\hline $11 / 17 / 118: 00$ & & 30.966 & 30.617 & 29.671 & 30.514 & & 29.904 & 30.999 & 30.47 & 30.306 & 28.895 & 26.581 & 32.077 & 36.506 & 33.104 & 17.011 & 16.087 & 20.659 & $\begin{array}{l}15.743 \\
1543\end{array}$ & $\begin{array}{l}10.945 \\
10.943\end{array}$ & $\begin{array}{l}32.253 \\
32.266\end{array}$ & $\begin{array}{l}32.978 \\
32.978\end{array}$ & .161 & 2.042 & 27.526 & $\begin{array}{l}34.402 \\
34.462\end{array}$ \\
\hline 11/17/11 9:00 & & 30.966 & 30.617 & 29.666 & 30.512 & & 29.911 & 30.999 & 30.467 & 30.303 & 28.891 & 26.576 & 32.074 & 36.508 & 33.104 & 17.009 & 16.087 & 20.652 & 15.755 & 10.938 & 32.257 & 32.976 & 30.154 & 32.037 & 27.528 & 34.462 \\
\hline 11/17/11 10:00 & & & 30.603 & 29.643 & & & & 31.001 & 30.467 & & 28.877 & & 32.067 & 36.504 & 33.097 & 17.006 & 16.08 & 20.647 & 15.778 & 10.933 & 32.234 & 32.969 & 30.131 & 32.035 & 27.526 & 34.457 \\
\hline 11/17/11 11:00 & & 30.955 & 30.592 & 29.622 & & & & 31.001 & 30.465 & & 28.867 & 26.535 & 32.06 & 36.501 & 33.088 & 17.006 & 16.078 & 20.64 & 15.788 & 10.922 & 32.222 & 32.962 & 30.117 & 32.027 & 27.526 & 34.457 \\
\hline 11/17/11 12:00 & & 30.945 & 30.571 & 29.582 & 30. & & & 30.999 & 30.46 & & 28.844 & 26.495 & 32.049 & 36.494 & 33.078 & 16.999 & 16.071 & 20.629 & 15.809 & 10.914 & 32.196 & $5 \quad 32.945$ & 30.077 & 32.013 & 27.528 & 34.452 \\
\hline $11 / 17 / 1113: 00$ & & 30.934 & 30.54 & 29.538 & & & 29.991 & 30.996 & 30.458 & 30.241 & 28.816 & 26.452 & 32.03 & 36.48 & 33.062 & 16.995 & 16.064 & 20.62 & 15.825 & 10.9 & 32.164 & 32.931 & 30.042 & 32.002 & 27.526 & 34.448 \\
\hline $11 / 17 / 1114: 00$ & & 30.927 & 30.517 & 29.507 & & & 29.998 & 30.989 & 30.453 & 30.217 & 28.795 & 26.425 & 32.014 & 36.471 & 33.05 & 16.988 & 16.059 & 20.613 & 15.832 & 10.886 & 32.143 & 32.914 & 30.009 & 31.983 & 27.528 & 34.443 \\
\hline $11 / 17 / 1115: 00$ & & 30.92 & 30.492 & 29.477 & 30. & & 30.003 & 30.982 & 30.448 & 30.199 & 28.772 & 26.399 & 32.002 & 36.459 & 33.034 & 16.981 & 16.054 & 20.601 & 15.837 & 10.87 & 32.117 & 32.902 & 29.983 & 31.966 & 27.526 & 34.441 \\
\hline $11 / 17 / 1116: 00$ & & 30.915 & 30.475 & 29.461 & 30.332 & & 29.991 & 30.977 & 30.448 & 30.183 & 28.758 & 26.387 & 31.988 & 36.45 & 33.022 & 16.974 & 16.05 & 20.597 & 15.832 & 10.858 & 32.108 & 32.888 & 29.964 & 31.952 & 27.526 & 34.436 \\
\hline 11/17/11 17:00 & & 30.913 & 30.461 & 29.454 & 30.316 & & 29.972 & 30.972 & 30.448 & 30.171 & 28.744 & 26.382 & 31.981 & 36.448 & 33.01 & 16.969 & 16.042 & 20.59 & 15.825 & 10.849 & 32.094 & 32.876 & 29.957 & 31.943 & 27.528 & 34.434 \\
\hline 11/17/11 18:00 & & 30.911 & 30.454 & 29.451 & 30.304 & & 29.951 & 30.97 & 30.448 & 30.159 & 28.737 & 26.385 & 32.002 & 36.44 & 33.039 & 16.962 & 16.047 & 20.587 & 15.816 & 10.875 & 32.103 & 32.902 & 29.955 & 31.964 & 27.528 & 34.434 \\
\hline 11/17/11 19:00 & & 30.906 & $\begin{array}{l}30.447 \\
\end{array}$ & 29.447 & 30.299 & & 29.932 & 30.965 & 30.443 & 30.15 & 28.728 & 26.385 & 31.981 & 36.44 & 33.015 & 16.957 & 16.04 & 20.585 & 15000 & 10.851 & 32.094 & 32 & .952 & 31.941 & 27.531 & 34.431 \\
\hline $\begin{array}{l}11 / 177 \\
117\end{array}$ & & 30.901 & 30.436 & 29.437 & 30 & & 29.92 & 30.96 & 30.443 & 30.139 & 28.718 & 26.382 & 31.969 & 36.431 & 33.001 & 16. & 16.03 & 20 & 15.799 & 10.842 & 32.082 & 32.867 & 43 & 31.929 & 27.531 & 34.429 \\
\hline 11/17 & & 30.896 & $\begin{array}{l}30.426 \\
30.426\end{array}$ & 29.426 & 30.28 & & 29.911 & 30.956 & 30.441 & 30.1 & 28.709 & 26.373 & 31.957 & 36.429 & 32.989 & 16.943 & $\begin{array}{l}16.028 \\
16.028\end{array}$ & 20 & 15.795 & 10.825 & 32.068 & 32.86 & 29 & 31.92 & 27.531 & 34.427 \\
\hline $\begin{array}{l}11 / 1 \\
11\end{array}$ & & $\begin{array}{l}30.8962 \\
30.892\end{array}$ & $\begin{array}{r}50.420 \\
30.41\end{array}$ & $\begin{array}{l}29.414 \\
29.414\end{array}$ & 30. & & $\begin{array}{l}29.906 \\
2911\end{array}$ & 30.951 & $\begin{array}{l}30.441 \\
30.441\end{array}$ & 30. & 28.693 & 26. & 31.948 & $\begin{array}{l}30.429 \\
36.429\end{array}$ & $\begin{aligned} 32.989 \\
32.98\end{aligned}$ & $\begin{array}{l}10.943 \\
16.939\end{array}$ & 16 & 20.571 & $\begin{array}{l}15.788 \\
15.788\end{array}$ & 10.816 & $\begin{array}{l}32.050 \\
32.059\end{array}$ & $\begin{array}{r}32.00 \\
32.845\end{array}$ & 26 & $\begin{array}{l}31.913 \\
31.913\end{array}$ & 27.528 & $\begin{array}{l}34.422 \\
34.422\end{array}$ \\
\hline & & 30 & 30. & 29.3 & & & 29 & 30. & & & 28 & 26 & & 36. & 32.966 & 16 & & 26 & 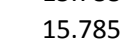 & 10 & 32 & & & 8 & 27.531 & 34.42 \\
\hline & & 30 & 30. & 29.3 & & & & & & & 28 & & & 36. & 32. & & & & & 10. & & & & 37 & 1 & \\
\hline & & & & 29.3 & & & & & & & & & & 0. & & & & & & & & & & 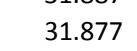 & & \\
\hline & & & & & & & & & & 30 & & & & & & & & & & & & & & & & \\
\hline & & & & & & & & & & & & & & 36 & & & & & & & & & & & & 34. \\
\hline & & & & 29. & & & & & & & & & & & & & & & & & & & & & & \\
\hline $11 /$ & & 30. & & 25 & & & & & 30 & & 28 & & & 36 & 32 & & & & & 1 & 31 & 32 & 29 & 31 & 27 & 34. \\
\hline 11/18/11 6:00 & & 30.848 & 30.314 & 29.309 & 30.176 & & 29.852 & 30.908 & 30.417 & 30.006 & 28.6 & 26.296 & 31.871 & 36.396 & 32.903 & 16.89 & 15.984 & 20.529 & 15.738 & 10.741 & 31.97 & 32.772 & 29.83 & 31.833 & 27.533 & 34.406 \\
\hline 11/18/11 7:00 & & 30.85 & 30.305 & 29.309 & 30.171 & & 29.836 & 30.905 & 30.417 & 29.997 & 28.595 & 26.301 & 31.864 & 36.389 & 32.895 & 16.888 & 15.977 & 20.532 & 15.722 & 10.736 & 31.968 & 32.763 & 29.828 & 31.826 & 27.535 & 34.403 \\
\hline
\end{tabular}




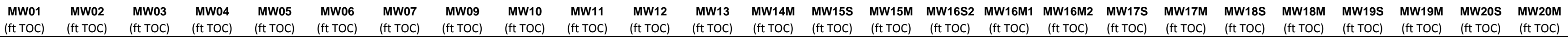

\begin{tabular}{|c|c|c|c|c|c|c|c|c|c|c|c|c|c|c|c|c|c|c|c|c|c|c|c|c|c|c|}
\hline Well & $\begin{array}{l}\text { (ft TOC) } \\
\text { (f) }\end{array}$ & $\begin{array}{l}\text { (ft TOC) } \\
\text { (f) }\end{array}$ & (ft TOC) & (ft TOC) & $\begin{array}{l}\text { (ft TOC) } \\
\text { (f) }\end{array}$ & $\begin{array}{l}\text { (ft ToC) } \\
\text { (f) }\end{array}$ & $\begin{array}{l}\text { (ft TOC) } \\
\text { (f) }\end{array}$ & $\begin{array}{l}\text { (ft TOC) } \\
\text { (ft) }\end{array}$ & $\begin{array}{l}\text { (ft TOC) } \\
\text { (f) }\end{array}$ & $\begin{array}{l}\text { (ft TOC) } \\
\text { (l) }\end{array}$ & $\begin{array}{l}\text { (ft TOC) } \\
\text { (n) }\end{array}$ & $\begin{array}{l}\text { ft TOC) } \\
\text { ft }\end{array}$ & (ft TOC) & $\begin{array}{l}\text { (ft toc) } \\
\text { (foc) }\end{array}$ & (ft TOC) & (ft TOC) & (ft TOC) & (ft TOC) & $\begin{array}{l}\text { (ft TOC) } \\
\text { (ft) }\end{array}$ & (ft TOC) & $\begin{array}{l}\text { (ft TOC) } \\
\text { (1) }\end{array}$ & (ft TOC) & $\begin{array}{l}\text { (ft TOC) } \\
\text { (n) }\end{array}$ & (ft TOC) & $\begin{array}{l}\text { (nt TOC) } \\
\text { (nt }\end{array}$ & (ft TOC) \\
\hline $11 / 18 / 118: 00$ & & 30.848 & 30.303 & 29.306 & 30.168 & & 29.824 & 30.903 & 30.415 & 29.99 & 28.591 & 26.308 & 31.855 & 36.389 & 32.888 & 16.885 & 15.975 & 20.546 & 15.717 & 10.726 & 31.965 & 32.755 & 29.828 & 31.821 & 27.533 & 34.401 \\
\hline $11 / 18 / 11$ 9:00 & & 30.838 & 30.298 & 29.295 & 30.162 & & 29.822 & 30.893 & 30.408 & 29.978 & 28.577 & 26.296 & 31.848 & 36.387 & 32.879 & 16.876 & 15.965 & 20.543 & 15.715 & 10.719 & 31.949 & 32.746 & 29.811 & 31.812 & 27.531 & 34.399 \\
\hline $11 / 18 / 1110: 00$ & & 30.843 & 30.296 & 29.304 & 30.159 & & 29.8 & 30.896 & 30.417 & 29.976 & 28.581 & 26.308 & 31.84 & 36.384 & 32.874 & 16.876 & 15.965 & 20.536 & 15.703 & 10.712 & 31.961 & 32.741 & 29.823 & 31.812 & 27.531 & 34.396 \\
\hline $8 / 1111: 00$ & & 30.831 & 30.284 & 29.283 & 30.1 & & 29.808 & 30.891 & 30.413 & 29.962 & 28.565 & 26.291 & 31.833 & 36.387 & 32.865 & 16.874 & 15.956 & 20.541 & 15.703 & 10.703 & 31.942 & 32.729 & 29.804 & 31.79 & 27.533 & 34.396 \\
\hline $11 / 18 / 1112: 00$ & & 30.829 & 30.279 & 29.276 & 30.14 & & 29.803 & 30.886 & 0.403 & 29.953 & 8.556 & 26.287 & 31.826 & 36.3 & 32.858 & 16.867 & 15.949 & 20.5 & 15.703 & 10.696 & 31.94 & 32.725 & & 31.795 & 27.538 & 34.391 \\
\hline $11 / 18 / 1113: 00$ & & 30.813 & 30.254 & 29.234 & 30.117 & & 29.819 & 30.879 & 30.401 & 29.923 & 28.532 & 26.253 & 31.812 & 36.377 & 32.846 & 16.862 & 15.942 & 20.543 & 15.712 & $\begin{array}{l}10.684 \\
\end{array}$ & 31.909 & 2.71 & 29.767 & 1.779 & 7.533 & 34.384 \\
\hline $11 / 18 / 1114: 00$ & & 30.817 & 30.242 & 29.229 & 30.108 & & 29.808 & 30.872 & 30.405 & 29.916 & 28.525 & 26.248 & 31.803 & 36.37 & 32.837 & 16.853 & 15.935 & 20.539 & 15.701 & 10.675 & 31.902 & 32.703 & 29.762 & 31.772 & 27.535 & 34.384 \\
\hline 11/18/11 15:00 & & 30.824 & 30.244 & 29.239 & 30.106 & & 29.784 & 30.869 & 30.403 & 29.911 & 28.525 & 26.268 & 31.801 & 36.373 & 32.83 & 16.853 & 15.933 & 20.527 & 15.687 & 10.668 & 31.907 & 32.701 & 29.771 & 31.765 & 27.535 & 34.382 \\
\hline 11/18/11 16:00 & & 30.822 & 30.24 & 29.246 & 30.108 & & 29.765 & 30.867 & 30.403 & 29.906 & 28.525 & 26.277 & 31.798 & 36.368 & 32.83 & 16.85 & 15.933 & 20.576 & 15.677 & 10.665 & 31.909 & 32.696 & 29.778 & 31.765 & 27.538 & 34.384 \\
\hline 11/18/11 17:00 & & 30.817 & 30.237 & 29.246 & 30.113 & & 29.751 & 30.864 & 30.403 & 29.906 & 28.523 & 26.282 & 31.796 & 36.368 & 32.823 & 16.846 & 15.928 & 20.571 & 15.67 & 10.661 & 31.907 & 32.694 & 29.776 & 31.76 & 27.535 & 34.38 \\
\hline 11/18/11 18:00 & & 30.82 & 30.24 & 29.255 & 30.117 & & 29.732 & 30.859 & 30.403 & 29.902 & 28.525 & 26.291 & 31.796 & 36.368 & 32.825 & 16.846 & 15.928 & 20.569 & 15.658 & 10.663 & 31.919 & 32.694 & 29.788 & 31.755 & 27.538 & 34.382 \\
\hline 11/18/11 19:00 & & 30.815 & 30.242 & 29.255 & 30.122 & & 29.723 & 30.857 & 30.401 & 29.899 & 28.523 & 26.294 & 31.796 & 36.365 & 32.825 & 16.841 & 15.923 & 20.578 & 15.649 & 10.658 & 31.916 & 32.694 & 29.785 & 31.753 & 27.538 & 34.382 \\
\hline $11 / 18 / 1120: 00$ & & 30.813 & 30.24 & 29.248 & 30.12 & & 29.714 & 30.857 & 30.405 & 29.897 & 28.521 & 26.296 & 31.791 & 36.365 & 32.823 & 16.839 & 15.921 & 20.576 & 15.637 & 10.658 & 31.914 & 32.689 & 29.788 & 31.753 & 27.54 & 34.382 \\
\hline 11/18/11 21:00 & & 30.81 & 30.235 & 29.246 & 30.117 & & 29.714 & 30.855 & 30.398 & 29.893 & 28.516 & 26.291 & 31.784 & 36.37 & 32.818 & 16.839 & 15.919 & 20.576 & 15.635 & 10.649 & 31.914 & 32.684 & 29.783 & 31.748 & 27.538 & 34.38 \\
\hline $11 / 18 / 1122: 00$ & & 30.806 & 30.23 & 29.234 & 30.115 & & 29.711 & 30.85 & 30.398 & 29.886 & 28.509 & 26.284 & 31.782 & 36.368 & 32.811 & 16.834 & 15.914 & 20.569 & 15.628 & 10.644 & 31.907 & 32.68 & 29.778 & 31.744 & 27.54 & 34.377 \\
\hline 11/18/11 23:00 & & 30.799 & 30.223 & 29.22 & 30.106 & & 29.714 & 30.848 & 30.391 & 29.876 & 28.5 & 26.272 & 31.773 & 36.363 & 32.804 & 16.832 & 15.907 & 20.557 & 15.626 & 10.642 & 31.891 & 32.672 & 29.767 & 31.739 & 27.54 & 34.373 \\
\hline $11 / 19 / 110: 00$ & & 30.794 & 30.207 & 29.194 & 30.089 & & 29.721 & 30.845 & 30.394 & 29.858 & 28.488 & 26.251 & 31.763 & 36.358 & 32.792 & 16.827 & 15.9 & 20.55 & 15.626 & 10.632 & 31.877 & 32.661 & 29.745 & 31.727 & 27.538 & 34.368 \\
\hline $11 / 19 / 11$ 1:00 & & 30.792 & 30.202 & 29.187 & 30.078 & & 29.714 & 30.841 & 30.389 & 29.846 & 28.479 & 26.246 & 31.756 & 36.356 & 32.788 & 16.825 & 15.898 & 20.546 & 15.619 & 10.628 & 31.872 & 32.656 & 29.738 & 31.722 & 27.538 & 34.366 \\
\hline $11 / 19 / 112: 00$ & & 30.789 & 30.191 & 29.171 & 30.066 & & 29.711 & 30.833 & 30.389 & 29.837 & 28.465 & 26.234 & 31.749 & 36.349 & 32.781 & 16.82 & $\begin{array}{l}15.0003 \\
15.893\end{array}$ & 20.534 & 15.616 & $\begin{array}{l}10.616 \\
\end{array}$ & 31.86 & 32.646 & 29.727 & 31.713 & 27.538 & 34.366 \\
\hline $11 / 19 / 113: 00$ & & 30.787 & 30.186 & 29.169 & 30.059 & & 29.706 & $\begin{array}{l}30.033 \\
30.833\end{array}$ & 30.386 & 29.828 & $\begin{array}{l}28.46 \\
28.46\end{array}$ & $\begin{array}{l}20.234 \\
26.234\end{array}$ & 31.74 & $\begin{array}{l}30.349 \\
36.351\end{array}$ & 32.776 & $\begin{array}{l}16.02 \\
16.818\end{array}$ & $\begin{array}{l}15.088 \\
15.88\end{array}$ & 20.53 & $\begin{array}{l}13.0100 \\
15.604\end{array}$ & 10.616 & $\begin{array}{l}31.00 \\
31.851\end{array}$ & $\begin{array}{l}32.642 \\
32.642\end{array}$ & 29.72 & 31.706 & 27.54 & 34.361 \\
\hline $11 / 19 / 114: 00$ & & 30.782 & 30.174 & 29.159 & 30.052 & & 29.697 & 30.829 & 30.386 & 29.821 & 28.451 & 26.227 & 31.735 & 36.351 & 32.769 & 16.815 & 15.884 & 20.527 & 15.6 & 10.607 & 31.842 & 32.637 & 29.715 & 31.701 & 27.54 & 34.361 \\
\hline $11 / 19 / 11$ 5:00 & & 30.782 & 30.174 & 29.164 & & & 29.683 & 30.824 & 30.384 & 29.816 & 28.451 & 26.234 & 31.733 & 36.347 & 32.764 & 16.813 & 15.884 & 20.523 & 15.59 & 10.604 & 851 & .635 & 29.715 & 31.699 & 27.54 & 34.361 \\
\hline $11 / 19 / 116: 00$ & & & & & & & 29.659 & 30.822 & 30.386 & 29.818 & 28.458 & 26.253 & 31.735 & 36.347 & & $\begin{array}{l}10.813 \\
16.813\end{array}$ & $\begin{array}{l}1.0004 \\
15.884\end{array}$ & 20.53 & 15.574 & 10.604 & 858 & 635 & 731 & 1.699 & 27.542 & 34.361 \\
\hline 11/19/11 7:00 & & & 30.177 & 76 & 30.061 & & 29.659 & 30.822 & 30.379 & 29.816 & 28.453 & 26.253 & 31.733 & 36.344 & 32.762 & 16.811 & 15.879 & 20.534 & 15.583 & 10.602 & 31.853 & .635 & .729 & 1.706 & 27.54 & 34.363 \\
\hline 11/19/11 8:00 & & 30.796 & 30.195 & 29.215 & & & 29.619 & 30.822 & 30.387 & 29.828 & 28.474 & 26.294 & 31.738 & 36.356 & 32.769 & 16.811 & 15.879 & 20.536 & 15.55 & 10.604 & 31.884 & .637 & .762 & 31.701 & 27.545 & 34.363 \\
\hline 11/19/11 9:00 & & 30.806 & 30.219 & 29.257 & 30.115 & & 29.587 & 30.824 & 30.391 & 29.841 & 28.493 & 26.337 & 31.749 & 36.365 & 32.776 & 16.815 & 15.881 & 20.532 & 15.532 & 10.614 & 31.912 & 32.649 & 29.807 & 31.708 & 27.545 & 34.363 \\
\hline 11/19/11 10:00 & & 30.81 & 30.235 & 29.288 & 30.145 & & 29.568 & & 30.396 & 29.858 & 28.514 & 26.37 & 31.759 & 36.375 & 32.788 & 16.818 & 15.884 & 20.532 & 15.522 & 10.623 & 31.935 & 32.656 & 29.839 & 31.72 & 27.547 & 34.366 \\
\hline 11/19/11 11:00 & & 30.824 & 30.279 & 29.36 & 30.194 & & 29.525 & 30.829 & 30.401 & 29.89 & 28.549 & 26.44 & 31.777 & 36.396 & 32.802 & 16.822 & 15.886 & 20.53 & 15.499 & 10.637 & 31.984 & 32.677 & 29.901 & 31.737 & 27.547 & 34.37 \\
\hline 11/19/11 12:00 & & 30.827 & 30.312 & 29.402 & 30.243 & & 29.518 & 30.838 & 30.396 & 29.916 & 28.577 & 26.478 & 31.794 & 36.41 & 32.82 & 16.829 & 15.891 & 20.529 & 15.504 & 10.656 & 32.01 & 32.696 & 29.936 & 31.758 & 27.547 & 34.375 \\
\hline $11 / 19 / 1113: 00$ & & 30.827 & 30.331 & 29.421 & 30.271 & & 29.528 & 30.843 & 30.396 & 29.937 & 28.595 & 26.495 & 31.829 & 36.419 & 32.856 & 16.836 & 15.895 & 20.527 & 15.508 & 10.689 & 32.04 & 32.729 & 29.957 & 31.793 & 27.549 & 34.377 \\
\hline $11 / 19 / 1114: 00$ & & 30.829 & 30.345 & 29.426 & 30.295 & & 29.544 & 30.845 & 30.396 & 29.951 & 28.607 & 26.497 & 32.231 & 36.433 & 33.107 & 16.843 & 15.902 & 20.525 & 15.513 & 10.945 & 32.199 & 33.125 & 29.973 & 32.199 & 27.547 & 34.375 \\
\hline $11 / 19 / 1115: 00$ & & 30.836 & 30.37 & 29.454 & 30.323 & & 29.542 & 30.853 & 30.401 & 29.971 & 28.628 & 26.524 & 31.969 & 36.44 & 33.001 & 16.85 & 15.923 & 20.532 & 15.506 & 10.837 & 32.131 & 32.869 & 30.009 & 31.927 & 27.547 & 34.377 \\
\hline $11 / 19 / 1116: 00$ & & 30.85 & 30.405 & 29.505 & 30.367 & & 29.525 & 30.86 & 30.406 & 30.004 & 28.66 & 26.567 & 31.927 & 36.457 & 32.956 & 16.86 & 15.933 & 20.583 & 15.501 & 10.79 & 32.138 & 32.829 & 30.056 & 31.887 & 27.549 & 34.384 \\
\hline 11/19/11 17:00 & & 30.852 & 30.431 & 29.531 & $\begin{array}{r}30.4 \\
\end{array}$ & & 29.533 & 30.869 & 30.403 & 30.029 & 28.684 & 26.591 & 31.927 & 36.464 & 32.952 & 16.869 & 15.94 & 20.583 & 15.506 & 10.79 & 32.15 & 32.829 & 30.084 & 31.887 & 27.552 & 34.391 \\
\hline 11 & & 30.857 & 30.45 & 29.55 & 30.4 & & 29.544 & 30.877 & 30.405 & 30.05 & 28.702 & 26.6 & 31.932 & 36.473 & 32.954 & $\begin{array}{l}16.878 \\
\end{array}$ & 15.949 & 20.587 & 15.511 & 10.792 & 32.168 & 32.834 & 30.1 & 31.889 & 27.552 & 34.396 \\
\hline $11 /$ & & 30.864 & 30.471 & 29.571 & 30. & & 29.554 & 30. & 30.408 & 30.071 & 28.721 & 26.617 & 31.941 & 36.487 & 32.963 & 16.885 & $\begin{array}{l}15.954 \\
\end{array}$ & 20.58 & 15.508 & 10.802 & 32.182 & 32.838 & 30.121 & 31.896 & 27.552 & 34.401 \\
\hline $11 /$ & & 30.869 & 30.487 & 29.589 & 30. & & 29.568 & $\begin{array}{l}30.890 \\
30.893\end{array}$ & 30.413 & $\begin{array}{l}30.094 \\
30.094\end{array}$ & 28.739 & 26.626 & $\begin{array}{r}31.95 \\
31.95\end{array}$ & $\begin{array}{l}36.492 \\
36.49\end{array}$ & 32.971 & $\begin{array}{l}10.000 \\
16.895\end{array}$ & $\begin{array}{l}15.964 \\
15.961\end{array}$ & 20.58 & 15.511 & $\begin{array}{l}10.811 \\
10.811\end{array}$ & 32.194 & $\begin{array}{l}2.030 \\
32.85\end{array}$ & 30.136 & 31.905 & 27.554 & 34.403 \\
\hline $11 /$ & & 30.871 & 30.501 & 29.596 & 30.48 & & 29.589 & 30.898 & 30.408 & 30.108 & 28.753 & 26.631 & 31.957 & $\begin{array}{l}36.504 \\
36.504\end{array}$ & 32.978 & $\begin{array}{l}10.906 \\
16.906\end{array}$ & 15.963 & 20.576 & 15.525 & $\begin{array}{l}10.816 \\
\end{array}$ & 32.203 & 32.86 & 77 & 31.92 & 27.554 & 34.408 \\
\hline 11/ & & 30. & 30.5 & 29.606 & $\begin{array}{l}30.4 c 5 \\
30.50\end{array}$ & & 29.603 & $\begin{array}{l}30.900 \\
30.908\end{array}$ & 30.413 & 30.125 & 28.767 & 26.636 & 31.965 & $\begin{array}{l}30.044 \\
36.506\end{array}$ & 32.987 & $\begin{array}{l}10.900 \\
16.913\end{array}$ & $\begin{array}{l}15.9303 \\
15.97\end{array}$ & 20.576 & 15.529 & 10.823 & 32.217 & $\begin{array}{l}32.800 \\
32.864\end{array}$ & 51 & 31.927 & $\begin{array}{l}27.554 \\
27.54\end{array}$ & .408 \\
\hline $11 /$ & & 30 & 30 & 29 & 3 & & & 30 & 30 & 30 & 28 & 26 & 31 & 36. & 32 & & 15 & $2 c$ & 15.548 & 10.828 & 32 & 7 & 3 & 31.927 & 27.556 & 406 \\
\hline & & & 30. & & 30.4 & & & 30. & 30. & 30 & 28 & 26 & 31 & 36. & 32 & 16. & 15.97 & 20 & 15.558 & 10.828 & 1 & 7 & 3 & 31.929 & 27.556 & 406 \\
\hline 11/20/11 1:00 & & 30.873 & 30.5 & 29.578 & & & 29.685 & & 30.41 & 30. & 28.772 & & 31.965 & 36.513 & 32.992 & & 15.97 & 20.559 & 15.569 & 10.83 & 32.199 & .867 & 33 & 31.927 & 27.556 & 403 \\
\hline $11 / 20 /$ & & 30. & 30.52 & 29.617 & & & 29.671 & 30.5 & & & 28.7 & 26.619 & 31.976 & 36.52 & 32.999 & 16.936 & 15.975 & 20.557 & 年 & 10.835 & & & & 列 & 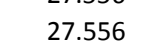 & 408 \\
\hline $11 / 20$ & & & 30.53 & 29.613 & & & & & 30 & & 28.7 & & 31 & 36.5 & & & & 20 & 15.574 & 10. & & & 7 & 8 & 27.559 & 34.406 \\
\hline 4:00 & & & 30.5 & 29.61 & & & & & 30. & & 2 & & & 36.5 & & & & 20.5 & 15.593 & & & & 30.159 & & & 34.406 \\
\hline $11 / 20 / 11$ 5:00 & & & 30.5 & 29.61 & & & & & 30.415 & 30.1 & 28.8 & & 31. & 36.523 & $33 . c$ & 16.9 & & & 15. & & & 32.883 & & 31.948 & 27.559 & 34.406 \\
\hline $11 / 20 / 116: 00$ & & 30.8 & 30.5 & 29.636 & & & 29.7 & & 30.42 & 30 & 28.8 & 26.6 & 31.997 & 36.532 & 33.02 & 16.955 & 15. & 20.5 & 15.604 & 10.858 & 32.234 & 32.895 & 30.171 & 31.959 & 27.561 & 34.41 \\
\hline 11/20/11 7:00 & & 30.8 & 30.5 & 29.634 & 30.5 & & 29. & 30.9 & 30.42 & 30.197 & 28.8 & 26.6 & 32.002 & 36.534 & 33.022 & 16.96 & 15.99 & 20.5 & 15.616 & 10.858 & 32.236 & 32.9 & 30.168 & 31.959 & 27.561 & 34.413 \\
\hline $11 / 20 / 118: 00$ & & 30.8. & 30.55 & 29.636 & 30.5 & & 29.744 & 30.953 & 30.42 & 30.201 & 28.823 & 26.60 & 32.002 & 36.532 & 33.027 & 16.964 & 15.998 & 20.5 & 15.63 & 10.863 & 32.234 & 32.905 & 30.166 & 31.969 & 27.561 & 34.413 \\
\hline 11/20/11 9:00 & & 30.906 & 30.559 & 29.65 & 30.533 & & 29.737 & 30.956 & 30.429 & 30.213 & 28.835 & 26.619 & 32.007 & 36.537 & 33.031 & 16.969 & 16.001 & 20.569 & 15.621 & 10.865 & 32.245 & 32.909 & 30.187 & 31.969 & 27.563 & 34.415 \\
\hline $11 / 20 / 1110: 00$ & & 30.903 & 30.561 & 29.645 & 30.533 & & 29.753 & 30.96 & 30.427 & 30.217 & 28.837 & 26.612 & 32.011 & 36.537 & 33.034 & 16.971 & 16 & 20.567 & 15.642 & 10.872 & 32.243 & 32.909 & 30.18 & 31.969 & 27.563 & 34.415 \\
\hline $11 / 20 / 1111: 00$ & & 30.908 & 30.566 & 29.657 & 30.54 & & 29.756 & 30.963 & 30.432 & 30.229 & 28.844 & 26.619 & 32.016 & 36.539 & 33.039 & 16.976 & 16.005 & 20.571 & 15.647 & 10.877 & 32.252 & 32.917 & 30.19 & 31.976 & 27.563 & 34.415 \\
\hline $11 / 20 / 1112: 00$ & & 30.903 & 30.559 & 29.641 & 30.528 & & 29.784 & 30.965 & 30.429 & 30.222 & 28.837 & 26.598 & 32.014 & 36.534 & 33.039 & 16.978 & 16.005 & 20.583 & 15.67 & 10.875 & 32.238 & .914 & 30.166 & 31.981 & 27.563 & 34.413 \\
\hline 11/20/11 13:00 & & 30.90 & 30.552 & 29.624 & 30.516 & & 29.798 & 30.967 & 30.424 & 30.22 & 28.83 & 26.581 & 32.009 & 36.532 & 33.036 & 16.978 & 16 & 20.594 & 15.682 & 10.87 & 32.227 & 32.912 & 30.159 & 31.974 & 27.563 & 34.408 \\
\hline $11 / 20 / 1114: 00$ & & 30.882 & 30.522 & 29.571 & 30.484 & & 29.848 & 30.965 & 30.41 & 30.199 & 28.805 & 26.521 & 32 & 36.523 & 33.024 & 16.976 & .998 & 20.624 & 15.717 & 10.858 & 32.185 & 32.898 & 30.11 & 31.962 & 27.561 & 34.403 \\
\hline $11 / 20 / 1115: 00$ & & 30.901 & 30.536 & 29.603 & 30.486 & & 29.817 & 30.967 & 30.424 & 30.208 & 28.818 & 26.555 & 32.002 & 36.525 & 33.027 & 16.981 & 16 & 20.668 & 15.698 & 10.86 & 32.213 & 32.902 & 30.136 & 31.964 & 27.566 & 34.406 \\
\hline
\end{tabular}




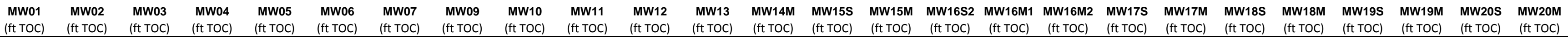

\begin{tabular}{|c|c|c|c|c|c|c|c|c|c|c|c|c|c|c|c|c|c|c|c|c|c|c|c|c|c|c|}
\hline well & $\begin{array}{l}\text { (ft TOC) } \\
\text { (f) }\end{array}$ & $\begin{array}{l}\text { (ft TOC) } \\
\text { (f) }\end{array}$ & $\begin{array}{l}\text { (ft TOC) } \\
\text { (f) }\end{array}$ & $\begin{array}{l}\text { (ft TOC) } \\
\text { (ft) }\end{array}$ & $\begin{array}{l}\text { (ft TOC) } \\
\text { (f) }\end{array}$ & $\begin{array}{l}\text { (ft toc) } \\
\text { (ftocts }\end{array}$ & (ft toc) & $\begin{array}{l}\text { (ift TOC) } \\
\text { (n) }\end{array}$ & $\begin{array}{l}\text { (ft TOC) } \\
\text { (ft) }\end{array}$ & $\begin{array}{l}\text { (ft TOC) } \\
\text { (f) }\end{array}$ & $\begin{array}{l}\text { (ft TOC) } \\
\text { (n) }\end{array}$ & $\begin{array}{l}\text { (ft TOC) } \\
\text { (f) }\end{array}$ & (ft TOC) & $\begin{array}{l}\text { (ft TOC) } \\
\end{array}$ & (ft TOC) & (ft TOC) & $\begin{array}{l}\text { (ft TOC) } \\
\text { (f) }\end{array}$ & (ft TOC) & $\begin{array}{l}\text { (ft TOC) } \\
\text { (ft) }\end{array}$ & (ft TOC) & $\begin{array}{l}\text { (ft TOC) } \\
\text { (f) }\end{array}$ & $\begin{array}{l}\text { (ft TOC) } \\
\text { (f) }\end{array}$ & $\begin{array}{l}\text { (ft TOC) } \\
\text { (n) }\end{array}$ & (ft TOC) & $\begin{array}{l}\text { (ft TOC) } \\
\text { (1) }\end{array}$ & (ft TOC) \\
\hline 00 & & 30.84 & 30.529 & 29.596 & 30.481 & & 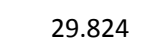 & 30.97 & 30.424 & 30. & 28.814 & 26 & 32.002 & 36.523 & 33. & 16.981 & 16.003 & 20.684 & 1571 & 10.867 & 32.203 & 32.905 & 30.124 & 69 & 27.566 & 34.406 \\
\hline $11 / 20 / 1117: 00$ & & 30.897 & 30.524 & 29.587 & 30.47 & & 29.824 & 30.967 & 30.427 & 30.201 & 8.809 & 26.533 & 32.002 & 36.5 & 33.029 & 16.981 & 16.005 & 20.7 & 15.7 & 10.863 & 32.199 & 32.902 & .211 & 1.959 & 68 & 34.406 \\
\hline $11 / 20 / 1118: 00$ & & 30.904 & 30.531 & 29.608 & 30.481 & & 29.81 & 30.972 & 30.429 & 30.21 & 28.816 & 26.555 & 32.079 & 36.53 & 33.104 & 16.983 & 16.01 & 20.786 & 15.715 & 10.94 & 32.222 & 32.983 & .136 & .039 & 7.566 & 4.41 \\
\hline 11/20/11 19:00 & & 30.906 & 30.531 & 29.617 & 30.486 & & 29.805 & 30.974 & 30.429 & 30.21 & 28.818 & 26.559 & 32.215 & 36.527 & 33.257 & 16.983 & 16.082 & 20.809 & 15.712 & 11.088 & 32.313 & 33.116 & 30.157 & 32.173 & 27.568 & 34.41 \\
\hline $11 / 20 / 1120: 00$ & & 30.906 & 30.531 & 29.617 & 30.495 & & 29.81 & 30.977 & 30.424 & 30.213 & 28.821 & 26.562 & 32.145 & 36.525 & 533.179 & 16.985 & 16.082 & 20.828 & 15.727 & 11.013 & 32.28 & 33.045 & 30.154 & 32.107 & 27.566 & 34.413 \\
\hline 11/20/11 21:00 & & 30.901 & 30.524 & 29.601 & 30.486 & & 29.824 & 30.975 & 30.427 & 30.208 & 28.812 & 26.54 & 32.112 & 36.53 & 33.142 & 16.985 & 16.075 & 20.844 & 15.736 & 10.978 & 32.259 & 33.011 & 30.14 & 32.072 & 27.568 & 34.413 \\
\hline $11 / 20 / 1122: 00$ & & 30.906 & 30.526 & 29.615 & 30.498 & & 29.815 & 30.979 & 30.427 & 30.213 & 28.818 & 26.555 & 32.098 & 36.53 & 333.125 & 16.988 & 16.073 & 20.872 & 15.734 & 10.959 & 32.259 & 32.995 & 30.15 & 32.06 & 27.568 & 34.415 \\
\hline $11 / 20 / 1123: 00$ & & 30.906 & 30.524 & 29.61 & 30.493 & & 29.822 & 30.982 & 30.427 & 30.21 & 28.814 & 26.55 & 32.082 & 36.527 & 33.109 & 16.985 & 16.068 & 20.895 & 15.743 & 10.95 & 32.252 & 32.985 & 30.147 & 32.046 & 27.568 & 34.413 \\
\hline 11/21/11 0:00 & & 30.901 & 30.517 & 29.594 & 30.484 & & 29.833 & 30.979 & 30.427 & 30.204 & 28.807 & 26.533 & 32.072 & 36.53 & $3 \quad 33.099$ & 16.988 & 16.061 & 20.913 & 15.752 & 10.936 & 32.236 & 32.971 & 30.133 & 32.035 & 27.568 & 34.408 \\
\hline 11/21/11 1:00 & & 30.904 & 30.517 & 29.596 & 30.481 & & 29.831 & 30.979 & 30.429 & 30.204 & 28.807 & 26.533 & 32.063 & 36.525 & 33.09 & 16.985 & 16.057 & 20.934 & 15.748 & 10.929 & 32.234 & 32.964 & 30.133 & 32.025 & 27.57 & 34.406 \\
\hline 11/21/11 2:00 & & 30.903 & 30.51 & 29.587 & 30.477 & & 29.833 & 30.982 & 30.432 & 30.201 & 28.805 & 26.528 & 32.053 & 36.525 & $5 \quad 33.083$ & 16.985 & 16.052 & 20.953 & 15.75 & 10.919 & 32.229 & 32.954 & 30.126 & 32.02 & 27.57 & 34.403 \\
\hline 11/21/11 3:00 & & 30.906 & 30.51 & 29.587 & 30.472 & & 29.833 & 30.982 & 30.429 & 30.199 & 28.8 & 26.524 & 32.049 & 36.523 & 333.076 & 16.985 & 16.05 & 20.971 & 15.75 & 10.912 & 32.222 & 32.95 & 30.129 & 32.011 & 27.57 & 34.401 \\
\hline 11/21/11 4:00 & & 30.906 & 30.508 & 29.589 & 30.472 & & 29.831 & 30.982 & 30.427 & 30.197 & 28.802 & 26.528 & 32.046 & 36.523 & $3 \quad 33.071$ & 16.988 & 16.047 & 20.99 & 15.757 & 10.907 & 32.227 & 32.947 & 30.121 & 32.013 & 27.57 & 34.399 \\
\hline $11 / 21 / 11$ 5:00 & & 30.903 & 30.506 & 29.585 & 30.467 & & 29.831 & 30.982 & 30.434 & 30.194 & 28.798 & 26.524 & 32.044 & 36.523 & 33.071 & 16.988 & 16.045 & 21.006 & 15.759 & 10.907 & 32.22 & 32.945 & 30.124 & 32.006 & 27.57 & 34.399 \\
\hline $11 / 21 / 116: 00$ & & 30.908 & 30.506 & 29.592 & 30.47 & & 29.824 & 30.984 & 30.432 & 30.197 & 28.8 & 26.531 & 32.044 & 36.523 & 33.071 & 16.985 & 16.045 & 21.04 & 15.752 & 10.907 & 32.222 & 32.945 & 30.131 & 32.006 & 27.573 & 34.399 \\
\hline 11/21/11 7:00 & & 30.913 & 30.512 & 29.608 & 30.479 & & 29.812 & 30.987 & 30.436 & 30.204 & 28.807 & 26.547 & 32.049 & 36.527 & 33.074 & 16.988 & 16.05 & 21.068 & 15.748 & 10.91 & 32.236 & 32.95 & 30.14 & 32.009 & 27.575 & 34.401 \\
\hline 11/21/11 8:00 & & 30.908 & 30.51 & 29.601 & 30.481 & & 29.822 & 30.989 & 30.434 & 30.199 & 28.805 & 26.54 & 32.049 & 36.53 & 33.076 & 16.988 & 16.05 & 21.091 & 15.759 & 10.914 & 32.229 & 32.947 & 30.136 & 32.011 & 27.573 & 34.401 \\
\hline 11/21/11 9:00 & & 30.913 & 30.51 & 29.606 & 30.484 & & 29.819 & 30.989 & 30.439 & 30.201 & 28.807 & 26.545 & 32.051 & 36.53 & 33.076 & 16.99 & 16.05 & 21.114 & 15.759 & 10.912 & 32.236 & 32.947 & 30.14 & 32.011 & 27.575 & 34.403 \\
\hline $11 / 21 / 11$ 10:00 & & 30.91 & 30.51 & 29.603 & 30.488 & & 29.829 & 30.989 & 30.432 & 30.201 & 28.805 & 26.543 & 32.053 & 36.534 & 33.078 & 16.99 & 16.05 & 21.138 & 15.771 & 10.912 & 32.227 & 32.95 & 30.136 & 32.018 & 27.573 & 34.403 \\
\hline 00 & & 30.911 & 30.503 & 29.594 & $\begin{array}{l}30.400 \\
30.479\end{array}$ & & 29.836 & $\begin{array}{l}30.9991 \\
3099\end{array}$ & $\begin{array}{l}30.436 \\
30.436\end{array}$ & $\begin{array}{l}30.197 \\
30.21\end{array}$ & $\begin{array}{l}28.802 \\
28.802\end{array}$ & 26.533 & $\begin{array}{l}32.049 \\
32.049\end{array}$ & $\begin{array}{l}\begin{array}{l}50.534 \\
36.53\end{array} \\
\end{array}$ & 33.071 & $\begin{array}{l}10.99 \\
16.99\end{array}$ & $\begin{array}{l}16.05 \\
16.05\end{array}$ & $\begin{array}{l}21.156 \\
21.156\end{array}$ & 15.771 & 10.912 & 32.227 & 32.947 & 30.136 & 32.013 & 27.575 & $\begin{array}{l}34.401 \\
34.401\end{array}$ \\
\hline bo & & 30.897 & 30.485 & 29.554 & 30.45 & & 29.864 & 30.984 & 30.429 & 30.18 & 28.781 & 26.492 & 32.037 & 36.523 & 33.067 & 16.988 & 16.045 & .172 & 15.792 & 10.9 & 32.199 & 32.94 & 30.1 & 32.002 & 27.575 & .396 \\
\hline 11 & & 30.892 & 30.464 & 29.517 & 30.43 & & 29.883 & 30.982 & 30.429 & 30.166 & 28.763 & 26.457 & 32.025 & 36.511 & 33.053 & 16.985 & 16.038 & 184 & 15.799 & 10.891 & 182 & 32.928 & 0.07 & 1.988 & 27.575 & .391 \\
\hline $11 / 21 / 1114: 00$ & & 30.88 & 30.443 & 29.482 & 30.4 & & 29.902 & 30.975 & 30.422 & 30.146 & 28.742 & 26.423 & 32.011 & 36.501 & 33.039 & 16.981 & 16.031 & 21.202 & 15.811 & 10.877 & 32.147 & 32.912 & .037 & 31.981 & 27.573 & 34.384 \\
\hline 11/21/11 15:00 & & 30.892 & 30.447 & 29.503 & 30.397 & & 29.866 & 30.97 & 30.429 & 30.148 & 28.746 & 26.452 & 32.011 & 36.499 & 33.036 & 16.981 & 16.031 & 21.216 & 15.783 & 10.875 & 32.164 & 32.909 & 30.053 & 31.971 & 27.575 & 34.384 \\
\hline 11/21/11 16:00 & & 30.89 & 30.44 & 29.498 & 30.39 & & 29.857 & 30.97 & 30.425 & 30.141 & 28.739 & 26.447 & 32.007 & 36.499 & 33.034 & 16.978 & 16.031 & 21.235 & 15.781 & 10.87 & 32.154 & 32.907 & 30.051 & 31.969 & 27.577 & 34.382 \\
\hline 11/21/11 17:00 & & 30.882 & 30.436 & 29.489 & 30.383 & & 29.857 & 30.965 & 30.427 & 30.134 & 28.735 & 26.437 & 32.002 & 36.49 & 33.031 & 16.974 & 16.031 & 21.253 & 15.783 & 10.867 & 32.147 & 32.905 & 30.039 & 31.966 & 27.575 & 34.382 \\
\hline $11 / 21 / 11$ 18:00 & & 30.871 & 30.417 & 29.456 & 30.362 & & 29.871 & 30.96 & 30.417 & 30.115 & 28.716 & 26.409 & 31.995 & 36.487 & 33.024 & 16.971 & 16.026 & 21.276 & 15.795 & 10.858 & 32.126 & 32.898 & 30.013 & 31.962 & 27.575 & 34.38 \\
\hline 11/21/11 19:00 & & 30.873 & 30.415 & 29.468 & 30.367 & & 29.855 & 30.958 & 30.415 & 30.118 & 28.716 & 26.425 & 31.997 & 36.485 & 33.029 & 16.971 & 16.029 & 21.295 & 15.792 & 10.863 & 32.133 & 32.898 & 30.02 & 31.966 & 27.575 & 34.384 \\
\hline $11 / 21 / 1120: 00$ & & 30.887 & 30.436 & 29.51 & 30.386 & & 29.805 & 30.96 & 30.425 & 30.127 & 28.735 & 26.468 & 32.007 & 36.494 & 33.036 & 16.974 & 16.038 & 21.323 & 15.75 & 10.87 & 32.164 & 32.907 & 30.06 & 31.969 & 27.58 & 34.387 \\
\hline 11/21/11 21:00 & & 30.869 & 30.415 & 29.458 & 30.367 & & 29.84 & 30.956 & 30.417 & 30.108 & 28.707 & 26.418 & 31.997 & 36.487 & 33.027 & 16.969 & 16.031 & 21.339 & 15.781 & 10.863 & 32.131 & 32.9 & 30.018 & 31.962 & 27.577 & 34.387 \\
\hline $11 / 21 / 1122: 00$ & & 30.878 & 30.422 & 29.486 & 30.376 & & 29.815 & 30.956 & 30.42 & 30.113 & 28.718 & 26.452 & 32.002 & 36.49 & 33.029 & 16.969 & 16.033 & 21.359 & 15.762 & 10.86 & 32.15 & 32.902 & 30.044 & 31.969 & 27.577 & 34.387 \\
\hline $11 / 21 / 1123: 00$ & & 30.885 & 30.422 & 29.498 & 30.386 & & 29.796 & 30.956 & 30.425 & 30.115 & 28.723 & 26.461 & 32.004 & 36.49 & 33.031 & 16.967 & 16.035 & 21.378 & 15.748 & 10.865 & 32.161 & 32.905 & 30.056 & 31.964 & 27.58 & 34.387 \\
\hline $11 / 22 / 110: 00$ & & 30.885 & 30.433 & 29.517 & 30.402 & & 29.782 & 30.956 & 30.427 & 30.12 & 28.732 & 26.485 & 32.007 & 36.497 & 33.031 & 16.967 & 16.035 & 21.401 & 15.743 & 10.867 & 32.171 & 32.909 & 30.074 & 31.971 & 27.58 & 34.387 \\
\hline 11/22/11 1:00 & & 30.88 & 30.429 & 29.507 & 30.402 & & 29.791 & 30.956 & 30.422 & 30.115 & 28.725 & 26.473 & 32.004 & 36.501 & 33.031 & 16.969 & 16.031 & 21.417 & 15.752 & 10.867 & 32.166 & 32.905 & 30.063 & 31.969 & 27.582 & 34.382 \\
\hline $11 / 22 / 112: 00$ & & 30.871 & 30.419 & 29.479 & 30. & & 29.812 & 30.951 & 30.417 & 30.106 & 28.714 & 26.447 & 31.995 & 36.494 & 33.022 & 16.964 & 16.024 & 21.4 & 15.757 & 10.858 & 32.145 & 32.898 & 30.042 & 31.957 & 27.582 & 34.375 \\
\hline $11 /$ & & 30.873 & 30.417 & 29.475 & 30. & & 29.808 & 30. & 30.417 & 30.101 & 28.712 & 26.447 & 31.99 & 36.492 & 33.017 & 16.967 & 16.019 & 21.443 & 15.748 & 10.851 & 32.143 & $\begin{array}{r}32.89 \\
32.89\end{array}$ & 30.044 & 31.955 & 27.582 & 34.37 \\
\hline $11 / 22 / 114: 00$ & & 30.869 & $\begin{array}{l}30.41 \\
30.41\end{array}$ & 29.47 & 30. & & 29.808 & 30.948 & 30.417 & 30.097 & 28.707 & 26.44 & 31.986 & $\begin{array}{l}36.497 \\
367\end{array}$ & 33.015 & $\begin{array}{l}16.964 \\
16.564\end{array}$ & 16.019 & 21.45 & 15.748 & 10.849 & 32.136 & 32.89 & 30.039 & 31.952 & 27.582 & 34.366 \\
\hline $11 / 22 / 11$ 5:00 & & 30.871 & 30.412 & 29.475 & 30. & & $\begin{array}{r}29.000 \\
29.8\end{array}$ & $\begin{array}{l}30.948 \\
3048\end{array}$ & 30.417 & 30.097 & 28.707 & 26.447 & $\begin{array}{l}31.988 \\
31.908\end{array}$ & $\begin{array}{l}36.492 \\
36.47\end{array}$ & 33.01 & $\begin{array}{l}10.074 \\
16.962\end{array}$ & 16.019 & 21.464 & 15.74 & $\begin{array}{l}10.849 \\
\end{array}$ & 32.143 & 32.886 & 42 & 31.952 & 27.585 & 1.366 \\
\hline & & 30.8 & 30. & 29.486 & 30 & & 29.789 & 30. & 30 & 30. & 28.712 & 26.459 & 31 & 36.49 & 33.015 & 16 & 16.019 & 7 & 15.731 & 10.849 & 7 & 8 & 1 & 31.952 & 27.585 & .366 \\
\hline & & 30. & 30. & 29 & & & & 30 & 30.422 & 30. & 28.714 & 26.46 & 31 & 36.494 & 33.02 & 16 & 1 & 1 & 15 & 10.856 & 54 & 32.893 & 8 & 31.957 & 27.585 & 34.368 \\
\hline & & 30.8 & 30. & & & & & 30 & 20 & & 28.723 & 26. & 32 & 36.504 & 33.027 & 16 & 16 & 21 & 15.722 & 10.865 & 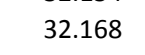 & & & 56 & 35 & 34.37 \\
\hline & & & 30. & & & & & & & & & 20 & 9 & 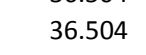 & & 16 & (5) & 21.5 & 15.7 & 10 & 178 & 32 & 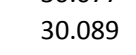 & 31.974 & 27.585 & 34.373 \\
\hline $11 / 2$ & & 30.8 & 30.4 & & & & & & & & 28.7 & & 32 & & & & & & 15.7 & & & & & & (1) & 73 \\
\hline & & 30 & 30. & 29. & & & & & 30. & 30 & 28.7 & & 32 & 36 & & & & 21. & 15. & 10 & & & 8 & 3 & 27.587 & 34.375 \\
\hline & & 30. & 30.4 & 29.524 & & & & & 30. & & 28 & & & & & & & 21. & 15. & 10 & & & & & 27.587 & 34.373 \\
\hline & & & 30. & 29.5 & & & & & & & & & & & & & & & & & & & & & & 34.37 \\
\hline $11 / 2$ & & 30.8 & 30.4 & 29.4 & & & 29.8 & & 30. & & 28.7 & 26.4 & 32.011 & 36.513 & 33. & 16. & 16. & 21.602 & 15. & 10.875 & 32.178 & & 30.072 & 31.976 & 27.589 & 34.368 \\
\hline $11 / 22 / 1115: 00$ & & $30 . \varepsilon$ & 30.4 & 29.4 & & & 29.8 & 30. & 30.417 & 30.1 & 28.7 & 26.4 & 32.011 & 36.5 & 33.039 & 16.9 & 16.029 & 21.651 & 15.7 & 10.872 & 32.168 & 32.909 & 30.077 & 31.976 & 27.589 & 34.366 \\
\hline $11 / 22 / 1116: 00$ & & 30.8 & 30.429 & 29.505 & 30. & & 29.796 & 30.9 & 30.422 & 30.1 & 28.723 & 26.47 & 32.014 & 36.513 & 33.041 & 16.978 & 16.0 & 21.725 & 15.743 & 10.877 & 32.178 & 32.912 & 30.084 & 31.974 & 27.589 & 34.363 \\
\hline $11 / 22 / 1117: 00$ & & 30.875 & 30.429 & 29.51 & 30.4 & & 29.793 & 30.953 & 30.422 & 30.106 & 28.725 & 26.483 & 32.014 & 36.518 & 33.041 & 16.978 & 16.0 & 21.771 & 15.748 & 10.879 & 32.182 & 32.917 & 30.086 & 31.978 & 27.589 & 34.366 \\
\hline $11 / 22 / 1118: 00$ & & 30.882 & 30.44 & 29.533 & 30.43 & & 29.775 & 30.953 & 30.42 & 30.115 & 28.735 & 26.504 & 32.023 & 36.516 & 33.05 & 16.981 & 16.04 & 21.783 & 15.736 & 10.884 & 32.199 & 32.924 & 30.105 & 31.985 & 27.589 & 34.368 \\
\hline $11 / 22 / 1119: 00$ & & 30.885 & 30.443 & 29.543 & 30.442 & & 29.768 & 30.958 & 30.425 & 30.118 & 28.739 & 26.514 & 32.03 & 36.52 & 33.055 & 16.983 & 16.045 & 21.787 & 15.729 & 10.889 & 32.201 & 32.931 & 30.117 & 31.995 & 27.592 & 34.37 \\
\hline $11 / 22 / 1120: 00$ & & 30.889 & 30.454 & 29.564 & 30.46 & & 29.756 & 30.963 & 30.429 & 30.129 & 28.751 & 26.535 & 32.042 & 36.534 & 33.067 & 16.988 & 16.054 & 21.799 & 15.724 & 10.9 & 32.22 & 32.943 & 30.133 & 32.004 & 27.592 & 34.377 \\
\hline $11 / 22 / 1121: 00$ & & 30.892 & 30.461 & 29.578 & 30.474 & & 29.753 & 30.965 & 30.427 & 30.136 & 28.758 & 26.547 & 32.051 & 36.537 & 33.076 & 16.99 & 16.057 & 21.808 & 15.719 & 10.912 & 32.231 & 32.952 & 30.15 & 32.013 & 27.592 & 34.38 \\
\hline $11 / 22 / 1122: 00$ & & 30.894 & 30.468 & 29.585 & 30.48 & & 29.756 & 30.967 & 30.429 & 30.141 & 28.765 & 26.552 & 32.06 & 36.532 & 33.078 & 16.995 & 16.064 & 21.817 & 15.722 & 10.919 & 32.243 & 32.959 & 30.154 & 32.02 & 27.594 & .382 \\
\hline $11 / 22 / 1123: 00$ & & 30.892 & 30.466 & 29.578 & 30.488 & & 29.77 & 30.972 & 30.429 & 30.143 & 28.765 & 26.543 & 32.06 & 36.537 & 33.083 & 16.997 & 16.066 & 21.82 & 15.727 & 10.922 & 32.236 & 32.959 & 30.152 & 32.025 & 27.594 & 2 \\
\hline
\end{tabular}




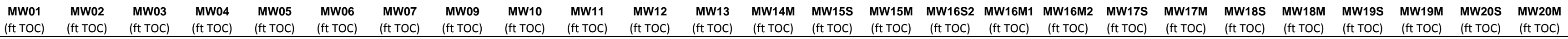

\begin{tabular}{|c|c|c|c|c|c|c|c|c|c|c|c|c|c|c|c|c|c|c|c|c|c|c|c|c|c|}
\hline well & $\begin{array}{l}\text { (ft TOC) } \\
\text { (f) }\end{array}$ & $\begin{array}{l}\text { (ft TOC) } \\
\text { (ft) }\end{array}$ & $\begin{array}{l}\text { (ft TOC) } \\
\text { (f) }\end{array}$ & $\begin{array}{l}\text { (ft TOC) } \\
\text { (ft) }\end{array}$ & $\begin{array}{ll}(\mathrm{ft} \text { TOC) } & \text { (ft TOC) }\end{array}$ & (ft toc) & $\begin{array}{l}\text { (ift TOC) } \\
\text { (n) }\end{array}$ & $\begin{array}{l}\text { (ft TOC) } \\
\text { (f) }\end{array}$ & $\begin{array}{l}\text { (ft TOC) } \\
\text { (f) }\end{array}$ & $\begin{array}{l}\text { (ft TOC) } \\
\text { (n) }\end{array}$ & $\begin{array}{l}\text { (ft TOC) } \\
\text { (ft }\end{array}$ & (ft TOC) & $\begin{array}{l}\text { (ft TOC) } \\
\end{array}$ & (ft TOC) & (ft TOC) & $\begin{array}{l}\text { (ft TOC) } \\
\text { (f) }\end{array}$ & (ft TOC) & $\begin{array}{l}\text { (ft TOC) } \\
\text { (ft) }\end{array}$ & (ft toc) & $\begin{array}{l}\text { (ft ToC) } \\
\text { (ft }\end{array}$ & $\begin{array}{l}\text { (ft TOC) } \\
\text { (f) }\end{array}$ & $\begin{array}{l}\text { (ft TOC) } \\
\text { (n) }\end{array}$ & (ft TOC) & $\begin{array}{l}\text { (ft TOC) } \\
\text { (1) }\end{array}$ & (ft TOC) \\
\hline bo & & 30.899 & 30.473 & 29.5 & 30. & 29.768 & 30. & 30.429 & 30.15 & 28.77 & 26 & 32.065 & 36.541 & 33.09 & 16.999 & 16.071 & 21.824 & 15.724 & 10. & 32.248 & 32.966 & 30.164 & 03 & 27.594 & 34.382 \\
\hline 11/23/11 1:00 & & 30.899 & 30.475 & 29.589 & 30.5 & 29.775 & 30.979 & 30.432 & 30.152 & 28.774 & 26.55 & 2.065 & 36.544 & 33.092 & 17.004 & 16.068 & 21.831 & .724 & 10.929 & 32.248 & .969 & .161 & 32.032 & 94 & .38 \\
\hline $11 / 23 / 112: 00$ & & 30.901 & 30.475 & 29.589 & 30.502 & 29.779 & 30.979 & 30.434 & 30.155 & 28.7 & 26.55 & 32.067 & 36.539 & 33.092 & 17.006 & 16.068 & 21.838 & 15.722 & 10.931 & 32.245 & 32.969 & 166 & דרטי & 1.596 & \\
\hline 11/23/11 3:00 & & 30.897 & 30.473 & 29.582 & 30.5 & 29.791 & 30.982 & 30.427 & 30.155 & 28.774 & 26.543 & 32.067 & 36.539 & 33.09 & 17.006 & 16.066 & 21.845 & 15.727 & 10.926 & 32.241 & 32.964 & 30.157 & 32.032 & 27.594 & 34.373 \\
\hline 11/23/11 4:00 & & 30.901 & 30.473 & 29.585 & 30.498 & 29.793 & 30.984 & 30.432 & 30.155 & 28.774 & 26.54 & 32.067 & 36.541 & 33.09 & 17.009 & 16.064 & 21.85 & 15.729 & 10.924 & 32.248 & 32.966 & 30.159 & 32.027 & 27.596 & 34.37 \\
\hline 11/23/11 5:00 & & 30.903 & 30.482 & 29.599 & 30.505 & 29.789 & 30.987 & 30.434 & 30.162 & 28.781 & 26.552 & 32.067 & 36.539 & 33.095 & 17.011 & 16.066 & 21.857 & 15.727 & 10.929 & 32.25 & 32.969 & 30.166 & 32.032 & 27.596 & 34.368 \\
\hline 11/23/11 6:00 & & 30.906 & 30.485 & 29.601 & 30.509 & 29.791 & 30.989 & 30.439 & 30.166 & 28.784 & 26.557 & 32.072 & 36.546 & 33.097 & 17.013 & 16.068 & 21.877 & 15.727 & 10.933 & 32.255 & 32.973 & 30.171 & 32.032 & 27.596 & 34.366 \\
\hline 11/23/11 7:00 & & 30.906 & 30.482 & 29.599 & 30.509 & 29.798 & 30.989 & 30.436 & 30.166 & 28.786 & 26.552 & 32.074 & 36.546 & 33.102 & 17.016 & 16.073 & 21.887 & 15.734 & 10.936 & 32.257 & 32.978 & 30.168 & 32.037 & 27.599 & 34.366 \\
\hline 11/23/11 8:00 & & 30.908 & 30.485 & 29.606 & 30.516 & 29.798 & 30.991 & 30.434 & 30.171 & 28.788 & 26.557 & 32.082 & 36.544 & 33.104 & 17.018 & 16.075 & 21.905 & 15.736 & 10.943 & 32.262 & 32.978 & 30.18 & 32.046 & 27.599 & 34.368 \\
\hline 11/23/11 9:00 & & 30.91 & 30.489 & 29.61 & 30.519 & 29.803 & 30.994 & 30.436 & 30.176 & 28.793 & 26.562 & 32.084 & 36.544 & 33.111 & 17.02 & 16.08 & 21.912 & 15.743 & 10.947 & 32.266 & 32.988 & 30.18 & 32.051 & 27.601 & 34.37 \\
\hline 11/23/11 10:00 & & 30.911 & 30.492 & 29.608 & 30.521 & 29.81 & 30.994 & 30.439 & 30.178 & 28.793 & 26.559 & 32.089 & 36.548 & 33.116 & 17.023 & 16.085 & 21.917 & 15.75 & 10.95 & 32.266 & 32.988 & 30.185 & 32.058 & 27.601 & 34.373 \\
\hline 11/23/11 11:00 & & 30.915 & 30.492 & 29.613 & 30.523 & 29.815 & 30.996 & 30.441 & 30.18 & 28.795 & 26.559 & 32.093 & 36.551 & 33.118 & 17.025 & 16.087 & 21.926 & 15.759 & 10.954 & 32.271 & 32.995 & 30.187 & 32.06 & 27.601 & 34.373 \\
\hline $11 / 23 / 1112: 00$ & & 30.906 & 30.485 & 29.589 & 30.514 & 29.836 & 30.996 & 30.439 & 30.173 & 28.786 & 26.538 & 32.091 & 36.544 & 33.116 & 17.025 & 16.089 & 21.94 & 15.773 & 10.957 & 32.259 & 32.99 & 30.168 & 32.058 & 27.601 & 34.373 \\
\hline $11 / 23 / 1113: 00$ & & 30.904 & 30.478 & 29.575 & 30.505 & 29.852 & 30.996 & 30.436 & 30.169 & 28.779 & 26.521 & 32.089 & 36.539 & 33.116 & 17.025 & 16.089 & 21.951 & 15.788 & 10.947 & 32.25 & 32.992 & 30.159 & 32.056 & 27.601 & 34.373 \\
\hline 11/23/11 14:00 & & 30.901 & 30.473 & 29.561 & 30.493 & 29.862 & 30.996 & 30.434 & 30.162 & 28.77 & 26.507 & 32.082 & 36.537 & 33.111 & 17.027 & 16.087 & 21.954 & 15.799 & 10.945 & 32.238 & 32.985 & 30.145 & 32.053 & 27.603 & 34.37 \\
\hline $11 / 23 / 1115: 00$ & & 30.899 & 30.464 & 29.545 & 30.479 & 29.866 & 30.991 & 30.432 & 30.155 & 28.765 & 26.495 & 32.079 & 36.527 & 33.107 & 17.027 & 16.085 & 21.965 & 15.802 & 10.938 & 32.231 & 32.983 & 30.129 & 32.046 & 27.603 & 34.366 \\
\hline $11 / 23 / 1116: 00$ & & 30.901 & 30.464 & 29.55 & 30.477 & 29.859 & 30.989 & 30.434 & 30.152 & 28.765 & 26.497 & 32.079 & 36.53 & 33.104 & 17.027 & 16.085 & 22.025 & 15.802 & 10.94 & 32.231 & 32.978 & 30.138 & 32.042 & 27.603 & 34.363 \\
\hline $11 / 23 / 1117: 00$ & & 30.903 & 30.461 & 29.55 & 30.474 & 29.85 & 30.989 & 30.436 & 30.152 & 28.76 & 26.5 & 32.079 & 36.53 & 33.104 & 17.027 & 16.087 & 22.03 & 15.799 & 10.94 & 32.234 & 32.976 & 30.133 & 32.042 & 27.603 & 34.361 \\
\hline $11 / 23 / 11$ 18:00 & & 30.906 & 30.464 & 29.559 & 30.477 & 29.838 & 30.991 & 30.436 & 30.155 & 28.765 & 26.509 & 32.082 & 36.532 & 33.107 & 17.027 & 16.092 & 22.046 & 15.787 & 10.943 & 32.241 & 32.983 & 30.143 & 32.044 & 27.603 & 34.361 \\
\hline & & 30.903 & 30.466 & 29.561 & 30.481 & 29.831 & 30.991 & 30.439 & 30.155 & 28.765 & 26.514 & 32.084 & 36.527 & 33.109 & 17.027 & 16.096 & 22.058 & 15.79 & 10.945 & 32.241 & 32.983 & 30.145 & 32.051 & 27.603 & 34.363 \\
\hline 10 & & 30.906 & 30.466 & 29.561 & 30.48 & 29.829 & 30.991 & 30.434 & 30.155 & 28.763 & 26.512 & 32.086 & 36.534 & 33.114 & 17.027 & 16.099 & 22.062 & 15.788 & 10.952 & 32.248 & 32.99 & 0.147 & 32.056 & 27.606 & .366 \\
\hline $11 / 2$ & & 30.908 & 30.471 & 29.573 & 30. & 29.819 & 30.991 & 30.439 & 30.159 & 28.77 & 26.524 & 32.096 & 36.537 & 33.123 & 17.03 & 16.108 & .072 & 15783 & 10.957 & 255 & 32.997 & 154 & 32.06 & 27.606 & 34.37 \\
\hline $11 / 23 / 1$ & & 30.908 & 30.471 & 29.573 & 30. & 29.817 & 30.991 & 30.441 & 30.157 & 28.77 & 26.524 & 32.098 & 36.537 & 33.125 & 17.032 & 16.113 & 22.081 & 15.778 & 10.959 & 255 & .999 & .157 & 2.06 & 27.606 & 34.373 \\
\hline 11/23/11 23:00 & & 30.906 & 30.466 & 29.561 & 30.488 & 29.824 & 30.991 & 30.439 & 30.155 & 28.765 & 26.514 & 32.1 & 36.541 & 33.125 & 17.032 & 16.115 & 22.088 & 15.781 & 10.961 & 32.255 & 33.002 & 152 & 32.065 & 27.608 & 34.373 \\
\hline 11/24/11 0:00 & & 30.906 & 30.466 & 29.559 & 30.488 & 29.826 & 30.994 & 30.441 & 30.152 & 28.763 & 26.509 & 32.1 & 36.539 & 33.125 & 17.034 & 16.115 & 22.095 & 15.783 & 10.961 & 32.25 & 32.999 & 30.145 & 32.063 & 27.606 & 34.373 \\
\hline 11/24/11 1:00 & & 30.903 & 30.461 & 29.547 & 30.479 & 29.831 & & 30.439 & 30.148 & 28.756 & 26.497 & 32.096 & 36.537 & 33.121 & 17.034 & 16.113 & 22.099 & 15.781 & 10.959 & 32.236 & 32.995 & 30.14 & 32.06 & 27.608 & 34.368 \\
\hline $11 / 24 / 112: 00$ & & 30.901 & 30.457 & 29.536 & 30.472 & 29.836 & 30.987 & 30.439 & 30.143 & 28.749 & 26.488 & 32.091 & 36.534 & 33.116 & 17.034 & 16.106 & 22.102 & 15.778 & 10.952 & 32.236 & 32.992 & 30.131 & 32.056 & 27.608 & 34.363 \\
\hline 11/24/11 3:00 & & 30.899 & 30.45 & 29.529 & 30.467 & 29.836 & 30.987 & 30.441 & 30.139 & 28.746 & 26.485 & 32.084 & 36.532 & 33.116 & 17.032 & 16.106 & 22.102 & 15.776 & 10.947 & 32.234 & 32.988 & 30.126 & 32.053 & 27.608 & 34.361 \\
\hline 11/24/11 4:00 & & 30.899 & 30.445 & 29.521 & 30.46 & 29.838 & 30.984 & 30.436 & 30.134 & 28.739 & 26.478 & 32.079 & 36.52 & 33.107 & 17.032 & 16.101 & 22.106 & 15.773 & 10.945 & 32.227 & 32.981 & 30.114 & 32.044 & 27.608 & 34.354 \\
\hline $11 / 24 / 11$ 5:00 & & 30.899 & 30.445 & 29.521 & 30.458 & 29.829 & 30.984 & 30.441 & 30.132 & 28.739 & 26.48 & 32.079 & 36.53 & 33.107 & 17.03 & 16.101 & 22.109 & 15.771 & 10.94 & 32.229 & 32.978 & 30.124 & 32.042 & 27.608 & 34.352 \\
\hline $11 / 24 / 116: 00$ & & 30.899 & 30.447 & 29.524 & 30.458 & 29.826 & 30.979 & 30.436 & 30.132 & 28.739 & 26.483 & 32.077 & 36.53 & 33.104 & 17.03 & 16.099 & 22.132 & 15.769 & 10.94 & 32.227 & 32.978 & 30.121 & 32.042 & 27.61 & 34.349 \\
\hline $11 / 24 / 11$ 7:00 & & 30.901 & 30.45 & 29.529 & 30.463 & 29.819 & 30.979 & 30.439 & 30.132 & 28.739 & 26.49 & 32.074 & 36.53 & 33.102 & 17.03 & 16.101 & 22.136 & 15.762 & 10.943 & 32.231 & 32.978 & 30.129 & 32.042 & 27.61 & 34.347 \\
\hline $11 / 24 / 118: 00$ & & 30.901 & 30.447 & 29.531 & 30.463 & 29.812 & 30.979 & 30.436 & 30.132 & 28.742 & 26.492 & 32.082 & 36.523 & 33.107 & 17.03 & 16.103 & 22.141 & 15.759 & 10.943 & 32.234 & 32.981 & 30.131 & 32.046 & 27.613 & 34.347 \\
\hline $11 / 24 / 119: 00$ & & 30.899 & 30.447 & 29.531 & 30.463 & 29.817 & 30.979 & 30.436 & 30.129 & 28.739 & 26.49 & 32.103 & 36.532 & 33.13 & 17.032 & 16.11 & 22.146 & 15.766 & 10.964 & 32.236 & 33.002 & 30.129 & 32.067 & 27.61 & 34.349 \\
\hline $11 / 24 / 1110: 00$ & & 30.894 & 30.443 & 29.521 & 30 & 29.824 & 30. & 30.432 & 30.125 & 28.735 & 26.48 & 32.093 & 36.532 & 33.123 & 17.032 & 16.113 & 22.157 & 15.769 & 10.959 & 32.231 & 32.992 & 30.124 & 32.06 & 27.613 & 34.349 \\
\hline $11 /$ & & 30.892 & 30. & 29.507 & 30.4 & 29.836 & & 30.434 & 30.12 & 28.728 & 26.471 & 32.093 & 36.53 & $\begin{array}{l}33.118 \\
33\end{array}$ & 17.03 & 16.113 & 22.1 & 15.7 & 10.954 & 32.222 & 32.99 & 30.112 & 32.056 & 27.613 & 34.349 \\
\hline $11 /$ & & 30.882 & $\begin{array}{l}30.450 \\
30.419\end{array}$ & 29.475 & 30.4 & 29.855 & 30.972 & $\begin{array}{l}30.429 \\
30.429\end{array}$ & $\begin{array}{l}30.106 \\
30.106\end{array}$ & 28.712 & 26.442 & 32.084 & 36.518 & $\begin{array}{l}33.111 \\
33.11\end{array}$ & 17.027 & $\begin{array}{l}16.113 \\
16.113\end{array}$ & 22.194 & $\begin{array}{l}15.792 \\
15.792\end{array}$ & $\begin{array}{l}10.95 \\
10.95\end{array}$ & 32.201 & 32.981 & 30.093 & 32.044 & 27.613 & 34.347 \\
\hline $11 /$ & & 30.876 & $\begin{array}{l}30.419 \\
30.41\end{array}$ & 29.449 & 30. & 29.871 & 30.967 & 30.427 & $\begin{array}{l}30.092 \\
30.092\end{array}$ & 28.695 & 26.418 & 32.072 & 36.516 & 33.104 & 17.027 & 16.11 & 22.215 & 15.804 & 10.936 & 32.187 & 32.971 & 55 & 32.035 & 27.613 & 34.345 \\
\hline $11 /$ & & 30 & 30.3 & 29 & & 29 & 30 & 30 & 30. & 28.681 & 26.399 & 32 & 36.508 & 33.09 & 17. & 1 & 22.233 & 15.802 & 10.931 & 8 & 4 & 3 & 32.027 & 27.615 & 34.34 \\
\hline & & 30. & 30 & 29 & & & 30. & 30 & 30. & 28.6 & 26 & 32 & 36.501 & 33. & 17 & 16 & 22.236 & 15 & 10 & 32 & 4 & 4 & 2 & 27.613 & 34.338 \\
\hline $11 /$ & & & 30 & & & 29. & & 30 & 30. & 28 & & 32 & 36. & 33. & 17 & 16 & 9 & 15. & 10 & 32 & 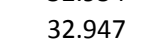 & 7 & 32 & 27.613 & .331 \\
\hline $11 /$ & & & & & & & & & & 28.6 & & & 36. & $33 . \mathrm{C}$ & & & 8 & 15.7 & 10. & 161 & & & 32.011 & 27.615 & \\
\hline & & 30 & 30.373 & & 30. & & & & & 28.6 & & 32.046 & 4 & 33.0 & & & 22.3 & 15.7 & & & & & & 27.615 & \\
\hline & & 30 & 30.37 & & & & & 30 & & 28.6 & 26.4 & 32. & 36.501 & 33. & 17. & & 22.3 & 15.769 & 10 & 32.175 & & 5 & 32.018 & 27.617 & 34.331 \\
\hline 11 & & & 30.3 & & 30. & & & 30.422 & 30 & 28. & 26 & 32. & & & & & 22 & 15.762 & & 32.178 & & 5 & 32.018 & 27.617 & 34.333 \\
\hline $11 /$ & & & 30.3 & & & & & 30.42 & & 28. & 26. & & & & & & 22.3 & 15. & & 32.18 & & & & 27.617 & 34.335 \\
\hline 22:00 & & $30 . \varepsilon$ & 30 & 29. & & 29. & & 30.42 & 30 & 28.665 & 26.418 & 32.056 & 36. & 33.083 & 17.0 & 16.115 & 22.319 & 15.7 & 10.919 & 32.175 & & 30.058 & 32.02 & 27.617 & 34.338 \\
\hline $11 / 24 / 1123: 00$ & & 30.857 & 30.36 & 29.414 & 30.3 & $29.7 \mathrm{C}$ & 30. & 30.417 & 30.6 & 28.6 & 26.406 & 32.056 & 36.4 & 33.6 & 17.0 & 16.117 & 22.331 & 15.7 & 10.919 & 32.171 & 32.957 & 30.046 & 32.02 & 27.617 & 34.338 \\
\hline 11/25/11 0:00 & & 30.855 & 30.356 & 29.412 & 30.3 & 29.793 & 30.9 & 30.42 & 30.6 & 28.651 & 26.401 & 32.051 & 36.497 & 33.083 & 17.013 & 16.117 & 22.34 & 15.75 & 10.914 & 32.166 & 32.952 & 30.044 & 32.016 & 27.617 & 34.338 \\
\hline 11/25/11 1:00 & & 30.85 & 30.352 & 29.398 & 30.36 & 29.793 & 30.927 & 30.413 & 30.029 & 28.642 & 26.392 & 32.046 & 36.494 & 33.076 & 17.013 & 16.115 & 22.34 & 15.752 & 10.91 & 32.154 & 32.95 & 30.035 & 32.013 & 27.62 & 34.335 \\
\hline $11 / 25 / 112: 00$ & & 30.843 & 30.34 & 29.379 & 30.358 & 29.803 & 30.924 & 30.413 & 30.018 & 28.63 & 26.373 & 32.042 & 36.49 & 33.067 & 17.009 & 16.113 & 22.335 & 15.747 & 10.905 & 32.147 & 32.943 & 30.025 & 32.006 & 27.617 & 34.333 \\
\hline $11 / 25 / 113: 00$ & & 30.843 & 30.333 & 29.372 & 30.351 & 29.796 & 30.92 & 30.413 & 30.011 & 28.628 & 26.368 & 32.032 & 36.487 & 33.064 & 17.009 & 16.11 & 22.331 & 15.738 & 10.907 & 32.138 & 32.936 & 30.016 & 31.997 & 27.617 & 34.328 \\
\hline $11 / 25 / 114: 00$ & & 30.838 & 30.321 & 29.358 & 30.339 & 29.796 & 30.92 & 30.408 & 30.002 & 28.614 & 26.356 & 32.023 & 36.485 & 33.055 & 17.006 & 16.101 & 22.328 & 15.738 & 10.893 & 32.124 & 32.924 & 30.002 & 31.988 & 27.62 & 34.319 \\
\hline $11 / 25 / 11$ 5:00 & & 30.834 & 30.314 & 29.353 & 30.33 & 29.791 & 30.912 & 30.406 & 29.99 & 28.607 & 26.351 & 32.016 & 36.483 & 33.046 & 17.004 & 16.099 & 22.321 & 15.729 & 10.882 & 32.122 & 32.914 & 29.997 & 31.983 & 27.622 & 34.317 \\
\hline 11/25/11 6:00 & & 30.836 & 30.314 & 29.356 & 30.327 & 29.777 & 30.908 & 30.41 & 29.988 & 28.605 & 26.361 & 32.014 & 36.478 & 33.043 & 17.004 & 16.094 & 22.324 & 15.717 & 10.882 & 32.122 & 32.914 & 30.002 & 31.981 & 27.622 & 34.312 \\
\hline /25/11 7:00 & & 30.829 & 30.307 & 29.346 & 30.323 & 29.777 & 30.905 & 30.403 & 29.981 & 28.6 & 26.356 & 32.009 & 36.478 & 33.039 & 16.997 & 16.092 & 22.324 & 15.722 & 10.875 & 32.108 & 32.907 & 29.988 & 31.974 & 27.62 & 34.305 \\
\hline
\end{tabular}




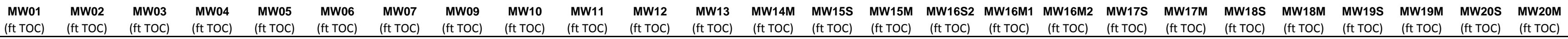

\begin{tabular}{|c|c|c|c|c|c|c|c|c|c|c|c|c|c|c|c|c|c|c|c|c|c|c|c|c|c|c|}
\hline Well & $\begin{array}{l}\text { (ft TOC) } \\
\text { (f) }\end{array}$ & $\begin{array}{l}\text { (ft TOC) } \\
\text { (f) }\end{array}$ & $\begin{array}{l}\text { (ft TOC) } \\
\text { (f) }\end{array}$ & (ft TOC) & (ft Toc) & $\begin{array}{l}\text { (ft ToC) } \\
\text { (f) }\end{array}$ & ft TOC) & (ft ToC) & $\begin{array}{l}\text { (ft TOC) } \\
\text { (f) }\end{array}$ & $\begin{array}{l}\text { (ft TOC) } \\
\text { (l) }\end{array}$ & $\begin{array}{l}\text { (ft TOC) } \\
\text { (n) }\end{array}$ & $\begin{array}{l}\text { ft TOC) } \\
\text { flats }\end{array}$ & (ft TOC) & (ft Toc) & (ft TOC) & (ft TOC) & (ft TOC) & (ft TOC) & $\begin{array}{l}\text { (ft TOC) } \\
\text { (ft) }\end{array}$ & (ft TOC) & $\begin{array}{l}\text { (ft TOC) } \\
\text { (1) }\end{array}$ & (ft TOC) & $\begin{array}{l}\text { (ft TOC) } \\
\text { (n) }\end{array}$ & (ft TOC) & $\begin{array}{l}\text { (nt TOC) } \\
\text { (nt }\end{array}$ & (ft TOC) \\
\hline $11 / 25 / 118: 00$ & & 30.831 & 30.312 & 29.36 & 30.325 & & 29.761 & 30.903 & 30.405 & 29.983 & 28.6 & 26.368 & 32.009 & 36.478 & $3 \quad 33.036$ & 16.999 & 16.089 & 22.331 & 15.71 & 10.87 & 32.117 & 32.907 & 29.997 & 31.974 & 27.62 & 34.307 \\
\hline $11 / 25 / 119: 00$ & & 30.827 & 30.31 & 29.36 & 30.327 & & 29.751 & 30.903 & 30.403 & 29.981 & 28.598 & 26.373 & 32.009 & 36.476 & 33.039 & 16.997 & 16.089 & 22.338 & 15.705 & 10.872 & 32.119 & 32.907 & 30.004 & 31.974 & 27.622 & 34.307 \\
\hline $11 / 25 / 1110: 00$ & & 30.829 & 30.312 & 29.365 & 30.334 & & 29.742 & 30.9 & 30.398 & 29.978 & 28.605 & 26.378 & 32.011 & 36.48 & $\begin{array}{l}3 \\
3\end{array}$ & 16.997 & 16.094 & 22.351 & 15.696 & 10.879 & 32.126 & 32.912 & 30.009 & 31.976 & 27.622 & 34.312 \\
\hline :00 & & 30.817 & 30.303 & 29.337 & 30.323 & & 29.761 & 30.898 & 30.394 & 29.969 & 28.591 & 26.356 & 32.007 & 36.478 & 33.034 & 16.992 & 16.094 & 22.361 & 15.708 & 10.87 & 201 & 32.907 & & 31.974 & & 34.307 \\
\hline$/ 1112: 00$ & & 30.817 & 30.3 & 29.33 & 30.31 & & 29.756 & 30.896 & 0.396 & 29.957 & 28.584 & 26.344 & 32.004 & 36.473 & 33.034 & 16.995 & 16.092 & 22.368 & 15.705 & 10.87 & 32.108 & 32.902 & 985 & 1.971 & & 34.307 \\
\hline $11 / 25 / 1113: 00$ & & 30.806 & 30.282 & 29.302 & 30.302 & & 29.77 & 30.893 & 30.389 & 29.944 & 28.57 & 26.325 & 31.995 & 36.469 & 33.029 & 16.99 & 16.089 & 22.372 & 15.717 & 10.865 & 32.089 & 32.898 & .959 & 1.964 & 27.622 & 34.303 \\
\hline $11 / 25 / 1114: 00$ & & 30.813 & 30.286 & 29.32 & 30.304 & & 29.749 & 30.891 & 30.391 & 29.946 & 28.572 & 26.344 & 31.997 & 36.469 & 33.024 & 16.992 & 16.092 & 22.377 & 15.701 & 10.863 & 32.098 & 32.895 & 29.973 & 31.964 & 27.624 & 34.305 \\
\hline $11 / 25 / 1115: 00$ & & 30.817 & 30.289 & 29.332 & 30.311 & & 29.732 & 30.886 & 30.394 & 29.948 & 28.577 & 26.358 & 31.997 & 36.476 & $5 \quad 33.027$ & 16.992 & 16.089 & 22.416 & 15.686 & 10.865 & 32.117 & 32.893 & 29.992 & 31.964 & 27.624 & 34.3 \\
\hline 11/25/11 16:00 & & 30.817 & 30.3 & 29.349 & 30.32 & & 29.714 & 30.889 & 30.391 & 29.953 & 28.584 & 26.378 & 32 & 36.469 & 33.029 & 16.99 & 16.094 & 22.421 & 15.682 & 10.867 & 32.117 & 32.9 & 30.002 & 31.969 & 27.624 & 34.303 \\
\hline & & 30.815 & 30.296 & 29.344 & 30.325 & & 29.714 & 30.889 & 30.391 & 29.951 & 28.584 & 26.375 & 32 & 36.473 & 333.029 & 16.99 & 16.089 & 22.421 & 15.68 & 10.86 & 32.119 & 32.9 & 30.004 & 31.966 & 27.624 & 34.3 \\
\hline $11 / 25 / 1118: 00$ & & 30.813 & 30.296 & 29.346 & 30.33 & & 29.709 & 30.884 & 30.386 & 29.953 & 28.584 & 26.378 & 32.002 & 36.466 & 33.029 & 16.99 & 16.092 & 22.474 & 15.677 & 10.867 & 32.119 & 32.902 & 30.006 & 31.969 & 27.624 & 34.298 \\
\hline $11 / 25 / 1119: 00$ & & 30.82 & 30.31 & 29.367 & 30.344 & & 29.688 & 30.886 & 30.394 & 29.957 & 28.595 & 26.397 & 32.009 & 36.485 & 33.034 & 16.992 & 16.099 & 22.511 & 15.656 & 10.872 & 32.14 & 32.909 & 30.032 & 31.974 & 27.627 & 34.298 \\
\hline $11 / 25 / 1120: 00$ & & 30.81 & 30.305 & 29.36 & 30.346 & & 29.699 & 30.884 & 30.382 & 29.957 & 28.588 & 26.392 & 32.004 & 36.483 & 33.031 & 16.992 & 16.087 & 22.502 & 15.67 & 10.865 & 32.129 & 32.905 & 30.016 & 31.969 & 27.627 & 34.293 \\
\hline $11 / 25 / 1121: 00$ & & 30.813 & 30.307 & 29.365 & 30.351 & & 29.69 & 30.881 & 30.389 & 29.962 & 28.593 & 26.397 & 32.007 & 36.485 & 33.034 & 16.995 & 16.092 & 22.497 & 15.661 & 10.872 & 32.138 & 32.907 & 30.025 & 31.974 & 27.627 & 34.293 \\
\hline $11 / 25 / 1122: 00$ & & 30.815 & 30.314 & 29.377 & 30.36 & & 29.683 & 30.881 & 30.389 & 29.964 & 28.598 & 26.406 & 32.011 & 36.487 & 33.041 & 16.997 & 16.099 & 22.492 & 15.654 & 10.877 & 32.143 & 32.917 & 30.037 & 31.978 & 27.629 & 34.296 \\
\hline 11/25/11 23:00 & & 30.815 & 30.314 & 29.377 & 30.36 & & 29.683 & 30.881 & 30.389 & 29.964 & 28.6 & 26.409 & 32.021 & 36.49 & 33.046 & 16.995 & 16.101 & 22.488 & 15.654 & 10.879 & 32.152 & 32.921 & 30.039 & 31.983 & 27.629 & 34.298 \\
\hline $11 / 26 / 110: 00$ & & 30.817 & 30.319 & 29.379 & 30.367 & & 29.676 & 30.893 & 30.389 & 29.967 & 28.605 & 26.413 & 32.018 & 36.492 & 33.041 & 16.999 & 16.099 & 22.483 & 15.64 & 10.875 & 32.159 & 32.917 & 30.044 & 31.983 & 27.631 & 34.296 \\
\hline 11/26/11 1:00 & & 30.815 & 30.324 & 29.386 & 30.367 & & 29.681 & 30.893 & 30.384 & 29.969 & 28.605 & 26.418 & 31.981 & $\begin{array}{l}36.494 \\
364\end{array}$ & 33.006 & 16.997 & $\begin{array}{l}16.071 \\
\end{array}$ & 22.455 & 15.64 & 10.839 & 32.133 & 32.879 & 30.042 & 31.945 & 27.627 & 34.275 \\
\hline $11 / 26 / 112: 00$ & & 30.808 & $\begin{array}{l}30.319 \\
30.319\end{array}$ & 29.372 & 30.362 & & 29.69 & 30.9 & $\begin{array}{l}30.384 \\
30.384\end{array}$ & 29.967 & 28.602 & 26.404 & $\begin{array}{l}31.979 \\
31.979\end{array}$ & $\begin{array}{l}36.494 \\
36.494\end{array}$ & 33.003 & 16.995 & 16.066 & 22.446 & $\begin{array}{l}15.647 \\
15.647\end{array}$ & $\begin{array}{l}10.842 \\
10.842\end{array}$ & 32.129 & 32.879 & $\begin{array}{r}30.03 \\
30.03\end{array}$ & 31.945 & 27.629 & 34.268 \\
\hline $11 / 26 / 113: 00$ & & 30.815 & 30.321 & 29.379 & $\begin{array}{l}30.02 \\
30.365\end{array}$ & & 29.681 & 30.896 & $\begin{array}{l}30.304 \\
30.389\end{array}$ & 29.969 & 28.607 & $\begin{array}{l}20.449 \\
26.409\end{array}$ & 31.983 & $\begin{array}{l}30.444 \\
36.494\end{array}$ & 33.006 & $\begin{array}{l}16.995 \\
1695\end{array}$ & $\begin{array}{l}10.000 \\
16.066\end{array}$ & 22.474 & $\begin{array}{l}15.628 \\
15.628\end{array}$ & 10.842 & 32.136 & 32.881 & 30.035 & $\begin{array}{l}31.948 \\
31.948\end{array}$ & 27.631 & $\begin{array}{l}34.265 \\
34.265\end{array}$ \\
\hline $11 / 26 / 114: 00$ & & $\begin{array}{l}30.817 \\
30.817\end{array}$ & 30.331 & 29.393 & $\begin{array}{l}30.303 \\
30.369\end{array}$ & & 29.671 & $\begin{array}{l}30.893 \\
30.893\end{array}$ & $\begin{array}{l}30.389 \\
30.389\end{array}$ & 29.976 & 28.614 & $\begin{array}{l}20.499 \\
26.425\end{array}$ & $\begin{array}{l}31.988 \\
31.988\end{array}$ & $\begin{array}{l}30.44 \\
36.497\end{array}$ & 33.013 & $\begin{array}{l}10.995 \\
16.995\end{array}$ & $\begin{array}{l}10.000 \\
16.064\end{array}$ & 22.469 & $\begin{array}{l}13.086 \\
15.626\end{array}$ & 10.846 & 32.145 & $\begin{array}{l}\begin{array}{r}3.281 \\
32.89\end{array} \\
\end{array}$ & $\begin{array}{l}30.043 \\
30.046\end{array}$ & $\begin{array}{l}31.948 \\
31.952\end{array}$ & 27.634 & 34.263 \\
\hline $11 / 26 / 11$ 5:00 & & 30.838 & 30.363 & 29.461 & 30.409 & & 29.624 & 30.896 & 30.398 & 30.002 & 28.649 & 26.49 & .002 & 36.513 & 33.027 & 16.999 & 16.071 & .472 & 15.593 & 10.865 & 32.192 & 32.902 & 0.098 & 31.966 & 27.634 & 34.261 \\
\hline $11 /$ & & & & 29.507 & 30. & & 29.608 & 30.903 & 30.401 & 30.027 & $\begin{array}{l}28.674 \\
28.074\end{array}$ & 26.535 & 018 & 36.525 & 33.043 & 17.006 & 16.078 & 472 & 15.593 & 10.879 & 32.215 & 919 & .129 & 31.983 & 27.634 & 263 \\
\hline $11 / 26 / 1$ & & & 88 & 29.524 & 30.463 & & 29.61 & 30.905 & 30.401 & 30.043 & 28.686 & 26.547 & 32.032 & 36.534 & 33.053 & 17.011 & & 474 & .595 & 10.893 & 32.231 & 933 & 147 & 1.995 & 27.634 & 34.263 \\
\hline 11/26/11 8:00 & & & 30.438 & 29.566 & 30.495 & & 29.598 & 30.91 & 30.408 & 30.067 & 28.714 & 26.588 & 32.051 & 36.544 & 33.071 & 17.02 & 16.087 & 474 & 15.593 & 10.907 & 32.257 & .954 & 176 & 32.013 & 27.636 & 34.268 \\
\hline 11/26/11 9:00 & & 30.869 & 30.471 & 29.613 & 30.533 & & 29.589 & 30.92 & 30.41 & 30.097 & 28.744 & 26.629 & 32.07 & 36.555 & 33.092 & 17.027 & 16.099 & 22.49 & 15.588 & 10.931 & 32.292 & 32.969 & .218 & 32.039 & 27.634 & 34.27 \\
\hline 11/26/11 10:00 & & 30.871 & 30.487 & 29.622 & 30.551 & & 29.605 & 30.924 & 30.408 & 30.113 & 28.765 & 26.636 & 32.086 & 36.562 & 33.107 & 17.032 & 16.106 & 22.497 & 15.609 & 10.947 & 32.303 & 985 & .225 & 32.046 & 27.634 & 34.272 \\
\hline 11/26/11 11:00 & & 30.871 & 30.489 & 29.622 & 30.561 & & 29.638 & 30.929 & 30.413 & 30.12 & 28.765 & 26.624 & 32.096 & 36.56 & 33.118 & 17.039 & 16.117 & 22.502 & 15.626 & 10.957 & 32.308 & 32.995 & 30.218 & 32.063 & 27.636 & 34.275 \\
\hline $11 / 26 / 1112: 00$ & & 30.871 & 30.492 & 29.608 & 30.556 & & 29.669 & 30.932 & 30.41 & 30.127 & 28.765 & 26.607 & 32.103 & 36.562 & 33.128 & 17.044 & 16.124 & 22.506 & 15.647 & 10.961 & 32.306 & 33.004 & 30.206 & 32.063 & 27.636 & 34.277 \\
\hline $11 / 26 / 1113: 00$ & & 30.875 & 30.496 & 29.615 & 30.561 & & 29.683 & 30.941 & 30.408 & 30.139 & 28.774 & 26.607 & 32.112 & 36.562 & 33.132 & 17.048 & 16.129 & 22.536 & 15.647 & 10.971 & 32.303 & 33.011 & 30.215 & 32.077 & 27.634 & 34.279 \\
\hline $11 / 26 / 1114: 00$ & & 30.878 & 30.499 & 29.62 & 30.558 & & 29.697 & 30.941 & 30.413 & 30.141 & 28.784 & 26.607 & 32.117 & 36.565 & 33.139 & 17.051 & 16.134 & 22.573 & 15.654 & 10.98 & 32.31 & 33.014 & 30.215 & 32.084 & 27.638 & 34.282 \\
\hline $11 / 26 / 1115: 00$ & & 30.885 & 30.512 & 29.641 & 30.57 & & 29.697 & 30.956 & 30.417 & 30.152 & 28.795 & 26.622 & 32.124 & 36.567 & 33.146 & 17.058 & 16.141 & 22.596 & 15.658 & 10.983 & 32.324 & 33.026 & 30.23 & 32.091 & 27.636 & 34.284 \\
\hline $11 / 26 / 1116: 00$ & & 30.894 & 30.529 & 29.659 & 30.582 & & 29.697 & 30.951 & 30.417 & 30.176 & 28.809 & 26.641 & 32.133 & 36.569 & 33.156 & 17.062 & 16.143 & 22.645 & 15.663 & 10.992 & 32.334 & 33.03 & 30.239 & 32.098 & 27.638 & 34.284 \\
\hline $11 / 26 / 1117: 00$ & & 30.901 & 30.545 & 29.683 & 30.6 & & 29.692 & 30.958 & 30.424 & 30.194 & 28.828 & 26.66 & 32.142 & 36.576 & 33.165 & 17.067 & 16.15 & 22.666 & 15.663 & 11.004 & 32.352 & 33.045 & 30.262 & 32.107 & 27.638 & 34.286 \\
\hline $11 /$ & & 30.908 & 30.566 & 29.708 & 30.617 & & 29.695 & 30.967 & 30.429 & 30.21 & 28.846 & 26.674 & 32.154 & 36.579 & 33.1 & 17.069 & 16.157 & 22.673 & 15.663 & 11.013 & 32.369 & 33.056 & 30.274 & 32.121 & 27.638 & 34.289 \\
\hline $11 / 2$ & & 30.915 & 30.5 & 29.725 & 30.6 & & 29.699 & 30. & 30.432 & 30.229 & 28.86 & 26.689 & 32.168 & 36.584 & 33.191 & 17.076 & 16.164 & 22.668 & 15.665 & 11.027 & 32.38 & 071 & 30.293 & 32.128 & 27.641 & 34.291 \\
\hline 20:00 & & 30.92 & 30.589 & 29.734 & 30.6 & & 29.714 & 30.982 & $\begin{array}{l}30.432 \\
30.432\end{array}$ & 30.243 & $\begin{array}{l}28.00 \\
28.87\end{array}$ & 26.693 & 32.175 & $\begin{array}{l}36.588 \\
36.58\end{array}$ & 33.2 & 17.078 & $\begin{array}{l}16.174 \\
16.171\end{array}$ & 22.666 & 15.673 & 11.041 & $\begin{array}{l}32.30 \\
32.394\end{array}$ & $\begin{array}{l}33.078 \\
3.178\end{array}$ & 30.302 & 32.14 & 27.638 & 34.293 \\
\hline $11 / 2$ & & 30.922 & 30.589 & 29.732 & 30.6 & & 29.73 & $\begin{array}{l}30.989 \\
309\end{array}$ & 30.439 & 30.25 & 28.877 & 26.681 & 32.184 & $\begin{array}{l}36.580 \\
36.58\end{array}$ & 33.21 & $\begin{array}{l}17.081 \\
\end{array}$ & 16.183 & 22.659 & 15.689 & 11.044 & 32.397 & .085 & 30.298 & 32.152 & 27.638 & 34.298 \\
\hline $11 / 2$ & & 30.924 & 30.594 & 29.732 & 30. & & 29.749 & 30.991 & 30.436 & 30.2 & 28.879 & $\begin{array}{l}20.001 \\
26.679\end{array}$ & 32 & $\begin{array}{l}30.000 \\
36.591\end{array}$ & 33.219 & $\begin{array}{l}17.083 \\
17.083\end{array}$ & 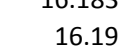 & $y$ & $\begin{array}{l}15.099 \\
15.694\end{array}$ & 11.053 & 397 & .097 & $\begin{array}{r}r 0.20 \\
30.3\end{array}$ & 32.157 & $\begin{array}{l}27.641 \\
27.641\end{array}$ & $\begin{array}{l}34.490 \\
34.3\end{array}$ \\
\hline & & 30 & 30 & 29. & & & 2 & 30 & 30 & 30 & 28 & 26 & 32 & 36. & 33 & 17 & 16 & 2 & 15.703 & 1 & 32.392 & 9 & 5 & 32. & 3 & 303 \\
\hline $11 / 27 / 11$ & & & 30 & & & & & & & 30 & 28. & & 32 & 36 & 33 & 17 & 4 & 22.67 & 15.705 & 11.067 & 32 & 6 & 3 & 1 & 27.643 & .305 \\
\hline $11 / 27 / 1$ & & 30.927 & 30.592 & 29.713 & & & & & & & 28.884 & & 32.208 & 36.598 & 33.233 & & & 6 & 15.719 & 11.072 & 32.39 & & 30.281 & 2.173 & 27.641 & 34.307 \\
\hline $11 / 2$ & & & $30.5 \varepsilon$ & 29.704 & & & & & & & 28.8 & & 32.208 & 36.5 & 33.231 & & & & 15.722 & 11.07 & 32.378 & & & (2) & 27.641 & 34.307 \\
\hline $11 / 2$ & & & 30. & 29.718 & & & & & & & & & 32.208 & 36. & & 17. & & 22.6 & 15.7 & 11 & 32.3 & & 1 & 8 & 27.643 & 34.307 \\
\hline $11 / 2$ & & & 30. & 29. & & & & & & & & & 32 & & & & & (n) & 15. & 11.072 & 32.385 & & 4 & 32.175 & 27.643 & 34.307 \\
\hline 11/27/11 5:00 & & & 30.5 & 29.7 & & & & & 30. & 30.2 & 28. & & 32.2 & 36.602 & 33. & & & 22.6 & 15. & 11.07 & 32.38 & & 30.272 & 32.171 & 27.643 & 34.303 \\
\hline 11/27/11 6:00 & & 30. & 30.5 & 29.716 & & & 29.8 & $31 . c$ & 30.446 & 30 & 28.8 & 26.6 & 32.208 & 36.598 & 33.233 & 17. & 16.2 & 22.6 & 15.7 & 11 & 32.38 & 33.106 & 30.267 & 32.171 & 27.645 & 34.3 \\
\hline 11/27/11 7:00 & & 30.9 & 30.5 & 29.718 & 30. & & 29.8 & 31.0 & 30.451 & 30.2 & 28.8 & 26.63 & 32.208 & 36.6 & 33.233 & 17.0 & 16.2 & 22.6 & 15.7 & 11.072 & 32.387 & 33.108 & 30.274 & 32.173 & 27.645 & 34.298 \\
\hline $11 / 27 / 118: 00$ & & 30.945 & 30.599 & 29.725 & 30.6 & & 29.836 & 31.011 & 30.453 & 30.289 & $28.8 \mathrm{~s}$ & 26.638 & 32.21 & 36.605 & 33.233 & 17.076 & 16.215 & 22.638 & 15.738 & 11.072 & 32.385 & 33.111 & 30.279 & 32.173 & 27.645 & 34.296 \\
\hline 11/27/11 9:00 & & 30.952 & 30.608 & 29.737 & 30.635 & & 29.836 & 31.015 & 30.455 & 30.299 & 28.905 & 26.65 & 32.213 & 36.598 & 33.24 & 17.074 & 16.22 & 22.654 & 15.736 & 11.077 & 32.392 & 33.116 & 30.284 & 32.18 & 27.645 & 34.298 \\
\hline $11 / 27 / 1110: 00$ & & 30.95 & 30.608 & 29.734 & 30.631 & & 29.848 & 31.018 & 30.46 & 30.301 & 28.907 & 26.643 & 32.215 & 36.607 & 33.242 & 17.071 & 16.222 & 22.654 & 15.755 & 11.079 & 32.39 & 33.116 & 30.288 & 32.182 & 27.645 & 34.296 \\
\hline $11 / 27 / 1111: 00$ & & 30.941 & 30.594 & 29.704 & 30.619 & & 29.878 & 31.02 & 30.455 & 30.294 & 28.895 & 26.612 & 32.213 & 36.6 & 33.24 & 17.067 & 16.225 & 22.657 & 15.773 & 11.077 & 32.376 & 33.116 & 30.262 & 32.178 & 27.645 & 34.296 \\
\hline $11 / 27 / 1112: 00$ & & 30.936 & 30.58 & 29.673 & 30.598 & & 29.902 & 31.02 & 30.455 & 30.28 & 28.881 & 26.581 & 32.208 & 36.595 & 33.235 & 17.065 & 16.225 & 22.67 & 15.792 & 11.074 & 32.355 & 106 & 30.239 & 32.175 & 27.648 & 34.296 \\
\hline 11/27/11 13:00 & & 30.934 & 30.568 & 29.648 & 30.5 & & 29.92 & 31.018 & 30.455 & 30.269 & 28.865 & 26.557 & 32.201 & 36.591 & 33.228 & 17.06 & 16.225 & 22.666 & 15.811 & 11.067 & 32.338 & 33.101 & 30.211 & 32.166 & 27.645 & 34.293 \\
\hline $11 / 27 / 1114: 00$ & & 30.931 & 30.557 & 29.627 & 30.556 & & 29.927 & 31.015 & 30.453 & 30.259 & 28.858 & 26.54 & 32.196 & 36.581 & 33.221 & 17.053 & 16.225 & 22.663 & 15.813 & 11.06 & 32.324 & 33.094 & 30.197 & 32.164 & 27.645 & 34.293 \\
\hline $11 / 27 / 1115: 00$ & & 30.934 & 30.547 & 29.622 & 30.544 & & 29.92 & 31.015 & 30.458 & 30.255 & 28.851 & 26.535 & 32.191 & 36.576 & 33.214 & 17.048 & 16.223 & 22.661 & 15.811 & 11.056 & 32.324 & 33.092 & 30.187 & 32.157 & 27.648 & 34.293 \\
\hline
\end{tabular}




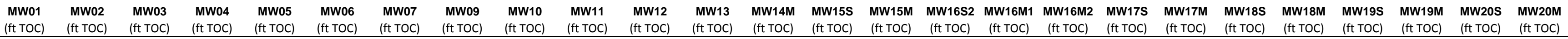

\begin{tabular}{|c|c|c|c|c|c|c|c|c|c|c|c|c|c|c|c|c|c|c|c|c|c|c|c|c|c|}
\hline well & $\begin{array}{l}\text { (ft TOC) } \\
\text { (f) }\end{array}$ & $\begin{array}{l}\text { (ft TOC) } \\
\end{array}$ & $\begin{array}{l}\text { (ft TOC) } \\
\end{array}$ & $\begin{array}{l}\text { (ft TOC) } \\
\text { (f) }\end{array}$ & $\begin{array}{l}\text { (ift TOC) } \\
\text { (ft TOC) }\end{array}$ & $\begin{array}{l}\text { (ft TOC) } \\
\end{array}$ & $\begin{array}{l}\text { (ift TOC) } \\
\end{array}$ & $\begin{array}{l}\text { (ft TOC) } \\
\text { (f) }\end{array}$ & $\begin{array}{l}\text { (ft TOC) } \\
\text { (f) }\end{array}$ & $\begin{array}{l}\text { (ft TOC) } \\
\text { (n) }\end{array}$ & $\begin{array}{l}\text { (ft TOC) } \\
\text { (ft) }\end{array}$ & (ft TOC) & $\begin{array}{l}\text { (ft TOC) } \\
\end{array}$ & (ft TOC) & (ft TOC) & (ft TOC) & (ft TOC) & $\begin{array}{l}\text { (ft TOC) } \\
\text { (n) }\end{array}$ & (ft TOC) & $\begin{array}{l}\text { (ft TOC) } \\
\text { (f) }\end{array}$ & (ft TOC) & $\begin{array}{l}\text { (ft TOC) } \\
\text { (n) }\end{array}$ & (ft TOC) & (ft TOC) & (ft TOC) \\
\hline 6:00 & & 30.934 & 30.5 & 29.6 & 30.5 & 29.9 & 31. & 30.451 & 30.25 & 28.846 & 26. & 32.187 & 36.574 & 33 & 17.041 & 16.222 & 22.742 & 15.818 & 11.051 & & 33.087 & & .152 & 48 & 1 \\
\hline 11/27/11 17:00 & & 30.931 & 30.538 & 29.613 & 30.526 & 29.916 & 31.013 & 30.453 & 30.243 & 28.839 & 26.526 & 32.18 & 36.574 & 33.207 & 17.039 & 16.22 & 22.758 & 5.816 & 11.046 & 32.306 & 33.078 & 176 & 107 & & 34.289 \\
\hline 11/27/11 18:00 & & 30.931 & 30.533 & 29.601 & 30.514 & 29.911 & 31.011 & 30.455 & 30.238 & 28.83 & 26.519 & 32.496 & 36.569 & 33.547 & 17.032 & 16.265 & 22.754 & 15.809 & 11.382 & 32.397 & & 176 & .457 & & 286 \\
\hline 11/27/11 19:00 & & 30.936 & 30.536 & 29.613 & 30.521 & 29.892 & 31.011 & 30.458 & 30.238 & 28.835 & 26.531 & 32.36 & 36.574 & 33.397 & 17.03 & 16.3 & 22.749 & 15.799 & 11.237 & 32.39 & 33.258 & 30.19 & 2.323 & 65 & 34.284 \\
\hline 11/27/11 20:00 & & 30.934 & 30.531 & 29.608 & 30.521 & 29.89 & 31.013 & 30.458 & 30.234 & 28.828 & 26.528 & 32.299 & 36.567 & 33.336 & 17.02 & 16.29 & 22.737 & 15.797 & 11.171 & 32.362 & 33.201 & 30.185 & 32.267 & 27.652 & 34.284 \\
\hline 11/27/11 21:00 & & 30.931 & 30.524 & 29.601 & 30.519 & 29.887 & 31.011 & 30.458 & 30.227 & 28.823 & 26.521 & 32.273 & 36.567 & 33.303 & 17.016 & 16.286 & 22.731 & 15.797 & 11.14 & 32.35 & 33.17 & 30.178 & 32.236 & 27.65 & 34.284 \\
\hline 11/27/11 22:00 & & 30.931 & 30.522 & 29.596 & 30.514 & 29.887 & 31.011 & 30.458 & 30.222 & 28.818 & 26.519 & 32.252 & 36.569 & 33.282 & 17.011 & 16.281 & 22.726 & 15.797 & 11.121 & 32.336 & 33.151 & 30.171 & 32.218 & 27.652 & 34.286 \\
\hline $11 / 27 / 1123: 00$ & & 30.929 & 30.515 & 29.58 & 30.507 & 29.892 & 31.008 & 30.455 & 30.215 & 28.812 & 26.507 & 32.238 & 36.565 & 33.271 & 17.006 & 16.279 & 22.724 & 15.797 & 11.103 & 32.324 & 33.139 & 30.161 & 32.203 & 27.65 & 34.286 \\
\hline 11/28/11 0:00 & & 30.929 & 30.512 & 29.58 & 30.502 & 29.887 & 31.006 & 30.455 & 30.21 & 28.807 & 26.507 & 32.229 & 36.565 & 33.259 & 17.002 & 16.276 & 22.719 & 15.792 & 11.093 & 32.322 & 33.127 & 30.159 & 32.192 & 27.653 & 34.286 \\
\hline 11/28/11 1:00 & & 30.924 & 30.503 & 29.568 & 30.495 & 29.885 & 31.006 & 30.458 & 30.206 & 28.8 & 26.497 & 32.217 & 36.567 & 33.25 & 16.999 & 16.274 & 22.719 & 15.792 & 11.086 & 32.306 & 33.12 & 30.15 & 32.185 & 27.653 & 34.289 \\
\hline 11/28/11 2:00 & & 30.924 & 30.496 & 29.559 & 30.486 & 29.885 & 31.003 & 30.455 & 30.199 & 28.793 & 26.49 & 32.21 & 36.56 & 33.238 & 16.992 & 16.269 & 22.714 & 15.788 & 11.074 & 32.299 & 33.108 & 30.147 & 32.175 & 27.655 & 34.286 \\
\hline 11/28/11 3:00 & & 30.92 & 30.487 & 29.547 & 30.474 & 29.89 & 30.996 & 30.451 & 30.187 & 28.781 & 26.478 & 32.199 & 36.56 & 33.226 & 16.988 & 16.265 & 22.707 & 15.788 & 11.067 & 32.289 & 33.097 & 30.126 & 32.164 & 27.653 & 34.286 \\
\hline 11/28/11 4:00 & & 30.92 & 30.482 & 29.543 & 30.467 & 29.885 & 30.996 & 30.451 & 30.183 & 28.779 & 26.476 & 32.189 & 36.558 & 33.219 & 16.983 & 16.26 & 22.696 & 15.783 & 11.056 & 32.278 & 33.09 & 30.121 & 32.157 & 27.652 & 34.284 \\
\hline $11 / 28 / 11$ 5:00 & & 30.917 & 30.471 & 29.529 & 30.458 & 29.885 & 30.994 & 30.453 & 30.173 & 28.767 & 26.466 & 32.177 & 36.553 & 33.207 & 16.978 & 16.255 & 22.701 & 15.781 & 11.046 & 32.269 & 33.08 & 30.114 & 32.145 & 27.653 & 34.279 \\
\hline $11 / 28 / 116: 00$ & & 30.92 & 30.475 & 29.543 & 30.46 & 29.869 & 30.991 & 30.455 & 30.176 & 28.772 & 26.48 & 32.173 & 36.555 & 33.2 & 16.974 & 16.248 & 22.696 & 15.771 & 11.039 & 32.273 & 33.073 & 30.119 & 32.14 & 27.653 & 34.277 \\
\hline 11/28/11 7:00 & & 30.915 & 30.468 & 29.529 & 30.451 & 29.873 & 30.989 & 30.453 & 30.169 & 28.76 & 26.471 & 32.161 & 36.553 & 33.193 & 16.967 & 16.241 & 22.693 & 15.769 & 11.03 & 32.257 & 33.063 & 30.107 & 32.128 & 27.655 & 34.272 \\
\hline 11/28/11 8:00 & & 30.917 & 30.468 & 29.533 & 30.449 & 29.864 & 30.989 & 30.453 & 30.164 & 28.763 & 26.473 & 32.159 & 36.553 & 33.189 & 16.962 & 16.236 & 22.698 & 15.759 & 11.025 & 32.259 & 33.059 & 30.11 & 32.124 & 27.655 & 34.268 \\
\hline 11/28/11 9:00 & & 30.917 & 30.471 & 29.54 & 30.453 & 29.855 & 30.987 & 30.451 & 30.164 & 28.763 & 26.485 & 32.154 & 36.555 & 33.184 & 16.96 & 16.234 & 22.689 & 15.759 & 11.02 & 32.259 & 33.054 & 30.114 & 32.121 & 27.655 & 34.27 \\
\hline $110: 00$ & & 30.92 & 30.471 & 29.545 & 30.453 & 29.852 & 30.987 & 30.455 & 30.164 & 28.765 & 26.49 & 32.152 & 36.555 & 33.182 & 16.955 & 16.232 & 22.691 & 15.764 & 11.018 & 32.262 & 33.052 & 30.117 & 32.119 & 27.657 & 34.268 \\
\hline 00 & & 30.915 & 30.468 & 29.536 & $\begin{array}{l}30.45 \\
30.4 \\
3\end{array}$ & 29.857 & $\begin{array}{l}30.987 \\
30.97\end{array}$ & $\begin{array}{l}30.453 \\
30.453\end{array}$ & $\begin{array}{l}30.154 \\
30.157\end{array}$ & 28.758 & $\begin{array}{l}26.485 \\
26.485\end{array}$ & 32.147 & 36.553 & $\begin{array}{l}33.177 \\
33.177\end{array}$ & $\begin{array}{l}16.953 \\
16.95\end{array}$ & 16.232 & 22.689 & 15.776 & $\begin{array}{l}11.018 \\
11.018\end{array}$ & $\begin{array}{l}32.252 \\
32.252\end{array}$ & $\begin{array}{l}33.049 \\
33.049\end{array}$ & 30.107 & 32.114 & 27.655 & $\begin{array}{l}34.268 \\
34.268\end{array}$ \\
\hline 10 & & 30.906 & 30.447 & 29.505 & 30.4 & 29.88 & 30.984 & 30.448 & 30.146 & 28.744 & 26.454 & 32.142 & 36.551 & 33.172 & 16.946 & 16.227 & 22.687 & 15.79 & 11.009 & 32.231 & .04 & .084 & 32.107 & 27.657 & 34.265 \\
\hline $11 / 2$ & & 30.899 & 30.431 & 29.475 & 30. & 29.895 & 30.979 & 30.443 & 30.129 & 28.725 & 26.428 & 32.131 & 36.544 & 33.163 & 16.941 & 16.225 & 22.684 & 15.797 & 10.997 & 32.215 & 03 & .063 & 2.098 & 27.655 & 34.261 \\
\hline $11 / 28 / 1114: 00$ & & 30.897 & 30.419 & 29.461 & 30. & 29.895 & 30.972 & 30.446 & 30.12 & 28.716 & 26.416 & 32.124 & 36.539 & 33.156 & 16.936 & 16.22 & .677 & 15.797 & 10.992 & 32.208 & 33.021 & .051 & 2.091 & 27.657 & 34.261 \\
\hline 11/28/11 15:00 & & 30.901 & 30.417 & 29.47 & 30.39 & 29.878 & 30.972 & 30.443 & 30.115 & 28.714 & 26.428 & 32.119 & 36.544 & 33.149 & 16.932 & 16.22 & 22.677 & 15.79 & 10.985 & 32.206 & 33.021 & .056 & 32.086 & 27.657 & 34.261 \\
\hline 11/28/11 16:00 & & 30.897 & 30.412 & 29.458 & 30.383 & 29.873 & 30.965 & 30.446 & 30.106 & 28.705 & 26.416 & 32.11 & 36.534 & 33.142 & 16.927 & 16.215 & 22.682 & 15.785 & 10.978 & 32.201 & 33.014 & 1049 & 32.079 & 27.66 & 34.258 \\
\hline 11/28/11 17:00 & & 30.901 & 30.419 & 29.484 & 30.395 & 29.845 & & 30.446 & 30.111 & 28.716 & 26.445 & 32.114 & 36.539 & 33.142 & 16.925 & 16.213 & 22.68 & 15.776 & 10.976 & 32.213 & 33.011 & .065 & 32.079 & 27.659 & 34.261 \\
\hline $11 / 28 / 1118: 00$ & & 30.903 & 30.426 & 29.5 & 30.404 & 29.824 & 30.967 & 30.444 & 30.115 & 28.723 & 26.466 & 32.112 & 36.544 & 33.142 & 16.92 & 16.213 & 22.694 & 15.764 & 10.976 & 32.217 & 33.016 & 30.082 & 32.079 & 27.66 & 34.258 \\
\hline 11/28/11 19:00 & & 30.906 & 30.433 & 29.519 & 30.418 & 29.81 & 30.967 & 30.446 & 30.12 & 28.73 & 26.483 & 32.117 & 36.544 & 33.144 & 16.92 & 16.213 & 22.721 & 15.752 & 10.983 & 32.234 & 33.018 & 30.089 & 32.084 & 27.662 & 34.258 \\
\hline $11 / 28 / 1120: 00$ & & 30.908 & 30.438 & 29.531 & 30.432 & 29.798 & 30.967 & 30.446 & 30.125 & 28.735 & 26.497 & 32.119 & 36.555 & 33.146 & 16.918 & 16.213 & 22.721 & 15.745 & 10.983 & 32.241 & 33.021 & 30.105 & 32.086 & 27.659 & 34.258 \\
\hline 11/28/11 21:00 & & 30.911 & 30.447 & 29.545 & 30.444 & 29.791 & 30.972 & 30.448 & 30.129 & 28.742 & 26.512 & 32.124 & 36.558 & 33.149 & 16.918 & 16.215 & 22.721 & 15.738 & 10.987 & 32.248 & 33.026 & 30.112 & 32.091 & 27.662 & 34.258 \\
\hline $11 / 28 / 1122: 00$ & & 30.915 & 30.457 & 29.554 & 30.456 & 29.786 & 30.972 & 30.451 & 30.136 & 28.749 & 26.521 & 32.128 & 36.567 & 33.158 & 16.918 & 16.22 & 22.724 & 15.731 & 10.992 & 32.257 & 33.028 & 30.133 & 32.096 & 27.662 & 34.261 \\
\hline $11 / 28 / 1123: 00$ & & 30.92 & 30.461 & 29.573 & 30.47 & 29.777 & 30.977 & 30.448 & 30.143 & 28.76 & 26.538 & 32.135 & 36.572 & 33.16 & 16.918 & 16.222 & 22.724 & 15.724 & 10.997 & 32.271 & 33.035 & 30.145 & 32.105 & 27.662 & 34.265 \\
\hline $11 / 29 / 110: 00$ & & 30.918 & 30.468 & 29.573 & 30.479 & 29.784 & 30.982 & 30.455 & 30.146 & 28.76 & 26.535 & 32.142 & 36.574 & 33.165 & 16.918 & 16.222 & 22.724 & 15.724 & 11.004 & 32.271 & 33.04 & 30.147 & 32.105 & 27.664 & 34.265 \\
\hline 11/29/11 1:00 & & 30.924 & 30.48 & 29.594 & 30.493 & 29.779 & 30.984 & 30.453 & 30.155 & 28.772 & 26.552 & 32.147 & 36.579 & 33.175 & 16.92 & 16.229 & 22.726 & 15.719 & 11.011 & 32.287 & 33.049 & 30.164 & 32.117 & 27.664 & 34.27 \\
\hline $11 / 29 / 112: 00$ & & 30.929 & 30.489 & 29.61 & 30. & 29.775 & 30.987 & 30.458 & 30.166 & 28.784 & 26.571 & 32.154 & 36.588 & 33.182 & 16.922 & 16.234 & 22.726 & 15.719 & 11.02 & 32.299 & 33.056 & 30.178 & 32.121 & 27.664 & 34.275 \\
\hline $11 /$ & & 30. & 30.506 & 29.638 & & 29.763 & 30.994 & $\begin{array}{l}30.462 \\
30.402\end{array}$ & 30.18 & 年 & 26.598 & 32.166 & 36.595 & 33.191 & 16.925 & $\begin{array}{l}16.239 \\
\end{array}$ & 22.724 & 15.715 & 11.027 & 32.32 & .068 & 30.211 & 32.133 & 27.664 & 34.277 \\
\hline $11 / 29 / 114: 00$ & & 30.938 & 30.51 & 29.643 & 30. & 29.777 & $\begin{array}{l}30.999 \\
309\end{array}$ & $\begin{array}{r}r .402 \\
30.46\end{array}$ & 30.187 & $\begin{aligned} 20.0 \\
28.805\end{aligned}$ & 26.6 & 32.17 & 36.598 & 33.198 & 16.927 & $\begin{array}{l}16.241 \\
16.24\end{array}$ & 22.724 & 15.717 & 11.034 & 32.327 & .071 & 30.213 & 32.14 & 27.667 & 34.277 \\
\hline $11 / 29 / 11$ 5:00 & & 30.938 & 30.517 & 29.648 & 30. & 29.789 & 31.003 & 30.463 & $\begin{array}{l}30.191 \\
30.192\end{array}$ & 28.812 & 26.598 & 32.173 & 36.602 & 33.203 & $\begin{array}{l}10.929 \\
16.929\end{array}$ & 16.241 & 22.721 & 15.726 & $\begin{array}{l}11.039 \\
11.039\end{array}$ & 32.329 & 5 & 11 & 14 & 27.667 & .277 \\
\hline & & 30.948 & 30 & 29. & 30 & 29 & 31 & 30 & 30 & 28 & 26 & 32 & 36 & 33 & 16 & 16 & 1 & 15.719 & 11.044 & 3 & 33.082 & 7 & 32.147 & 27.667 & 34.277 \\
\hline & & 30. & 30 & 29 & 30 & 29 & 31.015 & 30 & 30 & 28 & 26 & 32 & 36 & 33 & 16 & 16 & 22.738 & 15 & 11 & 2 & 3 & 8 & 32. & 27.667 & .277 \\
\hline & & & 30 & 29 & & 29 & 31.01 & & 30 & 28 & 26 & 5 & 36 & 33 & 16 & 16 & 22 & 15. & 11 & 373 & 7 & 7 & 54 & 59 & 34.277 \\
\hline & & & 30. & 29. & & & & & & 28 & & 32.201 & & & & 16.251 & 22.7 & 15. & 11.067 & 32.3 & 11 & 4 & 32.171 & 27.669 & 34.277 \\
\hline $11 / 2$ & & 30 & 30. & 29. & & & 31.0 & & & & 26 & 32.208 & 36.619 & & 16.946 & 16.25 & 22.7 & 15.743 & 11.067 & 32.38 & 8 & 4 & 32.175 & 27.667 & 34.275 \\
\hline & & 30 & 30.5 & & & & $31 . c$ & & & & & 32.21 & 36.616 & 33.233 & 16.95 & 16.253 & 22. & 15.757 & 11.072 & 32.376 & & 72 & 32.175 & 27.667 & 34.277 \\
\hline & & 30. & 30.5 & & & 29.8 & & & & & & & & & 16.9 & 16.255 & 22.7 & 15.778 & 11 & & & 1 & & 27.669 & 34.275 \\
\hline $11 /$ & & & 30 & & & & & & & & & 32.206 & 36. & & & & 22. & 15.792 & 11.067 & 32.348 & & & & 27.669 & 34.275 \\
\hline $11 / 29 / 1114: 00$ & & 30.948 & 30.5 & 29.6 & & 29.9 & 31.0 & 30. & & 28.832 & 26. & 32.201 & 36.605 & 33. & 16.95 & 16.255 & 22.733 & 15.806 & 11.065 & 32.331 & & 30.208 & 32.166 & 27.671 & 34.275 \\
\hline $11 / 29 / 1115: 00$ & & 30. & 30.5 & 29.6 & 30. & 29.9 & 31. & $30.4 \div<>$ & $30.2>-2$ & 28 & 26.5 & 32.199 & 36.6 & 33.224 & 16.948 & 16.255 & 22.733 & 15.802 & 11.063 & 32.336 & 33.099 & 30.208 & 32.166 & 27.671 & 34.272 \\
\hline 11/29/11 16:00 & & 30. & 30.5 & 29.638 & 30.5 & 29.9 & 31. & 30.474 & 30.229 & 28.83 & 26.5 & 32.196 & 36.6 & 33.224 & 16.948 & 16.25 & 22.781 & 15.806 & 11.063 & 32.327 & 33.097 & 30.204 & 32.166 & 27.671 & 34.275 \\
\hline $11 / 29 / 1117: 00$ & & 30.95 & 30.533 & 29.631 & 30.5 & 29.904 & 31.03 & 30.474 & 30.229 & 28.828 & 26.55 & 32.196 & 36.597 & 33.221 & 16.948 & 16.255 & 22.779 & 15.809 & 11.058 & 32.322 & 33.094 & 30.199 & 32.161 & 27.671 & 34.272 \\
\hline $11 / 29 / 1118: 00$ & & 30.952 & 30.533 & 29.641 & 30.54 & 29.895 & 31.03 & 30.477 & 30.229 & 28.828 & 26.562 & 32.196 & 36.602 & 33.224 & 16.95 & 16.255 & 22.779 & 15.804 & 11.06 & 32.327 & 33.097 & 30.208 & 32.161 & 27.674 & 34.272 \\
\hline 11/29/11 19:00 & & 30.955 & 30.533 & 29.636 & 30.544 & 29.895 & 31.03 & 30.477 & 30.227 & 28.825 & 26.559 & 32.194 & 36.598 & 33.221 & 16.946 & 16.255 & 22.772 & 15.806 & 11.053 & 32.327 & 33.092 & 30.201 & 32.161 & 27.671 & 34.27 \\
\hline $11 / 29 / 1120: 00$ & & 30.95 & 30.529 & 29.622 & 30.535 & 29.902 & 31.03 & 30.477 & 30.222 & 28.818 & 26.545 & 32.423 & 36.598 & 33.468 & 16.946 & 16.309 & 22.77 & 15.806 & 11.305 & 32.406 & 33.322 & 30.197 & 32.386 & 27.671 & 34.268 \\
\hline 11/29/11 21:00 & & 30.952 & 30.524 & 29.62 & 30.535 & 29.899 & 31.03 & 30.474 & 30.217 & 28.814 & 26.543 & 32.313 & 36.6 & 33.35 & 16.943 & 16.311 & 22.772 & 15.801 & 11.185 & 32.376 & 33.215 & 30.197 & 32.279 & 27.674 & 34.268 \\
\hline $11 / 29 / 1122: 00$ & & 30.95 & 30.522 & 29.615 & 30.535 & 29.899 & 31.027 & 30.479 & 30.217 & 28.814 & 26.543 & 32.28 & 36.595 & 33.308 & 16.943 & 16.304 & 22.77 & 15.799 & 11.15 & 32.357 & 33.18 & 30.192 & 32.243 & 27.674 & 34.265 \\
\hline $11 / 29 / 1123: 00$ & & 30.955 & 30.522 & 29.617 & 30.535 & 29.89 & 31.027 & 30.479 & 30.215 & 28.814 & 26.545 & 32.259 & 36.593 & 33.292 & 16.943 & 16.3 & 22.765 & 15.799 & 11.124 & 32.352 & 33.158 & 30.197 & 32.227 & 27.674 & 5 \\
\hline
\end{tabular}




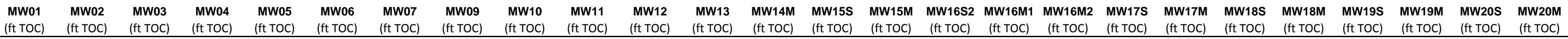

\begin{tabular}{|c|c|c|c|c|c|c|c|c|c|c|c|c|c|c|c|c|c|c|c|c|c|c|c|c|c|c|}
\hline Well & $\begin{array}{l}\text { (ft TOC) } \\
\text { (f) }\end{array}$ & $\begin{array}{l}\text { (ft TOC) } \\
\text { (ft) }\end{array}$ & $\begin{array}{l}\text { (ft TOC) } \\
\text { (f) }\end{array}$ & (ft TOC) & (ft Toc) & $\begin{array}{l}\text { (ft ToC) } \\
\text { (f) }\end{array}$ & $\begin{array}{l}\text { (ft TOC) } \\
\text { (n) }\end{array}$ & $\begin{array}{l}\text { (ft TOC) } \\
\text { (f) }\end{array}$ & $\begin{array}{l}\text { (ft TOC) } \\
\text { (f) }\end{array}$ & $\begin{array}{l}\text { (ft TOC) } \\
\text { (l) }\end{array}$ & $\begin{array}{l}\text { (ft TOC) } \\
\text { (n) }\end{array}$ & (ft TOC) & (ft TOC) & (ft Toc) & (ft TOC) & (ft TOC) & (ft TOC) & (ft TOC) & $\begin{array}{l}\text { (ft TOC) } \\
\text { (ft) }\end{array}$ & (ft TOC) & $\begin{array}{l}\text { (ft TOC) } \\
\text { (1) }\end{array}$ & (ft TOC) & $\begin{array}{l}\text { (ft TOC) } \\
\text { (n) }\end{array}$ & (ft TOC) & $\begin{array}{l}\text { (nt TOC) } \\
\text { (nt }\end{array}$ & (ft TOC) \\
\hline 11/30/11 0:00 & & 30.952 & 30.519 & 29.613 & 30.533 & & 29.895 & 31.03 & 30.477 & 30.213 & 28.809 & 26.54 & 32.245 & 36.598 & 33.278 & 16.939 & 16.3 & 22.763 & 15.801 & 11.112 & 32.345 & 33.149 & 30.19 & 32.215 & 27.674 & 34.268 \\
\hline $11 / 30 / 11$ 1:00 & & 30.945 & 30.506 & 29.582 & 30.514 & & 29.911 & 31.027 & 30.477 & 30.201 & 28.793 & 26.512 & 32.234 & 36.591 & 33.261 & 16.939 & 16.295 & 22.758 & 15.806 & 11.1 & 32.317 & 33.132 & 30.168 & 32.201 & 27.676 & 34.265 \\
\hline $11 / 30 / 112: 00$ & & 30.943 & 30.501 & 29.573 & 30.505 & & 29.909 & 31.023 & 30.474 & 30.192 & 28.788 & 26.502 & 32.222 & 36.588 & 33.254 & 16.934 & 16.29 & 22.758 & 15.801 & 11.088 & 32.313 & 33.125 & 30.154 & 32.187 & 27.676 & 34.265 \\
\hline $11 / 30 / 113: 00$ & & 30.941 & 30.494 & 29.564 & 30.493 & & 29.911 & 31.02 & 30.472 & 30.185 & 28.779 & 26.492 & 32.215 & 36.586 & 33.242 & 16.934 & 16.288 & 22.754 & 15.801 & 11.081 & 32.299 & & & 32.18 & & 34.263 \\
\hline $11 / 30 / 114: 00$ & & 30.934 & 30.478 & 29.54 & 30.47 & & 29.92 & 31.015 & 30.47 & 30.173 & 28.767 & 26.476 & 32.201 & 36.579 & 33.233 & 16.929 & 16.281 & 22.749 & 15.811 & & 32.278 & 33.101 & & 68 & & 34.261 \\
\hline $11 / 30 / 115: 00$ & & 30.931 & 30.468 & 29.526 & 30.463 & & 29.916 & 31.011 & 30.47 & 30.164 & 28.756 & 26.461 & 32.189 & 36.576 & 33.221 & 16.925 & 16.279 & 22.747 & 15.802 & 11.058 & 32.275 & 33.092 & 0.112 & 2.157 & 27.676 & 34.258 \\
\hline $11 / 30 / 116: 00$ & & 30.929 & 30.461 & 29.521 & 30.453 & & 29.911 & 31.008 & 30.467 & 30.157 & 28.749 & 26.459 & 32.18 & 36.569 & 33.212 & 16.922 & 16.272 & 22.742 & 15.794 & 11.048 & 32.266 & 33.082 & 30.107 & 32.147 & 27.676 & 34.256 \\
\hline 11/30/11 7:00 & & 30.929 & 30.454 & 29.512 & 30.446 & & 29.904 & 31.006 & 30.467 & 30.15 & 28.744 & 26.454 & 32.17 & 36.569 & 33.203 & 16.92 & 16.265 & 22.74 & 15.792 & 11.037 & 32.255 & 33.073 & 30.103 & 32.138 & 27.678 & 34.254 \\
\hline $11 / 30 / 11$ 8:00 & & 30.924 & 30.45 & 29.51 & 30.439 & & 29.899 & 31.001 & 30.467 & 30.146 & 28.737 & 26.454 & 32.166 & 36.567 & 33.193 & 16.915 & 16.262 & 22.74 & 15.79 & 11.032 & 32.25 & 33.066 & 30.098 & 32.133 & 27.676 & 34.249 \\
\hline 11/30/11 9:00 & & 30.927 & 30.45 & 29.512 & 30.435 & & & 30.999 & 30.465 & 30.141 & 28.735 & 26.454 & 32.159 & 36.567 & 33.189 & 16.915 & 16.255 & 22.74 & 15.785 & 11.025 & 32.25 & 33.059 & 30.096 & 32.124 & 27.678 & 34.247 \\
\hline $11 / 30 / 11$ 10:00 & & 30.92 & 30.44 & 29.503 & 30.428 & & 29.892 & 30.996 & 30.462 & 30.134 & 28.725 & 26.449 & 32.152 & 36.562 & 33.184 & 16.911 & 16.253 & 22.738 & 15.795 & 11.02 & 32.236 & 33.054 & 30.084 & 32.119 & 27.678 & 34.244 \\
\hline 11/30/11 11:00 & & 30.91 & 30.419 & 29.472 & 30.411 & & 29.909 & 30.989 & 30.458 & 30.115 & 28.709 & 26.423 & 32.14 & 36.56 & 33.172 & 16.906 & 16.248 & 22.731 & 15.806 & 11.009 & 32.213 & 33.04 & 30.063 & 32.107 & 27.678 & 34.242 \\
\hline $11 / 30 / 1112: 00$ & & 30.901 & 30.398 & 29.437 & 30.388 & & 29.923 & 30.982 & 30.455 & 30.097 & 28.691 & 26.392 & 32.128 & 36.553 & 33.16 & 16.899 & 16.239 & 22.724 & 15.82 & 10.997 & 32.192 & 33.026 & 30.032 & 32.091 & 27.676 & 34.237 \\
\hline $11 / 30 / 11$ 13:00 & & 30.894 & 30.375 & 29.407 & 30.358 & & 29.93 & 30.975 & 30.453 & 30.081 & 28.67 & 26.366 & 32.114 & 36.546 & 33.146 & 16.895 & 16.236 & 22.721 & 15.827 & 10.98 & 32.173 & 33.014 & 30.006 & 32.081 & 27.676 & 34.233 \\
\hline $11 / 30 / 1114: 00$ & & 30.887 & 30.354 & 29.381 & 30.334 & & 29.93 & 30.97 & 30.448 & 30.06 & 28.651 & 26.344 & 32.103 & 36.537 & 33.135 & 16.888 & 16.229 & 22.712 & 15.823 & 10.971 & 32.15 & 33.002 & 29.978 & 32.07 & 27.678 & 34.23 \\
\hline $11 / 30 / 1115: 00$ & & 30.88 & 30.335 & 29.36 & 30.311 & & 29.927 & 30.96 & 30.446 & 30.041 & 28.632 & 26.327 & 32.086 & 36.537 & 33.121 & 16.883 & 16.225 & 22.71 & 15.825 & 10.961 & 32.133 & 32.99 & 29.962 & 32.053 & 27.678 & 34.23 \\
\hline $11 / 30 / 1116: 00$ & & 30.88 & 30.328 & 29.363 & 30.299 & & 29.906 & 30.956 & 30.443 & 30.032 & 28.628 & 26.332 & 32.079 & 36.527 & 33.114 & 16.881 & 16.22 & 22.71 & 15.816 & 10.95 & 32.126 & 32.981 & 29.962 & 32.046 & 27.678 & 34.228 \\
\hline $11 / 30 / 1117: 00$ & & 30.878 & 30.324 & 29.36 & 30.295 & & 29.887 & 30.948 & 30.444 & 30.022 & 28.621 & 26.337 & 32.072 & 36.525 & 33.107 & $\begin{array}{l}10.016 \\
16.876\end{array}$ & 16.218 & 22.705 & 15.799 & 10.945 & 32.119 & 32.973 & 29.955 & 32.039 & 27.678 & 34.226 \\
\hline $11 / 30 / 1118: 00$ & & 30.878 & 30.321 & 29.367 & 30.292 & & 29.859 & $\begin{array}{l}30.948 \\
3048\end{array}$ & 30.444 & 30.018 & $\begin{array}{l}28.021 \\
28.616\end{array}$ & 26.346 & $\begin{array}{l}32.067 \\
3\end{array}$ & 36.52 & $\begin{array}{l}33.099 \\
3.09\end{array}$ & 16.871 & $\begin{array}{l}10.213 \\
16.213\end{array}$ & 22.703 & 15.785 & $\begin{array}{l}10.936 \\
\end{array}$ & 32.133 & 32.969 & 29.962 & 32.035 & 27.681 & 34.223 \\
\hline $11 / 30 / 1119: 00$ & & $\begin{array}{l}30.885 \\
30.85\end{array}$ & 30.335 & 29.402 & $\begin{array}{l}30.311 \\
30.311\end{array}$ & & 29.819 & 30.946 & $\begin{array}{l}30.444 \\
30.446\end{array}$ & 30.025 & $\begin{array}{l}28.603 \\
28.63\end{array}$ & $\begin{array}{l}20.340 \\
26.382\end{array}$ & 32.07 & $\begin{array}{l}36.522 \\
36.523\end{array}$ & 33.104 & $\begin{array}{l}10.869 \\
16.869\end{array}$ & $\begin{array}{l}10.215 \\
16.215\end{array}$ & 22.703 & 15.762 & 10.938 & 32.145 & $\begin{array}{l}32.971 \\
32.971\end{array}$ & $\begin{array}{l}29.995 \\
29.92\end{array}$ & 32.039 & $\begin{array}{l}27.681 \\
27.611\end{array}$ & 34.226 \\
\hline $11 / 30 / 1120: 00$ & & $\begin{array}{l}30.802 \\
30.882\end{array}$ & 30.347 & 29.419 & $\begin{array}{l}30.327 \\
30.327\end{array}$ & & 29.798 & 30.946 & $\begin{array}{l}30.440 \\
30.443\end{array}$ & $\begin{array}{l}30.029 \\
30.029\end{array}$ & $\begin{array}{l}28.037 \\
28.637\end{array}$ & $\begin{array}{l}20.302 \\
26.406\end{array}$ & 32.072 & $\begin{array}{l}30.53 \\
36.53\end{array}$ & $\begin{array}{l}33.104 \\
33.104\end{array}$ & $\begin{array}{l}1.0899 \\
16.867\end{array}$ & $\begin{array}{l}10.211 \\
16.211\end{array}$ & 22.703 & $\begin{array}{l}13.752 \\
15.752\end{array}$ & $\begin{array}{r}10.930 \\
10.94\end{array}$ & $\begin{array}{l}32.159 \\
32.45\end{array}$ & $\begin{array}{l}32.973 \\
32.973\end{array}$ & 30.006 & $\begin{array}{l}32.039 \\
32.039\end{array}$ & 27.681 & 34.226 \\
\hline $11 / 30 / 1121: 00$ & & 30.882 & 30.352 & 29.428 & 30.341 & & 29.786 & 30.944 & 30.439 & 30.032 & 28.642 & 26.418 & 32.077 & 36.525 & 33.104 & 16.867 & 16.211 & .705 & 15.743 & 10.943 & .166 & 973 & 30.018 & 32.042 & 27.683 & 34.226 \\
\hline $11 / 30$ & & $\begin{array}{l}3.002 \\
30.88\end{array}$ & 52 & & $\begin{array}{l}30.346 \\
30.446\end{array}$ & & 29.784 & 30.941 & 30.439 & $\begin{array}{l}30.032 \\
30.032\end{array}$ & $\begin{array}{l}28.639 \\
28.639\end{array}$ & 26.413 & 074 & 36.532 & 33.104 & $\begin{array}{l}10.0864 \\
16.864\end{array}$ & $\begin{array}{l}16.208 \\
16.21\end{array}$ & 712 & 15.733 & 10.94 & 32.161 & 976 & $\begin{array}{r}30.02 \\
30.02\end{array}$ & 2.042 & 27.683 & .223 \\
\hline $11 / 30 /$ & & 30.88 & 30.352 & 28 & 30.353 & & 29.779 & 30.941 & 30.439 & 30.029 & 28.642 & 26.421 & 32.072 & 36.534 & 33.104 & 16.862 & 16.208 & 714 & 15.729 & 10.94 & 32.166 & 976 & .02 & 2.042 & 27.683 & 34.226 \\
\hline 12/1/11 0:00 & & 30.878 & 30.352 & 29 & 30. & & 29.777 & 30.941 & 30.441 & 30.027 & 28.642 & 26.421 & 32.077 & 36.532 & 33.104 & 16.864 & 16.208 & 22.714 & 15.724 & 10.943 & 32.168 & .976 & 30.03 & 2.044 & 27.683 & 34.223 \\
\hline 12/1/11 1:00 & & 30.873 & 30.352 & 29.419 & 30.353 & & 29.782 & 30.936 & 30.436 & 30.025 & 28.637 & 26.413 & 32.074 & 36.53 & 33.099 & 16.862 & 16.208 & 22.714 & 15.719 & 10.94 & 32.168 & 32.973 & 30.02 & 32.042 & 27.683 & 34.223 \\
\hline 12/1/11 2:00 & & 30.875 & 30.352 & 29.421 & 30.355 & & 29.775 & 30.934 & 30.436 & 30.025 & 28.639 & 26.418 & 32.074 & 36.539 & 33.104 & 16.86 & 16.209 & 22.712 & 15.71 & 10.94 & 32.166 & 32.976 & 30.023 & 32.042 & 27.683 & 34.223 \\
\hline 12/1/11 3:00 & & 30.88 & 30.363 & 29.447 & 30.369 & & 29.756 & 30.936 & 30.436 & 30.032 & 28.651 & 26.445 & 32.079 & 36.539 & 33.109 & 16.862 & 16.211 & 22.714 & 15.698 & 10.943 & 32.187 & 32.981 & 30.049 & 32.046 & 27.685 & 34.226 \\
\hline 12/1/11 4:00 & & 30.882 & 30.375 & 29.463 & 30.388 & & 29.744 & 30.936 & 30.436 & 30.041 & 28.66 & 26.466 & 32.086 & 36.548 & 33.116 & 16.862 & 16.213 & 22.714 & 15.689 & 10.95 & 32.201 & 32.985 & 30.067 & 32.053 & 27.685 & 34.228 \\
\hline $12 / 1 / 115: 00$ & & 30.89 & 30.394 & 29.493 & 30.409 & & 29.728 & 30.941 & 30.441 & 30.053 & 28.677 & 26.49 & 32.093 & 36.548 & 33.118 & 16.867 & 16.215 & 22.717 & 15.677 & 10.957 & 32.22 & 32.995 & 30.093 & 32.063 & 27.688 & 34.23 \\
\hline $12 / 1 / 116: 00$ & & 30.897 & 30.41 & 29.521 & 30.435 & & 29.714 & 30.946 & 30.444 & 30.069 & 28.695 & 26.519 & 32.105 & 36.56 & 33.13 & 16.871 & 16.215 & 22.721 & 15.668 & 10.966 & 32.238 & 33.002 & 30.119 & 32.072 & 27.688 & 34.233 \\
\hline $12 / 1 / 117: 00$ & & 30.903 & 30.433 & 29.557 & 30.463 & & 29.702 & 30.951 & 30.448 & 30.085 & 28.716 & 26.552 & 32.114 & 36.574 & 33.139 & 16.876 & 16.22 & 22.721 & 15.658 & 10.976 & 32.269 & 33.016 & 30.147 & 32.081 & 27.688 & 34.235 \\
\hline $12 / 1 / 118: 00$ & & 30.904 & 30.443 & 29.566 & 30.484 & & 29.711 & 30.956 & 30.448 & 30.097 & 28.725 & 26.562 & 32.126 & 36.576 & 33.149 & 16.878 & 16.22 & 22.73 & 15.661 & 10.987 & 32.273 & 33.026 & 30.157 & 32.088 & 27.688 & 34.235 \\
\hline 12/1/11 9:00 & & 30.922 & 30.48 & 29.62 & 30.521 & & 29.688 & 30.965 & 30.455 & 30.125 & 28.753 & 26.612 & 32.138 & 36.59 & 33.16 & 16.885 & 16.229 & 22.728 & 15.647 & 10.999 & 32.313 & 33.04 & 30.211 & 32.107 & 27.69 & 34.237 \\
\hline 12/1/11 10:00 & & 30.927 & 30.501 & 29.648 & 30. & & 29.69 & 30.972 & 30.455 & 30.15 & 28.777 & 26.636 & 32.154 & 36.607 & 33.177 & $\begin{array}{l}16.892 \\
1692\end{array}$ & 16.234 & 22.733 & 15.649 & 11.016 & 32.336 & 33.054 & 30.232 & 32.119 & 27.688 & 34.242 \\
\hline $12 / 1 / 1$ & & 30.929 & 30.517 & 29.662 & 30. & & 29.706 & 30.982 & 30.455 & 30.162 & 28.788 & 26.646 & 32.168 & 36.614 & 33.189 & $\begin{array}{l}16.899 \\
1699\end{array}$ & $\begin{array}{l}16.239 \\
\end{array}$ & 22.735 & 15.668 & $\begin{array}{l}11.027 \\
\end{array}$ & 32.343 & 33.066 & 30.248 & 32.128 & 27.69 & 34.244 \\
\hline $12 / 1 / 1$ & & 30.931 & 30.529 & 29.671 & 30.5 & & 29.725 & $\begin{array}{l}30.989 \\
309\end{array}$ & 30.453 & 30.176 & 28.802 & 26.65 & 32.177 & 36.616 & $\begin{array}{r}3.109 \\
33.2\end{array}$ & $\begin{array}{l}10.904 \\
16.904\end{array}$ & $\begin{array}{l}16.246 \\
16.25\end{array}$ & 22.733 & $\begin{array}{l}15.000 \\
15.679\end{array}$ & 11.037 & 32.355 & $\begin{array}{l}33.075 \\
33.075\end{array}$ & $\begin{array}{l}30.255 \\
30.255\end{array}$ & 32.145 & 27.692 & 34.247 \\
\hline $12 / 1 / 1$ & & 30.938 & 30.538 & 29.678 & 30.6 & & 29.742 & 30.994 & 30.458 & $\begin{array}{l}30.187 \\
30.187\end{array}$ & $\begin{array}{l}28.8012 \\
28.812\end{array}$ & 26.65 & 32.184 & 36.621 & $\begin{array}{l}33.21 \\
33.21\end{array}$ & $\begin{array}{l}10.044 \\
16.911\end{array}$ & $\begin{array}{l}16.246 \\
16.46\end{array}$ & .733 & 15.684 & 11.046 & 32.362 & .087 & 30.265 & 32.149 & 27.69 & $\begin{array}{l}34.247 \\
3\end{array}$ \\
\hline $12 / 1 / 1$ & & 30.941 & 30.547 & $\begin{array}{l}29.0685 \\
29.685\end{array}$ & $\begin{array}{l}30.6 \\
30.6\end{array}$ & & 29.758 & $\begin{array}{l}30.999 \\
3099\end{array}$ & $\begin{array}{r}30.450 \\
30.462\end{array}$ & $\begin{array}{l}30.199 \\
30.199\end{array}$ & $\begin{array}{l}2.012 \\
28.821\end{array}$ & $\begin{array}{l}26.653 \\
26.653\end{array}$ & $\begin{array}{l}32.194 \\
32.191\end{array}$ & $\begin{array}{l}36.621 \\
36.621\end{array}$ & $\begin{array}{l}33.21 \\
33.217\end{array}$ & $\begin{array}{l}16.915 \\
16.915\end{array}$ & $\begin{array}{r}\begin{array}{r}1.240 \\
16.25\end{array}\end{array}$ & .738 & $\begin{array}{l}13.004 \\
15.698\end{array}$ & 11.056 & 32.371 & .094 & 30.272 & 32.159 & $\begin{array}{r}27.699 \\
27.692\end{array}$ & $\begin{array}{l}34.247 \\
34.247\end{array}$ \\
\hline & & $3 c$ & 30 & 29. & 30 & & 29 & 31 & & 30 & 28. & 26 & 32. & 36. & 33. & 16 & 1 & 22.735 & 15. & 11.067 & 9 & 33.104 & 30. & 32. & 2 & 251 \\
\hline $12 / 1 / 11$ & & 30. & & 29.741 & & & & 31 & & & & & 32 & 36. & 33. & 16 & 16 & 22.73 & 15.694 & 11.084 & 5 & 3 & 1 & 32 & 27.692 & 34.256 \\
\hline & & 30.969 & 30.61 & & 30 & & & & & & 28.879 & 26 & 32.231 & 36.642 & 33.257 & & 16.276 & & 15.698 & 11.095 & & .135 & .349 & 32.199 & 27.695 & 34.261 \\
\hline 8:00 & & 30.98 & 30.631 & & 30. & & & & & & 28.898 & & 32.25 & 36.6 & 33.273 & & 16.281 & & 15.698 & 11.107 & & & .371 & & 27.695 & 34.263 \\
\hline $12 /$ & & 30.9 & 30.6 & & & & & & & & 28. & & 32.259 & 36. & & & 16.288 & 22 & 15.712 & 11.121 & 32.464 & 33.163 & 2 & 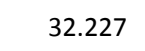 & 27.695 & 34.265 \\
\hline $12 /$ & & & 30. & & & & & & & & 28 & & 32.269 & & & & 16.29 & 22.7 & 15. & 11.128 & 32.469 & & 2 & 32.234 & 27.697 & 34.265 \\
\hline $12 /$ & & 30 & 30. & & & & & & & & 28 & & 32.273 & 36. & 33.2 & & 16.3 & 22.7 & 15.743 & 11.133 & 32.471 & & 30.382 & 32.241 & 27.697 & 34.268 \\
\hline $12 / 1 / 1122: 00$ & & 30.987 & 30. & 29. & & & 29.8 & & 30. & 30. & 28. & 26.7 & 32.28 & 36.656 & 33.3 & 16. & 16.302 & 22.77 & 15.736 & 11.14 & 32.483 & 33.182 & 30.387 & 32.246 & 27.697 & 34.268 \\
\hline 12/1/11 23:00 & & 30.99 & 30.6 & 29.811 & & & 29.8 & 31.0 & 30.4 & 30.3 & 28.9 & 26.72 & 32.287 & 36.661 & 33.31 & 16.98 & 16.309 & 22.774 & 15.74 & 11.145 & 32.481 & 33.189 & 30.392 & 32.255 & 27.697 & 34.268 \\
\hline $12 / 2 / 110: 00$ & & 30.985 & 30.659 & 29.793 & 30.738 & & 29.869 & 31.054 & 30.493 & 30.336 & 28.937 & 26.708 & 32.292 & 36.659 & 33.315 & 16.988 & 16.311 & 22.774 & 15.762 & 11.152 & 32.469 & 33.191 & 30.373 & 32.257 & 27.697 & 34.268 \\
\hline 12/2/11 1:00 & & 30.985 & 30.655 & 29.779 & 30.724 & & 29.89 & 31.056 & 30.498 & 30.334 & 28.935 & 26.689 & 32.29 & 36.661 & 33.315 & 16.99 & 16.311 & 22.777 & 15.766 & 11.152 & 32.471 & 33.191 & 30.373 & 32.257 & 27.697 & 34.265 \\
\hline 12/2/11 2:00 & & 30.983 & 30.65 & 29.767 & 30.717 & & 29.909 & 31.058 & 30.493 & 30.334 & 28.93 & 26.674 & 32.29 & 36.654 & 33.315 & 16.992 & 16.314 & 22.779 & 15.776 & 11.154 & 32.457 & 33.189 & 30.356 & 32.255 & 27.697 & 34.265 \\
\hline 12/2/11 3:00 & & 30.987 & 30.65 & 29.762 & 30.708 & & 29.916 & 31.063 & 30.496 & 30.334 & 28.93 & 26.665 & 32.292 & 36.651 & 33.318 & 16.997 & 16.316 & 22.777 & 15.778 & 11.157 & 32.455 & 33.191 & 30.356 & 32.26 & 27.699 & 34.268 \\
\hline 12/2/11 4:00 & & 30.98 & 30.638 & 29.739 & 30.691 & & 29.942 & 31.063 & 30.498 & 30.327 & 28.918 & 26.641 & 32.29 & 36.654 & 33.315 & 16.999 & 16.318 & 22.777 & 15.799 & 11.154 & 32.439 & 33.187 & 30.331 & 32.255 & 27.699 & 34.265 \\
\hline $12 / 2 / 115: 00$ & & 30.98 & 30.624 & 29.716 & 30.67 & & 29.953 & 31.063 & 30.496 & 30.317 & 28.907 & 26.617 & 32.283 & 36.647 & 33.31 & 16.997 & 16.316 & 22.772 & 15.806 & 11.145 & 32.425 & 33.184 & 30.312 & 32.25 & 27.699 & 34.263 \\
\hline 12/2/11 6:00 & & 30.978 & 30.615 & 29.694 & 30.652 & & 29.967 & 31.063 & 30.496 & 30.308 & 28.895 & 26.595 & 32.278 & 36.64 & 33.303 & 16.999 & 16.318 & 22.772 & 15.813 & 11.143 & 32.413 & 33.177 & 30.291 & 32.243 & 27.699 & 34.261 \\
\hline 12/2/11 7:00 & & 30.978 & 30.608 & 29.685 & 30.638 & & 29.97 & 31.066 & 30.496 & 30.301 & 28.888 & 26.581 & 32.271 & 36.635 & 33.299 & 16.997 & 16.316 & 22.772 & 15.816 & 11.135 & 32.404 & 33.17 & 30.279 & 32.236 & 27.702 & 34.261 \\
\hline
\end{tabular}




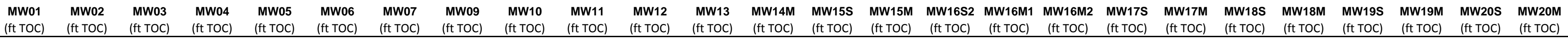

\begin{tabular}{|c|c|c|c|c|c|c|c|c|c|c|c|c|c|c|c|c|c|c|c|c|c|c|c|c|c|c|}
\hline Well & $\begin{array}{l}\text { (ft TOC) } \\
\text { (f) }\end{array}$ & $\begin{array}{l}\text { (ft TOC) } \\
\text { (f) }\end{array}$ & $\begin{array}{l}\text { (ft TOC) } \\
\text { (f) }\end{array}$ & (ft TOC) & $\begin{array}{l}\text { (ft TOC) } \\
\text { (n) }\end{array}$ & $\begin{array}{l}\text { (ft ToC) } \\
\text { (f) }\end{array}$ & $\begin{array}{l}\text { (ft TOC) } \\
\text { (f) }\end{array}$ & $\begin{array}{l}\text { (ft TOC) } \\
\text { (ft) }\end{array}$ & $\begin{array}{l}\text { (ft TOC) } \\
\text { (f) }\end{array}$ & $\begin{array}{l}\text { (ft TOC) } \\
\text { (l) }\end{array}$ & $\begin{array}{l}\text { (ft TOC) } \\
\text { (n) }\end{array}$ & (ft TOC) & (ft TOC) & $\begin{array}{l}\text { (ft TOC) } \\
\end{array}$ & (ft TOC) & (ft TOC) & (ft TOC) & (ft TOC) & $\begin{array}{l}\text { (ft TOC) } \\
\text { (ft) }\end{array}$ & (ft TOC) & $\begin{array}{l}\text { (ft TOC) } \\
\text { (1) }\end{array}$ & (ft TOC) & $\begin{array}{l}\text { (ft TOC) } \\
\text { (n) }\end{array}$ & (ft TOC) & $\begin{array}{l}\text { (nt TOC) } \\
\text { (nt }\end{array}$ & (ft TOC) \\
\hline /11 8:00 & & 30.973 & 30.599 & 29.673 & 30.621 & & 29.977 & 31.063 & 30.496 & 30.294 & 28.879 & 26.574 & 32.266 & 36.633 & 33.294 & 16.997 & 16.314 & 22.781 & 15.827 & 11.128 & 32.392 & 33.168 & 30.27 & 32.234 & 27.699 & 34.258 \\
\hline $12 / 2 / 119: 00$ & & 30.978 & 30.594 & 29.671 & 30.617 & & 29.967 & 31.061 & 30.498 & 30.289 & 28.877 & 26.571 & 32.264 & 36.623 & 33.292 & 16.997 & 16.314 & 22.777 & 15.823 & 11.126 & 32.387 & 33.161 & 30.267 & 32.229 & 27.702 & 34.256 \\
\hline $12 / 2 / 1110: 00$ & & 30.976 & 30.585 & 29.655 & 30.6 & & 29.977 & 31.063 & 30.496 & 30.282 & 28.867 & 26.559 & 32.257 & 36.626 & 33.282 & 16.992 & 16.314 & 22.777 & 15.834 & 11.119 & 32.376 & 33.156 & 30.246 & 32.222 & 27.702 & 34.254 \\
\hline $11111: 00$ & & 30.969 & 30.571 & 29.631 & 30.582 & & 29.991 & 31.058 & 30.491 & 30.271 & 28.851 & 26.533 & 32.248 & 36.619 & 33.271 & 16.99 & 16.309 & 22.774 & 15.846 & 11.112 & 32.362 & 33.146 & & 32.215 & 27.702 & 34.249 \\
\hline $12 / 2 / 1112: 00$ & & 30.964 & 30.557 & 29.61 & 30.563 & & 30 & 31.061 & 30.493 & 30.257 & 28.837 & 26.514 & 32.238 & 36.616 & 33.268 & 16.988 & 16.302 & 22.772 & 15.853 & 11.103 & 32.341 & 33.137 & & & 02 & 34.247 \\
\hline 12/2/11 13:00 & & 30.957 & 30.536 & 29.578 & 30.535 & & 30.007 & 31.054 & 30.489 & 30.238 & 28.818 & 26.488 & 32.227 & 36.609 & 33.257 & 16.983 & 16.302 & 22.77 & 15.862 & 11.093 & 32.317 & 33.125 & 176 & .194 & 27.704 & 34.242 \\
\hline $12 / 2 / 1114: 00$ & & 30.948 & 30.51 & 29.543 & 30.507 & & 30.019 & 31.044 & 30.486 & 30.217 & 28.793 & 26.452 & 32.213 & 36.598 & 33.245 & 16.976 & 16.297 & 22.763 & 15.872 & 11.084 & 32.292 & 33.111 & .147 & 2.178 & 27.699 & 34.237 \\
\hline $12 / 2 / 1115: 00$ & & 30.948 & 30.499 & 29.533 & 30.488 & & 30.007 & 31.039 & 30.484 & 30.206 & 28.784 & 26.447 & 32.206 & 36.591 & 33.235 & 16.974 & 16.293 & 22.751 & 15.858 & 11.072 & 32.28 & 33.104 & 30.136 & 32.171 & 27.702 & 34.237 \\
\hline 12/2/11 16:00 & & 30.941 & 30.48 & 29.512 & 30.467 & & 30.005 & 31.032 & 30.482 & 30.192 & 28.767 & 26.43 & 32.194 & 36.584 & 33.226 & 16.967 & 16.29 & 22.811 & 15.86 & 11.063 & 32.264 & 33.094 & 30.117 & 32.161 & 27.702 & 34.233 \\
\hline $12 / 2 / 1117: 00$ & & 30.938 & 30.471 & 29.503 & 30.453 & & 29.991 & 31.027 & 30.479 & 30.18 & 28.756 & 26.425 & 32.184 & 36.581 & 33.219 & 16.964 & 16.29 & 22.807 & 15.853 & 11.056 & 32.259 & 33.087 & 30.105 & 32.152 & 27.702 & 34.233 \\
\hline $12 / 2 / 1118: 00$ & & 30.936 & 30.461 & 29.496 & 30.439 & & 29.974 & 31.023 & 30.481 & 30.166 & 28.746 & 26.423 & 32.177 & 36.574 & 33.21 & 16.96 & 16.288 & 22.8 & 15.844 & 11.046 & 32.25 & 33.075 & 30.098 & 32.145 & 27.704 & 34.23 \\
\hline 12/2/11 19:00 & & 30.938 & 30.459 & 29.498 & 30.439 & & 29.951 & 31.018 & 30.479 & 30.166 & 28.744 & 26.433 & 32.175 & 36.574 & 33.207 & 16.955 & 16.286 & 22.788 & 15.83 & 11.044 & 32.255 & 33.075 & 30.1 & 32.14 & 27.704 & 34.23 \\
\hline $12 / 2 / 1120: 00$ & & 30.936 & 30.457 & 29.498 & 30.437 & & 29.934 & 31.015 & 30.484 & 30.159 & 28.739 & 26.44 & 32.173 & 36.574 & 33.203 & 16.95 & 16.283 & 22.791 & 15.823 & 11.039 & 32.257 & 33.071 & 30.107 & 32.14 & 27.704 & 34.23 \\
\hline 12/2/11 21:00 & & 30.927 & 30.445 & 29.479 & 30.428 & & 29.939 & 31.011 & 30.477 & 30.148 & 28.728 & 26.423 & 32.161 & 36.572 & 33.193 & 16.946 & 16.281 & 22.781 & 15.825 & 11.03 & 32.236 & 33.061 & 30.089 & 32.131 & 27.704 & 34.226 \\
\hline $12 / 2 / 1122: 00$ & & 30.929 & 30.44 & 29.486 & 30.425 & & 29.925 & 31.008 & 30.477 & 30.141 & 28.723 & 26.425 & 32.159 & 36.569 & 33.189 & 16.943 & 16.274 & 22.775 & 15.813 & 11.027 & 32.241 & 33.059 & 30.091 & 32.126 & 27.706 & 34.223 \\
\hline $12 / 2 / 1123: 00$ & & 30.927 & 30.436 & 29.486 & 30.423 & & 29.918 & 31.003 & 30.472 & 30.132 & 28.716 & 26.425 & 32.154 & 36.567 & 33.184 & 16.936 & 16.269 & 22.767 & 15.809 & 11.02 & 32.236 & 33.052 & 30.089 & 32.121 & 27.704 & 34.221 \\
\hline $12 / 3 / 110: 00$ & & 30.924 & 30.436 & 29.486 & 30.423 & & 29.906 & 31.001 & 30.472 & 30.129 & 28.714 & 26.433 & 32.149 & 36.565 & 33.179 & 16.934 & 16.267 & 22.761 & 15.806 & 11.013 & 32.234 & 33.052 & 30.091 & 32.117 & 27.706 & 34.219 \\
\hline 12/3/11 1:00 & & 30.913 & 30.419 & 29.456 & 30.409 & & 29.918 & 30.996 & 30.467 & 30.113 & 28.698 & 26.409 & 32.142 & $\begin{array}{r}36.56 \\
36\end{array}$ & 33.172 & $\begin{array}{l}16.929 \\
16.929\end{array}$ & 16.265 & 22.751 & 15.813 & 11.009 & 32.213 & 33.04 & 30.067 & 32.11 & 27.704 & 34.214 \\
\hline $12 / 3 / 112: 00$ & & 30.913 & 30.408 & 29.447 & $\begin{array}{l}30.397 \\
30.49\end{array}$ & & 29.916 & 30.994 & $\begin{array}{l}30.467 \\
30.407\end{array}$ & 30.104 & $\begin{array}{l}28.050 \\
28.68\end{array}$ & 26.399 & 32.131 & 36.558 & 33.158 & 16.925 & $\begin{array}{l}16.253 \\
16.253\end{array}$ & 22.742 & 15.804 & 10.997 & 32.203 & 33.028 & 30.053 & 32.098 & 27.706 & 34.207 \\
\hline $12 / 3 / 11$ 3:00 & & 30.915 & 30.408 & 29.456 & 30.397 & & 29.895 & $\begin{array}{l}30.989 \\
30.989\end{array}$ & $\begin{array}{r}30.401 \\
30.47\end{array}$ & $\begin{array}{l}30.099 \\
3099\end{array}$ & $\begin{array}{l}28.000 \\
28.691\end{array}$ & 26.411 & 32.126 & $\begin{aligned} 30.500 \\
36.56\end{aligned}$ & $\begin{array}{l}33.150 \\
33.156\end{array}$ & $\begin{array}{l}10.922 \\
16.922\end{array}$ & $\begin{array}{l}10.237 \\
16.248\end{array}$ & 22.735 & $\begin{array}{l}13.0 .74 \\
15.787\end{array}$ & 10.99 & 32.208 & $\begin{array}{l}53.020 \\
33.023\end{array}$ & 30.067 & $\begin{array}{l}32.093 \\
32.093\end{array}$ & 27.706 & 34.202 \\
\hline $12 / 3 / 114: 00$ & & $\begin{array}{l}30.913 \\
30.913\end{array}$ & $\begin{array}{l}30.408 \\
30.405\end{array}$ & $\begin{array}{l}29.450 \\
29.454\end{array}$ & $\begin{array}{l}30.397 \\
30.59\end{array}$ & & 29.887 & $\begin{array}{l}30.989 \\
30.984\end{array}$ & $\begin{array}{r}30.47 \\
30.465\end{array}$ & 30.094 & 28.684 & $\begin{array}{l}26.411 \\
26.413\end{array}$ & 32.121 & $\begin{array}{l}36.50 \\
3655\end{array}$ & $\begin{array}{l}33.151 \\
33.150\end{array}$ & $\begin{array}{l}10.922 \\
16.918\end{array}$ & $\begin{array}{l}10.248 \\
16.248\end{array}$ & 22.724 & $\begin{array}{l}13.171 \\
15.79\end{array}$ & $\begin{array}{l}10.99 \\
10.99\end{array}$ & 32.203 & $\begin{array}{l}33.021 \\
33.021\end{array}$ & 30.06 & $\begin{array}{l}32.088 \\
32.089\end{array}$ & 27.706 & $\begin{array}{r}34.202 \\
34.2\end{array}$ \\
\hline $12 / 3 / 115: 00$ & & 30.908 & 30.398 & 29.44 & 30.386 & & 29.887 & 30.984 & 30.462 & 30.087 & 28.674 & 26.404 & 117 & 36.553 & 33.144 & 16.913 & 16.243 & .728 & 15.788 & 10.98 & 32.196 & 016 & 30.049 & 32.084 & 27.706 & 4.198 \\
\hline 12/3/11 6:00 & & 30.897 & & 29.407 & 30. & & $\begin{array}{l}29.904 \\
2904\end{array}$ & $\begin{array}{l}30.984 \\
30.944\end{array}$ & 30.455 & 30.067 & 28.656 & 26.375 & 32.1 & 36.551 & & $\begin{array}{l}16.909 \\
16.909\end{array}$ & $\begin{array}{l}10.229 \\
16.229\end{array}$ & 721 & $\begin{array}{l}15.790 \\
15.792\end{array}$ & $\begin{array}{c}10.966 \\
10.966\end{array}$ & 32.168 & 999 & 0.023 & 32.07 & 27.704 & 186 \\
\hline $12 / 3 / 11$ & & & 30.368 & 29.395 & 30.348 & & 29.897 & 30.984 & 30.46 & 30.055 & 28.649 & 26.366 & 32.091 & 36.551 & & & & 712 & 15.783 & 10.954 & 32.159 & 988 & .006 & 32.056 & 27.709 & 34.181 \\
\hline $12 / 3 / 118: 00$ & & 30.892 & 30.359 & 29.388 & 30.337 & & 29.89 & 30.984 & 30.455 & 30.043 & 28.637 & 26.363 & 32.084 & 36.539 & 33.109 & 16.899 & 16.22 & .707 & 15.778 & 10.947 & 32.152 & 32.983 & 9.997 & 32.049 & 27.709 & 34.179 \\
\hline 12/3/11 9:00 & & 30.894 & 30.366 & 29.435 & 30.344 & & 29.864 & 30.912 & 30.443 & & 28.642 & 26.397 & 32.053 & 36.541 & 33.078 & 16.895 & 16.199 & 22.684 & 15.769 & 10.917 & 32.138 & 32.952 & 29.999 & 32.02 & 27.704 & 34.165 \\
\hline $12 / 3 / 1110: 00$ & & 30.885 & 30.356 & 29.613 & 30.334 & & 29.869 & 29.996 & 30.451 & 30.043 & 28.632 & 26.789 & 32.018 & 36.541 & 33.046 & 16.89 & 16.176 & 22.673 & 15.757 & 10.882 & 32.117 & 017 & 29.98 & 31.985 & 27.704 & 34.142 \\
\hline $12 / 3 / 1111: 00$ & & 30.871 & 30.321 & 29.493 & 30.29 & & 29.895 & 29.834 & 30.446 & 30.025 & 28.602 & 26.835 & 32.007 & 36.532 & 33.036 & 16.881 & 16.164 & 22.677 & 15.755 & 10.877 & 32.087 & 32.905 & 29.938 & 31.978 & 27.709 & 34.13 \\
\hline $12 / 3 / 1112: 00$ & & 30.873 & 30.319 & 29.152 & 30.276 & & 29.878 & 29.781 & 30.441 & 30.011 & 28.598 & 26.323 & 32.007 & 36.525 & 33.034 & 16.874 & 16.159 & 22.694 & 15.752 & 10.872 & 32.082 & 32.907 & 29.929 & 31.974 & 27.706 & 34.128 \\
\hline $12 / 3 / 1113: 00$ & & 30.871 & 30.312 & 29.117 & 30.264 & & 29.864 & 29.654 & 30.441 & 30.004 & 28.591 & 26.325 & 32 & 36.518 & 33.027 & 16.869 & 16.155 & 22.717 & 15.738 & 10.865 & 32.077 & 32.898 & 29.922 & 31.966 & 27.709 & 34.121 \\
\hline $12 / 3 / 1114: 00$ & & 30.866 & 30.307 & 29.269 & 30.253 & & 29.852 & 29.625 & 30.439 & 29.997 & 28.584 & 26.323 & 31.995 & 36.52 & 33.024 & 16.864 & 16.15 & 22.719 & 15.731 & 10.865 & 32.07 & 32.893 & 29.912 & 31.964 & 27.709 & 34.118 \\
\hline $12 / 3 / 1115: 00$ & & 30.873 & 30.312 & 29.264 & 30.257 & & 29.824 & 29.611 & 30.439 & 29.985 & 28.591 & 26.349 & 31.997 & 36.52 & 33.027 & 16.862 & 16.152 & 22.71 & 15.715 & 10.865 & 32.084 & 32.898 & 29.929 & 31.966 & 27.709 & 34.118 \\
\hline $12 / 3 / 1116: 00$ & & 30.885 & 30.335 & 29.384 & 30.283 & & 29.777 & 29.659 & 30.446 & 29.999 & 28.612 & 26.401 & 32.009 & 36.525 & 33.036 & 16.86 & 16.157 & 22.761 & 15.689 & 10.87 & 32.115 & 32.907 & 29.964 & 31.976 & 27.711 & 34.121 \\
\hline $12 / 3 / 1117: 00$ & & 30.89 & 30.354 & 29.43 & 30.306 & & 29.749 & 29.749 & 30.443 & 30.016 & 28.63 & 26.442 & 32.021 & 36.534 & 33.048 & 16.862 & 16.162 & 22.774 & 15.677 & 10.884 & 32.138 & 32.919 & 29.99 & 31.988 & 27.711 & 34.125 \\
\hline $12 / 3 / 1118: 00$ & & 30.899 & 30.38 & 29.479 & 30.3 & & 29.718 & 29.831 & 30.446 & 30.036 & 28.653 & 26.488 & 32.032 & 36.548 & 33.06 & 16.864 & 16.169 & 22.772 & 15.663 & 10.898 & 32.168 & 32.933 & 30.035 & 32.004 & 27.711 & 34.13 \\
\hline $12 / 3 / 1119: 00$ & & 30.894 & 30.391 & 29.496 & 30. & & 29.721 & 29.881 & 30.451 & 30.046 & 28.667 & 26.504 & 32.046 & 36.555 & 33.071 & $\begin{array}{l}10.049 \\
16.869\end{array}$ & 16.171 & 22.763 & 15.67 & 10.905 & 32.182 & .947 & 30.037 & 32.013 & 27.709 & 34.132 \\
\hline $12 / 3 / 1120: 00$ & & 30.906 & 30.415 & 29.531 & 30. & & 29.702 & 29.927 & 30.458 & 30.064 & 28.688 & 26.533 & 32.058 & 36.569 & 33.085 & $\begin{array}{l}10.079 \\
16.871\end{array}$ & 16.178 & 22.756 & 15.654 & $\begin{array}{l}10.919 \\
\end{array}$ & 32.206 & 962 & 30.063 & 32.025 & 27.711 & 34.139 \\
\hline $12 / 3 / 1$ & & 30.91 & 30.436 & 29.561 & 30.4 & & 29.699 & 29.979 & 30.451 & $\begin{array}{l}30.085 \\
30.085\end{array}$ & 28.709 & 26.562 & 32.074 & 36.579 & 33.097 & 16.876 & $\begin{array}{l}16.185 \\
16.18\end{array}$ & 22.749 & 15.656 & 10.931 & 32.227 & 973 & 30.091 & 32.039 & 27.711 & 34.142 \\
\hline $12 / 3 / 1$ & & 30.906 & $\begin{array}{r}50.4 \\
30 .\end{array}$ & 29.564 & $\begin{array}{l}30.4 \\
30.4\end{array}$ & & 29.718 & 30.02 & 30.453 & $\begin{array}{l}30.09 \\
30.09\end{array}$ & 28.714 & $\begin{array}{l}20.302 \\
26.557\end{array}$ & $\begin{array}{l}32.077 \\
32.074\end{array}$ & 36.586 & 33.104 & $\begin{array}{l}10.010 \\
16.878\end{array}$ & $\begin{array}{l}16.187 \\
16.187\end{array}$ & .149 & $\begin{array}{l}15.000 \\
15.663\end{array}$ & 10.938 & 32.224 & 6 & 30.086 & 32.046 & 27.711 & .142 \\
\hline $12 /$ & & 30 & 30 & 29 & 30 & & 29 & 30. & 30 & 30. & 2 & 26 & 32 & 36.593 & 33 & 16 & 16. & 22 & 15.656 & 10 & 3 & 8 & 1 & 32.056 & 27.711 & 142 \\
\hline & & 30 & 30 & 29 & & & & 30. & 30 & 30 & 28. & & 32 & 36.595 & 33. & 16 & 1 & 22.73 & 15.663 & 10.952 & 1 & & 3 & 32.058 & 27.711 & 34.142 \\
\hline 12/4/11 1:00 & & 30. & 30.466 & & & & & 30 & & & 28.739 & & 32.093 & $\begin{array}{r}36.6 \\
\end{array}$ & 33.116 & 16.888 & 16.192 & & 15.67 & 10.957 & 32.241 & 997 & 30.096 & 32.06 & 27.711 & 34.142 \\
\hline $12 / 4 /$ & & 30. & 30.47 & & & & & 30.175 & & & 28.744 & & 32.096 & 36.605 & 33.123 & 16.89 & & 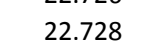 & 15.675 & 10.957 & & & & 32.065 & 27.711 & 34.142 \\
\hline & & 30 & 30.4 & & & & & 30.2 & & & 28.7 & & 32.1 & 36.612 & 33.1 & 16.8 & & 22.7 & 15.6 & 10.966 & 32.2 & & 30.11 & & 27.713 & 34.144 \\
\hline $12 / 4$ & & & 30. & & & & & & & & 28. & & 32.105 & & 33. & 16. & & 22.7 & 15.684 & 10.964 & 32 & & 30.103 & & 27.713 & 34.142 \\
\hline 12/4/11 5:00 & & & 30. & & & & & & 30. & 30. & 28 & & 32.103 & 36. & 33 & 16.8 & & 22.7 & 15.6 & 10.966 & 32.243 & & 30.091 & 32.072 & 27.713 & 34.142 \\
\hline $12 / 4 / 116: 00$ & & 30. & 30.4 & 29. & & & 29. & 30.3 & 30.458 & 30.1 & 28.7 & 26. & 32.107 & 36.616 & 33.132 & 16.895 & 16.202 & 22.719 & 15.6 & 10.973 & 32.25 & 33.009 & 30.1 & 32.074 & 27.711 & 34.144 \\
\hline 12/4/11 7:00 & & 30. & 30.4 & 29.6 & & & 29.791 & 30.3 & 30.46 & 30. & 28.7 & 26.5 & 32.112 & 36.616 & 33.137 & 16.895 & 16.204 & 22.719 & 15.689 & 10.973 & 32.255 & 33.014 & 30.105 & 32.079 & 27.713 & 34.146 \\
\hline $12 / 4 / 118: 00$ & & 30.931 & 30.506 & 29.627 & 30.4 & & 29.786 & 30.364 & 30.46 & 30.164 & 28.781 & 26.588 & 32.117 & 36.628 & 33.144 & 16.899 & 16.208 & 22.721 & 15.682 & 10.98 & 32.271 & 33.016 & 30.119 & 32.084 & 27.713 & 34.146 \\
\hline $12 / 4 / 119: 00$ & & 30.936 & 30.517 & 29.645 & 30.4 & & 29.786 & 30.398 & 30.465 & 30.176 & 28.793 & 26.602 & 32.124 & 36.626 & 33.149 & 16.899 & 16.211 & 22.721 & 15.684 & 10.987 & 32.275 & 33.026 & 30.133 & 32.093 & 27.713 & 34.149 \\
\hline $12 / 4 / 1110: 00$ & & 30.938 & 30.524 & 29.657 & 30.486 & & 29.793 & 30.427 & 30.465 & 30.187 & 28.802 & 26.612 & 32.131 & 36.635 & 33.156 & 16.902 & 16.215 & 22.719 & 15.694 & 10.992 & 32.282 & 33.03 & 30.147 & 32.1 & 27.713 & 34.151 \\
\hline 12/4/11 11:00 & & 30.931 & 30.522 & 29.641 & 30.481 & & 29.817 & 30.453 & 30.46 & 30.185 & 28.802 & 26.595 & 32.131 & 36.633 & 33.158 & 16.902 & 16.213 & 22.714 & 15.705 & 10.994 & 32.275 & 33.03 & 30.129 & 32.1 & 27.711 & 34.149 \\
\hline $12 / 4 / 1112: 00$ & & 30.929 & 30.512 & 29.617 & 30.47 & & 29.843 & 30.477 & 30.46 & 30.178 & 28.793 & 26.567 & 32.126 & 36.635 & 33.151 & 16.904 & 16.213 & 22.717 & 15.729 & 10.99 & 32.257 & 33.026 & 30.107 & 32.093 & 27.713 & 34.146 \\
\hline $12 / 4 / 1113: 00$ & & 30.924 & 30.503 & 29.596 & 30.451 & & 29.866 & 30.503 & 30.458 & 30.173 & 28.786 & 26.545 & 32.119 & 36.63 & 33.149 & 16.902 & 16.211 & 22.714 & 15.733 & 10.983 & 32.245 & 33.021 & 30.091 & 32.091 & 27.711 & 34.144 \\
\hline $12 / 4 / 1114: 00$ & & 30.924 & 30.496 & 29.582 & 30.435 & & 29.873 & 30.527 & 30.455 & 30.166 & 28.779 & 26.531 & 32.117 & 36.63 & 33.144 & 16.899 & 16.211 & 22.71 & 15.74 & 10.98 & 32.234 & 33.014 & 30.074 & 32.086 & 27.711 & 34.142 \\
\hline $12 / 4 / 1115: 00$ & & 30.924 & 30.496 & 29.585 & 30.43 & & 29.871 & 30.551 & 30.462 & 30.166 & 28.779 & 26.531 & 32.117 & 36.626 & 33.144 & 16.899 & 16.211 & 22.719 & 15.738 & 10.978 & 32.234 & 33.016 & 30.077 & 32.086 & 27.711 & 34.144 \\
\hline
\end{tabular}




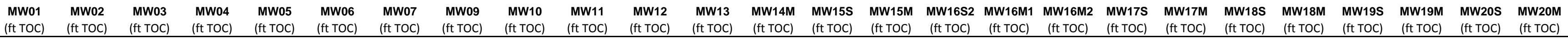

\begin{tabular}{|c|c|c|c|c|c|c|c|c|c|c|c|c|c|c|c|c|c|c|c|c|c|c|c|c|c|c|}
\hline Well & $\begin{array}{l}\text { (ft TOC) } \\
\text { (f) }\end{array}$ & $\begin{array}{l}\text { (ft TOC) } \\
\text { (f) }\end{array}$ & $\begin{array}{l}\text { (ft TOC) } \\
\text { (f) }\end{array}$ & (ft TOC) & (ft Toc) & $\begin{array}{l}\text { (ft ToC) } \\
\text { (f) }\end{array}$ & $\begin{array}{l}\text { (ft TOC) } \\
\text { (n) }\end{array}$ & $\begin{array}{l}\text { (ft TOC) } \\
\text { (f) }\end{array}$ & $\begin{array}{l}\text { (ft TOC) } \\
\text { (f) }\end{array}$ & $\begin{array}{l}\text { (ft TOC) } \\
\text { (l) }\end{array}$ & $\begin{array}{l}\text { (ft TOC) } \\
\text { (n) }\end{array}$ & (ft TOC) & (ft TOC) & $\begin{array}{l}\text { (ft ToC) } \\
\text { (ft }\end{array}$ & (ft TOC) & (ft TOC) & (ft TOC) & (ft TOC) & $\begin{array}{l}\text { (ft TOC) } \\
\text { (ft) }\end{array}$ & (ft TOC) & $\begin{array}{l}\text { (ft TOC) } \\
\text { (1) }\end{array}$ & (ft TOC) & $\begin{array}{l}\text { (ft TOC) } \\
\text { (n) }\end{array}$ & (ft TOC) & $\begin{array}{l}\text { (nt TOC) } \\
\text { (nt }\end{array}$ & (ft TOC) \\
\hline /11 16:00 & & 30.927 & 30.496 & 29.592 & 30.43 & & 29.864 & 30.57 & 30.463 & 30.169 & 28.779 & 26.538 & 32.114 & 36.63 & 33.142 & 16.895 & 16.213 & 22.721 & 15.74 & 10.978 & 32.234 & 33.016 & 30.077 & 32.084 & 27.711 & 34.144 \\
\hline $12 / 4 / 11$ 17:00 & & 30.934 & 30.503 & 29.613 & 30.437 & & 29.848 & 30.592 & 30.467 & 30.178 & 28.788 & 26.555 & 32.119 & 36.633 & 33.146 & 16.895 & 16.215 & 22.717 & 15.733 & 10.985 & 32.245 & 33.018 & 30.093 & 32.088 & 27.713 & 34.146 \\
\hline $12 / 4 / 1118: 00$ & & 30.936 & 30.51 & 29.627 & 30.446 & & 29.838 & 30.613 & 30.467 & 30.183 & 28.795 & 26.567 & 32.362 & 36.635 & 33.407 & 16.897 & 16.29 & 22.784 & 15.731 & 11.246 & 32.35 & 33.262 & 30.112 & 32.328 & 27.713 & 34.149 \\
\hline 12/4/11 19:00 & & 30.938 & 30.517 & 29.638 & 30.46 & & 29.831 & 30.628 & 30.47 & 30.192 & 28.805 & 26.581 & 32.266 & 36. & 33.301 & 16.895 & 16.293 & 22.781 & 15.729 & 11.138 & 32.327 & 33.165 & 26 & 32.234 & & 34.151 \\
\hline $12 / 4 / 1120: 00$ & & 30.945 & 30.529 & 29.657 & 30.474 & & 29.824 & 30.649 & 30.467 & 30.201 & 28.814 & 26.595 & 32.241 & 36.644 & 33.268 & 16.897 & 16.288 & 22. 2779 & 15.729 & 11.105 & 32.329 & 33.137 & 138 & .206 & & 34.153 \\
\hline 12/4/11 21:00 & & 30.95 & 30.54 & 29.68 & 30.495 & & 29.812 & 30.668 & 30.472 & 30.213 & 28.823 & 26.614 & 32.224 & 36.647 & 33.257 & 16.897 & 16.288 & 22.772 & 15.722 & 11.093 & 32.336 & 33.125 & .157 & 2.194 & 16 & 34.156 \\
\hline $12 / 4 / 1122: 00$ & & 30.955 & 30.554 & 29.699 & 30.512 & & 29.808 & 30.685 & 30.477 & 30.227 & 28.839 & 26.634 & 32.22 & 36.656 & 33.25 & 16.899 & 16.288 & 22.772 & 15.719 & 11.084 & 32.341 & 33.12 & .173 & 32.189 & 27.713 & 34.16 \\
\hline $12 / 4 / 1123: 00$ & & 30.952 & 30.554 & 29.699 & 30.521 & & 29.822 & 30.699 & 30.474 & 30.234 & 28.842 & 26.629 & 32.215 & 36.651 & 33.245 & 16.899 & 16.283 & 22.807 & 15.729 & 11.081 & 32.334 & 33.118 & 30.176 & 32.185 & 27.716 & 34.158 \\
\hline 12/5/11 0:00 & & 30.952 & 30.557 & 29.69 & 30.521 & & 29.836 & 30.716 & 30.477 & 30.231 & 28.842 & 26.619 & 32.208 & 36.663 & 33.235 & 16.899 & 16.281 & 22.802 & 15.729 & 11.074 & 32.336 & 33.111 & 30.164 & 32.175 & 27.716 & 34.158 \\
\hline 12/5/11 1:00 & & 30.952 & 30.552 & 29.683 & 30.516 & & 29.845 & 30.733 & 30.474 & 30.231 & 28.842 & 26.61 & 32.201 & 36.659 & 33.231 & 16.897 & 16.276 & 22.791 & 15.74 & 11.065 & 32.327 & 33.101 & 30.161 & 32.171 & 27.713 & 34.156 \\
\hline $12 / 5 / 112: 00$ & & 30.957 & 30.554 & 29.685 & 30.514 & & 29.85 & 30.747 & 30.477 & 30.236 & 28.846 & 26.607 & 32.196 & 36.656 & 33.226 & 16.899 & 16.274 & 22.781 & 15.731 & 11.063 & 32.327 & 33.097 & 30.159 & 32.166 & 27.718 & 34.153 \\
\hline 12/5/11 3:00 & & 30.962 & 30.564 & 29.699 & 30.523 & & 29.848 & 30.759 & 30.484 & 30.243 & 28.853 & 26.619 & 32.196 & 36.661 & 33.226 & 16.899 & 16.274 & 22.777 & 15.729 & 11.06 & 32.329 & 33.097 & 30.171 & 32.166 & 27.718 & 34.156 \\
\hline 12/5/11 4:00 & & 30.952 & 30.554 & 29.678 & 30.514 & & 29.876 & 30.771 & 30.474 & 30.238 & 28.844 & 26.602 & 32.191 & 36.663 & 33.217 & 16.899 & 16.269 & 22.775 & 15.757 & 11.058 & 32.31 & 33.092 & 30.152 & 32.161 & 27.716 & 34.153 \\
\hline $12 / 5 / 115: 00$ & & 30.976 & 30.589 & 29.739 & 30.547 & & 29.831 & 30.786 & 30.486 & 30.269 & 28.877 & 26.658 & 32.201 & 36.666 & 33.231 & 16.902 & 16.276 & 22.77 & 15.724 & 11.065 & 32.352 & 33.104 & 30.201 & 32.168 & 27.718 & 34.156 \\
\hline $12 / 5 / 116: 00$ & & 30.969 & 30.589 & 29.734 & 30.554 & & 29.852 & 30.798 & 30.481 & 30.273 & 28.879 & 26.648 & 32.203 & 36.666 & 33.228 & 16.904 & 16.279 & 22.779 & 15.745 & 11.07 & 32.345 & 33.104 & 30.197 & 32.178 & 27.713 & 34.16 \\
\hline 12/5/11 7:00 & & 30.983 & 30.608 & 29.765 & 30.57 & & 29.836 & 30.812 & 30.493 & 30.287 & 28.893 & 26.674 & 32.21 & $\begin{array}{l}36.67 \\
\end{array}$ & 33.238 & 16.909 & 16.281 & 22.784 & 15.731 & 11.079 & 32.369 & 33.111 & 30.223 & 32.178 & 27.718 & 34.16 \\
\hline $12 / 5 / 11$ 8:00 & & 30.983 & 30.62 & 29.779 & 30.586 & & 29.843 & 30.824 & 30.491 & 30.303 & 28.907 & 26.686 & 32.222 & 36.672 & 33.245 & 16.911 & 16.286 & 22.786 & 15.74 & 11.081 & 32.376 & 33.123 & 30.237 & 32.189 & 27.718 & 34.165 \\
\hline 12/5/11 9:00 & & 30.985 & 30.62 & 29.769 & 30.589 & & 29.862 & 30.836 & 30.493 & 30.301 & 28.909 & 26.677 & 32.222 & 36.673 & 33.247 & 16.911 & 16.288 & 22.786 & 15.755 & 11.088 & 32.376 & 33.12 & 30.227 & 32.189 & 27.718 & 34.165 \\
\hline 12/5/11 10:00 & & 30.987 & 30.62 & 29.769 & 30.589 & & 29.873 & 30.845 & 30.496 & 30.303 & 28.909 & 26.672 & 32.227 & 36.675 & $\begin{array}{lll} & \\
33.25 & & \end{array}$ & $\begin{array}{l}10.913 \\
16.913\end{array}$ & $\begin{array}{l}10.200 \\
16.288\end{array}$ & 22.779 & 15.757 & $\begin{array}{l}11.000 \\
11.084\end{array}$ & 32.373 & 33.123 & 30.23 & 32.194 & 27.718 & 34.165 \\
\hline 12/5/11 11:00 & & $\begin{array}{l}30.987 \\
30.987\end{array}$ & $\begin{array}{r}30.022 \\
30.622\end{array}$ & 29.767 & $\begin{array}{l}30.589 \\
30.589\end{array}$ & & 29.89 & $\begin{array}{l}30.857 \\
30.057\end{array}$ & $\begin{array}{l}30.490 \\
30.498\end{array}$ & 30.308 & 28.909 & 26.667 & 32.224 & 36.677 & $\begin{array}{l}33.25 \\
33.25\end{array}$ & $\begin{array}{l}16.913 \\
10.913\end{array}$ & $\begin{array}{l}10.200 \\
16.293\end{array}$ & 22.774 & 15.769 & 11.088 & 32.371 & 33.125 & 30.223 & 32.194 & 27.718 & $\begin{array}{l}34.165 \\
34.165\end{array}$ \\
\hline $\begin{array}{l}12 / 5 / 1112: 00 \\
12: 00\end{array}$ & & $\begin{array}{r}30.981 \\
30.98\end{array}$ & $\begin{array}{l}30.024 \\
30.61\end{array}$ & 29.741 & $\begin{array}{l}30.599 \\
30.577\end{array}$ & & $\begin{array}{l}29.913 \\
29.913\end{array}$ & 30.862 & $\begin{array}{l}30.496 \\
30.491\end{array}$ & $\begin{array}{l}30.301 \\
30.301\end{array}$ & 28.902 & $\begin{array}{l}26.007 \\
26.643\end{array}$ & $\begin{array}{l}32.222 \\
32.224\end{array}$ & $\begin{array}{l}36.673 \\
36.671\end{array}$ & $\begin{array}{l}33.25 \\
33.25\end{array}$ & $\begin{array}{l}10.915 \\
16.915\end{array}$ & 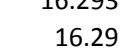 & 22.767 & $\begin{array}{l}13.799 \\
15.792\end{array}$ & 11.086 & 32.348 & $\begin{array}{l}3.123 \\
33.12\end{array}$ & $\begin{array}{l}30.225 \\
30.208\end{array}$ & $\begin{array}{l}32.194 \\
32.192\end{array}$ & 27.718 & 34.163 \\
\hline $12 / 5 / 1113: 00$ & & 30.973 & 30.592 & 29.704 & 30.549 & & 29.949 & 30.869 & 30.489 & 30.287 & 28.886 & 26.602 & 32.21 & 36.673 & 33.238 & 16.911 & 16.283 & .758 & 15.806 & 11.079 & 32.334 & 33.111 & 0.173 & 32.182 & 27.716 & 34.16 \\
\hline $12 / 5 / 1$ & & 30.969 & 75 & 29.676 & 30.52 & & 29.965 & 30.877 & 30.489 & 30.273 & 28.87 & 26.574 & 32.201 & 36.661 & & 16.906 & 16.279 & 758 & 15.818 & 11.07 & 32.313 & .104 & 147 & 32.171 & 27.718 & .156 \\
\hline $12 / 5 / 1$ & & 30.964 & 30.559 & 29.645 & 30.498 & & 29.981 & 30.879 & 30.486 & 30.257 & 28.853 & 26.547 & 32.187 & 36.649 & & 16.902 & 16.276 & 22.751 & 15.82 & 11.056 & 287 & 33.09 & 121 & 2.159 & 27.718 & 34.156 \\
\hline $12 / 5 / 1116: 00$ & & & 30.564 & 29.655 & 30.491 & & 29.96 & 30.884 & 30.493 & 30.257 & 28.851 & 26.555 & 32.184 & 36.649 & 33.214 & 16.902 & 16.276 & 22.8 & 15.809 & 11.051 & 32.296 & 33.087 & 126 & 32.154 & 27.72 & 34.153 \\
\hline 12/5/11 17:00 & & 30.976 & 30.568 & 29.676 & 30.5 & & 29.937 & 30.889 & 30.491 & 30.262 & 28.863 & 26.576 & 32.189 & 36.651 & 33.214 & 16.899 & 16.279 & 22.798 & 15.801 & 11.056 & 32.303 & 33.09 & 30.145 & 32.157 & 27.72 & 34.156 \\
\hline 12/5/11 18:00 & & 30.978 & 30.571 & 29.687 & 30.502 & & 29.925 & 30.891 & 30.493 & 30.266 & 28.867 & 26.586 & 32.191 & 36.656 & 33.219 & 16.899 & 16.281 & 22.798 & 15.794 & 11.058 & 32.31 & 33.092 & 30.15 & 32.161 & 27.72 & 34.16 \\
\hline 12/5/11 19:00 & & 30.983 & 30.582 & 29.711 & 30.516 & & 29.909 & 30.9 & 30.496 & 30.273 & 28.877 & 26.61 & 32.196 & 36.656 & 33.221 & 16.899 & 16.283 & 22.798 & 15.792 & 11.065 & 32.322 & 33.097 & 30.168 & 32.166 & 27.723 & 34.163 \\
\hline 12/5/11 20:00 & & 30.987 & 30.592 & 29.73 & 30.53 & & 29.899 & 30.908 & 30.498 & 30.28 & 28.884 & 26.629 & 32.203 & 36.666 & 33.233 & 16.899 & 16.29 & 22.788 & 15.794 & 11.067 & 32.334 & 33.104 & 30.185 & 32.173 & 27.723 & 34.167 \\
\hline $12 / 5 / 1121: 00$ & & 30.99 & 30.599 & 29.737 & 30.542 & & 29.899 & 30.915 & 30.498 & 30.287 & 28.891 & 26.634 & 32.21 & 36.675 & 33.235 & 16.899 & 16.293 & 22.793 & 15.792 & 11.077 & 32.345 & 33.108 & 30.201 & 32.18 & 27.723 & 34.167 \\
\hline $12 / 5 / 1122: 00$ & & 30.99 & 30.596 & 29.739 & 30.547 & & 29.904 & 30.917 & 30.5 & 30.287 & 28.893 & 26.636 & 32.21 & 36.68 & 33.235 & 16.897 & 16.293 & 22.791 & 15.801 & 11.074 & 32.341 & 33.113 & 30.192 & 32.182 & 27.72 & 34.17 \\
\hline $12 / 5 / 1123: 00$ & & 30.99 & 30.596 & 29.73 & 30.542 & & 29.918 & 30.924 & 30.503 & 30.287 & 28.891 & 26.624 & 32.21 & 36.673 & 33.238 & 16.897 & 16.29 & 22.788 & 15.804 & 11.077 & 32.343 & 33.111 & 30.187 & 32.18 & 27.723 & 34.17 \\
\hline $12 / 6 / 110: 00$ & & 30.987 & 30.587 & 29.711 & 30.533 & & 29.93 & 30.927 & 30.498 & 30.28 & 28.884 & 26.607 & 32.206 & 36.677 & 33.233 & 16.895 & 16.288 & 22.788 & 15.809 & 11.07 & 32.327 & 33.106 & 30.171 & 32.175 & 27.723 & 34.167 \\
\hline 12/6/11 1:00 & & 30.983 & 30.582 & 29.697 & 30.521 & & 29.944 & 30.934 & 30.496 & 30.273 & 28.874 & 26.593 & 32.199 & 36.668 & 33.226 & 16.892 & 16.283 & 22.784 & 15.82 & 11.065 & 32.315 & 33.099 & 30.154 & 32.168 & 27.723 & 34.163 \\
\hline 12/6/11 2:00 & & 30.978 & 30.568 & 29.673 & 30.5 & & 29.96 & 30.934 & 30.496 & 30.262 & 28.86 & 26.569 & 32.189 & 36.666 & 33.219 & 16.89 & 16.279 & 22.777 & $\begin{array}{l}15.825 \\
\end{array}$ & 11.058 & 32.296 & 33.09 & 30.136 & 32.161 & 27.723 & 34.158 \\
\hline 12/6/11 3:00 & & 30.98 & 30.566 & 29.669 & 30.4 & & 29.953 & 30. & 30.501 & 30.259 & 28.858 & 26.567 & 32.182 & 36.659 & 33.212 & 16.888 & $\begin{array}{l}16.279 \\
\end{array}$ & 22.77 & 15.816 & $\begin{array}{l}11.051 \\
\end{array}$ & 32.292 & 33.087 & 30.133 & 32.157 & 27.723 & 34.156 \\
\hline 12/6/11 4:00 & & 30.978 & 30.559 & 29.659 & 30.486 & & 29.958 & 30.939 & 30.493 & 30.255 & 28.851 & 26.559 & 32.18 & 36.659 & 33.207 & $\begin{array}{l}10.000 \\
16.883\end{array}$ & 16.274 & 22.768 & 15.82 & 11.048 & 32.287 & $\begin{array}{l}33.08 \\
33.08\end{array}$ & 30.124 & 32.149 & 27.723 & 34.153 \\
\hline $12 / 6 / 11$ 5:00 & & 30.973 & 30.547 & 29.641 & $\begin{array}{r}30.47 \\
30.47\end{array}$ & & 29.965 & 30.941 & 30.496 & 30.243 & 28.839 & 26.54 & 32.17 & 36.651 & 33.203 & $\begin{array}{l}10.003 \\
16.878\end{array}$ & $\begin{array}{l}16.269 \\
16.269\end{array}$ & 22.763 & $\begin{array}{l}15.82 \\
15.825\end{array}$ & $\begin{array}{l}11.039 \\
11.039\end{array}$ & 32.271 & $\begin{array}{l}33.071 \\
33.071\end{array}$ & $\begin{aligned} 30.11 \\
30.11\end{aligned}$ & 32.142 & 27.723 & 34.153 \\
\hline $12 / 6 / 11$ & & 30.976 & 30.547 & 29.643 & $\begin{array}{r}30.4 \\
30.4\end{array}$ & & 29.953 & 30.941 & $\begin{array}{l}30.490 \\
30.493\end{array}$ & $\begin{array}{l}30.238 \\
30.238\end{array}$ & $\begin{array}{l}28.039 \\
28.839\end{array}$ & $\begin{array}{l}26.543 \\
26.543\end{array}$ & 32.168 & 36.649 & 33.198 & $\begin{array}{l}10.010 \\
16.876\end{array}$ & $\begin{array}{l}10.259 \\
16.269\end{array}$ & 8 & 15.818 & 11.034 & 32.273 & 1 & 30.107 & 32.14 & 27.725 & .151 \\
\hline $12 / 6$ & & 30.9 & 30 & 29 & 30 & & & 30 & 30 & 30 & 28 & 26 & 32 & 36. & 33 & 16 & 16 & 22.754 & 15.806 & 11.037 & 32.278 & 1 & 9 & 14 & 27.725 & .151 \\
\hline $12 / 6$ & & 30 & 30 & 29. & 30. & & & 30 & 30 & 30 & 28. & 26 & 32 & 36. & 33 & 16 & 16 & 22.763 & 15.809 & 11.034 & 32 & 3 & 4 & 14 & 27.725 & 34.151 \\
\hline & & 30.9 & 30.5 & 29.655 & & & & & & & 28.8 & & & 36.654 & 33.198 & & & 1 & 15.811 & 11.039 & 32.275 & & & 32.14 & 27.725 & 34.151 \\
\hline $12 / 6 / 1110: 00$ & & 30. & 30.552 & 29.657 & & & & & & & 28.8 & 26.5 & 32.17 & 36.661 & 33.198 & 16.869 & & & 15.813 & 11.037 & & & & 32.138 & 27.725 & 34.153 \\
\hline 12/6/11 11:00 & & & 30.5 & 29.641 & 30.4 & & & & & & 28 & 26 & 32.163 & 36.656 & 33.1 & 16.8 & 16.272 & 24 & 15 & 11.032 & 32.2 & & & 8 & 27.725 & 34.151 \\
\hline $12 / 6$ & & & 30.5 & 29.608 & 30.4 & & & & & & 28.8 & & 32.1 & 36.649 & & 16.8 & 16.2 & 22.7 & 15.8 & 11.023 & & & & 32.128 & 27.725 & 34.146 \\
\hline 12/6/11 13:00 & & 30. & 30.5 & 29.58 & & & & & 30. & & 28 & 26.4 & 32.147 & 36.64 & & 16 & 16 & 22.7 & 15.844 & 11.011 & 32.229 & & 30.147 & 32.117 & 27.725 & 34.144 \\
\hline $12 / 6 / 1114: 00$ & & 30.9 & 30.4 & 29.566 & & & 29. & & 30. & 30. & 28.7 & 26 & 32.135 & 36.642 & 33.1 & 16.853 & 16.255 & 22.7. & 15.848 & 11.006 & 32.213 & 33.037 & 30.187 & 32.105 & 27.728 & 34.139 \\
\hline $12 / 6 / 1115: 00$ & & 30 & 30.4 & 29.547 & 30.3 & & 29.9 & 30. & 30.481 & 30.1 & 28.774 & 26.46 & 32.128 & 36.628 & 33.158 & 16.85 & 16.253 & 22.74 & 15.8 & 10.997 & 32.201 & 33.028 & 30.023 & 32.098 & 27.728 & 34.137 \\
\hline $12 / 6 / 1116: 00$ & & 30.945 & 30.478 & 29.536 & 30.374 & & 29.972 & 30.9 & 30.479 & 30.164 & 28.765 & 26.459 & 32.119 & 36.623 & 33.151 & 16.846 & 16.248 & 22.735 & 15.844 & 10.987 & 32.192 & 33.021 & 30.011 & 32.091 & 27.728 & 34.135 \\
\hline $12 / 6 / 1117: 00$ & & 30.945 & 30.471 & 29.536 & 30.365 & & 29.956 & 30.941 & 30.479 & 30.157 & 28.76 & 26.461 & 32.114 & 36.623 & 33.146 & 16.841 & 16.248 & 22.735 & 15.832 & 10.983 & 32.192 & 33.016 & 30.013 & 32.086 & 27.728 & 34.135 \\
\hline $12 / 6 / 1118: 00$ & & 30.95 & 30.478 & 29.557 & 30.372 & & 29.927 & 30.941 & 30.482 & 30.159 & 28.765 & 26.485 & 32.114 & 36.621 & 33.146 & 16.836 & 16.248 & 22.74 & 15.809 & 10.983 & 32.201 & 33.014 & 30.027 & 32.086 & 27.727 & 34.137 \\
\hline 12/6/11 19:00 & & 30.948 & 30.48 & 29.561 & 30.376 & & 29.911 & 30.944 & 30.482 & 30.159 & 28.763 & 26.492 & 32.117 & 36.626 & 33.146 & 16.836 & 16.248 & 22.742 & 15.804 & 10.983 & 32.203 & 33.016 & 30.032 & 32.088 & 27.728 & 34.137 \\
\hline $12 / 6 / 1120: 00$ & & 30.943 & 30.478 & 29.559 & 30.379 & & 29.906 & 30.944 & 30.477 & 30.157 & 28.763 & 26.492 & 32.117 & 36.63 & 33.149 & 16.834 & 16.25 & 22.742 & 15.799 & 10.978 & 32.206 & 33.021 & 30.037 & 32.088 & 27.73 & 34.139 \\
\hline 12/6/11 21:00 & & 30.945 & 30.478 & 29.559 & 30.38 & & 29.902 & 30.944 & 30.477 & 30.155 & 28.763 & 26.5 & 32.117 & 36.63 & 33.144 & 16.829 & 16.251 & 22.742 & 15.79 & 10.98 & 32.208 & 33.021 & 30.035 & 32.088 & 27.728 & 34.142 \\
\hline $12 / 6 / 1122: 00$ & & 30.943 & 30.475 & 29.557 & 30.383 & & 29.897 & 30.941 & 30.477 & 30.15 & 28.758 & 26.497 & 32.117 & 36.63 & 33.146 & 16.829 & 16.253 & 22.742 & 15.79 & 10.983 & 32.196 & 33.018 & 30.037 & 32.086 & 27.728 & 34.142 \\
\hline $12 / 6 / 1123: 00$ & & 30.936 & 30.468 & 29.543 & 30.376 & & 29.904 & 30.941 & 30.474 & 30.141 & 28.751 & 26.483 & 32.112 & 36.633 & 33.142 & 16.825 & 16.248 & 22.738 & 15.794 & 10.98 & 32.199 & 33.014 & 30.025 & 32.086 & 27.728 & 34.142 \\
\hline
\end{tabular}




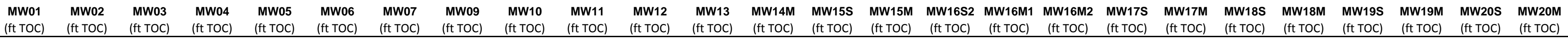

\begin{tabular}{|c|c|c|c|c|c|c|c|c|c|c|c|c|c|c|c|c|c|c|c|c|c|c|c|c|c|}
\hline well & $\begin{array}{l}\text { (ft TOC) } \\
\text { (f) }\end{array}$ & $\begin{array}{l}\text { (ft TOC) } \\
\text { (f) }\end{array}$ & $\begin{array}{l}\text { (ft TOC) } \\
\text { (f) }\end{array}$ & $\begin{array}{l}\text { (ft TOC) } \\
\text { (ft) }\end{array}$ & $\begin{array}{ll}(\mathrm{ft} \text { TOC) } & \text { (ft TOC) }\end{array}$ & (ft toc) & $\begin{array}{l}\text { (ift TOC) } \\
\text { (n) }\end{array}$ & $\begin{array}{l}\text { (ft TOC) } \\
\text { (ft) }\end{array}$ & $\begin{array}{l}\text { (ft TOC) } \\
\text { (f) }\end{array}$ & $\begin{array}{l}\text { (ft TOC) } \\
\text { (n) }\end{array}$ & $\begin{array}{l}\text { (ft TOC) } \\
\text { (f) }\end{array}$ & (ft TOC) & $\begin{array}{l}\text { (ft TOC) } \\
\end{array}$ & (ft TOC) & (ft TOC) & $\begin{array}{l}\text { (ft TOC) } \\
\text { (f) }\end{array}$ & (ft TOC) & $\begin{array}{l}\text { (ft TOC) } \\
\text { (ft) }\end{array}$ & (ft TOC) & $\begin{array}{l}\text { (ft TOC) } \\
\text { (f) }\end{array}$ & $\begin{array}{l}\text { (ft TOC) } \\
\text { (f) }\end{array}$ & $\begin{array}{l}\text { (ft TOC) } \\
\text { (n) }\end{array}$ & (ft TOC) & $\begin{array}{l}\text { (ft TOC) } \\
\text { (1) }\end{array}$ & (ft TOC) \\
\hline $.0: 00$ & & 30.934 & 30.464 & 29.533 & 30.3 & 29.904 & 30.9 & 30.472 & 30.1 & 28.744 & 26 & 32.107 & 36.621 & 33. & 16. & 16.244 & 22.735 & 15.787 & 10.976 & 32.189 & 33. & 30.018 & 79 & 3 & 34.139 \\
\hline $12 / 7 / 111: 00$ & & 30.934 & 30.464 & 29.533 & 30.367 & 29.899 & 30.939 & 30.47 & 30.132 & 28.742 & 26.478 & 32.103 & 36.623 & 33.132 & 16.82 & 16.241 & 22.73 & 5.783 & 10.966 & 32.187 & 33.002 & .016 & 32.074 & 73 & 34.137 \\
\hline $12 / 7 / 112: 00$ & & 30.927 & 30.45 & 29.51 & 30.353 & 29.909 & 30.934 & 30.47 & 30.122 & 28.728 & 26.459 & 32.093 & 36.626 & 33.125 & 16.818 & 16.232 & 22.726 & 15.783 & 10.964 & 32.175 & 32.992 & .995 & 32.065 & 3 & 34.132 \\
\hline 12/7/11 3:00 & & 30.924 & 30.447 & 29.507 & 30.348 & 29.902 & 30.932 & 30.467 & 30.115 & 28.723 & 26.459 & 32.089 & 36.626 & 33.118 & 16.815 & 16.229 & 22.719 & 15.78 & 10.954 & 32.166 & 32.988 & 29.992 & 32.06 & 73 & 34.13 \\
\hline $12 / 7 / 114: 00$ & & 30.918 & 30.433 & 29.489 & 30.334 & 29.909 & 30.927 & 30.465 & 30.104 & 28.714 & 26.442 & 32.077 & 36.614 & 33.109 & 16.811 & 16.22 & 22.714 & 15.78 & 10.945 & 32.154 & 32.978 & 29.973 & 32.049 & 27.73 & 34.125 \\
\hline 12/7/11 5:00 & & 30.917 & 30.429 & 29.484 & 30.327 & 29.902 & 30.924 & 30.462 & 30.097 & 28.707 & 26.442 & 32.074 & 36.609 & 33.104 & 16.808 & 16.218 & 22.71 & 15.778 & 10.938 & 32.145 & 32.971 & 29.971 & 32.044 & 27.73 & 34.123 \\
\hline 12/7/11 6:00 & & 30.915 & 30.422 & 29.475 & 30.318 & 29.902 & 30.922 & 30.46 & 30.087 & 28.698 & 26.433 & 32.065 & 36.612 & 33.097 & 16.804 & 16.215 & 22.719 & 15.776 & 10.933 & 32.14 & 32.966 & 29.966 & 32.037 & 27.732 & 34.121 \\
\hline $12 / 7 / 117: 00$ & & 30.915 & 30.419 & 29.477 & 30.313 & 29.888 & 30.92 & 30.462 & 30.085 & 28.695 & 26.437 & 32.065 & 36.616 & 33.095 & 16.801 & 16.215 & 22.721 & 15.764 & 10.929 & 32.143 & 32.964 & 29.969 & 32.035 & 27.732 & 34.121 \\
\hline 12/7/11 8:00 & & 30.911 & 30.417 & 29.472 & 30.311 & 29.883 & 30.917 & 30.46 & 30.078 & 28.691 & 26.437 & 32.06 & 36.609 & 33.09 & 16.799 & 16.211 & 22.724 & 15.764 & 10.929 & 32.138 & 32.964 & 29.962 & 32.032 & 27.732 & 34.121 \\
\hline 12/7/11 9:00 & & 30.913 & 30.422 & 29.491 & 30.32 & 29.862 & 30.917 & 30.46 & 30.083 & 28.698 & 26.457 & 32.063 & 36.609 & 33.092 & 16.797 & 16.213 & 22.719 & 15.755 & 10.931 & 32.15 & 32.964 & 29.98 & 32.035 & 27.735 & 34.118 \\
\hline $12 / 7 / 1110: 00$ & & 30.92 & 30.431 & 29.514 & 30.332 & 29.843 & 30.92 & 30.462 & 30.09 & 28.707 & 26.485 & 32.07 & 36.616 & 33.097 & 16.797 & 16.215 & 22.719 & 15.743 & 10.936 & 32.166 & 32.969 & 30.004 & 32.042 & 27.735 & 34.123 \\
\hline 12/7/111 11:00 & & 30.915 & 30.436 & 29.517 & 30.341 & 29.843 & 30.92 & 30.46 & 30.09 & 28.712 & 26.485 & 32.07 & 36.616 & 33.099 & 16.794 & 16.215 & 22.719 & 15.75 & 10.933 & 32.168 & 32.971 & 29.999 & 32.042 & 27.732 & 34.123 \\
\hline $12 / 7 / 1112: 00$ & & 30.908 & 30.429 & 29.5 & 30.339 & 29.855 & 30.92 & 30.455 & 30.083 & 28.705 & 26.473 & 32.067 & 36.623 & 33.097 & 16.792 & 16.213 & 22.712 & 15.759 & 10.936 & 32.161 & 32.969 & 29.997 & 32.039 & 27.732 & 34.121 \\
\hline $12 / 7 / 1113: 00$ & & 30.901 & 30.419 & 29.482 & 30.327 & 29.871 & 30.915 & 30.453 & 30.074 & 28.693 & 26.452 & 32.06 & 36.619 & 33.09 & 16.792 & 16.209 & 22.705 & 15.764 & 10.926 & 32.145 & 32.964 & 29.978 & 32.032 & 27.735 & 34.118 \\
\hline $12 / 7 / 1114: 00$ & & 30.899 & 30.415 & 29.472 & 30.323 & 29.871 & 30.912 & 30.448 & 30.069 & 28.686 & 26.447 & 32.058 & 36.614 & 33.088 & 16.79 & 16.204 & 22.705 & 15.769 & 10.924 & 32.147 & 32.957 & 29.969 & 32.027 & 27.732 & 34.116 \\
\hline 12/7/11 15:00 & & 30.901 & 30.415 & 29.472 & 30.32 & 29.864 & 30.91 & 30.451 & 30.064 & 28.686 & 26.449 & 32.056 & 36.612 & 33.083 & 16.787 & 16.204 & 22.7 & 15.757 & 10.922 & 32.138 & 32.954 & 29.971 & 32.027 & 27.737 & 34.114 \\
\hline $12 / 7 / 1116: 00$ & & 30.903 & 30.419 & 29.486 & 30.325 & 29.85 & 30.91 & 30.453 & 30.067 & 28.693 & 26.461 & 32.056 & 36.616 & 33.085 & 16.787 & 16.202 & 22.754 & 15.747 & 10.919 & 32.147 & 32.954 & 29.983 & 32.027 & 27.735 & 34.114 \\
\hline $12 / 7 / 1117: 00$ & & 30.903 & 30.422 & 29.498 & 30.33 & 29.838 & 30.912 & 30.451 & 30.069 & 28.695 & 26.473 & 32.056 & 36.621 & 33.088 & 16.787 & 16.204 & 22.754 & 15.743 & 10.922 & 32.157 & 32.957 & 29.988 & 32.03 & 27.737 & 34.114 \\
\hline $12 / 7 / 1118: 00$ & & 30.901 & 30.422 & 29.496 & 30.332 & 29.836 & 30.91 & 30.446 & 30.069 & 28.695 & 26.473 & 32.058 & 36.619 & 33.088 & 16.785 & 16.201 & 22.763 & 15.743 & 10.922 & 32.154 & 32.959 & 29.997 & 32.03 & 27.735 & 34.114 \\
\hline $12 / 7 /$ & & 30.908 & 30.438 & 29.524 & 30.35 & 29.81 & 30.912 & 30.451 & 30.081 & 28.709 & 26.502 & 32.067 & 36.623 & 33.097 & 16.787 & 16.208 & 22.781 & 15.726 & 10.929 & 32.175 & 32.969 & 30.018 & 32.039 & 27.737 & 34.118 \\
\hline $12 / 7 /$ & & 30.906 & 30.443 & 29.531 & 30.3 & 29.808 & 30.917 & 30.446 & 30.083 & 28.714 & 26.512 & 32.072 & 36.63 & 33.099 & 16.787 & 16.213 & 22.781 & 15.724 & 10.936 & 32.18 & 32.973 & 30.025 & 32.044 & 27.737 & .123 \\
\hline 12/7/11 21:00 & & 30.899 & 30.436 & 29.512 & 30. & 29.824 & 30.915 & 30.446 & 30.078 & 28.709 & 26.492 & 32.07 & 36.633 & 33.102 & 16.787 & 16.211 & 977 & 15.729 & 10.933 & 32.173 & 973 & 30.011 & 2.044 & 27.737 & .123 \\
\hline $12 / 7 / 1122: 00$ & & 30.904 & 30.44 & 29.526 & 30.372 & 29.815 & 30.917 & 30.451 & 30.083 & 28.714 & 26.507 & 32.074 & 36.637 & 33.107 & 16.787 & 16.213 & .768 & 15.719 & 10.94 & 32.182 & 976 & 1.025 & .049 & 27.737 & 34.125 \\
\hline 12/7/11 23:00 & & 30.903 & 30.445 & 29.531 & 30.374 & 29.815 & & 30.448 & 30.085 & 28.716 & 26.509 & 32.082 & 36.64 & 33.109 & 16.79 & 16.215 & 22.767 & 15.722 & 10.947 & 32.189 & .981 & .032 & 32.051 & 27.739 & 34.128 \\
\hline 12/8/11 0:00 & & 30.903 & 30.445 & 29.529 & 30.374 & 29.815 & 30.917 & 30.448 & 30.085 & 28.716 & 26.509 & 32.079 & 36.642 & 33.109 & 16.792 & 16.215 & 22.765 & 15.717 & 10.947 & 32.189 & 32.978 & .027 & 32.051 & 27.739 & 34.127 \\
\hline 12/8/11 1:00 & & 30.901 & 30.443 & 29.524 & 30.374 & 29.822 & 30.917 & 30.446 & 30.085 & 28.716 & 26.502 & 32.079 & 36.64 & 33.107 & 16.79 & 16.213 & 22.754 & 15.717 & 10.945 & 32.185 & 776 & 023 & 32.051 & 27.739 & 34.125 \\
\hline 12/8/11 2:00 & & 30.897 & 30.438 & 29.512 & 30.367 & 29.831 & 30.915 & 30.444 & 30.081 & 28.709 & 26.49 & 32.072 & 36.642 & 33.102 & 16.792 & 16.208 & 22.749 & 15.719 & 10.936 & 32.178 & 32.973 & 30.016 & 32.046 & 27.739 & 34.121 \\
\hline 12/8/11 3:00 & & 30.894 & 30.438 & 29.507 & 30.362 & 29.836 & 30.915 & 30.443 & 30.078 & 28.707 & 26.485 & 32.067 & 36.642 & 33.099 & 16.79 & 16.204 & 22.742 & 15.722 & 10.936 & 32.175 & 32.969 & 30.013 & 32.039 & 27.739 & 34.116 \\
\hline $12 / 8 / 114: 00$ & & 30.896 & 30.436 & 29.51 & 30.362 & 29.831 & 30.915 & 30.439 & 30.078 & 28.709 & 26.488 & 32.065 & 36.64 & 33.097 & 16.79 & 16.199 & 22.735 & 15.719 & 10.933 & 32.168 & 32.969 & 30.013 & 32.039 & 27.739 & 34.114 \\
\hline 12/8/11 5:00 & & 30.899 & 30.44 & 29.521 & 30.367 & 29.822 & 30.917 & 30.443 & 30.083 & 28.714 & 26.5 & 32.067 & 36.64 & 33.097 & 16.792 & 16.199 & 22.73 & 15.712 & 10.931 & 32.178 & 32.969 & 30.025 & 32.039 & 27.739 & 34.111 \\
\hline $12 / 8 / 116: 00$ & & 30.901 & 30.445 & 29.526 & 30.372 & 29.817 & 30.92 & 30.446 & 30.083 & 28.714 & 26.504 & 32.067 & 36.644 & 33.095 & 16.79 & 16.199 & 22.74 & 15.705 & 10.931 & 32.18 & 32.969 & 30.025 & 32.039 & 27.742 & 34.111 \\
\hline $12 / 8 / 117: 00$ & & 30.899 & 30.447 & 29.533 & 30.376 & 29.817 & 30.917 & 30.439 & 30.087 & 28.716 & 26.509 & 32.07 & 36.647 & 33.097 & 16.792 & 16.199 & 22.744 & 15.715 & 10.933 & 32.185 & 32.969 & 30.03 & 32.042 & 27.742 & 34.111 \\
\hline $12 / 8 / 118: 00$ & & 30.897 & 30.447 & 29.531 & 30.379 & 29.819 & 30.917 & 30.444 & 30.087 & 28.716 & 26.507 & 32.072 & 36.649 & 33.099 & 16.792 & 16.197 & 22.751 & 15.712 & 10.936 & 32.18 & 32.971 & 30.035 & 32.044 & 27.742 & 34.111 \\
\hline 12/8/11 9:00 & & 30.901 & 30.452 & 29.54 & 30.383 & 29.815 & 30.92 & 30.443 & 30.09 & 28.723 & 26.516 & 32.074 & 36.651 & 33.104 & 16.792 & 16.201 & 22.744 & 15.71 & 10.943 & 32.196 & 32.976 & 30.039 & 32.044 & 27.742 & 34.114 \\
\hline 12/8/11 10:00 & & 30. & 30.457 & 29.55 & 30. & 29.812 & 30.922 & 30.443 & 30.097 & 28.728 & 26.524 & 32.079 & 36.649 & 33.109 & 16.794 & 16.206 & 22.744 & 15.712 & 10.943 & 32.201 & 31 & 30.049 & 32.051 & 27.742 & 34.116 \\
\hline $12 / 8 / 1$ & & 30.894 & 30.452 & 29.536 & 30.3 & 29.829 & 30.922 & 30.439 & 30.092 & 28.721 & 26.512 & 32.082 & 36.656 & 33.107 & 16.797 & 16.206 & 22.74 & 15.722 & 10.945 & 32.196 & 881 & 30.032 & 32.051 & 27.742 & 34.116 \\
\hline $12 / 8 / 11$ & & 30.887 & $\begin{array}{l}r .042 \\
30.44\end{array}$ & 29.51 & 30. & 29.852 & $\begin{array}{l}30.92 \\
30.92\end{array}$ & 30.434 & 30.083 & 28.709 & 26.485 & 32.074 & 36.656 & 33.104 & 16.794 & 16.204 & 22.733 & 15.743 & 10.938 & 32.171 & 771 & 30.009 & 32.046 & 27.742 & 34.114 \\
\hline $12 / 8 / 11$ & & 30.88 & 30.426 & 29.482 & 30. & 29.871 & 30.915 & $\begin{array}{l}30.434 \\
30.434\end{array}$ & 30.071 & 28.698 & 26.459 & 32.065 & 36.647 & 33.097 & $\begin{array}{l}10.792 \\
16.792\end{array}$ & 16.201 & 22.731 & 15.752 & 10.931 & 32.154 & 56 & 92 & 32.037 & 27.742 & 34.111 \\
\hline $12 /$ & & 30.878 & 30 & 29. & 30. & 29 & 30 & 30 & 30.06 & 28.686 & 12 & 32 & 36.635 & 33.088 & 16.792 & 16.192 & 22.719 & 15.755 & 10.924 & 32.147 & 7 & 3 & 32.03 & 27.744 & .109 \\
\hline $12 / 8 /$ & & 30 & 30 & 29 & & 29. & 30 & $3 c$ & 30.057 & 28.684 & 26 & 32 & 36.637 & 33.083 & 16.79 & 1 & 22.717 & 15.745 & 10. & 32.14 & 4 & 3 & 25 & 27.746 & .107 \\
\hline $12 / 8 /$ & & & 30 & 29 & 30 & & & 30 & & 28. & 26 & 32. & 36. & & 16.79 & & 22.7 & 5 & 10. & & 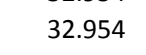 & 5 & 25 & 27.746 & 104 \\
\hline & & & & & & & 30. & & & 28. & & 32.0 & 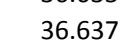 & & 16.7 & & & 15. & & & & 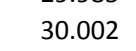 & 32.027 & & .107 \\
\hline $18: 00$ & & & 30.4 & & & & & & & 28. & & 32.058 & 36 & & & & & 15.7 & & & & & & & 1.107 \\
\hline 19:00 & & & 30.4 & 29 & & & & & & 28.698 & & 32.06 & 36.644 & 33.088 & 16.787 & & 22.7 & 15.722 & 10.929 & 32.166 & & 3 & & 27.746 & 34.109 \\
\hline 20:00 & & & 30.4 & & & & & & & 28.7 & & 32.067 & 36. & & 16. & & 22.7 & 15 & 10.931 & & & & & 27.749 & 34.114 \\
\hline $12 / 8 / 1121: 00$ & & & 30. & & & & & & & 28.7 & & 32.079 & 36. & 33. & 16. & & 22.7 & 15.712 & 10.94 & 32.203 & & & & 27.749 & 34.118 \\
\hline $12 / 8 / 1122: 00$ & & & 30.4 & 29. & & 29.7 & & & & 28 & & 32.089 & 36.666 & 33.111 & 16. & 16.208 & 22.7 & 15.708 & 10.95 & 32.21 & & 30.065 & 32.058 & 27.749 & 34.121 \\
\hline $12 / 8 / 1123: 00$ & & 30. & 30.4 & 29.5 & & 29 & & 30.4 & 30.1 & 28.7 & 26.5 & 32.093 & 36.675 & 33.118 & 16.7 & 16.215 & 22.7 & 15 & 10.957 & 32.227 & 32.997 & 30.077 & 32.067 & 27.749 & 34.123 \\
\hline 12/9/11 0:00 & & 30.8 & 30.475 & 29.582 & 30.4 & 29.784 & 30.5 & 30.434 & 30.111 & 28.742 & 26.5 & 32.1 & 36.67 & 33.125 & 16.799 & 16.218 & 22.784 & 15.715 & 10.961 & 32.229 & 33.002 & 30.086 & 32.07 & 27.749 & 34.125 \\
\hline 12/9/11 1:00 & & 30.8. & 30.48 & 29.585 & 30.4 & 29.789 & 30.932 & 30.436 & 30.113 & 28.746 & 26.55 & 32.1 & 36.677 & 33.128 & 16.801 & 16.215 & 22.779 & 15.715 & 10.964 & 32.227 & 32.999 & 30.086 & 32.072 & 27.749 & 34.125 \\
\hline 12/9/11 2:00 & & 30.899 & 30.48 & 29.587 & 30.4 & 29.798 & 30.934 & 30.434 & 30.118 & 28.749 & 26.557 & 32.1 & 36.677 & 33.132 & 16.806 & 16.215 & 22.774 & 15.719 & 10.966 & 32.234 & 33.002 & 30.089 & 32.072 & 27.749 & 34.123 \\
\hline 12/9/11 3:00 & & 30.908 & 30.494 & 29.61 & 30.449 & 29.786 & 30.939 & 30.441 & 30.129 & 28.76 & 26.579 & 32.105 & 36.68 & 33.132 & 16.808 & 16.218 & 22.77 & 15.715 & 10.969 & 32.248 & 33.007 & 30.107 & 32.077 & 27.751 & 34.121 \\
\hline 12/9/11 4:00 & & 30.904 & 30.492 & 29.603 & 30.451 & 29.803 & 30.939 & 30.434 & 30.132 & 28.76 & 26.567 & 32.105 & 36.68 & 33.135 & 16.811 & 16.213 & 22.761 & 15.726 & 10.971 & 32.243 & 33.007 & 30.1 & 32.079 & 27.751 & 34.118 \\
\hline 12/9/11 5:00 & & 30.906 & 30.496 & 29.61 & 30.456 & 29.805 & 30.944 & 30.439 & 30.134 & 28.767 & 26.574 & 32.107 & 36.677 & 33.132 & 16.813 & 16.213 & 22.761 & 15.729 & 10.971 & 32.248 & 33.009 & 30.105 & 32.079 & 27.751 & 34.116 \\
\hline $12 / 9 / 116: 00$ & & 30.911 & 30.501 & 29.615 & 30.46 & 29.805 & 30.946 & 30.436 & 30.141 & 28.772 & 26.579 & 32.11 & 36.684 & 33.139 & 16.815 & 16.213 & 22.763 & 15.729 & 10.976 & 32.252 & 33.009 & 30.112 & 32.084 & 27.751 & .114 \\
\hline 12/9/11 7:00 & & 30.92 & 30.519 & 29.648 & 30.479 & 29.791 & 30.951 & 30.446 & 30.155 & 28.788 & 26.605 & 32.117 & 36.687 & 33.146 & 16.82 & 16.218 & 22.772 & 15.722 & 10.983 & 32.271 & 33.018 & 30.143 & 32.091 & 27.753 & 6 \\
\hline
\end{tabular}




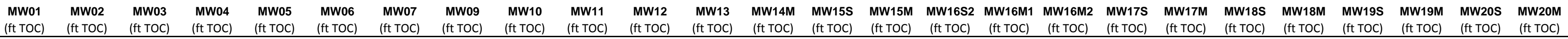

\begin{tabular}{|c|c|c|c|c|c|c|c|c|c|c|c|c|c|c|c|c|c|c|c|c|c|c|c|c|c|c|}
\hline Well & $\begin{array}{l}\text { MW01 } \\
\text { (ft TOC) }\end{array}$ & $\begin{array}{l}\text { MW02 } \\
\text { (ft TOC) }\end{array}$ & $\begin{array}{l}\text { MW03 } \\
\text { (ft Toc) }\end{array}$ & $\begin{array}{l}\begin{array}{l}\text { MW04 } \\
\text { (ft TOC) }\end{array}\end{array}$ & $\begin{array}{l}\text { MW05 } \\
\text { (ft Toc) }\end{array}$ & $\begin{array}{l}\text { MW06 } \\
\text { (ft TOC) }\end{array}$ & 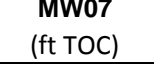 & $\begin{array}{l}\text { MW09 } \\
\text { (ft Toc) }\end{array}$ & $\begin{array}{l}\text { MW10 } \\
\text { (ft Toc) }\end{array}$ & $\begin{array}{l}\text { MW11 } \\
\text { (ft Toc) }\end{array}$ & $\begin{array}{l}\text { MW12 } \\
\text { (ft ToC) }\end{array}$ & $\begin{array}{l}\text { MW13 } \\
\text { (ft Toc) }\end{array}$ & $\begin{array}{l}\text { MW14M } \\
\text { (ft TOC) }\end{array}$ & $\begin{array}{l}\begin{array}{l}\text { MWW5S } \\
\text { (ft TOC) }\end{array} \\
\end{array}$ & $\begin{array}{l}\text { MW15M } \\
\text { (ft TOC) }\end{array}$ & $\begin{array}{l}\text { MW1652 } \\
\text { (ft TOC) }\end{array}$ & $\begin{array}{l}\text { MW16161 } \\
\text { (ft TOC) }\end{array}$ & $\begin{array}{l}\text { MW16m2 } \\
\text { (ft TOC) }\end{array}$ & $\begin{array}{l}\begin{array}{l}\text { MWW7S } \\
\text { (ft TOC) }\end{array} \\
\end{array}$ & $\begin{array}{l}\begin{array}{l}\text { MW17M } \\
\text { (ft TOC) }\end{array} \\
\end{array}$ & $\begin{array}{l}\begin{array}{l}\text { MWW18S } \\
\text { (ft TOC) }\end{array} \\
\end{array}$ & $\begin{array}{l}\text { MW18M } \\
\text { (ft Toc) }\end{array}$ & $\begin{array}{l}\begin{array}{l}\text { MWWigS } \\
\text { (ft TOC) }\end{array} \\
\end{array}$ & $\begin{array}{l}\text { MW19M } \\
\text { (ft Toc) }\end{array}$ & $\begin{array}{l}\text { MW20S } \\
\text { (ft Toc) }\end{array}$ & $\begin{array}{l}\begin{array}{l}\text { MW20M } \\
\text { (ft toc) }\end{array} \\
\text { (a) }\end{array}$ \\
\hline 12/9/11 8:00 & & 30.927 & 30.536 & 29.673 & 30.498 & & 29.784 & 30.953 & 30.446 & 30.173 & 28.805 & 26.629 & 32.128 & 36.689 & 33.156 & 16.825 & 16.225 & 22.774 & 15.724 & 10.992 & 32.287 & 33.03 & 30.161 & 32.1 & 27.753 & 34.118 \\
\hline 12/9/11 9:00 & & 30.924 & 30.54 & 29.68 & 30.509 & & 29.798 & 30.958 & 30.446 & 30.183 & 28.809 & 26.634 & 32.138 & 36.691 & 33.163 & 16.827 & 16.227 & 22.772 & 15.731 & 10.997 & 32.294 & 33.037 & 30.166 & 32.107 & 27.753 & 34.123 \\
\hline 12/9/11 10:00 & & 30.934 & 30.552 & 29.699 & 30.526 & & 29.796 & 30.963 & 30.451 & 30.199 & 28.825 & 26.648 & 32.149 & 36.698 & 33.175 & 16.834 & 16.234 & 22.772 & 15.736 & 11.011 & 32.31 & 33.047 & 30.183 & 32.119 & 27.753 & 34.125 \\
\hline 12/9/11 11:00 & & 30.934 & 30.561 & 29.701 & 30.535 & & 29.81 & 30.965 & 30.448 & 30.206 & 28.832 & 26.648 & 32.154 & 36.701 & 33.184 & 16.836 & 16.236 & 22.767 & 15.752 & 11.018 & 32.317 & 33.054 & 30.185 & 32.126 & 27.756 & 34.128 \\
\hline 12/9/11 12:00 & & 30.931 & 30.561 & 29.699 & 30.535 & & 29.829 & 30.965 & 30.448 & 30.208 & 28.832 & 26.638 & 32.156 & 36.696 & 33.184 & 16.839 & 16.239 & 22.765 & 15.762 & 11.02 & 32.315 & 33.059 & 30.187 & 32.128 & 27.753 & 34.128 \\
\hline 12/9/11 13:00 & & 30.931 & 30.559 & 29.687 & 30.533 & & 29.848 & 30.967 & 30.446 & 30.208 & 28.83 & 26.626 & 32.161 & 36.698 & 33.189 & 16.843 & 16.241 & 22.763 & 15.773 & 11.025 & 32.308 & 33.061 & 30.178 & 32.131 & 27.756 & 34.128 \\
\hline 12/9/11 14:00 & & 30.931 & 30.554 & 29.68 & 30.526 & & 29.862 & 30.97 & 30.451 & 30.208 & 28.828 & 26.614 & 32.159 & 36.698 & 33.184 & 16.846 & 16.243 & 22.754 & 15.783 & 11.025 & 32.296 & 33.061 & 30.164 & 32.133 & 27.756 & 34.128 \\
\hline 12/9/11 15:00 & & 30.931 & 30.55 & 29.671 & 30.521 & & 29.876 & 30.972 & 30.448 & 30.206 & 28.825 & 26.605 & 32.156 & 36.694 & 33.184 & 16.848 & 16.241 & 22.754 & 15.794 & 11.02 & 32.301 & 33.061 & 30.161 & 32.131 & 27.753 & 34.125 \\
\hline $12 / 9 / 1116: 00$ & & 30.936 & 30.557 & 29.685 & 30.526 & & 29.871 & 30.975 & 30.453 & 30.213 & 28.83 & 26.617 & 32.161 & 36.703 & 33.189 & 16.85 & 16.241 & 22.811 & 15.792 & 11.027 & 32.301 & 33.061 & 30.168 & 32.133 & 27.756 & 34.125 \\
\hline 12/9/11 17:00 & & 30.943 & 30.561 & 29.697 & 30.528 & & 29.864 & 30.977 & 30.458 & 30.217 & 28.837 & 26.622 & 32.163 & 36.698 & 33.191 & 16.855 & 16.243 & 22.807 & 15.785 & 11.025 & 32.31 & 33.063 & 30.173 & 32.133 & 27.756 & 34.125 \\
\hline $9 / 1118: 00$ & & 30.943 & 30.566 & 29.704 & 30.533 & & 29.859 & 30.979 & 30.46 & 30.224 & 28.842 & 26.631 & 32.17 & 36.703 & 33.198 & 16.855 & 16.248 & 22.8 & 15.792 & 11.03 & 32.31 & 33.068 & 30.183 & 32.138 & 27.758 & 34.125 \\
\hline 12/9/11 19:00 & & 30.948 & 30.571 & 29.711 & 30.54 & & 29.859 & 30.982 & 30.458 & 30.231 & 28.849 & 26.636 & 32.173 & 36.703 & 33.2 & 16.86 & 16.25 & 22.8 & 15.794 & 11.039 & 32.322 & .075 & 30.19 & 32.142 & 27.758 & 34.128 \\
\hline 12/9/11 20:00 & & 30.948 & 30.575 & 29.713 & 30.544 & & 29.864 & 30.984 & 30.46 & 30.236 & 28.851 & 26.638 & 32.177 & 36.703 & 33.207 & 16.862 & 16.255 & 22.8 & 15.797 & 11.044 & 32.331 & .082 & 30.192 & 2.149 & 27.758 & 7.13 \\
\hline 12/9/11 21:00 & & 30.95 & 30.575 & 29.72 & 30.549 & & 29.869 & 30.987 & 30.46 & 30.243 & 28.853 & 26.638 & 32.184 & 36.712 & 33.212 & 16.862 & 16.262 & 22.795 & 15.799 & 11.048 & 32.331 & .085 & 197 & 2.154 & 27.758 & 34.132 \\
\hline 12/9/11 22:00 & & 30.955 & 30.58 & 29.725 & 30.554 & & 29.869 & 30.989 & 30.465 & 30.245 & 28.858 & 26.641 & 32.191 & 36.705 & 33.217 & 16.867 & 16.267 & 22.793 & 15.792 & 11.053 & 32.334 & 33.09 & 30.197 & 32.164 & 27.76 & 34.137 \\
\hline 12/9/11 23:00 & & 30.955 & 30.58 & 29.73 & 30.556 & & 29.876 & 30.989 & 30.462 & 30.25 & 28.86 & 26.641 & 32.196 & 36.705 & 33.221 & 16.869 & 16.272 & 22.795 & 15.797 & 11.058 & 32.341 & 33.097 & 30.213 & 32.166 & 27.76 & 34.139 \\
\hline 12/10/11 0:00 & & 30.957 & 30.582 & 29.725 & 30.558 & & 29.883 & 30.994 & 30.467 & 30.252 & 28.86 & 26.638 & 32.199 & 36.698 & 33.224 & 16.871 & 16.274 & 22.793 & 15.804 & 11.06 & 32.341 & 33.099 & 208 & 32.168 & 27.76 & 34.139 \\
\hline 12/10/11 1:00 & & 30.959 & 30.582 & 29.727 & 30.561 & & 29.885 & 30.999 & 30.465 & 30.255 & 28.865 & 26.641 & 32.201 & 36.708 & 33.228 & 16.874 & 16.276 & 22.793 & 15.806 & 11.065 & 32.341 & 33.101 & .204 & 32.171 & 27.76 & 34.139 \\
\hline $12 / 10 / 112: 00$ & & 30.957 & 30.578 & 29.718 & 30.554 & & 29.897 & 30.999 & 30.47 & 30.252 & 28.86 & 26.624 & 32.199 & 36.708 & 33.224 & 16.874 & 16.274 & 22.786 & 15.811 & 11.063 & 32.331 & 33.099 & 30.197 & 32.171 & 27.76 & 34.137 \\
\hline $12 / 10 / 11$ 3:00 & & 30.959 & 30.578 & 29.718 & 30.554 & & 29.904 & 31.001 & 30.465 & 30.255 & 28.86 & 26.624 & 32.199 & 36.708 & 33.221 & 16.876 & 16.272 & 22.779 & 15.816 & 11.06 & 32.334 & 33.097 & 30.19 & 32.166 & 27.76 & 34.135 \\
\hline $12 / 10 / 114: 00$ & & 30.959 & 30.573 & 29.713 & 30.547 & & 29.913 & 31.001 & 30.47 & 30.25 & 28.858 & 26.617 & 32.191 & 36.705 & 33.219 & 16.876 & 16.272 & 22.774 & 15.82 & 11.056 & 32.322 & 33.092 & 30.183 & 32.164 & 27.76 & 34.13 \\
\hline $12 / 10 / 11$ 5:00 & & 30.955 & 30.566 & 29.694 & 30.537 & & 29.925 & 31.001 & 30.467 & 30.245 & 28.851 & 26.6 & 32.184 & 36.703 & 33.212 & 16.876 & 16.265 & 22.765 & 15.827 & 11.051 & 32.315 & 33.087 & 30.176 & 32.159 & 27.76 & 34.128 \\
\hline $12 / 10 / 116: 00$ & & 30.959 & 30.566 & 29.699 & 30.53 & & 29.92 & 31.006 & 30.47 & 30.245 & 28.851 & 26.6 & 32.184 & 36.701 & 33.212 & 16.876 & 16.265 & 22.763 & 15.827 & 11.048 & 32.315 & 33.087 & 30.173 & 32.154 & 27.763 & 34.125 \\
\hline $12 / 10 / 11$ 7:00 & & 30.962 & 30.568 & 29.699 & 30.53 & & 29.918 & 31.006 & 30.472 & 30.248 & 28.851 & 26.602 & 32.184 & 36.701 & 33.212 & 16.876 & 16.265 & 22.761 & 15.825 & 11.048 & 32.315 & 33.087 & 30.176 & 32.154 & 27.763 & 34.123 \\
\hline $12 / 10 / 118: 00$ & & 30.964 & 30.571 & 29.708 & 30.535 & & 29.913 & 31.006 & 30.47 & 30.25 & 28.856 & 26.612 & 32.187 & 36.705 & 33.212 & 16.876 & 16.267 & 22.758 & 15.83 & 11.053 & 32.315 & 33.087 & 30.178 & 32.157 & 27.763 & 34.123 \\
\hline $12 / 10 / 119: 00$ & & 30.966 & 30.571 & 29.708 & 30.535 & & 29.913 & 31.011 & 30.472 & 30.25 & 28.856 & 26.614 & 32.187 & 36.705 & 33.217 & 16.876 & 16.267 & 22.761 & 15.83 & 11.053 & 32.322 & 33.09 & 30.18 & 32.159 & 27.763 & 34.123 \\
\hline $12 / 10 / 1110: 00$ & & 30.966 & 30.575 & 29.716 & 30.537 & & 29.913 & 31.011 & 30.477 & 30.255 & 28.86 & 26.619 & 32.189 & 36.71 & 33.219 & 16.876 & $\begin{array}{l}16.269 \\
16.269\end{array}$ & 22.765 & $\begin{array}{l}15.837 \\
\end{array}$ & 11.056 & 32.327 & 33.092 & 30.19 & 32.161 & 27.765 & 34.128 \\
\hline $12 / 10 / 1111: 00$ & & $\begin{array}{l}30.960 \\
30.962\end{array}$ & 30.571 & 29.706 & $\begin{array}{l}30.535 \\
3.53\end{array}$ & & 29.927 & 31.013 & 30.472 & $\begin{array}{l}30.25 \\
30.25\end{array}$ & $\begin{array}{l}28.00 \\
28.853\end{array}$ & 26.607 & 32.191 & 36.71 & $\begin{array}{l}33.217 \\
33.217\end{array}$ & 16.878 & $\begin{array}{l}10.279 \\
16.274\end{array}$ & 22.77 & $\begin{array}{l}13.071 \\
15.848\end{array}$ & $\begin{array}{l}11.058 \\
11.058\end{array}$ & 32.317 & $\begin{array}{l}33.092 \\
33.092\end{array}$ & 30.178 & 32.161 & 27.765 & 34.128 \\
\hline 2:00 & & $\begin{array}{l}30.962 \\
30.962\end{array}$ & 30.566 & 29.697 & $\begin{array}{l}30.53 \\
30.53\end{array}$ & & 29.934 & 31.016 & $\begin{array}{l}30.474 \\
30.474\end{array}$ & $\begin{array}{r}30.253 \\
30.248\end{array}$ & $\begin{array}{l}28.851 \\
28.851\end{array}$ & $\begin{array}{r}20.017 \\
26.6\end{array}$ & $\begin{array}{l}32.1819 \\
32.189\end{array}$ & $\begin{array}{l}36.71 \\
36.705\end{array}$ & $\begin{array}{l}33.221 \\
33.221\end{array}$ & $\begin{array}{l}10.818 \\
16.876\end{array}$ & $\begin{array}{l}10.274 \\
16.274\end{array}$ & 22.767 & $\begin{array}{l}13.88 \\
15.855\end{array}$ & $\begin{array}{l}11.036 \\
11.056\end{array}$ & 32.315 & $\begin{array}{l}\begin{array}{r}3.3092 \\
33.09\end{array} \\
\end{array}$ & 30.176 & $\begin{array}{l}32.161 \\
32.161\end{array}$ & 27.765 & 34.128 \\
\hline 10 & & 7 & 30 & 29.671 & 30.5 & & 29.956 & 1 & 30.472 & 30 & 28.837 & 26.574 & 32.184 & 36.698 & 33.214 & 16 & 16.272 & 22.758 & 15.865 & 11.051 & 32.299 & 33.085 & 52 & 57 & 27.765 & 34.125 \\
\hline 0 & & 2 & 30 & 15 & & & 29.967 & 3 & 30.472 & 30.224 & 28.823 & 26.55 & 177 & 36.696 & 33.205 & 16.871 & 16.269 & 22.74 & 15.87 & 11.044 & 82 & 75 & 29 & 32.147 & 27.765 & .123 \\
\hline 0 & & & & 29.629 & & & 29.97 & 31.008 & 30.467 & 30.213 & 28.814 & 26.535 & 32.168 & 36.694 & 33.198 & & & 22.724 & 15.874 & 11.034 & 32.271 & 068 & .112 & 14 & 27.767 & 121 \\
\hline 0/11 16:00 & & 30.952 & 30.526 & 29.629 & 30.472 & & 29.96 & 31.008 & 30.47 & 30.21 & 28.812 & 26.538 & 32.163 & 36.687 & $\begin{array}{l}33.196 \\
33.196\end{array}$ & 16.869 & $\begin{array}{l}16.262 \\
16.262\end{array}$ & 22.703 & $\begin{array}{l}13.0 .84 \\
15.865\end{array}$ & $\begin{array}{l}1.043 \\
11.03\end{array}$ & 32.269 & 063 & 1112 & 32.135 & 27.767 & 34.118 \\
\hline 0/11 17:00 & & 30.95 & 30.524 & 29.627 & 30.46 & & 29.953 & 31.008 & 30.467 & 30.206 & 28.807 & 26.538 & 32.161 & 36.682 & 33.186 & 16.867 & 16.262 & 22.68 & 15.862 & 11.025 & 32.266 & 33.061 & 30.117 & 32.133 & 27.767 & 34.116 \\
\hline o/11 18:00 & & 30.95 & 30.522 & 29.629 & 30.4 & & 29.944 & 31.008 & 30.47 & 30.204 & 28.807 & 26.54 & 32.156 & 36.684 & 33.186 & 16.864 & 16.26 & 22.663 & 15.855 & 11.025 & 32.266 & & & 32.128 & 27.767 & 34.114 \\
\hline /111 19:00 & & 30.948 & 30.519 & 29.624 & 30 & & 29.942 & 31.008 & 30.47 & 30.199 & 28.802 & 26.538 & 32.154 & 36.68 & 33.184 & 16.862 & 16.26 & 22.64 & 15.855 & 11.02 & 32.259 & 33.056 & 5 & 32.126 & 27.77 & 34.114 \\
\hline 12/10/11 20:00 & & 30.948 & 30.519 & 29.624 & 30.4 & & 29.93 & 31.006 & 30.474 & 30.197 & 28.802 & 26.54 & 32.154 & 36.682 & 33.184 & 16.862 & 16.26 & 22.61 & 15.851 & 11.023 & 32.264 & 33.054 & 30.114 & 32.126 & 27.77 & 34.116 \\
\hline $12 / 10 / 1121: 00$ & & 30.948 & 30.517 & 29.622 & 30.4 & & 29.93 & 31.003 & 30.47 & 30.192 & 28.795 & 26.535 & 32.156 & 36.684 & 33.186 & 16.857 & 16.262 & 22.592 & 15.853 & 11.02 & 32.259 & 33.056 & 30.105 & 32.128 & 27.77 & 34.118 \\
\hline $12 / 10 / 1122: 00$ & & 30.945 & 30.51 & 29.613 & 30. & & 29.93 & 31.003 & 30.47 & 30.187 & 28.793 & 26.528 & 32.154 & 36.675 & 33.182 & 16.857 & 16.265 & 22.566 & 15.853 & 11.02 & 32.257 & 33.054 & 30.1 & 32.126 & 27.77 & 34.118 \\
\hline 12/10/11 23:00 & & 30.945 & 30.51 & 29.615 & 30.4 & & 29.923 & 31.001 & 30.47 & 30.185 & 28.791 & 26.533 & 32.154 & 36.68 & 33.184 & 16.855 & 16.267 & 22.564 & 15.846 & 11.016 & 32.255 & 33.052 & 30.105 & 32.126 & 27.77 & 34.121 \\
\hline $12 / 11 / 110: 00$ & & 30.945 & 30.508 & 29.61 & 30.4 & & 29.92 & 31.001 & 30.465 & 30.183 & 28.788 & 26.531 & 32.152 & 36.68 & 33.184 & 16.855 & 16.267 & 22.534 & 15.846 & 11.02 & 32.252 & 33.052 & 30.096 & 32.124 & 27.772 & 34.125 \\
\hline 12/11/11 1:00 & & 30.941 & 30.503 & 29.606 & 30.4 & & 29.92 & 30.999 & 30.467 & 30.178 & 28.784 & 26.526 & 32.149 & 36.68 & 33.177 & 16.853 & 16.267 & 22.502 & 15.848 & 11.016 & 32.255 & 33.049 & 30.096 & 32.121 & 27.77 & 34.123 \\
\hline $12 / 11 / 112: 00$ & & 30.941 & 30.499 & 29.599 & 30.4 & & 29.918 & 30.996 & 30.458 & 30.171 & 28.779 & 26.521 & 32.145 & 36.677 & 33.175 & 16.85 & 16.265 & 22.472 & 15.846 & 11.013 & 32.241 & 33.042 & 30.091 & 32.114 & 27.772 & 34.121 \\
\hline $12 / 11 / 113: 00$ & & 30.936 & 30.494 & 29.587 & 30. & & 29.925 & 30.996 & $\begin{array}{r}30.46 \\
30\end{array}$ & 30.166 & 28.772 & 26.514 & 32.138 & 36.677 & 33.17 & 16.848 & 16.26 & 22.439 & 15.844 & 11.001 & 32.234 & 33.035 & 30.079 & 32.11 & 27.772 & 34.118 \\
\hline $12 / 11 / 114: 00$ & & 30.931 & 30.485 & 29.573 & 30. & & 29.927 & 30.994 & 30.463 & 30.157 & 28.763 & 26.502 & 32.131 & 36.675 & 33.1 & 16.84 & 16.25 & 22.407 & $\begin{array}{l}15.8 \\
\end{array}$ & 10.994 & 32.21 & 6 & 5 & 32.1 & 27.772 & 34.111 \\
\hline $12 / 11 /$ & & 30.927 & 30.473 & 29.552 & 30.4 & & 29.937 & 30.989 & 30.458 & 30.146 & 28.749 & 26.48 & 32.117 & 36.668 & 33.151 & 16.843 & 16.246 & 22.374 & $\begin{array}{l}15.8 \\
15.8\end{array}$ & 10.987 & 32.208 & 018 & 46 & 32.091 & 27.772 & 34.107 \\
\hline $12 /$ & & 30.924 & 30.466 & 29.543 & 30.3 & & 29.932 & 30.984 & 30.458 & 30.136 & 28.742 & 26.473 & 32.11 & 36.666 & 33.139 & 16.836 & 16.239 & 22.342 & 15. & 10.976 & 32.199 & 9 & 37 & 32.081 & 27.774 & 34.102 \\
\hline 121 & & 30 & 30.4 & 29.9 & 30 & & & 30. & 30. & 30. & 28. & 26. & 32.1 & 36. & 33 & 16 & & 22 & 15 & 10.966 & 32.185 & 2 & 3 & 32 & 27.772 & 34.097 \\
\hline & & 30 & 30 & 29.5 & & & & & 30. & 30 & 28. & & 32. & 36. & 33. & & & 22 & 15 & 10 & & & 30 & 5 & 27.772 & 34.097 \\
\hline th & & 30.917 & 30. & 29. & & & & 30.9 & 30.4 & & & & 32.0 & & 33. & & & 22.2 & 15. & & & 32 & & 8 & 27.774 & . \\
\hline & & & & & & & & 30. & 30.4 & & & & 32. & & & & & & & & & & & & & \\
\hline & & & & 29.5 & & & & & & & & & & & & & & & & & & & & & & 34.097 \\
\hline & & & & 29 & & & & & & & & & & & & & & & & & & & & & 27. & 34. \\
\hline & & & 30. & 29. & & & & & & & & & 32 & & & & & 22 & 15 & & & & 8 & 32. & 27. & 34.6. \\
\hline 12/11/11 14:00 & & 30.896 & 30.408 & 29.468 & & & 29.916 & 30.956 & 30.441 & 30.071 & 28.684 & 26.423 & 32.063 & 36.642 & 33.092 & 16.813 & 16.215 & 22.127 & 15.837 & 10.929 & 32.136 & 32.962 & 29.971 & 32.03 & 27.774 & $34 . \mathrm{C}-\mathrm{r}-\mathrm{s}$ \\
\hline 12/11/11 15:00 & & 30.894 & 30.398 & 29.458 & 30.316 & & 29.913 & 30.949 & 30.441 & 30.062 & 28.677 & 26.411 & 32.051 & 36.637 & 33.085 & 16.808 & 16.213 & 22.099 & 15.827 & 10.922 & 32.131 & 32.952 & 29.959 & 32.023 & 27.774 & 34.086 \\
\hline
\end{tabular}




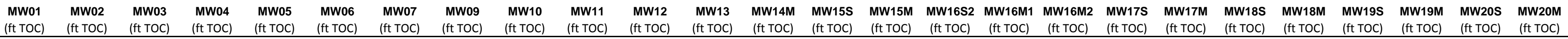

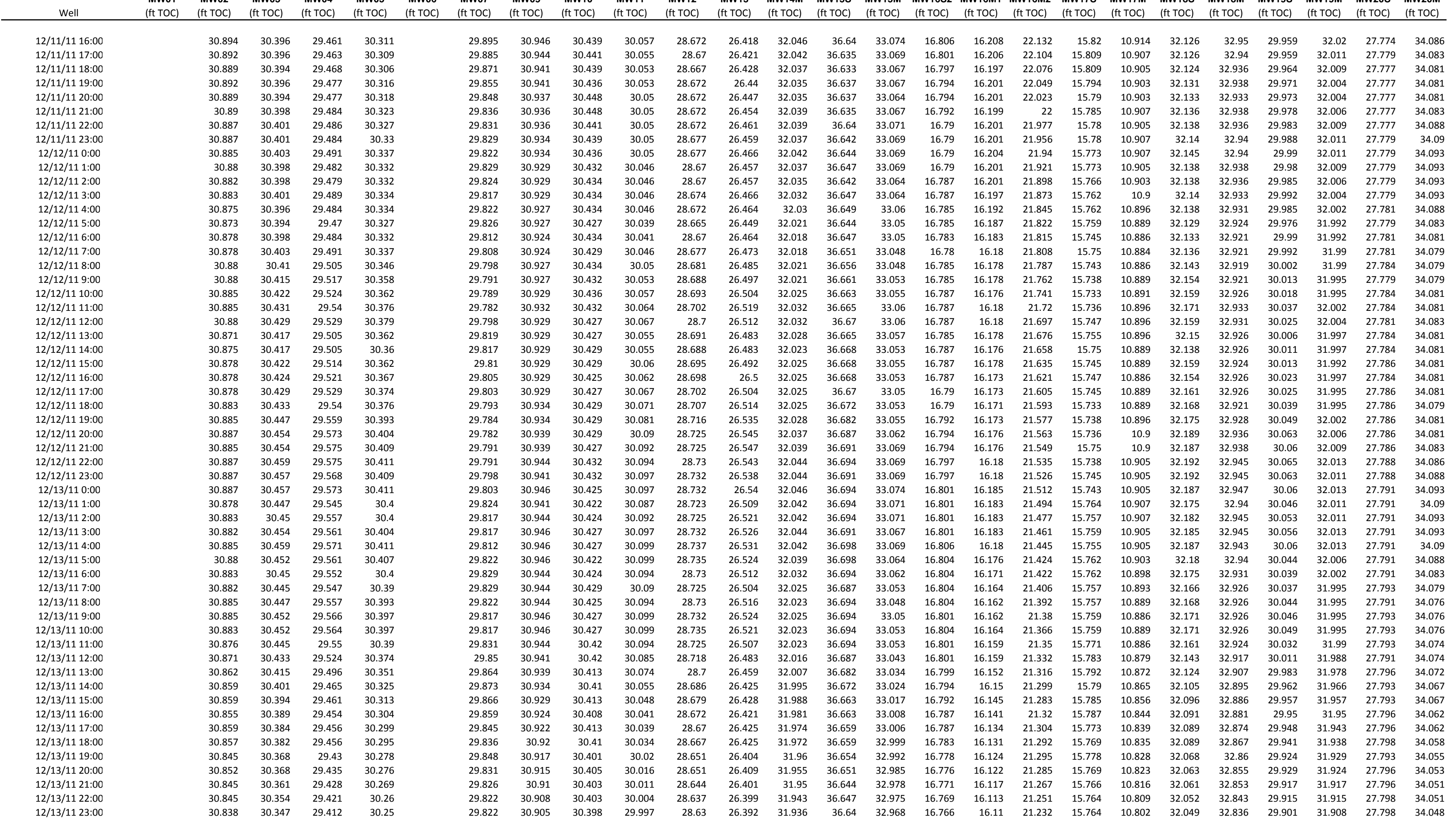




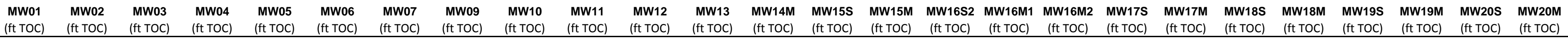

\begin{tabular}{|c|c|c|c|c|c|c|c|c|c|c|c|c|c|c|c|c|c|c|c|c|c|c|c|c|c|c|}
\hline Well & $\begin{array}{l}\text { (ft TOC) } \\
\text { (is }\end{array}$ & $\begin{array}{l}\text { (ft TOC) } \\
\text { (ft) }\end{array}$ & $\begin{array}{l}\text { (ft TOC) } \\
\text { (f) }\end{array}$ & (ft TOC) & $\begin{array}{l}\text { (ft toc) } \\
\text { (foc) }\end{array}$ & $\begin{array}{l}\text { (ft TOC) } \\
\text { (f) }\end{array}$ & (ft Toc) & $\begin{array}{l}\text { (ft TOC) } \\
\text { (f) }\end{array}$ & $\begin{array}{l}\text { (ft TOC) } \\
\text { (ft) }\end{array}$ & $\begin{array}{l}\text { (ft TOC) } \\
\text { (t) }\end{array}$ & $\begin{array}{l}\text { (ft TOC) } \\
\text { (f) }\end{array}$ & $\begin{array}{l}\text { (ft Toc) } \\
\text { (f) }\end{array}$ & (ft TOC) & $\begin{array}{l}\text { (ft TOC) } \\
\end{array}$ & (ft TOC) & (ft TOC) & $\begin{array}{l}\text { (ft TOC) } \\
\text { (f) }\end{array}$ & (ft TOC) & $\begin{array}{l}\text { (ft TOC) } \\
\text { (ft) }\end{array}$ & (ft TOC) & $\begin{array}{l}\text { (ft TOC) } \\
\end{array}$ & $\begin{array}{l}\text { (ft TOC) } \\
\text { (1) }\end{array}$ & $\begin{array}{l}\text { (ft TOC) } \\
\text { (n) }\end{array}$ & (ft TOC) & $\begin{array}{l}\text { (ft TOC) } \\
\end{array}$ & (ft TOC) \\
\hline $12 / 14 / 110: 00$ & & 30.834 & 30.34 & 29.395 & 30.239 & & 29.824 & 30.903 & 30.396 & 29.983 & 28.618 & 26.378 & 31.932 & 36.642 & 32.961 & $1 \quad 16.762$ & 16.108 & 21.214 & 15.766 & 10.797 & 32.033 & 32.831 & 29.889 & 31.901 & 27.798 & 34.046 \\
\hline $12 / 14 / 11$ 1:00 & & 30.831 & 30.328 & 29.384 & 30.227 & & 29.822 & 30.908 & 30.389 & 29.976 & 28.612 & 26.37 & 31.925 & 36.635 & 32.952 & $2 \quad 16.759$ & 16.099 & 21.198 & 15.764 & 10.792 & 32.028 & 32.822 & 29.872 & 31.894 & 27.798 & 34.046 \\
\hline $12 / 14 / 112: 00$ & & 30.827 & 30.319 & 29.372 & 30.215 & & 29.817 & 30.917 & 30.394 & 29.964 & 28.602 & 26.361 & 31.913 & 36.63 & 32.942 & $2 \quad 16.755$ & 16.096 & 21.182 & 15.757 & 10.778 & 32.014 & 32.812 & & & 27.8 & 34.039 \\
\hline $12 / 14 / 113: 00$ & & 30.824 & 30.314 & 29.365 & 30.206 & & 29.808 & 30.932 & 30.391 & 29.957 & 28.595 & 26.356 & 31.904 & 36.63 & 32.933 & 16.748 & 16.087 & 21.161 & 15.747 & 10.7 & 32.005 & 32.805 & 29.854 & & & \\
\hline $12 / 14 / 114: 00$ & & 30.817 & 30.305 & 29.346 & 30.189 & & 29.812 & 30.934 & 30.386 & 29.944 & 28.581 & 26.342 & 31.892 & 36.626 & 32.921 & 16.743 & 16.08 & 21.142 & 15.757 & 10.759 & 31.986 & 32.791 & 29.835 & .863 & 27.798 & 029 \\
\hline $12 / 14 / 115: 00$ & & 30.817 & 30.291 & 29.334 & 30.173 & & 29.808 & 30.936 & 30.384 & 29.93 & 28.57 & 26.33 & 31.878 & 36.619 & 32.912 & 16.741 & 16.071 & 21.124 & 15.755 & 10.745 & 31.975 & 32.779 & 29.816 & 1.851 & 27.8 & 34.025 \\
\hline $12 / 14 / 116: 00$ & & 30.81 & 30.279 & 29.318 & 30.154 & & 29.805 & 30.944 & 30.379 & 29.918 & 28.556 & 26.318 & 31.864 & 36.619 & 32.895 & $5 \quad 16.736$ & $\begin{array}{l}16.061 \\
\end{array}$ & 21.124 & 15.747 & 10.731 & 31.961 & 32.767 & 29.802 & 31.835 & 27.798 & 34.018 \\
\hline $12 / 14 / 117: 00$ & & 30.808 & 30.27 & 29.306 & 30.141 & & 29.798 & 30.956 & 30.375 & 29.904 & 28.546 & 26.306 & 31.84 & 36.612 & 32.874 & $4 \quad 16.729$ & 16.045 & 21.105 & 15.736 & 10.71 & 31.937 & 32.744 & 29.781 & 31.814 & 27.798 & 34.004 \\
\hline 12/14/11 8:00 & & 30.81 & 30.265 & 29.306 & 30.131 & & 29.782 & 30.951 & 30.384 & 29.899 & 28.542 & 26.311 & 31.838 & 36.607 & 32.867 & $7 \quad 16.725$ & 16.035 & 21.094 & 15.726 & 10.705 & 31.937 & 32.739 & 29.781 & 31.807 & 27.8 & 33.999 \\
\hline 12/14/11 9:00 & & 30.806 & 30.261 & 29.299 & 30.119 & & 29.775 & 30.934 & 30.377 & 29.893 & 28.535 & 26.308 & 31.829 & 36.607 & 32.863 & $\begin{array}{l}3 \quad 16.72 \\
\end{array}$ & 16.033 & 21.077 & 15.729 & 10.698 & 31.928 & 32.727 & 29.767 & 31.798 & 27.8 & 33.997 \\
\hline $12 / 14 / 1110: 00$ & & 30.803 & 30.254 & 29.299 & 30.113 & & 29.761 & 30.915 & 30.377 & 29.886 & 28.532 & 26.308 & 31.822 & 36.6 & 32.853 & $\begin{array}{l}316.715 \\
\end{array}$ & 16.026 & 21.066 & 15.729 & 10.691 & 31.919 & 32.725 & 29.755 & 31.793 & 27.8 & 33.994 \\
\hline 12/14/11 11:00 & & 30.803 & 30.251 & 29.299 & 30.11 & & 29.749 & 30.905 & 30.377 & 29.883 & 28.528 & 26.315 & 31.819 & 36.602 & 32.851 & $1 \quad 16.713$ & 16.024 & 21.052 & 15.726 & 10.686 & 31.916 & 32.72 & 29.762 & 31.79 & 27.8 & 33.994 \\
\hline $12 / 14 / 1112: 00$ & & 30.803 & 30.249 & 29.302 & 30.108 & & 29.739 & 30.893 & 30.377 & 29.879 & 28.525 & 26.32 & 31.817 & 36.595 & 32.846 & $6 \quad 16.708$ & 16.021 & 21.038 & 15.719 & 10.682 & 31.923 & 32.718 & 29.757 & 31.788 & 27.8 & 33.994 \\
\hline $12 / 14 / 1113: 00$ & & 30.794 & 30.24 & 29.285 & 30.091 & & 29.742 & 30.922 & 30.375 & 29.869 & 28.514 & 26.311 & 31.812 & 36.598 & 32.842 & 16.706 & 16.017 & 21.022 & 15.724 & 10.679 & 31.907 & 32.71 & 29.736 & 31.781 & 27.803 & 33.992 \\
\hline $12 / 14 / 1114: 00$ & & 30.792 & 30.235 & 29.281 & 30.085 & & 29.732 & 30.939 & 30.372 & 29.862 & 28.509 & 26.303 & 31.805 & 36.598 & 32.837 & 16.704 & 16.012 & 21.01 & 15.719 & 10.67 & 31.9 & 32.708 & 29.738 & 31.779 & 27.803 & 33.992 \\
\hline 12/14/11 15:00 & & 30.794 & 30.235 & 29.288 & 30.08 & & 29.718 & 30.934 & 30.372 & 29.86 & 28.509 & 26.315 & 31.803 & 36.593 & 32.832 & 16.699 & $\begin{array}{l}16.012 \\
\text {. }\end{array}$ & 20.99 & 15.71 & 10.668 & 31.902 & 32.701 & 29.734 & 31.774 & 27.803 & 33.992 \\
\hline $12 / 14 / 1116: 00$ & & 30.799 & 30.244 & 29.313 & 30.094 & & 29.688 & 30.929 & 30.372 & 29.867 & 28.521 & 26.342 & 31.803 & 36.595 & 32.832 & 16.699 & $\begin{array}{l}16.012 \\
\text {. }\end{array}$ & 21.04 & 15.698 & 10.67 & 31.916 & 32.706 & 29.757 & 31.774 & 27.803 & 33.994 \\
\hline 12/14/11 17:00 & & 30.801 & 30.251 & 29.332 & 30.108 & & 29.671 & 30.922 & 30.372 & 29.872 & 28.53 & 26.363 & 31.805 & 36.6 & 32.835 & 16.699 & $\begin{array}{l}16.007 \\
\end{array}$ & 21.026 & 15.684 & 10.672 & 31.928 & 32.706 & 29.774 & 31.776 & 27.803 & 33.994 \\
\hline $12 / 14 / 1118: 00$ & & 30.806 & 30.265 & 29.363 & 30.126 & & 29.645 & 30.906 & 30.375 & 29.883 & 28.546 & 26.394 & 31.81 & 36.607 & 32.835 & 16.699 & 16.01 & 21.013 & $\begin{array}{l}15.004 \\
15.67\end{array}$ & 10.675 & 31.944 & 32.708 & 29.795 & 31.779 & 27.803 & $\begin{array}{l}33.994 \\
3.994\end{array}$ \\
\hline $12 / 14 / 1119: 00$ & & $\begin{array}{l}30.000 \\
30.803\end{array}$ & $\begin{array}{l}30.203 \\
30.279\end{array}$ & 29.377 & 30.143 & & 29.636 & $\begin{array}{l}30.908 \\
30.908\end{array}$ & 30.377 & $\begin{array}{l}29.0003 \\
29.893\end{array}$ & $\begin{array}{l}28.540 \\
28.56\end{array}$ & $\begin{array}{l}20.544 \\
26.409\end{array}$ & $\begin{array}{l}31.81 \\
31.812\end{array}$ & 36.612 & 32.842 & 16.699 & $\begin{array}{l}16.001 \\
16.05\end{array}$ & $\begin{array}{l}21.003 \\
21.003\end{array}$ & $\begin{array}{l}15.668 \\
15.668\end{array}$ & 10.679 & 31.954 & 32.715 & 29.809 & 31.783 & 27.803 & $\begin{array}{l}33.994 \\
33.994\end{array}$ \\
\hline $12 / 14 / 1120: 00$ & & $\begin{array}{l}30.815 \\
30.815\end{array}$ & 30.3 & 29.416 & $\begin{array}{l}30.173 \\
30.171\end{array}$ & & $\begin{array}{r}29.030 \\
29.61\end{array}$ & $\begin{array}{l}30.877 \\
30.877\end{array}$ & $\begin{array}{l}30.379 \\
30.379\end{array}$ & 29.911 & $\begin{array}{l}28.5300 \\
28.59\end{array}$ & $\begin{array}{l}20.409 \\
26.449\end{array}$ & 31.876 & $\begin{array}{l}30.012 \\
36.621\end{array}$ & $\begin{array}{l}32.842 \\
32.907\end{array}$ & 16.701 & $\begin{array}{l}10.024 \\
16.024\end{array}$ & 20.992 & $\begin{array}{l}13.006 \\
15.647\end{array}$ & $\begin{array}{l}10.741 \\
10.79\end{array}$ & $\begin{array}{l}31.924 \\
32.003\end{array}$ & 32.777 & 29.847 & 31.844 & 27.805 & $\begin{array}{l}33.997 \\
33.997\end{array}$ \\
\hline $12 / 14 / 1121: 00$ & & 30.81 & 30.312 & 29.433 & 30.192 & & 29.612 & 30.874 & 30.372 & 29.923 & 28.591 & 26.468 & 31.862 & 36.635 & 32.891 & 16.704 & $\begin{array}{l}16.024 \\
\end{array}$ & 20.976 & 15.651 & 10.729 & 32.003 & 32.763 & .851 & 31.83 & 27.803 & 33.997 \\
\hline $12 / 14 / 1122: 00$ & & $\begin{array}{r}30.815 \\
30.815\end{array}$ & 30.331 & 29.451 & 30.2 & & 29.603 & 86 & 30.375 & 29.937 & 28.605 & 26.483 & 31.862 & 36.642 & 32.891 & 16.706 & $\begin{array}{l}10.024 \\
16.024\end{array}$ & 20.973 & 15.64 & 10.726 & 32.021 & 32.763 & .877 & 31.833 & 27.803 & $\begin{array}{l}33.999 \\
3.999\end{array}$ \\
\hline $12 / 14 / 1$ & & & 30.352 & 29.486 & 30.243 & & 29.591 & 343 & 30.379 & 29.957 & 28.625 & 26.516 & 31.871 & 36.651 & 32.898 & 16.711 & 16.028 & 20.969 & 15.635 & 10.733 & 2.04 & 32.774 & .91 & 842 & 27.803 & 34.004 \\
\hline 12/15/11 0:00 & & 30 & 30.37 & 29.51 & 30.266 & & & 30.841 & 30.379 & 29.974 & 28.644 & 26.538 & 31.88 & 36.661 & 32.905 & 16.715 & 16.031 & 20.959 & 15.628 & 10.745 & 32.059 & 32.779 & 29.931 & 1.849 & 27.805 & 34.011 \\
\hline 12/15/11 1:00 & & 30.834 & 30.391 & 29.538 & 30.29 & & 29.589 & 30.836 & 30.384 & & 28.663 & 26.559 & 31.892 & 36.67 & 32.917 & 16.72 & 16.038 & 20.953 & 15.618 & 10.752 & 32.077 & 32.793 & 29.957 & .861 & 27.805 & 34.015 \\
\hline $12 / 15 / 112: 00$ & & 30.834 & 30.405 & 29.547 & 30.309 & & 29.601 & 30.833 & 30.377 & & 28.677 & 26.564 & 31.899 & 36.68 & 32.924 & 16.725 & 16.04 & 20.943 & 15.632 & 10.762 & 32.089 & 32.803 & 29.966 & 31.868 & 27.803 & 34.018 \\
\hline 12/15/11 3:00 & & 30.843 & 30.422 & 29.568 & 30.327 & & 29.601 & 30.824 & 30.384 & 30.025 & 28.693 & 26.581 & 31.908 & 36.68 & 32.935 & 16.732 & 16.045 & 20.934 & 15.621 & 10.771 & 32.105 & 32.812 & 29.983 & 31.88 & 27.805 & 34.022 \\
\hline $12 / 15 / 11$ 4:00 & & 30.845 & 30.433 & 29.582 & 30.341 & & 29.612 & 30.834 & 30.389 & 30.036 & 28.707 & 26.588 & 31.918 & 36.694 & 32.942 & 16.736 & 16.047 & 20.925 & 15.625 & 10.778 & 32.117 & 32.817 & 29.997 & 31.884 & 27.805 & 34.022 \\
\hline $12 / 15 / 11$ 5:00 & & 30.845 & 30.447 & 29.592 & 30.355 & & 29.627 & 30.841 & 30.387 & 30.05 & 28.718 & 26.595 & 31.922 & 36.696 & 32.947 & 16.741 & 16.047 & 20.916 & 15.632 & 10.785 & 32.119 & 32.824 & 30.006 & 31.891 & 27.805 & 34.022 \\
\hline $12 / 15 / 116: 00$ & & 30.855 & 30.468 & 29.62 & 30.374 & & 29.624 & 30.846 & 30.387 & 30.071 & 28.737 & 26.617 & 31.934 & 36.701 & 32.956 & 16.746 & 16.05 & 20.925 & 15.625 & 10.792 & 32.136 & 32.831 & 30.03 & 31.898 & 27.805 & 34.025 \\
\hline $12 / 15 / 11$ 7:00 & & 30.857 & 30.482 & 29.636 & 30.393 & & 29.634 & 30.85 & 30.389 & 30.087 & 28.753 & 26.629 & 31.939 & 36.705 & 32.963 & 16.75 & 16.052 & 20.925 & 15.63 & 10.799 & 32.152 & 32.841 & 30.046 & 31.905 & 27.807 & 34.025 \\
\hline $12 / 15 / 118: 00$ & & 30.864 & 30.499 & 29.652 & 30.409 & & 29.641 & 30.86 & 30.396 & 30.106 & 28.767 & 26.641 & 31.946 & 36.717 & 32.971 & 16.757 & 16.054 & 20.922 & 15.632 & 10.804 & 32.161 & 32.845 & 30.056 & 31.915 & 27.807 & 34.027 \\
\hline $12 / 15 / 119: 00$ & & 30.871 & 30.515 & 29.673 & 30.425 & & 29.643 & 30.865 & 30.398 & 30.122 & 28.786 & 26.655 & 31.953 & 36.715 & 32.978 & 16.762 & 16.054 & 20.913 & 15.63 & 10.816 & 32.18 & 32.855 & 30.079 & 31.922 & 27.807 & 34.027 \\
\hline 12/15/11 10:00 & & 30.878 & 30.531 & 29.69 & 30.444 & & 29.657 & 30.872 & 30.398 & 30.143 & 28.802 & 26.667 & 31.965 & 36.724 & 32.989 & 16.766 & $\begin{array}{l}16.059 \\
\end{array}$ & 20.899 & 15.644 & 10.828 & 32.187 & 32.864 & .089 & 31.931 & 27.81 & 34.029 \\
\hline 11:00 & & 30.878 & 30.54 & 29.699 & 30. & & 29.676 & $\begin{array}{l}30.879 \\
3\end{array}$ & 30.398 & 30. & 28.814 & 26.669 & 31.972 & 36.724 & $\begin{array}{l}32.996 \\
\end{array}$ & 16.773 & 16.064 & 20.883 & 15.656 & $\begin{array}{l}10.837 \\
\end{array}$ & 32.196 & 32.872 & 30.096 & 31.938 & 27.81 & 34.032 \\
\hline $12: 00$ & & 30.88 & 30.545 & 29.701 & $\begin{array}{l}30.4 \\
30.4\end{array}$ & & 29.695 & 30.884 & 30.403 & 30.164 & 28.821 & 26.667 & 31.981 & 36.729 & 33.006 & 16.778 & $\begin{array}{l}10.006 \\
16.066\end{array}$ & 20.885 & 15.677 & $\begin{array}{l}10.839 \\
10.839\end{array}$ & 32.203 & 32.883 & 30.103 & 31.948 & 27.81 & 34.034 \\
\hline 12 & & $\begin{array}{l}30.800 \\
30.871\end{array}$ & 30.533 & 29.664 & 30.4 & & 29.742 & $\begin{array}{l}3.004 \\
30.917\end{array}$ & 30.396 & $\begin{array}{l}30.157 \\
30.157\end{array}$ & 28.809 & 26.626 & 31.979 & 36.729 & 33.003 & $\begin{array}{l}36.78 \\
3\end{array}$ & $\begin{array}{l}16.064 \\
16.064\end{array}$ & 20.872 & 15.703 & $\begin{array}{l}10.842 \\
10.852\end{array}$ & 32.182 & 32.881 & 30.07 & $\begin{array}{l}31.945 \\
31.94\end{array}$ & 7.81 & 34.034 \\
\hline 12 & & 30.875 & $\begin{array}{l}30.533 \\
30.529\end{array}$ & $\begin{array}{l}29.0064 \\
29.662\end{array}$ & 30. & & $\begin{array}{l}29.758 \\
\end{array}$ & 30.915 & $\begin{array}{l}30.398 \\
30.398\end{array}$ & 30. & $\begin{array}{l}28.0093 \\
28.812\end{array}$ & $\begin{array}{l}20.020 \\
26.614\end{array}$ & 31.979 & 36.726 & 33.003 & $\begin{array}{r}10.70 \\
3\end{array}$ & $\begin{array}{l}10.04 \\
16.068\end{array}$ & 20.865 & $10 . / 05$ & $\begin{array}{l}10.042 \\
10.839\end{array}$ & 32.175 & $\begin{array}{l}32.01 \\
32.879\end{array}$ & 30.063 & 45 & 7.81 & $\begin{array}{l}34.034 \\
34.034\end{array}$ \\
\hline & & 30 & 30 & 29 & & & & 30 & & & 28 & 26. & 3 & 36 & 33. & 16 & 16 & $2 c$ & 15.717 & 10.839 & 32.168 & 6 & 9 & 5 & 81 & 34.036 \\
\hline & & 30.8 & & 29.662 & & & 29 & & & & 28 & & & 36 & 33.008 & 16 & 16 & 26 & 15.722 & 10.842 & 32.178 & 32.883 & 55 & 8 & 81 & 34.036 \\
\hline & & 30 & & 29.669 & 30. & & & & & & 28.818 & & & 29 & 33.006 & $\begin{array}{ll}5.787 \\
5\end{array}$ & & & & 10.844 & 32.178 & & & 48 & 81 & 34.039 \\
\hline $12 / 1$ & & 30.8 & 30.536 & 29.68 & & & 29 & 30. & & & 28.8 & & & 36 & 33.006 & 16.79 & & & 29 & 10.849 & 32.185 & 32.883 & & & & 34.039 \\
\hline $12 / 1$ & & 30. & 30.54 & 29.692 & 30. & & & 30.9 & & & & & 31. & 36.734 & 33 & 16. & & 20.5 & 15. & 10.851 & 32.189 & & & & 12 & 34.039 \\
\hline $12 /$ & & 30. & 30.5 & 29. & & & & 30 & & & & & & 36.731 & & & & & 15. & 10.851 & 32. & & & & 17.812 & 34.039 \\
\hline $12 / 1$ & & 30. & 30.5 & & & & & & & & 28 & & 31.993 & 36.741 & 33.017 & 16 & 16. & 20.879 & 15.738 & 10.856 & 32.194 & 32.893 & & 31.959 & 27.812 & 34.039 \\
\hline 12/15/11 22:00 & & 30 & 30. & 29.706 & 30 & & & 30.9 & 30.408 & 30.2 & $28 . \varepsilon$ & 26. & 31.995 & 36.733 & 33.017 & 16.797 & 16. & 20.8 & 15. & 10.853 & 32.196 & 32.895 & 30.086 & 31.964 & 27.814 & 34.039 \\
\hline 12/15/11 23:00 & & 30.8 & 30.5 & 29.713 & & & 29. & 30. & 30.413 & 30 & 28.8. & 26.636 & & 36.74 & 33.6 & 16.797 & 16.078 & 20.862 & 15. & 10.863 & 32.199 & 32.898 & 30.093 & 31.966 & 27.814 & 34.041 \\
\hline $12 / 16 / 110: 00$ & & 30.89 & 30.554 & 29.716 & 30.4 & & 29.7 & 30.924 & 30.41 & 30.213 & 28.8 & 26.636 & 32.002 & 36.738 & 33.027 & 16.799 & 16.08 & 20.853 & 15.75 & 10.863 & 32.201 & 32.902 & 30.093 & 31.971 & 27.814 & 34.043 \\
\hline 12/16/11 1:00 & & 30.901 & 30.554 & 29.713 & 30.458 & & 29.796 & 30.924 & 30.413 & 30.215 & 28.858 & 26.631 & 32.004 & 36.738 & 33.029 & 16.799 & 16.082 & 20.842 & 15.755 & 10.865 & 32.203 & 32.905 & 30.091 & 31.971 & 27.814 & 34.046 \\
\hline $12 / 16 / 112: 00$ & & 30.906 & 30.554 & 29.713 & 30.456 & & 29.801 & 30.927 & 30.415 & 30.217 & 28.863 & 26.629 & 32.004 & 36.738 & 33.034 & 16.801 & 16.082 & 20.835 & 15.759 & 10.87 & 32.203 & 32.907 & 30.091 & 31.974 & 27.814 & 34.046 \\
\hline $12 / 16 / 113: 00$ & & 30.904 & 30.554 & 29.713 & 30.456 & & 29.81 & 30.929 & 30.413 & 30.222 & 28.863 & 26.626 & 32.007 & 36.74 & 33.034 & 16.801 & 16.087 & 20.828 & 15.764 & 10.87 & 32.199 & 32.907 & 30.089 & 31.976 & 27.814 & 34.046 \\
\hline $12 / 16 / 114: 00$ & & 30.906 & 30.552 & 29.708 & 30.451 & & 29.817 & 30.932 & 30.413 & 30.222 & 28.86 & 26.619 & 32.007 & 36.741 & 33.029 & 16.799 & 16.087 & 20.821 & 15.769 & 10.87 & 32.196 & 32.909 & 30.084 & 31.974 & 27.814 & 34.048 \\
\hline $12 / 16 / 115: 00$ & & 30.906 & 30.552 & 29.708 & 30.449 & & 29.822 & 30.934 & 30.415 & 30.222 & 28.863 & 26.617 & 32.004 & 36.74 & 33.034 & 16.799 & 16.087 & 20.811 & 15.773 & 10.867 & 32.194 & 32.907 & 30.082 & 31.974 & 27.814 & 34.046 \\
\hline 12/16/11 6:00 & & 30.913 & 30.559 & 29.727 & 30.458 & & 29.81 & 30.934 & 30.422 & 30.231 & 28.872 & 26.634 & 32.009 & 36.743 & 33.034 & 16.801 & 16.087 & 20.8 & 15.759 & 10.87 & 32.21 & 32.909 & 30.098 & 31.974 & 27.817 & 34.046 \\
\hline 12/16/11 7:00 & & 30.918 & 30.568 & 29.746 & 30.467 & & 29.805 & 30.939 & 30.42 & 30.243 & 28.881 & 26.648 & 32.011 & 36.75 & 33.039 & 16.804 & 16.087 & 20.795 & 15.764 & 10.872 & 32.213 & 32.914 & 30.107 & 31.978 & 27.817 & 34.048 \\
\hline
\end{tabular}




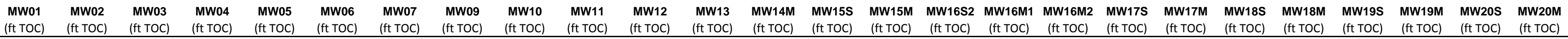

\begin{tabular}{|c|c|c|c|c|c|c|c|c|c|c|c|c|c|c|c|c|c|c|c|c|c|c|c|c|c|c|}
\hline Well & $\begin{array}{l}\text { MwOI } \\
\text { (ft TOC) }\end{array}$ & $\begin{array}{l}\text { MW02 } \\
\text { (ft TOC) }\end{array}$ & $\begin{array}{l}\text { (ft TOC) } \\
\end{array}$ & $\begin{array}{r}\text { MWva4 } \\
\text { (ft TOC) }\end{array}$ & $\begin{array}{r}\text { Mwos } \\
\text { (ft TOC) }\end{array}$ & $\begin{array}{l}\text { MWvo6 } \\
\text { (ft TOC) }\end{array}$ & $\begin{array}{l}\text { (ft TOC) } \\
\text { (ft }\end{array}$ & $\begin{array}{l}\text { Mwo9 } \\
\text { (ft ToC) }\end{array}$ & $\begin{array}{l}\text { MW10 } \\
\text { (ft ToC) }\end{array}$ & $\begin{array}{r}\text { MWII } \\
\text { (ft TOC) }\end{array}$ & $\begin{array}{r}\text { MW12 } \\
\text { (ft TOC) }\end{array}$ & $\begin{array}{l}\text { MWVI3 } \\
(\mathrm{ft} \text { TOC) }\end{array}$ & $\begin{array}{l}\text { (ft TOC) } \\
\end{array}$ & $\begin{array}{l}\text { MWvitss } \\
\text { (ft TOC) }\end{array}$ & $\begin{array}{l}\text { (ft TOC) } \\
\end{array}$ & $\begin{array}{l}\text { (ft TOC) } \\
\end{array}$ & $\begin{array}{l}\text { (ft TOC) } \\
\end{array}$ & $\begin{array}{l}\text { (ft TOC) } \\
\end{array}$ & (ft TOC) & (ft TOC) & $\begin{array}{l}\text { MWVIDS } \\
\text { (ft TOC) }\end{array}$ & $\begin{array}{l}\text { MWIsM } \\
\text { (ft TOC) }\end{array}$ & $\begin{array}{l}\text { (ft TOC) } \\
\end{array}$ & (ft TOC) & $\begin{array}{l}\text { MWUDS } \\
\text { (ft TOC) }\end{array}$ & $\begin{array}{l}\text { (ft TOC) } \\
\text { (at }\end{array}$ \\
\hline $12 / 16 / 118: 00$ & & 30.913 & 30.564 & 29.732 & 30.46 & & 29.824 & 30.951 & 30.42 & 30.241 & 28.879 & 26.634 & 32.009 & 36.743 & 33.036 & 16.801 & 16.082 & 20.795 & 15.778 & 10.875 & 32.201 & 32.909 & 30.096 & 31.978 & 27.817 & 34.043 \\
\hline 12/16/11 9:00 & & 30.915 & 30.559 & 29.73 & 30.458 & & 29.833 & 30.946 & 30.422 & 30.238 & 28.877 & 26.629 & 32.007 & 36.745 & 33.034 & 16.801 & 16.08 & 20.784 & 15.78 & 10.872 & 32.201 & 32.905 & 30.089 & 31.974 & 27.817 & 34.043 \\
\hline $12 / 16 / 1110: 00$ & & 30.917 & 30.559 & 29.732 & 30.458 & & 29.838 & 30.944 & 30.425 & 30.243 & 28.879 & 26.626 & 32.007 & 36.745 & 33.031 & 16.801 & 16.082 & 20.774 & 15.787 & 10.87 & 32.199 & 32.907 & 30.091 & 31.974 & 27.817 & 34.043 \\
\hline 12/16/11 11:00 & & 30.917 & 30.561 & 29.732 & 30.458 & & 29.845 & 30.953 & 30.424 & 30.243 & 28.879 & 26.629 & 32.007 & 36.771 & 33.031 & 16.799 & 16.082 & 20.765 & 15.801 & 10.867 & 32.199 & 32.905 & 30.093 & 31.974 & 27.819 & 34.043 \\
\hline $12 / 16 / 1112: 00$ & & 30.911 & 30.55 & 29.706 & 30.444 & & 29.866 & 30.977 & 30.422 & 30.234 & 28.87 & 26.602 & 32.002 & 36.832 & 33.029 & 16.799 & 16.08 & 20.751 & 15.82 & 10.863 & 32.187 & 32.905 & 30.067 & 31.971 & 27.817 & 34.041 \\
\hline 12/16/11 13:00 & & 30.911 & 30.538 & 29.683 & 30.425 & & 29.888 & 31.001 & 30.422 & 30.224 & 28.858 & 26.581 & 31.997 & 36.738 & 33.024 & 16.794 & 16.075 & 20.745 & 15.834 & 10.863 & 32.166 & 32.898 & 30.049 & 31.964 & 27.817 & 34.039 \\
\hline $12 / 16 / 1114: 00$ & & 30.906 & 30.526 & 29.662 & 30.409 & & 29.899 & 31.013 & 30.42 & 30.215 & 28.849 & 26.557 & 31.988 & 36.731 & 33.017 & 16.79 & 16.075 & 20.735 & 15.844 & 10.853 & 32.154 & 32.893 & 30.03 & 31.957 & 27.819 & 34.039 \\
\hline $12 / 16 / 1115: 00$ & & 30.901 & 30.515 & 29.645 & 30.39 & & 29.904 & 31.023 & 30.42 & 30.204 & 28.832 & 26.543 & 31.983 & 36.727 & 33.013 & 16.79 & 16.073 & 20.758 & 15.851 & 10.851 & 32.138 & 32.883 & 30.013 & 31.952 & 27.819 & 34.039 \\
\hline $12 / 16 / 1116: 00$ & & 30.901 & 30.51 & 29.641 & 30.379 & & 29.899 & 31.015 & 30.42 & 30.197 & 28.83 & 26.538 & 31.979 & 36.724 & 33.008 & 16.783 & 16.071 & 20.807 & 15.841 & 10.844 & 32.138 & 32.879 & 30.006 & 31.948 & 27.821 & 34.039 \\
\hline 12/16/11 17:00 & & 30.899 & 30.503 & 29.629 & 30.367 & & 29.895 & 31.02 & 30.417 & 30.19 & 28.823 & 26.531 & 31.974 & 36.719 & 33.001 & 16.78 & $\begin{array}{l}16.071 \\
\end{array}$ & 20.835 & 15.848 & 10.839 & 32.133 & 32.874 & 29.999 & 31.941 & 27.821 & 34.039 \\
\hline $12 / 16 / 1118: 00$ & & 30.901 & 30.503 & 29.645 & 30.369 & & 29.878 & 30.999 & 30.422 & 30.192 & 28.825 & 26.547 & 31.974 & 36.722 & 33.001 & 16.778 & 16.068 & 20.825 & 15.837 & 10.837 & 32.131 & 32.872 & 30.004 & 31.938 & 27.821 & 34.039 \\
\hline 6/11 19:00 & & 30.901 & 30.503 & 29.648 & 30.367 & & 29.866 & 30.989 & 30.42 & 30.19 & 28.825 & 26.55 & 31.969 & 36.719 & 32.994 & 16.776 & 16.068 & 20.809 & 15.83 & 10.835 & 32.129 & 32.869 & 30.006 & 31.936 & 27.821 & 34.036 \\
\hline 10 & & 30.901 & 30.503 & 29.65 & 30.36 & & 29.859 & 30.984 & 30.422 & 30.187 & 28.825 & 26.55 & 31.967 & 36.719 & 32.996 & 16.773 & 16.064 & 20.793 & 15.827 & 10.832 & 32.124 & 32.869 & 30.006 & 31.936 & 27.821 & 34.036 \\
\hline 10 & & 30.904 & 30.51 & 29.664 & 30.3 & & 29.845 & 30.968 & 30.424 & 30.194 & 28.832 & 26.569 & 31.967 & 36.722 & 32.994 & 16.771 & 16.064 & 20.779 & 15.823 & 10.835 & 32.14 & 32.869 & 30.02 & 31.938 & 27.821 & .036 \\
\hline $12 / 16 /$ & & 30.906 & 30.508 & 29.664 & 30.3 & & 29.843 & 30.958 & 30.425 & 30.194 & 28.832 & 26.571 & 31.969 & 36.729 & 32.994 & 16.769 & 16.064 & 20.765 & 15.818 & 10.83 & 32.14 & 867 & 30.027 & 31.936 & 27.824 & .036 \\
\hline 12/16/11 23:00 & & 30.906 & 30.508 & 29.669 & 30.383 & & 29.843 & 30.953 & 30.425 & 30.192 & 28.832 & 26.574 & 31.967 & 36.729 & 32.994 & 16.766 & 16.061 & .754 & 15.825 & 10.832 & 32.14 & .867 & 30.023 & 1.936 & 27.824 & .036 \\
\hline $12 / 17$ & & 30.908 & 30.513 & 29.68 & 30.39 & & 29.836 & 30.937 & 30.425 & 30.197 & 28.837 & 26.583 & 31.969 & 36.731 & 32.996 & 16.766 & 16.061 & 20.742 & 15.816 & 10.832 & 32.145 & 32.869 & 30.03 & 31.938 & 27.824 & 34.036 \\
\hline $12 / 17 / 1$ & & 30.904 & 30.508 & 29.666 & 30.383 & & 29.848 & 30.951 & 30.42 & 30.192 & 28.832 & 26.571 & 31.967 & 36.731 & 32.994 & 16.762 & 16.059 & 20.726 & 15.825 & 10.83 & 32.138 & 32.867 & 30.013 & 31.936 & 27.821 & 34.036 \\
\hline 12/17/11 2:00 & & 30.904 & 30.508 & 29.664 & 30.381 & & 29.848 & 30.946 & 30.424 & 30.187 & 28.83 & 26.567 & 31.967 & 36.734 & 32.992 & 16.762 & 16.059 & 20.712 & 15.818 & 10.83 & 32.138 & 32.867 & 30.023 & 31.934 & 27.824 & 34.036 \\
\hline 12/17/11 3:00 & & 30.908 & 30.508 & 29.671 & 30.383 & & 29.843 & 30.939 & 30.425 & 30.192 & 28.835 & 26.576 & 31.969 & 36.733 & 32.996 & 16.759 & 16.061 & 20.705 & 15.816 & 10.832 & 32.145 & 32.867 & 30.025 & 31.934 & 27.824 & 34.036 \\
\hline $12 / 17 / 114: 00$ & & 30.908 & 30.51 & 29.676 & 30.386 & & 29.841 & 30.941 & 30.427 & 30.192 & 28.835 & 26.581 & 31.967 & 36.738 & 32.996 & 16.759 & 16.061 & 20.694 & 15.813 & 10.835 & 32.143 & 32.869 & 30.03 & 31.936 & 27.826 & 34.039 \\
\hline 12/17/11 5:00 & & 30.906 & 30.508 & 29.673 & 30.386 & & 29.843 & 30.941 & 30.429 & 30.19 & 28.832 & 26.579 & 31.965 & 36.734 & 32.994 & 16.757 & 16.059 & 20.691 & 15.813 & 10.832 & 32.143 & 32.867 & 30.025 & 31.934 & 27.826 & 34.039 \\
\hline 12/17/11 6:00 & & 30.908 & 30.51 & 29.676 & 30.388 & & 29.841 & 30.941 & 30.429 & 30.19 & 28.835 & 26.581 & 31.967 & 36.736 & 32.994 & 16.757 & 16.059 & 20.696 & 15.809 & 10.83 & 32.145 & 32.867 & 30.025 & 31.931 & 27.826 & 34.039 \\
\hline $12 / 17 / 117: 00$ & & 30.911 & 30.51 & 29.678 & 30.388 & & 29.843 & 30.941 & 30.427 & 30.19 & 28.835 & 26.583 & 31.967 & 36.74 & 32.992 & 16.755 & 16.057 & 20.691 & 15.816 & 10.83 & 32.145 & 32.867 & 30.027 & 31.934 & 27.826 & 34.039 \\
\hline $12 / 17 / 118: 00$ & & 30.911 & 30.51 & 29.676 & 30.388 & & 29.843 & 30.949 & 30.427 & 30.19 & 28.837 & 26.581 & 31.965 & 36.741 & 32.992 & 16.755 & 16.052 & 20.682 & 15.815 & 10.828 & 32.143 & 32.864 & 30.027 & 31.934 & 27.826 & 34.036 \\
\hline $12 / 17 / 119: 00$ & & 30.91 & 30.51 & 29.68 & 30.39 & & 29.845 & 30.946 & 30.427 & 30.192 & 28.837 & 26.586 & 31.965 & 36.743 & 32.989 & 16.753 & 16.052 & 20.682 & 15.818 & 10.825 & 32.143 & 32.867 & 30.03 & 31.934 & 27.826 & 34.036 \\
\hline $12 / 17 / 1110: 00$ & & 30.913 & 30.51 & 29.685 & 30.393 & & 29.845 & 30.951 & 30.429 & 30.194 & 28.837 & 26.591 & 31.962 & 36.74 & 32.989 & 16.753 & 16.05 & 20.673 & 15.82 & 10.825 & 32.145 & 32.864 & 30.032 & 31.931 & 27.826 & 34.036 \\
\hline 12/17/11 11:00 & & 30.913 & 30.515 & 29.69 & 30.395 & & 29.85 & 30.956 & 30.429 & 30.197 & 28.839 & 26.593 & 31.962 & 36.745 & 32.989 & 16.75 & 16.05 & 20.666 & 15.827 & 10.825 & 32.15 & 32.864 & 30.032 & 31.929 & 27.828 & 34.034 \\
\hline 12:00 & & 30.906 & 30.501 & 29.662 & 30.3 & & 29.876 & 30.98 & 30.424 & 30.185 & 28.828 & 26.564 & 31.957 & 36.743 & 32.985 & 16.748 & 16.045 & 20.657 & 15.846 & 10.823 & 32.129 & 357 & 30.011 & 31.924 & 27.828 & 34.032 \\
\hline $12 / 1$ & & 30.901 & 30.487 & 29.634 & 30. & & 29.895 & 31.008 & 30.427 & 30.173 & 28.816 & 26.54 & 31.95 & 36.738 & 32.978 & 16.746 & 16.04 & 20.645 & $\begin{array}{l}15.0851 \\
\end{array}$ & 10.813 & 32.11 & 848 & 29.988 & 31.917 & 27.828 & .032 \\
\hline $12 /$ & & 30.899 & 30.482 & 29.622 & 30.3 & & 29.897 & 31.02 & 30.425 & 30.166 & 28.807 & 26.531 & 31.946 & 36.729 & 32.973 & 16.743 & 16.04 & 20.64 & $\begin{array}{l}15.082 \\
15.862\end{array}$ & $\begin{array}{l}10.809 \\
\end{array}$ & 32.103 & .843 & 29.978 & 31.913 & 27.828 & .032 \\
\hline 10 & & 30 & 30.471 & 8 & 30 & & 29.902 & 31.032 & 30 & 30.155 & 8 & 26.516 & 1 & 36.722 & 33 & 16.739 & 16.059 & 20.631 & 15.867 & 10.896 & 32.126 & 4 & 29.969 & 31.99 & 27.828 & .032 \\
\hline 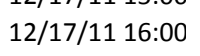 & & 30. & & 6 & & & & 5 & 30 & & & 26.519 & 88 & 36.722 & 33. & 16.739 & .059 & 5 & 15.858 & 10.853 & 15 & 8 & 3 & 95 & 27.831 & 032 \\
\hline $12 / 17 / 1117: 00$ & & 30.896 & 30.466 & 29.613 & 30.332 & & 29.878 & 31.016 & 30.425 & 30.15 & 28.795 & 26.524 & 31.972 & 36.719 & 33.003 & 16.734 & 057 & 668 & 5.855 & 10.839 & 32.11 & & 76 & 1.938 & 27.831 & .032 \\
\hline $12 / 17 / 1118: 00$ & & 30.892 & 30.464 & 29.61 & 30.33 & & 29.873 & 31.013 & 30.422 & 30.146 & 28.793 & 26.524 & 31.962 & 36.717 & 32.992 & 16.734 & 16.052 & 657 & 15.848 & 10.825 & 32.103 & 32.862 & 29.969 & 31.929 & 27.831 & 34.032 \\
\hline 12 & & 30.894 & 30.466 & 29.613 & 30.3 & & 4 & 31. & 30.427 & & 28.793 & 26.528 & 31.955 & 36.717 & 32.985 & 16.732 & 16.05 & 20.654 & 15.841 & 10.823 & 32.108 & 32.855 & 29.971 & 31.924 & 27.831 & 34.032 \\
\hline 20:00 & & 30.892 & 30.466 & 29.617 & 30 & & & & 30.422 & & 28.791 & 26.531 & 31.95 & 36.724 & 32.98 & 16.729 & 16.045 & 20.65 & 15.837 & 10.816 & 32.103 & & 29.973 & 31.92 & 27.831 & 34.032 \\
\hline $12 /$ & & 30.89 & 30.464 & 29.613 & 30 & & 29.852 & & 30.42 & & 28. & 26.533 & 31.943 & 36.724 & 32.973 & 16.727 & 16. & 20.636 & 15.832 & 10.813 & 32 & & 29.971 & & 27.831 & 34.029 \\
\hline 22:00 & & 30.887 & 30.459 & 29.606 & 30.3 & & 29.855 & 30.5 & 30.42 & 30.136 & 28.786 & 26.524 & 31.939 & 36.717 & 32.968 & 16.725 & 16.038 & 20.627 & 15.832 & 10.804 & 32.094 & 32.841 & 29.966 & 31.908 & 27.831 & 34.027 \\
\hline 12/17/11 23:00 & & 30.885 & 30.457 & 29.599 & 30. & & 29.855 & 30.9 & 30.417 & 30.132 & 28.779 & 26.519 & 31.932 & 36.717 & 32.963 & 16.72 & 16.031 & 20.617 & 15.827 & 10.797 & 32.084 & 32.834 & 29.955 & 31.901 & 27.833 & 34.027 \\
\hline 12/18/11 0:00 & & 30.885 & 30.452 & 29.594 & 30.3 & & 29.852 & 30.989 & 30.415 & 30.127 & 28.777 & 26.514 & 31.927 & 36.708 & 32.956 & 16.72 & 16.028 & 20.608 & 15.822 & 10.795 & 32.082 & 32.827 & 29.957 & 31.896 & 27.833 & 34.025 \\
\hline 12/18/11 1:00 & & 30.88 & 30.443 & 29.582 & 30.3 & & 29.859 & 30.996 & 30.417 & 30.12 & 28.77 & 26.504 & 31.922 & 36.712 & 32.952 & 16.718 & 16.024 & 20.596 & 15.82 & 10.785 & 32.07 & 32.822 & 29.941 & 31.889 & 27.831 & 34.022 \\
\hline $12 / 18 / 112: 00$ & & 30.876 & 30.431 & 29.566 & 30.2 & & 29.864 & 31.001 & 30.415 & 30.108 & 28.758 & 26.49 & 31.915 & 36.715 & 32.942 & 16.713 & 16.021 & 20.59 & 15.822 & 10.78 & 32.056 & 32.815 & 29.929 & 31.882 & 27.833 & 34.02 \\
\hline $12 / 18 / 113: 00$ & & 30.871 & 30.424 & 29.55 & 30.278 & & 29.866 & 31.003 & 30.413 & 30.099 & 28.751 & 26.476 & 31.908 & 36.719 & 32.935 & 16.708 & 16.014 & 20.58 & 15.823 & 10.771 & 32.049 & 32.803 & 29.915 & 31.873 & 27.833 & 34.018 \\
\hline $12 / 18 / 114: 00$ & & 30.866 & 30.412 & 29.536 & 30.266 & & 29.869 & 31.004 & 30.408 & 30.09 & 28.742 & 26.468 & 31.897 & 36.726 & 32.928 & 16.706 & 16.012 & 20.571 & 15.818 & 10.764 & 32.033 & 32.798 & 29.901 & 31.866 & 27.833 & 34.018 \\
\hline $12 / 18 / 11$ 5:00 & & 30.859 & 30.401 & 29.521 & 30.25 & & 29.873 & 30.999 & 30.408 & 30.078 & 28.728 & 26.449 & 31.89 & 36.743 & 32.919 & 16.701 & 16.007 & 20.559 & 15.822 & 10.757 & 32.021 & 1 & 29.889 & 31.856 & 27.833 & 34.015 \\
\hline $12 / 18 / 116: 00$ & & 30.857 & 30.394 & 29.514 & 30.2 & & 29.864 & 30.9 & 30.408 & 30.071 & 28.721 & 26.447 & 31.88 & 36.736 & 32.912 & 16.697 & 16.003 & 20.571 & 15.809 & 10.748 & 32.012 & 4 & 29.882 & 31.849 & 27.835 & 34.013 \\
\hline $12 / 18 / 11$ 7:00 & & 30.855 & 30.389 & 29.505 & 30.23 & & 29.859 & 30.989 & 30.403 & 30.062 & 28.714 & 26.44 & 31.873 & 36.743 & 32.907 & 16.694 & 15.998 & 20.585 & 15.813 & 10.743 & 32.012 & 4 & 29.872 & 31.842 & 27.835 & 34.013 \\
\hline $12 / 18 /$ & & 30.85 & 30.375 & 29.486 & 30. & & 29.862 & 30.987 & 30.401 & 30.05 & 28.702 & 26.428 & 31.866 & 36.687 & 32.898 & 16.69 & 15.993 & 20.615 & 15.811 & 10.731 & 31.991 & 7 & 29.856 & 31.835 & 27.835 & 34.008 \\
\hline & & 30.8 & 30. & 29.4 & & & & 30.5 & 30. & $30 . c$ & 28 & & 31.8 & 36.682 & 32.8 & 16. & & 20. & 15. & 10. & & & 29. & 31.823 & 27.835 & 34.006 \\
\hline & & & 30. & 29.4 & & & & & & $30 . c$ & & & & 36 & & & & & 15.7 & & & & & 31.816 & 27.835 & \\
\hline & & & & 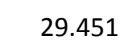 & & & & & & & & & & 36.6 & & & & & 15.8 & & & & & & & \\
\hline & & 30. & & 20 & & & & & & & & & & & & & & & & & & & & & & \\
\hline & & & 30. & & & & & & & & & & & & & & & & & & & & & & & 2 \\
\hline 12/18/11 14:00 & & 30.803 & 30.27 & 29.349 & & & 20000 & & 30.379 & 29.953 & & 26.306 & 31./994 & 36.601 & 32.825 & 16.657 & & & 15.832 & 1.003 & & & 29.727 & 31.762 & 27.838 & 33.99 \\
\hline 12/18/11 15:00 & & 30.803 & 30.263 & 29.346 & 30.082 & & 29.864 & 30.944 & 30.375 & 29.941 & 28.595 & 26.311 & 31.936 & 36.647 & 32.982 & 16.652 & 15.951 & 20.548 & 15.818 & 10.816 & 31.912 & 32.836 & 29.724 & 31.903 & 27.835 & 33.987 \\
\hline
\end{tabular}




\begin{tabular}{|c|c|c|c|c|c|c|c|c|c|c|c|c|c|c|c|c|c|c|c|c|c|c|c|c|c|c|}
\hline well & $\begin{array}{l}\text { (ft TOC) } \\
\text { (n) }\end{array}$ & $\begin{array}{l}\text { (ft TOC) } \\
\text { (f) }\end{array}$ & $\begin{array}{l}\text { (ft TOC) } \\
\text { (ft) }\end{array}$ & $\begin{array}{l}\text { (ft TOC) } \\
\text { (f) }\end{array}$ & $\begin{array}{l}\text { (ft TOC) } \\
\text { (n) }\end{array}$ & $\begin{array}{l}\text { (ft TOC) } \\
\text { (ft }\end{array}$ & $\begin{array}{l}\text { (ft TOC) } \\
\text { (f) }\end{array}$ & $\begin{array}{l}\text { (ft TOC) } \\
\text { (f) }\end{array}$ & $\begin{array}{l}\text { (ft TOC) } \\
\text { (f) }\end{array}$ & $\begin{array}{l}\text { (ft TOC) } \\
\text { (f) }\end{array}$ & $\begin{array}{l}\text { (ft TOC) } \\
\text { (f) }\end{array}$ & $\begin{array}{l}\text { (ft TOC) } \\
\text { (f) }\end{array}$ & (ft TOC) & $\begin{array}{l}\text { (ft TOC) } \\
\text { (f) }\end{array}$ & (ft TOC) & $\begin{array}{l}\text { (ft TOC) } \\
\end{array}$ & $\begin{array}{l}\text { (ft TOC) } \\
\end{array}$ & (ft TOC) & $\begin{array}{l}\text { (ft TOC) } \\
\end{array}$ & (ft TOC) & $\begin{array}{l}\text { (ft TOC) } \\
\end{array}$ & $\begin{array}{l}\text { (ft TOC) } \\
\text { (1) }\end{array}$ & $\begin{array}{l}\text { (ft TOC) } \\
\end{array}$ & (ft TOC) & $\begin{array}{l}\text { (ft TOC) } \\
\text { (1) }\end{array}$ & (ft TOC) \\
\hline 00 & & 30.803 & 30.261 & 29.356 & 30.085 & & 29.836 & 30 & 30 & 29.939 & 28 & 26.3 & 32. & 36. & 33.088 & 16.645 & $16 . c$ & 20. & 15 & 10.922 & 1 & 32.936 & 29.741 & 31.999 & 38 & \\
\hline 12/18/11 17:00 & & 30.801 & 30.261 & 29.358 & 30.087 & & 29.815 & 30.934 & 30.372 & 29.932 & 28.591 & 26.334 & 2.1 & 36.642 & 33.156 & 16.643 & 16.031 & 20.603 & 15.79 & 10.99 & 991 & 00 & 29.753 & 32.063 & .838 & 33.987 \\
\hline 12/18/11 18:00 & & 30.799 & 30.256 & 29.358 & 30.094 & & 29.796 & 30.932 & 30.372 & 29.927 & 28.588 & 26.339 & 32.03 & 36.642 & 33.076 & 16.638 & 16.064 & 20.592 & 15.783 & 10.91 & & 32.928 & 29.762 & 31.992 & .838 & 33.99 \\
\hline 12/18/11 19:00 & & 30.796 & 30.254 & 29.36 & 30.099 & & 29.779 & 30.927 & 30.372 & 29.923 & 28.586 & 26.344 & 31.96 & 36.64 & 33.001 & 16.634 & 16. & 20.603 & 15.769 & 10.837 & 31.979 & 32.857 & & 31.922 & .838 & 33.987 \\
\hline 12/18/11 20:00 & & 30.794 & 30.251 & 29.363 & 30.101 & & 29.765 & 30.922 & 30.368 & 29.916 & 28.584 & 26.349 & 31.918 & 36.642 & 32.956 & 16.631 & 16.035 & $5 \quad 20.596$ & 15.755 & 10.792 & 31.965 & 32.817 & 29.757 & 31.884 & 27.838 & 33.987 \\
\hline 12/18/11 21:00 & & 30.792 & 30.254 & 29.365 & 30.108 & & 29.751 & 30.92 & 30.37 & 29.916 & 28.581 & 26.356 & 31.892 & 36.637 & 32.928 & 16.627 & 16.024 & 20.59 & 15.745 & 10.764 & 31.958 & 32.791 & 29.767 & 31.856 & 27.838 & 33.987 \\
\hline 12/18/11 22:00 & & 30.789 & 30.251 & 29.365 & 30.11 & & 29.739 & 30.913 & 30.37 & 29.911 & 28.579 & 26.358 & 31.871 & 36.64 & 32.905 & 16.624 & 16.012 & $2 \quad 20.585$ & 15.738 & 10.741 & 31.947 & 32.77 & 29.764 & 31.837 & 27.84 & 33.985 \\
\hline 12/18/11 23:00 & & 30.789 & 30.254 & 29.372 & 30.117 & & 29.725 & 30.91 & 30.372 & 29.911 & 28.581 & 26.37 & 31.855 & 36.64 & 32.886 & 16.622 & 15.998 & $8 \quad 20.571$ & 15.724 & 10.726 & 31.947 & 32.753 & 29.771 & 31.823 & 27.84 & 33.983 \\
\hline 12/19/11 0:00 & & 30.785 & 30.249 & 29.365 & 30.115 & & 29.723 & 30.913 & 30.367 & 29.906 & 28.579 & 26.366 & 31.84 & 36.637 & 32.872 & 16.617 & 15.989 & $\begin{array}{l}9 \\
9\end{array}$ & 15.717 & 10.71 & 31.937 & 32.739 & 29.767 & 31.807 & 27.838 & 33.98 \\
\hline 12/19/11 1:00 & & 30.78 & 30.242 & 29.353 & 30.108 & & 29.725 & 30.91 & 30.363 & 29.897 & 28.57 & 26.356 & 31.824 & 36.635 & 32.858 & 16.615 & 15.977 & $7 \quad 20.546$ & 15.717 & 10.694 & 31.921 & 32.725 & 29.755 & 31.79 & 27.84 & 33.978 \\
\hline 12/19/11 2:00 & & 30.78 & 30.242 & 29.353 & 30.108 & & 29.716 & 30.913 & 30.37 & 29.895 & 28.572 & 26.358 & 31.812 & 36.635 & 32.846 & 16.613 & 15.968 & $8 \quad 20.534$ & 15.705 & 10.682 & 31.919 & 32.715 & 29.757 & 31.781 & 27.84 & 33.976 \\
\hline 12/19/11 3:00 & & 30.778 & 30.242 & 29.356 & 30.106 & & 29.706 & 30.915 & 30.36 & 29.893 & 28.57 & 26.363 & 31.805 & 36.63 & 32.837 & 16.61 & 15.961 & $1 \quad 20.522$ & 15.698 & 10.672 & 31.919 & 32.706 & 29.755 & 31.772 & 27.84 & 33.973 \\
\hline 12/19/11 4:00 & & 30.78 & 30.247 & 29.36 & 30.11 & & 29.697 & 30.915 & 30.363 & 29.893 & 28.572 & 26.373 & 31.801 & 36.637 & 32.83 & 16.608 & 15.956 & $6 \quad 20.509$ & 15.691 & 10.668 & 31.919 & 32.701 & 29.762 & 31.767 & 27.84 & 33.973 \\
\hline 12/19/11 5:00 & & 30.782 & 30.251 & 29.374 & 30.117 & & 29.683 & 30.908 & 30.365 & 29.897 & 28.579 & 26.39 & 31.798 & 36.637 & 32.83 & 16.608 & 15.951 & 20.502 & 15.679 & 10.668 & 31.926 & 32.699 & 29.776 & 31.765 & 27.84 & 33.973 \\
\hline $12 / 19 / 116: 00$ & & 30.782 & 30.256 & 29.379 & 30.127 & & 29.681 & 30.908 & 30.363 & 29.897 & 28.581 & 26.394 & 31.794 & 36.64 & 32.825 & 16.606 & 15.947 & 20.488 & 15.677 & 10.658 & 31.926 & 32.694 & 29.778 & 31.762 & 27.84 & 33.976 \\
\hline 12/19/11 7:00 & & 30.787 & 30.265 & 29.4 & 30.14 & & 29.664 & 30.889 & 30.365 & 29.904 & 28.593 & 26.413 & 31.794 & 36.644 & 32.823 & 16.608 & 15.944 & 20.488 & 15.663 & 10.661 & 31.937 & 32.696 & 29.802 & 31.76 & 27.842 & 33.978 \\
\hline 12/19/11 8:00 & & 30.789 & 30.277 & 29.421 & 30.154 & & 29.65 & 30.874 & 30.365 & 29.913 & 28.602 & 26.435 & 31.796 & 36.651 & 32.828 & 16.608 & 15.942 & 20.495 & 15.651 & 10.661 & 31.951 & 32.699 & 29.821 & 31.765 & 27.842 & 33.978 \\
\hline 12/19/11 9:00 & & 30.787 & 30.286 & 29.43 & 30.168 & & 29.65 & 30.87 & 30.363 & 29.92 & 28.609 & 26.447 & 31.798 & 36.651 & 32.828 & 16.61 & 15.94 & 20.497 & 15.658 & 10.665 & 31.956 & 32.701 & 29.828 & 31.765 & 27.842 & 33.978 \\
\hline 12/19/11 10:00 & & 30.787 & 30.291 & 29.435 & 30.176 & & 29.652 & 30.87 & 30.368 & 29.925 & 28.616 & 26.452 & 31.796 & 36.663 & 32.825 & 16.61 & 15.933 & 20.485 & 15.658 & 10.661 & 31.961 & 32.701 & 29.832 & 31.762 & 7.842 & 33.978 \\
\hline $12 / 19 /$ & & 30.785 & 30.296 & 29.437 & 30.183 & & 29.657 & 30.877 & 30.36 & 29.93 & 28.618 & 26.454 & 31.794 & 36.665 & 32.82 & 16.61 & 15.923 & 20.469 & 15.656 & 10.656 & 1.956 & 32.694 & 29.83 & 31.758 & 7.842 & 33.973 \\
\hline $12 /$ & & 30.782 & 30.293 & 29.423 & 30.18 & & 29.669 & 30.81 & 30.365 & 29.925 & 28.618 & 26.435 & 31.782 & 36.665 & 32.806 & 16.608 & 15.914 & 20.451 & 15.656 & 10.646 & 44 & 32.68 & 29.821 & 31.748 & 7.845 & 33.962 \\
\hline $12 / 19 / 1113: 00$ & & 30.78 & 30.298 & 29.433 & 30.183 & & 29.671 & 30.846 & 30.356 & 29.93 & 28.618 & 26.447 & 31.766 & 36.665 & 32.79 & 16.61 & 15.9 & 20.435 & 15.656 & 10.63 & 37 & 32.67 & 29.821 & .732 & .842 & 33.952 \\
\hline 12/19/11 14:00 & & 30.78 & 30.3 & 29.533 & 30.183 & & 29.669 & 30.833 & 30.358 & 29.934 & 28.621 & 26.447 & 31.752 & 36.665 & 32.776 & 16.608 & 15.884 & 20.411 & 15.651 & 10.616 & 31.93 & 32.651 & 29.821 & 31.718 & 842 & 33.945 \\
\hline 12/19/11 15:00 & & 30.78 & 30.3 & & 30.178 & & 29.674 & 30.851 & 30.358 & 29.944 & 28.623 & 26.44 & 31.723 & 36.666 & 32.748 & 16.606 & 15.863 & 20.388 & 15.64 & 10.585 & 31.921 & 32.62 & 29.823 & 31.694 & 7.845 & 33.927 \\
\hline 12/19/11 16:00 & & 30.778 & 30.289 & 29.603 & 30.159 & & 29.685 & 30.961 & 30.363 & 30.009 & 28.616 & 27.007 & 31.705 & 36.659 & 32.734 & 16.601 & 15.851 & 20.456 & 15.635 & 10.567 & 31.9 & 32.606 & 29.966 & 31.847 & 27.847 & 33.917 \\
\hline 12/19/11 17:00 & & 30.775 & 30.289 & 29.578 & 30.159 & & 29.688 & 30.963 & 30.351 & 30.018 & 28.614 & 26.914 & 31.712 & 36.661 & 32.741 & 16.599 & 15.849 & 20.465 & 15.644 & 576 & 31.898 & 32.616 & 29.875 & 31.767 & .845 & 33.915 \\
\hline 12/19/11 18:00 & & 30.796 & 30.314 & 29.655 & 30.173 & & 29.648 & 30.874 & 30.377 & 30.046 & 28.639 & 26.705 & 31.716 & 36.666 & 32.741 & 16.601 & 15.846 & $5 \quad 20.455$ & 15.607 & 10.581 & 31.93 & 32.62 & 30.046 & 31.905 & 27.847 & 33.91 \\
\hline 12/19/11 19:00 & & 30.782 & 30.31 & 29.624 & 30.169 & & 29.667 & 30.929 & 30.37 & 30.053 & 28.632 & 26.437 & 31.716 & 36.673 & 32.741 & 16.599 & 15.842 & 20.442 & 15.63 & 10.578 & 31.909 & 32.616 & 29.891 & 31.8 & 27.845 & 33.906 \\
\hline 12/19/11 20:00 & & 30.806 & 30.429 & 29.68 & 30.192 & & 29.622 & 30.944 & 30.403 & 30.083 & 28.66 & 26.507 & 31.723 & 36.68 & 32.745 & 16.603 & 15.839 & 20.432 & 15.602 & 10.581 & 31.942 & 32.62 & 29.847 & 31.76 & 27.84 & 33.903 \\
\hline 12/19/11 21:00 & & 30.794 & 30.436 & 29.622 & 30.192 & & 29.641 & 30.975 & 30.512 & 30.085 & 28.663 & 26.512 & 31.721 & 36.684 & 32.745 & 16.61 & 15.832 & 20.416 & 15.616 & 10.581 & 31.933 & 32.62 & 29.837 & 31.729 & 27.838 & 33.894 \\
\hline $12 / 19 / 1122: 00$ & & 30.78 & 30.403 & 29.533 & 30.166 & & 29.681 & 31.013 & 30.558 & 30.062 & 28.646 & 26.507 & 31.707 & 36.675 & 32.731 & 16.638 & 15.947 & 20.516 & 15.632 & 10.569 & 31.9 & 32.606 & 29.823 & 31.746 & 27.833 & 33.88 \\
\hline $12 / 19 / 1123: 00$ & & 30.782 & 30.396 & 29.22 & 30.148 & & 29.685 & 30.994 & 30.593 & 30.076 & 28.644 & 26.435 & 31.702 & 36.673 & 32.727 & 16.671 & 16.045 & 20.613 & 15.628 & 10.562 & 31.893 & 32.601 & 29.811 & 31.727 & 27.833 & 33.875 \\
\hline $12 / 20 / 110: 00$ & & 30.782 & 30.377 & 29.117 & 30.122 & & 29.695 & 30.989 & 30.607 & 30.071 & 28.635 & 26.423 & 31.691 & 36.668 & 32.717 & 16.673 & 16.04 & 20.599 & 15.628 & 10.555 & 31.879 & 32.594 & 29.828 & 31.751 & 27.828 & 33.871 \\
\hline 12/20/11 1:00 & & 30.771 & 30.405 & 29.07 & 30.096 & & 29.707 & 30.999 & 30.588 & 30.06 & 28.623 & 26.406 & 31.686 & 36.656 & 32.713 & 16.676 & 15.94 & 20.576 & 15.644 & 10.552 & 31.856 & 32.585 & 29.802 & 31.753 & 27.824 & 33.864 \\
\hline 12/20/11 2: & & 30.773 & 30.408 & 28.986 & & & & & 30.598 & 30.067 & 28.623 & 26.406 & 31.677 & 36.658 & 32.706 & 8 & 15. & 20.585 & 15.63 & 43 & 31.849 & 32.578 & 29.797 & 31.741 & 7.821 & 33.861 \\
\hline 12/20/11 3:00 & & & 30.394 & & & & 29.699 & & & 30.064 & 28.618 & 26.401 & 31.672 & 36.659 & 32.694 & 1 & 15.975 & 20.594 & 15.637 & .536 & 31.832 & 32.566 & 29.795 & 31.755 & 27.814 & 33.852 \\
\hline 12/20/11 4:00 & & 30.766 & 30.408 & 28.895 & 30.054 & & 29.699 & 30.994 & 30.596 & 30.05 & 28.616 & 26.394 & 31.663 & 36.656 & 32.692 & 16.694 & 15.986 & 20.596 & 15.637 & 10.531 & 31.83 & 32.561 & 29.762 & 31.734 & 27.81 & 33.847 \\
\hline 12/20/11 5:00 & & 30.771 & 30.412 & 28.888 & 30.045 & & 29.688 & 30.977 & 30.581 & 30.025 & 28.618 & 26.404 & 31.656 & 36.659 & 32.687 & 16.708 & 15.968 & 30.573 & 15.625 & 10.524 & 31.825 & 32.559 & 29.743 & 31.713 & 27.81 & 33.845 \\
\hline & & 30.773 & 30.38 & 28.869 & 30.04 & & 29.674 & 30.975 & 30.5 & 30.006 & 28.625 & 26.418 & 31.653 & 36.661 & 32.682 & 1 & 15.902 & 20.518 & 15.621 & 10.52 & 25 & 32.554 & 29.729 & 31.694 & 27.803 & 33.843 \\
\hline & & 30. & 30 & 28 & & & & & & 29 & & 26 & & & 32 & & 15. & & & 10 & & 32.552 & 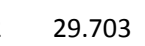 & & 18 & 33.84 \\
\hline & & 30 & & & & & & & & & & & & & & & 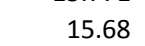 & & & & & 32. & & & & 33.84 \\
\hline & & & & & & & & & & & & 20 & & & & & 15 & & & & & 32. & 29 & 15 & 98 & 33.843 \\
\hline $12 / 20 / 1110: 00$ & & & 30 & & & & & & & & & 26 & 31.6 & & & & 15. & & & & & 32. & & & 93 & 33.838 \\
\hline .11:00 & & 30.766 & 30.38 & 29. & & & 29 & & 30. & 29.909 & 28 & 26.428 & 31 & & & 1 & 15. & 20. & 15. & & & 32.54 & 29.612 & & 91 & 33 \\
\hline $.12: 00$ & & & 30.368 & & & & 29. & & & 29 & & 26.401 & 31. & & & 1 & 15.732 & 20.268 & 15 & & 3 & 32.533 & & 31.596 & .788 & 31 \\
\hline $12 / 2$ & & & 30.356 & 29. & & & 29 & & & & & 26.382 & 31. & 36 & 32.649 & 16. & 15. & 20.254 & 15.628 & 10.489 & 31.765 & 32.518 & & & 7.786 & 33.829 \\
\hline 12/20/11 14:00 & & & 30.345 & & & & 29 & & & 29 & 28. & 26 & 31. & 36. & 32.64 & 16. & 15.722 & 20.243 & 15.625 & 10.48 & 31.751 & 32.509 & & 31.575 & 27.784 & 33.824 \\
\hline 12/20/11 15:00 & & 30. & 30.331 & & & & 29.6 & 30. & & 29. & 28. & 26. & 31. & 36. & 32.626 & 16.5 & 15.72 & 20.231 & 15.63 & 10.465 & 31.725 & 32.497 & 29.513 & 31.561 & 27.779 & 33.822 \\
\hline 12/20/11 16:00 & & 30.757 & 30.326 & 29. & 29. & & 29.6 & 30.791 & & 29.807 & 28. & 26.361 & 31.5 & 36.64 & 32.624 & 16.5 & 15.718 & 20.275 & 15.614 & 10.461 & 31.73 & 32.49 & 29.513 & 31.558 & 27.779 & 33.824 \\
\hline $12 / 20 / 1117: 00$ & & 30.752 & 30.314 & 29.334 & 29.891 & & 29.674 & 30.81 & 30.078 & 29.793 & 28.5 & 26.356 & 31.583 & 36.647 & 32.616 & 16.503 & 15.718 & 20.266 & 15.614 & 10.454 & 31.718 & 32.485 & 29.501 & 31.553 & 27.774 & 33.824 \\
\hline $12 / 20 / 1118: 00$ & & 30.752 & 30.31 & 29.341 & 29.884 & & 29.662 & 30.819 & 29.933 & 29.79 & 28.588 & 26.361 & 31.576 & 36.649 & 32.614 & 16.506 & 15.718 & 20.257 & 15.607 & 10.451 & 31.721 & 32.483 & 29.501 & 31.549 & 27.772 & 33.824 \\
\hline $12 / 20 / 1119: 00$ & & 30.757 & 30.305 & 29.36 & 29.884 & & 29.643 & 30.827 & 29.781 & 29.793 & 28.595 & 26.38 & 31.571 & 36.651 & 32.614 & 16.503 & 15.718 & 20.247 & 15.597 & 10.451 & 31.723 & 32.483 & 29.503 & 31.551 & 27.77 & 33.829 \\
\hline $12 / 20 / 1120: 00$ & & 30.757 & 30.303 & 29.37 & 29.886 & & 29.631 & 30.855 & 29.638 & 29.79 & 28.598 & 26.392 & 31.567 & 36.658 & 32.614 & 16.501 & 15.718 & 20.243 & 15.597 & 10.449 & 31.728 & 32.485 & 29.508 & 31.551 & 27.767 & 33.831 \\
\hline 12/20/11 21:00 & & 30.75 & 30.289 & 29.365 & 29.879 & & 29.636 & 30.901 & 29.491 & 29.821 & 28.595 & 26.385 & 31.564 & 36.656 & 32.609 & 16.499 & 15.718 & 20.231 & 15.597 & 10.449 & 31.716 & 32.481 & 29.494 & 31.544 & 27.765 & 33.833 \\
\hline 12/20/11 22:00 & & 30.745 & 30.268 & 29.356 & 29.867 & & 29.641 & 30.772 & 29.351 & 29.793 & 28.591 & 26.375 & 31.555 & 36.654 & 32.605 & 16.499 & 15.715 & 20.22 & 15.595 & 10.442 & 31.707 & 32.473 & 29.482 & 31.539 & 27.76 & 3.833 \\
\hline 12/20/11 23:00 & & 30.738 & 30.244 & 29.341 & 29.853 & & 29.648 & 30.779 & 29.225 & 29.811 & 28.584 & 26.358 & 31.543 & 36.654 & 32.598 & 16.494 & 15.711 & 20.213 & 15.599 & 10.435 & 31.693 & 32.464 & 29.461 & 31.535 & 27.76 & 29 \\
\hline
\end{tabular}




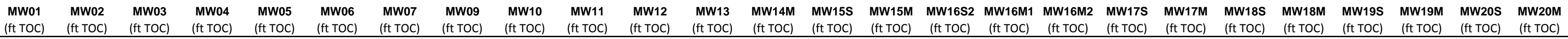

\begin{tabular}{|c|c|c|c|c|c|c|c|c|c|c|c|c|c|c|c|c|c|c|c|c|c|c|c|c|c|c|}
\hline Well & $\begin{array}{l}\text { (ft TOC) } \\
\text { (is }\end{array}$ & $\begin{array}{l}\text { (ft TOC) } \\
\text { (f) }\end{array}$ & $\begin{array}{l}\text { (ft TOC) } \\
\text { (f) }\end{array}$ & (ft TOC) & $\begin{array}{l}\text { (ft toc) } \\
\text { (foc) }\end{array}$ & $\begin{array}{l}\text { (ft TOC) } \\
\text { (f) }\end{array}$ & $\begin{array}{l}\text { (ft TOC) } \\
\text { (n) }\end{array}$ & $\begin{array}{l}\text { (ft TOC) } \\
\text { (f) }\end{array}$ & $\begin{array}{l}\text { (ft TOC) } \\
\text { (ft) }\end{array}$ & $\begin{array}{l}\text { (ft TOC) } \\
\text { (f) }\end{array}$ & $\begin{array}{l}\text { (ft TOC) } \\
\text { (f) }\end{array}$ & $\begin{array}{l}\text { (ft Toc) } \\
\text { (f) }\end{array}$ & (ft TOC) & $\begin{array}{l}\text { (ft TOC) } \\
\end{array}$ & (ft TOC) & (ft TOC) & $\begin{array}{l}\text { (ft TOC) } \\
\text { (f) }\end{array}$ & (ft TOC) & $\begin{array}{l}\text { (ft TOC) } \\
\text { (1) }\end{array}$ & (ft TOC) & $\begin{array}{l}\text { (ft TOC) } \\
\end{array}$ & $\begin{array}{l}\text { (ft TOC) } \\
\text { (1) }\end{array}$ & $\begin{array}{l}\text { (ft TOC) } \\
\text { (n) }\end{array}$ & (ft TOC) & $\begin{array}{l}\text { (ft TOC) } \\
\end{array}$ & (ft TOC) \\
\hline 12/21/11 0:00 & & 30.743 & 30.221 & 29.341 & 29.844 & & 29.641 & 30.762 & 29.118 & 29.809 & 28.581 & 26.361 & 31.532 & 36.647 & 32.588 & $8 \quad 16.492$ & 15.704 & 20.201 & 15.59 & 10.426 & 31.688 & 32.457 & 29.454 & 31.523 & 27.758 & 33.829 \\
\hline $12 / 21 / 111: 00$ & & 30.741 & 30.198 & 29.339 & 29.835 & & 29.636 & 30.752 & 29.028 & 29.809 & 28.579 & 26.358 & 31.539 & 36.649 & 32.579 & $\begin{array}{l}9 \\
9\end{array}$ & 15.697 & 20.194 & 15.583 & 10.418 & 31.676 & 32.447 & 29.452 & 31.518 & 27.756 & 33.824 \\
\hline $12 / 21 / 112: 00$ & & 30.741 & 30.167 & 29.332 & 29.823 & & 29.631 & 30.74 & 28.952 & 29.807 & 28.577 & 26.354 & 31.525 & 36.649 & 32.57 & $7 \quad 16.487$ & 15.692 & 20.185 & 15.578 & 10.407 & 31.672 & 32.438 & 29.433 & 31.507 & 27.756 & 33.822 \\
\hline $12 / 21 / 113: 00$ & & 30.736 & 30.142 & 29.325 & 29.814 & & 29.631 & 30.733 & 28.869 & 29.802 & 28.572 & 26.349 & 31.518 & 36.647 & 32.56 & 16.485 & 15.685 & 20.171 & 15.576 & 10.402 & 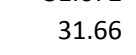 & & & & 27.753 & 33.817 \\
\hline $12 / 21 / 114: 00$ & & 30.727 & 30.107 & 29.313 & 29.797 & & 29.636 & 30.736 & 28.79 & 29.793 & 28.56 & 26.334 & 31.515 & 36.647 & 32.553 & 16.48 & 15.678 & 20.164 & 15.578 & 10.393 & 31.644 & 32.419 & 9.407 & 49 & & 33.812 \\
\hline $12 / 21 / 115: 00$ & & 30.729 & 30.07 & 29.313 & 29.793 & & 29.627 & 30.714 & 28.731 & 29.79 & 28.56 & 26.334 & 31.506 & 36.647 & 32.546 & $\begin{array}{ll}6 & 16.478\end{array}$ & 15.673 & 20.153 & 15.571 & 10.381 & 31.639 & 32.414 & 29.405 & 31.481 & 7.749 & 33.81 \\
\hline $12 / 21 / 116: 00$ & & 30.734 & 30.037 & 29.325 & 29.793 & & 29.61 & 30.685 & 28.669 & 29.793 & 28.563 & 26.349 & 31.508 & 36.642 & 32.541 & $1 \quad 16.475$ & 15.671 & 20.171 & 15.557 & $\begin{array}{l}10.379 \\
\end{array}$ & 31.641 & 32.412 & 29.407 & 31.476 & 27.746 & 33.81 \\
\hline 12/21/11 7:00 & & 30.731 & 30.002 & 29.327 & 29.788 & & 29.603 & 30.673 & 28.605 & 29.793 & 28.563 & 26.351 & 31.508 & 36.644 & 32.537 & $7 \quad 16.473$ & 15.669 & 20.169 & 15.557 & 10.371 & 31.641 & 32.407 & 29.409 & 31.471 & 27.746 & 33.812 \\
\hline 12/21/11 8:00 & & 30.729 & 29.972 & 29.332 & 29.79 & & 29.596 & 30.657 & 28.558 & 29.79 & 28.567 & 26.356 & 31.504 & 36.649 & 32.539 & $\begin{array}{l}9 \\
9\end{array} 16.468$ & 15.666 & 20.157 & 15.553 & 10.376 & 31.639 & 32.405 & 29.412 & 31.474 & 27.744 & 33.812 \\
\hline 12/21/11 9:00 & & 30.727 & 29.948 & 29.334 & 29.793 & & 29.591 & 30.645 & 28.51 & 29.79 & 28.567 & 26.361 & 31.504 & 36.651 & 32.537 & $7 \quad 16.468$ & 15.664 & 20.146 & 15.55 & 10.371 & 31.641 & 32.405 & 29.407 & 31.469 & 27.744 & 33.815 \\
\hline $12 / 21 / 1110: 00$ & & 30.731 & 29.925 & 29.341 & 29.795 & & 29.582 & 30.623 & 28.479 & 29.793 & 28.574 & 26.368 & 31.501 & 36.651 & 32.534 & $4 \quad 16.466$ & 15.664 & 20.136 & 15.541 & 10.369 & 31.648 & 32.402 & 29.421 & 31.467 & 27.744 & 33.817 \\
\hline 12/21/11 11:00 & & 30.722 & 30.149 & 29.339 & 29.793 & & 29.589 & 30.621 & 28.455 & 29.8 & 28.57 & 26.363 & 31.499 & 36.649 & 32.527 & $7 \quad 16.464$ & 15.662 & 20.127 & 15.548 & 10.369 & 31.639 & 32.4 & 29.409 & 31.464 & 27.739 & 33.815 \\
\hline $12 / 21 / 1112: 00$ & & 30.717 & 30.151 & 29.302 & 29.786 & & 29.591 & 30.618 & 28.448 & 29.832 & 28.567 & 26.356 & 31.494 & 36.649 & 32.525 & $5 \quad 16.461$ & 15.655 & 20.116 & 15.55 & 10.362 & 31.63 & 32.395 & 29.395 & 31.462 & 27.739 & 33.812 \\
\hline $12 / 21 / 1113: 00$ & & 30.708 & 30.144 & 29.295 & 29.767 & & 29.608 & 30.537 & 28.451 & 29.834 & 28.553 & 26.334 & 31.485 & 36.654 & 32.516 & 16.457 & 15.65 & 20.106 & 15.567 & 10.36 & 31.611 & 32.386 & 29.377 & 31.455 & 27.735 & 33.81 \\
\hline 12/21/11 14:00 & & 30.708 & 30.135 & 29.379 & 29.755 & & 29.61 & 30.575 & 28.463 & 29.783 & 28.546 & 26.323 & 31.475 & 36.644 & 32.509 & 16.452 & 15.645 & 20.095 & 15.564 & 10.348 & 31.602 & 32.376 & 29.365 & 31.446 & 27.737 & 33.808 \\
\hline 12/21/11 15:00 & & 30.708 & 30.132 & 29.465 & 29.748 & & 29.608 & 30.64 & 28.351 & 29.774 & 28.544 & 26.32 & 31.473 & 36.644 & 32.499 & 16.452 & $\begin{array}{l}15.641 \\
\end{array}$ & 20.085 & 15.56 & 10.336 & 31.595 & 32.369 & 29.367 & 31.441 & 27.735 & 33.805 \\
\hline 12/21/11 16:00 & & 30.715 & 30.139 & 29.472 & 29.751 & & 29.589 & 30.664 & 28.208 & 29.774 & 28.549 & 26.334 & 31.466 & 36.644 & 32.504 & 16.464 & 15.638 & 20.134 & 15.55 & 10.334 & 31.599 & 32.367 & 29.384 & 31.446 & 27.735 & 33.805 \\
\hline 12/21/11 17:00 & & 30.71 & 30.142 & 29.477 & 29.751 & & 29.582 & 30.647 & 28.071 & 29.769 & 28.549 & 26.342 & 31.464 & 36.642 & 32.497 & 16.457 & $\begin{array}{l}15.638 \\
\end{array}$ & 20.127 & 15.55 & 10.334 & 31.604 & 32.364 & 29.377 & 31.439 & 27.732 & 33.808 \\
\hline $12 / 21 / 1118: 00$ & & 30.715 & 30.153 & $\begin{array}{r}29.5 \\
29.5\end{array}$ & 29.76 & & 29.561 & 30.616 & 27.938 & 29.774 & 28.558 & $\begin{array}{l}20.342 \\
26.361\end{array}$ & 31.464 & 36.644 & 32.502 & 16.454 & $\begin{array}{l}15.030 \\
15.636\end{array}$ & 20.125 & 15.539 & $\begin{array}{l}10.34 \\
10.31\end{array}$ & 31.613 & 32.369 & 29.388 & 31.436 & 27.732 & $\begin{array}{l}33.808 \\
3.008\end{array}$ \\
\hline $12 / 21 / 1119: 00$ & & 30.72 & 30.17 & 29.517 & $\begin{array}{l}29.770 \\
\end{array}$ & & 29.54 & 30.583 & 27.817 & 29.783 & $\begin{array}{l}20.500 \\
28.57\end{array}$ & $\begin{array}{l}20.301 \\
26.385\end{array}$ & $\begin{array}{l}31.474 \\
31.471\end{array}$ & $\begin{array}{l}30.044 \\
36.656\end{array}$ & 32.504 & 16.454 & $\begin{array}{l}13.0030 \\
15.641\end{array}$ & 20.129 & 15.529 & $\begin{array}{l}10.31 \\
10.339\end{array}$ & 31.627 & $\begin{array}{l}32.374 \\
32.09\end{array}$ & 29.402 & $\begin{array}{l}31.400 \\
31.441\end{array}$ & 27.732 & $\begin{array}{l}33.812 \\
33.812\end{array}$ \\
\hline $12 / 21 / 1120: 00$ & & $\begin{array}{r}30.717 \\
30.717\end{array}$ & $\begin{array}{l}30.179 \\
30.179\end{array}$ & 29.372 & 29.788 & & 29.533 & $\begin{array}{l}50.503 \\
30.559\end{array}$ & 27.703 & 29.788 & $\begin{array}{r}28.51 \\
28.574\end{array}$ & $\begin{array}{l}20.383 \\
26.394\end{array}$ & $\begin{array}{l}31.478 \\
31.478\end{array}$ & $\begin{array}{l}30.050 \\
36.661\end{array}$ & $\begin{array}{l}32.544 \\
32.509\end{array}$ & $\begin{array}{l}10.434 \\
16.454\end{array}$ & $\begin{array}{l}13.044 \\
15.643\end{array}$ & 20.122 & $\begin{array}{l}13.524 \\
15.524\end{array}$ & 10.346 & $\begin{array}{l}31.627 \\
31.637\end{array}$ & $\begin{array}{l}32.381 \\
32.38\end{array}$ & $\begin{array}{l}29.402 \\
29.409\end{array}$ & $\begin{array}{l}31.441 \\
31.446\end{array}$ & 27.73 & $\begin{array}{l}33.012 \\
33.817\end{array}$ \\
\hline 21:00 & & 30.72 & 30.195 & 29.386 & 29.807 & & 29.518 & 30.525 & 27.6 & 29.797 & 28.588 & 26.416 & 48 & 36.665 & 32.518 & 16.454 & 15.645 & 20.118 & 5.517 & 10.35 & 31.651 & 32.388 & .431 & 1.448 & 7.73 & 33.822 \\
\hline $12 / 21 /$ & & 30.722 & 30.212 & 29.407 & 25 & & & 496 & 27.513 & & $\begin{array}{l}28.5002 \\
28.602\end{array}$ & & $\begin{array}{l}31.40 \\
31.49\end{array}$ & 36.68 & $\begin{array}{r}32.52 \\
32.52\end{array}$ & 16.454 & $\begin{array}{l}15.048 \\
15.648\end{array}$ & 20.116 & 513 & 10.357 & 31.669 & 395 & 454 & 1.46 & 7.73 & $\begin{array}{l}33.826 \\
33.826\end{array}$ \\
\hline 12/21/11 23:00 & & 30.72 & 30.226 & 29.426 & 29.844 & & 04 & 30.506 & 27.455 & 29.816 & 28.616 & 26.454 & 31.497 & 36.684 & 32.53 & 16.457 & 15.652 & 20.113 & 15.513 & 10.367 & 31.674 & 32.402 & .473 & 1.467 & 27.728 & 33.829 \\
\hline $12 / 22 / 1$ & & 30.713 & 30.223 & 29 & $2 \mathrm{c}$ & & 21 & 30.506 & 27.399 & 29.814 & 28.616 & 26.442 & 31.499 & 36.689 & 32.53 & 16.457 & 15.648 & 20.106 & 15.52 & 10.369 & 31.669 & 32.402 & 461 & 467 & 27.725 & 33.829 \\
\hline 12/22/11 1:00 & & 30.712 & 30.226 & 29.414 & 29.846 & & 29.533 & 30.504 & 27.356 & 29.816 & 28.616 & 26.437 & 31.499 & 36.689 & 32.527 & 16.454 & 15.645 & 20.099 & 15.524 & 10.367 & 31.669 & 32.402 & 461 & 467 & 27.723 & 33.826 \\
\hline $12 / 22 / 112: 00$ & & 30.717 & 30.233 & 29.421 & 29.851 & & 29.53 & 30.496 & 27.356 & 29.823 & 28.623 & 26.445 & 31.496 & 36.694 & 32.525 & 16.454 & 15.641 & 20.09 & 15.515 & 10.364 & 31.672 & 32.398 & 29.463 & 1.462 & 27.725 & 33.824 \\
\hline 12/22/11 3:00 & & 30.724 & 30.263 & 29.458 & 29.879 & & 29.507 & 30.499 & 27.337 & 29.844 & 28.644 & 26.483 & 31.504 & 36.703 & 32.532 & 16.457 & 15.641 & 20.083 & 15.51 & 10.364 & 31.693 & 32.405 & 29.494 & 31.469 & 27.725 & 33.824 \\
\hline $12 / 22 / 114: 00$ & & 30.72 & 30.268 & 29.356 & 29.884 & & 29.514 & 30.504 & 27.294 & 29.828 & 28.651 & 26.488 & 31.504 & 36.708 & 32.532 & 16.457 & 15.631 & 20.072 & 15.513 & 10.369 & 31.7 & 32.405 & 29.501 & 31.474 & 27.723 & 33.819 \\
\hline $12 / 22 / 115: 00$ & & 30.717 & 30.27 & 29.36 & 29.886 & & 29.533 & 30.499 & 27.268 & 29.811 & 28.653 & 26.478 & 31.504 & 36.71 & 32.53 & 16.457 & 15.631 & 20.062 & 15.527 & 10.369 & 31.693 & 32.405 & 29.496 & 31.469 & 27.72 & 33.817 \\
\hline $12 / 22 / 116: 00$ & & 30.726 & 30.293 & 29.407 & 29.907 & & 29.516 & 30.494 & 27.277 & 29.795 & 28.67 & 26.507 & 31.513 & 36.715 & 32.539 & 16.459 & 15.636 & 20.065 & 15.517 & 10.379 & 31.714 & 32.414 & 29.52 & 31.481 & 27.723 & 33.817 \\
\hline $12 / 22 / 11$ 7:00 & & 30.734 & 30.319 & 29.461 & 29.935 & & 29.5 & 30.487 & 27.292 & 29.786 & 28.693 & 26.54 & 31.525 & 36.727 & 32.551 & 16.461 & 15.641 & 20.069 & 15.508 & 10.383 & 31.739 & 32.426 & 29.555 & 31.485 & 27.723 & 33.824 \\
\hline $12 / 22 / 118: 00$ & & 30.731 & 30.331 & 29.486 & 29.947 & & 29.511 & 30.484 & 27.334 & 29.779 & 28.705 & 26.545 & 31.532 & 36.731 & 32.556 & 16.466 & 15.643 & 20.081 & 15.52 & 10.397 & 31.746 & 32.436 & 29.567 & 31.495 & 27.72 & 33.826 \\
\hline $12 / 22 / 119: 00$ & & 30.741 & 30.359 & 29.54 & 29.975 & & 29.502 & 30.487 & 27.415 & 29.858 & 28.728 & 26.579 & 31.546 & $\begin{array}{r}36.74 \\
\end{array}$ & 32.572 & 16.471 & 15.648 & 20.097 & 15.515 & 10.407 & 31.777 & 32.445 & 29.597 & 31.511 & 27.723 & 33.831 \\
\hline 12/22/11 10:00 & & 30.75 & 30.382 & 29.599 & 30.005 & & 29.497 & 30.508 & 27.486 & 29.876 & 28.751 & 26.605 & 31.56 & 36.743 & 32.588 & $\begin{array}{ll}36.475 \\
3\end{array}$ & $\begin{array}{l}15.655 \\
\end{array}$ & 20.09 & 15.515 & 10.423 & 31.802 & 32.462 & 29.626 & 31.521 & 27.723 & 33.838 \\
\hline 11:00 & & 30.747 & 30.398 & 29.622 & & & 29.514 & 30.5 & 27.534 & 29.869 & 28.765 & 26.612 & 31.571 & 36.752 & 32.595 & 16.478 & $\begin{array}{l}15.662 \\
1502\end{array}$ & 20.085 & 15.529 & $\begin{array}{l}10.435 \\
\end{array}$ & 31.809 & 32.471 & 29.635 & 31.532 & 27.723 & $\begin{array}{r}33.84 \\
3\end{array}$ \\
\hline 12:00 & & 30.747 & 30.398 & 29.617 & 30.0 & & 29.535 & 30.516 & 27.562 & 29.888 & 28.767 & 26.602 & 31.576 & 36.755 & r2. 32.6 & $\begin{array}{l}10.410 \\
16.48\end{array}$ & $\begin{array}{l}13.062 \\
15.659\end{array}$ & 20.076 & 15.545 & 10.44 & 31.807 & 32.478 & 29.63 & 31.539 & 27.72 & 33.843 \\
\hline 12 & & $\begin{array}{l}30.743 \\
30.74\end{array}$ & 30.375 & 29.603 & 30.0 & & 29.565 & 30.518 & 27.586 & $\begin{array}{l}29.000 \\
29.888\end{array}$ & 28.767 & 26.586 & 31.576 & 36.757 & $\begin{array}{r}32.602 \\
32.60\end{array}$ & 16.485 & $\begin{array}{l}15.659 \\
15.659\end{array}$ & 20.067 & 15.562 & $\begin{array}{l}10.444 \\
10.42\end{array}$ & 31.8 & 32.481 & 29.619 & 31.544 & 27.72 & $\begin{array}{r}r .045 \\
33.84\end{array}$ \\
\hline 12 & & $\begin{array}{l}30.743 \\
30.743\end{array}$ & $\begin{array}{l}30.39 \\
30.39\end{array}$ & 29.599 & 30.6 & & 29.584 & 30.5 & 27.603 & $\begin{array}{r}2.000 \\
29.89\end{array}$ & 28.765 & $\begin{array}{l}20.500 \\
26.574\end{array}$ & 31.576 & 36.762 & 32.605 & $\begin{array}{l}10.403 \\
16.487\end{array}$ & $\begin{array}{l}13.0599 \\
15.659\end{array}$ & 20.058 & $\begin{array}{l}13.002 \\
15.562\end{array}$ & $\begin{array}{l}10.442 \\
10.442\end{array}$ & $\begin{array}{l}31.00 \\
31.793\end{array}$ & $\begin{array}{l}32.481 \\
32.481\end{array}$ & 19 & 42 & 27.718 & $\begin{array}{l}53.04 \\
33.84\end{array}$ \\
\hline 12 & & 30 & 30 & 29 & & & & 30 & 27 & 29 & 28 & 26 & 3 & 36 & 32 & 16 & 15 & $2 c$ & 15.569 & 10. & 31.797 & 1 & 6 & 99 & 27.723 & 33.84 \\
\hline & & 30 & & & & & & 36 & 27.681 & & & & & 36 & 32 & 16 & 15 & 26 & 15 & 10.449 & 31.804 & 5 & & 6 & 27.72 & 33.843 \\
\hline & & 30 & & 29.627 & & & & & 27.7 & & 28.788 & & & 36 & 32.616 & & & 20.095 & 15.581 & 10.454 & 31.809 & 32.49 & 29.642 & 53 & 7.72 & 33.845 \\
\hline $12 / 2$ & & 30.752 & 30.419 & 29. & 30. & & & & 27.719 & & 28.79 & & & 36.769 & 32.624 & 16.496 & 15.669 & & 15585 & 10.461 & 31.818 & 32.497 & & 63 & 27.72 & 33.847 \\
\hline $12 / 2$ & & 30. & 30.4 & 29. & & & & 30. & 27.7 & & 28 & & & 36.769 & 32.628 & 16. & 15.676 & & 15. & 10.465 & 31.823 & 32.504 & 29.644 & 55 & 27.718 & 33.85 \\
\hline $12 / 2$ & & 30 & & & & & & 30. & 27. & & 28. & & & 36.773 & 32.638 & & 15 & & 15. & 10.473 & 31.825 & & 29.651 & 2 & 27.718 & 33.854 \\
\hline $12 / 2$ & & 30 & 30. & 29. & & & & 30 & 27.776 & & 28. & 26 & & 36.773 & 32.645 & 16.503 & 15. & & 15. & 10.48 & 31.839 & 32.518 & & 31.582 & 27.72 & 33.861 \\
\hline $12 / 22 / 1122: 00$ & & 30 & 30. & 29. & & & & 30.5 & 27 & & 28.8 & 26.612 & 31.6 & 36.776 & 32.652 & 16.506 & 15 & 20.0 & 15.602 & 10.487 & 31.844 & 32.523 & 29.673 & 31.589 & 27.718 & 33.866 \\
\hline 12/22/11 23:00 & & 30. & 30.4 & 29.678 & & & & 30.5 & 27.8 & $30 . c$ & 28.8 & 26.624 & 31.632 & 36.78 & 32.661 & 16.506 & 15.694 & 20.074 & 15.607 & 10.496 & 31.853 & 32.535 & 29.684 & 31.6 & 27.72 & 33.868 \\
\hline $12 / 23 / 110: 00$ & & 30.761 & 30.459 & 29.673 & 30.0 & & 29.638 & 30.523 & 27.84 & 30.0 & 28.835 & 26.612 & 31.632 & 36.776 & 32.663 & 16.51 & 15.694 & 20.067 & 15.623 & 10.498 & 31.851 & 32.537 & 29.68 & 31.598 & 27.72 & 33.868 \\
\hline 12/23/11 1:00 & & 30.761 & 30.452 & 29.662 & 30.068 & & 29.655 & 30.53 & 27.866 & 30.025 & 28.832 & 26.6 & 31.635 & 36.78 & 32.661 & 16.51 & 15.694 & 20.06 & 15.637 & 10.498 & 31.844 & 32.533 & 29.67 & 31.598 & 27.72 & 33.868 \\
\hline $12 / 23 / 112: 00$ & & 30.759 & 30.445 & 29.652 & 30.059 & & 29.671 & 30.52 & 27.888 & 30.02 & 28.828 & 26.583 & 31.628 & 36.773 & 32.656 & 16.508 & 15.69 & 20.053 & 15.635 & 10.494 & 31.837 & 32.528 & 29.661 & 31.593 & 27.72 & 33.866 \\
\hline $12 / 23 / 113: 00$ & & 30.759 & 30.445 & 29.648 & 30.054 & & 29.676 & 30.525 & 27.914 & 30.02 & 28.825 & 26.579 & 31.625 & 36.771 & 32.654 & 16.508 & 15.685 & 20.044 & 15.642 & 10.487 & 31.835 & 32.526 & 29.654 & 31.591 & 27.72 & 33.861 \\
\hline $12 / 23 / 114: 00$ & & 30.757 & 30.436 & 29.636 & 30.042 & & 29.69 & 30.523 & 27.945 & 30.016 & 28.818 & 26.564 & 31.618 & 36.78 & 32.647 & 16.506 & 15.68 & 20.037 & 15.651 & 10.484 & 31.818 & 32.521 & 29.642 & 31.584 & 27.72 & 33.859 \\
\hline $12 / 23 / 11$ 5:00 & & 30.757 & 30.436 & 29.641 & $\begin{array}{r}30.04 \\
304\end{array}$ & & 29.69 & 30.518 & 27.973 & 30.016 & 28.818 & 26.562 & 31.616 & 36.78 & 32.64 & 16.506 & 15.68 & 20.023 & 15.649 & 10.477 & 31.821 & 32.516 & 29.644 & 31.579 & 27.72 & 33.854 \\
\hline 12/23/11 6:00 & & 30.761 & 30.443 & 29.655 & 30.042 & & 29.681 & 30.52 & 27.999 & 30.025 & 28.825 & 26.576 & 31.616 & 36.78 & 32.645 & 16.506 & 15.678 & 20.023 & 15.653 & 10.484 & 31.825 & 32.518 & 29.647 & 31.584 & 27.72 & 33.854 \\
\hline 12/23/11 7:00 & & 30.757 & 30.436 & 29.645 & 30.038 & & 29.692 & 30.518 & 28.016 & 30.02 & 28.823 & 26.567 & 31.614 & 36.783 & 32.64 & 16.503 & 15.678 & 20.016 & 15.665 & 10.477 & 31.818 & 32.518 & 29.64 & 31.582 & 27.72 & 33.854 \\
\hline
\end{tabular}




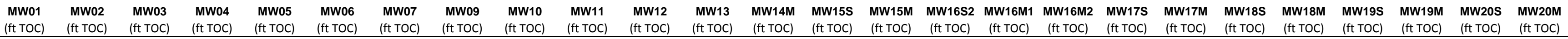

\begin{tabular}{|c|c|c|c|c|c|c|c|c|c|c|c|c|c|c|c|c|c|c|c|c|c|c|c|c|c|c|}
\hline Well & $\begin{array}{l}\text { (ft TOC) } \\
\text { (is }\end{array}$ & $\begin{array}{l}\text { (ft TOC) } \\
\text { (f) }\end{array}$ & $\begin{array}{l}\text { (ft TOC) } \\
\text { (f) }\end{array}$ & (ft TOC) & $\begin{array}{l}\text { (ft TOC) } \\
\text { (f) }\end{array}$ & $\begin{array}{l}\text { (ft TOC) } \\
\text { (f) }\end{array}$ & (ft Toc) & $\begin{array}{l}\text { (ft TOC) } \\
\text { (f) }\end{array}$ & $\begin{array}{l}\text { (ft TOC) } \\
\text { (ft) }\end{array}$ & $\begin{array}{l}\text { (ft TOC) } \\
\text { (f) }\end{array}$ & $\begin{array}{l}\text { (ft TOC) } \\
\text { (f) }\end{array}$ & $\begin{array}{l}\text { (ft Toc) } \\
\text { (f) }\end{array}$ & (ft TOC) & $\begin{array}{l}\text { (ft TOC) } \\
\end{array}$ & (ft TOC) & (ft TOC) & $\begin{array}{l}\text { (ft TOC) } \\
\text { (f) }\end{array}$ & (ft TOC) & $\begin{array}{l}\text { (ft TOC) } \\
\text { (ft) }\end{array}$ & (ft TOC) & $\begin{array}{l}\text { (ft TOC) } \\
\text { (f) }\end{array}$ & $\begin{array}{l}\text { (ft TOC) } \\
\text { (n) }\end{array}$ & $\begin{array}{l}\text { (ft TOC) } \\
\text { (n) }\end{array}$ & (ft TOC) & $\begin{array}{l}\text { (ft TOC) } \\
\end{array}$ & (ft TOC) \\
\hline $12 / 23 / 118: 00$ & & 30.755 & 30.429 & 29.631 & 30.028 & & 29.704 & 30.523 & 28.035 & 30.016 & 28.814 & 26.555 & 31.614 & 36.78 & 32.642 & $2 \quad 16.501$ & 15.678 & 20.016 & 15.67 & 10.482 & 31.809 & 32.511 & 29.628 & 31.579 & 27.72 & 33.854 \\
\hline 12/23/11 9:00 & & 30.754 & 30.429 & 29.631 & 30.024 & & 29.702 & 30.516 & 28.064 & 30.016 & 28.816 & 26.552 & 31.611 & 36.778 & 32.642 & $\begin{array}{l}16.499 \\
2\end{array}$ & 15.678 & 20.009 & 15.67 & 10.473 & 31.809 & 32.514 & 29.63 & 31.579 & 27.721 & 33.854 \\
\hline $12 / 23 / 1110: 00$ & & 30.752 & 30.422 & 29.629 & 30.021 & & 29.709 & 30.516 & 28.08 & 30.013 & 28.814 & 26.55 & 31.611 & 36.776 & 32.645 & $5 \quad 16.499$ & 15.68 & 20.002 & 15.677 & 10.48 & 31.804 & 32.511 & 29.628 & 31.579 & 27.723 & 33.857 \\
\hline $23 / 1111: 00$ & & 30.747 & 30.417 & 29.615 & 30.012 & & 29.723 & 30.527 & 28.092 & 30.006 & 28.805 & 26.54 & 31.609 & 36.776 & 32.64 & 16.494 & 15.678 & 19.995 & 15.698 & 10.477 & 31.795 & 32.509 & & & 27.723 & 33.857 \\
\hline $12 / 23 / 1112: 00$ & & 30.736 & 30.396 & 29.58 & 29.986 & & 29.749 & 30.556 & 28.099 & 29.99 & 28.784 & 26.516 & 31.599 & 36.773 & 32.631 & 16.492 & 15.673 & 19.986 & 15.719 & 10.47 & 31.772 & & 579 & & 27.72 & 33.857 \\
\hline 12/23/11 13:00 & & 30.729 & 30.38 & 29.547 & 29.961 & & 29.765 & 30.571 & 28.111 & 29.971 & 28.767 & 26.48 & 31.588 & 36.759 & 32.621 & 16.485 & 15.671 & 19.986 & 15.722 & 10.458 & 31.751 & 32.488 & 29.555 & 1.558 & 72 & 33.852 \\
\hline $12 / 23 / 1114: 00$ & & 30.724 & 30.363 & 29.521 & 29.94 & & 29.772 & 30.573 & 28.13 & 29.955 & 28.753 & 26.452 & 31.578 & 36.755 & 32.609 & $\begin{array}{ll}9 & 16.478\end{array}$ & $\begin{array}{l}15.664 \\
\end{array}$ & 19.975 & 15.731 & 10.449 & 31.735 & 32.481 & 29.532 & 31.549 & 27.72 & 33.85 \\
\hline 12/23/11 15:00 & & 30.722 & 30.356 & 29.507 & 29.919 & & 29.765 & 30.571 & 28.151 & 29.941 & 28.742 & 26.435 & 31.567 & 36.745 & 32.598 & $8 \quad 16.473$ & 15.659 & 19.968 & 15.726 & 10.437 & 31.721 & 32.469 & 29.518 & 31.535 & 27.72 & 33.845 \\
\hline 12/23/11 16:00 & & 30.717 & 30.345 & 29.5 & 29.907 & & 29.758 & 30.568 & 28.166 & 29.932 & 28.732 & 26.433 & 31.562 & 36.738 & 32.593 & $3 \quad 16.466$ & 15.655 & 19.956 & 15.724 & 10.43 & 31.711 & 32.459 & 29.513 & 31.528 & 27.72 & 33.843 \\
\hline 12/23/11 17:00 & & 30.713 & 30.333 & 29.486 & 29.895 & & 29.754 & 30.571 & 28.182 & 29.92 & 28.721 & 26.421 & 31.55 & 36.74 & 32.584 & $4 \quad 16.461$ & 15.65 & 19.956 & 15.722 & 10.418 & 31.7 & 32.455 & 29.496 & 31.516 & 27.72 & 33.843 \\
\hline $12 / 23 / 1118: 00$ & & 30.715 & 30.333 & 29.491 & 29.888 & & 29.732 & 30.554 & 28.206 & 29.916 & 28.716 & 26.425 & 31.548 & 36.733 & 32.579 & $\begin{array}{l}9 \\
9\end{array}$ & 15.648 & 19.961 & 15.712 & 10.418 & 31.702 & 32.447 & 29.499 & 31.514 & 27.72 & 33.843 \\
\hline 12/23/11 19:00 & & 30.712 & 30.333 & 29.498 & 29.888 & & 29.714 & 30.535 & 28.223 & 29.913 & 28.716 & 26.44 & 31.546 & 36.736 & 32.579 & $\begin{array}{l}9 \\
9\end{array}$ & 15.65 & 19.963 & 15.7 & 10.411 & 31.707 & 32.445 & 29.503 & 31.511 & 27.72 & 33.843 \\
\hline $12 / 23 / 1120: 00$ & & 30.71 & 30.326 & 29.496 & 29.888 & & 29.704 & 30.527 & 28.237 & 29.906 & 28.712 & 26.44 & 31.546 & 36.736 & 32.577 & $7 \quad 16.447$ & 15.652 & 19.954 & 15.698 & 10.416 & 31.702 & 32.447 & 29.506 & 31.511 & 27.723 & 33.847 \\
\hline $12 / 23 / 1121: 00$ & & 30.708 & 30.326 & 29.498 & 29.891 & & 29.697 & 30.518 & 28.254 & 29.904 & 28.712 & 26.447 & 31.546 & 36.736 & 32.579 & 16.445 & 15.65 & 19.956 & 15.693 & 10.416 & 31.704 & 32.447 & 29.515 & 31.514 & 27.723 & 33.85 \\
\hline $12 / 23 / 1122: 00$ & & 30.71 & 30.326 & 29.503 & 29.893 & & 29.683 & 30.499 & 28.275 & 29.904 & 28.712 & 26.449 & 31.548 & 36.736 & 32.581 & 16.443 & 15.655 & 19.949 & 15.689 & 10.416 & 31.711 & 32.447 & 29.51 & 31.516 & 27.723 & 33.854 \\
\hline 12/23/11 23:00 & & 30.703 & 30.319 & 29.491 & 29.888 & & 29.688 & 30.506 & 28.284 & 29.895 & 28.702 & 26.445 & 31.546 & 36.738 & 32.577 & 16.438 & 15.652 & 19.949 & 15.693 & 10.414 & 31.704 & 32.447 & 29.506 & 31.514 & 27.723 & 33.854 \\
\hline $12 / 24 / 110: 00$ & & 30.703 & 30.317 & 29.491 & 29.888 & & 29.681 & 30.496 & 28.299 & 29.89 & 28.7 & 26.447 & 31.546 & 36.74 & 32.577 & 16.436 & $\begin{array}{l}15.652 \\
\end{array}$ & 19.944 & 15.689 & 10.411 & 31.704 & 32.445 & 29.496 & 31.511 & 27.723 & 33.857 \\
\hline 12/24/11 1:00 & & 30.696 & 30.307 & 29.472 & 29.879 & & 29.69 & 30.506 & 28.308 & 29.881 & 28.691 & 26.428 & 31.539 & 36.733 & 32.567 & 16.431 & $\begin{array}{l}15.648 \\
\end{array}$ & 19.94 & 15.691 & 10.407 & 31.688 & 32.438 & $\begin{array}{l}29.494 \\
294\end{array}$ & 31.504 & 27.725 & 33.854 \\
\hline $12 / 24 / 112: 00$ & & $\begin{array}{l}30.699 \\
30.69\end{array}$ & 30.303 & 29.468 & 29.872 & & 29.685 & 30.501 & 28.325 & $\begin{array}{l}29.8016 \\
29.876\end{array}$ & 28.686 & 26.428 & 31.532 & 36.733 & 32.563 & 16.429 & $\begin{array}{l}15.040 \\
15.643\end{array}$ & 19.935 & 15.684 & $\begin{array}{l}10.497 \\
\end{array}$ & 31.686 & $\begin{array}{l}32.431 \\
32.40\end{array}$ & 29.489 & 31.497 & 27.725 & $\begin{array}{l}33.852 \\
33.852\end{array}$ \\
\hline $12 / 24 / 113: 00$ & & 30.703 & $\begin{array}{l}50.305 \\
30.307\end{array}$ & $\begin{array}{l}29.400 \\
29.47\end{array}$ & 29.879 & & 29.671 & 30.484 & 28.346 & 29.876 & $\begin{array}{l}28.680 \\
28.68\end{array}$ & $\begin{array}{l}20.420 \\
26.442\end{array}$ & 31.529 & 36.741 & 32.56 & 16.427 & $\begin{array}{l}13.045 \\
15.636\end{array}$ & 19.928 & $\begin{array}{l}13.004 \\
15.675\end{array}$ & 10.395 & $\begin{array}{l}51.000 \\
31.69\end{array}$ & $\begin{array}{l}32.428 \\
32.41\end{array}$ & 29.496 & 31.495 & 27.723 & $\begin{aligned} 53.052 \\
33.85\end{aligned}$ \\
\hline $12 / 24 / 114: 00$ & & 30.703 & $\begin{array}{l}30.301 \\
30.307\end{array}$ & $\begin{array}{l}29.482 \\
29.47\end{array}$ & 29.881 & & 29.662 & $\begin{array}{l}50.464 \\
30.477\end{array}$ & $\begin{array}{l}28.340 \\
28.356\end{array}$ & 29.874 & $\begin{array}{l}28.608 \\
28.688\end{array}$ & $\begin{array}{l}20.442 \\
26.447\end{array}$ & 31.522 & $\begin{array}{r}\begin{array}{r}30.141 \\
36.74\end{array} \\
\end{array}$ & $\begin{array}{l}32.50 \\
32.556\end{array}$ & $\begin{array}{l}10.427 \\
16.424\end{array}$ & $\begin{array}{l}13.030 \\
15.631\end{array}$ & 19.924 & $\begin{array}{l}13.672 \\
15.672\end{array}$ & $\begin{array}{l}10.393 \\
10.393\end{array}$ & $\begin{array}{r}31.09 \\
31.693\end{array}$ & $\begin{array}{l}32.48 \\
32.424\end{array}$ & $\begin{array}{l}29.490 \\
29.499\end{array}$ & $\begin{array}{r}31.459 \\
31.49\end{array}$ & 27.725 & $\begin{array}{r}33.047 \\
33.847\end{array}$ \\
\hline $12 / 24 / 115: 00$ & & 30.696 & 30.3 & 29.47 & & & & 30.48 & & 29.869 & & 26.435 & 31.515 & 36.733 & 32.548 & $3 \quad 16.42$ & 15.627 & 19.914 & 15.67 & 10.386 & 31.683 & 32.419 & 494 & 31.483 & 27.728 & 33.845 \\
\hline $12 / 24 / 116: 00$ & & 30 & 30.305 & 29.484 & 1 & & 29.655 & 30.465 & 28.389 & 29.869 & $\begin{array}{l}28.684 \\
28.686\end{array}$ & 26.449 & 31.515 & 7.745 & 32.546 & $\begin{array}{l}16.417 \\
16.417\end{array}$ & 15.624 & 9.91 & 15.663 & $\begin{array}{l}10.380 \\
10.383\end{array}$ & 31.688 & 417 & .501 & 31.483 & 27.728 & $\begin{array}{l}33.843 \\
33.043\end{array}$ \\
\hline 12/24/11 7:00 & & 30.706 & 30.312 & 29.5 & 29.895 & & 29.636 & 30.46 & 28.403 & 29.881 & 28.695 & 26.471 & 31.52 & 36.745 & 32.551 & 16.415 & 15.622 & .91 & $\begin{array}{l}13.053 \\
15.658\end{array}$ & 1.386 & 1.7 & 32.419 & 29.52 & 1.485 & 27.728 & $\begin{array}{l}53.045 \\
33.843\end{array}$ \\
\hline $12 / 24 / 1$ & & 30.701 & 30.31 & 29.496 & 29.895 & & 45 & 30.463 & 28.408 & 29.876 & 28.693 & 26.464 & 31.522 & 36.745 & 32.548 & 16.413 & 15.622 & 005 & 15.66 & 10.388 & 31.697 & 32.421 & 29.515 & 1.488 & 27.728 & 33.843 \\
\hline 12/24/11 9:00 & & 30.699 & 30.31 & 29.496 & 29.898 & & 29.643 & 30.458 & 28.42 & 29.879 & 28.693 & 26.471 & 31.522 & 36.75 & 32.553 & 16.415 & 15.62 & 19.901 & 15.66 & 10.386 & 31.7 & 32.424 & 29.52 & 1.488 & 27.73 & 33.84 \\
\hline 12/24/11 10:00 & & 30.706 & 30.321 & 29.519 & 29.912 & & 29.631 & 30.446 & 28.441 & 29.888 & 28.702 & 26.49 & 31.529 & 36.752 & 32.556 & 16.413 & 15.622 & 19.931 & 15.653 & 10.393 & 31.718 & 32.428 & 29.539 & 31.492 & 27.732 & 33.845 \\
\hline 12/24/11 11:00 & & 30.699 & 30.319 & 29.517 & 29.919 & & 29.638 & 30.463 & 28.446 & 29.89 & 28.702 & 26.5 & 31.529 & 36.757 & 32.56 & 16.413 & 15.624 & 19.956 & 15.665 & 10.395 & 31.716 & 32.431 & 29.541 & 31.5 & 27.73 & 33.847 \\
\hline $12 / 24 / 1112: 00$ & & 30.689 & 30.31 & 29.489 & 29.907 & & 29.667 & 30.487 & 28.436 & 29.879 & 28.691 & 26.476 & 31.527 & 36.755 & 32.558 & $3 \quad 16.41$ & 15.62 & 19.954 & 15.686 & 10.393 & 31.7 & 32.428 & 29.515 & 31.492 & 27.73 & 33.845 \\
\hline $12 / 24 / 1113: 00$ & & 30.685 & 30.3 & 29.47 & 29.893 & & 29.681 & 30.506 & 28.453 & 29.867 & 28.679 & 26.454 & 31.52 & 36.75 & 32.553 & 16.406 & 15.62 & 19.947 & 15.689 & 10.388 & 31.688 & 32.421 & 29.506 & 31.485 & 27.73 & 33.845 \\
\hline $12 / 24 / 1114: 00$ & & 30.682 & 30.286 & 29.444 & 29.872 & & 29.692 & 30.518 & 28.463 & 29.853 & 28.667 & 26.426 & 31.511 & 36.745 & 32.544 & 16.403 & 15.615 & 19.935 & 15.696 & 10.381 & 31.669 & 32.414 & 29.48 & 31.478 & 27.732 & 33.843 \\
\hline $12 / 24 / 1115: 00$ & & 30.685 & 30.277 & 29.435 & 29.86 & & 29.692 & 30.513 & 28.477 & 29.844 & 28.658 & 26.411 & 31.504 & 36.74 & 32.534 & 16.401 & 15.608 & 19.933 & 15.689 & 10.371 & 31.665 & 32.405 & 29.475 & 31.471 & 27.732 & 33.84 \\
\hline $12 / 24 / 1116: 00$ & & 30.687 & 30.279 & 29.442 & 29.86 & & 29.678 & 30.506 & 28.491 & 29.841 & 28.658 & 26.418 & 31.499 & 36.741 & 32.532 & 16.399 & 15.608 & 20 & 15.682 & 10.369 & 31.667 & 32.4 & 29.475 & 31.469 & 27.735 & 33.838 \\
\hline $12 / 24 / 1117: 00$ & & 30.692 & 30.284 & 29.458 & 29.865 & & 29.657 & 30.499 & 28.508 & 29.846 & 28.665 & 26.437 & 31.501 & 36.743 & 32.534 & 16.399 & 15.605 & 20.005 & 15.672 & 10.369 & 31.676 & 32.402 & 29.489 & 31.469 & 27.737 & 33.84 \\
\hline 12/24/11 18:00 & & 30.689 & 30.286 & 29.47 & 29.8 & & 29.645 & 30.487 & 28.517 & 29.848 & 28.67 & 26.452 & 31.504 & 36.748 & 32.532 & 16 & 15.603 & 19.993 & 15.67 & 10.369 & 31.679 & 32.405 & 29.501 & 31.469 & 27.735 & 33.84 \\
\hline 12/24/11 19:00 & & 30.687 & 30.289 & 29.475 & 29. & & 29.636 & 30.47 & 28.531 & 29.851 & 28 & 26.457 & 31.506 & 36.748 & 32.5 & 16.396 & $\begin{array}{l}15.603 \\
\end{array}$ & 19.982 & 15.658 & 10.364 & 31.683 & 32.405 & 29.506 & 31.471 & 27.737 & $\begin{array}{l}33.04 \\
33.84\end{array}$ \\
\hline $12 / 24 / 1120: 00$ & & 30.68 & 30.284 & 29.463 & 29.8 & & 29.645 & 30.477 & 28.527 & 29.846 & 28.663 & 26.452 & 31.504 & 36.748 & 32.532 & 16.394 & $\begin{array}{l}15.008 \\
15.608\end{array}$ & 19.977 & $\begin{array}{l}15.670 \\
15.672\end{array}$ & $\begin{array}{l}10.504 \\
10.369\end{array}$ & 31.679 & 32.407 & 29.494 & 31.471 & 27.737 & 33.843 \\
\hline $12 / 24 /$ & & $\begin{array}{l}30.60 \\
30.68\end{array}$ & $\begin{array}{l}30.204 \\
30.279\end{array}$ & 29.458 & 29.8 & & 29.645 & 30.47 & 28.539 & 29.841 & $\begin{array}{l}20.006 \\
28.66\end{array}$ & 26.445 & 31.506 & 36.755 & 32.537 & 16.392 & $\begin{array}{l}15.000 \\
15.608\end{array}$ & 19.968 & 15.67 & $\begin{array}{l}10.371 \\
\end{array}$ & 31.681 & 32.407 & 9.494 & 31.474 & 27.737 & $\begin{array}{l}33.843 \\
33.843\end{array}$ \\
\hline $12 / 24$ & & $\begin{array}{l}30.00 \\
30.68\end{array}$ & 30.279 & $\begin{array}{l}29.450 \\
29.456\end{array}$ & 29. & & $\begin{array}{l}29.643 \\
\end{array}$ & $\begin{array}{l}30.468 \\
30.468\end{array}$ & 28.548 & $\begin{array}{l}29.839 \\
29.839\end{array}$ & $\begin{array}{l}28.600 \\
28.658\end{array}$ & $\begin{array}{l}20.449 \\
26.442\end{array}$ & 31.508 & 36.748 & 32.537 & $\begin{array}{l}10.392 \\
16.392\end{array}$ & $\begin{array}{r}15.000 \\
15.61\end{array}$ & 19.958 & $\begin{array}{l}15.67 \\
15.67\end{array}$ & 10.374 & 31.683 & 32.409 & 4.496 & 78 & 27.737 & $\begin{array}{l}33.847 \\
33.047\end{array}$ \\
\hline 12 & & & 30 & 29 & & & 2 & 30 & $2 \varepsilon$ & 29 & 28 & 2 & 3 & 3 & 32 & & 1. & 19.949 & 8 & 10.376 & 31 & 9 & 9 & 6 & 27.739 & 33.85 \\
\hline & & & 30.279 & 29 & & & & 30 & & 29 & 2 & 26.449 & 3 & 36. & 32 & 16 & 15.612 & $1 \mathrm{~s}$ & 15.658 & 10.374 & 31.683 & 2 & 1 & 8 & 42 & 33.852 \\
\hline 12/25/11 1:00 & & 30.673 & 30.272 & 29.447 & 29 & & & 30.458 & & & 28.651 & 26.44 & & 36.752 & 32.539 & & 15.61 & & 15.67 & 10.374 & 31.679 & 32.409 & 34 & 76 & 27.742 & 33.854 \\
\hline 12/25/11 2:00 & & & & 29.454 & & & & & & & & 26.442 & & 36.752 & 32.539 & 16.387 & 15.608 & & 15.658 & 10.371 & 31.683 & 32.409 & .499 & 74 & 27.742 & 33.852 \\
\hline $12 / 2$ & & 30 & & 29 & & & & & & & & & & 36.755 & 32.537 & 16. & 15.605 & & 15.656 & 10.371 & 31.679 & 32.407 & .501 & 74 & 42 & 33.852 \\
\hline 1:00 & & & & 29 & & & & & & & & & & 36.752 & 32.532 & & & & 15 & 10.364 & 31.674 & 32.4 & & 31.469 & 27.744 & 33.847 \\
\hline 12/25/11 5:00 & & 30 & 30.2 & 29.433 & & & & 30. & & & 28 & 26.433 & & 36.752 & 32.525 & 16. & 15 & & 15.663 & 10.36 & 31.662 & & & & 27.742 & 33.843 \\
\hline 12/25/11 6:00 & & 30. & 30.2 & 29.435 & & & 29. & 30.4 & 28. & 29.8 & 28. & 26 & 31. & 36.7 & 32.523 & 16.3 & 15. & 19. & 15.6 & 10.355 & 31.662 & 32.391 & 29.487 & 31.457 & 27.746 & 33.84 \\
\hline 12/25/11 7:00 & & 30.675 & 30. & 29.451 & 29. & & 29.6 & 30.4 & 28.6 & 29 & 28. & 26.449 & 31.4 & 36.752 & 32.52 & 16.378 & 15. & 19.9 & 15.649 & 10.357 & 31.672 & 32.388 & 29.501 & 31.457 & 27.746 & 33.838 \\
\hline 12/25/11 8:00 & & 30.678 & 30.279 & 29.472 & 29.8 & & 29. & 30.444 & 28.655 & 29.841 & 28.6 & 26.464 & 31.492 & 36.755 & 32.525 & 16.38 & 15.584 & 19.894 & 15.644 & 10.36 & 31.69 & 32.395 & 29.513 & 31.462 & 27.746 & 33.84 \\
\hline 12/25/11 9:00 & & 30.68 & 30.291 & 29.489 & 29.9 & & 29.601 & 30.449 & 28.667 & 29.853 & 28.6 & 26.485 & 31.501 & 36.764 & 32.532 & 16.378 & 15.587 & 19.891 & 15.644 & 10.367 & 31.7 & 32.4 & 29.534 & 31.467 & 27.749 & 33.84 \\
\hline $12 / 25 / 1110: 00$ & & 30.68 & 30.303 & 29.5 & 29.916 & & 29.601 & 30.456 & 28.672 & 29.862 & 28.677 & 26.504 & 31.508 & 36.771 & 32.537 & 16.38 & 15.591 & 19.894 & 15.644 & 10.369 & 31.716 & 32.409 & 29.536 & 31.476 & 27.749 & 33.845 \\
\hline 12/25/11 11:00 & & 30.68 & 30.307 & 29.507 & 29.926 & & 29.605 & 30.458 & 28.684 & 29.867 & 28.681 & 26.509 & 31.513 & 36.776 & 32.544 & 16.38 & 15.594 & 19.912 & 15.653 & 10.381 & 31.711 & 32.414 & 29.56 & 31.483 & 27.749 & 33.847 \\
\hline $12 / 25 / 1112: 00$ & & 30.666 & 30.293 & 29.472 & 29.914 & & 29.641 & 30.489 & 28.672 & 29.855 & 28.667 & 26.48 & 31.511 & 36.773 & 32.539 & 16.382 & 15.591 & 19.919 & 15.682 & 10.379 & 31.697 & 32.412 & 29.522 & 31.476 & 27.751 & 33.847 \\
\hline 12/25/11 13:00 & & 30.661 & 30.282 & 29.451 & 29.8 & & 29.66 & 30.511 & 28.679 & 29.844 & 28.658 & 26.454 & 31.506 & 36.766 & 32.537 & 16.378 & 15.589 & 19.907 & 15.691 & 10.369 & 31.686 & 32.405 & 29.508 & 31.471 & 27.749 & 33.845 \\
\hline $12 / 25 / 1114: 00$ & & 30.657 & 30.268 & 29.426 & 29.879 & & 29.676 & 30.52 & 28.691 & 29.832 & 28.644 & 26.425 & 31.497 & 36.757 & 32.527 & 16.378 & 15.587 & 19.901 & 15.693 & 10.362 & 31.667 & 32.398 & 29.489 & 31.464 & 27.753 & 33.845 \\
\hline $12 / 25 / 11$ 15:00 & & 30.652 & 30.258 & 29.409 & 29.863 & & 29.681 & 30.518 & 28.698 & 29.821 & 28.632 & 26.406 & 31.49 & 36.75 & 32.52 & 16.373 & 15.584 & 19.894 & 15.7 & 10.357 & 31.653 & 32.391 & 29.471 & 31.453 & 27.753 & 33.843 \\
\hline
\end{tabular}




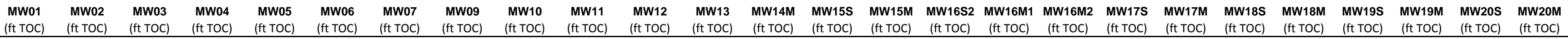

\begin{tabular}{|c|c|c|c|c|c|c|c|c|c|c|c|c|c|c|c|c|c|c|c|c|c|c|c|c|c|c|}
\hline Well & $\begin{array}{l}\text { (ft TOC) } \\
\text { (ft }\end{array}$ & $\begin{array}{l}\text { (ft TOC) } \\
\text { (f) }\end{array}$ & $\begin{array}{l}\text { (ft TOC) } \\
\text { (f) }\end{array}$ & $\begin{array}{l}\text { (ft TOC) } \\
\text { (f }\end{array}$ & $\begin{array}{l}\text { (ft TOC) } \\
\text { (f) }\end{array}$ & $\begin{array}{l}\text { (ft TOC) } \\
\text { (f) }\end{array}$ & $\begin{array}{l}\text { (ft TOC) } \\
\text { (ft }\end{array}$ & $\begin{array}{l}\text { (ft TOC) } \\
\text { (f) }\end{array}$ & $\begin{array}{l}\text { (ft TOC) } \\
\text { (ft) }\end{array}$ & $\begin{array}{l}\text { (ft TOC) } \\
\text { (f) }\end{array}$ & $\begin{array}{l}\text { (ft TOC) } \\
\text { (f) }\end{array}$ & $\begin{array}{l}\text { (ft TOC) } \\
\text { (f) }\end{array}$ & (ft TOC) & $\begin{array}{l}\text { (ft TOC) } \\
\end{array}$ & (ft TOC) & (ft TOC) & $\begin{array}{l}\text { (ft TOC) } \\
\text { (f) }\end{array}$ & (ft TOC) & $\begin{array}{l}\text { (ft TOC) } \\
\text { (1) }\end{array}$ & (ft TOC) & $\begin{array}{l}\text { (ft TOC) } \\
\end{array}$ & $\begin{array}{l}\text { (ft TOC) } \\
\text { (f) }\end{array}$ & $\begin{array}{l}\text { (ft TOC) } \\
\end{array}$ & (ft TOC) & $\begin{array}{l}\text { (ft TOC) } \\
\text { (1) }\end{array}$ & (ft TOC) \\
\hline 16:00 & & 30.652 & 30.251 & 29.4 & 29.851 & & 29.6 & 30.52 & 28. & $29 . \varepsilon$ & 28.623 & 26 & 31.482 & 36.75 & 32.516 & 16.371 & 15.58 & 19.935 & 15.696 & 10.348 & 31.651 & 32.383 & 29.468 & 48 & 53 & 38 \\
\hline $12 / 25 / 1117: 00$ & & 30.654 & 30.251 & 29.407 & 29.849 & & 29.664 & 30.511 & 28.722 & 29.811 & 28.623 & 26.404 & 31.48 & 36.743 & 32.509 & 16.368 & 15.575 & 19.933 & 15.689 & 0.343 & 31.648 & 32.381 & .466 & 1.446 & 7.753 & 33.838 \\
\hline 12/25/11 18:00 & & 30.661 & 30.251 & 29.419 & 29.853 & & 29.643 & 30.494 & 28.743 & 29.814 & 28.628 & 26.413 & 31.48 & 36.748 & 32.506 & 16.366 & 15.573 & 19.919 & 15.675 & 10.343 & 31.658 & 32.379 & 29.478 & .443 & 1.756 & 33.836 \\
\hline 12/25/11 19:00 & & 30.659 & 30.256 & 29.43 & 29.858 & & 29.629 & 30.48 & 28.752 & 29.816 & 28.63 & 26.425 & 31.48 & 36.75 & 32.511 & $1 \quad 16.366$ & 15.573 & 19.914 & 15.675 & 10.343 & 31.658 & 32.381 & 29.487 & 31.448 & 17.756 & 33.836 \\
\hline $12 / 25 / 1120: 00$ & & 30.652 & 30.247 & 29.412 & 29.851 & & 29.641 & 30.492 & 28.75 & 29.807 & 28.621 & 26.411 & 31.478 & 36.748 & 32.509 & $\begin{array}{l}9 \\
9\end{array}$ & 15.573 & 19.905 & 15.679 & 10.343 & 31.651 & 32.376 & 29.473 & 31.443 & 27.756 & 33.836 \\
\hline 12/25/11 21:00 & & 30.647 & 30.237 & 29.393 & 29.839 & & 29.645 & 30.499 & 28.752 & 29.797 & 28.612 & 26.401 & 31.473 & 36.745 & 32.504 & $\begin{array}{l}4 \quad 16.359 \\
\end{array}$ & 15.573 & 19.898 & 15.684 & 10.341 & 31.639 & 32.376 & 29.456 & 31.441 & 27.756 & 33.836 \\
\hline $12 / 25 / 1122: 00$ & & 30.643 & 30.228 & 29.381 & 29.83 & & 29.648 & 30.506 & 28.757 & 29.79 & 28.602 & 26.392 & 31.471 & 36.745 & 32.504 & $\begin{array}{l}4 \quad 16.359 \\
\end{array}$ & 15.57 & 19.896 & 15.682 & 10.336 & 31.632 & 32.369 & 29.449 & 31.436 & 27.756 & 33.838 \\
\hline 12/25/11 23:00 & & 30.64 & 30.219 & 29.37 & 29.821 & & 29.65 & 30.511 & 28.764 & 29.781 & 28.593 & 26.378 & 31.468 & 36.738 & 32.499 & $\begin{array}{l}9 \\
9\end{array}$ & 15.57 & 19.887 & 15.679 & 10.336 & 31.623 & 32.367 & 29.438 & 31.436 & 27.758 & 33.84 \\
\hline 12/26/11 0:00 & & 30.631 & 30.205 & 29.346 & 29.802 & & 29.66 & 30.525 & 28.764 & 29.767 & 28.579 & 26.368 & 31.464 & 36.731 & 32.492 & $2 \quad 16.352$ & 15.568 & 19.88 & 15.691 & 10.331 & 31.609 & 32.362 & 29.416 & 31.429 & 27.756 & 33.84 \\
\hline 12/26/11 1:00 & & 30.626 & 30.193 & 29.32 & 29.786 & & 29.667 & 30.532 & 28.771 & 29.751 & 28.563 & 26.339 & 31.452 & 36.724 & 32.485 & $5 \quad 16.347$ & 15.566 & 19.87 & 15.689 & 10.322 & 31.595 & 32.355 & 29.398 & 31.42 & 27.758 & 33.838 \\
\hline 12/26/11 2:00 & & 30.624 & 30.177 & 29.299 & 29.762 & & 29.669 & 30.535 & 28.774 & 29.737 & 28.546 & 26.318 & 31.443 & 36.719 & 32.473 & $\begin{array}{l}3 \\
16.34\end{array}$ & 15.559 & 19.866 & 15.684 & 10.31 & 31.576 & 32.343 & 29.377 & 31.41 & 27.758 & 33.836 \\
\hline 12/26/11 3:00 & & 30.626 & 30.167 & 29.297 & 29.755 & & 29.655 & 30.53 & 28.79 & 29.73 & 28.539 & 26.32 & 31.433 & 36.717 & 32.466 & $\begin{array}{ll}6 & 16.338\end{array}$ & 15.554 & 19.857 & 15.675 & 10.303 & 31.569 & 32.334 & 29.372 & 31.399 & 27.758 & 33.833 \\
\hline $12 / 26 / 114: 00$ & & 30.622 & 30.156 & 29.276 & 29.737 & & 29.655 & 30.52 & 28.795 & 29.711 & 28.525 & 26.299 & 31.419 & 36.71 & 32.452 & $2 \quad 16.333$ & 15.545 & 19.852 & 15.67 & 10.289 & 31.555 & 32.319 & 29.355 & 31.387 & 27.758 & 33.826 \\
\hline $12 / 26 / 11$ 5:00 & & 30.617 & 30.142 & 29.26 & 29.72 & & 29.65 & 30.537 & 28.8 & 29.7 & 28.512 & 26.289 & 31.408 & 36.705 & 32.443 & 16.326 & 15.535 & 19.84 & 15.67 & 10.277 & 31.536 & 32.308 & 29.334 & 31.375 & 27.758 & 33.822 \\
\hline 12/26/11 6:00 & & 30.617 & 30.137 & 29.264 & 29.716 & & 29.634 & 30.535 & 28.814 & 29.695 & 28.509 & 26.296 & 31.401 & 36.703 & 32.429 & 16.324 & 15.531 & 19.833 & 15.66 & 10.27 & 31.536 & 32.3 & 29.337 & 31.368 & 27.758 & 33.817 \\
\hline 12/26/11 7:00 & & 30.608 & 30.123 & 29.239 & 29.699 & & 29.638 & 30.532 & 28.812 & 29.679 & 28.493 & 26.279 & 31.387 & 36.698 & 32.422 & 16.315 & 15.521 & 19.824 & 15.66 & 10.254 & 31.52 & 32.289 & 29.313 & 31.354 & 27.758 & 33.81 \\
\hline 12/26/11 8:00 & & 30.605 & 30.111 & 29.227 & 29.685 & & 29.631 & 30.532 & 28.819 & 29.667 & 28.481 & 26.272 & 31.38 & 36.689 & 32.41 & 16.31 & 15.514 & 19.813 & 15.656 & 10.249 & 31.508 & 32.279 & 29.304 & 31.345 & 27.758 & 33.808 \\
\hline 12/26/11 9:00 & & 30.61 & 30.109 & 29.229 & 29.681 & & 29.612 & 30.52 & 28.836 & 29.66 & 28.479 & 26.272 & 31.37 & 36.691 & 32.403 & 16.305 & 15.51 & 19.813 & 15.642 & 10.24 & 31.506 & 32.272 & 29.306 & 31.338 & 27.76 & 33.805 \\
\hline $12 / 26 / 11$ 10:00 & & 30.608 & 30.109 & 29.243 & 29.685 & & 29.594 & 30.508 & 28.845 & 29.66 & 28.479 & 26.294 & 31.37 & 36.691 & 32.403 & 16.301 & 15.507 & 19.813 & 15.632 & 10.24 & 31.513 & 32.272 & 29.311 & 31.34 & 27.758 & 33.808 \\
\hline $12 / 26$ & & 30.596 & 30.098 & 29.222 & 29.674 & & 29.598 & 30.525 & 28.84 & 29.646 & 28.465 & 26.282 & 31.365 & 36.684 & 32.398 & 36.296 & 15.503 & 19.808 & 15.644 & 10.233 & 31.492 & 32.265 & .297 & 31.331 & 27.758 & 33.808 \\
\hline 12 & & 30.582 & 30.072 & 29.178 & 29.64 & & 29.62 & 30.516 & 28.833 & 29.623 & 28.442 & 26.241 & 31.354 & 36.68 & 32.387 & 16.291 & 15.496 & 19.833 & 15.656 & 10.219 & 31.466 & 32.253 & 259 & 31.319 & 27.758 & 33.803 \\
\hline $12 / 266$ & & 30.58 & 30.049 & 29.138 & 29.618 & & 29.627 & 30.504 & 28.84 & 29.598 & 28.419 & 26.208 & 31.337 & 36.672 & 32.37 & 16.28 & 15.489 & 19.827 & .653 & 10.205 & 31.443 & .237 & 231 & 1.305 & 27.758 & 33.798 \\
\hline $12 / 26 / 1114: 00$ & & 30.585 & 30.042 & 29.136 & 29.601 & & 29.608 & 30.496 & 28.859 & 29.586 & 28.407 & 26.198 & 31.328 & 36.668 & 32.361 & 16.275 & 15.484 & 19.852 & 15.639 & 10.197 & 31.434 & 32.227 & 226 & 1.293 & 27.76 & 33.796 \\
\hline $12 / 26 / 1115: 00$ & & 30.584 & 30.037 & 29.143 & 29.596 & & 29.584 & 30.511 & 28.874 & 29.581 & 28.405 & 26.208 & 31.319 & 36.665 & 32.354 & 16.271 & 15.479 & 19.847 & 15.63 & 10.188 & 31.434 & 32.218 & 231 & 1.288 & 27.76 & 33.796 \\
\hline 12/26/11 16:00 & & 30.578 & 30.032 & 29.147 & 29.597 & & 29.565 & 30.511 & 28.874 & 29.579 & 28.402 & 26.224 & 31.316 & 36.658 & 32.349 & 16.266 & 15.475 & 19.889 & 15.625 & 10.183 & 31.436 & 32.215 & 29.224 & 1.281 & 27.76 & 33.794 \\
\hline 12/26/11 17:00 & & 30.573 & 30.025 & 29.138 & 29.594 & & 29.554 & 30.499 & 28.881 & 29.568 & 28.393 & 26.21 & 31.305 & 36.656 & 32.34 & 16.259 & 15.468 & 19.894 & 15.614 & 10.174 & 31.429 & 32.208 & 29.221 & 1.274 & 27.76 & 33.789 \\
\hline $12 / 26 / 1118: 00$ & & 30.589 & 30.037 & 29.169 & 29.606 & & 29.514 & 30.458 & 28.912 & 29.575 & 28.405 & 26.244 & 31.307 & 36.658 & 32.34 & 16.257 & 15.465 & 19.891 & 15.585 & 10.176 & 31.448 & 32.208 & 29.25 & 31.274 & 27.76 & 33.789 \\
\hline 12/26/11 19:00 & & 30.58 & 30.035 & 29.166 & 29.613 & & 29.502 & 30.463 & 28.904 & 29.57 & 28.405 & 26.246 & 31.302 & 36.656 & 32.335 & 16.254 & 15.465 & 19.88 & 15.583 & 10.172 & 31.445 & 32.206 & 29.254 & 31.272 & 27.76 & 33.787 \\
\hline $12 / 26 / 1120: 00$ & & 30.577 & 30.039 & 29.183 & 29.625 & & 29.486 & 30.456 & 28.914 & 29.575 & 28.409 & 26.268 & 31.307 & 36.661 & 32.34 & 16.252 & 15.461 & 19.866 & 15.576 & 10.174 & 31.457 & 32.208 & 29.259 & 31.274 & 27.758 & 33.789 \\
\hline 12/26/11 21:00 & & 30.571 & 30.035 & 29.178 & 29.625 & & 29.481 & 30.46 & 28.919 & 29.572 & 28.405 & 26.26 & 31.305 & 36.656 & 32.337 & 16.25 & 15.458 & 19.857 & 15.567 & 10.174 & 31.455 & 32.208 & 29.257 & 31.272 & 27.763 & 33.789 \\
\hline $12 / 26 / 1122: 00$ & & 30.566 & 30.03 & 29.169 & 29.625 & & 29.481 & 30.47 & 28.923 & 29.568 & 28.402 & 26.26 & 31.305 & 36.658 & 32.333 & 16.245 & 15.456 & 19.838 & 15.564 & 10.174 & 31.448 & 32.206 & 29.257 & 31.272 & 27.76 & 33.791 \\
\hline $12 / 26 / 1123: 00$ & & 30.566 & 30.032 & 29.176 & 29.629 & & 29.467 & 30.463 & 28.93 & 29.568 & 28.405 & 26.265 & 31.307 & 36.658 & 32.34 & 16.245 & 15.458 & 19.836 & 15.555 & 10.174 & 31.457 & 32.208 & 29.266 & 31.27 & 27.763 & 33.791 \\
\hline $12 / 27 / 110: 00$ & & 30.568 & 30.039 & 29.19 & 29.641 & & 29.453 & 30.437 & 28.947 & 29.572 & 28.414 & 26.287 & 31.309 & 36.658 & 32.34 & 16.245 & 15.458 & 19.838 & 15.538 & 10.176 & 31.464 & 32.21 & 29.28 & 31.277 & 27.763 & 33.796 \\
\hline 12/27/11 1:00 & & 30.57 & 30.046 & 29.204 & 29.655 & & 29.439 & 30.425 & 28.954 & 29.579 & 28.423 & 26.299 & 31.316 & 36.661 & 32.347 & 16.243 & 15.463 & 19.831 & 15.529 & 10.181 & 31.48 & 32.215 & 29.299 & 31.284 & 27.763 & 33.798 \\
\hline $12 / 27 / 112: 00$ & & 30.571 & 30.053 & 29.213 & 29.6 & & 29.431 & 30.419 & 28.964 & 29.584 & 28.428 & 26.308 & 31 & 36.67 & 32.352 & 16.2 & 15.461 & 19.822 & 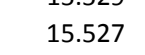 & 10.188 & 31.492 & 32.22 & 29.311 & 31.286 & 27.765 & 33.798 \\
\hline $12 / 27 / 113: 00$ & & 30.575 & 30.072 & 29.241 & 29 & & 29.41 & 30.391 & 28.978 & 29.598 & 28.444 & 26.337 & & 36.675 & 32.359 & 16.247 & $\begin{array}{l}15.465 \\
15.465\end{array}$ & $\begin{array}{l}19.817 \\
\end{array}$ & 3 & $\begin{array}{l}10.19 \\
0\end{array}$ & 31.506 & 32.229 & .332 & 31.293 & 27.767 & 33.803 \\
\hline $12 / 27 / 114: 00$ & & 30.571 & 30.084 & 29.25 & 29.706 & & 29.408 & 30.382 & 28.973 & 29.607 & $\begin{array}{l}20.444 \\
28.451\end{array}$ & 26.354 & 31.328 & 36.682 & 32.359 & 16.247 & $\begin{array}{l}15.461 \\
15.461\end{array}$ & 19.808 & 15.513 & 10.197 & 31.515 & 32.232 & .348 & 31.298 & 27.767 & 33.803 \\
\hline $12 / 27 /$ & & 30.568 & $\begin{array}{l}30.004 \\
30.091\end{array}$ & 29.257 & 29.718 & & 29.41 & 30.374 & 28.978 & 29.614 & 28.458 & 26.354 & 31.333 & $\begin{array}{l}30.082 \\
36.682\end{array}$ & 32.361 & 16.25 & $\begin{array}{l}15.458 \\
15.458\end{array}$ & $\begin{array}{l}19.000 \\
19.799\end{array}$ & 15.513 & 10.2 & 31.52 & 32.234 & .355 & 31.298 & 27.767 & 33.801 \\
\hline $12 / 27$ & & 30.568 & 30.1 & 29.262 & 29 & & 29. & 30. & 28 & 29 & 28. & 26.358 & 31. & 36 & 32.363 & 16.25 & 54 & 15 & 15.508 & 2 & 31.527 & 34 & 52 & 31.3 & 27.77 & 33.798 \\
\hline & & 30. & 30.11 & 29.278 & 29. & & & 30. & 28 & 29 & 28.474 & & & 36.6 & 32.368 & 3 16.254 & 56 & 4 & 1 & 10.205 & 31.536 & 39 & 4 & 31 & 27.77 & 33.798 \\
\hline & & & 30.12 & 29.283 & 29. & & & & & & 28.481 & & & 366 & 20 & & 15 & & 15 & 10.207 & 31.541 & 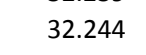 & 4 & 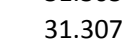 & 77 & 33.796 \\
\hline & & & 30 & 29 & & & & & & & 28 & & & & 32. & & 15 & & $15.5 \mathrm{~s}$ & 10. & 31.553 & 32.246 & 3 & 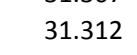 & 27.772 & 33.796 \\
\hline 12/27/11 10:00 & & & 30.146 & 29.313 & 29 & & & & & & 28 & & & 36.7 & 32. & & & & 15.5 & 9 & 31.569 & 32.253 & & & 27.774 & 33.798 \\
\hline $12 / 2$ & & & 30.15 & 29.318 & & & & & & & 28 & & & 36. & 32.389 & 16. & 15.458 & 19.785 & 15 & 10.226 & 31.574 & 32.263 & & 8 & 74 & 33.801 \\
\hline 12 & & & & 29. & & & & & & & 28 & & & 36. & 32.389 & 16 & & & & 10.223 & 31.55 & & & 24 & 27.772 & 33.801 \\
\hline $12 /$ & & & & 29.246 & & & & & & & & 26. & & & 32.382 & 16.261 & 15 & & 15. & 10.219 & 31.529 & & & & 27.772 & 33.798 \\
\hline $12 / 2$ & & & 30. & 29.234 & & & & 30. & 29.018 & 29. & 28. & 26.323 & 31. & 36.7 & 32.377 & 16.261 & 15.451 & 19.773 & 15.5 & 10.214 & 31.525 & 32.248 & & 31.314 & 27.777 & 33.798 \\
\hline $12 / 27 / 1115: 00$ & & 30 & 30. & 29.2 & 29. & & 29 . & 30. & 29.6 & & 28. & & 31.: & 36.6 & 32.377 & 16.261 & 15.454 & 19.769 & 15.5 & 10.212 & 31.525 & 32.248 & 29.358 & 31.312 & 27.774 & 33.801 \\
\hline $12 / 27 / 1116: 00$ & & 30.5 & 30.1 & 29.232 & & & 29. & 30.4 & 29.0 & 29.6 & 28.472 & 26.318 & 31.3. & 36.6 & 32.375 & 16.261 & 15.451 & 19.815 & 15.562 & 10.209 & 31.525 & 32.244 & 29.351 & 31.309 & 27.777 & 33.798 \\
\hline $12 / 27 / 1117: 00$ & & 30.547 & 30.107 & 29.234 & 29.72 & & 29.476 & 30.449 & 29.042 & 29.635 & 28.472 & 26.323 & 31.342 & 36.6 & 32.375 & 16.261 & 15.451 & 19.813 & 15.56 & 10.205 & 31.52 & 32.244 & 29.353 & 31.309 & 27.777 & 33.796 \\
\hline $12 / 27 / 1118: 00$ & & 30.545 & 30.105 & 29.229 & 29.725 & & 29.471 & 30.451 & 29.045 & 29.633 & 28.47 & 26.313 & 31.337 & 36.694 & 32.37 & 16.259 & 15.447 & 19.799 & 15.557 & 10.202 & 31.518 & 32.239 & 29.351 & 31.307 & 27.777 & 33.794 \\
\hline 12/27/11 19:00 & & 30.54 & 30.1 & 29.227 & 29.72 & & 29.467 & 30.453 & 29.049 & 29.63 & 28.467 & 26.315 & 31.337 & 36.698 & 32.368 & 16.257 & 15.442 & 19.796 & 15.56 & 10.2 & 31.513 & 32.239 & 29.346 & 31.302 & 27.777 & 33.791 \\
\hline $12 / 27 / 1120: 00$ & & 30.538 & 30.093 & 29.213 & 29.713 & & 29.474 & 30.453 & 29.054 & 29.623 & 28.46 & 26.301 & 31.33 & 36.694 & 32.363 & 16.254 & 15.44 & 19.785 & 15.56 & 10.195 & 31.506 & 32.232 & 29.33 & 31.298 & 27.777 & 33.789 \\
\hline 12/27/11 21:00 & & 30.538 & 30.091 & 29.211 & 29.711 & & 29.467 & 30.451 & 29.063 & 29.623 & 28.458 & 26.306 & 31.33 & 36.689 & 32.359 & 16.254 & 15.44 & 19.815 & 15.555 & 10.195 & 31.504 & 32.229 & 29.33 & 31.298 & 27.779 & 33.791 \\
\hline $12 / 27 / 1122: 00$ & & 30.536 & 30.084 & 29.208 & 29.704 & & 29.464 & 30.449 & 29.066 & 29.621 & 28.453 & 26.296 & 31.328 & 36.689 & 32.361 & 16.252 & 15.44 & 19.813 & 15.553 & 10.193 & 31.501 & 32.227 & 29.33 & 31.295 & 27.779 & 33.789 \\
\hline $12 / 27 / 1123: 00$ & & 30.543 & 30.088 & 29.218 & 29.706 & & 29.448 & 30.434 & 29.08 & 29.621 & 28.458 & 26.303 & 31.33 & 36.694 & 32.359 & 16.252 & 15.44 & 19.808 & 15.541 & 10.195 & 31.508 & 32.229 & 29.339 & 31.298 & 27.781 & 4 \\
\hline
\end{tabular}




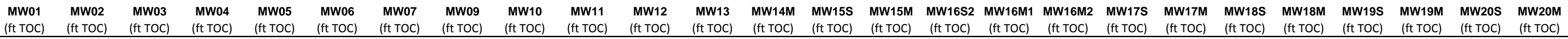

\begin{tabular}{|c|c|c|c|c|c|c|c|c|c|c|c|c|c|c|c|c|c|c|c|c|c|c|c|c|c|c|}
\hline well & $\begin{array}{l}\text { (ft TOC) } \\
\text { (f) }\end{array}$ & $\begin{array}{l}\text { (ft TOC) } \\
\text { (ft) }\end{array}$ & $\begin{array}{l}\text { (ft TOC) } \\
\text { (f) }\end{array}$ & $\begin{array}{l}\text { (ft TOC) } \\
\text { (ft) }\end{array}$ & $\begin{array}{l}\text { (ft TOC) } \\
\text { (f) }\end{array}$ & $\begin{array}{l}\text { (ft toc) } \\
\text { (ftocts }\end{array}$ & (ft toc) & $\begin{array}{l}\text { (ift TOC) } \\
\text { (n) }\end{array}$ & $\begin{array}{l}\text { (ft TOC) } \\
\text { (ft) }\end{array}$ & $\begin{array}{l}\text { (ft TOC) } \\
\text { (f) }\end{array}$ & $\begin{array}{l}\text { (ft TOC) } \\
\text { (n) }\end{array}$ & $\begin{array}{l}\text { (ft TOC) } \\
\text { (f) }\end{array}$ & (ft TOC) & $\begin{array}{l}\text { (ft TOC) } \\
\end{array}$ & (ft TOC) & (ft TOC) & $\begin{array}{l}\text { (ft TOC) } \\
\text { (f) }\end{array}$ & (ft TOC) & $\begin{array}{l}\text { (ft TOC) } \\
\text { (ft) }\end{array}$ & (ft TOC) & $\begin{array}{l}\text { (ft TOC) } \\
\text { (f) }\end{array}$ & $\begin{array}{l}\text { (ft TOC) } \\
\text { (f) }\end{array}$ & $\begin{array}{l}\text { (ft TOC) } \\
\text { (n) }\end{array}$ & (ft TOC) & $\begin{array}{l}\text { (ft TOC) } \\
\text { (1) }\end{array}$ & (ft TOC) \\
\hline 0 & & 30.529 & 30.081 & 29.208 & 29.702 & & ; & 30.4. & 29 & 29.61 & 28.451 & 26 & 31.328 & 36.691 & 32. & 16.25 & 15.44 & 19.803 & 15.555 & 10. & 31. & 32.232 & & 31.295 & 27.779 & 33.794 \\
\hline /11 1:00 & & 30.517 & 30.06 & 29.166 & 29.681 & & 29.481 & 30.453 & 29.064 & 9.6 & 28.432 & 26.268 & 31.3 & 36.684 & 32.352 & 16.245 & 15.435 & 19.792 & 15.569 & 10.188 & 31.476 & & 29.297 & 1.288 & 79 & \\
\hline $12 / 28 / 112: 00$ & & 30.524 & 30.056 & 29.162 & 29.671 & & 29.471 & 30.439 & 29.08 & 29.593 & 28.428 & 26.258 & 31.316 & 36.68 & 32.347 & 16.243 & 15.437 & 19.785 & 15.553 & 10.181 & 31.478 & 32.218 & 9.29 & 1.281 & 81 & 33.791 \\
\hline 12/28/11 3:00 & & 30.522 & 30.046 & 29.154 & 29.66 & & 29.467 & 30.439 & 29.087 & 29.586 & 28.419 & 26.248 & 31.307 & 36.672 & 32.342 & 16.238 & 15.433 & 19.778 & 15.548 & 10.176 & 31.469 & 32.21 & 29.285 & 31.274 & 81 & 33.791 \\
\hline 12/28/11 4:00 & & 30.533 & 30.058 & 29.187 & 29.674 & & 29.431 & 30.405 & 29.109 & 29.598 & 28.432 & 26.279 & 31.314 & 36.68 & $3 \quad 32.342$ & 16.238 & 15.433 & 19.773 & 15.529 & 10.179 & 31.492 & 32.213 & 29.313 & 31.281 & 27.784 & 33.794 \\
\hline 12/28/11 5:00 & & 30.517 & 30.044 & 29.162 & 29.662 & & 29.45 & 30.434 & 29.097 & 29.584 & 28.419 & 26.265 & 31.302 & 36.673 & $3 \quad 32.335$ & 16.233 & 15.428 & 19.764 & 15.541 & 10.169 & 31.466 & 32.203 & 29.29 & 31.272 & 27.781 & 33.787 \\
\hline 12/28/11 6:00 & & 30.524 & 30.056 & 29.185 & 29.671 & & 29.424 & 30.408 & 29.113 & 29.593 & 28.428 & 26.277 & 31.305 & 36.68 & $\begin{array}{ll}3 & 32.335 \\
\end{array}$ & 16.233 & 15.423 & 19.773 & 15.527 & 10.174 & 31.478 & 32.206 & 29.308 & 31.27 & 27.786 & 33.787 \\
\hline 12/28/11 7:00 & & 30.51 & 30.044 & 29.159 & 29.662 & & 29.439 & 30.432 & 29.102 & 29.581 & 28.414 & 26.263 & 31.298 & 36.675 & $5 \quad 32.328$ & 16.231 & 15.416 & 19.764 & 15.538 & 10.165 & 31.464 & 32.201 & 29.285 & 31.263 & 27.784 & 33.782 \\
\hline 12/28/11 8:00 & & 30.512 & 30.039 & 29.159 & 29.66 & & 29.431 & 30.429 & 29.109 & 29.579 & 28.414 & 26.26 & 31.291 & 36.675 & $5 \quad 32.323$ & 16.226 & 15.412 & 19.753 & 15.529 & 10.16 & 31.457 & 32.194 & 29.287 & 31.258 & 27.784 & 33.78 \\
\hline 12/28/11 9:00 & & 30.515 & 30.044 & 29.169 & 29.662 & & 29.417 & 30.42 & 29.121 & 29.581 & 28.416 & 26.275 & 31.291 & 36.672 & 32.323 & 16.226 & 15.412 & 19.746 & 15.522 & 10.158 & 31.469 & 32.191 & 29.297 & 31.258 & 27.784 & 33.78 \\
\hline 12/28/11 10:00 & & 30.519 & 30.056 & 29.192 & 29.674 & & 29.401 & 30.403 & 29.137 & 29.588 & 28.425 & 26.296 & 31.295 & 36.677 & 32.326 & 16.224 & 15.412 & 19.739 & 15.51 & 10.162 & 31.476 & 32.196 & 29.308 & 31.26 & 27.786 & 33.78 \\
\hline 12/28/11 11:00 & & 30.512 & 30.056 & 29.187 & 29.676 & & 29.403 & 30.405 & 29.135 & 29.588 & 28.425 & 26.299 & 31.298 & 36.67 & 32.323 & 16.224 & 15.409 & 19.746 & 15.52 & 10.16 & 31.478 & 32.199 & 29.308 & 31.263 & 27.786 & 33.78 \\
\hline $12 / 28 / 1112: 00$ & & 30.501 & 30.042 & 29.157 & 29.664 & & 29.427 & 30.439 & 29.125 & 29.575 & 28.414 & 26.272 & 31.291 & 36.677 & 32.319 & 16.222 & 15.409 & 19.743 & 15.534 & 10.158 & 31.462 & 32.194 & 29.287 & 31.255 & 27.786 & 33.78 \\
\hline $12 / 28 / 1113: 00$ & & 30.491 & 30.021 & 29.119 & 29.641 & & 29.453 & 30.458 & 29.118 & 29.558 & 28.393 & 26.239 & 31.279 & 36.668 & 32.314 & 16.217 & 15.402 & 19.734 & 15.55 & 10.15 & 31.429 & 32.182 & 29.252 & 31.248 & 27.786 & 33.777 \\
\hline $12 / 28 / 1114: 00$ & & 30.494 & 30.014 & 29.11 & 29.627 & & 29.446 & 30.451 & 29.135 & 29.551 & 28.386 & 26.224 & 31.277 & 36.663 & 32.307 & 16.215 & 15.402 & 19.729 & 15.548 & 10.141 & 31.424 & 32.18 & 29.25 & 31.241 & 27.786 & 33.775 \\
\hline $12 / 28 / 1115: 00$ & & 30.491 & 30.007 & 29.108 & 20.6 & & 29.441 & 30.427 & 29.14 & 29.544 & 28.381 & 26.222 & 31.274 & 36.661 & 32.305 & & 15.4 & 19.736 & 15.548 & 10.139 & 31.424 & 32.173 & 29.25 & 31.239 & 27.788 & 33.777 \\
\hline $12 / 28 / 1116: 00$ & & 30.496 & 30.009 & 29.119 & 29.62 & & 29.424 & 30.425 & 29.149 & 29.544 & 28.381 & 26.224 & 31.267 & 36.658 & 32.302 & 16.21 & 15.397 & 19.792 & 15.538 & 10.136 & 31.429 & 32.17 & 29.252 & 31.237 & 27.791 & 33.775 \\
\hline 8/11 17:00 & & 30.494 & 30.009 & 29.122 & 29.622 & & $\begin{array}{l}29.413 \\
29.47\end{array}$ & 30.432 & 29.151 & 29.542 & 28.381 & 26.234 & 31.27 & 36.661 & 32.298 & 16.208 & 15.393 & 19.796 & 15.529 & 10.134 & 31.427 & 32.168 & 29.254 & 31.234 & 27.789 & 33.775 \\
\hline 18:00 & & 30.491 & 30.009 & 29.131 & 29.629 & & 29.396 & 30.427 & 29.159 & 29.547 & 28.384 & 26.244 & 31.267 & 36.656 & 32.298 & 16.208 & 15.393 & 19.799 & 15.529 & 10.132 & 31.434 & 32.168 & 29.264 & 31.232 & 27.788 & 33.775 \\
\hline & & 30.491 & 30.009 & 29.133 & 29. & & 29.389 & 30.427 & 29.163 & 29.547 & 28.386 & 26.248 & 31.265 & 36.654 & 32.295 & 16.203 & 15.39 & 19.806 & 15.524 & 10.132 & 31.434 & 32.165 & 29.264 & 31.232 & 27.789 & 33.77 \\
\hline bo & & 30.482 & 30.004 & 29.122 & 29. & & 29.394 & 30.4 & 29.159 & 29.54 & 28.379 & 26.241 & 31.263 & 36.665 & 32.293 & 16.201 & 15.383 & 9.806 & 15.527 & 10.127 & 31.424 & .163 & 29.252 & 31.227 & 27.788 & 33.768 \\
\hline$: 00$ & & 30.484 & 30.002 & 29.119 & 29.622 & & 29.384 & 30.439 & 29.168 & 29.537 & 28.374 & 26.234 & 31.26 & 36.656 & 32.291 & 16.201 & 15.386 & 787 & 15522 & 10.127 & 424 & .158 & 29.25 & 31.225 & 27.791 & .768 \\
\hline $12 / 28 / 1$ & & 30.482 & 29.995 & 29.11 & 29.618 & & 29.384 & 30.439 & 29.175 & 29.533 & 28.372 & 26.227 & 31.256 & 36.658 & 32.286 & 16.198 & 15.381 & .783 & 15.513 & 10.118 & 31.422 & .156 & 29.252 & 31.223 & 27.791 & .768 \\
\hline 12/28/11 23:00 & & 30.48 & 29.997 & 29.117 & 29.618 & & 29.373 & & 29.18 & 29.533 & 28.374 & 26.236 & 31.26 & 36.656 & 32.288 & 16.196 & 15.381 & 8.778 & 15.506 & 10.124 & 31.424 & 32.154 & 29.25 & 31.22 & 27.791 & 33.768 \\
\hline 12/29/11 0:00 & & 30.47 & 29.983 & 29.096 & 29.608 & & 29.382 & & 29.173 & 29.523 & 28.36 & 26.22 & 31.253 & 36.654 & 32.284 & 16.194 & 15.381 & 19.764 & 15.515 & 10.118 & 31.41 & 32.156 & 29.233 & 31.22 & 27.788 & 33.768 \\
\hline 12/29/11 1:00 & & 30.466 & 29.972 & 29.075 & 29.592 & & 29.392 & & 29.173 & 29.51 & 28.349 & 26.201 & 31.244 & 36.647 & 32.277 & 16.191 & 15.374 & 19.755 & 15.515 & 10.113 & 31.396 & 32.144 & 29.214 & 31.211 & 27.788 & 33.766 \\
\hline 12/29/11 2:00 & & 30.468 & 29.969 & 29.075 & 29.587 & & 29.38 & 30.408 & 29.187 & 29.507 & 28.346 & 26.201 & 31.241 & 36.647 & 32.274 & 16.187 & 15.376 & 19.748 & 15.508 & 10.108 & 31.394 & 32.142 & 29.214 & 31.206 & 27.791 & 33.766 \\
\hline 12/29/11 3:00 & & 30.466 & 29.962 & 29.07 & 29.58 & & 29.375 & 30.408 & 29.192 & 29.5 & 28.342 & 26.193 & 31.237 & 36.649 & 32.269 & 16.187 & 15.372 & 19.736 & 15.499 & 10.106 & 31.392 & 32.139 & 29.214 & 31.204 & 27.793 & 33.766 \\
\hline 12/29/11 4:00 & & 30.461 & 29.953 & 29.063 & 29.571 & & 29.373 & 30.417 & 29.192 & 29.493 & 28.335 & 26.189 & 31.232 & 36.642 & 32.262 & 16.182 & 15.369 & 19.727 & 15.496 & 10.099 & 31.382 & 32.132 & 29.205 & 31.197 & 27.793 & 33.763 \\
\hline 12/29/11 5:00 & & 30.456 & 29.946 & 29.054 & 29.564 & & 29.37 & 30.432 & 29.192 & 29.486 & 28.328 & 26.184 & 31.225 & 36.642 & 32.258 & 16.18 & 15.365 & 19.716 & 15.499 & 10.094 & 31.375 & 32.128 & 29.196 & 31.192 & 27.791 & 33.761 \\
\hline $12 / 29 / 116: 00$ & & 30.459 & 29.946 & 29.059 & 29.566 & & 29.359 & 30.427 & 29.204 & 29.486 & 28.328 & 26.191 & 31.22 & 36.64 & 32.255 & 16.177 & 15.36 & 19.706 & 15.489 & 10.089 & 31.378 & 32.125 & 29.198 & 31.187 & 27.793 & 33.759 \\
\hline $12 / 29 / 11$ 7:00 & & 30.456 & 29.948 & 29.066 & 29.566 & & 29.347 & 30.422 & 29.208 & 29.486 & 28.328 & 26.196 & 31.22 & 36.637 & 32.251 & 16.177 & 15.355 & 19.699 & 15.482 & 10.085 & 31.378 & 32.12 & 29.2 & 31.185 & 27.793 & 33.756 \\
\hline $12 / 29 / 118: 00$ & & 30.459 & 29.948 & 29.068 & 29.568 & & 29.335 & 30.417 & 29.213 & 29.482 & 28.328 & 26.196 & 31.216 & 36.642 & 32.246 & 16.173 & 15.353 & 19.69 & 15.47 & 10.08 & 31.382 & 32.118 & 29.203 & 31.183 & 27.793 & 33.754 \\
\hline $12 / 29 / 119: 00$ & & 30.456 & 29.953 & 29.07 & 29.573 & & 29.326 & 30.413 & 29.218 & 29.484 & 28.328 & 26.21 & 31.213 & 36.635 & 32.244 & 16.17 & 15.348 & 19.679 & 15.473 & 10.08 & 31.378 & 32.116 & 29.207 & 31.18 & 27.793 & 33.754 \\
\hline$/ / 11$ 10:00 & & 30.449 & 29.951 & 29.07 & 29. & & 29.326 & 30. & 29.216 & 29.484 & 28.328 & 26.215 & 31.211 & 36.637 & 32.241 & 16.168 & 15.346 & 19.679 & 15.475 & 10.078 & 2 & 3 & 29.205 & 31.176 & 27.796 & 33.752 \\
\hline 12 & & 30.445 & 29.946 & 29.061 & & & 29.328 & & 29.218 & 29.479 & 28.3 & 26.205 & 31.211 & 36.6 & 32.241 & 16.17 & $\begin{array}{l}15.341 \\
\end{array}$ & 9 & 15.482 & 10.075 & 3 & 3 & 29.198 & 31.173 & 27.796 & 33.752 \\
\hline 12 & & 30.438 & 29.927 & 29.028 & 29. & & 29.347 & 30.386 & 29.211 & 29.461 & 28. & 26.172 & $\begin{array}{l}31.199 \\
31.199\end{array}$ & 36.633 & 32.232 & 16.163 & 15.339 & 19.672 & $\begin{array}{l}15.489 \\
15.489\end{array}$ & $\begin{array}{l}10.068 \\
\end{array}$ & 7 & 1 & 29.174 & 31.166 & 27.796 & $\begin{array}{l}33.749 \\
3\end{array}$ \\
\hline 12 & & 30.424 & 29.906 & 28.991 & 29. & & 29.37 & 30.405 & 29.201 & 29.44 & 28.284 & 26.148 & 31.192 & 36.626 & 32.225 & 16.159 & 15.334 & .662 & 15.513 & 10.056 & 4 & 12 & 29.144 & 31.157 & 27.793 & 33.747 \\
\hline 0 & & 30. & 29.8 & $\begin{array}{l}28.977 \\
28.977\end{array}$ & 29 & & 29.366 & 30. & 29.2 & $\begin{array}{r}29.428 \\
2928\end{array}$ & $\begin{array}{l}28.274 \\
28.274\end{array}$ & 26.126 & 31 & $\begin{array}{l}30.020 \\
36.626\end{array}$ & 32 & $\begin{array}{l}16.154 \\
1054\end{array}$ & $\begin{array}{l}15.354 \\
15.33\end{array}$ & 2 & $\begin{array}{l}15.503 \\
15.503\end{array}$ & $\begin{array}{l}10.049 \\
\end{array}$ & 4 & 5 & 29.134 & 31.148 & 27.796 & 1.747 \\
\hline 12 & & 30 & 29 & 28 & 29 & & 29 & 30 & 29 & 29 & 28 & 26 & 31 & 36 & 32 & 16 & 15 & .679 & 15.508 & 10. & 31.298 & 3 & 29. & 31.138 & 27.796 & 1742 \\
\hline & & 30. & & & & & & & 29.2 & & 28 & 26 & 31 & 36 & 32 & 16 & 3 & 19.734 & 15. & 10 & 31.294 & 1 & 29 & 31.129 & 3 & 3.74 \\
\hline $12 / 29 / 1117: 00$ & & 30. & 29. & 28.953 & & & 29. & 30. & 29.23 & & 28 & & & 36. & & & 15 & 19.7 & 15.499 & 10.026 & 9 & 9 & 29.099 & 31.124 & 27.796 & .738 \\
\hline 12/29/11 18:00 & & 30 & 29. & 28.932 & & & 25 & 30.377 & 29.227 & & 28.2 & & 31.146 & 36.605 & 32.18 & 16.135 & & 13 & 15.487 & 10.014 & & & 29.087 & 31.112 & 27.798 & $\begin{array}{l}33.735 \\
3.750\end{array}$ \\
\hline & & & 29 . & & & & & & 29.246 & 29. & 28.2 & & 31.148 & 36.607 & 32.18 & 16.135 & & 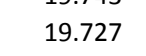 & 15.473 & 10.014 & 31. & & 29.111 & 31.115 & 27.798 & 33.738 \\
\hline 20:00 & & & & & & & & & 29 & & & & 31.155 & & 32.1 & 16. & & & 15.442 & & & & 29.158 & 31.124 & 27.796 & 33.738 \\
\hline 12 & & & & & & & & & 29.242 & 29.3 & & & 31 & & 32 & 16. & & & 15.463 & & & & 29.127 & 31.117 & 27.793 & 33.735 \\
\hline 12/29/11 22:00 & & 30. & 29. & 29. & & & 29. & 30. & 29 & 29 & 28. & 26.1 & 31.155 & 36.616 & 32.1 & 16. & 15. & 19.7 & 15. & 10.024 & 31. & & 29.167 & 31.122 & 27.8 & 33.738 \\
\hline 12/29/11 23:00 & & 30. & 29. & 29.063 & & & 29.1 & 30.3 & 29.2 & 29. & 28.2 & 26.2 & 31.167 & 36.623 & 32.1 & 16.135 & 15.3 & 19.6 & 15.4 & 10.031 & 31.354 & 32.066 & 29.2 & 31.131 & 27.8 & 33.74 \\
\hline $12 / 30 / 110: 00$ & & 30.438 & 29.9 & 29.112 & 29. & & 29.168 & 30 & 29.294 & 29.449 & 28.3 & 26.26 & 31.181 & 36.635 & 32.211 & 16.138 & 15.313 & 19.6 & 15.412 & 10.044 & 31.387 & 32.082 & 29.243 & 31.148 & 27.803 & 33.745 \\
\hline 12/30/11 1:00 & & 30.431 & 29.969 & 29.126 & 29.6 & & 29.171 & 30.295 & 29.284 & 29.463 & 28.328 & 26.282 & 31.192 & 36.644 & 32.223 & 16.142 & 15.316 & 19.6 & 15.421 & 10.056 & 31.403 & 32.094 & 29.259 & 31.159 & 27.803 & 33.749 \\
\hline $12 / 30 / 112: 00$ & & 30.433 & 29.986 & 29.14 & 29.6 & & 29.168 & 30.293 & 29.289 & 29.477 & 28.342 & 26.291 & 31.204 & 36.644 & 32.232 & 16.145 & 15.32 & 19.688 & 15.416 & 10.066 & 31.417 & 32.106 & 29.275 & 31.169 & 27.805 & 33.752 \\
\hline $12 / 30 / 113: 00$ & & 30.435 & 29.997 & 29.152 & 29.641 & & 29.173 & 30.295 & 29.289 & 29.489 & 28.353 & 26.296 & 31.211 & 36.656 & 32.241 & 16.149 & 15.325 & 19.683 & 15.416 & 10.078 & 31.431 & 32.113 & 29.29 & 31.176 & 27.805 & 33.752 \\
\hline $12 / 30 / 114: 00$ & & 30.438 & 30.023 & 29.183 & 29.667 & & 29.166 & 30.295 & 29.301 & 29.51 & 28.372 & 26.332 & 31.223 & 36.663 & 32.251 & 16.154 & 15.33 & 19.679 & 15.414 & 10.085 & 31.45 & 32.125 & 29.315 & 31.19 & 27.805 & 33.756 \\
\hline $12 / 30 / 115: 00$ & & 30.428 & 30.018 & 29.166 & 29.671 & & 29.194 & 30.315 & 29.289 & 29.512 & 28.37 & 26.315 & 31.225 & 36.67 & 32.255 & 16.159 & 15.327 & 19.674 & 15.428 & 10.092 & 31.438 & 32.128 & 29.301 & 31.194 & 27.803 & 33.754 \\
\hline 11 6:00 & & 30.435 & 30.03 & 29.18 & 29.683 & & 29.192 & 30.303 & 29.303 & 29.523 & 28.384 & 26.323 & 31.23 & 36.673 & 32.26 & 16.166 & 15.33 & 19.683 & 15.421 & 10.094 & 31.455 & 32.132 & 29.322 & 31.199 & 27.807 & 33.756 \\
\hline 12/30/11 7:00 & & 30.431 & 30.028 & 29.173 & 29.685 & & 29.208 & 30.31 & 29.303 & 29.528 & 28.386 & 26.311 & 31.234 & 36.67 & 32.26 & 16.166 & 15.33 & 19.674 & 15.426 & 10.099 & 31.459 & 32.135 & 29.318 & 31.202 & 27.807 & 33.754 \\
\hline
\end{tabular}




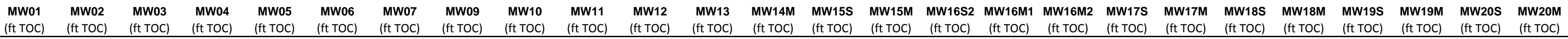

\begin{tabular}{|c|c|c|c|c|c|c|c|c|c|c|c|c|c|c|c|c|c|c|c|c|c|c|c|c|c|}
\hline well & $\begin{array}{l}\text { (ft TOC) } \\
\text { (f) }\end{array}$ & $\begin{array}{l}\text { (ft TOC) } \\
\text { (ft) }\end{array}$ & $\begin{array}{l}\text { (ft TOC) } \\
\text { (f) }\end{array}$ & $\begin{array}{l}\text { (ft TOC) } \\
\text { (ft) }\end{array}$ & $\begin{array}{ll}(\mathrm{ft} \text { TOC) } & \text { (ft TOC) }\end{array}$ & (ft toc) & $\begin{array}{l}\text { (ift TOC) } \\
\text { (n) }\end{array}$ & $\begin{array}{l}\text { (ft TOC) } \\
\text { (f) }\end{array}$ & $\begin{array}{l}\text { (ft TOC) } \\
\text { (f) }\end{array}$ & $\begin{array}{l}\text { (ft TOC) } \\
\text { (n) }\end{array}$ & $\begin{array}{l}\text { (ft TOC) } \\
\text { (f) }\end{array}$ & (ft TOC) & $\begin{array}{l}\text { (ft TOC) } \\
\end{array}$ & (ft TOC) & (ft TOC) & $\begin{array}{l}\text { (ft TOC) } \\
\text { (f) }\end{array}$ & (ft TOC) & $\begin{array}{l}\text { (ft TOC) } \\
\text { (ft) }\end{array}$ & $\begin{array}{l}\text { (ft toc) } \\
\text { (fol) }\end{array}$ & $\begin{array}{l}\text { (ft TOC) } \\
\text { (f) }\end{array}$ & $\begin{array}{l}\text { (ft TOC) } \\
\text { (f) }\end{array}$ & $\begin{array}{l}\text { (ft TOC) } \\
\text { (n) }\end{array}$ & (ft TOC) & $\begin{array}{l}\text { (ft TOC) } \\
\text { (1) }\end{array}$ & (ft TOC) \\
\hline 3:00 & & 30.433 & 30.046 & 29.197 & 29.699 & 29.201 & 30.3 & 29 & 29.5 & 28.4 & 26 & 31.241 & 36.6 & 32.2 & 16. & & 19.669 & 15. & 10.103 & 31. & 32.144 & 29.339 & 31.209 & 27.807 & 4 \\
\hline 0/11 9:00 & & 30.438 & 30.06 & 29.213 & 29.713 & 29.201 & 30.303 & 29.32 & 29.556 & 28.414 & 26.349 & 31.248 & 36.684 & 32.2 & 16. & 15.332 & 19.674 & 15.4 & 10.113 & 31.485 & & & 31.213 & 27.807 & 33.756 \\
\hline 12/30/11 10:00 & & 30.438 & 30.072 & 29.229 & 29.732 & 29.208 & 30.305 & 29.32 & 29.572 & 28.425 & 26.366 & 31.258 & 36.689 & 32.288 & 16.182 & 15.332 & 19.667 & 15.435 & 10.12 & 31.497 & 32.158 & 29.367 & 1.223 & 27.807 & 33.756 \\
\hline 12/30/11 11:00 & & 30.433 & 30.072 & 29.22 & 29.734 & 29.232 & 30.331 & 29.313 & 29.577 & 28.428 & 26.358 & 31.26 & 36.694 & 32.291 & 16.187 & 15.334 & 19.665 & 15.449 & 10.122 & 31.49 & 32.161 & 29.36 & 31.227 & 27.812 & 33.759 \\
\hline $12 / 30 / 11$ 12:00 & & 30.428 & 30.07 & 29.211 & 29.732 & 29.248 & 30.346 & 29.315 & 29.579 & 28.428 & 26.346 & 31.265 & 36.694 & 32.293 & 16.189 & 15.337 & 19.66 & 15.459 & 10.132 & 31.49 & 32.168 & 29.358 & 31.23 & 27.81 & 33.759 \\
\hline 12/30/11 13:00 & & 30.424 & 30.06 & 29.19 & 29.72 & 29.274 & 30.379 & 29.313 & 29.572 & 28.421 & 26.32 & 31.263 & 36.691 & 32.293 & 16.191 & 15.337 & 19.653 & 15.475 & 10.127 & 31.48 & 32.165 & 29.341 & 31.23 & 27.81 & 33.756 \\
\hline $12 / 30 / 11$ 14:00 & & 30.419 & 30.049 & 29.169 & 29.709 & 29.293 & 30.391 & 29.318 & 29.57 & 28.414 & 26.294 & 31.258 & 36.684 & 32.291 & 16.194 & 15.337 & 19.649 & 15.482 & 10.122 & 31.469 & 32.163 & 29.325 & 31.23 & 27.812 & 33.759 \\
\hline 12/30/11 15:00 & & 30.422 & 30.051 & 29.18 & 29.704 & 29.288 & 30.391 & 29.327 & 29.575 & 28.416 & 26.299 & 31.263 & 36.687 & 32.291 & 16.194 & 15.339 & 19.644 & 15.482 & 10.129 & 31.476 & 32.165 & 29.332 & 31.23 & 27.812 & 33.761 \\
\hline 12/30/11 16:00 & & 30.422 & 30.056 & 29.19 & 29.711 & 29.281 & 30.393 & 29.334 & 29.581 & 28.423 & 26.306 & 31.27 & 36.689 & 32.295 & 16.196 & 15.344 & 19.69 & 15.484 & 10.129 & 31.483 & 32.168 & 29.337 & 31.234 & 27.814 & 33.763 \\
\hline 12/30/11 17:00 & & 30.417 & 30.051 & 29.187 & 29.709 & 29.283 & 30.396 & 29.332 & 29.579 & 28.423 & 26.301 & 31.267 & 36.689 & 32.295 & 16.198 & 15.344 & 19.69 & 15.494 & 10.134 & 31.48 & 32.17 & 29.332 & 31.237 & 27.814 & 33.763 \\
\hline 12/30/11 18:00 & & 30.415 & 30.046 & 29.18 & 29.704 & 29.288 & 30.398 & 29.334 & 29.581 & 28.419 & 26.291 & 31.267 & 36.687 & 32.295 & 16.201 & 15.344 & 19.685 & 15.492 & 10.134 & 31.476 & 32.168 & 29.33 & 31.23 & 27.812 & 33.761 \\
\hline 12/30/11 19:00 & & 30.415 & 30.042 & 29.178 & 29.704 & 29.286 & 30.396 & 29.337 & 29.581 & 28.421 & 26.287 & 31.267 & 36.687 & 32.295 & 16.198 & 15.341 & 19.679 & 15.496 & 10.129 & 31.473 & 32.168 & 29.33 & 31.234 & 27.814 & 33.761 \\
\hline $12 / 30 / 1120: 00$ & & 30.412 & 30.042 & 29.173 & 29.699 & 29.288 & 30.393 & 29.341 & 29.581 & 28.421 & 26.279 & 31.265 & 36.684 & 32.293 & 16.201 & 15.339 & 19.667 & 15.496 & 10.132 & 31.469 & 32.165 & 29.322 & 31.23 & 27.814 & 33.759 \\
\hline $12 / 30 / 1121: 00$ & & 30.401 & 30.03 & 29.152 & 29.688 & 29.305 & 30.408 & 29.332 & 29.572 & 28.407 & 26.263 & 31.258 & 36.682 & 32.288 & 16.198 & 15.337 & 19.665 & 15.51 & 10.125 & 31.45 & 32.161 & 29.301 & 31.225 & 27.812 & 33.756 \\
\hline $12 / 30 / 1122: 00$ & & 30.396 & 30.016 & 29.129 & 29.669 & 29.314 & 30.408 & 29.332 & 29.563 & 28.395 & 26.239 & 31.251 & 36.677 & 32.284 & 16.196 & 15.337 & 19.655 & 15.515 & 10.118 & 31.438 & 32.154 & 29.285 & 31.218 & 27.814 & 33.754 \\
\hline $12 / 30 / 1123: 00$ & & 30.398 & 30.011 & 29.122 & 29.66 & 29.307 & 30.396 & 29.346 & 29.561 & 28.393 & 26.232 & 31.251 & 36.672 & 32.279 & 16.196 & 15.332 & 19.646 & 15.506 & 10.113 & 31.436 & 32.149 & 29.285 & 31.213 & 27.814 & 33.749 \\
\hline 12/31/11 0:00 & & 30.391 & 29.997 & 29.105 & 29.648 & 29.316 & 30.396 & 29.339 & 29.551 & 28.386 & 26.222 & 31.241 & 36.668 & 32.274 & 16.191 & 15.332 & 19.637 & 15.515 & 10.111 & 31.424 & 32.144 & 29.266 & 31.209 & 27.812 & 33.749 \\
\hline 12/31/11 1:00 & & 30.389 & 29.988 & 29.089 & 29.632 & 29.319 & 30.393 & 29.339 & 29.542 & 28.374 & 26.203 & 31.237 & 36.663 & 32.267 & 16.189 & 15.327 & 19.632 & 15.515 & 10.106 & 31.408 & 32.137 & 29.252 & 31.202 & 27.814 & 33.747 \\
\hline $12 / 31 / 112: 00$ & & 30.387 & 29.976 & 29.07 & 29.615 & 29.321 & 30.391 & 29.344 & 29.533 & 28.365 & 26.186 & 31.23 & 36.658 & 32.258 & 16.187 & 15.327 & 19.621 & 15.513 & 10.096 & 31.406 & 32.132 & 29.238 & 31.194 & 27.814 & 33.745 \\
\hline $12 / 31$ & & 30.382 & 29.965 & 29.061 & 29.603 & 29.314 & 30.389 & 29.349 & 29.523 & 28.356 & 26.179 & 31.225 & 36.649 & 32.253 & 16.182 & 15.323 & 19.616 & 15.51 & 10.092 & 31.389 & 32.125 & 29.224 & 31.187 & 27.814 & 33.745 \\
\hline $12 / 31$ & & 30.373 & 29.948 & 29.028 & 29.58 & 29.33 & 30.391 & 29.341 & 29.505 & 28.337 & 26.15 & 31.211 & 36.647 & 32.246 & 16.177 & 15.318 & 19.607 & 15.513 & 10.078 & 31.368 & 32.111 & 29.196 & 31.18 & 27.812 & 33.742 \\
\hline $12 / 31 / 1$ & & 30.37 & 29.927 & 29.003 & 29.557 & 29.333 & 30.384 & 29.346 & 29.489 & 28.321 & 26.124 & 31.202 & 36.635 & 32.23 & 16.17 & 15.311 & 19.6 & 15.515 & 10.068 & 31.35 & 32.101 & 29.17 & 31.166 & 814 & 738 \\
\hline $12 / 31 / 1$ & & 30.363 & 29.911 & 28.979 & 29.533 & 29.335 & 30.384 & 29.341 & 29.472 & 28.302 & 26.11 & 31.188 & 36.63 & 32.223 & 16.166 & 15.306 & 19.609 & 15.515 & 10.056 & 31.329 & 32.09 & 29.149 & 31.152 & 27.812 & 33.733 \\
\hline 12/31/11 7:00 & & 30.361 & 29.895 & 28.963 & 29.513 & 29.326 & 30.374 & 29.349 & 29.458 & 28.286 & 26.093 & 31.176 & 36.621 & 32.209 & 16.159 & 15.299 & 19.591 & 15.503 & 10.045 & 31.31 & 32.078 & 29.13 & 31.141 & 27.812 & 33.728 \\
\hline 12/31/11 8:00 & & 30.366 & 29.89 & 28.963 & 29.503 & 29.305 & 30.37 & 29.36 & 29.449 & 28.281 & 26.09 & 31.167 & 36.621 & 32.199 & 16.154 & 15.297 & 19.591 & 15.487 & 10.035 & 31.312 & 32.068 & 29.135 & 31.133 & 27.814 & 33.726 \\
\hline 12/31/11 9:00 & & 30.356 & 29.876 & 28.951 & 29.492 & 29.3 & 30.384 & 29.356 & 29.438 & 28.27 & 26.09 & 31.157 & 36.609 & 32.194 & 16.147 & 15.288 & 19.584 & 15.494 & 10.026 & 31.296 & 32.061 & 29.113 & 31.124 & 27.814 & 33.724 \\
\hline 12/31/11 10:00 & & 30.359 & 29.869 & 28.942 & 29.482 & 29.288 & 30.372 & 29.36 & 29.424 & 28.26 & 26.081 & 31.148 & 36.612 & 32.18 & 16.142 & 15.283 & 19.572 & 15.48 & 10.019 & 31.294 & 32.049 & 29.109 & 31.112 & 27.814 & 33.721 \\
\hline 12/31/11 11:00 & & 30.349 & 29.853 & 28.921 & 29.464 & 29.29 & 30.377 & 29.358 & 29.407 & 28.244 & 26.069 & 31.139 & 36.602 & 32.171 & 16.135 & 15.281 & 19.572 & 15.482 & 10.009 & 31.275 & 32.04 & 29.085 & 31.103 & 27.817 & 33.717 \\
\hline 12/31/11 12:00 & & 30.347 & 29.841 & 28.907 & 29.452 & 29.286 & 30.36 & 29.351 & 29.389 & 28.232 & 26.062 & 31.129 & 36.595 & 32.159 & 16.128 & 15.276 & 19.568 & 15.477 & 9.993 & 31.264 & 32.028 & 29.087 & 31.098 & 27.812 & 33.717 \\
\hline 12/31/11 13:00 & & 30.352 & 29.85 & 28.942 & 29.463 & 29.25 & 30.358 & 29.368 & 29.398 & 28.242 & 26.1 & 31.131 & 36.602 & 32.166 & 16.124 & 15.271 & 19.57 & 15.47 & 10.002 & 31.28 & 32.033 & 29.102 & 31.101 & 27.817 & 33.721 \\
\hline $12 / 31 / 1114: 00$ & & 30.356 & 29.85 & 28.953 & 29.471 & 29.225 & 30.358 & 29.382 & 29.398 & 28.246 & 26.098 & 31.131 & 36.6 & 32.164 & 16.122 & 15.271 & 19.565 & 15.459 & 9.998 & 31.289 & 32.033 & 29.118 & 31.098 & 27.819 & 33.719 \\
\hline $12 / 31 / 1115: 00$ & & 30.359 & 29.864 & 28.989 & 29.494 & 29.192 & 30.343 & 29.394 & 29.407 & 28.258 & 26.136 & 31.141 & 36.602 & 32.171 & 16.122 & 15.278 & 19.563 & 15.449 & 10.005 & 31.308 & 32.04 & 29.149 & 31.108 & 27.819 & 33.724 \\
\hline $12 / 31 / 1116: 00$ & & 30.37 & 29.899 & 29.054 & 29.536 & 29.142 & 30.295 & 29.42 & 29.438 & 28.291 & 26.201 & 31.157 & 36.616 & 32.183 & 16.122 & 15.281 & 19.561 & 15.423 & 10.019 & 31.352 & 32.054 & 29.2 & 31.124 & 27.821 & 33.731 \\
\hline $12 / 31 / 1117: 00$ & & 30.373 & 29.937 & 29.108 & 29.583 & 29.116 & 30.271 & 29.415 & 29.465 & 28.316 & 26.265 & 31.253 & 36.63 & 32.286 & 16.126 & 15.304 & 19.563 & 15.43 & 10.12 & 31.417 & 32.154 & 29.25 & 31.216 & 27.819 & 33.738 \\
\hline $12 / 31 / 1118: 00$ & & 30.377 & 29.958 & 29.131 & 29.6 & 29.109 & 30.267 & 29.417 & 29.479 & 28.337 & 26.282 & 31.227 & 36.644 & 32.262 & 16.128 & 15.309 & 19.586 & 15.421 & 10.096 & 31.429 & 32.128 & 29.283 & 31.194 & 27.819 & 33.74 \\
\hline $12 / 3$ & & 30.391 & 29.997 & 29.183 & 29. & 29.086 & 30.2 & 29.432 & 29.512 & 28.365 & 26.318 & 31.234 & 36.658 & 32.265 & 16.133 & 15.313 & 19.586 & 15.405 & 10.101 & 31.457 & 37 & 29.33 & $\begin{array}{l}31.199 \\
\end{array}$ & 27.824 & 33.745 \\
\hline $12 / 31 / 1120: 00$ & & 30.405 & 30.044 & 29.26 & 29 & 29.044 & 30.264 & 29.46 & 29.558 & 28.412 & 26.385 & 31.251 & 36.672 & 32.281 & 16.147 & 15.327 & $\begin{array}{l}19.5901 \\
19.591\end{array}$ & 15.381 & 10.118 & 515 & .154 & 29.393 & 31.216 & 27.824 & 33.747 \\
\hline 12/31/11 21:00 & & 30.414 & 30.098 & 29.316 & $29.7 .7 x-1$ & 29.037 & 30.276 & 29.46 & 29.595 & $\begin{array}{l}28.449 \\
28.449\end{array}$ & 26.447 & 31.274 & 36.694 & 32.305 & 16.156 & 15.332 & 19.595 & $\begin{array}{l}15.391 \\
15.391\end{array}$ & 10.141 & 31.553 & .175 & 29.447 & 31.241 & 27.824 & 33.752 \\
\hline & & 30.417 & 30. & 29.337 & 29 & 29 & 30. & 29.448 & 29.621 & 28.474 & 26.461 & 31 & 36.705 & 32.314 & 16.166 & 15.342 & 19.595 & 15.402 & 10.155 & 74 & 6 & 29.468 & 31.258 & 27.826 & 33.759 \\
\hline & & 30. & 30. & 29. & 29.8 & & 30. & 29.463 & 29. & 28.505 & 26 & 31 & 36.712 & 32.337 & 16.177 & & 19.593 & 15.402 & 10.174 & 31.609 & 3 & 29.508 & 31.281 & 27.828 & 33.761 \\
\hline & & & 30. & 29 & & & & 29.467 & & 28.535 & & 31.333 & 36.7 & 32.356 & 16.191 & & 19.5 & 15.414 & 10.193 & & 1 & 29.548 & 31.3 & 27.831 & \\
\hline $1 / 1 /$ & & & & 29 & 29.9 & & & 29 & 29. & 28. & & 31.349 & 36.7 & 32.375 & 16.2 & & 19.5 & 15 & 10.2 & 31.65 & 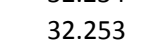 & 29. & 31.314 & 27.828 & 33.775 \\
\hline 1/1/12 2:00 & & 30.447 & 30. & 29. & & & & 29 & & 28. & 26.5 & 31.361 & 36 & 32.384 & 16.2 & & & & 10.2 & & & 29. & 31.326 & 27.831 & 33.777 \\
\hline 1/1/12 3:00 & & & 30. & 29.454 & & & 30 & 29.474 & 24 & 28. & 26 & 31.377 & 36.755 & 32.401 & 16.2 & & 19.5 & 15 & 10.237 & $31.6 \varepsilon$ & & 29.597 & & 27.833 & 33.78 \\
\hline $1 / 1 / 124$ & & & 30. & 29.475 & & & & 29.4 & & 28. & & 31.396 & 36.762 & 32.42 & 16.2 & & & 15.447 & 10.254 & & & 29.623 & 31.359 & 27.833 & 33.784 \\
\hline $1 / 1 / 125: C$ & & 30. & & & & & & 29. & & 28. & 26.5 & 31. & 36. & 32.431 & 16 & & & 15. & 10. & & & 29.644 & & 27. & 33.789 \\
\hline 1/1/12 6:00 & & 30 & 30.2 & 29.479 & & 29. & & 29.4 & & 28. & 26 & 31.417 & 36.773 & 32.443 & 16.2 & & 19. & 15. & 10.2 & 31.718 & 9 & 29.633 & 31.385 & 27.833 & 33.789 \\
\hline 1/1/12 7:00 & & 30.475 & 30.2 & 29.479 & & 29.2 & 30. & 29.4 & 29. & 28.6 & 26.538 & 31.426 & 36.7 & 32.45 & 16.2 & & 19.5 & 15.4 & 10.287 & 31.718 & 32. & 29.63 & 31.389 & 27.835 & 33.789 \\
\hline $1 / 1 / 128: 00$ & & 30.489 & 30. & 29.519 & 30.0 & 29.234 & 30.4 & 29.498 & 29.8 & 28.6 & 26.567 & 31.44 & 36.787 & 32.464 & 16.2 & & 19.595 & 15.487 & 10.299 & 31.755 & 32.343 & 29.67 & 31.403 & 27.838 & 33.794 \\
\hline 1/1/12 9:00 & & 30.501 & 30.335 & 29.557 & 30.073 & 29.234 & 30.427 & 29.51 & 29.888 & 28.698 & 26.6 & 31.459 & 36.794 & 32.483 & 16.291 & & 19.612 & 15.494 & 10.32 & 31.781 & 32.357 & 29.687 & 31.42 & 27.835 & 33.798 \\
\hline 1/1/12 10:00 & & 30.508 & 30.359 & 29.585 & 30.101 & 29.246 & 30.432 & 29.515 & 29.916 & 28.721 & 26.622 & 31.475 & 36.797 & 32.499 & 16.303 & & 19.616 & 15.51 & 10.334 & 31.8 & 32.376 & 29.731 & 31.441 & 27.838 & 33.803 \\
\hline 1/1/12 11:00 & & 30.508 & 30.363 & 29.585 & 30.11 & 29.279 & 30.441 & 29.505 & 29.927 & 28.73 & 26.619 & 31.487 & 36.804 & 32.506 & 16.312 & & 19.623 & 15.536 & 10.346 & 31.8 & 32.388 & 29.724 & 31.45 & 27.838 & 33.803 \\
\hline 1/1/12 12:00 & & 30.505 & 30.352 & 29.554 & 30.098 & 29.33 & 30.456 & 29.498 & 29.925 & 28.723 & 26.586 & 31.49 & 36.804 & 32.513 & 16.319 & & 19.642 & 15.567 & 10.35 & 31.788 & 32.391 & 29.703 & 31.45 & 27.838 & 33.805 \\
\hline $1 / 1 / 1213: 00$ & & 30.498 & 30.34 & 29.526 & 30.08 & 29.375 & 30.477 & 29.491 & 29.918 & 28.714 & 26.555 & 31.49 & 36.801 & 32.516 & 16.324 & & 19.66 & 15.597 & 10.352 & 31.765 & 32.388 & 29.68 & 31.453 & 27.838 & 33.803 \\
\hline 1/1/12 14:00 & & 30.498 & 30.331 & 29.503 & 30.061 & 29.403 & 30.489 & 29.503 & 29.913 & 28.705 & 26.516 & 31.487 & 36.794 & 32.513 & 16.329 & & 19.713 & 15.618 & 10.348 & 31.755 & 32.386 & 29.659 & 31.453 & 27.838 & 33.803 \\
\hline 1/1/12 15:00 & & 30.503 & 30.326 & 29.496 & 30.047 & 29.415 & 30.487 & 29.51 & 29.913 & 28.705 & 26.502 & 31.487 & 36.794 & 32.511 & 16.333 & & 19.706 & 15.632 & 10.35 & 31.751 & 32.388 & 29.654 & 31.45 & 27.838 & 13 \\
\hline
\end{tabular}




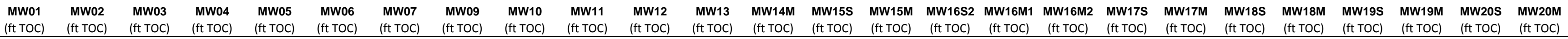

\begin{tabular}{|c|c|c|c|c|c|c|c|c|c|c|c|c|c|c|c|c|c|c|c|c|c|c|c|c|}
\hline Well & $\begin{array}{l}\text { (ft TOC) } \\
\text { (f) }\end{array}$ & $\begin{array}{l}\text { (ft TOC) } \\
\end{array}$ & $\begin{array}{l}\text { (ft TOC) } \\
\end{array}$ & $\begin{array}{l}\text { (ft TOC) } \\
\text { (f) }\end{array}$ & $\begin{array}{l}\text { (ift TOC) } \\
\text { (ft TOC) }\end{array}$ & $\begin{array}{l}\text { (ft TOC) } \\
\end{array}$ & $\begin{array}{l}\text { (ift TOC) } \\
\end{array}$ & $\begin{array}{l}\text { (ft TOC) } \\
\text { (f) }\end{array}$ & $\begin{array}{l}\text { (ft TOC) } \\
\text { (f) }\end{array}$ & $\begin{array}{l}\text { (ft TOC) } \\
\text { (n) }\end{array}$ & $\begin{array}{l}\text { (ft TOC) } \\
\text { (ft) }\end{array}$ & (ft TOC) & $\begin{array}{l}\text { (ft TOC) } \\
\end{array}$ & (ft TOC) & $\begin{array}{l}\text { (ft TOC) } \\
\text { (ft TOC) }\end{array}$ & (ft TOC) & $\begin{array}{l}\text { (nt TOC) } \\
\text { (ft }\end{array}$ & (ft TOC) & $\begin{array}{l}\text { (ft TOC) } \\
\text { (f) }\end{array}$ & (ft TOC) & $\begin{array}{l}\text { (ft TOC) } \\
\text { (n) }\end{array}$ & (ft TOC) & (ft TOC) & (ft TOC) \\
\hline 16:00 & & 30.505 & 30.321 & 29.498 & 30.045 & 29.422 & 30. & 29.51 & 29.9 & 28.702 & 26.49 & 31.48 & 36.7 & 32.5 & 16.3 & 19.6 & 15.6 & 10.35 & $31.7 .7>$ & 32.391 & 29.647 & 31.453 & 84 & 33.805 \\
\hline 1/1/12 17:00 & & 30.512 & 30.324 & 29.505 & 30.042 & 29.42 & 30.465 & 29.522 & 29.918 & 28.705 & 26.492 & 31.492 & 36.799 & 32.52 & 16.34 & 19.69 & 15.644 & 10.355 & 31.755 & 32.393 & .654 & 31.453 & .84 & 33.81 \\
\hline 1/1/12 18:00 & & 30.515 & 30.324 & 29.521 & 30.047 & 29.415 & 30.47 & 29.527 & 29.927 & 28.712 & 26.507 & 31.499 & 36.804 & 32.527 & 16.345 & 19.734 & 15.649 & 10.357 & 31.762 & 2. & 29.663 & 31.462 & 27.84 & 81 \\
\hline 1/1/12 19:00 & & 30.519 & 30.331 & 29.536 & 30.054 & 29.413 & 30.472 & 29.536 & 29.937 & 28.721 & 26.514 & 31.506 & 36.806 & 32.532 & 16.35 & 19.729 & 15.656 & 10.364 & 31.769 & 32.407 & 29.666 & 31.467 & 27.842 & 33.812 \\
\hline 1/1/12 20:00 & & 30.524 & 30.335 & 29.547 & 30.064 & 29.41 & 30.477 & 29.546 & 29.948 & 28.725 & 26.521 & 31.511 & 36.816 & 32.534 & 16.352 & 19.72 & 15.653 & 10.374 & 31.776 & 32.414 & 29.68 & 31.471 & 27.842 & 33.815 \\
\hline 1/1/12 21:00 & & 30.526 & 30.335 & 29.552 & 30.068 & 29.417 & 30.48 & 29.546 & 29.955 & 28.73 & 26.524 & 31.513 & 36.808 & 32.539 & 16.354 & 19.716 & 15.66 & 10.376 & 31.781 & 32.414 & 29.684 & 31.476 & 27.842 & 33.817 \\
\hline 1/1/12 22:00 & & 30.529 & 30.338 & 29.557 & 30.073 & 29.424 & 30.484 & 29.553 & 29.96 & 28.735 & 26.524 & 31.52 & 36.811 & 32.541 & 16.359 & 19.706 & 15.672 & 10.379 & 31.786 & 32.417 & 29.684 & 31.483 & 27.845 & 33.817 \\
\hline 1/1/12 23:00 & & 30.533 & 30.34 & 29.561 & 30.077 & 29.431 & 30.489 & 29.555 & 29.969 & 28.739 & 26.524 & 31.522 & 36.813 & 32.548 & 16.364 & 19.704 & 15.67 & 10.388 & 31.786 & 32.424 & 29.689 & 31.483 & 27.845 & 33.817 \\
\hline 1/2/12 0:00 & & 30.533 & 30.34 & 29.564 & 30.078 & 29.439 & 30.496 & 29.56 & 29.974 & 28.742 & 26.524 & 31.525 & 36.813 & 32.551 & 16.366 & 19.699 & 15.682 & 10.386 & 31.791 & 32.424 & 29.691 & 31.488 & 27.845 & 33.817 \\
\hline 1/2/12 1:00 & & 30.536 & 30.34 & 29.564 & 30.078 & 29.443 & 30.496 & 29.562 & 29.976 & 28.744 & 26.519 & 31.525 & 36.813 & 32.553 & 16.368 & 19.699 & 15.686 & 10.388 & 31.79 & 32.428 & 29.694 & 31.49 & 27.842 & 33.817 \\
\hline 1/2/12 2:00 & & 30.533 & 30.338 & 29.557 & 30.077 & 29.46 & 30.504 & 29.565 & 29.976 & 28.742 & 26.514 & 31.527 & 36.816 & 32.553 & 16.371 & 19.695 & 15.696 & 10.386 & 31.786 & 32.426 & 29.68 & 31.49 & 27.842 & 33.817 \\
\hline 1/2/12 3:00 & & 30.536 & 30.335 & 29.557 & 30.073 & 29.467 & 30.508 & 29.565 & 29.978 & 28.739 & 26.509 & 31.529 & 36.813 & 32.556 & 16.371 & 19.688 & 15.698 & 10.393 & 31.783 & 32.431 & 29.68 & 31.492 & 27.842 & 33.815 \\
\hline 1/2/12 4:00 & & 30.538 & 30.333 & 29.552 & 30.07 & 29.476 & 30.511 & 29.574 & 29.976 & 28.739 & 26.504 & 31.529 & 36.818 & 32.556 & 16.373 & 19.685 & 15.707 & 10.393 & 31.781 & 32.433 & 29.675 & 31.495 & 27.845 & 33.815 \\
\hline 1/2/12 5:00 & & 30.543 & 30.331 & 29.559 & 30.071 & 29.474 & 30.508 & 29.586 & 29.981 & 28.742 & 26.502 & 31.532 & 36.818 & 32.558 & 16.375 & 19.681 & 15.703 & 10.395 & 31.783 & 32.433 & 29.682 & 31.492 & 27.847 & 33.815 \\
\hline $1 / 2 / 126: 00$ & & 30.543 & 30.331 & 29.561 & 30.07 & 29.479 & 30.516 & 29.586 & 29.983 & 28.739 & 26.507 & 31.534 & 36.818 & 32.558 & 16.375 & 19.674 & 15.71 & 10.397 & 31.783 & 32.433 & 29.682 & 31.5 & 27.845 & 33.817 \\
\hline 1/2/12 7:00 & & 30.547 & 30.331 & 29.564 & 30.071 & 29.483 & 30.513 & 29.591 & 29.985 & 28.742 & 26.502 & 31.534 & 36.816 & 32.563 & 16.378 & 19.667 & 15.712 & 10.397 & 31.786 & 32.438 & 29.68 & 31.497 & 27.847 & 33.817 \\
\hline $1 / 2 / 128: 00$ & & 30.545 & 30.326 & 29.557 & 30.066 & 29.493 & 30.516 & 29.588 & 29.983 & 28.739 & 26.492 & 31.534 & 36.813 & 32.563 & 16.378 & 19.662 & 15.714 & 10.397 & 31.781 & 32.436 & 29.675 & 31.497 & 27.847 & 33.817 \\
\hline $1 / 2 / 129: 00$ & & 30.554 & 30.338 & 29.585 & 30.08 & 29.479 & 30.52 & 29.607 & 29.997 & 28.753 & 26.521 & 31.541 & 36.816 & 32.567 & 16.378 & 19.66 & 15.712 & 10.402 & 31.797 & 32.44 & 29.698 & 31.504 & 27.847 & 33.819 \\
\hline 1/2/12 10:00 & & 30.557 & 30.34 & 29.594 & 30.087 & 29.481 & 30.525 & 29.61 & 30.006 & 28.758 & 26.533 & 31.546 & 36.823 & 32.572 & 16.38 & 19.667 & 15.722 & 10.407 & 31.802 & 32.447 & 29.706 & 31.511 & 27.849 & 33.819 \\
\hline $1 / 2 / 1211: 00$ & & 30.547 & 30.335 & 29.573 & 30.08 & 29.511 & 30.539 & 29.596 & 29.999 & 28.751 & 26.521 & 31.546 & 36.82 & 32.572 & 16.38 & 19.685 & 15.747 & 10.409 & 31.795 & 32.447 & 29.687 & 31.511 & 27.847 & 33.817 \\
\hline 1/2/12 12:00 & & 30.538 & 30.314 & 29.531 & 30.056 & 29.547 & 30.575 & 29.591 & 29.981 & 28.73 & 26.478 & 31.536 & 36.813 & 32.567 & 16.378 & 19.681 & 15.773 & 10.4 & 31.767 & 32.436 & 29.654 & 31.507 & 27.847 & 33.817 \\
\hline 1/2/12 13:00 & & 30.533 & 30.293 & 29.491 & 30.024 & 29.575 & 30.594 & 29.591 & 29.96 & 28.709 & 26.437 & 31.525 & 36.799 & 32.553 & 16.375 & .695 & 15.79 & 10.393 & 31.742 & 428 & 29.623 & 31.495 & 27.849 & 33.812 \\
\hline 1/2/12 14:00 & & 30.529 & 30.272 & 29.465 & 29.998 & 29.589 & 30.604 & 29.593 & 29.944 & 28.691 & 26.404 & 31.515 & 36.792 & 32.548 & 16.373 & .685 & 15.799 & 10.381 & 31.723 & 417 & 29.597 & 31.483 & 27.849 & 33.81 \\
\hline 1/2/12 15:00 & & 30.526 & 30.258 & 29.447 & 29.977 & 29.591 & 30.609 & 29.603 & 29.93 & 28.677 & 26.382 & 31.506 & 36.78 & 32.539 & 16.368 & 19.679 & 15.801 & 10.371 & 31.707 & 32.409 & 29.576 & 31.476 & 27.849 & 33.81 \\
\hline 1/2/12 16:00 & & 30.526 & 30.247 & 29.437 & 29.961 & 29.587 & 30.604 & 29.605 & 29.918 & 28.665 & 26.373 & 31.501 & 36.778 & 32.532 & 16.364 & 19.725 & 15.801 & 10.367 & 31.702 & 32.402 & 29.565 & 31.464 & 27.849 & 33.81 \\
\hline 1/2/12 17:00 & & 30.524 & 30.24 & 29.433 & 29.949 & 29.577 & 30.599 & 29.615 & 29.911 & 28.658 & 26.373 & 31.497 & 36.776 & 32.527 & 16.359 & 19.727 & 15.799 & 10.364 & 31.69 & 32.398 & 29.557 & 31.46 & 27.849 & 33.808 \\
\hline 1/2/12 18:00 & & 30.519 & 30.223 & 29.414 & 29.933 & 29.577 & 30.599 & 29.61 & 29.897 & 28.644 & 26.354 & 31.49 & 36.766 & 32.518 & 16.354 & 19.718 & 15.799 & 10.357 & 31.681 & 32.391 & 29.548 & 31.453 & 27.849 & 33.808 \\
\hline 1/2/12 19:00 & & 30.517 & 30.216 & 29.409 & 29.926 & 29.565 & 30.592 & 29.619 & 29.888 & 28.637 & 26.351 & 31.485 & 36.766 & 32.516 & 16.35 & 19.709 & 15.785 & 10.348 & 31.674 & 32.386 & 29.534 & 31.448 & 27.852 & 33.808 \\
\hline 1/2/12 20:00 & & 30.515 & 30.202 & 29.395 & 29.909 & 29.563 & 30.592 & 29.622 & 29.876 & 28.623 & 26.342 & 31.478 & 36.759 & 32.509 & 16.345 & 19.699 & 15.783 & 10.346 & 31.669 & 32.379 & 29.529 & 31.443 & 27.852 & 33.805 \\
\hline 1/2/12 21:00 & & 30.51 & 30.188 & 29.377 & 29.895 & 29.563 & 30.592 & 29.619 & 29.862 & 28.609 & 26.325 & 31.471 & 36.755 & 32.499 & 16.338 & 19.688 & 15.785 & 10.334 & 31.651 & 32.372 & 29.51 & 31.434 & 27.852 & 33.803 \\
\hline 1/2/12 22:00 & & 30.508 & 30.179 & 29.374 & 29.888 & 29.551 & 30.583 & 29.622 & 29.853 & 28.602 & 26.327 & 31.464 & 36.75 & 32.49 & 16.331 & 19.679 & 15.775 & 10.331 & 31.644 & 32.364 & 29.508 & 31.429 & 27.849 & 33.803 \\
\hline $1 / 2 / 1223: 00$ & & 30.503 & 30.165 & 29.358 & 29.872 & 29.551 & 30.587 & 29.626 & 29.839 & 28.591 & 26.315 & 31.457 & 36.748 & 32.485 & 16.324 & 19.672 & 15.771 & 10.322 & 31.634 & 32.355 & 29.489 & 31.417 & 27.852 & 33.798 \\
\hline 1/3/12 0:00 & & 30.501 & 30.158 & 29.353 & 29.865 & 29.544 & 30.578 & 29.629 & 29.83 & 28.584 & 26.313 & 31.447 & 36.743 & 32.476 & 16.319 & 19.665 & 15.766 & 10.315 & 31.623 & 32.348 & 29.48 & 31.413 & 27.852 & 33.796 \\
\hline 1/3/12 1:00 & & 30.498 & 30.146 & 29.337 & 29.853 & 29.542 & 30.583 & 29.631 & 29.816 & 28.57 & 26.301 & 31.438 & 36.738 & 32.469 & 16.315 & 19.655 & 15.764 & 10.306 & 31.611 & 32.338 & 29.473 & 31.403 & 27.852 & 33.791 \\
\hline $1 / 3 / 122: 00$ & & 30.489 & 30.13 & 29.318 & 29.837 & 29.544 & 30.578 & 29.631 & 29.802 & 28.556 & 26.287 & 31.429 & 36.74 & 32.457 & 16.3 & 19.649 & 15.766 & 10.294 & 31.597 & 32.329 & 29.447 & 31.394 & 27.849 & 33.789 \\
\hline $\begin{array}{l}1 / 3 / 123: 00 \\
1 / 32\end{array}$ & & 30.487 & 30.116 & 29.306 & 29.8. & 29.54 & 30.571 & 29.629 & 29.79 & 28.544 & 26.277 & 31.417 & 36.736 & 32.445 & 16.3 & 19.637 & 15.759 & 10.287 & 31.585 & 32.319 & 29.438 & 31.385 & 27.849 & 33.787 \\
\hline $\begin{array}{l}1 / 3 / 124: 00 \\
1 / 320\end{array}$ & & 30.48 & 30.1 & 29.283 & 29.8 & 29.542 & 30.573 & 29.626 & 29.774 & 28.53 & 26.263 & 31.408 & 36.729 & 32.438 & $\begin{array}{l}16.5 \\
16.2\end{array}$ & 19.63 & 15.761 & 10.275 & 31.569 & 32.308 & $\begin{array}{l}29.419 \\
29.419\end{array}$ & 31.375 & 27.849 & 33.782 \\
\hline $\begin{array}{l}1 / 3 / 125: 00 \\
1 / 32\end{array}$ & & 30.473 & 30.079 & 29.26 & 29.783 & 29.544 & 30.556 & 29.626 & 29.753 & 28.512 & 26.236 & $\begin{array}{l}31.396 \\
31.396\end{array}$ & 36.724 & 32.429 & $\begin{array}{l}10.2 \\
16.2\end{array}$ & 19.621 & 15.759 & 10.266 & 31.548 & 296 & 29.393 & 31.361 & 27.852 & $\begin{array}{l}33.777 \\
3.762\end{array}$ \\
\hline $1 / 3 / 126: 00$ & & 30.473 & 30.067 & 29.255 & 29.7 & 29.53 & 30.549 & 29.638 & 29.742 & 28.502 & 26.229 & 31.386 & 36.722 & 32.417 & 16.2 & 19.625 & 15.743 & 10.256 & 31.541 & 289 & 29.386 & 31.352 & 27.852 & .775 \\
\hline $1 /$ & & 30.463 & 30.053 & 29.236 & 29.7 & 29.53 & 30.547 & 29.634 & 29.728 & 28.486 & 26.224 & 31.377 & 36.712 & 32.408 & 16.271 & 8 & 15.745 & 10.247 & 31.529 & 79 & 29.374 & 31.342 & 27.852 & 33.775 \\
\hline & & 30.459 & 30.04 & 29.227 & & 29.518 & 30.5 & 29.638 & 29.714 & 28.474 & 26.217 & 31.368 & 36.708 & 32.398 & 16.2 & 8 & 15.738 & 10.24 & 31.52 & 7 & 29.36 & 31.338 & 27.852 & 33.773 \\
\hline & & 30. & 30. & 29.229 & & 29.502 & & 29.6 & & 28.46 & & 31.363 & 36. & 32.391 & 16.2 & 19.6 & 15.7 & 10.233 & 31.51 & 3 & & 31.328 & 27.852 & 33.77 \\
\hline 1/3/12 10:00 & & 30.452 & 30.035 & 29.227 & 29. & 29.493 & 30 & 29.641 & & 28.46 & 26.2 & 31.358 & 36.7 & 32.389 & & & 15.7 & 10.226 & 31.513 & 8 & 29.353 & 31.324 & 27.849 & 33.77 \\
\hline 1/3/12 11:00 & & 30.438 & 30.007 & 29.19 & 29. & 29.511 & 30. & 29.636 & 29. & 28.439 & 26.208 & 31.342 & 36.698 & 32.375 & 16.2 & 8 & 15.743 & 10.214 & 31.4 & 6 & 29.325 & 31.314 & 27.852 & 33.766 \\
\hline $1 / 3 / 1212$ & & 30. & 29.972 & 29.138 & & 29.535 & & 29.626 & 29. & 28.407 & 26.16 & 31.326 & 36.691 & 32.359 & 16. & 3 & 15.757 & 10.2 & 31.445 & & 29.28 & 31.3 & 27.852 & 33.761 \\
\hline 1/3/12 13:00 & & 30.417 & 29.944 & 29.098 & & 29.54 & & 29.622 & & 28.381 & 26.124 & 31.305 & 36.677 & 32.34 & 16. & 19.584 & 15.752 & 10.176 & 31.42 & 32.206 & 29.247 & 31.279 & 27.852 & 33.756 \\
\hline 1/3/12 14:00 & & 30.412 & 29.9 & 29.08 & 29. & 29.53 & 30. & 29.626 & 29.591 & 28.36 & 26.1 & 31.288 & 36.668 & 32.321 & 16.2 & 19.575 & 15.743 & 10.16 & 31.403 & 32.191 & 29.226 & 31.26 & 27.854 & 33.752 \\
\hline 1/3/12 15:00 & & 30.415 & 29.91 & 29.091 & 29.6 & 29.497 & 30.5 & 29.634 & 29.584 & 28.356 & 26.112 & 31.284 & 36.663 & 32.314 & 16.2 & 19.57 & 15.726 & 10.153 & 31.403 & 32.182 & 29.236 & 31.253 & 27.854 & 33.749 \\
\hline 1/3/12 16:00 & & 30.405 & 29.911 & 29.089 & 29.6 & 29.479 & 30.4 & 29.634 & 29.572 & 28.349 & 26.119 & 31.274 & 36.661 & 32.305 & 16.19 & 19.563 & 15.717 & 10.146 & 31.401 & 32.177 & 29.228 & 31.244 & 27.854 & 33.749 \\
\hline $1 / 3 / 1217: 00$ & & 30.405 & 29.904 & 29.084 & 29.594 & 29.457 & 30.489 & 29.643 & 29.558 & 28.339 & 26.112 & 31.267 & 36.651 & 32.3 & 16.18 & 19.561 & 15.705 & 10.139 & 31.396 & 32.168 & 29.228 & 31.232 & 27.854 & 33.749 \\
\hline $1 / 3 / 1218: 00$ & & 30.412 & 29.913 & 29.119 & 29.611 & 29.413 & 30.475 & 29.655 & 29.565 & 28.349 & 26.145 & 31.272 & 36.651 & 32.3 & 16.187 & 19.558 & 15.677 & 10.139 & 31.413 & 32.173 & 29.252 & 31.234 & 27.856 & 33.752 \\
\hline 1/3/12 19:00 & & 30.405 & 29.913 & 29.122 & 29.618 & 29.396 & 30.482 & 29.655 & 29.561 & 28.346 & 26.155 & 31.267 & 36.654 & 32.3 & 16.18 & 19.558 & 15.67 & 10.136 & 31.417 & 32.17 & 29.257 & 31.232 & 27.857 & 33.752 \\
\hline $1 / 3 / 1220: 00$ & & 30.401 & 29.911 & 29.115 & 29.618 & 29.389 & 30.489 & 29.655 & 29.554 & 28.339 & 26.155 & 31.267 & 36.654 & 32.298 & 16.175 & 19.558 & 15.665 & 10.134 & 31.413 & 32.17 & 29.252 & 31.234 & 27.856 & 33.749 \\
\hline 1/3/12 21:00 & & 30.408 & 29.925 & 29.154 & 29.641 & 29.352 & 30.458 & 29.672 & 29.568 & 28.356 & 26.205 & 31.389 & 36.656 & 32.424 & 16.175 & 19.558 & 15.639 & 10.261 & 31.49 & 32.289 & 29.292 & 31.352 & 27.857 & 33.754 \\
\hline 1/3/12 22:00 & & 30.408 & 29.937 & 29.171 & 29.664 & 29.335 & 30.449 & 29.669 & 29.572 & 28.367 & 26.224 & 31.347 & 36.658 & 32.38 & 16.175 & 19.554 & 15.632 & 10.216 & 31.483 & 32.248 & 29.313 & 31.312 & 27.857 & 33.756 \\
\hline 1/3/12 23:00 & & 30.41 & 29.946 & 29.192 & 29.685 & 29.319 & 30.441 & 29.676 & 29.581 & 28.377 & 26.246 & 31.333 & 36.663 & 32.363 & 16.173 & 19.551 & 15.616 & 10.2 & 31.492 & 32.234 & 29.337 & 31.295 & 27.857 & 33.756 \\
\hline
\end{tabular}




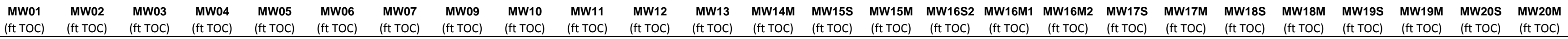

\begin{tabular}{|c|c|c|c|c|c|c|c|c|c|c|c|c|c|c|c|c|c|c|c|c|c|c|c|c|c|}
\hline Well & $\begin{array}{l}\text { (ft TOC) } \\
\text { (f) }\end{array}$ & $\begin{array}{l}\text { (ft TOC) } \\
\text { (f) }\end{array}$ & $\begin{array}{l}\text { (ft TOC) } \\
\text { (f) }\end{array}$ & $\begin{array}{l}\text { (ft TOC) } \\
\text { (ft) }\end{array}$ & $\begin{array}{l}\text { (ft TOC) } \\
\text { (f) }\end{array}$ & $\begin{array}{l}\text { (ft toc) } \\
\text { (ftocts }\end{array}$ & (ft toc) & $\begin{array}{l}\text { (ift TOC) } \\
\text { (n) }\end{array}$ & $\begin{array}{l}\text { (ft TOC) } \\
\text { (f) }\end{array}$ & $\begin{array}{l}\text { (ft TOC) } \\
\text { (f) }\end{array}$ & $\begin{array}{l}\text { (ft TOC) } \\
\text { (n) }\end{array}$ & $\begin{array}{l}\text { (ft TOC) } \\
\text { (f) }\end{array}$ & (ft TOC) & $\begin{array}{l}\text { (ft TOC) } \\
\end{array}$ & (ft TOC) & $\begin{array}{ll} & \text { (ft TTCC } \\
\text { (ft TOC) }\end{array}$ & (ft TOC) & $\begin{array}{l}\text { (ft TOC) } \\
\text { (ft) }\end{array}$ & $\begin{array}{l}\text { (ft toc) } \\
\text { (fol) }\end{array}$ & $\begin{array}{l}\text { (ft TOC) } \\
\text { (f) }\end{array}$ & $\begin{array}{l}\text { (ft TOC) } \\
\text { (f) }\end{array}$ & $\begin{array}{l}\text { (ft TOC) } \\
\text { (n) }\end{array}$ & (ft TOC) & (ft TOC) & (ft TOC) \\
\hline 20:00 & & 30.412 & 29.96 & 29.211 & 29.704 & & 29.305 & 30.427 & 29.681 & 29.588 & 28.386 & 26.26 & 31.328 & 36.67 & 32.3 & 16.1 & 19.554 & 15.5 & 10.193 & 31. & 32.229 & 29.353 & 31.291 & 27.857 & 33.756 \\
\hline 1/4/12 1:00 & & 30.412 & 29.965 & 29.215 & 29.718 & & 29.305 & 30.425 & 29.681 & 29.593 & 28.393 & 26.27 & 31.323 & 36.675 & 32.354 & 16.173 & 19.549 & 15.597 & 10.19 & 31.504 & 32.227 & .355 & 31.286 & 27.857 & 33.754 \\
\hline $1 / 4 / 122: 00$ & & 30.412 & 29.972 & 29.229 & 29.734 & & 29.3 & 30.415 & 29.683 & 29.602 & 28.402 & 26.284 & 31.323 & 36.677 & 32.349 & 16.175 & 19.549 & 15.59 & 10.188 & 31.518 & 32.222 & .377 & 31.286 & 27.857 & 33.754 \\
\hline 1/4/12 3:00 & & 30.419 & 29.993 & 29.255 & 29.755 & & 29.286 & 30.398 & 29.693 & 29.616 & 28.419 & 26.308 & 31.326 & 36.684 & 4. 32.354 & 16.175 & 19.547 & 15.578 & 10.19 & 31.529 & 32.229 & 29.402 & 31.291 & 27.859 & 33.754 \\
\hline 1/4/12 4:00 & & 30.417 & 30.004 & 29.274 & 29.776 & & 29.283 & 30.393 & 29.693 & 29.628 & 28.428 & 26.327 & 31.33 & 36.689 & $\begin{array}{l}92.359 \\
9\end{array}$ & 16.18 & 19.544 & 15.581 & 10.197 & 31.543 & 32.234 & 29.419 & 31.295 & 27.856 & 33.756 \\
\hline 1/4/12 5:00 & & 30.426 & 30.023 & 29.295 & 29.797 & & 29.281 & 30.393 & 29.695 & 29.642 & 28.444 & 26.342 & 31.337 & 36.703 & $3 \quad 32.366$ & 16.184 & 19.544 & 15.567 & 10.2 & 31.56 & 32.239 & 29.435 & 31.3 & 27.861 & 33.756 \\
\hline 1/4/12 6:00 & & 30.424 & 30.03 & 29.304 & 29.811 & & 29.286 & 30.403 & 29.695 & 29.651 & 28.451 & 26.351 & 31.342 & 36.705 & $5 \quad 32.37$ & 16.187 & 19.558 & 15.571 & 10.207 & 31.574 & 32.246 & 29.449 & 31.305 & 27.859 & 33.759 \\
\hline 1/4/12 7:00 & & 30.431 & 30.051 & 29.33 & 29.835 & & 29.281 & 30.405 & 29.707 & 29.667 & 28.47 & 26.375 & 31.351 & 36.71 & $1 \quad 32.377$ & 16.194 & 19.565 & 15.569 & 10.216 & 31.583 & 32.255 & 29.473 & 31.314 & 27.859 & 33.761 \\
\hline 1/4/12 8:00 & & 30.438 & 30.072 & 29.358 & 29.856 & & 29.276 & 30.405 & 29.712 & 29.686 & 28.488 & 26.397 & 31.363 & 36.722 & 32.387 & 16.198 & 19.563 & 15.557 & 10.228 & 31.609 & 32.265 & 29.499 & 31.326 & 27.861 & 33.763 \\
\hline 1/4/12 9:00 & & 30.442 & 30.086 & 29.374 & 29.879 & & 29.281 & 30.41 & 29.717 & 29.702 & 28.502 & 26.413 & 31.375 & 36.726 & $5 \quad 32.398$ & 16.203 & 19.561 & 15.564 & 10.235 & 31.623 & 32.277 & 29.51 & 31.338 & 27.861 & 33.766 \\
\hline 1/4/12 10:00 & & 30.442 & 30.095 & 29.379 & 29.893 & & 29.297 & 30.415 & 29.712 & 29.709 & 28.509 & 26.411 & 31.546 & 36.736 & 532.581 & 16.21 & 19.556 & 15.571 & 10.418 & 31.688 & 32.447 & 29.527 & 31.507 & 27.861 & 33.77 \\
\hline 1/4/12 11:00 & & 30.44 & 30.093 & 29.37 & 29.893 & & 29.326 & 30.434 & 29.705 & 29.714 & 28.512 & 26.401 & 31.466 & 36.736 & 532.499 & 16.212 & 19.549 & 15.597 & 10.336 & 31.665 & 32.369 & 29.518 & 31.441 & 27.859 & 33.77 \\
\hline $1 / 4 / 1212: 00$ & & 30.435 & 30.084 & 29.339 & 29.886 & & 29.363 & 30.47 & 29.698 & 29.707 & 28.502 & 26.368 & 31.443 & 36.74 & 32.469 & 16.217 & 19.544 & 15.618 & 10.308 & 31.634 & 32.343 & 29.496 & 31.413 & 27.861 & 33.768 \\
\hline $1 / 4 / 1213: 00$ & & 30.428 & 30.07 & 29.306 & 29.865 & & 29.394 & 30.499 & 29.693 & 29.693 & 28.488 & 26.332 & 31.422 & 36.729 & 32.452 & 16.217 & 19.538 & 15.637 & 10.284 & 31.609 & 32.322 & 29.475 & 31.392 & 27.861 & 33.763 \\
\hline $1 / 4 / 1214: 00$ & & 30.424 & 30.053 & 29.274 & 29.842 & & 29.417 & 30.511 & 29.695 & 29.679 & 28.474 & 26.299 & 31.403 & 36.719 & 32.436 & 16.219 & 19.531 & 15.644 & 10.27 & 31.59 & 32.305 & 29.454 & 31.373 & 27.864 & 33.761 \\
\hline 1/4/12 15:00 & & 30.422 & 30.046 & 29.267 & 29.828 & & 29.424 & 30.504 & 29.698 & 29.674 & 28.467 & 26.292 & 31.394 & 36.715 & 32.422 & 16.217 & 19.526 & 15.649 & 10.259 & 31.574 & 32.293 & 29.435 & 31.363 & 27.864 & 33.761 \\
\hline $1 / 4 / 1216: 00$ & & 30.419 & 30.035 & 29.25 & 29.811 & & 29.427 & 30.506 & 29.7 & 29.667 & 28.458 & 26.275 & 31.384 & 36.71 & 32.415 & 16.217 & 19.572 & 15.651 & 10.249 & 31.567 & 32.284 & 29.426 & 31.352 & 27.864 & 33.759 \\
\hline 1/4/12 17:00 & & 30.422 & 30.039 & 29.26 & 29.809 & & 29.413 & 30.501 & 29.71 & 29.667 & 28.46 & 26.282 & 31.38 & 36.708 & 32.41 & 16.219 & 19.575 & 15.644 & 10.245 & 31.571 & 32.279 & 29.433 & 31.342 & 27.864 & 33.761 \\
\hline 1/4/12 18:00 & & 30.424 & 30.042 & 29.274 & 29.814 & & 29.399 & 30.492 & 29.717 & 29.67 & 28.463 & 26.294 & 31.38 & 36.708 & 32.408 & 16.217 & 19.57 & 15.637 & 10.247 & 31.571 & 32.284 & 29.438 & 31.342 & 27.864 & 33.761 \\
\hline 1/4/12 19:00 & & 30.419 & 30.039 & 29.264 & 29.807 & & 29.399 & 30.501 & 29.717 & 29.667 & 28.46 & 26.284 & 31.375 & 36.715 & 32.403 & 16.215 & 19.568 & 15.639 & 10.237 & 31.569 & 32.277 & 29.428 & 31.34 & 27.864 & 33.761 \\
\hline 1/4/12 20:00 & & 30.419 & 30.037 & 29.257 & 29.804 & & 29.396 & 30.497 & 29.714 & 29.663 & 28.458 & 26.28 & 31.372 & 36.708 & 32.401 & 16.217 & 19.563 & 15.637 & 10.242 & 31.564 & 32.277 & 29.424 & 31.338 & 27.864 & 33.763 \\
\hline 1/4/12 21:00 & & 30.419 & 30.037 & 29.26 & 29.804 & & 29.387 & 30.489 & 29.719 & 29.663 & 28.458 & 26.284 & 31.37 & 36.708 & 32.401 & 16.215 & 19.561 & 15.63 & 10.24 & 31.56 & .274 & 29.431 & 31.338 & 27.864 & .763 \\
\hline 1/4/12 22:00 & & 30.412 & 30.03 & 29.253 & 29.8 & & 29.392 & 30.499 & 29.717 & 29.66 & 28.453 & 26.277 & 31.37 & 36.708 & 32.394 & 16.215 & .556 & 15.637 & 10.237 & 31.55 & .272 & 29.419 & 31.335 & 27.864 & 33.763 \\
\hline 1/4/12 23:00 & & 30.41 & 30.025 & 29.241 & 29.79 & & 29.394 & 30.501 & 29.717 & 29.656 & 28.446 & 26.265 & 31.363 & 36.701 & 32.389 & 16.215 & 19.554 & 15.632 & 10.23 & 31.548 & 32.265 & 29.412 & 31.328 & 27.866 & 33.763 \\
\hline 1/5/12 0:00 & & 30.405 & 30.014 & 29.22 & 29.776 & & 29.403 & 30.496 & 29.714 & 29.642 & 28.435 & 26.244 & 31.354 & 36.696 & 32.382 & 16.21 & 19.551 & 15.632 & 10.219 & 31.534 & 32.255 & 29.393 & 31.321 & 27.864 & 33.759 \\
\hline 1/5/12 1:00 & & 30.403 & 30.007 & 29.213 & 29.765 & & 29.401 & 30.501 & 29.719 & 29.637 & 28.425 & 26.239 & 31.347 & 36.694 & 32.375 & 16.208 & 19.54 & 15.628 & 10.212 & 31.52 & 32.246 & 29.384 & 31.312 & 27.866 & 33.756 \\
\hline 1/5/12 2:00 & & 30.401 & 29.997 & 29.204 & 29.755 & & 29.399 & 30.499 & 29.717 & 29.626 & 28.421 & 26.227 & 31.335 & 36.691 & 32.366 & 16.205 & 19.54 & 15.623 & 10.202 & 31.515 & 32.237 & 29.379 & 31.298 & 27.866 & 33.752 \\
\hline 1/5/12 3:00 & & 30.396 & 29.993 & 29.194 & 29.746 & & 29.399 & 30.506 & 29.717 & 29.621 & 28.414 & 26.222 & 31.328 & 36.687 & 32.359 & 16.203 & 19.533 & 15.628 & 10.195 & 31.504 & 32.229 & 29.365 & 31.295 & 27.866 & 33.749 \\
\hline 1/5/12 4:00 & & 30.394 & 29.983 & 29.183 & 29.734 & & 29.396 & 30.494 & 29.717 & 29.614 & 28.405 & 26.212 & 31.321 & 36.687 & 32.349 & 16.198 & 19.524 & 15.621 & 10.19 & 31.49 & 32.222 & 29.353 & 31.288 & 27.866 & 33.742 \\
\hline 1/5/12 5:00 & & 30.391 & 29.979 & 29.176 & 29.727 & & 29.392 & 30.487 & 29.719 & 29.607 & 28.4 & 26.208 & 31.314 & 36.682 & 32.345 & 16.196 & 19.514 & 15.616 & 10.179 & 31.49 & 32.218 & 29.348 & 31.281 & 27.866 & 33.742 \\
\hline 1/5/12 6:00 & & 30.391 & 29.974 & 29.183 & 29.725 & & 29.38 & 30.477 & 29.724 & 29.605 & 28.398 & 26.215 & 31.312 & 36.677 & 32.34 & 16.191 & 19.524 & 15.611 & 10.179 & 31.487 & 32.213 & 29.351 & 31.277 & 27.864 & 33.742 \\
\hline 1/5/12 7:00 & & 30.387 & 29.972 & 29.173 & 29.72 & & 29.38 & 30.484 & 29.721 & 29.6 & 28.393 & 26.21 & 31.307 & 36.675 & 32.338 & 16.189 & 19.521 & 15.609 & 10.172 & 31.483 & 32.21 & 29.341 & 31.274 & 27.866 & 33.738 \\
\hline 1/5/12 8:00 & & 30.387 & 29.967 & 29.166 & 29.713 & & 29.375 & 30.484 & 29.726 & 29.593 & 28.388 & 26.205 & 31.302 & 36.677 & 32.33 & 16.187 & 19.524 & 15.607 & 10.172 & 31.476 & 32.203 & 29.334 & 31.27 & 27.866 & 33.738 \\
\hline 1/5/12 9:00 & & 30.389 & 29.967 & 29.171 & 29.716 & & 29.361 & 30.47 & 29.726 & 29.595 & 28.388 & 26.212 & 31.302 & 36.675 & 32.328 & 16.182 & 19.524 & 15.599 & 10.167 & 31.483 & 32.203 & 29.339 & 31.267 & 27.868 & 33.738 \\
\hline 1/5/12 10:00 & & 30.384 & 29.965 & 29.173 & 29.7 & & 29.359 & 30.475 & 29.729 & 29.591 & 28.386 & 26.21 & 31.3 & 36.677 & 32.328 & 16.1 & 19.514 & 15.599 & 10.167 & 31.478 & 32.201 & 29.341 & 31.27 & 27.868 & 33.738 \\
\hline 1/5/12 11:00 & & 30.38 & 29.96 & 29.162 & 29.76 & & 29.366 & 30.48 & 29.724 & 29.586 & 28.379 & 26.205 & 31.295 & 36.672 & 32.323 & 16.1 & 19.508 & 15.602 & 10.162 & 31.471 & 32.196 & 29.332 & 31.267 & 27.868 & 33.74 \\
\hline 1/5/12 12:00 & & 30.37 & 29.944 & 29.126 & 29.688 & & 29.385 & 30.46 & 29.714 & 29.565 & 28.36 & 26.172 & 31.286 & 36.665 & 32.316 & 16.175 & 19.498 & 15.616 & 10.15 & 31.448 & 32.187 & 29.304 & 31.258 & 27.868 & 33.735 \\
\hline 1/5/12 13:00 & & 30.361 & 29.925 & 29.096 & 29.662 & & 29.401 & 30.463 & 29.712 & 29.547 & 28.342 & 26.141 & 31.27 & 36.663 & 32.305 & 16.168 & 19.491 & 15.625 & 10.139 & 31.422 & 173 & 29.275 & 31.244 & 27.868 & 33.733 \\
\hline 1/5/12 14:00 & & 30.359 & 29.904 & 29.068 & 29.6 & & 29.408 & $\begin{array}{l}30.463 \\
30.463\end{array}$ & 29.71 & 29.528 & $\begin{array}{l}28.342 \\
28.323\end{array}$ & 26.117 & 31.258 & 36.654 & 32.288 & 16.1 & 19.487 & 15.63 & 10.125 & 31.403 & 161 & 29.25 & 31.232 & 27.871 & 33.728 \\
\hline 1/5/12 15:00 & & 30.359 & 29.8 & 29. & 29 & & 29 & 30. & 29.714 & 29.519 & 28.314 & 26 & 31.248 & 36.649 & 32.279 & 16 & 19.48 & 15.621 & 10.115 & 31.401 & .149 & 29 & 31.218 & 27.868 & 33.724 \\
\hline $1 / 5 / 1216: 00$ & & 30.356 & 29 & 29. & 29 & & 29. & 30. & 29.717 & 29.507 & 28.307 & 26.112 & 31.241 & 36.644 & 32.272 & 16.154 & 19.475 & 15.616 & 10.108 & 31.392 & 142 & 29.236 & 31.209 & 27.868 & 33.721 \\
\hline & & 30.354 & 29.883 & 29.054 & & & 29.368 & 30.4 & 29.717 & 29 & $2 \varepsilon$ & 26.112 & 31.234 & 36.642 & 32.265 & 16.1 & 19.47 & 15.614 & 10.101 & 31.385 & 32.137 & 29.231 & 31.202 & 27.871 & 33.719 \\
\hline 1/5/12 18:00 & & 30.349 & 29.876 & 29.047 & 29. & & 29.356 & 30.4 & 29.719 & 29.491 & 28.293 & 26.11 & 31.23 & 36.64 & 32.26 & 16.14 & 19.468 & 15.609 & 10.096 & 31.38 & 32.132 & 29.224 & 31.194 & 27.868 & 33.719 \\
\hline 1/5/12 19:00 & & 30.349 & 29.871 & 29.054 & & & 29 & 30. & 29.719 & 29.4 & 28.288 & 26.114 & 31.227 & 36.635 & 32.26 & 16. & 19.466 & 15.597 & 10.094 & 31.378 & & 29.226 & 31.192 & 27.868 & 33.719 \\
\hline 1/5/12 20:00 & & 30. & 29.8 & 29.052 & & & 29.328 & & 29.719 & 29. & 28.2 & 26.117 & 31.227 & 36.64 & 32.255 & 16.1 & 19.466 & 15.59 & 10.094 & 31.378 & 32.128 & 29.226 & 31.19 & 27.871 & 33.721 \\
\hline 1/5/12 21:00 & & 30. & 29.8 & & & & 29.319 & & 29.719 & 29. & 28.279 & 26.112 & 31.225 & 36.63 & 32.248 & 16.1 & 19.468 & 15.583 & 10.089 & 31.375 & & 29.221 & 31.187 & 27.871 & 33.721 \\
\hline 1/5/12 22:00 & & 30.335 & 29.8 & 29.031 & & & 29.316 & 30.439 & 29.714 & 29.463 & 28.27 & 26.1 & 31.218 & 36.63 & 32.246 & 16.12 & 19.464 & 15.578 & 10.085 & 31.364 & 32.116 & 29.212 & 31.185 & 27.871 & 33.721 \\
\hline 1/5/12 23:00 & & 30.3 & 29.8 & 29.024 & & & 29.309 & 30.444 & 29.717 & 29.454 & 28.263 & 26.098 & 31.213 & 36.626 & 32.239 & 16.12 & 19.457 & 15.571 & 10.078 & 31.357 & 32.111 & 29.21 & 31.176 & 27.868 & 33.719 \\
\hline 1/6/12 0:00 & & 30.333 & 29.839 & 29.014 & 29.5 & & 29.305 & 30.437 & 29.714 & 29.445 & 28.256 & 26.09 & 31.209 & 36.626 & 32.23 & 16.11 & 19.457 & 15.562 & 10.071 & 31.347 & 32.104 & 29.196 & 31.169 & 27.871 & 33.717 \\
\hline 1/6/12 1:00 & & 30.328 & 29.832 & 29.005 & 29.55 & & 29.297 & 30.422 & 29.714 & 29.435 & 28.246 & 26.086 & 31.199 & 36.616 & 32.225 & 16.117 & 19.45 & 15.557 & 10.059 & 31.338 & 32.094 & 29.186 & 31.159 & 27.871 & 33.712 \\
\hline $\begin{array}{l}1 / 6 / 122: 00 \\
\text { lot }\end{array}$ & & 30.326 & 29.827 & 29.003 & 29.555 & & 29.286 & 30.437 & 29.714 & 29.428 & 28.242 & 26.083 & 31.19 & 36.619 & 32.216 & 16.112 & 19.447 & 15.545 & 10.052 & 31.336 & 32.087 & 29.181 & 31.152 & 27.871 & 33.71 \\
\hline $1 / 6 / 123: 00$ & & 30.326 & 29.827 & 29.007 & 29.552 & & 29.272 & 30.439 & 29.719 & 29.426 & 28.242 & 26.093 & 31.185 & 36.619 & 32.209 & 16.108 & 19.445 & 15.534 & 10.049 & 31.338 & 32.085 & 29.189 & 31.145 & 27.871 & 33.705 \\
\hline $1 / 6 / 124: 00$ & & 30.324 & 29.822 & 29.003 & 29.55 & & 29.265 & 30.398 & 29.719 & 29.421 & 28.237 & 26.095 & 31.178 & 36.609 & 32.204 & 16.103 & 19.438 & 15.527 & 10.042 & 31.333 & 32.075 & 29.181 & 31.143 & 27.871 & 33.702 \\
\hline $1 / 6 / 125: 00$ & & 30.321 & 29.815 & 29 & 29.548 & & 29.255 & 30.396 & 29.714 & 29.414 & 28.232 & 26.09 & 31.174 & 36.619 & 32.199 & 16.101 & 19.431 & 15.517 & 10.035 & 31.329 & 32.071 & 29.181 & 31.136 & 27.868 & 33.698 \\
\hline 1/6/12 6:00 & & 30.319 & 29.815 & 28.996 & 29.545 & & 29.246 & 30.389 & 29.717 & 29.407 & 28.228 & 26.086 & 31.169 & 36.612 & 32.194 & 16.098 & 19.438 & 15.506 & 10.031 & 31.326 & 32.066 & 29.179 & 31.129 & 27.871 & 33.695 \\
\hline $1 / 6 / 127: 00$ & & 30.321 & 29.82 & 29.01 & 29.55 & & 29.229 & 30.384 & 29.719 & 29.412 & 28.232 & 26.105 & 31.171 & 36.607 & 32.194 & 16.096 & 19.433 & 15.489 & 10.033 & 31.331 & 32.066 & 29.191 & 31.129 & 27.871 & 693 \\
\hline
\end{tabular}




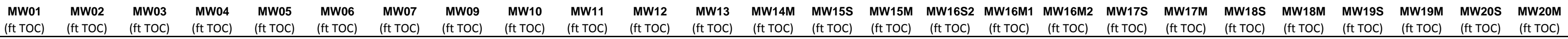

\begin{tabular}{|c|c|c|c|c|c|c|c|c|c|c|c|c|c|c|c|c|c|c|c|c|c|c|c|c|c|c|}
\hline Well & $\begin{array}{l}\text { (ft TOC) } \\
\text { (f) }\end{array}$ & $\begin{array}{l}\text { (ft TOC) } \\
\text { (f) }\end{array}$ & $\begin{array}{l}\text { (ft TOC) } \\
\text { (f) }\end{array}$ & (ft TOC) & (ft TOC) & $\begin{array}{l}\text { (ft ToC) } \\
\text { (f) }\end{array}$ & (ft ToC) & (ft ToC) & $\begin{array}{l}\text { (ft TOC) } \\
\text { (f) }\end{array}$ & $\begin{array}{l}\text { (ft TOC) } \\
\text { (t) }\end{array}$ & $\begin{array}{l}\text { (ft TOC) } \\
\text { (n) }\end{array}$ & $\begin{array}{l}\text { (ft TOC) } \\
\text { (f) }\end{array}$ & (ft TOC) & $\begin{array}{l}\text { (ft ToC) } \\
\text { (ft }\end{array}$ & (ft TOC) & (ft TOC) & (ft TOC) & (ft TOC) & $\begin{array}{l}\text { (ft TOC) } \\
\text { (f) }\end{array}$ & (ft TOC) & $\begin{array}{l}\text { (ft ToC) } \\
\text { (ft }\end{array}$ & $\begin{array}{l}\text { (ft TOC) } \\
\text { (f) }\end{array}$ & $\begin{array}{l}\text { (ft TOC) } \\
\text { (n) }\end{array}$ & (ft TOC) & $\begin{array}{l}\text { (nt TOC) } \\
\text { (nt }\end{array}$ & (ft TOC) \\
\hline $1 / 6 / 128: 00$ & & 30.317 & 29.813 & 28.998 & 29.548 & & 29.229 & 30.391 & 29.717 & 29.403 & 28.228 & 26.095 & 31.164 & 36.607 & 32.19 & 16.094 & & 19.44 & 15.492 & 10.031 & 31.322 & 32.061 & 29.181 & 31.124 & 27.871 & 33.693 \\
\hline $1 / 6 / 129: 00$ & & 30.331 & 29.829 & 29.042 & 29.571 & & 29.192 & 30.353 & 29.731 & 29.421 & 28.246 & 26.143 & 31.174 & 36.609 & 32.197 & 16.094 & & 19.436 & 15.47 & 10.033 & 31.354 & 32.071 & 29.219 & 31.136 & 27.873 & 33.698 \\
\hline $1 / 6 / 1210: 00$ & & 30.328 & 29.843 & 29.063 & 29.59 & & 29.18 & 30.35 & 29.736 & 29.431 & 28.258 & 26.165 & 31.181 & 36.621 & 32.204 & $\begin{array}{l}16.094 \\
16.094\end{array}$ & & 19.431 & 15.463 & 10.04 & 31.371 & 32.078 & 29.236 & 31.143 & 27.873 & 33.7 \\
\hline 1/6/12 11:00 & & 30.328 & 29.855 & 29.084 & 29.613 & & 29.173 & 30.355 & 29.736 & 29.44 & 28.27 & 26.186 & 31.188 & 36.626 & 32.209 & 16.096 & & 19.427 & 15.468 & 10.049 & 31.378 & 32.085 & 29.257 & 31.152 & 27.873 & 33.702 \\
\hline 1/6/12 12:00 & & 30.324 & 29.855 & 29.075 & 29.615 & & 29.185 & 30.362 & 29.729 & 29.44 & 28.27 & 26.179 & 31.19 & 36.633 & 32.213 & 16.096 & & 19.424 & 15.477 & 10.047 & 31.378 & 32.087 & 29.254 & 31.155 & 27.871 & 33.702 \\
\hline 1/6/12 13:00 & & 30.324 & 29.86 & 29.08 & 29.625 & & 29.187 & 30.374 & 29.731 & 29.447 & 28.274 & 26.181 & 31.192 & 36.63 & 32.213 & 16.098 & & 19.42 & 15.477 & 10.052 & 31.387 & 32.087 & 9.259 & 31.157 & 27.873 & 33.702 \\
\hline $1 / 6 / 1214: 00$ & & 30.326 & 29.867 & 29.087 & 29.634 & & 29.182 & 30.382 & 29.736 & 29.449 & 28.281 & 26.186 & 31.195 & 36.635 & 32.218 & 16.098 & & 19.417 & 15.473 & 10.054 & 31.389 & 32.094 & 29.264 & 31.155 & 27.875 & 33.702 \\
\hline 1/6/12 15:00 & & 30.328 & 29.883 & 29.112 & 29.653 & & 29.171 & 30.37 & 29.743 & 29.463 & 28.298 & 26.21 & 31.197 & 36.633 & $3 \quad 32.223$ & 16.103 & & 19.413 & 15.468 & 10.059 & 31.408 & 32.101 & 29.294 & 31.157 & 27.875 & 33.705 \\
\hline 1/6/12 16:00 & & 30.333 & 29.899 & 29.138 & 29.674 & & 29.156 & 30.36 & 29.752 & 29.479 & 28.312 & 26.234 & 31.209 & 36.64 & 32.232 & 16.105 & & 19.468 & 15.463 & 10.068 & 31.427 & 32.109 & 29.313 & 31.166 & 27.878 & 33.705 \\
\hline 1/6/12 17:00 & & 30.34 & 29.927 & 29.176 & 29.702 & & 29.138 & 30.336 & 29.757 & 29.498 & 28.332 & 26.268 & 31.223 & 36.656 & $5 \quad 32.239$ & 16.112 & & 19.468 & 15.459 & 10.082 & 31.455 & 32.12 & 29.344 & 31.178 & 27.875 & 33.71 \\
\hline 1/6/12 18:00 & & 30.345 & 29.948 & 29.204 & 29.73 & & 29.133 & 30.322 & 29.759 & 29.519 & 28.353 & 26.292 & 31.237 & 36.665 & $5 \quad 32.255$ & 16.117 & & 19.47 & 15.459 & 10.094 & 31.476 & 32.132 & 29.374 & 31.192 & 27.878 & 33.717 \\
\hline $1 / 6 / 1219: 00$ & & 30.349 & 29.974 & 29.232 & 29.758 & & 29.126 & 30.324 & 29.762 & 29.542 & 28.372 & 26.323 & 31.253 & 36.67 & 32.27 & 16.124 & & 19.47 & 15.466 & 10.108 & 31.499 & 32.149 & 29.4 & 31.211 & 27.878 & 33.724 \\
\hline $1 / 6 / 1220: 00$ & & 30.354 & 29.988 & 29.246 & 29.779 & & 29.135 & 30.331 & 29.764 & 29.556 & 28.386 & 26.332 & 31.265 & 36.682 & 32.286 & 16.131 & & 19.47 & 15.47 & 10.118 & 31.513 & 32.161 & 29.426 & 31.223 & 27.878 & 33.728 \\
\hline 1/6/12 21:00 & & 30.354 & 29.995 & 29.253 & 29.793 & & 29.147 & 30.338 & 29.759 & 29.565 & 28.398 & 26.332 & 31.277 & 36.684 & 32.295 & 16.138 & & 19.47 & 15.473 & 10.132 & 31.522 & 32.175 & 29.435 & 31.234 & 27.878 & 33.733 \\
\hline $1 / 6 / 1222: 00$ & & 30.359 & 30.004 & 29.26 & 29.807 & & 29.154 & 30.341 & 29.762 & 29.577 & 28.407 & 26.334 & 31.286 & 36.691 & 32.305 & 16.142 & & 19.477 & 15.482 & 10.146 & 31.534 & 32.184 & 29.442 & 31.244 & 27.878 & 33.735 \\
\hline $1 / 6 / 1223: 00$ & & 30.359 & 30.011 & 29.264 & 29.816 & & 29.171 & 30.35 & 29.762 & 29.588 & 28.416 & 26.337 & 31.295 & 36.701 & 32.314 & 16.149 & & 19.475 & 15.489 & 10.153 & 31.536 & 32.194 & 29.445 & 31.253 & 27.878 & 33.738 \\
\hline $1 / 7 / 120: 00$ & & 30.361 & 30.018 & 29.264 & 29.823 & & 29.18 & 30.353 & 29.759 & 29.595 & 28.421 & 26.335 & 31.3 & 36.701 & 32.319 & 16.156 & & 19.473 & 15.494 & 10.158 & 31.543 & 32.194 & 29.452 & 31.258 & 27.878 & 33.74 \\
\hline 1/7/12 1:00 & & 30.361 & 30.016 & 29.26 & 29.823 & & 29.199 & 30.36 & 29.762 & 29.6 & 28.423 & 26.325 & 31.305 & 36.703 & 32.321 & 16.161 & & 19.47 & 15.503 & 10.16 & 31.548 & 32.199 & 29.449 & 31.26 & 27.878 & 33.738 \\
\hline $1 / 7 / 122: 00$ & & 30.363 & 30.021 & 29.264 & 29.828 & & 29.203 & 30.358 & 29.764 & 29.607 & 28.428 & 26.325 & 31.305 & 36.705 & 32.323 & $\begin{array}{l}16.163 \\
16.163\end{array}$ & & 19.466 & 15.501 & 10.16 & 31.548 & 32.203 & 29.454 & 31.263 & 27.878 & $\begin{array}{l}33.733 \\
3.730\end{array}$ \\
\hline $1 / 7 / 123: 00$ & & 30.363 & 30.023 & 29.264 & 29.828 & & 29.215 & 30.362 & 29.767 & 29.614 & $\begin{array}{l}28.420 \\
28.435\end{array}$ & 26.323 & 31.307 & 36.708 & 32.328 & $\begin{array}{l}10.163 \\
16.168\end{array}$ & & $\begin{array}{l}19.400 \\
19.466\end{array}$ & $\begin{array}{l}13.506 \\
15.506\end{array}$ & 10.162 & $\begin{array}{l}31.546 \\
31.546\end{array}$ & 32.203 & $\begin{array}{l}29.444 \\
29.449\end{array}$ & 31.265 & 27.878 & 33.731 \\
\hline $1 / 7 / 124: 00$ & & $\begin{array}{l}30.303 \\
30.363\end{array}$ & 30.021 & $\begin{array}{r}29.284 \\
29.26\end{array}$ & 29.825 & & 29.229 & $\begin{array}{r}50.302 \\
30.37\end{array}$ & 29.764 & $\begin{array}{l}29.614 \\
29.614\end{array}$ & $\begin{array}{l}28.4535 \\
28.435\end{array}$ & $\begin{array}{l}20.323 \\
26.308\end{array}$ & $\begin{array}{l}31.305 \\
31.305\end{array}$ & 36.708 & $\begin{array}{l}52.326 \\
32.326\end{array}$ & $\begin{array}{l}10.108 \\
16.173\end{array}$ & & $\begin{array}{l}19.400 \\
19.459\end{array}$ & $\begin{array}{l}13.000 \\
15.51\end{array}$ & 10.162 & 31.543 & $\begin{array}{l}32.201 \\
32.201\end{array}$ & 29.447 & $\begin{array}{l}31.265 \\
31.265\end{array}$ & 27.878 & $\begin{array}{l}33.726 \\
33.726\end{array}$ \\
\hline $1 / 7 / 125: 00$ & & $\begin{array}{l}30.363 \\
30.363\end{array}$ & 30.018 & $\begin{array}{l}29.250 \\
2955\end{array}$ & 29.823 & & 29.236 & $\begin{array}{l}30.37 \\
30.37\end{array}$ & $\begin{array}{l}29.764 \\
29.764\end{array}$ & $\begin{array}{l}29.614 \\
29.619\end{array}$ & $\begin{array}{l}28.435 \\
28.435\end{array}$ & $\begin{array}{l}26.308 \\
26.303\end{array}$ & $\begin{array}{l}31.305 \\
31.305\end{array}$ & $\begin{array}{l}36.708 \\
36.705\end{array}$ & $\begin{array}{l}32.326 \\
32.323 \\
\end{array}$ & $\begin{array}{l}10.1 / 3 \\
16.175\end{array}$ & & $\begin{array}{l}19.459 \\
19.452\end{array}$ & $\begin{array}{r}15.51 \\
15.513\end{array}$ & $\begin{array}{r}10.102 \\
10.16\end{array}$ & $\begin{array}{l}31.343 \\
31.536\end{array}$ & $\begin{array}{l}32.201 \\
32.203\end{array}$ & $\begin{array}{r}29.44 \\
29.44\end{array}$ & $\begin{array}{l}51.205 \\
31.263\end{array}$ & 27.878 & $\begin{array}{l}33 . / 726 \\
33.724\end{array}$ \\
\hline $1 / 7 / 126: 00$ & & 30.37 & 30.03 & 29.276 & 29.832 & & 29.227 & 30.363 & 29.771 & 29.63 & 28.446 & 26.323 & 31.309 & 36.708 & & & & 19.452 & 15.508 & 10.167 & 31.555 & 32.208 & 29.459 & 31.27 & 27.88 & 33.724 \\
\hline 1/7/12 7:00 & & 30.366 & 30.028 & 29.269 & $\begin{array}{r}2.0028 \\
29.83\end{array}$ & & 29.241 & 30.374 & 29.769 & $\begin{array}{r}29.633 \\
29.633\end{array}$ & $\begin{array}{l}28.440 \\
28.444\end{array}$ & $\begin{array}{l}20.323 \\
26.313\end{array}$ & 31.312 & 36.71 & 32.33 & $\begin{array}{l}16.182 \\
162\end{array}$ & & $\begin{array}{l}19.452 \\
19.447\end{array}$ & $\begin{array}{l}13.000 \\
15.517\end{array}$ & 10.167 & 31.546 & $\begin{array}{l}2.22 .21 \\
32.21\end{array}$ & 29.454 & 31.272 & 27.88 & .721 \\
\hline $1 / 7 / 128: 00$ & & 30.37 & 30.035 & 29.278 & 29.837 & & 29.243 & 30.367 & 29.774 & 29.637 & 28.453 & & 31.314 & 36.715 & 32.335 & 16.187 & & 445 & 15.517 & 10.172 & 31.557 & 32.213 & 29.459 & 31.277 & 27.88 & 33.724 \\
\hline 1/7/12 9:00 & & 30.373 & 30.042 & 29.29 & 29.844 & & 29.246 & 30.377 & 29.774 & 29.646 & 28.46 & 26.325 & 31.323 & 36.719 & 32.342 & 16.189 & & 441 & 15.52 & 10.181 & 31.564 & 32.218 & 29.466 & 31.284 & 27.88 & 33.728 \\
\hline 1/7/12 10:00 & & 30.375 & 30.044 & 29.292 & 29.849 & & 29.25 & 30.389 & 29.776 & 29.656 & 28.463 & 26.327 & 31.328 & 36.719 & 32.349 & 16.194 & & 19.443 & 15.529 & 10.186 & 31.562 & 32.227 & 29.471 & 31.291 & 27.882 & 33.731 \\
\hline 1/7/12 11:00 & & 30.38 & 30.053 & 29.306 & 29.858 & & 29.25 & 30.391 & 29.781 & 29.663 & 28.472 & 26.334 & 31.333 & 36.717 & 32.356 & 16.196 & & 19.447 & 15.531 & 10.186 & 31.574 & 32.229 & 29.485 & 31.295 & 27.882 & 33.731 \\
\hline 1/7/12 12:00 & & 30.37 & 30.042 & 29.278 & 29.846 & & 29.281 & 30.42 & 29.769 & 29.656 & 28.463 & 26.306 & 31.333 & 36.724 & 32.352 & 16.198 & & 19.452 & 15.56 & 10.188 & 31.562 & 32.227 & 29.459 & 31.298 & 27.882 & 33.731 \\
\hline 1/7/12 13:00 & & 30.363 & 30.021 & 29.239 & 29.823 & & 29.312 & 30.451 & 29.759 & 29.642 & 28.444 & 26.268 & 31.321 & 36.712 & 32.345 & 16.198 & & 19.452 & 15.581 & 10.183 & 31.536 & 32.22 & 29.428 & 31.288 & 27.882 & 33.726 \\
\hline 1/7/12 14:00 & & 30.363 & 30.016 & 29.229 & 29.807 & & 29.316 & 30.444 & 29.767 & 29.635 & 28.439 & 26.256 & 31.316 & 36.708 & 32.34 & 16.196 & & 19.489 & 15.578 & 10.179 & 31.529 & 32.215 & 29.426 & 31.281 & 27.882 & 33.724 \\
\hline $1 / 7 / 1215: 00$ & & 30.366 & 30.014 & 29.229 & 29.802 & & 29.312 & 30.444 & 29.769 & 29.635 & 28.437 & 26.256 & 31.314 & 36.708 & 32.335 & 16.198 & & 19.517 & 15.581 & 10.172 & 31.527 & 32.21 & 29.424 & 31.274 & 27.882 & 33.724 \\
\hline 1/7/12 16:00 & & 30.368 & 30.016 & 29.243 & 29.804 & & 29.3 & 30.437 & 29.776 & 29.642 & 28.442 & 26.27 & 31.314 & 36.703 & 32.335 & 16.198 & & 19.54 & 15.578 & 10.176 & 31.536 & 32.213 & 29.431 & 31.277 & 27.885 & 33.721 \\
\hline 1/7/12 17:00 & & 30.37 & 30.023 & 29.255 & 29.809 & & 29.288 & 30.427 & 29.778 & 29.644 & 28.446 & 26.277 & 31.316 & 36.705 & 32.337 & 16.198 & & 19.538 & 15.571 & 10.176 & 31.539 & 32.213 & 29.438 & 31.274 & 27.882 & 33.721 \\
\hline 1/7/12 18:00 & & 30.366 & 30.018 & 29.248 & 29.807 & & 29.293 & 30.434 & 29.776 & 29.642 & 28.444 & 26.27 & 31.319 & 36.703 & 32.337 & 16.198 & & 19.584 & 15.583 & 10.179 & 31.534 & 32.215 & 29.431 & 31.277 & 27.882 & 33.721 \\
\hline 1/7/12 19:00 & & 30.37 & 30.023 & 29.262 & 29.816 & & 29.283 & 30.418 & 29.781 & 29.649 & 28.451 & 26.28 & 31.468 & 36.71 & 32.502 & $\begin{array}{l}16.198 \\
108\end{array}$ & & 19.577 & 15.571 & 10.336 & 31.609 & 32.367 & 29.447 & 31.424 & 27.882 & 33.724 \\
\hline $1 / 7 / 1220: 00$ & & 30.373 & 30.025 & 29.269 & 29.8 & & 29.276 & 30.408 & 29.786 & 29.653 & 28.453 & 26.287 & $\begin{array}{l}31.415 \\
31.400\end{array}$ & 36.715 & 32.441 & $\begin{array}{l}16.1201 \\
16.201\end{array}$ & & 19.565 & 15.574 & 10.28 & 31.595 & 32.312 & 29.456 & 31.37 & 27.885 & 33.726 \\
\hline 1/7/12 21:00 & & 30.375 & 30.032 & 29.278 & 29.83 & & 29.272 & 30.394 & 29.79 & 29.658 & 28.463 & 26.294 & 31.398 & 36.715 & 32.422 & 16.2 & & 19.551 & 15.569 & $\begin{array}{r}10.258 \\
10.258\end{array}$ & $\begin{array}{l}31.59 \\
31.59\end{array}$ & 32.296 & 29.471 & 31.352 & 27.885 & 33.731 \\
\hline $1 / 7 / 1222: 00$ & & 30.377 & 30.035 & $\begin{array}{r}29.210 \\
29.29\end{array}$ & 29.842 & & 29.267 & 30.391 & 29.79 & $\begin{array}{l}29.060 \\
29.665\end{array}$ & $\begin{array}{l}28.403 \\
28.465\end{array}$ & 26.301 & 31.391 & 36.719 & 32.413 & 16.2 & & $\begin{array}{l}19.549 \\
19.549\end{array}$ & $\begin{array}{l}15.5699 \\
15.569\end{array}$ & 10.252 & 31.595 & 32.291 & 29.475 & 31.347 & 27.887 & 33.733 \\
\hline $1 / 7 / 1223: 00$ & & $\begin{array}{l}30.382 \\
30.382\end{array}$ & 30.039 & $\begin{array}{l}29.392 \\
29.302\end{array}$ & 29.853 & & 29.265 & 30.393 & 29.793 & 29.672 & $\begin{array}{l}28.403 \\
28.47\end{array}$ & $\begin{array}{l}20.301 \\
26.311\end{array}$ & 31.391 & $\begin{array}{l}36.722 \\
36.72\end{array}$ & 32.415 & 16.2 & & $\begin{array}{l}19.049 \\
19.538\end{array}$ & $\begin{array}{l}13.5794 \\
15.574\end{array}$ & 10.252 & 31.602 & .289 & 29.482 & $\begin{array}{l}31.352 \\
31.352\end{array}$ & 27.887 & $\begin{array}{l}33.738 \\
3.738\end{array}$ \\
\hline $1 / 8 / 12$ & & 30. & 30.044 & $\begin{array}{l}29.302 \\
29.309\end{array}$ & $\begin{array}{r}29.893 \\
29.86\end{array}$ & & $\begin{array}{l}29.267 \\
29.267\end{array}$ & $\begin{array}{l}30.393 \\
30.394\end{array}$ & $\begin{array}{r}29.79 \\
29.79\end{array}$ & 29.677 & $\begin{array}{l}28.477 \\
28.477\end{array}$ & $\begin{array}{l}26.311 \\
26.315\end{array}$ & $\begin{array}{l}31.391 \\
31.389\end{array}$ & $\begin{array}{l}30.122 \\
36.726\end{array}$ & $\begin{array}{l}32.413 \\
32.408 \\
\end{array}$ & 16. & & 19 & $\begin{array}{l}13.5 / 4 \\
15.576\end{array}$ & $\begin{array}{l}10.252 \\
10.252\end{array}$ & $\begin{array}{l}31.002 \\
31.599\end{array}$ & 34 & $\begin{array}{l}29.482 \\
29.489\end{array}$ & $\begin{array}{l}31.322 \\
31.345\end{array}$ & 27.887 & $\begin{array}{r}3.138 \\
33.74\end{array}$ \\
\hline $1 / 8 / 12$ & & 30. & 30. & 29. & 29.8 & & 29 & 30 & 29.79 & 29.679 & 28.477 & 26.31 & 31 & 36.726 & 32.405 & 16. & & 15 & 15.581 & 10.242 & 31 & 2 & 29.492 & 31.342 & 27.887 & 33.74 \\
\hline 1/8/12 2: & & 30. & 30. & 29.3 & 29 & & & 30. & 29.795 & 29. & 28.481 & 26 & $\begin{array}{l}31.384 \\
31.384\end{array}$ & 36. & 32.408 & 16 & & 19.521 & $\begin{array}{l}15.581 \\
15.581\end{array}$ & 10.242 & 31. & 9 & 29.494 & 31.345 & 27.887 & 33.738 \\
\hline 1/8/12 3:00 & & 30.387 & 30.05 & 29.316 & 29.8 & & & & 29.795 & & 28.486 & 26.318 & 31.382 & 36.738 & 32.401 & 16.2 & & 19.512 & 15.583 & 10.24 & 31.604 & 32.279 & 29.496 & 31.34 & 27.887 & 33.735 \\
\hline $1 / 8 / 124: 00$ & & 30. & 30.0 & 29.309 & 29. & & 29 & & 29.793 & 29.688 & 28.484 & 26.313 & 31.375 & 36.729 & 32.396 & 16.2 & & & 15.592 & 10.235 & 31.599 & & 29.489 & 31.338 & 27.887 & 33.733 \\
\hline $1 / 8 / 125: 0$ & & & 30. & 29.304 & 29.8 & & & & 29.795 & & 28. & 26. & 31.373 & 36. & 32.3 & 16. & & 19.5 & 15.6 & 10.23 & 31. & & 29.489 & 31.333 & 27.887 & 33.728 \\
\hline $1 / 8 / 126$ & & & 30. & 29.311 & 29 & & & & 29.7 & 29. & 28. & 26. & 31.37 & 36. & 32. & 16. & & 19.4 & 15.5 & 10.233 & & 32. & 29.489 & 31.333 & 27.887 & 33.726 \\
\hline $1 / 8 / 127: \mathrm{C}$ & & 30. & $30 . c$ & 29.32 & & & 29. & & 29.8 & 29.698 & 28. & 26.3 & 31.37 & & & & & 19.4 & 15.592 & 10.233 & & & 29.506 & 31.331 & 27.889 & 33.726 \\
\hline $1 / 8 / 128: 00$ & & 30.3 & 30.0 & 29.323 & 29. & & 29.293 & 30.4 & 29.805 & 29.7 & 28.4 & 26 & 31.372 & 36.731 & 32.3 & 16.2 & & 19.4 & 15.592 & 10.235 & 31.606 & 32.272 & 29.503 & 31.333 & 27.889 & 33.726 \\
\hline 1/8/12 9:00 & & $30.3 \mathrm{~m}$ & 30.0 & 29.341 & 29.8 & & 29.283 & 30.4 & 29.814 & 29.711 & 28.5 & 26.3 & 31.382 & 36.736 & 32.401 & 16.2 & & 19.503 & 15.592 & 10.237 & 31.611 & 32.279 & 29.522 & 31.338 & 27.892 & 33.728 \\
\hline 1/8/12 10:00 & & 30.408 & 30.079 & 29.363 & 29.9 & & 29.276 & 30.413 & 29.816 & 29.725 & 28.518 & 26.354 & 31.386 & 36.738 & 32.41 & 16.2 & & 19.503 & 15.59 & 10.247 & 31.632 & 32.286 & 29.539 & 31.349 & 27.892 & 33.733 \\
\hline 1/8/12 11:00 & & 30.403 & 30.081 & 29.36 & 29.909 & & 29.29 & 30.417 & 29.812 & 29.728 & 28.518 & 26.351 & 31.394 & 36.743 & 32.41 & 16.224 & & 19.498 & 15.609 & 10.249 & 31.63 & 32.289 & 29.536 & 31.352 & 27.892 & 33.735 \\
\hline 1/8/12 12:00 & & 30.401 & 30.077 & 29.346 & 29.905 & & 29.314 & 30.439 & 29.802 & 29.725 & 28.514 & 26.339 & 31.393 & 36.745 & 32.413 & 16.229 & & 19.489 & 15.625 & 10.251 & 31.625 & 32.289 & 29.525 & 31.359 & 27.889 & 33.735 \\
\hline 1/8/12 13:00 & & 30.391 & 30.058 & 29.306 & 29.884 & & 29.347 & 30.47 & 29.793 & 29.711 & 28.495 & 26.299 & 31.384 & 36.738 & 32.405 & 16.226 & & 19.524 & 15.649 & 10.245 & 31.599 & 32.282 & 29.489 & 31.347 & 27.889 & 33.733 \\
\hline 14:00 & & 30.394 & 30.053 & 29.297 & 29.874 & & 29.352 & 30.475 & 29.797 & 29.707 & 28.493 & 26.289 & 31.38 & 36.733 & 32.403 & 16.226 & & 19.54 & 15.642 & 10.237 & 31.592 & 32.277 & 29.487 & 31.342 & 27.894 & 33.731 \\
\hline & & & 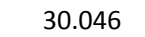 & 29 & & & & & & & & ד & 21,278 & & & ה & & & & & & & & & & \\
\hline
\end{tabular}




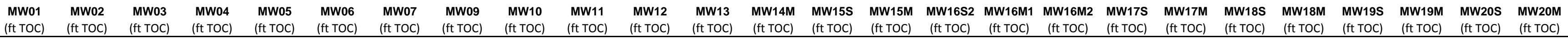

\begin{tabular}{|c|c|c|c|c|c|c|c|c|c|c|c|c|c|c|c|c|c|c|c|c|c|c|c|c|c|}
\hline Well & $\begin{array}{l}\text { (ft TOC) } \\
\text { (f) }\end{array}$ & $\begin{array}{l}\text { (ft TOC) } \\
\text { (ft) }\end{array}$ & $\begin{array}{l}\text { (ft TOC) } \\
\text { (f) }\end{array}$ & (ft TOC) & $\begin{array}{l}\text { (ft TOC) } \\
\text { (f) }\end{array}$ & $\begin{array}{l}\text { (ft ToC) } \\
\text { (f) }\end{array}$ & $\begin{array}{l}\text { (ft TOC) } \\
\text { (f) }\end{array}$ & (ft ToC) & $\begin{array}{l}\text { (ft TOC) } \\
\text { (f) }\end{array}$ & $\begin{array}{l}\text { (ft TOC) } \\
\text { (t) }\end{array}$ & $\begin{array}{l}\text { (ft TOC) } \\
\text { (n) }\end{array}$ & $\begin{array}{l}\text { (ft TOC) } \\
\text { (n) }\end{array}$ & (ft TOC) & $\begin{array}{l}\text { (ft ToC) } \\
\text { (ft }\end{array}$ & (ft TOC) & (ft TOC) (ft TOC) & (ft TOC) & $\begin{array}{l}\text { (ft TOC) } \\
\text { (ft) }\end{array}$ & (ft TOC) & $\begin{array}{l}\text { (ft TOC) } \\
\text { (1) }\end{array}$ & (ft TOC) & $\begin{array}{l}\text { (ft TOC) } \\
\text { (n) }\end{array}$ & (ft TOC) & $\begin{array}{l}\text { (nt TOC) } \\
\text { (nt }\end{array}$ & (ft TOC) \\
\hline $1 / 8 / 1216: 00$ & & 30.398 & 30.051 & 29.302 & 29.863 & & 29.34 & 30.463 & 29.805 & 29.705 & 28.491 & 26.289 & 31.373 & 36.731 & $\quad 32.394$ & 16.224 & 19.618 & 15.639 & 10.233 & 31.59 & 32.27 & 29.489 & 31.331 & 27.892 & 33.726 \\
\hline $1 / 8 / 1217: 00$ & & 30.398 & 30.053 & 29.316 & 29.867 & & 29.33 & 30.454 & 29.809 & 29.709 & 28.495 & 26.303 & 31.628 & 36.731 & 32.668 & 16.226 & 19.639 & 15.642 & 10.505 & 31.667 & 32.526 & 29.499 & 31.582 & 27.892 & 33.726 \\
\hline $1 / 8 / 1218: 00$ & & 30.403 & 30.063 & 29.332 & 29.881 & & 29.319 & 30.434 & 29.816 & 29.716 & 28.502 & 26.318 & 31.503 & 36.738 & 32.532 & 16.226 & 19.63 & 15.637 & 10.369 & 31.665 & 32.402 & 29.51 & 31.46 & 27.894 & 33.728 \\
\hline $\begin{array}{l}1 / 8 / 1219: 00 \\
\text { 1/8 }\end{array}$ & & 30.403 & 30.063 & 29.332 & 29.888 & & 29.321 & 30.432 & 29.814 & 29.716 & 28.505 & 26.318 & 31.468 & 36.743 & 32.495 & 16.229 & 19.614 & 15.639 & 10.331 & 31.648 & 32.364 & 29.515 & 31.429 & 27.894 & 33.728 \\
\hline $\begin{array}{l}1 / 8 / 1220: 00 \\
\text { 1/8 }\end{array}$ & & 30.403 & 30.065 & 29.337 & 29.893 & & 29.321 & 30.432 & 29.819 & 29.718 & 28.507 & 26.315 & 31.454 & 36.74 & 32.481 & 16.229 & 19.607 & 15.642 & 10.315 & 31.646 & 32.353 & 29.52 & 31.413 & 27.894 & 33.731 \\
\hline $1 / 8 / 1221: 00$ & & 30.405 & 30.063 & 29.332 & 29.893 & & 29.326 & 30.437 & 29.816 & 29.718 & 28.505 & 26.315 & 31.445 & 36.736 & 32.469 & 16.229 & 19.6 & 15.644 & 10.306 & 31.641 & 32.343 & 29.52 & 1.403 & 27.894 & 33.733 \\
\hline $1 / 8 / 1222: 00$ & & 30.41 & 30.07 & 29.344 & 29.9 & & 29.319 & 30.432 & 29.821 & 29.725 & 28.512 & 26.323 & 31.44 & 36.745 & 32.466 & 16.229 & 19.595 & 15.637 & 10.303 & 31.648 & 32.338 & 29.527 & 31.399 & 27.894 & 33.735 \\
\hline 1/8/12 23:00 & & 30.41 & 30.07 & 29.351 & 29.907 & & 29.319 & 30.437 & 29.819 & 29.73 & 28.514 & 26.327 & 31.443 & 36.745 & 32.464 & 16.233 & 19.593 & 15.642 & 10.303 & 31.644 & 32.338 & 29.532 & 31.401 & 27.894 & 33.74 \\
\hline 1/9/12 0:00 & & 30.408 & 30.067 & 29.339 & 29.905 & & 29.328 & 30.444 & 29.814 & 29.728 & 28.512 & 26.32 & 31.436 & 36.75 & 532.459 & 16.233 & 19.586 & 15.646 & 10.296 & 31.641 & 32.334 & 29.527 & 31.396 & 27.894 & 33.742 \\
\hline 1/9/12 1:00 & & 30.408 & 30.065 & 29.334 & 29.9 & & 29.337 & 30.444 & 29.816 & 29.725 & 28.507 & 26.311 & 31.431 & 36.745 & $5 \quad 32.455$ & 16.231 & 19.581 & 15.646 & 10.294 & 31.637 & 32.329 & 29.518 & 31.392 & 27.894 & 33.74 \\
\hline 1/9/12 2:00 & & 30.405 & 30.056 & 29.32 & 29.891 & & 29.347 & 30.449 & 29.814 & 29.718 & 28.5 & 26.299 & 31.424 & 36.738 & $3 \quad 32.445$ & 16.233 & 19.579 & 15.649 & 10.284 & 31.625 & 32.319 & 29.508 & 31.385 & 27.896 & 33.738 \\
\hline 1/9/12 3:00 & & 30.403 & 30.046 & 29.302 & 29.879 & & 29.359 & 30.458 & 29.809 & 29.709 & 28.493 & 26.282 & 31.415 & 36.738 & $3 \quad 32.441$ & 16.231 & 19.57 & 15.656 & 10.275 & 31.609 & 32.312 & 29.492 & 31.375 & 27.896 & 33.735 \\
\hline 1/9/12 4:00 & & 30.408 & 30.046 & 29.304 & 29.872 & & 29.354 & 30.449 & 29.819 & 29.707 & 28.488 & 26.275 & 31.405 & 36.731 & 32.429 & 16.231 & 19.561 & 15.646 & 10.268 & 31.604 & 32.305 & 29.489 & 31.366 & 27.896 & 33.731 \\
\hline 1/9/12 5:00 & & 30.405 & 30.049 & 29.309 & 29.874 & & 29.347 & 30.434 & 29.821 & 29.709 & 28.493 & 26.284 & 31.4 & 36.736 & 32.424 & 16.229 & 19.547 & 15.642 & 10.261 & 31.609 & 32.3 & 29.494 & 31.361 & 27.896 & 33.728 \\
\hline $1 / 9 / 126: 00$ & & 30.403 & 30.046 & 29.306 & 29.87 & & 29.347 & 30.437 & 29.821 & 29.707 & 28.488 & 26.282 & 31.398 & 36.736 & 32.42 & 16.229 & 19.561 & 15.642 & 10.259 & 31.604 & 32.296 & 29.489 & 31.356 & 27.896 & 33.726 \\
\hline 1/9/12 7:00 & & 30.41 & 30.051 & 29.32 & 29.874 & & 29.335 & 30.432 & 29.828 & 29.711 & 28.495 & 26.294 & 31.396 & 36.733 & 32.42 & 16.226 & 19.556 & 15.637 & 10.256 & 31.609 & 32.296 & 29.501 & 31.356 & 27.899 & 33.724 \\
\hline 1/9/12 8:00 & & 30.405 & 30.049 & 29.313 & 29.874 & & 29.345 & 30.439 & 29.824 & 29.709 & 28.491 & 26.291 & 31.393 & 36.733 & 32.417 & 16.229 & 19.554 & 15.642 & 10.254 & 31.606 & 32.291 & 29.494 & 31.356 & 27.899 & 33.721 \\
\hline 1/9/12 9:00 & & 30.408 & 30.051 & 29.318 & 29.874 & & 29.34 & 30.434 & 29.828 & 29.711 & 28.495 & 26.294 & 31.393 & 36.736 & 32.417 & 16.226 & 19.556 & 15.642 & 10.256 & 31.606 & 32.291 & $\begin{array}{l}29.499 \\
2999\end{array}$ & 31.356 & 27.899 & 33.724 \\
\hline 1/9/12 10:00 & & 30.405 & 30.042 & 29.306 & 29.867 & & 29.354 & 30.446 & 29.826 & 29.705 & 28.486 & 26.279 & 31.391 & 36.736 & 32.415 & $\begin{array}{l}16.224 \\
16.224\end{array}$ & 19.549 & 15.653 & 10.254 & 31.595 & 32.289 & 29.489 & 31.354 & 27.899 & $\begin{array}{l}33.724 \\
3.724\end{array}$ \\
\hline 1/9/12 11:00 & & 30.408 & 30.044 & 29.309 & $\begin{array}{r}29.87 \\
29.87\end{array}$ & & 29.354 & 30.444 & 29.824 & 29.705 & $\begin{array}{l}28.400 \\
28.488\end{array}$ & 26.284 & 31.391 & 36.734 & 32.415 & $\begin{array}{l}10.24 \\
16.226\end{array}$ & 19.542 & $\begin{array}{l}15.658 \\
15.658\end{array}$ & 10.254 & $\begin{array}{l}31.602 \\
31.602\end{array}$ & 32.291 & 29.494 & $\begin{array}{l}31.359 \\
31.359\end{array}$ & 27.899 & $\begin{array}{l}33.728 \\
3.728\end{array}$ \\
\hline $1 / 9 / 1212: 00$ & & 30.389 & 30.018 & 29.253 & 29.839 & & 29.396 & 30.494 & 29.8 & 29.679 & 28.463 & 26.229 & 31.382 & 36.724 & 32.406 & 16.222 & 19.542 & 15.693 & 10.24 & 31.564 & 32.282 & 29.445 & 31.352 & 27.899 & 33.724 \\
\hline 1/9/12 13:00 & & 30.38 & 29.995 & 29.208 & 29.804 & & 29.422 & 30.509 & 29.797 & 29.658 & 28.437 & 26.189 & 31.365 & 36.715 & 32.394 & 16.217 & 19.538 & 15.703 & 10.226 & 31.536 & 32.265 & 29.412 & 31.333 & 27.899 & 33.721 \\
\hline 1/9/12 14:00 & & 30.38 & 29.988 & 29.199 & 29.788 & & 29.415 & 30.509 & 29.8 & $\begin{array}{l}29.649 \\
29.69\end{array}$ & 28.428 & 26.181 & 31.358 & 36.701 & 32.382 & 16.212 & .531 & 15.698 & 10.221 & 31.525 & 255 & 29.398 & 31.319 & 27.901 & .719 \\
\hline 1/9/12 15:00 & & 30.377 & 29.983 & 29.201 & 29.781 & & 29.401 & 30.501 & 29.802 & 29.642 & 28.423 & 26.186 & 31.351 & 36.703 & 32.373 & 16.21 & 19.521 & 15.689 & 10.209 & 31.522 & 32.246 & 29.398 & 31.312 & 27.899 & 33.717 \\
\hline 1/9/12 16:00 & & 30.384 & 29.988 & 29.215 & 29.783 & & 29.377 & 30.487 & 29.814 & 29.644 & 28.425 & 26.201 & 31.347 & 36.703 & 32.37 & 16.21 & 19.57 & 15.684 & 10.209 & 31.529 & 246 & 29.409 & 31.309 & 27.899 & 33.714 \\
\hline 1/9/12 17:00 & & 30.387 & 29.99 & 29.229 & 29.788 & & 29.354 & 30.473 & 29.819 & 29.646 & 28.43 & 26.217 & 31.347 & 36.701 & 32.37 & 16.203 & 19.568 & 15.672 & 10.205 & 31.536 & 32.244 & 29.421 & 31.305 & 27.901 & 33.712 \\
\hline 1/9/12 18:00 & & 30.387 & 29.993 & 29.241 & 29.795 & & 29.34 & 30.465 & 29.819 & 29.649 & 28.432 & 26.229 & 31.344 & 36.701 & 32.368 & 16.203 & 19.556 & 15.663 & 10.205 & 31.546 & 32.244 & 29.421 & 31.302 & 27.901 & 33.712 \\
\hline 1/9/12 19:00 & & 30.382 & 29.993 & 29.236 & 29.797 & & 29.337 & 30.466 & 29.816 & 29.646 & 28.428 & 26.227 & 31.347 & 36.705 & 32.363 & 16.201 & 19.549 & 15.668 & 10.204 & 31.536 & 32.244 & 29.428 & 31.305 & 27.901 & 33.712 \\
\hline 1/9/12 20:00 & & 30.377 & 29.983 & 29.222 & 29.79 & & 29.342 & 30.472 & 29.816 & 29.637 & 28.421 & 26.215 & 31.342 & 36.703 & 32.366 & 16.196 & 19.542 & 15.663 & 10.205 & 31.529 & 32.239 & 29.416 & 31.305 & 27.901 & 33.71 \\
\hline 1/9/12 21:00 & & 30.37 & 29.972 & 29.197 & 29.774 & & 29.356 & 30.492 & 29.809 & 29.626 & 28.407 & 26.193 & 31.335 & 36.698 & 32.363 & 16.194 & 19.533 & 15.668 & 10.197 & 31.515 & 32.232 & 29.398 & 31.298 & 27.901 & 33.71 \\
\hline 1/9/12 22:00 & & 30.363 & 29.958 & 29.171 & 29.758 & & 29.366 & 30.494 & 29.805 & 29.609 & 28.393 & 26.165 & 31.33 & 36.691 & 32.354 & 16.189 & 19.528 & 15.667 & 10.19 & 31.501 & 32.225 & 29.372 & 31.288 & 27.901 & 33.71 \\
\hline 1/9/12 23:00 & & 30.363 & 29.951 & 29.171 & 29.748 & & 29.352 & 30.48 & 29.809 & 29.602 & 28.388 & 26.165 & 31.323 & 36.689 & 32.349 & 16.187 & 19.526 & 15.651 & 10.188 & 31.501 & 32.222 & 29.37 & 31.284 & 27.903 & 33.712 \\
\hline $1 / 10 / 120: 00$ & & 30.368 & 29.958 & 29.185 & 29.753 & & 29.328 & 30.456 & 29.816 & 29.605 & 28.393 & 26.186 & 31.326 & 36.689 & 32.349 & 16.184 & 19.526 & 15.632 & 10.188 & 31.506 & 32.222 & 29.388 & 31.286 & 27.903 & 33.714 \\
\hline 1/10/12 1:00 & & 30.366 & 29.955 & 29.19 & 29.755 & & 29.316 & 30.446 & 29.819 & 29.602 & 28.391 & 26.189 & 31.326 & 36.687 & 32.347 & 16.182 & 19.524 & 15.627 & 10.186 & 31.511 & 32.222 & 29.386 & 31.284 & 27.903 & 33.714 \\
\hline $1 / 10 / 122: 00$ & & 30.359 & 29.948 & 29.178 & 29.748 & & 29.323 & 30.454 & 29.812 & 29.595 & 28.384 & 26.181 & 31.321 & 36.689 & 32.347 & 16.18 & 19.519 & 15.63 & 10.181 & 31.497 & 32.215 & 29.377 & 31.279 & 27.903 & 33.714 \\
\hline 1/10/12 3:00 & & 30.359 & 29.946 & 29.176 & 29.746 & & 29.316 & 30.449 & 29.812 & 29.591 & 28.381 & 26.177 & 31.314 & 36.677 & 32.338 & 16.175 & 19.51 & 15.62 & 10.176 & 31.499 & 32.213 & 29.374 & 31.274 & 27.906 & 33.712 \\
\hline 1/10/12 4:00 & & 30.356 & 29.944 & 29.171 & 29.744 & & 29.312 & 30.441 & 29.814 & 29.588 & 28.377 & 26.179 & 31.307 & 36.684 & 32.335 & 16.175 & 19.503 & $\begin{array}{l}15.618 \\
15.618\end{array}$ & 10.172 & 31.492 & 32.206 & 29.372 & 31.27 & 27.901 & $\begin{array}{l}33.71 \\
33.71\end{array}$ \\
\hline 1/10/12 5:00 & & 30.352 & 29.934 & 29.159 & 29.737 & & 29.319 & 30.451 & 29.809 & $\begin{array}{l}29.500 \\
2979\end{array}$ & 28.37 & 26.165 & 31.302 & 36.682 & 32.326 & 16.17 & 19.491 & $\begin{array}{l}15.616 \\
15.616\end{array}$ & 10.164 & $\begin{aligned} 31.48 & \\
31.48 & \end{aligned}$ & $\begin{array}{l}32.199 \\
32\end{array}$ & 29.358 & 31.265 & 27.901 & 33.702 \\
\hline 1/10/12 6:00 & & 30.347 & 29.932 & 29.152 & 29.73 & & 29.314 & 30.451 & 29.809 & 29.572 & $\begin{array}{l}28.363 \\
28.363\end{array}$ & 26.162 & $\begin{array}{l}31.295 \\
31.295\end{array}$ & $\begin{array}{l}36.672 \\
36.672\end{array}$ & 32.319 & 16.1 & 19.498 & $\begin{array}{l}15.010 \\
15.613\end{array}$ & 10.153 & $\begin{array}{l}31.40 \\
31.476\end{array}$ & .189 & 29.355 & 31.255 & 27.901 & $\begin{array}{l}33.698 \\
3.698\end{array}$ \\
\hline $1 / 1$ & & 30 & 29 & 29. & 29. & & 29 & 30 & 29. & 29. & 28.36 & 26 & 31 & 36.67 & 32.314 & 16 & 4 & 15.607 & 10.148 & 31.476 & 9 & 29.355 & 31.251 & 27.903 & 33.695 \\
\hline $1 / 10 / 128: 00$ & & 30. & 29. & 29. & 29. & & 29 & 30. & 29.8 & 29. & 28.358 & 26 & 31.286 & 36.675 & 32.314 & 16. & 7 & 15.604 & 10.146 & 31.473 & 32.187 & 29.351 & 31.251 & 27.903 & 33.693 \\
\hline $1 / 10 / 129: 00$ & & 30.335 & 29.918 & 29.134 & 29.711 & & 29.312 & 30.446 & 29.807 & 29.554 & 28.349 & 26.148 & 31.281 & 36.672 & 32.305 & 16. & .501 & 15.607 & 10.141 & 31.457 & 32.18 & 29.337 & 31.241 & 27.903 & 33.691 \\
\hline $1 / 10 / 1210: 00$ & & 30.34 & 29.916 & 29.131 & 29.7 & & 29.305 & 30.439 & 29.812 & 29.551 & 28.344 & 26.148 & 31.281 & 36.672 & 32.302 & 16.156 & 19.494 & 15.602 & 10.141 & 31.457 & 32.175 & 29.339 & 31.241 & 27.906 & 33.691 \\
\hline 1/10/12 11:00 & & 30.338 & 29.918 & 29.145 & 29. & & 29.293 & 30.4 & 29.814 & 29 & 28.349 & 26.165 & 31.281 & 36.668 & 32.305 & 16.1 & 1 & 15.602 & 10.141 & 31.464 & 32.177 & 29.344 & 31.246 & 27.906 & 33.693 \\
\hline $1 / 10 / 1212: 00$ & & 30.326 & 29.904 & 29.11 & 29. & & 29.316 & & 29.805 & & 28.3 & & 31.274 & 36.668 & 32.298 & 16.1 & 19.482 & 15.623 & 10.136 & 31.443 & 32.17 & 29.32 & 31.241 & 27.906 & 33.693 \\
\hline $1 / 10 / 1213: 00$ & & 30.317 & 29.883 & 29.077 & 29.6 & & 29.333 & 30. & 29.795 & 29 . & 28.3 & 26. & 31.263 & 36.665 & 32.288 & 16.1 & 19.473 & 15.63 & 10.125 & 31.422 & 32.161 & 29.29 & 31.232 & 27.903 & 33.691 \\
\hline $1 / 10 / 1214: 00$ & & 30.31 & 29.864 & 29.047 & 29. & & 29.342 & 30.4 & 29.793 & 29.498 & 28.293 & 26.074 & 31.248 & 36.654 & 32.274 & 16.1 & 19.466 & 15.639 & 10.115 & 31.396 & 32.149 & 29.266 & 31.218 & 27.906 & 33.688 \\
\hline $1 / 10 / 1215: 00$ & & 30.305 & 29.85 & 29.028 & 29.6. & & 29.342 & 30.4 & 29.788 & 29.484 & 28.279 & 26.059 & 31.239 & 36.644 & 32.265 & 16.13 & 19.454 & 15.632 & 10.101 & 31.385 & 32.137 & 29.25 & 31.204 & 27.906 & 33.684 \\
\hline $1 / 10 / 1216: 00$ & & 30.3 & 29.836 & 29.014 & 29.611 & & 29.335 & 30.422 & 29.786 & 29.468 & 28.265 & 26.048 & 31.227 & 36.642 & 32.251 & 16.131 & 19.447 & 15.628 & 10.089 & 31.371 & 32.125 & 29.233 & 31.19 & 27.906 & 33.681 \\
\hline $1 / 10 / 1217: 00$ & & 30.305 & 29.834 & 29.021 & 29.604 & & 29.312 & 30.415 & 29.793 & 29.463 & 28.263 & 26.055 & 31.22 & 36.63 & 32.246 & 16.126 & 19.438 & 15.614 & 10.082 & 31.368 & 32.116 & 29.233 & 31.178 & 27.906 & 33.674 \\
\hline $1 / 10 / 1218: 00$ & & 30.305 & 29.834 & 29.033 & 29.606 & & 29.286 & 30.417 & 29.783 & 29.463 & 28.265 & 26.071 & 31.213 & 36.635 & 32.241 & 16.122 & 19.44 & 15.604 & 10.075 & 31.371 & 32.113 & 29.238 & 31.176 & 27.906 & 33.672 \\
\hline 1/10/12 19:00 & & 30.298 & 29.827 & 29.019 & 29.599 & & 29.279 & 30.425 & 29.783 & 29.454 & 28.256 & 26.067 & 31.209 & 36.633 & 32.232 & 16.119 & 19.433 & 15.595 & 10.073 & 31.366 & 32.104 & 29.231 & 31.169 & 27.906 & 33.67 \\
\hline $1 / 10 / 1220: 00$ & & 30.296 & 29.818 & 29.003 & 29.587 & & 29.274 & 30.415 & 29.783 & 29.44 & 28.244 & 26.055 & 31.202 & 36.628 & 32.227 & 16.115 & 19.433 & 15.592 & 10.064 & 31.354 & 32.097 & 29.221 & 31.164 & 27.906 & 33.667 \\
\hline 1/10/12 21:00 & & 30.293 & 29.813 & 29.005 & 29.585 & & 29.262 & 30.408 & 29.783 & 29.435 & 28.239 & 26.057 & 31.197 & 36.621 & 32.223 & 16. & 19.431 & 15.578 & 10.061 & 31.352 & 32.097 & 29.221 & 31.162 & 27.906 & 33.667 \\
\hline $1 / 10 / 1222: 00$ & & 30.291 & 29.808 & 29.007 & 29.585 & & 29.251 & 30.408 & 29.781 & 29.431 & 28.235 & 26.059 & 31.197 & 36.621 & 32.22 & 16.105 & 19.429 & 15.569 & 10.061 & 31.35 & 32.097 & 29.224 & 31.157 & 27.908 & 33.67 \\
\hline $1 / 10 / 1223: 00$ & & 30.291 & 29.804 & 29.003 & 29.58 & & 29.243 & 30.403 & 29.778 & 29.421 & 28.23 & 26.055 & 31.195 & 36.621 & 32.22 & 16.101 & 19.427 & 15.566 & 10.054 & 31.347 & 32.092 & 29.214 & 31.157 & 27.908 & 33.67 \\
\hline
\end{tabular}




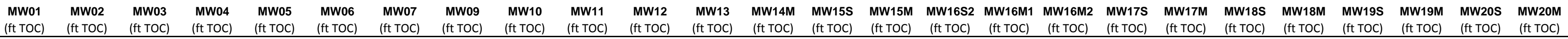

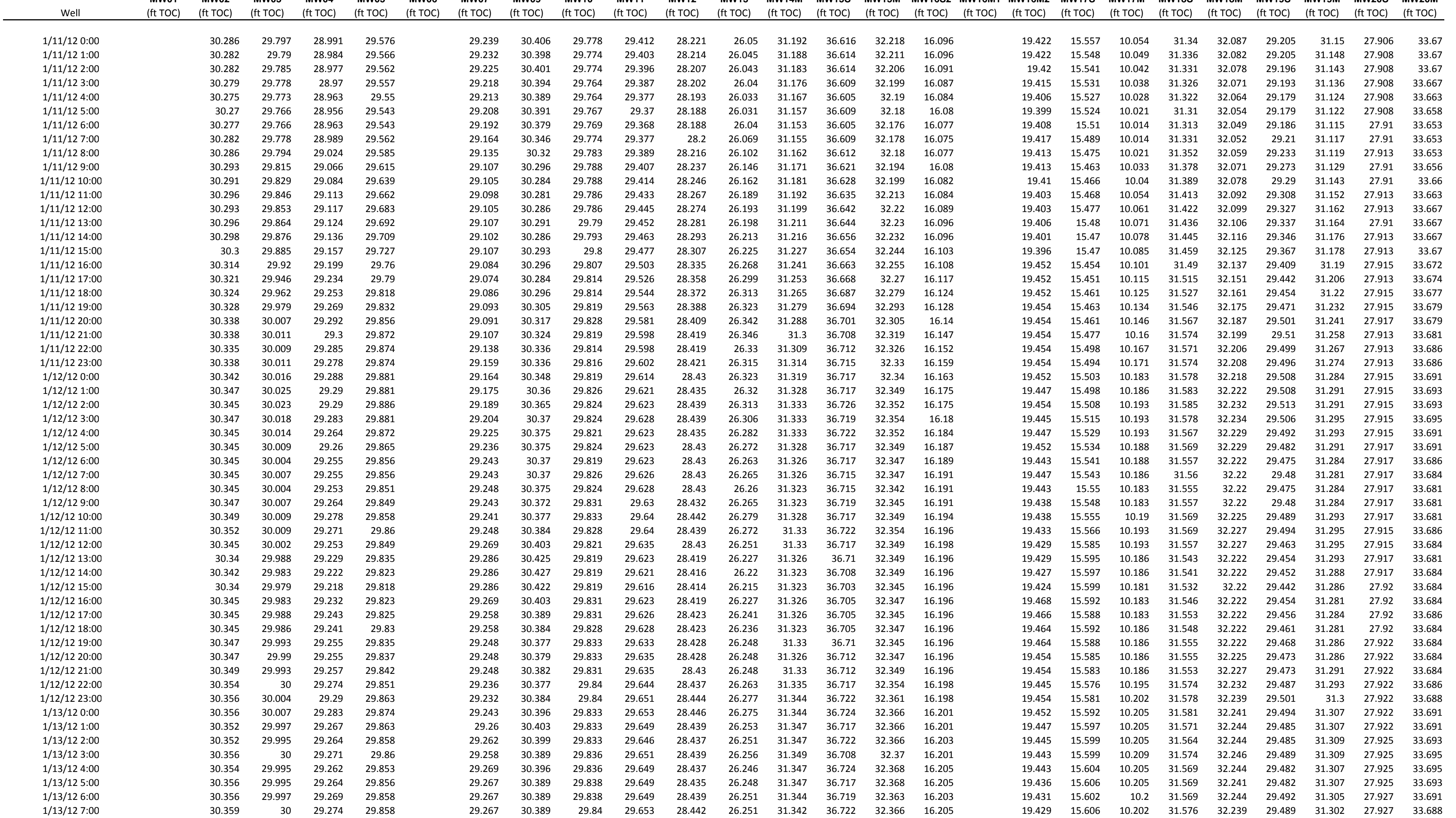




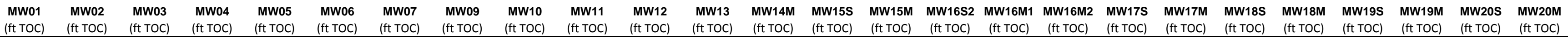

\begin{tabular}{|c|c|c|c|c|c|c|c|c|c|c|c|c|c|c|c|c|c|c|c|c|c|c|c|c|c|c|}
\hline Well & $\begin{array}{l}\text { (ft TOC) } \\
\text { (f) }\end{array}$ & $\begin{array}{l}\text { (ft TOC) } \\
\text { (f) }\end{array}$ & $\begin{array}{l}\text { (ft TOC) } \\
\text { (f) }\end{array}$ & (ft TOC) & (ft TOC) & $\begin{array}{l}\text { (ft ToC) } \\
\text { (f) }\end{array}$ & (ft ToC) & (ft ToC) & $\begin{array}{l}\text { (ft TOC) } \\
\text { (f) }\end{array}$ & $\begin{array}{l}\text { (ft TOC) } \\
\text { (t) }\end{array}$ & $\begin{array}{l}\text { (ft TOC) } \\
\text { (n) }\end{array}$ & $\begin{array}{l}\text { (ft TOC) } \\
\text { (f) }\end{array}$ & (ft TOC) & $\begin{array}{l}\text { (ft ToC) } \\
\text { (ft }\end{array}$ & (ft TOC) & (ft TOC) & (ft TOC) & (ft TOC) & $\begin{array}{l}\text { (ft TOC) } \\
\text { (f) }\end{array}$ & (ft TOC) & $\begin{array}{l}\text { (ft ToC) } \\
\text { (ft }\end{array}$ & (ft TOC) & $\begin{array}{l}\text { (ft TOC) } \\
\end{array}$ & (ft TOC) & $\begin{array}{l}\text { (nt TOC) } \\
\text { (nt }\end{array}$ & (ft TOC) \\
\hline $1 / 13 / 128: 00$ & & 30.356 & 29.995 & 29.267 & 29.853 & & 29.272 & 30.399 & $29.838 \mathrm{C}$ & 29.651 & 28.439 & 26.248 & 31.342 & 36.715 & $5 \quad 32.363$ & 16.203 & & 19.431 & 15.613 & 10.2 & 31.569 & 32.239 & 29.482 & 31.3 & 27.927 & 33.686 \\
\hline $1 / 13 / 129: 00$ & & 30.361 & 30.004 & 29.283 & 29.863 & & 29.262 & 30.394 & 29.847 & 29.658 & 28.446 & 26.263 & 31.347 & 36.722 & 32.368 & 16.203 & & 19.45 & 15.609 & 10.207 & 31.576 & 32.244 & 29.496 & 31.307 & 27.927 & 33.686 \\
\hline 1/13/12 10:00 & & 30.363 & 30.007 & 29.288 & 29.87 & & 29.265 & 30.399 & 29.845 & 29.66 & 28.451 & 26.265 & 31.349 & 36.724 & 32.368 & 16.205 & & 19.461 & 15.613 & 10.207 & 31.578 & 32.246 & 29.506 & 31.309 & 27.927 & 33.686 \\
\hline 1/13/12 11:00 & & 30.366 & 30.007 & 29.29 & 29.872 & & 29.267 & 30.399 & 29.843 & 29.663 & 28.451 & 26.268 & 31.354 & 36.726 & 32.37 & 16.208 & & 19.454 & 15.62 & 10.209 & 31.585 & 32.251 & 29.506 & 31.314 & 27.929 & 33.686 \\
\hline 1/13/12 12:00 & & 30.354 & 29.99 & 29.253 & 29.853 & & 29.302 & 30.434 & 29.828 & 29.649 & 28.437 & 26.232 & 31.349 & 36.722 & 32.368 & 16.205 & & 19.445 & 15.649 & 10.205 & 31.564 & 32.244 & & 31.312 & & 33.684 \\
\hline 1/13/12 13:00 & & 30.349 & 29.976 & 29.227 & 29.835 & & 29.321 & 30.451 & 29.826 & 29.637 & 28.421 & 26.201 & 31.34 & 36.705 & 32.363 & 16.203 & & 19.452 & 15.651 & 10.2 & 31.546 & 32.237 & .449 & 1.305 & 27.929 & 33.684 \\
\hline $1 / 13 / 1214: 00$ & & 30.347 & 29.969 & 29.215 & 29.821 & & 29.323 & 30.451 & 29.828 & 29.628 & 28.414 & 26.191 & 31.337 & 36.705 & 32.359 & 16.201 & & 19.466 & 15.656 & 10.195 & 31.539 & 32.234 & 29.442 & 31.302 & 27.929 & 33.684 \\
\hline $1 / 13 / 1215: 00$ & & 30.347 & 29.965 & 29.213 & 29.811 & & 29.316 & 30.449 & 29.831 & 29.626 & 28.409 & 26.193 & 31.335 & 36.703 & $3 \quad 32.359$ & 16.201 & & 19.459 & 15.651 & 10.195 & 31.536 & 32.232 & 29.44 & 31.298 & 27.932 & 33.684 \\
\hline 1/13/12 16:00 & & 30.347 & 29.965 & 29.211 & 29.809 & & 29.309 & 30.442 & 29.831 & 29.621 & 28.407 & 26.191 & 31.33 & 36.701 & 32.354 & 16.198 & & 19.507 & 15.646 & 10.19 & 31.534 & 32.227 & 29.438 & 31.293 & 27.929 & 33.681 \\
\hline 1/13/12 17:00 & & 30.349 & 29.965 & 29.225 & 29.814 & & 29.291 & 30.427 & 29.838 & 29.623 & 28.412 & 26.208 & 31.33 & 36.701 & 32.354 & 16.196 & & 19.507 & 15.637 & 10.195 & 31.546 & 32.229 & 29.449 & 31.293 & 27.932 & 33.684 \\
\hline $1 / 13 / 12$ 18:00 & & 30.347 & 29.965 & 29.225 & 29.816 & & 29.286 & 30.427 & 29.836 & 29.623 & 28.409 & 26.208 & 31.33 & 36.701 & 32.354 & 16.194 & & 19.496 & 15.635 & 10.19 & 31.541 & 32.229 & 29.445 & 31.288 & 27.929 & 33.679 \\
\hline 1/13/12 19:00 & & 30.345 & 29.962 & 29.22 & 29.811 & & 29.286 & 30.425 & 29.833 & 29.619 & 28.405 & 26.203 & 31.328 & 36.705 & 32.352 & 16.194 & & 19.477 & 15.635 & 10.186 & 31.534 & 32.225 & 29.447 & 31.284 & 27.932 & 33.677 \\
\hline $1 / 13 / 1220: 00$ & & 30.34 & 29.955 & 29.206 & 29.807 & & 29.291 & 30.43 & 29.828 & 29.612 & 28.4 & 26.191 & 31.323 & 36.694 & 32.345 & 16.191 & & 19.477 & 15.639 & 10.183 & 31.529 & 32.22 & 29.433 & 31.284 & 27.929 & 33.674 \\
\hline $1 / 13 / 1221: 00$ & & 30.342 & 29.953 & 29.204 & 29.802 & & 29.283 & 30.422 & 29.833 & 29.609 & 28.395 & 26.186 & 31.321 & 36.696 & 32.34 & 16.189 & & 19.473 & 15.627 & 10.179 & 31.525 & 32.218 & 29.431 & 31.281 & 27.929 & 33.672 \\
\hline $1 / 13 / 1222: 00$ & & 30.34 & 29.951 & 29.199 & 29.797 & & 29.281 & 30.415 & 29.828 & 29.605 & 28.393 & 26.186 & 31.316 & 36.694 & 32.337 & 16.187 & & 19.464 & 15.625 & 10.176 & 31.52 & 32.215 & 29.431 & 31.277 & 27.932 & 33.67 \\
\hline $1 / 13 / 1223: 00$ & & 30.335 & 29.941 & 29.18 & 29.786 & & 29.291 & 30.427 & 29.826 & 29.593 & 28.384 & 26.165 & 31.314 & 36.689 & 32.337 & 16.184 & & 19.459 & 15.632 & 10.174 & 31.513 & 32.21 & 29.414 & 31.277 & 27.932 & 33.67 \\
\hline $1 / 14 / 120: 00$ & & 30.333 & 29.937 & 29.176 & 29.781 & & 29.286 & 30.425 & 29.828 & 29.591 & 28.379 & 26.165 & 31.312 & 36.689 & 32.33 & 16.182 & & 19.452 & 15.632 & 10.172 & 31.506 & 32.206 & 29.412 & 31.272 & 27.932 & 33.67 \\
\hline 1/14/12 1:00 & & 30.333 & 29.937 & 29.18 & 29.779 & & 29.276 & 30.413 & 29.831 & 29.586 & 28.379 & 26.167 & 31.309 & 36.684 & 32.333 & 16.18 & & $\begin{array}{l}19.447 \\
\end{array}$ & 15.618 & 10.172 & 31.504 & 32.206 & 29.412 & 31.272 & 27.932 & 33.672 \\
\hline $1 / 14 / 122: 00$ & & 30.338 & 29.944 & 29.199 & 29.788 & & 29.258 & 30.391 & 29.838 & 29.593 & 28.386 & 26.189 & 31.316 & 36.687 & 32.335 & 16.18 & & $\begin{array}{l}19.447 \\
19.443\end{array}$ & $\begin{array}{l}15.609 \\
15.609\end{array}$ & 10.172 & 31.522 & 32.21 & 29.428 & 31.274 & 27.934 & $\begin{array}{l}33.672 \\
3.672\end{array}$ \\
\hline $1 / 14 / 123: 00$ & & $\begin{array}{r}30.350 \\
30.34\end{array}$ & 29.951 & 29.218 & 29.802 & & 29.246 & 30.372 & 29.838 & 29.598 & $\begin{array}{l}28.390 \\
28.395\end{array}$ & 26.203 & $\begin{array}{l}31.319 \\
31.319\end{array}$ & $\begin{array}{l}36.691 \\
36.691\end{array}$ & 32.34 & $\begin{array}{l}16.10 \\
16.182\end{array}$ & & 19.438 & 15.604 & 10.179 & $\begin{array}{l}31.536 \\
31.536\end{array}$ & 32.218 & 29.447 & 31.279 & $\begin{array}{l}27.932 \\
27.932\end{array}$ & $\begin{array}{l}33.674 \\
3.674\end{array}$ \\
\hline $1 / 14 / 124: 00$ & & $\begin{array}{r}30.344 \\
30.345\end{array}$ & 29.96 & 29.239 & 29.818 & & 29.232 & $\begin{array}{l}30.363 \\
30.363\end{array}$ & 29.845 & 29.609 & 28.402 & $\begin{array}{l}26.203 \\
26.227\end{array}$ & 31.323 & $\begin{array}{l}36.091 \\
36.698\end{array}$ & $\begin{array}{r}32.34 \\
32.347\end{array}$ & $\begin{array}{l}10.182 \\
16.182\end{array}$ & & $\begin{array}{l}19.436 \\
19.436\end{array}$ & $\begin{array}{l}13.004 \\
15.602\end{array}$ & 10.183 & 31.546 & $\begin{array}{l}32.222 \\
32.22\end{array}$ & 29.468 & 31.284 & 27.934 & $\begin{array}{l}33.074 \\
33.679\end{array}$ \\
\hline $1 / 14 / 125: 00$ & & 30.349 & 29.969 & 29.26 & 29.835 & & 29.222 & 30.367 & 29.847 & 29.619 & 28.414 & 26.246 & 31.33 & 36.703 & 32.352 & 16.184 & & 19.431 & 15.592 & 10.19 & 31.564 & 32.229 & 29.482 & 31.288 & 27.934 & 33.677 \\
\hline $1 / 14 / 126: 00$ & & 30.356 & 29.988 & 29.292 & 29.86 & & 29.204 & 30.372 & 29.855 & 29.635 & 28.43 & 26.277 & 31.34 & 36.715 & 32.363 & $\begin{array}{l}10.104 \\
16.187\end{array}$ & & $\begin{array}{l}19.431 \\
19.433\end{array}$ & 15.583 & 10.2 & 31.583 & .237 & 29.515 & 31.298 & 27.934 & $\begin{array}{l}33.679 \\
3.679\end{array}$ \\
\hline $1 / 14 / 127: 00$ & & 30.363 & 30.007 & 29.323 & $\begin{array}{r}29.800 \\
29.886\end{array}$ & & 29.196 & 30.379 & 29.859 & 29.656 & $\begin{array}{l}28.43 \\
28.449\end{array}$ & 26.304 & $\begin{array}{r}31.34 \\
31.351\end{array}$ & 36.722 & 32.37 & $\begin{array}{l}16.191 \\
161\end{array}$ & & $\begin{array}{l}19.438 \\
19.438\end{array}$ & $\begin{array}{l}15.583 \\
15.583\end{array}$ & $\begin{array}{r}10.209 \\
\end{array}$ & 31.602 & 248 & 29.534 & 31.307 & $\begin{array}{l}27.936 \\
27.936\end{array}$ & .681 \\
\hline 1/14/12 8:00 & & 30.375 & 30.03 & 29.36 & 29.919 & & 29.18 & 30.389 & 29.866 & 29.677 & & 26.337 & 31.361 & 36.733 & 32.384 & 16.196 & & .433 & 15.576 & 10.221 & 31.632 & 32.26 & .569 & 31.324 & 27.936 & 33.681 \\
\hline 1/14/12 9:00 & & 30.384 & 30.058 & 29.391 & 29.949 & & 29.175 & 30.398 & 29.874 & 29.7 & 28.491 & 26.366 & 31.377 & 36.738 & 32.394 & 16.203 & & .429 & 15.578 & 10.235 & 31.651 & 32.272 & .597 & 31.335 & 27.936 & 33.684 \\
\hline 1/14/12 10:00 & & 30.389 & 30.074 & 29.409 & 29.97 & & 29.187 & 30.408 & 29.874 & 29.716 & 28.507 & 26.38 & 31.389 & 36.745 & 32.406 & 16.205 & & 19.431 & 15.59 & 10.245 & 31.669 & 32.289 & 29.619 & 31.349 & 27.936 & 33.686 \\
\hline 1/14/12 11:00 & & 30.387 & 30.074 & 29.395 & 29.977 & & 29.222 & 30.415 & 29.862 & 29.718 & 28.507 & 26.366 & 31.396 & 36.748 & 32.417 & 16.212 & & 19.436 & 15.611 & 10.251 & 31.665 & 32.291 & 29.612 & 31.354 & 27.936 & 33.686 \\
\hline 1/14/12 12:00 & & 30.384 & 30.072 & 29.381 & 29.975 & & 29.251 & 30.432 & 29.852 & 29.718 & 28.505 & 26.349 & 31.401 & 36.752 & 32.417 & 16.217 & & 19.433 & 15.63 & 10.254 & 31.658 & 32.298 & 29.597 & 31.366 & 27.936 & 33.688 \\
\hline $1 / 14 / 1213: 00$ & & 30.382 & 30.063 & 29.36 & 29.965 & & 29.281 & 30.451 & 29.855 & 29.714 & 28.502 & 26.325 & 31.401 & 36.748 & 32.42 & 16.222 & & 19.429 & 15.646 & 10.254 & 31.653 & 32.298 & 29.59 & 31.363 & 27.936 & 33.691 \\
\hline $1 / 14 / 1214: 00$ & & 30.38 & 30.053 & 29.335 & 29.949 & & 29.309 & 30.478 & 29.847 & 29.707 & 28.491 & 26.299 & 31.398 & 36.752 & 32.417 & 16.222 & & 19.427 & 15.663 & 10.256 & 31.644 & 32.296 & 29.569 & 31.363 & 27.939 & 33.688 \\
\hline $1 / 14 / 1215: 00$ & & 30.38 & 30.049 & 29.325 & 29.94 & & 29.319 & 30.478 & 29.85 & 29.702 & 28.486 & 26.287 & 31.398 & 36.74 & 32.417 & 16.226 & & 19.433 & 15.665 & 10.254 & 31.63 & 32.293 & 29.557 & 31.359 & 27.939 & 33.691 \\
\hline 1/14/12 16:00 & & 30.382 & 30.044 & 29.316 & 29.928 & & 29.326 & 30.478 & 29.852 & 29.698 & 28.481 & 26.275 & 31.398 & 36.745 & 32.417 & 16.226 & & 19.445 & 15.674 & 10.254 & 31.627 & 32.293 & 29.553 & 31.356 & 27.939 & 33.693 \\
\hline $1 / 14 / 1217: 00$ & & 30.384 & 30.046 & 29.325 & 29.928 & & 29.319 & 30.466 & 29.857 & 29.702 & 28.484 & 26.279 & 31.398 & 36.74 & 32.42 & 16.229 & & 19.464 & 15.67 & 10.254 & 31.634 & 32.296 & 29.555 & 31.356 & 27.941 & 33.691 \\
\hline 1/14/12 18:00 & & 30.384 & 30.046 & 29.33 & 29.93 & & 29.316 & 30.458 & 29.859 & 29.705 & 28.486 & 26.284 & 31.398 & 36.74 & 32.422 & 16.231 & & 19.487 & 15.667 & 10.259 & 31.634 & 32.296 & 29.56 & 31.356 & 27.939 & 33.691 \\
\hline 1/14/12 19:00 & & 30.387 & 30.046 & 29.33 & 29.93 & & 29.314 & 30.449 & 29.855 & 29.705 & 28.486 & 26.279 & 31.401 & 36.748 & 32.42 & 16.231 & & 19.51 & 15.67 & 10.258 & 31.634 & 32.298 & 29.557 & 31.356 & 27.939 & 33.688 \\
\hline $1 / 14 / 1220: 00$ & & 30.387 & $\begin{array}{l}30.046 \\
30.040\end{array}$ & 29.325 & 29.928 & & 29.319 & $\begin{array}{l}30.449 \\
30.449\end{array}$ & 29.857 & 29.702 & $\begin{array}{l}28.480 \\
28.484\end{array}$ & 26.277 & 31.401 & 36.74 & 32.42 & $\begin{array}{l}10.233 \\
16.233\end{array}$ & & 19.533 & 15.672 & $\begin{array}{l}10.259 \\
10.259\end{array}$ & 31.627 & 32.298 & 29.557 & 31.359 & 27.939 & $\begin{array}{l}33.088 \\
33.68\end{array}$ \\
\hline 1/14/12 21:00 & & 30.384 & 30.037 & 29.314 & 29.91 & & 29.326 & 30.451 & 29.857 & 29.698 & $\begin{array}{l}28.477 \\
28.477\end{array}$ & 26.263 & 31.398 & 36.74 & 32.417 & 16.2 & & 19.549 & 15.679 & 10.256 & 31.625 & 32.293 & 29.543 & 31.359 & 27.939 & $\begin{array}{l}33.686 \\
3.686\end{array}$ \\
\hline $1 / 14 / 1222: 00$ & & $\begin{array}{l}30.304 \\
30.38\end{array}$ & 30.028 & 29.295 & 29.90 & & 29.34 & $\begin{array}{l}30.461 \\
30.463\end{array}$ & 29.847 & 29.688 & $\begin{array}{l}28.467 \\
28.467\end{array}$ & 26.246 & $\begin{array}{l}31.390 \\
31.396\end{array}$ & $\begin{array}{l}36.738 \\
36.738\end{array}$ & 32.415 & 16. & & $\begin{array}{l}19.549 \\
19.565\end{array}$ & 15.684 & $\begin{array}{l}10.250 \\
10.254\end{array}$ & 611 & 291 & 29.525 & 31.354 & 27.939 & $\begin{array}{l}33.080 \\
3.684\end{array}$ \\
\hline $1 / 14 / 1223: 00$ & & $\begin{array}{l}30.38 \\
30.38\end{array}$ & 30.021 & 29.285 & 29.89 & & $\begin{array}{l}29.344 \\
2932\end{array}$ & $\begin{array}{l}30.403 \\
30.463\end{array}$ & $\begin{aligned} 29.841 \\
29.85\end{aligned}$ & 29.684 & $\begin{array}{r}20.401 \\
28.46\end{array}$ & $\begin{array}{l}20.240 \\
26.237\end{array}$ & 31.389 & $\begin{array}{l}36.729 \\
36.750\end{array}$ & $\begin{array}{r}52.413 \\
32.41\end{array}$ & 16. & & $\begin{array}{l}19.003 \\
19.591\end{array}$ & $\begin{array}{l}13.044 \\
15.681\end{array}$ & 10.247 & 31.604 & 286 & 29.518 & $\begin{array}{l}31.352 \\
31.352\end{array}$ & 27.941 & $\begin{array}{l}33.084 \\
33.681\end{array}$ \\
\hline $1 / 15 / 120: 00$ & & $\begin{array}{c}30 . \\
30.3 y-1\end{array}$ & $\begin{array}{l}30.021 \\
30.009\end{array}$ & $\begin{array}{l}29.280 \\
29.262\end{array}$ & 29.8 & & $\begin{array}{l}29.342 \\
29.354\end{array}$ & $\begin{array}{l}30.463 \\
30.473\end{array}$ & $\begin{array}{l}29.845 \\
29.845\end{array}$ & $\begin{array}{r}29.084 \\
29.67\end{array}$ & $\begin{array}{l}28.40 \\
28.449\end{array}$ & $\begin{array}{l}26.231 \\
26.213\end{array}$ & $\begin{array}{l}31.389 \\
31.384\end{array}$ & $\begin{array}{l}30.729 \\
36.724\end{array}$ & $\begin{array}{r}32.41 \\
32.405 \\
\end{array}$ & 16. & & $\begin{array}{l}19.6 \\
19.6\end{array}$ & $\begin{array}{l}13.610 \\
15.689\end{array}$ & $\begin{array}{l}10.24 I \\
10.245\end{array}$ & $\begin{array}{l}31.004 \\
31.588 \\
\end{array}$ & 2 & 29.501 & $\begin{array}{l}31.322 \\
31.345\end{array}$ & $\begin{array}{l}27.939 \\
27.939\end{array}$ & 6.679 \\
\hline $1 / 15 / 121$ & & 30. & 29. & 29.2 & 29 & & 29 & 30. & 29.8 & 29.6 & 28.432 & 26 & 31 & 36.722 & 32.403 & 16 & & 19 & 15.689 & 10.24 & 31 & 32.274 & 29.48 & 31.34 & 27.939 & 677 \\
\hline $\begin{array}{l}1 / 15 / 122 \\
1 / 12\end{array}$ & & 30. & 29. & 29.22 & 29.8 & & 29. & $\begin{array}{l}30.4 \\
30.4\end{array}$ & 29.845 & 29.6 & 28.423 & 26 & 31. & 36 & 32.396 & 16. & & 19.655 & 15.686 & $\begin{array}{r}10.235 \\
\end{array}$ & 31.567 & 32.27 & 29.468 & 31.335 & 27.941 & 33.674 \\
\hline 1/15/12 3:00 & & 30.363 & 29.9 & 29.215 & 29.8 & & 29.356 & 30.47 & 29.845 & 29.64 & 28.414 & & 31.368 & 36.708 & 32.394 & & & & 15.684 & 10.23 & 31.56 & 32.267 & & 31.331 & 27.941 & 33.677 \\
\hline 1/15/12 4:00 & & 30.354 & 29.962 & 29.19 & 29.81 & & 29.366 & 30.48 & 29.836 & 29.623 & 28.398 & 26.153 & 31.361 & 36.701 & 32.384 & & & & 15.6 & 10.218 & 31.541 & 年 & 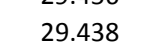 & 31.326 & 27.939 & 33.672 \\
\hline $1 / 15 / 125: 00$ & & 30. & 29. & 29.176 & 29 & & 29. & & 29.8 & & 28.3 & 26. & 31.356 & 36. & 32.3 & 16. & & 19.7 & 15.684 & 10.216 & 31.5 & 32. & 29.428 & 31.314 & 27.941 & 33.67 \\
\hline $1 / 15 / 126$ & & 30.3 & 29. & 29. & & & 29.363 & & 29. & & 28 & 26. & 31.344 & & & 16. & & 19. & 15. & 10. & & 1 & 29.409 & 31.309 & 27.941 & 33.665 \\
\hline $1 / 15 / 127: 00$ & & 30. & 29.5 & 29.1 & & & 29.356 & & 29.8 & & 28 & 26 & 31.34 & 36. & 32.3 & 16. & & 19. & 15.681 & & & & 29.395 & 31.298 & 27.943 & 33.663 \\
\hline $1 / 15 / 128: 00$ & & 30.34 & 29.9 & 29.143 & 29.7 & & 29.34 & 30.4 & 29.833 & 29.5 & 28.3 & 26.1 & 31.33 & 36.682 & 32.3 & 16.11 & & $19.7 \mathrm{r}-\mathrm{C}$ & 15.665 & 10.193 & 31.504 & 32.229 & 29.398 & 31.291 & 27.941 & 33.66 \\
\hline 1/15/12 9:00 & & 30.3 & 29. & 29.145 & 29. & & 29.328 & 30.4 & 29.836 & 29.575 & 28.3 & 26.119 & 31.33 & 36.677 & 32.3. & 16.1 & & 19.813 & 15.66 & 10.188 & 31.504 & 32.229 & 29.4 & 31.291 & 27.943 & 33.658 \\
\hline $1 / 15 / 1210: 00$ & & 30.335 & 29.92 & 29.148 & 29.76 & & 29.316 & 30.4 & 29.833 & 29.57 & 28.349 & 26.124 & 31.328 & 36.675 & 32.349 & 16.19 & & 19.831 & 15.656 & 10.186 & 31.504 & 32.225 & 29.398 & 31.291 & 27.943 & 33.656 \\
\hline 1/15/12 11:00 & & 30.331 & 29.916 & 29.143 & 29.76 & & 29.312 & 30.454 & 29.828 & 29.563 & 28.344 & 26.124 & 31.326 & 36.67 & 32.347 & 16.189 & & 19.887 & 15.656 & 10.188 & 31.501 & 32.222 & 29.4 & 31.293 & 27.943 & 33.658 \\
\hline $1 / 15 / 1212: 00$ & & 30.319 & 29.895 & 29.105 & 29.739 & & 29.333 & 30.451 & 29.819 & 29.544 & 28.325 & 26.09 & 31.319 & 36.668 & 32.34 & 16.184 & & 19.91 & 15.67 & 10.176 & 31.478 & 32.215 & 29.362 & 31.286 & 27.943 & 33.653 \\
\hline 1/15/12 13:00 & & 30.31 & 29.876 & 29.075 & 29.718 & & 29.342 & 30.442 & 29.812 & 29.526 & 28.307 & 26.067 & 31.305 & 36.665 & 32.33 & 16.177 & & 19.938 & 15.677 & 10.169 & 31.462 & 32.203 & 29.344 & 31.277 & 27.943 & 33.651 \\
\hline 1/15/12 14:00 & & 30.305 & 29.86 & 29.054 & 29.697 & & 29.345 & 30.442 & 29.812 & 29.505 & 28.291 & 26.045 & 31.3 & 36.656 & 32.323 & 16.173 & & 19.954 & 15.674 & 10.16 & 31.443 & 32.196 & 29.322 & 31.263 & 27.943 & 33.649 \\
\hline rata & & & & - & & & & & & & & & & & & & & & & & & & & & & \\
\hline
\end{tabular}




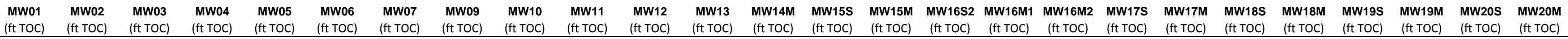

\begin{tabular}{|c|c|c|c|c|c|c|c|c|c|c|c|c|c|c|c|c|c|c|c|c|c|c|c|c|c|}
\hline Well & $\begin{array}{l}\text { (ft TOC) } \\
\text { (ft }\end{array}$ & $\begin{array}{l}\text { (ft TOC) } \\
\text { (f) }\end{array}$ & $\begin{array}{l}\text { (ft TOC) } \\
\text { (f) }\end{array}$ & $\begin{array}{l}\text { (ft TOC) } \\
\text { (f }\end{array}$ & $\begin{array}{l}\text { (ft TOC) } \\
\text { (f) }\end{array}$ & $\begin{array}{l}\text { (ft TOC) } \\
\text { (f) }\end{array}$ & $\begin{array}{l}\text { (ft TOC) } \\
\text { (ft }\end{array}$ & $\begin{array}{l}\text { (ft TOC) } \\
\text { (f) }\end{array}$ & $\begin{array}{l}\text { (ft TOC) } \\
\text { (ft) }\end{array}$ & $\begin{array}{l}\text { (ft TOC) } \\
\text { (f) }\end{array}$ & $\begin{array}{l}\text { (ft TOC) } \\
\text { (f) }\end{array}$ & $\begin{array}{l}\text { (ft TOC) } \\
\text { (f) }\end{array}$ & (ft TOC) & $\begin{array}{l}\text { (ft TOC) } \\
\end{array}$ & (ft TOC) & $\begin{array}{ll}\text { (ft TOC) } \\
\text { (ft TOC) }\end{array}$ & (ft TOC) & $\begin{array}{l}\text { (ft TOC) } \\
\text { (1) }\end{array}$ & (ft TOC) & $\begin{array}{l}\text { (ft TOC) } \\
\end{array}$ & (ft TOC) & $\begin{array}{l}\text { (ft TOC) } \\
\end{array}$ & (ft TOC) & $\begin{array}{l}\text { (ft TOC) } \\
\text { (1) }\end{array}$ & (ft TOC) \\
\hline 16:00 & & 30.298 & 29.843 & 29.045 & 29.678 & & 29.3 & 30.439 & 29.807 & 29.4 & 28.272 & 26.04 & 31.28 & 36.649 & 32.3 & 16.161 & $20 . c$ & 15.67 & 10. & 31. & 32 & 29.311 & 31.253 & 17.943 & 33.649 \\
\hline $1 / 15 / 12$ 17:00 & & 30.296 & 29.839 & 29.047 & 29.674 & & 29.302 & 30.432 & 29.812 & 29.479 & 28.267 & 26.05 & 31.286 & 36.644 & 32.309 & 16.156 & 20.053 & 15.653 & 10.148 & 31.436 & 32.182 & 29.308 & 1.244 & 27.946 & 33.649 \\
\hline $1 / 15 / 1218: 00$ & & 30.296 & 29.841 & 29.059 & 29.681 & & 29.276 & 30.415 & 29.812 & 29.479 & 28.27 & 26.064 & 31.555 & 36.642 & 32.602 & 16.154 & 20.069 & 15.642 & 10.437 & 31.52 & 32.455 & 29.325 & 1.511 & 17.946 & 33.649 \\
\hline 1/15/12 19:00 & & 30.296 & 29.846 & 29.07 & 29.69 & & 29.255 & 30.413 & 29.812 & 29.479 & 28.274 & 26.079 & 31.466 & 36.642 & 32.499 & 16.154 & 20.09 & 15.63 & 10.339 & 31.532 & 32.362 & 29.348 & 31.424 & 27.946 & 33.649 \\
\hline 1/15/12 20:00 & & 30.291 & 29.841 & 29.066 & 29.695 & & 29.251 & 30.43 & 29.809 & 29.472 & 28.267 & 26.079 & 31.41 & 36.647 & 32.441 & 16.147 & 20.111 & 15.627 & 10.277 & 31.506 & 52.308 & 29.344 & 31.368 & 27.946 & 33.646 \\
\hline 1/15/12 21:00 & & 30.286 & 29.832 & 29.047 & 29.69 & & 29.255 & 30.403 & 29.807 & 29.463 & 28.258 & 26.067 & 31.38 & 36.635 & 32.408 & $3 \quad 16.145$ & 20.125 & 15.627 & 10.245 & 31.49 & 32.274 & 29.332 & 31.338 & 27.946 & 33.642 \\
\hline 1/15/12 22:00 & & 30.279 & 29.825 & 29.031 & 29.678 & & 29.253 & 30.406 & 29.802 & 29.452 & 28.249 & 26.052 & 31.354 & 36.635 & 32.382 & 16.14 & 20.134 & 15.625 & 10.221 & 31.471 & 32.251 & 29.322 & 31.316 & 27.946 & 33.639 \\
\hline 1/15/12 23:00 & & 30.272 & 29.813 & 29.012 & 29.667 & & 29.258 & 30.411 & 29.795 & 29.438 & 28.232 & 26.036 & 31.335 & 36.626 & 32.361 & 16.135 & 20.143 & 15.623 & 10.2 & 31.457 & 32.232 & 29.306 & 31.298 & 27.946 & 33.635 \\
\hline 1/16/12 0:00 & & 30.268 & 29.806 & 29.003 & 29.66 & & 29.251 & 30.363 & 29.793 & 29.426 & 28.225 & 26.028 & 31.321 & 36.63 & 32.347 & 16.133 & 20.157 & 15.613 & 10.183 & 31.443 & 32.218 & 29.294 & 31.284 & 27.946 & 33.632 \\
\hline 1/16/12 1:00 & & 30.268 & 29.801 & 28.998 & 29.653 & & 29.241 & 30.367 & 29.795 & 29.419 & 28.216 & 26.024 & 31.312 & 36.621 & 32.335 & 16.128 & 20.164 & 15.606 & 10.174 & 31.434 & 32.206 & 29.287 & 31.272 & 27.946 & 33.63 \\
\hline $1 / 16 / 122: 00$ & & 30.265 & 29.792 & 28.991 & 29.643 & & 29.234 & 30.365 & 29.79 & 29.41 & 28.212 & 26.021 & 31.3 & 36.623 & 32.323 & 16.126 & 20.18 & 15.602 & 10.16 & 31.427 & 32.199 & 29.285 & 31.265 & 27.946 & 33.628 \\
\hline $1 / 16 / 123: 00$ & & 30.265 & 29.792 & 28.991 & 29.641 & & 29.22 & 30.365 & 29.786 & 29.403 & 28.209 & 26.026 & 31.293 & 36.616 & 32.319 & 16.122 & 20.194 & 15.588 & 10.155 & 31.424 & 32.187 & 29.285 & 31.255 & 27.946 & 33.628 \\
\hline $1 / 16 / 124: 00$ & & 30.258 & 29.78 & 28.977 & 29.634 & & 29.222 & 30.391 & 29.786 & 29.393 & 28.195 & 26.019 & 31.286 & 36.614 & 32.309 & 16.117 & 20.206 & 15.59 & 10.148 & 31.413 & 32.18 & 29.268 & 31.251 & 27.946 & 33.625 \\
\hline $\begin{array}{l}1 / 16 / 125: 00 \\
\text { s:1 }\end{array}$ & & 30.265 & 29.785 & 28.993 & 29.636 & & 29.199 & 30.346 & 29.797 & 29.391 & 28.2 & 26.033 & 31.281 & 36.616 & 32.305 & 16.119 & 20.217 & 15.571 & 10.141 & 31.424 & 32.177 & 29.287 & 31.241 & 27.948 & 33.625 \\
\hline 1/16/12 6:00 & & 30.263 & 29.785 & 28.998 & 29.643 & & 29.185 & 30.348 & 29.79 & 29.391 & 28.2 & 26.04 & 31.279 & 36.616 & 32.302 & 16.117 & 20.247 & 15.566 & 10.139 & 31.422 & 32.175 & 29.292 & 31.239 & 27.948 & 33.625 \\
\hline 1/16/12 7:00 & & 30.27 & 29.799 & 29.033 & 29.664 & & 29.156 & 30.329 & 29.797 & 29.403 & 28.214 & 26.078 & 31.281 & 36.619 & 32.305 & 16.115 & 20.264 & 15.548 & 10.141 & 31.445 & 52.177 & 29.322 & 31.244 & 27.948 & 33.628 \\
\hline $1 / 16 / 128: 00$ & & 30.277 & 29.815 & 29.063 & 29.688 & & 29.131 & 30.308 & 29.802 & 29.414 & 28.23 & 26.11 & 31.293 & 36.63 & 32.309 & 16.117 & 20.284 & 15.531 & 10.153 & 31.462 & 32.189 & 29.346 & 31.253 & 27.95 & 33.628 \\
\hline $1 / 16 / 129: 00$ & & 30.279 & 29.829 & 29.094 & 29.713 & & 29.112 & 30.288 & 29.807 & 29.431 & 28.246 & 26.141 & 31.3 & 36.633 & 32.319 & 16.119 & 20.298 & 15.522 & 10.155 & 31.485 & 32.194 & 29.379 & 31.258 & 27.95 & 33.628 \\
\hline $1 / 16 / 1210: 00$ & & 30.284 & 29.848 & 29.12 & 29.744 & & 29.102 & 30.277 & 29.812 & 29.445 & 28.26 & 26.165 & 31.307 & 36.644 & 32.328 & $3 \quad 16.122$ & 20.312 & 15.517 & 10.165 & 31.501 & 32.206 & 29.405 & 31.272 & 27.946 & 33.632 \\
\hline $1 / 16 / 1211: 00$ & & 30.279 & 29.848 & 29.115 & 29.751 & & 29.119 & 30.296 & 29.805 & 29.449 & 28.265 & 26.162 & 31.312 & 36.647 & 32.333 & 16.126 & 20.326 & 15.529 & 10.167 & 31.508 & 32.208 & 29.405 & 31.277 & 27.948 & 33.632 \\
\hline $1 / 16 / 1212: 00$ & & 30.279 & 29.855 & 29.117 & 29.76 & & 29.128 & 30.308 & 29.802 & 29.454 & 28.27 & 26.167 & 31.316 & 36.651 & 32.338 & $3 \quad 16.128$ & 20.34 & 15.534 & 10.172 & 31.511 & 32.213 & 29.409 & 31.284 & 27.95 & 33.63 \\
\hline $1 / 16 / 1213: 00$ & & 30.272 & 29.843 & 29.091 & 29.753 & & 29.156 & 30.353 & 29.793 & 29.447 & 28.26 & 26.136 & 31.316 & 36.649 & 32.337 & 16.126 & 20.354 & 15.548 & 10.172 & 31.497 & 32.213 & .391 & 31.281 & 27.95 & 33.632 \\
\hline $1 / 16 / 1214: 00$ & & 30.272 & 29.841 & 29.077 & 29.746 & & 29.166 & 30.341 & 29.795 & 29.442 & 28.258 & 26.117 & 31.314 & 36.647 & 32.335 & 16.131 & 20.363 & 15.548 & 10.169 & 31.49 & 32.208 & 9388 & 1.274 & 27.95 & 33.63 \\
\hline 1/16/12 15:00 & & 30.275 & 29.843 & 29.084 & 29.746 & & 29.164 & 30.341 & 29.8 & 29.449 & 28.258 & 26.134 & 31.316 & 36.642 & 32.335 & 16.133 & 20.381 & 15.548 & 10.172 & 31.497 & 32.213 & 29.391 & 31.281 & 27.95 & 33.632 \\
\hline 1/16/12 16:00 & & 30.27 & 29.841 & 29.08 & 29.744 & & 29.168 & 30.346 & 29.8 & 29.442 & 28.26 & 26.117 & 31.316 & 36.647 & 32.337 & 16.135 & 20.448 & 15.543 & 10.176 & 31.494 & 32.213 & 29.388 & 31.277 & 27.95 & 33.632 \\
\hline 1/16/12 17:00 & & 30.268 & 29.843 & 29.087 & 29.751 & & 29.166 & 30.358 & 29.793 & 29.449 & 28.26 & 26.129 & 31.321 & 36.647 & 32.34 & 16.135 & 20.465 & 15.548 & 10.178 & 31.494 & 32.215 & 29.386 & 31.284 & 27.95 & 33.635 \\
\hline $1 / 16 / 1218: 00$ & & 30.27 & 29.846 & 29.087 & 29.751 & & 29.164 & 30.355 & 29.795 & 29.452 & 28.26 & 26.134 & 31.326 & 36.649 & 32.342 & 16.138 & 20.476 & 15.548 & 10.179 & 31.501 & 32.222 & 29.398 & 31.286 & 27.953 & 33.635 \\
\hline 1/16/12 19:00 & & 30.265 & 29.843 & 29.084 & 29.755 & & 29.166 & 30.341 & 29.793 & 29.452 & 28.26 & 26.129 & 31.323 & 36.649 & 32.345 & 5 $\quad 16.14$ & 20.485 & 15.552 & 10.181 & 31.497 & 32.22 & 29.393 & 31.286 & 27.95 & 33.635 \\
\hline 1/16/12 20:00 & & 30.272 & 29.857 & 29.105 & 29.767 & & 29.147 & 30.336 & 29.802 & 29.463 & 28.274 & 26.143 & 31.328 & 36.649 & 32.352 & 16.145 & 20.499 & 15.529 & 10.19 & 31.522 & 32.225 & 29.419 & 31.291 & 27.955 & 33.637 \\
\hline $1 / 16 / 1221: 00$ & & 30.272 & 29.86 & 29.11 & 29.774 & & 29.142 & 30.336 & 29.802 & 29.465 & 28.279 & 26.148 & 31.335 & 36.651 & 32.352 & 16.147 & 20.506 & 15.529 & 10.193 & 31.525 & 32.229 & 29.416 & 31.295 & 27.953 & 33.637 \\
\hline $1 / 16 / 1222: 00$ & & 30.289 & 29.885 & 29.157 & 29.8 & & 29.109 & 30.296 & 29.819 & 29.486 & 28.3 & 26.189 & 31.342 & 36.658 & 32.361 & 16.149 & 20.518 & 15.496 & 10.2 & 31.555 & 32.239 & 29.459 & 31.295 & 27.957 & 33.637 \\
\hline $1 / 16 / 1223: 00$ & & 30.3 & 29.932 & 29.232 & 29.851 & & 29.07 & 30.293 & 29.828 & 29.523 & 28.339 & 26.268 & 31.363 & 36.675 & 32.382 & 16.159 & 20.543 & 15.489 & 10.223 & 31.597 & 32.258 & 29.52 & 31.324 & 27.953 & 33.642 \\
\hline $1 / 17 / 120: 00$ & & 30.3 & 29.948 & 29.253 & 29.879 & & 29.074 & 30.305 & 29.828 & 29.544 & 28.353 & 26.287 & 31.377 & 36.682 & 32.394 & 16.166 & 20.555 & 15.496 & 10.233 & 31.613 & 32.274 & 29.539 & 31.34 & 27.955 & 33.642 \\
\hline $\begin{array}{l}1 / 17 / 121: 00 \\
\text { 1/12 }\end{array}$ & & 30.321 & 29.993 & 29.309 & 29.93 & & 29.051 & 30.305 & 29.838 & 29.577 & 28.393 & 26.332 & 31.398 & 36.703 & 32.415 & 16.175 & 20.569 & 15.48 & 10.256 & 31.665 & 32.298 & 29.593 & 31.356 & 27.955 & 33.646 \\
\hline 1/17/12 2:00 & & 30.324 & 30.021 & 29.337 & 29.96 & & 29.058 & 30.324 & 29.833 & 29.605 & 28.416 & 26.359 & 31.417 & 36.708 & 32.434 & 16.1 & 20.585 & $\begin{array}{l}15.494 \\
\end{array}$ & 10.275 & 31.676 & 32.317 & 29.619 & 31.378 & 27.955 & 33.649 \\
\hline 1/17/12 3:00 & & 30.34 & 30.053 & 29.381 & 30.0 & & 29.051 & 30.334 & 29.85 & 29.635 & 28.446 & 26.394 & 31.44 & 36.724 & 32.452 & 16.196 & 20.606 & 15.487 & 10.294 & 31.716 & 32.336 & 29.661 & 31.401 & 27.953 & 33.653 \\
\hline 1/17/12 4:00 & & 30.347 & 30.074 & $\begin{array}{l}29.391 \\
29.393\end{array}$ & 30.031 & & 29.072 & $\begin{array}{l}30.344 \\
30.344\end{array}$ & 29.85 & 29.656 & $\begin{array}{l}20.440 \\
28.463\end{array}$ & 26.399 & $\begin{array}{l}31.454 \\
31.44\end{array}$ & 36.729 & 32.471 & 16.205 & 20.617 & 15.498 & 10.313 & 31.737 & 32.353 & 29.682 & 31.415 & 27.955 & $\begin{array}{l}33.656 \\
33.656\end{array}$ \\
\hline 1/17/12 5:00 & & 30.356 & 30.091 & 29.414 & 30.059 & & 29.088 & 30.358 & 29.855 & 29.679 & $\begin{array}{l}28.484 \\
28.484\end{array}$ & 26.416 & 31.473 & 36.74 & 32.488 & 16.215 & 20.629 & $\begin{array}{l}15.505 \\
15.505\end{array}$ & $\begin{array}{l}10.329 \\
\end{array}$ & 31.755 & 32.372 & 29.706 & 31.434 & 27.955 & $\begin{array}{r}3.050 \\
33.66\end{array}$ \\
\hline $1 / 17 / 126: 00$ & & 30.368 & 30.118 & 29.444 & 30.0 & & 29.098 & 30.37 & 29.864 & 29.707 & 28.509 & 26.435 & 31.492 & 36.75 & 32.506 & 16 & 20.645 & 15.496 & 10.348 & 31.779 & 32.391 & 29.736 & 31.448 & 27.957 & 33.665 \\
\hline $1 / 1$ & & 30.382 & 30.149 & 29.482 & 30 & & 29 & 30.382 & 29.876 & 29.739 & 28.535 & 26.464 & 31.513 & 36.755 & 32.525 & 16 & 20.661 & 15.501 & 10.367 & 31.814 & 32.409 & 29.769 & 31.471 & 27.957 & 33.667 \\
\hline & & 30. & 30.16 & 29.503 & 30. & & & 30.3 & 29.876 & & 28.553 & 26.478 & 31 & 36766 & 32.544 & 16 & 20.7 & 15.512 & 10.386 & 31.828 & 32.431 & 29.79 & 31.485 & 27.957 & 33.674 \\
\hline & & & 30.18 & 29.51 & 30. & & & 30.403 & 29.878 & 29.781 & 28.57 & 26.481 & 31.546 & 36.7 & 32.558 & 16. & 20. & 15 & 10.4 & 31.839 & 32.443 & 29.795 & 1.504 & 27.957 & 33.677 \\
\hline $1 / 17 / 1210: 00$ & & 30.403 & 30.198 & 29.526 & 30.1 & & 29 & 30.418 & 29.885 & 29.8 & 28.588 & 26.488 & 31.56 & 36.783 & 32.574 & 16. & & 15.543 & 10.411 & 31.856 & 32.455 & 29.811 & 1.521 & 27.957 & 33.681 \\
\hline 1/17/12 11:00 & & 30.408 & 30.207 & 29.529 & 30.1 & & 2 & 30.425 & 29.881 & 29.816 & 28.598 & 26.483 & 31.574 & 36.787 & 32.586 & 16.2 & 31 & 15.566 & 10.428 & 31.865 & 32.469 & 23 & 31.535 & 27.96 & 33.681 \\
\hline $1 / 17 / 1212: 00$ & & 30.405 & 30.2 & 29.512 & & & & 30.4 & 29.871 & & 28.598 & 26.457 & 31.578 & 36.79 & 32.593 & 16.2 & & 15.599 & 10.433 & 31.856 & 52.478 & 29.811 & 31.539 & 27.957 & 33.681 \\
\hline $1 / 17 / 1213: 00$ & & 30.398 & 30.188 & 29.47 & 30 & & & 30.4 & 29.859 & & 28.586 & 26. & 31.578 & 36.79 & 32.593 & 16.3 & 20.805 & 15.63 & 10.433 & 31.832 & 32.473 & 29.774 & 31.542 & 27.955 & 33.679 \\
\hline $1 / 17 / 1214: 00$ & & 30.401 & 30.17 & 29.454 & 30. & & & 30.487 & 29.864 & 29. & 28.579 & 26.392 & 31.576 & 36.794 & 32.595 & 16 & 20.816 & 15.651 & 10.435 & 31.823 & 32.473 & 29.76 & 31.542 & 27.957 & 33.681 \\
\hline 1/17/12 15:00 & & 30.408 & 30.1 & 29.458 & 30.1 & & 29. & 30.473 & 29.874 & $29.8 \mathrm{r}-\mathrm{r}$ & 28.584 & 26.385 & 31.583 & 36.79 & 32.6 & 16.3 & 20.825 & 15.653 & 10.437 & 31.832 & 32.478 & 29.767 & 31.542 & 27.96 & 33.681 \\
\hline $1 / 17 / 1216: 00$ & & 30.417 & 30.188 & 29.475 & 30.1 & & 29.319 & 30.458 & 29.883 & 29.821 & 28.591 & 26.402 & 31.59 & 36.797 & 32.605 & 16.3 & 20.892 & 15.656 & 10.444 & 31.84 & 32.485 & 29.776 & 31.549 & 27.962 & 33.684 \\
\hline $1 / 17 / 1217: 00$ & & 30.417 & 30.186 & 29.47 & 30.143 & & 29.33 & 30.461 & 29.876 & 29.821 & 28.591 & 26.39 & 31.595 & 36.797 & 32.612 & 16.326 & 20.902 & 15.674 & 10.451 & 31.835 & 32.49 & 29.769 & 31.556 & 27.957 & 33.686 \\
\hline $1 / 17 / 1218: 00$ & & 30.417 & 30.184 & 29.465 & 30.138 & & 29.34 & 30.463 & 29.878 & 29.821 & 28.588 & 26.38 & 31.597 & 36.797 & 32.616 & 16.331 & 20.906 & 15.677 & 10.454 & 31.835 & 32.492 & 29.764 & 31.556 & 27.96 & 33.688 \\
\hline 1/17/12 19:00 & & 30.422 & 30.181 & 29.47 & 30.138 & & 29.34 & 30.463 & 29.883 & 29.825 & 28.591 & 26.38 & 31.602 & 36.794 & 32.621 & 16.333 & 20.911 & 15.674 & 10.458 & 31.839 & 32.5 & 29.767 & 31.563 & 27.962 & 33.691 \\
\hline $1 / 17 / 1220: 00$ & & 30.426 & 30.188 & 29.477 & 30.141 & & 29.342 & 30.468 & 29.885 & 29.83 & 28.595 & 26.382 & 31.609 & 36.797 & 32.626 & 16.338 & 20.922 & 15.679 & 10.465 & 31.842 & 32.507 & 29.771 & 31.565 & 27.962 & 33.693 \\
\hline $1 / 17 / 1221: 00$ & & 30.426 & 30.181 & 29.468 & 30.134 & & 29.352 & 30.468 & 29.881 & 29.83 & 28.593 & 26.371 & 31.607 & 36.794 & 32.626 & 16.343 & 20.927 & 15.684 & 10.463 & 31.842 & 32.504 & 29.764 & 31.57 & 27.962 & 33.691 \\
\hline $1 / 17 / 1222: 00$ & & 30.424 & 30.177 & 29.454 & 30.127 & & 29.368 & 30.478 & 29.876 & 29.828 & 28.584 & 26.359 & 31.609 & 36.797 & 32.626 & 16.347 & 20.936 & 15.7 & 10.465 & 31.828 & $3 \quad 32.504$ & 29.748 & 31.572 & 27.96 & 33.691 \\
\hline $1 / 17 / 1223: 00$ & & 30.426 & 30.174 & 29.449 & 30.12 & & 29.373 & 30.475 & 29.878 & 29.823 & 28.579 & 26.349 & 31.607 & 36.792 & 32.624 & 16.347 & 20.943 & 15.703 & 10.463 & 31.823 & 32.504 & 29.741 & 31.57 & 27.96 & 33.688 \\
\hline
\end{tabular}




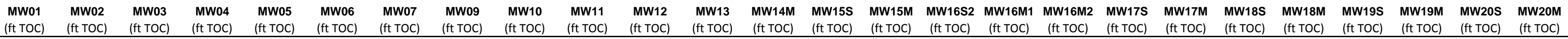

\begin{tabular}{|c|c|c|c|c|c|c|c|c|c|c|c|c|c|c|c|c|c|c|c|c|c|c|c|c|}
\hline Well & $\begin{array}{l}\text { (ft TOC) } \\
\text { (f) }\end{array}$ & $\begin{array}{l}\text { (ft TOC) } \\
\text { (ft) }\end{array}$ & $\begin{array}{l}\text { (ft TOC) } \\
\text { (f) }\end{array}$ & $\begin{array}{l}\text { (ft TOC) } \\
\text { (ft) }\end{array}$ & $\begin{array}{ll}(\mathrm{ft} \text { TOC) } & \text { (ft TOC) }\end{array}$ & (ft TOC) & $\begin{array}{l}\text { (ift TOC) } \\
\text { (n) }\end{array}$ & $\begin{array}{l}\text { (ft TOC) } \\
\text { (ft) }\end{array}$ & $\begin{array}{l}\text { (ft TOC) } \\
\text { (f) }\end{array}$ & $\begin{array}{l}\text { (ft TOC) } \\
\text { (n) }\end{array}$ & $\begin{array}{l}\text { (ft TOC) } \\
(\mathrm{f})\end{array}$ & (ft TOC) & $\begin{array}{l}\text { (ft TOC) } \\
\end{array}$ & (ft TOC) & $\begin{array}{ll} & \text { (ft TTCC } \\
\text { (ft TOC) }\end{array}$ & (ft TOC) & $\begin{array}{l}\text { (ft TOC) } \\
\text { (ft) }\end{array}$ & (ft TOC) & $\begin{array}{l}\text { (ft TOC) } \\
\text { (f) }\end{array}$ & $\begin{array}{l}\text { (ft TOC) } \\
\text { (f) }\end{array}$ & $\begin{array}{l}\text { (ft TOC) } \\
\text { (n) }\end{array}$ & (ft TOC) & (ft TOC) & (ft TOC) \\
\hline $20: 00$ & & 30.422 & 30.163 & 29.428 & 30.103 & 29.392 & 30.489 & 29.87 & 29.816 & 28. & 26 & 31.604 & 36.79 & 32.6 & 16 & 20.95 & 15.714 & 10.461 & 31.811 & & & 1.568 & 27.96 & 33.684 \\
\hline $1 / 18 / 121: 00$ & & 30.417 & 30.149 & 29.4 & 30.085 & 29.408 & 30.504 & 29.869 & 29.802 & 28.556 & 26.299 & 31.595 & 36.78 & 32.614 & 16.347 & 20.957 & 15.726 & 10.453 & 31.788 & 32.492 & .698 & 31.556 & 27.96 & 33.681 \\
\hline $1 / 18 / 122: 00$ & & 30.415 & 30.13 & 29.374 & 30.061 & 29.422 & 30.518 & 29.866 & 29.79 & 28.542 & 26.275 & 31.585 & 36.773 & 32.607 & 16.345 & 20.962 & 15.731 & 10.444 & 31.772 & 32.481 & 29.675 & 31.551 & 27.964 & 33.677 \\
\hline 1/18/12 3:00 & & 30.408 & 30.116 & 29.349 & 30.033 & 29.434 & 30.525 & 29.866 & 29.774 & 28.523 & 26.251 & 31.574 & 36.764 & $4 \quad 32.598$ & 16.34 & 20.964 & 15.735 & 10.435 & 31.753 & 32.473 & 29.647 & 31.539 & 27.962 & 33.672 \\
\hline $1 / 18 / 124: 00$ & & 30.412 & 30.105 & 29.334 & 30.019 & 29.424 & 30.516 & 29.871 & 29.763 & 28.514 & 26.234 & 31.569 & 36.759 & 32.588 & 16.34 & 20.966 & 15.733 & 10.426 & 31.746 & 32.464 & 29.644 & 31.53 & 27.964 & 33.67 \\
\hline 1/18/12 5:00 & & 30.405 & 30.088 & 29.318 & 30 & 29.432 & 30.523 & 29.864 & 29.753 & 28.502 & 26.224 & 31.562 & 36.75 & 32.584 & 16.336 & 20.969 & 15.745 & 10.423 & 31.732 & 32.457 & 29.623 & 31.528 & 27.962 & 33.665 \\
\hline 1/18/12 6:00 & & 30.408 & 30.084 & 29.32 & 29.993 & 29.417 & 30.516 & 29.871 & 29.749 & 28.498 & 26.227 & 31.557 & 36.745 & $5 \quad 32.579$ & 16.331 & 20.976 & 15.735 & 10.421 & 31.735 & 32.452 & 29.626 & 31.523 & 27.962 & 33.665 \\
\hline 1/18/12 7:00 & & 30.403 & 30.07 & 29.302 & 29.979 & 29.42 & 30.523 & 29.866 & 29.735 & 28.484 & 26.213 & 31.553 & 36.743 & $3 \quad 32.574$ & 16.329 & 20.98 & 15.742 & 10.414 & 31.716 & 32.447 & 29.604 & 31.518 & 27.964 & 33.663 \\
\hline 1/18/12 8:00 & & 30.405 & 30.065 & 29.304 & 29.972 & 29.406 & 30.513 & 29.871 & 29.732 & 28.479 & 26.212 & 31.548 & 36.74 & 32.57 & 16.326 & 20.994 & 15.735 & 10.411 & 31.718 & 32.445 & 29.607 & 31.514 & 27.964 & 33.663 \\
\hline 1/18/12 9:00 & & 30.401 & 30.06 & 29.297 & 29.97 & 29.401 & 30.513 & 29.869 & 29.723 & 28.474 & 26.21 & 31.548 & 36.736 & $5 \quad 32.565$ & 16.322 & 21.001 & 15.735 & 10.407 & 31.714 & 32.443 & 29.604 & 31.514 & 27.964 & 33.663 \\
\hline $1 / 18 / 1210: 00$ & & 30.403 & 30.058 & 29.3 & 29.968 & 29.394 & 30.511 & 29.871 & 29.721 & 28.47 & 26.217 & 31.546 & 36.733 & $\begin{array}{l}3 \\
32.57\end{array}$ & 16.319 & 21.008 & 15.738 & 10.404 & 31.716 & 32.443 & 29.602 & 31.514 & 27.964 & 33.663 \\
\hline 1/18/12 11:00 & & 30.396 & 30.042 & 29.274 & 29.951 & 29.408 & 30.528 & 29.864 & 29.707 & 28.456 & 26.191 & 31.539 & 36.731 & 32.563 & 16.315 & 21.015 & 15.747 & 10.397 & 31.695 & 32.433 & 29.579 & 31.507 & 27.962 & 33.66 \\
\hline $1 / 18 / 1212: 00$ & & 30.382 & 30.004 & 29.225 & 29.916 & 29.436 & 30.516 & 29.852 & 29.679 & 28.425 & 26.146 & 31.525 & 36.722 & 32.546 & 16.308 & 21.015 & 15.757 & 10.388 & 31.66 & 32.419 & 29.543 & 31.488 & 27.964 & 33.656 \\
\hline 1/18/12 13:00 & & 30.375 & 29.979 & 29.19 & 29.891 & 29.443 & 30.521 & 29.85 & 29.653 & 28.402 & 26.115 & 31.513 & 36.712 & 32.534 & 16.301 & 21.013 & 15.761 & 10.371 & 31.634 & 32.407 & 29.506 & 31.481 & 27.964 & 33.651 \\
\hline 1/18/12 14:00 & & 30.368 & 29.953 & 29.159 & 29.86 & 29.446 & 30.506 & 29.845 & 29.635 & 28.381 & 26.088 & 31.497 & 36.705 & 32.518 & 16.291 & 21.045 & 15.761 & 10.357 & 31.62 & 32.388 & 29.485 & 31.464 & 27.967 & 33.646 \\
\hline $1 / 18 / 1215: 00$ & & 30.363 & 29.944 & 29.152 & 29.842 & 29.429 & 30.499 & 29.85 & 29.619 & 28.372 & 26.083 & 31.487 & 36.698 & 32.511 & 16.287 & 21.047 & 15.75 & 10.348 & 31.604 & 32.383 & 29.478 & 31.455 & 27.967 & 33.644 \\
\hline $1 / 18 / 1216: 00$ & & 30.361 & 29.932 & 29.143 & 29.83 & 29.413 & 30.487 & 29.847 & 29.605 & 28.358 & 26.076 & 31.48 & 36.696 & 32.504 & 16.28 & 21.052 & 15.74 & 10.343 & 31.602 & 32.376 & 29.466 & 31.446 & 27.967 & 33.642 \\
\hline $1 / 18 / 1217: 00$ & & 30.354 & 29.918 & 29.138 & 29.818 & 29.403 & 30.494 & 29.845 & 29.593 & 28.346 & 26.079 & 31.471 & 36.682 & 32.495 & 16.273 & 21.059 & 15.742 & 10.334 & 31.592 & 32.369 & 29.456 & 31.434 & 27.967 & 33.642 \\
\hline $1 / 18 / 1218: 00$ & & 30.359 & 29.923 & 29.15 & 29.818 & 29.373 & 30.482 & 29.852 & 29.593 & 28.346 & 26.095 & 31.473 & 36.687 & 32.497 & 16.268 & 21.121 & 15.719 & 10.334 & 31.595 & 32.369 & 29.466 & 31.434 & 27.969 & 33.644 \\
\hline $1 / 18 / 12$ 19:00 & & 30.354 & 29.916 & 29.145 & 29.816 & 29.361 & 30.485 & 29.843 & 29.584 & 28.339 & 26.093 & 31.471 & 36.68 & 32.495 & 16.264 & 21.124 & 15.719 & 10.331 & 31.59 & 32.367 & 29.463 & 31.436 & 27.969 & 33.644 \\
\hline $1 / 18 / 1220: 00$ & & 30.347 & 29.904 & 29.127 & 29.80 & 29.361 & 30.485 & 29.838 & 29.57 & 28.325 & 26.081 & 31.466 & 36.682 & 32.49 & 16.2 & 21.128 & 15.714 & 10.327 & 31.583 & 32.362 & 29.452 & 31.427 & 27.967 & 33.642 \\
\hline $1 / 18 / 1221: 00$ & & 30.345 & 29.902 & 29.129 & 29.807 & 29.345 & 30.485 & 29.843 & 29.565 & 28.323 & 26.086 & 31.464 & 36.675 & 32.485 & 16.25 & 21.133 & 15.705 & 10.324 & 31.583 & 32.36 & 8.452 & 31.429 & 27.967 & 33.644 \\
\hline $1 / 18 / 1222: 00$ & & 30.34 & 29.89 & 29.115 & 29.797 & 29.342 & 30.47 & 29.843 & 29.554 & 28.312 & 26.078 & 31.457 & 36.672 & 32.478 & 16.25 & 21.133 & 15.698 & 10.315 & 31.574 & 32.353 & 29.442 & 31.422 & 27.967 & 33.642 \\
\hline 1/18/12 23:00 & & 30.335 & 29.881 & 29.103 & 29.79 & 29.34 & 30.475 & 29.838 & 29.54 & 28.302 & 26.071 & 31.45 & 36.668 & 32.471 & 16.245 & 21.137 & 15.691 & 10.31 & 31.567 & 32.346 & 29.433 & 31.413 & 27.967 & 33.639 \\
\hline 1/19/12 0:00 & & 30.335 & 29.881 & 29.103 & 29.783 & 29.326 & 30.478 & 29.84 & 29.535 & 28.298 & 26.074 & 31.445 & 36.658 & 32.466 & 16.238 & 21.137 & 15.684 & 10.308 & 31.562 & 32.341 & 29.428 & 31.41 & 27.967 & 33.637 \\
\hline 1/19/12 1:00 & & 30.328 & 29.874 & 29.096 & 29.776 & 29.319 & 30.468 & 29.838 & 29.523 & 28.291 & 26.069 & 31.436 & 36.658 & 32.457 & 16.233 & 21.142 & 15.679 & 10.296 & 31.555 & 32.331 & 29.428 & 31.399 & 27.967 & 33.632 \\
\hline 1/19/12 2:00 & & 30.326 & 29.867 & 29.087 & 29.774 & 29.312 & 30.463 & 29.833 & 29.514 & 28.281 & 26.069 & 31.429 & 36.656 & 32.452 & 16.229 & 21.142 & 15.672 & 10.294 & 31.548 & 32.327 & 29.421 & 31.396 & 27.967 & 33.628 \\
\hline 1/19/12 3:00 & & 30.331 & 29.871 & 29.108 & 29.781 & 29.288 & 30.418 & 29.843 & 29.516 & 28.286 & 26.083 & 31.431 & 36.654 & 32.45 & 16.224 & 21.142 & 15.651 & 10.287 & 31.56 & 32.322 & 29.433 & 31.389 & 27.969 & 33.625 \\
\hline 1/19/12 4:00 & & 30.333 & 29.881 & 29.124 & 29.795 & 29.265 & 30.401 & 29.847 & 29.521 & 28.295 & 26.11 & 31.431 & 36.658 & 32.455 & 16.224 & 21.147 & 15.635 & 10.289 & 31.576 & 32.327 & 29.454 & 31.394 & 27.971 & 33.623 \\
\hline 1/19/12 5:00 & & 30.338 & 29.892 & 29.152 & 29.814 & 29.239 & 30.377 & 29.85 & 29.53 & 28.307 & 26.138 & 31.436 & 36.656 & 32.455 & 16.224 & 21.147 & 15.618 & 10.296 & 31.592 & 32.334 & 29.475 & 31.396 & 27.971 & 33.625 \\
\hline 1/19/12 6:00 & & 30.342 & 29.906 & 29.178 & 29.837 & 29.222 & 30.358 & 29.855 & 29.54 & 28.319 & 26.158 & 31.445 & 36.663 & 32.462 & 16.224 & 21.17 & 15.616 & 10.303 & 31.609 & 32.341 & 29.499 & 31.406 & 27.974 & 33.625 \\
\hline 1/19/12 7:00 & & 30.354 & 29.93 & 29.227 & 29.872 & 29.189 & 30.353 & 29.869 & 29.563 & 28.344 & 26.21 & 31.459 & 36.677 & 32.476 & 16.229 & 21.188 & 15.588 & 10.317 & 31.644 & 32.355 & 29.543 & 31.417 & 27.971 & 33.63 \\
\hline $\begin{array}{l}1 / 19 / 128: 00 \\
\text { 1/12 }\end{array}$ & & 30.359 & 29.951 & 29.253 & 29.902 & 29.178 & 30.353 & 29.866 & 29.577 & 28.36 & 26.234 & 31.471 & 36.684 & 32.49 & 16.231 & 21.193 & 15.585 & 10.329 & 31.667 & 32.367 & 29.569 & 31.429 & 27.971 & 33.632 \\
\hline $\begin{array}{l}1 / 19 / 129: 00 \\
\text { 1/12 }\end{array}$ & & 30.368 & 29.976 & 29.295 & 29.937 & 29.161 & 30.365 & 29.874 & 29.605 & 28.384 & 26.28 & 31.49 & 36.698 & 32.506 & 16.236 & 21.202 & 15.583 & 10.346 & 31.697 & 32.383 & 29.604 & 31.453 & 27.976 & 33.639 \\
\hline 1/19/12 10:00 & & 30.368 & 29.988 & 29.304 & 29.963 & 29.178 & 30.367 & 29.874 & 29.614 & 28.398 & 26.284 & 31.501 & 36.708 & 32.52 & 16.24 & 21.205 & 15.592 & 10.36 & 31.711 & 32.395 & 29.626 & 31.469 & 27.974 & 33.642 \\
\hline 1/19/12 11:00 & & 30.368 & 29.99 & 29.3 & 29.972 & 29.201 & 30.384 & 29.869 & 29.619 & 28.4 & 26.28 & 31.508 & 36.708 & 32.523 & 16.24 & 21.211 & 15.609 & $\begin{array}{r}10.364 \\
\end{array}$ & 31.709 & 405 & 29.619 & 31.471 & 27.971 & 33.642 \\
\hline $1 / 19 / 1212: 00$ & & $\begin{array}{l}30.360 \\
30.363\end{array}$ & 29.988 & 29.285 & 29.972 & 29.234 & 30.408 & 29.862 & 29.619 & 28.395 & 26.263 & 31.511 & 36.71 & 32.53 & 16.25 & $\begin{array}{r}21.2 \\
21.2\end{array}$ & 15.632 & 10.371 & 31.704 & 32.405 & 29.609 & 31.478 & 27.969 & $\begin{array}{l}33.042 \\
33.642\end{array}$ \\
\hline $\begin{array}{l}1 / 19 / 12 ~ 13: 00 \\
1 / 12\end{array}$ & & 30.363 & 29.983 & 29.262 & 29.965 & 29.26 & 30.432 & 29.862 & 29.614 & 28.391 & 26.239 & 31.511 & 36.71 & 32.525 & 16.254 & 21.214 & $\begin{array}{l}15.639 \\
159\end{array}$ & 10.369 & 31.695 & 32.407 & 29.583 & $\begin{array}{l}31.478 \\
31.48\end{array}$ & 27.971 & $\begin{array}{l}33.639 \\
33.639\end{array}$ \\
\hline $1 / 19 / 1214: 00$ & & 30.366 & 29.974 & 29.243 & 29.949 & 29.276 & 30.449 & 29.862 & 29.607 & 28.381 & 26.213 & 31.506 & 36.705 & 32.525 & 16. & 21.214 & 15.639 & 10.362 & 31.683 & 32.402 & 29.583 & 31.469 & 27.976 & 33.637 \\
\hline & & 30. & 29.97 & 29.246 & & 29.274 & 30.4 & 29.869 & 29.607 & 28.384 & 26.213 & 31.506 & 36.705 & 32.527 & 16 & 21.218 & 15.632 & 10.367 & 31.693 & 32.4 & 29.59 & 31.467 & 27.974 & 33.635 \\
\hline & & & 29.5 & 29 & & & & 29.869 & & 28.3 & 26.213 & 31.513 & 36.71 & 32. & 16. & 7 & 15.63 & 10.367 & 31.69 & 32.405 & 29.595 & 31.467 & 27.974 & 33.637 \\
\hline & & 30. & 29.9 & 29 & & & & 29.864 & & 28. & 26.225 & 31.515 & 36.708 & 32.5 & 16.2 & 21.281 & 15.639 & 10.371 & 31.695 & 4 & 4 & 31.476 & 27.971 & 33.637 \\
\hline 1/19/12 18:00 & & 30. & 29.974 & 29.234 & & 29 & 30 & 29.857 & & 28. & 26.198 & 31.515 & 36.703 & 32.537 & 16.2 & 21.288 & 15.649 & 10.371 & & 32.409 & 29.583 & 31.474 & 27.974 & 33.637 \\
\hline 1/19/12 19:00 & & 30. & 29.983 & 29.257 & 29 & 29. & 30. & 29.871 & 29. & 28.3 & 26.22 & 31.522 & 36.708 & 32.541 & 16.268 & 21.29 & 15.63 & 10.376 & 31.704 & 32.417 & 29.602 & 31.481 & 27.974 & 33.642 \\
\hline 20:00 & & 30. & 29.988 & 29.267 & & 29.269 & & 29.876 & 29. & 28.398 & 26.229 & 31.529 & 36.708 & 32.546 & 16.2 & 21.297 & 15.627 & 10.386 & 31.709 & 32.419 & 29.609 & 31.488 & 27.978 & 33.642 \\
\hline 1/19/12 21:00 & & 30.373 & 29.9 & 29.269 & 29. & 29.272 & 30. & 29.871 & 29. & 28.398 & 26.237 & 31.534 & 36.715 & 32.551 & 16. & 21.302 & 15.639 & 10.392 & 31.714 & 32.431 & 29.612 & 31.502 & 27.974 & 33.646 \\
\hline $1 / 19 / 1222: 00$ & & 30.361 & 29.9 & 29.236 & 29.9 & 29.3 & 30.4 & 29.862 & 29.616 & 28.384 & 26.203 & 31.529 & 36.71 & 32.551 & 16.2 & 21.302 & 15.66 & 10.388 & 31.693 & 32.426 & 29.586 & 31.5 & 27.974 & 33.644 \\
\hline 1/19/12 23:00 & & 30.366 & 29.9 & 29.222 & 29. & 29.3 & $30.6>2>$ & 29.859 & 29.6 & 28.377 & 26.181 & 31.527 & 36.703 & 32.544 & 16.2 & 21.304 & 15.651 & 10.386 & 31.683 & 32.421 & 29.572 & 31.49 & 27.974 & 33.644 \\
\hline 1/20/12 0:00 & & 30.354 & 29.955 & 29.192 & 29.92 & 29.326 & 30.461 & 29.852 & 29.595 & 28.36 & 26.155 & 31.52 & 36.696 & 32.539 & 16.27 & 21.306 & 15.665 & 10.379 & 31.667 & 32.417 & 29.553 & 31.485 & 27.974 & 33.642 \\
\hline 1/20/12 1:00 & & 30.349 & 29.939 & 29.159 & 29.893 & 29.338 & 30.468 & 29.85 & 29.575 & 28.344 & 26.122 & 31.506 & 36.687 & 32.527 & 16.273 & 21.309 & 15.658 & 10.369 & 31.641 & 32.4 & 29.522 & 31.471 & 27.976 & 33.635 \\
\hline $1 / 20 / 122: 00$ & & 30.338 & 29.916 & 29.117 & 29.863 & 29.359 & 30.463 & 29.845 & 29.556 & 28.321 & 26.086 & 31.492 & 36.682 & 32.513 & 16.266 & 21.306 & 15.672 & 10.353 & 31.609 & 32.386 & 29.482 & 31.457 & 27.974 & 33.625 \\
\hline 1/20/12 3:00 & & 30.345 & 29.923 & 29.136 & 29.863 & 29.323 & 30.442 & 29.855 & 29.556 & 28.325 & 26.105 & 31.485 & 36.67 & 32.509 & 16.266 & 21.306 & 15.646 & 10.348 & 31.623 & 32.381 & 29.506 & 31.448 & 27.976 & 33.625 \\
\hline 1/20/12 4:00 & & 30.333 & 29.906 & 29.112 & 29.844 & 29.333 & 30.456 & 29.843 & 29.542 & 28.309 & 26.083 & 31.478 & 36.665 & 32.499 & 16.264 & 21.309 & 15.651 & 10.339 & 31.599 & 32.372 & 29.475 & 31.441 & 27.974 & 33.618 \\
\hline $1 / 20 / 125: 00$ & & 30.328 & 29.897 & 29.096 & 29.83 & 29.33 & 30.444 & 29.845 & 29.528 & 28.3 & 26.071 & 31.466 & 36.663 & 32.49 & 16.257 & 21.304 & 15.646 & 10.329 & 31.592 & 32.364 & 29.471 & 31.427 & 27.974 & 33.614 \\
\hline $1 / 20 / 12$ & & 30.338 & 29.909 & 29.126 & 29.839 & 29.295 & 30.422 & 29.855 & 29.537 & 28.307 & 26.105 & 31.473 & 36.668 & 32.49 & 16.257 & 21.327 & 15.627 & 10.329 & 31.613 & 32.367 & 29.489 & 31.436 & 27.976 & 33.616 \\
\hline $1 / 20 / 127: 00$ & & 30.34 & 29.916 & 29.15 & 29.853 & 29.269 & 30.411 & 29.859 & 29.542 & 28.316 & 26.126 & 31.473 & 36.665 & 32.495 & 16.257 & 21.339 & 15.611 & 10.331 & 31.625 & 32.372 & 29.506 & 31.434 & 27.978 & 616 \\
\hline
\end{tabular}




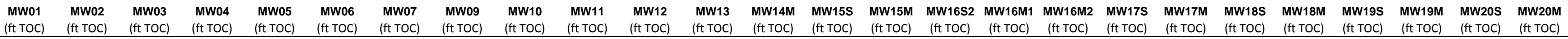

\begin{tabular}{|c|c|c|c|c|c|c|c|c|c|c|c|c|c|c|c|c|c|c|c|c|c|c|c|c|c|}
\hline Well & $\begin{array}{l}\text { MWOI } \\
\text { (ft ToC) }\end{array}$ & $\begin{array}{l}\text { Mwoz } \\
\text { (ft TOC) }\end{array}$ & $\begin{array}{l}\text { Mw03 } \\
\text { (ft TOC) }\end{array}$ & $\begin{array}{l}\text { Mivou } \\
\text { (ft TOC) }\end{array}$ & $\begin{array}{l}\text { Mwos } \\
\text { (ft TOC) }\end{array}$ & $\begin{array}{l}\text { Wwo6 } \\
\text { (ft TOC) }\end{array}$ & (ft TOC) & $\begin{array}{l}\text { Mw09 } \\
\text { (ft TOC) }\end{array}$ & $\begin{array}{l}\text { MWI0 } \\
\text { (ft TOC) }\end{array}$ & $\begin{array}{l}\text { MWII } \\
\text { (ft TOC) }\end{array}$ & $\begin{array}{l}\text { MWII2 } \\
(\mathrm{ft} \text { TOC) }\end{array}$ & $\begin{array}{l}\text { MWvis } \\
(\mathrm{ft} \text { Toc) }\end{array}$ & $\begin{array}{l}\text { MW14M } \\
(\mathrm{ft} \text { TOC) }\end{array}$ & $\begin{array}{l}\text { MWW } \\
\text { (ft TOC) }\end{array}$ & $\begin{array}{l}\text { MWW } \\
\text { (ft TOC) }\end{array}$ & 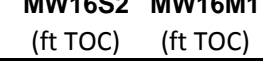 & $\begin{array}{l}\text { MWI6M2 } \\
\text { (ft TOC) }\end{array}$ & $\begin{array}{l}\text { MWW } \\
\text { (ft TOC) }\end{array}$ & (ft TOC) & $\begin{array}{l}\text { MWW } 185 \\
\text { (ft TOC) }\end{array}$ & $\begin{array}{l}\text { MWIIM } \\
\text { (ft TOC) } \\
\end{array}$ & $\begin{array}{l}\text { MWW } \\
\text { (ft TOC) }\end{array}$ & $\begin{array}{l}\text { MWVIM } \\
\text { (ft TOC) }\end{array}$ & $\begin{array}{l}\text { Mwwos } \\
\text { (ft Toc) }\end{array}$ & (ft TOC) \\
\hline $1 / 20 / 128: 00$ & & 30.333 & 29.913 & 29.143 & 29.856 & & 29.276 & 30.427 & 29.845 & 29.542 & 28.312 & 26.131 & 31.478 & 36.668 & 32.495 & $5 \quad 16.252$ & 21.341 & 15.63 & 10.334 & 31.616 & 32.372 & 29.499 & 31.446 & 27.971 & 33.616 \\
\hline $1 / 20 / 129: 00$ & & 30.328 & 29.904 & 29.124 & 29.846 & & 29.281 & 30.442 & 29.845 & 29.53 & 28.302 & 26.107 & 31.471 & 36.665 & 32.49 & 9 16.252 & 21.341 & 15.625 & 10.334 & 31.611 & $1 \quad 32.367$ & 29.492 & 31.436 & $5 \quad 27.976$ & 33.616 \\
\hline $1 / 20 / 1210: 00$ & & 30.335 & 29.913 & 29.143 & 29.858 & & 29.262 & 30.427 & 29.85 & 29.535 & 28.312 & 26.126 & 31.475 & 36.661 & 32.495 & 16.25 & 21.346 & 15.611 & 10.336 & 31.62 & $2 \quad 32.372$ & 29.506 & 31.441 & 27.978 & 333.616 \\
\hline $1 / 20 / 1211: 00$ & & 30.333 & 29.916 & 29.145 & 29.863 & & 29.26 & 30.432 & 29.85 & 29.537 & 28.309 & 26.134 & 31.478 & 36.672 & 32.499 & 16.25 & 21.346 & 15.613 & 10.336 & 31.623 & $\begin{array}{ll}3 & 32.372\end{array}$ & 29.513 & 31.446 & $5 \quad 27.976$ & 533.618 \\
\hline $1 / 20 / 1212: 00$ & & 30.333 & 29.916 & 29.15 & 29.867 & & 29.255 & 30.427 & 29.852 & 29.537 & 28.314 & 26.134 & 31.478 & 36.668 & 32.499 & 16.25 & 21.343 & 15.609 & 10.336 & 31.63 & $\begin{array}{l}3 \quad 32.376 \\
\end{array}$ & 29.515 & 31.443 & $\begin{array}{l}3 \quad 27.978 \\
\end{array}$ & 33.616 \\
\hline $1 / 20 / 1213: 00$ & & 30.331 & 29.916 & 29.145 & 29.867 & & 29.26 & 30.437 & 29.852 & 29.537 & 28.309 & 26.141 & 31.478 & 36.668 & 32.499 & 16.25 & 21.346 & 15.616 & 10.336 & 31.627 & $\begin{array}{l}7 \quad 32.374 \\
\end{array}$ & 29.515 & 31.446 & $5 \quad 27.978$ & 333.614 \\
\hline $1 / 20 / 1214: 00$ & & 30.333 & 29.916 & 29.15 & 29.872 & & 29.255 & 30.434 & 29.85 & 29.535 & 28.309 & 26.141 & 31.48 & 36.668 & 32.499 & 16.25 & 21.346 & 15.613 & 10.339 & 31.632 & $2 \quad 32.374$ & 29.518 & 31.443 & $\begin{array}{l}3 \quad 27.978 \\
\end{array}$ & 333.616 \\
\hline $1 / 20 / 1215: 00$ & & 30.342 & 29.937 & 29.19 & 29.891 & & 29.225 & 30.399 & 29.859 & 29.554 & 28.328 & 26.177 & 31.49 & 36.672 & 32.504 & 16.252 & 21.35 & 15.595 & 10.348 & 31.658 & $8 \quad 32.383$ & 29.548 & 31.448 & $\begin{array}{l}3 \quad 27.978 \\
\end{array}$ & 333.616 \\
\hline $1 / 20 / 1216: 00$ & & 30.349 & 29.953 & 29.225 & 29.916 & & 29.204 & 30.367 & 29.866 & 29.565 & 28.349 & 26.203 & 31.496 & 36.682 & 32.518 & $3 \quad 16.254$ & 21.355 & 15.583 & 10.353 & 31.676 & $6 \quad 32.393$ & 29.581 & 31.462 & 27.981 & 33.616 \\
\hline 1/20/12 17:00 & & 30.361 & 29.983 & 29.271 & 29.956 & & 29.178 & 30.363 & 29.881 & 29.593 & 28.372 & 26.258 & 31.515 & 36.694 & 32.532 & 16.261 & 21.357 & 15.571 & 10.371 & 31.711 & $1 \quad 32.409$ & 29.623 & 31.478 & $\begin{array}{l}37.981 \\
\end{array}$ & 33.621 \\
\hline $1 / 20 / 1218: 00$ & & 30.37 & 30.004 & 29.311 & 29.991 & & 29.164 & 30.37 & 29.883 & 29.614 & 28.398 & 26.287 & 31.532 & 36.705 & 32.549 & 16.266 & 21.364 & 15.566 & 10.388 & 31.746 & $\begin{array}{ll}6 \quad 32.428 \\
\end{array}$ & 29.659 & 31.492 & 27.983 & 33.625 \\
\hline $1 / 20 / 1219: 00$ & & 30.384 & 30.037 & 29.356 & 30.035 & & 29.142 & 30.375 & 29.895 & 29.64 & 28.425 & 26.32 & 31.553 & 36.722 & 32.567 & 16.275 & 21.373 & 15.555 & 10.409 & 31.783 & 32.445 & 29.706 & 31.518 & $\begin{array}{l}3 \quad 27.985 \\
\end{array}$ & 33.632 \\
\hline $1 / 20 / 1220: 00$ & & 30.391 & 30.065 & 29.393 & 30.073 & & 29.147 & 30.406 & 29.895 & 29.672 & 28.449 & 26.366 & 31.576 & 36.731 & 32.591 & 16.282 & 21.387 & 15.569 & 10.428 & 31.807 & 32.471 & 29.734 & 31.537 & 27.985 & 33.642 \\
\hline $1 / 20 / 1221: 00$ & & 30.398 & 30.084 & 29.409 & 30.101 & & 29.159 & 30.415 & 29.9 & 29.688 & 28.467 & 26.375 & 31.595 & 36.743 & 32.609 & 16.294 & 21.394 & 15.581 & 10.449 & 31.83 & 32.49 & 29.76 & 31.553 & $\begin{array}{l}37.983 \\
\end{array}$ & 33.646 \\
\hline $1 / 20 / 1222: 00$ & & 30.405 & 30.1 & 29.426 & 30.122 & & 29.175 & 30.427 & 29.9 & 29.709 & 28.481 & 26.39 & 31.609 & 36.745 & 32.624 & 16.301 & 21.403 & 15.592 & 10.468 & 31.847 & 32.509 & 29.776 & 31.572 & 27.985 & 33.653 \\
\hline $1 / 20 / 1223: 00$ & & 30.41 & 30.109 & 29.435 & 30.138 & & 29.192 & 30.434 & 29.902 & 29.718 & 28.498 & 26.387 & 31.623 & 36.757 & 32.638 & 16.31 & 21.41 & 15.599 & 10.477 & 31.853 & 32.516 & 29.785 & 31.586 & $\quad 27.981$ & 33.656 \\
\hline $1 / 21 / 120: 00$ & & 30.405 & 30.105 & 29.412 & 30.136 & & 29.236 & 30.446 & 29.89 & 29.721 & 28.491 & 26.368 & 31.623 & 36.759 & 32.642 & 16.317 & 21.417 & 15.627 & 10.477 & 31.842 & 32.521 & 29.769 & 1.589 & $\quad 27.983$ & 33.656 \\
\hline 1/21/12 1:00 & & 30.408 & 30.105 & 29.398 & 30.134 & & 29.262 & 30.475 & 29.885 & 29.723 & 28.493 & 26.342 & 31.627 & 36.755 & 32.647 & 16.324 & 21.424 & 15.637 & 10.484 & 31.842 & 32.523 & 9.762 & 1.591 & 27.985 & 33.653 \\
\hline $1 / 21 / 122: 00$ & & 30.419 & 30.109 & 29.409 & 30.136 & & 29.267 & 30.451 & 29.897 & 29.732 & 28.502 & 26.347 & 31.63 & 36.762 & 32.649 & 16.331 & 21.431 & 15.635 & 10.484 & 31.849 & 32.528 & 29.774 & 31.596 & $5 \quad 27.985$ & 33.653 \\
\hline 1/21/12 3:00 & & 30.412 & 30.105 & 29.391 & 30.127 & & 29.298 & 30.482 & 29.885 & 29.732 & 28.498 & 26.335 & 31.63 & 36.764 & 32.649 & 16.336 & 21.431 & 15.663 & 10.486 & 31.835 & 32.526 & 29.755 & 31.593 & $\begin{array}{l}3 \\
\quad 27.981\end{array}$ & 33.649 \\
\hline 1/21/12 4:00 & & 30.408 & 30.086 & 29.346 & 30.103 & & 29.338 & 30.506 & 29.878 & 29.711 & 28.479 & 26.272 & 31.621 & 36.755 & 32.64 & 16.338 & 21.426 & 15.67 & 10.477 & 31.811 & 32.516 & 29.72 & 31.584 & $\quad 27.981$ & 33.642 \\
\hline $1 / 21 / 125: 00$ & & 30.419 & 30.1 & 29.381 & 30.11 & & 29.314 & 30.47 & 29.89 & 29.732 & 28.493 & 26.313 & 31.625 & 36.762 & 32.645 & $5 \quad 16.343$ & 21.429 & 15.665 & 10.482 & 31.83 & 3232.523 & 29.745 & 31.591 & $1 \quad 27.985$ & 5 $\quad 33.644$ \\
\hline $1 / 21 / 126: 00$ & & 30.412 & 30.088 & 29.358 & 30.094 & & 29.34 & 30.492 & 29.883 & 29.723 & 28.484 & 26.287 & 31.623 & 36.757 & 32.642 & 16.345 & 21.436 & 15.689 & 10.477 & 31.811 & 32.518 & 29.72 & 31.584 & 27.983 & 33.637 \\
\hline $1 / 21 / 127: 00$ & & 30.419 & 30.095 & 29.372 & 30.099 & & 29.333 & 30.468 & 29.895 & 29.728 & 28.488 & 26.299 & 31.628 & 36.759 & 32.647 & 16.347 & 21.433 & 15.681 & 10.484 & 31.825 & 532.523 & 29.731 & 31.591 & 27.983 & 33.639 \\
\hline $1 / 21 / 128: 00$ & & 30.419 & 30.088 & 29.358 & 30.087 & & 29.345 & 30.48 & 29.89 & 29.725 & 28.484 & 26.28 & 31.628 & 36.752 & 32.647 & 16.35 & 21.44 & 15.691 & 10.482 & 31.814 & 32.523 & 29.722 & 31.593 & $\begin{array}{l}3 \\
3\end{array}$ & 33.639 \\
\hline 1/21/12 9:00 & & 30.417 & 30.081 & 29.339 & 30.075 & & 29.359 & 30.489 & 29.888 & 29.718 & 28.474 & 26.263 & 31.625 & 36.752 & 32.645 & $5 \quad 16.352$ & 21.457 & 15.705 & 10.484 & 31.802 & 32.523 & 29.708 & 31.589 & $\begin{array}{l}97.988 \\
\end{array}$ & $3 \quad 33.642$ \\
\hline 1/21/12 10:00 & & 30.419 & 30.081 & 29.349 & 30.075 & & 29.356 & 30.485 & 29.895 & 29.723 & 28.477 & 26.27 & 31.627 & 36.752 & 32.647 & 16.352 & 21.461 & 15.705 & 10.487 & 31.809 & 32.523 & 29.71 & 31.591 & $\begin{array}{l}17.988 \\
1\end{array}$ & 333.642 \\
\hline 1/21/12 11:00 & & 30.415 & 30.07 & 29.323 & 30.061 & & 29.377 & 30.511 & 29.885 & 29.711 & 28.467 & 26.244 & 31.625 & 36.745 & 32.647 & 16.352 & 21.463 & 15.721 & 10.484 & 31.795 & 32.521 & 29.691 & 31.586 & $\begin{array}{l}5 \\
5\end{array}$ & 33.639 \\
\hline $1 / 21 / 1212: 00$ & & 30.408 & 30.053 & 29.29 & 30.038 & & 29.401 & 30.525 & 29.878 & 29.695 & 28.449 & 26.213 & 31.616 & 36.74 & 32.635 & $5 \quad 16.352$ & 21.463 & 15.733 & 10.477 & 31.767 & 32.514 & 29.666 & 31.579 & $\begin{array}{l}27.983 \\
9\end{array}$ & 33.635 \\
\hline 1/21/12 13:00 & & 30.394 & 30.021 & 29.229 & 29.998 & & 29.436 & 30.533 & 29.869 & 29.667 & 28.419 & 26.155 & 31.599 & 36.726 & 32.624 & $\begin{array}{l}16.347 \\
7\end{array}$ & 21.459 & 15.757 & 10.461 & 31.732 & 32.497 & 29.616 & 31.565 & $\begin{array}{l}27.983 \\
5\end{array}$ & 33.63 \\
\hline 1/21/12 14:00 & & 30.382 & 29.993 & 29.183 & 29.961 & & 29.455 & 30.528 & 29.864 & 29.642 & 28.391 & 26.112 & 31.583 & 36.712 & 32.607 & 16.343 & 21.457 & 15.766 & 10.444 & 31.697 & 32.478 & 29.565 & 31.544 & $\begin{array}{r}47.983 \\
+4\end{array}$ & 33.621 \\
\hline $\begin{array}{l}1 / 21 / 12 ~ 15: 00 \\
\end{array}$ & & 30.377 & 29.967 & 29.148 & 29.921 & & 29.455 & 30.518 & 29.859 & 29.619 & 28.367 & 26.079 & 31.567 & 36.705 & 32.591 & 16.336 & 21.459 & 15.757 & 10.428 & 31.676 & $\begin{array}{l}52.462 \\
5\end{array}$ & 29.541 & 31.528 & $\begin{array}{l}27.988 \\
3\end{array}$ & 33.618 \\
\hline $\begin{array}{l}1 / 21 / 1216: 00 \\
\end{array}$ & & 30.37 & 29.944 & 29.124 & 29.888 & & 29.45 & 30.506 & 29.857 & 29.598 & 28.344 & 26.059 & 31.55 & 36.694 & $\begin{array}{l}32.574 \\
32.574\end{array}$ & $\begin{array}{l}16.350 \\
4.329\end{array}$ & $\begin{array}{l}21.459 \\
21.514\end{array}$ & 15.75 & $\begin{array}{l}10.460 \\
10.414\end{array}$ & 31.648 & $\begin{array}{l}32.402 \\
32.445 \\
3\end{array}$ & 29.513 & 31.514 & $\begin{array}{r}27.983 \\
+7\end{array}$ & 33.611 \\
\hline $1 / 21 / 1217: 00$ & & 30.361 & 29.918 & 29.098 & 29.86 & & 29.443 & 30.513 & 29.852 & 29.575 & 28.321 & 26.036 & 31.56 & 36.684 & 32.586 & $5 \quad 16.322$ & 21.517 & 15.745 & 10.43 & 31.632 & 32.457 & 29.482 & 31.523 & $\begin{array}{l}3 \\
27.985\end{array}$ & 33.604 \\
\hline $1 / 21 / 1218: 00$ & & 30.349 & 29.895 & 29.063 & 29 & & 29.436 & 30.489 & 29.845 & 29.549 & 28.298 & 26.009 & 31.592 & 36.672 & 32.621 & 16.312 & 21.51 & 15.742 & 10.461 & 31.637 & 32.485 & 9.454 & 31.553 & $\begin{array}{l}3 \quad 27.985 \\
\end{array}$ & 33.602 \\
\hline $1 / 21 / 1219: 00$ & & 30.345 & 29.871 & 29.042 & 29.8 & & & 30.487 & 29.845 & 29.523 & 28.277 & 25.99 & 31.553 & 36.665 & 32.581 & 16.305 & 21.514 & 15.726 & 10.418 & 31.611 & 32.45 & 29.442 & 31.516 & $\quad 27.988$ & 33.6 \\
\hline $1 / 21 / 1220: 00$ & & 30.342 & 29.864 & 29.047 & 29.793 & & 29.394 & 30.478 & 29.847 & 29.514 & 28.267 & 25.997 & 31.536 & 36.661 & 32.563 & $\begin{array}{l}36.296 \\
\end{array}$ & 21.517 & 15.705 & 10.402 & 31.602 & 32.431 & 29.435 & 31.495 & $\quad 27.986$ & 33.6 \\
\hline $1 / 21 / 1221: 00$ & & 30.333 & 29.85 & 29.033 & 29.783 & & 29.375 & 30.475 & 29.843 & 29.5 & 28.253 & 25.997 & 31.522 & 36.656 & 32.548 & $3 \quad 16.289$ & 21.551 & 15.705 & 10.388 & 31.588 & 32.419 & 29.424 & 31.483 & $3 \quad 27.985$ & 33.6 \\
\hline $1 / 21 / 1222: 00$ & & 30.335 & 29.846 & 29.04 & 29. & & 29.352 & 30.456 & 29.845 & 29.493 & 28.249 & 26.009 & 31.515 & 36.649 & 32.541 & 16.284 & 21.547 & 15.691 & 10.379 & 31.597 & 732.409 & 29.428 & 31.478 & 27.99 & 33.6 \\
\hline $1 / 21 / 1223: 00$ & & 30.324 & 29.832 & 29.019 & 29. & & 29.347 & 30.456 & 29.838 & 29.475 & 28.232 & 25.99 & 31.503 & 36.644 & 32.527 & 16.278 & 21.542 & 15.691 & 10.367 & 31.571 & 32.4 & 29.407 & 31.467 & 27.988 & 33.6 \\
\hline 1/22/12 0:00 & & 30.314 & 29.818 & & 29 . & & 29.342 & 30.468 & 29.833 & 29.454 & 28.214 & 25.978 & 31.489 & 36.635 & 32.518 & $\begin{array}{l}3 \\
3\end{array}$ & 21.533 & 15.681 & 10.357 & 31.553 & $3 \quad 32.386$ & 29.393 & 31.453 & $\begin{array}{l}3 \quad 27.985 \\
\end{array}$ & 33.597 \\
\hline 1/22/12 1:00 & & 30.303 & 29.792 & 28.965 & 29. & & 29.345 & 30.411 & 29.824 & 29.426 & 28.193 & 25.947 & 31.471 & 36.63 & 32.504 & 16.261 & 21.528 & 15.681 & 10.341 & 31.532 & 32.367 & 29.362 & 31.431 & 27.985 & 33.59 \\
\hline $1 / 22 / 122: 00$ & & 30.298 & 29.778 & 28.958 & 29. & & 29.33 & 30.408 & 29.819 & 29.412 & 28.179 & 25.947 & 31.459 & 36.626 & 32.485 & $5 \quad 16.252$ & 21.526 & 15.672 & 10.327 & 31.52 & 32.355 & 29.355 & 31.42 & 27.983 & 33.593 \\
\hline $1 / 22 / 123: 00$ & & 30.298 & 29.771 & 28.954 & 29.702 & & 29.309 & 30.413 & 29.826 & 29.4 & 28.172 & 25.952 & 31.447 & 36.621 & 32.473 & $3 \quad 16.245$ & 21.519 & 15.66 & 10.313 & 31.513 & 32.341 & 29.351 & 31.41 & $\begin{array}{l}1 \\
1\end{array}$ & $3 \quad 33.583$ \\
\hline $1 / 22 / 124: 00$ & & 30.291 & 29.757 & 28.942 & 29.688 & & 29.302 & 30.399 & 29.814 & 29.384 & 28.156 & 25.942 & 31.433 & 36.616 & 32.459 & $\begin{array}{l}9 \\
9\end{array}$ & 21.517 & 15.665 & 10.301 & 31.499 & 32.331 & 29.341 & 31.396 & $\quad 27.985$ & 33.579 \\
\hline $1 / 22 / 125: 00$ & & 30.279 & 29.741 & 28.916 & 29.669 & & 29.3 & 30.387 & 29.807 & 29.361 & 28.132 & 25.923 & 31.417 & 36.609 & 32.445 & $5 \quad 16.231$ & 21.507 & 15.667 & 10.284 & 31.476 & 32.312 & 29.313 & 31.38 & $\begin{array}{l}3 \quad 27.983 \\
\end{array}$ & 33.569 \\
\hline 1/22/12 6:00 & & 30.282 & 29.736 & 28.918 & 29.66 & & 29.279 & 30.387 & 29.812 & 29.354 & 28.13 & 25.93 & 31.408 & 36.604 & 32.434 & 16.222 & 21.503 & 15.649 & 10.275 & 31.476 & 32.305 & 29.315 & 31.373 & $\begin{array}{l}3 \quad 27.988 \\
\end{array}$ & 33.567 \\
\hline 1/22/12 7:00 & & 30.284 & 29.734 & 28.925 & 29.662 & & 29.251 & 30.387 & 29.816 & 29.347 & 28.13 & 25.937 & 31.403 & 36.6 & 32.427 & 16.217 & 21.5 & 15.632 & 10.263 & 31.478 & 32.296 & 29.327 & 31.37 & $\quad 27.99$ & 33.562 \\
\hline $1 / 22 / 128: 00$ & & 30.272 & 29.724 & 28.914 & 29.655 & & 29.246 & 30.375 & 29.809 & 29.335 & 28.116 & 25.935 & 31.393 & 36.593 & 32.42 & 16.21 & 21.503 & 15.635 & 10.259 & 31.464 & 32.289 & 29.313 & 31.356 & $\begin{array}{l}57.988 \\
\end{array}$ & 33.558 \\
\hline $1 / 22 / 129: 00$ & & 30.277 & 29.729 & 28.928 & 29.66 & & 29.218 & 30.372 & 29.812 & 29.333 & 28.119 & 25.949 & 31.394 & 36.6 & 32.417 & 16.208 & 21.51 & 15.625 & 10.259 & 31.473 & 32.289 & 29.32 & 31.359 & $\quad 27.988$ & 33.56 \\
\hline $1 / 22 / 1210: 00$ & & 30.277 & 29.736 & 28.944 & 29.671 & & 29.196 & 30.353 & 29.814 & 29.335 & 28.125 & 25.973 & 31.396 & 36.595 & 32.42 & 16.201 & 21.507 & 15.613 & 10.256 & 31.485 & 32.291 & 29.334 & 31.361 & 27.988 & 333.562 \\
\hline $1 / 22$ & & 30.272 & 29.738 & 28.954 & 29.6 & & 29.185 & 30.355 & 29.807 & 29.34 & 28.123 & 25.992 & 31.396 & 36.59 & 32.424 & 16.201 & 21.51 & 15.609 & 10.261 & 31.49 & 32.293 & 29.344 & 31.361 & 27.99 & 33.565 \\
\hline $1 / 2$ & & 30.2 & 29.73 & 28.937 & $x^{2}+2$ & & th & 30.3 & 年 & $202-3$ & 28.1 & 25.976 & 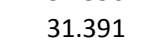 & 36.5 & 32.415 & -1 & -5 & ace & 10.2 & 31.483 & $a^{2}$ & - & anc & 27.99 & 33.565 \\
\hline & & 30. & 29.72 & 28. & & & & 30. & & & 28. & & & 36. & 32. & & 21.5 & 15. & 10 & 31. & & & & 9 & \\
\hline & & & 29.729 & 28 & & & & & & & 28.1 & & & 36.5 & 0 & & & 15.5 & & & & & & 93 & \\
\hline $215: 00$ & & 30.289 & 29.741 & 28.961 & 29.688 & & 29.133 & 30.288 & 29.826 & 29.319 & 28.125 & 25.985 & 31.387 & 36.59 & 32.408 & $\begin{array}{ll}3 & 16.189\end{array}$ & 21.544 & 15.573 & 10.247 & 31.506 & 32.284 & 29.372 & 31.354 & 8.004 & 33.56 \\
\hline
\end{tabular}




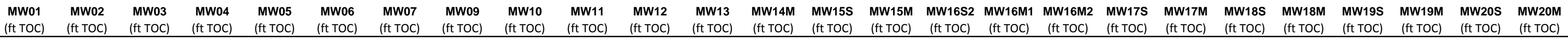

\begin{tabular}{|c|c|c|c|c|c|c|c|c|c|c|c|c|c|c|c|c|c|c|c|c|c|c|c|c|c|}
\hline Well & $\begin{array}{l}\text { (ft TOC) } \\
\text { (is }\end{array}$ & $\begin{array}{l}\text { (ft TOC) } \\
\text { (f) }\end{array}$ & $\begin{array}{l}\text { (ft TOC) } \\
\text { (f) }\end{array}$ & $\begin{array}{l}\text { (ft TOC) } \\
\text { (f }\end{array}$ & $\begin{array}{l}\text { (ft TOC) } \\
\text { (f) }\end{array}$ & $\begin{array}{l}\text { (ft TOC) } \\
\text { (f) }\end{array}$ & $\begin{array}{l}\text { (ft TOC) } \\
\text { (ft }\end{array}$ & $\begin{array}{l}\text { (ft TOC) } \\
\text { (f) }\end{array}$ & $\begin{array}{l}\text { (ft TOC) } \\
\text { (ft) }\end{array}$ & $\begin{array}{l}\text { (ft TOC) } \\
\text { (f) }\end{array}$ & $\begin{array}{l}\text { (ft TOC) } \\
\text { (f) }\end{array}$ & $\begin{array}{l}\text { (ft TOC) } \\
\text { (f) }\end{array}$ & (ft TOC) & $\begin{array}{l}\text { (ft TOC) } \\
\text { (f) }\end{array}$ & (ft TOC) & $\begin{array}{ll}\text { (ft TOC) } \\
\text { (ft TOC) }\end{array}$ & (ft TOC) & $\begin{array}{l}\text { (ft TOC) } \\
\text { (1) }\end{array}$ & (ft TOC) & $\begin{array}{l}\text { (ft TOC) } \\
\end{array}$ & $\begin{array}{l}\text { (ft TOC) } \\
\text { (f) }\end{array}$ & $\begin{array}{l}\text { (ft TOC) } \\
\text { (n) }\end{array}$ & (ft TOC) & $\begin{array}{l}\text { (ft TOC) } \\
\text { (1) }\end{array}$ & (ft TOC) \\
\hline 16:00 & & 30.293 & 29.773 & 29.031 & 29.73 & & 29.088 & 30.265 & 29.831 & 29.349 & 28.153 & 26.074 & 31.3 & 36.6 & 32.42 & 16. & 21.607 & 15.548 & 10.256 & 31. & 32.293 & 29.421 & 66 & 27.995 & 33.562 \\
\hline $1 / 22 / 1217: 00$ & & 30.303 & 29.813 & 29.094 & 29.783 & & 29.051 & 30.274 & 29.838 & 29.38 & 28.186 & 26.138 & 31.415 & 36.616 & 32.436 & 16.196 & 21.602 & 15.529 & 10.275 & 31.585 & 32.31 & 29.478 & 31.382 & 27.995 & 33.565 \\
\hline $1 / 22 / 1218: 00$ & & 30.31 & 29.843 & 29.138 & 29.83 & & 29.034 & 30.279 & 29.838 & 29.405 & 28.216 & 26.181 & 31.826 & 36.628 & 32.879 & 16.201 & 21.6 & 15.524 & 10.71 & 31.744 & 32.727 & 29.529 & 31.788 & 27.993 & 33.567 \\
\hline 1/22/12 19:00 & & 30.319 & 29.883 & 29.197 & 29.891 & & 29.008 & 30.281 & 29.85 & 29.445 & 28.251 & 26.239 & 31.681 & 36.647 & 32.71 & 16.208 & 21.591 & 15.505 & 10.55 & 31.762 & 32.575 & 29.588 & 31.64 & 27.993 & 33.567 \\
\hline 1/22/12 20:00 & & 30.331 & 29.923 & 29.241 & 29.944 & & 29.001 & 30.296 & 29.857 & 29.477 & 28.286 & 26.284 & 31.637 & 36.663 & 32.666 & $5 \quad 16.219$ & 21.591 & 15.503 & 10.503 & 31.776 & 32.535 & 29.635 & 31.603 & 27.99 & 33.572 \\
\hline 1/22/12 21:00 & & 30.335 & 29.944 & 29.26 & 29.982 & & 29.02 & 30.298 & 29.852 & 29.498 & 28.307 & 26.294 & 31.625 & 36.672 & 32.649 & 16.226 & 21.593 & 15.505 & 10.487 & 31.783 & 32.521 & 29.663 & 31.589 & 27.995 & 33.579 \\
\hline 1/22/12 22:00 & & 30.34 & 29.967 & 29.276 & 30.01 & & 29.032 & 30.327 & 29.855 & 29.521 & 28.323 & 26.308 & 31.625 & 36.687 & 32.645 & 16.236 & 21.602 & 15.515 & 10.484 & 31.8 & 32.518 & 29.684 & 31.589 & 27.995 & 33.586 \\
\hline 1/22/12 23:00 & & 30.314 & 29.939 & 29.211 & 29.993 & & 29.121 & 30.394 & 29.821 & 29.512 & 28.302 & 26.251 & 31.611 & 36.677 & 32.631 & 16.24 & 21.605 & 15.576 & 10.47 & 31.751 & 32.502 & 29.616 & 31.572 & 27.985 & 33.583 \\
\hline 1/23/12 0:00 & & 30.347 & 29.986 & 29.285 & 30.035 & & 29.072 & 30.344 & 29.857 & 29.547 & 28.349 & 26.308 & 31.62 & 36.696 & 32.64 & 16.252 & 21.609 & 15.527 & 10.482 & 31.804 & 32.516 & 29.694 & 31.586 & 27.995 & 33.593 \\
\hline 1/23/12 1:00 & & 30.354 & 30.002 & 29.302 & 30.057 & & 29.084 & 30.355 & 29.859 & 29.568 & 28.365 & 26.32 & 31.625 & 36.708 & 32.645 & $5 \quad 16.264$ & 21.616 & 15.534 & 10.484 & 31.816 & 32.523 & 29.717 & 31.591 & 27.995 & 33.597 \\
\hline 1/23/12 2:00 & & 30.363 & 30.025 & 29.327 & 30.08 & & 29.088 & 30.365 & 29.869 & 29.591 & 28.388 & 26.335 & 31.634 & 36.712 & 32.654 & 16.273 & 21.616 & 15.527 & 10.489 & 31.832 & 32.528 & 29.738 & 31.596 & 27.997 & 33.597 \\
\hline 1/23/12 3:00 & & 30.373 & 30.051 & 29.356 & 30.106 & & 29.093 & 30.377 & 29.874 & 29.616 & 28.409 & 26.361 & 31.642 & 36.719 & 32.659 & 16.284 & 21.618 & 15.536 & 10.498 & 31.853 & 32.537 & 29.762 & 31.607 & 27.997 & 33.602 \\
\hline 1/23/12 4:00 & & 30.375 & 30.058 & 29.356 & 30.117 & & 29.117 & 30.387 & 29.869 & 29.63 & 28.421 & 26.361 & 31.644 & 36.724 & 32.661 & 16.296 & 21.618 & 15.552 & 10.501 & 31.856 & 32.542 & 29.764 & 31.61 & 27.997 & 33.6 \\
\hline $1 / 23 / 125: 00$ & & 30.377 & 30.07 & 29.363 & 30.129 & & 29.138 & 30.396 & 29.874 & 29.644 & 28.432 & 26.361 & 31.649 & 36.731 & 32.668 & $\begin{array}{l}36.303 \\
3\end{array}$ & 21.614 & 15.564 & 10.505 & 31.863 & 32.547 & 29.776 & 31.612 & 27.995 & 33.597 \\
\hline $1 / 23 / 126: 00$ & & 30.382 & 30.077 & 29.365 & 30.136 & & 29.157 & 30.401 & 29.874 & 29.658 & 28.442 & 26.356 & 31.651 & 36.74 & 32.67 & 16.312 & 21.628 & 15.573 & 10.51 & 31.863 & 32.547 & 29.776 & 31.617 & 27.995 & 33.595 \\
\hline 1/23/12 7:00 & & 30.387 & 30.086 & 29.37 & 30.141 & & 29.173 & 30.406 & 29.881 & 29.667 & 28.451 & 26.351 & 31.653 & 36.738 & 32.673 & 16.322 & 21.635 & 15.583 & 10.512 & 31.872 & 32.552 & 29.781 & 31.622 & 27.997 & 33.595 \\
\hline $1 / 23 / 128: 00$ & & 30.394 & 30.093 & 29.381 & 30.148 & & 29.18 & 30.415 & 29.881 & 29.681 & 28.463 & 26.359 & 31.66 & 36.738 & 32.68 & $\begin{array}{l}36.329 \\
3\end{array}$ & 21.632 & 15.588 & 10.517 & 31.879 & 32.559 & 29.79 & 31.626 & 28 & 33.597 \\
\hline $1 / 23 / 129: 00$ & & 30.398 & 30.107 & 29.393 & 30.159 & & 29.189 & 30.42 & 29.888 & 29.695 & 28.474 & 26.366 & 31.67 & 36.745 & 32.687 & 16.338 & 21.637 & 15.595 & 10.524 & 31.881 & 32.566 & 29.804 & 31.636 & 27.997 & 33.6 \\
\hline $1 / 23 / 1210: 00$ & & 30.408 & 30.123 & 29.414 & 30.173 & & 29.194 & 30.427 & 29.893 & 29.716 & 28.491 & 26.38 & 31.684 & 36.747 & 32.701 & 16.347 & 21.639 & 15.602 & 10.541 & 31.905 & 32.58 & 29.825 & 31.645 & 28 & 33.604 \\
\hline $1 / 23 / 1211: 00$ & & 30.403 & 30.125 & 29.409 & 30.18 & & 29.22 & 30.444 & 29.888 & 29.723 & 28.495 & 26.375 & 31.691 & 36.752 & 32.708 & 16.354 & 21.642 & 15.625 & 10.543 & 31.902 & 32.585 & 29.816 & 31.652 & 27.997 & 33.607 \\
\hline $1 / 23 / 1212: 00$ & & 30.398 & 30.119 & 29.388 & 30.169 & & 29.255 & 30.466 & 29.881 & 29.723 & 28.488 & 26.351 & 31.691 & 36.757 & 32.708 & 16.361 & 21.644 & 15.649 & 10.545 & 31.891 & 32.585 & 9.797 & 31.654 & 27.997 & 33.609 \\
\hline $1 / 23 / 1213: 00$ & & 30.396 & 30.105 & 29.358 & 30.152 & & 29.288 & 30.489 & 29.878 & 29.714 & 28.479 & 26.315 & 31.688 & 36.75 & 32.706 & 16.366 & 21.642 & 15.667 & 10.543 & 31.874 & 32.582 & 9.781 & 31.652 & 28 & 33.609 \\
\hline $1 / 23 / 1214: 00$ & & 30.398 & 30.102 & 29.351 & 30.141 & & 29.302 & 30.499 & 29.881 & 29.714 & 28.477 & 26.301 & 31.684 & 36.75 & 32.706 & 16.371 & 21.642 & 15.674 & 10.54 & 31.87 & 32.58 & 29.771 & 1.647 & 28 & 33.607 \\
\hline $1 / 23 / 1215: 00$ & & 30.401 & 30.098 & 29.339 & 30.126 & & 29.312 & 30.499 & 29.888 & 29.711 & 28.472 & 26.287 & 31.681 & 36.748 & 32.701 & 16.375 & 21.639 & 15.681 & 10.536 & 31.858 & 32.575 & 29.76 & 31.645 & 28 & 33.604 \\
\hline 1/23/12 16:00 & & 30.401 & 30.091 & 29.328 & 30.117 & & 29.323 & 30.499 & 29.883 & 29.709 & 28.467 & 26.275 & 31.677 & 36.745 & 32.699 & 16.375 & 21.639 & 15.689 & 10.533 & 31.853 & 32.573 & 29.745 & 31.64 & 28 & 33.602 \\
\hline 1/23/12 17:00 & & 30.405 & 30.088 & 29.33 & 30.11 & & 29.319 & 30.497 & 29.888 & 29.709 & 28.47 & 26.27 & 31.674 & 36.74 & 32.694 & 16.378 & 21.646 & 15.691 & 10.531 & 31.851 & 32.571 & 29.745 & 31.638 & 28.002 & 33.602 \\
\hline $1 / 23 / 1218: 00$ & & 30.408 & 30.093 & 29.342 & 30.108 & & 29.309 & 30.482 & 29.89 & 29.716 & 28.472 & 26.28 & 31.674 & 36.74 & 32.694 & 16.382 & 21.678 & 15.691 & 10.531 & 31.851 & 32.571 & 29.75 & 31.64 & 28.002 & 33.6 \\
\hline 1/23/12 19:00 & & 30.415 & 30.102 & 29.363 & 30.122 & & 29.295 & 30.451 & 29.895 & 29.725 & 28.484 & 26.299 & 31.679 & 36.75 & 32.696 & 5 $\quad 16.385$ & 21.683 & 15.686 & 10.536 & 31.867 & 32.575 & 29.769 & 31.645 & 28 & 33.6 \\
\hline $1 / 23 / 1220: 00$ & & 30.415 & 30.107 & 29.372 & 30.129 & & 29.295 & 30.456 & 29.895 & 29.732 & 28.488 & 26.308 & 31.686 & 36.755 & 32.706 & 16.385 & 21.688 & 15.693 & 10.54 & 31.872 & 32.582 & 29.769 & 31.652 & 28 & 33.602 \\
\hline $1 / 23 / 1221: 00$ & & 30.415 & 30.105 & 29.36 & 30.127 & & 29.305 & 30.461 & 29.895 & 29.73 & 28.484 & 26.292 & 31.686 & 36.752 & 32.708 & $3 \quad 16.387$ & 21.692 & 15.705 & 10.545 & 31.872 & 32.585 & 29.767 & 31.652 & 28.002 & 33.604 \\
\hline $1 / 23 / 1222: 00$ & & 30.419 & 30.109 & 29.372 & 30.131 & & 29.3 & 30.454 & 29.902 & 29.737 & 28.491 & 26.301 & 31.695 & 36.755 & 32.713 & $3 \quad 16.392$ & 21.695 & 15.703 & 10.55 & 31.872 & 32.59 & 29.776 & 31.659 & 28.002 & 33.609 \\
\hline $1 / 23 / 1223: 00$ & & 30.422 & 30.111 & 29.374 & 30.134 & & 29.302 & 30.456 & 29.902 & 29.742 & 28.493 & 26.301 & 31.7 & 36.759 & 32.72 & 16.394 & 21.697 & 15.703 & 10.555 & 31.879 & 32.594 & 29.781 & 31.664 & 28.004 & 33.611 \\
\hline $1 / 24 / 120: 00$ & & 30.422 & 30.114 & 29.377 & 30.136 & & 29.307 & 30.458 & 29.904 & 29.744 & 28.495 & 26.301 & 31.702 & 36.752 & 32.724 & 16.399 & 21.695 & 15.703 & 10.559 & 31.879 & 32.601 & 29.778 & 31.668 & 28.002 & 33.614 \\
\hline 1/24/12 1:00 & & 30.422 & 30.114 & 29.377 & 30.138 & & 29.312 & 30.466 & 29.904 & 29.746 & 28.495 & 26.301 & 31.705 & 36.759 & 32.724 & 16.401 & 21.697 & 15.703 & 10.564 & 31.877 & 32.599 & 29.774 & 31.666 & 28.002 & 33.616 \\
\hline $1 / 24 / 122: 00$ & & 30.426 & 30.114 & 29.374 & 30.1 & & 29.316 & 30.466 & 29.902 & 29.749 & 28.498 & 26.299 & 31.707 & 36.759 & 32.727 & 16.4 & 21.697 & 15.707 & 10.566 & 31.877 & 32.601 & 29.778 & 31.671 & 28.002 & 33.616 \\
\hline 1/24/12 3:00 & & 30.426 & 30.112 & 29.374 & 30. & & 29.323 & 30.468 & 29.904 & 29.749 & 28.495 & 26.296 & 31.707 & 36.757 & 32.727 & 16.403 & 21.692 & 15.71 & 10.564 & 31.879 & 32.604 & 29.781 & 31.671 & 28.002 & 33.614 \\
\hline 1/24/12 4:00 & & 30.428 & 30.114 & 29.372 & 30.136 & & 29.328 & $\begin{array}{l}30.473 \\
30.473\end{array}$ & $\begin{array}{r}2.5044 \\
29.9\end{array}$ & 29.751 & 28.495 & 26.292 & 31.705 & 36.759 & 32.727 & 16.403 & 21.702 & 15.71 & $\begin{array}{l}10.564 \\
10.562\end{array}$ & 31.877 & 32.601 & 29.774 & 31.668 & 28.002 & 33.611 \\
\hline $1 / 24 / 125: 00$ & & 30.428 & 30.112 & 29.367 & 30.131 & & 29.33 & 30.473 & 29.904 & 29.749 & 28.495 & 26.287 & 31.702 & 36.757 & 32.722 & 16.4 & 21.702 & $\begin{array}{l}15.714 \\
15.714\end{array}$ & $\begin{array}{l}10.559 \\
10.559\end{array}$ & 31.874 & 32.599 & 29.771 & 31.666 & 28.004 & 33.607 \\
\hline $1 / 24 / 126: 00$ & & 30.428 & 30.109 & 29.367 & 30. & & 29.338 & 30.478 & 29.904 & 29.751 & 28.495 & 26.284 & 31 & 36.757 & 32.717 & 16. & 21.697 & 15.719 & 10.557 & 31.87 & 32.594 & 29.767 & 31.664 & 28.004 & 33.604 \\
\hline $1 / 24 / 127: C$ & & 30.435 & 30.116 & 29.3 & 30. & & 29 & 30.475 & 29.912 & 29. & 28.5 & 26.299 & 31 & 36.762 & 32.722 & 16. & 97 & 15.717 & 10.562 & 31.877 & 32.599 & 78 & 66 & 28.004 & 33.604 \\
\hline & & 30 & 30.12 & 29.3 & & & & 30.4 & 29.919 & & 28.507 & & 31.70 & 36.7 & 32.724 & 16 & 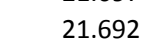 & 15.712 & 10.562 & 31.888 & 501 & 88 & 88 & 28.007 & 33.604 \\
\hline $1 / 24 / 12$ & & 30.438 & 30. & 29. & & & & 30.4 & 29 & & 28.509 & 26 & & 36.7 & 32.7 & & 21.69 & 15. & 10.5 & 31.888 & 604 & 88 & 31.673 & 28.007 & 33.604 \\
\hline $1 / 24 / 1210: 00$ & & 30.447 & 30.137 & 29.419 & 30.1 & & & 30.475 & 29.923 & & 28.5 & & & 36.764 & 32.736 & $\quad 16.415$ & & 15.7 & 10.573 & 31.902 & 32.613 & 29.816 & 31.68 & 28.007 & 33.609 \\
\hline 1/24/12 11:00 & & 30.442 & 30.139 & 29.421 & 30.1 & & 2 & 30.485 & 29.921 & 29. & 28.525 & 26.335 & 31 & 36.769 & 32.741 & 16.417 & 21.704 & 15.735 & 10.581 & 31.905 & 32.618 & 29.814 & 31.687 & 28.004 & 33.611 \\
\hline 1/24/12 12:00 & & 30.435 & 30.1 & 29.386 & & & & 30.5 & 29.904 & & 28.509 & 26.296 & 31.721 & 36.766 & 32.741 & 16.4 & & 15.752 & 10.581 & 31.886 & 32.618 & 29.781 & 85 & 28.007 & 33.614 \\
\hline $1 / 24 / 12$ 13:00 & & 30.426 & 30.1 & 29.346 & 30. & & & 30.542 & 29.9 & & 28.491 & & 31.714 & 36.759 & 32.736 & 516.4 & 21.704 & 15.773 & 10.574 & 31.865 & 32.609 & 29.75 & 31.678 & 28.004 & 33.609 \\
\hline $1 / 24 / 1214: 00$ & & 30.428 & 30.098 & 29.325 & 30. & & 29. & 30.547 & 29.9 & & 28.484 & 26.239 & 31.705 & 36.752 & 32.731 & 16 & 21.713 & 15.775 & 10.566 & 31.853 & 32.604 & 29.738 & 31.668 & 28.004 & 33.609 \\
\hline 1/24/12 15:00 & & 30.431 & 30.098 & 29.335 & 30.1 & & 29.3 & 30.5 & 29.907 & 29. & 28.484 & 26.246 & 31.705 & 36.75 & 32.727 & 16.4 & 21.736 & 15.768 & 10.564 & 31.856 & 32.601 & 29.743 & 31.668 & 28.007 & 33.607 \\
\hline $1 / 24 / 1216: 00$ & & 30.438 & 30.1 & 29.349 & 30.11 & & 29.38 & 30.521 & 29.912 & 29.749 & 28.488 & 26.258 & 31.705 & 36.75 & 32.727 & 16.4 & 21.787 & 15.761 & 10.562 & 31.86 & 32.601 & 29.757 & 31.668 & 28.009 & 33.609 \\
\hline $1 / 24 / 1217: 00$ & & 30.44 & 30.107 & 29.36 & 30.117 & & 29.368 & 30.504 & 29.916 & 29.751 & 28.493 & 26.268 & 31.707 & 36.747 & 32.727 & 16.417 & 21.783 & 15.757 & 10.564 & 31.867 & 32.601 & 29.767 & 31.671 & 28.009 & 33.607 \\
\hline $1 / 24 / 1218: 00$ & & 30.447 & 30.121 & 29.391 & 30.136 & & 29.349 & 30.482 & 29.919 & 29.767 & 28.505 & 26.299 & 31.714 & 36.759 & 32.729 & $\begin{array}{l}16.417 \\
\end{array}$ & 21.778 & 15.752 & 10.567 & 31.884 & 32.611 & 29.785 & 31.68 & 28.009 & 33.607 \\
\hline 1/24/12 19:00 & & 30.452 & 30.128 & 29.398 & 30.145 & & 29.345 & 30.485 & 29.926 & 29.772 & 28.512 & 26.304 & 31.719 & 36.762 & 32.736 & $5 \quad 16.42$ & 21.778 & 15.752 & 10.574 & 31.893 & 32.616 & 29.797 & 31.683 & 28.011 & 33.607 \\
\hline $1 / 24 / 1220: 00$ & & 30.456 & 30.137 & 29.416 & 30.159 & & 29.335 & 30.482 & 29.931 & 29.781 & 28.521 & 26.318 & 31.723 & 36.766 & 32.745 & $\quad 16.424$ & 21.776 & 15.747 & 10.581 & 31.909 & 32.62 & 29.811 & 31.69 & 28.014 & 33.609 \\
\hline $1 / 24 / 1221: 00$ & & 30.459 & 30.142 & 29.426 & 30.169 & & 29.338 & 30.485 & 29.931 & 29.79 & 28.528 & 26.332 & 31.731 & 36.769 & 32.75 & 16.427 & 21.78 & 15.754 & 10.588 & 31.916 & 32.63 & 29.823 & 31.697 & 28.011 & 33.611 \\
\hline $1 / 24 / 1222: 00$ & & 30.459 & 30.146 & 29.43 & 30.178 & & 29.342 & 30.482 & 29.931 & 29.795 & 28.532 & 26.332 & 31.74 & 36.776 & 32.76 & $\quad 16.427$ & 21.787 & 15.761 & 10.595 & 31.919 & 32.632 & 29.823 & 31.701 & 28.011 & 33.616 \\
\hline $1 / 24 / 1223: 00$ & & 30.466 & 30.158 & 29.451 & 30.192 & & 29.338 & 30.494 & 29.935 & 29.807 & 28.542 & 26.349 & 31.749 & 36.771 & 32.769 & 16.431 & 21.792 & 15.759 & 10.604 & 31.93 & 32.646 & 29.842 & 31.713 & 28.011 & 33.621 \\
\hline
\end{tabular}




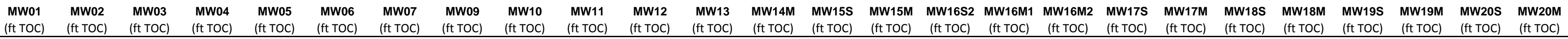

\begin{tabular}{|c|c|c|c|c|c|c|c|c|c|c|c|c|c|c|c|c|c|c|c|c|c|c|c|c|c|}
\hline Well & $\begin{array}{l}\text { (ft TOC) } \\
\text { (ft }\end{array}$ & $\begin{array}{l}\text { (ft TOC) } \\
\text { (f) }\end{array}$ & $\begin{array}{l}\text { (ft TOC) } \\
\text { (f) }\end{array}$ & $\begin{array}{l}\text { (ft TOC) } \\
\text { (f }\end{array}$ & $\begin{array}{l}\text { (ft TOC) } \\
\text { (f) }\end{array}$ & $\begin{array}{l}\text { (ft TOC) } \\
\text { (f) }\end{array}$ & $\begin{array}{l}\text { (ft TOC) } \\
\text { (ft }\end{array}$ & $\begin{array}{l}\text { (ft TOC) } \\
\text { (f) }\end{array}$ & $\begin{array}{l}\text { (ft TOC) } \\
\text { (ft) }\end{array}$ & $\begin{array}{l}\text { (ft TOC) } \\
\text { (f) }\end{array}$ & $\begin{array}{l}\text { (ft TOC) } \\
\text { (f) }\end{array}$ & $\begin{array}{l}\text { (ft TOC) } \\
\text { (f) }\end{array}$ & (ft TOC) & $\begin{array}{l}\text { (ft TOC) } \\
\end{array}$ & $\begin{array}{l}\text { (ft TOC) } \\
\end{array}$ & $\begin{array}{ll}\text { (ft TOC) } \\
\text { (ft TOC) }\end{array}$ & (ft TOC) & $\begin{array}{l}\text { (ft TOC) } \\
\text { (1) }\end{array}$ & (ft TOC) & $\begin{array}{l}\text { (ft TOC) } \\
\end{array}$ & (ft TOC) & $\begin{array}{l}\text { (ft TOC) } \\
\end{array}$ & (ft TOC) & $\begin{array}{l}\text { (ft TOC) } \\
\text { (1) }\end{array}$ & (ft TOC) \\
\hline 20:00 & & 30.463 & 30.163 & 29.461 & 30.203 & & 29.345 & 30.492 & 29.933 & 29.816 & 28.549 & 26.359 & 31.7 & 36. & 32. & 16. & 21. & 15.768 & 10.616 & 31.947 & 32 & 29.847 & 72 & 8.011 & 33.623 \\
\hline $1 / 25 / 121: 00$ & & 30.463 & 30.163 & 29.454 & 30.208 & & 29.356 & 30.494 & 29.931 & 29.816 & 28.549 & 26.351 & 31.761 & 36.783 & 32.781 & 16.438 & 21.796 & 15.773 & 10.616 & 31.942 & 32.661 & 29.847 & 31.725 & 28.011 & 33.625 \\
\hline $1 / 25 / 122: 00$ & & 30.461 & 30.16 & 29.437 & 30.201 & & 29.373 & 30.511 & 29.926 & 29.814 & 28.546 & 26.335 & 31.761 & 36.78 & 32.781 & 16.44 & 21.792 & 15.782 & 10.616 & 31.937 & 32.658 & 29.835 & 31.729 & 8.011 & 33.625 \\
\hline 1/25/12 3:00 & & 30.463 & 30.16 & 29.435 & 30.197 & & 29.382 & 30.513 & 29.933 & 29.811 & 28.544 & 26.325 & 31.761 & 36.78 & 32.783 & 3 16.441 & 21.792 & 15.775 & 10.616 & 31.935 & 32.658 & 29.837 & 31.725 & 28.014 & 33.623 \\
\hline 1/25/12 4:00 & & 30.456 & 30.151 & 29.414 & 30.185 & & 29.406 & 30.537 & 29.921 & 29.807 & 28.532 & 26.308 & 31.756 & 36.771 & 32.776 & $5 \quad 16.441$ & 21.787 & 15.803 & 10.616 & 31.919 & 32.656 & 29.814 & 31.722 & 28.011 & 33.618 \\
\hline 1/25/12 5:00 & & 30.461 & 30.151 & 29.414 & 30.183 & & 29.403 & 30.53 & 29.926 & 29.804 & 28.532 & 26.306 & 31.756 & 36.778 & 32.769 & 16.443 & 21.789 & 15.792 & 10.611 & 31.919 & 32.649 & 29.816 & 31.72 & 28.014 & 33.616 \\
\hline 1/25/12 6:00 & & 30.466 & 30.153 & 29.419 & 30.18 & & 29.399 & 30.523 & 29.928 & 29.807 & 28.535 & 26.308 & 31.752 & 36.776 & 32.774 & 16.445 & 21.801 & 15.789 & 10.609 & 31.919 & 32.651 & 29.811 & 31.715 & 28.014 & 33.614 \\
\hline 1/25/12 7:00 & & 30.468 & 30.149 & 29.412 & 30.176 & & 29.401 & 30.523 & 29.931 & 29.8 & 28.532 & 26.292 & 31.749 & 36.771 & 32.769 & 16.445 & 21.803 & 15.782 & 10.607 & 31.921 & 32.644 & 29.818 & 31.715 & 28.014 & 33.609 \\
\hline 1/25/12 8:00 & & 30.477 & 30.163 & 29.444 & 30.192 & & 29.38 & 30.492 & 29.95 & 29.816 & 28.546 & 26.327 & 31.756 & 36.78 & 32.774 & 16.447 & 21.803 & 15.768 & 10.611 & 31.935 & 32.651 & 29.842 & 31.72 & 28.014 & 33.614 \\
\hline 1/25/12 9:00 & & 30.48 & 30.17 & 29.458 & 30.201 & & 29.377 & 30.494 & 29.942 & 29.825 & 28.551 & 26.342 & 31.763 & 36.783 & 32.783 & 16.45 & 21.806 & 15.778 & 10.621 & 31.947 & 32.658 & 29.847 & 31.727 & 28.016 & 33.611 \\
\hline $1 / 25 / 1210: 00$ & & 30.477 & 30.172 & 29.456 & 30.206 & & 29.389 & 30.504 & 29.94 & 29.828 & 28.553 & 26.337 & 31.766 & 36.785 & 32.785 & 16.45 & 21.806 & 15.785 & 10.623 & 31.942 & 32.663 & 29.844 & 31.729 & 28.016 & 33.614 \\
\hline $1 / 25 / 1211: 00$ & & 30.468 & 30.167 & 29.437 & 30.199 & & 29.415 & 30.533 & 29.928 & 29.821 & 28.544 & 26.323 & 31.766 & 36.785 & 32.788 & $3 \quad 16.452$ & 21.801 & 15.808 & 10.623 & 31.933 & 32.663 & 29.83 & 31.732 & 28.016 & 33.616 \\
\hline $1 / 25 / 1212: 00$ & & 30.468 & 30.156 & 29.414 & 30.185 & & 29.434 & 30.559 & 29.928 & 29.811 & 28.532 & 26.296 & 31.766 & 36.78 & 32.785 & 16.452 & 21.787 & 15.815 & 10.623 & 31.914 & 32.661 & 29.814 & 31.732 & 28.014 & 33.616 \\
\hline $1 / 25 / 1213: 00$ & & 30.454 & 30.123 & 29.353 & 30.15 & & 29.476 & 30.609 & 29.914 & 29.788 & 28.507 & 26.244 & 31.754 & 36.769 & 32.776 & $5 \quad 16.45$ & 21.794 & 15.839 & 10.614 & 31.886 & 32.651 & 29.767 & 31.72 & 28.014 & 33.616 \\
\hline $1 / 25 / 1214: 00$ & & 30.445 & 30.088 & 29.3 & 30.108 & & 29.509 & 30.614 & 29.909 & 29.756 & 28.479 & 26.189 & 31.74 & 36.755 & 32.76 & 16.445 & 21.792 & 15.843 & 10.597 & 31.851 & 32.635 & 29.722 & 31.701 & 28.014 & 33.609 \\
\hline $1 / 25 / 1215: 00$ & & 30.449 & 30.081 & 29.295 & 30.089 & & 29.493 & 30.597 & 29.919 & 29.746 & 28.47 & 26.181 & 31.728 & 36.743 & 32.75 & 16.443 & 21.787 & 15.824 & 10.588 & 31.844 & 32.625 & 29.717 & 31.692 & 28.016 & 33.609 \\
\hline $1 / 25 / 1216: 00$ & & 30.452 & 30.079 & 29.304 & 30.082 & & 29.472 & 30.578 & 29.923 & 29.744 & 28.467 & 26.196 & 31.723 & 36.74 & 32.743 & 16.438 & 21.787 & 15.82 & 10.581 & 31.849 & 32.62 & 29.717 & 31.687 & 28.016 & 33.607 \\
\hline $1 / 25 / 1217: 00$ & & 30.452 & 30.081 & 29.309 & 30.08 & & 29.455 & 30.566 & 29.921 & 29.742 & 28.465 & 26.201 & 31.721 & 36.74 & 32.738 & $3 \quad 16.438$ & 21.78 & 15.813 & 10.578 & 31.846 & 32.616 & 29.72 & 31.685 & 28.018 & 33.604 \\
\hline $1 / 25 / 1218: 00$ & & 30.452 & 30.072 & 29.304 & 30.073 & & 29.446 & 30.561 & 29.921 & 29.735 & 28.458 & 26.201 & 31.716 & 36.738 & 32.738 & 3 16.431 & 21.783 & 15.806 & 10.574 & 31.837 & 32.609 & 29.715 & 31.678 & 28.018 & 33.6 \\
\hline $1 / 25 / 1219: 00$ & & 30.449 & 30.07 & 29.302 & 30.071 & & 29.439 & 30.554 & 29.923 & 29.73 & 28.453 & 26.198 & 31.709 & 36.733 & 32.731 & 16.431 & 21.815 & 15.804 & 10.569 & 31.835 & 32.606 & 29.71 & 31.676 & 28.016 & 33.597 \\
\hline $1 / 25 / 1220: 00$ & & 30.447 & 30.063 & 29.29 & 30.061 & & 29.436 & 30.552 & 29.921 & 29.721 & 28.444 & 26.189 & 31.705 & 36.736 & 32.729 & 16.42 & 21.808 & 15.799 & 10.564 & 31.825 & 32.601 & 29.703 & 1.668 & 28.016 & 33.595 \\
\hline $1 / 25 / 1221: 00$ & & 30.454 & 30.074 & 29.318 & 30.078 & & 29.403 & 30.513 & 29.933 & 29.728 & 28.456 & 26.222 & 31.709 & 36.733 & 32.729 & 16.424 & 21.806 & 15.778 & 10.567 & 31.846 & 32.606 & 29.729 & 1.676 & 28.018 & 33.597 \\
\hline $1 / 25 / 1222: 00$ & & 30.452 & 30.067 & 29.309 & 30.073 & & 29.408 & 30.518 & 29.928 & 29.723 & 28.449 & 26.213 & 31.709 & 36.731 & 32.731 & 16.42 & 21.803 & 15.78 & 10.567 & 31.842 & 32.606 & 29.72 & 1.673 & 28.018 & 33.597 \\
\hline 1/25/12 23:00 & & 30.447 & 30.058 & 29.285 & 30.061 & & 29.42 & 30.53 & 29.923 & 29.711 & 28.437 & 26.191 & 31.705 & 36.717 & 32.727 & 16.42 & 21.803 & 15.785 & 10.564 & 31.832 & 32.601 & 29.708 & 31.671 & 28.016 & 33.597 \\
\hline 1/26/12 0:00 & & 30.445 & 30.051 & 29.278 & 30.057 & & 29.417 & 30.533 & 29.921 & 29.705 & 28.432 & 26.189 & 31.705 & 36.726 & 32.724 & 16.417 & 21.803 & 15.778 & 10.564 & 31.823 & 32.599 & 29.701 & 31.668 & 28.018 & 33.597 \\
\hline 1/26/12 1:00 & & 30.447 & 30.051 & 29.281 & 30.054 & & 29.408 & 30.521 & 29.921 & 29.702 & 28.428 & 26.193 & 31.702 & 36.724 & 32.727 & 16.413 & 21.806 & 15.768 & 10.562 & 31.825 & 532.599 & 29.701 & 31.666 & 28.018 & 33.6 \\
\hline 1/26/12 2:00 & & 30.447 & 30.053 & 29.288 & 30.059 & & 29.399 & 30.509 & 29.933 & 29.702 & 28.432 & 26.201 & 31.702 & 36.722 & 32.724 & 16.415 & 21.808 & 15.759 & 10.559 & 31.83 & 32.599 & 29.708 & 31.668 & 28.018 & 33.6 \\
\hline 1/26/12 3:00 & & 30.442 & 30.039 & 29.267 & 30.047 & & 29.408 & 30.525 & 29.926 & 29.688 & 28.419 & 26.181 & 31.698 & 36.722 & 32.72 & 16.408 & 21.806 & 15.768 & 10.555 & 31.811 & 32.594 & 29.691 & 31.664 & 28.021 & 33.597 \\
\hline $1 / 26 / 124: 00$ & & 30.442 & 30.042 & 29.269 & 30.047 & & 29.401 & 30.513 & 29.926 & 29.688 & 28.419 & 26.186 & 31.695 & 36.722 & 32.717 & 16.408 & 21.801 & 15.754 & 10.552 & 31.821 & 32.592 & 29.689 & 31.661 & 28.018 & 33.595 \\
\hline $1 / 26 / 125: 00$ & & 30.445 & 30.037 & 29.271 & 30.047 & & 29.394 & 30.506 & 29.928 & 29.686 & 28.416 & 26.186 & 31.691 & 36.719 & 32.715 & 16.406 & 21.799 & 15.747 & 10.55 & 31.821 & 32.587 & 29.694 & 31.657 & 28.021 & 33.593 \\
\hline 1/26/12 6:00 & & 30.456 & 30.058 & 29.314 & 30.068 & & 29.359 & 30.461 & 29.942 & 29.698 & 28.435 & 26.229 & 31.698 & 36.724 & 32.717 & 16.408 & 21.815 & 15.721 & 10.557 & 31.839 & 32.592 & 29.727 & 31.664 & 28.021 & 33.593 \\
\hline 1/26/12 7:00 & & 30.454 & 30.067 & 29.332 & 30.087 & & 29.349 & 30.454 & 29.935 & 29.709 & 28.442 & 26.253 & 31.702 & 36.731 & 32.724 & 16.408 & 21.824 & 15.728 & 10.562 & 31.858 & $3 \quad 32.597$ & 29.738 & 31.664 & 28.021 & 33.593 \\
\hline $1 / 26 / 128: 00$ & & 30.466 & 30.081 & 29.353 & 30.101 & & 29.34 & 30.463 & 29.95 & 29.718 & 28.453 & 26.27 & 31.707 & 36.738 & 32.727 & 16.41 & 21.824 & 15.714 & 10.562 & 31.867 & 32.604 & 29.76 & 31.673 & 28.025 & 33.593 \\
\hline $\begin{array}{l}1 / 26 / 129: 00 \\
\text { 1/20 }\end{array}$ & & 30.466 & 30.091 & 29.37 & 30.12 & & 29.335 & 30.461 & 29.95 & 29.725 & 28.463 & 26.284 & 31.714 & 36.74 & 32.734 & 16.41 & 21.833 & 15.717 & 10.571 & 31.879 & 32.611 & 29.778 & 31.68 & 28.023 & 33.595 \\
\hline 1/26/12 10:00 & & 30.466 & 30.088 & 29.363 & 30.124 & & 29.354 & 30.475 & 29.94 & 29.728 & 28.463 & 26.277 & 31.719 & 36.75 & 32.738 & $\begin{array}{l}36.413 \\
3\end{array}$ & 21.836 & 15.728 & 10.576 & 31.884 & 32.613 & 29.774 & 31.683 & 28.023 & 33.595 \\
\hline 1/26/12 11:00 & & 30.466 & 30.091 & 29.36 & 30.126 & & 29.366 & 30.487 & 29.947 & 29.73 & 28.465 & 26.272 & 31.726 & 36.75 & 32.741 & 16.4 & 21.836 & 15.735 & 10.583 & 31.881 & 32.618 & 29.778 & 31.692 & 28.023 & 33.597 \\
\hline $\begin{array}{l}1 / 26 / 12 ~ 12: 00 \\
\end{array}$ & & 30.463 & 30.086 & 29.342 & 30.12 & & 29.389 & 30.516 & 29.942 & 29.721 & 28.456 & 26.256 & 31.726 & 36.748 & 32.745 & 16.4 & 21.84 & 15.747 & 10.58 & 31.872 & 32.618 & 29.753 & 31.683 & 28.021 & $\begin{array}{l}33.597 \\
3.597\end{array}$ \\
\hline $1 / 26 / 1213: 00$ & & 30.459 & 30.072 & 29.314 & 30.103 & & 29.415 & 30.549 & 29.933 & 29.707 & 28.442 & 26.227 & 31.716 & 36.743 & 32.741 & 16. & 21.838 & 15.761 & 10.578 & 31.856 & 32.613 & .741 & 1.685 & 28.023 & 33.6 \\
\hline /12 14:00 & & 30.459 & 30.074 & 29.309 & 30.0 & & 29.415 & 30.554 & 29.94 & 29.707 & 28 & 26.225 & 9 & 36.738 & 32.736 & 16 & 21.833 & 15.759 & 10.576 & 31.856 & 13 & 38 & 31.685 & 28.023 & 33.6 \\
\hline & & 30.459 & 30 & 29.302 & 30 & & 2 & 30.554 & 29.935 & 29 & 28 & 26 & 31 & 13 & 32.736 & 16 & 3 & 1 & 10.574 & 31.846 & 532 & 31 & 31.683 & 28.023 & 33.597 \\
\hline & & 30. & 30 & 29.309 & & & & 30 & 29.94 & 29 & 28 & 26 & 31 & 6 & 32.736 & 16 & 32 & 4 & 10.574 & 31.851 & 11 & 8 & 31.68 & 28.023 & 33.597 \\
\hline 1/26/12 17:00 & & 30. & 30.077 & 29.323 & & & 29. & 30.5 & 29.942 & 29 & & & 31 & 36.736 & 32.736 & 16 & 21 & 15.745 & 10.574 & 31.863 & 32.611 & 29.75 & 31.68 & 28.025 & 33.597 \\
\hline $1 / 26 / 1218: 00$ & & 30.466 & 30.079 & 29.33 & & & & 30.5 & 29.945 & 29.7 & 28.446 & & 31.716 & 36.74 & 32.736 & 16 & 21.868 & 15.75 & 10.574 & 31.865 & 32.613 & 29.755 & 31.683 & 28.023 & 33.597 \\
\hline $1 / 26 / 1219: 00$ & & 30.466 & 30.084 & 29.332 & 30. & & 2 & 30.506 & 29.945 & 29.711 & 28.444 & 26.244 & 31.719 & 36.743 & 32.736 & 16. & 21.861 & 15.747 & 10.576 & 31.865 & 32.613 & 29.755 & 31.683 & 28.025 & 33.593 \\
\hline $1 / 26 / 1220: 00$ & & 30.468 & 30. & 29.332 & & & & 30.5 & 29.95 & & 28.446 & 26.246 & & 36.743 & 32.738 & 36.4 & 21.863 & 15.745 & 10.574 & 31.87 & 32.611 & 29.753 & 31.683 & 28.025 & 33.593 \\
\hline $1 / 26 / 1221: 00$ & & 30.461 & 30. & 29.311 & & & & 30.518 & 29.938 & 29.705 & 28. & 26.227 & 31.716 & 36.74 & 32.736 & 16 & 21.857 & 15.754 & 10.569 & 31.856 & 532.611 & & 31.68 & 28.023 & 33.59 \\
\hline $1 / 26 / 1222: 00$ & & 30.468 & 30.072 & 29.311 & 30. & & & 30.5 & 29.945 & & 28.437 & 26.217 & 31.714 & 36.738 & 32.734 & 16. & 21.854 & 15.742 & 10.574 & 31.849 & 32.609 & 29.745 & 31.68 & 28.025 & 33.59 \\
\hline 1/26/12 23:00 & & 30.468 & 30.079 & 29.318 & 30. & & & 30.5 & 29.947 & 29.7 & 28.439 & 26.234 & 31.71 & 36.738 & 32.738 & 16.4 & 21.854 & 15.742 & 10.574 & 31.863 & 32.613 & 29.753 & 31.685 & 28.028 & 33.593 \\
\hline $1 / 27 / 120: 00$ & & 30.459 & 30.0 & 29.292 & 30. & & 29.4 & 30.52 & 29.938 & 29.693 & 28.428 & 26.208 & 31.716 & 36.738 & 32.736 & 16.4 & 21.854 & 15.752 & 10.574 & 31.844 & 32.611 & 29.727 & 31.68 & 28.023 & 33.593 \\
\hline 1/27/12 1:00 & & 30.454 & 30.046 & 29.267 & 30.071 & & 29.424 & 30.545 & 29.931 & 29.681 & 28.412 & 26.184 & 31.709 & 36.731 & 32.729 & 16.41 & 21.854 & 15.761 & 10.566 & 31.832 & 32.604 & 29.706 & 31.673 & 28.025 & 33.59 \\
\hline $1 / 27 / 122: 00$ & & 30.459 & 30.046 & 29.274 & 30.066 & & 29.408 & 30.525 & 29.942 & 29.679 & 28.414 & 26.186 & 31.707 & 36.729 & 32.729 & 16.4 & 21.852 & 15.742 & 10.564 & 31.839 & 32.601 & 29.715 & 31.671 & 28.03 & 33.593 \\
\hline $\begin{array}{l}1 / 27 / 123: 00 \\
\text { 1/20 }\end{array}$ & & 30.454 & 30.044 & 29.262 & 30.059 & & 29.413 & 30.533 & 29.935 & 29.672 & 28.402 & 26.179 & 31.705 & 36.726 & 32.724 & 16.415 & 21.847 & 15.747 & 10.564 & 31.823 & 32.601 & 29.701 & 31.668 & 28.025 & 33.59 \\
\hline 1/27/12 4:00 & & 30.449 & 30.032 & 29.25 & 30.047 & & 29.417 & 30.54 & 29.931 & 29.665 & 28.395 & 26.172 & 31.698 & 36.726 & 32.717 & 16.415 & 21.845 & 15.745 & 10.56 & 31.814 & 32.594 & 29.694 & 31.664 & 28.025 & 33.588 \\
\hline $1 / 27 / 125: 00$ & & 30.445 & 30.023 & 29.232 & 30.035 & & 29.422 & 30.547 & 29.931 & 29.653 & 28.384 & 26.153 & 31.693 & 36.719 & 32.713 & $\begin{array}{l}36.413 \\
3\end{array}$ & 21.85 & 15.745 & 10.552 & 31.802 & 32.587 & 29.675 & 31.654 & 28.028 & 33.586 \\
\hline $1 / 27 / 126: 00$ & & 30.445 & 30.018 & 29.234 & 30.028 & & 29.415 & 30.537 & 29.931 & 29.651 & 28.384 & 26.16 & 31.684 & 36.717 & 32.708 & $3 \quad 16.41$ & 21.845 & 15.738 & 10.548 & 31.8 & 32.58 & 29.668 & 31.65 & 28.025 & 33.581 \\
\hline $1 / 27 / 127: 00$ & & 30.449 & 30.016 & 29.232 & 30.026 & & 29.401 & 30.525 & 29.938 & 29.642 & 28.381 & 26.146 & 31.681 & 36.715 & 32.703 & 3 $\quad 16.408$ & 21.843 & 15.721 & 10.541 & 31.802 & 32.578 & 29.68 & 31.645 & 28.03 & 3.576 \\
\hline
\end{tabular}




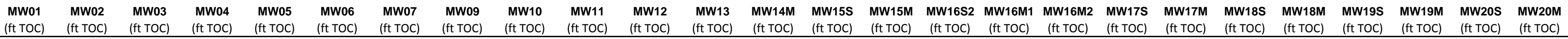

\begin{tabular}{|c|c|c|c|c|c|c|c|c|c|c|c|c|c|c|c|c|c|c|c|c|c|c|c|c|}
\hline Well & $\begin{array}{l}\text { (ft TOC) } \\
\text { (ft }\end{array}$ & $\begin{array}{l}\text { (ft TOC) } \\
\text { (f) }\end{array}$ & $\begin{array}{l}\text { (ft TOC) } \\
\text { (f) }\end{array}$ & $\begin{array}{l}\text { (ft TOC) } \\
\text { (ft) }\end{array}$ & $\begin{array}{ll}(\mathrm{ft} \text { TOC) } & \text { (ft TOC) }\end{array}$ & $\begin{array}{l}\text { (ft TOC) } \\
\text { (ft) }\end{array}$ & $\begin{array}{l}\text { (ft TOC) } \\
\text { (f) }\end{array}$ & $\begin{array}{l}\text { (ft TOC) } \\
\text { (ft) }\end{array}$ & $\begin{array}{l}\text { (ft TOC) } \\
\text { (f) }\end{array}$ & $\begin{array}{l}\text { (ft TOC) } \\
\text { (f) }\end{array}$ & $\begin{array}{l}\text { (ft TOC) } \\
\text { (f) }\end{array}$ & (ft TOC) & $\begin{array}{l}\text { (ft TOC) } \\
\text { (f) }\end{array}$ & (ft TOC) & $\begin{array}{ll}\text { (ft TOC) } \\
\text { (ft TOC) }\end{array}$ & (ft TOC) & $\begin{array}{l}\text { (ft TOC) } \\
\text { (1) }\end{array}$ & (ft TOC) & $\begin{array}{l}\text { (ft TOC) } \\
\end{array}$ & $\begin{array}{l}\text { (ft TOC) } \\
\text { (f) }\end{array}$ & $\begin{array}{l}\text { (ft TOC) } \\
\text { (n) }\end{array}$ & (ft TOC) & $\begin{array}{l}\text { (ft TOC) } \\
\text { (1) }\end{array}$ & (ft TOC) \\
\hline 8:00 & & 30.445 & 30.018 & 29.241 & 30.028 & 29.394 & 30.516 & 29.935 & 29.6 & 28.38 & 26.1 & 31.6 & 36.715 & 32.7 & 16. & 21.84 & 15.724 & 10.541 & 31.804 & 32. & 29.68 & 31.647 & 8.028 & 33.576 \\
\hline 1/27/12 9:00 & & 30.438 & 30.007 & 29.213 & 30.014 & 29.408 & 30.535 & 29.928 & 29.628 & 28.367 & 26.134 & 31.672 & 36.701 & 32.694 & 16.403 & 21.833 & 15.726 & 10.531 & 31.788 & 32.566 & 29.661 & 31.64 & 28.025 & 33.572 \\
\hline $1 / 27 / 1210: 00$ & & 30.442 & 30.011 & 29.232 & 30.021 & 29.387 & 30.516 & 29.935 & 29.637 & 28.372 & 26.165 & 31.677 & 36.705 & 32.699 & 16.401 & 21.843 & 15.724 & 10.536 & 31.798 & 32.573 & 29.675 & 31.643 & 28.028 & 33.576 \\
\hline 1/27/12 11:00 & & 30.447 & 30.018 & 29.246 & 30.031 & 29.373 & 30.499 & 29.938 & 29.64 & 28.379 & 26.174 & 31.679 & 36.71 & 32.701 & 16.403 & 21.843 & 15.707 & 10.536 & 31.809 & 32.573 & 29.689 & 31.645 & 28.03 & 33.579 \\
\hline 1/27/12 12:00 & & 30.442 & 30.021 & 29.248 & 30.035 & 29.375 & 30.499 & 29.933 & 29.642 & 28.379 & 26.181 & 31.681 & 36.715 & 32.703 & 36.401 & 21.854 & 15.717 & 10.538 & 31.809 & 32.578 & 29.691 & 31.647 & 28.03 & 33.579 \\
\hline 1/27/12 13:00 & & 30.442 & 30.018 & 29.241 & 30.035 & 29.38 & 30.509 & 29.933 & 29.637 & 28.377 & 26.177 & 31.681 & 36.717 & 32.701 & 16.399 & 21.875 & 15.719 & 10.538 & 31.804 & 32.575 & 29.689 & 31.647 & 28.03 & 33.581 \\
\hline 1/27/12 14:00 & & 30.459 & 30.039 & 29.29 & 30.061 & 29.342 & 30.458 & 29.954 & 29.656 & 28.398 & 26.222 & 31.693 & 36.715 & 32.713 & 36.401 & 21.873 & 15.693 & 10.548 & 31.846 & 32.587 & 29.734 & 31.661 & 28.032 & 33.583 \\
\hline 1/27/12 15:00 & & 30.47 & 30.072 & 29.36 & 30.108 & 29.295 & 30.413 & 29.966 & 29.686 & 28.428 & 26.287 & 31.712 & 36.736 & 32.729 & 16.408 & 21.875 & 15.681 & 10.569 & 31.881 & 32.609 & 29.785 & 31.676 & 28.035 & 33.59 \\
\hline 1/27/12 16:00 & & 30.482 & 30.105 & 29.409 & 30.152 & 29.276 & 30.401 & 29.971 & 29.716 & 28.46 & 26.332 & 31.73 & 36.745 & 32.745 & $5 \quad 16.413$ & 21.931 & 15.672 & 10.585 & 31.916 & 32.63 & 29.832 & 31.697 & 28.035 & 33.597 \\
\hline 1/27/12 17:00 & & 30.489 & 30.125 & 29.437 & 30.187 & 29.274 & 30.437 & 29.973 & 29.737 & 28.479 & 26.361 & 31.747 & 36.764 & 32.762 & 16.422 & 21.935 & 15.684 & 10.599 & 31.947 & 32.644 & 29.861 & 31.711 & 28.035 & 33.6 \\
\hline $1 / 27 / 1218: 00$ & & 30.501 & 30.153 & 29.48 & 30.227 & 29.265 & 30.518 & 29.983 & 29.765 & 28.507 & 26.394 & 31.766 & 36.778 & 32.781 & 16.429 & 21.935 & 15.67 & 10.613 & 31.975 & 32.658 & 29.901 & 31.727 & 28.035 & 33.602 \\
\hline $1 / 27 / 1219: 00$ & & 30.515 & 30.191 & 29.538 & 30.274 & 29.251 & 30.533 & 29.992 & 29.802 & 28.542 & 26.447 & 31.784 & 36.787 & 32.804 & 16.438 & 21.935 & 15.672 & 10.642 & 32.014 & 32.682 & 29.95 & 31.751 & 28.035 & 33.607 \\
\hline $1 / 27 / 1220: 00$ & & 30.517 & 30.219 & 29.571 & 30.313 & 29.258 & 30.537 & 29.99 & 29.832 & 28.57 & 26.476 & 31.805 & 36.797 & 32.818 & $3 \quad 16.447$ & 21.935 & 15.681 & 10.658 & 32.042 & 32.703 & 29.985 & 31.769 & 28.035 & 33.609 \\
\hline $1 / 27 / 1221: 00$ & & 30.515 & 30.237 & 29.582 & 30.339 & 29.281 & 30.545 & 29.983 & 29.853 & 28.584 & 26.483 & 31.822 & 36.804 & 32.837 & 16.457 & 21.933 & 15.7 & 10.677 & 32.054 & 32.715 & 29.997 & 31.786 & 28.035 & 33.611 \\
\hline $1 / 27 / 1222: 00$ & & 30.517 & 30.244 & 29.585 & 30.351 & 29.307 & 30.55 & 29.983 & 29.865 & 28.595 & 26.476 & 31.833 & 36.811 & 32.851 & 16.466 & 21.924 & 15.714 & 10.686 & 32.066 & 32.732 & 29.992 & 31.798 & 28.035 & 33.614 \\
\hline $1 / 27 / 1223: 00$ & & 30.519 & 30.251 & 29.587 & 30.365 & 29.33 & 30.559 & 29.99 & 29.876 & 28.607 & 26.473 & 31.845 & 36.815 & 32.858 & $3 \quad 16.473$ & 21.935 & 15.726 & 10.696 & 32.075 & 32.741 & 30.009 & 31.807 & 28.035 & 33.616 \\
\hline $1 / 28 / 120: 00$ & & 30.531 & 30.275 & 29.613 & 30.386 & 29.335 & 30.564 & 29.997 & 29.902 & 28.628 & 26.495 & 31.859 & 36.82 & 32.874 & 16.485 & 21.933 & 15.726 & 10.715 & 32.096 & 32.755 & 30.032 & 31.823 & 28.037 & 33.623 \\
\hline $1 / 28 / 121: 00$ & & 30.531 & 30.286 & 29.622 & 30.4 & 29.356 & 30.568 & 29.995 & 29.916 & 28.639 & 26.497 & 31.873 & 36.825 & 32.889 & 16.494 & 21.937 & 15.745 & 10.726 & 32.105 & 32.772 & 30.042 & 31.84 & 28.037 & 33.625 \\
\hline $1 / 28 / 122: 00$ & & 30.538 & 30.298 & 29.634 & 30.409 & 29.375 & 30.576 & 29.997 & 29.932 & 28.651 & 26.5 & 31.885 & 36.825 & 32.9 & 16.503 & 21.937 & 15.752 & 10.741 & 32.117 & 32.777 & 30.053 & 31.847 & 28.037 & 33.628 \\
\hline $1 / 28 / 123: 00$ & & 30.536 & 30.3 & 29.624 & 30.411 & 29.401 & 30.58 & 29.995 & 29.937 & 28.656 & 26.488 & 31.89 & 36.829 & 32.905 & 16.51 & 21.94 & 15.768 & 10.743 & 32.117 & 32.789 & 30.046 & 31.856 & 28.037 & 33.63 \\
\hline $1 / 28 / 124: 00$ & & 30.529 & 30.293 & 29.603 & 30.402 & 29.441 & 30.583 & 29.985 & 29.934 & 28.649 & 26.464 & 31.894 & 36.829 & 32.912 & 16.51 & 21.937 & 15.787 & 10.747 & 32.101 & 32.791 & .027 & 1.856 & 28.037 & 33.628 \\
\hline $1 / 28 / 125: 00$ & & 30.529 & 30.279 & 29.571 & 30.383 & 29.474 & 30.585 & 29.983 & 29.925 & 28.639 & 26.426 & 31.89 & 36.827 & 32.907 & 16.522 & 21.935 & 15.794 & 10.745 & 32.084 & 32.786 & .002 & 1.854 & 28.037 & 33.625 \\
\hline 1/28/12 6:00 & & 30.536 & 30.279 & 29.571 & 30.376 & 29.483 & 30.59 & 29.99 & 29.927 & 28.639 & 26.418 & 31.89 & 36.822 & 32.907 & 16.527 & 21.944 & 15.792 & 10.745 & 32.089 & 32.784 & .004 & 1.854 & 28.039 & 33.623 \\
\hline 1/28/12 7:00 & & 30.545 & 30.289 & 29.592 & 30.381 & 29.479 & 30.595 & 29.999 & 29.937 & 28.651 & 26.433 & 31.894 & 36.829 & 32.91 & 16.534 & 21.94 & 15.787 & 10.748 & 32.096 & 32.791 & 30.016 & 31.861 & 28.039 & 33.625 \\
\hline 1/28/12 8:00 & & 30.552 & 30.3 & 29.613 & 30.39 & 29.474 & 30.6 & 30.004 & 29.951 & 28.66 & 26.449 & 31.901 & 36.834 & 32.919 & 16.538 & 21.944 & 15.792 & 10.757 & 32.101 & 32.798 & 30.03 & 31.866 & 28.039 & 33.625 \\
\hline 1/28/12 9:00 & & 30.557 & 30.312 & 29.629 & 30.402 & 29.472 & 30.604 & 30.011 & 29.964 & 28.674 & 26.464 & 31.911 & 36.832 & 32.928 & $3 \quad 16.543$ & 21.944 & 15.792 & 10.766 & 32.122 & 32.805 & 30.044 & 31.873 & 28.039 & 33.628 \\
\hline 1/28/12 10:00 & & 30.559 & 30.321 & 29.643 & 30.411 & 29.481 & 30.609 & 30.011 & 29.976 & 28.681 & 26.471 & 31.918 & 36.839 & 32.935 & 16.548 & 21.942 & 15.803 & 10.768 & 32.126 & 32.812 & 30.053 & 31.882 & 28.039 & 33.63 \\
\hline 1/28/12 11:00 & & 30.561 & 30.331 & 29.657 & 30.425 & 29.49 & 30.614 & 30.009 & 29.988 & 28.693 & 26.483 & 31.922 & 36.839 & 32.942 & 16.552 & 21.944 & 15.81 & 10.783 & 32.138 & 32.819 & 30.067 & 31.889 & 28.042 & 33.635 \\
\hline 1/28/12 12:00 & & 30.55 & 30.319 & 29.622 & 30.411 & 29.535 & 30.616 & 29.999 & 29.981 & 28.679 & 26.447 & 31.925 & 36.837 & 32.945 & 16.557 & 21.942 & 15.846 & 10.783 & 32.119 & 32.819 & 30.037 & 31.891 & 28.039 & 33.635 \\
\hline 1/28/12 13:00 & & 30.543 & 30.3 & 29.578 & 30.383 & 29.575 & 30.616 & 29.99 & 29.964 & 28.658 & 26.402 & 31.922 & 36.832 & 32.94 & 16.557 & 21.947 & 15.869 & 10.776 & 32.089 & 32.817 & 30.004 & 31.889 & 28.039 & 33.632 \\
\hline $1 / 28 / 1214: 00$ & & 30.543 & 30.282 & 29.543 & 30.355 & 29.598 & 30.619 & 29.99 & 29.946 & 28.642 & 26.363 & 31.913 & 36.82 & 32.933 & 36.557 & 21.947 & 15.886 & 10.771 & 32.073 & 32.805 & 29.978 & 31.875 & 28.042 & 33.632 \\
\hline $1 / 28 / 1215: 00$ & & 30.543 & 30.27 & 29.524 & 30.334 & 29.61 & 30.616 & 29.992 & 29.934 & 28.63 & 26.344 & 31.906 & 36.811 & 32.928 & $3 \quad 16.557$ & 21.947 & 15.888 & 10.764 & 32.054 & 32.803 & 29.957 & 31.87 & 28.044 & 33.632 \\
\hline $1 / 28 / 1216: 00$ & & 30.543 & 30.256 & 29.505 & 30.311 & 29.615 & 30.614 & 29.992 & 29.925 & 28.618 & 26.323 & 31.899 & 36.806 & 32.921 & 16.555 & 21.947 & 15.895 & 10.759 & 32.045 & 32.796 & 29.931 & 31.866 & 28.042 & 33.63 \\
\hline 1/28/12 17:00 & & 30.545 & 30.249 & 29.498 & 30.297 & 29.61 & 30.614 & 29.995 & 29.916 & 28.609 & 26.313 & 31.894 & 36.804 & 32.914 & 16.555 & 21.944 & 15.89 & 10.752 & 32.038 & 32.786 & 29.926 & 31.859 & 28.042 & 33.628 \\
\hline $1 / 28 / 1218: 00$ & & 30.547 & 30.251 & 29.501 & 30.29 & 29.596 & 30.614 & 30.002 & 29.913 & 28.609 & 26.323 & 31.892 & 36.801 & 32.912 & 16.5 & 21.947 & 15.89 & 10.75 & 32.035 & 32.786 & 29.929 & 31.856 & 28.042 & 33.625 \\
\hline $1 / 28 / 1219: 00$ & & 30.552 & 30.249 & 29.508 & 30 & 29.584 & 30.614 & 30.007 & 29.913 & 28.605 & 26.325 & 31.89 & 36.794 & 32.907 & & 21.947 & 15.883 & 10.745 & 32.035 & 32.784 & 29.926 & 31.854 & 28.042 & 33.623 \\
\hline $\begin{array}{l}1 / 28 / 1220: 00 \\
1\end{array}$ & & 30.554 & $\begin{array}{l}30.249 \\
30.249\end{array}$ & 29.51 & 30.288 & 29.57 & $\begin{array}{l}30.616 \\
30.616\end{array}$ & 30.007 & 29.911 & 28.605 & 26.33 & 31.887 & 36.794 & 32.91 & 16 & 21.947 & $\begin{array}{l}1.000 \\
15.876\end{array}$ & 10.743 & 32.038 & 32.786 & 29.931 & 31.851 & 28.044 & 33.623 \\
\hline $1 / 28 / 1221: 00$ & & 30.554 & 30.244 & 29.503 & 30.283 & 29.575 & 30.616 & 30.004 & 29.906 & 28.6 & 26.325 & 31.885 & 36.797 & 32.903 & 16.55 & 21.947 & 15.879 & 10.741 & 32.033 & 32.781 & 29.919 & 31.849 & 28.044 & 33.621 \\
\hline $1 / 28 / 1222: 00$ & & 30.559 & 30.244 & 29.503 & 30. & 29.566 & 30.619 & 30.009 & 29.904 & 28.6 & 26.325 & 31.883 & 36.799 & 32.903 & 16. & 21.949 & 15.869 & 10.738 & 32.031 & 32.779 & 29.924 & 31.849 & 28.044 & 33.621 \\
\hline $1 / 28$ & & 30.559 & 30.2 & 29. & 30. & 29 & 30.616 & 30 & 29. & 28.598 & 26.33 & 31.883 & 36.794 & 32.905 & 16 & 21.954 & 15.871 & 10.743 & 32.031 & 32.781 & 26 & 47 & 28.044 & 33.621 \\
\hline & & & 30.2 & 29. & & & 30.6 & & & 28.595 & & 31.887 & 36794 & 32.9 & 16 & & 15.871 & 10.74 & 32.035 & .779 & 9 & 51 & 28.044 & 33.621 \\
\hline $1 / 29 / 121$ & & & 30. & 29. & & & 30. & & & 28.5 & 26 & 31.8 & 36.7 & 32.9 & 16 & & 15.8 & 10.738 & 32.019 & 9 & .915 & 17 & 28.044 & 33.621 \\
\hline 1/29/12 2:00 & & 30.557 & 30.223 & 29.477 & 30. & & 30. & & & 28.577 & 26. & 31.8 & 36.7 & 32 & 16. & & 15.8 & 10.736 & 32.014 & .774 & 29.903 & 44 & 28.044 & 33.618 \\
\hline 1/29/12 3:00 & & 30.559 & 30.223 & 29.484 & 30. & 2 & 30.6 & 30 & 29 & 28.579 & 26.313 & 31.878 & 36.794 & 32.9 & 16.541 & 21 & 15.869 & 10.736 & 32.021 & 32.777 & & 44 & 28.046 & 33.618 \\
\hline 1/29/12 4:00 & & 30.561 & 30.221 & 29.482 & & & 30. & & & 28.577 & & 31.878 & 36.794 & 32.896 & 16. & 21.949 & 15.86 & 10.733 & 32.017 & 32.772 & 29.905 & 31.842 & 28.044 & 33.618 \\
\hline 1/29/12 5:00 & & 30.559 & 30.214 & 29.475 & & & 30.619 & 30.011 & & 28.57 & 26. & 31.876 & 36.79 & 32.898 & 16. & 21.944 & 15.862 & 10.731 & 32.007 & 32.77 & & & 28.046 & 33.616 \\
\hline $1 / 29 / 126: 00$ & & 30.564 & 30.21 & 29.477 & 30. & & 30.616 & 30.014 & 29. & 28.57 & 26.308 & 31.873 & 36.785 & 32.893 & 16. & 21.963 & 15.855 & 10.731 & 32.014 & 32.77 & 29.908 & 31.835 & 28.046 & 33.616 \\
\hline 1/29/127:00 & & 30. & 30.214 & 29.48 & 30.2 & 29 . & 30.6 & $30.6 \mathrm{r}-\mathrm{x}$ & 29. & 28.57 & 26.308 & 31.871 & 36.792 & 32.891 & 16.5 & 21.961 & 15.855 & 10.731 & 32.012 & 32.765 & 29.905 & 31.835 & 28.046 & 33.614 \\
\hline 1/29/12 8:00 & & 30.568 & 30.219 & 29.484 & 30. & 29.551 & 30.619 & 30.016 & 29.876 & 28.57 & 26.313 & 31.873 & 36.79 & 32.893 & 16.5 & 21.961 & 15.846 & 10.726 & 32.019 & 32.767 & 29.908 & 31.837 & 28.046 & 33.614 \\
\hline 1/29/129:00 & & 30.568 & 30.223 & 29.496 & 30.267 & 29.547 & 30.621 & 30.021 & 29.879 & 28.572 & 26.33 & 31.873 & 36.792 & 32.893 & 16.534 & 21.972 & 15.848 & 10.731 & 32.021 & 32.77 & 29.919 & 31.84 & 28.046 & 33.614 \\
\hline 1/29/12 10:00 & & 30.566 & 30.219 & 29.487 & 30.264 & 29.556 & 30.621 & 30.018 & 29.876 & 28.57 & 26.32 & 31.873 & 36.792 & 32.893 & 16.534 & 21.968 & 15.862 & 10.729 & 32.017 & 32.77 & 29.912 & 31.835 & 28.049 & 33.614 \\
\hline 1/29/12 11:00 & & 30.566 & 30.216 & 29.482 & 30.262 & 29.563 & 30.621 & 30.023 & 29.872 & 28.565 & 26.315 & 31.873 & 36.79 & 32.893 & 16.531 & 21.965 & 15.857 & 10.731 & 32.017 & 32.767 & 29.917 & 31.84 & 28.049 & 33.616 \\
\hline 1/29/12 12:00 & & 30.559 & 30.207 & 29.468 & 30.255 & 29.58 & 30.619 & 30.011 & 29.867 & 28.556 & 26.306 & 31.869 & 36.79 & 32.891 & 16.531 & 21.958 & 15.878 & 10.729 & 32.005 & 32.767 & 29.894 & 31.835 & 28.049 & 33.616 \\
\hline 1/29/12 13:00 & & 30.557 & 30.193 & 29.444 & 30.241 & 29.594 & 30.619 & 30.009 & 29.851 & 28.544 & 26.28 & 31.866 & 36.785 & 32.884 & 16.529 & 21.956 & 15.878 & 10.724 & 31.996 & 32.76 & 29.882 & 31.833 & 28.051 & 33.614 \\
\hline $1 / 29 / 1214: 00$ & & 30.547 & 30.167 & 29.409 & 30.218 & 29.615 & 30.612 & 30.004 & 29.837 & 28.528 & 26.256 & 31.859 & 36.778 & 32.879 & 16.527 & 21.954 & 15.893 & 10.715 & 31.965 & 32.753 & 29.851 & 31.821 & 28.049 & 33.614 \\
\hline $1 / 29 / 1215: 00$ & & 30.55 & 30.156 & 29.393 & 30.199 & 29.615 & 30.607 & 30.007 & 29.823 & 28.514 & 26.237 & 31.852 & 36.776 & 32.87 & 16.522 & 21.947 & 15.888 & 10.71 & 31.963 & 32.746 & 29.835 & 31.816 & 28.051 & 3.614 \\
\hline
\end{tabular}




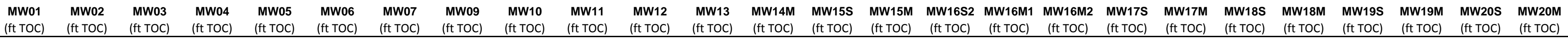

\begin{tabular}{|c|c|c|c|c|c|c|c|c|c|c|c|c|c|c|c|c|c|c|c|c|c|c|c|c|c|c|}
\hline well & $\begin{array}{l}\text { (ft TOC) } \\
\text { (is }\end{array}$ & $\begin{array}{l}\text { (ft TOC) } \\
\text { (ft) }\end{array}$ & $\begin{array}{l}\text { (ft TOC) } \\
\text { (f) }\end{array}$ & (ft TOC) & $\begin{array}{l}\text { (ft TOC) } \\
\text { (f) }\end{array}$ & $\begin{array}{l}\text { (ft TOC) } \\
\text { (f) }\end{array}$ & (ft toc) & $\begin{array}{l}\text { (ft TOC) } \\
\text { (ft }\end{array}$ & $\begin{array}{l}\text { (ft TOC) } \\
\text { (ft) }\end{array}$ & $\begin{array}{l}\text { (ft TOC) } \\
\text { (f) }\end{array}$ & $\begin{array}{l}\text { (ft TOC) } \\
\text { (f) }\end{array}$ & $\begin{array}{l}\text { (ft toc) } \\
\text { (foc) }\end{array}$ & $\begin{array}{l}\text { (ft TOC) } \\
\text { (n) }\end{array}$ & $\begin{array}{l}\text { (ft TOC) } \\
\end{array}$ & (ft TOC) & $\begin{array}{l}\text { (ft TOC) } \\
\text { (a) }\end{array}$ & (ft TOC) & (ft TOC) & $\begin{array}{l}\text { (ft TOC) } \\
\end{array}$ & (ft TOC) & $\begin{array}{l}\text { (ft TOC) } \\
\end{array}$ & $\begin{array}{l}\text { (ft TOC) } \\
\text { (1) }\end{array}$ & $\begin{array}{l}\text { (ft TOC) } \\
\end{array}$ & (ft TOC) & $\begin{array}{l}\text { (nt TOC) } \\
\text { (nt }\end{array}$ & (ft TOC) \\
\hline $1 / 29 / 1216: 00$ & & 30.55 & 30.146 & 29.391 & 30.192 & & 29.606 & 30.602 & 30.009 & 29.814 & 28.509 & 26.232 & 31.847 & 36.769 & 32.867 & 16.52 & & 22 & 15.881 & 10.703 & 31.956 & 32.741 & 29.832 & 31.812 & 28.051 & 33.611 \\
\hline $1 / 29 / 12 \quad 17: 00$ & & 30.55 & 30.144 & 29.388 & 30.185 & & 29.596 & 30.6 & 30.007 & 29.809 & 28.502 & 26.237 & 31.84 & 36.769 & 32.863 & $\begin{array}{l}36.517 \\
3\end{array}$ & & 22 & 15.881 & 10.7 & 31.954 & 32.734 & 29.83 & 31.807 & 28.051 & 33.611 \\
\hline $1 / 29 / 1218: 00$ & & 30.552 & 30.144 & 29.395 & 30.185 & & 29.582 & 30.597 & 30.014 & 29.807 & 28.5 & 26.241 & 32 & 36.764 & 33.041 & 16.515 & & 21.993 & 15.871 & 10.872 & 31.982 & 32.893 & 29.832 & 31.966 & 28.051 & 33.609 \\
\hline 1/29/12 19:00 & & 30.552 & 30.149 & 29.4 & 30.19 & & 29.568 & 30.597 & 30.016 & 29.804 & 28.5 & 26.248 & 31.967 & 36.762 & 32.994 & 16.513 & & 22 & 15.857 & 10.83 & 32.017 & 32.862 & 29.847 & 31.929 & 28.054 & 33.609 \\
\hline 1/29/12 20:00 & & 30.552 & 30.142 & 29.398 & 30.192 & & 29.561 & 30.592 & 30.014 & 29.804 & 28.498 & 26.251 & 31.925 & 36.761 & 32.949 & 16.51 & & 22 & 15.857 & 10.785 & 31.998 & 32.817 & 29.847 & & 28.051 & 33.607 \\
\hline 1/29/12 21:00 & & 30.547 & 30.137 & 29.384 & 30.185 & & 29.563 & 30.592 & 30.011 & 29.795 & 28.491 & 26.239 & 31.899 & 36.764 & 32.924 & 16.508 & & 21.991 & 15.86 & 10.759 & 31.982 & 32.793 & 29.837 & 1.863 & 28.053 & 33.604 \\
\hline $1 / 29 / 1222: 00$ & & 30.547 & 30.135 & 29.379 & 30.183 & & 29.561 & 30.59 & 30.014 & 29.788 & 28.484 & 26.237 & 31.883 & 36.757 & 32.907 & 16.506 & & 21.988 & 15.85 & 10.743 & 31.97 & 32.777 & 29.83 & 31.847 & 28.051 & 33.602 \\
\hline $1 / 29 / 1223: 00$ & & 30.547 & 30.128 & 29.372 & 30.176 & & 29.561 & 30.588 & 30.014 & 29.781 & 28.477 & 26.229 & 31.871 & 36.762 & 32.896 & 16.501 & & 21.981 & 15.85 & 10.731 & 31.965 & 32.765 & 29.821 & 31.835 & 28.051 & 33.6 \\
\hline $1 / 30 / 120: 00$ & & 30.545 & 30.121 & 29.365 & 30.171 & & 29.556 & 30.583 & 30.009 & 29.776 & 28.474 & 26.227 & 31.859 & 36.754 & 32.886 & $5 \quad 16.499$ & & 21.977 & 15.848 & 10.721 & 31.958 & 32.758 & 29.818 & 31.826 & 28.054 & 33.597 \\
\hline 1/30/12 1:00 & & 30.536 & 30.102 & 29.334 & 30.152 & & 29.573 & 30.578 & 30.004 & 29.758 & 28.456 & 26.201 & 31.85 & 36.745 & 32.872 & 16.496 & & 21.974 & 15.855 & 10.71 & 31.933 & 32.744 & 29.792 & 31.814 & 28.051 & 33.595 \\
\hline $1 / 30 / 122: 00$ & & 30.54 & 30.1 & 29.335 & 30.145 & & 29.558 & 30.576 & 30.011 & 29.753 & 28.453 & 26.201 & 31.84 & 36.75 & 32.863 & 3 16.494 & & 21.97 & 15.834 & 10.703 & 31.933 & 32.736 & 29.795 & 31.807 & 28.054 & 33.595 \\
\hline 1/30/12 3:00 & & 30.536 & 30.098 & 29.328 & 30.138 & & 29.556 & 30.571 & 30.004 & 29.746 & 28.444 & 26.198 & 31.836 & 36.745 & 32.856 & 5. 16.492 & & 21.968 & 15.836 & 10.694 & 31.926 & 32.725 & 29.783 & 31.8 & 28.051 & 33.593 \\
\hline $1 / 30 / 124: 00$ & & 30.533 & 30.088 & 29.321 & 30.131 & & 29.551 & 30.568 & 30.007 & 29.739 & 28.439 & 26.191 & 31.826 & 36.745 & 32.849 & 16.487 & & 21.965 & 15.825 & 10.689 & 31.912 & 32.722 & 29.783 & 31.793 & 28.053 & 33.593 \\
\hline $1 / 30 / 125: 00$ & & 30.531 & 30.084 & 29.316 & 30.124 & & 29.544 & 30.564 & 30.007 & 29.732 & 28.43 & 26.191 & 31.817 & 36.74 & 32.839 & 16.482 & & 21.956 & 15.822 & 10.682 & 31.912 & 32.713 & 29.769 & 31.783 & 28.053 & 33.59 \\
\hline $1 / 30 / 126: 00$ & & 30.531 & 30.079 & 29.311 & 30.12 & & 29.537 & 30.564 & 30.004 & 29.725 & 28.428 & 26.189 & 31.812 & 36.738 & 32.837 & 16.478 & & 21.97 & 15.817 & 10.672 & 31.907 & 32.708 & 29.771 & 31.779 & 28.056 & 33.588 \\
\hline $1 / 30 / 127: 00$ & & 30.529 & 30.079 & 29.309 & 30.117 & & 29.53 & 30.559 & 30.007 & 29.721 & 28.423 & 26.186 & 31.805 & 36.74 & 32.828 & 36.478 & & 21.977 & 15.806 & 10.665 & 31.905 & 32.701 & 29.767 & 31.774 & 28.054 & 33.586 \\
\hline $1 / 30 / 128: 00$ & & 30.533 & 30.081 & 29.325 & 30.122 & & 29.511 & 30.556 & 30.009 & 29.725 & 28.425 & 26.205 & 31.808 & 36.738 & 32.828 & $\begin{array}{l}36.475 \\
3\end{array}$ & & 21.972 & 15.794 & 10.665 & 31.912 & 32.701 & 29.781 & 31.772 & 28.056 & 33.586 \\
\hline 1/30/12 9:00 & & 30.533 & 30.088 & 29.332 & 30.134 & & 29.502 & 30.556 & 30.011 & 29.723 & 28.428 & 26.215 & 31.805 & 36.74 & 32.823 & 16.473 & & 21.965 & 15.787 & 10.66 & 31.909 & 32.699 & 29.79 & 31.769 & 28.056 & 33.586 \\
\hline 1/30/12 10:00 & & 30.529 & 30.086 & 29.323 & 30.129 & & 29.511 & 30.556 & 30.007 & 29.721 & 28.423 & 26.21 & 31.803 & 36.74 & 32.825 & 16.471 & & 21.961 & 15.794 & $\begin{array}{l}10.603 \\
10.63\end{array}$ & 31.909 & 32.699 & 29.781 & 31.767 & 28.056 & 33.586 \\
\hline 1/30/12 11:00 & & 30.529 & 30.081 & 29.316 & 30.124 & & 29.514 & 30.554 & 30.007 & 29.714 & $\begin{array}{l}20.420 \\
28.421\end{array}$ & $\begin{array}{l}26.214 \\
26.203\end{array}$ & 31.801 & $\begin{array}{l}36.733 \\
36.733\end{array}$ & 32.823 & $\begin{array}{l}16.471 \\
3\end{array}$ & & $\begin{array}{l}21.901 \\
21.961\end{array}$ & 15.794 & $\begin{array}{l}10.003 \\
10.611\end{array}$ & 31.907 & 32.694 & 29.778 & 31.765 & $\begin{array}{l}28.056 \\
28.056\end{array}$ & $\begin{array}{l}33.586 \\
33.580\end{array}$ \\
\hline $1 / 30 / 1212: 00$ & & $\begin{array}{l}50.529 \\
30.526\end{array}$ & $\begin{array}{l}30.081 \\
30.077\end{array}$ & $\begin{array}{l}29.307 \\
29.307\end{array}$ & $\begin{array}{l}50.144 \\
30.12\end{array}$ & & $\begin{array}{l}29.519 \\
29.519\end{array}$ & $\begin{array}{l}50.534 \\
30.552\end{array}$ & 30.007 & 29.707 & $\begin{array}{l}28.441 \\
28.414\end{array}$ & $\begin{array}{l}20.203 \\
26.193\end{array}$ & $\begin{array}{l}31.791 \\
31.794\end{array}$ & 36.729 & 32.816 & $\begin{array}{l}10.411 \\
16.468\end{array}$ & & $\begin{array}{l}21.901 \\
21.956\end{array}$ & $\begin{array}{l}15.799 \\
15.794\end{array}$ & $\begin{array}{l}10.001 \\
10.654\end{array}$ & 31.898 & $\begin{array}{r}32.094 \\
32.689 \\
3\end{array}$ & 29.774 & 31.76 & $\begin{array}{l}28.058 \\
28.058\end{array}$ & $\begin{array}{l}33.500 \\
33.586\end{array}$ \\
\hline $1 / 30 / 1213: 00$ & & 30.512 & 30.053 & 29.267 & 30.096 & & 29.547 & 30.547 & 29.992 & 29.688 & 28.393 & 26.165 & 31.787 & 36.733 & 32.806 & 16.464 & & 21.951 & 15.818 & 10.644 & 31.874 & 32.68 & 29.738 & 31.753 & 28.056 & 33.583 \\
\hline $1 / 30 / 1214: 00$ & & 30.508 & 30.032 & 29.234 & 30.071 & & 29.558 & 30.54 & 29.99 & 29.667 & 28.374 & 26.131 & 31.775 & 36.726 & 32.799 & 16.459 & & 21.944 & 15.827 & 10.637 & 31.851 & 32.668 & .708 & 31.739 & 28.056 & 33.581 \\
\hline $1 / 30 / 1215: 00$ & & 30.505 & 30.021 & 29.218 & 30.054 & & 29.556 & 30.533 & 29.985 & 29.653 & 28.363 & 26.117 & 31.763 & 36.719 & 32.79 & 16.457 & & 21.944 & $\begin{array}{l}15.82 \\
\end{array}$ & 10.625 & 31.837 & 32.656 & 698 & 31.732 & 28.056 & 33.576 \\
\hline $1 / 30 / 1216: 00$ & & 30.51 & 30.023 & 29.234 & 30.057 & & 29.528 & 30.528 & 29.995 & 29.653 & 28.363 & 26.136 & 31.763 & 36.719 & 32.785 & $\quad 16.452$ & & 21.995 & $\begin{array}{l}15.82 \\
15.803\end{array}$ & $\begin{array}{l}10.023 \\
10.623\end{array}$ & $\begin{array}{l}31.031 \\
31.851\end{array}$ & $\begin{array}{l}32.030 \\
32.656 \\
\end{array}$ & .713 & 1.727 & $\begin{array}{l}28.061 \\
28.061\end{array}$ & $\begin{array}{l}33.581 \\
33.58\end{array}$ \\
\hline $1 / 30 / 12$ 17:00 & & 30.503 & 30.021 & 29.234 & 30.052 & & 29.519 & 30.537 & 29.992 & 29.646 & 28.36 & 26.136 & 31.759 & 36.717 & 32.783 & $\begin{array}{l}36.45 \\
3\end{array}$ & & 21.988 & 15.806 & 10.623 & 31.844 & 32.654 & 29.71 & 31.725 & 28.058 & 33.581 \\
\hline $1 / 30 / 1218: 00$ & & 30.505 & 30.025 & 29.25 & 30.059 & & 29.495 & 30.528 & 29.997 & 29.649 & 28.363 & 26.153 & 31.759 & 36.715 & 32.783 & $\quad 16.448$ & & 21.984 & 15.796 & 10.618 & 31.851 & 32.651 & 29.722 & 1.725 & 28.056 & 33.581 \\
\hline 1/30/12 19:00 & & 30.51 & 30.035 & 29.269 & 30.071 & & 29.476 & 30.513 & 29.999 & 29.653 & 28.372 & 26.174 & 31.761 & 36.717 & 32.783 & 16.447 & & 21.984 & 15.775 & 10.621 & 31.865 & 32.656 & 29.741 & 31.727 & 28.058 & 33.581 \\
\hline $1 / 30 / 1220: 00$ & & 30.508 & 30.039 & 29.276 & 30.082 & & 29.469 & 30.511 & 29.997 & 29.656 & 28.372 & 26.186 & 31.761 & 36.722 & 32.785 & 16.445 & & 21.977 & 15.773 & 10.621 & 31.867 & 32.658 & 29.743 & 31.727 & 28.058 & 33.581 \\
\hline $1 / 30 / 1221: 00$ & & 30.512 & 30.046 & 29.283 & 30.089 & & 29.457 & 30.509 & 29.999 & 29.66 & 28.377 & 26.193 & 31.763 & 36.724 & 32.785 & 16.445 & & 21.977 & 15.766 & 10.621 & 31.874 & 32.658 & 29.75 & 31.729 & 28.058 & 33.581 \\
\hline $1 / 30 / 1222: 00$ & & 30.505 & 30.042 & 29.271 & 30.096 & & 29.462 & 30.521 & 29.992 & 29.656 & 28.372 & 26.184 & 31.761 & 36.722 & 32.781 & 16.445 & & 21.97 & 15.768 & 10.623 & 31.867 & 32.656 & 29.748 & 31.727 & 28.058 & 33.579 \\
\hline $1 / 30 / 1223: 00$ & & 30.503 & 30.039 & 29.262 & 30.089 & & 29.467 & 30.53 & 29.992 & 29.651 & 28.367 & 26.174 & 31.754 & 36.719 & 32.778 & $\begin{array}{l}36.443 \\
\end{array}$ & & 21.97 & 15.761 & 10.614 & 31.865 & 32.651 & 29.741 & 31.722 & 28.058 & 33.576 \\
\hline $1 / 31 / 120: 00$ & & 30.501 & 30.035 & 29.253 & 30.085 & & 29.467 & 30.537 & 29.992 & 29.646 & 28.363 & 26.174 & 31.754 & 36.717 & 32.776 & 16.443 & & 21.968 & 15.756 & 10.611 & 31.856 & 52.649 & 29.731 & 31.72 & 28.058 & 33.574 \\
\hline $1 / 31 / 121: 00$ & & 30.496 & 30.03 & 29.243 & 30.075 & & 29.472 & 30.547 & 29.99 & 29.64 & 28.356 & 26.16 & 31.747 & 36.717 & 32.771 & 16.44 & & 21.963 & 15.759 & 10.606 & 31.858 & 32.644 & 29.727 & 31.715 & 28.058 & 33.572 \\
\hline $1 / 31 / 122: 00$ & & 30.496 & 30.032 & 29.239 & 30.071 & & 29.469 & 30.542 & 29.99 & 29.635 & 28.351 & 26.158 & 31.747 & 36.708 & 32.771 & 16.44 & & 21.956 & 15.749 & 10.606 & 31.849 & 32.642 & 29.724 & 31.711 & 28.061 & 33.569 \\
\hline $1 / 31 / 123: 00$ & & 30.494 & 30.028 & 29.236 & 30.064 & & 29.464 & 30.545 & 29.99 & 29.633 & 28.349 & 26.155 & 31.744 & 36.71 & 32.767 & 16.438 & & 21.947 & 15.745 & $\begin{array}{l}10.602 \\
\end{array}$ & 31.842 & 32.639 & 29.724 & 31.711 & 28.061 & 33.569 \\
\hline $1 / 31 / 124: 00$ & & $\begin{array}{l}30.494 \\
30.494\end{array}$ & $\begin{array}{l}30.02 \\
30.03\end{array}$ & 29.236 & 30.0 & & $\begin{array}{l}29.404 \\
29.46\end{array}$ & 30.542 & 29.988 & 29.63 & $\begin{array}{l}20.049 \\
28.349\end{array}$ & 26.158 & 31.742 & 36.712 & 32.764 & $\begin{array}{r}16.430 \\
+736\end{array}$ & & 21.951 & 15.735 & $\begin{array}{l}10.602 \\
10.62\end{array}$ & $\begin{array}{l}31.837 \\
31.837\end{array}$ & 32.637 & 29.722 & 31.708 & 28.058 & $\begin{array}{l}33.567 \\
3.567\end{array}$ \\
\hline $1 / 31 / 125: 00$ & & 30.51 & 30.053 & 29.29 & $\begin{array}{l}30.0942 \\
30.092\end{array}$ & & 29.413 & 30.494 & $\begin{array}{l}30.000 \\
30.007\end{array}$ & 29.646 & $\begin{array}{l}20.347 \\
28.374\end{array}$ & 26.205 & 31.751 & 36.717 & 32.769 & 16.4 & & 21.951 & 15.707 & $\begin{array}{l}10.609 \\
10.09\end{array}$ & $\begin{array}{l}31.881 \\
31.881\end{array}$ & 32.649 & 29.767 & 31.718 & $\begin{array}{l}28.065 \\
28.065\end{array}$ & 33.572 \\
\hline $1 / 31 / 126: 00$ & & 30.505 & $\begin{array}{l}30.053 \\
30.058\end{array}$ & 29.29 & 30.101 & & 29.415 & $\begin{array}{l}30.492 \\
30.492\end{array}$ & 29.999 & $\begin{array}{l}29.650 \\
29.651\end{array}$ & $\begin{array}{l}20.574 \\
28.377\end{array}$ & 26.213 & 31.754 & 36.719 & 32.776 & $\begin{array}{l}10.450 \\
5.438\end{array}$ & & $\begin{array}{l}21.941 \\
21.947\end{array}$ & 15.714 & 10.613 & $\begin{array}{l}31.001 \\
31.884\end{array}$ & $\begin{array}{l}32.049 \\
32.649\end{array}$ & 29.771 & 31.722 & $\begin{array}{l}28.061 \\
28.061\end{array}$ & 33.572 \\
\hline $1 / 31 / 127: 00$ & & $\begin{array}{l}30.05 \\
30.51\end{array}$ & $\begin{array}{l}30.038 \\
30.067\end{array}$ & 29.309 & 30.117 & & 29.403 & $\begin{array}{r}30.452 \\
30.48\end{array}$ & 30.002 & $\begin{array}{l}29.658 \\
29.658\end{array}$ & 28.386 & $\begin{array}{l}20.213 \\
26.227\end{array}$ & $\begin{array}{l}31.749 \\
31.759\end{array}$ & 36.731 & 32.778 & $\begin{array}{l}10.4580 \\
36.443\end{array}$ & & $\begin{array}{l}21.941 \\
21.988\end{array}$ & $\begin{array}{l}15.705 \\
15.74\end{array}$ & $\begin{array}{l}10.013 \\
10.616\end{array}$ & $\begin{array}{l}11.084 \\
31.898\end{array}$ & $\begin{array}{l}32.049 \\
32.656\end{array}$ & 29.785 & 31.727 & $\begin{array}{l}28.061 \\
28.061\end{array}$ & $\begin{array}{l}33.574 \\
33.57\end{array}$ \\
\hline $1 / 31 / 12$ & & $\begin{array}{l}30.515 \\
30.515\end{array}$ & $\begin{array}{l}30.06 \% \\
30.084\end{array}$ & $\begin{array}{l}29.339 \\
29.339\end{array}$ & 30. & & $\begin{array}{l}29.403 \\
29.385\end{array}$ & $\begin{array}{l}30.488 \\
30.478\end{array}$ & $\begin{array}{l}30.002 \\
30.009\end{array}$ & $\begin{array}{l}29.678 \\
29.674\end{array}$ & $\begin{array}{l}28.380 \\
28.405\end{array}$ & $\begin{array}{r}20.247 \\
26.26\end{array}$ & $\begin{array}{l}31 . / 79 \\
31.768\end{array}$ & 36.731 & $\begin{array}{l}32.785 \\
32.785\end{array}$ & 16. & & $\begin{array}{l}21.988 \\
21.991\end{array}$ & $\begin{array}{l}13.1695 \\
15.695\end{array}$ & $\begin{array}{l}10.610 \\
10.623\end{array}$ & $\begin{array}{l}31.898 \\
31.919\end{array}$ & $\begin{array}{r}32.036 \\
32.665\end{array}$ & 29.814 & 31.737 & $\begin{array}{l}28.0063 \\
28.063\end{array}$ & $\begin{array}{l}33.574 \\
33.574\end{array}$ \\
\hline $1 /$ & & 30 & 30.09 & 29.356 & 30 & & 2 & 30.53 & 30 & 29 & 28. & 26.275 & 31 & 36.74 & 32. & 16 & & 36 & 15. & 10.635 & 31.933 & 2 & 2 & 6 & 28.061 & 33.576 \\
\hline $1 / 31 /$ & & 30. & 30 & 29 & & & 29 & 30.535 & 30 & 29. & 28. & 26. & 31 & 36.745 & 32.802 & 16 & & 21.984 & 15.695 & 10.639 & 31.944 & 32 & 44 & 51 & 28.061 & 33.579 \\
\hline 1/31/12 11:00 & & 30.524 & 30.118 & 29.384 & 30. & & & 30.54 & & 29.707 & 28.435 & 26.296 & & 36.752 & 32.811 & & & 21.984 & 15.702 & 10.649 & 31.951 & 32.689 & .856 & 58 & 28.065 & 33.581 \\
\hline $212: 00$ & & 30.529 & 30.128 & 29.391 & 30. & & 29.403 & 30.545 & & 29.7 & 28.446 & 26.304 & 31.801 & 36.766 & 32.818 & 16. & & 21.984 & 15.705 & 10.661 & 31.963 & & .868 & 67 & 28.063 & 33.583 \\
\hline 2 13:00 & & 30. & 30.1 & & & & & 30.5 & & & 28. & 26. & 31. & 36.759 & 32.825 & 16 & & 22. & 15.726 & 10. & 31.963 & 32. & 29.863 & 31.767 & 28.065 & 33.586 \\
\hline $1 / 31 / 1214$ & & 30. & 30 & 29. & & & & & & & 28 & & 31. & 36.761 & 32.828 & & & $22 . c$ & 15.738 & 10. & 31.956 & & & 31.776 & 28. & 33.586 \\
\hline $1 / 31 / 1215: 00$ & & 30.533 & 30.1 & 29.384 & 30. & & & & 30. & & 28. & 26. & 31. & 36.757 & 32.83 & & & 22.06 & 15. & 10.6 & 31.97 & 32.708 & 29.868 & 31.779 & 28.065 & 33.588 \\
\hline $1 / 31 / 1216: 00$ & & 30.536 & 30.1 & 29.393 & & & 29. & 30. & 30.021 & & 28. & 26.296 & 31.8 & 36.771 & 32.837 & 16 & & 22.115 & 15.735 & 10.6 & 31.972 & 32.718 & 29.877 & 31.783 & 28.068 & 33.59 \\
\hline $1 / 31 / 1217: 00$ & & 30.543 & 30.15 & 29.423 & 30.2 & & 29.427 & 30.561 & 30.03 & 29. & 28.474 & 26.323 & 31.82 & 36.773 & 32.849 & 16. & & 22.111 & 15.735 & 10.684 & 31.991 & 32.722 & 29.901 & 31.795 & 28.068 & 33.593 \\
\hline $1 / 31 / 1218: 00$ & & 30.55 & 30.172 & 29.444 & 30.2 & & 29.417 & 30.568 & 30.028 & 29.7 & 28.488 & 26.342 & 31.838 & 36.783 & 32.858 & 36. & & 22.122 & 15.735 & 10.694 & 32.012 & 32.736 & 29.922 & 31.807 & 28.065 & 33.595 \\
\hline $1 / 31 / 1219: 00$ & & 30.554 & 30.179 & 29.454 & 30.271 & & 29.422 & 30.576 & 30.033 & 29.774 & 28.495 & 26.349 & 31.85 & 36.783 & 32.865 & 16.492 & & 22.122 & 15.738 & 10.703 & 32.017 & 32.744 & 29.931 & 31.812 & 28.068 & 33.597 \\
\hline $1 / 31 / 1220: 00$ & & 30.557 & 30.191 & 29.463 & 30.283 & & 29.424 & 30.58 & 30.033 & 29.783 & 28.505 & 26.356 & 31.854 & 36.79 & 32.872 & 16.499 & & 22.113 & 15.74 & 10.707 & 32.031 & 32.751 & 29.943 & 31.821 & 28.068 & 33.6 \\
\hline $1 / 31 / 1221: 00$ & & 30.561 & 30.2 & 29.477 & 30.295 & & 29.429 & 30.588 & 30.037 & 29.795 & 28.514 & 26.363 & 31.861 & 36.794 & 32.881 & 16.503 & & 22.106 & 15.745 & 10.719 & 32.038 & 32.758 & 29.95 & 31.828 & 28.068 & 33.6 \\
\hline $1 / 31 / 1222: 00$ & & 30.566 & 30.212 & 29.487 & 30.306 & & 29.432 & 30.592 & 30.035 & 29.807 & 28.525 & 26.371 & 31.869 & 36.801 & 32.884 & 16.508 & & 22.099 & 15.752 & 10.724 & 32.042 & 32.765 & 29.962 & 31.833 & 28.068 & 33.602 \\
\hline & & & & & & & & & & & & & & & & & & & & & & & & & & \\
\hline
\end{tabular}




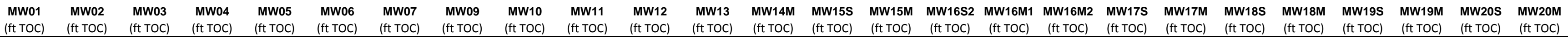

\begin{tabular}{|c|c|c|c|c|c|c|c|c|c|c|c|c|c|c|c|c|c|c|c|c|c|c|c|c|c|}
\hline Well & $\begin{array}{l}\text { (ft TOC) } \\
\text { (is }\end{array}$ & $\begin{array}{l}\text { (ft TOC) } \\
\text { (f) }\end{array}$ & $\begin{array}{l}\text { (ft TOC) } \\
\text { (f) }\end{array}$ & $\begin{array}{l}\text { (ft TOC) } \\
\text { (ft) }\end{array}$ & $\begin{array}{l}\text { (ft TOC) } \\
\text { (f) }\end{array}$ & $\begin{array}{l}\text { (ft TOC) } \\
\text { (f) }\end{array}$ & $\begin{array}{l}\text { (ft TOC) } \\
\text { (ft }\end{array}$ & $\begin{array}{l}\text { (ft TOC) } \\
\text { (f) }\end{array}$ & $\begin{array}{l}\text { (ft TOC) } \\
\text { (ft) }\end{array}$ & $\begin{array}{l}\text { (ft TOC) } \\
\text { (f) }\end{array}$ & $\begin{array}{l}\text { (ft TOC) } \\
\text { (f) }\end{array}$ & $\begin{array}{l}\text { (ft TOC) } \\
\text { (f) }\end{array}$ & (ft TOC) & $\begin{array}{l}\text { (ft TOC) } \\
\end{array}$ & (ft TOC) & $\begin{array}{ll}\text { (ft TOC) } \\
\text { (ft TOC) }\end{array}$ & (ft TOC) & $\begin{array}{l}\text { (ft TOC) } \\
\text { (1) }\end{array}$ & (ft TOC) & $\begin{array}{l}\text { (ft TOC) } \\
\end{array}$ & $\begin{array}{l}\text { (ft TOC) } \\
\text { (f) }\end{array}$ & $\begin{array}{l}\text { (ft TOC) } \\
\text { (n) }\end{array}$ & (ft TOC) & (ft TOC) & (ft TOC) \\
\hline 20:00 & & 30.573 & 30.221 & 29.498 & 30.318 & & 29.45 & 30.604 & 30.04 & 29.823 & 28.537 & 26.373 & 31.88 & 36.804 & 32.8 & 16 & 22. & $15.7 \mathrm{C}$ & 10.7 & 32.056 & 32. & 29.971 & 31.847 & 07 & 33.602 \\
\hline 2/1/12 1:00 & & 30.573 & 30.223 & 29.496 & 30.32 & & 29.462 & 30.607 & 30.045 & 29.828 & 28.542 & 26.371 & 31.883 & 36.806 & 32.903 & 16.524 & 22.083 & 15.768 & 10.736 & 32.059 & 32.779 & 29.971 & 31.847 & 28.068 & 33.6 \\
\hline 2/1/12 2:00 & & 30.573 & 30.228 & 29.494 & 30.323 & & 29.474 & 30.612 & 30.037 & 29.832 & 28.544 & 26.368 & 31.885 & 36.811 & 32.905 & 16.527 & 22.078 & 15.77 & 10.741 & 32.059 & 32.781 & 29.973 & 31.851 & 28.07 & 33.602 \\
\hline 2/1/12 3:00 & & 30.573 & 30.223 & 29.487 & 30.318 & & 29.488 & 30.614 & 30.033 & 29.83 & 28.542 & 26.354 & 31.887 & 36.808 & 32.905 & 16.531 & 22.074 & 15.78 & 10.74 & 32.054 & 32.781 & 29.964 & 31.851 & 28.068 & 33.6 \\
\hline 2/1/12 4:00 & & 30.575 & 30.226 & 29.484 & 30.313 & & 29.495 & 30.614 & 30.037 & 29.834 & 28.544 & 26.351 & 31.887 & 36.811 & 32.907 & 16.536 & 22.069 & 15.785 & 10.743 & 32.052 & 32.784 & 29.966 & 31.851 & 28.07 & 33.6 \\
\hline 2/1/12 5:00 & & 30.58 & 30.23 & 29.494 & 30.32 & & 29.495 & 30.619 & 30.04 & 29.841 & 28.549 & 26.356 & 31.892 & 36.813 & 32.91 & 16.538 & 22.065 & 15.782 & 10.745 & 32.063 & 32.789 & 29.969 & 31.856 & 28.07 & 33.6 \\
\hline 2/1/12 6:00 & & 30.578 & 30.23 & 29.491 & 30.318 & & 29.504 & 30.619 & 30.037 & 29.844 & 28.549 & 26.351 & 31.892 & 36.818 & 32.912 & 16.541 & 22.088 & 15.789 & 10.748 & 32.056 & 32.789 & 29.969 & 31.859 & 28.07 & 33.6 \\
\hline 2/1/12 7:00 & & 30.582 & 30.237 & 29.496 & 30.32 & & 29.502 & 30.621 & 30.045 & 29.846 & 28.553 & 26.354 & 31.894 & 36.815 & 32.917 & 16.545 & 22.088 & 15.785 & 10.752 & 32.063 & 32.791 & 29.976 & 31.861 & 28.07 & 33.6 \\
\hline 2/1/12 8:00 & & 30.585 & 30.244 & 29.512 & 30.332 & & 29.497 & 30.623 & 30.047 & 29.858 & 28.563 & 26.368 & 31.901 & 36.815 & 32.921 & 16.548 & 22.078 & 15.789 & 10.759 & 32.073 & 32.8 & 29.988 & 31.868 & 28.07 & 33.602 \\
\hline 2/1/12 9:00 & & 30.587 & 30.251 & 29.522 & 30.341 & & 29.5 & 30.626 & 30.049 & 29.865 & 28.567 & 26.378 & 31.906 & 36.82 & 32.926 & $\quad 16.552$ & 22.076 & 15.792 & 10.762 & 32.082 & 32.803 & 29.992 & 31.87 & 28.07 & 33.604 \\
\hline $2 / 1 / 1210: 00$ & & 30.596 & 30.263 & 29.543 & 30.353 & & 29.497 & 30.631 & 30.056 & 29.876 & 28.581 & 26.39 & 31.915 & 36.82 & 32.933 & 16.557 & 22.074 & 15.789 & 10.768 & 32.096 & 32.81 & 30.016 & 31.88 & $\begin{array}{l}3 \\
28.072\end{array}$ & 33.607 \\
\hline 2/1/12 11:00 & & 30.591 & 30.268 & 29.545 & 30.36 & & 29.511 & 30.628 & 30.052 & 29.886 & 28.586 & 26.394 & 31.92 & 36.825 & 32.94 & 16.559 & 22.069 & 15.808 & 10.778 & 32.101 & 32.815 & 30.016 & 31.884 & 28.072 & 33.609 \\
\hline $2 / 1 / 1212: 00$ & & 30.585 & 30.263 & 29.526 & 30.355 & & 29.537 & 30.633 & 30.042 & 29.881 & 28.581 & 26.375 & 31.922 & 36.825 & 32.94 & 16.564 & 22.067 & 15.829 & 10.773 & 32.087 & 32.817 & 29.999 & 31.889 & 28.072 & 33.611 \\
\hline 2/1/12 13:00 & & 30.58 & 30.249 & 29.496 & 30.337 & & 29.568 & 30.635 & 30.037 & 29.872 & 28.57 & 26.344 & 31.918 & 36.818 & 32.935 & 16.562 & 22.062 & 15.843 & 10.771 & 32.068 & 32.81 & 29.973 & 31.882 & 28.072 & 33.609 \\
\hline 2/1/12 14:00 & & 30.578 & 30.24 & 29.472 & 30.32 & & 29.587 & 30.633 & 30.037 & 29.862 & 28.556 & 26.318 & 31.908 & 36.818 & 32.931 & 16.562 & 22.058 & 15.855 & 10.766 & 32.045 & 32.803 & 29.955 & 31.877 & 28.075 & 33.607 \\
\hline $2 / 1 / 12$ 15:00 & & 30.58 & 30.233 & 29.463 & 30.306 & & 29.589 & 30.631 & 30.037 & 29.858 & 28.549 & 26.311 & 31.908 & 36.811 & 32.928 & $3 \quad 16.562$ & 22.053 & 15.853 & 10.766 & 32.045 & 32.803 & 29.945 & 31.873 & 328.075 & 33.609 \\
\hline 2/1/12 16:00 & & 30.582 & 30.233 & 29.465 & 30.302 & & 29.582 & 30.631 & 30.045 & 29.855 & 28.549 & 26.311 & 31.908 & 36.808 & 32.931 & 16.564 & 22.106 & 15.855 & 10.766 & 32.049 & 32.803 & 29.943 & 31.873 & 28.075 & 33.609 \\
\hline $2 / 1 / 1217: 00$ & & 30.589 & 30.235 & 29.475 & 30.304 & & 29.573 & 30.633 & 30.047 & 29.858 & 28.553 & 26.318 & 31.908 & 36.808 & 32.931 & 16.564 & 22.102 & 15.848 & 10.768 & 32.056 & 32.805 & 29.952 & 31.877 & 28.075 & 33.611 \\
\hline $2 / 1 / 1218: 00$ & & 30.591 & 30.242 & 29.484 & 30.309 & & 29.563 & 30.635 & 30.049 & 29.862 & 28.556 & 26.33 & 31.915 & 36.811 & 32.933 & 16.566 & 22.099 & 15.85 & 10.769 & 32.063 & 32.812 & 29.962 & 31.882 & 28.072 & 33.611 \\
\hline $2 / 1 / 1219: 00$ & & 30.598 & 30.251 & 29.508 & 30.323 & & 29.544 & 30.638 & 30.059 & 29.872 & 28.567 & 26.351 & 31.92 & 36.815 & 32.942 & 16.569 & 22.099 & 15.841 & 10.778 & 32.073 & 32.817 & 29.983 & 31.887 & 28.075 & 33.616 \\
\hline $2 / 1 / 1220: 00$ & & 30.598 & 30.258 & 29.522 & 30.334 & & 29.537 & 30.638 & 30.059 & 29.881 & 28.574 & 26.363 & 31.927 & 36.82 & 32.945 & 16.56 & 22.099 & 15.841 & 10.78 & 32.087 & 32.824 & .995 & 1.894 & 28.075 & 33.616 \\
\hline $2 / 1 / 1221: 00$ & & 30.596 & 30.261 & 29.519 & 30.339 & & 29.542 & 30.64 & 30.056 & 29.883 & 28.577 & 26.363 & 31.929 & 36.822 & 32.949 & 16.571 & 22.113 & 15.846 & 10.787 & 32.084 & 32.827 & 9.988 & 1.896 & 28.075 & 33.618 \\
\hline 2/1/12 22:00 & & 30.594 & 30.258 & 29.51 & 30.337 & & 29.556 & 30.643 & 30.054 & 29.879 & 28.572 & 26.349 & 31.929 & 36.825 & 32.947 & 16.573 & 22.127 & 15.853 & 10.785 & 32.08 & 32.824 & 29.985 & 1.891 & 28.075 & 33.618 \\
\hline 2/1/12 23:00 & & 30.594 & 30.254 & 29.503 & 30.33 & & 29.561 & 30.643 & 30.054 & 29.876 & 28.567 & 26.342 & 31.927 & 36.818 & 32.947 & 16.573 & 22.125 & 15.857 & 10.785 & 32.075 & 32.822 & 29.978 & 31.894 & 28.075 & 33.616 \\
\hline 2/2/12 0:00 & & 30.598 & 30.258 & 29.505 & 30.33 & & 29.561 & 30.647 & 30.059 & 29.879 & 28.57 & 26.339 & 31.927 & 36.82 & 32.947 & 16.573 & 22.118 & 15.853 & 10.785 & 32.077 & 32.822 & 29.98 & 31.894 & $\quad 28.077$ & 33.616 \\
\hline 2/2/12 1:00 & & 30.601 & 30.261 & 29.51 & 30.33 & & 29.558 & 30.645 & 30.059 & 29.881 & 28.57 & 26.344 & 31.927 & 36.818 & 32.947 & 16.573 & 22.109 & 15.843 & 10.785 & 32.077 & 32.822 & 29.983 & 31.894 & 28.077 & 33.614 \\
\hline 2/2/12 2:00 & & 30.603 & 30.268 & 29.517 & 30.334 & & 29.556 & 30.647 & 30.064 & 29.886 & 28.574 & 26.354 & 31.927 & 36.827 & 32.945 & 16.576 & 22.104 & 15.832 & 10.785 & 32.084 & 32.827 & 29.99 & 31.896 & $5 \quad 28.077$ & 33.614 \\
\hline 2/2/12 3:00 & & 30.601 & 30.265 & 29.522 & 30.339 & & 29.556 & 30.652 & 30.064 & 29.89 & 28.577 & 26.356 & 31.932 & 36.823 & 32.952 & 16.576 & 22.097 & 15.839 & 10.785 & 32.087 & 32.827 & 29.985 & 31.898 & $\begin{array}{l}3 \\
3\end{array}$ & 33.614 \\
\hline 2/2/12 4:00 & & 30.603 & 30.268 & 29.519 & 30.339 & & 29.563 & 30.65 & 30.061 & 29.888 & 28.579 & 26.354 & 31.932 & 36.822 & 32.949 & 16.576 & 22.085 & 15.841 & 10.785 & 32.084 & 32.824 & 29.99 & 31.896 & $\begin{array}{l}58.077 \\
5\end{array}$ & 33.611 \\
\hline 2/2/12 5:00 & & 30.61 & 30.275 & 29.533 & 30.346 & & 29.554 & 30.655 & 30.071 & 29.895 & 28.584 & 26.363 & 31.934 & 36.825 & 32.954 & 16.578 & 22.092 & 15.839 & 10.792 & 32.091 & 32.831 & 29.999 & 31.901 & 28.077 & 33.614 \\
\hline $2 / 2 / 126: 00$ & & 30.61 & 30.282 & 29.547 & 30.358 & & 29.551 & 30.657 & 30.071 & 29.902 & 28.593 & 26.378 & 31.941 & 36.827 & 32.959 & $\quad 16.58$ & 22.099 & 15.836 & 10.797 & 32.105 & 32.838 & 30.011 & 31.905 & $\quad 28.079$ & 33.614 \\
\hline $2 / 2 / 127: 00$ & & 30.61 & 30.282 & 29.547 & 30.36 & & 29.556 & 30.659 & 30.066 & 29.904 & 28.593 & 26.375 & 31.941 & 36.834 & 32.964 & 16.583 & 22.109 & 15.839 & 10.801 & 32.098 & 32.838 & 30.011 & 31.908 & $\begin{array}{l}3 \\
3\end{array}$ & 33.616 \\
\hline $2 / 2 / 128: 00$ & & 30.612 & 30.286 & 29.547 & 30.362 & & 29.563 & 30.659 & 30.071 & 29.909 & 28.595 & 26.375 & 31.946 & 36.832 & 32.966 & 16.583 & 22.102 & 15.841 & 10.802 & 32.103 & 32.843 & 30.011 & 31.91 & 28.079 & 33.616 \\
\hline 2/2/12 9:00 & & 30.608 & 30.284 & 29.538 & 30.36 & & 29.575 & 30.659 & 30.059 & 29.904 & 28.593 & 26.363 & 31.946 & 36.829 & 32.964 & 16.585 & 22.099 & 15.853 & 10.802 & 32.098 & 32.843 & 30.006 & 31.913 & $\begin{array}{l}3.079 \\
3\end{array}$ & 33.616 \\
\hline $2 / 2 / 1210: 00$ & & 30.612 & 30.284 & 29.533 & 30.3 & & 29.584 & 30.662 & 30.064 & 29.904 & 28.593 & 26.354 & 31.946 & 36.832 & 32.966 & 16. & 22.092 & 15.855 & 10.804 & 32.098 & 32.841 & 30.006 & 31.91 & 28.079 & 33.618 \\
\hline 2/2/12 11:00 & & 30.61 & 30.282 & 29.526 & 30.3 & & 29.594 & 30.664 & 30.066 & 29.904 & 28.588 & 26.354 & 31.946 & 36.827 & 32.966 & 16.5 & 22.088 & 15.862 & $\begin{array}{l}10.801 \\
\end{array}$ & 32.091 & 32.838 & 29.995 & 31.915 & $\begin{array}{l}28.079 \\
5\end{array}$ & 33.616 \\
\hline 2/2/12 12:00 & & 30.603 & 30.27 & 29.508 & 30.339 & & 29.615 & $\begin{array}{l}30.662 \\
30.62\end{array}$ & 30.061 & 29.895 & $\begin{array}{l}20.000 \\
28.577\end{array}$ & 26.337 & 31.941 & 36.822 & 32.964 & 16.5 & 22.083 & $\begin{array}{l}1.002 \\
15.883 \\
\end{array}$ & $\begin{array}{l}10.799 \\
10.799\end{array}$ & 32.08 & 32.838 & 29.98 & 31.913 & 28.077 & 33.616 \\
\hline 2/2/12 13:00 & & 30.598 & 30.251 & 29.47 & 30.316 & & 29.638 & 30.659 & 30.052 & 29.879 & 28.563 & 26.301 & 31.934 & $\begin{array}{l}30.02 \\
36.815\end{array}$ & 32.952 & 16.5 & 22.074 & $\begin{array}{l}1.000 \\
15.893 \\
\end{array}$ & 10.79 & 32.059 & 32.829 & $\begin{array}{l}29.950 \\
29.952\end{array}$ & 31.903 & $\begin{array}{l}28.077 \\
3\end{array}$ & 33.614 \\
\hline $2 / 2 / 1214: 00$ & & 30.594 & 30.228 & 29.435 & 30. & & 29.657 & 30.65 & 30.049 & 29.862 & 28.544 & 26.265 & 31.92 & 36.804 & 32.942 & 16.58 & 22.069 & 15.9 & 10.78 & 32.033 & 32.817 & 22 & 31.891 & 28.079 & 33.611 \\
\hline $2 / 2$ & & 30.591 & 30.216 & 29.416 & 30 & & 29 & 30.647 & 52 & 29 & 28.53 & 26.251 & 31 & 36.799 & 32.935 & 16. & 22 & 15.897 & 10.776 & 32.021 & 32.81 & 3 & 31.887 & 28.082 & 33.609 \\
\hline & & 30. & 30.2 & 29.407 & 30 & & 29.655 & 30.64 & 30 & 29 & 28.521 & 26 & 8 & 36.792 & 32.931 & 16. & 22 & 15.895 & 10.768 & 32.014 & 32.803 & 84 & 31.875 & $\quad 28.082$ & 33.609 \\
\hline $2 / 2 / 1$ & & 30 & 30.19 & 29.395 & & & & 30.638 & $3 c$ & 29. & 28.512 & & & 36.7 & 32.9 & 16. & 15 & 15.8 & 10.764 & 32.005 & 32.798 & 9.886 & 31.873 & $\begin{array}{l}38.082 \\
3\end{array}$ & 33.609 \\
\hline $2 / 2 / 1218: 00$ & & 30.591 & 30.193 & 29.398 & 30.236 & & 29.634 & 30.631 & 30.049 & 29.828 & 28.509 & 26.239 & 31.901 & 36.7 & 32.924 & 16.569 & 13 & 15.883 & 10.759 & 32.01 & 32.793 & 29.884 & 31.87 & 28.082 & 33.609 \\
\hline 2/2/12 19:00 & & 30.594 & 30.193 & 29.405 & 30.2 & & 29.617 & 30.6 & 30.056 & 29.823 & 28.509 & 26.241 & 31.901 & 36.783 & 32.924 & 16.564 & 22.109 & 15.871 & 10.759 & 32.014 & 32.793 & 29.891 & 31.863 & 8.084 & 33.611 \\
\hline $2 / 2 / 1220: 00$ & & 30.598 & 30.202 & 29.428 & 30. & & & 30. & & & 28.516 & 26.272 & 31.906 & 36.785 & 32.926 & 16.5 & 22.106 & 15.862 & 10.761 & 32.021 & 32.798 & 29.915 & 31.873 & $\quad 28.084$ & 33.616 \\
\hline 2/2/12 21:00 & & 30. & 30.19 & 29.405 & 30 & & & 30. & & & 28.502 & 26.251 & 31.901 & 36.78 & 32.924 & 16. & 22.099 & 15.874 & 10.759 & 32.01 & 32.796 & 29.894 & 31.873 & $3 \quad 28.082$ & 33.614 \\
\hline 2/2/12 22:00 & & 30. & 30.1 & 29.377 & 30. & & 29 & 30. & 30.049 & & 28.491 & 26.232 & 31.894 & 36.778 & 32.914 & 16. & 22.092 & 15.881 & 10.755 & 31.996 & 32.789 & 29.872 & 31.866 & $5 \quad 28.082$ & 33.614 \\
\hline $2 / 2 / 1223: 00$ & & 30.578 & 30.1 & 29.353 & 30. & & 29.6 & 30.6 & 30. & 29.793 & 28.472 & 26.21 & 31.883 & 36.771 & 32.903 & 16.5 & $22 . c$ & 15.881 & 10.738 & 31.977 & 32.774 & 29.847 & 31.854 & $\quad 28.084$ & 33.607 \\
\hline 2/3/12 0:00 & & 30.578 & 30.149 & 29.339 & 30.1 & & 29.617 & 30.612 & 30.052 & 29.779 & 28.467 & 26.189 & 31.866 & 36.769 & 32.889 & 16.5 & 22.067 & 15.862 & 10.724 & 31.958 & 32.762 & 29.842 & 31.828 & $3 \quad 28.084$ & 33.597 \\
\hline 2/3/12 1:00 & & 30.58 & 30.158 & 29.37 & 30.204 & & 29.591 & 30.65 & 30.049 & 29.788 & 28.474 & 26.225 & 31.845 & 36.771 & 32.86 & 16.5 & 22.044 & 15.848 & 10.7 & 31.958 & 32.741 & 29.847 & 31.814 & $\begin{array}{l}28.082 \\
7\end{array}$ & 33.574 \\
\hline $2 / 3 / 122: 00$ & & 30.58 & 30.214 & 29.409 & 30.199 & & 29.584 & 30.669 & 30.049 & 29.79 & 28.472 & 26.237 & 31.824 & 36.766 & 32.837 & 16.536 & 22.018 & 15.839 & 10.682 & 31.951 & 32.71 & 29.847 & 31.788 & $\begin{array}{l}3 \quad 28.084 \\
\end{array}$ & 33.56 \\
\hline 2/3/12 3:00 & & 30.587 & 30.251 & 29.753 & 30.204 & & 29.558 & 30.69 & 30.061 & 29.788 & 28.477 & 26.237 & 31.798 & 36.764 & 32.813 & $\quad 16.531$ & 21.995 & 15.817 & 10.658 & 31.949 & 32.687 & 29.854 & 31.758 & $\begin{array}{l}3 \quad 28.079 \\
\end{array}$ & 33.541 \\
\hline $2 / 3 / 124: 00$ & & 30.582 & 30.258 & 29.683 & 30.192 & & 29.568 & 30.698 & 30.056 & 29.828 & 28.472 & 26.225 & 31.789 & 36.761 & 32.806 & 16.524 & 21.979 & 15.82 & 10.654 & 31.933 & 32.68 & 29.844 & 31.751 & 28.082 & 33.527 \\
\hline 2/3/12 5:00 & & 30.582 & 30.256 & 29.606 & 30.197 & & 29.563 & 30.702 & 30.054 & 29.839 & 28.474 & 26.217 & 31.789 & 36.764 & 32.809 & 16.517 & 21.965 & 15.82 & 10.653 & 31.935 & 32.682 & 29.797 & 31.744 & $\begin{array}{r}28.063 \\
+\end{array}$ & 33.52 \\
\hline 2/3/12 6:00 & & 30.554 & 30.237 & 29.557 & 30.173 & & 29.587 & 30.707 & 30.014 & 29.862 & 28.449 & 26.198 & 31.791 & 36.757 & 32.816 & 16.51 & 21.97 & 15.864 & 10.691 & 31.898 & 32.675 & 29.729 & 31.753 & $3 \quad 28.054$ & 33.513 \\
\hline 2/3/12 7:00 & & 30.582 & 30.261 & 29.571 & 30.176 & & 29.57 & 30.688 & 30.049 & 29.881 & 28.463 & 26.217 & 31.794 & 36.754 & 32.811 & 16.51 & 21.968 & 15.803 & 10.651 & 31.926 & 32.682 & 29.797 & 31.755 & 28.068 & 3.516 \\
\hline
\end{tabular}




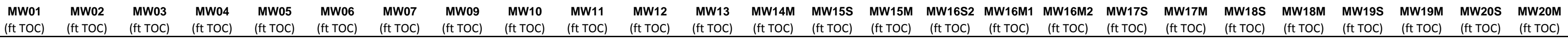

\begin{tabular}{|c|c|c|c|c|c|c|c|c|c|c|c|c|c|c|c|c|c|c|c|c|c|c|c|c|c|c|}
\hline Well & $\begin{array}{l}\text { (ft TOC) } \\
\text { (f) }\end{array}$ & $\begin{array}{l}\text { (ft TOC) } \\
\text { (f) }\end{array}$ & $\begin{array}{l}\text { (ft TOC) } \\
\text { (f) }\end{array}$ & (ft TOC) & (ft TOC) & $\begin{array}{l}\text { (ft ToC) } \\
\text { (f) }\end{array}$ & (ft ToC) & $\begin{array}{l}\text { (ft TOC) } \\
\text { (f) }\end{array}$ & $\begin{array}{l}\text { (ft TOC) } \\
\text { (f) }\end{array}$ & $\begin{array}{l}\text { (ft TOC) } \\
\text { (t) }\end{array}$ & $\begin{array}{l}\text { (ft TOC) } \\
\text { (n) }\end{array}$ & $\begin{array}{l}\text { (ft TOC) } \\
\text { (n) }\end{array}$ & (ft TOC) & $\begin{array}{l}\text { (ft ToC) } \\
\text { (ft }\end{array}$ & (ft TOC) & (ft TOC) & (ft TOC) & (ft TOC) & $\begin{array}{l}\text { (ft TOC) } \\
\text { (ft) }\end{array}$ & (ft TOC) & $\begin{array}{l}\text { (ft ToC) } \\
\text { (ft }\end{array}$ & $\begin{array}{l}\text { (ft TOC) } \\
\text { (f) }\end{array}$ & $\begin{array}{l}\text { (ft TOC) } \\
\text { (n) }\end{array}$ & (ft TOC) & $\begin{array}{l}\text { (nt TOC) } \\
\text { (nt }\end{array}$ & (ft TOC) \\
\hline $2 / 3 / 128: 00$ & & 30.594 & 30.286 & 29.561 & 30.183 & & 29.544 & 30.667 & 30.064 & 29.899 & 28.479 & 26.241 & 31.789 & 36.754 & $\begin{array}{l}42.804 \\
\end{array}$ & 16.508 & & 21.97 & 15.78 & 10.632 & 31.951 & 32.687 & 29.837 & 31.746 & 28.068 & 33.516 \\
\hline $2 / 3 / 129: 00$ & & 30.587 & 30.254 & 29.564 & 30.194 & & 29.53 & 30.65 & 30.059 & 29.918 & 28.481 & 26.275 & 31.812 & 36.762 & 32.823 & 16.508 & & 21.974 & 15.799 & 10.665 & 31.954 & 32.699 & 29.835 & 31.769 & 28.063 & 33.518 \\
\hline $2 / 3 / 1210: 00$ & & 30.596 & 28.379 & 29.589 & 30.201 & & 29.523 & 30.635 & 30.066 & 29.925 & 28.491 & 26.292 & 31.817 & 36.764 & 32.825 & 16.506 & & 21.968 & 15.785 & 10.661 & 31.968 & 32.703 & 29.844 & 31.772 & 28.065 & 33.52 \\
\hline 2/3/12 11:00 & & 30.598 & 26.419 & 29.638 & 30.206 & & 29.514 & 30.652 & 30.071 & 29.927 & 28.498 & 26.308 & 31.819 & 36.776 & $5 \quad 32.83$ & 16.506 & & 21.968 & 15.775 & 10.665 & 31.97 & 32.706 & 29.84 & 31.781 & 28.063 & 33.518 \\
\hline 2/3/12 12:00 & & 30.596 & 25.153 & 29.608 & 30.197 & & 29.533 & 30.657 & 30.059 & 29.923 & 28.495 & 26.277 & 31.808 & 36.776 & 532.818 & 16.503 & & 21.97 & 15.778 & 10.656 & 31.963 & 32.701 & 29.832 & 31.762 & 28.056 & 33.516 \\
\hline 2/3/12 13:00 & & 30.58 & 24.211 & 29.54 & 30.176 & & 29.561 & 30.631 & 30.049 & 29.918 & 28.474 & 26.239 & 31.815 & 36.771 & 32.823 & 16.499 & & 21.963 & 15.808 & 10.679 & 31.933 & 32.699 & 29.788 & 31.767 & 28.046 & 33.511 \\
\hline $2 / 3 / 1214: 00$ & & 30.58 & 23.22 & 29.484 & 30.155 & & 29.57 & 30.612 & 30.04 & 29.918 & 28.47 & 26.232 & 31.796 & 36.762 & 32.818 & 16.496 & & 21.954 & 15.801 & 10.66 & 31.916 & 32.687 & 29.771 & 31.758 & 28.039 & 33.509 \\
\hline 2/3/12 15:00 & & 30.587 & 22.306 & 29.447 & 30.145 & & 29.563 & 30.6 & 30.052 & 29.92 & 28.47 & 26.237 & 31.791 & 36.762 & 32.813 & 16.494 & & 21.944 & $\begin{array}{l}15.072 \\
15.792\end{array}$ & 10.651 & 31.916 & 32.687 & 29.774 & 31.755 & 28.037 & 33.509 \\
\hline 2/3/12 16:00 & & 30.58 & 20.499 & 29.372 & 30.141 & & 29.558 & 30.59 & 30.049 & 29.909 & 28.467 & 26.248 & 31.796 & 36.759 & 32.813 & 16.489 & & 22 & 15.796 & 10.656 & 31.914 & 32.684 & 29.769 & 31.76 & 28.032 & 33.509 \\
\hline 2/3/12 17:00 & & 30.587 & 16.387 & 29.377 & 30.136 & & 29.551 & 30.583 & 30.056 & 29.851 & 28.474 & 26.248 & 31.791 & 36.761 & 32.809 & 16.487 & & 21.998 & 15.778 & 10.651 & 31.919 & 32.687 & 29.767 & 31.758 & 28.032 & 33.511 \\
\hline 2/3/12 18:00 & & 30.589 & 13.577 & 29.659 & 30.138 & & 29.535 & 30.602 & 30.064 & 29.876 & 28.479 & 26.263 & 31.796 & 36.766 & 32.806 & 16.485 & & 22.009 & 15.768 & 10.642 & 31.921 & 32.68 & 29.722 & 31.748 & 28.028 & 33.506 \\
\hline 2/3/12 19:00 & & 30.594 & 11.976 & 29.711 & 30.145 & & 29.521 & 30.619 & 30.071 & 29.918 & 28.491 & 26.284 & 31.798 & 36.773 & $3 \quad 32.806$ & 16.482 & & 22.011 & 15.763 & 10.644 & 31.93 & 32.684 & 29.755 & 31.753 & 28.018 & 33.506 \\
\hline 2/3/12 20:00 & & 30.589 & 10.565 & 29.657 & 30.145 & & 29.516 & 30.64 & 30.075 & 29.944 & 28.491 & 26.296 & 31.817 & 36.778 & $\begin{array}{ll}3 & 32.811\end{array}$ & 16.478 & & 21.998 & 15.768 & 10.658 & 31.928 & 32.682 & 29.706 & 31.758 & 28.007 & 33.504 \\
\hline 2/3/12 21:00 & & 30.456 & 9.546 & 29.662 & 30.141 & & 29.528 & 30.64 & 30.064 & 29.944 & 28.495 & 26.282 & 31.796 & 36.778 & 32.806 & 16.475 & & 21.988 & 15.763 & 10.649 & 31.923 & 32.682 & 29.739 & 31.748 & 27.997 & 33.504 \\
\hline 2/3/12 22:00 & & 30.098 & 8.905 & 29.676 & 30.145 & & 29.523 & 30.616 & 30.026 & 29.951 & 28.5 & 26.296 & 31.805 & 36.778 & 32.811 & 16.473 & & 21.981 & 15.754 & 10.654 & 31.93 & 32.684 & 29.755 & 31.755 & 27.988 & 33.504 \\
\hline $2 / 3 / 1223: 00$ & & 29.772 & 8.378 & 29.671 & 30.145 & & 29.523 & 30.614 & 29.995 & 29.958 & 28.505 & 26.304 & 31.803 & 36.785 & 32.811 & 16.466 & & 21.974 & 15.752 & 10.653 & 31.93 & 32.687 & 29.757 & 31.755 & 27.983 & 33.506 \\
\hline $2 / 4 / 120: 00$ & & 29.509 & 7.949 & 29.629 & 30.143 & & 29.526 & 30.592 & 29.95 & 29.96 & 28.505 & 26.304 & 31.798 & 36.783 & 32.811 & 16.461 & & 21.965 & 15.752 & 10.651 & 31.926 & 32.687 & 29.76 & 31.755 & 27.978 & 33.506 \\
\hline 2/4/12 1:00 & & 29.292 & 7.59 & 29.617 & 30.143 & & 29.526 & 30.578 & 29.909 & 29.969 & 28.514 & 26.313 & 31.791 & 36.785 & 32.811 & 16.459 & & 21.958 & 15.742 & 10.651 & 31.93 & 32.687 & 29.767 & 31.755 & 27.976 & 33.504 \\
\hline 2/4/12 2:00 & & 29.099 & 7.287 & 29.603 & 30.148 & & 29.519 & 30.566 & 29.864 & 29.974 & 28.523 & 26.325 & 31.789 & 36.792 & 32.811 & $\begin{array}{l}16.457 \\
167\end{array}$ & & 21.951 & 15.735 & 10.646 & 31.937 & 32.684 & 29.764 & 31.758 & 27.969 & 33.502 \\
\hline 2/4/12 3:00 & & 28.91 & $\begin{array}{l}7.2013 \\
7.073\end{array}$ & 29.589 & 30.15 & & 29.521 & 30.559 & 29.821 & 29.978 & 28.53 & 26.335 & 31.791 & 36.799 & 32.811 & $\begin{array}{l}10.47 \\
16.452\end{array}$ & & $\begin{array}{l}21.949 \\
21.949\end{array}$ & 15.735 & 10.654 & 31.937 & $\begin{array}{l}32.687 \\
32.687\end{array}$ & 29.767 & 31.76 & 27.967 & $\begin{array}{l}33.502 \\
33.502\end{array}$ \\
\hline 2/4/12 4:00 & & 28.717 & 6.632 & 29.557 & $\begin{array}{r}30.152 \\
30.152\end{array}$ & & 29.516 & $\begin{array}{l}30.559 \\
30.552\end{array}$ & $\begin{array}{l}29.769 \\
29.769\end{array}$ & 29.971 & $\begin{array}{l}28.537 \\
28.537\end{array}$ & 26.344 & $\begin{array}{l}31.789 \\
31.789\end{array}$ & 36.806 & 32.813 & $\begin{array}{l}10.42 \\
16.447\end{array}$ & & $\begin{array}{l}21.949 \\
21.944\end{array}$ & $\begin{array}{l}13.728 \\
15.728\end{array}$ & $\begin{array}{l}10.034 \\
10.649\end{array}$ & 31.944 & $\begin{array}{l}32.689 \\
32.089\end{array}$ & 29.771 & 31.76 & 27.962 & $\begin{array}{l}33.502 \\
33.499\end{array}$ \\
\hline $2 / 4 / 125: 00$ & & 28.517 & $\begin{array}{l}0.032 \\
5.818\end{array}$ & 29.533 & $\begin{array}{l}30.152 \\
30.154\end{array}$ & & 29.514 & $\begin{array}{l}30.532 \\
30.545\end{array}$ & 29.719 & 29.969 & $\begin{array}{l}28.531 \\
28.546\end{array}$ & $\begin{array}{l}20.344 \\
26.351\end{array}$ & $\begin{array}{l}31.179 \\
31.784\end{array}$ & $\begin{array}{l}30.800 \\
36.808\end{array}$ & $\begin{array}{l}32.813 \\
32.816\end{array}$ & $\begin{array}{l}10.44 \prime \\
16.447\end{array}$ & & $\begin{array}{l}21.944 \\
21.937\end{array}$ & $\begin{array}{l}13 . / 78 \\
15.721\end{array}$ & $\begin{array}{l}10.649 \\
10.651\end{array}$ & $\begin{array}{l}31.944 \\
31.944\end{array}$ & $\begin{array}{l}32.609 \\
32.691\end{array}$ & 29.778 & $\begin{array}{r}31.76 \\
31.762\end{array}$ & $\begin{array}{l}27.957 \\
27.957\end{array}$ & $\begin{array}{l}33.499 \\
33.499\end{array}$ \\
\hline $2 / 4 / 126: 00$ & & 28.337 & 4.079 & 29.517 & 30.161 & & 29.511 & 30.54 & 29.672 & 29.964 & 28.558 & 26.368 & 31.787 & 36.811 & & 16.445 & & 21.933 & 15.719 & 10.649 & 31.947 & 694 & 29.783 & 31.765 & 27.953 & 33.502 \\
\hline 2/4/12 7:00 & & $\begin{array}{l}28.188 \\
28.188\end{array}$ & 1.96 & 29.529 & 30.165 & & 29.511 & 30.535 & 29.619 & $\begin{array}{l}29.958 \\
29.958\end{array}$ & $\begin{array}{l}28.567 \\
28.567\end{array}$ & $\begin{array}{l}20.300 \\
26.378\end{array}$ & $\begin{array}{l}31.784 \\
31.78\end{array}$ & 36.822 & 32.823 & $\begin{array}{l}10.445 \\
16.445\end{array}$ & & $\begin{array}{l}21.935 \\
21.94\end{array}$ & 15.714 & 10.656 & 31.961 & .699 & 29.79 & 31.769 & 27.95 & .504 \\
\hline $2 / 4 / 128: 00$ & & 28.063 & 0.169 & 29.526 & 30.18 & & 29.504 & 30.53 & 29.567 & 29.958 & 28.584 & 26.402 & 31.789 & .825 & & 16.443 & & 21.94 & $\begin{array}{l}15.709 \\
1509\end{array}$ & 10.658 & 31.975 & .706 & $\begin{array}{r}29.809 \\
29.80\end{array}$ & 31.776 & 27.946 & $\begin{array}{l}33.509 \\
33.509\end{array}$ \\
\hline 2/4/12 9:00 & & 27.955 & 0.137 & 29.505 & 30.192 & & 29.504 & 30.53 & 29.517 & 29.955 & 28.595 & 26.416 & 31.796 & 36.834 & 32.837 & 16.44 & & 21.942 & 15.709 & 10.668 & 31.984 & 32.713 & 29.818 & 31.783 & 27.941 & 33.511 \\
\hline 2/4/12 10:00 & & 27.86 & 0.106 & 29.512 & 30.211 & & 29.502 & 30.53 & 29.467 & 29.96 & 28.614 & 26.438 & 31.805 & 36.839 & 32.846 & 16.443 & & 21.951 & 15.7 & 10.677 & 31.996 & 32.722 & 29.837 & 31.79 & 27.939 & 33.513 \\
\hline 2/4/12 11:00 & & 27.765 & 0.085 & 29.517 & 30.22 & & 29.511 & 30.528 & 29.417 & 29.958 & 28.623 & 26.447 & 31.815 & 36.844 & 32.851 & 16.443 & & 21.958 & 15.709 & 10.684 & 32.007 & 32.725 & 29.844 & 31.8 & 27.934 & 33.518 \\
\hline 2/4/12 12:00 & & 27.674 & 0.071 & 29.522 & 30.22 & & 29.53 & 30.525 & 29.372 & 29.948 & 28.63 & 26.442 & 31.826 & 36.841 & 32.86 & 16.44 & & 21.961 & 15.721 & 10.698 & 32.005 & 32.732 & 29.835 & 31.805 & 27.929 & 33.518 \\
\hline $2 / 4 / 1213: 00$ & & 27.576 & 0.057 & 29.515 & 30.208 & & 29.556 & 30.518 & 29.327 & 29.937 & 28.625 & 26.421 & 31.824 & 36.848 & 32.856 & 16.44 & & 21.961 & 15.728 & 10.694 & 31.991 & 32.727 & 29.825 & 31.8 & 27.925 & 33.518 \\
\hline $2 / 4 / 1214: 00$ & & 27.487 & 0.048 & 29.58 & 30.197 & & 29.573 & 30.518 & 29.294 & 29.927 & 28.625 & 26.406 & 31.819 & 36.841 & 32.853 & 16.436 & & 21.961 & 15.735 & 10.694 & 31.977 & 32.727 & 29.804 & 31.798 & 27.918 & 33.518 \\
\hline 2/4/12 15:00 & & 27.399 & 0.039 & 29.594 & 30.199 & & 29.575 & 30.513 & 29.28 & 29.927 & 28.632 & 26.418 & 31.815 & 36.844 & 32.851 & 16.436 & & 21.986 & 15.728 & 10.689 & 31.986 & 32.727 & 29.807 & 31.795 & 27.915 & 33.518 \\
\hline 2/4/12 16:00 & & 27.327 & 0.057 & 29.608 & 30.201 & & 29.573 & 30.511 & 29.273 & 29.927 & 28.642 & 26.426 & 31.815 & 36.848 & 32.856 & 16.436 & & 22.032 & 15.724 & 10.691 & 31.989 & 32.727 & 29.821 & 31.8 & 27.913 & 33.52 \\
\hline 2/4/12 17:00 & & 27.248 & 0.05 & 29.61 & 30.201 & & 29.58 & 30.509 & 29.263 & 29.925 & 28.644 & 26.428 & 31.817 & 36.848 & 32.858 & 16.431 & & 22.028 & 15.728 & 10.698 & 31.986 & 32.734 & 29.816 & 31.802 & 27.91 & 33.52 \\
\hline 2/4/12 18:00 & & 27.187 & 0.034 & 29.627 & 30.211 & & 29.577 & 30.509 & 29.261 & 29.927 & 28.656 & 26.44 & 31.817 & 36.855 & 32.863 & 16.431 & & 22.028 & 15.724 & 10.698 & 32 & 32.736 & 29.825 & 31.807 & 27.908 & 33.527 \\
\hline 2/4/12 19:00 & & 27.133 & 0.025 & 29.631 & 30.213 & & 29.582 & 30.504 & 29.263 & 29.927 & 28.663 & 26.445 & 31.819 & 36.858 & 32.872 & 16.431 & & 22.018 & 15.721 & $\begin{array}{l}10.7 \\
\end{array}$ & 32.007 & 32.741 & 29.832 & 31.809 & 27.908 & 33.53 \\
\hline $2 / 4 / 1220: 00$ & & 27.073 & 0.015 & 29.643 & 30.2 & & 29.584 & 30.499 & 29.263 & 29.93 & 28.67 & $\begin{array}{l}26.4447 \\
\end{array}$ & 31.826 & 36.855 & 32.87 & $\begin{array}{l}16.429 \\
16.429\end{array}$ & & 22.011 & 15.712 & 10.707 & 32.003 & 32.746 & 29.837 & 31.819 & 27.906 & 33.534 \\
\hline 2/4/12 21:00 & & 27.017 & 0.009 & 29.648 & 30.2 & & 29.591 & 30.501 & 29.265 & 29.932 & 28.677 & 26.457 & $\begin{array}{l}31.820 \\
31.843\end{array}$ & 36.862 & 32.881 & 16.4 & & 22.011 & 15.724 & 10.714 & 32.012 & 32.751 & 29.842 & 31.823 & 27.903 & $\begin{array}{l}33.539 \\
3.539\end{array}$ \\
\hline 2/4/12 22:00 & & 26.966 & $\begin{aligned} 0.02 \\
0.02\end{aligned}$ & 29.638 & 30.2 & & 29.61 & 30.499 & 29.261 & 29.923 & 28.674 & 26.44 & $\begin{array}{l}31.045 \\
31.845\end{array}$ & 36.862 & 32.879 & $\begin{array}{l}10.472 \\
16.427\end{array}$ & & 22.007 & $\begin{array}{l}13.731 \\
15.731\end{array}$ & $\begin{array}{l}10.714 \\
10.719\end{array}$ & 32.005 & .753 & $\begin{array}{r}2.048 \\
29.83\end{array}$ & 31.823 & 27.901 & $\begin{array}{l}33.541 \\
3.541\end{array}$ \\
\hline 2/4/12 23:00 & & $\begin{array}{l}26.917 \\
26.917\end{array}$ & 0.029 & $\begin{array}{l}29.629 \\
29.629\end{array}$ & $\begin{array}{l}30.2 \\
30.2\end{array}$ & & $\begin{array}{r}29.621 \\
2.624\end{array}$ & $\begin{array}{l}30.499 \\
30.492\end{array}$ & 29.265 & 29.913 & $\begin{array}{l}28.672 \\
28.672\end{array}$ & $\begin{array}{l}20.44 \\
26.43\end{array}$ & $\begin{array}{l}31.845 \\
31.848\end{array}$ & $\begin{aligned} 50.002 \\
36.86\end{aligned}$ & 32.879 & $\begin{array}{l}10.427 \\
16.424\end{array}$ & & 22 & $\begin{array}{l}13.731 \\
15.731\end{array}$ & 10.717 & 31.998 & .748 & $\begin{array}{l}29.83 \\
29.821\end{array}$ & 31.823 & 27.901 & $\begin{array}{l}33.541 \\
33.541\end{array}$ \\
\hline $2 / 5 / 12$ & & 26.877 & 0.027 & 29.631 & 30. & & 29.627 & 30.489 & 29.28 & 29.911 & 28.674 & 26.428 & 31.85 & 36.862 & 32.8 & 16 & & 21.995 & 15.728 & 10.715 & 31 & 8 & 29.821 & 31.821 & 27.899 & 33.544 \\
\hline $2 / 5 / 12$ & & 26 & 0.0 & 29.6 & 30. & & 29 & 30. & 29.2 & 29. & 28.681 & 26 & 31 & 36.86 & 32 & 16 & & 21 & 15.726 & 10.712 & 31 & 1 & 29 & 31.821 & 27.899 & 1.546 \\
\hline $2 / 5 / 12$ & & 26.79 & 0.025 & 29.6 & 30. & & 29. & 30. & 29. & 29. & 28. & 26. & 31.845 & 36.862 & 32. & & & 21. & 15.726 & 10.712 & 31 & 6 & 29.818 & 31.816 & 27.899 & 33.541 \\
\hline 2/5/12 3:00 & & 26. & 0.01 & 29.643 & 30. & & 29.634 & 30.482 & & 29.902 & 28.686 & & 31.845 & $\begin{array}{r}36.86 \\
\end{array}$ & 32.872 & & & 21.977 & 15.724 & & 31.998 & & 29.821 & 31.816 & 27.896 & 33.541 \\
\hline $2 / 5 / 124: 00$ & & 26.738 & 0.02 & 29.638 & 30.2 & & & 30.48 & 29.341 & 29.895 & 28.686 & 26.433 & 31.845 & 36.865 & 32.872 & 16.4 & & 21.972 & 15.728 & 10.708 & & & 29.816 & 31.814 & 27.896 & 33.539 \\
\hline 2/5/12 5:00 & & 26.7 & 0.0 & 29.634 & & & & 30.4 & 29.356 & & 28.6 & 26.426 & 31.84 & 36.865 & 32.867 & 16 & & 21.965 & 15.731 & 10.705 & 31. & 32. & 29.814 & 31.814 & 27.894 & 33.537 \\
\hline 2/5/126: & & 26. & $0 . c$ & 29. & & & & & 29 & & 28 & 26. & 31.8 & 36.8 & 32.8 & 16. & & 21. & 15. & & & & 29.807 & 31.812 & 27. & 33.537 \\
\hline $2 / 5 / 127: C$ & & 26. & 0.0 & 29.6 & & & 29. & & 29. & & 28. & 26. & 31.836 & $36 . \varepsilon$ & 32 & 16. & & 21.954 & 15.719 & 10.703 & & & 29.809 & 31.809 & 27.894 & 33.537 \\
\hline $2 / 5 / 128: 00$ & & 26.7 & 0.02 & 29.641 & 30. & & 29.6 & 30.4 & 29.401 & 29.8 & 28.6 & 26.426 & 31.831 & 36.862 & 32.867 & 16.4 & & 21.951 & $15.7 \mathrm{C}$ & 10.705 & 31.984 & 32.739 & 29.814 & 31.812 & 27.892 & 33.539 \\
\hline $2 / 5 / 129: 00$ & & 26.8 & 0.01 & 29.645 & 30.1 & & 29.657 & 30.4 & 29.42 & 29.872 & 28.695 & 26.435 & 31.833 & 36.8 & 32.87 & 16.4 & & 21.965 & 15.719 & 10.71 & 31.986 & 32.741 & 29.816 & 31.814 & 27.894 & 33.541 \\
\hline 2/5/12 10:00 & & 26.947 & 0.013 & 29.652 & 30.201 & & 29.66 & 30.4 & 29.434 & 29.872 & 28.698 & 26.44 & 31.84 & 36.865 & 32.872 & 16.3 & & 21.968 & 15.728 & 10.705 & 31.993 & 32.746 & 29.823 & 31.816 & 27.892 & 33.546 \\
\hline 2/5/12 11:00 & & 26.991 & 0.013 & 29.657 & 30.204 & & 29.667 & 30.461 & 29.446 & 29.867 & 28.7 & 26.438 & 31.845 & 36.869 & 32.874 & 16.3 & & 21.963 & 15.726 & 10.71 & 31.991 & 32.744 & 29.821 & 31.819 & 27.892 & 33.548 \\
\hline 2/5/12 12:00 & & 26.97 & 0.034 & 29.638 & 30.194 & & 29.683 & 30.456 & 29.451 & 29.855 & 28.695 & 26.426 & 31.845 & 36.869 & 32.872 & 16.394 & & 21.986 & 15.742 & 10.71 & 31.979 & 32.744 & 29.807 & 31.816 & 27.889 & 33.548 \\
\hline 2/5/12 13:00 & & 26.898 & 0.081 & 29.603 & 30.176 & & 29.714 & 30.454 & 29.434 & 29.834 & 28.677 & 26.392 & 31.838 & 36.862 & 32.865 & 16.389 & & 21.986 & 15.756 & 10.703 & 31.958 & 32.734 & 29.783 & 31.809 & 27.889 & 33.548 \\
\hline 2/5/12 14:00 & & 26.859 & 0.12 & 29.582 & 30.155 & & 29.725 & 30.449 & 29.436 & 29.818 & 28.667 & 26.371 & 31.831 & 36.851 & 32.856 & 16.382 & & 21.972 & 15.761 & 10.696 & 31.944 & 32.725 & 29.762 & 31.802 & 27.889 & 33.546 \\
\hline & & & & 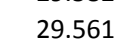 & & & & & & & & & & & & & & & & & & & & & & \\
\hline
\end{tabular}




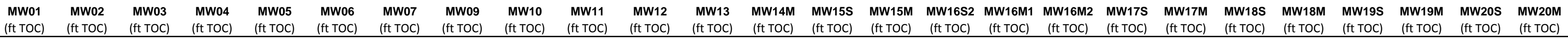

\begin{tabular}{|c|c|c|c|c|c|c|c|c|c|c|c|c|c|c|c|c|c|c|c|c|c|c|c|c|c|c|}
\hline Well & $\begin{array}{l}\text { Wwol } \\
\text { (ft TOC) }\end{array}$ & $\begin{array}{l}\text { Mwoz } \\
\text { (ft TOC) }\end{array}$ & $\begin{array}{r}\text { Mwo3 } \\
\text { (ft TOC) }\end{array}$ & $\begin{array}{r}\text { MWva4 } \\
\text { (ft TOC) }\end{array}$ & $\begin{array}{l}\text { MWOS } \\
\text { (ft TOC) }\end{array}$ & $\begin{array}{l}\text { MWvo6 } \\
\text { (ft TOC) }\end{array}$ & (ft TOC) & $\begin{array}{r}\text { Mw09 } \\
\text { (ft TOC) }\end{array}$ & $\begin{array}{l}\text { MW10 } \\
\text { (ft ToC) }\end{array}$ & $\begin{array}{r}\text { MWII } \\
\text { (ft TOC) }\end{array}$ & $\begin{array}{l}\text { MWII2 } \\
\text { (ft TOC) }\end{array}$ & $\begin{array}{l}\text { MWVI3 } \\
(\mathrm{ft} \text { TOC) }\end{array}$ & $\begin{array}{l}\text { MW14M } \\
\text { (ft TOC) }\end{array}$ & $\begin{array}{l}\text { MWvitss } \\
\text { (ft TOC) }\end{array}$ & $\begin{array}{l}\text { MW } \\
\text { (ft TOC) }\end{array}$ & $\begin{array}{l}\text { MWU } \\
(\mathrm{ft} \text { TOC) }\end{array}$ & $\begin{array}{c}\text { MWIBMI } \\
\text { (ft TOC) }\end{array}$ & $\begin{array}{l}\text { MWI } \\
\text { (ft TOC) }\end{array}$ & $\begin{array}{l}\text { MWd TOC } \\
\text { (ft TOC }\end{array}$ & (ft TOC) & $\begin{array}{l}\text { MWVIDS } \\
\text { (ft TOC) }\end{array}$ & $\begin{array}{l}\text { MWIIM } \\
\text { (ft TOC) } \\
\end{array}$ & $\begin{array}{l}\text { MWVI9S } \\
\text { (ft TOC) }\end{array}$ & $\begin{array}{l}\text { MWW } \\
\text { (ft TOC) }\end{array}$ & $\begin{array}{l}\text { MWwas } \\
\text { (ft TOC) }\end{array}$ & (ft TOC) \\
\hline 2/5/12 16:00 & & 26.912 & 0.204 & 29.545 & 30.12 & & 29.739 & 30.437 & 29.439 & 29.783 & 28.644 & 26.335 & 31.81 & 36.841 & 32.839 & 16.373 & & 22.016 & 15.766 & 10.677 & 31.914 & $\begin{array}{l}4 \quad 32.708 \\
\end{array}$ & 29.727 & 31.783 & 27.887 & 33.541 \\
\hline 2/5/12 17:00 & & 26.987 & 0.239 & 29.531 & 30.103 & & 29.739 & 30.432 & 29.444 & 29.767 & 28.632 & 26.327 & 31.883 & 36.839 & 32.917 & 16.366 & & 22.007 & 15.766 & 10.754 & 31.942 & $2 \quad 32.781$ & 29.713 & 31.851 & $\quad 27.887$ & 33.539 \\
\hline 2/5/12 18:00 & & 27.045 & 0.256 & 29.531 & 30.094 & & 29.728 & 30.427 & 29.453 & 29.756 & 28.63 & 26.323 & 31.845 & 36.827 & 32.872 & 16.361 & & 22.004 & 15.756 & 10.707 & 31.921 & $1 \quad 32.739$ & 29.713 & 31.809 & $\quad 27.887$ & 33.539 \\
\hline 2/5/12 19:00 & & 27.089 & 0.263 & 29.533 & 30.096 & & 29.711 & 30.422 & 29.472 & 29.751 & 28.628 & 26.335 & 31.833 & 36.837 & 32.86 & 16.354 & & 21.995 & 15.749 & 10.696 & 31.914 & $\begin{array}{l}4 \quad 32.727 \\
\end{array}$ & 29.717 & 31.795 & $\quad 27.885$ & $\quad 33.541$ \\
\hline 2/5/12 20:00 & & 27.129 & 0.267 & 29.538 & 30.096 & & 29.697 & 30.42 & 29.484 & 29.746 & 28.628 & 26.339 & 31.826 & 36.834 & 32.851 & 16.35 & & 21.988 & 15.738 & 10.686 & 31.909 & 32.72 & 29.724 & 31.788 & $\begin{array}{l}3 \quad 27.885 \\
\end{array}$ & $\quad 33.544$ \\
\hline 2/5/12 21:00 & & 27.166 & 0.274 & 29.536 & 30.096 & & 29.692 & 30.418 & 29.484 & 29.739 & 28.625 & 26.342 & 31.822 & 36.834 & 32.846 & $\quad 16.347$ & & 21.984 & 15.738 & 10.684 & 31.912 & $2 \quad 32.715$ & 29.72 & 31.786 & $5 \quad 27.882$ & 33.546 \\
\hline 2/5/12 22:00 & & 27.206 & 0.281 & 29.529 & 30.092 & & 29.692 & 30.413 & 29.486 & 29.73 & 28.621 & 26.337 & 32.065 & 36.834 & 33.116 & $5 \quad 16.343$ & & 21.993 & 15.728 & 10.95 & 31.977 & $7 \quad 32.962$ & 29.72 & 32.032 & 27.882 & 33.551 \\
\hline 2/5/12 23:00 & & 27.248 & 0.398 & 29.522 & 30.089 & & 29.692 & 30.411 & 29.484 & 29.721 & 28.614 & 26.33 & 32.009 & 36.837 & 33.048 & $3 \quad 16.336$ & & 22.002 & 15.726 & 10.886 & 31.989 & 32.9 & 29.727 & 31.971 & $\quad 27.882$ & 33.553 \\
\hline $2 / 6 / 120: 00$ & & 27.287 & 0.535 & 29.512 & 30.087 & & 29.693 & 30.406 & 29.489 & 29.709 & 28.607 & 26.325 & 31.941 & 36.832 & 32.973 & 3. 16.331 & & 21.995 & 15.728 & 10.808 & 31.958 & $8 \quad 32.836$ & 29.717 & 31.903 & 27.88 & 33.551 \\
\hline 2/6/12 1:00 & & 27.327 & 0.757 & 29.501 & 30.08 & & 29.693 & 30.399 & 29.491 & 29.7 & 28.6 & 26.315 & 31.904 & 36.829 & 32.935 & $\begin{array}{l}16.329 \\
5\end{array}$ & & 21.988 & 15.726 & 10.771 & 31.937 & $\begin{array}{l}7 \\
72.796\end{array}$ & 29.706 & 31.87 & 27.88 & 33.551 \\
\hline 2/6/12 2:00 & & 27.364 & 1.521 & 29.498 & 30.078 & & 29.688 & 30.399 & 29.498 & 29.693 & 28.595 & 26.315 & 31.878 & 36.827 & 32.907 & 16.322 & & 21.979 & 15.719 & 10.745 & 31.928 & $8 \quad 32.774$ & 29.703 & 31.842 & 27.88 & 33.548 \\
\hline 2/6/12 3:00 & & 27.397 & 2.363 & 29.487 & 30.071 & & 29.69 & 30.394 & 29.498 & 29.681 & 28.588 & 26.308 & 31.857 & 36.827 & 32.884 & 16.319 & & 21.972 & 15.709 & 10.722 & 31.912 & 32.751 & 29.696 & 31.821 & 27.88 & 33.544 \\
\hline $2 / 6 / 124: 00$ & & 27.434 & 3.298 & 29.475 & 30.059 & & 29.692 & 30.391 & 29.498 & 29.67 & 28.579 & 26.299 & 31.838 & 36.827 & 32.865 & 16.31 & & 21.965 & 15.709 & 10.703 & 31.895 & 32.729 & 29.68 & 31.802 & 27.88 & 33.539 \\
\hline $2 / 6 / 125: 00$ & & 27.473 & 4.165 & 29.47 & 30.054 & & 29.685 & 30.389 & 29.503 & 29.665 & 28.577 & 26.299 & 31.824 & 36.825 & 32.851 & 16.308 & & 21.958 & 15.7 & 10.686 & 31.891 & 32.717 & 29.68 & 31.79 & 27.88 & 33.537 \\
\hline $2 / 6 / 126: 00$ & & 27.511 & 4.988 & 29.47 & 30.052 & & 29.678 & 30.384 & 29.512 & 29.656 & 28.574 & 26.301 & 31.81 & 36.822 & 32.837 & 16.301 & & 21.963 & 15.693 & 10.677 & 31.884 & 32.708 & 29.675 & 31.779 & 27.88 & 33.534 \\
\hline 2/6/12 7:00 & & 27.546 & 5.769 & 29.472 & 30.05 & & 29.674 & 30.382 & 29.52 & 29.653 & 28.572 & 26.306 & 31.805 & 36.825 & 32.83 & 16.296 & & 21.958 & 15.691 & 10.67 & 31.881 & 32.696 & 29.677 & 31.772 & 27.878 & 33.534 \\
\hline 2/6/12 8:00 & & 27.578 & 6.508 & 29.475 & 30.052 & & 29.662 & 30.379 & 29.529 & 29.651 & 28.572 & 26.311 & 31.798 & 36.822 & 32.825 & 16.294 & & 21.956 & 15.686 & 10.663 & 31.881 & 32.694 & 29.682 & 31.765 & $\quad 27.878$ & 33.537 \\
\hline 2/6/12 9:00 & & 27.611 & 7.217 & 29.48 & 30.052 & & 29.66 & 30.379 & 29.534 & 29.651 & 28.572 & 26.318 & 31.796 & 36.822 & 32.821 & 16.289 & & 21.949 & 15.684 & 10.66 & 31.879 & 32.689 & 29.682 & 31.76 & $\quad 27.878$ & 33.539 \\
\hline $2 / 6 / 1210: 00$ & & 27.634 & 7.919 & 29.463 & 30.045 & & 29.674 & 30.377 & 29.527 & 29.64 & 28.56 & 26.304 & 31.789 & 36.82 & 32.813 & 16.284 & & 21.942 & 15.693 & 10.651 & 31.867 & 32.684 & 29.67 & 31.758 & $\begin{array}{l}3 \\
3\end{array}$ & 33.539 \\
\hline 2/6/12 11:00 & & 27.667 & 8.597 & 29.451 & 30.035 & & 29.678 & 30.377 & 29.529 & 29.63 & 28.556 & 26.292 & 31.782 & 36.818 & 32.809 & 16.28 & & 21.937 & 15.695 & 10.644 & 31.86 & 52.675 & 29.661 & 31.751 & 27.878 & $\quad 33.541$ \\
\hline 2/6/12 12:00 & & 27.697 & 9.278 & 29.426 & 30.019 & & 29.693 & 30.37 & 29.522 & 29.616 & 28.542 & 26.272 & 31.773 & 36.82 & 32.799 & 16.278 & & 21.931 & 15.702 & 10.635 & 31.844 & 32.665 & 29.644 & 31.744 & $\quad 27.878$ & 33.541 \\
\hline 2/6/12 13:00 & & 27.723 & 9.919 & 29.398 & 29.998 & & 29.709 & 30.365 & 29.508 & 29.598 & 28.523 & 26.248 & 31.761 & 36.811 & 32.785 & $\quad 16.268$ & & 21.921 & 15.709 & 10.623 & 31.818 & 3232.651 & 29.612 & 31.734 & $\quad 27.875$ & 33.539 \\
\hline 2/6/12 14:00 & & 27.748 & 10.539 & 29.363 & 29.975 & & 29.723 & 30.358 & 29.498 & 29.577 & 28.505 & 26.22 & 31.742 & 36.808 & 32.771 & 16.264 & & 21.91 & 15.709 & 10.609 & 31.798 & 32.635 & 29.59 & 31.715 & $5 \quad 27.873$ & 33.534 \\
\hline 2/6/12 15:00 & & 27.783 & 11.115 & 29.356 & 29.956 & & 29.714 & 30.355 & 29.51 & 29.563 & 28.493 & 26.21 & 31.73 & 36.799 & 32.757 & 16.259 & & 21.907 & 15.702 & 10.595 & 31.781 & 32.625 & 29.576 & 31.701 & 27.875 & $\quad 33.532$ \\
\hline 2/6/12 16:00 & & 27.813 & 11.663 & 29.353 & 29.949 & & 29.702 & 30.353 & 29.522 & 29.556 & 28.486 & 26.217 & 31.721 & 36.794 & 32.75 & $\quad 16.252$ & & 21.954 & 15.698 & 10.585 & 31.776 & 32.616 & 29.574 & 31.69 & $\quad 27.878$ & 33.53 \\
\hline 2/6/12 17:00 & & 27.841 & 12.183 & 29.353 & 29.942 & & 29.69 & 30.348 & 29.531 & 29.549 & 28.481 & 26.217 & 31.714 & 36.787 & 32.741 & 16.25 & & 21.949 & 15.688 & 10.576 & 31.776 & 32.606 & 29.569 & 31.683 & $\begin{array}{l}37.875 \\
\end{array}$ & 33.525 \\
\hline 2/6/12 18:00 & & 27.872 & 12.673 & 29.356 & 29.94 & & 29.671 & 30.348 & 29.539 & 29.542 & 28.479 & 26.22 & 31.707 & 36.794 & 32.734 & 16.243 & & 21.942 & 15.679 & 10.573 & 31.769 & 32.601 & 29.574 & 31.676 & $\quad 27.875$ & 33.527 \\
\hline 2/6/12 19:00 & & 27.9 & 13.139 & 29.363 & 29.944 & & 29.653 & 30.346 & 29.553 & 29.542 & 28.479 & 26.237 & 31.707 & 36.79 & 32.734 & 16.238 & & 21.937 & 15.672 & 10.569 & 31.776 & 32.599 & 29.581 & 31.673 & $\begin{array}{l}37.878 \\
\end{array}$ & 33.527 \\
\hline 2/6/12 20:00 & & 27.928 & 13.589 & 29.367 & 29.947 & & 29.641 & 30.341 & 29.56 & 29.54 & 28.479 & 26.241 & 31.705 & 36.792 & 32.731 & 16.236 & & 21.933 & 15.665 & 10.567 & 31.781 & 32.599 & 29.581 & 31.671 & 27.878 & 33.532 \\
\hline 2/6/12 21:00 & & 27.953 & 14.018 & 29.377 & 29.956 & & 29.624 & 30.341 & 29.572 & 29.542 & 28.481 & 26.253 & 31.709 & 36.797 & 32.731 & 16.231 & & 21.933 & 15.653 & 10.569 & 31.786 & 32.599 & 29.595 & 31.673 & $\begin{array}{l}37.875 \\
3\end{array}$ & 33.534 \\
\hline 2/6/12 22:00 & & 27.981 & 14.424 & 29.398 & 29.968 & & 29.606 & 30.341 & 29.584 & 29.549 & 28.491 & 26.277 & 31.712 & 36.804 & 32.736 & $5 \quad 16.231$ & & 21.928 & 15.646 & 10.576 & 31.795 & 32.606 & 29.614 & 31.678 & $\begin{array}{l}3 \quad 27.878 \\
\end{array}$ & 33.539 \\
\hline 2/6/12 23:00 & & 28.002 & $\begin{array}{l}14.429 \\
14.829\end{array}$ & $\begin{array}{l}29.390 \\
29.398\end{array}$ & 29.975 & & 29.603 & 30.341 & 29.586 & 29.549 & $\begin{array}{l}28.488 \\
28.47\end{array}$ & 26.28 & 31.714 & 36.801 & 32.736 & $\begin{array}{l}16.226 \\
5\end{array}$ & & 21.926 & 15.644 & 10.576 & 31.8 & 32.606 & 29.614 & $\begin{array}{r}31.68 \\
\end{array}$ & $\begin{array}{l}27.878 \\
3\end{array}$ & 33.541 \\
\hline $2 / 7 / 120: 00$ & & 28.032 & 15.209 & 29.407 & 29.984 & & 29.596 & 30.344 & 29.596 & 29.554 & 28.495 & 26.289 & 31.716 & 36.801 & 32.741 & 16.224 & & 21.921 & 15.639 & 10.578 & 31.809 & 32.608 & 29.628 & 31.683 & $\begin{array}{l}3 \\
3\end{array}$ & 33.546 \\
\hline 2/7/12 1:00 & & 28.053 & 15.585 & 29.405 & 29.989 & & 29.598 & 30.341 & 29.588 & 29.554 & 28.493 & 26.287 & 31.716 & 36.811 & 32.738 & 3. 16.224 & & 21.921 & 15.639 & 10.574 & 31.809 & 32.608 & 29.623 & 31.68 & $\begin{array}{l}3 \\
27.878\end{array}$ & 33.546 \\
\hline $2 / 7 / 122: 00$ & & 28.084 & 15.937 & 29.409 & 29. & & 29. & 30.341 & 29.598 & 29.556 & 28.5 & 26.294 & 31.709 & 36.811 & 32.736 & 16 & & 21.914 & 15.63 & 10.571 & 31.811 & 32.606 & .635 & 31.676 & $\quad 27.878$ & 33.544 \\
\hline 2/7/12 3:00 & & 28.102 & 16.296 & 29.405 & & & & 30.344 & 29.596 & 29.556 & 28.495 & 26.292 & 31.709 & 36.813 & 32.731 & 16.2 & & 21.914 & 15.634 & 10.569 & 31.811 & 32.604 & 9.626 & 1.678 & $\begin{array}{l}3 \quad 27.878 \\
\end{array}$ & 33.541 \\
\hline 2/7/12 4:00 & & 28.125 & 16.638 & 29.395 & 29.991 & & 29.606 & 30.344 & 29.591 & 29.551 & 28.491 & 26.282 & 31.705 & 36.811 & 32.727 & 16.21 & & 21.907 & 15.634 & 10.566 & 31.797 & 32.594 & 29.621 & 1.668 & $\begin{array}{l}37.878 \\
3\end{array}$ & 33.539 \\
\hline 2/7/12 5:00 & & 28.146 & 16.965 & 29.388 & 29.989 & & 29.606 & 30.339 & 29.591 & 29.551 & 28.488 & 26.282 & 31.7 & 36.811 & 32.722 & 16.215 & & 21.898 & 15.627 & 10.557 & 31.793 & 32.594 & 29.619 & 31.666 & $\quad 27.878$ & 33.537 \\
\hline 2/7/12 6:00 & & 28.174 & 17.272 & 29.395 & 29.991 & & 29.601 & 30.341 & 29.6 & 29.554 & 28.491 & 26.287 & 31.695 & 36.811 & 32.72 & 16.212 & & 21.91 & 15.625 & 10.555 & 31.79 & 32.59 & 29.626 & 31.661 & 27.878 & 33.534 \\
\hline 2/7/12 7:00 & & 28.205 & 17.566 & 29.412 & & & 29.584 & 30.346 & 29.615 & 29.558 & 28.5 & 26.296 & 31.695 & 36.815 & 32.72 & 16.215 & & 21.914 & 15.611 & 10.555 & 31.805 & 32.592 & 29.637 & 31.659 & 27.88 & 33.534 \\
\hline 2/7/12 8:00 & & 28.23 & 17.834 & 29.449 & 30.024 & & 29.563 & 30.351 & 29.636 & 29.584 & 28.518 & 26.337 & 31.705 & 36.825 & 32.729 & 16.215 & & 21.912 & 15.599 & 10.566 & 31.83 & 32.599 & 29.668 & 31.666 & $5 \quad 27.882$ & 33.539 \\
\hline 2/7/12 9:00 & & 28.251 & 18.107 & 29.472 & 30. & & 29.556 & 30.358 & 29.648 & 29.6 & 28.53 & 26.361 & 31.716 & 36.834 & 32.736 & 5 16.215 & & 21.905 & 15.604 & 10.574 & 31.849 & 32.609 & 29.689 & 31.68 & $\begin{array}{l}3 \quad 27.882 \\
\end{array}$ & 33.544 \\
\hline 2/7/12 10:00 & & 28.272 & 18.378 & 29.489 & 30.0 & & 29.556 & 30.363 & 29.65 & 29.614 & 28.542 & 26.375 & 31.726 & 36.841 & 32.748 & $\begin{array}{l}36.219 \\
\end{array}$ & & 21.905 & 15.604 & 10.585 & 31.86 & 32.618 & 29.706 & 31.687 & 27.882 & 33.546 \\
\hline $2 / 7 / 1211: 00$ & & 28.295 & 18.636 & 29.501 & 30. & & 29.558 & 30.372 & 29.653 & 29.626 & 28.551 & 26.387 & 31.733 & 36.848 & 32.752 & 16.222 & & 21.903 & 15.606 & 10.59 & 31.87 & 32.625 & 29.717 & 31.694 & $\begin{array}{l}27.882 \\
7\end{array}$ & 33.548 \\
\hline 2/7/12 12:00 & & 28.309 & 18.904 & 29.489 & 30.078 & & 29.577 & 30.372 & 29.641 & 29.628 & 28.549 & 26.378 & 31.733 & 36.851 & 32.755 & 16.222 & & 21.894 & 15.618 & 10.592 & 31.87 & 32.625 & 29.71 & 31.701 & 27.885 & 33.548 \\
\hline 2/7/12 13:00 & & 28.335 & 19.14 & 29.482 & 30.078 & & 29.594 & 30.375 & 29.636 & 29.626 & 28.546 & 26.363 & 31.73 & 36.855 & 32.753 & 16.224 & & 21.891 & 15.625 & 10.592 & 31.863 & 32.625 & 29.706 & 31.699 & $\quad 27.885$ & 33.548 \\
\hline 2/7/12 14:00 & & 28.358 & 19.347 & 29.477 & 30.075 & & 29.603 & 30.377 & 29.631 & 29.628 & 28.546 & 26.359 & 31.728 & 36.851 & 32.753 & 16.224 & & 21.882 & 15.63 & 10.59 & 31.856 & 32.62 & 29.698 & 31.692 & 27.885 & 33.544 \\
\hline 2/7/12 15:00 & & 28.379 & 19.557 & 29.482 & 30.075 & & 29.603 & 30.382 & 29.638 & 29.633 & 28.551 & 26.363 & 31.726 & 36.853 & 32.748 & $\begin{array}{l}36.224 \\
3\end{array}$ & & 21.88 & 15.627 & 10.59 & 31.858 & 32.62 & 29.706 & 31.692 & 27.885 & 33.541 \\
\hline 2/7/12 16:00 & & 28.405 & 19.783 & 29.489 & 30.08 & & 29.603 & 30.384 & 29.643 & 29.64 & 28.556 & 26.368 & 31.728 & 36.855 & 32.75 & 16.226 & & 21.875 & 15.63 & 10.588 & 31.863 & 32.62 & 29.708 & 31.69 & 27.887 & 33.539 \\
\hline 2/7/12 17:00 & & 28.428 & 20.028 & 29.494 & 30.085 & & 29.603 & 30.389 & 29.648 & 29.646 & 28.56 & 26.375 & 31.726 & 36.862 & 32.75 & 16.226 & & 21.868 & 15.63 & 10.59 & 31.863 & 32.623 & 29.713 & 31.694 & $\quad 27.887$ & 33.537 \\
\hline 2/7/12 18:00 & & 28.451 & 20.273 & 29.51 & 30.092 & & 29.599 & 30.394 & 29.66 & 29.656 & 28.57 & 26.385 & 31.73 & 36.86 & 32.753 & 16.229 & & 21.873 & 15.63 & 10.59 & 31.874 & 32.623 & 29.722 & 31.69 & $\quad 27.889$ & 33.539 \\
\hline 2/7/1219 & & 28.475 & 20.522 & 29.522 & 30.1 & & 29.601 & 30.399 & 29.664 & 29.67 & 28.577 & 26.397 & 31.737 & 36.867 & 32.76 & $\quad 16.229$ & & 21.875 & 15.627 & 10.595 & 31.881 & 32.63 & 29.729 & 31.699 & $\quad 27.889$ & 33.541 \\
\hline $2 / 7 / 12$ & & 28.49 & 20.749 & 29.531 & 30.1 & & or & 30.4 & 29.669 & 29.679 & 28.581 & 26.402 & 31.7. & 36.869 & 32.762 & 16. & & 21.877 & 15.632 & 10.604 & 31.888 & 32.637 & 29.738 & 31.706 & $\quad 27.889$ & 33.544 \\
\hline $2 / 7 / 1$ & & 28 & 20.977 & - & & & & 30.4 & - & & 28.5 & 26.406 & 31.7. & 36. & 32. & 16. & & 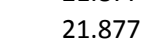 & $15 \mathrm{f} \mathrm{P}-\mathrm{s}$ & 10.6 & 31 & 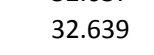 & 29.745 & 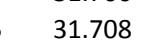 & 92 & 33.548 \\
\hline & & & 21.208 & & & & & & & & 28. & & & & & & & & & & & & & & 94 & \\
\hline $23: 00$ & & 28.563 & 21.418 & 29.552 & 30.129 & & 29.61 & 30.418 & 29.679 & 29.707 & 28.6 & 26.423 & 31.766 & 36.869 & 32.785 & 16.24 & & 21.889 & 15.646 & 10.623 & 31.909 & 32.658 & 29.757 & 31.729 & 27.892 & 33.558 \\
\hline
\end{tabular}




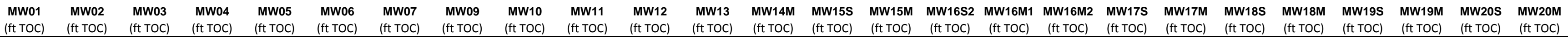

\begin{tabular}{|c|c|c|c|c|c|c|c|c|c|c|c|c|c|c|c|c|c|c|c|c|c|c|c|c|c|c|}
\hline Well & $\begin{array}{l}\text { (ft TOC) } \\
\text { (f) }\end{array}$ & $\begin{array}{l}\text { (ft TOC) } \\
\text { (f) }\end{array}$ & $\begin{array}{l}\text { (ft TOC) } \\
\text { (f) }\end{array}$ & (ft TOC) & (ft TOC) & $\begin{array}{l}\text { (ft ToC) } \\
\text { (f) }\end{array}$ & (ft ToC) & $\begin{array}{l}\text { (ft TOC) } \\
\text { (f) }\end{array}$ & $\begin{array}{l}\text { (ft TOC) } \\
\text { (f) }\end{array}$ & $\begin{array}{l}\text { (ft TOC) } \\
\text { (t) }\end{array}$ & $\begin{array}{l}\text { (ft TOC) } \\
\text { (n) }\end{array}$ & $\begin{array}{l}\text { (ft TOC) } \\
\text { (n) }\end{array}$ & (ft TOC) & $\begin{array}{l}\text { (ft ToC) } \\
\text { (ft }\end{array}$ & (ft TOC) & (ft TOC) & (ft TOC) & (ft TOC) & $\begin{array}{l}\text { (ft TOC) } \\
\text { (ft) }\end{array}$ & (ft TOC) & $\begin{array}{l}\text { (ft ToC) } \\
\text { (ft }\end{array}$ & $\begin{array}{l}\text { (ft TOC) } \\
\text { (f) }\end{array}$ & $\begin{array}{l}\text { (ft TOC) } \\
\text { (n) }\end{array}$ & (ft TOC) & $\begin{array}{l}\text { (nt TOC) } \\
\text { (nt }\end{array}$ & (ft TOC) \\
\hline $2 / 8 / 120: 00$ & & 28.584 & 21.632 & 29.545 & 30.129 & & 29.627 & 30.42 & 29.672 & 29.705 & 28.598 & 26.411 & 31,768 & 36.872 & $\begin{array}{l}2 \quad 32.788 \\
\end{array}$ & 16.243 & & 21.887 & 15.653 & 10.625 & 31.909 & 32.661 & 29.753 & 31.729 & 27.894 & 33.56 \\
\hline $2 / 8 / 121: 00$ & & 28.605 & 21.781 & 29.538 & 30.124 & & 29.636 & 30.425 & 29.669 & 29.707 & 28.598 & 26.402 & 31.766 & 36.869 & 32.788 & 16.245 & & 21.889 & 15.66 & 10.627 & 31.902 & 32.658 & 29.748 & 31.729 & 27.894 & 33.562 \\
\hline $2 / 8 / 122: 00$ & & 28.626 & 21.959 & 29.54 & 30.124 & & 29.643 & 30.43 & 29.672 & 29.709 & 28.6 & 26.402 & 31.768 & 36.869 & $\begin{array}{r}32.79 \\
9\end{array}$ & $\begin{array}{l}10.245 \\
16.245\end{array}$ & & 21.887 & 15.663 & 10.627 & 31.905 & 32.661 & 29.753 & 31.734 & 27.896 & 33.562 \\
\hline 2/8/12 3:00 & & 28.645 & 22.138 & 29.529 & 30.117 & & 29.655 & 30.427 & 29.669 & 29.707 & 28.595 & 26.387 & 31.763 & 36.872 & 32.788 & 16.245 & & 21.889 & 15.665 & 10.623 & 31.895 & 32.656 & 29.741 & 31.729 & 27.896 & 33.558 \\
\hline $2 / 8 / 124: 00$ & & 28.663 & 22.32 & 29.517 & 30.11 & & 29.667 & 30.427 & 29.664 & 29.707 & 28.588 & 26.38 & 31.761 & 36.874 & 32.783 & 16.245 & & 21.884 & 15.674 & 10.62 & 31.888 & 32.653 & 29.724 & 31.729 & 27.896 & 33.555 \\
\hline $2 / 8 / 125: 00$ & & 28.684 & 22.495 & 29.505 & 30.099 & & 29.674 & 30.427 & 29.662 & 29.702 & 28.581 & 26.363 & 31.751 & 36.862 & 32.774 & 16.245 & & 21.889 & 15.681 & 10.614 & 31.881 & 32.646 & 29.717 & 1.718 & 27.896 & 33.551 \\
\hline $2 / 8 / 126: 00$ & & 28.71 & 22.646 & 29.51 & 30.096 & & 29.671 & 30.43 & 29.674 & 29.705 & 28.584 & 26.366 & 31.749 & 36.865 & 32.771 & 16.245 & & 21.91 & 15.67 & 10.607 & 31.877 & 32.644 & 29.717 & 31.713 & 27.896 & 33.551 \\
\hline 2/8/12 7:00 & & 28.733 & 22.798 & 29.517 & 30.099 & & 29.662 & 30.432 & 29.681 & 29.709 & 28.588 & 26.373 & 31.749 & 36.869 & 32.771 & 16.243 & & 21.905 & 15.665 & 10.606 & 31.884 & 32.644 & 29.729 & 31.711 & 27.901 & 33.548 \\
\hline 2/8/12 8:00 & & 28.75 & 22.956 & 29.519 & 30.101 & & 29.664 & 30.432 & 29.686 & 29.711 & 28.588 & 26.38 & 31.749 & 36.867 & 32.774 & 16.243 & & 21.898 & 15.672 & 10.609 & 31.884 & 32.644 & 29.727 & 31.715 & 27.899 & 33.548 \\
\hline 2/8/12 9:00 & & 28.77 & 23.115 & 29.522 & 30.101 & & 29.664 & 30.439 & 29.688 & 29.716 & 28.588 & 26.38 & 31.754 & 36.869 & 32.776 & 16.243 & & 21.894 & 15.679 & 10.614 & 31.886 & 32.649 & 29.722 & 31.72 & 27.901 & 33.553 \\
\hline 2/8/12 10:00 & & 28.789 & 23.262 & 29.515 & 30.096 & & 29.671 & 30.439 & 29.688 & 29.714 & 28.586 & 26.373 & 31.752 & 36.867 & 32.776 & 16.24 & & 21.889 & 15.691 & 10.613 & 31.881 & 32.646 & 29.727 & 31.72 & 27.899 & 33.553 \\
\hline 2/8/12 11:00 & & 28.81 & 23.425 & 29.508 & 30.089 & & 29.681 & 30.439 & 29.683 & 29.709 & 28.581 & 26.363 & 31.752 & 36.869 & 32.774 & 16.24 & & 21.884 & 15.688 & 10.611 & 31.879 & 32.646 & 29.717 & 31.72 & 27.901 & 33.555 \\
\hline 2/8/12 12:00 & & 28.824 & 23.509 & 29.494 & 30.082 & & 29.693 & 30.437 & 29.681 & 29.707 & 28.574 & 26.351 & 31.751 & 36.867 & 32.774 & 16.238 & & 21.88 & 15.698 & 10.614 & 31.87 & 32.644 & 29.706 & 31.725 & 27.901 & 33.555 \\
\hline 2/8/12 13:00 & & 28.833 & 23.635 & 29.454 & 30.057 & & 29.721 & 30.432 & 29.662 & 29.691 & 28.553 & 26.318 & 31.742 & 36.858 & 32.764 & 16.236 & & 21.87 & 15.719 & 10.606 & 31.842 & 32.637 & 29.673 & 31.722 & 27.901 & 33.553 \\
\hline 2/8/12 14:00 & & 28.852 & 23.807 & 29.416 & 30.031 & & 29.737 & 30.425 & 29.648 & 29.672 & 28.535 & 26.28 & 31.73 & 36.846 & 32.755 & 16.229 & & 21.854 & 15.724 & 10.592 & 31.821 & 32.625 & 29.642 & 31.704 & 27.903 & 33.551 \\
\hline $2 / 8 / 1215: 00$ & & 28.871 & 24.052 & 29.398 & 30.01 & & 29.739 & 30.418 & 29.648 & 29.66 & 28.521 & 26.263 & 31.716 & 36.839 & 32.741 & 16.224 & & 21.831 & 15.724 & 10.578 & 31.807 & 32.611 & 29.626 & 31.685 & 27.901 & 33.546 \\
\hline 2/8/12 16:00 & & 28.892 & 24.248 & 29.395 & 29.998 & & 29.728 & 30.415 & 29.657 & 29.653 & 28.514 & 26.263 & 31.709 & 36.839 & 32.736 & 16.222 & & 21.863 & 15.717 & 10.574 & 31.8 & 32.604 & 29.621 & 31.678 & 27.903 & 33.544 \\
\hline 2/8/12 17:00 & & 28.906 & 24.36 & 29.386 & 29.986 & & 29.718 & 30.408 & 29.664 & 29.646 & 28.505 & 26.26 & 31.7 & 36.832 & 32.724 & 16.215 & & 21.833 & 15.714 & 10.562 & 31.786 & 32.594 & 29.609 & 31.666 & 27.903 & 33.539 \\
\hline 2/8/12 18:00 & & 28.927 & 24.465 & 29.386 & 29.979 & & 29.7 & 30.406 & 29.674 & 29.642 & 28.502 & 26.26 & 31.695 & 36.827 & 32.72 & 16.212 & & 21.81 & 15.705 & 10.555 & 31.786 & 32.587 & 29.616 & 31.657 & 27.906 & $\begin{array}{l}33.537 \\
3.53\end{array}$ \\
\hline $2 / 8 / 1219: 00$ & & $\begin{array}{l}28.943 \\
28.943\end{array}$ & 24.463 & $\begin{array}{l}29.300 \\
29.398\end{array}$ & 29.984 & & 29.685 & 30.406 & 29.683 & $\begin{array}{l}29.646 \\
29.646\end{array}$ & $\begin{array}{l}28.505 \\
28.505\end{array}$ & $\begin{array}{l}26.200 \\
26.272\end{array}$ & $\begin{array}{l}31.093 \\
31.693\end{array}$ & 36.827 & 32.715 & $\begin{array}{l}10.212 \\
16.208\end{array}$ & & $\begin{array}{l}21.01 \\
21.78\end{array}$ & $\begin{array}{r}13.153 \\
15.7\end{array}$ & 10.552 & $\begin{array}{l}31.788 \\
31.78\end{array}$ & $\begin{array}{l}32.585 \\
32.51\end{array}$ & 29.616 & 31.657 & 27.906 & $\begin{array}{l}33.537 \\
3.53\end{array}$ \\
\hline $2 / 8 / 1220: 00$ & & $\begin{array}{l}28.959 \\
28.959\end{array}$ & $\begin{array}{l}24.403 \\
24.518\end{array}$ & $\begin{array}{l}29.398 \\
29.395\end{array}$ & $\begin{array}{l}29.984 \\
29.982\end{array}$ & & 29.681 & $\begin{array}{l}30.406 \\
30.406\end{array}$ & 29.691 & $\begin{array}{l}29.0640 \\
29.642\end{array}$ & $\begin{array}{l}28.498 \\
28.498\end{array}$ & $\begin{array}{l}20.272 \\
26.272\end{array}$ & $\begin{array}{l}31.689 \\
31.688\end{array}$ & $\begin{array}{l}36.027 \\
36.827\end{array}$ & 32.715 & $\begin{array}{l}10.200 \\
16.203\end{array}$ & & 21.748 & $\begin{array}{l}13.698 \\
15.17\end{array}$ & 10.552 & 31.783 & $\begin{array}{l}32.583 \\
32.582\end{array}$ & 29.614 & $\begin{array}{l}31.657 \\
31.657\end{array}$ & 27.906 & $\begin{array}{l}33.531 \\
33.53\end{array}$ \\
\hline 2/8/12 21:00 & & $\begin{array}{l}28.959 \\
28.978\end{array}$ & $\begin{array}{l}24.318 \\
24.628\end{array}$ & 29.388 & 29.977 & & 29.674 & $\begin{array}{l}30.400 \\
30.403\end{array}$ & $\begin{array}{l}29.691 \\
29.693\end{array}$ & $\begin{array}{l}29.642 \\
29.637\end{array}$ & $\begin{array}{l}28.498 \\
28.493\end{array}$ & $\begin{array}{l}20.26 / 2 \\
26.27\end{array}$ & $\begin{array}{l}31.088 \\
31.688\end{array}$ & $\begin{array}{r}30.827 \\
36.82\end{array}$ & $\begin{array}{r}32 . .13 \\
32.71 \\
\end{array}$ & $\begin{array}{l}10.203 \\
16.201\end{array}$ & & 21.722 & $\begin{array}{r}13.068 \\
15.7\end{array}$ & $\begin{array}{l}10.532 \\
10.548\end{array}$ & $\begin{array}{l}31.783 \\
31.786\end{array}$ & $\begin{array}{l}32.522 \\
32.582\end{array}$ & $\begin{array}{l}29.614 \\
29.612\end{array}$ & $\begin{array}{l}31.65 / 1 \\
31.652\end{array}$ & $\begin{array}{l}27.906 \\
27.906\end{array}$ & $\begin{array}{l}33.531 \\
33.537\end{array}$ \\
\hline 2/8/12 22:00 & & 28.992 & 24.738 & 29.384 & & & 29.671 & 30.401 & 29.693 & 29.633 & 28.486 & 26.263 & $\begin{array}{l}31.600 \\
31.686\end{array}$ & 36.822 & 32.71 & $\begin{array}{l}16.194 \\
16.194\end{array}$ & & 21.692 & 15.7 & 10.548 & 31.781 & .578 & 29.604 & 31.654 & 27.908 & $\begin{array}{l}33.539 \\
3.53\end{array}$ \\
\hline $2 / 8 / 1223: 00$ & & 29.008 & 24.745 & 29.388 & 29.975 & & 29.66 & $\begin{array}{l}30.401 \\
30.401\end{array}$ & $\begin{array}{r}29.7 \\
29.7\end{array}$ & 29.633 & $\begin{array}{l}28.400 \\
28.488\end{array}$ & 26.27 & $\begin{array}{l}31.000 \\
31.686\end{array}$ & $\begin{aligned} 50.022 \\
36.82\end{aligned}$ & 32.706 & $\begin{array}{l}16.191 \\
161\end{array}$ & & .662 & $\begin{array}{l}15.693 \\
15.69\end{array}$ & 10.548 & 31.779 & 578 & 29.612 & $\begin{array}{l}31.652 \\
31.652\end{array}$ & $\begin{array}{l}27.908 \\
27.908\end{array}$ & $\begin{array}{l}33.544 \\
3.534\end{array}$ \\
\hline $2 / 9 / 120: 00$ & & 29.02 & 24.84 & 29.377 & 29.972 & & 29.662 & & 29.7 & 29.628 & 28.481 & 26.268 & 31.684 & 36.822 & 32.713 & 16.189 & & .632 & 15.695 & 10.548 & 31.779 & 578 & 29.604 & 31.654 & 27.906 & 33.546 \\
\hline 2/9/12 1:00 & & 29.031 & 24.933 & 29.36 & 29.961 & & 29.669 & 30.396 & 29.691 & 29.619 & 28.472 & 26.253 & 31.679 & 36.82 & 32.706 & 16.187 & & 21.605 & 15.7 & 10.538 & 31.769 & 32.571 & 29.588 & 31.647 & 27.908 & 33.546 \\
\hline 2/9/12 2:00 & & 29.052 & 25.024 & 29.363 & 29.958 & & 29.66 & 30.396 & 29.702 & 29.619 & 28.47 & 26.256 & 31.674 & 36.815 & 32.699 & 16.182 & & 21.57 & 15.691 & 10.538 & 31.765 & 32.568 & 29.59 & 31.643 & 27.908 & 33.544 \\
\hline 2/9/12 3:00 & & 29.064 & 25.036 & 29.346 & 29.949 & & 29.662 & 30.391 & 29.695 & 29.607 & 28.46 & 26.241 & 31.667 & 36.815 & 32.689 & 16.177 & & 21.54 & 15.693 & 10.529 & 31.751 & 32.559 & 29.581 & 31.636 & 27.91 & 33.541 \\
\hline 2/9/12 4:00 & & 29.076 & 25.132 & 29.332 & 29.937 & & 29.664 & 30.387 & 29.688 & 29.6 & 28.451 & 26.229 & 31.658 & 36.811 & 32.68 & 16.175 & & 21.505 & 15.691 & 10.517 & 31.744 & 32.549 & 29.562 & 31.626 & 27.91 & 33.537 \\
\hline $2 / 9 / 125: 00$ & & 29.09 & 25.232 & 29.328 & 29.93 & & 29.66 & 30.382 & 29.695 & 29.593 & 28.444 & 26.227 & 31.649 & 36.815 & 32.675 & 16.17 & & 21.473 & 15.686 & 10.512 & 31.732 & 32.542 & 29.56 & 31.617 & 27.91 & 33.534 \\
\hline $2 / 9 / 126: 00$ & & 29.103 & 25.32 & 29.328 & 29.926 & & 29.65 & 30.382 & 29.698 & 29.591 & 28.442 & 26.227 & 31.642 & 36.806 & 32.666 & 16.166 & & 21.457 & 15.677 & 10.503 & 31.73 & 32.535 & 29.555 & 31.607 & 27.91 & 33.53 \\
\hline $2 / 9 / 127: 00$ & & 29.117 & 25.409 & 29.328 & 29.923 & & 29.641 & 30.377 & 29.705 & 29.586 & 28.437 & 26.232 & 31.635 & 36.806 & 32.661 & 16.161 & & 21.436 & 15.677 & 10.498 & 31.728 & 32.528 & 29.555 & 31.603 & 27.91 & 33.53 \\
\hline 2/9/12 8:00 & & 29.131 & 25.486 & 29.33 & 29.926 & & 29.631 & 30.377 & 29.71 & 29.586 & 28.437 & 26.234 & 31.632 & 36.804 & 32.656 & 16.159 & & 21.403 & 15.665 & 10.493 & 31.728 & 32.526 & 29.56 & 31.598 & 27.915 & 33.527 \\
\hline 2/9/12 9:00 & & 29.148 & 25.563 & 29.33 & 29.921 & & 29.627 & 30.379 & 29.714 & 29.584 & 28.435 & 26.239 & 31.627 & 36.804 & 32.654 & 16.156 & & 21.371 & 15.665 & 10.489 & 31.73 & 32.523 & 29.555 & 31.598 & 27.913 & 33.527 \\
\hline 2/9/12 10:00 & & 29.155 & 25.656 & 29.33 & 29.923 & & 29.624 & 30.377 & 29.717 & 29.581 & 28.432 & 26.239 & 31.63 & 36.804 & 32.652 & 16.152 & & 21.346 & 15.665 & 10.489 & 31.725 & 32.521 & 29.555 & 31.6 & 27.913 & 33.53 \\
\hline 2/9/12 11:00 & & 29.171 & 25.631 & 29.325 & 29.921 & & 29.622 & 30.377 & 29.719 & 29.577 & 28.428 & 26.234 & 31.628 & 36.806 & 32.652 & 16.149 & & 21.316 & 15.663 & 10.489 & 31.723 & 32.521 & 29.557 & 31.596 & 27.915 & 33.532 \\
\hline 2/9/12 12:00 & & 29.173 & 25.516 & 29.295 & 29.905 & & 29.641 & 30.372 & 29.702 & 29.565 & $\begin{array}{l}28.420 \\
28.412\end{array}$ & 26.208 & 31.62 & 36.799 & 32.645 & 16.145 & & 21.283 & 15.67 & $\begin{array}{l}10.482 \\
10.452\end{array}$ & 31.709 & 32.511 & 29.532 & 31.593 & 27.915 & 33.532 \\
\hline 2/9/12 13:00 & & 29.178 & 25.53 & 29.255 & 29.87 & & 29.662 & 30.363 & 29.683 & 29.542 & $\begin{array}{l}28.412 \\
28.391\end{array}$ & 26.172 & 31.607 & 36.799 & 32.633 & 16.14 & & 21.26 & $\begin{array}{l}15.679 \\
\end{array}$ & $\begin{array}{l}10.47 \\
10.47\end{array}$ & 31.679 & 32.499 & 29.496 & 31.584 & 27.91 & $\begin{array}{l}33.527 \\
3.527\end{array}$ \\
\hline 2/9/12 14:00 & & 29.19 & 25.582 & 29.229 & 29.85 & & $\begin{array}{l}29.062 \\
29.667\end{array}$ & 30.355 & 29.676 & 29.528 & $\begin{array}{l}28.374 \\
28.374\end{array}$ & 26.153 & $\begin{array}{l}31.591 \\
31.592\end{array}$ & $\begin{array}{r}30.79 \\
36.79\end{array}$ & 32.621 & $\begin{array}{l}16.1 \\
16.1\end{array}$ & & 21.23 & 15.674 & $\begin{array}{l}10.465 \\
10.465\end{array}$ & 31.66 & 32.485 & 29.475 & 31.572 & 27.915 & $\begin{array}{l}33.527 \\
3.527\end{array}$ \\
\hline 2/9/12 15:00 & & 29.206 & $\begin{array}{l}25.582 \\
2584\end{array}$ & 29.227 & 29.8 & & 29.653 & $\begin{array}{l}30.353 \\
30.353\end{array}$ & 29.686 & 29.519 & $\begin{array}{l}28.367 \\
28.367\end{array}$ & $\begin{array}{l}20.135 \\
26.148\end{array}$ & $\begin{array}{l}31.585 \\
31.585\end{array}$ & 36.783 & $\begin{array}{l}52.021 \\
32.609\end{array}$ & $\begin{array}{l}10.135 \\
16.128\end{array}$ & & 21.2 & $\begin{array}{l}13.014 \\
15.667\end{array}$ & $\begin{array}{l}10.403 \\
10.451\end{array}$ & $\begin{array}{r}31.053 \\
31.653\end{array}$ & $\begin{array}{l}4.438 \\
.478\end{array}$ & 29.466 & 31.558 & 27.915 & $\begin{array}{l}33.525 \\
33.525\end{array}$ \\
\hline 2/9/12 16:00 & & $\begin{array}{r}29.200 \\
29.22\end{array}$ & $\begin{array}{l}25.584 \\
25.633\end{array}$ & $\begin{array}{l}29.229 \\
29.229\end{array}$ & 29.8 & & $\begin{array}{l}29.638 \\
29.638\end{array}$ & $\begin{array}{l}30.353 \\
30.348\end{array}$ & 29.691 & 29.514 & $\begin{array}{l}28.361 \\
28.363\end{array}$ & $\begin{array}{l}26.148 \\
26.153\end{array}$ & $\begin{array}{l}31.585 \\
31.578\end{array}$ & $\begin{array}{l}36.183 \\
36.783\end{array}$ & $\begin{array}{r}32.009 \\
32.6\end{array}$ & 16. & & 21.172 & $\begin{array}{l}13.067 \\
15.658\end{array}$ & $\begin{array}{l}10.451 \\
10.439\end{array}$ & $\begin{array}{l}31.653 \\
31.646\end{array}$ & 1 & 29.471 & 31.544 & 27.915 & $\begin{array}{l}33.523 \\
33.523\end{array}$ \\
\hline $2 / 9$ & & 29.2 & 25 . & 29.2 & 29 & & 29 & 30 & 29 & 29 & 28.358 & 26 & 31.569 & 36.776 & 32.595 & 16 & & 21 & 15.655 & 10.428 & 31 & 4 & 29.461 & 31.539 & 27.915 & 33.518 \\
\hline $2 / 9 / 1218$ & & 29. & 25.7 & 29.2 & 29.8 & & & 30. & 29. & 29. & 28. & 26.158 & 31.56 & 36. & 32.586 & 16. & & 21. & 15.641 & 10.426 & 31.644 & 32.452 & 29.463 & 31.53 & 27.918 & 33.516 \\
\hline 2/9/12 19:00 & & 29.253 & 25.614 & 29.236 & 29. & & & 30.341 & 29.705 & & 28.353 & 26.17 & 31.557 & 36.776 & 32.581 & 16 & & 2 & 15.634 & 10.421 & 31.641 & & 29.473 & 31.525 & 27.915 & \\
\hline $2 / 9 / 1220: 00$ & & 29.26 & 25.642 & 29.241 & 29.8 & & 29 & 30.341 & 29.712 & 29.503 & 28.356 & 26.174 & 31.555 & 36.773 & 32.579 & & & & 15.62 & 10.416 & & 32.445 & 29.475 & 31.518 & 27.918 & 33.513 \\
\hline 2/9/12 21:00 & & & 25.6 & 29.246 & & & & & 29.7 & 29. & 28.3 & & 31.553 & 36. & 32.5 & 16. & & 21.087 & 15.618 & 10.418 & 31. & 32. & 29.48 & 31.518 & 27.918 & 33.513 \\
\hline $2 / 9 / 1222$ & & & 25. & 29.2 & & & 29. & & 29. & 29.5 & 28. & & 31.553 & & & 16. & & 21.061 & 15 & 10. & & 32. & 29.48 & 31.521 & 27.918 & 33.516 \\
\hline $2 / 9 / 1223$ & & & 25. & 29.2 & & & 29. & & 29.7 & 5 & 28. & 26. & 31.55 & & & 16. & & 21.04 & 15.6 & 10.414 & & 32.443 & 29.475 & 31.516 & 27.92 & 33.516 \\
\hline 2/10/12 0:00 & & 29.3 & 25.7 & 29.234 & & & 29.556 & & 29.724 & 29.4 & 28. & 26.174 & 31.548 & 36.769 & 32.5 & 16. & & 21.015 & 15.6 & 10.409 & 31.646 & 32.44 & 29.473 & 31.509 & 27.92 & 33.518 \\
\hline 2/10/12 1:00 & & 29.3 & 25.7 & 29.232 & 29.8 & & 29.554 & 30.3 & 29.729 & 29.4 & 28.3 & 26.17 & 31.546 & 36.773 & 32. & 16. & & 20.994 & 15.606 & 10.411 & 31.637 & 32.44 & 29.466 & 31.516 & 27.92 & 33.52 \\
\hline 2/10/12 2:00 & & 29.327 & 25.742 & 29.239 & 29.8. & & 29.542 & 30.3 & 29.733 & 29.493 & 28.3 & 26.184 & 31.543 & 36.771 & 32.567 & 16.0 & & 20.973 & 15.59 & 10.406 & 31.646 & 32.435 & 29.482 & 31.507 & 27.925 & 33.52 \\
\hline 2/10/12 3:00 & & 29.334 & 25.766 & 29.243 & 29.839 & & 29.533 & 30.334 & 29.738 & 29.493 & 28.349 & 26.191 & 31.541 & 36.771 & 32.563 & 16.089 & & 20.953 & 15.585 & 10.404 & 31.646 & 32.438 & 29.485 & 31.504 & 27.92 & 33.518 \\
\hline 2/10/12 4:00 & & 29.346 & 25.787 & 29.253 & 29.846 & & 29.523 & 30.336 & 29.743 & 29.498 & 28.351 & 26.198 & 31.539 & 36.771 & 32.563 & 16.084 & & 20.927 & 15.576 & 10.402 & 31.651 & 32.435 & 29.494 & 31.502 & 27.922 & 33.518 \\
\hline 2/10/12 5:00 & & 29.36 & 25.78 & 29.276 & 29.86 & & 29.507 & 30.336 & 29.757 & 29.505 & 28.365 & 26.222 & 31.541 & 36.778 & 32.563 & 16.084 & & 20.904 & 15.564 & 10.402 & 31.665 & 32.431 & 29.508 & 31.5 & 27.927 & 33.518 \\
\hline 2/10/12 6:00 & & 29.374 & 25.789 & 29.314 & 29.891 & & 29.481 & 30.344 & 29.778 & 29.528 & 28.388 & 26.26 & 31.548 & 36.785 & 32.572 & 16.089 & & 20.881 & 15.552 & 10.409 & 31.686 & 32.443 & 29.541 & 31.507 & 27.925 & 33.518 \\
\hline & & & & . & & & & & & & & & & & & & & & & & & & & & & \\
\hline
\end{tabular}




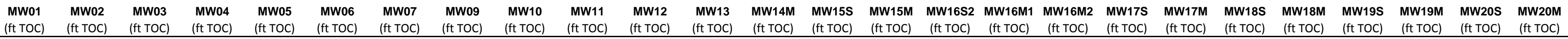

\begin{tabular}{|c|c|c|c|c|c|c|c|c|c|c|c|c|c|c|c|c|c|c|c|c|c|c|c|c|}
\hline well & $\begin{array}{l}\text { MW01 } \\
\text { (ft TOC) }\end{array}$ & $\begin{array}{l}\text { MW02 } \\
\text { (ft TOC) }\end{array}$ & $\begin{array}{l}\text { MW03 } \\
\text { (ft Toc) }\end{array}$ & $\begin{array}{l}\text { MWO04 } \\
\text { (ft TOC) }\end{array}$ & $\begin{array}{cc}\text { MW05 } & \text { MW06 } \\
\text { (ft TOC) } & \text { (ft TOC) } \\
\end{array}$ & $\begin{array}{l}\text { MWW7 } \\
\text { (ft TOC) }\end{array}$ & $\begin{array}{l}\text { MW09 } \\
\text { (ft ToC) }\end{array}$ & $\begin{array}{l}\text { MW10 } \\
\text { (ft TOC) }\end{array}$ & $\begin{array}{l}\text { MW11 } \\
\text { (ft TOC) }\end{array}$ & $\begin{array}{l}\text { MW12 } \\
\text { (ft TOC) }\end{array}$ & $\begin{array}{l}M W 13 \\
(\mathrm{ft} \text { Toc) } \\
\end{array}$ & $\begin{array}{l}\text { MW14M } \\
\text { (ft TOC) }\end{array}$ & $\begin{array}{l}\text { MW15S } \\
\text { (ft TOC) }\end{array}$ & $\begin{array}{l}\text { MW W } 15 \mathrm{M} \\
\text { (ft TOC) }\end{array}$ & $\begin{array}{cc}M W 1652 & \text { MW16M1 } \\
\text { (ft TOC) } & \text { (ft TOC) }\end{array}$ & $\begin{array}{c}\text { MW16M2 } \\
\text { (ft TOC) }\end{array}$ & $\begin{array}{l}\text { MWW7S } \\
\text { (ft TOC) }\end{array}$ & $\begin{array}{l}\text { MW17M } \\
\text { (ft Toc) }\end{array}$ & $\begin{array}{l}\text { MW18S } \\
\text { (ft TOC) }\end{array}$ & $\begin{array}{l}\text { MW18M } \\
\text { (ft TOC) }\end{array}$ & $\begin{array}{l}\text { MW19S } \\
\text { (ft TOC) }\end{array}$ & $\begin{array}{l}\text { MWW19M } \\
\text { (ft TOC) }\end{array}$ & $\begin{array}{l}\text { MWW20S } \\
\text { (ft TOC) }\end{array}$ & $\begin{array}{l}\mathrm{MW} 20 \mathrm{M} \\
\text { (ft Toc) }\end{array}$ \\
\hline $2 / 10 / 128: 00$ & & 29.392 & 25.829 & 29.342 & 29.921 & 29.474 & 30.353 & 29.783 & 29.549 & 28.407 & 26. & 31 & 36.801 & 32.579 & 16. & 20.853 & 5 & 10.416 & 1.704 & 32.452 & 29.567 & 16 & 7.927 & 33.52 \\
\hline $2 / 10 / 129: 00$ & & 29.406 & 25.868 & 29.363 & 29.944 & 29.472 & 30.358 & 29.793 & 29.561 & 28.421 & 26.301 & 31.562 & 36.806 & 32.586 & 16.094 & 20.853 & 15.548 & 10.428 & 31.718 & 32.457 & 29.588 & 31.523 & 27.929 & 33.52 \\
\hline 2/10/12 10:00 & & 29.416 & 25.917 & 29.379 & 29.961 & 29.472 & 30.365 & 29.795 & 29.575 & 28.43 & 26.318 & 31.574 & 36.82 & 32.593 & 16.096 & 20.867 & 15.555 & 10.43 & 31.739 & 32.469 & 29.604 & 31.532 & 27.929 & 33.525 \\
\hline 2/10/12 11:00 & & 29.432 & 25.931 & 29.414 & 29.986 & 29.462 & 30.375 & 29.809 & 29.593 & 28.453 & 26.347 & 31.583 & 36.825 & 32.607 & 16.103 & 20.916 & 15.55 & 10.442 & 31.76 & 32.478 & 29.635 & 31.544 & 27.932 & 33.534 \\
\hline $2 / 10 / 1212: 00$ & & 29.432 & 25.983 & 29.414 & 30 & 29.481 & 30.382 & 29.8 & 29.607 & 28.46 & 26.354 & 31.597 & 36.832 & 32.614 & 16.105 & 20.89 & 15.566 & 10.456 & 31.765 & 32.49 & 29.635 & 31.565 & 27.932 & 33.534 \\
\hline 2/10/12 13:00 & & 29.434 & 26.08 & 29.388 & 29.993 & 29.514 & 30.382 & 29.781 & 29.602 & 28.451 & 26.323 & 31.597 & 36.829 & 32.614 & 16.108 & 20.86 & 15.59 & 10.456 & 31.753 & 32.488 & 29.621 & 31.57 & 27.932 & 33.537 \\
\hline $2 / 10 / 1214: 00$ & & 29.448 & 26.153 & 29.384 & 29.991 & 29.528 & 30.387 & 29.776 & 29.602 & 28.451 & 26.313 & 31.597 & 36.832 & 32.619 & 16.11 & 20.842 & 15.594 & 10.456 & 31.755 & 32.49 & 29.616 & 31.565 & 27.934 & 33.539 \\
\hline $2 / 10 / 1215: 00$ & & 29.46 & 26.216 & 29.395 & 29.996 & 29.528 & 30.387 & 29.783 & 29.612 & 28.46 & 26.32 & 31.599 & 36.837 & 32.619 & 16.115 & 20.844 & 15.592 & 10.458 & 31.755 & 32.495 & 29.628 & 31.563 & 27.934 & 33.544 \\
\hline $2 / 10 / 1216: 00$ & & 29.479 & 26.244 & 29.412 & 30.007 & 29.521 & 30.394 & 29.795 & 29.626 & 28.472 & 26.337 & 31.602 & 36.832 & 32.624 & 16.119 & 20.879 & 15.59 & 10.461 & 31.769 & 32.497 & 29.637 & 31.561 & 27.936 & 33.544 \\
\hline 2/10/12 17:00 & & 29.485 & 26.265 & 29.426 & 30.017 & 29.519 & 30.399 & 29.802 & 29.635 & 28.481 & 26.349 & 31.607 & 36.841 & 32.628 & 16.122 & 20.851 & 15.592 & 10.465 & 31.779 & 32.5 & 29.651 & 31.563 & 27.939 & 33.544 \\
\hline 2/10/12 18:00 & & 29.495 & 26.283 & 29.442 & 30.033 & 29.512 & 30.406 & 29.807 & 29.646 & 28.495 & 26.359 & 31.614 & 36.848 & 32.633 & 16.126 & 20.823 & 15.594 & 10.47 & 31.793 & 32.507 & 29.668 & 31.561 & 27.939 & 33.541 \\
\hline $2 / 10 / 1219: 00$ & & 29.513 & $\begin{array}{r}20.263 \\
26.29\end{array}$ & $\begin{array}{l}29.477 \\
29.47\end{array}$ & $\begin{array}{l}30.054 \\
30.054\end{array}$ & 29.497 & $\begin{array}{l}30.400 \\
30.415\end{array}$ & 29.814 & $\begin{array}{r}29.040 \\
29.67\end{array}$ & $\begin{array}{l}28.4514 \\
28.514\end{array}$ & $\begin{array}{l}20.359 \\
26.392\end{array}$ & $\begin{array}{r}11.014 \\
31.62\end{array}$ & $\begin{array}{l}30.84 \\
36.855\end{array}$ & $\begin{array}{l}32.033 \\
32.642 \\
\end{array}$ & $\begin{array}{l}16.126 \\
26.131\end{array}$ & 20.8 & $\begin{array}{l}15.394 \\
15.585\end{array}$ & $\begin{array}{l}10.41 \\
10.479\end{array}$ & 31.811 & $\begin{array}{l}32.3014 \\
32.514\end{array}$ & 29.691 & 31.57 & $\begin{array}{l}27.939 \\
27.941\end{array}$ & $\begin{array}{l}53.341 \\
33.544 \\
\end{array}$ \\
\hline $2 / 10 / 1220: 00$ & & 29.52 & 26.323 & 29.489 & 30.071 & 29.5 & 30.422 & 29.819 & 29.684 & 28.523 & 26.404 & 31.63 & 36.862 & 32.647 & 16.135 & 20.774 & 15.592 & 10.484 & 31.821 & 32.523 & 29.701 & 31.589 & 27.943 & 33.546 \\
\hline $2 / 10 / 1221: 00$ & & 29.534 & 26.356 & 29.505 & 30.085 & 29.504 & 30.427 & 29.826 & 29.693 & 28.539 & 26.406 & 31.635 & 36.869 & 32.654 & 16.14 & 20.749 & 15.585 & 10.494 & 31.83 & 32.53 & 29.72 & 31.591 & 27.943 & 33.546 \\
\hline $2 / 10 / 1222: 00$ & & 29.541 & 26.391 & 29.508 & 30.096 & 29.516 & 30.437 & 29.819 & 29.705 & 28.544 & 26.404 & 31.642 & 36.869 & 32.661 & 16.147 & 20.728 & 15.599 & 10.501 & 31.842 & 32.535 & 29.724 & 31.6 & 27.943 & 33.548 \\
\hline $2 / 10 / 1223: 00$ & & 29.546 & 26.44 & 29.508 & 30.099 & 29.535 & 30.442 & 29.814 & 29.714 & 28.549 & 26.409 & 31.649 & 36.872 & 32.668 & 16.149 & 20.712 & 15.611 & 10.505 & 31.842 & 32.542 & 29.72 & 31.612 & 27.943 & 33.551 \\
\hline $2 / 11 / 120: 00$ & & 29.565 & 26.465 & 29.519 & 30.108 & 29.54 & 30.446 & 29.819 & 29.725 & 28.56 & 26.406 & 31.651 & 36.872 & 32.675 & 16.154 & 20.691 & 15.606 & 10.512 & 31.847 & 32.547 & 29.736 & 31.612 & 27.95 & 33.555 \\
\hline $2 / 11 / 121: 00$ & & 29.569 & 26.498 & 29.526 & 30.11 & 29.544 & 30.451 & 29.826 & 29.737 & 28.567 & 26.418 & 31.66 & 36.879 & 32.68 & 16.156 & 20.677 & 15.623 & 10.522 & 31.851 & 32.554 & 29.738 & 31.619 & 27.948 & 33.56 \\
\hline $2 / 11 / 122: 00$ & & 29.583 & 26.549 & 29.531 & 30.12 & 29.554 & 30.456 & 29.824 & 29.744 & 28.574 & 26.411 & 31.665 & 36.876 & 32.685 & 16.163 & 20.664 & 15.625 & 0.526 & 31.863 & 32.561 & 29.743 & 1.622 & 27.95 & 33.562 \\
\hline 2/11/12 3:00 & & 29.597 & 26.591 & 29.561 & 30.141 & 29.544 & 30.466 & 29.836 & 29.767 & 28.591 & 26.442 & 31.674 & 36.881 & 32.696 & 16.17 & 20.643 & 15.625 & 0.533 & 31.877 & 32.566 & 29.771 & 1.633 & 27.953 & 33.567 \\
\hline $2 / 11 / 124: 00$ & & 29.607 & $\begin{array}{l}20.391 \\
26.642\end{array}$ & 29.571 & 30.152 & 29.551 & $\begin{array}{l}30.400 \\
30.471\end{array}$ & 29.845 & 29.781 & $\begin{array}{r}20.591 \\
28.6\end{array}$ & $\begin{array}{l}20.447 \\
26.447\end{array}$ & $\begin{array}{l}31.684 \\
31.68\end{array}$ & $\begin{array}{l}30.01 \\
36.886\end{array}$ & 32.701 & $\begin{array}{l}16.11 \\
16.173\end{array}$ & 20.629 & $\begin{array}{l}15.027 \\
15.627\end{array}$ & 10.543 & $\begin{array}{l}31.811 \\
31.891\end{array}$ & 32.575 & 29.776 & 31.643 & 27.955 & $\begin{array}{l}33.567 \\
33.567\end{array}$ \\
\hline $2 / 11 / 125: 00$ & & 29.63 & 26.663 & 29.613 & 30.178 & 29.537 & 30.48 & 29.857 & 29.807 & 28.625 & 26.478 & 31.691 & 36.895 & 32.71 & $\begin{array}{r}10.1 / 3 \\
16.18\end{array}$ & 20.613 & $\begin{array}{l}13.027 \\
15.618\end{array}$ & $\begin{array}{l}10.545 \\
10.55\end{array}$ & $\begin{array}{l}31.091 \\
31.912\end{array}$ & $\begin{array}{l}32.5 / 3 \\
32.587\end{array}$ & 29.814 & $\begin{array}{l}31.043 \\
31.652\end{array}$ & $\begin{array}{l}27.953 \\
27.955\end{array}$ & $\begin{array}{l}33.50 \prime \\
33.569\end{array}$ \\
\hline 2/11/12 6:00 & & 29.632 & 26.717 & 29.622 & 30.194 & 29.549 & 30.485 & 29.862 & 29.823 & 28.637 & 26.49 & 31.702 & 36.893 & 32.717 & 16.184 & 20.608 & 15.639 & 10.559 & 31.921 & 32.594 & 29.818 & 31.661 & 27.955 & 33.572 \\
\hline 2/11/12 7:00 & & 29.641 & 26.768 & 29.631 & 30.204 & 29.563 & 30.494 & 29.857 & 29.839 & 28.649 & 26.497 & 31.707 & 36.895 & 32.722 & 16.191 & 20.592 & 15.653 & 10.564 & 31.926 & 32.599 & 29.828 & 31.671 & 27.957 & 33.572 \\
\hline $2 / 11 / 128: 00$ & & 29.651 & 26.831 & 29.627 & 30.208 & 29.584 & 30.499 & 29.852 & 29.841 & 28.651 & 26.485 & 31.712 & 36.897 & 32.729 & 16.196 & 20.571 & 15.663 & 10.569 & 31.926 & 32.606 & 29.828 & 31.673 & 27.96 & 33.569 \\
\hline $2 / 11 / 129: 00$ & & 29.665 & 26.88 & 29.641 & 30.218 & 29.591 & 30.499 & 29.855 & 29.858 & 28.663 & 26.49 & 31.716 & 36.902 & 32.736 & 16.201 & 20.557 & 15.663 & 10.576 & 31.94 & 32.611 & 29.839 & 31.678 & 27.962 & 33.572 \\
\hline $2 / 11 / 1210: 00$ & & 29.667 & 26.976 & 29.624 & 30.213 & 29.622 & 30.504 & 29.845 & 29.855 & 28.66 & 26.473 & 31.716 & 36.9 & 32.738 & $8 \quad 16.205$ & 20.546 & 15.686 & 10.578 & 31.93 & 32.608 & 29.825 & 31.68 & 27.96 & 33.574 \\
\hline 2/11/12 11:00 & & 29.679 & 27.083 & 29.624 & 30.213 & 29.636 & 30.509 & 29.847 & 29.86 & 28.663 & 26.471 & 31.721 & 36.905 & 32.741 & 16.208 & 20.539 & 15.698 & 10.578 & 31.926 & 32.618 & 29.828 & 31.69 & 27.962 & 33.574 \\
\hline $2 / 11 / 12$ 12:00 & & 29.681 & 27.197 & 29.606 & 30.199 & 29.662 & 30.513 & 29.831 & $\begin{array}{r}29.800 \\
2.858\end{array}$ & $\begin{array}{l}20.005 \\
28.656\end{array}$ & 26.454 & 31.721 & $\begin{array}{r}36.9 \\
36.9\end{array}$ & 32.743 & $\begin{array}{l}3 \quad 16.21 \\
3 \quad 1\end{array}$ & 20.527 & $\begin{array}{l}15.728 \\
15.78\end{array}$ & 10.583 & 31.919 & 32.616 & 29.816 & 31.697 & 27.962 & 33.579 \\
\hline 2/11/12 13:00 & & $\begin{array}{l}29.010 \\
29.69\end{array}$ & 27.286 & 29.587 & 30.185 & 29.685 & $\begin{array}{l}30.511 \\
30.511\end{array}$ & 29.819 & $\begin{array}{l}29.000 \\
29.848\end{array}$ & $\begin{array}{l}20.030 \\
28.646\end{array}$ & $\begin{array}{l}20.454 \\
26.428\end{array}$ & 31.721 & $\begin{array}{l}30.9 \\
36.9\end{array}$ & 32.741 & $\begin{array}{r}10.212 \\
1\end{array}$ & 20.511 & $\begin{array}{l}15.745 \\
15.740\end{array}$ & $\begin{array}{l}10.58 \\
10.58\end{array}$ & 31.905 & 32.613 & 29.795 & 31.69 & $\begin{array}{l}27.902 \\
27.962\end{array}$ & 33.581 \\
\hline $2 / 11 / 12$ 14:00 & & 29.695 & 27.379 & 29.566 & 30.166 & 29.704 & 30.511 & 29.814 & $\begin{array}{l}29.840 \\
29.81\end{array}$ & $\begin{array}{l}20.040 \\
28.639\end{array}$ & $\begin{array}{l}20.420 \\
26.402\end{array}$ & 31.719 & $\begin{array}{r}30.59 \\
36.893\end{array}$ & $\begin{array}{l}32.738 \\
32.738\end{array}$ & $\begin{array}{l}816.212 \\
8\end{array}$ & 20.497 & 15.756 & $\begin{array}{l}10.50 \\
10.578\end{array}$ & 31.893 & 32.611 & 29.774 & $\begin{array}{l}31.692 \\
31.692\end{array}$ & $\begin{array}{l}27.902 \\
27.962\end{array}$ & $\begin{array}{l}33.581 \\
33.581 \\
5\end{array}$ \\
\hline $2 / 11 / 12$ 15:00 & & $\begin{array}{r}29.095 \\
29.7\end{array}$ & 27.482 & 29.536 & $\begin{array}{l}30.160 \\
30.141\end{array}$ & 29.728 & $\begin{array}{l}30.511 \\
30.506\end{array}$ & $\begin{array}{r}29.014 \\
29.8\end{array}$ & $\begin{array}{l}29.825 \\
29.825\end{array}$ & $\begin{array}{l}28.059 \\
28.618\end{array}$ & $\begin{array}{l}20.402 \\
26.373\end{array}$ & 31.707 & $\begin{array}{l}30.891 \\
36.881\end{array}$ & $\begin{array}{l}32.738 \\
32.731\end{array}$ & $\begin{array}{r}16.212 \\
1\end{array}$ & 20.479 & 15.775 & 10.571 & $\begin{array}{l}31.093 \\
31.872\end{array}$ & $\begin{array}{l}32.011 \\
32.601\end{array}$ & 29.745 & $\begin{array}{l}31.092 \\
31.678\end{array}$ & $\begin{array}{l}27.962 \\
27.964\end{array}$ & $\begin{array}{l}33.501 \\
33.581 \\
5\end{array}$ \\
\hline $2 / 11 / 1216: 00$ & & 29.709 & $\begin{array}{l}27.482 \\
27.545\end{array}$ & $\begin{array}{l}29.515 \\
29.515\end{array}$ & $\begin{array}{l}30.141 \\
30.117\end{array}$ & 29.737 & $\begin{array}{l}30.506 \\
30.504\end{array}$ & 29.8 & $\begin{array}{l}29.814 \\
29.814\end{array}$ & $\begin{array}{l}28.018 \\
28.609\end{array}$ & $\begin{array}{l}20.373 \\
26.349\end{array}$ & 31.7 & $\begin{array}{l}30.81 \\
36.874\end{array}$ & $\begin{array}{r}32.131 \\
32.72\end{array}$ & $\begin{array}{l}10.21 \\
16.21\end{array}$ & $\begin{array}{l}20.465 \\
20.465\end{array}$ & $\begin{array}{l}15.778 \\
15.178\end{array}$ & $\begin{array}{l}10.562 \\
10.52\end{array}$ & $\begin{array}{l}31.872 \\
31.853 \\
\end{array}$ & $\begin{array}{l}32.001 \\
32.592 \\
\end{array}$ & 29.727 & $\begin{array}{l}31.678 \\
31.661\end{array}$ & $\begin{array}{l}27.964 \\
27.967\end{array}$ & $\begin{array}{l}33.581 \\
33.576\end{array}$ \\
\hline $2 / 11 / 1217: 00$ & & $\begin{array}{l}29.718 \\
29.718\end{array}$ & $\begin{array}{l}27.545 \\
27.566\end{array}$ & $\begin{array}{l}29.513 \\
29.496\end{array}$ & $\begin{array}{l}30.111 \\
30.099\end{array}$ & $\begin{array}{l}29.739 \\
29.739\end{array}$ & $\begin{array}{l}30.504 \\
30.499 \\
\end{array}$ & $\begin{array}{r}29.8 \\
29.797\end{array}$ & $\begin{array}{l}29.814 \\
29.804\end{array}$ & $\begin{array}{l}28.609 \\
28.598\end{array}$ & $\begin{array}{l}26.349 \\
26.335\end{array}$ & $\begin{array}{r}11.1 \\
31.688\end{array}$ & $\begin{array}{l}30.874 \\
36.872\end{array}$ & $\begin{array}{r}32.12 \\
32.713\end{array}$ & $\begin{array}{lr}3 & 16.21 \\
3 & 16.208\end{array}$ & $\begin{array}{l}20.465 \\
20.451\end{array}$ & $\begin{array}{l}15.778 \\
15.773\end{array}$ & $\begin{array}{l}10.562 \\
10.55\end{array}$ & $\begin{array}{l}31.853 \\
31.837\end{array}$ & $\begin{array}{l}32.592 \\
32.582\end{array}$ & 29.708 & $\begin{array}{r}31.601 \\
31.65\end{array}$ & $\begin{array}{l}27.961 \\
27.967\end{array}$ & $\begin{array}{l}33.5 / 6 \\
33.576\end{array}$ \\
\hline $2 / 11 / 1218: 00$ & & 29.728 & 27.568 & 29.494 & 30.085 & 29.73 & 30.499 & 29.807 & 29.797 & 28.591 & 26.325 & 31.681 & 36.862 & 32.703 & $3 \quad 16.203$ & 20.435 & 15.766 & 10.541 & 31.832 & 32.573 & 29.703 & 31.636 & 27.969 & 33.572 \\
\hline $2 / 11 / 12$ 19:00 & & 29.739 & 27.561 & 29.505 & 30.085 & 29.707 & 30.497 & 29.821 & 29.802 & 28.593 & 26.347 & 31.679 & 36.869 & 32.701 & $1 \quad 16.201$ & 20.421 & 15.761 & 10.541 & 31.837 & 32.571 & 29.708 & 31.636 & 27.969 & 33.572 \\
\hline & & 29. & 27.573 & 29. & 30. & 29 & 30.499 & 29.833 & 29.8 & 28.5 & 26.356 & 31.674 & 36 & 32.699 & $\begin{array}{ll}9 & 16 . \\
9\end{array}$ & 20.407 & 15.752 & $\begin{array}{l}10.54 \\
\end{array}$ & 31.842 & 32.571 & 29.717 & 38 & 27.969 & 33.569 \\
\hline $2 / 1$ & & 29. & 27.568 & 29. & 30. & 29.6 & 30.504 & 29 & 29. & 28 & 26.373 & 31.679 & 36.869 & 32.699 & $\begin{array}{ll}9 & 16 . \\
\end{array}$ & 20.395 & 2 & 10.538 & 31.846 & 32.571 & 1724 & 43 & 27.971 & 33.569 \\
\hline $2 / 11 / 122$ & & 29.762 & 27.591 & 29.5 & 30.08 & 29.69 & 30 & 29.836 & 29.8 & 28 & 26.354 & 31.672 & $\begin{array}{l}30.009 \\
36.869\end{array}$ & 32.696 & $\begin{array}{ll}16.190 \\
6\end{array}$ & 20.384 & 2 & 33 & $\begin{array}{l}31.040 \\
31.837\end{array}$ & 32.568 & .713 & 1.636 & 27.971 & 33.569 \\
\hline 2/11/12 23:00 & & 29.767 & 27.624 & 29.498 & $\begin{array}{l}30.0078 \\
30.078\end{array}$ & 29.697 & $\begin{array}{l}30.0049 \\
30.499\end{array}$ & 29.831 & 29.795 & $\begin{array}{l}20.598 \\
28.58\end{array}$ & $\begin{array}{l}20.534 \\
26.337\end{array}$ & 31.665 & $\begin{array}{l}30.009 \\
36.869\end{array}$ & $\begin{array}{l}32.090 \\
32.692 \\
\end{array}$ & $\begin{array}{ll}16.144 \\
2\end{array}$ & $\begin{array}{l}20.304 \\
20.37\end{array}$ & 15.756 & .527 & $\begin{array}{l}31.031 \\
31.825\end{array}$ & $\begin{array}{l}32.500 \\
32.561\end{array}$ & .696 & $\begin{array}{l}31.050 \\
31.629\end{array}$ & 27.971 & $\begin{array}{l}33.509 \\
33.567\end{array}$ \\
\hline $2 / 12 / 120: 00$ & & 29.772 & 27.661 & 29.48 & 30.066 & 29.707 & 30.497 & 29.824 & 29.786 & 28.572 & 26.323 & 31.663 & 36.865 & 32.682 & $\quad 16.184$ & 20.349 & 15.763 & 10.522 & 31.814 & 32.556 & 29.682 & 31.624 & 27.971 & 33.569 \\
\hline 2/12/12 1:00 & & 29.79 & 27.678 & 29.496 & 30.068 & 29 & 30.499 & 29.838 & 29.79 & 28.577 & 26.337 & 31.663 & 36.865 & 32.684 & $4 \quad 16.184$ & 20.338 & 15.756 & 10.526 & 31.821 & 32.556 & 29.694 & 31.626 & 27.974 & 33.572 \\
\hline 2/12/12 2:00 & & 29.795 & 27.701 & 29.496 & 30.066 & 29 & 30.499 & 29.843 & 29.788 & 28.577 & 26.339 & 31.66 & 36.862 & 32.684 & $4 \quad 16.182$ & 20.324 & 15.752 & 10.522 & 31.821 & 32.556 & 29.694 & 31.622 & 27.974 & 33.574 \\
\hline $2 / 12 / 123: 00$ & & 29.79 & 27.747 & 29.468 & 30.05 & 29.707 & 30.497 & 29.826 & 29.776 & 28.56 & 26.315 & 31.656 & 36.86 & 32.677 & $\begin{array}{l}7 \quad 16.177 \\
\end{array}$ & 20.31 & 15.773 & 10.517 & 31.807 & 32.547 & 29.668 & 31.622 & 27.974 & 33.572 \\
\hline $2 / 12 / 124: 00$ & & 29.795 & 27.792 & 29.43 & 30.024 & 29.725 & 30.489 & 29.812 & 29.758 & 28.539 & 26.28 & 31.642 & 36.858 & 32.666 & 16.17 & 20.298 & 15.773 & 10.505 & 31.776 & 32.537 & 29.637 & 31.61 & 27.974 & 33.567 \\
\hline 2/12/12 5:00 & & 29.802 & 27.824 & 29.409 & 30.003 & 29.73 & 30.482 & 29.809 & 29.742 & 28.525 & 26.26 & 31.628 & 36.846 & 32.654 & $\begin{array}{l}4 \quad 16.166 \\
\end{array}$ & 20.284 & 15.773 & 10.493 & 31.76 & 32.523 & 29.614 & 31.596 & 27.974 & 33.562 \\
\hline 2/12/12 6:00 & & 29.809 & 27.855 & 29.398 & 29.986 & 29.725 & 30.478 & 29.809 & 29.73 & 28.514 & 26.251 & 31.618 & 36.846 & 32.642 & $2 \quad 16.161$ & 20.284 & 15.773 & 10.484 & 31.748 & 32.514 & 29.6 & 31.584 & 27.976 & 33.56 \\
\hline $2 / 12 / 127: 00$ & & 29.818 & 27.883 & 29.388 & 29.975 & 29.718 & $\begin{array}{r}30.47 \\
30.47\end{array}$ & 29.812 & 29.721 & $\begin{array}{l}20.514 \\
28.507\end{array}$ & 26.244 & 31.609 & $\begin{array}{l}30.040 \\
36.837\end{array}$ & 32.633 & $\begin{array}{l}36.154 \\
3\end{array}$ & 20.268 & 15.763 & 10.473 & 31.737 & 32.502 & 29.6 & 31.572 & 27.976 & 33.558 \\
\hline $2 / 12 / 128: 00$ & & 29.823 & $\begin{array}{r}27.903 \\
27.92\end{array}$ & 29.384 & 29.965 & 29.707 & $\begin{array}{l}30.468 \\
30.468\end{array}$ & 29.821 & 29.714 & $\begin{array}{l}20.301 \\
28.498\end{array}$ & $\begin{array}{l}20.244 \\
26.246\end{array}$ & 31.602 & $\begin{array}{l}30.021 \\
36.829\end{array}$ & $\begin{array}{l}32.626 \\
32.626\end{array}$ & $\begin{array}{ll}6 & 16.1449 \\
5\end{array}$ & 20.25 & 15.768 & 10.465 & 31.73 & $\begin{array}{l}32.495 \\
32.405 \\
\end{array}$ & 29.583 & 31.568 & 27.976 & $\begin{array}{l}33.553 \\
33.553 \\
5\end{array}$ \\
\hline $2 / 12 / 129: 00$ & & 29.83 & 27.976 & 29.377 & 29.958 & 29.704 & $\begin{array}{l}30.408 \\
30.463 \\
\end{array}$ & $\begin{array}{l}29.821 \\
29.821\end{array}$ & $\begin{array}{l}29.1407 \\
29.707\end{array}$ & $\begin{array}{l}28.498 \\
28.491\end{array}$ & $\begin{array}{l}20.240 \\
26.241\end{array}$ & $\begin{array}{l}31.002 \\
31.595\end{array}$ & $\begin{array}{l}30.834 \\
36.834\end{array}$ & $\begin{array}{l}32.020 \\
32.619\end{array}$ & $\begin{array}{ll}0 \\
9\end{array}$ & $\begin{array}{l}20.245 \\
20.245\end{array}$ & $\begin{array}{l}15.7603 \\
15.763\end{array}$ & $\begin{array}{l}10.405 \\
10.458\end{array}$ & $\begin{array}{l}31.725 \\
31.725\end{array}$ & $\begin{array}{l}52.495 \\
32.488\end{array}$ & 29.581 & 31.561 & 27.976 & $\begin{array}{l}33.533 \\
33.548\end{array}$ \\
\hline $2 / 12 / 1210: 00$ & & 29.837 & 28.037 & 29.377 & 29.956 & 29.695 & 30.466 & 29.831 & 29.702 & 28.486 & 26.244 & 31.59 & 36.827 & 32.617 & $7 \quad 16.14$ & 20.229 & 15.759 & 10.454 & 31.721 & 32.483 & 29.581 & 31.558 & 27.978 & 33.551 \\
\hline $2 / 12 / 1211: 00$ & & 29.839 & 28.095 & 29.37 & 29.947 & 29.693 & 30.458 & 29.826 & 29.693 & 28.481 & 26.239 & 31.585 & 36.827 & 32.612 & $2 \quad 16.133$ & 20.213 & 15.761 & 10.449 & 31.716 & 32.478 & 29.569 & 31.553 & 27.978 & 33.551 \\
\hline $2 / 12 / 1212: 00$ & & 29.837 & 28.151 & 29.335 & 29.926 & 29.709 & 30.449 & 29.814 & 29.677 & 28.46 & 26.213 & 31.576 & 36.818 & 32.6 & $5 \quad 16.129$ & 20.201 & 15.773 & 10.442 & 31.693 & 32.471 & 29.541 & 31.544 & 27.978 & 33.548 \\
\hline $2 / 12 / 1213: 00$ & & 29.837 & 28.2 & 29.288 & 29.891 & 29.728 & 30.442 & 29.795 & 29.649 & 28.432 & 26.172 & 31.56 & 36.813 & 32.584 & $4 \quad 16.119$ & 20.185 & 15.778 & 10.426 & 31.662 & 32.454 & 29.506 & 31.532 & 27.976 & 33.546 \\
\hline $2 / 12 / 1214: 00$ & & 29.83 & 28.242 & 29.25 & 29.858 & 29.737 & 30.43 & 29.786 & 29.623 & 28.407 & 26.134 & 31.546 & 36.806 & 32.572 & 16.11 & 20.171 & 15.778 & 1011 & 31.634 & 32.438 & 29.468 & 31.516 & 27.976 & 33.541 \\
\hline $2 / 12 / 1215: 00$ & & 29.842 & 28.272 & 29.22 & 29.828 & 29.735 & 30.42 & 29.778 & 29.595 & 28.384 & 26.107 & 31.527 & 36.797 & 32.556 & 16.103 & 20.157 & 15.773 & 10.395 & 31.609 & 32.419 & 29.44 & 31.5 & 27.976 & 33.541 \\
\hline
\end{tabular}




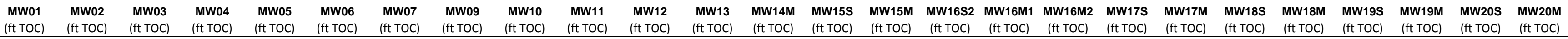

\begin{tabular}{|c|c|c|c|c|c|c|c|c|c|c|c|c|c|c|c|c|c|c|c|c|c|c|c|c|c|c|}
\hline well & 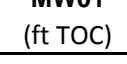 & $\begin{array}{l}\text { (ft TOC) } \\
\text { (f) }\end{array}$ & $\begin{array}{l}\text { (ft TOC) } \\
\text { (f) }\end{array}$ & (ft TOC) & $\begin{array}{l}\text { (ft TOC) } \\
\text { (f) }\end{array}$ & $\begin{array}{l}\text { (ft ToC) } \\
\text { (f) }\end{array}$ & (ft TOC) & $\begin{array}{l}\text { (ft TOC) } \\
\text { (f) }\end{array}$ & $\begin{array}{l}\text { (ft TOC) } \\
\text { (ft) }\end{array}$ & $\begin{array}{l}\text { (ft TOC) } \\
\text { (t) }\end{array}$ & $\begin{array}{l}\text { (ft TOC) } \\
\text { (f) }\end{array}$ & $\begin{array}{l}\text { (ft toc) } \\
\text { (foc) }\end{array}$ & $\begin{array}{l}\text { (ft TOC) } \\
\text { (t) }\end{array}$ & $\begin{array}{l}\text { (ft TOC) } \\
\end{array}$ & (ft TOC) & $\begin{array}{l}\text { (ft TOC) } \\
\text { (a) }\end{array}$ & (ft TOC) & (ft TOC) & $\begin{array}{l}\text { (ft TOC) } \\
\end{array}$ & (ft TOC) & $\begin{array}{l}\text { (ft TOC) } \\
\end{array}$ & (ft TOC) & $\begin{array}{l}\text { (ft TOC) } \\
\end{array}$ & $\begin{array}{l}\text { (ft TOC) } \\
\end{array}$ & $\begin{array}{l}\text { (ft TOC) } \\
\text { (1) }\end{array}$ & $\begin{array}{l}\text { (ft TOC) } \\
\end{array}$ \\
\hline $2 / 12 / 1216: 00$ & & 29.844 & 28.295 & 29.197 & 29.8 & & 29.73 & 30.411 & 29.776 & 29.577 & 28.363 & 26.093 & 31.513 & 36.785 & 32.539 & 16.094 & & 20.196 & 15.768 & 10.378 & 31.59 & 32.405 & 29.416 & 31.483 & 27.976 & 33.537 \\
\hline $2 / 12 / 1217: 00$ & & 29.846 & 28.312 & 29.176 & 29.776 & & 29.718 & 30.401 & 29.774 & 29.556 & 28.344 & 26.076 & 31.496 & 36.778 & 32.525 & 16.084 & & 20.183 & 15.763 & 10.369 & 31.569 & 32.388 & 29.393 & 31.467 & 27.974 & 33.534 \\
\hline $2 / 12 / 1218: 00$ & & 29.853 & 28.314 & 29.162 & 29.755 & & 29.702 & 30.391 & 29.778 & 29.537 & 28.33 & 26.064 & 31.48 & 36.773 & 32.509 & 16.075 & & 20.162 & 15.747 & 10.348 & 31.555 & 32.376 & 29.379 & 31.45 & 27.976 & 33.532 \\
\hline 2/12/12 19:00 & & 29.858 & 28.316 & 29.162 & 29.746 & & 29.681 & 30.382 & 29.783 & 29.523 & 28.321 & 26.067 & 31.468 & 36.766 & 32.497 & 16.068 & & 20.15 & 15.735 & 10.336 & 31.546 & 32.36 & 29.374 & 31.436 & 27.976 & 33.527 \\
\hline $2 / 12 / 1220: 00$ & & 29.86 & 28.319 & 29.152 & 29.734 & & 29.662 & 30.377 & 29.783 & 29.514 & 28.309 & 26.069 & 31.457 & 36.759 & 32.485 & 16.059 & & 20.134 & 15.728 & 10.324 & 31.534 & 32.35 & 29.365 & 31.427 & 27.976 & 33.525 \\
\hline 2/12/12 21:00 & & 29.863 & 28.323 & 29.148 & 29.725 & & 29.645 & 30.372 & 29.786 & 29.5 & 28.3 & 26.062 & 31.445 & 36.752 & 32.476 & 16.052 & & 20.111 & 15.714 & 10.313 & 31.522 & 32.338 & 29.36 & 31.41 & 27.976 & 33.52 \\
\hline $2 / 12 / 1222: 00$ & & 29.858 & 28.328 & 29.12 & 29.706 & & 29.643 & 30.367 & 29.776 & 29.477 & 28.279 & 26.048 & 31.429 & 36.75 & 32.455 & 16.04 & & 20.097 & 15.712 & 10.296 & 31.504 & 32.324 & 29.334 & 31.399 & 27.974 & 33.513 \\
\hline $2 / 12 / 1223: 00$ & & 29.865 & 28.33 & 29.103 & 29.69 & & 29.631 & 30.358 & 29.774 & 29.461 & 28.265 & 26.031 & 31.417 & 36.745 & 32.443 & $3 \quad 16.033$ & & 20.079 & 15.698 & 10.284 & 31.487 & 32.31 & 29.311 & 31.38 & 27.976 & 33.506 \\
\hline 2/13/12 0:00 & & 29.867 & 28.337 & 29.094 & 29.678 & & 29.617 & 30.353 & 29.776 & 29.445 & 28.251 & 26.026 & 31.405 & 36.738 & 32.431 & 16.026 & & 20.06 & 15.691 & 10.27 & 31.478 & 3232.298 & 29.308 & 31.368 & 27.976 & 33.504 \\
\hline $2 / 13 / 121: 00$ & & 29.865 & 28.351 & 29.08 & 29.664 & & 29.608 & 30.344 & 29.776 & 29.433 & 28.239 & 26.024 & 31.393 & 36.731 & 32.42 & 16.017 & & 20.044 & 15.686 & 10.263 & 31.464 & 32.286 & 29.297 & 31.363 & 27.976 & 33.502 \\
\hline 2/13/12 2:00 & & 29.865 & 28.37 & 29.049 & 29.639 & & 29.606 & 30.336 & 29.767 & 29.407 & 28.219 & 25.995 & 31.377 & 36.722 & 32.408 & $\begin{array}{l}3 \\
16.007\end{array}$ & & 20.032 & 15.679 & 10.249 & 31.443 & 32.272 & 29.273 & 31.349 & 27.974 & 33.499 \\
\hline 2/13/12 3:00 & & 29.867 & 28.384 & 29.033 & 29.622 & & 29.594 & 30.327 & 29.762 & 29.389 & 28.202 & 25.99 & 31.365 & 36.719 & 32.394 & 15.998 & & 20.016 & 15.67 & 10.235 & 31.434 & 32.258 & 29.257 & 31.335 & 27.976 & 33.497 \\
\hline $2 / 13 / 124: 00$ & & 29.86 & 28.407 & 29.003 & 29.599 & & 29.601 & 30.322 & 29.752 & 29.361 & 28.181 & 25.959 & 31.349 & 36.708 & 32.38 & $\begin{array}{l}35.991 \\
\end{array}$ & & 19.998 & 15.667 & 10.221 & 31.408 & $\begin{array}{ll}3 & 32.244\end{array}$ & 29.226 & 31.321 & 27.971 & 33.49 \\
\hline $2 / 13 / 125: 00$ & & 29.87 & 28.41 & 29.005 & 29.592 & & 29.568 & 30.317 & 29.762 & 29.352 & 28.174 & 25.971 & 31.34 & 36.708 & 32.366 & 15.984 & & 19.986 & 15.644 & 10.209 & 31.403 & 32.232 & 29.226 & 31.309 & 27.974 & 33.495 \\
\hline $2 / 13 / 126: 00$ & & 29.872 & 28.412 & 29.003 & 29.583 & & 29.549 & 30.31 & 29.762 & 29.342 & 28.163 & 25.976 & 31.328 & 36.698 & 32.359 & 15.973 & & 19.991 & 15.637 & 10.197 & 31.394 & 32.222 & 29.228 & 31.298 & 27.971 & 33.49 \\
\hline $2 / 13 / 127: 00$ & & 29.877 & 28.412 & 29.007 & 29.58 & & 29.528 & 30.305 & 29.767 & 29.335 & 28.163 & 25.983 & 31.319 & 36.694 & 32.347 & 15.968 & & 19.984 & 15.62 & 10.185 & 31.396 & 32.215 & 29.228 & 31.288 & 27.976 & 33.485 \\
\hline 2/13/12 8:00 & & 29.879 & 28.414 & 29.007 & 29.583 & & 29.507 & 30.303 & 29.774 & 29.328 & 28.158 & 25.988 & 31.314 & 36.691 & 32.338 & $\begin{array}{l}3.9063 \\
35.963\end{array}$ & & 19.965 & $\begin{array}{l}15.602 \\
\end{array}$ & 10.179 & 31.394 & 32.208 & 29.231 & 31.277 & 27.974 & 33.481 \\
\hline 2/13/12 9:00 & & 29.884 & 28.412 & 29.017 & 29.585 & & 29.483 & 30.3 & 29.774 & 29.326 & 28.158 & 26.002 & 31.309 & 36.694 & 32.333 & 15.956 & & 19.949 & 15.587 & 10.171 & 31.396 & 32.201 & 29.233 & 31.274 & 27.971 & 33.481 \\
\hline $2 / 13 / 12$ 10:00 & & 29.881 & $\begin{array}{l}28.424 \\
28.424\end{array}$ & 29.003 & 29.578 & & 29.481 & 30.293 & 29.771 & 29.317 & 28.149 & 25.995 & 31.3 & 36.686 & 32.326 & $\begin{array}{l}15.949 \\
5\end{array}$ & & 19.931 & 15.587 & 10.167 & 31.387 & 32.194 & 29.224 & 31.272 & 27.971 & $\begin{array}{l}33.478 \\
33.40\end{array}$ \\
\hline 2/13/12 11:00 & & 29.884 & $\begin{array}{l}20.424 \\
28.431\end{array}$ & 28.993 & 29.573 & & 29.474 & 30.291 & 29.767 & 29.305 & 28.139 & $\begin{array}{l}25.959 \\
25.99\end{array}$ & 31.293 & $\begin{array}{l}30.000 \\
36.686\end{array}$ & $\begin{array}{l}32.319 \\
32.319\end{array}$ & $\begin{array}{l}15.949 \\
15.945\end{array}$ & & 19.921 & 15.578 & 10.157 & 31.378 & 32.187 & 29.221 & 31.26 & 27.974 & $\begin{array}{l}33.476 \\
33.476\end{array}$ \\
\hline $2 / 13 / 1212: 00$ & & $\begin{array}{l}29.004 \\
29.886\end{array}$ & $\begin{array}{l}28.431 \\
28.438\end{array}$ & 28.982 & $\begin{array}{l}29.5 / 366 \\
29.566\end{array}$ & & 29.465 & 30.284 & 29.759 & 29.296 & $\begin{array}{l}28.139 \\
28.132\end{array}$ & 25.983 & 31.288 & $\begin{array}{l}30.060 \\
36.682\end{array}$ & $\begin{array}{l}32.314 \\
32.314\end{array}$ & $\begin{array}{r}15.343 \\
+\quad 15.94\end{array}$ & & $\begin{array}{l}19.921 \\
19.907\end{array}$ & $\begin{array}{l}13.569 \\
15.569\end{array}$ & 10.148 & $\begin{array}{l}11.518 \\
31.373\end{array}$ & 32.18 & $\begin{array}{r}29.21 \\
29.21\end{array}$ & $\begin{array}{r}31.20 \\
31.253\end{array}$ & 27.974 & $\begin{array}{l}33.470 \\
33.476\end{array}$ \\
\hline $2 / 13 / 1213: 00$ & & 29.884 & 28.442 & 28.968 & 29.557 & & 29.46 & 30.279 & 29.759 & 29.282 & 28.123 & 25.976 & 31.279 & 36.679 & 32.305 & $\quad 15.931$ & & 19.894 & 15.566 & 10.143 & 31.364 & 32.173 & 29.203 & 31.248 & 27.974 & 33.474 \\
\hline $2 / 13 / 1214: 00$ & & 29.884 & 28.454 & 28.954 & 29.543 & & 29.457 & 30.274 & 29.748 & 29.268 & 28.109 & 25.964 & 31.274 & 36.675 & 32.298 & $\begin{array}{l}15.926 \\
3\end{array}$ & & 19.884 & 15.562 & 10.136 & 31.347 & 32.163 & 29.186 & 31.241 & 27.971 & $\begin{array}{l}33.474 \\
33.47\end{array}$ \\
\hline 15:00 & & 29.893 & $\begin{array}{l}20.434 \\
28.447\end{array}$ & $\begin{array}{l}28.964 \\
28.965\end{array}$ & 29.55 & & 29.429 & 30.27 & 29.764 & $\begin{array}{l}29.260 \\
29.266\end{array}$ & 28.114 & 25.978 & 31.267 & 36.668 & 32.293 & $\begin{array}{l}15.920 \\
35.924\end{array}$ & & $\begin{array}{r}19.804 \\
19.87\end{array}$ & 15.533 & $\begin{array}{l}10.131 \\
10.131\end{array}$ & 31.364 & $\begin{array}{l}32.103 \\
32.161 \\
\end{array}$ & .198 & 31.232 & 27.974 & $\begin{array}{l}33.474 \\
33.474\end{array}$ \\
\hline $2 / 13 / 1216: 00$ & & 29.895 & 28.445 & 28.986 & 29.562 & & 29.401 & 30.27 & 29.778 & 29.273 & 28.121 & 26.002 & 31.269 & 36.672 & 32.293 & $\begin{array}{l}35.921 \\
3\end{array}$ & & 19.914 & $\begin{array}{l}15.517 \\
15.517\end{array}$ & $\begin{array}{l}10.131 \\
10.132\end{array}$ & $\begin{array}{l}11.504 \\
31.373\end{array}$ & 32.163 & 9.221 & $\begin{array}{l}31.232 \\
31.234\end{array}$ & 27.974 & $\begin{array}{l}33.474 \\
33.476\end{array}$ \\
\hline 2/13/12 17:00 & & 29.888 & 28.438 & 29.012 & 29.583 & & 29.375 & 30.267 & 29.786 & 29.28 & 28.132 & 26.036 & 31.272 & 36.668 & 32.295 & $\begin{array}{l}15.919 \\
5\end{array}$ & & 19.907 & 15.503 & 10.131 & 31.387 & 32.163 & 29.24 & 31.237 & 27.974 & 33.478 \\
\hline $2 / 13 / 1218: 00$ & & 29.872 & 28.414 & 29.052 & 29.611 & & 29.342 & 30.267 & 29.8 & 29.294 & 28.153 & 26.071 & 31.279 & 36.672 & 32.302 & 15.919 & & 19.907 & 15.482 & 10.141 & 31.413 & 32.172 & 29.275 & 31.241 & 27.976 & 33.483 \\
\hline 2/13/12 19:00 & & 29.858 & 28.393 & 29.075 & 29.636 & & 29.323 & 30.269 & 29.807 & 29.31 & 28.167 & 26.1 & 31.288 & 36.691 & 32.309 & 15.921 & & 19.919 & 15.475 & 10.146 & 31.431 & 32.184 & 29.297 & 31.246 & 27.976 & 33.483 \\
\hline $2 / 13 / 1220: 00$ & & 29.844 & 28.377 & 29.087 & 29.657 & & 29.316 & 30.269 & 29.809 & 29.319 & 28.179 & 26.11 & 31.288 & 36.694 & 32.312 & 15.921 & & 19.942 & 15.465 & 10.153 & 31.445 & 32.184 & 29.32 & 31.251 & 27.978 & 33.483 \\
\hline $2 / 13 / 1221: 00$ & & 29.821 & 28.349 & 29.096 & 29.671 & & 29.319 & 30.269 & 29.805 & 29.328 & 28.186 & 26.119 & 31.295 & 36.696 & 32.316 & $\quad 15.924$ & & 19.924 & 15.463 & 10.153 & 31.452 & 32.189 & 29.325 & 31.251 & 27.981 & 33.481 \\
\hline $2 / 13 / 1222: 00$ & & 29.809 & 28.323 & 29.105 & 29.685 & & 29.314 & 30.269 & 29.807 & 29.335 & 28.198 & 26.129 & 31.298 & 36.701 & 32.319 & 15.926 & & 19.907 & 15.458 & 10.158 & 31.459 & 32.194 & 29.337 & 31.258 & 27.978 & 33.481 \\
\hline $2 / 13 / 1223: 00$ & & 29.798 & 28.314 & 29.115 & 29.699 & & 29.312 & 30.272 & 29.805 & 29.347 & 28.205 & 26.138 & 31.302 & 36.705 & 32.323 & 15.926 & & 19.891 & 15.454 & 10.16 & 31.466 & 32.196 & 29.348 & 31.265 & 27.978 & 33.483 \\
\hline $2 / 14 / 120: 00$ & & 29.8 & 28.293 & 29.122 & 29.709 & & 29.309 & 30.274 & 29.805 & 29.352 & 28.214 & 26.141 & 31.307 & 36.708 & 32.328 & $\begin{array}{l}35.933 \\
\end{array}$ & & 19.88 & 15.447 & 10.167 & 31.478 & 32.201 & 29.353 & 31.267 & 27.983 & 33.483 \\
\hline 2/14/12 1:00 & & 29.804 & 28.284 & 29.124 & 29.716 & & 29.316 & 30.274 & 29.805 & 29.359 & 28.221 & 26.148 & 31.307 & 36.708 & 32.33 & 15.933 & & 19.866 & 15.447 & 10.165 & 31.476 & 32.203 & 29.36 & 31.265 & 27.978 & 33.483 \\
\hline 2/14/12 2:00 & & 29.804 & 28.277 & 29.131 & 29.725 & & 29.319 & 30.274 & 29.805 & 29.368 & 28.228 & 26.15 & 31.314 & 36.715 & 32.333 & $\quad 15.938$ & & 19.854 & 15.444 & 10.172 & 31.487 & 32.206 & 29.367 & 31.277 & 27.981 & 33.483 \\
\hline 2/14/12 3:00 & & 29.818 & 28.277 & 29.127 & 29.73 & & 29.328 & 30.277 & 29.802 & 29.37 & 28.23 & 26.148 & 31.314 & 36.717 & 32.335 & $\begin{array}{r}15.94 \\
5\end{array}$ & & 19.843 & 15.447 & 10.174 & 31.485 & 32.208 & 29.369 & 31.277 & 27.981 & 33.483 \\
\hline 2/14/12 4:00 & & 29.83 & 28.279 & 29.129 & 29.732 & & 29.331 & 30.274 & 29.797 & 29.375 & 28.235 & 26.153 & 31.314 & 36.719 & 32.335 & $\begin{array}{l}15.94 \\
15.94\end{array}$ & & $\begin{array}{l}19.045 \\
19.829\end{array}$ & $\begin{array}{l}15.451 \\
15.451\end{array}$ & 10.171 & 31.49 & 32.21 & 29.369 & 31.279 & 27.981 & $\begin{array}{l}33.485 \\
33.405\end{array}$ \\
\hline 2/14/12 5:00 & & 29.837 & 28.277 & 29.138 & 29.739 & & 29.326 & 30.277 & 29.805 & 29.382 & 28.242 & 26.155 & 31.319 & 36.722 & 32.34 & $\begin{array}{l}15.942 \\
4\end{array}$ & & 19.817 & 15.442 & 10.176 & 31.494 & 32.213 & 29.384 & 31.279 & 27.983 & $\begin{array}{l}33.485 \\
33.405\end{array}$ \\
\hline 2/14/12 6:00 & & 29.858 & 28.291 & 29.127 & 29.734 & & 29.342 & 30.277 & 29.793 & 29.384 & $\begin{array}{l}28.239 \\
28.239\end{array}$ & 26.146 & 31.319 & 36.717 & $\begin{array}{r}32.344 \\
32.342\end{array}$ & $\begin{array}{l}15.942 \\
25.945\end{array}$ & & 19.806 & $\begin{array}{l}15.442 \\
15.454\end{array}$ & 10.178 & $\begin{array}{l}31.485 \\
31.485\end{array}$ & 32.213 & $\begin{array}{l}29.304 \\
29.367\end{array}$ & 31.284 & 27.981 & $\begin{array}{l}33.485 \\
33.405\end{array}$ \\
\hline 2/14/12 7:00 & & 29.863 & $\begin{array}{l}28.291 \\
28.291\end{array}$ & 29.143 & 29.741 & & 29.328 & 30.279 & 29.802 & $\begin{array}{l}29.34 \\
29.391 \\
\end{array}$ & 28.251 & $\begin{array}{l}20.140 \\
26.158\end{array}$ & 31.321 & 36.717 & $\begin{array}{l}32.342 \\
32.342\end{array}$ & $\begin{array}{l}15.943 \\
15.947\end{array}$ & & $\begin{array}{l}19.8000 \\
19.794\end{array}$ & $\begin{array}{l}15.434 \\
15.444\end{array}$ & $\begin{array}{l}10.178 \\
10.178\end{array}$ & $\begin{array}{l}31.403 \\
31.497\end{array}$ & 32.215 & .384 & $\begin{array}{l}31.284 \\
31.284\end{array}$ & 27.986 & $\begin{array}{l}33.405 \\
33.485\end{array}$ \\
\hline $\begin{array}{l}2 / 414 / 128 \\
2 / 142\end{array}$ & & $\begin{array}{l}29.803 \\
29.872\end{array}$ & $\begin{array}{r}28.291 \\
28.3\end{array}$ & $\begin{array}{r}29.143 \\
29.15\end{array}$ & $\begin{array}{l}29.141 \\
29.748\end{array}$ & & $\begin{array}{l}29.328 \\
2931\end{array}$ & 30.279 & $\begin{array}{l}29.802 \\
29.805\end{array}$ & $\begin{array}{r}29.391 \\
29.4\end{array}$ & $\begin{array}{l}28.256 \\
28.256\end{array}$ & $\begin{array}{l}20.158 \\
26.162\end{array}$ & $\begin{array}{l}31.321 \\
31.323\end{array}$ & 36.726 & $\begin{array}{l}32.342 \\
32.342\end{array}$ & 15 & & $\begin{array}{l}19.194 \\
19.796\end{array}$ & $\begin{array}{l}15.444 \\
15.447\end{array}$ & $\begin{array}{l}10.181 \\
10.181\end{array}$ & 31.508 & $\begin{array}{l}32.213 \\
32.217\end{array}$ & 91 & $\begin{array}{l}31.284 \\
31.288\end{array}$ & $\begin{array}{l}27.986 \\
27.986\end{array}$ & $\begin{array}{l}33.485 \\
33.485\end{array}$ \\
\hline $2 / 1$ & & 29. & 28.33 & 29.155 & 29 & & 29. & 30. & 29.802 & 29.4 & 28. & 26 & 31 & 36.724 & 32 & 15 & & 15 & 15.449 & 10.183 & 31.508 & 32 & 5 & 3 & 27.983 & 33.483 \\
\hline $2 / 14 / 1$ & & 29 & 28.35 & 29.155 & 29 & & 29 & 30. & 29.8 & 29 & 28. & 26 & 31. & 36. & 32. & & & 19 & 15.456 & 10.185 & 31.513 & 32 & 29.402 & 31.293 & 27.986 & 33.483 \\
\hline 2/14/12 11:00 & & 30.002 & 28.431 & 29.15 & 29.7 & & 29.349 & 30.281 & 29.795 & 29.412 & 28.267 & 26.162 & 31.328 & 36.729 & 32.352 & & & & 15.465 & 10.188 & 31.506 & & 29.4 & 31.295 & 27.986 & 33.483 \\
\hline $2 / 14 / 1212: 00$ & & 30.044 & 28.494 & 29.145 & 29 & & 29.3 & $30.2 \varepsilon$ & 29.797 & 29.412 & 28.27 & 26.155 & 31.33 & 36.726 & 32.354 & & & & 15.47 & 10.19 & 31.508 & & 29.398 & & 27.988 & 33.483 \\
\hline 2 13:00 & & & 28. & & 29. & & & 30.2 & & & 28. & & 31. & 36.719 & 32.349 & & & 19 & 15.494 & 10.183 & 31.49 & 32.218 & 29.365 & 31.307 & 27.986 & 33.483 \\
\hline $2 / 14 / 121$ & & & 28. & & 29 & & & 30.2 & 29 & & 28 & & 31 & 36 & 32. & & & 19. & 15. & 10. & 31.476 & 532.213 & & 31.298 & 27.986 & 33.485 \\
\hline $2 / 14 / 1215: 00$ & & & 28. & 29 & 29 . & & & 30 & 29.7 & & 28. & & 31. & & 32.338 & & & 19. & 15.496 & 10.174 & 31.469 & & & 31.286 & 27.988 & 33.485 \\
\hline $2 / 14 / 1216: 00$ & & 30. & 28.6 & 29.084 & 29. & & 29. & 30.2 & 29.7 & & 28.2 & 26. & 31.3 & 36.708 & 32.338 & & & 19 & 15.496 & 10.174 & 31.471 & 32.206 & 29.346 & 31.281 & 27.988 & 33.485 \\
\hline $2 / 14 / 1217: 00$ & & 30.079 & 28.66 & 29.077 & 29.7 & & 29. & 30.2 & 29.776 & 29. & 28.2 & 26.086 & 31.30 & 36.708 & 32.335 & 15. & & 19.771 & 15.491 & 10.169 & 31.464 & 32.203 & 29.339 & 31.27 & 27.988 & 33.485 \\
\hline $2 / 14 / 1218: 00$ & & 30.035 & 28.638 & 29.094 & 29.711 & & 29.363 & 30.265 & 29.783 & 29.3 & 28.2 & 26.105 & 31.312 & 36.712 & 32.338 & $\quad 15.956$ & & 19.764 & 15.482 & 10.174 & 31.473 & 32.206 & 29.351 & 31.274 & 27.988 & 33.488 \\
\hline 2/14/12 19:00 & & 30 & 28.608 & 29.094 & 29.711 & & 29.359 & 30.265 & 29.786 & 29.393 & 28.244 & 26.105 & 31.309 & 36.712 & 32.333 & 15.954 & & 19.753 & 15.482 & 10.169 & 31.471 & 32.203 & 29.353 & 31.27 & 27.988 & 33.488 \\
\hline $2 / 14 / 1220: 00$ & & 29.984 & 28.587 & 29.098 & 29.716 & & 29.352 & 30.265 & 29.783 & 29.396 & 28.246 & 26.115 & 31.312 & 36.708 & 32.33 & 15.956 & & 19.743 & 15.482 & 10.171 & 31.476 & 32.203 & 29.353 & 31.274 & 27.988 & 33.488 \\
\hline $2 / 14 / 1221: 00$ & & 29.96 & 28.561 & 29.12 & 29.727 & & 29.331 & 30.265 & 29.795 & 29.405 & 28.256 & 26.124 & 31.314 & 36.715 & 32.338 & $\quad 15.956$ & & 19.732 & 15.465 & 10.174 & 31.485 & 32.208 & 29.379 & 31.272 & 27.99 & 33.488 \\
\hline $2 / 14 / 1222: 00$ & & 29.935 & 28.538 & 29.129 & 29.737 & & 29.326 & 30.267 & 29.795 & 29.41 & 28.263 & 26.138 & 31.316 & 36.717 & 32.338 & $\begin{array}{l}35.956 \\
3\end{array}$ & & 19.727 & 15.463 & 10.176 & 31.49 & 32.21 & 29.379 & 31.277 & 27.99 & 33.488 \\
\hline & & & 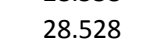 & & & & & & & & & & & & & & & & & & & & & & & \\
\hline
\end{tabular}




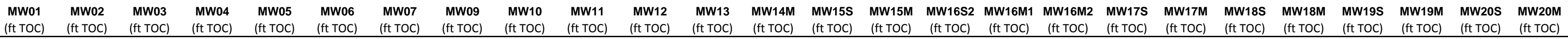

\begin{tabular}{|c|c|c|c|c|c|c|c|c|c|c|c|c|c|c|c|c|c|c|c|c|c|c|c|c|c|}
\hline Well & $\begin{array}{l}\text { (ft TOC) } \\
\text { (f) }\end{array}$ & $\begin{array}{l}\text { (ft TOC) } \\
\text { (f) }\end{array}$ & $\begin{array}{l}\text { (ft TOC) } \\
\text { (f) }\end{array}$ & $\begin{array}{l}\text { (ft TOC) } \\
\text { (ft) }\end{array}$ & $\begin{array}{l}\text { (ft TOC) } \\
\text { (f) }\end{array}$ & $\begin{array}{l}\text { (ft toc) } \\
\text { (ftocts }\end{array}$ & (ft toc) & $\begin{array}{l}\text { (ift TOC) } \\
\text { (n) }\end{array}$ & $\begin{array}{l}\text { (ft TOC) } \\
\text { (f) }\end{array}$ & $\begin{array}{l}\text { (ft TOC) } \\
\text { (f) }\end{array}$ & $\begin{array}{l}\text { (ft TOC) } \\
\text { (n) }\end{array}$ & $\begin{array}{l}\text { (ft TOC) } \\
\text { (ft }\end{array}$ & (ft TOC) & $\begin{array}{l}\text { (ft TOC) } \\
\end{array}$ & (ft TOC) & $\begin{array}{ll} & \text { (ft TTCC } \\
\text { (ft TOC) }\end{array}$ & (ft TOC) & $\begin{array}{l}\text { (ft TOC) } \\
\text { (n) }\end{array}$ & (ft TOC) & $\begin{array}{l}\text { (ft TOC) } \\
\text { (f) }\end{array}$ & $\begin{array}{l}\text { (ft TOC) } \\
\text { (f) }\end{array}$ & $\begin{array}{l}\text { (ft TOC) } \\
\text { (n) }\end{array}$ & (ft TOC) & (ft TOC) & (ft TOC) \\
\hline 20:00 & & 29.944 & 28.526 & 29.122 & 29.737 & & 29.333 & 30.265 & 29.78 & 29.41 & 28.265 & 26.12 & 31.312 & 36.722 & 32.3 & 15.95 & 19.706 & 15.47 & 10. & 31.487 & 32. & 29.377 & 77 & 27.99 & 33.483 \\
\hline $121: 00$ & & 29.974 & 28.554 & 29.103 & 29.73 & & 29.345 & 30.262 & 29.778 & 29.407 & 28.256 & 26.11 & 31.309 & 36.712 & 32.33 & 15.956 & 19.697 & 15.479 & 10.174 & 31.478 & 32.203 & 29.362 & 31.277 & 27.99 & 33.481 \\
\hline $2 / 15 / 122: 00$ & & 30 & 28.573 & 29.08 & 29.713 & & 29.359 & 30.26 & 29.769 & 29.396 & 28.244 & 26.088 & 31.3 & 36.71 & 32.326 & 15.954 & 19.685 & 15.477 & 10.16 & 31.462 & 32.194 & 29.341 & 31.263 & 27.99 & 33.478 \\
\hline 2/15/12 3:00 & & 29.998 & 28.568 & 29.098 & 29.716 & & 29.34 & 30.26 & 29.781 & 29.403 & 28.251 & 26.105 & 31.302 & 36.708 & 32.321 & 15.954 & 19.676 & 15.47 & 10.162 & 31.469 & 32.196 & 29.355 & 31.265 & 27.993 & 33.478 \\
\hline 2/15/12 4:00 & & 30.009 & 28.584 & 29.087 & 29.713 & & 29.347 & 30.255 & 29.774 & 29.4 & 28.246 & 26.098 & 31.3 & 36.705 & 32.319 & 15.954 & 19.665 & 15.475 & 10.155 & 31.462 & 32.191 & 29.346 & 31.265 & 27.99 & 33.476 \\
\hline 2/15/12 5:00 & & 30.019 & 28.587 & 29.075 & 29.704 & & 29.347 & 30.255 & 29.771 & 29.391 & 28.246 & 26.081 & 31.291 & 36.703 & 32.314 & 15.952 & 19.655 & 15.47 & 10.15 & 31.459 & 32.182 & 29.341 & 31.244 & 27.99 & 33.474 \\
\hline 2/15/12 6:00 & & 30.023 & 28.591 & 29.082 & 29.704 & & 29.34 & 30.253 & 29.774 & 29.393 & 28.244 & 26.091 & 31.295 & 36.705 & 32.314 & 15.949 & 19.667 & 15.468 & 10.15 & 31.459 & 32.187 & 29.344 & 31.258 & 27.993 & 33.474 \\
\hline 2/15/12 7:00 & & 30.014 & 28.594 & 29.098 & 29.711 & & 29.326 & 30.255 & 29.783 & 29.407 & 28.251 & 26.11 & 31.295 & 36.708 & 32.314 & 15.949 & 19.669 & 15.472 & 10.15 & 31.466 & 32.189 & 29.351 & 31.258 & 27.995 & 33.474 \\
\hline 2/15/12 8:00 & & 30.002 & 28.587 & 29.113 & 29.723 & & 29.314 & 30.255 & 29.788 & 29.412 & 28.258 & 26.124 & 31.298 & 36.712 & 32.319 & 15.952 & 19.655 & 15.458 & 10.155 & 31.476 & 32.191 & 29.365 & 31.26 & 27.995 & 33.476 \\
\hline 2/15/12 9:00 & & 29.993 & 28.589 & 29.131 & 29.737 & & 29.3 & 30.257 & 29.793 & 29.419 & 28.267 & 26.138 & 31.305 & 36.717 & 32.323 & 15.954 & 19.646 & 15.451 & 10.157 & 31.487 & 32.199 & 29.381 & 31.267 & 27.995 & 33.476 \\
\hline $2 / 15 / 1210: 00$ & & 29.998 & 28.601 & 29.138 & 29.744 & & 29.305 & 30.26 & 29.795 & 29.424 & 28.272 & 26.141 & 31.307 & 36.719 & 32.326 & 15.952 & 19.642 & 15.456 & 10.165 & 31.494 & 32.199 & 29.384 & 31.267 & 27.995 & 33.476 \\
\hline $2 / 15 / 1211: 00$ & & 30.005 & 28.61 & 29.148 & 29.753 & & 29.302 & 30.262 & 29.79 & 29.431 & 28.279 & 26.153 & 31.307 & 36.724 & 32.328 & 15.956 & 19.635 & 15.458 & 10.165 & 31.497 & 32.203 & 29.398 & 31.272 & 27.995 & 33.478 \\
\hline $2 / 15 / 1212: 00$ & & 30.023 & 28.629 & 29.152 & 29.76 & & 29.302 & 30.265 & 29.793 & 29.435 & 28.284 & 26.15 & 31.312 & 36.733 & 32.333 & 15.959 & 19.628 & 15.458 & 10.167 & 31.506 & 32.203 & 29.407 & 31.27 & 28 & 33.476 \\
\hline $2 / 15 / 1213: 00$ & & 30.042 & 28.654 & 29.145 & 29.762 & & 29.314 & 30.267 & 29.786 & 29.438 & 28.281 & 26.15 & 31.314 & 36.726 & 32.33 & 15.959 & 19.618 & 15.472 & 10.171 & 31.497 & 32.208 & 29.395 & 31.288 & 27.993 & 33.481 \\
\hline 2/15/12 14:00 & & 30.051 & 28.668 & 29.148 & 29.762 & & 29.316 & 30.265 & 29.788 & 29.442 & 28.284 & 26.15 & 31.312 & 36.729 & 32.333 & 15.959 & 19.609 & 15.477 & 10.171 & 31.501 & 32.208 & 29.398 & 31.281 & 28 & 33.478 \\
\hline 2/15/12 15:00 & & 30.035 & 28.661 & 29.164 & 29.767 & & 29.305 & 30.267 & 29.795 & 29.447 & 28.298 & 26.158 & 31.316 & 36.731 & 32.338 & 15.961 & 19.602 & 15.468 & 10.176 & 31.513 & 32.21 & 29.416 & 31.274 & 28.002 & 33.481 \\
\hline $2 / 15 / 1216: 00$ & & 30.016 & 28.654 & 29.18 & 29.783 & & 29.298 & 30.269 & 29.797 & 29.458 & 28.305 & 26.177 & 31.326 & 36.731 & 32.345 & 15.963 & 19.653 & 15.468 & 10.185 & 31.525 & 32.22 & 29.424 & 31.286 & $\begin{array}{r}28 \\
28\end{array}$ & 33.485 \\
\hline $2 / 15 / 1217: 00$ & & 30.005 & 28.65 & 29.197 & 29.795 & & 29.291 & 30.274 & 29.805 & 29.468 & 28.316 & 26.186 & 31.333 & 36.736 & 32.352 & 15.966 & 19.653 & 15.461 & 10.188 & 31.532 & 32.227 & 29.44 & 31.286 & 28.004 & 33.488 \\
\hline $2 / 15 / 1218: 00$ & & 29.958 & 28.619 & 29.257 & 29.832 & & 29.253 & 30.284 & 29.828 & 29.498 & 28.346 & 26.239 & 31.349 & 36.75 & 32.37 & 15.973 & 19.658 & 15.444 & 10.205 & 31.581 & 32.241 & 29.496 & 31.295 & 28.007 & 33.495 \\
\hline $2 / 15 / 12$ 19:00 & & 29.921 & 28.603 & 29.297 & 29.87 & & 29.241 & 30.296 & 29.838 & 29.523 & 28.37 & 26.28 & 31.368 & 36.754 & 32.382 & 15.979 & 19.649 & 15.447 & 10.221 & 31.609 & 32.263 & 29.532 & 31.319 & 28.004 & 33.502 \\
\hline $2 / 15 / 1220: 00$ & & 29.916 & 28.603 & 29.3 & 29.888 & & 29.26 & 30.303 & 29.828 & 29.535 & 28.377 & 26.282 & 31.377 & 36.766 & 32.391 & 15.98 & 19.649 & 15.468 & 10.228 & 31.611 & 32.27 & 29.529 & 31.333 & 28.004 & 33.502 \\
\hline 2/15/12 21:00 & & 29.909 & 28.594 & 29.321 & 29.909 & & 29.26 & 30.312 & 29.833 & 29.554 & 28.395 & 26.296 & 31.387 & 36.773 & 32.403 & 15.99 & 19.644 & 15.468 & 10.242 & 31.632 & 32.282 & 29.555 & 31.34 & 28.007 & 33.506 \\
\hline $2 / 15 / 1222: 00$ & & 29.895 & 28.575 & 29.337 & 29.928 & & 29.269 & 30.317 & 29.836 & 29.565 & 28.412 & 26.306 & 31.396 & 36.778 & 32.415 & 15.998 & 19.637 & 15.472 & 10.251 & 31.644 & 289 & 9.572 & 31.347 & 28.007 & 33.509 \\
\hline 2/15/12 23:00 & & 29.853 & 28.538 & 29.367 & 29.954 & & 29.262 & 30.327 & 29.843 & 29.591 & 28.432 & 26.33 & 31.56 & 36.785 & 32.584 & 16.005 & 19.632 & 15.472 & 10.421 & 31.714 & 32.452 & 29.602 & 31.497 & 28.009 & 33.511 \\
\hline $2 / 16 / 120: 00$ & & 29.858 & 28.531 & 29.367 & 29.965 & & 29.284 & 30.334 & 29.838 & 29.6 & 28.439 & 26.327 & 31.482 & 36.79 & 32.504 & 16.012 & 19.625 & 15.487 & 10.341 & 31.7 & 32.379 & 29.607 & 31.436 & 28.009 & 33.511 \\
\hline 2/16/12 1:00 & & 29.849 & 28.519 & 29.384 & 29.984 & & 29.293 & 30.344 & 29.84 & 29.619 & 28.456 & 26.342 & 31.471 & 36.799 & 32.49 & 16.021 & 19.618 & 15.494 & 10.327 & 31.702 & 32.367 & 29.623 & 31.422 & 28.011 & 33.511 \\
\hline 2/16/12 2:00 & & 29.87 & 28.528 & 29.358 & 29.979 & & 29.333 & 30.346 & 29.826 & 29.614 & 28.449 & 26.315 & 31.461 & 36.799 & 32.481 & 16.026 & 19.614 & 15.519 & 10.317 & 31.69 & 32.357 & 29.604 & 31.427 & 28.007 & 33.509 \\
\hline 2/16/12 3:00 & & 29.856 & 28.508 & 29.393 & 29.998 & & 29.319 & 30.356 & 29.845 & 29.637 & 28.467 & 26.337 & 31.466 & 36.806 & 32.483 & 16.033 & 19.609 & 15.508 & 10.322 & 31.707 & 32.36 & 29.628 & 31.422 & 28.011 & 33.511 \\
\hline $2 / 16 / 124: 00$ & & 29.867 & 28.508 & 29.391 & 30.003 & & 29.335 & 30.361 & 29.838 & 29.644 & 28.474 & 26.33 & 31.466 & 36.808 & 32.485 & 16.038 & 19.6 & 15.515 & 10.324 & 31.711 & 32.36 & 29.633 & 31.42 & 28.014 & 33.511 \\
\hline 2/16/12 5:00 & & 29.874 & 28.515 & 29.395 & 30.007 & & 29.347 & 30.365 & 29.843 & 29.651 & 28.481 & 26.332 & 31.471 & 36.811 & 32.488 & 16.045 & 19.593 & 15.522 & 10.322 & 31.711 & 32.362 & 29.635 & 31.42 & 28.014 & 33.511 \\
\hline $2 / 16 / 126: 00$ & & 29.879 & 28.515 & 29.409 & 30.014 & & 29.352 & 30.372 & 29.85 & 29.665 & 28.493 & 26.339 & 31.473 & 36.808 & 32.492 & 16.049 & 19.607 & 15.526 & 10.331 & 31.721 & 32.369 & 29.644 & 31.427 & 28.014 & 33.513 \\
\hline 2/16/12 7:00 & & 29.891 & 28.512 & 29.414 & 30.021 & & 29.361 & 30.377 & 29.85 & 29.674 & 28.5 & 26.344 & 31.478 & 36.818 & 32.497 & 16.056 & 19.612 & 15.545 & 10.334 & 31.723 & 32.372 & 29.649 & 31.436 & 28.014 & 33.516 \\
\hline $2 / 16 / 128: 00$ & & 29.902 & 28.519 & 29.423 & 30.028 & & 29.37 & 30.382 & 29.855 & 29.686 & 28.507 & 26.347 & 31.482 & 36.82 & 32.504 & 16.063 & 19.605 & 15.545 & 10.343 & 31.73 & 32.376 & 29.656 & 31.443 & 28.018 & 33.518 \\
\hline 2/16/12 9:00 & & 29.942 & 28.554 & 29.435 & 30.038 & & 29.378 & 30.389 & 29.855 & 29.698 & 28.518 & 26.354 & 31.489 & 36.825 & 32.509 & 16.068 & 19.598 & 15.557 & 10.345 & 31.744 & 32.383 & 29.666 & 31.45 & 28.018 & 33.52 \\
\hline $2 / 16 / 1210: 00$ & & 30.109 & 28.615 & 29.416 & 30.0 & & 29.41 & 30.394 & 29.845 & 29.698 & 28.512 & 26.335 & 31.489 & 36.825 & 32.509 & $\begin{array}{l}16.073 \\
\end{array}$ & 19.591 & 15.576 & 10.345 & 31.725 & 32.383 & 29.647 & 31.453 & 28.018 & 33.523 \\
\hline 2/16/12 11:00 & & 30.119 & 28.664 & 29.407 & 30.02 & & 29.427 & 30.396 & 29.85 & 29.693 & 28.509 & 26.313 & 31.489 & 36.827 & 32.506 & 16.075 & 19.581 & 15.58 & 10.343 & 31.725 & 32.381 & 29.647 & 31.448 & 28.021 & $\begin{array}{r}33.52 \\
33\end{array}$ \\
\hline 2/16/12 12:00 & & 30.121 & 28.748 & 29.412 & $\begin{array}{l}30.024 \\
30.24\end{array}$ & & 29.434 & 30.399 & 29.85 & 29.702 & 28.512 & 26.32 & $\begin{array}{l}31.492 \\
31.492\end{array}$ & 36.829 & 32.509 & 16.08 & 19.575 & $\begin{array}{l}15.597 \\
\end{array}$ & 10.346 & 31.723 & .381 & 29.642 & $\begin{aligned} 31.46 \\
31.46\end{aligned}$ & 28.021 & 33.523 \\
\hline $2 / 16 / 1213: 00$ & & 30.112 & 28.834 & 29.37 & 作 & & 29.472 & 30.396 & 29.838 & 29.686 & 28.495 & 26.28 & $\begin{array}{l}31.485 \\
31.45\end{array}$ & 36.827 & 32.499 & 16 & 19.568 & 15.625 & 10.341 & 31.697 & .376 & 29.614 & $\begin{array}{l}31.40 \\
31.46\end{array}$ & 28.021 & $\begin{array}{r}3.52 \\
33.52\end{array}$ \\
\hline $2 / 16 / 1214: 00$ & & 30.114 & $\begin{array}{l}20.034 \\
28.892\end{array}$ & 29.344 & 29.977 & & 29.495 & 30.392 & 29.828 & $\begin{array}{l}29.600 \\
29.674\end{array}$ & 28.484 & $\begin{array}{l}26.200 \\
26.256\end{array}$ & $\begin{array}{l}31.407 \\
31.473\end{array}$ & $\begin{aligned} 30.021 \\
36.82\end{aligned}$ & $\begin{array}{l}32.4995 \\
32.495\end{array}$ & 16 & $\begin{array}{l}19.561 \\
19.561\end{array}$ & $\begin{array}{l}13.0259 \\
15.639\end{array}$ & 10.329 & 31.679 & .367 & 29.588 & $\begin{array}{l}31.453 \\
31.453\end{array}$ & 28.021 & $\begin{array}{l}33.518 \\
33.518\end{array}$ \\
\hline :00 & & 30.117 & 28.9 & 29.321 & 29.9 & & 29.507 & 30. & 29.826 & 29.663 & 28.47 & 26.227 & 31.466 & 36.811 & 32.485 & 16 & 19 & 15.648 & 10.324 & 31.665 & 7 & 29.567 & 31.434 & 28.021 & .518 \\
\hline 6:00 & & 30. & 28.9 & 29.325 & & & 29 & 30. & 29.833 & 29. & 28. & 26.222 & 31.464 & 36.808 & 32.485 & 16. & 2 & 15.641 & 10.322 & 31 & 7 & 7 & 31.422 & 28.023 & 33.518 \\
\hline $2 / 16 / 1217: 00$ & & 30. & 28.957 & 29.318 & & & 29.495 & 30.384 & 29.833 & 29.658 & 28.463 & 26.225 & 31.461 & 36.804 & 32.483 & 16.0 & 8 & 15.653 & 10.319 & 31.655 & 3 & 77 & 31.422 & 28.023 & 33.518 \\
\hline $2 / 16 / 1218: 00$ & & 30.126 & 28.946 & 29.3 & 29 & & 29.497 & 30.382 & 29.826 & 29.649 & 28.451 & 26.21 & 31.457 & 36.799 & 32.476 & 16.0 & o & 15.658 & 10.31 & 31.644 & .348 & 9.543 & 31.417 & 28.023 & 33.518 \\
\hline 2/16/12 19:00 & & 30.131 & 28.911 & 29.295 & & & 29 & & 29.831 & 29. & 28.446 & 26.196 & 31.45 & 36.797 & 32.474 & 16 & 4 & 15.655 & 10.312 & 31.637 & 32.346 & 29.539 & 31.408 & 28.025 & 33.518 \\
\hline 2/16/12 20:00 & & 30.133 & 28.88 & 29.297 & & & 29.483 & 30. & 29.836 & 29. & 28.444 & 26.198 & 31.45 & 36.792 & 32.471 & 16. & & 15.653 & 10.308 & 31.641 & & 29.534 & 31.408 & 28.023 & 33.518 \\
\hline $2 / 16 / 1221: 00$ & & 30. & 28.8 & 29.307 & & & 29.467 & & 29.843 & 29. & 28.446 & 26.208 & 31.452 & 36.797 & 32.471 & 16 & 8 & 15.644 & 10.308 & 31.639 & 32.343 & 29.546 & 31.41 & 28.025 & 33.52 \\
\hline $2 / 16 / 1222: 00$ & & 30.142 & 28. & 29.311 & & & 29.457 & 30. & 29.847 & 29.644 & 28.446 & 26.213 & 31.45 & 36.799 & 32.469 & 16.0 & 19.563 & 15.641 & 10.305 & 31.646 & 32.343 & 29.55 & 31.406 & 28.028 & 33.52 \\
\hline $2 / 16 / 1223: 00$ & & 30.138 & 28.8 & 29.304 & & & 29.46 & 30.3 & 29.843 & 29.642 & 28.442 & 26.208 & 31.447 & 36.79 & 32.466 & 16.0 & 19.563 & 15.646 & 10.306 & 31.637 & 32.338 & 29.539 & 31.406 & 28.025 & 33.52 \\
\hline 2/17/120:00 & & 30.14 & 28.853 & 29.29 & 29. & & 29.469 & 30.375 & 29.836 & 29.633 & 28.432 & 26.196 & 31.438 & 36.797 & 32.459 & 16.0 & 19.554 & 15.648 & 10.296 & 31.627 & 32.331 & 29.525 & 31.401 & 28.028 & 33.518 \\
\hline $2 / 17 / 121: 00$ & & 30.138 & 28.848 & 29.276 & 29.8 & & 29.474 & 30.37 & 29.833 & 29.623 & 28.423 & 26.184 & 31.431 & 36.792 & 32.455 & 16.059 & 19.549 & 15.653 & 10.291 & 31.613 & 32.326 & 29.513 & 31.394 & 28.025 & 33.513 \\
\hline 2/17/12 2:00 & & 30.137 & 28.843 & 29.257 & 29.874 & & 29.479 & 30.365 & 29.828 & 29.612 & 28.412 & 26.165 & 31.422 & 36.785 & 32.443 & 16.056 & 19.535 & 15.648 & 10.284 & 31.599 & 32.315 & 29.494 & 31.382 & 28.028 & 33.509 \\
\hline 2/17/12 3:00 & & 30.142 & 28.834 & 29.25 & 29.863 & & 29.474 & 30.363 & 29.833 & 29.607 & 28.407 & 26.158 & 31.417 & 36.78 & 32.438 & 16.052 & 19.531 & 15.641 & 10.275 & 31.585 & 32.31 & 29.492 & 31.375 & 28.028 & 33.506 \\
\hline 2/17/12 4:00 & & 30.142 & 28.836 & 29.241 & 29.853 & & 29.474 & 30.361 & 29.828 & 29.6 & 28.398 & 26.153 & 31.408 & 36.778 & 32.431 & 16.047 & 19.517 & 15.639 & 10.265 & 31.585 & 32.3 & 29.478 & 31.37 & 28.028 & 33.502 \\
\hline 2/17/12 5:00 & & 30.144 & 28.836 & 29.232 & 29.844 & & 29.469 & 30.358 & 29.826 & 29.591 & 28.391 & 26.143 & 31.398 & 36.776 & 32.424 & 16.042 & 19.505 & 15.634 & 10.263 & 31.576 & 32.293 & 29.473 & 31.361 & 28.03 & 33.499 \\
\hline $2 / 17 / 126: 00$ & & 30.149 & 28.834 & 29.239 & 29.844 & & 29.457 & 30.351 & 29.836 & 29.588 & 28.391 & 26.15 & 31.4 & 36.776 & 32.424 & 16.04 & 19.526 & 15.627 & 10.259 & 31.581 & 32.291 & 29.48 & 31.356 & 28.028 & 33.502 \\
\hline $2 / 17 / 127: 00$ & & 30.149 & 28.848 & 29.241 & 29.839 & & 29.45 & 30.351 & 29.836 & 29.586 & 28.386 & 26.153 & 31.398 & 36.771 & 32.422 & 16.035 & 19.524 & 15.623 & 10.256 & 31.581 & 32.293 & 29.471 & 31.356 & 28.028 & 33.499 \\
\hline
\end{tabular}




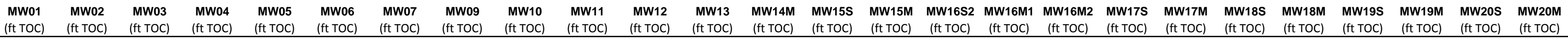

\begin{tabular}{|c|c|c|c|c|c|c|c|c|c|c|c|c|c|c|c|c|c|c|c|c|c|c|c|c|c|}
\hline Well & $\begin{array}{l}\text { (ft TOC) } \\
\text { (ft }\end{array}$ & $\begin{array}{l}\text { (ft TOC) } \\
\text { (f) }\end{array}$ & $\begin{array}{l}\text { (ft TOC) } \\
\text { (f) }\end{array}$ & $\begin{array}{l}\text { (ft TOC) } \\
\text { (ft) }\end{array}$ & $\begin{array}{l}\text { (ft TOC) } \\
\text { (f) }\end{array}$ & $\begin{array}{l}\text { (ft TOC) } \\
\text { (f) }\end{array}$ & $\begin{array}{l}\text { (ft TOC) } \\
\text { (ft }\end{array}$ & $\begin{array}{l}\text { (ft TOC) } \\
\text { (f) }\end{array}$ & $\begin{array}{l}\text { (ft TOC) } \\
\text { (ft) }\end{array}$ & $\begin{array}{l}\text { (ft TOC) } \\
\text { (f) }\end{array}$ & $\begin{array}{l}\text { (ft TOC) } \\
\text { (f) }\end{array}$ & $\begin{array}{l}\text { (ft TOC) } \\
\text { (f) }\end{array}$ & (ft TOC) & $\begin{array}{l}\text { (ft TOC) } \\
\text { (f) }\end{array}$ & (ft TOC) & $\begin{array}{ll}\text { (ft TOC) } \\
\text { (ft TOC) }\end{array}$ & (ft TOC) & $\begin{array}{l}\text { (ft TOC) } \\
\text { (1) }\end{array}$ & (ft TOC) & $\begin{array}{l}\text { (ft TOC) } \\
\end{array}$ & $\begin{array}{l}\text { (ft TOC) } \\
\text { (f) }\end{array}$ & $\begin{array}{l}\text { (ft TOC) } \\
\text { (n) }\end{array}$ & (ft TOC) & $\begin{array}{l}\text { (ft TOC) } \\
\text { (1) }\end{array}$ & (ft TOC) \\
\hline 8:00 & & 30.156 & 28.836 & 29.26 & 29.849 & & 29.429 & 30.353 & 29.84 & 29.5 & 28.395 & 26. & 31.398 & 36.773 & $32.4 \div>2$ & 16. & 19.512 & 15. & 10.258 & 85 & 32.293 & 29 & 56 & 03 & 33.504 \\
\hline /12 9:00 & & 30.158 & 28.862 & 29.271 & 29.858 & & 29.422 & 30.353 & 29.845 & 29.598 & 28.4 & 26.186 & & 36.778 & 32.424 & 16.035 & 19.519 & 15.611 & 10.261 & 31.595 & 32.296 & 29.499 & 31.363 & 28.032 & 33.506 \\
\hline $2 / 17 / 12$ 10:00 & & 30.156 & 28.911 & 29.264 & 29.86 & & 29.429 & 30.353 & 29.843 & 29.595 & 28.395 & 26.184 & 31.403 & 36.776 & 32.424 & 16.031 & 19.51 & 15.622 & 10.261 & 31.59 & 32.298 & 29.494 & 1.366 & 28.032 & 33.506 \\
\hline $2 / 17 / 1211: 00$ & & 30.158 & 28.969 & 29.262 & 29.858 & & 29.434 & 30.353 & 29.84 & 29.591 & 28.393 & 26.177 & 31.398 & 36.78 & 32.422 & 16.028 & 19.498 & 15.62 & 10.258 & 31.59 & 32.293 & 29.492 & 31.368 & 28.032 & 33.506 \\
\hline 2/17/12 12:00 & & 30.156 & 29.032 & 29.243 & 29.849 & & 29.446 & 30.349 & 29.833 & 29.584 & 28.386 & 26.16 & 31.393 & 36.773 & 32.417 & 16.026 & 19.491 & 15.63 & 10.254 & 31.581 & 32.286 & 29.482 & 31.363 & 28.032 & 33.504 \\
\hline 2/17/12 13:00 & & 30.156 & 29.076 & 29.227 & 29.839 & & 29.457 & 30.346 & 29.828 & 29.575 & 28.377 & 26.148 & 31.389 & 36.773 & 32.41 & 16.021 & 19.482 & 15.637 & 10.249 & 31.562 & 32.284 & 29.463 & 31.361 & 28.035 & 33.504 \\
\hline 2/17/12 14:00 & & 30.154 & 29.116 & 29.206 & 29.821 & & 29.469 & 30.341 & 29.821 & 29.563 & 28.363 & 26.127 & 31.379 & 36.764 & 32.403 & 316.021 & 19.466 & 15.641 & 10.24 & 31.55 & 32.274 & 29.445 & 31.349 & 28.035 & 33.499 \\
\hline 2/17/12 15:00 & & 30.156 & 29.139 & 29.197 & 29.811 & & 29.467 & 30.339 & 29.821 & 29.554 & 28.356 & 26.119 & 31.375 & 36.761 & 32.396 & $5 \quad 16.017$ & 19.468 & 15.637 & 10.233 & 31.543 & 32.265 & 29.435 & 31.34 & 28.035 & 33.499 \\
\hline 2/17/12 16:00 & & 30.158 & 29.144 & 29.192 & 29.804 & & 29.46 & 30.334 & 29.826 & 29.549 & 28.351 & 26.117 & 31.37 & 36.754 & 32.394 & 16.014 & 19.517 & 15.637 & 10.225 & 31.541 & 32.263 & 29.433 & 31.333 & 28.037 & 33.499 \\
\hline 2/17/12 17:00 & & 30.163 & 29.139 & 29.199 & 29.804 & & 29.443 & 30.334 & 29.831 & 29.547 & 28.353 & 26.124 & 31.368 & 36.761 & 32.389 & 16.01 & 19.514 & 15.63 & 10.225 & 31.543 & 32.26 & 29.447 & 31.328 & 28.037 & 33.497 \\
\hline $2 / 17 / 1218: 00$ & & 30.165 & 29.128 & 29.208 & 29.807 & & 29.432 & 30.334 & 29.828 & 29.547 & 28.353 & 26.134 & 31.368 & 36.759 & 32.389 & 16.007 & 19.505 & 15.618 & 10.228 & 31.548 & 32.263 & 29.44 & 31.331 & 28.037 & 33.499 \\
\hline $2 / 17 / 1219: 00$ & & 30.17 & 29.104 & 29.225 & 29.816 & & 29.415 & 30.332 & 29.838 & 29.549 & 28.358 & 26.148 & 31.373 & 36.761 & 32.396 & $5 \quad 16.007$ & 19.503 & 15.616 & 10.23 & 31.564 & 32.267 & 29.454 & 31.333 & 28.039 & 33.502 \\
\hline $2 / 17 / 1220: 00$ & & 30.172 & 29.086 & 29.234 & 29.823 & & 29.406 & 30.334 & 29.838 & 29.554 & 28.363 & 26.158 & 31.377 & 36.759 & 32.398 & $\begin{array}{l}3 \\
3\end{array}$ & 19.496 & 15.613 & 10.233 & 31.562 & 32.27 & 29.459 & 31.335 & 28.039 & 33.506 \\
\hline $2 / 17 / 1221: 00$ & & 30.175 & 29.065 & 29.25 & 29.837 & & 29.392 & 30.337 & 29.847 & 29.561 & 28.37 & 26.174 & 31.379 & 36.766 & 32.403 & 16.007 & 19.494 & 15.599 & 10.242 & 31.576 & 32.272 & 29.485 & 31.34 & 28.039 & 33.509 \\
\hline 2/17/12 22:00 & & 30.189 & 29.034 & 29.288 & 29.863 & & 29.363 & 30.341 & 29.862 & 29.577 & 28.388 & 26.21 & 31.391 & 36.776 & 32.41 & 16.01 & 19.494 & 15.585 & 10.247 & 31.602 & 32.284 & 29.515 & 31.345 & 28.042 & 33.513 \\
\hline 2/17/12 23:00 & & 30.186 & 29.009 & 29.304 & 29.881 & & 29.361 & 30.349 & 29.859 & 29.588 & 28.398 & 26.225 & 31.398 & 36.783 & 32.413 & 16.012 & 19.489 & 15.592 & 10.251 & 31.609 & 32.293 & 29.529 & 31.352 & 28.042 & 33.516 \\
\hline 2/18/12 0:00 & & 30.182 & 28.997 & 29.304 & 29.891 & & 29.37 & 30.351 & 29.855 & 29.593 & 28.4 & 26.227 & 31.4 & 36.785 & 32.417 & 16.014 & 19.475 & 15.597 & 10.261 & 31.611 & 32.291 & 29.527 & 31.356 & 28.042 & 33.516 \\
\hline 2/18/12 1:00 & & 30.186 & 28.983 & 29.304 & 29.898 & & 29.378 & 30.353 & 29.85 & 29.593 & 28.402 & 26.222 & 31.4 & 36.79 & 32.42 & 16.014 & 19.468 & 15.599 & 10.256 & 31.611 & 32.293 & 29.529 & 31.359 & 28.042 & 33.513 \\
\hline 2/18/12 2:00 & & 30.189 & 28.969 & 29.307 & 29.9 & & 29.38 & 30.356 & 29.855 & 29.598 & 28.407 & 26.225 & 31.401 & 36.794 & 32.42 & 16.014 & 19.459 & 15.599 & 10.256 & 31.613 & 32.293 & 29.534 & 31.356 & 28.042 & 33.511 \\
\hline $2 / 18 / 123: 00$ & & 30.189 & 28.948 & 29.304 & 29.9 & & 29.387 & 30.358 & 29.855 & 29.6 & 28.407 & 26.222 & 31.396 & 36.794 & 32.417 & 16.017 & 19.459 & 15.602 & 10.252 & 31.613 & 32.293 & 29.534 & 31.356 & 28.042 & 33.506 \\
\hline 2/18/12 4:00 & & 30.189 & 28.946 & 29.3 & 29.9 & & 29.396 & 30.361 & 29.847 & 29.598 & 28.407 & 26.21 & 31.394 & 36.794 & 32.415 & 5 16.019 & 19.452 & 15.601 & 10.254 & 31.611 & 32.291 & 29.525 & 31.354 & 28.044 & 33.502 \\
\hline 2/18/12 5:00 & & 30.191 & 28.943 & 29.302 & 29.9 & & 29.399 & 30.363 & 29.852 & 29.598 & 28.407 & 26.21 & 31.396 & 36.794 & 32.415 & 16.01 & 19.447 & 15.599 & 10.252 & 31.611 & 32.289 & .532 & 1.349 & 28.044 & 33.502 \\
\hline 2/18/12 6:00 & & 30.198 & 28.932 & 29.321 & 29.909 & & 29.389 & 30.365 & 29.859 & 29.609 & 28.418 & 26.229 & 31.4 & 36.794 & 32.422 & 16.021 & 19.447 & 15.599 & 10.256 & 31.625 & 32.293 & 29.546 & 31.356 & 28.044 & 33.504 \\
\hline 2/18/12 7:00 & & 30.203 & 28.918 & 29.339 & 29.921 & & 29.385 & 30.37 & 29.866 & 29.621 & 28.425 & 26.244 & 31.408 & 36.799 & 32.424 & 16.021 & 19.445 & 15.597 & 10.266 & 31.634 & 32.303 & 29.557 & 31.37 & 28.046 & 33.506 \\
\hline 2/18/12 8:00 & & 30.198 & 28.927 & 29.332 & 29.921 & & 29.399 & 30.372 & 29.855 & 29.621 & 28.425 & 26.239 & 31.41 & 36.804 & 32.431 & 16.026 & 19.445 & 15.611 & 10.27 & 31.63 & 32.305 & 29.548 & 31.37 & 28.044 & 33.506 \\
\hline 2/18/12 9:00 & & 30.203 & 28.927 & 29.339 & 29.93 & & 29.401 & 30.377 & 29.862 & 29.626 & 28.432 & 26.246 & 31.415 & 36.804 & 32.431 & 16.028 & 19.443 & 15.609 & 10.272 & 31.634 & 32.308 & 29.557 & 31.378 & 28.046 & 33.509 \\
\hline 2/18/12 10:00 & & 30.2 & 28.955 & 29.323 & 29.921 & & 29.42 & 30.377 & 29.852 & 29.621 & 28.425 & 26.222 & 31.415 & 36.806 & 32.434 & 16.028 & 19.47 & 15.616 & 10.27 & 31.627 & 32.308 & 29.548 & 31.373 & 28.049 & 33.511 \\
\hline 2/18/12 11:00 & & 30.2 & 29.013 & 29.309 & 29.914 & & 29.434 & 30.377 & 29.847 & 29.616 & 28.421 & 26.215 & 31.412 & 36.804 & 32.431 & 16.031 & 19.468 & 15.63 & 10.268 & 31.618 & 32.305 & 29.541 & 31.378 & 28.046 & 33.511 \\
\hline 2/18/12 12:00 & & 30.196 & 29.076 & 29.288 & 29.9 & & 29.457 & 30.372 & 29.838 & 29.607 & 28.409 & 26.191 & 31.408 & 36.797 & 32.427 & 16.028 & 19.459 & 15.641 & 10.263 & 31.606 & 32.3 & 29.52 & 31.375 & 28.049 & 33.509 \\
\hline 2/18/12 13:00 & & 30.196 & 29.128 & 29.267 & 29.881 & & 29.469 & 30.368 & 29.838 & 29.595 & 28.398 & 26.167 & 31.4 & 36.794 & 32.415 & 16.026 & 19.447 & 15.646 & 10.254 & 31.585 & 32.293 & 29.496 & 31.368 & 28.049 & 33.509 \\
\hline $2 / 18 / 1214: 00$ & & 30.196 & 29.177 & 29.236 & 29.86 & & 29.488 & 30.361 & 29.828 & 29.581 & 28.384 & 26.141 & 31.386 & 36.787 & 32.41 & 16.024 & 19.477 & 15.655 & 10.249 & 31.576 & 32.282 & 29.478 & 31.363 & 28.046 & 33.504 \\
\hline $2 / 18 / 1215: 00$ & & 30.2 & 29.191 & 29.241 & 29.851 & & 29.474 & 30.358 & 29.836 & 29.579 & 28.381 & 26.143 & 31.386 & 36.783 & 32.406 & 16.024 & 19.466 & 15.644 & 10.24 & 31.571 & 32.279 & 29.475 & 31.349 & 28.051 & 33.502 \\
\hline $2 / 18 / 1216: 00$ & & 30.198 & 29.202 & 29.227 & 29.839 & & 29.476 & 30.356 & 29.833 & 29.572 & 28.372 & 26.134 & 31.377 & 36.778 & 32.401 & 16.019 & 19.535 & 15.648 & 10.237 & 31.564 & 32.272 & 29.461 & 31.345 & 28.051 & 33.502 \\
\hline 2/18/12 17:00 & & 30.203 & 29.191 & 29.234 & 29.837 & & 29.46 & 30.353 & 29.84 & 29.57 & 28.372 & 26.138 & 31.377 & 36.773 & 32.398 & $\begin{array}{l}36.017 \\
3\end{array}$ & 19.526 & 15.639 & 10.235 & 31.56 & 32.272 & 29.468 & 31.335 & 28.051 & 33.499 \\
\hline $2 / 18 / 1218: 00$ & & 30.21 & 29.167 & 29.246 & 29.842 & & 29.441 & 30.351 & 29.843 & 29.572 & 28.377 & 26.153 & 31.377 & 36.771 & 32.398 & 16.0 & 19.512 & 15.63 & 10.233 & 31.567 & 32.27 & 29.475 & 31.335 & 28.051 & 33.502 \\
\hline $2 / 18 / 12$ 19:00 & & 30.212 & 29.142 & 29.255 & 29.846 & & 29.429 & 30.351 & 29.85 & 29.575 & 28.379 & 26.162 & 31.379 & 36.778 & 32.403 & 16.017 & 19.505 & 15.627 & 10.235 & 31.578 & 32.272 & 29.487 & 31.338 & 28.051 & 33.504 \\
\hline 2/18/12 20:00 & & 30.21 & 29.107 & 29.257 & 29.851 & & 29.425 & 30.351 & 29.847 & 29.575 & 28.379 & 26.165 & 31.382 & 36.776 & 32.403 & 16.014 & 19.494 & 15.625 & 10.24 & 31.576 & 32.277 & 29.487 & $\begin{array}{r}31.34 \\
31.34\end{array}$ & 28.054 & $\begin{array}{l}33.506 \\
33.506\end{array}$ \\
\hline 2/18/12 21:00 & & 30.214 & 29.081 & 29.255 & 29.851 & & 29.42 & 30.348 & 29.843 & 29.575 & 28.377 & 26.162 & 31.384 & 36.778 & 32.403 & 16.014 & 19.482 & 15.623 & 10.24 & 31.578 & 32.274 & 29.487 & $\begin{array}{l}31.344 \\
\end{array}$ & 28.054 & $\begin{array}{l}33.509 \\
3.509\end{array}$ \\
\hline 2/18/12 22:00 & & 30.212 & 29.055 & 29.253 & 29 & & 29.418 & 30.351 & 29.843 & 29.572 & 28 & 26.165 & 31.384 & 36.778 & 32.406 & 16. & 19.473 & 15.627 & 10.242 & 31.576 & 32.279 & 29.487 & 31.342 & 28.051 & 33.511 \\
\hline & & 30.2 & 29.03 & 29.248 & $2 \mathrm{~s}$ & & 2 & 30 & 29.84 & 2 & 28 & 26.16 & 31.384 & 36.78 & 32.403 & 1 & 34 & 15.622 & 10.237 & 31.571 & 32.277 & 32 & 31.342 & 28.054 & 33.511 \\
\hline & & 30. & 29.01 & 29.25 & 29 & & 25 & 36 & 29. & 29. & 28 & 26. & 31.382 & 36.776 & 32.401 & 16. & 9 & 15.616 & 10.24 & 31.571 & 32.272 & 35 & 31.338 & 28.054 & 33.511 \\
\hline $2 / 19 / 1$ & & & 28.95 & 29.248 & & & & 30 & 29 & 29 & 28.372 & 26. & 31 & 36.778 & 32.398 & 16 & 19.452 & 15.616 & 10.233 & 31.574 & 32.272 & 35 & 31.338 & 28.054 & 33.509 \\
\hline 2/19/12 2:00 & & 30.217 & 28.985 & 29.246 & 29.849 & & 29.413 & 30.349 & 29.843 & 29 & 28.37 & 26.158 & 31.375 & 36.769 & 32.396 & & 19.44 & 15.609 & 10.23 & 31.571 & 32.27 & 9.482 & 31.335 & 28.056 & $\begin{array}{l}33.506 \\
3.506\end{array}$ \\
\hline 2/19/12 3:00 & & 30.217 & 28.983 & 29.239 & 29.8. & & 29.415 & 30.344 & 29.836 & 29. & 28.365 & 26.15 & 31.37 & 36.776 & 32.391 & 16.007 & 19.429 & 15.611 & 10.228 & 31.56 & 32.263 & .475 & 31.333 & 28.054 & 33.502 \\
\hline 2/19/12 4:00 & & 30.214 & 28.98 & 29.222 & 29. & & & 30.3 & 29.833 & & 28.358 & 26.136 & 31.363 & 36.771 & 32.382 & 16.005 & 19. & 15.613 & 10.218 & 31.553 & 32.258 & 59 & 31.326 & 28.056 & 33.497 \\
\hline 2/19/12 5:00 & & 30.214 & 28.997 & 29.22 & 29. & & & 30.3 & 29.838 & & 28. & 26.131 & 31.356 & 36.769 & 32.382 & 16 & 19.408 & 15.609 & 10.219 & 31.55 & 32.251 & & 31.319 & 28.056 & 33.495 \\
\hline 2/19/12 6:00 & & 30.219 & 28.985 & 29.227 & 29.828 & & & 30.3 & 29.838 & & 28.356 & 26.141 & 31.356 & 36.771 & 32.375 & 16 & 19.401 & 15.602 & 10.214 & 31.548 & 32.251 & 29.459 & 31.316 & 28.056 & 33.492 \\
\hline $2 / 19 / 127: 00$ & & 30.221 & 28.98 & 29.234 & 29 & & 29. & 30.3 & 29.843 & 29.5 & 28.358 & 26.148 & 31.356 & 36.769 & 32.375 & $5 \quad 15.9$ & 19.394 & 15.597 & 10.214 & 31.555 & 32.251 & 29.461 & 31.316 & 28.058 & 33.492 \\
\hline 2/19/12 8:00 & & 30.226 & 28.988 & 29.246 & 29.8. & & 29.394 & 30.33 & 29.845 & 29.556 & 28.365 & 26.155 & 31.358 & 36.773 & 32.38 & 15.9 & 19.392 & 15.59 & 10.218 & 31.562 & 32.253 & 29.478 & 31.319 & 28.058 & 33.495 \\
\hline 2/19/129:00 & & 30.221 & 29.027 & 29.239 & 29.837 & & 29.403 & 30.337 & 29.838 & 29.554 & 28.36 & 26.15 & 31.361 & 36.771 & 32.382 & 15.998 & 19.413 & 15.602 & 10.218 & 31.557 & 32.258 & 29.471 & 31.319 & 28.056 & 33.497 \\
\hline 2/19/12 10:00 & & 30.221 & 29.062 & 29.234 & 29.835 & & 29.408 & 30.337 & 29.833 & 29.554 & 28.358 & 26.15 & 31.361 & 36.769 & 32.38 & $\begin{array}{l}35.998 \\
3\end{array}$ & 19.413 & 15.604 & 10.221 & 31.557 & 32.253 & 29.468 & 31.321 & 28.056 & 33.499 \\
\hline 2/19/12 11:00 & & 30.224 & 29.132 & 29.227 & 29.832 & & 29.415 & 30.337 & 29.836 & 29.547 & 28.351 & 26.143 & 31.361 & 36.776 & 32.382 & 15.996 & 19.408 & 15.609 & 10.219 & 31.553 & 32.251 & 29.461 & 31.328 & 28.058 & 33.502 \\
\hline 2/19/12 12:00 & & 30.221 & 29.195 & 29.22 & 29.825 & & 29.422 & 30.336 & 29.833 & 29.544 & 28.349 & 26.138 & 31.358 & 36.769 & 32.375 & 15.993 & 19.399 & 15.616 & 10.214 & 31.548 & 32.248 & 29.461 & 31.333 & 28.058 & 33.499 \\
\hline 2/19/12 13:00 & & 30.212 & 29.263 & 29.185 & 29.807 & & 29.448 & 30.332 & 29.821 & 29.53 & 28.332 & 26.112 & 31.344 & 36.769 & 32.37 & 15.991 & 19.424 & 15.63 & 10.207 & 31.525 & 32.236 & 29.428 & 31.326 & 28.056 & 33.497 \\
\hline 2/19/12 14:00 & & 30.212 & 29.317 & 29.157 & 29.783 & & 29.457 & 30.322 & 29.814 & 29.507 & 28.316 & 26.071 & 31.333 & 36.757 & 32.356 & 15.986 & 19.413 & 15.63 & 10.195 & 31.504 & 32.227 & 29.402 & 31.3 & 28.058 & 33.495 \\
\hline $2 / 19 / 12$ 15:00 & & 30.203 & 29.356 & 29.129 & 29.76 & & 29.472 & 30.313 & 29.807 & 29.493 & 28.3 & 26.055 & 31.321 & 36.752 & 32.345 & 15.984 & 19.401 & 15.639 & 10.183 & 31.483 & 32.215 & 29.374 & 31.288 & 28.058 & 33.488 \\
\hline
\end{tabular}




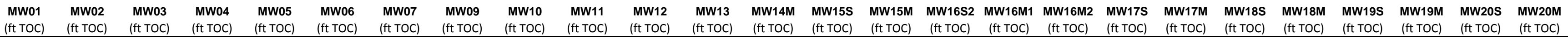

\begin{tabular}{|c|c|c|c|c|c|c|c|c|c|c|c|c|c|c|c|c|c|c|c|c|c|c|c|c|c|}
\hline Well & $\begin{array}{l}\text { MWO1 } \\
\text { (ft TOC) }\end{array}$ & $\begin{array}{l}\text { Mwoz } \\
\text { (ft TOC) }\end{array}$ & $\begin{array}{l}\text { Mw03 } \\
\text { (ft Toc) }\end{array}$ & $\begin{array}{l}\text { MWvis } \\
\text { (ft TOC) } \\
\end{array}$ & $\begin{array}{l}\text { MWOS } \\
\text { (ft TOC) }\end{array}$ & $\begin{array}{l}\text { MWvor } \\
\text { (ft TOC) }\end{array}$ & $\begin{array}{l}\text { Mwor } \\
\text { (ft ToC) }\end{array}$ & $\begin{array}{l}\text { Mw09 } \\
\text { (ft TOC) }\end{array}$ & $\begin{array}{l}\text { MW10 } \\
\text { (ft TOC) }\end{array}$ & $\begin{array}{l}\text { MWII } \\
\text { (ft TOC) }\end{array}$ & $\begin{array}{l}\text { MWII } \\
(\mathrm{ft} \text { TOC) }\end{array}$ & $\begin{array}{l}\text { MWVI3 } \\
(\mathrm{ft} \text { Toc) }\end{array}$ & $\begin{array}{l}\text { MW1 } \mathrm{ft} \text { TOC) } \\
\end{array}$ & $\begin{array}{l}\text { MWvitss } \\
\text { (ft TOC) }\end{array}$ & $\begin{array}{l}\text { MWW TOC) } \\
\text { (ft TOC) }\end{array}$ & $\begin{array}{cc}\text { (ft TOC) } & \text { (ft TOC) } \\
\end{array}$ & $\begin{array}{l}\text { MWI6M2 } \\
\text { (ft TOC) }\end{array}$ & $\begin{array}{l}\text { MWvirs } \\
\text { (ft TOC) }\end{array}$ & (ft TOC) & $\begin{array}{l}\text { MWVI } 185 \\
\text { (ft TOC) } \\
\end{array}$ & $\begin{array}{l}\text { MWWIMM } \\
\text { (ft TOC) }\end{array}$ & $\begin{array}{l}\text { MWV195 } \\
\text { (ft TOC) }\end{array}$ & $\begin{array}{l}\text { MWW } \\
\text { (ft TOC) }\end{array}$ & $\begin{array}{l}\text { Mwwes } \\
\text { (ft Toc) }\end{array}$ & (ft TOC) \\
\hline 2/19/12 16:00 & & 30.203 & 29.37 & 29.12 & 29.739 & & 29.465 & 30.308 & 29.807 & 29.482 & 28.286 & 26.04 & 31.312 & 36.743 & 32.338 & $3 \quad 15.977$ & 19.44 & 15.634 & 10.176 & 31.471 & 32.206 & 29.365 & 31.274 & 28.058 & 33.483 \\
\hline 2/19/12 17:00 & & 30.205 & 29.351 & 29.117 & 29.732 & & 29.448 & 30.306 & 29.812 & 29.475 & 28.284 & 26.043 & 31.305 & 36.738 & 32.328 & 3 15.975 & 19.429 & 15.62 & 10.164 & 31.466 & $\begin{array}{ll}6 & 32.199\end{array}$ & 29.358 & 31.265 & $5 \quad 28.058$ & 33.483 \\
\hline 2/19/12 18:00 & & 30.205 & 29.33 & 29.113 & 29.725 & & 29.441 & 30.303 & 29.812 & 29.468 & 28.274 & 26.043 & 31.335 & 36.74 & 32.375 & $5 \quad 15.97$ & 19.417 & 15.625 & 10.202 & 31.464 & $\begin{array}{l}4 \quad 32.225 \\
\end{array}$ & 29.355 & 31.305 & $5 \quad 28.061$ & 33.481 \\
\hline 2/19/12 19:00 & & 30.207 & 29.291 & 29.115 & 29.723 & & 29.42 & 30.298 & 29.814 & 29.463 & 28.272 & 26.043 & 31.695 & 36.733 & 32.753 & 15.966 & 19.41 & 15.611 & 10.583 & 31.595 & $5 \quad 32.587$ & 29.369 & 31.647 & 28.061 & 33.483 \\
\hline 2/19/12 20:00 & & 30.21 & 29.265 & 29.124 & 29.73 & & 29.401 & 30.296 & 29.819 & 29.461 & 28.272 & 26.055 & 31.651 & 36.733 & 32.692 & 15.963 & 19.401 & 15.599 & 10.531 & 31.627 & $7 \quad 32.544$ & 29.388 & 31.6 & $\quad 28.061$ & 33.485 \\
\hline 2/19/12 21:00 & & 30.207 & 29.258 & 29.127 & 29.744 & & 29.392 & 30.294 & 29.819 & 29.461 & 28.272 & 26.064 & 31.546 & 36.733 & 32.581 & 15.961 & 19.394 & 15.597 & 10.416 & 31.588 & $8 \quad 32.436$ & 29.395 & 31.5 & $\quad 28.058$ & 33.488 \\
\hline 2/19/12 22:00 & & 30.205 & 29.249 & 29.122 & 29.746 & & 29.385 & 30.291 & 29.814 & 29.458 & 28.267 & 26.064 & 31.492 & 36.731 & 32.523 & 15.959 & 19.387 & 15.597 & 10.36 & 31.569 & $\begin{array}{l}9 \\
92.386\end{array}$ & 29.388 & 31.45 & $\quad 28.061$ & 33.49 \\
\hline 2/19/12 23:00 & & 30.207 & 29.223 & 29.122 & 29.748 & & 29.375 & 30.288 & 29.814 & 29.452 & 28.267 & 26.062 & 31.457 & 36.733 & 32.488 & 3 15.956 & 19.38 & 15.583 & 10.324 & 31.548 & $8 \quad 32.348$ & 29.395 & 31.41 & 28.061 & 33.49 \\
\hline $2 / 20 / 120: 00$ & & 30.203 & 29.214 & 29.113 & 29.746 & & 29.378 & 30.284 & 29.807 & 29.445 & 28.263 & 26.052 & 31.429 & 36.726 & 32.457 & 15.954 & 19.376 & 15.583 & 10.299 & 31.541 & $1 \quad 32.322$ & 29.386 & 31.389 & 28.058 & 33.49 \\
\hline 2/20/12 1:00 & & 30.207 & 29.198 & 29.12 & 29.751 & & 29.363 & 30.284 & 29.809 & 29.447 & 28.263 & 26.06 & 31.412 & 36.726 & 32.441 & 15.952 & 19.371 & 15.573 & 10.277 & 31.539 & $\begin{array}{l}9 \\
92.305\end{array}$ & 29.391 & 31.368 & $\begin{array}{l}3.063 \\
3\end{array}$ & 33.488 \\
\hline $2 / 20 / 122: 00$ & & 30.21 & 29.184 & 29.124 & 29.753 & & 29.354 & 30.284 & 29.814 & 29.449 & 28.265 & 26.074 & 31.398 & 36.729 & 32.427 & 15.952 & 19.364 & 15.566 & 10.263 & 31.534 & $4 \quad 32.291$ & 29.395 & 31.356 & $5 \quad 28.063$ & 33.488 \\
\hline $2 / 20 / 123: 00$ & & 30.203 & 29.205 & 29.103 & 29.746 & & 29.368 & 30.279 & 29.802 & 29.44 & 28.253 & 26.059 & 31.379 & 36.724 & 32.403 & 15.947 & 19.357 & 15.576 & 10.244 & 31.508 & 32.272 & 29.372 & 31.345 & $\quad 28.058$ & 33.483 \\
\hline $2 / 20 / 124: 00$ & & 30.196 & 29.228 & 29.073 & 29.72 & & 29.385 & 30.272 & 29.788 & 29.424 & 28.237 & 26.033 & 31.356 & 36.722 & 32.384 & 15.945 & 19.346 & 15.58 & 10.223 & 31.487 & 32.248 & 29.346 & 31.324 & 28.058 & 33.476 \\
\hline $2 / 20 / 125: 00$ & & 30.191 & 29.237 & 29.052 & 29.704 & & 29.389 & 30.265 & 29.786 & 29.405 & 28.221 & 26.005 & 31.335 & 36.719 & 32.366 & 15.938 & 19.334 & 15.578 & 10.202 & 31.464 & 32.227 & 29.327 & 31.3 & 28.058 & 33.469 \\
\hline $2 / 20 / 126: 00$ & & 30.189 & 29.242 & 29.038 & 29.688 & & 29.387 & 30.262 & 29.788 & 29.393 & 28.212 & 26 & 31.321 & 36.712 & 32.347 & 15.933 & 19.341 & 15.571 & 10.183 & 31.45 & 32.215 & 29.311 & 31.286 & $\quad 28.061$ & 33.464 \\
\hline $2 / 20 / 127: 00$ & & 30.184 & 29.254 & 29.024 & 29.671 & & 29.385 & 30.26 & 29.778 & 29.382 & 28.202 & 25.985 & 31.307 & 36.71 & 32.335 & 15.92 & 19.334 & 15.566 & 10.172 & 31.434 & 32.199 & 29.297 & 31.27 & 28.058 & 33.464 \\
\hline $2 / 20 / 128: 00$ & & 30.191 & 29.244 & 29.042 & 29.676 & & 29.361 & 30.258 & 29.79 & 29.387 & 28.207 & 26.007 & 31.302 & 36.705 & 32.328 & 15.92 & 19.329 & 15.555 & 10.164 & 31.443 & 32.196 & 29.315 & 31.258 & $\begin{array}{l}3 \\
3\end{array}$ & 33.464 \\
\hline $2 / 20 / 129: 00$ & & 30.191 & 29.244 & 29.052 & 29.676 & & 29.342 & 30.255 & 29.8 & 29.384 & 28.207 & 26.014 & 31.293 & 36.705 & 32.321 & 15.924 & 19.327 & 15.536 & 10.162 & 31.443 & 32.189 & 29.318 & 31.253 & 8.063 & 33.464 \\
\hline 2/20/12 10:00 & & 30.189 & 29.247 & 29.052 & 29.676 & & 29.34 & 30.253 & 29.79 & 29.384 & 28.205 & 26.024 & 31.293 & 36.705 & 32.316 & 15.924 & 19.323 & 15.543 & 10.158 & 31.441 & 32.184 & 29.322 & 31.267 & 28.058 & 33.467 \\
\hline 2/20/12 11:00 & & 30.182 & 29.263 & 29.024 & 29.664 & & 29.352 & 30.248 & 29.778 & 29.368 & 28.191 & 26 & 31.284 & 36.705 & 32.307 & 15.917 & 19.313 & 15.552 & 10.146 & 31.424 & 32.177 & 29.297 & 31.255 & 28.061 & 33.464 \\
\hline 2/20/12 12:00 & & 30.179 & 29.284 & & 29.646 & & 29.356 & 30.248 & 29.771 & 29.347 & 28.177 & 25.964 & 31.269 & 36.691 & 32.298 & $3 \quad 15.917$ & 19.341 & 15.545 & 10.134 & 31.408 & $3 \quad 32.163$ & 29.278 & 31.23 & $\quad 28.065$ & 33.462 \\
\hline 2/20/12 13:00 & & 30.175 & 29.319 & 28.979 & 29.629 & & 29.363 & 30.243 & 29.764 & 29.333 & 28.16 & 25.961 & 31.26 & 36.696 & 32.284 & 15.912 & 19.339 & 15.552 & 10.122 & 31.387 & 32.151 & 29.254 & 31.237 & 28.063 & 33.46 \\
\hline $2 / 20 / 1214: 00$ & & 30.161 & 29.363 & 28.933 & 29.594 & & 29.385 & 30.234 & 29.748 & 29.308 & 28.13 & 25.923 & 31.237 & 36.689 & 32.265 & 15.903 & 19.323 & 15.566 & 10.103 & 31.354 & 32.132 & 29.219 & 31.218 & $3 \quad 28.061$ & 33.455 \\
\hline $2 / 20 / 1215: 00$ & & 30.149 & 29.393 & 28.907 & 29.566 & & 29.389 & 30.229 & 29.738 & 29.284 & 28.112 & 25.904 & 31.234 & 36.679 & 32.262 & 15.898 & 19.306 & 15.568 & 10.096 & 31.333 & 32.125 & 29.191 & 31.211 & 28.054 & 33.45 \\
\hline $2 / 20 / 1216: 00$ & & 30.163 & 29.344 & 28.949 & 29.578 & & 29.335 & 30.229 & 29.764 & 29.291 & 28.125 & 25.94 & 31.22 & 36.679 & 32.246 & 15.893 & 19.346 & 15.531 & 10.084 & 31.352 & 32.116 & 29.221 & 31.178 & $3 \quad 28.061$ & 33.446 \\
\hline $2 / 20 / 1217: 00$ & & 30.161 & 29.323 & 28.944 & 29.571 & & 29.326 & 30.227 & 29.759 & 29.284 & 28.119 & 25.935 & 31.211 & 36.67 & 32.234 & 15.891 & 19.332 & 15.522 & 10.068 & 31.345 & 32.104 & 29.212 & 31.173 & 28.063 & 33.443 \\
\hline $2 / 20 / 1218: 00$ & & 30.165 & 29.286 & 28.979 & 29.587 & & 29.286 & 30.224 & 29.776 & 29.291 & 28.13 & 25.966 & 31.209 & 36.675 & 32.232 & 15.886 & 19.325 & 15.5 & 10.071 & 31.361 & 32.101 & 29.245 & 31.166 & 28.065 & 33.443 \\
\hline $2 / 20 / 1219: 00$ & & 30.172 & 29.24 & 29.019 & 29.615 & & 29.246 & 30.224 & 29.795 & 29.305 & 28.146 & 26.012 & 31.213 & 36.679 & 32.237 & 15.886 & 19.32 & 15.477 & 10.075 & 31.385 & 32.106 & 29.283 & 31.176 & $\quad 28.065$ & 33.446 \\
\hline 2/20/12 20:00 & & 30.179 & 29.2 & 29.068 & 29.65 & & 29.211 & 30.229 & 29.807 & 29.328 & 28.172 & 26.059 & 31.225 & 36.686 & 32.248 & $\begin{array}{l}315.889 \\
3\end{array}$ & 19.311 & 15.463 & 10.087 & 31.415 & 32.12 & 29.32 & 31.187 & 28.065 & $\quad 33.45$ \\
\hline 2/20/12 21:00 & & 30.189 & 29.163 & 29.11 & 29.683 & & 29.185 & 30.236 & 29.814 & 29.347 & 28.195 & 26.1 & 31.239 & 36.703 & 32.26 & $\begin{array}{l}15.893 \\
5\end{array}$ & 19.304 & 15.451 & $\begin{array}{l}10.101 \\
\end{array}$ & 31.443 & 32.13 & 29.355 & 31.199 & 28.065 & 33.457 \\
\hline $2 / 20 / 1222: 00$ & & 30.2 & 29.118 & 29.159 & 29.727 & & 29.159 & 30.243 & 29.826 & 29.38 & 28.225 & 26.15 & 31.258 & 36.708 & 32.274 & 15.898 & 19.309 & 15.442 & 10.115 & 31.478 & 32.149 & 29.402 & 31.218 & $\begin{array}{l}3 \\
28.068\end{array}$ & 33.464 \\
\hline 2/20/12 23:00 & & 30.203 & 29.09 & 29.19 & 29.762 & & 29.154 & 30.251 & 29.828 & $\begin{array}{l}29.300 \\
2938\end{array}$ & 28.246 & 26.174 & 31.274 & 36.722 & 32.293 & $\begin{array}{l}15.905 \\
3\end{array}$ & 19.309 & 15.447 & 10.129 & 31.506 & 32.165 & 29.431 & 31.232 & $\begin{array}{l}28.068 \\
2806\end{array}$ & 33.471 \\
\hline $2 / 21 / 120: 00$ & & 30.207 & 29.058 & 29.206 & 29.793 & & 29.157 & 30.262 & 29.821 & 29.417 & 28.263 & 26.191 & 31.284 & 36.736 & 32.305 & 15.91 & 19.309 & 15.449 & 10.139 & 31.52 & 32.177 & 29.449 & 31.241 & 28.065 & 33.476 \\
\hline 2/21/12 1:00 & & 30.221 & 29.016 & 29.248 & 29.83 & & 29.145 & 30.27 & 29.836 & 29.447 & 28.293 & 26.227 & 31.3 & 36.745 & 32.321 & 15.921 & 19.306 & 15.44 & 10.157 & 31.555 & 32.196 & 29.494 & 31.26 & $\quad 28.068$ & 33.481 \\
\hline $2 / 21 / 122: 00$ & & 30.228 & 28.988 & 29.276 & 29 & & 29 & 30.279 & 29.838 & 29.47 & 28.314 & 26.251 & 31.314 & 36.754 & 32.335 & 15.9 & 19.306 & 15.437 & 10.172 & 31.574 & 32.208 & 29.52 & 31.272 & 28.07 & 33.485 \\
\hline 2/21/12 3:00 & & 30.221 & 28.995 & 29.253 & 29.8 & & 29.189 & 30.282 & 29.816 & 29.47 & 28.314 & 26.222 & 31.316 & 36.759 & 32.335 & 15.9 & 19.299 & 15.463 & 10.169 & 31.564 & 32.21 & 29.501 & 1.272 & 8.068 & 33.481 \\
\hline 2/21/12 4:00 & & 30.231 & 28.988 & 29.26 & 29.872 & & 29.199 & 30.289 & 29.819 & 29.482 & 28.323 & 26.225 & 31.321 & 36.761 & 32.34 & 15.942 & 19.292 & 15.461 & 10.174 & 31.571 & 32.215 & 29.51 & 1.279 & 28.072 & 33.478 \\
\hline $2 / 21 / 125: 00$ & & 30.231 & 28.995 & 29.257 & 29.874 & & 29.22 & 30.294 & 29.814 & 29.491 & 28.328 & 26.225 & 31.323 & 36.764 & 32.34 & 15.947 & 19.288 & 15.472 & 10.176 & 31.569 & 32.213 & 29.506 & 31.284 & 28.072 & 33.478 \\
\hline 2/21/12 6:00 & & 30.231 & 28.999 & 29.26 & 29.8 & & 29.234 & 30.298 & 29.814 & 29.5 & 28.335 & 26.222 & 31.328 & 36.771 & 32.347 & 15.954 & 19.295 & 15.477 & 10.185 & 31.578 & $3 \quad 32.217$ & 29.508 & 31.286 & $\quad 28.068$ & 33.476 \\
\hline 2/21/12 7:00 & & 30.228 & 29.018 & 29.243 & 29. & & 29.26 & 30.298 & 29.805 & 29.5 & 28.335 & 26.203 & 31.326 & 36.769 & 32.345 & 15.956 & 19.299 & 15.489 & 10.181 & 31.562 & 32.22 & 29.496 & 31.286 & 28.07 & 33.476 \\
\hline 2/21/12 8:00 & & 30.235 & 29.006 & 29.262 & 29 . & & 29.258 & 30.303 & 29.814 & 29.512 & 28.344 & 26.217 & 31.335 & 36.771 & 32.349 & 15.963 & 19.295 & 15.489 & 10.188 & 31.574 & t 32.227 & 29.513 & 31.293 & 28.07 & 33.478 \\
\hline 2/21/12 9:00 & & 30.238 & 29.039 & 29.264 & 29. & & 29.267 & 30.306 & 29.816 & 29.519 & 28.351 & 26.21 & 31.337 & 36.773 & 32.356 & 15.97 & 19.288 & 15.494 & 10.195 & 31.569 & 32.229 & 29.515 & 31.298 & $\begin{array}{l}3 \quad 28.072 \\
\end{array}$ & 33.481 \\
\hline $2 / 21 / 1210: 00$ & & 30.24 & 29.079 & 29.269 & 29. & & 29.279 & 30.313 & 29.814 & 29.526 & 28.356 & 26.215 & 31.342 & 36.773 & 32.361 & 15.973 & 19.286 & 15.505 & 10.195 & 31.583 & 32.234 & 29.52 & 31.305 & $\quad 28.075$ & 33.485 \\
\hline $2 / 21 / 1211: 00$ & & 30.24 & 29.132 & 29.267 & 29.8 & & 29.291 & 30.315 & 29.814 & 29.528 & 28.358 & 26.205 & 31.347 & 36.776 & 32.366 & 15.977 & 19.281 & 15.515 & 10.202 & 31.583 & 32.241 & 29.515 & 31.309 & $\quad 28.072$ & 33.485 \\
\hline $2 / 21 / 1212: 00$ & & 30.235 & 29.186 & 29.25 & 29.879 & & 29.312 & 30.315 & 29.805 & 29.528 & 28.356 & 26.191 & 31.347 & 36.776 & 32.363 & 315.979 & 19.276 & 15.531 & 10.204 & 31.576 & 32.236 & 29.503 & 31.314 & 28.075 & 33.49 \\
\hline $2 / 21 / 1213: 00$ & & 30.226 & 29.268 & 29.206 & 29.853 & & 29.352 & 30.313 & 29.788 & 29.512 & 28.332 & 26.148 & 31.337 & 36.769 & 32.359 & 15.979 & 19.269 & 15.557 & 10.195 & 31.548 & 32.232 & 29.468 & 31.298 & $\begin{array}{l}38.072 \\
\end{array}$ & 33.488 \\
\hline $2 / 21 / 1214: 00$ & & 30.219 & 29.344 & 29.164 & 29.823 & & 29.382 & 30.303 & 29.776 & 29.496 & 28.314 & 26.105 & 31.326 & 36.757 & 32.345 & 15.9 & 19.262 & 15.576 & 10.181 & 31.52 & 32.22 & 29.428 & 31.284 & $\begin{array}{r}28.072 \\
7\end{array}$ & 33.483 \\
\hline $2 / 21 / 1215: 00$ & & 30.219 & 29.393 & 29.136 & 29.795 & & 29.392 & 30.296 & 29.776 & 29.482 & 28.3 & 26.074 & 31.314 & 36.747 & 32.333 & 15.9 & 19.251 & 15.573 & $\begin{array}{l}10.172 \\
\text { L }\end{array}$ & 31.499 & 32.206 & 29.409 & 31.274 & $\begin{array}{l}28.075 \\
7\end{array}$ & 33.478 \\
\hline $2 / 21 / 1216: 00$ & & 30.219 & 29.41 & 29.138 & 29.783 & & 29.38 & 30.291 & 29.783 & 29.477 & 28.298 & 26.079 & 31.305 & 36.743 & 32.326 & 15.9 & 19.246 & 15.566 & 10.162 & 31.497 & 32.199 & 29.407 & 31.265 & $5 \quad 28.075$ & 33.476 \\
\hline $2 / 21 / 1217: 00$ & & 30.224 & 29.403 & 29.145 & 29.779 & & 29.363 & 30.286 & 29.79 & 29.477 & 28.295 & 26.083 & 31.3 & 36.74 & 32.321 & 15.975 & 19.244 & 15.559 & 10.16 & 31.499 & 32.191 & 29.409 & 31.263 & $\begin{array}{l}3 \\
28.077\end{array}$ & 33.474 \\
\hline $2 / 21 / 1218: 00$ & & 30.224 & 29.382 & 29.148 & 29.776 & & 29.352 & 30.286 & 29.79 & 29.477 & 28.298 & 26.086 & 31.3 & 36.736 & 32.319 & 15.975 & 19.242 & 15.554 & 10.155 & 31.501 & 32.189 & 29.412 & 31.258 & $\begin{array}{l}3 \\
28.075\end{array}$ & $\quad 33.471$ \\
\hline $2 / 21 / 1$ & & 30.224 & 29.342 & 29.155 & 29.77 & & 29.335 & 30.284 & 29.795 & 29.479 & 28.298 & 26.095 & 31.298 & 36.743 & 32.316 & $5 \quad 15.973$ & 19.242 & 15.555 & 10.155 & 31.499 & 32.191 & 29.409 & 31.258 & $\begin{array}{l}3 \quad 28.075 \\
\end{array}$ & 33.469 \\
\hline $2 / 2$ & & 30.2 & 29.31 & 29.15 & 29.7 & & 29.3 & $x_{-1}+2$ & 29.788 & $x_{1}+2$ & 28.2 & 26.088 & 3120 & 36.7 & 32.316 & 15 & & 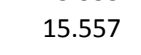 & 10.153 & 31.494 & 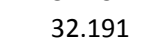 & o & 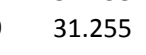 & 75 & 33.469 \\
\hline & & 30. & 29.2 & 29.1 & 29 & & & 30. & & & 28.2 & 26. & & 36. & 32.312 & & & & 10. & & & & 3 & & \\
\hline & & & 29.284 & & & & & & & & 28 & & & 36.7 & 32.309 & & & & & $31.4>2>$ & & & & 5 & \\
\hline 2 23:00 & & 30.212 & 29.268 & 29.096 & 29.739 & & 29.342 & 30.265 & 29.776 & 29.447 & 28.263 & 26.038 & 31.279 & 36.729 & 32.3 & 15.961 & 19.272 & 15.559 & 10.139 & 31.457 & 32.175 & 29.362 & 31.241 & 28.075 & 3.471 \\
\hline
\end{tabular}




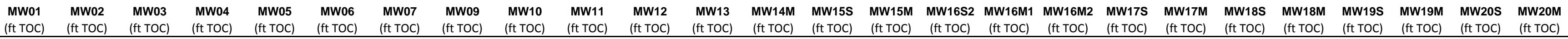

\begin{tabular}{|c|c|c|c|c|c|c|c|c|c|c|c|c|c|c|c|c|c|c|c|c|c|c|c|c|c|}
\hline Well & $\begin{array}{l}\text { (ft TOC) } \\
\text { (ft }\end{array}$ & $\begin{array}{l}\text { (ft TOC) } \\
\text { (f) }\end{array}$ & $\begin{array}{l}\text { (ft TOC) } \\
\text { (f) }\end{array}$ & $\begin{array}{l}\text { (ft TOC) } \\
\text { (f }\end{array}$ & $\begin{array}{l}\text { (ft TOC) } \\
\text { (f) }\end{array}$ & $\begin{array}{l}\text { (ft TOC) } \\
\text { (f) }\end{array}$ & $\begin{array}{l}\text { (ft TOC) } \\
\text { (ft }\end{array}$ & $\begin{array}{l}\text { (ft TOC) } \\
\text { (f) }\end{array}$ & $\begin{array}{l}\text { (ft TOC) } \\
\text { (ft) }\end{array}$ & $\begin{array}{l}\text { (ft TOC) } \\
\text { (f) }\end{array}$ & $\begin{array}{l}\text { (ft TOC) } \\
\text { (f) }\end{array}$ & $\begin{array}{l}\text { (ft TOC) } \\
\text { (f) }\end{array}$ & (ft TOC) & $\begin{array}{l}\text { (ft TOC) } \\
\end{array}$ & (ft TOC) & $\begin{array}{ll}\text { (ft TOC) } \\
\text { (ft TOC) }\end{array}$ & (ft TOC) & $\begin{array}{l}\text { (ft TOC) } \\
\text { (1) }\end{array}$ & (ft TOC) & $\begin{array}{l}\text { (ft TOC) } \\
\end{array}$ & $\begin{array}{l}\text { (ft TOC) } \\
\text { (f) }\end{array}$ & $\begin{array}{l}\text { (ft TOC) } \\
\text { (n) }\end{array}$ & (ft TOC) & $\begin{array}{l}\text { (ft TOC) } \\
\text { (1) }\end{array}$ & (ft TOC) \\
\hline :0:00 & & 30.21 & 29.258 & 29.082 & 29.725 & & 29.345 & 30.262 & 29.769 & 29.4 & 28.2 & $26.02 \varepsilon$ & 31.274 & 36.715 & 32.2 & 15.9 & 19.2 & 15.5 & 10.134 & 31. & 32.168 & 18 & 31.237 & 8.075 & 33.471 \\
\hline 2/22/12 1:00 & & 30.207 & 29.249 & 29.073 & 29.713 & & 29.338 & 30.258 & 29.771 & 29.431 & 28.249 & 26.024 & 31.269 & 36.7 & 32.293 & 15.954 & 19.26 & 15.552 & 10.131 & 31.445 & 32.163 & 29.337 & 1.23 & 28.075 & 33.471 \\
\hline 2/22/12 2:00 & & 30.207 & 29.24 & 29.068 & 29.706 & & 29.328 & 30.253 & 29.769 & 29.424 & 28.242 & 26.016 & 31.26 & 36.71 & 32.286 & 15.949 & 19.251 & 15.543 & 10.122 & 31.434 & 32.154 & 29.339 & 31.225 & 8.075 & 33.469 \\
\hline 2/22/12 3:00 & & 30.205 & 29.235 & 29.064 & 29.697 & & 29.323 & 30.251 & 29.767 & 29.417 & 28.237 & 26.014 & 31.258 & 36.705 & 32.277 & 15.947 & 19.242 & 15.538 & 10.113 & 31.431 & 32.149 & 29.33 & 31.216 & 28.075 & 33.464 \\
\hline 2/22/12 4:00 & & 30.198 & 29.247 & 29.04 & 29.683 & & 29.328 & 30.243 & 29.76 & 29.403 & 28.223 & 25.995 & 31.246 & 36.708 & 32.265 & 15.94 & 19.237 & 15.543 & 10.106 & 31.41 & 32.137 & 29.304 & 31.204 & 28.075 & 33.462 \\
\hline 2/22/12 5:00 & & 30.198 & 29.249 & 29.035 & 29.669 & & 29.321 & 30.239 & 29.757 & 29.396 & 28.214 & 25.988 & 31.234 & 36.698 & 32.255 & 15.938 & 19.225 & 15.529 & 10.096 & 31.403 & 32.125 & 29.306 & 31.197 & 28.075 & 33.455 \\
\hline 2/22/12 6:00 & & 30.198 & 29.251 & 29.035 & 29.664 & & 29.307 & 30.236 & 29.767 & 29.393 & 28.214 & 25.995 & 31.23 & 36.694 & 32.251 & 15.931 & 19.232 & 15.524 & 10.091 & 31.401 & 32.123 & 29.301 & 31.192 & 28.075 & 33.453 \\
\hline 2/22/12 7:00 & & 30.193 & 29.258 & 29.026 & 29.66 & & 29.307 & 30.231 & 29.759 & 29.382 & 28.207 & 25.985 & 31.223 & 36.696 & 32.246 & 5 $\quad 15.926$ & 19.23 & 15.519 & 10.082 & 31.392 & 32.118 & 29.285 & 31.187 & 28.072 & 33.453 \\
\hline 2/22/12 8:00 & & 30.193 & 29.261 & 29.031 & 29.657 & & 29.293 & 30.227 & 29.762 & 29.382 & 28.205 & 25.99 & 31.218 & 36.691 & 32.244 & 15.921 & 19.225 & 15.51 & 10.075 & 31.396 & 32.116 & 29.297 & 31.183 & 28.077 & 33.45 \\
\hline 2/22/12 9:00 & & 30.191 & 29.291 & 29.024 & 29.653 & & 29.293 & 30.224 & 29.757 & 29.375 & 28.198 & 25.985 & 31.216 & 36.694 & 32.239 & 15.919 & 19.218 & 15.512 & 10.075 & 31.389 & 32.111 & 29.285 & 31.178 & 28.075 & 33.45 \\
\hline $2 / 22 / 1210: 00$ & & 30.191 & 29.33 & 29.021 & 29.648 & & 29.286 & 30.222 & 29.759 & 29.37 & 28.195 & 25.988 & 31.213 & 36.691 & 32.237 & 15.914 & 19.214 & 15.505 & 10.073 & 31.387 & 32.104 & 29.292 & 31.176 & 28.077 & 33.453 \\
\hline $2 / 22 / 1211: 00$ & & 30.189 & 29.382 & 29.024 & 29.648 & & 29.279 & 30.222 & 29.755 & 29.368 & 28.193 & 25.997 & 31.216 & 36.691 & 32.239 & 15.912 & 19.207 & 15.508 & 10.073 & 31.387 & 32.109 & 29.292 & 31.176 & 28.077 & 33.455 \\
\hline $2 / 22 / 1212: 00$ & & 30.184 & 29.449 & 29.007 & 29.641 & & 29.288 & 30.219 & 29.752 & 29.359 & 28.184 & 25.981 & 31.211 & 36.686 & 32.232 & 15.91 & 19.2 & 15.515 & 10.068 & 31.38 & 32.101 & 29.278 & 31.173 & 28.077 & 33.457 \\
\hline $2 / 22 / 1213: 00$ & & 30.175 & 29.51 & 28.989 & 29.627 & & 29.298 & 30.215 & 29.741 & 29.345 & 28.17 & 25.964 & 31.204 & 36.682 & 32.227 & 15.905 & 19.193 & 15.524 & 10.061 & 31.361 & 32.097 & 29.264 & 31.169 & 28.075 & 33.455 \\
\hline 2/22/12 14:00 & & 30.175 & 29.564 & 28.963 & 29.606 & & 29.305 & 30.21 & 29.731 & 29.326 & 28.156 & 25.937 & 31.192 & 36.672 & 32.216 & $5 \quad 15.9$ & 19.184 & 15.529 & 10.056 & 31.347 & 32.087 & 29.243 & 31.157 & 28.077 & 33.455 \\
\hline $2 / 22 / 1215: 00$ & & 30.168 & 29.596 & 28.951 & 29.597 & & 29.302 & 30.207 & 29.736 & 29.312 & 28.144 & 25.928 & 31.183 & 36.67 & 32.204 & 15.896 & 19.175 & 15.529 & 10.042 & 31.336 & 32.075 & 29.228 & 31.148 & 28.077 & 33.45 \\
\hline $2 / 22 / 1216: 00$ & & 30.168 & 29.606 & 28.947 & 29.583 & & 29.293 & 30.205 & 29.733 & 29.303 & 28.135 & 25.925 & 31.174 & 36.672 & 32.195 & 15.891 & 19.223 & 15.524 & 10.033 & 31.329 & 32.066 & 29.217 & 31.136 & 28.077 & 33.443 \\
\hline $2 / 22 / 1217: 00$ & & 30.165 & 29.594 & 28.949 & 29.58 & & 29.279 & 30.203 & 29.733 & 29.301 & 28.135 & 25.93 & 31.167 & 36.67 & 32.19 & 15.886 & 19.228 & 15.522 & 10.026 & 31.322 & 32.061 & 29.224 & 31.129 & 28.077 & 33.443 \\
\hline $2 / 22 / 1218: 00$ & & 30.161 & 29.571 & 28.937 & 29.573 & & 29.272 & 30.198 & 29.731 & 29.289 & 28.123 & 25.921 & 31.162 & 36.663 & 32.183 & 15.882 & 19.223 & 15.519 & 10.016 & 31.317 & 32.056 & 29.212 & 31.119 & 28.075 & 33.439 \\
\hline $2 / 22 / 12$ 19:00 & & 30.158 & 29.529 & 28.937 & 29.566 & & 29.26 & 30.195 & 29.729 & 29.282 & 28.119 & 25.921 & 31.157 & 36.663 & 32.173 & 15.877 & 19.218 & 15.51 & 10.014 & 31.303 & 32.047 & 29.205 & 31.115 & 28.077 & 33.434 \\
\hline $2 / 22 / 1220: 00$ & & 30.154 & 29.491 & 28.933 & 29.562 & & 29.246 & 30.191 & 29.731 & 29.277 & 28.114 & 25.925 & 31.15 & 36.656 & 32.171 & 15.875 & 19.214 & 15.503 & 10.007 & 31.315 & 32.042 & 29.203 & 31.11 & 28.077 & 33.434 \\
\hline $2 / 22 / 1221: 00$ & & 30.144 & 29.473 & 28.907 & 29.543 & & 29.255 & 30.188 & 29.714 & 29.261 & 28.095 & 25.904 & 31.143 & 36.654 & 32.164 & 15.87 & 19.207 & 15.515 & 10 & 31.291 & .037 & 9.179 & 31.103 & 28.075 & 33.432 \\
\hline 2/22/12 22:00 & & 30.142 & 29.452 & 28.89 & 29.529 & & 29.251 & 30.183 & 29.712 & 29.243 & 28.086 & 25.887 & 31.134 & 36.656 & 32.157 & 15.865 & 19.202 & 15.498 & 9.993 & 31.282 & 32.028 & 29.163 & 31.094 & 28.075 & 33.432 \\
\hline 2/22/12 23:00 & & 30.133 & 29.433 & 28.862 & 29.51 & & 29.255 & 30.178 & 29.703 & 29.224 & 28.067 & 25.866 & 31.127 & 36.649 & 32.15 & $\quad 15.858$ & 19.193 & 15.505 & 9.988 & 31.263 & 32.016 & 29.144 & 31.087 & 28.075 & 33.429 \\
\hline 2/23/12 0:00 & & 30.126 & 29.424 & 28.837 & 29.485 & & 29.255 & 30.174 & 29.695 & 29.199 & 28.049 & 25.837 & 31.113 & 36.644 & 32.136 & $5 \quad 15.851$ & 19.186 & 15.493 & 9.974 & 31.238 & 32.007 & 29.123 & 31.072 & 28.075 & 33.427 \\
\hline 2/23/12 1:00 & & 30.135 & 29.393 & 28.858 & 29.485 & & 29.22 & 30.169 & 29.712 & 29.201 & 28.049 & 25.858 & 31.108 & 36.637 & 32.129 & 15.849 & 19.179 & 15.463 & 9.967 & 31.252 & 32.002 & 29.137 & 1.072 & 28.077 & 33.429 \\
\hline 2/23/12 2:00 & & 30.124 & 29.393 & 28.846 & 29.48 & & 29.218 & 30.167 & 29.705 & 29.189 & 28.039 & 25.856 & 31.103 & 36.635 & 32.124 & 15.844 & 19.172 & 15.475 & 9.957 & 31.24 & 31.995 & 29.125 & 31.063 & 28.072 & 33.427 \\
\hline 2/23/12 3:00 & & 30.121 & 29.37 & 28.848 & 29.478 & & 29.204 & 30.162 & 29.707 & 29.189 & 28.037 & 25.868 & 31.096 & 36.635 & 32.119 & 15.842 & 19.165 & 15.463 & 9.955 & 31.238 & 31.99 & 29.125 & 31.058 & 28.075 & 33.425 \\
\hline 2/23/12 4:00 & & 30.131 & 29.33 & 28.886 & 29.496 & & 29.159 & 30.16 & 29.724 & 29.199 & 28.049 & 25.897 & 31.099 & 36.633 & 32.117 & 15.84 & 19.161 & 15.433 & 9.957 & 31.263 & 31.992 & 29.149 & 31.056 & 28.075 & 33.422 \\
\hline 2/23/12 5:00 & & 30.119 & 29.335 & 28.853 & 29.48 & & 29.173 & 30.157 & 29.705 & 29.18 & 28.032 & 25.868 & 31.085 & 36.626 & 32.105 & 15.833 & 19.149 & 15.444 & 9.943 & 31.231 & 31.978 & 29.127 & 31.047 & 28.075 & 33.418 \\
\hline $2 / 23 / 126: 00$ & & 30.121 & 29.3 & 28.867 & 29.487 & & 29.152 & 30.152 & 29.712 & 29.18 & 28.037 & 25.882 & 31.082 & 36.626 & 32.105 & $\quad 15.83$ & 19.147 & 15.423 & 9.941 & 31.242 & 31.976 & 29.137 & 31.044 & 28.075 & 33.415 \\
\hline 2/23/12 7:00 & & 30.131 & 29.261 & 28.909 & 29.508 & & 29.114 & 30.152 & 29.736 & 29.194 & 28.056 & 25.918 & 31.087 & 36.63 & 32.108 & $3 \quad 15.833$ & 19.147 & 15.397 & 9.941 & 31.266 & 31.978 & 29.17 & 31.047 & 28.079 & 33.415 \\
\hline $2 / 23 / 128: 00$ & & 30.144 & 29.223 & 28.989 & 29.562 & & 29.055 & 30.16 & 29.769 & 29.233 & 28.091 & 26.009 & 31.106 & 36.649 & 32.124 & 15.835 & 19.175 & 15.376 & 9.965 & 31.317 & 32 & 29.236 & 31.065 & 28.077 & 33.42 \\
\hline 2/23/12 9:00 & & 30.151 & 29.223 & 29.031 & 29.601 & & 29.039 & 30.164 & 29.774 & 29.252 & 28.119 & 26.045 & 31.122 & 36.661 & 32.138 & $\begin{array}{l}35.84 \\
3\end{array}$ & 19.177 & 15.371 & 9.979 & 31.345 & 32.014 & 29.28 & 31.082 & 28.082 & 33.425 \\
\hline $2 / 23 / 12$ 10:00 & & 30.156 & 29.237 & 29.068 & 29.641 & & 29.025 & 30.169 & 29.778 & 29.277 & 28.144 & 26.086 & 31.141 & 36.668 & 32.157 & 15.844 & 19.2 & 15.369 & 9.995 & 31.38 & 32.03 & 29.313 & 31.101 & 28.082 & 33.434 \\
\hline 2/23/12 11:00 & & 30.168 & 29.237 & 29.105 & 29.6 & & 29.018 & 30.176 & 29.781 & 29.301 & 28.172 & 26.112 & 31.157 & 36.689 & 32.176 & 15.8 & 19.198 & 15.364 & 10.014 & 31.413 & 32.047 & 29.353 & 31.122 & 28.082 & $\begin{array}{l}33.436 \\
33.436\end{array}$ \\
\hline 2/23/12 12:00 & & 30.161 & 29.279 & 29.101 & 29.699 & & 29.046 & 30.183 & 29.764 & 29.312 & 28.177 & 26.119 & 31.171 & 36.684 & 32.187 & 15.8 & 19.193 & $\begin{array}{l}15.394 \\
15.392 \\
\end{array}$ & $\begin{array}{l}10.03 \\
10.04\end{array}$ & $\begin{array}{r}31.41 \\
31.41\end{array}$ & 32.061 & 29.36 & 31.133 & 28.079 & $\begin{array}{l}33.443 \\
33.450\end{array}$ \\
\hline 2/23/12 13:00 & & 30.161 & 29.298 & 29.117 & 29.723 & & 29.063 & 30.188 & 29.764 & 29.328 & 28.191 & 26.136 & 31.183 & $\begin{array}{l}30.694 \\
36.698\end{array}$ & 32.197 & 15.8 & 19.186 & 15.397 & 10.038 & 31.427 & 32.075 & $\begin{array}{l}29.300 \\
\end{array}$ & 31.143 & 28.079 & $\begin{array}{l}33.443 \\
33.443\end{array}$ \\
\hline bo & & 30 & 29.319 & 29.122 & 29 & & 4 & 30.193 & 29.764 & 29.34 & 28.205 & 26.129 & 31.19 & 36.703 & 32.206 & 15 & 19.181 & 15.4 & 10.047 & 31.434 & 32.082 & 34 & 31.152 & 28.079 & 33.448 \\
\hline $2 / 23 / 1$ & & 30. & 29.2 & 29 & 29 & & 29 & 30.205 & 29.786 & 29.368 & 28.237 & 26 & 6 & 12 & 32.22 & 15. & 19.181 & 15.381 & 10.063 & 31.476 & 099 & 29.435 & 31.171 & 28.082 & 33.453 \\
\hline $2 / 23 / 1216: 00$ & & 30.189 & 29.263 & 29 & 29. & & 25 & 30.21 & 29.793 & 29. & 28.26 & 26.201 & 8 & 36.722 & 32.237 & 15.8 & 1 & 15.385 & 10.073 & 31.499 & 32.113 & 466 & 31.18 & 28.079 & 33.455 \\
\hline $2 / 23 / 1217: 00$ & & 30.193 & 29.244 & 29.229 & 29 & & & 30.22 & 29.79 & 29.421 & 28.279 & 26.22 & 31.234 & 36.733 & 32.248 & 3 & 19.225 & 15.395 & 10.087 & 31.515 & 32.125 & 29.478 & 31.192 & 28.082 & 33.457 \\
\hline 2/23/12 18:00 & & 30.207 & 29.2 & 29.271 & 29.863 & & & 30.231 & 29.8 & 29.449 & 28.309 & 26.251 & 31.251 & 36.74 & 32.267 & 15 & & & 10.106 & 31.55 & & 29.522 & 31.213 & 2806 & 33.46 \\
\hline $2 / 23 / 12$ 19:00 & & 30.217 & 29.151 & 29.302 & 29.8. & & & 30.243 & 29.805 & & 28.337 & 26.268 & 31.267 & 36.754 & 32.281 & 15.9 & 19.221 & 15.393 & 10.117 & 31.574 & 32.158 & 29.55 & 25 & 28.086 & 33.462 \\
\hline $2 / 23 / 1220: 00$ & & 30.221 & 29.111 & 29.332 & 29. & & & 30.2 & 29.807 & & 28.363 & 26.301 & 31.286 & 36.759 & 32.3 & 15. & 19.218 & 15.404 & 10.143 & 31.597 & 32.182 & 29.581 & 31.246 & 28.082 & 33.464 \\
\hline $2 / 23 / 1221: 00$ & & 30.228 & $29 . c$ & 29.349 & 29. & & & 30.265 & 29.807 & & 28.379 & 26.32 & 31.307 & 36.766 & 32.319 & 15. & 19.218 & 15.418 & 10.157 & 31.616 & 32.196 & 29.597 & 31.263 & 28.086 & 33.469 \\
\hline $2 / 23 / 1222: 00$ & & 30.235 & 29.065 & 29.36 & 29 & & & 30.274 & 29.809 & & 28.398 & 26.313 & 31.321 & 36.771 & 32.333 & 15. & 19.218 & 15.423 & 10.172 & 31.632 & 32.213 & 29.616 & 31.279 & 28.086 & 33.474 \\
\hline 2/23/12 23:00 & & 30.24 & 29.0 & 29.367 & 29.9 & & $29.1>>>$ & $30.28 \mathrm{~F}$ & 29.805 & 29.5 & 28.409 & 26.318 & 31.333 & 36.776 & 32.347 & 15.9 & 19.218 & 15.437 & 10.183 & 31.639 & 32.227 & 29.623 & 31.295 & 28.084 & 33.478 \\
\hline 2/24/12 0:00 & & 30.245 & 29.044 & 29.379 & 29.9 & & 29.131 & 30.291 & 29.805 & 29.5 & 28.425 & 26.325 & 31.349 & 36.783 & 32.361 & 15.9 & 19.218 & 15.451 & 10.197 & 31.653 & 32.241 & 29.635 & 31.307 & 28.086 & 33.483 \\
\hline 2/24/12 1:00 & & 30.252 & 29.032 & 29.388 & 30.01 & & 29.147 & 30.298 & 29.807 & 29.602 & 28.437 & 26.325 & 31.358 & 36.792 & 32.375 & 15.98 & 19.218 & 15.463 & 10.212 & 31.66 & 32.253 & 29.647 & 31.321 & 28.086 & 33.488 \\
\hline 2/24/12 2:00 & & 30.259 & 29.023 & 29.407 & 30.026 & & 29.159 & 30.308 & 29.814 & 29.623 & 28.456 & 26.337 & 31.375 & 36.799 & 32.387 & 15.996 & 19.221 & 15.461 & 10.223 & 31.679 & 32.265 & 29.661 & 31.333 & 28.089 & 33.49 \\
\hline 2/24/12 3:00 & & 30.256 & 29.023 & 29.414 & 30.038 & & 29.18 & 30.315 & 29.814 & 29.637 & 28.465 & 26.337 & 31.382 & 36.806 & 32.396 & 16.005 & 19.216 & 15.477 & 10.233 & 31.683 & 32.274 & 29.666 & 31.342 & 28.089 & 33.492 \\
\hline $2 / 24 / 124: 00$ & & 30.261 & 29.034 & 29.412 & 30.045 & & 29.199 & 30.32 & 29.812 & 29.646 & 28.474 & 26.33 & 31.389 & 36.801 & 32.403 & $\begin{array}{l}36.014 \\
3\end{array}$ & 19.214 & 15.486 & 10.24 & 31.695 & 32.281 & 29.673 & 31.347 & 28.086 & 33.492 \\
\hline $2 / 24 / 125: 00$ & & 30.266 & 29.037 & 29.419 & 30.05 & & 29.215 & 30.327 & 29.809 & 29.66 & 28.481 & 26.33 & 31.396 & 36.813 & 32.406 & 16.024 & 19.212 & 15.496 & $\begin{array}{l}10.247 \\
\end{array}$ & 31.693 & 32.286 & 29.677 & 31.354 & 28.089 & 33.492 \\
\hline $2 / 24 / 126: 00$ & & 30.268 & 29.046 & 29.426 & 30.054 & & 29.229 & 30.334 & 29.814 & 29.67 & 28.493 & 26.327 & 31.4 & 36.813 & 32.417 & 16.031 & 19.205 & 15.503 & 10.256 & 31.704 & 32.293 & 29.677 & 31.361 & 28.089 & 33.492 \\
\hline $2 / 24 / 127: 00$ & & 30.28 & 29.051 & 29.447 & 30.071 & & 29.227 & 30.339 & 29.828 & 29.691 & 28.509 & 26.342 & 31.41 & 36.822 & 32.424 & 16.04 & 19.209 & 15.493 & 10.261 & 31.718 & 32.303 & 29.701 & 31.368 & 28.091 & 3.492 \\
\hline
\end{tabular}




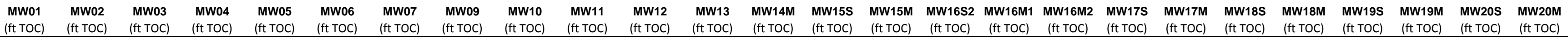

\begin{tabular}{|c|c|c|c|c|c|c|c|c|c|c|c|c|c|c|c|c|c|c|c|c|c|c|c|c|c|}
\hline Well & $\begin{array}{l}\text { Mwo1 } \\
\text { (ft TOC) }\end{array}$ & $\begin{array}{l}\text { Mwoz } \\
\text { (ft TOC) }\end{array}$ & $\begin{array}{l}\text { Mw03 } \\
\text { (ft Toc) }\end{array}$ & $\begin{array}{r}\text { MWva } \\
\text { (ft TOC) } \\
\end{array}$ & $\begin{array}{l}\text { MWOS } \\
\text { (ft TOC) }\end{array}$ & $\begin{array}{l}\text { MWvor } \\
\text { (ft TOC) }\end{array}$ & $\begin{array}{l}\text { Mwor } \\
\text { (ft ToC) }\end{array}$ & $\begin{array}{l}\text { Mw09 } \\
\text { (ft TOC) }\end{array}$ & $\begin{array}{l}\text { MW10 } \\
\text { (ft ToC) }\end{array}$ & $\begin{array}{l}\text { MWII } \\
\text { (ft TOC) }\end{array}$ & $\begin{array}{l}\text { MWII2 } \\
\text { (ft TOC) }\end{array}$ & $\begin{array}{l}\text { MWVI3 } \\
(\mathrm{ft} \text { Toc) }\end{array}$ & $\begin{array}{l}\text { (ft TOC) } \\
\end{array}$ & $\begin{array}{l}\text { MWvitss } \\
\text { (ft TOC) }\end{array}$ & $\begin{array}{l}\text { MWw TOC } \\
\text { (ft TOC) }\end{array}$ & 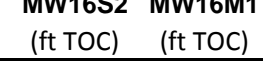 & $\begin{array}{l}\text { MWI6M2 } \\
\text { (ft TOC) }\end{array}$ & $\begin{array}{l}\text { MWvirs } \\
\text { (ft TOC) }\end{array}$ & (ft TOC) & $\begin{array}{l}\text { MWVIlos } \\
\text { (ft TOC) }\end{array}$ & $\begin{array}{l}\text { MWWIMM } \\
\text { (ft TOC) }\end{array}$ & $\begin{array}{l}\text { MWV195 } \\
\text { (ft TOC) }\end{array}$ & $\begin{array}{l}\text { MWW } \\
\text { (ft TOC) }\end{array}$ & $\begin{array}{l}\text { Mww } \\
\text { (ft Toc) }\end{array}$ & (ft TOC) \\
\hline 2/24/12 8:00 & & 30.284 & 29.065 & 29.468 & 30.087 & & 29.232 & 30.349 & 29.836 & 29.709 & 28.528 & 26.356 & 31.422 & 36.827 & 32.438 & $3 \quad 16.047$ & 19.195 & 15.507 & 10.275 & 31.735 & 32.317 & 29.722 & 31.382 & 28.091 & 33.497 \\
\hline 2/24/12 9:00 & & 30.284 & 29.114 & 29.494 & 30.106 & & 29.244 & 30.353 & 29.843 & 29.732 & 28.542 & 26.373 & 31.436 & 36.832 & 32.45 & 16.056 & 19.209 & 15.524 & 10.287 & 31.748 & $8 \quad 32.326$ & 29.736 & 31.396 & $5 \quad 28.091$ & 33.499 \\
\hline $2 / 24 / 1210: 00$ & & 30.287 & 29.191 & 29.494 & 30.113 & & 29.265 & 30.358 & 29.838 & 29.742 & 28.551 & 26.368 & 31.445 & 36.841 & 32.459 & 16.066 & 19.209 & 15.545 & 10.296 & 31.753 & $\begin{array}{l}32.336 \\
\end{array}$ & 29.736 & 31.406 & $5 \quad 28.089$ & 33.506 \\
\hline 2/24/12 11:00 & & 30.284 & 29.277 & 29.489 & 30.115 & & 29.293 & 30.368 & 29.833 & 29.749 & 28.553 & 26.361 & 31.452 & 36.844 & 32.469 & 16.07 & 19.205 & 15.564 & 10.308 & 31.755 & $5 \quad 32.345$ & 29.741 & 31.42 & 28.091 & 33.509 \\
\hline $2 / 24 / 1212: 00$ & & 30.287 & 29.349 & 29.487 & 30.113 & & 29.314 & 30.373 & 29.838 & 29.758 & 28.556 & 26.351 & 31.461 & 36.846 & 32.478 & 16.08 & 19.205 & 15.587 & 10.312 & 31.755 & $5 \quad 32.355$ & 29.734 & 31.427 & 28.091 & 33.513 \\
\hline $2 / 24 / 1213: 00$ & & 30.282 & 29.424 & 29.458 & 30.101 & & 29.349 & 30.372 & 29.817 & 29.751 & 28.551 & 26.325 & 31.461 & 36.848 & 32.476 & 16.084 & 19.202 & 15.618 & 10.315 & 31.746 & $\begin{array}{ll}6 & 32.357\end{array}$ & 29.713 & 31.431 & 28.091 & 33.513 \\
\hline $2 / 24 / 1214: 00$ & & 30.279 & 29.489 & 29.43 & 30.078 & & 29.385 & 30.375 & 29.812 & 29.739 & 28.537 & 26.287 & 31.459 & 36.844 & 32.476 & 16.087 & 19.198 & 15.639 & 10.312 & 31.728 & 32.35 & 29.687 & 31.422 & 28.091 & 33.513 \\
\hline $2 / 24 / 1215: 00$ & & 30.284 & 29.536 & 29.409 & 30.059 & & 29.401 & 30.375 & 29.807 & 29.732 & 28.523 & 26.263 & 31.452 & 36.839 & 32.471 & 16.091 & 19.193 & 15.653 & 10.31 & 31.709 & $\begin{array}{l}9 \\
9\end{array}$ & 29.673 & 31.413 & $3 \quad 28.091$ & 33.513 \\
\hline $2 / 24 / 1216: 00$ & & 30.287 & 29.552 & 29.402 & 30.047 & & 29.408 & 30.377 & 29.809 & 29.73 & 28.521 & 26.256 & 31.45 & 36.832 & 32.469 & 16.091 & 19.191 & 15.655 & 10.305 & 31.702 & $2 \quad 32.343$ & 29.668 & 31.41 & 28.091 & 33.511 \\
\hline $2 / 24 / 1217: 00$ & & 30.286 & 29.557 & 29.388 & 30.031 & & 29.418 & 30.375 & 29.809 & 29.721 & 28.512 & 26.234 & 31.443 & 36.834 & 32.462 & 16.091 & 19.195 & 15.662 & 10.296 & 31.695 & $5 \quad 32.336$ & 29.64 & 31.403 & $\begin{array}{l}28.093 \\
3\end{array}$ & 33.506 \\
\hline $2 / 24 / 1218: 00$ & & 30.294 & 29.531 & 29.395 & 30.028 & & 29.406 & 30.377 & 29.819 & 29.723 & 28.514 & 26.239 & 31.443 & 36.834 & 32.459 & 16.091 & 19.188 & 15.662 & 10.296 & 31.697 & $7 \quad 32.334$ & 29.651 & 31.396 & $5 \quad 28.093$ & 33.504 \\
\hline $2 / 24 / 1219: 00$ & & 30.3 & 29.475 & 29.421 & 30.038 & & 29.382 & 30.377 & 29.836 & 29.735 & 28.525 & 26.26 & 31.445 & 36.834 & 32.464 & 16.094 & 19.191 & 15.658 & 10.299 & 31.709 & 32.336 & 29.659 & 31.399 & 28.096 & $\quad 33.504$ \\
\hline $2 / 24 / 1220: 00$ & & 30.303 & 29.435 & 29.449 & 30.052 & & 29.366 & 30.387 & 29.843 & 29.751 & 28.537 & 26.284 & 31.452 & 36.841 & 32.471 & 16.096 & 19.188 & 15.653 & 10.308 & 31.723 & 32.343 & 29.684 & 31.408 & $\begin{array}{l}3 \\
28.093\end{array}$ & 33.506 \\
\hline $2 / 24 / 1221: 00$ & & 30.307 & 29.403 & 29.461 & 30.064 & & 29.361 & 30.389 & 29.847 & 29.758 & 28.544 & 26.294 & 31.459 & 36.848 & 32.474 & 16.098 & 19.188 & 15.658 & 10.312 & 31.73 & 32.35 & 29.696 & 31.413 & 28.096 & 33.506 \\
\hline 2/24/12 22:00 & & 30.31 & 29.372 & 29.473 & 30.073 & & 29.363 & 30.394 & 29.85 & 29.767 & 28.551 & 26.304 & 31.466 & 36.851 & 32.481 & 16.098 & 19.188 & 15.658 & 10.32 & 31.737 & 32.357 & 29.706 & 31.422 & 28.096 & 33.509 \\
\hline 2/24/12 23:00 & & 30.314 & 29.344 & 29.489 & 30.085 & & 29.356 & 30.399 & 29.855 & 29.779 & 28.56 & 26.315 & 31.473 & 36.851 & 32.49 & 16.103 & 19.186 & 15.658 & 10.327 & 31.753 & 32.369 & 29.717 & 31.427 & 28.096 & 33.513 \\
\hline $2 / 25 / 120: 00$ & & 30.314 & 29.335 & 29.494 & 30.094 & & 29.363 & 30.404 & 29.857 & 29.786 & 28.565 & 26.323 & 31.48 & 36.855 & 32.497 & 16.10 & 19.186 & 15.667 & 10.334 & 31.755 & 32.374 & 29.722 & 31.439 & 8.096 & 33.518 \\
\hline $2 / 25 / 121: 00$ & & 30.312 & 29.335 & 29.484 & 30.092 & & 29.382 & 30.406 & 29.847 & 29.786 & 28.563 & 26.311 & 31.485 & 36.858 & 32.499 & 16.108 & 19.186 & 15.681 & 10.341 & 31.753 & 32.379 & 29.715 & 1.443 & 8.096 & 33.518 \\
\hline $2 / 25 / 122: 00$ & & 30.321 & 29.328 & 29.496 & 30.099 & & 29.38 & 30.408 & 29.857 & 29.795 & 28.57 & 26.318 & 31.489 & 36.862 & 32.506 & 16.11 & 19.186 & 15.667 & 10.346 & 31.762 & 32.383 & 29.729 & 31.448 & $3 \quad 28.096$ & 33.518 \\
\hline 2/25/12 3:00 & & 30.312 & 29.347 & 29.47 & 30.087 & & 29.41 & 30.411 & 29.84 & 29.788 & 28.558 & 26.292 & 31.489 & 36.86 & 32.502 & 16.11 & 19.181 & 15.693 & 10.343 & 31.746 & 32.381 & 29.701 & 31.448 & $\begin{array}{l}3 \\
28.096\end{array}$ & 33.518 \\
\hline 2/25/12 4:00 & & 30.317 & 29.347 & 29.465 & 30.08 & & 29.418 & 30.411 & 29.847 & 29.783 & 28.556 & 26.282 & 31.485 & 36.855 & 32.502 & 16.115 & 19.181 & 15.693 & 10.336 & 31.742 & 32.379 & 29.701 & 31.441 & 28.096 & $\quad 33.518$ \\
\hline $2 / 25 / 125: 00$ & & 30.321 & 29.344 & 29.47 & 30.08 & & 29.418 & 30.413 & 29.85 & 29.788 & 28.558 & 26.287 & 31.487 & 36.855 & 32.502 & 16.115 & 19.179 & 15.693 & 10.341 & 31.744 & 32.379 & 29.701 & 31.446 & $5 \quad 28.096$ & 33.516 \\
\hline $2 / 25 / 126: 00$ & & 30.324 & 29.349 & 29.465 & 30.075 & & 29.425 & 30.416 & 29.852 & 29.786 & 28.558 & 26.28 & 31.485 & 36.853 & 32.499 & 16.115 & 19.186 & 15.693 & 10.341 & 31.739 & 32.376 & 29.701 & 31.443 & $3 \quad 28.096$ & 33.513 \\
\hline $2 / 25 / 127: 00$ & & 30.324 & 29.361 & 29.451 & 30.066 & & 29.441 & 30.418 & 29.843 & 29.779 & 28.549 & 26.263 & 31.48 & 36.853 & 32.497 & 16.115 & 19.186 & 15.7 & 10.336 & 31.732 & 32.374 & 29.682 & 31.439 & 28.096 & $\quad 33.511$ \\
\hline 2/25/12 8:00 & & 30.326 & 29.377 & 29.449 & 30.059 & & 29.443 & 30.42 & 29.85 & 29.776 & 28.544 & 26.258 & 31.475 & 36.848 & 32.495 & 16.115 & 19.181 & 15.709 & 10.331 & 31.728 & 32.372 & 29.684 & 31.436 & $\quad 28.098$ & 33.511 \\
\hline 2/25/12 9:00 & & 30.328 & 29.424 & 29.449 & 30.057 & & 29.448 & 30.423 & 29.855 & 29.776 & 28.544 & 26.258 & 31.478 & 36.851 & 32.497 & 16.115 & 19.179 & 15.714 & 10.334 & 31.728 & 32.369 & 29.677 & 31.436 & 28.098 & 33.511 \\
\hline 2/25/12 10:00 & & 30.324 & 29.491 & 29.426 & 30.042 & & 29.472 & 30.42 & 29.84 & 29.765 & 28.53 & 26.234 & 31.473 & 36.848 & 32.492 & 16.112 & 19.179 & 15.728 & 10.329 & 31.711 & 32.364 & 29.661 & 31.436 & 28.098 & 33.511 \\
\hline 2/25/12 11:00 & & 30.317 & 29.585 & 29.391 & 30.014 & & 29.495 & 30.418 & 29.828 & 29.746 & 28.514 & 26.201 & 31.468 & 36.841 & 32.485 & $5 \quad 16.11$ & 19.172 & 15.745 & 10.324 & 31.683 & 32.36 & 29.63 & 31.431 & 28.096 & 33.511 \\
\hline $2 / 25 / 1212: 00$ & & 30.31 & 29.671 & 29.342 & 29.982 & & 29.526 & 30.411 & 29.812 & 29.723 & 28.488 & 26.158 & 31.454 & 36.827 & 32.474 & 16.103 & 19.165 & 15.77 & 10.31 & 31.66 & 32.345 & 29.581 & 31.42 & 28.098 & 33.511 \\
\hline 2/25/12 13:00 & & 30.298 & 29.757 & 29.278 & 29.935 & & 29.561 & 30.399 & 29.79 & 29.686 & 28.451 & 26.098 & 31.433 & 36.811 & 32.457 & 16.096 & 19.158 & 15.78 & $\begin{array}{l}10.294 \\
\end{array}$ & 31.613 & 32.324 & 29.539 & 31.406 & $\begin{array}{l}28.093 \\
5\end{array}$ & 33.506 \\
\hline $2 / 25 / 1214: 00$ & & 30.289 & 29.822 & 29.222 & 29.886 & & 29.58 & 30.382 & 29.776 & 29.653 & 28.419 & 26.045 & 31.412 & 36.794 & 32.436 & 16.087 & 19.151 & 15.785 & 10.277 & 31.578 & 32.305 & 29.48 & 31.382 & 28.093 & 33.502 \\
\hline $2 / 25 / 12$ 15:00 & & 30.282 & 29.867 & 29.183 & 29.844 & & 29.582 & 30.37 & 29.774 & 29.621 & $\begin{array}{l}28.438 \\
28.38\end{array}$ & 26.012 & 31.394 & 36.783 & 32.417 & 16.08 & 19.149 & 15.784 & 10.256 & 31.543 & 32.284 & 29.447 & 31.352 & 28.098 & 33.495 \\
\hline $2 / 25 / 1216: 00$ & & 30.277 & 29.89 & 29.157 & 29.807 & & 29.575 & 30.356 & 29.776 & 29.598 & 28.363 & 25.988 & 31.377 & 36.773 & 32.398 & $3 \quad 16.068$ & 19.193 & 15.777 & 10.237 & 31.52 & 32.27 & 29.416 & 31.342 & 28.096 & 33.49 \\
\hline $2 / 25 / 1217: 00$ & & 30.266 & 29.895 & 29.113 & 29.772 & & 29.573 & 30.344 & 29.762 & 29.565 & 28.332 & 25.949 & 31.354 & 36.761 & 32.377 & 16.054 & 19.186 & 15.782 & 10.216 & 31.487 & 32.244 & 29.377 & 31.314 & 28.096 & 33.483 \\
\hline $2 / 25 / 1218: 00$ & & 30.263 & 29.878 & 29.098 & 29.7 & & 29.549 & 30.332 & 29.771 & 29.544 & 28.314 & 25.945 & 31.335 & 36.75 & 32.361 & 16.0 & 19.175 & 15.763 & 10.2 & 31.473 & 32.229 & .367 & 31.295 & 28.093 & 33.481 \\
\hline 2/25/12 19:00 & & 30.273 & 29.825 & 29.138 & 29. & & 29.488 & 30.329 & 29.79 & 29.549 & 28.319 & 25.983 & 31.328 & 36.745 & 32.356 & 16.035 & 19.168 & 15.733 & 10.19 & 31.485 & 32.222 & .391 & 1.288 & $\begin{array}{l}3 \quad 28.098 \\
\end{array}$ & 33.478 \\
\hline $2 / 25 / 1220: 00$ & & 30.266 & 29.794 & 29.136 & 29.744 & & 29.469 & 30.322 & 29.793 & 29.54 & 28.309 & 25.99 & 31.321 & 36.747 & 32.345 & 16.026 & 19.168 & 15.73 & 10.183 & 31.483 & 32.215 & 29.384 & 31.284 & 28.1 & 33.474 \\
\hline 2/25/12 21:00 & & 30.261 & 29.773 & 29.129 & 29.741 & & 29.45 & 30.315 & 29.788 & 29.528 & 28.298 & 25.988 & 31.314 & 36.74 & 32.335 & 16.017 & 19.163 & 15.719 & 10.178 & 31.473 & 32.208 & 29.379 & 31.279 & 28.096 & 33.471 \\
\hline 2/25/12 22:00 & & 30.263 & 29.75 & 29.129 & 29.739 & & 29.429 & 30.313 & 29.793 & 29.521 & 28.293 & 25.99 & 31.305 & 36.736 & 32.333 & 16.01 & 19.154 & 15.705 & 10.169 & 31.473 & 32.203 & 29.379 & 31.267 & 28.1 & . $\quad 33.474$ \\
\hline 2/25/12 23:00 & & 30.261 & 29.731 & 29.124 & 29.737 & & 29.415 & 30.306 & 29.786 & 29.51 & 28.286 & 25.99 & 31.302 & 36.733 & 32.326 & 16 & 19.144 & 15.698 & 10.167 & 31.471 & 32.199 & 29.377 & 31.263 & $\quad 28.098$ & 33.469 \\
\hline $2 / 26 / 120: 00$ & & 30.259 & 29.713 & 29.117 & 29.732 & & 29.408 & 30.301 & 29.786 & 29.505 & 28.277 & 25.995 & 31.3 & 36.731 & 32.321 & 15.993 & 19.138 & 15.695 & 10.16 & 31.462 & 32.191 & 29.369 & 31.26 & $\quad 28.098$ & 33.471 \\
\hline 2/26/12 1:00 & & 30.254 & 29.704 & 29.103 & 29.72 & & 29.401 & 30.291 & 29.774 & 29.484 & 28.265 & 25.978 & 31.291 & 36.726 & 32.312 & 15.986 & 19.131 & 15.684 & 10.153 & 31.452 & 32.184 & 29.36 & 31.251 & 28.1 & 33.471 \\
\hline 2/26/12 2:00 & & 30.275 & 29.641 & 29.166 & 29.7 & & 29.345 & 30.296 & 29.812 & 29.498 & 28.291 & 26.024 & 31.3 & 36.726 & 32.319 & 15.982 & 19.126 & 15.632 & 10.155 & 31.494 & 32.191 & 29.419 & 31.246 & $\quad 28.103$ & 33.476 \\
\hline $2 / 26 / 123: 00$ & & 30.275 & 29.592 & 29.208 & 29.783 & & 29.316 & 30.301 & 29.828 & 29.521 & 28.309 & 26.091 & 31.309 & 36.74 & 32.328 & $3 \quad 15.982$ & 19.119 & 15.632 & 10.167 & 31.518 & 32.203 & 29.442 & 31.267 & 28.103 & 33.478 \\
\hline 2/26/12 4:00 & & 30.28 & 29.543 & 29.236 & 29.814 & & 29.295 & 30.308 & 29.836 & 29.533 & 28.325 & 26.122 & 31.319 & 36.743 & 32.338 & $3 \quad 15.982$ & 19.119 & 15.622 & 10.174 & 31.536 & 32.21 & 29.471 & 31.277 & 28.1 & 33.478 \\
\hline 2/26/12 5:00 & & 30.28 & 29.51 & 29.236 & 29.828 & & 29.3 & 30.31 & 29.819 & 29.535 & 28.328 & 26.117 & 31.321 & 36.747 & 32.34 & 15.979 & 19.121 & 15.62 & 10.178 & 31.541 & 32.215 & 29.468 & 31.279 & 28.1 & 33.478 \\
\hline 2/26/12 6:00 & & 30.284 & 29.48 & 29.248 & 29.839 & & 29.3 & 30.313 & 29.824 & 29.542 & 28.335 & 26.129 & 31.326 & 36.752 & 32.345 & 15.9 & 19.128 & 15.611 & 10.179 & 31.553 & 32.217 & 29.496 & 31.281 & 28.103 & 33.478 \\
\hline 2/26/12 7:00 & & 30.289 & 29.456 & 29.26 & 29.853 & & 29.298 & 30.315 & 29.824 & 29.549 & 28.344 & 26.138 & 31.33 & 36.757 & 32.349 & 15.9 & 19.126 & 15.611 & 10.185 & 31.562 & 32.222 & 29.501 & 31.284 & 28.103 & 33.476 \\
\hline $2 / 26 / 128: 00$ & & 30.296 & 29.438 & 29.283 & 29.872 & & 29.288 & 30.32 & 29.828 & 29.558 & 28.353 & 26.158 & 31.337 & 36.764 & 32.354 & 15.9 & 19.119 & 15.597 & 10.19 & 31.578 & 32.227 & 29.522 & 31.291 & 28.105 & 33.478 \\
\hline 2/26/12 9:00 & & 30.298 & 29.459 & 29.3 & 29.891 & & 29.288 & 30.325 & 29.831 & 29.57 & 28.367 & 26.177 & 31.344 & 36.766 & 32.361 & 15.9 & 19.119 & 15.599 & 10.2 & 31.588 & 32.239 & 29.541 & 31.305 & $5 \quad 28.103$ & 33.481 \\
\hline $2 / 26 / 12$ 10:00 & & 30.298 & 29.51 & 29.304 & 29.902 & & 29.3 & 30.332 & 29.828 & 29.579 & 28.374 & 26.184 & 31.354 & 36.776 & 32.37 & 15.986 & 19.11 & 15.608 & 10.207 & 31.597 & 32.244 & 29.546 & 31.314 & 28.105 & 33.485 \\
\hline $2 / 26 / 1$ & & 30.298 & 29.571 & 29.297 & 29.9 & & 29.319 & 30.334 & 29.824 & 29.577 & 28.372 & 26.17 & 31.356 & 36.778 & 32.373 & $3 \quad 15.989$ & 19.112 & 15.618 & 10.207 & 31.597 & 32.246 & 29.548 & 31.326 & $\quad 28.103$ & 33.485 \\
\hline $2 / 2$ & & 30.34 & 29.63 & 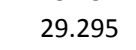 & 29.9 & & 30 & 30.3 & s & -5 & 28.3 & 26.172 & 21250 & 36.7 & 32.377 & 15. & & ace & 10.2 & 31.592 & anct & 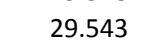 & 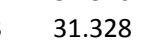 & 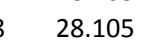 & 33.488 \\
\hline & & 30 & 29.6 & & & & & 30. & & 29 & 28. & & & 36. & 32 & & & & 10 & 31. & & & & & \\
\hline & & & 29.729 & & & & & $30 .>2>$ & & & 28 & & & & 32 & & 19 & & 0.219 & & & & 0 & 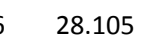 & \\
\hline 215:00 & & 30.303 & 29.757 & 29.283 & 29.902 & & 29.363 & 30.339 & 29.821 & 29.577 & 28.372 & 26.151 & 31.361 & 36.773 & 32.382 & 15.998 & 19.11 & 15.653 & 10.218 & 31.592 & 32.258 & 29.539 & 31.326 & 28.105 & 33.495 \\
\hline
\end{tabular}




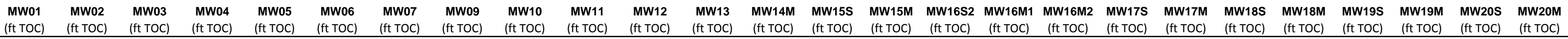

\begin{tabular}{|c|c|c|c|c|c|c|c|c|c|c|c|c|c|c|c|c|c|c|c|c|c|c|c|c|c|}
\hline Well & $\begin{array}{l}\text { (ft TOC) } \\
\text { (is }\end{array}$ & $\begin{array}{l}\text { (ft TOC) } \\
\text { (f) }\end{array}$ & $\begin{array}{l}\text { (ft TOC) } \\
\text { (f) }\end{array}$ & (ft TOC) & $\begin{array}{l}\text { (ft TOC) } \\
\text { (f) }\end{array}$ & $\begin{array}{l}\text { (ft TOC) } \\
\text { (f) }\end{array}$ & $\begin{array}{l}\text { (ft TOC) } \\
\text { (ft }\end{array}$ & $\begin{array}{l}\text { (ft TOC) } \\
\text { (f) }\end{array}$ & $\begin{array}{l}\text { (ft TOC) } \\
\text { (ft) }\end{array}$ & $\begin{array}{l}\text { (ft TOC) } \\
\text { (f) }\end{array}$ & $\begin{array}{l}\text { (ft TOC) } \\
\text { (f) }\end{array}$ & $\begin{array}{l}\text { (ft Toc) } \\
\text { (f) }\end{array}$ & $\begin{array}{l}\text { (ft TOC) } \\
\text { (t) }\end{array}$ & $\begin{array}{l}\text { (ft TOC) } \\
\end{array}$ & (ft TOC) & $\begin{array}{ll} & \text { (ft TTCC } \\
\text { (ft TOC) }\end{array}$ & (ft TOC) & $\begin{array}{l}\text { (ft TOC) } \\
\text { (ft) }\end{array}$ & (ft TOC) & $\begin{array}{l}\text { (ft TOC) } \\
\end{array}$ & $\begin{array}{l}\text { (ft TOC) } \\
\text { (1) }\end{array}$ & $\begin{array}{l}\text { (ft TOC) } \\
\text { (n) }\end{array}$ & (ft TOC) & $\begin{array}{l}\text { (ft TOC) } \\
\end{array}$ & (ft TOC) \\
\hline $2 / 26 / 1216: 00$ & & 30.31 & 29.755 & 29.304 & 29.914 & & 29.349 & 30.344 & 29.831 & 29.586 & 28.381 & 26.17 & 31.37 & 36.778 & 32.387 & 16 & 19.11 & 15.646 & 10.225 & 31.606 & 32.26 & 29.553 & 31.326 & 28.105 & 33.497 \\
\hline $2 / 26 / 1217: 00$ & & 30.317 & 29.727 & 29.325 & 29.928 & & 29.338 & 30.346 & 29.84 & 29.595 & 28.395 & 26.184 & 31.375 & 36.785 & 32.394 & 16.003 & 19.107 & 15.641 & 10.228 & 31.623 & 32.267 & 29.574 & 31.331 & 28.11 & 33.497 \\
\hline $2 / 26 / 1218: 00$ & & 30.326 & 29.673 & 29.363 & 29.954 & & 29.314 & 30.353 & 29.85 & 29.616 & 28.414 & 26.22 & 31.384 & 36.79 & 32.403 & 16.01 & 19.105 & $\begin{array}{l}15.032 \\
15.632\end{array}$ & 10.237 & 31.644 & 32.277 & 29.604 & 31.338 & 28.107 & 33.499 \\
\hline $2 / 26 / 1219: 00$ & & 30.333 & 29.606 & 29.398 & 29.982 & & 29.298 & 30.363 & 29.859 & 29.64 & 28.435 & 26.256 & 31.59 & 36.799 & 32.628 & 16.012 & 19.103 & 15.625 & 10.461 & 31.718 & 32.483 & 29.635 & 31.544 & 28.107 & 33.502 \\
\hline $2 / 26 / 1220: 00$ & & $\begin{array}{r}30.34 \\
\end{array}$ & 29.554 & 29.421 & 30.01 & & 29.295 & 30.37 & 29.864 & 29.656 & 28.451 & 26.275 & 31.665 & 36.808 & 32.694 & 16.019 & 19.103 & 15.63 & 10.529 & 31.797 & 32.559 & 29.67 & 31.612 & 28.107 & 33.502 \\
\hline $2 / 26 / 1221: 00$ & & 30.347 & 29.51 & 29.444 & 30.038 & & 29.293 & 30.382 & 29.869 & 29.672 & 28.467 & 26.292 & 31.585 & 36.813 & 32.607 & 16.026 & 19.105 & 15.627 & 10.442 & 31.781 & 32.476 & 29.694 & 1.535 & 28.107 & 33.504 \\
\hline $2 / 26 / 1222: 00$ & & 30.347 & 29.48 & 29.458 & 30.064 & & 29.302 & 30.389 & 29.866 & 29.688 & 28.481 & 26.304 & 31.557 & 36.818 & 32.577 & 16.033 & 19.107 & 15.637 & 10.414 & 31.788 & 32.447 & 29.71 & 31.509 & 28.107 & 33.504 \\
\hline 2/26/12 23:00 & & 30.352 & 29.452 & 29.472 & 30.082 & & 29.307 & 30.396 & 29.874 & 29.702 & 28.495 & 26.311 & 31.546 & 36.827 & 32.565 & 16.04 & 19.114 & 15.637 & 10.402 & 31.783 & 32.435 & 29.724 & 31.5 & 28.107 & 33.509 \\
\hline 2/27/12 0:00 & & 30.349 & 29.445 & 29.48 & 30.094 & & 29.326 & 30.404 & 29.866 & 29.714 & 28.502 & 26.315 & 31.539 & 36.832 & 32.556 & 16.047 & 19.114 & 15.646 & 10.393 & 31.783 & 32.431 & 29.727 & 31.492 & 28.11 & 33.511 \\
\hline 2/27/12 1:00 & & 30.361 & 29.412 & 29.505 & 30.115 & & 29.321 & 30.411 & 29.883 & 29.735 & 28.521 & 26.332 & 31.541 & 36.839 & 32.556 & 16.052 & 19.117 & 15.641 & 10.393 & 31.797 & 32.433 & 29.753 & 31.49 & 28.11 & 33.513 \\
\hline 2/27/12 2:00 & & 30.363 & 29.396 & 29.529 & 30.136 & & 29.326 & 30.418 & 29.888 & 29.753 & 28.539 & 26.354 & 31.543 & 36.844 & 32.563 & 16.061 & 19.124 & 15.644 & 10.395 & 31.816 & 32.435 & 29.771 & 31.495 & 28.11 & 33.518 \\
\hline $2 / 27 / 123: 00$ & & 30.361 & 29.403 & 29.522 & 30.138 & & 29.354 & 30.425 & 29.881 & 29.758 & 28.542 & 26.337 & 31.543 & 36.844 & 32.56 & 16.068 & 19.124 & 15.66 & 10.397 & 31.809 & 32.438 & 29.767 & 31.497 & 28.11 & 33.518 \\
\hline $2 / 27 / 124: 00$ & & 30.37 & 29.384 & 29.545 & 30.157 & & 29.354 & 30.43 & 29.888 & 29.779 & 28.558 & 26.359 & 31.548 & 36.853 & 32.563 & 16.075 & 19.11 & 15.658 & 10.404 & 31.825 & 32.44 & 29.79 & 31.5 & 28.11 & 33.523 \\
\hline $2 / 27 / 125: 00$ & & 30.37 & 29.372 & 29.557 & 30.173 & & 29.361 & 30.437 & 29.893 & 29.795 & 28.572 & 26.371 & 31.553 & 36.86 & 32.567 & 16.084 & 19.121 & 15.667 & 10.409 & 31.83 & 32.447 & 29.795 & 31.509 & 28.11 & 33.523 \\
\hline $2 / 27 / 126: 00$ & & 30.373 & 29.372 & 29.559 & 30.18 & & 29.38 & 30.44 & 29.895 & 29.802 & 28.579 & 26.363 & 31.555 & 36.865 & 32.572 & 16.089 & 19.126 & 15.674 & 10.409 & 31.835 & 32.447 & 29.804 & 31.507 & 28.112 & 33.525 \\
\hline $2 / 27 / 127: 00$ & & 30.373 & 29.384 & 29.557 & 30.183 & & 29.401 & 30.444 & 29.89 & 29.807 & 28.581 & 26.356 & 31.557 & 36.869 & 32.574 & 16.096 & 19.135 & 15.683 & 10.414 & 31.835 & 32.45 & 29.8 & 31.516 & 28.107 & 33.525 \\
\hline $2 / 27 / 128: 00$ & & 30.384 & 29.384 & 29.58 & 30.192 & & 29.399 & 30.449 & 29.902 & 29.825 & 28.595 & 26.371 & 31.562 & 36.869 & 32.579 & 16.105 & 19.135 & 15.676 & 10.418 & 31.853 & 32.454 & 29.816 & 31.518 & 28.115 & 33.525 \\
\hline 2/27/12 9:00 & & 30.384 & 29.438 & 29.582 & 30.199 & & 29.42 & 30.456 & 29.902 & 29.834 & 28.602 & 26.371 & 31.567 & 36.874 & 32.581 & 16.11 & 19.131 & 15.695 & 10.421 & 31.851 & 32.459 & 29.823 & 31.523 & 28.112 & 33.527 \\
\hline $2 / 27 / 12$ 10:00 & & $\begin{array}{l}30.304 \\
30.382\end{array}$ & 29.494 & 29.573 & 30.197 & & 29.446 & $\begin{array}{l}30.450 \\
30.456\end{array}$ & 29.89 & $\begin{array}{l}29.837 \\
29.837\end{array}$ & $\begin{array}{l}28.002 \\
28.602\end{array}$ & 26.363 & 31.569 & 36.874 & 32.586 & $\begin{array}{l}16.117 \\
16.117\end{array}$ & 19.128 & 15.714 & 10.423 & 31.846 & 32.464 & 29.816 & 31.53 & 28.112 & 33.53 \\
\hline 2/27/12 11:00 & & $\begin{array}{l}30.302 \\
30.382\end{array}$ & $\begin{array}{r}29.494 \\
29.55\end{array}$ & 29.561 & 30.19 & & 29.472 & $\begin{array}{l}30.450 \\
30.459\end{array}$ & 29.888 & 29.832 & $\begin{array}{r}20.006 \\
28.6\end{array}$ & $\begin{array}{l}20.303 \\
26.339\end{array}$ & 31.569 & 36.881 & 32.588 & $\begin{array}{l}10.111 \\
16.119\end{array}$ & 19.126 & $\begin{array}{l}13.726 \\
15.726\end{array}$ & 10.423 & $\begin{array}{l}31.840 \\
31.842\end{array}$ & $\begin{array}{l}32.444 \\
32.459\end{array}$ & 29.804 & 31.528 & 28.112 & 33.532 \\
\hline $2 / 27 / 1212: 00$ & & $\begin{array}{r}50.382 \\
30.38\end{array}$ & $\begin{array}{r}29.513 \\
29.613\end{array}$ & 29.538 & $\begin{array}{l}30.19 \\
30.173\end{array}$ & & $\begin{array}{r}29.412 \\
29.5\end{array}$ & $\begin{array}{l}30.4561 \\
30.461\end{array}$ & 29.876 & $\begin{array}{l}29.825 \\
29.825\end{array}$ & $\begin{array}{r}28.60 \\
28.591\end{array}$ & 26.318 & 31.567 & $\begin{array}{l}30.801 \\
36.874\end{array}$ & $\begin{array}{l}32.586 \\
32.586\end{array}$ & $\begin{array}{l}10.119 \\
16.122\end{array}$ & 19.158 & $\begin{array}{l}13.760 \\
15.745\end{array}$ & $\begin{array}{l}10.423 \\
10.421\end{array}$ & $\begin{array}{l}31.842 \\
31.828\end{array}$ & $\begin{array}{l}32.459 \\
32.459\end{array}$ & $\begin{array}{l}29.784 \\
29.783\end{array}$ & 31.53 & $\begin{array}{l}28.1107 \\
28.107\end{array}$ & $\begin{array}{l}53.532 \\
33.532\end{array}$ \\
\hline $2 / 27 / 1213: 00$ & & 30.377 & 29.669 & 29.505 & 30.15 & & 29.528 & 30.461 & 29.864 & 29.814 & 28.577 & 26.287 & 31.56 & 36.869 & 32.579 & 16.124 & 19.161 & 15.768 & 10.414 & 31.804 & 32.452 & 29.76 & 31.523 & 28.112 & 33.532 \\
\hline $2 / 27 / 12$ 14:00 & & 30.375 & 29.701 & & 30.124 & & 29.551 & 59 & 29.859 & 29.797 & 28.558 & 26.256 & 31.553 & $\begin{array}{r}36.86 \\
36.0\end{array}$ & 32.57 & 16.124 & 19.161 & 15.782 & $\begin{array}{l}10.411 \\
10.411\end{array}$ & 31.786 & 32.443 & 29.731 & 31.514 & 28.112 & 33.532 \\
\hline $2 / 27 / 1215: 00$ & & 30.373 & 29.734 & 29.447 & 30.099 & & 29.568 & 30.454 & 29.855 & 29.783 & 28.542 & 26.225 & 31.541 & 36.853 & 32.558 & 16.122 & 19.165 & 15.789 & 10.397 & 31.765 & 32.433 & 29.706 & 31.502 & 28.112 & 33.53 \\
\hline $2 / 27 / 1216: 00$ & & 30.37 & 29.743 & 29.43 & 30.075 & & 29.573 & 30.452 & 29.855 & 29.772 & 28.53 & 26.208 & 31.532 & 36.844 & 32.551 & 16.122 & 19.207 & 5.798 & 10.385 & 31.753 & 32.421 & 29.684 & 31.49 & 28.112 & 33.527 \\
\hline 2/27/12 17:00 & & 30.373 & 29.757 & 29.412 & 30.057 & & 29.575 & 30.447 & 29.855 & 29.76 & 28.516 & 26.194 & 31.522 & 36.841 & 32.542 & 16.117 & 19.209 & 15.796 & 10.379 & 31.735 & 32.414 & 29.668 & 31.485 & 28.112 & 33.527 \\
\hline 2/27/12 18:00 & & 30.37 & 29.757 & 29.398 & 30.038 & & 29.57 & 30.442 & 29.855 & 29.749 & 28.505 & 26.177 & 31.513 & 36.834 & 32.532 & 16.117 & 19.2 & 15.789 & 10.371 & 31.721 & 32.402 & 29.656 & 31.476 & 28.112 & 33.523 \\
\hline 2/27/12 19:00 & & 30.373 & 29.738 & 29.393 & 30.026 & & 29.563 & 30.44 & 29.857 & 29.742 & 28.498 & 26.174 & 31.503 & 36.825 & 32.525 & 16.112 & 19.223 & 15.787 & 10.362 & 31.714 & 32.398 & 29.644 & 31.467 & 28.112 & 33.52 \\
\hline $2 / 27 / 1220: 00$ & & 30.37 & 29.72 & 29.381 & 30.014 & & 29.556 & 30.435 & 29.855 & 29.735 & 28.488 & 26.167 & 31.501 & 36.829 & 32.516 & 16.108 & 19.218 & 15.789 & 10.357 & 31.702 & 32.39 & 29.635 & 31.46 & 28.112 & 33.518 \\
\hline $2 / 27 / 1221: 00$ & & 30.373 & 29.699 & 29.377 & 30.005 & & 29.549 & 30.432 & 29.859 & 29.723 & 28.481 & 26.158 & 31.49 & 36.82 & 32.511 & 16.103 & 19.207 & 15.78 & 10.35 & 31.697 & 32.383 & 29.63 & 31.448 & 28.115 & 33.516 \\
\hline $2 / 27 / 1222: 00$ & & 30.373 & 29.692 & 29.367 & 29.993 & & 29.544 & 30.43 & 29.857 & 29.716 & 28.472 & 26.153 & 31.48 & 36.813 & 32.502 & 16.098 & 19.198 & 15.78 & 10.341 & 31.688 & 32.374 & 29.616 & 31.439 & 28.115 & 33.511 \\
\hline $2 / 27 / 1223: 00$ & & 30.37 & 29.692 & 29.36 & 29.984 & & 29.54 & 30.425 & 29.859 & 29.709 & 28.465 & 26.151 & 31.478 & 36.811 & 32.497 & 16.094 & 19.188 & 15.78 & 10.334 & 31.679 & 32.369 & 29.614 & 31.439 & 28.114 & 33.513 \\
\hline $2 / 28 / 120: 00$ & & 30.368 & 29.713 & 29.339 & 29.968 & & 29.544 & 30.418 & 29.857 & 29.693 & 28.451 & 26.131 & 31.468 & 36.806 & 32.49 & 16.089 & 19.175 & 15.775 & 10.327 & 31.665 & 32.362 & 29.595 & 31.427 & 28.112 & 33.509 \\
\hline 2/28/12 1:00 & & 30.363 & 29.734 & 29.316 & 29.949 & & 29.551 & 30.413 & 29.843 & 29.679 & 28.435 & 26.107 & 31.457 & 36.804 & 32.478 & 16.082 & 19.168 & 15.782 & 10.317 & 31.646 & 32.35 & 29.569 & 31.417 & 28.114 & 33.506 \\
\hline 2/28/12 2:00 & & 30.361 & 29.748 & 29.297 & 29.93 & & 29.551 & 30.406 & 29.843 & 29.663 & 28.421 & 26.095 & 31.447 & 36.797 & 32.466 & 16.075 & 19.161 & 15.77 & 10.308 & 31.634 & 32.338 & 29.553 & 31.406 & 28.114 & 33.504 \\
\hline 2/28/12 3:00 & & 30.352 & 29.769 & 29.267 & 29.909 & & 29.561 & 30.401 & 29.836 & 29.644 & 28.4 & 26.069 & 31.436 & 36.792 & 32.455 & 16.07 & 19.156 & 15.787 & 10.296 & 31.609 & 32.329 & 29.527 & 31.401 & 28.112 & 33.502 \\
\hline 2/28/12 4:00 & & 30.347 & 29.78 & 29.243 & 29.886 & & 29.563 & 30.392 & 29.833 & 29.626 & $\begin{array}{l}20.4 \\
28.381 \\
\end{array}$ & 26.05 & 31.424 & 36.787 & 32.445 & $\begin{array}{l}16.061 \\
\end{array}$ & 19.147 & 15.78 & 10.284 & 31.592 & 32.315 & 29.506 & 31.389 & 28.115 & $\begin{array}{l}33.499 \\
33.02\end{array}$ \\
\hline 2/28/12 5:00 & & $\begin{array}{l}30.345 \\
30.341\end{array}$ & 29.79 & 29.229 & 29.867 & & 29.554 & 30.387 & 29.833 & 29.612 & $\begin{array}{l}28.367 \\
28.367\end{array}$ & 26.043 & 31.41 & 36.78 & 32.434 & 16.056 & 19.138 & $\begin{array}{l}15.775 \\
\end{array}$ & $\begin{array}{l}10.274 \\
10.272\end{array}$ & 31.578 & 32.303 & 29.489 & 31.378 & 28.115 & $\begin{array}{l}33.497 \\
33.49\end{array}$ \\
\hline $2 / 28 / 126: 00$ & & $\begin{array}{l}30.345 \\
30.342\end{array}$ & 29.794 & 29.218 & 29.851 & & 29.547 & 30.38 & 29.833 & 29.593 & 28.356 & $\begin{array}{l}20.049 \\
26.031\end{array}$ & $\begin{array}{r}11.41 \\
31.4\end{array}$ & $\begin{array}{l}36.769 \\
36.769\end{array}$ & $\begin{array}{l}32.422 \\
32.422\end{array}$ & 16.04 & 19.144 & 15.761 & $\begin{array}{l}10.258 \\
10.258\end{array}$ & 31.567 & 32.291 & 29.475 & $\begin{array}{l}31.359 \\
31.359\end{array}$ & 28.114 & $\begin{array}{l}33.492 \\
33.49\end{array}$ \\
\hline $2 / 28 / 12$ & & $3 c$ & 29.75 & 29.215 & 29 & & 2 & 30.375 & 29 & 29. & 28 & 26.033 & 31.391 & 36.764 & 32.415 & 16 & 4 & 15.756 & 10.249 & 31.562 & 86 & 3 & 31.352 & 28.115 & 33.49 \\
\hline 2/28/12 8:00 & & 30. & 29.79 & 29. & 29 & & 29. & 30.368 & 29.833 & 29.575 & 28.337 & 26.028 & 31 & 36.766 & 32.406 & 16. & 19.144 & 15.752 & 10.242 & 31.555 & 32.274 & 33 & 49 & 28.114 & 33.488 \\
\hline 2/28/12 9:00 & & 30.321 & 29.827 & 29.157 & 29. & & & 30.361 & 29.814 & 29.54 & 28.309 & 25.988 & 31.363 & 36.757 & 32.387 & 16.021 & 19.131 & 15.759 & 10.228 & 31.513 & 32.255 & 29.426 & 1.328 & 28.105 & 33.481 \\
\hline $2 / 28 / 1210: 00$ & & 30.321 & 29.843 & 29.141 & 29. & & 29.535 & 30.351 & 29.814 & 29.526 & 28.293 & 25.973 & 31.351 & 36.747 & 32.375 & 16.017 & 19.121 & 15.749 & 10.214 & 31.501 & 32.246 & 29.402 & 1.319 & 28.112 & 33.478 \\
\hline 2/28/12 11:00 & & 30.314 & 29.864 & 29.127 & 29. & & 29 & 30.3 & 29.814 & 29.5 & 28.2 & 25.966 & 31.34 & 36.745 & 32.366 & 16.005 & 19.117 & 15.745 & 10.202 & 31.483 & 32.234 & 29.386 & 31.302 & 28.112 & 33.474 \\
\hline $2 / 28 / 1212: 00$ & & 30.303 & 29.8 & 29.087 & 29. & & 29.535 & 30.3 & 29.795 & & 28.251 & 25.933 & 31.323 & 36.733 & 32.354 & 15. & 19.11 & 15.752 & 10.188 & 31.464 & 32.218 & 29.358 & 31.298 & 28.115 & 33.474 \\
\hline 2/28/12 13:00 & & 30.294 & 29.918 & 29.047 & 29.7 & & 29.54 & 30.3 & 29.79 & & 28.223 & 25.897 & 31.307 & 36.724 & 32.33 & 15. & 19.101 & 15.752 & 10.171 & 31.429 & 32.199 & 29.325 & 31.279 & 28.112 & 33.469 \\
\hline $2 / 28 / 1214: 00$ & & 30.277 & 29.946 & 29 & 29 & & 29.547 & 30.3 & 29.771 & 29. & 28.1 & 25.858 & 31.286 & 36.722 & 32.314 & 15. & 19.091 & 15.756 & 10.15 & 31.394 & 32.177 & 29.278 & 31.258 & 28.11 & 33.462 \\
\hline $2 / 28 / 1215: 00$ & & 30.273 & 29.965 & 28.97 & 29.6. & & 29.542 & 30.303 & 29.769 & 29.3 & 28.165 & 25.83 & 31.265 & 36.707 & 32.291 & 15.96 & 19.08 & 15.742 & 10.134 & 31.368 & 32.161 & 29.247 & 31.239 & 28.112 & 33.46 \\
\hline $2 / 28 / 1216: 00$ & & 30.266 & 29.993 & 28.951 & 29.601 & & 29.521 & 30.289 & 29.767 & 29.363 & 28.144 & 25.82 & 31.251 & 36.696 & 32.274 & 15.956 & 19.128 & 15.733 & 10.12 & 31.35 & 32.139 & 29.233 & 31.223 & 28.112 & 33.457 \\
\hline $2 / 28 / 1217: 00$ & & 30.259 & 30 & 28.94 & 29.578 & & 29.502 & 30.282 & 29.764 & 29.335 & 28.125 & 25.815 & 31.234 & 36.689 & 32.26 & 15.94 & 19.124 & 15.721 & 10.099 & 31.338 & 32.125 & 29.217 & 31.202 & 28.112 & 33.457 \\
\hline $2 / 28 / 1218: 00$ & & 30.252 & 29.997 & 28.919 & 29.557 & & 29.488 & 30.274 & 29.76 & 29.312 & 28.107 & 25.799 & 31.216 & 36.679 & 32.244 & 15.931 & 19.114 & 15.716 & 10.084 & 31.317 & 32.109 & 29.191 & 31.18 & 28.112 & 33.453 \\
\hline $2 / 28 / 1219: 00$ & & 30.249 & 29.988 & 28.907 & 29.543 & & 29.462 & 30.267 & 29.762 & 29.296 & 28.084 & 25.801 & 31.19 & 36.672 & 32.216 & 15.917 & 19.094 & 15.693 & 10.049 & 31.301 & 32.085 & 29.172 & 31.15 & 28.11 & 33.432 \\
\hline $2 / 28 / 1220: 00$ & & 30.268 & 29.86 & 29.243 & 29.552 & & 29.403 & 30.262 & 29.786 & 29.287 & 28.098 & 25.808 & 31.124 & 36.668 & 32.171 & 15.91 & 19.036 & 15.627 & 10 & 31.294 & 32.021 & 29.21 & 31.077 & 28.11 & 33.385 \\
\hline 2/28/12 21:00 & & 30.27 & 29.792 & 29.215 & 29.59 & & 29.328 & 30.262 & 29.816 & 29.317 & 28.123 & 25.916 & 31.152 & 36.668 & 32.178 & 15.898 & 19.036 & 15.604 & 10.016 & 31.338 & 32.045 & 29.261 & 31.112 & 28.112 & 33.39 \\
\hline $2 / 28 / 1222: 00$ & & 30.279 & 29.776 & 29 & 29.636 & & 29.272 & 30.267 & 29.836 & 29.34 & 28.153 & 25.978 & 31.169 & 36.672 & 32.187 & 15.893 & 19.031 & 15.583 & 10.014 & 31.375 & 32.061 & 29.32 & 31.124 & 28.115 & 33.394 \\
\hline $2 / 28 / 1223: 00$ & & 30.287 & 26.964 & 28.907 & 29.688 & & 29.232 & 30.272 & 29.847 & 29.37 & 28.184 & 26.045 & 31.192 & 36.684 & 32.209 & 15.891 & 19.029 & 15.571 & 10.04 & 31.42 & 32.087 & 29.374 & 31.148 & 28.112 & 33.397 \\
\hline
\end{tabular}




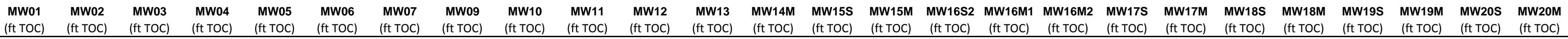

\begin{tabular}{|c|c|c|c|c|c|c|c|c|c|c|c|c|c|c|c|c|c|c|c|c|c|c|c|c|c|}
\hline Well & $\begin{array}{l}\text { (ft TOC) } \\
\text { (is }\end{array}$ & $\begin{array}{l}\text { (ft TOC) } \\
\text { (f) }\end{array}$ & $\begin{array}{l}\text { (ft TOC) } \\
\text { (f) }\end{array}$ & $\begin{array}{l}\text { (ft TOC) } \\
\text { (f }\end{array}$ & $\begin{array}{l}\text { (ft TOC) } \\
\text { (f) }\end{array}$ & $\begin{array}{l}\text { (ft TOC) } \\
\text { (f) }\end{array}$ & $\begin{array}{l}\text { (ft TOC) } \\
\text { (ft }\end{array}$ & $\begin{array}{l}\text { (ft TOC) } \\
\text { (f) }\end{array}$ & $\begin{array}{l}\text { (ft TOC) } \\
\text { (ft) }\end{array}$ & $\begin{array}{l}\text { (ft TOC) } \\
\text { (f) }\end{array}$ & $\begin{array}{l}\text { (ft TOC) } \\
\text { (f) }\end{array}$ & $\begin{array}{l}\text { (ft TOC) } \\
\text { (f) }\end{array}$ & (ft TOC) & $\begin{array}{l}\text { (ft TOC) } \\
\text { (f) }\end{array}$ & (ft TOC) & $\begin{array}{ll}\text { (ft TOC) } \\
\text { (ft TOC) }\end{array}$ & (ft TOC) & $\begin{array}{l}\text { (ft TOC) } \\
\text { (1) }\end{array}$ & (ft TOC) & $\begin{array}{l}\text { (ft TOC) } \\
\end{array}$ & $\begin{array}{l}\text { (ft TOC) } \\
\text { (f) }\end{array}$ & $\begin{array}{l}\text { (ft TOC) } \\
\text { (n) }\end{array}$ & (ft TOC) & $\begin{array}{l}\text { (ft TOC) } \\
\text { (1) }\end{array}$ & (ft TOC) \\
\hline 20:00 & & 30.289 & 21.182 & 29.003 & 29.72 & & 29.22 & 30.2 & 29.845 & 29.3 & 28 & 26.0 & 31.2 & 36.701 & 32.22 & 15.8 & 19.0 & 15.5 & 10.049 & 31.441 & 32 & 29.395 & 31.159 & 8.114 & 33.399 \\
\hline 2/29/12 1:00 & & 30.291 & 12.37 & 29.04 & 29.753 & & 29.215 & 30.282 & 29.845 & 29.403 & 28.219 & 26.091 & 31.216 & 36.708 & 32.234 & 15.893 & 19.017 & 15.554 & 10.066 & 31.459 & 32.109 & 29.419 & 31.176 & 28.114 & 33.401 \\
\hline 2/29/12 2:00 & & 30.291 & 2.356 & 29.11 & 29.776 & & 29.211 & 30.289 & 29.843 & 29.417 & 28.232 & 26.11 & 31.23 & 36.715 & 32.246 & 15.896 & 19.013 & 15.55 & 10.082 & 31.478 & 32.125 & 29.438 & 31.19 & 8.114 & 33.401 \\
\hline 2/29/12 3:00 & & 30.294 & 0.356 & 29.211 & 29.793 & & 29.222 & 30.294 & 29.84 & 29.426 & 28.244 & 26.115 & 31.239 & 36.722 & 32.253 & 15.9 & 19.01 & 15.545 & 10.089 & 31.483 & 32.127 & 29.44 & 31.194 & 28.117 & 33.404 \\
\hline 2/29/12 4:00 & & 30.298 & 0.286 & 29.218 & 29.807 & & 29.232 & 30.298 & 29.836 & 29.435 & 28.253 & 26.119 & 31.246 & 36.724 & 32.262 & 15.903 & 19.006 & 15.543 & 10.096 & 31.494 & 32.139 & 29.456 & 31.204 & 28.114 & 33.404 \\
\hline 2/29/12 5:00 & & 30.303 & 0.228 & 29.232 & 29.823 & & 29.232 & 30.298 & 29.838 & 29.447 & 28.265 & 26.131 & 31.253 & 36.729 & 32.27 & 15.907 & 18.999 & 15.536 & 10.103 & 31.508 & 32.149 & 29.463 & 31.211 & 28.115 & 33.404 \\
\hline 2/29/12 6:00 & & 30.303 & 0.179 & 29.251 & 29.837 & & 29.234 & 30.303 & 29.84 & 29.463 & 28.279 & 26.146 & 31.262 & 36.738 & 32.279 & 15.912 & 19.013 & 15.531 & 10.115 & 31.518 & 32.156 & 29.475 & 31.223 & 28.114 & 33.406 \\
\hline 2/29/12 7:00 & & 30.307 & 0.148 & 29.26 & 29.851 & & 29.239 & 30.308 & 29.845 & 29.475 & 28.293 & 26.155 & 31.269 & 36.738 & 32.286 & 5 $\quad 15.919$ & 19.015 & 15.524 & 10.122 & 31.529 & 32.161 & 29.489 & 31.227 & 28.114 & 33.408 \\
\hline 2/29/12 8:00 & & 30.312 & 0.111 & 29.281 & 29.865 & & 29.239 & 30.313 & 29.847 & 29.489 & 28.307 & 26.172 & 31.279 & 36.745 & 32.295 & 5 $\quad 15.921$ & 19.017 & 15.524 & 10.129 & 31.548 & 32.172 & 29.503 & 31.237 & 28.115 & 33.411 \\
\hline 2/29/12 9:00 & & 30.314 & 0.09 & 29.29 & 29.877 & & 29.253 & 30.315 & 29.84 & 29.5 & 28.319 & 26.181 & 31.286 & 36.75 & 32.307 & 15.926 & 19.013 & 15.526 & 10.141 & 31.553 & 32.177 & 29.518 & 31.246 & 28.11 & 33.411 \\
\hline $2 / 29 / 1210: 00$ & & 30.312 & 0.083 & 29.295 & 29.886 & & 29.267 & 30.32 & 29.845 & 29.514 & 28.323 & 26.186 & 31.295 & 36.754 & 32.309 & 15.935 & 19.01 & 15.536 & 10.15 & 31.56 & 32.187 & 29.527 & 31.263 & 28.112 & 33.413 \\
\hline 2/29/12 11:00 & & 30.319 & 0.078 & 29.297 & 29.891 & & 29.281 & 30.32 & 29.84 & 29.519 & 28.328 & 26.182 & 31.3 & 36.759 & 32.316 & 515.938 & 19.006 & 15.533 & 10.155 & 31.562 & 32.194 & 29.522 & 31.265 & 28.107 & 33.415 \\
\hline 2/29/12 12:00 & & 30.317 & 0.099 & 29.29 & 29.888 & & 29.298 & 30.322 & 29.836 & 29.521 & 28.337 & 26.172 & 31.305 & 36.759 & 32.323 & $\begin{array}{l}35.94 \\
3\end{array}$ & 19.01 & 15.54 & 10.157 & 31.56 & 32.194 & 29.518 & 31.272 & 28.105 & 33.418 \\
\hline 2/29/12 13:00 & & 30.314 & 0.144 & 29.269 & 29.879 & & 29.331 & 30.318 & 29.821 & 29.519 & 28.328 & 26.148 & 31.305 & 36.759 & 32.321 & 15.945 & 19.008 & 15.554 & 10.162 & 31.548 & 32.194 & 29.506 & 31.274 & 28.103 & 33.422 \\
\hline 2/29/12 14:00 & & 30.317 & 0.179 & 29.26 & 29.87 & & 29.34 & 30.315 & 29.824 & 29.514 & 28.328 & 26.131 & 31.302 & 36.754 & 32.321 & 15.945 & 19.003 & 15.552 & 10.158 & 31.543 & 32.196 & 29.496 & 31.265 & 28.1 & 33.42 \\
\hline $2 / 29 / 12$ 15:00 & & 30.314 & 0.223 & 29.258 & 29.865 & & 29.349 & 30.313 & 29.826 & 29.516 & 28.328 & 26.131 & 31.302 & 36.757 & 32.321 & 15.947 & 19.003 & 15.554 & 10.162 & 31.546 & 32.196 & 29.492 & 31.27 & 28.098 & 33.422 \\
\hline 2/29/12 16:00 & & 30.319 & 0.26 & 29.258 & 29.858 & & 29.354 & 30.308 & 29.826 & 29.519 & 28.328 & 26.129 & 31.305 & 36.754 & 32.326 & $\quad 15.947$ & 19.054 & 15.55 & 10.16 & 31.543 & 32.199 & 29.489 & 31.265 & 28.098 & 33.425 \\
\hline $2 / 29 / 1217: 00$ & & 30.317 & 0.302 & 29.253 & 29.856 & & 29.356 & 30.306 & 29.824 & 29.519 & 28.328 & 26.124 & 31.305 & 36.752 & 32.326 & 5 15.947 & 19.052 & 15.55 & 10.162 & 31.539 & 32.196 & 29.482 & 31.267 & 28.096 & 33.425 \\
\hline $2 / 29 / 1218: 00$ & & 30.314 & 0.344 & 29.241 & 29.846 & & 29.363 & 30.301 & 29.821 & 29.516 & 28.323 & 26.11 & 31.305 & 36.757 & 32.321 & 15.947 & 19.043 & 15.554 & 10.158 & 31.532 & 32.196 & 29.471 & 31.263 & 28.093 & 33.425 \\
\hline $2 / 29 / 1219: 00$ & & 30.317 & 0.363 & 29.246 & 29.846 & & 29.354 & 30.298 & 29.824 & 29.519 & 28.328 & 26.112 & 31.302 & 36.75 & 32.321 & 15.945 & 19.038 & 15.54 & 10.158 & 31.532 & 32.194 & 29.473 & 31.26 & 28.091 & 33.425 \\
\hline 2/29/12 20:00 & & 30.317 & 0.372 & 29.253 & 29.846 & & 29.347 & 30.294 & 29.824 & 29.523 & 28.33 & 26.119 & 31.3 & 36.745 & 32.319 & 15.9 & 19.031 & 15.54 & 10.157 & 31.536 & 32.196 & 29.473 & 31.263 & 28.091 & 33.425 \\
\hline 2/29/12 21:00 & & 30.319 & 0.379 & 29.253 & 29.844 & & 29.345 & 30.291 & 29.826 & 29.523 & 28.33 & 26.117 & 31.302 & 36.754 & 32.319 & 15.94 & 19.024 & 15.531 & 10.157 & 31.536 & 32.194 & 8.473 & 31.26 & 28.086 & 33.425 \\
\hline 2/29/12 22:00 & & 30.314 & 0.398 & 29.241 & 29.837 & & 29.352 & 30.286 & 29.819 & 29.521 & 28.328 & 26.105 & 31.298 & 36.75 & 32.316 & $5 \quad 15.942$ & 19.02 & 15.531 & 10.153 & 31.529 & 32.191 & .466 & 31.258 & 28.084 & 33.422 \\
\hline 2/29/12 23:00 & & 30.314 & 0.407 & 29.239 & 29.832 & & 29.354 & 30.282 & 29.819 & 29.519 & 28.323 & 26.103 & 31.295 & 36.743 & 32.312 & 15.94 & 19.015 & 15.524 & 10.153 & 31.522 & 32.187 & 29.456 & 31.255 & 28.084 & 33.42 \\
\hline 3/1/12 0:00 & & 30.31 & 0.437 & 29.208 & 29.814 & & 29.37 & 30.274 & 29.807 & 29.507 & 28.312 & 26.076 & 31.286 & 36.74 & 32.302 & 15.933 & 19.008 & 15.526 & 10.146 & 31.501 & 32.177 & 29.431 & 31.248 & 28.082 & 33.418 \\
\hline 3/1/12 1:00 & & 30.305 & 0.458 & 29.192 & 29.797 & & 29.378 & 30.267 & 29.802 & 29.498 & 28.302 & 26.059 & 31.276 & 36.733 & 32.295 & 15.931 & 19.001 & 15.524 & 10.136 & 31.487 & 32.173 & 29.416 & 31.239 & 28.079 & 33.413 \\
\hline 3/1/12 2:00 & & 30.301 & 0.643 & 29.166 & 29.779 & & 29.389 & 30.262 & 29.795 & 29.486 & 28.288 & 26.036 & 31.269 & 36.731 & 32.288 & $3 \quad 15.924$ & 18.997 & 15.526 & 10.125 & 31.473 & 32.161 & 29.393 & 31.232 & 28.077 & 33.411 \\
\hline 3/1/12 3:00 & & 30.294 & 0.878 & 29.138 & 29.755 & & 29.399 & 30.253 & 29.786 & 29.47 & 28.272 & 26.009 & 31.258 & 36.722 & 32.277 & 15.919 & 18.987 & 15.522 & 10.115 & 31.452 & 32.149 & 29.362 & 31.22 & 28.072 & 33.408 \\
\hline 3/1/12 4:00 & & 30.287 & 1.664 & 29.106 & 29.727 & & 29.408 & 30.246 & 29.776 & 29.454 & 28.253 & 25.981 & 31.246 & 36.715 & 32.262 & 15.91 & 18.978 & 15.519 & 10.106 & 31.424 & 32.135 & 29.337 & 31.209 & 28.072 & 33.404 \\
\hline 3/1/12 5:00 & & 30.282 & 2.554 & 29.085 & 29.704 & & 29.406 & 30.236 & 29.776 & 29.438 & 28.239 & 25.966 & 31.232 & 36.703 & 32.253 & 3. 15.903 & 18.969 & 15.51 & 10.096 & 31.413 & 32.123 & 29.315 & 31.197 & 28.068 & 33.401 \\
\hline 3/1/12 6:00 & & 30.282 & 3.38 & 29.092 & 29.695 & & 29.385 & 30.231 & 29.786 & 29.433 & 28.237 & 25.971 & 31.225 & 36.701 & 32.246 & 515.898 & 18.978 & 15.489 & 10.084 & 31.413 & 32.116 & 29.32 & 31.187 & 28.07 & 33.401 \\
\hline 3/1/12 7:00 & & 30.28 & 4.189 & 29.075 & 29.681 & & 29.38 & 30.224 & 29.779 & 29.419 & 28.225 & 25.952 & 31.216 & 36.694 & 32.239 & 15.889 & 18.98 & 15.484 & 10.078 & 31.396 & 32.109 & 29.306 & 31.176 & 28.068 & 33.399 \\
\hline 3/1/12 8:00 & & 30.273 & 4.963 & 29.071 & 29.671 & & 29.37 & 30.217 & 29.778 & 29.414 & 28.219 & 25.959 & 31.209 & 36.691 & 32.227 & 15.882 & 18.98 & 15.477 & 10.068 & 31.385 & 32.101 & 29.299 & 31.173 & 28.068 & 33.397 \\
\hline 3/1/12 9:00 & & 30.273 & 5.681 & 29.073 & 29.669 & & 29.356 & 30.21 & 29.781 & 29.407 & 28.216 & 25.959 & 31.204 & 36.689 & 32.227 & 15.875 & 18.971 & 15.463 & 10.063 & 31.389 & 32.097 & 29.301 & 31.166 & 28.065 & 33.397 \\
\hline $3 / 1 / 1210: 00$ & & 30.268 & 6.397 & 29.059 & 29.65 & & 29.359 & 30.205 & 29.778 & 29.398 & 28.207 & 25.95 & 31.197 & 36.691 & 32.218 & $3 \quad 15.8$ & 18.964 & 15.461 & 10.056 & 31.375 & 32.09 & 29.285 & 31.162 & 28.068 & 33.397 \\
\hline 3/1/12 11:00 & & 30.266 & 7.066 & 29.061 & 29.655 & & 29.347 & 30.203 & 29.779 & 29.393 & 28.205 & 25.959 & 31.192 & 36.684 & 32.216 & 15.8 & 18.957 & 15.456 & 10.049 & 31.375 & 32.085 & 29.292 & 31.162 & 28.061 & 33.397 \\
\hline $3 / 1 / 1212: 00$ & & 30.263 & 7.721 & 29.054 & 29.65 & & 29.342 & 30.195 & 29.776 & 29.389 & 28.2 & 25.957 & 31.188 & $\begin{array}{l}30.044 \\
36.682\end{array}$ & 32.209 & 15.8 & 18.953 & 15.449 & $\begin{array}{l}10.044 \\
\end{array}$ & 31.371 & 32.08 & 29.278 & 31.159 & 28.061 & 33.399 \\
\hline $3 / 1 / 1213: 00$ & & 30.256 & 8.404 & 29.028 & 29.634 & & 29.354 & 30.188 & 29.767 & 29.373 & $\begin{array}{r}28.184 \\
28.12\end{array}$ & 25.933 & 31.181 & $\begin{array}{l}30.02 \\
36.679\end{array}$ & 32.202 & 15.8 & 18.943 & 15.456 & 10.04 & 31.352 & 32.073 & 29.264 & 31.152 & 28.058 & $\begin{array}{l}33.397 \\
339\end{array}$ \\
\hline $3 / 1 / 12$ & & 30 & 9.033 & 29.012 & 29 & & 59 & 30.183 & 29.76 & 29.361 & 28 & 25.921 & 31 & 75 & 32.19 & 15. & 18.941 & 15.458 & 10.03 & 31.343 & 32.063 & 47 & 31.143 & 28.058 & 33.397 \\
\hline $3 / 1 /$ & & 30 & 9.607 & 29.021 & 29 & & 2 & 30.176 & 29.764 & 29.359 & 28 & 25.928 & 1 & 5 & 32.192 & 15 & $1 \varepsilon$ & 15.442 & 10.026 & 31.343 & 61 & 67 & 31.131 & 28.058 & 33.399 \\
\hline 3/1/12 16:00 & & 30 & 10.187 & 29.04 & 29 & & 2 & 30 & 29 & 29 & 28 & 25.947 & 9 & 36 & 32.19 & 15 & $1 \varepsilon$ & 15.432 & 10.026 & 31.352 & 32.059 & 66 & 31.129 & 28.058 & 33.401 \\
\hline $3 / 1 / 12$ 17:00 & & 30 & 10.721 & 29.035 & 29.62 & & 2 & 30.167 & 29.774 & 29.356 & 28.174 & 25.952 & 31.167 & 36.6 & 32.185 & 515. & 6 & 15.435 & 10.021 & 31.352 & 32.059 & 66 & 31.126 & 28.058 & 33.399 \\
\hline $3 / 1 / 1218: 00$ & & 30.254 & 11.197 & 29.066 & 29.6 & & 29.274 & 30.167 & 29.786 & 29.363 & 28.186 & 25.981 & 31.171 & 36.675 & 32.19 & 15.823 & 18.939 & 15.418 & 10.028 & 31.366 & 32.063 & 29.287 & 31.129 & 28.058 & 33.404 \\
\hline 3/1/12 19:00 & & 30.254 & 11.654 & 29.085 & 29. & & 29.255 & 30.167 & 29.79 & 29.373 & 28.195 & 26.007 & 31.176 & 36.677 & 32.195 & 15.8 & 18.936 & 15.414 & 10.033 & 31.382 & 32.071 & 29.306 & 31.138 & 28.056 & 33.404 \\
\hline 3/1/12 20:00 & & 30.254 & 12.106 & 29.084 & 29.6 & & 29 & 30.1 & 29.783 & 29.37 & 28.198 & 26 & 31.176 & 36.682 & 32.197 & & & 15.407 & 10.03 & 31.382 & 32.071 & 29.304 & 31.133 & 28.054 & 33.406 \\
\hline 3/1/12 21:00 & & 30.259 & 12.528 & 29.103 & 29.678 & & 29.2 & 30.162 & 29.79 & & 28.207 & 26.021 & 31.183 & 36.686 & 32.199 & 15. & & 15.395 & 10.04 & 31.396 & 32.073 & & 31.143 & 28.056 & 33.406 \\
\hline 3/1/12 22:00 & & 30.266 & 12.936 & 29.122 & 29. & & 29 & 30.162 & 29.8 & 29.387 & 28.219 & 26.031 & 31.181 & 36.689 & 32.202 & 15.8 & 18.943 & 15.376 & 10.037 & 31.403 & 32.075 & 29.339 & 31.133 & 28.056 & 33.408 \\
\hline 3/1/12 23:00 & & 30.245 & 13.384 & 29.08 & 29.6 & & 29.2 & 30.16 & 29.767 & 29 & 28.202 & 25.997 & 31.176 & 36.686 & 32.195 & 15.8 & 18.939 & 15.4 & 10.035 & 31.382 & 32.071 & 29.306 & 31.136 & 28.051 & 33.404 \\
\hline $3 / 2 / 120: 00$ & & 30.247 & 13.834 & 29.087 & 29.6 & & 29.246 & 30.155 & 29.776 & 29.377 & 28.202 & 26.012 & 31.178 & 36.686 & 32.197 & 15.8 & 18.939 & 15.39 & 10.035 & 31.382 & 32.068 & 29.308 & 31.136 & 28.051 & 33.404 \\
\hline 3/2/12 1:00 & & 30.245 & 14.214 & 29.08 & 29.678 & & 29.253 & 30.155 & 29.771 & 29.377 & 28.202 & 26.012 & 31.171 & 36.686 & 32.19 & 15.81 & 18.932 & 15.388 & 10.031 & 31.378 & 32.063 & 29.306 & 31.133 & 28.049 & 33.401 \\
\hline 3/2/12 2:00 & & 30.252 & 14.559 & 29.096 & 29.683 & & 29.236 & 30.152 & 29.776 & 29.373 & 28.209 & 26.007 & 31.174 & 36.684 & 32.19 & 15.807 & 18.932 & 15.367 & 10.028 & 31.387 & 32.063 & 29.322 & 31.126 & 28.049 & 33.401 \\
\hline 3/2/12 3:00 & & 30.254 & 14.899 & 29.108 & 29.692 & & 29.229 & 30.152 & 29.783 & 29.382 & 28.216 & 26.021 & 31.176 & 36.689 & 32.195 & 15.807 & 18.927 & 15.362 & 10.033 & 31.396 & 32.068 & 29.332 & 31.131 & 28.051 & 33.399 \\
\hline 3/2/12 4:00 & & 30.266 & 15.2 & 29.152 & 29.718 & & 29.199 & 30.155 & 29.805 & 29.398 & 28.242 & 26.057 & 31.183 & 36.703 & 32.204 & 15.81 & 18.925 & 15.338 & 10.042 & 31.429 & 32.078 & 29.369 & 31.136 & 28.049 & 33.404 \\
\hline 3/2/12 5:00 & & 30.259 & 15.533 & 29.152 & 29.725 & & 29.213 & 30.16 & 29.793 & 29.407 & 28.239 & 26.072 & 31.188 & 36.696 & 32.206 & 15.81 & 18.927 & 15.357 & 10.045 & 31.422 & 32.08 & 29.365 & 31.148 & 28.049 & 33.406 \\
\hline 3/2/12 6:00 & & 30.256 & 15.848 & 29.145 & 29.73 & & 29.225 & 30.157 & 29.786 & 29.405 & 28.242 & 26.055 & 31.19 & 36.705 & 32.206 & $\quad 15.81$ & 18.936 & 15.355 & 10.047 & 31.422 & 32.08 & 29.362 & 31.143 & 28.049 & 33.404 \\
\hline 3/2/12 7:00 & & 30.277 & 16.102 & 29.211 & 29.765 & & 29.178 & 30.167 & 29.814 & 29.435 & 28.274 & 26.112 & 31.204 & 36.719 & 32.223 & 15.814 & 18.939 & 15.322 & 10.059 & 31.469 & 32.097 & 29.424 & 31.15 & 28.056 & 3.408 \\
\hline
\end{tabular}




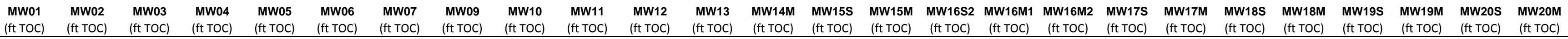

\begin{tabular}{|c|c|c|c|c|c|c|c|c|c|c|c|c|c|c|c|c|c|c|c|c|c|c|c|c|}
\hline Well & $\begin{array}{l}\text { (ft TOC) } \\
\text { (f) }\end{array}$ & $\begin{array}{l}\text { (ft TOC) } \\
\text { (ft) }\end{array}$ & $\begin{array}{l}\text { (ft TOC) } \\
\end{array}$ & $\begin{array}{l}\text { (ft TOC) } \\
\text { (f) }\end{array}$ & $\begin{array}{l}\text { (ift TOC) } \\
\text { (ft TOC) }\end{array}$ & $\begin{array}{l}\text { (ft TOC) } \\
\end{array}$ & $\begin{array}{l}\text { (ift TOC) } \\
\end{array}$ & $\begin{array}{l}\text { (ft TOC) } \\
\text { (f) }\end{array}$ & $\begin{array}{l}\text { (ft TOC) } \\
\text { (f) }\end{array}$ & $\begin{array}{l}\text { (ft TOC) } \\
\text { (n) }\end{array}$ & $\begin{array}{l}\text { (ft TOC) } \\
\text { (f) }\end{array}$ & (ft TOC) & $\begin{array}{l}\text { (ft TOC) } \\
\end{array}$ & (ft TOC) & $\begin{array}{l}\text { (ft TOC) } \\
\text { (ft TOC) }\end{array}$ & (ft TOC) & $\begin{array}{l}\text { (ft TOC) } \\
\text { (n) }\end{array}$ & (ft TOC) & $\begin{array}{l}\text { (ft TOC) } \\
\text { (f) }\end{array}$ & (ft TOC) & $\begin{array}{l}\text { (ft TOC) } \\
\text { (n) }\end{array}$ & (ft TOC) & (ft TOC) & (ft TOC) \\
\hline $8: 00$ & & 30.28 & 16.436 & 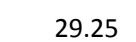 & 29.7 & 29.168 & 30.176 & 29.82 & 29.458 & 28.298 & 26.1 & 31.22 & 36.729 & 32.2 & $15 . \varepsilon$ & 18.948 & 15.327 & 10.073 & 31. & 32. & 4 & 1.173 & 28.051 & 33.413 \\
\hline 3/2/12 9:00 & & 30.282 & 16.734 & 29.258 & 29.816 & 29.18 & 30.181 & 29.821 & 29.47 & 28.309 & 26.162 & 31.232 & 36.731 & 32.244 & 15.823 & 18.948 & 15.338 & 10.084 & 31.501 & 2.12 & 29.461 & 31.183 & 28.051 & 33.418 \\
\hline 3/2/12 10:00 & & 30.287 & 17.028 & 29.281 & 29.839 & 29.187 & 30.188 & 29.817 & 29.489 & 28.323 & 26.179 & 31.241 & 36.743 & 32.255 & 15.828 & 18.948 & 15.343 & 10.094 & 31.518 & 32.132 & 29.478 & 31.204 & 8.051 & 33.42 \\
\hline $3 / 2 / 1211: 00$ & & 30.291 & 17.317 & 29.302 & 29.86 & 29.192 & 30.198 & 29.828 & 29.505 & 28.342 & 26.191 & 31.253 & 36.75 & 32.267 & 15.835 & 18.941 & 15.343 & 10.106 & 31.534 & 32.146 & 29.501 & 31.213 & 28.054 & 33.427 \\
\hline 3/2/12 12:00 & & 30.287 & 17.643 & 29.278 & 29.858 & 29.232 & 30.205 & 29.805 & 29.505 & 28.337 & 26.172 & 31.255 & 36.754 & 32.272 & 15.84 & 18.939 & 15.371 & 10.11 & 31.525 & 32.149 & 29.482 & 31.223 & 28.051 & 33.427 \\
\hline 3/2/12 13:00 & & 30.284 & 17.96 & 29.26 & 29.851 & 29.265 & 30.205 & 29.795 & 29.503 & 28.335 & 26.151 & 31.255 & 36.752 & 32.272 & 15.84 & 18.932 & 15.392 & 10.108 & 31.511 & 32.149 & 29.468 & 31.227 & 28.051 & 33.427 \\
\hline 3/2/12 14:00 & & 30.287 & 18.254 & 29.251 & 29.844 & 29.279 & 30.205 & 29.79 & 29.503 & 28.332 & 26.136 & 31.258 & 36.75 & 32.274 & 15.842 & 18.932 & 15.4 & 10.11 & 31.513 & 32.149 & 29.463 & 31.223 & 28.051 & 33.427 \\
\hline 3/2/12 15:00 & & 30.291 & 18.52 & 29.26 & 29.849 & 29.281 & 30.207 & 29.798 & 29.507 & 28.339 & 26.141 & 31.26 & 36.754 & 32.279 & 15.847 & 18.929 & 15.402 & 10.115 & 31.52 & 32.154 & 29.468 & 31.22 & 28.051 & 33.429 \\
\hline 3/2/12 16:00 & & 30.291 & 18.774 & 29.265 & 29.846 & 29.286 & 30.21 & 29.795 & 29.514 & 28.342 & 26.143 & 31.262 & 36.754 & 32.281 & 15.847 & 18.983 & 15.409 & 10.12 & 31.518 & 32.154 & 29.468 & 31.223 & 28.051 & 33.432 \\
\hline 3/2/12 17:00 & & 30.294 & 19.009 & 29.271 & 29.851 & 29.288 & 30.212 & 29.802 & 29.519 & 28.349 & 26.146 & 31.27 & 36.759 & 32.284 & 15.851 & 18.98 & 15.411 & 10.122 & 31.522 & 32.161 & 29.48 & 31.225 & 28.051 & 33.434 \\
\hline 3/2/12 18:00 & & 30.298 & 19.212 & 29.286 & 29.858 & 29.281 & 30.219 & 29.807 & 29.528 & 28.356 & 26.153 & 31.274 & 36.757 & 32.293 & 15.856 & 18.978 & 15.409 & 10.129 & 31.534 & 32.165 & 29.48 & 31.227 & 28.054 & 33.436 \\
\hline 3/2/12 19:00 & & 30.307 & 19.387 & 29.318 & 29.879 & 29.26 & 30.224 & 29.817 & 29.544 & 28.374 & 26.179 & 31.281 & 36.766 & 32.298 & 15.858 & 18.978 & 15.4 & 10.136 & 31.557 & 32.175 & 29.52 & 31.232 & 28.054 & 33.441 \\
\hline 3/2/12 20:00 & & 30.314 & 19.581 & 29.344 & 29.9 & 29.253 & 30.234 & 29.826 & 29.565 & 28.388 & 26.203 & 31.293 & 36.771 & 32.312 & 15.863 & 18.973 & 15.402 & 10.146 & 31.571 & 32.187 & 29.536 & 31.246 & 28.054 & 33.443 \\
\hline $3 / 2 / 1221: 00$ & & 30.314 & 19.779 & 29.356 & 29.914 & 29.255 & 30.241 & 29.824 & 29.577 & 28.4 & 26.215 & 31.305 & 36.773 & 32.319 & 15.865 & 18.978 & 15.409 & 10.157 & 31.578 & 32.196 & 29.546 & 31.258 & 28.054 & 33.448 \\
\hline $3 / 2 / 1222: 00$ & & 30.317 & 19.972 & 29.36 & 29.923 & 29.267 & 30.246 & 29.819 & 29.584 & 28.407 & 26.215 & 31.309 & 36.771 & 32.323 & 15.87 & 18.98 & 15.414 & 10.16 & 31.59 & 32.203 & 29.55 & 31.265 & 28.054 & 33.448 \\
\hline 3/2/12 23:00 & & 30.324 & 20.152 & 29.372 & 29.935 & 29.267 & 30.251 & 29.824 & 29.598 & 28.416 & 26.225 & 31.316 & 36.785 & 32.33 & 15.875 & 18.983 & 15.411 & 10.171 & 31.599 & 32.21 & 29.565 & 31.272 & 28.056 & 33.45 \\
\hline $3 / 3 / 120: 00$ & & 30.324 & 20.341 & 29.367 & 29.937 & 29.284 & 30.255 & 29.821 & 29.602 & 28.423 & 26.218 & 31.319 & 36.787 & 32.335 & 15.877 & 18.978 & 15.418 & 10.169 & 31.597 & 32.21 & 29.562 & 31.274 & 28.056 & 33.45 \\
\hline 3/3/12 1:00 & & 30.328 & 20.522 & 29.372 & 29.942 & 29.293 & 30.26 & 29.821 & 29.607 & 28.428 & 26.215 & 31.321 & 36.79 & 32.338 & 15.882 & 18.976 & 15.428 & 10.176 & 31.599 & 32.213 & 29.567 & 31.277 & 28.056 & 33.448 \\
\hline $3 / 3 / 122: 00$ & & 30.333 & 20.693 & 29.384 & 29.949 & 29.295 & 30.265 & 29.828 & 29.616 & 28.435 & 26.225 & 31.326 & 36.792 & 32.342 & 15.884 & 18.976 & 15.423 & 10.181 & 31.606 & 32.217 & 29.574 & 31.281 & 28.056 & 33.448 \\
\hline $3 / 3 / 12$ 3:00 & & 30.333 & 20.877 & 29.379 & 29.949 & 29.309 & 30.27 & 29.821 & 29.623 & 28.437 & 26.218 & 31.328 & 36.794 & 32.345 & 15.886 & 18.971 & 15.435 & 10.181 & 31.606 & 32.22 & 29.572 & 31.284 & 28.056 & 33.446 \\
\hline $3 / 3 / 124: 00$ & & 30.333 & 21.054 & 29.372 & 29.947 & 29.326 & 30.274 & 29.819 & 29.623 & 28.437 & 26.208 & 31.328 & 36.799 & 32.347 & 15.889 & 18.969 & 15.43 & 10.181 & 31.597 & 32.22 & 29.565 & 31.284 & 28.056 & 33.446 \\
\hline 3/3/12 5:00 & & 30.335 & 21.224 & 29.372 & 29.947 & 29.331 & 30.277 & 29.819 & 29.626 & 28.439 & 26.203 & 31.328 & 36.794 & 32.345 & 15.891 & .966 & 15.442 & 10.183 & 31.597 & 32.222 & 29.569 & 31.288 & 28.058 & 33.446 \\
\hline 3/3/12 6:00 & & 30.338 & 21.266 & 29.374 & 29.944 & 29.34 & 30.279 & 29.819 & 29.63 & 28.442 & 26.205 & 31.33 & 36.794 & 32.347 & 15.896 & 18.962 & 15.449 & 10.186 & 31.602 & 32.225 & 29.565 & 31.291 & 28.058 & 33.448 \\
\hline 3/3/12 7:00 & & 30.342 & 21.313 & 29.377 & 29.944 & 29.347 & 30.284 & 29.819 & 29.633 & 28.442 & 26.201 & 31.335 & 36.801 & 32.352 & 15.896 & 18.96 & 15.446 & 10.188 & 31.602 & 32.227 & 29.565 & 31.293 & 28.058 & 33.448 \\
\hline 3/3/12 8:00 & & 30.347 & 21.394 & 29.386 & 29.947 & 29.347 & 30.286 & 29.828 & 29.64 & 28.449 & 26.205 & 31.337 & 36.797 & 32.354 & 15.896 & 18.96 & 15.439 & 10.19 & 31.606 & 32.229 & 29.572 & 31.295 & 28.058 & 33.45 \\
\hline 3/3/12 9:00 & & 30.347 & 21.52 & 29.395 & 29.951 & 29.354 & 30.291 & 29.828 & 29.649 & 28.453 & 26.213 & 31.34 & 36.806 & 32.359 & 15.9 & 18.966 & 15.453 & 10.197 & 31.616 & 32.232 & 29.581 & 31.305 & 28.061 & 33.453 \\
\hline 3/3/12 10:00 & & 30.345 & 21.688 & 29.374 & 29.944 & 29.378 & 30.291 & 29.821 & 29.64 & 28.446 & 26.191 & 31.34 & 36.804 & 32.359 & 15.898 & 18.973 & 15.472 & 10.195 & 31.599 & 32.232 & 29.562 & 31.307 & 28.056 & 33.453 \\
\hline 3/3/12 11:00 & & 30.345 & 21.858 & 29.358 & 29.93 & 29.399 & 30.291 & 29.809 & 29.635 & 28.439 & 26.174 & 31.337 & 36.799 & 32.354 & 15.898 & 18.987 & 15.491 & 10.193 & 31.59 & 32.229 & 29.548 & 31.314 & 28.061 & 33.453 \\
\hline 3/3/12 12:00 & & 30.34 & 22.026 & 29.337 & 29.916 & 29.415 & 30.289 & 29.807 & 29.626 & 28.43 & 26.158 & 31.33 & 36.79 & 32.352 & 15.898 & 19.031 & 15.505 & 10.188 & 31.574 & 32.222 & 29.532 & 31.307 & 28.061 & 33.453 \\
\hline 3/3/12 13:00 & & 30.338 & 22.175 & 29.316 & 29.9 & 29.434 & 30.286 & 29.802 & 29.614 & 28.416 & 26.134 & 31.326 & 36.785 & 32.345 & 15.896 & 19.04 & 15.515 & 10.181 & 31.562 & 32.215 & 29.508 & 31.3 & 28.061 & 33.45 \\
\hline $3 / 3 / 1214: 00$ & & 30.331 & 22.306 & 29.286 & 29.877 & 29.45 & 30.282 & 29.795 & 29.6 & 28.405 & 26.105 & 31.316 & 36.78 & 32.335 & 15.891 & 19.036 & 15.524 & 10.171 & 31.543 & 32.203 & 29.482 & 31.286 & 28.061 & 33.448 \\
\hline $3 / 3 / 1215: 00$ & & 30.333 & 22.397 & 29.281 & 29.863 & 29.446 & 30.277 & 29.8 & 29.593 & 28.395 & 26.098 & 31.312 & 36.776 & 32.33 & 15.891 & 19.038 & 15.522 & 10.167 & 31.536 & 32.203 & 29.475 & 31.274 & 28.061 & 33.448 \\
\hline $3 / 3 / 1216: 00$ & & 30.333 & 22.458 & 29.283 & 29.853 & 29.434 & 30.272 & 29.802 & 29.593 & 28.395 & 26.103 & 31.305 & 36.771 & 32.326 & 15.886 & 19.103 & 15.522 & 10.162 & 31.529 & 32.199 & 29.475 & 31.267 & 28.061 & 33.448 \\
\hline $3 / 3 / 1217: 00$ & & 30.338 & 22.509 & 29.292 & 29.856 & 29.418 & 30.274 & 29.812 & 29.593 & 28.398 & 26.112 & 31.309 & 36.773 & 32.33 & 15.884 & 19.103 & 15.519 & 10.164 & 31.541 & 32.201 & 29.485 & 31.27 & 28.063 & 33.45 \\
\hline $3 / 3 / 1218: 00$ & & 30.34 & 22.486 & 29.309 & 29.865 & 29.401 & 30.277 & 29.816 & 29.598 & 28.402 & 26.126 & 31.312 & 36.775 & 32.33 & 15.8 & 19.089 & 15.515 & 10.169 & 31.55 & 32.201 & 29.494 & 31.267 & 28.063 & 33.453 \\
\hline 3/3/12 19:00 & & 30.34 & 22.483 & 29.316 & 29.8 & 29.392 & 30.277 & 29.817 & 29.6 & 28.405 & 26.131 & 31.314 & 36.776 & 32.33 & 15.879 & 19.082 & 15.517 & 10.169 & 31.553 & 32.206 & 29.496 & 31.27 & 28.063 & 33.455 \\
\hline $3 / 3 / 1220: 00$ & & 30.338 & 22.513 & 29.304 & 29.86 & 29.399 & 30.279 & 29.812 & $\begin{array}{l}29.593 \\
\end{array}$ & 28.4 & 26.122 & 31.314 & 36.78 & 32.33 & 15.877 & $\begin{array}{l}19.073 \\
19.02\end{array}$ & 15.524 & 10.171 & 31.548 & 32.206 & 29.489 & 31.272 & 28.063 & $\begin{array}{l}33.455 \\
3.45\end{array}$ \\
\hline $3 / 3 / 1221: 00$ & & 30.338 & 22.532 & 29.309 & 29.86 & 29.392 & 30.279 & 29.814 & 29.595 & $\begin{array}{r}28.4 \\
28.398\end{array}$ & 26.124 & $\begin{array}{l}31.314 \\
31.314\end{array}$ & 36.776 & 32.333 & 15.875 & 19.064 & 15.515 & 10.169 & 31.543 & 32.206 & 29.489 & 31.272 & 28.063 & $\begin{array}{l}33.455 \\
3.45\end{array}$ \\
\hline $3 / 3 / 1222: 00$ & & 30.34 & $\begin{array}{l}22.567 \\
22.567\end{array}$ & 29.302 & 29.8 & 29.392 & 30.279 & 29.812 & 29.591 & $\begin{array}{l}28.390 \\
28.395\end{array}$ & 26.119 & $\begin{array}{l}31.309 \\
31.34\end{array}$ & 36.778 & 32.33 & $\begin{array}{l}15.8 \\
15.8\end{array}$ & $\begin{array}{l}19.064 \\
19.061\end{array}$ & 15.512 & 10.169 & 31.543 & 32.203 & 29.485 & 31.267 & 28.065 & $\begin{array}{l}33.450 \\
33.457\end{array}$ \\
\hline 23:00 & & 30. & 22.6 & 29.2 & 29 & 29 & 30 & 29.8 & 29 & 28 & 26 & 31 & 36.773 & 32.328 & 15 & 2 & 15.512 & 10.162 & 31.536 & 1 & 29.48 & 31.265 & 28.063 & 455 \\
\hline 0:00 & & 30.335 & 22 & 29.283 & 29 & 29. & 30. & 29.805 & 29 & 28.384 & 26.107 & 31.307 & 36.771 & 32.321 & 15 & 5 & 15.51 & 10.16 & 534 & 6 & 29.468 & 31.26 & 28.07 & 33.455 \\
\hline 3/4/12 1:00 & & 30.338 & 22.707 & 29.286 & 29.8 & 29.394 & 30.2 & 29.807 & 29.579 & 28.384 & 26.103 & 31.3 & 36.768 & 32.319 & 15 & .036 & 15.505 & 10.155 & 31.529 & 191 & 29.473 & 31.26 & 28.063 & 33.453 \\
\hline $3 / 4 / 122: 00$ & & 30.335 & 22.763 & 29.281 & 29.8. & 29.394 & 30.274 & 29.807 & 29.575 & 28.379 & 26.1 & 31.295 & 36.766 & 32.314 & 15.8 & 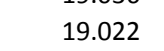 & 15.505 & 10.153 & 31.522 & 32.189 & 29.463 & 31.255 & 28.065 & 33.45 \\
\hline 3/4/12 3:00 & & 30.333 & 22.847 & 29.267 & 29 & 29.401 & 30. & 29.805 & 29 & 28.372 & 26.086 & 31.286 & 36.766 & 32.307 & 15.861 & 7 & 15.507 & 10.143 & 31.511 & 32.182 & 29.454 & 31.244 & 28.065 & 33.446 \\
\hline $3 / 4 / 124: 00$ & & 30.324 & 22.947 & 29.241 & 29.8 & 29. & & 29.795 & & 28.356 & 26.069 & 31.279 & 36.759 & 32.3 & 15.8 & 8 & 15.512 & 10.138 & 31.497 & & 29.419 & 31.246 & 28.063 & 33.443 \\
\hline 3/4/12 5:00 & & 30.328 & 23.038 & 29.241 & 29. & 29.408 & 30. & 29.798 & 29. & 28.351 & 26.062 & 31.274 & 36.761 & 32.293 & 15.8 & 19.001 & 15.498 & 10.131 & 31.492 & & 29.435 & 31.234 & 28.068 & 33.441 \\
\hline 3/4/12 6:00 & & 30.326 & 23.12 & 29.241 & 29.8 & 29.403 & 30.262 & 29.8 & 29.5 & 28.351 & 26.064 & 31.272 & 36.752 & 32.291 & 15.8 & 18.994 & 15.496 & 10.131 & 31.49 & 32.165 & 29.426 & 31.232 & 28.065 & 33.441 \\
\hline 3/4/122 7:00 & & 30.328 & 23.197 & 29.248 & 29.8 & 29.389 & 30.26 & 29.805 & 29.544 & 28.351 & 26.072 & 31.27 & 36.752 & 32.291 & 15.8 & 18.994 & 15.489 & 10.127 & 31.494 & 32.163 & 29.438 & 31.23 & 28.065 & 33.443 \\
\hline 3/4/12 8:00 & & 30.333 & 23.281 & 29.274 & 29.8 & 29.37 & 30.262 & 29.812 & 29.554 & 28.363 & 26.1 & 31.276 & 36.745 & 32.295 & 15.8 & 18.992 & 15.482 & 10.129 & 31.513 & 32.17 & 29.456 & 31.237 & 28.07 & 33.446 \\
\hline 3/4/12 9:00 & & 30.338 & 23.397 & 29.293 & 29.832 & 29.359 & 30.265 & 29.821 & 29.563 & 28.37 & 26.119 & 31.281 & 36.764 & 32.3 & 15.84 & 18.999 & 15.482 & 10.138 & 31.52 & 32.172 & 29.466 & 31.246 & 28.072 & 33.448 \\
\hline $3 / 4 / 1210: 00$ & & 30.338 & 23.53 & 29.307 & 29.844 & 29.356 & 30.272 & 29.814 & 29.568 & 28.377 & 26.131 & 31.286 & 36.769 & 32.305 & 15.844 & 18.994 & 15.482 & 10.141 & 31.532 & 32.18 & 29.482 & 31.253 & 28.07 & 33.45 \\
\hline $3 / 4 / 1211: 00$ & & 30.34 & 23.679 & 29.316 & 29.851 & 29.361 & 30.274 & 29.819 & 29.572 & 28.381 & 26.139 & 31.291 & 36.773 & 32.312 & 15.844 & 18.992 & 15.486 & 10.146 & 31.539 & 32.184 & 29.487 & 31.26 & 28.07 & 33.453 \\
\hline $3 / 4 / 1212: 00$ & & 30.335 & 23.831 & 29.311 & 29.858 & 29.37 & 30.277 & 29.821 & 29.572 & 28.381 & 26.136 & 31.293 & 36.778 & 32.312 & 15.844 & 18.992 & 15.493 & 10.15 & 31.534 & 32.187 & 29.489 & 31.265 & 28.072 & 33.455 \\
\hline 3/4/12 13:00 & & 30.338 & 23.98 & 29.309 & 29.856 & 29.382 & 30.279 & 29.814 & 29.572 & 28.377 & 26.129 & 31.291 & 36.778 & 32.309 & 15.844 & 18.985 & 15.51 & 10.148 & 31.532 & 32.184 & 29.482 & 31.267 & 28.07 & 33.453 \\
\hline 3/4/12 14:00 & & 30.335 & 24.113 & 29.293 & 29.849 & 29.396 & 30.274 & 29.809 & 29.565 & 28.37 & 26.115 & 31.288 & 36.775 & 32.307 & 15.844 & 18.987 & 15.519 & 10.146 & 31.525 & 32.18 & 29.475 & 31.258 & 28.072 & 33.45 \\
\hline 3/4/12 15:00 & & 30.34 & 24.234 & 29.3 & 29.851 & 29.392 & 30.277 & 29.814 & 29.565 & 28.374 & 26.117 & 31.286 & 36.773 & 32.307 & 15.844 & 18.983 & 15.515 & 10.146 & 31.527 & 32.18 & 29.478 & 31.248 & 28.072 & 53 \\
\hline
\end{tabular}




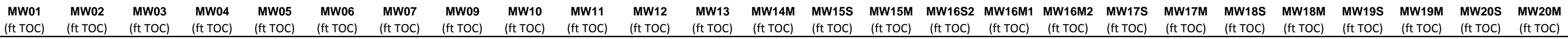

\begin{tabular}{|c|c|c|c|c|c|c|c|c|c|c|c|c|c|c|c|c|c|c|c|c|c|c|c|c|}
\hline Well & $\begin{array}{l}\text { (ft TOC) } \\
\text { (f) }\end{array}$ & $\begin{array}{l}\text { (ft TOC) } \\
\text { (ft) }\end{array}$ & $\begin{array}{l}\text { (ft TOC) } \\
\end{array}$ & $\begin{array}{l}\text { (ft TOC) } \\
\text { (f) }\end{array}$ & $\begin{array}{l}\text { (ift TOC) } \\
\text { (ft TOC) }\end{array}$ & $\begin{array}{l}\text { (ft TOC) } \\
\end{array}$ & $\begin{array}{l}\text { (ift TOC) } \\
\end{array}$ & $\begin{array}{l}\text { (ft TOC) } \\
\text { (f) }\end{array}$ & $\begin{array}{l}\text { (ft TOC) } \\
\text { (f) }\end{array}$ & $\begin{array}{l}\text { (ft TOC) } \\
\text { (n) }\end{array}$ & $\begin{array}{l}\text { (ft TOC) } \\
\text { (ft) }\end{array}$ & (ft TOC) & $\begin{array}{l}\text { (ft TOC) } \\
\end{array}$ & (ft TOC) & $\begin{array}{l}\text { (ft TOC) } \\
\text { (ft TOC) }\end{array}$ & (ft TOC) & $\begin{array}{l}\text { (nt TOC) } \\
\text { (ft }\end{array}$ & (ft TOC) & $\begin{array}{l}\text { (ft TOC) } \\
\text { (f) }\end{array}$ & (ft TOC) & $\begin{array}{l}\text { (ft TOC) } \\
\text { (n) }\end{array}$ & (ft TOC) & $\begin{array}{l}\text { (ft TOC) } \\
\text { (1) }\end{array}$ & (ft TOC) \\
\hline 16:00 & & 30.342 & 24.323 & 29.311 & 29.858 & 29.385 & 30.279 & 29.819 & 29.572 & 28.381 & 26.12 & 31.288 & 36.7 & 32. & 15.8 & 18.978 & 15.5 & 10.148 & 31. & 32.184 & 7 & 31.248 & 28.075 & 33.455 \\
\hline 3/4/12 17:00 & & 30.345 & 24.395 & 29.323 & 29.865 & 29.375 & 30.284 & 29.826 & 29.579 & 28.386 & 26.143 & 31.302 & 36.783 & 32.321 & 15.844 & 18.978 & 15.512 & 10.157 & 31.548 & 32.196 & 29.496 & 31.26 & 28.075 & 33.457 \\
\hline 3/4/12 18:00 & & 30.352 & 24.453 & 29.349 & 29.881 & 29.361 & 30.291 & 29.831 & 29.591 & 28.398 & 26.162 & 31.45 & 36.783 & 32.471 & 15.847 & 18.992 & 15.51 & 10.308 & 31.637 & 32.346 & 29.525 & 31.399 & 8.079 & 33.46 \\
\hline 3/4/12 19:00 & & 30.361 & 24.498 & 29.388 & 29.914 & 29.338 & 30.296 & 29.847 & 29.614 & 28.421 & 26.198 & 31.384 & 36.799 & 32.398 & 15.849 & 19.013 & 15.498 & 10.24 & 31.623 & 32.274 & 29.56 & 31.333 & 28.079 & 33.464 \\
\hline 3/4/12 20:00 & & 30.366 & 24.553 & 29.414 & 29.94 & 29.331 & 30.303 & 29.85 & 29.63 & 28.435 & 26.225 & 31.375 & 36.804 & 32.396 & 15.854 & 19.031 & 15.505 & 10.23 & 31.632 & 32.27 & 29.583 & 31.326 & 28.079 & 33.471 \\
\hline 3/4/12 21:00 & & 30.368 & 24.605 & 29.426 & 29.958 & 29.333 & 30.31 & 29.852 & 29.642 & 28.446 & 26.232 & 31.377 & 36.806 & 32.394 & 15.858 & 19.054 & 15.507 & 10.233 & 31.639 & 32.272 & 29.59 & 31.326 & 28.082 & 33.474 \\
\hline 3/4/12 22:00 & & 30.38 & 24.635 & 29.473 & 29.991 & 29.309 & 30.32 & 29.866 & 29.674 & 28.474 & 26.275 & 31.389 & 36.815 & 32.406 & 15.865 & 19.087 & 15.5 & 10.242 & 31.665 & 32.281 & 29.633 & 31.34 & 28.084 & 33.481 \\
\hline 3/4/12 23:00 & & 30.384 & 24.679 & 29.489 & 30.014 & 29.319 & 30.325 & 29.866 & 29.688 & 28.488 & 26.289 & 31.396 & 36.815 & 32.41 & 15.872 & 19.144 & 15.507 & 10.249 & 31.681 & 32.289 & 29.649 & 31.349 & 28.084 & 33.483 \\
\hline 3/5/12 0:00 & & 30.38 & 24.752 & 29.487 & 30.024 & 29.34 & 30.332 & 29.859 & 29.698 & 28.493 & 26.289 & 31.403 & 36.829 & 32.417 & 15.877 & 19.17 & 15.526 & 10.251 & 31.679 & 32.296 & 29.647 & 31.361 & 28.084 & 33.483 \\
\hline 3/5/12 1:00 & & 30.387 & 24.829 & 29.489 & 30.033 & 29.354 & 30.339 & 29.859 & 29.707 & 28.502 & 26.285 & 31.405 & 36.829 & 32.42 & 15.882 & 19.191 & 15.529 & 10.258 & 31.683 & 32.3 & 29.651 & 31.361 & 28.086 & 33.483 \\
\hline 3/5/12 2:00 & & 30.389 & 24.901 & 29.494 & 30.04 & 29.366 & 30.341 & 29.859 & 29.714 & 28.509 & 26.289 & 31.41 & 36.829 & 32.427 & 15.889 & 19.212 & 15.531 & 10.263 & 31.686 & 32.303 & 29.659 & 31.368 & 28.084 & 33.483 \\
\hline 3/5/12 3:00 & & 30.391 & 24.978 & 29.487 & 30.04 & 29.385 & 30.346 & 29.855 & 29.714 & 28.507 & 26.268 & 31.41 & 36.834 & 32.424 & 15.893 & 19.235 & 15.533 & 10.263 & 31.688 & 32.305 & 29.656 & 31.361 & 28.089 & 33.481 \\
\hline 3/5/12 4:00 & & 30.389 & 25.073 & 29.465 & 30.028 & 29.41 & 30.349 & 29.845 & 29.705 & 28.502 & 26.244 & 31.408 & 36.836 & 32.422 & 15.896 & 19.253 & 15.543 & 10.263 & 31.676 & 32.298 & 29.637 & 31.361 & 28.084 & 33.478 \\
\hline 3/5/12 5:00 & & 30.389 & 25.155 & 29.466 & 30.021 & 29.42 & 30.349 & 29.847 & 29.711 & 28.502 & 26.246 & 31.41 & 36.834 & 32.424 & 15.9 & 19.276 & 15.552 & 10.261 & 31.676 & 32.3 & 29.64 & 31.366 & 28.089 & 33.478 \\
\hline 3/5/12 6:00 & & 30.394 & 25.239 & 29.466 & 30.019 & 29.425 & 30.353 & 29.852 & 29.711 & 28.505 & 26.239 & 31.408 & 36.836 & 32.427 & 15.903 & 19.299 & 15.554 & 10.263 & 31.672 & 32.305 & 29.64 & 31.363 & 28.089 & 33.476 \\
\hline 3/5/12 7:00 & & 30.391 & 25.323 & 29.463 & 30.019 & 29.439 & 30.353 & 29.852 & 29.711 & 28.502 & 26.234 & 31.412 & 36.839 & 32.429 & 15.907 & 19.327 & 15.559 & 10.268 & 31.669 & 32.303 & 29.637 & 31.366 & 28.089 & 33.478 \\
\hline 3/5/12 8:00 & & 30.396 & 25.414 & 29.466 & 30.019 & 29.441 & 30.361 & 29.852 & 29.716 & 28.505 & 26.239 & 31.412 & 36.839 & 32.434 & 15.91 & 19.353 & 15.566 & 10.272 & 31.676 & 32.31 & 29.637 & 31.378 & 28.089 & 33.481 \\
\hline 3/5/12 9:00 & & 30.394 & 25.547 & 29.456 & 30.012 & 29.457 & 30.361 & 29.852 & 29.714 & 28.5 & 26.227 & 31.417 & 36.841 & 32.431 & 15.91 & 19.376 & 15.576 & 10.268 & 31.672 & 32.31 & 29.628 & 31.378 & 28.091 & 33.483 \\
\hline 3/5/12 10:00 & & 30.391 & 25.696 & 29.44 & 30.003 & 29.476 & 30.361 & 29.845 & 29.705 & 28.495 & 26.213 & 31.415 & 36.836 & 32.434 & 15.912 & 19.408 & 15.578 & 10.272 & 31.66 & 32.308 & 29.612 & 31.382 & 28.091 & 33.485 \\
\hline 3/5/12 11:00 & & 30.389 & 25.847 & 29.416 & 29.986 & 29.497 & 30.356 & 29.838 & 29.698 & 28.484 & 26.194 & 31.55 & 36.834 & 32.593 & 15.91 & 19.427 & 15.594 & 10.423 & 31.669 & 32.445 & 29.595 & 31.53 & 28.091 & 33.485 \\
\hline $3 / 5 / 1212: 00$ & & 30.384 & 25.994 & 29.393 & 29.968 & 29.516 & 30.356 & 29.833 & 29.684 & 28.467 & 26.167 & 31.513 & 36.827 & 32.539 & 15. & 19.443 & 15.606 & 10.376 & 31.681 & 32.405 & 29.586 & 31.483 & 28.091 & 33.483 \\
\hline 3/5/12 13:00 & & 30.373 & 26.141 & 29.344 & 29.937 & 29.549 & 30.349 & 29.812 & 29.66 & 28.446 & 26.122 & 31.536 & 36.818 & 32.565 & 15.905 & 19.461 & 15.625 & 10.402 & 31.667 & 431 & 29.548 & 31.514 & 28.089 & 33.478 \\
\hline 3/5/12 14:00 & & 30.363 & 26.283 & 29.29 & 29.9 & 29.58 & 30.334 & 29.805 & 29.633 & 28.414 & 26.072 & 31.473 & 36.799 & 32.497 & 15.903 & 19.484 & 15.639 & 10.338 & 31.611 & .362 & 29.496 & 31.443 & 28.091 & 33.471 \\
\hline 3/5/12 15:00 & & 30.356 & 26.4 & 29.26 & 29.87 & 29.582 & 30.322 & 29.798 & 29.609 & 28.393 & 26.043 & 31.438 & 36.792 & 32.462 & 15.896 & 19.501 & 15.634 & 10.303 & 31.581 & 32.329 & 29.471 & 31.403 & 28.089 & 33.467 \\
\hline 3/5/12 16:00 & & 30.354 & 26.489 & 29.239 & 29.844 & 29.577 & 30.313 & 29.798 & 29.591 & 28.374 & 26.024 & 31.41 & 36.775 & 32.434 & 15.889 & 19.572 & 15.639 & 10.277 & 31.56 & 32.3 & 29.452 & 31.378 & 28.091 & 33.464 \\
\hline 3/5/12 17:00 & & 30.347 & 26.554 & 29.211 & 29.816 & 29.573 & 30.301 & 29.795 & 29.572 & 28.353 & 26.007 & 31.389 & 36.771 & 32.41 & 15.882 & 19.593 & 15.632 & 10.251 & 31.534 & 32.281 & 29.428 & 31.354 & 28.091 & 33.46 \\
\hline 3/5/12 18:00 & & 30.349 & 26.589 & 29.211 & 29.802 & 29.551 & 30.294 & 29.8 & 29.561 & 28.342 & 26 & 31.377 & 36.754 & 32.401 & 15.875 & 19.605 & 15.62 & 10.237 & 31.527 & 32.27 & 29.421 & 31.333 & 28.091 & 33.457 \\
\hline 3/5/12 19:00 & & 30.342 & 26.614 & 29.211 & 29.795 & 29.526 & 30.289 & 29.805 & 29.551 & 28.337 & 26.009 & 31.365 & 36.757 & 32.389 & 15.865 & 19.623 & 15.608 & 10.225 & 31.515 & 32.26 & 29.419 & 31.326 & 28.093 & 33.46 \\
\hline 3/5/12 20:00 & & 30.34 & 26.642 & 29.199 & 29.783 & 29.516 & 30.282 & 29.8 & 29.54 & 28.325 & 26.002 & 31.356 & 36.754 & 32.382 & 15.861 & 19.632 & 15.601 & 10.218 & 31.515 & 32.251 & 29.409 & 31.316 & 28.091 & 33.46 \\
\hline 3/5/12 21:00 & & 30.335 & 26.684 & 29.199 & 29.779 & 29.495 & 30.279 & 29.805 & 29.533 & 28.323 & 26.007 & 31.351 & 36.754 & 32.373 & 15.854 & 19.649 & 15.594 & 10.211 & 31.513 & 32.241 & 29.407 & 31.312 & 28.093 & 33.46 \\
\hline 3/5/12 22:00 & & 30.333 & 26.731 & 29.19 & 29.772 & 29.486 & 30.27 & 29.805 & 29.521 & 28.312 & 26.002 & 31.344 & 36.752 & 32.37 & 15.849 & 19.667 & 15.59 & 10.207 & 31.501 & 32.236 & 29.402 & 31.307 & 28.093 & 33.462 \\
\hline 3/5/12 23:00 & & 30.328 & 26.785 & 29.185 & 29.765 & 29.474 & 30.265 & 29.802 & 29.512 & 28.305 & 25.995 & 31.337 & 36.743 & 32.361 & 15.844 & 19.679 & 15.585 & 10.195 & 31.494 & 32.229 & 29.398 & 31.298 & 28.093 & 33.462 \\
\hline $3 / 6 / 120: 00$ & & 30.324 & 26.831 & 29.169 & 29.758 & 29.472 & 30.26 & 29.793 & 29.5 & 28.293 & 25.988 & 31.328 & 36.743 & 32.347 & 15.837 & 19.692 & 15.583 & 10.19 & 31.483 & 32.217 & 29.384 & 31.291 & 28.091 & 33.46 \\
\hline 3/6/12 1:00 & & 30.321 & 26.876 & 29.159 & 29.746 & 29.465 & 30.255 & 29.795 & 29.489 & 28.284 & 25.978 & 31.316 & 36.736 & 32.342 & 15.833 & 19.706 & 15.576 & 10.181 & 31.473 & 32.208 & 29.374 & 31.279 & 28.091 & 33.455 \\
\hline 3/6/12 2:00 & & 30.314 & 26.924 & 29.141 & 29.734 & 29.462 & 30.251 & 29.788 & 29.477 & 28.267 & 25.973 & 31.305 & 36.738 & 32.328 & 15.8 & 19.716 & 15.571 & 10.169 & 31.462 & 32.196 & 29.362 & 31.272 & 28.091 & 33.45 \\
\hline 3/6/12 3:00 & & 30.314 & 26.966 & 29.127 & 29.72 & 29.457 & 30.246 & 29.783 & 29.463 & 28.256 & 25.962 & 31.293 & 36.731 & 32.319 & $\begin{array}{l}15.8 \\
15.8\end{array}$ & 19.729 & 15.564 & 10.155 & 31.45 & 32.189 & 29.346 & 31.258 & 28.091 & 33.448 \\
\hline $\begin{array}{l}3 / 6 / 124: 00 \\
3\end{array}$ & & 30.31 & 26.999 & 29.12 & 29.711 & 29.45 & 30.236 & 29.786 & 29.447 & 28.249 & 25.952 & 31.284 & 36.726 & 32.307 & 15.8 & 19.743 & 15.554 & 10.143 & 31.436 & 32.177 & 29.337 & 31.246 & 28.091 & $\begin{array}{l}33.440 \\
33.443\end{array}$ \\
\hline $\begin{array}{l}3 / 6 / 125: 00 \\
3\end{array}$ & & 30.307 & 27.027 & 29.122 & 29.704 & 29.432 & 30.234 & 29.788 & 29.442 & $\begin{array}{l}28.244 \\
28.244\end{array}$ & 25.957 & $\begin{array}{l}31.279 \\
31.279\end{array}$ & 36.719 & 32.298 & 15.8 & 19.755 & 15.54 & 10.136 & $\begin{array}{l}31.436 \\
31.43\end{array}$ & 32.17 & 29.334 & 31.237 & 28.089 & $\begin{array}{l}33.441 \\
33.441\end{array}$ \\
\hline 3/6/12 6:00 & & 30.307 & 27.048 & 29.134 & 29.7 & 29.408 & 30. & 29.795 & 29.44 & 28.246 & 25.973 & 31.274 & 36.722 & 32.3 & 15.803 & 19.771 & 15.524 & 10.136 & 31.441 & 32.168 & 29.348 & 31.234 & 28.091 & 33.439 \\
\hline $3 / 6$ & & 30.305 & 27.078 & 29. & 29.7 & 29.399 & 30. & 29.793 & 29.44 & 28.244 & 25.981 & 31 & 36.722 & 32.293 & 15. & 19.799 & 15.522 & 10.131 & 31.443 & 8 & 29.348 & 31.234 & 28.091 & 33.436 \\
\hline $3 / 6$ & & 30.307 & 27.111 & 29.145 & 29. & 29.385 & 30 & 29.798 & 29.435 & 28.244 & 25.99 & 31.274 & 36.719 & 32.295 & 15. & 7 & 15.51 & 10.134 & 31.445 & 32.168 & 29.355 & 31.234 & 28.091 & 33.441 \\
\hline 3/6/129: & & 30. & 27.14 & 29.155 & & 29.37 & 30. & 29.79 & 29 & 28.2 & 26.002 & 31.277 & 36.719 & 32.298 & 15.7 & 19.8. & 15.5 & 10.134 & 31.452 & 32.17 & 29.367 & 31.239 & 28.093 & 33.441 \\
\hline $3 / 6 / 1210: 00$ & & 30. & 27.214 & 29.141 & 29 & 29.378 & 30. & 29.793 & 29.431 & 28.242 & 25.992 & 31.274 & 36.722 & 32.298 & 15.7 & & 15.507 & 10.134 & 31.448 & 32.165 & 29.362 & 31.241 & 28.093 & 33.446 \\
\hline 3/6/12 11:00 & & 30. & 27.274 & 29.138 & 29 & 29.375 & 30. & 29.79 & 29.428 & 28.239 & 25.993 & 31.274 & 36.715 & 32.293 & 15.7 & 52 & 15.507 & 10.134 & 31.445 & 32.165 & 29.36 & 31.241 & 28.096 & 33.446 \\
\hline 3/6/12 12 & & 30.294 & 27.35 & 29.127 & & 29.382 & & 29.783 & & 28.2 & 25. & 31.272 & 36.715 & 32.291 & 15. & 7 & 15.51 & 10.129 & 31.436 & 32.163 & 29.351 & 31.244 & 28.096 & 33.45 \\
\hline 3/6/12 13: & & 30.287 & 27.4 & 29.099 & & 29.399 & 30.2 & 29.769 & 29. & 28.216 & 25.959 & 31.262 & 36.712 & 32.284 & 15. & 19.921 & 15.519 & 10.122 & 31.424 & 32.154 & 29.327 & 31.241 & 28.093 & 33.446 \\
\hline 3/6/12 14:00 & & 30.28 & 27.5 & 29.075 & & 29.406 & 30 & 29.764 & 29.387 & 28.202 & 25.94 & 31.251 & 36.708 & 32.274 & 15. & 19.94 & 15.517 & 10.113 & 31.406 & 32.142 & 29.306 & 31.223 & 28.089 & 33.439 \\
\hline 3/6/12 15:00 & & 30.275 & 27.57 & 29.063 & 29. & 29.406 & 30.205 & 29.762 & 29.3 & 28.193 & 25.935 & 31.241 & 36.7 & 32.267 & 15.7 & 19.947 & 15.517 & 10.101 & 31.396 & 32.137 & 29.299 & 31.211 & 28.093 & 33.441 \\
\hline $3 / 6 / 1216: 00$ & & 30.28 & 27.596 & 29.075 & 29.6 & 29.387 & 30.198 & 29.767 & 29.375 & 28.186 & 25.942 & 31.234 & 36.698 & 32.258 & 15.7 & 20.007 & 15.503 & 10.101 & 31.396 & 32.13 & 29.304 & 31.202 & 28.093 & 33.432 \\
\hline 3/6/12 17:00 & & 30.282 & 27.589 & 29.094 & 29.67 & 29.359 & 30.198 & 29.783 & 29.375 & 28.198 & 25.962 & 31.239 & 36.703 & 32.26 & 15.772 & 20.014 & 15.489 & 10.099 & 31.408 & 32.132 & 29.32 & 31.197 & 28.096 & 33.434 \\
\hline $3 / 6 / 1218: 00$ & & 30.277 & 27.591 & 29.092 & 29.678 & 29.352 & 30.198 & 29.779 & 29.373 & 28.195 & 25.962 & 31.234 & 36.696 & 32.258 & 15.768 & 20.042 & 15.484 & 10.099 & 31.413 & 32.13 & 29.322 & 31.192 & 28.093 & 33.432 \\
\hline $3 / 6 / 1219: 00$ & & 30.28 & 27.58 & 29.106 & 29.688 & 29.333 & 30.198 & 29.779 & 29.38 & 28.2 & 25.981 & 31.241 & $\begin{array}{r}36.7 \\
\end{array}$ & 32.262 & 15.768 & 20.044 & 15.477 & 10.099 & 31.417 & 32.137 & 29.33 & 31.199 & 28.093 & 33.432 \\
\hline $3 / 6 / 1220: 00$ & & 30.277 & 27.58 & 29.103 & 29.69 & 29.326 & 30.198 & 29.778 & 29.377 & 28.2 & 25.976 & 31.241 & 36.7 & 32.262 & 15.768 & 20.055 & 15.47 & 10.101 & 31.417 & 32.135 & 29.33 & 31.197 & 28.093 & 33.434 \\
\hline 3/6/12 21:00 & & 30.275 & 27.587 & 29.11 & 29.699 & 29.319 & 30.195 & 29.771 & 29.377 & 28.202 & 25.988 & 31.246 & 36.703 & 32.267 & 15.768 & 20.065 & 15.463 & 10.106 & 31.427 & 32.142 & 29.341 & 31.209 & 28.093 & 33.441 \\
\hline 3/6/12 22:00 & & 30.275 & 27.589 & 29.115 & 29.706 & 29.309 & 30.193 & 29.781 & 29.377 & 28.205 & 25.993 & 31.251 & 36.703 & 32.272 & 15.765 & 20.074 & 15.456 & 10.113 & 31.431 & 32.144 & 29.348 & 31.211 & 28.096 & 33.441 \\
\hline 3/6/12 23:00 & & 30.273 & 27.603 & 29.115 & 29.709 & 29.307 & 30.193 & 29.779 & 29.377 & 28.205 & 25.997 & 31.256 & 36.705 & 32.274 & 15.768 & 20.083 & 15.458 & 10.117 & 31.441 & 32.146 & 29.351 & 31.218 & 28.096 & 33.443 \\
\hline
\end{tabular}




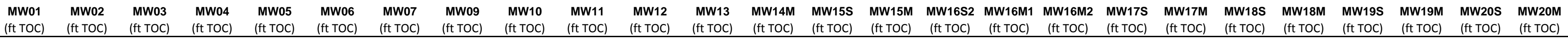

\begin{tabular}{|c|c|c|c|c|c|c|c|c|c|c|c|c|c|c|c|c|c|c|c|c|c|c|c|c|c|c|}
\hline Well & $\begin{array}{l}\text { (ft TOC) } \\
\text { (f) }\end{array}$ & $\begin{array}{l}\text { (ft TOC) } \\
\text { (f) }\end{array}$ & $\begin{array}{l}\text { (ft TOC) } \\
\text { (ft) }\end{array}$ & (ft TOC) & $\begin{array}{l}\text { (ft TOC) } \\
\text { (n) }\end{array}$ & $\begin{array}{l}\text { (ft TOC) } \\
\text { (f) }\end{array}$ & (ft toc) & $\begin{array}{l}\text { (ft TOC) } \\
\text { (a) }\end{array}$ & $\begin{array}{l}\text { (ft TOC) } \\
\text { (ft) }\end{array}$ & (ft TOC) & $\begin{array}{l}\text { (ft TOC) } \\
\text { (f) }\end{array}$ & (ft toc) & (ft TOC) & $\begin{array}{l}\text { (ft ToC) } \\
\text { (a) }\end{array}$ & (ft TOC) & (ft TOC) & (ft TOC) & (ft TOC) & $\begin{array}{l}\text { (ft TOC) } \\
\text { (a) }\end{array}$ & $\begin{array}{l}\text { (ft TOC) } \\
\text { (n) }\end{array}$ & $\begin{array}{l}\text { (ft TOC) } \\
\text { (f) }\end{array}$ & $\begin{array}{l}\text { (ift TOC) } \\
\text { (a) }\end{array}$ & (ft Toc) & $\begin{array}{l}\text { (ft TOC) } \\
\end{array}$ & $\begin{array}{l}\text { (nt Toc) } \\
\text { (ft }\end{array}$ & (ft TOC) \\
\hline 3/7/12 0:00 & & 30.268 & 27.624 & 29.11 & 29.711 & & 29.309 & 30.193 & 29.774 & 29.377 & 28.202 & 25.992 & 31.253 & 36.7 & 32.272 & $\quad 15.768$ & & 20.092 & 15.453 & 10.113 & 31.429 & 32.146 & 29.348 & 31.218 & 28.096 & 33.443 \\
\hline 3/7/12 1:00 & & 30.273 & 27.631 & 29.108 & 29.711 & & 29.305 & 30.188 & 29.779 & 29.375 & 28.205 & 25.993 & 31.253 & $\begin{array}{r}36.703 \\
\end{array}$ & 32.272 & 15.768 & & 20.104 & 15.444 & 10.113 & 31.436 & 32.144 & 29.353 & 31.216 & 28.096 & 33.441 \\
\hline 3/7/12 2:00 & & 30.27 & 27.638 & 29.117 & 29.716 & & 29.298 & 30.193 & 29.779 & 29.375 & 28.207 & 26.002 & 31.253 & 36.703 & 32.274 & 155.768 & & 20.116 & 15.437 & 10.11 & 31.438 & $\begin{array}{l}3 \\
32.146\end{array}$ & 29.362 & 31.218 & 28.096 & $\begin{array}{l}33.441 \\
33.439\end{array}$ \\
\hline 3/7/12 3:00 & & 30.266 & 27.661 & 29.096 & 29.709 & & 29.312 & 30.188 & 29.76 & 29.368 & 28.195 & 25.983 & 31.246 & 36.707 & 32.267 & 15.768 & & 20.122 & 15.442 & 10.108 & 31.422 & 32.142 & 29.344 & 31.218 & 28.098 & 33.436 \\
\hline 3/7/12 4:00 & & 30.263 & 27.673 & 29.099 & 29.706 & & 29.309 & 30.183 & 29.764 & 29.363 & 28.195 & 25.981 & 31.244 & 36.694 & 32.265 & $\quad 15.765$ & & 20.132 & 15.43 & 10.101 & 31.42 & 32.137 & 29.339 & 31.202 & 28.096 & 33.432 \\
\hline $3 / 7 / 125: 00$ & & 30.27 & 27.671 & 29.117 & 29.718 & & 29.291 & 30.186 & 29.776 & 29.373 & 28.205 & 25.997 & 31.246 & 36.707 & 32.262 & 15.763 & & 20.141 & 15.418 & 10.103 & 31.434 & 32.139 & 29.362 & 1.202 & 28.098 & 33.432 \\
\hline 3/7/12 6:00 & & 30.268 & 27.68 & 29.124 & 29.723 & & 29.284 & 30.188 & 29.776 & 29.373 & 28.207 & 26.009 & 31.251 & 36.705 & 32.267 & 15.77 & & 20.162 & 15.414 & 10.106 & 31.443 & 32.142 & 29.362 & 31.211 & 28.1 & 33.429 \\
\hline 3/7/12 7:00 & & 30.266 & 27.696 & 29.124 & 29.727 & & 29.281 & 30.188 & 29.776 & 29.377 & 28.209 & 26.012 & 31.248 & 36.71 & 32.267 & 15.768 & & 20.178 & 15.411 & 10.11 & 31.445 & 32.142 & 29.362 & 31.211 & 28.098 & $\begin{array}{l}33.429 \\
3.42\end{array}$ \\
\hline 3/7/12 8:00 & & 30.266 & 27.717 & 29.129 & 29.73 & & 29.284 & 30.191 & 29.774 & 29.375 & 28.212 & 26.009 & 31.253 & 36.705 & 32.272 & 15.77 & & 20.185 & 15.409 & 10.113 & 31.445 & 52.144 & 29.372 & 31.211 & 28.098 & 33.434 \\
\hline 3/7/12 9:00 & & 30.27 & 27.736 & 29.148 & 29.746 & & 29.269 & 30.191 & 29.781 & 29.387 & 28.225 & 26.028 & 31.26 & 36.705 & 32.279 & 15.772 & & 20.194 & 15.404 & 10.12 & 31.464 & 32.154 & 29.393 & 31.223 & 28.098 & 33.436 \\
\hline 3/7/12 10:00 & & 30.277 & 27.759 & 29.18 & 29.767 & & 29.253 & 30.198 & 29.795 & 29.403 & 28.239 & 26.062 & 31.274 & 36.722 & 32.291 & 15.777 & & 20.208 & 15.4 & 10.129 & 31.483 & 32.165 & 29.419 & 31.232 & 28.103 & 33.441 \\
\hline 3/7/12 11:00 & & 30.277 & 27.796 & 29.192 & 29.786 & & 29.255 & 30.2 & 29.795 & 29.414 & 28.246 & 26.076 & 31.281 & 36.722 & 32.302 & 15.779 & & 20.215 & 15.409 & 10.141 & 31.497 & 32.177 & 29.431 & 31.253 & 28.103 & 33.446 \\
\hline 3/7/12 12:00 & & 30.275 & 27.831 & 29.192 & 29.79 & & 29.269 & 30.203 & 29.79 & 29.414 & 28.251 & 26.069 & 31.288 & 36.731 & 32.307 & 15.782 & & 20.224 & 15.414 & 10.143 & 31.499 & 32.182 & 29.433 & 31.258 & 28.105 & 33.45 \\
\hline 3/7/12 13:00 & & 30.277 & 27.864 & 29.192 & 29.797 & & 29.272 & 30.205 & 29.783 & 29.414 & 28.253 & 26.062 & 31.293 & 36.731 & 32.309 & 15.784 & & 20.231 & 15.416 & 10.146 & 31.504 & 32.187 & 29.435 & 31.253 & 28.103 & 33.45 \\
\hline $3 / 7 / 1214: 00$ & & 30.277 & 27.883 & 29.206 & 29.809 & & 29.272 & 30.21 & 29.786 & 29.428 & 28.263 & 26.088 & 31.3 & 36.731 & 32.312 & 15.789 & & 20.238 & 15.418 & 10.155 & 31.508 & $3 \quad 32.191$ & 29.445 & 31.265 & 28.1 & 33.448 \\
\hline 3/7/12 15:00 & & 30.277 & 27.913 & 29.204 & 29.814 & & 29.279 & 30.21 & 29.783 & 29.431 & 28.267 & 26.071 & 31.298 & 36.731 & 32.314 & 15.791 & & 20.245 & 15.416 & 10.153 & 31.515 & 32.191 & 29.445 & 31.258 & 28.105 & 33.448 \\
\hline 3/7/12 16:00 & & 30.287 & 27.908 & 29.236 & 29.83 & & 29.262 & 30.217 & 29.795 & 29.447 & 28.284 & 26.105 & 31.307 & 36.743 & 32.319 & 15.796 & & 20.312 & 15.407 & 10.164 & 31.532 & 32.199 & 29.478 & 31.263 & 28.103 & 33.448 \\
\hline 3/7/12 17:00 & & 30.291 & 27.904 & 29.255 & 29.849 & & 29.258 & 30.222 & 29.8 & 29.458 & 28.295 & 26.119 & 31.314 & 36.747 & 32.328 & $\begin{array}{l}3.8 \\
3\end{array}$ & & 20.324 & 15.411 & $\begin{array}{l}10.167 \\
\end{array}$ & 31.546 & $\quad 32.21$ & 29.494 & 31.267 & 28.105 & $\begin{array}{l}33.448 \\
33.44\end{array}$ \\
\hline 3/7/12 18:00 & & 30.291 & 27.883 & 29.267 & 29.863 & & 29.258 & 30.229 & 29.8 & $\begin{array}{l}29.472 \\
29.42\end{array}$ & 28.305 & 26.134 & 31.323 & 36.75 & $\begin{array}{r}32.34 \\
\end{array}$ & $\begin{array}{r}15.807 \\
\end{array}$ & & 20.331 & 15.418 & 10.176 & 31.557 & 32.213 & 29.506 & 31.274 & 28.105 & $\begin{array}{l}33.440 \\
33.448\end{array}$ \\
\hline $\begin{array}{l}3 / 7 / 12190000 \\
3\end{array}$ & & 30.31 & 27.827 & 29.323 & $\begin{array}{r}29.003 \\
29.9\end{array}$ & & 29.225 & 30.241 & $\begin{array}{r}29.824 \\
2924\end{array}$ & $\begin{array}{r}29.47 \\
29.5\end{array}$ & 28.337 & $\begin{array}{l}20.1747 \\
26.177\end{array}$ & 31.34 & $\begin{array}{l}36.761 \\
36.761\end{array}$ & $\begin{array}{r}32.356 \\
32.356\end{array}$ & $\begin{array}{l}15.0012 \\
5\end{array}$ & & 20.342 & $\begin{array}{l}15.410 \\
15.392\end{array}$ & $\begin{array}{l}10.193 \\
10.193\end{array}$ & 31.595 & $\quad 32.234$ & 29.555 & 31.286 & 28.11 & $\begin{array}{r}r 3.440 \\
33.45\end{array}$ \\
\hline $\begin{array}{l}3 / 7 / 1220000 \\
3\end{array}$ & & $\begin{array}{r}30.31 \\
30.307\end{array}$ & 27.81 & $\begin{array}{l}29.339 \\
29.339\end{array}$ & 29.923 & & 29.232 & $\begin{array}{l}50.241 \\
30.251\end{array}$ & $\begin{array}{l}29.824 \\
29.821\end{array}$ & $\begin{array}{r}29.519 \\
29.519\end{array}$ & $\begin{array}{l}28.351 \\
28.349\end{array}$ & 26.198 & $\begin{array}{l}31.34 \\
31.356\end{array}$ & 36.771 & $\begin{array}{r}32.350 \\
32.37\end{array}$ & $\begin{array}{l}15.812 \\
15.819\end{array}$ & & $\begin{array}{l}20.342 \\
20.349\end{array}$ & $\begin{array}{l}15.411 \\
15.492\end{array}$ & 10.209 & 31.609 & $\begin{array}{r}32.254 \\
32.246\end{array}$ & 29.567 & $\begin{array}{l}31.3200 \\
31.314\end{array}$ & $\begin{array}{l}28.11 \\
28.105\end{array}$ & $\begin{array}{r}33.45 \\
33.455\end{array}$ \\
\hline 3/7/12 21:00 & & 30.312 & 27.801 & 29.353 & 29.94 & & 29.241 & 30.258 & 29.821 & 29.533 & 28.363 & 26.203 & 31.368 & 36.776 & 32.384 & $\begin{array}{l}15.828 \\
\end{array}$ & & 20.358 & 15.414 & 10.221 & 31.627 & 32.263 & 29.588 & 31.321 & 28.107 & 33.462 \\
\hline 3/7/122 22:00 & & 30.324 & 27.813 & 29.381 & 29.965 & & 29.239 & $\begin{array}{l}30.267 \\
30\end{array}$ & 29.826 & 29.551 & $\begin{array}{l}20.3838 \\
28.384\end{array}$ & 26.22 & 31.387 & 36.785 & 32.401 & $\begin{array}{l}15.8020 \\
15.835\end{array}$ & & 20.381 & $\begin{array}{l}15.414 \\
15.414\end{array}$ & $\begin{array}{l}10.235 \\
10.235\end{array}$ & 31.644 & 32.279 & .614 & 31.338 & 28.11 & $\begin{array}{l}33.402 \\
33.469\end{array}$ \\
\hline $\begin{array}{l}3 / 7 / 12232300 \\
3\end{array}$ & & 30.326 & 82 & $\begin{array}{r}29.501 \\
29.4\end{array}$ & 29.986 & & 29.244 & 30.279 & 29.826 & 29.57 & $\begin{array}{l}28.304 \\
28.395\end{array}$ & $\begin{array}{l}26.222 \\
26.239\end{array}$ & 31.403 & 36.794 & 32.415 & $\quad 15.844$ & & 20.393 & 5.423 & $\begin{array}{l}10.256 \\
10.256\end{array}$ & $\begin{array}{l}31.644 \\
31.669\end{array}$ & 32.293 & .626 & $\begin{array}{l}1.350 \\
1.359\end{array}$ & $\begin{array}{r}28.11 \\
28.112\end{array}$ & $\begin{array}{l}33.409 \\
33.474\end{array}$ \\
\hline 3/8/12 0:00 & & 30.326 & 27.817 & 29.398 & 29.996 & & & 30.286 & 29.819 & 29.581 & 28.402 & & 31.415 & 36.797 & 32.424 & $\begin{array}{r}15.849 \\
\end{array}$ & & 20.402 & $\begin{array}{l}15.439 \\
15.439\end{array}$ & $\begin{array}{l}10.200 \\
10.263\end{array}$ & 31.669 & $\begin{array}{l}32.293 \\
32.308\end{array}$ & .626 & 1.366 & 28.11 & $\begin{array}{l}53.474 \\
33.476\end{array}$ \\
\hline 3/8/12 1:00 & & 30.335 & 27.78 & 29.459 & 30.038 & & 29.246 & 30.298 & 29.843 & 29.63 & 28.437 & 26.323 & 31.426 & 36.804 & 32.438 & $\begin{array}{ll}3 & 15.863\end{array}$ & & 20.407 & 15.428 & 10.275 & 31.704 & $\quad 32.319$ & 29.673 & 1.387 & 28.103 & 33.476 \\
\hline $3 / 8 / 122: 00$ & & 30.345 & 27.75 & 29.473 & 30.064 & & 29.255 & 30.32 & 29.843 & 29.644 & 28.46 & 26.311 & 31.436 & 36.815 & $\begin{array}{l}32.452 \\
32\end{array}$ & $\begin{array}{l}\quad 15.87 \\
2\end{array}$ & & 20.409 & 15.43 & 10.287 & 31.718 & 32.326 & 29.696 & 1.385 & 28.107 & 33.474 \\
\hline 3/8/12 3:00 & & 30.349 & 27.778 & 29.48 & 30.075 & & 29.279 & 30.322 & 29.84 & 29.653 & 28.467 & 26.296 & 31.447 & 36.818 & 32.459 & $\quad 15.879$ & & 20.416 & 15.444 & 10.296 & 31.728 & $3 \quad 32.341$ & 29.701 & 31.408 & 28.117 & 33.471 \\
\hline 3/8/12 4:00 & & 30.356 & 27.785 & 29.505 & 30.099 & & 29.284 & 30.334 & 29.843 & 29.679 & 28.484 & 26.32 & 31.464 & 36.827 & 32.471 & 15.891 & & 20.43 & 15.456 & 10.315 & 31.748 & $3 \quad 32.355$ & 29.713 & 31.436 & 28.11 & 33.471 \\
\hline 3/8/12 5:00 & & 30.373 & 27.803 & 29.529 & 30.115 & & 29.288 & 30.339 & 29.852 & 29.695 & 28.505 & 26.328 & 31.468 & 36.832 & 32.483 & 15.898 & & 20.437 & 15.442 & 10.322 & 31.765 & 32.362 & 29.745 & 31.422 & 28.119 & 33.471 \\
\hline $3 / 8 / 126: 00$ & & 30.384 & 27.813 & 29.557 & 30.136 & & 29.293 & 30.349 & 29.862 & 29.716 & 28.53 & 26.347 & 31.482 & 36.843 & 32.497 & 15.907 & & 20.465 & 15.428 & 10.331 & 31.79 & 32.374 & 29.774 & 31.422 & 28.119 & 33.471 \\
\hline 3/8/12 7:00 & & 30.373 & 27.855 & 29.533 & 30.134 & & 29.342 & 30.353 & 29.845 & 29.721 & 28.523 & 26.33 & 31.489 & 36.846 & 32.506 & 15.917 & & 20.483 & 15.486 & 10.343 & 31.779 & 32.386 & 29.75 & $\quad 31.45$ & 28.115 & 33.471 \\
\hline $3 / 8 / 128: 00$ & & 30.394 & 27.85 & 29.571 & 30.157 & & 29.328 & 30.361 & 29.862 & 29.742 & 28.549 & 26.339 & 31.508 & 36.853 & 32.52 & 15.926 & & 20.495 & 15.465 & 10.359 & 31.804 & 32.4 & 29.783 & 31.462 & 28.122 & 33.476 \\
\hline 3/8/12 9:00 & & 30.391 & 27.894 & 29.573 & 30.164 & & 29.354 & 30.37 & 29.862 & 29.76 & 28.556 & 26.354 & 31.522 & 36.855 & 32.532 & $\begin{array}{l}15.935 \\
2\end{array}$ & & 20.506 & 15.493 & 10.371 & 31.809 & $\quad 32.414$ & 29.781 & 31.483 & 28.115 & 33.481 \\
\hline 3/8/12 10:00 & & 30.405 & 27.929 & 29.596 & 30.18 & & 29.359 & 30.377 & 29.869 & 29.779 & 28.574 & 26.359 & 31.536 & 36.858 & 32.549 & 15.947 & & 20.516 & 15.493 & $\begin{array}{l}10.388 \\
\end{array}$ & 31.83 & 32.428 & 29.809 & 31.492 & 28.119 & 33.488 \\
\hline 3/8/12 11:00 & & 30.412 & 27.995 & 29.608 & $\begin{array}{l}30.192 \\
30.1\end{array}$ & & 29.373 & 30.382 & 29.874 & 29.793 & 28.588 & 26.368 & 31.55 & 36.872 & 32.565 & 15.956 & & 20.522 & 15.496 & $\begin{array}{l}10.402 \\
1002\end{array}$ & 31.844 & 32.445 & 29.818 & 31.507 & 28.122 & $\begin{array}{l}33.495 \\
33.45\end{array}$ \\
\hline 3/8/12 12:00 & & $\begin{array}{l}30.401 \\
30.412\end{array}$ & 28.076 & 29.575 & 30.18 & & 29.422 & 30.387 & 29.859 & 29.788 & $\begin{array}{l}20.000 \\
28.579\end{array}$ & $\begin{array}{l}20.300 \\
2635\end{array}$ & 31.553 & 36.867 & 32.567 & 15.963 & & 20.532 & $\begin{array}{r}15.54 \\
154\end{array}$ & $\begin{array}{l}10.409 \\
109\end{array}$ & 31.825 & $\quad 32.445$ & 29.792 & 31.518 & 28.119 & $\begin{array}{l}33.495 \\
33.45\end{array}$ \\
\hline $\begin{array}{l}3 / 8 / 1213: 00 \\
3\end{array}$ & & 30.401 & 28.134 & 29.564 & 30.17 & & 29.448 & 30.389 & 29.855 & $\begin{array}{r}29.190 \\
299\end{array}$ & 28.572 & 26.32 & 31.555 & 36.872 & 32.572 & 15 & & 20.541 & $\begin{array}{l}15.559 \\
15.559\end{array}$ & 10.409 & 31.818 & 32.45 & 29.778 & 31.525 & 28.119 & $\begin{array}{l}33.499 \\
33.49\end{array}$ \\
\hline $\begin{array}{l}3 / 8 / 1214000 \\
3\end{array}$ & & 30.398 & $\begin{array}{l}28.197 \\
28.197\end{array}$ & 29.533 & 30.152 & & 29.483 & 30.389 & 29.843 & 29.779 & $\begin{array}{l}28.567 \\
28.567\end{array}$ & $\begin{array}{l}26.028 \\
26.285\end{array}$ & 31.553 & 36.865 & 32.57 & 15.975 & & 20.546 & 15.573 & 10.402 & $\begin{array}{l}31.010 \\
31.807\end{array}$ & 32.443 & 29.757 & 31.521 & 28.117 & $\begin{array}{l}33.497 \\
33.497\end{array}$ \\
\hline $\begin{array}{l}3 / 8 / 121.00 \\
35: 00\end{array}$ & & $\begin{array}{l}50.590 \\
30.405\end{array}$ & 28.249 & 29.543 & $\begin{array}{r}r .152 \\
30.15\end{array}$ & & 29.483 & $\begin{array}{l}50.309 \\
30.394\end{array}$ & $\begin{array}{r}29.845 \\
29.85\end{array}$ & 29.783 & $\begin{array}{l}28.301 \\
28.567\end{array}$ & $\begin{array}{l}20.283 \\
26.289\end{array}$ & 31.555 & $\begin{array}{l}30.800 \\
36.869\end{array}$ & $\begin{array}{l}32.51 \\
32.57\end{array}$ & $\begin{array}{l}15.975 \\
75.979\end{array}$ & & $\begin{array}{r}20.340 \\
20.55\end{array}$ & $\begin{array}{l}13.5 / 58 \\
15.58\end{array}$ & $\begin{array}{l}10.402 \\
10.411\end{array}$ & 31.807 & $\begin{array}{l}32.443 \\
32.447\end{array}$ & 29.757 & $\begin{array}{l}11.521 \\
31.518\end{array}$ & 28.122 & $\begin{array}{l}53.491 \\
33.497\end{array}$ \\
\hline $\begin{array}{l}3 / 8 / 121 \\
3 / 8 / 121\end{array}$ & & $\begin{array}{r}0.405 \\
30.41\end{array}$ & $\begin{array}{l}28.249 \\
28.288\end{array}$ & $\begin{array}{l}29.545 \\
29.545\end{array}$ & $\begin{array}{r}30.1 \\
30.1\end{array}$ & & $\begin{array}{l}29.483 \\
29.493\end{array}$ & $\begin{array}{l}30.394 \\
30.399\end{array}$ & $\begin{array}{l}29.857 \\
29.857\end{array}$ & $\begin{array}{l}29.178 \\
29.788\end{array}$ & $\begin{array}{r}28.501 \\
28.57\end{array}$ & $\begin{array}{l}\begin{array}{l}20.289 \\
26.292\end{array} \\
2\end{array}$ & $\begin{array}{l}31.535 \\
31.555\end{array}$ & $\begin{array}{l}30.869 \\
36.876\end{array}$ & $\begin{array}{r}32.571 \\
32.572\end{array}$ & 15. & & $\begin{array}{l}20.613 \\
20.613\end{array}$ & $\begin{array}{r}13.58 \\
15.594\end{array}$ & $\begin{array}{l}10.411 \\
10.409\end{array}$ & $\begin{array}{l}31.801 \\
31.809\end{array}$ & $\begin{array}{l}32.441 \\
32.447\end{array}$ & $\begin{array}{r}29.131 \\
29.76\end{array}$ & $\begin{array}{l}11.518 \\
31.523\end{array}$ & $\begin{array}{l}28.1119 \\
28.119\end{array}$ & $\begin{array}{l}33.491 \\
33.495\end{array}$ \\
\hline $3 / 8 / 121$ & & 30 & 28.307 & 29.543 & 30 & & 2 & 30 & 29.857 & 29 & 28. & 26. & 31 & 36.874 & 32.57 & 15 & & $2 c$ & 15.604 & 10.409 & 31.804 & 32 & 29.757 & 31.516 & 28.119 & $\begin{array}{l}33.495 \\
33.45\end{array}$ \\
\hline 3/8/12 18:00 & & 30. & 28.2 & 29. & 30. & & 29. & 30.404 & 29 & 29 & 28. & 26 & 31 & 36.872 & 32.584 & 15 & & 20 & $\begin{array}{l}15.608 \\
15\end{array}$ & 10.421 & 31.816 & 52 & 59 & 31.518 & 28.122 & $\begin{array}{l}33.492 \\
33.492\end{array}$ \\
\hline 3/8/12 19:00 & & 30. & 28.265 & 29.571 & 30. & & 29.488 & 30.406 & & 29.802 & 28.581 & 26.296 & 31.569 & 36.879 & 32.586 & & & & 15.604 & 10.421 & 31.823 & 32.462 & 29.778 & og & 28.122 & $\begin{array}{l}33.492 \\
\end{array}$ \\
\hline 3/8/12 20:00 & & 30.42 & 28.258 & 29.575 & 30. & & 29.49 & 30.408 & 29.878 & 29.811 & 28.586 & 26.306 & 31.576 & 36.879 & 32.591 & & & & 15.615 & 10.423 & 31.823 & 32.466 & 29.783 & 21 & 8.119 & $\begin{array}{l}33.492 \\
\end{array}$ \\
\hline 21:00 & & 30. & 28.246 & 29. & & & & 30.4 & 29.881 & & 28.5 & 26. & 31 & 36.881 & 32.595 & 16 & & 20. & 15.62 & 10.432 & 31.835 & 32.473 & 29.79 & 31.537 & 28.122 & 33.497 \\
\hline 3/8/12 22:00 & & 30. & 28.23 & 29. & & & & 30.4 & 29.89 & & 28 & 26 & 31. & 36.886 & 32. & & & 20. & 15 & 10. & 31.849 & & & 31.546 & 28.122 & 33.502 \\
\hline 3/8/12 23:00 & & 30.4 & 28.2 & 29.624 & & & & 30 & 29.9 & & 28. & & 31. & 36.888 & 32.619 & & & 20.731 & 15.611 & 10.453 & 31.863 & & & 31.556 & 28.124 & 33.506 \\
\hline 3/9/12 0:00 & & 30.433 & 28.209 & 29.625 & & & 29.493 & 30.4 & 29.895 & & 28.616 & 26. & 31.611 & 36.89 & 32.624 & 16. & & 20.728 & 15.634 & 10.463 & 31.865 & 32.504 & 29.823 & 31.572 & 28.119 & 33.509 \\
\hline 3/9/12 1:00 & & 30. & 28.211 & 29.625 & 30. & & 29.502 & 30.432 & 29.895 & 29.8 & 28.623 & 26.342 & 31.616 & 36.895 & 32.628 & $\begin{array}{l}3 \\
3\end{array}$ & & 20.737 & 15.637 & 10.47 & 31.872 & 32.509 & 29.828 & 31.575 & 28.122 & 33.511 \\
\hline 3/9/12 2:00 & & 30.44 & 28.218 & 29.622 & 30.2 & & 29.512 & 30.432 & 29.89 & 29.862 & 28.623 & 26.337 & 31.62 & 36.895 & 32.635 & 16. & & 20.742 & 15.646 & 10.47 & 31.87 & 32.514 & 29.825 & 31.579 & 28.122 & 33.511 \\
\hline 3/9/12 3:00 & & 30.431 & 28.239 & 29.594 & 30.187 & & 29.544 & 30.435 & 29.878 & 29.853 & 28.609 & 26.308 & 31.618 & 36.893 & 32.631 & 16.026 & & 20.742 & 15.658 & 10.47 & 31.853 & 32.511 & 29.797 & 31.579 & 28.119 & 33.509 \\
\hline 3/9/12 4:00 & & 30.436 & 28.258 & 29.58 & 30.178 & & 29.556 & 30.435 & 29.878 & 29.846 & 28.605 & 26.289 & 31.611 & 36.886 & 32.631 & 16.028 & & 20.772 & 15.658 & 10.468 & 31.844 & 32.504 & 29.79 & 31.57 & 28.122 & 33.504 \\
\hline $3 / 9 / 125: 00$ & & 30.44 & 28.267 & 29.587 & 30.176 & & 29.556 & 30.435 & 29.885 & 29.848 & 28.609 & 26.292 & 31.611 & 36.888 & 32.626 & 16.031 & & 20.779 & 15.66 & 10.463 & 31.849 & 32.502 & 29.79 & 31.568 & 28.124 & 33.504 \\
\hline $3 / 9 / 126: 00$ & & 30.44 & 28.279 & 29.582 & 30.169 & & 29.563 & 30.44 & 29.888 & 29.848 & 28.602 & 26.289 & 31.609 & 36.888 & 32.626 & 16.031 & & 20.777 & $\begin{array}{l}15.662 \\
\end{array}$ & 10.463 & 31.842 & 32.504 & 29.79 & 31.57 & 28.122 & 33.499 \\
\hline & & & & & & & 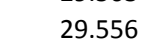 & & & & 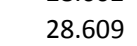 & & & & & & & & & & & & & & & \\
\hline
\end{tabular}




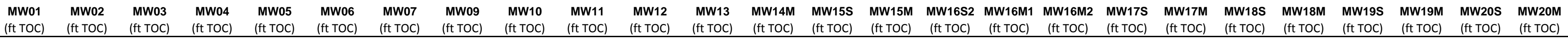

\begin{tabular}{|c|c|c|c|c|c|c|c|c|c|c|c|c|c|c|c|c|c|c|c|c|c|c|c|c|c|}
\hline Well & $\begin{array}{l}\text { (ft TOC) } \\
\text { (is }\end{array}$ & $\begin{array}{l}\text { (ft TOC) } \\
\text { (f) }\end{array}$ & $\begin{array}{l}\text { (ft TOC) } \\
\text { (f) }\end{array}$ & $\begin{array}{l}\text { (ft TOC) } \\
\text { (f }\end{array}$ & $\begin{array}{l}\text { (ft TOC) } \\
\text { (f) }\end{array}$ & $\begin{array}{l}\text { (ft TOC) } \\
\text { (f) }\end{array}$ & $\begin{array}{l}\text { (ft TOC) } \\
\text { (ft }\end{array}$ & $\begin{array}{l}\text { (ft TOC) } \\
\text { (f) }\end{array}$ & $\begin{array}{l}\text { (ft TOC) } \\
\text { (f) }\end{array}$ & $\begin{array}{l}\text { (ft TOC) } \\
\text { (f) }\end{array}$ & $\begin{array}{l}\text { (ft TOC) } \\
\text { (f) }\end{array}$ & $\begin{array}{l}\text { (ft TOC) } \\
\text { (f) }\end{array}$ & (ft TOC) & $\begin{array}{l}\text { (ft TOC) } \\
\end{array}$ & (ft TOC) & $\begin{array}{ll}\text { (ft TOC) } \\
\text { (ft TOC) }\end{array}$ & (ft TOC) & $\begin{array}{l}\text { (ft TOC) } \\
\text { (1) }\end{array}$ & (ft TOC) & $\begin{array}{l}\text { (ft TOC) } \\
\end{array}$ & (ft TOC) & $\begin{array}{l}\text { (ft TOC) } \\
\end{array}$ & (ft TOC) & $\begin{array}{l}\text { (ft TOC) } \\
\text { (1) }\end{array}$ & (ft TOC) \\
\hline 3/9/12 8:00 & & 30.45 & 28.309 & 29.608 & 30.18 & & 29.554 & 30.4 & 29.9 & 29.86 & 28.61 & 26. & 31.62 & 36.89 & 32.6 & 16.0 & 20.779 & 15.6 & 72 & 31. & 32. & 29.804 & 79 & 3.124 & 33.502 \\
\hline 3/9/12 9:00 & & 30.452 & 28.361 & 29.615 & 30.185 & & 29.559 & 30.449 & 29.907 & 29.865 & 28.616 & 26.318 & 31.625 & 36.895 & 32.642 & 16.035 & 20.786 & 15.672 & 10.477 & 31.867 & 32.518 & 29.811 & 31.586 & 28.126 & 33.506 \\
\hline $3 / 9 / 1210: 00$ & & 30.447 & 28.445 & 29.601 & 30.178 & & 29.58 & 30.452 & 29.897 & 29.86 & 28.609 & 26.304 & 31.63 & 36.897 & 32.642 & 16.038 & 20.791 & 15.693 & 10.482 & 31.856 & 32.521 & 29.802 & 31.6 & 8.124 & 33.511 \\
\hline 3/9/12 11:00 & & 30.447 & 28.528 & 29.587 & 30.173 & & 29.596 & 30.452 & 29.895 & 29.853 & 28.605 & 26.289 & 31.63 & 36.89 & 32.647 & 16.038 & 20.795 & 15.714 & 10.484 & 31.853 & 32.523 & 29.788 & 31.619 & 28.119 & 33.516 \\
\hline $3 / 9 / 12$ 12:00 & & 30.45 & 28.603 & 29.575 & 30.162 & & 29.613 & 30.452 & 29.888 & 29.848 & 28.598 & 26.275 & 31.63 & 36.888 & 32.647 & 16.04 & 20.798 & 15.726 & 10.484 & 31.846 & 52.526 & 29.776 & 31.619 & 28.124 & 33.52 \\
\hline 3/9/12 13:00 & & 30.443 & 28.682 & 29.545 & 30.143 & & 29.636 & 30.452 & 29.876 & 29.834 & 28.581 & 26.246 & 31.625 & 36.879 & 32.642 & 16.038 & 20.8 & 15.74 & 10.479 & 31.823 & 32.518 & 29.755 & 31.622 & 28.124 & 33.52 \\
\hline 3/9/12 14:00 & & 30.44 & 28.75 & 29.512 & 30.12 & & 29.657 & 30.449 & 29.866 & 29.816 & 28.563 & 26.217 & 31.616 & 36.872 & 32.635 & 5 16.033 & 20.818 & 15.766 & 10.472 & 31.804 & 32.509 & 29.722 & 31.591 & 28.124 & 33.518 \\
\hline 3/9/12 15:00 & & 30.438 & 28.79 & 29.487 & 30.099 & & 29.667 & 30.444 & 29.862 & 29.8 & 28.549 & 26.196 & 31.607 & 36.865 & 32.626 & $5 \quad 16.033$ & 20.835 & 15.768 & 10.461 & 31.786 & $5 \quad 32.497$ & 29.703 & 31.575 & 28.124 & 33.513 \\
\hline 3/9/12 16:00 & & 30.438 & 28.808 & 29.477 & 30.082 & & 29.664 & 30.442 & 29.864 & 29.79 & 28.537 & 26.186 & 31.599 & 36.86 & 32.617 & 16.028 & 20.897 & 15.768 & 10.451 & 31.779 & 32.49 & 29.701 & 31.565 & 28.126 & 33.511 \\
\hline 3/9/12 17:00 & & 30.436 & 28.804 & 29.463 & 30.066 & & 29.66 & 30.437 & 29.866 & 29.779 & 28.525 & 26.177 & 31.59 & 36.851 & 32.607 & 16.021 & 20.892 & 15.766 & 10.446 & 31.765 & 32.481 & 29.71 & 31.553 & 28.126 & 33.506 \\
\hline 3/9/12 18:00 & & 30.438 & 28.773 & 29.454 & 30.054 & & 29.65 & 30.432 & 29.869 & 29.772 & 28.516 & 26.172 & 31.581 & 36.848 & 32.6 & 16.019 & 20.885 & 15.759 & 10.435 & 31.753 & 32.473 & 29.706 & 31.544 & 28.126 & 33.502 \\
\hline $3 / 9 / 1219: 00$ & & 30.436 & 28.731 & 29.447 & 30.042 & & 29.638 & 30.43 & 29.869 & 29.76 & 28.509 & 26.165 & 31.574 & 36.841 & 32.593 & 36.014 & 20.879 & 15.754 & 10.43 & 31.746 & 32.469 & 29.68 & 31.532 & 28.126 & 33.497 \\
\hline $3 / 9 / 1220: 00$ & & 30.438 & 28.685 & 29.454 & 30.042 & & 29.62 & 30.425 & 29.876 & 29.758 & 28.507 & 26.172 & 31.571 & 36.839 & 32.588 & $3 \quad 16.01$ & 20.874 & 15.742 & 10.428 & 31.753 & 32.464 & 29.647 & 31.53 & 28.126 & 33.495 \\
\hline $3 / 9 / 1221: 00$ & & 30.438 & 28.657 & 29.456 & 30.04 & & 29.608 & 30.428 & 29.881 & 29.753 & 28.502 & 26.177 & 31.574 & 36.846 & 32.591 & 16.007 & 20.872 & 15.737 & 10.425 & 31.753 & 32.462 & 29.619 & 31.532 & 28.126 & 33.495 \\
\hline 3/9/12 22:00 & & 30.44 & 28.626 & 29.459 & 30.04 & & 29.596 & 30.425 & 29.883 & 29.751 & 28.5 & 26.182 & 31.571 & 36.834 & 32.588 & $3 \quad 16.003$ & 20.876 & 15.73 & 10.425 & 31.751 & 32.466 & 29.6 & 31.53 & 28.126 & 33.495 \\
\hline 3/9/12 23:00 & & 30.443 & 28.605 & 29.456 & 30.04 & & 29.589 & 30.425 & 29.888 & 29.749 & 28.498 & 26.184 & 31.571 & 36.834 & 32.591 & 16 & 20.876 & 15.723 & 10.43 & 31.755 & 32.466 & 29.583 & 31.532 & 28.126 & 33.497 \\
\hline $3 / 10 / 120: 00$ & & 30.443 & 28.591 & 29.456 & 30.04 & & 29.587 & 30.425 & 29.885 & 29.744 & 28.495 & 26.186 & 31.574 & 36.827 & 32.593 & 15.998 & 20.876 & 15.721 & 10.43 & 31.755 & 32.469 & 29.572 & 31.537 & 28.126 & 33.502 \\
\hline $3 / 10 / 121: 00$ & & 30.44 & 28.587 & 29.44 & 30.031 & & 29.591 & 30.42 & 29.881 & 29.735 & 28.484 & 26.17 & 31.569 & 36.834 & 32.591 & 15.993 & 20.879 & 15.721 & 10.428 & 31.744 & 32.464 & 29.661 & 31.532 & 28.126 & 33.499 \\
\hline $3 / 10 / 122: 00$ & & 30.436 & 28.58 & 29.423 & 30.024 & & 29.596 & 30.418 & 29.881 & 29.723 & 28.474 & 26.158 & 31.564 & 36.832 & 32.584 & 15.991 & 20.876 & 15.719 & 10.423 & 31.737 & 32.457 & 29.651 & 31.525 & 28.126 & 33.499 \\
\hline $3 / 10 / 123: 00$ & & 30.433 & 28.584 & 29.402 & 30.005 & & 29.606 & 30.413 & 29.871 & 29.709 & 28.46 & 26.141 & 31.557 & 36.827 & 32.579 & 15.984 & 20.879 & 15.716 & 10.416 & 31.723 & 32.45 & 29.628 & 31.523 & 28.126 & 33.497 \\
\hline 3/10/12 4:00 & & 30.431 & 28.589 & 29.386 & 29.993 & & 29.606 & 30.408 & 29.871 & 29.698 & 28.449 & 26.129 & 31.55 & 36.822 & 32.57 & 15.982 & 20.881 & 15.712 & 10.406 & 31.711 & 32.443 & 9.626 & 31.516 & 28.126 & 33.492 \\
\hline 3/10/12 5:00 & & 30.431 & 28.582 & 29.388 & 29.986 & & 29.596 & 30.406 & 29.876 & 29.693 & 28.444 & 26.129 & 31.543 & 36.818 & 32.565 & $\quad 15.977$ & 20.883 & 15.7 & 10.402 & 31.707 & 32.438 & 9.616 & 31.504 & 28.126 & 33.488 \\
\hline 3/10/12 6:00 & & 30.431 & 28.575 & 29.388 & 29.984 & & 29.587 & 30.404 & 29.878 & 29.686 & 28.442 & 26.136 & 31.541 & 36.818 & 32.558 & 3 15.975 & 20.885 & 15.695 & 10.397 & 31.704 & 32.433 & 9.604 & 31.502 & 28.129 & 33.485 \\
\hline 3/10/12 7:00 & & 30.433 & 28.566 & 29.391 & 29.984 & & 29.577 & 30.401 & 29.881 & 29.684 & 28.439 & 26.143 & 31.536 & 36.818 & 32.558 & $3 \quad 15.97$ & 20.888 & 15.679 & 10.392 & 31.704 & 32.426 & 29.595 & 31.497 & 28.126 & 33.483 \\
\hline 3/10/12 8:00 & & 30.433 & 28.577 & 29.4 & 29.989 & & 29.563 & 30.401 & 29.888 & 29.684 & 28.442 & 26.153 & 31.553 & 36.818 & 32.572 & 15.968 & 20.89 & 15.676 & 10.414 & 31.716 & $5 \quad 32.447$ & 29.609 & 31.516 & 28.129 & 33.485 \\
\hline 3/10/12 9:00 & & 30.436 & 28.626 & 29.409 & 29.993 & & 29.556 & 30.404 & 29.888 & 29.684 & 28.444 & 26.16 & 31.548 & 36.818 & 32.565 & 15.966 & 20.897 & 15.674 & 10.407 & 31.721 & 32.44 & 29.633 & 31.511 & 28.129 & 33.488 \\
\hline 3/10/12 10:00 & & 30.436 & 28.685 & 29.407 & 29.998 & & 29.559 & 30.401 & 29.888 & 29.681 & 28.439 & 26.162 & 31.548 & 36.818 & 32.567 & 15.963 & 20.904 & 15.679 & 10.402 & 31.721 & 32.44 & 29.677 & 31.525 & 28.129 & 33.49 \\
\hline 3/10/12 11:00 & & 30.429 & 28.75 & 29.395 & 29.993 & & 29.57 & 30.399 & 29.881 & 29.677 & 28.435 & 26.158 & 31.546 & 36.82 & 32.567 & 15.961 & 20.906 & 15.683 & 10.406 & 31.716 & 32.44 & 29.736 & 31.537 & 28.131 & 33.495 \\
\hline 3/10/12 12:00 & & 30.426 & 28.82 & 29.367 & 29.982 & & 29.589 & 30.396 & 29.871 & 29.66 & 28.421 & 26.129 & 31.543 & 36.811 & 32.565 & $\quad 15.959$ & 20.913 & 15.695 & 10.399 & 31.702 & 32.435 & 29.607 & 31.539 & 28.129 & 33.497 \\
\hline $3 / 10 / 1213: 00$ & & 30.424 & 28.874 & 29.349 & 29.965 & & 29.599 & 30.389 & 29.871 & 29.646 & 28.407 & 26.115 & 31.539 & 36.811 & 32.558 & $3 \quad 15.959$ & 20.92 & 15.7 & 10.395 & 31.693 & 32.431 & 29.59 & 31.525 & 28.129 & 33.497 \\
\hline $3 / 10 / 1214: 00$ & & 30.412 & 28.918 & 29.314 & 29.94 & & 29.617 & 30.385 & 29.857 & 29.626 & 28.386 & 26.081 & 31.525 & 36.804 & 32.546 & $5 \quad 15.949$ & 20.92 & 15.712 & 10.383 & 31.662 & 32.419 & 29.56 & 31.5 & 28.129 & 33.497 \\
\hline $3 / 10 / 1215: 00$ & & 30.408 & 28.946 & 29.288 & 29.919 & & 29.622 & 30.377 & 29.852 & 29.609 & 28.367 & 26.057 & 31.515 & 36.801 & 32.537 & 15.947 & 20.92 & 15.714 & 10.374 & 31.653 & 32.407 & 29.536 & 31.485 & 28.129 & 33.492 \\
\hline $3 / 10 / 1216: 00$ & & 30.405 & 28.957 & 29.274 & 29.9 & & 29.62 & 30.37 & 29.85 & 29.593 & 28.356 & 26.045 & 31.503 & 36.792 & 32.525 & 5 15.942 & 20.922 & 15.705 & 10.362 & 31.632 & 32.395 & 29.522 & 31.469 & 28.129 & 33.49 \\
\hline $3 / 10 / 12$ 17:00 & & 30.403 & 28.936 & 29.276 & 29.893 & & 29.601 & 30.365 & 29.852 & 29.588 & 28.351 & 26.052 & 31.496 & 36.787 & 32.518 & $\begin{array}{l}315.935 \\
3\end{array}$ & 20.922 & 15.688 & 10.355 & 31.634 & 32.388 & 29.522 & 31.46 & 28.131 & 33.485 \\
\hline $3 / 10 / 1218: 00$ & & 30.405 & 28.902 & 29.281 & 29.891 & & 29.58 & 30.365 & 29.862 & 29.584 & 28.349 & 26.06 & 31.492 & 36.787 & 32.506 & 15.9 & 20.92 & 15.679 & 10.348 & 31.627 & 32.381 & 29.525 & 31.453 & 28.133 & 33.483 \\
\hline 3/10/12 19:00 & & 30.403 & 28.86 & 29.288 & 29.893 & & 29.561 & 30.361 & 29.862 & 29.579 & 28.349 & 26.069 & 31.485 & 36.783 & 32.506 & 15. & 20.92 & 15.662 & $\begin{array}{l}10.343 \\
\end{array}$ & 31.637 & 32.379 & 29.529 & 31.443 & 28.129 & 33.478 \\
\hline 3/10/12 20:00 & & 30.398 & 28.834 & 29.274 & 29.886 & & 29.556 & 30.358 & 29.855 & 29.572 & 28.339 & 26.069 & 31.48 & 36.78 & 32.499 & 15.9 & 20.922 & $\begin{array}{l}15.662 \\
15.662\end{array}$ & $\begin{array}{l}10.336 \\
\end{array}$ & 31.62 & 32.372 & 29.52 & 31.446 & 28.131 & $\begin{array}{l}33.476 \\
33.46\end{array}$ \\
\hline $3 / 10 / 1221: 00$ & & 30.396 & $\begin{array}{l}20.034 \\
28.813\end{array}$ & 29.267 & 29.881 & & 29.549 & 30.351 & 29.857 & 29.563 & 28.33 & 26.06 & $\begin{array}{l}31.475 \\
31.475\end{array}$ & $\begin{array}{l}36.775 \\
36.75\end{array}$ & 32.497 & 15.9 & 20.918 & $\begin{array}{l}15.658 \\
15.658\end{array}$ & $\begin{array}{l}10.334 \\
\end{array}$ & $\begin{array}{l}31.62 \\
31.62\end{array}$ & 32.367 & 29.515 & 31.436 & 28.131 & $\begin{array}{l}33.471 \\
33.47\end{array}$ \\
\hline $3 / 1$ & & 30 & 28.797 & 29.274 & 29. & & 29.535 & 1 & 29.859 & 29.561 & 28.332 & 26.069 & 31.473 & 36.773 & 32.492 & 15 & 20.925 & 15.651 & 10.329 & 31.618 & 3232.364 & 29.52 & 38 & 28.131 & 33.471 \\
\hline $3 / 1$ & & 30 & 28.7 & 29 & 29 & & 2 & 9 & 2 & 29.551 & 28 & 26.062 & 31.471 & 36.775 & 32.49 & 15 & $2 c$ & 1 & 10.329 & 31.606 & .362 & 29 & 48 & 28.131 & 33.471 \\
\hline $3 / 11 / 120: 00$ & & & 28 & 29.246 & 29 & & 29 & 14 & 29 & 29.542 & 28 & 2 & 31.468 & 36.7 & 32.488 & 15 & 26 & 1 & 10.324 & 31.609 & 32.36 & 66 & 36 & 28.129 & 33.471 \\
\hline $3 / 11 / 121: 00$ & & 30.389 & 28.783 & 29.251 & & & & & 29 & 29 & & 26.057 & 31.466 & 36.768 & 32.485 & 15 & 29 & 15.637 & 10.324 & 31.606 & 52.357 & 29.499 & 29 & 28.133 & 33.474 \\
\hline 3/11/12 2:00 & & 30.387 & 28.776 & 29.251 & 29.863 & & 29.512 & 30.339 & 29.855 & 29.533 & 28.309 & 26.057 & 31.464 & 36.768 & 32.483 & 15.903 & & 15.625 & 10.319 & 31.602 & 32.355 & 29.506 & 31.424 & 28.131 & 33.474 \\
\hline 3/11/12 3:00 & & 30.382 & 28.766 & 29.241 & 29.8 & & & 30.334 & 29.855 & 29.528 & 28.302 & 26.057 & 31.459 & 36.766 & 32.478 & & 20.936 & 15.627 & 10.317 & 31.599 & 32.35 & 29.485 & 31.427 & 28.131 & 33.471 \\
\hline 3/11/12 4:00 & & 30.38 & 28.764 & 29.225 & 29 & & & 30.3 & 29.843 & & 28.293 & & 31.45 & 36.761 & 32.469 & 15.8 & & 15.615 & 10.308 & 31.588 & $3 \quad 32.343$ & 29.487 & 31.415 & 28.131 & 33.467 \\
\hline $3 / 11 / 125: 00$ & & 30.377 & 28.7 & 29.215 & 29. & & & 30.325 & 29.84 & & 28.286 & 26.028 & 31.44 & 36.759 & 32.462 & 15. & & 15.613 & 10.301 & 31.581 & 32.338 & 29.475 & 31.408 & 28.131 & 33.462 \\
\hline $3 / 11 / 126: 00$ & & 30.375 & 28.757 & 29.211 & 29.8 & & & 30.32 & 29 & 29. & 28.279 & 26.031 & 31.436 & 36.754 & 32.457 & 15. & 20.932 & 15.604 & 10.291 & 31.576 & 532.327 & 29.468 & 31.403 & 28.131 & 33.457 \\
\hline 3/11/127:00 & & 30.373 & 28.75 & 29.211 & 29 & & 29 . & 30. & 29 & 29.493 & 28.277 & 26.033 & 31.431 & 36.754 & 32.455 & 15.8 & 20.932 & 15.594 & 10.291 & 31.571 & 32.324 & 29.468 & 31.396 & 28.131 & 33.455 \\
\hline $3 / 11 / 128: 00$ & & 30.37 & 28.75 & 29.208 & 29.8. & & 29.486 & 30.315 & 29.84 & 29.489 & 28.272 & 26.033 & 31.426 & 36.745 & 32.448 & 15.8 & 20.934 & 15.587 & 10.284 & 31.571 & 32.322 & 29.461 & 31.392 & 28.133 & 33.453 \\
\hline 3/11/129:00 & & 30.366 & 28.762 & 29.199 & 29.821 & & 29.488 & 30.313 & 29.838 & 29.477 & 28.265 & 26.021 & 31.419 & 36.75 & 32.445 & 15.8 & 20.936 & 15.587 & 10.282 & 31.562 & 32.315 & 29.459 & 31.392 & 28.129 & 33.45 \\
\hline $3 / 11 / 1210: 00$ & & 30.363 & 28.771 & 29.185 & 29.809 & & 29.49 & 30.325 & 29.833 & 29.47 & 28.256 & 26.024 & 31.417 & 36.738 & 32.436 & 15.875 & 20.932 & 15.583 & 10.272 & 31.548 & 32.31 & 29.44 & 31.396 & 28.129 & 33.448 \\
\hline $3 / 11 / 12$ 11:00 & & 30.361 & 28.778 & 29.164 & 29.793 & & 29.493 & 30.313 & 29.828 & 29.449 & 28.244 & 25.988 & 31.405 & 36.747 & 32.434 & 15.868 & 20.932 & 15.573 & 10.268 & 31.536 & 32.3 & 29.435 & 31.373 & 28.129 & 33.446 \\
\hline $3 / 11 / 1212: 00$ & & 30.349 & 28.804 & 29.141 & 29.776 & & 29.5 & 30.306 & 29.817 & 29.438 & 28.228 & 25.978 & 31.4 & 36.738 & 32.424 & 15.865 & 20.936 & 15.585 & 10.263 & 31.525 & 32.296 & 29.409 & 31.38 & 28.133 & 33.448 \\
\hline 3/11/12 13:00 & & 30.345 & 28.813 & 29.134 & 29.765 & & 29.493 & 30.301 & 29.817 & 29.428 & 28.219 & 25.978 & 31.4 & 36.733 & 32.42 & 15.861 & 20.939 & 15.583 & 10.258 & 31.518 & 32.289 & 29.393 & 31.377 & 28.131 & 33.448 \\
\hline $3 / 11 / 12$ 14:00 & & 30.335 & 28.82 & 29.113 & 29.748 & & 29.493 & 30.294 & 29.809 & 29.414 & 28.205 & 25.964 & 31.391 & 36.729 & 32.415 & $\quad 15.858$ & 20.941 & 15.578 & 10.252 & 31.504 & 32.281 & 29.379 & 31.373 & 28.131 & 33.448 \\
\hline $3 / 11 / 12$ 15:00 & & 30.333 & 28.822 & 29.096 & 29.734 & & 29.49 & 30.289 & 29.805 & 29.4 & 28.193 & 25.952 & 31.379 & 36.722 & 32.403 & 15.851 & 20.943 & 15.571 & 10.242 & 31.49 & 32.272 & 29.367 & 31.356 & 28.131 & 33.446 \\
\hline
\end{tabular}


TABLE S1.1 Automatically recorded groundwater depths in monitoring wells, December 2009 to March 2013, measured from the top of the casing (ft TOC).

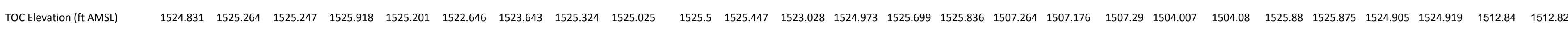

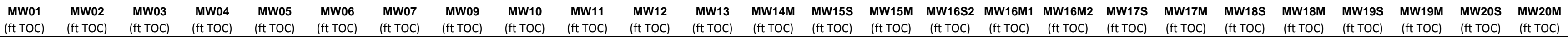

\begin{tabular}{|c|c|c|c|c|c|c|c|c|c|c|c|c|c|c|c|c|c|c|c|c|c|c|c|c|c|c|}
\hline Well & $\begin{array}{l}\text { (ft TOC) } \\
\text { (f) }\end{array}$ & $\begin{array}{l}\text { (ft TOC) } \\
\text { (f) }\end{array}$ & $\begin{array}{l}\text { (ft TOC) } \\
\text { (f) }\end{array}$ & $\begin{array}{l}\text { (ft TOC) } \\
\text { (f) }\end{array}$ & $\begin{array}{l}\text { (ft TOC) } \\
\text { (f) }\end{array}$ & $\begin{array}{l}\text { (ft TOC) } \\
\text { (ft }\end{array}$ & $\begin{array}{l}\text { (ift TOC) } \\
\text { (ft }\end{array}$ & $\begin{array}{l}\text { (ft TOC) } \\
\text { (f) }\end{array}$ & $\begin{array}{l}\text { (ft TOC) } \\
\text { (f) }\end{array}$ & $\begin{array}{l}\text { (ft TOC) } \\
\text { (f) }\end{array}$ & $\begin{array}{l}\text { (ft TOC) } \\
\text { (f) }\end{array}$ & $\begin{array}{l}\text { (ft TOC) } \\
\text { (f) }\end{array}$ & $\begin{array}{l}\text { (ft TOC) } \\
\text { (n) }\end{array}$ & $\begin{array}{l}\text { (ft TOC) } \\
\end{array}$ & $\begin{array}{l}\text { (ft TOC) } \\
\text { (1) }\end{array}$ & $\begin{array}{l}\text { (ft TOC) } \\
\end{array}$ & (ft TOC) & (ft TOC) & $\begin{array}{l}\text { (ft TOC) } \\
\text { (n) }\end{array}$ & $\begin{array}{l}\text { (ft TOC) } \\
\text { (f) }\end{array}$ & $\begin{array}{l}\text { (ft TOC) } \\
\text { (1) }\end{array}$ & $\begin{array}{l}\text { (ft TOC) } \\
\text { (1) }\end{array}$ & $\begin{array}{l}\text { (ft TOC) } \\
\end{array}$ & (ft TOC) & $\begin{array}{l}\text { (ft TOC) } \\
\text { (f) }\end{array}$ & (ft TOC) \\
\hline //12 16:00 & & 30.335 & 28.818 & 29.106 & 29.73 & & 29.467 & 30.284 & 29.814 & 29.396 & 28.193 & 25.959 & 31.375 & 5.724 & 32.398 & 15.847 & & 20.946 & 15.55 & 10.235 & .49 & 32.267 & 29.372 & 31.345 & 28.131 & 33.443 \\
\hline $3 / 11 / 12$ 17:00 & & 30.331 & 28.82 & 29.101 & 29.725 & & 29.455 & 30.279 & 29.812 & 29.389 & 28.186 & 25.957 & 31.368 & 36.717 & 32.389 & 15.844 & & 20.946 & 15.545 & 10.225 & 31.487 & 32.263 & 29.369 & 31.34 & 28.133 & 33.441 \\
\hline $3 / 11 / 12$ 18:00 & & 30.328 & 28.82 & 29.101 & 29.725 & & 29.443 & 30.274 & 29.814 & 29.384 & 28.184 & 25.964 & 31.363 & 36.715 & 32.384 & 15.84 & & 20.948 & 15.54 & 10.223 & 31.487 & 32.255 & 29.367 & 31.333 & 28.133 & 33.436 \\
\hline 3/11/12 19:00 & & 30.326 & 28.808 & 29.103 & 29.725 & & 29.432 & 30.27 & 29.817 & 29.38 & 28.181 & 25.969 & 31.695 & 36.715 & 32.75 & 15.835 & & 20.946 & 15.526 & 10.58 & 31.592 & 32.585 & 29.374 & 31.657 & 28.131 & 33.434 \\
\hline 3/11/12 20:00 & & 30.328 & 28.794 & 29.115 & 29.734 & & 29.411 & 30.272 & 29.817 & 29.382 & 28.186 & 25.981 & 31.77 & 36.707 & 32.828 & 15.833 & & 20.943 & 15.512 & 10.658 & 31.639 & 32.661 & 29.388 & 31.729 & 28.133 & 33.427 \\
\hline 3/11/12 21:00 & & 30.328 & 28.787 & 29.124 & 29.746 & & 29.396 & 30.289 & 29.824 & 29.38 & 28.188 & 25.99 & 31.646 & 36.707 & 32.677 & 15.83 & & 20.939 & 15.5 & 10.515 & 31.632 & 32.537 & 29.409 & 31.596 & 28.133 & 33.422 \\
\hline 3/11/12 22:00 & & 30.324 & 28.776 & 29.122 & 29.753 & & 29.392 & 30.286 & 29.821 & 29.382 & 28.186 & 25.997 & 31.567 & 36.71 & 32.595 & 15.826 & & 20.941 & 15.498 & 10.435 & 31.602 & 32.457 & 29.409 & 31.525 & 28.131 & 33.422 \\
\hline 3/11/12 23:00 & & 30.321 & 28.766 & 29.12 & 29.758 & & 29.385 & 30.277 & 29.817 & 29.377 & 28.186 & 25.995 & 31.525 & 36.707 & 32.553 & 15.826 & & 20.943 & 15.493 & 10.39 & 31.583 & 32.412 & 29.407 & 31.485 & 28.133 & 33.42 \\
\hline $3 / 12 / 120: 00$ & & 30.319 & 28.762 & 29.113 & 29.755 & & 29.387 & 30.268 & 29.814 & 29.375 & 28.184 & 25.993 & 31.494 & 36.707 & 32.523 & 15.826 & & 20.946 & 15.486 & 10.362 & 31.567 & 32.388 & 29.405 & 31.46 & 28.136 & 33.42 \\
\hline 3/12/12 1:00 & & 30.317 & 28.769 & 29.101 & 29.748 & & 29.387 & 30.265 & 29.809 & 29.366 & 28.177 & 25.985 & 31.475 & 36.705 & 32.499 & 15.821 & & 20.95 & 15.489 & 10.336 & 31.555 & 32.367 & 29.391 & 31.441 & 28.133 & 33.42 \\
\hline $3 / 12 / 122: 00$ & & 30.312 & 28.776 & 29.092 & 29.744 & & 29.385 & 30.26 & 29.805 & 29.361 & 28.167 & 25.978 & 31.454 & 36.7 & 32.481 & 15.819 & & 20.953 & 15.482 & 10.317 & 31.541 & 32.348 & 29.381 & 31.424 & 28.133 & 33.42 \\
\hline $3 / 12 / 123: 00$ & & 30.31 & 28.787 & 29.087 & 29.739 & & 29.387 & 30.255 & 29.802 & 29.352 & 28.165 & 25.973 & 31.44 & 36.7 & 32.466 & 15.817 & & 20.948 & 15.479 & 10.301 & 31.532 & 32.329 & 29.377 & 31.413 & 28.133 & 33.42 \\
\hline 3/12/12 4:00 & & 30.314 & 28.787 & 29.099 & 29.741 & & 29.371 & 30.253 & 29.809 & 29.354 & 28.17 & 25.983 & 31.431 & 36.7 & 32.452 & 15.817 & & 20.95 & 15.458 & 10.291 & 31.529 & 32.322 & 29.386 & 31.387 & 28.133 & 33.42 \\
\hline $3 / 12 / 125: 00$ & & 30.31 & 28.792 & 29.103 & 29.744 & & 29.363 & 30.248 & 29.809 & 29.356 & 28.172 & 25.997 & 31.422 & 36.701 & 32.445 & 15.814 & & 20.957 & 15.465 & 10.282 & 31.532 & 32.312 & 29.386 & 31.387 & 28.133 & 33.418 \\
\hline $3 / 12 / 126: 00$ & & 30.314 & 28.783 & 29.127 & 29.755 & & 29.338 & 30.248 & 29.821 & 29.363 & 28.181 & 26.017 & 31.417 & 36.703 & 32.438 & 15.817 & & 20.962 & 15.449 & 10.277 & 31.543 & 32.31 & 29.409 & 31.375 & 28.133 & 33.418 \\
\hline $3 / 12 / 12$ 7:00 & & 30.319 & 28.766 & 29.15 & 29.772 & & 29.319 & 30.248 & 29.831 & 29.373 & 28.193 & 26.038 & 31.415 & 36.71 & 32.441 & 15.819 & & 20.962 & 15.435 & 10.277 & 31.557 & 32.308 & 29.426 & 31.366 & 28.133 & 33.42 \\
\hline $3 / 12 / 128: 00$ & & 30.324 & 28.752 & 29.185 & 29.797 & & 29.295 & 30.253 & 29.84 & 29.391 & 28.214 & 26.072 & 31.422 & 36.717 & 32.441 & 15.819 & & 20.964 & 15.43 & 10.277 & 31.574 & 32.315 & 29.456 & 31.373 & 28.136 & 33.42 \\
\hline 3/12/12 9:00 & & 30.324 & 28.759 & 29.206 & 29.818 & & 29.291 & 30.255 & 29.838 & 29.405 & 28.225 & 26.093 & 31.426 & 36.722 & 32.445 & 15.823 & & 20.969 & 15.428 & 10.284 & 31.59 & 32.317 & 29.471 & 31.38 & 28.138 & 33.425 \\
\hline $3 / 12 / 1210: 00$ & & 30.326 & 28.79 & 29.215 & 29.832 & & 29.293 & 30.26 & 29.84 & 29.412 & 28.237 & 26.105 & 31.431 & 36.729 & 32.45 & 15.828 & & 20.971 & 15.43 & 10.284 & 31.599 & 32.324 & 29.485 & 31.399 & 28.136 & 33.427 \\
\hline 3/12/12 11:00 & & 30.328 & 28.829 & 29.229 & 29.851 & & 29.295 & 30.265 & 29.831 & 29.426 & 28.246 & 26.117 & 31.438 & 36.733 & 32.457 & 15.833 & & 20.976 & 15.439 & 10.301 & 31.609 & 32.331 & 29.496 & 31.417 & 28.138 & 33.434 \\
\hline $3 / 12 / 1212: 00$ & & 30.326 & 28.881 & 29.227 & 29.856 & & 29.314 & 30.265 & 29.826 & 29.428 & 28.251 & 26.115 & 31.443 & 36.738 & 32.459 & 15.837 & & 20.983 & 15.453 & 10.296 & 31.609 & 32.334 & 29.501 & 31.427 & 28.136 & 33.441 \\
\hline 3/12/12 13:00 & & 30.324 & 28.932 & 29.222 & 29.856 & & 29.328 & 30.267 & 29.824 & 29.431 & 28.251 & 26.105 & 31.445 & 36.74 & 32.464 & 15.84 & & 20.985 & 15.463 & 10.301 & 31.609 & 32.336 & 29.489 & 31.431 & 28.136 & 33.443 \\
\hline $3 / 12 / 1214: 00$ & & 30.321 & 28.983 & 29.204 & 29.851 & & 29.347 & 30.267 & 29.814 & 29.428 & 28.249 & 26.088 & 31.443 & 36.74 & 32.464 & 15.844 & & 20.987 & 15.475 & 10.298 & 31.599 & 32.334 & 29.494 & 31.429 & 28.138 & 33.448 \\
\hline 3/12/12 15:00 & & 30.324 & 29.018 & 29.206 & 29.851 & & 29.349 & 30.267 & 29.817 & 29.433 & 28.251 & 26.091 & 31.443 & 36.74 & 32.464 & 15.849 & & 20.994 & 15.477 & 10.298 & 31.606 & 32.338 & 29.492 & 31.417 & 28.138 & 33.45 \\
\hline $3 / 12 / 1216: 00$ & & 30.321 & 29.039 & 29.208 & 29.853 & & 29.352 & 30.27 & 29.817 & 29.435 & 28.253 & 26.091 & 31.443 & 36.74 & 32.464 & 15.851 & & 21.015 & 15.484 & 10.303 & 31.604 & 32.338 & 29.494 & 31.417 & 28.138 & 33.45 \\
\hline 3/12/12 17:00 & & 30.324 & 29.039 & 29.213 & 29.856 & & 29.349 & 30.267 & 29.819 & 29.44 & 28.258 & 26.093 & 31.445 & 36.74 & 32.462 & 15.854 & & 21.033 & 15.486 & 10.301 & 31.606 & 32.336 & 29.494 & 31.41 & 28.138 & 33.45 \\
\hline $3 / 12 / 1218: 00$ & & 30.331 & 29.009 & 29.229 & 29.867 & & 29.335 & 30.272 & 29.828 & 29.447 & 28.267 & 26.107 & 31.447 & 36.74 & 32.464 & 15.858 & & 21.047 & 15.477 & 10.301 & 31.613 & 32.338 & 29.508 & 31.403 & 28.138 & 33.45 \\
\hline $3 / 12 / 12$ 19:00 & & 30.333 & 28.967 & 29.251 & 29.881 & & 29.326 & 30.277 & 29.831 & 29.458 & 28.279 & 26.129 & 31.45 & 36.747 & 32.466 & 15.861 & & 21.05 & 15.47 & 10.305 & 31.63 & 32.343 & 29.529 & 31.403 & 28.138 & 33.45 \\
\hline 3/12/12 20:00 & & 30.34 & 28.922 & 29.283 & 29.902 & & 29.307 & 30.282 & 29.847 & 29.477 & 28.298 & 26.158 & 31.459 & 36.754 & 32.474 & 15.868 & & 21.045 & 15.463 & 10.31 & 31.646 & 32.35 & 29.548 & 31.408 & 28.14 & 33.45 \\
\hline $3 / 12 / 1221: 00$ & & 30.342 & 28.89 & 29.302 & 29.921 & & 29.3 & 30.289 & 29.845 & 29.493 & 28.314 & 26.174 & 31.464 & 36.766 & 32.483 & 15.872 & & 21.047 & 15.461 & 10.315 & 31.662 & 32.357 & 29.574 & 31.42 & 28.138 & 33.45 \\
\hline $3 / 12 / 1222: 00$ & & 30.345 & 28.867 & 29.314 & 29.937 & & 29.3 & 30.294 & 29.845 & 29.505 & 28.323 & 26.184 & 31.473 & 36.769 & 32.488 & 15.877 & & 21.047 & 15.468 & 10.327 & 31.672 & 32.364 & 29.583 & 31.431 & 28.138 & 33.45 \\
\hline $3 / 12 / 1223: 00$ & & 30.345 & 28.848 & 29.311 & 29.944 & & 29.314 & 30.298 & 29.84 & 29.51 & 28.328 & 26.177 & 31.475 & 36.773 & 32.492 & 15.882 & & 21.052 & 15.475 & 10.329 & 31.674 & 32.367 & 29.581 & 31.434 & 28.136 & 33.45 \\
\hline 3/13/12 0:00 & & 30.345 & 28.834 & 29.304 & 29.942 & & 29.331 & 30.306 & 29.833 & 29.512 & 28.325 & 26.17 & 31.478 & 36.775 & 32.495 & 15.886 & & 21.054 & 15.484 & 10.334 & 31.672 & 32.369 & 29.574 & 31.441 & 28.138 & 33.45 \\
\hline $3 / 13 / 121: 00$ & & 30.342 & 28.827 & 29.293 & 29.937 & & 29.342 & 30.306 & 29.824 & 29.512 & 28.325 & 26.155 & 31.475 & 36.768 & 32.495 & 15.891 & & 21.054 & 15.489 & 10.334 & 31.665 & 32.369 & 29.572 & 31.439 & 28.136 & 33.448 \\
\hline $3 / 13 / 122: 00$ & & 30.345 & 28.815 & 29.295 & 29.935 & & 29.349 & 30.306 & 29.828 & 29.514 & 28.33 & 26.151 & 31.48 & 36.775 & 32.495 & 15.893 & & 21.059 & 15.489 & 10.334 & 31.662 & 32.372 & 29.569 & 31.441 & 28.136 & 33.45 \\
\hline 3/13/12 3:00 & & 30.345 & 28.813 & 29.286 & 29.933 & & 29.359 & 30.308 & 29.824 & 29.516 & 28.325 & 26.146 & 31.48 & 36.773 & 32.497 & 15.898 & & 21.057 & 15.491 & 10.336 & 31.66 & 32.372 & 9.553 & 31.446 & 28.138 & 33.45 \\
\hline $3 / 13 / 124: 00$ & & 30.342 & 28.813 & 29.272 & 29.923 & & 29.373 & 30.306 & 29.817 & 29.514 & 28.323 & 26.129 & 31.475 & 36.768 & 32.495 & 15.9 & & 21.059 & 15.498 & 10.331 & 31.651 & 32.369 & 29.543 & 31.439 & 28.138 & 33.45 \\
\hline $3 / 13 / 125: 00$ & & 30.345 & 28.811 & 29.276 & 29.921 & & 29.373 & 30.306 & 29.821 & 29.514 & 28.323 & 26.126 & 31.478 & 36.773 & 32.492 & 15.903 & & 21.077 & 15.491 & 10.331 & 31.653 & 32.367 & 29.534 & 31.436 & 28.138 & 33.45 \\
\hline $3 / 13 / 126: 00$ & & 30.345 & 28.822 & 29.272 & 29.9 & & 29.375 & 30.306 & 29.819 & 29.517 & 28.323 & 26.131 & 31.473 & 36.766 & 32.492 & 15.905 & & 21.075 & 15.493 & 10.329 & 31.651 & 32.367 & 29.52 & 31.436 & 28.138 & 33.448 \\
\hline & & 30.3 & 28.82 & 29.276 & 29. & & 29 & 30. & 29.824 & & 28.328 & 26.127 & 31.471 & 36.768 & 32.49 & 15. & & 21.084 & 15.486 & 10.329 & 31.651 & 32.364 & 29.51 & 31.434 & 28.138 & 33.448 \\
\hline $3 / 13 / 128: 00$ & & 30.349 & 28.834 & 29.293 & 29. & & 29.363 & 30.308 & 29.826 & 29 & 28.332 & 26.148 & 31.475 & 36.775 & 32.4 & 15 & & 21.087 & 15.484 & 10.331 & 31.66 & 32.372 & 29.508 & 31.441 & 28.138 & 33.45 \\
\hline 3/13/12 9:00 & & 30.347 & 28.878 & 29.283 & 29.921 & & 29 & 30.31 & 29.824 & 29.523 & 28.332 & 26.131 & 31.473 & 36.771 & 32.495 & 15 & & 21. & 15.5 & 10.334 & 31.653 & 32.369 & 29.522 & 31. & 28.14 & 33.448 \\
\hline 3/13/12 10:00 & & 30.345 & 28.932 & & & & 29.389 & 30.31 & 29.819 & 29.521 & 28.325 & 26.124 & 31.478 & 36.768 & 32.495 & 15. & & 21.082 & 15.51 & 10.329 & 31.646 & 32.367 & 29.553 & 31.579 & 28.138 & 33.45 \\
\hline \multirow[t]{4}{*}{ 3/13/12 11:00 } & & 30.345 & & & & & 29.401 & 30.308 & 29.814 & & 28.325 & & 31.473 & 36.766 & 32.492 & 15.912 & & 21.077 & 15.515 & 10.329 & 31.648 & 32.367 & & & 28.14 & 33.453 \\
\hline & & & & & & & & & & & & & 31.471 & 36.761 & 32.495 & 15.912 & & 21.094 & 15.519 & 10.327 & 31.63 & 32.364 & & & 28.138 & 33.455 \\
\hline & & & & & & & & & & & & & 31.468 & 36.759 & 32.488 & 15.912 & & 21.096 & & & 31.625 & 32.362 & & & 28.14 & 33.457 \\
\hline & & & & & & & & & & & & & & & & 15.912 & & 21.098 & & & & & & & 28.14 & 33.457 \\
\hline
\end{tabular}


$\begin{array}{lllll}1500.266 & 1499.857 & 1500.648 & 1501.258 & 1501.095 \\ 1500152 & 1499823 & 1500515 & 1501.202 & 15003317\end{array}$ $\begin{array}{lllll}1500.152 & 1499.823 & 1500.515 & 1501.202 & 1500.317 \\ 1500.061 & 1499.799 & 1500.431 & 1501.195 & 1500.121\end{array}$ $\begin{array}{llllll}140.069 .982 & 1499.799 & 1500.431 & 1501.195 & 1500.121 \\ 1499.776 & 1500.366 & 1501.192 & 1500.07\end{array}$ $\begin{array}{llllll}1499.905 & 1499.755 & 1500.31 & 1501.192 & 1500.07 \\ 1498 & 1500.053\end{array}$ $\begin{array}{rrrrr}1499.851 & 1499.746 & 1500.278 & 1501.197 & 1500.063 \\ 1499778 & 1499732 & 1500.243 & 1501.202 & 1500.079\end{array}$ $\begin{array}{llllll}1499.798 & 1499.732 & 1500.243 & 1501.202 & 1500.079 \\ 1499.742 & 1499.718 & 1500.201 & 1501.176 & 1500.058\end{array}$ $\begin{array}{llllll}1499.742 & 1499.718 & 1500.201 & 1501.176 & 1500.058 \\ 1499.688 & 1499.704 & 1500.168 & 1501.155 & 1500.046\end{array}$ $\begin{array}{lllll}1499.634 & 1499.692 & 1500.133 & 1501.129 & 1500.023\end{array}$ $\begin{array}{lllll}1499.578 & 1499.676 & 1500.093 & 1501.087 & 1499.997 \\ 1499532 & 1499.662 & 1500.065 & 1501.069 & 1999.974\end{array}$ $\begin{array}{llllll}1499.532 & 1499.662 & 1500.065 & 1501.069 & 1499.974 \\ 1499.518 & 1499.655 & 1500.056 & 1501.113 & 1500.007\end{array}$ $\begin{array}{lllllll}1499.49 & 1499.65 & 1500.044 & 1501.108 & 1500.023\end{array}$ $\begin{array}{llllll}1499.48 & 1499.648 & 1500.044 & 1501.139 & 1500.051\end{array}$ $\begin{array}{llllll}1499.473 & 1499.646 & 1500.047 & 1501.16 & 1500.081\end{array}$ $\begin{array}{llllll}1499.462 & 1499.641 & 1500.042 & 1501.169 & 1500.098\end{array}$ $\begin{array}{llllll}1499.443 & 1499.634 & 1500.028 & 1501.148 & 1500.095\end{array}$ $\begin{array}{llllll}1499.422 & 1499.625 & 1500.016 & 1501.141 & 1500.088 \\ 1499.397 & 1499.613 & 1499.998 & 1501101 & 1500.063\end{array}$ $\begin{array}{llllllll}1499.38 & 149.613 & 1499.998 & 1501.101 & 1500.063\end{array}$ $\begin{array}{lllll}1499.38 & 1499.606 & 1499.991 & 1501.099 & 1500.053 \\ 1499.38 & 1499.606 & 1499.995 & 1501.132 & 1500.074\end{array}$

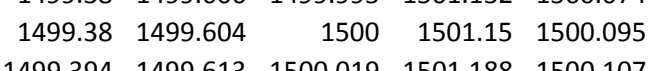
\begin{tabular}{lllll}
1499.394 & 1499.613 & 1500.019 & 1501.188 & 1500.107 \\
\hline
\end{tabular} $\begin{array}{llllll}1499.431 & 1499.636 & 1500.065 & 1501.274 & 1500.151 \\ 1499.425 & 1499.629 & 1500.063 & 1501.239 & 1500.13\end{array}$ $\begin{array}{rrrrr}1499.425 & 1499.629 & 1500.063 & 1501.239 & 1500.13 \\ 1499.431 & 1499.636 & 1500.075 & 1501.258 & 1500.126\end{array}$ $\begin{array}{llllll}1499.429 & 1499.629 & 1500.075 & 1501.249 & 1500.119\end{array}$ $\begin{array}{llllll}1499.415 & 1499.615 & 1500.054 & 1501.206 & 1500.088 \\ 1499.406 & 1499.611 & 1500.044 & 1501202 & 1500.065\end{array}$ $\begin{array}{llllll}1499.42 & 1499.62 & 1500.065 & 1501.242 & 1500.095\end{array}$ $\begin{array}{llllll}1499.406 & 1499.608 & 1500.054 & 1501.206 & 1500.077\end{array}$ $\begin{array}{llllll}1499.42 & 1499.618 & 1500.068 & 1501.209 & 1500.095\end{array}$ $\begin{array}{llllll}1499.431 & 1499.625 & 1500.089 & 1501.202 & 1500.133\end{array}$ $\begin{array}{llllll}1499.429 & 1499.622 & 1500.082 & 1501.185 & 1500.123\end{array}$ $\begin{array}{lllllll}1499.427 & 1499.608 & 1500.084 & 1501.178 & 1500.119 \\ 1499.427 & 1499.62 & 1500.084 & 1501.185 & 1500.121\end{array}$ $\begin{array}{lllllll}1499.427 & 1499.62 & 1500.084 & 1501.185 & 1500.121 \\ 1499.411 & 1499.611 & 1500.065 & 1501.204 & 1500.091\end{array}$ $\begin{array}{llllll}1499.411 & 1499.611 & 1500.065 & 1501.204 & 1500.091 \\ 1499.39 & 1499.594 & 1500.035 & 1501.167 & 1500.051\end{array}$ $\begin{array}{llllll}1499.38 & 1499.592 & 1500.026 & 1501.153 & 1500.035\end{array}$ $\begin{array}{lllllll}1499.364 & 1499.585 & 1500.012 & 1501.134 & 1500.009\end{array}$ $\begin{array}{llllll}1499.355 & 1499.588 & 1500.012 & 1501.141 & 1500.007\end{array}$ $\begin{array}{llllll}1499.383 & 1499.599 & 1500.035 & 1501.202 & 1500.063\end{array}$ $\begin{array}{llllll}1499.383 & 1499.594 & 1500.035 & 1501.192 & 1500.067 \\ 149908 & 1499.618 & 1500.072 & 1501.258 & 1500\end{array}$ $\begin{array}{rrrrrr}1499.408 & 1499.618 & 1500.072 & 1501.258 & 1500.112 \\ 1499.404 & 1499.608 & 1500.07 & 1501232 & 1500.114\end{array}$ $\begin{array}{llllll}1499.404 & 1499.608 & 1500.07 & 1501.232 & 1500.114 \\ 1499.408 & 1499.608 & 1500.065 & 1501228 & 1500102\end{array}$ $\begin{array}{lllll}1499.408 & 1499.608 & 1500.065 & 1501.228 & 1500.102 \\ 1499.415 & 1499.615 & 1500.077 & 1501251 & 1500.114\end{array}$ $\begin{array}{lllllll}1499.415 & 1499.613 & 1500.079 & 1501.237 & 1500.116\end{array}$ $\begin{array}{llllll}1499.411 & 1499.606 & 1500.068 & 1501.218 & 1500.091\end{array}$ $\begin{array}{llllll}1499.392 & 1499.597 & 1500.051 & 1501.183 & 1500.056\end{array}$ $\begin{array}{llllll}1499.38 & 1499.588 & 1500.037 & 1501.162 & 1500.037\end{array}$ $\begin{array}{lllll}1499.357 & 1499.569 & 1500.007 & 1501.113 & 1499.995 \\ 1499.324 & 1499.557 & 1499.974 & 1501.064 & 1499.939\end{array}$ 
$\begin{array}{rrrrr}1499.198 & 1499.501 & 1499.853 & 1500.919 & 1499.9794 \\ 1499.18 & 1499.49 & 1499.832 & 1500.905 & 1499.978\end{array}$ $\begin{array}{llllll}1499.152 & 1499.478 & 1499.811 & 1500.877 & 1499.745\end{array}$ $\begin{array}{llllll}1499.138 & 1499.471 & 1499.797 & 1500.882 & 1499.738\end{array}$ $\begin{array}{rrrrr}1499.126 & 1499.462 & 1499.79 & 1500.877 & 1499.741 \\ 1499.11 & 1499.452 & 1499.776 & 1500868 & 1499\end{array}$ $\begin{array}{llllll}1499.11 & 1499.452 & 1499.776 & 1500.868 & 1499.734 \\ 1499098 & 1499.445 & 1499.769 & 1500865 & 1499.722\end{array}$ $\begin{array}{llllll}1499.11 & 1499.441 & 1499.774 & 1500.898 & 1499.752\end{array}$ $\begin{array}{lllll}1499.093 & 1499.431 & 1499.762 & 1500.868 & 1499.736 \\ 149090 & 1499.422 & 1499.753 & 1500.858 & 149.72\end{array}$ $\begin{array}{lllll}1499.079 & 1499.422 & 1499.753 & 1500.858 & 1499.72 \\ 149.790 & 149.42 & 199.762 & 1500889 & 1499.741\end{array}$ $\begin{array}{llllll}1499.091 & 1499.42 & 1499.762 & 1500.889 & 1499.741 \\ 1499.096 & 1499.422 & 1499.774 & 1500.926 & 1499.769\end{array}$ $\begin{array}{rrrrrr}1499.096 & 1499.422 & 1499.774 & 1500.926 & 1499.769 \\ 1499.11 & 1499.427 & 1499.79 & 1500.956 & 1499.797\end{array}$ $\begin{array}{llllll}1499.154 & 1499.448 & 1499.839 & 1501.048 & 1499.881\end{array}$ $\begin{array}{lllll}1499.182 & 1499.459 & 1499.867 & 1501.08 & 1499.925 \\ 1499.208 & 1499.473 & 1499893 & 1501.111 & 1499.953\end{array}$ $\begin{array}{rrrrrr}1499.208 & 1499.473 & 1499.893 & 1501.111 & 1499.953 \\ 1499.247 & 1499.49 & 1499.93 & 1501.167 & 1500.007\end{array}$ $\begin{array}{lllll}1499.266 & 1499.501 & 1499.949 & 1501.171 & 1500.025 \\ 1499275 & 1499.504 & 1499.956 & 1501.164 & 1500.021\end{array}$ $\begin{array}{llllll}1499.275 & 1499.504 & 1499.956 & 1501.164 & 1500.021 \\ 1499.296 & 1499.511 & 1499.972 & 1501.127 & 1500.037\end{array}$ $\begin{array}{llllll}1499.296 & 1499.511 & 1499.972 & 1501.127 & 1500.037 \\ 1499.278 & 1499.499 & 1499.949 & 1501.127 & 1499.981\end{array}$

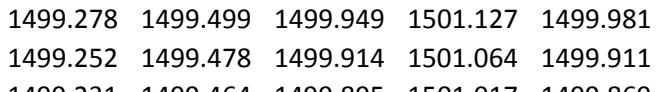
$\begin{array}{llllll}1499.231 & 1499.464 & 1499.895 & 1501.017 & 1499.869 \\ 1499.184 & 1499.431 & 1499.842 & 1500.928 & 1499794\end{array}$ $\begin{array}{llllll}1499.184 & 1499.431 & 1499.842 & 1500.928 & 1499.794 \\ 1499.145 & 1499.413 & 1499.804 & 1500879 & 1499.731\end{array}$ $\begin{array}{lllll}1499.142 & 1499.406 & 1499.797 & 1500.893 & 1499.734\end{array}$ $\begin{array}{llllll}1499.119 & 1499.394 & 1499.776 & 1500.868 & 1499.715\end{array}$ $\begin{array}{llllll}1499.103 & 1499.387 & 1499.765 & 1500.854 & 1499.699\end{array}$ $\begin{array}{lllllll}1499.096 & 1499.38 & 1499.762 & 1500.87 & 1499.715\end{array}$ $\begin{array}{llllll}1499.1 & 1499.378 & 1499.765 & 1500.891 & 1499.734\end{array}$ $\begin{array}{lllll}1499.089 & 1499.376 & 1499.753 & 1500.87 & 1499.717 \\ 1490.072 & 1499366 & 1499.739 & 1500839 & 1499699\end{array}$ $\begin{array}{llllll}1499.072 & 1499.366 & 1499.739 & 1500.839 & 1499.699\end{array}$ $\begin{array}{llllll}149.049 & 1499.357 & 1499.738 & 1500.804 & 1499.664 \\ 1499.03 & 1499.348 & 1499.706 & 1500.797 & 1499647\end{array}$ $\begin{array}{llllll}1499.042 & 1499.355 & 1499.718 & 1500.854 & 1499.673\end{array}$ $\begin{array}{llllll}1499.058 & 1499.359 & 1499.737 & 15000.854 & 14999.673 \\ 1490.717\end{array}$ $\begin{array}{llllll}1499.068 & 1499.359 & 1499.748 & 1500.914 & 1499.741\end{array}$ $\begin{array}{llllll}1499.117 & 1499.382 & 1499.797 & 1501.012 & 1499.827\end{array}$ $\begin{array}{rrrrr}1499.119 & 1499.383 & 1499.8 & 1500.984 & 1499.829 \\ 1499.11 & 1999.373 & 1499.788 & 1509054 & 1499801\end{array}$ $\begin{array}{rrrrrr}1499.11 & 1499.373 & 1499.788 & 1500.954 & 1499.801 \\ 1499.117 & 1499.373 & 1499.795 & 1500.959 & 1499.797\end{array}$ $\begin{array}{llllll}1499.117 & 1493.373 & 1499.795 & 1500.959 & 1499.797\end{array}$ $\begin{array}{llllll}1499.103 & 1499.364 & 1499.779 & 1500.926 & 1499.773 \\ 1499.096 & 1499.357 & 1499.772 & 1500.914 & 1499.75\end{array}$ $\begin{array}{lllll}1499.093 & 1499.35 & 1499.765 & 1500.9 & 1499.738\end{array}$ $\begin{array}{llllll}1499.072 & 1499.338 & 1499.741 & 1500.858 & 1499.703\end{array}$ $\begin{array}{lllll}1499.063 & 1499.336 & 1499.732 & 1500.858 & 1499.689 \\ 1499.051 & 1499329 & 1499.723 & 1500.849 & 1499.68\end{array}$ $\begin{array}{llllll}1499.051 & 1499.329 & 1499.723 & 1500.849 & 1499.68 \\ 1499.044 & 1499.324 & 1499.709 & 1500.835 & 1499.671\end{array}$ $\begin{array}{llllll}1499.044 & 1499.324 & 1499.709 & 1500.835 & 1499.671 \\ 1499.044 & 1499.327 & 1499.711 & 1500.844 & 1499.671\end{array}$ 

Well (ft AMSL) (ft AMSL) (ft AMSL) (ft AMSL) (ft AMSL) (ft AMSL) (ft AMSL) (ft AMSL) (ft AMSL) (ft AMSL) (ft AMSL) (ft AMsL) (ft AMSL) (ft AMSL) (ft AMSL) (ft AMSL) (ft AMSL) (ft AMSL) (ft AMSL) (ft AMSL) (ft AMSL) (ft AMSL) (ft AMSL) (ft AMSL) (ft AMSL) (ft AMSL)

$1 / 4 / 101600$ $1499.063 \quad 1499.334 \quad 1499.73 \quad 1500.898 \quad 1499.715$ $\begin{array}{lllll}1499.065 & 1499.329 & 1499.732 & 1500.889 & 1499.722 \\ 1499.063 & 1499329 & 1499.737 & 1500896 & 1499.72\end{array}$

$\begin{array}{rrrrr}1499.063 & 1499.329 & 1499.737 & 1500.896 & 1499.72 \\ 1499.07 & 1499.327 & 1499737 & 1500898 & 1499.722\end{array}$ $\begin{array}{lllll}1499.07 & 1499.327 & 1499.737 & 1500.898 & 1499.722 \\ 1499.07 & 1499.327 & 1499.741 & 1500.905 & 1499.729\end{array}$ $\begin{array}{lllllll}1499.075 & 1499.331 & 1499.751 & 1500.921 & 1499.738\end{array}$ 1/5/108:00 1/5/10 16:00 1/5/10 20:00 $1 / 6 / 100: 00$ 1/6/10 4:00 1/6/10 8:00 1/6/1012:00 $1 / 6 / 1020.00$ 1/6/10 20:00 1/7/10 4:00 $1 / 7 / 108: 00$ $1 / 7 / 1012: 00$ $1 / 7 / 1012600$
$1 / 7 / 1020: 00$
$1 / 8 / 1000$ $1 / 8 / 100: 00$ 1/8/100:00 1/8/10 8:00 1/8/10 12:00 1/8/10 16:00 1/8/10 20:00 1/9/100:00 $1 / 9 / 104: 00$ $1 / 9 / 108.00$ $1 / 9 / 1016: 00$ 1/9/10 20:00 $1 / 10 / 100: 00$ $1 / 10 / 104: 00$ 1/10/10 8:00 $1 / 10 / 1012: 00$ $1 / 10 / 1016: 00$ $1 / 11 / 100: 00$ 1/11/10 4:00 $1 / 11 / 108: 00$ 1/11/10 12:00 1/11/10 16:00 1/111/1020:00 $1 / 12 / 100: 00$ $1 / 12 / 108.00$ 1/12/10 12:00 $1 / 12 / 1016: 00$ $1 / 12 / 1020: 00$ $\begin{array}{llllllll}1499.107 & 1499.348 & 1499.781 & 15000.984 & 1499.792\end{array}$ $\begin{array}{llllll}1499.126 & 1499.362 & 1499.804 & 1501.015 & 1499.822\end{array}$ $\begin{array}{lllllll}1499.149 & 1499.371 & 1499.821 & 1501.029 & 1499.841\end{array}$ $\begin{array}{llllll}1499.173 & 1499.387 & 1499.846 & 1501.066 & 1499.876 \\ 1499.191 & 1499.397 & 1499.865 & 1501.083 & 149.902\end{array}$ $\begin{array}{lllllll}1499.191 & 1499.397 & 1499.865 & 1501.083 & 1499.902\end{array}$ $\begin{array}{lllll}1499.189 & 1499.387 & 1499.849 & 1501.029 & 1499.86\end{array}$ $\begin{array}{llllll}1499.131 & 1499.355 & 1499.79 & 1500.889 & 1499.743\end{array}$ $\begin{array}{llllll}1499.093 & 1499.334 & 1499.758 & 1500.846 & 1499.671\end{array}$ $\begin{array}{llllll}1499.082 & 1499.327 & 1499.746 & 1500.849 & 1499.657\end{array}$ $\begin{array}{llllll}149.063 & 149.313 & 1499.718 & 1500.816 & 1499.64\end{array}$ $\begin{array}{llllll}1499.037 & 1499.303 & 1499.695 & 1500.786 & 1499.608\end{array}$ $\begin{array}{llllll}1499.042 & 1499.301 & 1499.697 & 1500.821 & 1499.626 \\ 1499.019 & 1499.294 & 1499.678 & 1500786 & 1499.605\end{array}$ $\begin{array}{lllllll}149.019 & 149.294 & 1499.678 & 1500.786 & 1499.605\end{array}$ $\begin{array}{llllll}1498.995 & 1499.282 & 1499.653 & 1500.772 & 1499.586\end{array}$ $\begin{array}{llllll}1498.988 & 1499.278 & 1499.648 & 1500.779 & 1499.591\end{array}$ $\begin{array}{lllllll}1498.988 & 1499.28 & 1499.646 & 1500.79 & 1499.6\end{array}$ $\begin{array}{llllll}1499.016 & 1499.292 & 1499.676 & 1500.858 & 1499.654\end{array}$ $\begin{array}{llllll}1499.016 & 1499.287 & 1499.681 & 1500.856 & 1499.664\end{array}$ $\begin{array}{lllll}1499.014 & 1499.287 & 1499.681 & 1500.854 & 1499.659 \\ 1499009 & 1499.28 & 1999.674 & 1500837 & 149954\end{array}$ $\begin{array}{lllllll}1499 & 1499.273 & 1499.662 & 1500.837 & 1499.654\end{array}$ $\begin{array}{rrrrrr}1499 & 1499.273 & 1499.662 & 1500.811 & 1499.636 \\ 1498.986 & 1499.271 & 1499.65 & 15008 & 1499.615\end{array}$ $\begin{array}{llllll}1499.007 & 1499.275 & 1499.664 & 1500.832 & 1499.638\end{array}$ $\begin{array}{llllll}1499.005 & 1499.271 & 1499.662 & 1500.825 & 1499.638\end{array}$ $\begin{array}{lllll}1499.005 & 1499.266 & 1499.664 & 1500.83 & 1499.636\end{array}$ $\begin{array}{llllll}1499.016 & 1499.278 & 1499.681 & 1500.87 & 1499.666\end{array}$ $\begin{array}{lllllll}1499.03 & 1499.287 & 1499.697 & 1500.898 & 1499.696\end{array}$ $\begin{array}{llllll}1499.049 & 1499.299 & 1499.72 & 1500.94 & 1499.731 \\ 1499.098 & 1499.32 & 1499.762 & 1501.001 & 1499.797\end{array}$ $\begin{array}{llllll}149.098 & 1499.32 & 1499.762 & 15001.001 & 1499.731\end{array}$ $\begin{array}{lllllll}1499.096 & 149.31 & 1499.755 & 1500.061 & 1499.778 \\ 1499 & 1499.76 & 1500.956 & 1499.764\end{array}$ $\begin{array}{llllll}1499.096 & 1499.32 & 1499.765 & 1500.956 & 1499.762\end{array}$ $\begin{array}{llllll}1499.086 & 1499.308 & 1499.748 & 1500.914 & 1499.731\end{array}$ $\begin{array}{llllll}1499.068 & 1499.299 & 1499.73 & 1500.877 & 1499.692\end{array}$ $\begin{array}{lllll}1499.068 & 1499.292 & 1499.72 & 1500.865 & 1499.68\end{array}$ $\begin{array}{lllll}1499.04 & 1499.275 & 1499.695 & 1500.818 & 1499.638\end{array}$ $\begin{array}{llllll}1499.026 & 1499.266 & 1499.678 & 1500.807 & 1499.615 \\ 1499.019 & 1499.264 & 1499.674 & 1500816 & 1499.615\end{array}$

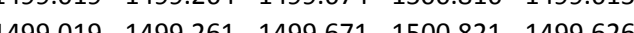
$\begin{array}{llllll}1499.019 & 1499.261 & 1499.671 & 1500.821 & 1499.626 \\ 1499.028 & 1499268 & 1499.683 & 1500.856 & 1499.647\end{array}$ $\begin{array}{llllll}1499.072 & 1499.296 & 1499.73 & 1500.949 & 1499.729\end{array}$ $\begin{array}{llllll}1499.082 & 1499.301 & 1499.744 & 1500.956 & 1499.757\end{array}$ $\begin{array}{rrrrr}1499.1 & 1499.31 & 1499.762 & 1500.977 & 1499.776 \\ 1499.124 & 1499.327 & 1499.786 & 1501.008 & 1499.804\end{array}$ $\begin{array}{rrrrrr}1499.147 & 1499.343 & 1499.804 & 1501.033 & 1499.839 \\ 1499.17 & 1499.355 & 1499.83 & 1501.066 & 1499.862\end{array}$ 
$\begin{array}{lllll}1499.208 & 1499.373 & 1499.863 & 1501.108 & 1499.913\end{array}$ $\begin{array}{lllll}1499.201 & 1499.371 & 1499.856 & 1501.057 & 1499.89 \\ 1499198 & 1499.373 & 1499.853 & 1501.048 & 149986\end{array}$ $\begin{array}{llllll}1499.148 & 1499.373 & 1499.853 & 1501.048 & 1499.86 \\ 1499.371 & 1499.849 & 1501026 & 1499.841\end{array}$ $\begin{array}{llllll}1499.173 & 1499.359 & 1499.823 & 1500.975 & 1499.797\end{array}$ $\begin{array}{lllllll}1499.147 & 1499.345 & 1499.797 & 1500.926 & 1499.741\end{array}$ $\begin{array}{llllll}1499.14 & 1499.341 & 1499.788 & 1500.924 & 1499.731\end{array}$ $\begin{array}{rrrrr}1499.11 & 1499.322 & 1499.755 & 1500.865 & 1499.685 \\ 1499.084 & 1499.308 & 1499.73 & 1500.837 & 1499.645\end{array}$ $\begin{array}{lllll}1499.084 & 1499.308 & 1499.73 & 1500.837 & 1499.645 \\ 1499.077 & 1499.301 & 1499.72 & 1500.844 & 1499.64\end{array}$ $\begin{array}{rrrrr}1499.058 & 1499.292 & 1499.704 & 1500.825 & 1499.624 \\ 149.047 & 1499.282 & 1499.69 & 1500814 & 1496.608\end{array}$ $\begin{array}{rrrrr}1499.047 & 1499.282 & 1499.69 & 1500.814 & 1499.608 \\ 1499.058 & 1499.289 & 1499.704 & 1500.865 & 1499.647\end{array}$ $\begin{array}{llllll}1499.058 & 1499.289 & 1499.711 & 1500.87 & 1499.664\end{array}$ $\begin{array}{llllll}1499.063 & 1499.287 & 1499.713 & 1500.882 & 1499.675\end{array}$ $\begin{array}{llllll}1499.07 & 1499.292 & 1499.72 & 1500.893 & 1499.687\end{array}$ $\begin{array}{llllll}1499.077 & 1499.296 & 1499.727 & 1500.912 & 1499.708\end{array}$ $\begin{array}{lllll}1499.082 & 1499.303 & 1499.737 & 1500.924 & 1499.713 \\ 1499.124 & 1499322 & 1499779 & 1501003 & 1499\end{array}$ $\begin{array}{rrrrrr}1499.124 & 1499.322 & 1499.779 & 1501.003 & 1499.776 \\ 1499.124 & 1499327 & 1499.783 & 1500.98 & 1499.783\end{array}$ $\begin{array}{llllll}149.124 & 1499.327 & 1499.783 & 1500.98 & 1499.783 \\ 1499.131 & 1499.334 & 1499.793 & 1500994 & 1499.792\end{array}$ $\begin{array}{rrrrr}1499.131 & 1499.334 & 1499.793 & 1500.994 & 1499.792 \\ 1499.147 & 1499.343 & 1499.807 & 1501.01 & 1499.804\end{array}$ $\begin{array}{llllll}1499.154 & 1499.348 & 1499.814 & 1501.005 & 1499.808\end{array}$ $\begin{array}{lllllll}1499.154 & 1499.35 & 1499.811 & 1500.998 & 1499.797\end{array}$ $\begin{array}{llllll}1499.173 & 1499.357 & 1499.828 & 1501.024 & 1499.818\end{array}$ $\begin{array}{llllll}1499.145 & 1499.343 & 1499.797 & 1500.954 & 1499.773\end{array}$ $\begin{array}{lllll}1499.135 & 1499.341 & 1499.79 & 1500.942 & 1499.745 \\ 1499.135 & 1999.341 & 1499.79 & 150952 & 1499741\end{array}$ $\begin{array}{llllll}1499.135 & 1499.341 & 1499.79 & 1500.952 & 1499.741 \\ 1499.131 & 1499336 & 1499.783 & 1500.94 & 1499.736\end{array}$ $\begin{array}{llllll}1499.131 & 1499.336 & 1499.783 & 1500.94 & 1499.736 \\ 1499.128 & 1499338 & 1499.781 & 1500.945 & 1499.729\end{array}$ $\begin{array}{llllll}1499.135 & 1499.341 & 1499.788 & 1500.963 & 1499.745\end{array}$ $\begin{array}{llllll}1499.126 & 1499.334 & 1499.774 & 1500.931 & 1499.727\end{array}$ $\begin{array}{llllll}1499.128 & 1499.338 & 1499.779 & 1500.945 & 1499.731\end{array}$ $\begin{array}{llllll}1499.135 & 1499.343 & 1499.788 & 1500.959 & 1499.75\end{array}$ $\begin{array}{lllll}1499.138 & 1499.345 & 1499.793 & 1500.949 & 1499.762\end{array}$ $\begin{array}{lllll}1499.145 & 1499.355 & 1499.807 & 1500.942 & 1499.778 \\ 149.187 & 1499373 & 1499842 & 1001.024 & 1499841\end{array}$ $\begin{array}{lllllll}1499.187 & 1499.373 & 1499.842 & 1501.024 & 1499.841 \\ 1499205 & 1499.385 & 1499.865 & 1501057 & 1499869\end{array}$ $\begin{array}{lllll}1499.205 & 1499.385 & 1499.865 & 1501.057 & 1499.869 \\ 1499.219 & 1499.397 & 1499.877 & 1501071 & 1499885\end{array}$ $\begin{array}{llllll}1499.217 & 1499.385 & 1499.858 & 1501.094 & 1499.855\end{array}$ $\begin{array}{lllllll}1499.187 & 1499.373 & 1499.832 & 1501.106 & 1499.9794\end{array}$ $\begin{array}{llllll}1499.18 & 1499.373 & 1499.828 & 1500.963 & 1499.778\end{array}$ $\begin{array}{llllll}1499.194 & 1499.382 & 1499.842 & 1501.026 & 1499.804\end{array}$ $\begin{array}{llllll}1499.191 & 1499.383 & 1499.842 & 1501.024 & 1499.801 \\ 1499.184 & 1499.38 & 149.832 & 1500998 & 1499.787\end{array}$ $\begin{array}{llllll}1499.184 & 1499.38 & 1499.832 & 1500.998 & 1499.787 \\ 1499.163 & 1499.366 & 1499.809 & 1500954 & 1499.759\end{array}$ $\begin{array}{llllll}1499.163 & 149.366 & 1499.809 & 1500.954 & 1499.759 \\ 1499.145 & 1499.359 & 1499.795 & 1500.931 & 1499.727\end{array}$ $\begin{array}{lllllll}1499.145 & 1499.359 & 1499.795 & 1500.931 & 1499.727 \\ 1499.133 & 1499.348 & 1499.776 & 1500.905 & 1499.692\end{array}$ $\begin{array}{lllll}1499.121 & 1499.338 & 1499.765 & 1500.903 & 1499.685\end{array}$ $\begin{array}{llllll}1499.098 & 1499.327 & 1499.737 & 1500.858 & 1499.654\end{array}$ $\begin{array}{rllll}1499.096 & 1499.322 & 1499.734 & 1500.879 & 1499.643 \\ 1499.1 & 1499.327 & 1499.741 & 1500.914 & 149.673 \\ 1499.1 & 1499.329 & 1499.746 & 1500.921 & 1499.692\end{array}$ $\begin{array}{rrrrrr}1499.1 & 1499.329 & 1499.746 & 1500.921 & 1499.692 \\ 1499.128 & 1499.348 & 1499.781 & 1500.989 & 1499.736\end{array}$ 

Well (ft AMSL) (ft AMSL) (ft AMSL) (ft AMSL) (ft AMSL) (ft AMSL) (ft AMSL) (ft AMSL) (ft AMSL) (ft AMSL) (ft AMSL) (ft AMSL) (ft AMSL) (ft AMSL) (ft AMSL) (ft AMSL) (ft AMSL) (ft AMSL) (ft AMSL) (ft AMSL) (ft AMSL) (ft AMSL) (ft AMSL) (ft AMSL) (ft AMSL) (ft AMS)

$1 / 22 / 101600$ $1499.173 \quad 1499.371 \quad 1499.823 \quad 1501.059 \quad 1499.82$ $\begin{array}{lllll}1499.168 & 1499.371 & 1499.823 & 1501.029 & 1499.815 \\ 1499.156 & 1499.348 & 1499.816 & 1500.987 & 1499.797\end{array}$ $\begin{array}{lllll}1499.156 & 1499.348 & 1499.816 & 1500.987 & 1499.797 \\ 1499205 & 1499336 & 1499.86 & 1501.073 & 1499.857\end{array}$ $\begin{array}{lllllll}1499.201 & 1499.35 & 1499.853 & 1501.078 & 1499.848\end{array}$ $\begin{array}{llllll}1499.184 & 1499.306 & 1499.842 & 1500.973 & 1499.815\end{array}$ $1 / 23 / 108: 00$ 1/23/10 16:00 1/23/10 20:00 $1 / 24 / 100: 00$ $1 / 24 / 104: 00$ $1 / 24 / 108: 00$
$1 / 24 / 1012: 00$ $1 / 24 / 1016: 00$ 1/24/1100 20:00 $1 / 25 / 100: 00$ 1/25/10 4:00 $1 / 25 / 108: 00$ 1/25/10 12:00 $1 / 25 / 1016: 00$ $1 / 26 / 100: 00$ $1 / 26 / 104: 00$ $1 / 26 / 108: 00$ $1 / 26 / 1012: 00$ $1 / 26 / 1016: 00$ 1/26/10 20:00 1/27/100:00 $1 / 27 / 104.00$ $1 / 27108.00$ $1 / 27 / 1016: 00$ $1 / 27 / 1020: 00$ $1 / 28 / 100: 00$ $1 / 28 / 104: 00$ $1 / 28 / 108: 00$ 1/28/10 12:00 $1 / 28 / 1016: 00$ $1 / 29 / 100: 00$ $1 / 29 / 104: 00$ $1 / 29 / 108: 00$ 1/29/10 12:00 $1 / 29 / 1016: 00$ $1 / 29 / 1020: 00$ $1 / 30 / 100: 00$ $1 / 30 / 104.00$ $1 / 30 / 1012: 00$ $1 / 30 / 1016: 00$ $1 / 30 / 1020: 00$

$\begin{array}{llllll}499.201 & 1499.378 & 1499.851 & 1500.919 & 1499.825\end{array}$ $\begin{array}{llllll}1499.18 & 1499.369 & 1499.83 & 1501.085 & 1499.787\end{array}$ $\begin{array}{llllll}1499.152 & 1499.355 & 1499.795 & 1501.113 & 1499.729\end{array}$ $\begin{array}{rrrrr}1499.121 & 1499.336 & 1499.765 & 1500.963 & 1499.68 \\ 149086 & 149932 & 1499.732 & 1500835 & 1499662\end{array}$ $\begin{array}{llllll}1499.086 & 1499.32 & 1499.732 & 1500.835 & 1499.626\end{array}$ $\begin{array}{lllllll}1499.072 & 1499.31 & 1499.716 & 1500.825 & 1499.603 \\ 1499.072 & 1499.308 & 1499.713 & 1500.851 & 1499.622\end{array}$ $\begin{array}{llllllll}1499.047 & 1499.296 & 1499.69 & 1500.814 & 1499.589\end{array}$ $\begin{array}{llllll}1499.037 & 1499.289 & 1499.676 & 1500.807 & 1499.572\end{array}$ $\begin{array}{llllll}1499.026 & 1499.282 & 1499.671 & 1500.807 & 1499.57\end{array}$ $\begin{array}{llllll}1499.005 & 1499.273 & 1499.648 & 1500.769 & 1499.551\end{array}$ $\begin{array}{lllll}1498.97 & 1499.255 & 1499.615 & 1500.718 & 1499.491\end{array}$ $\begin{array}{llllll}1498.96 & 1499.252 & 1499.604 & 1500.725 & 1499.489 \\ 1498.925 & 1499.238 & 1499.576 & 1500.674 & 1499.446\end{array}$ $\begin{array}{llllll}14989 & 1499.227 & 1499.546 & 1500.648 & 1499.446\end{array}$ $\begin{array}{lllll}1498.869 & 1499.213 & 1499.518 & 1500.62 & 1499.379\end{array}$ $\begin{array}{llllll}1498.837 & 1499.203 & 1499.49 & 1500.58 & 1499.346\end{array}$ $\begin{array}{llllll}1498.811 & 1499.189 & 1499.469 & 1500.568 & 1499.311\end{array}$ $\begin{array}{llllll}1498.839 & 1499.196 & 1499.497 & 1500.664 & 1499.383\end{array}$ $\begin{array}{llllll}1498.839 & 1499.189 & 1499.499 & 1500.666 & 1499.404\end{array}$ $\begin{array}{llllll}1498.846 & 1499.189 & 1499.511 & 1500.69 & 1499.425 \\ 1498.874 & 1499199 & 1499.538 & 1500.748 & 1499.472\end{array}$ $\begin{array}{lllll}1498.874 & 1499.199 & 1499.538 & 1500.748 & 1499.472 \\ 1498876 & 1499.196 & 1499.546 & 1500744 & 1499.496\end{array}$ $\begin{array}{lllllll}1498.86 & 1499.185 & 1499.524 & 1500.744 & 1499.496 \\ 1590 & 1499.458\end{array}$ $\begin{array}{llllll}1498.842 & 1499.173 & 1499.506 & 1500.657 & 1499.426\end{array}$ $\begin{array}{llllll}1498.788 & 1499.154 & 1499.445 & 1500.55 & 1499.327\end{array}$ $\begin{array}{llllll}1498.753 & 1499.136 & 1499.41 & 1500.51 & 1499.26\end{array}$ $\begin{array}{lllll}1498.727 & 1499.129 & 1499.392 & 1500.51 & 1499.236\end{array}$ $\begin{array}{llllll}1498.718 & 1499.119 & 1499.382 & 1500.508 & 1499.234\end{array}$ $\begin{array}{llllll}1498.723 & 1499.112 & 1499.385 & 1500.543 & 1499.253 \\ 1498734 & 1499.112 & 1499.401 & 1500585 & 1999299\end{array}$ $\begin{array}{lllllll}1498.734 & 1499.112 & 1499.401 & 1500.585 & 1499.299\end{array}$ $\begin{array}{llllll}1498.751 & 1499.11 & 1499.424 & 1500.617 & 149.33\end{array}$ $\begin{array}{llllll}1498.769 & 1499.115 & 1499.445 & 1500.662 & 1499.372\end{array}$ $\begin{array}{llllll}1498.783 & 1499.117 & 1499.459 & 1500.68 & 1499.404\end{array}$ $\begin{array}{lllll}1498.788 & 1499.112 & 1499.462 & 1500.674 & 1499.4\end{array}$ $\begin{array}{llllll}1498.816 & 1499.126 & 1499.494 & 1500.73 & 1499.449\end{array}$ $\begin{array}{lllll}1498.821 & 1499.126 & 1499.499 & 1500.718 & 1499.456 \\ 149823 & 1499.122 & 1499.497 & 150.713 & 1499.44\end{array}$ $\begin{array}{llllll}1498.823 & 1499.122 & 1499.497 & 1500.713 & 1499.444 \\ 1498.823 & 1499.119 & 1499.497 & 1500716 & 1499.437\end{array}$

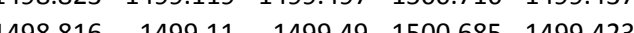
$\begin{array}{lllllll}1498.811 & 1499.108 & 1499.485 & 1500.681 & 1499.423 \\ 14902\end{array}$ $\begin{array}{llllll}1498.83 & 1499.117 & 1499.506 & 1500.722 & 1499.43\end{array}$ $\begin{array}{lllllll}1498.825 & 1499.11 & 1499.497 & 1500.699 & 1499.425\end{array}$ $\begin{array}{rrrrr}1498.823 & 1499.103 & 1499.492 & 1500.688 & 1499.412 \\ 1498.821 & 1499.103 & 1499.49 & 1500.69 & 1499.404\end{array}$ $\begin{array}{rrrrr}1498.821 & 1499.103 & 1499.49 & 1500.69 & 1499.404 \\ 1498.816 & 1499.098 & 1499.48 & 1500.669 & 1499.395\end{array}$ $\begin{array}{rrrrrr}1498.816 & 1499.098 & 1499.48 & 1500.669 & 1499.395 \\ 1498.807 & 1499.096 & 1499.473 & 1500.662 & 1499.379\end{array}$ 

Well (ft AMSL), (ft AMSL) (ft AMSL) (ft AMSL) (ft AMSL) (ft AMSL) (ft AMSL) (ft AMSL) (ft AMSL) (ft AMSL) (ft AMSL) (ft AMSL) (ft AMSL) (ft AMSL) (ft AMSL) (ft AMSL) (ft AMSL) (ft AMSL) (ft AMSL) (ft AMSL) (ft AMSL) (ft AMSL) (ft AMSL) (ft AMSL) (ft AMSL) (ft AMS)

$1 / 31 / 101600$

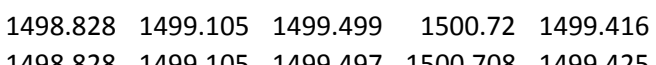
$\begin{array}{rrrrr}1498.828 & 1499.105 & 1499.497 & 1500.708 & 1499.425 \\ 1498.839 & 1499.108 & 1499.508 & 1500.727 & 1499.437\end{array}$ $\begin{array}{rrrrrr}1498.839 & 1499.108 & 1499.508 & 1500.727 & 1499.437 \\ 1498.846 & 1499.112 & 1499.52 & 1500.746 & 1499.451\end{array}$ $\begin{array}{llllll}1498.86 & 1499.119 & 1499.532 & 1500.758 & 1499.472\end{array}$ 2/1/10 8:00 2/1/10 16:00 2/1/10 20:00 $2 / 2 / 100: 00$ 2/2/10 4:00 $2 / 2 / 108: 00$ 2/2/1012:00 2/2/10 20:00 2/3/100:00 2/3/10 4:00 2/3/10 8:00 2/3/10 12:00 $2 / 3 / 1012600$ $2 / 3 / 1020: 00$ 2/4/100:00 2/4/10 8:00 2/4/10 12:00 2/4/10 16:00 2/4/10 20:00 2/5/100:00 2/5/104:00 2/5/1012:00 $2 / 5 / 1016: 00$ 2/5/10 20:00 2/6/100:00 $2 / 6 / 104: 00$ 2/6/10 8:00 2/6/10 12:00 $2 / 6 / 1016: 00$ 2/6/1020:00 2/7/10 4:00 2/7/108:00 2/7/10 12:00 2/7/10 16:00 2/7/1020:00 2/8/100:00 $2 / 8 / 104.00$ 2/8/10 12:00 2/8/10 16:00 $2 / 8 / 1020: 00$ 2/9/100:00 2/9/10 4:00 2/9/10 8:00 $\begin{array}{lllll}1498.865 & 1499.122 & 1499.539 & 1500.762 & 1499.477\end{array}$ $\begin{array}{llllll}1498.876 & 1499.126 & 1499.548 & 1500.776 & 1499.488\end{array}$ $\begin{array}{llllll}1498.865 & 1499.117 & 1499.532 & 1500.73 & 1499.458\end{array}$ $\begin{array}{llllll}1498.849 & 1499.105 & 1499.513 & 1500.697 & 1499.421\end{array}$ $\begin{array}{llllll}1498.839 & 1499.098 & 1499.501 & 1500.688 & 1499.397 \\ 1498.818 & 1499087 & 1499.473 & 1500.634 & 149936\end{array}$ $\begin{array}{llllll}1498.818 & 1499.087 & 1499.473 & 1500.634 & 1499.36 \\ 14988 & 1499.08 & 1499.455 & 1500.622 & 1499332\end{array}$ $\begin{array}{rrrrrr}1498.804 & 1499.082 & 1499.464 & 1500.652 & 1499.353\end{array}$ $\begin{array}{llllll}1498.8 & 1499.078 & 1499.455 & 1500.659 & 1499.353\end{array}$ $\begin{array}{llllll}1498.79 & 1499.075 & 1499.45 & 1500.722 & 1499.348\end{array}$ $\begin{array}{llllll}1498.795 & 1499.073 & 1499.455 & 1500.72 & 1499.362\end{array}$ $\begin{array}{llllll}1498.79 & 1499.068 & 1499.445 & 1500.69 & 1499.358\end{array}$ $\begin{array}{rrrrrr}1498.786 & 1499.066 & 1499.436 & 1500.659 & 1499.351 \\ 1498.788 & 1499.068 & 1499.5 & 1500.63 & 199367\end{array}$ $\begin{array}{llllll}1498.788 & 1499.068 & 1499.45 & 1500.603 & 1499.367\end{array}$ $\begin{array}{lllllll}1498.781 & 1499.059 & 1499.436 & 1500.622 & 1499.356\end{array}$ $\begin{array}{llllll}1498.781 & 1499.066 & 1499.448 & 1500.594 & 1499.369\end{array}$ $\begin{array}{llllll}1498.79 & 1499.07 & 1499.464 & 1500.592 & 1499.4\end{array}$ $\begin{array}{llllll}1498.8 & 1499.075 & 1499.478 & 1500.585 & 1499.423\end{array}$ $\begin{array}{llllll}1498.828 & 1499.096 & 1499.511 & 1500.554 & 1499.479\end{array}$ $\begin{array}{llllll}1498.837 & 1499.096 & 1499.515 & 1500.55 & 1499.486\end{array}$ $\begin{array}{rrrrr}1498.842 & 1499.096 & 1499.52 & 1500.552 & 1499.488 \\ 1498.851 & 1499.103 & 1499.536 & 1500552 & 1499.502\end{array}$ $\begin{array}{llllll}1498.851 & 1499.103 & 1499.536 & 1500.552 & 1499.502 \\ 1498.862 & 1499.108 & 1499.539 & 1500.545 & 1499.514\end{array}$ $\begin{array}{lllllll}1498.862 & 149.108 & 1499.101 & 1499.529 & 1500.451 & 1499.498\end{array}$ $\begin{array}{lllll}1498.846 & 1499.092 & 1499.513 & 1500.491 & 1499.47\end{array}$ $\begin{array}{llllll}1498.821 & 1499.071 & 1499.476 & 1500.599 & 1499.409\end{array}$ $\begin{array}{llllll}1498.793 & 1499.057 & 1499.45 & 1500.631 & 1499.367\end{array}$ $\begin{array}{llllll}1498.776 & 1499.05 & 1499.436 & 1500.662 & 1499.351\end{array}$ $\begin{array}{llllll}1498.753 & 1499.04 & 1499.41 & 1500.643 & 1499.325\end{array}$ $\begin{array}{lllll}1498.732 & 1499.017 & 1499.392 & 1500.503 & 1499.304 \\ 1489.706 & 1498994 & 1999.406 & 1500374 & 1499339\end{array}$ $\begin{array}{llllll}1498.706 & 1498.994 & 149.406 & 1500.374 & 1499.339\end{array}$ $\begin{array}{lllllll}1498.737 & 1498.996 & 1499.401 & 1500.496 & 1499.344 \\ 1498.734 & 1499.008 & 1499.403 & 1500.538 & 1499.351\end{array}$ $\begin{array}{llllll}1498.741 & 1499.015 & 1499.415 & 1500.559 & 1499.37\end{array}$ $\begin{array}{llllll}1498.741 & 1499.026 & 1499.415 & 1500.568 & 1499.381\end{array}$ $\begin{array}{llllll}1498.739 & 1499.031 & 1499.422 & 1500.585 & 1499.388\end{array}$ $\begin{array}{llllll}1498.762 & 1499.045 & 1499.45 & 1500.69 & 1499.437\end{array}$ $\begin{array}{rrrrr}1498.772 & 1499.04 & 1499.443 & 1500.716 & 1499.435 \\ 1489769 & 1499.043 & 1499.45 & 1500.727 & 1499.44\end{array}$ $\begin{array}{lllllll}1498.769 & 1499.043 & 1499.45 & 1500.727 & 1499.44 \\ 1498.769 & 1499.043 & 1499.452 & 1500722 & 1499.442\end{array}$ $\begin{array}{llllll}149.869 & 1499.013 & 149.452 & 1500.727 & 1499.44\end{array}$ $\begin{array}{llllll}1498.744 & 1499.024 & 1499.415 & 1500.664 & 1499.386\end{array}$ $\begin{array}{llllll}1498.732 & 1499.017 & 1499.399 & 1500.723 & 1499.358\end{array}$ $\begin{array}{llllll}1498.706 & 1499.005 & 1499.371 & 1500.718 & 1499.32\end{array}$ $\begin{array}{llllll}1498.699 & 1498.998 & 1499.357 & 1500.706 & 1499.302\end{array}$ $\begin{array}{llllll}1498.692 & 1498.998 & 1499.359 & 1500.715 & 1499.309\end{array}$ $\begin{array}{rrrrr}1498.676 & 1498.987 & 1499.34 & 1500.613 & 1499.297 \\ 1498.655 & 1498.977 & 1499.317 & 1500.402 & 1499.267\end{array}$ 

Well (ft AMSL) (ft AMSL) (ft AMSL) (ft AMSL) (ft AMSL) (ft AMSL) (ft AMSL) (ft AMSL) (ft AMSL) (ft AMSL) (ft AMSL) (ft AMSL) (ft AMSL) (ft AMSL) (ft AMSL) (ft AMSL) (ft AMSL) (ft AMSL) (ft AMSL) (ft AMSL) (ft AMSL) (ft AMSL) (ft AMSL) (ft AMSL) (ft AMSL) (ft AMS)

2/9/10 16:00 2/9/1020:00 (2/10/100000 2/10/10 8:00 2/10/10 12:00 2/10/10 16:00 2/10/10 20:00 2/11/100:00 2/11/10 4:00 2/11/108:00 $2 / 11 / 1016: 00$ 2/11/10 20:00 2/12/100:00 2/12/10 4:00 2/12/10 8:00 $2 / 12 / 1012: 00$ $2 / 12 / 1016: 00$ $2 / 13 / 10000$ 2/13/10 4:00 2/13/10 8:00 2/13/10 12:00 2/13/10 16:00 2/13/10 20:00 2/14/100:00 $2 / 14 / 104.00$ $2 / 14 / 1012 \cdot 00$ 2/14/10 16:00 2/14/10 20:00 2/15/100:00 2/15/10 4:00 2/15/10 8:00 2/15/10 12:00 $2 / 15 / 1020.00$ $2 / 16 / 100: 00$ $2 / 16 / 104: 00$ 2/16/10 8:00 2/16/10 12:00 2/16/10 16:00 2/16/10 20:00 2/17/100:00 $2 / 17 / 108.00$ 2/17/10 8:00 2/17/10 16:00 2/17/10 20:00 2/18/100:00 2/18/10 4:00 2/18/108:00 $\begin{array}{lllll}1498.657 & 1498.982 & 1499.329 & 1500.519 & 1499.288\end{array}$ $\begin{array}{rrrrrr}1498.653 & 1498.975 & 1499.322 & 1500.496 & 1499.288 \\ 1498.639 & 1498.97 & 1499.308 & 1500.479 & 1499.269\end{array}$ $\begin{array}{llllll}1498.639 & 1498.966 & 149931 & 1500503 & 1499.276\end{array}$ $\begin{array}{llllll}1498.639 & 1498.975 & 1499.312 & 1500.51 & 1499.276\end{array}$ $\begin{array}{llllll}1498.634 & 1498.975 & 1499.31 & 1500.503 & 1499.285\end{array}$ $\begin{array}{llllll}1498.667 & 1498.987 & 1499.345 & 1500.582 & 1499.339\end{array}$ $\begin{array}{rrrrrr}1498.676 & 1498.991 & 1499.357 & 1500.585 & 1499.36 \\ 1498.69 & 1498994 & 1499.375 & 1500.613 & 1499.383\end{array}$ $\begin{array}{llllll}1498.69 & 1498.994 & 1499.375 & 1500.613 & 1499.383\end{array}$ $\begin{array}{rrrrr}1498.713 & 1499.003 & 1499.392 & 1500.643 & 1499.411 \\ 1498.72 & 1499008 & 1499.406 & 1500.645 & 149.423\end{array}$ $\begin{array}{llllll}1498.72 & 1499.008 & 1499.406 & 1500.645 & 1499.423 \\ 1498.727 & 1499.01 & 1499.41 & 1500645 & 1499.423\end{array}$ $\begin{array}{rrrrrr}1498.727 & 1499.01 & 1499.41 & 1500.645 & 1499.423 \\ 1498.746 & 1499.019 & 1499.429 & 1500.676 & 1499.451\end{array}$ $\begin{array}{llllll}1498.741 & 1499.012 & 1499.42 & 1500.641 & 1499.43\end{array}$ $\begin{array}{lllllll}1498.734 & 1499.005 & 1499.415 & 1500.629 & 1499.414\end{array}$ $\begin{array}{llllll}1498.737 & 1499.008 & 1499.413 & 1500.627 & 1499.404\end{array}$ $\begin{array}{llllll}1498.725 & 1498.998 & 1499.401 & 1500.601 & 1499.388\end{array}$ $\begin{array}{llllll}1498.72 & 1498.989 & 1499.389 & 1500.582 & 1499.367\end{array}$ $\begin{array}{llllll}1498.741 & 1499.003 & 1499.413 & 1500.641 & 1499.402 \\ 1498739 & 1499.003 & 1499.413 & 1500.631 & 1499.409\end{array}$ $\begin{array}{lllllll}1498.739 & 1499.003 & 1499.413 & 1500.631 & 1499.409\end{array}$ $\begin{array}{rrrrr}1498.748 & 1499.008 & 1499.422 & 1500.65 & 1499.418 \\ 1498.774 & 1499.022 & 1499.445 & 1500.694 & 1499.453\end{array}$ $\begin{array}{lllllll}1498.769 & 1499.022 & 1499.443 & 1500.671 & 1499.456\end{array}$ $\begin{array}{llllll}1498.769 & 1499.019 & 1499.441 & 1500.664 & 1499.442\end{array}$ $\begin{array}{llllll}1498.779 & 1499.024 & 1499.45 & 1500.678 & 1499.453\end{array}$ $\begin{array}{llllll}1498.755 & 1499.012 & 1499.424 & 1500.613 & 1499.407\end{array}$ $\begin{array}{lllll}1498.727 & 1498.994 & 1499.387 & 1500.559 & 1499.341 \\ 1498702 & 1498.977 & 1999.359 & 1500.517 & 1499295\end{array}$ $\begin{array}{llllll}1498.702 & 1498.977 & 1499.359 & 1500.517 & 1499.295 \\ 1498.66 & 1498.956 & 1499315 & 1500447 & 1499.232\end{array}$ $\begin{array}{llllll}1498.66 & 1498.956 & 149.315 & 1500.447 & 1499.232 \\ 1498.648 & 1498.954 & 1499.301 & 1500.461 & 1499.22\end{array}$ $\begin{array}{llllll}1498.643 & 1498.952 & 1499.298 & 1500.472 & 1499.232\end{array}$ $\begin{array}{llllll}1498.629 & 1498.945 & 1499.282 & 1500.447 & 1499.225\end{array}$ $\begin{array}{llllll}1498.625 & 1498.942 & 1499.28 & 1500.461 & 1499.225\end{array}$ $\begin{array}{llllll}1498.636 & 1498.952 & 1499.296 & 1500.507 & 1499.255\end{array}$ $\begin{array}{llllll}1498.629 & 1498.947 & 1499.296 & 1500.493 & 1499.269\end{array}$

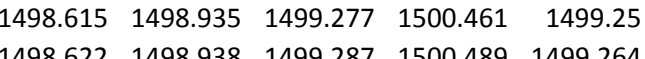
$\begin{array}{lllll}1498.622 & 1498.938 & 1499.287 & 1500.489 & 1499.264 \\ 1498.933 & 1499282 & 1500.482 & 1499.267\end{array}$ $\begin{array}{llllll}1498.62 & 1498.933 & 1499.282 & 1500.482 & 1499.26\end{array}$ $\begin{array}{llllll}1498.599 & 1498.926 & 1499.268 & 1500.461 & 1499.248\end{array}$ $\begin{array}{llllll}1498.583 & 1498.915 & 1499.252 & 1500.423 & 1499.227\end{array}$ $\begin{array}{llllll}1498.571 & 1498.912 & 1499.24 & 1500.419 & 1499.208\end{array}$ $\begin{array}{llllll}1498.59 & 1498.915 & 1499.259 & 1500.475 & 1499.243\end{array}$ $\begin{array}{llllll}1498.587 & 1498.912 & 1499.259 & 1500.468 & 1499.257\end{array}$ $\begin{array}{lllll}1498.585 & 1498.91 & 1499.256 & 1500.463 & 1499.255 \\ 1498.587 & 1498.91 & 1499.261 & 1500.468 & 1499.26\end{array}$ \begin{tabular}{lllllll}
1498.585 & 1498.91 & 149.256 & 1500.463 & 1499.255 \\
\hline
\end{tabular} $\begin{array}{llllll}1498.585 & 1498.905 & 1499261 & 1500.468 & 1499.25\end{array}$ $\begin{array}{llllll}1498.608 & 1498.917 & 1499.287 & 1500.526 & 1499.304\end{array}$ $\begin{array}{llllll}1498.613 & 1498.917 & 1499.294 & 1500.519 & 1499.316\end{array}$ $\begin{array}{llllll}1498.613 & 1498.915 & 1499.291 & 1500.51 & 1499.309\end{array}$ $\begin{array}{llllll}1498.62 & 1498.912 & 1499.296 & 1500.521 & 1499.318\end{array}$ $\begin{array}{rrrrr}1498.615 & 1498.91 & 1499.294 & 1500.51 & 1499.313 \\ 1498.634 & 1498.919 & 1499.312 & 1500.547 & 1499.332\end{array}$ 

Well (ft AMSL) (ft AMSL) (ft AMSL) (ft AMSL) (ft AMSL) (ft AMSL) (ft AMSL) (ft AMSL) (ft AMSL) (ft AMSL) (ft AMSL) (ft AMSL) (ft AMSL) (ft AMSL) (ft AMSL) (ft AMSL) (ft AMSL) (ft AMSL) (ft AMSL) (ft AMSL) (ft AMSL) (ft AMSL) (ft AMSL) (ft AMSL) (ft AMSL) (ft AMS)

W/18/1016:00 $1498.669 \quad 1498.941499 .347 \quad 1500.603 \quad 1499.391$

$\begin{array}{llllll}1498.676 & 1498.912 & 1499.357 & 1500.582 & 14999.4 \\ 1498 & 149.395\end{array}$ $\begin{array}{llllll}1498.676 & 1498.912 & 1499.357 & 1500.582 & 1499.395 \\ 1498.69 & 1498.924 & 1499.361 & 1500.578 & 1499.402\end{array}$ 2/19/10 16:00 2/19/10 20:00 2/20/100:00 2/20/10 4:00 2/20/108:00 $2 / 20 / 1016: 00$ 2/20/10 20:00 2/21/100:00 2/21/10 4:00 2/21/10 8:00 2/21/10 12:00 2/21/10 16:00 $2 / 22 / 100000$ 2/22/10 4:00 2/22/10 8:00 2/222/10 8:00 $12: 00$ 2/22/10 16:00 2/22/10 20:00 2/23/100:00 $2 / 23 / 104.00$ $2 / 23 / 1012.00$ 2/23/10 16:00 2/23/10 20:00 2/24/100:00 2/24/10 4:00 2/24/108:00 2/24/10 12:00 $2 / 24 / 1016: 00$ $2 / 25 / 100: 00$ 2/25/10 4:00 2/25/10 8:00 2/25/10 12:00 2/25/10 16:00 $2 / 25 / 1020: 00$ 2/26/100:00 $2 / 26 / 104.00$ 2/26/10 12:00 2/26/10 16:00 2/26/10 20:00

$\begin{array}{rrrrr}1498.664 & 1498.933 & 1499.347 & 1500.552 & 1499.376 \\ 1498.669 & 1498.928 & 1499.34 & 1500.535 & 1499.363\end{array}$ $\begin{array}{rrrrr}1498.65 & 1498.91 & 1499.315 & 1500.666 & 1499.323 \\ 1498.636 & 1498.905 & 1499.303 & 1500.687 & 149.292 \\ 149.632 & 1989 . & 1499296 & 1500.676 & 149.283\end{array}$ $\begin{array}{rrrrrr}1498.632 & 1498.9 & 1499.296 & 1500.676 & 1499.283 \\ 1498.62 & 1498.896 & 1499282 & 1500.685 & 1499.276\end{array}$ $\begin{array}{llllll}1498.62 & 1498.896 & 1499.282 & 1500.685 & 1499.276 \\ 1498.62 & 1498891 & 1499.282 & 1500.636 & 1499.281\end{array}$ $\begin{array}{lllllll}1498.634 & 1498.877 & 1499.303 & 1500.582 & 1499.316\end{array}$ $\begin{array}{llllll}1498.636 & 1498.877 & 1499298 & 1500.608 & 1499.325\end{array}$ $\begin{array}{llllll}1498.646 & 1498.87 & 1499.322 & 1500.594 & 1499.349\end{array}$ $\begin{array}{lllll}1498.657 & 1498.882 & 1499.333 & 1500.575 & 1499.372\end{array}$ $\begin{array}{llllll}1498.662 & 1498.915 & 1499.336 & 1500.571 & 1499.381\end{array}$ $\begin{array}{lllll}1498.657 & 1498.919 & 1499.324 & 1500.573 & 1499.362\end{array}$ $\begin{array}{llllll}1498.653 & 1498.917 & 1499.319 & 1500.582 & 1499.358 \\ 1498639 & 1498.903 & 1499303 & 1500.479 & 1499.325\end{array}$ $\begin{array}{llllll}1488.639 & 1498938 & 1499.303 & 1500.582 & 1499.358\end{array}$ $\begin{array}{lllllll}1498.618 & 1498.884 & 1499.277 & 1500.454 & 1499.292\end{array}$ $\begin{array}{llllll}1498.601 & 1498.879 & 1499.259 & 1500.421 & 1499.276\end{array}$ $\begin{array}{llllll}1498.592 & 1498.875 & 1499.249 & 1500.412 & 1499.267\end{array}$ $\begin{array}{llllll}1498.599 & 1498.882 & 1499.263 & 1500.353 & 1499.29\end{array}$ $\begin{array}{llllll}1498.599 & 1498.875 & 1499.254 & 1500.428 & 1499.285\end{array}$ $\begin{array}{llllll}1498.59 & 1498.873 & 1499.249 & 1500.496 & 1499.283 \\ 1498.594 & 1498875 & 1499.259 & 1500.498 & 1492.295\end{array}$ $\begin{array}{llllll}1498.594 & 1498.875 & 1499.259 & 1500.498 & 1499.295 \\ 1498.587 & 1498.863 & 1499.24 & 1500.884 & 1499.285\end{array}$ $\begin{array}{lllllll}1498.584 & 1498.856 & 1499.219 & 1500.083 & 1499.285 \\ 1499.25\end{array}$ $\begin{array}{cccccc}1498.562 & 1498.861 & 1499.221 & 1500.316 & 1499.257\end{array}$ $\begin{array}{llllll}1498.548 & 1498.847 & 1499.207 & 1500.428 & 1499.232\end{array}$ $\begin{array}{llllll}1498.538 & 1498.845 & 1499.198 & 1500.55 & 1499.215\end{array}$ $\begin{array}{llllll}1498.534 & 1498.842 & 1499.196 & 1500.587 & 1499.222\end{array}$ $\begin{array}{llllll}1498.529 & 1498.838 & 1499.193 & 1500.564 & 1499.225\end{array}$ $\begin{array}{llllll}1498.52 & 1498.826 & 1499.198 & 1500.318 & 1499.234 \\ 1498562 & 1498.859 & 1499.238 & 1500.164 & 1499.297\end{array}$ $\begin{array}{lllllll}1498.562 & 1498.856 & 1499.238 & 1500.164 & 1499.297\end{array}$ $\begin{array}{rrrrrr}1498.573 & 1498.863 & 1499.252 & 1500.36 & 1499.328 \\ 1498.585 & 1498.868 & 1499.261 & 1500.472 & 1499.346\end{array}$ $\begin{array}{lllllll}1498.599 & 1498.875 & 1499.277 & 1500.507 & 1499.358\end{array}$ $\begin{array}{llllll}1498.599 & 1498.875 & 1499.28 & 1500.512 & 1499.358\end{array}$ $\begin{array}{llllll}1498.608 & 1498.877 & 1499.282 & 1500.318 & 1499.36\end{array}$ $\begin{array}{llllll}1498.622 & 1498.886 & 1499.296 & 1500.218 & 1499.379\end{array}$ $\begin{array}{llllll}1498.601 & 1498.87 & 1499.273 & 1500.341 & 1499.344\end{array}$ $\begin{array}{llllll}1498.583 & 1498.861 & 1499.252 & 1500.384 & 1499.302 \\ 1498.585 & 1498856 & 1499.247 & 1500.405 & 1499295\end{array}$ $\begin{array}{llllll}1488.585 & 1498.856 & 1499.252 & 1500.384 & 1499.302\end{array}$ $\begin{array}{llllll}1498.562 & 1498.84 & 1499.219 & 1500.365 & 1499.26\end{array}$ $\begin{array}{llllll}1498.578 & 1498.852 & 1499.238 & 1500.428 & 1499.295\end{array}$ $\begin{array}{llllll}1498.578 & 1498.847 & 1499.233 & 1500.414 & 1499.302\end{array}$ $\begin{array}{lllll}1498.571 & 1498.84 & 1499.231 & 1500.405 & 1499.29 \\ 1498.566 & 149884 & 1499.224 & 1500.398 & 1499.288\end{array}$ $\begin{array}{llllll}1498.566 & 1498.84 & 1499.224 & 1500.398 & 1499.288 \\ 1498.562 & 1498.833 & 1499.217 & 1500.384 & 1499.281\end{array}$ $\begin{array}{rrrrr}1498.562 & 1498.833 & 1499.217 & 1500.384 & 1499.281 \\ 1498.548 & 1498.831 & 1499.2 & 1500.358 & 1499.255\end{array}$ 

Well (ft AMSL) (ft AMSL) (ft AMSL) (ft AMSL) (ft AMSL) (ft AMSL) (ft AMSL) (ft AMSL) (ft AMSL) (ft AMSL) (ft AMSL) (ft AMSL) (ft AMSL) (ft AMSL) (ft AMSL) (ft AMSL) (ft AMSL) (ft AMSL) (ft AMSL) (ft AMSL) (ft AMSL) (ft AMSL) (ft AMSL) (ft AMSL) (ft AMSL) (ft AMS)

W/27/1016.00 $1498.571 \quad 1498.8421499 .226 \quad 1500.4261499 .292$ $\begin{array}{llllll}1498.576 & 1498.845 & 1499.233 & 1500.428 & 1499.311 \\ 1498576 & 1498845 & 1499233 & 1500.426 & 1499316\end{array}$ $\begin{array}{llllll}1498.576 & 1498.845 & 1499.233 & 1500.426 & 1499.316 \\ 1498.576 & 1498842 & 1499.233 & 1500.421 & 1499311\end{array}$ $\begin{array}{llllll}1498.573 & 1498.838 & 1499.231 & 1500.412 & 1499.311\end{array}$ $\begin{array}{llllll}1498.578 & 1498.84 & 1499.233 & 1500.421 & 1499.311\end{array}$ 2/2/28/10 12:00 2/28/10 20:00 3/1/100:00 3/1/10 4:00 $3 / 1 / 108: 00$ $3 / 1 / 1016: 00$ 3/1/10 20:00 $3 / 2 / 100: 00$ $3 / 2 / 104: 00$ $3 / 2 / 108: 00$ $3 / 2 / 1012: 00$ $3 / 2 / 1016: 00$ $3 / 2 / 1020: 00$ 3/3/100:00 $3 / 3 / 108: 00$ $3 / 3 / 1012: 00$ 3/3/10 16:00 3/3/10 20:00 3/4/100:00 $3 / 4 / 104: 00$ $3 / 4 / 1012 \cdot 00$ $3 / 4 / 1016: 00$ $3 / 4 / 1020: 00$ 3/5/100:00 $3 / 5 / 104: 00$ $3 / 5 / 108: 00$ $3 / 5 / 1012: 00$ $\begin{array}{lllll}1498.597 & 1498.852 & 1499.256 & 1500.458 & 1499.341\end{array}$ $\begin{array}{llllll}1498.578 & 1498.84 & 1499.235 & 1500.4 & 1499.321 \\ \end{array}$ $\begin{array}{llllll}1498.564 & 1498.831 & 1499.217 & 1500.37 & 1499.276\end{array}$ $\begin{array}{llllll}1498.548 & 1498.821 & 1499.198 & 1500.353 & 1499.246 \\ 1498529 & 1498814 & 1499175 & 150032 & 1499.22\end{array}$ $\begin{array}{llllll}1498.529 & 1498.814 & 1499.175 & 1500.32 & 1499.22\end{array}$ $\begin{array}{llllll}1498.517 & 1498.805 & 1499.165 & 1500.33 & 1499208\end{array}$ $\begin{array}{lllllll}1498.508 & 1498.803 & 1499.161 & 1500.32 & 1499.213\end{array}$ $\begin{array}{llllll}1498.506 & 1498.803 & 1499.161 & 1500.325 & 1499.211\end{array}$ $\begin{array}{llllllll}1498.513 & 1498.803 & 1499.165 & 1500.348 & 1499.229\end{array}$ $\begin{array}{lllllll}1498.51 & 1498.8 & 1499.165 & 1500.346 & 1499.239 \\ & 1498.52 & 9887 & 149.17 & 150.372 & 1499.2502\end{array}$ $\begin{array}{llllll}1498.52 & 1498.807 & 1499.179 & 1500.372 & 1499.255 \\ 1488.55 & 1498824 & 1499.207 & 1500.426 & 149.302\end{array}$ $\begin{array}{llllll}1498.55 & 1498.824 & 1499.207 & 1500.426 & 1499.302 \\ 1498.555 & 1498.826 & 1499.214 & 1500.423 & 1499.314\end{array}$ $\begin{array}{llllll}1498548 & 1498821 & 1499207 & 1500398 & 1499.304\end{array}$ $\begin{array}{llllll}1498.543 & 1498.817 & 1499.203 & 1500.391 & 1499.825\end{array}$ $\begin{array}{llllll}1498.541 & 1498.81 & 1499.198 & 1500.377 & 1499.278\end{array}$ $\begin{array}{llllll}1498.534 & 1498.805 & 1499.186 & 1500.358 & 1499.257\end{array}$ $\begin{array}{lllll}1498.545 & 1498.814 & 1499.2 & 1500.4 & 1499.281\end{array}$ $\begin{array}{llllll}1498.55 & 1498.81 & 1499.2 & 1500.391 & 1499.288\end{array}$ $\begin{array}{lllll}1498.536 & 1498.807 & 1499.193 & 1500.372 & 1499.276 \\ 1495541 & 1498805 & 1499.193 & 1500374 & 1499.271\end{array}$ $\begin{array}{rrrrrr}1498.541 & 1498.805 & 1499.193 & 1500.374 & 1499.271 \\ 1498.531 & 14988 & 1499.184 & 1500348 & 1499.26\end{array}$ $\begin{array}{rrrrr}1498.531 & 1498.8 & 1499.184 & 1500.348 & 1499.26 \\ 1498.541 & 1498.803 & 1499189 & 1500372 & 1499.264\end{array}$ $\begin{array}{llllll}1498.569 & 1498.824 & 1499.224 & 1500.44 & 1499.316\end{array}$ $\begin{array}{lllll}1498.569 & 1498.821 & 1499.219 & 1500.416 & 1499.321\end{array}$ $\begin{array}{llllll}1498.562 & 1498.817 & 1499.212 & 1500.395 & 1499.306\end{array}$ $\begin{array}{llllll}1498.573 & 1498.824 & 1499.224 & 1500.423 & 1499.313\end{array}$ $\begin{array}{lllll}1498.571 & 1498.821 & 1499.221 & 1500.409 & 1499.313\end{array}$ $\begin{array}{llllll}1498.578 & 1498.826 & 1499.226 & 1500.419 & 1499.316 \\ 1498.604 & 1498.842 & 1499.252 & 1500.465 & 1499351\end{array}$ $\begin{array}{llllll}1498.604 & 1498.842 & 149.252 & 1500.465 & 1499.351\end{array}$ $\begin{array}{llllll}498.597 & 1498.838 & 1499.245 & 1500.426 & 1499.344 \\ 1498.573 & 1498.826 & 1499.219 & 1500.379 & 1499.306\end{array}$ $\begin{array}{llllll}1498.569 & 1498.824 & 1499.214 & 1500.384 & 1499.288\end{array}$ $\begin{array}{llllll}1498.55 & 1498.812 & 1499.198 & 1500.339 & 1499.262\end{array}$ $\begin{array}{llllll}1498.541 & 1498.803 & 1499.179 & 1500.325 & 1499.234\end{array}$ $\begin{array}{llllll}1498.555 & 1498.81 & 1499.196 & 1500.377 & 1499.26\end{array}$ $\begin{array}{lllll}1498.545 & 1498.803 & 1499.186 & 1500.348 & 1499.257\end{array}$ $\begin{array}{llllll}1498.529 & 1498.793 & 1499.165 & 1500.306 & 1499.229 \\ 1498.52 & 1498.791 & 1499.158 & 1500311 & 1499.218\end{array}$ $1498.51 \quad 1498789 \quad 1499.149 \quad 1500.302 \quad 1499.206$ $\begin{array}{llllll}1498.51 & 1498.789 & 1499.149 & 1500.302 & 1499.206 \\ 1498.515 & 1498.789 & 1499.154 & 1500.327 & 1499211\end{array}$ $\begin{array}{llllll}1498.534 & 1498.803 & 1499.175 & 1500.374 & 1499.25\end{array}$ $\begin{array}{llllll}1498.545 & 1498.81 & 1499.189 & 1500.393 & 1499.276\end{array}$ $\begin{array}{lllll}1498.559 & 1498.814 & 1499.2 & 1500.414 & 1499.285\end{array}$ $\begin{array}{llllll}1498.573 & 1498.824 & 1499.219 & 1500.433 & 1499.309\end{array}$ $\begin{array}{rrrrr}1498.583 & 1498.835 & 1499.233 & 1500.454 & 1499.327 \\ 1498599 & 1498.859 & 1499.27 & 1500.519 & 1499.379\end{array}$ 

Well (ft AMSL) (ft AMSL) (ft AMSL) (ft AMSL) (ft AMSL) (ft AMSL) (ft AMSL) (ft AMSL) (ft AMSL) (ft AMSL) (ft AMSL) (ft AMSL) (ft AMSL) (ft AMSL) (ft AMSL) (ft AMSL) (ft AMSL) (ft AMSL) (ft AMSL) (ft AMSL) (ft AMSL) (ft AMSL) (ft AMSL) (ft AMSL) (ft AMSL) (ft AMS)

$3 / 8 / 1016: 00$

$\begin{array}{lllll}1498.634 & 1498.838 & 1499.319 & 1500.519 & 1499.454\end{array}$

$3 / 9 / 100: 00$

$\begin{array}{llllll}1498.662 & 1498.854 & 1521.899 & 1500.442 & 1499.505 \\ 1498.681 & 1498.854 & 1525244 & 1500423 & 1499531\end{array}$

3/9/10 4:00

$3 / 9 / 1012: 00$

$3 / 9 / 1016: 00$

3/9/10 20:00

$3 / 10 / 100: 00$

$3 / 10 / 104: 00$

3/10/10 8:00

$3 / 10 / 1016: 00$

$3 / 10 / 1020: 00$

3/111/10 20:00

3/11/10 4:00

$3 / 11 / 108: 00$

$3 / 11 / 1012: 00$

$3 / 11 / 1010: 00$
$3 / 111102000$

$3 / 11 / 1020: 00$

$3 / 12 / 104: 00$

3/12/10 8:00

$3 / 12 / 1012: 00$

$3 / 12 / 1016: 00$

3/12/10 20:00

$3 / 13 / 100: 00$

$3 / 13 / 104: 00$

$3 / 13 / 108000$

$3 / 13 / 1016: 00$

$3 / 13 / 1020: 00$

3/14/100:00

$3 / 14 / 104: 00$

3/14/10 8:00

$3 / 14 / 1012: 00$

$3 / 14 / 1020.00$

$3 / 15 / 100: 00$

$3 / 15 / 104: 00$

3/15/10 8:00

$3 / 15 / 1012: 00$

3/15/10 16:00

$3 / 15 / 1020: 00$

3/16/100:00

$3 / 16 / 104.00$

3/16/10 12:00

$3 / 16 / 1016: 00$

$3 / 16 / 1020: 00$

$3 / 17 / 100: 00$

3/17/10 4:00

$3 / 17 / 108: 00$

149.563

$\begin{array}{rrrrr}1498.72 & 1498.879 & 1525.24 & 1500.414 & 1499.57 \\ \end{array}$

$\begin{array}{lllll}1498.657 & 1498.852 & 1525.23 & 1500.248 & 1499.544 \\ 1498716 & 1498.914 & 1525.23 & 1500.367 & 1499.528\end{array}$

$\begin{array}{rrrrrr}1498.716 & 1498.914 & 1525.23 & 1500.367 & 1499.528 \\ 1498.706 & 1498.907 & 1525.146 & 1500.47 & 1499.477\end{array}$

$\begin{array}{rrrrrr}1498.706 & 1498.907 & 1525.146 & 1500.47 & 1499.477 \\ 1498.697 & 1498.908 & 1522.209 & 1500.458 & 1499.451\end{array}$

$\begin{array}{lllrl}1498.697 & 1498.91 & 1518.623 & 1500.571 & 1499.444 \\ 149692 & 1498.91 & 1515832 & 150055 & 1499.435\end{array}$

$\begin{array}{lllll}4498.692 & 1498.91 & 1515.832 & 1500.55 & 1499.435 \\ 1498702 & 1498.917 & 1513594 & 1500.535 & 149.449\end{array}$

$\begin{array}{llllll}1498.716 & 1498.898 & 1511.778 & 1500.571 & 1499.468\end{array}$

$\begin{array}{llllll}1498.723 & 1498.898 & 1511.093 & 1500.634 & 1499.479\end{array}$

$\begin{array}{llllll}1498.718 & 1498.9 & 1513.14 & 1500.701 & 1499.463\end{array}$

$\begin{array}{llllll}1498.716 & 1498.898 & 1513.403 & 1500.753 & 1499.444\end{array}$

$\begin{array}{llllll}1498.706 & 1498.903 & 1513.606 & 1500.725 & 1499.409\end{array}$

$\begin{array}{llllll}1498.664 & 1498.9 & 1512.438 & 1500.652 & 1499.374\end{array}$

$\begin{array}{llllll}1498.676 & 1498.893 & 1510.836 & 1500.673 & 1499.367 \\ 1498671 & 1498886 & 1509.454 & 1500671 & 1999351\end{array}$

$\begin{array}{lllllll}1498.671 & 1498.886 & 1509.454 & 1500.671 & 1499.351\end{array}$

$\begin{array}{rrrrr}1498.662 & 1498.896 & 1507.3 & 1500.694 & 1499.384\end{array}$

$\begin{array}{lllll}1498.66 & 1498.896 & 1506.432 & 1500.69 & 1499.384\end{array}$

$\begin{array}{llllll}1498.636 & 1498.886 & 1505.67 & 1500.606 & 1499.363\end{array}$

$\begin{array}{llllll}1498.636 & 1498.88 & 1505.017 & 1500.603 & 1499.353\end{array}$

$\begin{array}{llllll}1498.604 & 1498.868 & 1504.567 & 1500.613 & 1499.318\end{array}$ $\begin{array}{llllll}1498.601 & 1498.868 & 1504.35 & 1500.627 & 1499.306 \\ 1498.608 & 1498872 & 1504.556 & 1500.643 & 149.332\end{array}$ $\begin{array}{llllll}1498.608 & 1498.872 & 1504.556 & 1500.643 & 1499.332 \\ 1498.597 & 1498.866 & 1504.488 & 1500.608 & 1499.323\end{array}$ $\begin{array}{lllllll}1498.583 & 1498.861 & 1504.488 & 1500.608 & 1499.323 \\ 1400 & 1500507 & 1499.311\end{array}$ $\begin{array}{llllll}1498.599 & 1498.87 & 1504.32 & 1500.381 & 1499.332\end{array}$ $\begin{array}{lllll}1498.59 & 1498.861 & 1504.171 & 1500.36 & 1499.33\end{array}$ $\begin{array}{llllll}1498.578 & 1498.852 & 1503.863 & 1500.339 & 1499.311\end{array}$ $\begin{array}{llllll}1498.566 & 1498.847 & 1503.551 & 1500.325 & 1499.297\end{array}$ $\begin{array}{llllll}1498.538 & 1498.831 & 1503.208 & 1500.271 & 1499.253\end{array}$ $\begin{array}{llllll}1498.51 & 1498.817 & 1502.865 & 1500.225 & 1499.215 \\ 149851 & 1498814 & 1502537 & 1500264 & 199.213\end{array}$ $\begin{array}{llllll}1498.51 & 1498.814 & 1502.537 & 1500.264 & 1499.213\end{array}$ $\begin{array}{llllll}1498.482 & 1498.803 & 152.203 & 1500.215 & 1499.185 \\ 1498.457 & 1498.791 & 1501.907 & 1500.192 & 1499.145\end{array}$ $\begin{array}{lllllll}1498.443 & 1498.784 & 1501.644 & 1500.204 & 1499.131\end{array}$ $\begin{array}{llllll}1498.419 & 1498.77 & 1501.387 & 1500.159 & 1499.108\end{array}$ $\begin{array}{llllll}1498.412 & 1498.768 & 1501.18 & 1500.183 & 1499.106\end{array}$ $\begin{array}{llllll}1498.422 & 1498.775 & 1501.003 & 1500.225 & 1499.141\end{array}$

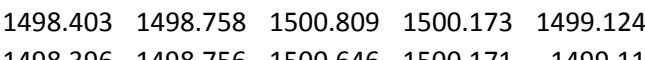
$\begin{array}{llllll}1498.396 & 1498.756 & 1500.646 & 1500.171 & 1499.11 \\ 1498.396 & 1498.752 & 1500.506 & 1500.192 & 1499.117\end{array}$ $\begin{array}{lllllll}1488.396 & 1498.752 & 1500.506 & 1500.192 & 1499.117\end{array}$ $\begin{array}{rrrrr}1498.394 & 1498.749 & 1500.373 & 1500.19 & 1499.127 \\ 1498.398 & 1498.751 & 1500.261 & 1500.208 & 1499.131\end{array}$ $\begin{array}{lllllll}1498.426 & 1498.763 & 1500.184 & 1500.271 & 1499.183\end{array}$ $\begin{array}{llllll}1498.436 & 1498.768 & 1500.096 & 1500.276 & 1499.208\end{array}$ $\begin{array}{lllll}1498.429 & 1498.761 & 1500.014 & 1500.26 & 1499.201\end{array}$ $\begin{array}{llllll}1498.44 & 1498.765 & 1499.94 & 1500.283 & 1499.211\end{array}$ $\begin{array}{rrrrr}1498.438 & 1498.761 & 1499.86 & 1500.262 & 1499.208 \\ 1498.44 & 1498.758 & 1499.79 & 1500.267 & 1499.204\end{array}$ 

Well (ft AMSL) (ft AMSL) (ft AMSL) (ft AMSL) (ft AMSL) (ft AMSL) (ft AMSL) (ft AMSL) (ft AMSL) (ft AMSL) (ft AMSL) (ft AMSL) (ft AMSL) (ft AMSL) (ft AMSL) (ft AMSL) (ft AMSL) (ft AMSL) (ft AMSL) (ft AMSL) (ft AMSL) (ft AMSL) (ft AMSL) (ft AMSL) (ft AMSL) (ft AMS)

$3 / 17 / 1016,00$ $\begin{array}{rrrrr}1498.461 & 1498.77 & 1499.744 & 1500.316 & 1499.236 \\ 1498.478 & 1498.777 & 1499.704 & 1500325 & 1499.26\end{array}$ $\begin{array}{rrrrr}1498.478 & 1498.777 & 1499.704 & 1500.325 & 1499.262 \\ 1498.485 & 1498.779 & 1499.66 & 150233 & 1499267\end{array}$ $\begin{array}{rrrrr}1498.485 & 1498.779 & 1499.66 & 1500.33 & 1499.267 \\ 1498.503 & 1498.789 & 1499.627 & 1500.358 & 1499292\end{array}$ $\begin{array}{llllll}1498.503 & 1498.789 & 1499.627 & 1500.358 & 1499.292 \\ 1498.513 & 1498.796 & 1499592 & 1500367 & 1499306\end{array}$ $\begin{array}{llllll}1498.541 & 1498.812 & 1499.573 & 1500.412 & 1499.341\end{array}$ 3/18/10 8:00 3/18/10 16:00 3/18/10 20:00 3/19/10 0:00 $3 / 19 / 104: 00$ 3/19/10 8:00 3/19/10 12:00 3/19/1900 20:00 3/120/10 20:00 3/20/10 4:00 3/20/10 8:00 3/20/10 12:00 3/20/10 16:00 3/20/10 20:00 $3 / 21 / 100: 00$ $3 / 21 / 108: 00$ 3/21/10 12:00 $3 / 21 / 1016: 00$ 3/21/10 20:00 $3 / 22 / 100: 00$ $3 / 22 / 104: 00$ $3 / 22 / 1012 \cdot 00$ $3 / 22 / 1016: 00$ $3 / 22 / 1020: 00$ $3 / 23 / 100: 00$ $3 / 23 / 104: 00$ 3/23/10 8:00 $3 / 23 / 1012: 00$ $3 / 23 / 1016: 00$ $3 / 24 / 100: 00$ $3 / 24 / 104: 00$ $3 / 24 / 108: 00$ $3 / 24 / 1012: 00$ 3/24/10 16:00 3/24/10 20:00 3/25/100:00 $3 / 25 / 104.00$ $3 / 25 / 1012: 00$ $3 / 25 / 1016: 00$ $3 / 25 / 1020: 00$ $\begin{array}{llllll}1498.587 & 1498.838 & 1499.573 & 1500.486 & 1499.409\end{array}$ $\begin{array}{lllll}1498.606 & 1498.849 & 1499.552 & 1500.482 & 1499.437\end{array}$ $\begin{array}{llllll}1498.62 & 1498.856 & 1499.529 & 1500.489 & 1499.442\end{array}$ $\begin{array}{llllll}1498.627 & 1498.859 & 1499.506 & 1500.486 & 1499.444 \\ 1498.627 & 1498859 & 1499.476 & 1500.458 & 1499.23\end{array}$ $\begin{array}{llllll}1498.627 & 1498.859 & 1499.476 & 1500.458 & 1499.423 \\ 1498.613 & 1498.854 & 1499.438 & 1500.419 & 1499.393\end{array}$ $\begin{array}{lllllll}1498.59 & 1498.84 & 1499.399 & 1500.37 & 1499.349\end{array}$ $\begin{array}{llllll}1498.571 & 1498.817 & 1499.338 & 1500.283 & 1499.271\end{array}$ $\begin{array}{lllll}1498.564 & 1498.803 & 1499.296 & 1500.255 & 1499.218\end{array}$ $\begin{array}{lllll}1498.55 & 1498.803 & 1499.28 & 1500.29 & 1499.227\end{array}$ $\begin{array}{llllll}1498.536 & 1498.786 & 1499.24 & 1500.239 & 1499.194\end{array}$ $\begin{array}{llllll}1498.52 & 1498.779 & 1499.224 & 1500.248 & 1499.192\end{array}$ $\begin{array}{llllll}1498.513 & 1498.782 & 1499.217 & 1500.269 & 1499.208 \\ 1498503 & 1498777 & 1499203 & 1500295 & 1499.222\end{array}$ $\begin{array}{llllll}1488.503 & 1498.777 & 1499.20 & 1500.295 & 1499.222\end{array}$ $\begin{array}{llllll}1498.529 & 1498.814 & 1499.196 & 1500.325 & 1499.271\end{array}$ $\begin{array}{llllll}498.531 & 1498.803 & 1499.186 & 1500.323 & 1499.285\end{array}$ $\begin{array}{lllllll}1498.538 & 1498.735 & 1499.177 & 1500.066 & 1499.281\end{array}$ $\begin{array}{llllll}1498.424 & 1498.817 & 1499.191 & 1500.091 & 1499.318\end{array}$ $\begin{array}{llllll}1498.543 & 1498.821 & 1499.184 & 1500.327 & 1499.325\end{array}$ $\begin{array}{rrrrr}1498.538 & 1498.828 & 1499.177 & 1500.454 & 1499.32 \\ 1498555 & 1988842 & 1999.184 & 150.5 & 1499.339\end{array}$ $\begin{array}{rrrrr}1498.555 & 1498.842 & 1499.184 & 1500.5 & 1499.339 \\ 1498.555 & 1498.842 & 1499.175 & 1500.402 & 1499.339\end{array}$ $\begin{array}{llllll}1498.58 & 1498.861 & 1499.198 & 1500.1117 & 1499.339 \\ 1499.37\end{array}$ $\begin{array}{llllll}1498.618 & 1498.882 & 1499.224 & 1500.482 & 1499.426\end{array}$ $\begin{array}{llllll}1498.625 & 1498.886 & 1499.221 & 1500.458 & 1499.435\end{array}$ $\begin{array}{llllll}1498.627 & 1498.889 & 1499.214 & 1500.449 & 1499.426\end{array}$ $\begin{array}{llllll}1498.646 & 1498.9 & 1499.226 & 1500.482 & 1499.437\end{array}$ $\begin{array}{llllll}1498.634 & 1498.893 & 1499.207 & 1500.428 & 1499.416\end{array}$ $\begin{array}{rrrrrr}1498.65 & 1498.907 & 1499.219 & 1500.463 & 1499.423 \\ 1498.667 & 1498.91 & 1499228 & 1500.477 & 1999.442\end{array}$ $\begin{array}{lllllll}1498.667 & 148.91 & 1499.228 & 1500.477 & 1499.442\end{array}$ $\begin{array}{llllll}1498.622 & 1498.891 & 1499.179 & 1500.372 & 1499.436\end{array}$ $\begin{array}{llllll}1498.634 & 1498.868 & 1499.186 & 1500.44 & 1499.365\end{array}$ $\begin{array}{lllllll}1498.629 & 1498.854 & 1499.165 & 1500.463 & 1499.342\end{array}$ $\begin{array}{lllllll}1498.618 & 1498.838 & 1499.161 & 1500.482 & 1499.332\end{array}$ $\begin{array}{lllllll}1498.613 & 1498.845 & 1499.158 & 1500.482 & 1499.339\end{array}$ $\begin{array}{lllllll}1498.62 & 1498.84 & 1499.147 & 1500.531 & 1499.325 \\ 1498.62 & 1498.828 & 1499.14 & 145036 & 1499321\end{array}$ $\begin{array}{llllll}1498.62 & 1498.828 & 1499.147 & 1500.386 & 1499.321 \\ 1498.632 & 1498.842 & 1499.149 & 1500.433 & 1499.327\end{array}$ $\begin{array}{lllllll}1498.632 & 1498.842 & 1499.147 & 1500.386 & 1499.321\end{array}$ $\begin{array}{lllll}1498.6557 & 1498.852 & 1499.1993 & 1500.175 & 1499.297\end{array}$ $\begin{array}{llllll}1498.58 & 1498.859 & 1499.11 & 1500.04 & 1499.29\end{array}$ $\begin{array}{lllll}1498.597 & 1498.863 & 1499.112 & 1500.393 & 1499.304\end{array}$ $\begin{array}{rrrrr}1498.587 & 1498.863 & 1499.105 & 1500.461 & 1499.304 \\ 1498594 & 1498872 & 1499.117 & 1500.449 & 1499.33\end{array}$ $\begin{array}{rrrrr}1498.594 & 1498.872 & 1499.117 & 1500.449 & 1499.33 \\ 1498.59 & 1498.877 & 1499.117 & 1500.377 & 1499.342\end{array}$ $\begin{array}{rrrrrr}1498.59 & 1498.877 & 1499.117 & 1500.377 & 1499.342 \\ 1498.629 & 1498.903 & 1499.151 & 1500.367 & 1499.395\end{array}$ 

Well (ft AMSL) (ft AMSL) (ft AMSL) (ft AMSL) (ft AMSL) (ft AMSL) (ft AMSL) (ft AMSL) (ft AMSL) (ft AMSL) (ft AMSL) (ft AMSL) (ft AMSL) (ft AMSL) (ft AMSL) (ft AMSL) (ft AMSL) (ft AMSL) (ft AMSL) (ft AMSL) (ft AMSL) (ft AMSL) (ft AMSL) (ft AMSL) (ft AMSL) (ft AMS)

$3 / 26 / 1016,00$

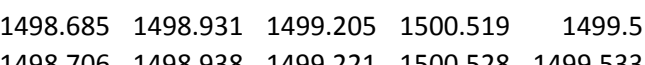
$\begin{array}{lllll}1498.706 & 1498.938 & 1499.221 & 1500.528 & 1499.533 \\ 1498.704 & 1498.905 & 1499219 & 1500505 & 1499521\end{array}$ $\begin{array}{llllll}1498.704 & 1498.905 & 1499.219 & 1500.505 & 1499.521 \\ 1498.723 & 1498.898 & 1499261 & 1500.533 & 1499.575\end{array}$ $\begin{array}{llllll}1498.723 & 1498.898 & 1499.261 & 1500.533 & 1499.575 \\ 1498.734 & 1498.919 & 1499256 & 1500.552 & 1499.575\end{array}$ $3 / 27 / 108: 00$ $3 / 27 / 1016: 00$ $3 / 27 / 1020: 00$ $3 / 28 / 100: 00$ $3 / 28 / 104: 00$ 3/28/10 8:00 $3 / 28 / 1016: 00$ $3 / 28 / 1020: 00$ $3 / 29 / 100: 00$ 3/29/10 4:00 $3 / 29 / 108: 00$ 3/29/10 12:00 $3 / 29 / 1016: 00$ $3 / 29 / 1020.00$ $3 / 30 / 104: 00$ 3/30/10 8:00 $3 / 30 / 1012: 00$ 3/30/10 16:00 3/30/10 20:00 $3 / 31 / 100: 00$ $3 / 31 / 104: 00$ $3 / 31 / 1012 \cdot 00$ $3 / 31 / 1016: 00$ $3 / 31 / 1020: 00$ 4/1/100:00 4/1/10 4:00 4/1/10 8:00 $4 / 1 / 1012: 00$ 4/1/1016:00 4/1/1/1020:00 $4 / 2 / 104: 00$ $4 / 2 / 108: 00$ $\begin{array}{lllll}1498.723 & 1498.903 & 1499.226 & 1500.594 & 1499.512 \\ 1498.706 & 1498.903 & 1499.193 & 1500.629 & 1499.433\end{array}$ $\begin{array}{llllll}1498.713 & 1498.868 & 1499.149 & 1500.687 & 1499.346\end{array}$ $\begin{array}{lllllll}1498.755 & 1498.872 & 1499.121 & 1500.543 & 1499.285\end{array}$ $\begin{array}{llllll}1498.683 & 1498.854 & 1499.107 & 1500.601 & 1499.264 \\ 1498.618 & 1498838 & 1999.082 & 1500.472 & 1499234\end{array}$ $\begin{array}{llllll}1498.618 & 1498.838 & 1499.082 & 1500.472 & 1499.234 \\ 1498.555 & 149884 & 1499.075 & 1500133 & 149.227\end{array}$ $\begin{array}{rrrrrr}149.555 & 1498.84 & 1499.075 & 1500.133 & 1499.227 \\ 1498.58 & 1498.854 & 1499.084 & 1500.138 & 1499.262\end{array}$ $\begin{array}{lllllll}1498.573 & 1498.859 & 1499.084 & 1500.327 & 1499.283\end{array}$ $\begin{array}{llllll}1498.576 & 1498.861 & 1499.084 & 1500.306 & 1499.295\end{array}$ $\begin{array}{lllllll}1498.585 & 1498.868 & 1499.093 & 1500.318 & 1499.318\end{array}$ $\begin{array}{llllll}1498.58 & 1498.866 & 1499.093 & 1500.264 & 1499.327\end{array}$ $\begin{array}{lllll}1498.606 & 1498.889 & 1499.119 & 1500.323 & 1499.365\end{array}$ $\begin{array}{llllll}1498.648 & 1498.914 & 1499.151 & 1500.426 & 1499.433 \\ 1498657 & 1498.921 & 1499.161 & 1500.423 & 1499.458\end{array}$ $\begin{array}{lllllll}1498.657 & 1498.921 & 1499.161 & 1500.423 & 1499.458\end{array}$ $\begin{array}{llllll}1498.69 & 1498.942 & 1499.196 & 1500.472 & 1499.493\end{array}$ $\begin{array}{llllll}1498.699 & 1498.952 & 1499.203 & 1500.461 & 1499.505\end{array}$ $\begin{array}{llllll}1498.72 & 1498.961 & 1499.224 & 1500.486 & 1499.524\end{array}$ $\begin{array}{llllll}1498.753 & 1498.977 & 1499.245 & 1500.538 & 1499.573\end{array}$ $\begin{array}{llllll}1498.767 & 1498.984 & 1499.256 & 1500.531 & 1499.589\end{array}$ $\begin{array}{lllll}1498.774 & 1498.987 & 1499.263 & 1500.535 & 1499.584 \\ 1498776 & 1988.994 & 1999266 & 1500.524 & 1499575\end{array}$ $\begin{array}{llllll}1498.776 & 1498.994 & 1499.266 & 1500.524 & 1499.575 \\ 1498.748 & 1498977 & 1492247 & 1500.456 & 1499528\end{array}$ $\begin{array}{llllll}1498.734 & 1498.973 & 1499.237 & 150.456 & 1499.528\end{array}$ $\begin{array}{llllll}1498.748 & 1498.982 & 1499.245 & 1500.47 & 1499.498\end{array}$ $\begin{array}{llllll}1498.751 & 1498.984 & 1499.247 & 1500.465 & 1499.502\end{array}$ $\begin{array}{lllllll}1498.744 & 1498.98 & 1499.238 & 1500.444 & 1499.486\end{array}$ $\begin{array}{llllll}1498.743 & 1498.982 & 1499.24 & 1500.447 & 1499.491\end{array}$ $\begin{array}{lllll}1498.727 & 1498.97 & 1499.224 & 1500.412 & 1499.465\end{array}$ $\begin{array}{llllll}1498.744 & 1498.982 & 1499.242 & 1500.458 & 1499.479 \\ 1498774 & 1498994 & 1499.273 & 1500526 & 1499.531\end{array}$ $\begin{array}{llllll}4498.774 & 1498.994 & 1499.273 & 1500.526 & 1499.531\end{array}$ $\begin{array}{lllll}1498.744 & 1498.996 & 1499.268 & 1500.5 & 1499.505 \\ 1498.767 & 1498.987 & 1499254 & 1500.463 & 1499.444\end{array}$ $\begin{array}{lllllll}1498.797 & 1498.982 & 1499.247 & 1500.62 & 1499.428\end{array}$ $\begin{array}{llllll}1498.795 & 1498.973 & 1499.231 & 1500.613 & 1499.409\end{array}$ $\begin{array}{llllll}1498.706 & 1498.954 & 1499.203 & 1500.325 & 1499.377\end{array}$ $\begin{array}{llllll}1498.72 & 1498.947 & 1499.198 & 1500.344 & 1499.372\end{array}$ $\begin{array}{llllll}1498.683 & 1498.924 & 1499.165 & 1500.302 & 1499.325\end{array}$ $\begin{array}{rrrrrr}1498.653 & 1498.9 & 1499.135 & 1500.262 & 1499.276 \\ 1498.648 & 1498.893 & 1499.126 & 1500274 & 1499.271\end{array}$ $\begin{array}{lllllll}1488.648 & 1498.883 & 1499.126 & 1500.274 & 1499.271\end{array}$ $\begin{array}{lllll}1498.629 & 1498.884 & 1499.112 & 1500.248 & 1499.253 \\ 1498.636 & 1498.886 & 1499.114 & 1500.299 & 1499.157\end{array}$ $\begin{array}{llllll}1498.664 & 1498.91 & 1499.138 & 1500.377 & 1499.185\end{array}$ $\begin{array}{llllll}1498.662 & 1498.907 & 1499.135 & 1500.365 & 1499.22\end{array}$ $\begin{array}{llllll}1498.655 & 1498.9 & 1499.128 & 1500.348 & 1499.243 \\ \end{array}$ $\begin{array}{lllllll}1498.65 & 1498.896 & 1499.119 & 1500.327 & 1499.257\end{array}$ $\begin{array}{lllll}1498.632 & 1498.884 & 1499.107 & 1500.311 & 1499.264 \\ 1498.641 & 1498.891 & 1499.121 & 1500.348 & 1499.299\end{array}$ 

Well

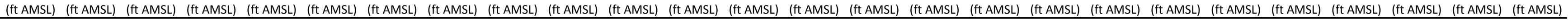

4/4/10 16:00

$\begin{array}{lllll}1498.618 & 1498.877 & 1499.105 & 1500.302 & 1499.297\end{array}$

$4 / 5 / 100: 00$

$\begin{array}{lllll}1498.592 & 1498.861 & 1499.082 & 1500.246 & 1499.243 \\ 1498.58 & 1498.859 & 1499.072 & 1500246 & 1499.173\end{array}$

$4 / 5 / 108: 00$

4/5/10 12:00

4/5/1016:00

4/5/10 20:00

$\begin{array}{lllllll}1498587 & 1498863 & 149902 & 1500295 & 1499.199\end{array}$

$\begin{array}{lllllll}1498.604 & 1498.875 & 1499.1 & 1500.341 & 1499.267\end{array}$

$\begin{array}{llllll}4998.632 & 1498.891 & 1499.124 & 1500.384 & 1499.33\end{array}$

$\begin{array}{llllll}1498.662 & 1498.91 & 1499.154 & 1500.442 & 1499.395\end{array}$

$\begin{array}{llllll}1498.678 & 1498.919 & 1499.168 & 1500.442 & 1499.428\end{array}$

$4 / 6 / 100: 00$

$\begin{array}{llllll}1498.701 & 1498.928 & 1499.182 & 1500.461 & 1499.449\end{array}$

$\begin{array}{rrrrr}1498.737 & 1498.942 & 1499.2 & 1500.489 & 1499.481 \\ 1498.741 & 1498.94 & 14992 & 1500.454 & 1499.437\end{array}$

4/6/10 8:00

$4 / 6 / 1016: 00$

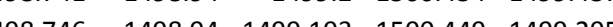

$\begin{array}{llllll}1498.774 & 1498.952 & 1499.205 & 1500.496 & 1499.306\end{array}$

$\begin{array}{lllllll}1498.723 & 1498.912 & 1499.151 & 1500.341 & 1499.22\end{array}$

4/6/10 20:00

4/7/10 4:00

4/7/10 8:00

4/7/10 12:00

4/7/10 16:00

4/7/10 20:00

4/8/100:00

4/8/10 8:00

4/8/108:00

4/8/10 16:00

$4 / 8 / 1020: 00$

4/9/10 0:00

4/9/10 4:00

$4 / 9 / 10 \cdot 12 \cdot 00$

$4 / 9 / 1016: 00$

$4 / 9 / 1020: 00$

$4 / 10 / 100: 00$

4/10/10 4:00

$4 / 10 / 108: 00$

$4 / 10 / 1012: 00$

4/10/10 16:00

$4 / 11 / 100: 00$

$4 / 11 / 104: 00$

$\begin{array}{lllll}4998.704 & 1498.896 & 1499.131 & 1500.323 & 1499.173\end{array}$

$\begin{array}{llllll}1498.695 & 1498.889 & 1499.121 & 1500.318 & 1499.178\end{array}$

$\begin{array}{llllll}1498.667 & 1498.868 & 1499.093 & 1500.278 & 1499.115\end{array}$

$\begin{array}{lllll}1498.622 & 1498.84 & 1499.047 & 1500.208 & 1498.959\end{array}$

\begin{tabular}{lllllll}
4498.571 & 1498.821 & 1499.009 & 1500.152 & 1498.905 \\
\hline
\end{tabular}

$\begin{array}{lllllll}1498.543 & 1498.807 & 1498.988 & 150014 & 1498.917\end{array}$

$\begin{array}{llllll}1498.508 & 1498.793 & 1498.97 & 1500.15 & 1498.98\end{array}$ $\begin{array}{lllll}1498.492 & 1498.791 & 1498.963 & 1500.143 & 1499.005\end{array}$ $\begin{array}{rrrrr}1498.496 & 1498.796 & 1498.97 & 1500.178 & 1499.045 \\ 1498515 & 1498.814 & 1498.993 & 1500.239 & 1499.11\end{array}$ $\begin{array}{lllll}1498.515 & 1498.814 & 1498.993 & 1500.239 & 1499.11 \\ 1498515 & 1498817 & 1498.991 & 1500232 & 1499.141\end{array}$ $\begin{array}{lllll}1498.515 & 1498.817 & 1498.991 & 1500.232 & 1499.141 \\ 1498.508 & 1498.812 & 1498.991 & 1500.232 & 1499.148\end{array}$ $\begin{array}{rrrrr}1498.508 & 1498.812 & 1498.991 & 1500.232 & 1499.148 \\ 1498.51 & 1498.812 & 1498.995 & 1500.241 & 1499.159\end{array}$ $\begin{array}{llllll}1498.51 & 1498.812 & 1498.995 & 1500.24 & 199159 \\ 1498.501 & 1498.807 & 1498.991 & 1500.222 & 1499.127\end{array}$ $\begin{array}{llllll}1498.508 & 1498.819 & 1498.995 & 1500.248 & 1499.129 \\ \end{array}$

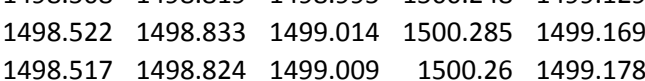
$\begin{array}{llllll}1498.499 & 1498.807 & 1498.991 & 1500.225 & 1499.152\end{array}$ $\begin{array}{llllll}1498.496 & 1498.8 & 1498.993 & 1500.229 & 1499.15\end{array}$ $\begin{array}{llllll}1498.466 & 1498.779 & 1499.035 & 1500.164 & 1499.12\end{array}$ $\begin{array}{lllllll}1498.473 & 1498.786 & 1498.977 & 1500.204 & 1499.12\end{array}$ $\begin{array}{llllll}1498.482 & 1498.784 & 1498.984 & 1500.222 & 1499.136 \\ 1498.461 & 1498.775 & 1498.967 & 1500.178 & 1499117\end{array}$ $\begin{array}{lllllll}1498.44 & 1498.763 & 1498.951 & 1500.145 & 1499.082\end{array}$ $\begin{array}{lllllll}1498.433 & 1498.758 & 1498.946 & 1500.145 & 1499.071\end{array}$ $\begin{array}{lllllll}1498.41 & 1498.747 & 1498.928 & 1500.105 & 1499.045\end{array}$ 12:00 4/11/1012.00 $4 / 11 / 1016: 00$ $\begin{array}{llllll}1498.415 & 1498.747 & 1498.937 & 1500.15 & 1499.054\end{array}$ $\begin{array}{llllll}1498.431 & 1498.754 & 1498.949 & 1500.197 & 1499.096\end{array}$ $\begin{array}{llllll}1498.429 & 1498.749 & 1498.949 & 1500.183 & 1499.106 \\ 1498.419 & 1498737 & 1498937 & 1500159 & 1499092\end{array}$ $\begin{array}{llllll}1498.419 & 1498.737 & 1498.937 & 1500.159 & 1499.092 \\ 1498.429 & 1498.742 & 1498.946 & 1500187 & 1499.106\end{array}$ $\begin{array}{llllllll}1488.429 & 1498.72 & 1498.946 & 1500.187 & 1499.106\end{array}$ $\begin{array}{llllll}1498.417 & 1498.735 & 1488.935 & 1500.157 & 1499.092 \\ 1498.429 & 1498.737 & 1498.946 & 1500.192 & 1499.106\end{array}$ $\begin{array}{llllll}1498.443 & 1498.744 & 1498.96 & 1500.22 & 1499.134\end{array}$ $\begin{array}{llllll}1498.45 & 1498.744 & 1498.967 & 1500.225 & 1499.152\end{array}$ $\begin{array}{llllll}1498.454 & 1498.742 & 1498.967 & 1500.227 & 1499.152\end{array}$ $\begin{array}{llllll}1498.457 & 1498.742 & 1498.972 & 1500.227 & 1499.157\end{array}$ $\begin{array}{rrrrrr}1498.447 & 1498.735 & 1498.967 & 1500.206 & 1499.148 \\ 1498.45 & 1498.733 & 1498.97 & 1500.215 & 1499.141\end{array}$ 

Well (ft AMSL) (ft AMSL) (ft AMSL) (ft AMSL) (ft AMSL) (ft AMSL) (ft AMSL) (ft AMSL) (ft AMSL) (ft AMSL) (ft AMSL) (ft AMSL) (ft AMSL) (ft AMSL) (ft AMSL) (ft AMSL) (ft AMSL) (ft AMSL) (ft AMSL) (ft AMSL) (ft AMSL) (ft AMSL) (ft AMSL) (ft AMSL) (ft AMSL) (ft AMS) $\begin{array}{lllll}1498.478 & 1498.749 & 1498.998 & 1500.271 & 1499.18\end{array}$ $\begin{array}{lllll}1498.473 & 1498.744 & 1498.988 & 1500.239 & 1499.183 \\ 1498.459 & 1498.733 & 1498974 & 1500204 & 14991157\end{array}$ $\begin{array}{rrrrr}1498.459 & 1498.733 & 1498.974 & 1500.204 & 1499.157 \\ 1498.45 & 1498.728 & 1498.967 & 1500.18 & 1499.129\end{array}$ $\begin{array}{llllll}1498.412 & 1498.716 & 1498.937 & 1500.112 & 1499.129\end{array}$ $\begin{array}{lllllll}1498.417 & 1498.716 & 1498.939 & 1500.145 & 1499.068\end{array}$ $4 / 14 / 1016: 00$ 4/14/10 20:00 $4 / 15 / 100: 00$ $4 / 15 / 104: 00$ 4/15/108:00 4/15/10 12:00 4/15/10 20:00 $4 / 16 / 100: 00$ 4/16/10 4:00 4/16/10 8:00 $4 / 16 / 1012: 00$ 4/16/10 16:00 $4 / 16 / 1020: 00$ $4 / 17 / 104: 00$ $4 / 17 / 108: 00$ 4/17/10 12:00 4/17/10 16:00 4/17/10 20:00 $4 / 18 / 100: 00$ $4 / 18 / 104.00$ $4 / 18 / 1012 \cdot 00$ $4 / 18 / 1016: 00$ $4 / 18 / 1020: 00$ $4 / 19 / 100: 00$ 4/19/10 4:00 $4 / 19 / 108: 00$ $4 / 19 / 1012: 00$ $4 / 19 / 1020.00$ 4/19/1020:00 $4 / 20 / 104: 00$ 4/20/10 8:00 4/20/10 12:00 $4 / 20 / 1016: 00$ $4 / 20 / 1020: 00$ $4 / 21 / 100: 00$ $\begin{array}{lllllll}1498.429 & 1498.723 & 1498.953 & 1500.199 & 1499.101\end{array}$ $\begin{array}{llllll}1498.426 & 1498.719 & 1498.946 & 1500.176 & 1499.038\end{array}$ $\begin{array}{lllllll}1498.41 & 1498.707 & 1498.925 & 1500.147 & 1498.905\end{array}$ $\begin{array}{llllll}1498.41 & 1498.705 & 1498.925 & 1500.161 & 1498.914 \\ 1498.394 & 1498.7 & 1498.907 & 1500.126 & 1498926\end{array}$ $\begin{array}{lrrrrr}1498.394 & 1498.7 & 1498.907 & 1500.126 & 1498.926\end{array}$ $\begin{array}{lllllll}1498.401 & 1498.7 & 1498.918 & 1500.178 & 1498.998\end{array}$ $\begin{array}{lllllll}1498.389 & 1498.691 & 1498.909 & 1500.143 & 1499.015\end{array}$ $\begin{array}{lllllll}1498.368 & 1498.686 & 1498.89 & 1500.098 & 1498.989\end{array}$ $\begin{array}{llllll}1498.366 & 1498.679 & 1498.888 & 1500.096 & 1498.989\end{array}$ $\begin{array}{llllll}1498.361 & 1498.649 & 1498.862 & 1500.047 & 1498.893\end{array}$ $\begin{array}{rrrrr}1498.317 & 1498.654 & 1498.853 & 1500.066 & 1498.767\end{array}$ $\begin{array}{llllll}1498.326 & 1498.663 & 1498.855 & 1500.112 & 1498.8\end{array}$ $\begin{array}{lllll}1498.347 & 1498.658 & 1498.858 & 1500.122 & 1498.858 \\ 1498335 & 1498658 & 1498858 & 1500115 & 1498898\end{array}$ $\begin{array}{llllll}1498.333 & 1498.663 & 1498.858 & 1500.126 & 1498.938\end{array}$ $\begin{array}{llllll}1498.326 & 1498.665 & 1498.853 & 1500.112 & 1498.959\end{array}$ $\begin{array}{llllll}1498.338 & 1498.675 & 1498.862 & 1500.14 & 1498.982\end{array}$ $\begin{array}{llllll}1498.366 & 1498.705 & 1498.895 & 1500.218 & 1499.047\end{array}$ $\begin{array}{lllllll}1498.366 & 1498.707 & 1498.897 & 1500.194 & 1499.066\end{array}$ $\begin{array}{llllll}1498.366 & 1498.707 & 1498.895 & 1500.178 & 1499.059 \\ 1498363 & 1498712 & 1498895 & 1500.171 & 149059\end{array}$ $\begin{array}{llllll}1498.363 & 1498.712 & 1488.895 & 1500.171 & 1499.059 \\ 1498352 & 1498705 & 1498881 & 1500.133 & 1498.975\end{array}$ $\begin{array}{llllll}1498.352 & 1498.70 & 1498.881 & 1500.133 & 1498.975 \\ 1498.349 & 1498707 & 1498.872 & 1500.15 & 1498849\end{array}$ $\begin{array}{lllllll}1498.359 & 1498.716 & 1498.883 & 1500.185 & 1498.886\end{array}$ $\begin{array}{lllllll}1498.354 & 1498.716 & 1498.876 & 1500.166 & 1498.919\end{array}$ $\begin{array}{lllllll}1498.347 & 1498.714 & 1498.874 & 1500.154 & 1498.94\end{array}$ $\begin{array}{llllll}1498.347 & 1498.716 & 1498.879 & 1500.164 & 1498.973\end{array}$ $\begin{array}{llllll}1498.34 & 1498.714 & 1498.869 & 1500.145 & 1498.98\end{array}$ $\begin{array}{lllll}1498.352 & 1498.726 & 1498.886 & 1500.182 & 1499.005 \\ 1498375 & 1988742 & 1498.907 & 1500.232 & 1499061\end{array}$ $\begin{array}{llllllll}1498.38 & 1498.742 & 1498.907 & 1500.232 & 1499.061\end{array}$ $\begin{array}{llllll}1498.38 & 1498.742 & 1498.911 & 1500.218 & 1499.075 \\ 1498.38 & 1498.74 & 1498.914 & 1500.208 & 1499.073\end{array}$ $\begin{array}{lllllll}1498.384 & 1498.74 & 1498.921 & 1500.218 & 1499.085\end{array}$ $\begin{array}{lllllll}1498.384 & 1498.74 & 1498.918 & 1500.206 & 1499.085\end{array}$ $\begin{array}{llllll}1498.398 & 1498.747 & 1498.93 & 1500.234 & 1499.096\end{array}$ $\begin{array}{llllll}1498.422 & 1498.763 & 1498.956 & 1500.276 & 1499.136\end{array}$ $\begin{array}{lllllll}1498.424 & 1498.761 & 1498.96 & 1500.264 & 1499.141 \\ & 1498.431 & 1498.761 & 1498.965 & 150.267 & 1499.146\end{array}$ $\begin{array}{llllll}1498.431 & 1498.761 & 1498.965 & 1500.267 & 1499.136 \\ 1498.44 & 1498.761 & 1498.972 & 1500.274 & 1499.148\end{array}$ $\begin{array}{lllllll}1498.44 & 1498.761 & 1488.972 & 1500.274 & 1499.148\end{array}$ $\begin{array}{lllllll}1498.447 & 1498.756 & 1498.981 & 1500.278 & 1499.143\end{array}$ $\begin{array}{llllll}1498.466 & 1498.765 & 1498.995 & 1500.304 & 1499.169\end{array}$ $\begin{array}{llllll}1498.475 & 1498.768 & 1499.005 & 1500.306 & 1499.18\end{array}$ $\begin{array}{lllll}1498.475 & 1498.765 & 1499.005 & 1500.292 & 1499.169 \\ 1498.500 & 1498.777 & 1499.028 & 1500.348 & 1499.206 \\ 1498501 & 1998.742 & 149.019 & 1500306 & 149.206\end{array}$ $\begin{array}{lllll}1498.501 & 1498.742 & 1499.019 & 1500.306 & 1499.206 \\ 1498.457 & 1498.709 & 1499.026 & 1500.313 & 1499.199\end{array}$ 

Well (ft AMSL) (ft AMSL) (ft AMSL) (ft AMSL) (ft AMSL) (ft AMSL) (ft AMSL) (ft AMSL) (ft AMSL) (ft AMSL) (ft AMSL) (ft AMSL) (ft AMSL) (ft AMSL) (ft AMSL) (ft AMSL) (ft AMSL) (ft AMSL) (ft AMSL) (ft AMSL) (ft AMSL) (ft AMSL) (ft AMSL) (ft AMSL) (ft AMSL) (ft AMS) $1498.3981498 .7231499 .061 \quad 1500.395 \quad 1499.248$ $\begin{array}{rrrrr}1498.51 & 1498.789 & 1499.058 & 1500.365 & 1499.26 \\ 1498515 & 1498737 & 1499082 & 1500.405 & 1499.278\end{array}$ $4 / 23 / 108: 00$ 4/23/10 12:00 $4 / 23 / 1020: 00$ $4 / 24 / 100: 00$ $4 / 24 / 104: 00$ $4 / 24 / 108: 00$
$4 / 24 / 1012: 00$ $4 / 24 / 1016: 00$ $4 / 24 / 1020: 00$ 4/25/100:00 4/25/10 4:00 4/25/10 8:00 4/25/10 12:00 4/25/10 16:00 $4 / 26 / 100 \cdot 00$ $4 / 26 / 104: 00$ $4 / 26 / 108: 00$ $4 / 26 / 1012: 00$ 4/26/10 16:00 4/26/10 20:00 4/27/100:00 $4 / 27 / 104.00$ $4 / 27 / 108.00$ 4/27/1012:00 $4 / 27 / 1020: 00$ $4 / 28 / 100: 00$ $4 / 28 / 104: 00$ $4 / 28 / 108: 00$ $4 / 28 / 1012: 00$ $4 / 28 / 1016.00$ $4 / 29 / 100: 00$ 4/29/10 4:00 $4 / 29 / 108: 00$ 4/29/10 12:00 4/29/10 16:00 $4 / 29 / 1020: 00$ $4 / 30 / 100: 00$ $4 / 30 / 104.00$ $4 / 30 / 1012: 00$ 4/30/10 16:00 4/30/10 20:00 $\begin{array}{lllll}1498.515 & 1498.737 & 1499.082 & 1500.405 & 1499.278 \\ 1498.531 & 1498.758 & 1499093 & 1500.414 & 1499.304\end{array}$ $\begin{array}{lllllll}1498.531 & 1498.742 & 1499.098 & 1500.409 & 1499.311\end{array}$ $\begin{array}{llllll}1498.51 & 1498.805 & 1499.107 & 1500.416 & 1499.318\end{array}$ $\begin{array}{llllll}1498.578 & 1498.826 & 1499.126 & 1500.444 & 1499.339\end{array}$ $\begin{array}{lllll}1498.578 & 1498.819 & 1499.121 & 1500.405 & 1499.33\end{array}$ $\begin{array}{llllll}1498.58 & 1498.821 & 1499.124 & 1500.405 & 1499.316\end{array}$ $\begin{array}{lllll}1498.58 & 1498.821 & 1499.117 & 1500.384 & 1499.316 \\ 149858 & 1498.824 & 1499117 & 1500379 & 1999313\end{array}$ $\begin{array}{rrrrrr}1498.58 & 1498.824 & 1499.117 & 1500.379 & 1499.313 \\ 1498.578 & 1498.824 & 1499.114 & 1500.37 & 1499.297\end{array}$ $\begin{array}{llllll}1498.55 & 1498765 & 1499.114 & 1500.369 & 1499.297\end{array}$ $\begin{array}{llllll}1498.592 & 1498.793 & 1499.086 & 1500.29 & 1499.246\end{array}$ $\begin{array}{llllll}1498.538 & 1498.777 & 1499.061 & 1500.246 & 1499.197\end{array}$ $\begin{array}{llllll}1498.517 & 1498.768 & 1499.044 & 1500.225 & 1499.169\end{array}$ $\begin{array}{llllll}1498.494 & 1498.758 & 1499.023 & 1500.192 & 1499.143\end{array}$ $\begin{array}{rrrrr}1498.485 & 1498.763 & 1499.019 & 1500.204 & 1499.131 \\ 1488.48 & 149877 & 1499016 & 1500.21 & 149.145\end{array}$ $\begin{array}{llllll}1498.48 & 1498.77 & 1499.016 & 1500.21 & 1499.145\end{array}$ $\begin{array}{lllllll}1498.452 & 1498.754 & 1498.986 & 1500.143 & 1499.115\end{array}$ $\begin{array}{rrrrr}1498.429 & 1498.74 & 1498.965 & 1500.117 & 1499.075 \\ 1498.401 & 1498.726 & 1498.946 & 1500.082 & 1499.043\end{array}$ $\begin{array}{llllll}1498.38 & 1498.719 & 1498.925 & 1500.068 & 1499.015\end{array}$ $\begin{array}{llllll}1498.384 & 1498.728 & 1498.935 & 1500.115 & 1499.031\end{array}$ $\begin{array}{llllll}1498.408 & 1498.747 & 1498.953 & 1500.18 & 1499.085\end{array}$ $\begin{array}{llllll}1498.391 & 1498.737 & 1498.939 & 1500.126 & 1499.087\end{array}$ $\begin{array}{llllll}1498.38 & 1498.73 & 1498.928 & 1500.105 & 1499.064 \\ 1498375 & 1498.728 & 1498.923 & 1500.098 & 1490.059\end{array}$ $\begin{array}{llllll}1498.375 & 1498.728 & 1498.923 & 1500.098 & 1499.059 \\ 1498.342 & 1498.709 & 1498.895 & 1500.038 & 1499.01\end{array}$ $\begin{array}{rrrrrr}1498.342 & 1498.709 & 1488.895 & 1500.038 & 1499.01 \\ 1498.335 & 1498709 & 1498.89 & 1500.059 & 1498.989\end{array}$ $\begin{array}{llllll}1498.361 & 1498.728 & 1498.914 & 1500.129 & 1499.036\end{array}$ $\begin{array}{lllllll}1498.373 & 1498.735 & 1498.918 & 1500.143 & 1499.068\end{array}$ $\begin{array}{lllllll}1498.384 & 1498.744 & 1498.93 & 1500.173 & 1499.087\end{array}$ $\begin{array}{llllll}1498.405 & 1498.754 & 1498.951 & 1500.206 & 1499.124\end{array}$ $\begin{array}{llllll}1498.433 & 1498.775 & 1498.972 & 1500.253 & 1499.169\end{array}$ $\begin{array}{llllll}1498.473 & 1498.805 & 1499.009 & 1500.313 & 1499.232 \\ 1498.515 & 1498.824 & 1499037 & 1500367 & 1499.295\end{array}$ $\begin{array}{llllllll}1498.515 & 1498.824 & 149.0037 & 1500.367 & 1499.295 \\ 1498545 & 1498.838 & 1490.065 & 1500391 & 1499.332\end{array}$ $\begin{array}{lllll}1498.545 & 1498.838 & 1499.065 & 1500.391 & 1499.332 \\ 1498.578 & 1498.859 & 1499.096 & 1500.435 & 1499.358\end{array}$ $\begin{array}{llllll}1498.597 & 1498.861 & 1499.107 & 1500.43 & 1499.383\end{array}$ $\begin{array}{llllll}1498.594 & 1498.861 & 1499.1 & 1500.4 & 1499.365\end{array}$ $\begin{array}{llllll}1498.604 & 1498.866 & 1499.117 & 1500.412 & 1499.351\end{array}$ $\begin{array}{llllll}1498.629 & 1498.87 & 1499.135 & 1500.449 & 1499.377\end{array}$ $\begin{array}{lllll}1498.648 & 1498.875 & 1499.147 & 1500.454 & 1499.395 \\ 1498634 & 1498.817 & 1499.107 & 150.327 & 149934\end{array}$ $\begin{array}{llllll}1498.634 & 1498.817 & 1499.107 & 1500.327 & 1499.344 \\ 1498.632 & 1498824 & 1499082 & 1500.503 & 1499.262\end{array}$ $\begin{array}{lllllll}1498.632 & 4988.824 & 1499.082 & 1500.503 & 1499.262\end{array}$ $\begin{array}{llllll}1498.536 & 1498.814 & 1499.075 & 1500.204 & 1499.225 \\ 1499239\end{array}$ $\begin{array}{lllllll}1498.573 & 1498.81 & 1499.077 & 1500.248 & 1499.248\end{array}$ $\begin{array}{lllllll}1498.531 & 1498.779 & 1499.037 & 1500.211 & 1499.185\end{array}$ $\begin{array}{llllll}1498.51 & 1498.768 & 1499.019 & 1500.187 & 1499.148 \\ 1498.489 & 1498756 & 1499.007 & 1500.171 & 1499.127\end{array}$ $\begin{array}{rrrrrr}1498.489 & 1498.756 & 1499.007 & 1500.171 & 1499.127 \\ 1498.468 & 1498.742 & 1498.988 & 1500.14 & 1499.103\end{array}$ $\begin{array}{lllll}14988.468 & 1498.742 & 1498.988 & 1500.14 & 1499.103 \\ 14998.749 & 1498.998 & 1500.157 & 1499.115\end{array}$ 

Well (ft AMSL) (ft AMSL) (ft AMSL) (ft AMSL) (ft AMSL) (ft AMSL) (ft AMSL) (ft AMSL) (ft AMSL) (ft AMSL) (ft AMSL) (ft AMSL) (ft AMSL) (ft AMSL) (ft AMSL) (ft AMSL) (ft AMSL) (ft AMSL) (ft AMSL) (ft AMSL) (ft AMSL) (ft AMSL) (ft AMSL) (ft AMSL) (ft AMSL) (ft AMSL)

5/1/10 16:00

$\begin{array}{lllll}1498.489 & 1498.758 & 1499.014 & 1500.236 & 1499.162\end{array}$

$5 / 2 / 100: 00$

$\begin{array}{rrrrr}1498.489 & 1498.754 & 1499.012 & 1500.225 & 1499.173 \\ 1498.485 & 1498.749 & 1499.009 & 1500.22 & 1499.176\end{array}$

$5 / 2 / 104: 00$
$5 / 2 / 108: 00$

$5 / 2 / 1012: 00$

$5 / 2 / 1016: 00$

5/2/10 20:00

5/3/100:00

$5 / 3 / 104: 00$

$5 / 3 / 108: 00$

$5 / 3 / 1012: 00$

$5 / 3 / 1020: 00$

5/3/10 20:00

5/4/10 4:00

5/4/10 8:00

5/4/10 12:00

$5 / 4 / 1016: 00$

$5 / 4 / 1020: 00$

5/5/10 0:00

5/5/10 8:00

5/5/10 8:00

5/5/10 16:00

5/5/10 20:00

5/6/100:00

5/6/10 4:00

$5 / 6 / 108.00$

$5 / 6 / 1016: 00$

5/6/10 20:00

5/7/100:00

5/7/10 4:00

5/7/10 8:00

5/7/10 12:00

$5 / 7 / 1016: 00$

5/8/100:00

5/8/10 4:00

5/8/10 8:00

5/8/10 12:00

5/8/10 16:00

$5 / 8 / 1020: 00$

5/9/100:00

$5 / 9 / 108000$

5/9/10 8:00

5/9/10 16:00

5/9/10 20:00

5/10/10 0:00

5/10/10 4:00

$5 / 10 / 108: 00$

$\begin{array}{lllll}1498.485 & 1498.749 & 1499.009 & 1500.22 & 1499.176\end{array}$

$\begin{array}{llllll}1498.473 & 1498.735 & 1499 & 1500.189 & 1499.166\end{array}$

$\begin{array}{llllll}1498.468 & 1498.737 & 1498.998 & 1500.183 & 1499.157\end{array}$

$\begin{array}{llllll}1498.468 & 1498.737 & 1498.998 & 1500.199 & 1499.162\end{array}$

$\begin{array}{rrrrrr}1498.445 & 1498.723 & 1498.977 & 1500.147 & 1499.124 \\ 1498.417 & 1498.7 & 1498.932 & 1500.22 & 1499.031 \\ 149829 & 1498.79 & & \end{array}$ $\begin{array}{rrrrr}1498.417 & 1498.7 & 1498.932 & 1500.22 & 1499.031 \\ 1498.429 & 1498.709 & 1498.93 & 1500.374 & 1499.019\end{array}$ $\begin{array}{rrrrr}1498.429 & 1498.709 & 1498.93 & 1500.374 & 1499.019 \\ 1498.377 & 1498.686 & 1498.9 & 1500.346 & 1498.907\end{array}$ $\begin{array}{rrrrr}1498.331 & 1498.682 & 1498.876 & 1499.928 & 1498.728 \\ 1498.34 & 1498.682 & 1498.876 & 1500.059 & 1498.737\end{array}$ $\begin{array}{llllll}1498.333 & 1498.675 & 1498.867 & 1500.068 & 1498.779\end{array}$ $\begin{array}{llllll}1498.333 & 1498.675 & 1498.869 & 1500.084 & 1498.83\end{array}$ $\begin{array}{llllll}1498.345 & 1498.679 & 1498.879 & 1500.119 & 1498.903\end{array}$ $\begin{array}{llllll}1498.352 & 1498.684 & 1498.89 & 1500.133 & 1498.956\end{array}$ $\begin{array}{llllll}1498.375 & 1498.693 & 1498.909 & 1500.176 & 1499.019\end{array}$ $\begin{array}{llllllll}1498.405 & 1498.712 & 1498.937 & 1500.243 & 1499.087 \\ 1498.41 & 1498.712 & 1498.939 & 1500.225 & 1499.113\end{array}$ $\begin{array}{lllllll}1498.41 & 1498.712 & 1498.939 & 1500.225 & 1499.113 \\ 1498375 & 1498684 & 1498909 & 1500.131 & 1499052\end{array}$ $\begin{array}{llllll}1498.342 & 1498.663 & 1498.879 & 1500.07 & 1498.989\end{array}$ $\begin{array}{llllll}1498.303 & 1498.654 & 1498.848 & 1500.012 & 1498.863\end{array}$ $\begin{array}{lllllll}1498.286 & 1498.644 & 1498.83 & 1500.012 & 1498.681\end{array}$ $\begin{array}{llllll}1498.284 & 1498.64 & 1498.823 & 1500.04 & 1498.678\end{array}$ $\begin{array}{llllll}1498.275 & 1498.633 & 1498.811 & 1500.035 & 1498.716\end{array}$ $\begin{array}{lllll}1498.263 & 1498.63 & 1498.809 & 1500.033 & 1498.751 \\ 1498279 & 1498637 & 1988.823 & 1500087 & 1488.823\end{array}$ $\begin{array}{llllll}1498.279 & 1498.637 & 1498.823 & 1500.087 & 1498.823 \\ 1498286 & 1498635 & 1498832 & 1500.105 & 1498877\end{array}$ $\begin{array}{llllll}1498.286 & 1498.635 & 148.832 & 1500.105 & 1498.877 \\ 1498.324 & 1498.656 & 1498.869 & 1500194 & 1498963\end{array}$ $\begin{array}{llllll}1498.368 & 1498.677 & 1498.904 & 1500.262 & 1499.061\end{array}$ $\begin{array}{llllll}1498.389 & 1498.684 & 1498.916 & 1500.257 & 1499.096\end{array}$ $\begin{array}{llllll}1498.415 & 1498.675 & 1498.914 & 1500.232 & 1499.103\end{array}$ $\begin{array}{llllll}1498.347 & 1498.64 & 1498.867 & 1500.08 & 1498.994\end{array}$ $\begin{array}{lllll}1498.266 & 1498.612 & 1498.804 & 1499.963 & 1498.804\end{array}$ $\begin{array}{llllll}1498.221 & 1498.595 & 1498.764 & 1499.935 & 1498.564 \\ 14982 & 1498584 & 1498.751 & 1499.935 & 1998527\end{array}$ $\begin{array}{llllll}1498.2 & 1498.584 & 1488.751 & 1499.935 & 1498.527\end{array}$ $\begin{array}{rrrrrr}1498.457 & 1498.549 & 1498.697 & 1499.892 & 1498.534 \\ 1498527\end{array}$ $\begin{array}{lllllll}1498.496 & 1498.54 & 1498.688 & 1499.876 & 1498.566\end{array}$ $\begin{array}{lllllll}1498.473 & 1498.526 & 1498.667 & 1499.836 & 1498.569\end{array}$ $\begin{array}{llllll}1498.482 & 1498.526 & 1498.674 & 1499.886 & 1498.611\end{array}$ $\begin{array}{lllllll}1498.501 & 1498.537 & 1498.699 & 1499.981 & 1498.667\end{array}$ $\begin{array}{llllll}1498.473 & 1498.533 & 1498.695 & 1499.967 & 1498.641 \\ 1498.64 & 1495523 & 1498685 & 149996 & 1498606\end{array}$ $\begin{array}{llllll}1498.464 & 1498.523 & 1498.685 & 1499.967 & 1498.606 \\ 1498.459 & 1498521 & 1498692 & 1499991 & 1498651\end{array}$ $\begin{array}{lllllll}1498.44 & 1498514 & 1498.681 & 1499.97 & 1498.62\end{array}$ $\begin{array}{llllll}1498.459 & 1498.533 & 1498.704 & 1500.01 & 1498.552\end{array}$ $\begin{array}{llllll}1498.461 & 1498.544 & 1498.72 & 1500.101 & 1498.583\end{array}$ $\begin{array}{lllll}1498.438 & 1498.542 & 1498.713 & 1500.08 & 1498.613\end{array}$ $\begin{array}{rrrrrr}1498.45 & 1498.544 & 1498.723 & 1500.115 & 1498.681 \\ 1498.461 & 1498.565 & 1498.757 & 1500.175 & 1498.791\end{array}$ $\begin{array}{rrrrrr}1498.461 & 1498.565 & 1498.757 & 1500.175 & 1498.791 \\ 1498.44 & 1498.584 & 1498.788 & 1500.166 & 1498.835\end{array}$ $\begin{array}{rrrrrr}1498.44 & 1498.584 & 1498.788 & 1500.166 & 1498.835 \\ 1498.349 & 1498.612 & 1498.82 & 1500.176 & 1498.809\end{array}$ 

Well (ft AMSL) (ft AMSL) (ft AMSL) (ft AMSL) (ft AMSL) (ft AMSL) (ft AMSL) (ft AMSL) (ft AMSL) (ft AMSL) (ft AMSL) (ft AMSL) (ft AMSL) (ft AMSL) (ft AMSL) (ft AMSL) (ft AMSL) (ft AMSL) (ft AMSL) (ft AMSL) (ft AMSL) (ft AMSL) (ft AMSL) (ft AMSL) (ft AMSL) (ft AMS) $\begin{array}{llllll}1498.233 & 1498.616 & 1498.841 & 1500.348 & 1498.893\end{array}$

$\begin{array}{llllll}1498.382 & 1498.537 & 1498.734 & 1500.033 & 1498.765\end{array}$ $\begin{array}{llllll}1498.342 & 1498.535 & 1498.739 & 1500.068 & 1498.779\end{array}$ $\begin{array}{lllllll}1498.405 & 1498.544 & 1498.762 & 1500.129 & 1498.851\end{array}$ $\begin{array}{llllll}1498.398 & 1498.542 & 1498.767 & 1500.126 & 1498.893\end{array}$ $\begin{array}{llllll}1498.405 & 1498.544 & 1498.783 & 1500.159 & 1498.926\end{array}$ $\begin{array}{lllll}1498.417 & 1498.553 & 1498.806 & 1500.199 & 1498.973 \\ 1498391 & 1498544 & 1498.799 & 1500.159 & 1498917\end{array}$ $\begin{array}{rrrrrr}4498.391 & 1498.544 & 1498.799 & 1500.159 & 1498.917 \\ 1498.398 & 1498546 & 1498.804 & 1500.19 & 1498.784\end{array}$ $\begin{array}{llrrrr}1498.398 & 1498.546 & 1498.804 & 1500.19 & 1498.784 \\ 1498.592 & 1498.693 & 1508.5 & 1500.143 & 1498.751\end{array}$ $\begin{array}{lllllll}1498.709 & 1498.863 & 1525.167 & 1500.409 & 1498.732\end{array}$ $\begin{array}{llllll}1498.75 & 1498.742 & 1525.226 & 1500.32 & 1498.777\end{array}$ $\begin{array}{llllll}1498.785 & 1498.756 & 1525.233 & 1500.379 & 1498.767\end{array}$ $\begin{array}{lllllll}1498.839 & 1498.733 & 1525.233 & 1500.386 & 1498.749\end{array}$ $\begin{array}{lllllll}1498.438 & 1498.411 & 1525.251 & 1500.009 & 1498.742\end{array}$ $\begin{array}{rrrrr}1498.389 & 1498.33 & 1525.195 & 1499.935 & 1498.776 \\ 1498352 & 1498.519 & 152426 & 1499.946 & 1498774\end{array}$ $\begin{array}{llllll}1488.352 & 1498.539 & 1524.26 & 1499.946 & 1498.774\end{array}$ $\begin{array}{llllll}1498.331 & 1498.621 & 1520.637 & 1499.923 & 1498.77 \\ 1498.623 & 1517.772 & 1499.928 & 1498.786\end{array}$ $\begin{array}{lllllll}1498.289 & 1498.595 & 1515.42 & 1499.886 & 1498.779\end{array}$ $\begin{array}{llllll}1498.282 & 1498.316 & 1513.464 & 1499.888 & 1498.788\end{array}$ $\begin{array}{llllll}1498.293 & 1498.195 & 1511.839 & 1499.97 & 1498.847\end{array}$ $\begin{array}{llllll}1498.272 & 1498.372 & 1510.438 & 1499.96 & 1498.874\end{array}$ $\begin{array}{rrrrr}1498.277 & 1498.449 & 1509.235 & 1499.989 & 1498.898 \\ 149828 & 1489.465 & 1508188 & 1500.012 & 1498931\end{array}$ $\begin{array}{llllll}1498.28 & 1498.465 & 1508.188 & 1500.012 & 1498.931 \\ 1498.259 & 1498.484 & 1507.262 & 1499.998 & 1498.938\end{array}$ $\begin{array}{llllll}1498.259 & 1498.484 & 1507.262 & 1499.998 & 1498.938 \\ 1498.203 & 1498.418 & 1506.453 & 1499918 & 1498.949\end{array}$ $\begin{array}{llllll}1498.217 & 1498.29 & 1505.754 & 1499.876 & 1498.991\end{array}$ $\begin{array}{llllll}1498.277 & 1498.393 & 1505.113 & 1499.949 & 1498.996\end{array}$ $\begin{array}{llllll}1498.31 & 1498.432 & 1504.546 & 1500.005 & 1498.994\end{array}$ $\begin{array}{llllll}1498.312 & 1498.43 & 1504.043 & 1500.028 & 1499.01\end{array}$ $\begin{array}{lllllll}1498.312 & 1498.451 & 1503.593 & 1500.035 & 1499.017\end{array}$ $\begin{array}{rrrrr}1498.149 & 1498.258 & 1503.187 & 1499.708 & 1499.026 \\ 1498265 & 149822 & 1502.83 & 1499.685 & 149905\end{array}$ $\begin{array}{llllll}1498.265 & 1498.22 & 1502.83 & 1499.685 & 1499.05 \\ 1498.307 & 1498.356 & 1502.495 & 1499846 & 1499.054\end{array}$ $\begin{array}{lllll}1498.307 & 1498.356 & 1502.495 & 1499.846 & 1499.054 \\ 1498.298 & 1498.495 & 1503.047 & 1499.979 & 1499.029\end{array}$ $\begin{array}{llllll}1498.303 & 1498.553 & 1503.0487 & 1499.979 & 1499.029 \\ 1500.105 & 1499.015\end{array}$ $\begin{array}{lllllll}1498.249 & 1498.533 & 1504.026 & 1500.054 & 1498.998\end{array}$ $\begin{array}{llllll}1498.254 & 1498.395 & 1503.845 & 1499.881 & 1498.987\end{array}$ $\begin{array}{lllllll}1498.265 & 1498.311 & 1503.567 & 1499.991 & 1499.005\end{array}$ $\begin{array}{lllll}1498.268 & 1498.367 & 1503.271 & 1500.096 & 1499.036 \\ 1498.252 & 1498.53 & 1502.945 & 1500023 & 1499.05\end{array}$ $\begin{array}{rrrrrr}1498.252 & 1498.53 & 1502.945 & 1500.023 & 1499.005 \\ 1498.273 & 1498.509 & 1502.667 & 1500049 & 1499026\end{array}$ $\begin{array}{lllll}1498.259 & 1498.500 & 152.667 & 1500.023 & 1499.026\end{array}$ $\begin{array}{rrrrr}1498.259 & 1498.493 & 1502.359 & 1499.979 & 1499.022 \\ 1498.28 & 1498.435 & 1502.101 & 1500.031 & 1499.05\end{array}$ $\begin{array}{llllll}1498.296 & 1498.453 & 1501.872 & 1500.115 & 1499.101\end{array}$ $\begin{array}{llllll}1498.305 & 1498.463 & 1501.648 & 1500.143 & 1499.136\end{array}$ $\begin{array}{rrrrr}1498.3 & 1498.465 & 1501.434 & 1500.108 & 1499.124 \\ 1498.317 & 1498.446 & 1501.266 & 1500.19 & 1499.169\end{array}$ 5/19/10 12:00 

Well (ft AMSL) (ft AMSL) (ft AMSL) (ft AMSL) (ft AMSL) (ft AMSL) (ft AMSL) (ft AMSL) (ft AMSL) (ft AMSL) (ft AMSL) (ft AMSL) (ft AMSL) (ft AMSL) (ft AMSL) (ft AMSL) (ft AMSL) (ft AMSL) (ft AMSL) (ft AMSL) (ft AMSL) (ft AMSL) (ft AMSL) (ft AMSL) (ft AMSL) (ft AMS)

$5 / 19 / 1016,00$ $1498.331 \quad 1498.4841500 .804 \quad 1500.11 \quad 1499.232$

$\begin{array}{llllll}1498.403 & 1498.488 & 1500.306 & 1500.229 & 1499.2218\end{array}$ $\begin{array}{llllll}1498.38 & 1498.477 & 1500.196 & 1500.176 & 1499.197\end{array}$ $\begin{array}{lllllll}1498.363 & 1498.495 & 1500.107 & 1500.124 & 1499.185\end{array}$ $\begin{array}{rrrrr}1498.382 & 1498.502 & 1500.016 & 1500.208 & 1499.166 \\ 1498.389 & 1498.498 & 1499.93 & 1500.309 & 1499.141\end{array}$ $\begin{array}{rrrrr}1498.389 & 1498.498 & 1499.93 & 1500.309 & 1499.141 \\ 1498.417 & 1498.493 & 1499.842 & 1500.43 & 1499.129\end{array}$ $\begin{array}{lllll}1498.478 & 1498.481 & 1499.753 & 1500.32 & 1499.099\end{array}$ $\begin{array}{lllll}1498.224 & 1498.486 & 1499.699 & 1499.853 & 1499.103 \\ 1498.347 & 1498.505 & 1499.664 & 1500.033 & 1499.159\end{array}$ $\begin{array}{llllll}1498.352 & 1498.514 & 1499.618 & 1500.15 & 1499.197\end{array}$ $\begin{array}{lllllll}1498.368 & 1498.526 & 1499.578 & 1500.154 & 1499.227\end{array}$ $\begin{array}{lllllll}1498.375 & 1498.537 & 1499.546 & 1500.173 & 1499.262\end{array}$ $\begin{array}{llllll}1498.382 & 1498.544 & 1499.506 & 1500.164 & 1499.267\end{array}$ $\begin{array}{llllll}1498.401 & 1498.558 & 1499.476 & 1500.192 & 1499.281\end{array}$ $\begin{array}{llllll}1498.412 & 1498.568 & 1499.45 & 1500.232 & 1499.304 \\ 1498.403 & 1498.563 & 1499.41 & 1500201 & 1499.297\end{array}$ $\begin{array}{llllll}1498.403 & 1498.563 & 1499.41 & 1500.232 & 1499.297\end{array}$ $\begin{array}{llllll}1498.403 & 1498.563 & 1499.371 & 1500.168 & 1499.264 \\ 1498.412 & 1498.567 & 1499.343 & 1500.183 & 1499.262\end{array}$ $\begin{array}{rrrrr}1498.389 & 1498.556 & 1499.291 & 1500.11 & 1499.222 \\ 1498.391 & 1498.549 & 1499.259 & 1500.096 & 1499.19\end{array}$ $\begin{array}{llllll}1498.398 & 1498.551 & 1499.231 & 1500.11 & 1499.187\end{array}$ $\begin{array}{llllll}1498.396 & 1498.551 & 1499.205 & 1500.105 & 1499.183\end{array}$ $\begin{array}{lllllll}1498.394 & 1498.542 & 1499.175 & 1500.084 & 1499.164 \\ 1498398 & 1498542 & 1499154 & 1500087 & 1499164\end{array}$ $\begin{array}{llllll}1498.398 & 1498.542 & 1499.154 & 1500.087 & 1499.164 \\ 1498.391 & 1498.535 & 1499.128 & 1500.068 & 1499.155\end{array}$ $\begin{array}{llllll}1498.391 & 1498.535 & 1499.128 & 1500.068 & 1499.155 \\ 1498398 & 1498.542 & 1499112 & 1500.089 & 1499.152\end{array}$ $\begin{array}{lllll}1498.405 & 1498.546 & 1499.1 & 1500.105 & 1499.176\end{array}$ $\begin{array}{llllll}1498.401 & 1498.542 & 1499.079 & 1500.091 & 1499.173\end{array}$ $\begin{array}{lllllll}1498.438 & 1498.5 & 1499.047 & 1500.208 & 1499.124\end{array}$ $\begin{array}{llllll}1498.482 & 1498.477 & 1500.858 & 1500.341 & 1499.117\end{array}$ $\begin{array}{llllll}1498.487 & 1498.467 & 1519.031 & 1500.377 & 1499.106\end{array}$ $\begin{array}{rrrrr}1498.27 & 1498.477 & 1522.761 & 1500 & 1499.099 \\ 1498.326 & 1498.498 & 1525.146 & 1499.841 & 1499\end{array}$ $\begin{array}{llllll}1498.326 & 1498.498 & 1525.146 & 1499.841 & 1499.127 \\ 1498.41 & 1498.526 & 1525.16 & 1500.103 & 1499.155\end{array}$ $\begin{array}{rrrrr}1498.41 & 1498.526 & 1525.16 & 1500.103 & 1499.155 \\ 1498.384 & 1498.63 & 1525.006 & 1500.087 & 1499.124\end{array}$ $\begin{array}{llllll}1498.377 & 1498.519 & 1523.367 & 1500.063 & 1499.085\end{array}$ $\begin{array}{llllll}1498.377 & 1498.519 & 1523.321 & 1499.977 & 1499.11\end{array}$ $\begin{array}{llllll}1498.231 & 1498.304 & 1521.95 & 1499.612 & 1499.103\end{array}$ $\begin{array}{llllll}1498.328 & 1498.507 & 1519.376 & 1499.699 & 1499.152\end{array}$ $\begin{array}{lllll}1498.363 & 1498.509 & 1517.08 & 1500.105 & 1499.171 \\ 1498363 & 1498502 & 1515.133 & 1500.047 & 1499.159\end{array}$ $\begin{array}{llllll}1498.363 & 1498.502 & 1515.133 & 1500.047 & 1499.159 \\ 1498.366 & 1498.505 & 1513.464 & 1500.031 & 1499.169\end{array}$ $\begin{array}{lllllll}1498.366 & 1498.500 & 1513.464 & 1500.031 & 1499.169\end{array}$ $\begin{array}{rrrrr}1498.361 & 1498.502 & 1512.009 & 1499.974 & 1499.164 \\ 1498.375 & 1498.512 & 1510.755 & 1499.993 & 1499.18\end{array}$ $\begin{array}{llllll}1498.387 & 1498.523 & 1509.666 & 1500.068 & 1499.22\end{array}$ $\begin{array}{llllll}1498.391 & 1498.528 & 1508.696 & 1500.07 & 1499.248\end{array}$ $\begin{array}{llllll}1498.389 & 1498.526 & 1507.817 & 1500.049 & 1499.239\end{array}$ $\begin{array}{llllll}1498.396 & 1498.53 & 1507.043 & 1500.056 & 1499.241\end{array}$ $\begin{array}{lllll}1498.396 & 1498.533 & 1506.346 & 1500.049 & 1499.243 \\ 1498.415 & 1498.542 & 1505.735 & 1500.077 & 1499.262\end{array}$ 

Well

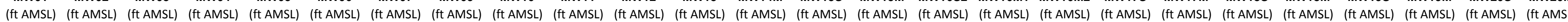

$5 / 28 / 1016,00$ $\begin{array}{lrrrr}1498.433 & 1498.56 & 1505.199 & 1500.136 & 1499.311\end{array}$ $\begin{array}{lllll}1498.438 & 1498.567 & 1504.712 & 1500.15 & 1499.344 \\ 1498.433 & 1498.567 & 1504.248 & 1500.122 & 1499332\end{array}$ $\begin{array}{lllll}1498.433 & 1498.567 & 1504.248 & 1500.122 & 1499.332 \\ 1498.447 & 1498.574 & 1503.845 & 1500.143 & 1499.346\end{array}$ $\begin{array}{lllllll}1498.447 & 1498.574 & 1503.462 & 1500.126 & 1499.339\end{array}$ $\begin{array}{lllllll}1498.473 & 1498.584 & 1503.133 & 1500.164 & 1499.358\end{array}$ $\begin{array}{lllllll}1498.482 & 1498.595 & 1502.844 & 1500.201 & 1499.391\end{array}$ $\begin{array}{llllll}1498.473 & 1498.598 & 1502.562 & 1500.187 & 1499.405\end{array}$ $\begin{array}{lllllll}1498.471 & 1498.598 & 1502.292 & 1500.164 & 1499.384\end{array}$ $\begin{array}{lllll}1498.485 & 1498.605 & 1502.287 & 1500.187 & 1499.393 \\ 149.454 & 1488595 & 1502.408 & 1500117 & 1499353\end{array}$ $\begin{array}{llllll}1498.454 & 1498.595 & 1502.408 & 1500.117 & 1499.353 \\ 1498.468 & 1498598 & 1502.593 & 1500.131 & 1499337\end{array}$ $\begin{array}{rrrrrr}1498.478 & 1498.6 & 1502.879 & 1500.14 & 1499.332\end{array}$ $\begin{array}{llllll}1498.48 & 1498.614 & 1503.113 & 1500.166 & 1499.297\end{array}$ $\begin{array}{llllll}1498.527 & 1498.7 & 1503.418 & 1500.318 & 1499.267\end{array}$ $\begin{array}{llllll}1498.51 & 1498.719 & 1503.479 & 1500.412 & 1499.269\end{array}$ $\begin{array}{llllll}1498.473 & 1498.686 & 1503.257 & 1500.346 & 1499.257\end{array}$ $\begin{array}{llllll}1498.349 & 1498.383 & 1503.01 & 1499.897 & 1499.276\end{array}$ $\begin{array}{llllll}1498.464 & 1498.416 & 1502.779 & 1500.094 & 1499.325 \\ 1498464 & 149856 & 1502.541 & 1500.18 & 1499351\end{array}$ $\begin{array}{llllll}1498.464 & 1498.56 & 1502.541 & 1500.18 & 1499.351 \\ 1498.471 & 1498.677 & 1502.297 & 1500136 & 1499.342\end{array}$ $\begin{array}{llllll}1498.478 & 1498.689 & 1502.075 & 1500.159 & 1499.363\end{array}$ $\begin{array}{llllll}1498.48 & 1498.665 & 1501.87 & 1500.138 & 1499.369\end{array}$ $\begin{array}{rrrrrr}1498.508 & 1498.423 & 1501.693 & 1500.18 & 1499.402\end{array}$ $\begin{array}{lllllll}1498.534 & 1498.46 & 1501.536 & 1500.257 & 1499.458\end{array}$ $\begin{array}{lllll}1498.538 & 1498.558 & 1501.383 & 1500.283 & 1499.44\end{array}$ $\begin{array}{rrrrr}1498.536 & 1498.719 & 1501.222 & 1500.255 & 1499.253 \\ 1498.52 & 1498.807 & 1501.056 & 1500.206 & 1499\end{array}$ $\begin{array}{lllll}1498.52 & 1498.807 & 1501.056 & 1500.206 & 1499.194 \\ 1498.51 & 1498.805 & 1500.902 & 1500.161 & 1499115\end{array}$ $\begin{array}{rrrrr}1498.51 & 1498.805 & 1500.902 & 1500.161 & 1499.115 \\ 1498.503 & 1498.626 & 1500.753 & 1500.143 & 1498.97\end{array}$ $\begin{array}{rrrrr}1498.508 & 1498.626 & 1500.627 & 1500.157 & 1498.989\end{array}$ $6 / 2 / 1012: 00$ $6 / 2 / 1020: 00$ 6/3/10 0:00 6/3/10 4:00 $6 / 3 / 108: 00$

$\begin{array}{llllll}1498.494 & 1498.614 & 1500.501 & 1500.124 & 1499.036\end{array}$ $\begin{array}{llllll}1498.499 & 1498.609 & 1500.392 & 1500.129 & 1499.075\end{array}$ $\begin{array}{llllll}1498.492 & 1498.602 & 1500.285 & 1500.117 & 1499.117\end{array}$ $\begin{array}{llllll}1498.48 & 1498.593 & 1500.194 & 1500.101 & 1499.134\end{array}$ $\begin{array}{lllll}1498.501 & 1498.602 & 1500.133 & 1500.145 & 1499.183 \\ 1498515 & 1498616 & 1500.075 & 1500.199 & 1499.246\end{array}$ $\begin{array}{llllll}1488.515 & 1498.616 & 1500.075 & 1500.199 & 1499.246\end{array}$ $\begin{array}{llllll}1498.503 & 1498.609 & 1499.935 & 1500.171 & 1499.239\end{array}$ $\begin{array}{llllll}1498.503 & 1498.609 & 1499.87 & 1500.164 & 1499.234\end{array}$ $\begin{array}{llllll}1498.499 & 1498.602 & 1499.8 & 1500.15 & 1499.155\end{array}$ $\begin{array}{llllll}1498.508 & 1498.605 & 1499.746 & 1500.173 & 1499.024\end{array}$ $\begin{array}{llllll}1498.515 & 1498.609 & 1499.702 & 1500.201 & 1499.05\end{array}$ $\begin{array}{rrrrr}1498.51 & 1498.609 & 1499.657 & 1500.192 & 1499.087\end{array}$ $\begin{array}{llllll}1498.506 & 1498.602 & 1499.608 & 1500.18 & 1499.066 \\ 1498.501 & 1498.602 & 1499.564 & 1500.187 & 1499.113\end{array}$ $\begin{array}{llllll}1488.501 & 1498.602 & 1499.564 & 1500.187 & 1499.113\end{array}$ $\begin{array}{llllll}1498.489 & 1498.588 & 1499.473 & 1500.157 & 1499.15\end{array}$ $\begin{array}{llllll}1498.494 & 1498.586 & 1499.434 & 1500.161 & 1499.143\end{array}$ $\begin{array}{llllll}1498.461 & 1498.565 & 1499.373 & 1500.091 & 1499.043\end{array}$ $\begin{array}{llllll}1498.443 & 1498.54 & 1499.312 & 1500.031 & 1498.926 \\ 1498.422 & 1498.519 & 1499.259 & 1499.991 & 1498.891\end{array}$ $\begin{array}{rrrrr}1498.422 & 1498.519 & 1499.259 & 1499.991 & 1498.891 \\ 1498.403 & 1498.5 & 1499.205 & 1499.96 & 1498.823\end{array}$ $\begin{array}{rrrrr}1498.403 & 1498.5 & 1499.205 & 1499.96 & 1498.823 \\ 1498.401 & 1498.493 & 1499.165 & 1499.972 & 1498.7\end{array}$ 

Well

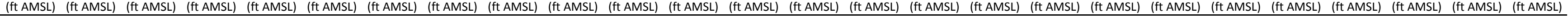

$6 / 6 / 1016: 00$

$\begin{array}{llllll}1498.412 & 1498.495 & 1499.142 & 1500.033 & 1498.702\end{array}$

6/7/100:00

$\begin{array}{rrrrr}1498.391 & 1498.484 & 1499.107 & 1500.024 & 1498.711 \\ 1498.375 & 1498.474 & 1499.07 & 1499.998 & 1498.718\end{array}$

6/7/10 8:00

6/7/10 12:00

6/7/10 16:00

6/7/10 20:00

6/8/100:00

6/8/10 4:00

6/8/10 8:00

6/8/10 12:00

$6 / 8 / 1020.00$

6/9/10 0:00

6/9/10 4:00

6/9/10 8:00

6/9/10 12:00

6/9/10 16:00

/

6/10/10 4:00

6/10/10 8:00

6/10/10 12:00

6/10/10 16:00

6/10/10 20:00

6/11/10 0:00

6/11/10 4:00

$6 / 11 / 1012.00$

6/11/10 16:00

6/11/10 20:00

6/12/100:00

6/12/104:00

6/12/10 8:00

6/12/10 12:00

6/12/10 16:00

$6 / 13 / 100: 00$

6/13/10 4:00

6/13/10 8:00

6/13/10 12:00

6/13/10 16:00

6/13/10 20:00

$6 / 14 / 100.00$

$6 / 14 / 104.00$

6/14/10 8:00

6/14/10 16:00

6/14/10 20:00

6/15/100:00

6/15/10 4:00

$6 / 15 / 108: 00$

$\begin{array}{llllll}1498.375 & 1498.474 & 1499.07 & 1499.998 & 1498.718 \\ 1498375 & 1498.433 & 1506.362 & 1500.063 & 1988772\end{array}$

$\begin{array}{lllll}1498.37 & 1498.509 & 1510.557 & 1500.094 & 1498.84\end{array}$

$\begin{array}{rrrrrr}1498.373 & 1498.519 & 1513.07 & 1500.161 & 1498.863 \\ 148.231 & 1498.36 & 1518.621 & 1499.874 & 1498.977\end{array}$

$\begin{array}{lllll}1498.231 & 1498.36 & 1518.621 & 1499.874 & 1498.977 \\ 14983 & 148.335 & 1520894 & 1999.965 & 1499.068\end{array}$

$\begin{array}{lllll}1498.389 & 1498.335 & 1520.894 & 1499.965 & 1499.068 \\ 1498391 & 1498.414 & 1522.262 & 1500.056 & 1499.11\end{array}$

$\begin{array}{rrrrr}1498.391 & 1498.414 & 1522.262 & 1500.056 & 1499.11 \\ 1498.391 & 1498.419 & 1521.687 & 1500.045 & 1499.169\end{array}$

$\begin{array}{llllll}1498.384 & 1498.47 & 1521.024 & 1500.019 & 1499.155 \\ 1498.338 & 1498.337 & 1519.689 & 1499.752 & 1499.185\end{array}$

$\begin{array}{llllll}1498.338 & 1498.337 & 1519.689 & 1499.752 & 1499.185 \\ 1498.391 & 1498.281 & 1517.441 & 1499.972 & 1499.218\end{array}$

$\begin{array}{lllllll}1498.37 & 1498.463 & 1515.511 & 1500.147 & 1499.199\end{array}$

$\begin{array}{llllll}1498.41 & 1498.602 & 1513.783 & 1500.133 & 1499.178\end{array}$

$\begin{array}{lllllll}1498.452 & 1498.605 & 1517.474 & 1500.131 & 1499.178\end{array}$

$\begin{array}{llllll}1498.487 & 1498.628 & 1517.525 & 1500.164 & 1499.152\end{array}$

$\begin{array}{lllll}1498.335 & 1498.446 & 1517.042 & 1499.865 & 1499.173\end{array}$

$\begin{array}{llllll}1498.377 & 1498.26 & 1518.427 & 1499.72 & 1499.243\end{array}$

$\begin{array}{llllll}1498.41 & 1498.388 & 1517.252 & 1500.133 & 1499.285\end{array}$ $\begin{array}{llllll}1498.436 & 1498.516 & 1513.825 & 1500.192 & 1499.351\end{array}$ $\begin{array}{llllll}1498.431 & 14985 & 1512.433 & 1500.178 & 1499.374\end{array}$ $\begin{array}{lllll}1498.452 & 1498.293 & 1511.226 & 1500.22 & 1499.412\end{array}$ $\begin{array}{llllll}1498.468 & 1498.344 & 1510.163 & 1500.267 & 1499.451\end{array}$ $\begin{array}{llllll}1498.459 & 1498.5 & 1509.214 & 1500.248 & 1499.47\end{array}$ $\begin{array}{lrrrr}1498.48 & 1498.598 & 1508.356 & 1500.267 & 1499.475 \\ 1498.478 & 1988.535 & 1507.591 & 1500267 & 1499.491\end{array}$ $\begin{array}{llllll}1498.478 & 1498.635 & 1507.591 & 1500.267 & 1499.491 \\ 1498.426 & 1498749 & 1506864 & 1500.154 & 1499.44\end{array}$ $\begin{array}{lllllll}1498.426 & 1498.749 & 1506.864 & 1500.154 & 1499.44 \\ 1498.468 & 1498.542 & 1506.234 & 1500211 & 1499.419\end{array}$ $\begin{array}{llllll}1498.475 & 1498.474 & 1505.67 & 1500.229 & 1499.435\end{array}$ $\begin{array}{llllll}1498.464 & 1498.57 & 1505.141 & 1500.192 & 1499.421\end{array}$ $\begin{array}{llllll}1498.475 & 1498.665 & 1504.672 & 1500.197 & 1499.409\end{array}$ $\begin{array}{llllll}1498.466 & 1498.719 & 1504.232 & 1500.173 & 1499.4\end{array}$ $\begin{array}{llllll}1498.443 & 1498.812 & 1503.796 & 1500.133 & 1499.342\end{array}$ $\begin{array}{llllll}1498.314 & 1498.817 & 1503.446 & 1499.778 & 1499.353 \\ 1498.443 & 1498.754 & 1503.133 & 1500.15 & 1499379\end{array}$

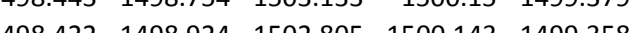
$\begin{array}{llllll}1498.422 & 1498.924 & 1502.805 & 1500.143 & 1499.358 \\ 1498.48 & 1499.087 & 1502.387 & 1500.225 & 1499.335\end{array}$ $\begin{array}{lllllll}1498.513 & 1499.094 & 1502.098 & 1499.967 & 1499.374\end{array}$ $\begin{array}{lllllll}1498.506 & 1499.154 & 1502.089 & 1499.869 & 1499.391\end{array}$ $\begin{array}{llllll}1498.391 & 1498.931 & 1502.206 & 1499.673 & 1499.437\end{array}$ $\begin{array}{llllll}1498.27 & 1498.756 & 1504.605 & 1499.879 & 1499.54\end{array}$

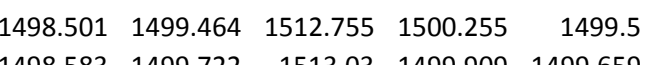
$\begin{array}{llllll}1498.583 & 1499.722 & 1513.03 & 1499.909 & 1499.659 \\ 1498.622 & 1499.639 & 1513.154 & 1500.185 & 1499.729\end{array}$

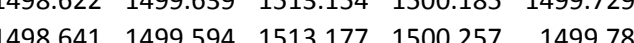
$\begin{array}{lllll}1498.587 & 1499.527 & 1512.981 & 1500.22 & 1499.78\end{array}$ $\begin{array}{llllll}1498.531 & 1499.406 & 1516.84 & 1500.185 & 1499.895\end{array}$ $\begin{array}{llllll}1498.594 & 1499.404 & 1520.74 & 1500.381 & 1499.96\end{array}$ $\begin{array}{rrrrr}1498.597 & 1499.466 & 1522.759 & 1500.521 & 1499.993 \\ 1498.641 & 1499.734 & 1523.74 & 1500.283 & 1499.974\end{array}$ $\begin{array}{rrrrrr}1498.641 & 1499.734 & 1523.74 & 1500.283 & 1499.974 \\ 1498.632 & 1499.694 & 1523.561 & 1500.344 & 1500.084\end{array}$ $\begin{array}{rrrrr}1498.632 & 1499.694 & 1523.561 & 1500.344 & 1500.084 \\ 1498.352 & 1499.445 & 1524.2 & 1500.215 & 1500.117\end{array}$ 

Well (ft AMSL) (ft AMSL) (ft AMSL) (ft AMSL) (ft AMSL) (ft AMSL) (ft AMSL) (ft AMSL) (ft AMSL) (ft AMSL) (ft AMSL) (ft AMSL) (ft AMSL) (ft AMSL) (ft AMSL) (ft AMSL) (ft AMSL) (ft AMSL) (ft AMSL) (ft AMSL) (ft AMSL) (ft AMSL) (ft AMSL) (ft AMSL) (ft AMSL) (ft AMS) $1498.454 \quad 1499.355 \quad 1525.128 \quad 1500.533 \quad 1500.184$ $\begin{array}{llllll}1498.578 & 1499.462 & 1525.139 & 1500.769 & 1500.24 \\ 1498597 & 1499.615 & 1525123 & 1500746 & 1500.259\end{array}$ $\begin{array}{rrrrrr}1498.597 & 1499.615 & 1525.123 & 1500.746 & 1500.259 \\ 1498.629 & 149962 & 1525.026 & 1500.793 & 1500296\end{array}$ $\begin{array}{llllll}1498.6295 & 1499.62 & 1525.006 & 1500.793 & 1500.296 \\ 1498.58 & 1524242 & 1500.809 & 1500.315\end{array}$ $\begin{array}{llllll}1498.688 & 1499.334 & 1522.23 & 1500.865 & 1500.369\end{array}$ $6 / 16 / 1012: 00$ 6/16/10 16:00 6/16/10 20:00 $6 / 17 / 100: 00$ $6 / 17 / 104: 00$ 6/17/10 8:00 6/17/10 12:00 $6 / 17 / 1020: 00$ $6 / 18 / 100: 00$ 6/18/10 4:00 $6 / 18 / 108: 00$ 6/18/10 12:00 6/18/10 16:00 $6 / 19 / 100 \cdot 00$ $6 / 19 / 104: 00$ $6 / 19 / 108: 00$ 6/19/10 12:00 6/19/10 16:00 6/19/10 20:00 $6 / 20 / 100: 00$ $6 / 20 / 104.00$ $6 / 20 / 1012 \cdot 00$ 6/20/10 16:00 6/20/10 20:00 6/21/100:00 $6 / 21 / 104: 00$ 6/21/10 8:00 $6 / 21 / 1012: 00$ $6 / 21 / 1016: 00$ 6/21/1020:00 6/22/10 4:00 6/22/10 8:00 $6 / 22 / 1012: 00$ 6/22/10 16:00 $6 / 22 / 1020: 00$ $6 / 23 / 100: 00$

$\begin{array}{llllll}1498.727 & 1499.397 & 1520.022 & 1500.956 & 1500.432\end{array}$ $\begin{array}{llllll}1498.76 & 1499.485 & 1518.031 & 1500.975 & 1500.471 \\ 1498.802 & 1499.592 & 1516287 & 1501.003 & 1500.471\end{array}$ $\begin{array}{llllll}1498.802 & 1499.592 & 1516.287 & 1501.003 & 1500.471\end{array}$ $\begin{array}{lllllll}1498.862 & 1499.583 & 1514.774 & 1501.052 & 1500.523 \\ 1498897 & 1499.564 & 1513.424 & 1501059 & 1500.525\end{array}$ $\begin{array}{lllll}1498.897 & 1499.564 & 1513.424 & 1501.059 & 1500.525 \\ 1498.951 & 1499.401 & 1512.24 & 1501.125 & 1500.558\end{array}$ $\begin{array}{llllll}1498.995 & 1499.371 & 1511.177 & 1501.125 & 1500.558 \\ 1439 & 1500.581\end{array}$ $\begin{array}{llllll}1499.035 & 1499.506 & 1510.221 & 1501.15 & 1500.595\end{array}$ $\begin{array}{lllllll}1499.058 & 1499.662 & 1509.351 & 1501.139 & 1500.572\end{array}$ $\begin{array}{llllll}1499.096 & 1499.576 & 1508.58 & 1501.174 & 1500.586\end{array}$ $\begin{array}{llllll}1499.114 & 1499.583 & 1507.871 & 1501.162 & 1500.581\end{array}$ $\begin{array}{llllll}1499.152 & 1499.594 & 1507.237 & 1501.185 & 1500.597\end{array}$ $\begin{array}{rrrrrr}1499.187 & 1499.608 & 1506.663 & 1501.211 & 1500.609 \\ 1499.208 & 1499.613 & 1506.136 & 1501.19 & 1500595\end{array}$ $\begin{array}{lllllll}149.208 & 1499.613 & 1506.136 & 1501.19 & 1500.595 \\ 1499219 & 1499.615 & 1505.64 & 1501.171 & 1500.562\end{array}$ $\begin{array}{rrrrr}1499.219 & 1499.615 & 1505.64 & 1501.171 & 1500.562 \\ 1499.226 & 1499.613 & 1505.183 & 1501.143 & 1500.539\end{array}$ $\begin{array}{llllll}1499.231 & 1499.615 & 1504.763 & 1501.127 & 1500.513\end{array}$ $\begin{array}{llllll}1499.25 & 1499.441 & 1504.392 & 1501.162 & 1500.513\end{array}$ $\begin{array}{lllll}1499.296 & 1499.383 & 1504.078 & 1501.223 & 1500.567\end{array}$ $\begin{array}{llllll}1499.329 & 1499.548 & 1503.777 & 1501.227 & 1500.597\end{array}$ $\begin{array}{llllll}1499.308 & 1499.636 & 1503.446 & 1501.122 & 1500.525 \\ 1499.327 & 1499.648 & 1503.185 & 1501.167 & 1500.523\end{array}$ $\begin{array}{llllll}1499.327 & 1499.648 & 1503.185 & 1501.167 & 1500.523 \\ 1499343 & 1499.653 & 1502.942 & 1501.164 & 1500.527\end{array}$ $\begin{array}{lllll}1499.343 & 1499.653 & 1502.942 & 1501.164 & 1500.527 \\ 1499.373 & 1499.667 & 1502.728 & 1501.209 & 1500551\end{array}$ $\begin{array}{llllll}1499.415 & 1499.685 & 1502.555 & 1501.269 & 1500.6\end{array}$ $\begin{array}{llllll}1499.422 & 1499.685 & 1502.35 & 1501.206 & 1500.588\end{array}$ $\begin{array}{llllll}1499.418 & 1499.685 & 1502.152 & 1501.197 & 1500.453\end{array}$ $\begin{array}{llllll}1499.443 & 1499.695 & 1502 & 1501.227 & 1500.483\end{array}$ $\begin{array}{llllll}1499.438 & 1499.69 & 1501.826 & 1501.164 & 1500.408\end{array}$ $\begin{array}{rrrrrr}1499.464 & 1499.706 & 1501.69 & 1501.216 & 1500.296 \\ 1499.49 & 1499.716 & 1501.581 & 1501.251 & 1500327\end{array}$ $\begin{array}{lllll}1499.49 & 1499.716 & 1501.581 & 1501.251 & 1500.327\end{array}$ $\begin{array}{lllllll}1499.493 & 1499.704 & 1501.529 & 1501.174 & 1500.385\end{array}$ $\begin{array}{llllll}1499.487 & 1499.711 & 1502.152 & 1501.185 & 1500.413\end{array}$ $\begin{array}{llllll}1499.492 & 1499.718 & 1502.705 & 1501.181 & 1500.441\end{array}$ $\begin{array}{llllll}1499.504 & 1499.718 & 1503.154 & 1501.19 & 1500.464\end{array}$ $\begin{array}{llllll}1499.532 & 1499.727 & 1503.465 & 1501.239 & 1500.506\end{array}$ $\begin{array}{rrrrr}1499.529 & 1499.725 & 1503.511 & 1501.195 & 1500.516 \\ 1499.52 & 1499.72 & 1503.432 & 150116 & 1500.48\end{array}$ $\begin{array}{rrrrrr}1499.52 & 1499.72 & 1503.432 & 1501.16 & 1500.488 \\ 1499.532 & 1499.725 & 150332 & 1501.171 & 1500506\end{array}$ $\begin{array}{llllll}149.532 & 1499.725 & 1503.32 & 1501.17 & 1500.506\end{array}$ $\begin{array}{llllll}1499.513 & 1499.713 & 1502.942 & 1501.122 & 1500.413 \\ 150245\end{array}$ $\begin{array}{llllll}1499.504 & 1499.706 & 1502.763 & 1501.113 & 1500.217\end{array}$ $\begin{array}{llllll}1499.494 & 1499.702 & 1502.574 & 1501.082 & 1500.25\end{array}$ $\begin{array}{llllll}1499.459 & 1499.676 & 1502.373 & 1501.024 & 1500.231\end{array}$ $\begin{array}{lllllll}1499.459 & 1499.676 & 1502.206 & 1501.05 & 1500.264\end{array}$ $\begin{array}{rrrrr}1499.45 & 1499.667 & 1502.038 & 1501.029 & 1500.278 \\ 1499.466 & 1499.674 & 1501.905 & 1501.087 & 1500.32\end{array}$ 
$\begin{array}{lllll}1499.492 & 1499.688 & 1501.777 & 1501.141 & 1500.359 \\ 1499511 & 1499699 & 1501.653 & 1501.157 & 1500\end{array}$

$\begin{array}{lllll}1499.511 & 1499.699 & 1501.653 & 1501.157 & 1500.399 \\ 1499.513 & 1499.699 & 1501.536 & 1501.143 & 1500378\end{array}$

6/25/10 8:00

$\begin{array}{lllll}1499.513 & 1499.699 & 1501.536 & 1501.143 & 1500.378 \\ 1499.529 & 1499.709 & 1501.429 & 1501.164 & 1500.404\end{array}$

$\begin{array}{lllll}1499.534 & 1499.711 & 1501.324 & 1501.16 & 1500.345\end{array}$

6/25/10 12:00

$6 / 25 / 1020: 00$

$6 / 26 / 100: 00$

$6 / 26 / 104: 00$

$6 / 26 / 108: 00$

$6 / 26 / 1016: 00$

$6 / 26 / 1020: 00$

6/27/100:00

6/27/10 4:00

6/27/10 8:00

6/27/10 12:00

6/27/10 16:00

6/27/10 20:00

6/28/100:00

6/28/10 8:00

6/28/10 12:00

6/28/10 16:00

6/28/10 20:00

6/29/10 0:00

6/29/10 4:00

$6 / 29 / 1012.00$

6/29/10 16:00

6/29/10 20:00

6/30/100:00

6/30/10 4:00

6/30/10 8:00

$6 / 30 / 1012: 00$

6/30/10 16:00

6/30/10 20:00

7/1/100:00

7/1/10 8:00

7/1/10 12:00

$7 / 1 / 1016: 00$

$7 / 1 / 1020: 00$

$7 / 2 / 100: 00$

$7 / 2 / 108000$

7/2/10 12:00

7/2/10 16:00

7/2/10 20:00

7/3/100:00

7/3/10 4:00

7/3/10 8:00

$\begin{array}{lllll}1499.55 & 1499.723 & 1501.231 & 1501.199 & 1500.229 \\ 1499.576 & 1499.737 & 1501.149 & 1501.241 & 1500.261\end{array}$

$\begin{array}{llllll}1499.583 & 1499.739 & 1501.068 & 1501.234 & 1500.324\end{array}$

$\begin{array}{llllll}1499.581 & 1499.737 & 1500.979 & 1501.211 & 1500.345\end{array}$

$\begin{array}{rrrrr}1499.585 & 1499.739 & 1500.912 & 1501.216 & 1500.38 \\ 149597 & 1499744 & 1500846 & 1501232 & 1500.411\end{array}$

$\begin{array}{llllll}499.597 & 1499.744 & 1500.846 & 1501.232 & 1500.411 \\ 1499.613 & 1499.753 & 1500.795 & 1501265 & 1500.439\end{array}$

$\begin{array}{llllll}1499.641 & 1499.762 & 1500751 & 1501.305 & 1500.49\end{array}$

$\begin{array}{llllll}1499.66 & 1499.767 & 1500.704 & 1501.309 & 1500.525\end{array}$

$\begin{array}{llllll}1499.655 & 1499.767 & 1500.648 & 1501.281 & 1500.513\end{array}$

$\begin{array}{lllll}1499.648 & 1499.762 & 1500.59 & 1501.248 & 1500.502\end{array}$

$\begin{array}{llllll}1499.634 & 1499.751 & 1500.532 & 1501.206 & 1500.399\end{array}$

$\begin{array}{llllll}1499.611 & 1499.739 & 1500.471 & 1501.171 & 1500.203\end{array}$

$\begin{array}{llllll}1499.618 & 1499.746 & 1500.436 & 1501.218 & 1500.203 \\ 1499.597 & 1499.732 & 1500.378 & 1501.164 & 1500.229\end{array}$

$\begin{array}{llllll}1499.569 & 1499.711 & 1500.327 & 1501.12 & 1500.224\end{array}$

$\begin{array}{lllll}1499.555 & 1499.702 & 1500.282 & 1501.108 & 1500.24\end{array}$

$\begin{array}{rrrrrr}1499.532 & 1499.685 & 1500.24 & 1501.085 & 1500.173 \\ 1499.525 & 1499.678 & 1500.201 & 1501.099 & 1500.037\end{array}$

$\begin{array}{llllll}1499.525 & 1499.674 & 1500.175 & 1501.122 & 1500.051\end{array}$

$\begin{array}{lllllll}1499.511 & 1499.664 & 1500.135 & 1501.101 & 1500.098\end{array}$ $\begin{array}{rrrrr}1499.485 & 1499.646 & 1500.1 & 1501.064 & 1500.105 \\ 1499.466 & 1499.634 & 1500.068 & 1501047 & 1500117\end{array}$ $\begin{array}{llllll}1499.466 & 1499.634 & 1500.068 & 1501.047 & 1500.117 \\ 1499.443 & 1499.618 & 1500.033 & 1501.01 & 1500.119\end{array}$ $\begin{array}{lllll}1499.443 & 1499.618 & 1500.033 & 1501.01 & 1500.119 \\ 1499.438 & 1499.613 & 1500.014 & 1501.043 & 1500.135\end{array}$ $\begin{array}{llllll}1499.45 & 1499.618 & 1500.002 & 1501.087 & 1500.18\end{array}$ $\begin{array}{llllll}1499.445 & 1499.608 & 1499.974 & 1501.054 & 1500.191\end{array}$ $\begin{array}{llllll}1499.425 & 1499.599 & 1499.946 & 1501.026 & 1500.159\end{array}$ $\begin{array}{llllll}1499.418 & 1499.592 & 1499.93 & 1501.029 & 1500.161\end{array}$ $\begin{array}{llllll}1499.397 & 1499.583 & 1499.895 & 1500.994 & 1500.075\end{array}$ $\begin{array}{llllll}1499.399 & 1499.581 & 1499.877 & 1501.031 & 1499.925 \\ 1499413 & 1499.588 & 1499.865 & 150108 & 1999.955\end{array}$ $\begin{array}{llllll}149.413 & 149.588 & 1499865 & 1501.08 & 1499.955\end{array}$ $\begin{array}{llllll}1499.408 & 1499.581 & 1499.846 & 1501.073 & 1499.986 \\ 1499.387 & 1499.564 & 1499.816 & 1501.024 & 1499.937\end{array}$ $\begin{array}{lllllll}1499.378 & 1499.56 & 1499.797 & 1501.031 & 1499.969\end{array}$ $\begin{array}{llllllll}1499.362 & 1499.553 & 1499.774 & 1501.001 & 1499.988\end{array}$ $\begin{array}{lllllll}1499.371 & 1499.553 & 1499.776 & 1501.057 & 1500.011\end{array}$ $\begin{array}{llllll}1499.387 & 1499.562 & 1499.774 & 1501.099 & 1500.072\end{array}$ $\begin{array}{llllll}1499.392 & 1499.56 & 1499.767 & 1501.094 & 1500.114 \\ 1499.38 & 149955 & 149.753 & 1501.078 & 1500.121\end{array}$ $\begin{array}{llllll}1499.38 & 1499.55 & 1499.753 & 1501.078 & 1500.121 \\ 1499.385 & 1499.553 & 1499.753 & 1501094 & 1500.14\end{array}$ $\begin{array}{lllllll}149.385 & 149.553 & 1499.753 & 1501.094 & 1500.14\end{array}$ $\begin{array}{llllll}1499.397 & 1499.555 & 1499.741 & 1501.134 & 1409.934\end{array}$ $\begin{array}{lllllll}1499.418 & 1499.567 & 1499.748 & 1501.185 & 1499.972\end{array}$ $\begin{array}{lllll}1499.425 & 1499.569 & 1499.744 & 1501.185 & 1500.044\end{array}$ $\begin{array}{llllll}1499.411 & 1499.557 & 1499.732 & 1501.16 & 1500.072\end{array}$ $\begin{array}{llllll}1499.429 & 1499.569 & 1499.739 & 1501.199 & 1500.135\end{array}$ $\begin{array}{rrrrr}1499.42 & 1499.56 & 1499.732 & 1501.169 & 1500.156 \\ 1499.429 & 1499.567 & 1499.737 & 1501.199 & 1500.191\end{array}$ 

Well (ft AMSL) (ft AMSL) (ft AMSL) (ft AMSL) (ft AMSL) (ft AMSL) (ft AMSL) (ft AMSL) (ft AMSL) (ft AMSL) (ft AMSL) (ft AMSL) (ft AMSL) (ft AMSL) (ft AMSL) (ft AMSL) (ft AMSL) (ft AMSL) (ft AMSL) (ft AMSL) (ft AMSL) (ft AMSL) (ft AMSL) (ft AMSL) (ft AMSL) (ft AMSL)

$7 / 3 / 1016: 00$

$\begin{array}{lllll}1499.441 & 1499.571 & 1499.746 & 1501.237 & 1500.222\end{array}$

$7 / 4 / 100: 00$

$\begin{array}{lllll}1499.452 & 1499.914 & 1499.748 & 1501.398 & 1500.25 \\ 1499.441 & 1499.902 & 1499.746 & 1501.431 & 1500.25\end{array}$

$7 / 4 / 108: 00$

$7 / 4 / 1012: 00$

$7 / 4 / 1016: 00$

7/4/10 20:00

$7 / 5 / 100: 00$

7/5/10 4:00

$7 / 5 / 108: 00$

$7 / 5 / 1016: 00$

$7 / 5 / 1020: 00$

$7 / 6 / 100: 00$

7/6/10 4:00

7/6/10 8:00

$7 / 6 / 1012: 00$

$7 / 6 / 1012600$

$7 / 7 / 1020: 00$

7/7/100:00

7/7/10 8:00

7/7/10 12:00

7/7/10 16:00

$7 / 7 / 1020: 00$

$7 / 8 / 100: 00$

$7 / 8 / 104: 00$

$7 / 8 / 108.000$

$7 / 8 / 1016: 00$

$7 / 8 / 1020: 00$

7/9/10 0:00

7/9/10 4:00

7/9/10 8:00

$7 / 9 / 1012: 00$

$7 / 9 / 1020.00$

$7 / 10 / 100: 00$

$7 / 10 / 104: 00$

$7 / 10 / 108: 00$

$7 / 10 / 1012: 00$

$7 / 10 / 1016: 00$

$7 / 10 / 1020: 00$

$7 / 11 / 100: 00$

$7 / 11 / 108.00$

$7 / 11 / 1012: 00$

$7 / 11 / 1016: 00$

$7 / 11 / 1020: 00$

$7 / 12 / 100: 00$

7/12/10 4:00

$7 / 12 / 108: 00$

$\begin{array}{lllll}499.441 & 1499.902 & 1499.746 & 1501.431 & 1500.25\end{array}$

$\begin{array}{llllll}1499.434 & 1500.449 & 1499.739 & 1501.485 & 1500.187\end{array}$

$\begin{array}{lllll}1499.42 & 1500.666 & 1499.732 & 1501.463 & 1500.047 \\ & & 1409\end{array}$

$\begin{array}{lllll}1499.385 & 1500.885 & 1499.585 & 1501.195 & 1499.99 \\ 1495 & 1500.743 & 199604 & 1501241 & 1500.117\end{array}$

$\begin{array}{llllll}1499.385 & 1500.743 & 1499.604 & 1501.241 & 1500.117 \\ 1499.411 & 1501311 & 1499.597 & 1501333 & 1500.203\end{array}$

$\begin{array}{lllll}1499.411 & 1501.311 & 1499.597 & 1501.333 & 1500.203 \\ 1499.429 & 1501.169 & 1499.634 & 1501.389 & 1500.289\end{array}$

$\begin{array}{llllll}1499.42 & 1501.518 & 1499.534 & 1501.174 & 1499.951\end{array}$

$\begin{array}{llllll}1499.408 & 1501.588 & 1499.532 & 1501.012 & 1499.718 \\ 1499.303 & 1501.32 & 1501.665 & 1500.727 & 1500.112\end{array}$

$\begin{array}{llllll}1499.364 & 1501.728 & 1506.115 & 1501.017 & 1500.259\end{array}$

$\begin{array}{lllll}1499.422 & 1501.639 & 1506.5 & 1501.23 & 1500.502\end{array}$

$\begin{array}{lllll}1499.473 & 1501.569 & 1506.833 & 1501.389 & 1500.773\end{array}$

$\begin{array}{llllll}1499.485 & 1501.483 & 1506.871 & 1501.337 & 1500.838\end{array}$

$\begin{array}{lllll}1499.404 & 1501.339 & 1508.978 & 1500.98 & 1500.906\end{array}$

$\begin{array}{llllll}1499.499 & 1501.29 & 1513.571 & 1501.503 & 1500.987 \\ 1499.532 & 1501.236 & 1512.429 & 1501.798 & 1501.041\end{array}$

$\begin{array}{llllll}1499.534 & 1501.236 & 1512.429 & 1501.798 & 1501.041\end{array}$

$\begin{array}{llllll}1499.56 & 1501.136 & 1510293 & 1501.835 & 1501.06\end{array}$

$\begin{array}{lllll}1499.564 & 1501.082 & 1509.407 & 1501.828 & 1501.053\end{array}$

\begin{tabular}{lllll}
1499.576 & 1501.034 & 1508.615 & 1501.838 & 1501.048 \\
\hline
\end{tabular} $\begin{array}{rrrrrr}1499.616 & 1500.996 & 1507.929 & 1501.931 & 1501.092 \\ 1499.637 & 1501.02 & 1507.286 & 1502.027 & 1501.104\end{array}$ $\begin{array}{rrrrrr}1499.637 & 1501.02 & 1507.286 & 1502.027 & 1501.104 \\ 1499.653 & 1501.346 & 1506.6 & 1502.277 & 1500.843\end{array}$ $\begin{array}{rrrrr}1499.653 & 1501.346 & 1506.6 & 1502.277 & 1500.843 \\ 1499.688 & 1501.236 & 1506.099 & 1502.361 & 1501.043\end{array}$ $\begin{array}{rrrrr}1499.688 & 1501.236 & 1506.099 & 1502.361 & 1501.043 \\ 149.711 & 1501.171 & 1506.26 & 1502.403 & 1501.123\end{array}$ $\begin{array}{lllll}1499.69 & 1501.059 & 1506.26 & 1502.34 & 1501.16 \\ \end{array}$ \begin{tabular}{rrrrr}
1499.702 & 1500.873 & 1506.973 & 1502.151 & 1501.221 \\
\hline
\end{tabular} $\begin{array}{rrrrr}1499.749 & 1500.899 & 1507.85 & 1502.139 & 1501.26 \\ 14903 & 1500.957 & 1507.262 & 152.118 & 1501.26\end{array}$

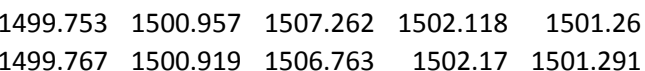
$\begin{array}{rrrrrr}1499.767 & 1500.919 & 1506.763 & 1502.17 & 1501.291 \\ 1499.777 & 1500.88 & 1506.353 & 1502.151 & 1501.288\end{array}$ $\begin{array}{rrrrr}1499.777 & 1500.88 & 1506.353 & 1502.151 & 1501.288 \\ 1499.798 & 1500.791 & 1506.05 & 1502.195 & 1501.305\end{array}$ \begin{tabular}{lllllll}
1499.835 & 1500.773 & 1505.803 & 1502.27 & 1501.351 \\
\hline
\end{tabular} $\begin{array}{lllll}1499.863 & 1500.754 & 1505.556 & 1502.291 & 1501.382 \\ 1499.877 & 1500.731 & 1505.297 & 1502.284 & 1501.372\end{array}$ $\begin{array}{llllll}1499.903 & 1500.715 & 1505.08 & 1502.317 & 1501.391\end{array}$ $\begin{array}{llllll}1499.917 & 1500.696 & 1504.861 & 1502.31 & 1501.396\end{array}$ $\begin{array}{llllll}1499.947 & 1500.684 & 1504.677 & 1502.357 & 1501.41\end{array}$ \begin{tabular}{llllll}
1499.994 & 1500.68 & 1504.535 & 1502.42 & 1501.459 \\
\hline
\end{tabular} $\begin{array}{rrrrr}1500.026 & 1500.673 & 1504.409 & 1502.438 & 1501.499 \\ 150061 & 1500.67 & 1504269 & 1502.466 & 1501.54\end{array}$ $\begin{array}{llllll}1500.061 & 1500.67 & 1504.269 & 1502.466 & 1501.51 \\ 1500.096 & 1500.67 & 1504.171 & 1502.499 & 1501.541\end{array}$ $\begin{array}{llllll}1500.061 & 1500.67 & 1504.269 & 1502.466 & 1501.51\end{array}$ $\begin{array}{llllll}1500.136 & 1500.656 & 1503.924 & 1502.478 & 1501.548\end{array}$ $\begin{array}{llllll}1500.171 & 1500.659 & 1503.838 & 1502.513 & 1501.566\end{array}$ $\begin{array}{llllll}1500.189 & 1500.654 & 1503.728 & 1502.494 & 1501.573\end{array}$ $\begin{array}{llllll}1500.187 & 1500.645 & 1503.677 & 1502.436 & 1501.529\end{array}$ $\begin{array}{llllll}1500.206 & 1500.642 & 1503.884 & 1502.436 & 1501.529\end{array}$ $\begin{array}{lllll}1500.215 & 1500.638 & 1503.938 & 1502.429 & 1501.531 \\ 1500.224 & 1500.635 & 1503.838 & 1502.401 & 1501.522\end{array}$ 
$\begin{array}{llllll}1500.271 & 1500.696 & 1503.101 & 1502.338 & 1501.508\end{array}$ $\begin{array}{llllll}1500.278 & 1500.687 & 1502.959 & 1502.329 & 1501.499\end{array}$ $\begin{array}{llllll}1500.299 & 1500.689 & 1502.837 & 1502.359 & 1501.513\end{array}$ $\begin{array}{llllll}1500.301 & 1500.68 & 1502.712 & 1502.329 & 1501.506\end{array}$ $\begin{array}{cccccc}1500.292 & 1500.663 & 1502.569 & 1502.277 & 1501.475 \\ 15002 & 150.69 & 1502.46 & 1502.24 & 1501.45\end{array}$ $\begin{array}{llllll}1500.29 & 1500.649 & 1502.446 & 1502.254 & 1501.45 \\ 1500283 & 1500.638 & 1502318 & 1502223 & 1501.31\end{array}$ $\begin{array}{llllll}1500.283 & 1500.638 & 1502.318 & 1502.223 & 1501.431 \\ 1500.297 & 1500.638 & 1502215 & 1502244 & 1501.438\end{array}$ $\begin{array}{llllll}1500.297 & 1500.638 & 1502.215 & 1502.244 & 1501.438 \\ 1500.313 & 1500.642 & 1502.119 & 1502.277 & 1501.445\end{array}$ $\begin{array}{lllll}1500.32 & 1500.635 & 1502.019 & 1502.256 & 1501.45\end{array}$ $\begin{array}{llllll}1500.308 & 1500.878 & 1501.879 & 1502.289 & 1501.372\end{array}$ $\begin{array}{lllllll}1500.294 & 1501.287 & 1501.814 & 1502.408 & 1501.356\end{array}$ $\begin{array}{lllllll}1500.269 & 1501.318 & 1501.718 & 1502.431 & 1501.344\end{array}$ $\begin{array}{llllll}1500.203 & 1501.241 & 1501.646 & 1502.104 & 1501.349\end{array}$ $\begin{array}{llllll}1500.264 & 1501.199 & 1501.588 & 1502.055 & 1501.379 \\ 1500.276 & 1501.166 & 1501.52 & 1502209 & 1501.405\end{array}$ $\begin{array}{lllllll}1500.27 & 1501.166 & 1501.52 & 1502.209 & 1501.405 \\ 1500283 & 1501.141 & 1501.462 & 1502167 & 1501.407\end{array}$ $\begin{array}{llllll}1500.283 & 1501.141 & 1501.462 & 1502.167 & 1501.407 \\ 1500.294 & 1501.124 & 1501.411 & 1502.179 & 1501.424\end{array}$ $\begin{array}{llllll}1500.299 & 1501.099 & 1501.357 & 1502.162 & 1501.429\end{array}$ $\begin{array}{llllll}1500.313 & 1501.08 & 1501.32 & 1502.184 & 1501.445\end{array}$ $\begin{array}{llllll}1500.346 & 1501.069 & 1501.294 & 1502.256 & 1501.487\end{array}$ $\begin{array}{lllll}1500.355 & 1501.05 & 1501.247 & 1502.24 & 1501.499\end{array}$ $\begin{array}{rrrrrr}1500.357 & 1501.031 & 1501.208 & 1502.219 & 1501.482 \\ 1500.35 & 1501.013 & 1501.161 & 1502172 & 1501.464\end{array}$ $\begin{array}{lllllll}1500.35 & 1501.013 & 1501.161 & 1502.172 & 1501.464 \\ 1500367 & 1500.996 & 1501.14 & 1502209 & 1501.485\end{array}$ $\begin{array}{llllll}1500.367 & 1500.996 & 1501.14 & 1502.209 & 1501.485 \\ 1500.383 & 1500.985 & 1501.119 & 1502.233 & 1501.494\end{array}$ $\begin{array}{llllll}1500.404 & 1500.975 & 1501.101 & 1502.277 & 1501.517\end{array}$ $\begin{array}{llllll}1500.425 & 1500.966 & 1501.082 & 1502.291 & 1501.541\end{array}$ $\begin{array}{llllll}1500.423 & 1500.954 & 1501.049 & 1502.249 & 1501.522\end{array}$ $\begin{array}{lllll}1500.42 & 1500.943 & 1501.026 & 1502.228 & 1501.51\end{array}$ $\begin{array}{lllll}1500.413 & 1500.927 & 1500.998 & 1502.219 & 1501.487\end{array}$ $\begin{array}{llllll}1500.423 & 1500.917 & 1500.986 & 1502.219 & 1501.489 \\ 1500.441 & 1500.912 & 1500.977 & 1502268 & 1501508\end{array}$ $\begin{array}{lllllll}1500.441 & 1500.912 & 1500.977 & 1502.268 & 1501.508\end{array}$ $\begin{array}{llllll}150.453 & 1500.903 & 1500.961 & 1502.263 & 1501.522 \\ 1500.441 & 1500.892 & 1500.94 & 1502.226 & 1501.499\end{array}$ $\begin{array}{lllll}1500.455 & 1500.885 & 1500.935 & 1502.256 & 1501.513\end{array}$ $\begin{array}{llllll}1500.46 & 1500.878 & 1500.919 & 1502.247 & 1501.51\end{array}$ $\begin{array}{llllll}1500.472 & 1500.871 & 1500.914 & 1502.263 & 1501.527\end{array}$ $\begin{array}{llllll}1500.49 & 1500.875 & 1500.916 & 1502.305 & 1501.55\end{array}$ $\begin{array}{lllll}1500.502 & 1500.866 & 1500.907 & 1502.296 & 1501.548 \\ 1500.493 & 1500.857 & 1500891 & 1502.256 & 1501.515\end{array}$ $\begin{array}{llllll}1500.493 & 1500.857 & 1500.891 & 1502.256 & 1501.515 \\ 1500.483 & 1500.847 & 1500.872 & 1502.226 & 1501.489\end{array}$ $\begin{array}{llllll}150.400 .476 & 1500.836 & 1500.86 & 1502.214 & 1501.489\end{array}$ $\begin{array}{llllll}1500.467 & 1500.826 & 1500.844 & 1502.188 & 1501.454\end{array}$ $\begin{array}{llllll}1500.472 & 1500.819 & 1500.842 & 1502.214 & 1501.471\end{array}$ $\begin{array}{lllll}1500.465 & 1500.81 & 1500.828 & 1502.177 & 1501.447\end{array}$ $\begin{array}{llllll}1500.432 & 1500.794 & 1500.793 & 1502.085 & 1501.382\end{array}$ $\begin{array}{llllll}1500.439 & 1500.787 & 1500.8 & 1502.17 & 1501.398\end{array}$ $\begin{array}{rlllll}1500.423 & 1500.777 & 1500.779 & 1502.167 & 1501.328 \\ 1500.43 & 1500773 & 1500.772 & 1502.16 & 1501.155\end{array}$ 

Well (ft AMSL) (ft AMSL) (ft AMSL) (ft AMSL) (ft AMSL) (ft AMSL) (ft AMSL) (ft AMSL) (ft AMSL) (ft AMSL) (ft AMSL) (ft AMSL) (ft AMSL) (ft AMSL) (ft AMSL) (ft AMSL) (ft AMSL) (ft AMSL) (ft AMSL) (ft AMSL) (ft AMSL) (ft AMSL) (ft AMSL) (ft AMSL) (ft AMSL) (ft AMS)

7/21/10 16:00 $7 / 21 / 1020: 00$ $7 / 22 / 100: 00$ $7 / 22 / 108: 00$ $7 / 22 / 1012: 00$ 7/22/10 16:00 $7 / 22 / 1020: 00$ $7 / 23 / 100: 00$ $7 / 23 / 104: 00$ $7 / 23 / 108: 00$
$7 / 23 / 1012: 00$ 7/23/10 12:00 $7 / 23 / 1020: 00$ 7/24/100:00 7/24/10 4:00 7/24/10 8:00 7/24/10 12:00 7/24/10 16:00 $7 / 25 / 100.00$ 7/25/10 4:00 $7 / 25 / 108: 00$ $7 / 25 / 1012: 00$ 7/25/10 16:00 $7 / 25 / 10020: 00$ 7/26/100:00 $7 / 26 / 104: 00$ $7 / 26 / 1012.00$ $7 / 26 / 1016: 00$ 7/26/10 20:00 7/27/100:00 $7 / 27 / 104: 00$ 7/27/10 8:00 7/27/10 12:00 7/27/10 16:00 $7 / 28 / 100: 00$ $7 / 28 / 104: 00$ $7 / 28 / 108: 00$ $7 / 28 / 10$ 12:00 7/28/10 16:00 $7 / 28 / 1020: 00$ $7 / 29 / 100: 00$ $7 / 29 / 10800$ 7/29/10 12:00 7/29/10 16:00 $7 / 29 / 1020: 00$ 7/30/10 0:00 7/30/10 4:00 $7 / 30 / 108: 00$
$7 / 30 / 1012: 00$

$\begin{array}{lllll}1500.448 & 1500.775 & 1500.779 & 1502.221 & 1501.169\end{array}$ $\begin{array}{rrrrr}1500.458 & 1500.768 & 1500.772 & 1502.223 & 1501.228 \\ 1500.441 & 1500.759 & 1500.753 & 1502.181 & 1501.23\end{array}$ $\begin{array}{lllllll}150.441 & 1500.759 & 1500.753 & 1502.181 & 1501.23\end{array}$ $\begin{array}{llllll}1500.448 & 1500.749 & 1500.751 & 1502207 & 1501.309\end{array}$ $\begin{array}{lllll}1500.465 & 1500.752 & 1500.758 & 1502.242 & 1501.349\end{array}$ $\begin{array}{llllll}1500.479 & 1500.752 & 1500.767 & 1502.146 & 1501.389\end{array}$ \begin{tabular}{llllll}
1500.481 & 1500.745 & 1500.763 & 1502.317 & 1501.372 \\
\hline
\end{tabular} $\begin{array}{lllllll}1500.472 & 1500.736 & 1500.751 & 1502.214 & 1501.323\end{array}$

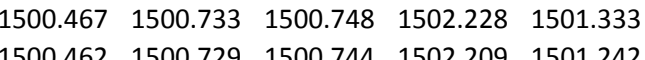
$\begin{array}{llllll}1500.462 & 1500.729 & 1500.744 & 1502.209 & 1501.242\end{array}$ $\begin{array}{lllllll}1500.462 & 1500.717 & 1500.728 & 1502.242 & 1501.088\end{array}$ $\begin{array}{llllll}1500.453 & 1500.71 & 1500.718 & 1502.212 & 1501.141\end{array}$ $\begin{array}{llllll}1500.423 & 1500.694 & 1500.7 & 1502.156 & 1501.151\end{array}$ $\begin{array}{llllll}1500.42 & 1500.689 & 1500.7 & 1502.179 & 1501.179\end{array}$ $\begin{array}{llllll}1500.399 & 1500.675 & 1500.683 & 1502.134 & 1501.183\end{array}$ $\begin{array}{llllll}1500.383 & 1500.666 & 1500.674 & 1502.116 & 1501.172\end{array}$ $\begin{array}{llllll}1500.381 & 1500.656 & 1500.669 & 1502.064 & 1501.015 \\ 1500.378 & 1500.919 & 1500.602 & 1502.371 & 150085\end{array}$ $\begin{array}{llllll}1500.378 & 1500.919 & 1500.602 & 1502.371 & 1500.85 \\ 1571 & 1501297 & 1500.611 & 1502.52 & 1500.871\end{array}$ $\begin{array}{rrrrr}1500.371 & 1501.297 & 1500.611 & 1502.52 & 1500.871 \\ 1500.402 & 1501.222 & 1500.609 & 1502.555 & 1500.943\end{array}$ $\begin{array}{llllll}1500.411 & 1501.141 & 1500.588 & 1502.56 & 1500.92\end{array}$ $\begin{array}{llllll}1500.329 & 1500.994 & 1500.581 & 1502.455 & 1500.934\end{array}$ $\begin{array}{llllll}1500.297 & 1500.826 & 1500.599 & 1502.326 & 1501.013\end{array}$ $\begin{array}{llllll}1500.297 & 1500.866 & 1500.602 & 1502.343 & 1501.078\end{array}$ $\begin{array}{llllll}1500.294 & 1500.924 & 1500.602 & 1502.375 & 1501.109 \\ 1500.299 & 1500.927 & 1500.609 & 1502.354 & 1501.148\end{array}$ $\begin{array}{llllll}1500.299 & 1500.927 & 1500.609 & 1502.354 & 1501.148 \\ 1500.292 & 1500.903 & 1500.604 & 1502.282 & 1501.155\end{array}$ $\begin{array}{llllllll}150.292 & 1500.950 & 150.604 & 1502.282 & 1501.155\end{array}$ $\begin{array}{lllll}1500.329 & 1500.687 & 1500.658 & 1501.831 & 1501.239\end{array}$ $\begin{array}{llllll}1500.334 & 1500.815 & 1500.662 & 1502.081 & 1501.263\end{array}$ $\begin{array}{llllll}1500.325 & 1500.94 & 1500.655 & 1502.268 & 1501.242\end{array}$ $\begin{array}{llllll}1500.334 & 1500.924 & 1500.665 & 1502.319 & 1501.258\end{array}$ $\begin{array}{lllll}1500.329 & 1500.894 & 1500.66 & 1502.275 & 1501.253\end{array}$ $\begin{array}{lllll}1500.332 & 1500.614 & 1500.66 & 1501.999 & 1501.253 \\ 1500.35 & 1500.631 & 1500.662 & 1501.908 & 1501263\end{array}$ $\begin{array}{lllllll}1500.35 & 1500.631 & 1500.662 & 1501.908 & 1501.263\end{array}$ $\begin{array}{llllllll}1500.334 & 1500.763 & 1500.658 & 1502.33 & 1501.267\end{array}$ $\begin{array}{llllll}1500.332 & 1500.903 & 1500.644 & 1502.354 & 1501.228\end{array}$ $\begin{array}{llllll}1500.313 & 1500.88 & 1500.63 & 1502.275 & 1501.204\end{array}$ $\begin{array}{llllll}1500.308 & 1500.575 & 1500.63 & 1501.971 & 1501.186\end{array}$ $\begin{array}{lllllll}1500.32 & 1500.568 & 1500.641 & 1501.877 & 1501.2\end{array}$ $\begin{array}{lllll}1500.311 & 1500.71 & 1500.634 & 1502.104 & 1501.188 \\ 1500294 & 1500.836 & 1500.623 & 1502.263 & 1501.153\end{array}$ $\begin{array}{llllll}1500.294 & 1500.836 & 1500.623 & 1502.263 & 1501.153 \\ 1500294 & 1500.829 & 1500.625 & 1502305 & 1501.158\end{array}$ $1500.287 \quad 1500.7781500 .623 \quad 1502.244 \quad 1501.158$ $\begin{array}{llllll}1500.287 & 1500.778 & 1500.623 & 1502.244 & 1501.158 \\ 1500.294 & 1500.484 & 1500.632 & 1501.978 & 1501.179\end{array}$ $\begin{array}{llllll}1500.313 & 1500.605 & 1500.648 & 1502.064 & 1501.223\end{array}$ $\begin{array}{lllll}1500.341 & 1500.719 & 1500.658 & 1502.226 & 1501.253\end{array}$ $\begin{array}{rrrrr}1500.336 & 1500.701 & 1500.662 & 1502.434 & 1501.251 \\ 1500.35 & 1500.691 & 1500.676 & 1502.548 & 1501.27\end{array}$ $\begin{array}{llllll}1500.35 & 1500.691 & 1500.676 & 1502.548 & 1501.27\end{array}$ $\begin{array}{rrrrr}1500.353 & 1500.677 & 1500.679 & 1502.476 & 1501.279 \\ 1500.364 & 1500.666 & 1500.69 & 1502.102 & 1501.291\end{array}$ 
$\begin{array}{lllll}1500.348 & 1500.608 & 1500.662 & 1502.326 & 1500.966 \\ 1500.334 & 1500.50 & 1500.58 & 150201 & 150.964\end{array}$

$7 / 31 / 1016: 00$ $\begin{array}{llllll}1500.339 & 1500.594 & 1500.655 & 1501.91 & 1500.819\end{array}$ $\begin{array}{llllll}1500.327 & 1500.582 & 1500.641 & 1502.165 & 1500.794\end{array}$

$8 / 1 / 10000$ $\begin{array}{llllll}1500.306 & 1500.573 & 1500.627 & 1502.336 & 1500.822\end{array}$ $\begin{array}{lllll}1500.292 & 1500.561 & 1500.623 & 1502.368 & 1500.882 \\ 1500.28 & 1500.552 & 1500.616 & 1502319 & 1500864\end{array}$ $\begin{array}{lllll}1500.28 & 1500.552 & 1500.616 & 1502.319 & 1500.864 \\ 1500.278 & 1500.547 & 1500.611 & 1502.006 & 1500.777\end{array}$ $\begin{array}{lllll}1500.278 & 1500.547 & 1500.611 & 1502.006 & 1500.777 \\ 1500.287 & 1500.545 & 1500.618 & 1501.877 & 1500.838\end{array}$ $\begin{array}{llllll}1500.28 & 1500.535 & 1500.616 & 1502.118 & 1500.91\end{array}$ $\begin{array}{rrrrr}1500.28 & 1500.535 & 1500.616 & 1502.118 & 1500.91 \\ 1500.264 & 1500.526 & 1500.609 & 1502.191 & 1500.929\end{array}$ $\begin{array}{llllll}1500.255 & 1500.519 & 1500.606 & 1502.188 & 1500.969\end{array}$ $8 / 2 / 108: 00$ $8 / 2 / 1012: 00$ $8 / 2 / 1016: 00$ $8 / 2 / 1020: 00$ $8 / 3 / 100: 00$
$8 / 3 / 104: 00$ 8/3/10 8:00 8/3/10 8:00 8/3/10 16:00 $8 / 3 / 1020: 00$ $8 / 4 / 100: 00$ 8/4/10 4:00 $8 / 4 / 1012 \cdot 00$ $8 / 4 / 1016: 00$ $8 / 4 / 1020: 00$ $8 / 5 / 100: 00$ 8/5/10 4:00 8/5/10 8:00 $8 / 5 / 1012: 00$ $8 / 5 / 1016: 00$ $\begin{array}{llllll}1500.243 & 1500.507 & 1500.599 & 1502.165 & 1500.917\end{array}$ $\begin{array}{lllll}1500.243 & 1500.503 & 1500.599 & 1502.181 & 1500.808\end{array}$ $\begin{array}{llllll}1500.243 & 1500.368 & 1500.604 & 1502.099 & 1500.857 \\ 1500234 & 1500.519 & 1500.597 & 1502.282 & 1500.915\end{array}$ $\begin{array}{lllllll}1500.234 & 1500.519 & 1500.597 & 1502.282 & 1500.915\end{array}$ $\begin{array}{llllll}1500.206 & 1500.477 & 1500.583 & 1502.427 & 1500.924 \\ 1500.194 & 1500.465 & 1500.581 & 1502.417 & 1500.948\end{array}$ $\begin{array}{llllll}1500.173 & 1500.454 & 1500.571 & 1502.106 & 1500.941\end{array}$ $\begin{array}{llllll}1500.175 & 1500.447 & 1500.576 & 1502.134 & 1500.955\end{array}$ $\begin{array}{lllll}1500.187 & 1500.459 & 1500.59 & 1502.181 & 1501.004\end{array}$ $\begin{array}{lllll}1500.187 & 1500.463 & 1500.592 & 1502.181 & 1501.039\end{array}$ $\begin{array}{llllll}1500.182 & 1500.456 & 1500.595 & 1502.172 & 1501.043 \\ 1500.152 & 1500.435 & 1500576 & 1502.085 & 150.1515\end{array}$ $\begin{array}{rrrrr}1500.152 & 1500.435 & 1500.576 & 1502.085 & 1501.015 \\ 1500.141 & 1500.428 & 1500.567 & 1502.083 & 1500.91\end{array}$ $\begin{array}{llllll}1500.141 & 1500.428 & 1500.567 & 1502.083 & 1500.91 \\ 1500.127 & 1500.419 & 1500.553 & 1502074 & 1500.728\end{array}$ $\begin{array}{llllll}1500.127 & 1500.417 & 1500.557 & 1502.118 & 1500.728\end{array}$ $\begin{array}{llllll}1500.12 & 1500.403 & 1500.548 & 1502.102 & 1500.784\end{array}$ $\begin{array}{llllll}1500.099 & 1500.391 & 1500.534 & 1502.074 & 1500.81\end{array}$ $\begin{array}{lllll}1500.092 & 1500.382 & 1500.532 & 1502.076 & 1500.845\end{array}$ $\begin{array}{llllll}1500.073 & 1500.372 & 1500.522 & 1502.057 & 1500.852\end{array}$ $\begin{array}{lllll}1500.068 & 1500.363 & 1500.525 & 1502.06 & 1500.873 \\ 1500.087 & 1500.365 & 1500.541 & 1502.132 & 1500896\end{array}$ $\begin{array}{lllllll}1500.087 & 1500.365 & 1500.541 & 1502.132 & 1500.896\end{array}$ $\begin{array}{lllllll}1500.082 & 1500.356 & 1500.534 & 1502.111 & 1500.791 \\ 1500.066 & 1500.347 & 1500.513 & 1502.085 & 1500.611\end{array}$ $\begin{array}{llllll}1500.059 & 1500.335 & 1500.508 & 1502.095 & 1500.637\end{array}$ $\begin{array}{llllll}1500.029 & 1500.321 & 1500.485 & 1502.036 & 1500.6\end{array}$ $\begin{array}{llllll}1500.024 & 1500.316 & 1500.483 & 1502.074 & 1500.518\end{array}$ $\begin{array}{llllll}1500.038 & 1500.314 & 1500.492 & 1502.125 & 1500.6\end{array}$ $\begin{array}{lllll}1500.031 & 1500.305 & 1500.492 & 1502.127 & 1500.693 \\ 1500.19 & 1500.29 & 1500.483 & 1502.106 & 1500.733\end{array}$ $\begin{array}{lllll}1500.019 & 1500.296 & 1500.483 & 1502.106 & 1500.733 \\ 1500.029 & 1500.293 & 1500.492 & 1502.142 & 1500.798\end{array}$ $\begin{array}{llllll}1500.036 & 1500.286 & 1500.504 & 1502.165 & 1500.843\end{array}$ $\begin{array}{llllll}1500.05 & 1500.291 & 1500.518 & 1502.205 & 1500.803\end{array}$ $\begin{array}{llllll}1500.045 & 1500.279 & 1500.506 & 1502.177 & 1500.674\end{array}$ $\begin{array}{lllll}1500.031 & 1500.27 & 1500.494 & 1502.139 & 1500.653 \\ 1500.031 & 1500.263 & 1500.494 & 1502.156 & 1500.709\end{array}$ $\begin{array}{lllll}1500.031 & 1500.263 & 1500.494 & 1502.156 & 1500.709 \\ 1500.008 & 1500.251 & 1500.492 & 1502.163 & 1500.682\end{array}$ $\begin{array}{lllll}150.008 & 1500.251 & 1500.492 & 1502.163 & 1500.682 \\ 1500.008 & 1500.251 & 1500.485 & 1502.137 & 1500.572\end{array}$ 

Well (ft AMSL) (ft AMSL) (ft AMSL) (ft AMSL) (ft AMSL) (ft AMSL) (ft AMSL) (ft AMSL) (ft AMSL) (ft AMSL) (ft AMSL) (ft AMSL) (ft AMSL) (ft AMSL) (ft AMSL) (ft AMSL) (ft AMSL) (ft AMSL) (ft AMSL) (ft AMSL) (ft AMSL) (ft AMSL) (ft AMSL) (ft AMSL) (ft AMSL) (ft AMSL)

$8 / 8 / 1016: 00$

$8 / 8 / 1020: 00$

$\begin{array}{rrrrr}1500.019 & 1500.249 & 1500.49 & 1502.193 & 1500.623 \\ 1500.012 & 1500.242 & 1500.487 & 1502.17 & 1500.606\end{array}$

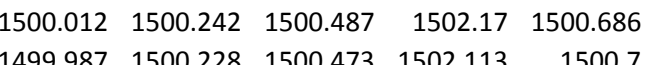

8/9/10 4:00

8/9/10 8:00

8/9/10 16:00

8/9/10 20:00

8/10/100:00

8/10/10 4:00

8/10/10 8:00

8/10/10 12:00

8/10/10 20:00

8/10/10 20:00

8/11/10 4:00

8/11/10 8:00

8/11/10 12:00

8/11/110 16:00

$8 / 11 / 1020: 00$

8/12/10 4:00

8/12/108:00

$8 / 12 / 10$ 12:00

8/12/10 16:00

8/12/10 20:00

$8 / 13 / 100: 00$

$8 / 13 / 104: 00$

$8 / 13 / 1012 \cdot 00$

$8 / 13 / 1016: 00$

8/13/10 20:00

8/14/100:00

$8 / 14 / 104: 00$

8/14/10 8:00

8/14/10 12:00

$8 / 14 / 1020.00$

$8 / 15 / 100: 00$

$8 / 15 / 104: 00$

8/15/10 8:00

$8 / 15 / 10$ 12:00

8/15/10 16:00

$8 / 15 / 1020: 00$

$8 / 16 / 100: 00$

$8 / 16 / 108: 00$

8/16/10 12:00

$8 / 16 / 1016: 00$

8/16/10 20:00

8/17/100:00

8/17/10 4:00

8/17/10 8:00

$\begin{array}{llllll}1499.975 & 1500.228 & 1500.473 & 1502.17 & 1500.686 & 1500.7\end{array}$

$\begin{array}{lllllll}1499.949 & 1500.209 & 1500.452 & 1502.067 & 1500.726\end{array}$

$\begin{array}{llllll}1499.94 & 1500.2 & 1500.443 & 1502.074 & 1500.499\end{array}$

$\begin{array}{lllll}1499.94 & 1500.095 & 1500.448 & 1502.109 & 1500.534\end{array}$

$\begin{array}{lllll}1499.928 & 1500.261 & 1500.438 & 1502.088 & 1500.595\end{array}$

$\begin{array}{llllll}1499.907 & 1500.174 & 1500.424 & 1502.05 & 1500.612\end{array}$

$\begin{array}{lllll}1499.912 & 1500.17 & 1500.429 & 1502.09 & 1500.665 \\ 1499891 & 1500.156 & 1500.417 & 1502.036 & 150066\end{array}$

\begin{tabular}{lllll}
149.891 & 1500.156 & 1500.417 & 1502.036 & 1500.66 \\
\hline
\end{tabular}

$\begin{array}{lllll}1499.9 & 1500.153 & 1500.434 & 1502.12 & 1500.726\end{array}$

$\begin{array}{lllll}1499.91 & 1500.284 & 1500.42 & 1502.508 & 1500.719\end{array}$

$\begin{array}{llllll}1499.884 & 1500.293 & 1500.417 & 1502.745 & 1500.712\end{array}$

$\begin{array}{llllll}1499.882 & 1500.277 & 1500.417 & 1502.845 & 1500.721\end{array}$

$\begin{array}{llllll}1499.861 & 1500.249 & 1500.403 & 1502.749 & 1500.628\end{array}$

$\begin{array}{llllll}1499.858 & 1500.23 & 1500.401 & 1501.915 & 1500.479\end{array}$

$\begin{array}{llllll}449.875 & 1499.988 & 1500.408 & 1502.097 & 1500.488\end{array}$

$\begin{array}{lllll}1499.856 & 1500.181 \quad 1500.394 \quad 1502.069 & 1500.56\end{array}$

$\begin{array}{lllll}1499.854 & 1500.216 & 1500.396 & 1502.081 & 1500.56\end{array}$ $\begin{array}{lllll}1499.844 & 1500.244 & 1500.389 & 1502.053 & 1500.614\end{array}$ $\begin{array}{llllll}1499.849 & 1499.904 & 1500.399 & 1502.083 & 1500.644 \\ & 149.875 & 1499.746 & 1500.422 & 1501.966 & 1500.709\end{array}$ $\begin{array}{llllll}1499.875 & 1499.746 & 1500.422 & 1501.966 & 1500.709 \\ 1499879 & 1500.021 & 1500.424 & 1502.174 & 1500.735\end{array}$ $\begin{array}{lllll}1499.879 & 1500.021 & 1500.424 & 1502.174 & 1500.735 \\ 1499.865 & 1500.23 & 1500.42 & 1502.343 & 1500.707\end{array}$ $\begin{array}{llllll}1499.872 & 1500.133 & 1500.431 & 1502.361 & 1500.726\end{array}$ $\begin{array}{llllll}4499.872 & 1500.126 & 1500.431 & 1502.303 & 1500.66 \\ 1499.877 & 1500.123 & 1500.431 & 1501.954 & 1500527\end{array}$ $\begin{array}{llllll}1499.893 & 1500.123 & 1500.441 & 1502.167 & 1500.56\end{array}$ $\begin{array}{llllll}1499.858 & 1500.102 & 1500.413 & 1502.291 & 1500.558\end{array}$ $\begin{array}{llllll}1499.816 & 1500.084 & 1500.385 & 1502.011 & 1500.516\end{array}$ $\begin{array}{llllll}1499.793 & 1500.072 & 1500.373 & 1501.992 & 1500.513\end{array}$ $\begin{array}{llllll}1499.772 & 1500.058 & 1500.359 & 1501.969 & 1500.504\end{array}$ $\begin{array}{llllll}1499.758 & 1500.051 & 1500.357 & 1501.976 & 1500.506 \\ 1499.753 & 1500.042 & 1500.357 & 1501994 & 1500525\end{array}$ $\begin{array}{llllll}1499.753 & 1500.042 \quad 1500.357 & 1501.994 \quad 1500.525\end{array}$ $\begin{array}{llllll}4499.718 & 1500.016 & 1500.327 & 1501.901 & 1500.474 \\ 1499.693 & 1500.007 & 1500.315 & 1501.898 & 1500.418\end{array}$ $\begin{array}{llllll}1499.66 & 1499.99 & 1500.287 & 1501.847 & 1500.387\end{array}$ $\begin{array}{llllll}1499.625 & 1499.97 & 1500.271 & 1501.81 & 1500.294\end{array}$ $\begin{array}{llllll}1499.606 & 1499.953 & 1500.247 & 1501.817 & 1500.128\end{array}$ $\begin{array}{llllll}1499.599 & 1500.044 & 1500.252 & 1501.962 & 1500.133\end{array}$ $\begin{array}{lllll}1499.611 & 1500.056 & 1500.238 & 1502.127 & 1500.205 \\ 1499588 & 1500.028 & 1500.229 & 1502.275 & 1500257\end{array}$ $\begin{array}{lllll}1499.588 & 1500.028 & 1500.229 & 1502.275 & 1500.257 \\ 1499.567 & 1499.993 & 1500.212 & 1502.366 & 1500.285\end{array}$ $\begin{array}{llllll}1499.555 & 1499.965 & 1500.201 & 1502.366 & 1500.285 \\ 1502338 & 1500.24\end{array}$ $\begin{array}{rrrrr}1499.555 & 1499.965 & 1500.201 & 1502.338 & 1500.24 \\ 1499.551 & 1499.944 & 1500.194 & 1502.046 & 1500.121\end{array}$ $\begin{array}{llllll}1499.572 & 1499.944 & 1500.208 & 1501.831 & 1500.198\end{array}$ $\begin{array}{llllll}1499.583 & 1499.937 & 1500.21 & 1501.924 & 1500.285\end{array}$ $\begin{array}{llllll}1499.567 & 1499.925 & 1500.198 & 1502.032 & 1500.322\end{array}$ $\begin{array}{lllll}1499.583 & 1500.13 & 1500.201 & 1502.13 & 1500.373\end{array}$ $\begin{array}{lllll}1499.576 & 1500.431 & 1500.205 & 1502.165 & 1500.427\end{array}$ 

Well

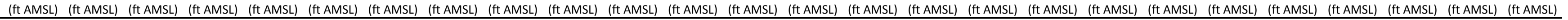

(8/17/1016:00 $1499.53 \quad 1500.412 \quad 1500.212 \quad 1502.046 \quad 1500.497$ $\begin{array}{llllll}1499.572 & 1500.349 & 1500.208 & 1502.106 & 1500.511 \\ 1499.56 & 1500.307 & 1500.203 & 1502.177 & 1500.504\end{array}$ $\begin{array}{rrrrrr}1499.56 & 1500.307 & 1500.203 & 1502.177 & 1500.504 \\ 1499.567 & 1500.27 & 1500.203 & 1502223 & 1500518\end{array}$ $\begin{array}{lllll}1499.567 & 1500.27 & 1500.203 & 1502.223 & 1500.518 \\ 1499.56 & 1500.244 & 1500.196 & 1502251 & 1500.485\end{array}$ 8/18/10 12:00 $8 / 18 / 1020: 00$ $8 / 19 / 100: 00$ $8 / 19 / 104: 00$ 8/19/10 8:00 8/19/10 16:00 8/19/10 20:00 $8 / 20 / 100: 00$ 8/20/10 4:00 8/20/10 8:00 8/20/10 12:00 8/20/10 16:00 $8 / 2011020: 00$
$8 / 21 / 100: 00$ $8 / 21 / 100: 00$ $8 / 21 / 108: 00$ 8/21/10 12:00 $8 / 21 / 1016: 00$ 8/21/10 20:00 $8 / 22 / 100: 00$ $8 / 22 / 104.00$ $8 / 22 / 1012 \cdot 00$ $8 / 22 / 1016: 00$ $8 / 22 / 1020: 00$ $8 / 23 / 100: 00$ $8 / 23 / 104: 00$ $8 / 23 / 108: 00$ $8 / 23 / 1012: 00$ $8 / 23 / 1016: 00$ $8 / 24 / 100: 00$ $8 / 24 / 104: 00$ $8 / 24 / 108: 00$ $8 / 24 / 1012: 00$ 8/24/10 16:00 $8 / 24 / 1020: 00$ $8 / 25 / 100: 00$ $8 / 25 / 104.00$ $8 / 25 / 1012: 00$ $8 / 25 / 1016: 00$ $8 / 25 / 1020: 00$ $8 / 26 / 100: 00$ $\begin{array}{llllll}1499.569 & 1500.226 & 1500.208 & 1502.015 & 1500.506\end{array}$ $\begin{array}{lllll}1499.597 & 1500.214 & 1500.229 & 1501.672 & 1500.565\end{array}$ $\begin{array}{llllll}1499.611 & 1500.2 & 1500.233 & 1501.992 & 1500.605\end{array}$ $\begin{array}{lllllll}1499.618 & 1500.184 & 1500.238 & 1501.976 & 1500.612\end{array}$ $\begin{array}{lllll}1499.639 & 1500.172 & 1500.254 & 1502.008 & 1500.649 \\ 1499.639 & 1500.156 & 1500.252 & 1501.969 & 1500644\end{array}$ $\begin{array}{llllll}1499.639 & 1500.156 & 1500.252 & 1501.969 & 1500.644\end{array}$ $\begin{array}{llllll}1499.655 & 1500.151 & 1500.268 & 1502.001 & 1500.663 \\ 1499.688 & 1500.151 & 1500.294 & 1501.976 & 1500.709\end{array}$ $\begin{array}{lllll}1499.704 & 1500.144 & 1500.306 & 1502.12 & 1500.738\end{array}$ $\begin{array}{lllll}1499.714 & 1500.135 & 1500.313 & 1502.23 & 1500.735\end{array}$ $\begin{array}{lllll}1499.721 & 1500.121 & 1500.32 & 1502.233 & 1500.742\end{array}$ $\begin{array}{llllll}1499.714 & 1500.2 & 1500.329 & 1502.165 & 1500.752\end{array}$ $\begin{array}{lllll}1499.725 & 1500.205 & 1500.338 & 1501.875 & 1500.756\end{array}$ $\begin{array}{llllll}1499.746 & 1500.209 & 1500.35 & 1502.004 & 1500.768 \\ 1499.728 & 1500.172 & 1500.334 & 1502.151 & 1500731\end{array}$ $\begin{array}{lllllll}149.728 & 1500.172 & 1500.334 & 1502.151 & 1500.731\end{array}$ $\begin{array}{lllll}1499.704 & 1500.133 & 1500.317 & 1501.94 & 1500.668 \\ 1499.688 & 1500.109 & 1500.308 & 1501.917 & 1500.637\end{array}$ $\begin{array}{llllll}1499.66 & 1500.086 & 1500.287 & 1501.854 & 1500.586\end{array}$ $\begin{array}{llllll}1499.644 & 1500.07 & 1500.278 & 1501.84 & 1500.553\end{array}$ $\begin{array}{llllll}1499.644 & 1500.06 & 1500.278 & 1501.868 & 1500.551\end{array}$ $\begin{array}{lllll}1499.632 & 1500.044 & 1500.266 & 1502.029 & 1500.49\end{array}$ $\begin{array}{rrrrr}1499.611 & 1500.028 & 1500.247 & 1501.812 & 1500.434 \\ 1499.604 & 1500.014 & 150024 & 1501.819 & 1500.446\end{array}$ $\begin{array}{rrrrrr}1499.604 & 1500.014 & 1500.24 & 1501.819 & 1500.446 \\ 1499.583 & 1499.995 & 1500.219 & 1501777 & 1500.338\end{array}$ $\begin{array}{llllll}1499.583 & 1499.995 & 1500.21 & 1501.777 & 1500.338 \\ 1499.586 & 1499.983 & 1500.212 & 1501817 & 1500.17\end{array}$ $\begin{array}{llllll}1499.59 & 1499.977 & 1500.212 & 1501.861 & 1500.212\end{array}$ $\begin{array}{lllll}1499.59 & 1499.965 & 1500.208 & 1501.866 & 1500.28\end{array}$ $\begin{array}{llllll}1499.576 & 1499.953 & 1500.201 & 1501.856 & 1500.324\end{array}$ $\begin{array}{llllll}1499.588 & 1499.944 & 1500.21 & 1501.884 & 1500.378\end{array}$ $\begin{array}{llllll}1499.579 & 1499.932 & 1500.198 & 1501.866 & 1500.329\end{array}$ $\begin{array}{llllll}1499.579 & 1499.925 & 1500.196 & 1501.889 & 1500.194 \\ 1499.59 & 1499.918 & 1500.198 & 1501919 & 1500.229\end{array}$ $\begin{array}{llllll}149.59 & 149.918 \quad 1500.198 \quad 1501.919 & 1500.229\end{array}$ $\begin{array}{llllll}1499.588 & 1499.911 & 1500.196 & 1501.915 & 1500.294 \\ 1499.551 & 1499.888 & 1500.17 & 1501.824 & 1500.289\end{array}$ $\begin{array}{llllll}4499.546 & 1500.13 & 1500.142 & 1501.908 & 1500.264\end{array}$ $\begin{array}{lllllll}1499.539 & 1500.5 & 1505.705 & 1502.074 & 1500.266\end{array}$ $\begin{array}{llllll}1499.45 & 1500.519 & 1514.548 & 1501.847 & 1500.273\end{array}$ $\begin{array}{llllll}1499.464 & 1500.438 & 1518.919 & 1501.76 & 1500.334\end{array}$ $\begin{array}{lllll}1499.462 & 1500.384 & 1516.835 & 1501.739 & 1500.38 \\ 1499.45 & 1500.344 & 1515075 & 1501.72 & 15039\end{array}$ $\begin{array}{llllll}1499.45 & 1500.344 & 1515.075 & 1501.721 & 1500.39 \\ 1499.448 & 1500.312 & 1513.587 & 1501.739 & 1500.42\end{array}$ $\begin{array}{lllll}1499.45 & 1500.344 & 1515.075 & 1501.721 & 1500.39\end{array}$ $\begin{array}{lllll}1499.441 & 1500.277 & 15132.294 & 1501.73 & 1500.348 \\ 1499.446 & 1500.249 & 1511.151 & 1501.768 & 1500.215\end{array}$ $\begin{array}{llllll}1499.464 & 1500.228 & 1510.165 & 1501.842 & 1500.266\end{array}$ $\begin{array}{llllll}1499.467 & 1500.202 & 1509.277 & 1501.84 & 1500.336\end{array}$

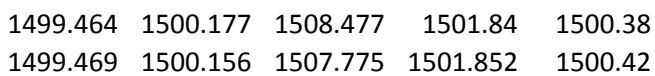

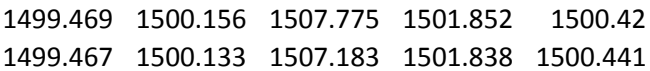
$\begin{array}{lllll}449.467 & 1500.133 & 1507.183 & 1501.838 & 1500.441 \\ 1499.476 & 1500.114 & 1506.736 & 1501.751 & 1500.467\end{array}$ 

Well (ft AMSL) (ft AMSL) (ft AMSL) (ft AMSL) (ft AMSL) (ft AMSL) (ft AMSL) (ft AMSL) (ft AMSL) (ft AMSL) (ft AMSL) (ft AMSL) (ft AMSL) (ft AMSL) (ft AMSL) (ft AMSL) (ft AMSL) (ft AMSL) (ft AMSL) (ft AMSL) (ft AMSL) (ft AMSL) (ft AMSL) (ft AMSL) (ft AMSL) (ft AMS)

$\begin{array}{lllll}1499.502 & 1500.102 & 1506.39 & 1501.927 & 1500.523\end{array}$

$8 / 26 / 1016.00$

$8 / 26 / 1020: 00$

$\begin{array}{rrrrrr}1499.518 & 1500.16 & 1506.136 & 1501.948 & 1500.565 \\ 1499.53 & 1500.226 & 1505889 & 1501952 & 1500.57\end{array}$

$\begin{array}{lllll}1499.53 & 1500.226 & 1505.889 & 1501.952 & 1500.57 \\ 1499.539 & 1500.202 & 1505.551 & 1501.966 & 1500.593\end{array}$

$\begin{array}{lllllll}1499.551 & 1500.165 & 1505.325 & 1501.957 & 1500.521\end{array}$

$8 / 27 / 108: 000$

8/27/10 16:00

$8 / 27 / 1020: 00$

$8 / 28 / 100: 00$

$8 / 28 / 104: 00$

$8 / 28 / 108: 00$

$8 / 28 / 1016: 00$

$8 / 28 / 1020: 00$

$8 / 29 / 100: 00$

8/29/10 4:00

$8 / 29 / 108: 00$

8/29/10 12:00

$8 / 29 / 1016: 00$

$8 / 30 / 100.00$

$8 / 30 / 104: 00$

$8 / 30 / 108: 00$

$8 / 30 / 1012: 00$

8/30/10 16:00

8/30/10 20:00

$8 / 31 / 100: 00$

$8 / 31 / 104.00$

$8 / 31 / 1012 \cdot 00$

$8 / 31 / 1016: 00$

$8 / 31 / 1020: 00$

9/1/100:00

9/1/10 4:00

9/1/10 8:00

$9 / 1 / 1012: 00$

$9 / 1 / 1016: 00$

$9 / 2 / 100: 00$

$9 / 2 / 104: 00$

9/2/10 8:00

$9 / 2 / 1012: 00$

$9 / 2 / 1012600$

9/2/10 20:00

$9 / 3 / 100: 00$
$9 / 3 / 104: 00$

$9 / 3 / 108.00$

9/3/10 12:00

$9 / 3 / 1016: 00$

$9 / 3 / 1020: 00$

9/4/100:00

9/4/10 4:00

9/4/10 8:00

$\begin{array}{llllll}1499.562 & 1499.869 & 1505.038 & 1501.887 & 1500.383\end{array}$

$\begin{array}{lllll}1499.593 & 1499.746 & 1505.09 & 1502.05 & 1500.432\end{array}$

$\begin{array}{lllll}49.600 & 1499.965 & 1504.903 & 1502.039 & 1500.497\end{array}$

$\begin{array}{llllll}1499.604 & 1500.107 & 1504.696 & 1502.02 & 1500.528\end{array}$

$\begin{array}{lllll}1499.611 & 1500.126 & 1504.509 & 1502.027 & 1500.56 \\ 149604 & 1500.114 & 1504327 & 150199 & 150.57\end{array}$

$\begin{array}{llllll}1499.604 & 1500.114 & 1504.327 & 1501.999 & 1500.57 \\ 1499.616 & 1499.83 & 1504.192 & 1502.034 & 1500.598\end{array}$

$\begin{array}{llllll}1499.616 & 1499.83 & 1504.192 & 1502.034 & 1500.598 \\ 1499.644 & 1499.723 & 1504.134 & 1502.079 & 1500.647\end{array}$

$\begin{array}{llllll}1499.646 & 1499.939 & 1504.054 & 1502.062 & 1500.665\end{array}$

$\begin{array}{llllll}1499.639 & 1500.088 & 1503.945 & 1502.032 & 1500.649\end{array}$

$\begin{array}{llllll}1499.641 & 1500.107 & 1503.786 & 1502.032 & 1500.647\end{array}$

$\begin{array}{llllll}1499.632 & 1500.079 & 1503.607 & 1501.997 & 1500.553\end{array}$

$\begin{array}{llllll}1499.632 & 1499.823 & 1503.458 & 1502.006 & 1500.399\end{array}$

$\begin{array}{rrrrrr}1499.641 & 1499.692 & 1503.32 & 1502.036 & 1500.418 \\ 1499.634 & 1499.876 & 1503.159 & 1502.015 & 1500.453\end{array}$

$\begin{array}{lllllll}149.634 & 149.872 & 1503.159 & 1502.015 & 1500.453\end{array}$

$\begin{array}{llllll}1499.604 & 1500.06 & 1502.819 & 1501.95 & 1500.481\end{array}$ $\begin{array}{rrrrr}1499.583 & 1500.044 & 1502.651 & 1501.915 & 1500.404 \\ 149.59 & 1499.76 & 1502.532 & 1501.962 & 1500.282\end{array}$ $\begin{array}{llllll}1499.59 & 1499.769 & 1502.532 & 1501.962 & 1500.282\end{array}$ $\begin{array}{llllll}1499.609 & 1499.895 & 1502.408 & 1501.863 & 1500.341 \\ 1499.607 & 1499888 & 1502.271 & 1502.013 & 1500.411\end{array}$ $\begin{array}{llllll}1499.607 & 1499.888 & 1502.271 & 1502.013 & 1500.411 \\ 1499.595 & 1499.879 & 1502.129 & 1502.104 & 1500.441\end{array}$ $\begin{array}{llllll}1499.593 & 1499.872 & 1502.01 & 1502.121 & 1500.476\end{array}$ $\begin{array}{lllll}1499.586 & 1499.862 & 1501.891 & 1502.088 & 1500.49 \\ 1499.586 & 1499.862 & 1501.795 & 1501.856 & 1500.521\end{array}$ $\begin{array}{llllll}1499.606 & 1499.862 & 1501.711 & 1501.721 & 1500.563\end{array}$ $\begin{array}{llllll}1499.595 & 1499.851 & 1501.609 & 1501.952 & 1500.57\end{array}$ $\begin{array}{llllll}1499.579 & 1499.841 & 1501.499 & 1502.079 & 1500.537\end{array}$ $\begin{array}{llllll}1499.583 & 1499.837 & 1501.432 & 1502.128 & 1500.539\end{array}$ $\begin{array}{llllll}1499.576 & 1499.834 & 1501.355 & 1502.139 & 1500.469\end{array}$ $\begin{array}{llllll}1499.586 & 1499.834 & 1501.292 & 1501.8 & 1500.324 \\ 1499597 & 1499.834 & 1501.233 & 1501.779 & 1500338\end{array}$ $\begin{array}{lllllll}1499.597 & 1499.834 & 1501.233 & 1501.779 & 1500.338\end{array}$ $\begin{array}{llllllll}1499.602 & 149983 & 1501.115 & 1502.0069 & 1500.487\end{array}$ $\begin{array}{lllllll}1499.588 & 1499.818 & 1501.047 & 1501.983 & 1500.458\end{array}$ $\begin{array}{llllll}1499.574 & 1499.811 & 1500.982 & 1501.943 & 1500.453\end{array}$ $\begin{array}{llllll}1499.541 & 1499.797 & 1500.909 & 1501.856 & 1500.425\end{array}$ $\begin{array}{lllllll}1499.499 & 1499.776 & 1500.825 & 1501.906 & 1500.357\end{array}$ $\begin{array}{lllll}1499.474 & 1499.767 & 1500.774 & 1502.008 & 1500.336 \\ 1499.432 & 1499.748 & 1500.702 & 1501.697 & 1500.289\end{array}$ $\begin{array}{llllll}1499.432 & 1499.748 & 1500.702 & 1501.697 & 1500.289 \\ 1499.411 & 1499.737 & 1500.662 & 1501702 & 1500271\end{array}$ $\begin{array}{lllllll}1499399 & 1499.725 & 1500.618 & 1501.602 & 1500.271\end{array}$ $\begin{array}{rrrrrr}1499.39 & 1499.72 & 1500.576 & 1501.712 & 1500.189\end{array}$ $\begin{array}{llllll}1499.415 & 1499.72 & 1500.569 & 1501.789 & 1500.1\end{array}$ $\begin{array}{llllll}1499.411 & 1499.711 & 1500.536 & 1501.791 & 1500.173\end{array}$ $\begin{array}{llllll}1499.399 & 1499.704 & 1500.494 & 1501.77 & 1500.203\end{array}$ $\begin{array}{llllll}1499.406 & 1499.702 & 1500.476 & 1501.796 & 1500.245\end{array}$ $\begin{array}{llllll}1499.406 & 1499.695 & 1500.448 & 1501.796 & 1500.273\end{array}$ 

Well (ft AMSL) (ft AMSL) (ft AMSL) (ft AMSL) (ft AMSL) (ft AMSL) (ft AMSL) (ft AMSL) (ft AMSL) (ft AMSL) (ft AMSL) (ft AMSL) (ft AMSL) (ft AMSL) (ft AMSL) (ft AMSL) (ft AMSL) (ft AMSL) (ft AMSL) (ft AMSL) (ft AMSL) (ft AMSL) (ft AMSL) (ft AMSL) (ft AMSL) (ft AMSL)

9/4/10 16:00

$9 / 4 / 1020: 00$ $\begin{array}{rrrrr}1499.471 & 1499.72 & 1500.462 & 1501.948 & 1500.408 \\ 1499.488 & 1499.725 & 1500.45 & 1501905 & 1500.45\end{array}$

$9 / 5 / 100: 00$ $\begin{array}{llllll}1499.488 & 1499.725 & 1500.45 & 1501.945 & 1500.453 \\ 1499.49 & 1499.718 & 1500.431 & 1501.936 & 1500.455\end{array}$

9/5/10 4:00

9/5/10 12:00

9/5/10 16:00

9/5/10 20:00

9/6/100:00

9/6/10 4:00

9/6/10 8:00

$9 / 6 / 1016: 00$

$9 / 6 / 1020.00$

$9 / 6 / 1020: 00$
$9 / 7 / 100: 00$

9/7/10 4:00

9/7/10 8:00

9/7/10 12:00

$9 / 7 / 1012600$

$9 / 7 / 1020: 00$

9/8/100:00

9/8/10 8:00

9/8/10 8:00

9/8/10 16:00

9/8/10 20:00

9/9/10 0:00

9/9/10 4:00

$9 / 9 / 1012.00$

$9 / 9 / 1016: 00$

9/9/10 20:00

9/10/10 0:00

$9 / 10 / 104: 00$

9/10/10 8:00

$9 / 10 / 1012: 00$

$9 / 10 / 1016: 00$

$9 / 11 / 100: 00$

$9 / 11 / 104: 00$

9/11/10 8:00

9/11/10 12:00

9/11/10 16:00

9/11/10 20:00

$9 / 12 / 100: 00$

$9 / 12 / 108: 00$

$9 / 12 / 1012: 00$

9/12/10 16:00

9/12/10 20:00

$9 / 13 / 100: 00$

9/13/10 4:00

$9 / 13 / 108: 00$

$\begin{array}{llllll}1499.49 & 1499.718 & 1500.431 & 1501.936 & 1500.455 \\ 1499.511 & 1499.725 & 1500.424 & 1501.966 & 1500.472\end{array}$

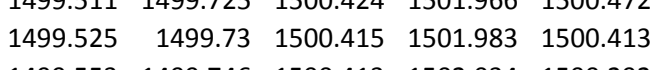

$\begin{array}{llllll}1499.553 & 1499.746 & 1500.413 & 1502.034 & 1500.292 \\ 149.586 & 149.76 & 1500.417 & 1502.069 & 1500.343\end{array}$

$\begin{array}{llllll}1499.586 & 1499.76 & 1500.417 & 1502.069 & 1500.343 \\ & 1490.59 & 149.758 & 150.45 & 152.03 & 15039\end{array}$

$\begin{array}{lllll}1499.59 & 1499.758 & 1500.401 & 1502.043 & 1500.39 \\ 1499.588 & 1499.753 & 1500.387 & 1502.025 & 1500.404\end{array}$

$\begin{array}{llllll}1499.59 & 1499.753 & 1500.383 & 1502.034 & 1500.432\end{array}$

$\begin{array}{llllll}1499.583 & 1499.744 & 1500.362 & 1502.001 & 1500.362 \\ 1499.574 & 1499.734 & 1500.345 & 1501.987 & 1500201\end{array}$

$\begin{array}{lllllll}1499.567 & 1499.762 & 1500.299 & 1502.086 & 1500.152\end{array}$

$\begin{array}{llllll}1499.471 & 1499.671 & 1500.245 & 1502.205 & 1500.077\end{array}$

$\begin{array}{llllll}1499.418 & 1499.653 & 1500.215 & 1501.704 & 1500.04\end{array}$

$\begin{array}{lllll}1499.39 & 1499.641 & 1500.196 & 1501.693 & 1500.056\end{array}$

$\begin{array}{rrrrr}1499.35 & 1499.625 & 1500.168 & 1501.646 & 1500.049 \\ 1499.329 & 1499.571 & 1500.154 & 1501.648 & 1500.056\end{array}$ $\begin{array}{lrrrrr}1499.329 & 1499.571 & 1500.154 & 1501.648 & 1500.056 \\ 1499.341 & 1499.49 & 1500.156 & 1501.721 & 1500.117\end{array}$ $\begin{array}{llllll}1499.331 & 1499.609 & 1500.138 & 1501.709 & 1500.145 \\ & 149.45 & 1500.133 & 1501.726 & 1500.184\end{array}$ $\begin{array}{lllll}1499.329 & 1499.595 & 1500.133 & 1501.726 & 1500.159 \\ 149.350 & 1500.128 & 1501.742 & 1500.184\end{array}$ $\begin{array}{lllllll}1499.324 & 1499.588 & 1500.114 & 1501.733 & 1500.107\end{array}$ $\begin{array}{lllllll}1499.336 & 1499.497 & 1500.114 & 1501.775 & 1499.974\end{array}$ $\begin{array}{llllll}1499.357 & 1499.553 & 1500.114 & 1501.884 & 1500.019\end{array}$ $\begin{array}{llllll}1499.373 & 1499.709 & 1500.105 & 1502.053 & 1500.084\end{array}$ $\begin{array}{rrrrr}1499.366 & 1499.727 & 1500.098 & 1502.135 & 1500.131 \\ 1499362 & 4499779 & 1500.117 & 1502.13 & 1500.205\end{array}$ $\begin{array}{rrrrr}1499.362 & 1499.779 & 1500.117 & 1502.13 & 1500.205 \\ 1499.35 & 1500.016 & 1500.117 & 1502.104 & 1500227\end{array}$ $\begin{array}{lllll}1499.35 & 1500.016 & 1500.117 & 1502.104 & 1500.247 \\ 1499.28 & 1500.007 & 1500.121 & 1501.985 & 1500273\end{array}$ $\begin{array}{llllll}1499.266 & 1499.911 & 1500.131 & 1501.817 & 1500.313\end{array}$ $\begin{array}{llllll}1499.404 & 1499.937 & 1500.131 & 1501.859 & 1500.324\end{array}$ $\begin{array}{lllll}1499.399 & 1499.979 & 1500.131 & 1501.952 & 1500.324\end{array}$ $\begin{array}{llllll}1499.42 & 1499.93 & 1500.145 & 1501.969 & 1500.357\end{array}$ $\begin{array}{lllll}1499.415 & 1499.914 & 1500.133 & 1501.938 & 1500.278\end{array}$ $\begin{array}{llllll}1499.408 & 1499.781 & 1500.119 & 1501.772 & 1500.112 \\ 1499.415 & 1499888 & 1500117 & 1501.873 & 1500.112\end{array}$ $\begin{array}{lllllll}1499.415 & 1499.888 & 1500.117 & 1501.873 & 1500.112\end{array}$ $\begin{array}{lllllll}1499.338 & 1499.83 & 1500.051 & 1501.714 & 1500.072\end{array}$ $\begin{array}{llllll}1499.296 & 1499.802 & 1500.021 & 1501.637 & 1500.03\end{array}$ $\begin{array}{lllllll}1499.264 & 1499.776 & 1500.009 & 1501.616 & 1500.007\end{array}$ $\begin{array}{llllll}4499.243 & 1499.755 & 1499.995 & 1501.592 & 1500.005\end{array}$ $\begin{array}{llllll}1499.25 & 1499.744 & 1500 & 1501.655 & 1500.054\end{array}$ $\begin{array}{rrrrr}1499.238 & 1499.723 & 1499.984 & 1501.63 & 1500.042\end{array}$ $\begin{array}{llllll}1499.217 & 1499.704 & 1499.965 & 1501.602 & 1500.009 \\ 1499.212 & 1499.685 & 1499.961 & 1501.609 & 1500.019\end{array}$ $\begin{array}{llllll}1499.196 & 1499.667 & 1499.944 & 1501.597 & 1499.937\end{array}$ $\begin{array}{llllll}1499.194 & 1499.655 & 1499.937 & 1501.613 & 1499.795\end{array}$ $\begin{array}{llllll}1499.219 & 1499.648 & 1499.944 & 1501.686 & 1499.848\end{array}$ $\begin{array}{llllll}1499.208 & 1499.63 & 1499.93 & 1501.655 & 1499.9\end{array}$ $\begin{array}{llllll}1499.208 & 1499.62 & 1499.926 & 1501.66 & 1499.939\end{array}$ $\begin{array}{llllll}1499.205 & 1499.606 & 1499.921 & 1501.66 & 1499.974\end{array}$ $\begin{array}{lllll}1499.196 & 1499.592 & 1499.909 & 1501.623 & 1499.918 \\ 1499205 & 1499.585 & 1499.907 & 1501.674 & 1499.804\end{array}$ 
$\begin{array}{lllll}1499.191 & 1499.404 & 1499.888 & 1501.651 & 1500.007 \\ 1499.21 & 149.222 & 1499.905 & 1501.712 & 1500.056\end{array}$

$9 / 14 / 1020: 00$

$\begin{array}{llllll}1499.201 & 149.26 & 149.905 & 1501.712 & 1500.056\end{array}$

$9 / 15 / 100.00$

$\begin{array}{llllll}1499.205 & 1499.581 & 1499.902 & 1501.695 & 1500.068\end{array}$ $\begin{array}{rrrrr}1499.212 & 1499.62 & 1499.905 & 1501.7 & 1500.089 \\ 1499.189 & 1499.555 & 14999 & 1501.707 & 1500\end{array}$

$9 / 15 / 108: 00$

$9 / 15 / 1012: 00$

$\begin{array}{rrrrr}1499.189 & 1499.555 & 1499.9 & 1501.707 & 1500 \\ 1499.196 & 1499.481 & 1499 & 1501637 & 1499874\end{array}$

$\begin{array}{rrrrr}1499.196 & 1499.481 & 1499.9 & 1501.637 & 1499.874 \\ 1499.238 & 1499.271 & 1499.921 & 1501.359 & 1499.939\end{array}$ $\begin{array}{lllllll}1499.191 & 1499.527 & 1499.879 & 1501.627 & 1499.93\end{array}$

$9 / 15 / 100: 00$

$9 / 16 / 104: 00$

9/16/10 8:00

$\begin{array}{rrrrr}1499.191 & 1499.527 & 1499.879 & 1501.627 & 1499.93 \\ 1499.171 & 1499.616 & 1499.867 & 1501.772 & 1499.918\end{array}$

$\begin{array}{lllll}1499.152 & 1499.616 & 1499.853 & 1501.826 & 1499.916\end{array}$

$\begin{array}{llllll}1499.129 & 1499.583 & 1499.835 & 1501.922 & 1499.895\end{array}$

$\begin{array}{llllll}1499.11 & 1499.469 & 1499.825 & 1501.768 & 1499.886\end{array}$

$9 / 16 / 1016: 00$

$9 / 16 / 1020: 00$

$\begin{array}{lllll}1499.126 & 1499.369 & 1499.839 & 1501.606 & 1499.939 \\ 1499.138 & 1499.425 & 1499842 & 1501.686 & 1499.984\end{array}$

$\begin{array}{llllll}1499.131 & 1499.497 & 1499.837 & 1501.736 & 14999.984 \\ 149.891\end{array}$

$\begin{array}{llllll}1499.133 & 1499.509 & 1499.837 & 1501.716 & 1499.993\end{array}$

$\begin{array}{llllll}1499.129 & 1499.481 & 1499.83 & 1501.658 & 1499.907\end{array}$

$9 / 17 / 1012: 00$

9/17/10 16:00

9/17/10 20:00

9/18/100:00

$9 / 18 / 104: 00$

$9 / 18 / 1012 \cdot 00$

9/18/1012:00

$9 / 18 / 1020: 00$

9/19/10 0:00

$9 / 19 / 104: 00$

9/19/10 8:00

$9 / 19 / 1012: 00$

$9 / 19 / 1016: 00$

$9 / 20 / 100: 00$

$9 / 20 / 104: 00$

9/20/10 8:00

9/20/10 12:00

9/20/10 16:00

$9 / 20 / 1020: 00$

9/21/100:00

$9 / 21 / 108: 00$

9/21/10 12:00

$9 / 21 / 1016: 00$

9/21/10 20:00

9/22/100:00

9/22/10 4:00

$9 / 22 / 108: 00$

$\begin{array}{llllll}1499.136 & 1499.355 & 1499.83 & 1501.599 & 1499.764 \\ 1499.159 & 1499.144 & 1499842 & 1501 & 1499.816\end{array}$

$\begin{array}{lllll}1499.159 & 1499.194 & 1499.842 & 1501.662 & 1499.816 \\ 1499.434 & 1499.83 & 1501.625 & 1499.862\end{array}$ $\begin{array}{rrrrr}1499.136 & 1499.584 & 1499.83 & 1501.625 & 1499.862 \\ 1499.818 & 1501.59 & 1499.874\end{array}$ $\begin{array}{lllll}1499.133 & 1499.592 & 1499.816 & 1501.59 & 1499.895 \\ 1499.115 & 1499.588 & 1499.802 & 1501.548 & 1499.89\end{array}$ $\begin{array}{lllll}1499.115 & 1499.588 & 1499.802 & 1501.548 & 1499.89 \\ 1499.117 & 1499297 & 1499809 & 1501.576 & 1499.907\end{array}$ $\begin{array}{llllll}1499.133 & 1499.143 & 1499.821 & 1501.62 & 1499.946\end{array}$ $\begin{array}{llllll}1499.11 & 1499.411 & 1499.797 & 1501.539 & 1499.925\end{array}$ $\begin{array}{llllll}1499.091 & 1499.578 & 1499.788 & 1501.515 & 1499.89\end{array}$ $\begin{array}{rrrrr}1499.08 & 1499.606 & 1499.779 & 1501.499 & 1499.876 \\ 1499.068 & 1499.574 & 1499.769 & 1501.492 & 1499.79\end{array}$ $\begin{array}{llllll}1499.068 & 1499.574 & 1499.769 & 1501.492 & 1499.79\end{array}$ $\begin{array}{llllll}1499.061 & 1499.371 & 1499.76 & 1501.499 & 1499.631 \\ 1499.091 & 1499.378 & 1499.781 & 1501.592 & 1499.701\end{array}$ $\begin{array}{llllll}449.091 & 1499.378 & 1499.781 & 1501.592 & 1499.701 \\ 1499.094 & 1499.369 & 1499.774 & 1501583 & 1499776\end{array}$ $\begin{array}{llllll}1499.094 & 1499.369 & 1499.774 & 1501.583 & 1499.776 \\ 1499.091 & 1499.362 & 1499.774 & 1501.581 & 1499.809\end{array}$ $\begin{array}{lllllll}1499.098 & 1499.357 & 1499.779 & 1501.602 & 1499.858\end{array}$ $\begin{array}{lllllll}1499.094 & 1499.35 & 1499.774 & 1501.578 & 1499.797\end{array}$ $\begin{array}{llllll}1499.103 & 1499.35 & 1499.774 & 1501.62 & 1499.68\end{array}$ $\begin{array}{llllll}1499.131 & 1499.362 & 1499.795 & 1501.683 & 1499.75\end{array}$ $\begin{array}{llllll}1499.136 & 1499.355 & 1499.795 & 1501.667 & 1499.827\end{array}$ $\begin{array}{llllll}1499.126 & 1499.346 & 1499.79 & 1501.644 & 1499.848 \\ 1499.129 & 1499.343 & 1499.795 & 1501.651 & 1499879\end{array}$ (4) $\begin{array}{lllllll}1499.119 & 1499.334 & 1499.795 & 1501.632 & 14999.9\end{array}$ $\begin{array}{lllllll}1499.124 & 1499.332 & 1499.804 & 1501.644 & 1499.939\end{array}$ $\begin{array}{llllll}1499.117 & 1499.325 & 1499.797 & 1501.616 & 1499.942\end{array}$ $\begin{array}{llllll}1499.096 & 1499.315 & 1499.781 & 1501.557 & 1499.907\end{array}$ $\begin{array}{lllllll}1499.08 & 1499.306 & 1499.779 & 1501.543 & 1499.883\end{array}$ $\begin{array}{lllll}1499.066 & 1499.299 & 1499.767 & 1501.527 & 1499.79 \\ 1499.068 & 1499.299 & 1499.762 & 1501.543 & 1499.638\end{array}$ 

Well

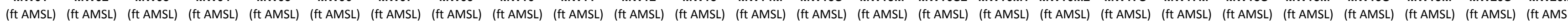

9/22/10 16:00

$\begin{array}{lllll}1499.08 & 1499.297 & 1499.767 & 1501.595 & 1499.682\end{array}$

$\begin{array}{llllll}1499.066 & 1499.287 & 1499.758 & 1501.557 & 1499.727 \\ 1499.059 & 1499283 & 1499748 & 1501546 & 1499.75\end{array}$

$\begin{array}{lllllll}1499.07 & 1499.28 & 1499.762 & 1501.546 & 1499.75\end{array}$

$\begin{array}{lllllll}149.07 & 1499.28 & 1499.762 & 151.546 & 1499.75 \\ 1499.061 & 1499.276 & 1499.76 & 1501.578 & 1499.834\end{array}$

$\begin{array}{llllll}1499.038 & 1499.262 & 1499.755 & 1501.517 & 1499.846\end{array}$

$9 / 23 / 108: 000$

9/23/10 16:00

9/23/10 20:00

$\begin{array}{lllllll}1499.035 & 1499.513 & 1499.762 & 1501.546 & 1499.869\end{array}$

$\begin{array}{llllll}1499.049 & 1499.758 & 1499.732 & 1501.683 & 1499.825\end{array}$

$\begin{array}{lllllll}1499.04 & 1499.842 & 1499.706 & 1501.819 & 1499.776\end{array}$

$9 / 24 / 104: 00$

$9 / 24 / 108: 00$
$9 / 24 / 1012: 00$

$9 / 24 / 1016: 00$

$9 / 24 / 1020: 00$

9/25/10 0:00

9/25/10 4:00

9/25/10 8:00

9/25/10 12:00

$9 / 25 / 1016: 00$

$9 / 25 / 1020: 00$

$9 / 26 / 104: 00$

$9 / 26 / 108: 00$

9/26/10 12:00

9/26/10 16:00

9/26/10 20:00

9/27/100:00

$9 / 27 / 104.00$

$9 / 27 / 1012 \cdot 00$

9/27/10 16:00

9/27/10 20:00

9/28/100:00

$9 / 28 / 104: 00$

9/28/10 8:00

$9 / 28 / 1012: 00$

$9 / 28 / 1016: 00$

$9 / 29 / 100: 00$

$9 / 29 / 104: 00$

9/29/10 8:00

9/29/10 12:00

9/29/10 16:00

9/29/10 20:00

9/30/100:00

$9 / 30 / 108: 00$

9/30/10 12:00

9/30/10 16:00

9/30/10 20:00

10/1/10 0:00

10/1/10 4:00

10/1/10 8:00

$\begin{array}{lllll}1499 & 1499.835 & 1499.683 & 1501.957 & 1499.732\end{array}$

$\begin{array}{llllll}1498.942 & 1499.755 & 1499.655 & 1502.025 & 1499.598 \\ 1498.919 & 1499.7 & 1499653 & 1501.644 & 1499.44\end{array}$

$\begin{array}{lllllll}1498.926 & 1499.669 & 1499.657 & 1501.45 & 1499.496\end{array}$

$\begin{array}{llllll}1498.916 & 1499.639 & 1499.643 & 1501.508 & 1499.563\end{array}$

$\begin{array}{lllllll}1498.909 & 1499.609 & 1499.639 & 1501.377 & 1499.608\end{array}$

$\begin{array}{llllll}1498.914 & 1499.583 & 1499.643 & 1501.408 & 1499.666\end{array}$

$\begin{array}{llllll}1498.909 & 1499.557 & 1499.634 & 1501.359 & 1499.689\end{array}$

$\begin{array}{llllll}1498.935 & 1499.546 & 1499.653 & 1501.438 & 1499.741\end{array}$

\begin{tabular}{llllll}
4498.947 & 1499.55 & 1499.653 & 1501.639 & 1499.788 \\
\hline
\end{tabular}

$\begin{array}{llllll}1498.914 & 149.532 & 1499.622 & 1501.994 & 1499.75 \\ \end{array}$

$\begin{array}{llllll}1498.905 & 1499.499 & 1499.606 & 1502.256 & 1499.741\end{array}$

$\begin{array}{lllllll}1498.888 & 1499.478 & 1499.583 & 1502.219 & 1499.652\end{array}$

$\begin{array}{llllll}1498.891 & 1499.464 & 1499.578 & 1501.805 & 1499.514\end{array}$ $\begin{array}{llllll}1498.919 & 1499.457 & 1499.604 & 1501.422 & 1499.573\end{array}$ $\begin{array}{llllll}1498.921 & 1499.439 & 1499.601 & 1501.445 & 1499.645\end{array}$ $\begin{array}{llllll}1498.921 & 1499.425 & 1499.602 & 1501.426 & 1499.689 \\ 1499828 & 1499.413 & 1499.608 & 1501.45 & 1499\end{array}$ $\begin{array}{llllll}1498.928 & 1499.413 & 1499.608 & 1501.445 & 1499.732 \\ 1498.928 & 1499.399 & 1499.604 & 1501.454 & 1499.682\end{array}$ $\begin{array}{lllllll}1498.928 & 1499.39 & 1499.604 & 1501.454 & 1499.682 \\ 1499.394 & 1499.611 & 1501.468 & 1499.568\end{array}$ $\begin{array}{lllll}1498.965 & 1499.39 & 1499.632 & 1501.506 & 1499.626\end{array}$ $\begin{array}{llllll}1498.972 & 1499.378 & 1499.636 & 1501.508 & 1499.692\end{array}$ $\begin{array}{llllll}1498.968 & 1499.364 & 1499.632 & 1501.487 & 1499.727\end{array}$ $\begin{array}{llllll}1498.965 & 1499.353 & 1499.632 & 1501.471 & 1499.755\end{array}$ $\begin{array}{llllll}1498.947 & 1499.341 & 1499.615 & 1501.454 & 1499.75 \\ \end{array}$ $\begin{array}{llllll}1498.949 & 1499.332 & 1499.623 & 1501.438 & 1499.764 \\ 1498.979 & 1499.334 & 1499.655 & 1501522 & 1499.832\end{array}$ $\begin{array}{llllll}1488.979 & 1499.334 & 1499.655 & 1501.522 & 1499.832\end{array}$ $\begin{array}{llllll}1498.984 & 1499.32 & 1499.662 & 1501.489 & 1499.862\end{array}$ $\begin{array}{lllllll}1498.993 & 1499.318 & 1499.671 & 1501.501 & 1499.876\end{array}$ $\begin{array}{llllll}1498.986 & 1499.306 & 1499.664 & 1501.473 & 1499.785\end{array}$ $\begin{array}{lllllll}1499.003 & 1499.304 & 1499.669 & 1501.506 & 1499.647\end{array}$ $\begin{array}{llllll}1499.01 & 1499.301 & 1499.674 & 1501.513 & 1499.671\end{array}$ $\begin{array}{llllll}1498.977 & 1499.283 & 1499.643 & 1501.433 & 1499.673 \\ 1499833 & 149262 & 1499.606 & 150135 & 1499629\end{array}$ $\begin{array}{llllll}1498.933 & 1499.262 & 1499.606 & 1501.335 & 1499.629 \\ 1498.905 & 1499.245 & 1499.587 & 1501.298 & 1499.615\end{array}$ $\begin{array}{llllll}1498.905 & 149.225 & 1499.587 & 1501.298 & 1499.615\end{array}$ $\begin{array}{llllll}1498.865 & 1499.22 & 1499.567 & 1501.277 & 1499.598\end{array}$ $\begin{array}{llllll}1498.877 & 1499.215 & 1499.587 & 1501.323 & 1499.661\end{array}$ $\begin{array}{llllll}1498.863 & 1499.204 & 1499.574 & 1501.286 & 1499.666\end{array}$ $\begin{array}{llllll}1498.853 & 1499.194 & 1499.562 & 1501.265 & 1499.654\end{array}$ $\begin{array}{lllllll}1498.846 & 1499.185 & 1499.56 & 1501.272 & 1499.659\end{array}$ $\begin{array}{llllll}1498.828 & 1499.173 & 1499.543 & 1501.244 & 1499.566\end{array}$ 

Well (ft AMSL) (ft AMSL) (ft AMSL) (ft AMSL) (ft AMSL) (ft AMSL) (ft AMSL) (ft AMSL) (ft AMSL) (ft AMSL) (ft AMSL) (ft AMSL) (ft AMSL) (ft AMSL) (ft AMSL) (ft AMSL) (ft AMSL) (ft AMSL) (ft AMSL) (ft AMSL) (ft AMSL) (ft AMSL) (ft AMSL) (ft AMSL) (ft AMSL) (ft AMS)

(10/1/1016:00

$1498.8561499 .1691499 .564 \quad 1501.342 \quad 1499.479$

$\begin{array}{rrrrr}1498.849 & 1499.157 & 1499.553 & 1501.321 & 1499.535 \\ 1498846 & 1499.15 & 149955 & 1501321 & 1499573\end{array}$

$\begin{array}{rrrrr}1498.846 & 1499.15 & 1499.55 & 1501.321 & 1499.573 \\ 1498.821 & 1499.136 & 1499.527 & 1501251 & 1499.568\end{array}$

$\begin{array}{lllllll}1498.779 & 1499.115 & 1499.49 & 1501.169 & 1499.524\end{array}$

10/2/1016:00

10/2/10 20:00

$\begin{array}{llllll}1498.767 & 1499.11 & 1499.483 & 1501.183 & 1499.512\end{array}$

$\begin{array}{llllll}1498.779 & 1499.103 & 1499.499 & 1501.235 & 1499.561\end{array}$

$\begin{array}{llllll}1498.765 & 1499.094 & 1499.487 & 1501.197 & 1499.573 \\ 1498.753 & 1499.082 & 1499.478 & 1501.176 & 1499.563 \\ 149.748 & 1499.075 & 1999.476 & 1501.88 & 149953\end{array}$

$1 / 3 / 10000$

$\begin{array}{lllll}1498.748 & 1499.075 & 1499.476 & 1501.188 & 1499.573 \\ 1498732 & 1499.064 & 1499.462 & 1501.148 & 149955\end{array}$

10/3/10 8:00

10/3/1012 16:00

$\begin{array}{lllll}1498.732 & 1499.064 & 1499.462 & 1501.148 & 1499.556 \\ 1498.744 & 1499061 & 1499.473 & 1501.197 & 1499.57\end{array}$

$10 / 3 / 1020: 00$

$\begin{array}{rrrrr}1498.744 & 1499.061 & 1499.473 & 1501.197 & 1499.57 \\ 1498.776 & 1499.071 & 1499.504 & 1501.272 & 1499.636\end{array}$

$\begin{array}{llllll}1498.779 & 1499.061 & 1499.501 & 1501.256 & 1499.652\end{array}$

$\begin{array}{llllll}1498.774 & 1499.052 & 1499.494 & 1501.235 & 1499.64\end{array}$

$10 / 4 / 104: 00$

10/4/10 8:00

$\begin{array}{llllll}1498.781 & 1499.048 & 1499.497 & 1501.239 & 1499.645\end{array}$

$\begin{array}{llllll}1498.769 & 1499.041 & 1499.49 & 1501.216 & 1499.631\end{array}$

$\begin{array}{lllll}1498.788 & 1499.043 & 1499.504 & 1501.249 & 1499.645\end{array}$

(10/4/10800

$10 / 4 / 1020: 00$

$\begin{array}{rrrrr}1498.814 & 1499.052 & 1499.525 & 1501.305 & 1499.694 \\ 1498811 & 1499.043 & 149952 & 150127 & 1499.69\end{array}$

$\begin{array}{llllll}1498.811 & 1499.043 & 1499.52 & 1501.27 & 1499.699\end{array}$

$\begin{array}{lllll}1498.809 & 1499.038 & 1499.515 & 1501.253 & 1499.678 \\ 1498.804 & 1499.034 & 1499.513 & 1501.239 & 1499.666\end{array}$

$\begin{array}{llllll}1498.793 & 1499.024 & 1499.506 & 1501.207 & 1499.645\end{array}$

10/5/108:00

10/5/10 16:00

10/5/10 20:00

10/6/10 0:00

10/6/10 4:00

$10 / 6 / 1012 \cdot 00$

0/6/10 16:00

10/6/10 20:00

10/7/100:00

$10 / 7 / 104: 00$

10/7/10 8:00

10/7/10 12:00

10/7/10 16:00

$10 / 8 / 100: 00$

$10 / 8 / 104: 00$

$10 / 8 / 108: 00$

0/8/10 12:00

10/8/10 16:00

10/8/10 20:00

10/9/10 0:00

$10 / 9 / 108: 00$

10/9/10 12:00

10/9/10 16:00

10/9/10 20:00

10/10/10 0:00

10/10/10 4:00

10/10/10 8:00

$\begin{array}{llllll} & & & \end{array}$ $\begin{array}{lllll}148.823 & 1499.034 & 1499.527 & 1501.279 & 1499.682\end{array}$ $\begin{array}{llllll}1498.816 & 1499.024 & 1499.52 & 1501.244 & 1499.675\end{array}$ $\begin{array}{lllll}1498.811 & 1499.017 & 1499.513 & 1501.232 & 1499.657 \\ 1988811 & 199.017 & 1999.518 & 1501.235 & 149657\end{array}$ $\begin{array}{lllll}498.811 & 1499.017 & 1499.518 & 1501.235 & 1499.657 \\ 1498814 & 1499.015 & 1499.515 & 1501.23 & 1499.654\end{array}$ $\begin{array}{lllll}4498.814 & 1499.015 & 1499.515 & 1501.23 & 1499.654 \\ 1498.825 & 1499.017 & 1499.525 & 1501.258 & 1499.666\end{array}$ $\begin{array}{llllll}1498.853 & 1499.031 & 1499.548 & 1501.312 & 1499.718\end{array}$ $\begin{array}{llllll}1498.853 & 1499.024 & 1499.546 & 1501.284 & 1499.715\end{array}$ $\begin{array}{llllll}1498.842 & 1499.017 & 1499.536 & 1501.258 & 1499.689\end{array}$ $\begin{array}{llllll}1498.849 & 1499.013 & 1499.539 & 1501.26 & 1499.682\end{array}$ $\begin{array}{llllll}1498.828 & 1499.006 & 1499.527 & 1501.214 & 1499.654\end{array}$ $\begin{array}{llllll}1498.835 & 1499.006 & 1499.536 & 1501.239 & 1499.647 \\ 1498.558 & 1499.02 & 1499.553 & 15013 & 1499.692\end{array}$ $\begin{array}{lllllll}1498.858 & 1499.02 & 1499.553 & 1501.239 & 1499.647\end{array}$ $\begin{array}{lllll}1498.86 & 1499.017 & 1499.553 & 1501.284 & 1499.703 \\ 1498.867 & 1499.015 & 1499.557 & 1501.291 & 1499.699\end{array}$ $\begin{array}{llllll}1498.872 & 1499.017 & 1499.564 & 1501.298 & 1499.706\end{array}$ $\begin{array}{llllll}1498.865 & 1499.013 & 1499.555 & 1501.267 & 1499.689\end{array}$ $\begin{array}{llllll}4998.877 & 1499.017 & 1499.564 & 1501.295 & 1499.696\end{array}$ $\begin{array}{llllll}1498.9 & 1499.029 & 1499.585 & 1501.342 & 1499.736\end{array}$ $\begin{array}{llllll}1498.898 & 1499.027 & 1499.578 & 1501.314 & 1499.722\end{array}$ $\begin{array}{lllllll}1498.893 & 1499.02 & 1499.574 & 1501.291 & 1499.659 \\ 1498.893 & 1499.017 & 1499.576 & 1501.298 & 1499.641\end{array}$ $\begin{array}{llllll}1498.893 & 1499.017 & 1499.574 & 1501.291 & 1499659 & 150.298\end{array}$ $\begin{array}{lllllll}1498.884 & 1499.01 & 1499.567 & 1501.279 & 1499.626\end{array}$ $\begin{array}{lllllll}1498.893 & 1499.013 & 1499.574 & 1501.296 & 1499.645\end{array}$ $\begin{array}{llllll}1498.879 & 1499.003 & 1499.564 & 1501.253 & 1499.627\end{array}$ $\begin{array}{rrrrr}1498.874 & 1499.001 & 1499.562 & 1501.256 & 1499.61 \\ 1498.872 & 1498.999 & 1499.56 & 1501.253 & 1499.61\end{array}$ $\begin{array}{rrrrr}1498.872 & 1498.999 & 1499.56 & 1501.253 & 1499.61 \\ 1498.863 & 1498.994 & 1499.553 & 1501.235 & 1499.603\end{array}$ $\begin{array}{rrrrr}1498.863 & 1498.994 & 1499.553 & 1501.235 & 1499.603 \\ 1498.858 & 1498.989 & 1499.55 & 1501.228 & 1499.594\end{array}$ 

Well (ft AMSL) (ft AMSL) (ft AMSL) (ft AMSL) (ft AMSL) (ft AMSL) (ft AMSL) (ft AMSL) (ft AMSL) (ft AMSL) (ft AMSL) (ft AMSL) (ft AMSL) (ft AMSL) (ft AMSL) (ft AMSL) (ft AMSL) (ft AMSL) (ft AMSL) (ft AMSL) (ft AMSL) (ft AMSL) (ft AMSL) (ft AMSL) (ft AMSL) (ft AMS)

10/10/10 16:00

$\begin{array}{llllll}1498.837 & 1498.996 & 1499.576 & 1501.181 & 1499.64 \\ 1498872 & 1498.999 & 1499.571 & 1501267 & 1499.647\end{array}$

10/11/100:00

$10 / 11 / 108: 00$

10/11/10 12:00

10/11/10 16:00

10/11/10 20:00

10/12/10 0:00

10/12/10 4:00

10/12/10 8:00

10/12/10 16:00

10/12/10 20:00

10/13/10 0:00

10/13/10 4:00

10/13/10 8:00

10/13/10 12:00

10/13/10 16:00

$10 / 14 / 100.00$

10/14/10 0:00

0/14/10 8:00

10/14/10 12:00

10/14/10 16:00

10/14/10 20:00

10/15/10 0:00

$10 / 15 / 104.00$

$10 / 15 / 108.00$

10/15/10 16:00

10/15/10 20:00

10/16/10 0:00

10/16/10 4:00

10/16/10 8:00

$10 / 16 / 1012: 00$
$10 / 16 / 1016: 00$

10/16/1016:00

10/17/10 0:00

10/17/10 4:00

10/17/10 8:00

10/17/10 12:00

10/17/10 16:00

10/17/10 20:00

10/18/10 0:00

$10 / 18 / 108.00$

0/18/10 12:00

10/18/10 16:00

10/18/10 20:00

10/19/10 0:00

10/19/10 4:00

10/19/10 8:00

$\begin{array}{lllll}4998.872 & 1498.999 & 1499.571 & 1501.267 & 1499.647 \\ 149887 & 1499.001 & 1999571 & 1501265 & 1499648\end{array}$

$\begin{array}{lllllll}1488877 & 1499.073 & 1499.576 & 1501.282 & 1499.648\end{array}$

$\begin{array}{lllllll}1498.874 & 1499.061 & 1499.574 & 1501256 & 1499.65\end{array}$

$\begin{array}{llllll}1498.872 & 1499.054 & 1499.571 & 1501.113 & 1499.636\end{array}$

$\begin{array}{llllll}1498.881 & 1499.052 & 1499.574 & 1501.066 & 1499.641\end{array}$

$\begin{array}{rrrrrr}1498.86 & 1499.038 & 1499.557 & 1501.181 & 1499.61 \\ 1498849 & 1499.024 & 1499.55 & 1501.288 & 1499.589\end{array}$

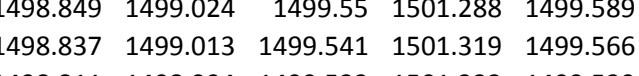

$\begin{array}{llllll}1498.811 & 1498.994 & 1499.522 & 1501.333 & 1499.529\end{array}$

$\begin{array}{llllll}1498.776 & 1498.975 & 1499.494 & 1501.09 & 1499.482\end{array}$

$\begin{array}{lllllll}1498.746 & 1498.959 & 1499.469 & 1501.048 & 1499.444\end{array}$

$\begin{array}{llllll}1498.713 & 1498.947 & 1499.441 & 1501.006 & 1499.393\end{array}$

$\begin{array}{llllll}1498.69 & 1498.936 & 1499.424 & 1500.982 & 1499.372\end{array}$

$\begin{array}{rrrrr}1498.66 & 1498.922 & 1499.399 & 1500.94 & 1499.339 \\ 1498.657 & 1498.917 & 1499.401 & 1500.975 & 1499.337\end{array}$

$\begin{array}{llllll}1498.678 & 1498.919 & 14999.413 & 1500.975 & 1499.043 & 1499.386 \\ 1408 & 140.02 & 149.393\end{array}$

$\begin{array}{llllll}149.69 & 1498.91 & 1499.403 & 1501.024 & 1499.393\end{array}$

$\begin{array}{lllllll}1498.678 & 1498.903 & 1499.401 & 1501.055 & 1499.414\end{array}$ $\begin{array}{llllll}1498.679 & 1498.901 & 1499.401 & 1501.057 & 1499.421\end{array}$ $\begin{array}{llllll}1498.713 & 1498.915 & 1499.424 & 1501.134 & 1499.47\end{array}$ $\begin{array}{llllll}1498.755 & 1498.933 & 1499.45 & 1501.2 & 1499.542\end{array}$ $\begin{array}{llllll}1498.751 & 1498.924 & 1499.443 & 1501.16 & 1499.498\end{array}$ $\begin{array}{llllll}1498.746 & 1498.917 & 1499.429 & 1501.148 & 1499.297 \\ 1498737 & 1498.903 & 1499.413 & 150112 & 1499258\end{array}$ $\begin{array}{llllll}1498.737 & 1498.903 & 1499.413 & 1501.12 & 1499.258 \\ 1498.716 & 1498891 & 1499389 & 1501.076 & 1499.183\end{array}$ $\begin{array}{lllllll}1498.716 & 1498.891 & 1499.389 & 1501.014 & 1499.183 \\ 1498.723 & 1498.894 & 1499.387 & 1501111 & 1499.069\end{array}$ $\begin{array}{lllllll}1498.741 & 1498.898 & 1499.399 & 1501.174 & 1499.141\end{array}$ $\begin{array}{llllll}1498.732 & 1498.887 & 1499.385 & 1501.146 & 1499.176\end{array}$ $\begin{array}{llllll}1498.727 & 1498.885 & 1499.382 & 1501.148 & 1499.211\end{array}$ $\begin{array}{llllll}1498.725 & 1498.88 & 1499.38 & 1501.151 & 1499.262\end{array}$ $\begin{array}{llllll}1498.709 & 1498.873 & 1499.371 & 1501.116 & 1499.286\end{array}$ $\begin{array}{llllll}1498.704 & 1498.873 & 1499.366 & 1501.118 & 1499.3 \\ 1498.713 & 1498.871 & 1499.373 & 1501.143 & 1499.342\end{array}$ $\begin{array}{llllll}1488.713 & 1498.871 & 1499.373 & 1501.143 & 1499.342\end{array}$ $\begin{array}{lllllll}1498.669 & 1498.908 & 1499.345 & 1501.057 & 1499.288\end{array}$ $\begin{array}{lllllll}1498.667 & 1498.91 & 1499.347 & 1501.066 & 1499.307\end{array}$ $\begin{array}{llllll}1498.655 & 1498.903 & 1499.336 & 1501.041 & 1499.216\end{array}$ $\begin{array}{llllll}1498.667 & 1498.905 & 1499.338 & 1501.09 & 1499.078\end{array}$ $\begin{array}{llllll}1498.692 & 1498.917 & 1499.354 & 1501.158 & 1499.141\end{array}$ $\begin{array}{rrrrrr}1498.692 & 1498.91 & 1499.352 & 1501.141 & 1499.202\end{array}$ $\begin{array}{llllll}1498.688 & 1498.901 & 1499.347 & 1501.132 & 1499.237 \\ 1498.685 & 1498.894 & 1499.347 & 1501.134 & 1499.276\end{array}$ $\begin{array}{llllll}1498.685 & 1498.894 & 1499347 & 1501.132 & 1499.237\end{array}$ $\begin{array}{llllll}1498.653 & 1498.871 & 1499.32 & 151.059 & 1499.262 \\ 1498.648 & 1498.859 & 1499322 & 1501.059 & 1499.258\end{array}$ $\begin{array}{llllll}1498.646 & 1498.854 & 1499.322 & 1501.064 & 1499.276\end{array}$ $\begin{array}{llllll}1498.623 & 1498.843 & 1499.303 & 1501.013 & 1499.26\end{array}$ $\begin{array}{llllll}1498.613 & 1498.836 & 1499.298 & 1501.006 & 1499.251\end{array}$ $\begin{array}{llllll}1498.602 & 1498.829 & 1499.291 & 1500.996 & 1499.251\end{array}$ $\begin{array}{lllll}1498.592 & 1498.822 & 1499.284 & 1500.973 & 1499.251 \\ 1498.59 & 1498.819 & 1499.289 & 1500.992 & 1499.253\end{array}$ 
$\begin{array}{lllll}1498.611 & 1498.824 & 1499.305 & 1501.052 & 1499.309\end{array}$

$\begin{array}{lllllll} & 1498.608 & 1498.817 & 1499.301 & 1501.034 & 1499.321 \\ & 1498.608 & 1498.815 & 1499.303 & 1501.034 & 1499.321\end{array}$

10/20/10 4:00

10/20/10 12:00

10/20/10 16:00

10/20/10 20:00

$\begin{array}{lllllll}1498.608 & 1498.815 & 1499.303 & 1501.034 & 1499.321\end{array}$

$\begin{array}{llllll}1498.595 & 1498.801 & 1499.289 & 1500.989 & 1499.302\end{array}$

$\begin{array}{lllll}1498.588 & 1498.798 & 1499.287 & 1500.989 & 1499.293 \\ 1498613 & 1498.805 & 1499.303 & 1501.043 & 1499.328\end{array}$

$\begin{array}{lllll}1498.613 & 1498.805 & 1499.303 & 1501.043 & 1499.328 \\ 148.30 & 1490 & \end{array}$

$\begin{array}{lllll}1498.606 & 1498.796 & 1499.301 & 1501.013 & 1499.328 \\ 1498.597 & 1498.789 & 1499291 & 1500.985 & 1499.304\end{array}$

$\begin{array}{lllll}1498.597 & 1498.789 & 1499.291 & 1500.985 & 1499.304 \\ 1498.599 & 1498.787 & 1499.296 & 1500.994 & 1499.307\end{array}$

$\begin{array}{llllll}1498.59 & 1498.78 & 1499.282 & 1500.964 & 1499.293 \\ 1498.599 & 1498.782 & 1499291 & 1500.96 & 149.302\end{array}$

$10 / 21 / 108.00$

$10 / 21 / 108.00$

$10 / 21 / 1016: 00$

$\begin{array}{lllll}1498.599 & 1498.782 & 1499.291 & 1500.996 & 1499.302 \\ 1498.629 & 1498.796 & 1499312 & 1501069 & 1499356\end{array}$

$\begin{array}{lllll}1498.615 & 1498.784 & 1499.301 & 1501.01 & 1499.342\end{array}$

10/21/1020:00

10/22/10 4:00

10/22/10 8:00

10/22/10 12:00

10/22/10 16:00

$10 / 23 / 100.00$

10/23/10 4:00

10/23/10 8:00

10/23/10 12:00

10/23/10 16:00

10/23/10 20:00

$\begin{array}{rrrrrrr}1488.623 & 1498.784 & 1499.301 & 1501.01 & 1499.342 \\ 14091.041 & 1499.346\end{array}$

$\begin{array}{llllll}1498.639 & 1498.789 & 1499.319 & 1501.062 & 1499.356\end{array}$

$\begin{array}{llllll}1498.632 & 1498.78 & 1499.31 & 1501.022 & 1499.353\end{array}$

$\begin{array}{lrrrr}1498.627 & 1498.78 & 1499.31 & 1501.017 & 1499.335\end{array}$

$\begin{array}{llllll}1498.651 & 1498.794 & 1499.329 & 1501.069 & 1499.372 \\ 1498.653 & 1498.789 & 1499.329 & 1501.062 & 1499.379\end{array}$

$\begin{array}{lllllll}1498.653 & 1498.789 & 149.329 & 1501.062 & 149.379\end{array}$

$\begin{array}{rrrrrr}1498.655 & 1498.798 & 1499.347 & 1501.08 & 1499.405\end{array}$ $\begin{array}{llllll}1498.664 & 1498.798 & 1499.347 & 1501.125 & 1499.407\end{array}$ $\begin{array}{rlllll}1498.679 & 1498.805 & 1499.359 & 1500.9 & 1499.414\end{array}$ $\begin{array}{lllll}1498.69 & 1498.715 & 1499.366 & 1500.896 & 1499.426\end{array}$ $\begin{array}{llllll}1498.681 & 1498.857 & 1499.357 & 1501.104 & 1499.395\end{array}$ $\begin{array}{lllllll}1498.672 & 1498.796 & 1499.35 & 1501.153 & 1499.367 \\ 1498.674 & 1498791 & 149935 & 1501.125 & 1499365\end{array}$

$\begin{array}{rrrrr}1498.674 & 1498.791 & 1499.35 & 1501.125 & 1499.365 \\ 149865 & 149878 & 1499331 & 1501.132 & 1999332\end{array}$ $\begin{array}{llllll}1498.65 & 1498.78 & 1499.331 & 1501.132 & 1499.332 \\ 1498.655 & 1498.782 & 1499.338 & 1501.001 & 1499.328\end{array}$ $\begin{array}{llllll}1498.681 & 1498.794 & 1499.352 & 1501.059 & 1499.365\end{array}$ $\begin{array}{llllll}1498.683 & 1498.794 & 1499.352 & 1501.055 & 1499.384\end{array}$ $\begin{array}{llllll}1498.709 & 1498.81 & 1499.373 & 1501.106 & 1499.416\end{array}$ $\begin{array}{llllll}1498.755 & 1498.833 & 1499.406 & 1501.188 & 1499.48\end{array}$ $\begin{array}{llllll}1498.786 & 1498.854 & 1499.427 & 1501.223 & 1499.536\end{array}$ $\begin{array}{rrrrrr}1498.851 & 1498.833 & 1499.478 & 1501.324 & 1499.62 \\ 1498.87 & 1498.812 & 1499.48 & 1501.293 & 1499.65\end{array}$ $\begin{array}{llllll}1498.87 & 1498.812 & 1499.48 & 1501.293 & 1499.65 \\ 1498.795 & 1498.859 & 1499.42 & 1501.104 & 1499512\end{array}$ $\begin{array}{rrrrrr}1498.795 & 1498.859 & 1499.42 & 1501.104 & 1499.512 \\ 1498.781 & 1498.847 & 1499.403 & 1501.153 & 1499.414\end{array}$ $\begin{array}{llllll}1498.739 & 1498.829 & 1499.382 & 1501.006 & 1499.339\end{array}$ $\begin{array}{llllll}1498.704 & 1498.808 & 1499.354 & 1500.95 & 1499.283\end{array}$ $\begin{array}{llllll}1498.688 & 1498.796 & 1499.347 & 1500.95 & 1499.258\end{array}$ $\begin{array}{llllll}1498.695 & 1498.801 & 1499.352 & 1500.987 & 1499.286\end{array}$ $\begin{array}{llllll}1498.662 & 1498.782 & 1499.326 & 1500.917 & 1499.253 \\ 149863 & 1498766 & 1499301 & 150086 & 1499.202\end{array}$ $\begin{array}{rrrrrr}1498.63 & 1498.766 & 1499.301 & 1500.868 & 1499.202 \\ 1498.604 & 1498.756 & 1499.28 & 1500837 & 1499.16\end{array}$ $\begin{array}{llllll}1498.55 & 1498735 & 1499.238 & 15000753 \quad 1499.16\end{array}$ $\begin{array}{llllll}1498.55 & 1498.735 & 1499.238 & 1500.753 & 1499.092 \\ 1498.731 & 1499.226 & 1500744 & 1499.05\end{array}$ $\begin{array}{lllllll}1498.499 & 1498.717 & 1499.21 & 1500.727 & 1499.036\end{array}$ $\begin{array}{llllll}1498.436 & 1498.691 & 1499.154 & 1500.618 & 1498.95\end{array}$ $\begin{array}{lllll}1498.399 & 1498.675 & 1499.138 & 1500.597 & 1498.898 \\ 1498.366 & 1498.661 & 1499.117 & 1500.576 & 1498.875\end{array}$ $\begin{array}{rrrrr}1498.366 & 1498.661 & 1499.117 & 1500.576 & 1498.875 \\ 1498.319 & 1498.642 & 1499.082 & 1500.524 & 1498.84\end{array}$ $\begin{array}{rrrrrr}1498.319 & 1498.642 & 1499.082 & 1500.524 & 1498.84 \\ 1498.31 & 1498.633 & 1499.082 & 1500.564 & 1498.842\end{array}$ 

$\begin{array}{lllll}1498.357 & 1498.677 & 1499.096 & 1500.723 & 1499.02\end{array}$ $\begin{array}{rrrrr}1498.394 & 1498.503 & 1499.119 & 1500.8 & 1499.071 \\ 1498.411 & 1498.531 & 1499.147 & 1500.865 & 1499.16\end{array}$ $\begin{array}{llllll}1498.441 & 1498.531 & 1499.147 & 1500.865 & 1499.16 \\ 1498.64 & 1498.61 & 1499.159 & 1500.877 & 1499.185\end{array}$ $\begin{array}{rrrrrr}1498.464 & 1498.61 & 1499.159 & 1500.877 & 1499.185 \\ 1498.487 & 1498.654 & 1499.175 & 1500.907 & 1499.206\end{array}$ $\begin{array}{rrrrr}1498.513 & 1498.673 & 1499.189 & 1500.933 & 1499.23 \\ 1495515 & 1488.724 & 1499.187 & 150091 & 1499225\end{array}$ $\begin{array}{llllll}1498.515 & 1498.724 & 1499.187 & 1500.91 & 1499.225 \\ 1498.515 & 1498619 & 1499.187 & 1500896 & 1499.199\end{array}$ $\begin{array}{lllll}1498.515 & 1498.619 & 1499.187 & 1500.896 & 1499.199 \\ 1498.52 & 1498.545 & 1499.189 & 1500.898 & 1499.197\end{array}$ $\begin{array}{rrrrr}1498.511 & 1498.689 & 1499.18 & 1500.861 & 1499.162\end{array}$ $\begin{array}{rrrrr}1498.499 & 1498.787 & 1499.166 & 1500.83 & 1499.134 \\ 149.52 & 1498.747 & 1499.182 & 1500.884 & 1499.146\end{array}$ $\begin{array}{lllll}1498.52 & 1498.747 & 1499.182 & 1500.884 & 1499.146 \\ 1498501 & 1498784 & 1499.163 & 1500.821 & 1499.127\end{array}$ $\begin{array}{rrrrr}1498.501 & 1498.784 & 1499.163 & 1500.821 & 1499.127 \\ 1498.506 & 1498.635 & 1499.166 & 1500.84 & 1499.113\end{array}$ $\begin{array}{rrrrr}1498.506 & 1498.635 & 1499.166 & 1500.84 & 1499.113 \\ 1498.49 & 1498.545 & 1499.154 & 1500.8 & 1499.106\end{array}$ $\begin{array}{llllll}1498.45 & 1498.745 & 1499.121 & 1500.711 & 1499.029 \\ & 498.438 & 1498.617 & 1499.117 & 1500.727 & 1499.001\end{array}$ $\begin{array}{lllll}1498.438 & 1498.617 & 1499.117 & 1500.727 & 1499.001 \\ 1498.422 & 1498.612 & 1499.103 & 1500.699 & 1498.982\end{array}$ $\begin{array}{llllll}1498.394 & 1498.596 & 1499.079 & 1500.646 & 1498.947\end{array}$ $\begin{array}{llllll}1498.38 & 1498.591 & 1499.07 & 1500.653 & 1498.931\end{array}$ $\begin{array}{llllll}1498.382 & 1498.586 & 1499.068 & 1500.667 & 1498.945\end{array}$ $\begin{array}{llllll}1498.347 & 1498.572 & 1499.042 & 1500.606 & 1498.91\end{array}$ $\begin{array}{rrrrr}1498.333 & 1498.565 & 1499.03 & 1500.601 & 1498.882 \\ 1498326 & 1498.558 & 1499.028 & 150.013 & 149887\end{array}$ $\begin{array}{lllll}1498.326 & 1498.558 & 1499.028 & 1500.613 & 1498.887 \\ 1498.308 & 1498.549 & 1499009 & 1500576 & 1498.873\end{array}$ $\begin{array}{lllllll}1498.308 & 1498.549 & 1499.009 & 1500.576 & 1498.873 \\ 1498.315 & 1498.554 & 1499.023 & 1500.629 & 1498887\end{array}$ $\begin{array}{llllll}1498.336 & 1498.554 & 1499.035 & 1500.674 & 1498.94\end{array}$ $\begin{array}{llllll}1498.333 & 1498.589 & 1499.028 & 1500.664 & 1498.947\end{array}$ $\begin{array}{llllll}1498.333 & 1498.645 & 1499.03 & 1500.671 & 1498.95\end{array}$ $\begin{array}{llllll}1498.338 & 1498.649 & 1499.03 & 1500.681 & 1498.964\end{array}$ $\begin{array}{lllllll}1498.322 & 1498.675 & 1499.012 & 1500.632 & 1498.94\end{array}$ $\begin{array}{llllll}1498.319 & 1498.484 & 1499.009 & 1500.634 & 1498.915 \\ 1488329 & 1498395 & 1999019 & 1500654 & 1498936\end{array}$ $\begin{array}{llllll}149831 & 14988.561 & 149.019 & 1500.664 & 1498.936\end{array}$ $\begin{array}{rrrrrr}1498.31 & 1498.561 & 1499 & 1500.615 & 1498.91 \\ 1498.296 & 1498.645 & 1498.988 & 1500.597 & 1498.887\end{array}$ $\begin{array}{llllll}1498.289 & 1498.656 & 1498.984 & 1500.597 & 1498.884\end{array}$ $\begin{array}{lllllll}1498.28 & 1498.666 & 1498.972 & 1500.576 & 1498.882\end{array}$ $\begin{array}{llllll}1498.298 & 1498.575 & 1498.988 & 1500.625 & 1498.898\end{array}$ $\begin{array}{llllll}1498.331 & 1498.454 & 1499.007 & 1500.737 & 1498.957\end{array}$ $\begin{array}{lllll}1498.317 & 1498.596 & 1498.993 & 1500.903 & 1498.943 \\ 1498308 & 1988.645 & 1488.986 & 1500.98 & 149822\end{array}$ $\begin{array}{llllll}1498.308 & 1498.645 & 1498.986 & 1500.98 & 1498.922 \\ 1498303 & 1498.619 & 1498.986 & 1500.992 & 1498922\end{array}$ $\begin{array}{llllll}1498.303 & 1498.619 & 1498.986 & 1500.992 & 1498.922 \\ 1498.282 & 1498.587 & 1498.965 & 1500.98 & 1498.894\end{array}$ $\begin{array}{lllllll}1498.282 & 1498.587 & 1498.965 & 1500.98 & 1498.894 \\ 1498.289 & 1498.423 & 1498.972 & 1500.578 & 1498.894\end{array}$ $\begin{array}{llllll}1498.312 & 1498.391 & 1498.993 & 1500.391 & 1498.95\end{array}$ $\begin{array}{llllll}1498.312 & 1498.521 & 1498.986 & 1500.639 & 1498.959\end{array}$ $\begin{array}{lllll}1498.305 & 1498.577 & 1498.984 & 1500.756 & 1498.945 \\ 1498.298 & 1498.591 & 1498.991 & 1500.807 & 1498.933\end{array}$ $\begin{array}{llllll}1498.298 & 1498.591 & 1498.991 & 1500.807 & 1498.933 \\ 1498.289 & 1498.575 & 1499.019 & 1500.781 & 1498.922\end{array}$ 

Well (ft AMSL) (ft AMSL) (ft AMSL) (ft AMSL) (ft AMSL) (ft AMSL) (ft AMSL) (ft AMSL) (ft AMSL) (ft AMSL) (ft AMSL) (ft AMSL) (ft AMSL) (ft AMSL) (ft AMSL) (ft AMSL) (ft AMSL) (ft AMSL) (ft AMSL) (ft AMSL) (ft AMSL) (ft AMSL) (ft AMSL) (ft AMSL) (ft AMSL) (ft AMS)

11/6/101600 $1498.3171498 .493 \quad 1498.988 \quad 1500.6671498 .971$

$\begin{array}{llllll}1498.303 & 1498.472 & 1498.974 & 1500.625 & 1498.945\end{array}$ $\begin{array}{llllll}1498.324 & 1498.484 & 1498.988 & 1500.662 & 1498.966\end{array}$ $\begin{array}{lllllll}1498.361 & 1498.51 & 1499.023 & 1500.749 & 1499.036\end{array}$ $\begin{array}{lllll}1498.366 & 1498.507 & 1499.021 & 1500.737 & 1499.052 \\ 1498378 & 1498512 & 1499028 & 1500.746 & 1499.05\end{array}$ $\begin{array}{rrrrr}1498.378 & 1498.512 & 1499.028 & 1500.746 & 1499.05 \\ 1498.387 & 1498.519 & 1499.04 & 1500.77 & 1499.066\end{array}$

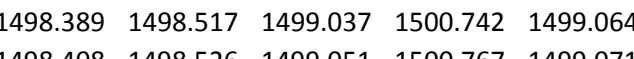
$\begin{array}{lllll}1498.408 & 1498.526 & 1499.051 & 1500.767 & 1499.071 \\ 1498.431 & 1498.538 & 1499.068 & 1500.802 & 1499.108\end{array}$ $\begin{array}{lllllll}1498.424 & 1498.533 & 1499.061 & 1500.777 & 1499.099\end{array}$ $\begin{array}{lllllll}1498.429 & 1498.538 & 1499.065 & 1500.772 & 1499.087\end{array}$ $\begin{array}{llllll}1498.441 & 1498.54 & 1499.075 & 1500.788 & 1499.094\end{array}$ $\begin{array}{lllllll}1498.438 & 1498.542 & 1499.072 & 1500.774 & 1499.09\end{array}$ $\begin{array}{lllll}1489.649 & 1489.826 & 1499.009 & 1500.784 & 1499.092\end{array}$ $\begin{array}{llllll}1490.423 & 1490.324 & 1487.84 & 1497.636 & 1480.652 \\ 1490.768 & 1490.639 & 1488.795 & 1500751 & 1496221\end{array}$ $\begin{array}{lllllll}1490.768 & 1490.639 & 1488.795 & 1400.751 & 1496.221\end{array}$ $\begin{array}{llllll}1491.312 & 1491.193 & 1489.618 & 1500.732 & 1498.826\end{array}$ $\begin{array}{llllll}1491.585 & 1491.454 & 1489.957 & 1500.627 & 1498.742\end{array}$ $\begin{array}{llllll}1491.855 & 1491.663 & 1490.281 & 1500.578 & 1496.898\end{array}$ $\begin{array}{llllll}1492.109 & 1491.875 & 1490.642 & 1500.571 & 1498.459\end{array}$ $\begin{array}{llllll}1492.301 & 1492.087 & 1491.118 & 1500.519 & 1498.571\end{array}$ $\begin{array}{lllll}1492.485 & 1492.299 & 1491.509 & 1500.489 & 1498.546 \\ 1492.664 & 1492516 & 1491.922 & 1500.489 & 1498546\end{array}$ $\begin{array}{rrrrrr}1492.664 & 1492.516 & 1491.922 & 1500.489 & 1498.546 \\ 1492832 & 1492.748 & 149229 & 1500.47 & 1498541\end{array}$ $\begin{array}{lllll}1492.832 & 1492.748 & 1492.29 & 1500.47 & 1498.541 \\ 1493.007 & 1492.972 & 1492.405 & 1500.489 & 1498555\end{array}$ $\begin{array}{lllll}1493.201 & 1493.165 & 1492.752 & 1500.517 & 1498.599\end{array}$ $\begin{array}{llllll}1493.378 & 1493.328 & 1492.983 & 1500.524 & 1498.616\end{array}$ $\begin{array}{lllllll}1493.571 & 1493.484 & 1493.22 & 1500.524 & 1498.639\end{array}$ $\begin{array}{llllll}1493.821 & 1493.659 & 1493.435 & 1500.639 & 1498.632\end{array}$ $\begin{array}{llllll}1493.998 & 1493.782 & 1493.712 & 1500.674 & 1498.648\end{array}$ $\begin{array}{llllll}1494.129 & 1493.957 & 1493.997 & 1500.695 & 1498.665 \\ 1494271 & 1494.108 & 1995575 & 1500.735 & 1988.747\end{array}$ \begin{tabular}{llllll}
1494.271 & 1494.108 & 1495.575 & 1500.735 & 1498.747 \\
\hline
\end{tabular} $\begin{array}{rrrrrr}1494.437 & 1494.32 & 1496.284 & 1500.793 & 1498.74 \\ 1494.469 & 1496.449 & 15008 & 1498.784\end{array}$ $\begin{array}{llllll}1494.714 & 1494.646 & 1496.536 & 1500.847 & 1498.807\end{array}$ $\begin{array}{llllll}1494.849 & 1494.844 & 1496.627 & 1500.896 & 1498.814\end{array}$ $\begin{array}{llllll}1494.903 & 1495 & 1496.547 & 1500.573 & 1498.866\end{array}$ $\begin{array}{llllll}1495.339 & 1495.175 & 1496.885 & 1500.526 & 1498.931\end{array}$ $\begin{array}{rrrrr}1495.528 & 1495.31 & 1497.021 & 1500.73 & 1498.931 \\ 1495.649 & 1495.436 & 1497.149 & 1500082 & 1498.936\end{array}$ $\begin{array}{llllll}1495.649 & 1495.436 & 1497.149 & 1500.802 & 1498.936 \\ 1495796 & 1495.557 & 1497.233 & 150084 & 1498.961\end{array}$ $\begin{array}{llllll}1495.796 & 1495.557 & 1497.233 & 1500.84 & 1498.961 \\ 1495.901 & 1495.673 & 1497.282 & 1500.837 & 1488.952\end{array}$ $\begin{array}{llllll}1496.057 & 1495.792 & 1497.14 & 1500.569 & 1498.9585\end{array}$ $\begin{array}{llllll}1496.225 & 1495.911 & 1497.405 & 1500.531 & 1499.045\end{array}$ $\begin{array}{llllll}1496.328 & 1496.011 & 1497.466 & 1500.646 & 1499.045\end{array}$ $\begin{array}{rrrrr}1496.461 & 1496.113 & 1497.555 & 1500.725 & 1499.069 \\ 1496.584 & 1496.207 & 1497.624 & 1500.76 & 1499.09\end{array}$ 

Well (ft AMSL) (ft AMSL) (ft AMSL) (ft AMSL) (ft AMSL) (ft AMSL) (ft AMSL) (ft AMSL) (ft AMSL) (ft AMSL) (ft AMSL) (ft AMSL) (ft AMSL) (ft AMSL) (ft AMSL) (ft AMSL) (ft AMSL) (ft AMSL) (ft AMSL) (ft AMSL) (ft AMSL) (ft AMSL) (ft AMSL) (ft AMSL) (ft AMSL) (ft AMS)

$\begin{array}{lllll}1496.841 & 1496.474 & 1497.722 & 1500.749 & 1499.069\end{array}$ $\begin{array}{lllll}1496.881 & 1496.558 & 1497.862 & 1500.709 & 1499.027 \\ 1496953 & 1496647 & 197.921 & 150072 & 149929\end{array}$ $\begin{array}{rrrrrr}1496.953 & 1496.647 & 1497.921 & 1500.72 & 1499.029 \\ 1497.037 & 1496728 & 1497.97 & 1500.727 & 1499.048\end{array}$ $\begin{array}{llllll}1497.09 & 1496.805 & 1498.021 & 1500.709 & 1499.048\end{array}$ $\begin{array}{llllll}1497.181 & 1496.88 & 1497.813 & 1500.727 & 1499.059\end{array}$ $\begin{array}{llllll}1497.293 & 1496.954 & 1498.002 & 1500.798 & 1499.113\end{array}$ $\begin{array}{rrrrr}1497.319 & 1497.01 & 1498.005 & 1500.749 & 1499.08 \\ 1497.366 & 1497.073 & 1498.028 & 1500.739 & 1499.062\end{array}$ $\begin{array}{llllll}1497.366 & 1497.073 & 1498.028 & 1500.739 & 1499.062\end{array}$ $\begin{array}{lrrrrr}1497.396 & 1497.122 & 1498.035 & 1500.72 & 1499.041 \\ 1497.314 & 1497.15 & 1497.998 & 1500.571 & 1498915\end{array}$ $\begin{array}{lllllll}1497.314 & 1497.15 & 1487.998 & 1500.571 & 1498.915 \\ 1497.284 & 1497.231 & 1498.079 & 1500564 & 1498789\end{array}$ $\begin{array}{llllll}1497.284 & 1497.231 & 1498.079 & 1500.564 & 1498.789 \\ 1497.324 & 1497.348 & 1498.151 & 1500.662 & 1498.728\end{array}$ $\begin{array}{llllll}1497.375 & 1497.408 & 1498.193 & 1500.798 & 1498.683\end{array}$ $\begin{array}{lllllll}1497.251 & 1497.322 & 1498.196 & 1500.891 & 1498.7\end{array}$ $\begin{array}{llllll}1497.291 & 1497.341 & 1498.179 & 1500.936 & 1498.725\end{array}$ $\begin{array}{lllllll}1497.324 & 1497.373 & 1498.179 & 1500.966 & 1498.732\end{array}$ $\begin{array}{llllll}1497.394 & 1497.408 & 1497.944 & 1500.653 & 1498.779\end{array}$ $\begin{array}{lllllll}1497.541 & 1497.476 & 1497.839 & 1500.592 & 1498.859 \\ 1497.634 & 1497.513 & 1498.007 & 1500749 & 1498.908\end{array}$ $\begin{array}{lllllll}1497.644 & 1497.513 & 1498.007 & 1500.749 & 1498.908\end{array}$ $\begin{array}{llllll}1497.832 & 1497.599 & 1498.119 & 1500.746 & 1499.031\end{array}$ $\begin{array}{llllll}1497.858 & 1497.627 & 1498.2 & 1500.73 & 1499.024\end{array}$ $\begin{array}{lllll}1497.888 & 1497.669 & 1498.079 & 1500.468 & 1499.006\end{array}$ $\begin{array}{llllll}1497.928 & 1497.704 & 1498.382 & 1500.473 & 1499.013\end{array}$ $\begin{array}{llllll}1497.904 & 1497.73 & 1498.354 & 1500.678 & 1498.964\end{array}$ $\begin{array}{llllll}1497.937 & 1497.767 & 1498.382 & 1500.718 & 1498.966 \\ 1497.981 & 1497.799 & 1498.424 & 1500716 & 149894\end{array}$ $\begin{array}{rrrrrr}1497.981 & 1497.799 & 1498.424 & 1500.716 & 1498.994 \\ 1497.981 & 1497.82 & 1498.417 & 1500711 & 1498975\end{array}$ $\begin{array}{lllllll}1498.081 & 1497.82 & 148.417 & 1500.711 & 1498.975 \\ 1499.853 & 1498.44 & 1500.475 & 1498.98\end{array}$ $\begin{array}{rrrrr}1498.049 & 1497.888 & 1498.48 & 1500.678 & 1499.001\end{array}$ $\begin{array}{llllll}1498.056 & 1497.914 & 1498.485 & 1500.653 & 1498.987\end{array}$ $\begin{array}{lllll}1498.1 & 1497.937 & 1498.529 & 1500.674 & 1499.01\end{array}$ $\begin{array}{llllll}1498.18 & 1497.974 & 1498.608 & 1500.73 & 1499.08\end{array}$ $\begin{array}{lllll}1498.173 & 1497.988 & 1498.601 & 1500.695 & 1499.057\end{array}$ $\begin{array}{rrrrrr}1498.196 & 1498.018 & 1498.625 & 1500.664 & 1499.052 \\ 1498.235 & 1498.042 & 1498.66 & 1500.739 & 1499.08\end{array}$ $\begin{array}{lllllll}1488.235 & 1498.042 & 1498.66 & 1500.739 & 149.08\end{array}$ $\begin{array}{llllll}1498.203 & 1498.056 & 1498.627 & 1500.674 & 1499.036 \\ 1498.254 & 1498.09 & 1498.676 & 1500.718 & 1499.064\end{array}$ $\begin{array}{lllllll}1498.284 & 1498.111 & 1498.704 & 1500.723 & 1499.09\end{array}$ $\begin{array}{llllll}1498.212 & 1498.114 & 1498.636 & 1500.634 & 1499.01\end{array}$ $\begin{array}{llllll}1498.161 & 1498.132 & 1498.585 & 1500.576 & 1498.947\end{array}$ $\begin{array}{llllll}1498.161 & 1498.139 & 1498.585 & 1500.583 & 1498.95\end{array}$ $\begin{array}{lllll}1498.098 & 1498.137 & 1498.527 & 1500.501 & 1498.887\end{array}$ $\begin{array}{rrrrrr}1498.079 & 1498.156 & 1498.51 & 1500.498 & 1498.866 \\ 1498.037 & 1498.16 & 1498.468 & 1500.449 & 1498824\end{array}$

$\begin{array}{llll}1498 & 1498165 \quad 1498431 \quad 1500414 \quad 1498793\end{array}$ $\begin{array}{llllll}1498 & 1498.165 & 1498.431 & 1500.414 & 1498.793 \\ 1498.023 & 1498.184 & 1498.452 & 1500.438 & 1498817\end{array}$ $\begin{array}{llllll}1498.117 & 1498.205 & 1498.548 & 1500.552 & 1498.924\end{array}$ $\begin{array}{llllll}1498.175 & 1498.221 & 1498.615 & 1500.59 & 1498.982\end{array}$ $\begin{array}{rrrrr}1498.27 & 1498.256 & 1498.711 & 1500.674 & 1499.069 \\ 1498.366 & 1498.281 & 1498.809 & 1500.746 & 1499.155 \\ 1498.417 & 1498.3 & 1498.865 & 1500.756 & 1499.185\end{array}$ $\begin{array}{rrrrrr}1498.417 & 1498.3 & 1498.865 & 1500.756 & 1499.185 \\ 1498.438 & 1498.319 & 1498.888 & 1500.746 & 1499.178\end{array}$ 

Well (ft AMSL) (ft AMSL) (ft AMSL) (ft AMSL) (ft AMSL) (ft AMSL) (ft AMSL) (ft AMSL) (ft AMSL) (ft AMSL) (ft AMSL) (ft AMSL) (ft AMSL) (ft AMSL) (ft AMSL) (ft AMSL) (ft AMSL) (ft AMSL) (ft AMSL) (ft AMSL) (ft AMSL) (ft AMSL) (ft AMSL) (ft AMSL) (ft AMSL) (ft AMS)

$1498.403 \quad 1498.305 \quad 1498.841 \quad 1500.674 \quad 1499.113$

$11 / 24 / 1020 \cdot 00$

$11 / 25 / 100: 00$

$\begin{array}{lllll}1498.322 & 1498.307 & 1498.746 & 1500.578 & 1498.994 \\ 1498266 & 1498.309 & 1498692 & 1500533 & 1498929\end{array}$

$\begin{array}{llllll}1498.21 & 1498.312 & 1498.639 & 1500.533 & 1498.929\end{array}$

$\begin{array}{llllll}1498.175 & 1498.309 & 1498.606 & 1500.452 & 1498854\end{array}$

$\begin{array}{llllll}1498.154 & 1498.326 & 1498.587 & 1500.426 & 1498.84\end{array}$

$1 / 25 / 1012: 00$

$11 / 25 / 1016: 00$

$11 / 25 / 1020: 00$

$\begin{array}{llllll}1498.18 & 1498.333 & 1498.615 & 1500.48 & 1498.877\end{array}$

$\begin{array}{llllll}1498.145 & 1498.333 & 1498.59 & 1500.433 & 1498.852\end{array}$

$\begin{array}{llllll}1498.126 & 1498.335 & 1498.571 & 1500.417 & 1498.831\end{array}$

$\begin{array}{llllll}1498.149 & 1498.344 & 1498.594 & 1500.442 & 1498.852 \\ 1988121 & 1988335 & 1498571 & 1500396 & 198831\end{array}$

$11 / 26 / 104: 00$

$11 / 26 / 108: 00$

$11 / 26 / 1016: 00$

$\begin{array}{llllll}1498.121 & 1498.335 & 1498.571 & 1500.396 & 1498.831 \\ 1498.131 & 1998347 & 198858 & 150.405 & 14988\end{array}$

$\begin{array}{rrrrr}1498.131 & 1498.347 & 1498.585 & 1500.405 & 1498.84 \\ 1498.17 & 1498.354 & 1498.627 & 1500.463 & 1498.882\end{array}$

$\begin{array}{llllll}1498.17 & 1498.358 & 1498.627 & 1500.449 & 1498.875\end{array}$

$11 / 26 / 1020: 00$

$11 / 27 / 100: 00$

$11 / 27 / 108: 00$

$11 / 27 / 1012: 00$

11/27/1016:00

$11 / 28 / 100: 00$

$11 / 28 / 100: 00$

$11 / 28 / 108: 00$

$11 / 28 / 1012: 00$

$11 / 28 / 1016: 00$

$11 / 28 / 1020: 00$

$11 / 29 / 100: 00$

$11 / 29 / 100: 00$

$11 / 29 / 108: 00$

$11 / 29 / 1016: 00$

$11 / 29 / 1020: 00$

$11 / 30 / 100: 00$

11/30/10 4:00

$11 / 30 / 108: 00$

$11 / 30 / 1012: 00$

11/30/10 16:00

$12 / 1 / 100: 00$

$12 / 1 / 104: 00$

$\begin{array}{lllll}1498.898 .361 & 1498.632 & 1500.442 & 1498.87\end{array}$

$\begin{array}{llllll}1498.173 & 1498.363 & 1498.634 & 1500.47 & 1498.866\end{array}$

$\begin{array}{llllll}1498.177 & 1498.368 & 1498.641 & 1500.505 & 1498.866\end{array}$

$\begin{array}{llllll}1498.205 & 1498.379 & 1498.671 & 1500.445 & 1498.891\end{array}$

$\begin{array}{llllll}1498.273 & 1498.393 & 1498.739 & 1500.543 & 1498.966\end{array}$

$\begin{array}{lllllll}1498.31 & 1498.398 & 1498.779 & 1500.559 & 1498.994\end{array}$

$\begin{array}{llllll}1498.399 & 1498.426 & 1498.876 & 1500.634 & 1499.066\end{array}$

$\begin{array}{llllll}1498.443 & 1498.44 & 1498.921 & 1500.66 & 1499.101\end{array}$

$\begin{array}{llllll}1498.494 & 1498.458 & 1498.97 & 1500.695 & 1499.143\end{array}$ $\begin{array}{llllll}1498.555 & 1498.472 & 1499.028 & 1500.744 & 1499.188\end{array}$ $\begin{array}{llllll}1498.541 & 1498.475 & 1499.016 & 1500.695 & 1499.136\end{array}$ $\begin{array}{llllll}1498.564 & 1498.491 & 1499.037 & 1500.713 & 1499.143 \\ 1498583 & 1498.498 & 1499.061 & 1500.73 & 1499.16\end{array}$ $\begin{array}{lllll}4498.583 & 1498.498 & 1499.061 & 1500.73 & 1499.16 \\ 1498.541 & 1498.491 & 1499.021 & 1500.667 & 1499.108\end{array}$ $\begin{array}{lllll}1498.541 & 1498.491 & 1499.021 & 1500.667 & 1499.108 \\ 1498.462 & 1498.479 & 1498.918 & 1500559 & 1499.001\end{array}$ $\begin{array}{llllll}1498.399 & 1498.465 & 1498.851 & 1500.503 & 1498.936\end{array}$ $\begin{array}{llllll}1498.31 & 1498.447 & 1498.76 & 1500.424 & 1498.849\end{array}$ $\begin{array}{llllll}1498.24 & 1498.433 & 1498.695 & 1500.379 & 1498.796\end{array}$ $\begin{array}{llllll}1498.228 & 1498.43 & 1498.685 & 1500.386 & 1498.805\end{array}$ $\begin{array}{llllll}1498.17 & 1498.414 & 1498.639 & 1500.339 & 1498.772\end{array}$ $\begin{array}{llllll}1498.166 & 1498.412 & 1498.629 & 1500.339 & 1498.772\end{array}$ $\begin{array}{llllll}4498.186 & 1498.419 & 1488.655 & 1500.382 & 1498.81\end{array}$ $\begin{array}{llllllll}1498.166 & 1498.41 & 1498.639 & 1500.0356 & 1498.896\end{array}$ $\begin{array}{llllll}1498.152 & 1498.407 & 1498.632 & 1500.342 & 1498.791\end{array}$ $\begin{array}{llllll}1498.131 & 1498.395 & 1498.611 & 1500.318 & 1498.768\end{array}$ $1 / 12: 00$ $12 / 1 / 1016: 00$ 12/1/10 20:00 $12 / 2 / 100: 00$

$\begin{array}{llllll}1498.156 & 1498.409 & 1498.639 & 1500.358 & 1498.793\end{array}$ $\begin{array}{llllll}1498.193 & 1498.419 & 1498.681 & 1500.407 & 1498.842\end{array}$ $\begin{array}{llllll}1498.214 & 1498.426 & 1498.709 & 1500.419 & 1498.859 \\ 1498238 & 1498.435 & 1488.734 & 150.433 & 149887\end{array}$ $\begin{array}{llllll}1498.238 & 1498.435 & 1498.734 & 1500.433 & 1498.877 \\ 1498242 & 1498.438 & 1498.737 & 1500.424 & 1498875\end{array}$ $\begin{array}{llllll}1498.242 & 1498.438 & 1498.734 & 1500.433 & 1498.877 \\ 1408.221 & 1498.431 & 1498709 & 1500382 & 14988845\end{array}$ $\begin{array}{llllll}1498.221 & 1498.431 & 1498.75 & 1500.382 & 1498.845 \\ 1498.198 & 1498.421 & 1498.685 & 1500.344 & 1498.81\end{array}$ $\begin{array}{lllllll}1498.214 & 1498.43 & 1498.709 & 1500.391 & 1498.831\end{array}$ $\begin{array}{lllll}1498.191 & 1498.421 & 1498.685 & 1500.351 & 1498.805\end{array}$ $\begin{array}{llllll}1498.214 & 1498.428 & 1498.711 & 1500.386 & 1498.826\end{array}$ $\begin{array}{lllllll}1498.242 & 1498.44 & 1498.744 & 1500.414 & 1498.859\end{array}$ $\begin{array}{llllll}1498.287 & 1498.456 & 1498.79 & 1500.456 & 1498.905\end{array}$ 

Well (ft AMSL) (ft AMSL) (ft AMSL) (ft AMSL) (ft AMSL) (ft AMSL) (ft AMSL) (ft AMSL) (ft AMSL) (ft AMSL) (ft AMSL) (ft AMSL) (ft AMSL) (ft AMSL) (ft AMSL) (ft AMSL) (ft AMSL) (ft AMSL) (ft AMSL) (ft AMSL) (ft AMSL) (ft AMSL) (ft AMSL) (ft AMSL) (ft AMSL) (ft AMS)

(2/3/1016:00 $\begin{array}{llllll}1498.408 & 1498.5 & 1498.909 & 1500.569 & 1499.029\end{array}$ $\begin{array}{llllll}1498.326 & 1498.472 & 1498.895 & 1500.519 & 1498.985\end{array}$ $\begin{array}{llllll}1498.326 & 1498.472 & 1498.83 & 1500.421 & 1498.894 \\ 1498.217 & 1498.445 & 1498.711 & 1500316 & 1498763\end{array}$ $\begin{array}{lllllll}1498.135 & 1498.419 & 1498.639 & 1500.258 & 1498.688\end{array}$ $\begin{array}{llllll}1498.068 & 1498.398 & 1498.578 & 1500.22 & 1498.634\end{array}$ $\begin{array}{llllll}1498.075 & 1498.391 & 1498.58 & 1500.26 & 1498.66\end{array}$ $\begin{array}{llllll}1498.026 & 1498.37 & 1498.541 & 1500.209 & 1498.62\end{array}$ $\begin{array}{llllll}1498 & 1498.358 & 1498.513 & 1500.192 & 1498.595\end{array}$ $12 / 5 / 100.00$ 12/5/108:00 $12 / 5 / 1016: 00$ $12 / 5 / 1020: 00$ $12 / 6 / 100: 00$ $12 / 6 / 104: 00$ $12 / 6 / 108: 00$ 12/6/10 12:00 $12 / 6 / 1016: 00$ (12/7/1020.00 12/7/10 4:00 $12 / 7 / 108: 00$ 12/7/10 12:00 12/7/10 16:00 $12 / 7 / 1020: 00$ 12/8/100:00 $12 / 8 / 104.00$ $12 / 8 / 1012 \cdot 00$ $12 / 8 / 1016: 00$ $12 / 8 / 1020: 00$ 12/9/10 0:00 $12 / 9 / 104: 00$ $12 / 9 / 108: 00$ $12 / 9 / 1012: 00$ $12 / 9 / 1020.00$ $12 / 10 / 100: 00$ $12 / 10 / 104: 00$ $12 / 10 / 108: 00$ $12 / 10 / 1012: 00$ 12/10/1016:00 12/10/10 20:00 $12 / 11 / 100: 00$ $12 / 11 / 108.00$ $\begin{array}{lllll}1498.016 & 1498.358 & 1498.538 & 1500.234 & 1498.627 \\ 149.988 & 1489347 & 1488515 & 150019 & 1498606\end{array}$ $\begin{array}{llllll}1497.988 & 1498.347 & 1498.515 & 1500.19 & 1498.606 \\ 1497.991 & 1498349 & 1498.515 & 1500209 & 1498606\end{array}$ $\begin{array}{llllll}1498.016 & 1498.351 & 1498.543 & 1500.239 & 14986441\end{array}$ $\begin{array}{llllll}1498.021 & 1498.344 & 1498.552 & 1500.237 & 1498.644\end{array}$ $\begin{array}{llllll}1498.033 & 1498.342 & 1498.562 & 1500.241 & 1498.651\end{array}$ $\begin{array}{llllll}1498.063 & 1498.349 & 1498.592 & 1500.274 & 1498.672\end{array}$ $\begin{array}{lllllll}1498.075 & 1498.349 & 1498.611 & 1500.274 & 1498.688\end{array}$ $\begin{array}{llllll}1498.131 & 1498.365 & 1498.66 & 1500.325 & 1498.732\end{array}$ $\begin{array}{llllll}1498.193 & 1498.389 & 1498.725 & 1500.398 & 1498.803\end{array}$ $\begin{array}{llllll}1488.228 & 1498.403 & 1498.758 & 1500.41 & 1498.826\end{array}$ $\begin{array}{llllll}1498.284 & 1498.423 & 1498.811 & 1500.442 & 1498.831 \\ 149852\end{array}$ $\begin{array}{lllllll}1498.273 & 1498.424 & 1498.807 & 1500.414 & 1498.831\end{array}$ $\begin{array}{llllll}1498.266 & 1498.421 & 1498.795 & 1500.386 & 1498.803\end{array}$ $\begin{array}{llllll}1498.254 & 1498.417 & 1498.788 & 1500.377 & 1498.786\end{array}$ $\begin{array}{llllll}1498.224 & 1498.407 & 1498.753 & 1500.339 & 1498.742\end{array}$ $\begin{array}{lllll}1498.217 & 1498.405 & 1498.739 & 1500.33 & 1498.725 \\ 1498.226 & 1498.407 & 1498753 & 1500344 & 1498737\end{array}$ $\begin{array}{llllll}1498.226 & 1498.407 & 1488.753 & 1500.344 & 1498.737 \\ 1498235 & 1498.407 & 149876 & 1500349 & 1498.747\end{array}$ $\begin{array}{lllllll}1498.235 & 1498.407 & 1498.76 & 1500.349 & 1498.747 \\ 1498.256 & 1498.417 & 1498.774 & 1500353 & 1498763\end{array}$ $\begin{array}{llllll}1498.282 & 1498.426 & 1498.804 & 1500.412 & 1498.798\end{array}$ $\begin{array}{llllll}1498.301 & 1498.433 & 1498.823 & 1500.424 & 1498.81\end{array}$ $\begin{array}{llllll}1498.331 & 1498.449 & 1498.848 & 1500.447 & 1498.838\end{array}$ $\begin{array}{llllll}1498.387 & 1498.468 & 1498.902 & 1500.503 & 1498.896\end{array}$ $\begin{array}{llllll}1498.408 & 1498.47 & 1498.921 & 1500.494 & 1498.912\end{array}$ $\begin{array}{llllll}1498.406 & 1498.477 & 1498.916 & 1500.473 & 1498.889\end{array}$ $\begin{array}{lllllll}1498.408 & 1498.477 & 1498.921 & 1500.482 & 1498.889\end{array}$ $\begin{array}{lllllll}1498.331 & 1498.463 & 1488.874 & 150.421 & 1988.821 \\ 1498338 & 1498.461 & 1498.853 & 1500.4 & 1498.786\end{array}$ $\begin{array}{llllll}1498.345 & 1498.463 & 1498.86 & 1500.414 & 1498.798\end{array}$ $\begin{array}{lllllll}1498.347 & 1498.468 & 1498.865 & 1500.421 & 1498.807\end{array}$ $\begin{array}{lllllll}1498.373 & 1498.477 & 1498.883 & 1500.435 & 1498.833\end{array}$ $\begin{array}{llllll}1498.438 & 1498.496 & 1498.944 & 1500.533 & 1498.915\end{array}$ $\begin{array}{rrrrrr}1498.431 & 1498.5 & 1498.942 & 1500.501 & 1498.901\end{array}$ $\begin{array}{llllll}1498.45 & 1498.496 & 1498.951 & 1500.482 & 1498.91 \\ 1498.427 & 1498.489 & 1498.928 & 1500.463 & 1498.87\end{array}$ $\begin{array}{lllllll}1498.427 & 1498.46 & 1488.928 & 1500.463 & 1498.87\end{array}$ $\begin{array}{rrrrr}1498.308 & 1498.456 & 148988 & 1500.337 & 1498.782\end{array}$ $\begin{array}{lllrrr}1498.261 & 1498.438 & 1498.758 & 1500.3 & 1498.679\end{array}$ $\begin{array}{llllll}1498.203 & 1498.416 & 1498.695 & 1500.248 & 1498.625\end{array}$ $\begin{array}{rrrrr}1498.154 & 1498.398 & 1498.648 & 1500.216 & 1498.59 \\ 1498.114 & 1498.382 & 1498.611 & 1500.19 & 1498.564\end{array}$ $\begin{array}{rrrrr}1498.114 & 1498.382 & 1498.611 & 1500.19 & 1498.564 \\ 1498.077 & 1498.365 & 1498.578 & 1500.166 & 1498.546\end{array}$ $\begin{array}{lllll}1498.077 & 1498.365 & 1498.578 & 1500.166 & 1498.546 \\ 1498.068 & 1498.368 & 1498.566 & 1500.18 & 1498.543\end{array}$ 
1498.0891498 .3651498 .5921500 .2181498 .583

$\begin{array}{lllllll} & 1498.089 & 1498.361 & 1498.601 & 1500.209 & 1498.595 \\ 12 / 13 / 100: 00 & 1498.112 & 1498.365 & 1498.627 & 1500241 & 149862\end{array}$

$12 / 13 / 104: 00$

$12 / 13 / 1012: 00$

12/13/10 16:00

$12 / 13 / 1020: 00$

$\begin{array}{llllll}1498.112 & 1498.365 & 1498.627 & 1500.241 & 1498.62 \\ 1498.135 & 1498372 & 1498.655 & 1500.269 & 1498651\end{array}$

$\begin{array}{llllll}1498.149 & 1498.372 & 1498.671 & 1500.272 & 1498.667\end{array}$

$\begin{array}{lllll}1498.166 & 1498.375 & 1498.685 & 1500.281 & 1498.674 \\ 149805 & 149838 & 1488.718 & 1500.328 & 1498583\end{array}$

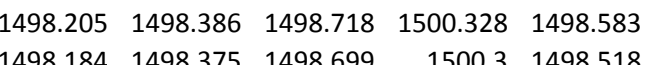

$\begin{array}{rrrrr}1498.184 & 1498.375 & 1498.699 & 1500.3 & 1498.518 \\ 1498.198 & 14983382 & 1498.709 & 1500307 & 1498.562\end{array}$

$12 / 14 / 104: 00$

$12 / 14 / 108: 00$

$\begin{array}{llllll}1498.221 & 1498.393 & 1498.734 & 1500.346 & 1498.623\end{array}$

$\begin{array}{lllll}1498.245 & 1498.398 & 1498.753 & 1500.351 & 1498.669\end{array}$

$\begin{array}{llllll}1498.275 & 1498.412 & 1498.783 & 1500.386 & 1498.714 \\ 1498.352 & 1498.438 & 1498.853 & 1500.468 & 1498.807\end{array}$

$\begin{array}{llllll}1498.343 & 1498.438 & 1498.848 & 1500.433 & 1498.796\end{array}$

$2 / 14 / 1016: 00$

$12 / 15 / 100: 00$

12/15/10 4:00

$12 / 15 / 108: 00$

12/15/10 12:00

$\begin{array}{lllllll}1498.399 & 1498.458 & 1498.888 & 1500.477 & 1498.835\end{array}$

$\begin{array}{lllllll}1498.441 & 1498.47 & 1498.928 & 1500.505 & 1498.877\end{array}$

$\begin{array}{llllll}1498.462 & 1498.482 & 1498.951 & 1500.512 & 1498.894\end{array}$

$\begin{array}{lllll}1498.492 & 1498.486 & 1498.977 & 1500.519 & 1498.912\end{array}$

$\begin{array}{llllll}1498.492 & 1498.484 & 1498.977 & 1500.491 & 1498.894 \\ 1498.415 & 1498.47 & 1498.907 & 1500396 & 1498.791\end{array}$

1/15/10 20:00

$12 / 16 / 100: 00$

$12 / 16 / 108: 00$

12/16/10 12:00

$12 / 16 / 1016: 00$

12/16/10 20:00

$12 / 17 / 100: 00$

$12 / 17 / 100: 00$

$12 / 17 / 108: 00$

$12 / 17 / 1016: 00$

12/17/10 20:00

$12 / 18 / 100: 00$

12/18/10 4:00

$12 / 18 / 108: 00$

12/18/10 12:00

$12 / 18 / 1020: 00$

$12 / 19 / 100: 00$

$12 / 19 / 104: 00$

$12 / 19 / 108: 00$

12/19/10 12:00

12/19/10 16:00

12/19/10 20:00

$12 / 20 / 100: 00$

$12 / 20 / 108.00$

2/2/20/10 12:00

12/20/10 16:00

$12 / 20 / 1020: 00$

$12 / 21 / 100: 00$

$12 / 21 / 104: 00$

$\begin{array}{llll} & \end{array}$

$\begin{array}{llllll}498.315 & 1498.44 & 1498.816 & 1500.321 & 1498.686\end{array}$

$\begin{array}{llllll}1498.256 & 1498.417 & 1498.76 & 1500.269 & 1498.639 \\ 1498.207 & 1498.407 & 1498.716 & 1500.232 & 1498.595\end{array}$ $\begin{array}{llllll}1498.196 & 1498.398 & 1498.706 & 1500.248 & 1498.604\end{array}$ $\begin{array}{lllllll}1498.159 & 1498.382 & 1498.671 & 1500.223 & 1498.581\end{array}$ $\begin{array}{llllll}1498.135 & 1498.372 & 1498.65 & 1500.204 & 1498.564 \\ 1998117 & 1988.363 & 1498.632 & 1500.197 & 1488555\end{array}$ $\begin{array}{llllll}1498.117 & 1498.363 & 1498.632 & 1500.197 & 1498.555 \\ 1498.063 & 1498344 & 1498583 & 1500.145 & 1498518\end{array}$ $\begin{array}{llllll}1498.035 & 1498.344 & 1488.559 & 1500.145 & 1498.518\end{array}$ $\begin{array}{lllllll}1498.056 & 1498.342 & 1498.578 & 1500.181 & 1498.534\end{array}$ $\begin{array}{llllll}1498.056 & 1498.337 & 1498.585 & 1500.173 & 1498.543\end{array}$ $\begin{array}{llllll}1498.068 & 1498.34 & 1498.601 & 1500.19 & 1498.56\end{array}$ $\begin{array}{lllll}1498.093 & 1498.342 & 1498.625 & 1500.22 & 1498.588\end{array}$ $\begin{array}{lllllll}1498.117 & 1498.347 & 1498.648 & 1500.241 & 1498.613\end{array}$ $\begin{array}{llllll}1498.147 & 1498.356 & 1498.676 & 1500.276 & 1498.641 \\ 1498.2 & 1498.372 & 1498.725 & 1500323 & 1498.02\end{array}$ $\begin{array}{llllll}1498.2 & 1498.372 & 1488.725 & 1500.323 & 1498.702\end{array}$ $\begin{array}{llllll}1498.2 & 1498.372 & 1498.732 & 1500.0302 & 1498.676\end{array}$ $\begin{array}{llllll}1498.219 & 1498.379 & 1498.748 & 1500.328 & 1498.695\end{array}$ $\begin{array}{lllll}1498.228 & 1498.386 & 1498.758 & 1500.325 & 1498.7\end{array}$ $\begin{array}{llllll}1498.254 & 1498.393 & 1498.783 & 1500.349 & 1498.718\end{array}$ $\begin{array}{lllllll}1498.28 & 1498.402 & 1498.807 & 1500.372 & 1498.744\end{array}$ $\begin{array}{lllllll}1498.289 & 1498.41 & 1498.814 & 1500.379 & 1498.742\end{array}$ $\begin{array}{llllll}1498.308 & 1498.414 & 1498.83 & 1500.389 & 1498.751 \\ 1498.373 & 1498.442 & 1498.883 & 1500.452 & 1498814\end{array}$ $\begin{array}{lllllll}1498.373 & 1498.442 & 1498.883 & 1500.389 & 1498.751\end{array}$ $\begin{array}{rrrrrr}1498.415 & 1498.456 & 1498.916 & 1500.452 & 1498.861\end{array}$ $\begin{array}{llllll}1498.382 & 1498.44 & 1498.876 & 1500.4 & 1498.522\end{array}$ $\begin{array}{llllll}1498.301 & 1498.417 & 1498.8 & 1500.309 & 1498.443\end{array}$ $\begin{array}{llllll}1498.247 & 1498.398 & 1498.746 & 1500.265 & 1498.438\end{array}$ $\begin{array}{lllllll}1498.207 & 1498.384 & 1498.711 & 1500.239 & 1498.452\end{array}$ $\begin{array}{rrrrrr}1498.135 & 1498.356 & 1498.65 & 1500.173 & 1498.422 \\ 1498.084 & 1498.34 & 1498.597 & 1500.141 & 1498.399\end{array}$ 

Well (ft AMSL) (ft AMSL) (ft AMSL) (ft AMSL) (ft AMSL) (ft AMSL) (ft AMSL) (ft AMSL) (ft AMSL) (ft AMSL) (ft AMSL) (ft AMSL) (ft AMSL) (ft AMSL) (ft AMSL) (ft AMSL) (ft AMSL) (ft AMSL) (ft AMSL) (ft AMSL) (ft AMSL) (ft AMSL) (ft AMSL) (ft AMSL) (ft AMSL) (ft AMS)

12/21/10 16:00 $12 / 21 / 1020: 00$ $12 / 22 / 10400$ $12 / 22 / 108: 00$ 12/22/10 12:00 $12 / 22 / 1016: 00$ 12/22/10 20:00 12/23/10 0:00 $12 / 23 / 104: 00$ 12/23/10 8:00 $12 / 23 / 1016: 00$ $12 / 23 / 1020: 00$ 12/24/100:00 $12 / 24 / 104: 00$ 12/24/10 8:00 12/24/10 12:00 12/24/10 16:00 $12 / 25 / 100000$ 12/25/10 0:00 12/25/10 8:00 $12 / 25 / 1012: 00$ 12/25/10 16:00

$\begin{array}{lllll}1498.044 & 1498.321 & 1498.564 & 1500.124 & 1498.403 \\ 1499.979 & 1498305 & 1498503 & 1500.078 & 1498334\end{array}$ $\begin{array}{lllll}1497.979 & 1498.305 & 1498.503 & 1500.078 & 1498.364 \\ 1497.911 & 1498.293 & 1498.443 & 1500.031 & 1498.322\end{array}$ $\begin{array}{lllll}1497.911 & 1498.293 & 1498.443 & 1500.031 & 1498.322 \\ 1497.879 & 1498.279 & 1498.417 & 1500.026 & 1498.317\end{array}$ $\begin{array}{lllllll}1497.83 & 1498.263 & 1498.371 & 1499.991 & 1498.317\end{array}$ $\begin{array}{lllllll}1497.76 & 1498.295 & 1498.268 & 1500.015 & 1497.995\end{array}$ $\begin{array}{llllll}1497.296 & 1497.979 & 1497.729 & 1500.033 & 1498.004\end{array}$ $\begin{array}{rrrrr}1497.347 & 1497.997 & 1497.811 & 1500.024 & 1498.149 \\ 1497.422 & 1498.023 & 1497.9 & 1500.064 & 1498.233\end{array}$ $\begin{array}{rrrrr}1497.422 & 1498.023 & 1497.9 & 1500.064 & 1498.233 \\ 1497.482 & 1498.039 & 1497.974 & 1500.075 & 1498.291\end{array}$ $\begin{array}{lllll}1497.482 & 1498.039 & 1497.974 & 1500.075 & 1498.291 \\ 1497.557 & 1498.053 & 1498.058 & 1500.117 & 1498.357\end{array}$ $\begin{array}{rrrrr}1497.65 & 1498.086 & 1498.154 & 1500.183 & 1498.431 \\ 1497.727 & 1498.086 & 1498.245 & 1500.197 & 1498.492\end{array}$ $\begin{array}{llllll}1497.767 & 1498.098 & 1498.294 & 1500.192 & 1498.506\end{array}$ $\begin{array}{llllll}1497.741 & 1498.107 & 14988.317 & 15000.113 & 1498.8097\end{array}$ $\begin{array}{lllllll}1497.739 & 1498.109 & 1498.333 & 1500.096 & 1498.485\end{array}$ $\begin{array}{llllll}1497.767 & 1498.13 & 1498.345 & 1500.092 & 1498.473\end{array}$ $\begin{array}{lllll}1497.708 & 1498.112 & 1498.357 & 1499.984 & 1498.466\end{array}$ $\begin{array}{rrrrrr}1497.792 & 1498.121 & 1498.371 & 1500.019 & 1498.466 \\ 1497.774 & 1498.116 & 149835 & 150008 & 1498413\end{array}$ $\begin{array}{lllllll}1497.792 & 1498.121 & 1498371 & 1500.019 & 1498.466\end{array}$ $\begin{array}{rrrrr}1497.753 & 1498.114 & 1498.336 & 1500.082 & 1498.38 \\ 1497.736 & 1498.107 & 1498.324 & 1500.057 & 1498.357\end{array}$ $\begin{array}{rrrrr}1497.718 & 1498.098 & 1498.315 & 1500.029 & 1498.34 \\ 1497.711 & 1498.093 & 1498308 & 1499.991 & 1498.333\end{array}$ $\begin{array}{llllll}1497.711 & 1498.093 & 1498.308 & 1499.991 & 1498.333 \\ 147.76 & 1498.10 & 148.359 & 1500.017 & 1498.389\end{array}$ $\begin{array}{llllll}1497.76 & 1498.104 & 1498.359 & 1500.017 & 1498.389 \\ 1497.769 & 1498.105 & 1498.371 & 1500.068 & 1498.396\end{array}$ $\begin{array}{llllll}1497.769 & 1498.105 & 1498.371 & 1500.068 & 1498.396 \\ 1497.804 & 1498.114 & 1498.406 & 1500.075 & 1498.424\end{array}$ $\begin{array}{lllll}1497.804 & 1498.114 & 1498.406 & 1500.075 & 1498.424 \\ 1497.837 & 1498.121 & 1498.441 & 1500.078 & 1498.452\end{array}$ $\begin{array}{llllll}1497.837 & 1498.121 & 1498.441 & 1500.078 & 1498.442 \\ 1497.846 & 1498.123 & 1498.452 & 1500.085 & 1498.452\end{array}$ $\begin{array}{llllll}1497.879 & 1498.139 & 1498.478 & 1500.057 & 1498.476\end{array}$ $\begin{array}{llllll}1497.935 & 1498.146 & 1498.534 & 1500.159 & 1498.534 \\ 1497.97 & 1488.151 & 1488.571 & 1500.173 & 1498.571\end{array}$ $\begin{array}{lllll}1497.97 & 1498.151 & 1498.571 & 1500.173 & 1498.571 \\ 1497 & 149816 & 1488.583 & 1500.176 & 1498.569\end{array}$ $\begin{array}{llllll}1497.991 & 1498.16 & 1498.583 & 1500.176 & 1498.569 \\ 1498.021 & 1498.17 & 1498.613 & 1500.199 & 1498.595\end{array}$ $\begin{array}{rrrrr}1498.021 & 1498.17 & 1498.613 & 1500.199 & 1498.595 \\ 1498.042 & 1498.174 & 1498.634 & 1500.202 & 1498.604\end{array}$ $\begin{array}{lllll}1498.061 & 1498.188 & 1498.65 & 1500.222 & 1498.613\end{array}$ $\begin{array}{llllll}1498.079 & 1498.188 & 1498.669 & 1500.216 & 1498.63 \\ 1498.061 & 1498.191 & 1498.655 & 1500.183 & 1498.592\end{array}$ $\begin{array}{lllllll}1498.054 & 1498.193 & 1498.646 & 1500.171 & 1498.576\end{array}$ $\begin{array}{lllllll}1498.047 & 1498.193 & 1498.646 & 1500.157 & 1498.569\end{array}$ $\begin{array}{llllll}1498.056 & 1498.2 & 1498.648 & 1500.166 & 1498.571\end{array}$ $\begin{array}{llllll}1498.072 & 1498.214 & 1498.662 & 1500.195 & 1498.585\end{array}$ $\begin{array}{llllll}1498.126 & 1498.221 & 1498.711 & 1500.246 & 1498.653\end{array}$ $\begin{array}{llllll}1498.156 & 1498.235 & 1498.739 & 1500.267 & 1498.679\end{array}$ $\begin{array}{llllll}1498.191 & 1498.249 & 1498.772 & 1500.295 & 1498.704 \\ 1498261 & 1498.268 & 1498832 & 1500353 & 1498.777\end{array}$ $\begin{array}{llllll}1498.261 & 1498.268 & 1488.832 & 1500.353 & 1498.777\end{array}$ $\begin{array}{llllll}1498.207 & 1498.293 & 1498.96 & 1500.225 & 1498.88\end{array}$ $\begin{array}{llllll}1498.261 & 1498.316 & 1499.044 & 1500.26 & 1498.952\end{array}$ $\begin{array}{llllll}1498.34 & 1498.335 & 1499.065 & 1500.269 & 1498.945\end{array}$ $\begin{array}{rrrrr}1498.317 & 1498.302 & 1499.107 & 1500.26 & 1498.973 \\ 1498.427 & 1498.347 & 1499.107 & 1500.283 & 1498.954\end{array}$ $\begin{array}{llllll}1498.427 & 1498.347 & 1499.107 & 1500.283 & 1498.954 \\ 1498.485 & 1498.363 & 1499.072 & 1500.316 & 1498.901\end{array}$ $\begin{array}{llllll}1498.485 & 1498.363 & 1499.072 & 1500.316 & 1498.901 \\ 1498.441 & 1498.365 & 1499.021 & 1500.403 & 1498.831\end{array}$ 

Well (ft AMSL) (ft AMSL) (ft AMSL) (ft AMSL) (ft AMSL) (ft AMSL) (ft AMSL) (ft AMSL) (ft AMSL) (ft AMSL) (ft AMSL) (ft AMSL) (ft AMSL) (ft AMSL) (ft AMSL) (ft AMSL) (ft AMSL) (ft AMSL) (ft AMSL) (ft AMSL) (ft AMSL) (ft AMSL) (ft AMSL) (ft AMSL) (ft AMSL) (ft AMS)

12/30/10 16:00 $12 / 30 / 1020: 00$

$12 / 31 / 104: 00$

$12 / 31 / 108: 00$

12/31/10 12:00

12/31/10 16:00

$12 / 31 / 1020: 00$

1/1/11 0:00

1/1/11 4:00

1/1/11 8:00

$1 / 1 / 1116: 00$

1/1/111 20:00

1/1/11 20:00

1/2/11 4:00

1/2/11 8:00

1/2/11 12:00

1/2/11 16:00

1/2/11 20:00

1/3/1110:00

1/3/11 8:00

1/3/1111 12:00

1/3/11 16:00

1/3/11 20:00

1/4/1110:00

$1 / 4 / 114: 00$

$1 / 4 / 1112000$

$1 / 4 / 1116: 00$

1/4/11 20:00

1/5/11 0:00

1/5/11 4:00

1/5/11 8:00

1/5/11 12:00

1/5/11 16:00

1/5/11 20:00

1/6/11 4:00

1/6/1/11 8:00

1/6/11 12:00

1/6/11 16:00

1/6/11 20:00

1/7/110:00

$1 / 7114.00$

1/7//11 12:00

1/7/11 16:00

1/7/11 20:00

1/8/11 0:00

1/8/11 4:00

1/8/11 8:00

$\begin{array}{rrrrr}1498.38 & 1498.358 & 1498.96 & 1500.33 & 1498.77 \\ 1498291 & 1498342 & 1498.879 & 1500248 & 1498672\end{array}$

$\begin{array}{rrrrr}1498.291 & 1498.342 & 1498.879 & 1500.248 & 1498.672 \\ 1498266 & 1498349 & 1498846 & 150026 & 1498655\end{array}$

$\begin{array}{llllll}149.2266 & 1498.351 & 1488.846 & 1500.26 & 1498.655\end{array}$

$\begin{array}{lllll}1498.224 & 1498.342 \quad 1498.804 \quad 1500216 \quad 1498.646\end{array}$

$\begin{array}{llllll}1498.126 & 1498.314 & 1498.706 & 1500.134 & 1498.543\end{array}$

$\begin{array}{llllll}1498.056 & 1498.295 & 1498.643 & 1500.115 & 1498.49\end{array}$

$\begin{array}{lllllll}1497.995 & 1498.272 & 1498.58 & 1500.061 & 1498.438\end{array}$

$\begin{array}{lllllll}1497.974 & 1498.258 & 1498.566 & 1500.057 & 1498.443\end{array}$

$\begin{array}{lllll}1497.946 & 1498.247 & 1498.541 & 1500.033 & 1498.431 \\ 197.888 & 1498226 & 1498.489 & 1499.986 & 198.392\end{array}$

$\begin{array}{lllllll}1497.888 & 1498.226 & 1488.489 & 1499.986 & 1498.392\end{array}$

$\begin{array}{llllll}1497.862 & 1498.216 & 1498.464 & 1499.986 & 1498.3599\end{array}$

$\begin{array}{llllll}1497.825 & 1498.198 & 1498.433 & 1499.951 & 1498.364\end{array}$

$\begin{array}{lllll}1497.834 & 1498.198 & 1498.443 & 1499.97 & 1498.378\end{array}$

$\begin{array}{llllll}1497.848 & 1498.195 & 1498.457 & 1499.989 & 1498.396\end{array}$

$\begin{array}{llllll}1497.86 & 1498.193 & 1498.466 & 1499.998 & 1498.413\end{array}$

$\begin{array}{llllll}1497.89 & 1498.193 & 1498.489 & 1500.031 & 1498.438\end{array}$

$\begin{array}{lllllll}1497.93 & 1498.205 & 1498.529 & 1500.064 & 1498.485 \\ 1497.937 & 1498205 & 1498.534 & 1500.049 & 1498473\end{array}$

$\begin{array}{llllll}1497.93 & 148.205 & 1498.529 & 1500.064 & 1498.485\end{array}$

$\begin{array}{llllll}1498 & 1498.221 & 1498.585 & 1500.12 & 1498.522\end{array}$

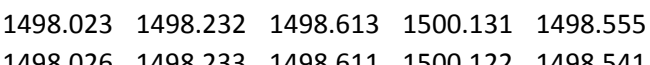

$\begin{array}{llllll}1498.026 & 1498.233 & 1498.611 & 1500.122 & 1498.541 \\ 1498.53 & 149823 & 148.613 & 1500.096 & 149856\end{array}$ $\begin{array}{rrrrrr}1498.019 & 1498.23 & 1498.613 & 1500.096 & 1498.536 \\ 1497.972 & 1498.212 & 1498.571 & 1500.031 & 1498.469\end{array}$ $\begin{array}{lrrrrr}1497.946 & 1498.2 & 1498.543 & 1500.008 & 1498.429\end{array}$ $\begin{array}{rrrrr}1497.935 & 1498.195 & 1498.527 & 1500.012 & 1498.422 \\ 1497.93 & 1988188 & 1498529 & 1500.005 & 1488.429\end{array}$ $\begin{array}{llllll}1497.93 & 1498.188 & 1498.529 & 1500.005 & 1498.429 \\ 1497.944 & 1498193 & 1498.531 & 1500.031 & 1498.441\end{array}$ $\begin{array}{lllllll}1498.002 & 1498.214 & 1498.585 & 1500.099 & 1498.515\end{array}$ $\begin{array}{llllll}1498.016 & 1498.219 & 1498.604 & 1500.099 & 1498.532\end{array}$ $\begin{array}{llllll}1498.047 & 1498.232 & 1498.627 & 1500.131 & 1498.55\end{array}$ $\begin{array}{llllll}1498.058 & 1498.237 & 1498.639 & 1500.134 & 1498.56\end{array}$ $\begin{array}{llllll}1498.056 & 1498.239 & 1498.639 & 1500.122 & 1498.553\end{array}$ $\begin{array}{llllll}1498.061 & 1498.242 & 1498.639 & 1500.129 & 1498.543 \\ 1498.056 & 1498.237 & 1488.639 & 1500101 & 1498536\end{array}$ $\begin{array}{lllll}149.056 & 1498.237 & 1488.639 & 1500.101 & 1498.536\end{array}$ $\begin{array}{lllllll}1498.019 & 1498226 & 1498.601 & 1500.004 & 1498.487\end{array}$ $\begin{array}{lllllll}1498.026 & 1498.228 & 1498.606 & 1500.073 & 1498.487\end{array}$ $\begin{array}{llllll}1498.026 & 1498.225 & 1498.608 & 1500.075 & 1498.492\end{array}$ $\begin{array}{llllll}1498.046 & 1498.232 & 1498.622 & 1500.101 & 1498.511\end{array}$ $\begin{array}{lllllll}1498.1 & 1498.253 & 1498.671 & 1500.159 & 1498.504\end{array}$ $\begin{array}{lllllll}1498.1 & 1498.256 & 1498.66 & 1500.152 & 1498.305\end{array}$ $\begin{array}{llllll}1498.117 & 1498.26 & 1498.664 & 1500.169 & 1498.354 \\ 1498.142 & 1498.27 & 1498.683 & 1500187 & 1498.436\end{array}$ $\begin{array}{lllllll}14981 & 1498.254 & 1498.653 & 1500.187 & 1498.436\end{array}$ $\begin{array}{llllll}1498.075 & 1498251 & 1498.632 & 1500.113 & 1498.42\end{array}$ $\begin{array}{llllll}1498.056 & 1498.237 & 1498.615 & 1500.082 & 1498.424\end{array}$ $\begin{array}{llllll}1498.019 & 1498.226 & 1498.587 & 1500.052 & 1498.406\end{array}$ $\begin{array}{llllll}1498.014 & 1498.221 & 1498.585 & 1500.061 & 1498.415\end{array}$ $\begin{array}{llllll}1498 & 1498.212 & 1498.573 & 1500.047 & 1498.415\end{array}$ $\begin{array}{lllll}1497.967 & 1498.195 & 1498.55 & 1500.008 & 1498.392\end{array}$ 

Well

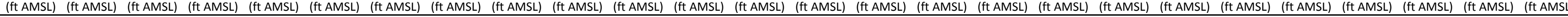

1/8/11 16:00 1/8/11 20:00 $1 / 9 / 110.00$ 1/9/11 8:00 1/9/11 12:00 1/9/11 16:00 1/9/11 20:00 1/10/11 0:00 1/10/11 4:00 1/10/11 8:00 $1 / 10 / 1116: 00$ 1/10/11 20:00 1/11/11 0:00 1/11/11 4:00 1/11/11 8:00 $1 / 11 / 1112: 00$ 1/11/11 16:00 1/12/11 0:00 1/12/11 8:00 1/12/11 12:00 1/12/11 16:00 1/12/111 20:00 1/13/11 0:00 $1 / 13 / 114.00$ $1 / 13 / 118.00$ 1/13/11 16:00 1/13/11 20:00 1/14/11 0:00 1/14/11 4:00 $1 / 14 / 118: 00$ 1/14/11 12:00 $1 / 14 / 1116: 00$ $1 / 15 / 110: 00$ 1/15/11 4:00 1/15/11 8:00 1/15/11 12:00 1/15/111 16:00 1/15/11 20:00 1/16/11 0:00 $1 / 16 / 118.00$ 1/16/11 12:00 1/16/11 16:00 1/16/11 20:00 1/17/11 0:00 1/17/11 4:00 1/177/11 8:00
$1 / 17 / 1112: 00$ $\begin{array}{llllll}1497.998 & 1498.207 & 1498.576 & 1500.078 & 1498.443\end{array}$ $\begin{array}{llllll}1497.986 & 1498.198 & 1498.566 & 1500.047 & 1498.417 \\ 1497.972 & 1498.191 & 1498.555 & 1500.033 & 1498.408\end{array}$ $\begin{array}{lllllll}1497.97 & 1498.188 & 1489.555 & 1500.033 & 1498.408\end{array}$ $\begin{array}{llllll}1497.958 & 1498.184 & 1498.543 & 1500.01 & 1498.408\end{array}$ $\begin{array}{llllll}1497.965 & 1498.191 & 1498.543 & 1500.035 & 1498.41\end{array}$ $\begin{array}{llllll}1497.956 & 1498.179 & 1498.538 & 1500.01 & 1498.415\end{array}$ $\begin{array}{rrrrr}1497.939 & 1498.172 & 1498.529 & 1499.996 & 1498.403 \\ 1497.914 & 1498.167 & 1498.508 & 1499.97 & 1498.38\end{array}$ $\begin{array}{llllll}1497.914 & 1498.167 & 1498.508 & 1499.97 & 1498.38\end{array}$ $\begin{array}{rrrrr}1497.916 & 1498.16 & 1498.494 & 1499.961 & 1498.364 \\ 1497.902 & 1498.153 & 1498.473 & 1499.965 & 14935\end{array}$ $\begin{array}{llllll}4497.902 & 1498.153 & 1488.473 & 1499.965 & 1498.35 \\ 1497.902 & 1498.151 & 1498.455 & 1499944 & 1498.338\end{array}$ $\begin{array}{llllll}1497.902 & 1498.151 & 1488.455 & 1499.944 & 1498.338 \\ 1497.9 & 1498.146 & 1498.413 & 1499.867 & 1498.303\end{array}$ $\begin{array}{llllll}1497.876 & 1498.144 & 1498.359 & 1499.82 & 1498.245\end{array}$ $\begin{array}{rrrrrr}1497.876 & 1498.144 & 1498.359 & 1499.82 & 1498.245 \\ 1497.883 & 1498.128 & 1498.333 & 1499.825 & 1498.231\end{array}$ $\begin{array}{lllllll}1497.89 & 1498.125 & 1498.287 & 1499.839 & 1498.191\end{array}$ $\begin{array}{llllll}1497.862 & 1498.109 & 1498.259 & 1499.79 & 1498.175\end{array}$ $\begin{array}{llllll}1497.86 & 1498.083 & 1498.242 & 1499.802 & 1498.168\end{array}$ $\begin{array}{llllll}1497.851 & 1498.086 & 1498.245 & 1499.813 & 1498.184 \\ 1497844 & 1498.074 & 1498242 & 1499797 & 1498.184\end{array}$ $\begin{array}{lllllll}4497.844 & 1498.074 & 1488.242 & 1499.797 & 1498.184\end{array}$ $\begin{array}{llllll}1497.839 & 1498.058 & 1498.224 & 1499.771 & 1498.163 \\ 1497.844 & 1498.046 & 1498.212 & 1499.755 & 1498.154\end{array}$ $\begin{array}{lllllll}1497.811 & 1498.037 & 1498.2 & 1499.746 & 1498.142\end{array}$ $\begin{array}{lllllll}1497.692 & 1498.037 & 1498.207 & 1499.783 & 1498.154\end{array}$ $\begin{array}{lllllll}1497.687 & 1498.042 & 1498.249 & 1499.825 & 1498.214\end{array}$ $\begin{array}{lllllll}1497.708 & 1498.049 & 1498.294 & 1499.867 & 1498.261\end{array}$ $\begin{array}{llllll}1497.75 & 1498.058 & 1498.331 & 1499.905 & 1498.298 \\ 1497.795 & 1498.069 & 1498371 & 1499937 & 1498355\end{array}$ $\begin{array}{lllllll}1497.795 & 1498.069 & 1498.37 & 1499.937 & 1498.345 \\ 1497.855 & 1498.091 & 1498.433 & 1499.993 & 1498.408\end{array}$ $\begin{array}{llllll}4497.855 & 1498.091 & 1498.433 & 1499.993 & 1498.408 \\ 1497.895 & 1498.111 & 1498.48 & 1500042 & 1498.448\end{array}$ $\begin{array}{llllll}1497.937 & 1498.123 & 1498.522 & 1500.04 & 1498.473\end{array}$ $\begin{array}{llllll}1497.942 & 1498.111 & 1498.536 & 1500.024 & 1498.473\end{array}$ $\begin{array}{lllllll}1497.942 & 1498.132 & 1498.55 & 1500.052 & 1498.471\end{array}$ $\begin{array}{lllllll}1497.939 & 1498.144 & 1498.564 & 1500.059 & 1498.476\end{array}$ $\begin{array}{llllll}1497.963 & 1498.151 & 1498.571 & 1500.049 & 1498.471\end{array}$ $\begin{array}{lllll}1497.949 & 1498.149 & 1498.552 & 1500.033 & 1498.429\end{array}$ $\begin{array}{llllll}4497.949 & 1498.153 & 1488.557 & 1500.012 & 1498.436\end{array}$ $\begin{array}{lllllll}1497.942 & 1498.153 & 1498.557 & 1499.991 & 1498.42\end{array}$ $\begin{array}{lllllll}1497.942 & 1498.156 & 1498.559 & 1500.024 & 1498.429\end{array}$ $\begin{array}{llllll}1497.932 & 1498.146 & 1498.536 & 1499.982 & 1498.392\end{array}$ $\begin{array}{llllll}1497.914 & 1498.135 & 1498.517 & 1499.947 & 1498.368\end{array}$ $\begin{array}{llllll}1497.909 & 1498.104 & 1498.548 & 1499.93 & 1498.417\end{array}$ $\begin{array}{rrrrr}1497.902 & 1498.13 & 1498.524 & 1499.905 & 1498.354 \\ 149.893 & 1498.125 & 1498.522 & 149989 & 1498352\end{array}$ $\begin{array}{llllll}1497.893 & 1498.125 & 1498.522 & 1499.898 & 1498.352 \\ 1497.888 & 1498132 & 1498.522 & 1499.905 & 1498359\end{array}$ $\begin{array}{llllll}1497.888 & 1498.132 & 1498.522 & 1498.8985 & 1498.352\end{array}$ $\begin{array}{llllll}1497.883 & 1498.146 & 1498.559 & 1499.891 & 1498.389 \\ 1498.417\end{array}$ $\begin{array}{llllll}1497.888 & 1498.135 & 1498.622 & 1499.853 & 1498.497\end{array}$ $\begin{array}{llllll}1497.895 & 1498.116 & 1498.66 & 1499.869 & 1498.527\end{array}$ $\begin{array}{llllll}1497.923 & 1498.172 & 1498.716 & 1499.933 & 1498.581 \\ 1497.984 & 1498.151 & 1498.783 & 1499.977 & 1498.639\end{array}$ $\begin{array}{rrrrr}1498.033 & 1498.107 & 1498.837 & 1500.01 & 1498.683 \\ 1498056 & 1498.111 & 1498.846 & 1499.991 & 1498.674\end{array}$ 
$\begin{array}{lllll}1498.058 & 1498.035 & 1498.844 & 1499.937 & 1498.655 \\ 149063 & 1489125 & 1498788 & 1500.043 & 1498571\end{array}$ $\begin{array}{lllll}1498.063 & 1498.125 & 1498.788 & 1500.043 & 1498.571 \\ 1498.023 & 1498.158 & 1498.697 & 1499.954 & 1498.459\end{array}$ $\begin{array}{llllll}1498.023 & 1498.158 & 1488.697 & 149.954 & 1498.459 \\ 1498.009 & 1498.188 & 1498.632 & 1499.933 & 1498389\end{array}$ $\begin{array}{llllll}1498.002 & 1498.146 & 1498.562 & 1500.054 & 1498336\end{array}$ $\begin{array}{rrrrr}1497.911 & 1498.13 & 1498.531 & 1500 & 1498.322 \\ 1497.907 & 1498.132 & 1488.531 & 1499.811 & 1498.347\end{array}$ $\begin{array}{llllll}1497.907 & 1498.132 & 1498.531 & 1499.811 & 1498.347 \\ & 1497.904 & 1498.128 & 1498.513 & 1499.884 & 1498.34\end{array}$ $\begin{array}{rrrrrr}1497.904 & 1498.128 & 1498.513 & 1499.884 & 1498.34 \\ 1497.907 & 1498.13 & 1498.51 & 1499.907 & 1498.345\end{array}$ $\begin{array}{rrrrrr}1497.902 & 1498.13 & 1498.508 & 1499.933 & 1498.347 \\ 1497.904 & 1498.128 & 1988.508 & 1999.951 & 1498359\end{array}$ $\begin{array}{lllll}1497.904 & 1498.128 & 1498.508 & 1499.951 & 1498.359 \\ 1497.939 & 1498146 & 1998534 & 1999.954 & 1988392\end{array}$ $\begin{array}{rrrrr}1497.939 & 1498.146 & 1498.534 & 1499.954 & 1498.392 \\ 1497.951 & 1498.144 & 1498.51 & 1499.94 & 1498.415\end{array}$ $\begin{array}{lllll}1497.869 & 1498.125 & 1498.636 & 1500.08 & 1498.319\end{array}$ $\begin{array}{rrrrr}1497.802 & 1498.105 & 1498.674 & 1500.18 & 1498.242 \\ 1497.776 & 148.095 & 148.639 & 1500.234 & 1498.221\end{array}$ $\begin{array}{lllll}1497.776 & 1498.095 & 1498.639 & 1500.234 & 1498.221 \\ 1477.75 & 1498.08 & 148.597 & 1500.262 & 1498.21\end{array}$ $\begin{array}{rrrrr}1497.75 & 1498.086 & 1498.597 & 1500.262 & 1498.21 \\ 1497.739 & 1498.069 & 1498.475 & 1500.113 & 1498.219\end{array}$ $\begin{array}{llllll}1497.739 & 1498.069 & 1498.475 & 1500.113 & 1498.219 \\ 1497.739 & 1497.981 & 1498.336 & 1500.035 & 1498.287\end{array}$ $\begin{array}{llllll}1497.839 & 1498.1 & 1498.396 & 1500.155 & 1498.343\end{array}$ $\begin{array}{rrrrrr}1497.928 & 1498.118 & 1498.35 & 1500101 & 1498.466\end{array}$ $\begin{array}{lllllll}1497.988 & 1498.144 & 1498.366 & 1500.029 & 1498.49\end{array}$ $\begin{array}{llllll}1497.963 & 1498.139 & 1498.371 & 1499.914 & 1498.471\end{array}$ $\begin{array}{lllllll}1497.991 & 1498.156 & 1498.566 & 1499.844 & 1498.459\end{array}$ $\begin{array}{llllll}1497.963 & 1498.144 & 1498.543 & 1499.977 & 1498.41\end{array}$ $\begin{array}{rrrrr}1497.974 & 1498.153 & 1498.55 & 1500.031 & 1498.42 \\ 1497.993 & 1498.163 & 1498.566 & 1500045 & 1498.488\end{array}$ $\begin{array}{llllll}1497.993 & 1498.163 & 1498.566 & 1500.045 & 1498.448 \\ 1498.033 & 1498.174 & 1498.601 & 1500.017 & 1498.85\end{array}$ $\begin{array}{lllllll}1498.033 & 1498.174 & 1498.601 & 1500.017 & 1498.485 \\ 1498079 & 1498193 & 1498.634 & 1499844 & 1498515\end{array}$ $\begin{array}{llllll}1498.119 & 1498.198 & 1488.676 & 1499.729 & 1488.553\end{array}$ $\begin{array}{lllll}1498.072 & 1498.186 & 1498.634 & 1500.033 & 1498.483\end{array}$ $\begin{array}{llllll}1498 & 1498.167 & 1498.576 & 1499.951 & 1498.394\end{array}$ $\begin{array}{lllllll}1497.909 & 1498.135 & 1498.489 & 1499.858 & 1498.289\end{array}$ $\begin{array}{llllll}1497.848 & 1498.114 & 1498.433 & 1499.825 & 1498.242\end{array}$ $\begin{array}{llllll}1497.848 & 1498.109 & 1498.422 & 1499.846 & 1498.252 \\ 1497876 & 1498114 & 1498.452 & 1998902 & 1488308\end{array}$ $\begin{array}{llllll}1497.876 & 1498.114 & 1498.452 & 1499.902 & 1498.308\end{array}$ $\begin{array}{llllll}1497.886 & 1498.116 & 1498.464 & 1499.912 & 1498.333 \\ 1497.921 & 1498.125 & 1498.492 & 1499.942 & 1498371\end{array}$ $\begin{array}{llllll}1497.925 & 1498.121 & 1498.496 & 1499.942 & 1498.375\end{array}$ $\begin{array}{lllllll}1497.909 & 1498.118 & 1498.482 & 1499.909 & 1498.354\end{array}$ $\begin{array}{llllll}1497.86 & 1498.1 & 1498.441 & 1499.841 & 1498.294\end{array}$ $\begin{array}{llllll}1497.846 & 1498.09 & 1498.424 & 1499.837 & 1498.273\end{array}$ $\begin{array}{llllll}1497.809 & 1498.076 & 1498.394 & 1499.813 & 1498.238\end{array}$ $\begin{array}{rlrrr}1497.799 & 1498.072 & 1498.385 & 1499.811 & 1498.233 \\ 1497.806 & 1498072 & 1498389 & 149982 & 1498245\end{array}$ $\begin{array}{llllllll}1497.806 & 1498.072 & 1498.389 & 1499.82 & 1498.245 \\ 1497.82 & 1498.072 & 1498.401 & 1498.841 & 1498273\end{array}$ $\begin{array}{rrrrr}1497.82 & 1498.072 & 1498.401 & 1499.841 & 1498.273 \\ 1497.855 & 1498.081 & 1498.429 & 1499.855 & 1498.308\end{array}$ $\begin{array}{llllll}1497.895 & 1498.093 & 1498.466 & 1499.933 & 1498.361\end{array}$ $\begin{array}{llllll}1497.893 & 1498.093 & 1498.468 & 1499.919 & 1498.354\end{array}$ $\begin{array}{llllll}1497.874 & 1498.086 & 1498.45 & 1499.884 & 1498.329\end{array}$ $\begin{array}{lllllll}1497.848 & 1498.076 & 1498.431 & 1499.858 & 1498.294\end{array}$ $\begin{array}{lllll}1497.818 & 1498.062 & 1498.403 & 1499.816 & 1498.259 \\ 1497.783 & 1498.058 & 1498.371 & 1499.783 & 1498.214\end{array}$ 

Well (ft AMSL) (ft AMSL) (ft AMSL) (ft AMSL) (ft AMSL) (ft AMSL) (ft AMSL) (ft AMSL) (ft AMSL) (ft AMSL) (ft AMSL) (ft AMSL) (ft AMSL) (ft AMSL) (ft AMSL) (ft AMSL) (ft AMSL) (ft AMSL) (ft AMSL) (ft AMSL) (ft AMSL) (ft AMSL) (ft AMSL) (ft AMSL) (ft AMSL) (ft AMS

1/26/11 16:00

1/26/11 20:00

$1 / 27 / 11400$

1/27/11 8:00

$1 / 27 / 1112: 00$

1/27/1116:00

1/27/11 20:00

1/28/11 0:00

$1 / 28 / 114: 00$

$1 / 28 / 118: 00$

$1 / 28 / 1116: 00$

1/28/11 20:00

1/28/11 20:00

1/29/11 4:00

1/29/11 8:00

1/29/11 12:00

$1 / 29 / 1116: 00$

1/29/11 20:00

1/30/11 4:00

$1 / 30 / 118: 00$

1/30/11 12:00

1/30/11 16:00

1/30/11 20:00

1/31/110:00

1/31/11 4:00

$1 / 31 / 118.00$

1/31/11 16:00

1/31/11 20:00

2/1/11 0:00

2/1/11 4:00

2/1/11 8:00

2/1/11 12:00

2/1/111 16:00

2/1/1/11 20:00

2/2/111 4:00

2/2/1/11 8:00

2/2/11 12:00

2/2/111 16:00

2/2/11120:00

2/3/11 0:00

$2 / 3 / 118.00$

2/3/11 12:00

2/3/11 16:00

2/3/11 20:00

2/4/11 0:00

2/4/11 4:00

2/4/11 8:00

$\begin{array}{rrrrr}1497.806 & 1498.06 & 1498.389 & 1499.832 & 1498.254 \\ 1497.799 & 1498.055 & 1498382 & 1499823 & 1498247\end{array}$

$\begin{array}{rrrrr}1497.799 & 1498.055 & 1498.382 & 1499.823 & 1498.247 \\ 1497.832 & 1498.069 & 1498.413 & 1499.87 & 1498284\end{array}$

$\begin{array}{lllllll}1497.832 & 1498.069 & 1498.413 & 1499.87 & 1498.284\end{array}$

$\begin{array}{llllll}1497.902 & 1498.086 & 1498.471 & 1499.926 & 1498.364\end{array}$

$\begin{array}{llllll}1497.893 & 1498.088 & 1498.461 & 1499.9 & 1498.345\end{array}$

$\begin{array}{lllllll}1497.904 & 1498.09 & 1498.475 & 1499.923 & 1498.354\end{array}$

$\begin{array}{rrrrr}1497.886 & 1498.083 & 1498.457 & 1499.893 & 1498.324 \\ 1497.883 & 1498.088 & 1498.459 & 1499.9 & 1498.317\end{array}$

$\begin{array}{rrrrrr}1497.883 & 1488.088 & 1498.459 & 1499.9 & 1498.347 \\ 1497.904 & 1498.095 & 1498.475 & 1499.926 & 1498.343\end{array}$

$\begin{array}{lllll}4497.907 & 1498.095 & 1498.478 & 1499.923 & 1498.35 \\ 1497.925 & 1498.104 & 1498.487 & 1499.928 & 1498.354\end{array}$

$\begin{array}{llllll}147.925 & 1498.104 & 1498.487 & 1499.928 & 1498.354 \\ 1497.951 & 1498.109 & 1498.51 & 1499.951 & 1498.382\end{array}$

$\begin{array}{lllllll}1497.914 & 1498.1 & 1498.485 & 1499.905 & 1498.338\end{array}$

$\begin{array}{lllllll}1497.904 & 1498.095 & 1498.473 & 1499.898 & 1498.319\end{array}$

$\begin{array}{lllllll}1497.907 & 1498.102 & 1498.478 & 1499.912 & 1498.326\end{array}$

$\begin{array}{llllll}1497.914 & 1498.097 & 1498.478 & 1499.912 & 1498.333\end{array}$

$\begin{array}{llllll}1497.946 & 1498.112 & 1498.51 & 1499.956 & 1498.366\end{array}$

$\begin{array}{llllll}1497.958 & 1498.114 & 1498.52 & 1499.951 & 1498.364 \\ 1497.865 & 1498.079 & 1498.436 & 149.813 & 1498224\end{array}$

$\begin{array}{lllllll}1497.958 & 1498114 & 1498.52 & 1499.951 & 1498.364 \\ 1497.799 & 1498.053 & 1498.436 & 149.813 & 1498.224 \\ 1498.378 & 1499.762 & 1498.151\end{array}$

$\begin{array}{lllllll}1497.769 & 1498.039 & 1498.345 & 1499.748 & 1498.137\end{array}$

$\begin{array}{llllll}1497.722 & 1498.032 & 1498.305 & 1499.725 & 1498.112\end{array}$

$\begin{array}{rrrrr}1497.72 & 1498.032 & 1498.298 & 1499.743 & 1498.123 \\ \end{array}$

$\begin{array}{rrrrrr}1497.771 & 1498.042 & 1498.345 & 1499.82 & 1498.184 \\ 1497.767 & 1498.032 & 1498.345 & 1499.806 & 1498.186\end{array}$

$\begin{array}{llllll}1497.767 & 1498.032 & 1498.345 & 1499.806 & 1498.186 \\ 1497.774 & 1498.03 & 1498.347 & 1499.802 & 1498.193\end{array}$

$\begin{array}{llllll}1497.788 & 1498.03 & 1498.347 & 1499.802 & 1498.193 \\ 1498.035 & 1498.359 & 1499.834 & 1498.214\end{array}$

$\begin{array}{llllll}4497.778 & 1498.03 & 1498.357 & 1499.813 & 1498.214\end{array}$

$\begin{array}{llllll}1497.769 & 1498.032 & 1498.343 & 1499.797 & 1498.196 \\ 1497.762 & 1498.021 & 1498.343 & 1499.792 & 1498.203\end{array}$

$\begin{array}{llllll}1497.713 & 1498.004 & 1498.298 & 1499.722 & 1498.137\end{array}$ $\begin{array}{llllll}1497.683 & 1497.995 & 1498.266 & 1499.701 & 1498.1\end{array}$ $\begin{array}{lllllll}1497.711 & 1498.007 & 1498.294 & 1499.771 & 1498.147\end{array}$ $\begin{array}{lllll}1497.699 & 1497.995 & 1498.27 & 1499.739 & 1498.144\end{array}$ $\begin{array}{rrrrrr}1497.676 & 1497.988 & 1498.259 & 1499.713 & 1498.126 \\ 1497.692 & 1497.983 & 149828 & 1499.736 & 1488147\end{array}$ $\begin{array}{lllllll}1497.676 & 1497.988 & 1498.259 & 1499.713 & 1498.126\end{array}$ $\begin{array}{lllll}1497.631 & 1497.965 & 1498.226 & 1499.661 & 1498.088 \\ 1497.564 & 1497.951 & 1498.165 & 1499612 & 1498.023\end{array}$ $\begin{array}{lllllll}1497.54 & 1497.941 & 1498.144 & 1499.619 & 1498.009\end{array}$ $\begin{array}{llllll}1497.506 & 1497.93 & 1498.112 & 1499.596 & 1497.988\end{array}$ $\begin{array}{llllll}1497.452 & 1497.916 & 1498.06 & 1499.563 & 1497.941\end{array}$ $\begin{array}{llllll}1497.475 & 1497.911 & 1498.081 & 1499.596 & 1497.981\end{array}$ $\begin{array}{rlllll}1497.477 & 1497.909 & 1498.086 & 1499.601 & 1497.995 \\ 1497491 & 1497.904 & 14981 & 149.615 & 1498006\end{array}$ $\begin{array}{rrrrrr}1497.491 & 1497.904 & 1498.1 & 1499.615 & 1498.006 \\ 1497.529 & 1497.906 & 1498.133 & 1499657 & 1498.053\end{array}$ $\begin{array}{llllllll}1497.529 & 1497.900 & 1498.133 & 1499.655 & 1498.053\end{array}$ $\begin{array}{llllll}1497.613 & 1497.92 & 1498.203 & 1499.727 & 1498.088\end{array}$ $\begin{array}{lllllll}1497.687 & 1497.944 & 1498.266 & 1499.797 & 1498.207\end{array}$ $\begin{array}{llllll}1497.708 & 1497.951 & 1498.287 & 1499.795 & 1498.219\end{array}$ $\begin{array}{llllll}1497.736 & 1497.962 & 1498.31 & 1499.818 & 1498.228\end{array}$ $\begin{array}{llllll}1497.776 & 1497.972 & 1498.352 & 1499.844 & 1498.261\end{array}$ $\begin{array}{rrrrr}1497.813 & 1497.986 & 1498.385 & 1499.867 & 1498.289 \\ 1497.86 & 1498.007 & 1498.427 & 1499.905 & 1498317\end{array}$ 

Well

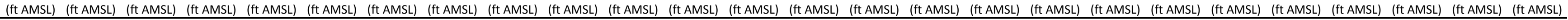

2/4/11 16:00 2/4/1120:00 $2 / 5 / 114: 00$ 2/5/11 8:00 2/5/11 12:00 2/5/11 16:00 2/5/11 20:00 2/6/11 0:00 2/6/11 4:00 2/6/11 8:00 $2 / 6 / 1116: 00$ $2 / 6 / 1120: 00$ 2/7/1110:00 2/7/11 4:00 2/7/11 8:00 2/7/11 12:00 2/7/111 16:00 2/7/11 20:00 2/8/11 0:00 2/8/11 8:00 2/8/11 8:00 2/8/11 16:00 2/8/11 20:00 2/9/11 0:00 2/9/11 4:00 2/9/11 12:00 2/9/11 16:00 $2 / 9 / 1120.00$ 2/10/11 0:00 2/10/11 4:00 2/10/11 8:00 2/10/11 12:00 2/10/1116:00 $2 / 11 / 110: 00$ $2 / 11 / 114: 00$ 2/11/11 8:00 2/11/11 12:00 2/11/11 16:00 2/11/11 20:00 2/12/11 0:00 $2 / 12 / 114.00$ 2/12/1112:00 2/12/11 16:00 2/12/11 20:00 2/13/11 0:00 2/13/11 4:00 2/13/111 8:00

$\begin{array}{lllll}1497.951 & 1498.032 & 1498.515 & 1499.944 & 1498.403\end{array}$ $\begin{array}{rrrrr}1497.988 & 1498.044 & 1498.55 & 1499.93 & 1498.422 \\ 1498.039 & 1498.065 & 1498.592 & 1500.026 & 1498.448\end{array}$ $\begin{array}{llllll}1498.039 & 1498.065 & 1498.592 & 1500.026 & 1498.448 \\ 1498.095 & 1498.079 & 1498.643 & 1499.996 & 1498.485\end{array}$ $\begin{array}{lllllll}1498.105 & 1498.086 & 1498.655 & 1499.958 & 1498.483\end{array}$ $\begin{array}{llllll}1498.121 & 1498.095 & 1498.664 & 1499.846 & 1498.476\end{array}$ $\begin{array}{llllll}1498.091 & 1497.997 & 1498.664 & 1499.713 & 1498.457\end{array}$ $\begin{array}{lllll}1498.049 & 1498.095 & 1498.683 & 1499.893 & 1498.38 \\ 1498 & 1498135 & 1498629 & 1499.949 & 149831\end{array}$ $\begin{array}{rrrrr}1498 & 1498.135 & 1498.629 & 1499.949 & 1498.31 \\ 1497.981 & 1498.093 & 1498.59 & 1500.014 & 1498.282\end{array}$ $\begin{array}{llllll}1497.914 & 1498.06 & 1498.538 & 1500.012 & 1498.245 \\ 1497.848 & 1498011 & 1498.466 & 1500.003 & 1498.182\end{array}$ $\begin{array}{lllllll}1497.82 & 1498.023 & 1498.452 & 1499.93 & 1498.179\end{array}$ $\begin{array}{llllll}1497.746 & 1498 & 1498.368 & 1500.117 & 1498.1\end{array}$ $\begin{array}{rrrrr}1497.746 & 1498 & 1498.368 & 1500.117 & 1498.1 \\ 1497.713 & 1497.986 & 1498.333 & 1500.166 & 1498.084\end{array}$ $\begin{array}{llllll}1497.704 & 1497.979 & 1498.315 & 1500.211 & 1498.088\end{array}$ $\begin{array}{llllll}1497.694 & 1497.976 & 1498.308 & 1500.227 & 1498.098\end{array}$ $\begin{array}{llllll}1497.694 & 1497.974 & 1498.305 & 1500.145 & 1498.112\end{array}$ $\begin{array}{llllll}1497.676 & 1497.993 & 1498.359 & 1500.003 & 1498.196 \\ 1497.748 & 1497.988 & 1498354 & 1500.183 & 1498193\end{array}$ $\begin{array}{llllll}4497.748 & 1497.988 & 1488.354 & 1500.183 & 1498.193\end{array}$ $\begin{array}{llllll}1497.673 & 1497.958 & 1498.289 & 1500.267 & 1498.102\end{array}$ $\begin{array}{llllll}1497.592 & 1497.937 & 1498.21 & 1500.283 & 1498.013\end{array}$ $\begin{array}{llllll}1497.559 & 1497.93 & 1498.17 & 1500.283 & 1497.985\end{array}$ $\begin{array}{llllll}1497.587 & 1497.927 & 1498.198 & 1500.265 & 1498.044\end{array}$ $\begin{array}{llllll}1497.559 & 1497.92 & 1488.172 & 1500.276 & 1498.02\end{array}$ $\begin{array}{lllll}1497.559 & 1497.913 & 1498.168 & 1500.286 & 1498.023 \\ 1997.582 & 1997.913 & 1488191 & 1500276 & 1488.058\end{array}$ $\begin{array}{llllll}1497.582 & 1497.913 & 1498.191 & 1500.276 & 1498.058 \\ 1497568 & 1497.906 & 1498177 & 1500276 & 1498.046\end{array}$ $\begin{array}{lllllll}1497.568 & 1497.906 & 1498.177 & 1500.276 & 1498.046 \\ 1497.578 & 1497.904 & 1498179 & 1500274 & 1498049\end{array}$ $\begin{array}{lllllll}1497.601 & 1497.906 & 1498.203 & 1500.253 & 1498.081\end{array}$ $\begin{array}{lllllll}1497.613 & 1497.904 & 1498.214 & 1500.244 & 1498.088\end{array}$ $\begin{array}{lllllll}1497.636 & 1497.909 & 1498.228 & 1500.229 & 1498.109\end{array}$ $\begin{array}{llllll}1497.687 & 1497.923 & 1498.268 & 1500.201 & 1498.156\end{array}$ $\begin{array}{lllllll}1497.711 & 1497.93 & 1498.294 & 1500.173 & 1498.182\end{array}$ $\begin{array}{llllll}1497.729 & 1497.939 & 1498.308 & 1500.143 & 1498.193 \\ 1497.783 & 1497.958 & 1488354 & 1500.08 & 1498.242\end{array}$ $\begin{array}{lllllll}1497.783 & 1497.958 & 1488.354 & 1500.08 & 1498.242\end{array}$ $\begin{array}{rrrrrr}1497.776 & 1497.96 & 1498.352 & 1490.021 & 1498.219 \\ 14972 & 1498.214\end{array}$ $\begin{array}{llllll}1497.774 & 1497.96 & 1498.347 & 1499.883 & 1498.203\end{array}$ $\begin{array}{llllll}1497.757 & 1497.951 & 1498.329 & 1499.844 & 1498.175\end{array}$ $\begin{array}{llllll}1497.753 & 1497.946 & 1498.308 & 1499.804 & 1498.144\end{array}$ $\begin{array}{llllll}1497.753 & 1497.941 & 1498.301 & 1499.785 & 1498.135\end{array}$ $\begin{array}{rlrrrr}1497.753 & 1497.927 & 1498.266 & 1499.806 & 1498.091\end{array}$ $\begin{array}{llllll}14497.75 & 1497.913 & 1498.24 & 1499.83 & 1498.058 \\ 1497.748 & 1497.911 & 1498242 & 149983 & 1480\end{array}$ $\begin{array}{llllll}1497.748 & 1497.911 & 1498.242 & 1499.834 & 1498.07 \\ 1497.75 & 1497.911 & 1498249 & 1499825 & 1498.093\end{array}$ $\begin{array}{llllll}1497.748 & 1497.918 & 1498.275 & 1499.788 & 1498.128\end{array}$ $\begin{array}{lllllll}1497.746 & 1497.923 & 1498.298 & 1499.757 & 1498.163\end{array}$ $\begin{array}{llllll}1497.729 & 1497.923 & 1498.294 & 1499.757 & 1498.147\end{array}$ $\begin{array}{llllll}1497.725 & 1497.909 & 1498.315 & 1499.746 & 1498.17\end{array}$ $\begin{array}{lllllll}1497.753 & 1497.886 & 1498.361 & 1499.725 & 1498.221\end{array}$ $\begin{array}{llllll}1497.781 & 1497.876 & 1498.385 & 1499.699 & 1498.247 \\ 1497.823 & 1497.876 & 1498.399 & 1499.668 & 1498.256\end{array}$ 
$1497.816 \quad 1497.853 \quad 1498.451499 .481 \quad 1498.305$

2/14/111 8:00

2/14/11112:00

2/14/11 20:00

2/15/11 0:00

2/15/11 8:00

2/15/111116:00

2/15/11 20:00

2/15/11 20:00

2/16/11 4:00

2/16/11 8:00

2/16/11 12:00

2/16/11 16:00

$2 / 16 / 1120: 00$

2/177/110:00

2/17/11 8:00

2/17/11 12:00

2/17/11 16:00

2/17/11 20:00

2/18/11 0:00

2/18/11 4:00

2/18/11 8:00

2/18/11 16:00

2/18/11 20:00

2/19/11 0:00

2/19/11 4:00

2/19/11 8:00

2/19/11 12:00

2/19/11 16:00

2/19/11 20:00

2/20/11 4:00

2/20/11 8:00

2/20/11 12:00

2/20/11 16:00

2/20/11 20:00

2/21/11 0:00

$2 / 21 / 114.00$

$\begin{array}{llllll}1497.781 & 1497.906 & 1498.389 & 1499.638 & 1498.217\end{array}$

$\begin{array}{llllll}1497.725 & 1497.993 & 1498.28 & 1499.713 & 1498.084 \\ 1497.657 & 1497.953 & 1498242 & 1499.781 & 1498.049\end{array}$

$\begin{array}{llllll}1497.657 & 1497.909 & 1498.193 & 1499.858 & 1498.023\end{array}$

$\begin{array}{llllll}1497.643 & 1497.876 & 1498.182 & 1499.549 & 1498.03\end{array}$

$\begin{array}{llllll}1497.592 & 1497.799 & 1504.56 & 1499.467 & 1498.119\end{array}$

$\begin{array}{llllll}1497.594 & 1497.899 & 1515.56 & 1499.631 & 1498.17\end{array}$

$\begin{array}{lllllll}1497.62 & 1497.911 & 1516.866 & 1499.725 & 1498.191\end{array}$

$\begin{array}{llllll}1497.741 & 1497.93 & 151313.907 & 1499.767 & 1498.263 \\ 149775 & 1497.932 & 1512114 & 1499762 & 148.266\end{array}$

$\begin{array}{llllll}1497.75 & 1497.932 & 1513.114 & 1499.762 & 1498.266\end{array}$

$\begin{array}{llllll}1497.725 & 1497.925 & 1510.69 & 1499.376 & 1498.261 \\ 1497.671 & 1497.948 & 1509.587 & 1499.248 & 1498.291\end{array}$

$\begin{array}{llllll}1497.755 & 1497.951 & 1508.594 & 1499.479 & 1498.263\end{array}$

$\begin{array}{lllllll}1497.783 & 1497.951 & 1507.705 & 1499.608 & 1498.249\end{array}$

$\begin{array}{llllll}1497.811 & 1497.969 & 1506.789 & 1499.643 & 1498.284\end{array}$

$\begin{array}{lllllll}4497.816 & 1497.972 & 1505.952 & 1499.671 & 1498.308\end{array}$

$\begin{array}{llll}1498.004 & 1505.204 & 1499.884 & 1498.345\end{array}$

$\begin{array}{llllllll}1498.023 & 1504.507 & 1499.937 & 1498.415 & 1498.005 & 1496.903 & 1498.286\end{array}$

$\begin{array}{rrrrrrr}1498.035 & 1503.854 & 1499.935 & 1498.424 & 1497.96 & 1496.908 & 1498.169 \\ 1498042 & 1503273 & 1499.923 & 1498.11 & 1497.912 & 1496912 & 1498138\end{array}$

$\begin{array}{llllllll}1498.042 & 1503.273 & 1492.775 & 1499.949 & 1498.429 & 1497.884 & 1496.919 & 1498.15\end{array}$

$\begin{array}{lllllllll}1498.042 & 1502.313 & 1499.897 & 1498.403 & 1497.827 & 1496.917 & 1498.138\end{array}$

$\begin{array}{lllllllll}1498.042 & 1501.893 & 1499.851 & 1498.336 & 1497.748 & 1496.912 & 1498.097\end{array}$

$\begin{array}{llllllll}1498.032 & 1501.525 & 1499.82 & 1498.308 & 1497.691 & 1496.912 & 1498.087\end{array}$

$\begin{array}{llllllll}1498.004 & 1501.154 & 1499.722 & 1498.207 & 1497.587 & 1496.894 & 1498.016\end{array}$

$\begin{array}{llllllll}1497.976 & 1500.814 & 1499.668 & 1498.121 & 1497.494 & 1496.877 & 1497.975 \\ 1497.951 & 1500.497 & 1499.629 & 1498.065 & 1497.406 & 1496863 & 149799\end{array}$

$\begin{array}{llllllll}1497.951 & 1500.497 & 1499.629 & 1498.065 & 1497.406 & 1496.863 & 1497.939\end{array}$

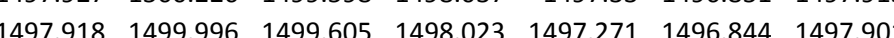

$\begin{array}{llllllll}1497.927 & 1499.853 & 1499.692 & 1498.107 & 1497.276 & 1496.858 & 1497.951\end{array}$

$\begin{array}{lllllllll}1497.925 & 1499.704 & 1499.703 & 1498.137 & 1497.257 & 1496.861 & 1497.951\end{array}$

$\begin{array}{lllllllll}1497.92 & 1499.564 & 1499.699 & 1498.137 & 1497.228 & 1496.861 & 1497.944\end{array}$

$\begin{array}{lllllllll}1497.927 & 1499.469 & 1499.739 & 1498.17 & 1497.228 & 1496.87 & 1497.965\end{array}$

$\begin{array}{llllllll}1497.927 & 1499.357 & 1499.732 & 1498.175 & 1497.207 & 1496.872 & 1497.956\end{array}$

$\begin{array}{llllllll}1497.934 & 1499.273 & 1499.764 & 1498.186 & 1497.207 & 1496.877 & 1497.963 \\ 1497.958 & 1499.24 & 1499.834 & 1498.261 & 1497245 & 1496898 & 1498.013\end{array}$

$\begin{array}{lllllllllll}1497.934 & 1499.273 & 1499.764 & 1498.24 & 149.834 & 148.261 & 1497.245 & 1496.898 & 1498.013\end{array}$

$\begin{array}{lllllllll}1497.988 & 1499.152 & 1499.888 & 1498.338 & 1497.29 & 1496.924 & 1498.059\end{array}$

$\begin{array}{llllllll}1498.021 & 1499.159 & 1499.965 & 1498.41 & 1497.342 & 1496.936 & 1498.102\end{array}$

$\begin{array}{llllllll}1498.028 & 1499.126 & 1499.956 & 1498.427 & 1497.363 & 1496.941 & 1498.102\end{array}$

$\begin{array}{lllllllll}1498.037 & 1499.082 & 1499.951 & 1498.413 & 1497.363 & 1496.943 & 1498.102\end{array}$

$\begin{array}{llllllll}1498.049 & 1499.058 & 1499.968 & 1488.422 & 1497.387 & 1496.95 & 1498.114\end{array}$

$\begin{array}{lllllll}1498.025 & 1498.942 & 1499.853 & 1498.317 & 1497.333 & 1496.936 & 1498.051\end{array}$

$\begin{array}{llllllll}1498.016 & 1498.842 & 1499.813 & 1498.245 & 1497.28 & 1496.929 & 1498.013 \\ 1498.002 & 1498762 & 1499.792 & 1498.214 & 1497.252 & 1496927 & 1497.996\end{array}$

$\begin{array}{lllllllll}1497.969 & 1498657 & 1499.696 & 1498.135 & 1497.19 & 1496908 & 1497.991\end{array}$

$\begin{array}{llllllll}1497.965 & 1498.59 & 1499.706 & 1498.112 & 1497.152 & 1496905 & 1497.934\end{array}$

$\begin{array}{llllllll}1497.955 & 1498.552 & 1499.715 & 1498.128 & 1497.147 & 1496.908 & 1497.941\end{array}$

$\begin{array}{llllllll}1497.937 & 1498.482 & 1499.661 & 1498.088 & 1497.102 & 1496.896 & 1497.901\end{array}$

$\begin{array}{lllllllll}1497.918 & 1498.424 & 1499.643 & 1498.06 & 1497.069 & 1496.889 & 1497.889\end{array}$

$\begin{array}{llllllllll}1497.916 & 1498.389 & 1499.654 & 1498.072 & 1497.057 & 1496.887 & 1497.894\end{array}$

$\begin{array}{lllllllll}1497.895 & 1498.338 & 1499.608 & 1498.046 & 1497.019 & 1496.877 & 1497.862\end{array}$

$1501.152 \quad 1500.33$

1500.9071500 .321

1500.8351500 .326

$1500.745 \quad 1500.283$

$1500.628 \quad 1500.204$

$1500.556 \quad 1500.187$

1500.4331500 .075

$\begin{array}{ll}1500.331 & 1500.027 \\ 1500.238 & 1499.986\end{array}$

$\begin{array}{ll}1500.238 & 1499.986 \\ 1500.168 & 1499.967\end{array}$

1500.1191499 .96

$1500.14 \quad 1500.075$

$1500.138 \quad 1500.082$

1500.1261500 .075

$1500.14 \quad 1500.113$

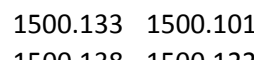

$\begin{array}{ll}1500.138 & 1500.122 \\ 1500.191 & 1500.204\end{array}$

15002191500.225

$\begin{array}{lll}1500.219 & 1500.225 \\ 1500.254 & 1500.259\end{array}$

$1500.31 \quad 1500.326$

$1500.333 \quad 1500.319$

$1500.333 \quad 1500.299$

$1500.359 \quad 1500.323$

$1500.294 \quad 1500.197$
1500.24

$\begin{array}{rr}1500.24 & 1500.142 \\ 1500.212 & 1500.13\end{array}$

$\begin{array}{lll}1500.212 & 1500.13 \\ 1500.147 \quad 1500.04\end{array}$

$1500.114 \quad 1500.048$

$1500.114 \quad 1500.077$

$1500.073 \quad 1500.022$

$1500.047 \quad 1500.012$

$1500.042 \quad 1500.034$

$\begin{array}{ll}1500.017 & 1499.988 \\ 1500.024 & 1500.039\end{array}$ 
$\begin{array}{lllllll}1497.934 & 1498.387 & 1499.769 & 1498.193 & 1497.078 & 1496.908 & 1497.946 \\ 1497.946 & 1498.401 & 1499.799 & 1498.217 & 1497.097 & 1496.917 & 1497.968\end{array}$ $\begin{array}{lllllllll}1497.946 & 1498.401 & 1499.79 & 148.217 & 1497.097 & 1496.917 & 1497.968 \\ 1497.962 & 1498.443 & 1499.844 & 1498.27 & 1497.133 & 1496.936 & 1498.004\end{array}$ $\begin{array}{llllllll}1497.96 & 1498.433 & 1499.813 & 1498.263 & 1497.133 & 1496.938 & 1497.989\end{array}$ $\begin{array}{llllllll}1497.962 & 1498.41 & 1499.806 & 1498.242 & 1497.126 & 1496.943 & 1497.98\end{array}$ $\begin{array}{lllllllll}1497.962 & 1498.389 & 1499.792 & 1498.217 & 1497.126 & 1496.945 & 1497.97\end{array}$ $\begin{array}{llllllll}1497.948 & 1498.343 & 1499.743 & 1498.163 & 1497.095 & 1496.934 & 1497.939\end{array}$ $\begin{array}{lllllllll}1497.939 & 1498.319 & 1499.727 & 1498.144 & 1497.081 & 1496.934 & 1497.929\end{array}$ $\begin{array}{rrrrrrrrr}1497.946 & 1498.326 & 1499.785 & 1498.184 & 1497.095 & 1496.943 & 1497.958\end{array}$ $\begin{array}{lllllllll}1497.916 & 1498.345 & 1499.813 & 1498.221 & 1497.112 & 1496.95 & 1497.97 \\ 1497.909 & 149834 & 1499.781 & 1498.226 & 1497.112 & 1496.955 & 1497.977\end{array}$

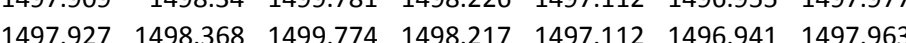
$\begin{array}{lllllllll}1497.927 & 1498.464 & 1499.853 & 1498.126 & 1497.057 & 1496.922 & 1497.908\end{array}$ $\begin{array}{llllllll}1497.904 & 1488.515 & 1499.933 & 1498.056 & 1497.002 & 1496.905 & 1497.862\end{array}$ $\begin{array}{llllllll}1497.888 & 1498.524 & 1499.897 & 1498.025 & 1496.962 & 1496.896 & 1497.843\end{array}$ $\begin{array}{lllllllll}1497.869 & 1498.506 & 1499.811 & 1498.002 & 1496.931 & 1496.884 & 1497.822\end{array}$ $\begin{array}{lllllllll}1497.869 & 1498.364 & 1499.664 & 1498.027 & 1496.924 & 1496.884 & 1497.838\end{array}$ $\begin{array}{llllllll}1497.886 & 1498.252 & 1499.652 & 1498.1 & 1496.96 & 1496.896 & 1497.886\end{array}$ $\begin{array}{llllllll}1497.881 & 1498.261 & 1499.685 & 1498.109 & 1496.953 & 1496.894 & 1497.882\end{array}$ $\begin{array}{lllllllll}1497.911 & 1498.142 & 1499.692 & 1498.212 & 1497.012 & 1496919 & 1497.944\end{array}$ $\begin{array}{llllllll}1497.918 & 1498.137 & 1499.729 & 1498.24 & 1497.04 & 1496.929 & 1497.958\end{array}$ $\begin{array}{lllllllll}1497.918 & 1498.079 & 1499.724 & 1498.275 & 1497.062 & 1496.938 & 1497.975\end{array}$ $\begin{array}{llllllll}1497.955 & 1498.336 & 1499.682 & 1498.322 & 1497.107 & 1496.959 & 1498.008\end{array}$ $\begin{array}{lllllllll}1497.965 & 1498.336 & 1499.717 & 1498.308 & 1497.112 & 1496.962 & 1497.999\end{array}$ $\begin{array}{lllllllll}1497.981 & 1498.375 & 1499.706 & 1498.331 & 1497.145 & 1496.971 & 1498.023\end{array}$ $\begin{array}{llllllllll}1497.995 & 1498.401 & 1499.701 & 1498.333 & 1497.171 & 1496.976 & 1498.032\end{array}$ $\begin{array}{llllllllll}1497.903 & 1498.403 & 1499.68 & 1488.33 & 1497.178 & 1496.974 & 1488.028\end{array}$ $\begin{array}{lllllllll}1498.018 & 1498.471 & 1499.762 & 1498.359 & 1497.245 & 1496985 & 1498.051\end{array}$ $\begin{array}{lllllllll}1497.981 & 1498.371 & 1499.722 & 1498.226 & 1497.181 & 1496.964 & 1497.977\end{array}$ $\begin{array}{llllllll}1497.96 & 1498.298 & 1499.689 & 1498.109 & 1497.114 & 1496.945 & 1497.917\end{array}$ $\begin{array}{lllllllll}1497.909 & 1498.158 & 1499.862 & 1497.964 & 1497.019 & 1496.915 & 1497.841\end{array}$ $\begin{array}{llllllll}1497.872 & 1498.058 & 1499.926 & 1497.855 & 1496.924 & 1496.887 & 1497.776\end{array}$ $\begin{array}{llllllll}1497.867 & 1497.904 & 1499.509 & 1497.841 & 1496.886 & 1496.877 & 1497.776 \\ 1497.865 & 1498007 & 1499.568 & 1497.894 & 1496888 & 1496877 & 1497.812\end{array}$ $\begin{array}{llllllll}1497.865 & 1498.007 & 1499.568 & 1497.894 & 1496.888 & 1496.877 & 1497.812\end{array}$ $\begin{array}{llllllllll}1497.86 & 1498.028 & 1499.608 & 1497.96 & 1496893 & 1496.877 & 1497.829\end{array}$ $\begin{array}{llllllll}1497.867 & 1498.058 & 1499.652 & 1498.006 & 1496.907 & 1496.884 & 1497.853\end{array}$ $\begin{array}{llllllll}1497.851 & 1498.051 & 1499.608 & 1497.997 & 1496.9 & 1496.88 & 1497.834\end{array}$ $\begin{array}{llllllll}1497.848 & 1498.039 & 1499.61 & 1497.985 & 1496.888 & 1496.875 & 1497.824\end{array}$ $\begin{array}{lllllllll}1497.855 & 1498.065 & 1499.647 & 1498.018 & 1496.907 & 1496.882 & 1497.843\end{array}$ $\begin{array}{rrrrrrr}1497.841 & 1498.042 & 1499.601 & 1498.002 & 1496.888 & 1496.875 & 1497.817\end{array}$ $\begin{array}{lllllllll}1497.832 & 1498.014 & 1499.563 & 1497.969 & 1496.86 & 1496.865 & 1497.791 \\ 1497.823 & 1497.984 & 149953 & 1497.943 & 1496831 & 1496856 & 1497.769\end{array}$ $\begin{array}{lllllllll}1497.823 & 1497.984 & 149.53 & 1497.943 & 1496.831 & 1496.856 & 1497.769\end{array}$ $\begin{array}{lllllllll}1497.82 & 1497.967 & 1499.563 & 1497.953 & 1496.805 & 1496.847 & 1497.781\end{array}$ $\begin{array}{llllllll}1497.844 & 1498.039 & 1499.666 & 1498.06 & 1496.858 & 1496.865 & 1497.85\end{array}$ $\begin{array}{llllllll}1497.839 & 1498.056 & 1499.657 & 1498.084 & 1496.872 & 1496.868 & 1497.85\end{array}$ $\begin{array}{rrrrrrrr}1497.855 & 1498.093 & 1499.706 & 1498.123 & 1496.9 & 1496.877 & 1497.879 \\ 1497.867 & 1498.13 & 1499.734 & 1498.165 & 1496.931 & 1496.891 & 1497.903\end{array}$ $\begin{array}{lllllllll}1497.867 & 1498.13 & 1499.734 & 1498.165 & 1496.931 & 1496.891 & 1497.903\end{array}$ $\begin{array}{rrrrrrr}1497.869 & 1498.142 & 1499.717 & 1498.168 & 1496.938 & 1496.894 & 1497.896 \\ 1497.888 & 1498.186 & 1499.778 & 1498.21 & 1496.976 & 1496.91 & 1497.934\end{array}$
$1500.098 \quad 1500.168$

$1500.11 \quad 1500.144$
$1500.135 \quad 1500.173$

$\begin{array}{rl}1500.135 & 1500.173 \\ 1500.18 & 1500.228\end{array}$

$\begin{array}{rl}1500.18 & 1500.228 \\ 1500.184 & 1500.192\end{array}$

$\begin{array}{rl}1500.18 & 1500.173 \\ 1500.175 & 1500.161\end{array}$

$1500.142 \quad 1500.106$

$1500.131 \quad 1500.106$
1500.152
15000

$1500.152 \quad 1500.158$
1500.17
1500.177

$\begin{array}{rl}1500.17 & 1500.177 \\ 1500.175 \quad 1500.18\end{array}$

$\begin{array}{lr}1500.175 & 1500.18 \\ 1500.168 & 1500.139\end{array}$

$1500.107 \quad 1500.055$

$1500.049 \quad 1499.996$

1500.0141499 .981

1499.9841499 .962

$1499.989 \quad 1500.005$

$\begin{array}{lll}1500.031 & 1500.087\end{array}$

$\begin{array}{ll}1500.063 & 1500.11 \\ 1500.112 & 1500.175\end{array}$

$\begin{array}{ll}1500.142 \quad 1500.192 \\ 1500.168 & 1500.209\end{array}$

$1500.219 \quad 1500.249$

$1500.219 \quad 1500.225$

$\begin{array}{lll}1500.249 & 1500.273 \\ 1500268 & 1500278\end{array}$

$1500.273 \quad 1500.252$

$1500.303 \quad 1500.28$

$1500.247 \quad 1500.137$

$1500.168 \quad 1500.055$

1500.0661499 .936

$\begin{array}{ll}1499.966 & 1499.859 \\ 1499.931 & 1499886\end{array}$

$1499947 \quad 1499.965$

$1499.973 \quad 1500.022$

$1500.003 \quad 1500.063$

$1500.003 \quad 1500.029$

$1499.998 \quad 1500.02$

$1500.021 \quad 1500.055$

$1499.952 \quad 1499.941$

$1499.921 \quad 1499.914$

$1499.935 \quad 1499.967$

$\begin{array}{lll}1500 & 1500.082\end{array}$

$1500.056 \quad 1500.113$

$1500.091 \quad 1500.14$

$\begin{array}{rr}1500.098 & 1500.12 \\ 1500.14 & 1500.18\end{array}$ 
$\begin{array}{llllllll}1497.883 & 1498.17 & 1499.675 & 1498.133 & 1496.976 & 1496.917 & 1497.889\end{array}$

$\begin{array}{lllllll}1497.883 & 1498.17 & 1499.675 & 1498.133 & 1496.976 & 1496.917 & 1497.889 \\ 1497.879 & 1498.135 & 1499.657 & 1498.079 & 1496.938 & 1496.908 & 1497.858\end{array}$ $\begin{array}{lllllll}1497.858 & 1498.102 & 1499.601 & 1498.046 & 1496.917 & 1496.896 & 1497.834\end{array}$

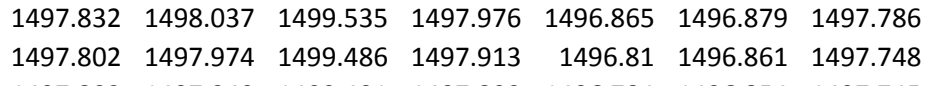
$3 / 5 / 110: 00$ 3/5/11 4:00 $\begin{array}{llllllll}1497.802 & 1497.949 & 1499.491 & 1497.899 & 1496.784 & 1496.854 & 1497.745 \\ 1497.783 & 1497.916 & 1499.465 & 1497.883 & 149.753 & 1496837 & 1497.728\end{array}$ $\begin{array}{llllllll}1497.783 & 1497.916 & 1499.465 & 1497.883 & 1496.753 & 1496.837 & 1497.728 \\ 1497.788 & 1497.914 & 1499.495 & 1497.897 & 1496.741 & 1496835 & 1497.736\end{array}$ $\begin{array}{llllllll}1497.788 & 1497.914 & 1499.495 & 1497.897 & 1496.741 & 1496.835 & 1497.736\end{array}$ $\begin{array}{lllllllll}1497.79 & 1497.97 & 1499.558 & 1497.992 & 1496.779 & 1496842 & 1497.783\end{array}$ $\begin{array}{lllllllll}1497.795 & 1497.988 & 1499.582 & 1498.006 & 1496.786 & 1496.844 & 1497.795\end{array}$ $\begin{array}{llllllll}1497.809 & 1498.035 & 1499.64 & 1498.065 & 1496.822 & 1496.856 & 1497.834\end{array}$ $\begin{array}{llllllll}1497.809 & 1498.044 & 1499.629 & 1498.074 & 1496.829 & 1496.856 & 1497.831\end{array}$ $\begin{array}{llllllll}1497.827 & 1498.086 & 1499.687 & 1498.112 & 1496.855 & 1496.865 & 1497.858\end{array}$ $\begin{array}{llllllllll}1497.848 & 1498.154 & 1499.741 & 1498.177 & 1496.915 & 1496.887 & 1497.905\end{array}$ $\begin{array}{llllllllll}1497.858 & 1498.172 & 1499.738 & 1488.1796 .1496 .936 & 1496.894 & 1497.908\end{array}$ $\begin{array}{llllllll}1497.874 & 1498.205 & 1499.755 & 1498.189 & 1496.964 & 1496.905 & 1497.92\end{array}$ $\begin{array}{rrrrrrr}1497.869 & 1498.2 & 1499.722 & 1498.175 & 1496.964 & 1496.908 & 1497.908 \\ 1497.881 & 1498.203 & 1499.743 & 1498.172 & 1496.974 & 1496.915 & 1497.917\end{array}$ $\begin{array}{llllllll}1497.892 & 1498.228 & 1499.764 & 1498.196 & 1496.993 & 1496.922 & 1497.932 \\ 1497881 & 1498207 & 149971 & 1488.161 & 1496976 & 1499.919 & 1497.908\end{array}$ $\begin{array}{lllllllll}1497.881 & 1498.207 & 1499.71 & 1498.161 & 1496.976 & 1496.919 & 1497.908\end{array}$ $\begin{array}{lllllll}1497.886 & 1498.203 & 1499.722 & 1498.151 & 1496.976 & 1496.924 & 1497.91 \\ 14978 & 1498221 & 1499746 & 1498.177 & 1496993 & 1469929 & 1497.925\end{array}$ $\begin{array}{lllllll}1497.89 & 1498.221 & 1499.746 & 1498.177 & 1496.993 & 1496.929 & 1497.925 \\ 1497.834 & 1498.231 & 1499.652 & 1488.182 & 1497.002 & 1496926 & 149.932\end{array}$ $\begin{array}{llllllll}1497.834 & 1498.231 & 1499.652 & 1498.182 & 1497.002 & 1496.926 & 1497.932 \\ 149782 & 1498.224 & 1499.615 & 1498165 & 1497.002 & 1496934 & 1497.92\end{array}$ $\begin{array}{llllllll}1497.813 & 1498.212 & 1499.584 & 1498.156 & 1496.991 & 1496.931 & 1497.91\end{array}$ $\begin{array}{llllllll}1497.818 & 1498.165 & 1499.566 & 1498.088 & 1496.953 & 1496.919 & 1497.865\end{array}$ $\begin{array}{llllllll}1497.841 & 1498.144 & 1499.549 & 1498.058 & 1496.929 & 1496.917 & 1497.853\end{array}$ $\begin{array}{lllllll}1497.839 & 1498.116 & 1499.542 & 1498.039 & 1496.912 & 1496.908 & 1497.836 \\ 1497.816 & 1498.067 & 1499.488 & 1497.988 & 1496869 & 1496.891 & 1497.8\end{array}$ $\begin{array}{llllllll}1497.816 & 1498.067 & 1499.488 & 1497.988 & 1496.869 & 1496.891 & 1497.8\end{array}$ $\begin{array}{llllllll}1497.806 & 1498.032 & 1499.292 & 1497.953 & 1496.841 & 1496.884 & 1497.779 \\ 1497.797 & 1498.019 & 1499514 & 1497.948 & 1496822 & 1496875 & 1497.774\end{array}$ $\begin{array}{rrrrrrrr}1497.797 & 1498.019 & 1499.514 & 1497.948 & 1496.822 & 1496.875 & 1497.774 \\ 1497.781 & 1497.972 & 1499.47 & 1497.904 & 1496.779 & 1496861 & 1497.738\end{array}$ $\begin{array}{llllllllll}1497.776 & 1497.944 & 1499.465 & 1497.885 & 1496.753 & 1496.849 & 1497.726\end{array}$ $\begin{array}{lllllllll}1497.762 & 1497.937 & 1499.46 & 1497.89 & 1496.741 & 1496.842 & 1497.726\end{array}$ $\begin{array}{llllllll}1497.755 & 1497.916 & 1499.451 & 1497.878 & 1496.717 & 1496.833 & 1497.716\end{array}$ $\begin{array}{llllllll}1497.769 & 1497.932 & 1499.505 & 1497.911 & 1496.725 & 1496.837 & 1497.74\end{array}$ $\begin{array}{llllllll}1497.785 & 1498 & 1499.594 & 1498.009 & 1496.777 & 1496.854 & 1497.805\end{array}$ $\begin{array}{lllllll}1497.792 & 1498.03 & 1499.608 & 1498.049 & 1496.801 & 1496.858 & 1497.819\end{array}$ $\begin{array}{llllllll}1497.804 & 1498.065 & 1499.638 & 1498.077 & 1496.829 & 1496.863 & 1497.836 \\ 1497.825 & 1498.105 & 1499.694 & 1498.128 & 1496862 & 149688 & 1497.877\end{array}$

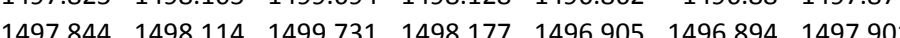
$\begin{array}{lllllllll}1497.855 & 1498.207 & 1499.748 & 1498.203 & 1496.938 & 1496.903 & 1497.922\end{array}$ $\begin{array}{lllllllll}1497.855 & 1498.207 & 1499.72 & 1498.191 & 1496.948 & 1496.903 & 1497.913\end{array}$ $\begin{array}{lllllll}1497.832 & 1498.149 & 1499.612 & 1498.091 & 1496.907 & 1496.891 & 1497.855\end{array}$ $\begin{array}{lllllll}1497.827 & 1498.114 & 1499.598 & 1498.034 & 1496.872 & 1496.882 & 1497.827 \\ 1497.823 & 1498.107 & 1499603 & 1498.032 & 1496869 & 1496882 & 1497.829\end{array}$

$1500.17 \quad 1500.19$ $\begin{array}{ll}1500.161 & 1500.154 \\ 1500.149 & 1500.139\end{array}$

$1500.152 \quad 1500.139$

$\begin{array}{lll}1500.152 & 1500.139 \\ 1500.112 & 1500.079\end{array}$

$1500.073 \quad 1500.034$

$1500.045 \quad 1500$

$1499.987 \quad 1499.933$

$1499.938 \quad 1499.89$

$1499.912 \quad 1499.898$
1499884
149988

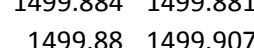

$1499.926 \quad 1499.991$

$1499.938 \quad 1499.986$

$1499.952 \quad 1500.003$

$1499.998 \quad 1500.07$

$\begin{array}{rr}1500.01 \quad 1500.051 \\ 1500.042 & 1500098\end{array}$

$\begin{array}{ll}1500.042 & 1500.098 \\ 1500.105 & 1500.161\end{array}$

$1500.105 \quad 1500.161$

$1500.128 \quad 1500.13$

$\begin{array}{ll}1500.128 & 1500.139 \\ 1500.145 & 1500.156\end{array}$

$\begin{array}{ll}1500.138 & 1500.12 \\ 1500.142 & 1500.137\end{array}$

$1500.161 \quad 1500.15$

$\begin{array}{rr}1500.14 & 1500.11 \\ 1500 & 15800 \\ 1500 & 150.159\end{array}$

$\begin{array}{ll}1500.138 & 1500.115 \\ 1500149 & 15000\end{array}$

1500.149
1500.154
1500.144

$1500.142 \quad 1500.127$

$1500.133 \quad 1500.108$

$1500.089 \quad 1500.034$

$1500.063 \quad 1500.029$

$1500.042 \quad 1500.005$

$1499.998 \quad 1499.95$

$\begin{array}{ll}1499.968 & 1499.936 \\ 1499.949 & 1499.933\end{array}$

$1499.949 \quad 1499.933$

$\begin{array}{lll}1499.889 & 1499.883\end{array}$

$1499.884 \quad 1499.895$

1499.8681499 .881

1499.8841499 .933

$1499.947 \quad 1500.029$

$1499.98 \quad 1500.043$

$\begin{array}{rr}1500.007 & 1500.067 \\ 1500.054 & 1500.12\end{array}$

$1500.054 \quad 1500.12$
$1500.103 \quad 1500.156$

$\begin{array}{ll}1500.103 & 1500.156 \\ 1500.133 & 1500.168\end{array}$

$1500.135 \quad 1500.134$

$1500.084 \quad 1500.029$

$1500.042 \quad 1499.99$

$1500.033 \quad 1500.005$

$\begin{array}{lll}1500.005 & 1499.962 \\ 1499.989 & 1499.967\end{array}$ 
$\begin{array}{llllllll}1497.767 & 1497.995 & 1499.493 & 1497.927 & 1496.777 & 1496.847 & 1497.75\end{array}$

$\begin{array}{llllllll}1497.76 & 1497.988 & 1499.505 & 1497.925 & 1496.763 & 1496.844 & 1497.748\end{array}$ $\begin{array}{rlllllll}1497.753 & 1497.993 & 1499.495 & 1497.922 & 1496.76 & 1496.837 & 1497.752\end{array}$ $\begin{array}{rrrrrrr}1497.743 & 1497.937 & 1499.528 & 1497.864 & 1496.725 & 1496.828 & 1497.714 \\ 1497.741 & 1497.937 & 1499.449 & 1497.88 & 1496.715 & 1496.825 & 1497.721\end{array}$ $\begin{array}{lllllllll}1497.741 & 1497.937 & 1499.449 & 1497.88 & 1496.715 & 1496.825 & 1497.721\end{array}$ $\begin{array}{rrrrrrr}1497.706 & 1497.939 & 1499.421 & 1497.892 & 1496.717 & 1496.823 & 1497.726 \\ 1497.678 & 1497.949 & 1499367 & 1497.88 & 1496698 & 1496814 & 1497.714\end{array}$ $\begin{array}{lllllllll}1497.678 & 1497.949 & 1499.367 & 1497.88 & 1496.698 & 1496.814 & 1497.714 \\ 1497.634 & 1497.83 & 1499.269 & 1497.897 & 1496.698 & 1496816 & 1497.726\end{array}$ $\begin{array}{llllllll}1497.634 & 1497.83 & 1499.269 & 1497.897 & 1496.698 & 1496.816 & 1497.726 \\ 1497.736 & 1497.846 & 1499.35 & 1497.976 & 1496.736 & 1496.83 & 1497.779\end{array}$ $\begin{array}{lllllllll}1497.739 & 1498.028 & 1499.516 & 1497.99 & 1496.744 & 1496.828 & 1497.781\end{array}$ $\begin{array}{llllllll}1497.741 & 1497.993 & 1499.61 & 1497.988 & 1496.741 & 1496.825 & 1497.774\end{array}$ $\begin{array}{lllllllll}1497.748 & 1498.035 & 1499.568 & 1498.023 & 1496.763 & 1496.835 & 1497.798\end{array}$ $\begin{array}{lllllllll}1497.741 & 1498.086 & 1499.519 & 1497.992 & 1496.748 & 1496.828 & 1497.767\end{array}$ $\begin{array}{llllllll}1497.755 & 1497.897 & 1499.577 & 1498.02 & 1496.772 & 1496.84 & 1497.798\end{array}$ $\begin{array}{lllllllll}1497.776 & 1497.939 & 1499.631 & 1498.079 & 1496.808 & 1496.851 & 1497.829\end{array}$ $\begin{array}{lllllllll}1497.762 & 1498.137 & 1499.561 & 1498.034 & 1496.789 & 1496.842 & 1497.798\end{array}$ $\begin{array}{lllllllll}1497.776 & 1498.135 & 1499.596 & 1498.046 & 1496805 & 1496847 & 1497812\end{array}$ $\begin{array}{lllllllll}1497.778 & 1498.074 & 1499.603 & 1498.063 & 1496.817 & 1496.851 & 1497.819\end{array}$ $\begin{array}{llllllll}1497.797 & 1497.965 & 1499.654 & 1498.102 & 1496.846 & 1496.863 & 1497.85\end{array}$ $\begin{array}{lllllllll}1497.823 & 1498.046 & 1499.724 & 1498.184 & 1496.905 & 1496.887 & 1497.898\end{array}$ $\begin{array}{llllllll}1497.83 & 1498.165 & 1499.703 & 1488.182 & 1496.915 & 1496.889 & 1497.891\end{array}$ $\begin{array}{llllllll}1497.839 & 1498.21 & 1499.71 & 1498.186 & 1496.936 & 1496.896 & 1497.901\end{array}$ $\begin{array}{llllllll}1497.853 & 1498.203 & 1499.75 & 1498.221 & 1496.967 & 1496.908 & 1497.929\end{array}$ $\begin{array}{rrrrrrrr}1497.841 & 1498.259 & 1499.668 & 1498.17 & 1496.953 & 1496.901 & 1497.891 \\ 1497.83 & 1498.165 & 1499.608 & 1498.1 & 1496.922 & 1496.891 & 1497.85\end{array}$ $\begin{array}{lllllllll}1497.82 & 1498.147 & 1499.577 & 1498.049 & 1496.893 & 1496.884 & 1497.829\end{array}$ $\begin{array}{llllllll}1497.809 & 1498.095 & 1499.537 & 1497.969 & 1496.855 & 1496.875 & 1497.795\end{array}$ $\begin{array}{llllllll}1497.783 & 1498.044 & 1499.479 & 1497.922 & 1496.817 & 1496.858 & 1497.762\end{array}$ $\begin{array}{llllllll}1497.776 & 1498.025 & 1499.488 & 1497.92 & 1496.801 & 1496.854 & 1497.757\end{array}$ $\begin{array}{llllllll}1497.746 & 1497.939 & 1499.39 & 1497.705 & 1496.736 & 1496.828 & 1497.695\end{array}$ $\begin{array}{llllllll}1497.734 & 1497.895 & 1499.416 & 1497.484 & 1496.71 & 1496.823 & 1497.688 \\ 1497.725 & 1497.904 & 1499.453 & 1497.575 & 1496.722 & 1496825 & 1497712\end{array}$ $\begin{array}{lllllllll}1497.734 & 1497.895 & 149.416 & 1497.484 & 1496.71 & 1496823 & 1497.688\end{array}$ $\begin{array}{llllllllll}1497.713 & 1497.874 & 1499.432 & 1497.698 & 1496.698 & 1496811 & 1497.683\end{array}$ $\begin{array}{lllllllll}1497.718 & 1497.907 & 1499.486 & 1497.785 & 1496.725 & 1496.816 & 1497.719\end{array}$ $\begin{array}{llrrrrrr}1497.713 & 1497.925 & 1499.5 & 1497.838 & 1496.732 & 1496.816 & 1497.736\end{array}$ $\begin{array}{llllllll}1497.676 & 1497.984 & 1499.451 & 1497.922 & 1496.763 & 1496.832 & 1497.762\end{array}$ $\begin{array}{lllllllll}1497.666 & 1498.023 & 1499.4 & 1497.995 & 1496.815 & 1496.851 & 1497.793\end{array}$ $\begin{array}{llllllll}1497.681 & 1498.037 & 1499.395 & 1498.009 & 1496.808 & 1496.844 & 1497.803\end{array}$ $\begin{array}{lllllllll}1497.676 & 1498.056 & 1499.379 & 1498.034 & 1496.827 & 1496.849 & 1497.819\end{array}$

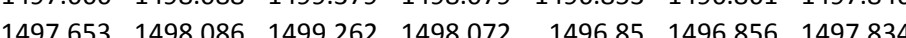
$\begin{array}{lllllllll}1497.734 & 1498.086 & 1499.383 & 1498.067 & 1496853 & 1496.858 & 1497.831\end{array}$ $\begin{array}{lllllllll}1497.757 & 1498.112 & 1499.498 & 1498.091 & 1496.872 & 1496.868 & 1497.85\end{array}$ $\begin{array}{llllllll}1497.746 & 1498.079 & 1499.573 & 1498.056 & 1496.85 & 1496.854 & 1497.817\end{array}$ $\begin{array}{lllllllll}1497.743 & 1498.056 & 1499.554 & 1498.018 & 1496.827 & 1496.849 & 1497.795\end{array}$ $\begin{array}{llllllll}1497.755 & 1498.074 & 1499.596 & 1498.053 & 1496.843 & 1496.858 & 1497.822\end{array}$ $\begin{array}{rrrrrrr}1497.753 & 1498.065 & 1499.573 & 1498.049 & 1496.839 & 1496.854 & 1497.812 \\ 1497.767 & 1498.086 & 1499.615 & 1498.077 & 1496.85 & 1496.863 & 1497.834\end{array}$

$1500.01 \quad 1500.01$ $\begin{array}{ll}1499.977 & 1499.941 \\ 1499.952 & 1499.919\end{array}$ 1499.9521499 .919 $\begin{array}{rr}1499.956 & 1499.95 \\ 1499.935 & 1499.917\end{array}$ $1499.924 \quad 1499.917$ 1499.9281499 .919 1499.8891499 .871 $1499.887 \quad 1499.89$ $\begin{array}{lll}1499.889 & 1499.898 \\ 1499875 & 1499874\end{array}$ $\begin{array}{lll}1499.875 & 1499.874 \\ 1499.877 & 1499.895\end{array}$ $\begin{array}{ll}1499.877 & 1499.895 \\ 1499.926 & 1499.974\end{array}$ $1499.938 \quad 1499.96$ 1499.94
1499.945 1499.9661499 .979 1499.9471499 .921 1499.9731499 .979 $1500.012 \quad 1500.029$
1499.991
1499.96 $\begin{array}{rr}1499.991 & 1499.96 \\ 1499.989 & 1499.967\end{array}$ 15001499.986 1500.0121499 .986 1500.041500 .036 $1500.103 \quad 1500.11$ $\begin{array}{rr}1500.11 \quad 1500.075 \\ 1500.119 & 1500082\end{array}$ $\begin{array}{ll}1500.119 & 1500.082 \\ 1500149 & 1500.115\end{array}$ 1500.1491500 .115 $1500.077 \quad 1499.974$ $1500.042 \quad 1499.941$ $1499.998 \quad 1499.893$ 1499.9541499 .852 $1499.935 \quad 1499.857$ $\begin{array}{rlr}1499.87 & 1499.771 \\ 1499845 & 1499775\end{array}$ $\begin{array}{lll}1499.845 & 1499.775 \\ 1499863 & 1499838\end{array}$ $\begin{array}{lll}1499.863 & 1499.838 \\ 1499.861 & 1499.826\end{array}$ $1499.852 \quad 1499.821$ $1499.882 \quad 1499.878$ $1499.903 \quad 1499.9$ $1499.938 \quad 1499.957$ $1500.003 \quad 1500.041$ $1499.994 \quad 1499.981$
1500.012
1499.991 $1500.012 \quad 1499.991$
$1500.042 \quad 1500.024$ $150.042 \quad 1400.024$ $1500.033 \quad 1499.974$ $1500.049 \quad 1500.003$ $1500.021 \quad 1499.938$ $\begin{array}{rl}1499.994 & 1499.905 \\ 1500.01 & 1499955\end{array}$ $1500.01 \quad 1499.955$ 1501
$1500.017 \quad 1499.939$ 
$\begin{array}{lllllllll}1497.865 & 1498.329 & 1499.795 & 1498.289 & 1497.04 & 1496.936 & 1497.951\end{array}$ $\begin{array}{lllllllll}1497.864 & 1498.354 & 1499.781 & 1498.303 & 1497.067 & 1496.938 & 1497.958\end{array}$ $\begin{array}{lllllll}1497.841 & 1498.28 & 1499.645 & 1498.198 & 1497.017 & 1496.915 & 1497.894 \\ 1497.839 & 1498.231 & 1499.629 & 1498.133 & 1496.981 & 1496.912 & 1497.874\end{array}$ $\begin{array}{llllllll}1497.839 & 1498.231 & 1499.629 & 1498.133 & 1496.981 & 1496.912 & 1497.874\end{array}$ $\begin{array}{lllllllll}1497.855 & 1498.196 & 1499.598 & 1498.098 & 1496.957 & 1496.908 & 1497.855\end{array}$ $\begin{array}{rrrrrrrr}1497.799 & 1498.109 & 1499.491 & 1498.013 & 1496.893 & 1496.882 & 1497.793 \\ 1497.79 & 149807 & 1499.491 & 1497.976 & 1496858 & 1466875 & 147.779\end{array}$ $\begin{array}{lllllllll}1497.781 & 1498.053 & 1499.491 & 1497.962 & 1496.843 & 1496.872 & 1497.781\end{array}$ $\begin{array}{lllllllll}1497.753 & 1497.984 & 1499.411 & 1497.906 & 1496.791 & 1496849 & 1497.731\end{array}$ $\begin{array}{lllllll}1497.732 & 1497.923 & 1499.374 & 1497.862 & 1496.739 & 1496.837 & 1497.7\end{array}$ $\begin{array}{llllllll}1497.725 & 1497.907 & 1499.395 & 1497.873 & 1496.72 & 1496.835 & 1497.702\end{array}$ $\begin{array}{lllllllll}1497.708 & 1497.872 & 1499.362 & 1497.857 & 1496.682 & 1496.821 & 1497.681\end{array}$ $\begin{array}{rrrrrrrrr}1497.725 & 1497.902 & 1499.453 & 1497.925 & 1496.706 & 1496.832 & 1497.719\end{array}$ $\begin{array}{lllllllll}1497.767 & 1498.014 & 1499.603 & 1498.07 & 1496.784 & 1496.863 & 1497.817\end{array}$ $\begin{array}{llllllll}1497.725 & 1498.037 & 1499.727 & 1498.102 & 1496.808 & 1496.861 & 1497.812\end{array}$ $\begin{array}{lllllllll}1497.788 & 1498.058 & 1499.774 & 1498.161 & 1496.846 & 1496.887 & 1497.86\end{array}$ $\begin{array}{llllllll}1497.767 & 1498137 & 1499.736 & 1498.091 & 1496.808 & 1496.863 & 1497.798\end{array}$ $\begin{array}{lllllllll}1497.774 & 1497.923 & 1499.554 & 1498.088 & 1496.812 & 1496.868 & 1497.805\end{array}$ $\begin{array}{llllllll}1497.785 & 1497.923 & 1499.589 & 1498.098 & 1496.834 & 1496.879 & 1497.834\end{array}$ $\begin{array}{llllllll}1497.783 & 1498.114 & 1499.556 & 1498.03 & 1496.82 & 1496.87 & 1497.81\end{array}$ $\begin{array}{lllllll}1497.79 & 1498.149 & 1499.591 & 1498.072 & 1496.839 & 1496.882 & 1497.836 \\ 149795 & 1498.17 & 149.603 & 148.109 & 146985 & 146891 & 149.85\end{array}$ $\begin{array}{lllllllll}1497.795 & 1498.17 & 1499.603 & 1498.109 & 1496.855 & 1496.891 & 1497.85\end{array}$ $\begin{array}{lrrrrrrrr}1497.785 & 1498.219 & 149.544 & 1498.084 & 1496.831 & 1496.875 & 1497.815 \\ 1497.774 & 1498.2 & 1499.507 & 1498.041 & 1496.803 & 1496.87 & 1497.788\end{array}$ $\begin{array}{lllllllll}1497.767 & 1498.168 & 1499.493 & 1498.037 & 1496.796 & 1496.863 & 1497.781\end{array}$ $\begin{array}{lllllllll}1497.75 & 1498.217 & 1499.442 & 1497.995 & 1496.765 & 1496.854 & 1497.755\end{array}$ $\begin{array}{llllllll}1497.757 & 1498.203 & 1499.484 & 1498.002 & 1496.76 & 1496.858 & 1497.762\end{array}$ $\begin{array}{llllllll}1497.743 & 1498.214 & 1499.446 & 1498.002 & 1496.748 & 1496.849 & 1497.75\end{array}$ $\begin{array}{lrllllll}1497.739 & 1498.2 & 1499.446 & 1497.99 & 1496.739 & 1496.847 & 1497.74\end{array}$ $\begin{array}{rrrrrrrr}1497.732 & 1498.135 & 1499.397 & 1498.002 & 1496.732 & 1496.847 & 1497.748 \\ 1497.743 & 1498.077 & 149939 & 1498.041 & 1496746 & 1496854 & 1497.769\end{array}$ $\begin{array}{llllllll}1497.743 & 1498.077 & 1499.39 & 1498.041 & 1496.746 & 1496.854 & 1497.769\end{array}$ $\begin{array}{lllllllll}14997.72 & 1498156 & 1499.428 & 1497.988 & 1496.7313 & 1496.832 & 1497.748\end{array}$ $\begin{array}{lllllllll}1497.727 & 1498.107 & 1499.409 & 1498.018 & 1496.725 & 1496.842 & 1497.75\end{array}$ $\begin{array}{llllllll}1497.72 & 1498.095 & 1499.386 & 1498.004 & 1496.713 & 1496.835 & 1497.736\end{array}$ $\begin{array}{llllllll}1497.727 & 1498.009 & 1499.308 & 1498.025 & 1496.72 & 1496.837 & 1497.748\end{array}$ $\begin{array}{lllllllll}1497.739 & 1497.937 & 1499.269 & 1498.07 & 1496.744 & 1496.847 & 1497.781\end{array}$ $\begin{array}{llllllll}1497.729 & 1497.993 & 1499.299 & 1498.046 & 1496.734 & 1496.835 & 1497.76\end{array}$ $\begin{array}{llllllll}1497.727 & 1498.007 & 1499.325 & 1498.02 & 1496.717 & 1496.832 & 1497.748 \\ 1497.722 & 1498.014 & 1499.334 & 1498.011 & 1496.708 & 1496825 & 1497.74\end{array}$ $\begin{array}{llllllllll}1497.706 & 1498042 & 1499.3294 & 1497.969 & 1496677 & 1496809 & 1497.707\end{array}$ $\begin{array}{llllllll}1497.706 & 1498.042 & 499.294 & 1497.969 & 1496.677 & 1496.809 & 1497.707 \\ 1497.708 & 1497.937 & 1499.18 & 1497.948 & 1496.66 & 1496.807 & 1497.7\end{array}$ $\begin{array}{llllllll}1497.704 & 1497.872 & 1499.241 & 1497.978 & 1496.67 & 1496.809 & 1497.719\end{array}$ $\begin{array}{lllllllll}1497.666 & 1497.974 & 1499.325 & 1497.946 & 1496.641 & 1496.792 & 1497.683\end{array}$ $\begin{array}{lllllllll}1497.673 & 1497.998 & 1499.346 & 1497.964 & 1496.649 & 1496.802 & 1497.704\end{array}$ $\begin{array}{llllllllll}1497.694 & 1498.002 & 1499.36 & 1497.997 & 1496.665 & 1496.807 & 1497.724\end{array}$ $\begin{array}{lllllll}1497.697 & 1497.993 & 1499.346 & 1497.999 & 1496.663 & 1496.802 & 1497.719 \\ 1497.706 & 1497.895 & 1499.217 & 1498.025 & 1496.682 & 1496.809 & 1497.743\end{array}$

$1500.061 \quad 1500.027$ $\begin{array}{ll}1500.077 & 1500.027 \\ 1500.098 & 1500.048\end{array}$

1500.1351500 .087

1500.1491500 .082

$1500.191 \quad 1500.132$

$1500.207 \quad 1500.115$

1500.141499 .972

1500.0941499 .943

1500.0591499 .919

$1499.947 \quad 1499.811$

$1499.935 \quad 1499.826$

1499.8821499 .761

$1499.833 \quad 1499.728$

$1499.821 \quad 1499.756$

1499.7941499 .735

$1499.952 \quad 1499.945$

$1499.952 \quad 1499.912$

$1500.005 \quad 1500$

$\begin{array}{lll}1499.961 & 1499.886 \\ 1499.963 & 1499.895\end{array}$

$1499.987 \quad 1499.941$

$1499.968 \quad 1499.888$

$1499.994 \quad 1499.933$
1500.01
1499.955

$1499.982 \quad 1499.888$

$1499.907 \quad 1499.792$

1499.91499 .819

$\begin{array}{lll}1499.889 & 1499.792 \\ 1499875 & 1499.785\end{array}$

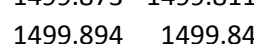

$\begin{array}{rl}149.894 & 1499.84 \\ 1499.88 & 1499.802\end{array}$

$1499.856 \quad 1499.766$

1499.8731499 .81

$1499.856 \quad 1499.802$

$1499.861 \quad 1499.792$

$\begin{array}{llll}1499.826 & 1499.737\end{array}$

$\begin{array}{lll}1499.81 & 1499.737\end{array}$

$1499.821 \quad 1499.77$

$\begin{array}{rrr}1499.81 & 1499.764 \\ 1499.828 & 1499.787\end{array}$

$\begin{array}{ll}1499.826 & 1499.775 \\ 1499852 & 1499811\end{array}$ 
$\begin{array}{llllllll}1497.76 & 1498.016 & 1499.526 & 1498.158 & 1496.782 & 1496.844 & 1497.827\end{array}$

$\begin{array}{llllllll}1497.788 & 1497.993 & 1499.432 & 1498.219 & 1496.841 & 1496.87 & 1497.874\end{array}$ $\begin{array}{llllllll}1497.806 & 1497.979 & 1499.629 & 1498.226 & 1496.855 & 1496.872 & 1497.879\end{array}$ $\begin{array}{lllllllll}1497.816 & 1498.016 & 1499.643 & 1498.235 & 1496.877 & 1496.882 & 1497.894\end{array}$ $\begin{array}{llllllll}1497.813 & 1498.189 & 1499.594 & 1498.198 & 1496.865 & 1496.87 & 1497.867\end{array}$ $\begin{array}{llllllll}1497.823 & 1498.249 & 1499.601 & 1498.182 & 1496.86 & 1496.872 & 1497.86\end{array}$ $\begin{array}{llllllll}1497.813 & 1498.305 & 1499.65 & 1498.156 & 1496.858 & 1496.872 & 1497.858\end{array}$ $\begin{array}{llllllll}1497.788 & 1498.336 & 1499.638 & 1488.098 & 1496.817 & 1496.854 & 1497.81 \\ 1497.788 & 1498105 & 1499.484 & 1498.058 & 1496796 & 1496.851 & 1497.798\end{array}$ $\begin{array}{lllllllll}1497.788 & 1498.105 & 1499.484 & 1498.058 & 1496.796 & 1496.851 & 1497.798 \\ 1497.781 & 1497.925 & 1499.474 & 1498.063 & 1496789 & 1496.851 & 1497.8\end{array}$ $\begin{array}{lllllllll}1497.774 & 1498107 & 1499.453 & 1498.041 & 1496.772 & 1496844 & 1497.781\end{array}$ $\begin{array}{llllllll}1497.769 & 1498.238 & 1499.442 & 1498.02 & 1496.758 & 1496.837 & 1497.771\end{array}$ $\begin{array}{lllllllll}1497.767 & 1498.27 & 1499.453 & 1498.034 & 1496.758 & 1496.842 & 1497.779\end{array}$ $\begin{array}{lllllllll}1497.76 & 1498.261 & 1499.425 & 1498.018 & 1496.741 & 1496.835 & 1497.762\end{array}$ $\begin{array}{llllllll}1497.781 & 1497.888 & 1499.519 & 1498.074 & 1496.772 & 1496.856 & 1497.81\end{array}$ $\begin{array}{llllllll}1497.806 & 1497.96 & 1499.61 & 1498.186 & 1496.834 & 496.884 & 1497.882\end{array}$ $\begin{array}{llllllllll}1497.825 & 1498.102 & 1499.631 & 1488.235 & 1496.874 & 1496.891 & 1497.901\end{array}$ $\begin{array}{lllllllll}1497.86 & 1498.163 & 1499.71 & 1498.324 & 1496.969 & 1496.948 & 1497.961\end{array}$ $\begin{array}{lllllll}1497.869 & 1498.179 & 1499.701 & 1498.324 & 1496.981 & 1496.962 & 1497.961 \\ 1497.888 & 1498.184 & 1499.738 & 1498.343 & 1497.017 & 1496.99 & 1497.97\end{array}$ $\begin{array}{lllllllll}1497.902 & 1498.268 & 1499.797 & 1498.406 & 1497.078 & 1497.035 & 1498.006\end{array}$ $\begin{array}{llllllll}1497.836 & 1498.578 & 1499.53 & 1498.224 & 1496.988 & 1496.973 & 1497.886\end{array}$ $\begin{array}{lllllllll}1497.813 & 1498.765 & 1499.435 & 1498.037 & 1496.879 & 1496.896 & 1497.803\end{array}$ $\begin{array}{rlrrrrr}1497.776 & 1498.795 & 1499.357 & 1497.955 & 1496.815 & 1496.861 & 1497.764 \\ 1497.781 & 1498.741 & 149932 & 1497.89 & 1496751 & 1496814 & 147.724\end{array}$

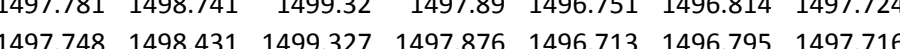
$\begin{array}{lllllllll}1497.748 & 1498.189 & 1499.364 & 1497.922 & 1496.717 & 1496.797 & 1497.74\end{array}$ $\begin{array}{lllllllll}1497.741 & 1498.326 & 1499.369 & 1497.922 & 1496.71 & 1496.795 & 1497.74\end{array}$ $\begin{array}{llllllll}1497.741 & 1498.387 & 1499.383 & 1497.936 & 1496.708 & 1496.795 & 1497.74\end{array}$ $\begin{array}{llllllll}1497.783 & 1498.359 & 1499.425 & 1497.985 & 1496.72 & 1496.807 & 1497.764\end{array}$ $\begin{array}{llllllll}1497.783 & 1498.273 & 1499.43 & 1498.006 & 1496.727 & 1496.814 & 1497.771\end{array}$ $\begin{array}{lllllllll}1497.639 & 1497.874 & 1499.498 & 1498.056 & 1496.753 & 1496.835 & 1497.807\end{array}$ $\begin{array}{lllllllllll}1497.799 & 1497.937 & 1499.586 & 1498.154 & 1496.817 & 1496.879 & 1497.867\end{array}$ $\begin{array}{lllllllll}1497.825 & 1498182 & 1499.61 & 1498.149 & 1496872 & 1496.922 & 1497.901\end{array}$ $\begin{array}{llllllll}1497.836 & 1498.226 & 1499.612 & 1498.177 & 1496.898 & 1496.943 & 1497.908\end{array}$ $\begin{array}{lllllllll}1497.813 & 1498.312 & 1499.505 & 1498.098 & 1496.855 & 1496.912 & 1497.843\end{array}$ $\begin{array}{llllllll}1497.799 & 1498.107 & 1499.446 & 1498.009 & 1496.805 & 1496.879 & 1497.8\end{array}$ $\begin{array}{lllllllll}1497.79 & 1497.916 & 1499.451 & 1498.002 & 1496.793 & 1496.875 & 1497.798\end{array}$ $\begin{array}{lllllll}1497.785 & 1498.063 & 1499.449 & 1497.995 & 1496.779 & 1496.865 & 1497.791 \\ 147.774 & 1498119 & 149.918 & 149.976 & 149676 & 149651 & 1497.776\end{array}$ $\begin{array}{llllllll}1497.774 & 1498.119 & 149.418 & 1497.976 & 1496.76 & 1496.851 & 1497.776 \\ 1497.781 & 1498.067 & 1499.47 & 1498.018 & 1496.779 & 1496863 & 1497.805\end{array}$ $\begin{array}{lllllllllll}1497.785 & 1498.07 & 1499.493 & 1498.051 & 1496791 & 1496872 & 1497.805\end{array}$ $\begin{array}{llllllllll}1497.804 & 1497.958 & 1499.35 & 14981 & 1496817 & 149689 & 1497.822\end{array}$ $\begin{array}{llllllll}1497.82 & 1497.979 & 1499.587 & 1498.156 & 1496.853 & 1496.924 & 1497.886\end{array}$ $\begin{array}{lllllllll}1497.802 & 1498.121 & 1499.509 & 1498.121 & 1496.841 & 1496.912 & 1497.858\end{array}$ $\begin{array}{rrrrrrr}1497.792 & 1498.091 & 1499.598 & 1498.063 & 1496.805 & 1496.889 & 1497.822 \\ 1497.79 & 1498.168 & 1499.622 & 1498.049 & 1496.801 & 1496.887 & 1497.812\end{array}$ $\begin{array}{llllllll}1497.79 & 1498.168 & 1499.622 & 1498.049 & 1496.801 & 1496.887 & 1497.812\end{array}$ $\begin{array}{lllllll}1497.771 & 1498.079 & 1499.556 & 1497.836 & 1496.765 & 1496.868 & 1497.776 \\ 1497.776 & 1497.855 & 1499.449 & 1497.687 & 1496.777 & 1496.875 & 1497.798\end{array}$

$1499.907 \quad 1499.883$ 1499.9211499 .864
1499.961
1499.917

1499.98714999936

$\begin{array}{ll}1499.987 & 1499.936 \\ 1500.019 & 1499.965\end{array}$

$1500.031 \quad 1499.95$

1500.0491499 .967

1500.0261499 .902

$1500.014 \quad 1499.893$

$1499.924 \quad 1499.792$

1499.9141499 .802

1499.8941499 .778

1499.8751499 .761

1499.8751499 .783

1499.99614999 .943

15000051499955

$1500.054 \quad 1500,008$

1500.0961500 .029

$\begin{array}{ll}1500.105 & 1500.008 \\ 1500.131 & 1500.036\end{array}$

$1500.182 \quad 1500.096$

$\begin{array}{rr}1500.07 & 1499.859 \\ 1499.942 & 1499.716\end{array}$

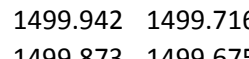

$\begin{array}{ll}1499.873 & 1499.675 \\ 1499.796 & 1499.622\end{array}$

$\begin{array}{lll}1499.796 & 1499.622 \\ 1499.763 & 1499.644\end{array}$

$1499.777 \quad 1499.704$
1499.78

$1499.78 \quad 1499.718$

$1499.782 \quad 1499.728$

$1499.805 \quad 1499.766$

$1499.821 \quad 1499.773$

$1499.854 \quad 1499.826$

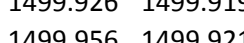

$1499.991 \quad 1499.938$

1500.011499 .931

1499.9561499 .819

$1499.898 \quad 1499.754$

$1499.884 \quad 1499.768$

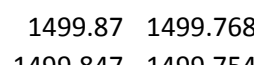

$\begin{array}{ll}1499.847 & 1499.754 \\ 1499.868 & 1499.804\end{array}$

$\begin{array}{lll}1499.868 & 1499.804 \\ 1499.887 & 1499.821\end{array}$

$\begin{array}{lll}1499.887 & 1499.821 \\ 1499.917 & 1499.888\end{array}$

$1499.956 \quad 1499.924$

$1499.94 \quad 1499.859$

1499.9031499 .797

$1499.894 \quad 1499.79$

$\begin{array}{lll}1499.856 & 1499.725 \\ 1499.866 & 1499.785\end{array}$ 
$\begin{array}{lllllllll}1497.755 & 1498.093 & 1499.437 & 1497.911 & 1496.77 & 1496.868 & 1497.771\end{array}$

4/9/11 12:00

4/9/11 20:00

$\begin{array}{llllllll}1497.755 & 1498.093 & 1499.437 & 1497.911 & 1496.77 & 1496.868 & 1497.771 \\ 1497.769 & 1497.867 & 1499.512 & 1497.976 & 1496.798 & 1496.889 & 1497.812\end{array}$

$\begin{array}{llllllll}1497.788 & 1497.678 & 1499.577 & 1498.06 & 1496.848 & 1496.922 & 1497.862\end{array}$

$\begin{array}{rrrrrrr}1497.785 & 1497.876 & 1499.544 & 1498.07 & 1496.853 & 1496.926 & 1497.858 \\ 1497.788 & 1498.014 & 1499.533 & 1498.051 & 1496839 & 1496.919 & 1497.843\end{array}$

4/10/111:00

4/10/11 8:00

4/10/11 12:00

4/10/111 20:00

4/11/11/11 20:00

4/11/11 4:00

4/11/11 8:00

4/11/11 12:00

4/11/111 16:00

4/11/11 20:00

4/12/11 0:00

4/12/11 8:00

4/12/11 12:00

4/12/11 16:00

4/12/11 20:00

4/13/11 0:00

4/13/11 4:00

4/13/11 8:00

4/13/11 16:00

4/13/11 20:00

4/14/11 0:00

4/14/11 4:00

4/14/11 8:00

4/14/11 12:00

4/14/11 16:00

4/14/11 20:00

4/15/11 4:00

4/15/11 8:00

4/15/11 12:00

4/15/11 16:00

4/15/11 20:00

4/16/11 0:00

4/16/114:00

4/16/11 12:00

4/16/11 16:00

4/16/11 20:00

4/17/11 0:00

4/17/11 4:00

$4 / 17 / 118: 00$

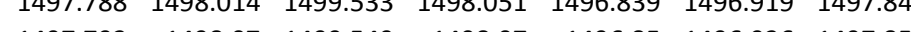

$\begin{array}{rrrrrrrr}1497.792 & 1498.07 & 1499.549 & 1498.07 & 1496.85 & 1496.926 & 1497.858\end{array}$

$\begin{array}{llllllll}1497.778 & 1498.135 & 1499.488 & 1498.037 & 1496.831 & 1496.91 & 1497.824 \\ 1497.769 & 1497.97 & 1499.453 & 1497.983 & 1496803 & 1496894 & 1497.8\end{array}$

$\begin{array}{rrrrrrrrr}1497.762 & 1497.851 & 1499.453 & 1497.967 & 1496801 & 1496.887 & 1497.798\end{array}$

$\begin{array}{lllllllll}1497.727 & 1498.123 & 1499.341 & 1497.878 & 1496.741 & 1496.844 & 1497.731\end{array}$

$\begin{array}{llllllll}1497.72 & 1497.928 & 1499.329 & 1497.843 & 1496.703 & 1496.816 & 1497.712\end{array}$

$\begin{array}{lllllllll}1497.739 & 1497.907 & 1499.325 & 1497.838 & 1496.679 & 1496.797 & 1497.702\end{array}$ $\begin{array}{llllllll}1497.69 & 1497.825 & 1499.245 & 1497.621 & 1496.622 & 1496.755 & 1497.649\end{array}$ $\begin{array}{lllllll}1497.687 & 1497.792 & 1499.276 & 1497.442 & 1496.603 & 1496.738 & 1497.652\end{array}$ $\begin{array}{llllllll}1497.676 & 1497.795 & 1499.299 & 1497.523 & 1496.599 & 1496.736 & 1497.659\end{array}$ $\begin{array}{lllllllll}1497.664 & 1497.774 & 1499.273 & 1497.577 & 1496.58 & 1496.72 & 1497.645\end{array}$ $\begin{array}{llllllll}1497.66 & 1497.746 & 1499.269 & 1497.654 & 1496.558 & 1496.694 & 1497.637\end{array}$ $\begin{array}{llllllll}1497.711 & 1497.734 & 1499.264 & 1497.668 & 1496534 & 1496677 & 1497.623\end{array}$ $\begin{array}{lllllllll}1497.639 & 1497.769 & 1499.329 & 1497.736 & 1496.551 & 1496.687 & 1497.657\end{array}$ $\begin{array}{llllllll}1497.669 & 1497.841 & 1499.409 & 1497.843 & 1496.603 & 1496.722 & 1497.716\end{array}$ $\begin{array}{llllllll}1497.664 & 1497.841 & 1499.39 & 1497.731 & 1496.611 & 1496.722 & 1497.707\end{array}$

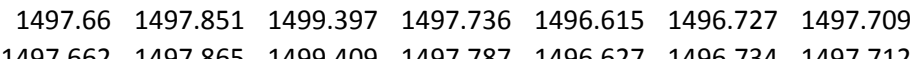
$\begin{array}{llllllllll}1497.662 & 1497.865 & 1499.409 & 1497.787 & 1496.627 & 1496.734 & 1497.712\end{array}$ $\begin{array}{llllllllll}1497.666 & 1497.876 & 149.425 & 1497.684 & 1496.634 & 1496.745 & 1497.719 \\ 1497.66 & 1497.86 & 1499.418 & 1497.519 & 1496.639 & 1496.745 & 1497.707\end{array}$ $\begin{array}{lllllllll}1497.666 & 1497.89 & 1499.456 & 1497.605 & 1496.672 & 1496.769 & 1497.731\end{array}$ $\begin{array}{llllllll}1497.66 & 1497.89 & 1499.435 & 1497.666 & 1496.675 & 1496.769 & 1497.719\end{array}$ $\begin{array}{lllllllll}1497.653 & 1497.872 & 1499.418 & 1497.689 & 1496.665 & 1496.755 & 1497.693\end{array}$ $\begin{array}{llllllll}1497.662 & 1497.914 & 1499.477 & 1497.773 & 1496.689 & 1496.778 & 1497.731\end{array}$ $\begin{array}{llllllll}1497.669 & 1497.949 & 1499.498 & 1497.834 & 1496.715 & 1496.802 & 1497.75\end{array}$ $\begin{array}{llllllll}1497.699 & 1498.042 & 1499.589 & 1497.943 & 1496.779 & 1496.844 & 1497.805 \\ 1497.708 & 1498.112 & 1499.591 & 1498018 & 1496834 & 1496879 & 1497.829\end{array}$ $\begin{array}{llllllllll}1497.708 & 1498.112 & 1499.591 & 1498.018 & 1496.834 & 1496.879 & 1497.829\end{array}$ $\begin{array}{lllllllllll}1497.722 & 1498.095 & 1499.596 & 1497.974 & 14968827 & 14968879 & 1497.815\end{array}$ $\begin{array}{lllllllll}1497.732 & 1498.109 & 1499.577 & 1498.004 & 1496.846 & 1496.894 & 1497.824\end{array}$ $\begin{array}{lllllllll}1497.687 & 1498.088 & 1499.57 & 1497.843 & 1496.843 & 1496.894 & 1497.812\end{array}$ $\begin{array}{llllllll}1497.804 & 1497.977 & 1499.549 & 1497.542 & 1496.77 & 1496.844 & 1497.719\end{array}$ $\begin{array}{llllllll}1497.797 & 1497.895 & 1499.549 & 1497.488 & 1496.715 & 1496.802 & 1497.673\end{array}$ $\begin{array}{rrrrrrrr}1497.629 & 1497.942 & 1499.54 & 1497.516 & 14966.677 & 1496.781 & 1497.645\end{array}$ $\begin{array}{llllllll}1497.622 & 1497.87 & 1499.294 & 1497.54 & 1496.646 & 1496.755 & 1497.618\end{array}$

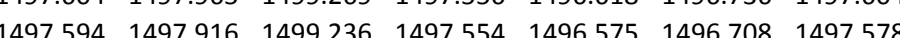
$\begin{array}{lllllllll}1497.597 & 1497.55 & 1499.273 & 1497.586 & 1496.565 & 1496.701 & 1497.597\end{array}$ $\begin{array}{llllllll}1497.606 & 1497.599 & 1499.343 & 1497.682 & 1496.601 & 1496.72 & 1497.647\end{array}$ $\begin{array}{llllllll}1497.601 & 1497.774 & 1499.341 & 1497.705 & 1496.603 & 1496.72 & 1497.647\end{array}$ $\begin{array}{lllllll}1497.599 & 1497.883 & 1499.348 & 1497.722 & 1496.608 & 1496.722 & 1497.647 \\ 1497.604 & 1497.916 & 1499376 & 1497.759 & 1496625 & 1496734 & 1497.666\end{array}$ $\begin{array}{lllllllll}1497.604 & 1497.916 & 1499.376 & 1497.759 & 1496.625 & 1496.734 & 1497.666\end{array}$ $\begin{array}{llllllll}1497.629 & 1497.622 & 1499.472 & 1497.533 & 1496.682 & 1496.774 & 1497.716\end{array}$

1499.8961499 .835

$\begin{array}{rl}1499.9 & 1499.838 \\ 14998 & 1499792\end{array}$

149.88 1499.792

$1499.861 \quad 1499.773$

$1499.896 \quad 1499.85$

$1499.947 \quad 1499.919$

$1499.954 \quad 1499.893$

$149.94 \quad 1499.866$

$1499894 \quad 1499.794$

1499.8891499 .792

1499.8241499 .694

$1499.784 \quad 1499.68$

$1499.763 \quad 1499.677$

1499.7051499 .589

1499.691
1499.71499 .646

$1499.694 \quad 1499.634$
1499.67
1499.613

$1499.68 \quad 1499.639$

$1499.666 \quad 1499.622$

1499.6981499 .687

$1499.763 \quad 1499.78$

$1499.773 \quad 1499.747$

$\begin{array}{rr}1499.787 & 1499.747 \\ 14998 & 1499.756\end{array}$

$\begin{array}{rl}1499.8 & 1499.756 \\ 1499814 & 1499.761\end{array}$

$\begin{array}{lll}1499.814 & 1499.761 \\ 1499.817 & 1499.737\end{array}$

$\begin{array}{lll}1499.852 & 1499.785\end{array}$

$1499.854 \quad 1499.764$

1499.8351499 .732

$1499.868 \quad 1499.797$

$1499.896 \quad 1499.826$

$\begin{array}{lll}1499.956 & 1499.909 \\ 1500.005 & 1499.928\end{array}$

1500.0051499 .928

$\begin{array}{lll}1499.984 & 1499.895\end{array}$

$1499.996 \quad 1499.89$

$1499.984 \quad 1499.852$

1499.8961499 .689

$1499.828 \quad 1499.632$

1499.789 1499.618

1499.7471499 .603
1499.7241499 .596

$1499.684 \quad 1499.56$

$\begin{array}{rrr}1499.684 & 1499.56 \\ 1499.684 & 1499.603\end{array}$

$1499.726 \quad 1499.692$

$1499.738 \quad 1499.68$

$1499.747 \quad 1499.692$

$1499.77 \quad 1499.716$

$\begin{array}{rr}1499.796 & 1499.739 \\ 1499.84 & 1499.79\end{array}$ 
$\begin{array}{llllllll}1497.599 & 1497.673 & 1499.399 & 1497.388 & 1496.675 & 1496.757 & 1497.657\end{array}$ $\begin{array}{llllllll}1497.608 & 1497.517 & 1499.458 & 1497.495 & 1496.708 & 1496.781 & 1497.688\end{array}$ $\begin{array}{llllllll}1497.601 & 1497.683 & 1499.437 & 1497.551 & 1496.708 & 1496.781 & 1497.681 \\ 1497.618 & 1497.75 & 1499.507 & 1497.652 & 1496.743 & 1496.804 & 1497.714\end{array}$ $\begin{array}{llllllll}1497.618 & 1497.75 & 1499.507 & 1497.652 & 1496.743 & 1496.804 & 1497.714\end{array}$ $\begin{array}{llllllll}1497.601 & 1497.862 & 1499.449 & 1497.696 & 1496.748 & 1496.809 & 1497.697 \\ 1497.576 & 1498 & 1499.355 & 1497.631 & 1496.696 & 1496767 & 1497.628\end{array}$ $\begin{array}{rrrrrrrr}1497.576 & 1498 & 1499.355 & 1497.631 & 1496.696 & 1496.767 & 1497.628 \\ 1497.566 & 1497.972 & 1499299 & 1497.577 & 1496646 & 1496.731 & 1497.585\end{array}$ $\begin{array}{rrrrrrr}1497.566 & 1497.972 & 1499.299 & 1497.577 & 1496.646 & 1496.731 & 1497.585 \\ 1497.557 & 1497.904 & 1499.276 & 1497.558 & 1496.61 & 1496.703 & 1497.563\end{array}$ $\begin{array}{llllllll}1497.534 & 1498.005 & 1499.184 & 1497.498 & 1496.553 & 1496.661 & 1497.525\end{array}$ $\begin{array}{lllllll}1497.534 & 1498.005 & 1499.184 & 1497.498 & 1496.553 & 1496.661 & 1497.525 \\ 1497.503 & 1498.067 & 1499.126 & 1497.453 & 1496.494 & 1496.619 & 1497.496\end{array}$ $\begin{array}{llllllll}1497.503 & 1498.044 & 1499.126 & 1497.446 & 1496.454 & 1496.59 & 1497.482\end{array}$ $\begin{array}{llllllll}1497.524 & 1498.002 & 1499.086 & 1497.25 & 1496.392 & 1496.546 & 1497.451\end{array}$ $\begin{array}{llllllll}1497.424 & 1497.778 & 1499.103 & 1497.08 & 1496.371 & 1496.524 & 1497.458\end{array}$ $\begin{array}{llllllll}1497.347 & 1497.52 & 1499.166 & 1497.185 & 1496.385 & 1496.534 & 1497.491\end{array}$ $\begin{array}{lllllllll}1497.434 & 1497.618 & 1499.175 & 1497.292 & 1496.39 & 1496.529 & 1497.494 \\ 1497.464 & 1497.69 & 1499.17 & 1497.35 & 1496378 & 1496517 & 1497.477\end{array}$ $\begin{array}{llllllll}1497.676 & 1497.657 & 1499.24 & 1497.446 & 1496.411 & 1496.536 & 1497.518\end{array}$ $\begin{array}{lllllllll}1497.476 & 1497.615 & 1499.271 & 1497.507 & 1496.428 & 1496.55 & 1497.537\end{array}$ $\begin{array}{llllllll}1497.497 & 1497.41 & 1499.355 & 1497.619 & 1496.477 & 1496.586 & 1497.59\end{array}$ $\begin{array}{llllllll}1497.513 & 1497.396 & 1499.395 & 1497.694 & 1496.518 & 1496.611 & 1497.621\end{array}$ $\begin{array}{llllllll}1497.513 & 1497.506 & 1499.388 & 1497.712 & 1496.542 & 1496.623 & 1497.625\end{array}$ $\begin{array}{llllllll}1497.531 & 1497.557 & 1499.435 & 1497.757 & 1496.575 & 1496.647 & 1497.659 \\ 1497.552 & 1497.566 & 1499.406 & 1497.841 & 1496.634 & 1496689 & 147.702\end{array}$ $\begin{array}{llllllllll}1497.552 & 1497.566 & 1499.406 & 1497.841 & 1496.634 & 1496.689 & 1497.702\end{array}$ $\begin{array}{llllllll}1497.508 & 1497.648 & 149.362 & 1497.796 & 1496.622 & 1496.677 & 1497.664 \\ 1497.492 & 1497.664 & 1499.184 & 1497.712 & 1496584 & 1496.649 & 1497.611\end{array}$ $\begin{array}{lllllllll}1497.529 & 1497.443 & 1499.371 & 1497.722 & 1496.594 & 1496.654 & 1497.63\end{array}$ $\begin{array}{lllllllll}1497.524 & 1497.645 & 1499.343 & 1497.682 & 1496.582 & 1496.644 & 1497.611\end{array}$ $\begin{array}{llllllll}1497.494 & 1497.883 & 1499.243 & 1497.591 & 1496.532 & 1496.611 & 1497.556\end{array}$ $\begin{array}{llllllll}1497.503 & 1497.893 & 1499.292 & 1497.614 & 1496.534 & 1496.602 & 1497.57\end{array}$ $\begin{array}{llllllll}1497.476 & 1497.911 & 1499.205 & 1497.551 & 1496.485 & 1496.628 & 1497.511\end{array}$ $\begin{array}{rrrrrrrr}1497.466 & 1497.809 & 1499.201 & 1497.528 & 1496.466 & 1496.6 & 1497.501 \\ 1497.473 & 1497.708 & 1499.25 & 1497.563 & 1496.473 & 1496555 & 1497527\end{array}$ $\begin{array}{llllllll}1497.473 & 1497.708 & 1499.25 & 1497.563 & 1496.473 & 1496.555 & 1497.527 \\ 1497.455 & 1497.788 & 1499.206 & 1497.554 & 1496.463 & 1496572 & 1497.513\end{array}$

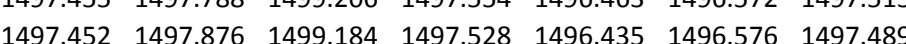
$\begin{array}{lllllllll}1497.455 & 1497.869 & 1499.21 & 1497.563 & 1496.444 & 1496.548 & 1497.508\end{array}$ $\begin{array}{lllllllll}1497.45 & 1497.82 & 1499.194 & 1497.565 & 1496.437 & 1496.564 & 1497.494\end{array}$ $\begin{array}{llllllll}1497.466 & 1497.482 & 1499.278 & 1497.621 & 1496.473 & 1496.508 & 1497.539\end{array}$ $\begin{array}{llllllll}1497.485 & 1497.366 & 1499.336 & 1497.682 & 1496.511 & 1496.532 & 1497.585\end{array}$ $\begin{array}{lllllll}1497.476 & 1497.555 & 1499.292 & 1497.675 & 1496.508 & 1496.583 & 1497.57\end{array}$ $\begin{array}{llllllll}1497.49 & 1497.648 & 1499.329 & 1497.684 & 1496.525 & 1496.562 & 1497.585 \\ 1497.459 & 1497.727 & 1499.395 & 1497.705 & 1496534 & 1496.63 & 1497.582\end{array}$ $\begin{array}{llllllll}1497.459 & 1497.727 & 149.395 & 1497.705 & 1496.534 & 1496.63 & 1497.582\end{array}$ $\begin{array}{lllllllll}1497.464 & 1497.652 & 1499.212 & 1497.792 & 1496608 & 1496.597 & 1497.657\end{array}$ $\begin{array}{llllllll}1497.494 & 1497.706 & 1499.257 & 1497.81 & 1496.627 & 1496.637 & 1497.666\end{array}$ $\begin{array}{llllllll}1497.527 & 1497.82 & 1499.315 & 1497.782 & 1496.62 & 1496.68 & 1497.647\end{array}$ $\begin{array}{llllllll}1497.534 & 1497.867 & 1499.334 & 1497.775 & 1496.627 & 1496.682 & 1497.652\end{array}$ $\begin{array}{lllllllll}1497.583 & 1497.928 & 1499.348 & 1497.778 & 1496.634 & 1496.689 & 1497.661\end{array}$ $\begin{array}{lllllll}1497.599 & 1497.93 & 1499.194 & 1497.764 & 1496.627 & 1496.689 & 1497.642 \\ 1497.296 & 1497.634 & 1499.395 & 1497.764 & 1496.639 & 1496.698 & 1497.657\end{array}$

1499.8841499 .823 $\begin{array}{ll}1499.873 & 1499.76 \\ 1499.838 & 1499.71\end{array}$ $\begin{array}{lll}1499.838 & 1499.711 \\ 1499.838 & 1499.718\end{array}$ $\begin{array}{ll}1499.838 & 1499.718 \\ 1499.814 & 1499.682\end{array}$ $1499.814 \quad 1499.696$ 1499.8491499 .754 1499.8521499 .744 $1499.887 \quad 1499.809$
149891149.975 $1499.891 \quad 1499.775$ $\begin{array}{rl}1499.828 & 1499.668 \\ 1499.77 & 1499.601\end{array}$ $1499.728 \quad 1499.57$ $1499.67 \quad 1499.51$ $1499.617 \quad 1499.481$ 1499.5771499 .462 $1499.524 \quad 1499.416$ $1499.512 \quad 1499.436$ $1499.542 \quad 1499.515$

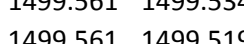
$1499.605 \quad 1499.591$ $1499.633 \quad 1499.62$ 1499.6961499 .696 $1499.747 \quad 1499.728$ $1499.768 \quad 1499.723$ $\begin{array}{ll}1499.803 & 1499.761 \\ 1499.863 & 1499.814\end{array}$ $\begin{array}{lll}1499.863 & 1499.814 \\ 1499.842 & 1499.728\end{array}$ $\begin{array}{lll}1499.842 & 1499.728 \\ 1499.994 & 1499.637\end{array}$ $\begin{array}{lll}1499.798 & 1499.673\end{array}$ $1499.78 \quad 1499.644$ $1499.721 \quad 1499.562$ $1499.724 \quad 1499.594$ $1499.663 \quad 1499.51$ $\begin{array}{rr}1499.64 & 1499.512 \\ 1499.649 & 1499.56\end{array}$ $\begin{array}{rrr}1499.649 & 1499.56 \\ 1499.642 & 1499.541\end{array}$ $\begin{array}{lll}1499.642 & 1499.541 \\ 1499.614 & 1499.512\end{array}$ $1499.626 \quad 1499.541$ $1499.621 \quad 1499.522$ 1499.6561499 .598 $1499.705 \quad 1499.668$ $1499.705 \quad 1499.62$ $\begin{array}{ll}1499.719 & 1499.641 \\ 1499.726 & 1499.625\end{array}$ 1499.7261499 .625
$1499.77 \quad 1499.675$ $\begin{array}{r}1499.803 \quad 1499.728 \\ \hline\end{array}$ $1499.824 \quad 1499.732$ $1499.812 \quad 1499.689$ $1499.81 \quad 1499.689$ $1499.814 \quad 1499.696$ $\begin{array}{lll}1499.803 & 1499.663 \\ 1499.805 & 1499.687\end{array}$ 
$\begin{array}{llllllll}1497.767 & 1497.981 & 1499.257 & 1497.642 & 1496.58 & 1496.656 & 1497.573\end{array}$ $\begin{array}{lllllllll}1497.666 & 1497.897 & 1499.212 & 1497.591 & 1496.544 & 1496.63 & 1497.539\end{array}$ $\begin{array}{llllllll}1497.571 & 1497.809 & 1499.199 & 1497.568 & 1496.518 & 1496.609 & 1497.523\end{array}$ $\begin{array}{rrrrrrr}1497.632 & 1497.893 & 1499.14 & 1497.521 & 1496.475 & 1496.579 & 1497.484 \\ 1497.713 & 1497.967 & 1499.145 & 1497.505 & 1496.449 & 1496.562 & 1497.475\end{array}$ $\begin{array}{lllllllll}1497.713 & 1497.967 & 1499.145 & 1497.505 & 1496.449 & 1496.562 & 1497.475\end{array}$ $\begin{array}{llllllll}1497.708 & 1497.977 & 1499.149 & 1497.514 & 1496.442 & 1496.557 & 1497.477 \\ 1497.683 & 1497.928 & 1499.093 & 1497.472 & 1496.401 & 1496532 & 1497.439\end{array}$ $\begin{array}{llllllllll}1497.683 & 1497.928 & 1499.093 & 1497.472 & 1496.401 & 1496.532 & 1497.439\end{array}$ $\begin{array}{llllllll}1497.266 & 1497.543 & 1499.142 & 1497.495 & 1496.401 & 1496.529 & 1497.465 \\ 1497.443 & 1497.314 & 1499.194 & 1497.551 & 1496.42 & 1496.539 & 1497.496\end{array}$ $\begin{array}{lllllllll}1497.441 & 1497.522 & 1499.189 & 1497.558 & 1496.425 & 1496541 & 1497.499\end{array}$ $\begin{array}{llllllll}1497.445 & 1497.685 & 1499.22 & 1497.572 & 1496.437 & 1466.548 & 1497.513\end{array}$ $\begin{array}{llllllll}1497.464 & 1497.739 & 1499.28 & 1497.638 & 1496.47 & 1496.576 & 1497.549\end{array}$ $\begin{array}{rrrrrrr}1497.48 & 1497.678 & 1499.318 & 1497.682 & 1496.499 & 1496.6 & 1497.573 \\ 1497.457 & 1497.387 & 1499.397 & 1497.771 & 1496.563 & 1496.649 & 1497.637\end{array}$ $\begin{array}{llllllll}1497.457 & 1497.387 & 1499.397 & 1497.771 & 1496.563 & 1496.649 & 1497.637 \\ 1497.212 & 1497.263 & 1499.453 & 1497.836 & 1496.62 & 1496.689 & 1497.669\end{array}$ $\begin{array}{llllllll}1497.334 & 1497.454 & 1499.46 & 1497.864 & 14966.62 & 1496.689 & 1497.669 \\ & 1496.715 & 1497.685\end{array}$ $\begin{array}{lllllllll}1497.531 & 1497.673 & 1499.425 & 1497.845 & 1496.67 & 1496.722 & 1497.678\end{array}$ $\begin{array}{llllllll}497.517 & 1497.911 & 1499.287 & 1497.729 & 1496.613 & 1496.68 & 199759\end{array}$ $\begin{array}{llllllll}1497.494 & 1498.005 & 1499.201 & 1497.589 & 1496.537 & 1496.628 & 1497.523 \\ 1497.485 & 1497.757 & 1499.201 & 1497.556 & 1496.511 & 1496.611 & 1497.518\end{array}$ $\begin{array}{llllllll}1497.382 & 1497.569 & 1499.201 & 1497.558 & 1496.504 & 1496.602 & 1497.513\end{array}$ $\begin{array}{lllllllll}1497.606 & 1497.83 & 1499.107 & 1497.409 & 1496.447 & 1496.562 & 1497.456\end{array}$ $\begin{array}{llllllll}1497.79 & 1497.988 & 1499.077 & 1497.336 & 1496.401 & 1496.527 & 1497.427 \\ 1497.778 & 147.993 & 1499065 & 1477.348 & 149375 & 14951 & 149.745\end{array}$ $\begin{array}{lllllll}1497.778 & 1497.993 & 1499.065 & 1497.348 & 1496.375 & 1496.51 & 1497.415 \\ 1497.729 & 1497.951 & 1499.053 & 1497.192 & 1463344 & 149.589 & 149.705\end{array}$ $\begin{array}{lllllllll}1497.729 & 1497.051 & 149.053 & 1497.192 & 1496.344 & 1496.489 & 1497.405 \\ 1497.583 & 1497.795 & 1499.044 & 1497.014 & 1496.321 & 1496.47 & 1497.393\end{array}$ $\begin{array}{lllllllll}1497.32 & 1497.487 & 1499.128 & 1497.131 & 1496.352 & 1496.487 & 1497.434\end{array}$ $\begin{array}{llllllll}1497.401 & 1497.622 & 1499.077 & 1497.196 & 1496.328 & 1496.517 & 1497.403\end{array}$ $\begin{array}{llllllll}1497.531 & 1497.711 & 1499.089 & 1497.229 & 1496.309 & 1496.475 & 1497.389\end{array}$ $\begin{array}{lllllll}1497.578 & 1497.771 & 1499.063 & 1497.259 & 1496.295 & 1496.489 & 1497.374 \\ 1497.587 & 1497.739 & 1498.988 & 1497.082 & 1496.245 & 1496.459 & 1497.338\end{array}$ $\begin{array}{lllllllll}1497.587 & 1497.739 & 1498.988 & 1497.082 & 1496.245 & 1496.459 & 1497.338\end{array}$ $\begin{array}{llllllll}1497.198 & 1497.352 & 1499.042 & 1496.933 & 1496.245 & 1496.395 & 1497.357\end{array}$ $\begin{array}{llllllllll}1497.082 & 1497.207 & 1499.063 & 1497.031 & 1496.257 & 1496.369 & 1497.367\end{array}$ $\begin{array}{llllllllll}1497.462 & 1497.531 & 1499.037 & 1497.147 & 1496.223 & 1496.393 & 1497.343\end{array}$ $\begin{array}{lllllllll}1497.527 & 1497.627 & 1499.042 & 1497.203 & 1496.226 & 1496.391 & 1497.343\end{array}$ $\begin{array}{llllllll}1497.501 & 1497.599 & 1499.018 & 1497.217 & 1496.209 & 1496.4 & 1497.322\end{array}$ $\begin{array}{lllllll}1497.101 & 1497.233 & 1499.07 & 1497.273 & 1496.228 & 1496.336 & 1497.35\end{array}$ $\begin{array}{llllllll}1496.956 & 1497.046 & 1499.124 & 1497.353 & 1496.257 & 1496.341 & 1497.386\end{array}$ $\begin{array}{llllllll}1497.187 & 1497.277 & 1499.098 & 1497.348 & 1496.261 & 1496.379 & 1497.381\end{array}$ $\begin{array}{lllllllll}1497.413 & 1497.48 & 1499.093 & 1497.339 & 1496.254 & 1496.391 & 1497.367 \\ 1497.471 & 1497.571 & 1499.121 & 1497.376 & 1496.271 & 1496379 & 1497384\end{array}$ $\begin{array}{lllllllllll}1497.41 & 1497.536 & 1499.114 & 1497.348 & 1496.271 & 1496.386 & 1497.384\end{array}$ $\begin{array}{llllllllll}1497.068 & 1497.198 & 1499.173 & 1497.117 & 1496299 & 1496362 & 1497.405\end{array}$ $\begin{array}{llllllll}1496.938 & 1497.032 & 1499.194 & 1497.201 & 1496.325 & 1496.383 & 1497.42\end{array}$ $\begin{array}{llllllll}1497.18 & 1497.282 & 1499.152 & 1497.25 & 1496.333 & 1496.426 & 1497.403\end{array}$ $\begin{array}{llllllll}1497.476 & 1497.531 & 1499.044 & 1497.192 & 1496.268 & 1496.414 & 1497.319\end{array}$ $\begin{array}{lllllllll}1497.483 & 1497.571 & 1499.079 & 1497.227 & 1496.266 & 1496.393 & 1497.326\end{array}$ $\begin{array}{rrrrrrrr}1497.438 & 1497.576 & 1499.075 & 1497.269 & 1496.271 & 1496.393 & 1497.334 \\ 1497.133 & 1497.3 & 1499.105 & 1497.304 & 1496.276 & 1496.362 & 1497.341\end{array}$

$1499.817 \quad 1499.699$ $\begin{array}{rr}1499.814 \quad 1499.68 \\ 199.796 & 1499.653\end{array}$ $1499.761 \quad 1499.606$ $1499.714 \quad 1499.543$ $1499.673 \quad 1499.503$ $1499.642 \quad 1499.491$ $1499.598 \quad 1499.452$ $\begin{array}{lll}1499.575 & 1499.455 \\ 1499.575 & 1499.474\end{array}$ $1499.533 \quad 1499.424$ $1499.542 \quad 1499.469$ $1499.568 \quad 1499.517$ $1499.582 \quad 1499.519$ $1499.598 \quad 1499.543$

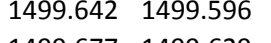
149.6771499 .629 1499.7521499 .716
1499.805
1499.751 $1499.84 \quad 1499.756$ $1499.842 \quad 1499.72$ $1499.768 \quad 1499.57$ $\begin{array}{rrr}1499.68 & 1499.462 \\ 1499.645 & 1499.474\end{array}$ $1499.631 \quad 1499.483$ $\begin{array}{lll}1499.521 & 1499.369 \\ 1499.496 & 1499.366\end{array}$ $1499.47 \quad 1499.357$ $1499.452 \quad 1499.335$ $1499.484 \quad 1499.397$ $1499.468 \quad 1499.376$ $\begin{array}{lll}1499.461 \quad 1499.371 \\ 149.412 & 149.304\end{array}$ $149.412 \quad 1499.304$ $1499.44 \quad 1499.381$ $\begin{array}{rrr}1499.42 & 149.381 \\ 1499.342\end{array}$ $1499.424 \quad 1499.354$ $1499.433 \quad 1499.361$ $1499.419 \quad 1499.33$ $\begin{array}{lll}1499.442 & 1499.385 \\ 149.482 & 149.443\end{array}$ 149.4821499 .443 $1499.5 \quad 1499.421$ $1499.507 \quad 1499.412$ $1499.568 \quad 1499.476$ $1499.561 \quad 1499.44$ 1499.4941499 .311 $149.484 \quad 1499.347$ 1499.487
$1499.489 \quad 1499.376$ 

Well

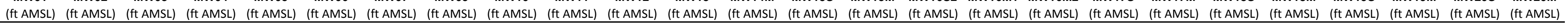

$5 / 5 / 1116: 00$

$5 / 5 / 1120: 00$

$5 / 6 / 114: 00$

5/6/114:00

5/6/11 8:00

5/6/11 12:00

5/6/11 20:00

5/7/11 0:00

$5 / 7 / 114: 00$

5/7/118:00

5/7/7111 16:00

5/7/11 20:00

5/8/11 0:00

5/8/11 4:00

5/8/11 8:00

5/8/11112:00

5/8/1116:00

5/8/11 20:00

5/9/11 0:00

5/9/11 8:00

5/9/11 8:00

5/9/11 16:00

5/9/11 20:00

5/10/110:00

5/10/11 4:00

$5 / 10 / 1112.00$

5/10/111 16:00

5/10/11 20:00

5/11/11 0:00

5/11/11 4:00

5/11/11 8:00

$5 / 11 / 1116: 00$

5/11/11 20:00

5/12/11 4:00

5/12/11 8:00

$5 / 12 / 11$ 12:00

5/12/11 16:00

$5 / 12 / 1120: 00$

5/13/11 0:00

$5 / 13 / 118.00$

5/13/111 8:00

5/13/11 16:00

5/13/11 20:00

5/14/11 0:00

5/14/111 4:00

$5 / 14 / 1118: 00$
$5 / 14 / 1112: 00$ $\begin{array}{lllllll}1497.026 & 1497.151 & 1499.147 & 1497.369 & 1496.306 & 1496.355 & 1497.374 \\ 1497.215 & 1497.377 & 1499.138 & 1497.327 & 1496311 & 1496386 & 1497.374\end{array}$ $\begin{array}{rrrrrrr}1497.215 & 1497.377 & 1499.138 & 1497.327 & 1496.311 & 1496.386 & 1497.374 \\ 1497.41 & 1497.566 & 1499.133 & 1497318 & 1496314 & 1496.4 & 1497365\end{array}$ $\begin{array}{rrrrrrr}1497.41 & 1497.566 & 1499.133 & 1497.318 & 1496.314 & 1496.4 & 1497.365 \\ 1497.441 & 1497.636 & 1499.184 & 1497.381 & 1496337 & 1496388 & 1497339\end{array}$ $\begin{array}{llllllll}1497.359 & 1497.571 & 1499.224 & 1497.28 & 1496.366 & 1496.388 & 1497.42\end{array}$ $\begin{array}{lllllllll}1497.001 & 1497.233 & 1499.276 & 1497.161 & 1496.413 & 1496.423 & 1497.463\end{array}$ $\begin{array}{lllllllll}1496.844 & 1497.072 & 1499.329 & 1497.273 & 1496.47 & 1496.461 & 1497.491\end{array}$ $\begin{array}{lllllllll}1497.082 & 1497.272 & 1499.306 & 1497.322 & 1496.482 & 1496.487 & 1497.487\end{array}$ $\begin{array}{llllllll}1497.32 & 1497.485 & 1499.285 & 1497.35 & 1496.48 & 1496.522 & 1497.465\end{array}$ $\begin{array}{lllllll}1497.434 & 1497.615 & 1499.269 & 1497.381 & 1496.48 & 1496.532 & 1497.458 \\ 1497.448 & 1497.638 & 1499231 & 1497.386 & 1496.47 & 146.546 & 147.432\end{array}$ $\begin{array}{llllllll}1497.448 & 1497.638 & 1499.231 & 1497.386 & 1496.47 & 1496.546 & 1497.432\end{array}$ $\begin{array}{llllllll}1497.012 & 1497.168 & 1499.238 & 1497.411 & 1496.463 & 1496.494 & 1497.424\end{array}$ $\begin{array}{lllllllll}1497.247 & 1497.424 & 1499.194 & 1497.397 & 1496.449 & 1496.508 & 1497.403\end{array}$ $\begin{array}{lllllllll}1497.441 & 1497.627 & 1499.208 & 1497.407 & 1496.442 & 1496.51 & 1497.396\end{array}$ $\begin{array}{llllllll}1497.322 & 1497.687 & 1499.248 & 1497.456 & 1496.468 & 1496.496 & 1497.422\end{array}$ $\begin{array}{llllllll}1497.322 & 1497.666 & 1499.243 & 1497.306 & 1496.468 & 1496.506 & 1497.422\end{array}$ $\begin{array}{lllllll}1497.336 & 1497.34 & 1499.301 & 1497.157 & 1496.511 & 1496.489 & 1497.458\end{array}$ $\begin{array}{lllllllll}1497.345 & 1497.149 & 1499.336 & 1497.217 & 1496.546 & 1496.51 & 1497.465\end{array}$ $\begin{array}{llllllllll}1497.334 & 1497.396 & 1499.283 & 1497.243 & 1496.542 & 1496.567 & 1497.446\end{array}$ $\begin{array}{lllllllll}1497.329 & 1497.694 & 1499.283 & 1497.313 & 1496.537 & 1496.564 & 1497.422\end{array}$ $\begin{array}{llllllll}1497.324 & 1497.697 & 1499.262 & 1497.187 & 1496.53 & 1496.567 & 1497.408\end{array}$ $\begin{array}{llllllll}1497.317 & 1497.429 & 1499.259 & 1497.026 & 1496.53 & 1496.546 & 1497.398\end{array}$ $\begin{array}{llllllll}1497.322 & 1497.235 & 1499.294 & 1497.108 & 1496.546 & 1496.51 & 1497.398\end{array}$ $\begin{array}{llllllll}1497.306 & 1497.457 & 1499.231 & 1497.126 & 1496.539 & 1496.562 & 1497.372\end{array}$ $\begin{array}{llllllll}1497.296 & 1497.627 & 1499.21 & 1497.11 & 1496.508 & 1496.543 & 1497.346 \\ 1497.285 & 1497.694 & 1499.201 & 1497.161 & 1496504 & 1496536 & 147.338\end{array}$ $\begin{array}{lllllllll}1497.285 & 1497.694 & 1499.201 & 1497.161 & 1496.504 & 1496.536 & 1497.338\end{array}$ $\begin{array}{lllllllll}1497.266 & 1497.729 & 1499.135 & 1497.15 & 1496.461 & 1496.501 & 1497.302 \\ 1497.271 & 1497.412 & 1499.163 & 1497.206 & 1496.458 & 1496.501 & 1497.317\end{array}$ $\begin{array}{lllllllll}1497.257 & 1497.41 & 1499.126 & 1497.224 & 1496.437 & 1496.482 & 1497.298\end{array}$ $\begin{array}{llllllll}1497.245 & 1497.569 & 1499.096 & 1497.22 & 1496.416 & 1496.463 & 1497.281\end{array}$ $\begin{array}{lllllllll}1497.245 & 1497.678 & 1499.096 & 1497.234 & 1496.399 & 1496.449 & 1497.283\end{array}$ $\begin{array}{lllllllll}1497.24 & 1497.708 & 1499.096 & 1497.257 & 1496.39 & 1496.445 & 1497.286\end{array}$ $\begin{array}{llllllll}1497.233 & 1497.659 & 1499.079 & 1497.108 & 1496.373 & 1496.43 & 1497.276\end{array}$ $\begin{array}{rrrrrrrr}1497.243 & 1497.501 & 1499.084 & 1496.898 & 1496.354 & 1496.367 & 1497.269 \\ 1497.238 & 1497394 & 1499119 & 1497.01 & 1496375 & 1496.423 & 1497.298\end{array}$ $\begin{array}{llllllllllllll}1497.238 & 1497.394 & 1499.119 & 1497.01 & 1496.375 & 1496.423 & 147.298\end{array}$ $\begin{array}{lllllllll}1497.212 & 1497.631 & 1499.051 & 1497.077 & 1496.33 & 1496.409 & 1497.243\end{array}$ $\begin{array}{llllllllll}1497.208 & 1497.604 & 1499.068 & 1497.143 & 1496.337 & 1496.388 & 1497.257\end{array}$ $\begin{array}{lllllllll}1497.191 & 1497.366 & 1499.004 & 1497.129 & 1496.297 & 1496.407 & 1497.219\end{array}$ $\begin{array}{lllllllll}1497.196 & 1497.375 & 1499.046 & 1497.173 & 1496.295 & 1496.351 & 1497.24\end{array}$ $\begin{array}{llllllll}1497.187 & 1497.38 & 1499.042 & 1497.189 & 1496.297 & 1496.353 & 1497.245\end{array}$ $\begin{array}{lllllll}1497.177 & 1497.356 & 1499.009 & 1497.073 & 1496.271 & 1496.376 & 1997.219\end{array}$ $\begin{array}{lllllllll}1497.17 & 1497.34 & 149.002 & 1497.049 & 1496.257 & 1496.369 & 1497.214\end{array}$ $\begin{array}{llllllllll}1497.138 & 1497.29 & 1499.058 & 1497.045 & 1496.211 & 1496.353 & 1497.159\end{array}$ $\begin{array}{lllllllll}1497.259 & 1497.21 & 1498.876 & 1496.739 & 1496.15 & 1496.296 & 1497.152\end{array}$ $\begin{array}{llllllll}1497.401 & 1497.196 & 1498.932 & 1496.825 & 1496.152 & 1496.264 & 1497.154\end{array}$ $\begin{array}{llllllll}1497.373 & 1497.165 & 1498.885 & 1496.874 & 1496.112 & 1496.268 & 1497.128\end{array}$ $\begin{array}{lllllllll}1497.34 & 1497.158 & 1498.909 & 1496.933 & 1496.095 & 1496.228 & 1497.133\end{array}$ $\begin{array}{llllllll}1497.308 & 1497.168 & 1498.923 & 1497.003 & 1496.095 & 1496.207 & 1497.14\end{array}$ $\begin{array}{lllllll}1497.273 & 1497.156 & 1498.899 & 1497.024 & 1496.076 & 1496.221 & 1497.118 \\ 1497.264 & 1497.186 & 1498.962 & 1497.091 & 1496.088 & 1496.162 & 1497.149\end{array}$
$1499.521 \quad 1499.429$

$\begin{array}{ll}1499.524 & 1499.416 \\ 1499.521 & 1499.404\end{array}$

149.5211499 .404

1499.5771499 .474

$1499.626 \quad 1499.529$

1499.6771499 .577

$1499.687 \quad 1499.555$

$1499.675 \quad 1499.515$

$\begin{array}{rrr}1499.666 & 1499.505 \\ 1499.64 & 1499.46\end{array}$

$1499.64 \quad 1499.46$
$1499.617 \quad 1499.436$

1499.6211499 .469

$1499.598 \quad 1499.426$

1499.5891499 .428

1499.6051499 .474

$1499.607 \quad 1499.462$

$1499.645 \quad 1499.512$

1499.6771499 .543

149.6631499 .495

$1499.647 \quad 1499.481$

$1499.633 \quad 1499.455$

1499.6261499 .448

$1499.642 \quad 1499.481$

$1499.617 \quad 1499.426$

$\begin{array}{lll}1499.584 & 1499.39 \\ 1499.568 & 1499.385\end{array}$

$\begin{array}{lll}1499.568 & 1499.385 \\ 1499.524 & 1499.333\end{array}$

$\begin{array}{lll}1499.524 & 1499.333 \\ 1499.519 & 1499.366\end{array}$

$1499.496 \quad 1499.335$

$1499.47 \quad 1499.309$

$1499.456 \quad 1499.318$

1499.452 1499.326

$1499.438 \quad 1499.311$

$1499.424 \quad 1499.297$

$\begin{array}{rr}1499.459 & 1499.34 \\ 1499.433 & 1499.287\end{array}$

$\begin{array}{rr}149.433 & 1499.287 \\ 1499.41 & 1499.275\end{array}$

1499.4191499 .304

$1499.384 \quad 1499.249$

$1499.389 \quad 1499.285$

$1499.394 \quad 1499.292$

$1499.375 \quad 1499.254$

$1499.368 \quad 1499.251$
$1499.324 \quad 1499.194$

$\begin{array}{lll}1499.291 & 1499.163\end{array}$

$\begin{array}{r}1499.27 \quad 1499.146 \\ \hline\end{array}$

$1499.273 \quad 1499.177$

$1499.247 \quad 1499.144$

$1499.242 \quad 1499.16$

$1499.252 \quad 1499.187$

$\begin{array}{ll}1499.242 & 1499.165 \\ 1499.268 & 1499.218\end{array}$ 
$\begin{array}{llllllll}1497.191 & 1497.244 & 1498.927 & 1497.175 & 1496.078 & 1496.191 & 1497.137\end{array}$ $\begin{array}{lllllllll}1497.194 & 1497.093 & 1498.96 & 1497.201 & 1496.086 & 1496.162 & 1497.149\end{array}$ $\begin{array}{llllllll}1497.182 & 1497.128 & 1498.955 & 1497.213 & 1496.086 & 1496.16 & 1497.152\end{array}$ $\begin{array}{rrrrrrr}1497.173 & 1497.284 & 1498.934 & 1497.206 & 1496.081 & 1496.181 & 1497.137 \\ 1497.17 & 1497.361 & 1498.923 & 1497.194 & 1496.069 & 1496.186 & 1497.126\end{array}$ $\begin{array}{rrrrrrr}1497.17 & 1497.361 & 1498.923 & 1497.194 & 1496.069 & 1496.186 & 1497.126 \\ 1497.161 & 1497.368 & 1498.937 & 1497.213 & 1496.081 & 1496.17 & 1497.14\end{array}$ $\begin{array}{llllllll}1497.157 & 1497.345 & 1498.925 & 1497.208 & 1496.069 & 1496.167 & 1497.13\end{array}$ $\begin{array}{llllllll}1497.164 & 1497.121 & 1498.972 & 1497.238 & 1496.081 & 1496.125 & 1497.152 \\ 1497.175 & 1497.189 & 1499.046 & 1497.318 & 1496.124 & 1496144 & 1497.207\end{array}$ $\begin{array}{lllllllll}1497.168 & 1497.361 & 1499.028 & 1497.329 & 1496.131 & 1496.186 & 1497.207\end{array}$ $\begin{array}{lllllllll}1497.161 & 1497.543 & 1499.016 & 1497.28 & 1496.126 & 1496.193 & 1497.197\end{array}$ $\begin{array}{llllllll}1497.159 & 1497.489 & 1499.028 & 1497.301 & 1496.14 & 1496.202 & 1497.204\end{array}$ $\begin{array}{llllllll}1497.152 & 1497.431 & 1499.009 & 1497.294 & 1496.135 & 1496.212 & 1497.19\end{array}$ $\begin{array}{lllllll}1497.168 & 1497.251 & 1499.068 & 1497.343 & 1496.164 & 1496.172 & 1497.226\end{array}$ $\begin{array}{rllllll}1497.187 & 1497.317 & 1499.138 & 1497.425 & 1496.216 & 1496.21 & 1497.276 \\ 1497.18 & 1497.452 & 1499.096 & 1497.428 & 1496.219 & 1496257 & 1497.257\end{array}$ $\begin{array}{llllllll}1497.18 & 1497.452 & 1499.096 & 1497.428 & 1496.219 & 1496.257 & 1497.257\end{array}$ $\begin{array}{lllllllll}1497.157 & 1497.638 & 1499.142 & 1497.444 & 1496.245 & 1496.264 & 1497.276\end{array}$ $\begin{array}{rrrrrrr}1497.229 & 1497.648 & 1499.096 & 1497.425 & 1496.245 & 1496.285 & 1497.264 \\ 1497.236 & 1497.62 & 1499.06 & 1497.43 & 1966254 & 1496285 & 1497271\end{array}$ $\begin{array}{llllllll}1497.236 & 1497.62 & 1499.06 & 1497.43 & 1496.254 & 1496.285 & 1497.271 \\ 1497238 & 1497.56 & 1499.023 & 147.246 & 1496276 & 149628 & 1497.288\end{array}$ $\begin{array}{lllllll}1497.238 & 1497.569 & 1499.023 & 1497.446 & 1496.276 & 1496.28 & 1497.288 \\ 1497231 & 1497.576 & 1499018 & 1497.439 & 1496268 & 1496311 & 1497.276\end{array}$ $\begin{array}{llllllll}1497.231 & 1497.576 & 1499.018 & 1497.439 & 1496.268 & 1496.311 & 1497.276 \\ 1497.219 & 1497.557 & 1499.023 & 1497.446 & 1496.278 & 1496.301 & 1497.286\end{array}$ $\begin{array}{llllllll}1497.219 & 1477.557 & 1499.023 & 1497.446 & 1496.278 & 1496.301 & 1497.286 \\ 1497.215 & 1497.534 & 1498.983 & 1497.467 & 1496.297 & 1496.311 & 1497.298\end{array}$ $\begin{array}{llllllll}1497.225 & 1499.734 & 1498.983 & 1479.467 & 1496.297 & 1496.311 & 1497.298 \\ 1497.229 & 1497.557 & 1488.972 & 1497.421 & 1496.273 & 1496.343 & 1497.264\end{array}$ $\begin{array}{llllllll}1497.166 & 1497.459 & 1498.775 & 1497.432 & 1496.285 & 1496.315 & 1497.276\end{array}$ $\begin{array}{llllllll}1497.264 & 1497.436 & 1498.941 & 1497.467 & 1496.318 & 1496.304 & 1497.314\end{array}$ $\begin{array}{llllllll}1497.271 & 1497.527 & 1499.093 & 1497.442 & 1496.297 & 1496.334 & 1497.272\end{array}$ $\begin{array}{lllllllll}1497.259 & 1497.604 & 1499.044 & 1497.402 & 1496.276 & 1496.348 & 1497.248\end{array}$ $\begin{array}{lllllllll}1497.266 & 1497.611 & 1499.037 & 1497.393 & 1496.271 & 1496.315 & 1497.185\end{array}$ $\begin{array}{llllllll}1497.387 & 1497.622 & 1499.039 & 1497.393 & 1496.254 & 1496.322 & 1497.2\end{array}$ $\begin{array}{llllllll}1497.424 & 1497.503 & 1498.796 & 1497.404 & 1496.249 & 1496.311 & 1497.264\end{array}$ $\begin{array}{llllllllll}1497.462 & 1497.366 & 1488.843 & 1497.453 & 1496.276 & 1496.278 & 1497.296\end{array}$ $\begin{array}{llllllllll}1497.429 & 1497.613 & 1499.018 & 1497.437 & 1496261 & 1496329 & 1497.262\end{array}$ $\begin{array}{lllllllll}1497.415 & 1497.613 & 1499.039 & 1497.458 & 1496.271 & 1496.313 & 1497.267\end{array}$ $\begin{array}{llllllll}1497.392 & 1497.604 & 1499.004 & 1497.432 & 1496.247 & 1496.329 & 1497.241\end{array}$ $\begin{array}{llllllll}1497.387 & 1497.366 & 1499.056 & 1497.47 & 1496.268 & 1496.275 & 1497.269\end{array}$ $\begin{array}{lllllllll}1497.378 & 1497.272 & 1499.063 & 1497.5 & 1496.283 & 1496.287 & 1497.284\end{array}$ $\begin{array}{rrrrrrr}1497.364 & 1497.445 & 1499.044 & 1497.491 & 1496.278 & 1496.322 & 1497.269\end{array}$ $\begin{array}{llllllll}1497.354 & 1497.624 & 1499.046 & 1497.49 & 1496.28 & 1496.336 & 1497.264 \\ 1497.343 & 1497.676 & 1499049 & 1497.505 & 149629 & 1496336 & 1497.271\end{array}$

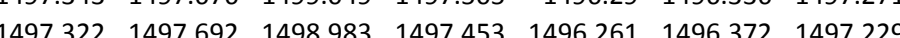
$\begin{array}{llllllllll}1497.315 & 1497347 & 1498.99 & 1497.437 & 1496252 & 1496329 & 1497.221\end{array}$ $\begin{array}{llllllll}1497.31 & 1497.361 & 1499.009 & 1497.451 & 1496.261 & 1496.299 & 1497.233\end{array}$ $\begin{array}{llllllll}1497.308 & 1497.522 & 1499.025 & 1497.474 & 1496.271 & 1496.308 & 1497.243\end{array}$ $\begin{array}{llllllll}1497.285 & 1497.478 & 1498.948 & 1497.423 & 1496.238 & 1496.362 & 1497.197\end{array}$ $\begin{array}{lllllllll}1497.285 & 1497.548 & 1498.974 & 1497.435 & 1496.247 & 1496.332 & 1497.209\end{array}$ $\begin{array}{rrrrrrr}1497.278 & 1497.552 & 1498.96 & 1497.423 & 1496.233 & 1496.336 & 1497.193 \\ 1497.275 & 1497.361 & 1498.958 & 1497.413 & 1496.233 & 1496.306 & 1497.19\end{array}$

1499.3011499 .261 $\begin{array}{rl}1499.284 & 1499.199 \\ 1499.28 & 1499.196\end{array}$

1499.28
1499.2941499 .196
1499.218

$1499.284 \quad 1499.189$ $\begin{array}{ll}1499.296 & 1499.213 \\ 1499.303 & 1499.213\end{array}$ $1499.291 \quad 1499.187$ 1499.2771499 .175
$1499284 \quad 1499.192$ $1499.277 \quad 1499.18$ $1499.294 \quad 1499.218$ $1499.345 \quad 1499.294$ $1499.356 \quad 1499.273$ 1499.3491499 .249 $1499.359 \quad 1499.256$ 1499.3491499 .227 1499.377
$1499.433 \quad 1499.354$ 1499.4311499306 $1499.431 \quad 1499302$ $1499.445 \quad 1499.323$ $1499.433 \quad 1499.297$ $1499.435 \quad 1499.299$ $1499.449 \quad 1499.316$ 149.441499 .292 $1499.456 \quad 1499.314$ $1499.426 \quad 1499.256$ 1499.431 1499.282 1499.459 1499.347 $1499.431 \quad 1499.27$ $\begin{array}{lll}1499.401 & 1499.225 \\ 149.387 & 1499.23\end{array}$ $1499.387 \quad 1499.23$ $149.363 \quad 1499.223$ $1499.382 \quad 1499.259$ 1499.3681499232 $1499.356 \quad 1499.215$ 1499.359 1499.235 $1499.338 \quad 1499.18$ 1499.3611499 .249 1499.3771499 .266 $1499363 \quad 1499.232$ $1499331 \quad 1499.175$ $1499.319 \quad 1499.18$ $\begin{array}{ll}1499.326 & 1499.208 \\ 1499.335 & 1499.225\end{array}$ 1499.2981499 .146 149.3031499 .192 $1499.282 \quad 1499.16$ 
$\begin{array}{llllllll}1497.264 & 1497.408 & 1499.032 & 1497.467 & 1496.261 & 1496.278 & 1497.231\end{array}$ $\begin{array}{llllllll}1497.303 & 1497.527 & 1499.205 & 1497.6 & 1496.347 & 1496.36 & 1497.305\end{array}$ $\begin{array}{lllllll}1497.257 & 1497.655 & 1499.397 & 1497.516 & 1496.295 & 1496.369 & 1497.181 \\ 1497.289 & 1497.699 & 1499.397 & 1497.542 & 1496335 & 1496.355 & 1497.279\end{array}$ $\begin{array}{llllllll}1497.289 & 1497.699 & 1499.397 & 1497.542 & 1496.335 & 1496.355 & 1497.279\end{array}$ $\begin{array}{llllllll}1497.415 & 1497.704 & 1499.336 & 1497.575 & 1496.359 & 1496.36 & 1497.303 \\ 1497.622 & 1497.727 & 1499.234 & 1497.556 & 1496349 & 1496398 & 1497.262\end{array}$ $\begin{array}{llllllll}1497.622 & 1497.727 & 1499.234 & 1497.556 & 1496.349 & 1496.398 & 1497.262\end{array}$ $\begin{array}{llllllll}1497.648 & 1497.683 & 1499.021 & 1497.474 & 1496.302 & 1496.454 & 1497.236 \\ 1497.676 & 1497.587 & 1499.084 & 1497.362 & 1496.23 & 1496.407 & 1497.164\end{array}$ $\begin{array}{llllllll}1497.783 & 1497.482 & 1499.077 & 1497.269 & 1496.159 & 1496.358 & 1497.101\end{array}$ $\begin{array}{lllllllll}1497.732 & 1497.424 & 1498.799 & 1497.245 & 1496.121 & 1496.318 & 1497.085\end{array}$ $\begin{array}{llllllll}1497.664 & 1497.419 & 1498.815 & 1497.264 & 1496.102 & 1496.287 & 1497.087\end{array}$ $\begin{array}{llllllll}1497.613 & 1497.412 & 1498.761 & 1497.229 & 1496.062 & 1496.28 & 1497.056\end{array}$ $\begin{array}{llllllll}1497.331 & 1497.119 & 1498.838 & 1497.276 & 1496.067 & 1496.195 & 1497.092\end{array}$ \begin{tabular}{llllllllll}
1497.166 & 1497.189 & 1498.93 & 1497.369 & 1496.114 & 1496.144 & 1497.152 \\
\hline
\end{tabular} $\begin{array}{llllllllll}1497.334 & 1497.361 & 1498.916 & 1497.397 & 1496.119 & 1496.195 & 1497.152\end{array}$ $\begin{array}{llllllll}1497.441 & 1497.524 & 1498.967 & 1497.453 & 1496.154 & 1496.21 & 1497.183\end{array}$ $\begin{array}{llllllll}1497.385 & 1497.492 & 1498972 & 1497.47 & 1496.166 & 1496.233 & 1497.188\end{array}$ $\begin{array}{lllllllll}1497.191 & 1497.317 & 1499.007 & 1497.498 & 1496.19 & 1496.226 & 1497.209\end{array}$ $\begin{array}{llllllll}1497.103 & 1497.331 & 1499.023 & 1497.518 & 1496.209 & 1496.231 & 1497.224\end{array}$ $\begin{array}{lllllllll}1497.233 & 1497.468 & 1498.96 & 1497.484 & 1496.197 & 1496.296 & 1497.195\end{array}$ $\begin{array}{lllllllll}1497.361 & 1497.585 & 1498.986 & 1497.467 & 1496.197 & 1496.287 & 1497.19\end{array}$ $\begin{array}{lllllll}1497.368 & 1497.601 & 1499.002 & 1497.498 & 1496.216 & 1496.282 & 1497.209 \\ 1497.371 & 1497.597 & 1498.976 & 1497.479 & 1496202 & 1496315 & 149.788\end{array}$ $\begin{array}{llllllllll}1497.208 & 1497.44 & 1498.997 & 1497.488 & 1496223 & 1496.3282 & 1497.188\end{array}$ $\begin{array}{lllllllll}1497.18 & 1497.417 & 1498.997 & 1497.493 & 1496.221 & 1496.292 & 1497.209\end{array}$ $\begin{array}{llllllll}1497.266 & 1497.489 & 1498.965 & 1497.472 & 1496.216 & 1496.32 & 1497.193\end{array}$ $\begin{array}{llllllll}1497.266 & 1497.499 & 1499.051 & 1497.518 & 1496.252 & 1496.273 & 1497.236\end{array}$ $\begin{array}{llllllll}1497.252 & 1497.508 & 1499.084 & 1497.57 & 1496.29 & 1496.278 & 1497.262\end{array}$ $\begin{array}{llllllll}1497.254 & 1497.500 & 1499.044 & 1497.551 & 1496.29 & 1496.334 & 1497.25\end{array}$ $\begin{array}{llllllll}1496.998 & 1497.415 & 1499.03 & 1497.495 & 1496.287 & 1496.339 & 1497.241 \\ 1496.942 & 1497.424 & 1499.032 & 1497.486 & 1496287 & 1496332 & 1497.238\end{array}$ $\begin{array}{llllllllll}1496.942 & 1497.424 & 1499.032 & 1497.486 & 1496.287 & 1496.332 & 1497.238\end{array}$ $\begin{array}{lllllllllll}1497.392 & 1497.627 & 1498.953 & 1497.399 & 1496.275 & 1496.386 & 1497.176\end{array}$ $\begin{array}{lllllllll}1497.443 & 1497.608 & 1498.934 & 1497.385 & 1496.233 & 1496.374 & 1497.162\end{array}$ $\begin{array}{lllllllll}1497.438 & 1497.552 & 1498.885 & 1497.185 & 1496.202 & 1496.379 & 1497.128\end{array}$ $\begin{array}{lllllllll}1497.203 & 1497.354 & 1498.899 & 1496.991 & 1496.204 & 1496.318 & 1497.126\end{array}$ $\begin{array}{lllllllll}1497.17 & 1497.289 & 1498.916 & 1497.056 & 1496.204 & 1496.287 & 1497.123\end{array}$ $\begin{array}{llllllll}1497.24 & 1497.373 & 1498.876 & 1497.11 & 1496.197 & 1496.313 & 1497.102\end{array}$ $\begin{array}{llllllll}1497.457 & 1497.466 & 1498.768 & 1497.063 & 1496.124 & 1496.322 & 1497.013 \\ 1497.594 & 1497.296 & 1498.953 & 1497.003 & 1496045 & 1496.273 & 1496.98\end{array}$

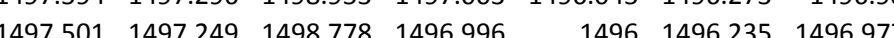
$\begin{array}{lllllllll}1497.294 & 1497.065 & 1498.672 & 1497.005 & 1495.957 & 1496193 & 1496.973\end{array}$ $\begin{array}{lllllllll}1497.119 & 1496.948 & 1498.715 & 1497.063 & 1495.955 & 1496.115 & 1497.001\end{array}$ $\begin{array}{lllllllll}1497.257 & 1497.105 & 1498.705 & 1497.096 & 1495.943 & 1496.118 & 1496.996\end{array}$ $\begin{array}{rrrrrrrr}1497.187 & 1497.196 & 1498.708 & 1497.11 & 1495.926 & 1496.113 & 1496.987 \\ 1497.173 & 1497.233 & 1498.759 & 1497.166 & 1495.938 & 1496.073 & 1497.008\end{array}$ $\begin{array}{lllllllll}1497.173 & 1497.233 & 1498.759 & 1497.166 & 1495.938 & 1496.073 & 1497.008\end{array}$ $\begin{array}{lllllll}1497.159 & 1497.151 & 1498.759 & 1497.185 & 1495.934 & 1496.066 & 1497.004 \\ 1497.157 & 1497.016 & 1498.822 & 1497.243 & 1495.957 & 1496.024 & 1497.035\end{array}$

1499.3011499 .201 $\begin{array}{rl}1499.294 & 1499.175 \\ 149928 & 1499.168\end{array}$

$\begin{array}{rl}1499.28 & 1499.168 \\ 1499.294 & 1499.194\end{array}$

$1499.303 \quad 1499.201$

$1499.324 \quad 1499.23$

1499.4121499 .37

$1499.349-1499.17$

149.3891499 .271

1499.3941499 .249

14993381499.151

1499.2541499 .036

1499.1751498 .981

1499.1351498 .993

1499.1281499 .014

1499.0891498 .981

1499.1871499 .137

$\begin{array}{rr}1499.21 & 1499.153 \\ 1499.238 & 1499.182\end{array}$

$1499.256 \quad 1499.18$

$1499.282 \quad 1499.208$

$1499.305 \quad 1499.22$

$\begin{array}{rr}1499.28 & 1499.176\end{array}$

$1499.28 \quad 1499.139$

$1499.296 \quad 1499.182$

$1499.289 \quad 1499.14$

$1499.354 \quad 1499.218$
$1499347 \quad 1499.199$

$1499.345 \quad 1499.201$

14993171499148

1499.2631499 .093

$1499.224 \quad 1499.041$

$1499.214 \quad 1499.06$

$1499.003 \quad 1498.866$

$1498.97 \quad 1498.87$

$\begin{array}{lll}498.98 & 1498.926\end{array}$

$1498.998 \quad 1498.974$

$\begin{array}{rr}1499.01 & 1498.971 \\ 1499.047 & 1499.022\end{array}$ 


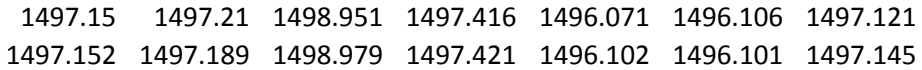
$\begin{array}{lllllll}1497.147 & 1497.314 & 1498.969 & 1497.353 & 1496.119 & 1496.148 & 1497.145\end{array}$ $\begin{array}{lllllll}1497.131 & 1497.473 & 1498.92 & 1497.297 & 1496.093 & 1496.21 & 1497.102\end{array}$ $\begin{array}{lllllll}1497.133 & 1497.485 & 1498.962 & 1497.353 & 1496.124 & 1496.172 & 1497.128 \\ 1497.117 & 1497.515 & 1498.895 & 1497.329 & 14961 & 1496.221 & 1497.087\end{array}$ $\begin{array}{llllllll}1497.117 & 1497.515 & 1498.895 & 1497.329 & 1496.1 & 1496.221 & 1497.087\end{array}$ $\begin{array}{llllllllll}1497.117 & 1497.182 & 1498.927 & 1497.339 & 1496.109 & 1496.158 & 1497.095\end{array}$ $\begin{array}{llllllllll}1497.108 & 1497.307 & 1498.911 & 1497.318 & 1496.102 & 1496.181 & 1497.083\end{array}$ $\begin{array}{llllllll}1497.094 & 1497.447 & 1498.815 & 1497.185 & 1496.048 & 1496.205 & 1497.013\end{array}$ $\begin{array}{llllllll}1497.082 & 1497.473 & 1498.799 & 1497.094 & 1496.029 & 1496.16 & 1496.994\end{array}$ $\begin{array}{llllllll}1497.073 & 1497.223 & 1498.785 & 1497.045 & 1496.012 & 1496.155 & 1496.977\end{array}$ $\begin{array}{llllllll}1497.068 & 1497.23 & 1498.792 & 1497.084 & 1496.01 & 1496.12 & 1496.98\end{array}$ $\begin{array}{lllllll}1497.061 & 1497.2242 & 1498.808 & 1497.143 & 1496.017 & 1496.092 & 1496.992 \\ 1497049 & 1497.24 & 1498.96 & 1497.173 & 1496017 & 196115 & 19698\end{array}$ $\begin{array}{llllllllll}1497.049 & 1497.24 & 1498.796 & 1497.173 & 1496.017 & 1496.115 & 1496.984\end{array}$ $\begin{array}{rrrrrrrrr}1497.035 & 1497.217 & 1498.78 & 1497.15 & 1495.993 & 1496.111 & 1496.968\end{array}$ $\begin{array}{llllllllll}1497.028 & 1497.212 & 1498.782 & 1497.017 & 1495.988 & 1496.097 & 1496.97\end{array}$ $\begin{array}{lllllllll}1497.028 & 1497.212 & 1498.81 & 1496.865 & 1496.005 & 1496.059 & 1496.977\end{array}$ $\begin{array}{llllllll}1497.028 & 1497.251 & 1498.869 & 1496.961 & 1496.038 & 1496.036 & 1497.006\end{array}$ $\begin{array}{lllllllll}1497.017 & 1497.256 & 1498.862 & 1496.956 & 1496.05 & 1496.08 & 1497.006\end{array}$ $\begin{array}{llllllll}1497.012 & 1497.242 & 1498.848 & 1496.968 & 1496.048 & 1496.115 & 1496.982\end{array}$ $\begin{array}{rlllllll}1497.005 & 1497.249 & 1498.857 & 1497.042 & 1496.052 & 1496.12 & 1496.982\end{array}$ $\begin{array}{llllllllll}1496.998 & 1497.235 & 1488.84 & 1497.042 & 1496.052 & 1496.12 & 149692\end{array}$ $\begin{array}{llllllllll}1497.001 & 1497.293 & 1498.925 & 1496.925 & 1496097 & 1496.057 & 1497.011\end{array}$ $\begin{array}{lllllllll}1496.987 & 1497.298 & 1498.899 & 1497.012 & 1496.105 & 1496.118 & 1496.999\end{array}$ $\begin{array}{llllllll}1496.982 & 1497.272 & 1498.873 & 1497.01 & 1496.09 & 1496.16 & 1496.965\end{array}$ $\begin{array}{llllllll}1496.975 & 1497.277 & 1498.885 & 1497.035 & 1496.097 & 1496.148 & 1496.973\end{array}$ $\begin{array}{llllllll}1496.975 & 1497.277 & 1498.89 & 1497.045 & 1496.102 & 1496.139 & 1496.968\end{array}$ $\begin{array}{rlrrrrrr}1496.977 & 1497.319 & 1498.937 & 1497.094 & 1496.121 & 1496.099 & 1497.001 \\ 1496977 & 1497.349 & 1498.958 & 1497.143 & 149615 & 1496106 & 1497.02\end{array}$ $\begin{array}{llllllllllll}149.977 & 1497.39 & 1498.958 & 1497.143 & 1496.15 & 1496.106 & 1497.02\end{array}$ $\begin{array}{lllllllll}1496.956 & 1497.298 & 1498.876 & 1497.07 & 1496.119 & 1496.162 & 1496.996 & 1496.956\end{array}$ $\begin{array}{lllllllll}1496.959 & 1497.3 & 1498.895 & 1497.112 & 1496.124 & 1496.174 & 1496.963\end{array}$ $\begin{array}{llllllll}1496.945 & 1497.282 & 1498.873 & 1496.986 & 1496.119 & 1496.174 & 1496.956\end{array}$ $\begin{array}{llllllll}1496.945 & 1497.265 & 1498.873 & 1496.825 & 1496.112 & 1496.146 & 1496.946\end{array}$ $\begin{array}{lllllllll}1496.942 & 1497.282 & 1498.902 & 1496.898 & 1496.131 & 1496.111 & 1496.963\end{array}$ $\begin{array}{llllllll}1496.91 & 1497.235 & 1498.808 & 1496.87 & 1496.097 & 1496.193 & 1496.915\end{array}$ $\begin{array}{lrrrrrrr}1496.919 & 1497.214 & 1498.81 & 1496.851 & 1496.081 & 1496.177 & 1496.906\end{array}$ $\begin{array}{lllllllll}1496.914 & 1497.2 & 1498.803 & 14968.851 & 1496.081 & 1496.177 & 1496.906\end{array}$ $\begin{array}{lllllllll}1496.905 & 1497.228 & 1498.827 & 1496.989 & 1496.067 & 1496 & 1496.917\end{array}$ $\begin{array}{llllllll}1496.91 & 1497.27 & 1498.881 & 1496.835 & 1496.093 & 1496.05 & 1496.944\end{array}$ $\begin{array}{llllllll}1496.877 & 1497.203 & 1498.766 & 1496.622 & 1496.05 & 1496.144 & 1496.87\end{array}$ $\begin{array}{lllllll}1496.872 & 1497.156 & 1498.736 & 1496.603 & 1496.007 & 1496.132 & 1496.841 \\ 1496.861 & 1497.161 & 149874 & 1496.697 & 1496005 & 1496.094 & 1496.846\end{array}$ $\begin{array}{llllllllll}1496.861 & 1497.161 & 1498.74 & 1496.697 & 1496.005 & 1496.094 & 1496.846\end{array}$ $\begin{array}{lllllll}1496.84 & 1497.116 & 1498.684 & 1496.585 & 1495.964 & 1496.108 & 1496.807 \\ 1496.84 & 1497.128 & 1498.724 & 1496.475 & 1495.962 & 1496.033 & 1496.819\end{array}$

$1499.119 \quad 1499.113$ $\begin{array}{ll}1499.142 & 1499.086 \\ 1499.126 & 1499.031\end{array}$ $1499.131 \quad 1499.026$ $\begin{array}{rr}1499.131 & 1499.026 \\ 1499.18 & 1499.113\end{array}$ 1499.1911499 .103 $1499.226 \quad 1499.132$ $1499.238 \quad 1499.125$ $1499.205 \quad 1499.041$ $1499.228 \quad 1499.105$ $\begin{array}{lll}1499.196 & 1499.034 \\ 1499.184 & 1499.048\end{array}$ $1499.196 \quad 1499.062$ 1499.1911499 .046 $1499.124 \quad 1498.931$ $1499.096 \quad 1498.928$ $1499.075 \quad 1498.921$ $1499.07 \quad 1498.938$
1490977149859 1499.0771498 .959
1499.075
1498.957 149.0751498 .957 $1499.052 \quad 1498.938$ $1499.054 \quad 1498.94$ $1499.066 \quad 1498.955$ $1499.103 \quad 1499.012$ $1499.117 \quad 1499.003$ $\begin{array}{rrr}1499.11 & 1498.979 \\ 1499.114 & 1498983\end{array}$ $\begin{array}{llll}1499.114 & 1498.983 \\ 1499.107 & 1498.964\end{array}$ $\begin{array}{lll}1499.107 & 1498.964 \\ 1499.124 & 1498.988\end{array}$ $1499.156 \quad 1499.038$ $1499.161 \quad 1499.022$ $1499.138 \quad 1498.974$ $1499.138 \quad 1498.988$ $1499.135 \quad 1498.98$ $\begin{array}{lll}1499.161 & 1499.031\end{array}$ $1499.173 \quad 1499.014$ $\begin{array}{lll}1499.135 & 1498.957\end{array}$ $1499.133 \quad 1498.98$ $1499.121 \quad 1498.962$ $1499.112 \quad 1498.945$ $\begin{array}{lll}1499.124 & 1498.981 \\ 1499.089 & 1498909\end{array}$ $1499.089 \quad 1498.909$ $1499.066 \quad 1498.905$ $1499.049 \quad 1498.892$ $1499.052 \quad 1498.936$ $1499.082 \quad 1498.974$ $1499.033 \quad 1498.861$ $1498.991 \quad 1498.823$ 1498.9871498 .847 $\begin{array}{lll}1498.947 & 1498.804 \\ 1498.952 & 1498.825\end{array}$ 

$\begin{array}{llllllll}1496.779 & 1497.147 & 1498.705 & 1496.835 & 1495.898 & 1495.932 & 1496.786\end{array}$ $\begin{array}{llllllll}1496.782 & 1497.193 & 1498.754 & 1496.886 & 1495.917 & 1495.899 & 1496.815\end{array}$ $\begin{array}{lllllll}1496.782 & 1497.23 & 1498.787 & 1496.933 & 1495.943 & 1495.918 & 1496.839 \\ 1496.714 & 1497.24 & 1498.724 & 1496.947 & 1459.941 & 1495.599 & 1496.836 \\ 1496777 & 149.24 & 1998.768 & 1469.991 & 1495.95 & 149937 & 149655\end{array}$ $\begin{array}{lllllll}1496.777 & 1497.24 & 1498.768 & 1496.991 & 1495.95 & 1495.937 & 1496.855\end{array}$ $\begin{array}{lllllll}1496.947 & 1497.158 & 1498.731 & 1496.762 & 1495.886 & 1496.028 & 1496.767 \\ 1496.94 & 1497.186 & 1498357 & 1496.645 & 1495.914 & 1495.932 & 1496819\end{array}$ $\begin{array}{lllllll}1496.94 & 1497.186 & 1498.357 & 1496.645 & 1495.914 & 1495.932 & 1496.819 \\ 1496.938 & 1497.198 & 1498.773 & 1496.734 & 1495.929 & 1495.92 & 1496.824\end{array}$ $\begin{array}{lllllllll}1496.914 & 1497.191 & 1498.759 & 1496.809 & 1495.929 & 1495.958 & 1496.815\end{array}$ $\begin{array}{lllllllll}1496.898 & 1497.191 & 1498.773 & 1496.86 & 1495.934 & 1495.967 & 1496.805\end{array}$ $\begin{array}{llllllll}1496.884 & 1497.214 & 1498.796 & 1496.94 & 1495.955 & 1495.956 & 1496.827\end{array}$ $\begin{array}{llllllll}1496.875 & 1497.2 & 1498.789 & 1496.846 & 1495.957 & 1495.977 & 1496.82\end{array}$ $\begin{array}{lllllll}1496.865 & 1497.196 & 1498.803 & 1496.722 & 1495.964 & 1495.963 & 1496.819\end{array}$ $\begin{array}{llllllll}1496.863 & 1497.226 & 1498.845 & 1496.816 & 1496 & 1495.934 & 1496.836\end{array}$ $\begin{array}{llllllllll}1496.844 & 1497.186 & 148.792 & 1496.858 & 1495.983 & 1496.003 & 1496.803\end{array}$ $\begin{array}{llllllll}1496.824 & 1497.175 & 1498.775 & 1496.944 & 1495.976 & 1496.024 & 1496.791\end{array}$ $\begin{array}{llllllll}1496.81 & 1497.149 & 1498.745 & 1496.942 & 1495.96 & 1496.028 & 1496.769\end{array}$ $\begin{array}{llllllll}1496.807 & 1497.135 & 1498.738 & 1496.951 & 1495.953 & 1496.003 & 1496.767\end{array}$ $\begin{array}{llllllll}1496.803 & 1497.156 & 1498.768 & 1496.996 & 1495.96 & 1495.96 & 1496.784\end{array}$ $\begin{array}{llllllll}1496.793 & 1497.13 & 1498.726 & 1496.956 & 1495.941 & 1495.993 & 1496.757\end{array}$ $\begin{array}{lllllll}1496.777 & 1497.102 & 1498.677 & 1496.933 & 1495.912 & 1496.017 & 1496.733\end{array}$ $\begin{array}{llllllll}1496.761 & 1497.091 & 1498.661 & 1496.933 & 1495.9 & 1496.003 & 1496.726 \\ 1496749 & 1497.056 & 1498633 & 1496767 & 1495862 & 1495.984 & 1496700\end{array}$ $\begin{array}{llllllllll}1496.751 & 1497.063 & 1498.665 & 149662 & 1495862 & 1495.916 & 1496.709\end{array}$ \begin{tabular}{llllllllll}
1496.749 & 1497.102 & 1498.722 & 1496.722 & 1495.888 & 1495.866 & 1496.75 \\
\hline
\end{tabular} $\begin{array}{llllllll}1496.742 & 1497.119 & 1498.736 & 1496.821 & 1495.903 & 1495.895 & 1496.757\end{array}$ $\begin{array}{llllllll}1496.744 & 1497.14 & 1498.764 & 1496.87 & 1495.919 & 1495.89 & 1496.774\end{array}$ $\begin{array}{llllllll}1496.74 & 1497.151 & 1498.771 & 1496.93 & 1495.934 & 1495.909 & 1496.783\end{array}$ $\begin{array}{llllllll}1496.709 & 1497.112 & 1498.682 & 1496.912 & 1495.907 & 1495.991 & 1496.731\end{array}$ $\begin{array}{llllllll}1496.74 & 1497.142 & 1498.768 & 1496.956 & 1495.929 & 1495.92 & 1496.76 \\ 1496744 & 1497.172 & 1498808 & 1497.026 & 1495.953 & 1495.904 & 1496.784\end{array}$ $\begin{array}{lllllllll}1496.744 & 1497.172 & 1488.808 & 1497.026 & 1495.929 & 1495.92 & 1496.76 & 1496.904 & 1496.784\end{array}$ $\begin{array}{lllllllll}1496.716 & 1497.196 & 1498.71 & 1497.052 & 1495.991 & 1495.89 & 1496.824\end{array}$ $\begin{array}{llllllll}1496.723 & 1497.161 & 1498.733 & 1497.054 & 1495.962 & 1495.979 & 1496.779\end{array}$ $\begin{array}{llllllll}1496.721 & 1497.137 & 1498.619 & 1496.893 & 1495.95 & 1495.967 & 1496.764\end{array}$ $\begin{array}{llllllll}1496.714 & 1497.121 & 1498.743 & 1496.734 & 1495.945 & 1495.951 & 1496.757\end{array}$ $\begin{array}{lllllllll}1496.709 & 1497.114 & 1498.75 & 1496.774 & 1495.95 & 1495.939 & 1496.748\end{array}$ $\begin{array}{llllllll}1496.807 & 1497.105 & 1498.717 & 1496.811 & 1495.929 & 1495.986 & 1496.719\end{array}$ $\begin{array}{lllllll}1497.019 & 1497.081 & 1498.993 & 1496.851 & 1495.922 & 1495.956 & 1500.37\end{array}$ $\begin{array}{lllllllll}1496.956 & 1497.051 & 1488.976 & 1496.893 & 1495.922 & 1495.956 & 1500.37\end{array}$ $\begin{array}{llllllllll}1496.886 & 1497.023 & 1498.665 & 1496.918 & 1495.867 & 1495902 & 1496961\end{array}$ $\begin{array}{llllllll}1496.782 & 1497.037 & 1498.694 & 1496.975 & 1495.877 & 1495.876 & 1496.858\end{array}$ $\begin{array}{lllllllll}1496.875 & 1497.056 & 1498.698 & 1497.019 & 1495.893 & 1495.892 & 1496.817\end{array}$ $\begin{array}{llllllll}1496.898 & 1497.056 & 1498.757 & 1497.021 & 1495.862 & 1495.925 & 1496.745\end{array}$ $\begin{array}{llllllll}1496.844 & 1497.079 & 1498.708 & 1497.063 & 1495.891 & 1495.942 & 1496.774\end{array}$ $\begin{array}{rrrrrrr}1496.835 & 1497.095 & 1498.743 & 1496.963 & 1495.91 & 1495.92 & 1496.786 \\ 1496.833 & 1497.128 & 1498.799 & 1496.86 & 1495.945 & 1495.897 & 1496.819\end{array}$

1498.9661498 .861 $\begin{array}{lll}1498.956 & 1498.837 \\ 1498.921 & 1498.794\end{array}$

$1498.907 \quad 1498.794$

1498.9051498 .802

$1498.919 \quad 1498.837$

$1498.952 \quad 1498.885$

$\begin{array}{lll}1498.982 & 1498.912\end{array}$

$\begin{array}{lll}1498.975 & 1498.892 \\ 1498.996 & 1498924\end{array}$

$\begin{array}{lll}1498.924 & 1498.775\end{array}$

$1498961 \quad 1498.861$

$1498.977 \quad 1498.883$

$1498.982 \quad 1498.871$

$1498.984 \quad 1498.864$

$1499.007 \quad 1498.902$

$\begin{array}{lll}1499.007 & 1498.885\end{array}$

$1499.014 \quad 1498.883$

$1499024 \quad 1498876$

1499.0191498873

$1499.01 \quad 1498.85$

$\begin{array}{rr}1498.987 & 1498.83 \\ 1498.973 & 1498.828\end{array}$

$1498.98 \quad 1498.854$

$1498.954 \quad 1498.806$

$\begin{array}{rr}1498.926 & 1498.775 \\ 1498.905 & 1498.77\end{array}$

$\begin{array}{rr}1498.905 & 1498.77 \\ 1498.87 & 1498.735\end{array}$

$\begin{array}{lll}1498.87 & 1498.735 \\ 1498.87 & 1498.751\end{array}$

$1498.905 \quad 1498.818$

$1498.926 \quad 1498.833$

$1498.942 \quad 1498.866$

$1498.961 \quad 1498.869$

$1498.928 \quad 1498.792$

$\begin{array}{rr}1498.952 & 1498.842 \\ 1498.984 & 14989\end{array}$

$\begin{array}{rr}1498.984 & 1498.9 \\ 1498.949 & 1498.77\end{array}$

$\begin{array}{rr}1489.949 & 1498.77 \\ 1499.01 & 1498.967\end{array}$

$1498.975 \quad 1498.833$

1498.9541498 .816

$1498.952 \quad 1498.806$

$1498.947 \quad 1498.804$

$\begin{array}{rll}1498.924 & 1498.797 \\ 149891 & 149875\end{array}$

$1498.91 \quad 1498.775$
1498.882
1498.768

$\begin{array}{lll}1498.882 & 1498.768 \\ 1498.831 & 1498.677\end{array}$

$\begin{array}{lll}1498.831 & 1498.677 \\ 1498.854 & 1498.751\end{array}$

$1498.866 \quad 1498.778$

$1498.884 \quad 1498.797$

$1498.856 \quad 1498.68$

$\begin{array}{lll}1498.891 & 1498.802\end{array}$

$1498.91 \quad 1498.828$
$1498.952 \quad 1498.876$ 
$\begin{array}{llrrrrrr}1496.807 & 1497.196 & 1498.82 & 1496.949 & 1496.021 & 1496.036 & 1496.822\end{array}$

$\begin{array}{llllllll}1496.803 & 1497.175 & 1498.817 & 1496.811 & 1496.021 & 1496.028 & 1496.812\end{array}$

$\begin{array}{lllllllll}1496.791 & 1497.17 & 1498.813 & 1496.862 & 1496.024 & 1496.01 & 1496.817\end{array}$ $\begin{array}{rrrrrrr}1496.77 & 1497.116 & 1498.715 & 1496.863 & 1495.991 & 1496.099 & 1496.738 \\ 1496.765 & 1497.098 & 1498.726 & 1496.876 & 1495.983 & 1496.05 & 1496.731\end{array}$ $\begin{array}{lllllll}1496.742 & 1497.037 & 1498.649 & 1496.865 & 1495.938 & 1496.061 & 1496.678\end{array}$ $\begin{array}{lrrrrrrr}1496.728 & 1497 & 1498.621 & 1496.853 & 1495.905 & 1496.024 & 1496.649\end{array}$ $\begin{array}{lllllllll}1496.716 & 1496.979 & 1488.607 & 1496.855 & 1495.884 & 1495.974 & 1496.643 \\ 1496.707 & 1496.969 & 1498.612 & 1496.886 & 1495.867 & 1495.932 & 1496.64\end{array}$ $\begin{array}{lllllllll}1496.688 & 1496.93 & 1498.56 & 1496.86 & 1495.824 & 1495.953 & 1496.606\end{array}$ $\begin{array}{llllllll}1496.674 & 1496.911 & 1498.546 & 1496.853 & 1495.796 & 1495.937 & 1466.592\end{array}$ $\begin{array}{llllllll}1496.663 & 1496.892 & 1498.528 & 1496.851 & 1495.765 & 1495.92 & 1496.575\end{array}$ $\begin{array}{lllllllll}1496.647 & 1496.881 & 1498.521 & 1496.711 & 1495.744 & 1495.88 & 1496.573\end{array}$ $\begin{array}{lllllll}1496.658 & 1496.916 & 1498.598 & 1496.606 & 1495.758 & 1495.789 & 1496.611\end{array}$ $\begin{array}{llllllllll}1496.642 & 1496.934 & 1498.602 & 1496.685 & 145.7777 & 1495.782 & 1496.628\end{array}$ $\begin{array}{llllllllllll}1496.626 & 1496.925 & 1488.574 & 1496.737 & 1495.758 & 145.819 & 1496.604\end{array}$ $\begin{array}{lllllllll}1496.609 & 1496.897 & 1498.521 & 1496.771 & 1495.713 & 1495.838 & 1496.564\end{array}$ $\begin{array}{llllllll}1496.6 & 1496.895 & 1498.516 & 1496.783 & 1495.698 & 1495.815 & 1496.552\end{array}$ $\begin{array}{rrrrrrr}1496.605 & 1496.925 & 1498.57 & 1496.806 & 1495.71 & 1495.749 & 1496.58\end{array}$ $\begin{array}{llllllll}1496.605 & 1496.974 & 1498.614 & 1496.876 & 1495.736 & 1495.721 & 1496.619 \\ 1496.602 & 1497 & 1498626 & 1496925 & 1495751 & 1495749 & 1496.63\end{array}$ $\begin{array}{rrrrrrr}1496.602 & 1497 & 1498.626 & 1496.925 & 1495.751 & 1495.749 & 1496.63 \\ 1496.598 & 1496.993 & 1498.612 & 1496.933 & 1495.741 & 1495.782 & 1496.619\end{array}$ $\begin{array}{lrrrrrr}1496.598 & 1496.993 & 1498.612 & 1496.933 & 1495.741 & 1495.782 & 1496.619 \\ 1496.591 & 1496.995 & 1498.595 & 1496.94 & 1495.744 & 1495.805 & 1496.609\end{array}$ $\begin{array}{rrrrrrrr}1496.591 & 1496.999 & 1498.595 & 1496.94 & 1495.744 & 1495.805 & 1496.609 \\ 1496.595 & 1497 & 1498.609 & 1496.816 & 1495.746 & 1495.786 & 1496.619\end{array}$ $\begin{array}{llllllll}1496.595 & 1497 & 1498.6093 & 1496.816 & 1459.746 & 1455.786 & 1496.61 \\ 1496.591 & 1497 & 1498.633 & 1496.652 & 1495.753 & 1495.768 & 1496.621\end{array}$ $\begin{array}{lllllllll}1496.6 & 1497.044 & 1498.689 & 1496.741 & 1495.791 & 1495.739 & 1496.657\end{array}$ $\begin{array}{lllllllll}1496.637 & 1497.049 & 1498.691 & 1496.823 & 1495.808 & 1495.782 & 1496.662\end{array}$ $\begin{array}{llllllll}1496.626 & 1497.03 & 1498.661 & 1496.848 & 1495.798 & 1495.824 & 1496.638\end{array}$ $\begin{array}{llllllll}1496.626 & 1497.049 & 1498.686 & 1496.905 & 1495.82 & 1495.824 & 1496.652\end{array}$ $\begin{array}{llllllll}1496.593 & 1496.986 & 1498.588 & 1496.842 & 1495.77 & 1495.876 & 1496.585\end{array}$ $\begin{array}{lllllllll}1496.595 & 1497.004 & 1498.64 & 1496.872 & 1495.786 & 4495.798 & 1496.607\end{array}$ $\begin{array}{lllllllll}1496.588 & 1497.004 & 1498.64 & 1466.902 & 1495.789 & 1495.796 & 1496.611\end{array}$ $\begin{array}{lllllllll}14966.574 & 1496.967 & 1498.607 & 1496.564 & 1495.774 & 1495819 & 1496.583\end{array}$ $\begin{array}{lllllllll}1496.572 & 1496.969 & 1498.621 & 1496.498 & 1495.784 & 1495.805 & 1496.59\end{array}$ $\begin{array}{llllllll}1496.565 & 1496.953 & 1498.612 & 1496.412 & 1495.779 & 1495.803 & 1496.576\end{array}$ $\begin{array}{llllllll}1496.563 & 1496.958 & 1498.644 & 1496.328 & 1495.793 & 1495.777 & 1496.588\end{array}$ $\begin{array}{llllllll}1496.567 & 1496.997 & 1498.696 & 1496.412 & 1495.834 & 1495.742 & 1496.619\end{array}$ $\begin{array}{llllllll}1496.551 & 1496.988 & 1498.677 & 1496.524 & 1495.839 & 1495.812 & 1496.609\end{array}$ $\begin{array}{llllllll}1496.546 & 1496.974 & 1488.673 & 1496.601 & 1495.839 & 1495.833 & 1496.592\end{array}$ $\begin{array}{llllllllllll}1496.495 & 1496.897 & 1498.516 & 1496.571 & 1495.789 & 145.933 & 1496.592 & 149.518\end{array}$ $\begin{array}{llllllllll}1496.509 & 1496755 & 1498.427 & 1496.164 & 1495.653 & 1495 & 1463 & 1496.418\end{array}$ $\begin{array}{llllllll}1496.507 & 1496.748 & 1498.443 & 1496.249 & 1495.639 & 1495.718 & 1496.427\end{array}$ $\begin{array}{llllllll}1496.486 & 1496.718 & 1498.399 & 1496.272 & 1495.603 & 1495.735 & 1496.399\end{array}$ $\begin{array}{llllllll}1496.472 & 1496.694 & 1498.39 & 1496.274 & 1495.563 & 1495.699 & 1496.41\end{array}$ $\begin{array}{llllllll}1496.458 & 1496.704 & 1498.392 & 1496.342 & 1495.556 & 1495.652 & 1496.406\end{array}$ $\begin{array}{lllllll}1496.439 & 1496.701 & 1498.376 & 1496.393 & 1495.527 & 1495.655 & 1496.394 \\ 1496.444 & 1496.767 & 1498.462 & 1496.508 & 1495.556 & 1495.58 & 1496.401\end{array}$

$1499.007 \quad 1498.926$ 1499.0311498 .924

1499.0281498 .892

$1499.024 \quad 1498.852$

$1499.01 \quad 1498.837$

1499.0191498 .861

$1498.961 \quad 1498.747$

1498.9451498 .763

$1498.826 \quad 1498.67$

$1498.81 \quad 1498.675$

$\begin{array}{lll}1498.768 & 1498.639 \\ 1498742 & 1498627\end{array}$

1498.7191498 .605

$1498.703 \quad 1498.608$

$1498.733 \quad 1498.668$

1498.7541498 .694
1498.747
1498.665

$\begin{array}{llll}1498.747 & 1498.665 \\ 1498.726 & 1498.624\end{array}$

$\begin{array}{rr}1498.726 & 1498.61\end{array}$

$\begin{array}{ll}1498.696 & 1498.608 \\ 1498.719 & 1498.658\end{array}$

$1498.754 \quad 1498.716$

$\begin{array}{rrr}1498.777 \quad 1498.72 \\ 198977 & 1498696\end{array}$

1498.771498 .696
$149877 \quad 1498.682$

$\begin{array}{lll}1498.77 & 1498.682 \\ 1498.78 & 1498.684\end{array}$

1498.781498 .684
1498789
1498687

$1498.833 \quad 1498.744$

$1498.847 \quad 1498.742$

$1498.833 \quad 1498.708$

$1498.849 \quad 1498.739$

$\begin{array}{lll}1498.789 & 1498.629 \\ 148805 & 149889\end{array}$

$\begin{array}{ll}1498.805 & 1498.689 \\ 1498.808 & 1498.687\end{array}$

1498.8081498 .687
1498.787
1498.644

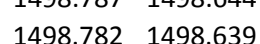

1498.7891498 .658

$1498.784 \quad 1498.634$

$1498.796 \quad 1498.663$

$1498.835 \quad 1498.715$

$1498.842 \quad 1498.699$

$\begin{array}{lll}1498.835 & 1498.689 \\ 1498.775 & 1498.572\end{array}$

1498.775
$1498.666 \quad 1498.44$

$1498.621 \quad 1498.443$

$1498.612 \quad 1498.483$

$1498.577 \quad 1498.455$

$1498.545 \quad 1498.44$

$1498.549 \quad 1498.479$

$\begin{array}{ll}1498.526 & 1498.447 \\ 1498568 & 1498538\end{array}$ 
$\begin{array}{llllllll}1496.435 & 1496.832 & 1498.509 & 1496.624 & 1495.592 & 1495.589 & 1496.442 \\ 1496.425 & 1496.82 & 1498.49 & 1496.592 & 1495573 & 1495.634 & 1496.422\end{array}$

$\begin{array}{lllllllll}1496.432 & 1496.82 & 1498.542 & 1496.659 & 1495.608 & 1495.608 & 1496.422\end{array}$

$\begin{array}{llllllllll}1496.414 & 1496.836 & 1498.49 & 1496.655 & 1495.587 & 1495.636 & 1496.432\end{array}$ $\begin{array}{lllllllll}1496.421 & 1496.862 & 1498.537 & 1496.702 & 1495.606 & 1495.603 & 1496.454\end{array}$ $\begin{array}{llllllll}1496.428 & 1496.902 & 1498.584 & 1496.755 & 1495.634 & 1495.594 & 1496.485\end{array}$ $\begin{array}{llllllll}1496.414 & 1496.876 & 1498.551 & 1496.512 & 1495.637 & 1495.643 & 1496.475\end{array}$ $\begin{array}{llllllll}1496.404 & 1496.843 & 1498.525 & 1496.349 & 1495.622 & 1495.652 & 1496.451\end{array}$ $\begin{array}{lllllll}1496.395 & 1496.825 & 1498.521 & 1496.403 & 1495.62 & 1495.657 & 1496.432\end{array}$ $\begin{array}{llllllll}1496.383 & 1496.792 & 1498.486 & 1496.335 & 1495.603 & 1495.657 & 1496.401 \\ 1496381 & 1496.792 & 1498504 & 1496.157 & 1495606 & 1495.612 & 1496.403\end{array}$ $\begin{array}{llllllllll}1496.374 & 1496.811 & 1498.539 & 1496.237 & 1495.625 & 1495.605 & 1496.422\end{array}$ $\begin{array}{llllllll}1496.355 & 1496.788 & 1498.504 & 1496.298 & 1495.622 & 1495.627 & 1496.401\end{array}$ $\begin{array}{lllllll}1496.351 & 1496.755 & 1498.476 & 1496.295 & 1495.603 & 1495.65 & 1496.367\end{array}$ $\begin{array}{lllllllll}1496.337 & 1496.748 & 1498.469 & 1496.365 & 1495.599 & 1495.641 & 1496.365\end{array}$ $\begin{array}{llllllll}1496.33 & 1496.72 & 1498.439 & 1496.27 & 1495.575 & 1495.631 & 1496.355\end{array}$ $\begin{array}{llllllll}1496.325 & 1496.718 & 1498.455 & 1496.092 & 1495.575 & 1495.584 & 1496.353\end{array}$ $\begin{array}{lllllllll}1496.311 & 1496.718 & 1498.453 & 1496.148 & 1495.58 & 1495.57 & 1496.351\end{array}$ $\begin{array}{lllllllll}1496.295 & 1496.694 & 1498.42 & 196.148 & 1495.58 & 1495.57 & 1496.351\end{array}$ $\begin{array}{llllllll}1496.269 & 1496641 & 1498.371 & 1496.263 & 1495511 & 1495.575 & 1496.31\end{array}$ $\begin{array}{lllllllll}1496.258 & 1496.61 & 1498.348 & 1496.288 & 1495.478 & 1495.537 & 1496.296\end{array}$ $\begin{array}{lllllllll}1496.258 & 1496.643 & 1498.394 & 1496.333 & 1495.485 & 1495.483 & 1496.293\end{array}$ $\begin{array}{llllllll}1496.367 & 1496.671 & 1498.752 & 1496.351 & 1495.497 & 1495.483 & 1496.298\end{array}$ $\begin{array}{llllllll}1496.328 & 1496.666 & 1498.864 & 1496.37 & 1495.494 & 1495.514 & 1496.296\end{array}$ $\begin{array}{lllllll}1496.304 & 1496.678 & 1498.432 & 1496.426 & 1495.497 & 1495.509 & 1496.296\end{array}$ $\begin{array}{lllllll}1496.286 & 1496.683 & 1498.418 & 1496.468 & 1455.497 & 1495.495 & 1496.298\end{array}$ $\begin{array}{lllllllll}1496.272 & 1496.664 & 1498.39 & 1496.377 & 1455.475 & 1495.49 & 1496.286 \\ 1496.274 & 1496.68 & 1498.509 & 1496.26 & 1495.492 & 1495.464 & 1496291\end{array}$ $\begin{array}{llllllll}1496.286 & 1496.715 & 1498.577 & 1496.37 & 1495.516 & 1495.483 & 1496.31\end{array}$ $\begin{array}{llllllll}1496.328 & 1496.711 & 1498.544 & 1496.452 & 1495.516 & 1495.507 & 1496.329\end{array}$ $\begin{array}{llllllll}1496.316 & 1496.685 & 1498.5 & 1496.484 & 1495.497 & 1495.521 & 1496.322\end{array}$ $\begin{array}{lllllllll}1496.302 & 1496.685 & 1498.439 & 1496.533 & 1495.499 & 1495.518 & 1496.317\end{array}$ $\begin{array}{llllllll}1496.307 & 1496.666 & 1498.394 & 1496.442 & 1495.485 & 1495.514 & 1496.303\end{array}$ $\begin{array}{llllllll}1496.234 & 1496.659 & 1498.408 & 1496.333 & 1495.485 & 1495.481 & 1496.293\end{array}$ $\begin{array}{llllllllll}1496.036 & 1496.687 & 1498.448 & 1496.421 & 1495.500 & 1495.457 & 1496.298\end{array}$ $\begin{array}{llllllll}1496.26 & 1496.655 & 1498.383 & 1496.501 & 1495.482 & 1495.526 & 1496.284\end{array}$ $\begin{array}{lllllllll}1496.246 & 1496.657 & 1498.397 & 1496.543 & 1495.48 & 1495.509 & 1496.279\end{array}$ $\begin{array}{llllllll}1496.227 & 1496.648 & 1498.376 & 1496.557 & 1495.47 & 1495.504 & 1496.276\end{array}$ $\begin{array}{llllllll}1496.227 & 1496.657 & 1498.401 & 1496.592 & 1495.47 & 1495.464 & 1496.272\end{array}$ $\begin{array}{llllllll}1496.234 & 1496.685 & 1498.432 & 1496.643 & 1495.487 & 1495.45 & 1496.281\end{array}$ $\begin{array}{lllllll}1496.227 & 1496.678 & 1498.394 & 1496.617 & 1495.478 & 1495.488 & 1496.291\end{array}$ $\begin{array}{llllllll}1496.227 & 1496.657 & 1498.383 & 1496.554 & 1495.456 & 1495.471 & 1496.274 \\ 1496.176 & 1496587 & 1498.259 & 1496.489 & 1495.409 & 1495.467 & 1496.26\end{array}$ $\begin{array}{llllllllllll}1496.175 & 1496.587 & 1498.259 & 1496.554 & 1495.456 & 1495.471 & 1496.274\end{array}$ $\begin{array}{lllllllll}1496.195 & 1496.615 & 1498.369 & 1496.265 & 1495418 & 1495.415 & 1496.257\end{array}$ $\begin{array}{llllllll}1496.202 & 1496.645 & 1498.418 & 1496.347 & 1495.442 & 1495.392 & 1496.26\end{array}$ $\begin{array}{llllllll}1496.19 & 1496.657 & 1498.413 & 1496.442 & 1495.451 & 1495.434 & 1496.267\end{array}$ $\begin{array}{lllllllll}1496.199 & 1496.68 & 1498.45 & 1496.524 & 1495.47 & 1495.431 & 1496.269\end{array}$ $\begin{array}{lllllllll}1496.167 & 1496.657 & 1498.436 & 1496.536 & 1495.447 & 1495.467 & 1496.262\end{array}$ $\begin{array}{rrrrrrr}1496.204 & 1496.659 & 1498.289 & 1496.573 & 1495.454 & 1495.478 & 1496.265 \\ 1496.23 & 1496.655 & 1498394 & 1496589 & 1495.454 & 1495.453 & 1496.255\end{array}$
$1498.603 \quad 1498.582$ $\begin{array}{ll}1498.628 & 1498.589 \\ 1498.614 & 1498.546\end{array}$

$1498.654 \quad 1498.601$

1498.6381498 .555

$1498.661 \quad 1498.58$

1498.6981498 .634

1498.6961498 .591

$1498.68 \quad 1498.548$

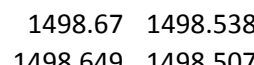

$\begin{array}{lll}1498.649 & 1498.507 \\ 1498.649 & 1498.519\end{array}$

$\begin{array}{lll}1498.649 & 1498.519 \\ 1498.677 & 1498.558\end{array}$

$1498.663 \quad 1498.531$

1498.6351498 .495

1498.6311498 .498

$1498.605 \quad 1498.464$

$\begin{array}{ll}1498.603 & 1498.476 \\ 1498.605 & 1498.486\end{array}$

$\begin{array}{rr}1498.605 & 1498.486 \\ 1498591 & 1498.46\end{array}$

$\begin{array}{rr}1498.591 & 1498.46 \\ 1498.556 & 1498.419\end{array}$

$1498.538 \quad 1498.414$

$1498.501 \quad 1498.38$

$1498.519 \quad 1498.44$

$1498.535 \quad 1498.483$

1498.5421498 .462
$1498.547 \quad 1498.479$

$1498.524 \quad 1498.424$

$1498.547 \quad 1498.464$

$\begin{array}{lll}1498.58 & 1498.51\end{array}$

$1498.584 \quad 1498.49$

1498.5471498 .438

$1498.575 \quad 1498.486$

1498581498.481

$1498.547 \quad 1498.426$

$1498.547 \quad 1498.44$

$1498.533 \quad 1498.426$

$1498.538 \quad 1498.445$

1498.5561498 .479

$\begin{array}{lll}1498.552 & 1498.455 \\ 1498.526 & 1498.409\end{array}$

$1498.47 \quad 1498.373$

$1498.494 \quad 1498.409$

$1498.521 \quad 1498.4$

$1498.538 \quad 1498.455$

$1498.556 \quad 1498.479$

$\begin{array}{rr}1498.533 & 1498.409 \\ 1498.54 & 1498.424\end{array}$ 
$7 / 7 / 1116: 00$ $7 / 7 / 1120: 00$ $7 / 8 / 114: 00$ 7/8/114:00 7/8/11 8:00 7/8/11 16:00 7/8/11 20:00 7/9/11 0:00 7/9/11 4:00 7/9/11 8:00 $7 / 9 / 1116: 00$ 7/9/11 20:00 7/9/11 20:00 7/10/11 4:00 7/10/11 8:00 7/10/11 12:00 $7 / 10 / 1116: 00$ //10/11 20:00 7/111/11 0:00 7/11/11 8:00 7/11/11 12:00 7/11/11 16:00 7/11/11 20:00 7/12/11 0:00 7/12/11 4:00 $7 / 12 / 1112.00$ 7/12/111 16:00 $7 / 12 / 1120: 00$ 7/13/11 0:00 7/13/11 4:00 $7 / 13 / 118: 00$ 7/13/111 12:00 7/13/11 16:00 7/114/11 0:00 7/14/11 4:00 7/14/11 8:00 7/14/11 12:00 7/14/11 16:00 $7 / 14 / 1120: 00$ 7/15/110:00 $7 / 15 / 118.00$ 7/15/11 8:00 7/15/11 16:00 7/15/11 20:00 7/16/11 0:00 7/16/11 4:00 $7 / 16 / 118: 00$
$7 / 16 / 1112: 00$ $\begin{array}{lllllll}1496.251 & 1496.676 & 1498.427 & 1496.638 & 1495.47 & 1495.431 & 1496.26\end{array}$ $\begin{array}{rrrrrrrr}1496.244 & 1496.676 & 1498.406 & 1496.655 & 1495.47 & 1495.464 & 1496.269 \\ 1496.232 & 1496.657 & 1498.38 & 1496.638 & 1495.454 & 1495.483 & 1496257\end{array}$ $\begin{array}{rrrrrrr}1496.232 & 1496.657 & 1498.38 & 1496.638 & 1495.454 & 1495.483 & 1496.25 \\ 1496218 & 1496.655 & 1498.373 & 1496.641 & 1495.451 & 1495.46 & 1496255\end{array}$ $\begin{array}{lllllllll}1496.209 & 1496.613 & 1498.355 & 1496.484 & 1495.435 & 1495.455 & 1496.245\end{array}$ $\begin{array}{llllllll}1496.423 & 1496.641 & 1498.392 & 1496.27 & 1495.447 & 1495.422 & 1496.248\end{array}$ $\begin{array}{lllllllll}1496.423 & 1496.673 & 1498.436 & 1496.354 & 1495.48 & 1495.415 & 1496.26\end{array}$ $\begin{array}{rrrrrrr}1496.4 & 1496.666 & 1498.415 & 1496.431 & 1495.482 & 1495.455 & 1496.265 \\ 1496372 & 1496.65 & 1498.406 & 1496.47 & 1495.478 & 1495.471 & 1496.253\end{array}$ $\begin{array}{llllllll}1496.372 & 1496.65 & 1498.406 & 1496.47 & 1495.478 & 1495.471 & 1496.253\end{array}$ $\begin{array}{lllllll}1496.337 & 1496.638 & 1498.378 & 1496.505 & 1495.473 & 1495.49 & 1496.248 \\ 1496323 & 1496.638 & 1498392 & 1496531 & 1495.466 & 1495.448 & 1492.43\end{array}$ $\begin{array}{llllllll}1496.323 & 1496.638 & 1488.392 & 1496.531 & 1495.466 & 1495.448 & 1496.243\end{array}$ $\begin{array}{lllllllll}1496.314 & 1496.704 & 1498.472 & 1496.643 & 1495.523 & 1495.434 & 1496.272\end{array}$ $\begin{array}{llllllll}1496.3 & 1496.701 & 1498.45 & 1496.662 & 1495.523 & 1495.481 & 1496.281\end{array}$ $\begin{array}{lllllllll}1496.279 & 1496.669 & 1498.397 & 1496.624 & 1495.501 & 1495.507 & 1496.269\end{array}$ $\begin{array}{llllllll}1496.265 & 1496.657 & 1498.38 & 1496.615 & 1495.489 & 1495.504 & 1496.25\end{array}$ $\begin{array}{llllllll}1496.248 & 1496.627 & 1498.345 & 1466.449 & 1495.47 & 1495.483 & 1496.238\end{array}$ $\begin{array}{lllllll}1496.248 & 1496.615 & 1498.366 & 1496.263 & 1495.466 & 1495.453 & 1496.233\end{array}$ $\begin{array}{lllllllllll}1496.241 & 1496.624 & 1498.39 & 1496.321 & 1495.485 & 1495.431 & 1496.233\end{array}$ $\begin{array}{llllllllll}1496.223 & 1496.606 & 1498.359 & 1466.323 & 1455.47 & 1495.481 & 1496.231\end{array}$ $\begin{array}{llllllll}1496.19 & 1496522 & 1498.259 & 1496.256 & 1495.399 & 1495.434 & 1496.217\end{array}$ $\begin{array}{llllllll}1496.181 & 1496.505 & 1498.271 & 1496.197 & 1495.39 & 1495.417 & 1496.195\end{array}$ $\begin{array}{lllllllll}1496.178 & 1496.524 & 1498.32 & 1496.113 & 1495.404 & 1495.377 & 1496.19\end{array}$ $\begin{array}{llllllll}1496.176 & 1496.557 & 1498.355 & 1496.209 & 1495.418 & 1495.366 & 1496.19\end{array}$ $\begin{array}{llllllll}1496.158 & 1496.559 & 1498.338 & 1496.249 & 1495.423 & 1495.401 & 1496.186\end{array}$ $\begin{array}{lllllll}1496.148 & 1496.524 & 1498.294 & 1496.223 & 1495.397 & 1495.417 & 1496.176 \\ 196141 & 1496524 & 1498299 & 1496.279 & 1495394 & 145.401 & 149.169\end{array}$ $\begin{array}{lllllllll}1496.141 & 1496.524 & 1498.299 & 1496.279 & 1495.394 & 1495.401 & 1496.169\end{array}$ $\begin{array}{llllllllll}1496.111 & 1496517 & 1498296 & 1496335 & 1495371 & 1495356 & 1496.159\end{array}$ $\begin{array}{lllllllll}1496.137 & 1496.55 & 1498.331 & 1496.431 & 1495.387 & 1495.347 & 1496.155\end{array}$ $\begin{array}{llllllll}1496.123 & 1496.54 & 1498.294 & 1496.382 & 1495.38 & 1495.396 & 1496.152\end{array}$ $\begin{array}{llllllll}1496.116 & 1496.515 & 1498.278 & 1496.279 & 1495.359 & 1495.37 & 1496.142\end{array}$ $\begin{array}{lllllll}1496.106 & 1496.519 & 1498.289 & 1496.328 & 1495.359 & 1495.373 & 1496.14\end{array}$ $\begin{array}{llllllll}1496.102 & 1496.503 & 1498.266 & 1496.244 & 1495.345 & 1495.361 & 1496.133\end{array}$ $\begin{array}{lllllllll}1496.097 & 1496.512 & 148.264 & 1496.123 & 1495.352 & 1495.33 & 1496.128\end{array}$ $\begin{array}{lllllllll}149.0991 & 1496.545 & 1488.338 & 1496.209 & 1495.352 & 1495.33 & 1496.128 & 149.316 & 1496.133\end{array}$ $\begin{array}{lllllllll}1496.076 & 1496.505 & 1498.271 & 146.253 & 1495.368 & 1495.375 & 1496.126\end{array}$ $\begin{array}{llllllll}1496.071 & 1496.508 & 1498.282 & 1496.309 & 1495.352 & 1495.359 & 1496.116\end{array}$ $\begin{array}{llllllll}1496.069 & 1496.522 & 1498.301 & 1496.365 & 1495.359 & 1495.349 & 1496.116\end{array}$ $\begin{array}{llllllll}1496.067 & 1496.524 & 1498.303 & 1496.379 & 1495.359 & 1495.342 & 1496.111\end{array}$ $\begin{array}{llllllll}1496.076 & 1496.552 & 1498.343 & 1496.386 & 1495.383 & 1495.326 & 1496.121\end{array}$ $\begin{array}{lllllll}1496.067 & 1496.552 & 1498.327 & 1496.41 & 1495.38 & 1495.37 & 1496.121\end{array}$ $\begin{array}{llllllll}1496.06 & 1496.533 & 1498.301 & 1496.403 & 1495.368 & 1495.375 & 1496.111 \\ 1496.055 & 1496.533 & 1498296 & 1496.426 & 1495.371 & 1495.377 & 1496.111\end{array}$ $\begin{array}{llllllllll}149605 & 149651 & 1498273 & 1496309 & 1495356 & 1495355 & 1496105\end{array}$ $\begin{array}{lllllllll}1496.053 & 1496.51 & 1498296 & 1496.165 & 1495361 & 1495333 & 1496.102\end{array}$ $\begin{array}{llllllll}1496.05 & 1496.531 & 1498.327 & 1496.214 & 1495.378 & 1495.319 & 1496.107\end{array}$ $\begin{array}{llllllll}1496.036 & 1496.512 & 1498.289 & 1496.228 & 1495.373 & 1495.37 & 1496.102\end{array}$ $\begin{array}{llllllll}1496.022 & 1496.454 & 1498.217 & 1496.183 & 1495.328 & 1495.345 & 1496.088\end{array}$ $\begin{array}{llllllll}1496.018 & 1496.452 & 1498.231 & 1496.23 & 1495.321 & 1495.337 & 1496.08\end{array}$ $\begin{array}{rrrrrrr}1496.013 & 1496.431 & 1498.21 & 1496.244 & 1495.299 & 1495.316 & 1496.071 \\ 1496.011 & 1496.431 & 1498.231 & 1496.211 & 1495.299 & 1495.293 & 1496.066\end{array}$
$1498.552 \quad 1498.46$

1498.5491498 .445

$1498.521 \quad 1498.426$

1498.5081498 .385

1498.5191498 .407

$1498.552 \quad 1498.46$

$1498.552 \quad 1498.438$

$1498.542 \quad 1498.41$

$1498.531 \quad 1498.412$

$\begin{array}{lll}1498.524 & 1498.409 \\ 1498.561 & 1498.471\end{array}$

1498.5821498 .486

$1498.58 \quad 1498.457$

$1498.547 \quad 1498.397$

$1498.528 \quad 1498.39$

$1498.501 \quad 1498.359$

$1498.498 \quad 1498.369$

$\begin{array}{lll}1498.514 & 1498.395 \\ 1498503 & 1498.371\end{array}$

$\begin{array}{lll}1498.503 & 1498.371 \\ 1498.456 & 1498294\end{array}$

\begin{tabular}{rr}
$1498.419 \quad 1498.27$ \\
\hline
\end{tabular}

$1498.405 \quad 1498.285$

$1498.421 \quad 1498.326$

$1498.447 \quad 1498.366$

$1498.454 \quad 1498.361$

$\begin{array}{lll}1498.424 & 1498.302 \\ 1498.424 & 1498.314\end{array}$

$\begin{array}{lll}1498.424 & 1498.314 \\ 1498.396 & 1498.28\end{array}$

$\begin{array}{ll}1498.396 & 1498.28 \\ 1498.405 & 1498.318\end{array}$

$1498.426 \quad 1498.354$

$1498.414 \quad 1498.316$

$1498.396 \quad 1498.287$

$1498.405 \quad 1498.311$

$1498.384 \quad 1498.273$

$\begin{array}{lll}1498.396 & 1498.304 \\ 1498.421 & 1498.342\end{array}$

1498.4211498 .342

$\begin{array}{lll}1498.417 & 1498.311 \\ 1498.394 & 1498.268\end{array}$

$1498.396 \quad 1498.285$

$1498.405 \quad 1498.309$

$1498.405 \quad 1498.309$

$\begin{array}{lll}1498.431 & 1498.347\end{array}$

$1498.431 \quad 1498.326$

$\begin{array}{ll}1498.412 & 1498.29 \\ 1498.412 & 1498.299\end{array}$

$\begin{array}{ll}1498.412 & 1498.299 \\ 1498.391 & 1498.268\end{array}$

$\begin{array}{lr}1498.398 & 1498.28\end{array}$

$1498.419 \quad 1498.316$

$1498.405 \quad 1498.285$

1498.352 1498.204

1498.3471498 .223

$\begin{array}{lll}1498.321 & 1498.201 \\ 1498.319 & 1498.223\end{array}$ 

Well

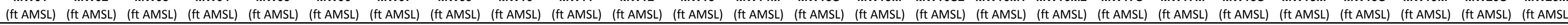

$7 / 16 / 1116: 00$ 7/16/11 20:00 7/17/11 0:00 $7 / 17 / 118: 00$ $7 / 17 / 1112: 00$ 7/17/11112:00 $7 / 17 / 1120: 00$ 7/18/110:00 $7 / 18 / 114: 00$ $7 / 18 / 118: 00$
$7 / 18 / 1112: 00$ $7 / 18 / 1112: 00$ 7/18/111 20:00 7/18/11120:00 7/19/11 4:00 7/19/11 8:00 7/19/11 12:00 $7 / 19 / 1116: 00$ 7/19/11 20:00 7/20/110:00 7/20/11 8:00 7/20/11 12:00 7/20/11 16:00 7/20/11 20:00 $7 / 21 / 110: 00$ 7/21/11 4:00 $7 / 21 / 118.00$ 7/21/11 16:00 7/21/11 20:00 7/22/110:00 7/22/11 4:00 $7 / 22 / 1118: 00$ 7/22/111 12:00 7/22/11 16:00 7/22/11 20:00 7/23/11 4:00 7/23/11 8:00 7/23/11 12:00 7/23/11 16:00 $7 / 23 / 1120: 00$ 7/24/110:00 $7 / 24 / 118.00$ 7/24/11 8:00 7/24/11 16:00 $7 / 24 / 1120: 00$ 7/25/11 0:00 7/25/11 4:00 7/25/11 8:00 $\begin{array}{rrrrrrr}1496.002 & 1496.438 & 1498.235 & 1496.174 & 1495.297 & 1495.286 & 1496.059\end{array}$ $\begin{array}{rrrrrrr}1495.99 & 1496.417 & 1498.198 & 1496.162 & 1495.283 & 1495.3 & 1496.054 \\ 1495.983 & 1496.389 & 1498.177 & 1496.127 & 1495.254 & 1495.276 & 1496042\end{array}$ $\begin{array}{rrrrrrrr}1495.983 & 1496.389 & 1498.177 & 1496.127 & 1495.254 & 1495.276 & 1496.042 \\ 1495.971 & 1496.382 & 1498.175 & 1496.162 & 1495.24 & 1495269 & 1496032\end{array}$ $\begin{array}{lllllllll}1495.962 & 1496.352 & 1498.142 & 1496.064 & 1495.212 & 1495.243 & 1496.023\end{array}$ $\begin{array}{llllllll}1495.96 & 1496.349 & 1498.161 & 1495.924 & 1495.207 & 1495.22 & 1496.011\end{array}$ $\begin{array}{llllllll}1495.955 & 1496.373 & 1498.191 & 1496.003 & 1495.216 & 1495.194 & 1496.009\end{array}$ $\begin{array}{lllllllll}1495.948 & 1496.375 & 1498.177 & 1496.078 & 1495.214 & 1495.236 & 1495.999\end{array}$ $\begin{array}{lllllllll}1495.939 & 1496.359 & 1498.158 & 1496.113 & 1495.197 & 1495.218 & 1495.994\end{array}$ $\begin{array}{lllllll}1495.927 & 1496.356 & 1498.147 & 1496.15 & 1495.185 & 1495.215 & 1495.987\end{array}$ $\begin{array}{llllllll}1495.925 & 1496.342 & 1498.137 & 1496.059 & 1495.174 & 1495.192 & 1495.98 \\ 1495.929 & 149637 & 1498191 & 1495.931 & 1495.188 & 1495.156 & 1495.978\end{array}$ $\begin{array}{lllllllll}1495.927 & 1496.398 & 1498.219 & 1496.006 & 1495.207 & 1495.156 & 1495.978\end{array}$ $\begin{array}{llllllll}1495.918 & 1496.401 & 1498.212 & 1496.09 & 1495.212 & 1495.201 & 1495.978\end{array}$ $\begin{array}{llllllll}1495.911 & 1496.375 & 1498.175 & 1496.116 & 1495.197 & 1495.208 & 1495.973\end{array}$ $\begin{array}{llllllll}1495.911 & 1496.382 & 1498.189 & 1496.167 & 1495.195 & 1495.203 & 1495.966\end{array}$ $\begin{array}{llllllll}1495.892 & 1496.377 & 1498.17 & 1466.186 & 1495.193 & 1495.196 & 1495.961\end{array}$ $\begin{array}{lllllll}1495.969 & 1496.41 & 1498.226 & 1496.171 & 1495.209 & 1495.159 & 1495.966\end{array}$ $\begin{array}{llllllll}1495.992 & 1496.438 & 1498.252 & 1496.188 & 1495.235 & 1495.168 & 1495.978 \\ 1495.988 & 1496.447 & 1498261 & 1496.211 & 1495.245 & 1495.203 & 1495.99\end{array}$

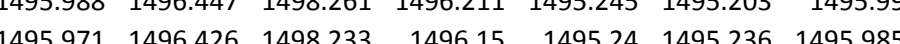
$\begin{array}{lllllll}1495.957 & 1466.417 & 1488.228 & 1496.104 & 1495.242 & 1455.239 & 1495.98\end{array}$ $\begin{array}{llllllll}1495.939 & 1496387 & 1498.207 & 1495.987 & 1495.231 & 1495.222 & 1495.963\end{array}$ $\begin{array}{llllllll}1495.934 & 1496.403 & 1498.247 & 1495.903 & 1495.252 & 1495.194 & 1495.963\end{array}$ $\begin{array}{llllllll}1495.927 & 1496.424 & 1498.273 & 1495.992 & 1495.278 & 1495.196 & 1495.966\end{array}$ $\begin{array}{llllllll}1495.911 & 1496.41 & 1498.247 & 1495.994 & 1495.28 & 1495.243 & 1495.97\end{array}$ $\begin{array}{lllllll}1495.892 & 1496.366 & 1498.198 & 1495.924 & 1495.252 & 1495.234 & 1495.956\end{array}$ $\begin{array}{llllllll}1495.885 & 1496.338 & 1498.17 & 1495.961 & 1495.235 & 1495.218 & 1495.947 \\ 1495.881 & 1496.331 & 1498177 & 1496.029 & 1495.233 & 1495.208 & 1495.939\end{array}$ $\begin{array}{llllllllll}1495.88 & 1468 & 1496342 & 1498.184 & 1496.092 & 14955235 & 1495.208 & 1495.939\end{array}$ $\begin{array}{llllllll}1495.88 & 1496377 & 1498.231 & 1496.172 & 1495.259 & 1495.175 & 1495.939\end{array}$ $\begin{array}{llllllll}1495.911 & 1496.361 & 1498.186 & 1496.09 & 1495.25 & 1495.229 & 1495.937\end{array}$ $\begin{array}{llllllll}1495.89 & 1496.321 & 1498.147 & 1495.957 & 1495.223 & 1495.208 & 1495.927\end{array}$ $\begin{array}{llllllll}1495.873 & 1496.3 & 1498.135 & 1495.971 & 1495.204 & 1495.187 & 1495.918\end{array}$ $\begin{array}{llllllll}1495.864 & 1496.293 & 1498.144 & 1495.919 & 1495.202 & 1495.18 & 1495.913\end{array}$ $\begin{array}{llllllll}1495.862 & 1496.3 & 1498.165 & 1495.812 & 1495.207 & 1495.163 & 1495.908\end{array}$ $\begin{array}{lllllllllll}1455.864 & 1496.338 & 1498.212 & 1455.912 & 1495.233 & 1495.145 & 1455.911\end{array}$ $\begin{array}{lllllllll}1495.834 & 1496.272 & 1498.116 & 1495.919 & 1495.19 & 1495.173 & 1495.894\end{array}$ $\begin{array}{llllllll}1495.825 & 1496.258 & 1498.109 & 1495.966 & 1495.181 & 1495.159 & 1495.884\end{array}$ $\begin{array}{llllllll}1495.815 & 1496.237 & 1498.086 & 1495.987 & 1495.155 & 1495.138 & 1495.877\end{array}$ $\begin{array}{llllllll}1495.815 & 1496.247 & 1498.119 & 1496.025 & 1495.155 & 1495.114 & 1495.872\end{array}$ $\begin{array}{llllllll}1495.813 & 1496.277 & 1498.149 & 1496.039 & 1495.169 & 1495.105 & 1495.87\end{array}$ $\begin{array}{lllllll}1495.792 & 1496.244 & 1498.083 & 1495.992 & 1495.14 & 1495.121 & 1495.865\end{array}$ $\begin{array}{llllllll}1495.785 & 1496.219 & 1498.06 & 1455.982 & 1495.114 & 1495.098 & 1495.853\end{array}$ $\begin{array}{lllllllll}1495769 & 1496191 & 1498044 & 1495.891 & 1495081 & 1495069 & 1495.844\end{array}$ $\begin{array}{llllllll}1495.769 & 1496.198 & 1498.074 & 1495.735 & 1495081 & 1495.053 & 1495.827\end{array}$ $\begin{array}{llllllll}1495.757 & 1496.219 & 1498.093 & 1495.812 & 1495.088 & 1495.06 & 1495.825\end{array}$ $\begin{array}{llllllll}1495.731 & 1496.186 & 1498.016 & 1495.845 & 1495.062 & 1495.058 & 1495.82\end{array}$ $\begin{array}{llllllll}1495.748 & 1496.184 & 1498.081 & 1495.894 & 1495.055 & 1495.001 & 1495.81\end{array}$ $\begin{array}{llllllll}1495.741 & 1496.179 & 1498.055 & 1495.94 & 1495.048 & 1495.008 & 1495.805\end{array}$ $\begin{array}{rrrrrrrr}1495.72 & 1496.17 & 1498.027 & 1495.866 & 1495.033 & 1495.02 & 1495.796 \\ 1495.724 & 1496.181 & 1498.058 & 1495.735 & 1495.038 & 1495.001 & 1495.793\end{array}$
1498.3241498 .232 $\begin{array}{ll}1498.305 & 1498.201 \\ 1498.277 & 1498.168\end{array}$

$\begin{array}{rl}1498.277 & 1498.168 \\ 1498.27 & 1498.177\end{array}$

$1498.242 \quad 1498.144$

$1498.24 \quad 1498.151$

1498.2561498 .192

$1498.256 \quad 1498.18$

$1498.242 \quad 1498.163$

$\begin{array}{lll}1498.235 & 1498.158 \\ 1498.221 & 1498.139\end{array}$

1498.2211498 .139

1498.271498 .211

1498.2821498 .208

1498.2611498 .163

1498.2661498 .175

1498.2561498 .168

1498311498239

14983241498242

14983121498204

$1498.31 \quad 1498.194$

$1498.294 \quad 1498.16$

$1498.312 \quad 1498.19$

$1498.333 \quad 1498.21$

$\begin{array}{rl}1498.296 & 1498.137 \\ 1498.27 & 1498.113\end{array}$

$1498266 \quad 1498.137$

1498.266 1498.15

$1498.291 \quad 1498.194$

1498.2771498 .156

1498.2491498 .11

1498.2281498 .089

$1498.221 \quad 1498.105$
14982281498.117

$1498.259 \quad 1498.172$

$1498.245 \quad 1498.122$

$1498.212 \quad 1498.077$

$1498.196 \quad 1498.074$

$1498.168 \quad 1498.053$

$1498.191 \quad 1498.115$

$1498.166 \quad 1498.048$

$1498.135 \quad 1498.05$

$1498.108 \quad 1498.014$

$1498.115 \quad 1498.034$

$1498.108 \quad 1498.012$

$1498.103 \quad 1498.026$ 
$\begin{array}{lllllll}1495.72 & 1496.242 & 1498.119 & 1495.933 & 1495.083 & 1495.032 & 1495.805 \\ 1495.713 & 1496.226 & 1498.095 & 1495.919 & 1995074 & 1495046 & 1495796\end{array}$ $\begin{array}{llllllll}1495.713 & 1496.226 & 1498.095 & 1495.919 & 1495.074 & 1495.046 & 1495.796 \\ 1495.71 & 1496237 & 1498.114 & 1495973 & 1495083 & 1495048 & 1495796\end{array}$ $\begin{array}{rlllllll}1495.706 & 1496.233 & 1498.102 & 1456.006 & 1495.081 & 1495.044 & 1495.791 \\ 149571 & 1496258 & 1498.142 & 1496.025 & 1495.102 & 1495502 & 1495.798\end{array}$ $\begin{array}{llllllll}1495.71 & 1496.258 & 1498.142 & 1496.025 & 1495.102 & 1495.022 & 1495.798\end{array}$ $\begin{array}{lllllllll}1495.72 & 1496.298 & 1498.186 & 1496.104 & 1495.136 & 1495.025 & 1495.815\end{array}$ $\begin{array}{rrrrrrrr}1495.72 & 1496.31 & 1498.186 & 1496.078 & 1495.155 & 1495.069 & 1495.827 \\ 1495.71 & 1496.284 & 1498154 & 1496013 & 1495.14 & 1495.088 & 1495.822\end{array}$ $\begin{array}{rrrrrrr}1495.71 & 1496.284 & 1498.154 & 1496.013 & 1495.14 & 1495.088 & 1495.822 \\ 1495.706 & 1496.275 & 1498.149 & 1496.043 & 1495.14 & 1495.095 & 1495.82\end{array}$ $\begin{array}{lllllllll}1495.701 & 1496.254 & 1498.121 & 1495.947 & 1495.126 & 1495.081 & 1495.813\end{array}$ $\begin{array}{llllllll}1495.699 & 1496.242 & 1498.13 & 1455.807 & 1495.131 & 1495.069 & 1495.801 \\ 1495.699 & 1496.256 & 1498.151 & 1495.866 & 1495.15 & 1495.062 & 1495803\end{array}$ $\begin{array}{llllllll}1495.682 & 1496.214 & 1498.083 & 1495.875 & 1495.131 & 1495.084 & 1495.789\end{array}$

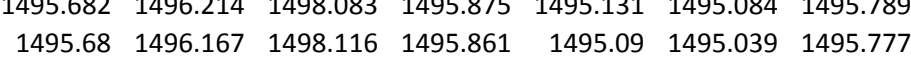
$\begin{array}{llllllll}1495.659 & 1496.13 & 1497.978 & 1495.861 & 1495.052 & 1495.022 & 1495.762\end{array}$ $\begin{array}{lllllllll}1495.652 & 1496.109 & 1497.974 & 1455.877 & 1495.029 & 1495.008 & 1495.755\end{array}$ $\begin{array}{lllllll}1495.65 & 1496.123 & 1498.002 & 1495.924 & 1495.029 & 1494.997 & 1495.748\end{array}$ $\begin{array}{llllllll}1495.657 & 1496.158 & 1498.058 & 1455.959 & 1495.05 & 1494.973 & 1495.75\end{array}$ $\begin{array}{lllllllll}1495.638 & 1496.128 & 1498.997 & 1455.938 & 1495.024 & 1494.976 & 1495.741\end{array}$ $\begin{array}{llllllllll}1495.624 & 1496.09 & 1497.957 & 1495.926 & 1494.972 & 1494.947 & 1495.722\end{array}$ $\begin{array}{llllllll}1495.617 & 1496.072 & 1497.948 & 1495.817 & 1494.955 & 1494.931 & 149571\end{array}$ $\begin{array}{lllllllll}1495.61 & 1496.072 & 1497.962 & 1495.67 & 1494.953 & 1494.917 & 1495.705\end{array}$ $\begin{array}{llllllll}1495.61 & 1496.088 & 1497.978 & 1495.709 & 1494.957 & 1494.91 & 1495.702\end{array}$ $\begin{array}{llllllll}1495.589 & 1496.072 & 1497.943 & 1495.66 & 1494.941 & 1494.926 & 1495.698\end{array}$ $\begin{array}{llllllll}1495.587 & 1496.055 & 1497.939 & 1495.658 & 1494.931 & 1494.905 & 1495.688\end{array}$ $\begin{array}{llllllll}1495.582 & 1496.065 & 1497.953 & 1495.733 & 1494.929 & 1494.903 & 1495.683 \\ 1495.573 & 1496055 & 1497.936 & 1495.77 & 1494.917 & 1494888 & 1495674\end{array}$ $\begin{array}{llllllllll}14595.573 & 1496.065 & 1497.95 & 1495.77 & 1494912 & 1494.867 & 1495.674\end{array}$ $\begin{array}{llllllll}1495.578 & 1496.116 & 1498.011 & 1495.833 & 1494.948 & 1494.851 & 1495.676\end{array}$ $\begin{array}{llllllll}1495.559 & 1496.093 & 1497.96 & 1495.819 & 1494.931 & 1494.896 & 1495.669\end{array}$ $\begin{array}{llllllll}1495.555 & 1496.06 & 1497.925 & 1495.775 & 1494.903 & 1494.872 & 1495.662\end{array}$ $\begin{array}{rrrrrrr}1495.543 & 1496.055 & 1497.92 & 1495.807 & 1494.9 & 1494.87 & 1495.657 \\ 1495.536 & 1496.03 & 1497.899 & 1495.7 & 1494.879 & 1494.853 & 1495.647\end{array}$ $\begin{array}{llllllll}1495.536 & 1496.03 & 1497.899 & 1495.7 & 1494.879 & 1494.853 & 1495.647\end{array}$ $\begin{array}{llllllll}1495.536 & 1496.03 & 1497.918 & 1495.501 & 1494.877 & 1494.841 & 1495.643\end{array}$ $\begin{array}{lllllllll}1455.541 & 1496.005 & 1497.976 & 145.536 & 1494.903 & 1494.823 & 1495.643\end{array}$ $\begin{array}{llllllll}1495.519 & 1496041 & 1497.929 & 1495.67 & 1494.891 & 1494.848 & 1495.631\end{array}$ $\begin{array}{llllllll}1495.513 & 1496.046 & 1497.932 & 1495.726 & 1494.891 & 1494.851 & 1495.628\end{array}$ $\begin{array}{llllllll}1495.51 & 1496.039 & 1497.932 & 1495.665 & 1494.886 & 1494.842 & 1495.624\end{array}$ $\begin{array}{llllllll}1495.515 & 1496.06 & 1497.969 & 1495.537 & 1494.903 & 1494.827 & 1495.624\end{array}$ $\begin{array}{lllllllll}1495.515 & 1496.1 & 1498.023 & 1495.607 & 1494.931 & 1494.818 & 1495.633\end{array}$ $\begin{array}{lllllll}1495.508 & 1496.095 & 1498.006 & 1495.684 & 1494.938 & 1494.884 & 1495.638\end{array}$ $\begin{array}{llllllllll}1495.499 & 1496.065 & 1497.974 & 1495.702 & 1494.924 & 1494.872 & 1495.626\end{array}$ $\begin{array}{llllllllll}149.496 & 1496.069 & 147.985 & 1495.702 & 1494.924 & 1494.872 & 1495.626 \\ 1495.489 & 1496.048 & 1497.953 & 1495.763 & 1494917 & 1494.865 & 1495.6216\end{array}$ $\begin{array}{lllllllll}1495.492 & 1496058 & 1497.974 & 1495.777 & 1494.922 & 1494.856 & 1495.616\end{array}$ $\begin{array}{llllllll}1495.496 & 1496.088 & 1498.009 & 1495.845 & 1494.95 & 1494.851 & 1495.621\end{array}$ $\begin{array}{llllllll}1495.475 & 1496.055 & 1497.946 & 1495.833 & 1494.929 & 1494.87 & 1495.616\end{array}$ $\begin{array}{rrrrrrr}1495.473 & 1496.023 & 1497.904 & 1495.798 & 1494.9 & 1494.837 & 1495.607 \\ 1495.461 & 1496.007 & 1497.88 & 1495.803 & 1494.884 & 1494837 & 1495.602\end{array}$ $\begin{array}{lllllllll}1495.461 & 1496.007 & 1497.88 & 1495.803 & 1494.884 & 1494.837 & 1495.602\end{array}$ $\begin{array}{rrrrrrr}1495.459 & 1495.969 & 1497.85 & 1495.667 & 1494.851 & 1494.804 & 1495.59 \\ 1495.466 & 1495.986 & 1497.918 & 1495.548 & 1494.865 & 1494.79 & 1495.59\end{array}$
$1498.135 \quad 1498.093$ $1498.154 \quad 1498.086$

$\begin{array}{lll}1498.152 & 1498.067\end{array}$

1498.1491498 .058

$\begin{array}{lll}14988.173 & 1498.058 \\ 1498.089\end{array}$

$1498.205 \quad 1498.129$

$\begin{array}{lll}1498.224 & 1498.127\end{array}$

$1498.203 \quad 1498.074$

$\begin{array}{rl}1498.196 & 1498.065 \\ 1498.18 & 1498031\end{array}$

$\begin{array}{rrr}1498.18 & 1498.031 \\ 1498.177 & 1498.036\end{array}$

$1488.187 \quad 1498.065$

$1498.156 \quad 1498$

$\begin{array}{rr}1498.156 & 1498 \\ 1498.11 & 1497.957\end{array}$

$1498.063 \quad 1497.919$

$1498.042 \quad 1497.916$

$\begin{array}{lll}1498.047 & 1497.957 \\ 1498.073 & 1498019\end{array}$

$\begin{array}{lll}1498.073 & 1498.019 \\ 1498.047 & 1497.957\end{array}$

14980121499.912

$\begin{array}{lll}1498.012 & 1497.912 \\ 1498.001 & 1497.916\end{array}$

1497.984 1497.909

$1497.987 \quad 1497.921$

$\begin{array}{lll}1497.998 & 1497.926\end{array}$

$\begin{array}{lll}1497.989 & 1497.909 \\ 1497977 & 1497.792\end{array}$

$\begin{array}{ll}1497.977 & 1497.892 \\ 1497.984 & 1497.912\end{array}$

$\begin{array}{lll}1497.984 & 1497.912 \\ 1497.973 & 1497.897\end{array}$

$\begin{array}{lll}1497.973 & 1497.897 \\ 1497.973 & 1497.909\end{array}$

\begin{tabular}{l}
$1498.017 \quad 1497.974$ \\
\hline
\end{tabular}

$\begin{array}{lll}1498.003 & 1497.926\end{array}$

$1497.975 \quad 1497.878$

$1497.973 \quad 1497.89$

$\begin{array}{ll}1497.952 & 1497.861 \\ 1497.952 & 1497.866\end{array}$

$\begin{array}{lll}1497.952 & 1497.866 \\ 1497.984 & 1497.924\end{array}$

$\begin{array}{lll}1497.984 & 1497.924 \\ 1497.984 & 1497.907\end{array}$

$\begin{array}{rrr}1497.984 & 1497.907 \\ 1497.973 & 1497.88\end{array}$

$1497.975 \quad 1497.888$

$\begin{array}{lll}1497.973 & 1497.885\end{array}$

$1497.991 \quad 1497.907$

$\begin{array}{lll}1498.028 & 1497.959 \\ 148.035 & 147.95\end{array}$

$1498.035 \quad 1497.95$

$\begin{array}{ll}1498.017 & 1497.902 \\ 1498.024 & 1497.916\end{array}$

1498.0011499878

$\begin{array}{lll}1498.001 & 1499.788 \\ 14808 & 147.897\end{array}$

$1498.031 \quad 1497.938$

$1498.008 \quad 1497.883$

$1497.968 \quad 1497.823$

$\begin{array}{lll}1497.952 & 1497.828 \\ 1497.917 & 1997.78\end{array}$

$\begin{array}{ll}1497.917 & 1497.78 \\ 1497.933 & 1497.842\end{array}$ 

$\begin{array}{lllllll}1496.542 & 1495.974 & 1498.193 & 1495.831 & 1494.858 & 1494.839 & 1495.604\end{array}$

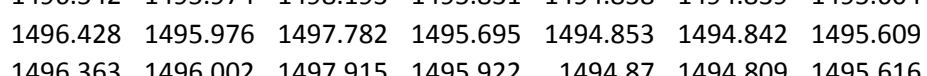
$\begin{array}{llllllll}1496.363 & 1496.002 & 1497.915 & 1455.922 & 1494.87 & 1494.809 & 1495.616\end{array}$

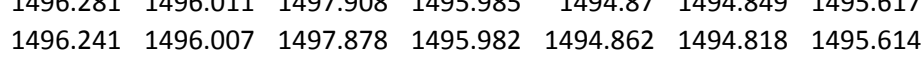
$\begin{array}{rrrrrrr}1496.206 & 1495.988 & 1497.859 & 1495.866 & 1494.846 & 1494.86 & 1495.6\end{array}$ $\begin{array}{lllllll}1496.165 & 1495.976 & 1497.861 & 1495.709 & 1494.846 & 1494.842 & 1495.592 \\ 1496.141 & 1495.995 & 1497.899 & 1495.768 & 1494.858 & 1494.813 & 1495.588\end{array}$ $\begin{array}{lllllllll}1496.106 & 1496.009 & 1497.904 & 1495.856 & 1494.872 & 1494.839 & 1495.592\end{array}$ $\begin{array}{llllllll}1496.078 & 1495.997 & 1497.88 & 1495.884 & 1494.862 & 1494.865 & 1495.585\end{array}$ $\begin{array}{llllllll}1495.999 & 1495.983 & 1497.815 & 1495.896 & 1494.848 & 1494.818 & 1495.576\end{array}$ $\begin{array}{rrrrrrr}1495.96 & 1495.981 & 1497.761 & 1495.931 & 1494.848 & 1494.853 & 1495.58 \\ 1496.004 & 1495.969 & 1497.41 & 1495.938 & 1494.832 & 1494.846 & 1495.571\end{array}$ $\begin{array}{rrrrrrr}1496.004 & 1495.969 & 1497.41 & 1495.938 & 1494.832 & 1494.846 & 1495.571 \\ 1495.995 & 1496.004 & 1497.904 & 1496.013 & 1494.86 & 1494.799 & 1495.583\end{array}$ $\begin{array}{rrrrrrr}1495.995 & 1496.004 & 1497.904 & 1496.013 & 1494.86 & 1494.799 & 1495.583 \\ 1495.971 & 1496.016 & 1497.887 & 1496.043 & 1494.865 & 1494.844 & 1495.59\end{array}$ $\begin{array}{llllllll}1495.962 & 1496.014 & 1497.882 & 1496.052 & 1494.862 & 1494.86 & 1495.59\end{array}$ $\begin{array}{llllllll}1495.936 & 1496.007 & 1497.854 & 1496.048 & 1494.853 & 1494.884 & 1495.585\end{array}$ $\begin{array}{llllllll}1495.927 & 1495.995 & 1497.847 & 1495.929 & 1494.841 & 1494.874 & 1495.578\end{array}$ $\begin{array}{llllllll}1495.913 & 1495.99 & 1497.861 & 1495.775 & 1494.851 & 1494.834 & 1495.576 \\ 1495.878 & 1496.037 & 1479.915 & 1495.84 & 1498.877 & 149802 & 149553\end{array}$ $\begin{array}{llllllll}1495.904 & 1496.023 & 1497.915 & 1495.84 & 1494.877 & 1494.802 & 1495.583 \\ 1495878 & 1496037 & 1497.904 & 1495.912 & 1498889 & 1494.86 & 1495.59\end{array}$ $\begin{array}{llllllll}1495.878 & 1496.037 & 1497.904 & 1495.912 & 1494.889 & 1494.86 & 1495.59 \\ 1495.857 & 1496.011 & 1497.859 & 1495.924 & 1494.877 & 1494.91 & 1495.583\end{array}$ $\begin{array}{rrrrrrr}1495.857 & 1496.011 & 1497.859 & 1495.924 & 1494.877 & 1494.91 & 1495.583 \\ 1495.85 & 1496.011 & 1497.861 & 1495.947 & 1494.872 & 1494.912 & 1495.578\end{array}$ $\begin{array}{rrrrrrrr}1495.85 & 1466.011 & 1497.861 & 1495.947 & 1494.872 & 1494.912 & 1495.578 \\ 1495.822 & 1495.953 & 1497.773 & 1495.957 & 1494.832 & 1494.834 & 1495.564\end{array}$ $\begin{array}{lllllllll}1495.813 & 1495.995 & 1497.871 & 1495.607 & 1494.874 & 1494.867 & 1495.573\end{array}$ $\begin{array}{llllllll}1495.806 & 1495.993 & 1497.878 & 1495.775 & 1494.881 & 1494.872 & 1495.568\end{array}$ $\begin{array}{lllllll}1495.773 & 1495.983 & 1497.836 & 1495.828 & 1494.872 & 1494.865 & 1495.568\end{array}$ $\begin{array}{llllllll}1495.778 & 1495.962 & 1497.826 & 1495.842 & 1494.853 & 1494.849 & 1495.554 \\ 1495752 & 195.95 & 1497.815 & 145.877 & 149.846 & 1498442 & 1495549\end{array}$ $\begin{array}{rrrrrrr}1495.752 & 1495.955 & 1497.815 & 1495.877 & 1494.846 & 1494.842 & 1495.549 \\ 1495.736 & 1495.93 & 1497.777 & 1495.863 & 1494.817 & 1494.82 & 1495.54\end{array}$ $\begin{array}{rrrrrrr}1495.736 & 1495.93 & 1497.777 & 1495.863 & 1494.817 & 1494.82 & 1495.54 \\ 1495.729 & 1495.941 & 1497.819 & 1495.87 & 1494.827 & 1494.827 & 1495.535\end{array}$ $\begin{array}{rrrrrrrr}1495.729 & 1495.941 & 1497.819 & 1495.87 & 1494.827 & 1494.827 & 1495.535 \\ 1495.722 & 1495.993 & 1497.88 & 1495.959 & 1494.86 & 1494.853 & 1495545\end{array}$ $\begin{array}{lllllll}149.722 & 1495.993 & 1497.88 & 1495.957 & 1494.86 & 1494.853 & 1455.545\end{array}$ $\begin{array}{llllllllll}1495.892 & 1495.967 & 1497.882 & 1495.961 & 1494.834 & 1494.832 & 1495.54\end{array}$ $\begin{array}{lllllllll}1496.153 & 1495.92 & 1497.939 & 1495.915 & 1494.794 & 1494.809 & 1495.531\end{array}$ $\begin{array}{llllllll}1496.402 & 1495.93 & 1497.887 & 1495.901 & 1494.794 & 1494.799 & 1495.533\end{array}$ $\begin{array}{llllllll}1496.325 & 1495.932 & 1497.518 & 1495.744 & 1494.801 & 1494.806 & 1495.549\end{array}$ $\begin{array}{lllllllll}1496.304 & 1495.934 & 1497.674 & 1495.779 & 1494.798 & 1494.809 & 1495.554\end{array}$ $\begin{array}{lllllll}1496.251 & 1495.93 & 1497.78 & 1495.866 & 1494.798 & 1494.806 & 1495.554 \\ 1196206 & 1495.911 & 1497745 & 1495896 & 1494.777 & 1494.709 & 145554\end{array}$ $\begin{array}{llllllll}1496.206 & 1495.911 & 1497.745 & 1495.896 & 1494.777 & 1494.79 & 1495.542 \\ 1496.188 & 1495.946 & 1497.815 & 1495.992 & 1494801 & 1494.809 & 1495549\end{array}$ $\begin{array}{lllllllllll}1496.188 & 1495.946 & 1497.815 & 1495.992 & 1494.801 & 1494.899 & 1495.549\end{array}$ $\begin{array}{llllllllll}1496.116 & 1495.79 & 1497.789 & 1496.032 & 1494.782 & 1494.794 & 1495.54\end{array}$ $\begin{array}{llllllll}1496.088 & 1495.834 & 1497.831 & 1496.109 & 1494.81 & 1494.778 & 1495.557\end{array}$ $\begin{array}{lllllll}1496.062 & 1496.011 & 1497.829 & 1496.139 & 1494.824 & 1494.809 & 1495.564\end{array}$ $\begin{array}{llllllll}1496.046 & 1496.158 & 1497.812 & 1496.132 & 1494.813 & 1494.83 & 1495.564\end{array}$ $\begin{array}{lllllllll}1496.004 & 1496.097 & 1497.768 & 1496.127 & 1494.81 & 1494.846 & 1495.566\end{array}$ $\begin{array}{lllllll}1495.953 & 1496.079 & 1497.726 & 1496.031 & 1494.808 & 1494.825 & 1495.557 \\ 1496.036 & 1495.974 & 1497.56 & 1495.868 & 1494.808 & 1494.804 & 1495.557\end{array}$

$1497.968 \quad 1497.902$ $\begin{array}{rr}1497.977 & 1497.9 \\ 1497.947 & 1497.849 \\ 1 & r 97.938\end{array}$

$\begin{array}{lll}1497.947 & 1497.849 \\ 1497.938 & 1497.842\end{array}$

1479.9281497 .835

1497.9211497 .825

$1497.942 \quad 1497.866$

$1497.947 \quad 1497.876$

$1497.94 \quad 1497.857$
1499.933
149.823

1497.9121497 .804

14979151497.816

$1497.931 \quad 1497.854$

1497.9471497 .871

$1497.935 \quad 1497.845$

$1497.919 \quad 1497.833$

1497.91714997 .828

$1497.935 \quad 1497.857$

1497.9241497 .833

$1497.912 \quad 1497.809$

$\begin{array}{lll}1497.921 & 1497.828\end{array}$

$\begin{array}{lll}1497.952 & 1497.88\end{array}$

$\begin{array}{ll}1497.945 & 1497.835 \\ 1497.935 & 1497.813\end{array}$

$\begin{array}{lll}1497.935 & 1497.813 \\ 1497.887 & 1497.725\end{array}$

$1497.928 \quad 1497.821$

$1497.905 \quad 1497.794$

$\begin{array}{lll}1497.919 & 1497.869\end{array}$

$1497.894 \quad 1497.79$

$1497.845 \quad 1497.682$

$1497.847 \quad 1497.739$

$\begin{array}{lll}1497.854 & 1497.77 \\ 1497.863 & 1497.785\end{array}$

$\begin{array}{lll}1497.863 & 1497.785 \\ 1497.861 & 1497.782\end{array}$

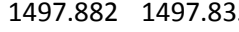

$1497.896 \quad 1497.837$

$\begin{array}{llll}1497.887 & 1497.806 \\ 1497.884 & 1497.797\end{array}$

$\begin{array}{llll}1497.884 & 1497.797 \\ 1497.882 & 1497.794\end{array}$

$\begin{array}{ll}1497.882 & 1497.794 \\ 1497.778\end{array}$ 
$\begin{array}{lllllllll}1495.957 & 1495.906 & 1497.693 & 1496.006 & 1494.751 & 1494.809 & 1495.513\end{array}$

$\begin{array}{llllllll}1495.941 & 1495.904 & 1497.705 & 1496.02 & 1494.741 & 1494.787 & 1495.507 \\ 1495.932 & 1495.927 & 1497.742 & 1496.069 & 1494.753 & 1494.762 & 1495.506\end{array}$ $\begin{array}{lllllllll}1495.906 & 1495.932 & 1497.724 & 1496.092 & 1494.753 & 1494.783 & 1495.509\end{array}$ $\begin{array}{llllllll}1495.888 & 1495.913 & 1497.688 & 1496.064 & 1494.729 & 1494.809 & 1495.502\end{array}$ $\begin{array}{lllllll}1495.869 & 1495.913 & 1497.688 & 1496.071 & 1494.725 & 1444.799 & 1495.494 \\ 1495.85 & 1495909 & 1479.684 & 1495.912 & 1494.722 & 1494787 & 1495\end{array}$ $\begin{array}{llllllll}1495.85 & 1495.909 & 1497.684 & 1495.912 & 1494.722 & 1494.787 & 1495.492\end{array}$ $\begin{array}{llllllll}1495.846 & 1495.927 & 1497.742 & 145.838 & 1494.739 & 1494.743 & 1495.495 \\ 1495.836 & 1495.972 & 1497.803 & 1495.919 & 1494.777 & 1494.75 & 1495511\end{array}$ $\begin{array}{llllllllll}1495.813 & 1495.988 & 1497.805 & 1496.013 & 1494.794 & 1494.818 & 1495.523\end{array}$ $\begin{array}{lllllllll}1495.811 & 1495.997 & 1497.817 & 1496.067 & 1494.803 & 1494.818 & 1495.531\end{array}$ $\begin{array}{llllllll}1495.787 & 1495.997 & 1497.791 & 1496.097 & 1494.81 & 1494.83 & 1495.535 \\ & 149585 & 14959 & & 149.85 & 149.50 & \end{array}$ $\begin{array}{lllllll}1495.785 & 1495.997 & 1497.805 & 1496.006 & 1494.813 & 1494.823 & 1495.535 \\ 1495.783 & 1496.027 & 1497.857 & 1495.901 & 1494848 & 1494816 & 1495.547\end{array}$ $\begin{array}{llllllll}1495.783 & 1496.027 & 1497.857 & 1495.901 & 1494.848 & 1494.816 & 1495.547\end{array}$ $\begin{array}{llllllll}1495.769 & 1496.058 & 1497.885 & 1495.94 & 1494.881 & 1494.872 & 1495.562\end{array}$ $\begin{array}{lllllllllll}1495.755 & 1496.048 & 1497.864 & 1495.994 & 1494.886 & 1494.881 & 1455.569\end{array}$ $\begin{array}{lllllllll}1495.736 & 1496.025 & 1497.838 & 1496.064 & 1494.884 & 1494.889 & 1495.559\end{array}$ $\begin{array}{lrrrrrr}1495.729 & 1496.025 & 1497.836 & 1496.095 & 1494.881 & 1494.886 & 1495.557 \\ 149.71 & 149 & 1977.789 & 1496.05 & 1498.867 & 1494891 & 1495.555\end{array}$ $\begin{array}{rrrrrrr}1495.71 & 1496 & 1497.789 & 1496.05 & 1494.867 & 1494.891 & 1495.545 \\ 14954 & 146 & 1477.798 & 1496.071 & 149.872 & 144.86 & 1455.54\end{array}$ $\begin{array}{rrrrrrrr}1495.704 & 1496 & 1497.798 & 1496.071 & 1494.872 & 1494.86 & 1495.54 \\ 1495.671 & 1495.96 & 1497.712 & 1496.043 & 1494846 & 1494.893 & 1495.523\end{array}$ $\begin{array}{rrrrrrrr}1495.671 & 1495.96 & 1497.712 & 1496.043 & 1494.846 & 1494.893 & 1495.523 \\ 1495.664 & 1495.918 & 1497.665 & 1496.004 & 1494.803 & 1494.863 & 1495.499\end{array}$ $\begin{array}{lllllll}1495.664 & 1495.918 & 1497.665 & 1496.004 & 1494.803 & 1494.863 & 1495.499 \\ 1495.659 & 1495.911 & 1497.672 & 1496.003 & 1494.782 & 1494.842 & 1495.487\end{array}$ $\begin{array}{llllllll}1495.659 & 1495.911 & 1497.672 & 1496.003 & 1494.782 & 1494.842 & 1495.487 \\ 1495.634 & 1495.883 & 1497.63 & 1495.877 & 1494.758 & 1494.827 & 1495.48\end{array}$

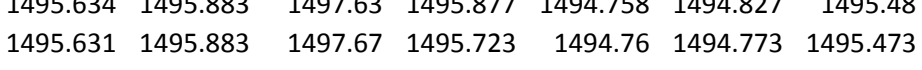
$\begin{array}{llllllll}1495.629 & 1495.916 & 1497.728 & 1495.803 & 1494.777 & 1494.766 & 1495.473\end{array}$ $\begin{array}{llllllll}1495.61 & 1495.92 & 1497.719 & 1495.889 & 1494.786 & 1494.806 & 1495.483\end{array}$ $\begin{array}{lllllllll}1495.603 & 1495.913 & 1497.7 & 1495.924 & 1494.782 & 1494.818 & 1495.473\end{array}$ $\begin{array}{llllllll}1495.601 & 1495.941 & 1497.742 & 1495.99 & 1494.803 & 1494.804 & 1495.475\end{array}$ $\begin{array}{llllllll}1495.596 & 1495.948 & 1497.747 & 1496.029 & 1494.805 & 1494.804 & 1495.483\end{array}$ $\begin{array}{llllllll}1495.594 & 1495.972 & 1497.777 & 1496.076 & 1494.822 & 1494.797 & 1495.492\end{array}$ $\begin{array}{llllllllll}1455.606 & 1496.032 & 1497.854 & 1456.165 & 1494.872 & 1494.816 & 145.516\end{array}$ $\begin{array}{llllllllll}1495.599 & 1496.053 & 1497.826 & 1496.188 & 1494886 & 1494.914 & 1495.542\end{array}$ $\begin{array}{lllllllll}1495.592 & 1496.039 & 1497.808 & 1496.167 & 1494.881 & 1494.886 & 1495.545\end{array}$ $\begin{array}{lllllllll}1495.582 & 1496.02 & 1497.784 & 1496.041 & 1494.877 & 1494.898 & 1495.54\end{array}$ $\begin{array}{llllllll}1495.582 & 1496.02 & 1497.801 & 1495.884 & 1494.884 & 1494.865 & 1495.54\end{array}$ $\begin{array}{lllllllll}1495.582 & 1496.037 & 1497.843 & 1495.947 & 1494.91 & 1494.867 & 1495.55\end{array}$ $\begin{array}{lllllll}1495.552 & 1496 & 1497.754 & 1495.966 & 1494.893 & 1494.919 & 1495.545\end{array}$ $\begin{array}{llllllll}1495.559 & 1495.948 & 1497.747 & 1495.924 & 1494.846 & 1494.849 & 1495.513\end{array}$ $\begin{array}{lllllllll}149.536 & 1495.923 & 147.645 & 1455.924 & 1494.846 & 1494.849 & 1495.513 \\ 1495.517 & 1495.916 & 1497.705 & 1495.943 & 149481 & 1494.823 & 145.495\end{array}$ $\begin{array}{lllllllll}1495564 & 1495.916 & 1497.607 & 1495.966 & 1494.81 & 149482 & 149.468\end{array}$ $\begin{array}{llllll}1495.58 & 1497.63 & 1494.82 & 1494.825 & 1495.466\end{array}$ $\begin{array}{llllllll}1495.595 & 1495.937 & 1497.728 & 1495.901 & 1494.813 & 1494.839 & 1495.471\end{array}$ $\begin{array}{rrrrrrr}1495.564 & 1495.905 & 1497.681 & 1495.878 & 1494.789 & 1494.82 & 1495.464 \\ 1495539 & 1495907 & 1497.686 & 1495.856 & 1494.779 & 1494813 & 1495.459\end{array}$ $\begin{array}{llllllll}1495.539 & 1495.907 & 1497.686 & 1495.856 & 1494.779 & 1494.813 & 1455.459\end{array}$ $\begin{array}{rrrrrrr}1495.518 & 1495.884 & 1497.66 & 1495.8 & 1494.76 & 1494.802 & 1495.452 \\ 1495.499 & 1495.793 & 1497.639 & 1495.586 & 1494.767 & 1494.804 & 1495.449\end{array}$

1497.9051497 .825 $\begin{array}{rr}1497.889 & 1497.79 \\ 1497.85 & 1497.71\end{array}$ $1497.845 \quad 1497.717$ $\begin{array}{ll}1497.838 & 1497.73 \\ 1497.815 & 1497.703\end{array}$ $1497.803 \quad 1497.715$ $\begin{array}{lll}1497.821 & 1497.758 \\ 1497.82 & 1497776\end{array}$ $1497.824 \quad 1497.756$ $\begin{array}{ll}1497.798 & 1497.715 \\ 1497.796 & 1497.715\end{array}$ $\begin{array}{lll}1497.796 & 1497.715 \\ 1497.789 & 1497708\end{array}$ $\begin{array}{lll}1497.789 & 1497.708 \\ 1497.819 & 1497.758\end{array}$ $\begin{array}{lll}1497.819 & 1497.758 \\ 1497.859 & 1497.816\end{array}$ $\begin{array}{lr}1497.882 & 1497.83\end{array}$ $\begin{array}{lll}1497.882 & 1497.83 \\ 1497.891 & 1497.821\end{array}$ $1497.898 \quad 1497.797$ $1497.898 \quad 1497.804$ \begin{tabular}{ll}
1497.935 & 1497.847 \\
1497.968 & 1497.885 \\
\hline
\end{tabular} $\begin{array}{lll}1497.968 & 1497.885 \\ 1497.968 & 1497.859\end{array}$ $\begin{array}{lll}1497.968 & 1477.859 \\ 1497.949 & 1497.818\end{array}$ $1497.949 \quad 1497.823$ $\begin{array}{lll}1497.945 & 1497.816\end{array}$ $1497.921 \quad 1497.778$ $\begin{array}{rrr}1497.921 & 1497.794 \\ 1497.88 & 1497.72\end{array}$ $\begin{array}{rll}1497.88 \quad 1497.72 \\ 1497.83 & 149767\end{array}$ \begin{tabular}{rr}
1497.833 & 1497.675 \\
1497.812 & 1497.68 \\
\hline
\end{tabular} $\begin{array}{rr}1497.812 & 1497.68 \\ 1497.782 & 1497.648\end{array}$ $\begin{array}{lll}1497.782 & 1497.688 \\ 1497.787 & 1497.682\end{array}$ $\begin{array}{lll}1497.812 & 1497.735\end{array}$ $\begin{array}{lll}1497.826 & 1497.747 \\ 1497.82 & 149725\end{array}$ 1497.8191497 .725

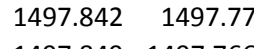
$\begin{array}{rr}1497.849 & 1497.766 \\ 1497.868 & 1497.79\end{array}$ $\begin{array}{lll}1497.868 & 1497.79 \\ 1497.924 & 1497.864\end{array}$ $\begin{array}{ll}1497.924 & 1497.864 \\ 1497.954 & 1497.883\end{array}$ $1497.935 \quad 1497.813$ $\begin{array}{lll}1497.921 & 1497.792\end{array}$ $1497.903 \quad 1497.758$ $1497.908 \quad 1497.77$ 1497.9351497 .811 $\begin{array}{rl}1497.91 & 1497.763 \\ 1497842 & 1497.689\end{array}$ $\begin{array}{ll}1497.842 & 1497.689 \\ 1497.815 & 1497.648\end{array}$ $\begin{array}{lll}1497.815 & 1497.648 \\ 1497.801 & 1497.651\end{array}$ $\begin{array}{ll}1497.801 & 1497.651 \\ 1497.794 & 1497.67\end{array}$ $\begin{array}{lll}1497.808 & 1497.72\end{array}$ $\begin{array}{lll}1497.805 & 1497.715\end{array}$ $1497.773 \quad 1497.668$ $1497.763 \quad 1497.672$ $\begin{array}{lll}1497.745 & 1497.651 \\ 1497.759 & 1497.677\end{array}$ 
$\begin{array}{lllllllll}1495.443 & 1495.886 & 1497.646 & 1495.728 & 1494.77 & 1494.799 & 1495.442\end{array}$ $\begin{array}{llllllll}1495.436 & 1495.851 & 1497.665 & 1495.516 & 1494.765 & 1494.799 & 1495.437\end{array}$ $\begin{array}{lllllllll}1495.432 & 1495.793 & 1497.726 & 1495.523 & 1494.794 & 1494.816 & 1495.442\end{array}$ $\begin{array}{lllllll}1495.441 & 1495.816 & 1497.731 & 1495.656 & 1494.805 & 1494.827 & 1495.452 \\ 1495.427 & 1495882 & 1497.731 & 1495.707 & 1494.805 & 1494.82 & 1495.452\end{array}$ $\begin{array}{llllllll}1495.427 & 1495.882 & 1497.731 & 1495.707 & 1494.805 & 1494.82 & 1495.452\end{array}$ $\begin{array}{lllllll}1495.429 & 1495.893 & 1497.759 & 1495.77 & 1494.829 & 1494.809 & 1495.461 \\ 1495.42 & 1495907 & 1497.74 & 1495807 & 1494832 & 14948 & 1495.461\end{array}$ $\begin{array}{llllllll}1495.42 & 1495.907 & 1497.74 & 1495.807 & 1494.832 & 1494.83 & 1495.461 \\ 1495.425 & 1495842 & 1497.784 & 1495.908 & 1494.848 & 1494.799 & 1495.468\end{array}$ $\begin{array}{llllllll}1495.425 & 1495.842 & 1497.784 & 1455.908 & 1494.848 & 1494.799 & 1495.468\end{array}$ $\begin{array}{lllllllll}1495.429 & 1495.933 & 1497.761 & 1495.94 & 1494.862 & 1494.87 & 1495.492\end{array}$ $\begin{array}{llllllll}1495.415 & 1495.9 & 1497.747 & 1495.884 & 1494.851 & 1494.86 & 1495.485\end{array}$ $\begin{array}{llllllll}1495.413 & 1495.898 & 1497.74 & 1495.88 & 1494.851 & 1494.858 & 1495.483\end{array}$ $\begin{array}{rrrrrrr}1495.404 & 1495.882 & 1497.693 & 1495.861 & 1494.829 & 1494.879 & 1495.471 \\ 1495.397 & 1495.849 & 1497.663 & 1495.8 & 1494.81 & 1494.86 & 1495.454\end{array}$ $\begin{array}{llllllll}1495.397 & 1495.849 & 1497.663 & 1495.8 & 1494.81 & 1494.86 & 1495.454\end{array}$ $\begin{array}{llllllll}1495.394 & 1495.842 & 1497.665 & 1495.775 & 1494.803 & 1494.827 & 1495.444\end{array}$ $\begin{array}{llllllllllll}1495.378 & 1495.821 & 1497.593 & 1455.814 & 1494.793 & 1494.872 & 1495.432\end{array}$ $\begin{array}{lllllllll}1495.364 & 1495.774 & 1497.581 & 1495.751 & 1494.72 & 1494.832 & 1495.416\end{array}$ $\begin{array}{lllllllll}1495.352 & 1495.746 & 1497.555 & 1495.719 & 1494.694 & 1494.813 & 1495.404\end{array}$ $\begin{array}{llllllll}1495.355 & 1495.749 & 1497.595 & 1495.714 & 1494.696 & 1494.745 & 1495.401\end{array}$ $\begin{array}{lllllllll}1495.364 & 1495.791 & 1497.667 & 1495.772 & 1494.725 & 1494.701 & 1495.411\end{array}$ $\begin{array}{lllllllll}1495.364 & 1495.819 & 1497.646 & 1495.817 & 1494.729 & 1494.747 & 1495.418\end{array}$ $\begin{array}{lllllll}1495.35 & 1495.802 & 1497.614 & 1495.793 & 1494.708 & 1494.78 & 1495.408\end{array}$ $\begin{array}{lllllllll}1495.35 & 1495.805 & 1497.637 & 1495.789 & 1494.715 & 1494.762 & 1495.408\end{array}$ $\begin{array}{llllllllll}1455.343 & 1495.8 & 1497.593 & 1459.782 & 1494.696 & 1494.78 & 1495.401\end{array}$ $\begin{array}{lllllllll}1495.357 & 1495.84 & 1497.698 & 1495.814 & 1494.744 & 1494.686 & 1495.416\end{array}$ $\begin{array}{llllllll}1495.352 & 1495.844 & 1497.623 & 1495.828 & 1494.725 & 1494.795 & 1495.418\end{array}$ $\begin{array}{llllllll}1495.341 & 1495.823 & 1497.63 & 1495.791 & 1494.722 & 1494.778 & 1495.411\end{array}$ $\begin{array}{llllllll}1495.334 & 1495.819 & 1497.621 & 1495.784 & 1494.715 & 1494.776 & 1495.406 \\ 1495.327 & 1495805 & 1497.604 & 1495.763 & 1494703 & 1494.771 & 1495.397\end{array}$ $\begin{array}{lllllllll}1495.327 & 1495.805 & 1497.604 & 1495.763 & 1494.703 & 1494.771 & 1495.397\end{array}$ $\begin{array}{llllllll}1495.332 & 1495.805 & 1497.644 & 1495.758 & 1494.715 & 1494.729 & 1495.397\end{array}$ $\begin{array}{lllllllllll}1495.332 & 1495.805 & 1497.644 & 1495.758 & 1494.715 & 1494.729 & 1495.397\end{array}$ $\begin{array}{llllllllll}1495.334 & 1495.84 & 1497.658 & 1495.793 & 1494.739 & 1494.771 & 1495.418\end{array}$ $\begin{array}{lllllllll}1495.332 & 1495.84 & 1497.665 & 1495.791 & 1494.739 & 1494.769 & 1495.418\end{array}$ $\begin{array}{lllllllll}1495.329 & 1495.84 & 1497.684 & 1495.793 & 1494.753 & 1494.757 & 1495.42\end{array}$ $\begin{array}{lllllllll}1495.332 & 1495.847 & 1497.705 & 1495.665 & 1494.765 & 1494.747 & 1495.428\end{array}$ $\begin{array}{lllllllll}1495.343 & 1495.875 & 1497.733 & 1495.53 & 1494.789 & 1494.752 & 1495.437\end{array}$ $\begin{array}{lllllll}1495.336 & 1495.865 & 1497.7 & 1495.555 & 1494.786 & 1494.795 & 1495.437\end{array}$ $\begin{array}{llllllll}1495.318 & 1495.833 & 1497.644 & 1455.555 & 1494.763 & 1494.839 & 1495.418\end{array}$ $\begin{array}{llllllllllll}1495.313 & 1495.807 & 1497.653 & 1455.567 & 1494.763 & 1494.806 & 1495.404\end{array}$ $\begin{array}{llllllllll}1495.306 & 1495.749 & 1497.628 & 149531 & 1494.737 & 1494.726 & 1495.385\end{array}$ $\begin{array}{lllllllll}1495.311 & 1495.779 & 1497.684 & 1495.331 & 1494.767 & 1494.715 & 1495.389\end{array}$ $\begin{array}{lllllllll}1495.304 & 1495.798 & 1497.658 & 1495.478 & 1494.767 & 1494.771 & 1495.387\end{array}$ $\begin{array}{lllllllll}1495.299 & 1495.784 & 1497.674 & 1495.53 & 1494.772 & 1494.771 & 1495.385\end{array}$ $\begin{array}{llllllll}1495.297 & 1495.802 & 1497.551 & 1495.59 & 1494.753 & 1494.79 & 1495.38\end{array}$ $\begin{array}{lllllll}1495.296 & 1495.779 & 1497.562 & 1495.593 & 1494.763 & 1494.773 & 1495.377 \\ 1495.278 & 1495.774 & 1497.668 & 1495.614 & 1494.765 & 1494.75 & 1495.375\end{array}$

$1497.782 \quad 1497.718$ $\begin{array}{rll}1497.784 & 1497.708 \\ 1497.77 & 1497.677\end{array}$ $\begin{array}{rl}1497.77 & 1497.677 \\ 1497.777 & 1497.694\end{array}$ $\begin{array}{rr}1497.777 & 1497.694 \\ 1497.759 & 1497.66\end{array}$ $1497.759 \quad 1497.668$ 1497.7891497 .716 $1497.81 \quad 1497.737$ $\begin{array}{rr}1497.81 & 1497.725 \\ 1497.831 & 1497.747\end{array}$ $\begin{array}{ll}1497.831 & 1497.747 \\ 1497.828 & 1497.727\end{array}$ $\begin{array}{lll}1497.828 & 1497.727 \\ 1497.847 & 1497.761\end{array}$ $\begin{array}{lll}1497.847 & 1497.76 \\ 1497.873 & 1497.794\end{array}$ 1497.8561497 .747 $1497.835 \quad 1497.708$ $\begin{array}{lll}1497.826 & 1497.703 \\ 1497.871 & 1497.65\end{array}$

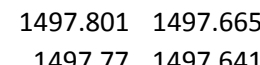
$\begin{array}{rr}1497.77 & 1497.641 \\ 1497.763 & 1497.651 \\ 1\end{array}$ $\begin{array}{lll}1497.763 & 1497.651 \\ 1497.719 & 1497.596\end{array}$ $\begin{array}{lll}1497.719 & 1497.596 \\ 1497.694 & 1497.586\end{array}$ $\begin{array}{lll}1497.694 & 1497.586 \\ 1497.673 & 1497.577\end{array}$ $1497.647 \quad 1497.562$ $\begin{array}{llll}1497.652 & 1497.598\end{array}$ $1497.691 \quad 1497.672$ $\begin{array}{rr}1497.701 & 1497.66 \\ 1497.687 & 1497.617\end{array}$ $\begin{array}{lll}1497.687 & 1497.617 \\ 1497.694 & 1497.636\end{array}$ $\begin{array}{lll}1497.694 & 1497.636 \\ 1497.677 & 1497.601\end{array}$ $\begin{array}{llll}1497.677 & 1497.601 \\ 1497.687 & 1497.632\end{array}$ $\begin{array}{lll}1497.731 & 1497.703\end{array}$ $\begin{array}{lll}1497.705 & 1497.648\end{array}$ $1497.701 \quad 1497.622$ $1497.694 \quad 1497.61$ $\begin{array}{ll}1497.677 & 1497.591 \\ 1497.689 & 1497.629\end{array}$ $\begin{array}{lll}1497.689 & 1497.629 \\ 1497.715 & 1497.67\end{array}$ $\begin{array}{ll}1497.715 & 1497.67 \\ 1497.719 & 1497.66\end{array}$ $\begin{array}{rr}1497.719 & 1497.66 \\ 1497.715 & 1497.639\end{array}$ $1497.717 \quad 1497.639$ $1497.724 \quad 1497.656$ $\begin{array}{lll}1497.738 & 1497.665 \\ 1497763 & 1497.677\end{array}$ $\begin{array}{lll}1497.763 & 1497.677 \\ 1497.756 & 1497.651\end{array}$ $\begin{array}{lll}1497.756 \quad 1497.651 \\ 1497724 & 149759\end{array}$ $\begin{array}{lll}1497.724 & 1497.589 \\ 1497.717 & 1497.598\end{array}$ $\begin{array}{lll}1497.717 & 1497.598 \\ 1497.677 & 1497.531\end{array}$ $\begin{array}{lll}1497.677 & 1497.531 \\ 1497.579\end{array}$ $1497.715 \quad 1497.639$ $1497.715 \quad 1497.629$ $1497.717 \quad 1497.632$

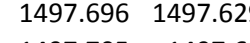
$\begin{array}{lll}1497.705 & 1497.61 \\ 1497.708 & 1497.613\end{array}$ 
$\begin{array}{llllllll}1495.257 & 1495.735 & 1497.602 & 1495.544 & 1494.727 & 1494.769 & 1495.358\end{array}$ $\begin{array}{lllllllll}1495.257 & 1495.711 & 1497.623 & 1495.317 & 1494.727 & 1494.731 & 1495.353\end{array}$ $\begin{array}{llllllll}1495.264 & 1495.732 & 1497.663 & 1495.317 & 1494.744 & 1494.724 & 1495.353\end{array}$ $\begin{array}{llllllll}1495.252 & 1495.735 & 1497.623 & 1495.406 & 1494.734 & 1494.776 & 1495.349\end{array}$ $\begin{array}{lllllll}1495.238 & 1495.693 & 1497.583 & 1495.415 & 1494.708 & 1494.733 & 1495.344\end{array}$ $\begin{array}{lllllll}1495.231 & 1495.676 & 1497.567 & 1495.439 & 1494.694 & 1494.75 & 1495.332\end{array}$ $\begin{array}{llllllll}1495.224 & 1495.66 & 1497.569 & 1495.457 & 1494.684 & 1494.729 & 1495.325 \\ 1495.224 & 1495.66 & 1497.597 & 1495.483 & 1494.682 & 1494.684 & 1495322\end{array}$ $\begin{array}{lllllllll}1495.231 & 1495.7 & 1497.637 & 1495.516 & 1494.703 & 1494.693 & 1495.327\end{array}$ $\begin{array}{lllllllll}1495.22 & 1495.697 & 1497.588 & 1495.53 & 1494.684 & 1494.729 & 1495.318\end{array}$ $\begin{array}{llllllll}1495.208 & 1495.669 & 1497.569 & 1495.509 & 1494.668 & 1494.689 & 1495.313\end{array}$ $\begin{array}{llllllll}1495.203 & 1495.665 & 1497.562 & 1495.516 & 1494.658 & 1494.696 & 1495.306\end{array}$ $\begin{array}{lllllllll}1495.194 & 1495.644 & 1497.562 & 1495.457 & 1494.646 & 1494.686 & 1495.301\end{array}$ $\begin{array}{lllllll}1495.283 & 1495.637 & 1497.593 & 1495.203 & 1494.651 & 1494.644 & 1495.296\end{array}$ $\begin{array}{llllllll}1495.255 & 1495.676 & 1497.628 & 1495.236 & 1494.665 & 1494.628 & 1495.298\end{array}$ $\begin{array}{lllllllll}1495.234 & 1495.679 & 1497.588 & 1495.336 & 1494.663 & 1494.682 & 1495.294\end{array}$ $\begin{array}{lllllllll}1495.199 & 1495.641 & 1497.562 & 1495.404 & 1494.646 & 1494.686 & 1495.284\end{array}$ $\begin{array}{llllllllll}1495.185 & 1495.63 & 1497.569 & 1495.422 & 1494.642 & 1494.665 & 1495279\end{array}$ $\begin{array}{lllllllll}1495.171 & 1495.623 & 1497.565 & 1495.441 & 1494.63 & 1494.656 & 1495.272\end{array}$ $\begin{array}{llllllll}1495.173 & 1495.648 & 1497.595 & 1495.481 & 1494.646 & 1494.616 & 1495.272\end{array}$ $\begin{array}{lllllll}1495.136 & 1495.606 & 1497.448 & 1495.427 & 1494.577 & 1494.588 & 1495.267\end{array}$ $\begin{array}{rrrrrrr}1495.12 & 1495.546 & 1497.434 & 1495.341 & 1494.53 & 1494.531 & 1495.253 \\ 1495113 & 1495532 & 1497.42 & 1459336 & 1494.509 & 14945 & 1495253\end{array}$ $\begin{array}{lllllll}1495.113 & 1495.532 & 1497.42 & 1495.336 & 1494.509 & 1494.52 & 1495.243 \\ 1495.096 & 1495.492 & 1497.408 & 1495.252 & 1494.478 & 1494.449 & 1495227\end{array}$ $\begin{array}{lllllll}1495.096 & 1455.492 & 1497.408 & 1455.252 & 1494.478 & 1494.449 & 1495.227 \\ 1495.096 & 1495.478 & 1497.445 & 1495.039 & 1494.478 & 1494.409 & 1495.217\end{array}$ $\begin{array}{llllllll}1495.099 & 1495.506 & 1497.487 & 1495.093 & 1494.499 & 1494.388 & 1495.217\end{array}$ $\begin{array}{llllllll}1495.08 & 1495.502 & 1497.438 & 1495.17 & 1494.48 & 1494.44 & 1495.207\end{array}$ $\begin{array}{lllllll}1495.068 & 1495.476 & 1497.415 & 1495.177 & 1494.459 & 1494.444 & 1495.198\end{array}$ $\begin{array}{llllllll}1495.064 & 1495.481 & 1497.436 & 1495.222 & 1494.459 & 1494.414 & 1495.193\end{array}$ $\begin{array}{llllllll}1495.054 & 1495.476 & 1497.408 & 1495.198 & 1494.442 & 1494.414 & 1495.184\end{array}$ $\begin{array}{lllllll}1495.052 & 1495.481 & 1497.462 & 1495.007 & 1494.456 & 1494.35 & 1495.179\end{array}$ $\begin{array}{llllllll}1495.064 & 1495.541 & 1497.532 & 1455.081 & 1494.494 & 1494.313 & 1495.191 \\ 1495.054 & 1495.557 & 1497.497 & 1495.191 & 1494.492 & 1494.386 & 1495.191\end{array}$ $\begin{array}{llllllll}1495.038 & 1495.527 & 1497.462 & 1495.21 & 1494.471 & 1494.421 & 1495.176\end{array}$ $\begin{array}{llllllll}1495.031 & 1495.52 & 1497.464 & 1495.245 & 1494.473 & 1494.409 & 1495.172\end{array}$ $\begin{array}{llllllll}1495.017 & 1495.502 & 1497.417 & 1495.254 & 1494.449 & 1494.418 & 1495.164\end{array}$ $\begin{array}{llllllll}1495.017 & 1495.497 & 1497.457 & 1495.264 & 1494.454 & 1494.357 & 1495.157\end{array}$ $\begin{array}{llllllll}1495.029 & 1495.543 & 1497.504 & 1495.313 & 1494.475 & 1494.324 & 1495.169\end{array}$ $\begin{array}{lllllll}1495.017 & 1495.546 & 1497.438 & 1495.327 & 1494.461 & 1494.402 & 1495.162\end{array}$ $\begin{array}{lllllll}1494.999 & 1495.511 & 1497.399 & 1495.296 & 1494.437 & 1494.421 & 1495.15 \\ 1494987 & 1495.485 & 1497.396 & 1495287 & 1494.425 & 1494 . & 1495.145\end{array}$ \begin{tabular}{llllllllll}
1494.98 & 1495.462 & 1497.357 & 1495.224 & 1494.425 & 1494.4 & 1495.145 \\
\hline
\end{tabular} $\begin{array}{rrrrrrrr}1494.975 & 1495.427 & 1497.38 & 1494.979 & 1494.387 & 1444.341 & 1455.129\end{array}$ $\begin{array}{llllllll}1494.982 & 1495.455 & 1497.438 & 1495.011 & 1494.414 & 1494.289 & 1495.129\end{array}$ $\begin{array}{llllllll}1494.971 & 1495.471 & 1497.408 & 1495.119 & 1494.409 & 1494.32 & 1495.124\end{array}$ $\begin{array}{llllllll}1494.961 & 1495.45 & 1497.389 & 1495.154 & 1494.395 & 1494.339 & 1495.119\end{array}$ $\begin{array}{llllllll}1494.95 & 1495.441 & 1497.389 & 1495.184 & 1494.39 & 1494.329 & 1495.109\end{array}$ $\begin{array}{rrrrrrr}1494.947 & 1495.448 & 1497.371 & 1495.224 & 1494.383 & 1494.329 & 1495.107 \\ 1494.95 & 1495.446 & 1497.422 & 1495.243 & 1494.39 & 1494.369 & 1495.1\end{array}$

\section{$\begin{array}{ll}1497.724 & 1497.641 \\ 1497.705 & 1497.593\end{array}$} $\begin{array}{rr}1497.705 & 1497.593 \\ 1497.684 & 1497.57\end{array}$

$\begin{array}{rr}1497.684 & 1497.57 \\ 1497.675 & 1497.569\end{array}$

$\begin{array}{ll}1497.675 & 1497.569 \\ 1497.652 & 1497.541\end{array}$

$\begin{array}{lll}1497.652 & 1497.5 .51 \\ 1497.649 & 1497.55\end{array}$

1497.6731497 .596

$\begin{array}{ll}1497.663 & 1497.569 \\ 1497.633 & 1497.519\end{array}$

$\begin{array}{lll}1497.633 & 1497.519 \\ 1497.612 & 1497.507\end{array}$

$\begin{array}{lr}1497.601 & 1497.51 \\ 1497.605 & 1497.538\end{array}$

$\begin{array}{ll}1497.605 & 1497.538 \\ 1497.633 & 1497.591\end{array}$

$1497.615 \quad 1497.543$

$\begin{array}{lll}1497.615 & 1497.543 \\ 1497.596 & 1497.515\end{array}$

$1497.587 \quad 1497.51$

$1497.575 \quad 1497.5$

$1497.589 \quad 1497.519$

$\begin{array}{rr}1497.612 & 1497.56 \\ 1497.605 & 1497.529\end{array}$

$\begin{array}{ll}1497.605 & 1497.569 \\ 1497.589 & 1497.507\end{array}$

$\begin{array}{lll}1497.589 & 1497.507 \\ 1497.584 & 1497.503\end{array}$

$1497.58 \quad 1497.50$

$\begin{array}{lll}1497.58 & 1497.505 \\ 1497.57 & 1497.493\end{array}$

$1497.587 \quad 1497.541$

$\begin{array}{rrrr}1497.515 & 1497.424 & 1492.979\end{array}$

$\begin{array}{rrrr}1497.47 & 1497.395 & 1493.04\end{array}$

1497.454 $1497.409 \quad 1493.077$

$\begin{array}{llll}1497.419 & 1497.371 & 1489.816 \\ 1497.426 & 1497.416 & 1490.017\end{array}$

$\begin{array}{llll}1497.461 & 1497.476 & 1491.725\end{array}$

$\begin{array}{llll}1497.447 & 1497.436 & 1491.84\end{array}$

$\begin{array}{llll}1497.433 & 1497.407 & 1492.394\end{array}$

$\begin{array}{llll}1497.438 & 1497.433 & 1492.614\end{array}$

$\begin{array}{llll}1497.426 & 1497.409 & 1489.448\end{array}$ $\begin{array}{llll}1497.445 & 1497.448 & 1489.565\end{array}$ $\begin{array}{llll}1497.482 & 1497.45 & 1492.359\end{array}$ $\begin{array}{llll}1497.454 & 1497.407 & 1492.68\end{array}$ $\begin{array}{llll}1497.475 & 1497.443 & 1492.715 \\ 1497.445 & 1497.397 & 149286\end{array}$

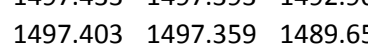
$\begin{array}{lllll}1497.396 & 1497.359 & 1489.867\end{array}$ $\begin{array}{rrrr}1497.415 & 1497.385 & 1492.383 \\ 1497.412 & 1497.39 & 1492.565\end{array}$ $\begin{array}{llll}1497.412 & 1497.39 & 1492.565 \\ 1497.405 & 1497.383 & 1492.69\end{array}$ $\begin{array}{lll}1497.405 & 1497.383 & 1492.691 \\ 1497.422 & 1497.424 & 1492.771\end{array}$ $\begin{array}{lll}1492.826 & 1490.839\end{array}$ $\begin{array}{ll}1492.892 & 1490.843 \\ 1492.932 & 1490.855\end{array}$ 1492.932
14900.854
1490
1490.855 1490.0641490 .855
1489.699
1490.86 $1491.511 \quad 1490.876$ $\begin{array}{ll}1491.511 & 1490.876 \\ 1491.623 & 1490.881\end{array}$ $1492.219 \quad 1490.876$ $\begin{array}{lll}1492.446 & 1490.878\end{array}$ $1489.682 \quad 1490.876$ $1489.232 \quad 1490.874$ $\begin{array}{rr}1491.246 & 1490.89 \\ 149186 & 1490897\end{array}$ $\begin{array}{rr}1491.86 & 1490.897 \\ 1492.181 & 1490.89\end{array}$ $\begin{array}{ll}1492.181 & 1490.89 \\ 1492.388 & 1490.89\end{array}$ 1492.5191490 .888 $1492.092 \quad 1490.883$ $1492.099 \quad 1490.902$ $\begin{array}{lll}1492.552 & 1490.904\end{array}$ $\begin{array}{lll}1492.681 & 1490.885 \\ 1492.756 & 1900874\end{array}$ $\begin{array}{ll}1492.756 & 1490.874 \\ 1489.905 & 1490.862\end{array}$ $\begin{array}{lll}1489.9051 & 1490.862 \\ 1489.551 & 1490.846\end{array}$

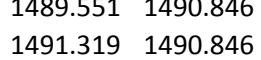
$1491.916 \quad 1490.848$ $1492.21 \quad 1490.841$ $\begin{array}{ll}1492.404 & 1490.834 \\ 1492.528 & 1490.832\end{array}$ $\begin{array}{ll}1492.528 & 1490.832 \\ 1492.613 & 1490.827\end{array}$
1484.427 1484.436 1484.455
1484.45 1484.45
1484.446 1484.439 1484.439
1484.404 1484.409 1484.425 1484.425
1484.423
1484.432 1484.432 1484.402 1484.286 1484.284
1484.302 1484.27 1484.291 1484.316 1484.27 1484.284
1484.307 1484.288 1484.288 1484.302 1484.252 1484.279 1484.291 1484.282
1484.256 $\begin{array}{lllll}1492.827 & 1494.028 & 1493.003 & 1495.427 & 1492.988\end{array}$ $\begin{array}{rrrrr}1492.89 & 1494.014 & 1493.067 & 1495.363 & 1493.05 \\ 1492.93 & 149038 & 143.105 & 149537 & 143\end{array}$ $\begin{array}{llllll}1492.93 & 1494.038 & 1493.105 & 1495.37 & 1493.087 \\ 1490.063 & 1492.725 & 1489.802 & 1495.173 & 1489.84\end{array}$ $\begin{array}{lllll}1490.063 & 1492.725 & 1489.802 & 1495.173 & 1489.847 \\ 1489.705 & 1492.629 & 1490.079 & 1494.994 & 1490.055\end{array}$ $\begin{array}{llllll}1491.518 & 1493.364 & 1491.762 & 1495.159 & 1491.743\end{array}$ $\begin{array}{lllllll}1491.621 & 1493.459 & 1491.878 & 1495.203 & 1491.862\end{array}$ $\begin{array}{lllllll}1492.216 & 1493.66 & 1492.423 & 1495.213 & 1492.407\end{array}$ $\begin{array}{lllllll}1492.444 & 1493.783 & 1492.643 & 1495.264 & 1492.623\end{array}$ $\begin{array}{llllll}1489.677 & 1492.571 & 1489.44 & 1495.126 & 1489.478\end{array}$ $\begin{array}{llllll}1489.233 & 1492.473 & 1489.627 & 1494.954 & 1489.607 \\ 1491248 & 1493261 & 1491.508 & 1495.142 & 149.489\end{array}$ $\begin{array}{llllll}1491.248 & 1493.261 & 1491.508 & 1495.142 & 1491.489 \\ 1491.861 & 1493.545 & 1492.086 & 1495.234 & 1492.069\end{array}$ $\begin{array}{llllll}1492.181 & 1493.662 & 1492.39 & 1495.238 & 1492.372\end{array}$ $\begin{array}{llllll}1492.385 & 1493.765 & 1492.584 & 1495.274 & 1492.56\end{array}$ $\begin{array}{lllllll}1492.519 & 1493.814 & 1492.71 & 1495.267 & 1492.693\end{array}$ $\begin{array}{llllll}1492.089 & 1493.718 & 1492.328 & 1495.281 & 1492.311\end{array}$ $\begin{array}{llllll}1492.101 & 1493.706 & 1492.333 & 1495.328 & 1492.311\end{array}$ $\begin{array}{llllll}1492.548 & 1493.881 & 1492.747 & 1495.344 & 1492.726\end{array}$ $\begin{array}{rrrrrr}1492.675 & 1493.9 & 1492.868 & 1495.307 & 1492.843 \\ 1492.755 & 1493.928 & 1492.939 & 1495.297 & 1492.914\end{array}$ $\begin{array}{llllll}142.755 & 1493.928 & 1492.939 & 1495.297 & 1492.914 \\ 1489.898 & 1492.645 & 1489.648 & 1495.128 & 1489.687\end{array}$ $\begin{array}{llllll}1489.548 & 1492.519 & 1489.93 & 1494.912 & 1489.903\end{array}$ $\begin{array}{llllll}1491.314 & 1493.238 & 1491.57 & 1495.062 & 1491.55\end{array}$ $\begin{array}{llllll}1491.913 & 1493.52 & 1492.141 & 1495.161 & 1492.116\end{array}$ $\begin{array}{llllll}1492.207 & 1493.648 & 1492.416 & 1495.18 & 1492.397\end{array}$ $\begin{array}{llllll}1492.397 & 1493.741 & 1492.596 & 1495.208 & 1492.576\end{array}$ $\begin{array}{rrrrr}1492.524 & 1493.811 & 1492.719 & 1495.248 & 1492.698 \\ 1492.607 & 1493.855 & 1492.797 & 1495.267 & 1492.78\end{array}$ 


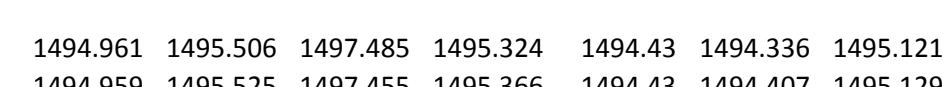
$\begin{array}{llllllll}1494.9595 & 1495.525 & 1497.455 & 1495.366 & 1494.43 & 1494.407 & 1495.129\end{array}$ $\begin{array}{lllllll}1494.947 & 1495.506 & 1497.45 & 1495.343 & 1494.428 & 1494.425 & 1495.121 \\ 1494.952 & 1495511 & 1497.457 & 1495359 & 1494.433 & 1494223 & 1495.121\end{array}$ $\begin{array}{lllllll}1494.952 & 1495.511 & 1497.457 & 1495.359 & 1494.433 & 1494.423 & 1495.121 \\ 1494.947 & 1495.504 & 1497.438 & 1495.303 & 1494.428 & 1494.433 & 1495.114\end{array}$ $\begin{array}{lllllll}1494.95 & 1495.502 & 1497.483 & 1495.081 & 1494.449 & 1494.383 & 1495.119\end{array}$ $\begin{array}{lllllllll}1494.968 & 1495.56 & 1497.532 & 1495.123 & 1494.487 & 1494.376 & 1495.136\end{array}$ $\begin{array}{lllllllll}1494.957 & 1495.546 & 1497.483 & 1495.159 & 1494.478 & 1494.454 & 1495.131\end{array}$ $\begin{array}{lllllll}1494.945 & 1495.518 & 1497.455 & 1495.156 & 1494.466 & 1494.473 & 1495.112\end{array}$

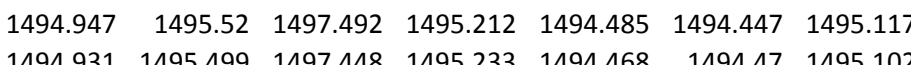
$\begin{array}{lllllll}1494.931 & 1495.499 & 1497.448 & 1495.233 & 1494.468 & 1494.47 & 1495.102 \\ 1494.929 & 1495.478 & 1497.441 & 1495.235 & 1494.461 & 1494.449 & 1495.095\end{array}$ $\begin{array}{lllllll}1494.929 & 1495.478 & 1497.441 & 1495.235 & 1494.461 & 1494.449 & 1495.095 \\ 1494.94 & 1495.504 & 1497.483 & 1495.296 & 1494.485 & 1494.416 & 1495.105\end{array}$ $\begin{array}{lllllllll}1494.936 & 1495.504 & 1497.434 & 1495.322 & 1494.471 & 1494.465 & 1495.102\end{array}$ $\begin{array}{llllllll}1494.936 & 1495.504 & 1497.434 & 1455.322 & 1494.471 & 1494.465 & 1495.102 \\ 1494.922 & 1495.471 & 1497.403 & 1495.289 & 1494.456 & 1494.475 & 1495.09\end{array}$ $\begin{array}{lllllllll}1494.922 & 1495.462 & 1497.394 & 1495.289 & 1494.447 & 1494.463 & 1495.085\end{array}$ $\begin{array}{llllllll}1494.908 & 1495.436 & 1497.364 & 1495.219 & 1494.43 & 1494.461 & 1495.078\end{array}$ $\begin{array}{lllllll}1494.917 & 1495.42 & 1497.413 & 1494.995 & 1494.437 & 1494.39 & 1495.076\end{array}$ $\begin{array}{rrrrrrr}1494.922 & 1495.455 & 1497.455 & 1495.037 & 1494.459 & 1494.364 & 1495.088 \\ 149.915 & 1495.46 & 149.415 & 149.123 & 149.452 & 1494.425 & 1495\end{array}$ $\begin{array}{lllllllll}1494.915 & 1495.46 & 1497.415 & 1495.123 & 1494.452 & 1494.425 & 1495.081 \\ 1494.901 & 1495.436 & 1497.413 & 1495.147 & 1494.449 & 1494.433 & 1495073\end{array}$ $\begin{array}{lllllllll}1494.903 & 1495.441 & 1497.422 & 1495.194 & 1494.454 & 1494.428 & 1495.069\end{array}$ $\begin{array}{llllllll}1494.898 & 1495.434 & 1497.41 & 1495.17 & 1494.452 & 1494.425 & 1495.069\end{array}$ $\begin{array}{llllllll}1494.908 & 1495.453 & 1497.481 & 1495.002 & 1494.485 & 1494.369 & 1495.078\end{array}$ $\begin{array}{llllllll}1494.922 & 1495.509 & 1497.546 & 1495.039 & 1494.53 & 1494.355 & 1495.102\end{array}$ $\begin{array}{llllllll}1494.919 & 1495.513 & 1497.497 & 1495.081 & 1494.528 & 1494.444 & 1495.102\end{array}$ $\begin{array}{lllllll}1494.908 & 1495.49 & 1497.483 & 1495.114 & 1494.525 & 1494.473 & 1495.09\end{array}$ $\begin{array}{lllllll}1494.905 & 1495.49 & 1497.476 & 1455.17 & 1494.525 & 1494.484 & 1495.088\end{array}$ $\begin{array}{llllllll}1494.884 & 1495.448 & 1497.401 & 1495.163 & 1494.49 & 1494.517 & 1495.067 \\ 1494.877 & 1495.408 & 1497396 & 1495.105 & 1494.473 & 1494.463 & 1495.054\end{array}$ $\begin{array}{llllllll}1494.884 & 1495.42 & 1497.42 & 1495.156 & 1494.482 & 1494.423 & 1495.057\end{array}$ $\begin{array}{llllllll}1494.877 & 1495.415 & 1497.368 & 1495.184 & 1494.463 & 1494.465 & 1495.05\end{array}$ $\begin{array}{llllllll}1494.861 & 1495.376 & 1497.361 & 1495.149 & 1494.442 & 1494.461 & 1495.042\end{array}$ $\begin{array}{lllllllll}1494.873 & 1495.383 & 1497.319 & 1495.182 & 1494.428 & 1494.461 & 1495.035\end{array}$ $\begin{array}{llllllll}1494.842 & 1495.331 & 1497.282 & 1495.081 & 1494.39 & 1494.458 & 1495.023\end{array}$ $\begin{array}{lllllll}1494.901 & 1495.292 & 1497.284 & 1494.361 & 1494.414 & 1495.014 \\ 1494898 & 1495 & & 1494.93 & 1494.366 & 1494348 & 195000\end{array}$ $\begin{array}{llllllll}1494.898 & 1495.303 & & 1494.93 & 1494.366 & 1494.348 & 1495.006\end{array}$ $\begin{array}{llllllll}1494.866 & 1495.28 & 1497.256 & 1494.925 & 1494.328 & 1494.393 & 1494.994 \\ 1494.836 & 1495.236 & 1497.249 & 1494.843 & 1494297 & 1494.371 & 1494.98\end{array}$ $\begin{array}{llllllll}1494.822 & 1495.236 & 1497.235 & 1494.888 & 1494.278 & 1494.35 & 1494.971\end{array}$ $\begin{array}{llllllll}1494.803 & 1495.217 & 1497.242 & 1494.902 & 1494.264 & 1494.315 & 1494.961\end{array}$ $\begin{array}{llllllll}1494.78 & 1495.203 & 1497.258 & 1494.92 & 1494.259 & 1494.268 & 1494.956\end{array}$ $\begin{array}{lllllll}1494.791 & 1495.252 & 1497.35 & 1495.011 & 1494.209 & 1494.968\end{array}$

1494.796
1494.796

$1494.791 \quad 1495.285 \quad 1497.1351495 .067$ $\begin{array}{lllll}1494.794 & 1495.287 & 1497.13 & 1495.072\end{array}$ $1494.796 \quad 1495.292 \quad 1497.121 \quad 1495.079$ $\begin{array}{llllll}1494.789 & 1495.287 & 1497.121 & 1495.077\end{array}$ $\begin{array}{llll}1494.791 & 1495.289 & 1497.121 & 1495.077 \\ 1494.784 & 1495282 & 1497.123 & 1495074\end{array}$

\begin{tabular}{|c|c|c|c|c|c|}
\hline 1497.47 & 3 & 1492.888 & 728 & 1490.841 & 1484.231 \\
\hline 1497.468 & 1497.464 & 1492.818 & 1492.693 & 1490.85 & 1484.166 \\
\hline 1497.463 & 1497.438 & 1492.979 & 1492.824 & 1490.846 & 1484.191 \\
\hline 1497.468 & 1497.448 & 1493.033 & 1492.878 & 1490.846 & 1484.226 \\
\hline 1497.459 & 1497.421 & 1489.774 & 1490.017 & 1490.843 & 1484.221 \\
\hline 1497.477 & 1497.448 & 1489.97 & 1489.649 & 1490.834 & 1484.224 \\
\hline 1497.519 & 1497.51 & 1490.92 & 1490.648 & 1490.848 & 1484.228 \\
\hline 1497.505 & 1497.452 & 1491.236 & 1491.049 & 1490.848 & 1484.187 \\
\hline 1497.482 & 1497.412 & 1492.069 & 1491.877 & 1490.836 & 1484.221 \\
\hline 1497.501 & 1497.452 & 1492.399 & 1492.221 & 1490.832 & 1484.249 \\
\hline 1497.475 & 1497.404 & 1492.584 & 1492.416 & 1490.825 & 1484.242 \\
\hline 1497.459 & 1497.402 & 1492.694 & 1492.533 & 1490.811 & 1484.245 \\
\hline 1497.482 & 1497.455 & 1492.769 & 1492.603 & 1490.816 & 1484.249 \\
\hline 1497.463 & 1497.414 & 1492.876 & 1492.718 & 1490.816 & 1484.178 \\
\hline 1497.44 & 1497.378 & 1492.916 & 1492.765 & 1490.804 & 1484.198 \\
\hline 1497.426 & 1497.376 & 1492.961 & 1492.807 & 1490.797 & 1484.231 \\
\hline 1497.403 & 1497.342 & 1489.703 & 1489.949 & 1490.785 & 1484.217 \\
\hline 1497.412 & 1497.381 & 1489.902 & 1489.581 & 1490.771 & 1484.222 \\
\hline 1497.442 & 1497.433 & 1491.477 & 1491.253 & 1490.778 & 1484.242 \\
\hline 1497.435 & 1497.404 & 1492.099 & 1491.909 & 1490.778 & 1484.187 \\
\hline 1497.428 & 1497.393 & 1492.376 & 1492.202 & 1490.767 & 1484.196 \\
\hline 1497.433 & 1497.4 & 1492.567 & 1492.397 & 1490.767 & 1484.208 \\
\hline 1497.426 & 1497.383 & 1489.392 & 1489.621 & 1490.76 & 1484.194 \\
\hline 1497.461 & 1497.443 & 1489.652 & 1489.316 & 1490.76 & 1484.212 \\
\hline 1497.515 & 1497.505 & 1489.685 & 1489.295 & 1490.771 & 1484.231 \\
\hline 1497.505 & 1497.452 & 1491.379 & 1491.147 & 1490.776 & 1484.187 \\
\hline 1497.496 & 1497.426 & 1491.926 & 1491.729 & 1490.767 & 1484.203 \\
\hline 1497.491 & 1497.419 & 1492.238 & 1492.055 & 1490.764 & 1484.21 \\
\hline 1497.445 & 1497.349 & 1492.071 & 1491.855 & 1490.748 & 1484.201 \\
\hline 1497.422 & 1497.34 & 1492.294 & 1492.116 & 1490.729 & 1484.208 \\
\hline 1497.431 & 1497.381 & 1492.511 & 1492.341 & 1490.732 & 1484.21 \\
\hline 1497.41 & 1497.352 & 1492.642 & 1492.484 & 1490.727 & 1484.136 \\
\hline 1497.384 & 1497.318 & 1492.703 & 1492.545 & 1490.711 & 1484.134 \\
\hline 1497.363 & 1497.309 & 1492.769 & 1492.613 & 1490.708 & 1484.157 \\
\hline 1497.326 & 1497.256 & 1489.504 & 1489.741 & 1490.69 & 1484.143 \\
\hline 1497.298 & 1497.239 & 1489.512 & 1489.18 & 1490.669 & 1484.157 \\
\hline 1497.31 & 1497.282 & 1491.306 & 1491.082 & 1490.662 & 1484.159 \\
\hline 1497.273 & 1497.232 & 1491.875 & 1491.689 & 1490.648 & 1484.12 \\
\hline 1497.247 & 1497.206 & 1492.144 & 1491.968 & 1490.629 & 1484.122 \\
\hline 1497.233 & 1497.22 & 1492.326 & 1492.155 & 1490.625 & 1484.138 \\
\hline & 1497.225 & 1492.45 & 1492.287 & 1490.613 & 1484.136 \\
\hline & & 1492.532 & 1492.371 & 1490.604 & 1484.145 \\
\hline
\end{tabular}

$\begin{array}{lllll}1492.719 & 1493.965 & 1492.909 & 1495.363 & 1492.89\end{array}$ $\begin{array}{llllll}1492.684 & 1493.974 & 1492.842 & 1495.382 & 1492.824 \\ 1492818 & 1493.998 & 1493.001 & 1495.351 & 1492.982\end{array}$ $\begin{array}{llllll}1492.872 & 1494.035 & 1493.053 & 1495.377 & 1493.936\end{array}$ $\begin{array}{llllll}1490.009 & 1492.752 & 1489.753 & 1495.21 & 1489.798\end{array}$ $\begin{array}{lllll}1489.647 & 1492.645 & 1490.023 & 1495.013 & 1490.006\end{array}$ $\begin{array}{llllll}1490.644 & 1493.077 & 1490.959 & 1495.142 & 1490.945\end{array}$ $\begin{array}{llllll}1491.046 & 1493.235 & 1491.269 & 1495.17 & 1491.259\end{array}$ $\begin{array}{lllll}1491.871 & 1493.531 & 1492.096 & 1495.182 & 1492.083\end{array}$ $\begin{array}{rrrrrr}1492.214 & 1493.704 & 1492.423 & 1495.245 & 1492.404 \\ 1492.409 & 1493.774 & 1492.605 & 1495.25 & 1492.59\end{array}$ $\begin{array}{rrrrrr}1492.409 & 1493.774 & 1492.605 & 1495.25 & 1492.59 \\ 1492.527 & 1493.82 & 1492.717 & 1495.255 & 1492.7 \\ & \end{array}$ $\begin{array}{rrrrr}1492.527 & 1493.82 & 1492.717 & 1495.25 & 1492.59 \\ 1492.6 & 1493.897 & 1492.79 & 1495.328 & 1492.775\end{array}$ $\begin{array}{llllll}1492.71 & 1493.944 & 1492.897 & 1495.339 & 1492.883\end{array}$ $\begin{array}{lllllll}1492.755 & 1493.935 & 1492.935 & 1495.297 & 1492.92\end{array}$ $\begin{array}{llllll}1492.802 & 1493.96 & 1492.98 & 1495.307 & 1492.965\end{array}$ $\begin{array}{lllllll}1489.932 & 1492.666 & 1489.679 & 1495.123 & 1489.727\end{array}$ $\begin{array}{lllll}1489.581 & 1492.556 & 1489.954 & 1494.921 & 1489.938\end{array}$ $\begin{array}{lllllll}1491.243 & 1493.237 & 1491.506 & 1495.083 & 1491.492\end{array}$ $\begin{array}{llllll}1491.901 & 1493.522 & 1492.124 & 1495.156 & 1492.111 \\ 1492.198 & 1493.638 & 1492.399 & 1495.168 & 1492383\end{array}$ $\begin{array}{lllllll}1492.39 & 1493.741 & 1492.584 & 1495.222 & 1492.571\end{array}$ $\begin{array}{llllll}1489.61 & 1492.535 & 1489.374 & 1495.086 & 1489.42\end{array}$ $\begin{array}{llllll}1489.313 & 1492.472 & 1489.708 & 1494.933 & 1489.685\end{array}$ $\begin{array}{llllll}1489.292 & 1492.659 & 1489.731 & 1495.043 & 1489.727\end{array}$ $\begin{array}{llllll}1491.142 & 1493.223 & 1491.404 & 1495.107 & 1491.393\end{array}$ $\begin{array}{rrrrrr}1491.723 & 1493.45 & 1491.949 & 1495.133 & 1491.935 \\ 1492.05 & 1493.608 & 1492.259 & 1495.196 & 14922.3\end{array}$ $\begin{array}{llllll}1492.05 & 1493.608 & 1492.259 & 1495.196 & 1492.243\end{array}$ $\begin{array}{llllll}1491.848 & 1493.575 & 1492.089 & 1495.161 & 1492.081 \\ 1492.106 & 1493.582 & 1492.314 & 1495.116 & 1492.301\end{array}$ $\begin{array}{llllll}1492.336 & 1493.713 & 1492.53 & 1495.182 & 1492.517\end{array}$ $\begin{array}{lllllll}1492.48 & 1493.781 & 1492.665 & 1495.201 & 1492.651\end{array}$ $\begin{array}{llllll}1492.539 & 1493.774 & 1492.722 & 1495.156 & 1492.707\end{array}$ $\begin{array}{llllll}1492.607 & 1493.837 & 1492.788 & 1495.201 & 1492.773\end{array}$ $\begin{array}{lllll}1489.737 & 1492.512 & 1489.483 & 1494.978 & 1489.528\end{array}$ $\begin{array}{llllll}1489.182 & 1492.305 & 1489.57 & 1494.735 & 1489.55 \\ 1491.079 & 1493.039 & 1491.333 & 1494.862 & 149132\end{array}$ $\begin{array}{llllll}1491.0795 & 1493.039 & 1491.333 & 1494.862 & 1491.32\end{array}$ $\begin{array}{rrrrrr}1491.685 & 1493.284 & 141.902 & 1494.909 & 1491.888 \\ 1491.96 & 1493.389 & 1492.165 & 1494.916 & 1492.153\end{array}$ $\begin{array}{llllll}1492.151 & 1493.498 & 1492.345 & 1494.961 & 1492.332\end{array}$ $\begin{array}{llllll}1492.283 & 1493.557 & 1492.47 & 1494.982 & 1492.45\end{array}$ $\begin{array}{llllll}1492.367 & 1493.599 & 1492.553 & 1494.996 & 1492.534\end{array}$
$1494.226 \quad 1494.883 \quad 1495.406$

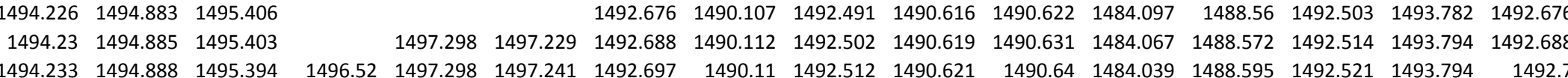
$\begin{array}{llllllllllllllllll}1494.244 & 1494.885 & 1495.387 & 1496.492 & 1497.296 & 1497.227 & 1492.707 & 1490.112 & 1492.523 & 1490.621 & 1490.65 & 1484.041 & 1488.6 & 1492.533 & 1493.805 & 1492.709 & 1495.131 & 1492.692\end{array}$ $\begin{array}{lllllllllllllllllll}1494.249 & 1494.888 & 1455.382 & 1496.469 & 1497.296 & 1497.22 & 1492.714 & 1490.117 & 1492.53 & 1490.623 & 1490.659 & 1484.053 & 1488.609 & 1492.545 & 1493.815 & 1492.717 & 1495.138 & 1492.700\end{array}$ $\begin{array}{llllllllllllllllll}1494.263 & 1494.885 & 1495.37 & 1496.441 & 1497.294 & 1497.208 & 1492.718 & 1490.107 & 1492.537 & 1490.623 & 1490.666 & 1484.055 & 1488.638 & 1422.552 & 1493.808 & 1492.724 & 1495.127 & 1492.708\end{array}$

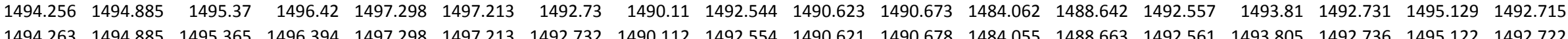


Well

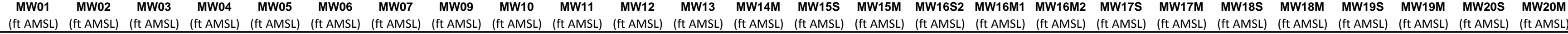

9/15/11 22:00 9/15/11 22:30 $9 / 15 / 1123: 30$ 9/15/1123:110:00 $9 / 16 / 110: 30$ 9/16/111:00 9/16/111:30 9/16/112:00 9/16/11 2:30 9/16/11 3:00 $9 / 16 / 114: 00$ 9/16/114:00 9/16/11 5:00 9/16/11 5:30 9/16/11 6:00 9/16/116:30 9/16/117:00 $9 / 16 / 117: 30$ 9/16/11 8:00 9/16/11 9:00 9/16/11 9:30 9/16/11 10:00 9/16/11 10:30 9/16/11 11:00 9/16/11 11:30 9/16/11 12:00 9/16/11 13:00 9/16/11 13:30 9/16/11 14:00 9/16/11 14:30 9/16/11 15:00 9/16/11 15:30 $9 / 16 / 11116: 30$ 9/16/11 16:30 9/16/11 17:30 9/16/11 18:00 9/16/11 18:30 9/16/11 19:00 9/16/11 19:30 9/16/11 20:00 (16/112100 9/16/11 21:00 9/16/11 22:00 9/16/11 22:30 9/16/11 23:00 9/16/1123:30 9/17/11 0:00 $\begin{array}{lllll}1494.791 & 1495.287 & 1497.128 & 1495.079 \\ 1494.787 & 1495.285 & 1497.121 & 1495.079\end{array}$ $\begin{array}{llll}1494.787 & 1495.285 & 1497.121 & 1495.079\end{array}$ $\begin{array}{rrrrr}1494.787 & 1495.287 & 1497.126 & 1495.081 \\ 1494.789 & 1495292 & 1497.13 & 1495086\end{array}$ $\begin{array}{rrrr}1494.784 & 1495.289 & 1497.142 & 1495.084\end{array}$ $\begin{array}{lllll}1494.787 & 1495.292 & 1497.144 & 1495.086\end{array}$ $\begin{array}{lllll}1494.787 & 1495.296 & 1497.154 & 1495.0091\end{array}$ $\begin{array}{lllll}1494.789 & 1495.301 & 1497.163 & 1495.098\end{array}$ $\begin{array}{lllll}1494.791 & 1495.308 & 1497.168 & 1495.105\end{array}$ $\begin{array}{lllll}1494.794 & 1495.313 & 1497.172 & 1495.114\end{array}$ $\begin{array}{rrrr}1494.796 & 1495.32 & 1497.17 & 1495.121 \\ 1494.796 & 1495.324 & 1497.156 & 1495.128\end{array}$ $\begin{array}{llrl}1494.796 & 1495.324 & 1497.156 & 1495.128 \\ 1494.796 & 1495.329 & 1497.158 & 1495.135\end{array}$ $\begin{array}{llllll}1494.794 & 1495.329 & 1497.161 & 1495.135\end{array}$ $\begin{array}{llll}1494.789 & 1495.324 & 1497.161 & 1455.135\end{array}$ $\begin{array}{lllll}1494.789 & 1495.327 & 1497.158 & 1495.135\end{array}$ $\begin{array}{lllll}1494.794 & 1495.329 & 1497.154 & 1495.137\end{array}$ $\begin{array}{llll}1494.794 & 1495.329 & 1497.147 & 1495.135\end{array}$ $\begin{array}{lllll}1494.791 & 1495.327 & 1497.151 & 1495.121\end{array}$ $\begin{array}{rrrrr}1494.791 & 1495.327 & 1497.149 & 1495.098 \\ 1494.787 & 1495.32 & 1497.151 & 1495.07\end{array}$ $\begin{array}{rrrrr}1494.789 & 1495.324 & 1497.147 & 1495.042\end{array}$ $\begin{array}{lllll}1494.789 & 1495.322 & 1497.144 & 1495.014\end{array}$ $\begin{array}{lllll}1494.789 & 1495.324 & 1497.147 & 1494.983\end{array}$ $\begin{array}{lllll}1494.784 & 1495.32 & 1497.151 & 1494.951\end{array}$ $\begin{array}{llll}1494.787 & 1495.32 & 1497.154 & 1494.92\end{array}$ $\begin{array}{lllll}1494.784 & 1495.317 & 1497.161 & 1494.892\end{array}$ $\begin{array}{lllll}1494.787 & 1495.32 & 14997.17 & 1494.867\end{array}$ $\begin{array}{rrrr}1494.787 & 1495.32 & 1497.179 & 1494.846\end{array}$ $\begin{array}{lllll}1494.789 & 1495.327 & 1497.196 & 1494.832\end{array}$ $\begin{array}{lllll}1494.791 & 1495.334 & 1497.205 & 1494.839\end{array}$ $1494.791 \quad 1495.341 \quad 1497.205 \quad 1494.843$ $\begin{array}{lllll}1494.794 & 1495.345 & 1497.207 & 1494.855\end{array}$ $\begin{array}{lllll}1494.794 & 1495.35 & 1497.207 & 1494.867\end{array}$ $\begin{array}{lllll}1494.796 & 1495.355 & 1497.212 & 1494.881\end{array}$ $\begin{array}{lllll}1494.796 & 1495.359 & 1497.207 & 1494.895\end{array}$ $\begin{array}{llll}1494.796 & 1455.362 & 1497.203 & 14494.92\end{array}$ $\begin{array}{lllll}1494.794 & 1495.362 & 1497.198 & 1494.93\end{array}$ $\begin{array}{lllll}1494.794 & 1495.359 & 1497.196 & 1494.939\end{array}$ $\begin{array}{llllll}1494.794 & 1495.362 & 1497.196 & 1494.946\end{array}$ $\begin{array}{lllll}1494.789 & 1495.357 & 1497.191 & 1494.953\end{array}$ $\begin{array}{llll}1494.789 & 1495.355 & 1497.175 & 1494.962\end{array}$ $1494.791 \quad 1495.357 \quad 1497.1651494 .972$ $\begin{array}{lllll}1494.784 & 1495.352 & 1497.161 & 1494.972 \\ 1494.782 & 1495348 & 1497.156 & 1494974\end{array}$ $\begin{array}{llll}1494.782 & 1455.343 & 1497.165 & 1494.974\end{array}$ $\begin{array}{llllll}1494.782 & 1495.341 & 1497.158 & 1494.976\end{array}$ $\begin{array}{llll}1494.777 & 1495.334 & 1497.161 & 1494.976\end{array}$ $\begin{array}{lllll}1494.782 & 1495.338 & 1497.177 & 1494.988\end{array}$ $\begin{array}{llll}1494.777 & 1495.336 & 1497.182 & 1494.988\end{array}$ $\begin{array}{rrrr}1494.78 & 1495.336 & 1497.175 & 1494.995 \\ 1494.784 & 1495.341 & 1497.175 & 1495.004\end{array}$ $\begin{array}{llllllllllllllllll}1494.266 & 1494.885 & 1495.363 & 1496.38 & 1497.298 & 1497.213 & 1492.739 & 1490.105 & 1492.559 & 1490.623 & 1490.683 & 1484.058 & 1488.661 & 1492.571 & 1493.815 & 1492.743 & 1495.129 & 1492.729\end{array}$ $\begin{array}{lllllllllllllllllll}1494.263 & 1494.888 & 1495.353 & 1496.36 & 1497.301 & 1497.217 & 1492.744 & 1490.105 & 1492.563 & 1490.623 & 1490.682 & 1484.062 & 1488.68 & 1492.575 & 1493.817 & 1492.747 & 1495.127 & 1492.729\end{array}$

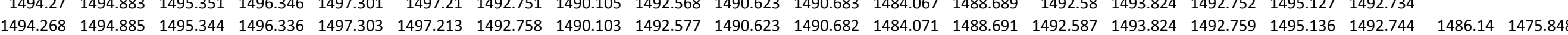
$\begin{array}{llllllllllllllllllll}1494.256 & 1494.888 & 1495.346 & 1496.318 & 1497.312 & 1497.217 & 1492.76 & 1490.103 & 1492.58 & 1490.626 & 1490.687 & 1484.074 & 1488.71 & 1492.589 & 1493.822 & 1492.762 & 1495.122 & 1492.746 & 1486.14 & 1476.378\end{array}$ $\begin{array}{lllllllllllllllllllll}1494.256 & 1494.888 & 1495.342 & 1496.306 & 1497.315 & 1497.229 & 1492.77 & 1490.103 & 1492.587 & 1490.626 & 1490.692 & 1484.076 & 1488.715 & 1492.594 & 1493.833 & 1492.769 & 1495.134 & 1492.753 & 1486.14 & 1476.708\end{array}$ \begin{tabular}{lllllllllllllllllllll}
1494.252 & 1494.89 & 1495.342 & 1496.297 & 1497.319 & 1497.229 & 1492.774 & 1490.1 & 1492.591 & 1490.628 & 1490.694 & 1484.065 & 1488.72 & 1492.603 & 1493.84 & 1492.773 & 1495.145 & 1492.758 & 1486.14 & 1476.925 \\
\hline
\end{tabular}

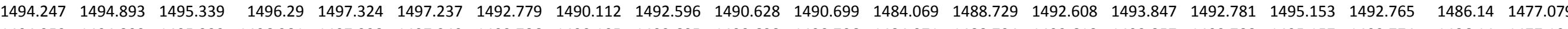

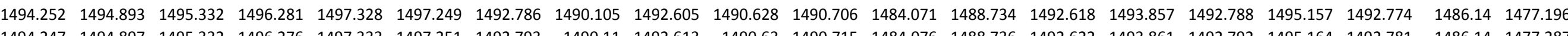

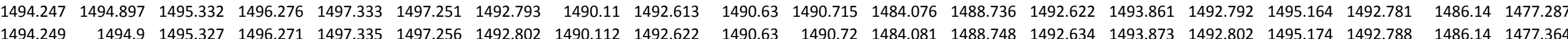
$\begin{array}{llllllllllllllllllll}1494.249 & 1494.9 & 1495.327 & 1496.271 & 1497.335 & 1497.256 & 1492.802 & 1490.112 & 1492.622 & 1490.63 & 1490.72 & 1484.081 & 1488.748 & 1492.634 & 1493.873 & 1492.802 & 1495.174 & 1492.788 & 1486.14 & 1477.364 \\ 1494.261 & 1494.897 & 1495323 & 1496264 & 1497.333 & 1497.253 & 149281 & 1490114 & 1492.631 & 1490633 & 1490727 & 1484088 & 1488748 & 1492641 & 1493878 & 1492814 & 1495181 & 1492.798 & 1486.14 & 1477.429\end{array}$ $\begin{array}{lllllllllllllllllllllll}1494.266 & 1494.897 & 1495.318 & 1496.257 & 1497.333 & 1497.239 & 1492.817 & 1490.11 & 1492.636 & 1490.635 & 1490.734 & 1484.09 & 1488.762 & 1492.648 & 1493.889 & 1492.818 & 1495.185 & 1492.802 & 1486.14 & 1477.488\end{array}$ \begin{tabular}{llllllllllllllllllllll}
1494.27 & 1494.897 & 1495.318 & 1496.248 & 1497.335 & 1497.241 & 1492.824 & 1490.119 & 1492.641 & 1490.635 & 1490.743 & 1484.095 & 1488.769 & 1492.653 & 1493.887 & 1492.823 & 1495.183 & 1492.809 & 1486.14 & 1477.537 \\
\hline
\end{tabular} $\begin{array}{lllllllllllllllllllll}1494.27 & 1494.9 & 1495.313 & 1496.232 & 1497.338 & 1497.239 & 1492.828 & 1490.117 & 1492.648 & 1490.633 & 1490.748 & 1484.097 & 1488.788 & 1492.662 & 1493.882 & 1492.828 & 1495.176 & 1492.812 & 1486.14 & 1477.581\end{array}$

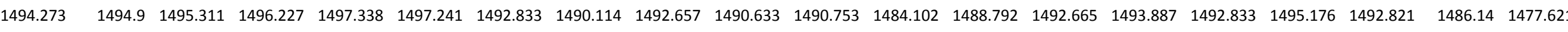
\begin{tabular}{lllllllllllllllllllll}
1494.277 & 1494.9 & 1495.308 & 1496.218 & 1497.335 & 1497.237 & 1492.023 & 1490.112 & 1492.448 & 1490.635 & 1490.753 & 1484.106 & 1488.795 & 1492.458 & 1493.719 & 1491.883 & 1495.181 & 1492.002 & 1486.143 & 1477.658 \\
\hline
\end{tabular}

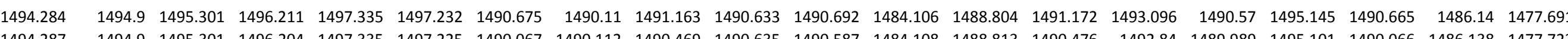

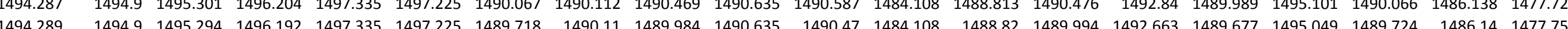

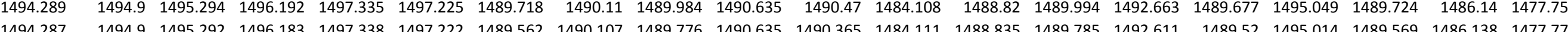
$\begin{array}{lllllllllllllllllllll}1494.289 & 1494.898 & 1495.289 & 1496.176 & 1497.335 & 1497.225 & 1489.026 & 1490.107 & 1489.377 & 1490.633 & 1490.264 & 1484.115 & 1488.835 & 1489.386 & 1492.422 & 1488.959 & 1494.983 & 1489.036 & 1486.14 & 1477.8\end{array}$ \begin{tabular}{llllllllllllllllllll}
1494.292 & 1494.897 & 1495285 & 1496.169 & 1497.335 & 1497.215 & 1488.605 & 1490.105 & 1488.981 & 1490.533 & 1490.157 & 1484.12 & 1488.839 & 1488.993 & 1492.25 & 1488.535 & 1494.953 & 1488.616 & 1486.141 & 1477.824 \\
\hline
\end{tabular} \begin{tabular}{lllllllllllllllllllllll}
1494.287 & 1494.898 & 1495.285 & 1496.16 & 1497.338 & 1497.213 & 1488.251 & 1490.105 & 1488.61 & 1490.633 & 1490.047 & 1484.12 & 1488.844 & 1488.622 & 1492.103 & 1488.189 & 1494.92 & 1488.269 & 1486.138 & 1477.845 \\
\hline
\end{tabular}

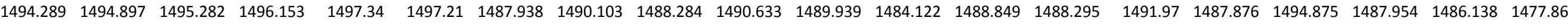

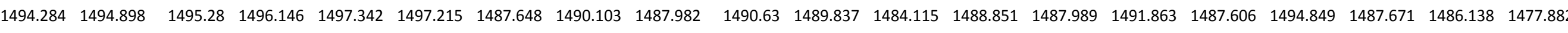

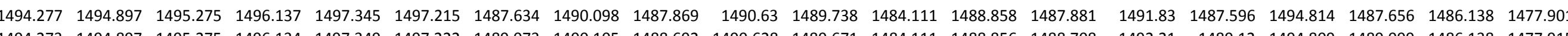

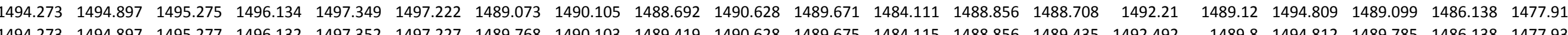

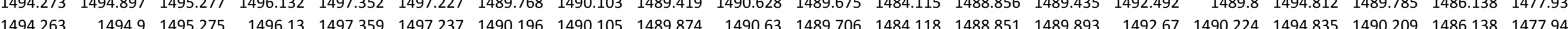
$\begin{array}{llllllllllllllllllll}1494.261 & 1494.902 & 1495.273 & 1496.125 & 1497.363 & 1497.246 & 1490.505 & 1490.107 & 1490.207 & 1490.63 & 1489.743 & 1484.118 & 1488.863 & 1490.218 & 1492.789 & 1490.527 & 1494.849 & 1490.514 & 1486.143 & 1477.961 \\ \end{array}$ $\begin{array}{llllllllllllllllllllll}1494.259 & 1494.905 & 1495.27 & 1496.125 & 1497.368 & 1497.253 & 1490.748 & 1490.11 & 1490.465 & 1490.63 & 1489.78 & 1484.115 & 1488.849 & 1490.481 & 1492.898 & 1490.767 & 1494.873 & 1490.754 & 1486.141 & 1477.971\end{array}$

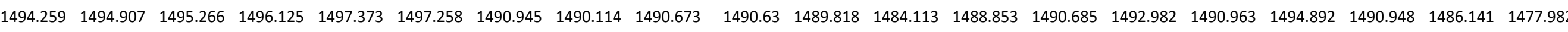
$\begin{array}{llllllllllllllllllllll}1494.256 & 1494.909 & 1495.268 & 1496.125 & 1497.375 & 1497.263 & 1491.108 & 1490.112 & 1490.849 & 1490.633 & 1489.858 & 1484.111 & 1488.858 & 1490.859 & 1493.052 & 1491.122 & 1494.91 & 1491.11 & 1486.141 & 1477.997\end{array}$

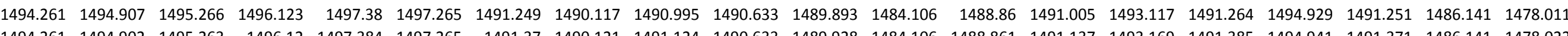

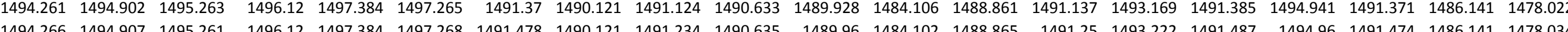

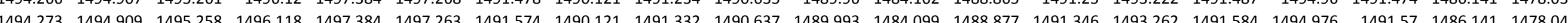

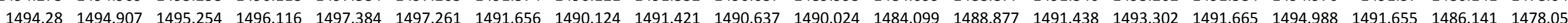
$\begin{array}{lllllllllllllllllllllll}1494.284 & 1494.907 & 1495.254 & 1496.109 & 1497.384 & 1497.256 & 1491.731 & 1490.124 & 1491.501 & 1490.635 & 1490.051 & 1484.097 & 1488.886 & 1491.508 & 1493.334 & 1491.738 & 1494.993 & 1491.725 & 1486.141 & 1478.067\end{array}$ $\begin{array}{llllllllllllllllllll}1494.292 & 1494.912 & 1495.254 & 1496.104 & 1497.387 & 1497.251 & 1491.773 & 1490.121 & 1491.546 & 1490.635 & 1490.084 & 1484.085 & 1488.889 & 1491.56 & 1493.36 & 1491.781 & 1494.997 & 1491.77 & 1486.138 & 1478.076\end{array}$ \begin{tabular}{llllllllllllllllllllll}
1494.296 & 1494.909 & 1495.251 & 1496.1 & 1497.384 & 1497.249 & 1491.85 & 1490.128 & 1491.628 & 1490.635 & 1490.108 & 1484.088 & 1488.889 & 1491.64 & 1493.393 & 1491.856 & 1495.009 & 1491.845 & 1486.141 & 1478.085 \\
\hline
\end{tabular}

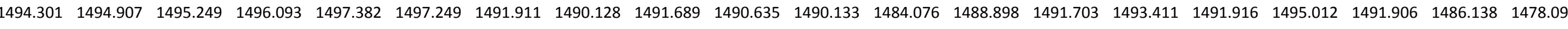

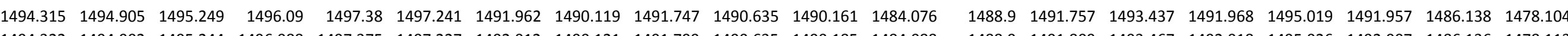
$\begin{array}{llllllllllllllllllll}1494.322 & 1494.902 & 1495.244 & 1496.088 & 1497.375 & 1497.227 & 1492.012 & 1490.121 & 1491.799 & 1490.635 & 1490.185 & 1484.088 & 1488.9 & 1491.809 & 1493.467 & 1492.018 & 1495.026 & 1492.007 & 1486.136 & 1478.113 \\ 1494331 & 14949 & 1495242 & 1496079 & 1497.37 & 1497217 & 1492.056 & 1490117 & 1491843 & 1490633 & 1490208 & 1484092 & 1488912 & 1491854 & 1493.472 & 1492.06 & 1495019 & 1492047 & 1486136 & 147812\end{array}$

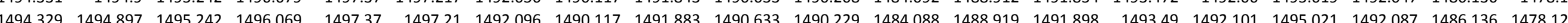
$\begin{array}{lllllllllllllllllllll}1494.329 & 1494.897 & 1495.242 & 1496.062 & 1497.373 & 1497.208 & 1492.131 & 1490.114 & 1491.923 & 1490.63 & 1490.248 & 1484.09 & 1488.926 & 1491.936 & 1493.509 & 1492.134 & 1495.019 & 1492.124 & 1486.136 & 1478.134\end{array}$ $\begin{array}{lllllllllllllllllllll}1494.322 & 1494.897 & 1495.237 & 1496.055 & 1497.37 & 1497.217 & 1492.166 & 1490.107 & 1491.958 & 1490.628 & 1490.269 & 1484.092 & 1488.926 & 1491.971 & 1493.518 & 1492.169 & 1495.019 & 1492.155 & 1486.136 & 1478.139\end{array}$ $\begin{array}{llllllllllllllllllllll}1494.329 & 1494.895 & 1495.239 & 1496.046 & 1497.368 & 1497.208 & 1492.196 & 1490.107 & 1491.991 & 1490.628 & 1490.288 & 1484.095 & 1488.94 & 1492 & 1493.532 & 1492.202 & 1495.021 & 1492.187 & 1486.134 & 1478.146\end{array}$

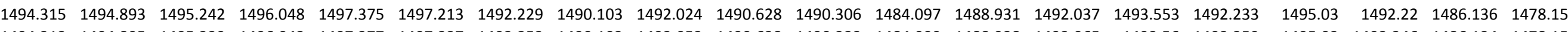
$\begin{array}{llllllllllllllllllll}1494.313 & 1494.895 & 1495.239 & 1496.042 & 1497.377 & 1497.227 & 1492.253 & 1490.103 & 1492.052 & 1490.628 & 1490.323 & 1484.099 & 1488.938 & 1492.065 & 1493.56 & 1492.259 & 1495.03 & 1492.246 & 1486.134 & 1478.158 \\ \end{array}$

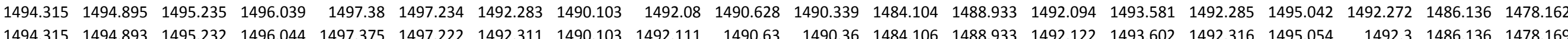


Well

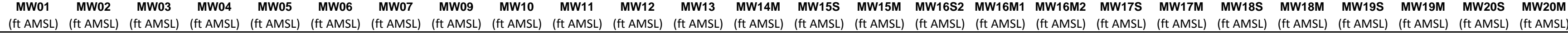

9/17/11 1:00 $9 / 17 / 111: 30$ $9 / 17 / 112.30$ 9/17/112:30 $9 / 17 / 113: 00$ 9/17/11 4:00 9/17/11 4:30 9/17/11 5:00 9/17/11 5:30 9/17/11 6:00 / $/ 17 / 1117: 00$ 9/17/117:30 9/17/11 8:00 9/17/11 8:30 9/17/11 9:00 9/17/11 9:30 9/17/11 10:00 /17/11 10:30 9/177/11 11:00 (17/11112:00 9/17/11 12:30 9/17/11 13:00 9/17/11 13:30 9/17/11 14:00 9/17/11 14:30 9/17/11 15:00 9/17/11 16:00 9/17/11 16:30 9/17/11 17:00 9/17/11 17:30 9/17/111 18:00 9/17/11 18:30 9/17/11 19:00 9/17/11 19:30 9/17/11 20:30 9/17/11 21:00 9/17/11 21:30 9/17/11 22:00 9/17/11 22:30 9/17/11 23:00 $9 / 18 / 110.00$ 9/18/110:00 9/18/110:30 9/18/11 1:30 9/18/112:00 9/18/11 2:30 9/18/11 3:00 $\begin{array}{llll}1494.784 & 1495.343 & 1497.184 & 1495.014 \\ 1494.782 & 1495.341 & 1497.193 & 1495.018\end{array}$ $\begin{array}{rrrr}1494.782 & 1495.341 & 1497.193 & 1495.018 \\ 1494.78 & 1953341 & 1977.193 & 195.023\end{array}$ $\begin{array}{llll}1494.78 & 1495.341 & 1497.193 & 1495.023\end{array}$ $\begin{array}{lllll}1494.782 & 1495.35 & 1497.196 & 1495.042\end{array}$ $\begin{array}{lllll}1494.782 & 1495.35 & 1497.186 & 1495.049\end{array}$ $\begin{array}{llllll}1494.787 & 1495.355 & 1497.196 & 1495.058\end{array}$ $\begin{array}{lllll}1494.78 & 1495.355 & 1497.191 & 1495.06\end{array}$ $\begin{array}{llll}1494.78 & 1495.352 & 1497.212 & 1495.063\end{array}$ $\begin{array}{rrrr}1494.784 & 1495.357 & 1497.233 & 1495.07 \\ 1499.784 & 1495357 & 1497.235 & 1495077\end{array}$ $\begin{array}{llll}1494.784 & 1495.357 & 1497.235 & 1495.077 \\ 1494.794 & 1495373 & 1497.186 & 1955093\end{array}$ $\begin{array}{llll}1494.794 & 1495.373 & 1497.186 & 1495.093 \\ 1494.798 & 1495.383 & 1497.179 & 1495.109\end{array}$ $\begin{array}{lllll}1494.789 & 1495.383 & 1497.184 & 1495.114\end{array}$ $\begin{array}{lllll}1494.775 & 1495.362 & 1497.196 & 1455.114 \\ 1495.1\end{array}$ $\begin{array}{rlrrr}1494.777 & 1495.359 & 1497.191 & 1495.098\end{array}$ $\begin{array}{lllll}1494.782 & 1495.364 & 1497.191 & 1495.102\end{array}$ $\begin{array}{llll}1494.784 & 1495.366 & 1497.198 & 1495.107\end{array}$ $\begin{array}{lllll}1494.782 & 1495.364 & 1497.198 & 1495.107\end{array}$ $\begin{array}{llll}1494.784 & 1495.364 & 1497.205 & 1495.114 \\ 1494.784 & 1495369 & 1497.212 & 1495.119\end{array}$ $\begin{array}{lllll}1494.784 & 1455.366 & 1497.217 & 1495.121\end{array}$ $\begin{array}{lllll}1494.787 & 1495.371 & 1497.221 & 1495.128\end{array}$ $\begin{array}{lllll}1494.789 & 1495.373 & 1497.226 & 1495.135\end{array}$ $\begin{array}{lllll}1494.789 & 1495.38 & 1497.231 & 1495.14\end{array}$ $\begin{array}{llll}1494.791 & 1495.378 & 1497.238 & 1495.144\end{array}$ $\begin{array}{lllll}1494.794 & 1495.383 & 1497.24 & 1495.151\end{array}$ $\begin{array}{llll}1494.794 & 1495.385 & 1497.242 & 1495.158\end{array}$ $\begin{array}{lllll}1494.794 & 1495.39 & 1497.249 & 1495.163 \\ 1494.794 & 1495394 & 1497.261 & 1495.168\end{array}$ $\begin{array}{llll}1494.796 & 1495.397 & 1497.261 & 1495.173\end{array}$ $\begin{array}{llll}1494.798 & 1495.406 & 1497.256 & 1495.18\end{array}$ $\begin{array}{llllll}1494.803 & 1495.411 & 1497.278 & 1495.194\end{array}$ $\begin{array}{lllll}1494.803 & 1495.415 & 1497.278 & 1495.198\end{array}$ $\begin{array}{lllll}1494.801 & 1495.415 & 1497.254 & 1495.2\end{array}$ $\begin{array}{llll}1494.808 & 1495.425 & 1497.235 & 1495.212 \\ 1498.805 & 1495.425 & 1497.231 & 195217\end{array}$ $\begin{array}{llll}1494.805 & 1495.425 & 1497.231 & 1495.217\end{array}$ $\begin{array}{rrrrr}1494.801 .791 & 1495.42 & 1497.242 & 1495.215 \\ 1411 & 1497.242 & 1495203\end{array}$ $\begin{array}{lllll}1494.796 & 1495.411 & 1497.247 & 1495.203\end{array}$ $\begin{array}{lllll}1494.803 & 1495.415 & 1497.233 & 1495.212\end{array}$ $\begin{array}{lllll}1494.803 & 1495.413 & 1497.233 & 1495.21\end{array}$ $\begin{array}{lllll}1494.803 & 1495.415 & 1497.24 & 1495.215\end{array}$ $\begin{array}{lllll}1494.798 & 1495.413 & 1497.226 & 1495.208\end{array}$ $1494.798 \quad 1495.408$ 1497.221 1495.208 $\begin{array}{rrrr}1494.798 & 1495.408 & 1497.214 & 1495.21 \\ 1494.798 & 1495.408 & 1497.196 & 1495.205\end{array}$ $\begin{array}{llllll}1494.798 & 1495.404 & 1497.214 & 1495.201\end{array}$ $\begin{array}{llll}1494.789 & 1495.394 & 1497.214 & 1495.194\end{array}$ $\begin{array}{llllll}1494.787 & 1495.385 & 1497.207 & 1495.184\end{array}$ $\begin{array}{llllll}1494.801 & 1495.399 & 1497.207 & 1495.196\end{array}$ $\begin{array}{lllll}1494.798 & 1495.397 & 1497.203 & 1495.196\end{array}$ $\begin{array}{llll}1494.794 & 1495.392 & 1497.151 & 1495.189 \\ 1494.798 & 1495.392 & 1497.128 & 1495.191\end{array}$

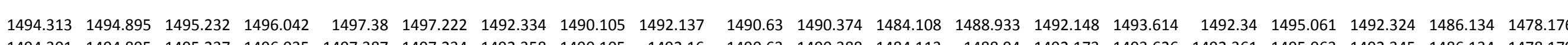

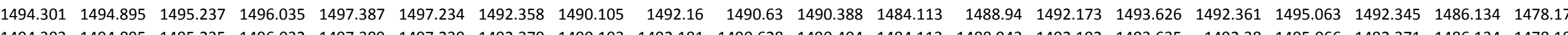

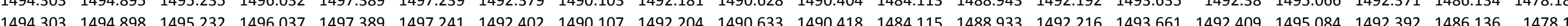

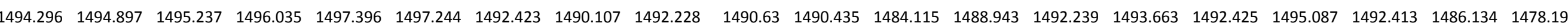
$\begin{array}{llllllllllllllllllllll}1494.31 & 1494.897 & 1495228 & 1496035 & 1497.387 & 1497244 & 1492.442 & 1490107 & 1492.251 & 1490.633 & 1490.449 & 1484.106 & 1488.947 & 1492.263 & 1493.677 & 1492.444 & 1495.091 & 1492.431 & 1486.134 & 14782\end{array}$ $\begin{array}{lllllllllllllllllllllll}1494.313 & 1494.898 & 1495.232 & 1496.037 & 1497.389 & 1497.232 & 1492.463 & 1490.107 & 1492.27 & 1490.633 & 1490.465 & 1484.106 & 1488.938 & 1492.279 & 1493.696 & 1492.465 & 1495.101 & 1492.45 & 1486.136 & 1478.204\end{array}$ $\begin{array}{llllllllllllllllllllll}1494.31 & 1494.9 & 1495.228 & 1496.032 & 1497.408 & 1497.244 & 1492.482 & 1490.11 & 1492.289 & 1490.633 & 1490.477 & 1484.108 & 1488.952 & 1492.298 & 1493.693 & 1492.484 & 1495.103 & 1492.469 & 1486.134 & 1478.2096\end{array}$

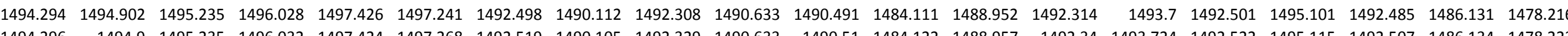

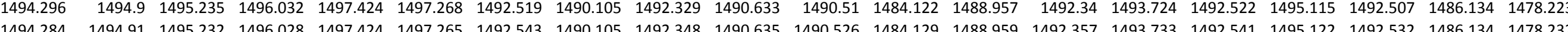
$\begin{array}{llllllllllllllllllllll}1494.284 & 1494.91 & 1495.232 & 1496.028 & 1497.424 & 1497.265 & 1492.543 & 1490.105 & 1492.348 & 1490.635 & 1490.526 & 1484.129 & 1488.959 & 1492.357 & 1493.733 & 1492.541 & 1495.122 & 1492.532 & 1486.134 & 1478.232 \\ 1494.313 & 1494.902 & 1495.223 & 1496039 & 1497.428 & 1497.263 & 1492568 & 1490114 & 1492376 & 1490637 & 1490549 & 1484143 & 1488.952 & 1492385 & 1493761 & 1492.57 & 1495.148 & 1492556 & 1486136 & 1478246\end{array}$

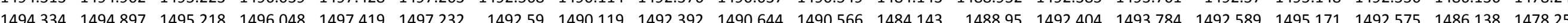
$\begin{array}{lllllllllllllllllllll}1494.336 & 14949 & 149522 & 1496044 & 1497.41 & 1497.217 & 1492.601 & 1490121 & 1492413 & 1490644 & 1490.577 & 1484145 & 1488961 & 1492.423 & 1493.78 & 1492.605 & 1495.162 & 1492.591 & 1486.136 & 1478.26\end{array}$ $\begin{array}{lllllllllllllllllllll}1494.324 & 1494.902 & 1495.228 & 1496.028 & 1497.382 & 1497.206 & 1492.608 & 1490.117 & 1492.423 & 1490.642 & 1490.584 & 1484.145 & 1488.989 & 1492.432 & 1493.763 & 1492.61 & 1495.122 & 1492.593 & 1486.129 & 1478.263\end{array}$

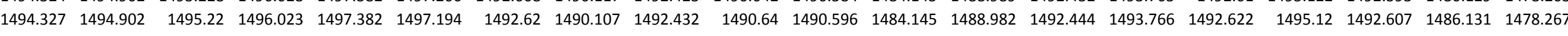
$\begin{array}{lllllllllllllllllllllll}1494.327 & 1494.902 & 1495.218 & 1496.023 & 1497.37 & 1497.237 & 1492.634 & 1490.112 & 1492.446 & 1490.637 & 1490.61 & 1484.148 & 1488.98 & 1492.458 & 1493.777 & 1492.636 & 1495.138 & 1492.622 & 1486.131 & 1478.272 & \end{array}$

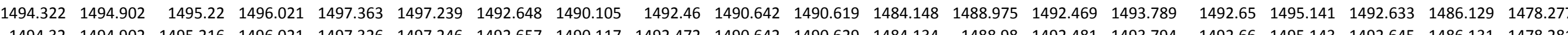

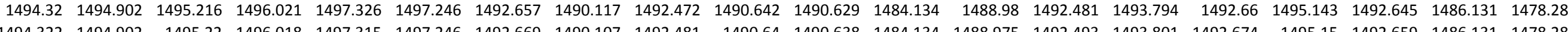

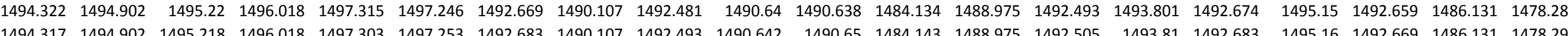
\begin{tabular}{llllllllllllllllllllll}
1494.306 & 1494.907 & 1495.22 & 1496.016 & 1497.298 & 1497.258 & 1492.559 & 1490.11 & 1492366 & 1490.644 & 1490.655 & 1484.143 & 1488.98 & 1492.376 & 1493.796 & 1492558 & 1495.155 & 1492.549 & 1486.131 & 14782293 \\
\hline
\end{tabular} $\begin{array}{lllllllllllllllllllll}1494.31 & 1494.907 & 1495216 & 1496018 & 1497291 & 1497263 & 1492533 & 1490.105 & 1492333 & 1490.644 & 1490.629 & 1484145 & 1488973 & 1492345 & 1493763 & 1492.532 & 1495.167 & 1492.521 & 1486.131 & 1478295\end{array}$ $\begin{array}{llllllllllllllllllllll}1494.308 & 1494.909 & 1495.216 & 1496.021 & 1497.296 & 1497.265 & 1492.599 & 1490.11 & 1492.406 & 1490.647 & 1490.631 & 1484.15 & 1488.968 & 1492.413 & 1493.789 & 1492.598 & 1495.169 & 1492.586 & 1486.131 & 1478.3\end{array}$

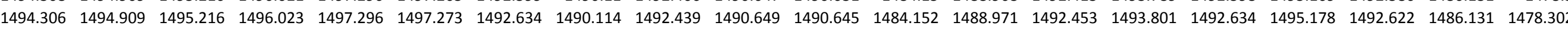

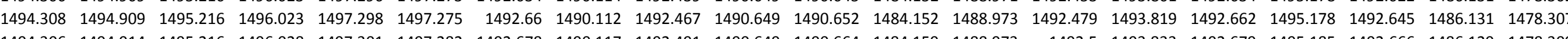

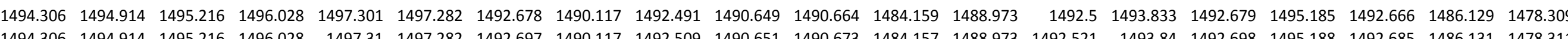

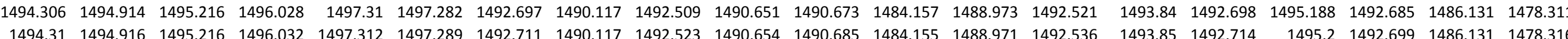
$\begin{array}{llllllllllllllllllll}1494.31 & 1494.916 & 1495.216 & 1496.032 & 1497.312 & 1497.289 & 1492.711 & 1490.117 & 1492.523 & 1490.654 & 1490.685 & 1484.155 & 1488.971 & 1492.536 & 1493.85 & 1492.714 & 1495.2 & 1492.699 & 1486.131 & 1478.316 \\ 1494.303 & 1494.919 & 1495213 & 1496.035 & 1497315 & 1497292 & 1492714 & 1490.117 & 1492.526 & 1490.654 & 1490.697 & 1484.148 & 1488.973 & 1492.54 & 1493.854 & 1492714 & 1495202 & 1492.701 & 1486.129 & 1478319\end{array}$ $\begin{array}{llllllllllllllllllllll}1494.306 & 1494.921 & 1495213 & 1496.037 & 1497.322 & 1497.304 & 1492.735 & 1490.121 & 1492.547 & 1490.656 & 1490.704 & 1484.145 & 1488.971 & 1492.559 & 1493.866 & 1492.733 & 1495.209 & 1492.718 & 1486.131 & 1478.323\end{array}$ $\begin{array}{lllllllllllllllllllll}1494.31 & 1494.921 & 1495.213 & 1496.039 & 1497.331 & 1497.306 & 1492.749 & 1490.119 & 1492.563 & 1490.658 & 1490.715 & 1484.092 & 1488.973 & 1492.573 & 1493.878 & 1492.75 & 1495.216 & 1492.737 & 1486.131 & 1478.328\end{array}$ $\begin{array}{llllllllllllllllllllllll}1494.313 & 1494.924 & 1495.211 & 1496.046 & 1497.331 & 1497.296 & 1492.765 & 1490.126 & 1492.58 & 1490.66 & 1490.724 & 1484.053 & 1488.968 & 1492.587 & 1493.894 & 1492.764 & 1495.23 & 1492.751 & 1486.131 & 1478.333 \\ \end{array}$ $\begin{array}{llllllllllllllllllllll}1494.306 & 1494.928 & 1495.216 & 1496.048 & 1497.356 & 1497.318 & 1492.777 & 1490.133 & 1492.591 & 1490.663 & 1490.736 & 1484.053 & 1488.978 & 1492.601 & 1493.899 & 1492.778 & 1495.235 & 1492.765 & 1486.127 & 1478.335\end{array}$

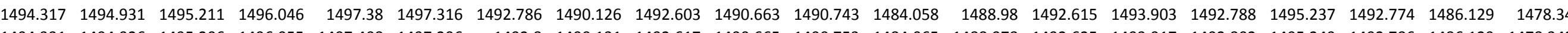

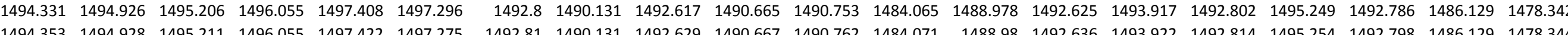

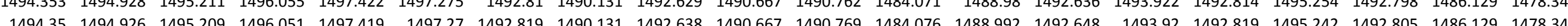

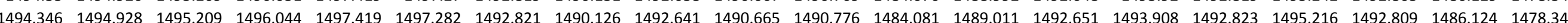
$\begin{array}{llllllllllllllllllllllll}1494.348 & 1494.929 & 1495.204 & 1496.042 & 1497.419 & 1497.282 & 1492.831 & 1490.121 & 1492.65 & 1490.665 & 1490.781 & 1484.085 & 1489.001 & 1492.662 & 1493.913 & 1492.83 & 1495.223 & 1492.814 & 1486.127 & 1478.351 \\ \end{array}$ $\begin{array}{llllllllllllllllllll}1494.346 & 1494.931 & 1495.209 & 1496.044 & 1497.422 & 1497.282 & 1492.84 & 1490.131 & 1492.657 & 1490.667 & 1490.792 & 1484.095 & 1488.992 & 1492.669 & 1493.929 & 1492.842 & 1495.239 & 1492.826 & 1486.129 & 1478.358\end{array}$

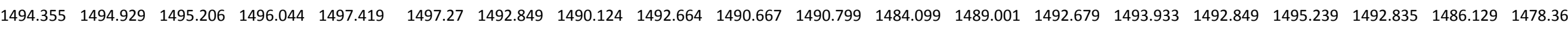

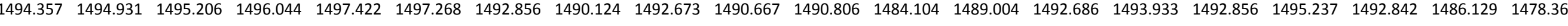

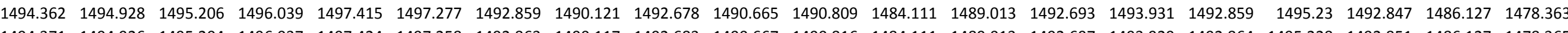

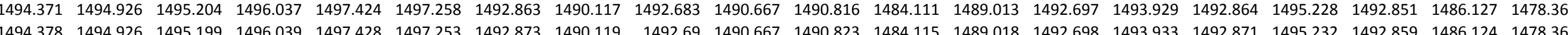

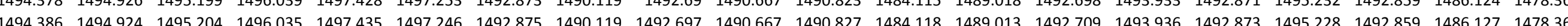
$\begin{array}{lllllllllllllllllllllll}1494.386 & 1494.922 & 1495.209 & 1496.03 & 1497.426 & 1497.22 & 1492.877 & 1490.114 & 1492.699 & 1490.667 & 1490.832 & 1484.106 & 1489.025 & 1492.709 & 1493.931 & 1492.878 & 1495.221 & 1492.863 & 1486.124 & 1478.368\end{array}$ $\begin{array}{lllllllllllllllllllll}1494.369 & 1494.924 & 1495.204 & 1496.023 & 1497.428 & 1497.246 & 1492.877 & 1490.11 & 1492.704 & 1490.663 & 1490.837 & 1484.104 & 1489.036 & 1492.712 & 1493.917 & 1492.88 & 1495.204 & 1492.866 & 1486.122 & 1478.368\end{array}$ \begin{tabular}{llllllllllllllllllllll}
1494.378 & 1494.921 & 1495.199 & 1496.016 & 1497.438 & 1497.249 & 1492.88 & 1490.107 & 1492.704 & 1490.663 & 1490.839 & 1484.104 & 1489.048 & 1492.712 & 1493.913 & 1492.88 & 1495.19 & 1492.866 & 1486.122 & 1478.37 \\
\hline
\end{tabular} \begin{tabular}{lllllllllllllllllllll}
1494.383 & 1494.924 & 1495.197 & 1496.023 & 1497.442 & 1497.237 & 1492.887 & 1490.11 & 1492.711 & 1490.663 & 1490.844 & 1484.108 & 1489.018 & 1492.721 & 1493.936 & 1492.89 & 1495.221 & 1492.875 & 1486.124 & 1478.372 \\
\hline
\end{tabular}

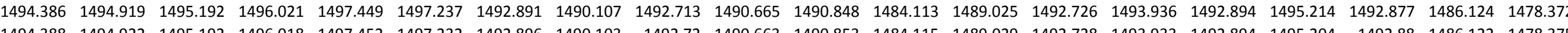

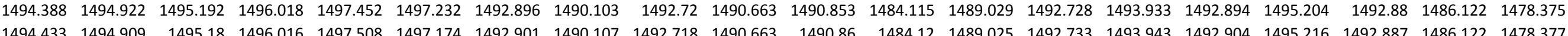


Well

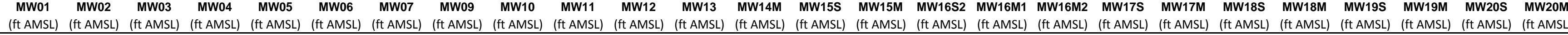

9/18/11 4:00 $9 / 18 / 114: 30$ $9 / 18 / 115: 00$ 9/18/115:30 9/18/116:30 9/18/116:30 9/18/117:30 9/18/11 8:00 $9 / 18 / 118: 30$ 9/18/11 9:00 9/18/11 10:00 9/18/11 10:00 9/18/111 10:30 9/18/11 11:30 9/18/11 12:00 /18/11 13:00 9/18/1113:30 9/18/11 14:00 /18/11 15:00 9/18/11 15:00 9/18/11 16:00 9/18/11 16:30 9/18/11 17:00 9/18/1117:30 9/18/11 18:00 9/18/1119:00 9/18/11 19:30 9/18/11 20:00 9/18/11 20:30 9/18/11 21:00 9/18/1121:30 9/18/11 22:00 9/18/11 22:30 9/18/11 23:00 9/19/110:00 9/19/11 0:30 9/19/111:00 9/19/11 1:30 //19/11 2:30 9/19/11 3:00 9/19/11 4:00 9/19/11 4:30 9/19/11 5:00 9/19/11 5:30 9/19/11 6:00 $\begin{array}{rrrr}1494.794 & 1495.39 & 1497.151 & 1495.184 \\ 1494.768 & 1495.357 & 1497.149 & 1495.154\end{array}$ $\begin{array}{llll}1494.768 & 1495.357 & 1497.149 & 1495.154 \\ 1494777 & 1459355 & 1497.196 & 1455.147\end{array}$ $\begin{array}{rrrr}1494.777 & 1495.355 & 1497.196 & 1495.147\end{array}$ $\begin{array}{rrrr}1494.787 & 1495.359 & 1497.198 & 1495.154\end{array}$ $\begin{array}{llll}1494.808 & 1495.387 & 1497.205 & 1495.175\end{array}$ $\begin{array}{lllll}1494.791 & 1495.378 & 1497.137 & 1495.161\end{array}$ $\begin{array}{lllll}1494.798 & 1495.378 & 1497.13 & 1495.149\end{array}$ $\begin{array}{llll}1494.794 & 1495.38 & 1497.128 & 1495.128\end{array}$ $\begin{array}{rrrr}1494.773 & 1495.35 & 1497.13 & 1495.072\end{array}$ $\begin{array}{rrrr}1494.777 & 1495.345 & 1497.126 & 1495.039 \\ 1194.78 & 1495338 & 1497.123 & 1455.004\end{array}$ $\begin{array}{llll}1494.78 & 1495.338 & 1497.123 & 1495.004 \\ 1494.78 & 1495.338 & 1497.125 & 1494.972\end{array}$ $\begin{array}{lllll}1494.775 & 1495.331 & 1497.126 & 1494.972 .934\end{array}$ $\begin{array}{rrrrr}1494.775 & 1495.329 & 1497.13 & 1494.904\end{array}$ $\begin{array}{lllll}1494.777 & 1495.327 & 1497.123 & 1494.876\end{array}$ $\begin{array}{lllll}1494.775 & 1495.324 & 1497.121 & 1494.855\end{array}$ $\begin{array}{rrrrr}1494.777 & 1495.324 & 1497.126 & 1494.841 \\ 149477 & 1495317 & 1497.126 & 149.825\end{array}$ $\begin{array}{lllll}1494.77 & 1495.317 & 1497.126 & 1494.825\end{array}$ $\begin{array}{rrrrr}1494.77 & 1495.317 & 1497.128 & 1494.822 \\ 1494.773 & 1495.315 & 1497.13 & 1494822\end{array}$ $\begin{array}{rrrrr}1494.773 & 1495.315 & 1497.128 & 14944.827\end{array}$ $\begin{array}{lllll}1494.77 & 1495.313 & 1497.128 & 1494.832\end{array}$ $\begin{array}{lllll}1494.77 & 1495.31 & 1497.13 & 1494.839\end{array}$ $\begin{array}{lllll}1494.77 & 1495.31 & 1497.126 & 1494.846\end{array}$ $\begin{array}{lllll}1494.77 & 1495.31 & 1497.126 & 1494.857\end{array}$ $\begin{array}{lllll}1494.768 & 1495.31 & 1497.119 & 1494.867\end{array}$ $\begin{array}{lllll}1494.766 & 1495.303 & 1497.116 & 1494.876\end{array}$ $\begin{array}{lllll}1494.763 & 1495.306 & 1497.112 & 1494.883 \\ 1494.761 & 1495299 & 1497.105 & 1494.89\end{array}$ $\begin{array}{lllll}1494.763 & 1495.299 & 1497.095 & 1494.897\end{array}$ $\begin{array}{lllll}1494.761 & 1495.299 & 1497.086 & 1494.906\end{array}$ $\begin{array}{lllll}1494.756 & 1495.292 & 1497.072 & 1494.911\end{array}$ $1494.756 \quad 1495.287 \quad 1497.067 \quad 1494.913$ $\begin{array}{lllll}1494.752 & 1495.282 & 1497.072 & 1494.913\end{array}$ $1494.747 \quad 1495.275 \quad 1497.0671494 .916$ $\begin{array}{lllll}1494.749 & 1495.27 & 1497.069 & 1494.916\end{array}$ $\begin{array}{lllll}1494.749 & 1455.268 & 1497.072 & 1494.923 \\ 1494.752 & 1495268 & 1497.069 & 1494.927\end{array}$ $\begin{array}{llll}1494.749 & 1495.266 & 1497.062 & 1494.93\end{array}$ $\begin{array}{lllll}1494.749 & 1495.266 & 1497.053 & 1494.937\end{array}$ $\begin{array}{lllll}1494.747 & 1495.264 & 1497.053 & 1494.941\end{array}$ $1494.745 \quad 1495.259 \quad 1497.055 \quad 1494.944$ $1494.745 \quad 1495.257 \quad 1497.051 \quad 1494.946$ $1494.745 \quad 1495.254 \quad 1497.0461494 .948$ $\begin{array}{lllll}1494.742 & 1495.254 & 1497.041 & 1494.953\end{array}$ $\begin{array}{lllll}1494.74 & 1495.245 & 1497.039 & 1494.955\end{array}$ $\begin{array}{lllll}1494.738 & 1495.243 & 1497.039 & 1494.96\end{array}$ $1494.735 \quad 1495.24 \quad 1497.0391494 .958$ $\begin{array}{lllll}1494.738 & 1495.238 & 1497.032 & 1494.962\end{array}$ $\begin{array}{lllll}1494.738 & 1495.24 & 1497.032 & 1494.967\end{array}$ $\begin{array}{llll}1494.735 & 1495.238 & 1497.023 & 1494.969 \\ 1494.733 & 1495.233 & 1497.016 & 1494.967\end{array}$ $\begin{array}{llllllllllllllllllll}1494.407 & 1494.909 & 1495.197 & 1496.011 & 1497.538 & 1497.177 & 1492.903 & 1490.1 & 1492.727 & 1490.66 & 1490.863 & 1484.122 & 1489.032 & 1492.738 & 1493.933 & 1492.901 & 1495.207 & 1492.889 & 1486.124 & 1478.377\end{array}$ $\begin{array}{lllllllllllllllllllllll}1494.404 & 1494.907 & 1495.192 & 1495.99 & 1497.542 & 1497.17 & 1492.903 & 1490.089 & 1492.732 & 1490.654 & 1490.872 & 1484.134 & 1489.079 & 1492.74 & 1493.892 & 1492.906 & 1495.153 & 1492.889 & 1486.117 & 1478.377 \\ 1494.386 & 1494.909 & 1495204 & 1495.984 & 1497524 & 1497.174 & 1492927 & 1490.084 & 1492.751 & 1490.654 & 14909 & 1484.162 & 1489.072 & 1492.761 & 1493908 & 1492.925 & 1495.164 & 149291 & 1486.122 & 1478388\end{array}$

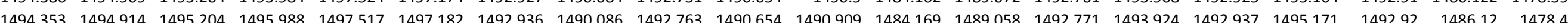
$\begin{array}{llllllllllllllllllllll}1494.374 & 1494.912 & 1495.194 & 1495.986 & 1497.528 & 1497.194 & 1492.11 & 1490.089 & 1492.54 & 1490.656 & 1490.916 & 1484.169 & 1489.043 & 1492.547 & 1493.763 & 1491.975 & 1495.181 & 1492.087 & 1486.122 & 1478.414\end{array}$ $\begin{array}{lllllllllllllllllllll}1494.355 & 1494.914 & 1495201 & 1496.002 & 1497.519 & 1497.203 & 1490.778 & 1490.098 & 1491.274 & 1490.663 & 1490.862 & 1484.178 & 1489.015 & 1491.28 & 1493.171 & 1490.674 & 1495.19 & 1490.768 & 1486.129 & 1478.421\end{array}$

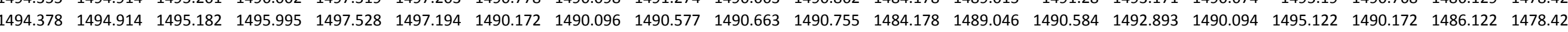
$\begin{array}{lllllllllllllllllllll}1494.409 & 1494.909 & 1495.182 & 1495.997 & 1497.512 & 1497.179 & 1489.826 & 1490.098 & 1490.097 & 1490.663 & 1490.645 & 1484.178 & 1489.034 & 1490.105 & 1492.73 & 1489.786 & 1495.096 & 1489.832 & 1486.122 & 1478.426\end{array}$

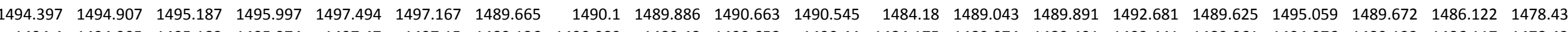

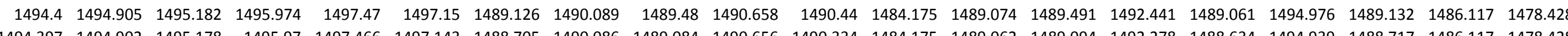
$\begin{array}{llllllllllllllllllll}1494.397 & 1494.902 & 1495.178 & 1495.97 & 1497.466 & 1497.143 & 1488.705 & 1490.086 & 1489.084 & 1490.656 & 1490.334 & 1484.175 & 1489.062 & 1489.094 & 1492.278 & 1488.634 & 1494.939 & 1488.717 & 1486.117 & 1478.428 \\ 1494.395 & 1494902 & 1495.178 & 1495.965 & 1497.428 & 1497.127 & 1488352 & 1490.082 & 1488716 & 1490656 & 1490.225 & 1484.178 & 1489.06 & 1488.723 & 1492.124 & 1488.291 & 1494.901 & 1488.367 & 1486117 & 1478.431\end{array}$

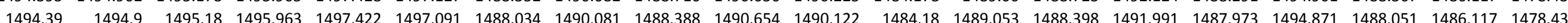

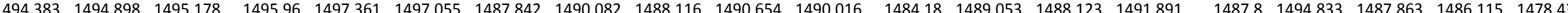
$\begin{array}{llllllllllllllllllllll}1494.376 & 1494.897 & 1495.175 & 1495.956 & 1497.268 & 1497.153 & 1487.758 & 1490.082 & 1487.998 & 1490.649 & 1489.918 & 1484.182 & 1489.048 & 1488.008 & 1491.86 & 1487.717 & 1494.802 & 1487.781 & 1486.117 & 1478.438\end{array}$

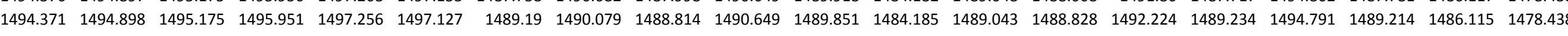
$\begin{array}{lllllllllllllllllllll}1494.371 & 1494.893 & 1495.178 & 1495.951 & 1497.208 & 1497.15 & 1489.873 & 1490.075 & 1489.532 & 1490.651 & 1489.851 & 1484.173 & 1489.036 & 1489.548 & 1492.516 & 1489.904 & 1494.798 & 1489.893 & 1486.115 & 1478.44\end{array}$

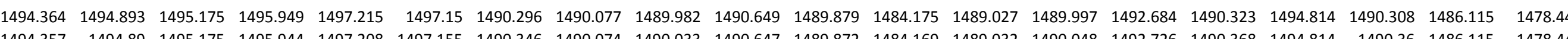

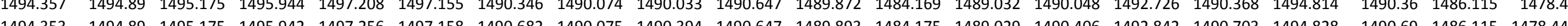
$\begin{array}{lllllllllllllllllllll}1494.353 & 1494.89 & 1495.175 & 1495.942 & 1497.256 & 1497.158 & 1490.682 & 1490.075 & 1490.394 & 1490.647 & 1489.893 & 1484.175 & 1489.029 & 1490.406 & 1492.842 & 1490.703 & 1494.828 & 1490.69 & 1486.115 & 1478.442 \\ 1494.348 & 1494.888 & 1495.173 & 1495.939 & 1497.322 & 1497.162 & 1490912 & 1490.075 & 1490638 & 1490649 & 1489.928 & 1484187 & 1489.02 & 1490648 & 1492928 & 1490928 & 1494842 & 1490913 & 1486115 & 1478.442\end{array}$ $\begin{array}{lllllllllllllllllllllll}1494.346 & 1494.89 & 1495.173 & 1495.937 & 1497.322 & 1497.165 & 1491.092 & 1490.075 & 1490.826 & 1490.647 & 1489.967 & 1484.194 & 1489.018 & 1490.841 & 1493.003 & 1491.105 & 1494.859 & 1491.092 & 1486.115 & 1478.445\end{array}$ \begin{tabular}{lllllllllllllllllllllll}
1494.343 & 1494.888 & 1495.17 & 1495.935 & 1497.317 & 1497.165 & 1491.239 & 1490.075 & 1490.988 & 1490.647 & 1490.003 & 1484.208 & 1489.013 & 1490.998 & 1493.066 & 1491.255 & 1494.868 & 1491.239 & 1486.115 & 1478.445 \\
\hline
\end{tabular} $\begin{array}{llllllllllllllllllllll}1494.341 & 1494.885 & 1495.17 & 1495.932 & 1497.289 & 1497.167 & 1491.368 & 1490.075 & 1491.119 & 1490.647 & 1490.035 & 1484.222 & 1489.011 & 1491.132 & 1493.122 & 1491.38 & 1494.882 & 1491.369 & 1486.115 & 1478.447\end{array}$

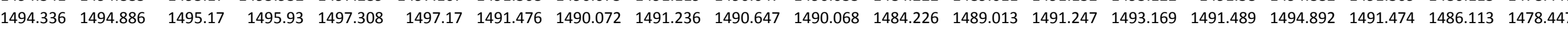
$\begin{array}{llllllllllllllllllllll}1494.341 & 1494.885 & 1495.166 & 1495.93 & 1497.356 & 1497.165 & 1491.572 & 1490.072 & 1491.337 & 1490.647 & 1490.101 & 1484.196 & 1489.004 & 1491.353 & 1493.215 & 1491.582 & 1494.91 & 1491.57 & 1486.113 & 1478.449\end{array}$

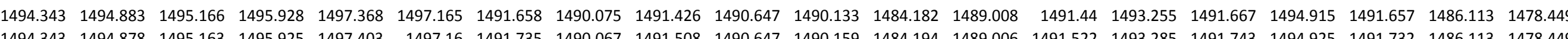

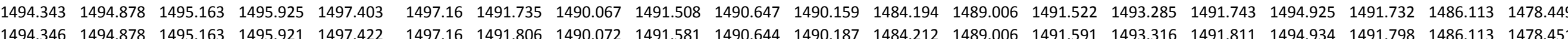

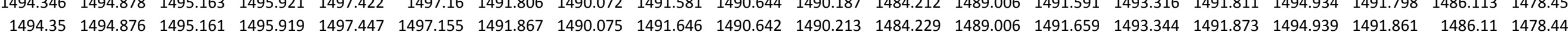
$\begin{array}{lllllllllllllllllllll}1494.355 & 1494.876 & 1495.161 & 1495.916 & 1497.48 & 1497.15 & 1491.923 & 1490.07 & 1491.705 & 1490.642 & 1490.239 & 1484.245 & 1489.008 & 1491.72 & 1493.372 & 1491.928 & 1494.948 & 1491.918 & 1486.11 & 1478.452\end{array}$ $\begin{array}{llllllllllllllllllllll}1494.36 & 1494.873 & 1495.156 & 1495.914 & 1497.508 & 1497.143 & 1491.974 & 1490.072 & 1491.759 & 1490.642 & 1490.262 & 1484.263 & 1489.013 & 1491.772 & 1493.395 & 1491.98 & 1494.95 & 1491.969 & 1486.11 & 1478.454\end{array}$ $\begin{array}{llllllllllllllllllllll}1494.367 & 1494.869 & 1495.154 & 1495.909 & 1497.531 & 1497.134 & 1492.023 & 1490.068 & 1491.811 & 1490.64 & 1490.285 & 1484.275 & 1489.008 & 1491.821 & 1493.416 & 1492.029 & 1494.955 & 1492.016 & 1486.11 & 1478.456\end{array}$ $\begin{array}{lllllllllllllllllllllll}1494.371 & 1494.866 & 1495.154 & 1495.905 & 1497.547 & 1497.119 & 1492.065 & 1490.068 & 1491.855 & 1490.64 & 1490.309 & 1484.293 & 1489.011 & 1491.868 & 1493.43 & 1492.07 & 1494.953 & 1492.056 & 1486.11 & 1478.4596\end{array}$

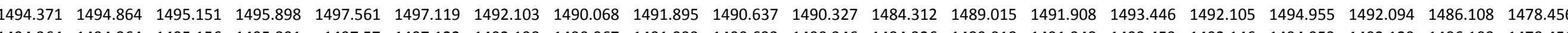

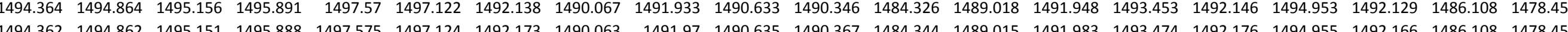

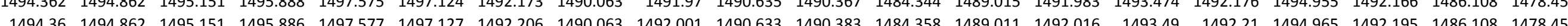
$\begin{array}{lllllllllllllllllllll}1494.357 & 1494.859 & 1495.151 & 1495.881 & 1497.575 & 1497.129 & 1492.234 & 1490.058 & 1492036 & 1490.63 & 14904 & 1484.374 & 1489011 & 1492047 & 1493.507 & 1492.24 & 1494965 & 1492.225 & 1486108 & 1478.459\end{array}$ \begin{tabular}{lllllllllllllllllllllll}
1494.357 & 1494.859 & 1495.151 & 1495.879 & 1497.575 & 1497.127 & 1492.264 & 1490.058 & 1492.066 & 1490.628 & 1490.416 & 1484.388 & 1489.011 & 1492.075 & 1493.523 & 1492.269 & 1494.969 & 1492.253 & 1486.108 & 1478.461 \\
\hline
\end{tabular}

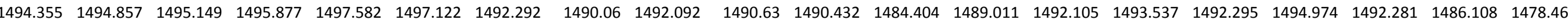
$\begin{array}{lllllllllllllllllllll}1494.357 & 1494.857 & 1495.147 & 1495.874 & 1497.594 & 1497.117 & 1492.316 & 1490.06 & 1492.118 & 1490.628 & 1490.449 & 1484.404 & 1489.011 & 1492.129 & 1493.546 & 1492.321 & 1494.983 & 1492.305 & 1486.108 & 1478.46\end{array}$ $\begin{array}{llllllllllllllllllllll}1494.355 & 1494.854 & 1495.144 & 1495.872 & 1497.603 & 1497.117 & 1492.339 & 1490.058 & 1492.144 & 1490.628 & 1490.463 & 1448.418 & 1489.008 & 1492.155 & 1493.556 & 1492.342 & 1494.988 & 1492.328 & 1486.108 & 1478.463\end{array}$

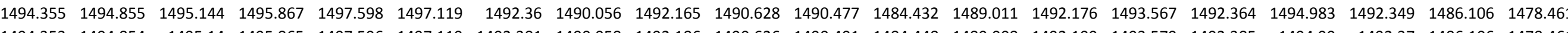

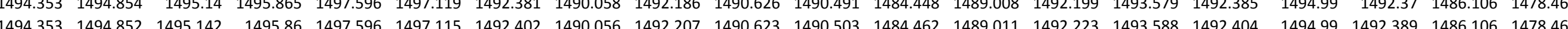

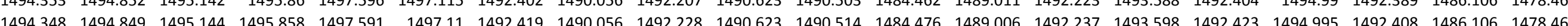
$\begin{array}{llllllllllllllllllllll}1494.35 & 1494.847 & 1495.142 & 1495.854 & 1497.377 & 1497.107 & 1492.437 & 1490.049 & 1492.247 & 1490.621 & 1490.528 & 1484.492 & 1489.008 & 1492.256 & 1493.602 & 1492.442 & 1494.993 & 1492.424 & 1486.106 & 1478.463\end{array}$ $\begin{array}{llllllllllllllllllllll}1494.343 & 1494.849 & 1495.14 & 1495.851 & 1497.326 & 1497.11 & 1492.456 & 1490.053 & 1492.263 & 1490.619 & 1490.54 & 1484.506 & 1489.006 & 1492.275 & 1493.607 & 1492.456 & 1494.993 & 1492.441 & 1486.103 & 1478.461\end{array}$ $\begin{array}{llllllllllllllllllll}1494.341 & 1494.847 & 1495.14 & 1495.849 & 1497.322 & 1497.112 & 1492.47 & 1490.046 & 1492.28 & 1490.619 & 1490.549 & 1484.52 & 1489.006 & 1492.293 & 1493.619 & 1492.473 & 1494.993 & 1492.457 & 1486.103 & 1478.463\end{array}$

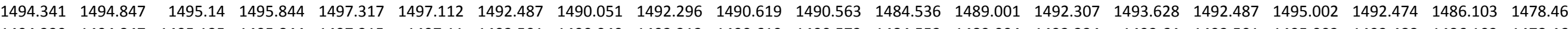
$\begin{array}{lllllllllllllllllllllll}1494.339 & 1494.847 & 1495.135 & 1495.844 & 1497.315 & 1497.11 & 1492.501 & 1490.049 & 1492.312 & 1490.619 & 1490.573 & 1484.552 & 1489.004 & 1492.324 & 1493.64 & 1492.501 & 1495.002 & 1492.488 & 1486.103 & 1478.463\end{array}$

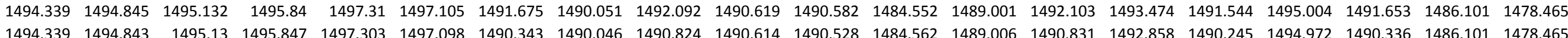


Well

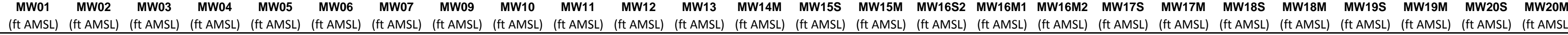

9/19/11 7:00 $9 / 19 / 117: 30$ $9 / 19 / 118: 00$ 9/19/118:30 9/19/11 9:30 9/19/11 10:00 9/19/11 10:30 9/19/11 11:00 9/19/11 11:30 9/19/11 12:00 9/19/11 13:00 9/19/1111 13:00 9/19/1111 13:30 9/19/11 14:30 9/19/11 15:00 /19/11 16:00 9/19/11 16:30 9/19/11 17:00 9/19/11 17:30 9/19/11 18:00 9/19/11 19:00 9/19/11 19:30 9/19/11 20:00 9/19/11 20:30 9/19/11 21:00 9/19/1122:00 9/19/11 22:30 9/19/11 23:00 9/19/11 23:30 9/20/11 0:00 9/20/11 0:30 9/20/11 1:00 9/20/111:30 9/20/11 2:30 9/20/11 3:00 9/20/11 3:30 9/20/11 4:00 9/20/11 4:30 9/20/11 5:00 /20/116:00 9/20/11 6:00 9/20/116:30 9/20/117:30 9/20/11 8:00 9/20/11 8:30 9/20/11 9:00 $\begin{array}{llll}1494.728 & 1495.229 & 1497.013 & 1494.958 \\ 1494.728 & 1495.224 & 1497.013 & 1494.934\end{array}$ $\begin{array}{llll}1494.728 & 1495.224 & 1497.013 & 1494.934 \\ 1499726 & 1459219 & 1497.013 & 1449\end{array}$ $\begin{array}{lllll}1494.726 & 1495.219 & 1497.013 & 1494.911\end{array}$ $\begin{array}{llll}1494.726 & 1495.215 & 1497.009 & 1494.86\end{array}$ $\begin{array}{rrrr}1494.726 & 1495.21 & 1497.009 & 1494.834\end{array}$ $\begin{array}{lllll}1494.724 & 1495.205 & 1497.016 & 1494.801\end{array}$ $\begin{array}{lllll}1494.721 & 1495.203 & 1497.02 & 1494.771\end{array}$ $\begin{array}{llll}1494.724 & 1495.201 & 1497.025 & 1494.745\end{array}$ $\begin{array}{lllll}1494.724 & 1495.198 & 1497.041 & 1494.72\end{array}$ $\begin{array}{llll}1494.728 & 1495.198 & 1497.051 & 1494.701 \\ 1494.728 & 1495.201 & 1497.062 & 1494.692\end{array}$ $\begin{array}{lllll}1494.728 & 1495.201 & 1497.062 & 1494.692 \\ 1494.733 & 1495.203 & 1497.079 & 1494.694\end{array}$ $\begin{array}{lllll}1494.731 & 1495.21 & 1497.093 & 1494.701\end{array}$ $\begin{array}{lllll}1494.733 & 1495.212 & 1497.107 & 1494.71\end{array}$ $\begin{array}{rllll}1494.735 & 1495.222 & 1497.116 & 1494.727\end{array}$ $\begin{array}{lllll}1494.74 & 1495.231 & 1497.123 & 1494.748\end{array}$ $\begin{array}{lrrrr}1494.74 & 1495.24 & 1497.13 & 1494.766\end{array}$ $\begin{array}{llll}1494.74 & 1495.247 & 1497.128 & 1494.79\end{array}$ $\begin{array}{llll}1494.742 & 1495.252 & 1497.133 & 1494.808 \\ 1494.742 & 1495259 & 1497.135 & 1494827\end{array}$ $\begin{array}{llll}1494.74 & 1495.259 & 1497.135 & 1494.843\end{array}$ $\begin{array}{lllll}1494.74 & 1495.266 & 1497.133 & 1494.86\end{array}$ $\begin{array}{rrrrr}1494.74 & 1455.266 & 1497.133 & 1494.86 \\ 1494.74 & 1495.266 & 1497.135 & 1494.876\end{array}$ $\begin{array}{llllll}1494.738 & 1495.268 & 1497.133 & 1494.888\end{array}$ $\begin{array}{llll}1494.738 & 1495.268 & 1497.123 & 1494.902\end{array}$ $\begin{array}{lllll}1494.738 & 1495.268 & 1497.114 & 1494.913\end{array}$ $\begin{array}{llll}1494.735 & 1495.268 & 1497.109 & 1494.923\end{array}$ $\begin{array}{lllll}1494.733 & 1495.266 & 1497.112 & 1494.927 \\ 1494.731 & 1495259 & 1497.116 & 1494.932\end{array}$ $\begin{array}{lllll}1494.731 & 1495.261 & 1497.121 & 1494.937\end{array}$ $\begin{array}{lllll}1494.733 & 1495.264 & 1497.123 & 1494.948\end{array}$ $\begin{array}{lllll}1494.733 & 1495.264 & 1497.126 & 1494.955\end{array}$ $\begin{array}{lllll}1494.733 & 1495.264 & 1497.123 & 1494.965\end{array}$ $\begin{array}{llll}1494.733 & 1495.266 & 1497.123 & 1494.974\end{array}$ $\begin{array}{llll}1494.733 & 1495.273 & 1497.128 & 1494.983 \\ 1499.733 & 1459271 & 1497.13 & 14949\end{array}$ $\begin{array}{lllll}1494.733 & 1455.27 & 1497.13 & 1494.99\end{array}$ $\begin{array}{rrrrr}1494.733 & 1455.275 & 1497.13 & 1495.904\end{array}$ $\begin{array}{lllll}1494.733 & 1495.278 & 1497.133 & 1495.014\end{array}$ $\begin{array}{lllll}1494.731 & 1495.28 & 1497.135 & 1495.021\end{array}$ $\begin{array}{lllll}1494.731 & 1495.278 & 1497.133 & 1495.028\end{array}$ $\begin{array}{lllll}1494.731 & 1495.28 & 1497.128 & 1495.035\end{array}$ $\begin{array}{llll}1494.731 & 1495.282 & 1497.135 & 1495.039\end{array}$ $\begin{array}{llrl}1494.731 & 1495.282 & 1497.13 & 1495.044\end{array}$ $\begin{array}{llll}1494.731 & 1495.282 & 1497.128 & 1495.046 \\ 1494.731 & 1495285 & 1497.135 & 1495.056\end{array}$ $\begin{array}{llllll}1494.728 & 1495.282 & 1497.144 & 1495.058\end{array}$ $\begin{array}{lllll}1494.731 & 1495.282 & 1497.121 & 1495.063\end{array}$ $\begin{array}{lllll}1494.731 & 1495.285 & 1497.114 & 1495.067\end{array}$ $\begin{array}{llll}1494.728 & 1495.287 & 1497.105 & 1495.072\end{array}$ $\begin{array}{llll}1494.724 & 1495.282 & 1497.086 & 1495.07\end{array}$ $\begin{array}{llll}1494.724 & 1495.278 & 1497.088 & 1495.067 \\ 1494.724 & 1495.275 & 1497.098 & 1495.065\end{array}$

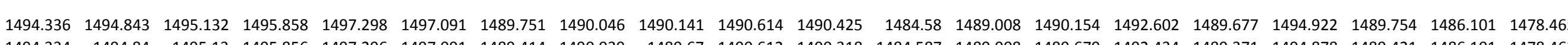
$\begin{array}{llllllllllllllllllll}1494.334 & 1494.84 & 1495.13 & 1495.856 & 1497.296 & 1497.091 & 1489.414 & 1490.039 & 1489.67 & 1490.612 & 1490.318 & 1484.587 & 1489.008 & 1489.679 & 1492.434 & 1489.371 & 1494.878 & 1489.421 & 1486.101 & 1478.463\end{array}$

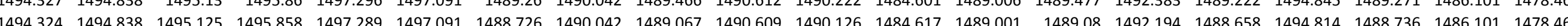
$\begin{array}{llllllllllllllllllllll}1494.32 & 1494.838 & 1495.125 & 1495833 & 1497.284 & 1497.084 & 1488312 & 1490.042 & 1488.678 & 1490.609 & 1490.028 & 1484.633 & 1488.999 & 1488.69 & 1492.028 & 1488.243 & 1494.777 & 1488.325 & 1486101 & 1478.465\end{array}$ $\begin{array}{llllllllllllllllllllll}1494.317 & 1494.838 & 1495 & 125 & 1495 & 791 & 1497.282 & 1497.084 & 1487.942 & 1490.039 & 1488.296 & 1490.607 & 1489.925 & 1484.649 & 1488.994 & 1488.304 & 1491.881 & 1487.881 & 1494.744 & 1487.961 & 1486.099 & 1478.4655\end{array}$ $\begin{array}{lllllllllllllllllllllll}1494.306 & 1494.835 & 1495.123 & 1495.749 & 1497.282 & 1497.084 & 1487.648 & 1490.042 & 1487.987 & 1490.607 & 1489.825 & 1484.663 & 1488.992 & 1487.996 & 1491.756 & 1487.589 & 1494.713 & 1487.666 & 1486.099 & 1478.465\end{array}$ $\begin{array}{lllllllllllllllllllll}1494.299 & 1494.835 & 1495.128 & 1495.809 & 1497.282 & 1497.088 & 1487.477 & 1490.039 & 1487.726 & 1490.607 & 1489.724 & 1484.675 & 1488.987 & 1487.74 & 1491.658 & 1487.437 & 1494.678 & 1487.497 & 1486.099 & 1478.468\end{array}$

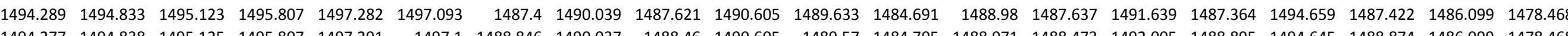

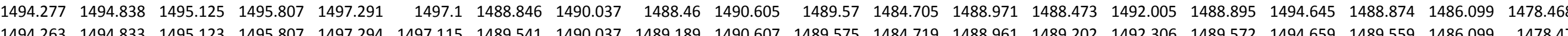

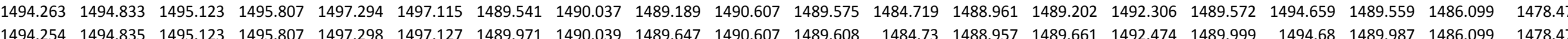

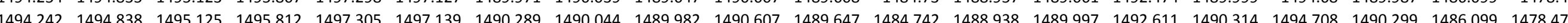
$\begin{array}{lllllllllllllllllllllll}1494.228 & 1494.838 & 1495.128 & 1495814 & 1497.31 & 1497.155 & 1490.533 & 1490.046 & 1490.242 & 1490.609 & 1489.692 & 1484.754 & 1488.933 & 1490.258 & 1492.714 & 1490.553 & 1494.73 & 1490.54 & 1486.096 & 1478.475\end{array}$ $\begin{array}{llllllllllllllllllllll}1494.219 & 1494.84 & 1495.128 & 1495.816 & 1497.319 & 1497.172 & 1490.736 & 1490.044 & 1490.458 & 1490.612 & 1489.731 & 1484.767 & 1488.926 & 1490.472 & 1492.807 & 1490.755 & 1494.758 & 1490.74 & 1486.099 & 1478.475 \\ \end{array}$ $\begin{array}{llllllllllllllllllllll}1494.214 & 1494.845 & 1495.123 & 1495.821 & 1497.324 & 1497.184 & 1490.907 & 1490.051 & 1490.638 & 1490.612 & 1489.773 & 1484.781 & 1488.914 & 1490.65 & 1492.886 & 1490.923 & 1494.791 & 1490.909 & 1486.099 & 1478.477 \\ \end{array}$ $\begin{array}{lllllllllllllllllllllll}1494.212 & 1494.847 & 1495.123 & 1495.826 & 1497.331 & 1497.196 & 1491.055 & 1490.053 & 1490.793 & 1490.616 & 1489.816 & 1484.795 & 1488.91 & 1490.808 & 1492.961 & 1491.068 & 1494.809 & 1491.054 & 1486.099 & 1478.482\end{array}$

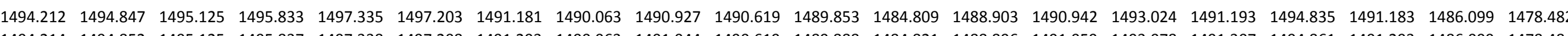

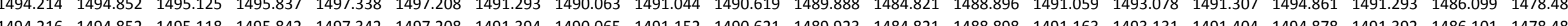

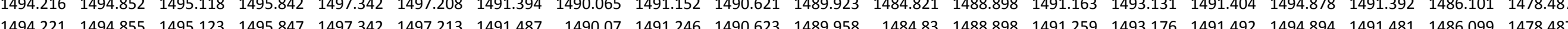
$\begin{array}{lllllllllllllllllllll}1494.226 & 1494.854 & 1495.121 & 1495.849 & 1497.345 & 1497.213 & 1491.565 & 1490.07 & 1491.33 & 1490.623 & 1489.988 & 1484.846 & 1488.896 & 1491.348 & 1493.215 & 1491.572 & 1494.908 & 1491.561 & 1486.099 & 1478.489\end{array}$ \begin{tabular}{llllllllllllllllllllll}
1494.237 & 1494.857 & 1495.118 & 1495.856 & 1497.347 & 1497.213 & 1491.707 & 1490.075 & 1491.475 & 1490.628 & 1490.047 & 1484.869 & 1488.9 & 1491.494 & 1493.285 & 1491.714 & 1494.936 & 1491.702 & 1486.096 & 1478.491 \\
\hline
\end{tabular} $\begin{array}{llllllllllllllllllllll}1494.247 & 1494.859 & 1495.121 & 1495.856 & 1497.349 & 1497.213 & 1491.766 & 1490.075 & 1491.541 & 1490.63 & 1490.08 & 1484.881 & 1488.903 & 1491.558 & 1493.313 & 1491.774 & 1494.948 & 1491.765 & 1486.096 & 1478.494\end{array}$

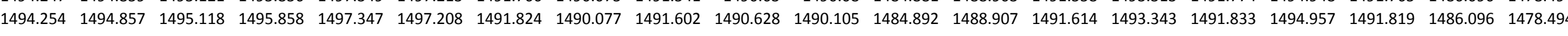

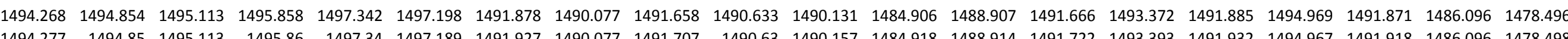

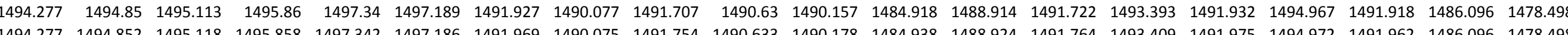
$\begin{array}{lllllllllllllllllllll}1494.277 & 1494.852 & 1495.118 & 1495.858 & 1497.342 & 1497.186 & 1491.969 & 1490.075 & 1491.754 & 1490.633 & 1490.178 & 1484.938 & 1488.924 & 1491.764 & 1493.409 & 1491.975 & 1494.972 & 1491.962 & 1486.096 & 1478.498 \\ 1494.277 & 1494.85 & 1495.113 & 1495.856 & 1497342 & 1497189 & 1492009 & 1490.067 & 1491.797 & 1490.63 & 1490201 & 1484.943 & 1488.931 & 1491.811 & 1493.423 & 1492.013 & 1494.969 & 1492.004 & 1486096 & 1478.496\end{array}$

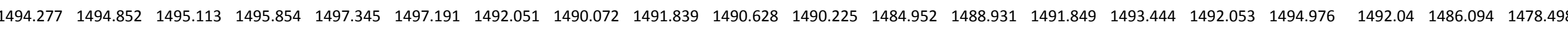
$\begin{array}{lllllllllllllllllllllll}1494.275 & 1494.85 & 1495.116 & 1495.856 & 1497.347 & 1497.194 & 1492.086 & 1490.07 & 1491.876 & 1490.63 & 1490.243 & 1484.964 & 1488.931 & 1491.889 & 1493.469 & 1492.091 & 1494.99 & 1492.08 & 1486.096 & 1478.501\end{array}$

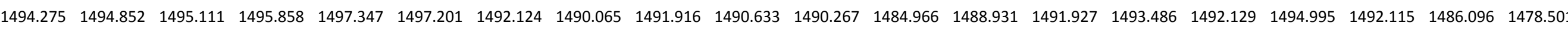
$\begin{array}{lllllllllllllllllllll}1494.28 & 1494.852 & 4495.116 & 1495.858 & 1497.347 & 1497.201 & 1492.157 & 1490.07 & 1491.949 & 1490.633 & 1490.288 & 1484.975 & 1488.928 & 1491.962 & 1493.504 & 1492.16 & 1495.009 & 1492.148 & 1486.096 & 1478.501\end{array}$

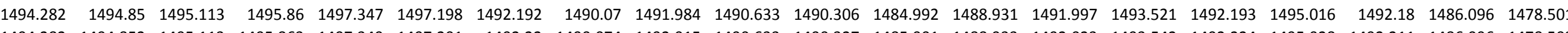

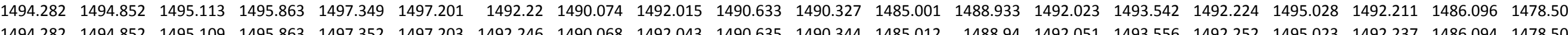

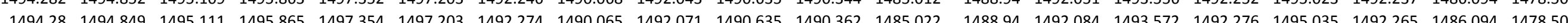

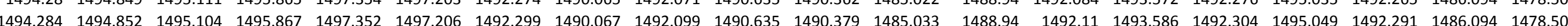
$\begin{array}{llllllllllllllllllllll}1494.287 & 1494.852 & 1495.109 & 1495.87 & 1497.354 & 1497.203 & 1492.325 & 1490.077 & 1492.125 & 1490.637 & 1490.397 & 1485.045 & 1488.943 & 1492.136 & 1493.602 & 1492.33 & 1495.051 & 1492.314 & 1486.094 & 1478.505\end{array}$ \begin{tabular}{llllllllllllllllllllll}
1494.287 & 1494.854 & 1495.106 & 1495.87 & 1497.354 & 1497.206 & 1492.349 & 1490.068 & 1492.146 & 1490.637 & 1490.411 & 1485.054 & 1488.947 & 1492.162 & 1493.614 & 1492.352 & 1495.061 & 1492.338 & 1486.094 & 1478.505 \\
\hline
\end{tabular} $\begin{array}{lllllllllllllllllllll}1494.289 & 1494.852 & 1495.106 & 1495.87 & 1497.356 & 1497.208 & 1492.37 & 1490.068 & 1492.172 & 1490.637 & 1490.425 & 1485.068 & 1488.95 & 1492.183 & 1493.626 & 1492.375 & 1495.061 & 1492.361 & 1486.094 & 1478.505 \\ \end{array}$

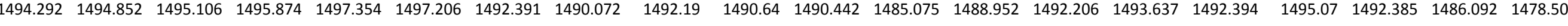

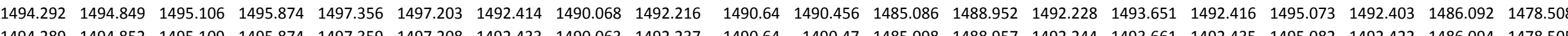

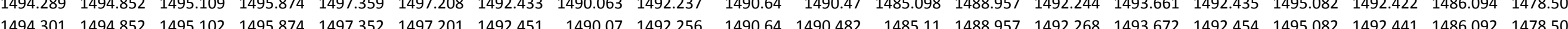

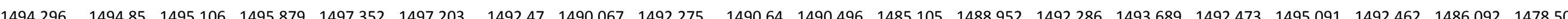
$\begin{array}{llllllllllllllllllllll}1494.294 & 1494.852 & 1495.106 & 1495.879 & 1497.359 & 1497.206 & 1492.487 & 1490.068 & 1492.291 & 1490.637 & 1490.51 & 1485.11 & 1488.961 & 1492.305 & 1493.686 & 1492.489 & 1495.084 & 1492.476 & 1486.092 & 1478.508\end{array}$ $\begin{array}{llllllllllllllllllllll}1494.301 & 1494.852 & 1495.099 & 1495.879 & 1497.354 & 1497.21 & 1492.505 & 1490.065 & 1492.31 & 1490.64 & 1490.524 & 1485.119 & 1488.957 & 1492.322 & 1493.7 & 1492.508 & 1495.094 & 1492.493 & 1486.094 & 1478.508 \\ \end{array}$ $\begin{array}{llllllllllllllllllll}1494.308 & 1494.849 & 1495.099 & 1495.881 & 1497.352 & 1497.191 & 1492.522 & 1490.065 & 1492.326 & 1490.642 & 1490.535 & 1485.121 & 1488.954 & 1492.336 & 1493.71 & 1492.522 & 1495.106 & 1492.511 & 1486.092 & 1478.51\end{array}$ $\begin{array}{lllllllllllllllllllllll}1494.31 & 1494.847 & 1495.097 & 1495.884 & 1497.347 & 1497.184 & 1492.54 & 1490.065 & 1492.343 & 1490.642 & 1490.547 & 1485.126 & 1488.959 & 1492.357 & 1493.724 & 1492.541 & 1495.106 & 1492.528 & 1486.092 & 1478.51 & \end{array}$ $\begin{array}{lllllllllllllllllllll}1494.315 & 1494.847 & 1495.097 & 1495.877 & 1497.342 & 1497.174 & 1492.552 & 1490.06 & 1492.357 & 1490.64 & 1490.556 & 1485.137 & 1488.964 & 1492.369 & 1493.719 & 1492.553 & 1495.089 & 1492.542 & 1486.092 & 1478.507\end{array}$

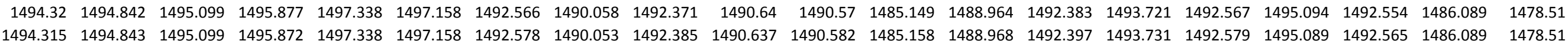


Well

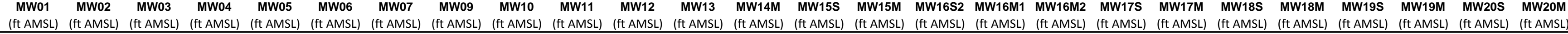

9/20/11 10:00 9/20/11 10:30 $9 / 20 / 1111: 00$ 9/20/11 11:30 9/20/11 12:00 9/20/11 13:00 9/20/11 13:30 9/20/11 14:00 $9 / 20 / 1114: 30$ 9/20/11 15:00 9/20/11 15:30 9/20/1116:00 9/20/11 16:30 9/20/11 17:30 9/20/11 18:00
9/20/11 18:30 9/20/11 19:00 9/20/11 19:30 9/20/11 20:00 9/20/1121:00 9/20/11 21:00 9/20/11 22:00 9/20/11 22:30 9/20/11 23:00 $9 / 20 / 1123: 30$
$9 / 21 / 110: 00$
$9 / 21 / 110: 30$ 9/21/110:30 9/21/11 1:30 9/21/112:30 9/21/11 3:00 9/21/113:30 $9 / 21 / 114: 00$ 9/21/114:30 9/21/11 5:00 9/21/11 6:00 9/21/116:30 9/21/11 7:00 9/21/11 7:30 9/21/11 8:00 $9 / 21 / 119.00$ 9/21/11 9:00 $9 / 21 / 1110: 00$ 9/21/11 10:30 9/21/11 11:00 9/21/11 12:00 $\begin{array}{rrrr}1494.717 & 1495.266 & 1497.114 & 1495.06 \\ 1494.719 & 1495.266 & 1497.112 & 1495.058\end{array}$ $\begin{array}{llll}1494.719 & 1495.266 & 1497.112 & 1495.058 \\ 1494.724 & 1495271 & 1497.112 & 1995067\end{array}$ $\begin{array}{llll}1494.724 & 1495.271 & 1497.112 & 1495.067 \\ 1494.728 & 1495275 & 1497116 & 1495074\end{array}$ $\begin{array}{rrrr}1494.728 & 1495.275 & 1497.116 & 1495.074 \\ 1494.726 & 1495.275 & 1497.13 & 1495.079\end{array}$ $\begin{array}{lllll}1494.728 & 1495.275 & 1497.142 & 1495.084\end{array}$ $\begin{array}{lllll}1494.728 & 1495.28 & 1497.149 & 1495.088\end{array}$ $\begin{array}{lllll}1494.733 & 1495.282 & 1497.158 & 1495.093\end{array}$ $\begin{array}{llll}1494.735 & 1495.289 & 1497.163 & 1495.098\end{array}$

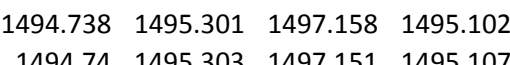
$\begin{array}{rrrr}1494.74 & 1495.303 & 1497.151 & 1495.107 \\ 1494.738 & 1495.308 & 1497.144 & 1495.114\end{array}$ $\begin{array}{rrrr}1494.738 & 1495.308 & 1497.144 & 1495.114 \\ 1494.735 & 1495.306 & 1497.13 & 1495.114\end{array}$ $\begin{array}{lllll}1494.733 & 1495.306 & 1497.123 & 1495.112\end{array}$ $\begin{array}{lllll}1494.731 & 1495.301 & 1497.105 & 1495.112\end{array}$ $\begin{array}{lllll}1494.731 & 1495.296 & 1497.088 & 1495.109\end{array}$ $\begin{array}{lllll}1494.726 & 1495.294 & 1497.076 & 1495.102\end{array}$ $\begin{array}{llll}1494.721 & 1495.282 & 1497.072 & 1495.093\end{array}$ 1494.7191495 .2751497 .0721495 .088 $\begin{array}{rrrr}1494.719 & 1495.273 & 1497.06 & 1495.079 \\ 1494.721 & 1495271 & 1497.046 & 1495.079\end{array}$ $\begin{array}{lllll}1494.717 & 1495.266 & 1497.034 & 1495.074\end{array}$ $\begin{array}{lllll}1494.712 & 1495.259 & 1497.025 & 1495.067\end{array}$ $\begin{array}{lllll}1494.71 & 1495.252 & 1497.018 & 1495.058\end{array}$ $\begin{array}{llll}1494.707 & 1495.245 & 1497.009 & 1495.053\end{array}$ $\begin{array}{lllll}1494.705 & 1495.238 & 1497.004 & 1495.046\end{array}$ $\begin{array}{lllll}1494.705 & 1495.233 & 1496.995 & 1495.042\end{array}$ $\begin{array}{llll}1494.705 & 1495.226 & 1496.992 & 1495.035\end{array}$ $\begin{array}{rrrr}1494.705 & 1495.222 & 1496.995 & 1495.03 \\ 1494.703 & 1495.217 & 1496.997 & 1495.023\end{array}$ $\begin{array}{lllll}1494.7 & 1495.212 & 1496.99 & 1495.018\end{array}$ $\begin{array}{rrrr}1494.705 & 1495.212 & 1496.997 & 1455.018\end{array}$ $\begin{array}{lllll}1494.705 & 1495.21 & 1496.971 & 1495.021\end{array}$ $\begin{array}{lllll}1494.7 & 1495.208 & 1496.967 & 1495.018\end{array}$ $\begin{array}{lllll}1494.703 & 1495.208 & 1496.974 & 1495.018\end{array}$ $\begin{array}{llll}1494.689 & 1495.184 & 1496.969 & 1495\end{array}$ $\begin{array}{llll}1494.696 & 1495.191 & 1496.992 & 1495.002 \\ 1494698 & 1495189 & 1496.992 & 1495.002\end{array}$ $\begin{array}{llll}1494.698 & 1455.189 & 1496.992 & 1495.002 \\ 1494.7 & 1495.187 & 1496971 & 1495004\end{array}$ $\begin{array}{rrrr}1494.71 & 1495.196 & 1496.995 & 1495.014\end{array}$ $\begin{array}{llll}1494.7 & 1495.191 & 1496.988 & 1495.014\end{array}$ $\begin{array}{rrrr}1494.696 & 1495.184 & 1496.978 & 1495.007\end{array}$ $\begin{array}{lllll}1494.705 & 1495.191 & 1496.964 & 1495.016\end{array}$ $\begin{array}{lllll}1494.705 & 1495.189 & 1496.957 & 1495.021\end{array}$ $\begin{array}{lllll}1494.698 & 1495.182 & 1496.962 & 1495.016\end{array}$ $\begin{array}{lllll}1494.691 & 1495.175 & 1496.964 & 1495.007 \\ 1494.689 & 1495.173 & 1496.957 & 1495.004\end{array}$ $\begin{array}{llllll}1494.693 & 1495.173 & 1496.95 & 1495.004\end{array}$ $\begin{array}{lllll}1494.693 & 1495.17 & 1496.953 & 1495.007\end{array}$ 1494.6861495 .1661496 .9621495 .002 $1494.686 \quad 1495.161 \quad 1496.96 \quad 1495$ $1494.6891495 .163 \quad 1496.948 \quad 1495.002$ $\begin{array}{llll}1494.691 & 1495.166 & 1496.948 & 1495.007 \\ 1494.686 & 1495.161 & 1496.957 & 1495.004\end{array}$

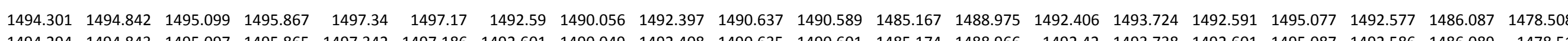
$\begin{array}{lllllllllllllllllllllll}1494.294 & 1494.843 & 1495.097 & 1495.865 & 1497.342 & 1497.186 & 1492.601 & 1490.049 & 1492.408 & 1490.635 & 1490.601 & 1485.174 & 1488.966 & 1492.42 & 1493.738 & 1492.601 & 1495.087 & 1492.586 & 1486.089 & 1478.51 \\ 1494.294 & 1494843 & 149509 & 1495867 & 1497345 & 1497.182 & 1492618 & 1490.044 & 1492.423 & 1490.637 & 1490.612 & 1485.186 & 1488.959 & 1492.432 & 1493747 & 1492.62 & 1495094 & 1492.603 & 1486089 & 147851\end{array}$

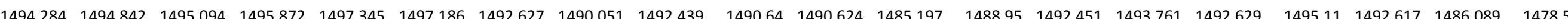
$\begin{array}{llllllllllllllllllllll}1494.277 & 1494.845 & 1495 & 099 & 1495872 & 1497.347 & 1497.189 & 1492288 & 1490.049 & 1492057 & 1490.637 & 1490.626 & 1485207 & 1488952 & 1492.068 & 1493.668 & 1492.29 & 1495103 & 1492281 & 1486089 & 1478.512\end{array}$ $\begin{array}{llllllllllllllllllllll}1494.268 & 1494.847 & 1495.102 & 1495.874 & 1497.356 & 1497.201 & 1492.138 & 1490.051 & 1491.893 & 1490.64 & 1490.619 & 1485.221 & 1488.95 & 1491.906 & 1493.612 & 1492.143 & 1495.106 & 1492.134 & 1486.087 & 1478.512\end{array}$

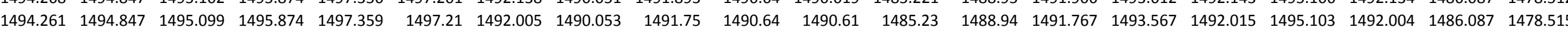
$\begin{array}{llllllllllllllllllllll}1494.254 & 1494.852 & 1495.099 & 1495.879 & 1497.363 & 1497.222 & 1491.976 & 1490.053 & 1491.724 & 1490.642 & 1490.596 & 1485.234 & 1488.933 & 1491.739 & 1493.549 & 1491.984 & 1495.108 & 1491.974 & 1486.089 & 1478.517\end{array}$

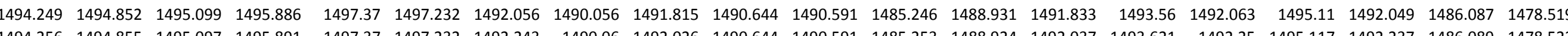

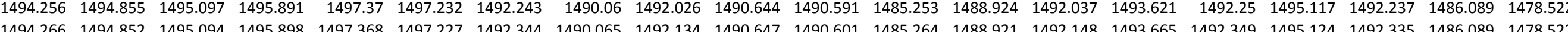
$\begin{array}{lllllllllllllllllllll}1494.266 & 1494.852 & 1495.094 & 1495.898 & 1497.368 & 1497.227 & 1492.344 & 1490.065 & 1492.134 & 1490.647 & 1490.601 & 1485.264 & 1488.921 & 1492.148 & 1493.665 & 1492.349 & 1495.124 & 1492.335 & 1486.089 & 1478.522 \\ 1494.27 & 1494.855 & 1495.092 & 1495.9 & 1497366 & 149722 & 1492.414 & 1490063 & 1492207 & 1490649 & 1490608 & 1485276 & 1488924 & 1492218 & 1493698 & 1492.418 & 1495134 & 1492.403 & 1486087 & 1478524\end{array}$

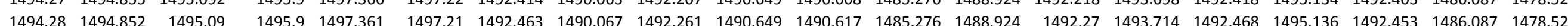
$\begin{array}{llllllllllllllllllllll}1494.289 & 1494.852 & 1495.09 & 1495.9 & 1497.359 & 1497.198 & 1492.505 & 1490.07 & 1492305 & 1490.649 & 1490.626 & 1485.244 & 1488.926 & 1492317 & 1493.731 & 1492508 & 1495.134 & 1492.495 & 1486.087 & 1478.526\end{array}$ $\begin{array}{llllllllllllllllllll}1494.303 & 1494.849 & 1495.085 & 1495.898 & 1497.349 & 1497.189 & 1492.536 & 1490.063 & 1492.338 & 1490.647 & 1490.636 & 1485.23 & 1488.933 & 1492.35 & 1493.74 & 1492.539 & 1495.129 & 1492.525 & 1486.087 & 1478.526\end{array}$

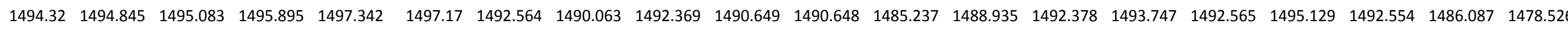
$\begin{array}{llllllllllllllllllllllll}1494.327 & 1494.842 & 1495.085 & 1495.891 & 1497.338 & 1497.153 & 1492.587 & 1490.058 & 1492.392 & 1490.647 & 1490.657 & 1485.248 & 1488.947 & 1492.404 & 1493.747 & 1492.589 & 1495.117 & 1492.575 & 1486.085 & 1488.526\end{array}$

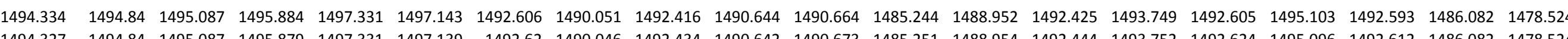

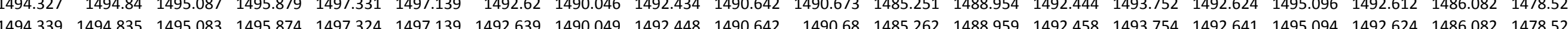

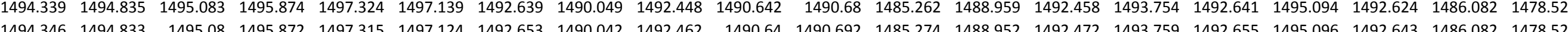

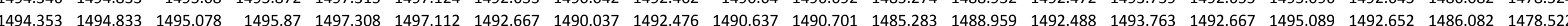
$\begin{array}{lllllllllllllllllllllll}1494.355 & 1494.83 & 1495.078 & 1495.858 & 1497.294 & 1497.093 & 1492.688 & 1490.035 & 1492.5 & 1490.633 & 1490.713 & 1485.304 & 1488.971 & 1492.512 & 1493.759 & 1492.688 & 1495.068 & 1492.676 & 1486.08 & 1478.521 \\ & & & \end{array}$

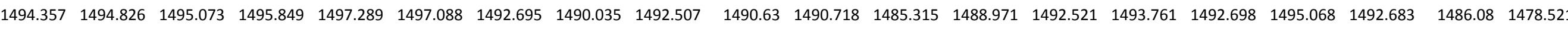

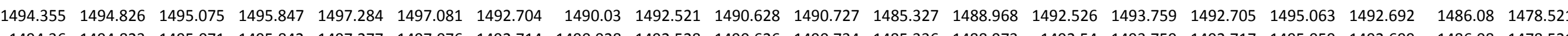

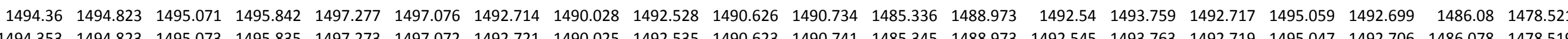

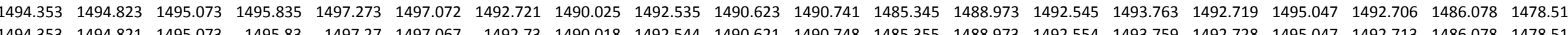

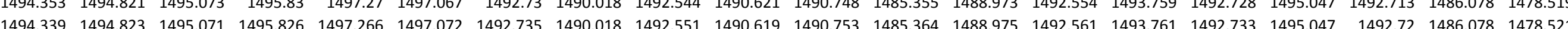
$\begin{array}{llllllllllllllllllll}1494.339 & 1494.819 & 1495.071 & 1495.819 & 1497.261 & 1497.074 & 1492.739 & 1490.016 & 1492.554 & 1490.616 & 1490.76 & 1485.371 & 1488.978 & 1492.568 & 1493.759 & 1492.738 & 1495.04 & 1492.727 & 1486.078 & 1478.519\end{array}$ $\begin{array}{lllllllllllllllllllllll}1494.327 & 1494.818 & 1495.075 & 1495.816 & 1497.261 & 1497.069 & 1492.746 & 1490.018 & 1492.563 & 1490.616 & 1490.764 & 1485.378 & 1488.971 & 1492.575 & 1493.768 & 1492.747 & 1495.049 & 1492.734 & 1486.078 & 1478.519\end{array}$

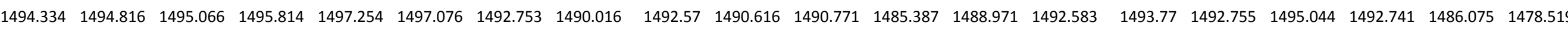
$\begin{array}{lllllllllllllllllllllll}1494.346 & 1494.814 & 1495.068 & 1495.809 & 1497.242 & 1497.045 & 1492.758 & 1490.014 & 1492.575 & 1490.614 & 1490.778 & 1485.396 & 1488.971 & 1492.585 & 1493.768 & 1492.759 & 1495.04 & 1492.746 & 1486.075 & 1478.519\end{array}$

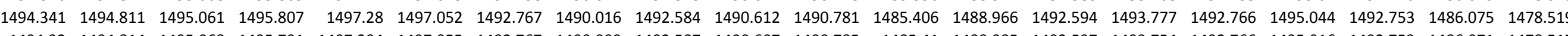

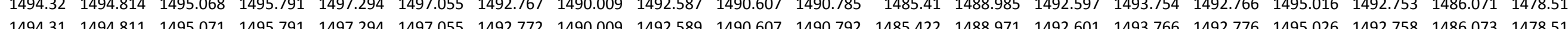

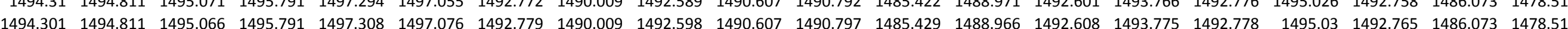

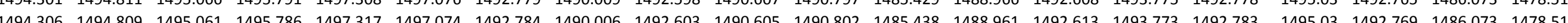
$\begin{array}{lllllllllllllllllllllll}1494.299 & 1494.806 & 1495.068 & 1495.793 & 1497.308 & 1497.057 & 1492.791 & 1490.011 & 1492.612 & 1490.605 & 1490.809 & 1485.447 & 1488.95 & 1492.622 & 1493.791 & 1492.792 & 1495.054 & 1492.781 & 1486.075 & 1478.519\end{array}$

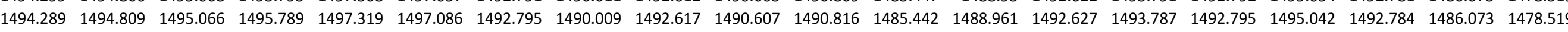

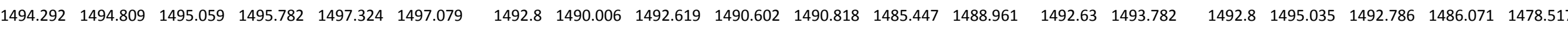

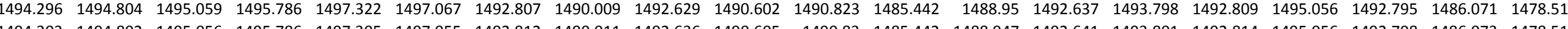

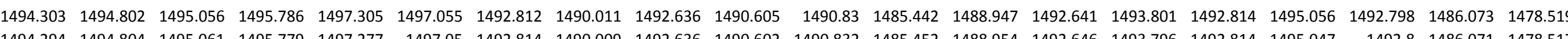

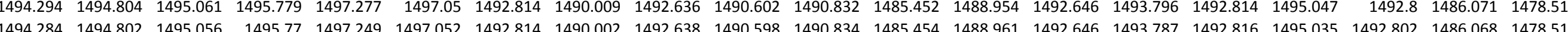

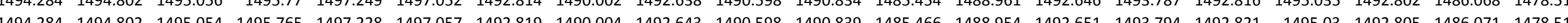
$\begin{array}{lllllllllllllllllllll}1494.284 & 1494.799 & 1495.052 & 1495.768 & 1497.212 & 1497.05 & 1492.821 & 1490 & 1492.645 & 1490.595 & 1490.844 & 1485.475 & 1488.945 & 1492.655 & 1493.791 & 1492.821 & 1495.033 & 1492.809 & 1486.071 & 1478.517\end{array}$ $\begin{array}{lllllllllllllllllllllll}1494.277 & 1494.797 & 1495.054 & 1495.765 & 1497.219 & 1497.045 & 1492.828 & 1490.004 & 1492.648 & 1490.595 & 1490.848 & 1485.484 & 1488.94 & 1492.662 & 1493.798 & 1492.826 & 1495.042 & 1492.814 & 1486.071 & 1478.514\end{array}$ $\begin{array}{llllllllllllllllllllllll}1494.268 & 1494.8 & 1495.056 & 1495.758 & 1497.194 & 1497.048 & 1492.831 & 1489.997 & 1492.652 & 1490.593 & 1490.853 & 1485.493 & 1488.95 & 1492.662 & 1493.787 & 1492.83 & 1495.026 & 1492.816 & 1486.068 & 1478.514\end{array}$ $\begin{array}{llllllllllllllllllll}1494.266 & 1494.797 & 1495.052 & 1495.754 & 1497.217 & 1497.057 & 1492.833 & 1490.002 & 1492.657 & 1490.593 & 1490.855 & 1485.5 & 1488.94 & 1492.667 & 1493.791 & 1492.835 & 1495.03 & 1492.819 & 1486.068 & 1478.515 \\ \end{array}$

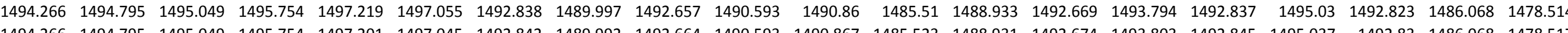

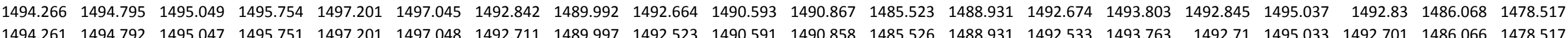


Well

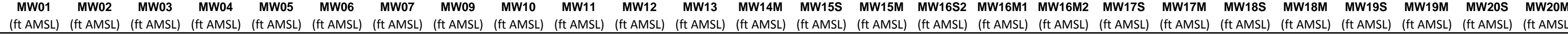

9/21/11 13:00 $9 / 21 / 1113: 30$ $9 / 21 / 1114: 30$ 9/21/11 14:30 9/21/11 15:30 9/21/11 16:00 9/21/11 16:30 9/21/11 17:00 9/21/11 17:30 9/21/11 18:00 9/21/11 18:30 9/21/11 19:30 9/21/1120:30 9/21/11 21:00 9/21/11 22:00 9/21/11 22:30 9/21/11 23:00 9/22/11 0:00 9/22/11 0:30 9/22/111:30 9/22/112:00 $9 / 22 / 112: 30$ 9/22/113:00 9/22/11 4:00 9/22/11 4:30 9/22/11 5:00 9/22/115:30 9/22/11 6:00 9/22/11 6:30 $9 / 22 / 117: 30$
$9 / 22 / 118: 00$ 9/22/111 8:00 9/22/119:00 9/22/11 9:30 9/22/11 10:00 9/22/11 10:30 9/22/11 11:00 9/22/11 11:30 (22/11 13:00 9/22/11 13:30 9/22/11 14:00 9/22/11 15:00 $\begin{array}{llll}1494.684 & 1495.156 & 1496.969 & 1495\end{array}$ $\begin{array}{rrrr}1494.684 & 1495.156 & 1496.988 & 1495 \\ 1494.689 & 1495.159 & 1496995 & 1495002\end{array}$ $\begin{array}{llll}1494.689 & 1495.159 & 1496.995 & 1495.002 \\ 1494.691 & 1495.161 & 1497.006 & 1495011\end{array}$ $\begin{array}{lllll}1494.7 & 1495.17 & 1497.011 & 1495.023\end{array}$ $\begin{array}{rllll}1494.698 & 1495.175 & 1497.009 & 1455.035\end{array}$ $\begin{array}{llll}1494.698 & 1495.175 & 1497.009 & 1495.035 \\ 1494.7 & 1495.182 & 1497.006 & 1495.042\end{array}$ $\begin{array}{lllll}1494.696 & 1495.18 & 1497.011 & 1495.049\end{array}$ $\begin{array}{llll}1494.696 & 1495.182 & 1497.009 & 1495.051\end{array}$ $\begin{array}{lllll}1494.698 & 1495.184 & 1496.999 & 1495.058\end{array}$ $\begin{array}{rrrr}1494.696 & 1495.184 & 1496.997 & 1495.06\end{array}$ $\begin{array}{rrrr}1494.696 & 1495.187 & 1496.997 & 1495.065 \\ 1494.691 & 1495.182 & 1496.995 & 1495.06\end{array}$ $\begin{array}{lllll}1494.691 & 1495.182 & 1496.988 & 1495.065\end{array}$ $\begin{array}{lllll}1449.691 & 1495.182 & 1496.981 & 1495.067\end{array}$ $\begin{array}{lllll}1494.691 & 1495.18 & 1496.976 & 1495.065\end{array}$ $1494.6891495 .177 \quad 1496.971 \quad 1495.063$ $\begin{array}{lllll}1494.684 & 1495.175 & 1496.967 & 1495.063\end{array}$ $\begin{array}{lllll}1494.684 & 1495.175 & 1496.974 & 1495.06\end{array}$ $\begin{array}{lllll}1494.682 & 1495.17 & 1496.974 & 1495.056\end{array}$ $\begin{array}{llll}1494.686 & 1455.173 & 1496.99 & 1495.06\end{array}$ $\begin{array}{lllll}1494.686 & 1495.173 & 1497.004 & 1495.06\end{array}$ $\begin{array}{lllll}1494.684 & 1495.173 & 1496.999 & 1495.063\end{array}$ $\begin{array}{lllll}1494.691 & 1495.18 & 1497.009 & 1495.072\end{array}$ $\begin{array}{lllll}1494.693 & 1495.187 & 1497.013 & 1495.081\end{array}$ $\begin{array}{lllll}1494.691 & 1495.184 & 1497.023 & 1495.081\end{array}$ $\begin{array}{llllll}1494.693 & 1495.191 & 1497.006 & 1495.088\end{array}$ $\begin{array}{lllll}1494.696 & 1495.194 & 1497.006 & 1495.098 \\ 1494.693 & 1495.196 & 1497.006 & 1495.102\end{array}$ $\begin{array}{lllll}1494.686 & 1495.191 & 1497.009 & 1495.098\end{array}$ $\begin{array}{lllll}1494.691 & 1495.194 & 1496.997 & 1495.1\end{array}$ $\begin{array}{llll}1494.691 & 1495.194 & 1497.002 & 1495.102\end{array}$ $1494.689 \quad 1495.196 \quad 1496.985 \quad 1495.102$ $\begin{array}{lllll}1494.686 & 1495.191 & 1496.981 & 1495.102\end{array}$ $1494.6891495 .191 \quad 1496.9691495 .102$ $\begin{array}{lllll}1494.682 & 1495.187 & 1496.964 & 1495.093 \\ 1494.679 & 1495.184 & 1496967 & 1950.091\end{array}$ $\begin{array}{lllll}1494.679 & 1495.184 & 1496.967 & 1495.091 \\ 1494.679 & 1495182 & 1496.974 & 1495086\end{array}$ $\begin{array}{lllll}1494.679 & 1495.177 & 1496.971 & 1495.081\end{array}$ $\begin{array}{llllll}1494.682 & 1495.18 & 1496.974 & 1495.081\end{array}$ $\begin{array}{llllll}1494.682 & 1495.182 & 1496.971 & 1495.084\end{array}$ $\begin{array}{lllll}1494.682 & 1495.18 & 1496.978 & 1495.084\end{array}$ $\begin{array}{llll}1494.682 & 1495.18 & 1496.985 & 1495.084\end{array}$ $\begin{array}{lllll}1494.679 & 1495.18 & 1496.997 & 1495.084\end{array}$ $\begin{array}{llllll}1494.684 & 1495.182 & 1497.011 & 1495.088\end{array}$ $\begin{array}{rrrr}1494.691 & 1455.194 & 1497.032 & 1495.102\end{array}$ $\begin{array}{lllll}1494.696 & 1495.198 & 1497.046 & 1495.112\end{array}$ $\begin{array}{lllll}1494.696 & 1495.203 & 1497.06 & 1495.119\end{array}$ $\begin{array}{llll}1494.696 & 1495.215 & 1497.069 & 1495.128\end{array}$ $1494.703 \quad 1495.224 \quad 1497.0791495 .142$ $\begin{array}{llll}1494.703 & 1495.233 & 1497.086 & 1495.154 \\ 1494.705 & 1495.245 & 1497.093 & 1495.161\end{array}$

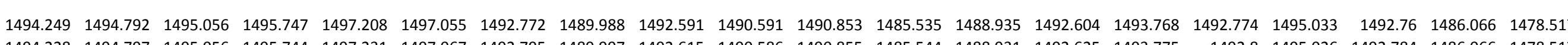

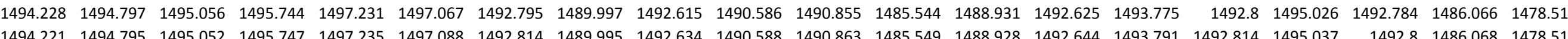

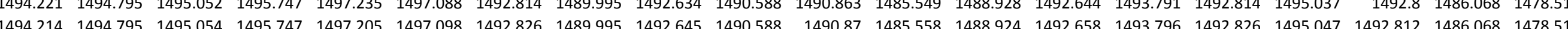

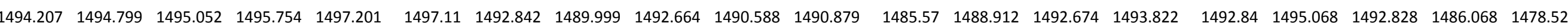
$\begin{array}{llllllllllllllllllllll}1494.207 & 1494.797 & 1495.049 & 1495.758 & 1497.219 & 1497.112 & 1492.852 & 1490.004 & 1492.673 & 1490.591 & 1490.888 & 1485.579 & 1488.912 & 1492.681 & 1493.831 & 1492.854 & 1495.07 & 1492.84 & 1486.066 & 1478.528\end{array}$

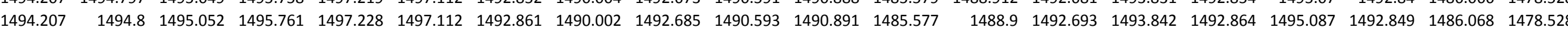

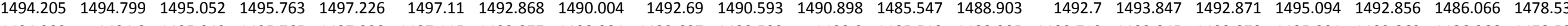

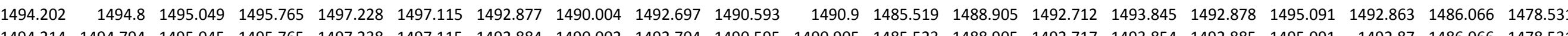

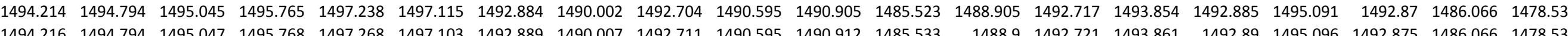

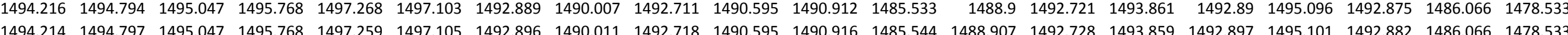

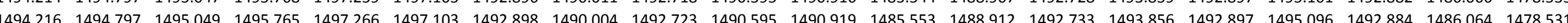

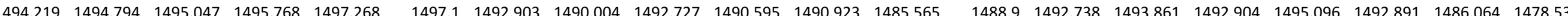
$\begin{array}{llllllllllllllllllllll}1494.223 & 1494.792 & 1495.042 & 1495.765 & 1497.284 & 1497.095 & 1492.908 & 1489.999 & 1492.732 & 1490.595 & 1490.928 & 1485.574 & 1488.905 & 1492.742 & 1493.866 & 1492.909 & 1495.096 & 1492.891 & 1486.064 & 1478.531\end{array}$ $\begin{array}{lllllllllllllllllllllll}1494.226 & 1494.792 & 1495.042 & 1495.763 & 1497.303 & 1497.088 & 1492.91 & 1490.004 & 1492.734 & 1490.593 & 1490.933 & 1485.584 & 1488.905 & 1492.745 & 1493.866 & 1492.913 & 1495.094 & 1492.896 & 1486.064 & 1478.531 \\ \end{array}$

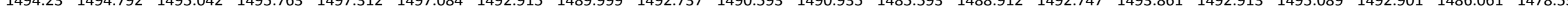

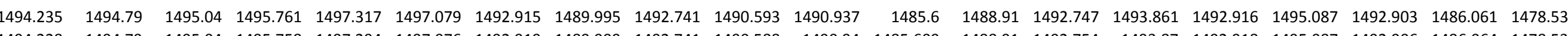

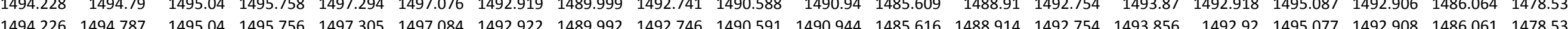

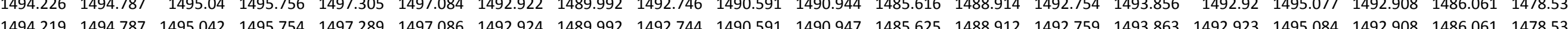
$\begin{array}{llllllllllllllllllllll}1494.212 & 1494.788 & 1495.042 & 1495.756 & 1497.268 & 1497.086 & 1492.929 & 1489.992 & 1492.753 & 1490.588 & 1490.951 & 1485.632 & 1488.905 & 1492.764 & 1493.868 & 1492.928 & 1495.089 & 1492.913 & 1486.061 & 1478.531\end{array}$ \begin{tabular}{llllllllllllllllllllll}
1494.202 & 1494.79 & 1495.045 & 1495.756 & 1497.252 & 1497.103 & 1492.931 & 1489.99 & 1492.758 & 1490.591 & 1490.956 & 1485.641 & 1488903 & 1492.766 & 1493.87 & 1492.932 & 1495.094 & 1492.917 & 1486.061 & 1478.533 \\
\hline
\end{tabular}

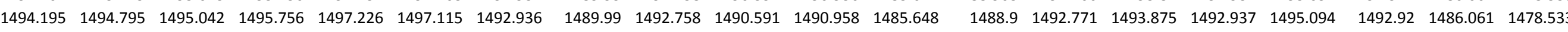

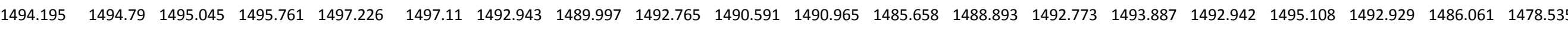

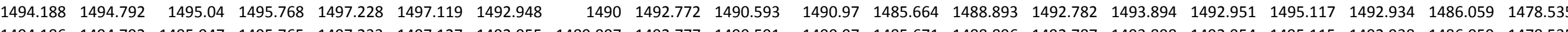

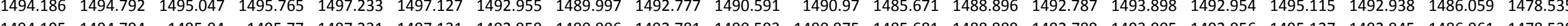

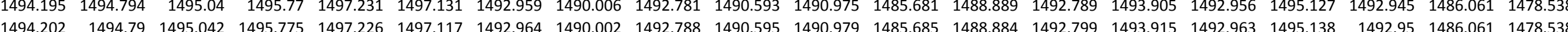

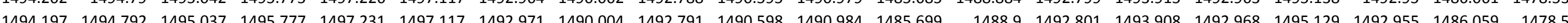
$\begin{array}{llllllllllllllllllllll}1494.193 & 1494.792 & 1495.042 & 1495.772 & 1497.233 & 1497.117 & 1492.966 & 1490.002 & 1492.793 & 1490.595 & 1490.986 & 1485.706 & 1488.91 & 1492.803 & 1493.905 & 1492.97 & 1495.124 & 1492.955 & 1486.056 & 1478.538\end{array}$ $\begin{array}{llllllllllllllllllllll}1494.2 & 1494.792 & 1495.037 & 1495.775 & 1497.233 & 1497.119 & 1492.973 & 1490.004 & 1492.798 & 1490.595 & 1490.991 & 1485.708 & 1488.898 & 1492.803 & 1493.915 & 1492.973 & 1495.129 & 1492.957 & 1486.059 & 147854\end{array}$ $\begin{array}{llllllllllllllllllll}1494.205 & 1494.792 & 1495.033 & 1495.775 & 1497.228 & 1497.11 & 1492.976 & 1489.999 & 1492.802 & 1490.598 & 1490.991 & 1485.715 & 1488.898 & 1492.811 & 1493.915 & 1492.977 & 1495.134 & 1492.962 & 1486.059 & 1478.54\end{array}$

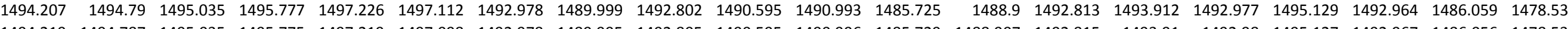

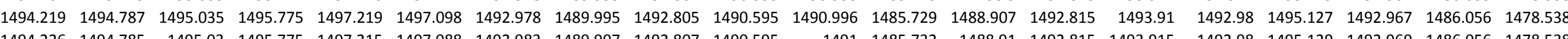

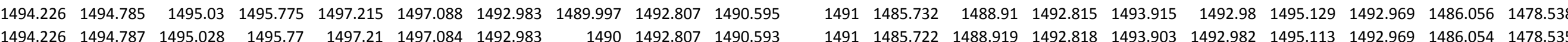

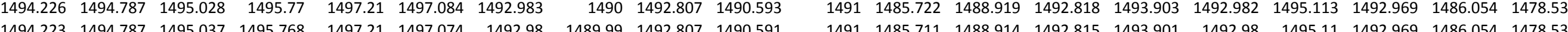

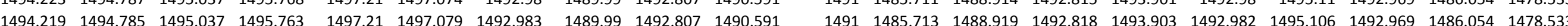
$\begin{array}{lllllllllllllllllllll}1494.216 & 1494.783 & 1495.03 & 1495.761 & 1497.212 & 1497.086 & 1492.983 & 1489.988 & 1492.807 & 1490.591 & 1491.003 & 1485.722 & 1488.919 & 1492.815 & 1493.898 & 1492.982 & 1495.106 & 1492.967 & 1486.054 & 1478.533\end{array}$ \begin{tabular}{llllllllllllllllllllll}
1494.209 & 1494.782 & 1495.033 & 1495.761 & 1497.212 & 1497.084 & 1492.985 & 1489.988 & 1492.809 & 1490.591 & 1491.005 & 1485.732 & 1488.912 & 1492.82 & 1493.898 & 1492.984 & 1495.106 & 1492.969 & 1486.054 & 1478.531 \\
\hline
\end{tabular}

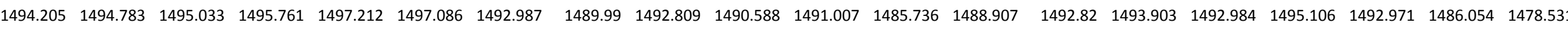
$\begin{array}{llllllllllllllllllllll}1494.2 & 1494.78 & 1495.033 & 1495.761 & 1497.21 & 1497.088 & 1492.99 & 1489.988 & 1492.814 & 1490.588 & 1491.01 & 1485.743 & 1488.896 & 1492.822 & 1493.905 & 1492.989 & 1495.113 & 1492.976 & 1486.054 & 1478.531\end{array}$

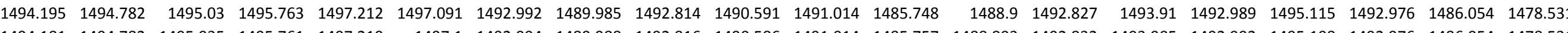

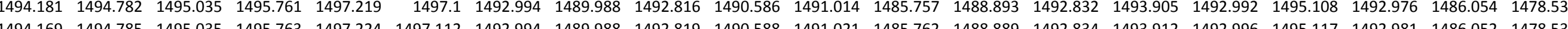

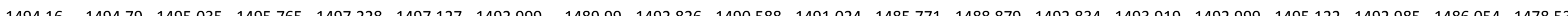
$\begin{array}{llllllllllllllllllllll}1494.15 & 1494.79 & 1495.035 & 1495.77 & 1497.235 & 1497.136 & 1493.006 & 1489.992 & 1492.828 & 1490.591 & 1491.028 & 1485.778 & 1488.865 & 1492.839 & 1493.926 & 1493.006 & 1495.136 & 1492.992 & 1486.054 & 1478.533\end{array}$

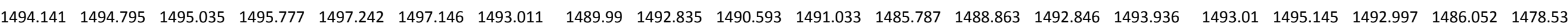
$\begin{array}{llllllllllllllllllll}1494.134 & 1494.797 & 1495.042 & 1495.782 & 1497.252 & 1497.162 & 1493.018 & 1489.999 & 1492.84 & 1490.595 & 1491.04 & 1485.794 & 1488.856 & 1492.848 & 1493.947 & 1493.018 & 1495.157 & 1493.002 & 1486.054 & 1478.538\end{array}$

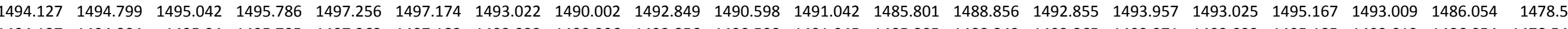
\begin{tabular}{lllllllllllllllllllll}
1494.127 & 1494.804 & 1495.04 & 1495.795 & 1497.263 & 1497.182 & 1493.032 & 1490.006 & 1492.856 & 1490.598 & 1491.045 & 1485.805 & 1488.842 & 1492.865 & 1493.971 & 1493.032 & 1495.185 & 1493.013 & 1486.054 & 1478.545 \\
\hline
\end{tabular}

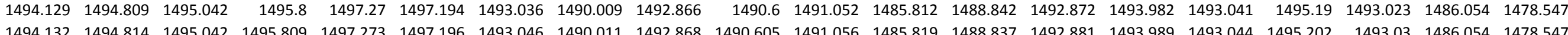


Well

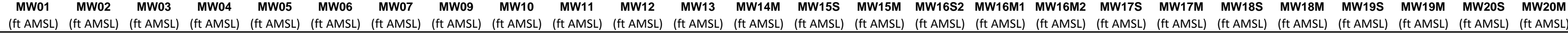

9/22/11 16:00 $9 / 22 / 1116: 30$ $9 / 22 / 1117 \cdot 30$ 9/22/11 17:30 9/22/11 18:30 9/22/11 19:00 9/22/11 19:30 9/22/11 20:00 9/22/11 20:30 9/22/11 21:00 9/22/1121:30 9/22/1122:00 9/22/1122:30 9/22/11 23:30 9/23/11 0:00 9/23/111:00 $9 / 23 / 111: 30$ 9/23/112:00 9/23/11 3:00 9/23/11 3:30 9/23/11: $1: 00$ 9/23/11 4:30 9/23/11 5:00 /23/11 6:00 9/23/11 6:30 9/23/11 7:30 9/23/11 8:00 9/23/11 8:30 9/23/119:00 9/23/119:30 $9 / 23 / 1110: 00$ $9 / 23 / 1110: 30$ 9/23/11111:30 9/23/11 12:00 9/23/11 12:30 9/23/11 13:00 9/23/11 13:30 9/23/11 14:00 9/23/11 14:30 $9 / 23 / 1115: 30$
$9 / 23 / 1116: 00$ 9/23/11 16:30 9/23/11 17:00 9/23/11 18:00 $\begin{array}{lrrr}1494.707 & 1495.25 & 1497.095 & 1495.175 \\ 1494.707 & 1495.254 & 1497.088 & 1495.18\end{array}$ $\begin{array}{llll}1494.707 & 1495.254 & 1497.088 & 1495.18 \\ 194.712 & 1955261 & 1497.088 & 1495.191\end{array}$ $\begin{array}{lllll}1494.712 & 1495.261 & 1497.088 & 1495.191 \\ 1494.71 & 1495264 & 1497.088 & 1495196\end{array}$ $\begin{array}{lllll}1494.71 & 1495.264 & 1497.086 & 1495.196\end{array}$ $\begin{array}{lllll}1494.707 & 1495.266 & 1497.086 & 1495.201\end{array}$ $\begin{array}{lllll}1494.707 & 1495.268 & 1497.088 & 1495.203\end{array}$ $\begin{array}{lllll}1494.71 & 1495.266 & 1497.083 & 1495.203\end{array}$ $\begin{array}{lllll}1494.71 & 1495.268 & 1497.076 & 1495.205\end{array}$

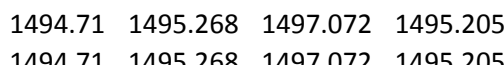
$\begin{array}{llll}1494.71 & 1495.268 & 1497.072 & 1495.205 \\ 1494.71 & 1495268 & 1497.065 & 1495203\end{array}$ $\begin{array}{rrrrr}1494.71 & 1495.268 & 1497.065 & 1495.203 \\ 1494.71 & 1495.266 & 1497.06 & 1495.201\end{array}$ $\begin{array}{lllll}1494.707 & 1495.266 & 1497.06 & 1495.201\end{array}$ $\begin{array}{llll}1494.707 & 1495.264 & 1497.065 & 1495.196\end{array}$ $\begin{array}{lllll}1494.707 & 1495.264 & 1497.067 & 1495.191\end{array}$ $\begin{array}{lllll}1494.712 & 1495.261 & 1497.074 & 1495.194\end{array}$ $\begin{array}{llll}1494.71 & 1495.264 & 1497.074 & 1495.194\end{array}$ $\begin{array}{llll}1494.712 & 1495.268 & 1497.074 & 1495.196\end{array}$ $\begin{array}{llll}1494.714 & 1495.268 & 1497.072 & 1495.198 \\ 1494.714 & 1495271 & 1497.074 & 1495.198\end{array}$ $\begin{array}{lllll}1494.714 & 1495.271 & 1497.072 & 1495.201\end{array}$ $\begin{array}{llllll}1494.714 & 1495.271 & 1497.072 & 1495.201\end{array}$ $\begin{array}{lllll}1494.717 & 1495.271 & 1497.072 & 1495.201\end{array}$ $\begin{array}{llllll}1494.719 & 1495.271 & 1497.072 & 1495.201\end{array}$ $\begin{array}{llll}1494.714 & 1495.273 & 1497.069 & 1495.201\end{array}$ $\begin{array}{llllll}1494.717 & 1495.275 & 1497.067 & 1495.203\end{array}$ $\begin{array}{lllll}1494.717 & 1495.273 & 1497.062 & 1495.203\end{array}$ $\begin{array}{lllll}1494.717 & 1495.271 & 1497.062 & 1495.198 \\ 1494.714 & 1495.273 & 1497.051 & 1495.198\end{array}$ $\begin{array}{lllll}1494.714 & 1495.271 & 1497.046 & 1495.198\end{array}$ $\begin{array}{lllll}1494.717 & 1495.271 & 1497.053 & 1455.196\end{array}$ $\begin{array}{lllll}1494.714 & 1495.266 & 1497.051 & 1495.191\end{array}$ $\begin{array}{lllll}1494.714 & 1495.266 & 1497.046 & 1495.189\end{array}$ $\begin{array}{llll}1494.717 & 1495.266 & 1497.039 & 1495.189\end{array}$ $\begin{array}{lllll}1494.714 & 1495.264 & 1497.032 & 1495.187\end{array}$ $\begin{array}{rrrrr}1494.714 & 1495.264 & 1497.034 & 1495.184 \\ 1494.714 & 1495259 & 1497.037 & 1495.18\end{array}$ $\begin{array}{rrrr}1494.714 & 1455.259 & 1497.037 & 1495.18 \\ 1494.712 & 1495.259 & 1497.037 & 1495.175\end{array}$ $\begin{array}{lllll}1494.714 & 1495.261 & 1497.046 & 1495.175\end{array}$ $1494.712 \quad 1495.259 \quad 1497.051 \quad 1495.173$ $\begin{array}{llllll}1494.714 & 1495.259 & 1497.067 & 1495.175\end{array}$ $\begin{array}{lllll}1494.719 & 1495.264 & 1497.081 & 1495.177\end{array}$ $\begin{array}{lllll}1494.721 & 1495.266 & 1497.091 & 1495.182\end{array}$ $\begin{array}{rrrrr}1494.726 & 1495.271 & 1497.095 & 1495.189 \\ 1494.731 & 1495.28 & 1497.1 & 1495201\end{array}$ $\begin{array}{rrrrr}1494.731 & 1495.28 & 1497.1 & 1495.201 \\ 1494.731 & 1495287 & 1497.102 & 1495.21\end{array}$ $\begin{array}{lllll}1494.731 & 1495.289 & 1497.095 & 1495.212\end{array}$ $\begin{array}{lllll}1494.731 & 1495.294 & 1497.093 & 1495.219\end{array}$ $\begin{array}{lllll}1494.731 & 1495.296 & 1497.091 & 1495.224\end{array}$ $\begin{array}{llll}1494.731 & 1495.296 & 1497.091 & 1495.224 \\ 1494.731 & 1495.296 & 1497.086 & 1495.226\end{array}$ $1494.731 \quad 1495.296 \quad 1497.0861495 .226$ $\begin{array}{llll}1494.731 & 1495.299 & 1497.091 & 1495.229 \\ 1494.731 & 1495.296 & 1497.093 & 1495.226\end{array}$ $\begin{array}{llllllllllllllllllll}1494.134 & 1494.816 & 1495.04 & 1495.812 & 1497.28 & 1497.203 & 1493.053 & 1490.016 & 1492.875 & 1490.609 & 1491.061 & 1485.829 & 1488.835 & 1492.886 & 1493.999 & 1493.055 & 1495.211 & 1493.035 & 1486.054 & 1478.549\end{array}$

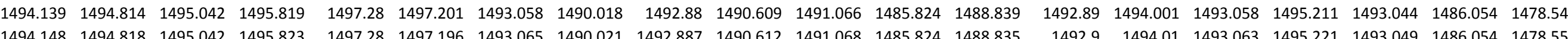

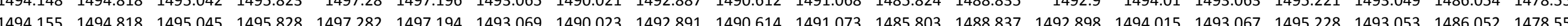

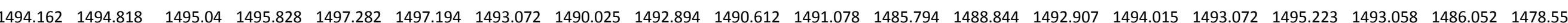
$\begin{array}{llllllllllllllllllllll}1494.167 & 1494.823 & 1495.042 & 1495.83 & 1497.284 & 1497.191 & 1493.074 & 1490.018 & 1492.901 & 1490.616 & 1491.08 & 1485.799 & 1488846 & 1492.909 & 1494.01 & 1493.077 & 1495.228 & 1493.058 & 1486.052 & 1478.554\end{array}$ $\begin{array}{llllllllllllllllllll}1494.174 & 1494.821 & 1495.04 & 1495.833 & 1497.284 & 1497.186 & 1493.079 & 1490.025 & 1492.903 & 1490.616 & 1491.082 & 1485.805 & 1488.849 & 1492.914 & 1494.015 & 1493.077 & 1495.228 & 1493.065 & 1486.052 & 1478.554 \\ \end{array}$

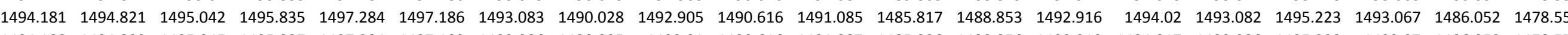

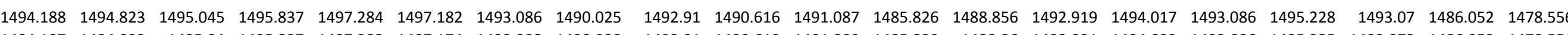

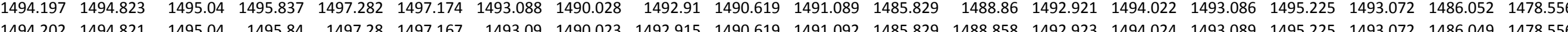

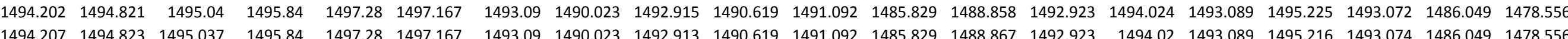

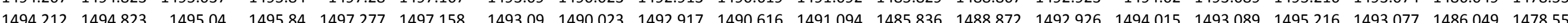
$\begin{array}{lllllllllllllllllllll}1494.214 & 1494.823 & 1495.037 & 1495.84 & 1497.277 & 1497.155 & 1493.093 & 1490.023 & 1492.92 & 1490.619 & 1491.096 & 1485.845 & 1488.877 & 1492.928 & 1494.015 & 1493.093 & 1495.214 & 1493.077 & 1486.049 & 1478.556\end{array}$ $\begin{array}{llllllllllllllllllll}1494.212 & 1494.823 & 1495.035 & 1495.84 & 1497.28 & 1497.155 & 1493.097 & 1490.014 & 1492.92 & 1490.616 & 1491.101 & 1485.849 & 1488.879 & 1492.93 & 1494.013 & 1493.096 & 1495.209 & 1493.079 & 1486.049 & 1478.554 \\ \end{array}$

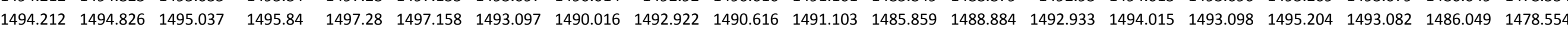
$\begin{array}{lllllllllllllllllllll}1494.209 & 1494.826 & 1495.04 & 1495.842 & 1497.282 & 1497.16 & 1493.097 & 1490.011 & 1492.924 & 1490.616 & 1491.106 & 1485.863 & 1488.879 & 1492.935 & 1494.015 & 1493.098 & 1495.209 & 1493.082 & 1486.049 & 1478.5569\end{array}$

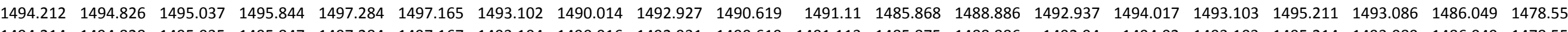

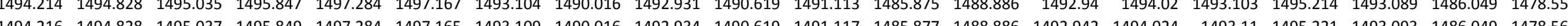

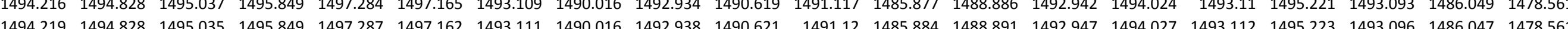
$\begin{array}{lllllllllllllllllllllll}1494.221 & 1494.831 & 1495.035 & 1495.851 & 1497.284 & 1497.162 & 1493.114 & 1490.014 & 1492.938 & 1490.621 & 1491.122 & 1485.889 & 1488.891 & 1492.949 & 1494.027 & 1493.115 & 1495.218 & 1493.1 & 1486.049 & 1478.561\end{array}$ $\begin{array}{lllllllllllllllllllll}1494.216 & 1494.828 & 1495.037 & 1495.851 & 1497.287 & 1497.162 & 1493.116 & 1490.014 & 1492.941 & 1490.621 & 1491.127 & 1485.896 & 1488.896 & 1492.952 & 1494.029 & 1493.117 & 1495.218 & 1493.1 & 1486.047 & 1478563 & \end{array}$ $\begin{array}{llllllllllllllllllllll}1494.223 & 1494.828 & 1495.037 & 1495.854 & 1497.284 & 1497.16 & 1493.121 & 1490.011 & 1492.945 & 1490.623 & 1491.129 & 1485.903 & 1488.898 & 1492.956 & 1494.031 & 1493.122 & 1495.218 & 1493.103 & 1486.047 & 1478.563\end{array}$ $\begin{array}{llllllllllllllllllll}1494.223 & 1494.83 & 1495.037 & 1495.854 & 1497.287 & 1497.162 & 1493.123 & 1490.018 & 1492.945 & 1490.621 & 1491.131 & 1485.91 & 1488.896 & 1492.956 & 1494.036 & 1493.122 & 1495.223 & 1493.107 & 1486.047 & 1478.563\end{array}$

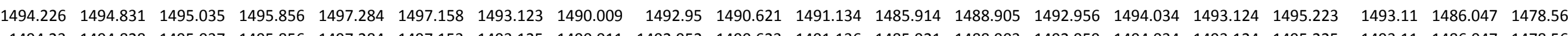

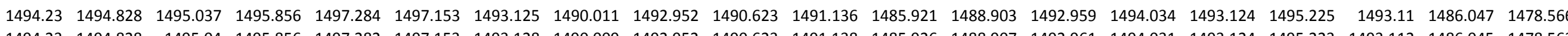

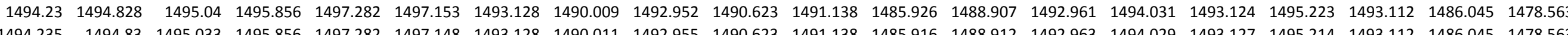

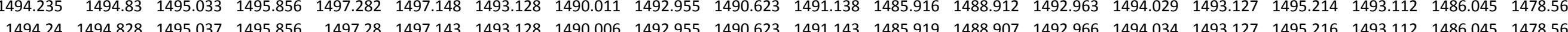
$\begin{array}{llllllllllllllllllllll}1494.24 & 1494.828 & 1495.033 & 1495.858 & 1497.277 & 1497.136 & 1493.13 & 1490.004 & 1492.955 & 1490.623 & 1491.141 & 1485.921 & 1488.91 & 1492.963 & 1494.034 & 1493.129 & 1495.214 & 1493.112 & 1486.045 & 1478.563\end{array}$ $\begin{array}{llllllllllllllllllll}1494.24 & 1494.828 & 1495.035 & 1495.856 & 1497.275 & 1497.131 & 1493.128 & 1490.004 & 1492.955 & 1490.621 & 1491.141 & 1485.912 & 1488.912 & 1492.961 & 1494.024 & 1493.127 & 1495.209 & 1493.112 & 1486.045 & 1478.563\end{array}$ $\begin{array}{lllllllllllllllllllllll}1494.235 & 1494.826 & 1495.03 & 1495.856 & 1497.275 & 1497.136 & 1493.125 & 1490.002 & 1492.957 & 1490.621 & 1491.141 & 1485.916 & 1488.914 & 1492.966 & 1494.024 & 1493.127 & 1495.207 & 1493.112 & 1486.045 & 1478.561\end{array}$ $\begin{array}{lllllllllllllllllllll}1494.237 & 1494.828 & 1495.03 & 1495.854 & 1497.275 & 1497.131 & 1493.128 & 1489.997 & 1492.955 & 1490.619 & 1491.141 & 1485.91 & 1488.914 & 1492.963 & 1494.022 & 1493.127 & 1495.202 & 1493.112 & 1486.045 & 1478.559\end{array}$

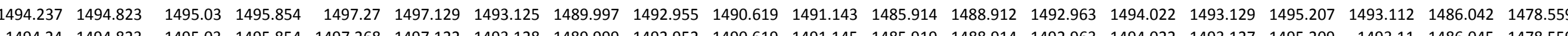

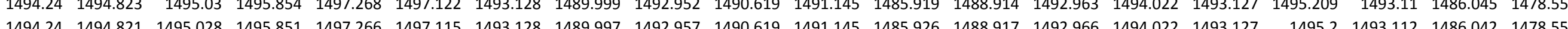

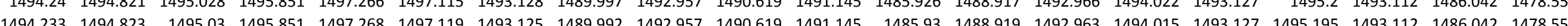

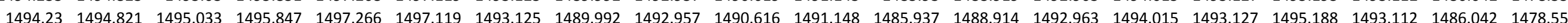
$\begin{array}{lllllllllllllllllllll}1494.221 & 1494.821 & 1495.03 & 1495.849 & 1497.268 & 1497.119 & 1493.046 & 1489.99 & 1492.868 & 1490.614 & 1491.15 & 1485.944 & 1488.912 & 1492.876 & 1494.008 & 1493.046 & 1495.195 & 1493.035 & 1486.042 & 1478.556\end{array}$

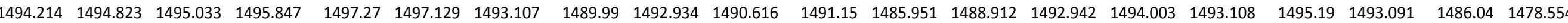

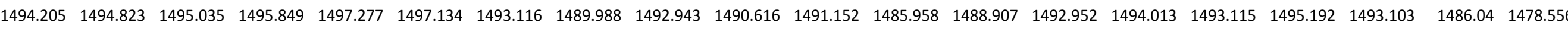
$\begin{array}{llllllllllllllllllll}1494.193 & 1494.828 & 1495.037 & 1495.851 & 1497.284 & 1497.15 & 1493.123 & 1489.988 & 1492.95 & 1490.616 & 1491.155 & 1485.96 & 1488.896 & 1492.961 & 1494.022 & 1493.127 & 1495.2 & 1493.11 & 1486.04 & 1478.559 \\ \end{array}$

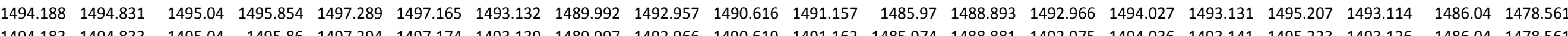

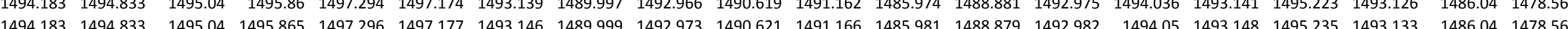

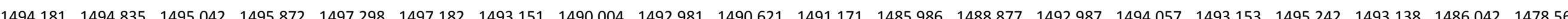
$\begin{array}{llllllllllllllllllllllll}1494.186 & 1494.838 & 1495.04 & 1495.874 & 1497.301 & 1497.184 & 1493.158 & 1490.006 & 1492.981 & 1490.623 & 1491.176 & 1485 & 995 & 1488.877 & 1492.989 & 1494.062 & 1493.157 & 1495.244 & 1493.143 & 1486.04 & 1478.568\end{array}$ $\begin{array}{llllllllllllllllllllllll}1494.195 & 1494.838 & 1495.042 & 1495.877 & 1497.298 & 1497.174 & 1493.16 & 1490.011 & 1492.988 & 1490.626 & 1491.18 & 1485.997 & 1488.872 & 1492.996 & 1494.066 & 1493.162 & 1495.251 & 1493.145 & 1486.04 & 1478.57\end{array}$ $\begin{array}{llllllllllllllllllllll}1494.195 & 1494.84 & 1495.04 & 1495.879 & 1497.301 & 1497.174 & 1493.163 & 1490.011 & 1492.992 & 1490.626 & 1491.183 & 1485.965 & 1488.879 & 1493.003 & 1494.066 & 1493.164 & 1495.251 & 1493.15 & 1486.04 & 1478.573\end{array}$ $\begin{array}{lllllllllllllllllllll}1494.2 & 1494.84 & 1495.04 & 1495.879 & 1497.301 & 1497.172 & 1493.165 & 1490.011 & 1492.995 & 1490.626 & 1491.185 & 1485.94 & 1488.879 & 1493.001 & 1494.069 & 1493.167 & 1495.251 & 1493.152 & 1486.04 & 1478.573 & \end{array}$

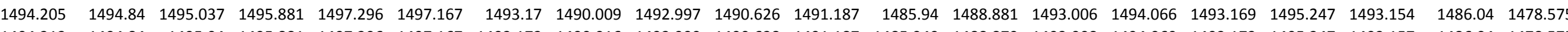

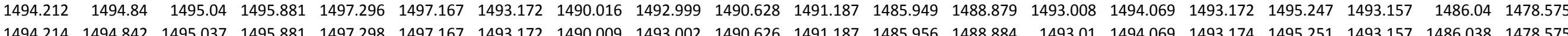


Well

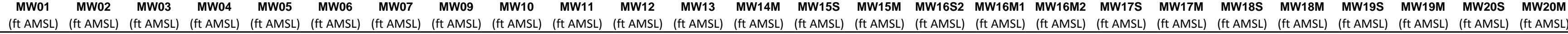

$9 / 23 / 11$ 19:00 $9 / 23 / 1119: 30$ $9 / 23 / 1120: 30$ $9 / 23 / 1120: 30$ $9 / 23 / 1121: 00$ 9/23/11 22:00 9/23/11 22:30 9/23/11 23:00 9/23/11 23:30 9/24/11 0:00 $9 / 24 / 111.00$ 9/24/111:00 9/24/111:30 9/24/11 2:30 9/24/11 3:00 9/24/11 :3:30 9/24/114:00 9/24/11 4:30 9/24/11 5:00 9/24/11 6:00 9/24/11 6:30 9/24/111:300 9/24/117:30 9/24/11 8:00 9/24/11 8:30 $9 / 24 / 119 \cdot 30$ 9/24/11 10:00 9/24/11 10:30 9/24/11 11:00 9/24/11 11:30 9/24/11 12:00 9/24/11 12:30 9/24/1113:00 9/24/11 13:30 9/244/11 14:30 9/24/11 15:00 9/24/11 15:30 9/24/11 16:00 $9 / 24 / 1116: 30$ $9 / 24 / 1117: 00$
$9 / 24 / 1117: 30$ 9/24/11 17:30 海/24/11 18:30 9/24/11 19:30 9/24/11 20:00 9/24/11 21:00 $\begin{array}{llll}1494.731 & 1495.296 & 1497.095 & 1495.226 \\ 1494.733 & 1495.296 & 1497.098 & 1495.229\end{array}$ $\begin{array}{llll}1494.733 & 1495.296 & 1497.098 & 1495.229 \\ 1494.733 & 1495301 & 1497.086 & 1495231\end{array}$ $\begin{array}{llll}1494.733 & 1495.301 & 1497.086 & 1495.231 \\ 1494.735 & 1495301 & 1497.081 & 1495231\end{array}$ $\begin{array}{lllll}1494.735 & 1495.303 & 1497.083 & 1495.231\end{array}$ $\begin{array}{lllll}1494.733 & 1495.296 & 1497.086 & 1495.226\end{array}$ $\begin{array}{lllll}1494.731 & 1495.296 & 1497.086 & 1495.224\end{array}$ $\begin{array}{lllll}1494.733 & 1495.296 & 1497.088 & 1495.226\end{array}$ $\begin{array}{lllll}1494.738 & 1495.301 & 1497.091 & 1495.229\end{array}$ $\begin{array}{lllll}1494.738 & 1495.303 & 1497.095 & 1495.229\end{array}$ $\begin{array}{llll}1494.738 & 1495.303 & 1497.1 & 1495.226 \\ 1494.738 & 1495.303 & 1497.1 & 1495229\end{array}$ $\begin{array}{rrrr}1494.738 & 1495.303 & 1497.1 & 1495.229 \\ 1494.74 & 1495.306 & 1497.107 & 1495.233\end{array}$ $\begin{array}{llll}1494.74 & 1455.306 & 1497.107 & 1495.233 \\ 1494.742 & 1495.313 & 1497.114 & 1495.236\end{array}$ $\begin{array}{lllll}1494.742 & 1495.742 & 1497.114 & 1495.236 \\ 1495.31 & 1497.114 & 1495.236\end{array}$ $\begin{array}{lllll}1494.747 & 1495.317 & 1497.116 & 1495.24\end{array}$ $\begin{array}{llll}1494.747 & 1495.322 & 1497.116 & 1495.245\end{array}$ $\begin{array}{llll}1494.747 & 1495.322 & 1497.116 & 1495.247\end{array}$ $1494.747 \begin{array}{llll}1495.324 & 1497.116 & 1495.25 \\ 149.749 & 1455324 & 1497.116 & 1495254\end{array}$ $\begin{array}{llll}1494.749 & 1495.324 & 1497.116 & 1495.254 \\ 1494.749 & 1495.324 & 1497.107 & 1495254\end{array}$ $\begin{array}{lllll}1494.749 & 1495.324 & 1497.107 & 1495.254 \\ 1494.749 & 1495.327 & 1497.107 & 1495.254\end{array}$ $\begin{array}{lllll}1494.749 & 1495.327 & 1497.107 & 1459.254\end{array}$

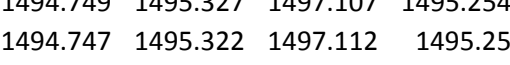
$\begin{array}{lllll}1494.747 & 1495.32 & 1497.114 & 1495.25 \\ 149.752 & 49532 & 147.11 & 145.25\end{array}$ 1494.752 $1495.327 \quad 1497.119 \quad 1495.252$ $\begin{array}{rrrr}1494.754 & 1495.329 & 1497.13 & 1495.254 \\ 1494754 & 1495329 & 1497.137 & 1455.254\end{array}$ $\begin{array}{llll}1494.754 & 1495.329 & 1497.137 & 1495.254 \\ 1494.756 & 1459.329 & 1497.133 & 145255\end{array}$ $\begin{array}{llll}1494.756 & 1495.329 & 1497.133 & 1495.257 \\ 1494.759 & 1495.338 & 1497.137 & 1495.264\end{array}$ $\begin{array}{llrr}1494.761 & 1495.343 & 1497.14 & 1495.266\end{array}$ $\begin{array}{lllll}1494.759 & 1495.341 & 1497.149 & 1495.268\end{array}$ $\begin{array}{lllll}1494.761 & 1495.345 & 1497.158 & 1495.271\end{array}$ $\begin{array}{lllll}1494.763 & 1495.348 & 1497.168 & 1495.275\end{array}$ $\begin{array}{lllll}1494.766 & 1495.352 & 1497.186 & 1495.28\end{array}$ $\begin{array}{llll}1494.773 & 1495.364 & 1497.189 & 1495.289 \\ 1494.775 & 1455373 & 1497.207 & 19529\end{array}$ $\begin{array}{rrrrr}1494.775 & 1495.373 & 1497.207 & 1495.299 \\ 1494.78 & 149538 & 1497.217 & 1955308\end{array}$ $\begin{array}{rrrr}1494.78 & 1495.38 & 1497.217 & 1495.308 \\ 1494.782 & 1495.39 & 1497.219 & 1495.315\end{array}$ $\begin{array}{rrrrr}1494.782 & 1495.39 & 1497.219 & 1495.315 \\ 1494.787 & 1495.401 & 1497.217 & 1495.327\end{array}$ $\begin{array}{lllll}1494.787 & 1495.406 & 1497.217 & 1495.338\end{array}$ $\begin{array}{llllll}1494.787 & 1495.411 & 1497.214 & 1495.343\end{array}$ $\begin{array}{lllll}1494.784 & 1495.415 & 1497.207 & 1495.35\end{array}$ $\begin{array}{llll}1494.787 & 1495.415 & 1497.2 & 1495.35\end{array}$ $\begin{array}{rrrr}1494.784 & 1495.415 & 1497.193 & 1495.352 \\ 149.787 & 145.413 & 1497.179 & 149535\end{array}$

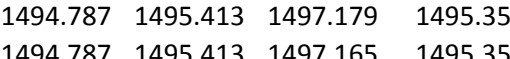
$\begin{array}{lllll}1494.784 & 1495.411 & 1497.154 & 1495.345\end{array}$ $\begin{array}{lllll}1494.78 & 1495.406 & 1497.128 & 1495.336\end{array}$ $1494.777 \quad 1495.399 \quad 1497.109 \quad 1495.327$ $\begin{array}{lllll}1494.775 & 1495.394 & 1497.098 & 1495.317\end{array}$ $\begin{array}{lllll}1494.768 & 1495.378 & 1497.088 & 1495.299\end{array}$ $\begin{array}{llll}1494.766 & 1495.369 & 1497.086 & 1495.282 \\ 1494.768 & 1495.364 & 1497.088 & 1495.271\end{array}$

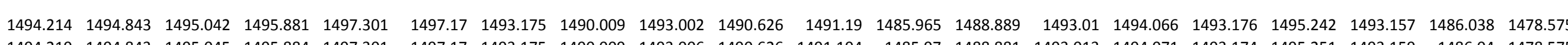

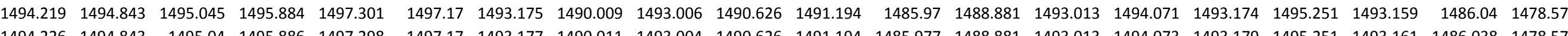

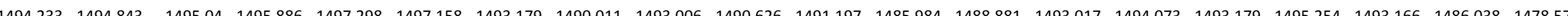

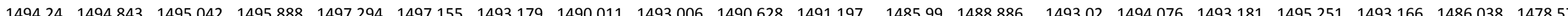
$\begin{array}{lllllll} & \end{array}$

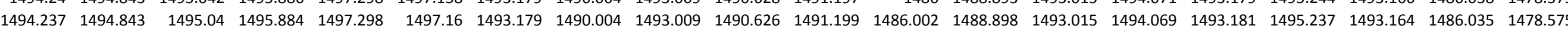
$\begin{array}{lllllllllllllllllllll}1494.24 & 1494.845 & 1495.04 & 1495.888 & 1497.301 & 1497.158 & 1493.182 & 1490.002 & 1493.009 & 1490.628 & 1491.199 & 1486.009 & 1488.896 & 1493.02 & 1494.066 & 1493.179 & 1495.239 & 1493.164 & 1486.038 & 1478.573 & \end{array}$

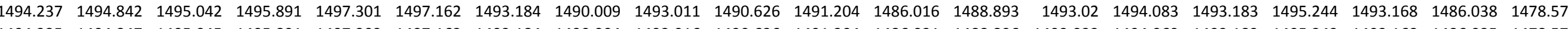

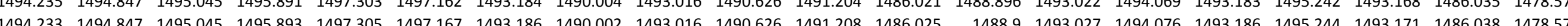
$\begin{array}{llllllllllllllllllll}1494.233 & 1494.847 & 1495.045 & 1495.893 & 1497.305 & 1497.167 & 1493.186 & 1490.002 & 1493.016 & 1490.626 & 1491.208 & 1486.025 & 1488.9 & 1493.027 & 1494.076 & 1493.186 & 1495.244 & 1493.171 & 1486.038 & 1478.575 \\ 1494.235 & 1494.847 & 1495.045 & 1495893 & 1497308 & 1497.17 & 1493191 & 1490.006 & 1493018 & 1490.626 & 1491208 & 1486.032 & 1488.902 & 1493024 & 1494076 & 1493188 & 1495251 & 1493.173 & 1486038 & 1478577\end{array}$

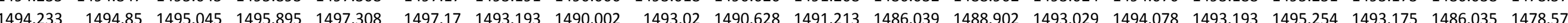
$\begin{array}{llllllllllllllllllllll}1494.23 & 1494.85 & 1495.047 & 1495.898 & 1497.315 & 1497.177 & 1493.196 & 1490.009 & 1493.023 & 1490.628 & 1491.218 & 1486.046 & 1488.905 & 1493.034 & 1494.087 & 1493.195 & 1495.256 & 1493.18 & 1486.035 & 1478.577\end{array}$ $\begin{array}{llllllllllllllllllllll}1494.228 & 1494.852 & 1495.047 & 1495.9 & 1497.317 & 1497.182 & 1493.198 & 1490.009 & 1493.025 & 1490.628 & 1491.218 & 1486.053 & 1488.905 & 1493.032 & 1494.087 & 1493.198 & 1495.251 & 1493.182 & 1486.035 & 1478.58\end{array}$

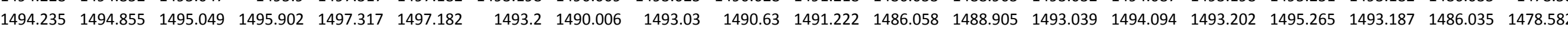
$\begin{array}{llllllllllllllllllllll}1494.235 & 1494.852 & 1495.049 & 1495.907 & 1497.317 & 1497.182 & 1493.207 & 1490.011 & 1493.034 & 1490.63 & 1491.227 & 1486.062 & 1488.903 & 1493.043 & 1494.099 & 1493.205 & 1495.275 & 1493.192 & 1486.038 & 1478.584 & \end{array}$ $\begin{array}{llllllllllllllllllll}1494.24 & 1494.857 & 1495.042 & 1495.907 & 1497.319 & 1497.182 & 1493.21 & 1490.018 & 1493.037 & 1490.633 & 1491.229 & 1486.069 & 1488.909 & 1493.046 & 1494.101 & 1493.207 & 1495.268 & 1493.192 & 1486.035 & 1478.584\end{array}$

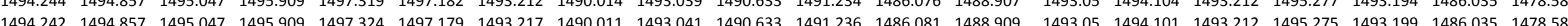

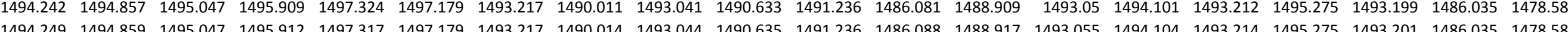
$\begin{array}{llllllllllllllllllllllll}1494.261 & 1494.857 & 1495.042 & 1495.914 & 1497.315 & 1497.172 & 1493.219 & 1490.011 & 1493.046 & 1490.635 & 1491.241 & 1486.085 & 1488.914 & 1493.05 & 1494.108 & 1493.217 & 1495.275 & 1493.204 & 1486.035 & 1478.589\end{array}$

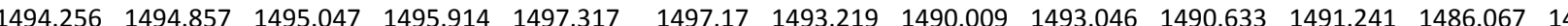
$\begin{array}{llllllllllllllllllllllllll}1494.254 & 1494.859 & 1495.049 & 1495.914 & 1497.319 & 1497.167 & 1493.219 & 1490.014 & 1493.048 & 1490.633 & 1491.243 & 1486.069 & 1488.926 & 1493.074 & 1494.097 & 1493.217 & 1495.265 & 1493.201 & 1486.033 & 1478.589\end{array}$ $\begin{array}{llllllllllllllllllllll}1494.252 & 1494.859 & 1495.052 & 1495.914 & 1497.319 & 1497.172 & 1493.219 & 1490.009 & 1493.046 & 1490.633 & 1491.244 & 1486.074 & 1488.926 & 1493.079 & 1494.097 & 1493.219 & 1495.268 & 1493.201 & 1486.033 & 1478.587\end{array}$ $\begin{array}{llllllllllllllllllll}1494.254 & 1494.859 & 1495.049 & 1495.914 & 1497.322 & 1497.174 & 1493.221 & 1490.009 & 1493.048 & 1490.633 & 1491.241 & 1486.078 & 1488.924 & 1493.076 & 1494.101 & 1493.219 & 1495.268 & 1493.201 & 1486.033 & 1478.589 \\ \end{array}$

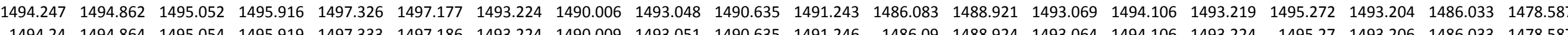

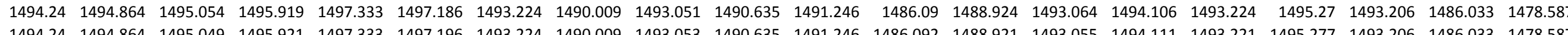

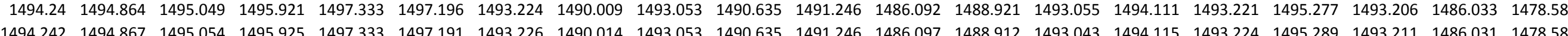
$\begin{array}{lllllllllllllllllllll}1494.237 & 1494.869 & 1495.054 & 1495.928 & 1497.335 & 1497.196 & 1493.228 & 1490.011 & 1493.053 & 1490.637 & 1491.248 & 1486.099 & 1488.914 & 1493.027 & 1494.122 & 1493.228 & 1495.296 & 1493.213 & 1486.033 & 1478.584\end{array}$ \begin{tabular}{llllllllllllllllllllll}
1494.235 & 1494.869 & 1495.054 & 1495.93 & 1497.34 & 1497.198 & 1493.231 & 1490.014 & 1493.056 & 1490.637 & 1491.248 & 1486.104 & 1488.921 & 1493.029 & 1494.115 & 1493.226 & 1495.289 & 1493.213 & 1486.031 & 1478.584 \\
\hline
\end{tabular}

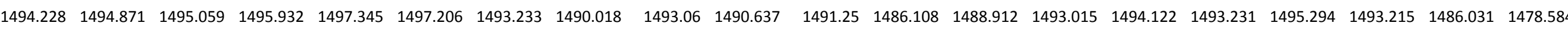
$\begin{array}{llllllllllllllllllllll}1494.221 & 1494.876 & 1495.059 & 1495.935 & 1497.354 & 1497.215 & 1493.235 & 1490.014 & 1493.06 & 1490.637 & 1491.255 & 1486.108 & 1488.914 & 1493.01 & 1494.127 & 1493.233 & 1495.296 & 1493.218 & 1486.033 & 1478.584\end{array}$

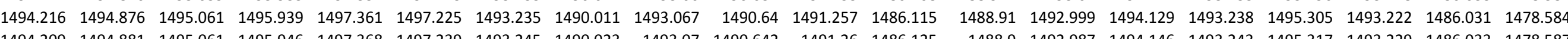

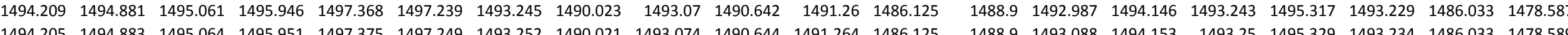

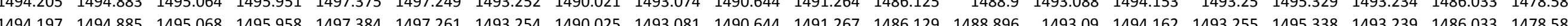

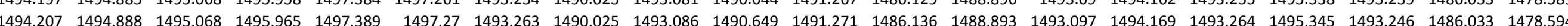
$\begin{array}{llllllllllllllllllllllll}1494.207 & 1494.89 & 1495.068 & 1495.974 & 1497.396 & 1497.268 & 1493.268 & 1490.032 & 1493.093 & 1490.651 & 1491.278 & 1486.138 & 1488.889 & 1493.102 & 1494.183 & 1493.269 & 1495.359 & 1493.253 & 1486.033 & 1478.596\end{array}$

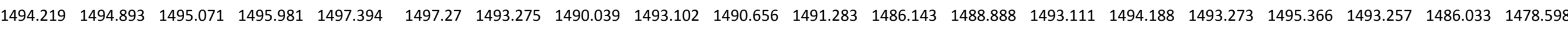
\begin{tabular}{llllllllllllllllllllllll}
1494.223 & 1494.895 & 1495.068 & 1495.984 & 1497.394 & 1497.265 & 1493.28 & 1490.039 & 1493.109 & 1490.656 & 1491.288 & 1486.148 & 1488.888 & 1493.114 & 1494.192 & 1493.278 & 1495.373 & 1493.262 & 1486.033 & 1478.601 \\
\hline
\end{tabular}

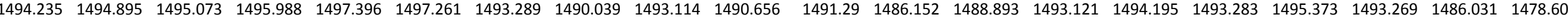

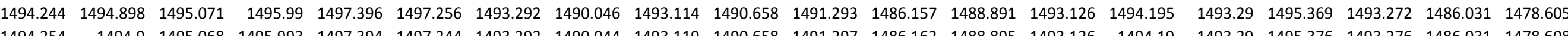

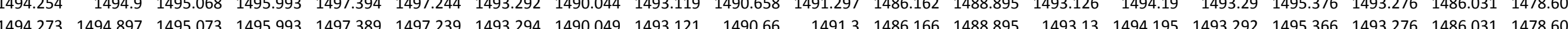

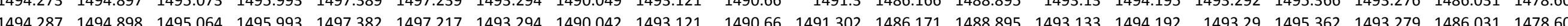
$\begin{array}{llllllllllllllllllllll}1494.299 & 1494.895 & 1495.068 & 1495.99 & 1497.375 & 1497.203 & 1493.296 & 1490.042 & 1493.124 & 1490.66 & 1491.302 & 1486.148 & 1488.905 & 1493.133 & 1494.183 & 1493.295 & 1495.352 & 1493.281 & 1486.028 & 1478.808\end{array}$ $\begin{array}{lllllllllllllllllllllll}1494.322 & 1494.895 & 1495.056 & 1495.988 & 1497.361 & 1497.191 & 1493.292 & 1490.039 & 1493.121 & 1490.658 & 1491.302 & 1486.141 & 1488.917 & 1493.128 & 1494.174 & 1493.292 & 1495.341 & 1493.276 & 1486.028 & 1478.608\end{array}$ $\begin{array}{lllllllllllllllllllll}1494.343 & 1494.89 & 1495.064 & 1495.984 & 1497.352 & 1497.16 & 1493.289 & 1490.035 & 1493.119 & 1490.656 & 1491.304 & 1486.129 & 1488.919 & 1493.126 & 1494.169 & 1493.29 & 1495.331 & 1493.272 & 1486.028 & 1478.608\end{array}$

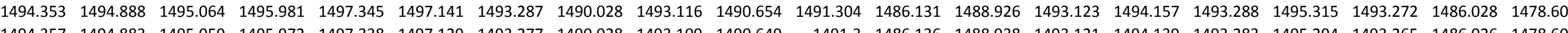
$\begin{array}{llllllllllllllllllll}1494.357 & 1494.883 & 1495.059 & 1495.972 & 1497.338 & 1497.129 & 1493.277 & 1490.028 & 1493.109 & 1490.649 & 1491.3 & 1486.136 & 1488.938 & 1493.121 & 1494.139 & 1493.283 & 1495.294 & 1493.265 & 1486.026 & 1478.603\end{array}$

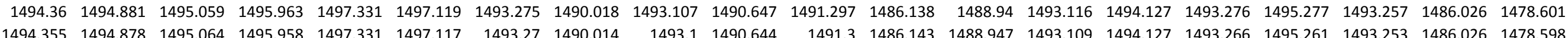


Well

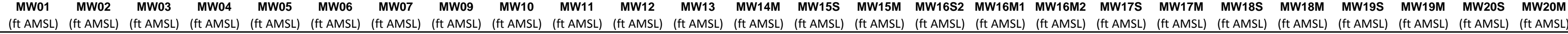

9/24/11 22:00 9/24/1122:30 $9 / 24 / 1123: 00$ 9/24/1123:110:00 9/25/110:30 $9 / 25 / 1110: 30$ 9/25/111:30 9/25/112:00 9/25/11 2:30 9/25/11 3:00 9/25/113:30 9/25/114:30 9/25/11 5:00 9/25/11 5:30 9/25/11 6:00 9/25/11 6:30 9/25/117:00 $9 / 25 / 117: 30$ 9/25/11 8:00 9/25/118:30 9/25/11 9:00 9/25/11 10:00 9/25/11 10:30 9/25/11 11:00 9/25/11 11:30 9/25/11 12:00 9/25/11 13:00 $9 / 25 / 1113: 30$ 9/25/11 14:00 9/25/11 14:30 9/25/11 15:00 9/25/11 15:30 $9 / 25 / 1116.00$ 9/25/11 16:30 9/25/1117:00 9/255/11 18:00 9/25/11 18:30 9/25/11 19:00 9/25/11 19:30 9/25/11 20:00 9/25/11 21:00 $9 / 25 / 1122: 00$ 9/25/11 22:30 9/25/11 23:00 9/25/11 23:30 9/26/11 0:00 $\begin{array}{llll}1494.768 & 1495.357 & 1497.091 & 1495.259 \\ 1494.768 & 1495.352 & 1497.093 & 1495.252\end{array}$ $\begin{array}{rrrr}1494.768 & 1495.352 & 1497.093 & 1495.252 \\ 1494.77 & 1495.35 & 1497.1 & 1495247\end{array}$ $\begin{array}{lllll}1494.77 & 1495.35 & 1497.1 & 1495.247 \\ 1494.773 & 1495.352 & 1497.1 & 1495243\end{array}$ $\begin{array}{lllll}1494.773 & 1495.352 & 1497.109 & 1495.243\end{array}$ $\begin{array}{lllll}1494.775 & 1495.352 & 1497.107 & 1459.243\end{array}$ $\begin{array}{lllll}1494.773 & 1495.355 & 1497.102 & 1495.243\end{array}$ $\begin{array}{lllll}1494.777 & 1495.357 & 1497.098 & 1495.243\end{array}$ $\begin{array}{lllll}1494.777 & 1495.357 & 1497.098 & 1495.245\end{array}$ $\begin{array}{llll}1494.773 & 1495.352 & 1497.098 & 1495.24\end{array}$ $\begin{array}{rrrr}1494.777 & 1495.352 & 1497.1 & 1495.24 \\ 1494.775 & 149535 & 1497.098 & 1495.238\end{array}$ $\begin{array}{rrrr}1494.775 & 1495.35 & 1497.098 & 1495.238 \\ 1494.777 & 1495.352 & 1497.093 & 1495.238\end{array}$ $\begin{array}{lllll}1494.775 & 1495.352 & 1497.083 & 1495.238\end{array}$ $\begin{array}{llll}1494.775 & 1495.35 & 1497.072 & 1495.236\end{array}$ $\begin{array}{lllll}1494.773 & 1495.345 & 1497.067 & 1495.233\end{array}$ $\begin{array}{lllll}1494.773 & 1495.343 & 1497.06 & 1495.229\end{array}$ $1494.768 \quad 1495.336 \quad 1497.055 \quad 1495.219$ $\begin{array}{llll}1494.768 & 1495.329 & 1497.051 & 1495.215\end{array}$ $\begin{array}{lllll}1494.768 & 1495.331 & 1497.041 & 1495.212 \\ 1494.768 & 1495324 & 1497.041 & 1495205\end{array}$ $\begin{array}{lllll}1444.766 & 1495.32 & 1497.037 & 1495.201\end{array}$ $\begin{array}{lllll}1494.766 & 1495.315 & 1497.032 & 1495.194\end{array}$ $\begin{array}{lllll}1494.766 & 1495.315 & 1497.034 & 1995.191\end{array}$ $\begin{array}{lllll}1494.763 & 1495.31 & 1497.039 & 1495.184\end{array}$ $\begin{array}{lllll}1494.768 & 1495.308 & 1497.048 & 1495.184\end{array}$ $1494.766 \quad 1495.308 \quad 1497.055 \quad 1495.18$ $\begin{array}{rrrr}1494.768 & 1495.308 & 1497.062 & 1495.18 \\ 1494.77 & 1955315 & 1997.074 & 1495.184\end{array}$ $\begin{array}{llll}1494.77 & 1495.315 & 1497.074 & 1495.184 \\ 1494.77 & 1495315 & 1497.083 & 1495.189\end{array}$ $\begin{array}{lllll}1494.773 & 1495.322 & 1497.095 & 1495.191\end{array}$ $\begin{array}{lllll}1494.777 & 1495.322 & 1497.105 & 1459.198\end{array}$ $\begin{array}{lllll}1494.777 & 1495.329 & 1497.107 & 1495.203\end{array}$ $\begin{array}{lllll}1494.782 & 1495.336 & 1497.109 & 1495.21\end{array}$ $\begin{array}{lllll}1494.782 & 1495.343 & 1497.116 & 1495.217\end{array}$ $\begin{array}{llll}1494.78 & 1495.343 & 1497.116 & 1495.222\end{array}$ $\begin{array}{llll}1494.782 & 1495.345 & 1497.112 & 1495.226 \\ 1494.784 & 1495348 & 1497.107 & 1955231\end{array}$ $\begin{array}{lllll}1494.782 & 1455.348 & 1497.102 & 14952.231\end{array}$ $\begin{array}{lllll}1494.784 & 1495.35 & 1497.095 & 1495.233\end{array}$ $\begin{array}{lllll}1494.78 & 1495.348 & 1497.091 & 1495.233\end{array}$ $\begin{array}{lllll}1494.782 & 1495.345 & 1497.086 & 1495.233\end{array}$ $\begin{array}{lllll}1494.78 & 1495.345 & 1497.079 & 1995.229\end{array}$ $\begin{array}{llll}1494.78 & 1495.341 & 1497.06 & 1495.226\end{array}$ $\begin{array}{rrrr}1494.78 & 1495.338 & 1497.055 & 1495.222 \\ 1494.775 & 1495334 & 1497.06 & 1495217\end{array}$ $\begin{array}{lllll}1494.775 & 1495.334 & 1497.06 & 1495.217\end{array}$ $\begin{array}{lllll}1494.773 & 1495.324 & 1497.06 & 1495.203\end{array}$ $\begin{array}{lllll}1494.777 & 1495.327 & 1497.062 & 1495.203\end{array}$ $1494.775 \quad 1495.327 \quad 1497.067 \quad 1495.201$ $\begin{array}{llll}1494.775 & 1495.327 & 1497.067 & 1495.201 \\ 1494.777 & 1495.327 & 1497.069 & 1495.198\end{array}$ $\begin{array}{rlll}1494.777 & 1495.327 & 1497.069 & 1495.198 \\ 1494.78 & 1495327 & 1497.072 & 1495.198\end{array}$ $\begin{array}{rrrr}1494.78 & 1495.327 & 1497.072 & 1495.198 \\ 1494.777 & 1495.329 & 1497.074 & 1495.198\end{array}$

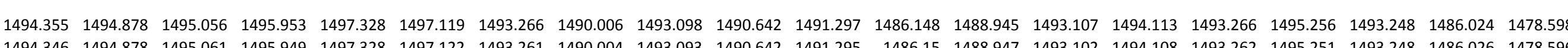

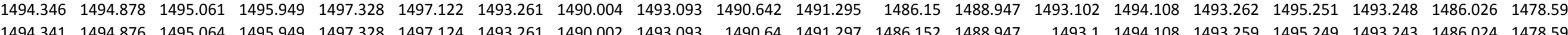

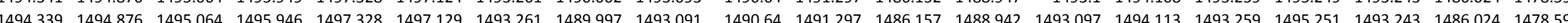
$\begin{array}{lllllllllllllllllllllll}1494.331 & 1494.876 & 1495.064 & 1495.946 & 1497.328 & 1497.131 & 1493.259 & 1489.997 & 1493093 & 1490.637 & 1491.3 & 1486.162 & 1488942 & 14931 & 1494.108 & 1493259 & 1495254 & 1493243 & 1486.024 & 1478.596\end{array}$ $\begin{array}{llllllllllllllllllllll}1494.329 & 1494.876 & 1495064 & 1495946 & 1497.328 & 1497.139 & 1493.261 & 1489.997 & 1493.091 & 1490.637 & 1491.3 & 1486.168 & 1488.938 & 14931 & 1494.113 & 1493.262 & 1495.251 & 1493.246 & 1486.026 & 1478.596\end{array}$ $\begin{array}{lllllllllllllllllllll}1494.327 & 1494.878 & 1495.064 & 1495.946 & 1497.328 & 1497.139 & 1493.261 & 1489.99 & 1493.093 & 1490.64 & 1491.302 & 1486.173 & 1488.938 & 1493.1 & 1494.113 & 1493.262 & 1495.256 & 1493.243 & 1486.024 & 1478.596\end{array}$ $\begin{array}{llllllllllllllllllllll}1494.331 & 1494.874 & 1495.064 & 1495.946 & 1497.324 & 1497.134 & 1493.266 & 1489.995 & 1493.095 & 1490.637 & 1491.302 & 1486.175 & 1488.94 & 1493.102 & 1494.115 & 1493.266 & 1495.265 & 1493.246 & 1486.024 & 1478.598\end{array}$

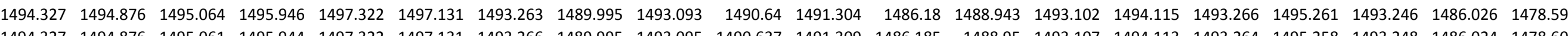

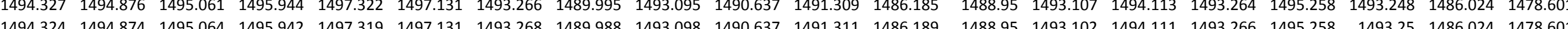

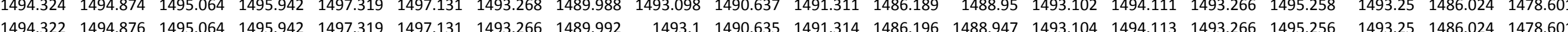

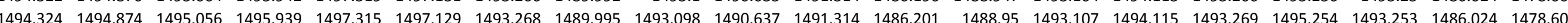

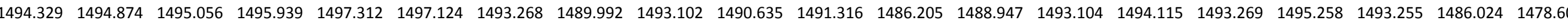
$\begin{array}{lllllllllllllllllllll}1494.334 & 1494.871 & 1495.056 & 1495.937 & 1497.305 & 1497.117 & 1493.27 & 1489.992 & 1493.1 & 1490.633 & 1491.318 & 1486.226 & 1488.954 & 1493.109 & 1494.113 & 1493.271 & 1495.254 & 1493.253 & 1486.024 & 1478.608\end{array}$

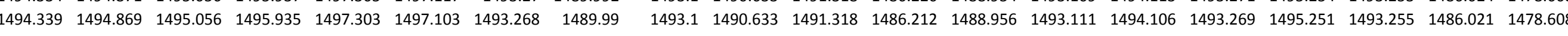
$\begin{array}{llllllllllllllllllllll}1494.339 & 1494.866 & 1495.052 & 1495.93 & 1497.296 & 1497.1 & 1493.266 & 1489.988 & 1493.1 & 1490.63 & 1491.318 & 1486.201 & 1488.961 & 1493.107 & 1494.104 & 1493.266 & 1495.239 & 1493.25 & 1486.021 & 1478.6005\end{array}$

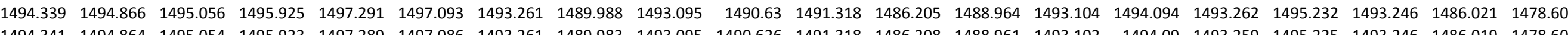

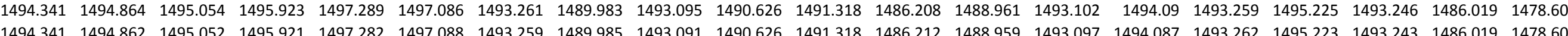
$\begin{array}{lllllllllllllllllllll}1494.341 & 1494.862 & 1495.052 & 1495.921 & 1497.282 & 1497.088 & 1493.259 & 1489.985 & 1493.091 & 1490.626 & 1491.318 & 1486.212 & 1488.959 & 1493.097 & 1494.087 & 1493.262 & 1495.223 & 1493.243 & 1486.019 & 1478.605 \\ 1494.341 & 1494.859 & 1495.052 & 1495.916 & 149728 & 1497.079 & 1493256 & 1489.983 & 1493088 & 1490623 & 1491316 & 1486215 & 1488959 & 1493097 & 1494083 & 1493252 & 1495214 & 1493241 & 1486019 & 1478603\end{array}$ $\begin{array}{llllllllllllllllllllll}1494.339 & 1494.857 & 1495.049 & 1495.912 & 1497.277 & 1497.079 & 1493.254 & 1489.983 & 1493.084 & 1490.623 & 1491.316 & 1466.212 & 1488.961 & 1493.093 & 1494.083 & 1493.252 & 1495.214 & 1493.234 & 1486.019 & 1478.601\end{array}$

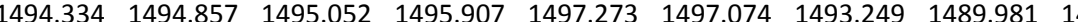
$\begin{array}{llllllllllllllllllllll}1494.324 & 1494.855 & 1495.052 & 1495.905 & 1497.273 & 1497.076 & 1493.247 & 1489.978 & 1493.074 & 1490.619 & 1491.311 & 1486.173 & 1488.959 & 1493.088 & 1494.071 & 1493.245 & 1495.202 & 1493.229 & 1486.017 & 1478.596\end{array}$

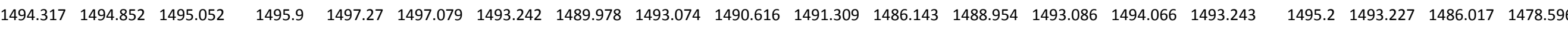

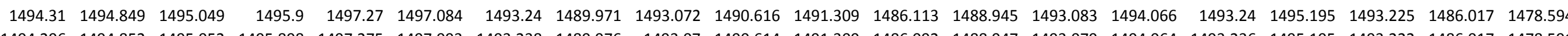

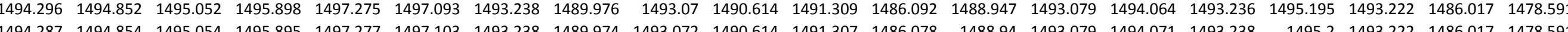

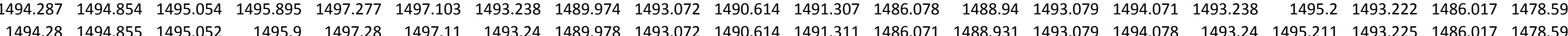

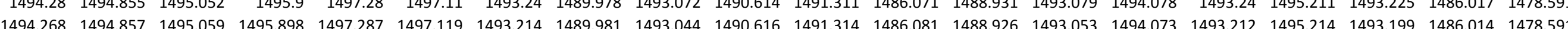

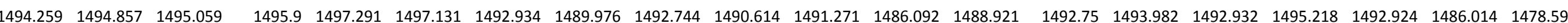
$\begin{array}{lllllllllllllllllllll}1494.247 & 1494.859 & 1495.061 & 1495.902 & 1497.296 & 1497.146 & 1493.055 & 1489.985 & 1492.875 & 1490.616 & 1491.25 & 1486.097 & 1488.917 & 1492.886 & 1494.008 & 1493.055 & 1495.218 & 1493.042 & 1486.014 & 1478.594\end{array}$ $\begin{array}{llllllllllllllllllllll}1494.247 & 1494.862 & 1495.059 & 1495.907 & 1497.301 & 1497.153 & 1493.107 & 1489.978 & 1492.929 & 1490.616 & 1491.257 & 1486.099 & 1488.912 & 1492.937 & 1494.031 & 1493.105 & 1495.228 & 1493.093 & 1486.017 & 1478.596\end{array}$ $\begin{array}{llllllllllllllllllllll}1494.247 & 1494.864 & 1495.059 & 1495.912 & 1497.301 & 1497.155 & 1493.137 & 1489.992 & 1492.962 & 1490.621 & 1491.262 & 1486.099 & 1488.907 & 1492.966 & 1494.052 & 1493.136 & 1495.235 & 1493.124 & 1486.017 & 1478.598\end{array}$ $\begin{array}{llllllllllllllllllll}1494.247 & 1494.867 & 1495.056 & 1495.914 & 1497.303 & 1497.158 & 1493.158 & 1489.99 & 1492.981 & 1490.621 & 1491.267 & 1486.099 & 1488.907 & 1492.987 & 1494.064 & 1493.157 & 1495.239 & 1493.145 & 1486.014 & 1478.601 \\ \end{array}$

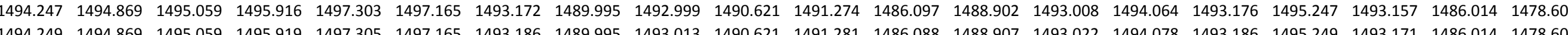

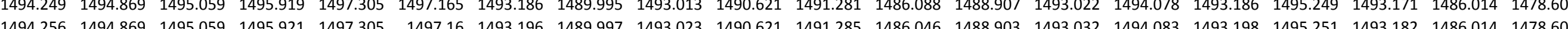

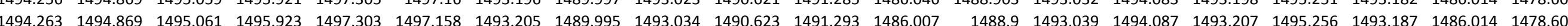
$\begin{array}{lllllllllllllllllllllll}1494.273 & 1494.869 & 1495.061 & 1495.923 & 1497.301 & 1497.15 & 1493.212 & 1489.997 & 1493.037 & 1490.623 & 1491.295 & 1485.988 & 1488.903 & 1493.048 & 1494.09 & 1493.212 & 1495.251 & 1493.196 & 1486.014 & 1478.81\end{array}$ \begin{tabular}{llllllllllllllllllllll}
1494.277 & 1494.869 & 1495.059 & 1495.921 & 1497.301 & 1497.141 & 1493.217 & 1489.995 & 1493.046 & 1490.623 & 1491.297 & 1485.981 & 1488.905 & 1493.05 & 1494.087 & 1493.217 & 1495.254 & 1493.199 & 1486.014 & 1478.61 \\
\hline
\end{tabular}

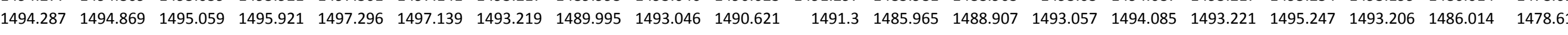
$\begin{array}{llllllllllllllllllll}1494.292 & 1494.869 & 1495.056 & 1495.919 & 1497.291 & 1497.131 & 1493.221 & 1489.992 & 1493.048 & 1490.621 & 1491.3 & 1485.958 & 1488.909 & 1493.06 & 1494.09 & 1493.221 & 1495.242 & 1493.208 & 1486.014 & 1478.612\end{array}$

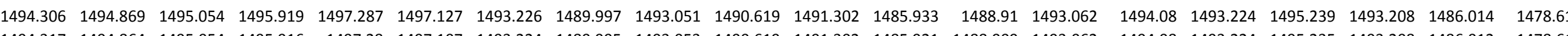

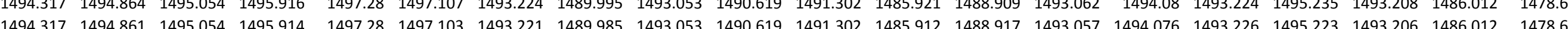

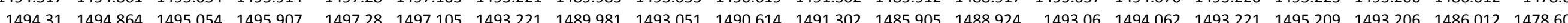
$\begin{array}{llllllllllllllllllllll}1494.31 & 1494.864 & 1495.054 & 1495.905 & 1497.277 & 1497.11 & 1493.219 & 1489.981 & 1493.048 & 1490.614 & 1491.3 & 1485.903 & 1488.919 & 1493.057 & 1494.064 & 1493.217 & 1495.209 & 1493.204 & 1486.01 & 1478.605 \\ \end{array}$ \begin{tabular}{llllllllllllllllllllll}
1494.308 & 1494.859 & 1495.056 & 1495.905 & 1497.28 & 1497.11 & 1493.217 & 1489.981 & 1493.046 & 1490.614 & 1491.302 & 1485.893 & 1488.912 & 1493.06 & 1494.069 & 1493.219 & 1495.216 & 1493.204 & 1486.012 & 1478.605 \\
\hline
\end{tabular}

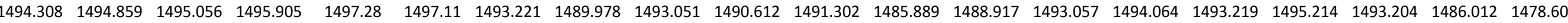

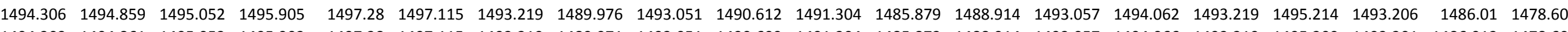
$\begin{array}{lllllllllllllllllllll}1494.303 & 1494.861 & 1495.052 & 1495.902 & 1497.28 & 1497.115 & 1493.219 & 1489.971 & 1493.051 & 1490.609 & 1491.304 & 1485.873 & 1488.914 & 1493.057 & 1494.066 & 1493.219 & 1495.209 & 1493.201 & 1486.012 & 1478.601\end{array}$

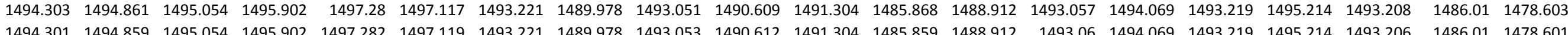


Well

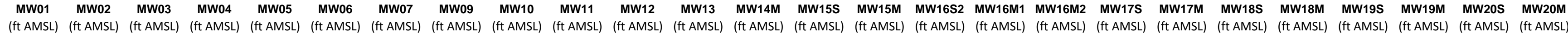

$9 / 26 / 111: 00$ $9 / 26 / 111: 30$ $9 / 26 / 112: 00$ 9/26/112:30 9/26/11 :3:30 9/26/114:00 9/26/11 4:30 9/26/115:00 9/26/11 5:30 9/26/11 6:00 9/26/11 6:30 9/26/117:30 $9 / 26 / 117: 30$
$9 / 26 / 118: 00$ $9 / 26 / 118: 30$ 9/26/11 9:00 9/26/11 9:30 9/26/11 10:00

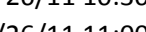
9/26/11 11:00 源/26/1111:30 9/26/11 12:30 9/26/11 13:00 9/26/11 13:30 9/26/11 14:00 9/26/11 14:30 9/26/11 15:00 9/26/11 16:00 9/26/11 16:30 9/26/11 17:00 9/26/11 17:30 9/26/11 18:00 9/26/11 18:30 9/26/11 19:00 9/26/11 19:30 9/266/1120:30 9/26/1121:00 9/26/11 21:30 9/26/11 22:00 9/26/11 22:30 9/26/11 23:00 9/26/11 23:30 9/27/11 0:00 9/27/11 1:00 9/27/11 1:30 9/27/112:30 9/27/11 3:00 $\begin{array}{lllll}1494.78 & 1495.331 & 1497.069 & 1495.198 \\ 149478 & 1495331 & 1497.067 & 1495.201\end{array}$ $\begin{array}{llll}1494.78 & 1495.331 & 1497.067 & 1495.201 \\ 149478 & 1495331 & 1997.067 & 1955.201\end{array}$ $\begin{array}{lllll}1494.78 & 1495.331 & 1497.067 & 1495.201 \\ 1494.777 & 1495327 & 1497.069 & 1495.198\end{array}$ $\begin{array}{lllll}1494.78 & 1495.329 & 1497.055 & 1495.201\end{array}$ $\begin{array}{lllll}1494.78 & 1495.329 & 1497.051 & 1455.201\end{array}$ $\begin{array}{lllll}1494.777 & 1495.329 & 1497.044 & 1495.198\end{array}$ $\begin{array}{lllll}1494.775 & 1495.324 & 1497.039 & 1495.194\end{array}$ $\begin{array}{llll}1494.775 & 1495.32 & 1497.032 & 1495.191\end{array}$ $\begin{array}{rrrr}1494.773 & 1495.32 & 1497.027 & 1495.184 \\ 1994.773 & 1495.317 & 1997.009 & 1955.182\end{array}$ $\begin{array}{rrrrr}1494.773 & 1495.317 & 1497.009 & 1495.182 \\ 1494.77 & 1495.31 & 1496.999 & 1495.175\end{array}$ $\begin{array}{rrrr}1494.77 & 1495.31 & 1496.999 & 1495.175 \\ 1494.768 & 1495.306 & 1496.99 & 1495.168\end{array}$ $\begin{array}{rrrr}1494.768 & 1495.306 & 1496.99 & 1495.168 \\ 1494.763 & 1495.299 & 1496.988 & 1495.161\end{array}$ $\begin{array}{lllll}1494.763 & 1495.289 & 1496.978 & 1495.151\end{array}$ $1494.761 \quad 1495.285 \quad 1496.971 \quad 1495.142$ $\begin{array}{lllll}1494.759 & 1495.28 & 1496.976 & 1495.137\end{array}$ $1494.759 \begin{array}{llll}1495.278 & 1496.99 & 1495.13\end{array}$ $\begin{array}{llll}1494.756 & 1495.271 & 1496.999 & 1495.121\end{array}$ $\begin{array}{rrrrr}1494.759 & 1495.271 & 1497.009 & 1495.116 \\ 1494.763 & 1495.28 & 1497.013 & 1495.123\end{array}$ $\begin{array}{llll}1494.763 & 1495.28 & 1497.02 & 1495.126\end{array}$ $\begin{array}{llll}1494.766 & 1495.282 & 1497.027 & 1495.13\end{array}$ $\begin{array}{llll}1494.766 & 1495.287 & 1497.039 & 1495.135\end{array}$ $\begin{array}{lllll}1494.766 & 1495.289 & 1497.048 & 1495.137\end{array}$ $\begin{array}{lllll}1494.77 & 1495.296 & 1497.058 & 1495.144\end{array}$ $\begin{array}{llll}1494.77 & 1495.301 & 1497.062 & 1495.154\end{array}$ $1494.7751495 .306 \quad 1497.0671495 .163$ $\begin{array}{rrrrr}1494.777 & 1495.31 & 1497.072 & 1495.168 \\ 1494.777 & 1495315 & 1497.074 & 1495.177\end{array}$ $\begin{array}{llll}1494.777 & 1495.317 & 1497.076 & 1995.182\end{array}$ $\begin{array}{lllll}1494.777 & 1495.322 & 1497.072 & 1495.189\end{array}$ $\begin{array}{lllll}1494.777 & 1495.324 & 1497.072 & 1495.191\end{array}$ $\begin{array}{lllll}1494.775 & 1495.324 & 1497.069 & 1495.198\end{array}$ $\begin{array}{lllll}1494.777 & 1495.324 & 1497.062 & 1495.196\end{array}$ $1494.7751495 .324 \quad 1497.055 \quad 1495.198$ $\begin{array}{lllll}1494.775 & 1495.324 & 1497.051 & 1495.198\end{array}$ $\begin{array}{lllll}1494.773 & 145.322 & 1497.044 & 1495.196 \\ 1494.773 & 1495.32 & 1497.037 & 1495.194\end{array}$ $\begin{array}{lllll}1494.773 & 1495.32 & 1497.032 & 1495.189\end{array}$ $\begin{array}{lllll}1494.77 & 1495.315 & 1497.03 & 1495.184\end{array}$ $\begin{array}{llll}1494.768 & 1495.31 & 1497.02 & 1495.18\end{array}$ $\begin{array}{llll}1494.77 & 1495.31 & 1497.018 & 1495.175\end{array}$ $\begin{array}{llll}1494.768 & 1495.306 & 1497.016 & 1495.17\end{array}$ $\begin{array}{llll}1494.768 & 1495.301 & 1497.013 & 1495.163 \\ 1494.766 & 1495301 & 1497.009 & 1955.159\end{array}$ 1494.7661495 .3011497 .0091495 .159 $1494.7661495 .296 \quad 1497.0091495 .151$ $1494.766 \quad 1495.294 \quad 1496.997 \quad 1495.147$ $1494.766 \quad 1495.292 \quad 1496.988 \quad 1495.144$ $1494.763 \quad 1495.292 \quad 1496.99 \quad 1495.14$ $1494.7591495 .2851496 .985 \quad 1495.135$ $\begin{array}{llll}1494.759 & 1495.28 & 1496.974 & 1495.128 \\ 1494.759 & 1495.28 & 1496.971 & 1495.128\end{array}$ $\begin{array}{lllllllllllllllllllll}1494.301 & 1494.861 & 1495.054 & 1495.905 & 1497.282 & 1497.122 & 1493.221 & 1489.976 & 1493.056 & 1490.612 & 1491.307 & 1485.852 & 1488.914 & 1493.06 & 1494.071 & 1493.221 & 1495.218 & 1493.206 & 1486.01 & 1478.603 \\ 1494301 & 1494859 & 1495052 & 1495905 & 149728 & 1497.117 & 1493226 & 1489.976 & 1493056 & 1490612 & 1491311 & 1485845 & 1488914 & 1493062 & 1494073 & 1493224 & 1495.218 & 1493211 & 148601 & 1478.603\end{array}$

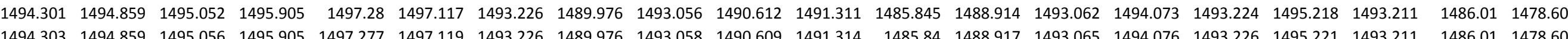

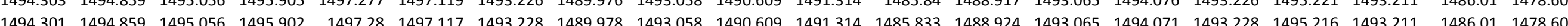
$\begin{array}{llllllllllllllllllllll}1494.31 & 1494.862 & 1495.052 & 1495.902 & 1497.275 & 1497.117 & 1493.233 & 1489.978 & 1493.06 & 1490.607 & 1491.316 & 1485.826 & 1488.921 & 1493.069 & 1494.076 & 1493.231 & 1495.221 & 1493.215 & 1486.01 & 1478.608\end{array}$

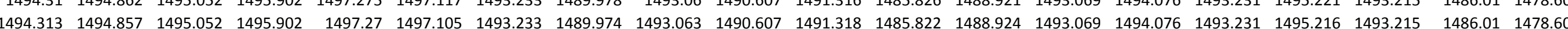

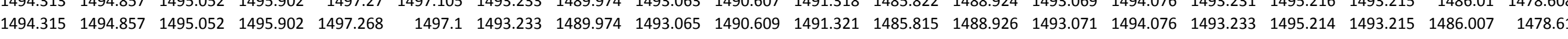
$\begin{array}{llllllllllllllllllllll}1494.317 & 1494.857 & 1495.049 & 1495.898 & 1497.266 & 1497.093 & 1493.231 & 1489.974 & 1493.063 & 1490.605 & 1491.321 & 1485.805 & 1488.931 & 1493.069 & 1494.069 & 1493.231 & 1495.204 & 1493.215 & 1486.007 & 1478.612\end{array}$

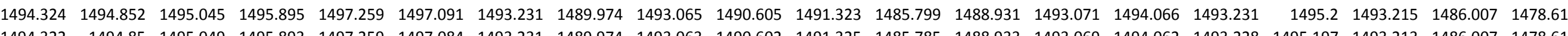

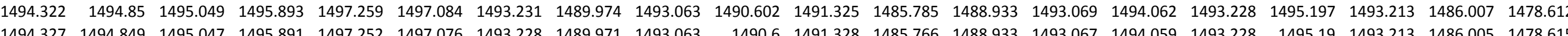
$\begin{array}{llllllllllllllllllll}1494.327 & 1494.849 & 1495.047 & 1495.891 & 1497.252 & 1497.076 & 1493.228 & 1489.971 & 1493.063 & 1490.6 & 1491.328 & 1485.766 & 1488.933 & 1493.067 & 1494.059 & 1493.228 & 1495.19 & 1493.213 & 1486.005 & 1478.615 \\ 1494.336 & 1494.845 & 1495.042 & 1495886 & 1497.242 & 1497.062 & 1493228 & 1489.969 & 149306 & 1490.6 & 1491328 & 1485757 & 1488933 & 1493067 & 1494057 & 1493226 & 149519 & 1493213 & 1486005 & 1478615\end{array}$

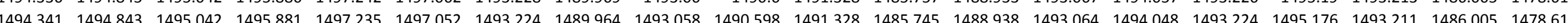

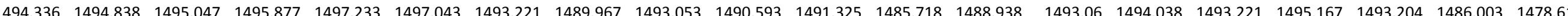
$\begin{array}{llllllllllllllllllllllll}1494.341 & 1494.835 & 1495.042 & 1495.87 & 1497.226 & 1497.043 & 1493.217 & 1489.967 & 1493.051 & 1490.593 & 1491.323 & 1485.711 & 1488.938 & 1493.055 & 1494.031 & 1493.217 & 1495.153 & 1493.201 & 1486.003 & 1478.61\end{array}$

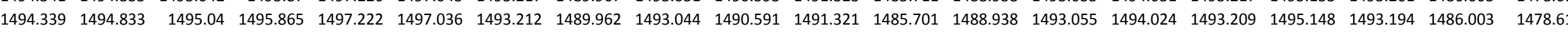

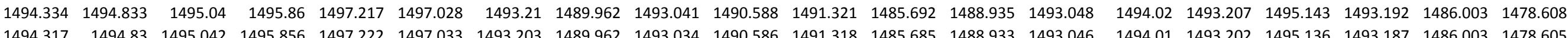

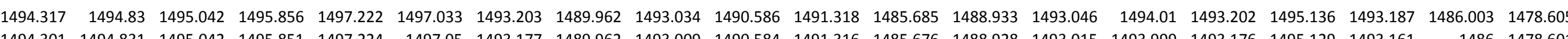

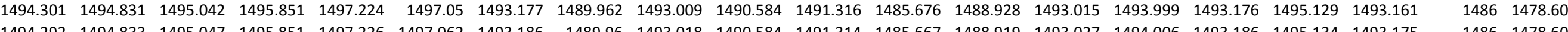

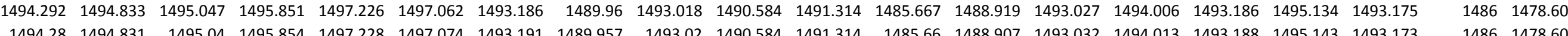
$\begin{array}{llllllllllllllllllll}1494.27 & 1494.83 & 1495.045 & 1495.854 & 1497.233 & 1497.081 & 1493.191 & 1489.953 & 1493.023 & 1490.584 & 1491.311 & 1485.651 & 1488902 & 1493.029 & 1494.015 & 1493.191 & 1495.148 & 1493.175 & 1486.003 & 1478.598\end{array}$ $\begin{array}{llllllllllllllllllllll}1494.254 & 1494.833 & 1495.045 & 1495.858 & 1497.24 & 1497.095 & 1493.196 & 1489.955 & 1493.027 & 1490.584 & 1491.314 & 1485.63 & 1488.895 & 1493.036 & 1494.027 & 1493.195 & 1495.16 & 1493.18 & 1486 & 1478.598\end{array}$ \begin{tabular}{llllllllllllllllllllllll}
1494.247 & 1494.835 & 1495.045 & 1495.86 & 1497.245 & 1497.105 & 1493.196 & 1489.964 & 1493.025 & 1490.584 & 1491.311 & 1485.623 & 1488.891 & 1493.034 & 1494.029 & 1493.191 & 1495.164 & 1493.178 & 1486 & 1478.601 \\
\hline
\end{tabular}

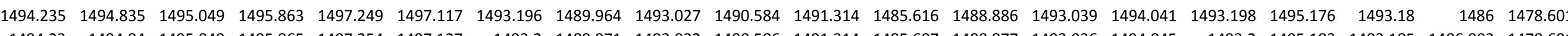

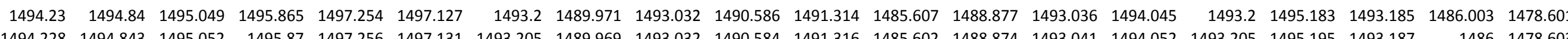

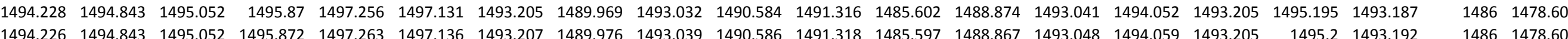
$\begin{array}{llllllllllllllllllllll}1494.226 & 1494.843 & 1495.052 & 1495.872 & 1497.263 & 1497.136 & 1493.207 & 1489.91 & 1493.039 & 1490.586 & 1491.318 & 1485.597 & 1488.867 & 1493.048 & 1494.059 & 1493.205 & 1495.2 & 1493.192 & 1486 & 1478.605 \\ 1494.228 & 1494.845 & 1495.052 & 1495.874 & 1497266 & 1497.141 & 149321 & 1489.974 & 1493.041 & 1490588 & 1491.323 & 1485588 & 1488.867 & 1493.048 & 1494.062 & 1493212 & 1495207 & 1493.194 & 1486 & 1478608\end{array}$ \begin{tabular}{llllllllllllllllllllllll}
1494.228 & 1494.847 & 1495.049 & 1495.879 & 1497.266 & 1497.143 & 1493.214 & 1489.976 & 1493.046 & 1490.588 & 1491.325 & 1485.584 & 1488.867 & 1493.055 & 1494.064 & 1493.214 & 1495.214 & 1493.199 & 1486 & 1478.61 \\
\hline
\end{tabular} $\begin{array}{lllllllllllllllllllll}1494.233 & 1494.845 & 1495.049 & 1495.879 & 1497.266 & 1497.143 & 1493.217 & 1489.978 & 1493.046 & 1490.591 & 1491.33 & 1485.537 & 1488.863 & 1493.06 & 1494.073 & 1493.217 & 1495.216 & 1493.204 & 1486 & 1478.612\end{array}$ \begin{tabular}{llllllllllllllllllllllll}
1494.237 & 1494.847 & 1495.052 & 1495.884 & 1497.268 & 1497.141 & 1493.224 & 1489.978 & 1493.051 & 1490.593 & 1491.328 & 1485.503 & 1488.86 & 1493.06 & 1494.073 & 1493.219 & 1495.225 & 1493.206 & 1486 & 1478.612 \\
\hline
\end{tabular}

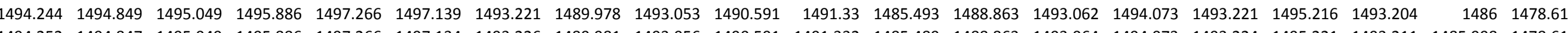

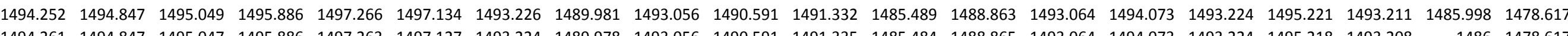

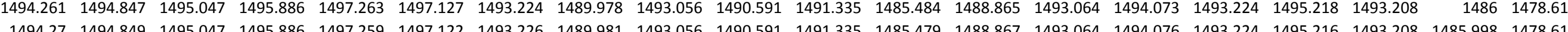

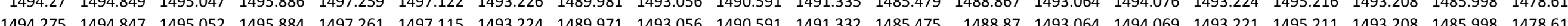

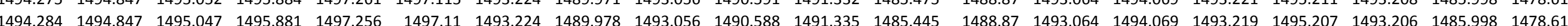
$\begin{array}{lllllllllllllllllllll}1494.292 & 1494.845 & 1495.045 & 1495.881 & 1497.252 & 1497.1 & 1493.219 & 1489.981 & 1493.053 & 1490.586 & 1491.332 & 1485.424 & 1488.872 & 1493.062 & 1494.064 & 1493.219 & 1495.202 & 1493.201 & 1485.998 & 1478.617\end{array}$ $\begin{array}{llllllllllllllllllll}1494.294 & 1494.847 & 1495.045 & 1495.879 & 1497.249 & 1497.098 & 1493.217 & 1489.971 & 1493.051 & 1490.586 & 1491.332 & 1485.412 & 1488.874 & 1493.058 & 1494.057 & 1493.217 & 1495.195 & 1493.204 & 1485.998 & 1478.615 \\ \end{array}$ \begin{tabular}{llllllllllllllllllllll}
1494.299 & 1494.843 & 1495.042 & 1495.877 & 1497.245 & 1497.093 & 1493.214 & 1489.974 & 1493.046 & 1490.586 & 1491.328 & 1485.389 & 1488.881 & 1493.055 & 1494.045 & 1493.214 & 1495.185 & 1493.199 & 1485.996 & 1478.612 \\
\hline
\end{tabular} $\begin{array}{llllllllllllllllllll}1494.303 & 1494.842 & 1495.042 & 1495.877 & 1497.242 & 1497.084 & 1493.21 & 1489.969 & 1493.044 & 1490.584 & 1491.328 & 1485.364 & 1488.881 & 1493.051 & 1494.05 & 1493.209 & 1495.183 & 1493.199 & 1485.996 & 1478.61 \\ \end{array}$ $\begin{array}{lllllllllllllllllllll}1494.306 & 1494.838 & 1495.042 & 1495.874 & 1497.24 & 1497.081 & 1493.207 & 1489.967 & 1493.041 & 1490.581 & 1491.328 & 1485.359 & 1488.879 & 1493.051 & 1494.043 & 1493.212 & 1495.183 & 1493.192 & 1485.996 & 1478.612\end{array}$

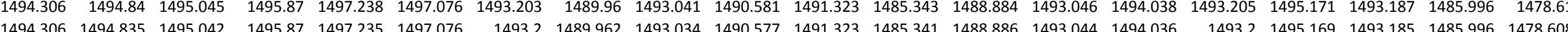

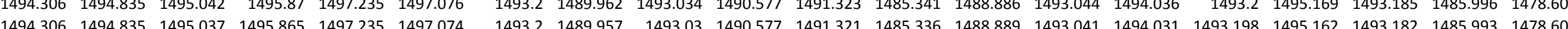
$\begin{array}{llllllllllllllllllllll}1494.303 & 1494.833 & 1495.04 & 1495.865 & 1497.233 & 1497.074 & 1493.196 & 1489.96 & 1493.03 & 1490.577 & 1491.321 & 1485.336 & 1488.891 & 1493.039 & 1494.029 & 1493.198 & 1495.16 & 1493.182 & 1485.993 & 1478.603\end{array}$ $\begin{array}{llllllllllllllllllllllll}1494.306 & 1494.833 & 1495.04 & 1495.863 & 1497.231 & 1497.072 & 1493.193 & 1489.953 & 1493.027 & 1490.574 & 1491.321 & 1485.332 & 1488.886 & 1493.036 & 1494.027 & 1493.193 & 1495.16 & 1493.18 & 1485.993 & 1478.603\end{array}$

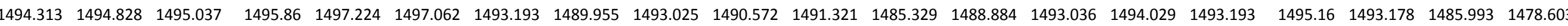
\begin{tabular}{llllllllllllllllllll}
1494.31 & 1494.828 & 1495.037 & 1495.86 & 1497.224 & 1497.052 & 1493.193 & 1489.955 & 1493.025 & 1490.572 & 1491.321 & 1485.327 & 1488.891 & 1493.034 & 1494.024 & 1493.193 & 1495.155 & 1493.178 & 1485.991 & 1478.603 \\
\hline
\end{tabular}

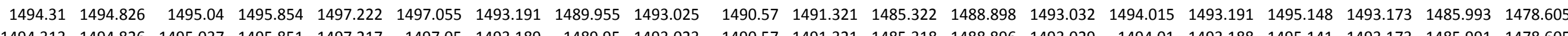

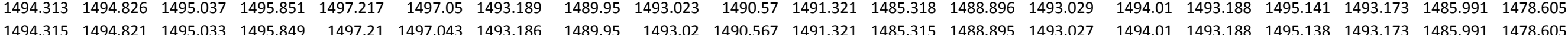


Well

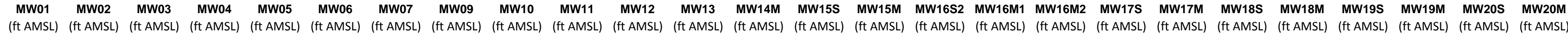

$9 / 27 / 114: 00$ $9 / 27 / 114: 30$ 9/27/11 5:00 9/27/115:30 $9 / 27116: 00$ $9 / 27 / 116: 30$ 9/27/11 6:30 9/27/117:30 9/27/11 8:00 9/27/11 8:30 9/27/119:00 $9 / 27 / 1110: 00$ $9 / 27 / 1110: 30$ 9/27/11 11:00 9/27/11 11:30 9/27/11 12:00 $9 / 27 / 11$ 13:00 (2/27/11 13:30 9/27/11 14:00 $9 / 27 / 1115: 00$ 9/27/11 15:30 9/27/11 16:00 9/27/11 16:30 9/27/11 17:00 9/27/11 17:30 9/27/11 18:00 9/27/11 19:00 9/27/11 19:30 9/27/11 20:00 9/27/11 20:30 9/27/1121:00 9/27/1121:30 $9 / 27 / 1122: 00$
$9 / 27 / 1122: 30$ 9/27/11 22:30 9/27/11 23:00 9/28/110:00 9/28/110:30 9/28/111:00 $9 / 28 / 111: 30$ $9 / 28 / 112: 00$
$9 / 28 / 112: 30$

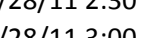
9/28/113:00 9/28/113:30 9/28/11 4:30 9/28/11 5:00 9/28/115:30 9/28/11 6:00 $\begin{array}{llll}1494.756 & 1495.278 & 1496.974 & 1495.123\end{array}$ $1494.754 \quad 1495.273 \quad 1496.974 \quad 1495.114$ $\begin{array}{llll}1494.754 & 1495.268 & 1496.971 & 1495.109 \\ 1494.754 & 1495.268 & 1496964 & 1495.109\end{array}$ $\begin{array}{lllll}1494.754 & 1495.266 & 1496.957 & 1495.107\end{array}$ $\begin{array}{llll}1494.754 & 1495.266 & 1496.948 & 1495.107\end{array}$ $\begin{array}{rrrr}1494.754 & 1495.266 & 1496.948 & 1495.107 \\ 1494.747 & 1495.261 & 1496.939 & 1495.1\end{array}$ $\begin{array}{llll}1494.745 & 1495.257 & 1496.936 & 1495.095\end{array}$ $\begin{array}{lllll}1494.742 & 1495.25 & 1496.927 & 1495.088\end{array}$ $\begin{array}{llll}1494.742 & 1495.245 & 1496.92 & 1495.081 \\ 1494.738 & 1495243 & 1496.92 & 195077\end{array}$ $\begin{array}{rrrr}1494.738 & 1495.243 & 1496.92 & 1495.077 \\ 1494.735 & 1495233 & 1496.922 & 1955.065\end{array}$ $\begin{array}{llll}1494.735 & 1495.233 & 1496.922 & 1495.065 \\ 1494.735 & 1495.229 & 1496.932 & 1495.058\end{array}$ $\begin{array}{lllll}1494.735 & 1495.226 & 1466.941 & 14595.051\end{array}$ $\begin{array}{llll}1494.735 & 1495.226 & 1496.95 & 1495.046\end{array}$ $\begin{array}{lllll}1494.74 & 1495.229 & 1496.962 & 1495.039\end{array}$ $\begin{array}{lllll}1494.74 & 1495.231 & 1496.974 & 1495.037\end{array}$ $\begin{array}{llll}1494.742 & 1495.236 & 1496.988 & 1495.035\end{array}$ $\begin{array}{llllll}1494.745 & 1495.24 & 1496.997 & 1495.037\end{array}$ $\begin{array}{rrrrr}1494.745 & 1495.243 & 1497.013 & 1495.039 \\ 1494745 & 1495.25 & 1497.018 & 1495046\end{array}$ $\begin{array}{rrrr}1494.747 & 1495.254 & 1497.018 & 1495.056\end{array}$ $\begin{array}{lllll}1494.752 & 1495.264 & 1497.018 & 1495065\end{array}$ $\begin{array}{lllll}1494.749 & 1495.266 & 1497.023 & 1495.072\end{array}$ $1494.747 \quad \begin{array}{llll}1495.266 & 1497.025 & 1495.077\end{array}$ $\begin{array}{lllll}1494.749 & 1495.268 & 1497.02 & 1495.084\end{array}$ $\begin{array}{lllll}1494.749 & 1495.271 & 1497.016 & 1495.088\end{array}$ $\begin{array}{lllll}1494.747 & 1495.273 & 1497.016 & 1495.093 \\ 1494747 & 1495271 & 1497.011 & 1950.093\end{array}$ $\begin{array}{llll}1494.747 & 1495.271 & 1497.011 & 1495.093 \\ 1494.745 & 1495.271 & 1497.013 & 1495095\end{array}$ $\begin{array}{lllll}1494.747 & 1495.271 & 1497.011 & 1495.098\end{array}$ $\begin{array}{llll}1494.745 & 1495.268 & 1497.011 & 1495.098\end{array}$ $\begin{array}{lllll}1494.747 & 1495.271 & 1497.011 & 1495.1\end{array}$ $\begin{array}{lllll}1494.745 & 1495.268 & 1497.011 & 1495.1\end{array}$ $\begin{array}{lllll}1494.745 & 1495.271 & 1497.011 & 1495.1\end{array}$ $\begin{array}{lllll}1494.745 & 1495.273 & 1497.009 & 1495.1\end{array}$

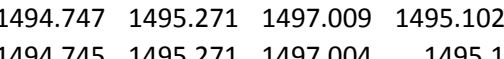
$\begin{array}{llll}1494.745 & 1495.271 & 1497.004 & 1495.1 \\ 1494.745 & 1495268 & 1497.004 & 1495.1\end{array}$ $\begin{array}{lllll}1494.745 & 1495.268 & 1497.006 & 1495.1\end{array}$ $\begin{array}{llll}1494.742 & 1495.268 & 1497.002 & 1495.1\end{array}$ $\begin{array}{llll}1494.742 & 1495.268 & 1497.004 & 1495.1\end{array}$ $\begin{array}{lllll}1494.742 & 1495.266 & 1497.004 & 1495.1\end{array}$ $\begin{array}{lllll}1494.74 & 1495.264 & 1497.009 & 1495.1\end{array}$ $\begin{array}{llll}1494.742 & 1495.264 & 1497.011 & 1495.1 \\ 194.742 & 1952.268 & 1977.016 & 145.5\end{array}$ $\begin{array}{lllll}1494.742 & 1495.268 & 1497.016 & 1495.1 \\ 1494.742 & 1495271 & 1497.018 & 1495.1\end{array}$ $\begin{array}{lllll}1494.745 & 1495.271 & 1497.023 & 1495.105\end{array}$ $\begin{array}{llll}1494.745 & 1495.273 & 1497.027 & 1495.107\end{array}$ $\begin{array}{lllll}1494.742 & 1495.273 & 1497.032 & 1495.107\end{array}$ $\begin{array}{llll}1494.745 & 1495.278 & 1497.032 & 1495.112\end{array}$ $\begin{array}{lllll}1494.747 & 1495.28 & 1497.032 & 1495.116\end{array}$ $\begin{array}{rrrrr}1494.747 & 1495.282 & 1497.03 & 1495.121 \\ 1494.747 & 1495.28 & 1497.023 & 1495.123\end{array}$

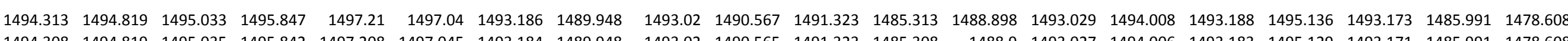
$\begin{array}{lllllllllllllllllllllll}1494.308 & 1494.819 & 1495.035 & 1495.842 & 1497.208 & 1497.045 & 1493.184 & 1489.948 & 1493.02 & 1490.565 & 1491.323 & 1485.308 & 1488.9 & 1493.027 & 1494.006 & 1493.183 & 1495.129 & 1493.171 & 1485.991 & 1478.608 \\ 1494.303 & 1494816 & 1495033 & 1495837 & 1497208 & 1497.043 & 1493184 & 1489.95 & 1493018 & 1490.56 & 1491325 & 1485311 & 1488.898 & 1493.025 & 1494001 & 1493.183 & 1495.124 & 1493.168 & 1485991 & 147861\end{array}$ $\begin{array}{lllllllllllllllllllllll}144.306 & 1494.816 & 1495.033 & 1495.837 & 1497205 & 1497.043 & 1493.186 & 1489.943 & 1493.02 & 1490.563 & 1491.325 & 14885304 & 1488.898 & 1493.024 & 1494.003 & 1493.183 & 1495.127 & 1493.168 & 1485.991 & 1478.61\end{array}$

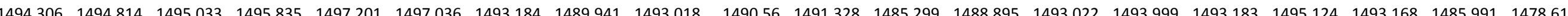
$\begin{array}{lllllllllllllllllllll}1494.313 & 1494.811 & 1495028 & 1495833 & 1497.194 & 1497.028 & 1493.184 & 1489.941 & 1493.016 & 1490.56 & 1491.325 & 1485.297 & 1488.898 & 1493.027 & 1493.999 & 1493.183 & 1495.124 & 1493.168 & 1485.989 & 1478.612\end{array}$ $\begin{array}{lllllllllllllllllllllll}1494.317 & 1494.807 & 1495.026 & 1495.828 & 1497.187 & 1497.019 & 1493.182 & 1489.946 & 1493.016 & 1490.558 & 1491.323 & 1485.283 & 1488.9 & 1493.022 & 1493.989 & 1493.179 & 1495.115 & 1493.164 & 1485.989 & 1478.615 \\ \end{array}$ $\begin{array}{lllllllllllllllllllllll}1494.313 & 1494.806 & 1495.028 & 1495.823 & 1497.184 & 1497.012 & 1493.179 & 1489.943 & 1493.011 & 1490.556 & 1491.325 & 1485.272 & 1488.902 & 1493.022 & 1493.985 & 1493.174 & 1495.108 & 1493.161 & 1485.989 & 1478.612\end{array}$ \begin{tabular}{llllllllllllllllllll}
1494.313 & 1494.802 & 1495.026 & 1495.819 & 1497.18 & 1497.009 & 1493.175 & 1489.941 & 1493.011 & 1490.553 & 1491.323 & 1485.26 & 1488.905 & 1493.024 & 1493.975 & 1493.172 & 1495.094 & 1493.159 & 1485.986 & 1478.612 \\
\hline
\end{tabular}

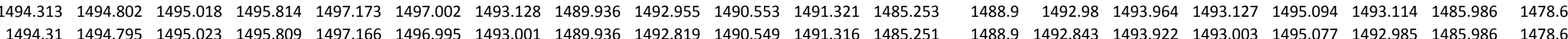

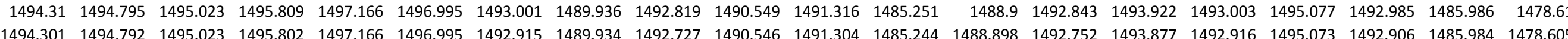
$\begin{array}{llllllllllllllllllllllll}1494.289 & 1494.792 & 1495.026 & 1495.798 & 1497.168 & 1496.997 & 1492.833 & 1489.931 & 1492.631 & 1490.544 & 1491.29 & 1485.239 & 1488.895 & 1492.663 & 1493.845 & 1492.835 & 1495.061 & 1492.821 & 1485.986 & 1478.605\end{array}$ $\begin{array}{lllllllllllllllllllll}1494.263 & 1494.792 & 1495.026 & 1495.793 & 1497.173 & 1497.017 & 1492.363 & 1489.929 & 1492.12 & 1490.542 & 1491.241 & 1485.202 & 1488.884 & 1492.131 & 1493.665 & 1492.366 & 1495.042 & 1492.359 & 1485.984 & 1478.601\end{array}$ $\begin{array}{llllllllllllllllllllll}1494.249 & 1494.795 & 1495.026 & 1495.793 & 1497.177 & 1497.026 & 1492.381 & 1489.927 & 1492.153 & 1490.539 & 1491.211 & 1485.147 & 1488.874 & 1492.148 & 1493.633 & 1492.385 & 1495.033 & 1492.378 & 1485.984 & 1478.601\end{array}$ $\begin{array}{llllllllllllllllllllll}1494.237 & 1494.795 & 1495.026 & 1495.795 & 1497.18 & 1497.038 & 1492.615 & 1489.931 & 1492.413 & 1490.542 & 1491.197 & 1485.11 & 1488.867 & 1492.392 & 1493.71 & 1492.617 & 1495.035 & 1492.608 & 1485.984 & 1478.598 & \end{array}$

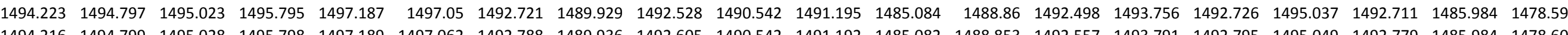

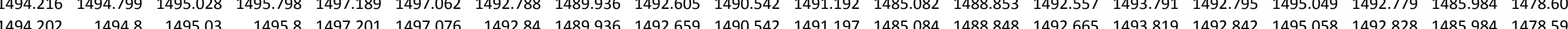

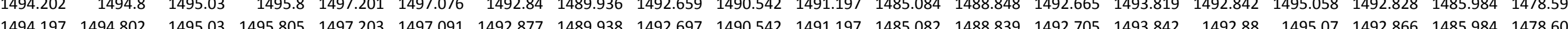

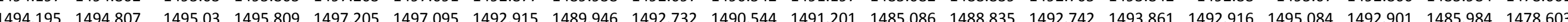

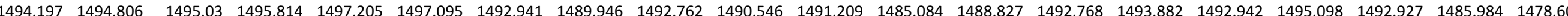

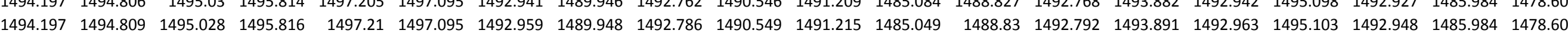

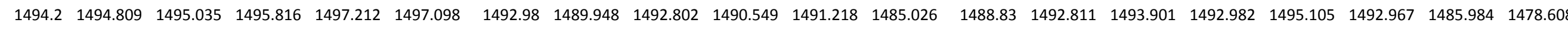

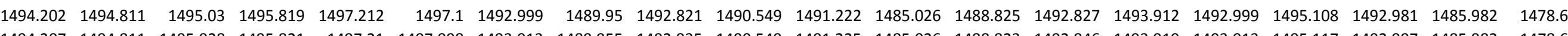

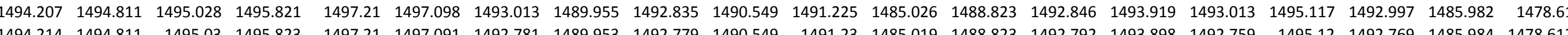

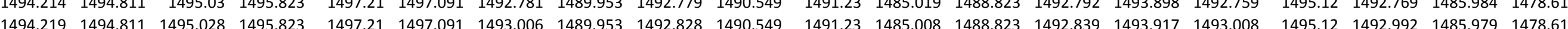

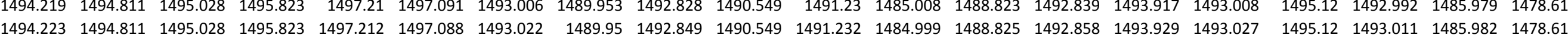

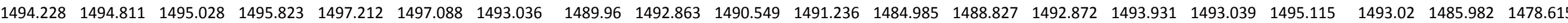
$\begin{array}{llllllllllllllllllllll}1494.23 & 1494.811 & 1495.028 & 1495.823 & 1497.212 & 1497.088 & 1493.025 & 1489.95 & 1492.852 & 1490.546 & 1491.234 & 1484.973 & 1488.83 & 1492.86 & 1493.933 & 1493.022 & 1495.12 & 1493.011 & 1485.982 & 1478.615 \\ \end{array}$

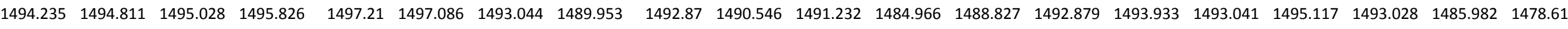
$\begin{array}{llllllllllllllllllll}1494.237 & 1494.814 & 1495.026 & 1495.823 & 1497.212 & 1497.086 & 1493.051 & 1489.95 & 1492.877 & 1490.546 & 1491.229 & 1484.964 & 1488.83 & 1492.888 & 1493.938 & 1493.051 & 1495.117 & 1493.037 & 1485.982 & 1478.615\end{array}$

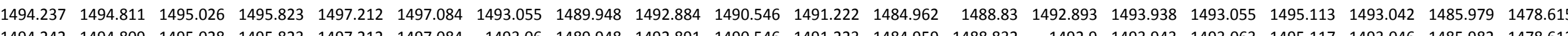

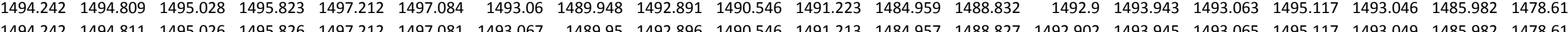

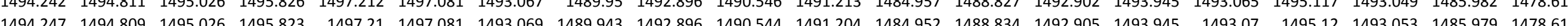

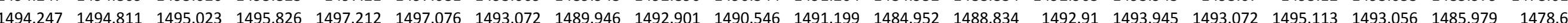
$\begin{array}{lllllllllllllllllllllll}1494.247 & 1494.809 & 1495.023 & 1495.826 & 1497.212 & 1497.079 & 1493.074 & 1489.943 & 1492.905 & 1490.544 & 1491.19 & 1484.948 & 1488.834 & 1492.914 & 1493.95 & 1493.072 & 1495.117 & 1493.058 & 1485.979 & 1478.608\end{array}$ \begin{tabular}{lllllllllllllllllllllll}
1494.249 & 1494.811 & 1495.023 & 1495.823 & 1497.21 & 1497.079 & 1493.074 & 1489.943 & 1492.903 & 1490.542 & 1491.187 & 1484.945 & 1488.841 & 1492.914 & 1493.943 & 1493.074 & 1495.113 & 1493.06 & 1485.979 & 1478.605 \\
\hline
\end{tabular}

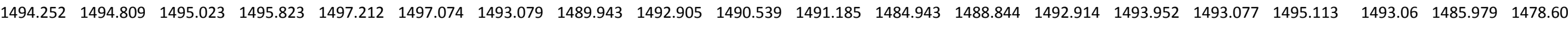

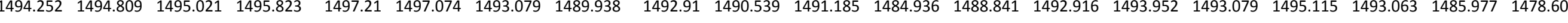

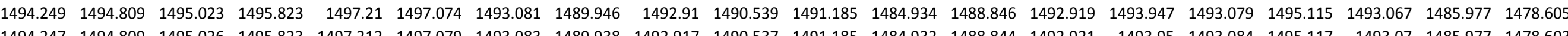

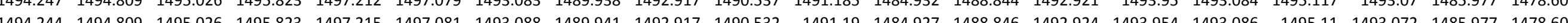

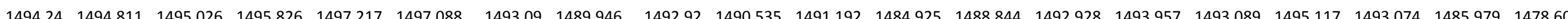

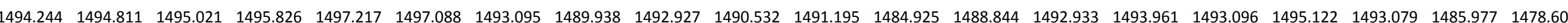
$\begin{array}{lllllllllllllllllllllll}1494.242 & 1494.809 & 1495.023 & 1495.828 & 1497.219 & 1497.091 & 1493.097 & 1489.946 & 1492.931 & 1490.53 & 1491.197 & 1484.925 & 1488.844 & 1492.938 & 1493.961 & 1493.096 & 1495.127 & 1493.084 & 1485.977 & 1478.608\end{array}$

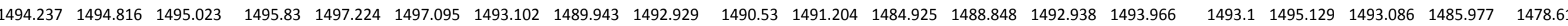

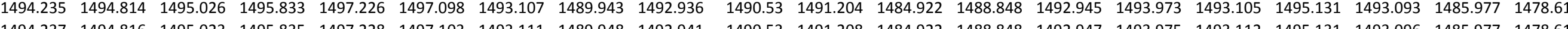

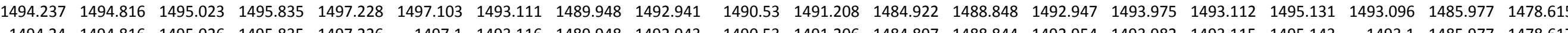

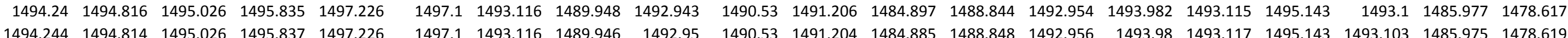


Well

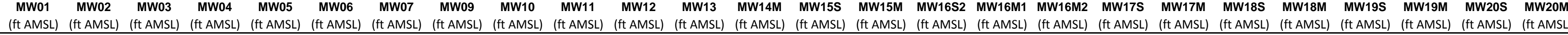

9/28/117:00 $9 / 28 / 117: 30$ $9 / 28 / 118: 00$ 9/28/118:30 9/28/11 9:30 9/28/11 10:00 9/28/11 10:30 $9 / 28 / 1111: 00$ $9 / 28 / 1111: 30$ 9/28/11 12:00 9/28/11 13:00 9/28/1113:00 9/28/11 13:30 9/28/11 14:30 9/28/11 15:00 /28/11 16:00 9/28/11 16:30 9/28/11 17:00 /28/11 17:30 9/28/11 18:30 9/28/11 19:00 9/28/11 19:30 9/28/11 20:00 9/28/11 20:30 9/28/11 21:00 9/28/1121:30 9/28/11 22:30 9/28/11 23:00 9/28/11 23:30 9/29/110:00 9/29/11 0:30 9/29/11 1:00 9/29/111:30 9/29/11 2:30 9/29/11 3:00 9/29/11 3:30 9/29/11 4:00 9/29/11 4:30 9/29/11 5:00 /2/12/116.00 9/29/11 6:00 9/29/117:00 9/29/11 7:30 9/29/11 8:00 9/29/11 8:30 9/29/11 9:00 $\begin{array}{rrrr}1494.747 & 1495.285 & 1497.02 & 1495.123 \\ 1494.745 & 1495.282 & 1497.018 & 1495.126\end{array}$ $\begin{array}{rrrr}1494.745 & 1495.282 & 1497.018 & 1495.126\end{array}$ $\begin{array}{lllll}1494.742 & 1495.28 & 1497.02 & 1495.123 \\ 1494.742 & 1495.278 & 1497.023 & 1495.121\end{array}$ $\begin{array}{lllll}1494.742 & 1495.278 & 1497.02 & 1495.119\end{array}$ $\begin{array}{lllll}1494.745 & 1495.278 & 1497.037 & 1495.121\end{array}$ $\begin{array}{lrrrr}1494.745 & 1495.28 & 1497.034 & 1495.121\end{array}$ $\begin{array}{lllll}1494.742 & 1495.28 & 1497.041 & 1495.119\end{array}$ $\begin{array}{llll}1494.749 & 1495.287 & 1497.046 & 1495.126\end{array}$ $1494.749 \quad 1495.287 \quad 1497.055 \quad 1495.126$ $\begin{array}{rrrr}1494.749 & 1495.289 & 1497.06 & 1495.126 \\ 1494.749 & 1495294 & 1497.069 & 1495.133\end{array}$ $\begin{array}{llrll}1494.749 & 1495.294 & 1497.069 & 1495.133 \\ 1494.754 & 1495.296 & 1497.074 & 1495.137\end{array}$ $1494.754 \quad 1495.303 \quad 1497.0791495 .142$ $\begin{array}{lllll}1494.756 & 1495.306 & 1497.079 & 1495.144\end{array}$ $\begin{array}{llllll}1494.759 & 1495.31 & 1497.079 & 1495.149\end{array}$ $\begin{array}{llll}1494.759 & 1495.315 & 1497.081 & 1495.151\end{array}$ $1494.759 \begin{array}{llll}1495.315 & 1497.083 & 1495.151\end{array}$ $\begin{array}{lllll}1494.756 & 1495.315 & 1497.081 & 1495.156\end{array}$ $\begin{array}{lllll}1494.759 & 1495.317 & 1497.081 & 1495.159 \\ 1494.759 & 1495322 & 1497.072 & 1495.159\end{array}$ $\begin{array}{lllll}1494.759 & 1495.32 & 1497.062 & 1495.156\end{array}$ $\begin{array}{lllll}1494.759 & 1495.32 & 1497.058 & 1495.154\end{array}$ $\begin{array}{lllll}1494.756 & 1495.317 & 1497.048 & 1995.154\end{array}$ $\begin{array}{lllll}1494.752 & 1495.31 & 1497.041 & 1495.144\end{array}$ $\begin{array}{lllll}1494.754 & 1495.31 & 1497.027 & 1495.142\end{array}$ $\begin{array}{llll}1494.749 & 1495.306 & 1497.023 & 1495.135\end{array}$ 1494.7471495 .2991497 .0181495 .126 $\begin{array}{llll}1494.747 & 1495.294 & 1497.013 & 1495.119 \\ 1494.747 & 1495.294 & 1497.006 & 1495.114\end{array}$ $1494.747 \quad 1495.289 \quad 1497.004 \quad 1495.109$ $\begin{array}{lllll}1494.745 & 1495.285 & 1496.999 & 1455.102\end{array}$ $\begin{array}{lllll}1494.745 & 1495.28 & 1496.99 & 1495.095\end{array}$ $\begin{array}{lllll}1494.742 & 1495.278 & 1496.983 & 1495.091\end{array}$ $\begin{array}{lllll}1494.74 & 1495.273 & 1496.978 & 1495.088\end{array}$ $\begin{array}{llll}1494.738 & 1495.273 & 1496.978 & 1495.079\end{array}$ $\begin{array}{rrrr}1494.735 & 1495.268 & 1496.978 & 1495.074 \\ 1494.738 & 1495264 & 1496.978 & 1495.07\end{array}$ $\begin{array}{llll}1494.738 & 1495261 & 1469.978 & 1495.07\end{array}$ $\begin{array}{lllll}1494.735 & 1495.261 & 1496.976 & 1495.065\end{array}$ $\begin{array}{lllll}1494.735 & 1495.259 & 1496.981 & 1495.06\end{array}$ $\begin{array}{lllll}1494.738 & 1495.257 & 1496.976 & 1495.058\end{array}$ $\begin{array}{lllll}1494.735 & 1495.257 & 1496.969 & 1495.06\end{array}$ $1494.7351495 .259 \quad 1496.967 \quad 1495.058$ $\begin{array}{rrrr}1494.731 & 1495.254 & 1496.95 & 1495.058 \\ 1494.728 & 1495252 & 1496948 & 1495053\end{array}$ $\begin{array}{llll}1494.728 & 1495.252 & 1496.948 & 1495.053 \\ 1494.728 & 1495.247 & 1496892 & 1495051\end{array}$ $\begin{array}{lllll}1494.726 & 1495.243 & 1496.894 & 1495.042\end{array}$ $\begin{array}{llll}1494.721 & 1495.238 & 1496.878 & 1495.037\end{array}$ $\begin{array}{lllll}1494.7 & 1495.208 & 1496.864 & 1495.009\end{array}$ $\begin{array}{lllll}1494.707 & 1495.21 & 1496.859 & 1495.002\end{array}$ $\begin{array}{lllll}1494.705 & 1495.198 & 1496.852 & 1494.988\end{array}$ $\begin{array}{rrrr}1494.7 & 1495.189 & 1496.845 & 1494.974 \\ 1494.698 & 1495.18 & 1496.845 & 1494.96\end{array}$ $\begin{array}{llllllllllllllllllll}1494.252 & 1494.814 & 1495.023 & 1495.84 & 1497.222 & 1497.091 & 1493.118 & 1489.946 & 1492.95 & 1490.528 & 1491.201 & 1484.883 & 1488.856 & 1492.957 & 1493.982 & 1493.122 & 1495.141 & 1493.105 & 1485.975 & 1478.619\end{array}$

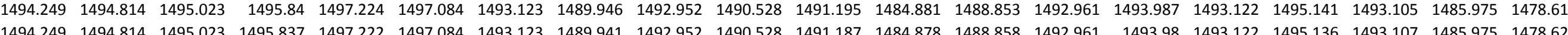

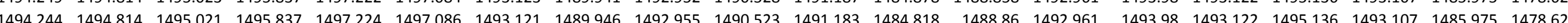

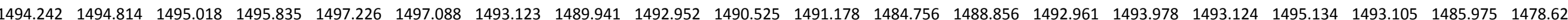
$\begin{array}{lllllllllllllllllllll}1494.24 & 1494814 & 495.026 & 1495837 & 1497.226 & 1497.086 & 1492.966 & 1489.946 & 1492.788 & 1490.521 & 1491.15 & 1484.652 & 1488853 & 1492.794 & 1493922 & 1492.968 & 1495.131 & 1492.955 & 1485.972 & 1478.622\end{array}$ $\begin{array}{llllllllllllllllllll}1494.233 & 1494.816 & 1495.023 & 1495.84 & 1497.231 & 1497.1 & 1493.025 & 1489.943 & 1492.849 & 1490.518 & 1491.143 & 1484.58 & 1488.849 & 1492.858 & 1493.94 & 1493.025 & 1495.131 & 1493.011 & 1485.975 & 1478.622\end{array}$ $\begin{array}{llllllllllllllllllll}1494.23 & 1494.816 & 1495.026 & 1495.84 & 1497.233 & 1497.098 & 1493.046 & 1489.934 & 1492.873 & 1490.518 & 1491.141 & 1484.573 & 1488.851 & 1492.881 & 1493.947 & 1493.046 & 1495.127 & 1493.032 & 1485.972 & 1478.619\end{array}$

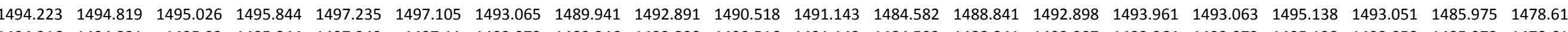

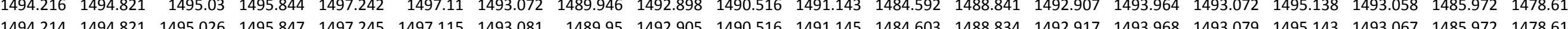
$\begin{array}{lllllllllllllllllllll}1494.214 & 1494.821 & 1495.026 & 1495.847 & 1497.245 & 1497.115 & 1493.081 & 1489.95 & 1492.905 & 1490.516 & 1491.145 & 1484.603 & 1488.834 & 1492.917 & 1493.968 & 1493.079 & 1495.143 & 1493.067 & 1485.972 & 1478.617 \\ 1494.207 & 1494.823 & 1495.028 & 1495851 & 1497.249 & 1497.122 & 1493086 & 1489.941 & 1492912 & 1490.516 & 1491.148 & 1484.61 & 1488.837 & 1492.921 & 1493.98 & 1493086 & 1495148 & 1493072 & 1485.972 & 1478617\end{array}$

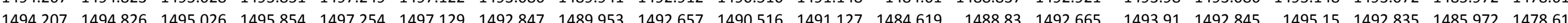
$\begin{array}{lllllllllllllllllllll}1494,202 & 1494828 & 1495.03 & 1495858 & 1497.261 & 1497.134 & 149295 & 1489.955 & 1492769 & 1490516 & 1491108 & 1484.626 & 1488827 & 1492.778 & 1493.922 & 1492949 & 1495.155 & 1492.936 & 1485.972 & 1478615\end{array}$ $\begin{array}{llllllllllllllllllllll}1494.202 & 1494.831 & 1495.028 & 1495.86 & 1497.261 & 1497.139 & 1492.959 & 1489.953 & 1492.777 & 1490.516 & 1491.115 & 1484.636 & 1488.825 & 1492.785 & 1493.931 & 1492.958 & 1495.157 & 1492.945 & 1485.97 & 1478.615\end{array}$

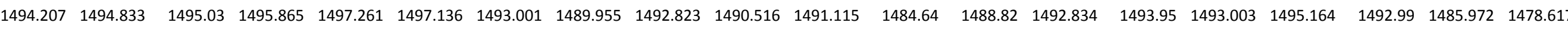
$\begin{array}{llllllllllllllllllllll}1494.207 & 1494.833 & 1495.03 & 1495.87 & 1497.266 & 1497.136 & 1493.027 & 1489.955 & 1492.849 & 1490.516 & 1491.122 & 1484.647 & 1488.816 & 1492.858 & 1493.964 & 1493.025 & 1495.164 & 1493.013 & 1485.972 & 1478.617\end{array}$

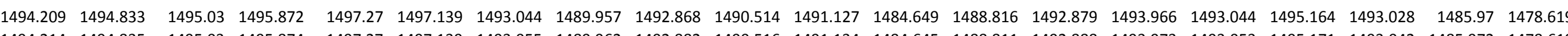

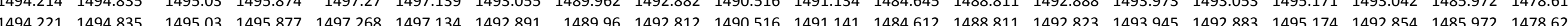

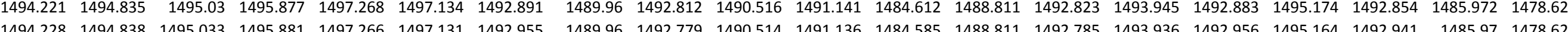

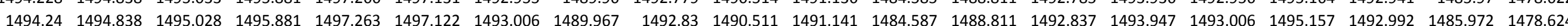
$\begin{array}{lllllllllllllllllllll}1494.249 & 1494.835 & 1495.028 & 1495.881 & 1497.261 & 1497.11 & 1493.029 & 1489.96 & 1492.856 & 1490.514 & 1491.145 & 1484.594 & 1488.811 & 1492.865 & 1493.959 & 1493.032 & 1495.169 & 1493.016 & 1485.97 & 1478.624\end{array}$ $\begin{array}{llllllllllllllllllllll}1494.256 & 1494.835 & 1495.028 & 1495.881 & 1497.256 & 1497.105 & 1493.046 & 1489.962 & 1492.873 & 1490.511 & 1491.148 & 1484.596 & 1488.818 & 1492.879 & 1493.964 & 1493.046 & 1495.153 & 1493.032 & 1485.97 & 1478.626\end{array}$

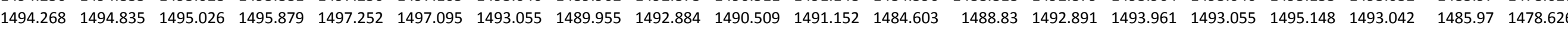

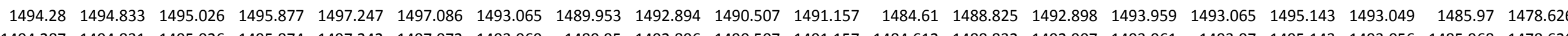

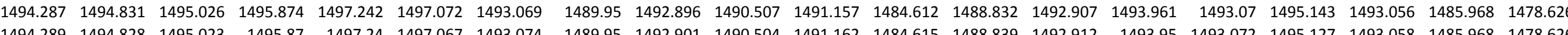

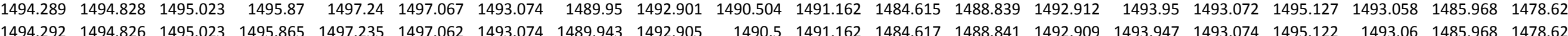

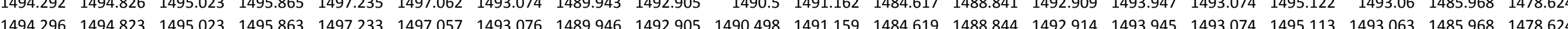

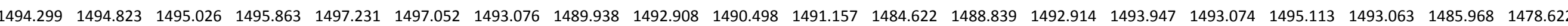
$\begin{array}{llllllllllllllllllllll}1494.301 & 1494.821 & 1495.018 & 1495.858 & 1497.224 & 1497.048 & 1493.076 & 1489.938 & 1492.908 & 1490.498 & 1491.15 & 1484.619 & 1488.851 & 1492.914 & 1493.94 & 1493.077 & 1495.103 & 1493.063 & 1485.968 & 1478.622\end{array}$ $\begin{array}{llllllllllllllllllll}1494.303 & 1494.816 & 1495.021 & 1495.85 & 1497.222 & 1497.043 & 1493.076 & 1489.936 & 1492.905 & 1490.493 & 1491.145 & 1484.622 & 1488.844 & 1492.914 & 1493.94 & 1493.079 & 1495.101 & 1493.063 & 1485.965 & 1478.617\end{array}$ $\begin{array}{llllllllllllllllllllll}1494.306 & 1494.816 & 1495.018 & 1495.854 & 1497.215 & 1497.036 & 1493.072 & 1489.934 & 1492.905 & 1490.493 & 1491.141 & 1484.62 & 1488.848 & 1492.91 & 1493.94 & 1493.074 & 1495.089 & 1493.058 & 1485.965 & 1478.617\end{array}$

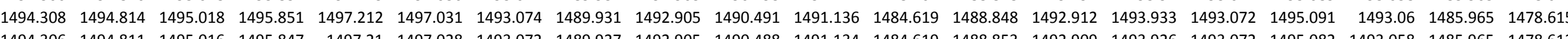

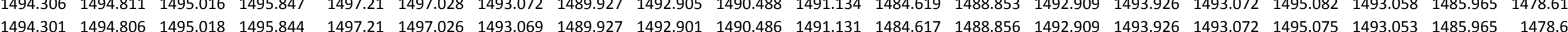

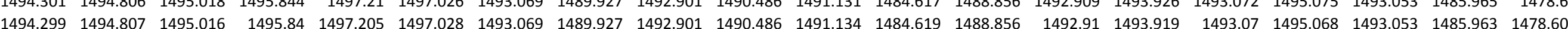

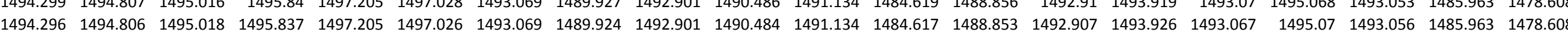
$\begin{array}{llllllllllllllllllll}1494.287 & 1494.804 & 1495.018 & 1495.835 & 1497.208 & 1497.031 & 1493.069 & 1489.924 & 1492.901 & 1490.479 & 1491.134 & 1484.617 & 1488.858 & 1492.91 & 1493.917 & 1493.07 & 1495.07 & 1493.053 & 1485.963 & 1478.608\end{array}$

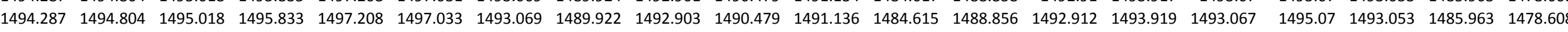

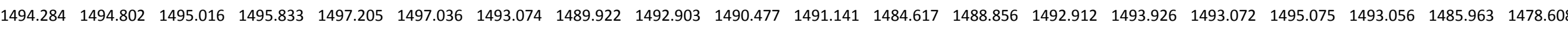
$\begin{array}{llllllllllllllllllll}1494.292 & 1494.797 & 1495.011 & 1495.833 & 1497.198 & 1497.031 & 1493.074 & 1489.915 & 1492.905 & 1490.477 & 1491.143 & 1484.617 & 1488.86 & 1492.912 & 1493.919 & 1493.07 & 1495.075 & 1493.058 & 1485.963 & 1478.608 \\ \end{array}$ $\begin{array}{llllllllllllllllllll}1494.292 & 1494.797 & 1495.011 & 1495.828 & 1497.196 & 1497.026 & 1493.072 & 1489.92 & 1492.908 & 1490.474 & 1491.145 & 1484.619 & 1488.858 & 1492.914 & 1493.922 & 1493.074 & 1495.073 & 1493.06 & 1485.963 & 1478.608\end{array}$ $\begin{array}{llllllllllllllllllllll}1494.294 & 1494.794 & 1495.014 & 1495.826 & 1497.191 & 1497.024 & 1493.074 & 1489.924 & 1492.908 & 1490.474 & 1491.15 & 1484.62 & 1488.865 & 1492.912 & 1493.917 & 1493.074 & 1495.066 & 1493.06 & 1485.96 & 1478.61 \\ 1494.294 & 1494.792 & 1495.011 & 1495823 & 1497.187 & 1497.012 & 1493.074 & 1489.922 & 1492.008 & 1490.47 & 1491.15 & 1484.613 & 1488.865 & 1492.914 & 1493917 & 1493.074 & 1495.061 & 149306 & 148596 & 147861\end{array}$

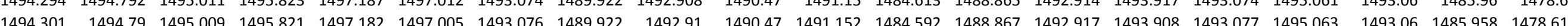
$\begin{array}{llllllllllllllllllllll}1494.334 & 1494.783 & 1495.009 & 1495.816 & 1497.159 & 1496.95 & 1493.074 & 1489.917 & 1492.908 & 1490.47 & 1491.157 & 1484.587 & 1488.87 & 1492.914 & 1493.912 & 1493.074 & 1495.054 & 1493.06 & 1485.96 & 1478.612\end{array}$ $\begin{array}{llllllllllllllllllll}1494.331 & 1494.783 & 1495.004 & 1495.812 & 1497.149 & 1496.954 & 1493.074 & 1489.915 & 1492.91 & 1490.467 & 1491.157 & 1484.58 & 1488.872 & 1492.917 & 1493.905 & 1493.072 & 1495.042 & 1493.06 & 1485.958 & 1478.615 \\ \end{array}$

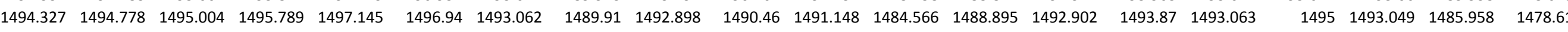

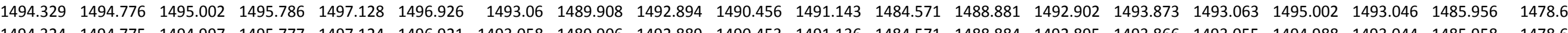
$\begin{array}{lllllllllllllllllllll}1494.324 & 1494.775 & 1494.997 & 1495.777 & 1497.124 & 1496.921 & 1493.058 & 1489.906 & 1492.889 & 1490.453 & 1491.136 & 1484.571 & 1488.884 & 1492.895 & 1493.866 & 1493.055 & 1494.988 & 1493.044 & 1485.958 & 1478.61 \\ \end{array}$

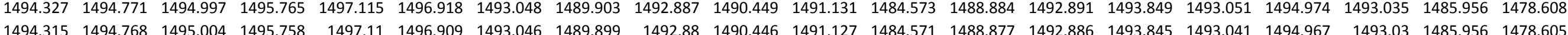


Well

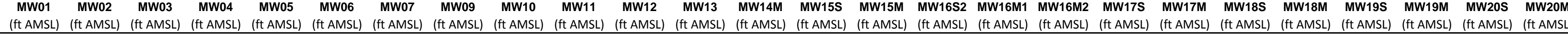

$9 / 29 / 1110: 00$ 9/29/11 10:30 $9 / 29 / 1111 \cdot 30$ 9/29/11 11:30 9/29/11 12:00 9/29/11 13:00 9/29/11 13:30 9/29/11 14:00 $9 / 29 / 1114: 30$ 9/29/11 15:00 9/29/11 15:30 9/29/1116:00 9/29/11 16:30 9/29/11 17:30 $9 / 29 / 1118: 00$
$9 / 29 / 1118: 30$ 9/29/11 19:00 9/29/11 19:30 9/29/11 20:00 9/29/11 21:00 9/29/11 21:00 9/29/11 22:00 9/29/11 22:30 9/29/11 23:00 9/29/11 23:30 9/30/11 0:30 /30/111 1:30 9/30/111:30 9/30/112:30 9/30/11 3:00 9/30/113:30 9/30/114:00 9/30/11 4:30 9/30/115:30 9/30/11 6:00 9/30/11 6:30 9/30/11 7:00 9/30/117:30 9/30/11 8:00 9/30/11 9:00 $9 / 30 / 1110: 00$ 9/30/11 10:30 9/30/11 11:00 9/30/11 12:00 $\begin{array}{lllll}1494.696 & 1495.17 & 1496.843 & 1494.951 \\ 1494.693 & 1495.166 & 1496.845 & 1494.939\end{array}$ $\begin{array}{rrrr}1494.693 & 1495.166 & 1496.845 & 1494.939 \\ 1494.691 & 1495.156 & 149684 & 1994934\end{array}$ $\begin{array}{rrrr}1494.691 & 1495.156 & 1496.84 & 1494.934 \\ 1494.693 & 1495.154 & 1496843 & 1494932\end{array}$ $\begin{array}{lllll}1494.689 & 1495.152 & 1496.847 & 1494.923\end{array}$ $\begin{array}{lllll}1494.686 & 1495.145 & 1496.854 & 1494.92\end{array}$ $\begin{array}{lllll}1494.686 & 1495.142 & 1496.866 & 1494.916\end{array}$ $\begin{array}{lllll}1494.689 & 1495.145 & 1496.866 & 1494.918\end{array}$ $\begin{array}{lllll}1494.689 & 1495.147 & 1496.868 & 1494.918\end{array}$ $1494.691 \quad 1495.149 \quad 1496.868 \quad 1494.923$ $\begin{array}{llll}1494.689 & 1495.147 & 1496.866 & 1494.923\end{array}$ $\begin{array}{lllll}1494.686 & 1495.147 & 1496.864 & 1494.923 \\ 1494.684 & 1495.147 & 1496.866 & 1494.927\end{array}$ $\begin{array}{lllll}1494.684 & 1495.147 & 1496.864 & 1494.925\end{array}$ $\begin{array}{lllll}1494.682 & 1495.145 & 1496.857 & 1494.925\end{array}$ $\begin{array}{llllll}1494.682 & 1495.142 & 1496.857 & 1494.927\end{array}$ $\begin{array}{lllll}1494.677 & 1495.14 & 1496.847 & 1494.927\end{array}$ $\begin{array}{llll}1494.673 & 1495.133 & 1496.843 & 1494.923 \\ 1496.673 & 1459.133 & 1496836 & 149.923\end{array}$ $\begin{array}{llll}1494.673 & 1495.133 & 1496.836 & 1494.923\end{array}$ $\begin{array}{lllll}1494.67 & 1495.128 & 1496.826 & 1494.918\end{array}$ $\begin{array}{llll}1494.668 & 1495.124 & 1496.822 & 1494.916 \\ 1494.666 & 1495.117 & 1496.812 & 1494.909\end{array}$ $\begin{array}{lllll}1494.663 & 1495.112 & 1496.812 & 1494.902\end{array}$ $\begin{array}{lllll}1494.663 & 1495.108 & 1496.808 & 1494.897\end{array}$ $\begin{array}{lllll}1494.661 & 1495.103 & 1496.808 & 1494.892\end{array}$ $\begin{array}{lllll}1494.661 & 1495.101 & 1496.808 & 1494.89\end{array}$ $\begin{array}{llll}1494.663 & 1495.098 & 1496.808 & 1494.883\end{array}$ $\begin{array}{lllll}1494.661 & 1495.094 & 1496.808 & 1494.878\end{array}$ $\begin{array}{lllll}1494.661 & 1495.094 & 1496.801 & 1494.876 \\ 1494.661 & 1495.091 & 1496.798 & 1494876\end{array}$ $\begin{array}{llll}1494.661 & 1455.087 & 1496.789 & 1494.874\end{array}$ $\begin{array}{llllll}1494.656 & 1495.084 & 1496.787 & 1494.869\end{array}$ $\begin{array}{lllll}1494.654 & 1495.08 & 1496.791 & 1494.864\end{array}$ $\begin{array}{lllll}1494.652 & 1495.073 & 1496.798 & 1494.857\end{array}$ $\begin{array}{lllll}1494.654 & 1495.073 & 1496.801 & 1494.855\end{array}$ $\begin{array}{llll}1494.654 & 1495.07 & 1496.796 & 1494.853\end{array}$ $\begin{array}{lllll}1494.656 & 1495.073 & 1496.794 & 1494.86\end{array}$ $\begin{array}{lllll}1494.656 & 1455.073 & 1466.801 & 1494.857 \\ 1494.652 & 1495.07 & 1496805 & 1494.855\end{array}$ $\begin{array}{lllll}1494.652 & 1495.068 & 1496.803 & 1494.855\end{array}$ $\begin{array}{lllll}1494.656 & 1495.07 & 1496.812 & 1494.86\end{array}$ $\begin{array}{lllll}1494.654 & 1495.07 & 1496.81 & 1494.862\end{array}$ $\begin{array}{llll}1494.652 & 1495.07 & 1496.808 & 1494.86\end{array}$ $\begin{array}{llll}1494.654 & 1495.073 & 1496.798 & 1494.867\end{array}$ $\begin{array}{lllll}1494.652 & 1495.07 & 1496.805 & 1494.869\end{array}$ 1494.6471495 .06814960 .8031494 .864 $1494.649 \begin{array}{llll}1495.068 & 1496.81 & 1494.864\end{array}$ $1494.649 \quad 1495.066 \quad 1496.815 \quad 1494.867$ $1494.652 \quad 1495.07 \quad 1496.824 \quad 1494.871$ $1494.6471495 .073 \quad 1496.833 \quad 1494.871$ $1494.649 \quad 1495.071496 .843 \quad 1494.876$ $\begin{array}{rrrr}1494.654 & 1495.075 & 1496.85 & 1494.883 \\ 1494.654 & 1495.082 & 1496.868 & 1494.89\end{array}$ $\begin{array}{lllllllllllllllllllll}1494.308 & 1494.766 & 1495.002 & 1495.751 & 1497.103 & 1496.914 & 1493.039 & 1489.899 & 1492.877 & 1490.442 & 1491.124 & 1484.559 & 1488.881 & 1492.883 & 1493.84 & 1493.039 & 1494.955 & 1493.025 & 1485.953 & 1478.603\end{array}$

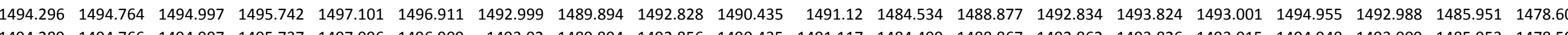

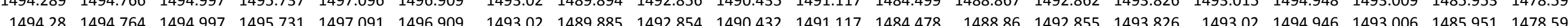
\begin{tabular}{llllllllllllllllllllll}
1494.27 & 1494.764 & 1494.999 & 1495.728 & 1497.091 & 1496.911 & 1493.015 & 1489.887 & 1492.852 & 1490.428 & 1491.11 & 1484.469 & 1488.856 & 1492.86 & 1493.819 & 1493.015 & 1494.934 & 1493.004 & 1485.951 & 1478.596 \\
\hline
\end{tabular} $\begin{array}{llllllllllllllllllll}1494.254 & 1494761 & 1495002 & 1495.724 & 1497.091 & 1496.918 & 1493011 & 1489.887 & 1492847 & 1490.428 & 1491.118 & 1484.467 & 1488848 & 1492855 & 1493.817 & 1493.018 & 1494.939 & 1493.002 & 1485.951 & 1478.596\end{array}$ $\begin{array}{lllllllllllllllllllllll}1494.237 & 1494.763 & 1494.999 & 1495.719 & 1497.096 & 1496.928 & 1493.008 & 1489.885 & 1492.845 & 1490.423 & 1491.108 & 1484.465 & 1488.848 & 1492.851 & 1493.817 & 1493.008 & 1494.936 & 1492.997 & 1485.951 & 1478.591 \\ \end{array}$ $\begin{array}{llllllllllllllllllllll}1494.228 & 1494.761 & 1494.999 & 1495.719 & 1497.096 & 1496.94 & 1493.008 & 1489.889 & 1492.845 & 1490.423 & 1491.106 & 1484.462 & 1488.834 & 1492.846 & 1493.819 & 1493.01 & 1494.941 & 1492.997 & 1485.949 & 1478.589\end{array}$ $\begin{array}{llllllllllllllllllll}1494.226 & 1494.759 & 1494.999 & 1495.719 & 1497.094 & 1496.94 & 1493.006 & 1489.88 & 1492.842 & 1490.423 & 1491.106 & 1484.411 & 1488.832 & 1492.848 & 1493.821 & 1493.01 & 1494.948 & 1492.999 & 1485.949 & 1478.589\end{array}$

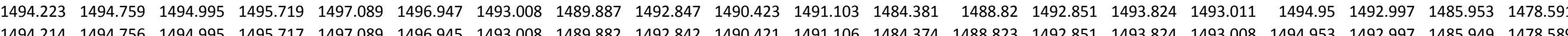
$\begin{array}{llllllllllllllllllll}1494.214 & 1494.756 & 1494.995 & 1495.717 & 1497.089 & 1496.945 & 1493.008 & 1489.882 & 1492.842 & 1490.421 & 1491.106 & 1484.374 & 1488.823 & 1492.851 & 1493.824 & 1493.008 & 1494.953 & 1492.997 & 1485.949 & 1478.589 \\ 1494.216 & 1494.759 & 1494.992 & 1495714 & 1497.089 & 1496.945 & 1493008 & 1489.885 & 1492.845 & 1490.418 & 1491.108 & 1484.374 & 1488818 & 1492846 & 1493833 & 1493.008 & 1494955 & 1492.997 & 1485.949 & 1478589\end{array}$ $\begin{array}{llllllllllllllllllllllll}1494.207 & 1494.759 & 1494.992 & 1495.712 & 1497.089 & 1496.945 & 1493.008 & 1489.882 & 1492.842 & 1490.421 & 1491.106 & 1484.374 & 1488.816 & 1492846 & 1493.826 & 1493.008 & 1494.953 & 1492.995 & 1485.949 & 14788589\end{array}$ $\begin{array}{llllllllllll} & 0 \\ \end{array}$ $\begin{array}{llllllllllllllllllll}1494.209 & 1494.751 & 1494.99 & 1495.71 & 1497.08 & 1496.945 & 1493.008 & 1489.882 & 1492.842 & 1490.414 & 1491.11 & 1484.37 & 1488.809 & 1492.846 & 1493.826 & 1493.008 & 1494.953 & 1492.992 & 1485.949 & 1478.589\end{array}$

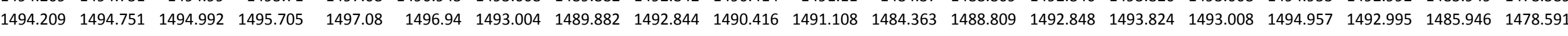

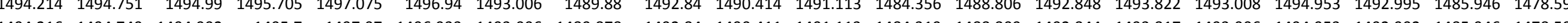

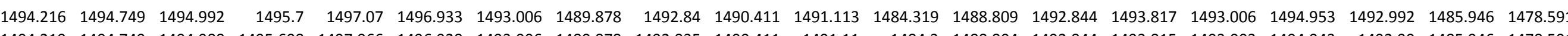

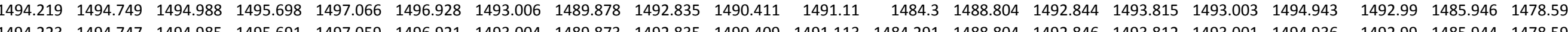

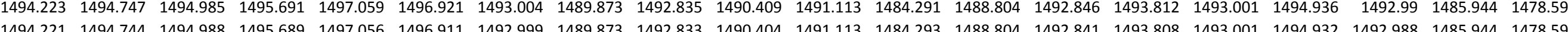
$\begin{array}{lllllllllllllllllllll}1494.223 & 1494.744 & 1494.983 & 1495.684 & 1497.052 & 1496.906 & 1492.994 & 1489.875 & 1492.83 & 1490.404 & 1491.113 & 1484.298 & 1488.804 & 1492.837 & 1493.8 & 1492.996 & 1494.925 & 1492.981 & 1485.944 & 1478.589\end{array}$ $\begin{array}{lllllllllllllllllllllll}1494.223 & 1494.742 & 1494.983 & 1495.677 & 1497.047 & 1496.902 & 1492.99 & 1489875 & 1492.826 & 14904 & 1491.113 & 1484.3 & 1488804 & 1492.837 & 1493.798 & 1492.992 & 1494.917 & 1492.978 & 1485.944 & 1478.589\end{array}$ $\begin{array}{lllllllllllllllllllllll}1494.221 & 1494.74 & 1494.985 & 1495.67 & 1497.042 & 1496.902 & 1492.987 & 1489.866 & 1492.823 & 1490.4 & 1491.108 & 1484.303 & 1488.811 & 1492.83 & 1493.789 & 1492.987 & 1494.91 & 1492.974 & 1485.944 & 1478.587\end{array}$

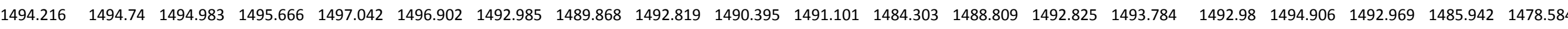

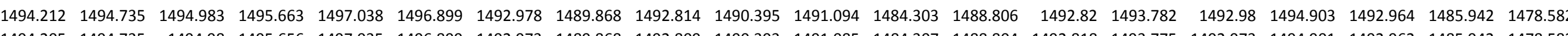

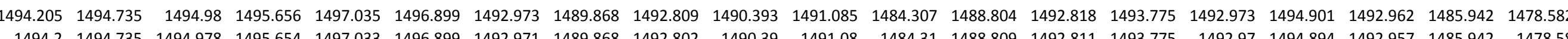

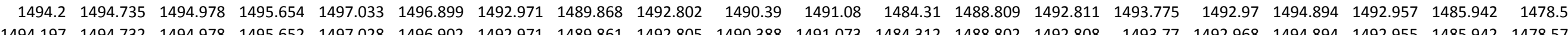
$\begin{array}{lllllllllllllllllllll}1494.197 & 1494.732 & 1494.978 & 1495.652 & 1497.028 & 1496.902 & 1492.971 & 1489.861 & 1492.805 & 1490.388 & 1491.073 & 1484.312 & 1488.802 & 1492.808 & 1493.77 & 1492.968 & 1494.894 & 1492.955 & 1485.942 & 1478.575 \\ 1494.197 & 1494.732 & 1494.978 & 1495.649 & 1497.026 & 1496894 & 1492.964 & 1489.859 & 1492.798 & 1490388 & 1491.068 & 1484.314 & 14887999 & 1492804 & 1493.775 & 1492.965 & 1494.894 & 1492.95 & 1485.942 & 1478575\end{array}$ \begin{tabular}{llllllllllllllllllllll}
1494.2 & 1494.73 & 1494.976 & 1495.647 & 1497.022 & 1496.892 & 1492.959 & 1489.861 & 1492.798 & 1490.386 & 1491.066 & 1484.319 & 1488.804 & 1492.804 & 1493.768 & 1492.963 & 1494.894 & 1492.948 & 1485.942 & 1478.57 \\
\hline
\end{tabular} $\begin{array}{lllllllllllllllllllllll}1494.2 & 1494.73 & 1494.978 & 1495.642 & 1497.017 & 1496.883 & 1492.957 & 1489.861 & 1492.791 & 1490.383 & 1491.061 & 1484.319 & 1488.797 & 1492.797 & 1493.766 & 1492.956 & 1494.887 & 1492.943 & 1485.942 & 1478.57\end{array}$ $\begin{array}{llllllllllllllllllllllll}1494.193 & 1494.73 & 1494.978 & 1495.638 & 1497.015 & 1496.883 & 1492.955 & 1489.856 & 1492.791 & 1490.381 & 1491.061 & 1484.319 & 1488.802 & 1492.799 & 1493.756 & 1492.954 & 1494.88 & 1492.938 & 1485.939 & 1478.568\end{array}$ $\begin{array}{lllllllllllllllllllllll}1494.183 & 1494.728 & 1494.976 & 1495.631 & 1497.017 & 1496.887 & 1492.952 & 1489.854 & 1492.784 & 1490.376 & 1491.059 & 1484.317 & 1488.802 & 1492.792 & 1493.752 & 1492.949 & 1494.878 & 1492.936 & 1485.939 & 1478.566\end{array}$

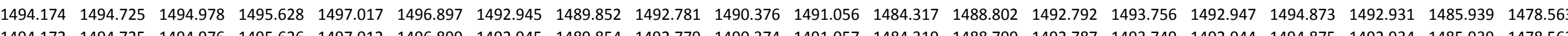

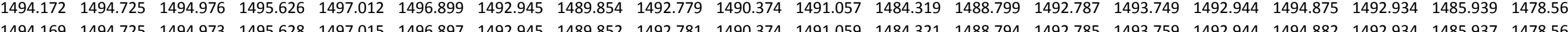

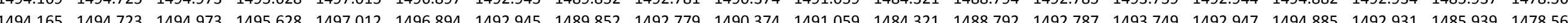

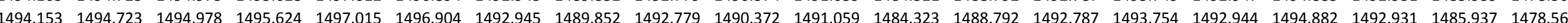
$\begin{array}{llllllllllllllllllllllll}1494.15 & 1494.723 & 1494.973 & 1495.621 & 1497.012 & 1496.911 & 1492.943 & 1489.849 & 1492.776 & 1490.369 & 1491.059 & 1484.323 & 1488.797 & 1492.785 & 1493.752 & 1492.942 & 1494.873 & 1492.931 & 1485.939 & 1478.566\end{array}$ $\begin{array}{llllllllllllllllllll}1494.148 & 1494.718 & 1494.973 & 1495.624 & 1497.015 & 1496.906 & 1492.945 & 1489.852 & 1492.781 & 1490.372 & 1491.054 & 1484.31 & 1488.787 & 1492.787 & 1493.761 & 1492.944 & 1494.887 & 1492.931 & 1485.937 & 1478.563\end{array}$

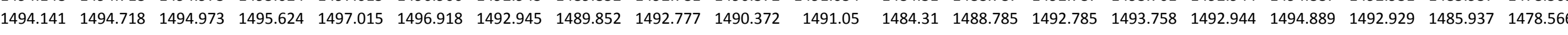

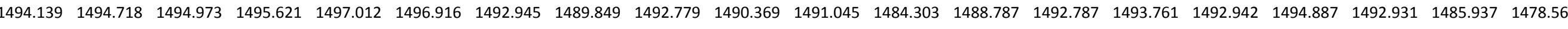

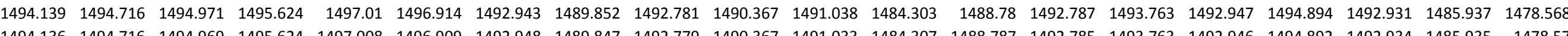

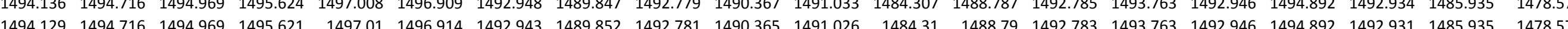

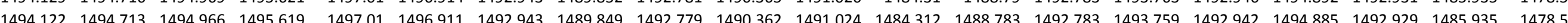
$\begin{array}{llllllllllllllllllllll}1494.113 & 1494.716 & 1494.969 & 1495.619 & 1497.012 & 1496.918 & 1492.945 & 1489.849 & 1492.779 & 1490.362 & 1491.019 & 1484.314 & 1488.778 & 1492.785 & 1493.766 & 1492.944 & 1494.896 & 1492.931 & 1485.932 & 1478.568\end{array}$ $\begin{array}{lllllllllllllllllllllll}1494.106 & 1494.713 & 1494.971 & 1495.619 & 1497.015 & 1496.921 & 1492.943 & 1489.849 & 1492.777 & 1490.362 & 1491.019 & 1484.317 & 1488.771 & 1492.783 & 1493.763 & 1492.939 & 1494.896 & 1492.929 & 1485.935 & 1478.568\end{array}$

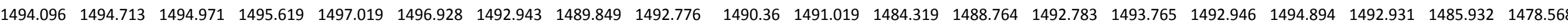

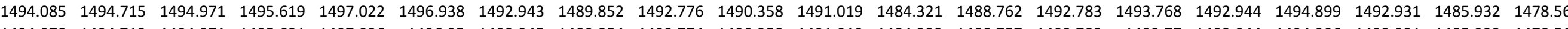

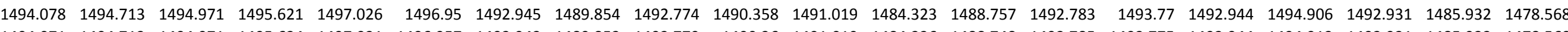

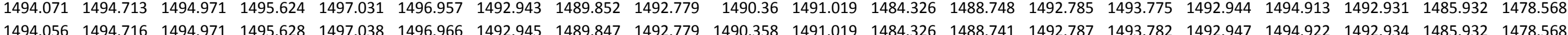


Well

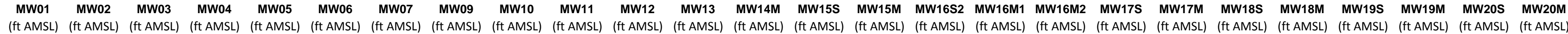

9/30/11 13:00 $9 / 30 / 1113: 30$ $9 / 30 / 1114: 00$ 9/30/111 14:30 9/30/11 15:00 $9 / 30 / 1116: 00$ 9/30/11 16:30 9/30/11 17:00 9/30/11 17:30 9/30/11 18:00 $9 / 30 / 1118: 30$ 9/30/11 19:30 9/30/11 20:00 9/30/11 20:30 9/30/11 21:00 9/30/11 22:00 /30/11 22:30 9/30/11 23:00 10/1/1110:00 $10 / 1 / 1110: 00$
$10 / 1 / 110: 30$ 10/1/11 1:00 10/1/111:30 10/1/112:00 $10 / 1 / 112: 30$ 10/1/11 3:00 10/1/113:30 10/1/11 4:30 10/1/11 5:00 10/1/11 5:30 10/1/11 6:00 10/1/11 6:30 10/1/117:30 10/1/111 8:00 10/1/11 9:00 10/1/11 9:30 10/1/11 10:00 10/1/11 10:30 10/1/11 11:00 10/1/11 11:30 10/1/11 12:00 10/1/11 13:00 10/1/11 14:00 10/1/111 15:00 $\begin{array}{llll}1494.654 & 1495.084 & 1496.882 & 1494.895\end{array}$ $\begin{array}{llll}1494.656 & 1495.091 & 1496.892 & 1494.904\end{array}$ $\begin{array}{rrrrr}1494.659 & 1495.098 & 1496.903 & 1494.918 \\ 1494.663 & 1495.108 & 1496913 & 1494.93\end{array}$ $\begin{array}{rrrr}1494.663 & 1455.108 & 1496.913 & 1494.93 \\ 1494.666 & 1495.115 & 1496.922 & 1494.939\end{array}$ $\begin{array}{lllll}1494.666 & 1495.119 & 1496.929 & 1444.939\end{array}$ $\begin{array}{rrrrr}1494.666 & 1455.119 & 1466.929 & 1494.948 \\ 1494.666 & 1495.128 & 1496.939 & 1494.96\end{array}$ $\begin{array}{lllll}1494.668 & 1495.135 & 1496.941 & 1494.969\end{array}$ $\begin{array}{llll}1494.668 & 1495.142 & 1496.941 & 1494.981\end{array}$ $\begin{array}{llll}1494.67 & 1495.145 & 1496.939 & 1494.988\end{array}$ $\begin{array}{rrrr}1494.668 & 1495.149 & 1496.939 & 1494.993 \\ 1494.67 & 1495.154 & 1496934 & 1494.993\end{array}$ $\begin{array}{rrrr}1494.67 & 1495.154 & 1496.934 & 1494.993 \\ 1494.668 & 1495.156 & 1496.925 & 1494.993\end{array}$ $\begin{array}{lllll}1494.668 & 1495.159 & 1496.922 & 1494.993\end{array}$ $\begin{array}{rrrrr}1494.666 & 1495.156 & 1496.915 & 1494.983\end{array}$ $\begin{array}{lllll}1494.666 & 1495.156 & 1496.915 & 1494.983 \\ 1494.666 & 1495.156 & 1496.911 & 1494.976\end{array}$ $\begin{array}{llllll}1494.663 & 1495.154 & 1496.911 & 1494.974\end{array}$ $\begin{array}{lllll}1494.661 & 1455.149 & 1496.911 & 1494.969\end{array}$ $\begin{array}{lllll}1494.661 & 1495.149 & 1496.899 & 1494.962\end{array}$ $\begin{array}{rrrr}1494.661 & 1495.149 & 1496.896 & 1494.962 \\ 1494.659 & 1495.149 & 1496894 & 1494.96\end{array}$ $\begin{array}{rrrrr}1494.656 & 1495.145 & 1496.889 & 1494.955\end{array}$ $\begin{array}{llllll}1494.654 & 1495.142 & 1496.889 & 1494.951\end{array}$ $\begin{array}{lllll}1494.656 & 1495.142 & 1496.889 & 1494.948\end{array}$ $\begin{array}{llllll}1494.654 & 1495.14 & 1496.885 & 1494.946\end{array}$ $\begin{array}{lllll}1494.654 & 1495.14 & 1496.875 & 1494.946\end{array}$ $\begin{array}{rrrr}1494.654 & 1495.14 & 1496.88 & 1494.941\end{array}$ $\begin{array}{llll}1494.652 & 1495.135 & 1496.885 & 1494.939\end{array}$ $\begin{array}{lllll}1494.649 & 1495.135 & 1496.882 & 1494.934 \\ 1494.652 & 1495.133 & 1496878 & 1494.939\end{array}$ $\begin{array}{lllll}1494.652 & 1495.135 & 1496.875 & 1994.939\end{array}$ $\begin{array}{lllll}1494.649 & 1495.135 & 1496.873 & 1494.937\end{array}$ $\begin{array}{lllll}1494.647 & 1495.135 & 1496.868 & 1494.937\end{array}$ $\begin{array}{llllll}1494.647 & 1495.133 & 1496.864 & 1494.934\end{array}$ $\begin{array}{lllll}1494.647 & 1495.133 & 1496.854 & 1494.934\end{array}$ $\begin{array}{llll}1494.642 & 1495.128 & 1496.843 & 1494.93 \\ 1496.642 & 1455.126 & 149684 & 1494927\end{array}$ $\begin{array}{llll}1494.642 & 1495.126 & 1496.84 & 1494.927 \\ 1494.638 & 1495.122 & 1496833 & 1494923\end{array}$ $\begin{array}{llll}1494.638 & 1495.122 & 1496.833 & 1494.923 \\ 1494.635 & 1495.117 & 1496829 & 1494.916\end{array}$ $\begin{array}{lllll}1494.638 & 1495.115 & 1496.826 & 1494.911\end{array}$ $\begin{array}{lllll}1494.635 & 1495.112 & 1496.829 & 1494.904\end{array}$ $\begin{array}{lllll}1494.633 & 1495.108 & 1496.829 & 1494.902\end{array}$ $\begin{array}{lllll}1494.635 & 1495.105 & 1496.831 & 1494.899\end{array}$ $\begin{array}{llll}1494.638 & 1495.105 & 1496.845 & 1494.897\end{array}$ $\begin{array}{llll}1494.64 & 1495.108 & 1496.852 & 1494.897 \\ 1494.64 & 195.108 & 1496859 & 194.899\end{array}$ $\begin{array}{rrrrr}1494.64 & 1495.108 & 1496.859 & 1494.899\end{array}$ $\begin{array}{lllll}1494.645 & 1495.112 & 1496.878 & 1494.906\end{array}$ $\begin{array}{lllll}1494.645 & 1495.119 & 1496.892 & 14494.913\end{array}$ $\begin{array}{lllll}1494.645 & 1495.124 & 1496.903 & 1494.92\end{array}$ $\begin{array}{lllll}1494.647 & 1495.133 & 1496.906 & 1494.93\end{array}$ 1494.6521495 .1421496 .9111494 .939 $\begin{array}{llll}1494.652 & 1495.149 & 1496.911 & 1494.948 \\ 1494.649 & 1495.152 & 1496.913 & 1494.953\end{array}$

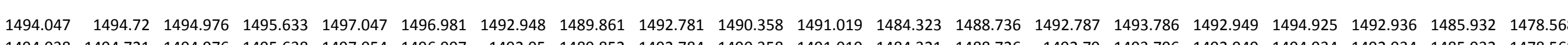

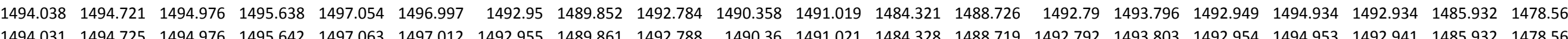

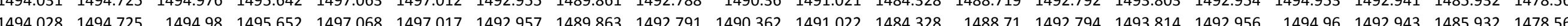
$\begin{array}{lllllllllllllllllllllll}1494.024 & 1494.73 & 1494.978 & 1495659 & 1497.075 & 1497.026 & 1492.964 & 1489.875 & 1492.793 & 1490.362 & 1491.026 & 1484.323 & 1488.708 & 1492801 & 1493.821 & 1492.961 & 1494.974 & 1492.948 & 1485932 & 147857\end{array}$ $\begin{array}{llllllllllllllllllllll}1494.021 & 1494.735 & 1494.978 & 1495.663 & 1497.082 & 1497.036 & 1492.966 & 1489.868 & 1492.795 & 1490.367 & 1491.031 & 1484.321 & 1488.71 & 1492.806 & 1493.831 & 1492.968 & 1494.983 & 1492.952 & 1485.932 & 147857 \\ \end{array}$

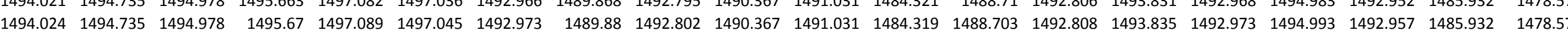
$\begin{array}{lllllllllllllllllllll}1494.024 & 1494.737 & 1494.98 & 1495.677 & 1497.094 & 1497.05 & 1492.973 & 1489.878 & 1492.805 & 1490.369 & 1491.036 & 1484.319 & 1488.698 & 1492.811 & 1493.842 & 1492.973 & 1495.007 & 1492.959 & 1485.932 & 1478.573\end{array}$ $\begin{array}{llllllllllllllllllll}1494.031 & 1494.74 & 1494.978 & 1495.682 & 1477.098 & 1497.052 & 1492.98 & 1489.882 & 1492.809 & 1490.369 & 1491.04 & 1484.317 & 1488.694 & 1492.818 & 1493.852 & 1492.977 & 1495.012 & 1492.967 & 1485.932 & 1478.575 \\ \end{array}$

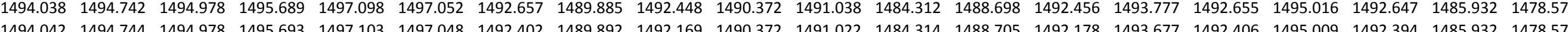

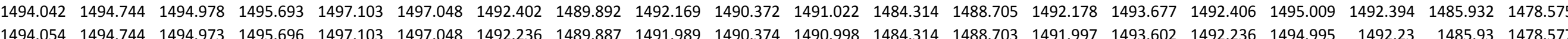

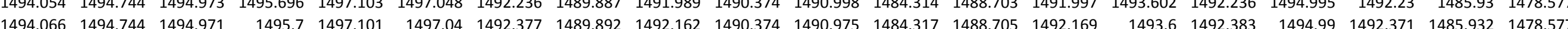
$\begin{array}{lllllllllllllllllllll}1494.075 & 1494.747 & 1494.973 & 1495.703 & 1497.103 & 1497.031 & 1492.55 & 1489.892 & 1492.35 & 1490.374 & 1490.968 & 1484.317 & 1488.701 & 1492359 & 1493.668 & 1492.556 & 1494.986 & 1492.542 & 1485.932 & 1478.577\end{array}$ $\begin{array}{llllllllllllllllllllll}1494.085 & 1494.744 & 1494.973 & 1495.703 & 1497.098 & 1497.026 & 1492.639 & 1489.889 & 1492.446 & 1490.374 & 1490.968 & 1484.314 & 1488.712 & 1492.456 & 1493.696 & 1492.641 & 1494.983 & 1492.626 & 1485.93 & 1478.577\end{array}$ \begin{tabular}{lllllllllllllllllllllll}
1494.092 & 1494.742 & 1494.969 & 1495.7 & 1497.101 & 1497.019 & 1492.695 & 1489.885 & 1492.509 & 1490.374 & 1490.968 & 1484.319 & 1488.717 & 1492.517 & 1493.714 & 1492.698 & 1494.974 & 1492.683 & 1485.93 & 1478.58 \\
\hline
\end{tabular}

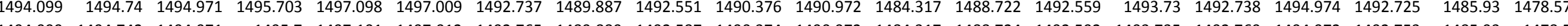

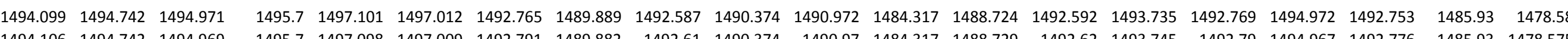

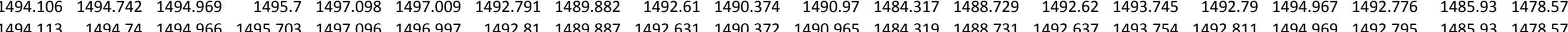

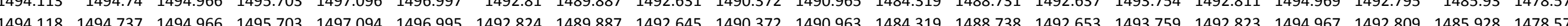
\begin{tabular}{llllllllllllllllllllll}
1494.12 & 1494.733 & 1494.966 & 1495.703 & 1497.094 & 1496.99 & 1492.833 & 1489.878 & 1492.659 & 1490.369 & 1490.958 & 1484.319 & 1488.741 & 1492.665 & 1493.759 & 1492.837 & 1494.967 & 1492.821 & 1485.93 & 14785573 \\
\hline
\end{tabular}

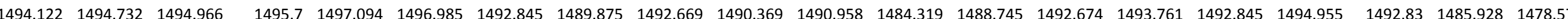

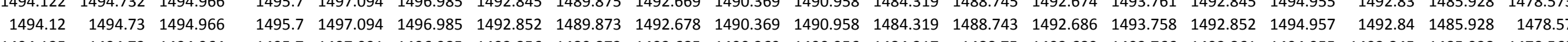

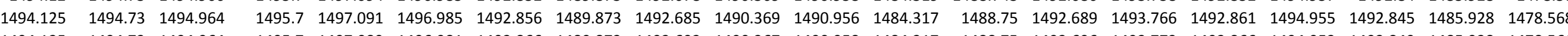

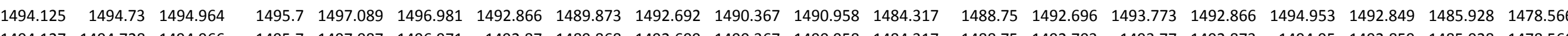

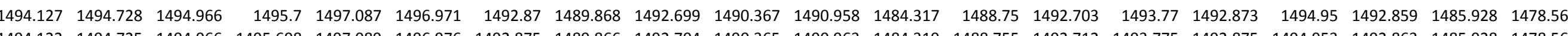

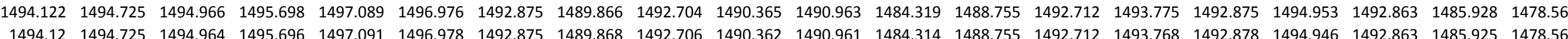

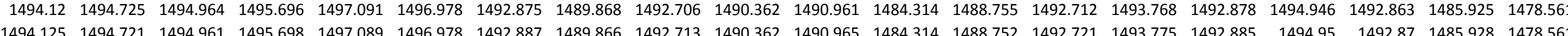

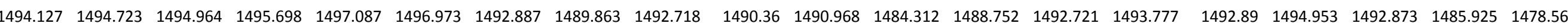
$\begin{array}{llllllllllllllllllllll}1494.125 & 1494.723 & 1494.961 & 1495.698 & 1497.084 & 1496.971 & 1492.891 & 1489.863 & 1492.723 & 1490.362 & 1490.972 & 1484.314 & 1488.75 & 1492.729 & 1493.779 & 1492.892 & 1494.948 & 1492.88 & 1485.925 & 1478.561 \\ \end{array}$

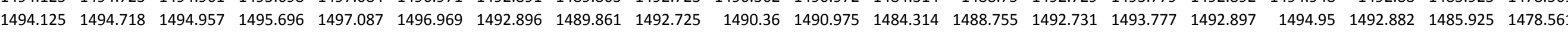
$\begin{array}{lllllllllllllllllllllll}1494.129 & 1494.718 & 1494.959 & 1495.696 & 1497.084 & 1496.964 & 1492.898 & 1489.861 & 1492.73 & 1490.36 & 1490.98 & 1484.317 & 1488.75 & 1492.733 & 1493.78 & 1492.899 & 1494.943 & 1492.884 & 1485.925 & 1478.561\end{array}$

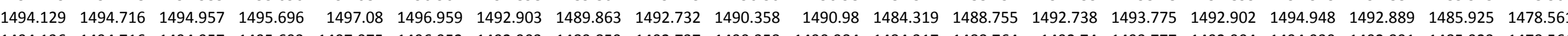

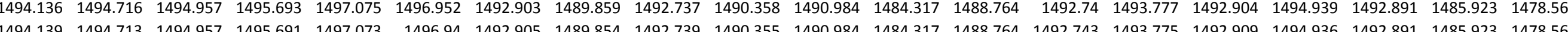

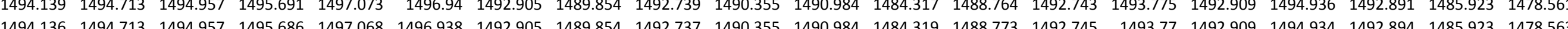

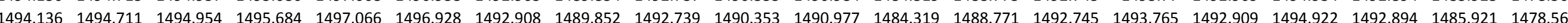
$\begin{array}{lllllllllllllllllllllll}1494.134 & 1494.711 & 1494.954 & 1495.682 & 1497.066 & 1496.926 & 1492.91 & 1489.849 & 1492.739 & 1490.351 & 1490.975 & 1484.317 & 1488.769 & 1492.745 & 1493.768 & 1492.909 & 1494.922 & 1492.894 & 1485.921 & 1478.561\end{array}$ $\begin{array}{llllllllllllllllllllll}1494.127 & 1494.709 & 1494.954 & 1495.677 & 1497.061 & 1496.926 & 1492.908 & 1489.849 & 1492.739 & 1490.348 & 1490.972 & 1484.312 & 1488.771 & 1492.75 & 1493.761 & 1492.909 & 1494.913 & 1492.894 & 1485.921 & 1478.561 \\ \end{array}$

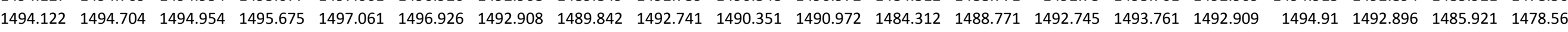

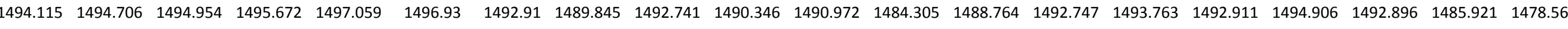

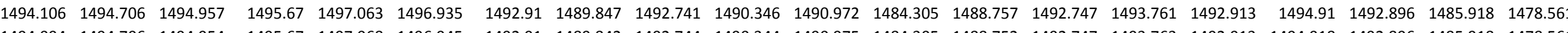

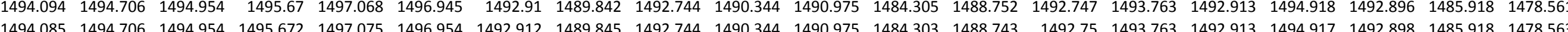

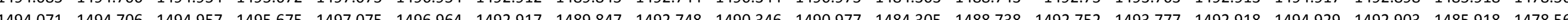
$\begin{array}{lllllllllllllllllllllll}1494.066 & 1494.711 & 1494.957 & 1495.677 & 1497.08 & 1496.971 & 1492.917 & 1489.849 & 1492.748 & 1490.332 & 1490.98 & 1484.303 & 1488.729 & 1492.754 & 1493.779 & 1492.918 & 1494.927 & 1492.903 & 1485.918 & 1478.561\end{array}$ $\begin{array}{lllllllllllllllllllllll}1494.054 & 1494.711 & 1494.957 & 1495.679 & 1497.084 & 1496.981 & 1492.922 & 1489.847 & 1492.751 & 1490.346 & 1490.982 & 1484.305 & 1488.726 & 1492.761 & 1493.789 & 1492.923 & 1494.939 & 1492.908 & 1485.918 & 1478.561 \\ & & & \end{array}$

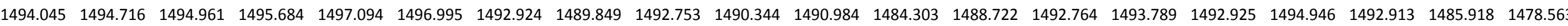
$\begin{array}{lllllllllllllllllllll}1494.04 & 1494.718 & 1494.961 & 1495.686 & 1497.098 & 1497.007 & 1492.927 & 1489.859 & 1492.758 & 1490.346 & 1490.987 & 1484.307 & 1488.715 & 1492.764 & 1493.796 & 1492.93 & 1494.96 & 1492.913 & 1485.918 & 1478.5633\end{array}$ $\begin{array}{lllllllllllllllllllll}1494.042 & 1494.721 & 1494.959 & 1495.693 & 1497.101 & 1497.009 & 1492.934 & 1489.859 & 1492.762 & 1490.348 & 1490.991 & 1484.307 & 1488.708 & 1492.768 & 1493.81 & 1492.932 & 1494.967 & 1492.917 & 1485.918 & 1478.563\end{array}$

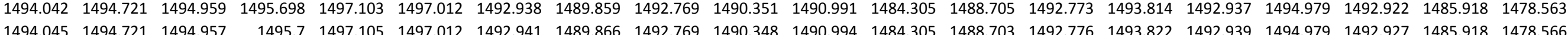


Well

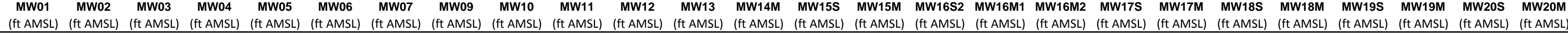

10/1/11 16:00 10/1/11 16:30 10/1/11 17:00 10/1/111 18:00 $10 / 1 / 11118: 00$ 10/1/11 19:00 10/1/11 19:30 10/1/1120:00 10/1/11 20:30 10/1/1121:00 $10 / 1 / 1122: 00$ 10/1/1122:00 0/1/11 23:00 $10 / 1 / 1123: 30$ 10/2/11 0:00 10/2/11 0:30 10/2/11 1:00 $10 / 2 / 1112: 00$
$10 / 2 / 112: 30$ 10/2/111 2:30 10/2/11 3:30 10/2/111 4:00 10/2/11 4:30 10/2/1115:00 10/2/115:30 $10 / 2 / 116: 00$ 10/2/11 6:30 10/2/1117:00 10/2/11 8:00 10/2/11 8:30 10/2/119:00 10/2/11 9:30 10/2/11110.30 10/2/11 10:30 10/2/11111:30 10/2/111 12:00 10/2/111 12:30 10/2/11 13:00 10/2/11 13:30 10/2/11 14:00 10/2/111 15:00 $10 / 2 / 11$ 15:30 10/2/111 16:30 10/2/11 17:00 10/2/11 17:30 10/2/11 18:00 $\begin{array}{llll}1494.652 & 1495.156 & 1496.913 & 1494.96\end{array}$ 1494.6491495 .1591496 .9181494 .962 $\begin{array}{lllll}1494.649 & 1495.161 & 1496.913 & 1494.969 \\ 1494.652 & 1495.163 & 1496913 & 1494.972\end{array}$ $\begin{array}{lllll}1494.652 & 1495.166 & 1496.913 & 1494.974\end{array}$ $\begin{array}{lllll}1494.649 & 1495.166 & 1496.911 & 1449.976\end{array}$ $\begin{array}{llllll}1494.647 & 1495.166 & 1496.906 & 1494.974\end{array}$ $\begin{array}{lllll}1494.649 & 1495.166 & 1496.903 & 1494.976\end{array}$ $\begin{array}{lllll}1494.647 & 1495.166 & 1496.894 & 1494.976\end{array}$ $\begin{array}{lllll}1494.645 & 1495.163 & 1496.892 & 1494.976\end{array}$ $\begin{array}{llll}1494.645 & 1495.161 & 1496.892 & 1494.972 \\ 1494.642 & 1495.159 & 1496.887 & 1494.969\end{array}$ $\begin{array}{lllll}1494.642 & 1495.159 & 1496.887 & 1494.969 \\ 1494.642 & 1495.156 & 1496.885 & 1494.965\end{array}$ $\begin{array}{lllll}1494.642 & 1495.156 & 1496.885 & 1494.965\end{array}$ $\begin{array}{lllll}1494.642 & 1455.152 & 1496.887 & 1494.962\end{array}$ $\begin{array}{lllll}1494.64 & 1495.154 & 1496.885 & 1494.962\end{array}$ $\begin{array}{lllll}1494.64 & 1495.152 & 1496.887 & 1494.958\end{array}$ $\begin{array}{llll}1494.64 & 1495.154 & 1496.878 & 1494.96\end{array}$ $\begin{array}{llll}1494.64 & 1495.154 & 1496.873 & 1494.958\end{array}$ $\begin{array}{llll}1494.64 & 1495.152 & 1496.871 & 1494.955 \\ 1494.635 & 1495.149 & 1496873 & 1494.953\end{array}$ $\begin{array}{llll}1494.635 & 1495.147 & 1496.878 & 1494.948\end{array}$ $\begin{array}{lllll}1494.635 & 1495.145 & 1496.873 & 1494.946\end{array}$ $\begin{array}{lllll}1494.635 & 1495.145 & 1496.871 & 1494.944\end{array}$ $1494.638 \quad 1495.147 \quad 1496.866 \quad 1494.946$ $\begin{array}{lllll}1494.635 & 1495.145 & 1496.864 & 1494.944\end{array}$ $\begin{array}{lllll}1494.633 & 1495.142 & 1496.857 & 1494.941\end{array}$ $\begin{array}{rrrr}1494.633 & 1495.138 & 1496.859 & 1494.939\end{array}$ $\begin{array}{rrrrr}1494.631 & 1495.14 & 1496.852 & 1494.937 \\ 1494.628 & 1495.135 & 1496.85 & 1494.932\end{array}$ $\begin{array}{lllll}1494.631 & 1495.135 & 1496.847 & 1494.93\end{array}$ $\begin{array}{lllll}1494.628 & 1495.131 & 1496.845 & 1494.923\end{array}$ $\begin{array}{lllll}1494.628 & 1495.131 & 1496.843 & 1494.92\end{array}$ $\begin{array}{lllll}1494.628 & 1495.128 & 1496.84 & 1494.916\end{array}$ $\begin{array}{llll}1494.628 & 1495.128 & 1496.845 & 1494.913\end{array}$ $\begin{array}{llll}1494.628 & 1495.122 & 1496.852 & 1494.909\end{array}$ $\begin{array}{llll}1494.631 & 1495.124 & 1496.861 & 1494.906\end{array}$ $\begin{array}{lllll}1494.631 & 1495.124 & 1496.873 & 1494.906 \\ 1494.633 & 1495.133 & 1496887 & 1494.899\end{array}$ $\begin{array}{llll}1494.635 & 1495.135 & 1496.901 & 1494.885\end{array}$ $\begin{array}{lllll}1494.64 & 1495.14 & 1496.911 & 1494.867\end{array}$ $\begin{array}{lllll}1494.64 & 1495.147 & 1496.929 & 1494.85\end{array}$ $\begin{array}{lllll}1494.642 & 1495.156 & 1496.943 & 1494.832\end{array}$ $\begin{array}{llll}1494.645 & 1495.161 & 1496.955 & 1494.811\end{array}$ $1494.649 \quad 1495.1751496 .957 \quad 1494.797$ 1494.6491495 .1821496 .9551494 .78 $1494.649 \quad 1495.191 \quad 1496.955 \quad 1494.738$ $1494.649 \quad 1495.194 \quad 1496.953 \quad 1494.717$ $1494.6471495 .191 \quad 1496.946 \quad 1494.701$ $1494.6471495 .1941496 .948 \quad 1494.689$ $1494.645 \quad 1495.189 \quad 1496.943 \quad 1494.687$ $\begin{array}{llll}1494.645 & 1495.187 & 1496.939 & 1494.682 \\ 1494.645 & 1495.189 & 1496.934 & 1494.685\end{array}$

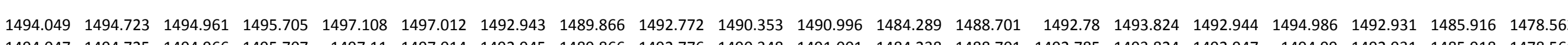

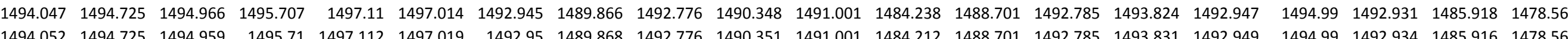

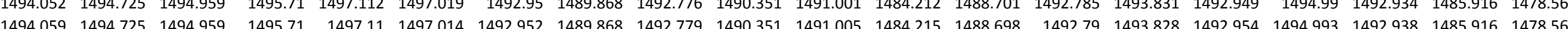
$\begin{array}{lllllllllllllllllllll}1494.063 & 1494.725 & 1494.957 & 1495.714 & 1497.112 & 1497.009 & 1492.955 & 1489.87 & 1492.786 & 1490351 & 1491.008 & 1484.215 & 1488.701 & 1492.792 & 1493.835 & 1492.954 & 1494.995 & 1492.941 & 1485.916 & 1478.566\end{array}$ $\begin{array}{llllllllllllllllllllll}1494.068 & 1494.725 & 1494.957 & 1495.714 & 1497.112 & 1497.009 & 1492.957 & 1489.87 & 1492.788 & 1490.353 & 1491.01 & 1484.222 & 1488.705 & 1492.792 & 1493.831 & 1492.956 & 1494.993 & 1492.941 & 1485.916 & 1478.568\end{array}$

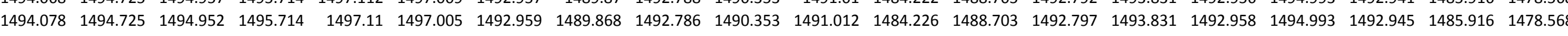
$\begin{array}{lllllllllllllllllllllll}1494.085 & 1494.725 & 1494.952 & 1495.717 & 1497.11 & 1497 & 1492.948 & 1489.87 & 1492.781 & 1490.353 & 1491.015 & 1484.226 & 1488.705 & 1492.785 & 1493.833 & 1492.949 & 1494.993 & 1492.934 & 1485.916 & 1478.568\end{array}$

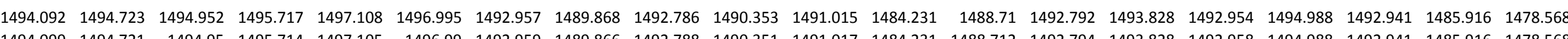

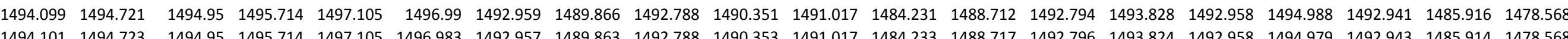

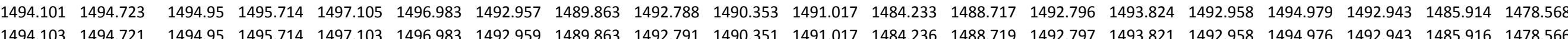

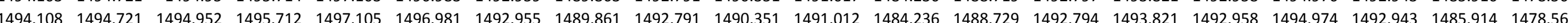
$\begin{array}{llllllllllllllllllllll}1494.108 & 1494.721 & 1494.95 & 1495.712 & 1497.103 & 1496.976 & 1492.959 & 1489.859 & 1492.788 & 1490.348 & 1491.01 & 1484.238 & 1488.724 & 1492.797 & 1493.822 & 1492.958 & 1494.974 & 1492.943 & 1485.914 & 1478.566\end{array}$ $\begin{array}{llllllllllllllllllll}1494.106 & 1494.718 & 1494.954 & 1495.712 & 1497.105 & 1496.976 & 1492.959 & 1489.861 & 1492.788 & 1490.348 & 1491.003 & 1484.24 & 1488.726 & 1492.794 & 1493.817 & 1492.958 & 1494.969 & 1492.943 & 1485.914 & 1478.566\end{array}$ $\begin{array}{lllllllllllllllllllll}1494.106 & 1494.716 & 1494.954 & 1495.712 & 1497.105 & 1496.978 & 1492.955 & 1489.854 & 1492.788 & 1490.348 & 1491.001 & 1484.24 & 1488.729 & 1492.794 & 1493.814 & 1492.956 & 1494.969 & 1492.941 & 1485.914 & 1478.566\end{array}$ $\begin{array}{llllllllllllllllllllll}1494.108 & 1494.716 & 1494.95 & 1495.712 & 1497.103 & 1496.976 & 1492.955 & 1489.856 & 1492.786 & 1490.348 & 1490.998 & 1484.236 & 1488.733 & 1492.792 & 1493.815 & 1492.956 & 1494.964 & 1492.941 & 1485.914 & 1478.563\end{array}$

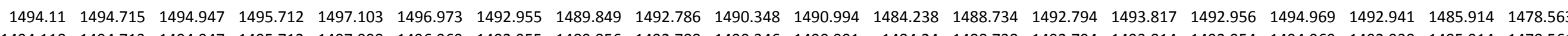

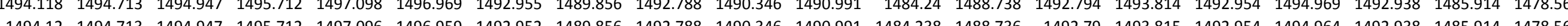

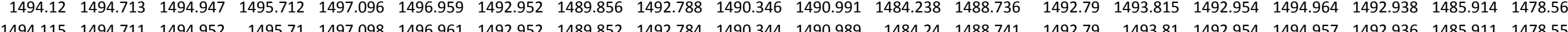
$\begin{array}{lllllllllllllllllllll}1494.113 & 1494.711 & 1494.95 & 1495.707 & 1497.098 & 1496.961 & 1422.948 & 1489.854 & 1492.781 & 1490.342 & 1490.991 & 1484.236 & 1488.743 & 1492.79 & 1493.805 & 1492.954 & 1494.957 & 1492.934 & 1485.911 & 1478.559\end{array}$ $\begin{array}{llllllllllllllllllllll}1494.11 & 1494.709 & 1494.95 & 1495.705 & 1497.098 & 1496966 & 1492.948 & 1489.849 & 1492.784 & 1490.342 & 1490.991 & 1484236 & 1488743 & 1492.787 & 1493.805 & 1492.951 & 1494.953 & 1492.934 & 1485.911 & 1478.556\end{array}$ $\begin{array}{lllllllllllllllllllllll}1494.113 & 1494.708 & 1494.945 & 1495.705 & 1497.096 & 1496.964 & 1492.95 & 1489.849 & 1492.781 & 1490.342 & 1490.991 & 1484.238 & 1488.736 & 1492.787 & 1493.803 & 1492.949 & 1494.96 & 1492.936 & 1485.911 & 1478.556\end{array}$

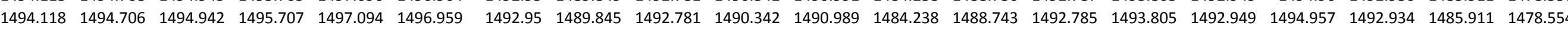

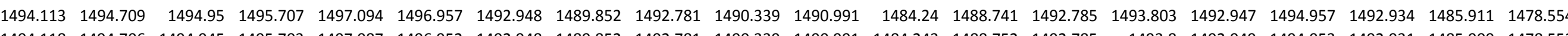

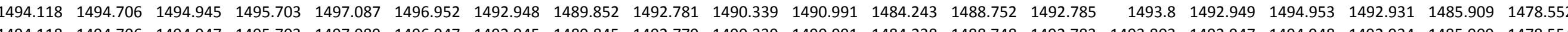

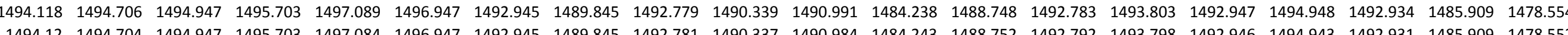

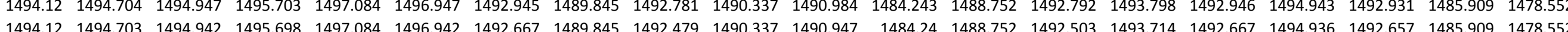

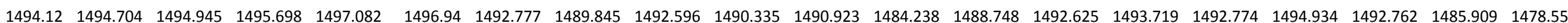
$\begin{array}{llllllllllllllllllll}1494.12 & 1494.701 & 1494.94 & 1495.696 & 1497.08 & 1496.938 & 1492.817 & 1489.84 & 1492.64 & 1490.335 & 1490.916 & 1484.238 & 1488.762 & 1492.674 & 1493.733 & 1492.814 & 1494.922 & 1492.802 & 1485.909 & 1478.552\end{array}$

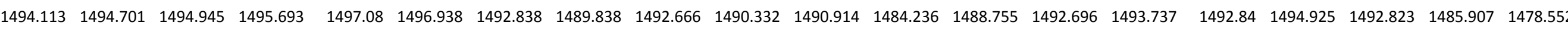
$\begin{array}{llllllllllllllllllllll}1494.11 & 1494.701 & 1494.94 & 1495.693 & 1497.077 & 1496.935 & 1492.852 & 1489.835 & 1492.68 & 1490.332 & 1490.909 & 1484.233 & 1488.757 & 1492.71 & 1493.747 & 1492.852 & 1494.915 & 1492.837 & 1485.909 & 1478.552\end{array}$

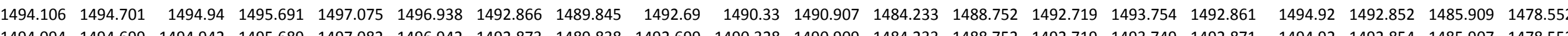

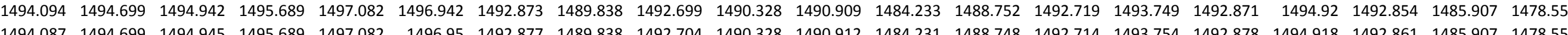

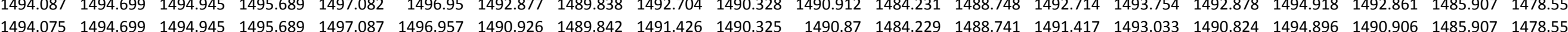

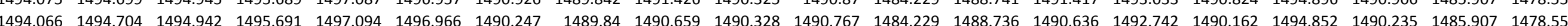
$\begin{array}{lllllllllllllllllllll}1494.049 & 1494.706 & 1494.947 & 1495.693 & 1497.103 & 1496.981 & 1489.754 & 1489.842 & 1490.139 & 1490.325 & 1490.659 & 1484.231 & 1488.726 & 1490.091 & 1492.548 & 1489.684 & 1494.816 & 1489.75 & 1485.907 & 1478.556\end{array}$ $\begin{array}{lllllllllllllllllllllll}1494.045 & 1494.708 & 1494.947 & 1495.696 & 1497.105 & 1496.993 & 1489.679 & 1489.845 & 1489.904 & 1490.325 & 1490.557 & 1484.231 & 1488.724 & 1489.842 & 1492.499 & 1489.639 & 1494.786 & 1489.679 & 1485.907 & 1478.559\end{array}$

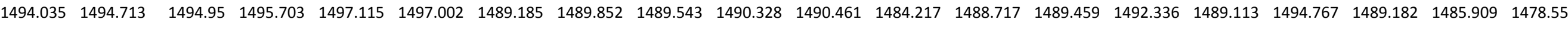

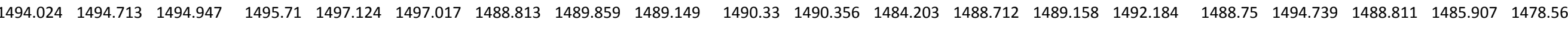

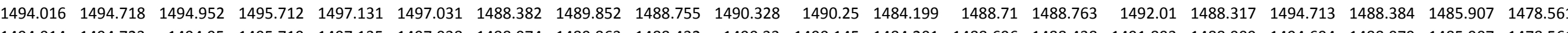

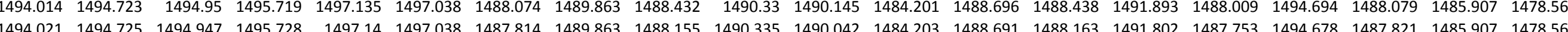

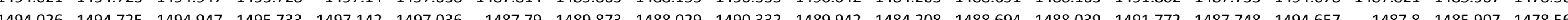
$\begin{array}{lllllllllllllllllllllll}1494.033 & 1494.725 & 1494.95 & 1495.735 & 1497.142 & 1497.033 & 1488.977 & 1489.873 & 1488.612 & 1490.337 & 1489.865 & 1484.206 & 1488.696 & 1488.617 & 1492.04 & 1489.023 & 1494.635 & 1489.008 & 1485.907 & 1478.563\end{array}$ $\begin{array}{llllllllllllllllllllll}1494.04 & 1494.728 & 1494.942 & 1495.737 & 1497.145 & 1497.031 & 1489.772 & 1489.873 & 1489.433 & 1490.337 & 1489.853 & 1484.208 & 1488.694 & 1489.44 & 1492.38 & 1489.807 & 1494.643 & 1489.789 & 1485.907 & 1478.566\end{array}$ $\begin{array}{llllllllllllllllllll}1494.049 & 1494.728 & 1494.942 & 1495.735 & 1497.142 & 1497.026 & 1490.231 & 1489.88 & 1489.916 & 1490.337 & 1489.876 & 1484.206 & 1488.689 & 1489.922 & 1492.56 & 1490.257 & 1494.65 & 1490.24 & 1485.904 & 1478.566\end{array}$

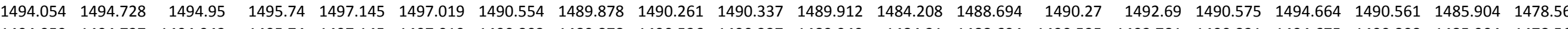

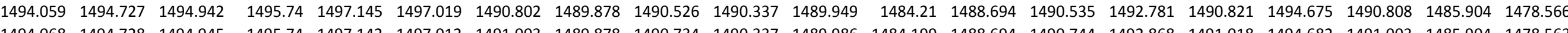
$\begin{array}{llllllllllllllllllll}1494.068 & 1494.728 & 1494.945 & 1495.74 & 1497.142 & 1497.012 & 1491.003 & 1489.878 & 1490.734 & 1490.337 & 1489.986 & 1484.199 & 1488.694 & 1490.744 & 1492.868 & 1491.018 & 1494.682 & 1491.003 & 1485.904 & 1478.563 \\ 1494.078 & 1494.725 & 149494 & 149574 & 1497.14 & 1497.007 & 1491165 & 1489885 & 1490.913 & 1490337 & 1490.019 & 1484.196 & 1488.698 & 1490.921 & 1492.935 & 1491.181 & 1494.692 & 1491.164 & 1485.904 & 1478.566\end{array}$ 
Well

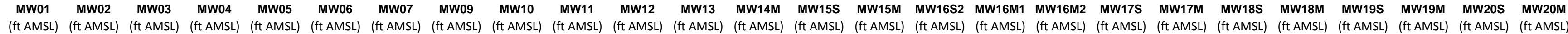

10/2/11 19:00 10/2/11 19:30 $10 / 2 / 1120: 30$ 10/2/1121:00 10/2/1121:30 10/2/111 22:00 10/2/11 22:30 10/2/11 23:00 10/2/11 23:30 10/3/11 0:00 10/3/111:00 10/3/1111:00 10/3/111 2:00 10/3/11 2:30 10/3/11 3:00 10/3/11 3:30 10/3/11 4:00 10/3/11 4:30 10/3/11 5:00 10/3/111 5:30 10/3/111 6:30 10/3/11 7:00 10/3/11 7:30 10/3/11 8:00 10/3/111 8:30 10/3/119:30 10/3/11 10:00 10/3/11 10:30 10/3/111 10:30 10/3/11 11:30 10/3/11 12:00 10/3/11 12:30 10/3/11 13:00 10/3/11 13:30 10/3/11 14:30 10/3/11 14:30 10/3/11 15:30 10/3/11 16:00 10/3/11 16:30 10/3/11117:00 10/3/1118:00 10/3/11 18:00 10/3/11 18:30 10/3/11 19:30 10/3/11 20:00 10/3/11 20:30 10/3/11 21:00 $\begin{array}{llll}1494.642 & 1495.187 & 1496.929 & 1494.685 \\ 1494.64 & 1495.182 & 1496925 & 1494.687\end{array}$ $\begin{array}{rrrr}1494.64 & 1495.182 & 1496.925 & 1494.687\end{array}$ $\begin{array}{rrrrr}1494.638 & 1495.18 & 1496.92 & 1494.692 \\ 1494.638 & 1495.175 & 1496918 & 1494.696\end{array}$ $\begin{array}{lllll}1494.635 & 1495.173 & 1496.913 & 1494.701\end{array}$ $\begin{array}{lllll}1494.635 & 1495.17 & 1496.903 & 1494.701\end{array}$ $\begin{array}{rrrrr}1494.635 & 1495.17 & 1496.903 & 1494.703 \\ 1494.635 & 1495.168 & 1496.901 & 1494.71\end{array}$ $\begin{array}{rrrrr}1494.631 & 1495.163 & 1496.899 & 1494.715\end{array}$ $\begin{array}{lllll}1494.628 & 1495.161 & 1496.901 & 1494.715\end{array}$ $\begin{array}{llll}1494.626 & 1495.154 & 1496.896 & 1494.72\end{array}$ $\begin{array}{llll}1494.628 & 1495.154 & 1496.892 & 1494.727 \\ 1494.626 & 1495.154 & 1996892 & 1494.734\end{array}$ $\begin{array}{llll}1494.626 & 1495.154 & 1496.892 & 1494.734 \\ 1494.624 & 1495.149 & 1496.887 & 1494.736\end{array}$ $\begin{array}{lllll}1494.624 & 1495.149 & 1496.887 & 1494.736 \\ 1494.624 & 1495.147 & 1496.892 & 1494.741\end{array}$ $\begin{array}{lllll}1494.621 & 1495.145 & 1496.894 & 1494.744\end{array}$ $\begin{array}{llllll}1494.624 & 1495.142 & 1496.896 & 1494.748\end{array}$ $\begin{array}{lllll}1494.624 & 1495.142 & 1496.894 & 1494.752\end{array}$ $\begin{array}{lllll}1494.624 & 1495.142 & 1466.896 & 1494.759\end{array}$ $\begin{array}{lllll}1494.626 & 1495.147 & 1496.899 & 1494.766\end{array}$ $\begin{array}{lllll}1494.624 & 1495.145 & 1496.901 & 1494.769 \\ 1494.624 & 1495.142 & 1496.901 & 1494.776\end{array}$ $\begin{array}{lllll}1494.624 & 1495.142 & 1496.901 & 1494.776 \\ 1494.621 & 1495.142 & 1496.899 & 1494.783\end{array}$ $\begin{array}{lllll}1494.624 & 1495.145 & 1496.894 & 1494.787\end{array}$ $\begin{array}{llllll}1494.621 & 1495.145 & 1496.892 & 1494.787\end{array}$ $\begin{array}{lllll}1494.621 & 1495.145 & 1496.882 & 1494.78\end{array}$ $\begin{array}{lllll}1494.619 & 1495.14 & 1496.887 & 1494.764\end{array}$ $\begin{array}{llll}1494.617 & 1495.138 & 1496.88 & 1494.738\end{array}$ $\begin{array}{lllll}1494.617 & 1495.133 & 1496.88 & 1494.713\end{array}$ $\begin{array}{rrrrr}1494.617 & 1495.131 & 1496.871 & 1494.692 \\ 1494.614 & 1495.128 & 149688 & 1494.659\end{array}$ $\begin{array}{llll}1494.614 & 1495.126 & 1496.88 & 1494.633\end{array}$ $\begin{array}{lllll}1494.614 & 1495.122 & 1496.887 & 1494.608\end{array}$ $\begin{array}{lllll}1494.617 & 1495.122 & 1496.894 & 1494.582\end{array}$ $\begin{array}{lllll}1494.614 & 1495.122 & 1496.901 & 1494.554\end{array}$ $\begin{array}{lllll}1494.617 & 1495.126 & 1496.908 & 1494.54\end{array}$ $\begin{array}{llll}1494.617 & 1495.126 & 1496.915 & 1494.526 \\ 1496.619 & 1495.128 & 1496929 & 194526\end{array}$ $\begin{array}{lllll}1494.619 & 1495.128 & 1496.929 & 1494.526 \\ 1494.619 & 1495.135 & 1496934 & 1494526\end{array}$ $\begin{array}{lllll}1494.621 & 1459.138 & 1496.934 & 1494.526 \\ 1496.936 & 1494.535\end{array}$ $\begin{array}{lllll}14494.624 & 1495.145 & 1496.934 & 1494.547\end{array}$ $\begin{array}{lllll}1494.624 & 1495.149 & 1496.936 & 1494.559\end{array}$ $\begin{array}{lllll}1494.621 & 1495.152 & 1496.934 & 1494.57\end{array}$ $\begin{array}{llll}1494.621 & 1495.149 & 1496.936 & 1494.58\end{array}$ $\begin{array}{llll}1494.619 & 1495.152 & 1496.934 & 1494.591\end{array}$ $\begin{array}{llll}1494.619 & 1495.149 & 1496.932 & 1494.603 \\ 1494.619 & 1495149 & 1496927 & 1494612\end{array}$ $\begin{array}{lllll}1494.619 & 1495.149 & 1496.927 & 1494.612\end{array}$ $\begin{array}{lllll}1494.614 & 1495.147 & 1496.922 & 1494.633\end{array}$ $\begin{array}{lllll}1494.612 & 1495.145 & 1496.911 & 1494.643\end{array}$ $\begin{array}{lllll}1494.61 & 1495.145 & 1496.901 & 1494.652\end{array}$ $\begin{array}{lllll}1494.607 & 1495.14 & 1496.892 & 1494.654\end{array}$ $\begin{array}{lllll}1494.605 & 1495.135 & 1496.882 & 1494.657\end{array}$ $\begin{array}{rrrrr}1494.603 & 1495.128 & 1496.88 & 1494.659 \\ 1494.6 & 1495.124 & 1496.878 & 1494.659\end{array}$

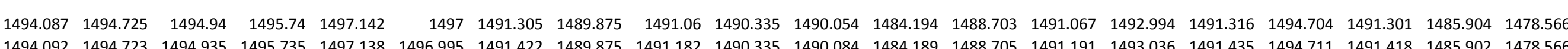

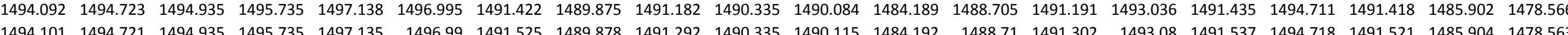

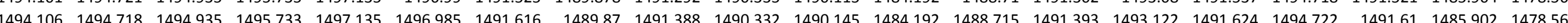
$\begin{array}{llllllllllllllllllllll}1494.11 & 1494.718 & 1494.933 & 1495.731 & 1497.133 & 1496.983 & 1491.693 & 1489.868 & 1491.473 & 1490332 & 1490.171 & 1484.189 & 1488.717 & 1491.478 & 1493.152 & 1491.703 & 1494.727 & 1491.688 & 1485.902 & 1478.563\end{array}$ $\begin{array}{lllllllllllllllllllllllllll}1494.118 & 1494.713 & 1494933 & 1495.731 & 1497.131 & 1496.978 & 1491.766 & 1489.868 & 1491.548 & 1490.332 & 1490.197 & 1484.194 & 1488.722 & 1491.555 & 1493.185 & 1491.776 & 1494.732 & 1491.76 & 1485.902 & 1478.563\end{array}$ $\begin{array}{llllllllllllllllllllll}1494.12 & 1494.711 & 1494.931 & 1495.728 & 1497.128 & 1496.969 & 1491.831 & 1489.866 & 1491.611 & 1490.33 & 1490.215 & 1484.192 & 1488.722 & 1491.621 & 1493.215 & 1491.835 & 1494.739 & 1491.824 & 1485.902 & 1478.563\end{array}$ $\begin{array}{lllllllllllllllllllll}1494.122 & 1494.709 & 1494.935 & 1495.726 & 1497.126 & 1496.964 & 1491.888 & 1489.861 & 1491.675 & 1490.328 & 1490.232 & 1484.189 & 1488.726 & 1491.682 & 1493.234 & 1491.892 & 1494.741 & 1491.878 & 1485.902 & 1478.563\end{array}$

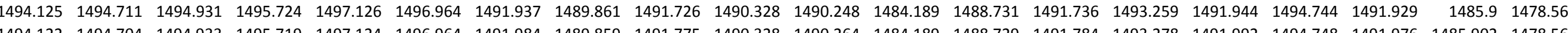

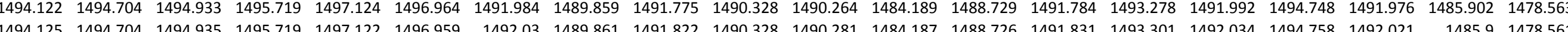

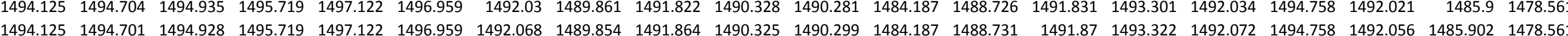

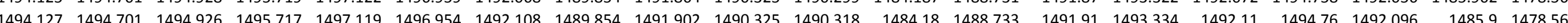

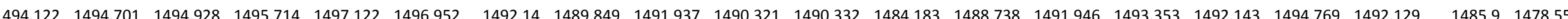
$\begin{array}{llllllllllllllllllllll}1494.122 & 1494.699 & 1494.928 & 1495.712 & 1497.119 & 1496.954 & 1492.171 & 1489.847 & 1491.972 & 1490.321 & 1490.349 & 1484.182 & 1488.738 & 1491.979 & 1493.362 & 1492.174 & 1494.769 & 1492.159 & 1485.9 & 14778.556\end{array}$ $\begin{array}{lllllllllllllllllllllll}1494.118 & 1494.699 & 1494.928 & 1495.712 & 1497.124 & 1496.957 & 1492.199 & 1489.849 & 1492.003 & 1490.321 & 1490.365 & 1484.183 & 1488.738 & 1492.009 & 1493.381 & 1492.202 & 1494.772 & 1492.187 & 1485.9 & 1478.556\end{array}$

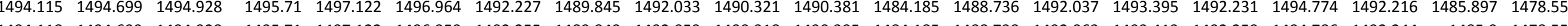

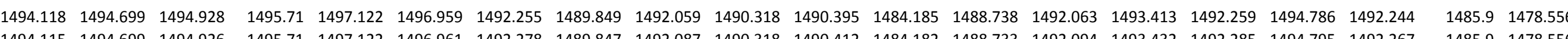

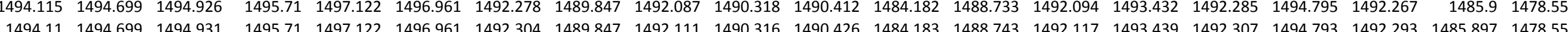

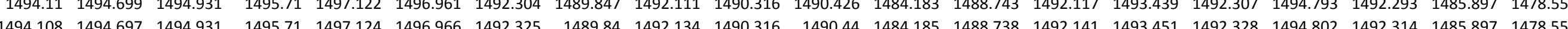

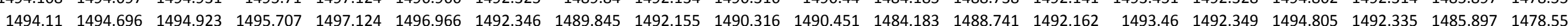
$\begin{array}{lllllllllllllllllllllll}1494.11 & 1494.694 & 1494.926 & 149571 & 1497.122 & 1496961 & 1491.436 & 1489852 & 1491867 & 1490316 & 1490.458 & 1484.166 & 1488738 & 1491.875 & 1493271 & 1491312 & 1494.812 & 1491.397 & 1485897 & 1478554\end{array}$ $\begin{array}{lllllllllllllllllllllll}1494.115 & 1494.697 & 1494.926 & 1495.71 & 1497.119 & 1496.957 & 1490.177 & 1489.849 & 1490.648 & 1490.318 & 1490.393 & 1484.166 & 1488.741 & 1490.648 & 1492.679 & 1490.079 & 1494.774 & 1490.163 & 1485.897 & 1478.554 \\ \end{array}$

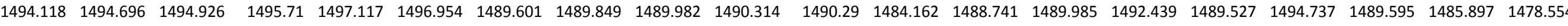

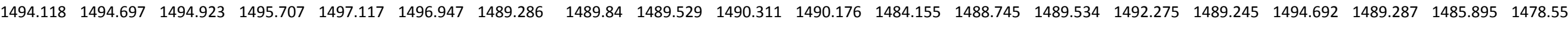

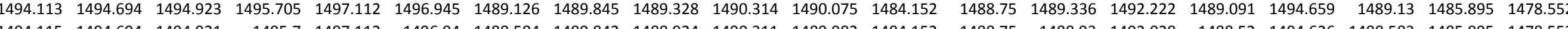

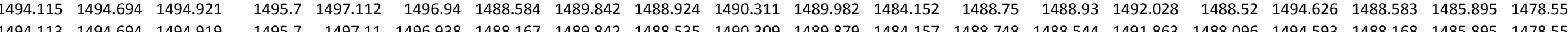

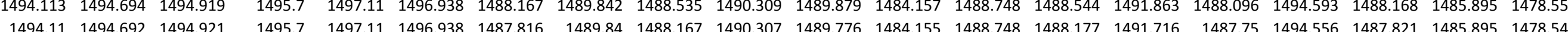
\begin{tabular}{llllllllllllllllllll}
1494.103 & 1494.694 & 1494.921 & 1495.698 & 1497.11 & 1496.94 & 1487.512 & 1489.84 & 1487.841 & 1490.307 & 1489.675 & 1484.157 & 1488.743 & 1487.851 & 1491.592 & 1487.456 & 1494.525 & 1487.523 & 1485.904 & 1478.545 \\
\hline
\end{tabular} $\begin{array}{lllllllllllllllllllll}1494.096 & 1494.692 & 1494.921 & 1495.696 & 1497.11 & 1496.935 & 1487.367 & 1489.838 & 1487.614 & 1490.302 & 1489.577 & 1484.159 & 1488.743 & 1487.62 & 1491.501 & 1487.329 & 1494.494 & 1487.38 & 1485.904 & 1478.545 \\ \end{array}$

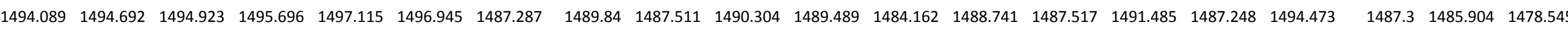
$\begin{array}{llllllllllllllllllllll}1494.082 & 1494.692 & 1494.921 & 1495.693 & 1497.119 & 1496.95 & 1488.764 & 1489.842 & 1488.385 & 1490.302 & 1489.425 & 1484.159 & 1488.733 & 1488.394 & 1491.863 & 1488.809 & 1494.457 & 1488.792 & 1485.904 & 1478.542\end{array}$

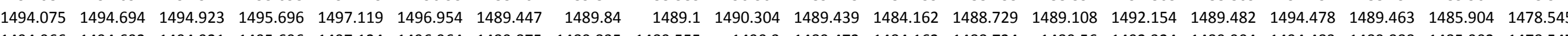
$\begin{array}{llllllllllllllllllllll}1494.066 & 1494.692 & 1494.921 & 1495.696 & 1497.124 & 1496.964 & 1489.87 & 1489.835 & 1489.555 & 1490.3 & 1489.472 & 1484.162 & 1488.724 & 1489.56 & 1492.324 & 1489.904 & 1494.483 & 1489.888 & 1485.902 & 1478.547 \\ 1494.061 & 1494.694 & 1494.921 & 1495.698 & 1497.131 & 1496.971 & 1490.189 & 1489845 & 1489888 & 1490302 & 1489.514 & 1484.164 & 1488717 & 1489.893 & 1492.453 & 1490215 & 1494.511 & 1490.198 & 1485.902 & 1478547\end{array}$

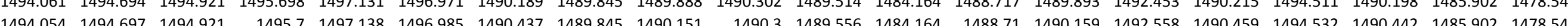

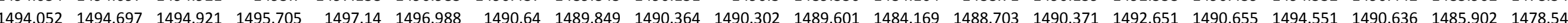
$\begin{array}{lllllllllllllllllllllll}1494.054 & 1494.697 & 1494.921 & 1495.707 & 1497.138 & 1496.99 & 1490.809 & 1489.856 & 1490.544 & 1490.302 & 1489.64 & 1484.169 & 1488.698 & 1490.549 & 1492.73 & 1490.824 & 1494.577 & 1490.805 & 1485.904 & 1478.549\end{array}$ $\begin{array}{lllllllllllllllllllll}1494.056 & 1494.699 & 1494.921 & 1495.71 & 1497.142 & 1496.99 & 1490.954 & 1489.852 & 1490.694 & 1490.304 & 1489.682 & 1484.169 & 1488.698 & 1490.705 & 1492.791 & 1490.966 & 1494.596 & 1490.951 & 1485.904 & 1478.552 \\ \end{array}$

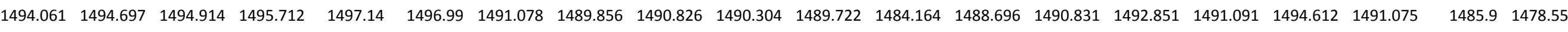

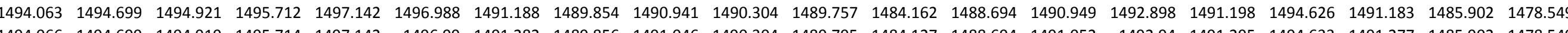
$\begin{array}{llllllllllllllllllll}1494.066 & 1494.699 & 1494.919 & 1495.714 & 1497.142 & 1496.99 & 1491.282 & 1489.856 & 1491.046 & 1490.304 & 1499.795 & 1484.127 & 1488.694 & 1491.052 & 1492.94 & 1491.295 & 1494.633 & 1491.277 & 1485.902 & 1478.549\end{array}$

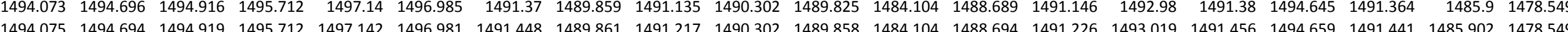

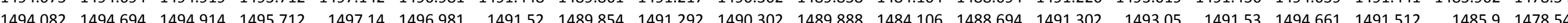

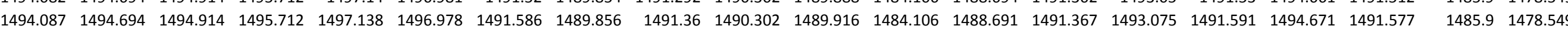
$\begin{array}{llllllllllllllllllll}1494.099 & 1494.692 & 1494.914 & 1495.71 & 1497.133 & 1496.971 & 1491.642 & 1489.854 & 1491.419 & 1490.297 & 1489.942 & 1484.104 & 1488.698 & 1491.431 & 1493.099 & 1491.65 & 1494.678 & 1491.634 & 1485.9 & 1478.549\end{array}$ $\begin{array}{llllllllllllllllllll}1494.106 & 1494.692 & 1494.912 & 1495.71 & 1497.131 & 1496.959 & 1491.551 & 1489.854 & 1491.325 & 1490.3 & 1489.958 & 1484.106 & 1488.701 & 1491.335 & 1493.092 & 1491.56 & 1494.687 & 1491.545 & 1485.9 & 1478.549 \\ \end{array}$

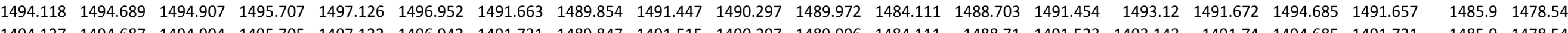

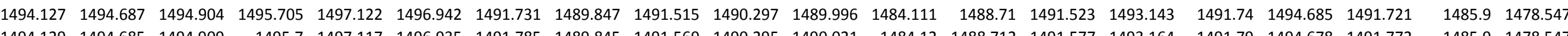

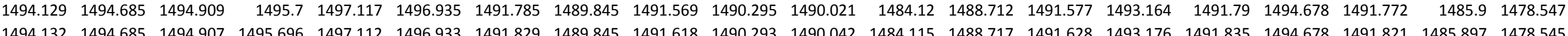


Well

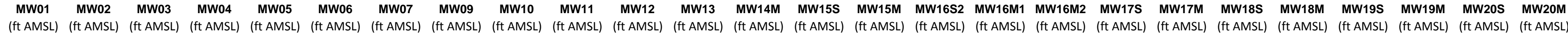

10/3/11 22:00 $10 / 3 / 1122: 30$ 10/3/112 23:00 10/4/1110:00 10/4/110:30 10/4/1110:30 10/4/11 1:30 10/4/112:00 10/4/11 2:30 10/4/111 3:00 $10 / 4 / 114: 00$ 10/4/4114:00 10/4/114:3:30
10/4/11 5:00 10/4/11 5:30 10/4/11 6:00 10/4/11 6:30 10/4/11 7:00 10/4/117:30 10/4/11 8:00 10/4/118:30 10/4/11 9:30 10/4/11 10:00 10/4/11 10:30 10/4/11111:00 10/4/11 11:30 10/4/11 12:00 10/4/11 13:00 10/4/11 13:30 10/4/11 14:00 10/4/11 14:30 10/4/11 15:00 10/4/11 15:30 $10 / 4 / 1116: 30$ 10/4/11 16:30 10/4/11 17:30 10/4/11 18:00 10/4/11 18:30 10/4/11 19:00 10/4/11 19:30 $10 / 4 / 1120: 00$
$10 / 4 / 1120: 30$ 10/4/11 20:30 10/4/11 21:00 10/4/11 21:30 10/4/11 22:30 10/4/11 23:00 10/4/11 23:30 10/5/11 0:00
10/5/11 0:30 $\begin{array}{lllll}1494.6 & 1495.119 & 1496.88 & 1494.661 \\ 1494.6 & 1495.115 & 1496.88 & 1494.664\end{array}$ $\begin{array}{rrrrr}1494.6 & 1495.115 & 1496.88 & 1494.664 \\ 1494.6 & 1495.1115 & 1496.875 & 1494.664\end{array}$ $\begin{array}{llll}1494.6 & 1495.115 & 1496.875 & 1494.664\end{array}$ $\begin{array}{lllll}1494.6 & 1495.112 & 1496.873 & 1494.675\end{array}$ $\begin{array}{lllll}1494.598 & 1495.108 & 1496.873 & 1494.678\end{array}$ \begin{tabular}{r|rrr}
1494.6 & 1495.108 & 1496.866 & 1494.68
\end{tabular} $\begin{array}{llllll}1494.6 & 1495.108 & 1496.868 & 1494.687\end{array}$ $\begin{array}{llll}1494.596 & 1495.103 & 1496.873 & 1494.692\end{array}$ $\begin{array}{lllll}1494.596 & 1495.101 & 1496.873 & 1494.692\end{array}$ $\begin{array}{llll}1494.598 & 1495.098 & 1496.868 & 1494.699\end{array}$ $\begin{array}{llll}1494.598 & 1495.101 & 1496.861 & 1494.706 \\ 1494.596 & 1495.098 & 1496.854 & 1494.708\end{array}$ $\begin{array}{llll}1494.596 & 1495.096 & 1496847 & 1494.713\end{array}$ $\begin{array}{lllll}1494.589 & 1495.094 & 1496.84 & 14494.71\end{array}$ $\begin{array}{llll}1494.589 & 1495.089 & 1496.836 & 1494.71\end{array}$ $\begin{array}{lllll}1494.586 & 1495.087 & 1496.829 & 1494.71\end{array}$ $\begin{array}{llll}1494.582 & 1495.08 & 1496.822 & 1494.708\end{array}$ $\begin{array}{lllll}1494.582 & 1495.077 & 1496.822 & 1494.708\end{array}$ $\begin{array}{lllll}1494.584 & 1495.073 & 1496.812 & 1494.706\end{array}$ $\begin{array}{lllll}1494.579 & 1495.063 & 1496.805 & 1494.701\end{array}$ $\begin{array}{lllll}1494.577 & 1495.059 & 1496808 & 1494.696\end{array}$ $\begin{array}{lllll}1494.577 & 1495.054 & 1496.81 & 1494.689\end{array}$ $\begin{array}{lllll}1494.577 & 1495.052 & 1496.815 & 1494.687\end{array}$ $\begin{array}{lllll}1494.579 & 1495.052 & 1496.824 & 1494.682\end{array}$ $\begin{array}{llll}1494.582 & 1495.049 & 1496.833 & 1494.678\end{array}$ $\begin{array}{rrrr}1494.584 & 1495.052 & 1496.843 & 1494.678\end{array}$ $\begin{array}{rrrr}1494.584 & 1495.052 & 1496.85 & 1494.678 \\ 1494.584 & 1495.056 & 1496.864 & 1494.685\end{array}$ $\begin{array}{lllll}1494.586 & 1495.061 & 1496.88 & 1494.692\end{array}$ $\begin{array}{llllll}1494.589 & 1495.068 & 1496.887 & 1494.699\end{array}$ $\begin{array}{lllll}1494.591 & 1495.077 & 1496.894 & 1494.71\end{array}$ $\begin{array}{lllll}1494.593 & 1495.089 & 1496.894 & 1494.727\end{array}$ $\begin{array}{lllll}1494.593 & 1495.091 & 1496.901 & 1494.738\end{array}$ $1494.591 \quad 1495.096 \quad 1496.9061494 .745$ $\begin{array}{lllll}1494.593 & 1455.098 & 1496.906 & 1494.755\end{array}$ $\begin{array}{llll}1494.593 & 1495.105 & 1496.906 & 1494.766 \\ 1494.593 & 1495108 & 1496.903 & 1494.776\end{array}$ $\begin{array}{lllll}1494.591 & 1495.108 & 1496.894 & 1494.78\end{array}$ $\begin{array}{lllll}1494.589 & 1495.108 & 1496.892 & 1494.785\end{array}$ $\begin{array}{lllll}1494.589 & 1495.108 & 1496.88 & 1494.79\end{array}$ $\begin{array}{lllll}1494.586 & 1495.105 & 1496.873 & 1494.79\end{array}$ $\begin{array}{lllll}1494.584 & 1495.101 & 1496.866 & 1494.792\end{array}$ $\begin{array}{llll}1494.579 & 1495.098 & 1496.861 & 1494.79\end{array}$ $\begin{array}{lllll}1494.579 & 1495.096 & 1496.857 & 1494.785\end{array}$ $\begin{array}{llll}1494.577 & 1495.087 & 1496.854 & 1494.778\end{array}$ $\begin{array}{llllll}1494.575 & 1495.087 & 1496.854 & 1494.776\end{array}$ $\begin{array}{lllll}1494.575 & 1495.084 & 1496.85 & 1494.776\end{array}$ $\begin{array}{lllll}1494.575 & 1495.082 & 1496.852 & 1494.773\end{array}$ $\begin{array}{lllll}1494.572 & 1495.077 & 1496.854 & 1494.773\end{array}$ $\begin{array}{llll}1494.575 & 1495.08 & 1496.857 & 1494.771 \\ 1494.575 & 1495.08 & 1496.857 & 1494.771\end{array}$

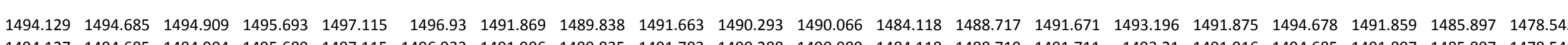

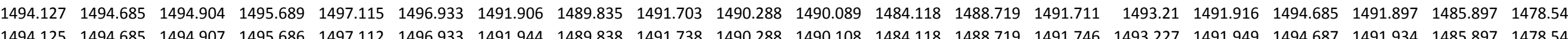
$\begin{array}{llllllllllllllllllllll}1494.125 & 1494.685 & 1494.907 & 1495.686 & 1497.112 & 1496.933 & 1491.944 & 1489.838 & 1491.738 & 1490.288 & 1490.108 & 1484.118 & 1488.719 & 1491.746 & 1493.227 & 1491.949 & 1494.687 & 1491.934 & 1485.897 & 1478.545 \\ 1494.127 & 1494.682 & 1494.904 & 1495.689 & 1497.11 & 1496.928 & 1491.977 & 1489.838 & 1491.773 & 1490 & 1490.129 & 1484.115 & 1488.715 & 1491.779 & 1493245 & 1491.982 & 1494697 & 1491.965 & 1485897 & 1478545 \\ \end{array}$

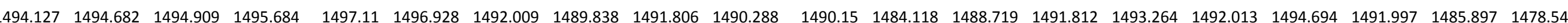
$\begin{array}{lllllllllllllllllllll}1494.122 & 1494.68 & 1494.904 & 1495.682 & 1497.11 & 1496.93 & 1492.037 & 1489.835 & 1491.836 & 1490.286 & 1490.169 & 1484.12 & 1488.717 & 1491.845 & 1493.28 & 1492.044 & 1494.701 & 1492.026 & 1485.897 & 1478.542\end{array}$ $\begin{array}{llllllllllllllllllllll}1494.129 & 1494.68 & 1494.904 & 1495.679 & 1497.108 & 1496.93 & 1492.065 & 1489.833 & 1491.867 & 1490.286 & 1490.187 & 1484.122 & 1488.719 & 1491.87 & 1493.292 & 1492.07 & 1494.711 & 1492.054 & 1485.895 & 1478.542\end{array}$ $\begin{array}{lllllllllllllllllllll}1494.127 & 1494.68 & 1494.902 & 1495.679 & 1497.105 & 1496.923 & 1492.089 & 1489.831 & 1491.89 & 1490.286 & 1490.204 & 1484.12 & 1488.719 & 1491.899 & 1493.306 & 1492.094 & 1494.708 & 1492.08 & 1485.895 & 1478.542 & \end{array}$

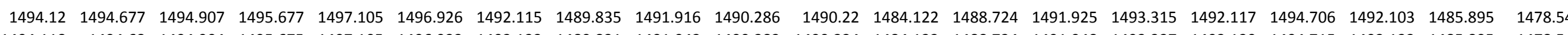

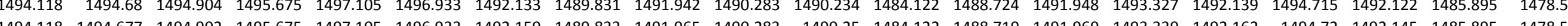
$\begin{array}{llllllllllllllllllll}1494.118 & 1494.677 & 1494.902 & 1495.675 & 1497.105 & 1496.933 & 1492.159 & 1489.833 & 1491.965 & 1490.283 & 1490.25 & 1484.122 & 1488.719 & 1491.969 & 1493.339 & 1492.162 & 1494.72 & 1492.145 & 1485.895 & 1478.54 \\ 1494.122 & 1494.675 & 1494.902 & 1495.675 & 1497.101 & 1496.928 & 1492.178 & 1489.831 & 1491.986 & 1490.283 & 1490.267 & 1484.122 & 1488.722 & 1491.995 & 1493.355 & 1492.184 & 1494.732 & 1492.166 & 1485895 & 147854\end{array}$

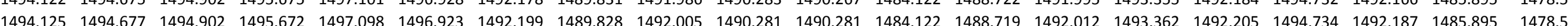
$\begin{array}{lllllllllllllllllllllll}1494.129 & 1494.673 & 1494.895 & 1495.67 & 1497.094 & 1496.916 & 1492218 & 1489.835 & 1492.024 & 1490.283 & 1490.292 & 1484.125 & 1488.726 & 1492.03 & 1493.369 & 1492.219 & 1494.732 & 1492.204 & 1485.895 & 1478.538\end{array}$ \begin{tabular}{lllllllllllllllllllllll}
1494.129 & 1494.67 & 1494.9 & 1495.668 & 1497.091 & 1496.911 & 1492.232 & 1489.831 & 1492.043 & 1490.283 & 1490.306 & 1484.125 & 1488.731 & 1492.047 & 1493.374 & 1492.236 & 1494.732 & 1492.22 & 1485.893 & 1478.535 \\
\hline
\end{tabular}

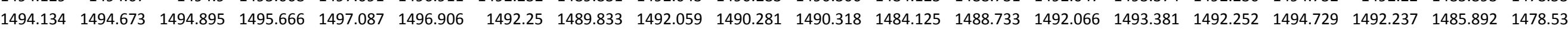

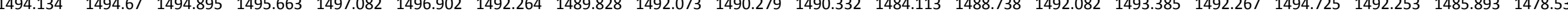

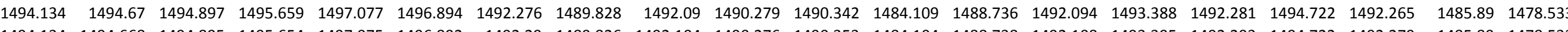

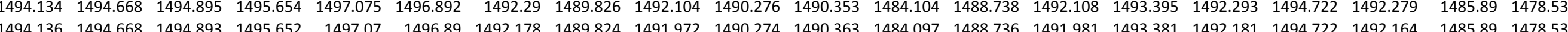
$\begin{array}{lllllllllllllllllllllll}1494.136 & 1494.668 & 1494.893 & 1495.652 & 1497.07 & 1496.89 & 1492.178 & 1489.824 & 1491.972 & 1490.274 & 1490.363 & 1484.097 & 1488.736 & 1491.981 & 1493.381 & 1492.181 & 1494.722 & 1492.164 & 1485.89 & 1478.531 \\ 1494.134 & 1494.663 & 149489 & 1495647 & 1497.063 & 1496883 & 1491.925 & 1489821 & 1491693 & 1490276 & 1490363 & 1484.097 & 1488738 & 1491704 & 149329 & 1491.928 & 1494.711 & 1491.918 & 148589 & 1478528\end{array}$ $\begin{array}{lllllllllllllllllllllll}1494.129 & 1494.663 & 1494.888 & 1495.645 & 1497.061 & 1496.88 & 1491.796 & 1489.821 & 1491.571 & 1490.272 & 1490.356 & 1484.099 & 1488.743 & 1491.572 & 1493.231 & 1491.8 & 1494.706 & 1491.796 & 1485.89 & 1478.528\end{array}$ $\begin{array}{lllllllllllllllllllll}1494.12 & 1494.663 & 1494.893 & 1495.64 & 1497.061 & 1496878 & 1491.726 & 1489.817 & 1491.482 & 1490272 & 1490.344 & 1484.099 & 1488741 & 1491.492 & 1493.194 & 1491.733 & 1494.692 & 1491.721 & 1485.89 & 1478.528\end{array}$ $\begin{array}{lllllllllllllllllllll}1494.115 & 1494.661 & 1494.893 & 1495.635 & 1497.059 & 1496.883 & 1491.668 & 1489.821 & 1491.424 & 1490.272 & 1490.335 & 1484.099 & 1488.738 & 1491.431 & 1493.173 & 1491.677 & 1494.682 & 1491.664 & 1485.89 & 1478.526\end{array}$

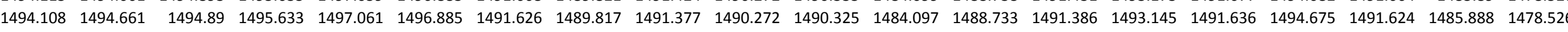
$\begin{array}{lllllllllllllllllllll}1494.096 & 1494.661 & 1494.895 & 1495.633 & 1497.061 & 1496.89 & 1491.628 & 1489.819 & 1491.384 & 1490.269 & 1490.316 & 1484.099 & 1488.729 & 1491.393 & 1493.141 & 1491.636 & 1494.68 & 1491.627 & 1485.888 & 1478.528 \\ \end{array}$

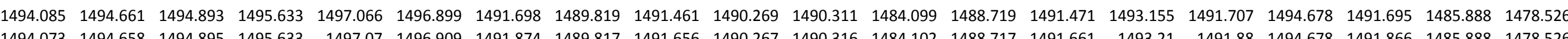

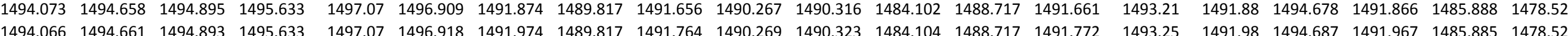

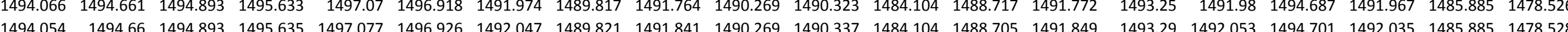
$\begin{array}{lllllllllllllllllllll}1494.042 & 1494.665 & 1494.895 & 1495.638 & 1497.084 & 1496.94 & 1492.103 & 1489.819 & 1491.897 & 1490.272 & 1490.351 & 1484.106 & 1488.703 & 1491.908 & 1493.318 & 1492.105 & 1494.713 & 1492.091 & 1485.885 & 1478.528\end{array}$ $\begin{array}{llllllllllllllllllllll}1494.031 & 1494.666 & 1494.895 & 1495.64 & 1497.091 & 1496.954 & 1492147 & 1489.819 & 1491.946 & 1490.274 & 1490.36 & 1484.106 & 1488.691 & 1491.955 & 1493.341 & 1492.15 & 1494.725 & 1492.138 & 1485.885 & 1478.531\end{array}$ $\begin{array}{llllllllllllllllllllll}1494.028 & 1494.668 & 1494.895 & 1495.645 & 1497.096 & 1496.966 & 1492.187 & 1489.824 & 1491.991 & 1490.274 & 1490.377 & 1484.109 & 1488.687 & 1491.997 & 1493.374 & 1492.193 & 1494.741 & 1492.178 & 1485.888 & 1478.533\end{array}$ \begin{tabular}{llllllllllllllllllllll}
1494.028 & 1494.668 & 1494.895 & 1495.652 & 1497.098 & 1496.969 & 1492.225 & 1489.828 & 1492.026 & 1490.276 & 1490.391 & 1484.111 & 1488.67 & 1492.037 & 1493.399 & 1492.229 & 1494.76 & 1492.213 & 1485.888 & 1478.535 \\
\hline
\end{tabular}

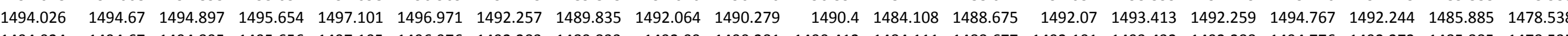

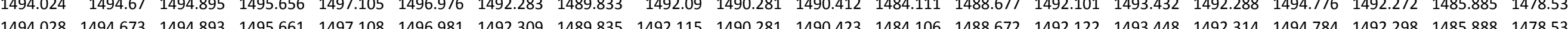

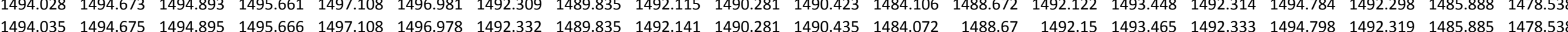

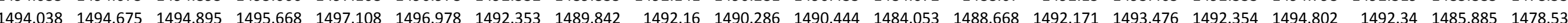
$\begin{array}{lllllllllllllllllllll}1494.045 & 1494.675 & 1494.893 & 1495.67 & 1497.108 & 1496.973 & 1492.372 & 1489.842 & 1492.179 & 1490.288 & 1490.454 & 1484.053 & 1488.67 & 1492.188 & 1493.481 & 1492.373 & 1494.805 & 1492.359 & 1485.885 & 1478.538\end{array}$

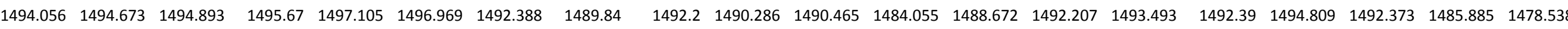

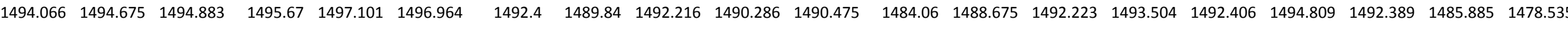

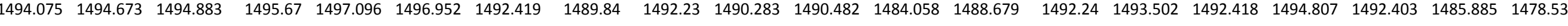

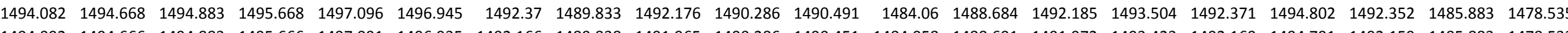

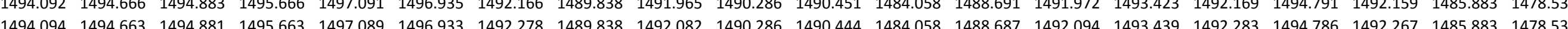

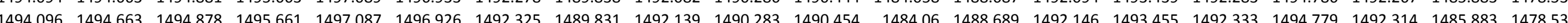
$\begin{array}{llllllllllllllllllll}1494.096 & 1494.661 & 1494.883 & 1495.659 & 1497.089 & 1496.928 & 1492.36 & 1489.826 & 1492.174 & 1490.281 & 1490.465 & 1484.06 & 1488.691 & 1492.178 & 1493.465 & 1492.361 & 1494.779 & 1492.347 & 1485.881 & 1478.531\end{array}$

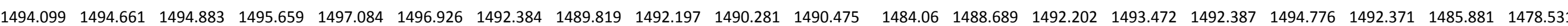

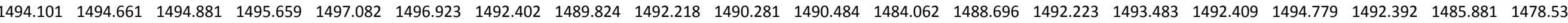
$\begin{array}{llllllllllllllllllll}1494.096 & 1494.658 & 1494.881 & 1495.656 & 1497.082 & 1496.921 & 1492.421 & 1489.826 & 1492.237 & 1490.281 & 1490.493 & 1484.065 & 1488.696 & 1492.242 & 1493.488 & 1492.423 & 1494.779 & 1492.408 & 1485.881 & 1478.531 \\ \end{array}$

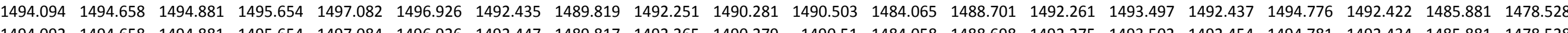

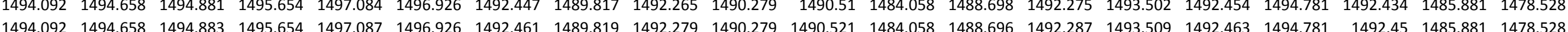


Well

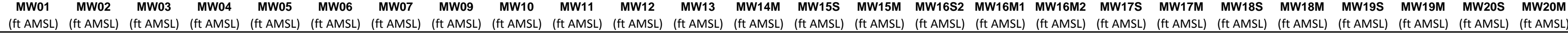

10/5/11 1:00 10/5/11 1:30 10/5/112:00 10/5/112:30 10/5/1113:00 10/5/113:30 10/5/11 4:30 10/5/11 5:00 10/5/11 5:30 10/5/11 6:00 $10 / 5 / 117: 00$ 10/5/117:00 10/5/117:30 10/5/11 8:30 10/5/11 9:00 10/5/11 9:30 0/5/11 10:00 (0/5/1111.00 10/5/11 11:00 10/5/11 12:00 10/5/11 12:30 10/5/11 13:00 10/5/11 13:30 10/5/11 14:00 10/5/11 14:30 10/5/11 15:00 10/5/11 16:00 10/5/11 16:30 10/5/11 17:00 10/5/11 17:30 10/5/11 18:00 10/5/11 18:30 10/5/11 19:00 10/5/11 19:30 10/5/11 20:30 10/5/11 21:00 10/5/11 21:30 10/5/11 22:00 10/5/11 22:30 10/5/11 23:00 10/6/11 0:00 10/6/11 0:30 10/6/11::30 10/6/11 2:00 10/6/11 2:30 10/6/11 3:00 $\begin{array}{llll}1494.575 & 1495.082 & 1496.859 & 1494.773 \\ 1494.575 & 1495.08 & 1496861 & 1494.778\end{array}$ $\begin{array}{llll}1494.575 & 1495.08 & 1496.861 & 1494.778 \\ 1494.575 & 149508 & 1496861 & 1494.78\end{array}$ $\begin{array}{llll}1494.575 & 1495.08 & 1496.861 & 1494.78\end{array}$ $\begin{array}{lllll}1494.572 & 1495.082 & 1496.864 & 1494.785\end{array}$ $\begin{array}{lllll}1494.572 & 1495.082 & 1496.861 & 1494.787\end{array}$ $\begin{array}{rrrrr}1494.572 & 1495.082 & 1496.861 & 1494.787 \\ 1494.575 & 1495.08 & 1496.857 & 1494.79\end{array}$ $\begin{array}{lllll}1494.572 & 1495.082 & 1496.859 & 1494.794\end{array}$ $\begin{array}{lllll}1494.572 & 1495.082 & 1496.859 & 1494.794\end{array}$ $\begin{array}{llll}1494.572 & 1495.08 & 1496.857 & 1494.794 \\ 1494.57 & 1495.08 & 146854 & 194.797\end{array}$ $\begin{array}{llll}1494.57 & 1495.08 & 1496.854 & 1494.797 \\ 1494.57 & 1495.08 & 1996854 & 1494.797\end{array}$ $\begin{array}{rrrrr}1494.57 & 1495.08 & 1496.854 & 1494.797 \\ 1494.568 & 1495.077 & 1496.852 & 1494.78\end{array}$ $\begin{array}{lllll}1494.568 & 1495.077 & 1496.85 & 1494.762\end{array}$ $\begin{array}{llll}1494.568 & 1495.077 & 1496.85 & 1494.738\end{array}$ $\begin{array}{lllll}1494.565 & 1495.073 & 1496.852 & 1494.71\end{array}$ $\begin{array}{lllll}1494.565 & 1495.07 & 1496.857 & 1494.685\end{array}$ $\begin{array}{lllll}1494.568 & 1495.073 & 1496.866 & 1494.657\end{array}$ $\begin{array}{llll}1494.568 & 1495.073 & 1496.864 & 1494.629\end{array}$ $\begin{array}{llll}1494.568 & 1495.075 & 1496.871 & 1494.601 \\ 1494.572 & 1495.075 & 1996882 & 1494.575\end{array}$ $\begin{array}{lllll}1494.568 & 1495.075 & 1496.889 & 1494.547\end{array}$ $\begin{array}{lllll}1494.572 & 1495.08 & 1496.906 & 1494.533\end{array}$ $\begin{array}{lllll}1494.572 & 1495.084 & 1496.925 & 1494.524\end{array}$ $1494.575 \quad 1495.0891496 .929 \quad 1494.524$ $\begin{array}{llll}1494.582 & 1495.098 & 1496.929 & 1494.528\end{array}$ $\begin{array}{llll}1494.582 & 1495.105 & 1496.927 & 1494.54\end{array}$ $\begin{array}{llll}1494.582 & 1495.112 & 1496.929 & 1494.552\end{array}$ $\begin{array}{lllll}1494.577 & 1495.112 & 1496.941 & 1494.561 \\ 1494.579 & 1495115 & 1496.946 & 1494568\end{array}$ $\begin{array}{lllll}1494.579 & 1495.117 & 1496.946 & 1494.58\end{array}$ $\begin{array}{llll}1494.584 & 1495.124 & 1496.934 & 1494.598\end{array}$ $\begin{array}{lllll}1494.579 & 1495.124 & 1496.932 & 1494.612\end{array}$ $\begin{array}{lllll}1494.579 & 1495.126 & 1496.934 & 1494.622\end{array}$ $\begin{array}{llll}1494.575 & 1495.126 & 1496.925 & 1494.629\end{array}$ $\begin{array}{lllll}1494.575 & 1495.124 & 1496.922 & 1494.638\end{array}$ $\begin{array}{llll}1494.572 & 1495.119 & 1496.913 & 1494.647 \\ 1494572 & 1495.119 & 1496911 & 1494.652\end{array}$ $\begin{array}{lllll}1494.572 & 1455.119 & 1496.911 & 1494.652 \\ 1494.57 & 1495.115 & 1496.901 & 1494.657\end{array}$ $\begin{array}{lllll}1494.565 & 1495.112 & 1496.892 & 1494.659\end{array}$ $\begin{array}{llllll}1494.568 & 1495.11 & 1496.894 & 1494.664\end{array}$ $\begin{array}{lllll}1494.563 & 1495.103 & 1496.896 & 1494.661\end{array}$ $\begin{array}{lllll}1494.561 & 1495.098 & 1496.889 & 1494.664\end{array}$ $1494.563 \quad 1495.098 \quad 1496.906 \quad 1494.666$ $1494.561 \quad 1495.098 \quad 1496.9011494 .673$ $\begin{array}{lllll}1494.561 & 1495.096 & 1496.903 & 1494.675\end{array}$ $\begin{array}{llll}1494.563 & 1455.098 & 1466.908 & 1494.692\end{array}$ $\begin{array}{lllll}1494.563 & 1495.098 & 1496.911 & 1494.696\end{array}$ $\begin{array}{lllll}1494.563 & 1495.098 & 1496.915 & 1494.703\end{array}$ $\begin{array}{lllll}1494.563 & 1495.098 & 1496.925 & 1494.713\end{array}$ $\begin{array}{lllll}1494.565 & 1495.103 & 1496.929 & 1494.717\end{array}$ $\begin{array}{rrrr}1494.563 & 1495.105 & 1496.939 & 1494.724 \\ 1494.565 & 1495.11 & 1496.929 & 1494.734\end{array}$ $\begin{array}{llllllllllllllllllll}1494.089 & 1494.656 & 1494.878 & 1495.652 & 1497.082 & 1496.928 & 1492.475 & 1489.817 & 1492.293 & 1490.281 & 1490.528 & 1484.06 & 1488.694 & 1492.298 & 1493.516 & 1492.477 & 1494.788 & 1492.462 & 1485.881 & 1478.528 \\ 1494087 & 1494656 & 1494883 & 1495654 & 1497.884 & 1496928 & 1492.84 & 1489817 & 1492303 & 1490281 & 1490535 & 1484.065 & 1488698 & 1492312 & 1493528 & 1492.487 & 1494.791 & 1492.471 & 1485881 & 1478528\end{array}$

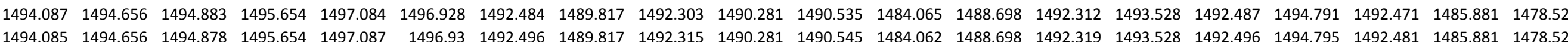

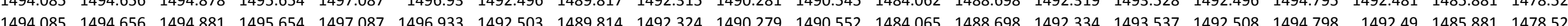

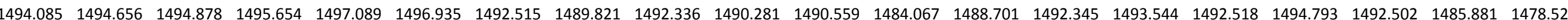
$\begin{array}{lllllllllllllllllllllll}1494.085 & 1494.656 & 1494.878 & 1495.654 & 1497.087 & 1496.935 & 1492.524 & 148981 & 1492.343 & 1490.279 & 1490.566 & 1484.069 & 1488.703 & 1492.348 & 1493.546 & 1492.527 & 1494.802 & 1492.511 & 1485.878 & 1478.528\end{array}$ \begin{tabular}{llllllllllllllllllllll}
1494.087 & 1494.656 & 1494.878 & 1495.654 & 1497.087 & 1496.933 & 1492.531 & 1489.821 & 1492.352 & 1490.281 & 1490.57 & 1484.069 & 1488.701 & 1492.362 & 1493.549 & 1492.532 & 1494.8 & 1492.516 & 1485.878 & 1478.528 \\
\hline
\end{tabular} $\begin{array}{lllllllllllllllllllll}1494.087 & 1494.656 & 1494.876 & 1495.654 & 1497.084 & 1496.93 & 1492.54 & 1489.817 & 1492.361 & 1490.281 & 1490.578 & 1484.072 & 1488.703 & 1492.369 & 1493.556 & 1492.539 & 1494.809 & 1492.525 & 1485.878 & 1478.528\end{array}$

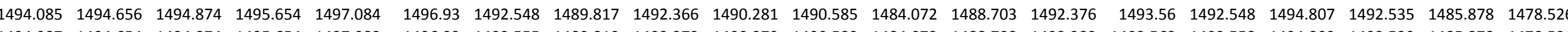

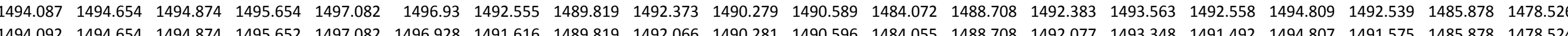

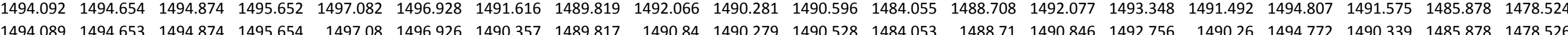

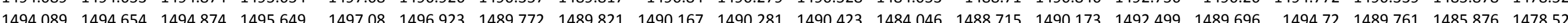
$\begin{array}{llllllllllllllllllll}1494.089 & 1494.654 & 1494876 & 1495649 & 1497.08 & 1496918 & 1499.456 & 1489812 & 148971 & 1490281 & 1490311 & 1484042 & 1488712 & 1489715 & 1492.341 & 1489.416 & 1494.68 & 1489.456 & 1485.878 & 1478.521\end{array}$ $\begin{array}{lllllllllllllllllllllll}1494.087 & 1494.654 & 1494.871 & 1495.649 & 1497.077 & 1496.918 & 1489.332 & 1489.817 & 1489.541 & 1490.279 & 1490.211 & 1484.041 & 1488.712 & 1489.546 & 1492.303 & 1489.295 & 1494.645 & 1489.332 & 1485.876 & 1478.521 \\ \end{array}$

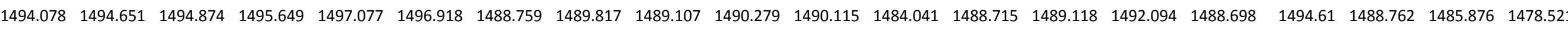
$\begin{array}{llllllllllllllllllllllll}1494.077 & 1494.651 & 1494.871 & 1495.647 & 1497.08 & 1496.921 & 1488.326 & 1489.814 & 1488.711 & 1490.279 & 1490.01 & 1484.044 & 1488.712 & 1488.718 & 1491.921 & 1488.257 & 1494.574 & 1488.33 & 1485.876 & 1478.519 & \end{array}$

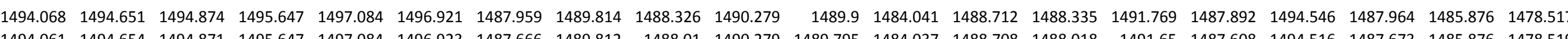

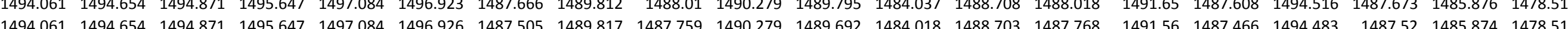

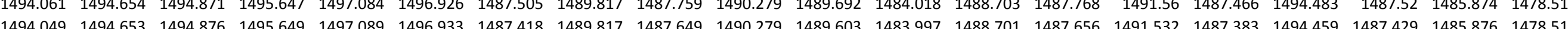
$\begin{array}{lllllllllllllllllllllll}1494.045 & 1494.651 & 1494.871 & 1495.649 & 1497.094 & 1496.942 & 1488.893 & 1489.819 & 1488.523 & 1490.279 & 1489.533 & 1483.986 & 1488.696 & 1488.528 & 1491.914 & 1488.937 & 1494.45 & 1488.917 & 1485.874 & 1478.517\end{array}$ $\begin{array}{llllllllllllllllllllll}1494.035 & 1494.656 & 1494874 & 1495.654 & 1497.101 & 1496.95 & 1489.564 & 1489819 & 1489.227 & 1490281 & 1489.538 & 1483.981 & 1488694 & 1489.231 & 1492.201 & 1489.601 & 1494.466 & 1489.583 & 1485.874 & 1478.519\end{array}$ $\begin{array}{llllllllllllllllllllllll}1494.021 & 1494.656 & 1494.878 & 1495.659 & 1497.112 & 1496.964 & 1489.985 & 1489.826 & 1489.668 & 1490.281 & 1489.57 & 1483.977 & 1488.682 & 1489.68 & 1492.376 & 1490.008 & 1494.483 & 1489.998 & 1485.874 & 1478.519 \\ \end{array}$

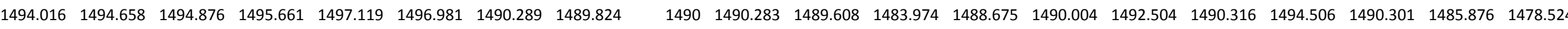

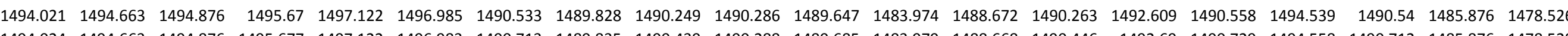

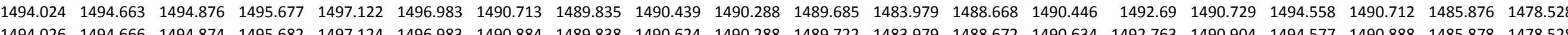

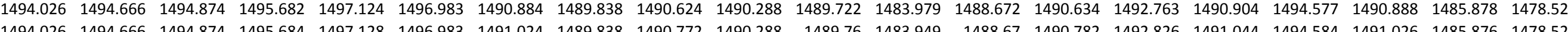

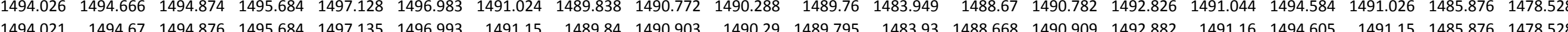

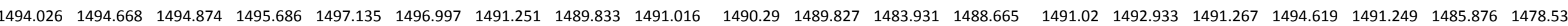
$\begin{array}{llllllllllllllllllllll}1494.038 & 1494.67 & 1494.881 & 1495.691 & 1497.135 & 1496.997 & 1491.347 & 1489.84 & 1491.114 & 1490.293 & 1489.86 & 1483.931 & 1488.661 & 1491.125 & 1492.977 & 1491.359 & 1494.64 & 1491.345 & 1485.876 & 1478.531 \\ \end{array}$ \begin{tabular}{llllllllllllllllllll}
1494.045 & 1494.668 & 1494.871 & 1495.696 & 1497.135 & 1496.988 & 1491.434 & 1489.842 & 1491.201 & 1490.293 & 1489.89 & 1483.935 & 1488.665 & 1491.21 & 1493.019 & 1491.444 & 1494.65 & 1491.43 & 1485.874 & 1478.533 \\
\hline
\end{tabular} $\begin{array}{llllllllllllllllllllll}1494.054 & 1494.67 & 4994.874 & 1495.698 & 1497.135 & 1496.985 & 1491.506 & 1489.847 & 1491.283 & 1490.295 & 1489.919 & 1483.937 & 1488.67 & 1491.29 & 1493.05 & 1491.518 & 1494.659 & 1491.502 & 1485.876 & 1478.533 & \end{array}$

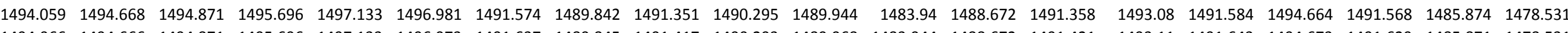

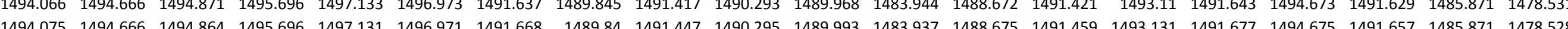

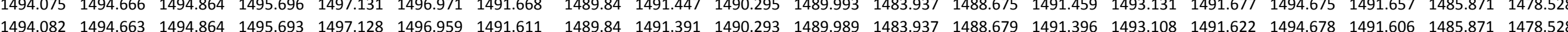
$\begin{array}{llllllllllllllllllllll}1494.087 & 1494.661 & 1494.866 & 1495.693 & 1497.126 & 1496.959 & 1491.703 & 1489.84 & 1491.485 & 1490.295 & 1490.01 & 1483.942 & 1488.682 & 1491.494 & 1493.138 & 1491.71 & 1494.675 & 1491.697 & 1485.871 & 1478.526\end{array}$ $\begin{array}{lllllllllllllllllllll}1494.101 & 1494.661 & 1494.862 & 1495.689 & 1497.119 & 1496.947 & 1491.761 & 1489.833 & 1491.55 & 1490.29 & 1490.031 & 1483.944 & 1488.687 & 1491.56 & 1493.162 & 1491.771 & 1494.675 & 1491.756 & 1485.871 & 1478.526\end{array}$

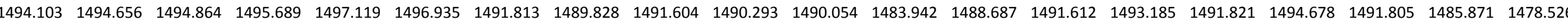

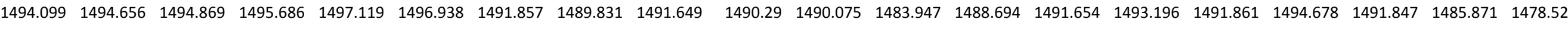

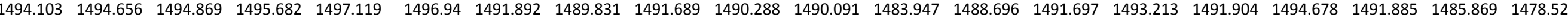

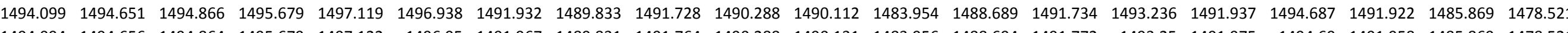

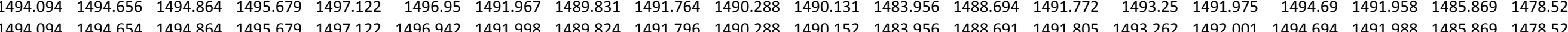

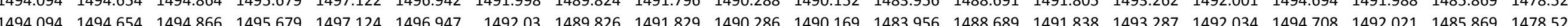
$\begin{array}{llllllllllllllllllll}1494.092 & 1494.654 & 1494.862 & 1495.679 & 1497.124 & 1496.952 & 1492.056 & 1489.826 & 1491.86 & 1490.288 & 1490.19 & 1483.956 & 1488.691 & 1491.866 & 1493.301 & 1492.06 & 1494.715 & 1492.044 & 1485.869 & 1478.521 \\ \end{array}$

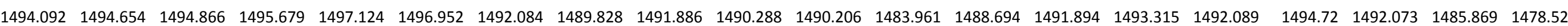

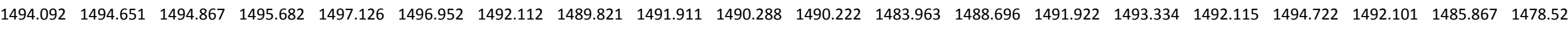

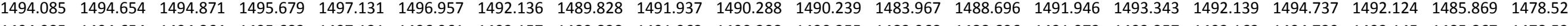

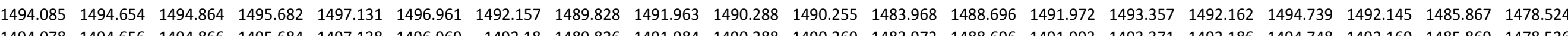

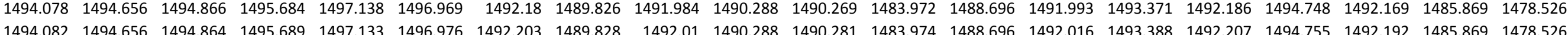


Well

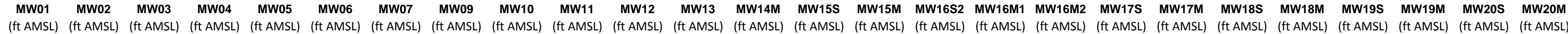

10/6/11 4:00 10/6/114:30 10/6/11 5:00 10/6/115:30 10/6/11 6:30 10/6/11 7:00 10/6/11 7:30 10/6/11 8:00 10/6/11 8:30 10/6/11 9:00 10/6/11 10:00 10/6/11 10:30 10/6/11 10:30 10/6/11 11:30 10/6/11 12:00 10/6/11 12:30 10/6/11 13:00 10/6/11 13:30 10/6/11 14:00 0/6/11 15:00 10/6/11 15:00 10/6/11 16:00 10/6/11 16:30 10/6/11 17:00 10/6/11 17:30 10/6/11 18:00 10/6/11 19:00 10/6/11 19:30 10/6/11 20:00 10/6/11 20:30 10/6/11 21:00 10/6/1121:30 $10 / 6 / 1122: 00$ 10/6/11 22:30 10/6/111 23:30 10/7/1110:00 10/7/11 0:30 10/7/11 1:00 10/7/11 1:30 10/7/112:00 10/7/11 2:30 10/7/11 3:00 10/7/11 4:00 10/7/11 4:30 10/7/11 5:00 10/7/11 5:30 10/7/11 6:00 $\begin{array}{llll}1494.565 & 1495.115 & 1496.932 & 1494.743 \\ 1494565 & 1495.119 & 1496929 & 149475\end{array}$ $\begin{array}{rrrr}1494.565 & 1495.119 & 1496.929 & 1494.75 \\ 1494.565 & 1495117 & 1496925 & 1494.755\end{array}$ $\begin{array}{llll}1494.565 & 1495.117 & 1496.925 & 1494.755 \\ 1494.565 & 1495.117 & 1496.922 & 1494.762\end{array}$ $\begin{array}{lllll}1494.563 & 1495.117 & 1496.925 & 1494.764\end{array}$ $\begin{array}{lllll}1494.563 & 1495.117 & 1496.92 & 1494.764 .766\end{array}$ $\begin{array}{lllll}1494.561 & 1495.112 & 1496.913 & 1494.769\end{array}$ $\begin{array}{llllll}1494.558 & 1495.112 & 1496.908 & 1494.773\end{array}$ $\begin{array}{lllll}1494.558 & 1495.11 & 1496.906 & 1494.771\end{array}$ $\begin{array}{llll}1494.558 & 1495.108 & 1496.901 & 1494.771\end{array}$ $\begin{array}{llll}1494.558 & 1495.103 & 1496.896 & 1494.771 \\ 1494.556 & 1495.101 & 1496.899 & 1494.769\end{array}$ $\begin{array}{llll}1494.556 & 1495.101 & 1496.899 & 1494.769 \\ 1494.554 & 1495.101 & 1496.911 & 1494.773\end{array}$ $\begin{array}{lllll}1494.551 & 1495.098 & 1496.915 & 1494.769\end{array}$ $\begin{array}{lllll}1494.554 & 1495.098 & 1496.913 & 1494.769\end{array}$ $1494.561 \quad 1495.103 \quad 1496.929 \quad 1494.778$ $\begin{array}{lllll}1494.558 & 1495.105 & 1496.946 & 1494.78\end{array}$ $\begin{array}{llll}1494.561 & 1495.105 & 1496.964 & 1494.783\end{array}$ 1494.5631495 .1121496 .9761494 .797 $\begin{array}{lllll}1494.572 & 1495.124 & 1496.976 & 1494.808 \\ 1494.575 & 1495.133 & 1496978 & 194.825\end{array}$ $\begin{array}{lllll}1494.575 & 1495.14 & 1496.983 & 1494.832\end{array}$ $\begin{array}{lllll}1494.572 & 1495.145 & 1496.988 & 1494.841\end{array}$ $\begin{array}{rrrrr}1494.577 & 1495.147 & 1496.99 & 1494.853\end{array}$ $\begin{array}{lllll}1494.575 & 1495.154 & 1496.981 & 1494.857\end{array}$ $1494.575 \quad 1495.156 \quad 1496.976 \quad 1494.864$ $\begin{array}{lllll}1494.575 & 1495.156 & 1496.974 & 1494.867\end{array}$ $\begin{array}{llll}1494.572 & 1495.159 & 1496.969 & 1494.871 \\ 149457 & 1955.156 & 1966.957 & 194.874\end{array}$ $\begin{array}{llll}1494.57 & 1495.156 & 1496.957 & 1494.874 \\ 1494.57 & 1495.152 & 1496946 & 1494.871\end{array}$ $\begin{array}{lllll}1494.568 & 1495.154 & 1496.936 & 1494.871\end{array}$ $\begin{array}{lllll}1494.568 & 1495.147 & 1496.922 & 1994.867\end{array}$ $\begin{array}{lllll}1494.563 & 1495.14 & 1496.915 & 1494.862\end{array}$ $\begin{array}{lllll}1494.561 & 1495.135 & 1496.913 & 1494.857\end{array}$ $\begin{array}{llll}1494.561 & 1495.133 & 1496.908 & 1494.85\end{array}$ $\begin{array}{lllll}1494.556 & 1495.126 & 1496.8999 & 1494.841\end{array}$ $\begin{array}{llllll}1494.561 & 1495.124 & 1496.896 & 1494.836 \\ 1494.556 & 1495.122 & 1496.903 & 194834\end{array}$ $\begin{array}{lllll}1494.556 & 1455.122 & 1496.903 & 1494.834 \\ 1494.556 & 1495.117 & 1496.913 & 1494.827\end{array}$ $\begin{array}{lllll}1494.554 & 1495.117 & 1496.911 & 1494.825\end{array}$ $\begin{array}{lllll}1494.556 & 1495.115 & 1496.918 & 1494.827\end{array}$ $\begin{array}{llllll}1494.556 & 1495.117 & 1496.913 & 1494.827\end{array}$ $\begin{array}{lllll}1494.556 & 1495.119 & 1496.915 & 1494.829\end{array}$ $\begin{array}{lllll}1494.558 & 1495.117 & 1496.913 & 1494.832\end{array}$ $\begin{array}{llll}1494.556 & 1495.115 & 1496.903 & 1494.832 \\ 1494.556 & 1495.115 & 1996896 & 1494834\end{array}$ $\begin{array}{lllll}1494.556 & 1495.115 & 1496.896 & 1494.834 \\ 1494.556 & 1495.119 & 1496892 & 1494.834\end{array}$ $\begin{array}{llll}1494.551 & 1495.112 & 1496.889 & 1494.829\end{array}$ $\begin{array}{lllll}1494.549 & 1495.108 & 1496.882 & 1494.829\end{array}$ $\begin{array}{lllll}1494.551 & 1495.103 & 1496.878 & 1494.827\end{array}$ $\begin{array}{lllll}1494.547 & 1495.101 & 1496.873 & 1494.822\end{array}$ $\begin{array}{lllll}1494.547 & 1495.101 & 1496.868 & 1494.815\end{array}$ $\begin{array}{llll}1494.544 & 1495.096 & 1496.866 & 1494.813 \\ 1494.544 & 1495.094 & 1496.854 & 1494.806\end{array}$

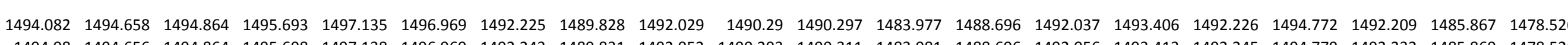
$\begin{array}{rrrrrrrrrrrrrrrrrrrrr}1494.08 & 1494.656 & 1494.864 & 1495.698 & 1497.138 & 1496.969 & 1492.243 & 1489.831 & 1492.052 & 1490.293 & 1490.311 & 1483.981 & 1488.696 & 1492.056 & 1493.413 & 1492.245 & 1494.779 & 1492.232 & 1485.869 & 1478.526 \\ 1494.089 & 1494.656 & 1494.864 & 1495.696 & 1497.135 & 1496.969 & 1492.26 & 1489.826 & 1492.068 & 1490.29 & 1490.323 & 1483.981 & 1488.701 & 1492.07 & 1493.425 & 1492.264 & 1494.774 & 1492.249 & 1485.867 & 1478.526\end{array}$

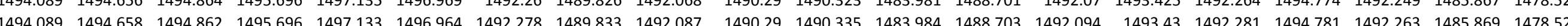

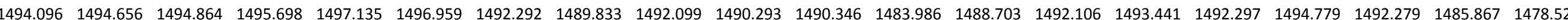
$\begin{array}{llllllllllllllllllllll}1494.099 & 1494.656 & 1494.862 & 1495.696 & 1497.133 & 1496.959 & 1492.309 & 1489.828 & 1492.118 & 1490.29 & 1490.358 & 1483.979 & 1488.705 & 1492.124 & 1493.448 & 1492.309 & 1494.781 & 1492.293 & 1485.867 & 1478.524\end{array}$

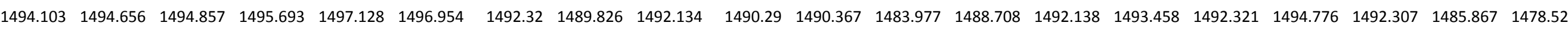
$\begin{array}{lllllllllllllllllllllll}1494.103 & 1494.654 & 1494.859 & 1495.696 & 1497.126 & 1496.95 & 1492.33 & 1489.828 & 1492.143 & 1490.29 & 1490.377 & 1483.974 & 1488.71 & 1492.153 & 1493.46 & 1492.338 & 1494.781 & 1492.319 & 1485.864 & 1478.521\end{array}$

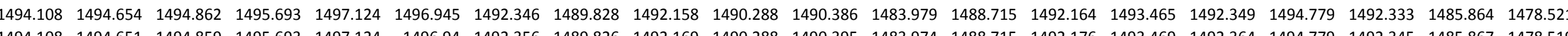

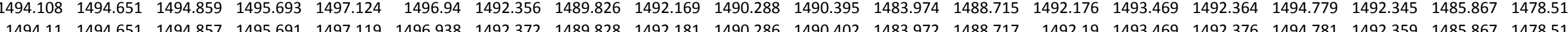

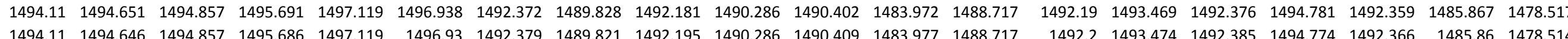

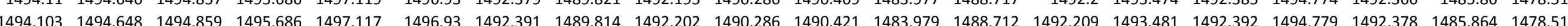

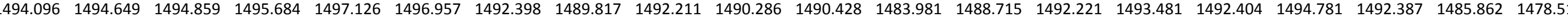
$\begin{array}{llllllllllllllllllllll}1494.089 & 1494.651 & 1494.859 & 1495.684 & 1497.122 & 1496.947 & 1492.409 & 1489.817 & 1492.223 & 1490.286 & 1490.435 & 1483.981 & 1488.715 & 1492.228 & 1493.49 & 1492.411 & 1494.781 & 1492.399 & 1485.862 & 1478.514\end{array}$

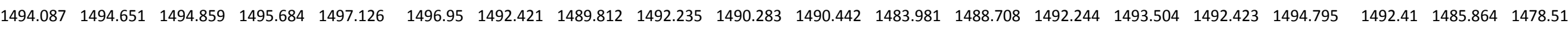
$\begin{array}{lllllllllllllllllllllll}1494.075 & 1494.654 & 1494.864 & 1495.689 & 1497.135 & 1496.961 & 1492.433 & 1489.814 & 1492.247 & 1490.286 & 1490.449 & 1483.981 & 1488.708 & 1492.251 & 1493.507 & 1492.437 & 1494.802 & 1492.427 & 1485.862 & 1478.512 & \end{array}$

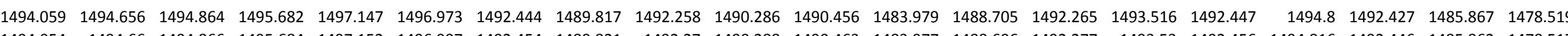

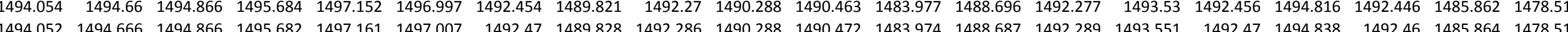
$\begin{array}{llllllllllllllllllllll}1494.052 & 1494.666 & 1494.866 & 1495.682 & 1497.161 & 1497.007 & 1492.47 & 1489.828 & 1492.286 & 1490.288 & 1490.472 & 1483.974 & 1488.687 & 1492.289 & 1493.551 & 1492.47 & 1494.838 & 1492.46 & 1485.864 & 1478.519 \\ 1494.054 & 1494.666 & 1494.866 & 1495.684 & 1497.156 & 1497 & 1492.484 & 1489828 & 1492298 & 149029 & 1490.479 & 1483972 & 1488682 & 1492305 & 1493572 & 1492.492 & 1494849 & 1492.476 & 1485867 & 1478524\end{array}$ $\begin{array}{llllllllllllllllllllll}1494.054 & 1494.668 & 1494.869 & 1495.686 & 1497.163 & 1497.007 & 1492.498 & 1489.828 & 1492.312 & 1490.293 & 1490.489 & 1483.972 & 1488.684 & 1492.312 & 1493.579 & 1492.501 & 1494.863 & 1492.486 & 1485.867 & 1478.526\end{array}$ $\begin{array}{lllllllllllllllllllll}1494.049 & 1494.67 & 1494.869 & 1495.686 & 1497.175 & 1497.012 & 1492.508 & 1489.833 & 1492.324 & 1490.295 & 1490.498 & 1483.968 & 1488.684 & 1492.327 & 1493.581 & 1492.508 & 1494.861 & 1492.495 & 1485.864 & 1478.526\end{array}$ $\begin{array}{llllllllllllllllllllll}1494.052 & 1494.675 & 1494.869 & 1495.689 & 1497.17 & 1497.017 & 1492.519 & 1489.838 & 1492.331 & 1490.297 & 1490.505 & 1483.965 & 1488.689 & 1492.343 & 1493.595 & 1492.522 & 1494.875 & 1492.509 & 1485.862 & 1478.526\end{array}$

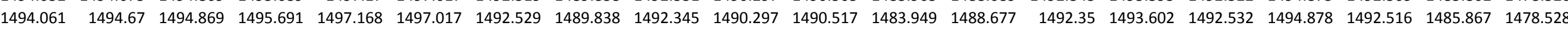

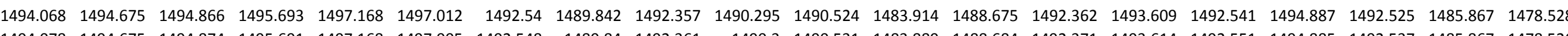

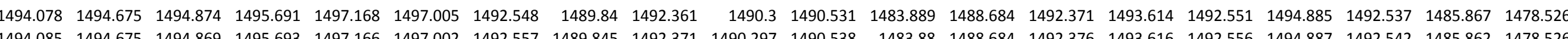

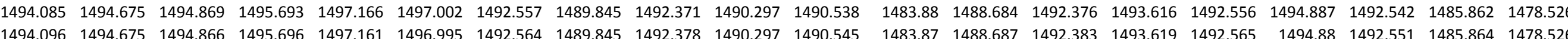

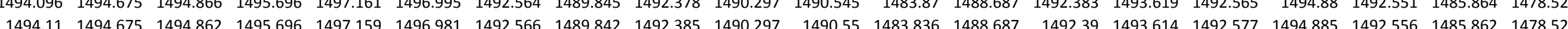
$\begin{array}{lllllllllllllllllllll}1494.122 & 1494.673 & 1494.859 & 1495.696 & 1497.149 & 1496.969 & 1492.573 & 1489.842 & 1492.392 & 1490.297 & 1490.554 & 1483.817 & 1488.689 & 1492.399 & 1493.614 & 1492.575 & 1494.878 & 1492.561 & 1485.86 & 1478.524\end{array}$ $\begin{array}{llllllllllllllllllllll}1494.129 & 1494.668 & 1494.855 & 1495.693 & 1497.145 & 1496.959 & 1492.578 & 1489.838 & 1492.397 & 1490.295 & 1490.559 & 1483.817 & 1488.698 & 1492.404 & 1493.614 & 1492.579 & 1494.863 & 1492.563 & 1485.862 & 1478.521\end{array}$

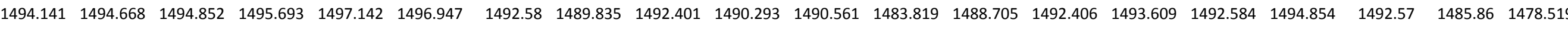

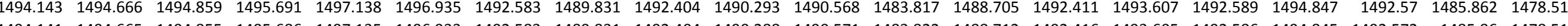
$\begin{array}{llllllllllllllllllll}1494.141 & 1494.665 & 1494.855 & 1495.686 & 1497.135 & 1496.933 & 1492.583 & 1489.831 & 1492.404 & 1490.288 & 1490.571 & 1483.822 & 1488.712 & 1492.416 & 1493.605 & 1492.586 & 1494.845 & 1492.572 & 1485.86 & 1478.517\end{array}$

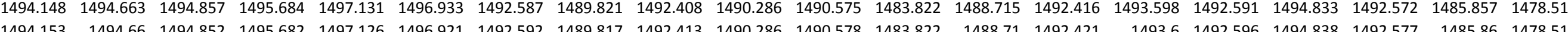

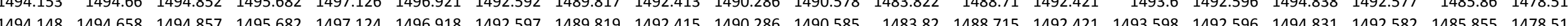

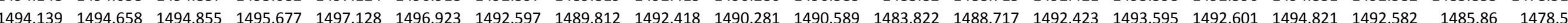
$\begin{array}{llllllllllllllllllllll}1494.134 & 1494.656 & 1494.857 & 1495.675 & 1497.128 & 1496.933 & 1492.601 & 1489.817 & 1492.422 & 1490.279 & 1490.592 & 1483.82 & 1488.715 & 1492.428 & 1493.595 & 1492.603 & 1494.821 & 1492.584 & 1485.857 & 1478.51 \\ & & \end{array}$

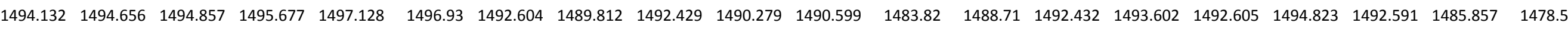

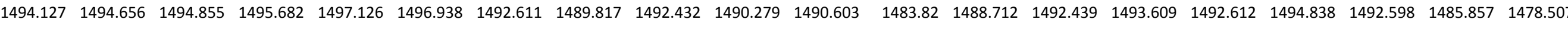
\begin{tabular}{llllllllllllllllllll}
1494.127 & 1494.656 & 1494.852 & 1495.679 & 1497.124 & 1496.933 & 1492.613 & 1489.814 & 1492.437 & 1490.281 & 1490.608 & 1483.817 & 1488.112 & 1492.444 & 1493.612 & 1492.62 & 1494.833 & 1492.605 & 1485.855 & 1478.51 \\
\hline
\end{tabular}

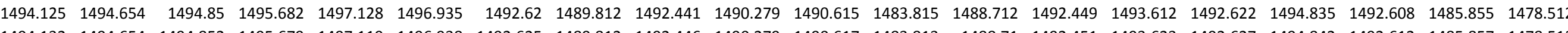

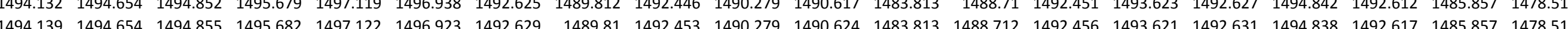

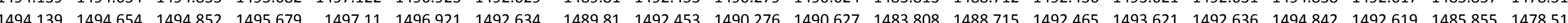

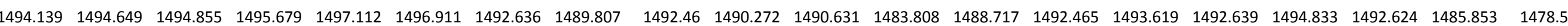

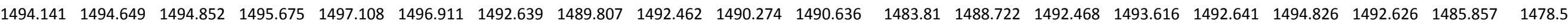

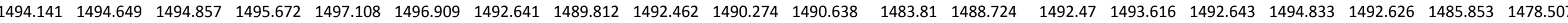
$\begin{array}{llllllllllllllllllllll}1494.141 & 1494.649 & 1494.852 & 1495.67 & 1497.101 & 1496.904 & 1492.643 & 1489.807 & 1492.465 & 1490.274 & 1490.643 & 1483.803 & 1488.729 & 1492.472 & 1493.612 & 1492.646 & 1494.821 & 1492.631 & 1485.853 & 1478.507 \\ \end{array}$ \begin{tabular}{lllllllllllllllllllll}
1494.139 & 1494.646 & 1494.847 & 1495.672 & 1497.096 & 1496.902 & 1492.641 & 1489.807 & 1492.469 & 1490.272 & 1490.643 & 1483.801 & 1488.724 & 1492.472 & 1493.609 & 1492.646 & 1494.819 & 1492.626 & 1485.855 & 1478.507 \\
\hline
\end{tabular}

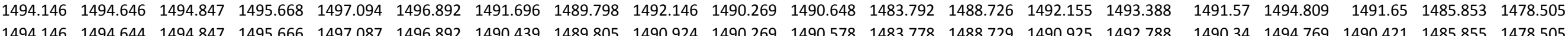


Well

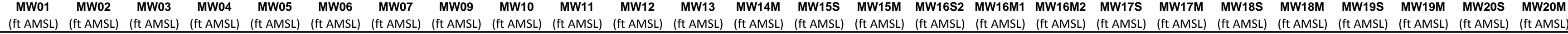

10/7/117:00 10/7/11 7:30 $10 / 7 / 118: 30$ 10/7/11/11:30:00 10/7/11 9:30 10/7/11 10:00 10/7/11 10:30 10/7/11 11:00 10/7/11 11:30 10/7/11 12:00 10/7/11 12:30
$10 / 7 / 1113: 00$ 10/7/111 13:30 10/7/7/11 14:00 10/7/11 14:30 10/7/11 15:00 0/7/11116:00 0/7/11 16:30 10/7/11117:00 10/7/11 18:00 10/7/11 18:00 10/7/11 19:00 10/7/11 19:30 10/7/11 20:00 10/7/1120:30 0/7/11 21:00 10/7/11 22:00 10/7/11 22:30 10/7/11 23:00 10/7/11 23:30 10/8/11 0:00 10/8/11 0:30 10/8/111:00 10/8/111:30 10/8/11 2:30 10/8/11 3:00 10/8/11 3:30 10/8/11 4:00 10/8/11 4:30 10/8/11 5:00 10/8/116.00 10/8/11 6:00 10/8/116:30 10/8/11 7:00 10/8/11 8:00 10/8/11 8:30 10/8/11 9:00 $\begin{array}{rrrr}1494.542 & 1495.087 & 1496.84 & 1494.792\end{array}$ $\begin{array}{llll}1494.542 & 1495.082 & 1496.829 & 1494.769 \\ 1494.54 & 1495.08 & 1496824 & 1494.756\end{array}$ $\begin{array}{rrrr}1494.54 & 1495.08 & 1496.824 & 1494.736 \\ 1494.535 & 1495.07 & 1496819 & 1494.703\end{array}$ $\begin{array}{lllll}1494.533 & 1495.063 & 1496.817 & 1494.666\end{array}$ $\begin{array}{llllll}1494.533 & 1495.059 & 149681 & 1494.631\end{array}$ $\begin{array}{ccccc}1494.533 & 1495.054 & 1496.808 & 1494.594\end{array}$ $\begin{array}{llllll}1494.533 & 1495.049 & 1496.81 & 1494.559\end{array}$ $\begin{array}{lllll}1494.533 & 1495.042 & 1496.81 & 1494.526\end{array}$ $\begin{array}{llll}1494.533 & 1495.038 & 1496.819 & 1494.496\end{array}$ $\begin{array}{llll}1494.533 & 1495.033 & 1496.824 & 1494.47 \\ 1494535 & 1495033 & 1966831 & 1494.456\end{array}$ $\begin{array}{llll}1494.535 & 1495.033 & 1496.831 & 1494.456 \\ 1494.537 & 1495.033 & 1496.843 & 1494.446\end{array}$ $\begin{array}{lllll}4494.537 & 1495.033 & 1496.843 & 1494.446 \\ 1494.537 & 1495.031 & 1496.843 & 1494.442\end{array}$ $\begin{array}{lllll}1494.537 & 1495.031 & 1496.843 & 1494.442 \\ 1494.54 & 1495.031 & 1496.854 & 1494.451\end{array}$ $\begin{array}{lllll}1494.54 & 1495.035 & 1496.854 & 1494.456\end{array}$ $\begin{array}{llll}1494.537 & 1495.035 & 1496.857 & 1494.465\end{array}$ $\begin{array}{lllll}1494.542 & 1495.038 & 1496.857 & 1494.477\end{array}$ $\begin{array}{llll}1494.537 & 1495.038 & 1496.857 & 1494.491\end{array}$ $\begin{array}{llrr}1494.537 & 1455.035 & 1496.857 & 1494.5\end{array}$ $\begin{array}{llll}1494.535 & 1495.033 & 1496.854 & 1494.521\end{array}$ $\begin{array}{lllll}1494.533 & 1495.033 & 149685 & 1494.531\end{array}$ $\begin{array}{lllll}1494.533 & 1495.033 & 1496.84 & 1494.542\end{array}$ $1494.533 \quad 1495.033 \quad 1496.838 \quad 1494.552$ $\begin{array}{lllll}1494.528 & 1495.028 & 1496.84 & 1494.559\end{array}$ $\begin{array}{llll}1494.526 & 1495.026 & 1496.838 & 1494.563\end{array}$ $\begin{array}{rrrr}1494.526 & 1495.024 & 1496.838 & 1494.568\end{array}$ $\begin{array}{rrrr}1494.526 & 1495.021 & 1496.84 & 1494.577 \\ 1494.526 & 1495.021 & 1496.838 & 1494.582\end{array}$ $\begin{array}{llll}1494.528 & 1495.021 & 1496.84 & 1494.588\end{array}$ $\begin{array}{llll}1494.528 & 1495.024 & 1496.84 & 1494.594\end{array}$ $\begin{array}{llll}1494.528 & 1495.021 & 1496.847 & 1494.605\end{array}$ $\begin{array}{lllll}1494.526 & 1495.019 & 1496.852 & 1494.61\end{array}$ $\begin{array}{lllll}1494.526 & 1495.019 & 1496.843 & 1494.615\end{array}$ $\begin{array}{llll}1494.53 & 1495.026 & 1496.838 & 1494.624 \\ 1499.526 & 1450.024 & 146982 & 194.629\end{array}$ $\begin{array}{lllll}1494.526 & 1495.024 & 1496.829 & 1494.629\end{array}$ $\begin{array}{lllll}1494.523 & 1455.024 & 1496.829 & 1494.633 \\ 1494.523 & 1495.021 & 1496831 & 1494.638\end{array}$ $\begin{array}{lllll}1494.519 & 1495.017 & 1496.829 & 1494.638\end{array}$ $\begin{array}{lllll}1494.519 & 1495.017 & 1496.833 & 1494.64\end{array}$ $\begin{array}{lllll}1494.521 & 1495.014 & 1496.836 & 1494.645\end{array}$ $\begin{array}{lllll}1494.519 & 1495.012 & 1496.824 & 1494.65\end{array}$ $\begin{array}{llll}1494.521 & 1495.014 & 1496.808 & 1494.654\end{array}$ $\begin{array}{llll}1494.521 & 1495.014 & 1496.796 & 1494.659 \\ 1494.516 & 1495014 & 1496.798 & 1494.657\end{array}$ $\begin{array}{lllll}1494.516 & 1455.014 & 1496.798 & 1494.657\end{array}$ $\begin{array}{rrrrr}1494.51 & 1495.003 & 1496.801 & 1494.65 \\ 1494.509 & 1495 & 1496.803 & 1494.647\end{array}$ $\begin{array}{lllll}1494.512 & 1494.998 & 1496.787 & 1494.65\end{array}$ $\begin{array}{lllll}1494.512 & 1494.998 & 1496.759 & 1494.652\end{array}$ $\begin{array}{lllll}1494.509 & 1494.991 & 1496.747 & 1494.65\end{array}$ $\begin{array}{lllll}1494.505 & 1494.986 & 1496.754 & 1494.643\end{array}$ $\begin{array}{rrrrr}1494.496 & 1494.975 & 1496.719 & 1494.631 \\ 1494.5 & 1494.968 & 1496.714 & 1494.626\end{array}$

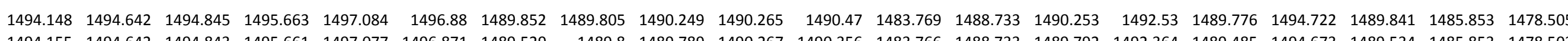

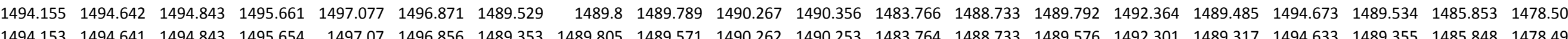

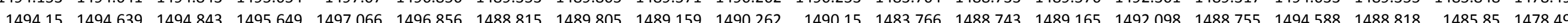
\begin{tabular}{llllllllllllllllllllll}
1494.148 & 1494.637 & 1494.84 & 1495.642 & 1497.061 & 1496.847 & 1488.38 & 1489.8 & 1488.765 & 1490.258 & 1490.04 & 1483.776 & 1488.736 & 1488.772 & 1491.921 & 1488.31 & 1494.541 & 1488.386 & 148585 & 1478.493 \\
\hline
\end{tabular} $\begin{array}{llllllllllllllllllll}1494.146 & 1494.634 & 1494838 & 1495.635 & 1497.054 & 1496.844 & 1488.006 & 1489.793 & 1488.378 & 1490.255 & 1489.928 & 1483.771 & 1488.736 & 1488.382 & 1491.762 & 1487.94 & 1494.504 & 1488.015 & 1485.853 & 1478.491\end{array}$ $\begin{array}{lllllllllllllllllllllll}1494.143 & 1494.632 & 1494.838 & 1495.633 & 1497.054 & 1496.842 & 1487.708 & 1489.793 & 1488.054 & 1490.253 & 1489.818 & 1483.771 & 1488.733 & 1488.062 & 1491.632 & 1487.648 & 1494.466 & 1487.72 & 1485.848 & 1478.491 \\ \end{array}$ $\begin{array}{lllllllllllllllllllllll}1494.141 & 1494.634 & 1494.84 & 1495.628 & 1497.054 & 1496.839 & 1487.545 & 1489.791 & 1487.801 & 1490.253 & 1489.711 & 1483.773 & 1488.729 & 1487.808 & 1491.532 & 1487.504 & 1494.429 & 1487.56 & 1485.85 & 1478.489\end{array}$

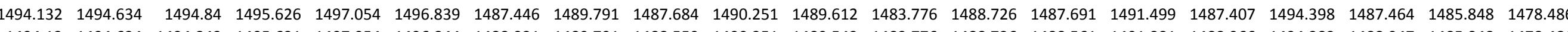

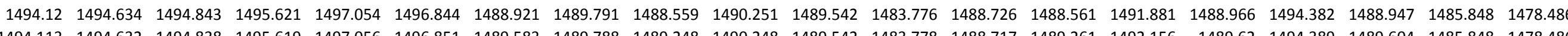

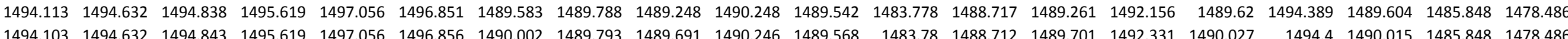

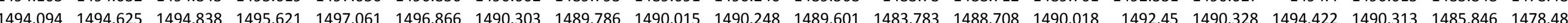
$\begin{array}{llllllllllllllllllllll}1494.087 & 1494.629 & 1494.838 & 1495.617 & 1497.063 & 1496878 & 1490.54 & 1489791 & 1490.265 & 1490.246 & 1489.638 & 1483.787 & 1488.703 & 1490.272 & 1492.546 & 1490.558 & 1494.433 & 1490.543 & 1485.848 & 1478.489\end{array}$ $\begin{array}{llllllllllllllllllllll}1494.082 & 1494.63 & 1494.843 & 1495.621 & 1497.061 & 1496.878 & 1490.729 & 1489.788 & 1490.465 & 1490.246 & 1489.673 & 1483.789 & 1488.696 & 1490.474 & 1492.635 & 1490.748 & 1494.457 & 1490.735 & 1485.846 & 1478.486\end{array}$

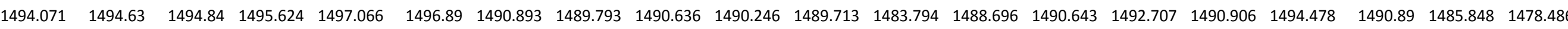
$\begin{array}{llllllllllllllllllllllll}1494.071 & 1494.63 & 1494.838 & 1495.624 & 1497.068 & 1496.892 & 1491.024 & 1489.791 & 1490.781 & 1490.246 & 1489.746 & 1483.796 & 1488.694 & 1490.784 & 1492.767 & 1491.039 & 1494.49 & 1491.026 & 1485.846 & 1478.489 & \end{array}$ $\begin{array}{llllllllllllllllllll}1494.071 & 1494.63 & 1494.84 & 1495.626 & 1497.066 & 1496.894 & 1491.141 & 1489.795 & 1490.905 & 1490.248 & 1489.781 & 1483.803 & 1488.682 & 1490.914 & 1492.826 & 1491.158 & 1494.52 & 1491.146 & 1485.846 & 1478.489\end{array}$

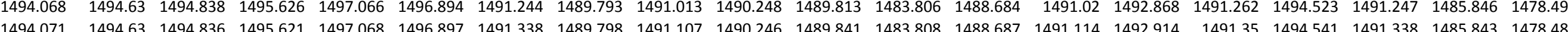
$\begin{array}{lllllllllllllllllllllll}1494.071 & 1494.63 & 1494.836 & 1495.621 & 1497.068 & 1496.897 & 1491.338 & 1489.798 & 1491.107 & 1490.246 & 1489.841 & 1483.808 & 1488.687 & 1491.114 & 1492.914 & 1491.35 & 1494.541 & 1491.338 & 1485.843 & 1478.489 \\ 1494.073 & 1494.627 & 1494.833 & 1495626 & 1497.066 & 1496897 & 1491.417 & 1489.798 & 1491.192 & 1490248 & 1489.869 & 1483.813 & 1488684 & 1491.198 & 1492.947 & 1491.43 & 1494546 & 1491.411 & 1485.843 & 1478.489\end{array}$

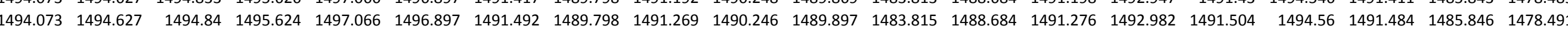

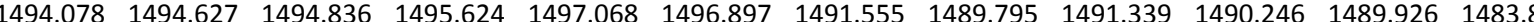
$\begin{array}{llllllllllllllllllllllll}1494.08 & 1494.627 & 1494.833 & 1495.624 & 1497.059 & 1496.89 & 1491.544 & 1489.795 & 1491.323 & 1490.244 & 1489.947 & 1483.817 & 1488.679 & 1491.327 & 1493.031 & 1491.553 & 1494.579 & 1491.542 & 1485.846 & 1478.489\end{array}$ $\begin{array}{llllllllllllllllllllllll}1494.085 & 1494.625 & 1494.833 & 1495.624 & 1497.061 & 1496.883 & 1491.644 & 1489.791 & 1491.426 & 1490.244 & 1489.968 & 1483.817 & 1488.679 & 1491.433 & 1493.059 & 1491.655 & 1494.589 & 1491.638 & 1485.846 & 1478.491\end{array}$ $\begin{array}{lllllllllllllllllllll}1494.082 & 1494.622 & 1494.833 & 1495.621 & 1497.059 & 1496.883 & 1491.7 & 1489.798 & 1491.489 & 1490.244 & 1489.991 & 1483.815 & 1488.684 & 1491.494 & 1493.085 & 1491.71 & 1494.591 & 1491.692 & 1485.843 & 1478.486\end{array}$

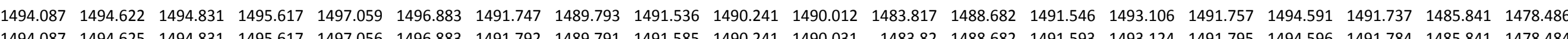
$\begin{array}{lllllllllllllllllllll}1494.087 & 1494.625 & 1494.831 & 1495.617 & 1497.056 & 1496.883 & 1491.792 & 1489.791 & 1491.585 & 1490.241 & 1490.031 & 1483.82 & 1488.682 & 1491.593 & 1493.124 & 1491.795 & 1494.596 & 1491.784 & 1485.841 & 1478.484 \\ 1494.082 & 1494.62 & 1494.833 & 1495.614 & 1497.059 & 1496883 & 1491.831 & 1489.788 & 1491.625 & 1490239 & 1490.049 & 1483817 & 1488682 & 1491633 & 1493143 & 1491838 & 1494.6 & 1491821 & 1485841 & 1478.882\end{array}$

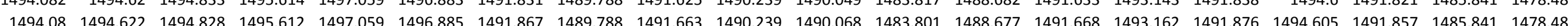
$\begin{array}{llllllllllllllllllllll}1494.082 & 1494.625 & 1494.831 & 1495.612 & 1497.059 & 1496.885 & 1491.899 & 1489.791 & 1491.698 & 1490.239 & 1490.087 & 1483.792 & 1488.677 & 1491.706 & 1493.18 & 1491.909 & 1494.612 & 1491.89 & 1485.841 & 1478.479\end{array}$ $\begin{array}{llllllllllllllllllllll}1494.082 & 1494.622 & 1494.831 & 1495.614 & 1497.056 & 1496.887 & 1491.934 & 1489.788 & 1491.733 & 1490.239 & 1490.105 & 1483.776 & 1488.677 & 1491.744 & 1493.201 & 1491.94 & 1494.624 & 1491.925 & 1485.843 & 1478.479 \\ \end{array}$ $\begin{array}{llllllllllllllllllllllll}1494.078 & 1494.62 & 1494.831 & 1495.612 & 1497.059 & 1496.887 & 1491.963 & 1489.791 & 1491.764 & 1490.237 & 1490.119 & 1483.759 & 1488.677 & 1491.774 & 1493.215 & 1491.968 & 1494.626 & 1491.955 & 1485.843 & 1478.477\end{array}$ $\begin{array}{llllllllllllllllllllll}1494.073 & 1494.62 & 1494.831 & 1495.61 & 1497.061 & 1496.894 & 1491.991 & 1489.791 & 1491.794 & 1490.237 & 1490.136 & 1483.757 & 1488.67 & 1491.802 & 1493.229 & 1491.999 & 1494.633 & 1491.981 & 1485.841 & 1478.479 & \\ \end{array}$

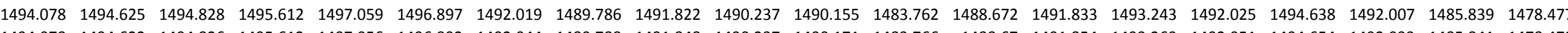

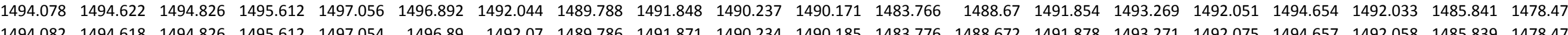

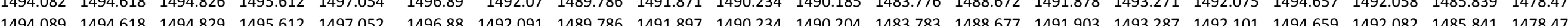

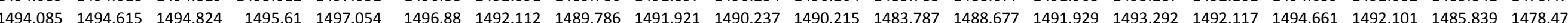
$\begin{array}{lllllllllllllllllllllll}1494.085 & 1494.615 & 1494.824 & 1495.607 & 1497.052 & 1496.885 & 1492.133 & 1489.781 & 1491.942 & 1490.232 & 1490.229 & 1483.789 & 1488.679 & 1491.948 & 1493.299 & 1492.136 & 1494.652 & 1492.119 & 1485.839 & 1478.477 \\ \end{array}$ \begin{tabular}{llllllllllllllllllllll}
1494.085 & 1494.617 & 1494.826 & 1495.603 & 1497.049 & 1496.883 & 1492.152 & 1489.784 & 1491.958 & 1490.232 & 1490.243 & 1483.794 & 1488.677 & 1491.969 & 1493.313 & 1492.155 & 1494.664 & 1492.141 & 1485.841 & 1478.477 \\
\hline
\end{tabular} $\begin{array}{llllllllllllllllllll}1494.08 & 1494.618 & 1494.824 & 1495.605 & 1497.052 & 1496.885 & 1492.171 & 1489.786 & 1491.982 & 1490.232 & 1490.257 & 1483.801 & 1488.677 & 1491.983 & 1493.318 & 1492.176 & 1494.668 & 1492.159 & 1485.839 & 1478.475 \\ \end{array}$

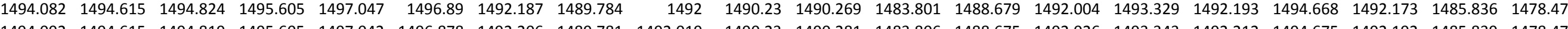

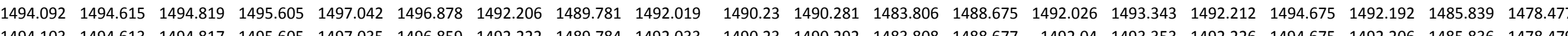

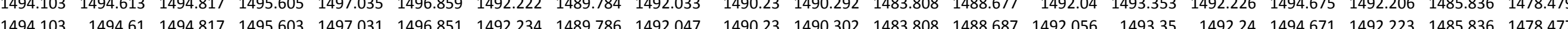

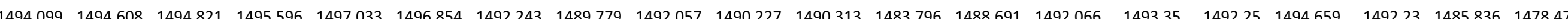

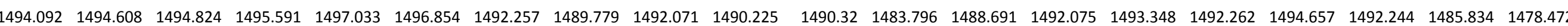

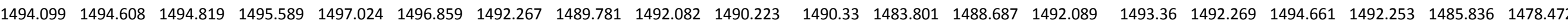

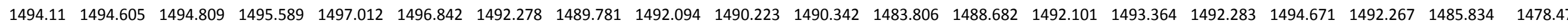

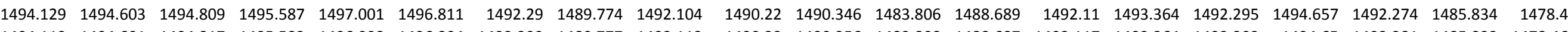
$\begin{array}{llllllllllllllllllllll}1494.113 & 1494.601 & 1494.817 & 1495.582 & 1496.998 & 1496.804 & 1492.299 & 1489.777 & 1492.113 & 1490.22 & 1490.356 & 1483.808 & 1488.687 & 1492.117 & 1493.364 & 1492.302 & 1494.65 & 1492.281 & 1485.832 & 1478.468\end{array}$

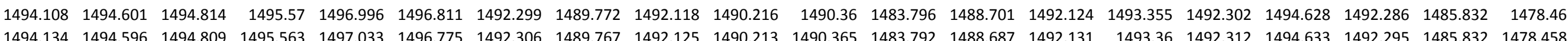


Well

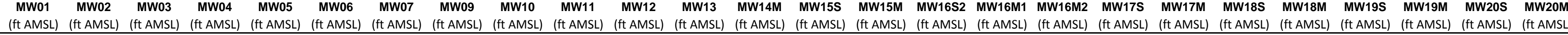

10/8/11 10:00 10/8/11 10:30 $10 / 8 / 1111: 30$ 10/8/11112:00 10/8/11 12:00 10/8/11 13:00 10/8/11 13:30 10/8/11 14:00 10/8/11 14:30 10/8/11 15:00 10/8/11 15:30 10/8/11 16:30 10/8/11 16:30 10/8/11 17:30 10/8/11 18:00 0/8/11 19:00 10/8/11 19:30 10/8/11 20:30 0/8/11 21:00 $10 / 8 / 1121: 00$
$10 / 8 / 1121: 30$ 10/8/11 22:00 10/8/11 22:30 10/8/11 23:00 10/8/1123:30 10/9/11 0:00 10/9/11 0:30 10/9/11 1:00 10/9/111:30 10/9/11 2:30 10/9/11 3:00 10/9/113:30 10/9/11 4:00 10/9/11 4:30 10/9/115:00 10/9/11 6:00 10/9/11 6:30 10/9/11 7:00 10/9/11 7:30 10/9/11 8:00 10/9/11 8:30 10/9/11 9:00 10/9/11 10:00 10/9/11 10:30 10/9/11 11:00 10/9/11 11:30 10/9/11 12:00 $\begin{array}{rrrr}1494.5 & 1494.965 & 1496.719 & 1494.619\end{array}$ $\begin{array}{llll}1494.484 & 1494.947 & 1496.724 & 1494.603 \\ 1494.491 & 1494942 & 1496719 & 1494601\end{array}$ $\begin{array}{lllll}1494.491 & 1494.942 & 1496.719 & 1494.601\end{array}$ $\begin{array}{rrrrr}1494.493 & 1494.94 & 1496.731 & 1494.594 \\ 1494.493 & 1494.937 & 1496.747 & 1494.594\end{array}$ $\begin{array}{lllll}1494.498 & 1494.937 & 1496.759 & 1494.594\end{array}$ $\begin{array}{lllll}1494.498 & 1494.94 & 1496.768 & 1494.601\end{array}$ $\begin{array}{llll}1494.505 & 1494.942 & 1496.766 & 1494.608\end{array}$ $\begin{array}{lllll}1494.505 & 1494.947 & 1496.768 & 1494.619\end{array}$ $\begin{array}{rrrr}1494.505 & 1494.949 & 1496.791 & 1494.626\end{array}$ $\begin{array}{rrrrr}1494.5 & 1494.947 & 1496.787 & 1494.629 \\ 1494.505 & 1494.949 & 1496789 & 149464\end{array}$ $\begin{array}{rrrr}1494.505 & 1494.949 & 1496.789 & 1494.64 \\ 1494.507 & 1494.958 & 1496.808 & 1494.652\end{array}$ $\begin{array}{rrrr}1494.498 & 1494.954 & 1496.784 & 1494.65 \\ 1494.503 & 1494.956 & 1496.777 & 1494.664\end{array}$ $\begin{array}{llllll}1494.505 & 1494.963 & 1496.787 & 1494.673\end{array}$ $\begin{array}{lllll}1494.496 & 1494.956 & 1496.754 & 1494.668\end{array}$ $1494.496 \quad 1494.951 \quad 1496.749 \quad 1494.673$ $\begin{array}{lllll}1494.493 & 1494.954 & 1496.749 & 1494.673\end{array}$ $\begin{array}{lllll}1494.489 & 1494.944 & 1496.749 & 1494.668\end{array}$ $\begin{array}{lllll}1494.486 & 1494.94 & 1496.745 & 1494.664\end{array}$ $\begin{array}{llllll}1494.489 & 1494.94 & 1496.731 & 1494.664\end{array}$ $1494.4891494 .937 \quad 1496.731 \quad 1494.664$ $\begin{array}{lllll}1494.482 & 1494.933 & 1496.74 & 1494.659\end{array}$ $\begin{array}{lllll}1494.482 & 1494.926 & 1496.738 & 1494.657\end{array}$ $\begin{array}{llll}1494.484 & 1494.928 & 1496.752 & 1494.657\end{array}$ $1494.486 \quad 1494.9281496 .763 \quad 1494.659$ $\begin{array}{rrrrr}1494.486 & 1494.928 & 1496.749 & 1494.664 \\ 1494.486 & 1494.93 & 149674 & 1494.671\end{array}$ $\begin{array}{llll}1494.489 & 1494.933 & 1496.747 & 1494.675\end{array}$ $\begin{array}{lllll}1494.482 & 1494.928 & 1496.754 & 1494.675\end{array}$ $\begin{array}{lllll}1494.484 & 1494.93 & 1496.763 & 1494.675\end{array}$ $\begin{array}{lllll}1494.484 & 1494.93 & 1496.768 & 1494.678\end{array}$ $1494.486 \quad 1494.933 \quad 1496.768 \quad 1494.685$ $\begin{array}{lllll}1494.489 & 1494.937 & 1496.773 & 1494.692\end{array}$ $\begin{array}{rrrrr}1494.486 & 1494.942 & 1496.766 & 1494.699\end{array}$ $\begin{array}{rrrr}1494.486 & 1494.94 & 1496.77 & 1494.703 \\ 1494.486 & 1494.947 & 1496.768 & 1494.708\end{array}$ $\begin{array}{lllll}1494.482 & 1494.942 & 1496.773 & 1494.708\end{array}$ $\begin{array}{lllll}1494.484 & 1494.942 & 1496.773 & 1494.713\end{array}$ $\begin{array}{llllll}1494.482 & 1494.944 & 1496.777 & 1494.71\end{array}$ $\begin{array}{lllll}1494.484 & 1494.944 & 1496.77 & 1494.701\end{array}$ $\begin{array}{lllll}1494.486 & 1494.951 & 1496.78 & 1494.687\end{array}$ $1494.4841494 .951 \quad 1496.7771494 .668$ $\begin{array}{lllll}1494.482 & 1494.949 & 1496.766 & 1494.64\end{array}$ $\begin{array}{llllll}1494.479 & 1494.949 & 1496.766 & 1494.589\end{array}$ $\begin{array}{lllll}1494.477 & 1494.944 & 1496.768 & 14494.556\end{array}$ $\begin{array}{lllll}1494.475 & 1494.942 & 1496.768 & 1494.521\end{array}$ $1494.477 \quad 1494.942 \quad 1496.759 \quad 1494.493$ $\begin{array}{lllll}1494.479 & 1494.942 & 1496.766 & 1494.47\end{array}$ $\begin{array}{llll}1494.472 & 1494.937 & 1496.759 & 1494.442 \\ 1494.475 & 1494.935 & 1496.775 & 1494.428\end{array}$

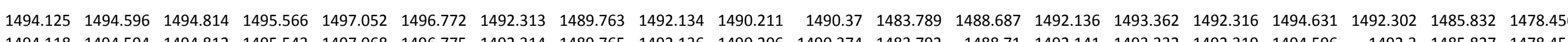
$\begin{array}{rlllllllllllllllllllll}1494.118 & 1494.594 & 1494.812 & 1495.543 & 1497.068 & 1496.775 & 1492.314 & 1489.765 & 1492.136 & 1490.206 & 1490.374 & 1483.792 & 1488.71 & 1492.141 & 1493.332 & 1492.319 & 1494.596 & 1492.3 & 1485.827 & 1478.451 \\ 1494.108 & 1494591 & 1494812 & 1495542 & 1497.059 & 1496782 & 1492323 & 1489.763 & 1492146 & 1490204 & 1490384 & 1483796 & 1488.696 & 1492.153 & 1493.346 & 1492326 & 14946 & 1492.307 & 1485.829 & 147851 \\ \end{array}$

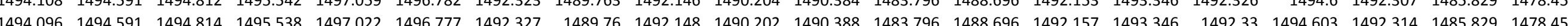

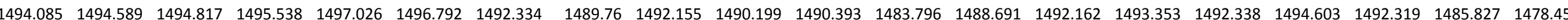
$\begin{array}{llllllllllllllllllllllll}1494.071 & 1494.591 & 1494817 & 495536 & 1497.026 & 1496808 & 1492344 & 1489.758 & 1492.165 & 1490.199 & 1490.4 & 1483.801 & 1488.689 & 1492.174 & 1493.36 & 1492.347 & 1494.61 & 1492.328 & 1485.829 & 1478.451\end{array}$

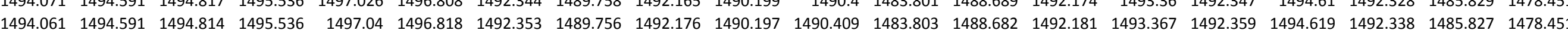
$\begin{array}{lllllllllllllllllllllll}1494.056 & 1494.591 & 1494.812 & 1495.538 & 1497.047 & 1496.83 & 1492.363 & 1489.76 & 1492.181 & 1490.199 & 1490.416 & 1483.808 & 1488.677 & 1492.193 & 1493.381 & 1492.368 & 1494.626 & 1492.352 & 1485.827 & 1478.451\end{array}$

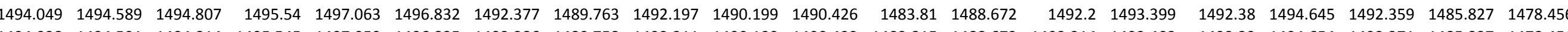

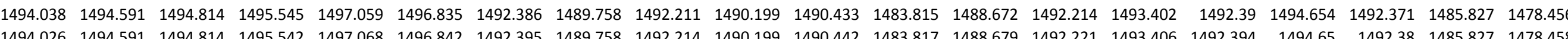
$\begin{array}{llllllllllllllllllll}1494.026 & 1494.591 & 1494.814 & 1495.542 & 1497.068 & 1496.842 & 1492.395 & 1489.758 & 1492.214 & 1490.199 & 1490.442 & 1483.817 & 1488.679 & 1492.221 & 1493.406 & 1492.394 & 1494.65 & 1492.38 & 1485.827 & 1478.458 \\ 1494.038 & 1494.591 & 1494.814 & 1495.545 & 1497.07 & 1496.854 & 1492.405 & 1489.76 & 1492.23 & 1490.199 & 1490.454 & 1483.822 & 1488.661 & 1492.235 & 1493.425 & 1492.409 & 1494.673 & 1492.392 & 1485829 & 1478.46\end{array}$ $\begin{array}{lllllllllllllllllllllll}1494.021 & 1494.594 & 1494.814 & 1495.552 & 1497.091 & 1496.861 & 1492.419 & 1489.767 & 1492.24 & 1490.202 & 1490.461 & 14883829 & 1488.656 & 1492.249 & 1493.437 & 1492.423 & 1494.682 & 1492.403 & 1485.829 & 1478.463\end{array}$ $\begin{array}{llllllllllllllllllllllll}1494.028 & 1494.591 & 1494.812 & 1495.549 & 1497.103 & 1496.883 & 1492.423 & 1489.765 & 1492.247 & 1490.202 & 1490.47 & 1483.787 & 1488.675 & 1492.254 & 1493.432 & 1492.43 & 1494.675 & 1492.41 & 1485.827 & 1478.463\end{array}$ $\begin{array}{llllllllllllllllllllll}1494.033 & 1494.589 & 1494.809 & 1495.549 & 1497.119 & 1496.873 & 1492.435 & 1489.765 & 1492.258 & 1490.202 & 1490.477 & 1483.776 & 1488.663 & 1492.261 & 1493.446 & 1492.437 & 1494.687 & 1492.42 & 1485.827 & 1478.468\end{array}$ $\begin{array}{lllllllllllllllllllll}1494.033 & 1494.589 & 1494.812 & 1495.556 & 1497.122 & 1496.875 & 1492.444 & 1489.767 & 1492.27 & 1490.204 & 1490.486 & 1483.78 & 1488.663 & 1492.275 & 1493.458 & 1492.451 & 1494.699 & 1492.434 & 1485.827 & 1478.47\end{array}$ $\begin{array}{lllllllllllllllllllllll}1494.049 & 1494.587 & 1494.805 & 1495.549 & 1497.147 & 1496.875 & 1492.451 & 1489.763 & 1492.275 & 1490.199 & 1490.489 & 1483.778 & 1488.67 & 1492.28 & 1493.462 & 1492.456 & 1494.68 & 1492.436 & 1485.825 & 1478.468\end{array}$

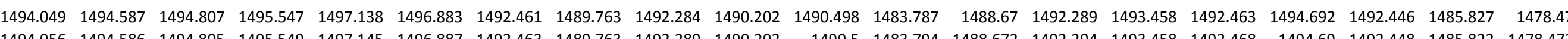

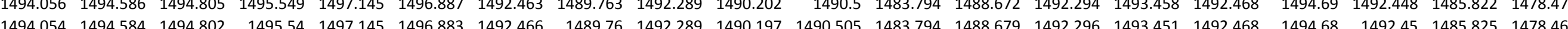
$\begin{array}{llllllllllllllllllll}1494.054 & 1494.584 & 1494.802 & 1495.54 & 1497.145 & 1496.883 & 1492.466 & 1489.76 & 1492.289 & 1490.197 & 1490.505 & 1483.794 & 1488.679 & 1492.296 & 1493.451 & 1492.468 & 1494.68 & 1492.45 & 1485.825 & 1478.468 \\ 1494.052 & 1494.584 & 1494.805 & 1495.536 & 1497.135 & 149689 & 1492.47 & 1489.758 & 1492.291 & 1490197 & 1490.51 & 1483796 & 1488687 & 1492298 & 1493.448 & 1492.47 & 1494.675 & 1492.45 & 1485825 & 1478.468\end{array}$ $\begin{array}{lllllllllllllllllllll}1494.042 & 1494.582 & 1494.807 & 1495.536 & 1497.14 & 1496.904 & 1492.473 & 1489.751 & 1492.296 & 1490.195 & 1490.514 & 1483.799 & 1488.677 & 1492.301 & 1493.451 & 1492.475 & 1494.671 & 1492.455 & 1485.825 & 1478.465\end{array}$

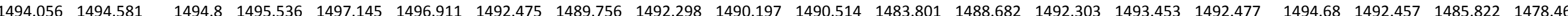
$\begin{array}{llllllllllllllllllllll}1494.054 & 1494.582 & 1494.802 & 1495.533 & 1497.14 & 1496.904 & 1492.475 & 1489.751 & 1492.303 & 1490.195 & 1490.519 & 1483.803 & 1488.672 & 1492.305 & 1493.458 & 1492.477 & 1494.675 & 1492.464 & 1485.822 & 1478.463\end{array}$

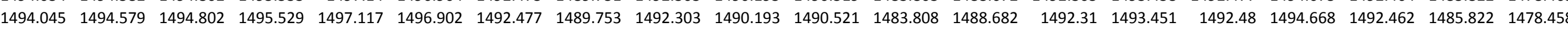

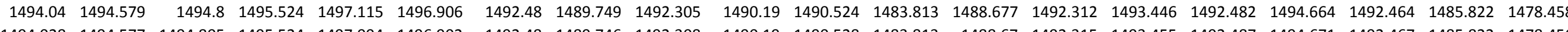

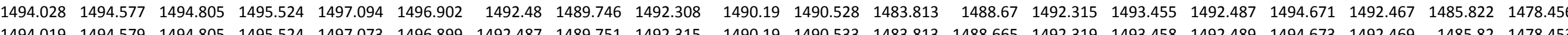

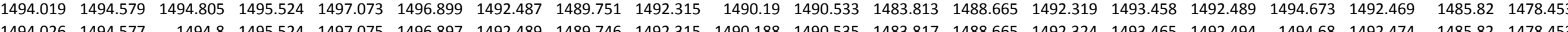

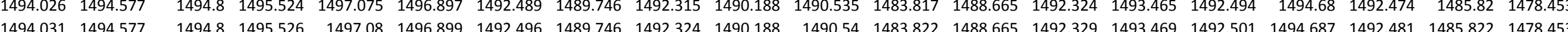
$\begin{array}{lllllllllllllllllllllll}1494.024 & 1494.575 & 1494.798 & 1495.529 & 1497.073 & 1496.894 & 1492.503 & 1489.751 & 1492.331 & 1490.19 & 1490.547 & 1483.829 & 1488.665 & 1492.336 & 1493.476 & 1492.503 & 1494.692 & 1492.488 & 1485.82 & 1478.453\end{array}$ $\begin{array}{llllllllllllllllllllll}1494.019 & 1494.572 & 1494.802 & 1495.526 & 1497.068 & 1496.899 & 1492.505 & 1489.749 & 1492.331 & 1490.188 & 1490.547 & 1483.824 & 1488.668 & 1492.343 & 1493.472 & 1492.508 & 1494.685 & 1492.49 & 1485.82 & 1478.453\end{array}$ \begin{tabular}{lllllllllllllllllllllll}
1494.009 & 1494.577 & 1494.802 & 1495.524 & 1497.056 & 1496.897 & 1492.51 & 1489.749 & 1492.338 & 1490.188 & 1490.557 & 1483.822 & 1488.665 & 1492.345 & 1493.476 & 1492.513 & 1494.692 & 1492.495 & 1485.82 & 1478.453 \\
\hline
\end{tabular} $\begin{array}{llllllllllllllllllllll}1494.002 & 1494.575 & 1494.802 & 1495.524 & 1497.049 & 1496.897 & 1492.515 & 1489.746 & 1492.343 & 1490.186 & 1490.559 & 1483.824 & 1488.658 & 1492.35 & 1493.483 & 1492.52 & 1494.697 & 1492.5 & 1485.82 & 1478.4566\end{array}$

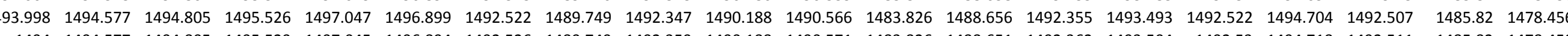
$\begin{array}{llllllllllllllllllllll}1494 & 1494.577 & 1494.805 & 1495.529 & 1497.045 & 1496.894 & 1492.526 & 1489.749 & 1492.359 & 1490.188 & 1490.571 & 1483.826 & 1488.651 & 1492.362 & 1493.504 & 1492.53 & 1494.718 & 1492.511 & 1485.82 & 1478.458\end{array}$

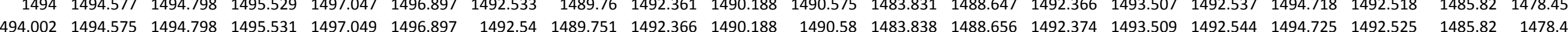
$\begin{array}{llllllllllllllllllllllll}1493.998 & 1494.575 & 1494.798 & 1495533 & 1497.038 & 1496894 & 1492.545 & 1489.753 & 1492373 & 1490.19 & 1490.585 & 148384 & 1488651 & 1492.378 & 1493551 & 1492548 & 1494.725 & 1492.528 & 1485818 & 1478.46\end{array}$ $\begin{array}{lllllllllllllllllllllll}1494 & 1494.575 & 1494.802 & 1495.531 & 1497.033 & 1496.892 & 1492.548 & 1489.749 & 1492.378 & 1490.188 & 1490.587 & 1483.843 & 1488.654 & 1492.383 & 1493.514 & 1492.548 & 1494.725 & 1492.53 & 1485.818 & 1478.458\end{array}$ $\begin{array}{llllllllllllllllllllllll}1494 & 1494.577 & 1494.802 & 1495.531 & 1497.028 & 1496.89 & 1491.59 & 1489.751 & 1492.043 & 1490.188 & 1490.589 & 1483.838 & 1488.651 & 1492.056 & 1493.294 & 1491.468 & 1494.727 & 1491.549 & 1485.815 & 1478.458\end{array}$ \begin{tabular}{llllllllllllllllllllll}
1493.988 & 1494.575 & 1494.798 & 1495.531 & 1497.026 & 1496.89 & 1490.341 & 1489.751 & 1490.83 & 1490.188 & 1490.524 & 1483.836 & 1488.651 & 1490.839 & 1492.702 & 1490.245 & 1494.69 & 1490.327 & 1485.818 & 1478.458 \\
\hline
\end{tabular}

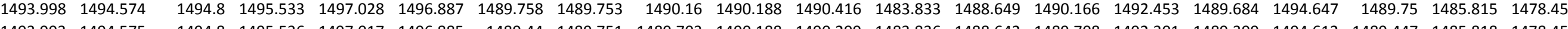

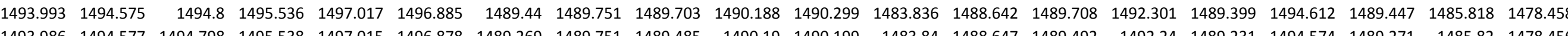

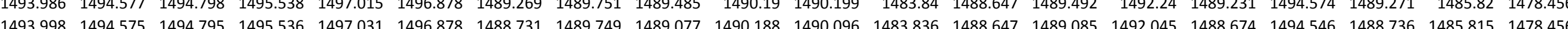

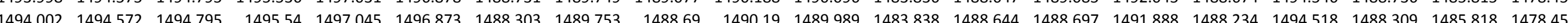
$\begin{array}{llllllllllllllllllllll}1494.002 & 1494.572 & 1494.798 & 1495.538 & 1497.061 & 1496.875 & 1487.938 & 1489.751 & 1488.305 & 1490.19 & 1489.881 & 1483.84 & 1488.649 & 1488.316 & 1491.723 & 1487.874 & 1494.476 & 1487.95 & 1485.815 & 1478.456\end{array}$ $\begin{array}{llllllllllllllllllllllll}1493.998 & 1494.572 & 1494.798 & 1495.536 & 1497.056 & 1496.866 & 1487.641 & 1489.749 & 1487.991 & 1490.188 & 1489.774 & 1483.845 & 1488.654 & 1487.999 & 1491.59 & 1487.582 & 1494.433 & 1487.654 & 1485.815 & 1478.453 \\ \end{array}$ $\begin{array}{lllllllllllllllllllllll}1493.993 & 1494.572 & 1494.79 & 1495.533 & 1497.061 & 1496.859 & 1487.479 & 1489.753 & 1487.743 & 1490.188 & 1489.671 & 1483.845 & 1488.654 & 1487.745 & 1491.497 & 1487.442 & 1494.403 & 1487.499 & 1485.815 & 1478.458\end{array}$

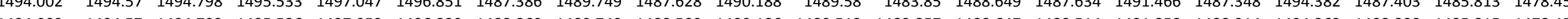
$\begin{array}{llllllllllllllllllll}1494.002 & 1494.57 & 1494.793 & 1495.536 & 1497.052 & 1496.839 & 1488.869 & 1489.749 & 1488.509 & 1490.186 & 1489.512 & 1483.857 & 1488.647 & 1488.514 & 1491.858 & 1488.914 & 1494.363 & 1488.898 & 1485.815 & 1478.458\end{array}$

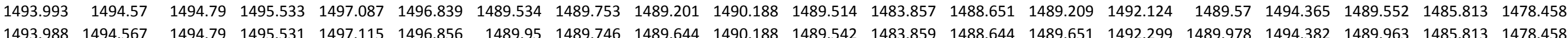


Well

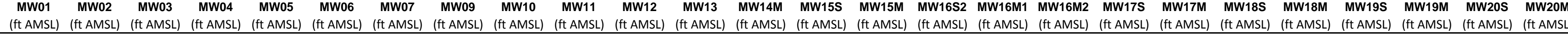

10/9/11 13:00 10/9/11 13:30 10/9/1114:30 10/9/11 15:00 10/9/11 15:30 10/9/11 16:00 10/9/11 16:30 10/9/11 17:00 10/9/11 17:30 10/9/11 18:00 10/9/11 18:30 10/9/11 19:30 10/9/11 19:30 10/9/11 20:30 10/9/11 21:00 0/9/11 22:00 0/9/11 22:30 10/9/11 23:00 10/10/11 0:00 10/10/11 0:30 10/10/11 1:00 10/10/11 1:30 10/10/11 2:00 $10 / 10 / 112: 30$ 10/10/11 3:00 10/10/11 4:00 10/10/11 4:30 10/10/11 5:00 10/10/11 5:30 10/10/11 6:00 10/10/11 6:30 10/10/11 7:00 10/10/11 7:30 10/10/11 8:30 10/10/11 9:00 10/10/11 9:30 10/10/11 10:00 10/10/11 10:30 10/10/11 11:00 10/10/11 11:30 10/10/11 12:00 10/10/11 12:30 10/10/11 13:30 10/10/11 14:00 10/10/11 14:30 10/10/11 15:00
10/10/11 15:30 $\begin{array}{llll}1494.477 & 1494.94 & 1496.775 & 1494.421 \\ 1494.479 & 1494.94 & 1496.766 & 1494.421\end{array}$ $\begin{array}{rrrr}1494.479 & 1494.94 & 1496.766 & 1494.421\end{array}$ $\begin{array}{lllll}1494.479 & 1494.944 & 1496.763 & 1494.423 \\ 1494.477 & 1494.942 & 1496.759 & 1494.428\end{array}$ $\begin{array}{llll}1494.472 & 1494.937 & 1496.756 & 1494.432\end{array}$ $\begin{array}{llll}1494.468 & 1494.935 & 1496.761 & 1494.439\end{array}$ $\begin{array}{rrrrr}1494.468 & 1494.935 & 1496.761 & 1494.439 \\ 1494.47 & 1494.935 & 1496.763 & 1494.449\end{array}$ $\begin{array}{lllll}1494.465 & 1494.93 & 1496.761 & 1494.456\end{array}$ $\begin{array}{lllll}1494.472 & 1494.933 & 1496.759 & 1494.468\end{array}$ $\begin{array}{rrrrr}1494.47 & 1494.935 & 1496.745 & 1494.482\end{array}$ $\begin{array}{rrrr}1494.468 & 1494.93 & 1496.747 & 1494.491 \\ 1494.468 & 1494.93 & 1496.763 & 149.5\end{array}$ $\begin{array}{rrrr}1494.468 & 1494.93 & 1496.763 & 1494.5 \\ 1494.461 & 1494.926 & 1496.759 & 1494.505\end{array}$ $\begin{array}{llll}1494.461 & 1494.926 & 1496.759 & 1494.505 \\ 1494.465 & 1494.926 & 1496.756 & 1494.519\end{array}$ $\begin{array}{llll}1494.47 & 1444.93 & 1496.766 & 1494.531\end{array}$ $\begin{array}{lllll}1494.468 & 1494.93 & 1496.768 & 1494.54\end{array}$ $\begin{array}{llll}1494.465 & 1494.928 & 1496.759 & 1494.547\end{array}$ $\begin{array}{lllll}1494.47 & 1494.935 & 1496.752 & 1494.561\end{array}$ $\begin{array}{llll}1494.468 & 1494.935 & 1496.74 & 1494.568\end{array}$ $\begin{array}{llll}1494.468 & 1494.937 & 1496.742 & 1494.577 \\ 1494.456 & 1494.926 & 1496754 & 1494.575\end{array}$ $\begin{array}{llll}1494.461 & 1494.928 & 1496.756 & 1494.584\end{array}$ $\begin{array}{lllll}1494.458 & 1494.923 & 1496.766 & 1494.589\end{array}$ $\begin{array}{lllll}1494.47 & 1494.935 & 1496.768 & 1494.64\end{array}$ $\begin{array}{lllll}1494.463 & 1494.93 & 1496.768 & 1494.615\end{array}$ $1494.465 \quad 1494.933 \quad 1496.759 \quad 1494.626$ $\begin{array}{llll}1494.463 & 1494.937 & 1496.756 & 1494.64\end{array}$ $\begin{array}{lllll}1494.461 & 1494.937 & 1496.754 & 1494.647\end{array}$ $\begin{array}{lllll}1494.461 & 1494.933 & 1496.752 & 1494.652 \\ 1494.458 & 1494.933 & 1496.752 & 1494.664\end{array}$ $\begin{array}{lllll}1494.456 & 1494.933 & 1496.749 & 1494.668\end{array}$ $\begin{array}{lllll}1494.456 & 1494.93 & 1496.747 & 1494.678\end{array}$ $\begin{array}{llllll}1494.458 & 1494.93 & 1496.742 & 1494.682\end{array}$ $\begin{array}{lllll}1494.454 & 1494.93 & 1496.747 & 1494.687\end{array}$ $\begin{array}{lllll}1494.454 & 1494.93 & 1496.749 & 1494.694\end{array}$ $1494.454 \quad 1494.928 \quad 1496.747 \quad 1494.699$ $\begin{array}{rrrr}1494.454 & 1494.926 & 1496.74 & 1494.694 \\ 1494.454 & 1494.928 & 1496738 & 194969\end{array}$ $\begin{array}{llll}1494.454 & 1494.928 & 1496.738 & 1494.689 \\ 1494.454 & 1494.928 & 1496.731 & 1494.685\end{array}$ $\begin{array}{lllll}14494.451 & 1494.926 & 1496.731 & 1494.68\end{array}$ $1494.447 \quad 1494.923 \quad 1496.731 \quad 1494.678$ $\begin{array}{lllll}1494.447 & 1494.919 & 1496.733 & 1494.675\end{array}$ $1494.4491494 .919 \quad 1496.735 \quad 1494.68$ $1494.447 \quad 1494.919 \quad 1496.742 \quad 1494.682$ $\begin{array}{llll}1494.449 & 1494.921 & 1496.749 & 1494.687 \\ 1494.451 & 1494.919 & 1496.759 & 1494.692\end{array}$ 1494.4511494 .9191496 .7591494 .692

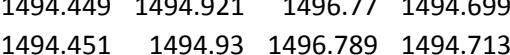
$1494.456 \quad 1494.935 \quad 1496.803 \quad 1494.722$ $1494.458 \quad 1494.944 \quad 1496.81 \quad 1494.736$ $1494.461 \quad 1494.9541496 .8191494 .752$ $1494.4651494 .963 \quad 1496.8171494 .769$ $\begin{array}{llll}1494.465 & 1494.972 & 1496.819 & 1494.783 \\ 1494.465 & 1494.979 & 1496.826 & 1494.797\end{array}$

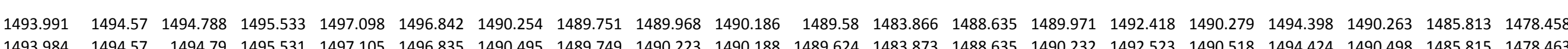

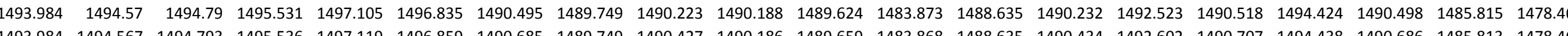

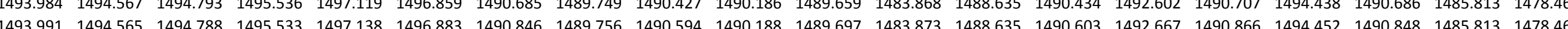
$\begin{array}{llllllllllllllllllllll}1493.998 & 1494.565 & 1494.783 & 1495.531 & 1497.156 & 1496892 & 1490.984 & 1489749 & 1490.744 & 1490.188 & 1489.734 & 1483875 & 1488647 & 1490.747 & 1492.725 & 1491.004 & 1494.462 & 1490.984 & 1485813 & 1478.468\end{array}$ $\begin{array}{llllllllllllllllllllll}1494 & 1494.563 & 1494.78 & 1495.529 & 1497.161 & 1496.902 & 1491.101 & 1489.749 & 1490.866 & 1490.186 & 1489.767 & 1483.882 & 1488.644 & 1490.874 & 1492.77 & 1491.117 & 1494.473 & 1491.099 & 1485.81 & 1478.47\end{array}$

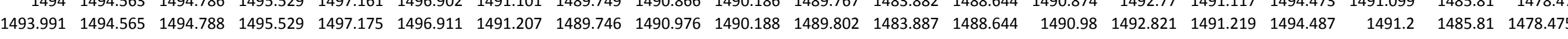

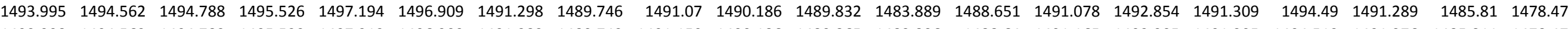

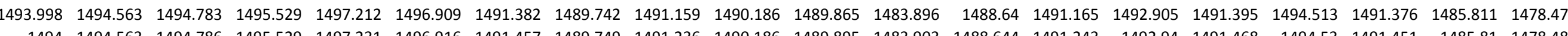
$\begin{array}{llllllllllllllllllll}1494 & 1494.563 & 1494.786 & 1495.529 & 1497.231 & 1496.916 & 1491.457 & 1489.749 & 1491.236 & 1490.186 & 1489.895 & 1483.903 & 1488.644 & 1491.243 & 1492.94 & 1491.468 & 1494.53 & 1491.451 & 1485.81 & 1478.482 \\ 1494.002 & 1494.575 & 1494.783 & 1495.526 & 1497.235 & 1496.916 & 1491.525 & 1499746 & 1491.309 & 1490.186 & 1489.921 & 1483905 & 1488647 & 1491313 & 1492.97 & 1491.534 & 1494.537 & 1491516 & 14581 & 147834\end{array}$

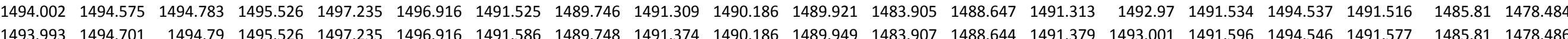

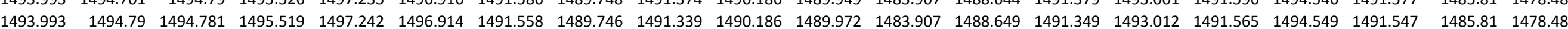

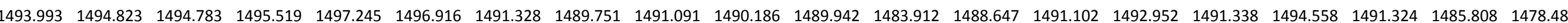
$\begin{array}{lllllllllllllllllllll}1493.988 & 1494.835 & 1494.783 & 1495.524 & 1497.261 & 1496.918 & 1491.27 & 1489.744 & 1491.032 & 1490.186 & 1489.9 & 1483.907 & 1488.64 & 1491.038 & 1492.924 & 1491.281 & 1494.572 & 1491.265 & 1485.811 & 1478.486\end{array}$

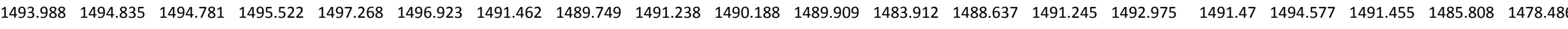
$\begin{array}{lllllllllllllllllllll}1493.986 & 1494.855 & 1494.781 & 1495.522 & 1497.282 & 1496.926 & 1491.56 & 1489.751 & 1491.353 & 1490.186 & 1489.937 & 1483.914 & 1488.64 & 1491.356 & 1493.017 & 1491.579 & 1494.581 & 1491.559 & 1485.808 & 1478.486\end{array}$

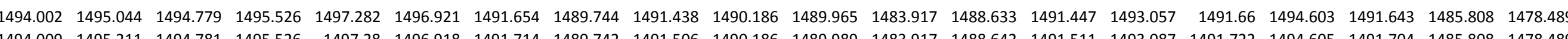

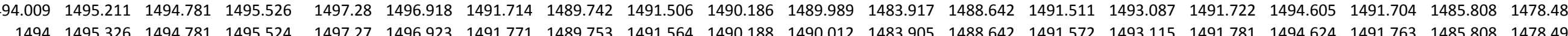
$\begin{array}{llllllllllllllllllll}1494 & 1495.326 & 1494.781 & 1495.524 & 1497.27 & 1496.923 & 1491.771 & 1489.753 & 1491.564 & 1490.188 & 1490.012 & 1483.905 & 1488.642 & 1491.572 & 1493.115 & 1491.781 & 1494.624 & 1491.763 & 1485.808 & 1478.491 \\ \end{array}$ $\begin{array}{llllllllllllllllllll}1493.984 & 1455.312 & 1444.781 & 1455.517 & 1497.238 & 1496.921 & 1491.852 & 1489.749 & 1491.653 & 1490.186 & 1490.054 & 1483.886 & 1488.64 & 1491.659 & 1493.148 & 1491.861 & 1494.628 & 1491.843 & 1485.808 & 1478.486\end{array}$

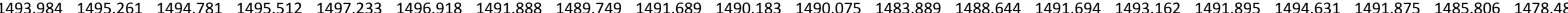
$\begin{array}{llllllllllllllllllllll}1493.977 & 1495.209 & 1494.783 & 1495.519 & 1497.233 & 1496.918 & 1491.925 & 1489.744 & 1491.728 & 1490.186 & 1490.096 & 1483.894 & 1488.628 & 1491.732 & 1493.196 & 1491.932 & 1494.659 & 1491.915 & 1485.808 & 1478.486\end{array}$ $\begin{array}{llllllllllllllllllllll}1493.977 & 1495.166 & 1494.781 & 1495.519 & 1497.231 & 1496.921 & 1491.955 & 1489.746 & 1491.759 & 1490.183 & 1490.112 & 1483.896 & 1488.637 & 1491.765 & 1493.206 & 1491.963 & 1494.666 & 1491.943 & 1485.806 & 1478.486\end{array}$ $\begin{array}{lllllllllllllllllllll}1493.986 & 1495.132 & 1494.776 & 1495.522 & 1497.226 & 1496.916 & 1491.988 & 1489.746 & 1491.792 & 1490.186 & 1490.131 & 1483.9 & 1488.637 & 1491.8 & 1493.229 & 1491.994 & 1494.68 & 1491.976 & 1485.803 & 1478.486\end{array}$

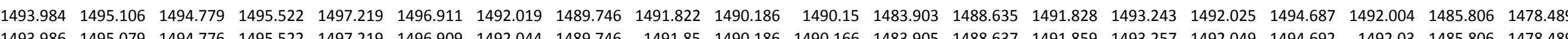

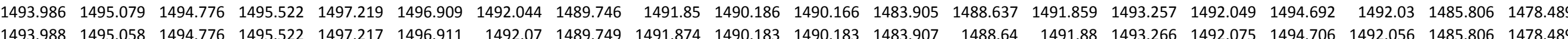
$\begin{array}{lllllllllllllllllllll}1493.988 & 1495.058 & 1494.776 & 1495.522 & 1497.217 & 1496.911 & 1492.07 & 1489.749 & 1491.874 & 1490.183 & 1490.183 & 1483.907 & 1488.64 & 1491.88 & 1493.266 & 1492.075 & 1494.706 & 1492.056 & 1485.806 & 1478.489 \\ 1493.984 & 1495.034 & 1494.779 & 1495.522 & 1497212 & 1496911 & 1492.094 & 1489.746 & 1491.902 & 1490186 & 1490201 & 1483.91 & 1488.642 & 1491.908 & 1493285 & 1492.098 & 1494.706 & 1492.08 & 1485803 & 1478.489\end{array}$ $\begin{array}{llllllllllllllllllllllll}1493.986 & 1495.012 & 1494.776 & 1495.519 & 1497.212 & 1496.914 & 1492.115 & 1489.742 & 1491.923 & 1490.183 & 1490.215 & 1483.912 & 1488.644 & 1491.932 & 1493.297 & 1492.12 & 1494.715 & 1492.101 & 1485.803 & 1478.489\end{array}$ $\begin{array}{llllllllllllllllllllll}1493.986 & 1494.993 & 1494.774 & 1495.519 & 1497.224 & 1496.921 & 1492.136 & 1489.742 & 1491.946 & 1490.186 & 1490.229 & 1483.914 & 1488.635 & 1491.953 & 1493.311 & 1492.143 & 1494.722 & 1492.124 & 1485.803 & 1478.491 \\ \end{array}$ $\begin{array}{lllllllllllllllllllllll}1493.993 & 1494.972 & 1494.771 & 1495.519 & 1497.249 & 1496.935 & 1492.159 & 1489.742 & 1491.97 & 1490.183 & 1490.246 & 1483.917 & 1488.64 & 1491.974 & 1493.322 & 1492.165 & 1494.734 & 1492.143 & 1485.803 & 1478.491 \\ & 1493 & \end{array}$

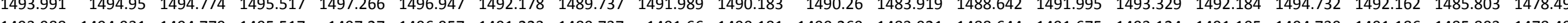

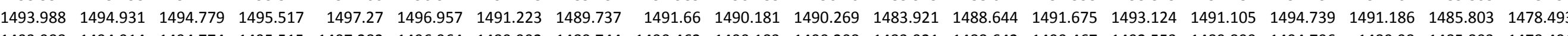

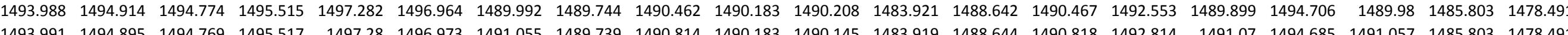

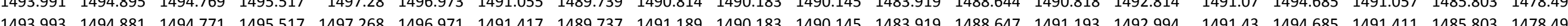

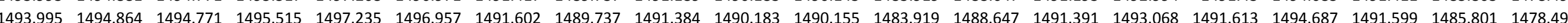
$\begin{array}{lllllllllllllllllllllll}1493.993 & 1494.85 & 1494.769 & 1495.512 & 1497.182 & 1496.933 & 1491.726 & 1489.739 & 1491.515 & 1490.181 & 1490.166 & 1483.919 & 1488.644 & 1491.52 & 1493.124 & 1491.738 & 1494.69 & 1491.718 & 1485.801 & 1478.489\end{array}$

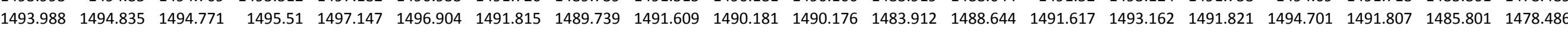

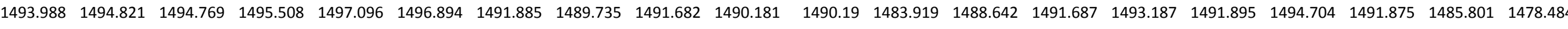

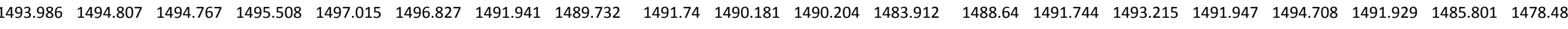

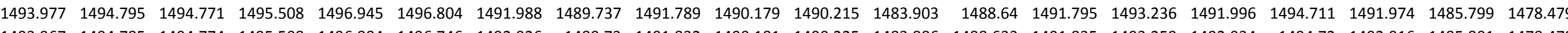

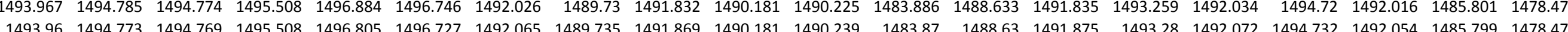

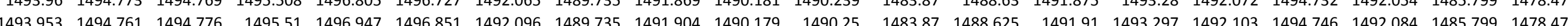
\begin{tabular}{llllllllllllllllllllll}
1493.939 & 1494.756 & 1494.774 & 1495.515 & 1496.959 & 1496.863 & 1492.126 & 1489.737 & 1491.935 & 1490.181 & 1490.262 & 1483.873 & 1488.618 & 1491.941 & 1493.32 & 1492.134 & 1494.758 & 1492.112 & 1485.799 & 1478.474 \\
\hline
\end{tabular} $\begin{array}{lllllllllllllllllllll}1493.929 & 1494.744 & 1494.774 & 1495.515 & 1496.97 & 1496.88 & 1492.157 & 1489.737 & 1491.963 & 1490.181 & 1490.274 & 1483.875 & 1488.616 & 1491.969 & 1493.341 & 1492.162 & 1494.772 & 1492.143 & 1485.799 & 1478.475\end{array}$ $\begin{array}{lllllllllllllllllllll}1493.925 & 1494.737 & 1494.774 & 1495.522 & 1496.973 & 1496.892 & 1492.185 & 1489.737 & 1491.991 & 1490.183 & 1490.29 & 1483.87 & 1488.607 & 1492 & 1493.367 & 1492.191 & 1494.788 & 1492.171 & 1485.799 & 1478.475 \\ \end{array}$

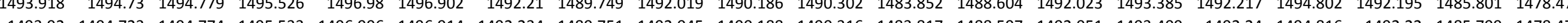

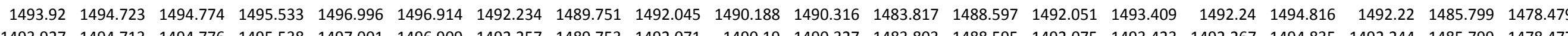

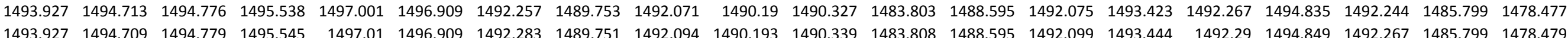


Well

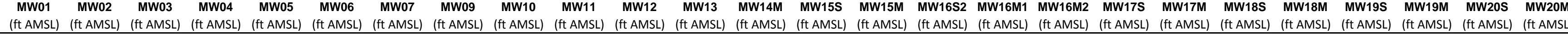

10/10/11 16:00 10/10/11 16:30 10/10/11 17:00 10/10/1111 18:00 10/10/11118:00 10/10/11 18:30 10/10/11 19:30 10/10/11 20:00 10/10/11 20:30 10/10/11 21:00 10/10/11 21:30 10/10/11 22:00 10/10/11 22:30 10/10/11 23:30 10/11/11 0:00 10/11/11 1:00 10/11/11 1:30 10/11/11 2:00 10/11/11 3:00 10/11/11 3:30 10/11/11 4:30 10/11/11 5:00 $10 / 11 / 115: 30$
$10 / 11 / 116: 00$
$10 / 11 / 116: 30$ 10/11/11 7:00 10/11/117:30 10/11/11 8:30 10/11/119:00 10/11/11 9:30 10/11/11 10:00 10/11/11 10:30 10/11/11 11:30 10/11/11 12:00 10/11/11 12:30 10/11/11 13:00 10/11/11 13:30 10/11/1114:00 10/11/11 14:30 10/11/11 15:00 10/11/11 16:00 10/11/11 16:30 10/11/11 17:00 10/11/11 18:00 $\begin{array}{llll}1494.463 & 1494.979 & 1496.831 & 1494.804\end{array}$ $\begin{array}{llll}1494.465 & 1494.982 & 1496.836 & 1494.813 \\ 1494.465 & 1494.989 & 1496836 & 1494822\end{array}$ $\begin{array}{llll}1494.465 & 1444.989 & 1496.836 & 1494.822\end{array}$ $\begin{array}{rrrrr}1494.47 & 1494.993 & 1496.838 & 1494.836 \\ 1494.468 & 1494.998 & 1496.831 & 1494.843\end{array}$ $\begin{array}{rrrrr}1494.468 & 144.998 & 1496.831 & 1494.843 \\ 1494.468 & 1495 & 1496.829 & 1494.848\end{array}$ $\begin{array}{llll}1494.468 & 1495 & 1496.829 & 1494.848 \\ 1494.468 & 1495 & 1496.824 & 1494.857\end{array}$ $\begin{array}{rrrrr}1494.468 & 1495.003 & 1496.819 & 1494.862\end{array}$ $\begin{array}{lrrrr}1494.465 & 1495 & 1496.812 & 1494.862\end{array}$ $\begin{array}{rrrr}1494.465 & 1495 & 1496.808 & 1494.864\end{array}$ $\begin{array}{lllll}1494.463 & 1494.998 & 1496.808 & 1494.864 \\ 1494.463 & 1494.996 & 1496808 & 1494867\end{array}$ $\begin{array}{rrrr}1494.463 & 1494.996 & 1496.808 & 1494.867 \\ 1494.463 & 1494.996 & 1496.81 & 1494.867\end{array}$ $\begin{array}{rrrrr}1494.463 & 1494.996 & 1496.81 & 1494.867 \\ 1494.463 & 1494.996 & 1496.812 & 1494.864\end{array}$ $\begin{array}{llrr}1494.463 & 1494.996 & 1496.812 & 1494.864 \\ 1494.461 & 1494.996 & 1496.819 & 1494.867\end{array}$ $\begin{array}{lllll}1494.461 & 1494.996 & 1496.819 & 1494.867 \\ 1494.463 & 1494.996 & 1496.826 & 1494.871\end{array}$ $\begin{array}{lllll}1494.465 & 1494.998 & 1496.833 & 1494.876\end{array}$ $\begin{array}{lllll}1494.468 & 1495.003 & 1466.833 & 1494.881\end{array}$ $\begin{array}{llll}1494.47 & 1495.007 & 1496.831 & 1494.885\end{array}$ $\begin{array}{rrrrr}1494.47 & 1495.007 & 1496.831 & 1494.892 \\ 1494.468 & 1495.01 & 1496.84 & 1494895\end{array}$ $\begin{array}{rrrr}1494.468 & 1495.01 & 1496.84 & 1494.895 \\ 1494.47 & 1495.012 & 1496.84 & 1494.902\end{array}$ $\begin{array}{rrrr}1494.468 & 1495.012 & 1496.845 & 1494.904\end{array}$ $\begin{array}{lllll}1494.472 & 1495.019 & 1496.843 & 1494.911\end{array}$ $\begin{array}{llllll}1494.472 & 1495.019 & 1496.84 & 1494.913\end{array}$ $\begin{array}{llll}1494.475 & 1495.021 & 1496.84 & 1494.918\end{array}$ $\begin{array}{llll}1494.472 & 1495.021 & 1496.838 & 1494.92\end{array}$ $\begin{array}{llll}1494.475 & 1495.021 & 1496.84 & 1494.925 \\ 1494.472 & 1495021 & 1496843 & 1994.925\end{array}$ $\begin{array}{rrrrr}1494.472 & 1495.021 & 1496.843 & 1494.925 \\ 1494.475 & 1495.021 & 1496.84 & 1494.927\end{array}$ $\begin{array}{llll}1494.475 & 1495.026 & 1496.84 & 1494.93\end{array}$ $\begin{array}{rrrrr}1494.475 & 1495.024 & 1496.843 & 1494.932\end{array}$ $\begin{array}{lllll}1494.475 & 1495.026 & 1496.847 & 1494.937\end{array}$ $\begin{array}{lllll}1494.475 & 1495.026 & 1496.852 & 1494.937\end{array}$ $\begin{array}{lllll}1494.477 & 1495.028 & 1466.859 & 1494.939\end{array}$ $\begin{array}{lllll}1494.479 & 1495.031 & 1496.861 & 1494.944\end{array}$ $\begin{array}{rrrrr}1494.482 & 1495.035 & 1496.861 & 1494.946\end{array}$ $\begin{array}{lllll}1494.482 & 1495.04 & 1496.866 & 1494.953 \\ 1494.482 & 1495.045 & 1496.868 & 1494.955\end{array}$ $\begin{array}{lllr}1494.484 & 1495.045 & 1496.88 & 1494.96\end{array}$ $\begin{array}{lllll}1494.484 & 1495.047 & 1496.894 & 1494.965\end{array}$ $\begin{array}{llllll}1494.489 & 1495.052 & 1496.903 & 1494.967\end{array}$ $\begin{array}{lllll}1494.491 & 1495.061 & 1496.915 & 1494.976\end{array}$ $\begin{array}{lllll}1494.498 & 1495.07 & 1496.918 & 1494.986\end{array}$ $\begin{array}{rrrr}1494.498 & 1495.077 & 1496.92 & 1494.995 \\ 1494.503 & 1495.087 & 1496.922 & 1995007\end{array}$ $\begin{array}{rrrr}1494.503 & 1495.087 & 1496.922 & 1495.007 \\ 14945 & 1495.091 & 1496925 & 1495011\end{array}$ $\begin{array}{lllll}1494.503 & 1495.094 & 1496.927 & 1495.016\end{array}$ $\begin{array}{lllll}1494.503 & 1495.096 & 1496.934 & 1495.023\end{array}$ $\begin{array}{lllll}1494.505 & 1495.098 & 1496.927 & 1495.028\end{array}$ $\begin{array}{lllll}1494.507 & 1495.103 & 1496.925 & 1495.032\end{array}$ $\begin{array}{lllll}1494.509 & 1495.11 & 1496.918 & 1495.039\end{array}$ $\begin{array}{llll}1494.505 & 1495.103 & 1496.911 & 1495.037 \\ 1494.507 & 1495.105 & 1496.903 & 1495.039\end{array}$

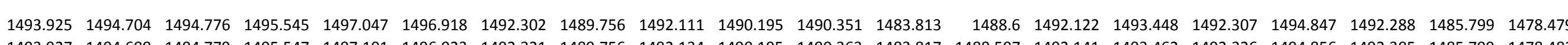

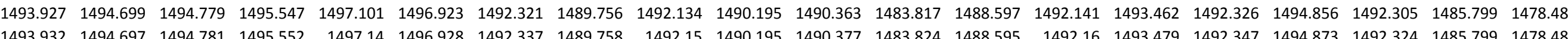

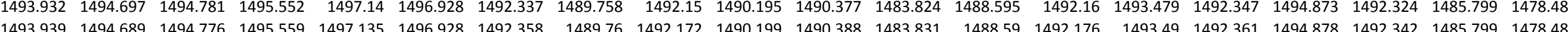

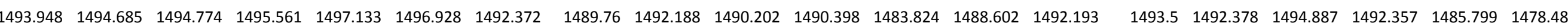
$\begin{array}{lllllllllllllllllllll}1493.955 & 1494.682 & 1494.781 & 1495.563 & 1497.131 & 1496.921 & 1492.388 & 1489.763 & 1492.202 & 1490.206 & 1490.407 & 1483.792 & 1488.6 & 1492.209 & 1493.516 & 1492.394 & 1494.892 & 1492.373 & 1485.799 & 1478.482 & 1484\end{array}$ \begin{tabular}{lllllllllllllllllllllll}
1493.962 & 1494.677 & 1494.776 & 1495.566 & 1497.128 & 1496.918 & 1492.402 & 1489.765 & 1492.216 & 1490.206 & 1490.416 & 1483.78 & 1488.6 & 1492.225 & 1493.521 & 1492.409 & 1494.889 & 1492.387 & 1485.799 & 1478.484 \\
\hline
\end{tabular}

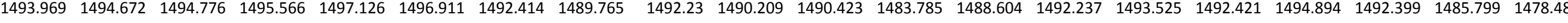

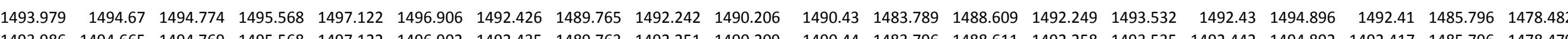

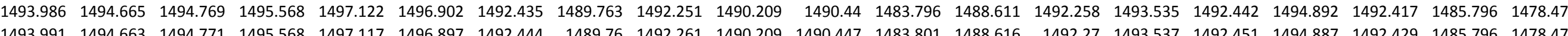

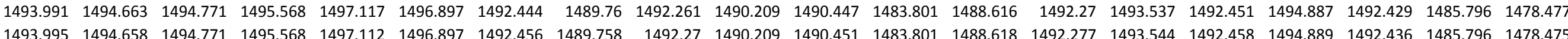

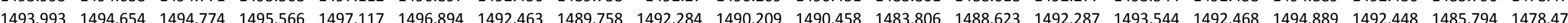

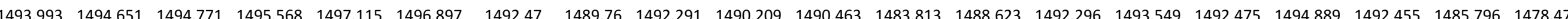
$\begin{array}{llllllllllllllllllllll}1493.988 & 1494.649 & 1494.776 & 1495.568 & 1497.117 & 1496.899 & 1492.48 & 1489.749 & 1492.298 & 1490.209 & 1490.47 & 1483.817 & 1488.623 & 1492.301 & 1493.549 & 1492.484 & 1494.892 & 1492.467 & 1485.794 & 14748.472\end{array}$ $\begin{array}{llllllllllllllllllll}1493.984 & 1494.646 & 1494.774 & 1495.568 & 1497.119 & 1496.906 & 1492.487 & 1489.756 & 1492.308 & 1490.209 & 1490.477 & 1483.82 & 1488.625 & 1492.312 & 1493.558 & 1492.492 & 1494.892 & 1492.471 & 1485.794 & 1478.47\end{array}$ $\begin{array}{lllllllllllllllllllllll}1493.984 & 1494.644 & 1494.769 & 1495.573 & 1497.119 & 1496.914 & 1492.496 & 1489.751 & 1492.317 & 1490.211 & 1490.484 & 1483.824 & 1488.625 & 1492.322 & 1493.567 & 1492.501 & 1494.903 & 1492.478 & 1485.794 & 1478.47\end{array}$

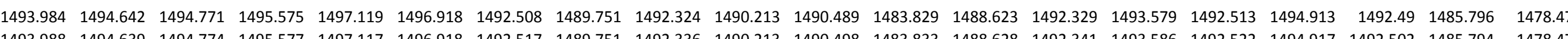

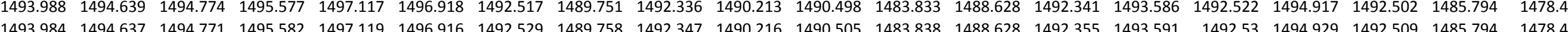
$\begin{array}{llllllllllllllllllllll}1493.984 & 1494.637 & 1494.771 & 1495.582 & 1497.119 & 1496.916 & 1492.529 & 1489.758 & 1492.347 & 1490.216 & 1490.505 & 1483.838 & 1488.628 & 1492.355 & 1493.591 & 1492.53 & 1494.929 & 1492.509 & 1485.794 & 1478.47 \\ 1493.988 & 1494.634 & 1494.774 & 1495582 & 1497.119 & 1496916 & 1492536 & 1489.751 & 1492354 & 1490218 & 1490512 & 1483838 & 1488635 & 1492362 & 1493593 & 1492541 & 1494925 & 1492521 & 1485794 & 1478.47\end{array}$ $\begin{array}{llllllllllllllllllllll}1493.986 & 1494.634 & 1494.776 & 1495.584 & 1497.122 & 1496.923 & 1492.545 & 1489.756 & 1492.366 & 1490.216 & 1490.519 & 1483.838 & 1488.635 & 1492.371 & 1493.605 & 1492.548 & 1494.929 & 1492.53 & 1485.794 & 1478.47\end{array}$

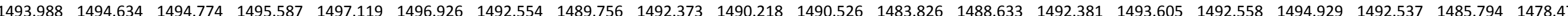

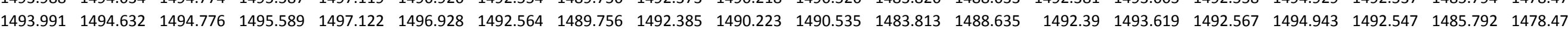

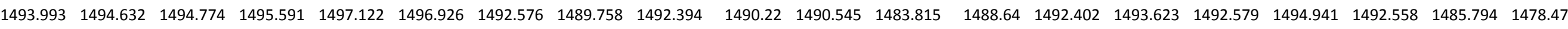

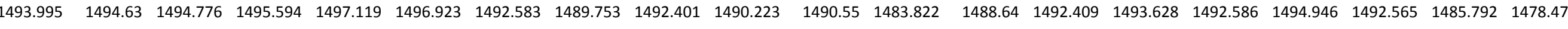

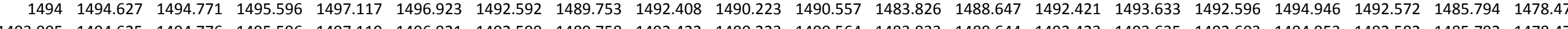

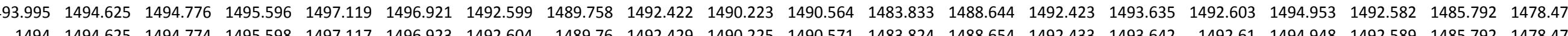
$\begin{array}{llllllllllllllllllll}1494 & 1494.625 & 1494.774 & 1495.598 & 1497.117 & 1496.923 & 1492.604 & 1489.76 & 1492.429 & 1490.225 & 1490.571 & 1483.824 & 1488.654 & 1492.433 & 1493.642 & 1492.61 & 1494.948 & 1492.589 & 1485.792 & 1478.479 \\ \end{array}$ $\begin{array}{llllllllllllllllllllll}1494.002 & 1494.622 & 1494.776 & 1495.601 & 1497.117 & 1496.923 & 1492.62 & 1489.751 & 1492.446 & 1490.227 & 1490.585 & 1483.831 & 1488.651 & 1492.446 & 1493.649 & 1492.624 & 1494.955 & 1492.605 & 1485.792 & 1478.479\end{array}$ $\begin{array}{lllllllllllllllllllllll}1494.005 & 1494.622 & 1494.776 & 1495.603 & 1497.119 & 1496.923 & 1492.625 & 1489.756 & 1492.448 & 1490.227 & 1490.587 & 1483.836 & 1488.654 & 1492.451 & 1493.658 & 1492.629 & 1494.957 & 1492.61 & 1485.792 & 1478.482 \\ \end{array}$ $\begin{array}{lllllllllllllllllllllll}1494.002 & 1494.622 & 1494.774 & 1495.603 & 1497.119 & 1496.926 & 1492.632 & 1489.756 & 1492.453 & 1490.227 & 1490.594 & 1483.84 & 1488.654 & 1492.461 & 1493.656 & 1492.636 & 1494.96 & 1492.615 & 1485.792 & 1478.479\end{array}$ $\begin{array}{lllllllllllllllllllll}1494 & 1494.622 & 1494.776 & 1495.605 & 1497.122 & 1496.928 & 1492.636 & 1489.758 & 1492.46 & 1490.23 & 1490.596 & 1483.843 & 1488.656 & 1492.465 & 1493.661 & 1492.641 & 1494.96 & 1492.619 & 1485.792 & 1478.479\end{array}$

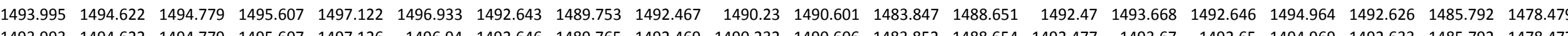

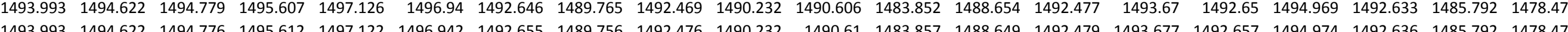

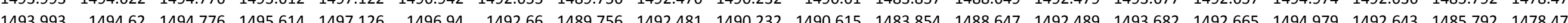

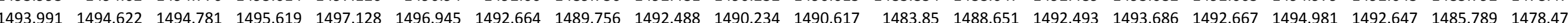
$\begin{array}{lllllllllllllllllllllll}1493.988 & 1494.62 & 1494.783 & 1495.621 & 1497.128 & 1496.95 & 1492.672 & 1489.756 & 1492.493 & 1490.237 & 1490.622 & 1483.852 & 1488.649 & 1492.498 & 1493.691 & 1492.674 & 1494.99 & 1492.652 & 1485.789 & 1478.475 \\ \end{array}$ $\begin{array}{lllllllllllllllllllll}1493.977 & 1494.625 & 1494.783 & 1495.621 & 1497.138 & 1496.957 & 1492.676 & 1489.76 & 1492.497 & 1490.237 & 1490.624 & 1483.852 & 1488.649 & 1492.505 & 1493.693 & 1492.681 & 1494.993 & 1492.659 & 1485.789 & 1478.472\end{array}$ $\begin{array}{llllllllllllllllllll}1493.974 & 1494.625 & 1494.786 & 1495.626 & 1497.142 & 1496.969 & 1492.683 & 1489.756 & 1492.505 & 1490.239 & 1490.629 & 1483.852 & 1488.647 & 1492.51 & 1493.7 & 1492.686 & 1494.993 & 1492.664 & 1485.789 & 1478.47 \\ \end{array}$

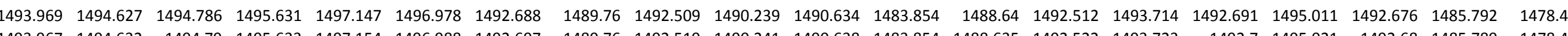

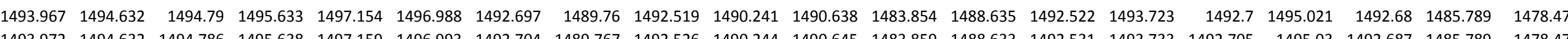
$\begin{array}{llllllllllllllllllllll}1493.972 & 1494.632 & 1494.786 & 1495.638 & 1497.159 & 1496.993 & 1492.704 & 1489.767 & 1492.526 & 1490.244 & 1490.645 & 1483.85 & 1488.633 & 1492.531 & 1493.733 & 1492.705 & 1495.03 & 1492.687 & 1485.789 & 1478.47 \\ 1493.974 & 1494.634 & 1494.786 & 1495.64 & 1497159 & 1496993 & 1492711 & 1489.774 & 1492533 & 1490248 & 1490652 & 1483859 & 1488628 & 1492536 & 1493744 & 1492717 & 1495.037 & 1492.694 & 1485789 & 1478.872\end{array}$

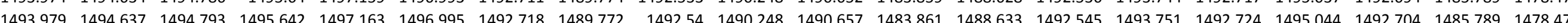
$\begin{array}{llllllllllllllllllllll}1493.981 & 1494.639 & 1494.79 & 1495.645 & 1497.166 & 1496.997 & 1492.728 & 1489.772 & 1492.547 & 1490.248 & 1490.664 & 1483.866 & 1488.63 & 1492.557 & 1493.751 & 1492.729 & 1495.049 & 1492.708 & 1485.787 & 1478.475\end{array}$ $\begin{array}{llllllllllllllllllllll}1493.986 & 1494.634 & 1494.79 & 1495.649 & 1497.168 & 1497 & 1492.732 & 1489.774 & 1492.554 & 1490.253 & 1490.666 & 1483.866 & 1488.628 & 1492.559 & 1493.758 & 1492.738 & 1495.051 & 1492.718 & 1485.789 & 1478.474\end{array}$ \begin{tabular}{lllllllllllllllllllllll}
1493.991 & 1494.642 & 1494.788 & 1495.652 & 1497.168 & 1497.002 & 1492.739 & 1489.772 & 1492.561 & 1490.253 & 1490.671 & 1483.831 & 1488.633 & 1492.566 & 1493.761 & 1492.745 & 1495.056 & 1492.725 & 1485.789 & 1478.477 \\
\hline
\end{tabular}

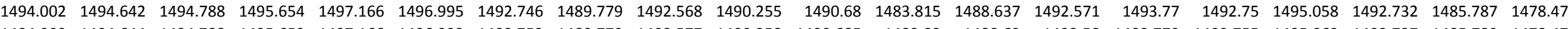

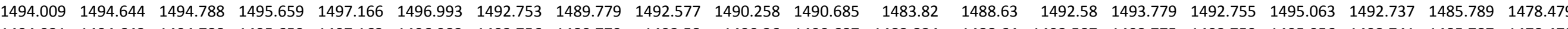

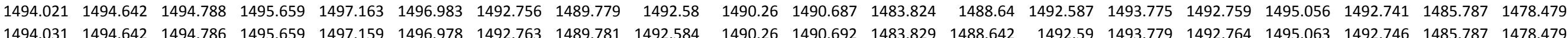


Well

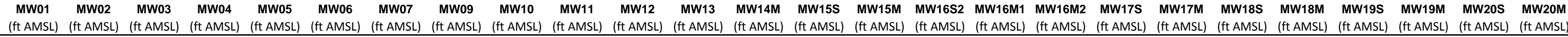
10/11/11 19:00 10/11/11 19:30 10/11/11 20:00 10/11/11 20:30 10/11/1121:30 10/11/1122:00 10/11/11 22:30 10/11/11 23:00 10/11/11 23:30 10/12/11 0:00 10/12/11 0:30 10/12/11 1:00 10/12/11 1:30 10/12/1112:00 10/12/11 3:00 0/12/11 4:00 0/12/11 4:30 10/12/11 5:00 0/12/11 5:30 $10 / 12 / 116: 30$ 10/12/11 7:00 10/12/117:30 10/12/11 8:00 10/12/11 8:30 0/12/11 9:30 10/12/111 10:30 10/12/11 11:00 10/12/11 11:30 10/12/1112:30 10/12/11 13:00 10/12/11 13:30 10/12/11 14:00 10/12/11 15:00 10/12/11 15:30 10/12/11 16:00 10/12/11 16:30 10/12/11 17:00 10/12/1117:30 10/12/11 18:00 10/12/11 19:00 10/12/11 19:30 $10 / 12 / 11120: 00$
$10 / 12 / 1120: 30$ 10/12/11 21:00
10/12/11 21:30 $\begin{array}{llll}1494.505 & 1495.103 & 1496.903 & 1495.037\end{array}$ $\begin{array}{lllll}1494.507 & 1495.103 & 1496.892 & 1495.032 \\ 1494.505 & 1495.101 & 1496887 & 1495.03\end{array}$ $\begin{array}{llll}1494.503 & 1495.096 & 1496.889 & 1495.025\end{array}$ $\begin{array}{lllll}1494.505 & 1495.096 & 1496.892 & 1459.025\end{array}$ $\begin{array}{llll}1494.505 & 1495.096 & 1496.892 & 1495.021 \\ 1494.505 & 1495.094 & 1496.901 & 1495.016\end{array}$ $\begin{array}{llll}1494.514 & 1495.101 & 1496.922 & 1495.023\end{array}$ $\begin{array}{rrrr}1494.514 & 1495.105 & 1496.925 & 1495.028 \\ 149.519 & 1455105 & 149692 & 1955028\end{array}$ $\begin{array}{llll}1494.519 & 1495.112 & 1496.913 & 1495.039\end{array}$ $\begin{array}{lllll}1494.519 & 1495.112 & 1496.903 & 1495.039\end{array}$ $\begin{array}{lllll}1494.519 & 1495.11 & 1496.899 & 1495.037\end{array}$ $\begin{array}{llll}1494.516 & 1495.11 & 1496.894 & 1495.035\end{array}$ $\begin{array}{llll}1494.514 & 1495.101 & 1496.892 & 1495.025 \\ 1494519 & 1495.098 & 1496892 & 1495025\end{array}$ $\begin{array}{lllll}1494.519 & 1495.101 & 1496.892 & 1495.021\end{array}$ $\begin{array}{lllll}1494.519 & 1495.098 & 1496.894 & 1495.018\end{array}$ $\begin{array}{lllll}1494.521 & 1495.101 & 1496.892 & 1495.021\end{array}$ $\begin{array}{lllll}1494.521 & 1495.098 & 1496.885 & 1495.021\end{array}$ $\begin{array}{lllll}1494.523 & 1495.101 & 1496.878 & 1495.023\end{array}$ $\begin{array}{llll}1494.523 & 1495.098 & 1496.878 & 1495.023\end{array}$ $\begin{array}{llll}1494.519 & 1495.096 & 1496.871 & 1495.018\end{array}$ $\begin{array}{lllll}1494.521 & 1495.096 & 1496.866 & 1495.014 \\ 1494.521 & 1495.096 & 1496.868 & 1495.011\end{array}$ $\begin{array}{lllll}1494.519 & 1495.091 & 1496.871 & 1495.007\end{array}$ $\begin{array}{lllll}1494.521 & 1495.087 & 1496.873 & 1495.002\end{array}$ $1494.523 \quad 1495.0891496 .882 \quad 1495.002$ $\begin{array}{lllll}1494.526 & 1495.091 & 1496.873 & 1495.002\end{array}$ $\begin{array}{lllll}1494.528 & 1495.091 & 1496.871 & 1495.004\end{array}$ $\begin{array}{llll}1494.526 & 1495.094 & 1496.878 & 1495.004\end{array}$ $\begin{array}{rrrr}1494.528 & 1495.094 & 1496.882 & 1495.004\end{array}$ $\begin{array}{llll}1494.53 & 1495.096 & 1496.868 & 1495.004\end{array}$ $1494.528 \quad 1495.094 \quad 1496.847 \quad 1495.007$ $\begin{array}{llll}1494.523 & 1495.087 & 1496.843 & 1495\end{array}$ $\begin{array}{lllll}1494.526 & 1495.084 & 1496.836 & 1494.997\end{array}$ $\begin{array}{llll}1494.521 & 1495.077 & 1496.812 & 1494.983 \\ 1494.523 & 1495.073 & 1496.798 & 1494979\end{array}$ $\begin{array}{lllll}1494.516 & 1495.068 & 1496.789 & 1494.969\end{array}$ $\begin{array}{lllll}1494.514 & 1495.059 & 1496.782 & 1494.96\end{array}$ $\begin{array}{lllll}1494.514 & 1495.052 & 1496.777 & 1494.953\end{array}$ $\begin{array}{lllll}1494.512 & 1495.04 & 1496.754 & 1494.937\end{array}$ $\begin{array}{llll}1494.514 & 1495.035 & 1496.747 & 1494.93 \\ 1494.507 & 1495.031 & 1496.745 & 1494.918\end{array}$ $\begin{array}{llll}1494.507 & 1495.103 & 1496.897 & 1495.035\end{array}$ $\begin{array}{lllll}1494.507 & 1495.094 & 1496.911 & 1495.016\end{array}$ $\begin{array}{lllll}1494.509 & 1495.098 & 1496.915 & 1495.018\end{array}$ $\begin{array}{rrrr}1494.519 & 1495.105 & 1496.92 & 1495.028 \\ 1494.521 & 1495.11 & 1496.915 & 1495.039\end{array}$ $\begin{array}{llll}1494.516 & 1495.105 & 1496.887 & 1495.032\end{array}$ 1494.5161495 .1051496 .88551495 .03 $1494.5231495 .0891496 .88 \quad 1495$ $1494.53 \quad 1495.096 \quad 1496.861 \quad 1495.009$ $1494.521 \quad 1495.08 \quad 1496.826 \quad 1494.99$ 1494.5121495 .0471496 .7681494 .944

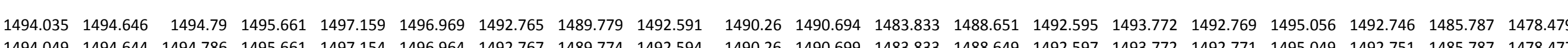

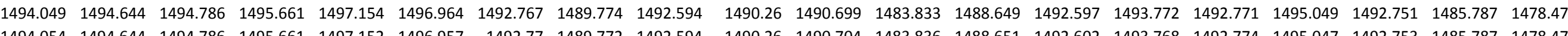

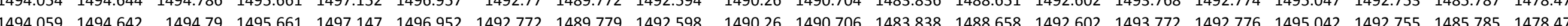

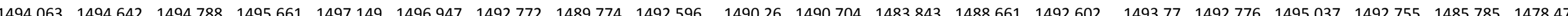

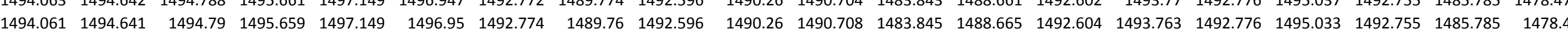
$\begin{array}{lllllllllllllllllllll}1494.056 & 1494.644 & 1494.79 & 1495.661 & 1497.149 & 1496.954 & 1492.774 & 1489.772 & 1492.601 & 1490.26 & 1490.708 & 1483.84 & 1488.665 & 1492.606 & 1493.763 & 1492.774 & 1495.03 & 1492.755 & 1485.785 & 1478.47\end{array}$ $\begin{array}{lllllllllllllllllllllll}1494.052 & 1494.646 & 1494.79 & 1495.661 & 1497.152 & 1496.959 & 1492.777 & 1489.763 & 1492.603 & 1490.26 & 1490.711 & 1483.831 & 1488.668 & 1492.609 & 1493.768 & 1492.778 & 1495.03 & 1492.76 & 1485.785 & 1478.468\end{array}$

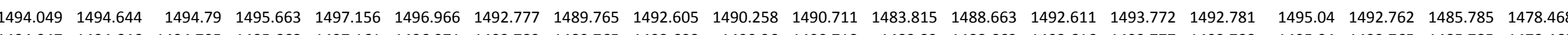

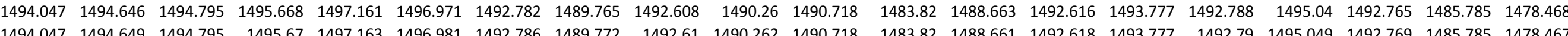

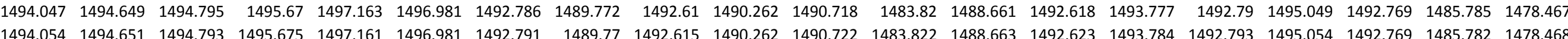
$\begin{array}{llllllllllllllllllllllll}1494.056 & 1494.651 & 1494.793 & 1495.677 & 1497.159 & 1496.976 & 1492.796 & 1489.77 & 1492.619 & 1490.265 & 1490.725 & 1483.822 & 1488.663 & 1492.625 & 1493.796 & 14928 & 1495.061 & 1492.776 & 1485.782 & 1478.4656\end{array}$ $\begin{array}{llllllllllllllllllllll}1494.063 & 1494.651 & 1494.798 & 1495.682 & 1497.159 & 1496.969 & 1492.803 & 1489.767 & 1492.626 & 1490265 & 1490727 & 1483826 & 1488.668 & 1492.632 & 1493.791 & 14928 & 1495.061 & 1492.781 & 1485.785 & 1478.468\end{array}$ $\begin{array}{lllllllllllllllllllllll}1494.071 & 1494.651 & 1494.793 & 1495.682 & 1497.154 & 1496.966 & 1492.8 & 1489.77 & 1492.629 & 1490.267 & 1490.732 & 1483.829 & 1488.67 & 1492.632 & 1493.791 & 1492.807 & 1495.056 & 1492.786 & 1485.782 & 1478.466\end{array}$ $\begin{array}{llllllllllllllllllllllll}1494.078 & 1494.651 & 1494.793 & 1495.679 & 1497.154 & 1496.957 & 1492.807 & 1489.767 & 1492.631 & 1490.267 & 1490.734 & 1483.833 & 1488.679 & 1492.637 & 1493.793 & 1492.807 & 1495.056 & 1492.788 & 1485.782 & 1478.468\end{array}$ $\begin{array}{llllllllllllllllllll}1494.078 & 1494.651 & 1494.795 & 1495.682 & 1497.149 & 1496.952 & 1492.81 & 1489.77 & 1492.633 & 1490.265 & 1490.739 & 1483.84 & 1488.684 & 1492.637 & 1493.791 & 1492.811 & 1495.049 & 1492.788 & 1485.782 & 1478.468\end{array}$

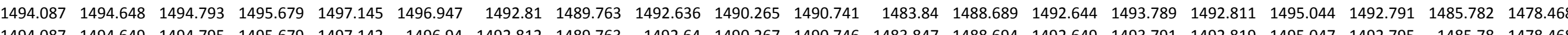

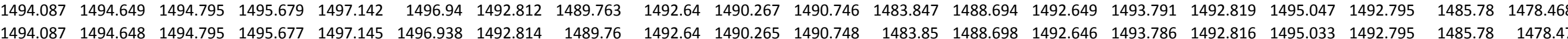
$\begin{array}{lllllllllllllllllllllll}1494.087 & 1494.648 & 1494.795 & 1495.677 & 1497.145 & 1496.938 & 1492.814 & 1489.76 & 1492.64 & 1490.265 & 1490.748 & 1483.85 & 1488.698 & 1492.646 & 1493.786 & 1492.816 & 1495.033 & 1492.795 & 1485.78 & 1478.47 \\ 1494.094 & 1494.649 & 1494.795 & 1495677 & 1497.14 & 1496942 & 1492819 & 1489.758 & 1492645 & 1490265 & 1490753 & 1483854 & 1488698 & 1492651 & 1493784 & 1492819 & 1495.037 & 14928 & 148578 & 1478.47\end{array}$ $\begin{array}{llllllllllllllllllllllll}1494.087 & 1494.651 & 1494.793 & 1495.677 & 1497.14 & 1496.945 & 1492.819 & 1489.753 & 1492.648 & 1490.262 & 1490.757 & 1483.859 & 1488.698 & 1492.651 & 1493.782 & 1492.823 & 1495.033 & 1492.8 & 1485.78 & 1478.47\end{array}$

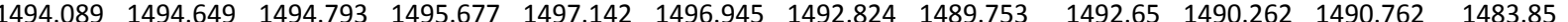

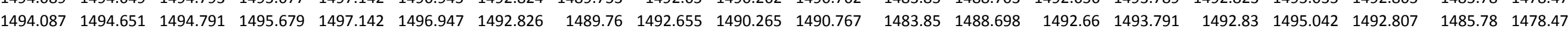

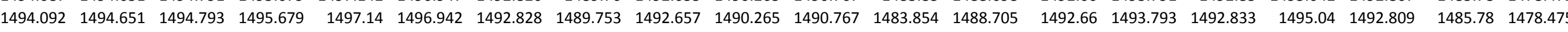

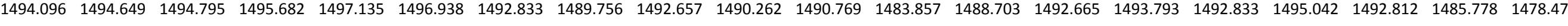

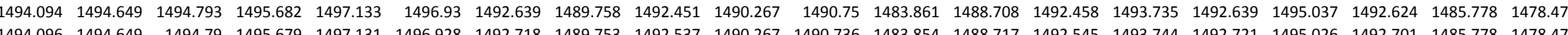

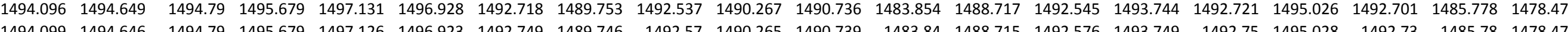

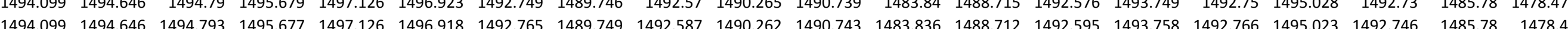
$\begin{array}{lllllllllllllllllllllll}1494.092 & 1494.646 & 1494.795 & 1495.677 & 1497.124 & 1496.921 & 1492.774 & 1489.749 & 1492.601 & 1490.262 & 1490.743 & 1483.831 & 1488.71 & 1492.602 & 1493.758 & 1492.778 & 1495.021 & 1492.755 & 1485.778 & 1478.47\end{array}$ $\begin{array}{lllllllllllllllllllll}1494.085 & 1494.646 & 1494.795 & 1495.672 & 1497.126 & 1496.921 & 1492.772 & 1489.746 & 1492.598 & 1490.262 & 1490.743 & 1483.831 & 1488.715 & 1492.604 & 1493.758 & 1492.778 & 1495.009 & 1492.753 & 1485.778 & 1478.467\end{array}$ $\begin{array}{llllllllllllllllllllll}1494.075 & 1494.648 & 1494.795 & 1495.675 & 1497.128 & 1496.926 & 1492.782 & 1489.739 & 1492.605 & 1490.26 & 1490.748 & 1483.833 & 1488.708 & 1492.613 & 1493.758 & 1492.785 & 1495.011 & 1492.762 & 1485.775 & 1478.468\end{array}$ $\begin{array}{llllllllllllllllllllllll}1494.08 & 1494.649 & 1494.793 & 1495.675 & 1497.126 & 1496.933 & 1492.788 & 1489.746 & 1492.615 & 1490.262 & 1490.746 & 1483.838 & 1488.701 & 1492.62 & 1493.763 & 1492.793 & 1495.019 & 1492.772 & 1485.778 & 1478.4653 & \end{array}$

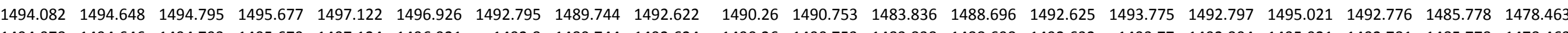

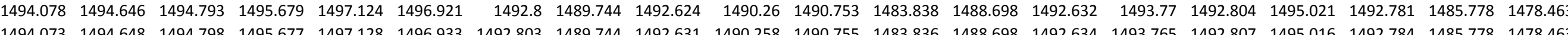

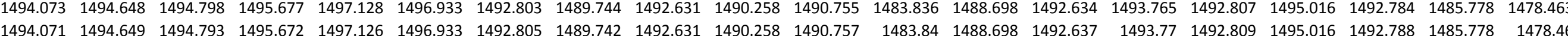
$\begin{array}{lllllllllllllllllllll}1494.071 & 1494.649 & 14948 & 1495679 & 1497.124 & 1496.933 & 1492812 & 1489.741 & 1492.636 & 1490.26 & 1490.76 & 1483843 & 1488.694 & 1492.644 & 1493.777 & 1492814 & 1495.026 & 1492.791 & 1485.578 & 1478.458\end{array}$ $\begin{array}{llllllllllllllllllll}1494.075 & 1494.646 & 1494.795 & 1495.679 & 1497.124 & 1496.933 & 1492.812 & 1489.749 & 1492.64 & 1490.258 & 1490.762 & 1483.845 & 1488.689 & 1492.646 & 1493.779 & 1492.819 & 1495.023 & 1492.795 & 1485.778 & 1478.458\end{array}$ $\begin{array}{llllllllllllllllllllll}1494.085 & 1494.648 & 1494.793 & 1495.682 & 1497.117 & 1496.921 & 1492.819 & 1489.746 & 1492.645 & 1490.26 & 1490.765 & 1483.847 & 1488.684 & 1492.651 & 1493.782 & 1492.821 & 1495.028 & 1492.802 & 1485.778 & 1478.458\end{array}$

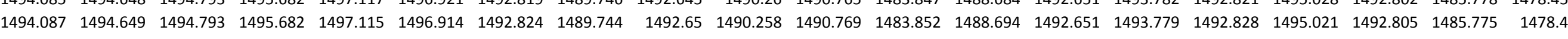

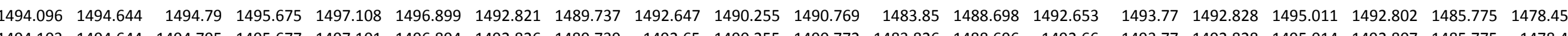

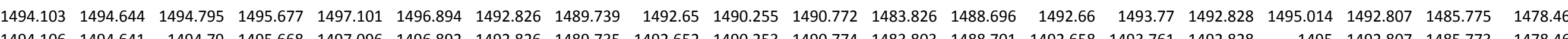
$\begin{array}{llllllllllllllllllll}1494.106 & 1494.641 & 1494.79 & 1495.6688 & 1497.096 & 1496.892 & 1492.826 & 1489.735 & 1492.652 & 1490.253 & 1490.774 & 1483.803 & 1488.701 & 1492.658 & 1493.761 & 1492.828 & 1495 & 1492.807 & 1485.773 & 1478.46 \\ 1494.115 & 1494.637 & 1494.788 & 1495.666 & 1497.089 & 1496.88 & 1492824 & 1489.735 & 1492.652 & 1490255 & 1490776 & 1483796 & 1488701 & 1492658 & 1493758 & 1492828 & 1494997 & 1492807 & 1485775 & 1478.46\end{array}$

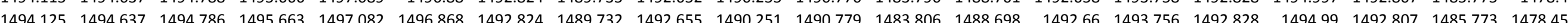
$\begin{array}{llllllllllllllllllllll}1494.132 & 1494.632 & 1494.783 & 1495.659 & 1497.073 & 1496.854 & 1492.821 & 1489.73 & 1492.655 & 1490.251 & 1490.781 & 1483.801 & 1488.703 & 1492.658 & 1493.749 & 1492.83 & 1494.981 & 1492.805 & 1485.771 & 1478.458\end{array}$ $\begin{array}{llllllllllllllllllllll}1494.134 & 1494.629 & 1494.786 & 1495.652 & 1497.068 & 1496.847 & 1492.821 & 1489.725 & 1492.65 & 1490.246 & 1490.781 & 1483.806 & 1488.71 & 1492.658 & 1493.74 & 1492.826 & 1494.962 & 1492.802 & 1485.771 & 1478.456\end{array}$ \begin{tabular}{llllllllllllllllllll}
1494.134 & 1494.627 & 1494.781 & 1495.647 & 1497.063 & 1496.842 & 1492.821 & 1489.727 & 1492.648 & 1490.244 & 1490.781 & 1483.81 & 1488.708 & 1492.653 & 1493.737 & 1492.823 & 1494.957 & 1492.802 & 1485.771 & 1478.453 \\
\hline
\end{tabular}

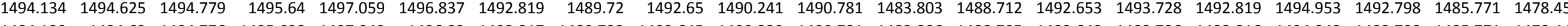
$\begin{array}{lllllllllllllllllllll}1494.139 & 1494.62 & 1494.776 & 1495.638 & 1497.049 & 1496.83 & 1492.817 & 1489.723 & 1492.645 & 1490.239 & 1490.781 & 1483.806 & 1488.705 & 1492.649 & 1493.726 & 1492.816 & 1494.943 & 1492.798 & 1485.771 & 1478.449\end{array}$ $\begin{array}{llllllllllllllllllll}1494.143 & 1494.62 & 1494.781 & 1495.635 & 1497.045 & 1496.813 & 1492.814 & 1489.72 & 1492.645 & 1490.237 & 1490.783 & 1483.801 & 1488.708 & 1492.646 & 1493.719 & 1492.816 & 1494.943 & 1492.793 & 1485.771 & 1478.444 \\ 1494139 & 1494.618 & 1494.781 & 1495.626 & 1497.038 & 1496811 & 1492812 & 1489.716 & 1492.64 & 1490.234 & 1490.781 & 1483.799 & 1488.71 & 1492646 & 1493714 & 1492.811 & 1494925 & 1492.791 & 1485.768 & 1478.442\end{array}$ 
Well

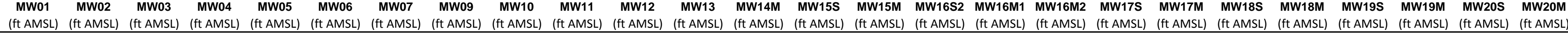

10/12/11 22:00 $10 / 12 / 1122: 30$ 10/12/1123:00 10/12/11/11 0:00 $10 / 13 / 110: 30$
$10 / 13 / 111: 00$ 10/13/11 1:30 10/13/11 2:00 10/13/11 2:30 10/13/11 3:00 10/13/11 4:00 10/13/11 5:00 10/13/11 5:30 10/13/11 6:00 10/13/117:00 10/13/117:30 $10 / 13 / 118: 00$
$10 / 13 / 118: 30$ 10/13/11 9:00 10/13/11 10:00 10/13/11 10:30 10/13/11 11:00 10/13/11 11:30 10/13/11 12:00 10/13/11 12:11 13:00 10/13/11 13:11 13:30 10/13/11 14:00 10/13/11 14:30 10/13/11 15:30 10/13/11 16:00 10/13/11 16:30 10/13/11 17:00 10/13/11 18:00 10/13/11 18:30 10/13/11 19:00 10/13/11 19:30 10/13/11 20:00 10/13/11 20:30 10/13/11 21:00 10/13/11 21:30 10/13/11 22:30 10/13/11 23:00 10/13/11 23:30 10/14/11 0:00
10/14/11 0:30 $\begin{array}{llll}1494.505 & 1495.021 & 1496.742 & 1494.911 \\ 1494.507 & 1495.019 & 1496.738 & 1494.904\end{array}$ $\begin{array}{llll}1494.507 & 1495.019 & 1496.738 & 1494.904 \\ 1494.505 & 1495017 & 1496735 & 1494.99\end{array}$ $\begin{array}{lllll}1494.505 & 1495.017 & 1496.735 & 1494.899 \\ 1494.505 & 1495.012 & 1496735 & 1494895\end{array}$ $\begin{array}{llllll}1494.503 & 1495.01 & 1496.738 & 1494.89\end{array}$ $\begin{array}{lllll}1494.503 & 1495.01 & 1496.745 & 1494.883\end{array}$ $\begin{array}{lllll}1494.505 & 1495.007 & 1496.749 & 1494.883\end{array}$ $\begin{array}{lllll}1494.505 & 1495.005 & 1496.745 & 1494.885\end{array}$ $\begin{array}{lllll}1494.505 & 1495.007 & 1496.742 & 1494.885\end{array}$ $\begin{array}{lllll}1494.505 & 1495.012 & 1496.74 & 1494.888\end{array}$ $\begin{array}{llll}1494.503 & 1495.007 & 1496.733 & 1494.885 \\ 1494.507 & 1495007 & 1496.733 & 1994888\end{array}$ $\begin{array}{llll}1494.507 & 1495.007 & 1496.733 & 1494.888 \\ 1494.503 & 1495.005 & 1496.728 & 1494.885\end{array}$ $\begin{array}{lllll}1494.503 & 1495.003 & 1496.726 & 1494.883\end{array}$ $\begin{array}{lllll}1494.5 & 1494.998 & 1496.724 & 1494.883\end{array}$ $\begin{array}{llll}1494.498 & 14494.998 & 1496.724 & 1494.878\end{array}$ $\begin{array}{lllll}1494.5 & 1494.996 & 1496.721 & 1494.878\end{array}$ $\begin{array}{lllll}1494.498 & 1494.993 & 1496.716 & 1494.876\end{array}$ $\begin{array}{lllll}1494.498 & 1494.993 & 1496.719 & 1494.874\end{array}$ $\begin{array}{lllll}1494.498 & 1494.993 & 1496.705 & 1494.874\end{array}$ $\begin{array}{llll}1494.496 & 1494.989 & 1496.695 & 1494.871\end{array}$ $\begin{array}{llllll}1494.491 & 1494.982 & 1496.691 & 1494.862\end{array}$ $\begin{array}{lllll}1449.491 & 1449.979 & 1496.691 & 1494.857\end{array}$ $1494.4891494 .975 \quad 1496.698 \quad 1494.855$ $\begin{array}{lllll}1494.489 & 1494.972 & 1496.707 & 1494.85\end{array}$ $1494.489 \quad 1494.97 \quad 1496.719 \quad 1494.848$ $\begin{array}{lllll}1494.491 & 1494.97 & 1496.726 & 1494.853\end{array}$ $\begin{array}{lllll}1494.493 & 1494.975 & 1496.747 & 1494.853 \\ 1494.496 & 1494.982 & 1496763 & 1494.86\end{array}$ $\begin{array}{llll}1494.496 & 1494.986 & 1496.78 & 1494.867\end{array}$ $\begin{array}{lllll}1494.503 & 1494.991 & 1496.796 & 1494.878\end{array}$ $\begin{array}{lllll}1494.505 & 1495.003 & 1496.81 & 1494.89\end{array}$ $\begin{array}{lllll}1494.509 & 1495.012 & 1496.815 & 1494.902\end{array}$ $\begin{array}{llll}1494.512 & 1495.019 & 1496.81 & 1494.916\end{array}$ $\begin{array}{llll}1494.514 & 1495.028 & 1496.812 & 1494.925 \\ 1494.514 & 1955.033 & 196812 & 1994937\end{array}$ $\begin{array}{llll}1494.514 & 1495.033 & 1496.812 & 1494.937 \\ 1494514 & 1495.035 & 1496812 & 1994.944\end{array}$ $\begin{array}{lllll}1494.512 & 1495.038 & 14698815 & 1494.944\end{array}$ $\begin{array}{lllll}1494.514 & 1495.035 & 1496.812 & 1494.951\end{array}$ $\begin{array}{llllll}1494.514 & 1495.04 & 1496.803 & 1494.953\end{array}$ $\begin{array}{lllll}1494.514 & 1495.04 & 1496.796 & 1494.958\end{array}$ $\begin{array}{lllll}1494.514 & 1495.04 & 1496.789 & 1494.958\end{array}$ $\begin{array}{llll}1494.512 & 1495.035 & 1496.791 & 1494.953\end{array}$ $\begin{array}{rrrr}1494.509 & 1495.033 & 1496.791 & 1494.951 \\ 1494.51 & 1495.031 & 1496.789 & 1994.946\end{array}$ $\begin{array}{lllll}1494.51 & 1495.031 & 1496.789 & 1494.946\end{array}$ $\begin{array}{lllll}1444.512 & 1455.035 & 1496.787 & 1494.946\end{array}$ $\begin{array}{lllll}1494.509 & 1495.031 & 1496.787 & 1494.944\end{array}$ $\begin{array}{lllll}1494.514 & 1495.033 & 1496.78 & 1494.941\end{array}$ $\begin{array}{lllll}1494.512 & 1495.033 & 1496.782 & 1494.941\end{array}$ $\begin{array}{lllll}1494.512 & 1495.031 & 1496.784 & 1494.939\end{array}$ $\begin{array}{rrrrr}1494.512 & 1495.031 & 1496.78 & 1494.939 \\ 1494.514 & 1495.033 & 1496.768 & 1494.937\end{array}$ $\begin{array}{llllllllllllllllllll}1494.136 & 1494.615 & 1494.781 & 1495.619 & 1497.033 & 1496.808 & 1492.807 & 1489.716 & 1492.633 & 1490.232 & 1490.776 & 1483.808 & 1488.712 & 1492.644 & 1493.707 & 1492.809 & 1494.92 & 1492.786 & 1485.768 & 1478.439\end{array}$

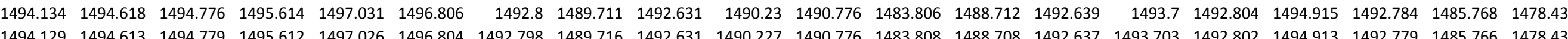

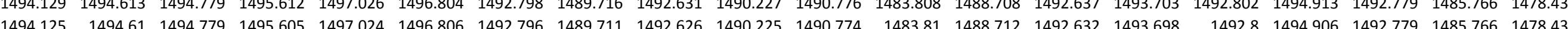
$\begin{array}{lllllllllllllllllllllll}1494.115 & 1494.608 & 1494.779 & 1495603 & 1497.022 & 1496.806 & 1492795 & 1489.706 & 1492.624 & 1490.223 & 1490.774 & 1483808 & 1488.712 & 1492.632 & 1493.693 & 1492.8 & 1494.908 & 1492.776 & 1485.766 & 1478.43\end{array}$ $\begin{array}{llllllllllllllllllllll}1494.106 & 1494.608 & 1494.779 & 1495.601 & 1497.024 & 1496.813 & 1492.793 & 1489.711 & 1492.624 & 1490.223 & 1490.774 & 1483.808 & 1488.708 & 1492.63 & 1493.691 & 1492.797 & 1494.901 & 1492.774 & 1485.766 & 1478.428\end{array}$ $\begin{array}{llllllllllllllllllll}1494.101 & 1494.605 & 1494.781 & 1495.598 & 1497.024 & 1496.818 & 1492.791 & 1489.704 & 1492.624 & 1490.22 & 1490.774 & 1483.813 & 1488.701 & 1492.627 & 1493.696 & 1492.793 & 1494.908 & 1492.774 & 1485.766 & 1478.428 \\ & & & \end{array}$ $\begin{array}{llllllllllllllllllllll}1494.094 & 1494.606 & 1494.776 & 1495.598 & 1497.022 & 1496.825 & 1492.793 & 1489.704 & 1492.624 & 1490.22 & 1490.774 & 1483.81 & 1488.694 & 1492.627 & 1493.696 & 1492.797 & 1494.913 & 1492.774 & 1485.768 & 1478.425\end{array}$

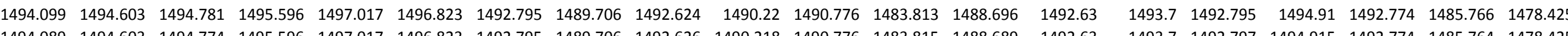

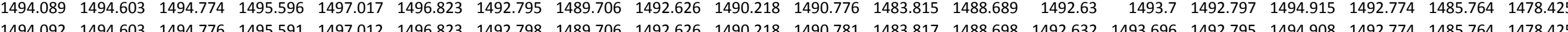

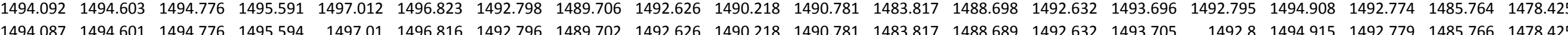

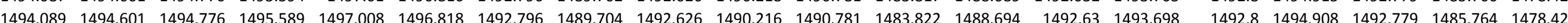
$\begin{array}{llllllllllllllllllll}1494.087 & 1494.596 & 1494.774 & 1495587 & 1497.005 & 1496813 & 1492.798 & 1489.704 & 1492.629 & 1490213 & 1490.783 & 1483.824 & 1488.694 & 1492.63 & 1493.7 & 1492.802 & 1494.908 & 1492.779 & 1485.764 & 1478.425\end{array}$ $\begin{array}{lllllllllllllllllllll}1494.087 & 1494.596 & 1494.776 & 1495.584 & 1497.001 & 1496.816 & 1492.8 & 1489.699 & 1492.629 & 1490.211 & 1490.788 & 1483.82 & 1488.696 & 1492.634 & 1493.698 & 1492.804 & 1494.903 & 1492.779 & 1485.764 & 1478.425\end{array}$

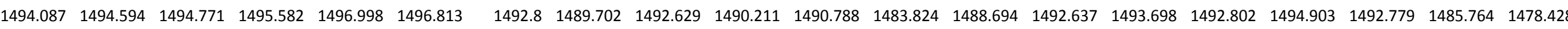
$\begin{array}{lllllllllllllllllllllllll}1494.08 & 1494.594 & 1494.774 & 1495.58 & 1496.998 & 1496.813 & 1492.8 & 1489.702 & 1492.633 & 1490.211 & 1490.79 & 1483.813 & 1488.698 & 1492.63 & 1493.696 & 1492.802 & 1494.899 & 1492.781 & 1485.761 & 1478.428\end{array}$

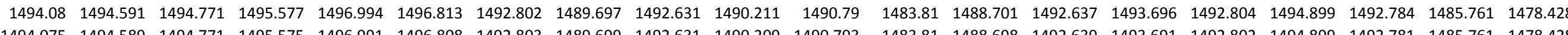

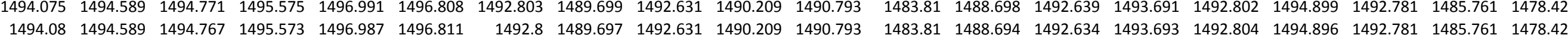
$\begin{array}{llllllllllllllllllllll}1494.08 & 1494.589 & 1494.767 & 1495.573 & 1496.987 & 1496.811 & 1492.8 & 1489.697 & 1492.631 & 1490.209 & 1490.793 & 1483.81 & 1488.694 & 1492.634 & 1493.693 & 1492.804 & 1494.896 & 1492.781 & 1485.761 & 1478.428 \\ 1494.082 & 1494.587 & 1494.769 & 1495.57 & 149698 & 1496801 & 1492.8 & 1489697 & 1492629 & 1490206 & 1490795 & 1483815 & 1488694 & 1492639 & 1493693 & 1492802 & 1494896 & 1492781 & 1485 & 1461 & 1478.428\end{array}$ $\begin{array}{lllllllllllllllllllll}14494.078 & 1444.584 & 1444.771 & 1495.568 & 1496.977 & 1496.792 & 1492.798 & 1489.692 & 1492.631 & 1490.204 & 1490.793 & 1483.815 & 1488.691 & 1492.637 & 1493.691 & 1492.802 & 1494.894 & 1492.781 & 1485.761 & 1478.428\end{array}$ \begin{tabular}{llllllllllllllllllllll}
1494.075 & 1494.579 & 1494.764 & 1495.561 & 1496.973 & 1496.792 & 1492.798 & 1489.695 & 1492.629 & 1490.204 & 1490.79 & 1483815 & 1488694 & 1492.637 & 1493.684 & 1492.802 & 1494.882 & 1492.779 & 1485.759 & 1478.425 \\
\hline
\end{tabular}

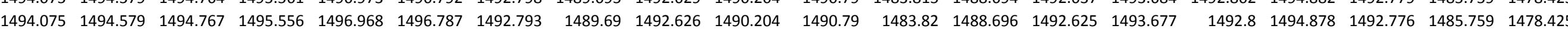

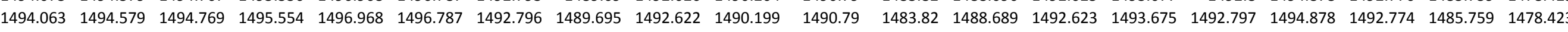
\begin{tabular}{lllllllllllllllllllll}
1494.054 & 1494.577 & 1494.771 & 1495.549 & 1496.97 & 1496.794 & 1492.777 & 1489.69 & 1492.61 & 1490.197 & 1490.79 & 1483.82 & 1488.687 & 1492.611 & 1493.67 & 1492.781 & 1494.87 & 1492.758 & 1485.759 & 1478.42 \\
\hline
\end{tabular}

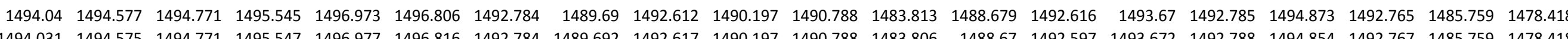

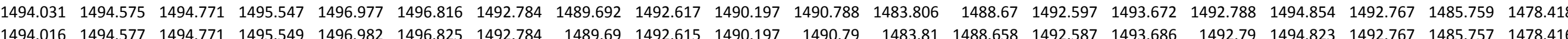

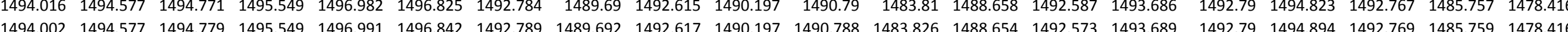
$\begin{array}{llllllllllllllllllll}1493.991 & 1494.579 & 1494.776 & 1495.552 & 1496.998 & 1496.861 & 1492.791 & 1489.69 & 1492.619 & 1490.197 & 1490.79 & 1483.817 & 1488.642 & 1492.625 & 1493.693 & 1492.795 & 1494.899 & 1492.772 & 1485.759 & 1478.416\end{array}$ $\begin{array}{lllllllllllllllllllll}1493.981 & 1494.579 & 1494.779 & 1495.559 & 1497.005 & 1496.878 & 1492.795 & 1489.697 & 1492.624 & 1490.197 & 1490.79 & 1483.82 & 1488.633 & 1492.627 & 1493.705 & 1492.797 & 1494.915 & 1492.774 & 1485.759 & 1478.416\end{array}$

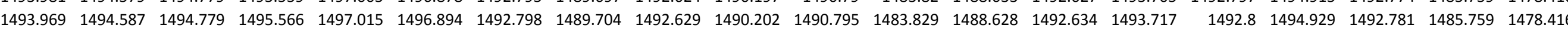
$\begin{array}{llllllllllllllllllll}1493.967 & 1494.589 & 1494.783 & 1495.57 & 1497.019 & 1496.906 & 1492.807 & 1489.704 & 1492.63 & 1490.202 & 1490.193 & 1483.84 & 1488.625 & 1492.642 & 1493.73 & 1492.804 & 1494.943 & 1492.788 & 1485.759 & 1478.418\end{array}$

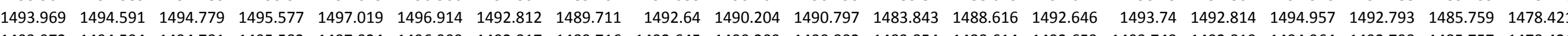

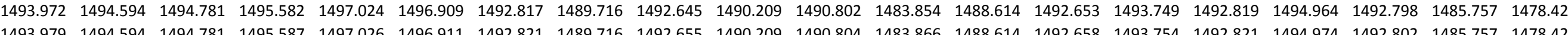

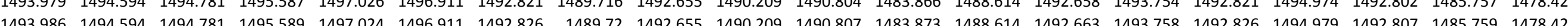

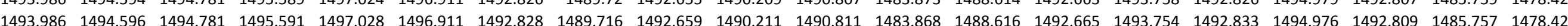
$\begin{array}{llllllllllllllllllllll}1493.993 & 1494.599 & 1494.779 & 1495.594 & 1497.028 & 1496.911 & 1492.831 & 1489.718 & 1492.657 & 1490.211 & 1490.809 & 1483.875 & 1488.616 & 1492.67 & 1493.758 & 1492.833 & 1494.974 & 1492.814 & 1485.757 & 1478.425\end{array}$ $\begin{array}{llllllllllllllllllllll}1494.005 & 1494.599 & 1494.779 & 1495.596 & 1497.026 & 1496.909 & 1492.835 & 1489.727 & 1492.664 & 1490.211 & 1490.814 & 1483.884 & 1488.614 & 1492.67 & 1493.761 & 1492.838 & 1494.983 & 1492.819 & 1485.757 & 1478.428 \\ \end{array}$

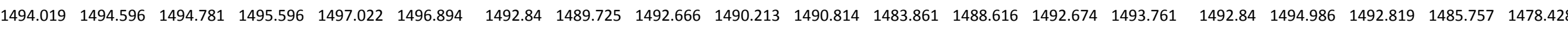

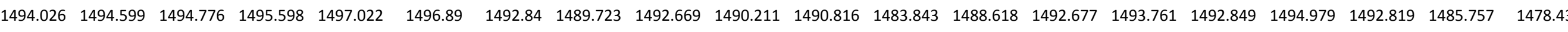

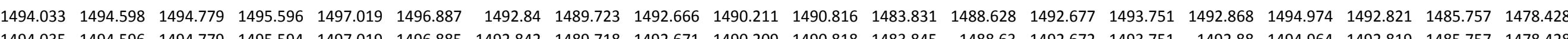

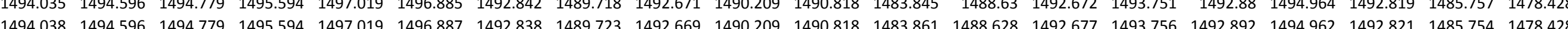

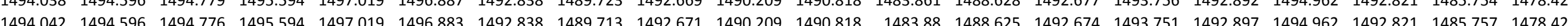
$\begin{array}{llllllllllllllllllll}1494.042 & 1494.599 & 1494.776 & 1495.596 & 1497.017 & 1496.88 & 1492.84 & 1489.718 & 1492.671 & 1490.209 & 1490.818 & 1483.896 & 1488.628 & 1492.677 & 1493.758 & 1492.906 & 1494.962 & 1492.823 & 1485.754 & 1478.428\end{array}$ $\begin{array}{llllllllllllllllllllll}1494.042 & 1494.596 & 1494.779 & 1495.594 & 1497.022 & 1496.88 & 1492.838 & 1489.718 & 1492.671 & 1490.209 & 1490.818 & 1483.912 & 1488.63 & 1492.677 & 1493.751 & 1492.911 & 1494.957 & 1492.821 & 1485.754 & 1478.425\end{array}$ $\begin{array}{llllllllllllllllllllll}1494.047 & 1494.596 & 1494.779 & 1495.596 & 1497.017 & 1496.88 & 1492.84 & 1489.711 & 1492.669 & 1490.206 & 1490.818 & 1483.931 & 1488.633 & 1492.677 & 1493.754 & 1492.909 & 1494.96 & 1492.821 & 1485.754 & 1478.425 \\ \end{array}$ \begin{tabular}{llllllllllllllllllll}
1494.049 & 1494.599 & 1494.776 & 1495.596 & 1497.015 & 1496.873 & 1492.84 & 1489.713 & 1492.671 & 1490.206 & 1490.816 & 1483.944 & 1488.633 & 1492.674 & 1493.749 & 1492.913 & 1494.955 & 1492.819 & 1485.754 & 1478.423 \\
\hline
\end{tabular} $\begin{array}{lllllllllllllllllllll}1494.052 & 1494.596 & 1494.776 & 1495.594 & 1497.017 & 1496.875 & 1492.84 & 1489.713 & 1492.669 & 1490.206 & 1490.816 & 1483.961 & 1488.635 & 1492.677 & 1493.751 & 1492.916 & 1494.957 & 1492.821 & 1485.752 & 1478.421 \\ \end{array}$

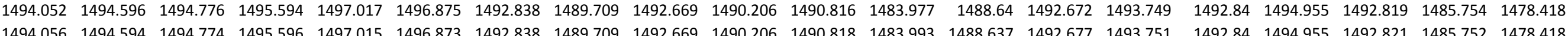


Well

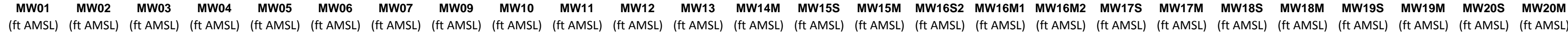

10/14/11 1:00 10/14/11 1:30 $10 / 14 / 112 \cdot 30$ 10/14/11 3:300 10/14/11 3:30 10/14/11 4:00 10/14/11 4:30 10/14/11 5:00 10/14/11 5:30 10/14/11 6:00 10/14/11 6:30 10/14/11 7:30 10/14/11 7:30 10/14/11 8:30 10/14/11 9:00 10/14/11 9:30 10/14/11 10:00 10/14/11 10:30 10/14/11 11:30 0/14/11 12:00 10/14/11 12:30 10/14/11 13:00 10/14/11 13:30 10/14/11 14:00 10/14/11 14:30 10/14/11 15:00 10/14/11 16:00 0/14/11 16:30 10/14/11 17:00 10/14/11 17:30 10/14/11 18:00 10/14/11 18:30 10/14/11 19:00 10/14/11 19:30 10/14/11 20:30 10/14/11 21:00 10/14/11 21:30 10/14/11 22:00 10/14/11 22:30 10/14/11 23:00 0/14/11 23:30 10/15/11 0:00 10/15/11 1:00 10/15/11 1:30 10/15/11 2:00 10/15/11 3:00 $\begin{array}{llll}1494.514 & 1495.033 & 1496.759 & 1494.939 \\ 1494.512 & 1495.031 & 1496738 & 1494.934\end{array}$ $\begin{array}{llll}1494.512 & 1495.031 & 1496.738 & 1494.934 \\ 1494.507 & 1495.024 & 1496.733 & 1994.927\end{array}$ $\begin{array}{lllll}1494.507 & 1495.024 & 1496.733 & 1494.927 \\ 1494.498 & 1495012 & 1496738 & 1494913\end{array}$ $\begin{array}{lllll}1494.503 & 1495.012 & 1496.74 & 1494.909\end{array}$ $\begin{array}{lllll}1494.503 & 1495.01 & 1496.735 & 1494.909\end{array}$ $\begin{array}{llll}1494.505 & 1495.01 & 1496.733 & 1494.902\end{array}$ $\begin{array}{lllll}1494.505 & 1495.012 & 1496.726 & 1494.899\end{array}$ $\begin{array}{lllll}1494.505 & 1495.007 & 1496.719 & 1494.897\end{array}$ $\begin{array}{rrrrr}1494.503 & 1495.005 & 1496.709 & 1494.892 \\ 1494.5 & 1495.003 & 1496.698 & 1494.888 \\ 1494.5 & 1494.998 & 1496.688 & 1494.883\end{array}$ $\begin{array}{rrrr}1494.5 & 1494.998 & 1496.688 & 1494.883 \\ 1494.498 & 1494.993 & 1496.681 & 1494.876\end{array}$ $\begin{array}{lllll}1494.493 & 1494.984 & 1496.667 & 1494.855\end{array}$ $\begin{array}{lllll}1494.493 & 1494.982 & 1496.66 & 1494.853\end{array}$ $\begin{array}{lllll}1494.489 & 1494.977 & 1496.656 & 1494.801\end{array}$ $\begin{array}{lllll}1494.486 & 1494.968 & 1496.653 & 1494.762\end{array}$ $\begin{array}{llll}1494.484 & 1494.958 & 1496.656 & 1494.727\end{array}$ $1494.482 \quad 1494.954 \quad 1496.656 \quad 1494.694$ $\begin{array}{rrrr}1494.484 & 1494.949 & 1496.66 & 1494.671 \\ 1494.482 & 1494944 & 1496.672 & 1994.654\end{array}$ $\begin{array}{llll}1494.482 & 1494.944 & 1496.672 & 1494.654 \\ 1494.479 & 1494.944 & 1496.677 & 1494.645\end{array}$ $\begin{array}{lllll}1494.484 & 1494.944 & 14966.679 & 1499.643\end{array}$ $\begin{array}{rlrrr}1494.484 & 1494.947 & 1496.684 & 14944.64\end{array}$ $\begin{array}{lllll}1494.484 & 1494.944 & 1496.693 & 1494.633\end{array}$ 1494.484 $1494.9491496 .707 \quad 1494.622$ $1494.484 \quad 1494.947 \quad 1496.709 \quad 1494.605$ $1494.484 \quad 1494.949 \quad 1496.707 \quad 1494.591$ $\begin{array}{lllll}1494.486 & 1494.949 & 1496.705 & 1494.577 \\ 1494.486 & 1494.954 & 1496.709 & 1494.566\end{array}$ $\begin{array}{llll}1494.482 & 1449.949 & 1496.702 & 1494.556\end{array}$ $\begin{array}{lllll}1494.479 & 1494.949 & 1496.698 & 1494.552\end{array}$ 1494.479 1494.9491496 .688 1494.554 $1494.477 \quad 1494.947 \quad 1496.686 \quad 1494.549$ $\begin{array}{lllll}1494.475 & 1494.942 & 1496.677 & 1494.538\end{array}$

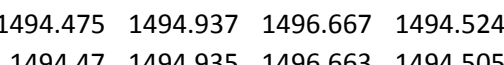
$\begin{array}{llll}1494.47 & 1494.935 & 1496.663 & 1494.505 \\ 1494.468 & 1494.926 & 199663 & 1494.477\end{array}$ $\begin{array}{lllll}1494.465 & 1494.921 & 14696656 & 1494.479\end{array}$ $\begin{array}{lllll}1494.465 & 1494.919 & 1496.653 & 1494.425\end{array}$ $1494.463 \quad 1494.914 \quad 1496.644 \quad 1494.407$ $\begin{array}{lllll}1494.461 & 1494.907 & 1496.639 & 1494.39\end{array}$ $\begin{array}{llll}1494.458 & 1494.902 & 1496.635 & 1494.376\end{array}$ $\begin{array}{lllll}1494.454 & 1494.895 & 1496.642 & 1494.367\end{array}$ $\begin{array}{llll}1494.451 & 1494.888 & 1496.644 & 1494.365\end{array}$ $\begin{array}{lllll}1494.451 & 1494.886 & 1496.651 & 1494.365 \\ 1494.451 & 1494.884 & 1496.653 & 1494372\end{array}$ $\begin{array}{llll}14494.451 & 1494.886 & 1496.646 & 1494.379\end{array}$ $\begin{array}{lllll}1494.454 & 1494.888 & 1496.644 & 1494.395\end{array}$ $\begin{array}{lllll}1494.451 & 1494.888 & 1496.639 & 1494.404\end{array}$ $\begin{array}{lllll}1494.444 & 1494.881 & 1496.632 & 1494.411\end{array}$ $\begin{array}{llll}1494.442 & 1494.874 & 1496.635 & 1494.418\end{array}$ $\begin{array}{rrrrr}1494.442 & 1494.872 & 1496.642 & 1494.43 \\ 1494.437 & 1494.872 & 1496.637 & 1494.432\end{array}$

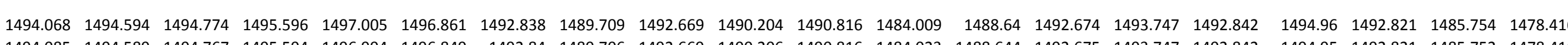

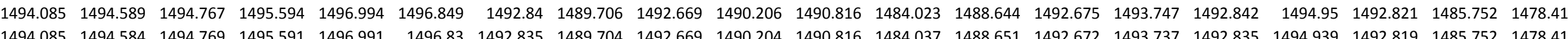

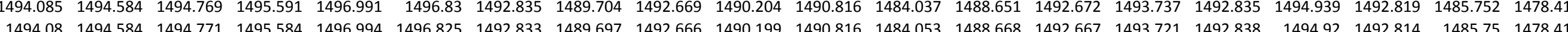
$\begin{array}{lllllllllllllllllllllll}1494.078 & 1494.582 & 1494.769 & 1495.58 & 1496.991 & 1496.83 & 1492833 & 1489.697 & 1492.664 & 1490.197 & 1490816 & 1484.067 & 1488.658 & 1492.67 & 1493.719 & 1492.833 & 1494.915 & 1492.812 & 148575 & 1478.411\end{array}$ $\begin{array}{lllllllllllllllllllllll}1494.073 & 1494.584 & 1494.779 & 1495577 & 1496991 & 1496.83 & 1492.831 & 1489.699 & 1492.664 & 1490.197 & 1490.814 & 1484.081 & 1488.656 & 1492.667 & 1493.719 & 1492.835 & 1494.91 & 1492.814 & 1485.752 & 1478.411\end{array}$

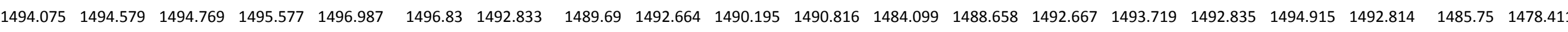

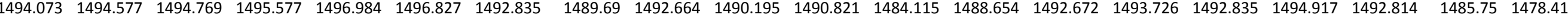

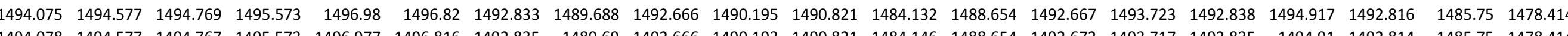

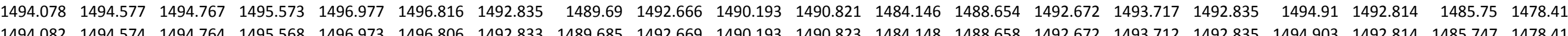
$\begin{array}{lllllllllllllllllllll}1494.082 & 1494.574 & 1494.764 & 1495.568 & 1496.973 & 1496.806 & 1492.833 & 1489.685 & 1492.669 & 1490.193 & 1490.823 & 1484.148 & 1488.658 & 1492.672 & 1493.712 & 1492.835 & 1494.903 & 1492.814 & 1485.747 & 1478.416 \\ 1494.085 & 1494.572 & 1494.762 & 1495.566 & 1496.966 & 1496.794 & 1492.835 & 1489.688 & 1492.666 & 1490.19 & 1490.825 & 1484.155 & 1488.656 & 1492.672 & 1493.714 & 1492.835 & 1494.901 & 1492.814 & 1485.747 & 1478.414\end{array}$

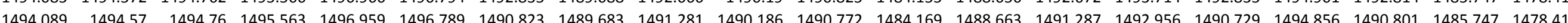
$\begin{array}{lllllllllllllllllllllll}1494.092 & 1494.565 & 1494.76 & 1495.556 & 1496.952 & 1496.78 & 1490.388 & 1489.685 & 1490.671 & 1490.188 & 1490.669 & 1484.183 & 1488.675 & 1490.674 & 1492.746 & 1490.335 & 1494.805 & 1490.378 & 1485.745 & 1478.414\end{array}$ $\begin{array}{lllllllllllllllllllll}1494.094 & 1494.565 & 1494.757 & 1495.552 & 1496.945 & 1496.765 & 1489.669 & 1489.683 & 1490.113 & 1490.186 & 1490.564 & 1484.201 & 1488.672 & 1490.129 & 1492.497 & 1489.617 & 1494.76 & 1489.637 & 1485.745 & 1478.414\end{array}$

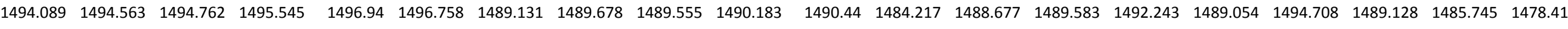
$\begin{array}{llllllllllllllllllllll}1494.087 & 1494.558 & 1494.762 & 1495.54 & 1496.935 & 1496.751 & 1488.907 & 1489.681 & 1489.22 & 1490.181 & 1490.32 & 1484.215 & 1488.677 & 1489.245 & 1492.129 & 1488.852 & 1494.657 & 1488.912 & 1485.745 & 1478.411\end{array}$

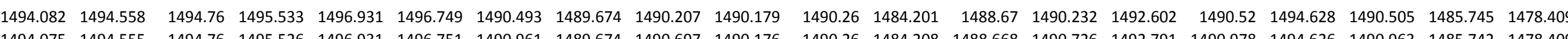

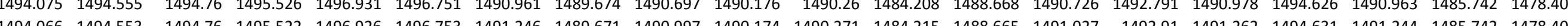

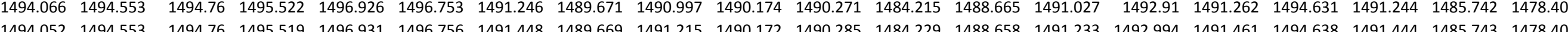
$\begin{array}{lllllllllllllllllllll}1494.042 & 1494.553 & 1494.757 & 1495.517 & 1496.931 & 1496.768 & 1491.597 & 1489.671 & 1491.374 & 1490.172 & 1490.299 & 1484.245 & 1488.651 & 1491.389 & 1493.057 & 1491.61 & 1494.64 & 1491.594 & 1485.743 & 1478.402\end{array}$ $\begin{array}{lllllllllllllllllllll}1494.024 & 1494.551 & 1494.76 & 1495.517 & 1496.935 & 1496.777 & 1489.669 & 1489.669 & 1490.057 & 1490.169 & 1490.248 & 1484.277 & 1488.64 & 1490.044 & 1492.401 & 1489.598 & 1494.633 & 1489.665 & 1485.743 & 1478.4\end{array}$

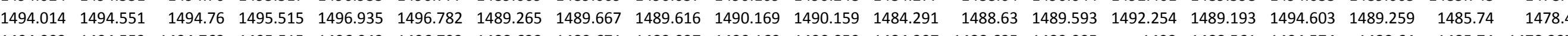

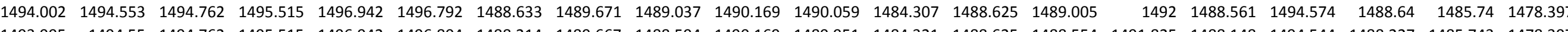

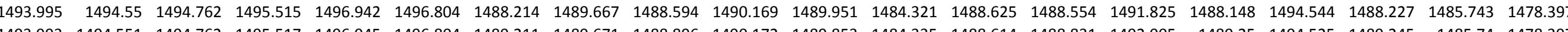

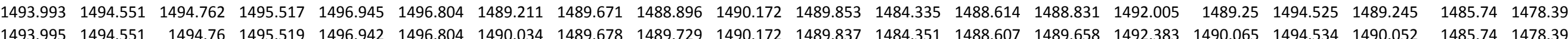
$\begin{array}{lllllllllllllllllllllll}149.995 & 1494.551 & 1494.76 & 1495.519 & 1496.942 & 1496.804 & 1490.034 & 1489.678 & 1489.729 & 1490.172 & 1489.837 & 1484.351 & 1488.607 & 1489.658 & 1492.383 & 1490.065 & 1494.534 & 1490.052 & 1485.74 & 1478.397 \\ 1493.993 & 1494.548 & 1494.76 & 1495.522 & 1496947 & 1496801 & 1490.458 & 1489.681 & 1490.174 & 1490.172 & 1489.86 & 1484.368 & 1488.607 & 1490.105 & 1492.558 & 1490.485 & 1494.544 & 1490.47 & 1485.74 & 1478.4\end{array}$ $\begin{array}{llllllllllllllllllllllll}1493.993 & 1494.548 & 1494.757 & 1495.519 & 1496.945 & 1496.806 & 1490.746 & 1489.678 & 1490.479 & 1490.172 & 1489.886 & 1484.374 & 1488.604 & 1490.415 & 1492.669 & 1490.767 & 1494.551 & 1490.751 & 1485.74 & 1478.397 \\ \end{array}$ $\begin{array}{llllllllllllllllllllll}1493.998 & 1494.548 & 1494.75 & 1495.519 & 1496.942 & 1496.801 & 1490.615 & 1489.667 & 1490.544 & 1490.169 & 1489.916 & 1484.351 & 1488.604 & 1490.484 & 1492.693 & 1490.615 & 1494.56 & 1490.606 & 1485.738 & 1478397\end{array}$ $\begin{array}{lllllllllllllllllllllll}1494.007 & 1494.546 & 1494.755 & 1495.517 & 1496.935 & 1496.794 & 1490.273 & 1489.678 & 1490.202 & 1490.169 & 1489.912 & 1484.349 & 1488.604 & 1490.14 & 1492.527 & 1490.271 & 1494.57 & 1490.275 & 1485.738 & 1478.4\end{array}$ \begin{tabular}{llllllllllllllllllll}
1494.007 & 1494.546 & 1494.755 & 1495.515 & 1496.935 & 1496.787 & 1488.694 & 1489.673 & 1489.262 & 1490.169 & 1489.865 & 1484.361 & 1488.602 & 1489.207 & 1492.026 & 1488.58 & 1494.541 & 1488.689 & 1485.738 & 1478.4 \\
\hline
\end{tabular} \begin{tabular}{lllllllllllllllllllll}
1494.014 & 1494.546 & 1494.752 & 1495.512 & 1496.933 & 1496.784 & 1488.172 & 1489.673 & 1488.561 & 1490.169 & 1489.76 & 1484.377 & 1488.611 & 1488.523 & 1491.76 & 1488.106 & 1494.502 & 1488.184 & 1485.738 & 1478.4 \\
\hline
\end{tabular}

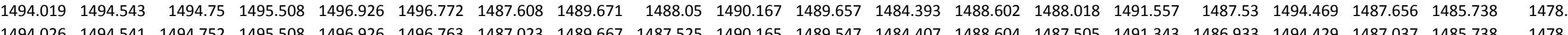

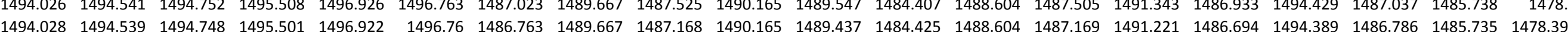
$\begin{array}{lllllllllllllllllllllll}1444.026 & 1494.539 & 1494.75 & 1495.498 & 1496.919 & 1496.758 & 1488013 & 1489.667 & 1487.872 & 1490.162 & 1489.355 & 1484.43 & 1488607 & 1487.884 & 1491.543 & 1488.025 & 1494.363 & 1488.046 & 1485.535 & 1478397\end{array}$ $\begin{array}{llllllllllllllllllllll}1494.028 & 1494.536 & 1494.748 & 1495.494 & 1496.919 & 1496.753 & 1488.427 & 1489.667 & 1488.296 & 1490.162 & 1489.32 & 1484.441 & 1488.602 & 1488.326 & 1491.72 & 1488.435 & 1494.349 & 1488.45 & 1485.735 & 1478.397 \\ \end{array}$ \begin{tabular}{lllllllllllllllllllll}
1494.033 & 1494.534 & 1494.743 & 1495.491 & 1496.912 & 1496.749 & 1488.953 & 1489.664 & 1488.638 & 1490.16 & 1489.299 & 1484.453 & 1488.602 & 1488.671 & 1491.846 & 1488.994 & 1494.349 & 1488.987 & 1485.735 & 1478.395 \\
\hline
\end{tabular} \begin{tabular}{lllllllllllllllllllll}
1494.035 & 1494.532 & 1494.743 & 1495.487 & 1496.91 & 1496.739 & 1489.569 & 1489.657 & 1489.243 & 1490.158 & 1489.323 & 1484.465 & 1488.604 & 1489.294 & 1492.105 & 1489.598 & 1494.351 & 1489.585 & 1485.735 & 1478.395 \\
\hline
\end{tabular}

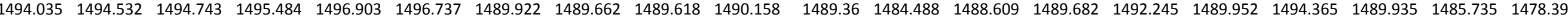

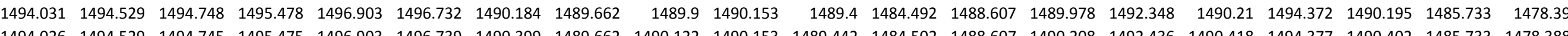

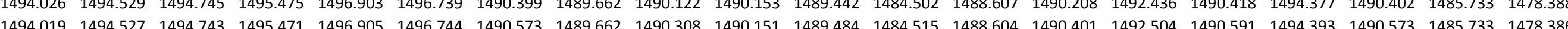

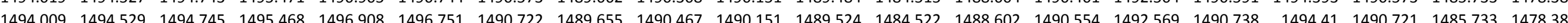

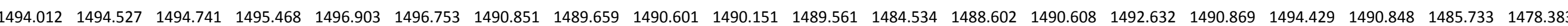
$\begin{array}{llllllllllllllllllllll}1494.012 & 1494.524 & 1494.741 & 1495.471 & 1496.901 & 1496.746 & 1490.966 & 1489.657 & 1490.723 & 1490.151 & 1489.598 & 1484.548 & 1488.593 & 1490.728 & 1492.69 & 1490.982 & 1494.455 & 1490.963 & 1485.733 & 1478.383\end{array}$

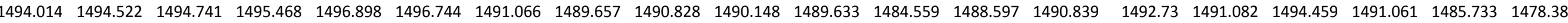

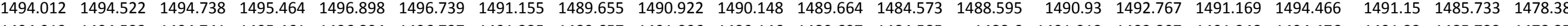

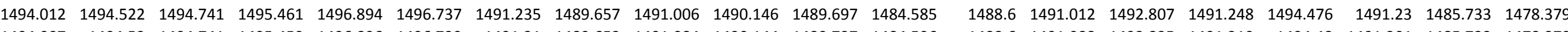

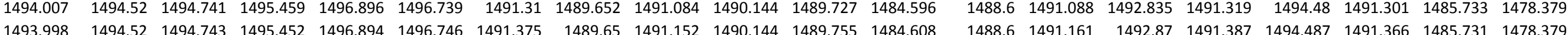


Well

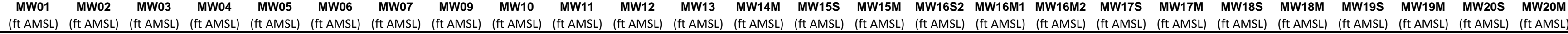

10/15/11 4:00 10/15/11 4:30 10/15/115:00 10/15/115:30 10/15/11 6:30 10/15/11 7:00 10/15/11 7:30 10/15/11 8:00 10/15/11 8:30 10/15/11 9:00 10/15/11 10:00 10/15/1111 10:00 10/15/11 10:30 0/15/11 11:30 10/15/11 12:00 10/15/11 12:30 10/15/11 13:00 10/15/11 13:30 10/15/11 14:00 10/15/11 15:00 10/15/11 15:00 10/15/11 16:00 10/15/11 16:30 10/15/11 17:00 10/15/11 17:30 0/15/11 18:00 10/15/11 18:30 0.15/11 19:30 10/15/11 20:00 10/15/11 20:30 10/15/11 21:00 10/15/11 21:30 10/15/11 22:00 10/15/11 22:30 10/15/11 23:30 10/16/11 0:00 10/16/11 0:30 10/16/11 1:00 10/16/11 1:30 10/16/11 2:00 10/16/11 3:00 10/16/11 3:30 10/16/11 4:30 10/16/11 5:00 10/16/11 6:00 $\begin{array}{rrrr}1494.44 & 1494.87 & 1496.642 & 1494.444 \\ 14944 & 1494.867 & 1496.651 & 1494.456\end{array}$ $\begin{array}{lllll}1494.44 & 1494.867 & 1496.651 & 1494.456\end{array}$ $\begin{array}{lllll}1494.44 & 1494.867 & 1496.663 & 1494.465 \\ 1494.442 & 1494.87 & 1496663 & 1494.475\end{array}$ $\begin{array}{lllll}1494.444 & 1494.87 & 1496.67 & 1494.491\end{array}$ $\begin{array}{lrrr}1494.444 & 1494.874 & 1496.67 & 1494.503\end{array}$ $\begin{array}{rrrrr}1494.442 & 1494.874 & 1496.658 & 1494.498\end{array}$ $\begin{array}{lllll}1494.444 & 1494.877 & 1496.644 & 1494.491\end{array}$ $\begin{array}{llll}1494.44 & 1494.874 & 1496.635 & 1494.475\end{array}$ $\begin{array}{rrrr}1494.435 & 1494.87 & 1496.642 & 1494.446 \\ 1494.43 & 1494.86 & 1496.663 & 1494.418\end{array}$ $\begin{array}{rrrr}1494.43 & 1494.86 & 1496.66 & 1494.418\end{array}$ $\begin{array}{rrrr}1494.428 & 1494.858 & 1496.663 & 1494.4 \\ 1494.435 & 1494.858 & 1496.674 & 1494.388\end{array}$ $\begin{array}{lllll}1494.44 & 1494.863 & 1496.691 & 1494.388\end{array}$ $\begin{array}{rrrrr}1494.44 & 1494.863 & 1496.691 & 1494.388 \\ 1494.437 & 1494.863 & 1496.7 & 1494.383\end{array}$ $\begin{array}{rrrrr}1494.437 & 1494.863 & 1496.7 & 1494.383 \\ 1494.44 & 1494.867 & 1496.705 & 1494.388\end{array}$ $\begin{array}{lllll}1494.444 & 1494.877 & 1496.716 & 1494.393\end{array}$ $\begin{array}{lllll}1494.444 & 1494.879 & 1496.738 & 1494.397\end{array}$ $\begin{array}{lllll}1494.444 & 1494.884 & 1496.752 & 1494.402\end{array}$ $\begin{array}{lllll}1494.447 & 1494.891 & 1496.754 & 1494.402 \\ 1494.449 & 1494.905 & 1496761 & 1494.407\end{array}$ $\begin{array}{llll}1444.451 & 1494.912 & 1496.773 & 1494.402\end{array}$ $\begin{array}{lllll}1494.449 & 1494.916 & 1496.787 & 1494.395\end{array}$ $\begin{array}{lllll}1494.449 & 1494.921 & 1496.794 & 1494.393\end{array}$ $\begin{array}{lllll}1494.454 & 1494.93 & 1496.796 & 1494.402\end{array}$ $\begin{array}{lllll}1494.454 & 1494.94 & 1496.794 & 1494.411\end{array}$ 1494.454 $1494.947 \quad 1496.794 \quad 1494.423$ $1494.451 \quad 1494.9491496 .79814994 .435$ $\begin{array}{llll}1494.451 & 1494.947 & 1496.798 & 1494.439 \\ 1494.454 & 1494.954 & 1496.796 & 1494.442\end{array}$ $\begin{array}{llll}1494.454 & 1494.954 & 1496.784 & 1494.439\end{array}$ $\begin{array}{llllll}1494.451 & 1494.954 & 1496.791 & 1494.43\end{array}$ $1494.447 \quad 1494.949 \quad 1496.784 \quad 1494.418$ $\begin{array}{lllll}1494.444 & 1494.947 & 1496.784 & 1494.407\end{array}$ 1494.447 $1494.949 \quad 1496.784 \quad 1494.402$ $\begin{array}{lllll}1494.444 & 1494.947 & 1496.787 & 1494.4\end{array}$ $\begin{array}{llll}1494.442 & 1494.944 & 1496.784 & 1494.402 \\ 1494.444 & 1494.947 & 1496.789 & 1494.407\end{array}$ $\begin{array}{lllll}1494.444 & 1494.947 & 1496.789 & 1494.407 \\ 1494.444 & 1494949 & 1496.794 & 1494.414\end{array}$ $\begin{array}{lllll}1494.442 & 1494.947 & 1496.801 & 1494.425\end{array}$ $\begin{array}{lllll}1494.442 & 1494.949 & 1496.808 & 1494.437\end{array}$ $\begin{array}{llllll}1494.444 & 1494.954 & 1496.805 & 1494.451\end{array}$ $\begin{array}{lllll}1494.447 & 1494.958 & 1496.805 & 1494.465\end{array}$ $\begin{array}{lllll}1494.444 & 1494.961 & 1496.808 & 1494.482\end{array}$ $\begin{array}{rrrr}1494.442 & 1494.958 & 1496.805 & 1494.493 \\ 1494.444 & 1494.961 & 149681 & 1994505\end{array}$ $\begin{array}{rrrrr}1494.444 & 1494.961 & 1496.81 & 1494.505 \\ 1494.442 & 1494.963 & 1496812 & 1494521\end{array}$ $\begin{array}{lllll}1494.442 & 1494.963 & 1496.817 & 1494.528\end{array}$ $\begin{array}{lllll}1494.442 & 1494.965 & 1496.81 & 1494.545\end{array}$ $\begin{array}{lllll}1494.44 & 1494.965 & 1496.794 & 1494.556\end{array}$ $\begin{array}{llllll}1494.442 & 1494.97 & 1496.735 & 1494.568\end{array}$ $\begin{array}{lllll}1494.44 & 1494.965 & 1496.749 & 1494.577\end{array}$ $\begin{array}{rrrr}1494.428 & 1494.958 & 1496.766 & 1494.575 \\ 1494.43 & 1494.949 & 1496.745 & 1494.568\end{array}$

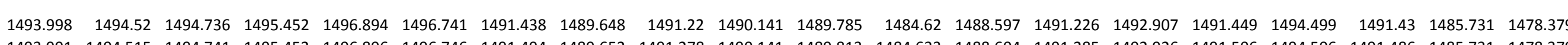

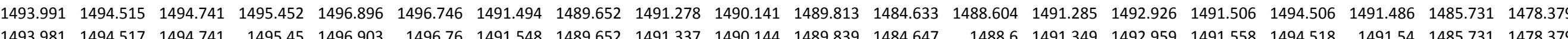

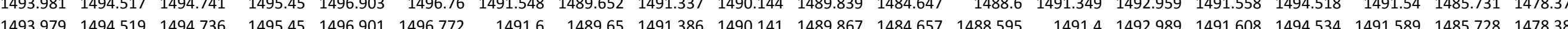
\begin{tabular}{lllllllllllllllllllll}
1493.977 & 1494.517 & 1494.736 & 1495.452 & 1496.903 & 1496.772 & 1490.442 & 1489.65 & 1491.002 & 1490.141 & 1489.89 & 1484.668 & 1488.593 & 1491.02 & 1492.744 & 1490.288 & 1494.541 & 1490.399 & 1485.731 & 1478.381 \\
\hline
\end{tabular} \begin{tabular}{lllllllllllllllllllll}
1493.969 & 1494.515 & 1494.736 & 1495.454 & 1496.908 & 1496.777 & 1489.056 & 1489.655 & 1489.644 & 1490.141 & 1489.821 & 1484.682 & 1488.588 & 1489.649 & 1492.108 & 1488.935 & 1494.518 & 1489.048 & 1485.731 & 1478.383 \\
\hline
\end{tabular} $\begin{array}{llllllllllllllllllllllll}1493.974 & 1494.517 & 1494.731 & 1495.454 & 1496.903 & 1496.78 & 1488.464 & 1489.659 & 1488.981 & 1490.141 & 1489.715 & 1484.694 & 1488.595 & 1488.991 & 1491.886 & 1488.355 & 1494.48 & 1488.461 & 1485.731 & 1478.383\end{array}$ $\begin{array}{llllllllllllllllllll}1493.981 & 1494.515 & 1494.736 & 1495.457 & 1496.898 & 1496.768 & 1487.753 & 1489.652 & 1488.298 & 1490.144 & 1489.601 & 1484.707 & 1488.59 & 1488.3 & 1491.606 & 1487.648 & 1494.45 & 1487.76 & 1485.728 & 1478.386 \\ \end{array}$ $\begin{array}{llllllllllllllllllll}1493.991 & 1494.512 & 1494.731 & 1495.457 & 1496.894 & 1496.753 & 1487.465 & 1489.657 & 1487.829 & 1490.144 & 1489.484 & 1484.714 & 1488.595 & 1487.837 & 1491.448 & 1487.407 & 1494.408 & 1487.485 & 1485.728 & 1478.386\end{array}$

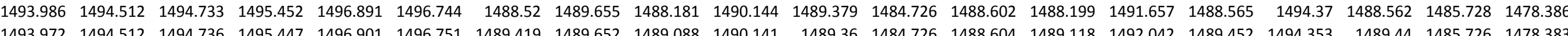

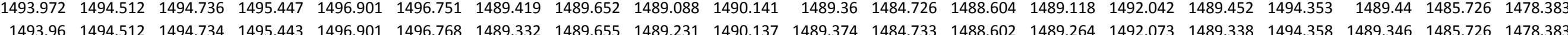
$\begin{array}{llllllllllllllllllllll}1493.95 & 1494.512 & 1494.733 & 1495.445 & 1496.905 & 1496.768 & 1489.342 & 1489.648 & 1489.246 & 1490.139 & 1489.372 & 1484.733 & 1488.588 & 1489.259 & 1492.075 & 1489.343 & 1494.368 & 1489.351 & 1485.528 & 1478.383\end{array}$ $\begin{array}{llllllllllllllllllllll}1493.941 & 1494.51 & 1494738 & 1495.447 & 1496915 & 1496.782 & 148937 & 1489.648 & 1489.278 & 1490.139 & 1489.367 & 1484.737 & 1488.579 & 1489.271 & 1492.091 & 1489.376 & 1494.375 & 1489.381 & 1485.726 & 1478.383\end{array}$ $\begin{array}{lllllllllllllllllllllll}1493.932 & 1494.512 & 1494.738 & 1495.447 & 1496.917 & 1496.796 & 1489.396 & 1489.652 & 1489.304 & 1490.139 & 1489.362 & 1484.747 & 1488.576 & 1489.292 & 1492.101 & 1489.399 & 1494.37 & 1489.409 & 1485.726 & 1478.383\end{array}$

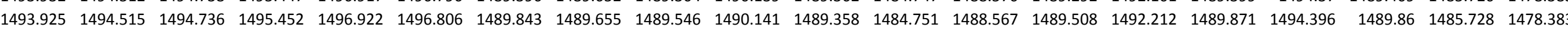

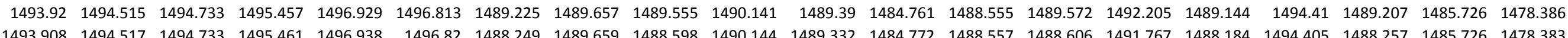

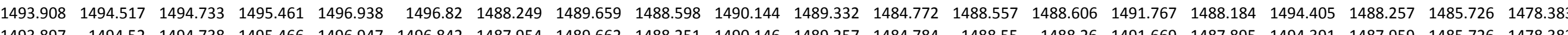
$\begin{array}{lllllllllllllllllllll}1493.897 & 1494.52 & 1494.738 & 1495.466 & 1496.947 & 1496.842 & 1487.954 & 1489.662 & 1488.251 & 1490.146 & 1489.257 & 1484.784 & 1488.55 & 1488.26 & 1491.669 & 1487.895 & 1494.391 & 1487.959 & 1485.726 & 1478.383 \\ 1493892 & 1494.522 & 1494.738 & 1495.468 & 1496954 & 1496854 & 1487.414 & 1489674 & 1487.757 & 1490146 & 1489.18 & 1484793 & 1488541 & 1487.766 & 1491.464 & 1487.352 & 1494372 & 1487.429 & 1485726 & 1478381\end{array}$

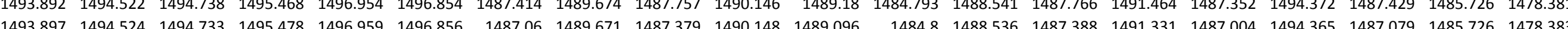
$\begin{array}{llllllllllllllllllll}1493.894 & 1494.527 & 1494.738 & 1495.482 & 1496.966 & 1496.863 & 1488.113 & 1489.678 & 1487.752 & 1490.151 & 1489.021 & 1484.8 & 1488.529 & 1487.75 & 1491.515 & 1488.165 & 1494.353 & 1488.159 & 1485.726 & 1478.386\end{array}$ $\begin{array}{lllllllllllllllllllll}1493.89 & 1494.526 & 1494.738 & 1495.484 & 1496.973 & 1496.873 & 1488.993 & 1489.681 & 1488.641 & 1490.153 & 1489.021 & 1484.804 & 1488.527 & 1488.648 & 1491.902 & 1489.03 & 1494.363 & 1489.017 & 1485.726 & 1478.386\end{array}$ $\begin{array}{lllllllllllllllllllll}1493.885 & 1494.534 & 1494.741 & 1495.489 & 1496.98 & 1496.885 & 1489.463 & 1489.683 & 1489.138 & 1490.153 & 1489.059 & 1484.814 & 1488.529 & 1489.144 & 1492.096 & 1489.499 & 1494.382 & 1489.48 & 1485.724 & 1478.386\end{array}$

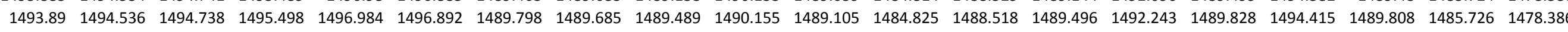

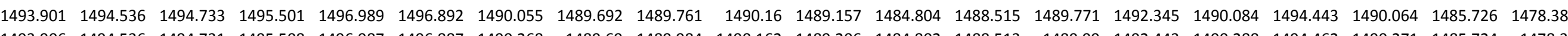

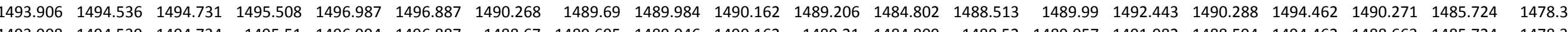

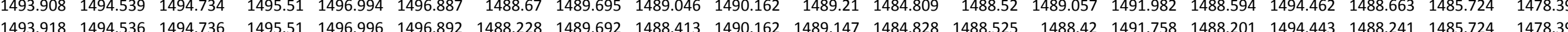

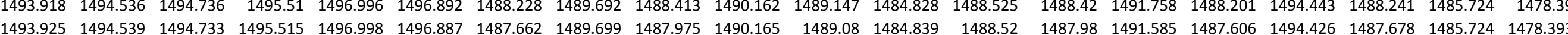
$\begin{array}{llllllllllllllllllllll}1493.939 & 1494.538 & 1494.729 & 1495.519 & 1496.996 & 1496.883 & 1487.17 & 1489.699 & 1487.501 & 1490.165 & 1489 & 1484.853 & 1488.525 & 1487.51 & 1491.401 & 1487.115 & 1494.403 & 1487.19 & 1485.724 & 1478.395 \\ \end{array}$ $\begin{array}{llllllllllllllllllllll}1493.948 & 1494.536 & 1494.731 & 1495.519 & 1496.996 & 1496.871 & 1487.011 & 1489.702 & 1487.236 & 1490.167 & 1488.918 & 1484.86 & 1488.527 & 1487.242 & 1491.315 & 1486.975 & 1494.386 & 1487.035 & 1485.724 & 1478.395\end{array}$

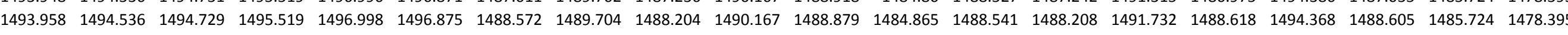
$\begin{array}{lllllllllllllllllllll}1493.958 & 1494.534 & 1494.731 & 1495.517 & 1496.998 & 1496.868 & 1489.169 & 1489.704 & 1488.826 & 1490.167 & 1488.907 & 1484.876 & 1488.539 & 1488.831 & 1491.977 & 1489.203 & 1494.37 & 1489.189 & 1485.724 & 1478.395 \\ \end{array}$

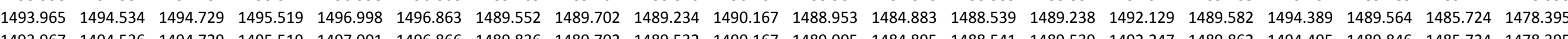

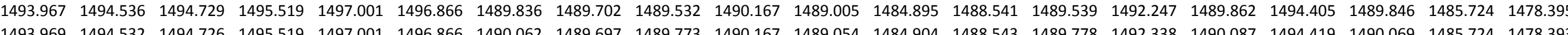

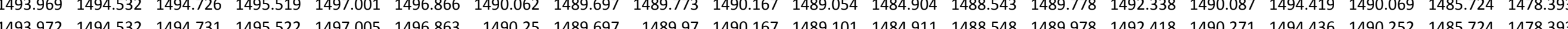

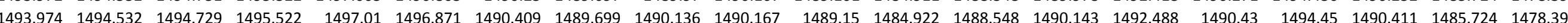
$\begin{array}{lllllllllllllllllllll}1493.969 & 1494.532 & 1494.729 & 1495.522 & 1497.01 & 1496.873 & 1490.545 & 1489.699 & 1490.282 & 1490.167 & 1489.192 & 1484.934 & 1488.55 & 1490.286 & 1492.546 & 1490.565 & 1494.464 & 1490.545 & 1485.721 & 1478.39\end{array}$

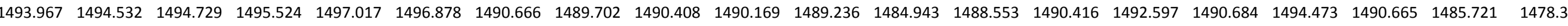
$\begin{array}{llllllllllllllllllll}1493.969 & 1494.532 & 1494.726 & 1495.526 & 1497.019 & 1496.883 & 1490.776 & 1489.699 & 1490.523 & 1490.169 & 1489.278 & 1484.952 & 1488.543 & 1490.528 & 1492.653 & 1490.79 & 1494.499 & 1490.773 & 1485.724 & 1478.39 \\ \end{array}$

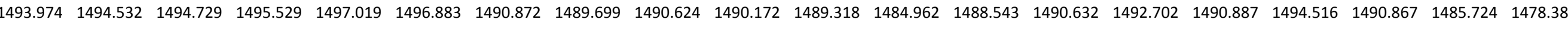

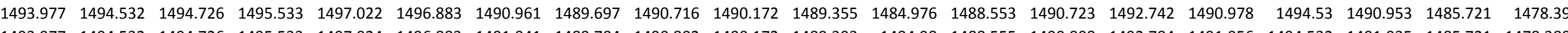
$\begin{array}{llllllllllllllllllll}1493.977 & 1494.532 & 1494.726 & 1455.533 & 1497.024 & 1466.883 & 1491.041 & 1489.704 & 1490.802 & 1400.172 & 1489.393 & 1484.98 & 1488.555 & 1490.808 & 1492.784 & 1491.056 & 1494.532 & 1491.035 & 1485.721 & 1478.388\end{array}$

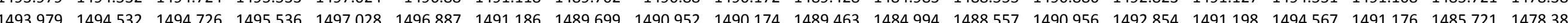
$\begin{array}{llllllllllllllllllllll}1493.981 & 1494.534 & 1494.726 & 1495.538 & 1497.031 & 1496.887 & 1491.251 & 1489.702 & 1491.02 & 1490.174 & 1489.496 & 1485.003 & 1488.56 & 1491.022 & 1492.889 & 1491.262 & 1494.577 & 1491.24 & 1485.719 & 1478.386\end{array}$ $\begin{array}{lllllllllllllllllllll}1493.981 & 1494.529 & 1494.726 & 1495.538 & 1497.033 & 1496.89 & 1491.312 & 1489.704 & 1491.081 & 1490.174 & 1489.531 & 1485.015 & 1488.562 & 1491.083 & 1492.919 & 1491.323 & 1494.591 & 1491.298 & 1485.721 & 1478.388\end{array}$

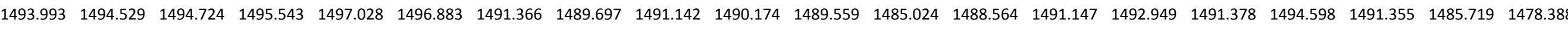

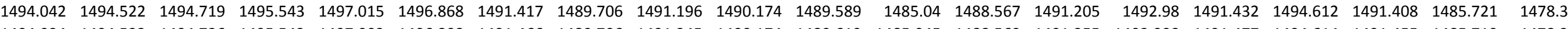

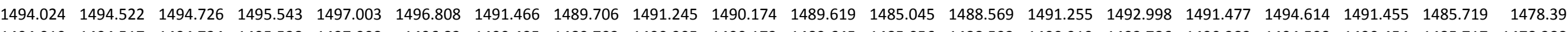

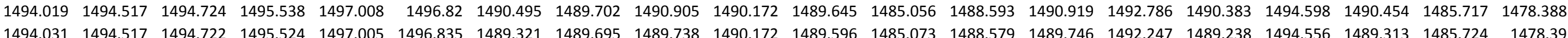


Well

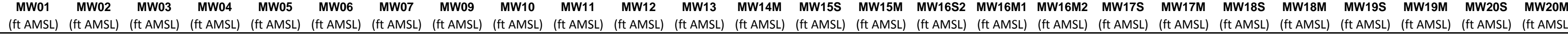

10/16/11 7:00 10/16/11 7:30 $10 / 16 / 118: 30$ 10/16/11 9:00 10/16/11 9:30 10/16/11 10:00 10/16/11 10:30 10/16/11 11:00 10/16/11 11:30 10/16/11 12:00 10/16/11 13:00 10/16/111113:00 10/16/11 13:3:0 10/16/11 14:30 10/16/11 15:00 10/16/11 15:30 10/16/11 16:00 10/16/11 16:30 10/16/11 17:00 10/16/11 18:00 10/16/11 18:00 10/16/11 19:00 10/16/11 19:30 10/16/11 20:00 10/16/11 20:30 10/16/11 21:00 10/16/11 21:30 10/16/11 22:30 10/16/11 23:00 10/16/11 23:30 10/17/11 0:00 10/17/11 0:30 10/17/11 1:00 10/17/111 1:30 10/17/11 2:30 10/17/1113:00 10/17/11 3:30 10/17/11 4:00 10/17/11 4:30 10/17/11 5:00 10/17/11 6:00 10/17/11 6:30 10/17/11 7:30 10/17/11 8:00 10/17/11 9:00 $\begin{array}{llll}1494.423 & 1494.935 & 1496.724 & 1494.549\end{array}$ $\begin{array}{llll}1494.428 & 1494.94 & 1496.698 & 1494.542\end{array}$ $\begin{array}{lrlll}1494.423 & 1494.93 & 1496.681 & 1494.521\end{array}$ $\begin{array}{llll}1494.414 & 1494.919 & 1496.665 & 1494.486 \\ 1494.407 & 1494.905 & 1496.653 & 1494.451\end{array}$ $\begin{array}{lllll}1494.405 & 1494.893 & 1496.642 & 1494.451 \\ 1494.421\end{array}$ $\begin{array}{lllll}1494.405 & 1494.893 & 1496.642 & 1494.421 \\ 1494.402 & 1494.881 & 1496.632 & 1494.397\end{array}$ $\begin{array}{llll}1494.402 & 1494.872 & 1496.632 & 1494.397 \\ 1486\end{array}$ $\begin{array}{lllll}1494.4 & 1494.865 & 1496.628 & 1494.372\end{array}$ $1494.405 \quad 1494.858 \quad 1496.637 \quad 1494.367$ $\begin{array}{rrrr}1494.405 & 1494.858 & 1496.649 & 1494.367 \\ 1494.4 & 1498846 & 1496653 & 1943652\end{array}$ $\begin{array}{rrrr}1494.4 & 1494.846 & 1496.653 & 1494.362 \\ 1494.407 & 1494.851 & 1496.665 & 1494.36\end{array}$ $\begin{array}{lllll}1494.412 & 1494.853 & 1496.667 & 1494.355\end{array}$ $\begin{array}{llll}1494.412 & 1494.851 & 1496.66 & 1494.346\end{array}$ $\begin{array}{rrrrr}1494.412 & 1494.851 & 1496.66 & 1494.346 \\ 1494.414 & 1494.856 & 1496.658 & 1494.337\end{array}$ $\begin{array}{lllll}1494.409 & 1494.853 & 1496.658 & 1494.32\end{array}$ $\begin{array}{llll}1494.405 & 1494.849 & 1496.658 & 1494.309\end{array}$ $\begin{array}{lllll}1494.405 & 1494.846 & 1496.651 & 1494.306\end{array}$ $\begin{array}{rrrrr}1494.402 & 1494.842 & 1496.658 & 1494.302 \\ 1494.4 & 1994.842 & 14966 & 1494306\end{array}$ $\begin{array}{lllll}1494.4 & 1494.842 & 1496.66 & 1494.306 \\ 1494.4 & 1494.837 & 1496.656 & 1494.311\end{array}$ $\begin{array}{lllll}1494.4 & 1494.837 & 1496.644 & 1494.316\end{array}$ $\begin{array}{lllll}1494.402 & 1494.837 & 1496.639 & 1494.313\end{array}$ $\begin{array}{llllll}1494.4 & 1494.835 & 1496.639 & 1494.302\end{array}$ $\begin{array}{llll}1494.393 & 1494.828 & 1496.637 & 1494.283\end{array}$ $\begin{array}{lllll}1494.391 & 1494.821 & 1496.644 & 1494.267\end{array}$ $\begin{array}{lllll}1494.393 & 1494.821 & 1496.649 & 1494.255\end{array}$ $\begin{array}{rrrr}1494.393 & 1494.821 & 1496.651 & 1494.248 \\ 1494.393 & 1494.818 & 1496.656 & 1494.25\end{array}$ $\begin{array}{lllll}1494.395 & 1494.818 & 1496.656 & 1494.253\end{array}$ $\begin{array}{llllll}1494.393 & 1494.816 & 1496.653 & 1494.26\end{array}$ $\begin{array}{lllll}1494.398 & 1494.818 & 1496.651 & 1494.267\end{array}$ $\begin{array}{lllll}1494.391 & 1494.816 & 1496.653 & 1494.276\end{array}$ $\begin{array}{lllll}1494.391 & 1494.816 & 1496.663 & 1494.288\end{array}$ $\begin{array}{lllll}1494.389 & 1494.814 & 1496.663 & 1494.297\end{array}$ $\begin{array}{lllll}1494.388 & 1494.814 & 1496.66 & 1494.309\end{array}$ $\begin{array}{llll}1494.395 & 1494.823 & 1496.658 & 1494.327 \\ 1494.391 & 1494.821 & 1496.663 & 1494.337\end{array}$ $\begin{array}{lllll}1494.386 & 1494.818 & 1496.665 & 1494.348\end{array}$ $\begin{array}{llllll}1494.388 & 1494.818 & 1496.679 & 1494.358\end{array}$ $\begin{array}{llllll}1494.388 & 1494.821 & 1496.684 & 1494.372\end{array}$ $\begin{array}{llllll}1494.391 & 1494.823 & 1496.7 & 1494.386\end{array}$ $\begin{array}{lllll}1494.393 & 1494.83 & 1496.716 & 1494.402\end{array}$ $\begin{array}{llll}1494.395 & 1494.837 & 1496.712 & 1494.418 \\ 1494.395 & 1494846 & 1496.719 & 1944.437\end{array}$ $\begin{array}{rllll}1494.395 & 1494.846 & 1496.719 & 1494.437\end{array}$ $\begin{array}{rrrrr}1494.395 & 1494.858 & 1496.728 & 1494.468\end{array}$ $\begin{array}{lllll}1494.395 & 1494.86 & 1496.724 & 1494.47\end{array}$ $\begin{array}{lllll}1494.395 & 1494.863 & 1496.716 & 1494.468\end{array}$ $\begin{array}{lllll}1494.398 & 1494.865 & 1496.707 & 1494.458\end{array}$ $\begin{array}{lllll}1494.393 & 1494.865 & 1496.698 & 1494.439\end{array}$ $\begin{array}{llll}1494.391 & 1494.863 & 1496.719 & 1494.423 \\ 1494.388 & 1494.858 & 1496.716 & 1494.407\end{array}$

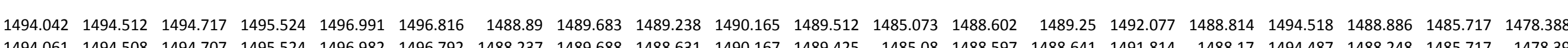

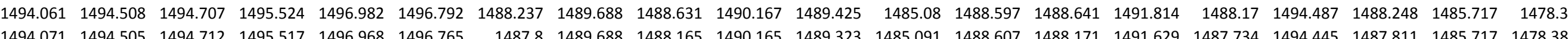

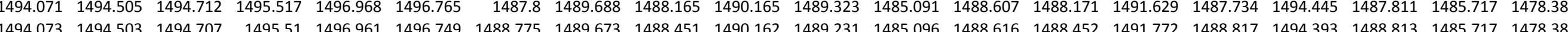

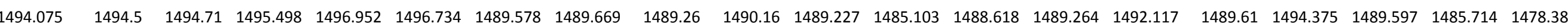
\begin{tabular}{llllllllllllllllllllll}
1494.073 & 1494.496 & 1494.703 & 1495.489 & 1496.945 & 1496.727 & 1489.99 & 1489.662 & 1489.693 & 1490.155 & 1489.252 & 1485.107 & 1488.621 & 1489.699 & 1492.275 & 1490.018 & 1494.363 & 1490.001 & 1485.714 & 1478.383 \\
\hline
\end{tabular} $\begin{array}{lllllllllllllllllllll}1494.073 & 1494.493 & 1494.705 & 1495.48 & 1496.935 & 1496.713 & 1490.268 & 1489.655 & 1489.989 & 1490.153 & 1489.288 & 1485.107 & 1488.618 & 1489.992 & 1492.378 & 1490.29 & 1494.368 & 1490.273 & 1485.714 & 1478.383\end{array}$ $\begin{array}{lllllllllllllllllllllll}1494.061 & 1494.493 & 1494.707 & 1495.471 & 1496.935 & 1496.708 & 1490.477 & 1489.65 & 1490.207 & 1490.148 & 1489.323 & 1485.098 & 1488.621 & 1490.216 & 1492.457 & 1490.497 & 1494.365 & 1490.482 & 1485.712 & 1478.381\end{array}$

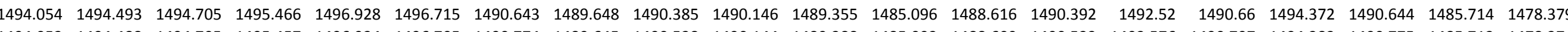

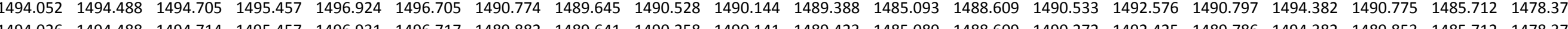
$\begin{array}{lllllllllllllllllllll}1494.026 & 1494.488 & 1494.714 & 1495.457 & 1496.931 & 1496.717 & 1489.882 & 1489.641 & 1490.258 & 1490.141 & 1489.423 & 1485.089 & 1488.609 & 1490.272 & 1492.425 & 1489.786 & 1494.382 & 1489.853 & 1485.712 & 1478.374 \\ 1494.016 & 1494.486 & 1494.71 & 1495.447 & 1496.933 & 1496.732 & 1488.757 & 1489.638 & 1489.147 & 1490.139 & 1489.367 & 1485.077 & 1488.602 & 1489.156 & 1491.912 & 1488.684 & 1494356 & 1488.759 & 1485712 & 1478374\end{array}$

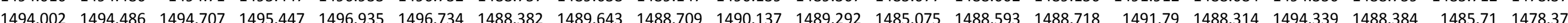
$\begin{array}{llllllllllllllllllllll}1493.988 & 1494.488 & 1494.707 & 1495.45 & 1496.935 & 1496.746 & 1487.767 & 1489.641 & 1488136 & 1490.139 & 1489.201 & 1485.061 & 1488586 & 1488.142 & 1491.548 & 1487.701 & 1494.325 & 1487.781 & 1485.712 & 1478.374\end{array}$ $\begin{array}{lllllllllllllllllllllll}1493.988 & 1494.484 & 1494.705 & 1495.447 & 1496.935 & 1496.751 & 1487.36 & 1489.641 & 1487.703 & 1490.137 & 1489.11 & 1485.043 & 1488.583 & 1487.712 & 1491.389 & 1487.302 & 1494.297 & 1487.38 & 1485.71 & 1478.374\end{array}$

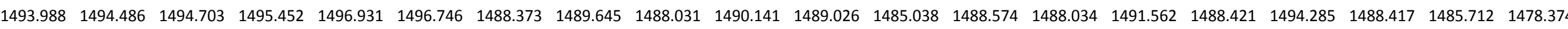
$\begin{array}{llllllllllllllllllllll}1493.984 & 1494.484 & 4494.707 & 1495.45 & 1496.928 & 1496.744 & 1489.211 & 1489.641 & 1488.875 & 1490.141 & 1489.021 & 1485.038 & 1488.572 & 1488.878 & 1491.928 & 1489.243 & 1494.285 & 1489.233 & 1485.71 & 1478.372\end{array}$

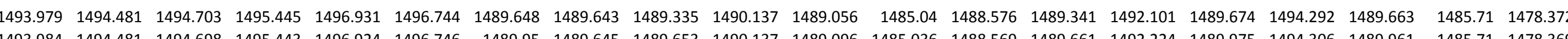

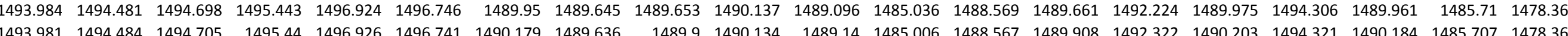

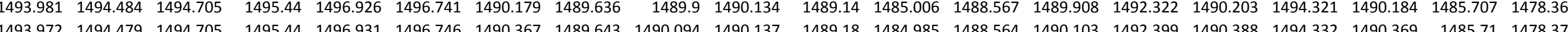
$\begin{array}{lllllllllllllllllllll}1493.972 & 1494.476 & 1494.7 & 1495.438 & 1496.928 & 1496.751 & 1488.75 & 1489.641 & 1489.14 & 1490.134 & 1489.185 & 1484.98 & 1488.562 & 1489.151 & 1491.933 & 1488.672 & 1494.33 & 1488.745 & 1485.71 & 1478.369\end{array}$ $\begin{array}{lllllllllllllllllllllll}1493.986 & 1494.479 & 1494.695 & 1495.436 & 1496.919 & 1496.734 & 1487.687 & 1489.641 & 1488.019 & 1490.132 & 1489.038 & 1484.98 & 1488.56 & 1488.03 & 1491.506 & 1487.627 & 1494.295 & 1487.701 & 1485.71 & 1478.372 \\ \end{array}$ $\begin{array}{lllllllllllllllllllll}1493.986 & 1494.474 & 1494.7 & 1495.436 & 1496.917 & 1496.732 & 1487.203 & 1489.638 & 1487.541 & 1490.134 & 1488.953 & 1484.985 & 1488.562 & 1487.55 & 1491.315 & 1487.144 & 1494.267 & 1487.222 & 1485.707 & 1478.372\end{array}$

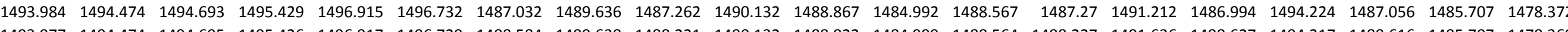

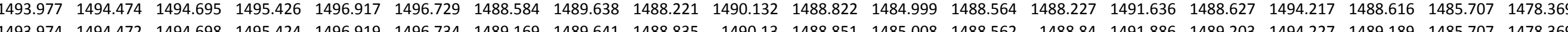

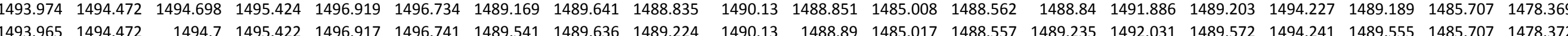

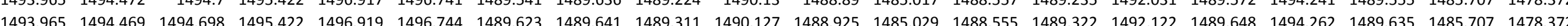
$\begin{array}{llllllllllllllllllllll}1493.96 & 1494.469 & 1494.696 & 1495.422 & 1496.924 & 1496.751 & 1489.791 & 1489.636 & 1489.489 & 1490.13 & 1488.923 & 1485.045 & 1488.553 & 1489.496 & 1492.161 & 1489.814 & 1494.274 & 1489.801 & 1485.705 & 1478.369\end{array}$ $\begin{array}{lllllllllllllllllllll}1493.962 & 1494.469 & 1494.693 & 1495.422 & 1496.919 & 1496.753 & 1490.051 & 1489.636 & 1489.768 & 1490.127 & 1488.965 & 1485.045 & 1488.555 & 1489.774 & 1492.254 & 1490.075 & 1494.283 & 1490.057 & 1485.705 & 1478.369\end{array}$

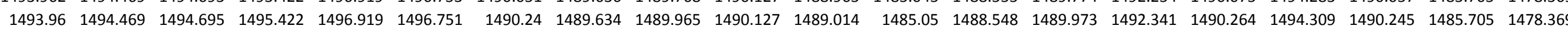

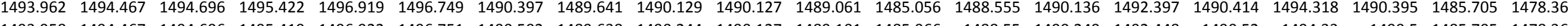

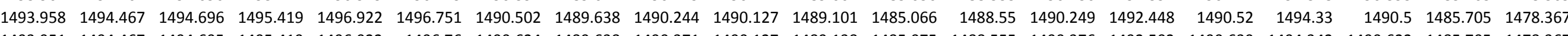

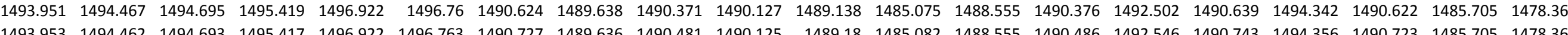

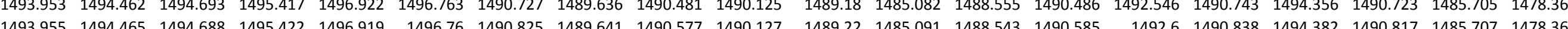

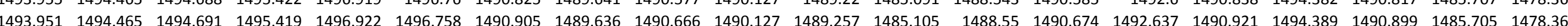
$\begin{array}{llllllllllllllllllllllll}1493.944 & 1494.465 & 1494.691 & 1495.417 & 1496.924 & 1496.765 & 1490.982 & 1489.638 & 1490.746 & 1490.125 & 1489.292 & 1485.112 & 1488.555 & 1490.752 & 1492.667 & 1490.994 & 1494.393 & 1490.974 & 1485.703 & 1478.362\end{array}$ $\begin{array}{llllllllllllllllllll}1493.937 & 1494.465 & 1494.691 & 1495.417 & 1496.931 & 1496.768 & 1491.052 & 1489.638 & 1490.821 & 1490.125 & 1489.327 & 1485.121 & 1488.548 & 1490.815 & 1492.709 & 1491.063 & 1494.412 & 1491.042 & 1485.705 & 1478.362\end{array}$ $\begin{array}{lllllllllllllllllllll}1493.932 & 1494.467 & 1494.693 & 1495.419 & 1496.933 & 1496.78 & 1491.113 & 1489.636 & 1490.887 & 1490.127 & 1489.36 & 1485.13 & 1488.553 & 1490.888 & 1492.739 & 1491.127 & 1494.422 & 1491.108 & 1485.703 & 1478.362\end{array}$

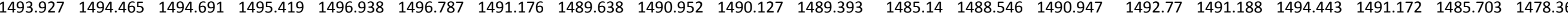

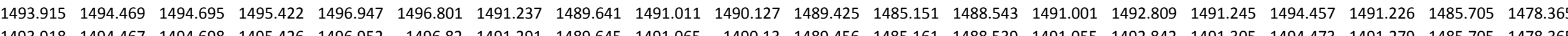

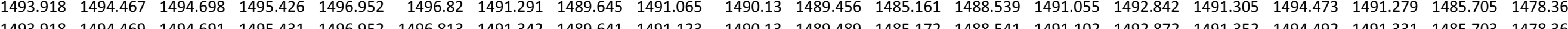

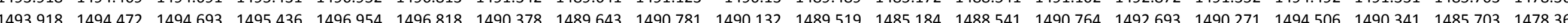
$\begin{array}{lllllllllllllllllllll}1493.918 & 1494.472 & 1494.691 & 1495.438 & 1496.956 & 1496.823 & 1489.213 & 1489.652 & 1489.625 & 1490.132 & 1489.472 & 1485.191 & 1488.543 & 1489.6 & 1492.163 & 1489.127 & 1494.49 & 1489.205 & 1485.705 & 1478.367\end{array}$ $\begin{array}{llllllllllllllllllll}1493.918 & 1494.474 & 1494.691 & 1495.44 & 1496.959 & 1496.827 & 1488.776 & 1489.655 & 1489.133 & 1490.132 & 1489.393 & 1485.202 & 1488.546 & 1489.111 & 1492.014 & 1488.698 & 1494.464 & 1488.769 & 1485.703 & 1478.369\end{array}$

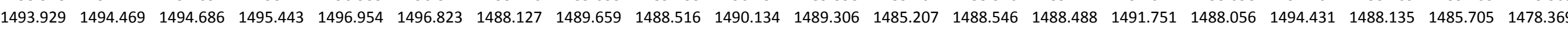

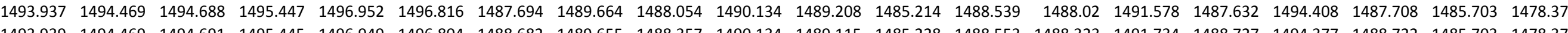

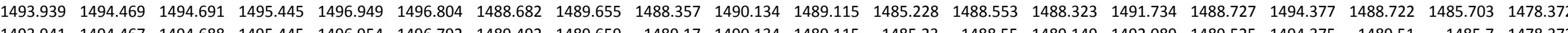

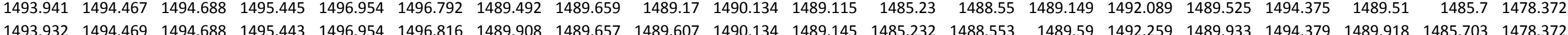


Well

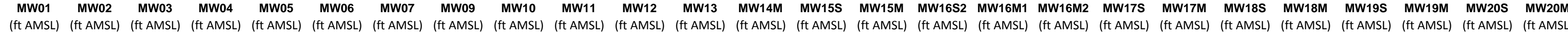

10/17/11 10:00 $10 / 17 / 1110: 30$ $10 / 17 / 1111 \cdot 30$ 10/17/111112:00 10/17/11 12:30 10/17/11 13:00 10/17/11 13:30 10/17/1114:00 10/17/11 14:30 10/17/11 15:00 10/17/11 15:30 10/17/11 16:30 10/17/11 16:30 10/17/11 17:30 10/17/11 18:00 10/17/11 19:00 10/17/11 19:30 10/17/11 20:00 10/17/11 20:30 10/17/11 21:30 10/17/11 22:00 10/17/11 22:30 10/17/11 23:00 10/17/11 23:30 10/18/11 0:00 10/18/11 1:00 $10 / 18 / 111: 30$ 10/18/11 2:00 10/18/11 2:30 10/18/11 3:00 10/18/11 3:30 10/18/11 4:30 10/18/11 5:30 10/18/11 6:30 10/18/11 7:00 10/18/11 7:30 10/18/11 8:00 10/18/11 8:30 10/18/11 9:00 10/18/11 10:00 10/18/11 10:30 10/18/11 11:00 10/18/11 11:30 10/18/11 12:00
10/18/11 12:30 $\begin{array}{llll}1494.384 & 1494.851 & 1496.719 & 1494.395 \\ 1494.388 & 1494.858 & 1496.745 & 1494.397\end{array}$ $\begin{array}{llll}1494.388 & 1494.858 & 1496.745 & 1494.397\end{array}$ $\begin{array}{rrrr}1494.391 & 1494.863 & 1496.752 & 1494.4\end{array}$ $\begin{array}{lllll}1494.398 & 1494.879 & 1496.766 & 1494.425\end{array}$ $\begin{array}{lllll}1494.398 & 1494.886 & 1496.759 & 14494.435\end{array}$ $\begin{array}{llll}1494.398 & 1494.886 & 1496.759 & 1494.435 \\ 1494.398 & 1494.888 & 1496.756 & 1494.442\end{array}$ $\begin{array}{llllll}1494.395 & 1494.893 & 1496.745 & 1494.437\end{array}$ $\begin{array}{lllll}1494.393 & 1494.895 & 1496.728 & 1494.43\end{array}$ $\begin{array}{llll}1494.393 & 1494.888 & 1496.702 & 1494.416\end{array}$ $\begin{array}{llll}1494.391 & 1494.886 & 1496.681 & 1494.397\end{array}$ $\begin{array}{llll}1494.381 & 1494.874 & 1496.667 & 1494.374 \\ 1494.377 & 1494.863 & 1496.656 & 1494.355\end{array}$ $\begin{array}{lllll}1494.37 & 1494.853 & 1496.642 & 1494.341\end{array}$ $\begin{array}{rrrrr}1494.37 & 1494.853 & 1496.642 & 1494.341 \\ 1494.367 & 1494.842 & 1496.618 & 1494.332\end{array}$ $\begin{array}{llllll}1494.363 & 1494.837 & 1496.607 & 1494.323\end{array}$ $\begin{array}{lllll}1494.358 & 1494.823 & 1496.583 & 1494.311\end{array}$ $\begin{array}{llll}1494.354 & 1494.814 & 1496.579 & 1494.29\end{array}$ $\begin{array}{lllll}1494.351 & 1494.802 & 1496.583 & 1494.267\end{array}$ $\begin{array}{lllll}1494.346 & 1494.79 & 1496.593 & 1494.239\end{array}$ $\begin{array}{llll}1494.356 & 1494.781 & 1496.593 & 1494.211\end{array}$ $\begin{array}{lllll}1494.36 & 1494.781 & 1496.572 & 1494.208\end{array}$ $\begin{array}{rllll}1494.363 & 1494.781 & 1496.572 & 1494.208 \\ 14964.358 & 1494.213\end{array}$ $\begin{array}{lllll}1494.351 & 1494.774 & 1496.558 & 1494.211\end{array}$ $\begin{array}{lllll}1494.344 & 1494.762 & 1496.562 & 1494.208\end{array}$ $\begin{array}{rrrr}1494.342 & 1494.755 & 1496.558 & 1494.206 \\ 1494.34 & 1494748 & 1496558 & 194.21\end{array}$ $\begin{array}{llll}1494.34 & 1494.748 & 1496.558 & 1494.211\end{array}$ $\begin{array}{llll}1494.344 & 1494.746 & 1496.567 & 1494.215 \\ 1494.342 & 1494.741 & 1496565 & 1494.222\end{array}$ $\begin{array}{lllll}1494.342 & 1494.739 & 1496.562 & 1494.232\end{array}$ $\begin{array}{lllll}1494.344 & 1494.741 & 1496.56 & 1499.243\end{array}$ $\begin{array}{lllll}1494.34 & 1494.737 & 1496.574 & 1494.253\end{array}$ $\begin{array}{lllll}1494.34 & 1494.732 & 1496.586 & 1494.262\end{array}$ $\begin{array}{lllll}1494.34 & 1494.73 & 1496.593 & 1494.271\end{array}$ $\begin{array}{llll}1494.344 & 1494.734 & 1496.6 & 1494.288\end{array}$ $\begin{array}{llll}1494.346 & 1494.739 & 1496.611 & 1494.306\end{array}$ $\begin{array}{llllll}1494.344 & 1494.744 & 14966.604 & 1494.318\end{array}$ $\begin{array}{lllll}1494.346 & 1494.748 & 1496.604 & 1494.351\end{array}$ $\begin{array}{lllll}1494.34 & 1494.744 & 1496.604 & 1494.358\end{array}$ $\begin{array}{rrrr}1494.337 & 1494.744 & 1496.6 & 1494.367\end{array}$ $\begin{array}{lllll}1494.337 & 1494.744 & 1496.597 & 1494.367\end{array}$ $\begin{array}{llllll}1494.335 & 1494.741 & 1496.586 & 1494.358\end{array}$ $\begin{array}{rrrrr}1494.333 & 1494.739 & 1496.576 & 1494.351 \\ 1494.33 & 1494.739 & 1496567 & 1494344\end{array}$ $\begin{array}{lllll}1494.33 & 1494.739 & 1496.567 & 1494.344 \\ 1494.328 & 1494.734 & 1496562 & 1494337\end{array}$ $\begin{array}{lllll}1494.323 & 1494.725 & 1496.558 & 1494.33\end{array}$ $\begin{array}{llll}1494.319 & 1494.723 & 1496.558 & 1494.332\end{array}$ $\begin{array}{lllll}1494.321 & 1494.718 & 1496.567 & 1494.33\end{array}$ $\begin{array}{lllll}1494.321 & 1494.716 & 1496.579 & 1494.33\end{array}$ $\begin{array}{lllll}1494.323 & 1494.714 & 1496.588 & 1494.337\end{array}$ $\begin{array}{llll}1494.323 & 1494.718 & 1496.597 & 1494.344 \\ 1494.328 & 1494.718 & 1496.607 & 1494.353\end{array}$

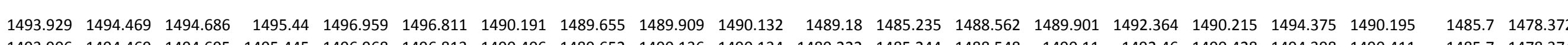
$\begin{array}{lllllllllllllllllllll}1493.906 & 1494.469 & 1494.695 & 1495.445 & 1496.968 & 1496.813 & 1490.406 & 1489.652 & 1490.136 & 1490.134 & 1489.222 & 1485.244 & 1488.548 & 1490.11 & 1492.46 & 1490.428 & 1494.398 & 1490.411 & 1485.7 & 1478.374 \\ 1493.911 & 1494.472 & 1494695 & 1495.445 & 1496975 & 1496837 & 1490582 & 1489657 & 1490319 & 1490.134 & 1489259 & 1495.253 & 1488546 & 1490284 & 1492.537 & 1490601 & 1494.424 & 149058 & 14857 & 147834\end{array}$

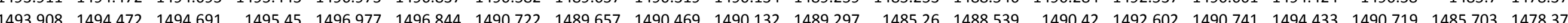

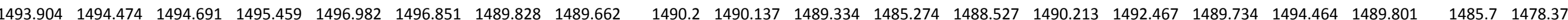
$\begin{array}{lllllllllllllllllllll}1493.908 & 1494.474 & 1494.688 & 1495.464 & 1496.982 & 1496.856 & 1488.71 & 1489.664 & 1489.098 & 1490.139 & 1489.283 & 1485.274 & 1488.529 & 1489.102 & 1491.975 & 1488.636 & 1494.452 & 1488.712 & 1485.698 & 1478.376\end{array}$ $\begin{array}{llllllllllllllllllllllll}1493.908 & 1494.476 & 1494.688 & 1495.461 & 1496.987 & 1496.849 & 1488.324 & 1489.667 & 1488.655 & 1490.139 & 1489.21 & 1485.283 & 1488.529 & 1488.662 & 1491.842 & 1488.25 & 1494.436 & 1488.323 & 1485.7 & 1478.376 \\ \end{array}$ $\begin{array}{llllllllllllllllllll}1493.918 & 1494.474 & 1494.681 & 1495.459 & 1496.984 & 1496.847 & 1487.716 & 1489.667 & 1488.078 & 1490.141 & 1489.119 & 1485.285 & 1488.532 & 1488.084 & 1491.595 & 1487.653 & 1494.41 & 1487.729 & 1485.7 & 1478.37\end{array}$

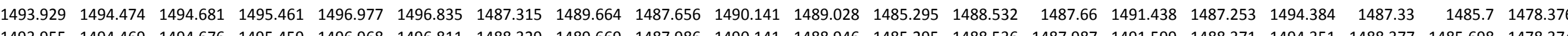

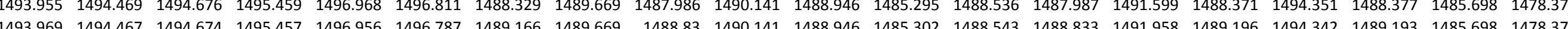
$\begin{array}{lllllllllllllllllllll}1493.969 & 1494.467 & 1494.674 & 1495.457 & 1496.956 & 1496.787 & 1489.166 & 1489.669 & 1488.83 & 1490.141 & 1488.946 & 1485.302 & 1488.543 & 1488.833 & 1491.958 & 1489.196 & 1494.342 & 1489.193 & 1485.698 & 1478.374 \\ 1493.984 & 1494.46 & 1494.672 & 1495.452 & 1496.947 & 1496.765 & 1489.609 & 1489.659 & 1489.29 & 1490.139 & 1488.979 & 1485.309 & 1488.555 & 1489.294 & 1492119 & 1489.651 & 1494332 & 1489.623 & 1485696 & 1478372\end{array}$ $\begin{array}{lllllllllllllllllllllll}1493.991 & 1494.46 & 1494.674 & 1495.443 & 1496.942 & 1496.749 & 1489.908 & 1489.655 & 1489.614 & 1490.137 & 1489.019 & 1485.311 & 1488.557 & 1489.616 & 1492.226 & 1489.968 & 1494.332 & 1489.916 & 1485.696 & 1478369\end{array}$ $\begin{array}{lllllllllllllllllllll}1494 & 1494.455 & 1494.672 & 1495.436 & 1496.933 & 1496.739 & 1490.133 & 1489.65 & 1489.855 & 1490.132 & 1489.061 & 1485.285 & 1488.562 & 1489.861 & 1492.308 & 1490.219 & 1494.323 & 1490.141 & 1485.696 & 1478367\end{array}$ $\begin{array}{llllllllllllllllllll}1494.007 & 1494.455 & 1494.672 & 1495.429 & 1496.924 & 1496.722 & 1490.32 & 1489.645 & 1490.05 & 1490.132 & 1489.101 & 1485.272 & 1488.564 & 1490.051 & 1492.376 & 1490.411 & 1494.328 & 1490.322 & 1485.696 & 1478.367\end{array}$ $\begin{array}{llllllllllllllllllll}1494.014 & 1494.45 & 1494.667 & 1495.424 & 1496.915 & 1496.701 & 1488.698 & 1489.641 & 1489.086 & 1490.127 & 1489.108 & 1485.269 & 1488.564 & 1489.094 & 1491.9 & 1488.712 & 1494.318 & 1488.691 & 1485.696 & 1478.365 \\ \end{array}$ $\begin{array}{lllllllllllllllllllllll}1494.021 & 1494.45 & 4494.667 & 1495.415 & 1496.905 & 1496.694 & 1488.209 & 1489.638 & 1488.404 & 1490.125 & 1489.03 & 1485.26 & 1488.572 & 1488.413 & 1491.641 & 1488.288 & 1494.276 & 1488.224 & 1485.696 & 1478.365\end{array}$

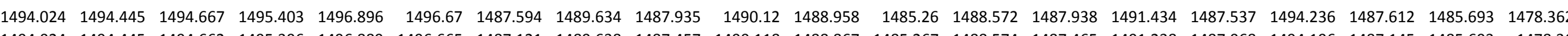

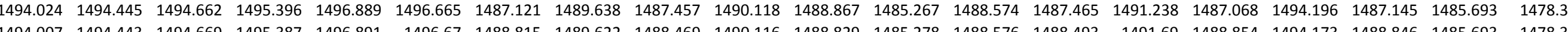

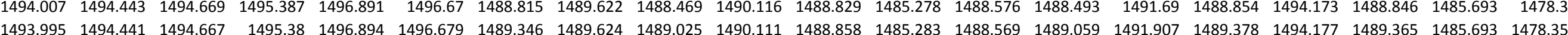
$\begin{array}{llllllllllllllllllll}1493.986 & 1444.441 & 1444.667 & 1495.38 & 1496.891 & 1496.694 & 1489.688 & 1489.62 & 1489.388 & 1490.111 & 1488.9 & 1485.292 & 1488.557 & 1489.419 & 1492.056 & 1489.717 & 1494.194 & 1489.7 & 1485.693 & 1478.358\end{array}$ $\begin{array}{llllllllllllllllllll}1494 & 1494.438 & 1494.66 & 1495.38 & 149688 & 1496.679 & 1489.941 & 1489.62 & 1489.656 & 1490.109 & 1488.946 & 1485.299 & 1488.56 & 1489.698 & 1492.166 & 1489.968 & 1494.213 & 1489.951 & 1485.693 & 1478362\end{array}$ $\begin{array}{llllllllllllllllllllll}1494.007 & 1494.436 & 1494.653 & 1495.382 & 1496.868 & 1496.665 & 1490.147 & 1489.62 & 1489.869 & 1490.109 & 1488.991 & 1485.304 & 1488.55 & 1489.917 & 1492.252 & 1490.167 & 1494.238 & 1490.151 & 1485.691 & 1478.362\end{array}$ $\begin{array}{llllllllllllllllllll}1494.002 & 1494.433 & 1494.662 & 1495.375 & 1496.868 & 1496.65 & 1490.306 & 1489.617 & 1490.038 & 1490.106 & 1489.035 & 1485.311 & 1488.562 & 1490.114 & 1492.31 & 1490.328 & 1494.234 & 1490.308 & 1485.693 & 1478.36\end{array}$ $\begin{array}{lllllllllllllllllllll}1494 & 1494.431 & 1494.66 & 1495.365 & 1496.863 & 1496.648 & 1490.444 & 1489.617 & 1490.186 & 1490.104 & 1489.073 & 1485.318 & 1488.569 & 1490.286 & 1492.355 & 1490.461 & 1494.231 & 1490.444 & 1485.691 & 1478.358\end{array}$

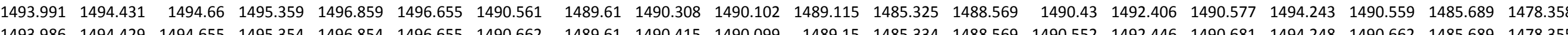

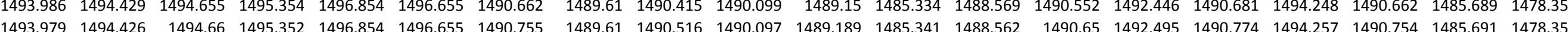
$\begin{array}{lllllllllllllllllllllll}1493.979 & 1494.426 & 1494.66 & 1495.352 & 1496.854 & 1496.655 & 1490.755 & 1489.61 & 1490.516 & 1490.097 & 1489.189 & 1485.341 & 1488.562 & 1490.65 & 1492.495 & 1490.774 & 1494.257 & 1490.754 & 1485.691 & 1478.335 \\ 1493.965 & 1494.426 & 1494.66 & 1495348 & 1496856 & 1496665 & 1490842 & 1489603 & 1490605 & 1490.097 & 1489.224 & 1485348 & 1488.562 & 1490.611 & 1492.53 & 1490.854 & 1494.269 & 1490.836 & 1485.691 & 1478355\end{array}$ $\begin{array}{llllllllllllllllllllll}1493.965 & 1494.421 & 1494.657 & 1495.345 & 1496.852 & 1496.665 & 1490.919 & 1489.608 & 1490.683 & 1490.095 & 1489.259 & 1485.352 & 1488.56 & 1490.691 & 1492.567 & 1490.93 & 1494.283 & 1490.913 & 1485.689 & 1478.353\end{array}$

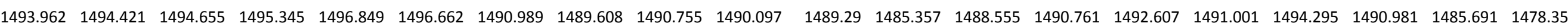
$\begin{array}{llllllllllllllllllll}1493.951 & 1494.421 & 1494.655 & 1495.343 & 1496.852 & 1496.662 & 1491.05 & 1489.605 & 1490.821 & 1490.092 & 1489.323 & 1485.362 & 1488.56 & 1490.829 & 1492.635 & 1491.063 & 1494.304 & 1491.045 & 1485.689 & 1478.351 \\ \end{array}$ $\begin{array}{llllllllllllllllllllll}1493.937 & 1494.419 & 1494.657 & 1495.341 & 1496.854 & 1496.677 & 1491.108 & 1489.606 & 1490.884 & 1490.092 & 1489.353 & 1485.366 & 1488.557 & 1490.89 & 1492.658 & 1491.122 & 1494.314 & 1491.099 & 1485.686 & 1478.351\end{array}$

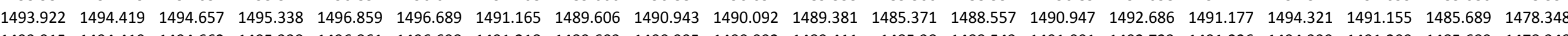

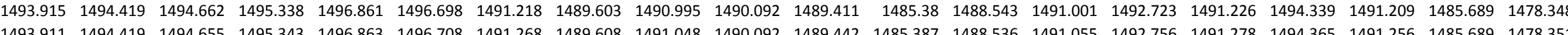

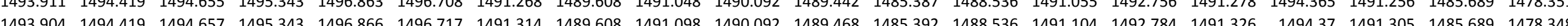

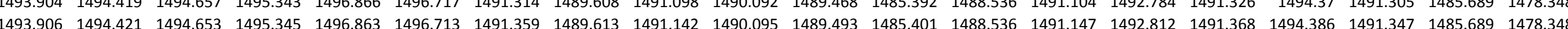
$\begin{array}{llllllllllllllllllllll}1493.904 & 1494.419 & 1494.655 & 1495.35 & 1496.863 & 1496.717 & 1491.401 & 1489.613 & 1491.189 & 1490.095 & 1489.519 & 1485.41 & 1488.532 & 1491.191 & 1492.842 & 1491.413 & 1494.41 & 1491.39 & 1485.689 & 1478.348\end{array}$

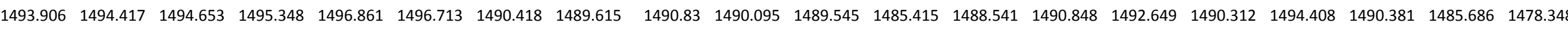

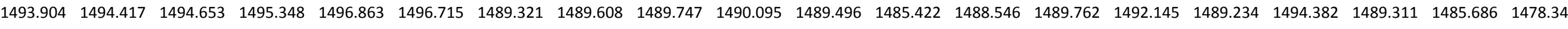

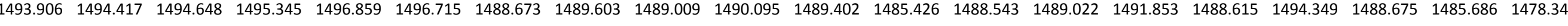

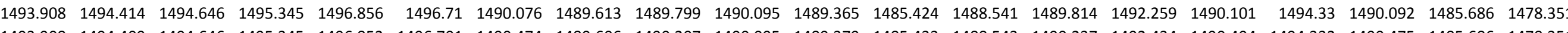

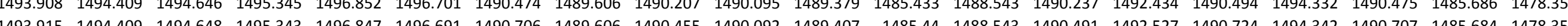

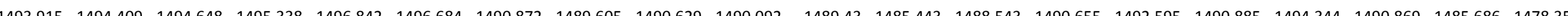
$\begin{array}{llllllllllllllllllll}1493.915 & 1494.407 & 1494.648 & 1495.334 & 1496.842 & 1496.677 & 1490.994 & 1489.606 & 1490.758 & 1490.09 & 1489.456 & 1485.45 & 1488.55 & 1490.801 & 1492.642 & 1491.008 & 1494.349 & 1490.989 & 1485.684 & 1478.351\end{array}$ $\begin{array}{llllllllllllllllllllll}1493.911 & 1494.407 & 1494.643 & 1495.329 & 1496.84 & 1496.677 & 1491.097 & 1489.601 & 1490.868 & 1490.085 & 1489.484 & 1485.454 & 1488.548 & 1490.912 & 1492.679 & 1491.11 & 1494.349 & 1491.087 & 1485.684 & 1478.351 \\ \end{array}$ $\begin{array}{lllllllllllllllllllllll}1493.901 & 1494.405 & 1494.646 & 1495.327 & 1496.84 & 1496.677 & 1491.181 & 1489.601 & 1490.955 & 1490.088 & 1489.507 & 1485.47 & 1488.546 & 1491.001 & 1492.718 & 1491.191 & 1494.356 & 1491.174 & 1485.684 & 1478.348\end{array}$

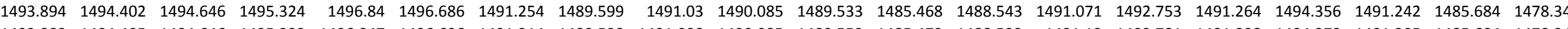
$\begin{array}{lllllllllllllllllllll}1493.882 & 1494.405 & 1494.646 & 1495.322 & 1496.847 & 1496.696 & 1491.314 & 1489.598 & 1491.098 & 1490.085 & 1489.559 & 1485.473 & 1488.532 & 1491.13 & 1492.781 & 1491.328 & 1494.372 & 1491.305 & 1485.684 & 1478.348\end{array}$

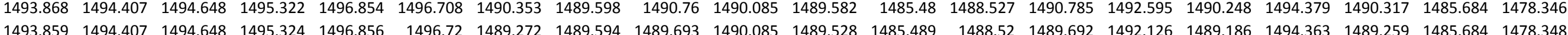


Well

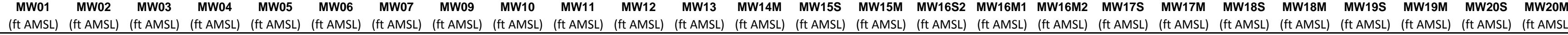

10/18/11 13:00 10/18/1113:30 10/18/11 14:00 10/18/1111 15:00 10/18/11 15:00 10/18/11 16:00 10/18/11 16:30 10/18/11 17:00 10/18/11 17:30 10/18/11 18:00 10/18/11 18:30 10/18/111 19:00 10/18/11 19:30 10/18/11 20:30 10/18/11 21:00
10/18/11 21:30 10/18/11 21:30 10/18/11 22:00 10/18/11 22:30 10/18/11 23:00 $10 / 19 / 110: 00$
$10 / 19 / 110: 30$ 10/19/11 1:00 $10 / 19 / 111: 30$
$10 / 19 / 112: 00$ 10/19/11 2:00 10/19/11 2:30 10/19/11 3:00 10/19/11 4:00 10/19/114:30 10/19/11 5:30 10/19/11 6:00 10/19/11 6:30 $10 / 19 / 117: 00$
$10 / 19 / 117: 30$
$10 / 19 / 118: 00$ 10/19/11 8:30 10/19/11 9:00 10/19/11 9:30 10/19/11 10:00 10/19/11 11:00 10/19/11 11:30 10/19/11 12:00 10/19/11 12:30 10/19/11 13:30 10/19/11 14:00 $10 / 19 / 1114: 30$
$10 / 19 / 1115: 00$ $10 / 19 / 1115: 00$
$10 / 19 / 1115: 30$ $\begin{array}{rrrrr}1494.33 & 1494.725 & 1496.614 & 1494.355 \\ 1494.333 & 1494.73 & 1496.618 & 1494.353\end{array}$ $\begin{array}{rrrr}1494.333 & 1494.73 & 1496.618 & 1494.353\end{array}$ $\begin{array}{lllll}1494.33 & 1494.734 & 1496.616 & 1494.353 \\ 1494.328 & 1494.739 & 1496.618 & 1494.353\end{array}$ $\begin{array}{lllll}1494.33 & 1494.741 & 1496.616 & 1494.355\end{array}$ $\begin{array}{lllll}1494.328 & 1494.741 & 1496.618 & 1494.36\end{array}$ $\begin{array}{lllll}1494.326 & 1494.744 & 1496.614 & 1494.365\end{array}$ $\begin{array}{lllll}1494.323 & 1494.746 & 1496.611 & 1494.3659\end{array}$ $\begin{array}{lllll}1494.321 & 1494.746 & 1496.607 & 1494.379\end{array}$ $\begin{array}{llll}1494.321 & 1494.746 & 1496.597 & 1494.383\end{array}$ $\begin{array}{llll}1494.321 & 1494.744 & 1496.59 & 1494.383 \\ 1494314 & 1494.741 & 1496581 & 194.376\end{array}$ $\begin{array}{rrrr}1494.314 & 1494.741 & 1496.581 & 1494.376 \\ 1494.312 & 1494.737 & 1496.576 & 1494.36\end{array}$ $\begin{array}{lllll}1494.309 & 1494.732 & 1496.576 & 1494.346\end{array}$ $\begin{array}{llll}1494.307 & 1494.727 & 1496.579 & 1494.332\end{array}$ $\begin{array}{lllll}1494.307 & 1494.727 & 1496.579 & 1494.323\end{array}$ $\begin{array}{llll}1494.309 & 1494.725 & 1496.583 & 1494.311\end{array}$ $1494.307 \quad 1494.725 \quad 1496.583 \quad 1494.304$ $\begin{array}{llrl}1494.309 & 1494.723 & 1496.588 & 1494.299\end{array}$ $\begin{array}{llll}1494.312 & 1494.725 & 1496.59 & 1494.297\end{array}$ $\begin{array}{llll}1444.309 & 1494.725 & 1496.602 & 1494.302\end{array}$ $\begin{array}{lllll}1494.312 & 1494.732 & 1496609 & 1494.313\end{array}$ $\begin{array}{rrrr}1494.309 & 1494.734 & 1496.6 & 1494.32\end{array}$ $\begin{array}{lllll}1494.309 & 1494.734 & 1496.595 & 1494.33\end{array}$ $1494.309 \quad 1494.739 \quad 1496.593 \quad 1494.344$ $\begin{array}{llll}1494.305 & 1494.734 & 1496.595 & 1494.351 \\ 1494302 & 1494.734 & 1496597 & 1494358\end{array}$ $\begin{array}{rrrr}1494.302 & 1494.734 & 1496.597 & 1494.358\end{array}$ $\begin{array}{rrrr}1494.3 & 1494.732 & 1496.6 & 1494.365 \\ 1494305 & 1494.735 & 1496.597 & 1494.376\end{array}$ $\begin{array}{lllll}1494.302 & 1494.737 & 1496.593 & 1494.383\end{array}$ $\begin{array}{lllll}1494.302 & 1494.739 & 1496.59 & 1494.393\end{array}$ $\begin{array}{lllll}1494.302 & 1494.737 & 1496.586 & 1494.4\end{array}$ $1494.298 \quad 1494.739 \quad 1496.588 \quad 1494.407$ $\begin{array}{lllll}1494.295 & 1494.734 & 1496.59 & 1494.411\end{array}$ $\begin{array}{rlll}1494.295 & 1494.732 & 1496.579 & 1494.416\end{array}$ $\begin{array}{rllll}1494.3 & 1494.734 & 1496.567 & 1494.414\end{array}$ $\begin{array}{llll}1494.295 & 1494.734 & 1496.553 & 1494.402 \\ 1494.293 & 1494.727 & 1496.551 & 1494.386\end{array}$ $\begin{array}{llll}1494.286 & 1494.723 & 1496.544 & 1494.372\end{array}$ $1494.284 \quad 1494.716 \quad 1496.548 \quad 1494.365$ $\begin{array}{lllll}1494.286 & 1494.716 & 1496.548 & 1494.355\end{array}$ $\begin{array}{lllll}1494.284 & 1494.707 & 1496.548 & 1494.351\end{array}$ $1494.2881494 .709 \quad 1496.565 \quad 1494.353$ $\begin{array}{llll}1494.288 & 1494.707 & 1496.572 & 1494.353\end{array}$ $\begin{array}{lllll}1494.288 & 1494.709 & 1496.581 & 1494.36\end{array}$ $\begin{array}{lllll}1494.291 & 1494.716 & 1496.609 & 1494.376\end{array}$ $\begin{array}{lllll}1494.293 & 1494.721 & 1496.625 & 1494.376\end{array}$ $\begin{array}{lllll}1494.298 & 1494.734 & 1496.63 & 1494.381\end{array}$ $\begin{array}{lllll}1494.298 & 1494.741 & 1496.635 & 1494.379\end{array}$ $\begin{array}{llllll}1494.3 & 1494.748 & 1496.642 & 1494.383\end{array}$ $\begin{array}{rrrr}1494.298 & 1494.753 & 1496.642 & 1494.388 \\ 1494.3 & 1494.76 & 1496.639 & 1494.395\end{array}$

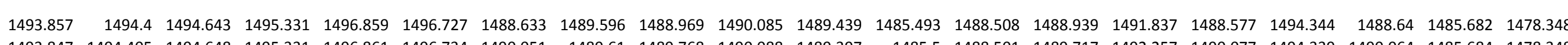

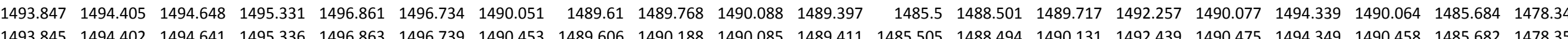

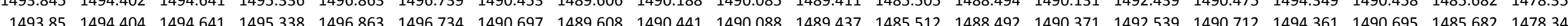
$\begin{array}{llllllllllllllllllll}1493.847 & 1494.405 & 1494.646 & 1495.338 & 1496.868 & 1496.739 & 1490.87 & 1489.605 & 1490.624 & 1490.088 & 1489.46 & 1485.519 & 1488.492 & 1490.547 & 1492.621 & 1490.883 & 1494.372 & 1490.862 & 1485.686 & 1478348\end{array}$ $\begin{array}{llllllllllllllllllll}1493.847 & 1494.405 & 1494.638 & 1495.338 & 1496.866 & 1496.737 & 1490.998 & 1489.61 & 1490.76 & 1490.09 & 1489.489 & 1485.524 & 1488.489 & 1490.683 & 1492.674 & 1491.018 & 1494.379 & 1490.993 & 1485.682 & 1478.348\end{array}$ $\begin{array}{lllllllllllllllllllll}1493.852 & 1494.402 & 1494.638 & 1495.341 & 1496.866 & 1496.739 & 1491.106 & 1489.61 & 1490.873 & 1490.092 & 1489.514 & 1485.521 & 1488.489 & 1490.789 & 1492.721 & 1491.12 & 1494.398 & 1491.099 & 1485.682 & 1478.348\end{array}$

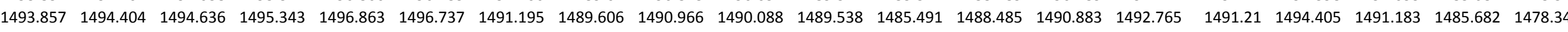
$\begin{array}{llllllllllllllllllll}1493.859 & 1494.402 & 1494.641 & 1495.341 & 1496.866 & 1496.737 & 1491.272 & 1489.613 & 1491.048 & 1490.09 & 1489.566 & 1485.48 & 1488.487 & 1490.961 & 1492.802 & 1491.283 & 1494.412 & 1491.263 & 1485.679 & 1478.348\end{array}$

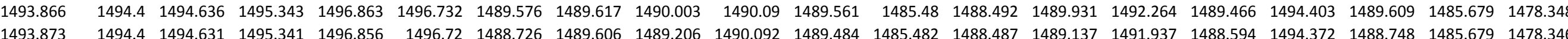

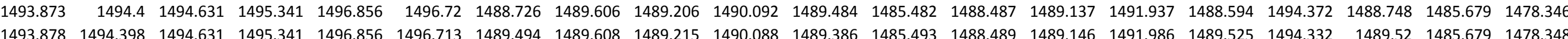

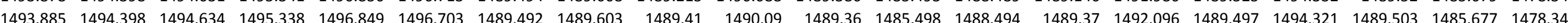

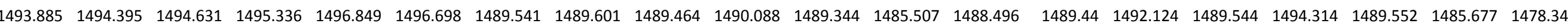
$\begin{array}{lllllllllllllllllllll}1493.885 & 1494.395 & 1494.631 & 1495.331 & 1496.849 & 1496.698 & 1489.552 & 1489.601 & 1489.473 & 1490.085 & 1489.327 & 1485.514 & 1488.496 & 1489.471 & 1492.117 & 1489.556 & 1494.297 & 1489.557 & 1485.677 & 1478.344\end{array}$

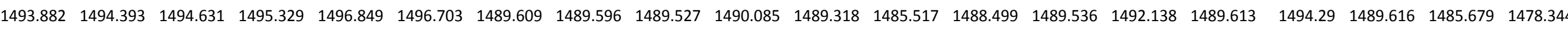
$\begin{array}{llllllllllllllllllllll}1493.878 & 1494.39 & 1494.639 & 1495.329 & 1496.849 & 1496.701 & 1489.597 & 1489.596 & 1489.515 & 1490.085 & 1489.306 & 1485.521 & 1488.499 & 1489.534 & 1492.126 & 1489.596 & 1494.29 & 1489.604 & 1485.677 & 1478.344\end{array}$

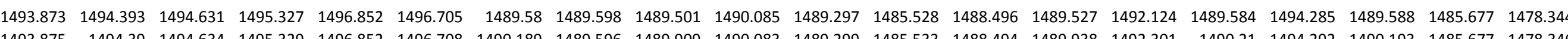

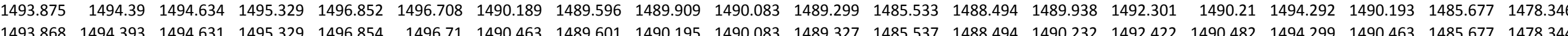

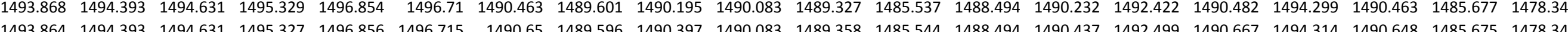
$\begin{array}{lllllllllllllllllllllll}1493.859 & 1494.39 & 1494.631 & 1495.329 & 1496.859 & 1496.722 & 1490.795 & 1489.598 & 1490.549 & 1490.083 & 1489.388 & 1485.551 & 1488.494 & 1490.589 & 1492.562 & 1490.812 & 1494.328 & 1490.791 & 1485.675 & 1478.344\end{array}$

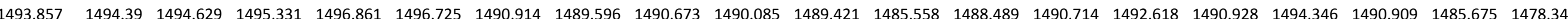
$\begin{array}{lllllllllllllllllllllll}1493.857 & 1494.39 & 1494.627 & 1495.334 & 1496.861 & 1496.732 & 1491.015 & 1489.598 & 1490.781 & 1490.085 & 1489.449 & 1485.565 & 1488.485 & 1490.82 & 1492.674 & 1491.027 & 1494.358 & 1491.007 & 1485.675 & 1478.344\end{array}$ $\begin{array}{llllllllllllllllllllll}1493.864 & 1494.39 & 1494.629 & 1495.334 & 1496.859 & 1496.725 & 1491.101 & 1489.599 & 1490.873 & 1490.085 & 1489.474 & 1485.572 & 1488.487 & 1490.914 & 1492.711 & 1491.115 & 1494.372 & 1491.094 & 1485.677 & 1478.344\end{array}$ $\begin{array}{llllllllllllllllllll}1493.864 & 1494.388 & 1494.627 & 1495.336 & 1496.856 & 1496.72 & 1491.179 & 1489.601 & 1490.952 & 1490.088 & 1489.503 & 1485.577 & 1488.487 & 1490.996 & 1492.749 & 1491.191 & 1494.386 & 1491.169 & 1485.675 & 1478.344\end{array}$

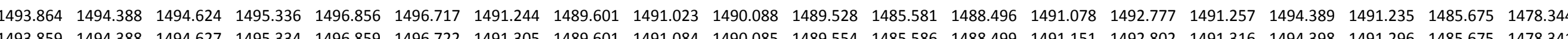

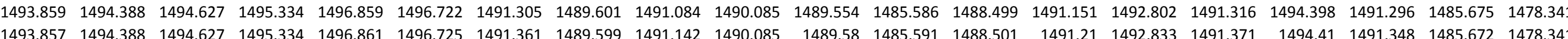

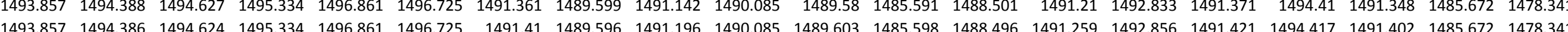
$\begin{array}{lllllllllllllllllllllll}1493.857 & 1494.388 & 1494.629 & 1495.334 & 1496.859 & 1496.725 & 1491.457 & 1489.594 & 1491.243 & 1490.083 & 1489.626 & 1485.604 & 1488.496 & 1491.313 & 1492.886 & 1491.468 & 1494.424 & 1491.448 & 1485.672 & 1478.339\end{array}$ $\begin{array}{llllllllllllllllllllll}1493.861 & 1494.385 & 1494.624 & 1495.336 & 1496859 & 1496.72 & 1491.504 & 1489.601 & 1491.288 & 1490.085 & 1489.647 & 1485.607 & 1488.492 & 1491.356 & 1492.917 & 1491.511 & 1494.433 & 1491.488 & 1485.672 & 1478.339\end{array}$

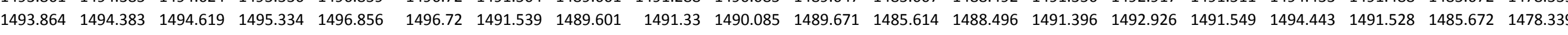

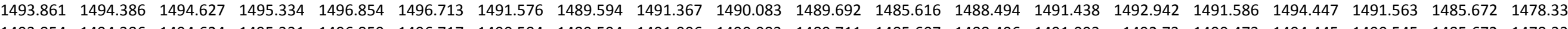

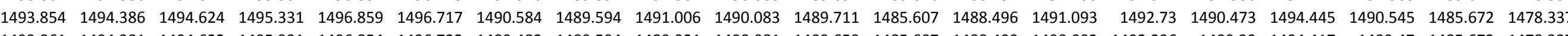

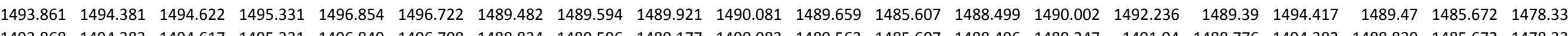

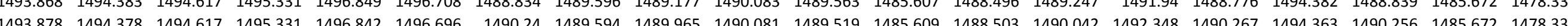

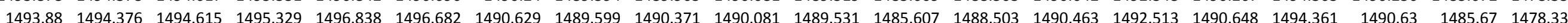
$\begin{array}{llllllllllllllllllllll}1493.878 & 1494.376 & 1494.615 & 1495.322 & 1496.835 & 1496.682 & 1490.694 & 1489.594 & 1490.437 & 1490.078 & 1489.54 & 1485.611 & 1488.51 & 1490.545 & 1492.574 & 1490.71 & 1494.356 & 1490.695 & 1485.67 & 1478.334\end{array}$ \begin{tabular}{lllllllllllllllllllll}
1493.875 & 1494.376 & 1494.617 & 1495.317 & 1496.833 & 1496.677 & 1490.87 & 1489.589 & 1490.624 & 1490.078 & 1489.531 & 1485.614 & 1488.508 & 1490.735 & 1492.611 & 1490.885 & 1494.353 & 1490.871 & 1485.67 & 1478.334 \\
\hline
\end{tabular}

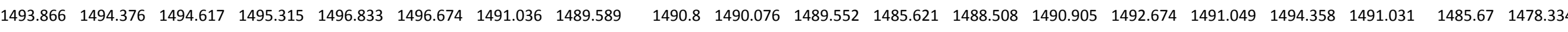

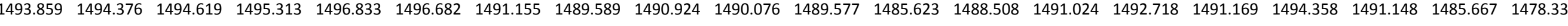

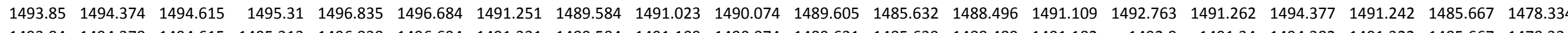

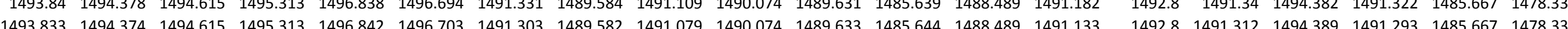

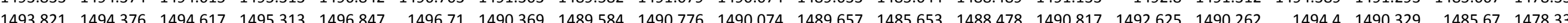
$\begin{array}{llllllllllllllllllllll}1493.807 & 1494.378 & 1494.617 & 1495.317 & 1496.856 & 1496.727 & 1489.304 & 1489.587 & 1489.726 & 1490.074 & 1489.605 & 1485.66 & 1488.473 & 1489.748 & 1492.152 & 1489.217 & 1494.384 & 1489.297 & 1485.667 & 1478.334\end{array}$ $\begin{array}{llllllllllllllllllllll}1493.795 & 1494.381 & 1494.619 & 1495.32 & 1496.863 & 1496.739 & 1488.682 & 1489.587 & 1489.011 & 1490.076 & 1489.51 & 1485.66 & 1488.471 & 1489.012 & 1491.872 & 1488.622 & 1494.361 & 1488.687 & 1485.67 & 1478.339\end{array}$ $\begin{array}{llllllllllllllllllll}1493.793 & 1494.381 & 1494.617 & 1495.324 & 1496.868 & 1496.753 & 1490.109 & 1489.589 & 1489.827 & 1490.078 & 1489.472 & 1485.669 & 1488.457 & 1489.79 & 1492.299 & 1490.134 & 1494.356 & 1490.125 & 1485.67 & 1478.339\end{array}$

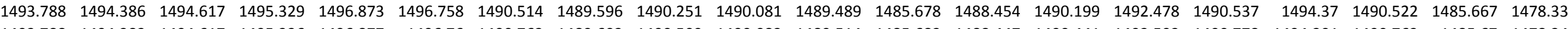
\begin{tabular}{lllllllllllllllllllllll}
1493.788 & 1494.383 & 1494.617 & 1495.336 & 1496.877 & 1496.76 & 1490.762 & 1489.603 & 1490.509 & 1490.083 & 1489.514 & 1485.683 & 1488.447 & 1490.441 & 1492.593 & 1490.778 & 1494.391 & 1490.763 & 1485.67 & 1478.341 \\
\hline
\end{tabular} $\begin{array}{lllllllllllllllllllllll}1493.791 & 1494.388 & 1494.615 & 1495.338 & 1496.877 & 1496.768 & 1490.938 & 1489.601 & 1490.694 & 1490.083 & 1489.54 & 1485.69 & 1488.449 & 1490.611 & 1492.667 & 1490.956 & 1494.41 & 1490.934 & 1485.667 & 1478.341 \\ 1493.795 & 1494.386 & 1494.615 & 1495.343 & 1496.88 & 1496.768 & 1491.073 & 1489.608 & 1490.835 & 1490.085 & 1489.568 & 1485.692 & 1488.447 & 1490.742 & 1492.735 & 1491.084 & 1494.422 & 1491.066 & 1485.667 & 1478.341\end{array}$ 
Well

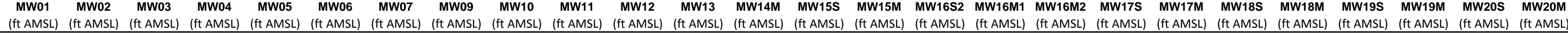

10/19/11 16:00 10/19/11 16:30 10/19/11 17:00 10/19/11 18:00 10/19/11 18:30 10/19/11 18:30 10/19/11 19:30 10/19/11 20:00 10/19/11 20:30 10/19/11 21:00 10/19/11 21:30 10/19/1111 22:00 10/19/11 22:30 10/19/11 23:30 10/20/11 0:00 10/20/11 1:00 10/20/11 1:30 10/20/11 2:00 10/20/11 3:00 10/20/11 3:30 10/20/11 4:30 10/20/11 5:00 $10 / 20 / 115: 30$
$10 / 20 / 116: 00$
$10 / 20 / 116: 30$ 10/20/11 7:00 10/20/11 7:30 10/20/11 8:30 10/20/11 9:00 10/20/11 10:00 10/20/11 10:30 10/20/1111:00 10/20/11 12:00 10/20/11 12:30 10/20/11 13:00 10/20/11 13:30 10/20/11 14:00 10/20/11 14:30 10/20/11 15:00 10/20/11 15:30 10/20/11 16:30 10/20/11 17:00 10/20/11 17:30 $10 / 20 / 1118: 00$
$10 / 20 / 1118: 30$ $\begin{array}{rllll}1494.3 & 1494.762 & 1496.639 & 1494.402\end{array}$ $\begin{array}{llll}1494.295 & 1494.762 & 1496.637 & 1494.409\end{array}$ $\begin{array}{llll}1494.295 & 1494.765 & 1496.637 & 1494.418 \\ 1494.295 & 1494.765 & 1496.635 & 1494.425\end{array}$ $\begin{array}{lllll}1494.295 & 1494.767 & 1496.63 & 1494.428\end{array}$ $\begin{array}{rrrr}1494.295 & 1494.767 & 1496.63 & 1494.428 \\ 1494.291 & 1494.765 & 1496.625 & 1494.421\end{array}$ $\begin{array}{llll}1494.291 & 1494.765 & 1496.623 & 14944.421 \\ 1411\end{array}$ $\begin{array}{lllll}1494.288 & 1494.765 & 1496.621 & 1494.4\end{array}$ $1494.286 \quad 1494.76 \quad 1496.618 \quad 1494.393$ $\begin{array}{llll}1494.286 & 1494.76 & 1496.621 & 1494.393\end{array}$ $\begin{array}{llll}1494.284 & 1494.758 & 1496.616 & 1494.393 \\ 1494284 & 1494.758 & 1496.611 & 1494395\end{array}$ $\begin{array}{rrrr}1494.284 & 1494.758 & 1496.611 & 1494.395 \\ 1494.284 & 1494.758 & 1496.609 & 1494.4\end{array}$ $\begin{array}{lllll}1494.281 & 1494.755 & 1496.604 & 1494.402\end{array}$ $\begin{array}{llll}1494.281 & 1494.755 & 1496.604 & 1494.402 \\ 1494.279 & 1494.751 & 1496.611 & 1494.409\end{array}$ $\begin{array}{lllll}1499.479 & 1494.451 & 1496.611 & 1494.409 \\ 1494.279 & 1494.753 & 1496.609 & 1494.414\end{array}$ $\begin{array}{lllll}1494.277 & 1494.751 & 1496.602 & 1494.416\end{array}$ $\begin{array}{llrrr}1494.281 & 1494.753 & 1496.6 & 1494.425 \\ 149.279 & 1494753 & 1496597 & 1494.432\end{array}$ $\begin{array}{llll}1494.279 & 1494.753 & 1496.597 & 1494.432\end{array}$ $\begin{array}{rrrr}1494.277 & 1494.748 & 1496.6 & 1494.437 \\ 1494.274 & 1494.748 & 1496602 & 194.439\end{array}$ $\begin{array}{llll}1494.274 & 1494.748 & 1496.602 & 1494.439 \\ 1494.279 & 1494.748 & 1496.609 & 1494.446\end{array}$ $\begin{array}{llllll}1494.277 & 1494.748 & 1496.614 & 1499.453\end{array}$ $\begin{array}{lllll}1494.277 & 1494.751 & 1496.609 & 1494.458\end{array}$ $\begin{array}{lllll}1494.279 & 1494.753 & 1496.607 & 1494.47\end{array}$ $\begin{array}{llll}1494.279 & 1494.758 & 1496.6 & 1494.479\end{array}$ $\begin{array}{llll}1494.277 & 1494.755 & 1496.595 & 1494.482\end{array}$ $\begin{array}{llll}1494.274 & 1494.755 & 1496.588 & 1494.484\end{array}$ $\begin{array}{rrrrr}1494.272 & 1494.751 & 1496.593 & 1494.486 \\ 1494.27 & 1494.751 & 1496.581 & 1494.484\end{array}$ $\begin{array}{lllll}1494.27 & 1494.746 & 1496.574 & 1494.477\end{array}$ $\begin{array}{lllll}1494.27 & 1494.746 & 1496.56 & 1494.463\end{array}$ $\begin{array}{rrrrr}1494.265 & 1494.741 & 1496.551 & 1494.446\end{array}$ $\begin{array}{llllll}1494.265 & 1494.737 & 1496.546 & 1494.432\end{array}$ $\begin{array}{lllll}1494.26 & 1494.73 & 1496.548 & 1494.421\end{array}$ $\begin{array}{rrrr}1494.258 & 1494.725 & 1496.553 & 1494.409 \\ 1494.26 & 1494.721 & 14965 & 1944.47\end{array}$ $\begin{array}{llll}1494.26 & 1494.721 & 1496.56 & 1494.407 \\ 1494.26 & 1494.721 & 1496.569 & 194.404\end{array}$ $\begin{array}{rrrr}1494.26 & 1494.721 & 1496.569 & 1494.404 \\ 1494.263 & 1494.723 & 1496.579 & 1494.409\end{array}$ $\begin{array}{lllll}1494.267 & 1494.725 & 1496.588 & 1494.409\end{array}$ $\begin{array}{lllll}1494.265 & 1494.725 & 1496.609 & 1494.423\end{array}$ $\begin{array}{lllll}1494.27 & 1494.727 & 1496.621 & 1494.43\end{array}$ $\begin{array}{lllll}1494.272 & 1494.737 & 1496.632 & 1494.428\end{array}$ $\begin{array}{llll}1494.277 & 1494.751 & 1496.644 & 1494.43\end{array}$ $1494.2791494 .755 \quad 1496.644 \quad 1494.428$ $\begin{array}{lllll}1494.281 & 1494.765 & 1496.642 & 1494.432 \\ 1494279 & 1494.772 & 1496.644 & 1494.437\end{array}$ $\begin{array}{llll}1494.277 & 1494.772 & 1496.637 & 1494.439\end{array}$ $\begin{array}{lllll}1494.277 & 1494.774 & 1496.63 & 1494.444\end{array}$ $1494.277 \quad 1494.774 \quad 1496.623 \quad 1494.451$ $\begin{array}{lllll}1494.274 & 1494.772 & 1496.616 & 1494.458\end{array}$ $\begin{array}{llllll}1494.27 & 1494.769 & 1496.604 & 1494.458\end{array}$ $\begin{array}{rrrr}1494.267 & 1494.767 & 1496.6 & 1494.451 \\ 1494.267 & 1494.762 & 1496.588 & 1494.439\end{array}$

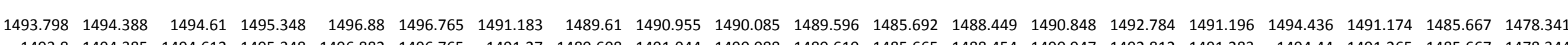

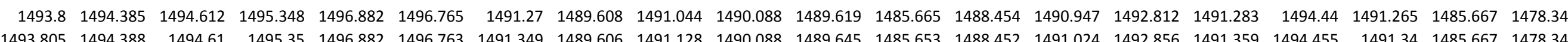

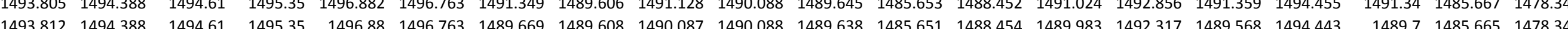

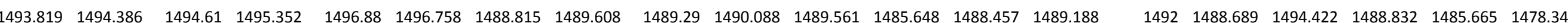

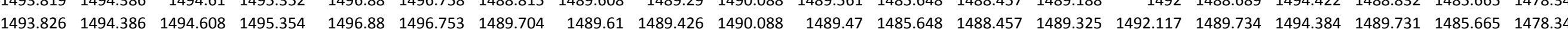
$\begin{array}{llllllllllllllllllllll}1493.833 & 1494.385 & 1494.605 & 1495.352 & 1496.877 & 1496.746 & 1490.322 & 1489.606 & 1490.045 & 1490.088 & 1489.474 & 1485.646 & 1488.461 & 1489.967 & 1492.408 & 1490.345 & 1494.379 & 1490.329 & 1485.663 & 1478.341 \\ \end{array}$ $\begin{array}{lllllllllllllllllllll}1493.835 & 1494.38 & 1494.605 & 1495.352 & 1496.877 & 1496.744 & 1490.626 & 1489.61 & 1490.369 & 1490.088 & 1489.498 & 1485.641 & 1488.461 & 1490.3 & 1492.53 & 1490.643 & 1494.384 & 1490.629 & 1485.665 & 1478.341\end{array}$ $\begin{array}{llllllllllllllllllll}1493.84 & 1494.381 & 1494.605 & 1495.35 & 1496.875 & 1496.741 & 1490.828 & 1489.606 & 1490.582 & 1490.085 & 1489.521 & 1485.635 & 1488.468 & 1490.535 & 1492.613 & 1490.845 & 1494.384 & 1490.827 & 1485.663 & 1478.339 \\ \end{array}$

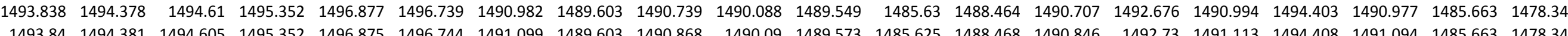

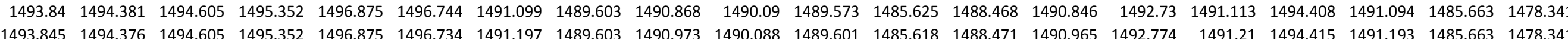

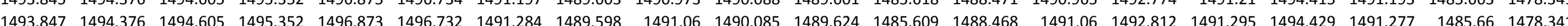

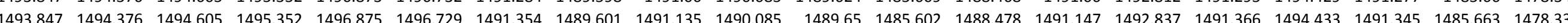
$\begin{array}{llllllllllllllllllllll}1493.85 & 1494.373 & 1494.603 & 1495.352 & 1496.875 & 1496.725 & 1491.417 & 1489.601 & 1491.201 & 1490.085 & 1489.675 & 1485.593 & 1488.48 & 1491.226 & 1492.868 & 1491.428 & 1494.433 & 1491.409 & 1485.663 & 1478.339\end{array}$ $\begin{array}{llllllllllllllllllll}1493.845 & 1494.373 & 1494.605 & 1495.35 & 1496.875 & 1496.732 & 1491.478 & 1489.594 & 1491.262 & 1490.085 & 1489.697 & 1485.588 & 1488.478 & 1491.29 & 1492.898 & 1491.485 & 1494.44 & 1491.465 & 1485.663 & 1478.339\end{array}$

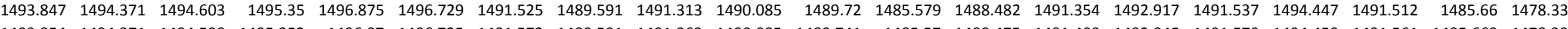

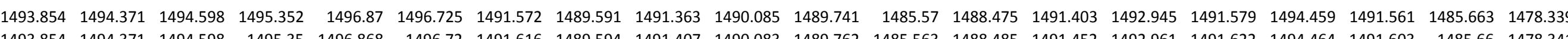

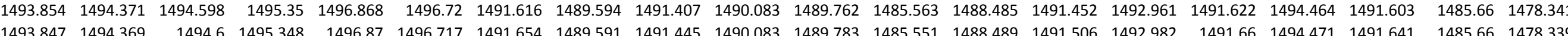
$\begin{array}{llllllllllllllllllllll}1493.847 & 1494.369 & 1494.6 & 1495.348 & 1496.87 & 1496.717 & 1491.654 & 1489.591 & 1491.445 & 1490.083 & 1489.783 & 1485.551 & 1488.489 & 1491.506 & 1492.982 & 1491.66 & 1494.471 & 1491.641 & 1485.66 & 1478.339 \\ 1493847 & 1494.369 & 1494.603 & 1495348 & 149687 & 1496.722 & 1491.686 & 1489591 & 1491.482 & 1490.083 & 1489802 & 1485544 & 1488.92 & 1491551 & 1492994 & 1491693 & 1494.471 & 1491676 & 148566 & 1478339\end{array}$ $\begin{array}{llllllllllllllllllllll}1493845 & 1494.371 & 1494.601 & 1495.348 & 1496.873 & 1496.722 & 1491.717 & 1489.587 & 1491.517 & 1490.083 & 1489.818 & 1485.537 & 1488.485 & 1491.586 & 1493.019 & 1491.729 & 1494.48 & 1491.707 & 1485.66 & 1478.339\end{array}$ $\begin{array}{llllllllllllllllllllllll}1493.838 & 1494371 & 1494.6 & 1495348 & 1496875 & 1496732 & 1491.747 & 1489587 & 1491.548 & 1490081 & 1489839 & 1485528 & 1488485 & 1491.626 & 1493.033 & 1491.757 & 1494.478 & 1491.737 & 148566 & 1478337\end{array}$ $\begin{array}{lllllllllllllllllllllll}1493.838 & 1494.369 & 1494.596 & 1495.35 & 1496.875 & 1496.737 & 1491.78 & 1489.587 & 1491.581 & 1490.081 & 1489.853 & 1485.517 & 1488.489 & 1491.654 & 1493.045 & 1491.786 & 1494.492 & 1491.765 & 1485.658 & 1478.339\end{array}$

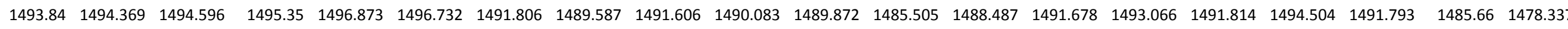

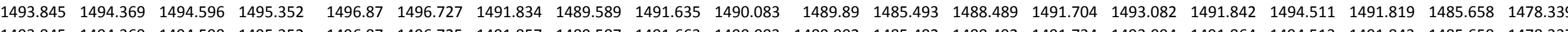

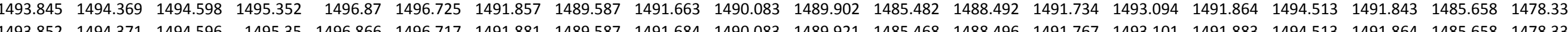

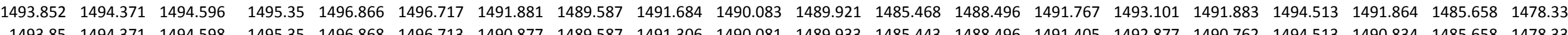

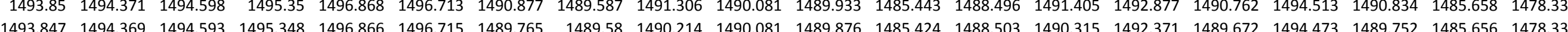
$\begin{array}{lllllllllllllllllllllll}1493.857 & 1494.366 & 1494.596 & 1495.345 & 1496.861 & 1496.703 & 1489.105 & 1489.587 & 1489.461 & 1490.078 & 1489.774 & 1485.408 & 1488.501 & 1489.56 & 1492.07 & 1489.039 & 1494.433 & 1489.109 & 1485.656 & 1478.332\end{array}$ $\begin{array}{llllllllllllllllllllll}1493.859 & 1494.364 & 1494.591 & 1495.345 & 1496.854 & 1496.696 & 1490.502 & 1489.584 & 1490.24 & 1490.078 & 1489.727 & 1485.396 & 1488.499 & 1490.335 & 1492.481 & 1490.52 & 1494.412 & 1490.51 & 1485.656 & 1478.332\end{array}$ $\begin{array}{llllllllllllllllllllll}1493.866 & 1494.362 & 1494.589 & 1495.341 & 1496.847 & 1496.684 & 1490.877 & 1489.582 & 1490.631 & 1490.078 & 1489.732 & 1485.389 & 1488.51 & 1490.745 & 1492.642 & 1490.892 & 1494.41 & 1490.881 & 1485.656 & 1478.332\end{array}$ $\begin{array}{llllllllllllllllllllll}1493.864 & 1494.359 & 1494.589 & 1495.341 & 1496.842 & 1496.677 & 1491.094 & 1489.587 & 1490.859 & 1490.078 & 1489.746 & 1485.38 & 1488.51 & 1490.977 & 1492.728 & 1491.11 & 1494.408 & 1491.089 & 1485.656 & 1478.33\end{array}$

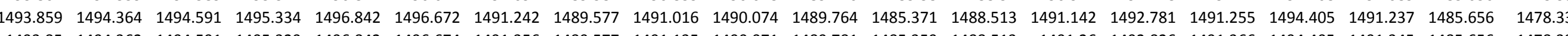

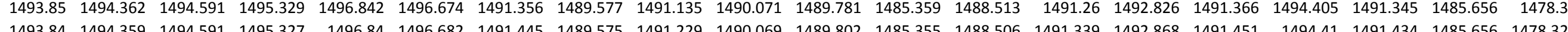

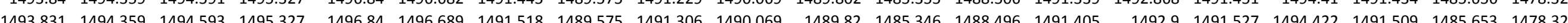

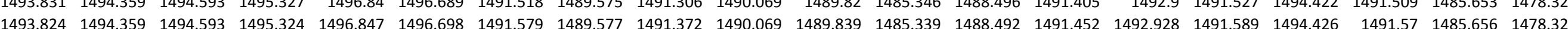
$\begin{array}{lllllllllllllllllllll}1493.814 & 1494.362 & 1494.591 & 1495.327 & 1496.847 & 1496.708 & 1491.637 & 1489.575 & 1491.428 & 1490.071 & 1489.86 & 1485.332 & 1488.485 & 1491.485 & 1492.963 & 1491.643 & 1494.445 & 1491.624 & 1485.656 & 1478.327\end{array}$ \begin{tabular}{llllllllllllllllllllll}
1493.8 & 1494.362 & 1494.593 & 1495.329 & 1496.854 & 1496.715 & 1490.659 & 1489.577 & 1491.079 & 1490.071 & 1489.876 & 1485.325 & 1488.48 & 1491.128 & 1492.763 & 1490.549 & 1494.447 & 1490.62 & 1485.653 & 1478.33 \\
\hline
\end{tabular} $\begin{array}{lllllllllllllllllllllllll}1493.786 & 1494.364 & 1494.598 & 1495.331 & 1496.863 & 1496.734 & 1489.571 & 1489.58 & 1490.012 & 1490.071 & 1489.823 & 1485.318 & 1488.475 & 1490.03 & 1492.28 & 1489.48 & 1494.431 & 1489.562 & 1485.653 & 1478.33 \\ \end{array}$

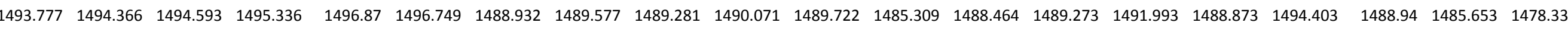

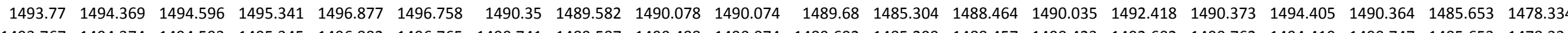

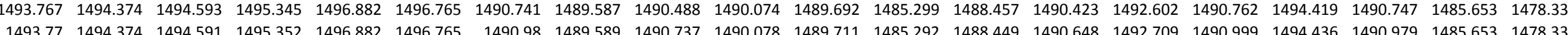

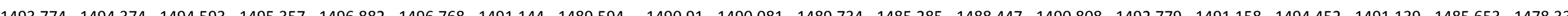
$\begin{array}{llllllllllllllllllllllll}1493.779 & 1494.376 & 1494.593 & 1495.357 & 1496.884 & 1496.765 & 1491.265 & 1489.594 & 1491.041 & 1490.081 & 1489.755 & 1485.278 & 1488.452 & 1490.93 & 1492.835 & 1491.278 & 1494.459 & 1491.265 & 1485.651 & 1478.339\end{array}$ $\begin{array}{llllllllllllllllllllll}1493.786 & 1494.373 & 1494.589 & 1495.359 & 1496.884 & 1496.76 & 1491.366 & 1489.601 & 1491.145 & 1490.081 & 1489.776 & 1485.267 & 1488.449 & 1491.029 & 1492.879 & 1491.378 & 1494.469 & 1491.359 & 1485.651 & 1478.339\end{array}$

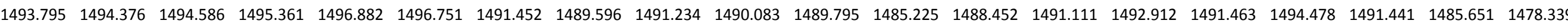
$\begin{array}{llllllllllllllllllll}1493.803 & 1494.371 & 1494.584 & 1495.361 & 1496.877 & 1496.746 & 1491.518 & 1489.596 & 1491.304 & 1490.083 & 1489.816 & 1485.2 & 1488.452 & 1491.186 & 1492.94 & 1491.53 & 1494.48 & 1491.512 & 1485.651 & 1478.337 \\ \end{array}$ $\begin{array}{lllllllllllllllllllll}1493.812 & 1494.371 & 1494.584 & 1495.359 & 1496.875 & 1496.739 & 1489.836 & 1489.594 & 1490.256 & 1490.081 & 1489.804 & 1485.191 & 1488.457 & 1490.145 & 1492.399 & 1489.743 & 1494.462 & 1489.862 & 1485.651 & 1478.339\end{array}$

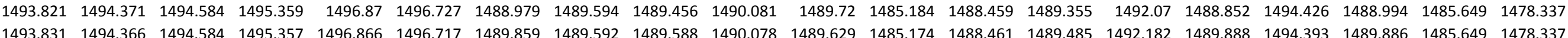


Well

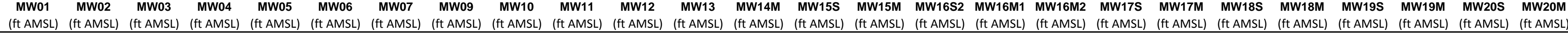

10/20/11 19:00 10/20/11 19:30 10/20/11 20:00 10/20/11 21:00 10/20/11 21:30 10/20/11 22:00 10/20/11 22:30 10/20/11 23:00 10/20/11 23:30 10/21/11 0:00 10/21/11 0:30 10/21/111:30 10/21/11 2:00 10/21/11 2:30 10/21/11 3:00 10/21/11 4:00 10/21/11 4:30 10/21/11 5:00 10/21/11 6:00 10/21/11 6:30 10/21/11 7:00 10/21/11 7:30 10/21/11 8:30 10/21/11 9:00 10/21/11 9:30 10/21/11 10:00 10/21/11 11:00 10/21/11 11:30 10/21/11 12:00 10/21/11 12:30 10/21/11 13:00 10/21/11 13:30 10/21/11 14:00 10/21/11 15:00 10/21/11 15:30 10/21/11 16:00 10/21/11 16:30 10/21/11 17:00 10/21/11 17:30 10/21/11 18:00 10/21/11 18:30 10/21/11 19:30 10/21/11 20:00 10/21/11 20:30 10/21/11 21:00
10/21/11 21:30 $\begin{array}{rrrr}1494.265 & 1494.758 & 1496.583 & 1494.425 \\ 1494.26 & 1494.753 & 1496586 & 1494.409\end{array}$ $\begin{array}{rrrr}1494.26 & 1494.753 & 1496.586 & 1494.409 \\ 1499.258 & 1499748 & 1496583 & 144.397\end{array}$ $\begin{array}{lllll}1494.258 & 1494.748 & 1496.583 & 1494.397 \\ 1494256 & 1494.744 & 1496579 & 1494.39\end{array}$ $\begin{array}{lllll}1494.258 & 1494.744 & 1496576 & 1494.39\end{array}$ $\begin{array}{llll}1494.258 & 1494.744 & 1496574 & 1494.39\end{array}$ $\begin{array}{lllll}1494.256 & 1444.739 & 1496.576 & 1494.39\end{array}$ $\begin{array}{llllll}1494.253 & 1494.737 & 1496.579 & 1494.395\end{array}$ $\begin{array}{llll}1494.256 & 1494.739 & 1496.576 & 1494.4\end{array}$ $\begin{array}{llll}1494.256 & 1494.735 & 1496.572 & 1494.402\end{array}$ $\begin{array}{llll}1494.253 & 1494.737 & 1496.567 & 1494.407 \\ 1494.253 & 1494.732 & 1496565 & 194.411\end{array}$ $\begin{array}{rrrrr}1494.253 & 1494.732 & 1496.565 & 1494.411 \\ 1494.253 & 1494.73 & 1496.56 & 1494.418\end{array}$ $\begin{array}{lllll}1494.249 & 1494.73 & 1496.56 & 1494.421\end{array}$ $\begin{array}{lllll}1494.251 & 1494.727 & 1496.572 & 1494.423\end{array}$ $\begin{array}{lllll}1494.249 & 1494.727 & 1496.562 & 1494.428\end{array}$ $\begin{array}{lllll}1494.249 & 1494.727 & 1496.565 & 1494.432\end{array}$ $\begin{array}{lllll}1494.256 & 1494.727 & 1496.567 & 1494.444\end{array}$ $\begin{array}{lllll}1494.251 & 1494.723 & 1496.569 & 1494.442\end{array}$ $\begin{array}{llll}1494.251 & 1494.725 & 1496.574 & 1494.451 \\ 1494251 & 1494.725 & 1496.572 & 1494.456\end{array}$ $\begin{array}{lllll}1494.251 & 1494.727 & 14965567 & 1494.463\end{array}$ $\begin{array}{llll}1494.251 & 1494.73 & 1496.56 & 1494.47\end{array}$ $\begin{array}{llll}1494.249 & 1494.727 & 1496.555 & 1494.47\end{array}$ $\begin{array}{lllll}1494.246 & 1494.723 & 1496.544 & 1494.456\end{array}$ $\begin{array}{lllll}1494.244 & 1494.723 & 1496.539 & 1494.444\end{array}$ $\begin{array}{lllll}1494.242 & 1494.718 & 1496.532 & 1494.428\end{array}$ $1494.237 \quad 1494.711 \quad 1496.534 \quad 1494.411$ $\begin{array}{lllll}1494.237 & 1494.709 & 1496.534 & 1494.402 \\ 1494.237 & 1494.707 & 1496541 & 1494395\end{array}$ $\begin{array}{llll}1494.239 & 1494.707 & 1496.544 & 1494.393\end{array}$ $\begin{array}{lllll}1494.239 & 1494.702 & 1496.551 & 1494.393\end{array}$ $\begin{array}{llllll}1494.242 & 1494.704 & 1496.553 & 1494.397\end{array}$ $\begin{array}{lllll}1494.242 & 1494.704 & 1496.567 & 1494.402\end{array}$ $\begin{array}{llll}1494.244 & 1494.704 & 1496.581 & 1494.407\end{array}$ $\begin{array}{llll}1494.244 & 1494.709 & 1496.593 & 1494.411 \\ 1492.246 & 1494.716 & 1496597 & 194.414\end{array}$ $\begin{array}{llll}1494.246 & 1494.716 & 1496.597 & 1494.414\end{array}$ $\begin{array}{lllll}1494.251 & 1494.725 & 1496.609 & 1494.409 \\ 1494.73 & 1496.609 & 1494.407\end{array}$ $\begin{array}{lllll}1494.251 & 1494.737 & 1496.607 & 1494.407\end{array}$ $\begin{array}{llllll}1494.253 & 1494.744 & 1496.609 & 1494.411\end{array}$ $\begin{array}{llllll}1494.251 & 1494.744 & 1496.611 & 1494.414\end{array}$ $\begin{array}{lllll}1494.251 & 1494.744 & 1496.611 & 1494.418\end{array}$ $\begin{array}{lllll}1494.249 & 1494.746 & 1496.611 & 1494.425\end{array}$ $\begin{array}{llll}1494.249 & 1494.748 & 1496.602 & 1494.43 \\ 1494249 & 1494.748 & 1496.593 & 1494.437\end{array}$ $\begin{array}{llll}1494.249 & 1494.748 & 1496.593 & 1494.437 \\ 1494246 & 1494.746 & 1496583 & 1494.435\end{array}$ $\begin{array}{lllll}1494.244 & 1494.744 & 1496579 & 1494.423\end{array}$ $1494.242 \quad 1494.739 \quad 1496.576 \quad 1494.407$ 1494.2391494 .7351496 .5741494 .393 $1494.2391494 .7321496 .574 \quad 1494.386$ $1494.237 \quad 1494.732 \quad 1466.569 \quad 1494.381$ $\begin{array}{rrrr}1494.237 & 1494.73 & 1496.565 & 1494.379 \\ 1494.235 & 1494.727 & 1496.56 & 1494.379\end{array}$ $\begin{array}{lllllllllllllllllllll}1493.835 & 1494.364 & 1494.581 & 1495.354 & 1496.863 & 1496.708 & 1490.465 & 1489.587 & 1490.197 & 1490.078 & 1489.626 & 1485.167 & 1488.464 & 1490.119 & 1492.46 & 1490.487 & 1494.384 & 1490.472 & 1485.649 & 1478.334\end{array}$ $\begin{array}{llllllllllllllllllllll}1493.838 & 1494.362 & 1494.584 & 1495.352 & 1496.861 & 1496.701 & 1490.762 & 1489.591 & 1490.512 & 1490.076 & 1489.643 & 1485.161 & 1488.466 & 1490.453 & 1492.586 & 1490.781 & 1494.384 & 1490.766 & 1485.649 & 1478.334 \\ 1493.833 & 1494362 & 1494584 & 1495348 & 1496863 & 1496703 & 1490.556 & 1489.584 & 1490718 & 1490.074 & 1489664 & 1485.151 & 1488.468 & 1490681 & 1492.66 & 1490.97 & 1494382 & 1490.956 & 1485649 & 1478332\end{array}$

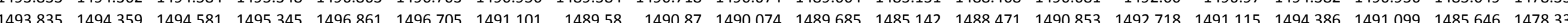
$\begin{array}{llllllllllllllllllllll}1493.838 & 1494.359 & 1494.584 & 1495.345 & 1496.856 & 1496.701 & 1491.216 & 1489.575 & 1490.988 & 1490.071 & 1489.708 & 1485.13 & 1488.464 & 1490.98 & 1492.77 & 1491.229 & 1494.396 & 1491.211 & 1485.649 & 1478.332\end{array}$ $\begin{array}{lllllllllllllllllllll}1493.838 & 1494357 & 1494.584 & 1495343 & 1496.859 & 1496.698 & 149131 & 1489.577 & 1491.091 & 1490.071 & 1489.729 & 1485.121 & 1488.466 & 1491.088 & 1492.809 & 1491.323 & 1494.405 & 1491.303 & 1485.649 & 1478.332\end{array}$ $\begin{array}{lllllllllllllllllllllll}1493.835 & 1494.354 & 1494.582 & 1495.341 & 1496.856 & 1496.694 & 1491.387 & 1489.58 & 1491.17 & 1490.069 & 1489.753 & 1485.114 & 1488.475 & 1491.184 & 1492.842 & 1491.399 & 1494.41 & 1491.38 & 1485.649 & 1478.332\end{array}$ $\begin{array}{lllllllllllllllllllllll}1493.833 & 1494.354 & 1494.581 & 1495.341 & 1496.856 & 1496.698 & 1491.455 & 1489.575 & 1491.241 & 1490.069 & 1489.774 & 1485.105 & 1488.468 & 1491.269 & 1492.87 & 1491.466 & 1494.412 & 1491.444 & 1485.649 & 1478.332\end{array}$

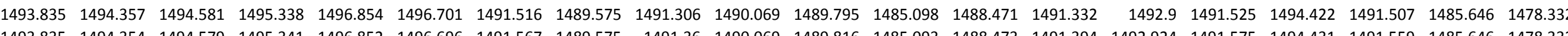

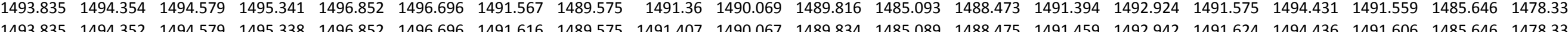
$\begin{array}{llllllllllllllllllll}1493.835 & 1494.352 & 1494.579 & 1495.338 & 1496.852 & 1496.696 & 1491.616 & 1489.575 & 1491.407 & 1490.067 & 1489.834 & 1485.089 & 1488.475 & 1491.459 & 1492.942 & 1491.624 & 1494.436 & 1491.606 & 1485.646 & 1478.332 \\ 1493.835 & 1494.352 & 1494.577 & 1495.336 & 1496.849 & 1496.691 & 1491.658 & 1489.573 & 1491.452 & 1490.067 & 1489.853 & 1485.082 & 1488.475 & 1491.506 & 1492.968 & 1491.667 & 1494.44 & 1491.645 & 1485.644 & 1478332\end{array}$ $\begin{array}{llllllllllllllllllll}1493.835 & 1494.354 & 1494.579 & 1495.336 & 1496.847 & 1496.691 & 1491.696 & 1489.57 & 1491.492 & 1490.064 & 1489.872 & 1485.077 & 1488.478 & 1491.553 & 1492.989 & 1491.707 & 1494.445 & 1491.685 & 1485.646 & 1478332\end{array}$ $\begin{array}{llllllllllllllllllllll}1493.835 & 1494352 & 1494577 & 1495334 & 1496845 & 1496686 & 1491.731 & 1489.568 & 1491.527 & 1490.064 & 1489888 & 1485.068 & 1488.48 & 1491.596 & 1493.005 & 1491.738 & 1494.447 & 1491.721 & 1485.646 & 147833\end{array}$ $\begin{array}{lllllllllllllllllllll}1493.831 & 1494.352 & 1494.577 & 1495.334 & 1496.847 & 1496.689 & 1491.766 & 1489.573 & 1491.567 & 1490.064 & 1489.907 & 1485.059 & 1488.485 & 1491.64 & 1493.019 & 1491.771 & 1494.452 & 1491.751 & 1485.644 & 1478.332\end{array}$

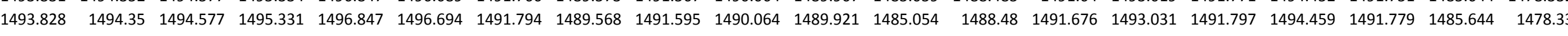
$\begin{array}{lllllllllllllllllllllll}1493.824 & 1494.352 & 1494.577 & 1495.331 & 1496.847 & 1496.691 & 1491.822 & 1489.568 & 1491.625 & 1490.062 & 1489.937 & 1485.047 & 1488.482 & 1491.706 & 1493.047 & 1491.828 & 1494.459 & 1491.807 & 1485.644 & 1478.332\end{array}$

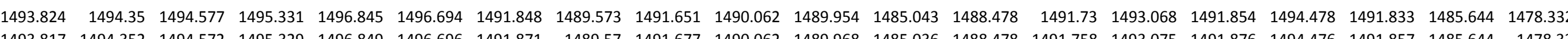

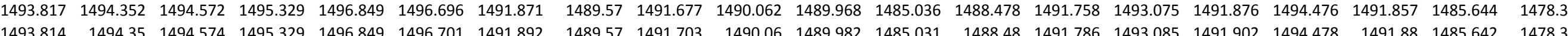
$\begin{array}{lllllllllllllllllllll}1493.814 & 1494.35 & 1494.574 & 1495.329 & 1496.849 & 1496.701 & 1491.892 & 1489.57 & 1491.703 & 1490.06 & 1489.982 & 1485.031 & 1488.48 & 1491.786 & 1493.085 & 1491.902 & 1494.478 & 1491.88 & 1485.642 & 1478.33 \\ 1493814 & 1494.347 & 1494.577 & 1495327 & 1496849 & 1496708 & 1491.916 & 1489.57 & 1491.724 & 1490.062 & 1489996 & 1485022 & 1488475 & 1491805 & 1493101 & 1491.923 & 1494.487 & 1491.901 & 1485.444 & 147833\end{array}$ $\begin{array}{lllllllllllllllllllllll}1493.817 & 1494.347 & 1494.572 & 1495.331 & 1496847 & 1496.705 & 1491.937 & 1489.57 & 1491.745 & 1490.06 & 1490.01 & 1485.515 & 1488.473 & 1491.821 & 1493.117 & 1491.942 & 1494.497 & 1491.922 & 1485.642 & 1478.33\end{array}$ $\begin{array}{lllllllllllllllllllll}1493.824 & 1494.35 & 1494572 & 1495331 & 1496845 & 1496.696 & 1490926 & 1489.575 & 1491363 & 1490.06 & 1490.021 & 1485008 & 1488478 & 1491.452 & 1492891 & 1490812 & 1494.497 & 1490.881 & 1485.644 & 147833\end{array}$ $\begin{array}{lllllllllllllllllllllll}1493.824 & 1494.347 & 1494.572 & 1495.331 & 1496.84 & 1496.691 & 1489.814 & 1489.563 & 1490.27 & 1490.06 & 1489.961 & 1484.999 & 1488.485 & 1490.357 & 1492.387 & 1489.722 & 1494.464 & 1489.801 & 1485.642 & 1478.33\end{array}$

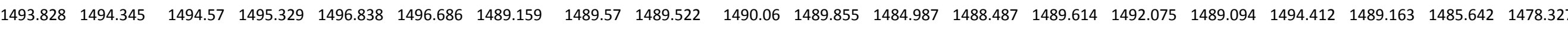

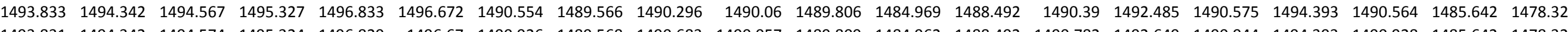

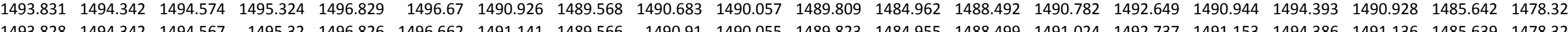

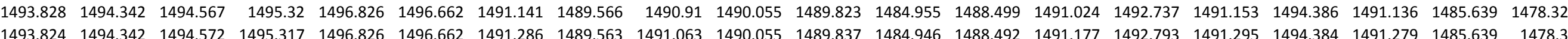
$\begin{array}{llllllllllllllllllllll}1493.824 & 1494.342 & 1494.572 & 1495.317 & 1496.826 & 1496.662 & 1491.286 & 1489.563 & 1491.063 & 1490.055 & 1489.837 & 1484.946 & 1488.492 & 1491.177 & 1492.793 & 1491.295 & 1494.384 & 1491.279 & 1485.639 & 1478.32 \\ 1493.817 & 1494.345 & 1494.57 & 1495.313 & 1496824 & 1496.667 & 1491396 & 1489.563 & 1491.18 & 1490.053 & 1489853 & 1484.939 & 1488.489 & 1491283 & 1492837 & 1491.409 & 1494391 & 1491.39 & 1485639 & 1478.32\end{array}$ $\begin{array}{llllllllllllllllllllll}1493.807 & 1494.342 & 1494.57 & 1495.313 & 1496.829 & 1496.672 & 1491.483 & 1489.561 & 1491.274 & 1490.05 & 1489.869 & 1484.934 & 1488.487 & 1491.36 & 1492.875 & 1491.494 & 1494.396 & 1491.477 & 1485.639 & 1478.32\end{array}$ $\begin{array}{lllllllllllllllllllll}1493.803 & 1494.342 & 1494.572 & 1495.31 & 1496.829 & 1496.677 & 1491.558 & 1489.561 & 1491.349 & 1490.05 & 1489.888 & 1484.899 & 1488.482 & 1491.424 & 1492.91 & 1491.57 & 1494.408 & 1491.547 & 1485.639 & 1478.318\end{array}$

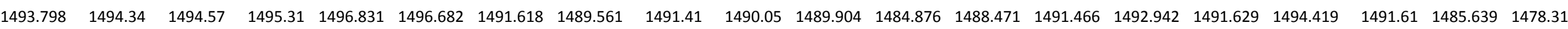

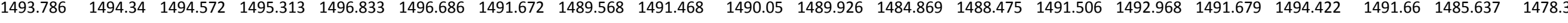

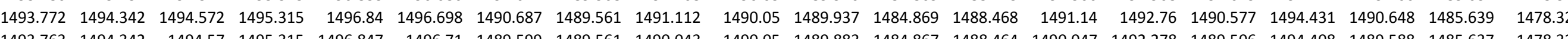

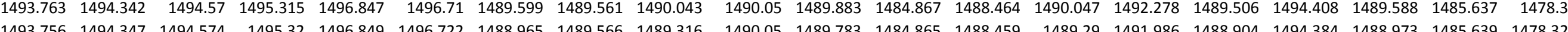

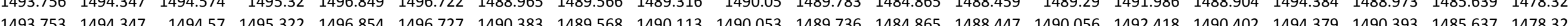

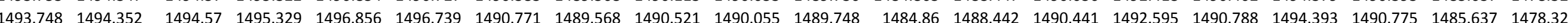
$\begin{array}{llllllllllllllllllllllll}1493.753 & 1494.35 & 1494.57 & 1495.329 & 1496.861 & 1496.737 & 1491.005 & 1489.575 & 1490.765 & 1490.055 & 1489.764 & 1484.858 & 1488.438 & 1490.672 & 1492.697 & 1491.02 & 1494.403 & 1491.005 & 1485.637 & 1478.33\end{array}$ $\begin{array}{lllllllllllllllllllllll}1493.751 & 1494.352 & 1494.567 & 1495.336 & 1496.863 & 1496.739 & 1491.169 & 1489.575 & 1490.936 & 1490.057 & 1489.788 & 1484.855 & 1488.431 & 1490.822 & 1492.765 & 1491.181 & 1494.419 & 1491.165 & 1485.637 & 1478.33\end{array}$

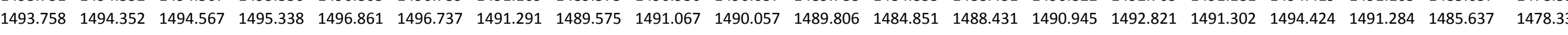

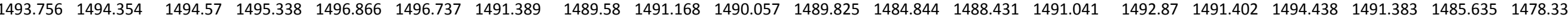

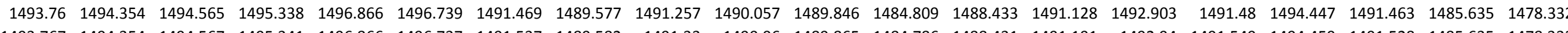

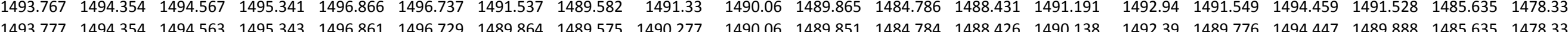

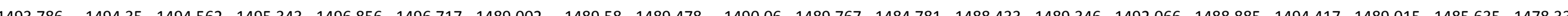
\begin{tabular}{lllllllllllllllllllllll}
1493.793 & 1494.35 & 1494.56 & 1495.341 & 1496.852 & 1496.708 & 1489.88 & 1489.575 & 1489.611 & 1490.057 & 1489.673 & 1484.774 & 1488.435 & 1489.478 & 1492.18 & 1489.909 & 1494.375 & 1489.904 & 1485.635 & 1478.33 \\
\hline
\end{tabular} $\begin{array}{llllllllllllllllllllll}1493.798 & 1494.347 & 1494.562 & 1495.338 & 1496.852 & 1496.703 & 1490.484 & 1489.573 & 1490.221 & 1490.055 & 1489.666 & 1484.77 & 1488.438 & 1490.11 & 1492.462 & 1490.504 & 1494.365 & 1490.491 & 1485.632 & 1478.327\end{array}$

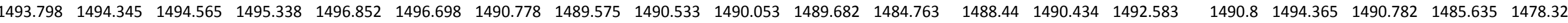
$\begin{array}{lllllllllllllllllllllllll}1493.8 & 1494.345 & 1494.563 & 1495.336 & 1496.849 & 1496.698 & 1490.975 & 1489.568 & 1490.734 & 1490.053 & 1489.704 & 1484.758 & 1488.442 & 1490.658 & 1492.665 & 1490.989 & 1494.37 & 1490.974 & 1485.635 & 1478.327\end{array}$

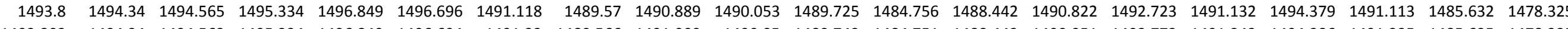

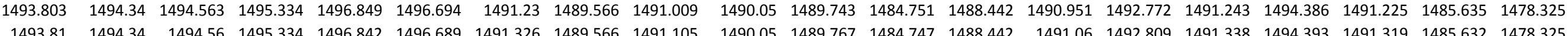


Well

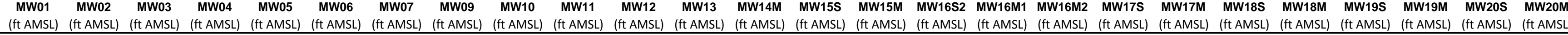

10/21/11 22:00 10/21/11 22:30

10/21/1123:00

10/22/11 0:00

10/22/11 0:30

10/22/11 1:00

$10 / 22 / 111: 30$
$10 / 22 / 112: 00$

10/22/11 2:00

10/222/113:00

10/22/11 3:30

10/22/11 4:00

10/22/11 5:00

10/22/11 5:30

10/22/11 6:00

10/22/11 7:00

10/22/117:30

10/22/11 8:00

10/22/11 9:00

10/22/1119:30

10/22/11 10:30

10/22/11 11:00

10/22/11 11:30

10/22/11 12:00

10/22/1111 13:30

10/22/11 13:30

10/22/11 14:00

10/22/11 14:30

10/22/11 15:30

10/22/11 16:00

10/22/11 16:30

10/22/11 17:30

10/22/11 18:00

10/22/12/11 18:00

10/22/11 19:00

10/22/11 19:30

10/22/11 20:00

10/22/11 20:30

10/22/11 21:00

10/22/11 22:00

10/22/11 22:30

10/22/1123:00

10/22/1123:30

10/23/11 0:00 $\begin{array}{rrrr}1494.237 & 1494.725 & 1496.555 & 1494.381 \\ 1494.23 & 1494.721 & 1496.553 & 1494.381\end{array}$ $\begin{array}{llll}1444.23 & 1494.721 & 1496.553 & 1494.381 \\ 1494.23 & 1494.718 & 1496558 & 1994383\end{array}$ $\begin{array}{rrrrr}1494.23 & 1494.718 & 1496.558 & 1494.383 \\ 1494.232 & 1494.718 & 149656 & 1494.386\end{array}$ $\begin{array}{lllll}1494.23 & 1494.714 & 1496.565 & 1494.388\end{array}$ $\begin{array}{rrrrr}1494.235 & 1494.718 & 1496.565 & 1494.397\end{array}$ $\begin{array}{lllll}1494.232 & 1494.716 & 1496.565 & 1494.404\end{array}$ $\begin{array}{lllll}1494.235 & 1494.718 & 1496.567 & 1494.411\end{array}$ $\begin{array}{lllll}1494.232 & 1494.716 & 1496.572 & 1494.418\end{array}$ $\begin{array}{lllll}1494.232 & 1494.718 & 1496.576 & 1494.425\end{array}$ $\begin{array}{llll}1494.235 & 1494.721 & 1496.576 & 1494.432 \\ 1494235 & 1994.725 & 1996569 & 1494.442\end{array}$ $\begin{array}{lllll}1494.235 & 1494.725 & 1496.569 & 1494.442 \\ 1494.235 & 1494.725 & 1496.565 & 1494.451\end{array}$ $\begin{array}{lllll}1494.232 & 1494.725 & 1496.56 & 1494.456\end{array}$ $\begin{array}{rrrrr}1494.23 & 1494.725 & 1496.562 & 1494.458\end{array}$ $\begin{array}{lllll}1494.23 & 1494.721 & 1496.569 & 1494.463\end{array}$ $\begin{array}{lllll}1494.228 & 1494.721 & 1496.565 & 1494.463\end{array}$ $\begin{array}{lllll}1494.23 & 1494.723 & 1496.567 & 1494.465\end{array}$ $\begin{array}{lllll}1494.232 & 1494.723 & 1496.56 & 1494.463\end{array}$ $\begin{array}{llll}1494.232 & 1494.721 & 1496.555 & 1494.449\end{array}$ $\begin{array}{lllll}1494.225 & 1494.718 & 1496.553 & 1494.421\end{array}$ $\begin{array}{lllll}1494.223 & 1494.716 & 1496.553 & 1494.414\end{array}$ $\begin{array}{lllll}1494.223 & 1494.714 & 1496.555 & 1944.407\end{array}$ $\begin{array}{lllll}1494.228 & 1494.714 & 1496.555 & 1494.409\end{array}$ $\begin{array}{llll}1494.228 & 1494.711 & 1496.558 & 1494.407\end{array}$ $\begin{array}{llll}1494.225 & 1494.714 & 1496.567 & 1494.411\end{array}$ $\begin{array}{llll}1494.225 & 1494.711 & 1496.572 & 1494.414 \\ 1494225 & 1494.714 & 149658 & 194.421\end{array}$ $\begin{array}{rrrrr}1494.225 & 1494.714 & 1496.583 & 1494.421 \\ 1494.23 & 1494.723 & 1496.588 & 1494.425\end{array}$ $\begin{array}{lllll}1494.23 & 1494.723 & 1496.595 & 1494.423\end{array}$ $\begin{array}{lllll}1494.232 & 1494.73 & 1496.588 & 1494.416\end{array}$ $\begin{array}{llll}1494.232 & 1494.735 & 1496.593 & 1494.407\end{array}$ $\begin{array}{lllll}1494.235 & 1494.737 & 1496.597 & 1494.4\end{array}$ $\begin{array}{llll}1494.232 & 1494.737 & 1496.597 & 1494.39\end{array}$ $\begin{array}{llll}1494.23 & 1494.737 & 1496.609 & 1494.381 \\ 1994.23 & 1494.739 & 1496.64 & 19436\end{array}$ $\begin{array}{lllll}1494.23 & 1494.739 & 1496.614 & 1494.369 \\ 1494.232 & 1494.741 & 1496644 & 1494367\end{array}$ $\begin{array}{llll}1494.235 & 1494.748 & 1496649 & 1494.367\end{array}$ $\begin{array}{lllll}1494.239 & 1494.753 & 1496.639 & 1494.372\end{array}$ $\begin{array}{llllll}1494.242 & 1494.767 & 1496.637 & 1494.379\end{array}$ $\begin{array}{llllll}1494.242 & 1494.772 & 1496.623 & 1494.376\end{array}$ $\begin{array}{lllll}1494.239 & 1494.769 & 1496.6 & 1494.365\end{array}$ $1494.2371494 .767 \quad 1496.586 \quad 1494.355$ $\begin{array}{lllll}1494.23 & 1494.76 & 1496.579 & 1494.344\end{array}$ $\begin{array}{llll}1494.228 & 1494.751 & 1496.583 & 1494.332 \\ 1494225 & 1494.746 & 1496.586 & 1494325\end{array}$ $\begin{array}{lllll}1494.225 & 1494.741 & 1496.581 & 1494.32\end{array}$ $\begin{array}{lllll}1494.225 & 1494.739 & 1496.581 & 1494.323\end{array}$ $\begin{array}{llllll}1494.225 & 1494.739 & 1496.588 & 1494.327\end{array}$ $\begin{array}{lllll}1494.223 & 1494.737 & 1496.588 & 1494.332\end{array}$ $\begin{array}{lllll}1494.223 & 1494.737 & 1496.59 & 1494.337\end{array}$ $\begin{array}{llll}1494.225 & 1494.739 & 1496.593 & 1494.346 \\ 1494.223 & 1494.737 & 1496.588 & 1494.351\end{array}$

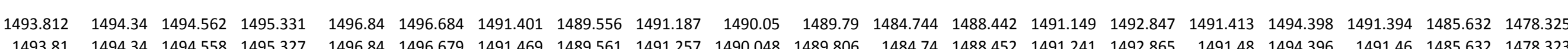

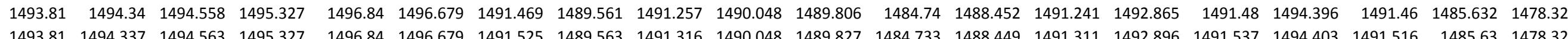

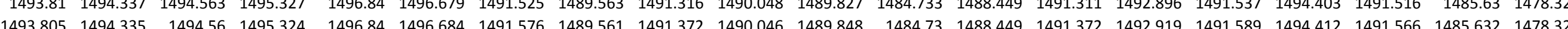
$\begin{array}{llllllllllllllllllllll}1493.8 & 1494.337 & 1494.558 & 1495322 & 1496842 & 1496.686 & 1491.623 & 1489.561 & 1491.421 & 1490.043 & 1489.867 & 1484.728 & 1488.452 & 1491.433 & 1492.94 & 1491.629 & 1494.412 & 1491.613 & 1485.63 & 1478323\end{array}$ $\begin{array}{lllllllllllllllllllll}1493.798 & 1494.335 & 1494.56 & 1495.322 & 1496.842 & 1496.691 & 1491.668 & 1489.554 & 1491.466 & 1490.043 & 1489.883 & 1484.724 & 1488.452 & 1491.478 & 1492.968 & 1491.674 & 1494.422 & 1491.655 & 1485.632 & 1478.325 \\ \end{array}$ $\begin{array}{llllllllllllllllllllllll}1493.798 & 1494.335 & 1494.56 & 1495.324 & 1496.842 & 1496.694 & 1491.703 & 1489.559 & 1491.503 & 1490.043 & 1489.902 & 1484.721 & 1488.452 & 1491.518 & 1492.982 & 1491.714 & 1494.431 & 1491.692 & 1485.63 & 1478.325 \\ \end{array}$ $\begin{array}{lllllllllllllllllllll}1493.795 & 1494.335 & 1494.56 & 1495.324 & 1496.845 & 1496.691 & 1491.743 & 1489.556 & 1491.543 & 1490.046 & 1489.921 & 1484.721 & 1488.454 & 1491.563 & 1493.003 & 1491.748 & 1494.44 & 1491.728 & 1485.63 & 1478.325 \\ \end{array}$

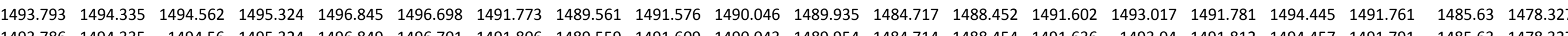

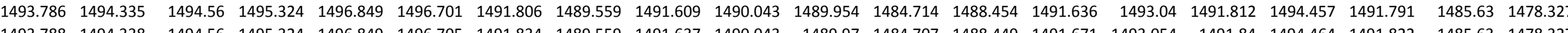

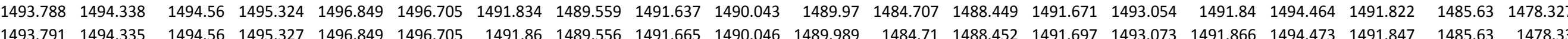
$\begin{array}{lllllllllllllllllllll}1493.795 & 1494.335 & 1494.558 & 1495.329 & 1496845 & 1496.698 & 1491.888 & 1489.561 & 1491.696 & 1490.043 & 1490.003 & 1484.698 & 1488.452 & 1491.722 & 1493.087 & 1491.892 & 1494.48 & 1491.871 & 1485.63 & 1478.33\end{array}$ $\begin{array}{lllllllllllllllllllllll}1493.8 & 1494.335 & 1494.555 & 1495.329 & 1496.84 & 1496.694 & 1491.909 & 1489.563 & 1491.717 & 1490.043 & 1490.014 & 1484.694 & 1488.457 & 1491.753 & 1493.096 & 1491.921 & 1494.48 & 1491.894 & 1485.628 & 1478.33\end{array}$ $\begin{array}{lllllllllllllllllllll}1493.8 & 1494.333 & 1494.555 & 1495.329 & 1496.84 & 1496.689 & 1491.927 & 1489.561 & 1491.742 & 1490.046 & 1490.028 & 1484.689 & 1488.457 & 1491.786 & 1493.103 & 1491.947 & 1494.483 & 1491.915 & 1485.63 & 1478.33\end{array}$

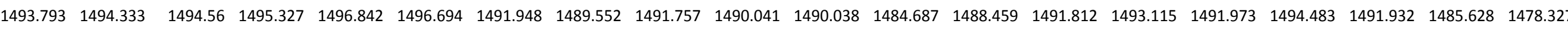

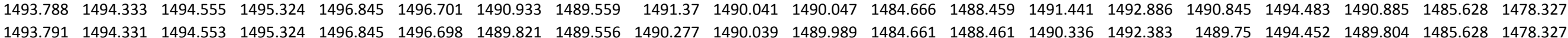

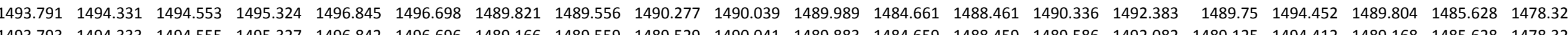

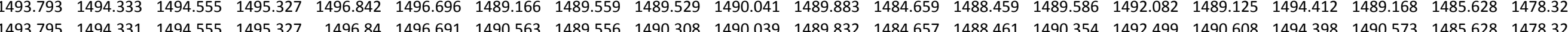

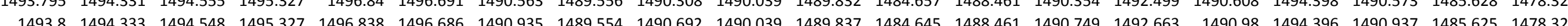

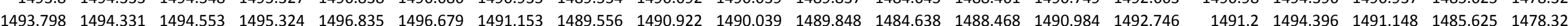
$\begin{array}{lllllllllllllllllllll}1493.788 & 149433 & 1494553 & 1495324 & 1496838 & 1496682 & 1491298 & 1489552 & 1491074 & 1490036 & 1489.867 & 1484633 & 1488468 & 1491144 & 1492807 & 1491.35 & 1494.396 & 1491293 & 1485625 & 1478323\end{array}$ $\begin{array}{llllllllllllllllllllll}1493.791 & 1494.328 & 1494.555 & 1495.322 & 1496.835 & 1496.684 & 1491.408 & 1489.552 & 1491.191 & 1490.036 & 1489.879 & 1484.631 & 1488.464 & 1491.252 & 1492.856 & 1491.454 & 1494.4 & 1491.401 & 1485.625 & 1478.32\end{array}$ $\begin{array}{lllllllllllllllllllll}1493.784 & 1494.331 & 1494.555 & 1495.322 & 1496.835 & 1496.684 & 1491.495 & 1489.554 & 1491.285 & 1490.036 & 1489.897 & 1484.626 & 1488.457 & 1491.33 & 1492.896 & 1491.53 & 1494.417 & 1491.491 & 1485.625 & 1478.318\end{array}$ $\begin{array}{llllllllllllllllllll}1493.781 & 1494.331 & 1494.553 & 1495.322 & 1496.838 & 1496.686 & 1491.567 & 1489.556 & 1491.363 & 1490.036 & 1489.912 & 1484.624 & 1488.468 & 1491.398 & 1492.928 & 1491.591 & 1494.422 & 1491.561 & 1485.625 & 1478.318\end{array}$

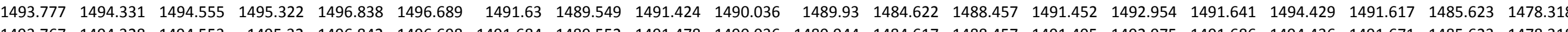

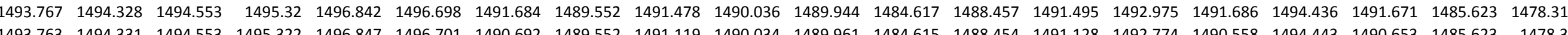

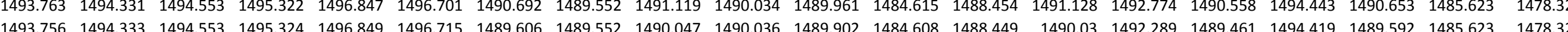
$\begin{array}{llllllllllllllllllllll}1493.751 & 1494.335 & 1494.553 & 1495.327 & 1496.854 & 1496.715 & 1488.764 & 1489.556 & 1489.271 & 1490.036 & 1489.804 & 1484.606 & 1488.442 & 1489.236 & 1491.963 & 1488.587 & 1494.386 & 1488.759 & 1485.623 & 1478.32\end{array}$ $\begin{array}{lllllllllllllllllllllll}1493.751 & 1494.335 & 1494.553 & 1495.331 & 1496.854 & 1496.722 & 1489.679 & 1489.554 & 1489.607 & 1490.039 & 1489.727 & 1484.603 & 1488.442 & 1489.55 & 1492.191 & 1489.591 & 1494.37 & 1489.691 & 1485.623 & 1478.323\end{array}$ \begin{tabular}{lllllllllllllllllllllll}
1493.751 & 1494.335 & 1494.551 & 1495.334 & 1496.856 & 1496.715 & 1489.812 & 1489.556 & 1489.74 & 1490.036 & 1489.697 & 1484.599 & 1488.44 & 1489.67 & 1492.261 & 1489.71 & 1494.361 & 1489.822 & 1485.623 & 1478.323 \\
\hline
\end{tabular} $\begin{array}{llllllllllllllllllllll}1493.748 & 1494.335 & 1494.551 & 1495.336 & 1496.859 & 1496.717 & 1489.875 & 1489.563 & 1489.794 & 1490.039 & 1489.673 & 1484.599 & 1488.433 & 1489.713 & 1492.289 & 1489.878 & 1494.361 & 1489.881 & 1485.623 & 1478.325\end{array}$

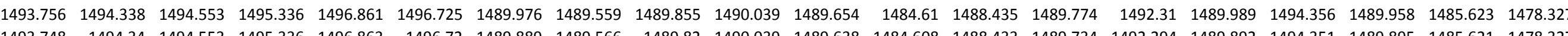

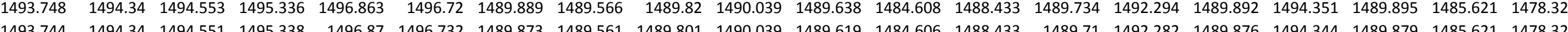

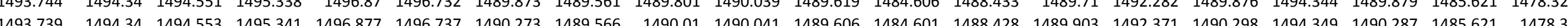

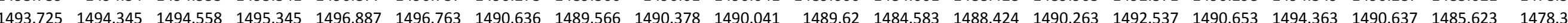
$\begin{array}{llllllllllllllllllllllll}1493.734 & 1494.347 & 1494.553 & 1495.348 & 1496.889 & 1496.768 & 1489.105 & 1489.566 & 1489.466 & 1490.041 & 1489.606 & 1484.571 & 1488.421 & 1489.344 & 1492.079 & 1489.037 & 1494.363 & 1489.137 & 1485.623 & 1478.33\end{array}$

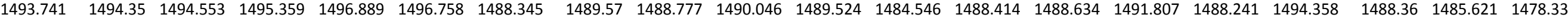
$\begin{array}{llllllllllllllllllllll}1493.756 & 1494.35 & 1494.548 & 1495.361 & 1496.884 & 1496.753 & 1489.3 & 1489.57 & 1488.995 & 1490.046 & 1489.435 & 1484.532 & 1488.417 & 1488.841 & 1491.951 & 1489.335 & 1494.328 & 1489.327 & 1485.621 & 1478.332\end{array}$ $\begin{array}{lllllllllllllllllllll}1493.777 & 1494.347 & 1494.543 & 1495.361 & 1496.877 & 1496.737 & 1489.646 & 1489.575 & 1489.339 & 1490.046 & 1489.393 & 1484.52 & 1488.424 & 1489.196 & 1492.173 & 1489.672 & 1494.323 & 1489.667 & 1485.621 & 1478.332\end{array}$

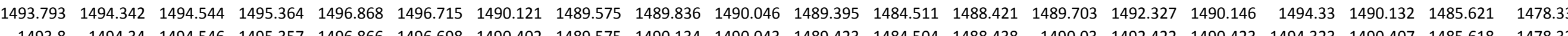
$\begin{array}{lllllllllllllllllllll}1493.8 & 1494.34 & 1494.546 & 1495.357 & 1496.866 & 1496.698 & 1490.402 & 1489.575 & 1490.134 & 1490.043 & 1489.423 & 1484.504 & 1488.438 & 1490.03 & 1492.422 & 1490.423 & 1494.323 & 1490.407 & 1485.618 & 1478.33 \\ 1493.798 & 1494337 & 1494546 & 1495352 & 1496866 & 1496694 & 1490.605 & 1489.563 & 149035 & 1490.039 & 1489.53 & 1484.499 & 1488.447 & 1490277 & 1492.492 & 1490.622 & 1494316 & 1490.606 & 1485.618 & 1478327\end{array}$

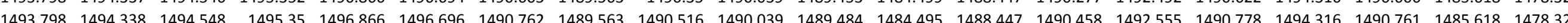
$\begin{array}{llllllllllllllllllllll}1493.8 & 1494.335 & 1494.548 & 1495.345 & 1496861 & 1496.696 & 1490.888 & 1489.566 & 1490.65 & 1490.039 & 1489.517 & 1484.488 & 1488.447 & 1490.608 & 1492.604 & 1490.906 & 1494.323 & 1490.888 & 1485.618 & 1478.323\end{array}$ $\begin{array}{lllllllllllllllllllllll}1493.798 & 1494.333 & 1494.548 & 1495.345 & 1496.863 & 1496.691 & 1490.998 & 1489.561 & 1490.767 & 1490.039 & 1489.545 & 1484.488 & 1488.445 & 1490.726 & 1492.655 & 1491.013 & 1494.337 & 1490.996 & 1485.618 & 1478.323\end{array}$ $\begin{array}{llllllllllllllllllllllll}1493.798 & 1494.331 & 1494.546 & 1495.345 & 1496.866 & 1496.694 & 1491.094 & 1489.561 & 1490.866 & 1490.036 & 1489.575 & 1484.485 & 1488.445 & 1490.836 & 1492.697 & 1491.105 & 1494.344 & 1491.087 & 1485.618 & 1478.32\end{array}$ \begin{tabular}{llllllllllllllllllll}
1493.793 & 1494.331 & 1494.546 & 1495.343 & 1496.866 & 1496.698 & 1491.176 & 1489.556 & 1490.955 & 1490.036 & 1489.601 & 1484.481 & 1488.447 & 1490.928 & 1492.728 & 1491.188 & 1494.351 & 1491.169 & 1485.618 & 1478.323 \\
\hline
\end{tabular}

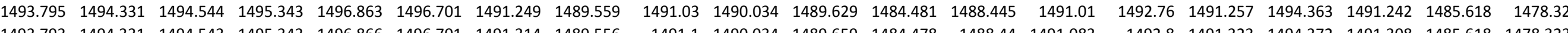

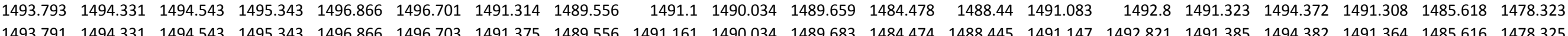


Well

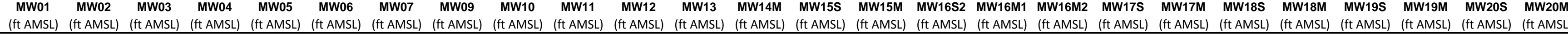

10/23/11 1:00 10/23/11 1:30 $10 / 23 / 112 \cdot 30$ 10/23/1113:00 10/23/11 3:00 10/23/11 4:00 10/23/11 4:30 10/23/11 5:00 10/23/11 5:30 $10 / 23 / 116: 00$ 10/23/11 6:30 $10 / 23 / 1177: 00$ 10/23/117:30 10/23/11 8:30 10/23/11 9:00 0/23/11 10:00 10/23/11 10:30 10/23/11 11:00 10/23/11 12:00 10/23/11 12:30 10/23/11 13:00 10/23/1113:30 10/23/11 14:00 10/23/1114:30 10/23/11 15:00 10/23/11 16:00 $10 / 23 / 1116: 30$ 10/23/11 17:00 10/23/11 17:30 10/23/11 18:00 10/23/11 18:30 10/23/11 19:00 10/23/11 19:30 10/23/11 20:30 10/23/11 21:00 10/23/11 21:30 10/23/11 22:00 10/23/11 22:30 10/23/11 23:00 $10 / 24 / 110: 00$ 10/24/11 0:00 10/24/11 1:00 10/24/11 1:30 10/24/11 2:00 10/24/11 3:00 $\begin{array}{rrrr}1494.223 & 1494.739 & 1496.59 & 1494.362 \\ 1494.223 & 1494.737 & 1496593 & 1494.372\end{array}$ $1494.223 \quad 1494.737 \quad 1496.593 \quad 1494.372$ $\begin{array}{lllll}1494.223 & 1494.737 & 1496.595 & 1494.379\end{array}$ $\begin{array}{lllll}1494.223 & 1494.737 & 1496.6 & 1494.395\end{array}$ $\begin{array}{lllll}1494.223 & 1494.737 & 1496.595 & 1494.395\end{array}$ $\begin{array}{lllll}1494.223 & 1494.741 & 1496.583 & 1494.411\end{array}$ $\begin{array}{lllll}1494.223 & 1494.739 & 1496.583 & 1494.421\end{array}$ $\begin{array}{llll}1494.219 & 1494.737 & 1496.583 & 1494.423\end{array}$ $\begin{array}{llll}1494.216 & 1494.73 & 1496.59 & 1494.425\end{array}$ $\begin{array}{llll}1494.216 & 1494.732 & 1496.586 & 1494.43 \\ 1494221 & 1494.734 & 1496581 & 1494.432\end{array}$ $\begin{array}{llll}1494.221 & 1494.734 & 1496.581 & 1494.432 \\ 1494.218 & 1494.734 & 1496.581 & 1494.428\end{array}$ $\begin{array}{lllll}1494.218 & 1494.732 & 1496.572 & 1494.414\end{array}$ $\begin{array}{lllll}1494.219 & 1494.73 & 1496.562 & 1494.402\end{array}$ $\begin{array}{lllll}1494.216 & 1494.727 & 1496.558 & 1494.39\end{array}$ $\begin{array}{lllll}1494.211 & 1494.723 & 1496.558 & 1494.383\end{array}$ $\begin{array}{llrl}1494.209 & 1494.718 & 1496.56 & 1494.374\end{array}$ $\begin{array}{rrrr}1494.211 & 1494.716 & 1496.565 & 1494.372\end{array}$ $\begin{array}{llll}1494.211 & 1494.716 & 1496.56 & 1494.372\end{array}$ $\begin{array}{lllll}1494.214 & 1494.714 & 1496.572 & 1494.379\end{array}$ $\begin{array}{lllll}1494.211 & 1494.716 & 1496588 & 1494.383\end{array}$ $\begin{array}{llrl}1494.216 & 1494.714 & 1496.6 & 1494.388\end{array}$ $\begin{array}{lllll}1494.218 & 1494.721 & 1496.602 & 1494.386\end{array}$ $\begin{array}{lllll}1494.221 & 1494.728 & 1496.614 & 1494.381\end{array}$ $\begin{array}{lllll}1494.223 & 1494.732 & 1496.616 & 1494.379\end{array}$ $\begin{array}{lllll}1494.225 & 1494.737 & 1496.621 & 1494.376\end{array}$ $\begin{array}{llll}1494.225 & 1494.744 & 1496.628 & 1494.376 \\ 1494.225 & 1494.748 & 1496.625 & 1494.376\end{array}$ $\begin{array}{lllll}1494.225 & 1494.751 & 1496.625 & 1494.374\end{array}$ $\begin{array}{lllll}1494.228 & 1494.751 & 1496.618 & 1494.374\end{array}$ $\begin{array}{lllll}1494.225 & 1494.755 & 1496.604 & 1494.372\end{array}$ $\begin{array}{lllll}1494.223 & 1494.751 & 1496.59 & 1494.365\end{array}$ $\begin{array}{lllll}1494.221 & 1494.748 & 1496.576 & 1494.353\end{array}$ $\begin{array}{llll}1494.216 & 1494.741 & 1496.562 & 1494.332 \\ 1492.214 & 1494.734 & 1496548 & 194306\end{array}$ $\begin{array}{lllll}1494.214 & 1494.734 & 1496.548 & 1494.306\end{array}$ $\begin{array}{llll}1494.209 & 1494.727 & 1496.541 & 1494.283 \\ 1494.207 & 1494.721 & 1496.529 & 1494.262\end{array}$ $\begin{array}{llll}1494.202 & 1494.716 & 1496.515 & 1494.248\end{array}$ $\begin{array}{lllll}1494.2 & 1494.711 & 1496.506 & 1494.241\end{array}$ $\begin{array}{llllll}1494.197 & 1494.7 & 1496.506 & 1494.229\end{array}$ $1494.193 \quad 1494.693 \quad 1496.506 \quad 1494.225$ $\begin{array}{lllll}1494.195 & 1494.686 & 1496.501 & 1494.22\end{array}$ $\begin{array}{llll}1494.195 & 1494.683 & 1496.504 & 1494.222\end{array}$ $\begin{array}{lllll}1494.195 & 1494.681 & 1496.506 & 1494.225 \\ 1494.195 & 1494.676 & 1496504 & 1494.227\end{array}$ $\begin{array}{llll}1494.195 & 1494.676 & 1496.506 & 1494.234\end{array}$ $\begin{array}{lllll}1494.195 & 1494.672 & 1496.508 & 1494.236\end{array}$ $\begin{array}{lllll}1494.195 & 1494.672 & 1496.513 & 1494.243\end{array}$ $\begin{array}{llll}1494.195 & 1494.669 & 1496.515 & 1494.25\end{array}$ $\begin{array}{lllll}1494.197 & 1494.672 & 1496.518 & 1494.262\end{array}$ $\begin{array}{llll}1494.195 & 1494.667 & 1496.513 & 1494.269 \\ 1494.195 & 1494.669 & 1496.511 & 1494.278\end{array}$

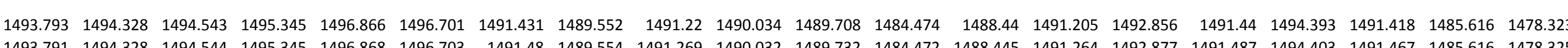

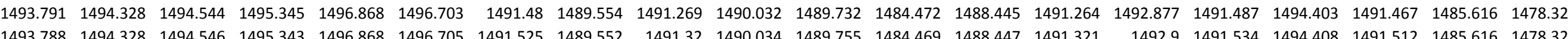

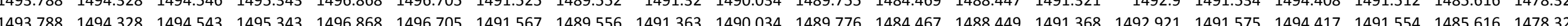
\begin{tabular}{llllllllllllllllllllll}
1493.788 & 1494.331 & 1494.546 & 1495345 & 149687 & 1496.708 & 1491.609 & 1489.554 & 1491.402 & 1490.034 & 1489.797 & 1484.465 & 1488.449 & 1491.41 & 1492.942 & 1491.613 & 1494.431 & 1491.596 & 1485.616 & 1478.327 \\
\hline
\end{tabular} $\begin{array}{lllllllllllllllllllll}1493.788 & 1494.328 & 1494.539 & 1495345 & 1496.868 & 1496.71 & 1491.642 & 1489.556 & 1491.44 & 1490.032 & 1489.818 & 1484.465 & 1488.454 & 1491.45 & 1492.961 & 1491.653 & 1494.431 & 1491.629 & 1485.614 & 1448.33\end{array}$ $\begin{array}{lllllllllllllllllllllll}1493.798 & 1494.328 & 1494.543 & 1495.345 & 1496.863 & 1496.705 & 1491.679 & 1489.559 & 1491.48 & 1490.032 & 1489.839 & 1484.462 & 1488.449 & 1491.488 & 1492.982 & 1491.686 & 1494.443 & 1491.667 & 1485.616 & 1478.33\end{array}$ $\begin{array}{llllllllllllllllllll}1493.8 & 1494.328 & 1494.541 & 1495.348 & 1496.861 & 1496.696 & 1491.71 & 1489.556 & 1491.51 & 1490.034 & 1489.858 & 1484.46 & 1488.454 & 1491.52 & 1492.998 & 1491.719 & 1494.45 & 1491.697 & 1485.614 & 1478.332\end{array}$ \begin{tabular}{lllllllllllllllllllll}
1493.798 & 1494.328 & 1494.541 & 1495.345 & 1496.863 & 1496.694 & 1491.738 & 1489.554 & 1491.543 & 1490.034 & 1489.874 & 1484.46 & 1488.459 & 1491.565 & 1493.01 & 1491.745 & 1494.447 & 1491.725 & 1485.614 & 1478.332 \\
\hline
\end{tabular}

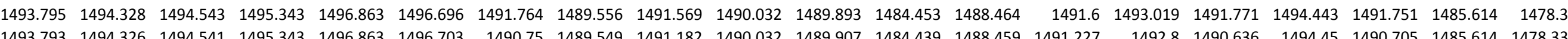

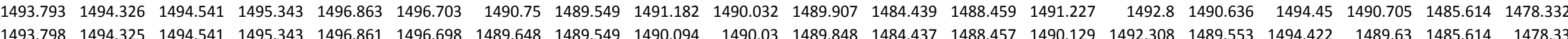

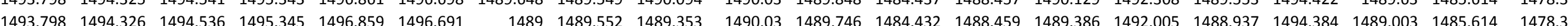
$\begin{array}{lllllllllllllllllllllll}1493.805 & 1494.323 & 1494.536 & 1495.341 & 1496.852 & 1496.682 & 1490.781 & 1489.552 & 1490.535 & 1490.03 & 1489.706 & 1484.428 & 1488.457 & 1490.578 & 1492.578 & 1490.8 & 1494.365 & 1490.784 & 1485.611 & 1478.327\end{array}$

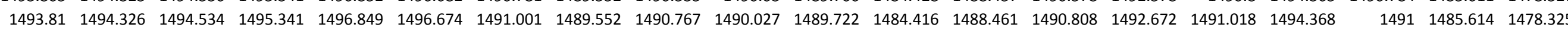
$\begin{array}{llllllllllllllllllllll}1493.803 & 1494.323 & 1494.534 & 1495.338 & 1496.849 & 1496.672 & 1491.153 & 1489.547 & 1490.924 & 1490.027 & 1489.739 & 1444.404 & 1488.464 & 1490.968 & 1492.732 & 1491.167 & 1494.365 & 1491.146 & 1485.611 & 1478.323\end{array}$

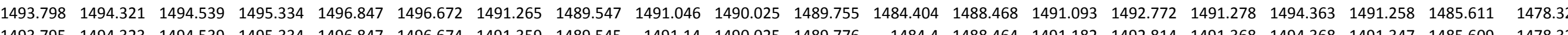

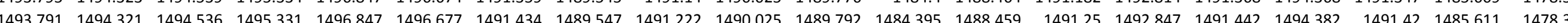
$\begin{array}{llllllllllllllllllllllll}1493.791 & 1494.321 & 1494.536 & 1495.331 & 1496.847 & 1496.677 & 1491.434 & 1489.547 & 1491.222 & 1490.025 & 1489.792 & 1484.395 & 1488.459 & 1491.25 & 1492.847 & 1491.442 & 1494.382 & 1491.42 & 1485.611 & 1478.32 \\ 1493.786 & 1494319 & 1494.539 & 1495331 & 1496847 & 1496.677 & 1491.497 & 1489547 & 1491288 & 1490.025 & 1489.811 & 1484.393 & 1488.457 & 1491.304 & 1492877 & 1491.506 & 1494.389 & 1491.486 & 1485.609 & 1478338\end{array}$ $\begin{array}{llllllllllllllllllll}1493.779 & 1494.321 & 1494.534 & 1495.329 & 1496.849 & 1496.682 & 1491.551 & 1489.545 & 1491.344 & 1490.02 & 1489.832 & 1484.391 & 1488.454 & 1491.351 & 1492.907 & 1491.56 & 1494.393 & 1491.54 & 1485.609 & 1478.318\end{array}$ (492.697 $1490.447 \quad 1494.408 \quad 1490.51514856091478318$ $\begin{array}{lllllllllllllllllllllll}1493.756 & 1494.323 & 1494.539 & 1495.329 & 1496.861 & 1496.703 & 1489.477 & 1489.545 & 1489.914 & 1490.023 & 1489.79 & 1484.388 & 1488.44 & 1489.894 & 1492.215 & 1489.385 & 1494.377 & 1489.461 & 1485.609 & 1478.318 \\ \end{array}$

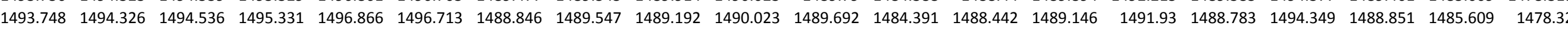
$\begin{array}{llllllllllllllllllll}1493.746 & 1494.328 & 1494.536 & 1495.336 & 1496.868 & 1496.717 & 1490.271 & 1489.554 & 1490 & 1490.023 & 1489.647 & 1484.388 & 1488.431 & 1489.922 & 1492.352 & 1490.293 & 1494.342 & 1490.28 & 1485.607 & 1478.32\end{array}$

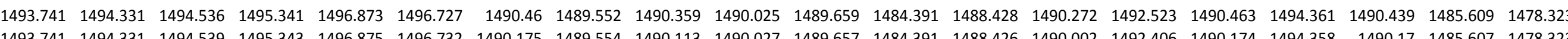

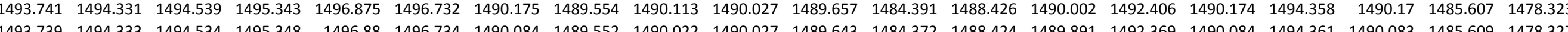

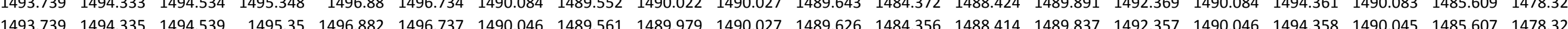

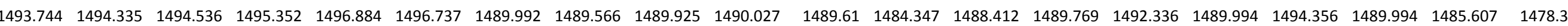
$\begin{array}{lllllllllllllllllllll}1493.753 & 1494.335 & 1494.534 & 1495354 & 1496.882 & 1496.734 & 1489.945 & 1489.561 & 1489.876 & 1490.027 & 1489.594 & 1484.3 & 1488.414 & 1489.713 & 1492.317 & 1489.947 & 1494.358 & 1489.947 & 1485.607 & 1478.327\end{array}$ $\begin{array}{llllllllllllllllllllll}1493.76 & 1494.335 & 1494.532 & 1495.357 & 1496.88 & 1496.727 & 1489.903 & 1489.561 & 1489.836 & 1490.03 & 1489.575 & 1484.266 & 1488.417 & 1489.673 & 1492.299 & 1489.907 & 1494.351 & 1489.909 & 1485.607 & 1478.33\end{array}$ $\begin{array}{llllllllllllllllllllll}1493.777 & 1494.333 & 1494.529 & 1495.354 & 1496.873 & 1496.113 & 1488.368 & 1489.566 & 1488.887 & 1490.03 & 1489.519 & 1484.243 & 1488.414 & 1488.725 & 1491.795 & 1488.262 & 1494.321 & 1488.391 & 1485.607 & 1478.33\end{array}$

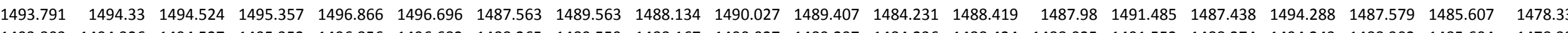
$\begin{array}{llllllllllllllllllll}1493.803 & 1494.326 & 1494.527 & 1495.352 & 1496.856 & 1496.682 & 1488.265 & 1489.559 & 1488.167 & 1490.027 & 1489.297 & 1484.236 & 1488.424 & 1488.025 & 1491.552 & 1488.274 & 1494.243 & 1488.302 & 1485.604 & 1478.33 \\ 149381 & 1494.321 & 1494.527 & 1495348 & 1496852 & 1496667 & 1489311 & 1489556 & 1488995 & 1490.025 & 1489259 & 1484238 & 1488431 & 1488876 & 1491928 & 1489345 & 1494.224 & 1489337 & 1485604 & 1478327\end{array}$

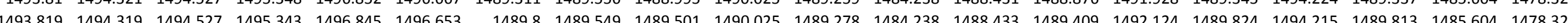

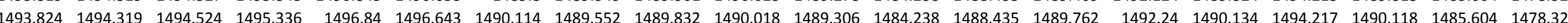
$\begin{array}{llllllllllllllllllllll}1493.831 & 1494.316 & 1494.524 & 1495.331 & 1496.831 & 1496.636 & 1490.339 & 1489.542 & 1490.075 & 1490.02 & 1489.337 & 1484.24 & 1488.44 & 1490.032 & 1492.334 & 1490.357 & 1494.22 & 1490.343 & 1485.604 & 1478.32\end{array}$ $\begin{array}{llllllllllllllllllll}1493.833 & 1494.314 & 1494.525 & 1495.327 & 1496.826 & 1496.624 & 1490.519 & 1489.54 & 1490.263 & 1490.016 & 1489.367 & 1484.238 & 1488.44 & 1490.234 & 1492.399 & 1490.537 & 1494.222 & 1490.519 & 1485.604 & 1478.318\end{array}$

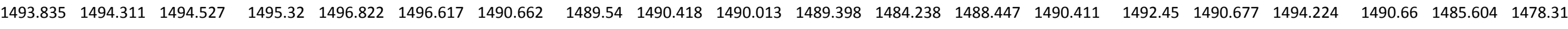

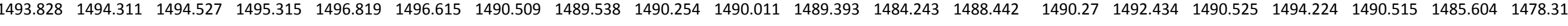

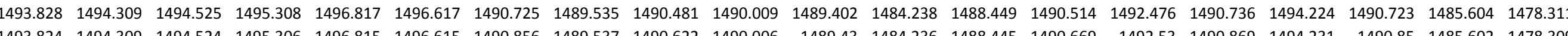

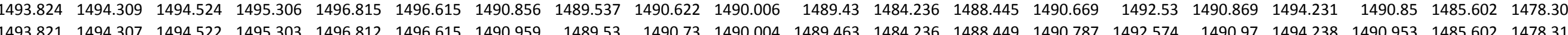

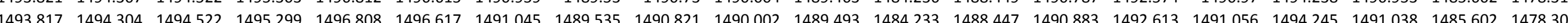

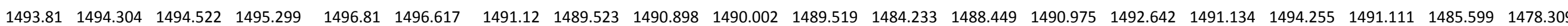
$\begin{array}{llllllllllllllllllllll}1493.807 & 1494.307 & 1494.52 & 1495.296 & 1496.81 & 1496.622 & 1491.188 & 1489.53 & 1490.971 & 1490.002 & 1489.549 & 1484.233 & 1488.445 & 1491.048 & 1492.672 & 1491.198 & 1494.264 & 1491.181 & 1485.602 & 1478.309\end{array}$

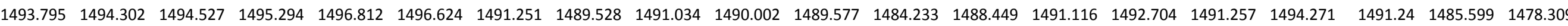
$\begin{array}{lllllllllllllllllllll}1493.798 & 1494.304 & 1494.52 & 1495.294 & 1496.81 & 1496.631 & 1491.305 & 1489.523 & 1491.093 & 1489.999 & 1489.601 & 1484.233 & 1488.449 & 1491.18 & 1492.73 & 1491.312 & 1494.281 & 1491.294 & 1485.599 & 1478.313 \\ \end{array}$ $\begin{array}{llllllllllllllllllll}1493.793 & 1494.302 & 1494.52 & 1495.292 & 1496.81 & 1496.631 & 1491.354 & 1489.528 & 1491.145 & 1489.999 & 1489.627 & 1484.238 & 1488.447 & 1491.229 & 1492.763 & 1491.366 & 1494.297 & 1491.345 & 1485.602 & 1478.313\end{array}$

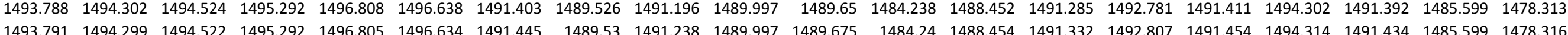


Well

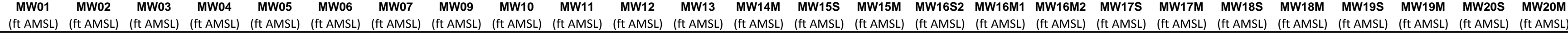

10/24/11 4:00 10/24/11 4:30

$10 / 24 / 115: 30$

10/24/111 6:300

10/24/11 6:00

10/24/117:30

10/24/11 8:00

10/24/11 8:30

10/24/119:00

10/24/119:30

10/24/11 10:00

10/24/11 10:30

10/24/11 11:30

10/24/11 12:00

10/24/11 12:30

10/24/11 13:00

10/24/11 13:30

10/24/11 14:00

10/24/11 15:00

10/24/11 15:00

10/24/11 16:00

10/24/11 16:30

10/24/11 17:00

10/24/11 17:30

10/24/11 18:00

10/24/11 19:00

10/24/11 19:30

10/24/11 20:00

10/24/11 20:30

10/24/11 21:00

10/24/11 21:30

10/24/11 22:00

10/24/11 22:30

10/24/11 23:30

10/24/11 23:11 000

10/25/11 0:30

10/25/11 1:00

10/25/111 1:30

10/25/11 2:00

10/25/11 3:00

10/25/113:30

10/25/11 4:30

10/25/11 5:00

10/25/11 6:00 $\begin{array}{rrrr}1494.197 & 1494.669 & 1496.508 & 1494.29 \\ 1494.193 & 1494.665 & 1496.506 & 1494.292\end{array}$ $\begin{array}{rrrr}1494.193 & 1494.665 & 1496.506 & 1494.292 \\ 1494.191 & 1494.66 & 1496.504 & 1494.297\end{array}$ $\begin{array}{lllll}1494.191 & 1494.66 & 1496.504 & 1494.297\end{array}$ $\begin{array}{lllll}1494.19 & 1494.66 & 1496.497 & 1494.309\end{array}$ $\begin{array}{lllll}1494.19 & 1494.658 & 1496.492 & 1494.313\end{array}$ $\begin{array}{lllll}1494.19 & 1494.658 & 1496.492 & 1494.39 \\ 1496.494 & 1494.309\end{array}$ $\begin{array}{lllll}1494.183 & 1494.648 & 1496.501 & 1494.292\end{array}$ $\begin{array}{lllll}1494.183 & 1494.648 & 1496.499 & 1494.278\end{array}$ $\begin{array}{llll}1494.186 & 1494.651 & 1496.501 & 1494.271\end{array}$ $\begin{array}{llll}1494.188 & 1494.646 & 1496.506 & 1494.267\end{array}$ $\begin{array}{llll}1494.188 & 1494.646 & 1496.513 & 1494.264 \\ 1494.188 & 1494.646 & 1496.518 & 1494.267\end{array}$ $\begin{array}{llll}1494.188 & 1494.646 & 1496.518 & 1494.267 \\ 1494.188 & 1494.648 & 1496.532 & 1494.269\end{array}$ $\begin{array}{lllll}1494.19 & 1444.646 & 1496.544 & 1494.276\end{array}$ $\begin{array}{lllll}1494.193 & 1494.651 & 1496.56 & 1494.283\end{array}$ $\begin{array}{lllll}1494.198 & 1494.658 & 1496.581 & 1494.297\end{array}$ $\begin{array}{lllll}1494.197 & 1494.665 & 1496.597 & 1494.309\end{array}$ $\begin{array}{lrlll}1494.2 & 1494.676 & 1496.621 & 1494.316\end{array}$ $\begin{array}{llll}1494.207 & 1494.69 & 1496.632 & 1494.318 \\ 1494209 & 14947 & 1496.639 & 1494323\end{array}$ $\begin{array}{rrrr}1494.209 & 1494.7 & 1496.639 & 1494.323 \\ 1494.214 & 1494.716 & 1496.639 & 1494.332\end{array}$ $\begin{array}{lllll}1494.214 & 1494.723 & 1496.646 & 1494.344\end{array}$ $\begin{array}{lllll}1494.214 & 1494.732 & 1496.653 & 1494.355\end{array}$ $\begin{array}{lllll}1494.214 & 1494.737 & 1496.658 & 1494.362\end{array}$ $\begin{array}{lllll}1494.216 & 1494.744 & 1496.642 & 1494.376\end{array}$ $1494.2181494 .748 \quad 1496.649 \quad 1494.388$ $\begin{array}{llll}1494.218 & 1494.753 & 1496.644 & 1494.395 \\ 194.216 & 194.753 & 146.637 & 1494.39\end{array}$ $\begin{array}{rrrr}1494.216 & 1494.753 & 1496.637 & 1494.39 \\ 1494.216 & 1494.751 & 1496.63 & 1494.379\end{array}$ $\begin{array}{lllll}1494.216 & 1494.751 & 1496.628 & 1494.362\end{array}$ $\begin{array}{lllll}1494.214 & 1494.746 & 1496.625 & 1494.362\end{array}$ $\begin{array}{lllll}1494.212 & 1494.741 & 1496.625 & 1994.332\end{array}$ $\begin{array}{llllll}1494.212 & 1494.739 & 1496.628 & 1494.323\end{array}$ $\begin{array}{lllll}1494.211 & 1494.737 & 1496.63 & 1494.318\end{array}$ $\begin{array}{llll}1494.211 & 1494.739 & 1496.635 & 1494.318\end{array}$ $\begin{array}{lllll}1494.214 & 1494.739 & 1496.639 & 1494.318\end{array}$ $\begin{array}{lllll}1494.214 & 1494.741 & 1496.656 & 1494.33\end{array}$ $\begin{array}{lllll}1494.216 & 1494.744 & 1496.665 & 1494.341\end{array}$ $\begin{array}{llllll}1494.218 & 1494.748 & 1496.67 & 1494.353\end{array}$ $\begin{array}{lllll}1494.218 & 1494.753 & 1496.674 & 1494.365\end{array}$ $\begin{array}{llllll}1494.221 & 1494.76 & 1496.681 & 1494.376\end{array}$ $\begin{array}{llll}1494.221 & 1494.765 & 1496.681 & 1494.39\end{array}$ $\begin{array}{llll}1494.223 & 1494.769 & 1496.684 & 1494.402 \\ 1494.221 & 1494.774 & 1496686 & 1494.416\end{array}$ $1494.221 \quad 1494.7741496 .6861494 .416$ $1494.225 \quad 1494.7761496 .698 \quad 1494.439$ $1494.223 \quad 1494.781 \quad 1496.705 \quad 1494.449$ $1494.228 \quad 1494.788 \quad 1496.707 \quad 1494.46$ $1494.228 \quad 1494.788 \quad 1496.714 \quad 1494.47$ $1494.232 \quad 1494.795 \quad 1496.714 \quad 1494.484$ $\begin{array}{rrrr}1494.23 & 1494.797 & 1496.707 & 1494.496 \\ 1494.232 & 1494.804 & 1496.709 & 1494.5\end{array}$

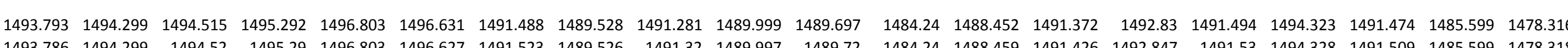

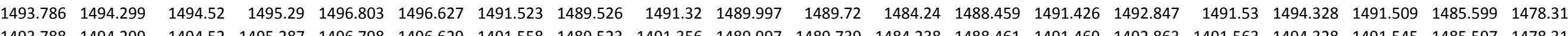

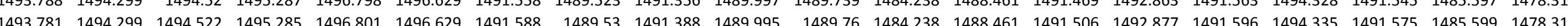
$\begin{array}{lllllllllllllllllllll}1493.779 & 1494.299 & 1494.52 & 1495.283 & 1496.798 & 1496.634 & 1490.563 & 1489.523 & 1490.992 & 1489.995 & 1489.776 & 1484.229 & 1488.461 & 1491.121 & 1492.658 & 1490.451 & 1494.339 & 1490.519 & 1485.597 & 1478.318\end{array}$ $\begin{array}{llllllllllllllllllllll}1493.788 & 1494295 & 1494515 & 1495283 & 1496.794 & 1496.622 & 1489.477 & 1489.526 & 1489.916 & 1489.995 & 1489.718 & 1484.224 & 1488.459 & 1490.044 & 1492.161 & 1489.385 & 1494.309 & 1489.463 & 1485.597 & 1478.318\end{array}$ $\begin{array}{lllllllllllllllllllll}1493.786 & 1494.297 & 1494.52 & 1495.283 & 1496.789 & 1496.617 & 1488.836 & 1489.528 & 1489.185 & 1489.995 & 1489.617 & 1484.224 & 1488.464 & 1489.311 & 1491.865 & 1488.772 & 1494.271 & 1488.839 & 1485.597 & 1478.318\end{array}$ $\begin{array}{llllllllllllllllllllll}1493.777 & 1494.294 & 1494.52 & 1495.276 & 1496.794 & 1496.619 & 1490.24 & 1489.521 & 1489.977 & 1489.992 & 1489.568 & 1484.213 & 1488.466 & 1490.11 & 1492.275 & 1490.264 & 1494.245 & 1490.254 & 1485.597 & 1478.316\end{array}$

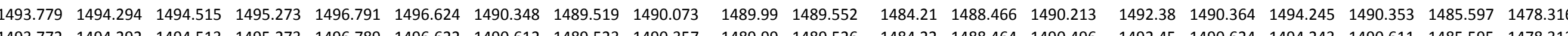

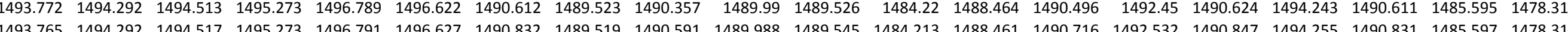
$\begin{array}{llllllllllllllllllll}1493.765 & 1494.292 & 1494.517 & 1495.273 & 1496.791 & 1496.627 & 1490.832 & 1489.519 & 1490.591 & 1489.988 & 1489.545 & 1484.213 & 1488.461 & 1490.716 & 1492.532 & 1490.847 & 1494.255 & 1490.831 & 1485.597 & 1478.313 \\ 1493.756 & 1494292 & 1494.517 & 1495273 & 1496794 & 1496.631 & 1490.98 & 1489519 & 1490751 & 1489.9 & 1489568 & 1484208 & 1488454 & 1490862 & 1492597 & 1490996 & 1494262 & 1490977 & 1485597 & 1478311\end{array}$

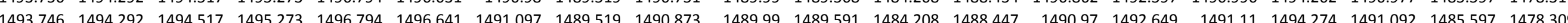
$\begin{array}{lllllllllll} & \end{array}$ $\begin{array}{llllllllllllllllllll}1493.727 & 1494.297 & 1494.517 & 1495.276 & 1496.805 & 1496.655 & 1491.268 & 1489.521 & 1491.053 & 1489.988 & 1489.638 & 1484.208 & 1488.44 & 1491.109 & 1492.73 & 1491.278 & 1494.297 & 1491.261 & 1485.595 & 14778.306\end{array}$

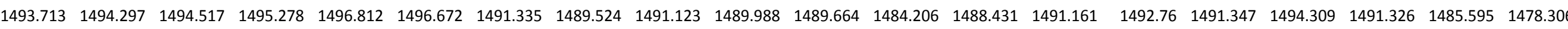
$\begin{array}{llllllllllllllllllllll}1493.704 & 1494.299 & 1494.517 & 1495.28 & 1496.822 & 1496.684 & 1490.348 & 1489.521 & 1490.76 & 1489.988 & 1489.683 & 1484.208 & 1488.424 & 1490.778 & 1492.571 & 1490.238 & 1494.328 & 1490.306 & 1485.595 & 1478.306\end{array}$

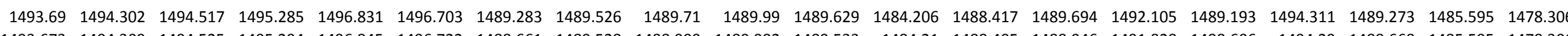

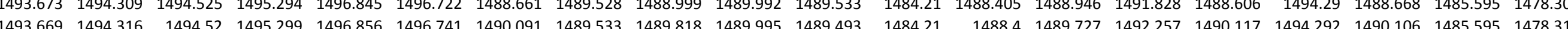

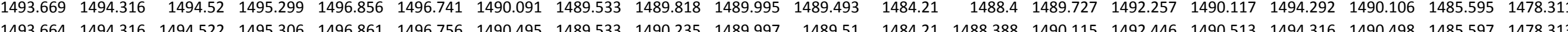
$\begin{array}{llllllllllllllllllllll}1493.669 & 1494.319 & 1494.517 & 1495.317 & 1496.866 & 1496.76 & 1490.739 & 1489.547 & 1490.49 & 1490.002 & 1489.533 & 1484.21 & 1488.381 & 1490.338 & 1492.558 & 1490.755 & 1494.337 & 1490.74 & 1485.597 & 1478316 & 1437\end{array}$ $\begin{array}{llllllllllll} & \end{array}$ $\begin{array}{llllllllllllllllllllll}1493.676 & 1494.323 & 1494.524 & 1495.324 & 1496.877 & 1496.768 & 1491.043 & 1489.559 & 1490.809 & 1490.006 & 1489.587 & 1484.213 & 1488.379 & 1490.62 & 1492.697 & 1491.056 & 1494.379 & 1491.04 & 1485.595 & 1478.32\end{array}$

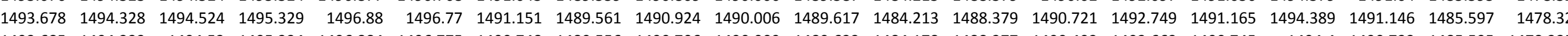

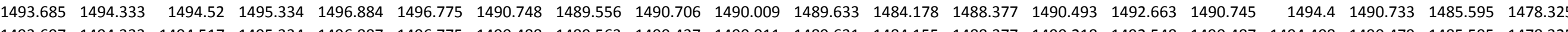

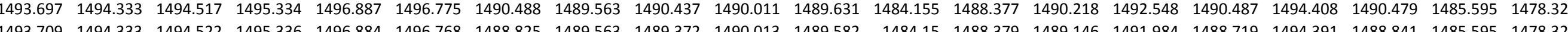

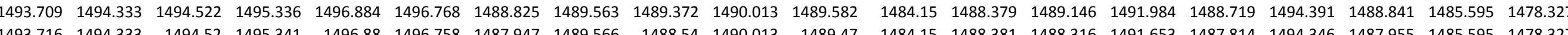

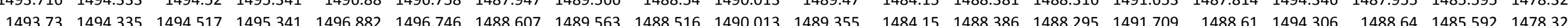

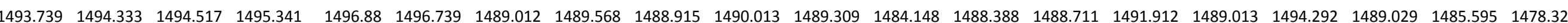
$\begin{array}{llllllllllllllllllllll}1493.746 & 1494.33 & 1494.513 & 1495.343 & 1496.882 & 1496.732 & 1489.704 & 1489.568 & 1489.41 & 1490.011 & 1489.292 & 1484.155 & 1488.393 & 1489.217 & 1492.119 & 1489.736 & 1494.281 & 1489.726 & 1485.592 & 1478.325\end{array}$ $\begin{array}{llllllllllllllllllllll}1493.753 & 1494.331 & 1494.515 & 1495.338 & 1496.877 & 1496.729 & 1490.109 & 1489.563 & 1489.827 & 1490.011 & 1489.316 & 1484.155 & 1488.398 & 1489.656 & 1492.287 & 1490.132 & 1494.283 & 1490.116 & 1485.592 & 1478.323\end{array}$ $\begin{array}{lllllllllllllllllllllll}1493.756 & 1494.333 & 1494.51 & 1495.341 & 1496.882 & 1496.727 & 1490.367 & 1489.563 & 1490.104 & 1490.011 & 1489.344 & 1484.155 & 1488.398 & 1489.938 & 1492.387 & 1490.388 & 1494.292 & 1490.369 & 1485.592 & 1478.318\end{array}$

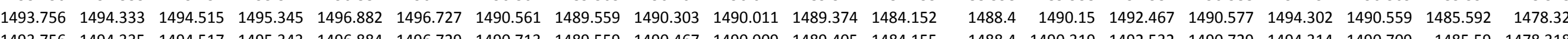

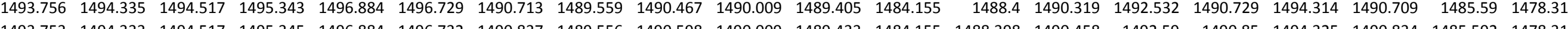

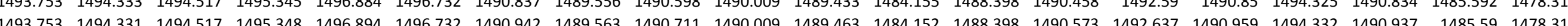

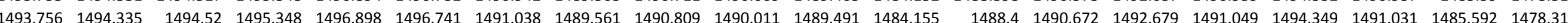
$\begin{array}{lllllllllllllllllllllll}1493.751 & 1494.333 & 1494.517 & 1495.352 & 1496.896 & 1496.753 & 1491.118 & 1489.556 & 1490.891 & 1490.013 & 1489.517 & 1484.155 & 1488.393 & 1490.756 & 1492.718 & 1491.127 & 1494.363 & 1491.111 & 1485.59 & 1478.316\end{array}$ $\begin{array}{lllllllllllllllllllllll}1493.746 & 1494.337 & 1494.515 & 1495.354 & 1496.905 & 1496.758 & 1491.19 & 1489.556 & 1490.969 & 1490.009 & 1489.545 & 1484.157 & 1488.398 & 1490.829 & 1492.767 & 1491.207 & 1494.379 & 1491.186 & 1485.595 & 1478.318\end{array}$

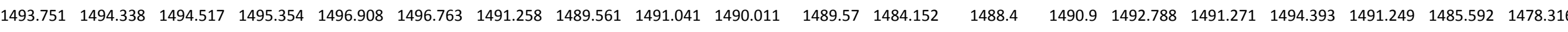
$\begin{array}{lllllllllllllllllllll}1493.748 & 1494.338 & 1494.517 & 1495.357 & 1496.912 & 1496.765 & 1491.321 & 1489.568 & 1491.105 & 1490.013 & 1489.594 & 1484.15 & 1488.395 & 1490.959 & 1492.826 & 1491.331 & 1494.41 & 1491.31 & 1485.59 & 1478.318 \\ \end{array}$

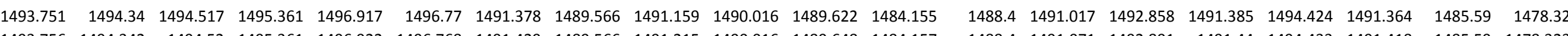

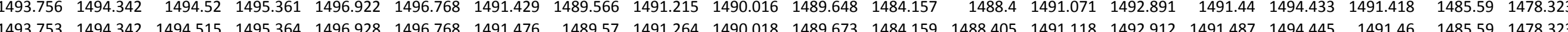

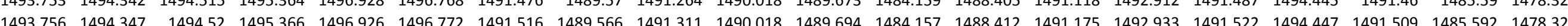
$\begin{array}{lllllllllllllllllllll}1493.76 & 1494.347 & 1494.52 & 1495.368 & 1496.928 & 1496.78 & 1491.562 & 1489.57 & 1491.353 & 1490.018 & 1489.72 & 1484.157 & 1488.407 & 1491.217 & 1492.961 & 1491.57 & 1494.469 & 1491.547 & 1485.59 & 1478.327\end{array}$ $\begin{array}{lllllllllllllllllllllll}1493.758 & 1494.35 & 1494.517 & 1495.371 & 1496.935 & 1496.782 & 1491.597 & 1489.573 & 1491.393 & 1490.018 & 1489.743 & 1484.157 & 1488.414 & 1491.257 & 1492.975 & 1491.61 & 1494.478 & 1491.587 & 1485.59 & 1478.33\end{array}$

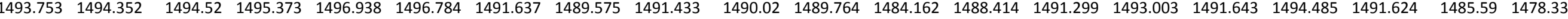

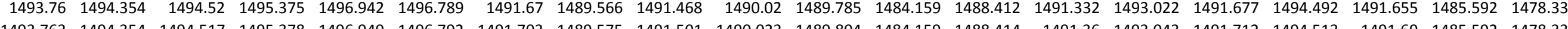
$\begin{array}{llllllllllllllllllll}1493.763 & 1494.354 & 1494.517 & 1495.378 & 1496.949 & 1496.792 & 1491.703 & 1489.575 & 1491.501 & 1490.023 & 1489.804 & 1484.159 & 1488.414 & 1491.36 & 1493.043 & 1491.712 & 1494.513 & 1491.69 & 1485.592 & 1478.337 \\ 14937 & & \end{array}$

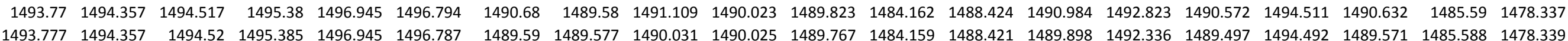


Well

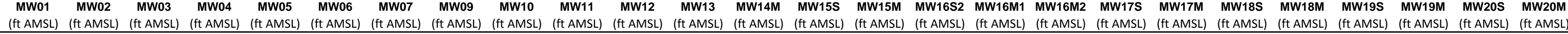

10/25/117:00 10/25/11 7:30 $10 / 25 / 118: 30$ 10/25/11 9:300 10/25/119:00 10/25/11 10:00 10/25/11 10:30 10/25/1111:00 10/25/11 11:30 10/25/11 12:00 10/25/11 12:30 10/25/11 13:30 10/25/11 13:30 10/25/11 14:30 10/25/11 15:00 10/25/11 15:30 10/25/11 16:00 10/25/11 16:30 10/25/11 17:00 10/25/11 18:00 10/25/11 18:30 10/25/11 19:00 10/25/11 19:30 10/25/11 20:00 10/25/11 20:30 10/25/11 21:00 10/25/11 22:00 10/25/11 22:00 10/25/11 23:00 10/25/11 23:30 10/26/11 0:00 10/26/11 0:30 $10 / 26 / 111: 00$
$10 / 26 / 111: 30$
$10 / 26 / 112: 00$ 10/26/11 2:30 10/26/113:00 10/26/11 4:00 10/26/11 4:30 10/26/11 5:00 $10 / 26 / 116.00$ 10/26/11 6:00 10/26/11 6:30 10/26/11 7:30 10/26/11 8:00 10/26/11 9:00 $\begin{array}{rrrr}1494.232 & 1494.804 & 1496.719 & 1494.498\end{array}$ $\begin{array}{llll}1494.23 & 1494.802 & 1496.712 & 1494.486\end{array}$ $\begin{array}{llll}1494.232 & 1494.804 & 1496.709 & 1494.479\end{array}$ $\begin{array}{llll}1494.232 & 1494.802 & 1496.693 & 1494.47\end{array}$ $\begin{array}{rrrr}1494.232 & 1494.807 & 14967 & 1494.468\end{array}$ $\begin{array}{rrrrr}1494.23 & 1494.8 & 1496.698 & 1494.46\end{array}$ $\begin{array}{lllll}1494.23 & 1494.797 & 1496.688 & 1494.463\end{array}$ $\begin{array}{lllll}1494.235 & 1494.8 & 1496.672 & 1494.468\end{array}$ $\begin{array}{llll}1494.232 & 1494.802 & 1496.66 & 1494.468\end{array}$ $1494.228 \quad 1494.793 \quad 1496.663 \quad 1494.468$ $\begin{array}{rrrr}1494.223 & 1494.786 & 1496.653 & 1494.46 \\ 1494.221 & 1494.779 & 1496.644 & 1494.444\end{array}$ $\begin{array}{lllll}1494.225 & 1494.781 & 1496.642 & 1494.43\end{array}$ $\begin{array}{rrrr}1494.223 & 1494.774 & 1496.642 & 1494.416\end{array}$ $\begin{array}{llll}1494.221 & 1494.772 & 1496.637 & 1494.4\end{array}$ $\begin{array}{llll}1494.218 & 1494.765 & 1496.623 & 1494.39\end{array}$ $\begin{array}{llll}1494.223 & 1494.765 & 1496.614 & 1494.39\end{array}$ $1494.216 \quad 1494.76 \quad 1496.604 \quad 1494.381$ $\begin{array}{lllll}1494.214 & 1494.753 & 1496.597 & 1494.376\end{array}$ $\begin{array}{llll}1494.209 & 1494.746 & 1496.586 & 1494.372 \\ 1494.209 & 1494.741 & 1496.576 & 1494.372\end{array}$ $\begin{array}{lllll}1494.207 & 1494.737 & 1496.56 & 1494.36\end{array}$ $\begin{array}{llll}1499.202 & 1494.727 & 1496.551 & 1494.341\end{array}$ $\begin{array}{lllll}1494.2 & 1494.721 & 1496.536 & 1494.32\end{array}$ $1494.197 \quad 1494.711 \quad 1496.522 \quad 1494.304$ $\begin{array}{llll}1494.193 & 1494.702 & 1496.522 & 1494.285\end{array}$ $\begin{array}{rrrr}1494.188 & 1494.69 & 1496.515 & 1494.269\end{array}$ $\begin{array}{lllll}1494.188 & 1494.688 & 1496.522 & 1494.262 \\ 1494.184 & 1494.683 & 1496.525 & 1494.255\end{array}$ $\begin{array}{lllll}1494.188 & 1494.681 & 1496.525 & 1494.255\end{array}$ $\begin{array}{lllll}1494.183 & 1494.679 & 1496.518 & 1494.257\end{array}$ $\begin{array}{lllll}1494.186 & 1494.679 & 1496.529 & 1494.262\end{array}$ $\begin{array}{lllll}1494.181 & 1494.674 & 1496.515 & 1494.264\end{array}$ $\begin{array}{lllll}1494.183 & 1494.672 & 1496.522 & 1494.269\end{array}$ $\begin{array}{lllll}1494.183 & 1494.674 & 1496.511 & 1494.276\end{array}$ $\begin{array}{lllll}1494.181 & 1494.669 & 1496.529 & 1494.281 \\ 1494.186 & 1494.674 & 149652 & 149429\end{array}$ $\begin{array}{rrrr}1494.186 & 1494.674 & 1496.52 & 1494.29 \\ 1494.179 & 1494.667 & 1496.515 & 1494.292\end{array}$ $\begin{array}{lllll}1494.188 & 1494.672 & 1496.501 & 1494.306\end{array}$ $\begin{array}{lllll}1494.177 & 1494.66 & 1496.518 & 1494.304\end{array}$ $\begin{array}{lllll}1494.174 & 1494.66 & 1496.422 & 1494.304\end{array}$ $\begin{array}{lllll}1494.174 & 1494.655 & 1496.469 & 1494.311\end{array}$ $\begin{array}{llll}1494.174 & 1494.655 & 1496.459 & 1494.316\end{array}$ $\begin{array}{rrrr}1494.167 & 1494.634 & 1496.445 & 1494.295 \\ 1494.165 & 1494.63 & 1496.455 & 1494292\end{array}$ $\begin{array}{rrrrr}1494.165 & 1494.63 & 1496.455 & 1494.292 \\ 1494.165 & 1494.625 & 1496.443 & 1494.297\end{array}$ $\begin{array}{lllll}1444.158 & 1494.618 & 1496.441 & 1494.288\end{array}$ $\begin{array}{lllll}1494.167 & 1494.618 & 1496.434 & 1494.281\end{array}$ $\begin{array}{lllll}1494.163 & 1494.613 & 1496.438 & 1494.262\end{array}$ 1494.158 $1494.609 \quad 1496.438 \quad 1494.246$ $\begin{array}{lllll}1494.158 & 1494.604 & 1496.424 & 1494.232\end{array}$ $\begin{array}{rrrr}1494.16 & 1494.604 & 1496.441 & 1494.227 \\ 1494.158 & 1494.599 & 1496.452 & 1494.22\end{array}$

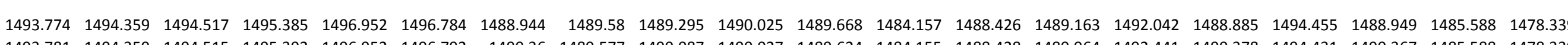

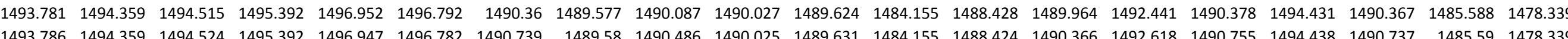

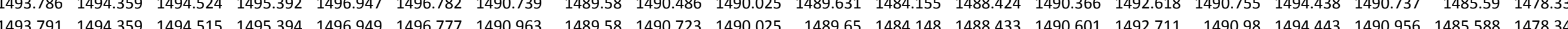
$\begin{array}{llllllllllllllllllllll}1493.805 & 1494361 & 1494517 & 1495394 & 1496945 & 1496.775 & 1491.115 & 1489.58 & 1490884 & 1490.027 & 1489.668 & 1484146 & 1488438 & 1490.768 & 1492.77 & 1491.129 & 1494.447 & 1491.111 & 1485585 & 1478.337\end{array}$ $\begin{array}{llllllllllllllllllllll}1493.8 & 1494.359 & 1494.515 & 1495399 & 1496947 & 149676 & 1491.235 & 1489.582 & 1491.011 & 1490.027 & 1489.69 & 1484.143 & 1488.431 & 1490893 & 1492828 & 1491.248 & 1494.462 & 1491.226 & 14855588 & 1478.337\end{array}$ $\begin{array}{llllllllllllllllllllll}1493.8 & 1494.361 & 1494.513 & 1495.396 & 1496.947 & 1496.765 & 1491.326 & 1489.577 & 1491.109 & 1490.027 & 1489.708 & 1484.134 & 1488.445 & 1491.001 & 1429.856 & 1491.338 & 14944.455 & 1491.319 & 1485.583 & 1478.332\end{array}$ $\begin{array}{llllllllllllllllllllll}1493.807 & 1494.359 & 1494.513 & 1495.396 & 1496.942 & 1496.763 & 1491.399 & 1489.58 & 1491.189 & 1490.025 & 1489.727 & 1484.132 & 1488.44 & 1491.086 & 1492.889 & 1491.416 & 1494.464 & 1491.392 & 1485.583 & 1478.33 \\ \end{array}$

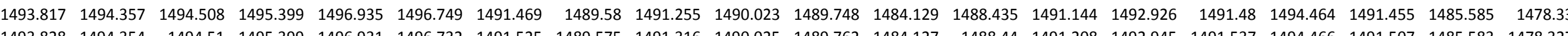
$\begin{array}{lllllllllllllllllllll}1493.828 & 1494.354 & 1494.51 & 1495.399 & 1496.931 & 1496.732 & 1491.525 & 1489.575 & 1491.316 & 1490.025 & 1489.762 & 1484.127 & 1488.44 & 1491.208 & 1492.945 & 1491.537 & 1494.466 & 1491.507 & 1485.583 & 1478.327 \\ 1493831 & 1494354 & 1494.51 & 1495399 & 1496928 & 1496717 & 1490523 & 1489573 & 1490938 & 1490.023 & 1489.781 & 1484.129 & 1488.442 & 1490.85 & 1492.73 & 1490.418 & 1494.459 & 1490.475 & 1485583 & 1478327\end{array}$ $\begin{array}{llllllllllllllllllll}1493.831 & 1494.354 & 1494.51 & 1495.399 & 1496.928 & 1496.717 & 1490.523 & 1489.573 & 1490.938 & 1490.023 & 1489.781 & 1484.129 & 1488.442 & 1490.85 & 1492.73 & 1490.418 & 1494.459 & 1490.475 & 1485.583 & 1478.327 \\ 1493.826 & 1494.354 & 1494.508 & 1495394 & 1496928 & 1496.72 & 1489.44 & 1489.563 & 1489874 & 1490.02 & 1489.72 & 1484.115 & 1488.454 & 1489.788 & 1492.24 & 1489.35 & 1494.422 & 1489.423 & 1485.583 & 1478325\end{array}$

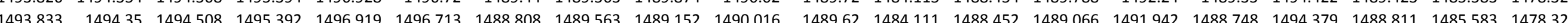
$\begin{array}{llllllllllllllllllllll}1493.835 & 1494.347 & 1494.508 & 1495.389 & 1496.917 & 1496.701 & 1490.619 & 1489.563 & 1490.369 & 1490.016 & 1489.587 & 1484.109 & 1488.452 & 1490.277 & 1492.525 & 1490.641 & 1494.361 & 1490.622 & 1485.581 & 1478.323 \\ \end{array}$ \begin{tabular}{llllllllllllllllllllll}
1493.828 & 1494.347 & 1494.51 & 1495.385 & 1496.91 & 1496.701 & 1490.849 & 1489.556 & 1490.605 & 1490.011 & 1489.606 & 1484.109 & 1488.449 & 1490.526 & 1492.613 & 1490.869 & 1494.358 & 1490.845 & 1485.583 & 1478.323 \\
\hline
\end{tabular} $\begin{array}{lllllllllllllllllllllll}1493.838 & 1494.342 & 1494.505 & 1495.385 & 1496.905 & 1496.694 & 1491.012 & 1489.549 & 1490.779 & 1490.013 & 1489.629 & 1484.109 & 1488.449 & 1490.7 & 1492.679 & 1491.03 & 1494.363 & 1491.007 & 1485.583 & 1478.325 & \end{array}$

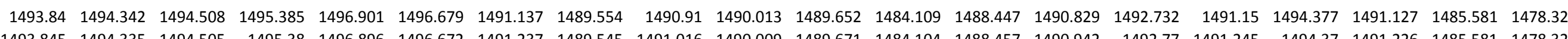

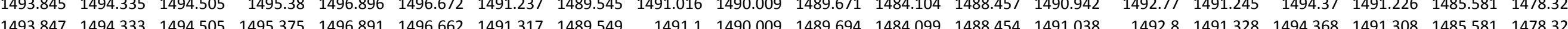

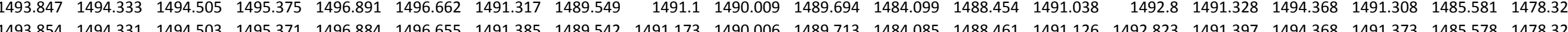

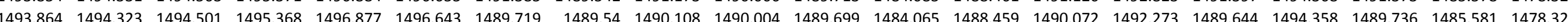
$\begin{array}{lllllllllllllllllllllll}1493.871 & 1494.314 & 1494.498 & 1495.359 & 1496.859 & 1496.617 & 1489.73 & 1489.53 & 1489.461 & 1489.997 & 1489.521 & 1484.062 & 1488.473 & 1489.452 & 1492.061 & 1489.757 & 1494.264 & 1489.757 & 1485.578 & 1478.325 \\ \end{array}$ \begin{tabular}{lllllllllllllllllllll}
1493.878 & 1494.311 & 1494.501 & 1495.352 & 1496.852 & 1496.61 & 1490.329 & 1489.526 & 1490.066 & 1489.997 & 1489.517 & 1484.065 & 1488.471 & 1490.082 & 1492.336 & 1490.352 & 1494.262 & 1490.339 & 1485.578 & 1478.323 \\
\hline
\end{tabular} \begin{tabular}{lllllllllllllllllllll}
1493.885 & 1494.307 & 1494.498 & 1495.345 & 1496.84 & 1496.595 & 1490.463 & 1489.521 & 1490.204 & 1489.992 & 1489.503 & 1484.06 & 1488.473 & 1490.235 & 1492.404 & 1490.48 & 1494.252 & 1490.468 & 1485.578 & 1478.32 \\
\hline
\end{tabular}

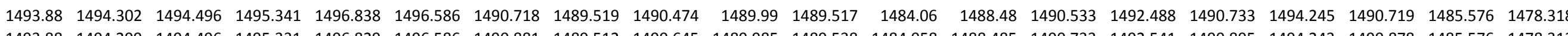

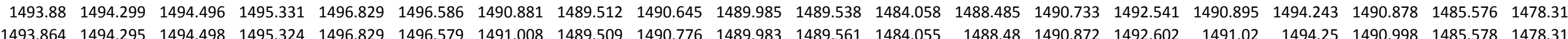
$\begin{array}{lllllllllllllllllllll}1493.864 & 1494.29 & 1494.498 & 149.324 & 1496.829 & 1496.579 & 1491.008 & 1489.509 & 1490.776 & 1489.983 & 1489.561 & 1484.055 & 1488.48 & 1490.872 & 1492.602 & 1491.02 & 1494.25 & 1490.998 & 1485.578 & 1478.311 \\ 1493.859 & 1494.292 & 1494.503 & 1495.32 & 1496829 & 1496591 & 1491.106 & 1489.516 & 1490.884 & 1489.983 & 1489.58 & 1484.055 & 1488.482 & 1490.992 & 1492.639 & 1491.115 & 1494.25 & 1491.099 & 1485.576 & 1478300\end{array}$ $\begin{array}{lllllllllllllllllllllll}1493.847 & 1494.292 & 1494.503 & 1495.317 & 1496.829 & 1496.593 & 1491.186 & 1489.514 & 1490.969 & 1489.981 & 1489.601 & 1484.053 & 1488.475 & 1491.081 & 1492.676 & 1491.198 & 1494.26 & 1491.179 & 1485.576 & 1478.306\end{array}$ $\begin{array}{lllllllllllllllllllll}1493.852 & 1494.29 & 1494.496 & 1495.313 & 1496.822 & 1496.598 & 1491.258 & 1489.507 & 1491.044 & 1489.978 & 1489.624 & 1484.051 & 1488.475 & 1491.156 & 1492.716 & 1491.271 & 1494.264 & 1491.249 & 1485.576 & 1478.304 \\ \end{array}$ $\begin{array}{llllllllllllllllllll}1493.833 & 1494.29 & 1494.503 & 1495.313 & 1496.826 & 1496.591 & 1491.319 & 1489.507 & 1491.107 & 1489.976 & 1489.643 & 1484.048 & 1488.475 & 1491.224 & 1492.739 & 1491.333 & 1494.278 & 1491.31 & 1485.576 & 1478.302\end{array}$ $\begin{array}{lllllllllllllllllllll}1493.843 & 1494.287 & 1494.496 & 1495.31 & 1496.817 & 1496.605 & 1491.375 & 1489.509 & 1491.163 & 1489.976 & 1489.664 & 1484.048 & 1488.478 & 1491.292 & 1492.758 & 1491.383 & 1494.274 & 1491.366 & 1485.574 & 1478.302\end{array}$

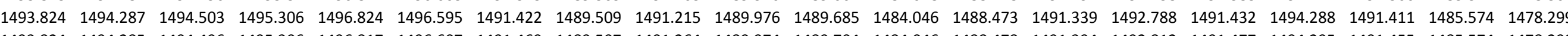

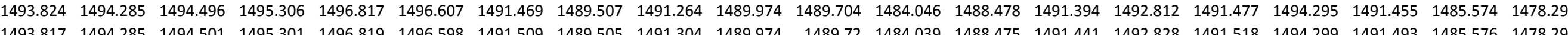

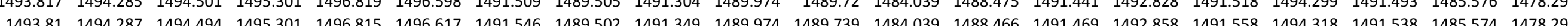

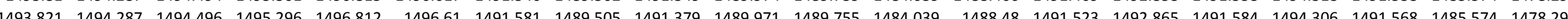
$\begin{array}{lllllllllllllllllllll}1493.824 & 1494.283 & 1494.496 & 1495.301 & 1496.808 & 1496.607 & 1491.616 & 1489.507 & 1491.419 & 1489.971 & 1489.776 & 1484.039 & 1488.466 & 1491.542 & 1492.891 & 1491.624 & 1494.335 & 1491.603 & 1485.571 & 1478.299\end{array}$

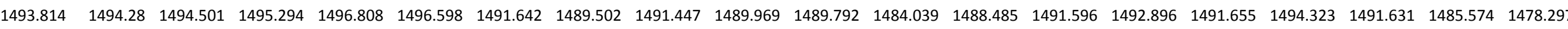

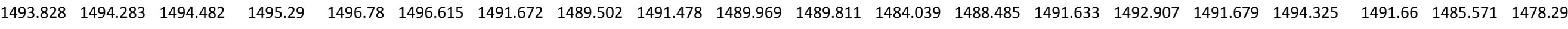

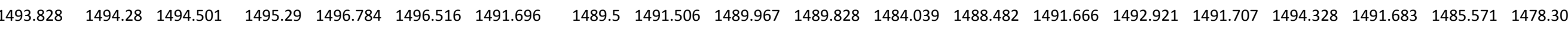
$\begin{array}{llllllllllllllllllll}1493.835 & 1494.275 & 1494.496 & 1495.287 & 1496.777 & 1496.569 & 1491.724 & 1489.502 & 1491.531 & 1489.967 & 1489.846 & 1484.044 & 1488.489 & 1491.697 & 1492.933 & 1491.733 & 1494.33 & 1491.709 & 1485.569 & 1478.304\end{array}$

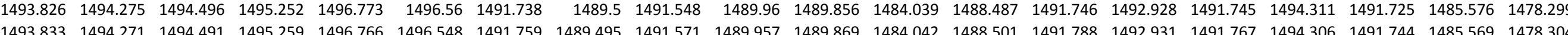

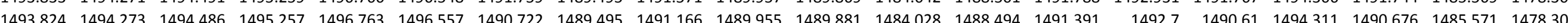

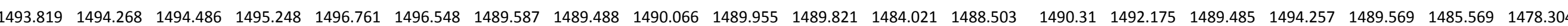
$\begin{array}{llllllllllllllllllllll}1493.824 & 1494.268 & 1494.486 & 1495.25 & 1496.752 & 1496.545 & 1488.972 & 1489.495 & 1489.33 & 1489.953 & 1489.718 & 1484.014 & 1488.492 & 1489.558 & 1491.888 & 1488.911 & 1494.229 & 1488.975 & 1485.569 & 1478.304\end{array}$ $\begin{array}{lllllllllllllllllllllll}1493.812 & 1494.266 & 1494.491 & 1495.243 & 1496.756 & 1496.538 & 1490.376 & 1489.495 & 1490.122 & 1489.953 & 1489.666 & 1484.005 & 1488.492 & 1490.352 & 1492.301 & 1490.397 & 1494.208 & 1490.39 & 1485.569 & 1478.304 \\ \end{array}$

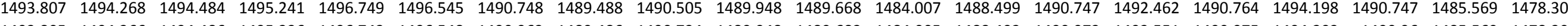
$\begin{array}{llllllllllllllllllll}1493.805 & 1494.266 & 1494.486 & 1495.236 & 1496.742 & 1496.548 & 1490.963 & 1489.486 & 1490.734 & 1489.948 & 1489.683 & 1484.005 & 1488.492 & 1490.973 & 1492.551 & 1490.975 & 1494.203 & 1490.96 & 1485.569 & 1478.302\end{array}$ $\begin{array}{llllllllllllllllllll}1493.795 & 1494.261 & 1494.489 & 1495.234 & 1496.745 & 1496.536 & 1491.111 & 1489.481 & 1490.889 & 1489.948 & 1489.701 & 1484.007 & 1488.485 & 1491.121 & 1492.621 & 1491.122 & 1494.213 & 1491.108 & 1485.569 & 1478.302 \\ 1493.781 & 1494.264 & 1494.489 & 1495.231 & 1496747 & 1496.55 & 1491.221 & 1489.488 & 1491.004 & 1489.946 & 1489.718 & 1484005 & 1488489 & 1491236 & 1492.665 & 1491.231 & 1494.215 & 1491.211 & 1485.567 & 1478.299\end{array}$ 
Well

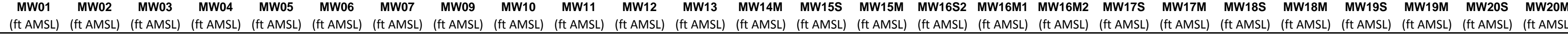

10/26/11 10:00 $10 / 26 / 1110: 30$ 10/26/1111:00 10/26/1111 12:00 10/26/11 12:30 10/26/11 13:00 10/26/11 13:30 10/26/111 14:00 10/26/11 14:30 10/26/11 15:00 10/26/11 15:30 10/26/11 16:30 10/26/11 16:30 10/26/11 17:00 10/26/11 18:00 10/26/11 18:30 10/26/11 19:00 10/26/11 19:30 10/26/11 20:00 10/26/11 21:00 10/26/11 21:30 10/26/11 22:00 10/26/11 22:30 10/26/11 23:00 10/26/11 23:30 10/27/11 0:00 10/27/11 1:00 $10 / 27 / 111: 30$ $10 / 27 / 112: 00$
$10 / 27 / 112: 30$ 10/27/11 2:30 10/27/11 3:00 10/27/11 4:00 10/27/11 4:30 10/27/115:00 10/27/11 6:00 10/27/11 6:30 10/27/11 7:00 10/27/11 7:30 $10 / 27 / 118: 00$
$10 / 27 / 118: 30$ 10/27/11 9:00 10/27/11 $19: 30$ 10/27/11 10:30 10/27/11 11:00 $10 / 27 / 1111: 30$
$10 / 27 / 1112: 00$ 10/27/11 12:00 $\begin{array}{llll}1494.158 & 1494.595 & 1496.455 & 1494.218 \\ 1494.165 & 1494.599 & 1496.448 & 1494.222\end{array}$ $\begin{array}{llll}1494.165 & 1494.599 & 1496.448 & 1494.222\end{array}$ $\begin{array}{lllll}1494.163 & 1494.599 & 1496.455 & 1494.229 \\ 1494.163 & 1494.602 & 1496.464 & 1494.236\end{array}$ $\begin{array}{lllll}1494.16 & 1494.597 & 1466.473 & 1494.239\end{array}$ $\begin{array}{lllll}1494.16 & 1494.597 & 1496.497 & 1494.243\end{array}$ $\begin{array}{rllll}1494.163 & 1494.599 & 1496.511 & 1494.243\end{array}$ $\begin{array}{llll}1494.17 & 1494.609 & 1496.511 & 1494.239\end{array}$ $\begin{array}{llll}1494.172 & 1494.613 & 1496.506 & 1494.243\end{array}$ $\begin{array}{lllll}1494.174 & 1494.623 & 1496.501 & 1494.246\end{array}$ $\begin{array}{llll}1494.172 & 1494.623 & 1496.492 & 1494.25\end{array}$ $\begin{array}{llll}1494.165 & 1494.618 & 1496.473 & 1494.246 \\ 1494.163 & 1494.613 & 1496.457 & 1494.248\end{array}$ $\begin{array}{lllll}1494.16 & 1494.611 & 1496.459 & 1494.25\end{array}$ $\begin{array}{rrrrr}1494.16 & 1494.611 & 1496.459 & 1494.25 \\ 1494.151 & 1494.602 & 1496.469 & 1494.243\end{array}$ $\begin{array}{lllll}1494.149 & 1494.597 & 1496.469 & 1494.241\end{array}$ $\begin{array}{lllll}1494.153 & 1494.595 & 1496.452 & 1494.239\end{array}$ $\begin{array}{llll}1494.156 & 1494.597 & 1496.441 & 1494.232\end{array}$ 1494.158 1494.599 $1496.438 \quad 1494.225$ $\begin{array}{llll}1494.144 & 1494.585 & 1496.434 & 1494.201 \\ 1494.149 & 1494.585 & 1496.434 & 1494.197\end{array}$ $\begin{array}{llll}1494.149 & 1494.585 & 1496.434 & 1494.197 \\ 1494.144 & 1494.581 & 1496.422 & 1494.187\end{array}$ $\begin{array}{lllll}1494.144 & 1494.578 & 1496.42 & 1494.185\end{array}$ $\begin{array}{rrrr}1494.139 & 1494.571 & 1496.417 & 1494.18\end{array}$ $\begin{array}{lllll}1494.142 & 1494.567 & 1496.427 & 1494.183\end{array}$ $\begin{array}{llll}1494.139 & 1494.564 & 1496.422 & 1494.183\end{array}$ $\begin{array}{llll}1494.139 & 1494.562 & 1496.422 & 1494.183\end{array}$ $\begin{array}{rrrrr}1494.144 & 1494.564 & 1496.415 & 1494.192 \\ 1494.142 & 1494.56 & 1496.415 & 1494.197\end{array}$ $\begin{array}{llll}1494.142 & 1494.56 & 1496.415 & 1494.197 \\ 1494.135 & 1494.557 & 1496.427 & 1494.197\end{array}$ $\begin{array}{lllll}1494.142 & 1494.557 & 1496.429 & 1494.204\end{array}$ $\begin{array}{llll}1494.139 & 1494.555 & 1496.445 & 1494.208\end{array}$ $\begin{array}{lllll}1494.139 & 1494.555 & 1496.443 & 1494.215\end{array}$ $\begin{array}{lllll}1494.139 & 1494.557 & 1496.457 & 1494.227\end{array}$ $\begin{array}{lllll}1494.146 & 1494.567 & 1496.452 & 1494.243\end{array}$ $\begin{array}{llll}1494.144 & 1494.567 & 1496.45 & 1494.25 \\ 1494.146 & 149.569 & 1496.448 & 149426\end{array}$ $\begin{array}{llll}1494.146 & 1494.569 & 1496.448 & 1494.262 \\ 1494.144 & 1494.571 & 1496.448 & 194.269\end{array}$ $\begin{array}{llll}1494.144 & 1494.571 & 1496.448 & 1494.269 \\ 1494.569 & 1496.457 & 1494.278\end{array}$ $\begin{array}{lllll}1494.137 & 1494.567 & 1496.452 & 1494.281\end{array}$ $\begin{array}{lllll}1494.137 & 1494.564 & 1496.441 & 1494.283\end{array}$ \begin{tabular}{llllll}
1494.142 & 1494.571 & 1496.415 & 1494.295 \\
\hline
\end{tabular} $\begin{array}{lllll}1494.137 & 1494.567 & 1496.406 & 1494.283\end{array}$ $\begin{array}{llll}1494.132 & 1494.562 & 1496.399 & 1494.269\end{array}$ $\begin{array}{rrrr}1494.125 & 1494.553 & 1496.396 & 1494.243 \\ 1494.125 & 1494548 & 149641 & 194422\end{array}$ $\begin{array}{rrrr}1494.125 & 1494.548 & 1496.41 & 1494.229 \\ 1494.125 & 1494.543 & 1496.413 & 1494215\end{array}$ $\begin{array}{lllll}1494.125 & 1494.541 & 1496.422 & 1494.208\end{array}$ $\begin{array}{lllll}1494.135 & 1494.543 & 1496.424 & 1994.215\end{array}$ $\begin{array}{lllll}1494.13 & 1494.543 & 1496.42 & 1494.215\end{array}$ $1494.132 \quad 1494.548 \quad 1496.42 \quad 1494.222$ $1494.13 \quad 1494.546 \quad 1496.427 \quad 1494.225$ $\begin{array}{llll}1494.13 & 1494.546 & 1496.441 & 1494.232 \\ 1494.13 & 1494.546 & 1496.441 & 1494.232\end{array}$

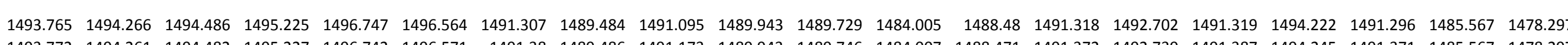

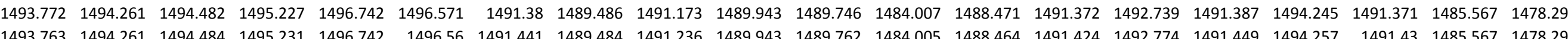

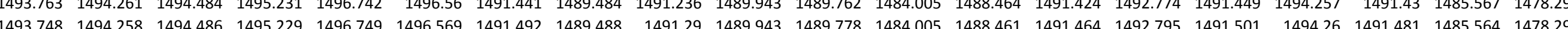

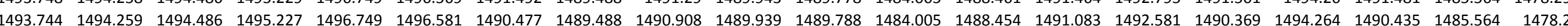
$\begin{array}{lllllllllllllllllllll}1493.723 & 1494.259 & 1494.489 & 1495.227 & 1496.759 & 1496.591 & 1489.398 & 1489.486 & 1489.846 & 1489.941 & 1489.727 & 1484.005 & 1488.449 & 1489.992 & 1492.105 & 1489.307 & 1494.241 & 1489.386 & 1485.567 & 1478.229\end{array}$ $\begin{array}{llllllllllllllllllllllll}1493.706 & 1494.264 & 1494.489 & 1495.229 & 1496.768 & 1496.612 & 1488.78 & 1489.486 & 1489.126 & 1489.939 & 1489.627 & 1484.002 & 1488.445 & 1489.247 & 1491.816 & 1488.717 & 1494.213 & 1488.785 & 1485.564 & 1478.29\end{array}$ $\begin{array}{lllllllllllllllllllll}1493.706 & 1494.266 & 1494.486 & 1495.231 & 1496.77 & 1496.627 & 1490.203 & 1489.479 & 1489.935 & 1489.943 & 1489.58 & 1484.005 & 1488.428 & 1490.025 & 1492.24 & 1490.224 & 1494.205 & 1490.214 & 1485.567 & 1478.29\end{array}$

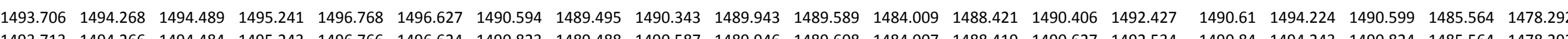

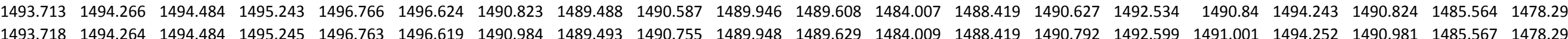
$\begin{array}{lllllllllllllllllllll}1493.718 & 1494.264 & 1494.484 & 1495.245 & 1496.763 & 1496.619 & 1490.984 & 1489.493 & 1490.755 & 1489.948 & 1489.629 & 1484.009 & 1488.419 & 1490.792 & 1492.599 & 1491.001 & 1494.252 & 1490.981 & 1485.567 & 1478.292 \\ 1493727 & 1494261 & 1494.477 & 1495243 & 1496756 & 14966 & 1491108 & 1489.995 & 1490887 & 1489948 & 1489648 & 1484011 & 1488.426 & 1490921 & 1492646 & 1491122 & 1494252 & 1491.01 & 1485564 & 1478295\end{array}$

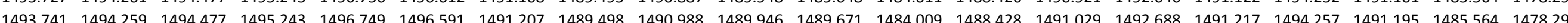
\begin{tabular}{llllllllllllllllllllll}
1493.739 & 1494.256 & 1494.482 & 1495.234 & 1496.749 & 1496.579 & 1491.349 & 1489.495 & 1491.145 & 1489.943 & 1489.708 & 1483.951 & 1488.435 & 1491.208 & 1492.737 & 1491.364 & 1494.252 & 1491.343 & 1485.562 & 1478.295 \\
\hline
\end{tabular}

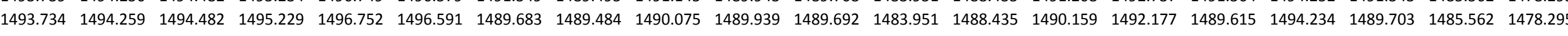
$\begin{array}{llllllllllllllllllllll}1493.734 & 1494.256 & 1494.479 & 1495.227 & 1496.749 & 1496.593 & 1488.815 & 1489.484 & 1489.278 & 1489.936 & 1489.606 & 1483.947 & 1488.431 & 1489.365 & 1491.863 & 1488.719 & 1494.205 & 1488.823 & 1485.562 & 1478.295 & \end{array}$

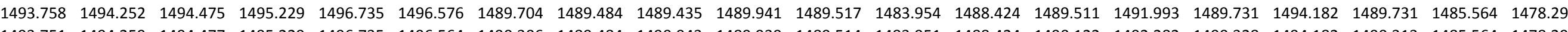

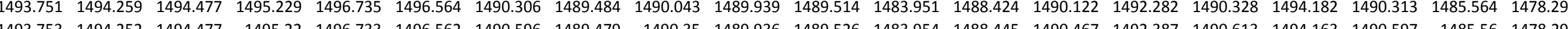

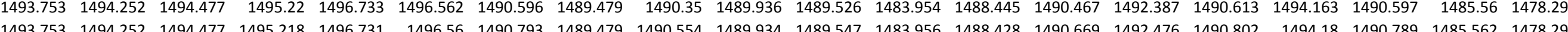

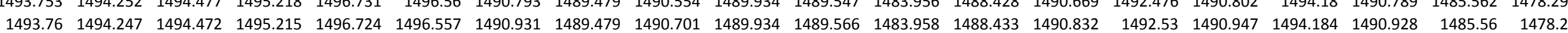
$\begin{array}{llllllllllllllll} & \end{array}$ \begin{tabular}{lllllllllllllllllllllll}
1493.753 & 1494.247 & 1494.475 & 1495.208 & 1496.719 & 1496.545 & 1491.134 & 1489.47 & 1490.915 & 1489.929 & 1489.601 & 1483.951 & 1488.44 & 1491.064 & 1492.611 & 1491.143 & 1494.187 & 1491.122 & 1485.557 & 1478.285 \\
\hline
\end{tabular}

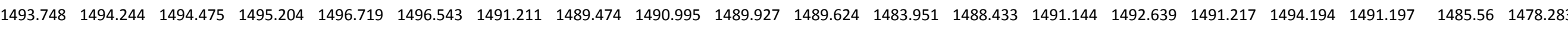

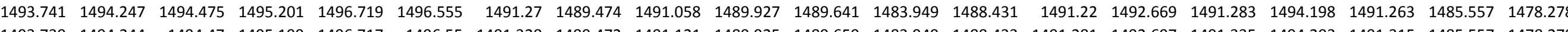

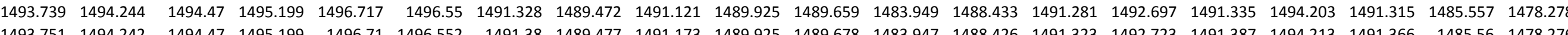

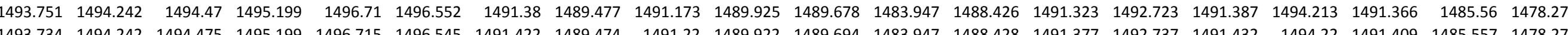
$\begin{array}{lllllllllllllllllllllll}1493.734 & 1494.242 & 1494.475 & 1495.199 & 1496.715 & 1496.545 & 1491.422 & 1489.474 & 1491.22 & 1489.922 & 1489.694 & 1483.947 & 1488.428 & 1491.377 & 1492.737 & 1491.432 & 1494.22 & 1491.409 & 1485.557 & 1478.273 \\ 1493.727 & 149424 & 1494.477 & 1495.194 & 1496.715 & 1496.55 & 1491.462 & 1489.472 & 1491.259 & 1489.922 & 1489.706 & 1483947 & 1488.44 & 1491.431 & 1492.76 & 1491.47 & 1494.215 & 1491.448 & 1485557 & 1478274\end{array}$ $\begin{array}{llllllllllllllllllll}1493.73 & 1494.24 & 1494.475 & 1495.192 & 1496.712 & 1496.56 & 1491.499 & 1489.467 & 1491.299 & 1489.922 & 1489.727 & 1483.947 & 1488.424 & 1491.464 & 1492.784 & 1491.508 & 1494.231 & 1491.486 & 1485.557 & 1478.271 \\ \end{array}$ $\begin{array}{llllllllllllllllllllll}1493.709 & 1494.242 & 1494.477 & 1495.19 & 1496.719 & 1496.562 & 1491.534 & 1489.467 & 1491.337 & 1489.92 & 1489.739 & 1483.947 & 1488.428 & 1491.502 & 1492.798 & 1491.539 & 1494.229 & 1491.521 & 1485.557 & 1478.269\end{array}$ $\begin{array}{llllllllllllllllllllll}1493.704 & 1494.242 & 1494.472 & 1495.192 & 1496.719 & 1496.579 & 1491.567 & 1489.472 & 1491.37 & 1489.92 & 1489.757 & 1483.949 & 1488.426 & 1491.532 & 1492.816 & 1491.575 & 1494.245 & 1491.552 & 1485.557 & 1478.271\end{array}$

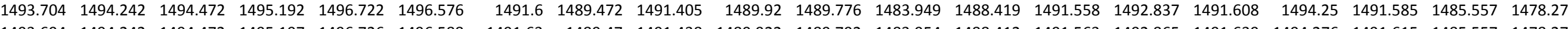

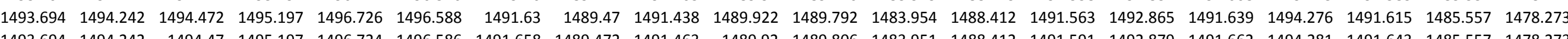

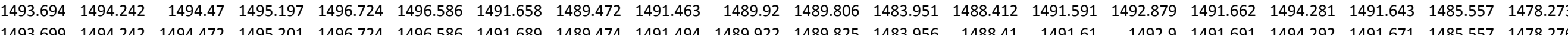

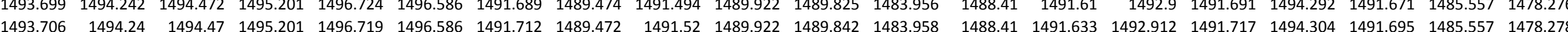

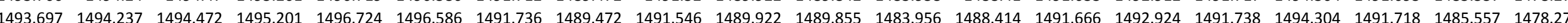

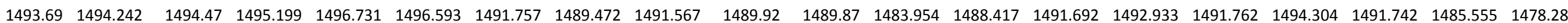

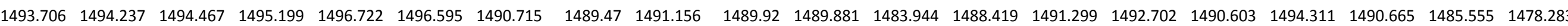

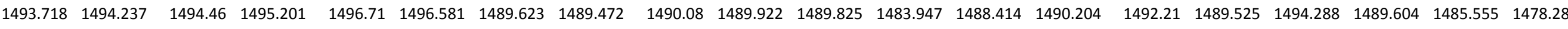

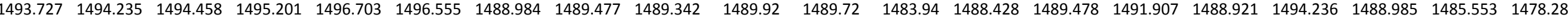

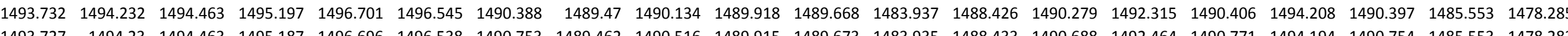

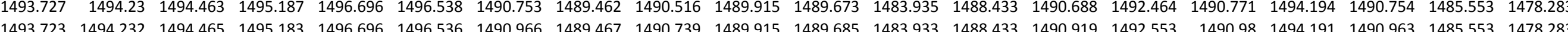

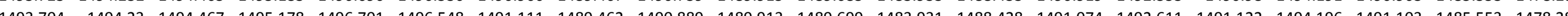
$\begin{array}{llllllllllllllllllllll}1493.704 & 1494.23 & 1494.465 & 1495.173 & 1496.701 & 1496.552 & 1491.223 & 1489.463 & 1491.006 & 1489.911 & 1489.715 & 1483.928 & 1488.421 & 1491.187 & 1492.656 & 1491.231 & 1494.205 & 1491.214 & 1485.553 & 1478.278\end{array}$ $\begin{array}{lllllllllllllllllllllll}1493.685 & 1494.23 & 1494.467 & 1495.178 & 1496.708 & 1496.557 & 1491.31 & 1489.465 & 1491.098 & 1489.911 & 1489.732 & 1483.928 & 1488.41 & 1491.245 & 1492.707 & 1491.321 & 1494.217 & 1491.298 & 1485.553 & 1478.278\end{array}$

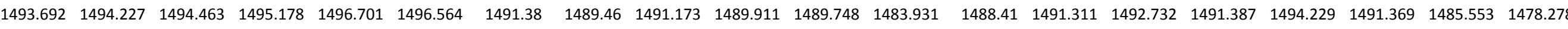
$\begin{array}{lllllllllllllllllllll}1493.687 & 1494.23 & 1494.463 & 1495.18 & 1496.701 & 1496.562 & 1491.443 & 1489.463 & 1491.236 & 1489.911 & 1489.762 & 1483.931 & 1488.395 & 1491.346 & 1492.767 & 1491.449 & 1494.243 & 1491.427 & 1485.553 & 1478.276 \\ \end{array}$

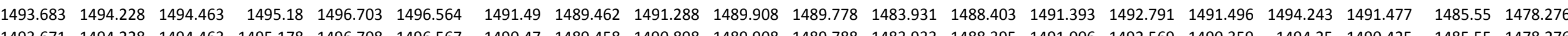

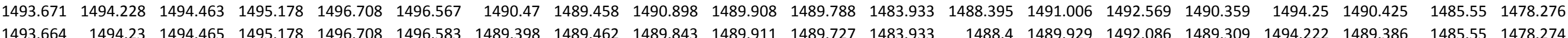


Well

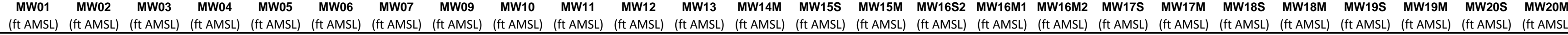

10/27/11 13:00 10/27/11 13:30 10/27/11 14:00 10/27/711 15:00 10/27/1111 15:00 10/27/11 16:00 10/27/11 16:30 10/27/11 17:00 10/27/111 17:30 10/27/11 18:00 10/27/11 18:30 10/27/11 19:30 10/27/11 19:30 10/27/11 20:30 10/27/11 21:00
10/27/121 21:30
$10 / 27 / 112200$ 10/27/11 22:00 10/27/11 22:30 10/27/11 23:00 10/28/11 0:00 $10 / 28 / 110: 30$
10/28/11 1:00 $10 / 28 / 111: 30$
$10 / 28 / 112: 00$ 10/28/11 2:00 10/28/11 2:30 10/28/113:00 10/28/11 4:00 10/28/114:30 10/28/11 5:30 10/28/11 6:00 10/28/11 6:30 10/28/11 7:00 10/28/11 8:30 10/28/11 9:00 $10 / 28 / 119: 30$
$10 / 28 / 1110: 00$ 10/28/11 10:00 10/28/11 10:30 10/28/11 11:00 10/28/11 12:00 10/28/11 12:00 10/28/11 13:00 10/28/11 13:30 10/28/11 14:00 10/28/11 14:30 $10 / 28 / 1115: 00$
$10 / 28 / 1115: 30$ $\begin{array}{rrrr}1494.13 & 1494.546 & 1496.452 & 1494.232 \\ 1494.135 & 1494.55 & 1496.469 & 1494.225\end{array}$ $\begin{array}{rrrr}1494.135 & 1494.55 & 1496.469 & 1494.225\end{array}$ $\begin{array}{rrrr}1494.132 & 1494.553 & 1496.471 & 1494.218 \\ 1494.137 & 1494.56 & 1496.471 & 1494.22\end{array}$ $\begin{array}{rrrrr}1494.139 & 1494.569 & 1496.469 & 1494.222\end{array}$ $\begin{array}{lllll}1494.137 & 1494.571 & 1496.466 & 1494.225\end{array}$ $\begin{array}{lllll}1494.135 & 1494.574 & 1496.462 & 14944.229\end{array}$ $\begin{array}{lllll}1494.132 & 1494.571 & 1496.459 & 1494.239\end{array}$ $1494.131494 .569 \quad 1496.451494 .239$ $\begin{array}{llll}1494.13 & 1494.574 & 1496.441 & 1494.243\end{array}$ $\begin{array}{lllll}1494.128 & 1494.571 & 1496.434 & 1494.241 \\ 1494.123 & 1494564 & 1496.422 & 1994.229\end{array}$ $\begin{array}{rrrrr}1494.123 & 1494.564 & 1496.422 & 1494.229 \\ 1494.121 & 1494.56 & 1496.42 & 1494.215\end{array}$ $\begin{array}{lllll}1494.118 & 1494.555 & 1496.415 & 1494.199\end{array}$ $\begin{array}{lllll}1494.116 & 1494.553 & 1466.417 & 1494.19\end{array}$ $\begin{array}{rrrrr}1494.118 & 1494.55 & 1496.413 & 1494.185\end{array}$ $\begin{array}{lllll}1494.116 & 1494.548 & 1496.408 & 1494.183\end{array}$ $1494.118 \quad 1494.543 \quad 1496.403 \quad 1494.183$ $\begin{array}{lllll}1494.116 & 1494.543 & 1496.399 & 1494.183\end{array}$ $\begin{array}{llll}1494.111 & 1494.539 & 1496.401 & 1494.183 \\ 1494.114 & 1494536 & 1496.408 & 1494185\end{array}$ $\begin{array}{lllll}1494.111 & 1494.534 & 1496.413 & 1494.185\end{array}$ $\begin{array}{lllll}1494.111 & 1494.532 & 1496.408 & 1494.192\end{array}$ $\begin{array}{llll}1494.116 & 1494.536 & 1496.406 & 1494.201\end{array}$ $\begin{array}{lllll}1494.114 & 1494.536 & 1496.401 & 1494.208\end{array}$ $\begin{array}{llll}1494.114 & 1494.536 & 1496.399 & 1494.215\end{array}$ $\begin{array}{llll}1494.109 & 1494.534 & 1496.399 & 1494.215\end{array}$ 1494.109 1494.529 $1496.401 \quad 1494.218$ $\begin{array}{lllll}1494.107 & 1494.529 & 1496.401 & 1494.222 \\ 1494.109 & 1494.529 & 1496.401 & 1494.227\end{array}$ $1494.109 \quad 1494.529 \quad 1496.396 \quad 1494.234$ $1494.109 \quad 1494.529 \quad 1496.399 \quad 1494.236$ $1494.109 \quad 1494.529 \quad 1496.399 \quad 1494.243$ $1494.107 \quad 1494.527 \quad 1496.399 \quad 1494.248$ 1494.107 $1494.529 \quad 1496.394 \quad 1494.25$ $\begin{array}{llll}1494.104 & 1494.529 & 1496.394 & 1494.255\end{array}$ $\begin{array}{llll}1494.104 & 1494.527 & 1496.382 & 1494.246\end{array}$ $\begin{array}{rrrr}1494.102 & 1494.525 & 1496.385 & 1494.232 \\ 1494.525 & 1496.375 & 1494.22\end{array}$ $\begin{array}{llll}1494.097 & 1494.518 & 1496.368 & 1494.206\end{array}$ $\begin{array}{lllll}1494.097 & 1494.515 & 1496.361 & 1494.199\end{array}$ $\begin{array}{lllll}1494.095 & 1494.511 & 1496.366 & 1494.192\end{array}$ $\begin{array}{lllll}1494.093 & 1494.508 & 1496.371 & 1494.185\end{array}$ $\begin{array}{llll}1494.093 & 1494.504 & 1496.382 & 1494.183\end{array}$ $\begin{array}{cccc}1494.093 & 1494.504 & 1496.387 & 1494.183 \\ 1494.097 & 1494.506 & 1496394 & 14941\end{array}$ $\begin{array}{llll}4494.097 & 1494.506 & 1496.394 & 1494.19 \\ 1494.1 & 1494511 & 149641 & 1494.197\end{array}$ $\begin{array}{lllll}1494.102 & 1494.513 & 14996.42 & 1494.204\end{array}$ $\begin{array}{lllll}1494.102 & 1494.518 & 1496.431 & 1494.199\end{array}$ $\begin{array}{llllll}1494.107 & 1494.529 & 1496.441 & 1494.199\end{array}$ 1494.107 $1494.534 \quad 1496.441 \quad 1494.194$ $\begin{array}{lllll}1494.109 & 1494.541 & 1496.443 & 1494.197\end{array}$ $\begin{array}{rrrrr}1494.109 & 1494.546 & 1496.443 & 1494.199 \\ 1494.107 & 1494.55 & 1496.441 & 1494.201\end{array}$

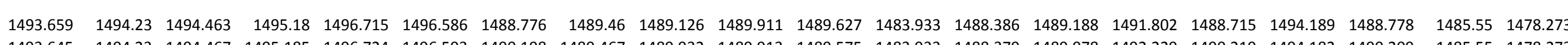

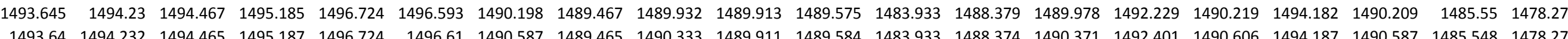

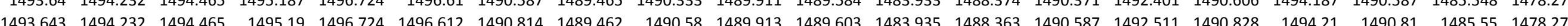

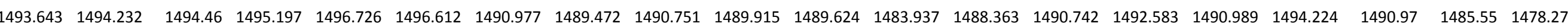
$\begin{array}{lllllllllllllllllllll}1493.65 & 1494.232 & 1494.463 & 1495.199 & 1496.726 & 1496.61 & 1491.101 & 1489.47 & 1490.88 & 1489.915 & 1489.643 & 1483.942 & 1488.358 & 1490.862 & 1492.635 & 1491.113 & 1494.236 & 1491.092 & 1485.55 & 1478.276\end{array}$ $\begin{array}{lllllllllllllllllllllll}1493.652 & 1494.232 & 1494.46 & 1495.201 & 1496.726 & 1496.605 & 1491.197 & 1489.472 & 1490.98 & 1489.915 & 1489.662 & 1483.944 & 1488.36 & 1490.959 & 1492.676 & 1491.21 & 1494.248 & 1491.19 & 1485.548 & 1478.278\end{array}$ $\begin{array}{llllllllllllllllllll}1493.654 & 1494.23 & 1494.463 & 1495.201 & 1496.729 & 1496.603 & 1491.279 & 1489.474 & 1491.067 & 1489.915 & 1489.685 & 1483.942 & 1488.363 & 1491.046 & 1492.716 & 1491.29 & 1494.255 & 1491.27 & 1485.548 & 1478.276\end{array}$ $\begin{array}{llllllllllllllllllll}1493.662 & 1494.23 & 1494.458 & 1495.201 & 1496.724 & 1496.6 & 1491.347 & 1489.474 & 1491.138 & 1489.913 & 1489.704 & 1483.944 & 1488.365 & 1491.121 & 1492.739 & 1491.357 & 1494.255 & 1491.333 & 1485.548 & 1478.278\end{array}$

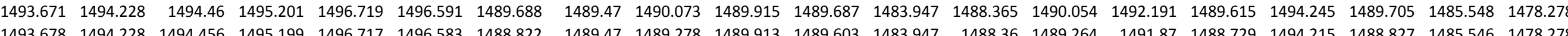

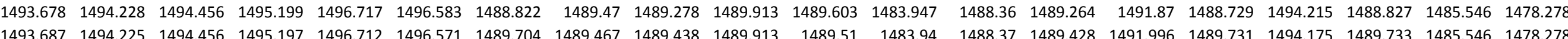
$\begin{array}{llllllllllllllllllllll}1493.69 & 1494.225 & 1494.451 & 1495.194 & 149671 & 1496.562 & 1490.303 & 1489.467 & 1490.04 & 1489.911 & 1489.507 & 1483.917 & 1488.372 & 1490.047 & 1492.273 & 1490.321 & 1494.173 & 1490.308 & 1485.546 & 1478278\end{array}$ $\begin{array}{lllllllllllllllllllllll}1493.69 & 1494.225 & 1494.453 & 1495.192 & 1496.708 & 1496.56 & 1490.594 & 1489.465 & 1490.347 & 1489.911 & 1489.521 & 1483.889 & 1488.377 & 1490.385 & 1492.394 & 1490.61 & 1494.17 & 1490.594 & 1485.546 & 1478.278\end{array}$ $\begin{array}{llllllllllllllllllllll}1493.69 & 1494.223 & 1494.451 & 1495.187 & 1496.705 & 1496.555 & 1490.788 & 1489.458 & 1490.551 & 1489.906 & 1489.54 & 1483.868 & 1488.379 & 1490.606 & 1492.469 & 1490.802 & 1494.168 & 1490.784 & 1485.546 & 1478.276\end{array}$

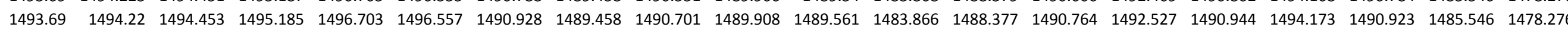
$\begin{array}{lllllllllllllllllllllll}1493.694 & 1494.223 & 1494.451 & 1495.185 & 1496.701 & 1496.555 & 1491.041 & 1489.458 & 1490.819 & 1489.906 & 1489.582 & 1483.866 & 1488.381 & 1490.895 & 1492.576 & 1491.053 & 1494.18 & 1491.033 & 1485.546 & 1478.273\end{array}$

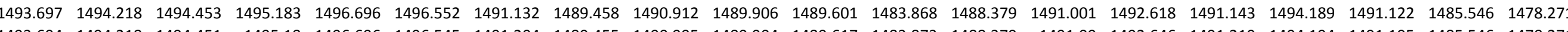

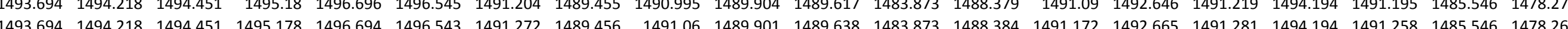

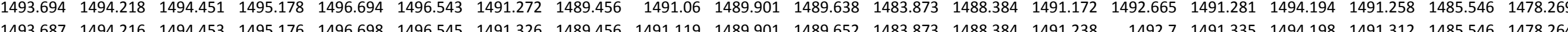
$\begin{array}{llllllllllllllllllllll}1493.678 & 1494.215 & 1494.451 & 1495.173 & 1496.701 & 1496.555 & 1491.375 & 1489.453 & 1491.168 & 1489.899 & 1489.669 & 1483.875 & 1488.386 & 1491.299 & 1492.716 & 1491.385 & 1494.208 & 1491.362 & 1485.543 & 1478.264\end{array}$ $\begin{array}{lllllllllllllllllllllll}1493.678 & 1494.218 & 1494.448 & 1495.173 & 1496.698 & 1496.557 & 1491.42 & 1489.455 & 1491.215 & 1489.899 & 1489.687 & 1483.875 & 1488.381 & 1491.346 & 1492.742 & 1491.428 & 1494.213 & 1491.404 & 1485.543 & 1478.262\end{array}$ $\begin{array}{llllllllllllllllllllll}1493.678 & 1494.213 & 1494.451 & 1495.176 & 1496.696 & 1496.555 & 1491.459 & 1489.456 & 1491.259 & 1489.899 & 1489.704 & 1483.873 & 1488.377 & 1491.384 & 1492.77 & 1491.468 & 1494.222 & 1491.446 & 1485.543 & 1478.259\end{array}$

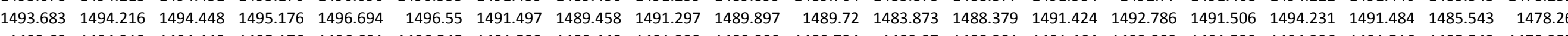

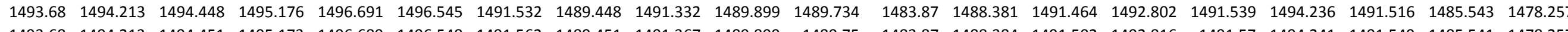

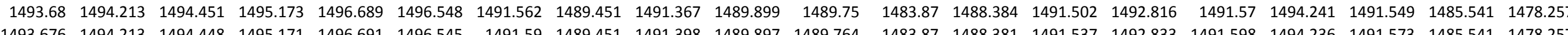

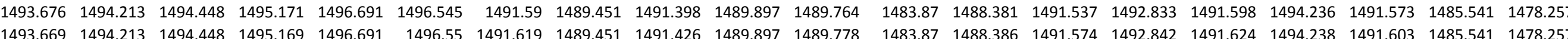

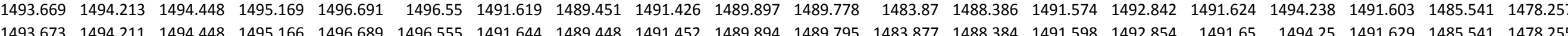

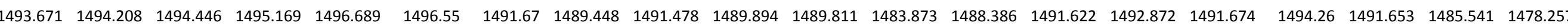
$\begin{array}{lllllllllllllllllllll}1493.669 & 1494.211 & 1494.448 & 1495.166 & 1496.689 & 1496.548 & 1491.696 & 1489.451 & 1491.501 & 1489.894 & 1489.825 & 1483.877 & 1488.384 & 1491.654 & 1492.886 & 1491.698 & 1494.262 & 1491.676 & 1485.541 & 1478.259\end{array}$ $\begin{array}{llllllllllllllllllll}1493.666 & 1494.208 & 1494.448 & 1495.169 & 1496.687 & 1496.552 & 1491.714 & 1489.446 & 1491.527 & 1489.892 & 1489.839 & 1483.875 & 1488.388 & 1491.675 & 1492.896 & 1491.719 & 1494.269 & 1491.7 & 1485.541 & 1478.259\end{array}$ $\begin{array}{llllllllllllllllllllll}1493.666 & 1494.208 & 1494.446 & 1495.166 & 1496.687 & 1496.552 & 1491.738 & 1489.453 & 1491.548 & 1489.892 & 1489.853 & 1483.875 & 1488.388 & 1491.701 & 1492.907 & 1491.74 & 1494.267 & 1491.721 & 1485.541 & 1478.259\end{array}$ $\begin{array}{llllllllllllllllllll}1493.666 & 1494.208 & 1494.441 & 1495.166 & 1496.687 & 1496.55 & 1490.692 & 1489.455 & 1491.131 & 1489.892 & 1489.865 & 1483.854 & 1488.386 & 1491.297 & 1492.672 & 1490.577 & 1494.276 & 1490.639 & 1485.539 & 1478.262\end{array}$

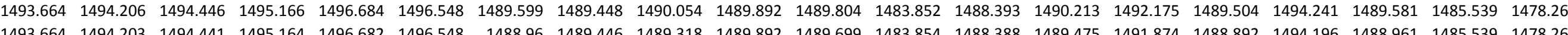

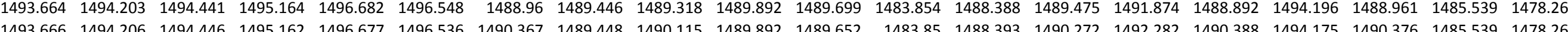
$\begin{array}{llllllllllllllllllllll}1493.669 & 1494.204 & 1494.441 & 1495.162 & 1496.673 & 1496.536 & 1490.739 & 1489.448 & 1490.5 & 1489.89 & 1489.657 & 1483847 & 1488393 & 1490.655 & 1492.453 & 1490.752 & 1494.177 & 1490.735 & 1485539 & 1478.266\end{array}$ $\begin{array}{lllllllllllllllllllll}1493.669 & 1494.204 & 1494.444 & 1495.157 & 1496.67 & 1496.528 & 1490.952 & 1489.448 & 1490.723 & 1489.89 & 1489.671 & 1483.852 & 1488.393 & 1490.9 & 1492.541 & 1490.961 & 1494.175 & 1490.944 & 1485.536 & 1478.266\end{array}$

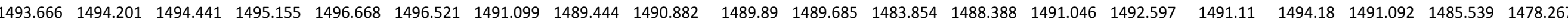

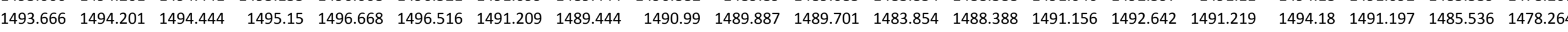

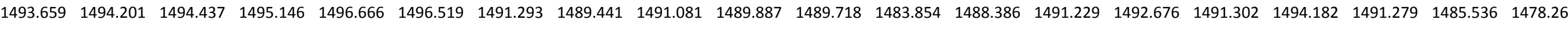

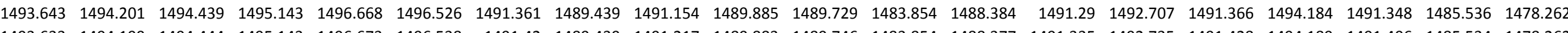

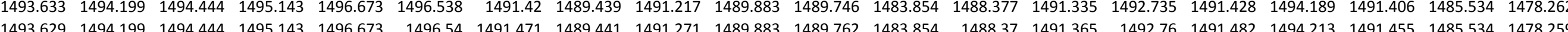

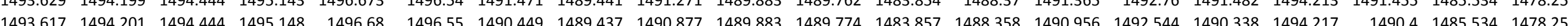
$\begin{array}{llllllllllllllllllllll}1493.603 & 1494.201 & 1494.444 & 1495.148 & 1496.684 & 1496.564 & 1489.379 & 1489.441 & 1489.82 & 1489.883 & 1489.713 & 1483.857 & 1488.356 & 1489.875 & 1492.065 & 1489.286 & 1494.194 & 1489.362 & 1485.534 & 1478.259\end{array}$ $\begin{array}{llllllllllllllllllllll}1493.596 & 1494.203 & 1494.444 & 1495.15 & 1496.689 & 1496.571 & 1488.757 & 1489.441 & 1489.102 & 1489.883 & 1489.608 & 1483.859 & 1488.344 & 1489.132 & 1491.779 & 1488.696 & 1494.163 & 1488.759 & 1485.534 & 1478.257\end{array}$

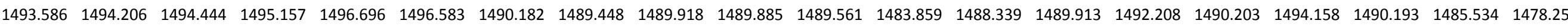
\begin{tabular}{llllllllllllllllllll}
1493.589 & 1494.206 & 1494.441 & 1495.16 & 1496.698 & 1496.593 & 1490.57 & 1489.446 & 1490.319 & 1489.887 & 1489.568 & 1483.863 & 1488.332 & 1490.296 & 1492.385 & 1490.584 & 1494.173 & 1490.569 & 1485.534 & 1478.259 \\
\hline
\end{tabular}

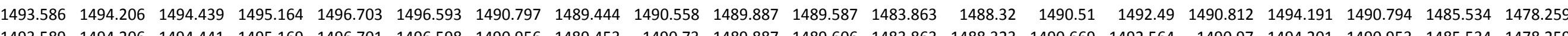

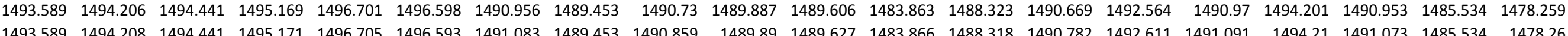


Well

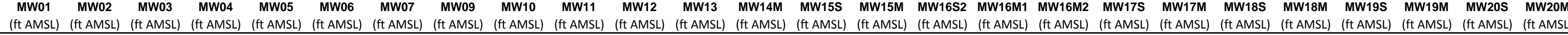

10/28/11 16:00 10/28/11 16:30 10/28/11 17:00 10/28/111 18:00 10/28/11 18:30 10/28/11 19:00 10/28/11 19:30 10/28/11 20:00 10/28/11 20:30 10/28/11 21:00 10/28/11 21:30 10/28/1122:00 10/28/11 22:30 10/28/11 23:30 10/29/11 0:00 10/29/11 1:00 10/29/11 1:30 $10 / 29 / 112: 00$
$10 / 29 / 112: 30$ 10/29/11 3:00 10/29/11 3:30 10/29/11 4:30 10/29/11 5:00 10/29/11 5:30 10/29/11 6:00 10/29/11 7:00 $10 / 29 / 117: 30$ 10/29/11 8:00 10/29/11 8:30 10/29/11 9:00 10/29/11 10:00 10/29/11 10:30 10/29/1111:00 10/29/11 12:00 10/29/11 12:30 10/29/11 13:00 10/29/11 13:30 10/29/11 14:00 10/29/11 14:30 10/29/11 15:00 10/29/11 16:00 10/29/11 16:30 10/29/11 17:00 10/29/11 17:30 10/29/11 18:00
10/29/11 18:30 $\begin{array}{lllll}1494.104 & 1494.553 & 1496.441 & 1494.206 \\ 1494.104 & 1494.55 & 1496.436 & 1494.213\end{array}$ $\begin{array}{rrrr}1494.104 & 1494.55 & 1496.436 & 1494.213 \\ 1494.102 & 1494.553 & 1496.431 & 1494.22\end{array}$ $\begin{array}{rrrr}1494.102 & 1494.553 & 1496.431 & 1494.22 \\ 1494.102 & 1494.55 & 1496.422 & 1494.225\end{array}$ $\begin{array}{rrrr}1494.102 & 1494.55 & 1496.422 & 1494.225 \\ 1499.1 & 1444.553 & 1496.413 & 1444.222 \\ 1494.1 & 1494.548 & 1496.41 & 1494.213\end{array}$ $\begin{array}{rrrrr}1494.095 & 1494.546 & 1496.41 & 1994.194\end{array}$ $\begin{array}{lllll}1494.09 & 1494.541 & 1496.41 & 1494.183\end{array}$ $1494.093 \quad 1494.539 \quad 1496.417 \quad 1494.176$ $\begin{array}{llll}1494.093 & 1494.536 & 1496.42 & 1494.171\end{array}$ $\begin{array}{llll}1494.095 & 1494.541 & 1496.424 & 1494.176 \\ 1499.095 & 1494543 & 1496.429 & 194.178\end{array}$ $\begin{array}{llll}1494.095 & 1494.543 & 1496.429 & 1494.178 \\ 1494.097 & 1494.546 & 1496.431 & 1494.185\end{array}$ $\begin{array}{lllll}1494.093 & 1494.548 & 1496.429 & 1494.192\end{array}$ $\begin{array}{llll}1494.095 & 1494.55 & 1496.438 & 1494.199\end{array}$ $\begin{array}{llllll}1494.093 & 1494.553 & 1496.441 & 1494.206\end{array}$ $\begin{array}{lllll}1494.095 & 1494.553 & 1496.445 & 1494.218\end{array}$ $\begin{array}{lllll}1494.097 & 1494.56 & 1496.45 & 1494.227\end{array}$ $\begin{array}{llll}1494.097 & 1494.564 & 1496.455 & 1494.236\end{array}$ $\begin{array}{llllll}1494.097 & 1494.567 & 1496.455 & 1494.246\end{array}$ $\begin{array}{lllll}1494.097 & 1444.574 & 1496.473 & 1494.267\end{array}$ $\begin{array}{lllll}1494.1 & 1494.576 & 1496.483 & 1494.274\end{array}$ $\begin{array}{llllll}1494.1 & 1494.583 & 1496.487 & 1494.288\end{array}$ $\begin{array}{llllll}1494.1 & 1494.59 & 1496.483 & 1494.297\end{array}$ $\begin{array}{lllll}1494.102 & 1494.597 & 1496.485 & 1494.311\end{array}$ $\begin{array}{llll}1494.102 & 1494.599 & 1496.48 & 1494.32\end{array}$ $\begin{array}{rrrr}1494.102 & 1494.602 & 1496.483 & 1494.327\end{array}$ $\begin{array}{lllll}1494.1 & 1494.602 & 1496.485 & 1494.332 \\ 1494.1 & 1494.604 & 1496 & 1494334\end{array}$ $\begin{array}{lllll}1494.1 & 1494.604 & 1496.476 & 1494.33\end{array}$ $\begin{array}{lllll}1494.1 & 1494.602 & 1496.471 & 1494.318\end{array}$ $\begin{array}{lllll}14994.1 & 1494.602 & 1496.476 & 1494.304\end{array}$ $\begin{array}{lllll}1494.097 & 1494.602 & 1496.478 & 1494.292\end{array}$ $\begin{array}{llll}1494.095 & 1494.597 & 1496.487 & 1494.288\end{array}$ $\begin{array}{llll}1494.097 & 1494.599 & 1496.501 & 1494.285 \\ 14941 & 1494.602 & 1496511 & 194.28\end{array}$ $\begin{array}{rrrrr}1494.1 & 1494.602 & 1496.511 & 1494.288 \\ 1494.102 & 1494.604 & 1496525 & 1494.292\end{array}$ $\begin{array}{llll}1494.102 & 1494.604 & 1496.525 & 1494.292 \\ 1494.107 & 1494.618 & 1496.532 & 1494.304\end{array}$ $\begin{array}{lllll}1494.107 & 1494.625 & 1496.541 & 1494.316\end{array}$ $\begin{array}{lllll}1494.109 & 1494.63 & 1496.558 & 1494.327\end{array}$ $\begin{array}{lllll}1494.109 & 1494.639 & 1496.581 & 1494.334\end{array}$ $\begin{array}{lllll}1494.116 & 1494.646 & 1496.604 & 1494.339\end{array}$ $1494.121 \quad 1494.662 \quad 1496.616 \quad 1494.341$ $\begin{array}{rrrr}1494.128 & 1494.676 & 1496.625 & 1494.344 \\ 1494.128 & 1494.688 & 1496.63 & 1494353\end{array}$ $\begin{array}{rrrrr}1494.128 & 1494.688 & 1496.63 & 1494.353 \\ 1494.13 & 1494.7 & 1496.632 & 1494.36\end{array}$ $\begin{array}{rrrr}1494.135 & 1494.709 & 1496.632 & 1494.374\end{array}$ $\begin{array}{llllll}1494.132 & 1494.714 & 1496.623 & 1494.379\end{array}$ $\begin{array}{lllll}1494.132 & 1494.718 & 1496.621 & 1494.388\end{array}$ $\begin{array}{lllll}1494.135 & 1494.718 & 1496.611 & 1494.395\end{array}$ $\begin{array}{lllll}1494.13 & 1494.718 & 1496.595 & 1494.395\end{array}$ $\begin{array}{rrrr}1494.132 & 1494.721 & 1496.586 & 1494.395 \\ 1494.13 & 1494.714 & 1496.581 & 1494.383\end{array}$

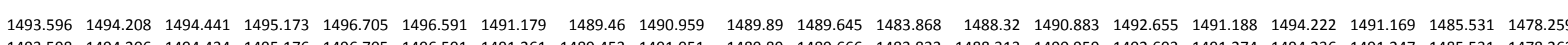
$\begin{array}{lllllllllllllllllllll}1493.598 & 1494.206 & 1494.434 & 1495.176 & 1496.705 & 1496.591 & 1491.261 & 1489.453 & 1491.051 & 1489.89 & 1489.666 & 1483.833 & 1488.313 & 1490.959 & 1492.693 & 1491.274 & 1494.236 & 1491.247 & 1485.531 & 1478.262 \\ 1493.605 & 1494206 & 1494.439 & 1495 & 1496703 & 1496.588 & 1491331 & 1489.456 & 1491119 & 148989 & 1489685 & 1483815 & 148832 & 1491031 & 1492723 & 1491338 & 1492.236 & 1491315 & 1485531 & 1478262\end{array}$

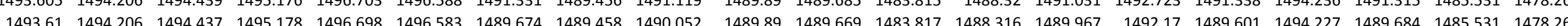
$\begin{array}{llllllllllllllllllllll}1493.624 & 1494201 & 1494.437 & 1495.178 & 1496.696 & 1496.574 & 1488.79 & 1489.455 & 1489.243 & 1489.89 & 1489.584 & 1483.815 & 148832 & 1489.163 & 1491.844 & 1488.698 & 1494.191 & 1488.792 & 1485.529 & 1478.262\end{array}$ $\begin{array}{llllllllllllllllllll}1493.631 & 1494.201 & 1494.439 & 1495.176 & 1496.691 & 1496562 & 1489.679 & 1489.453 & 1489.414 & 1489.89 & 1489.489 & 1483.817 & 1488.32 & 1489.33 & 1491.977 & 1489.707 & 1494.158 & 1489.705 & 1485.531 & 1478.264\end{array}$

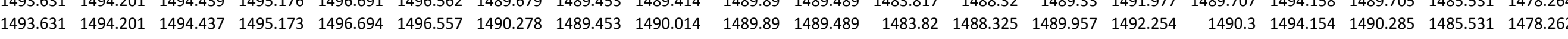

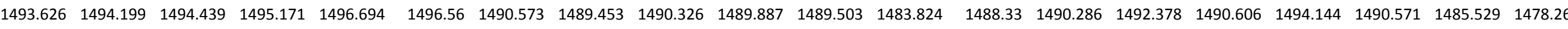

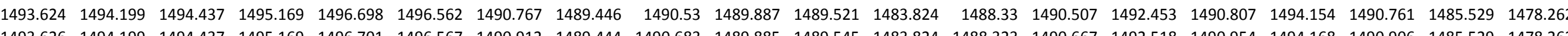
$\begin{array}{llllllllllllllllllll}1493.626 & 1494.199 & 1494.437 & 1495.169 & 1496.701 & 1496.567 & 1490.912 & 1489.444 & 1490.683 & 1489.885 & 1489.545 & 1483.824 & 1488.323 & 1490.667 & 1492.518 & 1490.954 & 1494.168 & 1490.906 & 1485.529 & 1478.262 \\ 1493622 & 1494.197 & 1494.434 & 1495171 & 1496701 & 1496569 & 1491024 & 1489.446 & 14908 & 1489885 & 1489563 & 1483826 & 1488327 & 1490789 & 1492569 & 1491072 & 1494175 & 1491017 & 1485529 & 1478262\end{array}$ $\begin{array}{llllllllllllllllllll}1493.622 & 1494.197 & 1494.434 & 1495.171 & 1496.701 & 1496.569 & 1491.024 & 1489.446 & 1490.8 & 1489.885 & 1489.563 & 1483.826 & 1488.327 & 1490.789 & 1492.569 & 1491.072 & 1494.175 & 1491.017 & 1485.529 & 1478.262 \\ 1493.622 & 1494.199 & 1494.437 & 1495.171 & 1496.703 & 1496.576 & 1491.113 & 1489.444 & 1490.896 & 1489.885 & 1489.587 & 1483.831 & 1488.327 & 1490.895 & 1492.611 & 1491.162 & 1494.187 & 1491106 & 1485.529 & 1478262\end{array}$

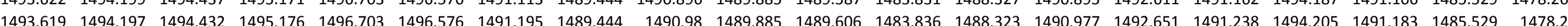

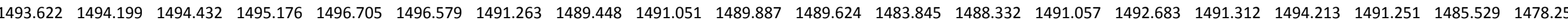
$\begin{array}{lllllllllllllllllllllll}1493.617 & 1494.196 & 1494.437 & 1495.176 & 1496.708 & 1496.579 & 1491.321 & 1489.448 & 1491.114 & 1489.885 & 1489.643 & 1483.852 & 1488.325 & 1491.118 & 1492.711 & 1491.371 & 1494.227 & 1491.308 & 1485.529 & 1478.257\end{array}$

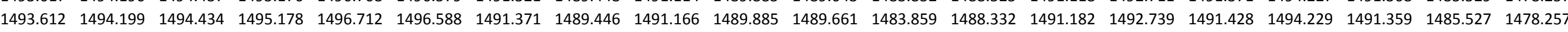
$\begin{array}{lllllllllllllllllllllll}1493.612 & 1494.199 & 1494.434 & 1495.178 & 1496.715 & 1496.591 & 1491.417 & 1489.448 & 1491.215 & 1489.885 & 1489.678 & 1483.868 & 1488.33 & 1491.231 & 1492.76 & 1491.473 & 1494.238 & 1491.404 & 1485.529 & 1478.255\end{array}$

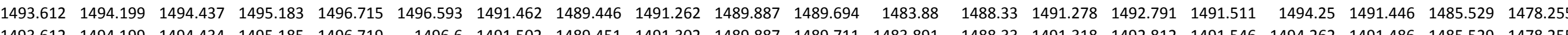

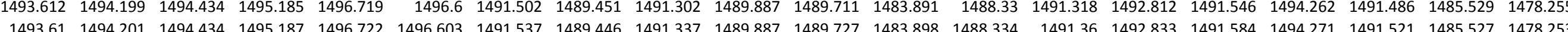

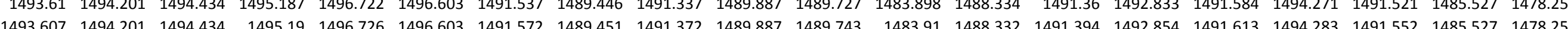
\begin{tabular}{lllllllllllllllllllll}
1493.6 & 1494.206 & 1494.434 & 1495.194 & 1496.731 & 1496.612 & 14916 & 1498.448 & 1491.405 & 1489.887 & 1489.76 & 1483.921 & 1488.332 & 1491.424 & 1492.87 & 1491.641 & 1494.29 & 1491.585 & 1485.527 & 1478.252 \\
\hline
\end{tabular} $\begin{array}{llllllllllllllllllllllll}1493.598 & 1494.206 & 1494.437 & 1495197 & 1496738 & 1496617 & 1491.63 & 1489451 & 1491.435 & 1489.887 & 1489.776 & 1483935 & 1488332 & 1491.457 & 1492.886 & 1491.672 & 1494.292 & 1491.615 & 1485.527 & 1478.252\end{array}$ $\begin{array}{lllllllllllllllllllllll}1493.596 & 1494.208 & 1494.437 & 1495.199 & 1496.74 & 1496.631 & 1491.658 & 1489.458 & 1491.466 & 1489.89 & 1489.792 & 1483.947 & 1488.332 & 1491.476 & 1492.91 & 1491.691 & 1494.314 & 1491.643 & 1485.527 & 1478.253 \\ \end{array}$

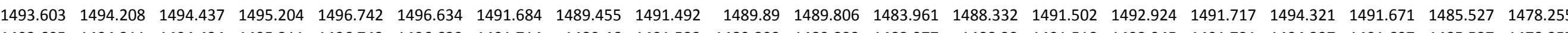

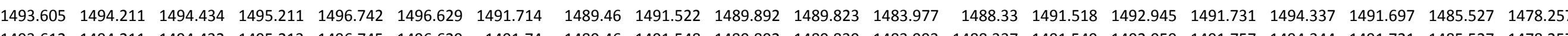

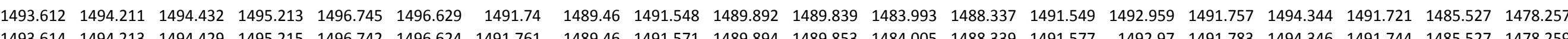

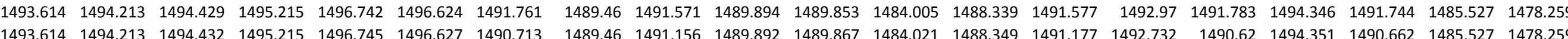

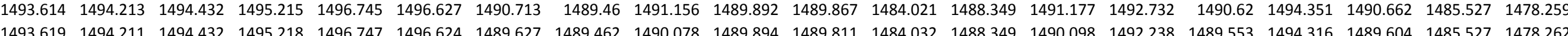
$\begin{array}{lllllllllllllllllllll}1493.622 & 1494.213 & 1494.427 & 1495.213 & 1496.745 & 1496.622 & 1488.986 & 1489.465 & 1489.344 & 1489.894 & 1489.704 & 1484.053 & 1488.349 & 1489.365 & 1491.944 & 1488.947 & 1494.281 & 1488.989 & 1485.524 & 1478.264\end{array}$ $\begin{array}{llllllllllllllllllllll}1493.629 & 1494.213 & 1494.432 & 1495.218 & 1496.742 & 1496.617 & 1490.399 & 1489.46 & 1490.143 & 1489.897 & 1489.654 & 1484.067 & 1488.356 & 1490.162 & 1492.355 & 1490.442 & 1494.26 & 1490.407 & 1485.524 & 1478.264\end{array}$ $\begin{array}{lllllllllllllllllllll}1493.631 & 1494.213 & 1494.434 & 1495.225 & 1496.745 & 1496.607 & 1490.767 & 1489.47 & 1490.528 & 1489.894 & 1489.664 & 1484.085 & 1488.358 & 1490.554 & 1492.52 & 1490.814 & 1494.257 & 1490.768 & 1485.527 & 1478.266\end{array}$ $\begin{array}{llllllllllllllllllllll}1493.631 & 1494.213 & 1494.432 & 1495.234 & 1496.745 & 1496.61 & 1490.984 & 1489.46 & 1490.758 & 1489.894 & 1489.678 & 1444.095 & 1488.358 & 1490.787 & 1492.611 & 1491.034 & 1494.26 & 1490.981 & 1485.524 & 1478.2667\end{array}$

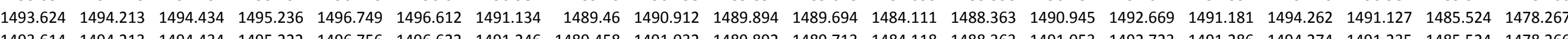

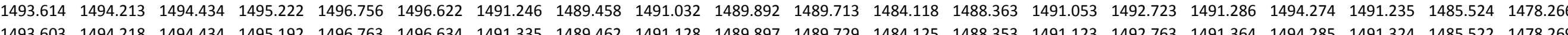

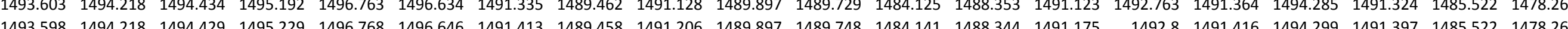
$\begin{array}{rlllllllllllllllllll}14393.596 & 1494.22 & 1494.432 & 1495.231 & 1496.773 & 1496.655 & 1491.473 & 1489.465 & 1491.271 & 1489.897 & 1489.769 & 1484.152 & 1488.332 & 1491.215 & 1492.84 & 1491.456 & 1494.316 & 1491.463 & 1485.524 & 1478.269\end{array}$

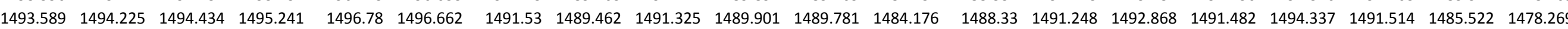
$\begin{array}{llllllllllllllllllllllll}1493.579 & 1494.227 & 1494.441 & 1495.245 & 1496.794 & 1496.674 & 1490.509 & 1489.467 & 1490.934 & 1489.904 & 1489.799 & 1484.189 & 1488.327 & 1490.846 & 1492.66 & 1490.309 & 1494.344 & 1490.461 & 1485.522 & 1478.269\end{array}$ $\begin{array}{lllllllllllllllllllll}1493.57 & 1494.235 & 1494.439 & 1495.199 & 1496.805 & 1496.689 & 1489.438 & 1489.474 & 1489.881 & 1489.901 & 1489.739 & 1484.203 & 1488.323 & 1489.762 & 1492.187 & 1489.231 & 1494.323 & 1489.419 & 1485.522 & 1478.269 & \end{array}$ $\begin{array}{llllllllllllllllllll}1493.556 & 1494.24 & 1494.439 & 1495.257 & 1496.815 & 1496.708 & 1488.82 & 1489.474 & 1489.163 & 1489.904 & 1489.636 & 1484.208 & 1488.318 & 1489.015 & 1491.902 & 1488.76 & 1494.302 & 1488.825 & 1485.522 & 1478.269\end{array}$

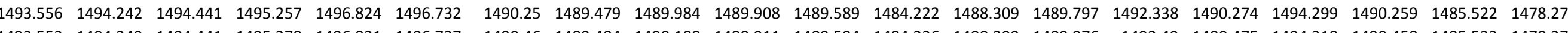

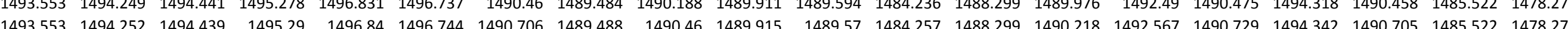

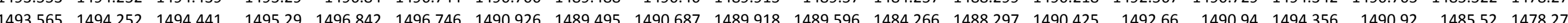
$\begin{array}{llllllllllllllllllllll}1493.572 & 1494.261 & 1494.441 & 1495.266 & 1496.847 & 1496.749 & 1491.076 & 1489.495 & 1490.844 & 1489.918 & 1489.617 & 1484.277 & 1488.295 & 1490.568 & 1492.728 & 1491.089 & 1494.379 & 1491.071 & 1485.522 & 1478.278\end{array}$ $\begin{array}{llllllllllllllllllllll}1493.589 & 1494.259 & 1494.439 & 1495.313 & 1496.845 & 1496.746 & 1491.193 & 1489.498 & 1490.964 & 1489.925 & 1489.641 & 1484.294 & 1488.297 & 1490.686 & 1492.781 & 1491.203 & 1494.386 & 1491.186 & 1485.524 & 1478.278\end{array}$ $\begin{array}{lllllllllllllllllllllll}1493.6 & 1494.261 & 1494.439 & 1495.315 & 1496.847 & 1496.734 & 1491.286 & 1489.502 & 1491.067 & 1489.925 & 1489.666 & 1484.312 & 1488.304 & 1490.785 & 1492.819 & 1491.297 & 1494.398 & 1491.277 & 1485.522 & 1478.278\end{array}$

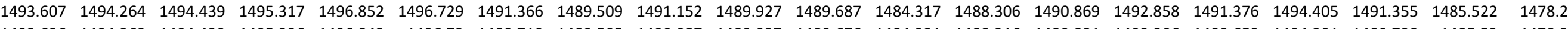

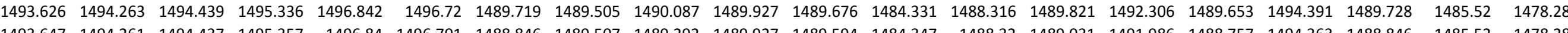
$\begin{array}{llllllllllllllllllll}1493.647 & 1494.261 & 1494.437 & 1495.357 & 1496.84 & 1496.701 & 1488.846 & 1489.507 & 1489.292 & 1489.927 & 1489.594 & 1484.347 & 1488.32 & 1489.031 & 1491.986 & 1488.757 & 1494.363 & 1488.846 & 1485.52 & 1478.28 \\ 1493.657 & 1494.261 & 1494.437 & 149538 & 1496835 & 1496689 & 1489.742 & 1489505 & 1489.473 & 1489.927 & 1489.503 & 1484358 & 1488325 & 1489219 & 149211 & 1489.767 & 1494.316 & 1489.766 & 1485.52 & 1478.283\end{array}$ 
Well

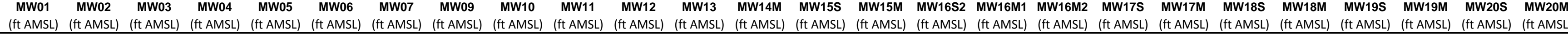

10/29/11 19:00 10/29/11 19:30 10/29/11 20:00 10/29/11 20:30 $21: 00$ 10/29/11 21:30 10/29/11 22:00 10/29/11 22:30 10/29/11 23:00 10/29/11 23:30 10/30/11 0:00 10/30/11 0:30 10/30/111:30 $10 / 30 / 112: 00$
$10 / 30 / 112: 30$
$10 / 30 / 113: 00$ 10/30/11 3:00 10/30/11 4:00 10/30/11 4:30 10/30/11 5:00 10/30/11 6:00 10/30/11 6:30 10/30/11 7:30 10/30/11 8:00 10/30/11 8:30 10/30/11 $10: 30$ 10/30/11 10:00 10/30/11 11:00 10/30/11 11:30 10/30/11 12:30 10/30/11 13:30 10/30/11 14:00 10/30/11 15:00 10/30/11 15:30 10/30/11 16:00 10/30/11 16:30 10/30/11 17:00 10/30/11 17:30 10/30/11 18:00 10/30/11 18:30 10/30/11 19:30 $10 / 30 / 1120: 00$
$10 / 30 / 1120: 30$ $10 / 30 / 1120: 30$
$10 / 30 / 1121: 00$ $10 / 30 / 1121: 00$
$10 / 30 / 1121: 30$ $\begin{array}{lllll}1494.123 & 1494.704 & 1496.579 & 1494.362 \\ 1494.125 & 1494.702 & 1496.581 & 1494.351\end{array}$ $\begin{array}{rrrrr}1494.125 & 1494.702 & 1496.581 & 1494.351 \\ 1494.125 & 1494.7 & 1496586 & 1494.339\end{array}$ $\begin{array}{llll}1494.125 & 1494.7 & 1496.586 & 1494.339\end{array}$ $\begin{array}{rrrrr}1494.128 & 1494.7 & 1496.583 & 1494.334 \\ 1494.13 & 1494.702 & 1496.574 & 1494.334\end{array}$ $\begin{array}{rrrrr}1494.13 & 1494.702 & 146.574 & 1494.334 \\ 1494.135 & 1494.704 & 1496.574 & 1494.344\end{array}$ $\begin{array}{rrrr}1494.135 & 1494.704 & 1496.574 & 1494.344 \\ 1494.128 & 1494.7 & 1496.572 & 1494.337\end{array}$ $\begin{array}{llllll}1494.128 & 1494.695 & 1496.576 & 1494.339\end{array}$ $\begin{array}{lllll}1494.125 & 1494.69 & 1496.581 & 1494.341\end{array}$ $\begin{array}{rrrr}1494.13 & 1494.693 & 1496.574 & 1494.346 \\ 1494.128 & 1494.693 & 1496572 & 1494.351\end{array}$ $\begin{array}{rrrrr}1494.128 & 1494.693 & 1496.572 & 1494.351 \\ 1494.13 & 1494.693 & 1496562 & 149436\end{array}$ $\begin{array}{rrrr}1494.13 & 1494.693 & 1496.562 & 1494.36 \\ 1494.128 & 1494.69 & 1496.555 & 1494.365\end{array}$ $\begin{array}{lllll}1494.125 & 1494.688 & 1496.553 & 1494.362\end{array}$ $\begin{array}{lllll}1494.125 & 1494.688 & 1496.544 & 1494.367\end{array}$ $\begin{array}{lllll}1494.123 & 1494.681 & 1496.527 & 1494.367\end{array}$ $\begin{array}{lllll}1494.123 & 1494.679 & 1496.518 & 1494.367\end{array}$ $\begin{array}{llll}1494.118 & 1494.672 & 1496.511 & 1494.362\end{array}$ $\begin{array}{rrrr}1494.116 & 1494.665 & 1496.497 & 1494.362\end{array}$ $\begin{array}{rrrr}1494.116 & 1494.66 & 1496.48 & 1494.36 \\ 1494111 & 1494.655 & 1496.462 & 1494.355\end{array}$ $\begin{array}{llll}1494.107 & 1494.646 & 1496.448 & 1494.346\end{array}$ $\begin{array}{llllll}1494.102 & 1494.637 & 1496.427 & 1494.339\end{array}$ $\begin{array}{lllll}1494.102 & 1494.63 & 1496.417 & 1494.327\end{array}$ $\begin{array}{lllll}1494.095 & 1494.618 & 1496.406 & 1494.304\end{array}$ $\begin{array}{llll}1494.095 & 1494.606 & 1496.392 & 1494.278\end{array}$ $\begin{array}{llll}1494.09 & 1494.597 & 1496.38 & 1494.25\end{array}$ $\begin{array}{llll}1494.086 & 1494.588 & 1496.373 & 1494.229\end{array}$ $\begin{array}{lllll}1494.081 & 1494.578 & 1496.368 & 1494.211 \\ 1494.081 & 1494.571 & 1496366 & 1494.199\end{array}$ $\begin{array}{lllll}1494.079 & 1494.564 & 1496.361 & 1494.19\end{array}$ $\begin{array}{llll}1494.076 & 1494.557 & 1496.357 & 1494.18\end{array}$ $\begin{array}{lllll}1494.076 & 1494.555 & 1496.366 & 1494.178\end{array}$ $\begin{array}{llllll}1494.076 & 1494.548 & 1496.378 & 1494.176\end{array}$ $\begin{array}{lllll}1494.074 & 1494.543 & 1496.389 & 1494.173\end{array}$ $1494.081 \quad 1494.548 \quad 1496.396 \quad 1494.178$ $\begin{array}{rrrrr}1494.086 & 1494.55 & 1496.403 & 1494.176 \\ 1494.086 & 1494.555 & 1496.41 & 1494.169\end{array}$ $\begin{array}{rrrr}1494.086 & 1494.555 & 1496.41 & 1494.169 \\ 1494.088 & 1494.557 & 1496.417 & 1494.166\end{array}$ $\begin{array}{lllll}1494.086 & 1494.56 & 1496.422 & 1494.162\end{array}$ $\begin{array}{llllll}1494.088 & 1494.56 & 1496.424 & 1494.164\end{array}$ $\begin{array}{llllll}1494.088 & 1494.56 & 1496.431 & 1494.169\end{array}$ $\begin{array}{lllll}1494.088 & 1494.564 & 1496.427 & 1494.171\end{array}$ $\begin{array}{lllll}1494.086 & 1494.564 & 1496.422 & 1494.171\end{array}$ $\begin{array}{llll}1494.086 & 1494.567 & 1496.42 & 1494.176\end{array}$ $\begin{array}{llll}1494.083 & 1494.567 & 1496.41 & 1494.171\end{array}$ $\begin{array}{rrrrr}1494.079 & 1494.56 & 1496.406 & 1494.143\end{array}$ $\begin{array}{lllll}1494.079 & 1494.555 & 1496.408 & 1494.122\end{array}$ $1494.076 \quad 1494.553 \quad 1496.406 \quad 1494.103$ $\begin{array}{lllll}1494.074 & 1494.55 & 1496.406 & 1494.087\end{array}$ $\begin{array}{lllll}1494.076 & 1494.55 & 1496.399 & 1494.078\end{array}$ $\begin{array}{llll}1494.076 & 1494.548 & 1496.394 & 1494.066 \\ 1494.074 & 1494.543 & 1496.389 & 1494.054\end{array}$

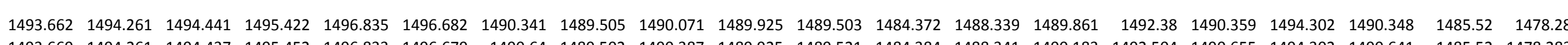
$\begin{array}{llllllllllllllllllll}1493.669 & 1494.261 & 1494.437 & 1495.452 & 1496.833 & 1496.679 & 1490.64 & 1489.502 & 1490.387 & 1489.925 & 1489.521 & 1484.384 & 1488.341 & 1490.183 & 1492.504 & 1490.655 & 1494.302 & 1490.641 & 1485.52 & 1478.283 \\ 1493.669 & 1494.264 & 1494.437 & 1495338 & 1496838 & 1496.679 & 1490839 & 1489.498 & 1490.598 & 1489.925 & 1489.545 & 1484.398 & 1488344 & 1490409 & 1492585 & 1490852 & 1494309 & 1490836 & 14852 & 1478283\end{array}$

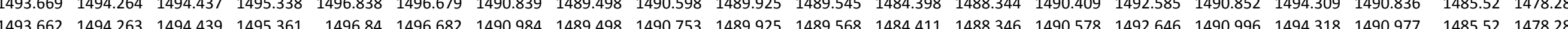
$\begin{array}{lllllllllllllllllllllll}1493.68 & 1494.264 & 1494.429 & 1495.375 & 1496.833 & 1496.679 & 1491.101 & 1489.5 & 1490.875 & 1489.925 & 1489.591 & 1484.425 & 1488344 & 1490.705 & 1492.697 & 1491.115 & 1494.323 & 1491.094 & 1485.52 & 147828\end{array}$ $\begin{array}{lllllllllllllllllllll}1493.683 & 1494.264 & 1494.437 & 1495.382 & 1496.835 & 1496.665 & 1491.197 & 1489.502 & 1490.976 & 1489.925 & 1489.615 & 1484.448 & 1488.344 & 1490.806 & 1492.742 & 1491.212 & 1494.346 & 1491.19 & 1485.52 & 1478.28\end{array}$ $\begin{array}{lllllllllllllllllllllll}1493.692 & 1494.261 & 1494.434 & 1495.392 & 1496.831 & 1496.667 & 1491.279 & 1489.502 & 1491.058 & 1489.925 & 1489.634 & 1484.448 & 1488.356 & 1490.909 & 1492.77 & 1491.288 & 1494.339 & 1491.265 & 1485.515 & 1478.28\end{array}$ \begin{tabular}{llllllllllllllllllllll}
1493.683 & 1494.261 & 1494.437 & 1495.403 & 1496.835 & 1496.665 & 1491.347 & 1489.5 & 1491.131 & 1489.925 & 1489.654 & 1484.441 & 1488.36 & 1490.996 & 1492.798 & 1491.357 & 1494.339 & 1491.333 & 1485.52 & 1478.28 \\
\hline
\end{tabular}

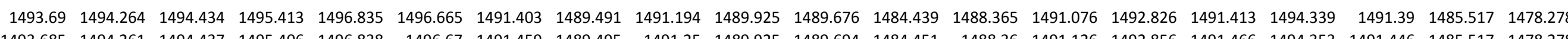

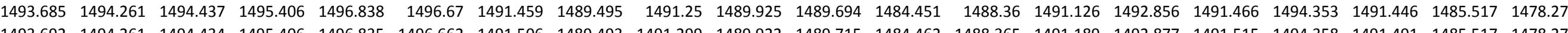

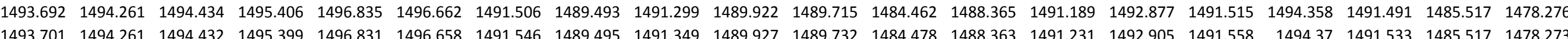

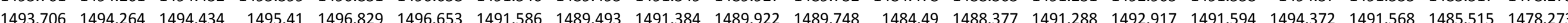

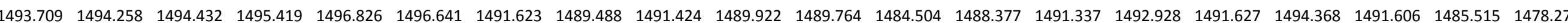

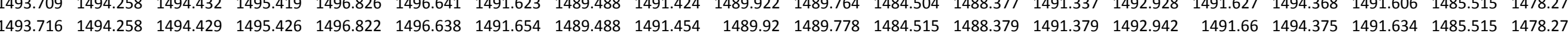
$\begin{array}{llllllllllllllllllll}1493.725 & 1494.254 & 1494.425 & 1495.364 & 1496.812 & 1496.627 & 1491.682 & 1489.486 & 1491.485 & 1489.922 & 1489.795 & 1484.527 & 1488.386 & 1491.426 & 1492.954 & 1491.688 & 1494.37 & 1491.667 & 1485.515 & 1478.269\end{array}$ $\begin{array}{lllllllllllllllllllllll}1493.73 & 1494.254 & 4494.225 & 1495.378 & 1496.808 & 1496.615 & 1491.71 & 1489.481 & 1491.513 & 1489.92 & 1489.809 & 1484.536 & 1488.388 & 1491.469 & 1492.966 & 1491.714 & 1494.37 & 1491.69 & 1485.515 & 1478.269\end{array}$

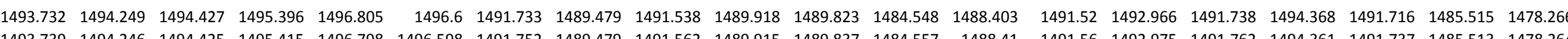

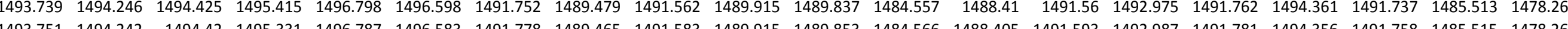

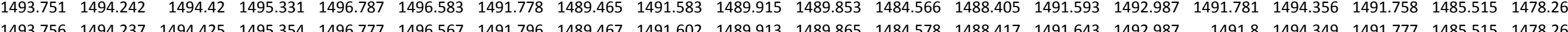

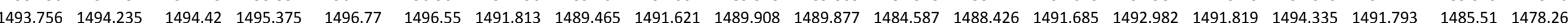
$\begin{array}{llllllllllllllllllllllll}1493.77 & 1494.223 & 1494.422 & 1495.396 & 1496.749 & 1496.516 & 1489.662 & 1489.455 & 1490.118 & 1489.901 & 1489.825 & 1484.61 & 1488.428 & 1490.211 & 1492.226 & 1489.563 & 1494.288 & 1489.642 & 1485.513 & 1478.262 \\ \end{array}$

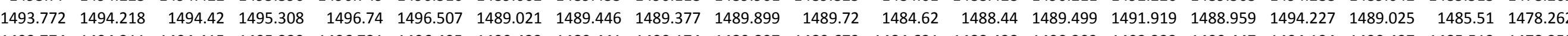

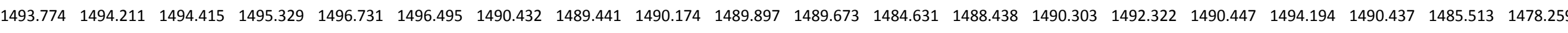

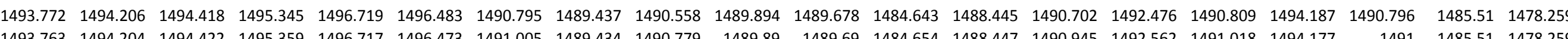

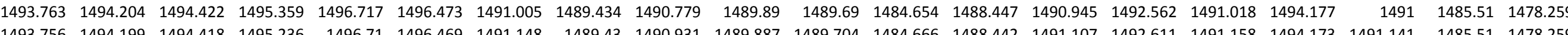

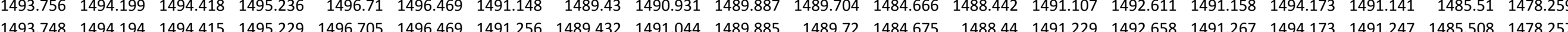
$\begin{array}{llllllllllllllllllllll}1493.746 & 1494.197 & 1494.415 & 1495.22 & 1496.698 & 1496.469 & 1491.342 & 1489.427 & 1491.133 & 1489.883 & 1489.736 & 1484.684 & 1488.433 & 1491.311 & 1492.69 & 1491.35 & 1494.177 & 1491.331 & 1485.508 & 1478.257\end{array}$ $\begin{array}{llllllllllllllllllllll}1493.737 & 1494.191 & 1494.415 & 1495.218 & 1496.696 & 1496.464 & 1491.408 & 1489.425 & 1491.206 & 1489.88 & 1489.753 & 1484.694 & 1488.431 & 1491.377 & 1492.718 & 1491.418 & 1494.18 & 1491.397 & 1485.51 & 1478.257\end{array}$ $\begin{array}{llllllllllllllllllllll}1493.723 & 1494.192 & 1494.413 & 1495.211 & 1496.694 & 1496.466 & 1491.466 & 1489.427 & 1491.264 & 1489.878 & 1489.769 & 1484.705 & 1488.428 & 1491.436 & 1492.744 & 1491.473 & 1494.173 & 1491.453 & 1485.508 & 1478.255 \\ \end{array}$ $\begin{array}{llllllllllllllllllllll}1493.706 & 1494.189 & 1494.415 & 1495.206 & 1496.694 & 1496.476 & 1491.516 & 1489.425 & 1491.316 & 1489.878 & 1489.783 & 1484.714 & 1488.421 & 1491.476 & 1492.758 & 1491.522 & 1494.18 & 1491.505 & 1485.508 & 1478.255\end{array}$

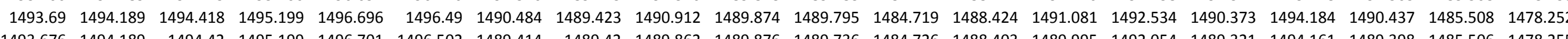

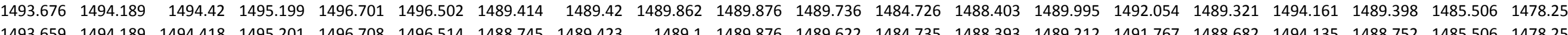

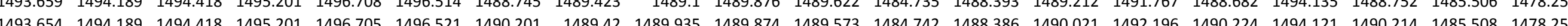

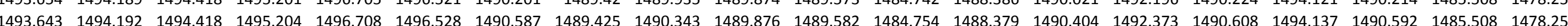

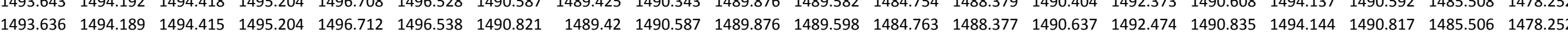
$\begin{array}{llllllllllllllllllllll}1493.629 & 1494.189 & 1494.42 & 1495.204 & 1496.715 & 1496.543 & 1490.797 & 1489.425 & 1490.704 & 1489.876 & 1489.62 & 1484.774 & 1488.365 & 1490.745 & 1492.541 & 1490.802 & 1494.163 & 1490.761 & 1485.508 & 1478.252\end{array}$ $\begin{array}{llllllllllllllllllllll}1493.624 & 1494.192 & 1494.418 & 1495.206 & 1496.717 & 1496.548 & 1490.409 & 1489.423 & 1490.357 & 1489.878 & 1489.619 & 1484.786 & 1488.363 & 1490.373 & 1492.383 & 1490.404 & 1494.17 & 1490.397 & 1485.506 & 1478.255 \\ \end{array}$

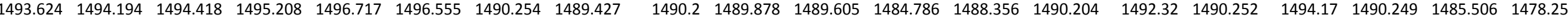

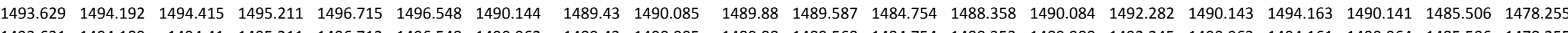

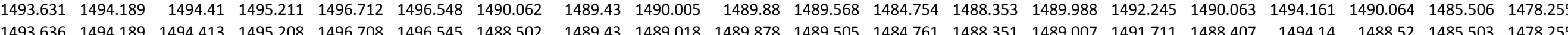

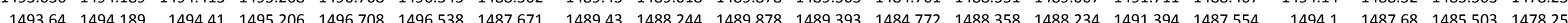
$\begin{array}{lllllllllllllllllllll}1493.643 & 1494.185 & 1494.408 & 1495.204 & 1496.705 & 1496.536 & 1488.153 & 1489.425 & 1488.054 & 1489.878 & 1489.274 & 1484.784 & 1488.356 & 1488.046 & 1491.436 & 1488.158 & 1494.064 & 1488.189 & 1485.503 & 1478.255\end{array}$ $\begin{array}{lllllllllllllllllllllll}1493.645 & 1494.185 & 1494.41 & 1495.201 & 1496.703 & 1496.531 & 1488.387 & 1489.427 & 1488.263 & 1489.878 & 1489.175 & 1484.798 & 1488.36 & 1488.274 & 1491.569 & 1488.388 & 1494.043 & 1488.41 & 1485.503 & 1478.255 \\ \end{array}$

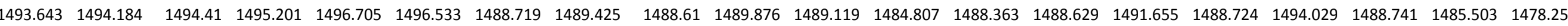

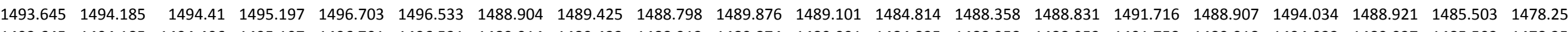

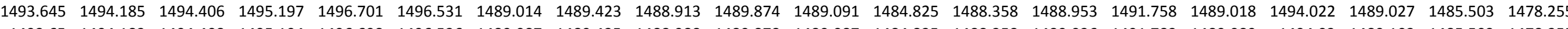

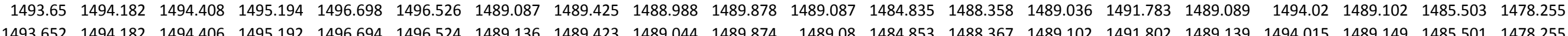


Well

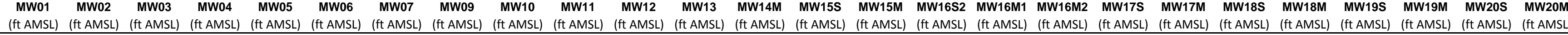

10/30/11 22:00 10/30/11 22:30 10/30/1123:00 10/30/11 23:30 10/31/11 0:30 10/31/11 1:00 $10 / 31 / 111: 30$
$10 / 31 / 112: 00$ 10/31/11 2:00 10/31/11 3:00 10/31/11 3:30 10/31/11 4:00 10/31/11 5:00 10/31/11 5:30 10/31/11 6:00 10/31/117:00 10/31/117:30 10/31/11 8:00 10/31/11 9:00 10/31/11 9:30 10/31/11 10:30 10/31/11 11:00 10/31/1111:30 10/31/11 12:00 10/31/11 12:30 10/31/1111 13:00 10/31/11 14:00 10/31/11 14:30 10/31/11 15:30 $10 / 31 / 1116: 00$ 10/31/11 16:30 10/31/1117:00 10/31/11 18:00 10/31/11 18:30 10/31/11 19:00 10/31/11 19:30 10/31/11 20:00 10/31/11 20:30 10/31/11 21:00 10/31/1121:30 10/31/11 22:30 10/31/11 23:00 10/31/11 23:30 $11 / 1 / 110: 00$
$11 / 1 / 110: 30$ $\begin{array}{llll}1494.072 & 1494.539 & 1496.394 & 1494.045 \\ 1494.069 & 1494.536 & 1496.394 & 1494.038\end{array}$ $1494.069 \quad 1494.536 \quad 1496.394 \quad 1494.038$ $\begin{array}{lllll}1494.072 & 1494.534 & 1496.399 & 1494.033 \\ 1494.072 & 1494.536 & 1496399 & 1494.029\end{array}$ $\begin{array}{lllll}1494.072 & 1494.532 & 1496.401 & 1494.026\end{array}$ $\begin{array}{lllll}1494.072 & 1494.534 & 1496.403 & 14494.029\end{array}$ $\begin{array}{lllll}1494.072 & 1494.534 & 1496.403 & 1494.031\end{array}$ $\begin{array}{llllll}1494.072 & 1494.534 & 1496.41 & 1494.033\end{array}$ $\begin{array}{lllll}1494.072 & 1494.534 & 1496.415 & 1494.04\end{array}$ $\begin{array}{lllll}1494.072 & 1494.532 & 1496.424 & 1494.052\end{array}$ $\begin{array}{lllll}1494.074 & 1494.534 & 1496.429 & 1494.064 \\ 1494074 & 1994536 & 1996.334 & 1494.075\end{array}$ $\begin{array}{llll}1494.074 & 1494.536 & 1496.434 & 1494.075 \\ 1494.074 & 1494.541 & 1496.438 & 1494.092\end{array}$ $\begin{array}{lllll}1494.076 & 1494.543 & 1496.438 & 1494.092\end{array}$ $\begin{array}{rrrrr}1494.074 & 1494.543 & 1496.438 & 1494.12\end{array}$ $\begin{array}{rrrr}1494.074 & 1494.546 & 1496.441 & 1494.131 \\ 14964 & 1494.13\end{array}$ $\begin{array}{llllll}1494.074 & 1494.548 & 1496.441 & 1494.145\end{array}$ $\begin{array}{lllll}1494.074 & 1494.548 & 1496.441 & 1494.152\end{array}$ $\begin{array}{llll}1494.072 & 1494.55 & 1496.436 & 1494.152\end{array}$ $\begin{array}{llll}1494.072 & 1494.55 & 1496.436 & 1494.145 \\ 1494.069 & 1494548 & 1496.429 & 1494.138\end{array}$ $\begin{array}{llll}1494.069 & 1494.548 & 1496.429 & 1494.138 \\ 1494.548 & 1496.431 & 1494.134\end{array}$ $\begin{array}{llllll}1494.067 & 1494.548 & 1496.436 & 1494.131\end{array}$ $\begin{array}{lllll}1494.065 & 1494.541 & 1496.441 & 1494.129\end{array}$ $\begin{array}{lllll}1494.069 & 1494.546 & 1496.438 & 1494.136\end{array}$ $\begin{array}{lllll}1494.069 & 1494.546 & 1496.443 & 1494.143\end{array}$ $\begin{array}{rrrr}1494.072 & 1494.55 & 1496.45 & 1494.152\end{array}$ $\begin{array}{rrrr}1494.069 & 1494.553 & 1496.462 & 1494.157\end{array}$ $\begin{array}{rrrrr}1494.069 & 1494.55 & 1496.476 & 1494.166 \\ 1494.072 & 1494.557 & 1496.494 & 1494.176\end{array}$ $\begin{array}{lllll}1494.076 & 1494.567 & 1496.506 & 1494.18\end{array}$ $\begin{array}{llll}1494.079 & 1494.574 & 1496.515 & 1494.18\end{array}$ $1494.083 \quad 1494.585 \quad 1496.525 \quad 1494.183$ $\begin{array}{lllll}1494.083 & 1494.595 & 1496.529 & 1494.19\end{array}$ $\begin{array}{lllll}1494.086 & 1494.599 & 1496.532 & 1494.199\end{array}$ $\begin{array}{llll}1494.086 & 1494.606 & 1496.532 & 1494.208\end{array}$ $\begin{array}{lllll}1494.083 & 1494.606 & 1496.534 & 1494.213\end{array}$ $\begin{array}{lllll}1494.086 & 1494.616 & 1496.534 & 1494.225 \\ 1494.088 & 1494.618 & 1496.536 & 1494.234\end{array}$ $\begin{array}{lllll}1494.086 & 1494.618 & 1496.539 & 1494.243\end{array}$ $\begin{array}{lllll}1494.086 & 1494.62 & 1496.52 & 1494.246\end{array}$ $\begin{array}{lllll}1494.09 & 1494.625 & 1496.518 & 1494.246\end{array}$ $\begin{array}{lllll}1494.083 & 1494.623 & 1496.522 & 1494.236\end{array}$ $\begin{array}{llll}1494.081 & 1494.616 & 1496.508 & 1494.22\end{array}$ $\begin{array}{lllll}1494.083 & 1494.616 & 1496.497 & 1494.22\end{array}$ $\begin{array}{lllll}1494.081 & 1494.613 & 1496.501 & 1494.218\end{array}$ $\begin{array}{lllll}1494.079 & 1494.604 & 1496.494 & 1494.208\end{array}$ $\begin{array}{lllll}1494.076 & 1494.606 & 1496.497 & 1494.213\end{array}$ $\begin{array}{lllll}1494.076 & 1494.602 & 1496.494 & 1494.215\end{array}$ $\begin{array}{lllll}1494.076 & 1494.602 & 1496.506 & 1494.22\end{array}$ $\begin{array}{lllll}1494.076 & 1494.602 & 1496.511 & 1494.225\end{array}$ $\begin{array}{llll}1494.076 & 1494.599 & 1496.515 & 1494.234 \\ 1494.079 & 1494.606 & 1496.522 & 1494.246\end{array}$

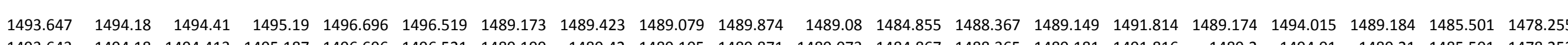

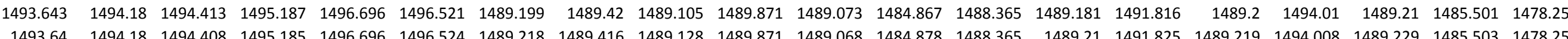

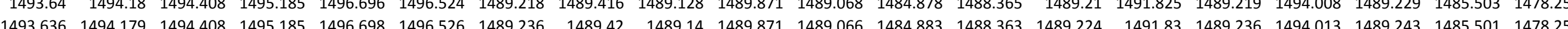
$\begin{array}{lllllllllllllllllllllll}1493.636 & 1494.177 & 1494.403 & 1495.183 & 1496.696 & 1496.531 & 1489.765 & 1489.423 & 1489.485 & 1489.871 & 1489.068 & 1484.895 & 1488.363 & 1489.569 & 1491.875 & 1489.788 & 1494.01 & 1489.778 & 1485.501 & 1478.25\end{array}$ $\begin{array}{lllllllllllllllllllllll}1493.636 & 1494.175 & 1494.408 & 1495.183 & 1496.698 & 1496.528 & 1490.074 & 1489.418 & 1489.799 & 1489.871 & 1489.098 & 1484.904 & 1488.36 & 1489.887 & 1492.115 & 1490.094 & 1494.027 & 1490.078 & 1485.501 & 1478.25\end{array}$ $\begin{array}{lllllllllllllllllllllllll}1493.631 & 1494.177 & 1494.406 & 1495.183 & 1496.696 & 1496.531 & 1490.278 & 1489.418 & 1490.019 & 1489.871 & 1489.133 & 1484.911 & 1488.363 & 1490.11 & 1492.194 & 1490.295 & 1494.036 & 1490.277 & 1485.501 & 1478.25 & 149.25\end{array}$ \begin{tabular}{lllllllllllllllllllllll}
1493.629 & 1494.177 & 1494.406 & 1495.183 & 1496.701 & 1496.536 & 1490.434 & 1489.42 & 1490.188 & 1489.871 & 1489.168 & 1484.92 & 1488.36 & 1490.279 & 1492.271 & 1490.449 & 1494.048 & 1490.435 & 1485.501 & 1478.248 \\
\hline
\end{tabular}

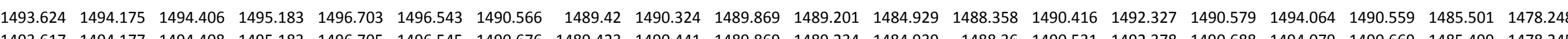

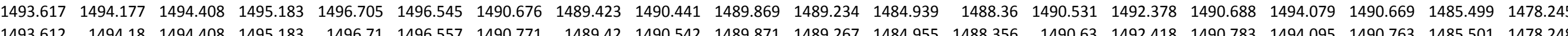

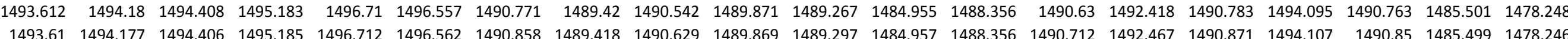

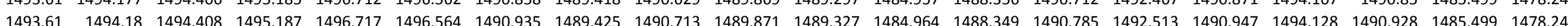
$\begin{array}{llllllllllllllllllll}1493.607 & 1494.177 & 1494.403 & 1495.19 & 1496715 & 1496.569 & 1491.005 & 1489.425 & 1490784 & 1489.871 & 1489355 & 1484.973 & 1488349 & 1490.853 & 1492.546 & 1491.013 & 1494.144 & 1490.996 & 1485.499 & 1478.248\end{array}$ $\begin{array}{llllllllllllllllllllll}1493.607 & 1494.177 & 1494.401 & 1495.192 & 1496.715 & 1496.571 & 1491.069 & 1489.427 & 1490.849 & 1489.871 & 1489.386 & 1484.985 & 1488.356 & 1490.921 & 1492.583 & 1491.077 & 1494.156 & 1491.054 & 1485.499 & 1478.248\end{array}$ $\begin{array}{lllllllllllllllllllll}1493.61 & 1494.177 & 1494.406 & 1495.192 & 1496.719 & 1496.571 & 1491.127 & 1489.427 & 1490.91 & 1489.871 & 1489.414 & 1484.987 & 1488.356 & 1490.977 & 1492.611 & 1491.136 & 1494.168 & 1491.115 & 1485.499 & 1478.248\end{array}$

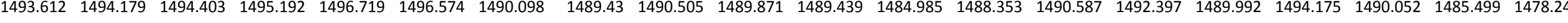

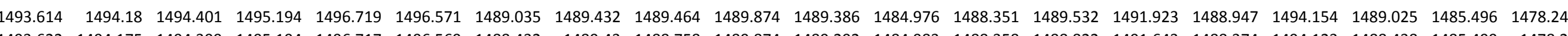

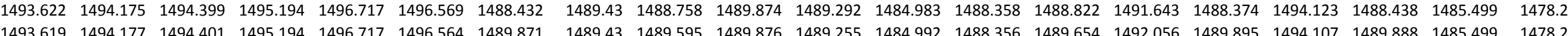
$\begin{array}{llllllllllllllllllllll}1493.619 & 1494.177 & 1494.401 & 1495.194 & 1496.717 & 1496.564 & 1489.871 & 1489.43 & 1489.595 & 1489.87 & 1489.255 & 1484.992 & 1488.356 & 1489.654 & 1492.056 & 1489.895 & 1494.107 & 1489.888 & 1485.499 & 1478.25 \\ 1493.624 & 1494.177 & 1494.401 & 1495.194 & 1496715 & 1496564 & 1490264 & 1489.43 & 1490005 & 1489871 & 1489274 & 1485.001 & 148836 & 1490072 & 1492233 & 1490283 & 1494.109 & 1490271 & 1485.499 & 147825\end{array}$ $\begin{array}{lllllllllllllllllllll}1493.624 & 1494.177 & 1494.401 & 1495.194 & 1496.715 & 1496.56 & 1490.505 & 1489.425 & 1490.256 & 1489.871 & 1489.299 & 1485.01 & 1488.36 & 1490.326 & 1492.329 & 1490.518 & 1494.119 & 1490.503 & 1485.499 & 1478.252 \\ \end{array}$ \begin{tabular}{llllllllllllllllllllll}
1493.619 & 1494.175 & 1494.406 & 1495.192 & 1496.719 & 1496.56 & 1490.673 & 1489.432 & 1490.434 & 1489.871 & 1489.327 & 1485.022 & 148836 & 1490.496 & 1492.399 & 1490.688 & 1494.126 & 1490.669 & 1485.496 & 1478.252 \\
\hline
\end{tabular} $\begin{array}{llllllllllllllllllllll}1493.614 & 1494.175 & 1494.399 & 1495.192 & 1496.719 & 1496.564 & 1490.804 & 1489.427 & 1490.573 & 1489.874 & 1489.355 & 1485.031 & 1488.36 & 1490.625 & 1492.457 & 1490.819 & 1494.135 & 1490.796 & 1485.496 & 1478.252\end{array}$

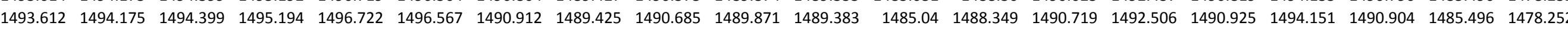

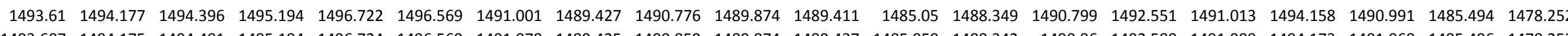

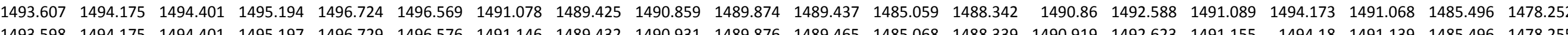

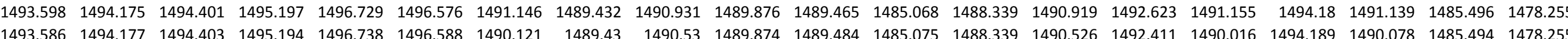

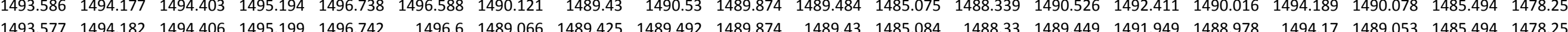
$\begin{array}{lllllllllllllllllllll}1493.57 & 1494.185 & 1494.403 & 1495.206 & 1496.752 & 1496.617 & 1488.467 & 1489.432 & 1488.793 & 1489.876 & 1489.337 & 1485.096 & 1488.32 & 1488.723 & 1491.674 & 1488.409 & 1494.149 & 1488.475 & 1485.494 & 1478.257\end{array}$ $\begin{array}{llllllllllllllllllllll}1493.563 & 1494.187 & 1494.403 & 1495.211 & 1496.759 & 1496.631 & 1489.91 & 1489.441 & 1489.632 & 1489.876 & 1489.297 & 1485.1 & 1488.311 & 1489.529 & 1492.1 & 1489.935 & 1494.149 & 1489.926 & 1485.492 & 1478.257\end{array}$ $\begin{array}{lllllllllllllllllllllll}1493.563 & 1494.191 & 1494.406 & 1495.218 & 1496.766 & 1496.636 & 1490.311 & 1489.437 & 1490.047 & 1489.878 & 1489.316 & 1485.107 & 1488.302 & 1489.915 & 1492.289 & 1490.326 & 1494.168 & 1490.313 & 1485.494 & 1478.259\end{array}$ $\begin{array}{lllllllllllllllllllll}1493.563 & 1494.191 & 1494.401 & 1495.225 & 1496.77 & 1496.646 & 1490.552 & 1489.444 & 1490.301 & 1489.885 & 1489.341 & 1485.119 & 1488.297 & 1490.148 & 1492.397 & 1490.568 & 1494.189 & 1490.55 & 1485.494 & 1478.259\end{array}$ $\begin{array}{llllllllllllllllllll}1493.565 & 1494.197 & 1494.403 & 1495.231 & 1496.773 & 1496.646 & 1490.722 & 1489.451 & 1490.483 & 1489.885 & 1489.369 & 1485.124 & 1488.292 & 1490.31 & 1492.474 & 1490.741 & 1494.205 & 1490.719 & 1485.492 & 1478.262\end{array}$

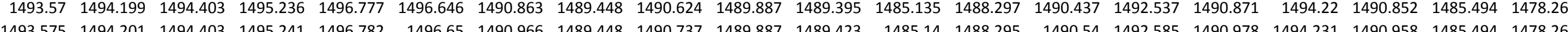

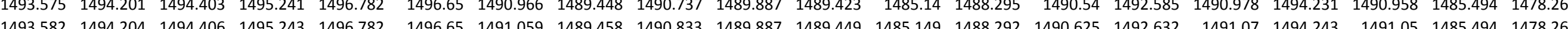

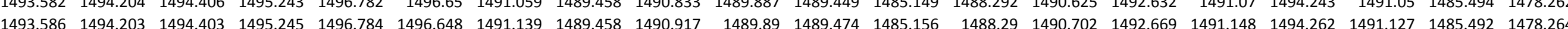
$\begin{array}{lllllllllllllllllllll}1493.589 & 1494.206 & 1494.403 & 1495.25 & 1496.787 & 1496.65 & 1489.489 & 1489.455 & 1489.846 & 1489.892 & 1489.463 & 1485.158 & 1488.295 & 1489.635 & 1492.131 & 1489.428 & 1494.25 & 1489.503 & 1485.494 & 1478.264\end{array}$

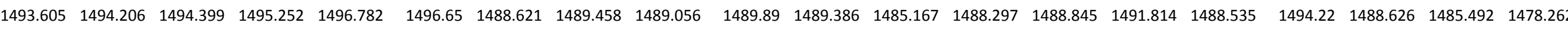

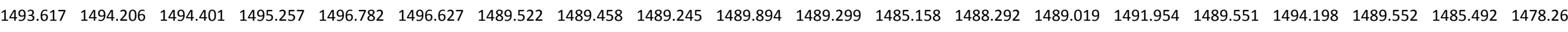

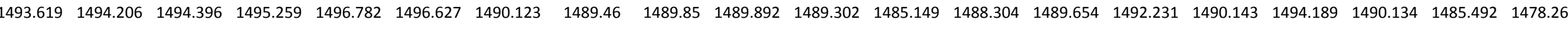

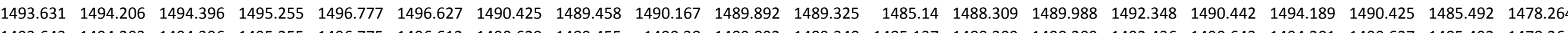
$\begin{array}{llllllllllllllllllll}1493.643 & 1494.203 & 1494.396 & 1495.255 & 1496.775 & 1496.612 & 1490.629 & 1489.455 & 1490.38 & 1489.892 & 1489.348 & 1485.137 & 1488.309 & 1490.209 & 1492.436 & 1490.643 & 1494.201 & 1490.627 & 1485.492 & 1478.264 \\ 1493.647 & 1494201 & 1494.401 & 1495255 & 1496775 & 1496 & 1490781 & 1489455 & 1490542 & 1489892 & 1489376 & 1485.14 & 1488311 & 149038 & 1492502 & 1490795 & 149421 & 1490775 & 1485.492 & 1478264\end{array}$

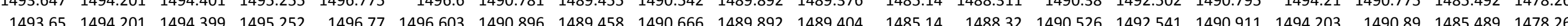
$\begin{array}{llllllllllllllllllll}1493.655 & 1494.199 & 1494.396 & 1495.25 & 1496.773 & 1496.598 & 1490.996 & 1489.448 & 1490.769 & 1489.89 & 1489.43 & 1485.151 & 1488.323 & 1490.648 & 1492.581 & 1491.006 & 1494.213 & 1490.986 & 1485.489 & 1478.264\end{array}$ $\begin{array}{llllllllllllllllllllll}1493.654 & 1494.199 & 1494.394 & 1495.25 & 1496.77 & 1496.598 & 1491.078 & 1489.446 & 1490.856 & 1489.89 & 1489.453 & 1485.161 & 1488.327 & 1490.74 & 1492.62 & 1491.091 & 1494.22 & 1491.068 & 1485.489 & 1478.264\end{array}$

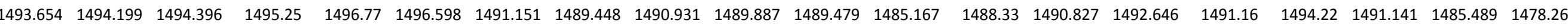

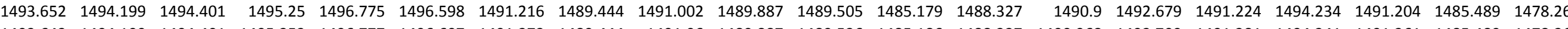
$\begin{array}{llllllllllllllllllll}1493.643 & 1494.199 & 1494.401 & 1495.252 & 1496.777 & 1496.607 & 1491.272 & 1489.444 & 1491.06 & 1489.887 & 1489.526 & 1485.186 & 1488.327 & 1490.968 & 1492.709 & 1491.281 & 1494.241 & 1491.261 & 1485.489 & 1478.264\end{array}$

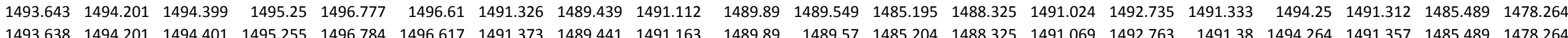


Well

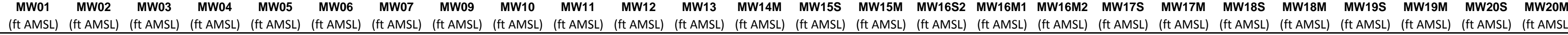

11/1/11 1:00 $11 / 1 / 111: 30$ $11 / 1 / 112: 00$ 11/1/11 3:00 11/1/11113:00 $11 / 1 / 114: 00$ 11/1/11 4:30 11/1/11 5:00 $11 / 1 / 115: 30$ 11/1/111 6:00 $11 / 1 / 117 \cdot 00$ 11/1/11 7:30 11/1/1111 8:00 $11 / 1 / 118: 30$ 11/1/11 9:00 11/1/1119:30 11/1/11 10:00 11/1/11111:00 11/1/11 11:00 11/1/11 12:00 11/1/111 12:30 11/1/11 13:00 11/1/11 13:30 11/1/111 14:00 11/1/11 14:30 11/1/11 15:00 11/1/11 16:00 11/1/11 16:30 11/1/11 17:00 11/1/11 17:30 11/1/11 18:00 11/1/111 18:30 $11 / 1 / 1119: 00$ 11/1/1111 $19: 30$ $11 / 1 / 1120: 30$ 11/1/111 21:00 11/1/11 21:30 11/1/11 22:00 11/1/11 22:30 11/1/11 23:00 $11 / 2 / 110.00$ 11/2/11 0:00 $11 / 2 / 110: 30$ 11/2/111:30 11/2/112:00 11/2/11 2:30 11/2/11 3:00 $\begin{array}{llll}1494.079 & 1494.606 & 1496.527 & 1494.255\end{array}$ $\begin{array}{llll}1494.081 & 1494.611 & 1496.532 & 1494.264\end{array}$ $\begin{array}{lllll}1494.081 & 194.613 & 1996.534 & 1494.276\end{array}$ $\begin{array}{llllll}1494.081 & 1494.62 & 1496.546 & 1494.295\end{array}$ $\begin{array}{lllll}1494.083 & 1494.623 & 1496.544 & 1494.304\end{array}$ $\begin{array}{lllll}1494.086 & 1494.627 & 1496.5444 & 1494.316\end{array}$ $\begin{array}{lllll}1494.088 & 1494.63 & 1496.536 & 1494.325\end{array}$ $\begin{array}{lllll}1494.086 & 1494.63 & 1496.536 & 1494.332\end{array}$ $\begin{array}{rrrr}1494.086 & 1494.634 & 1496.539 & 1494.341 \\ 1494083 & 1494.63 & 1466536 & 194344\end{array}$ $\begin{array}{llll}1494.083 & 1494.63 & 1496.536 & 1494.344\end{array}$ $\begin{array}{llll}1494.083 & 1494.627 & 1496.532 & 1494.344 \\ 1494.086 & 1494.632 & 1496.522 & 1494.341\end{array}$ $\begin{array}{lllll}1494.086 & 1494.63 & 1496.515 & 1494.327\end{array}$ $\begin{array}{lllll}1494.083 & 1494.627 & 1496.513 & 1494.311\end{array}$ $\begin{array}{llllll}1494.081 & 1494.623 & 1496.513 & 1494.299\end{array}$ $\begin{array}{lllll}1494.079 & 1494.618 & 1496.508 & 1494.29\end{array}$ $\begin{array}{llll}1494.079 & 1494.616 & 1496.511 & 1494.285\end{array}$ $\begin{array}{lllll}1494.079 & 1494.616 & 1496.513 & 1494.281\end{array}$ $\begin{array}{llll}1494.079 & 1494.613 & 1496.518 & 1494.281 \\ 1494.081 & 1494.616 & 149652 & 1994.283\end{array}$ $\begin{array}{rrrr}1494.081 & 1494.616 & 1496.52 & 1494.283 \\ 1494.079 & 1494.616 & 1496.527 & 1494.288\end{array}$ $\begin{array}{llllll}1494.081 & 1494.618 & 1496.534 & 1494.295\end{array}$ $\begin{array}{lllll}1494.083 & 1494.618 & 1496.546 & 1494.297\end{array}$ $\begin{array}{lllll}1494.083 & 1494.618 & 1496.544 & 1494.295\end{array}$ $\begin{array}{lllll}1494.083 & 1494.623 & 1496.548 & 1494.288\end{array}$ $\begin{array}{llll}1494.088 & 1494.627 & 1496.551 & 1494.285\end{array}$ $\begin{array}{lllll}1494.086 & 1494.63 & 1496.558 & 1494.281\end{array}$ $\begin{array}{rrrr}1494.088 & 1494.63 & 1496.56 & 1494.281 \\ 1494.09 & 1494.632 & 1496555 & 1494285\end{array}$ $\begin{array}{lllll}1494.09 & 1494.637 & 1496.551 & 1494.29\end{array}$ $\begin{array}{lllll}1494.088 & 1494.639 & 1496548 & 1494.295\end{array}$ $\begin{array}{llllll}1494.088 & 1494.637 & 1496.541 & 1494.297\end{array}$ $\begin{array}{lllll}1494.088 & 1494.637 & 1496.539 & 1494.302\end{array}$ $\begin{array}{lllll}1494.088 & 1494.634 & 1496.525 & 1494.299\end{array}$ $\begin{array}{llllll}1494.086 & 1494.632 & 1496.515 & 1494.288\end{array}$ $\begin{array}{lllll}1494.083 & 1494.63 & 1496.508 & 1494.274\end{array}$ $\begin{array}{lllll}1494.081 & 1494.625 & 1496.494 & 1494.253 \\ 1494.079 & 1494.618 & 1496.494 & 1494236\end{array}$ $\begin{array}{lllll}1494.076 & 1494.613 & 1496.49 & 1494.22\end{array}$ $\begin{array}{lllll}1494.074 & 1494.606 & 1496.483 & 1494.211\end{array}$ $\begin{array}{lllll}1494.076 & 1494.606 & 1496.485 & 1494.208\end{array}$ $1494.072 \quad 1494.599 \quad 1496.483 \quad 1494.204$ $\begin{array}{lllll}1494.072 & 1494.597 & 1496.49 & 1494.199\end{array}$ $\begin{array}{llll}1494.074 & 1494.599 & 1496.49 & 1494.204\end{array}$ $\begin{array}{llll}1494.074 & 1494.597 & 1496.499 & 1494.208\end{array}$ $\begin{array}{lllll}1444.074 & 1494.597 & 1496.497 & 1494.222\end{array}$ $\begin{array}{lllll}1494.074 & 1494.595 & 1496.487 & 1494.229\end{array}$ $\begin{array}{lllll}1494.076 & 1494.599 & 1496.494 & 1494.239\end{array}$ $\begin{array}{lllll}1494.072 & 1494.595 & 1496.499 & 1494.243\end{array}$ $\begin{array}{lllll}1494.072 & 1494.597 & 1496.485 & 1494.25\end{array}$ $\begin{array}{rrrr}1494.076 & 1494.599 & 1496.483 & 1494.26 \\ 1494.074 & 1494.595 & 1496.48 & 1494.264\end{array}$

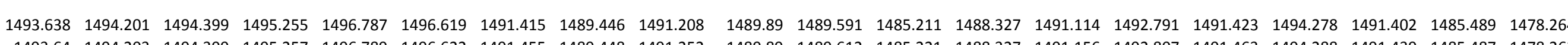

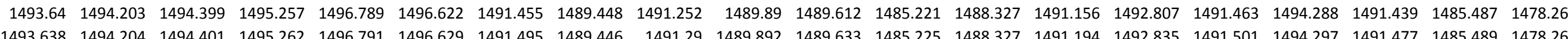

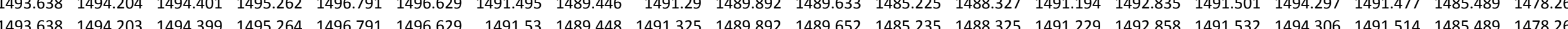
$\begin{array}{lllllllllllllllllllll}1493.636 & 1494.206 & 1494.399 & 1495.266 & 1496.796 & 1496.636 & 1491.562 & 1489.48 & 1491.358 & 1489.892 & 1489.668 & 1485241 & 1488.327 & 1491.262 & 1492.872 & 1491.57 & 1494.318 & 1491.545 & 1485.489 & 1478.264\end{array}$ $\begin{array}{llllllllllllllllllllllll}1493.636 & 1494208 & 1494.401 & 1495269 & 1496.798 & 1496.638 & 1491.59 & 1489.453 & 1491393 & 1489.892 & 1489.689 & 1485251 & 148833 & 1491295 & 1492.898 & 1491.601 & 1494.325 & 1491.577 & 1485.487 & 1478.264\end{array}$ $\begin{array}{lllllllllllllllllllllll}1493.64 & 1494.208 & 1494.399 & 1495.273 & 1496.798 & 1496.638 & 1491.623 & 1489.451 & 1491.424 & 1489.894 & 1489.706 & 1485.258 & 1488.327 & 1491.323 & 1492.914 & 1491.629 & 1494.337 & 1491.606 & 1485.489 & 1478.264\end{array}$ $\begin{array}{lllllllllllllllllllll}1493.643 & 1494.211 & 1494.399 & 1495.278 & 1496.798 & 1496.634 & 1491.654 & 1489.453 & 1491.454 & 1489.897 & 1489.725 & 1485.265 & 1488.327 & 1491.351 & 1492.933 & 1491.658 & 1494.349 & 1491.634 & 1485.487 & 1478.264\end{array}$

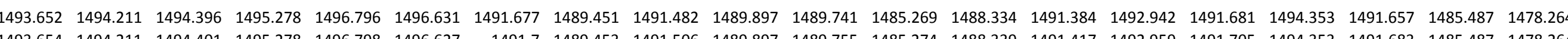

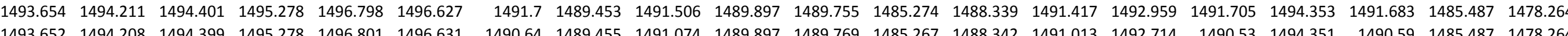

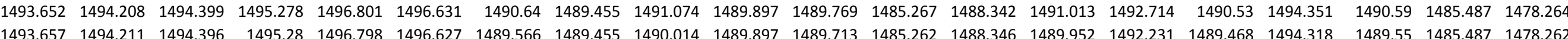

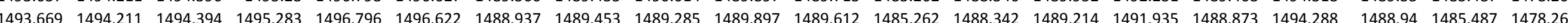
$\begin{array}{llllllllllllllllllll}1493.673 & 1494.211 & 1494.391 & 1495283 & 1496789 & 149661 & 1490348 & 1489.453 & 1490.9 & 1489894 & 1489.568 & 1485272 & 1488353 & 1490.023 & 1492348 & 1490.369 & 1494.269 & 1490.364 & 1485.485 & 1478264\end{array}$ $\begin{array}{llllllllllllllllllllll}1493.678 & 1494.208 & 1494.394 & 1495.283 & 1496.787 & 1496.603 & 1490.722 & 1489.448 & 1490.476 & 1489.894 & 1489.577 & 1485.278 & 1488.356 & 1490.429 & 1492.506 & 1490.738 & 1494.26 & 1490.723 & 1485.485 & 1478.264\end{array}$ $\begin{array}{llllllllllllllllllll}1493.678 & 1494.208 & 1494.396 & 1495.278 & 1496.787 & 1496.598 & 1490.938 & 1489.448 & 1490.706 & 1489.892 & 1489.591 & 1485.285 & 1488.358 & 1490.667 & 1492.592 & 1490.952 & 1494.262 & 1490.935 & 1485.485 & 1478.264\end{array}$ $\begin{array}{llllllllllllllllllllll}1493.68 & 1494.208 & 1494.391 & 1495.278 & 1496.78 & 1496.598 & 1491.092 & 1489.446 & 1490.863 & 1489.892 & 1489.612 & 1485.295 & 1488.36 & 1490.829 & 1492.653 & 1491.098 & 1494.262 & 1491.08 & 1485.485 & 1478.264\end{array}$

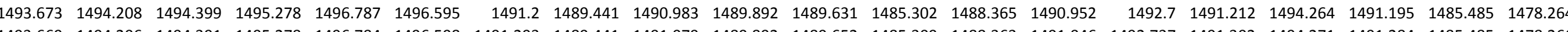

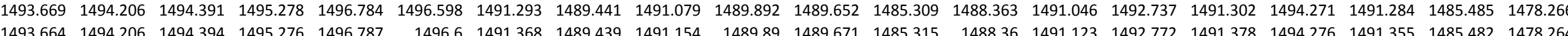

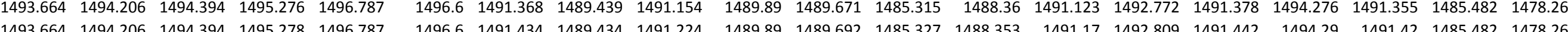
$\begin{array}{lllllllllllllllllllll}1493.659 & 1494.206 & 1494.394 & 1495278 & 1496.789 & 1496.605 & 1491.485 & 1489.437 & 1491.281 & 1489.892 & 1489.711 & 1485.343 & 1488.356 & 1491.227 & 1492.826 & 1491.494 & 1494.295 & 1491.472 & 1485.482 & 1478.266\end{array}$ $\begin{array}{lllllllllllllllllllll}1493.647 & 1494.211 & 1494.396 & 1495.28 & 1496.798 & 1496.615 & 1489.396 & 1489.439 & 1489.836 & 1489.892 & 1489.668 & 1485.348 & 1488.351 & 1489.762 & 1492.147 & 1489.302 & 1494.278 & 1489.381 & 1485.482 & 1478.266\end{array}$

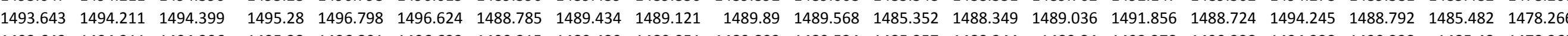

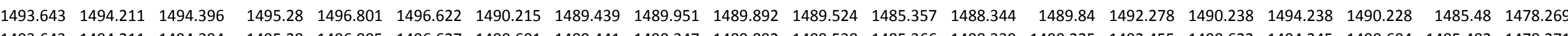

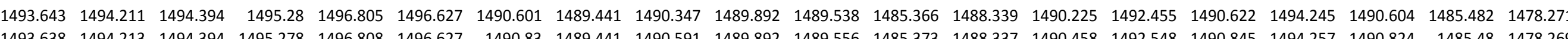

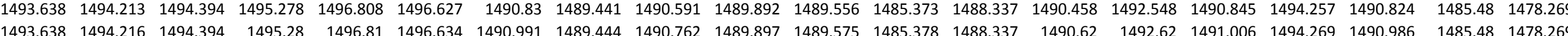
$\begin{array}{llllllllllllllllllll}1493.638 & 1494.216 & 1494.394 & 1495.28 & 1496.81 & 1496.634 & 1490.991 & 1489.444 & 1490.762 & 1489.897 & 1489.57 & 1485.37 & 1488.337 & 1490.62 & 1492.62 & 1491.006 & 1494.269 & 1490.986 & 1485.48 & 1478.269 \\ 1493.643 & 1494.215 & 1494.396 & 1495.283 & 1496.812 & 1496.636 & 1491.115 & 1489.446 & 1490.891 & 1489.897 & 1489.601 & 1485387 & 1488.327 & 1490.738 & 1492.679 & 1491.127 & 1494.285 & 1491.108 & 1485.48 & 1478271\end{array}$

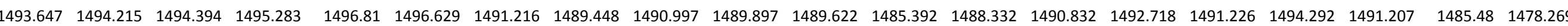
$\begin{array}{lllllllllllllllllllll}1493.654 & 1494.218 & 1494.394 & 1495.283 & 1496.808 & 1496.624 & 1491.298 & 1489.451 & 1491.081 & 1489.899 & 1489.645 & 1485.357 & 1488.33 & 1490.921 & 1492.763 & 1491.309 & 1494.299 & 1491.287 & 1485.48 & 1478.271 \\ & 1435 & & \end{array}$

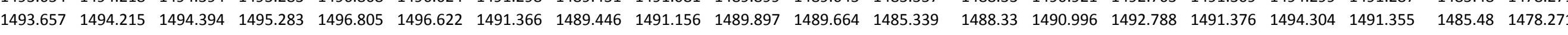
$\begin{array}{lllllllllllllllllllll}1493.666 & 1494.216 & 1494.391 & 1495.285 & 1496.805 & 1496.615 & 1489.737 & 1489.448 & 1490.08 & 1489.897 & 1489.65 & 1485.341 & 1488.334 & 1489.931 & 1492.24 & 1489.677 & 1494.29 & 1489.75 & 1485.478 & 1478.271 \\ \end{array}$

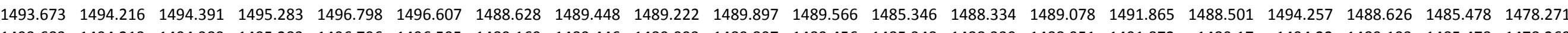

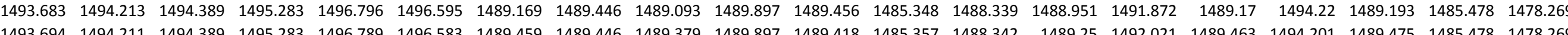

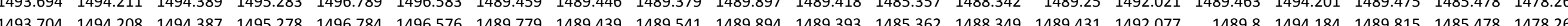

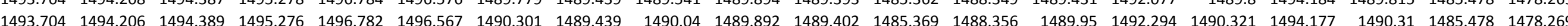
$\begin{array}{llllllllllllllllllllll}1493.706 & 1494.203 & 1494.387 & 1495.273 & 1496.777 & 1496.564 & 1490.559 & 1489.432 & 1490.31 & 1489.89 & 1489.425 & 1485.378 & 1488.356 & 1490.244 & 1492.397 & 1490.575 & 1494.18 & 1490.561 & 1485.475 & 1478.266\end{array}$ $\begin{array}{lllllllllllllllllllll}1493.706 & 1494.201 & 1494.391 & 1495.269 & 1496.775 & 1496.557 & 1490.741 & 1489.43 & 1490.497 & 1489.89 & 1489.451 & 1485.385 & 1488.358 & 1490.446 & 1492.469 & 1490.752 & 1494.18 & 1490.737 & 1485.478 & 1478.266\end{array}$

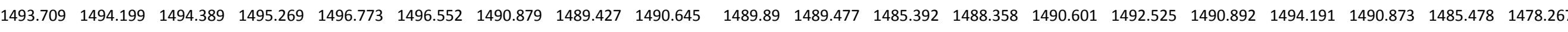
$\begin{array}{llllllllllllllllllll}1493.704 & 1494.199 & 1494.387 & 1495.264 & 1496.77 & 1496.555 & 1490.989 & 1489.43 & 1490.765 & 1489.887 & 1489.503 & 1485.396 & 1488.36 & 1490.738 & 1492.574 & 1491.001 & 1494.194 & 1490.984 & 1485.475 & 1478.266\end{array}$

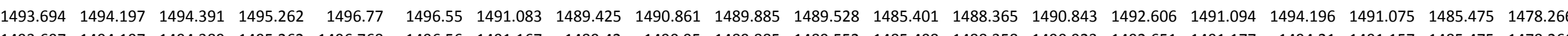

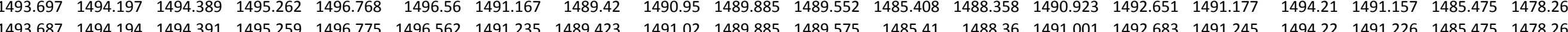

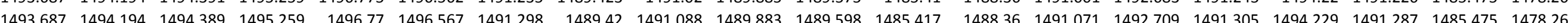
$\begin{array}{llllllllllllllllllll}1493.685 & 1494.194 & 1494.389 & 1495.259 & 1496.773 & 1496.567 & 1491.354 & 1489.427 & 1491.147 & 1489.883 & 1489.619 & 1485.424 & 1488.358 & 1491.128 & 1492.742 & 1491.364 & 1494.236 & 1491.343 & 1485.475 & 1478.266\end{array}$ $\begin{array}{llllllllllllllllllllll}1493.692 & 1494.191 & 1494.389 & 1495.262 & 1496.768 & 1496.567 & 1491.403 & 1489.423 & 1491.196 & 1489.883 & 1489.64 & 1485.426 & 1488.356 & 1491.177 & 1492.765 & 1491.413 & 1494.248 & 1491.392 & 1485.473 & 1478.266\end{array}$

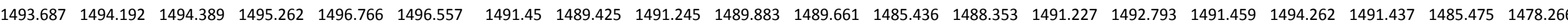
$\begin{array}{llllllllllllllllllll}1493.683 & 1494.192 & 1494.391 & 1495.259 & 1496.77 & 1496.564 & 1491.49 & 1489.423 & 1491.29 & 1489.883 & 1489.68 & 1485.44 & 1488.37 & 1491.283 & 1492.805 & 1491.499 & 1494.26 & 1491.479 & 1485.473 & 1478.264 \\ \end{array}$

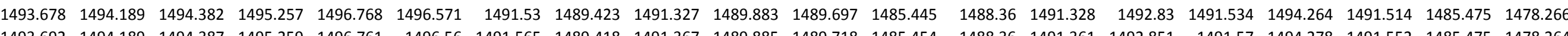

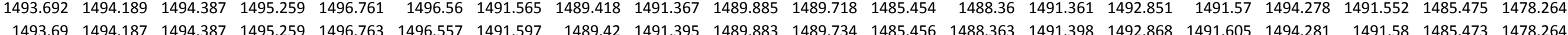


Well

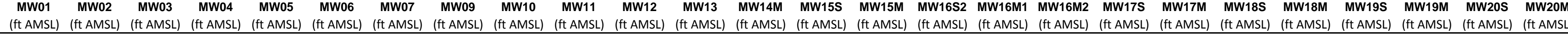

11/2/11 4:00 $11 / 2 / 114: 30$ $11 / 2 / 115: 00$ 11/2/1116:00 11/2/11116:00 11/2/11 6:30 11/2/117:30 11/2/11 8:00 $11 / 2 / 118: 30$ 11/2/11 9:00 $11 / 2 / 1110: 00$ $11 / 2 / 1110: 30$ 11/2/1111:00 11/2/11 11:30 $11 / 2 / 1112: 00$ 11/2/111 12:30 11/2/11 13:00 11/2/11 13:30 $11 / 2 / 1114: 30$ 11/2/11 15:00 $11 / 2 / 1116: 00$ 11/2/11 16:30 11/2/111 17:00 $11 / 2 / 1117: 30$ $11 / 2 / 1118: 00$ $11 / 2 / 1119: 00$ 11/2/11 19:30 11/2/11 20:00 11/2/11 20:30 11/2/11 21:00 11/2/1121:30 11/2/1122:00 $11 / 2 / 1122: 30$ 11/2/2/11 23:30 $11 / 3 / 110: 00$ $11 / 3 / 110: 30$ 11/3/11 1:00 11/3/11 1:30 $11 / 3 / 112: 00$
$11 / 3 / 112: 30$ 11/3/113:00 $11 / 3 / 113: 30$ $11 / 3 / 114: 30$ $11 / 3 / 115: 00$ $11 / 3 / 115: 30$ 11/3/11 6:00 $\begin{array}{rrrr}1494.065 & 1494.59 & 1496.473 & 1494.267 \\ 1494.069 & 1494.592 & 1496.464 & 1494.274\end{array}$ $1494.0691494 .592 \quad 1496.464 \quad 1494.274$ $\begin{array}{llll}1494.067 & 1494.588 & 1496.459 & 1494.276 \\ 1494.067 & 1494583 & 1496.457 & 1494.278\end{array}$ $\begin{array}{lllll}1494.062 & 1494.578 & 1496.441 & 1494.276\end{array}$ $\begin{array}{lllll}1494.062 & 1494.578 & 1496.436 & 1994.274\end{array}$ $\begin{array}{lllll}1494.06 & 1494.576 & 1496.417 & 1494.264\end{array}$ $\begin{array}{lllll}1494.058 & 1494.574 & 1496.403 & 1494.253\end{array}$ $\begin{array}{lllll}1494.055 & 1494.564 & 1496.399 & 1494.236\end{array}$ $\begin{array}{lllll}1494.048 & 1494.553 & 1496.392 & 1494.222\end{array}$ $\begin{array}{llll}1494.048 & 1494.546 & 1496.375 & 1494.211 \\ 1494.051 & 1494.543 & 1996373 & 1494.201\end{array}$ $\begin{array}{llll}1494.051 & 1494.543 & 1496.373 & 1494.201 \\ 1494.048 & 1494.541 & 1496354 & 1494.199\end{array}$ $\begin{array}{lllll}1494.044 & 1494.532 & 1496.359 & 1494.194\end{array}$ $\begin{array}{llll}1494.046 & 1494.529 & 1496.349 & 1494.19\end{array}$ $\begin{array}{rrrrr}1494.046 & 1494.529 & 1496.349 & 1494.19 \\ 1494.041 & 1494.518 & 1496.345 & 1494.183\end{array}$ $\begin{array}{lllll}1494.046 & 1494.515 & 1496.349 & 1494.185\end{array}$ $\begin{array}{lllll}1494.041 & 1494.511 & 1466.357 & 1494.183\end{array}$ $\begin{array}{llll}1494.042 & 1494.504 & 1496.347 & 1494.178\end{array}$ $\begin{array}{llll}1494.046 & 1494.508 & 1496.342 & 1494.192 \\ 1494.051 & 1494508 & 1496329 & 1494.192\end{array}$ $\begin{array}{rrrr}1494.051 & 1494.508 & 1496.329 & 1494.192 \\ 1494.041 & 1494.501 & 1496.317 & 1494.19\end{array}$ $\begin{array}{lllll}1494.044 & 1494.501 & 1496.3 & 1494.192\end{array}$ $\begin{array}{lllll}1494.034 & 1494.49 & 1496.298 & 1494.185\end{array}$ $\begin{array}{lllll}1494.03 & 1494.476 & 1496.289 & 1494.178\end{array}$ $\begin{array}{lllll}1494.034 & 1494.473 & 1496.272 & 1494.176\end{array}$ $\begin{array}{llll}1494.032 & 1494.469 & 1496.272 & 1494.171\end{array}$ $\begin{array}{lllll}1494.032 & 1494.464 & 1496.258 & 1494.169\end{array}$ $\begin{array}{rrrrr}1494.023 & 1494.45 & 1496.244 & 1494.159 \\ 1494.023 & 1494.445 & 1496.244 & 1494.157\end{array}$ $\begin{array}{lllll}1494.021 & 1494.438 & 1496.233 & 1494.145\end{array}$ $\begin{array}{llllll}1494.013 & 1494.424 & 1496.221 & 1494.141\end{array}$ $\begin{array}{lllll}1494.02 & 1494.42 & 1496.209 & 1494.141\end{array}$ $\begin{array}{lllll}1494.013 & 1494.41 & 1496.214 & 1494.131\end{array}$ $\begin{array}{llll}1494.011 & 1494.406 & 1496.209 & 1494.124\end{array}$ $\begin{array}{llll}1494.009 & 1494.394 & 1496.212 & 1494.12\end{array}$ $\begin{array}{lllll}1494.016 & 1494.396 & 1496.223 & 1494.122\end{array}$ $\begin{array}{rrrr}1494.009 & 1494.387 & 1466.228 & 1494.115 \\ 1494.016 & 1494.387 & 1496228 & 1494.12\end{array}$ $\begin{array}{llll}1494.018 & 1494.389 & 1496.235 & 1494.124\end{array}$ $\begin{array}{lllll}1494.018 & 1494.387 & 1496.249 & 1494.129\end{array}$ $\begin{array}{rllll}1494.016 & 1494.387 & 1496.242 & 1494.134\end{array}$ $\begin{array}{lllll}1494.02 & 1494.392 & 1496.251 & 1494.143\end{array}$ $\begin{array}{llll}1494.02 & 1494.392 & 1496.256 & 1494.15\end{array}$ $\begin{array}{llll}1494.018 & 1494.392 & 1496.258 & 1494.155 \\ 1494.018 & 1494.394 & 1496261 & 1494.164\end{array}$ $\begin{array}{lllll}1494.02 & 1494.394 & 146.261 & 1494.164\end{array}$ $\begin{array}{rrrr}1494.016 & 1494.399 & 1496.265 & 1494.178\end{array}$ $\begin{array}{llllll}1494.018 & 1494.401 & 1496.247 & 1494.185\end{array}$ $\begin{array}{lllll}1494.021 & 1494.403 & 1496.247 & 1494.194\end{array}$ $\begin{array}{lllll}1494.013 & 1494.399 & 1496.242 & 1494.199\end{array}$ $\begin{array}{lllll}1494.007 & 1494.394 & 1496.24 & 1494.194\end{array}$ $\begin{array}{llll}1494.009 & 1494.392 & 1496.235 & 1494.194 \\ 1494.009 & 1494389 & 1496237 & 1494.199\end{array}$

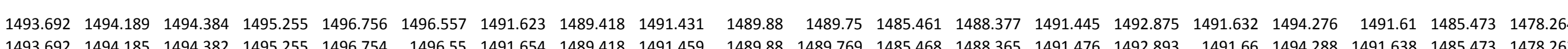

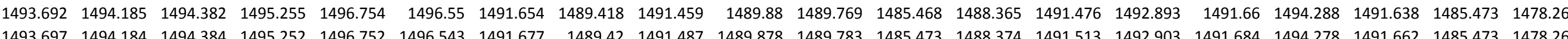

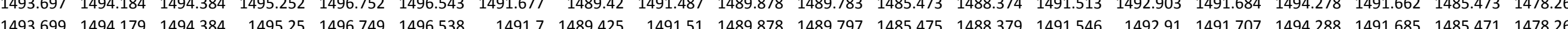

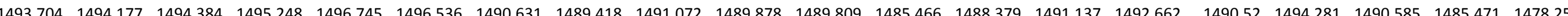
$\begin{array}{lllllllllllllllllllllll}1493.711 & 1494.172 & 1494.38 & 1495.241 & 1496.729 & 1496.516 & 1490.594 & 1489.413 & 1490.359 & 1489.876 & 1489.685 & 1485.459 & 1488.381 & 1490.413 & 1492.394 & 1490.613 & 1494.222 & 1490.608 & 1485.471 & 1478.259\end{array}$ $\begin{array}{llllllllllllllllllllll}1493.723 & 1494.173 & 1494.38 & 1495.236 & 1496.722 & 1496.504 & 1490.959 & 1489.416 & 1490.732 & 1489.874 & 1489.682 & 1485.466 & 1488.381 & 1490.801 & 1492.557 & 1490.975 & 1494.215 & 1490.965 & 1485.468 & 1478.257\end{array}$

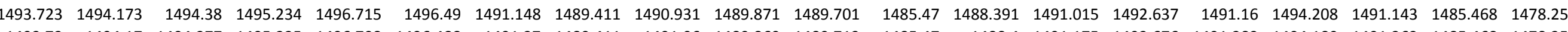

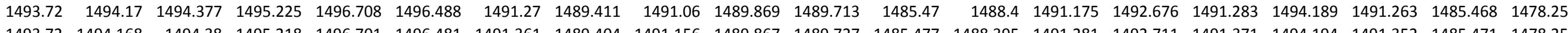

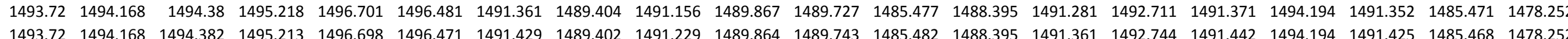

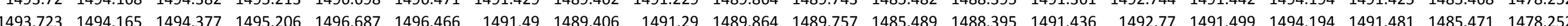
$\begin{array}{llllllllllllllllllllll}1493.723 & 1494.16 & 1494.38 & 1495.201 & 1496.682 & 1496.452 & 1491.539 & 1489.402 & 1491.344 & 1489.86 & 1489.771 & 1485.493 & 1488.4 & 1491.504 & 1492.774 & 1491.549 & 1494.191 & 1491.528 & 1485.466 & 1478.252\end{array}$ $\begin{array}{llllllllllllllllllll}1493.713 & 1494.161 & 1494.377 & 1495.194 & 1496.682 & 1496.461 & 1491.581 & 1489.402 & 1491.388 & 1489.86 & 1489.79 & 1485.5 & 1488.395 & 1491.558 & 1492.8 & 1491.591 & 1494.191 & 1491.573 & 1485.468 & 1478.255\end{array}$ $\begin{array}{llllllllllllllllllll}1493.716 & 1494.163 & 1494.375 & 1495.187 & 1496.675 & 1496.454 & 1491.619 & 1489.397 & 1491.424 & 1489.855 & 1489.806 & 1485.507 & 1488.403 & 1491.614 & 1492.805 & 1491.627 & 1494.187 & 1491.608 & 1485.466 & 1478.25\end{array}$ $\begin{array}{lllllllllllllllllllllll}1493.711 & 1494.16 & 1494.38 & 1495.187 & 1496.675 & 1496.449 & 1491.651 & 1489.397 & 1491.459 & 1489.855 & 1489.823 & 1485.51 & 1488.398 & 1491.652 & 1492.83 & 1491.66 & 1494.194 & 1491.636 & 1485.466 & 1478.252\end{array}$

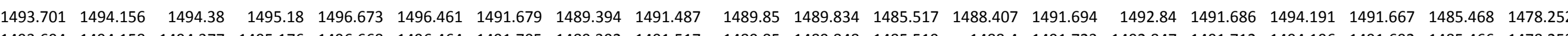

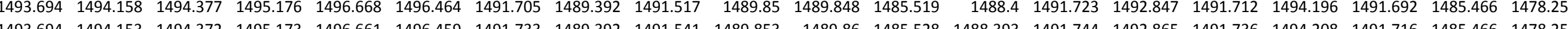
$\begin{array}{llllllllllllllllllllll}1493.694 & 1494.153 & 1494.372 & 1495.173 & 1496.661 & 1496.459 & 1491.733 & 1489.392 & 1491.541 & 1489.853 & 1489.86 & 1485.528 & 1488.393 & 1491.744 & 1492.865 & 1491.736 & 1494.208 & 1491.716 & 1485.4666 & 1478.252 \\ 1493.692 & 1494.156 & 1494.375 & 1495.173 & 1496.656 & 1496.459 & 1491.759 & 1489.392 & 1491.567 & 1489.85 & 1489879 & 1485.533 & 1488388 & 1491765 & 1492879 & 1491.762 & 149422 & 1491.739 & 1485.466 & 1478252\end{array}$

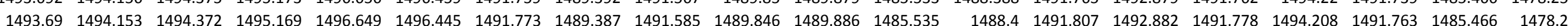
$\begin{array}{lllllllllllllllllllll}1493.709 & 1494148 & 149437 & 1495 & 964 & 1496.64 & 1496435 & 1491.794 & 1489392 & 1491.606 & 1489846 & 1489.897 & 1485542 & 1488391 & 1491821 & 1492.898 & 1491.8 & 1494.213 & 1491.784 & 1485.464 & 1478.252\end{array}$ $\begin{array}{llllllllllllllllllllll}1493.706 & 1494.146 & 1494.368 & 1495.157 & 1496.631 & 1496.418 & 1491.81 & 1489.385 & 1491.623 & 1489.846 & 1489.904 & 1485.547 & 1488.41 & 1491.871 & 1492.886 & 1491.812 & 1494.191 & 1491.793 & 1485.466 & 1478.25 \\ \end{array}$

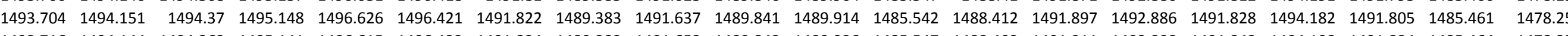
$\begin{array}{llllllllllllllllllll}1493.716 & 1494.144 & 1494.363 & 1495.141 & 1496.615 & 1496.433 & 1491.834 & 1489.383 & 1491.653 & 1489.843 & 1489.926 & 1485.547 & 1488.403 & 1491.911 & 1492.898 & 1491.842 & 1494.198 & 1491.824 & 1485.464 & 1478.25 \\ \end{array}$

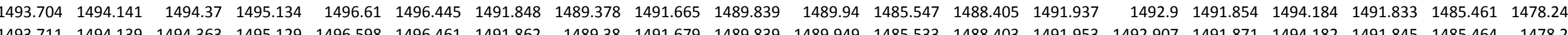

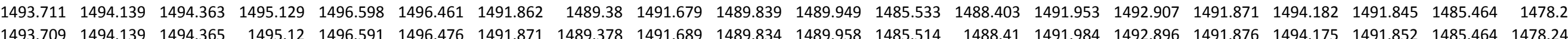
$\begin{array}{lllllllllllllllllllll}1493.709 & 1494.139 & 1494.365 & 1495.12 & 1496.591 & 1496.476 & 1491.87 & 1489.37 & 1491.689 & 1489.83 & 1489.958 & 1485.514 & 1488.41 & 1491.984 & 1492.896 & 1491.876 & 1494.175 & 1491.852 & 1485.464 & 1478.243 \\ 1493.704 & 1494.137 & 1494.37 & 1495.111 & 1496584 & 1496.497 & 1491.885 & 1489.376 & 1491.703 & 1489.832 & 1489.963 & 1485514 & 1488.41 & 1492 & 1492.9 & 1491.885 & 1494.17 & 1491.866 & 1485.464 & 1478248\end{array}$ $\begin{array}{llllllllllllllllllllll}1493.701 & 1494.134 & 1494.363 & 1495.104 & 1496.577 & 1496.512 & 1491.892 & 1489.371 & 1491.714 & 1489.829 & 1489.972 & 1485.521 & 1488.41 & 1492.024 & 1492.896 & 1491.897 & 1494.158 & 1491.875 & 1485.464 & 1478.248\end{array}$ $\begin{array}{lllllllllllllllllllll}1493.697 & 1494.13 & 1494.363 & 1495.095 & 1496.568 & 1496.361 & 1491.899 & 1489.371 & 1491.717 & 1489.827 & 1489.982 & 1485.524 & 1488.414 & 1492.052 & 1492.898 & 1491.904 & 1494.151 & 1491.88 & 1485.461 & 1478.228\end{array}$

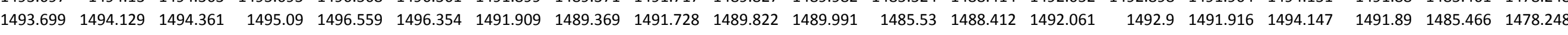

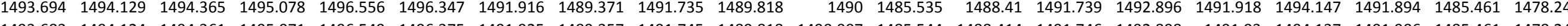

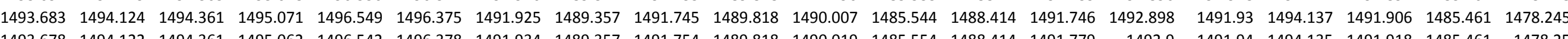

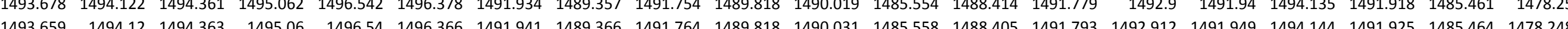

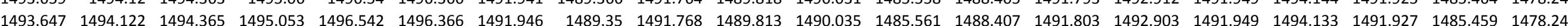

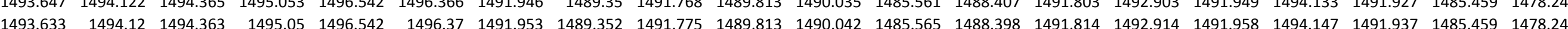
$\begin{array}{lllllllllllllllllllllll}1493.626 & 1494.12 & 1494.365 & 1495.053 & 1496.54 & 1496.387 & 1491.963 & 1489.352 & 1491.785 & 1489.811 & 1490.049 & 1455.572 & 1488.391 & 1491.821 & 1492.921 & 1491.966 & 1494.154 & 1491.944 & 1485.459 & 1478.248\end{array}$ $\begin{array}{llllllllllllllllllll}1493.61 & 1494.117 & 1494.363 & 1495.05 & 1496.545 & 1496.399 & 1491.97 & 1489.355 & 1491.792 & 1489.811 & 1490.056 & 1485.575 & 1488.384 & 1491.831 & 1492.938 & 1491.975 & 1494.161 & 1491.951 & 1485.456 & 1478.245\end{array}$ \begin{tabular}{llllllllllllllllllllll}
1493.603 & 1494.117 & 1494.361 & 1495.048 & 1496.547 & 1496.411 & 1491.977 & 1489.355 & 1491.799 & 1489.811 & 1490.063 & 1485.581 & 1488.381 & 1491.847 & 1492.935 & 1491.98 & 1494.166 & 1491.96 & 1485.459 & 1478.248 \\
\hline
\end{tabular}

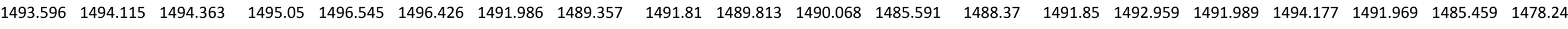

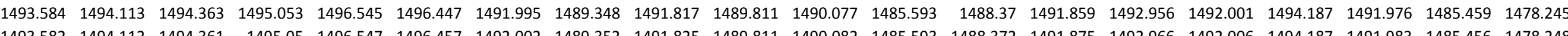

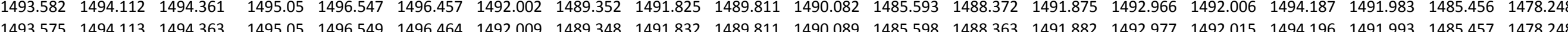
$\begin{array}{lllllllllllllllllllll}1493.565 & 1494.112 & 1494.358 & 1495.053 & 1496.552 & 1496.478 & 1492.019 & 148935 & 1491.843 & 1489811 & 1490.096 & 1485.504 & 1488363 & 1491.897 & 1492.977 & 1492.023 & 1494.203 & 1491.998 & 1485.456 & 1478.245\end{array}$

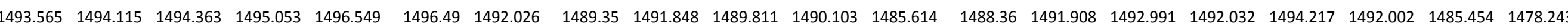
$\begin{array}{lllllllllllllllllllll}1493.572 & 1494.113 & 1494.358 & 1495.05 & 1496.542 & 1496.497 & 1492.033 & 1489.35 & 1491.855 & 1489.811 & 1490.108 & 1485.614 & 1488.353 & 1491.92 & 1492.998 & 1492.039 & 1494.222 & 1492.014 & 1485.456 & 1478.245 \\ \end{array}$ $\begin{array}{lllllllllllllllllllll}1493.577 & 1494.113 & 1494.356 & 1495.055 & 1496.535 & 1496.502 & 1492.044 & 1489.352 & 1491.864 & 1489.811 & 1490.11 & 1485.623 & 1488.353 & 1491.932 & 1493.005 & 1492.046 & 1494.229 & 1492.021 & 1485.456 & 1478.245 \\ \end{array}$

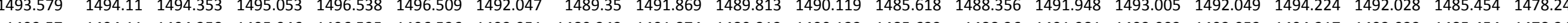

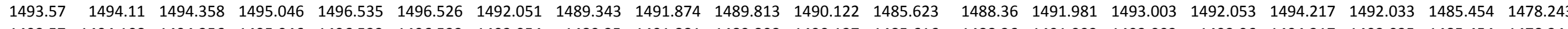

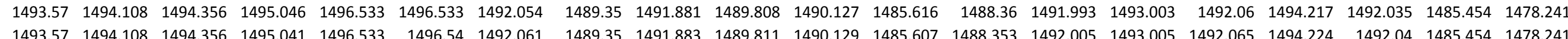


Well

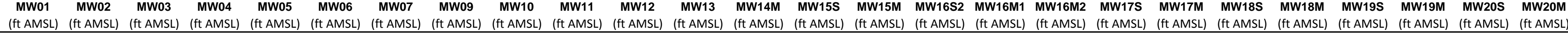

11/3/11 7:00 $11 / 3 / 117: 30$ 11/3/11 8:00 11/3/1119:00 $11 / 3 / 119: 30$ $11 / 3 / 1110: 00$ 11/3/111 10:30 11/3/11 11:00 11/3/11 11:30 $11 / 3 / 1112: 00$ 11/3/11 13:00 11/3/1111 13:30 $11 / 3 / 1113: 30$ 11/3/11 14:30 11/3/11 15:00 $11 / 3 / 1116: 00$ $11 / 3 / 11116: 30$ 11/3/11117:00 $11 / 3 / 1118: 00$ 11/3/11 18:00 11/3/11 19:00 11/3/11 19:30 11/3/11120:00 11/3/11 20:30 $11 / 3 / 1121: 00$ $11 / 3 / 1122: 00$ $11 / 3 / 1122: 30$ 11/3/11 23:00 11/3/11 23:30 11/4/11 0:00 $11 / 4 / 110: 30$
$11 / 4 / 111: 00$
$11 / 4 / 111: 30$ 11/4/111:30 11/4/11 2:30 11/4/11 3:00 11/4/11 3:30 11/4/11 4:00 $11 / 4 / 114: 30$ 11/4/11 5:00 11/4/115:30 11/4/11 6:00 111/4/116:30 $11 / 4 / 117: 30$ 11/4/11 8:00 11/4/11 8:30 11/4/11 9:00 $\begin{array}{lllll}1494.007 & 1494.387 & 1496.221 & 1494.197 \\ 1494.007 & 1494.387 & 1496207 & 1494.197\end{array}$ $\begin{array}{llll}1494.007 & 1494.387 & 1496.207 & 1494.197 \\ 1494.007 & 1494387 & 1496.195 & 1494.197\end{array}$

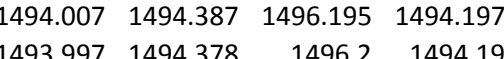
$\begin{array}{llll}1493.997 & 1494.373 & 1496207 & 1494.185\end{array}$ $\begin{array}{lllll}1493.995 & 1494.366 & 1496221 & 1494.185\end{array}$ $\begin{array}{llll}1493.997 & 1494.366 & 1496.221 & 1494.18\end{array}$ $\begin{array}{lllll}1494.002 & 1494.368 & 1496.235 & 1494.183\end{array}$ $\begin{array}{lllll}1494.007 & 1494.373 & 1496.249 & 1494.192\end{array}$ $\begin{array}{llll}1494.002 & 1494.373 & 1496.258 & 1494.197\end{array}$ $\begin{array}{llll}1494.009 & 1494.378 & 1496.27 & 1494.204\end{array}$ $\begin{array}{llll}1494.011 & 1494.385 & 1496.282 & 1494.215 \\ 1494.011 & 1494.392 & 1496298 & 1494.225\end{array}$ $\begin{array}{llll}1494.013 & 1494.396 & 1496.312 & 1494.236\end{array}$ $\begin{array}{lllll}1494.014 & 1494.403 & 1496.321 & 1494.236\end{array}$ $\begin{array}{lllll}1494.014 & 1499.403 & 1496.321 & 1494.248 \\ 1494.018 & 1494.413 & 1496.326 & 1494.262\end{array}$ $\begin{array}{lllll}1494.02 & 1494.422 & 1496.329 & 1494.278\end{array}$ $\begin{array}{llll}1494.021 & 1494.429 & 1496.333 & 1494.29\end{array}$ $\begin{array}{llll}1494.02 & 1494.431 & 1496.338 & 1494.302\end{array}$ $\begin{array}{llll}1494.018 & 1494.436 & 1496.326 & 1494.311\end{array}$ $\begin{array}{llll}1494.02 & 1494.438 & 1496.324 & 1494.318 \\ 1494.02 & 1494.445 & 1496.319 & 1494.325\end{array}$ $\begin{array}{llll}1494.016 & 1494.441 & 1496.317 & 1494.323\end{array}$ $\begin{array}{lllll}1494.016 & 1494.441 & 1496.31 & 1494.325\end{array}$ $\begin{array}{lllll}1494.016 & 1494.441 & 1496.317 & 1494.33\end{array}$ $\begin{array}{lllll}1494.016 & 1494.441 & 1496.321 & 1494.327\end{array}$ $\begin{array}{lllll}1494.014 & 1494.441 & 1496.319 & 1494.327\end{array}$ $\begin{array}{lllll}1494.016 & 1494.443 & 1496.328 & 1494.33 \\ 194.014 & 194.445 & 1966328 & 1494332\end{array}$ $\begin{array}{lllll}1494.014 & 1494.445 & 1496.328 & 1494.332 \\ 1494.018 & 1494.448 & 1496319 & 1494.339\end{array}$ $\begin{array}{lllll}1494.016 & 1494.45 & 1496.31 & 1494.341\end{array}$ $\begin{array}{llll}1494.016 & 1494.455 & 1496.31 & 1494.346\end{array}$ $\begin{array}{rrrrr}1494.011 & 1494.452 & 1496.314 & 1494.344\end{array}$ $\begin{array}{lllll}1494.011 & 1494.45 & 1496.319 & 1494.342\end{array}$ $\begin{array}{llll}1494.016 & 1494.452 & 1496.326 & 1494.346\end{array}$ $\begin{array}{llll}1494.016 & 1494.452 & 1496.333 & 1494.344 \\ 1494.016 & 1494.457 & 149634 & 194351\end{array}$ $\begin{array}{lllll}1494.016 & 1494.457 & 1496.34 & 1494.351 \\ 1494.02 & 1499.462 & 1496343 & 194.358\end{array}$ $\begin{array}{rrrr}1494.02 & 1494.462 & 1496.343 & 1494.358 \\ 1494.021 & 1494.466 & 1496354 & 1494.36\end{array}$ $\begin{array}{rrrrr}1494.023 & 1494.469 & 1496.352 & 1494.367\end{array}$ $\begin{array}{llllll}1494.025 & 1494.476 & 1496.349 & 1494.374\end{array}$ $\begin{array}{llllll}1494.023 & 1494.478 & 1496.357 & 1494.379\end{array}$ $\begin{array}{llllll}1494.023 & 1494.48 & 1496.368 & 1494.383\end{array}$ $\begin{array}{lllll}1494.023 & 1494.483 & 1496.364 & 1494.386\end{array}$ $\begin{array}{rrrr}1494.023 & 1494.485 & 1496.359 & 1494.39\end{array}$ $\begin{array}{lllll}1494.027 & 1494.49 & 1496.359 & 1494.397\end{array}$ $\begin{array}{llll}1494.025 & 1494.492 & 1496354 & 1494.4\end{array}$ $\begin{array}{lllll}1494.025 & 1494.492 & 1496.347 & 1494.402\end{array}$ $\begin{array}{llllll}1494.025 & 1494.494 & 1496.342 & 1494.402\end{array}$ $\begin{array}{lllll}1494.025 & 1494.494 & 1496.34 & 1494.404\end{array}$ $\begin{array}{lllll}1494.023 & 1494.492 & 1496.347 & 1494.4\end{array}$ $\begin{array}{rrrr}1494.023 & 1494.492 & 1496.357 & 1494.397 \\ 1494.023 & 1494.49 & 1496.364 & 1494.395\end{array}$

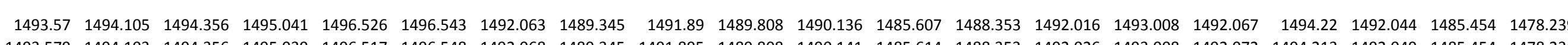

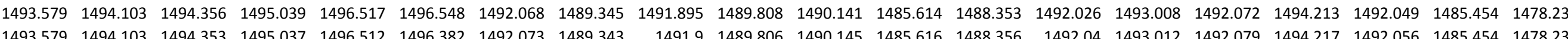

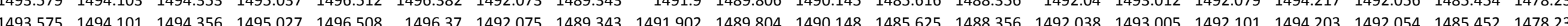
$\begin{array}{llllllllllllllllllllll}1493.565 & 1494.101 & 1494.356 & 149502 & 149651 & 1496378 & 1492.077 & 1489338 & 1491.904 & 1489.801 & 1490.152 & 1485.625 & 1488356 & 1492.047 & 1493.001 & 1492.115 & 1494.198 & 1492.059 & 1485.452 & 1478.234\end{array}$ $\begin{array}{lllllllllllllllllllll}1493.551 & 1494.098 & 1494.358 & 1495.011 & 1496512 & 1496387 & 1492.08 & 1489.336 & 1491.907 & 1489.801 & 1490.155 & 1485.628 & 1488.353 & 1492.054 & 1493.001 & 1492.134 & 1494.191 & 1492.059 & 1485.452 & 1478.231 & \end{array}$ $\begin{array}{llllllllllllllllllllllll}1493.542 & 1494.096 & 1494.358 & 1495.013 & 1496.512 & 1496.399 & 1492.084 & 1489.341 & 1491.911 & 1489.799 & 1490.157 & 1485.635 & 1488.346 & 1492.047 & 1493.005 & 1492.139 & 1494.201 & 1492.066 & 1485.452 & 1478.229\end{array}$ $\begin{array}{lllllllllllllllllllll}1493.535 & 1494.098 & 1494.356 & 1495.011 & 1496.515 & 1496.402 & 1492.089 & 1489.338 & 1491.916 & 1489.797 & 1490.166 & 1485.637 & 1488.337 & 1492.026 & 1493.01 & 1492.139 & 1494.203 & 1492.068 & 1485.449 & 1478.231\end{array}$

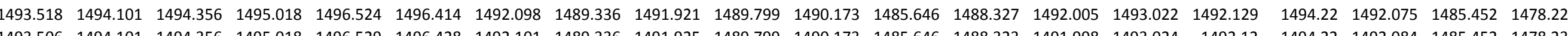

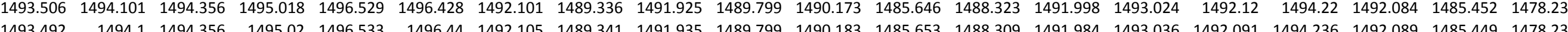

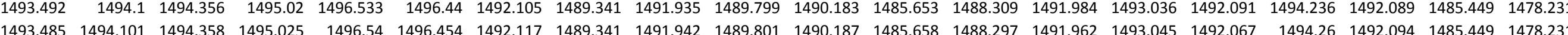
$\begin{array}{llllllllllllllllllllll}1493.473 & 1494.106 & 1494.361 & 1495.03 & 1496.547 & 1496.466 & 1492.122 & 1489.343 & 1491.951 & 1489.804 & 1490.194 & 1485.669 & 1488.29 & 1491.948 & 1493.059 & 1492.044 & 1494.262 & 1492.105 & 1485.447 & 1478.234\end{array}$

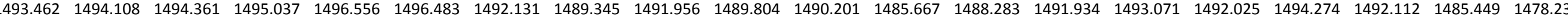
$\begin{array}{lllllllllllllllllllll}1493.457 & 1494.11 & 1494.358 & 1495.041 & 1496.561 & 1496.497 & 1492.138 & 1489.348 & 1491.965 & 1489.808 & 1490.206 & 1485.672 & 1488.276 & 1491.92 & 1493.082 & 1492.018 & 1494.285 & 1492.12 & 1485.449 & 1478.236\end{array}$

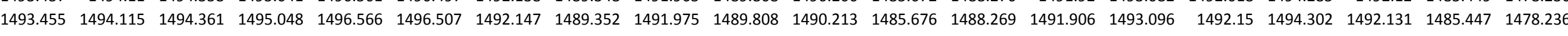
$\begin{array}{llllllllllllllllllllll}1493.452 & 1494.118 & 1494.358 & 1495.055 & 1496.57 & 1496.514 & 1492.159 & 1489.359 & 1491.984 & 1489.813 & 1490.22 & 1485.683 & 1488.264 & 1491.894 & 1493.112 & 1492.16 & 1494.316 & 1492.141 & 1485.447 & 1478.238\end{array}$

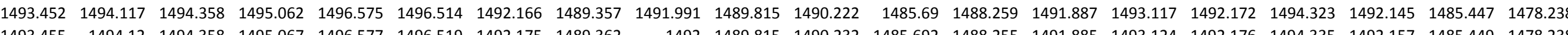

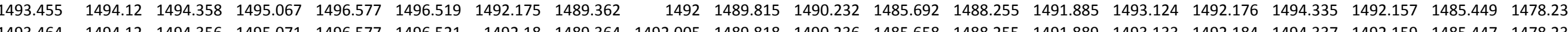

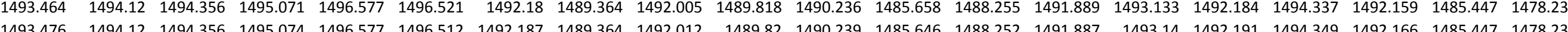
$\begin{array}{llllllllllllllllllllll}1493.48 & 1494.122 & 1494.353 & 1495.078 & 1496.577 & 1496.507 & 1492.192 & 1489.366 & 1492.019 & 1489.82 & 1490.243 & 1485.651 & 1488.252 & 1491.894 & 1493.143 & 1492.195 & 1494.351 & 1492.173 & 1485.447 & 1478.238\end{array}$

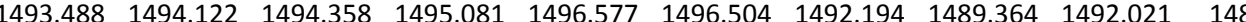
$\begin{array}{lllllllllllllllllllllll}1493.497 & 1494.12 & 1494.358 & 1495.078 & 1496.577 & 1496.502 & 1492.199 & 1489.364 & 1492.026 & 1489.82 & 1490.25 & 1485.662 & 1488.257 & 1491.927 & 1493.14 & 1492.203 & 1494.344 & 1492.181 & 1485.447 & 1478.236\end{array}$

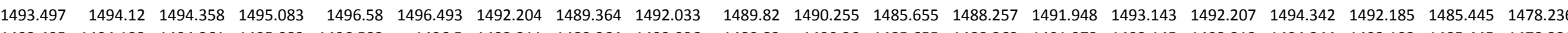

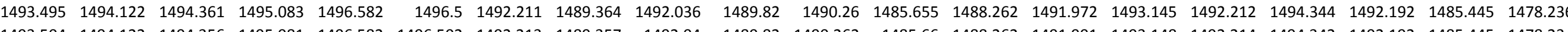

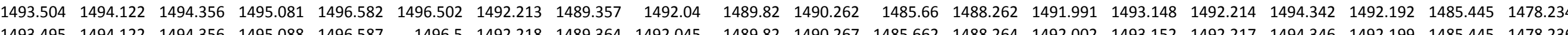
$\begin{array}{lllllllllllllllllllll}1493.495 & 1494.122 & 1494.356 & 1495.088 & 1496.587 & 1496.5 & 1492.218 & 1489.364 & 1492.045 & 1489.82 & 1490.267 & 1485.662 & 1488.264 & 1492.002 & 1493.152 & 1492.217 & 1494.346 & 1492.199 & 1485.445 & 1478.236 \\ 1493.495 & 1494.125 & 1494.358 & 149509 & 1496591 & 1496514 & 149222 & 1489362 & 1492.05 & 148982 & 1490269 & 1485669 & 1488266 & 1492016 & 1493157 & 1492224 & 1494349 & 1492202 & 1485.445 & 1478234\end{array}$ $\begin{array}{llllllllllllllllllll}143.45 & 1494.125 & 1494.358 & 1495.09 & 1496.591 & 1496.514 & 1492.22 & 1489.362 & 1492.05 & 1489.82 & 1490.269 & 1485.669 & 1488.266 & 1492.016 & 1493.157 & 1492.224 & 1494.349 & 1492.202 & 1485.445 & 1478.234 \\ 1493.502 & 1494.122 & 1494.353 & 1495.09 & 1496589 & 1496514 & 1492.225 & 1489364 & 1492.054 & 1489822 & 1490274 & 1485.674 & 1488262 & 1492.024 & 1493.166 & 1492229 & 1494.356 & 1492206 & 1485.445 & 1478.234\end{array}$ $\begin{array}{llllllllllllllllllll}1493.513 & 1494.122 & 1494.351 & 1495.095 & 1496.587 & 1496.502 & 1492.232 & 1489.362 & 1492.059 & 1489.822 & 1490.279 & 1485.681 & 1488.266 & 1492.033 & 1493.164 & 1492.233 & 1494.358 & 1492.213 & 1485.445 & 1478.234\end{array}$ $\begin{array}{llllllllllllllllllllll}1493.516 & 1494.122 & 1494.351 & 1495.097 & 1496.584 & 1496.493 & 1492.236 & 1489.362 & 1492.066 & 1489.825 & 1490.283 & 1485.69 & 1488.269 & 1492.047 & 1493.171 & 1492.238 & 1494.358 & 1492.216 & 1485.442 & 1478.236\end{array}$ $\begin{array}{llllllllllllllllllll}1493.513 & 1494.122 & 1494.356 & 1495.097 & 1496.587 & 1496.493 & 1492.239 & 1489.355 & 1492.066 & 1489.825 & 1490.285 & 1485.69 & 1488.28 & 1492.075 & 1493.166 & 1492.243 & 1494.353 & 1492.22 & 1485.442 & 1478.234 \\ \end{array}$ $\begin{array}{lllllllllllllllllllll}1493.513 & 1494.12 & 1494.356 & 1495.095 & 1496.589 & 1496.495 & 1492.246 & 1489.362 & 1492.073 & 1489.822 & 1490.29 & 1485.69 & 1488.28 & 1492.089 & 1493.171 & 1492.243 & 1494.349 & 1492.223 & 1485.442 & 1478.234\end{array}$

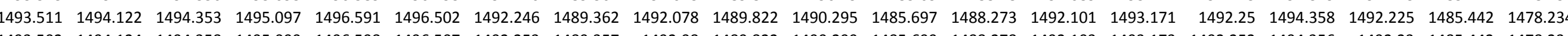

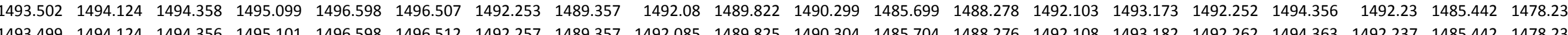

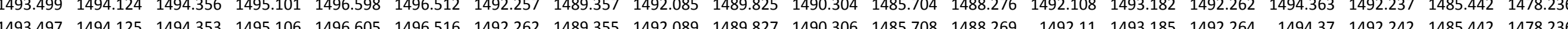

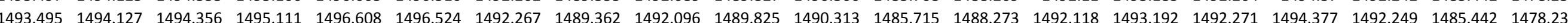
$\begin{array}{lllllllllllllllllllllll}1493.497 & 1494.129 & 1494.353 & 1495.113 & 1496.61 & 1496.533 & 1492.274 & 1489.359 & 1492.099 & 1489.829 & 1490.318 & 1485.722 & 1488.271 & 1492.115 & 1493.203 & 1492.276 & 1494.384 & 1492.253 & 1485.442 & 1478.238\end{array}$

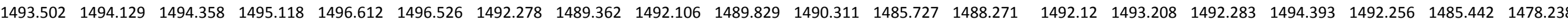

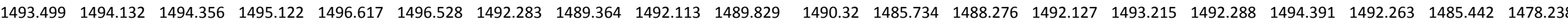

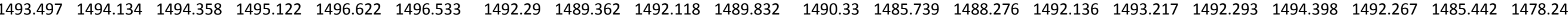

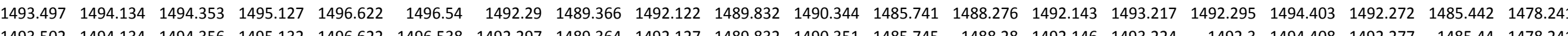

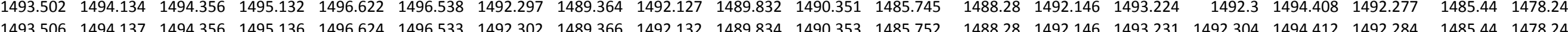

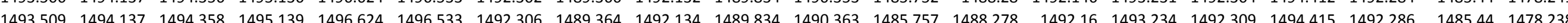
$\begin{array}{lllllllllllllllllllllll}1493.513 & 1494.134 & 1494.353 & 1495.139 & 1496.622 & 1496.528 & 1492.311 & 1489.366 & 1492.139 & 1489.834 & 1490.377 & 1485.748 & 1488.283 & 1492.174 & 1493.234 & 1492.312 & 1494.41 & 1492.291 & 1485.44 & 1478.238\end{array}$ $\begin{array}{llllllllllllllllllllll}1493.52 & 1494.139 & 1494.353 & 1495.141 & 1496.622 & 1496.526 & 1492.316 & 1489.364 & 1492.141 & 1489.834 & 1490.386 & 1485.748 & 1488.285 & 1492.183 & 1493.231 & 1492.314 & 1494.41 & 1492.293 & 1485.44 & 1478.238\end{array}$ $\begin{array}{lllllllllllllllllllll}1493.525 & 1494.134 & 1494.356 & 1495.139 & 1496.622 & 1496.516 & 1492.316 & 1489.36 & 1492.143 & 1489.834 & 1490.395 & 1485.75 & 1488.288 & 1492.195 & 1493.231 & 1492.319 & 1494.412 & 1492.293 & 1485.44 & 1478.238\end{array}$

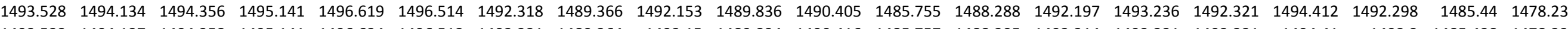
$\begin{array}{llllllllllllllllllllll}1493.523 & 1494.137 & 1494.358 & 1495.141 & 1496.624 & 1496.512 & 1492.321 & 1489.364 & 1492.15 & 1489.834 & 1490.416 & 1485.757 & 1488.295 & 1492.214 & 1493.231 & 1492.321 & 1494.41 & 1492.3 & 1485.438 & 1478.236 \\ \end{array}$

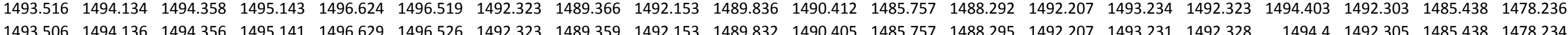


Well

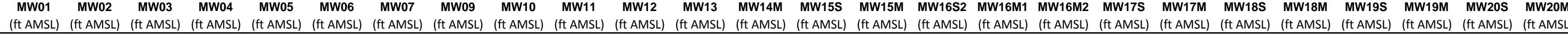

11/4/11 10:00 $11 / 4 / 1110: 30$ $11 / 4 / 1111: 00$ 11/4/11111:30 11/4/111 12:30 11/4/11 12:30 11/4/111 13:30 11/4/1114:00 11/4/11 14:30 11/4/11 15:00 11/4/111 16:300 11/4/1116:00 11/4/1116:30 11/4/11 17:30 11/4/11 18:00 11/4/111 18:30 $11 / 4 / 1119: 00$ 11/4/11 19:30 11/4/1120:30 11/4/11 21:00 $11 / 4 / 1121: 00$
$11 / 4 / 1121: 30$ 11/4/11 22:00 11/4/11 22:30 11/4/11 23:00 11/4/11 23:30 11/5/11 0:00 11/5/11 0:30 11/5/111:30 $11 / 5 / 112: 00$ 11/5/11 2:30 11/5/11 3:00 11/5/11 3:30 11/5/11 4:00 11/5/11 4:30 $11 / 5 / 11$ : 5:00 11/5/11 6:00 $11 / 5 / 116: 30$ 11/5/11 7:00 11/5/117:30 11/5/11 8:00 $11 / 5 / 118: 30$ 11/5/11 9:00 11/5/11 10:00 11/5/11 10:30 11/5/11 11:00 11/5/11 11:30 11/5/11 12:00 $\begin{array}{rrrr}1494.028 & 1494.497 & 1496.373 & 1494.4\end{array}$ $\begin{array}{rrrr}1494.03 & 1494.501 & 1496.38 & 1494.407\end{array}$ $\begin{array}{llll}1494.032 & 1494.508 & 1496.392 & 1494.414\end{array}$ $\begin{array}{lllll}1494.037 & 1494.515 & 1496.417 & 1494.423\end{array}$ $\begin{array}{llll}1494.039 & 1494.525 & 1496.434 & 1494.433\end{array}$ $\begin{array}{lllll}1494.044 & 1494.536 & 1496.445 & 1494.444\end{array}$ $\begin{array}{llll}1494.046 & 1494.546 & 1496.457 & 1494.456\end{array}$ $\begin{array}{lllll}1494.051 & 1494.557 & 1496.462 & 1494.468\end{array}$ $\begin{array}{lllll}1494.055 & 1494.569 & 1466.478 & 1494.479\end{array}$ $\begin{array}{rrrr}1494.056 & 1494.576 & 1496.487 & 1494.491 \\ 149.058 & 1494585 & 1496.94 & 1494.5\end{array}$ $\begin{array}{rrrr}1494.058 & 1494.585 & 1496.494 & 1494.5 \\ 1494.062 & 1494.602 & 1496.494 & 1494.514\end{array}$ $\begin{array}{llll}1494.062 & 1494.602 & 1496.494 & 1494.514 \\ 1494.065 & 1494.606 & 1496.497 & 1494.524\end{array}$ $\begin{array}{lllll}1494.067 & 1494.611 & 1496.492 & 1494.533\end{array}$ $\begin{array}{lllll}1494.065 & 1494.611 & 1496.494 & 1494.538\end{array}$ $\begin{array}{lllll}1494.067 & 1494.62 & 1496.483 & 1494.545\end{array}$ $\begin{array}{rrrrr}1494.069 & 1494.62 & 1496.48 & 1494.547\end{array}$ $\begin{array}{llll}1494.072 & 1494.627 & 1496.48 & 1494.552 \\ 1494.067 & 1494.625 & 1496.483 & 194547\end{array}$ $\begin{array}{rrrr}1494.067 & 1494.625 & 1496.483 & 1494.547 \\ 1494.067 & 1494.62 & 1496476 & 194.545\end{array}$ $\begin{array}{lrrrr}1494.067 .069 & 1494.623 & 1496.476 & 1494.545 .48 & 1494.547\end{array}$ $\begin{array}{lllll}1494.072 & 1494.625 & 1496.476 & 14494.547\end{array}$ $\begin{array}{llll}1494.072 & 1494.625 & 1496.478 & 1494.545\end{array}$ $\begin{array}{lllll}1494.072 & 1494.627 & 1496.478 & 1494.542\end{array}$ $\begin{array}{llll}1494.074 & 1494.627 & 1496.49 & 1494.542\end{array}$ $\begin{array}{lllll}1494.076 & 1494.63 & 1496.494 & 1494.531\end{array}$ $\begin{array}{lllll}1494.079 & 1494.63 & 1496.501 & 1494.535\end{array}$ $\begin{array}{lllll}1494.083 & 1494.637 & 1496.502 & 1494.545 \\ 1494.083 & 1494.639 & 1496.504 & 1494.547\end{array}$ $\begin{array}{lllll}1494.083 & 1494.639 & 1496.506 & 1494.554\end{array}$ $\begin{array}{lllll}1494.086 & 1494.646 & 1496.513 & 1494.559\end{array}$ $\begin{array}{lllll}1494.086 & 1494.648 & 1496.511 & 1494.561\end{array}$ $\begin{array}{lllll}1494.088 & 1494.651 & 1496.515 & 1494.566\end{array}$ $\begin{array}{llll}1494.09 & 1494.653 & 1496.525 & 1494.568\end{array}$ $\begin{array}{llll}1494.093 & 1494.653 & 1496.532 & 1494.57 \\ 1491.095 & 1494.655 & 1496525 & 1494577\end{array}$ $\begin{array}{llll}14941 & 1494.655 & 1966.525 & 1494.577\end{array}$ $\begin{array}{lllll}1494.1 & 1494.665 & 1496.527 & 1494.582 \\ 1494.1 & 1494.669 & 1496.525 & 1494.587\end{array}$ $\begin{array}{lllll}1494.097 & 1494.667 & 1496.536 & 1494.589\end{array}$ $\begin{array}{lllll}1494.1 & 1494.672 & 1496.539 & 1494.594\end{array}$ $\begin{array}{lllll}1494.102 & 1494.672 & 1496.539 & 1494.594\end{array}$ $1494.107 \quad 1494.676 \quad 1496.541 \quad 1494.598$ $1494.104 \quad 1494.679 \quad 1496.544 \quad 1494.598$ $\begin{array}{lllll}1494.107 & 1494.681 & 1496.551 & 1494.598\end{array}$ $\begin{array}{lllll}1494.111 & 1494.683 & 149656 & 1494.601\end{array}$ $\begin{array}{llllll}1494.116 & 1494.688 & 1496.558 & 1494.61\end{array}$ $\begin{array}{rrrrr}1494.114 & 1494.69 & 1496.567 & 1494.612\end{array}$ $1494.118 \quad 1494.697 \quad 1496.581 \quad 1494.615$ $1494.121 \quad 1494.7 \quad 1496.586 \quad 1494.619$ $\begin{array}{lllll}1494.123 & 1494.707 & 1496.595 & 1494.626\end{array}$ $\begin{array}{rrrr}1494.128 & 1494.714 & 1496.6 & 1494.633 \\ 1494.13 & 1494.718 & 1496.611 & 1494.638\end{array}$

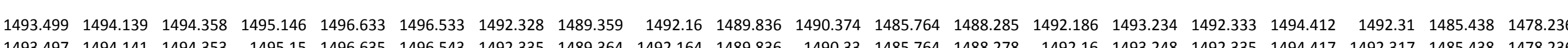

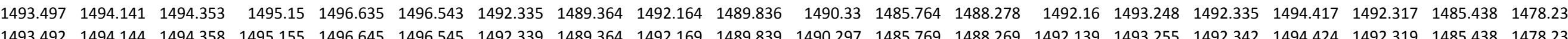
$\begin{array}{lllllllllllllllllllll}1493.492 & 1494.144 & 1494.358 & 1495.155 & 1496.645 & 1496.545 & 1492.339 & 1489.364 & 1492.169 & 1489.839 & 1490.297 & 1485.769 & 1488.269 & 1492.139 & 1493.255 & 1492.342 & 1494.424 & 1492.319 & 1485.438 & 1478.236 \\ 1493.483 & 1494.146 & 1494.361 & 1495.16 & 1496.652 & 149656 & 1492.346 & 1489.362 & 1492.174 & 1489836 & 1490272 & 1485.776 & 1488.269 & 1492.127 & 1493262 & 1492.349 & 1494.433 & 1492326 & 1485.438 & 1478236\end{array}$ $\begin{array}{lllllllllllllllllllllll}1493.471 & 1494.149 & 1494.361 & 1495.164 & 1496.661 & 1496.571 & 1492.351 & 1489.366 & 1492.179 & 1489.839 & 1490.241 & 1485.778 & 1488.259 & 1492.11 & 1493.266 & 1492.354 & 1494.44 & 1492.331 & 1485.438 & 1478.236\end{array}$

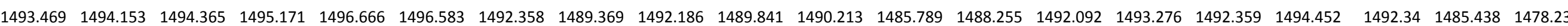
\begin{tabular}{lllllllllllllllllllll}
1493.462 & 1494.156 & 1494.363 & 1495.178 & 1496.677 & 1496.595 & 1492.365 & 1489.369 & 1492.193 & 1489.843 & 1490.178 & 1485.789 & 1488.25 & 1492.071 & 1493.29 & 1492.366 & 1494.469 & 1492.345 & 1485.438 & 1478.241 \\
\hline
\end{tabular} $\begin{array}{lllllllllllllllllllll}1493.457 & 1494.16 & 1494.365 & 1495.185 & 1496.689 & 1496.61 & 1492.372 & 1489.378 & 1492.197 & 1489.846 & 1490.157 & 1485.794 & 1488.248 & 1492.054 & 1493.304 & 1492.371 & 1494.478 & 1492.352 & 1485.438 & 1478.241\end{array}$

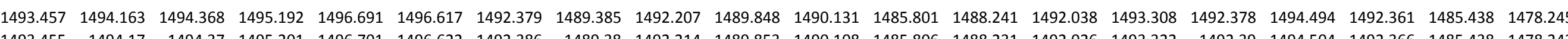

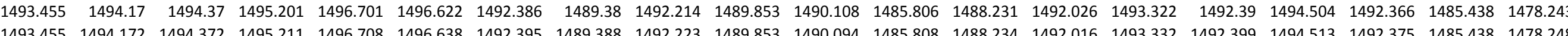

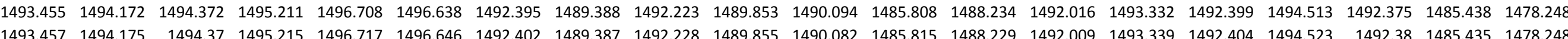

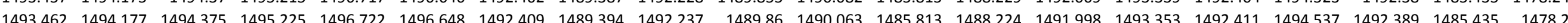
$\begin{array}{llllllllllllllllllllll}1493.471 & 1494.179 & 1494.37 & 1495.231 & 1496.726 & 1496.65 & 1492.416 & 1489.402 & 1492.242 & 1489.864 & 1490.061 & 1485.815 & 1488.229 & 1491.998 & 1493.36 & 1492.418 & 1494.541 & 1492.396 & 1485.435 & 1478.252\end{array}$ $\begin{array}{lllllllllllllllllllll}1493.483 & 1494.182 & 1494.37 & 1495.238 & 1496.724 & 1496.653 & 1492.423 & 1489.397 & 1492.249 & 1489.864 & 1490.061 & 1485.815 & 1488.231 & 1491.998 & 1493.364 & 1492.425 & 1494.549 & 1492.403 & 1485.438 & 1478.252\end{array}$

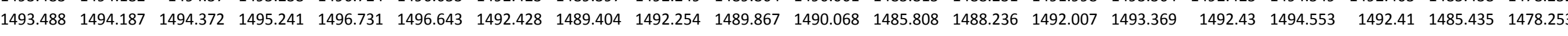
$\begin{array}{lllllllllllllllllllllll}1493.506 & 1494.189 & 1494.368 & 1495.248 & 1496.724 & 1496.646 & 1492.433 & 1489.402 & 1492.261 & 1489.869 & 1490.084 & 1485.803 & 1488.238 & 1492.014 & 1493.374 & 1492.435 & 1494.556 & 1492.413 & 1485.435 & 1478.252\end{array}$ $\begin{array}{llllllllllllllllllll}1493.518 & 1494.187 & 1494.37 & 1495.248 & 1496.726 & 1496.631 & 1492.435 & 1489.404 & 1422.265 & 1489.869 & 1490.098 & 1485.803 & 1488.238 & 1492.019 & 1493.378 & 1492.437 & 1494.56 & 1492.417 & 1485.435 & 1478.252 \\ 1\end{array}$

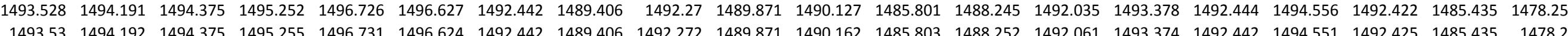

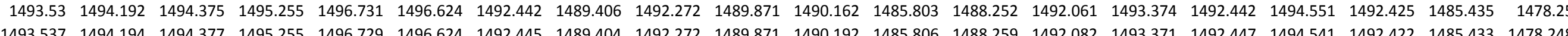

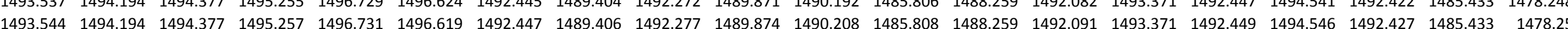
$\begin{array}{llllllllllllllllllllll}1493.551 & 1494194 & 1494375 & 1495259 & 1496731 & 1496617 & 1492.449 & 1489.404 & 1492.277 & 1489871 & 1490229 & 148581 & 1488266 & 1492106 & 1493.374 & 1492.451 & 1494.549 & 1492.429 & 1485.433 & 147825\end{array}$ $\begin{array}{llllllllllllllllllllllll}1493.556 & 1494.197 & 1494.375 & 1495.262 & 1496.731 & 1496.615 & 1492.452 & 1489.406 & 1492.282 & 1489.874 & 1490.255 & 1485.81 & 1488.271 & 1492.125 & 1493.376 & 1492.456 & 1494.544 & 1492.432 & 1485.433 & 1478.25\end{array}$

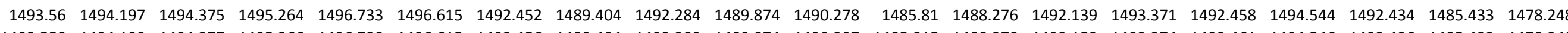

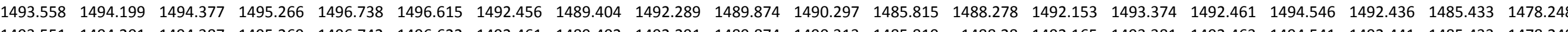

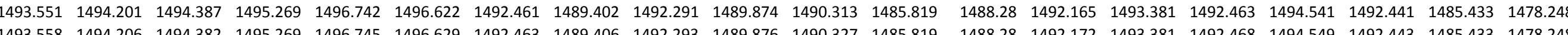

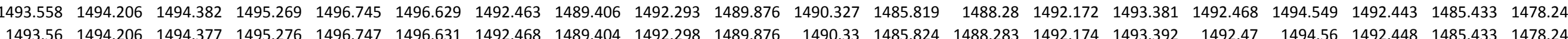
$\begin{array}{llllllllllllllllllllll}1493.56 & 1494.206 & 1494.37 & 1495.276 & 1496.747 & 1496.631 & 1492.468 & 1489.404 & 1492.298 & 1489.876 & 1490.33 & 1485.824 & 1488.283 & 1492.14 & 1493.392 & 1492.47 & 1494.56 & 1492.448 & 1485.433 & 1478.2248 \\ 1493.56 & 1494.208 & 1494.38 & 1495278 & 1496.752 & 1496.629 & 1492.475 & 1489.404 & 1492.305 & 1489.878 & 1490.344 & 1485.829 & 1488.288 & 1492.186 & 1493.388 & 1492.477 & 1494.56 & 1492.455 & 1485.433 & 147825\end{array}$ $\begin{array}{llllllllllllllllllllll}1493.563 & 1494.208 & 1494.38 & 1495.283 & 1496.754 & 1496.634 & 1492.48 & 1489.406 & 1492.308 & 1489.878 & 1490.356 & 1485.833 & 1488.295 & 1492.19 & 1493.395 & 1492.482 & 1494.563 & 1492.457 & 1485.431 & 1478.252\end{array}$ $\begin{array}{llllllllllllllllllllll}1493.563 & 1494.211 & 1494.382 & 1495.285 & 1496.756 & 1496.631 & 1492.482 & 1489.409 & 1492.312 & 1489.88 & 1490.367 & 1485.84 & 1488.295 & 1492.202 & 1493.399 & 1492.485 & 1494.567 & 1492.46 & 1485.433 & 1478.255 \\ \end{array}$ \begin{tabular}{lllllllllllllllllllllll}
1493.565 & 1494.213 & 1494.38 & 1495.29 & 1496.759 & 1496.638 & 1492.487 & 1489.411 & 1492.317 & 1489.88 & 1490.377 & 1485.84 & 1488.297 & 1492.212 & 1493.404 & 1492.489 & 1494.57 & 1492.467 & 1485.431 & 1478.255 \\
\hline
\end{tabular} $\begin{array}{lllllllllllllllllllllll}1493.57 & 1494.213 & 1494.384 & 1495.292 & 1496.761 & 1496.636 & 1492.494 & 1489.409 & 1492.319 & 1489.883 & 1490.384 & 1485.843 & 1488.299 & 1492.21 & 1493.409 & 1492.494 & 1494.574 & 1492.471 & 1485.431 & 1478.255 & \end{array}$

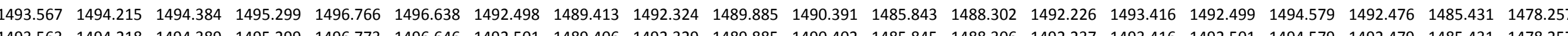

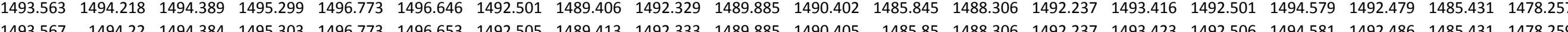

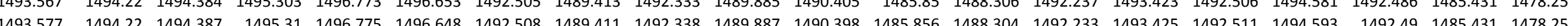

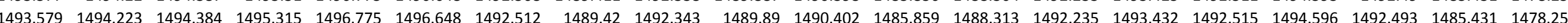
$\begin{array}{llllllllllllllllllllll}1493.582 & 1494.223 & 1494.389 & 1495.315 & 1496.78 & 1496.643 & 1492.517 & 1489.416 & 1492.35 & 1489.89 & 1490.409 & 1485.863 & 1488.309 & 1492.247 & 1493.43 & 1492.52 & 1494.593 & 1492.495 & 1485.431 & 1478.259\end{array}$

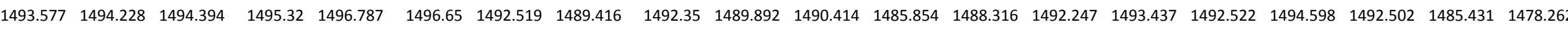
$\begin{array}{lllllllllllllllllllll}1493.584 & 1494.228 & 1494.389 & 1495.322 & 1496.782 & 1496.653 & 1492.522 & 1489.418 & 1492.35 & 1489.892 & 1490.419 & 1485.859 & 1488.318 & 1492.249 & 1493.434 & 1492.522 & 1494.598 & 1492.502 & 1485.431 & 1478.262\end{array}$

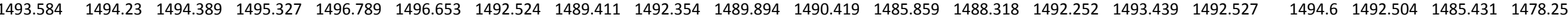

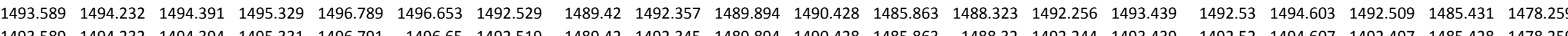

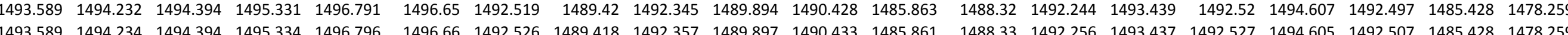

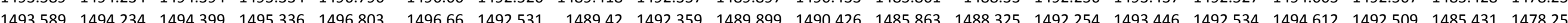
$\begin{array}{llllllllllllllllllllll}1493.589 & 1494.239 & 1494.399 & 1495.343 & 1496.803 & 1496.667 & 1492.538 & 1489.42 & 1492.366 & 1489.897 & 1490.407 & 1485.866 & 1488.32 & 1492.24 & 1493.451 & 1492.541 & 1494.619 & 1492.518 & 1485.431 & 1478.262\end{array}$

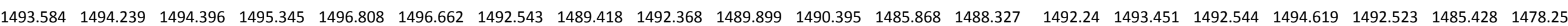
\begin{tabular}{lllllllllllllllllllll}
1493.584 & 1494.246 & 1494.399 & 1495.352 & 1496.817 & 1496.674 & 1492.545 & 1489.42 & 1492.371 & 1489.901 & 1490.377 & 1485.868 & 1488.327 & 1492.233 & 1493.46 & 1492.549 & 1494.621 & 1492.528 & 1485.431 & 1478.262 \\
\hline
\end{tabular} $\begin{array}{llllllllllllllllllll}1493.589 & 1494.244 & 1494.396 & 1495.354 & 1496.822 & 1496.682 & 1492.552 & 1489.42 & 1492.38 & 1489.899 & 1490.36 & 1485.87 & 1488.323 & 1492.226 & 1493.465 & 1492.551 & 1494.631 & 1492.535 & 1485.431 & 1478.259 \\ \end{array}$ $\begin{array}{llllllllllllllllllll}1493.572 & 1494.246 & 1494.406 & 1495.361 & 1496.824 & 1496.686 & 1492.557 & 1489.42 & 1492.385 & 1489.901 & 1490.337 & 1485.875 & 1488.318 & 1492.214 & 1493.472 & 1492.556 & 1494.638 & 1492.54 & 1485.428 & 1478.259\end{array}$ $\begin{array}{llllllllllllllllllll}1493.579 & 1494.249 & 1494.401 & 1495.368 & 1496.838 & 1496.696 & 1492.564 & 1489.425 & 1492.39 & 1489.904 & 1490.325 & 1485.882 & 1488.318 & 1492.209 & 1493.493 & 1492.563 & 1494.647 & 1492.547 & 1485.431 & 1478.264 \\ 1493577 & 1494252 & 1494.403 & 1495375 & 1496835 & 1496.698 & 1492566 & 1489.425 & 1492397 & 1489906 & 1490304 & 1485887 & 1488318 & 1492193 & 1493.488 & 149257 & 1494.654 & 1492551 & 1485.431 & 1478.262\end{array}$ 
Well

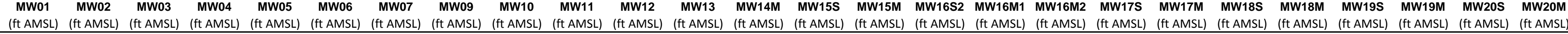

11/5/11 13:00 11/5/11 13:30

$11 / 5 / 1114: 30$

11/5/1114:30

11/5/111 15:30

11/5/11 16:00

11/5/11 16:30

11/5/111 17:00

11/5/11 17:30

$11 / 5 / 1118: 00$

$11 / 5 / 1118: 30$
$11 / 5 / 1119: 00$

11/5/11 19:30

11/5/11 20:00

11/5/11 20:30

11/5/11 21:00

11/5/11 21:30

11/5/11 22:00

11/5/11 22:30

11/5/11 23:30

11/6/11 0:00

11/6/11 0:00

11/6/111:00

11/6/111:30

11/6/112:00

11/6/11 2:30

11/6/11 3:00

$11 / 6 / 114: 00$

$11 / 6 / 114: 30$

11/6/11 5:00

11/6/11 5:30

11/6/11 6:00

11/6/11 6:30

$11 / 6 / 117: 00$

11/6/117:30

$11 / 6 / 6 / 11$ 8:00

11/6/11 9:00

11/6/11 9:30

11/6/11 10:00

$11 / 6 / 1110: 30$

11/6/11 11:00

$11 / 6 / 1111.30$

11/6/11 12:00

$11 / 6 / 1113: 00$

11/6/11 13:30

11/6/11 14:00

11/6/11 14:30

11/6/11 15:00 $\begin{array}{llll}1494.132 & 1494.725 & 1496.639 & 1494.645 \\ 1494.132 & 1494.732 & 1496.651 & 1494.652\end{array}$ $\begin{array}{llll}1494.132 & 1494.732 & 1496.651 & 1494.652\end{array}$ $\begin{array}{llll}1494.142 & 1494.741 & 1496.656 & 1494.664\end{array}$ $\begin{array}{rrrrr}1494.149 & 1494.769 & 1496.684 & 1494.692\end{array}$ $\begin{array}{lllll}1494.156 & 1494.774 & 1496.693 & 1494.701\end{array}$ $\begin{array}{lllll}1494.156 & 1494.783 & 1496.681 & 1494.71\end{array}$ $\begin{array}{lllll}1494.163 & 1494.8 & 1496.672 & 1494.727\end{array}$ $\begin{array}{lllll}1494.165 & 1494.807 & 1496.658 & 1494.738\end{array}$ $\begin{array}{lllll}1494.153 & 1494.804 & 1496.658 & 1494.734\end{array}$ $\begin{array}{rrrr}1494.146 & 1494.8 & 1496.639 & 1494.727 \\ 1494.151 & 1494802 & 1966.19 & 1494729\end{array}$ $\begin{array}{llll}1494.151 & 1494.802 & 1496.618 & 1494.729 \\ 1494.149 & 1494.797 & 1496.609 & 1494.724\end{array}$ $\begin{array}{lllll}1494.142 & 1494.793 & 1496.604 & 1494.724\end{array}$ $\begin{array}{llll}1494.144 & 1494.786 & 1496.607 & 1494.703\end{array}$ $\begin{array}{lllll}1494.142 & 1494.779 & 1496.614 & 1494.692\end{array}$ $\begin{array}{lllll}1494.144 & 1494.776 & 1496.616 & 1494.687\end{array}$ $\begin{array}{lllll}1494.151 & 1494.781 & 1496.614 & 1494.682\end{array}$ $\begin{array}{llllll}1494.153 & 1494.781 & 1496.607 & 1494.685\end{array}$ $\begin{array}{llll}1494.156 & 1494.786 & 1496.611 & 1494.685 \\ 1494.151 & 1494.779 & 1496609 & 194.678\end{array}$ $\begin{array}{lllll}1494.156 & 1444.776 & 1496.604 & 1494.673\end{array}$ $\begin{array}{lllll}1494.158 & 1494.779 & 1496593 & 1494.678\end{array}$ $\begin{array}{lllll}1494.16 & 1494.779 & 1496.588 & 1494.671\end{array}$ $\begin{array}{lllll}1494.156 & 1494.779 & 1496.574 & 1494.668\end{array}$ $\begin{array}{llll}1494.156 & 1494.772 & 1496.56 & 1494.661\end{array}$ $\begin{array}{lllll}1494.156 & 1494.769 & 1496.548 & 1494.657\end{array}$ $\begin{array}{lllll}1494.151 & 1494.76 & 1496.525 & 1494.645\end{array}$ $\begin{array}{lllll}1494.149 & 1494.755 & 1496.515 & 1494.638 \\ 1494.146 & 1494.744 & 1496.511 & 1494.624\end{array}$ $\begin{array}{lllll}1494.139 & 1494.727 & 1496.504 & 1494.61\end{array}$ $\begin{array}{lllll}1494.142 & 1494.723 & 1496.492 & 1494.598\end{array}$ $\begin{array}{lllll}1494.146 & 1494.721 & 1496.487 & 1494.594\end{array}$ $\begin{array}{lllll}1494.144 & 1494.716 & 1496.469 & 1494.582\end{array}$ $\begin{array}{llll}1494.142 & 1494.704 & 1496.457 & 1494.57\end{array}$ $1494.142 \quad 1494.702 \quad 1496.448 \quad 1494.563$ $\begin{array}{rrrr}1494.139 & 1494.688 & 1496.434 & 1494.552 \\ 1494.137 & 1494.683 & 1496.42 & 1494.54\end{array}$ $\begin{array}{rrrr}1494.135 & 1494.672 & 1496.401 & 1494.531\end{array}$ $\begin{array}{lllll}1494.13 & 1494.658 & 1496.403 & 1494.514\end{array}$ $\begin{array}{lllll}1494.13 & 1494.651 & 1496.387 & 1494.505\end{array}$ $\begin{array}{lllll}1494.128 & 1494.639 & 1496.378 & 1494.491\end{array}$ $\begin{array}{lllll}1494.13 & 1494.637 & 1496.382 & 1494.484\end{array}$ $\begin{array}{llll}1494.123 & 1494.623 & 1496.378 & 1494.468\end{array}$ $\begin{array}{llll}1494.123 & 1494.616 & 1496.38 & 1494.458\end{array}$ $\begin{array}{lllll}1494.128 & 1494.613 & 1496.399 & 1494.453 \\ 1494.123 & 1494.611 & 1496.406 & 1494.451\end{array}$ $\begin{array}{lllll}1494.13 & 1494.611 & 1496.417 & 1494.451\end{array}$ $\begin{array}{lllll}1494.135 & 1494.616 & 1496.42 & 1994.453\end{array}$ $\begin{array}{lllll}1494.137 & 1494.62 & 1496.422 & 1494.458\end{array}$ $1494.137 \quad 1494.623 \quad 1496.42 \quad 1494.465$ $\begin{array}{lllll}1494.137 & 1494.627 & 1496.42 & 1494.47\end{array}$ $\begin{array}{rrrr}1494.135 & 1494.627 & 1496.42 & 1494.472 \\ 1494.135 & 1494.625 & 1496.413 & 1494.475\end{array}$

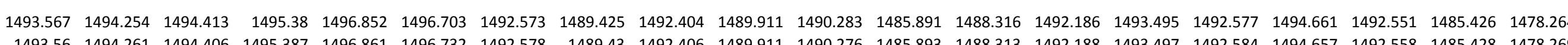

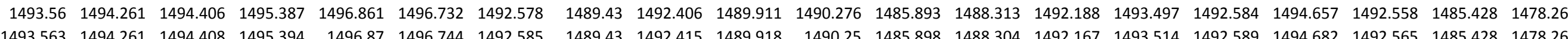

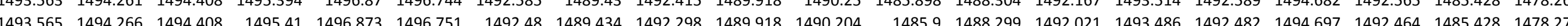
$\begin{array}{lllllllllllllllllllll}1493.563 & 1494.27 & 1494.413 & 1495.422 & 1496891 & 1496.758 & 1492.529 & 1489.439 & 1492352 & 1489.92 & 1490.197 & 1485.903 & 1488302 & 1492.066 & 1493.504 & 1492.53 & 1494.708 & 1492.511 & 1485.428 & 1478.273\end{array}$ $\begin{array}{lllllllllllllllllllll}1493.556 & 1494.271 & 1494.408 & 1495.429 & 1496.898 & 1496.772 & 1492.555 & 1489.439 & 1492.378 & 1489.925 & 1490.183 & 1485.907 & 1488.299 & 1492.078 & 1493.523 & 1492.556 & 1494.715 & 1492.537 & 1485.426 & 1478.278\end{array}$ $\begin{array}{llllllllllllllllllllllll}1493.572 & 1494.273 & 1494.408 & 1495.436 & 1496.898 & 1496.777 & 1492.571 & 1489.451 & 1492.399 & 1489.925 & 1490.183 & 1485.912 & 1488.299 & 1492.092 & 1493.535 & 1492.577 & 1494.722 & 1492.554 & 1485.431 & 1478.278\end{array}$ $\begin{array}{lllllllllllllllllllllll}1493.593 & 1494.278 & 1494.413 & 1495.45 & 1496.896 & 1496.763 & 1492.587 & 1489.444 & 1492.411 & 1489.929 & 1490.164 & 1485.882 & 1488.29 & 1492.08 & 1493.558 & 1492.594 & 1494.748 & 1492.57 & 1485.428 & 1478.283\end{array}$

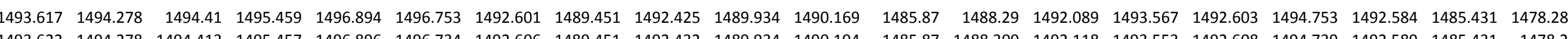

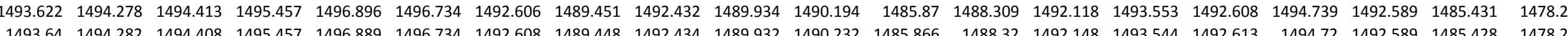

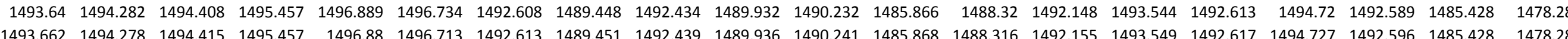

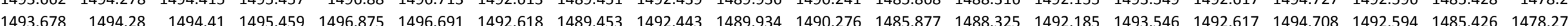

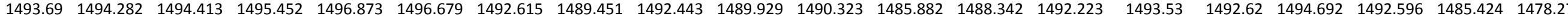
$\begin{array}{llllllllllllllllllll}1493.694 & 1494.28 & 1494.413 & 1495.447 & 1496.87 & 1496.672 & 1492.615 & 1489.446 & 1492.446 & 1489.932 & 1490.349 & 1485.882 & 1488.342 & 1492.237 & 1493.53 & 1492.615 & 1494.685 & 1492.596 & 1485.428 & 1478.273\end{array}$

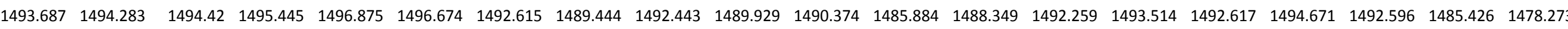
$\begin{array}{llllllllllllllllllllll}1493.694 & 1494.283 & 1494.42 & 1495.445 & 1496.875 & 1496.677 & 1492.615 & 1489.441 & 1492.446 & 1489.927 & 1490.4 & 1485.891 & 1488.353 & 1492.27 & 1493.516 & 1492.615 & 1494.666 & 1492.594 & 1485.426 & 1478.271\end{array}$

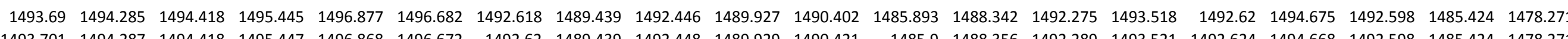

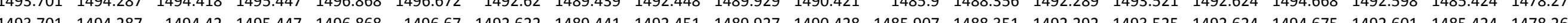

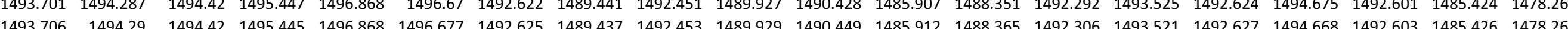
$\begin{array}{lllllllllllllllllllll}1493.709 & 1494.29 & 1494.422 & 1495.445 & 1496.868 & 1496.67 & 1492.625 & 1489.432 & 1492.453 & 1489.927 & 1490.458 & 1485.917 & 1488.363 & 1492.317 & 1493.521 & 1492.627 & 1494.668 & 1492.605 & 1485.426 & 1478.269\end{array}$

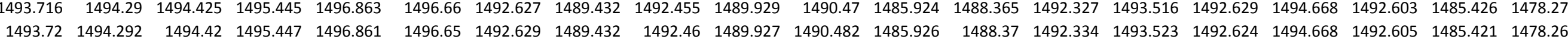
$\begin{array}{llllllllllllllllllll}1493.73 & 1494.29 & 1494.42 & 1495.445 & 1496.854 & 1496.646 & 1492.629 & 1489.434 & 1492.462 & 1489.927 & 1490.503 & 1485.93 & 1488.379 & 1492.346 & 1493.511 & 1492.631 & 1494.661 & 1492.612 & 1485.424 & 1478.271 \\ \end{array}$

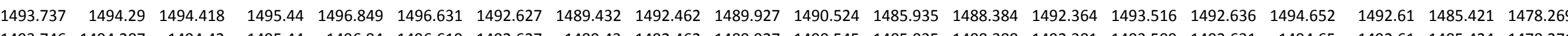

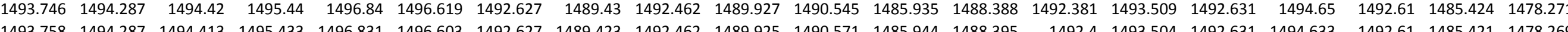

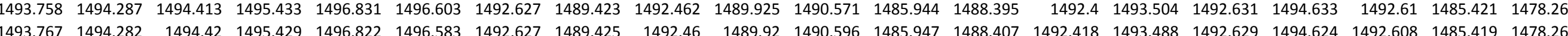
$\begin{array}{lllllllllllllllllllll}1493.767 & 1494.282 & 1494.42 & 1495.429 & 1496.822 & 1496.583 & 1492.627 & 1489.425 & 1492.46 & 1489.92 & 1490.596 & 1485.947 & 1488.407 & 1492.418 & 1493.488 & 1492.629 & 1494.624 & 1492.608 & 1485.419 & 1478.269 \\ 1493.767 & 1494.28 & 1494.42 & 1495.424 & 1496.815 & 1496.571 & 1492.625 & 1489.418 & 1492.46 & 1489.918 & 1490.62 & 1485.947 & 1488.412 & 1492.437 & 1493.483 & 1492.627 & 1494.607 & 1492.605 & 1485.421 & 1478269\end{array}$ $\begin{array}{llllllllllllllllllllll}1493.765 & 1494.278 & 1494.42 & 1495.415 & 1496.81 & 1496.569 & 1492.62 & 1489.413 & 1492.455 & 1489.915 & 1490.655 & 1485.949 & 1488.419 & 1492.468 & 1493.465 & 1492.622 & 1494.588 & 1492.601 & 1485.419 & 1478.266\end{array}$ $\begin{array}{lllllllllllllllllllll}1493.77 & 1494.278 & 1494.418 & 1495408 & 1496803 & 1496.56 & 1492.618 & 1489.409 & 1492.453 & 1489.913 & 1490.678 & 1485.954 & 1488.421 & 1492.482 & 1493.46 & 1492.622 & 1494.579 & 1492.596 & 1485.419 & 1478.266\end{array}$ $\begin{array}{llllllllllllllllllll}1493.77 & 1494.278 & 1494.42 & 1495.403 & 1496.798 & 1496.55 & 1492.618 & 1489.406 & 1492.451 & 1489.913 & 1490.69 & 1485.958 & 1488.421 & 1492.482 & 1493.46 & 1492.62 & 1494.577 & 1492.596 & 1485.419 & 1478.266\end{array}$ $\begin{array}{llllllllllllllllllllll}1493.777 & 1494.275 & 1494.415 & 1495.396 & 1496.787 & 1496.545 & 1492.613 & 1489.404 & 1492.45 & 1489.911 & 1490.713 & 1485.961 & 1488.421 & 1492.498 & 1493.451 & 1492.615 & 1494.57 & 1492.596 & 1485.417 & 1478.264\end{array}$ \begin{tabular}{llllllllllllllllllll}
1493.779 & 1494.271 & 1494.418 & 1495.392 & 1496.777 & 1496.528 & 1492.611 & 1489.399 & 1492.448 & 1489.908 & 1490.736 & 1485.965 & 1488.428 & 1492.515 & 1493.441 & 1492.612 & 1494.56 & 1492.589 & 1485.417 & 1478.264 \\
\hline
\end{tabular}

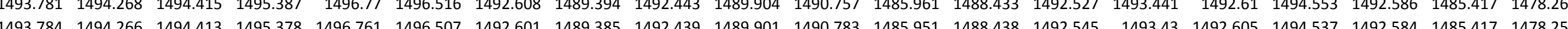

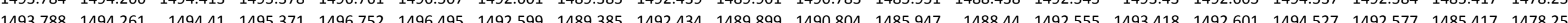

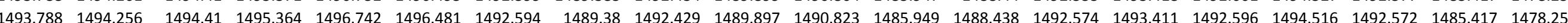
$\begin{array}{lllllllllllllllllllll}1493.784 & 1494.252 & 1494.413 & 1495.354 & 1496.735 & 1496.466 & 1492.587 & 1489.373 & 1492.422 & 1489.892 & 1490.853 & 1485.951 & 1488.449 & 1492.592 & 1493.395 & 1492.591 & 1494.502 & 1492.57 & 1485.417 & 1478.255\end{array}$ \begin{tabular}{lllllllllllllllllllll}
1493.777 & 1494.251 & 1494.41 & 1495.345 & 1496.729 & 1496.469 & 1492.583 & 1489.369 & 1492.42 & 1489.892 & 1490.858 & 1485.954 & 1488.447 & 1492.595 & 1493.39 & 1492.584 & 1494.49 & 1492.563 & 1485.414 & 1478.252 \\
\hline
\end{tabular}

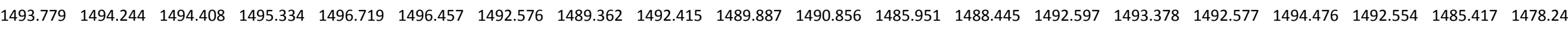
$\begin{array}{llllllllllllllllllll}1493.763 & 1494.242 & 1494.418 & 1495.331 & 1496.715 & 1496.447 & 1422.573 & 1489.357 & 1492.408 & 1489.883 & 1490.83 & 1485.951 & 1488.442 & 1492.583 & 1493.381 & 1492.577 & 1494.476 & 1492.551 & 1485.414 & 1478.245 \\ \end{array}$

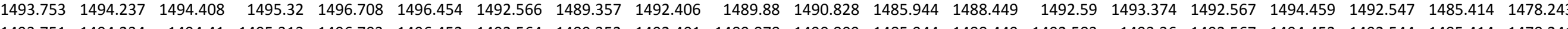

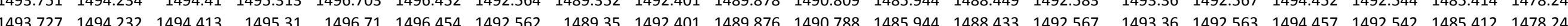

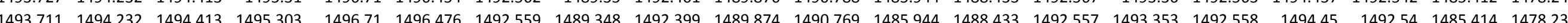

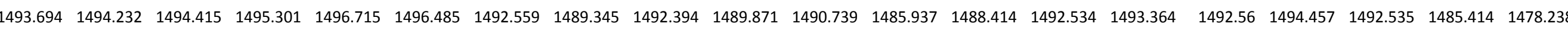
$\begin{array}{lllllllllllllllllllllll}1493.685 & 1494.234 & 1494.415 & 1495.303 & 1496.715 & 1496.497 & 1492.559 & 1489.348 & 1492.394 & 1489.871 & 1490.704 & 1485.928 & 1488.41 & 1492.51 & 1493.371 & 1492.563 & 1494.469 & 1492.54 & 1485.412 & 1478.241 \\ \end{array}$

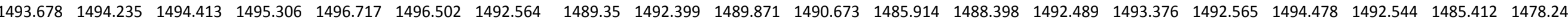

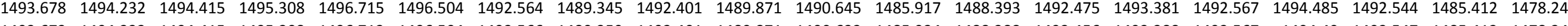
\begin{tabular}{llllllllllllllllllllll}
1493.673 & 1494.232 & 1494.415 & 1495.308 & 1496.712 & 1496.504 & 1492.566 & 1489.359 & 1492.401 & 1489.871 & 1490.622 & 1485.924 & 1488.388 & 1492.456 & 1493.388 & 1492.567 & 1494.49 & 1492.547 & 1485.412 & 1478.243 \\
\hline
\end{tabular}

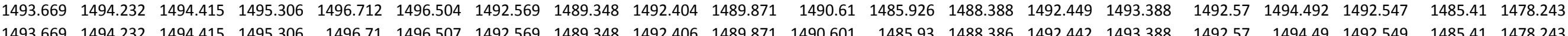


Well

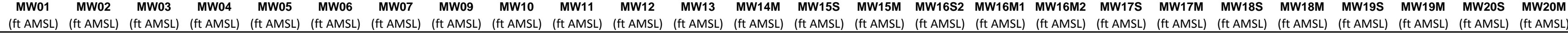

11/6/11 16:00 11/6/11 16:30 $11 / 6 / 1117: 00$ 11/6/11117:30 11/6/111 18:30 $11 / 6 / 1118: 30$
$11 / 6 / 1119: 00$ 11/6/11 19:30 11/6/11 20:00 $11 / 6 / 1120: 30$ $11 / 6 / 1121: 00$ 11/6/1121:30 11/6/1122:30 $11 / 6 / 1122: 30$ 11/6/11 23:30 11/7/110:00 11/7/11 0:30 11/7/11 1:00 11/7/112:00 11/7/112:30 11/7/11 3:30 11/7/114:00 11/7/11 4:30 $11 / 7 / 115: 30$ 11/7/11 6:00 11/7/11 6:30 11/7/7/117:00 11/7/11 8:00 11/7/11 8:30 11/7/119:00 11/7/119:30 11/7/11 10:00 11/7/1110:30 11/7/11 11:30 11/7/711 12:00 11/7/11 12:30 11/7/11 13:00 $11 / 7 / 1113: 30$ 11/7/11114:00 11/7/1114:30 11/7/1115:30 $11 / 7 / 1116: 30$ 11/7/111 17:00 11/7/11 17:30 11/7/11 18:00 $\begin{array}{llll}1494.137 & 1494.625 & 1496.408 & 1494.477 \\ 1494.135 & 1494.625 & 1496.03 & 1494.477\end{array}$ $\begin{array}{lllll}1494.135 & 1494.625 & 1496.403 & 1494.477\end{array}$ $\begin{array}{llll}1494.132 & 1494.62 & 1496.396 & 1494.475 \\ 194.132 & 1494.62 & 1963385 & 194.475\end{array}$ $\begin{array}{lllll}1494.132 & 1494.618 & 1496.371 & 1494.47\end{array}$ $\begin{array}{lllll}1494.13 & 1494.613 & 1496.364 & 1494.465\end{array}$ $\begin{array}{rrrr}1494.13 & 1494.613 & 1496.364 & 1494.465 \\ 1494.128 & 1494.609 & 1496.359 & 1494.458\end{array}$ $\begin{array}{lllll}1494.125 & 1494.602 & 1496.357 & 1494.449\end{array}$ $\begin{array}{lllll}1494.125 & 1494.595 & 1496.347 & 1494.439\end{array}$ $\begin{array}{llll}1494.123 & 1494.592 & 1496.338 & 1494.432\end{array}$ $\begin{array}{lllll}1494.125 & 1494.59 & 1496.333 & 1494.428\end{array}$ $\begin{array}{llll}1494.121 & 1494.583 & 1496.326 & 1494.416 \\ 1494.118 & 1494.574 & 1496.328 & 1494.409\end{array}$ $\begin{array}{lllll}1494.116 & 1494.574 & 1496.326 & 1494.409\end{array}$ $\begin{array}{lllll}1494.1118 & 1494.569 & 1496.335 & 1494.394\end{array}$ $\begin{array}{llll}1494.118 & 1494.569 & 1496.335 & 1444.397 \\ 1494.397\end{array}$ $\begin{array}{lllll}1494.116 & 1494.567 & 1496.342 & 1494.393\end{array}$ $\begin{array}{llll}1494.121 & 1494.571 & 1496.342 & 1494.397\end{array}$ $\begin{array}{llll}1494.121 & 1494.574 & 1496.345 & 1494.4\end{array}$ $\begin{array}{rrrr}1494.121 & 1494.574 & 1496.338 & 1494.4 \\ 1494.121 & 1494.574 & 1496.333 & 1494.402\end{array}$ $\begin{array}{llll}1494.121 & 1494.574 & 1496.331 & 1494.402\end{array}$ $\begin{array}{lllll}1494.118 & 1494.574 & 1496333 & 1494.402\end{array}$ $\begin{array}{llll}1494.116 & 1494.567 & 1496.338 & 1494.4\end{array}$ $\begin{array}{lllll}1494.116 & 1494.569 & 1496.335 & 1494.4\end{array}$ $\begin{array}{lllr}1494.118 & 1494.569 & 1496.326 & 1494.402\end{array}$ $\begin{array}{llll}1494.118 & 1494.571 & 1496.324 & 1494.404\end{array}$ $\begin{array}{lllll}1494.118 & 1494.571 & 1496.324 & 1494.407\end{array}$ $\begin{array}{rrrr}1494.111 & 1494.564 & 1496.324 & 1494.4 \\ 1494.118 & 1494.567 & 1496324 & 1494.404\end{array}$ $\begin{array}{rrrr}1494.118 & 1494.567 & 1496.324 & 1494.404 \\ 1494.114 & 1494.562 & 1496.345 & 1494.4 \\ 1494.116 & 1494.564 & 1496.338 & 1494.402\end{array}$ $\begin{array}{llll}1494.116 & 1494.569 & 1496.328 & 1494.402\end{array}$ $\begin{array}{llllll}1494.123 & 1494.578 & 1496.331 & 1494.414\end{array}$ $\begin{array}{lllll}1494.114 & 1494.569 & 1496.342 & 1494.409\end{array}$ $\begin{array}{llll}1494.114 & 1494.569 & 1496.342 & 1494.407 \\ 1494.116 & 1494571 & 1496359 & 194.400\end{array}$ $\begin{array}{lllll}1494.116 & 1494.571 & 1496.359 & 1494.409\end{array}$ $\begin{array}{llll}1494.118 & 1494.574 & 1496.364 & 1494.414 \\ 1494.121 & 1494.576 & 1496366 & 1494.416\end{array}$ $\begin{array}{lllll}1494.128 & 1494.588 & 1496.387 & 1494.43\end{array}$ $\begin{array}{lllll}1494.121 & 1494.585 & 1496.403 & 1494.43\end{array}$ $\begin{array}{lllll}1494.123 & 1494.59 & 1496.41 & 1494.437\end{array}$ $\begin{array}{lllll}1494.139 & 1494.606 & 1496.403 & 1494.456\end{array}$ $\begin{array}{lllll}1494.137 & 1494.613 & 1496.455 & 1494.465\end{array}$ $\begin{array}{lllll}1494.128 & 1494.611 & 1496.459 & 1494.47\end{array}$ $\begin{array}{rrrrr}1494.142 & 1494.62 & 1496.413 & 1494.486 \\ 1494.144 & 1494.634 & 1496.455 & 1494505\end{array}$ $\begin{array}{lllll}1494.142 & 1494.641 & 1496.434 & 1494.517\end{array}$ $1494.135 \quad 1494.627 \quad 1496.455 \quad 1494.507$ $1494.149 \quad 1494.646 \quad 1496.4551494 .531$ $1494.1391494 .644 \quad 1496.471 \quad 1494.533$ $1494.1491494 .6511496 .45 \quad 1494.542$ $\begin{array}{llll}1494.149 & 1494.653 & 1496.441 & 1494.552 \\ 1494.151 & 1494.662 & 1496.427 & 1494.563\end{array}$

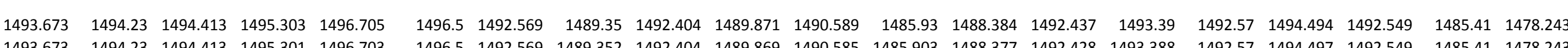

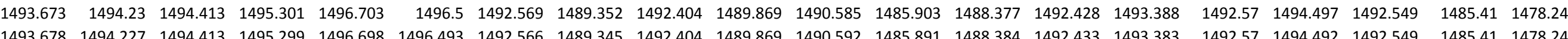

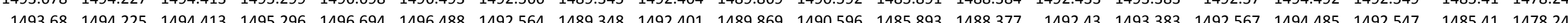

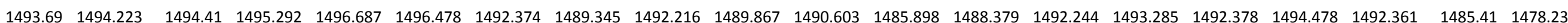

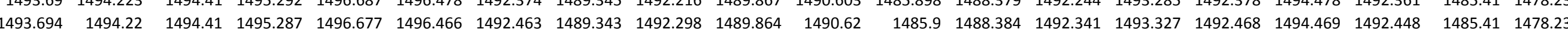
$\begin{array}{lllllllllllllllllllll}1493.694 & 1494.216 & 1494.408 & 1495.283 & 1496.677 & 1496.459 & 1492.227 & 1489.343 & 1492.038 & 1489.862 & 1490.622 & 1485.898 & 1488.386 & 1492.101 & 1493.266 & 1492.231 & 1494.455 & 1492.211 & 1485.407 & 1478.236\end{array}$ $\begin{array}{lllllllllllllllllllll}1493.694 & 1494.213 & 1494.41 & 1495.276 & 1496.673 & 1496.457 & 1492.255 & 1489.341 & 1492.075 & 1489.86 & 1490.606 & 1485.903 & 1488.388 & 1492.153 & 1493.234 & 1492.257 & 1494.436 & 1492.244 & 1485.41 & 1478.234\end{array}$

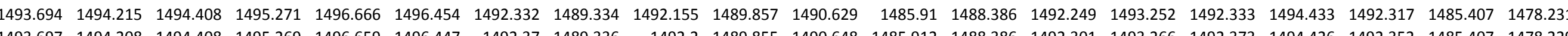

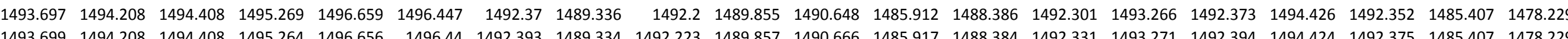

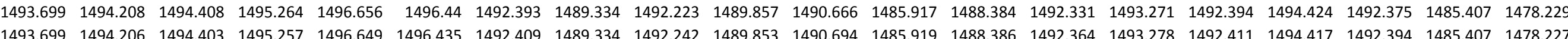

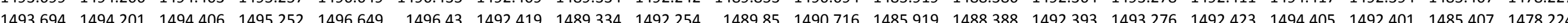
$\begin{array}{lllllllllllllllllllll}1493.69 & 1494.201 & 1494.406 & 1495248 & 1496647 & 1496.433 & 1492.431 & 1489.326 & 1492.263 & 1489.848 & 1490.732 & 1485.921 & 1488.388 & 1492.407 & 1493.266 & 1492.435 & 1494.403 & 1492.408 & 1485.407 & 1478.222\end{array}$ $\begin{array}{lllllllllllllllllllllll}1493.683 & 1494.199 & 1494.406 & 1495.241 & 1496.645 & 1496.433 & 1492.435 & 1489.326 & 1492.27 & 1489.846 & 1490.743 & 1485.926 & 1488.388 & 1492.428 & 1493.278 & 1492.44 & 1494.403 & 1492.42 & 1485.407 & 1478.222 \\ \end{array}$ $\begin{array}{lllllllllllllllllllll}1493.671 & 1494.199 & 1494.408 & 1495.238 & 1496.647 & 1496.442 & 1492.442 & 1489.324 & 1492.277 & 1489.843 & 1490.75 & 1485.926 & 1488.384 & 1492.428 & 1493.276 & 1492.442 & 1494.405 & 1492.425 & 1485.405 & 1478.222 \\ \end{array}$ $\begin{array}{lllllllllllllllllllll}1493.666 & 1494.196 & 1494.408 & 1495.236 & 1496.647 & 1496.454 & 1492.445 & 1489.329 & 1492.282 & 1489.843 & 1490.762 & 1485.928 & 1488.386 & 1492.444 & 1493.278 & 1492.449 & 1494.403 & 1492.427 & 1485.405 & 1478.222\end{array}$

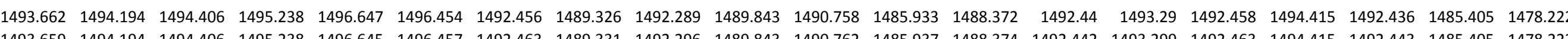

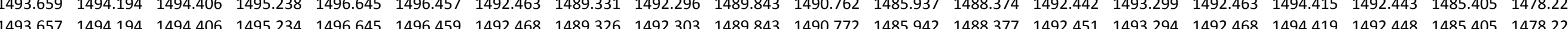

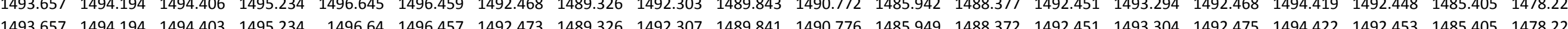
$\begin{array}{lllllllllllllllllllll}1493.659 & 1494.191 & 1494.403 & 1495.236 & 1496.638 & 1496.452 & 1492.475 & 1489.322 & 1492.312 & 1489.841 & 1490.783 & 1485.951 & 1488.372 & 1492.458 & 1493.304 & 1492.48 & 1494.424 & 1492.455 & 1485.405 & 1478.227\end{array}$ $\begin{array}{lllllllllllllllllllll}1493.652 & 1494.189 & 1494.403 & 1495.227 & 1496.638 & 1496.454 & 1492.482 & 1489.326 & 1492.317 & 1489.839 & 1490.8 & 1485.958 & 1488.374 & 1492.477 & 1493.301 & 1492.482 & 1494.412 & 1492.464 & 1485.403 & 1478.227\end{array}$

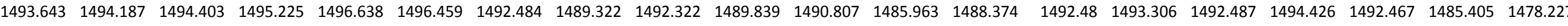

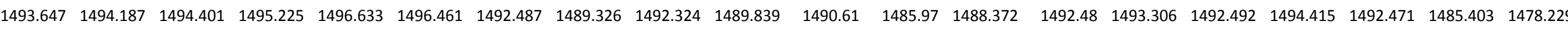

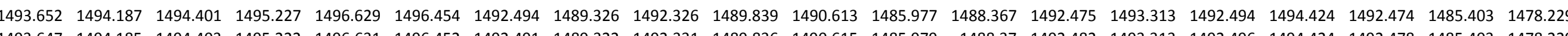

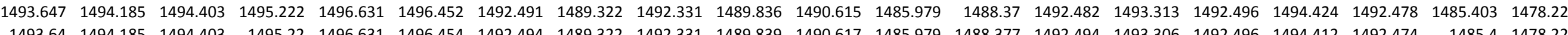
$\begin{array}{lllllllllllllllllllll}1493.64 & 1494.185 & 1494.403 & 1495.22 & 1496.631 & 1496.454 & 1492.494 & 1489.322 & 1492.331 & 1489.839 & 1490.617 & 1485.979 & 1488.377 & 1492.494 & 1493.306 & 1492.496 & 1494.412 & 1492.474 & 1485.4 & 1478.229 \\ 1493.636 & 1494.184 & 1494.401 & 1495.218 & 1496.629 & 1496.454 & 1492.496 & 1489.324 & 1492333 & 1489836 & 1490617 & 1485984 & 1488367 & 1492.489 & 1493311 & 1492.499 & 1494.422 & 1492.479 & 1485.403 & 14782229\end{array}$ $\begin{array}{llllllllllllllllllllll}1493.626 & 1494.182 & 1494.406 & 1495.215 & 1496.633 & 1496.457 & 1492.498 & 1489.32 & 1492.333 & 1489.834 & 1490.617 & 1485.984 & 1488.367 & 1492.491 & 1493.306 & 1492.499 & 1494.417 & 1492.479 & 1485.4 & 1478.229\end{array}$ $\begin{array}{llllllllllllllllllll}1493.619 & 1494.184 & 1494.406 & 1495.215 & 1496.635 & 1496.476 & 1492.496 & 1489.322 & 1492.333 & 1489.834 & 1490.617 & 1485.979 & 1488.36 & 1492.484 & 1493.318 & 1492.501 & 1494.424 & 1492.479 & 1485.4 & 1478.227 \\ \end{array}$ $\begin{array}{llllllllllllllllllllll}1493.633 & 1494.182 & 1494.399 & 1495.215 & 1496.626 & 1496.473 & 1492.498 & 1489.324 & 1492.333 & 1489.834 & 1490.62 & 1485.972 & 1488.356 & 1492.477 & 1493.315 & 1492.501 & 1494.424 & 1492.481 & 1485.403 & 1478.227 \\ \end{array}$ $\begin{array}{llllllllllllllllllllllll}1493.624 & 1494.184 & 1494.399 & 1495.22 & 1496.626 & 1496.461 & 1492.503 & 1489.327 & 1492.34 & 1489.836 & 1490.624 & 1485.974 & 1488.346 & 1492.461 & 1493.334 & 1492.508 & 1494.438 & 1492.486 & 1485.4 & 1478.227\end{array}$

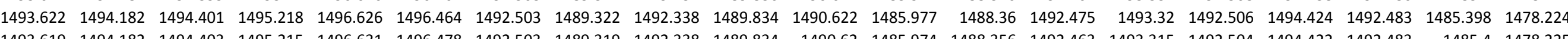
$\begin{array}{llllllllllllllllllllll}1493.619 & 1494.182 & 1494.403 & 1495.215 & 1496.631 & 1496.478 & 1492.503 & 1489.319 & 1492.338 & 1489.834 & 1490.62 & 1485.974 & 1488.356 & 1492.463 & 1493.315 & 1492.504 & 1494.422 & 1492.483 & 1485.4 & 1478.225 \\ 1493.605 & 1494.182 & 1494.406 & 1495213 & 1496.638 & 1496.478 & 1492503 & 1489.319 & 1492.34 & 1489.834 & 1490.622 & 1485.979 & 1488353 & 1492.456 & 1493318 & 1492.506 & 1494.426 & 1492.486 & 14854 & 1478222\end{array}$

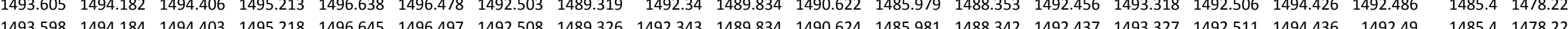

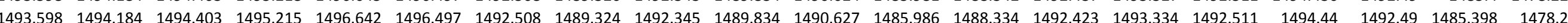
$\begin{array}{lllllllllllllllllllllll}1493.593 & 1494.184 & 1494.403 & 1495.222 & 1496.647 & 1496.504 & 1492.512 & 1489.326 & 1492.352 & 1489.834 & 1490.631 & 1485.988 & 1488.323 & 1492.393 & 1493.35 & 1492.515 & 1494.459 & 1492.495 & 1485.398 & 1478.224\end{array}$ \begin{tabular}{lllllllllllllllllllll}
1493.567 & 1494.189 & 1494.413 & 1495.222 & 1496.663 & 1496.524 & 1492.515 & 1489.324 & 1492.35 & 1489.836 & 1490.631 & 1485.988 & 1488.334 & 1492.395 & 1493.339 & 1492.515 & 1494.455 & 1492.497 & 1485.398 & 1478.222 \\
\hline
\end{tabular}

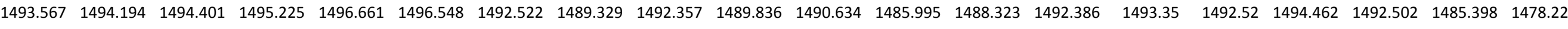
$\begin{array}{llllllllllllllllllll}1493.584 & 1494.197 & 1494.403 & 1495.234 & 1496.659 & 1496.55 & 1492.533 & 1489.334 & 1422.368 & 1489.841 & 1490.643 & 1485.993 & 1488.302 & 1492.355 & 1493.381 & 1492.534 & 1494.494 & 1492.514 & 1485.403 & 1478.231 \\ \end{array}$

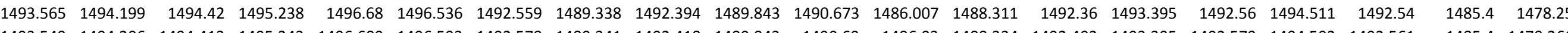

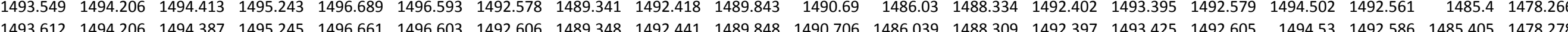

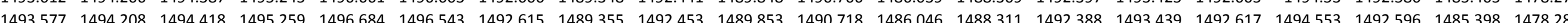
$\begin{array}{llllllllllllllllllllll}1493.563 & 1494.218 & 1494.413 & 1495.266 & 1496.694 & 1496.591 & 1492.634 & 1489.359 & 1492.472 & 1489.86 & 1490.727 & 1486.062 & 1488.323 & 1492.409 & 1493.453 & 1492.636 & 1494.556 & 1492.612 & 1485.4 & 1478.295\end{array}$ $\begin{array}{lllllllllllllllllllll}1493.589 & 1494.223 & 1494.41 & 1495.255 & 1496.689 & 1496.569 & 1492.643 & 1489.352 & 1492.483 & 1489.857 & 1490.739 & 1486.074 & 1488.327 & 1492.449 & 1493.451 & 1492.646 & 1494.541 & 1492.624 & 1485.403 & 1478.299\end{array}$ $\begin{array}{lllllllllllllllllllllll}1493.572 & 1494.23 & 1494.413 & 1495.269 & 1496.701 & 1496.588 & 1492.648 & 1489.364 & 1492.486 & 1489.862 & 1490.743 & 1486.046 & 1488.313 & 1492.418 & 1493.472 & 1492.65 & 1494.574 & 1492.631 & 1485.4 & 1478.304\end{array}$

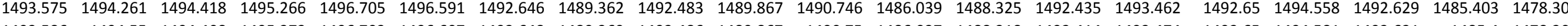
\begin{tabular}{lllllllllllllllllllll}
1493.586 & 1494.55 & 1494.408 & 1495.273 & 1496.703 & 1496.607 & 1492.648 & 1489.369 & 1492.486 & 1489.867 & 1490.75 & 1486.037 & 1488.318 & 1492.414 & 1493.474 & 1492.65 & 1494.581 & 1492.631 & 1485.4 & 1478.309 \\
\hline
\end{tabular}

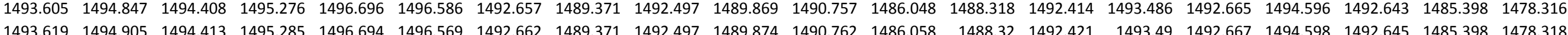


Well

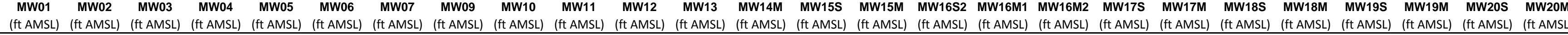

11/7/11 19:00 $11 / 7 / 1119: 30$ $11 / 7 / 1120: 00$ $11 / 7 / 1121: 00$ $11 / 7 / 1121: 30$ 11/7/11 22:00 11/7/11122:30 11/7/11 23:00 $11 / 7 / 1123: 30$ 11/8/11 0:00 11/8/11 0:30 11/8/111:30 $11 / 8 / 1112: 00$ $11 / 8 / 112: 30$ 11/8/11 3:00 11/8/11 3:30 11//8/11 4:00 11/8/11 4:30 11/8/115:00 $11 / 8 / 116: 00$ 1/8/11 6:30 11/8/11 7:00 11/8/11 7:30 $11 / 8 / 118: 00$ $11 / 8 / 118: 30$ 11/8/119.00 11/8/11 10:00 11/8/11 10:30 11/8/11 11:00 11/8/11 11:30 11/8/11 12:00 $11 / 8 / 1112: 30$ $11 / 8 / 1113.00$ 11/8/11 13:30 11/8/11 14:30 11//8/11 15:00 11/8/11 15:30 11/8/11 16:00 11/8/11 16:30 11/8/11 17:00 $11 / 8 / 1117.30$ 11/8/11 18:00 11/8/11 18:30 11//8/11 19:30 11/8/11 20:00 $11 / 8 / 1120: 30$ 11/8/11 21:00 $\begin{array}{llll}1494.139 & 1494.653 & 1496.429 & 1494.559\end{array}$ $1494.137 \quad 1494.646 \quad 1496.445 \quad 1494.549$ $\begin{array}{lllll}1494.132 & 1494.639 & 1496.445 & 1494.545 \\ 1494.144 & 1494.648 & 1496.415 & 1494554\end{array}$ $1494.149 \quad 1494.655 \quad 1496.413 \quad 1494.561$ $\begin{array}{lllll}1494.149 & 1494.655 & 1496.429 & 1494.563\end{array}$ $\begin{array}{lllll}1494.135 & 1494.639 & 1496.422 & 1494.554\end{array}$ $\begin{array}{lllll}1494.142 & 1494.644 & 1496.422 & 1494.554\end{array}$ $\begin{array}{lllll}1494.149 & 1494.651 & 1496.438 & 1494.559\end{array}$ $\begin{array}{llll}1494.149 & 1494.651 & 1496.424 & 1494.561 \\ 1494.151 & 1494.651 & 1496.408 & 1494.566\end{array}$ $\begin{array}{rrrr}1494.151 & 1494.651 & 1496.408 & 1494.566 \\ 1494.156 & 1494.658 & 1496.41 & 1494.575\end{array}$ $\begin{array}{rrrr}1494.156 & 1494.658 & 1496.41 & 1494.575 \\ 1494.146 & 1494.646 & 1496.441 & 1494.568\end{array}$ $\begin{array}{lllll}1494.139 & 1494.639 & 1496.483 & 1494.563\end{array}$ $\begin{array}{lllll}1494.153 & 1494.651 & 1496.436 & 1494.54 .57\end{array}$ $\begin{array}{lllll}1494.165 & 1494.665 & 1496.413 & 1494.589\end{array}$ $\begin{array}{lllll}1494.17 & 1494.674 & 1496.42 & 1494.605\end{array}$ $\begin{array}{llll}1494.146 & 1494.655 & 1496.427 & 1494.594\end{array}$ $\begin{array}{llll}1494.144 & 1494.648 & 1496.422 & 1494.582\end{array}$ $\begin{array}{llll}1494.144 & 1494.648 & 1496.42 & 1494.584 \\ 1494.153 & 1494.653 & 1496.417 & 1994591\end{array}$ $\begin{array}{lllll}1494.153 & 1494.653 & 1496.413 & 1494.591\end{array}$ $\begin{array}{llllll}1494.151 & 1494.655 & 1496.417 & 1494.594\end{array}$ $\begin{array}{lllll}1494.156 & 1494.653 & 1496.413 & 1494.594\end{array}$ $\begin{array}{lllll}1494.151 & 1494.648 & 1496.417 & 1494.594\end{array}$ 1494.156 $1494.653 \quad 1496.403 \quad 1494.596$ $\begin{array}{llll}1494.153 & 1494.651 & 1496.385 & 1494.596\end{array}$ $\begin{array}{llll}1494.153 & 1494.651 & 1496.387 & 1494.594\end{array}$ $\begin{array}{llll}1494.151 & 1494.646 & 1496.392 & 1494.591 \\ 1494.153 & 1494.641 & 1496.394 & 1494.584\end{array}$ $\begin{array}{lllll}1494.153 & 1494.641 & 1496.403 & 1494.584\end{array}$ $\begin{array}{lllll}1494.156 & 1494.641 & 1496.399 & 1494.587\end{array}$ $\begin{array}{lllll}1494.158 & 1494.646 & 1496.415 & 1494.589\end{array}$ $\begin{array}{lllll}1494.158 & 1494.646 & 1496.422 & 1494.596\end{array}$ $\begin{array}{lllll}1494.156 & 1494.646 & 1496.45 & 1494.596\end{array}$ $\begin{array}{llll}1494.165 & 1494.653 & 1496.459 & 1494.605 \\ 1494.174 & 1494.655 & 1466.427 & 194.622\end{array}$ $\begin{array}{lllll}1494.174 & 1494.665 & 1496.427 & 1494.622 \\ 1494.181 & 1994.679 & 1496.55 & 1494.638\end{array}$ $\begin{array}{rrrr}1494.181 & 1494.679 & 1496.45 & 1494.638 \\ 1494.177 & 1494.676 & 1496.436 & 1494.645\end{array}$ $\begin{array}{lllll}1494.158 & 1494.665 & 1496.443 & 1494.633\end{array}$ $\begin{array}{lllll}1494.17 & 1494.681 & 1496.448 & 1494.652\end{array}$ $\begin{array}{lllll}1494.167 & 1494.676 & 1496.434 & 1494.65\end{array}$ $\begin{array}{lllll}1494.172 & 1494.679 & 1496.403 & 1494.659\end{array}$ $\begin{array}{llll}1494.17 & 1494.681 & 1496.401 & 1494.659\end{array}$ $\begin{array}{llll}1494.165 & 1494.676 & 1496.378 & 1494.657\end{array}$ $\begin{array}{llll}1494.153 & 1494.66 & 1496.38 & 1494.643 \\ 1494.156 & 1494.655 & 1496349 & 1494.643\end{array}$ $\begin{array}{lllll}1494.153 & 1494.648 & 1496.331 & 1494.631\end{array}$ $\begin{array}{lllll}1494.156 & 1494.646 & 1496.314 & 1494.631\end{array}$ $\begin{array}{lllll}1494.146 & 1494.63 & 1496.298 & 1494.615\end{array}$ $\begin{array}{lllll}1494.146 & 1494.625 & 1496.289 & 1494.605\end{array}$ $\begin{array}{lllll}1494.144 & 1494.611 & 1496.277 & 1494.596\end{array}$ $\begin{array}{rrrrr}1494.139 & 1494.602 & 1496.27 & 1494.587 \\ 1494.142 & 1494.592 & 1496.256 & 1494.577\end{array}$

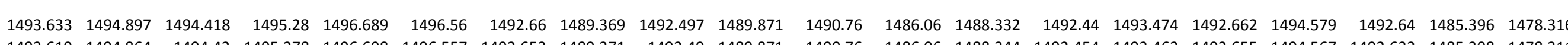
$\begin{array}{lllllllllllllllllllll}1493.619 & 1494.864 & 1494.42 & 1495.278 & 1496.698 & 1496.557 & 1492.653 & 1489.371 & 1492.49 & 1489.871 & 1490.76 & 1486.06 & 1488.344 & 1492.454 & 1493.462 & 1492.655 & 1494.567 & 1492.633 & 1485.398 & 1478.311 \\ 1493.619 & 1494821 & 1494.415 & 1495273 & 1496701 & 1496.571 & 1492648 & 1489369 & 1492.488 & 1489874 & 1490.76 & 1486.062 & 1488356 & 1492.48 & 1493.451 & 1492.653 & 1494551 & 1492.631 & 1485396 & 1478311\end{array}$

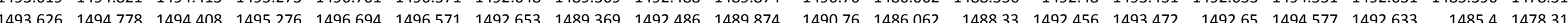

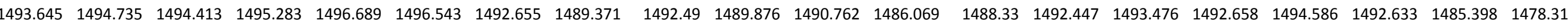
$\begin{array}{lllllllllllllllllllllll}1493.64 & 1494.701 & 1494.413 & 1495.283 & 1496.694 & 1496.538 & 1492.65 & 1489.371 & 1492.488 & 1489.876 & 1490.757 & 1486.069 & 1488.33 & 1492.449 & 1493.472 & 1492.653 & 1494.591 & 1492.636 & 1485.398 & 1478.309\end{array}$ $\begin{array}{llllllllllllllllllllllll}1493.643 & 1494.68 & 1494.408 & 1495.276 & 1496.691 & 1496.55 & 1492.646 & 1489.364 & 1492.486 & 1489.874 & 1490.757 & 1486.071 & 1488.353 & 1492.484 & 1493.453 & 1492.65 & 1494.563 & 1492.629 & 1485.396 & 1478.306\end{array}$ $\begin{array}{lllllllllllllllllllllll}1493.643 & 1494.656 & 1494.418 & 1495.276 & 1496.691 & 1496.545 & 1492.646 & 1489.362 & 1492.486 & 1489.874 & 1490.76 & 1486.069 & 1488.342 & 1492.473 & 1493.462 & 1492.648 & 1494.579 & 1492.626 & 1485.398 & 1478.304\end{array}$ $\begin{array}{llllllllllllllllllll}1493.643 & 1494.636 & 1494.422 & 1495.28 & 1496.701 & 1496.545 & 1492.648 & 1489.364 & 1492.486 & 1489.874 & 1490.76 & 1486.074 & 1488.332 & 1492.468 & 1493.467 & 1492.653 & 1494.586 & 1492.631 & 1485.398 & 1478.306\end{array}$

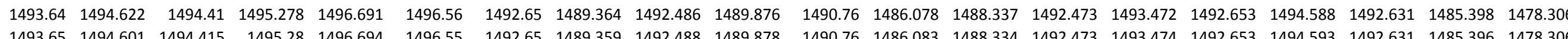

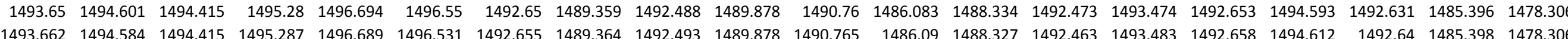

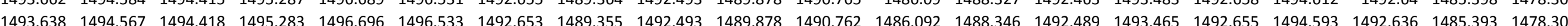

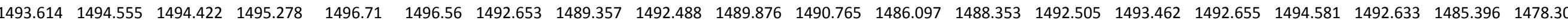
$\begin{array}{llllllllllllllllllllll}1493.624 & 1494.541 & 1494.418 & 1495.278 & 1496.708 & 1496.6 & 1492.655 & 1489.355 & 1492.493 & 1489.88 & 1490.769 & 1486.099 & 1488.337 & 1492.487 & 1493.474 & 1492.66 & 1494.603 & 1492.64 & 1485.396 & 1478.306\end{array}$ $\begin{array}{llllllllllllllllllll}1493.652 & 1494.524 & 1494.41 & 1495.29 & 1496.694 & 1496.557 & 1492.665 & 1489.359 & 1492.502 & 1489.883 & 1490.774 & 1486.108 & 1488.32 & 1492.461 & 1493.5 & 1492.667 & 1494.645 & 1492.647 & 1485.398 & 1478.311\end{array}$ $\begin{array}{llllllllllllllllllllll}1493.654 & 1494.51 & 1494.425 & 1495.303 & 1496.694 & 1496.531 & 1492.674 & 1489.366 & 1492.509 & 1489.887 & 1490.181 & 1486.115 & 1488.32 & 1492.456 & 1493.514 & 1492.674 & 1494.652 & 1492.657 & 1485.396 & 1478.318\end{array}$

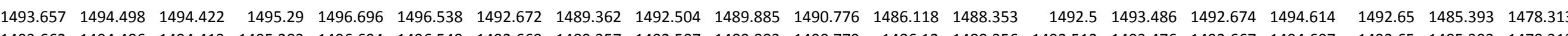

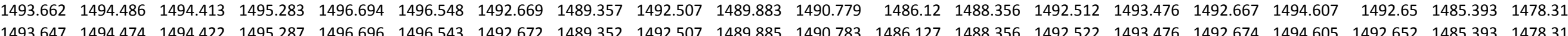
$\begin{array}{llllllllllllllllllll}1493.647 & 1494.474 & 1494.422 & 1495.287 & 1496.696 & 1496.543 & 1492.672 & 1489.352 & 1492.507 & 1489.885 & 1490.783 & 1486.127 & 1488.356 & 1492.522 & 1493.476 & 1492.674 & 1494.605 & 1492.652 & 1485.393 & 1478.313 \\ 1493.662 & 1494.464 & 1494.42 & 1495287 & 1496691 & 1496543 & 1492674 & 1489357 & 1492507 & 1489885 & 1490786 & 1486129 & 1488351 & 1492515 & 1493.888 & 1492679 & 1494614 & 1492655 & 1485393 & 1478318\end{array}$ $\begin{array}{llllllllllllllllllllllll}1493.662 & 1494.457 & 1494.418 & 1495.287 & 1496.694 & 1496.536 & 1492.676 & 1489.352 & 1492.511 & 1489.887 & 1490.786 & 1486.134 & 1488.349 & 1492.515 & 1493.488 & 1492.676 & 1494.624 & 1492.657 & 1485.391 & 1478.318\end{array}$ $\begin{array}{llllllllllllll} & \end{array}$ $\begin{array}{llllllllllllllllllllllll}1493.664 & 1494.44 & 1494.415 & 1495.287 & 1496.689 & 1496.54 & 1492.679 & 1489.357 & 1492.514 & 1489.887 & 1490.788 & 1486.129 & 1488.346 & 1492.524 & 1493.495 & 1492.681 & 1494.621 & 1492.662 & 1485.393 & 1478.318\end{array}$

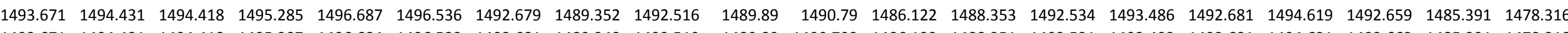

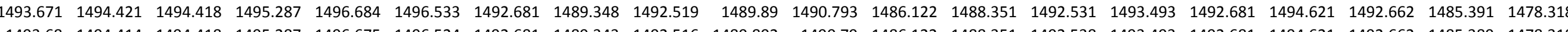

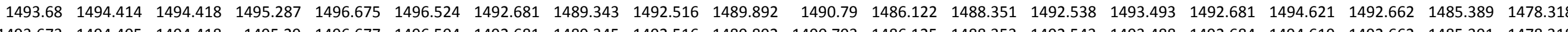

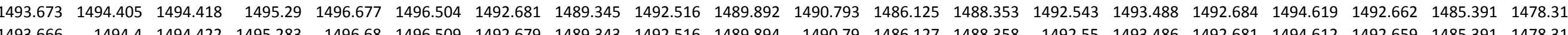
$\begin{array}{llllllllllllllllllll}1493.666 & 1494.4 & 1494.422 & 1495.283 & 1496.68 & 1496.509 & 1492.679 & 1489.343 & 1492.516 & 1489.894 & 1490.79 & 1486.127 & 1488.358 & 1492.55 & 1493.486 & 1492.681 & 1494.612 & 1492.659 & 1485.391 & 1478.316 \\ 1493.671 & 1494.39 & 1494.427 & 1495.278 & 1496.682 & 1496516 & 1492.676 & 1489.341 & 1492511 & 1489.894 & 1490.79 & 1486.132 & 1488.353 & 1492552 & 1493.481 & 1492.681 & 1494.612 & 1492.657 & 1485391 & 1478313\end{array}$ $\begin{array}{llllllllllllllllllll}1493.647 & 1494.385 & 1494.437 & 1495.278 & 1496.691 & 1496.514 & 1492.679 & 1489.341 & 1492.514 & 1489.894 & 1490.793 & 1486.136 & 1488.356 & 1492.552 & 1493.483 & 1492.679 & 1494.61 & 1492.659 & 1485.391 & 1478.313\end{array}$ $\begin{array}{lllllllllllllllllllll}1493.659 & 1494.383 & 1494.425 & 1495.278 & 1496.682 & 1496.526 & 1492.679 & 1489.334 & 1492.516 & 1489.897 & 1490.793 & 1486.139 & 1488.358 & 1492.559 & 1493.481 & 1492.679 & 1494.61 & 1492.662 & 1485.389 & 1478.316\end{array}$

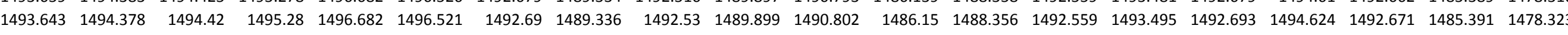

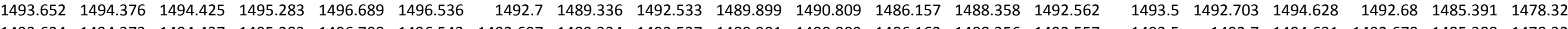

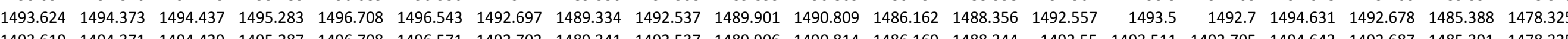
$\begin{array}{llllllllllllllllllll}1493.619 & 1494.371 & 1494.429 & 1495.287 & 1496.708 & 1496.571 & 1492.702 & 1489.341 & 1492.537 & 1489.906 & 1490.814 & 1486.169 & 1488.344 & 1492.55 & 1493.511 & 1492.705 & 1494.643 & 1492.687 & 1485.391 & 1478.325 \\ 1493.622 & 1494.371 & 1494.429 & 1495292 & 1496712 & 1496581 & 1492709 & 1489345 & 1492.547 & 1489.911 & 1490818 & 1486173 & 1488.33 & 149252 & 1493532 & 1492.712 & 1494.671 & 1492.692 & 1485393 & 1478327\end{array}$

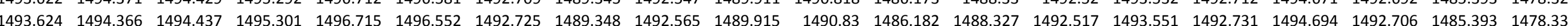
$\begin{array}{lllllllllllllllllllll}1493.633 & 1494.366 & 1494.429 & 1495.306 & 1496.708 & 1496571 & 1492.728 & 1498.352 & 1492561 & 1489.918 & 1490.828 & 1486.185 & 1488.339 & 1492.52 & 1493.549 & 1492.729 & 1494.69 & 1492.706 & 1485.396 & 1478.337\end{array}$ $\begin{array}{llllllllllllllllllllll}1493.645 & 1494361 & 1494.427 & 1495296 & 1496703 & 1496557 & 1492723 & 1489348 & 1492561 & 1489918 & 149083 & 1486185 & 1488356 & 1492543 & 1493535 & 1492724 & 1494.668 & 1492.704 & 1485.391 & 1478334\end{array}$ $\begin{array}{lllllllllllllllllllll}1493.64 & 1494.361 & 1494.427 & 1495.308 & 1496.708 & 1496.562 & 1492.732 & 1489.352 & 1492.568 & 1489.925 & 1490.837 & 1486.196 & 1488.342 & 1492.529 & 1493.556 & 1492.736 & 1494.697 & 1492.713 & 1485.396 & 1478.341\end{array}$

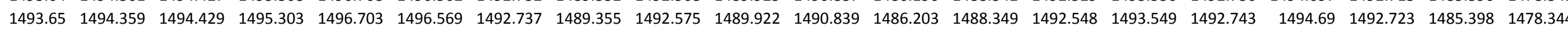
$\begin{array}{llllllllllllllllllll}1493.669 & 1494.357 & 1494.427 & 1495.308 & 1496.696 & 1496.557 & 1492.746 & 1489.35 & 1492.582 & 1489.927 & 1490.835 & 1486.206 & 1488.344 & 1492.55 & 1493.556 & 1492.748 & 1494.699 & 1492.727 & 1485.4 & 1478.348 \\ 143 & & & \end{array}$

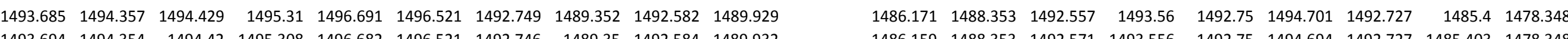

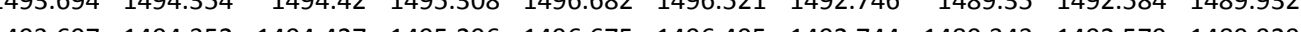

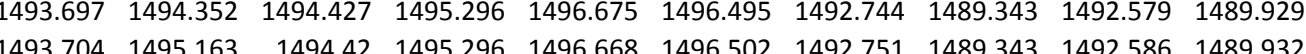
$\begin{array}{llllllllll}1493.725 & 1495.819 & 1494.422 & 1495.287 & 1496.654 & 1496.471 & 1492.756 & 1489.336 & 1492.594 & 1489.932\end{array}$ $\begin{array}{llllllllllll}1493.727 & 1496.082 & 1494.422 & 1495.29 & 1496.647 & 1496.457 & 1492.76 & 1489.336 & 1492.596 & 1489.934\end{array}$ $\begin{array}{llllllllllll}1493.734 & 1496.544 & 1494.418 & 1495.273 & 1496.638 & 1496.445 & 1492.753 & 1489.329 & 1492.591 & 1489.932\end{array}$ $\begin{array}{lllllllllll}1493.739 & 1498.314 & 1494.422 & 1495.266 & 1496.629 & 1496.428 & 1492.746 & 1489.322 & 1492.586 & 1489.929\end{array}$ $\begin{array}{lllllllllll}1493.739 & 1498.724 & 1494.42 & 1495.259 & 1496.622 & 1496.418 & 1492.746 & 1489.319 & 1492.584 & 1489.929\end{array}$ $\begin{array}{llllllllllll}1493.739 & 1498.812 & 1494.418 & 1455.252 & 1466.62 & 1466.40 & 1492.746 & 1489.31 & 1492.584 & 1489.929\end{array}$

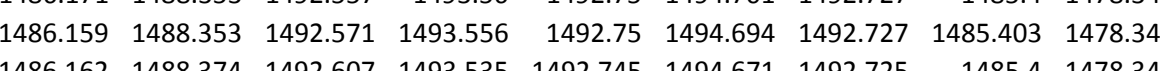

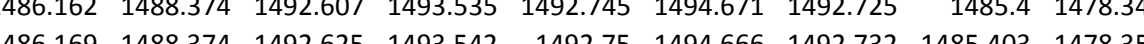
$\begin{array}{lllllllllll}1486.173 & 1488.379 & 1492.646 & 1493.532 & 1492.757 & 1494.661 & 1492.734 & 1485.405 & 1478.355\end{array}$ $\begin{array}{lllllllllll}1486.18 & 1488.379 & 1492.663 & 1493.528 & 1492.762 & 1494.661 & 1492.739 & 1485.407 & 1478.358\end{array}$ $\begin{array}{lllllllll}1486.182 & 1488.393 & 1492.691 & 1493.516 & 1492.752 & 1494.638 & 1492.734 & 1485.407 & 1478.35\end{array}$ $\begin{array}{llllllllll}1486.187 & 1488.393 & 1492.703 & 1493.509 & 1492.75 & 1494.635 & 1492.727 & 1485.405 & 1478.358\end{array}$ $\begin{array}{lllllllllll}1486.194 & 1488.398 & 1492.729 & 1493.5 & 1492.748 & 1494.626 & 1492.727 & 1485.405 & 1478.358\end{array}$ $\begin{array}{llllllllll}1486.201 & 1488.407 & 1492.755 & 1493.493 & 1492.748 & 1494.617 & 1492.723 & 1485.407 & 1478.358 \\ 148621 & 1488.407 & 1492.773 & 1493.488 & 1492.745 & 1494.617 & 1492.725 & 1485.41 & 1478.365\end{array}$ 
Well

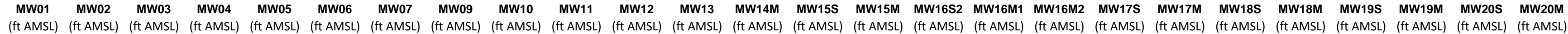

$11 / 8 / 1122: 00$ $11 / 8 / 1122: 30$ $11 / 8 / 1123.30$ 11/8/11110:00 11/9/11 0:00 11/9/11 0:30 11/9/11 1:30 11/9/112:00 11/9/112:30 11/9/11 3:00 11/9/11 3:30 11/9/111 4:30 11/9/11/11 5:00 11/9/11 5:30 11/9/11 6:00 11/9/11 6:30 11/9/11 7:00 11/9/11 7:30 11/9/11 8:00 11/9/11 9:00 11/9/11 9:30 11/9/11 10:00 11/9/11 10:30 11/9/11 11:00 11/9/11 11:30 11/9/11 12:00 11/9/11 13:00 11/9/11 13:30 11/9/11 14:00 11/9/11 14:30 11/9/11 15:00 11/9/11 15:30 $11 / 9 / 1116.00$ 11/9/11 16:30 $11 / 9 / 1117: 30$ 11////11 18:00 11/9/11 18:30 11/9/11 19:00 11/9/11 19:30 $11 / 9 / 1120: 00$ (11///1121:00 11/9/11 21:00 $11 / 9 / 1122: 00$ 11///11 22:30 11/9/11 23:00 11/9/11 23:30 11/10/11 0:00 $\begin{array}{rrrr}1494.139 & 1494.583 & 1496.249 & 1494.57 \\ 1494.137 & 1494.576 & 1496.247 & 1494.563\end{array}$ $\begin{array}{lllll}1494.137 & 1494.576 & 1496.247 & 1494.563\end{array}$ $\begin{array}{llll}1494.137 & 1494.564 & 1496.249 & 1494.561\end{array}$ $\begin{array}{lrrrr}1494.139 & 1494.56 & 1496.242 & 1494.554\end{array}$ $\begin{array}{lllll}1494.139 & 1494.557 & 1496.244 & 1494.55\end{array}$ $\begin{array}{lllll}1494.137 & 1494.553 & 1496.244 & 1494.554\end{array}$ $\begin{array}{lllll}1494.137 & 1494.55 & 1496.23 & 1494.556\end{array}$ $\begin{array}{llll}1494.137 & 1494.55 & 1496.221 & 1494.559\end{array}$ $\begin{array}{rrrr}1494.137 & 1494.55 & 1496.216 & 1494.563 \\ 1494.132 & 1494.539 & 1469.212 & 1494.556\end{array}$ $\begin{array}{rrrr}1494.132 & 1494.539 & 1496.212 & 1494.556 \\ 1194.13 & 1494.532 & 1496214 & 1494.554\end{array}$ $\begin{array}{llll}1494.13 & 1494.532 & 1496.214 & 1494.554 \\ 1494.13 & 1494.532 & 1496.202 & 1494.556\end{array}$ $\begin{array}{lllll}1494.13 & 1494.525 & 1496.2 & 1494.556\end{array}$ $\begin{array}{llll}1494.13 & 1494.525 & 1496.2 & 1494.556 \\ 1494.13 & 1494.522 & 1496.2 & 1494.559\end{array}$ $\begin{array}{lllll}1494.125 & 1494.515 & 1496.2 & 1494.556\end{array}$ $\begin{array}{llll}1494.125 & 1494.515 & 1496.198 & 1494.556\end{array}$ $\begin{array}{lllll}1494.125 & 1494.513 & 1496.191 & 1494.563\end{array}$ $\begin{array}{llll}1494.125 & 1494.508 & 1496.181 & 1494.561\end{array}$ $\begin{array}{lllll}1494.125 & 1494.508 & 1496.167 & 1494.566\end{array}$ $\begin{array}{llll}1494.121 & 1494.497 & 1496.137 & 1494.563\end{array}$ $\begin{array}{lllll}1494.114 & 1494.485 & 1496.132 \quad 1494.556\end{array}$ $\begin{array}{lllll}1494.111 & 1494.476 & 1496.132 & 1494.549\end{array}$ $\begin{array}{lllll}1494.109 & 1494.464 & 1496.135 & 1494.542\end{array}$ $\begin{array}{lllll}1494.111 & 1494.462 & 1496.134 & 1494.538\end{array}$ $1494.109 \quad 1494.457 \quad 1496.139 \quad 1494.54$ $\begin{array}{rrrr}1494.109 & 1494.455 & 1496.151 & 1494.54 \\ 194.111 & 194.455 & 149616 & 149454\end{array}$ $\begin{array}{rrrr}1494.111 & 1494.455 & 1496.16 & 1494.54 \\ 1494.111 & 1494.455 & 1496.172 & 1494.545\end{array}$ $\begin{array}{lllll}1494.114 & 1494.459 & 1496.181 & 1494.552\end{array}$ $\begin{array}{lllll}1494.114 & 1494.464 & 1496.186 & 1494.561\end{array}$ $\begin{array}{lllll}1494.118 & 1494.469 & 1496.193 & 1494.57\end{array}$ $\begin{array}{lllll}1494.118 & 1494.473 & 1496.193 & 1494.58\end{array}$ $\begin{array}{lllll}1494.118 & 1494.473 & 1496.198 & 1494.587\end{array}$ $\begin{array}{llll}1494.116 & 1494.48 & 1496.2 & 1494.594\end{array}$ $\begin{array}{rrrr}1494.116 & 1494.478 & 1496.2 & 1494.603 \\ 1494.118 & 1494.485 & 1496.202 & 1494.61\end{array}$ $\begin{array}{llll}1494.118 & 1494.485 & 1496.202 & 1494.61 \\ 1494.118 & 1496 & 1496.198 & 1494.615\end{array}$ $\begin{array}{lllll}1494.121 & 1494.487 & 1496.198 & 1494.619\end{array}$ $\begin{array}{lllll}1494.118 & 1494.49 & 1496.2 & 1494.626\end{array}$ $1494.118 \quad 1494.487 \quad 1496.2 \quad 1494.629$ $1494.118 \quad 1494.49 \quad 1496.2 \quad 1494.631$ $1494.121 \quad 1494.49 \quad 1496.198 \quad 1494.633$ $1494.1181494 .492 \quad 1496.19514994 .636$

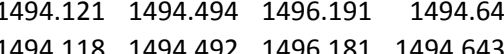
$1494.1161494 .492 \quad 1496.1811494 .638$ $1494.114 \quad 1494.492 \quad 1496.181 \quad 14494.638$ $1494.114 \quad 1494.49 \quad 1496.186 \quad 1494.636$ $1494.116 \quad 1494.49 \quad 1496.184 \quad 1494.636$ $1494.116 \quad 1494.49 \quad 1496.186 \quad 1494.636$ $\begin{array}{llll}1494.116 & 1494.492 & 1496.188 & 1494.638 \\ 1494.116 & 1494.492 & 1496.202 & 1494.638\end{array}$ $\begin{array}{rrrrrrrrrr}1493.739 & 1498.525 & 1494.42 & 1495.234 & 1496.598 & 1496.39 & 1492.744 & 1489.312 & 1492.582 & 1489.929 \\ 1493.734 & 1500391 & 1494.415 & 1495.227 & 1496.594 & 1496385 & 1492737 & 1489.308 & 1492.577 & 1489\end{array}$

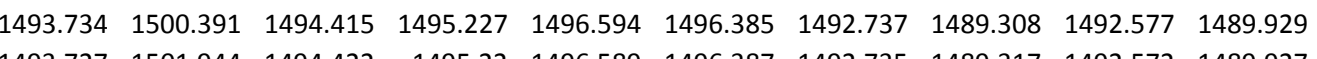
$\begin{array}{llllllllll}143.727 & 1501.944 & 1494.422 & 1495.22 & 1496.589 & 1496.387 & 1492.735 & 1489.317 & 1492.572 & 1489.927\end{array}$ $\begin{array}{llllllllllll}1493.711 & 1503.715 & 1494.418 & 1495.208 & 1496.582 & 1496.392 & 1492.732 & 1489.303 & 1492.57 & 1489.932\end{array}$ $\begin{array}{llllllllll}1493.704 & 1503.725 & 1494.418 & 1495.206 & 1496.58 & 1496.387 & 1492.728 & 1489.301 & 1492.568 & 1489.932\end{array}$ $\begin{array}{lllllllllll}1493.694 & 1503.543 & 1494.415 & 1495.201 & 1496.577 & 1496.392 & 1492.728 & 1489.303 & 1492.568 & 1489.932\end{array}$ $\begin{array}{lllllllllll}1493.692 & 1502.978 & 1494.413 & 1495.197 & 1496.57 & 1496.392 & 1492.725 & 1489.301 & 1492.565 & 1489.932\end{array}$ $\begin{array}{llllllllll}1493.697 & 1501.928 & 1494.413 & 1495.197 & 1496.563 & 1496.385 & 1492.725 & 1489.301 & 1492.563 & 1489.934\end{array}$ $\begin{array}{llllllllll}1493.697 & 1501.028 & 1494.415 & 1495.194 & 1496.559 & 1496.38 & 1492.725 & 4489.298 & 1492.563 & 1489.936\end{array}$ $\begin{array}{llllllllll}1493.685 & 1500.286 & 1494.415 & 1495.185 & 1496.556 & 1496.375 & 1492.723 & 1489.294 & 1492.561 & 1489.936 \\ 1493.685 & 1499.659 & 1494.415 & 1495.178 & 1496549 & 1496.373 & 1492721 & 1489.296 & 1492558 & 1489936\end{array}$ $\begin{array}{llllllllllll}1493.676 & 1499.121 & 1494.415 & 1495.173 & 1496.547 & 1496.375 & 1492.721 & 1489.294 & 1492.558 & 1489.936\end{array}$ $\begin{array}{llllllllllll}1493.68 & 1498.652 & 1494.413 & 1495.169 & 1496.542 & 1496.37 & 1492.721 & 1489.287 & 1492.556 & 1489.936\end{array}$ $\begin{array}{lllllllllll}1493.673 & 1498.245 & 1494.415 & 1495.166 & 1496.538 & 1496.366 & 1492.718 & 1489.289 & 1492.556 & 1489.939\end{array}$ $\begin{array}{llllllllllll}1493.666 & 1497.886 & 1494.413 & 1495.157 & 1496.535 & 1496.368 & 1492.718 & 1489.284 & 1492.556 & 1489.939\end{array}$ $\begin{array}{lllllllllll}1493.662 & 1497.57 & 1494.41 & 1495.153 & 1496.531 & 1496.368 & 1492.718 & 1489.289 & 1492.556 & 1489.939\end{array}$ $\begin{array}{llllllllll}1493.657 & 1497.293 & 1494.41 & 1495.153 & 1496.529 & 1496.37 & 1492.721 & 1489.28 & 1492.554 & 1489.941\end{array}$

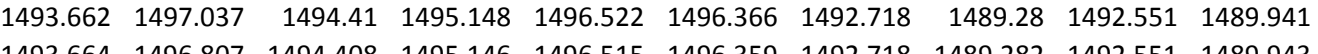
$\begin{array}{lllllllllll}1493.664 & 1496.807 & 1494.408 & 1495.146 & 1496.515 & 1496.359 & 1492.718 & 1489.282 & 1492.551 & 1489.943 \\ 1493.669 & 1496599 & 1494.408 & 1495.139 & 1496.501 & 1496347 & 1492.714 & 1489.28 & 1492549 & 1489943\end{array}$ $\begin{array}{lllllllllllll}1493.669 & 1496.41 & 1494.406 & 1495.132 & 1496.494 & 1496.33 & 1492.716 & 1489.282 & 1492.551 & 1489.941\end{array}$ $\begin{array}{llllllllll}1493.666 & 1496.226 & 1494.406 & 1495.122 & 1496487 & 1496325 & 1492.709 & 1489.277 & 1492.544 & 1489.913\end{array}$ $\begin{array}{llllllllllll}1493.659 & 1496.175 & 1494.403 & 1495.113 & 1496.484 & 1496.318 & 1492.704 & 1489.273 & 1492.54 & 1489.943\end{array}$ $\begin{array}{lllllllllll}1493.647 & 1496.134 & 1494.408 & 1495.101 & 1496.48 & 1496.318 & 1492.693 & 1489.275 & 1492.528 & 1489.943\end{array}$ $\begin{array}{llllllllll}1493.643 & 1496.094 & 1494.403 & 1495.097 & 1496.473 & 1496.323 & 1492.69 & 1489.27 & 1492.528 & 1489.941\end{array}$ $\begin{array}{lllllllllll}1493.629 & 1496.055 & 1494.403 & 1495.092 & 1496.475 & 1496.323 & 1492.69 & 1489.266 & 1492.528 & 1489.943\end{array}$ $\begin{array}{llllllllll}1493.614 & 1496.02 & 1494.408 & 1495.09 & 1496.477 & 1496.332 & 1492.688 & 1489.266 & 1492.526 & 1489.943 \\ 1493.603 & 1495.982 & 1494.403 & 1495.088 & 1496477 & 1496344 & 1492688 & 1489266 & 1492523 & 149943\end{array}$ $\begin{array}{llllllllll}1493.603 & 1495.982 & 1494.403 & 1495.088 & 1496.477 & 1496.344 & 1492.688 & 1489.266 & 1492.523 & 1489.943 \\ 1493.591 & 1495.945 & 1494.408 & 1495085 & 1496.482 & 1496354 & 1492688 & 1489261 & 1492528 & 1489.946\end{array}$ $\begin{array}{lllllllllll}1493.575 & 1495.912 & 1494.408 & 1495.088 & 1496.484 & 1496.368 & 1492.693 & 1489.268 & 1492.526 & 1489.948\end{array}$ $\begin{array}{lllllllllll}1493.57 & 1495.881 & 1494.408 & 1495.088 & 1496.489 & 1496.38 & 1492.693 & 1489.266 & 1492.53 & 1489.948\end{array}$ $\begin{array}{llllllllllll}1493.563 & 1495.85 & 1494.406 & 1495.092 & 1496.491 & 1496.385 & 1492.695 & 1489.266 & 1492.533 & 1489.95\end{array}$ $\begin{array}{lllllllllll}1493.558 & 1495.819 & 1494.406 & 1495.097 & 1496.491 & 1496.39 & 1492.697 & 1489.266 & 1492.535 & 1489.955\end{array}$ $\begin{array}{lllllllllll}1493.551 & 1495.787 & 1494.406 & 1495.095 & 1496.494 & 1496.397 & 1492.704 & 1489.268 & 1492.537 & 1489.955\end{array}$ $\begin{array}{lllllllllll}1493.551 & 1495.759 & 1494.406 & 1495.099 & 1496.496 & 1496.402 & 1492.707 & 1489.27 & 1492.537 & 1489.962 \\ 1493549 & 1495.73 & 1494.408 & 1495099 & 1496.496 & 1496404 & 1492.707 & 1489268 & 1492542 & 148962\end{array}$

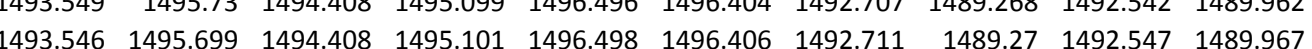
$\begin{array}{lllllllllllll}1493.549 & 1495.673 & 1494.406 & 1495.104 & 1496.496 & 1496.411 & 1492.711 & 1489.273 & 1492.549 & 1489.969\end{array}$ $\begin{array}{lllllllllll}1493.551 & 1495.649 & 1494.406 & 1495.104 & 1496.491 & 1496.409 & 1492.718 & 1489.273 & 1492.549 & 1489.9691\end{array}$ $\begin{array}{llllllllll}1493.553 & 1495.622 & 1494.406 & 1495.106 & 1496.494 & 1496.406 & 1492.718 & 1489.268 & 1492.554 & 1489.974\end{array}$ $\begin{array}{lllllllllll}1493.551 & 1495.596 & 1494.41 & 1495.104 & 1496.496 & 1496.411 & 1492.721 & 1489.266 & 1492.554 & 1489.976\end{array}$ $\begin{array}{llllllllll}1493.551 & 1495.565 & 1494.406 & 1495.106 & 1496.496 & 1496.414 & 1492.62 & 1489.27 & 1492.436 & 1489.978\end{array}$ $\begin{array}{llllllllll}1493.551 & 1495.548 & 1494.403 & 1495.106 & 1496.496 & 1496.414 & 1492.508 & 1489.263 & 1492.331 & 1489.978\end{array}$ $\begin{array}{lllllllllll}1493.556 & 1455.524 & 1494.406 & 1495.108 & 1496.496 & 1496.41 & 1492.583 & 1489.27 & 1492.408 & 1489.983\end{array}$ $\begin{array}{lllllllllllll}1493.563 & 1495.5 & 1494.408 & 1455.108 & 1496.494 & 1496.411 & 1492.615 & 1489.263 & 1492.443 & 1489.985\end{array}$ $\begin{array}{llllllllllll}1493.565 & 1495.455 & 1494.406 & 1495.106 & 1496.489 & 1496.399 & 1492.648 & 1489.261 & 1492.476 & 1489.988\end{array}$ $\begin{array}{llllllllllll}1493.567 & 1495.431 & 1494.403 & 1495.104 & 1496.489 & 1496.397 & 1492.655 & 1489.263 & 1492.486 & 1489.99\end{array}$ $\begin{array}{lllllllllll}1493.563 & 1495.412 & 1494.406 & 1495.099 & 1496.489 & 1496.399 & 1492.66 & 1489.263 & 1492.493 & 1489.992\end{array}$ $\begin{array}{lllllllllll}1493.56 & 1495.39 & 1494.403 & 1495.099 & 1496.487 & 1496.404 & 1492.665 & 1489.261 & 1492.497 & 1489.992\end{array}$ $\begin{array}{llllllllllll}1493.558 & 1495.371 & 1494.406 & 1495.101 & 1496.489 & 1496.402 & 1492.672 & 1489.259 & 1492.502 & 1489.995\end{array}$ $\begin{array}{llllllllll}1493.56 & 1495.35 & 1494.408 & 1495.101 & 1496.489 & 1496.406 & 1492.676 & 1489.261 & 1492.509 & 1489.995\end{array}$ $\begin{array}{lrrrrrrrr}1486.219 & 1488.41 & 1492.795 & 1493.49 & 1492.748 & 1494.614 & 1492.725 & 1485.407 & 1478.369\end{array}$ $\begin{array}{lrrrrrrrrrr}1486.224 & 1488.412 & 1492.804 & 1493.481 & 1492.738 & 1494.607 & 1492.718 & 1485.41 & 1478.367 \\ 1486222 & 1488.41 & 1492811 & 1493.476 & 1492.733 & 1494603 & 1492.713 & 1485.41 & 4478367\end{array}$

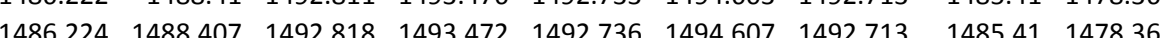
$\begin{array}{lllllllllll}1486.229 & 1488.4 & 1492813 & 1493.472 & 1492.731 & 1494.605 & 1492.711 & 1485412 & 1478.365\end{array}$ $\begin{array}{lllllllllllll}1486.231 & 1488395 & 1492809 & 1493.474 & 1492731 & 149461 & 1492.7108 & 1485.412 & 1478365\end{array}$ $\begin{array}{lllllllllll}1486.236 & 1488.398 & 1492.806 & 1493.472 & 1492.729 & 1494.614 & 1492.708 & 1485.412 & 1478.362\end{array}$ $\begin{array}{llllllllll}1486.238 & 1488.393 & 1492.806 & 1493.476 & 1492.729 & 1494.614 & 1492.706 & 1485.412 & 1478.362\end{array}$ $\begin{array}{lllllllll}1486.243 & 1488.393 & 1492.802 & 1493.476 & 1492.726 & 1494.619 & 1492.704 & 1485.412 & 1478.362\end{array}$ $\begin{array}{lllllllll}1486.245 & 1488.388 & 1492.802 & 1493.479 & 1492.726 & 1494.624 & 1492.706 & 1485.414 & 1478.365\end{array}$ $\begin{array}{lllllllllll}1486.25 & 1488.395 & 1492.816 & 1493.472 & 1492.724 & 1494.619 & 1492.701 & 1485.412 & 14788.365 \\ 1486.25 & 1488395 & 149282 & 1493.469 & 1492.724 & 1494614 & 1492.701 & 1485.412 & 1478.362\end{array}$ $\begin{array}{lllllllll}1486.25 & 1488.339 & 1492.82 & 1493.469 & 1492.724 & 1494.614 & 1429.701 & 1485.412 & 1488.362\end{array}$ $\begin{array}{lllllllllll}1486.256 & 1488.391 & 1492.825 & 1493.462 & 1492.721 & 1494.626 & 1492.701 & 1485.412 & 1478.365\end{array}$ $\begin{array}{llllllllll}1486.261 & 1488.388 & 1492.827 & 1493.469 & 1492.721 & 1494.624 & 1492.699 & 1485.412 & 1478.365\end{array}$ $\begin{array}{lllllllllll}1486.266 & 1488.393 & 1492.832 & 1493.465 & 1492.719 & 1494.621 & 1492.699 & 1485.41 & 1478.365\end{array}$ $\begin{array}{llllllllll}1486.268 & 1488.386 & 1492.83 & 1493.467 & 1492.721 & 1494.626 & 1492.697 & 1485.41 & 1478.3656\end{array}$ $\begin{array}{lllllllll}1486.266 & 1488.381 & 1492.825 & 1493.472 & 1492.719 & 1494.628 & 1492.699 & 1485.41 & 1478.365\end{array}$ $\begin{array}{lllllllll}1486.259 & 1488.381 & 1492.825 & 1493.465 & 1492.719 & 1494.633 & 1492.697 & 1485.41 & 1478.365\end{array}$ $\begin{array}{lllllllllll}1486.247 & 1488.37 & 1492.82 & 1493.472 & 1492.717 & 1494.638 & 1492.699 & 1485.407 & 1478.365\end{array}$ $\begin{array}{llllllllll}1486.247 & 1488.379 & 1492.832 & 1493.462 & 1492.714 & 1494.626 & 1492.692 & 1485.405 & 1478.36\end{array}$ $\begin{array}{llllllllll}1486.25 & 1488.384 & 1492839 & 1493.451 & 1492.712 & 1494.617 & 1492.69 & 1485.4 & 1478.358\end{array}$ $\begin{array}{lllllllll}1486.252 & 1488.381 & 1492.839 & 1493.448 & 1492.703 & 1494.612 & 1492.685 & 1485.398 & 1478.35\end{array}$ $\begin{array}{llllllllll}1486.252 & 1488.377 & 1492.827 & 1493.434 & 1492.695 & 1494.605 & 1492.673 & 1485.398 & 1478.353\end{array}$ $\begin{array}{llllllllll}1486.254 & 1488.372 & 1492.809 & 1493.434 & 1492.695 & 1494.603 & 1492.673 & 1485.398 & 1478.353\end{array}$ $\begin{array}{llllllllll}1486.259 & 1488.36 & 1492.792 & 1493.432 & 1492.693 & 1494.61 & 1492.669 & 1485.398 & 1478.346\end{array}$ $\begin{array}{lllllllll}1486.261 & 1488.358 & 1492.783 & 1493.434 & 1492.691 & 1494.607 & 1492.671 & 1485.396 & 1478.348\end{array}$ $\begin{array}{llllllllll}1486.261 & 1488.349 & 1492.766 & 1493.434 & 1492.688 & 1494.612 & 1492.669 & 1485.398 & 1478.344 \\ 1486.263 & 1488.342 & 1492745 & 1493.437 & 1492.691 & 1494.619 & 1492.673 & 1485396 & 1478344\end{array}$ $\begin{array}{lllllllllll}4866.263 & 1488.332 & 1492.726 & 1493.446 & 1492.695 & 1494.621 & 1492.673 & 1485.398 & 1478.341\end{array}$ $\begin{array}{llllllllll}1486.266 & 1488.327 & 1492.708 & 1493.455 & 1492.693 & 1494.633 & 1492.676 & 1485.396 & 1478.344\end{array}$ $\begin{array}{lllllllllll}1486.27 & 1488.313 & 1492.686 & 1493.467 & 1492.698 & 1494.65 & 1492.676 & 1485.398 & 1478.344\end{array}$ $\begin{array}{lllllllll}1486.273 & 1488.309 & 1492.67 & 1493.469 & 1492.703 & 1494.659 & 1422.678 & 1485.398 & 1478.34\end{array}$ $\begin{array}{lllllllll}1486.273 & 1488.306 & 1492.658 & 1493.474 & 1492.705 & 1494.666 & 1492.683 & 1485.398 & 1478.344\end{array}$ $\begin{array}{llllllllll}1486.277 & 1488.302 & 1492.649 & 1493.483 & 1492.707 & 1494.675 & 1492.687 & 1485.398 & 1478.344\end{array}$

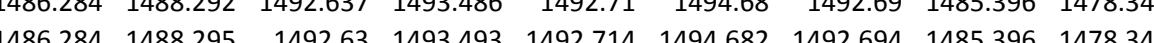
$\begin{array}{llllllllll}1486.287 & 1488.295 & 1492.625 & 1493.493 & 14292.714 & 1494.69 & 1492.692 & 1485.396 & 1478.344\end{array}$ $\begin{array}{llllllllll}1486.291 & 1488.29 & 1492.621 & 1493.495 & 1492.717 & 1494.697 & 1492.699 & 1485.396 & 1478.344\end{array}$ $\begin{array}{llllllllll}1486.293 & 1488.29 & 1492.623 & 1493.5 & 1492.719 & 1494.697 & 1492.699 & 1485.393 & 1478.344\end{array}$ $\begin{array}{llllllllll}1486.298 & 1488.285 & 1492.625 & 1493.5 & 1492.721 & 1494.701 & 1492.699 & 1485.393 & 1478.344\end{array}$ $\begin{array}{lllllllllll}1486.296 & 1488.288 & 1492.517 & 1493.493 & 1492.624 & 1494.697 & 1492.596 & 1485.393 & 1478.34\end{array}$ $\begin{array}{llllllllll}1486.298 & 1488.283 & 1492.407 & 1493.425 & 1492.511 & 1494.699 & 1492.495 & 1485.393 & 1478.341\end{array}$ $\begin{array}{lllllllll}1486.3 & 1488.283 & 1492.491 & 1493.444 & 1492.584 & 1494.697 & 1492.568 & 1485.393 & 1478.341 \\ 1486303 & 148828 & 1492531 & 1493.62 & 1492.617 & 1494.699 & 1492.598 & 1485393 & 1478339\end{array}$

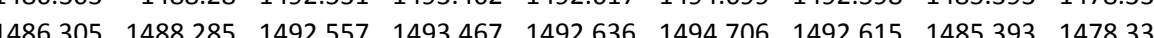
$\begin{array}{lllllllllll}1486.307 & 1488.288 & 1492.585 & 1493.469 & 1492.648 & 1494.701 & 1492.626 & 1485.391 & 1478.334\end{array}$ $\begin{array}{lllllllll}1486.31 & 1488.29 & 1492.602 & 1493.469 & 1492.658 & 1494.697 & 1492.636 & 1485391 & 1478.334\end{array}$ $\begin{array}{lllllllll}1486.31 & 1488.29 & 1492.618 & 1493.469 & 1492.662 & 1494.692 & 1492.645 & 1485.391 & 1478.33\end{array}$ $\begin{array}{lllllllll}1486.31 & 1488.288 & 1492.63 & 1493.474 & 1492.667 & 1494.694 & 1492.65 & 1485.388 & 1478.3\end{array}$ $\begin{array}{lllllllllll}1486.31 & 1488.283 & 1492.635 & 1493.476 & 1492.672 & 1494.697 & 1492.652 & 1485.391 & 1478.327\end{array}$ $\begin{array}{llllllllll}1486.31 & 1488.283 & 1492.639 & 1493.479 & 1492.679 & 1494.699 & 1492.659 & 1485.388 & 1478.327 \\ 1486312 & 1488.285 & 1492.649 & 1493.483 & 1492.681 & 1494.699 & 1492.659 & 1485.389 & 1478.327\end{array}$ 
Well

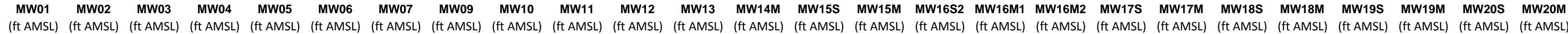

11/10/11 1:00 11/10/11 1:30 $11 / 10 / 112: 30$ $11 / 10 / 113: 00$ $11 / 10 / 113: 30$ $11 / 10 / 114: 00$ 11/10/11 4:30 11/10/11 5:00 11/10/11 5:30 $11 / 10 / 116: 00$ 11/10/11 6:30 $11 / 10 / 117: 30$ 11/10/11 7:30 $8: 00$ $11 / 10 / 118: 30$ 11/10/11 9:00 11/10/11 9:30 11/10/11 10:00 11/10/11 10:30 11/10/11 11:30 $1 / 10 / 1112: 00$ $11 / 10 / 1112: 00$ 1/10/11 13:00 11/10/11 13:30 11/10/11 14:00 $11 / 10 / 1114: 30$ $11 / 10 / 11$ 15:00 11/10/11 16:00 $11 / 10 / 1116: 30$ 11/10/11 17:00 $11 / 10 / 11$ 17:30 $11 / 10 / 11$ 18:00 $11 / 10 / 1118: 30$ $11 / 10 / 1119: 00$ $11 / 10 / 1119: 30$
$11 / 10 / 1120: 00$ $11 / 10 / 1120: 00$ 11/10/11 21:00 $11 / 10 / 1121: 30$ 11/10/11 22:00 $11 / 10 / 1122: 30$ 11/10/11 23:00 11/10/11 23:30 11/11/11 0:00 11/11/11 1:00 11/11/11 1:30 11/11/11 2:00 11/11/11 3:00 $\begin{array}{llll}1494.116 & 1494.494 & 1496.205 & 1494.64 \\ 1494.118 & 1494.497 & 1496207 & 1494.645\end{array}$ $\begin{array}{llll}1494.118 & 1494.497 & 1496.207 & 1494.645 \\ 149.123 & 1494.504 & 149620 & 1494.64\end{array}$ $\begin{array}{lllll}1494.123 & 1494.504 & 1496.209 & 1494.654\end{array}$ $\begin{array}{lllll}1494.121 & 1494.506 & 1496.226 & 1494.659\end{array}$ $\begin{array}{lllll}1494.123 & 1494.511 & 1496.23 & 1494.668\end{array}$ $\begin{array}{lllll}1494.123 & 1494.515 & 1496.237 & 1494.673\end{array}$ $\begin{array}{rrrr}1494.13 & 1494.518 & 1496.24 & 1494.685 \\ 1494.128 & 1494.525 & 1496.24 & 1494.692\end{array}$ $\begin{array}{lllll}1494.128 & 1494.529 & 1496.244 & 1494.696\end{array}$ $\begin{array}{rrrr}1494.128 & 1494.529 & 1496.244 & 1494.701 \\ 149413 & 194.534 & 1966242 & 1994.706\end{array}$ $\begin{array}{rrrr}1494.13 & 1494.534 & 1496.242 & 1494.706 \\ 1494.132 & 1494.536 & 1496.24 & 1494.71\end{array}$ $\begin{array}{llll}1494.132 & 1494.536 & 1496.237 & 1494.713\end{array}$ $\begin{array}{lllll}1494.13 & 1494.536 & 1496.233 & 1494.715\end{array}$ $\begin{array}{lllll}1494.13 & 1494.536 & 1496.23 & 1494.715\end{array}$ $\begin{array}{lllll}1494.13 & 1494.536 & 1496.235 & 1494.715\end{array}$ $\begin{array}{lllll}1494.13 & 1494.536 & 1496.237 & 1494.713\end{array}$ $\begin{array}{lllll}1494.132 & 1494.539 & 1496.242 & 1494.713\end{array}$ $\begin{array}{rrrr}1494.135 & 1494.541 & 1496.256 & 1494.717 \\ 1494.135 & 1494.541 & 1496.263 & 1494.72\end{array}$ $\begin{array}{lllll}1494.137 & 1494.548 & 1496.284 & 1494.724\end{array}$ $\begin{array}{llllll}1494.142 & 1494.555 & 1496303 & 1494.731\end{array}$ $\begin{array}{llllll}1494.144 & 1494.56 & 1496.321 & 1494.741\end{array}$ $\begin{array}{lllll}1494.151 & 1494.569 & 1496.34 & 1494.752\end{array}$ $\begin{array}{lllll}1494.158 & 1494.585 & 1496.352 & 1494.766\end{array}$ $\begin{array}{llll}1494.158 & 1494.592 & 1496.364 & 1494.78\end{array}$ $\begin{array}{lllll}1494.163 & 1494.602 & 1496.375 & 1494.797\end{array}$ $\begin{array}{rrrrr}1494.165 & 1494.613 & 1496.389 & 1494.811 \\ 1494.167 & 1494.62 & 1496.403 & 1494.822\end{array}$ $\begin{array}{lllll}1494.174 & 1494.63 & 1496.408 & 1494.836\end{array}$ $\begin{array}{lllll}1494.174 & 1494.644 & 1496.413 & 1494.848\end{array}$ $\begin{array}{llllll}1494.179 & 1494.651 & 1496.415 & 1494.862\end{array}$ $\begin{array}{lllll}1494.179 & 1494.655 & 1496.415 & 1494.871\end{array}$ $\begin{array}{lllll}1494.181 & 1494.662 & 1496.415 & 1494.878\end{array}$ $\begin{array}{llll}1494.183 & 1494.667 & 1496.42 & 1494.888\end{array}$ $\begin{array}{lllll}1494.183 & 1494.672 & 1496.422 & 1494.89\end{array}$ $\begin{array}{llll}1494.184 & 1494.674 & 1496.434 & 1494.892 \\ 1494.186 & 1494.679 & 1496.443 & 1494.899\end{array}$ $\begin{array}{lllll}1494.19 & 1494.683 & 1496.448 & 1494.904\end{array}$ $\begin{array}{lllll}1494.193 & 1494.69 & 1496.45 & 1494.909\end{array}$ $\begin{array}{llll}1494.197 & 1494.697 & 1496.455 & 1494.918\end{array}$ $1494.195 \quad 1494.702 \quad 1496.462 \quad 1494.923$ $1494.195 \quad 1494.704 \quad 1496.473 \quad 1494.927$ $1494.198 \quad 1494.709 \quad 1496.483 \quad 1494.932$ $\begin{array}{llll}1494.202 & 1494.718 & 1496.49 & 1494.939\end{array}$ $1494.207 \quad 1494.732 \quad 1496.494 \quad 1494.953$ $1494.205 \quad 1494.7391496 .508 \quad 1494.96$ $1494.207 \quad 1494.744 \quad 1496.516 \quad 1494.965$ $1494.2111494 .746 \quad 1496.527 \quad 1494.969$ $\begin{array}{llll}1494.216 & 1494.758 & 1496.532 & 1494.979\end{array}$ $\begin{array}{llll}1494.218 & 1494.762 & 1496.536 & 1494.986 \\ 1494.221 & 1494.772 & 1496.541 & 1494.995\end{array}$ $\begin{array}{llllllllll}1493.544 & 1495.309 & 1494.406 & 1495.101 & 1496.498 & 1496.423 & 1492.683 & 1489.263 & 1492.516 & 1490.002\end{array}$

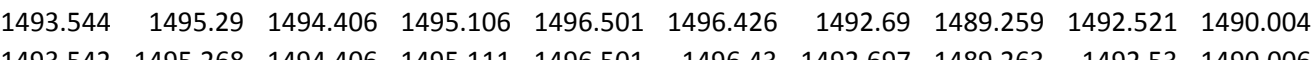
$\begin{array}{lllllllllll}1493.542 & 1495.268 & 1494.406 & 1495.111 & 1496.501 & 1496.43 & 1492.697 & 1489.263 & 1492.53 & 1490.006 \\ 1493.537 & 1495.249 & 1494.408 & 1495113 & 1496.505 & 1496433 & 1492702 & 1489263 & 1492535 & 1490009\end{array}$ $\begin{array}{llllllllllll}1493.53 & 1495.23 & 1494.41 & 1495.115 & 1496.51 & 1496.44 & 1492.707 & 1489.261 & 1492.54 & 1490.011\end{array}$ $\begin{array}{llllllllll}1493.528 & 1495.208 & 1494.408 & 1495.118 & 1496.515 & 1496.449 & 1492.714 & 1489.266 & 1492.544 & 1490.016\end{array}$ $\begin{array}{lllllllllll}1493.528 & 1495.192 & 1494.413 & 1495.122 & 1496.517 & 1496.454 & 4492.721 & 1489.266 & 1492.549 & 1490.018\end{array}$ $\begin{array}{lllllllllll}1493.523 & 1495.173 & 1494.41 & 1495.127 & 1496.522 & 1496.461 & 1492.725 & 1489.268 & 1492.558 & 1490.02\end{array}$ $\begin{array}{llllllllll}1493.53 & 1495.156 & 1494.41 & 1495.132 & 1496.519 & 1496.464 & 1492.732 & 1489.268 & 1492.563 & 1490.025\end{array}$ $\begin{array}{llllllllllll}1493.528 & 1495.141 & 1494.41 & 1455.136 & 1496.524 & 1496.461 & 1492.739 & 1489.27 & 1492.568 & 1490.027\end{array}$ $\begin{array}{llllllllll}1493.53 & 1495.125 & 1494.41 & 1495.139 & 1496.526 & 1496.466 & 1492.744 & 1489.273 & 1492.579 & 1490.032 \\ 1493.532 & 1495.108 & 1494.41 & 1495.141 & 1496526 & 1496466 & 1492.746 & 1489.27 & 1492579 & 1490.036\end{array}$ $\begin{array}{lllllllllll}1493.532 & 1495.108 & 1494.41 & 1495.141 & 446.526 & 1496.466 & 1492.746 & 1489.27 & 1492.579 & 1490.036 \\ 1493.535 & 1495.096 & 1494.41 & 1495.143 & 1496.526 & 1496.466 & 1492.751 & 1489.27 & 1492.584 & 1490.039\end{array}$

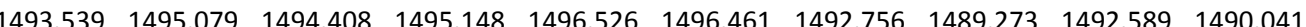
$\begin{array}{llllllllllll}1493.544 & 1495.065 & 1494.41 & 1495.15 & 1496.526 & 1496.459 & 1492.76 & 1489.273 & 1492.591 & 1490.043\end{array}$ $\begin{array}{lllllllllll}1493.546 & 1495.048 & 1494.413 & 1495.15 & 1496.522 & 1496.454 & 1492.76 & 1489.273 & 1492.594 & 1490.046\end{array}$ $\begin{array}{llllllllll}1493.542 & 1495.034 & 1494.41 & 1495.15 & 1496.526 & 1496.452 & 1492.76 & 1489.27 & 1492.594 & 1490.048\end{array}$ $\begin{array}{llllllllll}1493.542 & 1495.019 & 1494.41 & 1495.15 & 1496.529 & 1496.459 & 1492.763 & 1489.266 & 1492.596 & 1490.048\end{array}$ $\begin{array}{lllllllllll}1493.537 & 1495.005 & 1494.413 & 1495.15 & 1466.531 & 1466.459 & 1492.767 & 1489.268 & 1492.596 & 1490.05\end{array}$ $\begin{array}{lllllllllll}1493.528 & 1494.993 & 1494.418 & 1495.155 & 1496.535 & 1496.464 & 1492.767 & 1489.266 & 1492.598 & 1490.053 \\ 1493523 & 1494.976 & 1494.415 & 1495.157 & 1496542 & 1496476 & 1492767 & 1489266 & 1492601 & 1490055\end{array}$ $\begin{array}{llllllllllll}1493.513 & 1494.962 & 1494.418 & 1495.16 & 1496.552 & 1496.483 & 1492.772 & 1489.268 & 1492.603 & 1490.06\end{array}$ $\begin{array}{llllllllll}1493.499 & 1494.948 & 1494.42 & 1495.166 & 1496.561 & 1496.5 & 1492.774 & 1489.268 & 1492.608 & 1490.062\end{array}$ $\begin{array}{lllllllllll}1493.49 & 1494.938 & 1494.425 & 1495.171 & 1496.573 & 1496.519 & 1492.782 & 1489.27 & 1492.61 & 1490.064\end{array}$ $\begin{array}{lllllllllll}1493.483 & 1494.921 & 1494.427 & 1495.18 & 1496.582 & 1496.538 & 1492.784 & 1489.27 & 1492.617 & 1490.071\end{array}$ $\begin{array}{lrlrllllll}1493.478 & 1494.912 & 1494.422 & 1495.192 & 1496.594 & 1496.555 & 1492.793 & 1489.277 & 1492.624 & 1490.074\end{array}$ $\begin{array}{llllllllll}1493.473 & 1494.9 & 1494.429 & 1495.204 & 1496.601 & 1496.564 & 1492.8 & 1489.284 & 1492.631 & 1490.078\end{array}$ $\begin{array}{llllllllll}1493.473 & 1494.888 & 1494.427 & 1495.213 & 1496.608 & 1496.579 & 1492.81 & 1489.289 & 1492.64 & 1490.083 \\ 1493.469 & 1494878 & 1494.432 & 149522 & 1496.622 & 1496588 & 1492817 & 1489296 & 1492.645 & 1490088\end{array}$ $\begin{array}{rrrrrrrrrr}1493.469 & 1494.878 & 1494.432 & 1495.22 & 1496.622 & 1496.588 & 1492.817 & 1489.296 & 1492.645 & 1490.088 \\ 1493.473 & 1494.869 & 1494.429 & 1495.231 & 1496.629 & 1496.6 & 1492.824 & 1489296 & 1492.655 & 1490.095\end{array}$ $\begin{array}{llllllllllll}1493.473 & 1494.857 & 1494.432 & 1495.241 & 1496.635 & 1496.612 & 1492.831 & 1489.301 & 1492.661 & 1490.099\end{array}$ $\begin{array}{llllllllll}1493.483 & 1494.845 & 1494.434 & 1495.248 & 1496.642 & 1496.615 & 1492.842 & 1489.308 & 1492.669 & 1490.106\end{array}$ $\begin{array}{llllllllllll}1493.492 & 1494.835 & 1494.434 & 1495.259 & 1496.647 & 1496.619 & 1492.849 & 1489.308 & 1492.68 & 1490.111\end{array}$ $\begin{array}{llllllllll}1493.499 & 1494.823 & 1494.439 & 1495.266 & 1496.654 & 1496.619 & 1492.856 & 1489.317 & 1492.687 & 1490.113\end{array}$ $\begin{array}{llllllllllll}1493.511 & 1494.811 & 1494.439 & 1495.273 & 1496.654 & 1496.619 & 1492.859 & 1489.319 & 1492.69 & 1490.118\end{array}$ $\begin{array}{llllllllll}1493.52 & 1494.802 & 1494.439 & 1495.278 & 1496.661 & 1496.615 & 1492.868 & 1489.319 & 1492.697 & 1490.123\end{array}$ $\begin{array}{llllllllll}1493.528 & 1494.792 & 1494.439 & 1495.285 & 1496.663 & 1496.617 & 1492.87 & 1489.319 & 1492.699 & 1490.125 \\ 1493532 & 1494.783 & 1494.441 & 1495287 & 1496.67 & 1496619 & 1492877 & 1489322 & 1492704 & 1490.13\end{array}$ $\begin{array}{lllllllllllll}1493.535 & 1494.77 & 1494.441 & 1495.294 & 1496.68 & 1496.624 & 1492.882 & 1489.322 & 1492.7111 & 1490.134\end{array}$ $\begin{array}{lllllllllll}1493.537 & 1494.761 & 1494.446 & 1495.299 & 1496.684 & 1496.631 & 1492.884 & 1489.326 & 1492.715 & 1490.139\end{array}$ $\begin{array}{lllllllllll}1493.546 & 1494.754 & 1494.446 & 1495.308 & 1496.687 & 1496.636 & 1492.889 & 1489.326 & 1492.72 & 1490.144\end{array}$ $\begin{array}{llllllllll}1493.553 & 1494.747 & 1494.444 & 1495.315 & 1496.694 & 1496.636 & 1492.894 & 1489.331 & 1492.723 & 1490.146\end{array}$ $\begin{array}{lllllllllll}1493.558 & 1494.737 & 1494.448 & 1495.322 & 1496.698 & 1496.638 & 1492.896 & 1489.331 & 1492.727 & 1490.151\end{array}$ $\begin{array}{lllllllllll}1493.553 & 1494.73 & 1494.451 & 1495.324 & 1496.71 & 1496.646 & 1492.901 & 1489.331 & 1492.729 & 1490.151\end{array}$ $\begin{array}{llllllllll}1493.56 & 1494.723 & 1494.448 & 1495.331 & 1496.715 & 1496.658 & 4492.903 & 1489.336 & 1492.732 & 1490.155 \\ 1993.56 & 1494.713 & 1494.453 & 1995338 & 1496722 & 149666 & 1492908 & 148336 & 149273 & 140.162\end{array}$

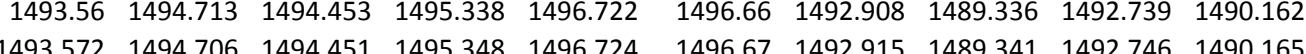
$\begin{array}{llllllllllll}1493.572 & 1494.701 & 1494.453 & 1495.357 & 1496.731 & 1496.665 & 1492.92 & 1489.343 & 1492.751 & 1490.169\end{array}$ $\begin{array}{llllllllllll}1493.579 & 1494.694 & 1494.456 & 1495.364 & 1496.735 & 1496.67 & 1492.924 & 1489.345 & 1492.753 & 1490.174\end{array}$ $\begin{array}{llllllllll}1493.577 & 1494.692 & 1494.456 & 1495.366 & 1496.745 & 1496.679 & 1492.929 & 1489.343 & 1492.758 & 1490.174\end{array}$ $\begin{array}{llllllllllll}1493.579 & 1494.684 & 1494.458 & 1495.373 & 1496.754 & 1496.684 & 1492.934 & 1489.345 & 1492.765 & 1490.181\end{array}$ $\begin{array}{llllllllllll}1493.579 & 1494.68 & 1494.46 & 1495.382 & 1496.761 & 1496.691 & 1492.943 & 1489.352 & 1492.772 & 1490.186\end{array}$ $\begin{array}{llllllllll}1493.586 & 1494.672 & 1494.463 & 1495.389 & 1496.766 & 1496.696 & 1492.95 & 1489.348 & 1492.776 & 1490.188\end{array}$ $\begin{array}{lllllllll}1486.314 & 1488.288 & 1492.656 & 1493.483 & 1492.686 & 1494.708 & 1492.666 & 1485.388 & 1478.327\end{array}$ $\begin{array}{llllllllll}1486.317 & 1488.276 & 1492.654 & 1493.495 & 1492.691 & 1494.713 & 1492.673 & 1485.389 & 1478.327 \\ 1486319 & 1488276 & 1492654 & 1493504 & 1492698 & 1494722 & 1492.678 & 1485388 & 1478327\end{array}$ $\begin{array}{lllllllll}1486.321 & 1488.276 & 1492.654 & 1493.504 & 1492.698 & 1494.722 & 1492.678 & 1485.388 & 1488.327\end{array}$ $\begin{array}{lllllllllll}1486.326 & 1488.278 & 1492.661 & 1493.514 & 1492.71 & 1494.732 & 1492.687 & 1485.389 & 1478.33\end{array}$ $\begin{array}{lllllllllll}1486.328 & 1488.278 & 1492.663 & 1493.523 & 1492.714 & 1494.737 & 1492.694 & 1485.386 & 1478.33\end{array}$ $\begin{array}{lllllllllll}1486.333 & 1488.276 & 1492.665 & 1493.53 & 1492.719 & 1494.741 & 1492.701 & 1485.386 & 1478.332\end{array}$ $\begin{array}{llllllllll}1486.335 & 1488.271 & 1492.661 & 1493.539 & 1492.726 & 1494.758 & 1492.708 & 1485.386 & 1478.33\end{array}$ $\begin{array}{lllllllll}1486.34 & 1488.269 & 1492.663 & 1493.542 & 1492.733 & 1494.76 & 1492.713 & 1485.386 & 1478.337\end{array}$ $\begin{array}{llllllllll}1486.344 & 1488.276 & 1492.668 & 1493.544 & 1492.74 & 1494.765 & 1492.718 & 1485.386 & 1478.339\end{array}$ $\begin{array}{llllllllll}1486.347 & 1488.276 & 1492.675 & 1493.553 & 1492.745 & 1494.767 & 1492.723 & 1485.386 & 1478.341 \\ 1486354 & 1488276 & 1492675 & 1493.551 & 1492.75 & 1494769 & 1492.727 & 1485386 & 1478.341\end{array}$ $\begin{array}{lllllllllll}1486.334 & 1488.27 & 1492.67 & 143.551 & 1492.75 & 4494.79 & 1492.727 & 1458.386 & 1478.341 \\ 1486354 & 1488.278 & 1492.677 & 1493.56 & 1492.755 & 1494.779 & 1492.734 & 1485.386 & 1478.341\end{array}$ $\begin{array}{llllllllll}1486347 & 1488278 & 1492.679 & 1493.56 & 1492.757 & 1494.779 & 1492.737 & 1485.386 & 1478.344\end{array}$ $\begin{array}{llllllllll}1486.347 & 1488.278 & 1492.684 & 1493.563 & 1492.759 & 1494.776 & 1492.739 & 1485.384 & 1478.34\end{array}$ $\begin{array}{llllllllll}1486.349 & 1488.28 & 1492.689 & 1493.563 & 1492.759 & 1494.776 & 1492.741 & 1485.386 & 1478.341\end{array}$ $\begin{array}{lllllllllll}1486.349 & 1488.28 & 1492.691 & 1493.563 & 1492.759 & 1494.774 & 1492.744 & 1485.386 & 1478.34 & \end{array}$ $\begin{array}{lllllllll}1486.354 & 1488.278 & 1492.686 & 1493.56 & 1492.764 & 1494.774 & 1492.741 & 1485.384 & 1478.341\end{array}$ $\begin{array}{lllllllllll}1486.351 & 1488.276 & 1492.672 & 1493.565 & 1492.766 & 1494.772 & 1492.744 & 1485.384 & 1478.339\end{array}$ $\begin{array}{lllllllllll}1486.351 & 1488.269 & 1492.651 & 1493.567 & 492.767 & 1494.72 & 1492.748 & 1485.384 & 1478.339\end{array}$ $\begin{array}{llllllllll}1486.335 & 1488.255 & 1492.623 & 1493.577 & 1492.771 & 1494.784 & 1492.753 & 1455.384 & 1478.337\end{array}$

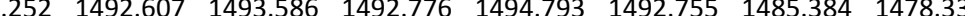
$\begin{array}{llllllllll}1486.324 & 1488.243 & 1492.59 & 1493.595 & 1492.781 & 1494.812 & 1492.76 & 1485.384 & 1478.337\end{array}$ $\begin{array}{llllllllll}1486.331 & 1488.234 & 1492.564 & 1493.605 & 1492.785 & 1494.821 & 1492.767 & 1485.384 & 1478.339\end{array}$ $\begin{array}{lllllllllll}1486.333 & 1488.224 & 1492.536 & 1493.621 & 1492.795 & 1494.842 & 1492.774 & 1485.384 & 1478.339\end{array}$ $\begin{array}{llllllllll}1486.34 & 1488.222 & 142.517 & 1493.63 & 1429.802 & 1494.856 & 1492.784 & 1485.384 & 1478.339\end{array}$ $\begin{array}{lllllllllll}1486.344 & 1488.217 & 1492.501 & 1493.649 & 1492.812 & 1494.873 & 1492.788 & 1485.384 & 1478.341\end{array}$ $\begin{array}{rlllllllll}1486.349 & 1488.215 & 1492.487 & 1493.66 & 1492.81 & 1494.885 & 1492.8 & 1485.384 & 1478.344\end{array}$ $\begin{array}{lllllllllll}1486.363 & 1488.21 & 1492.463 & 1493.681 & 1492.833 & 1494.908 & 1492.814 & 1485.384 & 1478.348\end{array}$ $\begin{array}{llllllllll}1486.333 & 1488.205 & 1492.458 & 1493.691 & 1492.84 & 1494.917 & 1492.821 & 1485.384 & 1478.35\end{array}$ $\begin{array}{llllllllll}1486.321 & 1488.208 & 1492.451 & 1493.7 & 1492.852 & 1494.934 & 1492.828 & 1485.384 & 1478.353\end{array}$ $\begin{array}{llllllllll}1486.324 & 1488.21 & 1492.451 & 1493.705 & 1492.857 & 1494.936 & 1422.838 & 1485.384 & 1478.353\end{array}$ $\begin{array}{llllllllll}1486.328 & 1488.208 & 1492.456 & 1493.714 & 1422.864 & 1494.943 & 1492.842 & 1485.381 & 1478.355\end{array}$

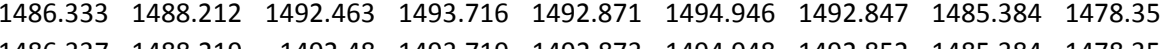
$\begin{array}{llllllllll}1486344 & 148822 & 1492.48 & 1493.79 & 1492.873 & 1499948 & 142.852 & 1455.384 & 1478.358\end{array}$ $\begin{array}{lllllllllll}1486.347 & 1488224 & 1492.505 & 1493.726 & 1492.88 & 1494.95 & 1492.861 & 1485.384 & 148.358\end{array}$ $\begin{array}{lllllllllll}1486.354 & 1488.226 & 1492.515 & 1493.733 & 1492.885 & 1494.967 & 1492.863 & 1485.384 & 1478.358\end{array}$ $\begin{array}{lllllllll}1486.36 & 1488.229 & 1492.522 & 1493.737 & 1492.89 & 1494.964 & 1492.868 & 1485.381 & 1478.358\end{array}$ $\begin{array}{lllllllll}1486.363 & 1488.229 & 1492.522 & 1493.749 & 1492.897 & 1494.974 & 1492.873 & 1485.384 & 1478.35\end{array}$ $\begin{array}{lllllllllll}4486.367 & 1488.238 & 1492.531 & 1493.747 & 1492.899 & 1494.976 & 1492.877 & 1485.381 & 1478.355\end{array}$ $\begin{array}{lllllllllll}1486.372 & 1488.243 & 1492.545 & 1493.751 & 1422.902 & 1494.979 & 1492.877 & 1485.381 & 1478.355\end{array}$ $\begin{array}{llllllllll}1486.379 & 1488.248 & 1492.552 & 1493.754 & 1492.904 & 1494.983 & 1492.884 & 1485.381 & 1478.355\end{array}$

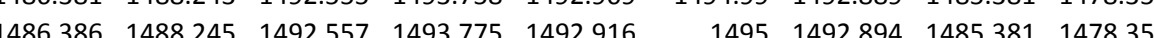

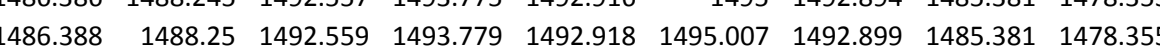
$\begin{array}{llllllllll}1486.393 & 1488.259 & 1492.574 & 1493.779 & 1492.923 & 1495.011 & 1492.903 & 1485.381 & 1478.355\end{array}$ $\begin{array}{lllllllll}1486.395 & 1488.259 & 1492.576 & 1493.789 & 1492.932 & 1495.016 & 1492.91 & 1485.381 & 1478.355\end{array}$ $\begin{array}{llllllllll}1486.4 & 1488.262 & 1492.585 & 1493.791 & 1492.937 & 1495.021 & 1492.913 & 1485.381 & 1478.358\end{array}$ $\begin{array}{lllllllllll}1486.397 & 1488.262 & 1492.583 & 1493.807 & 1492.939 & 1495.03 & 1492.922 & 1485.381 & 1478.36\end{array}$ $\begin{array}{lllllllll}1486.404 & 1488.264 & 1492.585 & 1493.812 & 1492.949 & 1495.042 & 1492.929 & 1485.381 & 1478.362 \\ 1486.407 & 1488266 & 1492.592 & 1493817 & 1492.956 & 1495049 & 1492936 & 1485381 & 1478.365\end{array}$ 
Well

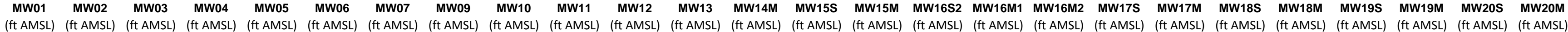

11/11/11 4:00 $11 / 11 / 114: 30$ $11 / 11 / 115.00$ 11/111/11 6:00 11/11/1116:00 $11 / 11 / 11$ 7:00 $11 / 11 / 117: 30$ 11/11/11 8:00 $11 / 11 / 118: 30$ $11 / 11 / 119: 00$ $11 / 11 / 1110: 00$ 11/111/11 10:30 11/11/11 11:00 $11 / 11 / 1111: 30$ $11 / 11 / 1112: 00$ $11 / 11 / 1112: 30$ 11/11/11 13:00 11/11/11 13:30 $11 / 11 / 1114: 30$ $1 / 11 / 1115: 00$ 11/11/1115:00 11/111/11 16:00 $11 / 11 / 1116: 30$ $11 / 11 / 1117: 00$ 11/11/11 17:30 $11 / 11 / 1118: 00$
$11 / 11 / 1119: 00$ 11/111/111 20:00 1/11/11 21:00 11/11/11 22:00 $11 / 11 / 1123: 00$ $11 / 12 / 110: 00$ 11/12/11 1:00 $11 / 12 / 112: 00$ 11/12/11 3:00 $11 / 12 / 114: 00$ 11/12/12/11 6:00 11/12/11 7:00 11/12/11 8:00 11/12/11 9:00 11/12/11 10:00 $11 / 12 / 1111: 00$ 11/12/11112:00 $11 / 12 / 1114: 00$ $11 / 12 / 1115: 00$ 11/12/11 16:00 11/12/11 17:00 11/12/11 18:00 $\begin{array}{rrrr}1494.221 & 1494.776 & 1496.544 & 1495 \\ 1494221 & 1494781 & 1496541 & 1495004\end{array}$ $\begin{array}{llll}1494.221 & 1494.781 & 1496.541 & 1495.004\end{array}$ $\begin{array}{llll}194.223 & 1944.786 & 1496.539 & 1495.009\end{array}$ $\begin{array}{lllll}1494.225 & 1994.793 & 1496.548 & 1495.016\end{array}$ $\begin{array}{lllll}494.223 & 1494.793 & 1496.541 & 1455.016\end{array}$ $\begin{array}{lllll}1494.228 & 1494.8 & 1496.534 & 1495.021\end{array}$ $\begin{array}{lllll}1494.228 & 1494.802 & 1496.534 & 1495.023\end{array}$ $\begin{array}{lllll}1494.223 & 1494.802 & 1496.529 & 1495.021\end{array}$ $\begin{array}{llll}1494.223 & 1494.8 & 1496.532 & 1495.018\end{array}$ $\begin{array}{rrrr}1494.228 & 1494.807 & 1496.522 & 1495.016 \\ 149423 & 194.804 & 149653 & 1995016\end{array}$ $\begin{array}{lllll}1494.23 & 1494.804 & 1496.53 & 1495.016 \\ 1494.23 & 1494.804 & 1496.532 & 1495.016\end{array}$ $\begin{array}{llll}1494.225 & 1494.802 & 1496.532 & 14595.011\end{array}$ $\begin{array}{llll}1494.23 & 1494.807 & 1496.539 & 1495.009\end{array}$ $\begin{array}{lllll}1494.237 & 1494.804 & 1496.544 & 1495.011\end{array}$ $\begin{array}{lllll}1494.232 & 1494.809 & 1496.551 & 1495.011\end{array}$ $1494.242 \quad 1494.814 \quad 1496.565 \quad 1495.014$ 1494.2421494 .8161496 .5721495 .016 $\begin{array}{lllll}1494.244 & 1494.823 & 1496.574 & 1495.021 \\ 1494249 & 1494.828 & 1965581 & 1995.028\end{array}$ $\begin{array}{llll}1494.249 & 1494.828 & 1496.581 & 1495.028 \\ 1494.251 & 1494.835 & 1496583 & 1495035\end{array}$ $\begin{array}{lll}1494.251 & 1494.837 & 1495.037\end{array}$ $\begin{array}{lll}1494.253 & 1494.842 & 1495.042\end{array}$ $\begin{array}{llll}1494.251 & 1494.844 & 1496.623 & 1495.035\end{array}$ $1494.251 \quad 1494.844$ $1494.253 \quad 1494.846 \quad 1496.623 \quad 1495.035$ 494.256

$\begin{array}{lllll}4994.249 & 1494.844 & 1496.609 & 1495.028\end{array}$ $\begin{array}{llll}4494.256 & 1494.846 & 1496.607 & 1495.023 \\ 1494.26 & 1494.846 & 1496.609 & 1495.023\end{array}$ $\begin{array}{rrrrr}1494.26 & 1494.846 & 1496.609 & 1495.023 \\ 1494.263 & 1494.849 & 1496.612 & 1495.021\end{array}$ $\begin{array}{lllll}1494.267 & 1494.851 & 1496.621 & 1495.023\end{array}$ $\begin{array}{lllll}1494.281 & 1494.87 & 1496.649 & 1495.039\end{array}$ $\begin{array}{llll}1494.284 & 1494.881 & 1496.663 & 1495.051\end{array}$ $\begin{array}{llll}1494.286 & 1494.886 & 1496.675 & 1495.067 \\ 1491.291 & 1494.902 & 1469693 & 195084\end{array}$ $\begin{array}{rrrr}1494.291 & 1494.902 & 1496.693 & 1495.084 \\ 14943 & 1494916 & 1496.719 & 1495.105\end{array}$ $\begin{array}{rllll}1494.3 & 1494.916 & 1496.719 & 1495.105 \\ 1494.307 & 1494.935 & 1496.742 & 1495.126\end{array}$ $\begin{array}{lllll}1494.305 & 1494.944 & 1496.747 & 1495.142\end{array}$ $\begin{array}{lllll}1494.307 & 1494.951 & 1496.754 & 1495.151\end{array}$ $1494.3121494 .958 \quad 1496.759 \quad 1495.161$ $1494.321 \quad 1494.975 \quad 1496.778 \quad 1495.173$ $1494.3141494 .972 \quad 1496.7611495 .168$ $1494.321 \quad 1494.9771496 .7661495 .173$

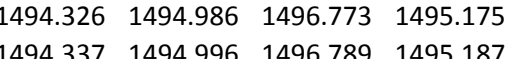
$1494.333 \quad 1494.996 \quad 1496.785 \quad 1495.182$ $\begin{array}{llll}1494.337 & 1495 & 1496.785 & 1495.184\end{array}$ $1494.33 \quad 1494.996 \quad 1496.768 \quad 1495.177$ $1494.33 \quad 1494.984 \quad 1496.747 \quad 1495.161$ $1494.333 \quad 1494.982 \quad 1496.735 \quad 1495.149$ $\begin{array}{rrrr}1494.33 & 1494.97 & 1496.714 & 1495.133 \\ 1494.337 & 1494.968 & 1496.719 & 1495.128\end{array}$ $\begin{array}{rrrrrrrrrr}1493.6 & 1494.66 & 1494.463 & 1495.403 & 1496.773 & 1496.701 & 1492.962 & 1489.357 & 1492.79 & 1490.197 \\ 1493.607 & 1499.658 & 1494.465 & 1495.41 & 1496.775 & 1496.698 & 1492.969 & 1489.359 & 1492.988 & 1490202\end{array}$ $\begin{array}{llllllllll}1493.607 & 1494.658 & 1494.465 & 1495.41 & 1496.775 & 1496.698 & 1492.969 & 1489.359 & 1492.798 & 1490.202\end{array}$ $\begin{array}{llllllllll}1493.619 & 1494.653 & 1494.465 & 1495.417 & 1496.777 & 1496.696 & 1492.973 & 1489.359 & 1492.802 & 1490.206 \\ 1493.626 & 1494.648 & 1494.467 & 1495.424 & 1496.784 & 1496.694 & 1492.98 & 1489355 & 1492809 & 1490209\end{array}$ $\begin{array}{llllllllllll}1493.631 & 1494.641 & 1494.467 & 1495.426 & 1496.787 & 1496.694 & 1492.983 & 1489.364 & 1492.816 & 1490.211\end{array}$ $\begin{array}{lllllllllll}1493.638 & 1494.636 & 1494.467 & 1495.431 & 1496.789 & 1496.696 & 1492.99 & 1489.359 & 1492.819 & 1490.213\end{array}$ $\begin{array}{lllllllllll}1493.659 & 1494.632 & 1494.46 & 1495.433 & 1496.782 & 1496.686 & 1492.994 & 1489.362 & 1492.823 & 1490.22\end{array}$ $\begin{array}{llllllllllll}1493.664 & 1494.625 & 1494.467 & 1495.44 & 1496.782 & 1496.679 & 1492.997 & 1489.366 & 1492.828 & 1490.223\end{array}$ $\begin{array}{lllllllllll}1493.664 & 1494.622 & 1494.467 & 1495.44 & 1496.787 & 1496.677 & 1493.001 & 1489.371 & 1492.83 & 1490.225\end{array}$ $\begin{array}{lllllllllll}1493.671 & 1494.615 & 1494.467 & 1495.443 & 1496.789 & 1496.672 & 1493.001 & 1489.366 & 1492.83 & 1490.227 \\ 1493676 & 1494.61 & 1494.472 & 195.445 & 196.787 & 14966 & 1493.004 & 148936 & 1492837 & 149027\end{array}$ $\begin{array}{llllllllll}1493.676 & 1494.61 & 1494.472 & 1495.445 & 1496.787 & 1496.67 & 1493.004 & 1489.366 & 1492.837 & 1490.227 \\ 1493.678 & 1494.606 & 1494.472 & 1495.447 & 1496.787 & 1496.66 & 1493.004 & 1489.362 & 1492.837 & 1490232\end{array}$ $\begin{array}{lllllllllllll}1493.683 & 1494.601 & 1494.472 & 1495.45 & 1496.787 & 1496.667 & 1493.006 & 1489.364 & 1492.837 & 1490.234\end{array}$ $\begin{array}{lllllllllll}1493.68 & 1494.596 & 1494.472 & 1495.45 & 1496.791 & 1496.665 & 1493.006 & 1489.357 & 1492.835 & 1490.237\end{array}$ $\begin{array}{rrrrrrrrrr}1493.685 & 1494.593 & 1494.477 & 1495.454 & 1496.791 & 1496.662 & 1493.006 & 1489.364 & 1492.837 & 1490.237\end{array}$ $\begin{array}{llllllllllll}1493.678 & 1494.589 & 1494.479 & 1495.457 & 1496.798 & 1496.667 & & 1489.359 & 1492.84 & 1490.239\end{array}$ $\begin{array}{lllllllllll}1493.68 & 1494.584 & 1494.477 & 1495.459 & 1496.801 & 1496.674 & 1493.016 & 1489.362 & 1492.842 & 1490.241\end{array}$ $\begin{array}{lllllllllll}1493.676 & 1494.581 & 1494.482 & 1495.464 & 1496.808 & 1496.677 & & 1489.359 & 1492.844 & 1490.246\end{array}$

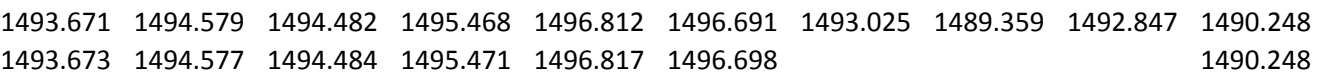
$\begin{array}{llllllllll}1493.673 & 1494.577 & 1494.484 & 1495.471 & 1496.817 & 1496.698 & & & 1490.248 \\ 1493.673 & 1494.572 & 1494.482 & 1495.48 & 1496.822 & 1496.696 & 1493.032 & 1489.366 & 1492.861 & 1490.258\end{array}$ $\begin{array}{lllllllllll}1495.066 & 1492.997 & 1485.377 & 1478.376\end{array}$ $\begin{array}{rlll}1495.08 & 1493.009\end{array}$

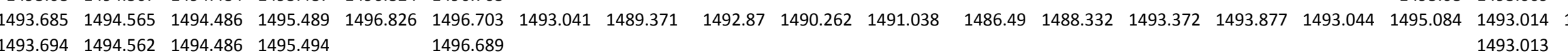

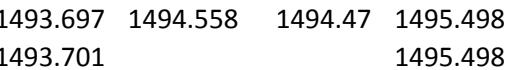

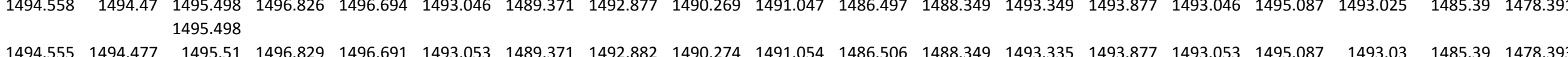
$\begin{array}{lllllllllllllllllllllll}1493.732 & 1494.546 & 1494.477 & 1495.508 & 1496.824 & 1496.674 & 1493.053 & 1489.371 & 1492.884 & 1490.279 & 1491.054 & 1486.506 & 1488.363 & 1493.354 & 1493.87 & 1493.056 & 1495.077 & 1493.032 & 1485.388 & 1478.393\end{array}$

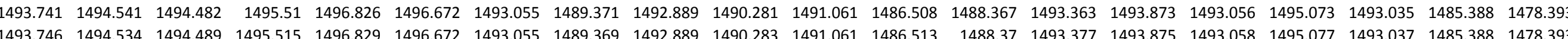
\begin{tabular}{lllllllllllllllllllll}
1493.756 & 1494.529 & 1494.486 & 1495.517 & 1496.831 & 1496.67 & 1493.055 & 1489.362 & 1492.891 & 1490.288 & 1491.063 & 1486.515 & 1488.384 & 1493.386 & 1493.875 & 1493.058 & 1495.07 & 1493.035 & 1485.388 & 1478.391 \\
\hline
\end{tabular} $\begin{array}{lllllllllllllllllllll}1493.756 & 1494.526 & 1494.494 & 1495.522 & 1496.838 & 1496.677 & 1493.058 & 1489.369 & 1492.891 & 1490.295 & 1491.063 & 1486.515 & 1488.384 & 1493.389 & 1493.875 & 1493.06 & 1495.08 & 1493.037 & 1485.388 & 1478.388\end{array}$ $\begin{array}{llllllllllllllllllllll}1493.741 & 1494.524 & 1494.498 & 1495.536 & 1496.852 & 1496.703 & 1493.065 & 1489.366 & 1492.896 & 1490.302 & 1491.066 & 1486.522 & 1488.384 & 1493.37 & 1493.898 & 1493.067 & 1495.105 & 1493.046 & 1485.388 & 1478.388\end{array}$

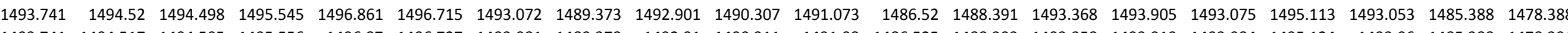

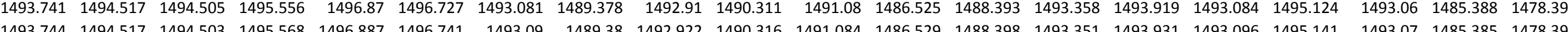

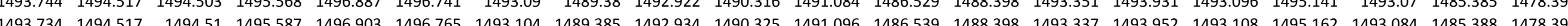

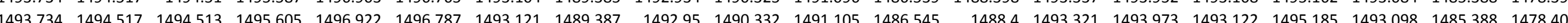
$\begin{array}{lllllllllllllllllllll}1493.748 & 1494.514 & 1494.508 & 1495.617 & 1496.931 & 1496.789 & 1493.133 & 1489.397 & 1492.962 & 1490.337 & 1491.115 & 1486.552 & 1488.405 & 1493.318 & 1493.985 & 1493.134 & 1495.195 & 1493.112 & 1485.388 & 1478.412\end{array}$ $\begin{array}{lllllllllllllllllllll}1493.76 & 1494.517 & 1494.513 & 1495.628 & 1496.942 & 1496.792 & 1493.142 & 1489.397 & 1492.973 & 1490.346 & 1491.124 & 1486.559 & 1488.417 & 1493.321 & 1493.992 & 1493.146 & 1495.202 & 1493.124 & 1485.385 & 1478.414\end{array}$ \begin{tabular}{llllllllllllllllllllll}
1493.774 & 1494.514 & 1494.517 & 1495.635 & 1496.947 & 1496.792 & 1493.151 & 1489.402 & 1492.98 & 1490.351 & 1491.134 & 1486.573 & 1488.417 & 1493.316 & 1493.999 & 1493.155 & 1495.211 & 1493.133 & 1485.385 & 1478.416 \\
\hline
\end{tabular} $\begin{array}{lllllllllllllllllllll}1493.774 & 1494.517 & 1494.52 & 1495.654 & 1496.963 & 1496.811 & 1493.163 & 1489.406 & 1492.994 & 1490.36 & 1491.141 & 1486.576 & 1488.412 & 1493.295 & 1494.015 & 1493.167 & 1495.228 & 1493.143 & 1485.388 & 1478.423\end{array}$

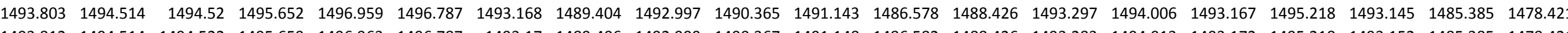

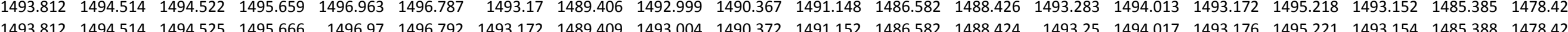

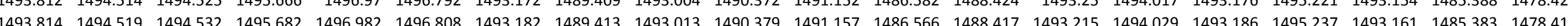
$\begin{array}{lllllllllllllllllllllll}1493.833 & 1494.519 & 1494.529 & 1495.682 & 1496.982 & 1496.794 & 1493.186 & 1489.411 & 1493.016 & 1490.381 & 1491.159 & 1486.566 & 1488.426 & 1493.205 & 1494.027 & 1493.188 & 1495.23 & 1493.164 & 1485.383 & 1478.419\end{array}$ $\begin{array}{lllllllllllllllllllllllll}1493.843 & 1494.519 & 1494.534 & 1495.686 & 1496.987 & 1496.794 & 1493.189 & 1489.413 & 1493.02 & 1490.388 & 1491.166 & 1486.576 & 1488.426 & 1493.189 & 1494.024 & 1493.191 & 1495.23 & 1493.168 & 1485.383 & 1478.419\end{array}$

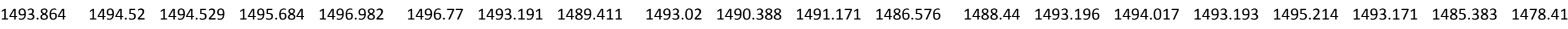

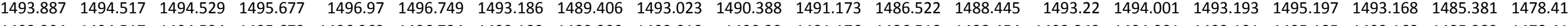
$\begin{array}{lllllllllllllllllllll}1493.904 & 1494.517 & 1494.534 & 1495.672 & 1496.963 & 1496.734 & 1493.189 & 1489.399 & 1493.018 & 1490.39 & 1491.176 & 1486.518 & 1488.454 & 1493.243 & 1494.001 & 1493.191 & 1495.185 & 1493.168 & 1485.383 & 1478.419\end{array}$ $\begin{array}{lllllllllllllllllllll}1493.922 & 1494.517 & 1494.534 & 1495.666 & 1496.956 & 1496.713 & 1493.186 & 1489.394 & 1493.02 & 1490.39 & 1491.178 & 1486.527 & 1488.464 & 1493.285 & 1493.98 & 1493.186 & 1495.162 & 1493.164 & 1485.381 & 1478.419 \\ 1493.925 & 1494.514 & 1494532 & 1495663 & 1496952 & 1496715 & 1493.182 & 148939 & 1493018 & 1490393 & 1491.18 & 1486534 & 1488.468 & 1493314 & 149398 & 1493.186 & 1495.16 & 1493.164 & 1485.381 & 1478.419\end{array}$ 
Well

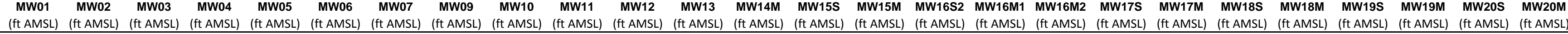

$11 / 12 / 1120: 00$ $11 / 12 / 1121: 00$ $11 / 12 / 1122: 00$ 11/13/11 0:00 11/13/11:00 $11 / 13 / 113: 00$
$11 / 13 / 114: 00$ 11/13/11 4:00 $11 / 13 / 116: 00$ 11/13/11 7:00 11/13/11 9:00 $11 / 13 / 1110: 00$ 11/13/11 11:00 11/13/11 12:00 $11 / 13 / 1114: 00$ $11 / 13 / 11155: 00$ 11/13/11 11 16:00 11/13/11 17:00 11/13/11 18:00 11/13/11 20:00 11/13/11 21:00 $11 / 13 / 1122: 00$
$11 / 13 / 1123: 00$ $11 / 13 / 1123: 00$
$11 / 14 / 110: 00$ $11 / 14 / 110: 00$
$11 / 14 / 111: 00$ 11/14/11 2:00 11/14/11 3:00 11/14/11 4:00 11/14/11 5:00 11/14/11 6:00 11/14/11 8:00 11/14/11 9:00 11/14/11111:00 11/14/111 12:00 11/14/11 13:00 11/14/11 14:00 11/14/11 15:00 11/14/111 16:00 11/14/11 17:00 11/11/11111 18:00 11/14/11 19:00 11/14/11 21:00 11/14/11 22:00 11/14/11 23:00 11/15/11 1:00 $\begin{array}{llll}1494.346 & 1494.972 & 1496.728 & 1495.126 \\ 1494.358 & 1494.982 & 1496.749 & 1495.135\end{array}$ $\begin{array}{llll}1494.358 & 1494.982 & 1496.749 & 1495.135 \\ 1494363 & 1499.993 & 1496.775 & 1455.154\end{array}$ $\begin{array}{rrrrr}1494.363 & 1494.993 & 1496.775 & 1495.154 \\ 1494.375 & 1495.017 & 1496813 & 1495.18\end{array}$ $\begin{array}{lllll}1494.367 & 1495.024 & 1496.817 & 1495.194\end{array}$ $\begin{array}{llll}1494.374 & 1495.031 & 1496.822 & 1495.201\end{array}$ $\begin{array}{lllll}1494.368 & 1495.028 & 1496.806 & 1495.194\end{array}$ $\begin{array}{lllll}1494.37 & 1495.028 & 1496.801 & 1495.194\end{array}$ $\begin{array}{llll}1494.354 & 1494.998 & 1496.742 & 1495.161\end{array}$ $\begin{array}{lllll}1494.354 & 1494.977 & 1496.712 & 1495.133\end{array}$ $\begin{array}{llll}1494.353 & 1494.951 & 1496.684 & 1495.105 \\ 1494.356 & 1494.935 & 1496.655 & 1495084\end{array}$ $\begin{array}{rrrr}1494.356 & 1494.935 & 1496.665 & 1495.084 \\ 1494.354 & 1494.919 & 1496.644 & 1495.06\end{array}$ $\begin{array}{rrrr}1494.351 & 1494.895 & 1496.619 & 1495.035\end{array}$ $\begin{array}{llll}1494.36 & 1494.898 & 1496.63 & 1495.03\end{array}$ $1494.36 \quad 1494.886 \quad 1496.6191495 .016$ $\begin{array}{lllll}1494.367 & 1494.886 & 1496.628 & 1495.014\end{array}$ $\begin{array}{llll}1494.379 & 1494.9 & 1496.658 & 1495.03\end{array}$ $1494.3771494 .898 \quad 1496.6541495 .032$ $\begin{array}{lllll}1494.379 & 1494.902 & 1496.654 & 1495.032\end{array}$ $\begin{array}{lllll}1494.386 & 1494.905 & 1496.661 & 1495.037 \\ 1494.381 & 1494.898 & 1496.647 & 1495.035\end{array}$ $\begin{array}{llllll}1494.391 & 1494.909 & 1496.665 & 1459.045\end{array}$ $\begin{array}{lllll}1494.393 & 1494.912 & 1496.672 & 1995.049\end{array}$ $\begin{array}{lllll}1494.395 & 1494.916 & 1496.679 & 1495.053\end{array}$ $\begin{array}{rrrrr}1494.405 & 1494.93 & 1496.696 & 1495.065\end{array}$ $\begin{array}{llll}1494.4 & 1494.935 & 1496.703 & 1495.074\end{array}$ $\begin{array}{lllll}1494.407 & 1494.944 & 1496.714 & 1495.081\end{array}$ $\begin{array}{llll}1494.407 & 1494.949 & 1496.721 & 1495.088 \\ 1494.405 & 1494.949 & 1496.714 & 1495.091\end{array}$ $\begin{array}{rrrr}1494.407 & 1494.949 & 1496.71 & 1495.091\end{array}$ $\begin{array}{lllll}1494.402 & 1494.937 & 1496.691 & 1495.079\end{array}$ $\begin{array}{lllll}1494.389 & 1494.902 & 1496.633 & 1495.046\end{array}$ $\begin{array}{lllll}1494.379 & 1494.872 & 1496.588 & 1495.009\end{array}$ $\begin{array}{lllll}1494.379 & 1494.842 & 1496.551 & 1494.972\end{array}$ $\begin{array}{rrrr}1494.384 & 1494.837 & 1496.548 & 1494.958 \\ 1493.377 & 1498.814 & 196552 & 194934\end{array}$

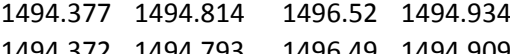
$\begin{array}{rrrr}1494.372 & 1494.793 & 1496.49 & 1494.909 \\ 1494.372 & 1494.783 & 1496.485 & 1494895\end{array}$ $\begin{array}{lllll}1494.372 & 1494.774 & 1496.478 & 1494.881\end{array}$ $\begin{array}{lllll}1494.384 & 1494.783 & 1496.504 & 1494.89\end{array}$ $\begin{array}{lllll}1494.389 & 1494.793 & 1496.523 & 1494.904\end{array}$ $\begin{array}{lllll}1494.388 & 1494.8 & 1496.532 & 1494.911\end{array}$ $\begin{array}{lllll}1494.395 & 1494.811 & 1496.555 & 1494.927\end{array}$ $\begin{array}{llll}1494.398 & 1494.821 & 1496.562 & 1494.941 \\ 1 & 1494.4948 & 149658 & 149494\end{array}$ $\begin{array}{llll}1494.4 & 1494.821 & 1496.558 & 1494.944 \\ 1494.393 & 1494.814 & 1496539 & 1494939\end{array}$ $\begin{array}{llll}1494.393 & 1494.814 & 1496.539 & 1494.939 \\ 1494.4 & 1494.818 & 1496.541 & 1494.939\end{array}$ $\begin{array}{llll}1494.402 & 1494.823 & 1496.548 & 1494.946\end{array}$ $\begin{array}{lllll}149.402 .4 & 1494.823 & 1496.548 & 1494.946 \\ 149494.944\end{array}$ $\begin{array}{lllll}1494.405 & 1494.825 & 1496.539 & 1494.941\end{array}$ $1494.407 \quad 1494.828 \quad 1496.544 \quad 1494.946$ $\begin{array}{llll}1494.412 & 1494.835 & 1496.555 & 1494.953 \\ 1494.419 & 1494.849 & 1496.579 & 1494.969\end{array}$ $\begin{array}{lllllllllllllllllllll}1493.918 & 1494.517 & 1494.544 & 1495.666 & 1496.956 & 1496.725 & 1493.184 & 1489.392 & 1493.016 & 1490.395 & 1491.183 & 1486.539 & 1488.464 & 1493.318 & 1493.982 & 1493.186 & 1495.169 & 1493.166 & 1485.381 & 1478.419\end{array}$

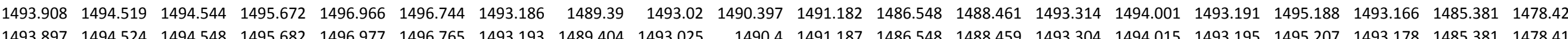

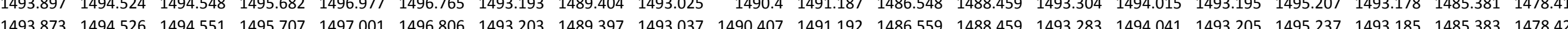

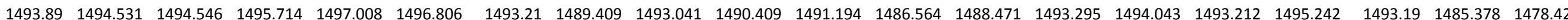
$\begin{array}{llllllllllllllllllllll}1493.897 & 1494.536 & 1494.555 & 1495719 & 1497.012 & 1496.808 & 1493.217 & 1489.409 & 1493.046 & 1490.411 & 1491.199 & 1486551 & 1488.473 & 14933 & 1494.048 & 1493.219 & 1495.246 & 1493.197 & 1485.378 & 1487.421\end{array}$

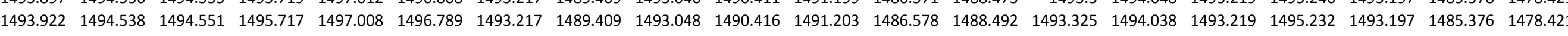

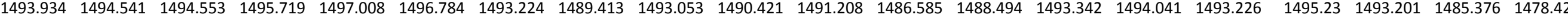

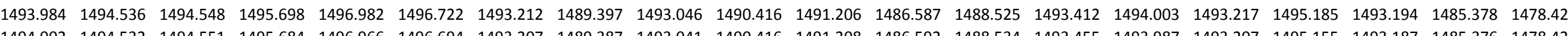

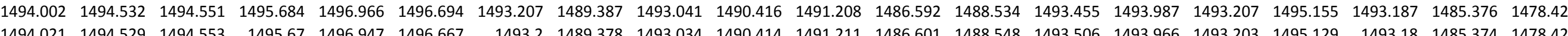
$\begin{array}{llllllllllllllllllll}1494.021 & 1494.529 & 1494.553 & 1495.67 & 1496.947 & 1496.667 & 1493.2 & 1489.378 & 1493.034 & 1490.414 & 1491.211 & 1486.601 & 1488.548 & 1493.506 & 1493.966 & 1493.203 & 1495.129 & 1493.18 & 1485.374 & 1478.421 \\ 1494.026 & 1494.524 & 1494.553 & 1495.659 & 1496.933 & 1496.648 & 1493.191 & 1489.364 & 1493.03 & 1490.414 & 1491.215 & 1486.603 & 1488.553 & 1493.537 & 1493.952 & 1493.193 & 1495.108 & 1493.173 & 1485374 & 1478.421\end{array}$

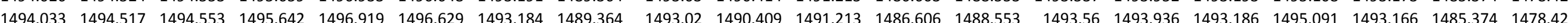
$\begin{array}{lllllllllllllllllllll}1494.038 & 1494.514 & 1494.558 & 1495.626 & 1496.901 & 1496.605 & 1493.175 & 1489.35 & 1493.013 & 1490.404 & 1491.21 & 1486.599 & 1488.557 & 1493.544 & 1493.919 & 1493.174 & 1495.068 & 1493.157 & 1485.374 & 1478.416\end{array}$ $\begin{array}{llllllllllllllllllllll}1494.012 & 1494.517 & 1494.563 & 1495.624 & 1496.901 & 1496.619 & 1493.172 & 1489.341 & 1493.006 & 1490.404 & 1491.213 & 1486.601 & 1488.543 & 1493.499 & 1493.919 & 1493.174 & 1495.07 & 1493.152 & 1485.371 & 1478.446\end{array}$

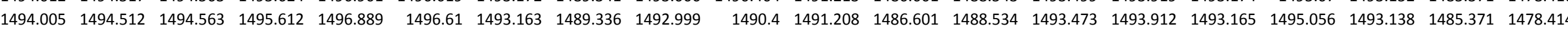
$\begin{array}{lllllllllllllllllllllll}1493.986 & 1494.514 & 1494.565 & 1495.61 & 1496.889 & 1496.622 & 1493.158 & 1489.334 & 1492.992 & 1490.397 & 1491.208 & 1486.606 & 1488.525 & 1493.436 & 1493.917 & 1493.162 & 1495.07 & 1493.14 & 1485.371 & 1478.412\end{array}$

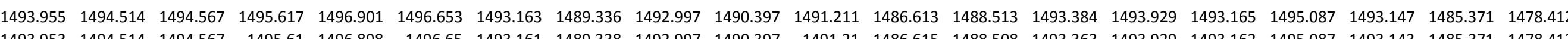

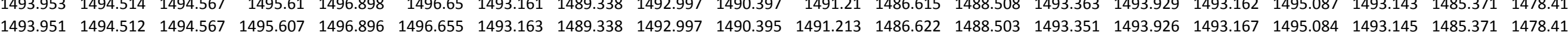

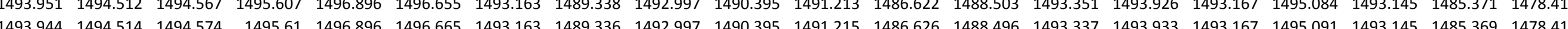
$\begin{array}{llllllllllllllllllll}1493.955 & 1494.512 & 1494.572 & 1495.603 & 1496889 & 1496.653 & 1493.163 & 1498.334 & 142.999 & 1490395 & 1491.215 & 1486.631 & 1488.506 & 1493.358 & 1493.926 & 1493.167 & 1495.082 & 1493.147 & 1485.369 & 1478.414\end{array}$ $\begin{array}{llllllllllllllllllllll}1493.944 & 1494.514 & 1494.577 & 1495.607 & 1496.898 & 1496.682 & 1493.044 & 1489.334 & 1492.87 & 1490.397 & 1491.173 & 1486.582 & 1488.499 & 1493.238 & 1493.884 & 1493.046 & 1495.098 & 1493.03 & 1485.369 & 1478.419\end{array}$ $\begin{array}{llllllllllllllllllll}1493.941 & 1494.519 & 1494.579 & 1495.61 & 1496.903 & 1496.686 & 1493.088 & 1489.338 & 1492.917 & 1490.4 & 1491.18 & 1486.582 & 1488.494 & 1493.29 & 1493.91 & 1493.094 & 1495.103 & 1493.07 & 1485.369 & 1478.419\end{array}$

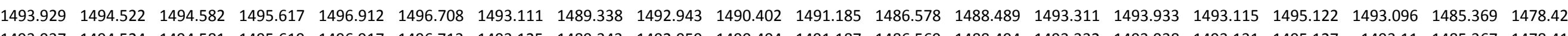

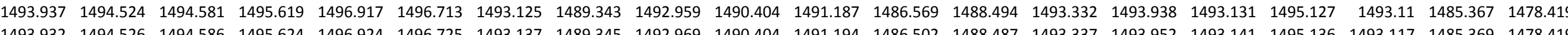

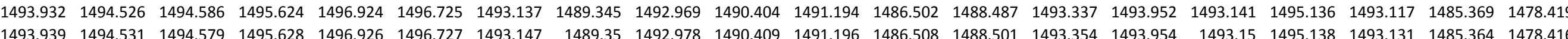

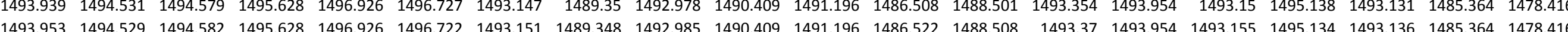

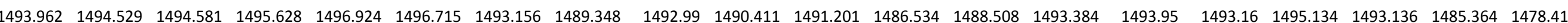
$\begin{array}{llllllllllllllllllllllll}1493.984 & 1494.534 & 1494.584 & 1495.624 & 1496.917 & 1496.698 & 1493.158 & 1489.343 & 1492.99 & 1490.411 & 1491.204 & 1486.545 & 1488.522 & 1493.419 & 1493.945 & 1493.16 & 1495.12 & 1493.14 & 1485.364 & 1478.414 \\ \end{array}$

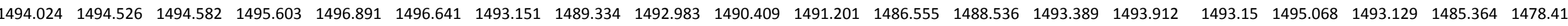
$\begin{array}{llllllllllllllllllllll}1494.054 & 1494.519 & 1494.574 & 1495.582 & 1496.866 & 1496.598 & 1493.137 & 1489.322 & 1492.976 & 1490.404 & 1491.203 & 1486.564 & 1488.558 & 1493.382 & 1493.882 & 1493.141 & 1495.033 & 1493.126 & 1485.362 & 1478.4097\end{array}$ $\begin{array}{llllllllllllllllllll}1494.066 & 1494.512 & 1494.581 & 1495.559 & 1496.842 & 1496.562 & 1493.128 & 1489.312 & 1492.964 & 1490.4 & 1491.199 & 1486.578 & 1488.555 & 1493.372 & 1493.854 & 1493.129 & 1494.997 & 1493.112 & 1485.362 & 1478.407\end{array}$

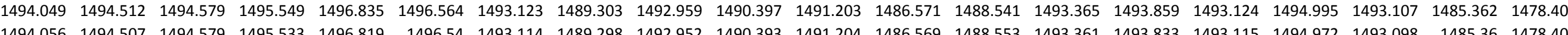

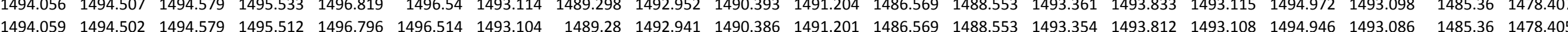
$\begin{array}{lllllllllllllllllllll}1494.042 & 14945 & 1494.584 & 1495501 & 1496787 & 1496512 & 1493.095 & 1489.284 & 1492.933 & 1490.379 & 1491.196 & 1486573 & 1488539 & 1493.339 & 1493814 & 1493.098 & 1494.934 & 1493.077 & 1485367 & 1478.402\end{array}$ $\begin{array}{llllllllllllllllllllll}1494.031 & 1494.495 & 1494.584 & 1495.489 & 1496.777 & 1496.509 & 1493.086 & 1489.27 & 1492.924 & 1490.376 & 1491.194 & 1486.58 & 1488.529 & 1493.335 & 1493.796 & 1493.089 & 1494.929 & 1493.07 & 1485.357 & 14784\end{array}$ $\begin{array}{lllllllllllllllllllll}1493.988 & 1494.495 & 1494.593 & 1495.491 & 1496.787 & 1496.54 & 1493.088 & 1489.273 & 1492.926 & 1490.374 & 1491.194 & 1486.589 & 1488.501 & 1493.332 & 1493.817 & 1493.091 & 1494.95 & 1493.065 & 1485.36 & 1478.402\end{array}$

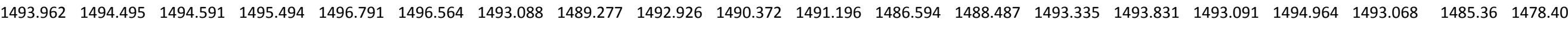

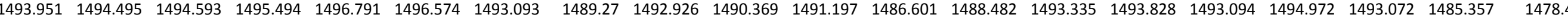

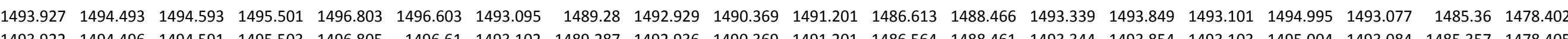
$\begin{array}{llllllllllllllllllll}1493.922 & 1494.496 & 1494.591 & 1495.503 & 1496.805 & 1496.61 & 1493.102 & 1489.287 & 1492.936 & 1490.369 & 1491.201 & 1486.564 & 1488.461 & 1493.344 & 1493.854 & 1493.103 & 1495.004 & 1493.084 & 14855.357 & 1478.405 \\ 1493.927 & 1494.493 & 1494.593 & 1495.498 & 1496803 & 1496.61 & 1493.102 & 148928 & 1492936 & 1490369 & 1491201 & 1486573 & 1488.461 & 1493347 & 1493854 & 1493.105 & 1495 & 1493.086 & 1485355 & 1478.405\end{array}$

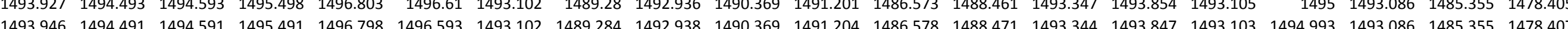
$\begin{array}{lllllllllllllllllllllll}1493.941 & 1494.493 & 1494.593 & 1495.494 & 1496.798 & 1496.598 & 1493.104 & 1489.282 & 1492.938 & 1490.367 & 1491.203 & 1486.587 & 1488.466 & 1493.347 & 1493.847 & 1493.105 & 1494.993 & 1493.086 & 1485.355 & 1478.407\end{array}$ $\begin{array}{llllllllllllllllllllll}1493.939 & 1494.491 & 1494.596 & 1495.494 & 1496.801 & 1496.605 & 1493.104 & 1489.275 & 1492.941 & 1490.369 & 1491.206 & 1486.594 & 1488.461 & 1493.347 & 1493.852 & 1493.11 & 1494.997 & 1493.086 & 1485.355 & 1478.407\end{array}$ $\begin{array}{lllllllllllllllllllll}1493.944 & 1494.488 & 1494.593 & 1495.491 & 1496.798 & 1496.6 & 1493.102 & 1489.277 & 1492.936 & 1490.369 & 1491.206 & 1486.599 & 1488.459 & 1493.344 & 1493.852 & 1493.103 & 1494.995 & 1493.084 & 1485.353 & 1478.407\end{array}$ \begin{tabular}{llllllllllllllllllll}
1493.941 & 1494.488 & 1494.598 & 1495.491 & 1496.796 & 1496.6 & 1493.1 & 1489.28 & 1492.936 & 1490.367 & 1491.201 & 1486.606 & 1488.454 & 1493.344 & 1493.859 & 1493.103 & 1495.002 & 1493.084 & 1485.353 & 1478.405 \\
\hline
\end{tabular} \begin{tabular}{lllllllllllllllllllll}
1493.937 & 1494.486 & 1494.6 & 1495.491 & 1496.798 & 1496.607 & 1493.1 & 1489.275 & 1492.934 & 1490.369 & 1491.201 & 1486.603 & 1488.452 & 1493.342 & 1493.854 & 1493.103 & 1494.997 & 1493.079 & 1485.353 & 1478.402 \\
\hline
\end{tabular}

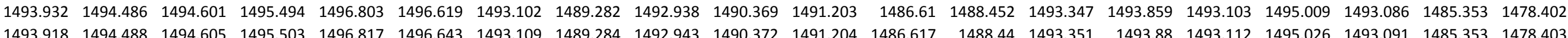


Well

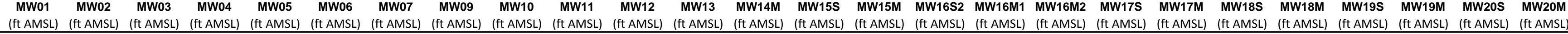

$11 / 15 / 112: 00$ 11/15/11 3:00

$11 / 15 / 115.00$

11/15/115:00

11/15/11 6:00

11/15/117:00

11/15/11 9:00

11/15/11 10:00

11/15/11 11:00

11/15/1112:00

111/15/11 14:00

11/15/11 14:00

11/15/11 16:00

11/15/11 17:00

11/15/11 18:00

11/15/11 19:00

11/15/11 20:00

11/15/11 21:00

11/15/11 22:00

$11 / 16 / 110: 00$

11/16/110:00

11/16/111 2:00

11/16/11 3:00

11/16/11 4:00

$11 / 16 / 115: 00$

$1 / 16 / 116: 00$

11/16/117 7:00

$11 / 16 / 119: 00$

11/16/11 10:00

11/16/11 11:00

11/16/11 12:00

$11 / 16 / 1113: 00$
$11 / 16 / 1114: 00$

11/16/11 14:00

11/16/11 15:00

$11 / 16 / 11$ 17:00

11/16/11 18:00

11/16/11 19:00

11/16/11 20:00

11/16/11 21:00

11/16/11 22:00

$11 / 16 / 1123: 00$

11/17/11 0:00

11/17/11 2:00

$11 / 17 / 11$ 3:00

11/17/11 4:00

11/17/11 5:00

11/17/11 6:00 $\begin{array}{rrrrr}1494.419 & 1494.858 & 1496.584 & 1494.976 \\ 1494.421 & 1494.86 & 1496.588 & 1494.983\end{array}$ $\begin{array}{llll}1494.421 & 1494.86 & 1496.588 & 1494.983\end{array}$ $\begin{array}{rrrr}1494.423 & 1494.87 & 1496.598 & 1494.99 \\ 1494.398 & 1494.849 & 1496551 & 1494.969\end{array}$ $\begin{array}{lllll}1494.409 & 1494.846 & 1496.548 & 1494.967\end{array}$ $\begin{array}{lllll}1494.405 & 1494.844 & 1496.537 & 1494.96\end{array}$ $\begin{array}{lllll}1494.409 & 1494.839 & 14966.53 & 1494.951\end{array}$ $\begin{array}{lllll}1494.405 & 1494.83 & 1496.516 & 1494.939\end{array}$ $\begin{array}{lllll}1494.405 & 1494.821 & 1496.497 & 1494.925\end{array}$ $1494.405 \quad 1494.814 \quad 1496.495 \quad 1494.918$ 1494.4091494 .8181496 .49914994 .916 $\begin{array}{llll}1494.416 & 1494.821 & 1496.513 & 1494.923 \\ 1494.416 & 1494.828 & 1496.516 & 1494.925\end{array}$ $\begin{array}{lllll}1494.412 & 1494.825 & 1496.506 & 1494.923\end{array}$ $\begin{array}{lllll}1494.409 & 1494.816 & 1496.488 & 1494.911\end{array}$ $\begin{array}{llllll}1494.407 & 1494.807 & 1496.476 & 1494.904\end{array}$ $\begin{array}{llllll}1494.398 & 1494.79 & 1496.446 & 1494.883\end{array}$ $\begin{array}{llll}1494.391 & 1494.765 & 1496.408 & 1494.855\end{array}$ $\begin{array}{llll}1494.391 & 1494.751 & 1496.397 & 1494.836\end{array}$ $\begin{array}{llllll}1494.384 & 1494.737 & 1496.375 & 1494.818\end{array}$ $\begin{array}{lllll}1494.381 & 1494.721 & 1496.361 & 1494.792\end{array}$ $\begin{array}{lllll}1494.377 & 1494.716 & 1496.357 & 1494.783\end{array}$ $\begin{array}{lllll}1494.375 & 1494.709 & 1496.352 & 1494.776\end{array}$ $\begin{array}{lllll}1494.377 & 1494.709 & 1496.354 & 1494.776\end{array}$ $\begin{array}{lllll}1494.367 & 1494.697 & 1496.338 & 1494.769\end{array}$ $\begin{array}{lllll}1494.37 & 1494.695 & 1496.333 & 1494.759\end{array}$ $\begin{array}{lllll}1494.372 & 1494.7 & 1496.347 & 1494.766\end{array}$ $\begin{array}{rrrr}1494.358 & 1494.681 & 1496.312 & 1494.745 \\ 1494.354 & 1494.66 & 1496.28 & 1494.722\end{array}$ $\begin{array}{lllll}1494.342 & 1494.637 & 1496.247 & 1494.696\end{array}$ $\begin{array}{lllll}1494.34 & 1494.618 & 1496.224 & 1494.673\end{array}$ $\begin{array}{lllll}1494.333 & 1494.604 & 1496.21 & 1494.657\end{array}$ $1494.323 \quad 1494.588 \quad 1496.188 \quad 1494.636$ $\begin{array}{llll}1494.333 & 1494.595 & 1496.21 & 1494.638\end{array}$ $\begin{array}{llll}1494.33 & 1494.602 & 1496.228 & 1494.647 \\ 1494.326 & 1494.595 & 1496219 & 194.645\end{array}$ $\begin{array}{lllll}1494.326 & 1494.595 & 1496.219 & 1494.645\end{array}$ $\begin{array}{lllll}1494.326 & 1494.597 & 1496.217 & 1494.645 \\ 1494.319 & 1494.595 & 1496.212 & 1494.643\end{array}$ $\begin{array}{lllll}1494.312 & 1494.588 & 1496.195 & 1494.633\end{array}$ $\begin{array}{lllll}1494.307 & 1494.581 & 1496.184 & 1494.624\end{array}$ $\begin{array}{llllll}1494.307 & 1494.583 & 1496.188 & 1494.626\end{array}$ $\begin{array}{lllll}1494.305 & 1494.578 & 1496.186 & 1494.624\end{array}$ $\begin{array}{llll}1494.302 & 1494.578 & 1496.181 & 1494.619\end{array}$ $\begin{array}{llll}1494.305 & 1494.581 & 1496.193 & 1494.624 \\ 1494.305 & 1494.588 & 1496.207 & 1494.631\end{array}$ $\begin{array}{rrrrr}1494.305 & 1494.588 & 1496.207 & 1494.631 \\ 1494.3 & 149459 & 1496207 & 1494636\end{array}$ $\begin{array}{rrrrr}1494.298 & 1494.592 & 1496.202 & 1494.633\end{array}$ $\begin{array}{lllll}1494.298 & 1494.595 & 1496.21 & 1494.638\end{array}$ $\begin{array}{lllll}1494.298 & 1494.599 & 1496.217 & 1494.643\end{array}$ $\begin{array}{lllll}1494.302 & 1494.611 & 1496.235 & 1494.657\end{array}$ $\begin{array}{lllll}1494.305 & 1494.623 & 1496.254 & 1494.673\end{array}$ $\begin{array}{rrrrr}1494.302 & 1494.627 & 1496.261 & 1494.687 \\ 1494.302 & 1494.63 & 1496.261 & 1494.692\end{array}$ $\begin{array}{lllllllllllllllllllll}1493.92 & 1494.488 & 1494.603 & 1495.508 & 1496.819 & 1496.646 & 1493.109 & 1489.287 & 1492.945 & 1490.374 & 1491.204 & 1486.626 & 1488.445 & 1493.351 & 1493.88 & 1493.112 & 1495.035 & 1493.093 & 1485.35 & 1478.402\end{array}$ $\begin{array}{llllllllllllllllllll}1493.92 & 1494.491 & 1494.605 & 1495.508 & 1496.824 & 1496.65 & 1493.114 & 1489.289 & 1492.945 & 1490.374 & 1491.208 & 1486.631 & 1488.442 & 1493.354 & 1493.884 & 1493.117 & 1495.042 & 1493.096 & 1485.35 & 1478.405 \\ 1493.92 & 1494.491 & 1494.605 & 1495.515 & 1496.829 & 1496.66 & 1493.118 & 1489.289 & 1492.952 & 1490.376 & 1491.208 & 1486.64 & 1488.44 & 1493358 & 1493.894 & 1493.12 & 1495.047 & 1493.103 & 148535 & 1478.405\end{array}$

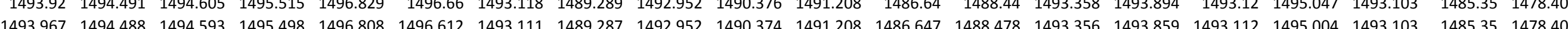
$\begin{array}{lllllllllllllllllllll}1493.96 & 1494.486 & 1494.596 & 1495.498 & 1496808 & 1496.612 & 1493.114 & 1489287 & 1492.5 & 1490.376 & 1491.213 & 1486.65 & 1488.464 & 1493356 & 1493866 & 1493.117 & 1495009 & 1493098 & 1485348 & 1478.405\end{array}$ $\begin{array}{llllllllllllllllllllll}1493.967 & 1494.486 & 1494.598 & 1495.494 & 1496.805 & 1496.605 & 1493.114 & 1489.277 & 1492.95 & 1490.374 & 1491.213 & 1486.643 & 1488.466 & 1493.356 & 1493.856 & 1493.117 & 1495.004 & 1493.098 & 1485.348 & 1478.407\end{array}$

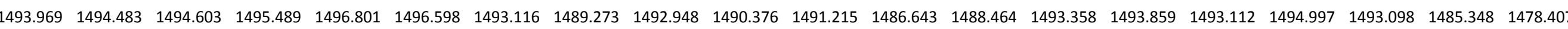
$\begin{array}{lllllllllllllllllllllll}1493.977 & 1494.481 & 1494.605 & 1495.482 & 1496.791 & 1496.583 & 1493.111 & 1489.27 & 1492.945 & 1490.376 & 1491.215 & 1486.643 & 1488.461 & 1493.354 & 1493.847 & 1493.11 & 1494.983 & 1493.093 & 1485.348 & 1478.4097\end{array}$ $\begin{array}{llllllllllllllllllll}1493.979 & 1494.479 & 1494.603 & 1495.473 & 1496.782 & 1496.567 & 1493.104 & 1489.263 & 14292.941 & 1490.372 & 1491.215 & 1486.645 & 1488.459 & 1493.349 & 1493.838 & 1493.108 & 1494.969 & 1493.086 & 1485.346 & 1478.407\end{array}$

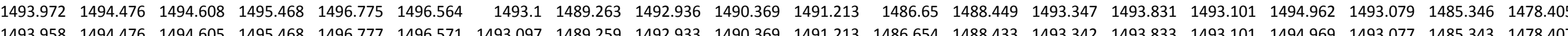
$\begin{array}{lllllllllllllllllllll}1493.958 & 1494.476 & 1494.605 & 1495.468 & 1496.777 & 1496.571 & 1493.097 & 1489.259 & 1492.933 & 1490.369 & 1491.213 & 1486.654 & 1488.433 & 1493.342 & 1493.833 & 1493.101 & 1494.969 & 1493.077 & 1485.343 & 1478.407 \\ 1493.941 & 1494.476 & 1494.61 & 1495.468 & 1496.782 & 1496.586 & 1493.095 & 1489.266 & 1492931 & 1490369 & 1491.21 & 1486.661 & 1488419 & 1493344 & 1493842 & 1493103 & 1494981 & 1493.079 & 1485343 & 1478.402\end{array}$

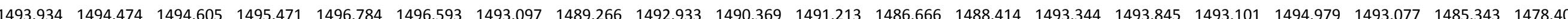

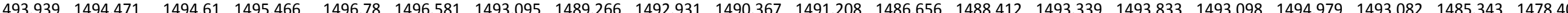
$\begin{array}{lllllllllllllllllllll}1493.948 & 1494.471 & 1494.605 & 1495.459 & 1496.773 & 1496.569 & 1493.09 & 1489.263 & 1492.929 & 1490.365 & 1491.208 & 1486.631 & 1488.41 & 1493.337 & 1493.826 & 1493.094 & 1494.962 & 1493.077 & 1485.343 & 1478.402\end{array}$

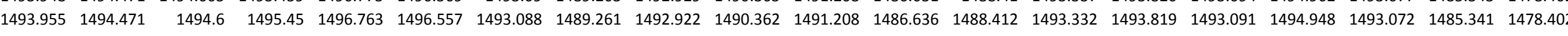
$\begin{array}{llllllllllllllllllllll}1493.977 & 1494.467 & 1494.603 & 1495.433 & 1496.747 & 1496.528 & 1493.081 & 1489.259 & 1492.917 & 1490.362 & 1491.206 & 1486.638 & 1488.421 & 1493.323 & 1493.796 & 1493.082 & 1494.925 & 1493.068 & 1485.339 & 1478.4\end{array}$ $\begin{array}{llllllllllllllllllll}1493.995 & 1494.464 & 1494.601 & 1495.413 & 1496.726 & 1496.493 & 1493.069 & 1489.249 & 1492.905 & 1490.358 & 1491.201 & 1486.643 & 1488.428 & 1493.314 & 1493.775 & 1493.072 & 1494.894 & 1493.053 & 1485.339 & 1478.398\end{array}$

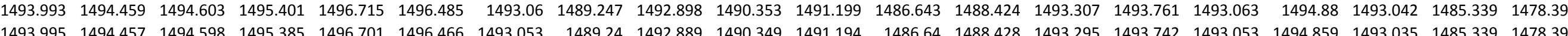

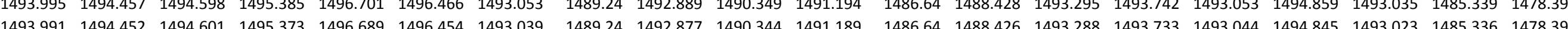
$\begin{array}{lllllllllllllllllllllll}1493.979 & 1494.45 & 1494.598 & 1495.364 & 1496.682 & 1496.459 & 1493.032 & 1489.233 & 1492.873 & 1490.339 & 1491.187 & 1486.64 & 1488.419 & 1493.281 & 1493.73 & 1493.034 & 1494.842 & 1493.018 & 1485.336 & 1478.386\end{array}$ $\begin{array}{llllllllllllllllllll}1493.969 & 1494.447 & 1494.598 & 1495.357 & 1496.675 & 1496.454 & 1493.025 & 1489.23 & 1492865 & 1490.335 & 1491.183 & 1486.643 & 1488.14 & 1493.271 & 1493.723 & 1493.027 & 1494.835 & 1493.009 & 1485.334 & 1478.386\end{array}$ $\begin{array}{llllllllllllllllllllllll}1493.958 & 1494.445 & 1494.596 & 1495.35 & 1496.67 & 1496.457 & 1493.018 & 1489.228 & 1492.858 & 1490.33 & 1491.178 & 1486.647 & 1488.417 & 1493.264 & 1493.716 & 1493.02 & 1494.828 & 1493.004 & 1485.334 & 1478.381\end{array}$

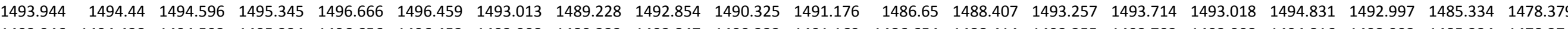

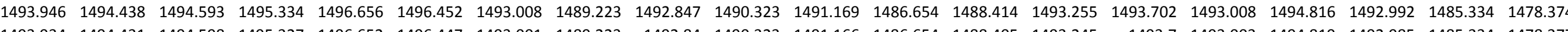

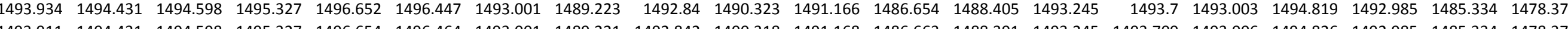

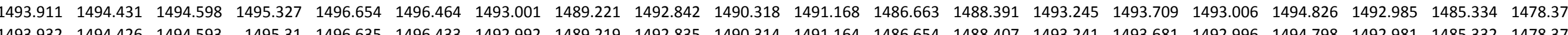

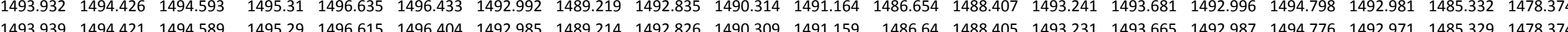
$\begin{array}{llllllllllllllllllll}1493.948 & 1494.414 & 1494.586 & 1495.266 & 1496.596 & 1496.375 & 1492.973 & 1489.209 & 1492.812 & 1490.302 & 1491.157 & 1486.631 & 1488.41 & 1493.22 & 1493.646 & 1492.973 & 1494.751 & 1492.957 & 1485.329 & 1478.37 \\ \end{array}$ $\begin{array}{llllllllllllllllllll}1493.944 & 1494.409 & 1494.584 & 1495.248 & 1496.577 & 1496359 & 1492.959 & 1489.202 & 1492.802 & 1490.297 & 1491.15 & 1486.631 & 1488.4 & 1493.208 & 1493.628 & 1492.963 & 1494.722 & 1492.945 & 1485.329 & 1478.37\end{array}$

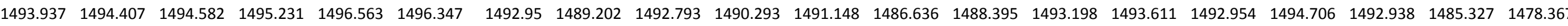
$\begin{array}{lllllllllllllllllllllll}1493.927 & 1494.397 & 1494.574 & 1495.213 & 1496.547 & 1496.335 & 1492.941 & 1489.198 & 1492.781 & 1490.283 & 1491.143 & 1486.636 & 1488.393 & 1493.187 & 1493.6 & 1492.942 & 1494.694 & 1492.924 & 1485.327 & 1478.363 & \end{array}$

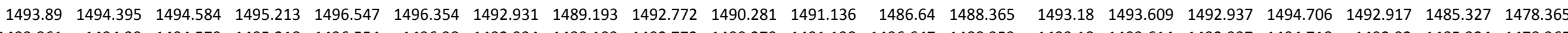

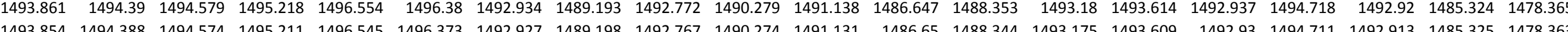

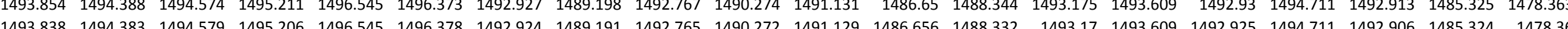

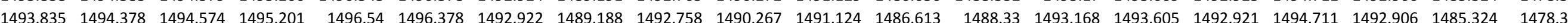
$\begin{array}{lllllllllllllllllllll}1493.838 & 1494.373 & 1494.57 & 1495.19 & 1496.531 & 1496.366 & 1492.915 & 1489.186 & 1492.753 & 1490.262 & 1491.122 & 1486.603 & 1488.332 & 1493.161 & 1493.595 & 1492.916 & 1494.697 & 1492.901 & 1485.322 & 1478.36\end{array}$ $\begin{array}{llllllllllllllllllllll}1493.835 & 1494.368 & 1494.565 & 1495.178 & 1496.522 & 1496.356 & 1492.91 & 1489.183 & 1492.746 & 1490.26 & 1491.117 & 1486.61 & 1488.334 & 1493.154 & 1493.586 & 1492.911 & 1494.69 & 1492.894 & 1485.322 & 1478.358\end{array}$ $\begin{array}{llllllllllllllllllllll}1493.821 & 1494.364 & 1494.57 & 1495.176 & 1496.522 & 1496.366 & 1492.906 & 1489.186 & 1492.744 & 1490.258 & 1491.117 & 1486.615 & 1488.316 & 1493.149 & 1493.586 & 1492.906 & 1494.685 & 1492.889 & 1485.322 & 1478.358\end{array}$ $\begin{array}{lllllllllllllllllllll}1493.814 & 1494.361 & 1494.567 & 1495.171 & 1496.517 & 1496.366 & 1492.901 & 1489.176 & 1492.737 & 1490.255 & 1491.115 & 1486.622 & 1488.313 & 1493.147 & 1493.586 & 1492.906 & 1494.69 & 1492.887 & 1485.322 & 1478.358 & 1486\end{array}$ $\begin{array}{llllllllllllllllllll}1493.81 & 1494.359 & 1494.565 & 1495.164 & 1496.512 & 1496.366 & 1492.899 & 1489.181 & 1492.737 & 1490.253 & 1491.11 & 1486.626 & 1488.313 & 1493.147 & 1493.581 & 1492.899 & 1494.682 & 1492.882 & 1485.322 & 1478.358\end{array}$

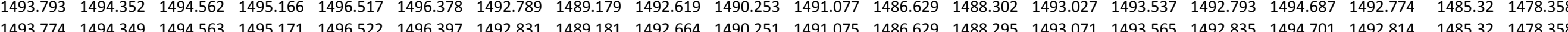

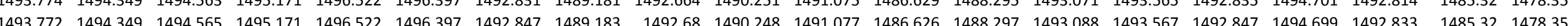
$\begin{array}{llllllllllllllllllllll}1493.772 & 1494.342 & 1494.56 & 1495.169 & 1496.519 & 1496.397 & 1492.854 & 1489.181 & 1492.687 & 1490.248 & 1491.075 & 1486.629 & 1488.295 & 1493.095 & 1493.565 & 1492.854 & 1494.697 & 1492.838 & 1485.317 & 1478.356\end{array}$ $\begin{array}{lllllllllllllllllllllll}1493.76 & 1494.337 & 1494.563 & 1495.169 & 1496.524 & 1496.404 & 1492.859 & 1489.183 & 1492.694 & 1490.246 & 1491.075 & 1486.636 & 1488.285 & 1493.104 & 1493.574 & 1492.861 & 1494.704 & 1492.842 & 1485.317 & 1478.353\end{array}$ $\begin{array}{lllllllllllllllllllll}1493.751 & 1494.335 & 1494.56 & 1495.173 & 1496.526 & 1496.414 & 1492.863 & 1489.179 & 1492.699 & 1490.246 & 1491.075 & 1486.638 & 1488.278 & 1493.107 & 1493.586 & 1492.864 & 1494.708 & 1492.847 & 1485.317 & 1478.353\end{array}$ \begin{tabular}{llllllllllllllllllllll}
1493.734 & 1494.333 & 1494.563 & 1495.18 & 1496.538 & 1496.435 & 1492.87 & 1489.179 & 1492.704 & 1490.248 & 1491.077 & 1486.643 & 1488.266 & 1493.116 & 1493.598 & 1492.873 & 1494.727 & 1492.856 & 1485.317 & 1478.353 \\
\hline
\end{tabular} $\begin{array}{llllllllllllllllllll}1493.723 & 1494.335 & 1494.563 & 1495.187 & 1496.547 & 1496.452 & 1492.88 & 1489.186 & 1492.713 & 1490.251 & 1491.082 & 1486.65 & 1488.259 & 1493.121 & 1493.614 & 1492.88 & 1494.746 & 1492.861 & 1485.317 & 1478.356\end{array}$

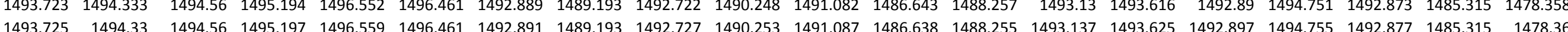


Well

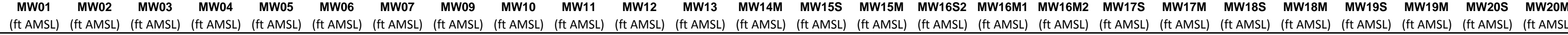

11/17/11 8:00 11/17/119:00 $11 / 17 / 11$ 10:00 11/177/11 12:00 11/17/11 12:00 11/17/11 14:00 11/17/11 15:00 $11 / 17 / 1116: 00$ $11 / 17 / 1117: 00$ 11/17/11 18:00 11/17/11 19:00 11/17/11 21:00 11/177/11 21:00 11/17/11 23:00 11/18/11 0:00 $11 / 18 / 111: 00$ 11/18/11 2:00 $11 / 18 / 113: 00$ 11/18/11 4:00 11/18/11 6:00 11/18/11 6:00 11/18/1118:00 $11 / 18 / 119: 00$ 11/18/11 10:00 11/18/11 11:00 11/18/11 12:00 11/18/18/11 14:00 $11 / 18 / 1115: 00$ 11/18/11 16:00 11/18/11 17:00 11/18/11 18:00 11/18/11 19:00 11/18/11 20:00 11/18/11 21:00 11/18/11 22:00 11/19/11 0:00 11/19/11 1:00 11/19/11 2:00 11/19/11 3:00 11/19/11 4:00 $11 / 19 / 115.00$ 11/19/11 6:00 $11 / 19 / 118: 00$ $11 / 19 / 119: 00$ 11/19/11 10:00 11/19/11 11:00 $11 / 19 / 1112: 00$
$11 / 19 / 1113: 00$ $\begin{array}{llll}1494.298 & 1494.63 & 1496.247 & 1494.687 \\ 1494.298 & 1494.63 & 1496252 & 1494.689\end{array}$ $\begin{array}{lllll}1494.298 & 1494.63 & 1496.252 & 1494.689\end{array}$ $\begin{array}{lllll}1494.307 & 1494.644 & 1496.275 & 1494.703\end{array}$ $\begin{array}{lllll}1494.319 & 1494.676 & 1496.336 & 1494.748\end{array}$ $\begin{array}{llll}1494.33 & 1494.707 & 1496.38 & 1494.785\end{array}$ $\begin{array}{rrrrr}1494.33 & 1494.707 & 1496.38 & 1494.785 \\ 1494.337 & 1494.73 & 1496.411 & 1494.815\end{array}$ $\begin{array}{llllll}1494.344 & 1494.755 & 1496.441 & 1494.846\end{array}$ $1494.3491494 .772 \quad 1496.457 \quad 1494.869$ $\begin{array}{llll}1494.351 & 1494.786 & 1496.464 & 1494.885\end{array}$ $\begin{array}{rrrrr}1494.353 & 1494.793 & 1496.467 & 1494.897 \\ 1494.358 & 1494.8 & 1496.471 & 1494902\end{array}$ $\begin{array}{rrrrr}1494.358 & 1494.8 & 1496.471 & 1494.902 \\ 1494.363 & 1494.811 & 1496.481 & 1494.911\end{array}$ $\begin{array}{lllll}1494.368 & 1494.821 & 1496.492 & 1494.919\end{array}$ $\begin{array}{lllll}1494.372 & 1494.837 & 1496.504 & 1494.919 \\ 1497\end{array}$ $\begin{array}{rrrrr}1494.472 & 1494.837 & 1496.504 & 1494.927 \\ 1494.379 & 1494.849 & 1496.52 & 1494.944\end{array}$ $\begin{array}{lllll}1494.386 & 1494.865 & 1496.541 & 1494.958\end{array}$ $\begin{array}{llllll}1494.389 & 1494.874 & 1496.551 & 1494.969\end{array}$ $\begin{array}{llll}1494.391 & 1494.881 & 1496.553 & 1494.979\end{array}$ $\begin{array}{lllll}1494.398 & 1494.891 & 1496.565 & 1494.988\end{array}$ $\begin{array}{llll}1444.409 & 1494.919 & 1496.598 & 1495.014\end{array}$ $\begin{array}{lllll}1494.416 & 1494.933 & 1496609 & 1495.025\end{array}$ $\begin{array}{lllll}1494.414 & 1449.942 & 1496.609 & 1495.05\end{array}$ $1494.416 \quad 1494.944 \quad 1496.612 \quad 1495.033$ $\begin{array}{lllll}1494.426 & 1494.949 & 1496.623 & 1495.039\end{array}$ $\begin{array}{lllll}1494.421 & 1494.951 & 1496.614 & 1495.042\end{array}$ $\begin{array}{llll}1494.433 & 1494.963 & 1496.635 & 1495.051 \\ 1494.435 & 1494.968 & 1496.642 & 1495.058\end{array}$ $\begin{array}{llll}1494.435 & 1494.968 & 1496.642 & 1495.058 \\ 1494.451 & 1494993 & 1496.684 & 1495084\end{array}$ $\begin{array}{llll}1494.447 & 1495.005 & 1496.689 & 1495.093\end{array}$ $\begin{array}{lllll}1494.44 & 1495.003 & 1496.679 & 1495.095\end{array}$ $\begin{array}{lllll}1494.442 & 1495.007 & 1496.672 & 1495.093\end{array}$ $\begin{array}{lllll}1494.447 & 1495.01 & 1496.672 & 1495.088\end{array}$ $\begin{array}{lllll}1494.444 & 1495.007 & 1496.663 & 1495.084\end{array}$ $\begin{array}{rrrr}1494.449 & 1495.005 & 1496.663 & 1495.079 \\ 1494.451 & 1495007 & 1496.67 & 19508\end{array}$ $\begin{array}{lllll}1494.451 & 1495.007 & 1496.67 & 1495.081 \\ 1494.454 & 1495.012 & 1496.672 & 1955.084\end{array}$ $\begin{array}{rrrr}1494.454 & 1495.012 & 1496.672 & 1495.084 \\ 1494.458 & 1495.017 & 1496.684 & 1495.086\end{array}$ $\begin{array}{lllll}1494.465 & 1495.024 & 1496.698 & 1495.095\end{array}$ $\begin{array}{llllll}1494.47 & 1495.04 & 1496.724 & 1495.112\end{array}$ $\begin{array}{lllll}1494.472 & 1495.045 & 1496.731 & 1495.123\end{array}$ $\begin{array}{lllll}1494.475 & 1495.056 & 1496.747 & 1495.135\end{array}$ $\begin{array}{lllll}1494.477 & 1495.061 & 1496.749 & 1495.142\end{array}$ $\begin{array}{llll}1494.482 & 1495.073 & 1496.759 & 1495.149 \\ 1494.482 & 1495.073 & 1496.754 & 1495149\end{array}$ $\begin{array}{llllll}1494.482 & 1495.073 & 1496.754 & 1495.149\end{array}$ $\begin{array}{llll}1494.482 & 1495.07 & 1496.742 & 1495.14\end{array}$ $\begin{array}{lllll}1494.468 & 1495.052 & 1496.703 & 1495.119\end{array}$ $1494.458 \quad 1495.028 \quad 1496.661 \quad 1495.086$ $\begin{array}{lllll}1494.454 & 1495.012 & 1496.63 & 1495.056\end{array}$ $\begin{array}{lllll}1494.44 & 1494.968 & 1496.558 & 1495.007\end{array}$ $\begin{array}{llll}1494.437 & 1494.935 & 1496.516 & 1494.958 \\ 1494.437 & 1494.916 & 1496.497 & 1494.93\end{array}$ $\begin{array}{llllllllllllllllllll}1493.739 & 1494.325 & 1494.555 & 1495.194 & 1496.552 & 1496.447 & 1492.896 & 1489.193 & 1492.732 & 1490.253 & 1491.089 & 1486.631 & 1488.264 & 1493.137 & 1493.614 & 1492.897 & 1494.744 & 1492.877 & 1485.315 & 1478.36\end{array}$ $\begin{array}{lllllllllllllllllllllll}1493.732 & 1494.325 & 1494.558 & 1495.197 & 1496.556 & 1496.452 & 1492.899 & 1489.191 & 1492.732 & 1490.255 & 1491.089 & 1486.638 & 1488.252 & 1493.142 & 1493.623 & 1492.899 & 1494.751 & 1492.882 & 1485.313 & 1478.36 \\ 1493.711 & 1494323 & 1494558 & 1495 & 1408 & 14957 & 1496473 & 1492906 & 1489.195 & 1492.739 & 1490258 & 1491.096 & 1486643 & 1488229 & 1493147 & 1493646 & 1492906 & 1494774 & 1492884 & 1485315 & 1478365\end{array}$

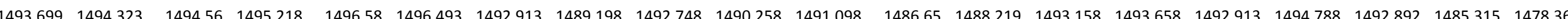
$\begin{array}{lllllllllllllllllllll}1493.671 & 1494.325 & 1494.565 & 1495.238 & 1496.603 & 1496.533 & 1492.924 & 1489.205 & 1492.758 & 1490.265 & 1491.105 & 1486.661 & 1488.198 & 1493.166 & 1493.684 & 1492.93 & 1494.828 & 1492.906 & 1485.313 & 1478.37\end{array}$ $\begin{array}{lllllllllllllllllllllll}1493.652 & 1494.328 & 1494.567 & 1495.259 & 1496.631 & 1496.576 & 1492.943 & 1489.219 & 1492.774 & 1490.269 & 1491.112 & 1486.67 & 1488.182 & 1493.18 & 1493.716 & 1492.944 & 1494.863 & 1492.917 & 1485.315 & 1478.374\end{array}$ $\begin{array}{lllllllllllllllllllll}1493.645 & 1494.335 & 1494.572 & 1495.283 & 1496.652 & 1496.603 & 1492.959 & 1489.228 & 1492.786 & 1490.276 & 1491.117 & 1486.677 & 1488.175 & 1493.194 & 1493.737 & 1492.961 & 1494.896 & 1492.936 & 1485.313 & 1478.379\end{array}$ $\begin{array}{lllllllllllllllllllll}1493.64 & 1494.342 & 1494.577 & 1495.301 & 1496.675 & 1496.629 & 1492.971 & 1489.24 & 1492.802 & 1490.283 & 1491.122 & 1486.689 & 1488.17 & 1493.21 & 1493.76 & 1492.973 & 1494.922 & 1492.953 & 1485.315 & 1478.381\end{array}$

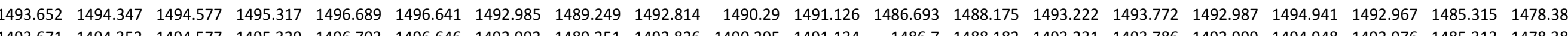

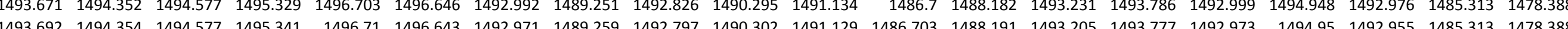
$\begin{array}{llllllllllllllllllll}1493.692 & 1494.354 & 1494.577 & 1495.341 & 1496.71 & 1496.643 & 1492.971 & 1489.259 & 1492.797 & 1490.302 & 1491.129 & 1486.703 & 1488.191 & 1493.205 & 1493.777 & 1492.973 & 1494.95 & 1492.955 & 1485.313 & 1478.388 \\ 1493.711 & 1494.359 & 1494.582 & 1495.35 & 1496.719 & 1496.643 & 1492.992 & 1489259 & 1492.821 & 1490.307 & 1491.136 & 1486.705 & 1488.201 & 1493229 & 1493786 & 1492.992 & 1494.953 & 1492.978 & 148531 & 1478391\end{array}$

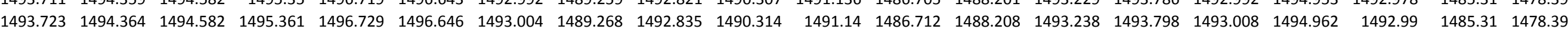

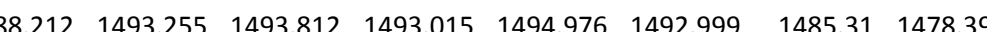
$\begin{array}{llllllllllllllllllllll}1493.737 & 1494.373 & 1494.584 & 1495.387 & 1496.754 & 1496.662 & 1493.025 & 1489.27 & 1492.856 & 1490.325 & 1491.152 & 1486.719 & 1488.219 & 1493.264 & 1493.821 & 1493.03 & 1494.979 & 1493.006 & 1485.313 & 1478.4\end{array}$

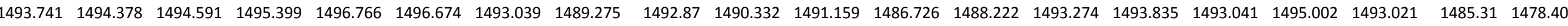
$\begin{array}{lllllllllllllllllllllll}1493.744 & 1494.385 & 1494.593 & 1495.417 & 1496.78 & 1496.689 & 1493.051 & 1489.282 & 1492.882 & 1490.337 & 1491.164 & 1486.728 & 1488.224 & 1493.285 & 1493.856 & 1493.051 & 1495.016 & 1493.032 & 1485.31 & 1478.407\end{array}$

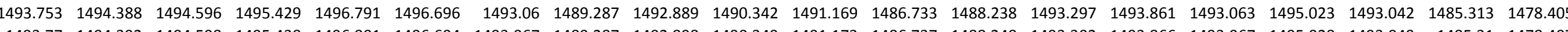

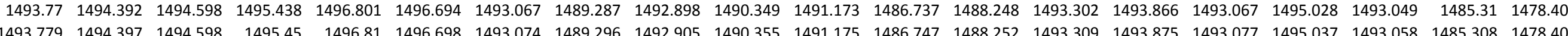

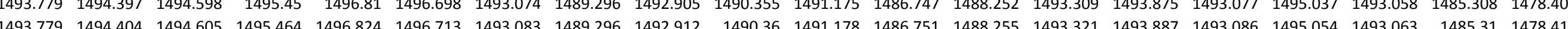

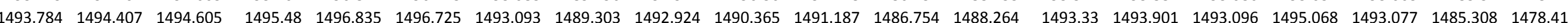
$\begin{array}{lllllllllllllllllllllll}1493.807 & 1494.419 & 1494.608 & 1495.503 & 1496.852 & 1496.727 & 1493.109 & 1489.31 & 1492.941 & 1490.376 & 1491.199 & 1486.758 & 1488.285 & 1493.344 & 1493.912 & 1493.112 & 1495.077 & 1493.093 & 1485.306 & 1478.419\end{array}$

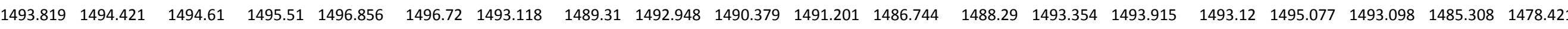

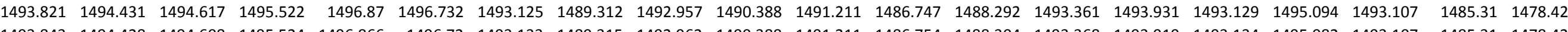

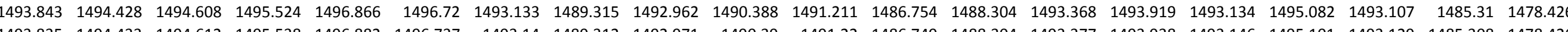

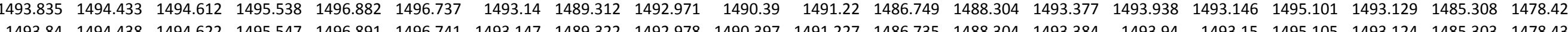

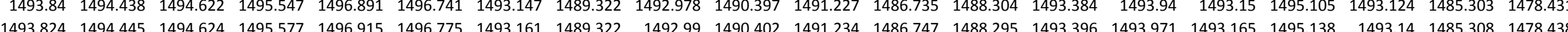

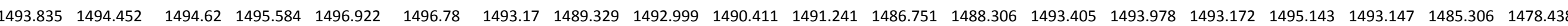
$\begin{array}{llllllllllllllllllllll}1493.859 & 1494.455 & 1494.622 & 1495.589 & 1496.922 & 1496.76 & 1493.172 & 1489.326 & 1493.006 & 1490.411 & 1491.243 & 1486.763 & 1488.32 & 1493.412 & 1493.973 & 1493.174 & 1495.134 & 1493.154 & 1485.306 & 1478.44\end{array}$

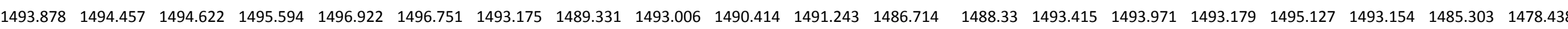
$\begin{array}{llllllllllllllllllllll}1493.892 & 1494.46 & 1494.622 & 1495.594 & 1496.924 & 1496.746 & 1493.177 & 1489.331 & 1493.013 & 1490.418 & 1491.248 & 1486.719 & 1488.337 & 1493.419 & 1493.973 & 1493.181 & 1495.129 & 1493.159 & 1485.306 & 1478.442\end{array}$

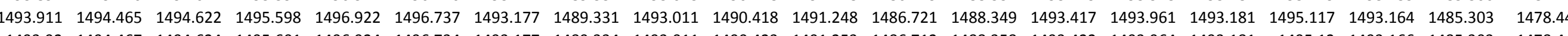
$\begin{array}{lllllllllllllllllllll}1493.92 & 1494.467 & 1494.624 & 1495.601 & 1496.924 & 1496.734 & 1493.177 & 1489.334 & 1493.011 & 1490.423 & 1491.253 & 1486.712 & 1488.358 & 1493.422 & 1493.964 & 1493.181 & 1495.12 & 1493.166 & 1485.303 & 1478.44 \\ 1493.929 & 1494.467 & 1494.62 & 1495603 & 1496926 & 1496.732 & 1493.182 & 1489334 & 1493013 & 1490.425 & 1491.255 & 1486714 & 148837 & 1493.422 & 1493966 & 1493.186 & 1495.117 & 1493.166 & 1485301 & 1478.44\end{array}$

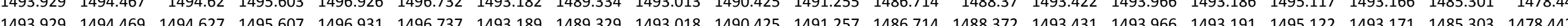

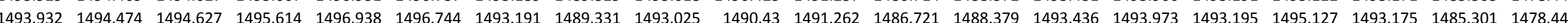
$\begin{array}{llllllllllllllllllllll}1493.929 & 1494.476 & 1494.634 & 1495.624 & 1496.947 & 1496.756 & 1493.2 & 1489.336 & 1493.032 & 1490.432 & 1491.269 & 1486.733 & 1488.381 & 1493.438 & 1493.989 & 1493.203 & 1495.138 & 1493.18 & 1485.301 & 1478.449\end{array}$

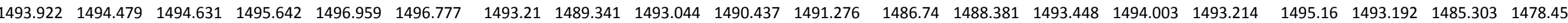
$\begin{array}{lllllllllllllllllllll}1493.929 & 1494.483 & 1494.636 & 1495.654 & 1496.968 & 1496.782 & 1493.217 & 1489.343 & 1493.048 & 1490.439 & 1491.278 & 1486.744 & 1488.388 & 1493.452 & 1494.008 & 1493.219 & 1495.167 & 1493.197 & 1485.303 & 1478.456\end{array}$ $\begin{array}{llllllllllllllllllllll}1493.932 & 1494.491 & 1494.636 & 1495.663 & 1496.982 & 1496.794 & 1493.224 & 1489.35 & 1493.055 & 1490.444 & 1491.283 & 1486.756 & 1488.391 & 1493.464 & 1494.02 & 1493.229 & 1495.178 & 1493.206 & 1485.303 & 1478.456\end{array}$

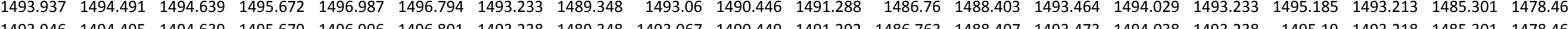

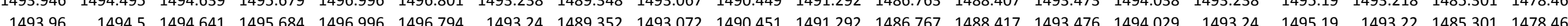

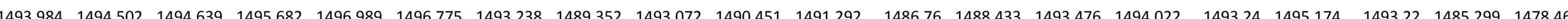
\begin{tabular}{lllllllllllllllllllllll}
1493.984 & 1494.502 & 1494.646 & 1495.684 & 1496.994 & 1496.775 & 1493.24 & 1489.355 & 1493.074 & 1490.453 & 1491.297 & 1486.756 & 1488.424 & 1493.478 & 1494.027 & 1493.24 & 1495.176 & 1493.213 & 1485.301 & 1478.459 \\
\hline
\end{tabular} $\begin{array}{llllllllllllllllllllll}1494.024 & 1494.502 & 1494.638 & 1495.672 & 1496.973 & 1496.734 & 1493.235 & 1489.343 & 1493.067 & 1490.453 & 1491.297 & 1486.754 & 1488.457 & 1493.476 & 1493.996 & 1493.238 & 1495.143 & 1493.218 & 1485.296 & 1478.459\end{array}$

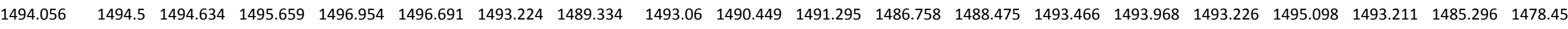

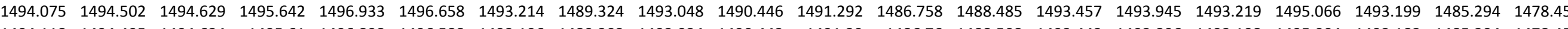

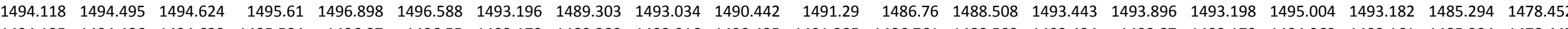
$\begin{array}{llllllllllllllllllllll}1494.125 & 1494.486 & 1494.629 & 1495.584 & 1496.87 & 1496.55 & 1493.179 & 1489.289 & 1493.016 & 1490.435 & 1491.285 & 1486.761 & 1488.503 & 1493.424 & 1493.87 & 1493.179 & 1494.969 & 1493.161 & 1485.294 & 1478.447 \\ 1494.115 & 1494.481 & 1494629 & 1495563 & 1496852 & 1496533 & 1493.144 & 1489.28 & 1492.98 & 1490.428 & 1491281 & 1486.763 & 1488.499 & 1493.391 & 1493.84 & 1493.146 & 1494.948 & 1493.126 & 1485.292 & 1478.445\end{array}$ 
Well

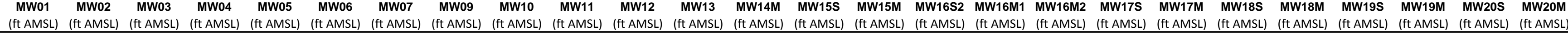

11/19/11 14:00 11/19/11 15:00 11/19/111 16:00 11/19/1111 18:00 11/19/11 18:00 11/19/11 20:00 11/19/11 21:00 11/19/11 22:00 11/19/11 23:00 11/20/110:00 11/20/11 1:00 11/20/11 3:00 $11 / 20 / 114: 00$
$11 / 20 / 115: 00$ 11/20/11 6:00 11/20/11 7:00 11/20/11 9:00 11/20/11 10:00 11/20/11 11:00 11/20/11 12:00 11/20/11 14:00 11/20/11 15:00 11/20/11 16:00 11/20/11 17:00 11/20/11 18:00 11/20/11 19:00 11/20/11 20:00 11/20/11 22:00 11/20/11 23:00 11/21/11 0:00 11/21/11 1:00 $11 / 21 / 112: 00$
$11 / 21 / 113: 00$ 11/21/11 3:00 11/21/11 5:00 11/21/11 11:00 11/21/11 8:00 11/21/11 9:00 $11 / 21 / 1110: 00$
$11 / 21 / 1111: 00$
$11 / 21 / 1112 \cdot 00$ 11/21/11 12:00 11/21/111 13:00 11/21/11 15:00 $11 / 21 / 1116: 00$
$11 / 21 / 1117: 00$ 11/21/11 18:00 $\begin{array}{llll}1494.435 & 1494.902 & 1496.492 & 1494.906 \\ 1494.428 & 1494.877 & 1496.464 & 1494.878\end{array}$ $\begin{array}{lllll}1494.428 & 1494.877 & 1496.464 & 1494.878\end{array}$ $\begin{array}{lllll}1494.414 & 1494.842 & 1496.413 & 1494.834 \\ 1494.412 & 1494.816 & 1496387 & 1494.801\end{array}$ $\begin{array}{lllll}1494.407 & 1494.797 & 1496.368 & 1494.776\end{array}$ $\begin{array}{lllll}1494.4 & 1494.776 & 1496.347 & 1494.75\end{array}$ $\begin{array}{rrrrr}1494.395 & 1494.76 & 1496.329 & 1494.729\end{array}$ $\begin{array}{llllll}1494.393 & 1494.746 & 1496.322 & 1494.713\end{array}$ $\begin{array}{llll}1494.388 & 1494.732 & 1496.312 & 1494.699\end{array}$ $\begin{array}{llll}1494.393 & 1494.732 & 1496.326 & 1494.701\end{array}$ $\begin{array}{rrrr}1494.391 & 1494.732 & 1496.331 & 1494.706 \\ 1493.391 & 1494.737 & 149634 & 194.713\end{array}$ $\begin{array}{rrrrr}1494.391 & 1494.737 & 1496.34 & 1494.713 \\ 1494.377 & 1494.718 & 1496.301 & 1494.696\end{array}$ $\begin{array}{lllll}1494.377 & 1494.716 & 1496.305 & 1494.694\end{array}$ $\begin{array}{llll}1494.377 & 1494.714 & 1496.308 & 1494.694\end{array}$ $\begin{array}{lllll}1494.377 & 1494.716 & 1496.308 & 1494.696\end{array}$ $\begin{array}{llllll}1494.367 & 1494.7 & 1496.282 & 1494.682\end{array}$ $\begin{array}{lllll}1494.37 & 1494.7 & 1496.284 & 1494.678\end{array}$ $1494.365 \quad 1494.697 \quad 1496.282 \quad 1494.675$ $\begin{array}{llll}1494.358 & 1494.688 & 1496.268 & 1494.668 \\ 1494361 & 1494.686 & 1496273 & 1494.668\end{array}$ $\begin{array}{lllll}1494.356 & 1494.681 & 1496.261 & 14944.668\end{array}$ $\begin{array}{llllll}1494.361 & 1494.688 & 1496.277 & 1494.673\end{array}$ $\begin{array}{lllll}1494.363 & 1494.695 & 1496.294 & 1494.685\end{array}$ $\begin{array}{lllll}1494.463 & 1449.695 & 1496.294 & 1444.685 \\ 1494.382 & 1494.725 & 1496.347 & 1494.717\end{array}$ $\begin{array}{llll}1494.363 & 1494.711 & 1496.315 & 1494.715\end{array}$ $\begin{array}{llll}1494.368 & 1494.718 & 1496.322 & 1494.72\end{array}$ $\begin{array}{rrrr}1494.367 & 1494.723 & 1496.331 & 1494.731 \\ 1494.36 & 1494.716 & 149631 & 1494.725\end{array}$ $\begin{array}{rrrr}1494.36 & 1494.716 & 1496.31 & 1494.72 \\ 1494.358 & 1494.716 & 1496.301 & 1494.715\end{array}$ $\begin{array}{lllll}1494.358 & 1494.716 & 1496.301 & 1494.706\end{array}$ $\begin{array}{lllll}1494.363 & 1494.723 & 1496.317 & 1494.715\end{array}$ $\begin{array}{lllll}1494.358 & 1494.721 & 1496.303 & 1494.703\end{array}$ $\begin{array}{lllll}1494.358 & 1494.723 & 1496.308 & 1494.708\end{array}$ $\begin{array}{llll}1494.363 & 1494.73 & 1496.324 & 1494.717\end{array}$ $\begin{array}{rrrr}1494.36 & 1494.73 & 1496.322 & 1494.72\end{array}$ $\begin{array}{llll}1494.361 & 1494.737 & 1496.331 & 1494.724 \\ 1494358 & 1494.737 & 1496331 & 1494.729\end{array}$ $\begin{array}{lllll}1494.358 & 1494.737 & 1496.331 & 1494.729 \\ 1494.358 & 1494.739 & 1496.329 & 1494.729\end{array}$ $\begin{array}{lllll}1494.361 & 1494.741 & 1496.333 & 1494.734\end{array}$ $\begin{array}{lllll}1494.356 & 1494.741 & 1496.326 & 1494.731\end{array}$ $\begin{array}{lllll}1494.351 & 1494.735 & 1496.31 & 1494.722\end{array}$ $\begin{array}{lllll}1494.356 & 1494.737 & 1496.317 & 1494.72\end{array}$ $\begin{array}{llll}1494.351 & 1494.737 & 1496.312 & 1494.717\end{array}$ $\begin{array}{llll}1494.354 & 1494.737 & 1496.315 & 1494.713\end{array}$ $\begin{array}{lllll}1494.353 & 1494.744 & 1496.324 & 1494.722 \\ 1494.367 & 1494.762 & 1496364 & 1494.745\end{array}$ $\begin{array}{llllll}1494.372 & 1494.783 & 1496.401 & 1494.771\end{array}$ $\begin{array}{lllll}1494.384 & 1494.804 & 1496.436 & 1494.801\end{array}$ $\begin{array}{lllll}1494.372 & 1494.8 & 1496.415 & 1494.804\end{array}$ $\begin{array}{rrrrr}1494.374 & 1494.807 & 1496.42 & 1494.811 \\ 1494.382 & 1494.811 & 1496.429 & 1494.818\end{array}$ $\begin{array}{lllll}1494.382 & 1494.811 & 1496.429 & 1494.818\end{array}$ $\begin{array}{rrrrr}1494.393 & 1494.83 & 1496.462 & 1494.839 \\ 1494.391 & 1494.832 & 1496.45 & 1494.834\end{array}$ $\begin{array}{llllllllllllllllllll}1494.099 & 1494.479 & 1494.629 & 1495.549 & 1496.84 & 1496.531 & 1492.742 & 1489.266 & 1492.729 & 1490.421 & 1491.274 & 1486.765 & 1488.494 & 1493.135 & 1493.681 & 1492.75 & 1494.932 & 1492.72 & 1485.294 & 1478.447\end{array}$ $\begin{array}{llllllllllllllllllllll}1494.101 & 1494.471 & 1494.624 & 1495.529 & 1496.819 & 1496.504 & 1493.004 & 1489.259 & 1492.835 & 1490.414 & 1491.253 & 1486.758 & 1488.501 & 1493.243 & 1493.749 & 1493.006 & 1494.896 & 1492.992 & 1485.294 & 1478.445 \\ 1494.118 & 1494.464 & 1494619 & 1495.96 & 1496787 & 1496461 & 1493.046 & 1489242 & 149288 & 1490.04 & 1491243 & 1486707 & 1488506 & 149329 & 1493742 & 1493046 & 1494849 & 1493032 & 1485292 & 1478438\end{array}$

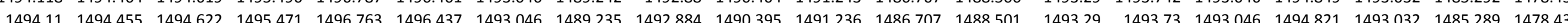
$\begin{array}{llllllllllllllllllllll}1494.099 & 1494.447 & 1494.62 & 1495.45 & 1496.745 & 1496.428 & 1493.041 & 1489.226 & 1492.822 & 1490.386 & 1491.227 & 1486.703 & 1488.496 & 1493.288 & 1493.712 & 1493.041 & 1494.805 & 1493.03 & 1485289 & 1478.426\end{array}$

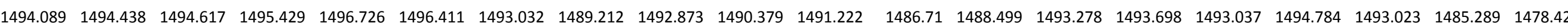
$\begin{array}{lllllllllllllllllllllll}1494.075 & 1494.431 & 1494.612 & 1495.406 & 1496.708 & 1496.402 & 1493.023 & 1489.207 & 1492.865 & 1490.369 & 1491.215 & 1486.71 & 1488.496 & 1493.269 & 1493.686 & 1493.025 & 1494.769 & 1493.014 & 1485.287 & 1478.419\end{array}$ $\begin{array}{lllllllllllllllllllllll}1494.054 & 1494.426 & 1494.617 & 1495.392 & 1496.694 & 1496.397 & 1493.016 & 1489.195 & 1492.858 & 1490.358 & 1491.213 & 1486.714 & 1488.482 & 1493.264 & 1493.677 & 1493.015 & 1494.758 & 1492.999 & 1485.287 & 1478.414\end{array}$

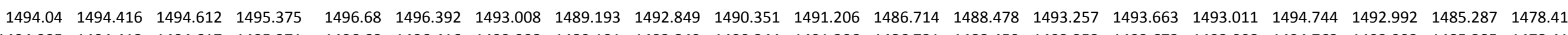

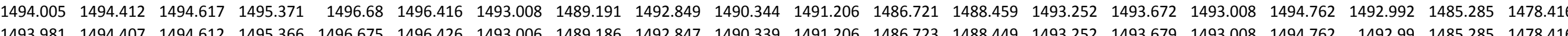

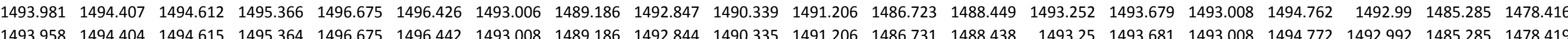
$\begin{array}{llllllllllllllllllllllll}14393.972 & 1494.4 & 1494.608 & 1495.343 & 1496.656 & 1496.409 & 1492.997 & 1489.179 & 1492.837 & 1490.328 & 1491.201 & 1486.733 & 1488.447 & 1493.245 & 1493.663 & 1492.999 & 1494.746 & 1492.983 & 1485.285 & 1478.414\end{array}$ $\begin{array}{llllllllllllllllllllllll}1493.953 & 1494.392 & 1494.608 & 1495338 & 1496654 & 1496.418 & 1492.997 & 1489.176 & 1492.835 & 1490.323 & 1491.199 & 148673 & 1488433 & 1493.243 & 1493658 & 1492999 & 1494.748 & 1492.981 & 1485.282 & 1478.416\end{array}$ $\begin{array}{llllllllllllllllllllll}1493.937 & 1494.388 & 1494.61 & 1495.331 & 1496.647 & 1496.423 & 1492.99 & 1489.172 & 1492.828 & 1490.318 & 1491.194 & 1486.728 & 1488.414 & 1493.236 & 1493.663 & 1492.992 & 1494.746 & 1492.976 & 1485.282 & 14748.416\end{array}$ $\begin{array}{lllllllllllllllllllll}1493.92 & 1494.385 & 1494.61 & 1495.327 & 1496.645 & 1496.43 & 1492.99 & 1489.176 & 1492.828 & 1490.316 & 1491.192 & 1486.73 & 1488.403 & 1493.231 & 1493.665 & 1492.992 & 1494.755 & 1492.971 & 1485.282 & 1478.416\end{array}$ $\begin{array}{llllllllllllllllllllll}1493.927 & 1494.38 & 1494.605 & 1495.31 & 1496.631 & 1496.409 & 1492.976 & 1489.167 & 1492.816 & 1490.309 & 1491.187 & 1486.721 & 1488.403 & 1493.222 & 1493.646 & 1492.98 & 1494.734 & 1492.96 & 1485.28 & 1478.412\end{array}$

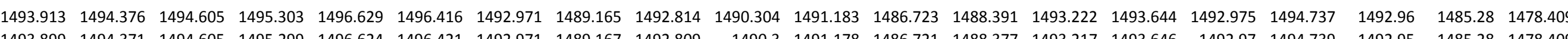

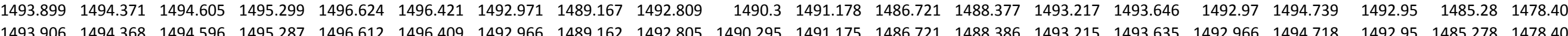
$\begin{array}{lllllllllllllllllllll}1493.906 & 1494.368 & 1494.596 & 1495.287 & 1496.612 & 1496.409 & 1492.966 & 1489.162 & 1492.805 & 1490.295 & 1491.175 & 1486.721 & 1488.386 & 1493.215 & 1493.635 & 1492.966 & 1494.718 & 1492.95 & 1485.278 & 1478.407 \\ 149389 & 1494.364 & 1494.598 & 1495283 & 149661 & 1496416 & 1492962 & 1489.162 & 1492802 & 1490293 & 1491.176 & 1486723 & 1488365 & 1493208 & 1493637 & 1492966 & 1494.725 & 1492.95 & 1485278 & 1478.407\end{array}$

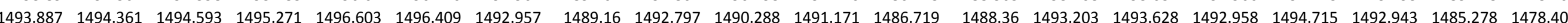
$\begin{array}{llllllllllllllllllll}1493.859 & 1494359 & 1494596 & 1495278 & 1466.1 & 149643 & 1492959 & 1489165 & 1492797 & 1490.286 & 1491.171 & 1486707 & 1488337 & 1493205 & 1493642 & 1492.961 & 1494739 & 1492938 & 1485278 & 1478.409\end{array}$

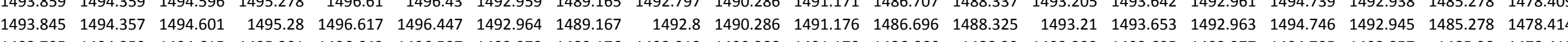

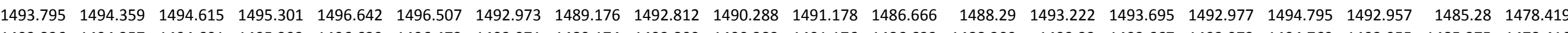

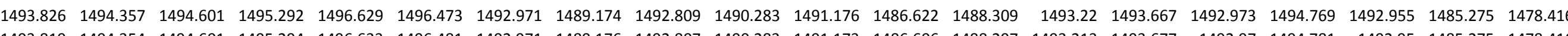

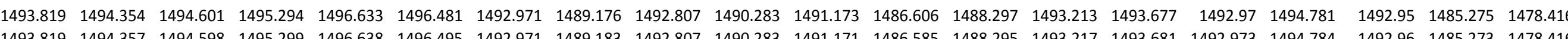

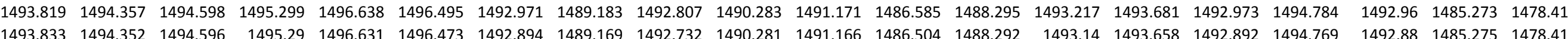
$\begin{array}{llllllllllllllllllll}1493.833 & 1494.352 & 1494.596 & 1495.29 & 1496.631 & 1496.473 & 1492.894 & 1489.169 & 1492.732 & 1490.281 & 1491.166 & 1486.504 & 1488.292 & 1493.14 & 1493.658 & 1492.892 & 1494.769 & 1492.88 & 1485.275 & 1478.412 \\ 1493.838 & 1494.35 & 1494.596 & 149529 & 1496629 & 1496.469 & 1492.758 & 1489.172 & 1492579 & 1490281 & 1491.094 & 1486481 & 1488295 & 1492992 & 1493567 & 1492759 & 1494.748 & 1492746 & 1485273 & 1478.412\end{array}$ $\begin{array}{llllllllllllllllllllll}1493.833 & 1494.347 & 1494.601 & 1495.287 & 1496.626 & 1496.466 & 1492.828 & 1489.174 & 1492.657 & 1490.279 & 1491.094 & 1486.462 & 1488.28 & 1493.067 & 1493.6 & 1492.83 & 1494.751 & 1492.812 & 1485.275 & 1478.409\end{array}$ $\begin{array}{lllllllllllllllllllll}1493.819 & 1494.349 & 1494.598 & 1495.292 & 1496.635 & 1496.488 & 1492.861 & 1489.169 & 1492.694 & 1490.279 & 1491.101 & 1486.446 & 1488.271 & 1493.102 & 1493.621 & 1492.864 & 1494.765 & 1492.847 & 1485.273 & 1478.409\end{array}$

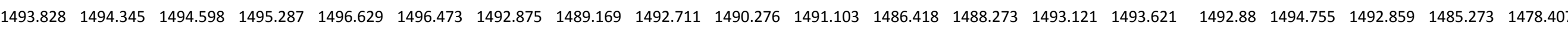

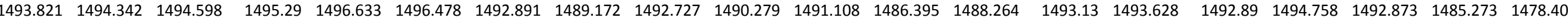

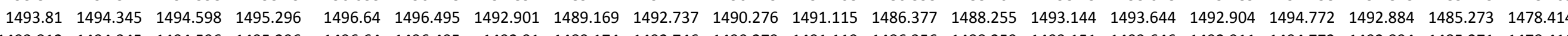

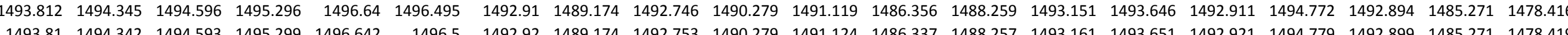

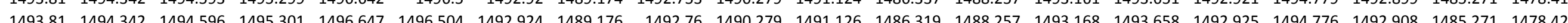

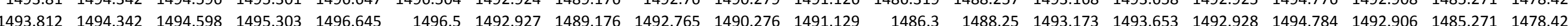
$\begin{array}{lllllllllllllllllllll}1493.812 & 1494.342 & 1494.591 & 1495.306 & 1496.649 & 1496.504 & 1492.929 & 1489.176 & 1492.765 & 1490.276 & 1491.131 & 1486.284 & 1488.248 & 1493.173 & 1493.66 & 1492.93 & 1494.781 & 1492.913 & 1485.271 & 1478.423\end{array}$ $\begin{array}{llllllllllllllllllllll}1493.819 & 1494.34 & 1494.593 & 1495.303 & 1496.647 & 1496.497 & 1492.929 & 1489.176 & 1492.765 & 1490.279 & 1491.131 & 1486.25 & 1488.255 & 1493.173 & 1493.658 & 1492.93 & 1494.774 & 1492.913 & 1485.268 & 1478.423 \\ \end{array}$ \begin{tabular}{lllllllllllllllllllll}
1493.831 & 1494.337 & 1494.589 & 1495.296 & 1496.64 & 1496.481 & 1492.924 & 1489.172 & 1492.762 & 1490.276 & 1491.126 & 1486.222 & 1488.259 & 1493.17 & 1493.644 & 1492.925 & 1494.765 & 1492.91 & 1485.266 & 1478.421 \\
\hline
\end{tabular} $\begin{array}{llllllllllllllllllll}1493.821 & 1494.335 & 1494.591 & 1495.301 & 1496.642 & 1496.488 & 1492.924 & 1489.169 & 1492.76 & 1490.276 & 1491.126 & 1486.199 & 1488.248 & 1493.166 & 1493.651 & 1492.928 & 1494.769 & 1492.908 & 1485.268 & 1478.421\end{array}$

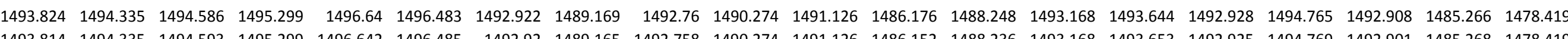

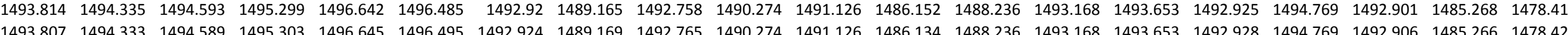

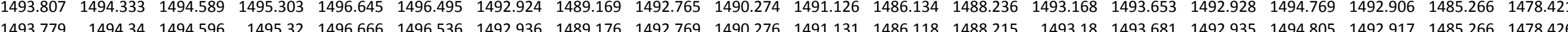
$\begin{array}{llllllllllllllllllll}1493.76 & 1494.342 & 1494.596 & 1495.334 & 1496.684 & 1496.571 & 1492.948 & 1489.188 & 1492.783 & 1490.279 & 1491.138 & 1486.106 & 1488.208 & 1493.189 & 1493.698 & 1492.947 & 1494.835 & 1492.931 & 1485.266 & 1478.431\end{array}$ $\begin{array}{llllllllllllllllllllll}1493.741 & 1494.349 & 1494.603 & 1495.354 & 1496.705 & 1496.605 & 1492.962 & 1489.198 & 1492.797 & 1490.283 & 1491.145 & 1486.088 & 1488.196 & 1493.203 & 1493.733 & 1492.963 & 1494.868 & 1492.938 & 1485.268 & 1478.438\end{array}$ $\begin{array}{lllllllllllllllllllll}1493.777 & 1494.354 & 1494.596 & 1495.352 & 1496.701 & 1496.576 & 1492.962 & 1489.2 & 1492.8 & 1490.283 & 1491.145 & 1486.074 & 1488.224 & 1493.205 & 1493.716 & 1492.966 & 1494.852 & 1492.948 & 1485.266 & 1478.438\end{array}$

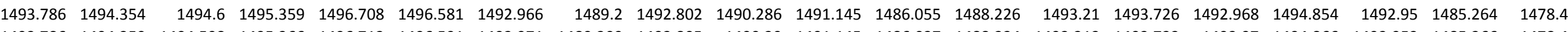
$\begin{array}{llllllllllllllllllll}1493.786 & 1494.359 & 1494.598 & 1495.366 & 1496.712 & 1496.591 & 1492.971 & 1489.209 & 1492.805 & 1490.29 & 1491.145 & 1486.037 & 1488.224 & 1493.213 & 1493.733 & 1492.97 & 1494.866 & 1492.953 & 1485.266 & 1478.44\end{array}$

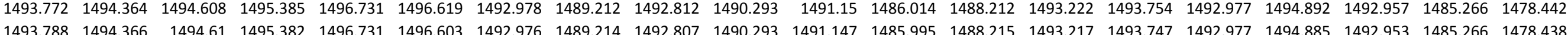


Well

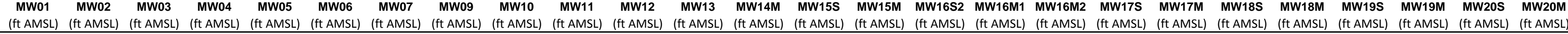

$11 / 21 / 1120: 00$ 11/21/11 21:00

$11 / 21 / 1122: 00$

$11 / 22 / 110: 00$

$11 / 22 / 111: 00$

11/22/11 2:00

11/22/11 3:00

11/22/11 4:00

11/22/11 6:00

$11 / 22 / 117: 00$

$11 / 22 / 11$ 8:00
$11 / 22 / 11$ 9:00
$11 / 22 / 1110: 00$

11/22/11 11:00

11/22/11 12:00

11/22/11 14:00

$11 / 22 / 1115: 00$

11/22/11 16:00

11/22/11 18:00

11/22/11 18:00

11/22/11 20:00

11/22/11 21:00

11/22/11 22:00

11/22/11 23:00

$11 / 23 / 110: 00$
$11 / 23 / 111: 00$

11/23/11 1:00

11/23/11 3:00

11/23/11 4:00

$11 / 23 / 115: 00$
$11 / 23 / 116: 00$

11/23/11 7:00

11/23/11 8:00

11/23/11 9:00

11/23/111 10:00

11/23/11 12:00

11/23/111112:00

11/23/11 14:00

11/23/11 16:00

$11 / 23 / 1117: 00$

11/23/11 18:00

11/23/11 19:00

11/23/11 21:00

11/23/11 22:00

$11 / 23 / 1123: 00$
$11 / 24 / 11000$

11/24/11 0:00 $\begin{array}{rrrr}1494.377 & 1494.811 & 1496.408 & 1494.815 \\ 1494.395 & 1494.832 & 1496.46 & 1494.834\end{array}$ $\begin{array}{rrrr}1494.395 & 1494.832 & 1496.46 & 1494.834 \\ 1494.386 & 1494.825 & 1496.432 & 1994825\end{array}$ $\begin{array}{rrrr}1494.386 & 1494.825 & 1496.432 & 1494.825 \\ 1494.379 & 1494.825 & 1496.42 & 1494.815\end{array}$ $\begin{array}{rrrrr}1494.379 & 1494.814 & 1496.401 & 1494.799\end{array}$ $\begin{array}{lllll}1494.384 & 1494.818 & 1496.411 & 1494.799\end{array}$ $\begin{array}{lllll}1494.384 & 1494.818 & 1496.411 & 1494.799 \\ 1494.393 & 1494.828 & 1496.439 & 1494.811\end{array}$ $\begin{array}{llllll}1494.391 & 1494.83 & 1496.443 & 1494.818\end{array}$ $\begin{array}{llll}1494.395 & 1494.837 & 1466.448 & 1494.822\end{array}$ $\begin{array}{llll}1494.393 & 1494.835 & 1496.443 & 1494.82\end{array}$ $\begin{array}{llll}1494.391 & 1494.83 & 1496.432 & 1494.815 \\ 1493.391 & 1494.828 & 1496.425 & 194808\end{array}$ $\begin{array}{llll}1494.391 & 1494.828 & 1496.425 & 1494.808 \\ 1494.386 & 1494.818 & 1496.404 & 1494.794\end{array}$ $\begin{array}{llll}1494.384 & 1494.814 & 1496.392 & 1494.783\end{array}$ $\begin{array}{lllll}1444.377 & 1494.802 & 1496.375 & 1494.769\end{array}$ $\begin{array}{lllll}1494.437 & 1494.802 & 1496.375 & 1494.769 \\ 1494.384 & 1494.807 & 1496.385 & 1494.769\end{array}$ $\begin{array}{lllll}1494.386 & 1494.809 & 1496.394 & 1494.769\end{array}$ $\begin{array}{llll}1494.393 & 1494.818 & 1496.418 & 1494.78\end{array}$ $\begin{array}{llll}1494.389 & 1494.821 & 1496.42 & 1494.783\end{array}$ $\begin{array}{llll}1494.391 & 1494.823 & 1496.42 & 1494.785\end{array}$ $\begin{array}{llll}1494.389 & 1494.818 & 1496.408 & 1494.78\end{array}$ $\begin{array}{llllll}1494.382 & 1494.807 & 1496.385 & 1494.766\end{array}$ $\begin{array}{lllll}1494.379 & 1494.804 & 1496.375 & 1494.766\end{array}$ $\begin{array}{lllll}1494.375 & 1494.793 & 1496.354 & 1494.741\end{array}$ $\begin{array}{rrrr}1494.372 & 1494.786 & 1496.34 & 1494.727 \\ 1494.37 & 149.779 & 1496333 & 194.75\end{array}$ $\begin{array}{rrrr}1494.37 & 1494.779 & 1496.333 & 1494.715 \\ 1494372 & 1494.781 & 149634 & 1494.713\end{array}$ $\begin{array}{rrrr}1494.372 & 1494.781 & 1496.34 & 1494.713\end{array}$ $\begin{array}{llll}1494.365 & 1494.774 & 1496.331 & 1494.703 \\ 1494.365 & 1494.772 & 1496.329 & 1494.701\end{array}$ $\begin{array}{lllll}1494.363 & 1494.772 & 1496.329 & 1494.699\end{array}$ $\begin{array}{lllll}1494.367 & 1494.774 & 1496.336 & 1494.701\end{array}$ $\begin{array}{llllll}1494.363 & 1494.774 & 1496.333 & 1494.703\end{array}$ $\begin{array}{lllll}1494.361 & 1494.765 & 1496.319 & 1494.696\end{array}$ $\begin{array}{lllll}1494.358 & 1494.762 & 1496.317 & 1494.692\end{array}$ $\begin{array}{llll}1494.358 & 1494.765 & 1496.319 & 1494.692\end{array}$ $\begin{array}{lllll}1494.356 & 1494.762 & 1496.312 & 1494.685 \\ 1494354 & 1494.758 & 1496308 & 194.682\end{array}$ $\begin{array}{lllll}1494.354 & 1494.758 & 1464.308 & 1494.682 \\ 1494.35 & 149631 & 1494.68\end{array}$ $\begin{array}{llll}1494.349 & 1494.755 & 1496.305 & 1494.678\end{array}$ $\begin{array}{lllll}1494.358 & 1494.762 & 1496.329 & 1494.687\end{array}$ $\begin{array}{llll}1494.36 & 1494.769 & 1496.343 & 1494.696\end{array}$ $\begin{array}{lllll}1494.363 & 1494.774 & 1496.357 & 1494.708\end{array}$ $\begin{array}{llll}1494.365 & 1494.783 & 1496.373 & 1494.722\end{array}$ $\begin{array}{llll}1494.363 & 1494.783 & 1496.368 & 1494.724\end{array}$ $\begin{array}{lllll}1494.361 & 1494.786 & 1496.368 & 1494.727\end{array}$ $\begin{array}{lllll}1494.361 & 1494.781 & 1496357 & 1494.72\end{array}$ $\begin{array}{lllll}1494.358 & 1494.781 & 1496.357 & 1494.72\end{array}$ $\begin{array}{lllll}1494.356 & 1494.776 & 1496.345 & 1494.71\end{array}$ $\begin{array}{lllll}1494.356 & 1494.776 & 1496.345 & 1494.71\end{array}$ $\begin{array}{lllll}1494.358 & 1494.781 & 1496.357 & 1494.713\end{array}$ $\begin{array}{llll}1494.358 & 1494.781 & 1496.359 & 1494.713 \\ 1494.361 & 1494.786 & 1496.371 & 1494.722\end{array}$

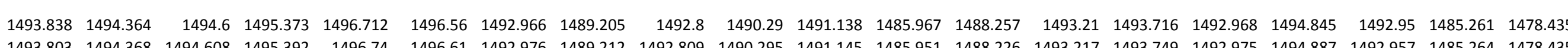

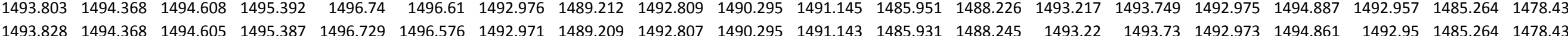

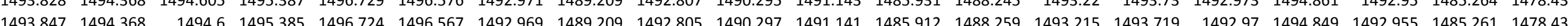
$\begin{array}{lllllllllllllllllllll}1493.861 & 1494.368 & 1494.598 & 149538 & 1496.715 & 1496543 & 1492.966 & 1489202 & 1492805 & 1490.297 & 1491.141 & 1485889 & 1488264 & 1493213 & 1493709 & 1492.966 & 1494.831 & 1492.948 & 1485261 & 1478.435\end{array}$ $\begin{array}{llllllllllllllllllllll}1493.852 & 1494.368 & 1494.603 & 1495.385 & 1496.722 & 1496.555 & 1492.969 & 1489.198 & 1492.805 & 1490.295 & 1491.145 & 1485.873 & 1488.255 & 1493.213 & 1493.714 & 1492.97 & 1494.842 & 1492.95 & 1485.259 & 1478.44\end{array}$ $\begin{array}{lllllllllllllllllllllll}1493.831 & 1494.373 & 1494.608 & 1495.394 & 1496.733 & 1496.581 & 1492.978 & 1489.205 & 1492.814 & 1490.3 & 1491.152 & 1485.863 & 1488.25 & 1493.222 & 1493.735 & 1492.977 & 1494.863 & 1492.962 & 1485.259 & 1478.447\end{array}$ $\begin{array}{llllllllllllllllllll}1493.835 & 1494.373 & 1494.608 & 1495.399 & 1496.735 & 1496.581 & 1492.983 & 1489.207 & 1492.819 & 1490.297 & 1491.157 & 1485.847 & 1488.259 & 1493.229 & 1493.737 & 1492.985 & 1494.861 & 1492.964 & 1485.259 & 1478.452\end{array}$

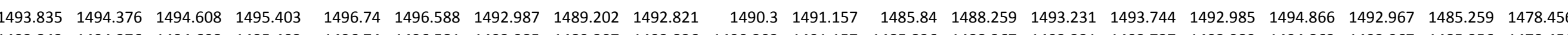

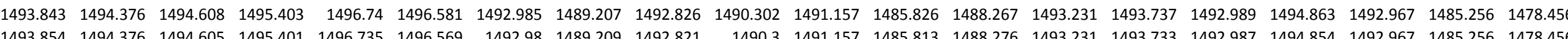
$\begin{array}{lllllllllllllllllllll}1493.854 & 1494.376 & 1494.605 & 1495.401 & 1496.735 & 1496.569 & 1492.98 & 1489.209 & 1492.821 & 1490.3 & 1491.157 & 1485.813 & 1488.276 & 1493.231 & 1493.733 & 1492.987 & 1494.854 & 1492.967 & 1485.256 & 1478.456 \\ 1493.859 & 1494378 & 1494.603 & 1495.401 & 1496.733 & 1496.56 & 1492.978 & 1489.205 & 1492816 & 1490.302 & 1491.155 & 1485.789 & 1488.276 & 1493.224 & 1493.726 & 1492.982 & 1494847 & 1492.962 & 1485256 & 1478.454\end{array}$ $\begin{array}{lllllllllllllllllllllll}1493.878 & 1494.373 & 1494.605 & 1495.392 & 1496.724 & 1496.538 & 1492.971 & 1489.195 & 1492.809 & 1490.297 & 1491.152 & 1485.766 & 1488.285 & 1493.215 & 1493.712 & 1492.975 & 1494.828 & 1492.953 & 1485.256 & 1478.452\end{array}$

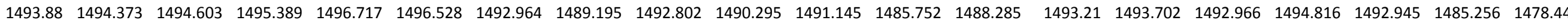
$\begin{array}{lllllllllllllllllllll}1493.89 & 1494.371 & 1494.596 & 1495.38 & 1496.708 & 1496.512 & 1492.957 & 1489.188 & 1492.797 & 1490.293 & 1491.141 & 1485.741 & 1488.292 & 1493.203 & 1493.686 & 1492.958 & 1494.798 & 1492.943 & 1485.257 & 1478.449\end{array}$

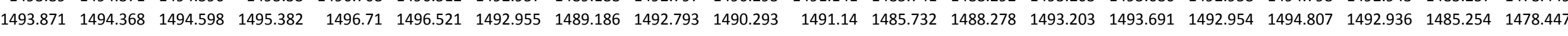
$\begin{array}{lllllllllllllllllllll}1493.859 & 1494.368 & 1494.603 & 1495.387 & 1496.715 & 1496.531 & 1492.957 & 1489.183 & 1492.79 & 1490.293 & 1491.141 & 1485.715 & 1488.271 & 1493.201 & 1493.695 & 1492.958 & 1494.812 & 1492.931 & 1485.254 & 1478.449\end{array}$ $\begin{array}{llllllllllllllllllll}1493.838 & 1494.368 & 1494.605 & 1495.394 & 1496.726 & 1496.555 & 1492.962 & 1489.186 & 1492.795 & 1490.29 & 1491.145 & 1485.702 & 1488.259 & 1493.203 & 1493.707 & 1492.961 & 1494.826 & 1492.941 & 1485.254 & 1478.452\end{array}$

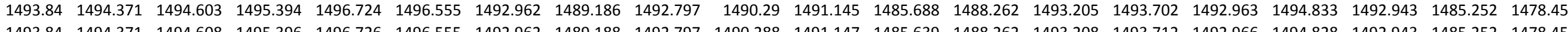

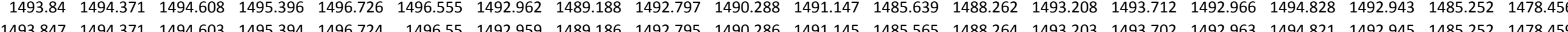
$\begin{array}{lllllllllllllllllllllll}1493.85 & 1494.371 & 1494.603 & 1495.394 & 1496.722 & 1496.545 & 1492.959 & 1489.181 & 1492.795 & 1490.286 & 1491.143 & 1485.519 & 1488.259 & 1493.201 & 1493.698 & 1492.958 & 1494.819 & 1492.941 & 1485.252 & 1478.456\end{array}$

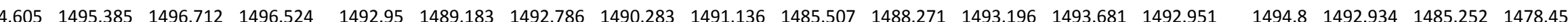
\begin{tabular}{llllllllllllllllllllll}
1493.875 & 1494.366 & 1494.6 & 1495.382 & 1496.708 & 1496.514 & 1492.943 & 1489.179 & 1492.781 & 1490.281 & 1491.131 & 1485.503 & 1488.278 & 1493.191 & 1493.679 & 1492.944 & 1494.788 & 1492.924 & 1485.249 & 1478.452 \\
\hline
\end{tabular}

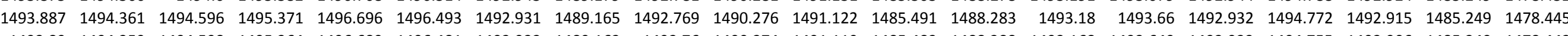

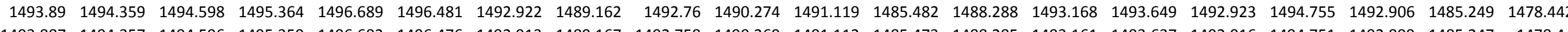

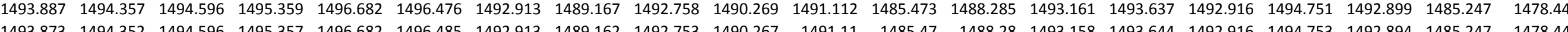
$\begin{array}{lllllllllllllllllllll}1493.873 & 1494.352 & 1494.596 & 1495.357 & 1496.682 & 1496.485 & 1492.913 & 1489.162 & 1492.753 & 1490.267 & 1491.11 & 1485.47 & 1488.28 & 1493.158 & 1493.644 & 1492.916 & 1494.753 & 1492.894 & 1485.247 & 1478.44 \\ 1493.875 & 1494.347 & 1494.596 & 149535 & 1496677 & 1496.476 & 1492908 & 1489.158 & 1492746 & 1490265 & 1491105 & 1485466 & 1488283 & 1493158 & 1493632 & 1492909 & 1494741 & 1492889 & 1485247 & 1478.44\end{array}$

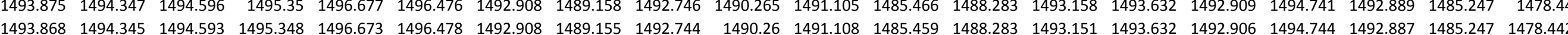
\begin{tabular}{lllllllllllllllllllll}
1493.864 & 1494.345 & 1494.591 & 1495.345 & 1496.67 & 1496.478 & 1492.906 & 1489.16 & 1492.744 & 1490.258 & 1491.108 & 1485.452 & 1488.285 & 1493.149 & 1493.635 & 1492.906 & 1494.739 & 1492.892 & 1485.245 & 1478.445 \\
\hline
\end{tabular} $\begin{array}{llllllllllllllllllll}1493.852 & 1494.342 & 1494.598 & 1495.345 & 1496.673 & 1496.485 & 1492.906 & 1489.16 & 1492.746 & 1490.258 & 1491.11 & 1485.445 & 1488.28 & 1493.154 & 1493.639 & 1492.911 & 1494.748 & 1492.887 & 1485.247 & 1478.449\end{array}$

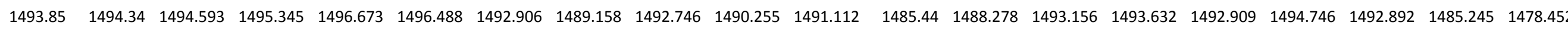

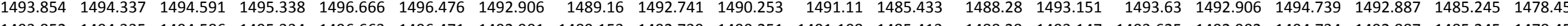

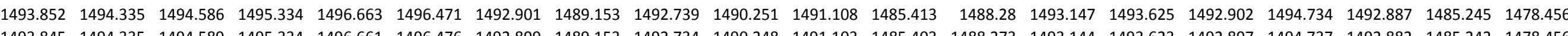

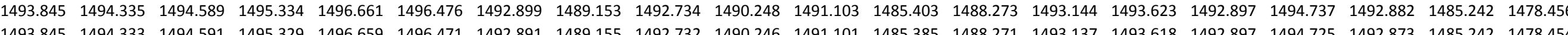

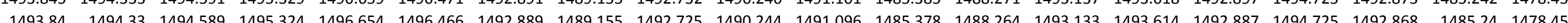

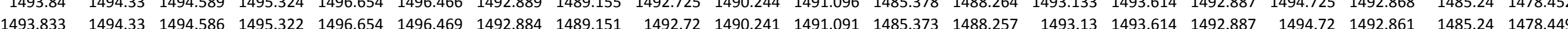
$\begin{array}{lllllllllllllllllllll}1493.828 & 1494.328 & 1494.584 & 1495.32 & 1496.652 & 1496.469 & 1492.88 & 1489.148 & 1492.718 & 1490.239 & 1491.089 & 1485.364 & 1488.248 & 1493.126 & 1493.609 & 1492.88 & 1494.718 & 1492.859 & 1485.24 & 1478.449\end{array}$

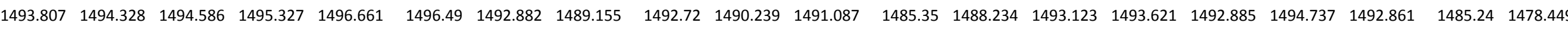

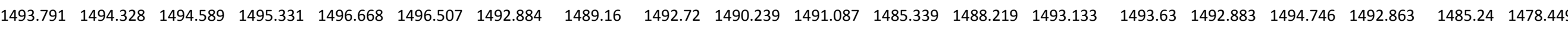
$\begin{array}{lllllllllllllllllllll}1493.781 & 1494.328 & 1494.591 & 1495.338 & 1496.677 & 1496.521 & 1492.891 & 1489.162 & 1492.725 & 1490.237 & 1491.089 & 1485.336 & 1488.208 & 1493.135 & 1493.642 & 1492.89 & 1494.76 & 1492.866 & 1485.238 & 1478.452 \\ \end{array}$

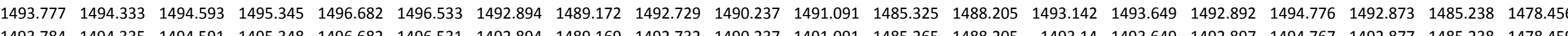

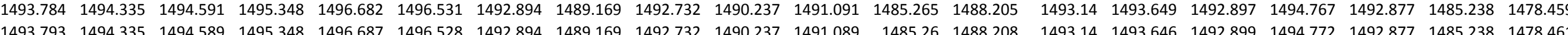

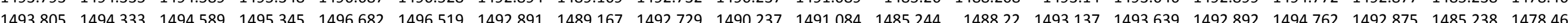
$\begin{array}{llllllllllllllllllllll}1493.812 & 1494.333 & 1494.586 & 1495.345 & 1496.682 & 1496.514 & 1492.889 & 1489.172 & 1492.727 & 1490.237 & 1491.08 & 1485.232 & 1488.217 & 1493.135 & 1493.639 & 1492.892 & 1494.76 & 1492.868 & 1485.238 & 1478.459\end{array}$ $\begin{array}{llllllllllllllllllllll}1493.814 & 1494.333 & 1494.591 & 1495.345 & 1496.684 & 1496.516 & 1492.887 & 1489.165 & 1492.722 & 1490.237 & 1491.077 & 1485.228 & 1488.219 & 1493.128 & 1493.632 & 1492.885 & 1494.758 & 1492.863 & 1485.235 & 1478.456\end{array}$ $\begin{array}{llllllllllllllllllll}1493.824 & 1494.333 & 1494.586 & 1495.341 & 1496.677 & 1496.504 & 1492.877 & 1489.162 & 1492.713 & 1490.234 & 1491.068 & 1485.218 & 1488.224 & 1493.123 & 1493.625 & 1492.878 & 1494.751 & 1492.859 & 1485.235 & 1478.452\end{array}$

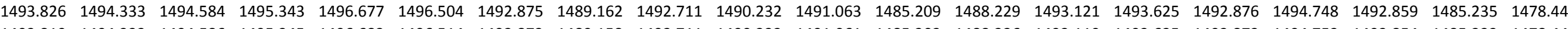
$\begin{array}{llllllllllllllllllllll}1493.819 & 1494.333 & 1494.586 & 1495.345 & 1496.682 & 1496.514 & 1492.873 & 1489.158 & 1492.711 & 1490.232 & 1491.061 & 1485.202 & 1488.226 & 1493.119 & 1493.625 & 1492.873 & 1494.753 & 1492.854 & 1485.233 & 1478.449\end{array}$

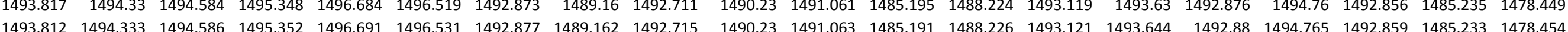


Well

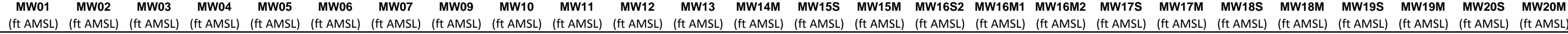

$11 / 24 / 112: 00$ $11 / 24 / 113: 00$

$11 / 24 / 114: 00$

11/24/115:00

11/24/11117:00

11/24/11 7:00

11/24/11 9:00

11/24/11 10:00

$11 / 24 / 1111: 00$

11/24/1112:00

11/24/1111 14:00

$11 / 24 / 11$ 15:00

11/24/11 16:00

$11 / 24 / 1117: 00$

$11 / 24 / 1118: 00$

11/24/11 19:00

11/24/11 20:00

11/24/11 21:00

11/24/11/11 22:00

$11 / 25 / 110: 00$

11/25/110:00

11/25/11 2:00

$11 / 25 / 113: 00$

$11 / 25 / 11$ 4:00

11/25/11 5:00

11/25/11 7:00

11/25/111 8:00

11/25/11 10:00

11/25/11 11:00

$11 / 25 / 1112: 00$

$11 / 25 / 1113: 00$
$11 / 25 / 1114: 00$

$11 / 25 / 1114: 00$

11/25/11 15:00

$11 / 25 / 11$ 17:00

11/25/11 18:00

11/25/11 19:00

11/25/11 20:00

11/25/11 21:00

$11 / 25 / 1122: 00$

$11 / 26 / 110.00$

11/26/11 0:00

11/26/11 1:00

11/26/11 3:00

11/26/11 4:00

$11 / 26 / 11$ 5:00

11/26/11 6:00 $\begin{array}{llll}1494.363 & 1494.79 & 1496.382 & 1494.729 \\ 1494.365 & 1494.797 & 1496.389 & 1494.734\end{array}$ $\begin{array}{llll}1494.365 & 1494.797 & 1496.389 & 1494.734\end{array}$ $\begin{array}{lllll}1494.365 & 1494.802 & 1496.397 & 1494.741 \\ 1494.365 & 1494802 & 1496397 & 1494.743\end{array}$ $\begin{array}{lllll}1494.365 & 1494.8 & 1496394 & 1494.743\end{array}$ $\begin{array}{lllll}1494.363 & 1494.797 & 1496389 & 1494.738\end{array}$ $\begin{array}{lllll}1494.363 & 1494.8 & 1496.387 & 1494.738\end{array}$ $\begin{array}{llllll}1494.365 & 1494.8 & 1496.387 & 1494.738\end{array}$ $\begin{array}{rrrrr}1494.37 & 1494.804 & 1496.397 & 1494.741\end{array}$ $\begin{array}{lllll}1494.372 & 1494.811 & 1496.411 & 1494.745\end{array}$ $\begin{array}{llll}1494.382 & 1494.828 & 1496.443 & 1494.764 \\ 1494.388 & 1494846 & 196.469 & 1494785\end{array}$ $\begin{array}{llll}1494.388 & 1494.846 & 1496.469 & 1494.785 \\ 1494.393 & 1494.863 & 1496.485 & 1494.804\end{array}$ $\begin{array}{lllll}1494.393 & 1494.867 & 1496.492 & 1494.813\end{array}$ $\begin{array}{lllll}1494.395 & 1494.877 & 1496.497 & 1494.822\end{array}$ $\begin{array}{llllll}1494.398 & 1494.877 & 1496.497 & 1494.825\end{array}$ $\begin{array}{lllll}1494.395 & 1494.874 & 1496.485 & 1494.822\end{array}$ $\begin{array}{lllll}1494.393 & 1494.87 & 1496.474 & 1494.811\end{array}$ $\begin{array}{llllll}1494.395 & 1494.87 & 1496.471 & 1494.808\end{array}$ $\begin{array}{llll}1494.4 & 1494.874 & 1496.481 & 1494.808\end{array}$ $\begin{array}{llll}1494.407 & 1494.884 & 1496.504 & 1494.818\end{array}$ $\begin{array}{lllll}1494.409 & 1494.891 & 1496.506 & 1494.822\end{array}$ $\begin{array}{llll}1494.414 & 1494.895 & 1496.52 & 1494.832\end{array}$ $\begin{array}{lllll}1494.421 & 1494.907 & 1496.539 & 1494.843\end{array}$ $1494.421 \quad 1494.914 \quad 1496.546 \quad 1494.85$ $\begin{array}{lllll}1494.426 & 1494.926 & 1496.56 & 1494.862\end{array}$ $\begin{array}{llll}14444.43 & 1494.933 & 1496.565 & 1494.871 \\ 1494.428 & 1994.933 & 1966562 & 1949874\end{array}$ $\begin{array}{lllll}1494.428 & 1494.933 & 1496.562 & 1494.874 \\ 1494.435 & 149494 & 1496572 & 1494.878\end{array}$ $\begin{array}{lllll}1494.433 & 1494.935 & 1496.558 & 1494.876\end{array}$ $\begin{array}{llll}1494.437 & 1494.937 & 1496.558 & 1494.874\end{array}$ $\begin{array}{lllll}1494.435 & 1494.935 & 1496.553 & 1494.867\end{array}$ $\begin{array}{lllll}1494.447 & 1494.944 & 1496.581 & 1494.878\end{array}$ $1494.447 \quad \begin{array}{lll}1494.947 & 1496.588 & 1494.888\end{array}$ $1494.4581494 .9651496 .616 \quad 1494.899$ $\begin{array}{rrrr}1494.451 & 1494.961 & 1496.598 & 1494.897 \\ 1494.447 & 1494.958 & 1496586 & 1494.89\end{array}$ $\begin{array}{lllll}1494.447 & 1494.958 & 1496.586 & 1494.89\end{array}$ $\begin{array}{lllll}1494.449 & 1494.951 & 1496.574 & 1494.876\end{array}$ $\begin{array}{lllll}1494.451 & 1494.951 & 1496.572 & 1494.871\end{array}$ $\begin{array}{lllll}1494.444 & 1494.937 & 1496.551 & 1494.857\end{array}$ $\begin{array}{lllll}1494.454 & 1494.942 & 1496.558 & 1494.855\end{array}$ $\begin{array}{llll}1494.451 & 1494.94 & 1496.553 & 1494.85\end{array}$ $\begin{array}{lllll}1494.449 & 1494.933 & 1496.541 & 1494.841 \\ 1494.449 & 1499933 & 1496541 & 194841\end{array}$ $\begin{array}{lllll}1494.449 & 1494.933 & 1496.541 & 1494.841\end{array}$ $1494.449 \quad 1494.923 \quad 1496.532 \quad 1494.834$ $\begin{array}{lllll}1494.456 & 1494.928 & 1496.546 & 1494.839\end{array}$ 1494.449 $1494.926 \quad 1496.5391494 .836$ $1494.447 \quad 1494.916 \quad 1496.525 \quad 1494.832$ $\begin{array}{lllll}1494.426 & 1494.884 & 1496.457 & 1494.792\end{array}$ $\begin{array}{llll}1494.421 & 1494.853 & 1496.411 & 1494.759 \\ 1494.419 & 1494.839 & 1496.394 & 1494.738\end{array}$

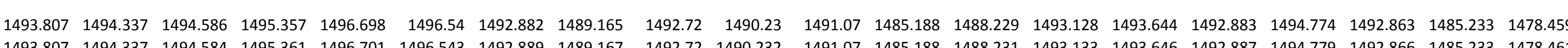
$\begin{array}{lllllllllllllllllllll}1493.807 & 1494.337 & 1494.584 & 1495.361 & 1496.701 & 1496.543 & 1492.889 & 1489.167 & 1492.72 & 1490.232 & 1491.07 & 1485.188 & 1488.231 & 1493.133 & 1493.646 & 1492.887 & 1494.779 & 1492.866 & 1485.233 & 1478.461 \\ 1493805 & 149434 & 1494589 & 1495366 & 1496708 & 149655 & 1492894 & 1489.179 & 1492.729 & 1490232 & 1491.075 & 1485.184 & 1488234 & 1493135 & 1493653 & 1492894 & 1494791 & 1492875 & 1485233 & 147848\end{array}$

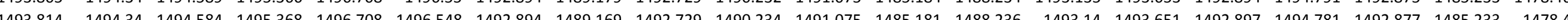
$\begin{array}{llllllllllllllllllllll}1493.817 & 1494345 & 1494.589 & 1495368 & 1496708 & 1496545 & 1492896 & 1489169 & 1492.732 & 1490.234 & 1491.077 & 1485158 & 1488238 & 1493.14 & 1493653 & 1492897 & 1494.784 & 1492877 & 1485231 & 1478.473\end{array}$ $\begin{array}{llllllllllllllllllllll}1493.824 & 1494.345 & 1494.586 & 1495368 & 1496708 & 1496.538 & 1492.899 & 1489.169 & 1492.734 & 1490.234 & 1491.075 & 1485.154 & 1488.245 & 1493.137 & 1493.649 & 1492.897 & 1494.776 & 1492.877 & 1485.231 & 1478.475\end{array}$ $\begin{array}{llllllllllllllllllllll}1493.831 & 1494.345 & 1494.589 & 1495.368 & 1496.705 & 1496.536 & 1492.891 & 1489.176 & 1492.729 & 1490.234 & 1491.073 & 1485.149 & 1488.248 & 1493.137 & 1493.646 & 1492.894 & 1494.774 & 1492.873 & 1485.228 & 1478.475 \\ \end{array}$

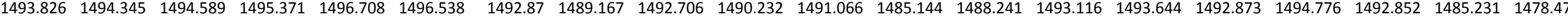

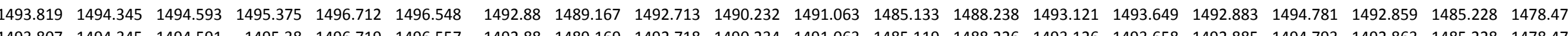

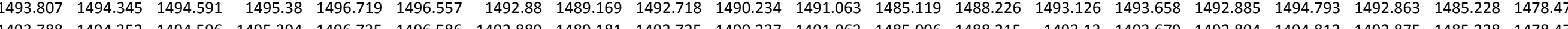
$\begin{array}{llllllllllllllllllll}1493.788 & 1494.352 & 1494.596 & 1495.394 & 1496.735 & 1496.586 & 1492.889 & 1489.181 & 1492.725 & 1490.237 & 1491.063 & 1485.096 & 1488.215 & 1493.13 & 1493.679 & 1492.894 & 1494.812 & 1492.875 & 1485.228 & 1478.475 \\ 1493.772 & 1494.357 & 1494.598 & 1495.408 & 1496752 & 1496 & 1492.901 & 1489183 & 1492.732 & 1490.237 & 1491.066 & 1485.075 & 1488.203 & 1493.144 & 1493.693 & 1492.904 & 149484 & 1492884 & 1485228 & 1478.477\end{array}$ $\begin{array}{llllllllllllllllllllllll}1493.772 & 1494.364 & 1494.598 & 1495.422 & 1496.766 & 1496.629 & 1492.91 & 1489.191 & 1492.746 & 1490.241 & 1491.066 & 1485.057 & 1488.205 & 1493.149 & 1493.7 & 14929.911 & 1494.852 & 1492.892 & 1485.2268 & 1478.482\end{array}$

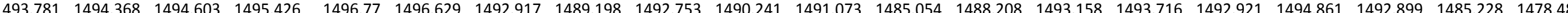
$\begin{array}{llllllllllllllllllll}1493.791 & 1494.371 & 1494.603 & 1495.433 & 1496.777 & 1496.634 & 1492.924 & 1489.2 & 1492.758 & 1490.241 & 1491.075 & 1485.001 & 1488.21 & 1493.163 & 1493.719 & 1492.928 & 1494.868 & 1492.91 & 1485.228 & 1478.491\end{array}$

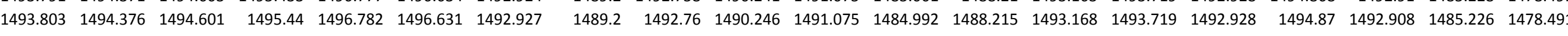
$\begin{array}{llllllllllllllllllllll}1493.826 & 1494.378 & 1494.601 & 1495.44 & 1496.78 & 1496.615 & 1492.927 & 1489.205 & 1492.76 & 1490.248 & 1491.073 & 1484.987 & 1488.234 & 1493.166 & 1493.712 & 1492.928 & 1494.854 & 1492.91 & 1485.226 & 1478.491\end{array}$

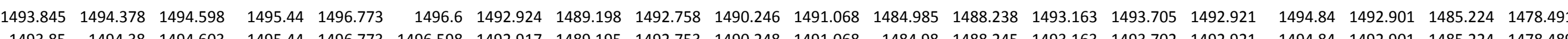

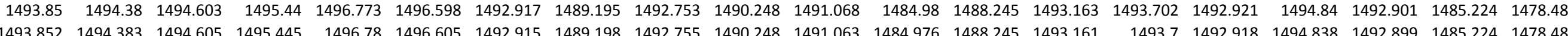

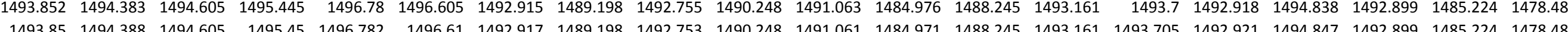
$\begin{array}{llllllllllllllllllllllll}1493.847 & 1494.388 & 1494.608 & 1495.457 & 1496.794 & 1496.622 & 1492.917 & 1489.2 & 1492.751 & 1490.251 & 1491.059 & 1484.959 & 1488248 & 1493.161 & 1493.709 & 1492.918 & 1494.859 & 1492.899 & 1485.224 & 1478.484\end{array}$ $\begin{array}{llllllllllllllllllllll}1493.85 & 1494.397 & 1494.612 & 1495.471 & 1496.805 & 1496.636 & 1492.927 & 1489.205 & 1492.76 & 1490.251 & 1491.061 & 1484.95 & 1488.255 & 1493.17 & 1493.726 & 1492.925 & 1494.87 & 1492.906 & 1485.221 & 1478.487\end{array}$

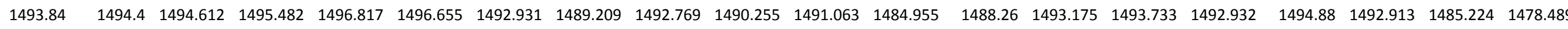
\begin{tabular}{llllllllllllllllllll}
1493.847 & 1494.404 & 1494.612 & 1495.489 & 1496.819 & 1496.66 & 1492.941 & 1489.212 & 1492.772 & 1490.255 & 1491.066 & 1484.959 & 1488.269 & 1493.173 & 1493.742 & 1492.939 & 1494.889 & 1492.922 & 1485.224 & 1478.454 \\
\hline
\end{tabular}

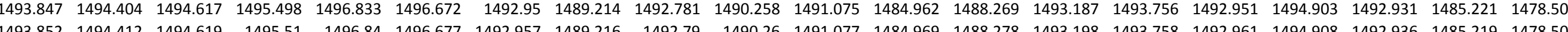

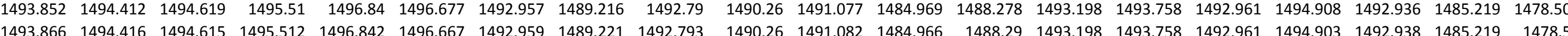

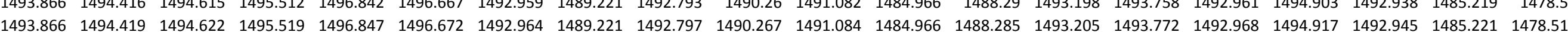

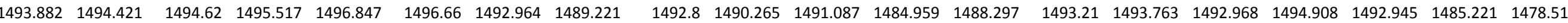
$\begin{array}{lllllllllllllllllllll}1493.892 & 1494.421 & 1494.622 & 1495.519 & 1496.849 & 1496.655 & 1492.964 & 1489.223 & 1492.797 & 1490.267 & 1491.087 & 1484.952 & 1488.302 & 1493.208 & 1493.761 & 1492.968 & 1494.901 & 1492.945 & 1485.219 & 1478.515\end{array}$

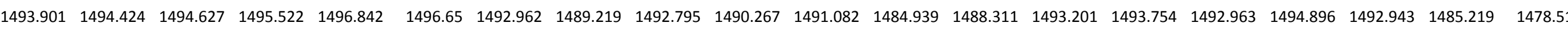

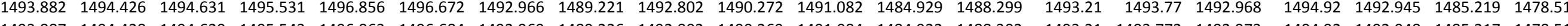

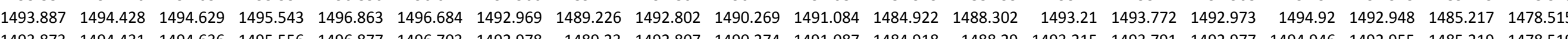

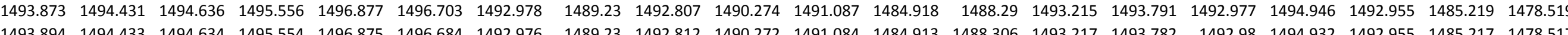

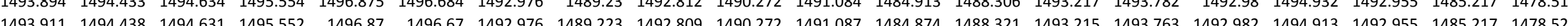

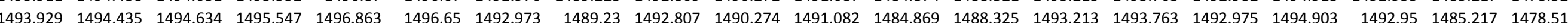
$\begin{array}{llllllllllllllllllllll}1493.929 & 1494.435 & 1494.634 & 1495.549 & 1496.863 & 1496.653 & 1492.973 & 1489.226 & 1492.807 & 1490.274 & 1491.087 & 1484.869 & 1488.327 & 1493.22 & 1493.761 & 1492.975 & 1494.901 & 1492.953 & 1485.217 & 1478.522\end{array}$

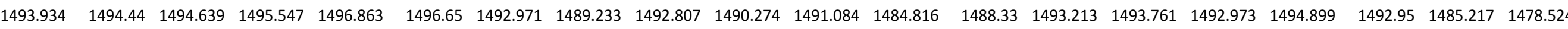

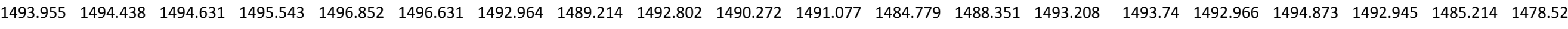
\begin{tabular}{llllllllllllllllllllll}
1493.944 & 1494.44 & 1494.643 & 1495.543 & 1496.859 & 1496.636 & 1492.969 & 1489.216 & 1492.805 & 1490.272 & 1491.089 & 1484.788 & 1488.337 & 1493.215 & 1493.751 & 1492.97 & 1494.889 & 1492.95 & 1485.214 & 1478.529 \\
\hline
\end{tabular}

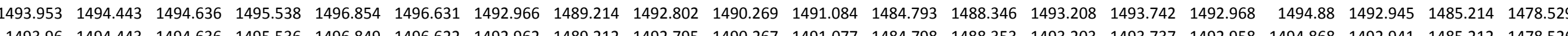

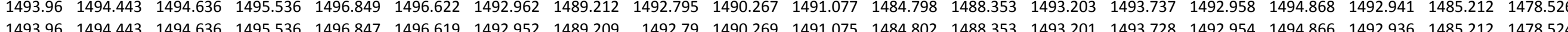

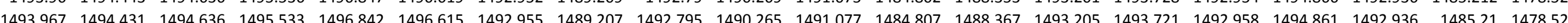
$\begin{array}{lllllllllllllllllllll}1493.962 & 1494.431 & 1494.641 & 1495.531 & 1496.842 & 1496.61 & 1492.992 & 1489.205 & 1492.83 & 1490.267 & 1491.105 & 1484.835 & 1488.367 & 1493.241 & 1493.747 & 1492.996 & 1494.863 & 1492.974 & 1485.214 & 1478.547\end{array}$ $\begin{array}{lllllllllllllllllllll}1493.953 & 1494.424 & 1494.641 & 1495.533 & 1496.845 & 1496.624 & 1492.994 & 1489.205 & 1492.833 & 1490.269 & 1491.11 & 1484.844 & 1488.36 & 1493.238 & 1493.751 & 1492.996 & 1494.875 & 1492.974 & 1485.212 & 1478.554 \\ \end{array}$ $\begin{array}{llllllllllllllllllllll}1493.962 & 1494.428 & 1494.636 & 1495.531 & 1496.84 & 1496.619 & 1492.99 & 1489.205 & 1492.83 & 1490.269 & 1491.11 & 1484.816 & 1488.379 & 1493.238 & 1493.744 & 1492.994 & 1494.87 & 1492.971 & 1485.21 & 1478.557\end{array}$ \begin{tabular}{llllllllllllllllllll}
1493.972 & 1494.431 & 1494.636 & 1495.524 & 1496.833 & 1496.603 & 1492.985 & 1489.202 & 1492.823 & 1490.269 & 1491.112 & 1484.821 & 1488.381 & 1493.234 & 1493.735 & 1492.985 & 1494.859 & 1492.967 & 1485.207 & 1478.559 \\
\hline
\end{tabular} $\begin{array}{llllllllllllllllllll}1494.019 & 1494.428 & 1494.627 & 1495.498 & 1496.798 & 1496.538 & 1492.971 & 1489.186 & 1492.809 & 1490.265 & 1491.105 & 1484.818 & 1488.414 & 1493.215 & 1493.688 & 1492.973 & 1494.807 & 1492.953 & 1485.207 & 1478.561\end{array}$ $\begin{array}{llllllllllllllllllll}1494.035 & 1494.421 & 1494.624 & 1495.473 & 1496.773 & 1496.493 & 1492.955 & 1489.174 & 1492.793 & 1490.258 & 1491.098 & 1484.818 & 1488.414 & 1493.201 & 1493.665 & 1492.956 & 1494.776 & 1492.936 & 1485.207 & 1478.559 \\ 1494033 & 1494.419 & 1494.624 & 1495.457 & 1496761 & 1496.481 & 1492941 & 1489.165 & 1492.783 & 1490253 & 1491.098 & 1484.816 & 1488.412 & 1493.187 & 1493649 & 1492942 & 1494.758 & 1492.924 & 1485207 & 1478.559\end{array}$ 
Well

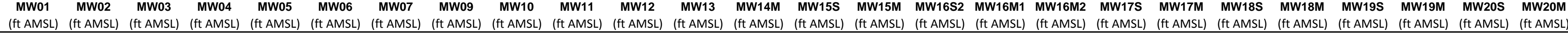

11/26/11 8:00 $11 / 26 / 119: 00$
$11 / 26 / 1110 \cdot 00$ $11 / 26 / 11$ 10:00 11/26/1111:00 $1 / 26 / 1113: 00$ $11 / 26 / 1114: 00$ 11/26/11 15:00 11/26/11 16:00 $11 / 26 / 1117: 00$ $11 / 26 / 11$ 18:00 11/26/11 19:00 $11 / 26 / 1121: 00$ $11 / 26 / 1121: 00$ 11/26/11 23:00 11/27/11 0:00 11/27/11 1:00 $11 / 27 / 112: 00$ 11/27/11 3:00

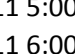
11/27/11 6:00 11/27/11 8:00 11/27/11 9:00 11/27/11 10:00 $11 / 27 / 1111: 00$ 11/27/111 12:00 11/27//111 14:00 $11 / 27 / 1114: 00$ 11/27/11 16:00 11/27/11 17:00 11/27/11 18:00 11/27/11 19:00 11/27/11 20:00 11/27/11 21:00 11/27/11 22:00 11/28/11 0:00 11/28/11 1:00 $11 / 28 / 112: 00$ $11 / 28 / 113: 00$ 11/28/11 4:00 $11 / 28 / 115.00$ 11/28/11 6:00 $11 / 28 / 118: 00$ $11 / 28 / 119: 00$ $11 / 28 / 1110: 00$ $1 / 28 / 11$ 12:00 $\begin{array}{llll}1494.407 & 1494.809 & 1496.352 & 1494.706 \\ 1494.395 & 1494.776 & 1496.305 & 1494.668\end{array}$ $\begin{array}{lllll}1494.395 & 1494.776 & 1496.305 & 1494.668\end{array}$ $\begin{array}{rrrr}1494.393 & 1494.76 & 1496.296 & 1494.65\end{array}$ $\begin{array}{lllll}1494.393 & 1494.755 & 1496.31 & 1494.645\end{array}$ $\begin{array}{lllll}1494.389 & 1494.751 & 1496.303 & 1494.64\end{array}$ $\begin{array}{llll}1494.386 & 1494.748 & 1496.298 & 1494.643\end{array}$ $\begin{array}{lllll}1494.379 & 1494.735 & 1496.277 & 1494.631\end{array}$ $\begin{array}{lllll}1494.37 & 1494.718 & 1496.259 & 1494.619\end{array}$ $1494.363 \quad 1494.702 \quad 1496.2351494 .601$ $\begin{array}{rrrr}1494.356 & 1494.681 & 1496.21 & 1494.584 \\ 1494349 & 1494.669 & 1496.193 & 194.568\end{array}$ $\begin{array}{llll}1494.349 & 1494.669 & 1496.193 & 1494.568 \\ 1494.344 & 1494.658 & 1496.184 & 1494.556\end{array}$ $\begin{array}{lllll}1494.342 & 1494.658 & 1496.186 & 1494.556\end{array}$ $\begin{array}{llll}4494.342 & 1494.658 & 1496.186 & 1494.556 \\ 1494.34 & 1494.653 & 1496.186 & 1494.552\end{array}$ $\begin{array}{lllll}1494.34 & 1494.653 & 1496.193 & 1494.554\end{array}$ $\begin{array}{lllll}1494.333 & 1494.648 & 1496.188 & 1494.552\end{array}$ $\begin{array}{llll}1494.337 & 1494.655 & 1496.205 & 1494.563\end{array}$ $\begin{array}{lllll}1494.337 & 1494.66 & 1496.214 & 1494.573\end{array}$ $\begin{array}{rrrr}1494.33 & 1494.655 & 1496.2 & 1494.57 \\ 149433 & 1494.653 & 1496207 & 1494.573\end{array}$ $\begin{array}{lllll}1494.328 & 1494.655 & 1496.212 & 1494.58\end{array}$ $\begin{array}{lllll}1494.326 & 1494.653 & 1496.202 & 1494.577\end{array}$ $\begin{array}{lllll}1494.319 & 1494.651 & 1496.2 & 1494.58\end{array}$ $\begin{array}{lllll}1494.319 & 1494.648 & 1496.193 & 1494.575\end{array}$ $\begin{array}{lllll}1494.312 & 1494.639 & 1496.181 & 1494.566\end{array}$ $\begin{array}{lllll}1494.314 & 1494.639 & 1496.184 & 1494.57\end{array}$ $\begin{array}{lllll}1494.323 & 1494.653 & 1496.214 & 1494.582\end{array}$ $\begin{array}{rrrrr}1494.328 & 1494.667 & 1496.245 & 1494.603 \\ 1494.33 & 1494.679 & 1496.27 & 1494626\end{array}$ $\begin{array}{lllll}1494.333 & 1494.69 & 1496291 & 1494.645\end{array}$ $\begin{array}{lllll}1494.33 & 1494.7 & 1496.296 & 1494.657\end{array}$ $\begin{array}{lllll}1494.33 & 1494.702 & 1496.301 & 1494.666\end{array}$ $\begin{array}{lllll}1494.333 & 1494.709 & 1496.305 & 1494.675\end{array}$ $\begin{array}{lllll}1494.333 & 1494.714 & 1496.317 & 1494.687\end{array}$ $\begin{array}{rrrr}1494.328 & 1494.711 & 1496.305 & 1494.68 \\ 1994.33 & 1494.716 & 149631 & 14948\end{array}$ $\begin{array}{llll}1494.33 & 1494.716 & 1496.31 & 1494.68 \\ 1494.333 & 1494.723 & 1496317 & 1494.682\end{array}$ $\begin{array}{lllll}1494.333 & 1494.725 & 1496.322 & 1944.687\end{array}$ $\begin{array}{lllll}1494.335 & 1494.732 & 1496.338 & 1494.694\end{array}$ $\begin{array}{lllll}1494.335 & 1494.735 & 1496.338 & 1494.699\end{array}$ $\begin{array}{lllll}1494.34 & 1494.744 & 1496.35 & 1494.706\end{array}$ $\begin{array}{lllll}1494.34 & 1494.751 & 1496.359 & 1494.715\end{array}$ $\begin{array}{lllll}1494.344 & 1494.76 & 1496.371 & 1494.727\end{array}$ $\begin{array}{llll}1494.344 & 1494.765 & 1496.375 & 1494.734\end{array}$ $\begin{array}{lllll}1494.347 & 194.776 & 1466.389 & 1494.743\end{array}$ $\begin{array}{lllll}1494.349 & 1494.779 & 1496.389 & 1494.75\end{array}$ $\begin{array}{lllll}1494.347 & 1494.779 & 1496.385 & 1494.752\end{array}$ $\begin{array}{llll}1494.347 & 1494.776 & 1496.378 & 1494.748\end{array}$ $1494.344 \quad \begin{array}{lllll}1494.776 & 1496.373 & 1494.748\end{array}$ $\begin{array}{lllll}1494.349 & 1494.779 & 1496.382 & 1494.75\end{array}$ $\begin{array}{rrrr}1494.358 & 1494.8 & 1496.413 & 1494.764 \\ 1494.365 & 1494.816 & 1496.443 & 1494.787\end{array}$

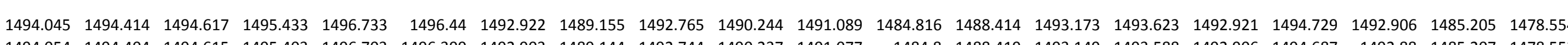

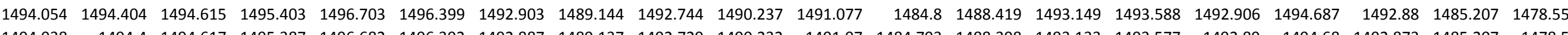

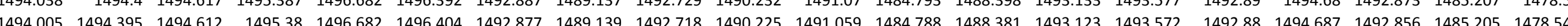
$\begin{array}{lllllllllllllllllllllll}1493.974 & 1494392 & 1494.615 & 1495373 & 1496.682 & 1496.421 & 149287 & 1489137 & 1492708 & 1490.22 & 1491.052 & 1484.784 & 148836 & 1493119 & 1493.574 & 1492.871 & 1494.699 & 1492856 & 1485205 & 1478.545\end{array}$ \begin{tabular}{llllllllllllllllllllll}
1493.96 & 1494.383 & 1494.617 & 1495.361 & 1496.673 & 1496.421 & 1492.861 & 1489.137 & 1492.704 & 1490.216 & 1491.047 & 1484.754 & 1488.36 & 1493.109 & 1493.577 & 1492.864 & 1494.69 & 1492.842 & 1485.207 & 1478.543 \\
\hline
\end{tabular} $\begin{array}{llllllllllllllllllllllll}1493.946 & 1494.383 & 1494.612 & 1495.359 & 1496.663 & 1496.421 & 1492.856 & 1489.134 & 1492.697 & 1490.213 & 1491.042 & 1484.717 & 1488.353 & 1493.1 & 1493.57 & 1492.861 & 1494.69 & 1492.835 & 1485.203 & 1478.54 \\ \end{array}$

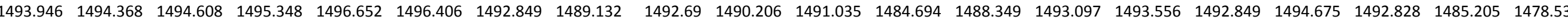

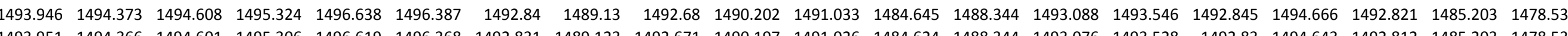

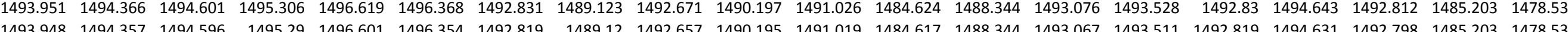
$\begin{array}{llllllllllllllllllll}1493.948 & 1494.357 & 1494.596 & 1495.29 & 1496.601 & 1496.354 & 1492.819 & 1489.12 & 1492.657 & 1490.195 & 1491.019 & 1484.617 & 1488.344 & 1493.067 & 1493.511 & 1492.819 & 1494.631 & 1492.798 & 1485.203 & 1478.533 \\ 1493.944 & 1494.347 & 1494.593 & 1495271 & 1496587 & 1496339 & 1492805 & 1489115 & 1492645 & 1490188 & 1491012 & 1484622 & 1488342 & 1493053 & 1493.5 & 1492804 & 1494612 & 1492.791 & 14852 & 1478531\end{array}$

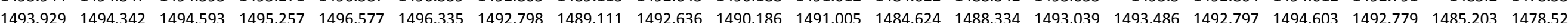
$\begin{array}{llllllllllllllllllllll}1493.913 & 1494.335 & 1494.586 & 149525 & 149657 & 1496347 & 1492.789 & 1489.111 & 1492.626 & 1490183 & 1490.993 & 1484.631 & 1488.318 & 1493.036 & 1493.483 & 1492.79 & 1494.607 & 1492.767 & 1485.203 & 1478524\end{array}$ $\begin{array}{llllllllllllllllllll}1493.894 & 1494.333 & 1494.589 & 1495.243 & 1496.568 & 1496.349 & 1492.782 & 1489.108 & 1492.617 & 1490.181 & 1490.986 & 1484.631 & 1488.313 & 1493.027 & 1493.483 & 1492.778 & 1494.605 & 1492.762 & 14852 & 1478.522\end{array}$ $\begin{array}{lllllllllllllllllllll}1493.873 & 1494.33 & 1494.586 & 1495.241 & 1496.566 & 1496.363 & 1492.774 & 1489.106 & 1492.61 & 1490.179 & 1490.979 & 1484.615 & 1488.304 & 1493.02 & 1493.488 & 1492.776 & 1494.61 & 1492.755 & 1485.198 & 1478.519\end{array}$ $\begin{array}{llllllllllllllllllllll}1493.864 & 1494.323 & 1494.586 & 1495.234 & 1496.559 & 1496.361 & 1492.765 & 1489.104 & 1492.603 & 1490.179 & 1490.972 & 1484.617 & 1488.302 & 1493.013 & 1493.483 & 1492.769 & 1494.612 & 1492.748 & 1485.198 & 1478.517\end{array}$

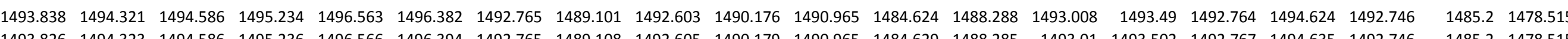

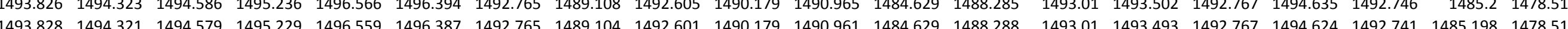

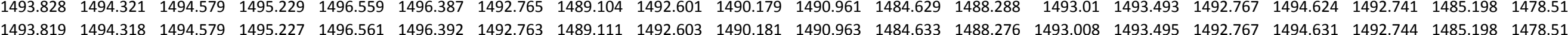

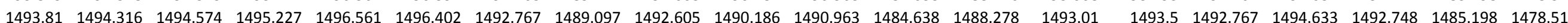
$\begin{array}{lllllllllllllllllllllll}1493.807 & 1494.316 & 1494.579 & 149522 & 1496556 & 1496397 & 1492765 & 1489101 & 1492603 & 1490183 & 1490.963 & 1484.647 & 1488276 & 149301 & 1493.5 & 1492.769 & 1494.638 & 1492748 & 1485196 & 1478.522\end{array}$ \begin{tabular}{lllllllllllllllllllllll}
1493.81 & 1494.311 & 1494.574 & 1495.218 & 1496.552 & 1496.394 & 1492.765 & 1489.099 & 1492.603 & 1490.186 & 1490.963 & 1484.645 & 1488.281 & 1493.008 & 1493.493 & 1492.767 & 1494.631 & 1492.746 & 1485.196 & 1478.524 \\
\hline
\end{tabular} $\begin{array}{llllllllllllllllllll}1493.807 & 1494.313 & 1494.572 & 1495.211 & 1496.549 & 1496.39 & 1492.763 & 1489.094 & 1492.603 & 1490.188 & 1490.961 & 1484.652 & 1488.269 & 1493.008 & 1493.495 & 1492.764 & 1494.626 & 1492.746 & 1485.196 & 1478.526 \\ & & 14945 & & & & \end{array}$

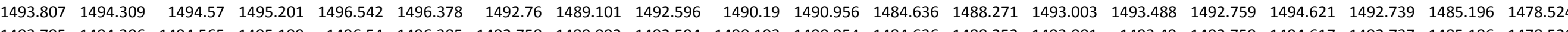

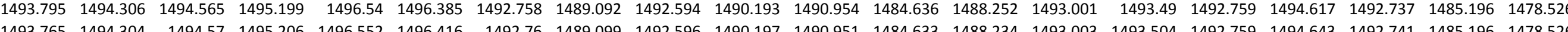

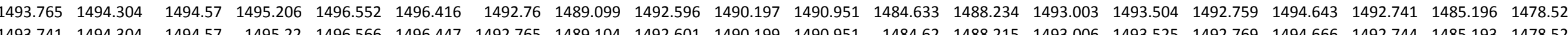

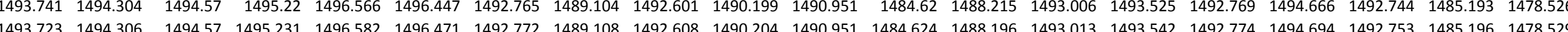
$\begin{array}{lllllllllllllllllllll}1493.716 & 1494.309 & 1494.572 & 1495.241 & 1496.589 & 1496.488 & 1492.777 & 1489.118 & 1492.615 & 1490.211 & 1490.951 & 1484.627 & 1488.194 & 1493.02 & 1493.556 & 1492.781 & 1494.708 & 1492.755 & 1485.196 & 1478.529 \\ 1453 & & & \end{array}$ $\begin{array}{lllllllllllllllllllll}1493.723 & 1494.309 & 1494.567 & 1495.245 & 1496.596 & 1496.493 & 1492.782 & 1489.123 & 1492.622 & 1490.216 & 1490.953 & 1484.629 & 1488.196 & 1493.024 & 1493.556 & 1492.783 & 1494.718 & 1492.762 & 1485.193 & 1478.529\end{array}$ $\begin{array}{lllllllllllllllllllllll}1493.725 & 1494.309 & 1494.574 & 1495.25 & 1496.601 & 1496.497 & 1492.786 & 1489.125 & 1492.624 & 1490.223 & 1490.954 & 1484.548 & 1488.189 & 1493.029 & 1493.563 & 1492.788 & 1494.715 & 1492.767 & 1485.193 & 1478.531 \\ \end{array}$ $\begin{array}{lllllllllllllllllllll}1493.727 & 1494.311 & 1494.572 & 1495.257 & 1496.608 & 1496.502 & 1492.793 & 1489.125 & 1492.629 & 1490.225 & 1490.956 & 1484.532 & 1488.191 & 1493.034 & 1493.574 & 1492.797 & 1494.729 & 1492.772 & 1485.191 & 1478.533\end{array}$

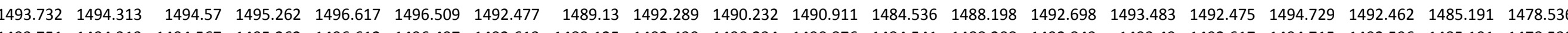
$\begin{array}{lllllllllllllllllllllll}1493.751 & 1494.313 & 1494.567 & 1495.262 & 1496.612 & 1496.497 & 1492.613 & 1489.125 & 1492.439 & 1490.234 & 1490.876 & 1484.541 & 1488.208 & 1492.843 & 1493.49 & 1492.617 & 1494.715 & 1492.596 & 1485.191 & 1478.538 \\ 1493.753 & 1494311 & 1494567 & 1495266 & 1496.619 & 14965 & 1492.674 & 1489.132 & 1492.5 & 1490244 & 1490886 & 1484553 & 148821 & 1492.909 & 1493.518 & 1492.674 & 1494.72 & 1492.652 & 1485.189 & 147858\end{array}$

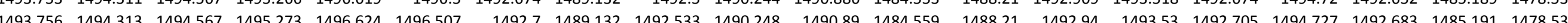

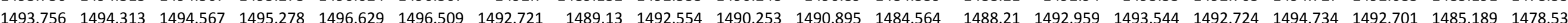
$\begin{array}{lllllllllllllllllllll}1493.751 & 1494.316 & 1494.57 & 1495.285 & 1496.635 & 1496.521 & 1492.735 & 1489.134 & 1492.565 & 1490.258 & 1490.897 & 1484.566 & 1488.21 & 1492.977 & 1493.556 & 1492.736 & 1494.744 & 1492.716 & 1485.191 & 1478.536\end{array}$ $\begin{array}{lllllllllllllllllllll}1493.756 & 1494.318 & 1494.57 & 1495.29 & 1496.64 & 1496.521 & 1492.744 & 1489.134 & 1492.577 & 1490.262 & 1490.9 & 1484.571 & 1488.215 & 1492.987 & 1493.558 & 1492.748 & 1494.746 & 1492.727 & 1485.188 & 1478.536\end{array}$ $\begin{array}{llllllllllllllllllll}1493.758 & 1494.318 & 1494.567 & 1495.294 & 1496.647 & 1496.531 & 1492.756 & 1489.132 & 1492.586 & 1490.265 & 1490.902 & 1484.571 & 1488.215 & 1492.994 & 1493.574 & 1492.755 & 1494.755 & 1492.734 & 1485.188 & 1478.533 \\ \end{array}$ $\begin{array}{lllllllllllllllllllll}1493.758 & 1494.321 & 1494.57 & 1495.301 & 1496.654 & 1496.338 & 142.763 & 1489.139 & 142.598 & 1490.272 & 1490.907 & 1484.576 & 1488.219 & 1493.006 & 1493.581 & 1492.767 & 1494.758 & 1492.744 & 1485.186 & 1478.536\end{array}$

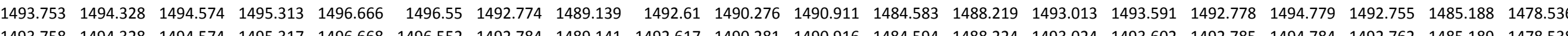

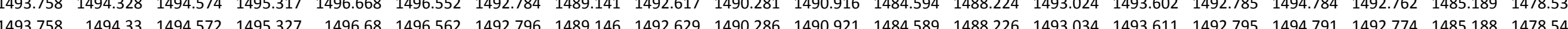

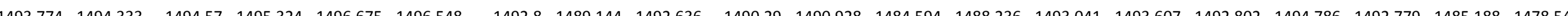

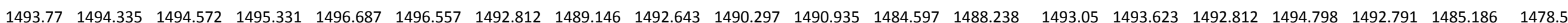
$\begin{array}{lllllllllllllllllllll}1493.779 & 1494.335 & 1494.572 & 1495.336 & 1496.684 & 1496.555 & 1492.814 & 1489.146 & 1492.647 & 1490.302 & 1490.94 & 1484.592 & 1488.248 & 1493.055 & 1493.621 & 1492.816 & 1494.795 & 1492.795 & 1485.186 & 1478.554\end{array}$

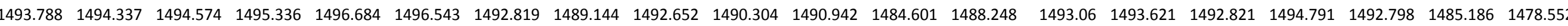
\begin{tabular}{lllllllllllllllllllll}
1493.791 & 1494.337 & 1494.57 & 1495.336 & 1496.682 & 1496.538 & 1492.821 & 1489.144 & 1492.654 & 1490.309 & 1490.944 & 1484.599 & 1488.243 & 1493.062 & 1493.618 & 1492.823 & 1494.788 & 1492.8 & 1485.184 & 1478.554 & 1454 \\
\hline
\end{tabular} $\begin{array}{lllllllllllllllllllll}1493.786 & 1494.337 & 1494.572 & 1495.343 & 1496.689 & 1496.543 & 1492.826 & 1489.146 & 1492.659 & 1490.314 & 1490.944 & 1484.601 & 1488.231 & 1493.062 & 1493.628 & 1492.826 & 1494.798 & 1492.805 & 1485.186 & 1478.554\end{array}$ $\begin{array}{llllllllllllllllllllllllll}1493.763 & 1494.34 & 1494.577 & 1495.354 & 1496.703 & 1496.574 & 1492.831 & 1489.148 & 1492.664 & 1490.318 & 1490.949 & 1484.603 & 1488.217 & 1493.071 & 1493.649 & 1492.835 & 1494.821 & 1492.812 & 1485.184 & 1478.557 \\ 1493.748 & 1494.345 & 1494.582 & 1495.371 & 1496.722 & 1496.6 & 1492.842 & 1489.155 & 1492.673 & 1490.323 & 1490.951 & 1484.606 & 1488.21 & 1493.083 & 1493.665 & 1492.845 & 1494.842 & 1492.821 & 1485.186 & 1478.561\end{array}$ 
Well

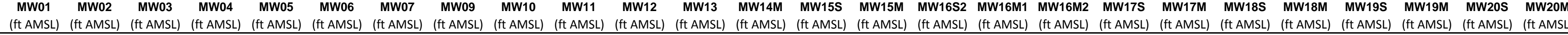

$11 / 28 / 1114: 00$ 11/28/11 15:00 $11 / 28 / 1116: 00$ 11/28/1111 18:00 11/28/11 18:00 $11 / 28 / 1120: 00$ 11/28/11 21:00 $11 / 28 / 1122: 00$ 11/28/11 23:00 11/29/110:00 11/29/11 1:00 11/29/11 2:00 $11 / 29 / 114: 00$
$11 / 29 / 115: 00$ 11/29/11 6:00 11/29/11 7:00 11/29/11 8:00 11/29/11 10:00 11/29/11 10:00 11/29/11 12:00 11/29/11 13:00 $11 / 29 / 11$ 15:00 $11 / 29 / 1116: 00$ 11/29/11 17:00 11/29/11 18:00 11/29/11 19:00 11/29/111120:00 11/29/11 22:00 11/29/11 23:00 11/30/11 0:00 11/30/11 1:00 $11 / 30 / 112: 00$
$11 / 30 / 113: 00$ 11/30/11 3:00 11/30/11 5:00 11/30/11 7:00 11/30/11 8:00 11/30/11 9:00 $11 / 30 / 1110: 00$ $11 / 30 / 1111: 00$ 11/30/11 12:00 11/30/11 13:00 $11 / 30 / 1115: 00$ 11/30/11 16:00 11/30/11 17:00 11/30/11 18:00 $\begin{array}{lllll}1494.367 & 1494.828 & 1496.457 & 1494.806 \\ 1494.363 & 1494.83 & 1496.448 & 1494.811\end{array}$ $\begin{array}{rrrr}1494.363 & 1494.83 & 1496.448 & 1494.811\end{array}$ $\begin{array}{lllll}1494.367 & 1494.835 & 1496.46 & 1494.818\end{array}$ $\begin{array}{llll}1494.361 & 1494.821 & 1496.418 & 1494.797\end{array}$ $\begin{array}{llllll}1494.358 & 1494.814 & 1496.399 & 1494.783\end{array}$ $\begin{array}{lllll}1494.358 & 1494.814 & 1496.399 & 1494.783 \\ 1494.356 & 1494.809 & 1496.387 & 1494.769\end{array}$ $\begin{array}{lrrrr}1494.353 & 1494.8 & 1496.373 & 1494.757\end{array}$ $\begin{array}{lrrr}1494.349 & 1494.79 & 1496.364 & 1494.745\end{array}$ $\begin{array}{llll}1494.344 & 1494.786 & 1496.345 & 1494.731 \\ 1493.346 & 1494779 & 1496345 & 194.722\end{array}$ $\begin{array}{rrrr}1494.346 & 1494.779 & 1496.345 & 1494.722 \\ 1494.34 & 1494.767 & 1996324 & 1494.708\end{array}$ $\begin{array}{rrrr}1494.34 & 1494.767 & 1496.324 & 1494.708 \\ 1494.335 & 1494.758 & 1496.308 & 1494.692\end{array}$ $\begin{array}{lllll}1494.328 & 1494.741 & 1496.28 & 1494.668\end{array}$ $\begin{array}{rrrrr}1494.328 & 1494.741 & 1496.28 & 1494.668 \\ 1494.326 & 1494.737 & 1496.275 & 1494.657\end{array}$ $\begin{array}{rrrrr}1494.426 & 1494.437 & 1496.275 & 1494.657 \\ 1494.326 & 1494.73 & 1496.27 & 1494.65\end{array}$ $\begin{array}{lllll}1494.316 & 1494.716 & 1496.252 & 1494.633\end{array}$ $\begin{array}{lllll}1494.309 & 1494.7 & 1496.221 & 1494.612\end{array}$ $\begin{array}{llll}1494.305 & 1494.688 & 1496.21 & 1494.598\end{array}$ $\begin{array}{llll}1494.305 & 1494.681 & 1496.2 & 1494.587 \\ 1494.302 & 1494.676 & 14962 & 149458\end{array}$ $\begin{array}{rrrr}1494.305 & 1494.674 & 1496.205 & 1494.58\end{array}$ $\begin{array}{lllll}1494.309 & 1494.681 & 1496.231 & 1494.594\end{array}$ $\begin{array}{lllll}1494.314 & 1494.697 & 1496.259 & 1494.615\end{array}$ $\begin{array}{lllll}1494.316 & 1494.709 & 1496.28 & 1494.633\end{array}$ $1494.314 \quad 1494.709 \quad 1496.282 \quad 1494.643$ $\begin{array}{llll}1494.314 & 1494.711 & 1496.28 & 1494.647\end{array}$ $\begin{array}{llll}1494.314 & 1494.714 & 1496.287 & 1494.652\end{array}$ $\begin{array}{llll}1494.312 & 1494.714 & 1496.277 & 1494.654 \\ 1494.309 & 1494.714 & 1496.282 & 1494.657\end{array}$ $\begin{array}{lllll}1494.314 & 1494.718 & 1496.296 & 1494.666\end{array}$ $\begin{array}{lllll}1494.312 & 1494.723 & 1496.298 & 14494.666\end{array}$ $\begin{array}{lllll}1494.314 & 1494.725 & 1496.303 & 14944.666\end{array}$ $\begin{array}{lllll}1494.309 & 1494.725 & 1496.301 & 1494.666\end{array}$ $1494.312 \quad 1494.728 \quad 1496.305 \quad 1494.668$ $\begin{array}{lllll}1494.319 & 1494.741 & 1496.336 & 1494.687\end{array}$ $\begin{array}{llll}1494.321 & 1494.746 & 1496.345 & 1494.696\end{array}$ $\begin{array}{rrrrr}1494.323 & 1494.753 & 1466.354 & 1494.708 \\ 1494.33 & 1494.769 & 1496.378 & 1494.724\end{array}$ $\begin{array}{lllll}1494.333 & 1494.779 & 1496.392 & 1494.738\end{array}$ $\begin{array}{lllll}1494.335 & 1494.786 & 1496.397 & 1494.748\end{array}$ $\begin{array}{lllll}1494.335 & 1494.793 & 1496.406 & 1494.755\end{array}$ $\begin{array}{lllll}1494.34 & 1494.797 & 1496.408 & 1494.762\end{array}$ $1494.337 \quad 1494.797 \quad 1496.406 \quad 1494.766$ $\begin{array}{llll}1494.344 & 1494.807 & 1496.415 & 1494.773\end{array}$ $\begin{array}{lllll}1494.354 & 1494.828 & 1496.446 & 1494.79\end{array}$ $\begin{array}{lllll}1494.37 & 1494.872 & 1496.511 & 1494.843\end{array}$ $\begin{array}{llll}1494.377 & 1494.893 & 1496.537 & 1494.867\end{array}$ $\begin{array}{lllll}1494.384 & 1494.912 & 1496.558 & 1494.89\end{array}$ $1494.384 \quad 1494.919 \quad 1496.555 \quad 1494.902$ $\begin{array}{lllll}1494.386 & 1494.923 & 1496.558 & 1494.906\end{array}$

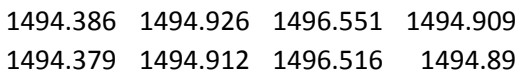

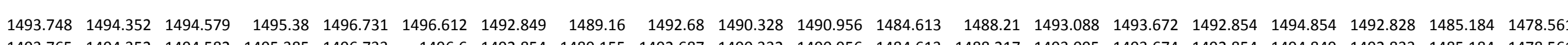

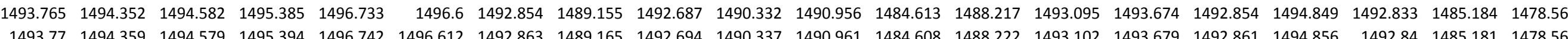

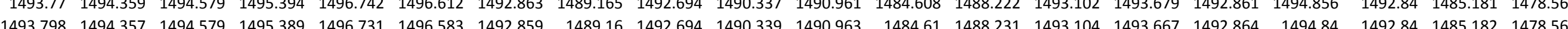

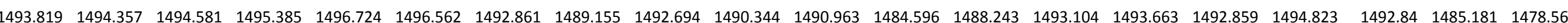
$\begin{array}{llllllllllllllllllllll}1493.833 & 1494.357 & 1494.579 & 1495.38 & 1496.717 & 1496.545 & 1492.856 & 1489.155 & 1492.692 & 1490.344 & 1490.963 & 1484.569 & 1488.255 & 1493.097 & 1493.646 & 1492.857 & 1494.816 & 1492.835 & 1485.179 & 1478.564\end{array}$ $\begin{array}{llllllllllllllllllllllll}1493.845 & 1494.357 & 1494.579 & 1495.375 & 1496.712 & 1496.531 & 1492.854 & 1489.144 & 1492.69 & 1490.346 & 1490.963 & 1484.569 & 1488.262 & 1493.097 & 1493.639 & 1492.854 & 1494.8 & 1492.833 & 1485.182 & 1478.564\end{array}$ $\begin{array}{lllllllllllllllllllll}1493.852 & 1494.352 & 1494.577 & 1495.371 & 1496.705 & 1496.516 & 1492.849 & 1489.141 & 1492.687 & 1490.346 & 1490.961 & 1484.569 & 1488.269 & 1493.093 & 1493.632 & 1492.849 & 1494.793 & 1492.828 & 1485.179 & 1478.564 & \end{array}$

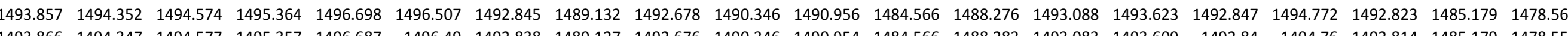

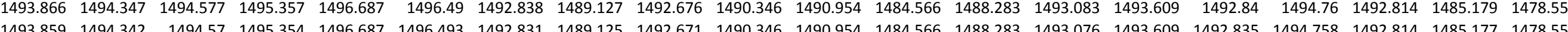
$\begin{array}{llllllllllllllllllll}1493.859 & 1494.342 & 1494.57 & 1495.354 & 1496.687 & 1496.493 & 1492.831 & 1489.125 & 1492.671 & 1490.346 & 1490.954 & 1484.566 & 1488.283 & 1493.076 & 1493.609 & 1492.835 & 1494.758 & 1492.814 & 1485.177 & 1478.55 \\ 1493.864 & 1494.34 & 1494.572 & 1495.345 & 1496.675 & 1496.476 & 1492.826 & 1489.12 & 1492.661 & 1490.344 & 1490.947 & 1484.564 & 1488.288 & 1493.069 & 1493.593 & 1492826 & 1494.741 & 1492.802 & 1485.177 & 1478552\end{array}$ $\begin{array}{lllllllllllllllllllllll}1493.868 & 1494.337 & 1494.567 & 1495.334 & 1496.663 & 1496.457 & 1492.819 & 1489.111 & 1492.654 & 1490.342 & 1490.942 & 1484.564 & 1488.288 & 1493.06 & 1493.581 & 1492.819 & 1494.727 & 1492.798 & 1485.577 & 1477.547\end{array}$ $\begin{array}{llllllllllllllllllllll}1493.88 & 1494.33 & 1494.563 & 1495.32 & 1496.647 & 1496.43 & 1492.807 & 1489104 & 1492.645 & 1490.339 & 1490.937 & 1484.566 & 1488.292 & 1493.053 & 1493.56 & 1492.807 & 1494.694 & 1492.786 & 1485.177 & 1478.545\end{array}$ $\begin{array}{lllllllllllllllllllll}1493.866 & 1494.325 & 1494.565 & 1495.313 & 1496.642 & 1496.428 & 1492.803 & 1489.101 & 1492.638 & 1490.337 & 1490.935 & 1484.566 & 1488.29 & 1493.046 & 1493.553 & 1492.804 & 1494.692 & 1492.779 & 1485.174 & 1478.545\end{array}$

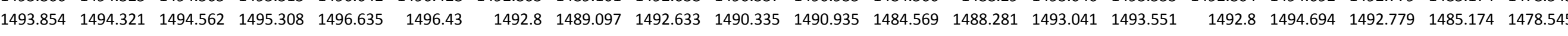
$\begin{array}{lllllllllllllllllllllll}1493.861 & 1494.316 & 1494.56 & 1495.292 & 1496.622 & 1496.411 & 1492.791 & 1489.094 & 1492.629 & 1490.33 & 1490.935 & 1484.569 & 1488.288 & 1493.036 & 1493.537 & 1492.793 & 1494.668 & 1492.772 & 1485.174 & 1478.545 & \\ \end{array}$

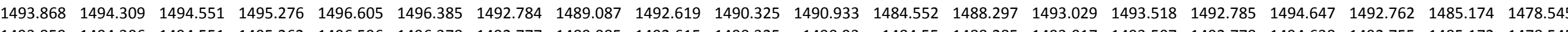

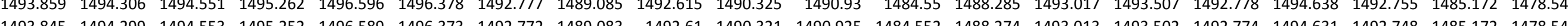

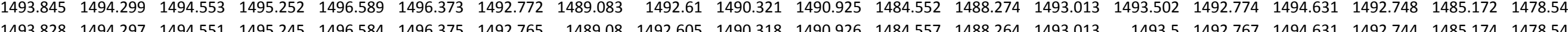

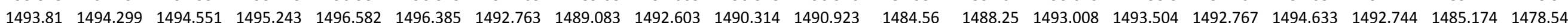

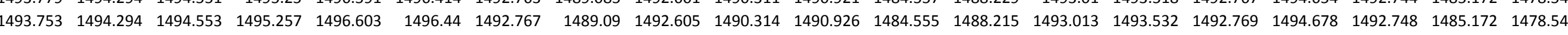
$\begin{array}{llllllllllllllllllll}1493.734 & 1494.297 & 1494.553 & 1495.266 & 1496.615 & 1496.464 & 1492.772 & 1489.094 & 1492.61 & 1490.314 & 1490.921 & 1484.557 & 1488.201 & 1493.015 & 1493.549 & 1492.776 & 1494.697 & 1492.753 & 1485.17 & 1478.547\end{array}$

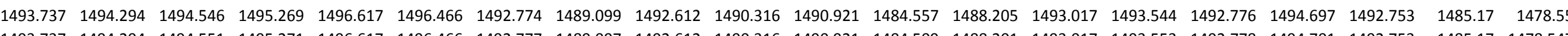

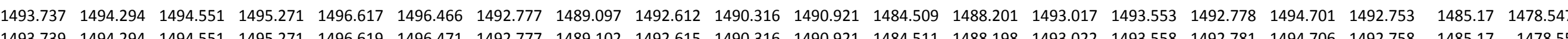

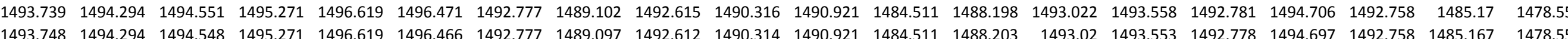
$\begin{array}{llllllllllllllllllll}1493.748 & 1494.294 & 1494.548 & 1495.27 & 1496.61 & 1496.466 & 1492.777 & 1489.097 & 1492.612 & 1490.314 & 1490.921 & 1484.511 & 1488.203 & 1493.02 & 1493.553 & 1492.778 & 1494.697 & 1492.758 & 1485.167 & 1478.55 \\ 1493.748 & 1494.294 & 1494.548 & 1495.273 & 1496.622 & 1496.469 & 1492.779 & 1489.101 & 1492.615 & 1490318 & 1490.921 & 1484.518 & 1488201 & 1493.027 & 1493553 & 1492783 & 1494.704 & 1492.758 & 1485.17 & 1478.552\end{array}$ $\begin{array}{lllllllllllllllllllll}1493.741 & 1494.294 & 1494.548 & 1495.278 & 1496.629 & 1496.483 & 1492.55 & 1489.101 & 1492.368 & 1490.318 & 1490.867 & 1484.52 & 1488.201 & 1492.775 & 1493.474 & 1492.553 & 1494.708 & 1492.533 & 1485.17 & 1478.554\end{array}$ $\begin{array}{llllllllllllllllllllll}1493.744 & 1494.294 & 1494.551 & 1495.283 & 1496.633 & 1496.485 & 1492.66 & 1489.099 & 1492.486 & 1490.321 & 1490.865 & 1484.518 & 1488.206 & 1492.895 & 1493.504 & 1492.66 & 1494.708 & 1492.64 & 1485.167 & 1478.554 \\ \end{array}$ $\begin{array}{lllllllllllllllllllll}1493.744 & 1494.297 & 1494.546 & 1495.283 & 1496.633 & 1496.485 & 1492.693 & 1489.104 & 1492.528 & 1490.321 & 1490.872 & 1484.52 & 1488.208 & 1492.93 & 1493.523 & 1492.695 & 1494.713 & 1492.676 & 1485.167 & 1478.557\end{array}$ $\begin{array}{lllllllllllllllllllll}1493.753 & 1494.297 & 1494.546 & 1495.285 & 1496.633 & 1496.483 & 1492.714 & 1489.106 & 1492.544 & 1490.321 & 1490.876 & 1484.525 & 1488.208 & 1492.956 & 1493.528 & 1492.717 & 1494.708 & 1492.692 & 1485.167 & 1478.557 & \end{array}$

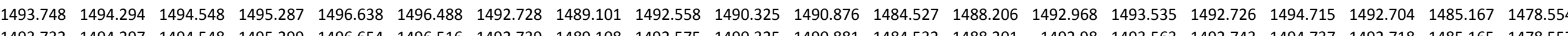

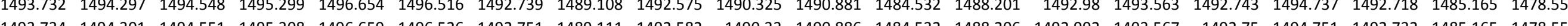

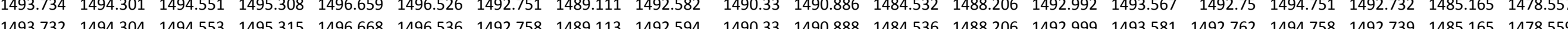

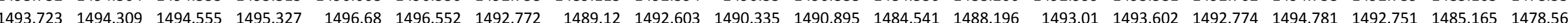
$\begin{array}{lllllllllllllllllllllll}1493.727 & 1494.313 & 1494.555 & 1495.336 & 1496.691 & 1496.567 & 1492.784 & 1489.123 & 1492.615 & 1490.339 & 1490.897 & 1484.543 & 1488.205 & 1493.022 & 1493.605 & 1492.783 & 1494.793 & 1492.762 & 1485.165 & 1478.564\end{array}$ $\begin{array}{llllllllllllllllllll}1493.732 & 1494.316 & 1494.558 & 1495.343 & 1496.698 & 1496.569 & 1492.793 & 1489.13 & 1492.624 & 1490.342 & 1490.904 & 1484.548 & 1488.213 & 1493.032 & 1493.614 & 1492.793 & 1494.798 & 1492.772 & 1485.165 & 1478.566\end{array}$

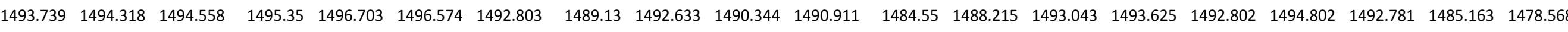
$\begin{array}{lllllllllllllllllllll}1493.744 & 1494.323 & 1494.558 & 1495.354 & 1496.71 & 1496.574 & 1492.807 & 1489.132 & 1492.643 & 1490.349 & 1490.914 & 1484.55 & 1488.217 & 1493.048 & 1493.63 & 1492.809 & 1494.807 & 1492.786 & 1485.165 & 1478.573 \\ \end{array}$

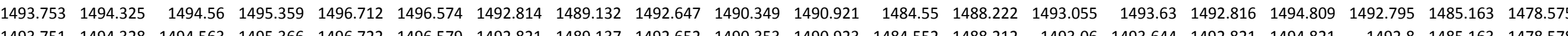

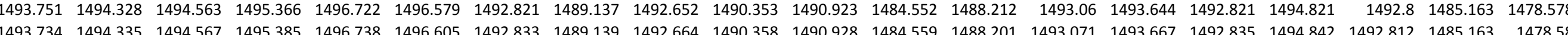

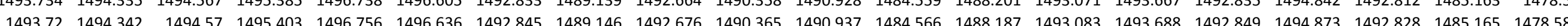
$\begin{array}{llllllllllllllllllllll}1493.713 & 1494.349 & 1494.572 & 1495.419 & 1496.777 & 1496.662 & 1492.859 & 1489.153 & 1492.69 & 1490.369 & 1490.94 & 1484.569 & 1488.18 & 1493.1 & 1493.707 & 1492.861 & 1494.899 & 1492.838 & 1485.165 & 1478.589\end{array}$ $\begin{array}{llllllllllllllllllllllll}1493.713 & 1494.354 & 1494.577 & 1495.44 & 1496.796 & 1496.684 & 1492.87 & 1489.162 & 1492.701 & 1490.376 & 1490.947 & 1484.578 & 1488.184 & 1493.109 & 1493.73 & 1492.873 & 1494.927 & 1492.849 & 1485.163 & 1478.592 \\ \end{array}$

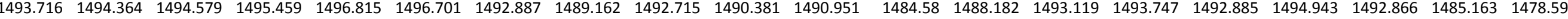
\begin{tabular}{llllllllllllllllllll}
1493.737 & 1494.368 & 1494.582 & 1495.468 & 1496.819 & 1496.696 & 1492.894 & 1489.172 & 1492.722 & 1490.383 & 1490.956 & 1484.58 & 1488.191 & 1493.13 & 1493.754 & 1492.894 & 1494.943 & 1492.873 & 1485.163 & 1478.594 \\
\hline
\end{tabular}

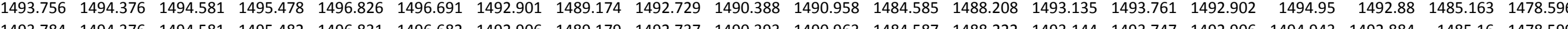

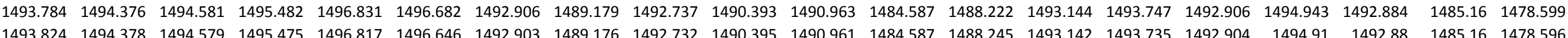


Well

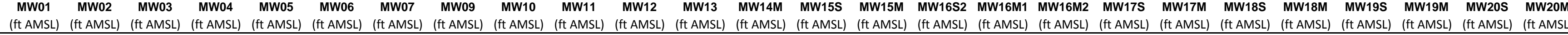

$11 / 30 / 1120: 00$ $11 / 30 / 1121: 00$ $11 / 30 / 1122: 00$ 11/30/11 23:00 $12 / 1 / 11111: 00$
$12 / 1 / 112: 00$ $12 / 1 / 112: 00$ $12 / 1 / 113: 00$
$12 / 1 / 11$ 4:00 12/1/111:00 2/1/111 6:00 12/1/1111:00 12/1/119:00 $12 / 1 / 1110: 00$ 12/1/11 11:00 12/1/11 12:00 12/1/1114:00 $12 / 1 / 1115: 00$ 12/1/11 16:00 12/1/11 18:00 $12 / 1 / 1119: 00$ 12/1/11 20:00 12/1/1121:00 12/1/1122:00 $-12 / 1 / 1123: 00$
$12 / 2 / 110: 00$ 12/2/1110:00 12/2/1111:00 12/2/11 3:00 12/2/11 5:00 12/2/11 6:00 $12 / 2 / 118: 00$ $12 / 2 / 119: 00$ 12/2/11 11:00 12/2/2/11 11:00 12/2/11 13:00 12/2/11 14:00 12/2/11 15:00 12/2/111 16:00 $12 / 2 / 1118.00$ 12/2/111 18:00 $12 / 2 / 1120: 00$ 12/2/11 21:00 12/2/11 22:00 12/2/1123:00 12/3/11 0:00 $\begin{array}{lrrr}1494.382 & 1494.9 & 1496.499 & 1494.874\end{array}$ $\begin{array}{rrrr}1494.382 & 1494.895 & 1496.49 & 1494.86 \\ 1494.384 & 1494.895 & 1496.492 & 1494.855\end{array}$ $\begin{array}{llll}1494.384 & 1494.895 & 1496.492 & 1494.855 \\ 1494.384 & 1494.895 & 1496.49 & 1494848\end{array}$ $\begin{array}{lllll}1494.386 & 1494.895 & 1496.492 & 1494.846\end{array}$ $\begin{array}{lllll}1494.391 & 1494.895 & 1496.499 & 1449.846\end{array}$ $\begin{array}{llll}1494.389 & 1494.895 & 1496.497 & 1494.846\end{array}$ $\begin{array}{llllll}1494.384 & 1494.884 & 1496.471 & 1494.832\end{array}$ $\begin{array}{lllll}1494.382 & 1494.872 & 1496.455 & 1494.813\end{array}$ $\begin{array}{llll}1494.374 & 1494.853 & 1496.425 & 1494.792\end{array}$ $\begin{array}{lllll}1494.367 & 1494.837 & 1496.397 & 1494.766\end{array}$ $\begin{array}{llll}1494.361 & 1494.814 & 1496.361 & 1494.738 \\ 1494.36 & 1494.804 & 1496.352 & 1494.717\end{array}$ $\begin{array}{rrrr}1494.342 & 1494.804 & 1496.352 & 1994.747 \\ 1494.337 & 1949.767 & 1496.298 & 1494.68 \\ 1496.27 & 1494.645\end{array}$ $\begin{array}{lllll}1494.335 & 1494.73 & 1496.256 & 1494.624\end{array}$ $\begin{array}{lllll}1494.333 & 1494.718 & 1496.247 & 1494.608\end{array}$ $\begin{array}{llll}1494.326 & 1494.709 & 1496.24 & 1494.598\end{array}$ $\begin{array}{llll}1494.323 & 1494.7 & 1496.233 & 1494.589\end{array}$ $\begin{array}{lllll}1494.314 & 1494.683 & 1496.214 & 1494.573 \\ 1494.302 & 149466 & 1496177 & 1494547\end{array}$ $\begin{array}{rrrrr}1494.302 & 1494.66 & 1496.177 & 1494.547 \\ 1494.295 & 1494.637 & 1496.146 & 1494.521\end{array}$ $\begin{array}{llllll}1494.284 & 1494.616 & 1496.121 & 14494.493\end{array}$ $\begin{array}{lllll}1494.286 & 1494.606 & 1496.116 & 1494.479\end{array}$ $\begin{array}{lllll}1494.284 & 1494.599 & 1496.114 & 1494.472\end{array}$ $\begin{array}{lllll}1494.284 & 1494.592 & 1496.114 & 1494.468\end{array}$ $1494.277 \quad \begin{array}{llll}1494.592 & 1496.116 & 1494.468\end{array}$ $\begin{array}{llll}1494.274 & 1494.583 & 1496.107 & 1494.458\end{array}$ $\begin{array}{llll}1494.279 & 1494.588 & 1496.125 & 1494.463 \\ 1494.279 & 1494.592 & 1496.139 & 1494.477\end{array}$ $\begin{array}{lllll}1494.281 & 1494.597 & 1496.151 & 1494.484\end{array}$ $\begin{array}{lllll}1494.277 & 1494.597 & 1496.156 & 1494.493\end{array}$ $\begin{array}{lllll}1494.284 & 1494.609 & 1496.179 & 1494.51\end{array}$ $\begin{array}{lllll}1494.284 & 1494.623 & 1496.202 & 1494.531\end{array}$ $\begin{array}{llll}1494.286 & 1494.632 & 1496.224 & 1494.549\end{array}$ 1494.2861494 .6391496 .2331494 .563 $\begin{array}{lllll}1494.291 & 1494.648 & 1496.245 & 1494.58\end{array}$ $\begin{array}{llll}1494.286 & 1494.653 & 1496.247 & 1494.584 \\ 1494.288 & 1494.662 & 1496.263 & 1494.601\end{array}$ $\begin{array}{llll}1494.295 & 1494.676 & 1496.287 & 1494.6169\end{array}$ $\begin{array}{lllll}1494.3 & 1494.69 & 1496.308 & 1494.638\end{array}$ $\begin{array}{lllll}1494.307 & 1494.711 & 1496.34 & 1494.666\end{array}$ $\begin{array}{lllll}1494.316 & 1494.737 & 1496.375 & 1494.694\end{array}$ $\begin{array}{lllll}1494.316 & 1494.748 & 1496.385 & 1494.713\end{array}$ $\begin{array}{llll}1494.323 & 1494.767 & 1496.406 & 1494.734\end{array}$ $\begin{array}{llll}1494.326 & 1494.776 & 1496.415 & 1494.748 \\ 1494.328 & 1494.786 & 1496.422 & 1494.762\end{array}$ $\begin{array}{lllll}1494.326 & 1494.788 & 1496.42 & 1494.762\end{array}$ $\begin{array}{llll}1494.328 & 1494.79 & 1496.42 & 1494.764\end{array}$ $\begin{array}{lllll}1494.337 & 1494.802 & 1496.439 & 1494.773\end{array}$ $1494.3351494 .8071496 .432 \quad 1494.776$ $1494.3371494 .811 \quad 1496.4321494 .778$ $\begin{array}{rrrr}1494.34 & 1494.811 & 1496.432 & 1494.778 \\ 1494.351 & 1494.828 & 1496.462 & 1494.792\end{array}$

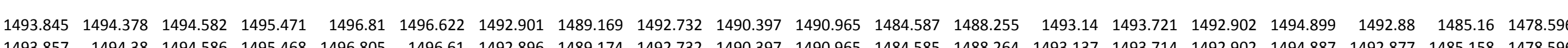

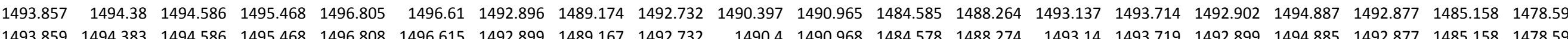

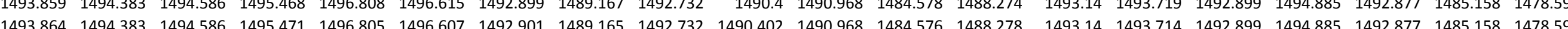

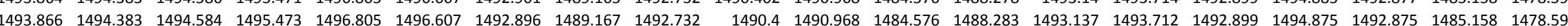
$\begin{array}{lllllllllllllllllllll}1493.861 & 1494.388 & 1494.589 & 1495.475 & 1496.81 & 1496.615 & 1492.899 & 1489.169 & 1492.737 & 1490.402 & 1490.968 & 1484.576 & 1488288 & 1493.14 & 1493.712 & 1492.902 & 1494.885 & 1492.877 & 1485.158 & 1478.599\end{array}$

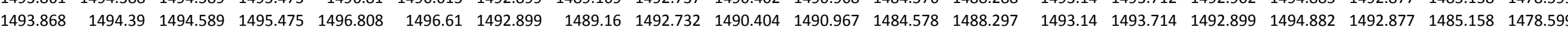
$\begin{array}{llllllllllllllllllll}1493.887 & 1494.388 & 1494.589 & 1495.468 & 1496.796 & 1496.583 & 1492.894 & 1489.16 & 1492.727 & 1490.402 & 1490.965 & 1484.576 & 1488.309 & 1493.137 & 1493.693 & 1492.894 & 1494.856 & 1492.873 & 1485.156 & 1478.5964\end{array}$

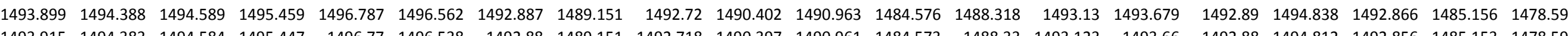

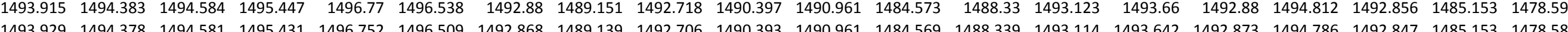
$\begin{array}{llllllllllllllllllll}1493.929 & 1494.378 & 1494.581 & 1495.431 & 1496.752 & 1496.509 & 1492.868 & 1489.139 & 1492.706 & 1490.393 & 1490.961 & 1484.569 & 1488.339 & 1493.114 & 1493.642 & 1492.873 & 1494.786 & 1492.847 & 1485.153 & 1478.589 \\ 1493.941 & 1494373 & 1494577 & 1495.415 & 1496.731 & 1496476 & 1492.859 & 1489.125 & 1492.697 & 1490.388 & 1490.956 & 1484.569 & 1488349 & 1493.104 & 1493.611 & 1492.859 & 1494.758 & 1492.838 & 1485.153 & 1478588\end{array}$

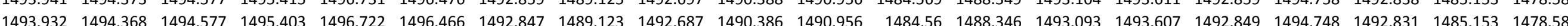

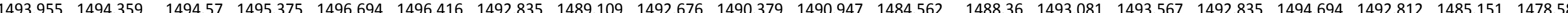
$\begin{array}{lllllllllllllllllllll}1493.953 & 1494.352 & 1494.57 & 1495.35 & 1496.67 & 1496.392 & 1492.819 & 1489.092 & 1492.659 & 1490.372 & 1490.942 & 1484.557 & 1488.358 & 1493.064 & 1493.544 & 1492.821 & 1494.673 & 1492.8 & 1485.153 & 1478.58\end{array}$

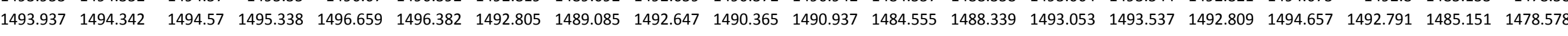
$\begin{array}{lllllllllllllllllllllll}1493.918 & 1494.335 & 1494.572 & 1495.324 & 1496.645 & 1496.378 & 1492.796 & 1489.083 & 1492.636 & 1490.36 & 1490.93 & 1484.557 & 1488.328 & 1493.043 & 1493.525 & 1492.8 & 1494.65 & 1492.774 & 1485.149 & 1478.575 & \\ \end{array}$

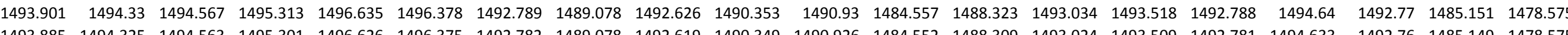

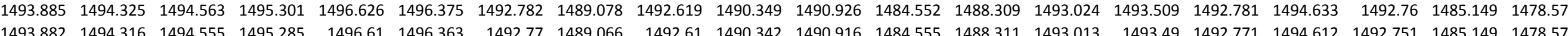

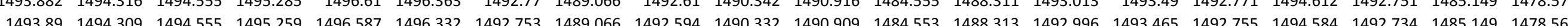
\begin{tabular}{llllllllllllllllllllll}
1493.89 & 1494.297 & 1494.546 & 1495.238 & 1496.568 & 1496306 & 1492.742 & 1489.057 & 1492.579 & 1490.323 & 14909 & 1484.548 & 1488309 & 1492.985 & 1493.444 & 1492.74 & 1494.556 & 1492.72 & 1485.146 & 1478.561 \\
\hline
\end{tabular}

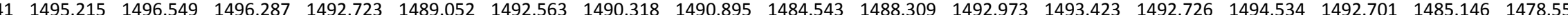
$\begin{array}{lllllllllllllllllllllll}1493.871 & 1494.287 & 1494.539 & 1495.201 & 1496.538 & 1496.287 & 1492.714 & 1489.052 & 1492.551 & 1490.309 & 1490.888 & 1484.541 & 1488.295 & 1492.959 & 1493.416 & 1492.712 & 1494.523 & 1492.692 & 1485.146 & 1478.557\end{array}$

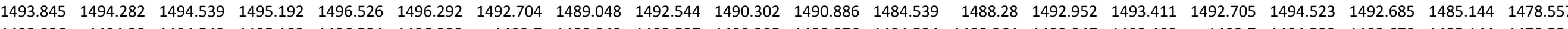

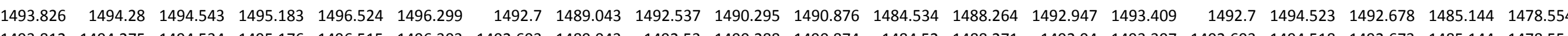

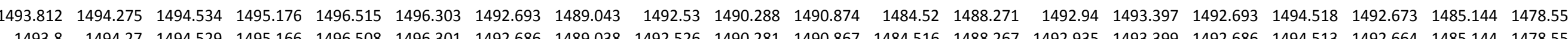

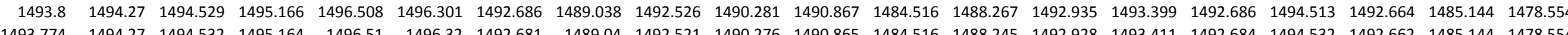
$\begin{array}{llllllllllllllllllll}143.774 & 1494.27 & 1494.532 & 1495.164 & 1496.51 & 1496.32 & 1492.681 & 1489.04 & 1492.521 & 1490.27 & 1490.865 & 1484.516 & 1488.245 & 1492.928 & 1493.411 & 1492.684 & 1494.532 & 1492.662 & 1485.144 & 1478.554 \\ 1493.753 & 1494.268 & 1494.527 & 1495.166 & 1496.512 & 1496339 & 1492.683 & 1489.038 & 1492.521 & 1490274 & 1490865 & 1484.513 & 1488241 & 1492928 & 1493.409 & 1492.684 & 1494.532 & 1492.662 & 1485.144 & 1478557\end{array}$

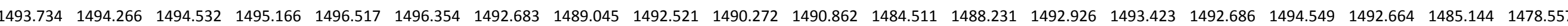
$\begin{array}{lllllllllllllllllllll}1493.727 & 1494.261 & 1494.529 & 1495.166 & 1496.517 & 1496.363 & 1492.681 & 1489.048 & 1492.518 & 1490.267 & 1490.86 & 1484.513 & 1488.229 & 1492.923 & 1493.425 & 1492.684 & 1494.549 & 1492.659 & 1485.142 & 1478.554\end{array}$

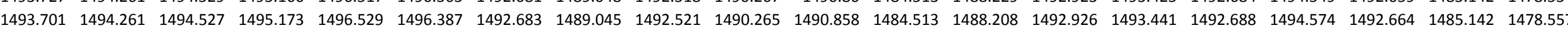
$\begin{array}{lllllllllllllllllllllll}1493.69 & 1494.261 & 1494.529 & 1495.183 & 1496.54 & 1496.411 & 1492.69 & 1489.052 & 1492.526 & 1490.267 & 1490.86 & 1484.518 & 1488.201 & 1492.935 & 1493.455 & 1492.691 & 1494.593 & 1492.669 & 1485.142 & 1478.559 & \end{array}$

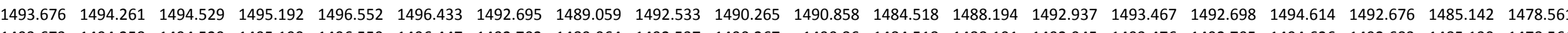

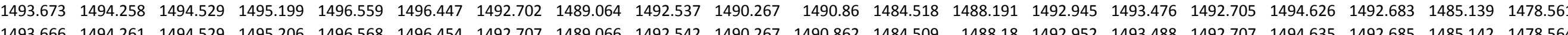

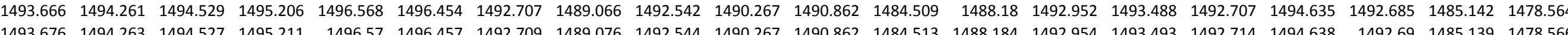

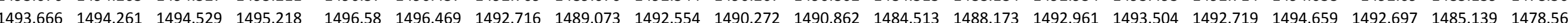
$\begin{array}{llllllllllllllllllllll}1493.652 & 1494.266 & 1494.534 & 1495.229 & 1496.596 & 1496.495 & 1492.725 & 1489.08 & 1492.565 & 1490.274 & 1490.867 & 1484.516 & 1488.161 & 1492.968 & 1493.518 & 1492.729 & 1494.678 & 1492.704 & 1485.139 & 1478.573\end{array}$

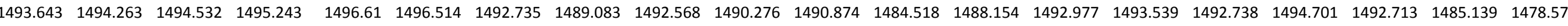
$\begin{array}{llllllllllllllllllllll}1493.636 & 1494.27 & 1494.536 & 1495.262 & 1496.629 & 1496.54 & 1492.746 & 1489.09 & 1492.579 & 1490.281 & 1490.874 & 1484.52 & 1488.145 & 1492.987 & 1493.563 & 1492.75 & 1494.729 & 1492.725 & 1485.137 & 1478.58 \\ \end{array}$

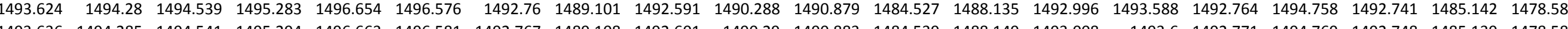

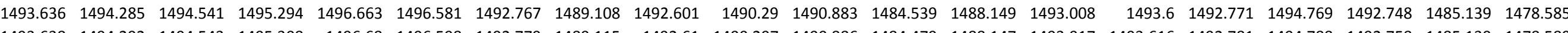

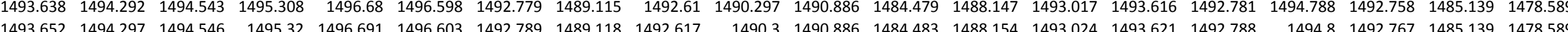

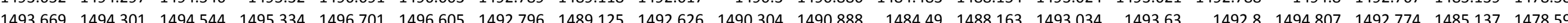
$\begin{array}{lllllllllllllllllllll}1493.692 & 1494.306 & 1494.546 & 1495.334 & 1496.703 & 1496.595 & 1492.798 & 1489.125 & 1492.629 & 1490.309 & 1490.89 & 1484.502 & 1488.177 & 1493.036 & 1493.625 & 1492.8 & 1494.805 & 1492.779 & 1485.137 & 1478.592\end{array}$ $\begin{array}{llllllllllllllllllllllll}1493.709 & 1494.309 & 1494.541 & 1495.341 & 1496.708 & 1496.588 & 1492.8 & 1489.125 & 1492.633 & 1490.314 & 1490.893 & 1484.499 & 1488.184 & 1493.041 & 1493.623 & 1492.804 & 1494.798 & 1492.779 & 1485.137 & 1478.592\end{array}$

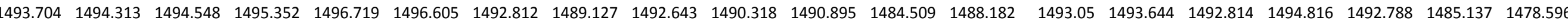
$\begin{array}{llllllllllllllllllll}1493.718 & 1494.316 & 1494.548 & 1495.359 & 1496.724 & 1496.603 & 1492.814 & 1489.13 & 1492.647 & 1490.321 & 1490.902 & 1484.515 & 1488.194 & 1493.053 & 1493.639 & 1492.816 & 1494.814 & 1492.793 & 1485.135 & 1478.599 \\ \end{array}$

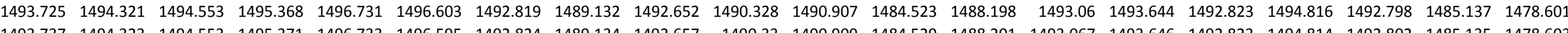

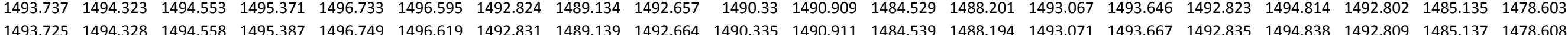


Well

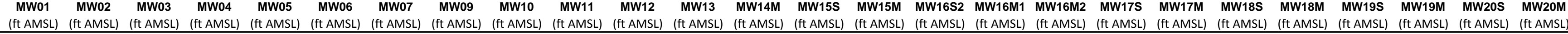

$12 / 3 / 112: 00$ $12 / 3 / 113: 00$ 12/3/11 4:00 12/3/11116:00 12/3/111 6:00 $12 / 3 / 117: 00$ 12/3/11 9:00 12/3/11 10:00 12/3/11 11:00 12/3/11 12:00 12/3/11 14:00 $12 / 3 / 1115: 00$ 12/3/1111 16:00 12/3/11 17:00 12/3/11 18:00 12/3/111 19:00 12/3/1120:00 2/3/1121:00 $12 / 3 / 1123: 00$ 12/4/11 0:00 12/4/1110:00 12/4/11 2:00 12/4/11 3:00 12/4/11 4:00

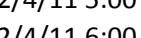
12/4/11 6:00 12/4/117:00 12/4/11 9:00 $12 / 4 / 1110: 00$ 12/4/11 11:00 12/4/111 12:00 12/4/11 13:00 12/4/11 15:00 $12 / 4 / 1116: 00$
$12 / 4 / 1117: 00$ 12/4/11 18:00 12/4/111 19:00 12/4/11 20:00 12/4/11 21:00 12/4/11 22:00

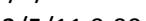
12/5/11 0:00 12/5/111:00 12/5/11 3:00 12/5/11 4:00 $12 / 5 / 115: 00$ 12/5/11 6:00 $\begin{array}{llll}1494.351 & 1494.839 & 1496.471 & 1494.804\end{array}$ 1494.3491494 .8391496 .4621494 .804 $\begin{array}{lllll}1494.351 & 1494.842 & 1496.464 & 1494.806 \\ 1494.356 & 1494.849 & 1496478 & 1494815\end{array}$ $\begin{array}{lllll}1494.367 & 1494.87 & 1496.511 & 1494.834\end{array}$ $\begin{array}{lllll}1494.37 & 1494.879 & 1496.523 & 1494.853\end{array}$ $\begin{array}{rrrrr}1494.372 & 1994.888 & 14966.53 & 1494.864\end{array}$ $\begin{array}{lllll}1494.37 & 1494.881 & 1496.483 & 1494.857\end{array}$ $\begin{array}{lllll}1494.379 & 1494.891 & 1496.305 & 1494.867\end{array}$ $\begin{array}{lllll}1494.393 & 1494.926 & 1496.425 & 1494.911\end{array}$ $\begin{array}{llll}1494.391 & 1494.928 & 1496.766 & 1494.925 \\ 1494393 & 1494935 & 1496801 & 1494.937\end{array}$ $\begin{array}{rrrr}1494.393 & 1494.935 & 1496.801 & 1494.937 \\ 1494.398 & 1494.94 & 1496.649 & 1494.948\end{array}$ $\begin{array}{lllll}1494.391 & 1494.935 & 1496.654 & 1494.944\end{array}$ $\begin{array}{lllll}1494.379 & 1494.912 & 1496.534 & 1494.918\end{array}$ $\begin{array}{llllll}1494.374 & 1494.893 & 1496.488 & 1494.895\end{array}$ $\begin{array}{llll}1494.365 & 1494.867 & 1496.439 & 1494.86\end{array}$ $\begin{array}{llll}1494.37 & 1494.856 & 1496.422 & 1494.839\end{array}$

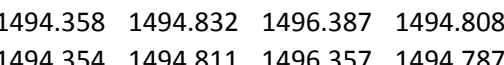
$\begin{array}{lllll}1494.354 & 194.811 & 1496.357 & 1494.787\end{array}$ $\begin{array}{lllll}1494.358 & 1494.807 & 1496.354 & 1494.776 \\ 1494.349 & 1494.788 & 1496.336 & 1494.759\end{array}$ $\begin{array}{lllll}1494.349 & 1494.783 & 1496.336 & 1494.755\end{array}$ $\begin{array}{lllll}1494.349 & 1494.781 & 1496.34 & 1494.757\end{array}$ $\begin{array}{rrrr}1494.347 & 1494.774 & 1496.329 & 1494.752\end{array}$ $\begin{array}{lllll}1494.344 & 1494.765 & 1496.317 & 1494.745\end{array}$ $\begin{array}{llll}1494.346 & 1494.765 & 1496.322 & 1494.748\end{array}$ $\begin{array}{rlll}1494.347 & 1494.769 & 1496.333 & 1494.755\end{array}$ $\begin{array}{rrrrr}1494.342 & 1494.76 & 1496.319 & 1494.752 \\ 1494.337 & 1494.755 & 149631 & 1494.75\end{array}$ $\begin{array}{lllll}1494.333 & 1494.741 & 1496.291 & 1494.736\end{array}$ $\begin{array}{lllll}1494.328 & 1494.73 & 1496.273 & 1494.724\end{array}$ $\begin{array}{lllll}1494.326 & 1494.723 & 1496.261 & 1494.715\end{array}$ $\begin{array}{lllll}1494.333 & 1494.725 & 1496.277 & 1494.72\end{array}$ $\begin{array}{llll}1494.335 & 1494.735 & 1496.301 & 1494.731\end{array}$ $\begin{array}{llll}1494.34 & 1494.744 & 1496.322 & 1494.75 \\ 1194.34 & 1994.751 & 1996336 & 1494.766\end{array}$ $\begin{array}{llll}1494.34 & 1494.751 & 1496.336 & 1494.766\end{array}$ $\begin{array}{rrrrr}1494.34 & 1494.751 & 1496.333 & 1494.771 \\ 1494.337 & 1494.751 & 1496.326 & 1494.771\end{array}$ $\begin{array}{lllll}1494.33 & 1494.744 & 1496.305 & 1494.764\end{array}$ $\begin{array}{lllll}1494.328 & 1494.737 & 1496.291 & 1494.755\end{array}$ $\begin{array}{llllll}1494.326 & 1494.73 & 1496.28 & 1494.741\end{array}$ $\begin{array}{lllll}1494.319 & 1494.718 & 1496.261 & 1494.727\end{array}$ $\begin{array}{lllll}1494.314 & 1494.707 & 1496.238 & 1494.706\end{array}$ $\begin{array}{llll}1494.309 & 1494.693 & 1496.219 & 1494.689\end{array}$ $\begin{array}{lllll}1494.312 & 1494.693 & 1496.219 & 1494.68\end{array}$ $\begin{array}{llll}1494.312 & 1494.695 & 1496.235 & 1494.685\end{array}$ $\begin{array}{lllll}1494.307 & 1494.693 & 1496.233 & 1494.687\end{array}$ $\begin{array}{lllll}1494.302 & 1494.683 & 1496.219 & 1494.678\end{array}$ $\begin{array}{lllll}1494.312 & 1494.693 & 1496.24 & 1494.687\end{array}$ $\begin{array}{lllll}1494.288 & 1494.658 & 1496.179 & 1494.654\end{array}$ $\begin{array}{llll}1494.295 & 1494.658 & 1496.184 & 1494.647 \\ 1494.281 & 1494.639 & 1496.153 & 1494.631\end{array}$

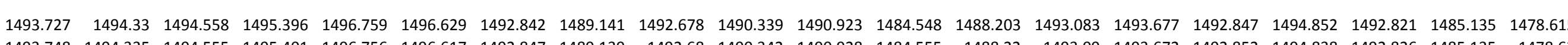
$\begin{array}{llllllllllllllllllllll}1493.748 & 1494.335 & 1494.555 & 1495.401 & 1496.756 & 1496.617 & 1492.847 & 1489.139 & 1492.68 & 1490.342 & 1490.928 & 1484.555 & 1488.22 & 1493.09 & 1493.672 & 1492.852 & 1494.838 & 1492.826 & 1485.135 & 1478.62 \\ 1493.756 & 149434 & 149456 & 1495.06 & 1496763 & 1496615 & 1492852 & 1489.144 & 1492.685 & 1490346 & 1490928 & 1484566 & 1488.217 & 149309 & 1493677 & 1492854 & 1494845 & 1492831 & 1485135 & 1478622\end{array}$

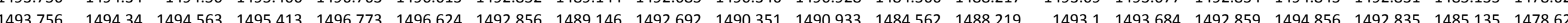
$\begin{array}{llllllllllllllllllllll}1493.739 & 1494.34 & 1494.57 & 1495.433 & 1496791 & 1496.653 & 1492873 & 1489.148 & 1492.708 & 1490.355 & 1490.947 & 1484.569 & 1488215 & 1493114 & 1493.712 & 1492876 & 1494.882 & 1492849 & 1485137 & 1478.636\end{array}$ \begin{tabular}{lllllllllllllllllllll}
1493.753 & 1494.34 & 1494.57 & 1495.457 & 1496.81 & 1496.665 & 1492.889 & 1489.16 & 1492.727 & 1490.365 & 1490.956 & 1484.583 & 1488.229 & 1493.133 & 1493.728 & 1492.892 & 1494.908 & 1492.87 & 1485.132 & 1478.643 \\
\hline
\end{tabular} $\begin{array}{lllllllllllllllllllll}1493.779 & 1494.412 & 1494.582 & 1495.452 & 1496.805 & 1496.631 & 1492.92 & 1489.158 & 1492.758 & 1490.369 & 1490.977 & 1484.606 & 1488.238 & 1493.163 & 1493.742 & 1492.923 & 1494.906 & 1492.899 & 1485.137 & 1478.657\end{array}$

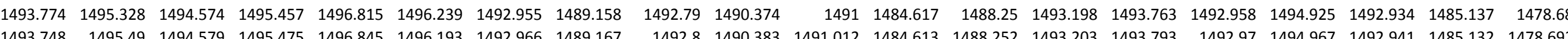
$\begin{array}{llllllllllllllllllll}1493.748 & 1495.49 & 1494.579 & 1495.475 & 1496.845 & 1496.193 & 1492.966 & 1489.167 & 1492.8 & 1490.383 & 1491.012 & 1484.613 & 1488.252 & 1493.203 & 1493.793 & 1492.97 & 1494.967 & 1492.941 & 1485.132 & 1478.692 \\ 1493765 & 1495543 & 1494584 & 1495.489 & 1496849 & 1496705 & 1492966 & 1489.174 & 1492.802 & 149039 & 1491.017 & 1484.596 & 1488255 & 1493208 & 1493798 & 1492968 & 1494.976 & 1492.945 & 1485.135 & 1478694\end{array}$ \begin{tabular}{lllllllllllllllllllll}
1493.765 & 1495.543 & 1494.584 & 1495.489 & 1496.849 & 1496.705 & 1492.966 & 1489.174 & 1492.802 & 1490.39 & 1491.017 & 1484.596 & 1488.255 & 1493.208 & 1493.798 & 1492.968 & 1494.976 & 1492.945 & 1485.135 & 1478.694 \\
1493.779 & 1495.67 & 1494.584 & 1495.496 & 1496.856 & 1496.703 & 1492.973 & 1489.181 & 1492.809 & 1490.395 & 1491.021 & 1484.573 & 1488.269 & 1493.215 & 1493.803 & 1492.977 & 1494.983 & 1492.953 & 1485.132 & 1478.70 \\
\hline
\end{tabular}

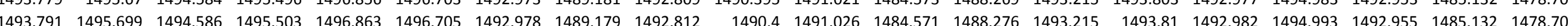
$\begin{array}{llllllllllllllllll} & 1488292 & 1493215 & 1493796 & 1492977 & 1494.976 & 1492.953 & 1485.132 & 1478704\end{array}$ $\begin{array}{lllllllllllllllllllll}1493.866 & 1495.665 & 1494.579 & 1495.501 & 1496.835 & 1496.627 & 1492.964 & 1489.174 & 1492.8 & 1490.404 & 1491.019 & 1484.529 & 1488.318 & 1493.21 & 1493.765 & 1492.968 & 1494.941 & 1492.943 & 1485.13 & 1478.701\end{array}$ $\begin{array}{llllllllllllllllllll}1493.894 & 1495.575 & 1494.582 & 1495.484 & 1496.817 & 1496.586 & 1492.952 & 1489.165 & 1492.788 & 1490.402 & 1491.014 & 1484.516 & 1488.33 & 1493.196 & 1493.742 & 1492.956 & 1494.915 & 1492.931 & 1485.13 & 1478.697\end{array}$ $\begin{array}{lllllllllllllllllllll}1493.925 & 1495.493 & 1494.579 & 1495.464 & 1496.794 & 1496.54 & 1492.941 & 1489.151 & 1492.776 & 1490.4 & 1491.007 & 1484.518 & 1488.344 & 1493.182 & 1493.712 & 1492.942 & 1494.87 & 1492.915 & 1485.13 & 1478.692\end{array}$

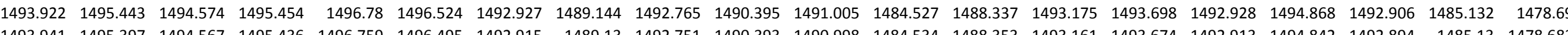

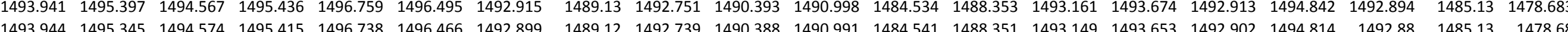

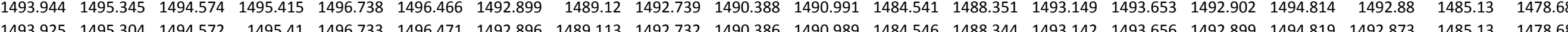
$\begin{array}{lllllllllllllllllllllll}1493.929 & 1495.263 & 1494.57 & 1495.394 & 1496.717 & 1496.449 & 1492.887 & 1489.106 & 1492.727 & 1490.381 & 1490.989 & 1484.552 & 1488.351 & 1493.133 & 1493.637 & 1492.887 & 1494.805 & 1492.863 & 1485.13 & 1478.68\end{array}$ $\begin{array}{llllllllllllllllllllllll}1493.911 & 1495223 & 1494572 & 1495387 & 1496712 & 1496457 & 1492882 & 1489104 & 149272 & 1490381 & 1490.986 & 1484559 & 1488344 & 1493128 & 1493639 & 1492885 & 1494.802 & 1492.861 & 1485.13 & 1478.68\end{array}$ $\begin{array}{lllllllllllllllllllllll}1493.894 & 1495.184 & 1494.57 & 1495.385 & 1496.708 & 1496.464 & 1492.88 & 1489.099 & 1492.72 & 1490.376 & 1490.984 & 1484.564 & 1488.337 & 1493.123 & 1493.639 & 1492.878 & 1494.809 & 1492.859 & 1485.13 & 1478.68\end{array}$

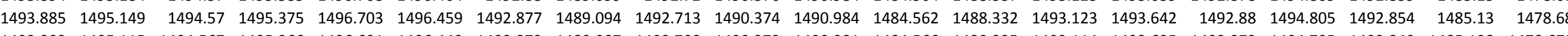

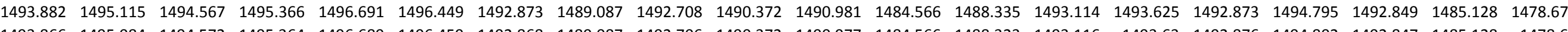

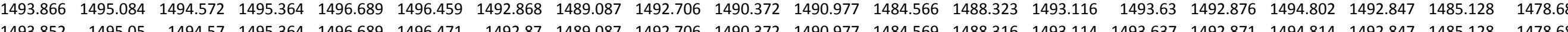
$\begin{array}{lllllllllllllllllllllll}1493.852 & 1495.05 & 1494.57 & 1495.364 & 1496.689 & 1496.471 & 1492.87 & 1489.087 & 1492.706 & 1490.37 & 1490.977 & 1484.569 & 1488.316 & 1493.114 & 1493.637 & 1492.871 & 1494.814 & 1492.847 & 1485.128 & 1478.68 \\ 1493.852 & 1495.022 & 1494.567 & 1495354 & 1496682 & 1496459 & 1492866 & 1489083 & 1492704 & 1490369 & 1490974 & 1484571 & 1488323 & 1493107 & 1493.63 & 1492866 & 1494805 & 1492845 & 1485.13 & 1478678\end{array}$

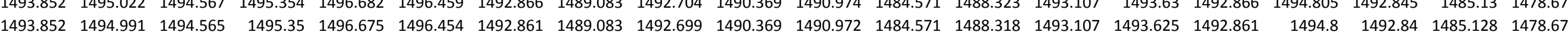
\begin{tabular}{llllllllllllllllllllll}
1493.857 & 1494.96 & 1494.565 & 1495.336 & 1496.666 & 1496.44 & 1492.856 & 1489.071 & 1492.692 & 1490.365 & 1490.968 & 1484.569 & 1488.325 & 1493.1 & 1493.609 & 1492.859 & 1494.786 & 1492.835 & 1485.128 & 1478.676 \\
\hline
\end{tabular} \begin{tabular}{llllllllllllllllllll}
1493.857 & 1494.926 & 1494.56 & 1495.324 & 1496.654 & 1496.426 & 1492.849 & 1489.073 & 1492.687 & 1490.365 & 1490.965 & 1484.569 & 1488.323 & 1493.093 & 1493.605 & 1492.849 & 1494.772 & 1492.826 & 1485.128 & 1478.673 \\
\hline
\end{tabular} \begin{tabular}{lllllllllllllllllllllll}
1493.85 & 1494.897 & 1494.56 & 1495.313 & 1496.645 & 1496.416 & 1492.842 & 1489.064 & 1492.68 & 1490.362 & 1490.961 & 1484.571 & 1488.313 & 1493.088 & 1493.598 & 1492.845 & 1494.758 & 1492.819 & 1485.128 & 1478.671 \\
\hline
\end{tabular} $\begin{array}{lllllllllllllllllllllll}1493.826 & 1494.871 & 1494.565 & 1495.315 & 1496.645 & 1496.433 & 1492.842 & 1489.066 & 1492.678 & 1490.362 & 1490.963 & 1484.576 & 1488.302 & 1493.086 & 1493.605 & 1492.845 & 1494.776 & 1492.819 & 1485.13 & 1478.673\end{array}$

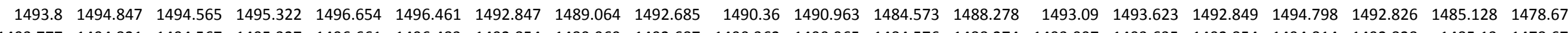

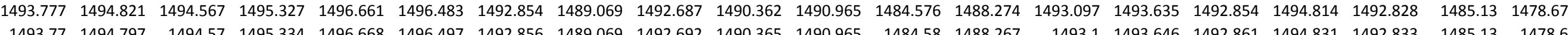

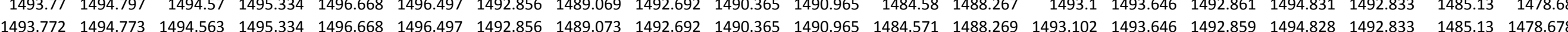

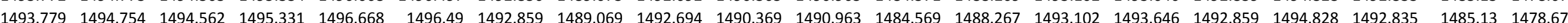
$\begin{array}{llllllllllllllllllllll}1493.795 & 1494.732 & 1494.558 & 1495.322 & 1496.659 & 1496.473 & 1492.854 & 1489.066 & 1492.69 & 1490.369 & 1490.961 & 1484.573 & 1488.274 & 1493.095 & 1493.635 & 1492.857 & 1494.812 & 1492.831 & 1485.128 & 1478.676\end{array}$ $\begin{array}{lllllllllllllllllllll}1493.805 & 1494.711 & 1494.558 & 1495.317 & 1496.652 & 1496.461 & 1492.611 & 1489.064 & 1492.429 & 1490.367 & 1490.886 & 1484.506 & 1488.276 & 1492.834 & 1493.53 & 1492.613 & 1494.793 & 1492.591 & 1485.128 & 1478.673 \\ \end{array}$

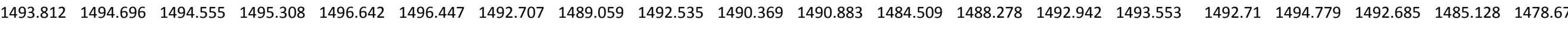

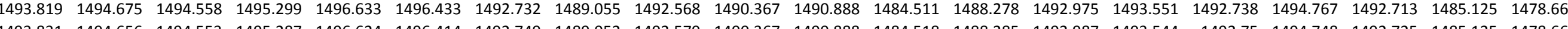

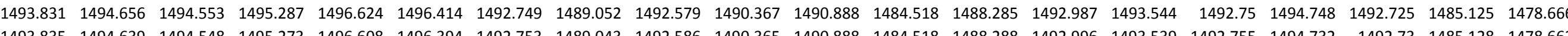

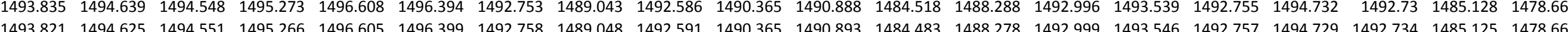

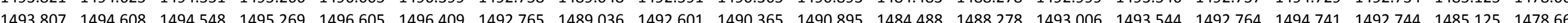

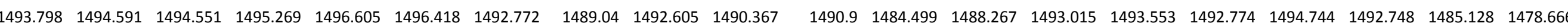
$\begin{array}{llllllllllllllllllllll}1493.793 & 1494.577 & 1494.548 & 1495.264 & 1496.601 & 1496.421 & 1492.777 & 1489.043 & 1492.61 & 1490.365 & 1490.902 & 1484.509 & 1488.276 & 1493.017 & 1493.553 & 1492.778 & 1494.746 & 1492.753 & 1485.123 & 1478.669\end{array}$

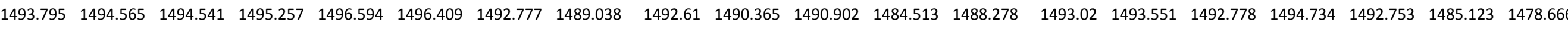

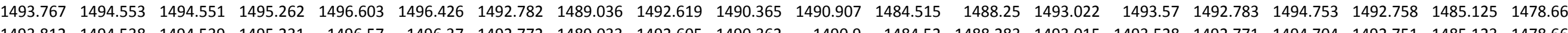

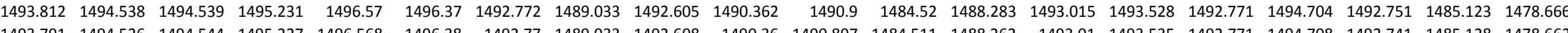
$\begin{array}{llllllllllllllllllllll}1493.791 & 1494.526 & 1494.544 & 1495.227 & 1496.568 & 1496.38 & 1492.77 & 1489.033 & 1492.608 & 1490.36 & 1490.897 & 1484.511 & 1488.262 & 1493.01 & 1493.535 & 1492.771 & 1494.708 & 1492.741 & 1485.128 & 1478.662 \\ 1493807 & 1494.512 & 1494.532 & 1495213 & 1496554 & 1496354 & 1492.763 & 1489.029 & 1492598 & 1490355 & 1490895 & 1484.506 & 1488.276 & 1493.001 & 1493.511 & 1492.764 & 1494.682 & 1492.741 & 1485.123 & 1478.662\end{array}$ 
Well

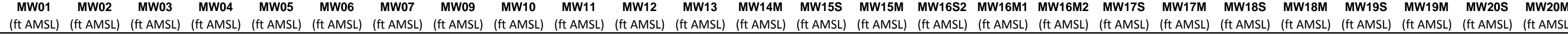

$12 / 5 / 118: 00$ 12/5/11 9:00 12/5/11 10:00 12/5/11112:00 12/5/11112:00 $12 / 5 / 1114: 00$ 12/5/11 15:00 12/5/11 16:00 12/5/11 17:00 12/5/11 18:00 12/5/11 20:00 12/5/11120:00 $21: 00$ 2/5/5/11 22:00 12/5/111 23:00 12/6/11 0:00 12/6/111:00 12/6/11 2:00 12/6/11 4:00 12/6/11 5:00 $12 / 6 / 116: 00$ 12/6/11 8:00 12/6/11 9:00 12/6/11 10:00 12/6/1111:00 $12 / 6 / 1112: 00$ 12/6/11 14:00 12/6/11 15:00 12/6/11 16:00 12/6/11 17:00 12/6/11 18:00 12/6/11 19:00 12/6/1120:00 12/6/11 21:00 $12 / 6 / 1123: 00$ 12/7/11 0:00 12/7/111:00 12/7/112:00 12/7/11 3:00 12/7/1114:00 12/7/11 6:00 12/7/1117:00 12/7/11 9:00 12/7/111 10:00 12/7/11 11:00 12/7/11 12:00 $\begin{array}{llll}1494.281 & 1494.627 & 1496.139 & 1494.615\end{array}$ $\begin{array}{llll}1494.279 & 1494.627 & 1496.149 & 1494.612 \\ 1494.277 & 1494.627 & 1496.149 & 1494.612\end{array}$ $\begin{array}{llll}1494.277 & 1494.627 & 1496.149 & 1494.612 \\ 1494.277 & 1994.625 & 1496151 & 1494612\end{array}$ $\begin{array}{llll}1494.284 & 1494.637 & 1496.177 & 1494.624\end{array}$ $\begin{array}{lllll}1494.291 & 1494.655 & 1496214 & 1494.652\end{array}$ $\begin{array}{llll}1494.291 & 1494.655 & 1496.214 & 1494.652 \\ 1494.295 & 1494.672 & 1496.242 & 1494.675\end{array}$ $\begin{array}{lllll}1494.3 & 1494.688 & 1496.273 & 1494.703\end{array}$ $\begin{array}{llll}1494.293 & 1494.683 & 1496.263 & 1494.71\end{array}$ $\begin{array}{llll}1494.288 & 1494.679 & 1496.242 & 1494.701\end{array}$ $\begin{array}{llll}1494.286 & 1494.676 & 1496.231 & 1494.699 \\ 1494281 & 1494665 & 1496207 & 1994.685\end{array}$ $\begin{array}{llll}1494.281 & 1494.665 & 1496.207 & 1494.685 \\ 1494.277 & 1494.655 & 1496.188 & 1494.671\end{array}$ $\begin{array}{lllll}1494.274 & 1494.648 & 1496.181 & 1494.659\end{array}$ $\begin{array}{lllll}1494.274 & 1494.651 & 1496.179 & 1494.654\end{array}$ $\begin{array}{llllll}1494.274 & 1494.651 & 1496.188 & 1494.659\end{array}$ $\begin{array}{lllll}1494.277 & 1494.66 & 1496.207 & 1494.668\end{array}$ $\begin{array}{llll}1494.281 & 1494.665 & 1496.221 & 1494.68\end{array}$ 1494.2861494 .6791496 .245 1494.699 $\begin{array}{lllll}1494.284 & 1494.681 & 1496.249 & 1494.706\end{array}$ $\begin{array}{lllll}1444.291 & 1494.7 & 1496.277 & 1494.731\end{array}$ $\begin{array}{lllll}1494.288 & 1494.7 & 1496.275 & 1494.736\end{array}$ $\begin{array}{lllll}1494.286 & 1494.695 & 1496.261 & 1494.734\end{array}$ $\begin{array}{llllll}1494.284 & 1494.695 & 1496.259 & 1494.729\end{array}$ $\begin{array}{lllll}1494.286 & 1494.695 & 1496.263 & 1494.731\end{array}$ $\begin{array}{lllll}1494.286 & 1494.695 & 1496.261 & 1494.731\end{array}$ $\begin{array}{lllll}1494.293 & 1494.704 & 1496.277 & 1494.738\end{array}$ $\begin{array}{rrrr}1494.3 & 1494.723 & 1496.31 & 1494.759 \\ 1494.305 & 1494.735 & 1496.338 & 1494.78\end{array}$ $\begin{array}{lllll}1494.312 & 1494.748 & 1496.352 & 1494.797\end{array}$ $\begin{array}{lllll}1494.314 & 1494.762 & 1496.371 & 1494.815\end{array}$ $\begin{array}{lllll}1494.319 & 1494.769 & 1496.382 & 1494.827\end{array}$ $\begin{array}{llllll}1494.319 & 1494.776 & 1496.382 & 1494.836\end{array}$ $\begin{array}{lllll}1494.314 & 1494.769 & 1496.361 & 1494.829\end{array}$ $\begin{array}{lllll}1494.316 & 1494.767 & 1496.357 & 1494.825\end{array}$ $\begin{array}{rrrr}1494.321 & 1494.769 & 1496.359 & 1494.822 \\ 1494319 & 1494.769 & 1496359 & 1494.82\end{array}$ $\begin{array}{llll}1494.321 & 1494.772 & 1496.361 & 1494.818\end{array}$ $\begin{array}{lllll}1494.328 & 1494.779 & 1496.375 & 1494.825\end{array}$ $\begin{array}{lllll}1494.33 & 1494.783 & 1496.385 & 1494.832\end{array}$ $\begin{array}{lllll}1494.33 & 1494.783 & 1496.385 & 1494.834\end{array}$ $\begin{array}{lllll}1494.337 & 1494.797 & 1496.408 & 1494.848\end{array}$ $\begin{array}{llll}1494.34 & 1494.8 & 1496.411 & 1494.853\end{array}$ $\begin{array}{llll}1494.346 & 1494.814 & 1496.429 & 1494.867\end{array}$ $\begin{array}{lllll}1494.349 & 1494.825 & 1466.434 & 1494.874\end{array}$ $1494.349 \begin{array}{lllll}1494.828 & 1496.441 & 1494.888\end{array}$ $\begin{array}{lllll}1494.353 & 1494.83 & 1496.446 & 1494.89\end{array}$ $\begin{array}{lllll}1494.351 & 1494.825 & 1496.427 & 1494.881\end{array}$ $\begin{array}{llllll}1494.344 & 1494.816 & 1496.404 & 1494.869\end{array}$ $\begin{array}{lllll}1494.349 & 1494.811 & 1496.401 & 1494.86\end{array}$ $\begin{array}{llll}1494.356 & 1494.818 & 1496.418 & 1494.862 \\ 1494.363 & 1494.828 & 1496.436 & 1494.874\end{array}$ $\begin{array}{lrlllllllllllllllllll}1493.8 & 1494.5 & 1494.534 & 1495.197 & 1496.54 & 1496.342 & 1492.751 & 1489.027 & 1492.591 & 1490.353 & 1490.89 & 1484.504 & 1488.267 & 1492.999 & 1493.504 & 1492.752 & 1494.668 & 1492.73 & 1485.123 & 1478.657 \\ 1493.781 & 1494.88 & 1494532 & 1495.199 & 1496538 & 1496351 & 1492751 & 1489026 & 1492589 & 1490353 & 1490888 & 1484504 & 1488252 & 1492.992 & 1493504 & 1492755 & 1494678 & 1492.73 & 1485.123 & 1478657\end{array}$

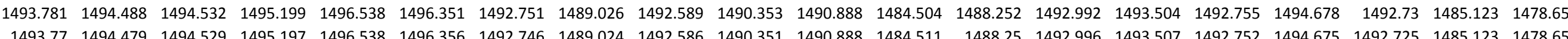

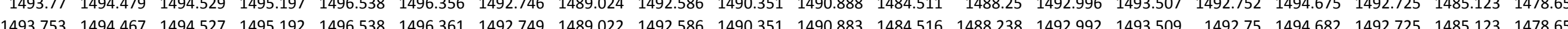
$\begin{array}{lllllllllllllllllllllll}1493.73 & 1494.462 & 1494.534 & 1495.199 & 1496.545 & 1496.385 & 1492.751 & 1489.026 & 1492.586 & 1490.349 & 1490.886 & 1484.523 & 1488215 & 1492994 & 1493.532 & 1492.755 & 1494.697 & 1492.727 & 1485.123 & 1478.659\end{array}$

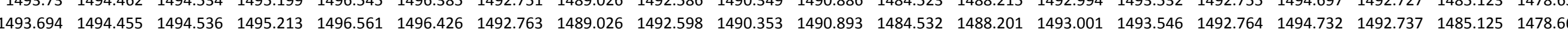
$\begin{array}{lllllllllllllllllllllll}1493.678 & 1494.447 & 1494.536 & 1495.227 & 1496.577 & 1496.454 & 1492.772 & 1489.038 & 1492.608 & 1490.358 & 1490.897 & 1484.532 & 1488.189 & 1493.01 & 1493.567 & 1492.771 & 1494.758 & 1492.748 & 1485.123 & 1478.666\end{array}$ $\begin{array}{llllllllllllllllllllll}1493.662 & 1494.445 & 1494.539 & 1495.243 & 1496.594 & 1496.481 & 1492.786 & 1489.05 & 1492.617 & 1490.362 & 1490.9 & 1484.539 & 1488.187 & 1493.024 & 1493.593 & 1492.785 & 1494.784 & 1492.76 & 1485.123 & 1478.666\end{array}$

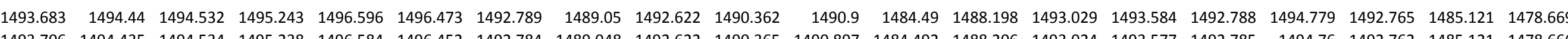

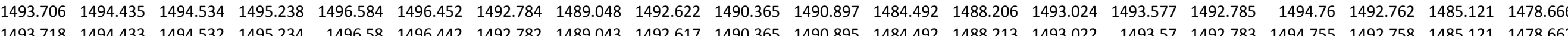
$\begin{array}{llllllllllllllllllll}1493.718 & 1494.433 & 1494.532 & 1495.234 & 1496.58 & 1496.442 & 1492.782 & 1489.043 & 1492.617 & 1490.365 & 1490.895 & 1484.492 & 1488.213 & 1493.022 & 1493.57 & 1492.783 & 1494.755 & 1492.758 & 1485.121 & 1478.662 \\ 1493.734 & 1494.424 & 1494.529 & 1495.227 & 149657 & 1496418 & 1492777 & 1489.043 & 1492615 & 1490365 & 1490893 & 1484.492 & 1488215 & 1493.015 & 1493558 & 1492778 & 1494737 & 1492.753 & 1485.118 & 1478659\end{array}$

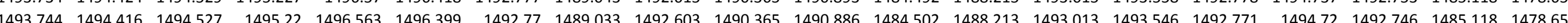
$\begin{array}{lllllllllllllllllllllll}1493.744 & 1494.409 & 1494.527 & 1495213 & 1496556 & 1496394 & 1492.763 & 1489.024 & 1492601 & 1490365 & 1490883 & 1484.497 & 1488.215 & 1493.003 & 1493.535 & 1492767 & 1494.704 & 1492.739 & 1485.118 & 1478.655\end{array}$ $\begin{array}{lllllllllllllllllllllll}1493.739 & 1494.407 & 1494.525 & 1495.213 & 1496.554 & 1496.392 & 1492.763 & 1489.019 & 1492.601 & 1490.367 & 1490.883 & 1484.499 & 1488.206 & 1493.006 & 1493.539 & 1492.762 & 1494.713 & 1492.737 & 1485.121 & 1478.652\end{array}$

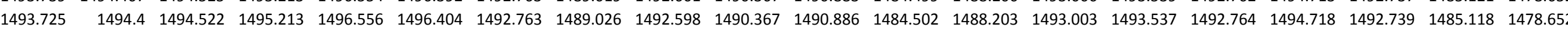
$\begin{array}{llllllllllllllllllllll}1493.713 & 1494.397 & 1494.527 & 1495.22 & 1496.563 & 1496.421 & 1492.767 & 1489.022 & 1492.603 & 1490.369 & 1490.888 & 1484.502 & 1488.198 & 1493.01 & 1493.553 & 1492.769 & 1494.734 & 1492.744 & 1485.118 & 1478.6559\end{array}$

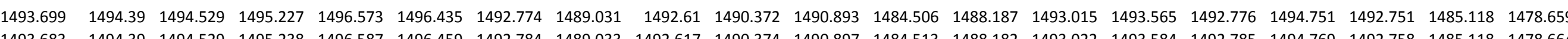

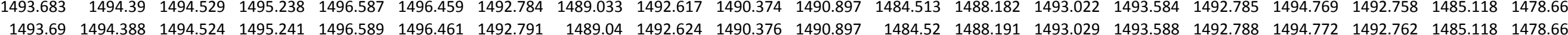

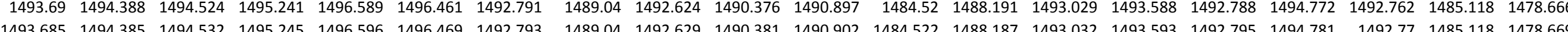
$\begin{array}{llllllllllllllllllllllll}1493.678 & 1494.383 & 1494.529 & 1495.257 & 1496.608 & 1496.488 & 1492.803 & 1489.048 & 1492.633 & 1490.386 & 1490.907 & 1484.527 & 1488.182 & 1493.041 & 1493.609 & 1492.804 & 1494.795 & 1492.777 & 1485.118 & 1478.6696\end{array}$ $\begin{array}{llllllllllllllllllll}1493.69 & 1494383 & 1494532 & 1495262 & 1496608 & 1496485 & 1492.805 & 1489.05 & 1492.638 & 1490.388 & 1490.907 & 1484.532 & 1488189 & 1493.046 & 1493607 & 1492.804 & 1494.798 & 1492.779 & 1485116 & 1478671\end{array}$ $\begin{array}{llllllllllllllllllllll}1493.706 & 1494.378 & 1494.529 & 1495.259 & 1496.605 & 1496.469 & 1492.803 & 1489.043 & 1492.638 & 1490.388 & 1490.902 & 1484.536 & 1488.201 & 1493.043 & 1493.602 & 1492.804 & 1494.786 & 1492.779 & 1485.116 & 1478.671 \\ \end{array}$

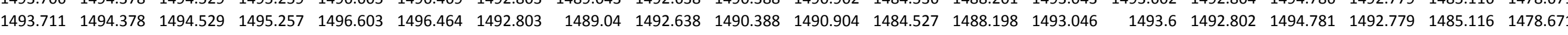

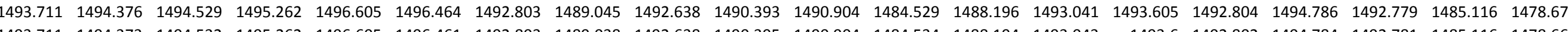

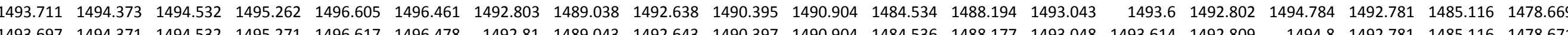

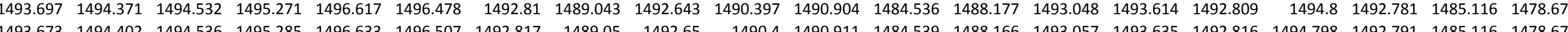
$\begin{array}{llllllllllllllllllll}1493.673 & 1494.402 & 1494.536 & 1495.285 & 1496.63 & 1496.507 & 1492.817 & 1489.05 & 1492.65 & 1490.4 & 490.911 & 1484.539 & 1488.166 & 1493.057 & 1493.635 & 1492.816 & 1494.798 & 1492.791 & 1485.116 & 1478.676 \\ 1493.666 & 1494.373 & 1494.539 & 1495301 & 1496.647 & 1496533 & 1492826 & 1489.059 & 1492.659 & 1490.404 & 1490.916 & 1484.546 & 1488.163 & 1493.069 & 1493.651 & 1492.828 & 1494.758 & 1492.802 & 1485.116 & 1478678\end{array}$

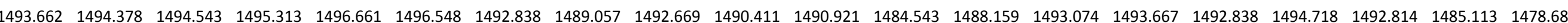
$\begin{array}{llllllllllllllllllllll}1493.666 & 1494.378 & 1494.544 & 1495.327 & 1496.673 & 1496.56 & 1492.845 & 1489.071 & 1492.678 & 1490.414 & 1490.923 & 1484.55 & 1488.163 & 1493.083 & 1493.679 & 1492.847 & 1494.882 & 1492.821 & 1485.113 & 1478.685\end{array}$

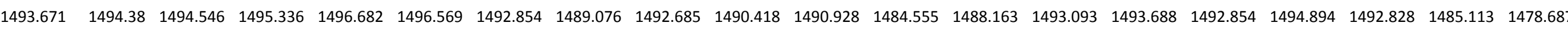
$\begin{array}{lllllllllllllllllllll}1493.687 & 1494.383 & 1494.546 & 1495.343 & 1496.687 & 1496.567 & 1492.859 & 1489.076 & 1492.69 & 1490.423 & 1490.928 & 1484.555 & 1488.175 & 1493.097 & 1493.688 & 1492.859 & 1494.892 & 1492.833 & 1485.113 & 1478.687\end{array}$

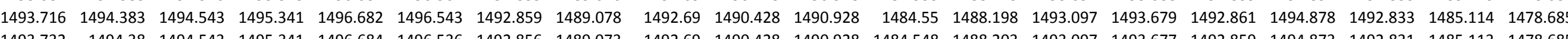
$\begin{array}{llllllllllllllllllllllll}1493.732 & 1494.38 & 1494.543 & 1495.341 & 1496.684 & 1496.536 & 1492.856 & 1489.073 & 1492.69 & 1490.428 & 1490.928 & 1484.548 & 1488.203 & 1493.097 & 1493.677 & 1492.859 & 1494.878 & 1492.831 & 1485.113 & 1478.685 \\ 1493.737 & 149438 & 1494.548 & 1495343 & 1496684 & 1496536 & 1492856 & 1489069 & 1492.687 & 1490.43 & 1490926 & 1484548 & 1488208 & 1493102 & 1493674 & 1492854 & 1494868 & 1492831 & 1485111 & 1478683\end{array}$

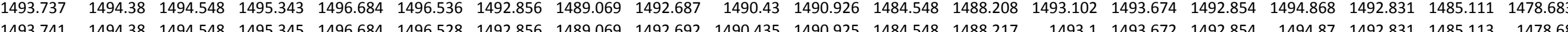

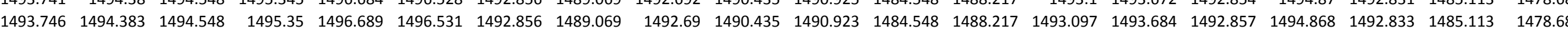
$\begin{array}{lllllllllllllllllllllll}1493.739 & 1494.383 & 1494.551 & 1495.359 & 1496.696 & 1496.545 & 1492.861 & 1489.066 & 1492.694 & 1490.439 & 1490.928 & 1484.552 & 1488.213 & 1493.1 & 1493.681 & 1492.861 & 1494.88 & 1492.833 & 1485.113 & 1478.68\end{array}$

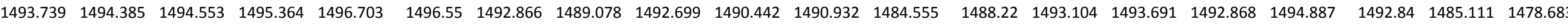
\begin{tabular}{llllllllllllllllllllll}
1493.744 & 1494.385 & 1494.555 & 1495.368 & 1496.705 & 1496.55 & 1492.87 & 1489.076 & 1492.704 & 1490.444 & 1490.935 & 1484.56 & 1488.224 & 1493.114 & 1493.693 & 1492.873 & 1494.889 & 1492.845 & 1485.111 & 1478.685 \\
\hline
\end{tabular} $\begin{array}{lllllllllllllllllllll}1493.734 & 1494.39 & 1494.555 & 1495.378 & 1496.719 & 1496.569 & 1492.88 & 1489.073 & 1492.711 & 1490.446 & 1490.944 & 1484.564 & 1488.224 & 1493.116 & 1493.705 & 1492.883 & 1494.91 & 1492.854 & 1485.111 & 1478.69\end{array}$

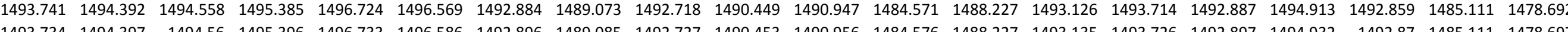

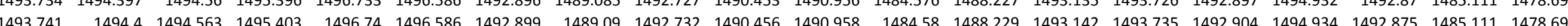

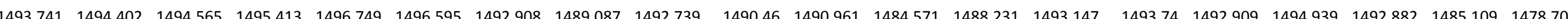
$\begin{array}{llllllllllllllllllllll}1493.755 & 1494.404 & 1494.563 & 1495.415 & 1496.752 & 1496.591 & 1492.908 & 1489.083 & 1492.741 & 1490.463 & 1490.961 & 1484.569 & 1488.243 & 1493.151 & 1493.737 & 1492.911 & 1494.936 & 1492.884 & 1485.109 & 1478.701\end{array}$

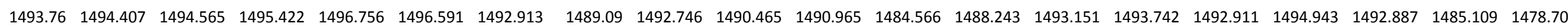

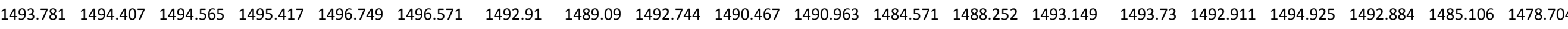
$\begin{array}{lllllllllllllllllllll}1493.8 & 1494.404 & 1494.563 & 1495.41 & 1496.74 & 1496.543 & 1492.903 & 1489.083 & 1492.739 & 1490.467 & 1490.961 & 1484.571 & 1488.264 & 1493.144 & 1493.714 & 1492.906 & 1494.901 & 1492.877 & 1485.106 & 1478.699 & \end{array}$ $\begin{array}{lllllllllllllllllllll}1493.8 & 1494.404 & 1494.565 & 1495.41 & 1496.735 & 1496.543 & 1492.903 & 1489.083 & 1492.737 & 1490.47 & 1490.961 & 1484.571 & 1488.257 & 1493.147 & 1493.712 & 1492.904 & 1494.906 & 1492.877 & 1485.109 & 1478.699\end{array}$

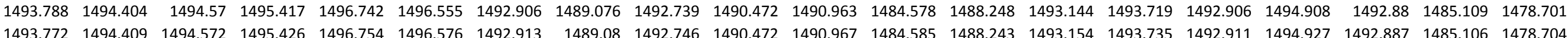


Well

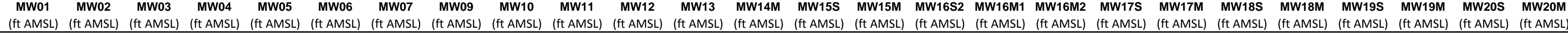

12/7/11 14:00 12/7/11 15:00 12/7/11 16:00 12/7/111117:00 12/7/11 18:00 12/7/11 20:00 12/7/1121:00 12/7/11122:00 12/7/11 23:00 12/8/11 0:00 2/8/111:00 12/8/1112:00 2/2/8/11 4:00 12/8/11 5:00 12/8/11 6:00 12/8/117:00 12/8/11 8:00 12/8/11 9:00 $12 / 8 / 1111: 00$ 12/8/1111:00 12/8/11 12:00 12/8/111 14:00 12/8/11 15:00 12/8/111 16:00 $12 / 8 / 1117: 00$ $12 / 8 / 1118: 00$ $12 / 8 / 1120: 00$ $12 / 8 / 1121: 00$ $12 / 8 / 1121: 00$
$12 / 8 / 1122: 00$ 12/8/11 23:00 12/9/11 0:00 2/9/111:00 12/9/11 2:00 12/9/11 3:00 12/9/11 5:00 12/9/11 6:00 12/9/11 7:00 12/9/11 8:00 12/9/11 9:00 12/9/11 10:00 12/9/1112.00 12/9/11 12:00 12/9/11 13:00 12/9/11 15:00 12/9/11 16:00 12/9/11 17:00 12/9/11 18:00 $\begin{array}{llll}1494.365 & 1494.832 & 1496.446 & 1494.878\end{array}$ $\begin{array}{lllll}1494.363 & 1494.832 & 1496.446 & 1494.881\end{array}$ $\begin{array}{lllll}1494.361 & 1494.828 & 1496.432 & 1494.876 \\ 1494.361 & 1494.825 & 1496.42 & 1494.871\end{array}$ $\begin{array}{lllll}1494.363 & 1494.825 & 1496.422 & 1494.869\end{array}$ $\begin{array}{lllll}1494.356 & 1494.809 & 1496.394 & 1494.848\end{array}$ $\begin{array}{llllll}1494.358 & 1494.804 & 1496.387 & 1494.836\end{array}$ $\begin{array}{lllll}1494.365 & 1494.811 & 1496.406 & 1494.841\end{array}$ $\begin{array}{lllll}1494.36 & 1494.807 & 1496.392 & 1494.829\end{array}$ $1494.361 \quad 1494.802 \quad 1496.387 \quad 1494.827$ $\begin{array}{llll}1494.361 & 1494.802 & 1496.389 & 1494.827 \\ 1494.363 & 1494.804 & 1496394 & 1494.827\end{array}$ $\begin{array}{llll}1494.363 & 1494.804 & 1496.394 & 1494.827 \\ 1494.367 & 1494.809 & 1496.406 & 1494.834\end{array}$ $\begin{array}{lllll}1494.37 & 1494.809 & 1496.411 & 1494.839\end{array}$ $\begin{array}{rrrrr}1494.37 & 1494.809 & 1496.411 & 1494.839 \\ 1494.368 & 1494.811 & 1496.408 & 1494.839\end{array}$ $\begin{array}{lllll}1494.365 & 1494.807 & 1496.397 & 1494.834\end{array}$ $\begin{array}{lllll}1494.363 & 1494.802 & 1496.392 & 1494.829\end{array}$ $\begin{array}{lllll}1494.365 & 1494.8 & 1496.385 & 1494.825\end{array}$ $\begin{array}{lllll}1494.367 & 1494.8 & 1496.387 & 1494.822\end{array}$ $\begin{array}{rrrr}1494.363 & 1494.795 & 1496.378 & 1494.818 \\ 1494363 & 149479 & 1496368 & 1494811\end{array}$ $\begin{array}{rrrrr}1494.363 & 1494.79 & 1496.368 & 1494.811 \\ 1494.37 & 1494.795 & 1496.382 & 1494.813\end{array}$ $\begin{array}{lllll}1494.377 & 1494.807 & 1496.408 & 1494.827\end{array}$ $\begin{array}{lllll}1494.384 & 1494.821 & 1496.436 & 1494.848\end{array}$ $\begin{array}{lllll}1494.386 & 1494.832 & 1496.453 & 1494.862\end{array}$ $\begin{array}{lllll}1494.384 & 1494.835 & 1496.453 & 1494.871\end{array}$ $\begin{array}{llll}1494.382 & 1494.832 & 1496.441 & 1494.871\end{array}$ $\begin{array}{lllll}1494.379 & 1494.823 & 1496.422 & 1494.864\end{array}$ $\begin{array}{rrrrr}1494.379 & 1494.821 & 1496.415 & 1494.855 \\ 1494.379 & 1494.818 & 1496.415 & 1494.85\end{array}$ $\begin{array}{lllll}1494.372 & 1494.804 & 1496.38 & 1494.829\end{array}$ $\begin{array}{llll}1494.37 & 1494.795 & 1496.366 & 1494.813\end{array}$ $\begin{array}{rrrr}1494.367 & 1494.786 & 1496.35 & 1494.799\end{array}$ $\begin{array}{lllll}1494.363 & 1494.774 & 1496.336 & 1494.783\end{array}$ $1494.368 \quad 1494.772 \quad 1496.3361494 .773$ $\begin{array}{lllll}1494.365 & 1494.767 & 1496.333 & 1494.769\end{array}$ $\begin{array}{llll}1494.365 & 1494.767 & 1496.331 & 1494.764 \\ 194.356 & 1494.753 & 1996308 & 1994.752\end{array}$

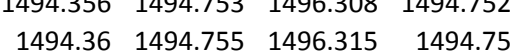
$\begin{array}{lllll}1494.358 & 1494.751 & 1496.308 & 1494.745\end{array}$ $\begin{array}{lllll}1494.353 & 1494.746 & 1496.303 & 1494.741\end{array}$ $\begin{array}{lllll}1494.344 & 1494.728 & 1496.27 & 1494.722\end{array}$ $\begin{array}{lllll}1494.337 & 1494.711 & 1496.245 & 1494.703\end{array}$ $\begin{array}{llll}1494.34 & 1494.707 & 1496.238 & 1494.692\end{array}$ $\begin{array}{llll}1494.33 & 1494.695 & 1496.219 & 1494.675 \\ 149433 & 194.686 & 196217 & 194.666\end{array}$ $\begin{array}{lllll}1494.33 & 1494.686 & 1496.217 & 1494.666 \\ 1494.333 & 1494.686 & 1496219 & 1494.666\end{array}$ $\begin{array}{lllll}1494.333 & 1494.688 & 1496231 & 1494.668\end{array}$ $\begin{array}{lllll}1494.333 & 1494.693 & 1496.238 & 1494.675\end{array}$ $\begin{array}{llllll}1494.333 & 1494.697 & 1496.247 & 1494.68\end{array}$ $\begin{array}{llll}1494.328 & 1494.69 & 1496.233 & 1494.675\end{array}$ $\begin{array}{lllll}1494.321 & 1494.686 & 1496.221 & 1494.673\end{array}$ $\begin{array}{llll}1494.321 & 1494.681 & 1496.214 & 1494.668 \\ 1494.316 & 1494.676 & 1496.207 & 1494.661\end{array}$

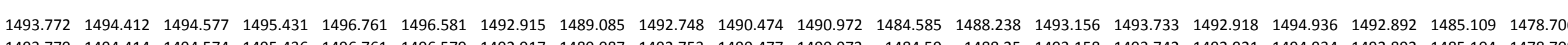
$\begin{array}{lllllllllllllllllllll}1493.779 & 1494.414 & 1494.574 & 1495.436 & 1496.761 & 1496.579 & 1492.917 & 1489.087 & 1492.753 & 1490.477 & 1490.972 & 1484.59 & 1488.25 & 1493.158 & 1493.742 & 1492.921 & 1494.934 & 1492.892 & 1485.104 & 1478.708 \\ 1493.793 & 1494.414 & 1494.572 & 1495.433 & 1496.754 & 1496567 & 1492.917 & 1489.083 & 1492751 & 1490.477 & 1490.974 & 1484.536 & 1488.26 & 1493.161 & 1493.733 & 1492.921 & 1494.922 & 1492892 & 1485.106 & 1478708\end{array}$

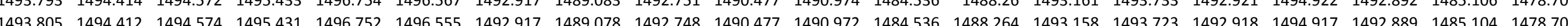
$\begin{array}{lllllllllllllllllllll}1493.807 & 1494.414 & 1494.579 & 1495.431 & 1496.752 & 1496.555 & 1492.915 & 1489.08 & 1492.748 & 1490.479 & 1490.975 & 1484.527 & 1488.264 & 1493.158 & 1493.726 & 1492.916 & 1494.908 & 1492.889 & 1485.106 & 1478.708\end{array}$ \begin{tabular}{lllllllllllllllllllllll}
1493.833 & 1494.412 & 1494.574 & 1495.419 & 1496.738 & 1496.526 & 1492.906 & 1489.076 & 1492.739 & 1490.477 & 1490.968 & 1484.509 & 1488.281 & 1493.151 & 1493.705 & 1492.906 & 1494.887 & 1492.88 & 1485.104 & 1478.704 \\
\hline
\end{tabular} $\begin{array}{lllllllllllllllllllllll}1493.835 & 1494.407 & 1494.579 & 1495.417 & 1496.733 & 1496.516 & 1492.901 & 1489.069 & 1492.737 & 1490.477 & 1490.963 & 1484.509 & 1488.283 & 1493.144 & 1493.7 & 1492.902 & 1494.88 & 1492.875 & 1485.104 & 1478.699\end{array}$ $\begin{array}{llllllllllllllllllllll}1493.819 & 1494.409 & 1494.579 & 1495.422 & 1496.738 & 1496.536 & 1492.903 & 1489.066 & 1492.734 & 1490.477 & 1490.965 & 1484.513 & 1488.278 & 1493.147 & 1493.707 & 1492.902 & 1494.894 & 1492.875 & 1485.104 & 1478.6997\end{array}$

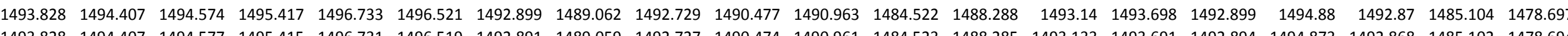

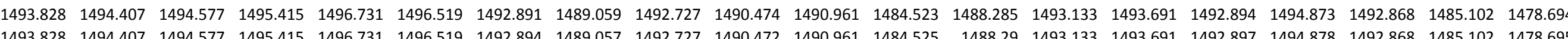

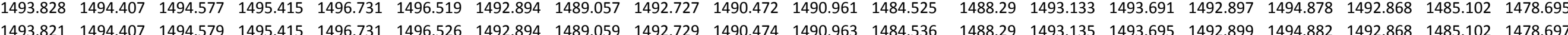

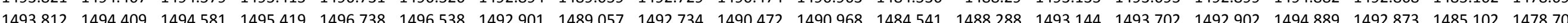
$\begin{array}{lllllllllllllllllllll}1493.807 & 1494.409 & 1494.582 & 1495422 & 149674 & 1496543 & 1492.906 & 1489057 & 1492.737 & 1490474 & 1490.972 & 1484.548 & 1488285 & 1493.144 & 1493705 & 1492.906 & 1494.892 & 149288 & 1485.102 & 1478706\end{array}$ $\begin{array}{lllllllllllllllllllll}1493.812 & 1494.409 & 1494.586 & 1495.422 & 1496.738 & 1496.54 & 1492.908 & 1489.059 & 1492.739 & 1490.474 & 1490.977 & 1484.555 & 1488.288 & 1493.147 & 1493.712 & 1492.906 & 1494.892 & 1492.88 & 1485.102 & 1478.708\end{array}$

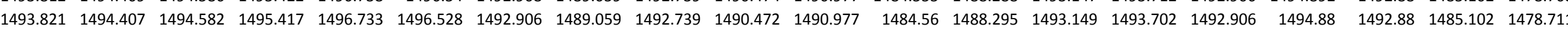
$\begin{array}{llllllllllllllllllllllll}1493.826 & 1494.404 & 1494.579 & 1495.417 & 1496.733 & 1496.524 & 1492.906 & 1489.055 & 1492.741 & 1490.474 & 1490.977 & 1484.55 & 1488.302 & 1493.149 & 1493.7 & 1492.906 & 1494.88 & 1492.88 & 1485.099 & 1478.711\end{array}$

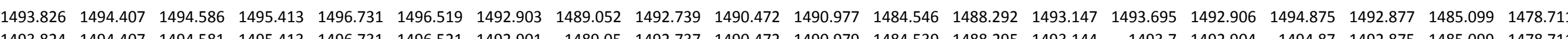

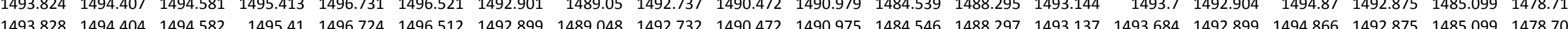
$\begin{array}{lllllllllllllllllllll}1493.828 & 1494.404 & 1494.582 & 1495.41 & 1496.724 & 1496.512 & 1492.899 & 1489.048 & 1492.732 & 1490.472 & 1490.975 & 1484.546 & 1488.297 & 1493.137 & 1493.684 & 1492.899 & 1494.866 & 1492.875 & 1485.099 & 1478.708 \\ 1493831 & 1494.402 & 1494.582 & 1495.403 & 1496719 & 1496504 & 1492894 & 1489.05 & 1492.727 & 1490.47 & 1490.97 & 1484546 & 1488295 & 1493 & 137 & 1493679 & 1492894 & 1494856 & 1492868 & 1485099 & 1478.706\end{array}$ $\begin{array}{llllllllllllllllllllll}1493.814 & 1494.402 & 1494.586 & 1495.408 & 1496.726 & 1496.516 & 1492.891 & 1489.043 & 1492.729 & 1490.467 & 1490.97 & 1484.55 & 1488.285 & 1493.135 & 1493.684 & 1492.894 & 1494.873 & 1492.868 & 1485.099 & 1478.706\end{array}$

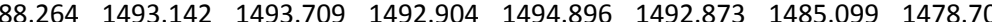
$\begin{array}{lllllllllllllllllllllll}1493.772 & 1494.409 & 1494.591 & 1495.429 & 1496.749 & 1496.569 & 1492.908 & 1489.052 & 1492.739 & 1490.472 & 1490.975 & 1484.559 & 1488.255 & 1493.149 & 1493.726 & 1492.909 & 1494.913 & 1492.882 & 1485.099 & 1478.711 \\ \end{array}$

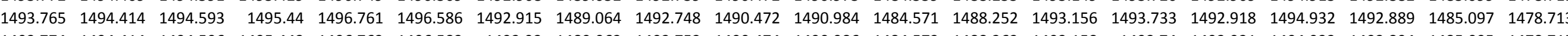

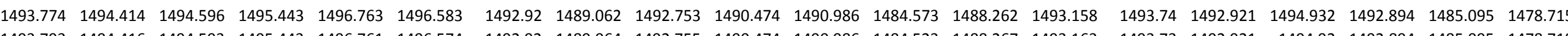

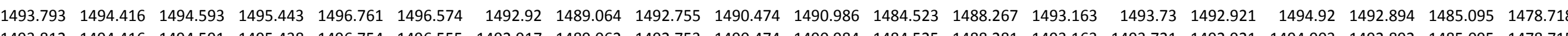

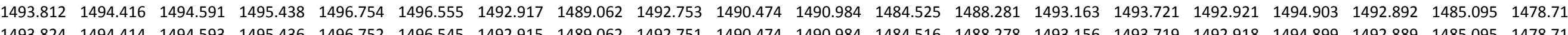

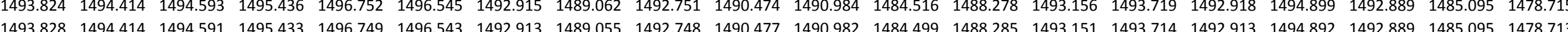
$\begin{array}{llllllllllllllllllll}1493.854 & 1494.412 & 1494.589 & 1495.422 & 1496.735 & 1496.514 & 1492.906 & 1489.05 & 1492.739 & 1490.472 & 1490.975 & 1484.502 & 1488.297 & 1493.149 & 1493.693 & 1492.902 & 1494.87 & 1492.877 & 1485.092 & 1478.708\end{array}$ $\begin{array}{llllllllllllllllllllll}1493.861 & 1494.409 & 1494.591 & 1495.413 & 1496.726 & 1496.497 & 1492.894 & 1489.045 & 1492.729 & 1490.47 & 1490.97 & 1484.504 & 1488.295 & 1493.14 & 1493.677 & 1492.897 & 1494.849 & 1492.868 & 1485.092 & 1478.704\end{array}$ $\begin{array}{lllllllllllllllllllll}1493.868 & 1494.402 & 1494.591 & 1495.406 & 1496.717 & 1496.481 & 1492.884 & 1489.033 & 1492.725 & 1490.467 & 1490.968 & 1484.502 & 1488.299 & 1493.13 & 1493.67 & 1492.89 & 1494.84 & 1492.861 & 1485.092 & 1478.701 \\ \end{array}$ $\begin{array}{llllllllllllllllllllll}1493.873 & 1494.402 & 1494.589 & 1495.394 & 1496.705 & 1496.469 & 1492.88 & 1489.024 & 1492.718 & 1490.465 & 1490.961 & 1484.504 & 1488.297 & 1493.123 & 1493.653 & 1492.878 & 1494.828 & 1492.852 & 1485.092 & 1478.6997 & \end{array}$

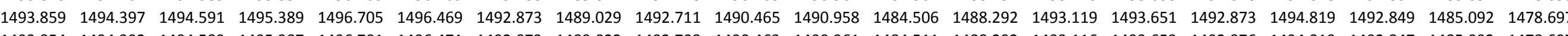

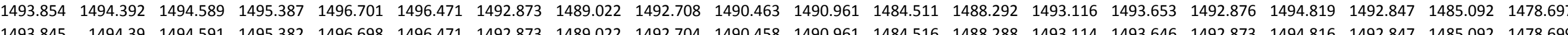

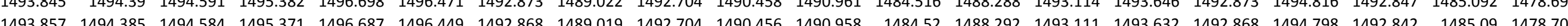
\begin{tabular}{llllllllllllllllllllllll}
1493.84 & 1494.385 & 1494.591 & 1495.368 & 1496.687 & 1496.461 & 1492.868 & 1489.019 & 1492.701 & 1490.453 & 1490.963 & 1484.529 & 1488.281 & 1493.109 & 1493.637 & 1492.868 & 1494.805 & 1492.84 & 1485.09 & 1478.704 \\
\hline
\end{tabular} $\begin{array}{lllllllllllllllllllllll}1493.838 & 1494.38 & 1494.586 & 1495.366 & 1496.68 & 1496.454 & 1492.866 & 1489.022 & 1492.704 & 1490.451 & 1490.963 & 1484.529 & 1488.278 & 1493.109 & 1493.632 & 1492.866 & 1494.8 & 1492.84 & 1485.09 & 1478.706\end{array}$

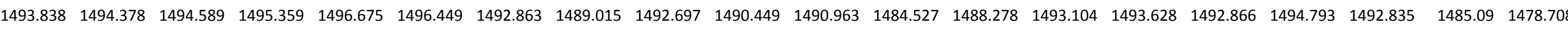

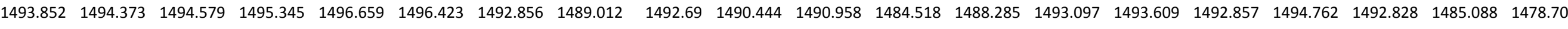
\begin{tabular}{llllllllllllllllllllll}
1493.859 & 1494.371 & 1494.579 & 1495.327 & 1496.642 & 1496.399 & 1492.845 & 1489.01 & 1492.68 & 1490.439 & 1490.951 & 1484.516 & 1488.283 & 1493.088 & 1493.593 & 1492.845 & 1494.744 & 1492.819 & 1485.088 & 1478.704 \\
\hline
\end{tabular}

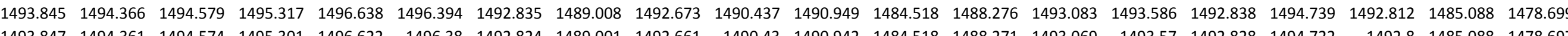

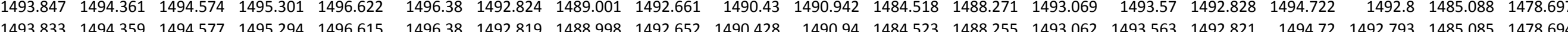

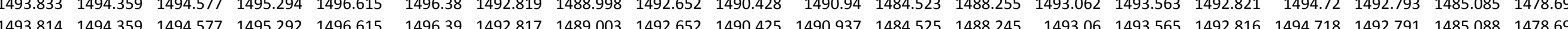
$\begin{array}{lllllllllllllllllllll}1493.795 & 1494.357 & 1494.579 & 1495.292 & 1496.617 & 1496.402 & 1492.812 & 1489.001 & 1492.647 & 1490.421 & 1490.935 & 1484.527 & 1488.234 & 1493.055 & 1493.572 & 1492.814 & 1494.727 & 1492.788 & 1485.085 & 1478.694\end{array}$ $\begin{array}{lllllllllllllllllllllll}1493.781 & 1494.354 & 1494.574 & 1495.292 & 1496.619 & 1496.414 & 1492.814 & 1489.001 & 1492.652 & 1490.418 & 1490.933 & 1484.536 & 1488.224 & 1493.055 & 1493.584 & 1492.814 & 1494.741 & 1492.786 & 1485.085 & 1478.694 \\ \end{array}$

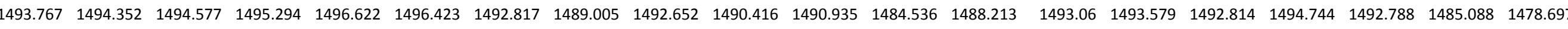

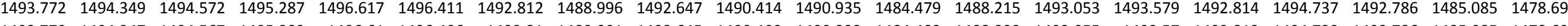

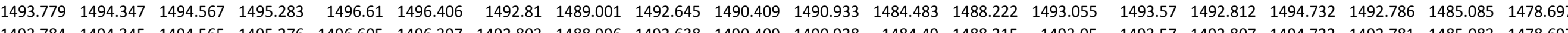

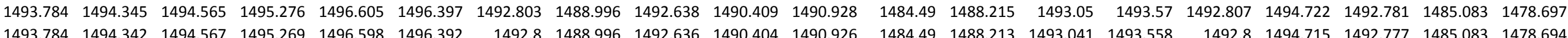


Well

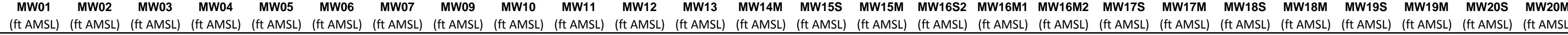

12/9/11 20:00 12/9/11 21:00 $12 / 9 / 1123.00$ 12/10/11 0:00 $12 / 10 / 111: 00$ 12/10/11 2:00 12/10/11 3:00 $12 / 10 / 114: 00$ 12/10/11 5:00 12/10/11 6:00 $12 / 10 / 117: 00$
$12 / 10 / 118: 00$ $12 / 10 / 119: 00$ $12 / 10 / 1110: 00$ 2/10/11 11:00 12/10/11 12:00 12/10/11 13:00 12/10/11 14:00 $12 / 10 / 11$ 15:00 12/10/10/11 17:00 1/12/1118:00 12/10/11 19:00 $12 / 10 / 1120: 00$ 12/10/11 21:00 12/10/11 22:00 12/10/11 23:00 $12 / 11 / 110: 00$ 12/11/11 2:00 12/11/11 3:00 12/11/11 5:00 12/11/11 6:00 2/11/118:00 12/11/11 9:00 12/11/11 11:00 12/11/11 12:00 12/11/11 13:00 12/11/11 14:00 $12 / 11 / 1115: 00$ 12/11/111 16:00 $12 / 11 / 1117.00$ 12/11/111 18:00 12/11/11 19:00 12/11/11 21:00 12/11/11 22:00 12/12/11 0:00 $\begin{array}{llll}1494.316 & 1494.672 & 1496.205 & 1494.657\end{array}$ $\begin{array}{llll}1494.314 & 1494.672 & 1496.198 & 1494.652\end{array}$ $\begin{array}{lllll}1494.309 & 1494.667 & 1496.193 & 1494.647 \\ 1494.309 & 1494.667 & 1496.188 & 1494.645\end{array}$ $\begin{array}{llll}1494.307 & 1944.665 & 1496.193 & 1494.645\end{array}$ $\begin{array}{rrrrr}1494.305 & 1494.665 & 1496.191 & 1494.64\end{array}$ $\begin{array}{rrrrr}1494.307 & 1494.669 & 1496.2 & 1494.647\end{array}$ $\begin{array}{lllll}1494.305 & 1494.669 & 1496.2 & 1494.647\end{array}$ $\begin{array}{llll}1494.305 & 1494.674 & 1496.205 & 1494.654\end{array}$ $\begin{array}{llll}1494.309 & 1494.681 & 1496.224 & 1494.664\end{array}$ $\begin{array}{llll}1494.305 & 1494.681 & 1496.219 & 1494.671 \\ 1494.302 & 1494.679 & 1496219 & 1494671\end{array}$ $\begin{array}{lllll}1494.3 & 1494.676 & 1496.21 & 1944.666\end{array}$ $\begin{array}{lllll}1494.298 & 1494.676 & 1496.21 & 1944.666\end{array}$ $\begin{array}{lllll}1494.298 & 1494.672 & 1496.202 & 1494.664\end{array}$ $\begin{array}{lllll}1494.302 & 1494.676 & 1496.212 & 1494.666\end{array}$ $\begin{array}{lllll}1494.302 & 1494.681 & 1496.221 & 1494.671\end{array}$ $\begin{array}{lllll}1494.307 & 1494.695 & 1496.247 & 1494.687\end{array}$ $\begin{array}{llll}1494.312 & 1494.709 & 1496.273 & 1494.708\end{array}$ $\begin{array}{llll}1494.314 & 1494.718 & 1496.289 & 1494.722 \\ 1494312 & 1494.721 & 1496289 & 1494.729\end{array}$ $\begin{array}{lllll}1494.312 .314 & 1494.721 & 1496.723 & 1496.291 & 1494.729 \\ 1494.736\end{array}$ $\begin{array}{llllll}1494.314 & 1494.725 & 1496.289 & 1494.738\end{array}$ $\begin{array}{lllll}1494.316 & 1494.728 & 1496.294 & 1494.741\end{array}$ $\begin{array}{llllll}1494.316 & 1494.728 & 1496.294 & 1494.743\end{array}$ $\begin{array}{lllll}1494.316 & 1494.73 & 1496.296 & 1494.745\end{array}$ $\begin{array}{lllll}1494.319 & 1494.737 & 1496.305 & 1494.748\end{array}$ $\begin{array}{llll}1494.319 & 1494.737 & 1496.303 & 1494.75\end{array}$ $\begin{array}{lllll}1494.319 & 1494.739 & 1496.308 & 1494.752 \\ 1494.323 & 1494.744 & 1496.312 & 1494.757\end{array}$ $\begin{array}{lllll}1494.323 & 1494.748 & 1496.319 & 1494.764\end{array}$ $\begin{array}{llllll}1494.328 & 1494.753 & 1496331 & 1494.769\end{array}$ $\begin{array}{lllll}1494.333 & 1494.762 & 1496.345 & 1494.78\end{array}$ $\begin{array}{llllll}1494.337 & 1494.774 & 1496.366 & 1494.794\end{array}$ $\begin{array}{llll}1494.34 & 1494.781 & 1496.375 & 1494.806\end{array}$ $\begin{array}{llll}1494.344 & 1494.795 & 1496.389 & 1494.82 \\ 1494.344 & 1494.797 & 1469392 & 1498.827\end{array}$ $\begin{array}{llll}1494.344 & 1494.797 & 1496.392 & 1494.827\end{array}$ $\begin{array}{lllll}1494.344 & 1494.8 & 1494.3 & 149638 & 1494.832 \\ 1494827\end{array}$ $\begin{array}{rrrrr}1494.349 & 1494.8 & 1496.385 & 1494.827\end{array}$ $\begin{array}{lllll}1494.358 & 1494.811 & 1496.408 & 1494.841\end{array}$ $\begin{array}{llllll}1494.368 & 1494.832 & 1496.441 & 1494.862\end{array}$ $\begin{array}{lllll}1494.368 & 1494.839 & 1496.45 & 1494.874\end{array}$ $\begin{array}{llll}1494.37 & 1494.849 & 1496.46 & 1494.885\end{array}$ $\begin{array}{llll}1494.37 & 1494.851 & 1496.457 & 1494.89\end{array}$ $\begin{array}{lllll}1494.372 & 1494.851 & 1496.455 & 1494.892\end{array}$ $\begin{array}{lllll}1494.372 & 1494.851 & 1496.441 & 1494.885\end{array}$ $\begin{array}{lllll}1494.375 & 1494.853 & 1496.441 & 1494.883\end{array}$ $\begin{array}{lllll}1494.374 & 1494.849 & 1496.434 & 1494.878\end{array}$ $\begin{array}{lllll}1494.377 & 1494.846 & 1496.432 & 1494.874\end{array}$ $\begin{array}{llllll}1494.377 & 1494.846 & 1496.434 & 1494.871\end{array}$ $\begin{array}{llll}1494.379 & 1494.844 & 1496.427 & 1494.864 \\ 1494.384 & 1494.849 & 1496.436 & 1494.869\end{array}$

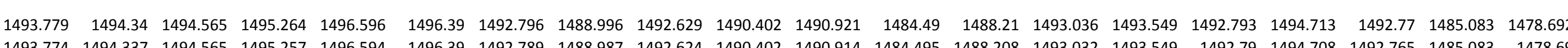

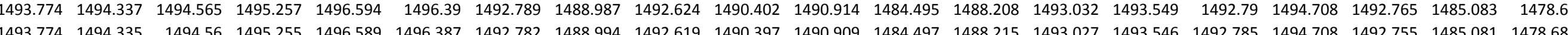
$\begin{array}{llllllllllllllllllll}1493767 & 1494335 & 1494563 & 149525 & 1496587 & 1496387 & 1492777 & 1488994 & 1492615 & 1490395 & 1490904 & 1484495 & 148821 & 1493022 & 1493539 & 1492778 & 1494692 & 1492753 & 1485081 & 1478683\end{array}$ $\begin{array}{lllllllllllllllllllllll}1493.76 & 1494.33 & 1494.558 & 1495248 & 1496587 & 149639 & 1492774 & 1489001 & 1492.612 & 1490.393 & 1490.902 & 1484.497 & 1488203 & 149302 & 1493.539 & 1492.776 & 1494.697 & 1492.751 & 1485081 & 1478.683\end{array}$ $\begin{array}{lllllllllllllllllllllll}1493.758 & 1494.325 & 1494.56 & 1495245 & 1496582 & 1496387 & 1492772 & 1488991 & 1492.608 & 1490.39 & 1490.9 & 1484.497 & 1488201 & 1493015 & 1493539 & 1492.774 & 1494.701 & 1492.748 & 1485081 & 1478.683\end{array}$

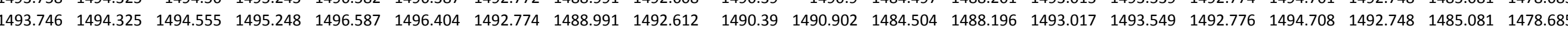
$\begin{array}{llllllllllllllllllllll}1493.739 & 1494.323 & 1494.56 & 1495.245 & 1496.587 & 1496.404 & 1492.774 & 1488.991 & 1492.615 & 1490.388 & 1490.904 & 1484.511 & 1488.191 & 1493.02 & 1493.546 & 1492.778 & 1494.715 & 1492.753 & 1485.081 & 1478.687\end{array}$

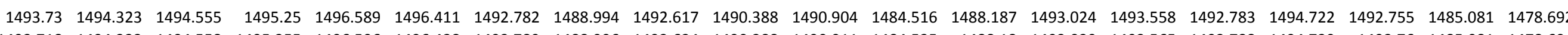

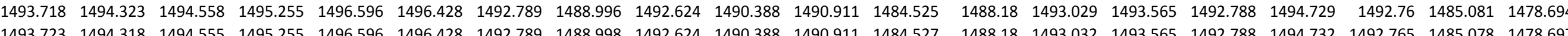
$\begin{array}{llllllllllllllllllll}1493.723 & 1494.318 & 1494.555 & 1495.255 & 1496.596 & 1496.428 & 1492.789 & 1488.998 & 1492.624 & 1490.388 & 1490.911 & 1484.527 & 1488.18 & 1493.032 & 1493.565 & 1492.788 & 1494.732 & 1492.765 & 1485.078 & 1478.697 \\ 1493.725 & 1494.318 & 1494.553 & 1495.252 & 1496.596 & 1496.426 & 1492.789 & 1488.998 & 1492.624 & 1490388 & 1490.911 & 1484.529 & 1488182 & 1493.032 & 1493.565 & 1492.788 & 1494729 & 1492.765 & 1485078 & 1478699\end{array}$ $\begin{array}{llllllllllllllllllllllll}1493.73 & 1494.318 & 1494.555 & 1495.25 & 1496591 & 1496416 & 1492.786 & 1488.994 & 1492.624 & 1490388 & 1490.909 & 1484.532 & 1488177 & 1493027 & 1493.565 & 1492.788 & 1494727 & 1492.762 & 1485078 & 1478699\end{array}$ $\begin{array}{llllllllllllllllllllllll}1493.73 & 1494.313 & 1494.553 & 1495.25 & 1496.591 & 1496.414 & 1492.786 & 1488.994 & 1492.619 & 1490.388 & 1490.909 & 1484.529 & 1488.177 & 1493.027 & 1493.558 & 1492.785 & 1494.725 & 1492.76 & 1485.078 & 1478.699\end{array}$ $\begin{array}{llllllllllllllllllllll}1493.73 & 1494.313 & 1494.548 & 1495.245 & 1496.587 & 1496.409 & 1492.784 & 1488.989 & 1492.617 & 1490.388 & 1490.907 & 1484.525 & 1488.17 & 1493.024 & 1493.553 & 1492.783 & 1494.715 & 1492.758 & 1485.076 & 1478.6994\end{array}$

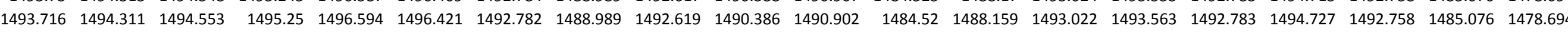
$\begin{array}{llllllllllllllllllllll}1493.709 & 1494.308 & 1494.551 & 1495.252 & 1496.596 & 1496.428 & 1492.784 & 1488.994 & 1492.615 & 1490.388 & 1490.902 & 1484.523 & 1488.152 & 1493.024 & 1493.565 & 1492.785 & 1494.729 & 1492.758 & 1485.076 & 1478.694\end{array}$

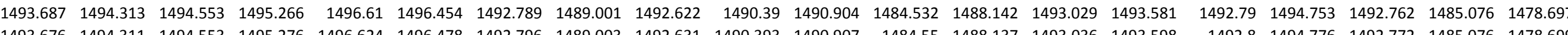

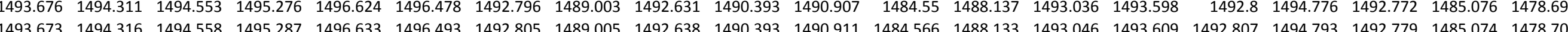

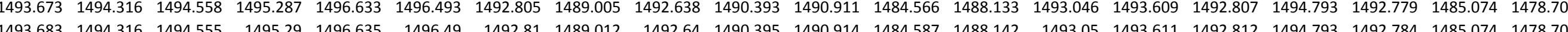
$\begin{array}{llllllllllllllllllllllll}1493.69 & 1494.316 & 1494.558 & 1495.294 & 1496.64 & 1496.49 & 1492.812 & 1489.017 & 1492.65 & 1490.397 & 1490.914 & 1484.61 & 1488.145 & 1493.055 & 1493.614 & 1492.814 & 1494.788 & 1492.786 & 1485.074 & 1478.706\end{array}$ $\begin{array}{llllllllllllllllllllll}1493.701 & 1494.316 & 1494.555 & 1495.301 & 1496.645 & 1496.49 & 1492.819 & 1489.019 & 1492.652 & 1490.402 & 1490.916 & 1484.65 & 1488.152 & 1493.06 & 1493.621 & 1492.819 & 1494.8 & 1492.793 & 1485.071 & 1478.708\end{array}$

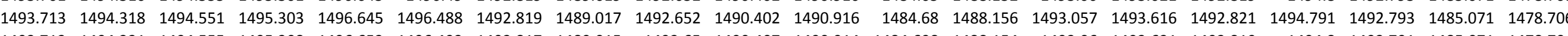
$\begin{array}{lllllllllllllllllllll}1493.713 & 1494.321 & 1494.555 & 1495.308 & 1496.652 & 1496.493 & 1492.817 & 1489.015 & 1492.65 & 1490.407 & 1490.914 & 1484.698 & 1488.154 & 1493.06 & 1493.621 & 1492.819 & 1494.8 & 1492.791 & 1485.071 & 1478.704\end{array}$

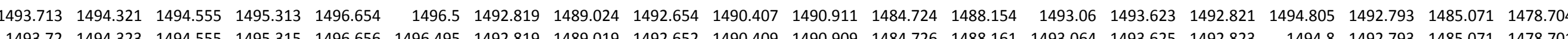

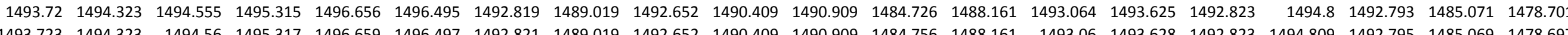
$\begin{array}{llllllllllllllllllll}1493.723 & 1494.323 & 1494.56 & 1495.317 & 1496.659 & 1496.497 & 1492.821 & 1489.019 & 1492.652 & 1490.409 & 1490.909 & 1484.756 & 1488.161 & 1493.06 & 1493.628 & 1492.823 & 1494.809 & 1492.795 & 1485.069 & 1478.697 \\ 1493.723 & 1494.325 & 1494.558 & 1495322 & 1496.663 & 1496502 & 1492824 & 1489019 & 1492659 & 1490.411 & 1490.909 & 1484.788 & 1488.159 & 1493064 & 1493625 & 1492826 & 1494.809 & 1492.798 & 1485.071 & 1478699\end{array}$ $\begin{array}{lllllllllllllllllllll}1493.725 & 1494.328 & 1494.567 & 1495.329 & 1496.668 & 1496.507 & 1492.828 & 1489.022 & 1492.661 & 1490.414 & 1490.911 & 1484.818 & 1488.161 & 1493.067 & 1493.639 & 1492.833 & 1494.814 & 1492.805 & 1485.069 & 1478.701\end{array}$ $\begin{array}{lllllllllllllllllllllll}1493.718 & 1494.328 & 1494.565 & 1495.334 & 1496.675 & 1496.514 & 1492.835 & 1489.022 & 1492.666 & 1490.416 & 1490.916 & 1484.851 & 1488.163 & 1493.079 & 1493.646 & 1492.84 & 1494.826 & 1492.809 & 1485.069 & 1478.704\end{array}$ $\begin{array}{llllllllllllllllllllll}1493.716 & 1494.33 & 1494.562 & 1495.343 & 1496.684 & 1496.526 & 1492.842 & 1489.024 & 1492.678 & 1490.418 & 1490.926 & 1484.883 & 1488.161 & 1493.086 & 1493.67 & 1492.849 & 1494.84 & 1492.819 & 1485.069 & 1478.711\end{array}$ $\begin{array}{llllllllllllllllllllll}1493.706 & 1494.335 & 1494.567 & 1495.354 & 1496.698 & 1496.548 & 1492.856 & 1489.031 & 1492.685 & 1490.421 & 1490.93 & 1444.916 & 1488.154 & 1493.093 & 1493.672 & 1492.857 & 1494.859 & 1492.828 & 1485.069 & 1478.71\end{array}$

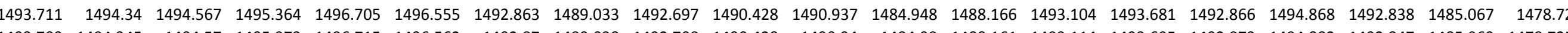

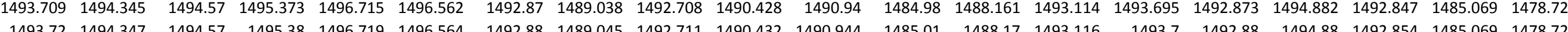

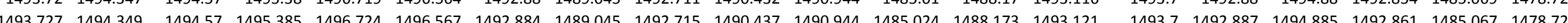

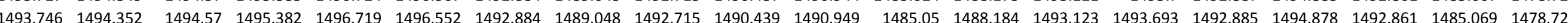
$\begin{array}{llllllllllllllllllllll}1493.748 & 1494.354 & 1494.574 & 1495.387 & 1496.722 & 1496.55 & 1492.887 & 1489.038 & 1492.718 & 1490.442 & 1490.947 & 1485.082 & 1488.18 & 1493.123 & 1493.695 & 1492.89 & 1494.885 & 1492.859 & 1485.067 & 1478.725\end{array}$ \begin{tabular}{lllllllllllllllllllll}
1493.737 & 1494.357 & 1494.579 & 1495.401 & 1496.738 & 1496.574 & 1492.894 & 1489.048 & 1492.727 & 1490.444 & 1490.951 & 1485.105 & 1488.17 & 1493.133 & 1493.716 & 1492.894 & 1494.899 & 1492.87 & 1485.067 & 1478.727 \\
\hline
\end{tabular} $\begin{array}{llllllllllllllllllll}1493.72 & 1494.364 & 1494.582 & 1495.417 & 1496.754 & 1496.6 & 1492.906 & 1489.05 & 1492.737 & 1490.451 & 1490.956 & 1485.135 & 1488.163 & 1493.142 & 1493.735 & 1492.906 & 1494.925 & 1492.88 & 1485.067 & 1478.729 \\ \end{array}$ \begin{tabular}{llllllllllllllllllll}
1493.727 & 1494.368 & 1494.584 & 1495.429 & 1496.763 & 1496.605 & 1492.91 & 1489.057 & 142.744 & 1490.451 & 1490.961 & 1485.163 & 1488.17 & 1493.151 & 1493.744 & 1492.913 & 1494.934 & 1492.889 & 1485.067 & 1478.732 \\
\hline
\end{tabular}

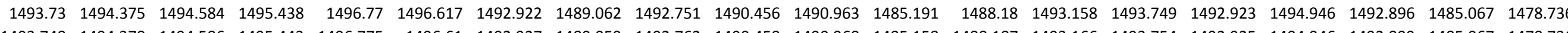

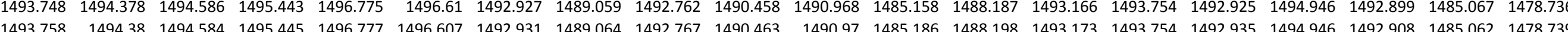

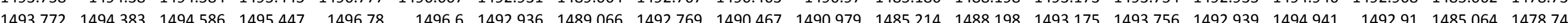
$\begin{array}{llllllllllllllllllll}1493.788 & 1494.383 & 1494.589 & 1495.447 & 1496.775 & 1496.588 & 1492.938 & 1489.062 & 1492.769 & 1490.47 & 1490.975 & 1485241 & 1488.213 & 1493.177 & 1493.749 & 1492.937 & 1494.934 & 1492.915 & 1485.064 & 1478.741\end{array}$ $\begin{array}{llllllllllllllllllllll}1493.795 & 1494.387 & 1494.577 & 1495.45 & 1496.775 & 1496.581 & 1492.938 & 1489.062 & 1492.772 & 1490.47 & 1490.975 & 1485.267 & 1488.217 & 1493.177 & 1493.747 & 1492.942 & 1494.932 & 1492.915 & 1485.064 & 1478.741 \\ \end{array}$

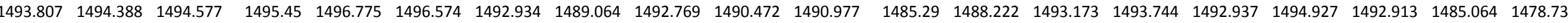

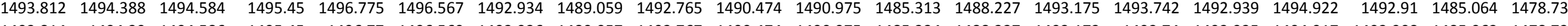

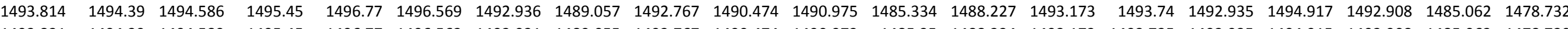

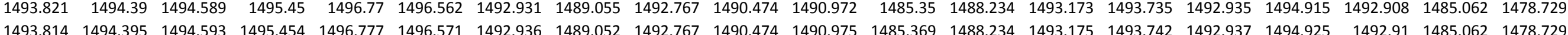


Well

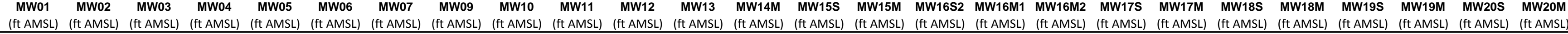

$12 / 12 / 112: 00$ $12 / 12 / 1113: 00$ $12 / 12 / 115 \cdot 00$ 12/12/11 5:00 12/12/11 6:00 $12 / 12 / 11$ 8:00 12/12/11 9:00 12/12/1110:00 2/12/11 11:00 12/12/11 12:00 12/12/111 14:00 $12 / 12 / 1115: 00$ 12/112/11 16:00 $12 / 12 / 11116: 00$ 12/12/11 18:00 12/12/11 19:00 2/12/111 20:00 2/12/11 21:00 12/12/12/111 22:00 12/13/11 0:00 12/13/11 1:00 12/13/11 2:00 12/13/11 3:00 12/13/11 4:00 12/13/11 5:00 12/13/11 8:00 12/13/11 9:00 12/13/11 10:00 12/13/11 11:00 12/13/11 12:00 12/13/111 13:00 2/13/1114:00 $12 / 13 / 1115: 00$ $12 / 13 / 11$ 17:00 $12 / 13 / 11$ 18:00 12/13/11 19:00 12/13/11 20:00 12/13/11 21:00 $12 / 13 / 1122: 00$
$12 / 13 / 1123: 00$ $12 / 14 / 110.00$ 12/14/11 0:00 $12 / 14 / 11$ 2:00 12/14/11 3:00 12/14/11 4:00 12/14/11 5:00 12/14/11 6:00 $\begin{array}{llll}1494.382 & 1494.849 & 1496.439 & 1494.869\end{array}$ $\begin{array}{llll}1494.381 & 1494.846 & 1496.429 & 1494.867 \\ 1494.389 & 1494.851 & 1496.434 & 1994.867\end{array}$ $\begin{array}{lllll}1494.389 & 1494.851 & 1496.434 & 1494.867 \\ 1494.391 & 1494.853 & 1496.448 & 1494.874\end{array}$ $\begin{array}{lllll}1494.386 & 1494.849 & 1496.434 & 1494.869\end{array}$ $\begin{array}{lllll}1494.386 & 1494.844 & 1496.427 & 1494.864\end{array}$ $\begin{array}{lllll}1494.384 & 1494.837 & 1496.413 & 1494.855\end{array}$ $\begin{array}{lllll}1494.384 & 1494.832 & 1496.401 & 1494.843\end{array}$ $\begin{array}{lllll}1494.379 & 1494.825 & 1496.394 & 1494.839\end{array}$ $\begin{array}{lllll}1494.379 & 1494.816 & 1496.378 & 1494.825\end{array}$ $\begin{array}{rrrr}1494.384 & 1494.818 & 1496.389 & 1494.822\end{array}$ $\begin{array}{llll}1494.393 & 1494.83 & 1496.413 & 1494.839 \\ 1494.389 & 1494.83 & 1496.413 & 1494.841\end{array}$ $\begin{array}{llll}1494.386 & 1494.825 & 1496.404 & 1494.839\end{array}$ $\begin{array}{lllll}1494.386 & 1494.823 & 1496.397 & 1494.834\end{array}$ $\begin{array}{lllll}1494.386 & 1494.818 & 1496.389 & 1494.827\end{array}$ $\begin{array}{lllll}1494.381 & 1494.814 & 1496.378 & 1494.825\end{array}$ $\begin{array}{lllll}1494.379 & 1494.8 & 1496.359 & 1494.808\end{array}$ $\begin{array}{lllll}1494.377 & 1494.793 & 1496.345 & 1494.797\end{array}$ $\begin{array}{lllll}1494.37 & 194.793 & 1496.343 & 1494.792\end{array}$ $\begin{array}{lllll}1494.377 & 1494.79 & 1496.35 & 1494.792\end{array}$ $\begin{array}{llllll}1494.377 & 1494.79 & 1496345 & 1494.79\end{array}$ $\begin{array}{lllll}1494.386 & 1494.8 & 1496.373 & 1494.801\end{array}$ $\begin{array}{lllll}1494.381 & 1494.797 & 1496.361 & 1494.801\end{array}$ $\begin{array}{lllll}1494.382 & 1494.793 & 1496.357 & 1494.797\end{array}$ $\begin{array}{lllll}1494.379 & 1494.788 & 1496.347 & 1494.79\end{array}$ $\begin{array}{llll}1494.384 & 1494.795 & 1496.357 & 1494.794\end{array}$ $\begin{array}{lllll}1494.381 & 1494.797 & 1496.366 & 1494.801 \\ 1494.382 & 1494.802 & 1496.371 & 1494811\end{array}$ $\begin{array}{llllll}1494.379 & 1494.8 & 1496.361 & 1494.808\end{array}$ $\begin{array}{llllll}1494.379 & 1494.795 & 1496.352 & 1494.804\end{array}$ $\begin{array}{lllll}1494.381 & 1494.795 & 1496.354 & 1494.804\end{array}$ $\begin{array}{lllll}1494.388 & 1494.802 & 1496.368 & 1494.811\end{array}$ $\begin{array}{llll}1494.393 & 1494.814 & 1496.394 & 1494.827\end{array}$ $\begin{array}{llll}1494.402 & 1494.832 & 1496.422 & 1494.85\end{array}$ $\begin{array}{llll}1494.405 & 1494.846 & 1496.453 & 1494.876\end{array}$ $\begin{array}{lllll}1494.405 & 1494.853 & 1496.457 & 1494.888 \\ 1494.409 & 1494.858 & 1496.464 & 1494.897\end{array}$ $\begin{array}{lllll}1494.405 & 1494.863 & 1496.462 & 1494.902\end{array}$ $\begin{array}{lllll}1494.407 & 1494.865 & 1496.462 & 1494.906\end{array}$ $\begin{array}{lllll}1494.419 & 1494.879 & 1496.488 & 1494.923\end{array}$ $1494.412 \quad 1494.8791496 .483 \quad 1494.925$ $\begin{array}{llll}1494.419 & 1494.886 & 1496.49 & 1494.932\end{array}$ $\begin{array}{llll}1494.419 & 1494.893 & 1496.497 & 1494.941 \\ 1494.426 & 1494.9 & 1496.506 & 1494.951\end{array}$ $\begin{array}{lllll}1494.426 & 1494.9 & 1496.506 & 1494.951 \\ 1494.43 & 1494.907 & 1496523 & 1494962\end{array}$ $\begin{array}{rrrr}1494.43 & 1494.907 & 1496.523 & 1494.962 \\ 1494.433 & 1494.919 & 1496.534 & 1494.974\end{array}$ $\begin{array}{lllll}1494.437 & 1494.928 & 1496.546 & 1494.986\end{array}$ $\begin{array}{lllll}1494.44 & 1494.933 & 1496.553 & 1494.995\end{array}$ $1494.447 \quad \begin{array}{llll}1494.942 & 1496.572 & 1495.012\end{array}$ $\begin{array}{lllll}1494.447 & 1494.956 & 1496.584 & 1495.028\end{array}$ $\begin{array}{rrrr}1494.454 & 1494.968 & 1496.6 & 1495.047 \\ 1494.456 & 1494.977 & 1496.612 & 1495.06\end{array}$ $\begin{array}{llllllllllllllllllll}1493.819 & 1494.395 & 1494.591 & 1495.454 & 1496.777 & 1496.571 & 1492.938 & 1489.057 & 1492.772 & 1490.477 & 1490.975 & 1485.392 & 1488.241 & 1493.177 & 1493.742 & 1492.939 & 1494.92 & 1492.913 & 1485.062 & 1478.729\end{array}$

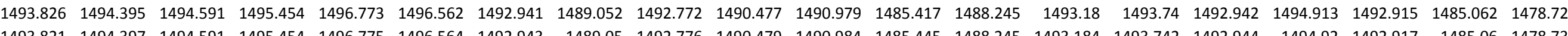

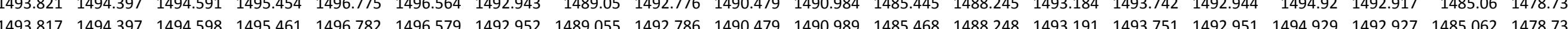
$\begin{array}{lllllllllllllllllllllll}1493831 & 1494.4 & 1494.591 & 1495.459 & 1496777 & 1496564 & 1492955 & 1489.052 & 1492786 & 1490.481 & 1490.993 & 1485475 & 1488262 & 1493194 & 1493747 & 1492.954 & 1494.915 & 1492.927 & 148506 & 1478.741\end{array}$ $\begin{array}{lllllllllllllllllllll}1493.835 & 1494.4 & 1494.596 & 1495.454 & 1496.77 & 1496555 & 1492.955 & 1489.048 & 1492.788 & 1490.484 & 1490.996 & 1485482 & 1488.257 & 1493.196 & 1493.744 & 1492.954 & 1494.913 & 1492.929 & 148506 & 1478.743\end{array}$ $\begin{array}{llllllllllllllllllll}1493.845 & 1494.397 & 1494.591 & 1495.45 & 1496.766 & 1496.543 & 1492.952 & 1489.043 & 1492.788 & 1490.479 & 1490.998 & 1485.503 & 1488.264 & 1493.194 & 1493.737 & 1492.956 & 1494.903 & 1492.929 & 1485.057 & 1478.743\end{array}$

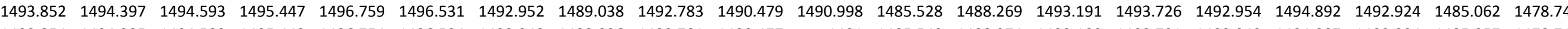

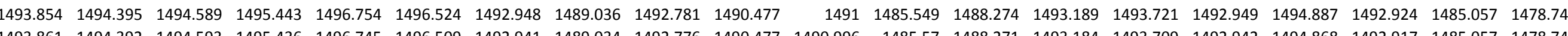

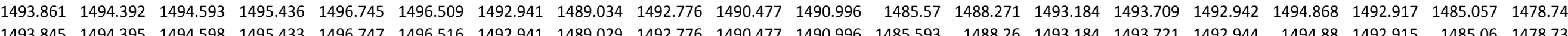
$\begin{array}{llllllllllllllllllll}1493.845 & 1494.395 & 1494.598 & 1495.433 & 1496.747 & 1496.516 & 1492.941 & 1489.029 & 1492.776 & 1490.477 & 1490.996 & 1485.593 & 1488.26 & 1493.184 & 1493.721 & 1492.944 & 1494.88 & 1492.915 & 1485.06 & 1478.739 \\ 1493.824 & 1494.395 & 1494.598 & 1495.445 & 1496.756 & 1496.545 & 1492.945 & 1489.034 & 1492.779 & 1490.479 & 1490.998 & 1485.614 & 1488.252 & 1493.184 & 1493.73 & 1492.949 & 1494.899 & 1492.922 & 1485.057 & 1478.741 \\ \end{array}$ $\begin{array}{lllllllllllllllllllllll}1493.826 & 1494.395 & 1494.596 & 1495.445 & 1496.759 & 1496.545 & 1492.95 & 1489.031 & 1492.783 & 1490.477 & 1491 & 1485.632 & 1488.257 & 1493.191 & 1493.742 & 1492.949 & 1494.894 & 1492.922 & 1485.557 & 1478.741\end{array}$ $\begin{array}{lllllllllllllllllllll}1493.838 & 1494.395 & 1494.6 & 1495.438 & 1496.749 & 1496.528 & 1492.948 & 1489.031 & 1492.783 & 1490.477 & 1491.003 & 1485.669 & 1488.26 & 1493.194 & 1493.726 & 1492.949 & 1494.882 & 1492.922 & 1485.057 & 1478.741\end{array}$

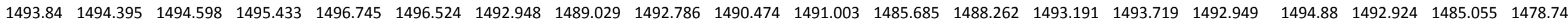
$\begin{array}{llllllllllllllllllllllll}1493.85 & 1494.39 & 1494.596 & 1495.429 & 1496.74 & 1496.514 & 1492.948 & 1489.027 & 1492.783 & 1490.474 & 1491.005 & 1485.697 & 1488.274 & 1493.191 & 1493.712 & 1492.954 & 1494.866 & 1492.924 & 1485.055 & 1478.743 & \end{array}$

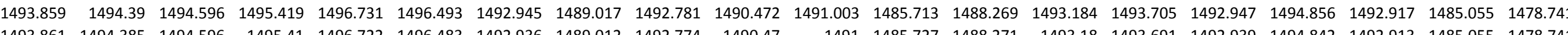

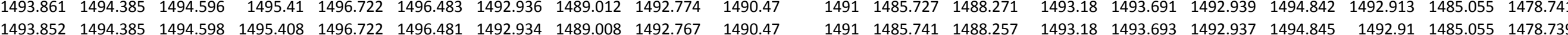

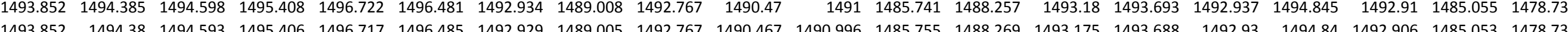
$\begin{array}{lllllllllllllllllllll}1493.845 & 1494.383 & 1494.593 & 1495.403 & 1496.715 & 1496.49 & 1492.929 & 1489.008 & 1492.767 & 1490.467 & 1490.996 & 1485.764 & 1488.262 & 1493.175 & 1493.688 & 1492.93 & 1494.842 & 1492.908 & 1485.053 & 1478.734\end{array}$ \begin{tabular}{llllllllllllllllllllll}
1493.84 & 1494.378 & 1494.6 & 1495.403 & 1496.715 & 1496.488 & 1492.927 & 1489.005 & 1492.762 & 1490.463 & 1490.991 & 1485.778 & 1488264 & 1493.175 & 1493.693 & 1492.928 & 1494.845 & 1492.906 & 1485.05 & 1478.729 \\
\hline
\end{tabular} $\begin{array}{llllllllllllllllllllllll}1493.819 & 1494.383 & 1494.603 & 1495.413 & 1496.724 & 1496.519 & 1492.931 & 1489.005 & 1492.765 & 1490.463 & 1490.993 & 1485.796 & 1488.243 & 1493.173 & 1493.705 & 1492.935 & 1494.859 & 1492.908 & 1485.05 & 1478.732\end{array}$ $\begin{array}{llllllllllllllllllll}1493.826 & 1494.38 & 1494.601 & 1495.408 & 1496.722 & 1496.507 & 1492.931 & 1489.005 & 1492.765 & 1490.463 & 1490.993 & 1485.813 & 1488.25 & 1493.173 & 1493.698 & 1492.93 & 1494.852 & 1492.908 & 1485.05 & 1478.729\end{array}$

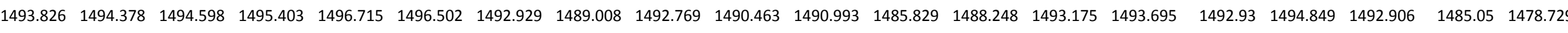

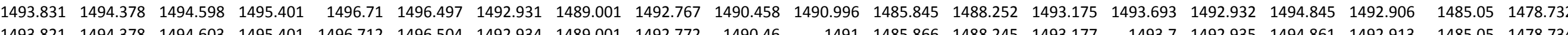

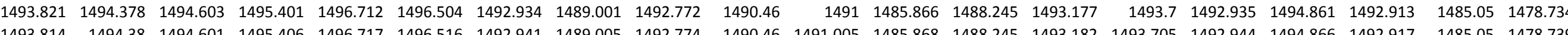

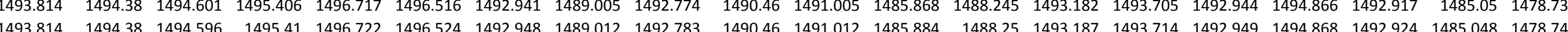

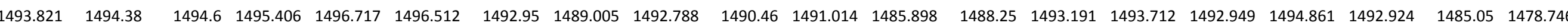
$\begin{array}{llllllllllllllllllllll}1493.826 & 1494.378 & 1494.598 & 1495.401 & 1496.715 & 1496.504 & 1492.948 & 1489.005 & 1492.786 & 1490.463 & 1491.014 & 1485.91 & 1488.248 & 1493.194 & 1493.709 & 1492.949 & 1494.859 & 1492.924 & 1485.048 & 1478.746\end{array}$

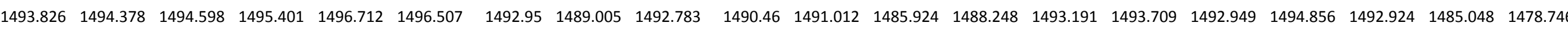
$\begin{array}{llllllllllllllllllll}1493.812 & 1494.38 & 1494.605 & 1495.406 & 1496.722 & 1496.521 & 1492.95 & 1489.005 & 1492.783 & 1490.463 & 1491.017 & 1485.94 & 1488.236 & 1493.194 & 1493.719 & 1492.951 & 1494.873 & 1492.929 & 1485.048 & 1478.748\end{array}$

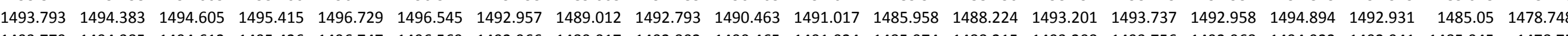

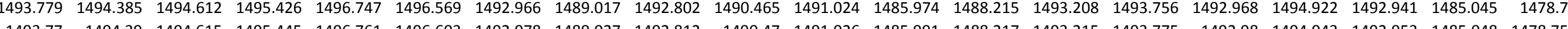

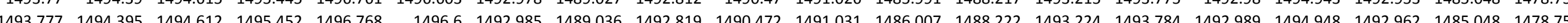
$\begin{array}{lllllllllllllllllllllllll}1493.784 & 1494.4 & 1494.617 & 1495.459 & 1496.775 & 1496.607 & 1492.992 & 1489.036 & 1492.828 & 1490.477 & 1491.035 & 148597 & 148822 & 1493236 & 1493.789 & 1492.994 & 1494.955 & 1492.969 & 1485045 & 147876\end{array}$ $\begin{array}{llllllllllllllllllllll}1493.798 & 1494.402 & 1494.612 & 1495.461 & 1496.777 & 1496.603 & 1492.999 & 1489.04 & 1492.83 & 1490.477 & 1491.042 & 1485.986 & 1488.234 & 1493.241 & 1493.791 & 1493.001 & 1494.957 & 1492.976 & 1485.045 & 1478.76\end{array}$

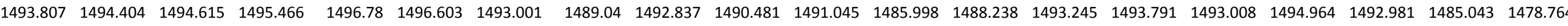
$\begin{array}{lllllllllllllllllllll}1493.795 & 1494.407 & 1494.624 & 1495.48 & 1496.796 & 1496.624 & 1493.013 & 1489.045 & 1492.844 & 1490.486 & 1491.052 & 1485.995 & 1488.229 & 1493.252 & 1493.812 & 1493.015 & 1494.981 & 1492.99 & 1485.048 & 1478.767\end{array}$

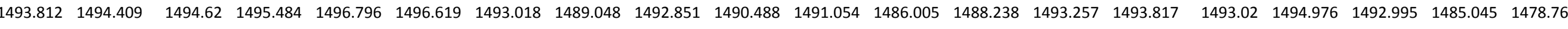

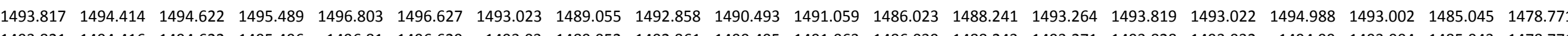

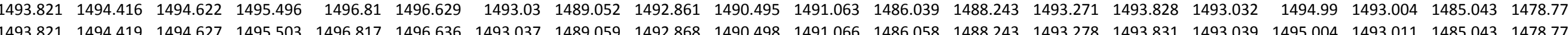

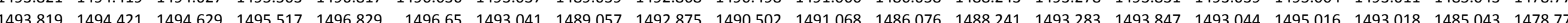
$\begin{array}{lllllllllllllllllllll}1493.821 & 1494.416 & 1494.636 & 1495.524 & 1496.835 & 1496.658 & 1493.048 & 1489.064 & 1492.884 & 1490.505 & 1491.077 & 1486.092 & 1488.243 & 1493.288 & 1493.852 & 1493.053 & 1495.033 & 1493.025 & 1485.043 & 1478.776\end{array}$ \begin{tabular}{llllllllllllllllllllll}
1493.826 & 1494.407 & 1494.631 & 1495.536 & 1496.845 & 1496.667 & 1493.06 & 1489.069 & 1492.894 & 1490.509 & 1491.08 & 1486.108 & 1488.25 & 1493.302 & 1493.866 & 1493.063 & 1495.035 & 1493.037 & 1485.041 & 1478.783 \\
\hline
\end{tabular} $\begin{array}{lllllllllllllllllllllll}1493.835 & 1494.392 & 1494.634 & 1495.543 & 1496.852 & 1496.672 & 1493.069 & 1489.069 & 1492.903 & 1490.516 & 1491.089 & 1486.129 & 1488.26 & 1493.311 & 1493.875 & 1493.07 & 1495.051 & 1493.046 & 1485.043 & 1478.788\end{array}$

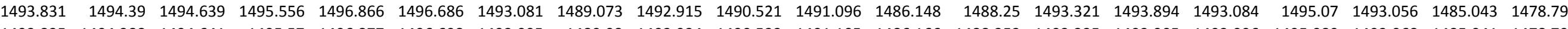

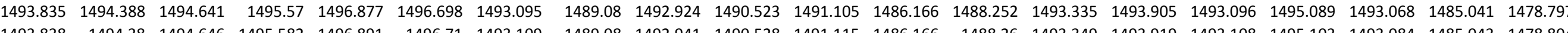

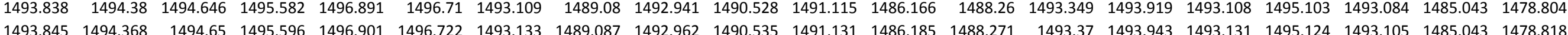


Well

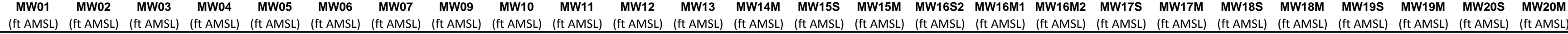

12/14/11 8:00 $12 / 14 / 119: 00$ 12/14/11 10:00 12/114/11 12:00 $12 / 14 / 1113: 00$ 12/14/11 14:00 $12 / 14 / 1115: 00$ 12/14/11 16:00 12/14/11 17:00 12/14/11 18:00 12/14/111 19:00 12/14/11 21:00 12/14/11122:00 12/14/11 23:00 $12 / 15 / 11$ 0:00 $12 / 15 / 111: 00$ $12 / 15 / 112: 00$
$12 / 15 / 113: 00$
$12 / 15 / 114: 00$ 12/15/11 4:00 12/15/11 6:00 $12 / 15 / 117: 000$ 12/15/11 9:00 12/15/11 10:00 12/15/11 11:00 12/15/111 12:00 12/15/11 14:00 $12 / 15 / 1115: 00$ 12/15/11 16:00 12/15/11 17:00 12/15/11 18:00 12/15/11 19:00 $12 / 15 / 1120: 00$ 12/15/11 21:00 12/15/11 22:00 12/16/11 0:00 12/16/111:00 12/16/11 2:00 12/16/11 3:00 12/16/11 4:00 $12 / 16 / 116.00$ 12/16/11 6:00 12/16/11 8:00 $12 / 16 / 119: 00$ 12/16/11 10:00 12/16/1111:00 12/16/11 12:00 $\begin{array}{cccc}1494.454 & 1494.982 & 1496.612 & 1495.07 \\ 1494.458 & 1494.986 & 1496619 & 1495.082\end{array}$ $1494.458 \quad 1494.986 \quad 1496.61914955 .082$ $\begin{array}{llll}1494.461 & 1494.993 & 1496.619 & 1495.088 \\ 1494.461 & 1494.996 & 1496619 & 1495091\end{array}$ $\begin{array}{llll}1494.461 & 1494.998 & 1496.616 & 1495.093\end{array}$ $\begin{array}{lllll}1494.47 & 1495.007 & 1496.633 & 1455.093 .11\end{array}$ $\begin{array}{rrrrr}1494.47 & 1495.007 & 1496.633 & 1495.11 \\ 1494.472 & 1495.012 & 1496.637 & 1495.116\end{array}$ $\begin{array}{llllll}1494.47 & 1495.012 & 1496.63 & 1495.121\end{array}$ $\begin{array}{llll}1494.465 & 1495.003 & 1496.605 & 1495.107\end{array}$ $1494.463 \quad 1494.996 \quad 1496.58614995 .093$ $\begin{array}{llll}1494.458 & 1494.982 & 1496.555 & 1495.075 \\ 1494.461 & 1499968 & 1496541 & 1495058\end{array}$ $\begin{array}{cccc}1494.461 & 1494.968 & 1496.541 & 1495.058 \\ 1494.449 & 1494.947 & 1496.502 & 1495.03\end{array}$ $\begin{array}{llll}1494.454 & 1494.935 & 1496.485 & 1495.009\end{array}$ $\begin{array}{llll}1494.449 & 1494.916 & 1496.467 & 1494.988\end{array}$ $\begin{array}{lllll}1494.44 & 1494.895 & 1496.432 & 1494.958\end{array}$ $\begin{array}{lllll}1494.435 & 1494.877 & 1496.408 & 1494.935\end{array}$ $\begin{array}{llll}1494.43 & 1494.856 & 1496.38 & 1494.911\end{array}$ $\begin{array}{lllll}1494.43 & 1494.842 & 1496.371 & 1494.892\end{array}$ $\begin{array}{rrrr}1494.421 & 1494.825 & 1496.35 & 1494.874\end{array}$ $\begin{array}{rrrrr}1494.419 & 1494.814 & 1496.336 & 1494.86 \\ 1494.419 & 1494.8 & 1496.326 & 1494.846\end{array}$ $\begin{array}{lllll}1494.409 & 1494.779 & 1496.298 & 1494.827\end{array}$ $\begin{array}{lllll}1494.407 & 1494.765 & 1496.282 & 1494.828\end{array}$ $\begin{array}{llllll}1494.4 & 1494.748 & 1496.266 & 1494.792\end{array}$ $\begin{array}{lllll}1494.393 & 1494.732 & 1496.245 & 1494.776\end{array}$ $\begin{array}{llll}1494.386 & 1494.716 & 1496.228 & 1494.757\end{array}$ $\begin{array}{lllll}1494.386 & 1494.707 & 1496.219 & 1494.745\end{array}$ $\begin{array}{lllll}1494.384 & 1494.702 & 1496.217 & 1494.738 \\ 1494.393 & 1494.714 & 1496254 & 1494.757\end{array}$ $\begin{array}{lllll}1494.389 & 1494.718 & 1496.256 & 1494.766\end{array}$ $\begin{array}{lllll}1494.391 & 1494.728 & 1496.275 & 1494.78\end{array}$ $\begin{array}{lllll}1494.381 & 1494.718 & 1496.256 & 1494.773\end{array}$ $\begin{array}{lllll}1494.384 & 1494.716 & 1496.249 & 1494.776\end{array}$ $\begin{array}{lllll}1494.377 & 1494.711 & 1496.238 & 1494.771\end{array}$ $\begin{array}{rrrr}1494.374 & 1494.704 & 1496.226 & 1494.764 \\ 1494.372 & 14947 & 1469219 & 1494.757\end{array}$ $\begin{array}{rrrrr}1494.372 & 1494.7 & 1496.219 & 1494.757 \\ 1494.37 & 1494.7 & 1496217 & 1494.755\end{array}$ $\begin{array}{rrrrr}1494.37 & 1494.7 & 1496.217 & 1494.755 \\ 1494.367 & 1494.697 & 1496.212 & 1494.752\end{array}$ $\begin{array}{lllll}1494.365 & 1494.693 & 1496.205 & 1494.748\end{array}$ $\begin{array}{llllll}1494.365 & 1494.693 & 1496.202 & 1494.743\end{array}$ $\begin{array}{llllll}1494.363 & 1494.693 & 1496.205 & 1494.743\end{array}$ $\begin{array}{lllll}1494.358 & 1494.693 & 1496.205 & 1494.745\end{array}$ $\begin{array}{llll}1494.36 & 1494.693 & 1496.205 & 1494.745\end{array}$ $\begin{array}{llll}1494.358 & 1494.695 & 1496.21 & 1494.75 \\ 1494358 & 1494.695 & 1496.21 & 1494.752\end{array}$ $\begin{array}{llll}1494.358 & 1494.695 & 1496.21 & 1494.752\end{array}$ $\begin{array}{lllll}1494.346 & 1494.679 & 1496.172 & 1494.734\end{array}$ $\begin{array}{lllll}1494.351 & 1494.683 & 1496.186 & 1494.741\end{array}$ $\begin{array}{lllll}1494.349 & 1494.688 & 1496.188 & 1494.743\end{array}$ $\begin{array}{lllll}1494.347 & 1494.688 & 1496.186 & 1494.743\end{array}$ $\begin{array}{llllll}1494.347 & 1494.686 & 1496.186 & 1494.743\end{array}$ $\begin{array}{llll}1494.353 & 1494.697 & 1496.212 & 1494.757 \\ 1494.353 & 1494.709 & 1496.235 & 1494.776\end{array}$

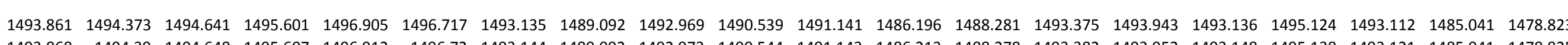

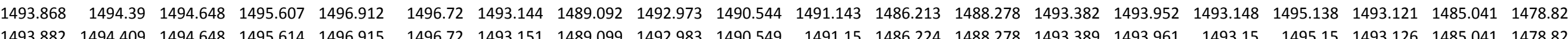

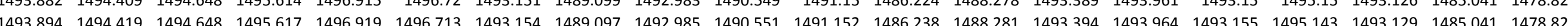
$\begin{array}{lllllllllllllllllllll}1493.904 & 1494.431 & 1494.648 & 1495.621 & 1496.922 & 1496.708 & 1493156 & 1489.104 & 149299 & 1490.556 & 1491.155 & 1486252 & 1488288 & 1493.398 & 1493.957 & 1493.157 & 1495.148 & 1493.131 & 1485.041 & 1478.828\end{array}$ $\begin{array}{llllllllllllllllllll}1493.901 & 1494.402 & 1494.65 & 1495.631 & 1496.933 & 1496.717 & 1493.161 & 1489.101 & 1492.994 & 1490.558 & 1491.159 & 1486.268 & 1488.283 & 1493.401 & 1493.973 & 1493.165 & 1495.169 & 1493.138 & 1485.038 & 147883\end{array}$ $\begin{array}{lllllllllllllllllllllll}1493.911 & 1494.385 & 1494.653 & 1495.638 & 1496.938 & 1496.725 & 1493.168 & 1489.101 & 1492.999 & 1490.56 & 1491.164 & 1486.28 & 1488.288 & 1493.41 & 1493.98 & 1493.167 & 1495.167 & 1493.14 & 1485.038 & 1478.83 \\ \end{array}$

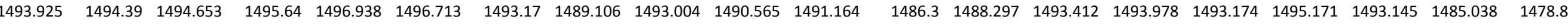

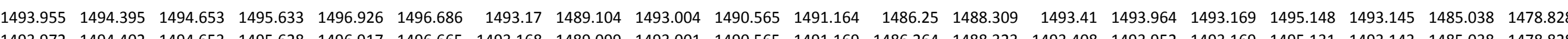

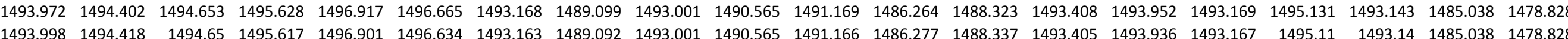
$\begin{array}{llllllllllllllllllll}1493.998 & 1494.418 & 1494.65 & 1495.617 & 1496.901 & 1496.634 & 1493.163 & 1489.092 & 1493.001 & 1490.565 & 1491.166 & 1486.277 & 1488.337 & 1493.405 & 1493.936 & 1493.167 & 1495.11 & 1493.14 & 1485.038 & 1478.828 \\ 1494.007 & 1494.416 & 1494648 & 1495607 & 1496891 & 1496.619 & 1493.161 & 1489.087 & 1492.994 & 1490.565 & 1491.171 & 1486.287 & 1488.339 & 1493.401 & 1493.926 & 1493.16 & 1495.096 & 1493.136 & 1485.038 & 1478828\end{array}$

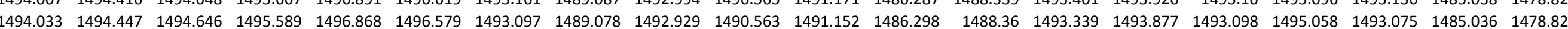
$\begin{array}{lllllllllllllllllllll}1494.031 & 1494.45 & 1494.653 & 1495577 & 1496856 & 1496.56 & 1493.111 & 1489.064 & 1492.945 & 1490.56 & 1491.152 & 1486314 & 1488.356 & 1493.351 & 1493.877 & 1493.112 & 1495.054 & 1493.089 & 1485.038 & 1478.825\end{array}$ $\begin{array}{lllllllllllllllllllllll}1494.04 & 1494.464 & 1494.65 & 1495.563 & 1496.842 & 1496.545 & 1493.111 & 1489.057 & 1492.945 & 1490.558 & 1491.152 & 1486.317 & 1488.367 & 1493.354 & 1493.859 & 1493.112 & 1495.028 & 1493.086 & 1485.038 & 1478.823\end{array}$ \begin{tabular}{llllllllllllllllllllll}
1494.052 & 1494.481 & 1494.646 & 1495.543 & 1496.822 & 1496.512 & 1493.102 & 1489.048 & 1492.938 & 1490.553 & 1491.148 & 1486.321 & 1488.372 & 1493.347 & 1493.84 & 1493.101 & 1494.995 & 1493.077 & 1485.038 & 1478.818 \\
\hline
\end{tabular} $\begin{array}{lllllllllllllllllllllll}1494.054 & 1494.483 & 1494.646 & 1495.526 & 1496.803 & 1496.49 & 1493.093 & 1489.038 & 1492.931 & 1490.549 & 1491.145 & 1486.331 & 1488.379 & 1493.335 & 1493.821 & 1493.096 & 1494.974 & 1493.07 & 1485.036 & 1478.8811 & \end{array}$

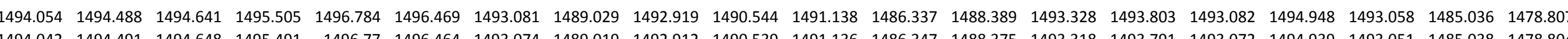

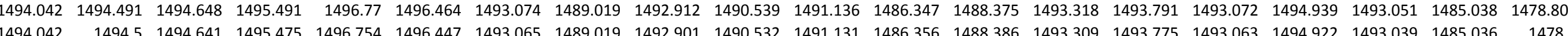

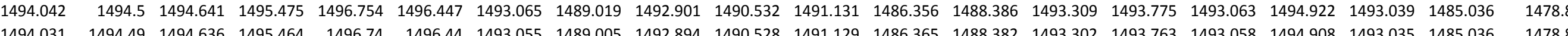
$\begin{array}{lllllllllllllllllllll}14494.016 & 1494.483 & 1444.638 & 1495.45 & 1496.729 & 1496.433 & 1493.051 & 1489.003 & 1492.889 & 1490.523 & 1491.129 & 1486.374 & 1488.375 & 1493.295 & 1493.761 & 1493.051 & 1494.899 & 1493.028 & 1485.036 & 1478.8\end{array}$ $\begin{array}{lllllllllllllllll} & 0\end{array}$

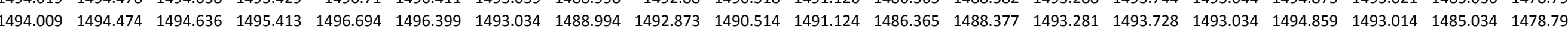
$\begin{array}{llllllllllllllllllllll}1494.002 & 1494.464 & 1494.629 & 1495.394 & 1496.68 & 1496.387 & 1493.027 & 1488.982 & 1492.865 & 1490.507 & 1491.122 & 1486.368 & 1488.375 & 1493.276 & 1493.719 & 1493.03 & 1494.849 & 1493.004 & 1485.034 & 1478.795\end{array}$ $\begin{array}{lllllllllllllllllllllll}1494 & 1494.459 & 1494.627 & 1495.378 & 1496.661 & 1496.373 & 1493.02 & 1488.984 & 1492.858 & 1490.502 & 1491.122 & 1486.377 & 1488.377 & 1493.264 & 1493.7 & 1493.02 & 1494.826 & 1492.997 & 1485.034 & 1478.795\end{array}$

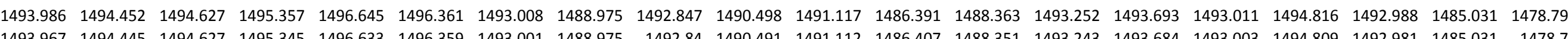

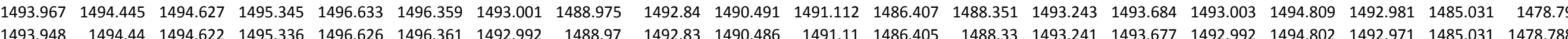

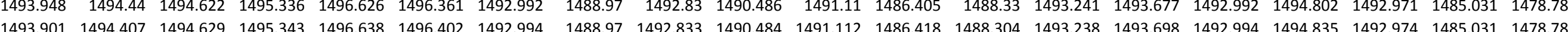
$\begin{array}{llllllllllllllllllllllll}1493.885 & 1494.409 & 1494.627 & 1495.341 & 1496.635 & 1496.414 & 1492.994 & 1488.973 & 1492.833 & 1490.481 & 1491.108 & 1486.425 & 1488.295 & 1493.241 & 1493.705 & 1492.996 & 1494.842 & 1492.974 & 1485.031 & 1478.788\end{array}$ $\begin{array}{lllllllllllllllllllll}1493.866 & 1494.399 & 1494.627 & 1495.348 & 1496.642 & 1496.433 & 1492.999 & 1488.977 & 1492.835 & 1490.479 & 1491.108 & 1486.435 & 1488.29 & 1493.241 & 1493.712 & 1492.999 & 1494.856 & 1492.974 & 1485.031 & 1478.786\end{array}$ \begin{tabular}{llllllllllllllllllllll}
1493.875 & 1494.426 & 1494.622 & 1495.336 & 1496.631 & 1496.418 & 1492.992 & 1488.972 & 1492.828 & 1490.477 & 1491.105 & 1486.388 & 1488.285 & 1493.238 & 1493.702 & 1492.992 & 1494.84 & 1492.971 & 1485.031 & 1478.786 \\
\hline
\end{tabular} $\begin{array}{llllllllllllllllllllll}1493.873 & 1494.428 & 1494.622 & 1495.334 & 1496.629 & 1496.418 & 1492.992 & 1488.97 & 1492.83 & 1490.477 & 1491.103 & 1486.397 & 1488.281 & 1493.236 & 1493.702 & 1492.994 & 1494.845 & 1492.971 & 1485.031 & 1478.783\end{array}$

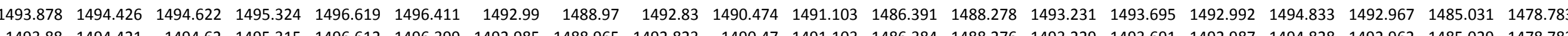

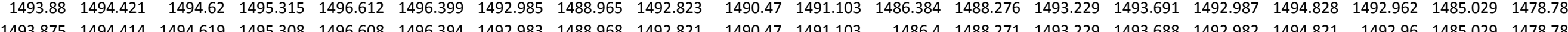

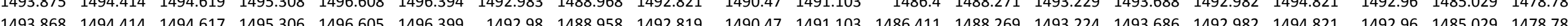

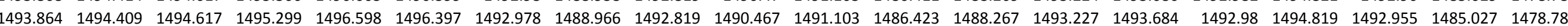
$\begin{array}{lllllllllllllllllllllll}1493.864 & 1494.404 & 1494.612 & 1495.29 & 1496.591 & 1496.392 & 1492.973 & 1488.959 & 1492.814 & 1490.467 & 1491.098 & 1486.428 & 1488.267 & 1493.217 & 1493.681 & 1492.977 & 1494.812 & 1492.953 & 1485.027 & 1478.781 \\ \end{array}$ $\begin{array}{lllllllllllllllllllll}1493.857 & 1494.4 & 1494.615 & 1495.287 & 1496.587 & 1496.392 & 1492.971 & 1488.961 & 1492.809 & 1490.465 & 1491.096 & 1486.437 & 1488.257 & 1493.217 & 1493.679 & 1492.973 & 1494.812 & 1492.948 & 1485.027 & 1478.779\end{array}$ $\begin{array}{llllllllllllllllllllll}1493.847 & 1494.4 & 1494.612 & 1495.285 & 1496.589 & 1496.397 & 1492.969 & 1488.961 & 1492.807 & 1490.465 & 1491.094 & 1486.448 & 1488.252 & 1493.215 & 1493.677 & 1492.97 & 1494.814 & 1492.948 & 1485.027 & 1478.776 \\ \end{array}$

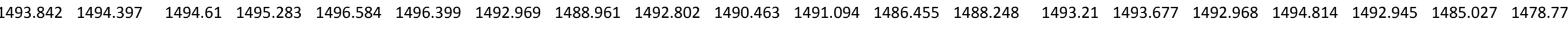

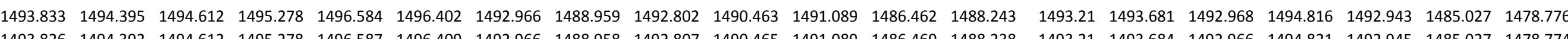

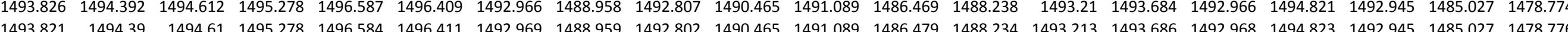

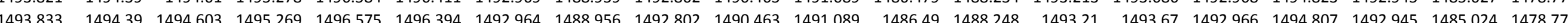
$\begin{array}{lllllllllllllllllllll}1493.838 & 1494.385 & 1494.605 & 1495.257 & 1496.566 & 1496.38 & 1492.962 & 1488.949 & 1492.797 & 1490.46 & 1491.089 & 1486.495 & 1488.243 & 1493.208 & 1493.667 & 1492.961 & 1494.798 & 1492.941 & 1485.024 & 1478.774\end{array}$ $\begin{array}{lllllllllllllllllllll}1493.819 & 1494.373 & 1494.605 & 1495.259 & 1496.568 & 1496.394 & 1492.964 & 1488.956 & 1492.8 & 1490.463 & 1491.094 & 1486.495 & 1488.229 & 1493.205 & 1493.679 & 1492.966 & 1494.809 & 1492.941 & 1485.024 & 1478.779\end{array}$

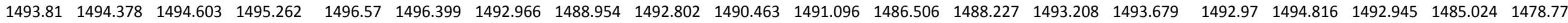

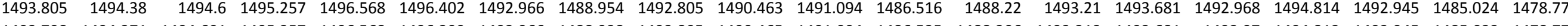

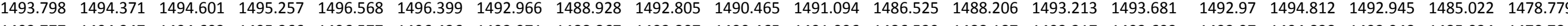
$\begin{array}{llllllllllllllllllllllll}1493.777 & 1494.347 & 1494.603 & 1495.266 & 1496.577 & 1496.426 & 1492.971 & 1488.867 & 1492.807 & 1490.465 & 1491.096 & 1486.539 & 1488.187 & 1493.217 & 1493.693 & 1492.97 & 1494.838 & 1492.948 & 1485.024 & 1478.781 \\ 1493.755 & 1494.323 & 1494.603 & 1495.276 & 1496.589 & 1496.447 & 1492.976 & 1488.961 & 1492.812 & 1490.47 & 1491.101 & 1486.545 & 1488.173 & 1493.217 & 1493.714 & 1492.977 & 1494.856 & 1492.955 & 1485.024 & 1478.783\end{array}$ 
Well

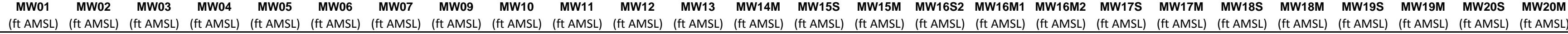

$12 / 16 / 1114: 00$ $12 / 16 / 1115: 00$ 12/16/11 16:00 12/16/11 18:00 12/16/11 18:00 12/16/11 20:00 12/16/11 21:00 12/16/11 22:00 12/16/11 23:00 12/17/110:00 $12 / 17 / 111: 00$
$12 / 17 / 112: 00$ 12/17/11 3:00 12/17/11 4:00 $12 / 17 / 115: 00$
$12 / 17 / 116: 00$ 12/17/11 6:00 12/17/11 8:00 12/17/11 9:00 12/17/11 10:00 12/17/11 12:00 12/17/11 12:00 12/17/11 14:00 12/17/11 15:00 12/17/11 16:00 12/17/11 17:00 12/17/11 18:00 12/17/7111 19:00 $20: 00$ 12/17/11 21:00 12/17/11 22:00 12/17/11 23:00 12/18/11 0:00 12/18/11 1:00 $12 / 18 / 112: 00$
$12 / 18 / 113: 00$ 12/18/11 3:00 12/18/11 5:00 12/18/117:00 12/18/11 8:00 12/18/11 9:00 12/18/11 10:00 $12 / 18 / 1111: 00$ 12/18/11112:00 12/18/11 13:00 12/18/11 15:00 12/18/11 16:00 $12 / 18 / 1118: 00$
$12 / 18 / 1119: 00$ $\begin{array}{lllll}1494.358 & 1494.721 & 1496.256 & 1494.792 \\ 1494.363 & 1494.732 & 1496.273 & 1494.811\end{array}$ $\begin{array}{llll}1494.363 & 1494.732 & 1496.273 & 1494.811 \\ 1494.363 & 1494.737 & 1469277 & 1494.822\end{array}$ $\begin{array}{lllll}1494.363 & 1494.737 & 1496.277 & 1494.822 \\ 1494.365 & 1494.744 & 1496289 & 1494.834\end{array}$ $\begin{array}{llll}1494.363 & 1494.744 & 1496.273 & 1494.832\end{array}$ $\begin{array}{llll}1494.363 & 1494.744 & 1496.27 & 1494.834\end{array}$ $\begin{array}{rrrrr}1494.363 & 1494.744 & 1496.268 & 1994.834\end{array}$ $\begin{array}{llllll}1494.36 & 1494.737 & 1496.254 & 1494.825\end{array}$ $\begin{array}{lllll}1494.358 & 1494.739 & 1496.254 & 1494.822\end{array}$ $\begin{array}{llll}1494.358 & 1494.739 & 1496.249 & 1494.818 \\ 1494356 & 1494.734 & 1496238 & 194811\end{array}$ $\begin{array}{rrrr}1494.356 & 1494.734 & 1496.238 & 1494.811 \\ 1494.36 & 1494.739 & 1496252 & 1494819\end{array}$ $\begin{array}{rrrr}1494.36 & 1494.739 & 1496.252 & 1494.818 \\ 1494.36 & 1494.739 & 1496.254 & 1494.82\end{array}$ $\begin{array}{rrrrr}1494.356 & 1494.739 & 1496.247 & 1494.818\end{array}$ $\begin{array}{lllll}1444.356 & 1494.737 & 1496.242 & 1494.815\end{array}$ $\begin{array}{lllll}1494.358 & 1494.739 & 1496.245 & 1494.815\end{array}$ $\begin{array}{lllll}1494.356 & 1494.737 & 1496.242 & 1494.813\end{array}$ $\begin{array}{llll}1494.353 & 1494.737 & 1496.24 & 1494.813\end{array}$ $\begin{array}{llll}1494.353 & 1494.737 & 1496.242 & 1494.813\end{array}$ $\begin{array}{lllll}1494.354 & 1494.737 & 1496.238 & 1494.811\end{array}$ $\begin{array}{lllll}1494.351 & 1494.732 & 1496.228 & 1494.806\end{array}$ $\begin{array}{lllll}1494.358 & 1494.746 & 1496.256 & 1494.82\end{array}$ $\begin{array}{lllll}1499.363 & 1494.76 & 1496.284 & 1494.841\end{array}$ $\begin{array}{lllll}1494.365 & 1494.765 & 1496.296 & 1494.85\end{array}$ $\begin{array}{lllll}1494.37 & 1494.776 & 1496.31 & 1494.867\end{array}$ $\begin{array}{lllll}1494.367 & 1494.779 & 1496.312 & 1494.871\end{array}$ $\begin{array}{llll}1494.368 & 1494.781 & 1496.305 & 1494.869\end{array}$ $\begin{array}{lllll}1494.372 & 1494.783 & 1496.308 & 1494.871 \\ 1494.37 & 1494.781 & 1496305 & 1494874\end{array}$ $\begin{array}{lllll}1494.372 & 1494.781 & 1496.301 & 1494.871\end{array}$ $\begin{array}{lllll}1494.374 & 1494.783 & 1496.305 & 1494.871\end{array}$ $\begin{array}{lllll}1494.377 & 1494.788 & 1496.312 & 1494.876\end{array}$ $\begin{array}{lllll}1494.379 & 1494.79 & 1496.319 & 1494.881\end{array}$ $\begin{array}{lllll}1494.379 & 1494.795 & 1496.324 & 1494.888\end{array}$ $\begin{array}{lllll}1494.384 & 1494.804 & 1496.336 & 1494.895\end{array}$ $\begin{array}{lllll}1494.388 & 1494.816 & 1496.352 & 1494.906\end{array}$ $\begin{array}{llll}1494.393 & 1494.823 & 1496.368 & 1494.923 \\ 1494.398 & 1494.835 & 1496.382 & 1494.935\end{array}$ $1494.405 \quad 1494.846 \quad 1496.397 \quad 1494.951$ $\begin{array}{llll}1494.407 & 1494.853 & 1496.404 & 1494.96\end{array}$ $\begin{array}{rrrr}1494.409 & 1494.858 & 1496.413 & 1494.969\end{array}$ $\begin{array}{lllll}1494.414 & 1494.872 & 1496.432 & 1494.984\end{array}$ $\begin{array}{llll}1494.419 & 1494.881 & 1496.446 & 1494.995 \\ 1494.421 & 1498.888 & 1496.446 & 1455004\end{array}$ $1494.421 \quad 1494.888 \quad 1496.44671495 .004$ $\begin{array}{llll}1494.43 & 1494.902 & 1496.467 & 1495.018 \\ 1494.442 & 1494.923 & 1496.502 & 1495.042\end{array}$ $\begin{array}{lllll}1444.454 & 1444.954 & 1496.544 & 1495.075\end{array}$ $\begin{array}{lllll}1494.461 & 1494.977 & 1496.569 & 1495.103\end{array}$ $\begin{array}{lllll}1494.461 & 1494.984 & 1496.572 & 1495.119\end{array}$ $1494.461 \quad 1494.986 \quad 1496.562 \quad 1495.116$ $\begin{array}{lllll}1494.463 & 1494.986 & 1496.56 & 1495.114\end{array}$ $\begin{array}{rrrr}1494.465 & 1494.991 & 1496.56 & 1495.107 \\ 1494.468 & 1494.993 & 1496.558 & 1495.102\end{array}$

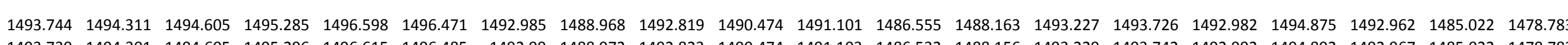

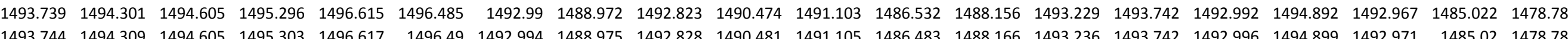
$\begin{array}{lllllllllllllllllllll}1493.744 & 1494.309 & 1494.605 & 1495.303 & 1496.617 & 1496.49 & 1492.994 & 1488.975 & 1492.828 & 1490.481 & 1491.105 & 1486.483 & 1488.166 & 1493.236 & 1493.742 & 1492.996 & 1494.899 & 1492.971 & 1485.02 & 1478.783 \\ 1493.748 & 1494.304 & 1494.608 & 149531 & 1496.624 & 1496.497 & 1492999 & 1488.98 & 1492835 & 1490.484 & 1491.105 & 1486.455 & 1488.159 & 1493241 & 1493747 & 1493.001 & 1494.906 & 1492978 & 148502 & 1478783\end{array}$ $\begin{array}{lllllllllllllllllllllll}1493.765 & 1494.325 & 1494.603 & 1495.308 & 1496.622 & 1496.481 & 1492.999 & 1488.977 & 1492.835 & 1490.486 & 1491.108 & 1486.465 & 1488.17 & 1493.243 & 1493.749 & 1493.003 & 1494.901 & 1492.981 & 148502 & 1478783\end{array}$ $\begin{array}{lllllllllllllllllllll}1493.777 & 1494.335 & 1494.605 & 1495.31 & 1496.622 & 1496.478 & 1493.004 & 1488.98 & 1492.842 & 1490.488 & 1491.108 & 1486.481 & 1488.177 & 1493.245 & 1493.751 & 1493.006 & 1494.899 & 1492.983 & 1485.02 & 1478.786\end{array}$ \begin{tabular}{lllllllllllllllllllllll}
1493.784 & 1494.34 & 1494.603 & 1495.313 & 1496.622 & 1496.478 & 1493.006 & 1488.98 & 1492.84 & 1490.491 & 1491.112 & 1486.497 & 1488.18 & 1493.248 & 1493.756 & 1493.006 & 1494.899 & 1492.983 & 1485.02 & 1478.786 \\
\hline
\end{tabular} $\begin{array}{lllllllllllllllllllll}1493.798 & 1494.356 & 1494.601 & 1495.306 & 1496.615 & 1496.459 & 1493.006 & 1488.977 & 1492.842 & 1490.493 & 1491.112 & 1486.511 & 1488.184 & 1493.245 & 1493.74 & 1493.006 & 1494.885 & 1492.981 & 1485.02 & 1478.786\end{array}$ $\begin{array}{llllllllllllllllllll}1493.8 & 1494.366 & 1494.6 & 1495.306 & 1496.615 & 1496.457 & 1493.004 & 1488.97 & 1492.842 & 1490.495 & 1491.112 & 1486.525 & 1488.189 & 1493.25 & 1493.74 & 1493.008 & 1494.878 & 1492.983 & 1485.017 & 1478.786\end{array}$

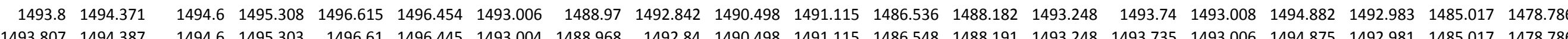

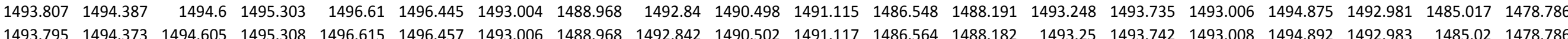

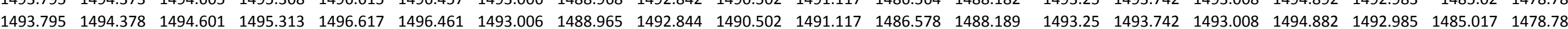
$\begin{array}{llllllllllllllllllllll}1493.8 & 1494.385 & 1494.6 & 1495.308 & 1496.612 & 1496.452 & 1493.004 & 1488.966 & 1492.84 & 1490.505 & 1491.115 & 1486.585 & 1488.191 & 1493.248 & 1493.735 & 1493.008 & 1494.88 & 1492.985 & 1485.017 & 1478.786\end{array}$ $\begin{array}{lllllllllllllllllllll}1493.802 & 1494.383 & 1494.598 & 1495.308 & 1496.612 & 1496.447 & 1493.006 & 1488.961 & 1492.84 & 1490.505 & 1491.115 & 1486.596 & 1488.194 & 1493.245 & 1493.737 & 1493.006 & 1494.875 & 1492.983 & 1485.015 & 1478.783\end{array}$ $\begin{array}{lllllllllllllllllllllll}1493.8 & 1494.383 & 1494.596 & 1495.31 & 1496.615 & 1496.449 & 1493.008 & 1488.965 & 1492.842 & 1490.507 & 1491.117 & 1486.599 & 1488.194 & 1493.248 & 1493.737 & 1493.008 & 1494.88 & 1492.985 & 1485.015 & 1478.783\end{array}$ $\begin{array}{lllllllllllllllllllll}1493.802 & 1494.383 & 1494.596 & 1495.31 & 1496.612 & 1496.447 & 1493.006 & 1488.963 & 1492.842 & 1490.507 & 1491.117 & 1486.594 & 1488.198 & 1493.25 & 1493.735 & 1493.008 & 1494.88 & 1492.988 & 1485.015 & 1478.783 & \end{array}$ $\begin{array}{llllllllllllllllllll}1493.8 & 1494.383 & 1494.598 & 1495.31 & 1496.612 & 1496.445 & 1493.006 & 1488.959 & 1492.844 & 1490.509 & 1491.119 & 1486.599 & 1488.191 & 1493.25 & 1493.735 & 1493.008 & 1494.878 & 1492.985 & 1485.015 & 1478.783\end{array}$

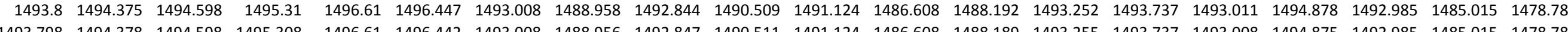

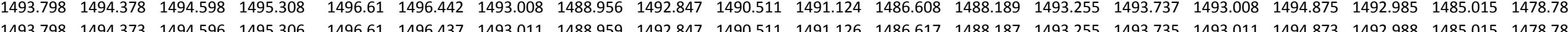
$\begin{array}{llllllllllllllllllllll}1493.793 & 1494.368 & 1494.596 & 1495.303 & 1496.608 & 1496.435 & 1493.011 & 1488.954 & 1492.847 & 1490.514 & 1491.126 & 1486.624 & 1488.18 & 1493.255 & 1493.73 & 1493.011 & 1494.873 & 1492.99 & 1485.013 & 1478.788\end{array}$

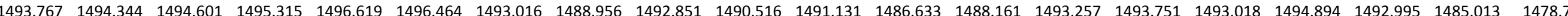
$\begin{array}{lllllllllllllllllllllllll}1493.748 & 1494.316 & 1494.598 & 1495.327 & 1496.631 & 1496.488 & 1493.023 & 1488.961 & 1492.858 & 1490.518 & 1491.136 & 1486.645 & 1488.156 & 1493.267 & 1493.77 & 1493.027 & 1494.917 & 1493.002 & 1485.013 & 1478.79\end{array}$ \begin{tabular}{lllllllllllllllllllllll}
1493.746 & 1494.304 & 1494.6 & 1495.334 & 1496.64 & 1496.497 & 1493.027 & 1488.97 & 1492.863 & 1490.521 & 1491.136 & 1486.65 & 1488.145 & 1493.271 & 1493.777 & 1493.032 & 1494.927 & 1493.006 & 1485.013 & 1478.79 \\
\hline
\end{tabular} \begin{tabular}{llllllllllllllllllll}
1493.741 & 1494.292 & 1494.603 & 1495.345 & 1496.647 & 1496.512 & 1492.952 & 1488.977 & 1492.776 & 1490.525 & 1491.117 & 1486.659 & 1488.14 & 1493.184 & 1493.754 & 1492.951 & 1494.936 & 1492.929 & 1485.013 & 1478.79 \\
\hline
\end{tabular}

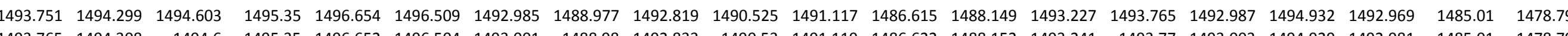

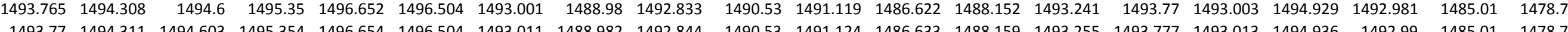
$\begin{array}{lllllllllllllllllllll}1493.77 & 1494.311 & 1494.603 & 1495.354 & 1496.654 & 1496.504 & 1493.011 & 1488.982 & 1492.844 & 1490.53 & 1491.124 & 1486.633 & 1488.159 & 1493.255 & 1493.777 & 1493.013 & 1494.936 & 1492.99 & 1485.01 & 1478.79 \\ 1493.779 & 1494.32 & 1494.598 & 1495354 & 1496.654 & 1496.5 & 1493.018 & 1488.982 & 1492851 & 1490532 & 1491.126 & 1486.636 & 1488.166 & 1493257 & 1493.772 & 1493.02 & 1494.934 & 1492995 & 1485.01 & 1478.79\end{array}$ $\begin{array}{llllllllllllllllllllll}1493.786 & 1494.328 & 1494.603 & 1495.357 & 1496.656 & 1496.497 & 1493.023 & 1488.975 & 1492.856 & 1490.535 & 1491.131 & 1486.64 & 1488.17 & 1493.264 & 1493.777 & 1493.025 & 1494.932 & 1492.999 & 1485.01 & 1478.79\end{array}$ $\begin{array}{lllllllllllllllllllllll}1493.791 & 1494.332 & 1494.605 & 1495.359 & 1496.656 & 1496.495 & 1493.03 & 1488.975 & 1492.863 & 1490.537 & 1491.131 & 1486.654 & 1488.175 & 1493.267 & 1493.779 & 1493.032 & 1494.934 & 1493.009 & 1485.01 & 1478.793 \\ \end{array}$ $\begin{array}{llllllllllllllllllllllll}1493.788 & 1494.332 & 1494.605 & 1495.364 & 1496.661 & 1496.504 & 1493.034 & 1488.982 & 1492.868 & 1490.539 & 1491.138 & 1486.663 & 1488.175 & 1493.276 & 1493.786 & 1493.034 & 1494.939 & 1493.011 & 1485.01 & 1478.795 & \end{array}$ $\begin{array}{llllllllllllllllllll}1493.788 & 1494.332 & 1494.608 & 1495.368 & 1496.668 & 1496.509 & 1493.041 & 1488.982 & 1492.873 & 1490.544 & 1491.145 & 1486.673 & 1488.18 & 1493.283 & 1493.796 & 1493.041 & 1494.95 & 1493.018 & 1485.008 & 1488.795\end{array}$

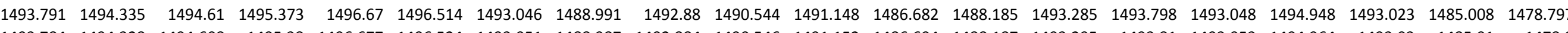

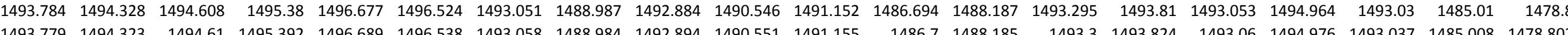

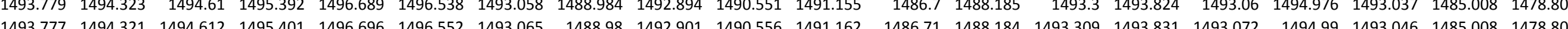

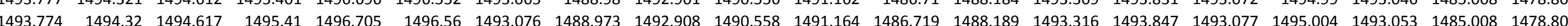
$\begin{array}{llllllllllllllllllllll}1493.77 & 1494.325 & 1494.617 & 1495.422 & 1496.719 & 1496.579 & 1493.083 & 1488.956 & 1492.917 & 1490.563 & 1491.169 & 1486.731 & 1488.185 & 1493.323 & 1493.859 & 1493.084 & 1495.016 & 1493.063 & 1485.008 & 1478.807\end{array}$ $\begin{array}{lllllllllllllllllllllll}1493.779 & 1494.335 & 1494.617 & 1495.429 & 1496.726 & 1496.581 & 1493.093 & 1488.963 & 1492.924 & 1490.567 & 1491.173 & 1486.719 & 1488.198 & 1493.332 & 1493.868 & 1493.091 & 1495.023 & 1493.07 & 1485.006 & 1478.809\end{array}$

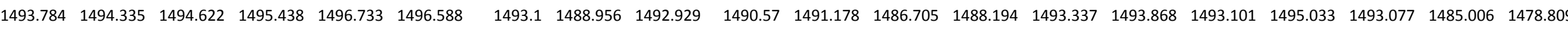

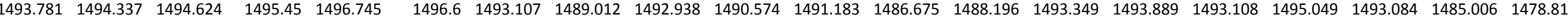

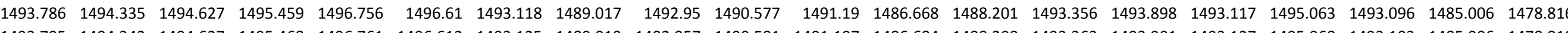

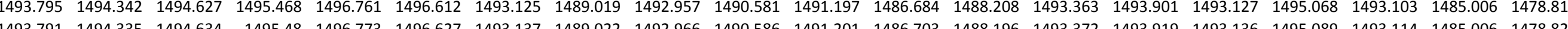

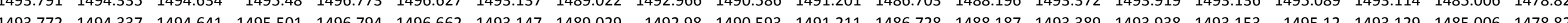
$\begin{array}{llllllllllllllllllllll}1493.758 & 1494.363 & 1494.643 & 1495.526 & 1496.822 & 1496.696 & 1493.165 & 1489.036 & 1492.997 & 1490.6 & 1491.215 & 1486.733 & 1488.18 & 1493.405 & 1493.973 & 1493.167 & 1495.152 & 1493.143 & 1485.006 & 1478.83\end{array}$ \begin{tabular}{llllllllllllllllllllll}
1493.755 & 1494.375 & 1494.646 & 1495.547 & 1496.84 & 1496.722 & 1493.179 & 1489.038 & 1493.011 & 1490.607 & 1491.227 & 1486.751 & 1488.175 & 1493.417 & 1493.996 & 1493.181 & 1495.178 & 1493.157 & 1485.003 & 1478.832 \\
\hline
\end{tabular} $\begin{array}{lllllllllllllllllllll}1493.779 & 1494.38 & 1494.65 & 1495.559 & 1496.852 & 1496.717 & 1493.037 & 1489.052 & 1492.854 & 1490.612 & 1491.225 & 1486.742 & 1488.189 & 1493.264 & 1493.968 & 1493.039 & 1495.181 & 1493.016 & 1485.006 & 1478.835 \\ \end{array}$ $\begin{array}{llllllllllllllllllll}1493.807 & 1494.385 & 1494.648 & 1495.561 & 1496.854 & 1496.703 & 1492.938 & 1489.052 & 1492.748 & 1490.619 & 1491.148 & 1486.682 & 1488.21 & 1493.158 & 1493.889 & 1492.939 & 1495.164 & 1492.92 & 1485.003 & 1478.832 \\ \end{array}$ \begin{tabular}{llllllllllllllllllll}
1493.828 & 1494.39 & 1494.653 & 1495.568 & 1496.856 & 1496.694 & 1492.873 & 1489.057 & 1492.68 & 1490.621 & 1491.145 & 1486.687 & 1488.217 & 1493.09 & 1493.889 & 1492.876 & 1495.152 & 1492.856 & 1485.003 & 1478.835 \\
\hline
\end{tabular}

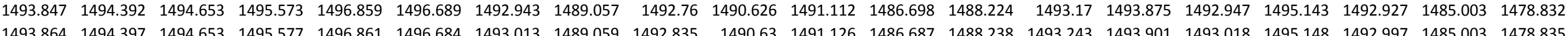


Well

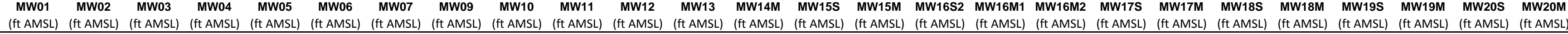

$12 / 18 / 1120: 00$ $12 / 18 / 1121: 00$ $12 / 18 / 1122: 00$ 12/19/110:00 12/19/11 0:00 12/19/11 2:00 12/19/11 3:00 12/19/11 4:00 12/19/11 5:00 12/19/11 6:00 12/19/11 8:00 12/19/11 1900 12/19/11 11:00 $12 / 19 / 1112: 00$
$12 / 19 / 1113: 00$ 12/19/11 14:00 $12 / 19 / 1115: 00$ 12/19/11 16:00 $12 / 19 / 1118: 00$ $12 / 19 / 1118: 00$
$12 / 19 / 1119: 00$ 12/19/11 20:00 12/19/11 21:00 12/19/11 22:00 12/19/11 23:00 12/20/11 0:00 12/20/11 1:00 12/20/11 3:00 $12 / 20 / 115: 00$
$12 / 20 / 116: 00$
$12 / 20 / 11700$ 12/20/11 6:00 12/20/11 7:00 $12 / 20 / 118: 00$
$12 / 20 / 119: 00$ 12/20/11 9:00 12/20/11 11:00 12/20/11 12:00 12/20/11 13:00 12/20/11 14:00 12/20/11 15:00 12/20/11 17:00 12/20/11 18:00 12/20/11 19:00 12/20/11 21:00 12/20/11 22:00 12/20/11 23:00 12/21/11 0:00 $\begin{array}{rrrr}1494.47 & 1494.996 & 1496.555 & 1495.1 \\ 1494.472 & 1494.993 & 1496.553 & 1495.093\end{array}$ $\begin{array}{llll}1494.472 & 1494.993 & 1496.553 & 1495.093 \\ 1494.475 & 1494996 & 1496553 & 1495091\end{array}$ $\begin{array}{lllll}1494.475 & 1494.996 & 1496.553 & 1495.091 \\ 1494.475 & 1494.993 & 1496.546 & 1495084\end{array}$ $1494.479 \quad 1494.998 \quad 1496.553 \quad 1495.086$ $\begin{array}{lllll}1494.484 & 1495.005 & 1496.565 & 1455.093\end{array}$ $\begin{array}{lllll}14994.484 & 1495.005 & 1496.565 & 1495.093\end{array}$ $\begin{array}{lllll}1494.486 & 1495.005 & 1496.562 & 1495.095\end{array}$ $\begin{array}{llllll}1494.484 & 1495 & 1496.558 & 1495.091\end{array}$ $1494.482 \quad 1494.996 \quad 1496.544 \quad 1495.084$ $\begin{array}{llll}1494.482 & 1494.991 & 1496.539 & 1495.074 \\ 1494.477 & 1494.982 & 1496518 & 1495061\end{array}$ $\begin{array}{rrrrr}1494.477 & 1494.982 & 1496.518 & 1495.061 \\ 1494.475 & 1494.97 & 1496.497 & 1495.047\end{array}$ $\begin{array}{lllll}1494.477 & 1494.961 & 1496.488 & 1455.033\end{array}$ $\begin{array}{lllll}1494.477 & 1494.956 & 1496.483 & 1495.025\end{array}$ $\begin{array}{lllll}1494.477 & 1494.956 & 1496.483 & 1495.025 \\ 1494.479 & 1494.951 & 1496.481 & 1495.018\end{array}$ $\begin{array}{lllll}1494.482 & 1494.954 & 1496.495 & 1495.021\end{array}$ $\begin{array}{lllll}1494.484 & 1494.949 & 1496.485 & 1495.018\end{array}$ $\begin{array}{lllll}1494.484 & 1494.947 & 1496.385 & 1495.018\end{array}$ $\begin{array}{lllll}1494.484 & 1494.947 & 1496.291 & 1495.023 \\ 1494.486 & 1494.958 & 1496315 & 1495.042\end{array}$ $\begin{array}{llll}1494.489 & 1444.958 & 1496.34 & 1495.042\end{array}$ $\begin{array}{lllll}1494.468 & 1494.933 & 1496263 & 1495.028\end{array}$ $\begin{array}{lllll}1494.482 & 1494.937 & 1496.294 & 1495.032\end{array}$ $\begin{array}{llll}1494.458 & 1494.818 & 1496.238 & 1495.009\end{array}$ $\begin{array}{lllll}1494.47 & 1494.811 & 1496.296 & 1495.009\end{array}$ $\begin{array}{llll}1494.484 & 1494.844 & 1496.385 & 1495.035\end{array}$ $\begin{array}{lllll}1494.482 & 1494.851 & 1496.698 & 1495.053\end{array}$ $\begin{array}{rrrrr}1494.482 & 1494.87 & 1496.801 & 1495.079 \\ 1494.493 & 1494.842 & 1496.848 & 1495.105\end{array}$ $1494.491 \quad 1494.839 \quad 1496.932 \quad 1495.114$ $\begin{array}{lllll}1494.498 & 1494.853 & 1496.997 & 1495.133\end{array}$ $\begin{array}{lllll}1494.498 & 1494.839 & 1497.023 & 1495.147\end{array}$ $\begin{array}{lllll}1494.493 & 1494.835 & 1497.03 & 1495.156\end{array}$ $\begin{array}{lllll}1494.491 & 1494.867 & 1497.049 & 1495.159\end{array}$ $\begin{array}{llll}1494.491 & 1494.9 & 1497.039 & 1495.161\end{array}$ $\begin{array}{lllll}1494.491 & 1494.856 & 1496.691 & 1495.17 \\ 1494.489 & 1494.853 & 1496572 & 1495.173\end{array}$ $\begin{array}{lllll}1494.489 & 1494.853 & 1496.572 & 1495.173 \\ 1494.491 & 1494.858 & 1496.532 & 1495.182\end{array}$ $\begin{array}{lllll}1494.498 & 1494.867 & 1496.53 & 1495.194\end{array}$ $\begin{array}{lllll}1494.507 & 1494.879 & 1496.548 & 1495.219\end{array}$ $\begin{array}{lllll}1494.507 & 1494.891 & 1496.56 & 1495.24\end{array}$ $\begin{array}{lllll}1494.507 & 1494.902 & 1496.569 & 1495.264\end{array}$ $1494.516 \quad 1494.916 \quad 1496.593 \quad 1495.289$ $\begin{array}{lllll}1494.507 & 1494.921 & 1496.717 & 1495.299\end{array}$ $\begin{array}{rrrrr}1494.512 & 1494.933 & 1496.584 & 1495.31 \\ 1494.512 & 1494.937 & 1496577 & 1495317\end{array}$ $\begin{array}{lllll}1494.507 & 1494.942 & 1496.558 & 1495.317\end{array}$ $\begin{array}{lllll}1494.507 & 1494.944 & 1496.548 & 1495.315\end{array}$ $\begin{array}{lllll}1494.514 & 1494.958 & 1496.553 & 1495.322\end{array}$ $\begin{array}{lllll}1494.519 & 1494.979 & 1496.562 & 1495.334\end{array}$ $\begin{array}{lllll}1494.526 & 1495.003 & 1496.577 & 1495.348\end{array}$ $\begin{array}{llll}1494.521 & 1495.026 & 1496.577 & 1495.357 \\ 1494.523 & 1495.049 & 1496.579 & 1495.366\end{array}$

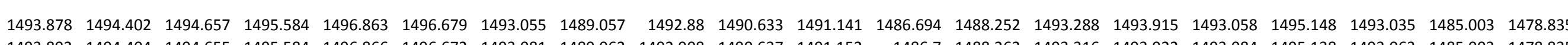

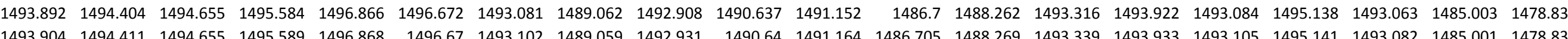

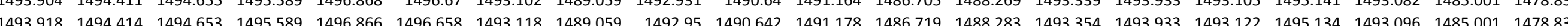
$\begin{array}{llllllllllllllllllllll}1493.92 & 1494.411 & 1494.658 & 1495.594 & 1496.868 & 1496.662 & 1493.133 & 1489.062 & 1492.964 & 1490.647 & 1491.187 & 1486.728 & 148829 & 1493.37 & 1493.943 & 1493.136 & 1495.138 & 1493.112 & 1485.003 & 1478.842\end{array}$ $\begin{array}{llllllllllllllllllllll}1493.918 & 1494.414 & 1494.662 & 1495603 & 1496.877 & 14966672 & 1493.149 & 1489.064 & 1492.978 & 1490.649 & 1491.199 & 1486744 & 1488.29 & 1493386 & 1493.959 & 1493.15 & 1495.15 & 1493.129 & 1485.001 & 14788.844\end{array}$ $\begin{array}{lllllllllllllllllllll}1493.927 & 1494.411 & 1494.655 & 1495.605 & 1496.875 & 1496.67 & 1493.161 & 1489.064 & 1492.99 & 1490.651 & 1491.208 & 1486.756 & 1488.302 & 1493.398 & 1493.961 & 1493.16 & 1495.148 & 1493.138 & 1485.001 & 1478.846\end{array}$ $\begin{array}{llllllllllllllllllllll}1493.937 & 1494.409 & 1494.665 & 1495.607 & 1496.877 & 1496.665 & 1493.168 & 1489.069 & 1492.999 & 1490.654 & 1491.215 & 1486.68 & 1488.309 & 1493.408 & 1493.961 & 1493.169 & 1495.15 & 1493.147 & 1485.001 & 1478.849\end{array}$

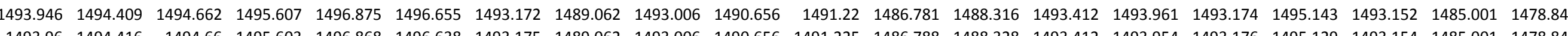

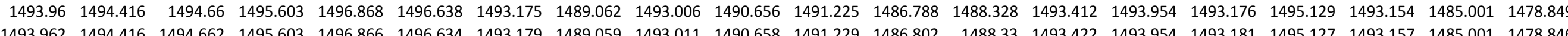

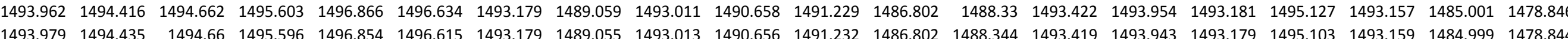

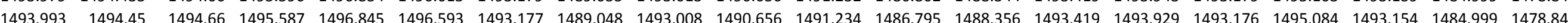

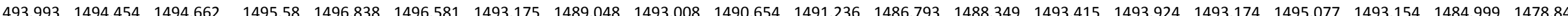
$\begin{array}{llllllllllllllllllllll}1493.991 & 1494.454 & 1494.657 & 1495.575 & 1496.831 & 1496.576 & 1493.177 & 1489.036 & 1493.011 & 1490.654 & 1491.243 & 1486.805 & 1488.349 & 1493.419 & 1493.919 & 1493.174 & 1495.073 & 1493.157 & 1484.999 & 1478.844\end{array}$ $\begin{array}{lllllllllllllllllllllllll}1493.986 & 1494.447 & 1494.665 & 1495.57 & 1496.829 & 1496.574 & 1493.179 & 1489.034 & 1493.016 & 1490.654 & 1491.253 & 1486.821 & 1488.351 & 1493.424 & 1493.924 & 1493.181 & 1495.075 & 1493.161 & 1484.999 & 1478.849\end{array}$ $\begin{array}{lllllllllllllllllllll}1493.974 & 1494.514 & 1494.66 & 1495.575 & 1496.829 & 1496.593 & 1493.191 & 1489.034 & 1493.03 & 1490.656 & 1491.262 & 1486.839 & 1488.351 & 1493.434 & 1493.936 & 1493.195 & 1495.084 & 1493.171 & 1484.996 & 1478.867\end{array}$

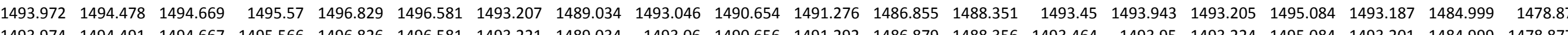

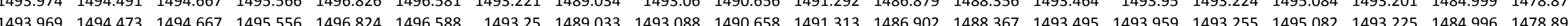
$\begin{array}{lllllllllllllllllllll}1493.969 & 1494.473 & 1494.667 & 1495.556 & 1496.824 & 1496.588 & 1493.25 & 1489.03 & 1493.088 & 1490.658 & 1491.313 & 1486.902 & 1488.367 & 1493.495 & 1493.959 & 1493.255 & 1495.082 & 1493.225 & 1484.996 & 1478.895 \\ 1493.958 & 1494.363 & 1494.662 & 1495.491 & 1496831 & 1496021 & 1493268 & 1489.04 & 1493.102 & 1490663 & 1491325 & 1486834 & 1488372 & 1493513 & 149398 & 1493269 & 1494939 & 1493072 & 1484994 & 1478905\end{array}$ $\begin{array}{llllllllllllllllllll}1493.955 & 1494.361 & 1494.674 & 1495.482 & 1496833 & 1496.114 & 1493.261 & 1489.038 & 1493.095 & 1490.665 & 1491.327 & 1486.825 & 1488.363 & 1493.504 & 1493.982 & 1493.259 & 1495.03 & 1493.152 & 1484.996 & 1478.907\end{array}$ $\begin{array}{llllllllllllllllllll}1493.995 & 1494.45 & 1494.648 & 1495.454 & 1496808 & 1496.323 & 1493257 & 1489.033 & 1493.095 & 1490.663 & 1491.33 & 1486.835 & 1488.4 & 1493.499 & 1493.95 & 1493.255 & 1494.859 & 1493.014 & 1484.994 & 1478912\end{array}$ $\begin{array}{lllllllllllllllllllllll}1493.976 & 1494.395 & 1494.655 & 1495.447 & 1496.815 & 1496.591 & 1493.257 & 1489.026 & 1493.095 & 1490.665 & 1491.334 & 1486.848 & 1488.377 & 1493.502 & 1493.971 & 1493.259 & 1495.014 & 1493.119 & 1484.996 & 1478.916\end{array}$

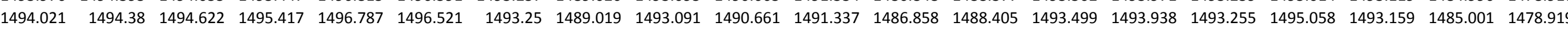
$\begin{array}{llllllllllllllllllll}1494.002 & 1494.349 & 1494.513 & 1495.415 & 1496.784 & 1496.516 & 1493.252 & 1489.015 & 1493.091 & 1490.654 & 1491.344 & 1486.874 & 1488.391 & 1493.499 & 1493.947 & 1493.255 & 1495.068 & 1493.19 & 1485.003 & 1478.928 \\ & & & & \end{array}$

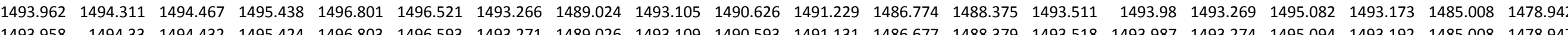

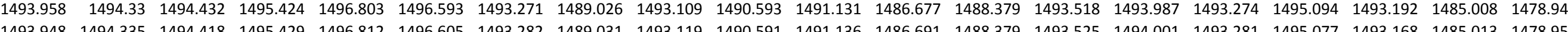
$\begin{array}{lllllllllllllllllllll}1493.948 & 1494.335 & 1494.418 & 1495.429 & 1496.812 & 1496.605 & 1493.282 & 1489.031 & 1493.119 & 1490.591 & 1491.136 & 1486.691 & 1488.379 & 1493.525 & 1494.001 & 1493.281 & 1495.077 & 1493.168 & 1485.013 & 1478.951 \\ 1493.936 & 1494.325 & 1494.437 & 1495.44 & 1496.824 & 1496.622 & 1493.287 & 1489.043 & 1493.123 & 1490588 & 1491.236 & 1486714 & 1488.363 & 1493.528 & 1494.024 & 1493.29 & 1495.103 & 1493.166 & 1485.017 & 1478958\end{array}$

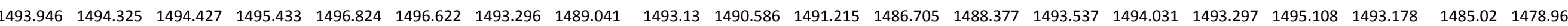
$\begin{array}{llllllllllllllllllllll}1493.944 & 1494.325 & 1494.432 & 1495.436 & 1496.829 & 1496.627 & 1493.301 & 1489.04 & 1493.142 & 1490.581 & 1491.201 & 1486.696 & 1488.37 & 1493.544 & 1494.048 & 1493.309 & 1495.11 & 1493.164 & 1485.027 & 1478.97\end{array}$ \begin{tabular}{llllllllllllllllllllll}
1493.944 & 1494.33 & 1494.429 & 1495.45 & 1496.831 & 1496.634 & 1493.31 & 1489.043 & 1493.144 & 1490.57 & 1491.19 & 1486.694 & 1488.37 & 1493.549 & 1494.05 & 1493.314 & 1495.143 & 1493.185 & 1485.031 & 1478.975 \\
\hline
\end{tabular} $\begin{array}{lllllllllllllllllllll}1493.955 & 1494.347 & 1494.444 & 1495.475 & 1496.829 & 1496.624 & 1493.317 & 1489.04 & 1493.149 & 1490.556 & 1491.208 & 1486.717 & 1488.382 & 1493.556 & 1494.055 & 1493.316 & 1495.162 & 1493.206 & 1485.031 & 1478.977\end{array}$

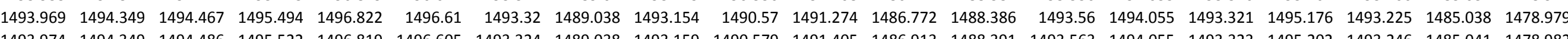

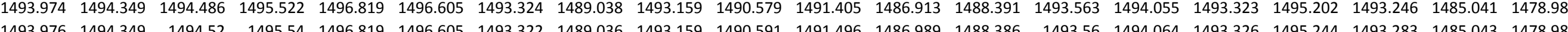

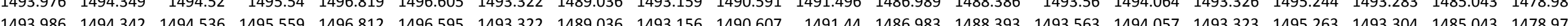

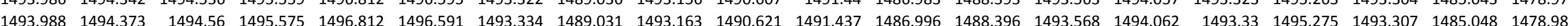
$\begin{array}{llllllllllllllllllll}1493.976 & 1494.395 & 1494.586 & 1495.591 & 1496.815 & 1496.6 & 1493.343 & 1489.034 & 1493.168 & 1490.635 & 1491.44 & 1487.013 & 1488.386 & 1493.572 & 1494.078 & 1493.335 & 1495.293 & 1493.314 & 1485.05 & 1478.989\end{array}$ $\begin{array}{llllllllllllllllllllll}1493.96 & 1494.397 & 1494.605 & 1495.61 & 1496.829 & 1496.627 & 1493.348 & 1489.033 & 1493.177 & 1490.658 & 1491.444 & 1487.022 & 1488.377 & 1493.586 & 1494.097 & 1493.342 & 1495.324 & 1493.323 & 1485.053 & 1478.991 \\ \end{array}$ $\begin{array}{llllllllllllllllllll}1493.953 & 1494.409 & 1494.62 & 1495.624 & 1496.838 & 1496.646 & 1493.355 & 1489.04 & 1493.187 & 1490.679 & 1491.449 & 1487.036 & 1488.379 & 1493.591 & 1494.115 & 1493.357 & 1495.343 & 1493.337 & 1485.055 & 1478.993 \\ \end{array}$

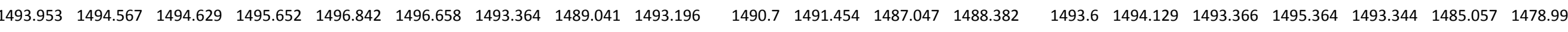

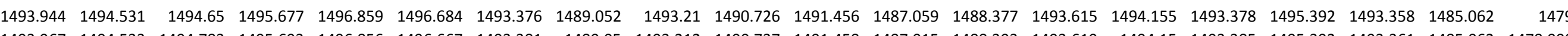
$\begin{array}{lllllllllllllllllllll}1493.967 & 1494.533 & 1494.783 & 1495.693 & 1496.856 & 1496.667 & 1493.381 & 1489.05 & 1493.212 & 1490.737 & 1491.458 & 1487.01 & 1488.393 & 1493.619 & 1494.15 & 1493.385 & 1495.392 & 1493.361 & 1485.062 & 1478.998 \\ 1493.969 & 1494.514 & 1494.947 & 1495.707 & 1496.861 & 1496.672 & 149339 & 1489.052 & 149322 & 1490.761 & 1491.458 & 1487.024 & 1488393 & 1493.626 & 1494.162 & 1493.39 & 1495.404 & 1493.366 & 1485067 & 1478998\end{array}$

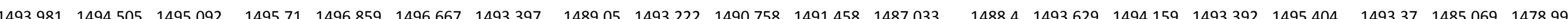
$\begin{array}{llllllllllllllllllllll}1494 & 1494.497 & 1495.244 & 1495.707 & 1496.852 & 1496.648 & 1493.402 & 1489.048 & 1493.222 & 1490.761 & 1491.458 & 1487.043 & 1488.41 & 1493.629 & 1494.157 & 1493.392 & 1495.402 & 1493.368 & 1485.071 & 1478.993\end{array}$ $\begin{array}{lllllllllllllllllllllll}1494.012 & 1494.469 & 1495.387 & 1495.71 & 1496.849 & 1496.636 & 1493.406 & 1489.041 & 1493.222 & 1490.763 & 1491.458 & 1487.047 & 1488.41 & 1493.631 & 1494.152 & 1493.39 & 1495.397 & 1493.368 & 1485.074 & 1478.991 \\ \end{array}$ $\begin{array}{llllllllllllllllllll}1494.007 & 1494.423 & 1495.534 & 1495.679 & 1496.852 & 1496.643 & 1493.409 & 1489.043 & 1493.227 & 1490.765 & 1491.458 & 1487.059 & 1488.41 & 1493.631 & 1494.164 & 1493.394 & 1495.411 & 1493.375 & 1485.076 & 1478.989\end{array}$ $\begin{array}{llllllllllllllllllllll}1494.002 & 1494.552 & 1495.674 & 1495.707 & 1496.856 & 1496.653 & 1493.418 & 1489.045 & 1493.231 & 1490.765 & 1491.461 & 1487.07 & 1488.412 & 1493.638 & 1494.173 & 1493.402 & 1495.423 & 1493.38 & 1485.081 & 1478.989 \\ \end{array}$

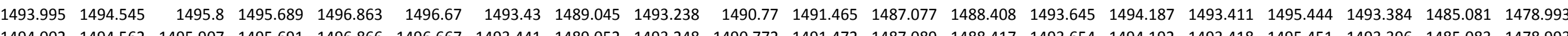

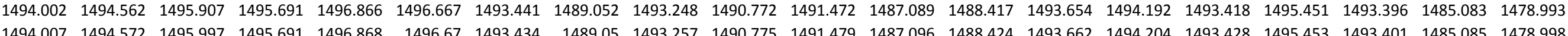


Well

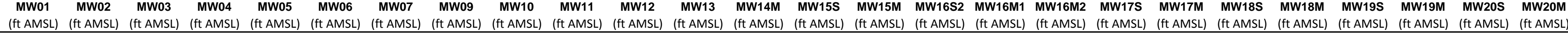

$12 / 21 / 112: 00$ $12 / 21 / 113: 00$ $12 / 21 / 115.00$ 12/21/111 6:00 $12 / 21 / 116: 00$ $12 / 21 / 118: 00$ 12/21/11 9:00 $12 / 21 / 1110: 00$ $12 / 21 / 1111: 00$ 12/21/11 12:00 12/21/111 14:00 $12 / 21 / 1115: 00$ $12 / 21 / 1116: 00$ $12 / 21 / 1117: 00$ $12 / 21 / 1118: 00$ 12/21/11 19:00 2/21/11 20:00 2/21/1121:00 12/21/21/11 23:00 $12 / 22 / 110: 00$ 12/22/110:00 12/22/112:00 12/22/11 3:00 12/22/11 4:00 12/22/11 5:00 $12 / 22 / 118: 00$ 12/22/11 9:00 12/22/11 11:00 $12 / 22 / 1113: 00$ 2/22/111 14:00 12/22/11 15:00 $12 / 22 / 1117: 00$ 12/22/11 18:00 12/22/11 19:00 12/22/11 20:00 $12 / 22 / 1121: 00$ 12/22/11122:00 $12 / 23 / 110.00$ 12/23/11 0:00 12/23/11 2:00 12/23/11 4:00 12/23/11 5:00 12/23/11 6:00 $\begin{array}{lrrrr}1494.523 & 1495.08 & 1496.586 & 1495.378 \\ 1494.528 & 1495.105 & 1496.593 & 1495.387\end{array}$ $\begin{array}{rrrr}1494.528 & 1495.105 & 1496.593 & 1495.387 \\ 194.537 & 149514 & 1496605 & 195.404\end{array}$ $\begin{array}{lllll}1494.537 & 1495.14 & 1496.605 & 1495.404 \\ 1494.535 & 1495.177 & 1496605 & 1495.408\end{array}$ $\begin{array}{lllll}1494.53 & 1495.21 & 1496.593 & 1495.408\end{array}$ $\begin{array}{lllll}1494.533 & 1495.245 & 1496591 & 1455.408\end{array}$ $\begin{array}{lllll}1494.535 & 1495.275 & 1496.586 & 1495.411\end{array}$ $\begin{array}{lllll}1494.537 & 1495.299 & 1496.584 & 1495.408\end{array}$ $\begin{array}{llll}1494.533 & 1495.322 & 1496.577 & 1495.406\end{array}$ $\begin{array}{lllll}1494.542 & 1495.098 & 1496.579 & 1495.408\end{array}$ $\begin{array}{llll}1494.547 & 1495.096 & 1496.616 & 1495.415 \\ 1494556 & 1495103 & 1496.623 & 1955.434\end{array}$ $\begin{array}{llll}1494.556 & 1495.103 & 1496.623 & 1495.434 \\ 1494.556 & 1495.112 & 1496.539 & 1495.446\end{array}$ $\begin{array}{llll}1494.556 & 1495.112 & 1496.539 & 1495.446 \\ 1494.556 & 1495.115 & 1496.453 & 1495.453\end{array}$ $\begin{array}{rrrr}1494.556 & 1495.115 & 1496.453 & 1495.453 \\ 1494.549 & 1495.108 & 1496.446 & 1495.45\end{array}$ $\begin{array}{llll}1494.554 & 1495.105 & 1496.441 & 14955.45 \\ 1495 & 1495\end{array}$ $\begin{array}{llll}1494.549 & 1495.094 & 1496.418 & 1495.441\end{array}$ $\begin{array}{lllll}1494.544 & 1495.077 & 1466.401 & 1495.425\end{array}$ $\begin{array}{llll}1494.547 & 1495.068 & 1496.546 & 1495.413\end{array}$ $\begin{array}{llll}1494.544 & 1495.052 & 1496.532 & 1495.394 \\ 1494542 & 1495035 & 1496511 & 1495376\end{array}$ $\begin{array}{lllll}1494.542 & 1495.035 & 1496.511 & 1495.376 \\ 1494.544 & 1495.021 & 1496.492 & 1495.357\end{array}$ $\begin{array}{llll}1494.551 & 1495.024 & 1496.504 & 1495.357\end{array}$ $\begin{array}{lllll}1494.552 & 1495.021 & 1496.504 & 1495.355\end{array}$ $\begin{array}{lllll}1494.547 & 1495.014 & 1496.497 & 1495.35\end{array}$ $\begin{array}{lllll}1494.54 & 1494.984 & 1496.46 & 1495.322\end{array}$ $\begin{array}{lllll}1494.544 & 1494.979 & 1496.562 & 1495.317\end{array}$ $\begin{array}{lllll}1494.547 & 1494.977 & 1496.558 & 1495.315 \\ 1494.538 & 1494.954 & 1496511 & 195294\end{array}$ $\begin{array}{lllll}1494.538 & 1494.954 & 1496.511 & 1495.294 \\ 1494.53 & 1494.928 & 1496.457 & 1495.266\end{array}$ $\begin{array}{lllll}1494.533 & 1494.916 & 1496.432 & 1495.254\end{array}$ $\begin{array}{llllll}1494.523 & 1494.888 & 1496.378 & 1495.226\end{array}$ 1494.514 $1494.865 \quad 1496.319 \quad 1495.196$ $\begin{array}{lllll}1494.517 & 1494.849 & 1496.296 & 1495.182\end{array}$ $\begin{array}{llll}1494.517 & 1494.849 & 1496.301 & 1495.175\end{array}$ $\begin{array}{llll}1494.521 & 1494.872 & 1496.315 & 1495.18\end{array}$ $\begin{array}{llll}1494.521 & 1494.856 & 1496.319 & 1495.182 \\ 1494.519 & 1494.853 & 1496322 & 1495.184\end{array}$ $\begin{array}{lllll}1494.514 & 1494.842 & 1496298 & 1495.184\end{array}$ $\begin{array}{lllll}1494.514 & 1494.835 & 1496.291 & 1495.17\end{array}$ $\begin{array}{lllll}1494.512 & 1494.828 & 1496.284 & 1495.163\end{array}$ $\begin{array}{rrrrr}1494.512 & 1494.823 & 1496.28 & 1495.161\end{array}$ $\begin{array}{lllll}1494.509 & 1494.818 & 1496.273 & 1495.156\end{array}$ $\begin{array}{lllll}1494.505 & 1494.804 & 1496.254 & 1495.145\end{array}$ $\begin{array}{rrrr}1494.503 & 1494.8 & 1496.249 & 1495.135 \\ 1494.498 & 1494.79 & 1496.24 & 1495.126\end{array}$ $\begin{array}{rrrrr}1494.498 & 1494.79 & 1496.24 & 1495.126 \\ 1494.503 & 1494.788 & 1496245 & 1495.126\end{array}$ $\begin{array}{llll}1444.503 & 1494.795 & 1496.256 & 1495.133\end{array}$ $\begin{array}{lllll}1494.505 & 1494.802 & 1496.266 & 1495.142\end{array}$ $\begin{array}{lllll}1494.505 & 1494.802 & 1496.27 & 1495.147\end{array}$ $\begin{array}{lllll}1494.507 & 1494.811 & 1496.282 & 1495.159\end{array}$ $\begin{array}{lllll}1494.507 & 1494.811 & 1496.277 & 1495.161\end{array}$ $\begin{array}{llll}1494.503 & 1494.804 & 1496.263 & 1495.159 \\ 1494.507 & 1494.811 & 1496.273 & 1495.163\end{array}$

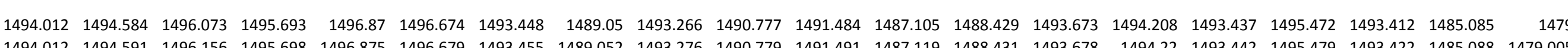

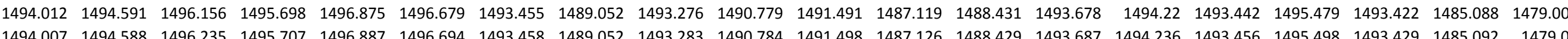

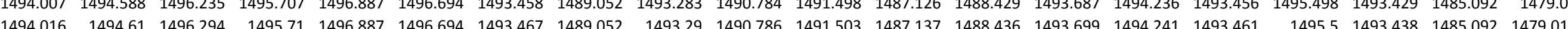

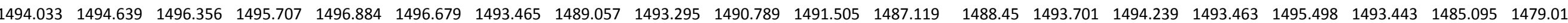
$\begin{array}{llllllllllllllllllllll}1494.04 & 1494.651 & 1496.42 & 1495.707 & 1496.884 & 1496.677 & 1493.465 & 1489.055 & 1493.299 & 1490.791 & 1491.507 & 1487.121 & 1488.45 & 1493.709 & 1494.239 & 1493.468 & 1495.496 & 1493.448 & 1485.095 & 14979.01\end{array}$

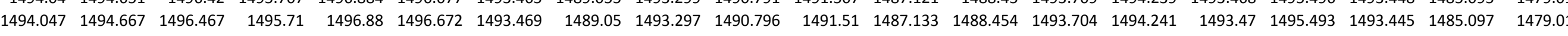
$\begin{array}{llllllllllllllllllllll}1494.052 & 1494.679 & 1496.515 & 1495.71 & 1496.88 & 1496.667 & 1493.469 & 1489.048 & 1493.299 & 1490.796 & 1491.512 & 1487.144 & 1488.457 & 1493.709 & 1494.239 & 1493.47 & 1495.498 & 1493.45 & 1485.097 & 1479.007\end{array}$

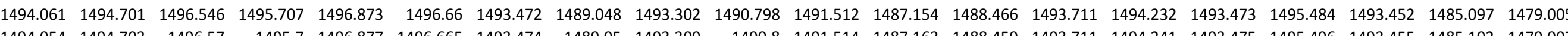

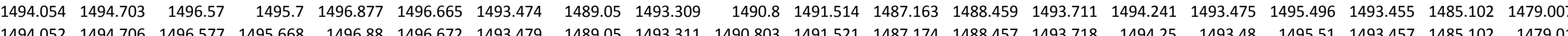

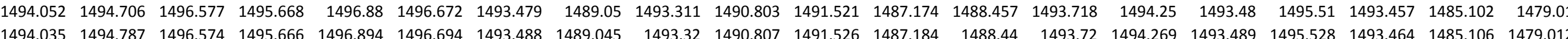

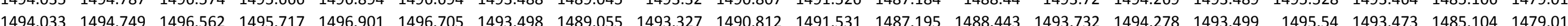

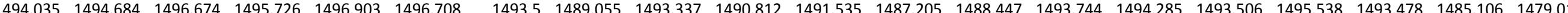
$\begin{array}{llllllllllllllllllllll}1494.054 & 1494.66 & 1496.817 & 1495.726 & 1496.898 & 1496.694 & 1493.507 & 1489.055 & 1493.332 & 1490.8 & 1491.538 & 1487.156 & 1488.457 & 1493.746 & 1494.281 & 1493.508 & 1495.521 & 1493.473 & 1485.106 & 1479.017\end{array}$

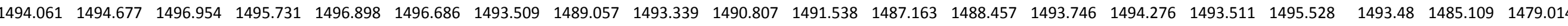
$\begin{array}{llllllllllllllllllllll}1494.082 & 1494.708 & 1497.087 & 1495.726 & 1496.889 & 1496.667 & 1493.509 & 1489.055 & 1493.334 & 1490.81 & 1491.54 & 1487.165 & 1488.468 & 1493.749 & 1494.267 & 1493.506 & 1495.517 & 1493.483 & 1485.109 & 1499.014 & 1493\end{array}$

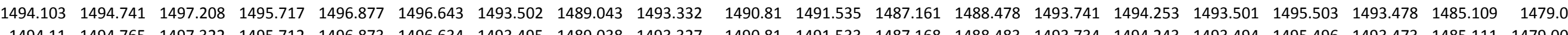

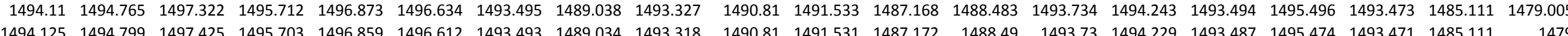

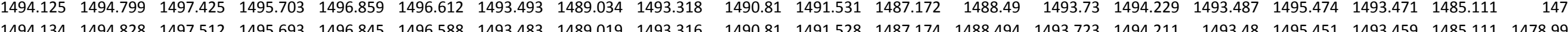

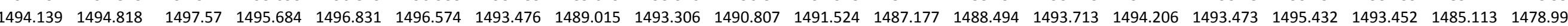
$\begin{array}{llllllllllllll}4 & \end{array}$ $\begin{array}{llllllllllllllllllllll}1494.11 & 1494.82 & 1497.669 & 1495.684 & 1496.831 & 1496.591 & 1493.474 & 1489.01 & 1493.309 & 1490.81 & 1491.531 & 1487.191 & 1488.483 & 1493.713 & 1494.211 & 1493.473 & 1495.444 & 1493.452 & 1485.118 & 1478.996\end{array}$ $\begin{array}{llllllllllllllllllllll}1494.113 & 1494.828 & 1497.669 & 1495.677 & 1496.824 & 1496.583 & 1493.477 & 1489.005 & 1493.311 & 1490.81 & 1491.535 & 1487.2 & 1488.492 & 1493.716 & 1494.208 & 1493.477 & 1495.442 & 1493.457 & 1485.116 & 1478.998\end{array}$ $\begin{array}{llllllllllllllllllll}1494.136 & 1494.825 & 1497.688 & 1495.656 & 1496.803 & 1496.545 & 1493.469 & 1488.996 & 1493.304 & 1490.807 & 1491.535 & 1487.207 & 1488.497 & 1493.716 & 1494.187 & 1493.47 & 1495.411 & 1493.45 & 1485.116 & 1478.998\end{array}$

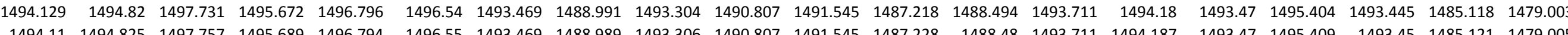

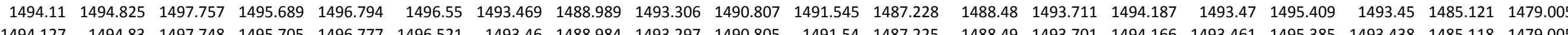

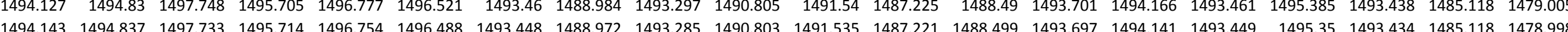
$\begin{array}{lllllllllllllllllllll}1494.132 & 1494.84 & 1497.691 & 1495.721 & 1496.742 & 1496.483 & 1493.441 & 1488.968 & 1493.28 & 1490.798 & 1491.533 & 1487.209 & 1488.487 & 1493.683 & 1494.134 & 1493.439 & 1495.338 & 1493.424 & 1485.121 & 1478.996\end{array}$ \begin{tabular}{lllllllllllllllllllllll}
1494.141 & 1494.837 & 1497.61 & 1495.642 & 1496.719 & 1496.449 & 1493.427 & 1488.959 & 1493.264 & 1490.793 & 1491.528 & 1487.193 & 1488.492 & 1493.673 & 1494.103 & 1493.43 & 1495.308 & 1493.408 & 1485.118 & 1478.991 \\
\hline
\end{tabular}

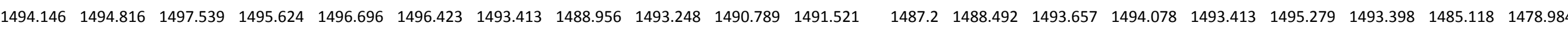
$\begin{array}{llllllllllllllllllllllllll}1494.129 & 1494.813 & 1497.491 & 1495.631 & 1496.682 & 1496.416 & 1493.402 & 1488.947 & 1493.241 & 1490.786 & 1491.514 & 1487.205 & 1488.478 & 1493.645 & 1494.071 & 1493.404 & 1495.27 & 1493.387 & 1485.118 & 1478.982\end{array}$ $\begin{array}{llllllllllllllllllll}1494.108 & 1494.808 & 1497.463 & 1495.612 & 1496.68 & 1496.426 & 1493.397 & 1488.944 & 1493.236 & 1490.784 & 1491.517 & 1487.214 & 1488.462 & 1493.64 & 1494.073 & 1493.397 & 1495.275 & 1493.38 & 1485.121 & 1478.979\end{array}$

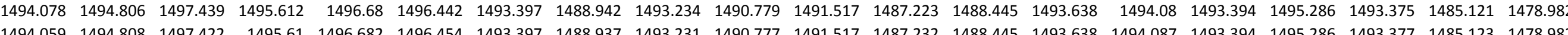

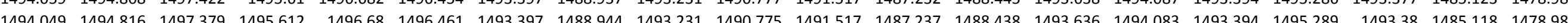

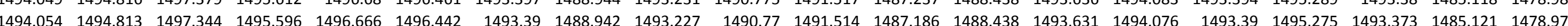
\begin{tabular}{lllllllllllllllllllllll}
1494.047 & 1494.811 & 1497.325 & 1495.538 & 1496.659 & 1496.44 & 1493.381 & 1488.935 & 1493.22 & 1490.77 & 1491.51 & 1487.195 & 1488.426 & 1493.626 & 1494.071 & 1493.385 & 1495.263 & 1493.366 & 1485.121 & 1478.977 \\
\hline
\end{tabular}

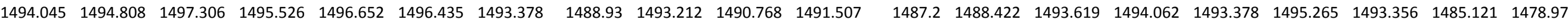

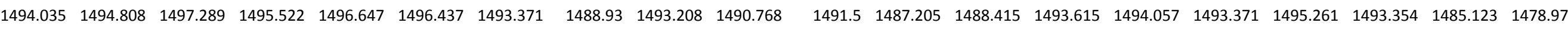

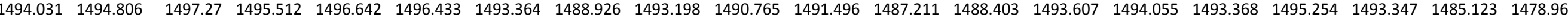

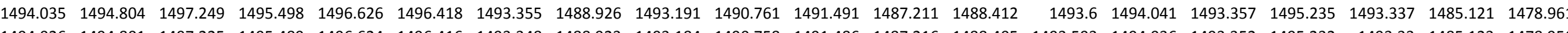

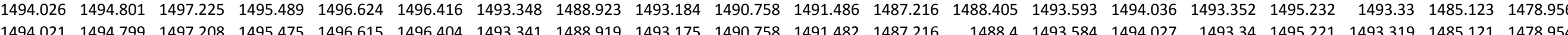

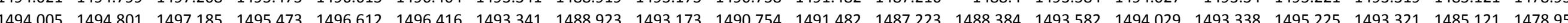

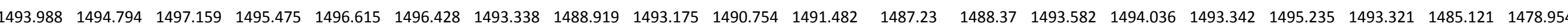
$\begin{array}{llllllllllllllllllll}1493.972 & 1494.804 & 1497.137 & 1495.48 & 1496.619 & 1496.445 & 1493.345 & 1488.926 & 1493.18 & 1490.756 & 1491.486 & 1487.237 & 1488.372 & 1493.586 & 1494.043 & 1493.347 & 1495.244 & 1493.326 & 1485.121 & 1478.956\end{array}$

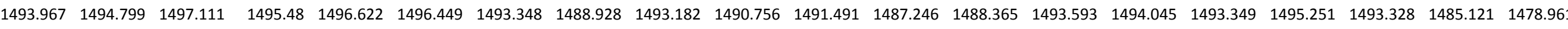
\begin{tabular}{llllllllllllllllllll}
1493.953 & 1494.801 & 1497.08 & 1495.484 & 1496.629 & 1496.464 & 1493.355 & 1488.919 & 1493.189 & 1490.758 & 1491.496 & 1487.253 & 1488.356 & 1493.596 & 1494.062 & 1493.354 & 1495.263 & 1493.335 & 1485.121 & 1478.963 \\
\hline
\end{tabular} $\begin{array}{lllllllllllllllllllll}1493.953 & 1494.806 & 1497.052 & 1495.484 & 1496.629 & 1496.466 & 1493.357 & 1488.919 & 1493.196 & 1490.758 & 1491.496 & 1487.267 & 1488.358 & 1493.603 & 1494.059 & 1493.359 & 1495.261 & 1493.34 & 1485.121 & 1478.968\end{array}$

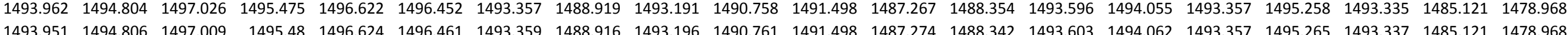


Well

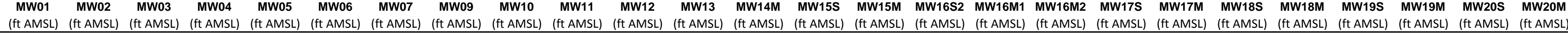

12/23/11 8:00 $12 / 23 / 119: 00$ $12 / 23 / 1110: 00$ 12/23/111112:00 $12 / 23 / 11113: 00$ $12 / 23 / 1114: 00$ 12/23/11 15:00 $12 / 23 / 1116: 00$ $12 / 23 / 1117: 00$ $12 / 23 / 1118: 00$ 12/23/11 19:00 $12 / 23 / 1121: 00$ $12 / 23 / 1122: 00$ 12/23/11 23:00 12/24/11 0:00 12/24/11 1:00 12/24/11 2:00 12/24/11 4:00 12/24/11 6:00 12/22/1117:00 $12 / 24 / 119: 00$ 12/24/11 10:00 $12 / 24 / 1111: 00$ 12/24/24/11 12:00 12/24/11 14:00 12/24/111 15:00 $12 / 24 / 1116: 00$ 12/24/11 17:00 12/24/1118:00 12/24/1119:00 $12 / 24 / 1120: 00$ 12/24/11121:00 12/24/11 22:00 12/24/11 23:00 12/25/111:00 12/25/11 2:00 12/25/11 3:00 12/25/11 4:00 12/25/11 5:00 12/25/11 6:00 $12 / 25 / 119: 00$ 12/25/11 10:00 12/25/11 11:00 12/25/11 12:00 $\begin{array}{rrrr}1494.509 & 1494.818 & 1496.287 & 1495.173 \\ 1494.51 & 1494.818 & 1496.287 & 1495.177\end{array}$ $\begin{array}{rrrr}1444.51 & 1494.818 & 1496.287 & 1495.177 \\ 1494.512 & 1494825 & 1496289 & 1495.18\end{array}$ $\begin{array}{rrrrr}1494.512 & 1494.825 & 1496.289 & 1495.18 \\ 1494.517 & 149483 & 1496303 & 1495.189\end{array}$ $\begin{array}{lllll}1494.528 & 1494.851 & 1496.338 & 1495.215\end{array}$ $\begin{array}{lllll}1494.535 & 1494.867 & 1496.371 & 1455.24\end{array}$ $\begin{array}{lllll}1494.54 & 1494.884 & 1496.397 & 1495.261\end{array}$ $\begin{array}{llllll}1494.542 & 1494.891 & 1496.411 & 1495.282\end{array}$ $\begin{array}{llll}1494.547 & 1494.902 & 1496.418 & 1495.294\end{array}$ $\begin{array}{llll}1494.551 & 1494.914 & 1496.432 & 1495.306\end{array}$ $\begin{array}{rrrr}1494.549 & 1494.914 & 1496.427 & 1495.313 \\ 1494.552 & 1494.914 & 1496.42 & 1495313\end{array}$ $\begin{array}{rrrr}1494.552 & 1494.914 & 1496.42 & 1495.313 \\ 1494.554 & 1494.921 & 1496.422 & 1495.313\end{array}$ $\begin{array}{rrrr}1494.554 & 1494.921 & 1496.422 & 1495.313 \\ 1494.556 & 1499.921 & 1496.42 & 1495.31 \\ 1494.554 & 1494.921 & 1496.415 & 1495.308\end{array}$ $\begin{array}{lllll}1494.561 & 1494.928 & 1496.427 & 1495.313\end{array}$ $\begin{array}{lllll}1494.561 & 1494.93 & 1496.427 & 1495.313\end{array}$ $\begin{array}{lllll}1494.568 & 1494.94 & 1496.446 & 1495.322\end{array}$ $\begin{array}{llll}1494.565 & 1494.944 & 1496.45 & 1495.329\end{array}$ $\begin{array}{lllll}1494.561 & 1494.94 & 1496.441 & 1495.322 \\ 1494.561 & 1494.94 & 1496.436 & 1495.32\end{array}$ $\begin{array}{llll}1494.568 & 1494.947 & 1466.448 & 1495.324\end{array}$ $\begin{array}{lllll}1494.563 & 1494.942 & 1496.434 & 1495.32\end{array}$ $\begin{array}{llll}1494.558 & 1494.935 & 1496.418 & 1495.306\end{array}$ $\begin{array}{llllll}1494.563 & 1494.937 & 1496.422 & 1495.306\end{array}$ $\begin{array}{llll}1494.565 & 1494.937 & 1496.422 & 1495.303\end{array}$ $\begin{array}{llll}1494.558 & 1494.926 & 1496.399 & 1495.289\end{array}$ $\begin{array}{lllll}1494.565 & 1494.928 & 1496.401 & 1495.282\end{array}$ $\begin{array}{llll}1494.575 & 1494.937 & 1496.429 & 1495.294 \\ 1494.579 & 1494.947 & 1496.448 & 1495308\end{array}$ $\begin{array}{lllll}1494.582 & 1494.961 & 1496.474 & 1495.329\end{array}$ $\begin{array}{llll}1494.579 & 1494.97 & 1496.483 & 1495.341\end{array}$ $\begin{array}{lllll}1494.577 & 1494.968 & 1496.476 & 1495.341\end{array}$ $\begin{array}{lllll}1494.572 & 1494.963 & 1496.46 & 1495.336\end{array}$ $\begin{array}{llll}1494.575 & 1494.961 & 1496.448 & 1495.327\end{array}$ $\begin{array}{llll}1494.577 & 1494.958 & 1496.443 & 1495.32\end{array}$ $\begin{array}{llll}1494.584 & 1494.963 & 1496.455 & 1495.327\end{array}$ $\begin{array}{rrrrr}1494.584 & 1494.968 & 1496.46 & 1495.324 \\ 1494.584 & 1494.968 & 1496.462 & 1495.327\end{array}$ $\begin{array}{lllll}1494.584 & 1494.97 & 1496.464 & 1495.327\end{array}$ $\begin{array}{lllll}1494.584 & 1494.968 & 1496.457 & 1495.322\end{array}$ $\begin{array}{llllll}1494.591 & 1494.975 & 1496.471 & 1495.327\end{array}$ $1494.5891494 .975 \quad 1496.464 \quad 1495.324$ $\begin{array}{lllll}1494.589 & 1494.97 & 1496.462 & 1495.32\end{array}$ $\begin{array}{llll}1494.593 & 1494.975 & 1496.469 & 1495.324 \\ 149.596 & 1494982 & 1466.485 & 1455334\end{array}$ $\begin{array}{lllll}1494.596 & 1494.982 & 1496.485 & 1495.334\end{array}$ $\begin{array}{llllll}1494.589 & 1494.977 & 1496.467 & 1495.327\end{array}$ $1494.586 \quad 1494.968 \quad 1496.446 \quad 1495.313$ 1494.5841494 .9561496 .4291495 .296 $1494.5841494 .944 \quad 1496.418 \quad 1495.285$ $1494.584 \quad 1494.94 \quad 1496.411 \quad 1495.275$ $\begin{array}{llll}1494.598 & 1494.954 & 1496.446 & 1495.287 \\ 1494.603 & 1494.965 & 1496.467 & 1495.303\end{array}$ $\begin{array}{llllllllllllllllllll}1493.939 & 1494.801 & 1496.99 & 1495.484 & 1496.633 & 1496.473 & 1493.359 & 1488.919 & 1493.194 & 1490.763 & 1491.498 & 1487.274 & 1488.337 & 1493.598 & 1494.071 & 1493.364 & 1495.277 & 1493.34 & 1485.121 & 1478.968 \\ 1493.941 & 1494.808 & 1496.961 & 1495.484 & 1496.631 & 1496.476 & 1493362 & 1488.921 & 1493.194 & 1490.765 & 1491.498 & 1487.281 & 1488337 & 1493607 & 1494.071 & 1493361 & 1495275 & 1493.34 & 1485.12 & 1478.968\end{array}$

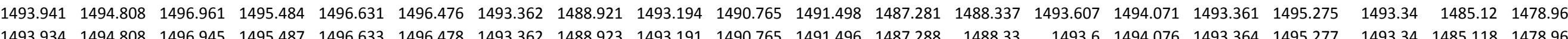

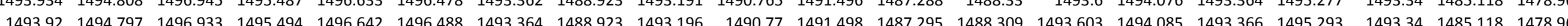
$\begin{array}{llllllllllllllllllllll}1493.894 & 1494.768 & 1496.926 & 1495.51 & 1496.663 & 1496.512 & 1493.374 & 1488.926 & 1493.205 & 1490.772 & 1491.503 & 1487.304 & 1488288 & 1493.61 & 1494.108 & 1493.375 & 1495.326 & 1493.347 & 1485.121 & 1478.965\end{array}$ $\begin{array}{llllllllllllllllllllll}1493.878 & 1494.753 & 1496.914 & 1495529 & 1496.68 & 1496548 & 1493.385 & 1488.94 & 1493215 & 1490.779 & 1491.505 & 1487.304 & 1488285 & 1493.622 & 1494.129 & 1493.387 & 1495.35 & 1493.361 & 1485.121 & 1478.97\end{array}$

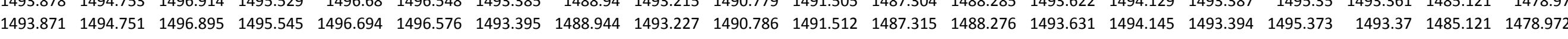
$\begin{array}{llllllllllllllllllll}1493.878 & 1494.753 & 1496.874 & 1495.559 & 1496.705 & 1496.593 & 1493.406 & 1488.954 & 1493.238 & 1490.791 & 1491.517 & 1487.322 & 1488.281 & 1493.643 & 1494.159 & 1493.406 & 1495.387 & 1493.384 & 1485.121 & 1478.977 \\ \end{array}$

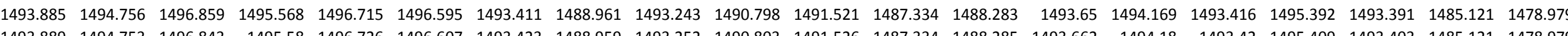

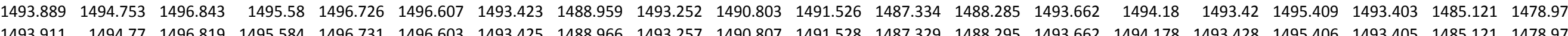
$\begin{array}{lllllllllllllllllllll}1493.911 & 1494.77 & 1496.819 & 1495.584 & 1496.731 & 1496.603 & 1493.425 & 1488.966 & 1493.257 & 1490.807 & 1491.528 & 1487.329 & 1488.295 & 1493.662 & 1494.178 & 1493.428 & 1495.406 & 1493.405 & 1485.121 & 1478.979 \\ 1493.929 & 1494.789 & 1496.802 & 1495.587 & 1496.731 & 1496.588 & 1493.427 & 1488.963 & 1493.257 & 1490.812 & 1491.526 & 1487.327 & 1488.307 & 1493.669 & 1494.173 & 1493.43 & 1495.402 & 1493.408 & 1485.121 & 1478979\end{array}$

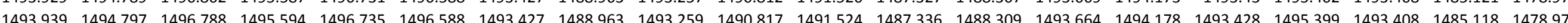
$\begin{array}{llllllllllllllllllll}1493.946 & 1494.806 & 1496.771 & 1495.596 & 1496.735 & 1496.581 & 1493.427 & 1488.963 & 1493.257 & 1490.819 & 1491.526 & 1487.334 & 1488.314 & 1493.664 & 1494.176 & 1493.428 & 1495.39 & 1493.405 & 1485.118 & 1478.972\end{array}$ $\begin{array}{llllllllllllllllllllll}1493.96 & 1494.825 & 1496.75 & 1495.596 & 1496.735 & 1496.579 & 1493.425 & 1488.963 & 1493.255 & 1490.821 & 1491.521 & 1487.341 & 1488.318 & 1493.664 & 1494.169 & 1493.428 & 1495.395 & 1493.403 & 1485.118 & 1478.968\end{array}$

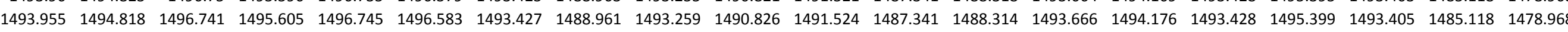
$\begin{array}{lllllllllllllllllllllll}1493.962 & 1494.828 & 1496.726 & 1495.61 & 1496.747 & 1496.581 & 1493.427 & 1488.959 & 1493.259 & 1490.828 & 1491.524 & 1487.346 & 1488.318 & 1493.669 & 1494.176 & 1493.43 & 1495.409 & 1493.408 & 1485.118 & 1478.9658 & \end{array}$

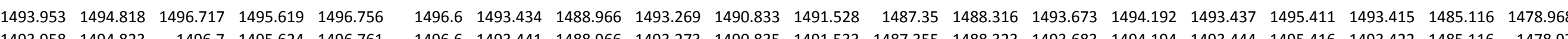

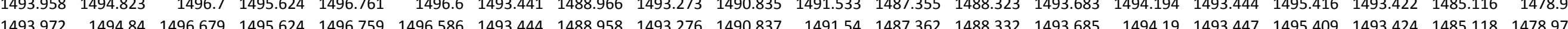

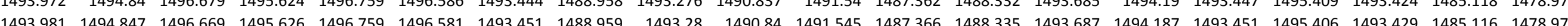
$\begin{array}{lllllllllllllllllllll}14393.976 & 1494.844 & 1496.655 & 1495.631 & 1496.763 & 1496.593 & 1493.458 & 1488.966 & 1493.288 & 1490.844 & 1491.549 & 1487.376 & 1488.337 & 1493.694 & 1494.197 & 1493.456 & 1495.411 & 1493.436 & 1485.113 & 1478.977\end{array}$ $\begin{array}{llllllllllllllllllllll}1494.007 & 1494.864 & 1496.622 & 1495.619 & 1496.752 & 1496.557 & 1493.453 & 1488.954 & 1493.285 & 1490.849 & 1491.554 & 1487.38 & 1488.349 & 1493.694 & 1494.18 & 1493.456 & 1495.385 & 1493.434 & 1485.113 & 1478.979 \\ \end{array}$

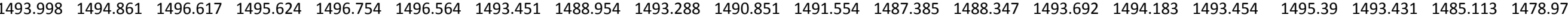
$\begin{array}{llllllllllllllllllll}1494 & 1494.866 & 1496.605 & 1495.621 & 1496.754 & 1496.557 & 1493.451 & 1488.949 & 1493.283 & 1490.849 & 1491.556 & 1487.389 & 1488.347 & 1493.694 & 1494.18 & 1493.451 & 1495.385 & 1493.431 & 1485.111 & 1478.982\end{array}$ $\begin{array}{llllllllllllllllllll}1494.012 & 1494.878 & 1496.584 & 1495.612 & 1496.745 & 1496.538 & 1493.444 & 1488.947 & 1493.28 & 1490.851 & 1491.554 & 1487.359 & 1488.354 & 1493.687 & 1494.162 & 1493.447 & 1495.366 & 1493.427 & 1485.109 & 1478.977\end{array}$ $\begin{array}{llllllllllllllllllllllll}1494.005 & 1494.861 & 1496.579 & 1495.61 & 1496.745 & 1496.528 & 1493.444 & 1488.942 & 1493.28 & 1490.851 & 1491.552 & 1487.334 & 1488.342 & 1493.685 & 1494.164 & 1493.444 & 1495.364 & 1493.419 & 1485.111 & 1478.975 \\ 1493.976 & 1494837 & 1496589 & 1495.621 & 1496756 & 1496.552 & 1493.446 & 1488944 & 1493278 & 1490854 & 1491.556 & 1487.336 & 1488321 & 1493687 & 1494.18 & 1493.447 & 149539 & 1493.427 & 1485.111 & 1478977\end{array}$

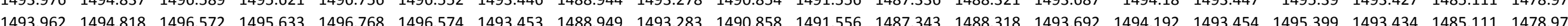
$\begin{array}{lllllllllllllllllllll}1493.951 & 1494.806 & 1496.562 & 1495.647 & 1496.78 & 1496.602 & 1493.462 & 1488.954 & 1493.292 & 1490.861 & 1491.561 & 1487.355 & 1488.311 & 1493.699 & 1494.211 & 1493.461 & 1495.425 & 1493.441 & 1485.109 & 1478.979\end{array}$ $\begin{array}{llllllllllllllllllllllll}1493.951 & 1494.811 & 1496.548 & 1495.656 & 1496.789 & 1496.617 & 1493.469 & 1488.959 & 1493.302 & 1490.863 & 1491.568 & 1487.357 & 1488.318 & 1493.709 & 1494.215 & 1493.47 & 1495.43 & 1493.448 & 1485.109 & 1478.982\end{array}$ $\begin{array}{llllllllllllllllllll}1493.965 & 1494.818 & 1496.534 & 1495.659 & 1496.789 & 1496.61 & 1493.474 & 1488.958 & 1493.304 & 1490.865 & 1491.568 & 1487.29 & 1488.325 & 1493.711 & 1494.213 & 1493.475 & 1495.43 & 1493.45 & 1485.106 & 1478.984 \\ \end{array}$

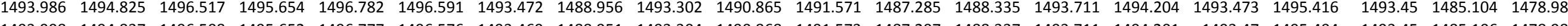

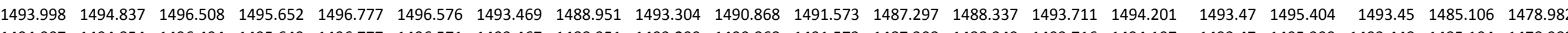

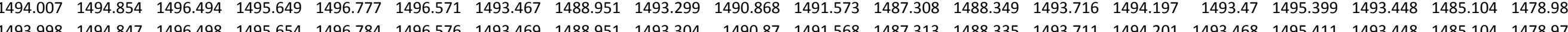

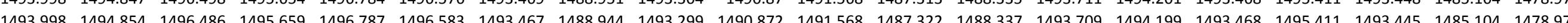

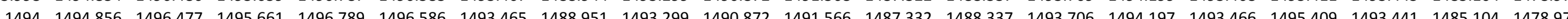
$\begin{array}{lllllllllllllllllllll}1494 & 1494.861 & 1496.463 & 1495.663 & 1496791 & 1496.588 & 1493.462 & 1488.949 & 1493.297 & 1490.875 & 1491.566 & 1487.341 & 1488.339 & 1493.704 & 1494.199 & 1493.466 & 1495.406 & 1493.443 & 1485.102 & 1478.972\end{array}$ $\begin{array}{llllllllllllllllllllll}1494.009 & 1494.875 & 1496.458 & 1495.661 & 1496.787 & 1496.579 & 1493.462 & 1488.944 & 1493.297 & 1490.875 & 1491.564 & 1487.346 & 1488.349 & 1493.706 & 1494.197 & 1493.463 & 1495.404 & 1493.441 & 1485.099 & 1478.97\end{array}$ $\begin{array}{llllllllllllllllllllll}1494 & 1494.866 & 1496.451 & 1495.666 & 1496.796 & 1496.588 & 1493.465 & 1488.947 & 1493.297 & 1490.877 & 1491.566 & 1487.355 & 1488.337 & 1493.706 & 1494.201 & 1493.466 & 1495.411 & 1493.443 & 1485.099 & 1478.968\end{array}$ $\begin{array}{llllllllllllllllllllll}1494.007 & 1494.875 & 1496.439 & 1495.66 & 1496.791 & 1496.586 & 1493.467 & 1488.947 & 1493.297 & 1490.877 & 1491.568 & 1487.364 & 1488.349 & 1493.709 & 1494.197 & 1493.466 & 1495.406 & 1493.445 & 1485.099 & 1478.97\end{array}$

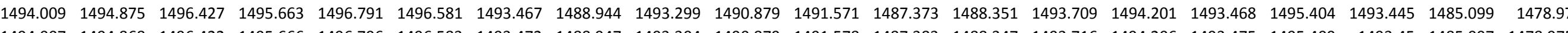

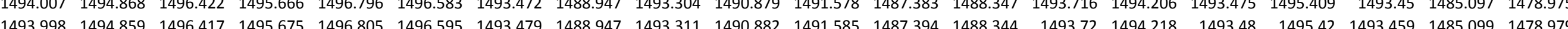

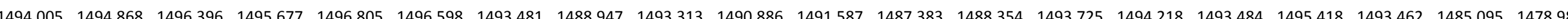
$\begin{array}{lllllllllllllllllllllll}1494.019 & 1494.88 & 1496.384 & 1495.67 & 1496.798 & 1496.579 & 1493.481 & 1488.947 & 1493.316 & 1490.886 & 1491.589 & 1487.387 & 1488.358 & 1493.723 & 1494.208 & 1493.487 & 1495.404 & 1493.462 & 1485.095 & 1478.984\end{array}$ $\begin{array}{lllllllllllllllllllllll}1494.033 & 1494.88 & 1496.37 & 1495.659 & 1496.789 & 1496.564 & 1493.481 & 1488.944 & 1493.311 & 1490.884 & 1491.592 & 1487.396 & 1488.363 & 1493.72 & 1494.19 & 1493.48 & 1495.392 & 1493.457 & 1485.095 & 1478.982 \\ \end{array}$

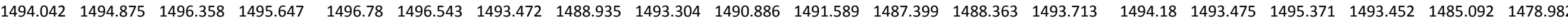

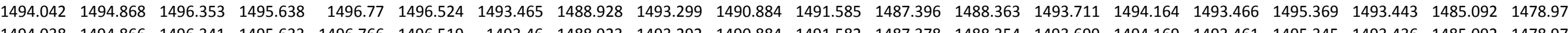
$\begin{array}{llllllllllllllllllllll}1494.038 & 1494.866 & 1496.341 & 1495.633 & 1496.766 & 1496.519 & 1493.46 & 1488.923 & 1493.292 & 1490.884 & 1491.582 & 1487.378 & 1488.354 & 1493.699 & 1494.169 & 1493.461 & 1495.345 & 1493.436 & 1485.092 & 1478.975 \\ \end{array}$ $\begin{array}{llllllllllllllllllll}1494.002 & 1494.835 & 1496.353 & 1495.645 & 1496.78 & 1496.548 & 1493.462 & 1488.926 & 1493.297 & 1490.882 & 1491.585 & 1487.371 & 1488.325 & 1493.701 & 1494.183 & 1493.463 & 1495.383 & 1493.443 & 1485.09 & 1478.975 \\ 1493.983 & 1494.813 & 1496.346 & 1495.656 & 1496.789 & 1496.574 & 1493.467 & 1488.933 & 1493.299 & 1490.886 & 1491.587 & 1487.383 & 1488.316 & 1493.711 & 1494.194 & 1493.47 & 1495.397 & 1493.448 & 1485.092 & 1478.977\end{array}$ 
Well

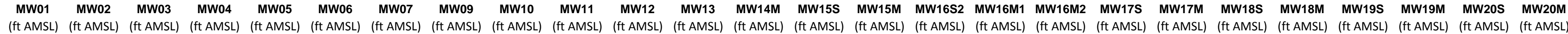

$\begin{array}{llll}1494.607 & 1494.979 & 1496.492 & 1495.322 \\ 1494.612 & 1494.989 & 1496509 & 1495338\end{array}$ $\begin{array}{rrrr}1494.612 & 1494.989 & 1496.509 & 1495.338 \\ 1494.612 & 1494.996 & 1496516 & 149535\end{array}$ $\begin{array}{llll}1494.612 & 1494.996 & 1496.516 & 1495.35 \\ 1494.61 & 1494.996 & 1496511 & 1495352\end{array}$ $\begin{array}{rrrr}1494.61 & 1494.996 & 1496.511 & 1495.352 \\ 1494.603 & 1494.996 & 1496.499 & 1495.348\end{array}$ $\begin{array}{rrrrr}1494.603 & 1494.996 & 1496.499 & 1495.348 \\ 1494.605 & 1494.991 & 1496.488 & 1495.343 \\ 1494.612 & 1495 & 1496.506 & 1495.35\end{array}$ $\begin{array}{rrrr}1494.612 & 1495 & 1466.506 & 1495.35 \\ 149.617 & 1455.01 & 1496525 & 1495362\end{array}$ $\begin{array}{rrrrr}1494.617 & 1495.01 & 1496.525 & 1495.362 \\ 1494.621 & 1495.019 & 1496.537 & 1495.371\end{array}$ $\begin{array}{lrrrr}1494.621 & 1495.019 & 1496.537 & 1495.371 \\ 1494.624 & 1495.028 & 1496.548 & 1495.38\end{array}$ $\begin{array}{rrrr}1494.624 & 1495.028 & 1496.548 & 1495.38 \\ 1494.633 & 1495.042 & 1496.572 & 1495.399\end{array}$ $\begin{array}{llll}1494.633 & 1495.042 & 1496.572 & 1495.399 \\ 1494.638 & 1495.054 & 1496.598 & 1495.415 \\ & 149507 & 1496.619 & 1495.439\end{array}$ $\begin{array}{lrrrr}1494.64 & 1495.07 & 1496.619 & 1495.439\end{array}$ $\begin{array}{rrrr}1494.638 & 1945.08 & 1496.621 & 1495.446 \\ 149642 & 1495.091 & 1496.642 & 1955.464\end{array}$ $\begin{array}{lllll}1494.642 & 1495.091 & 1469.642 & 1459.464 \\ 1494.647 & 1495.105 & 1496.658 & 1495.481\end{array}$ $\begin{array}{lllll}1494.647 & 1495.11 & 1496.654 & 1495.485\end{array}$ $\begin{array}{llll}1494.656 & 1495.124 & 1496.679 & 1495.502 \\ 1494.659 & 1455.136 & 1496.591 & 1455.516\end{array}$ 1494.6591495 .1361496 .6911495 .516 $\begin{array}{rrrr}1494.654 & 1495.138 & 1496.689 & 1495.52 \\ 1494.656 & 1495.138 & 1496.675 & 1495516\end{array}$ $\begin{array}{lllll}1494.658 & 1495.138 & 1496.675 & 1495.516 \\ 1496.696 & 1495.527\end{array}$ $\begin{array}{lllll}1494.682 & 1495.175 & 1496.74 & 1495.555\end{array}$ $\begin{array}{llll}1494.684 & 1495.198 & 14966.78 & 1995.5583\end{array}$ $\begin{array}{rrrrr}1494.679 & 1495.205 & 1496.782 & 1495.6\end{array}$ $\begin{array}{llll}1494.68 & 1495.21 & 1496.775 & 1495.605\end{array}$ $\begin{array}{rrrr}1494.686 & 1495.215 & 1496.771 & 1495.604\end{array}$ $\begin{array}{rrrr}1494.691 & 1495.222 & 1496.78 & 1495.607 \\ 1494.675 & 1495.21 & 1496749 & 1455595\end{array}$ $\begin{array}{llll}1494.675 & 1495.21 & 1496.749 & 1495.595 \\ 1494.684 & 1495212 & 1496.752 & 1495.588\end{array}$ $\begin{array}{lllll}1494.684 & 1495.212 & 1496.752 & 1495.588 \\ 1494.687 & 1495.208 & 1496.735 & 1495.576\end{array}$ $\begin{array}{rrrrr}1494.687 & 1495.208 & 1496.735 & 1495.576 \\ 1495.212 & 1496.74 & 1495.576\end{array}$ $\begin{array}{lllll}1494.698 & 1495.217 & 1496.749 & 14955.576\end{array}$ $\begin{array}{lllll}1494.698 & 1495.215 & 1496.742 & 1495.572\end{array}$ $\begin{array}{llll}1494.696 & 1495.208 & 1496.728 & 1495.56\end{array}$ $\begin{array}{llll}1494.694 & 1495.201 & 1496.714 & 1495.546 \\ 1494.693 & 1495.194 & 1496.705 & 1495.532\end{array}$ $\begin{array}{lllll}1494.693 & 1495.194 & 1496.705 & 1495.532 \\ 1494.689 & 1495.175 & 1496.677 & 1995.511\end{array}$ $\begin{array}{llll}1494.689 & 1495.175 & 1496.677 & 1495.511 \\ 1494.693 & 1495.163 & 1496.668 & 1495.495\end{array}$ $\begin{array}{llll}1494.693 & 1495.163 & 1496.668 & 1495.495 \\ 1494.696 & 1495.156 & 1496.661 & 1495.483\end{array}$ $\begin{array}{lllll}1494.696 & 1495.147 & 1496.656 & 14955.474\end{array}$ $\begin{array}{rrrr}1494.696 & 1495.133 & 1496.64 & 1495.46\end{array}$ $\begin{array}{rrrr}1494.698 & 1495.126 & 1496.635 & 1495.45\end{array}$ $\begin{array}{rrrr}1494.696 & 1495.117 & 1496.628 & 1495.441 \\ 1499.693 & 1459.101 & 1496.605 & 1455.425\end{array}$ $\begin{array}{rrrr}1494.693 & 1495.101 & 1496.605 & 1495.425 \\ 1494.696 & 1495.094 & 1496.6 & 1495.413\end{array}$ $\begin{array}{rrrr}1494.696 & 1495.094 & 1496.6 & 1495.413 \\ 1494.71 & 1495.11 & 1496.64 & 1995.427\end{array}$ $\begin{array}{rrrr}1494.71 & 1495.11 & 1496.64 & 1495.427 \\ 1494.719 & 1495.126 & 1496.672 & 1495.448\end{array}$ $\begin{array}{lllll}1494.717 & 1495.133 & 1496.684 & 1495.46\end{array}$ $\begin{array}{lllll}1494.712 & 1495.133 & 1496.677 & 1495.464\end{array}$ $\begin{array}{lllll}1494.717 & 1495.14 & 1496.686 & 1495.471\end{array}$ $\begin{array}{lllll}1494.717 & 1495.14 & 1496.684 & 1495.474\end{array}$ $\begin{array}{llll}1494.719 & 1495.142 & 1496.689 & 1495.476 \\ 1494.724 & 1495.147 & 1496.691 & 1495.481\end{array}$

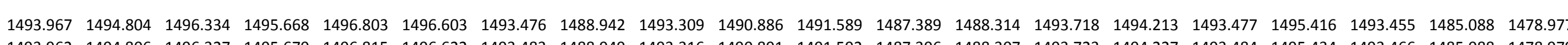

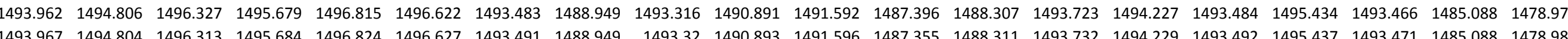

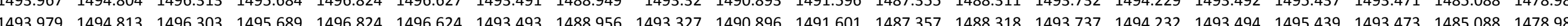
$\begin{array}{lllllllllllllllllllll}1494 & 1494.83 & 1496.282 & 1495.686 & 1496.819 & 1496.615 & 1493.493 & 1488.951 & 1493.33 & 1490.898 & 1491.603 & 1487371 & 1488.332 & 1493.737 & 1494.222 & 1493.496 & 1495.427 & 1493.476 & 1485.085 & 1478986\end{array}$ $\begin{array}{llllllllllllllllllllll}1494.014 & 1494.844 & 1496.273 & 1495.684 & 1496.817 & 1496.603 & 1493.493 & 1488.949 & 1493.325 & 1490.898 & 1491.603 & 1487.376 & 1488.332 & 1493.737 & 1494.222 & 1493.494 & 1495.418 & 1493.471 & 1485.085 & 1478.986\end{array}$

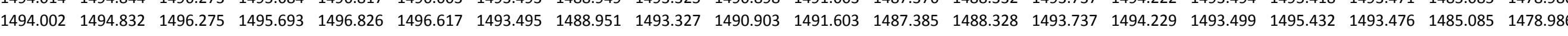
\begin{tabular}{llllllllllllllllllllll}
1493.998 & 1494.825 & 1496.273 & 1495.703 & 1496.835 & 1496.627 & 1493.5 & 1488.954 & 1493.332 & 1490.905 & 1491.603 & 1487.392 & 1488.323 & 1493.739 & 1494.241 & 1493.499 & 1495.449 & 1493.478 & 1485.085 & 1478.986 \\
\hline
\end{tabular}

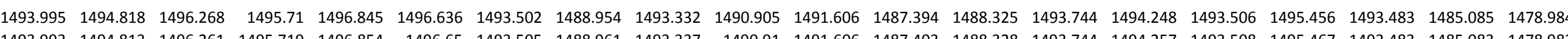

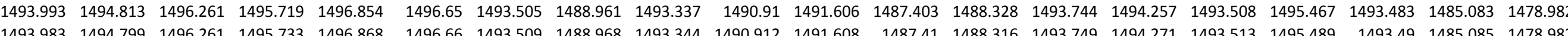

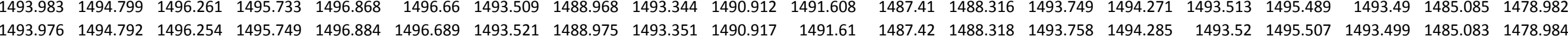

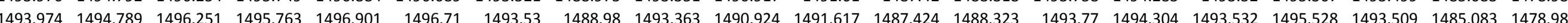
$\begin{array}{lllllllllllllllllllllll}1493.988 & 1494.794 & 1496.235 & 1495.77 & 1496.908 & 1496.708 & 1493.54 & 1488.982 & 1493.37 & 1490.926 & 1491.622 & 1487.433 & 1488.332 & 1493.777 & 1494.311 & 1493.541 & 1495.533 & 1493.52 & 1485.083 & 1478.999\end{array}$ $\begin{array}{lllllllllllllllllllllll}1493.988 & 1494.804 & 1496.23 & 1495.789 & 1496.922 & 1496.729 & 1493.554 & 1488.989 & 1493.384 & 1490.931 & 1491.631 & 1487.438 & 1488.337 & 1493.791 & 1494.325 & 1493.556 & 1495.55 & 1493.532 & 1485.083 & 1478.999\end{array}$

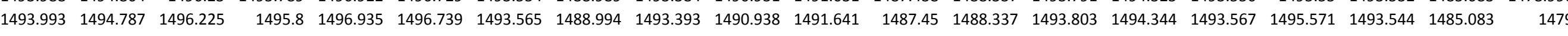
\begin{tabular}{llllllllllllllllllllll}
1494.009 & 1494.789 & 1496.211 & 1495.805 & 1496.938 & 1496.732 & 1493.572 & 1488.996 & 1493.407 & 1490.94 & 1491.645 & 1487.457 & 1488.347 & 1493.81 & 1494.344 & 1493.575 & 1495.568 & 1493.551 & 1485.083 & 1479.005 \\
\hline
\end{tabular}

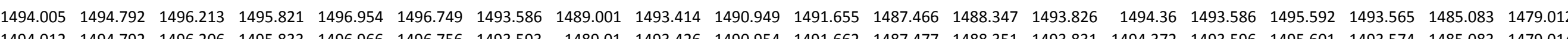

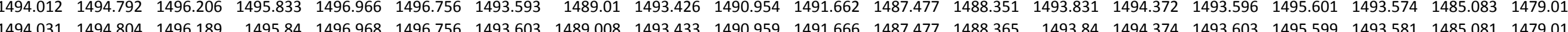

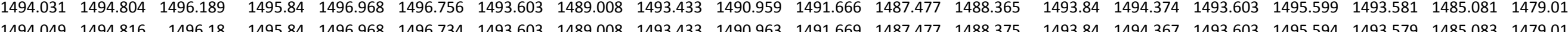
$\begin{array}{llllllllllllllllllllll}1494.045 & 1494.799 & 1496.185 & 1495.854 & 1496.982 & 1496.746 & 1493.608 & 1489.015 & 1493.438 & 1490.968 & 1491.673 & 1487.482 & 1488.363 & 1493.847 & 1494.388 & 1493.61 & 1495.608 & 1493.588 & 1485.083 & 1479.014\end{array}$ \begin{tabular}{lllllllllllllllllllllll}
1494.023 & 1494.808 & 1496.192 & 1495.877 & 1497.005 & 1496.787 & 1493.619 & 1489.019 & 1493.449 & 1490.973 & 1491.68 & 1487.457 & 1488.351 & 1493.861 & 1494.414 & 1493.622 & 1495.646 & 1493.6 & 1485.083 & 1479.019 \\
\hline
\end{tabular} $\begin{array}{llllllllllllllllllllllll}1494.016 & 1494.82 & 1496.185 & 1495.902 & 1497.028 & 1496.82 & 1493.636 & 1489.027 & 1493.466 & 1490.984 & 1491.687 & 1487.463 & 1488.354 & 1493.875 & 1494.437 & 1493.638 & 1495.674 & 1493.614 & 1485.083 & 1479.024\end{array}$ $\begin{array}{lllllllllllllllllllllll}1494.035 & 1494.828 & 1496.166 & 1495.914 & 1497.04 & 1496.83 & 1493.645 & 1489.031 & 1493.475 & 1490.989 & 1491.692 & 1487.438 & 1488.368 & 1493.883 & 1494.446 & 1493.648 & 1495.679 & 1493.626 & 1485.081 & 1479.026\end{array}$

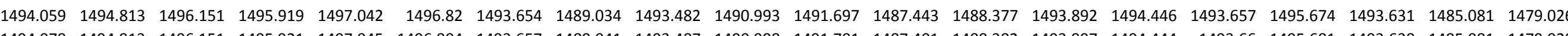

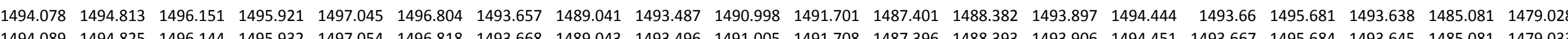

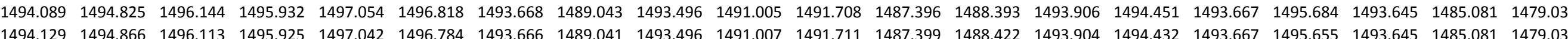
$\begin{array}{llllllllllllllllllll}1494.129 & 1494.866 & 1496.113 & 1495.925 & 1497.042 & 1496.784 & 1493.6666 & 1489.041 & 1493.496 & 1491.007 & 1491.711 & 1487.399 & 1488.422 & 1493.904 & 1494.432 & 1493.667 & 1495.655 & 1493.645 & 1485.081 & 1479.033 \\ 1494.141 & 1494.861 & 1496.121 & 1495.93 & 1497.042 & 1496.782 & 1493.671 & 1489043 & 1493501 & 1491.01 & 1491711 & 1487.41 & 1488.424 & 1493908 & 1494.435 & 1493669 & 1495.651 & 1493.647 & 1485081 & 1479035\end{array}$ $\begin{array}{lllllllllllllllllllll}1494.157 & 1494.868 & 1496.111 & 1495.925 & 1497.038 & 1496.76 & 1493.666 & 1489.038 & 1493.496 & 1491.012 & 1491.715 & 1487.424 & 1488.431 & 1493.906 & 1494.423 & 1493.667 & 1495.646 & 1493.645 & 1485.083 & 1479.033\end{array}$ $\begin{array}{llllllllllllllllllllll}1494.162 & 1494.864 & 1496.106 & 1495.928 & 1497.042 & 1496.768 & 1493.668 & 1489.043 & 1493.499 & 1491.014 & 1491.718 & 1487.433 & 1488.44 & 1493.906 & 1494.425 & 1493.667 & 1495.648 & 1493.647 & 1485.078 & 1479.033\end{array}$ $\begin{array}{lllllllllllllllllllllll}1494.162 & 1494.854 & 1496.102 & 1495.932 & 1497.045 & 1496.768 & 1493.668 & 1489.041 & 1493.503 & 1491.019 & 1491.72 & 1487.452 & 1488.443 & 1493.906 & 1494.432 & 1493.669 & 1495.648 & 1493.647 & 1485.081 & 1479.031 \\ \end{array}$ $\begin{array}{lllllllllllllllllllllll}1494.176 & 1494.861 & 4496.095 & 1495.932 & 1497.042 & 1496.763 & 1493.666 & 1489.041 & 1493.496 & 1491.019 & 1491.718 & 1487.454 & 1488.452 & 1493.906 & 1494.423 & 1493.667 & 1495.639 & 1493.649 & 1485.078 & 1479.031\end{array}$

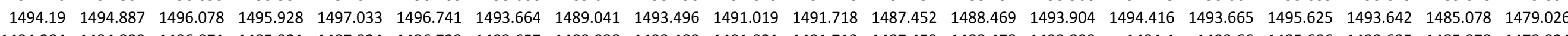

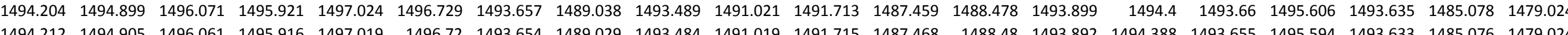

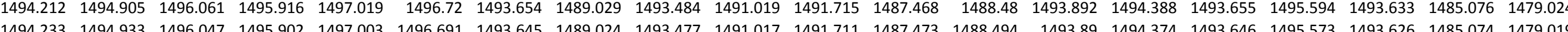
$\begin{array}{lllllllllllllllllllll}1494.235 & 1494.942 & 1496.052 & 1495.893 & 1496.996 & 1496.674 & 1493.645 & 1489.017 & 1493.477 & 1491.017 & 1491.715 & 1487.782 & 1488.494 & 1493.883 & 1494.365 & 1493.643 & 1495.557 & 1493.621 & 1485.074 & 1479.019\end{array}$ $\begin{array}{llllllllllllllllllllll}1494.233 & 1494.95 & 1496.047 & 1495.886 & 1496.989 & 1496.674 & 1493.64 & 1489.017 & 1493.475 & 1491.014 & 1491.718 & 1487.491 & 1488.494 & 1493.88 & 1494.36 & 1493.641 & 1495.55 & 1493.621 & 1485.074 & 1479.021\end{array}$ $\begin{array}{llllllllllllllllllll}1494.23 & 1494.95 & 1496.037 & 1495.879 & 1496.982 & 1496.67 & 1493.638 & 1489.012 & 1493.473 & 1491.014 & 1491.722 & 1487.489 & 1488.499 & 1493.88 & 1494.353 & 1493.641 & 1495.543 & 1493.619 & 1485.071 & 1479.024\end{array}$

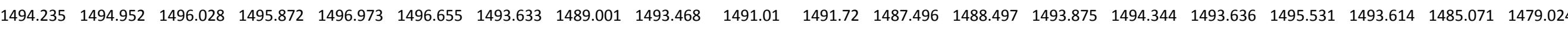

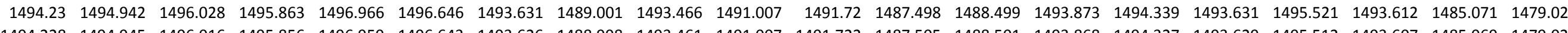

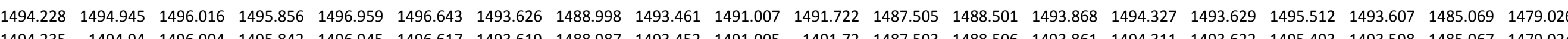

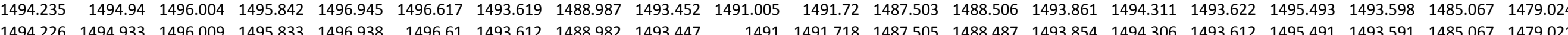

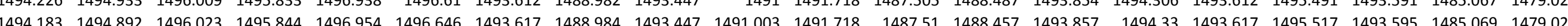
$\begin{array}{lllllllllllllllllllll}1494.155 & 1494.871 & 1496.016 & 1495.858 & 1496.966 & 1496.682 & 1493.619 & 1488.994 & 1493.454 & 1491.003 & 1491.722 & 1487.51 & 1488.445 & 1493.861 & 1494.351 & 1493.62 & 1495.543 & 1493.603 & 1485.069 & 1479.024\end{array}$ $\begin{array}{lllllllllllllllllllll}1494.153 & 1494.871 & 1496.007 & 1495.865 & 1496.973 & 1496.705 & 1493.626 & 1488.996 & 1493.459 & 1491.003 & 1491.725 & 1487.517 & 1488.445 & 1493.866 & 1494.355 & 1493.627 & 1495.552 & 1493.605 & 1485.064 & 1479.024\end{array}$

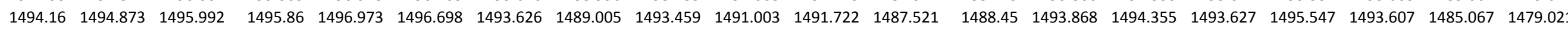
$\begin{array}{llllllllllllllllllll}1494.16 & 1494.871 & 1495.99 & 1495.865 & 1496.975 & 1496.71 & 1493.629 & 1489.003 & 1493.461 & 1491.003 & 1491.725 & 1487.475 & 1488.445 & 1493.871 & 1494.355 & 1493.631 & 1495.554 & 1493.61 & 1485.064 & 1479.024 \\ \end{array}$ $\begin{array}{llllllllllllllllllll}1494.167 & 1494.875 & 1495.983 & 1495.865 & 1496.975 & 1496.705 & 1493.631 & 1489.003 & 1493.461 & 1491.003 & 1491.725 & 1487.477 & 1488.447 & 1493.875 & 1494.36 & 1493.631 & 1495.552 & 1493.61 & 1485.064 & 1479.026\end{array}$

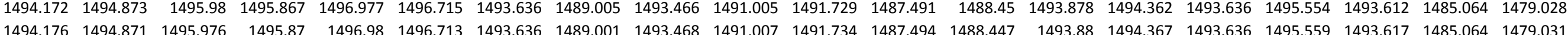


Well

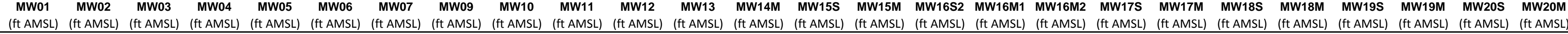
12/27/11 20:00 $12 / 27 / 1121: 00$ $12 / 27 / 1122: 00$ 12/28/11 0:00 $12 / 28 / 111: 00$
$12 / 28 / 112: 00$ 12/28/113:00 12/28/11 4:00 12/28/11 5:00 12/28/11 6:00 12/28/11 7:00 12/28/11 9:00 12/28/11 10:00 12/28/11 11:00 12/28/11 12:00 12/28/11 14:00 12/28/11 15:00 12/28/11 16:00 12/28/11 18:00 $12 / 28 / 1118: 00$
$12 / 28 / 1119: 00$ 12/28/11120:00 12/28/11 21:00 12/28/11 22:00 12/28/11 23:00 12/29/11 0:00 12/29/11 1:00 12/29/11 3:00 12/29/11 5:00 12/29/11 6:00 12/29/11 7:00 12/29/11 8:00 12/29/11 9:00 12/29/11 11:00 12/29/11 12:00 12/29/11 14:00 12/29/11 15:00 12/29/11 16:00 12/29/11 17:00 12/29/11 18:00 12/29/11 19:00 12/29/11 21:00 12/29/11 22:00 12/30/11 0:00

$\begin{array}{llll}1494.726 & 1495.154 & 1496.705 & 1495.488\end{array}$ $\begin{array}{rrrr}1494.726 & 1495.156 & 1496.707 & 1495.49 \\ 1494.728 & 1495.163 & 149671 & 1495.497\end{array}$ $\begin{array}{rrrr}1494.728 & 1495.163 & 1496.71 & 1495.497\end{array}$ $\begin{array}{lllll}1494.735 & 1495.166 & 1496.71 & 1455.495\end{array}$ $\begin{array}{rrrr}1494.735 & 1495.166 & 1496.71 & 1495.499 \\ 1494.747 & 1495.187 & 1496.752 & 1495.52\end{array}$ $\begin{array}{lllll}1494.74 & 1495.191 & 1496.756 & 1495.53\end{array}$ $\begin{array}{lllll}1494.742 & 1495.201 & 1496.764 & 1495.541\end{array}$ $\begin{array}{lllll}1494.731 & 1495.189 & 1496.731 & 1495.527\end{array}$ $\begin{array}{llll}1494.747 & 1495.203 & 1496.756 & 1495.539\end{array}$ $\begin{array}{rrrr}1494.74 & 1495.191 & 1496.733 & 1495.53 \\ 1494.754 & 1495.203 & 1496759 & 1495.539\end{array}$ $\begin{array}{llll}1494.754 & 1495.203 & 1496.759 & 1495.539 \\ 1494.752 & 1495.208 & 1496.759 & 1495.541\end{array}$ $\begin{array}{lllll}1494.749 & 1495.203 & 1496.749 & 1495.539\end{array}$ $\begin{array}{lllll}1494.745 & 1495.191 & 1496.726 & 1495.527\end{array}$ $\begin{array}{llllll}1494.752 & 1495.191 & 1496.731 & 1495.525\end{array}$ $\begin{array}{lllll}1494.763 & 1495.205 & 1496.761 & 1495.537\end{array}$ $\begin{array}{rrrrr}1494.773 & 1495.226 & 1496.799 & 1495.56 \\ 149477 & 1495233 & 1496808 & 1495.574\end{array}$ $1494.77 \quad 1495.233 \quad 1496.8081495 .574$ $\begin{array}{rrrr}1494.773 & 1495.24 & 1496.81 & 1495.581 \\ 1494.768 & 1495.238 & 1496.799 & 1495.581\end{array}$ $\begin{array}{llll}1494.768 & 1495.238 & 1496.799 & 1495.581 \\ 1494.77 & 1495.238 & 1496.796 & 1495.579\end{array}$ $\begin{array}{llllll}1494.773 & 1495.238 & 1496.787 & 1495.572\end{array}$ $\begin{array}{lllll}1494.773 & 1495.238 & 1496.785 & 1495.572\end{array}$ $\begin{array}{lllll}1494.782 & 1495.243 & 1496.796 & 1495.576\end{array}$ $\begin{array}{lllll}1494.78 & 1495.245 & 1496.799 & 1495.579\end{array}$ $\begin{array}{lllll}1494.782 & 1495.252 & 1496.808 & 1495.583\end{array}$ $\begin{array}{rrrr}1494.784 & 1495.25 & 1496.801 & 1495.583 \\ 1494.794 & 1495264 & 1496822 & 1995.593\end{array}$ $\begin{array}{llll}1494.794 & 1495.264 & 1496.822 & 1495.593 \\ 1494.798 & 1495.275 & 1496.843 & 1495.609\end{array}$ $\begin{array}{llll}1494.796 & 1495.278 & 1496.843 & 1495.614\end{array}$ $\begin{array}{llll}1494.798 & 1495.285 & 1496.848 & 1495.621\end{array}$ $\begin{array}{lllll}1494.803 & 1495.294 & 1496.855 & 1495.63\end{array}$ $\begin{array}{lllll}1494.808 & 1495.301 & 1496.864 & 1495.637\end{array}$ $\begin{array}{llll}1494.805 & 1495.301 & 1496.859 & 1495.635\end{array}$ $\begin{array}{rrrr}1494.808 & 1495.299 & 1496.852 & 1495.635 \\ 1498.805 & 1495299 & 1496.85 & 195633\end{array}$ $\begin{array}{llll}1494.805 & 1455.299 & 1496.85 & 1495.633\end{array}$ $\begin{array}{llll}1494.808 & 1495.294 & 1496.848 & 1495.628 \\ 1494.815 & 1495.296 & 1496.848 & 1495.628\end{array}$ $\begin{array}{llll}1494.819 & 1495.301 & 1496.857 & 1495.628 \\ 1495.63\end{array}$ $\begin{array}{lllll}1494.826 & 1495.32 & 1496.89 & 1495.653\end{array}$ $\begin{array}{lllll}1494.84 & 1495.341 & 1496.927 & 1495.679\end{array}$ $\begin{array}{lllll}1494.838 & 1495.355 & 1496.941 & 1495.695\end{array}$ $\begin{array}{llll}1494.842 & 1495.369 & 1496.965 & 1495.716\end{array}$ $\begin{array}{llll}1494.845 & 1495.378 & 1496.972 & 1495.731 \\ 1494847 & 1495383 & 1496965 & 1495.733\end{array}$ $\begin{array}{lllll}1494.847 & 1495.383 & 1496.965 & 1495.733 \\ 1494847 & 1495394 & 1496.986 & 1495749\end{array}$ $\begin{array}{llll}1494.847 & 1495.394 & 1496.986 & 1495.749 \\ 1494.84 & 1495.385 & 1496.951 & 1495.735\end{array}$ $\begin{array}{llll}1494.822 & 1495.355 & 1496.894 & 14955.707\end{array}$ $\begin{array}{lllll}1494.847 & 1495.371 & 1496.932 & 1495.714\end{array}$ $\begin{array}{lllll}1494.833 & 1495.35 & 1496.892 & 1495.691\end{array}$ $\begin{array}{lllll}1494.831 & 1495.324 & 1496.855 & 1495.661\end{array}$ $\begin{array}{rrrr}1494.826 & 1495.292 & 1496.806 & 1495.625 \\ 1494.833 & 1495.278 & 1496.792 & 1495.6\end{array}$

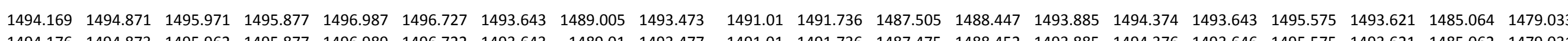

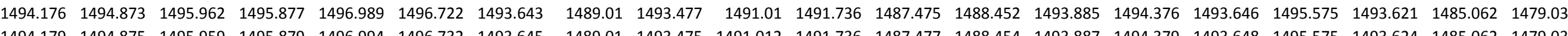

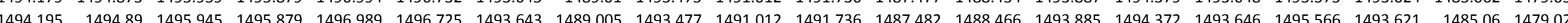
$\begin{array}{llllllllllllllllllllll}1494.188 & 1494.88 & 1495.952 & 1495.881 & 1496.996 & 1496.722 & 1493.645 & 1489.008 & 1493.475 & 1491.014 & 1491.736 & 1487.487 & 1488.452 & 1493.883 & 1494.379 & 1493.643 & 1495.575 & 1493.624 & 1485.062 & 1479.028\end{array}$ $\begin{array}{lllllllllllllllllllll}1494.162 & 1494.871 & 1495961 & 14959 & 1497.015 & 1496.76 & 1493.65 & 1489.015 & 1493.484 & 1491019 & 1491.741 & 1487.498 & 1488.438 & 1493.892 & 1494.404 & 1493.655 & 1495.608 & 1493.631 & 1485.062 & 1479.028\end{array}$ $\begin{array}{lllllllllllllllllllll}1494.172 & 1494.885 & 1495.945 & 1495.907 & 1497.019 & 1496.77 & 1493.657 & 1489.019 & 1493.489 & 1491.021 & 1491.739 & 1487.505 & 1488.454 & 1493.899 & 1494.402 & 1493.657 & 1495.615 & 1493.638 & 1485.06 & 1479.031 \\ \end{array}$ $\begin{array}{lllllllllllllllllllllll}1494.176 & 1494.885 & 1495.938 & 1495.914 & 1497.028 & 1496.78 & 1493.666 & 1489.027 & 1493.494 & 1491.026 & 1491.743 & 1487.512 & 1488.459 & 1493.904 & 1494.411 & 1493.665 & 1495.62 & 1493.645 & 1485.06 & 1479.031\end{array}$

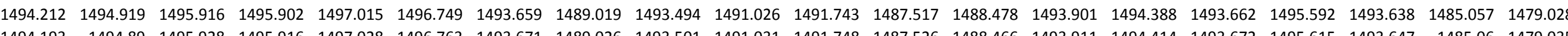

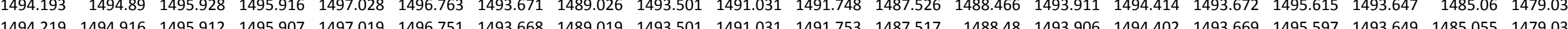
$\begin{array}{llllllllllllllllllll}1494.219 & 1494.916 & 1495.912 & 1495.907 & 1497.019 & 1496.751 & 1493.668 & 1489.019 & 1493.501 & 1491.031 & 1491.753 & 1487.517 & 1488.48 & 1493.906 & 1494.402 & 1493.669 & 1495.597 & 1493.649 & 1485.055 & 1479.035 \\ 1494.204 & 1494.892 & 1495.923 & 1495.919 & 1497.033 & 1496.765 & 1493.675 & 1489.024 & 1493.508 & 1491.033 & 1491.76 & 1487.526 & 1488.469 & 1493.915 & 1494.416 & 1493.674 & 1495.62 & 1493.656 & 1485.057 & 147904\end{array}$

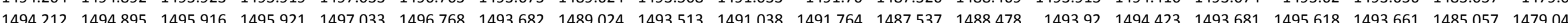
$\begin{array}{llllllllllllllllllllll}1494.226 & 1494.904 & 1495.904 & 1495.919 & 1497.031 & 1496.753 & 1493.682 & 1489.027 & 1493513 & 1491.038 & 1491.764 & 1487.544 & 1488.485 & 1493.922 & 1494.411 & 1493.684 & 1495.608 & 1493.661 & 1485.057 & 1479.042\end{array}$ $\begin{array}{llllllllllllllllllllll}1494.242 & 1494.921 & 1495.888 & 1495.912 & 1497.022 & 1496.732 & 1493.678 & 1489.022 & 1493.51 & 1491.04 & 1491.764 & 1487.551 & 1488.497 & 1493.918 & 1494.404 & 1493.679 & 1495.597 & 1493.659 & 1485.055 & 1479.042\end{array}$ $\begin{array}{lllllllllllllllllllll}1494.24 & 1494.919 & 1495.89 & 1495.912 & 1497.022 & 1496.729 & 1493.675 & 1489.029 & 1493.513 & 1491.04 & 1491.767 & 1487.544 & 1488.487 & 1493.92 & 1494.402 & 1493.676 & 1495.597 & 1493.656 & 1485.055 & 1479.042\end{array}$ $\begin{array}{llllllllllllllllllll}1494.216 & 1494.885 & 1495.9 & 1495.925 & 1497.033 & 1496.756 & 1493.682 & 1489.022 & 1493.517 & 1491.042 & 1491.767 & 1487.547 & 1488.473 & 1493.922 & 1494.418 & 1493.681 & 1495.618 & 1493.664 & 1485.055 & 1479.042\end{array}$

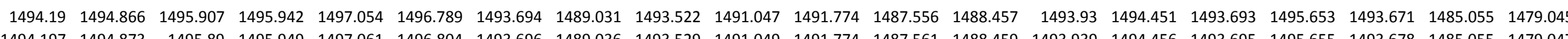

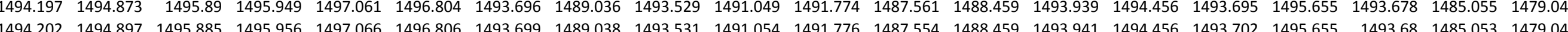

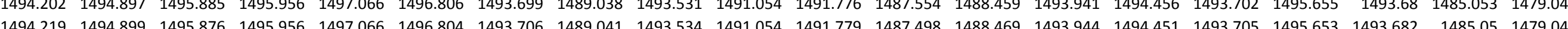
$\begin{array}{llllllllllllllllllllllll}1494.23 & 1494.892 & 1495.874 & 1495.958 & 1497.066 & 1496.794 & 1493.703 & 1489.038 & 1493.538 & 1491.056 & 1491.783 & 1487.494 & 1488.478 & 1493.946 & 1494.453 & 1493.707 & 1495.651 & 1493.685 & 1485.052 & 1479.047\end{array}$ \begin{tabular}{|llllllllllllllllllllll}
1495866 & 1495.953 & 1497.063 & 1496784 & 1493706 & 1489.043 & 1493538 & 1491.056 & 1491.783 & 1487.491 & 1488.478 & 1493.948 & 1494.446 & 1493.707 & 1495.641 & 1493.687 & 1485.053 & 1479.047
\end{tabular} $\begin{array}{lllllllllllllllllllllllll}1494.254 & 1494.897 & 1495.862 & 1495.953 & 1497.061 & 1496.78 & 1493.708 & 1489.045 & 1493.541 & 1491.061 & 1491.786 & 1487.484 & 1488.483 & 1493.948 & 1494.446 & 1493.71 & 1495.641 & 1493.687 & 1485.052 & 1479.052 \\ \end{array}$

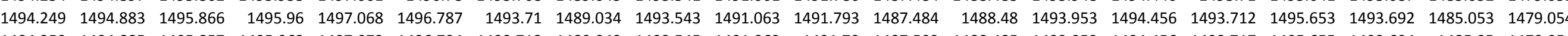

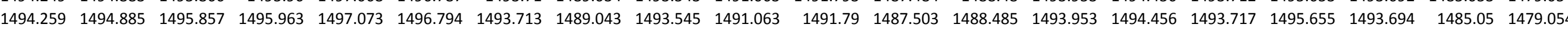

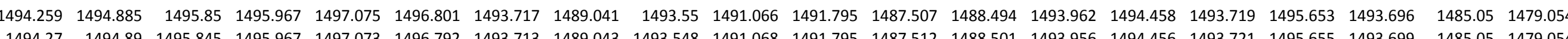

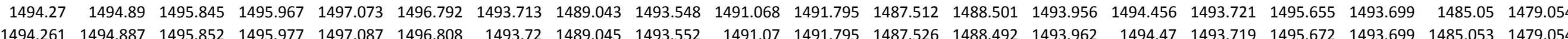

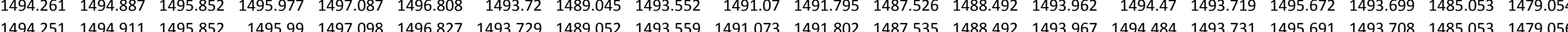
$\begin{array}{llllllllllllllllllllll}1494.263 & 1494.916 & 1495.838 & 1495.993 & 1497.101 & 1496.827 & 1493.732 & 1489.052 & 1493.562 & 1491.077 & 1491.8 & 1487.542 & 1488.499 & 1493.972 & 1494.486 & 1493.733 & 1495.691 & 1493.713 & 1485.05 & 1479.056\end{array}$ $\begin{array}{lllllllllllllllllllll}1494.268 & 1494.916 & 1495.833 & 1496 & 1497.105 & 1496.835 & 1493.736 & 1489.05 & 1493.567 & 1491.077 & 1491.804 & 1487.554 & 1488.508 & 1493.974 & 1494.488 & 1493.736 & 1495.691 & 1493.715 & 1485.048 & 1479.056\end{array}$

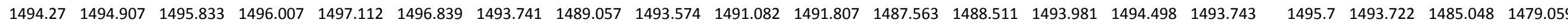
\begin{tabular}{llllllllllllllllllllll}
1494.273 & 1494.892 & 1495.833 & 1496.014 & 1497.119 & 1496.844 & 1493.748 & 1489.057 & 1493.578 & 1491.084 & 4491.811 & 1487.574 & 1488.508 & 1493.986 & 1494.505 & 1493.747 & 1495.709 & 1493.727 & 1485.05 & 1479.061 \\
\hline
\end{tabular}

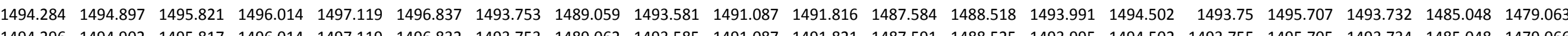

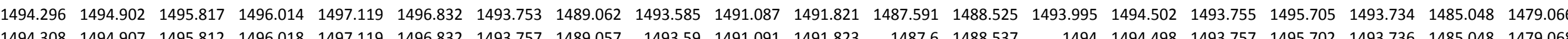

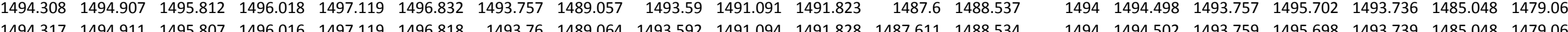

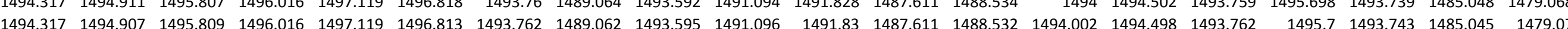
$\begin{array}{llllllllllllllllllllllll}1494.315 & 1494.897 & 1495.807 & 1496.021 & 1497.124 & 1496.823 & 1493.762 & 1489.062 & 1493.595 & 1491.094 & 1491.835 & 1487.611 & 1488.525 & 1494.005 & 1494.507 & 1493.762 & 1495.707 & 1493.746 & 1485.045 & 1479.07\end{array}$ $\begin{array}{lllllllllllllllllllllll}1494.296 & 1494.938 & 1495.814 & 1496.039 & 1497.138 & 1496.856 & 1493.774 & 1489.066 & 1493.604 & 1491.101 & 1491.837 & 1487.618 & 1488.518 & 1494.012 & 1494.523 & 1493.774 & 1495.731 & 1493.753 & 1485.045 & 1479.073\end{array}$ \begin{tabular}{llllllllllllllllllllll}
1494.273 & 1494.919 & 1495.824 & 1496.06 & 1497.163 & 1496.88 & 1493.781 & 1489.073 & 1493.611 & 1491.105 & 1491.842 & 1487.628 & 1488.494 & 1494.024 & 1494.556 & 1493.783 & 1495.761 & 1493.762 & 1485.048 & 1479.075 \\
\hline
\end{tabular} $\begin{array}{llllllllllllllllllll}1494.277 & 1494.916 & 1495.807 & 1496.072 & 1497.173 & 1496.902 & 1493.792 & 1489.073 & 1493.623 & 1491.11 & 1491.846 & 1487.637 & 1488.504 & 1494.031 & 1494.558 & 1493.79 & 1495.771 & 1493.771 & 1485.045 & 1479.075 \\ \end{array}$

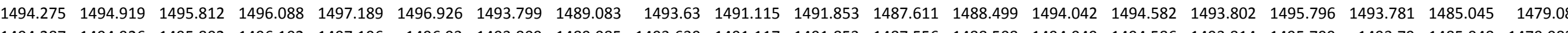

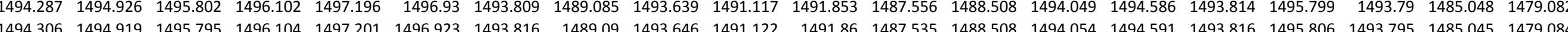

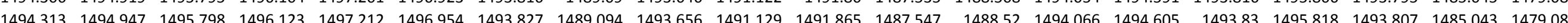
$\begin{array}{lllllllllllllllllllll}1494.353 & 1494.954 & 1495.779 & 1496.113 & 1497.203 & 1496.911 & 1493.825 & 1489.092 & 1493.656 & 1491.129 & 1491.867 & 1487.563 & 1488.534 & 1494.066 & 1494.582 & 1493.826 & 1495.794 & 1493.804 & 1485.043 & 1479.084\end{array}$ $\begin{array}{lllllllllllllllllllllll}1494.402 & 1494.986 & 1495.755 & 1496.09 & 1497.18 & 1496.844 & 1493.818 & 1489.083 & 1493.649 & 1491.129 & 1491.865 & 1487.572 & 1488.565 & 1494.056 & 1494.556 & 1493.819 & 1495.747 & 1493.795 & 1485.045 & 1479.084\end{array}$

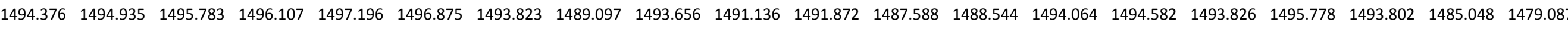

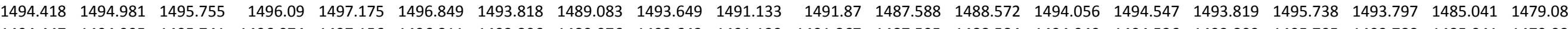
\begin{tabular}{lllllllllllllllllllll}
1494.447 & 1494.995 & 1495.741 & 1496.074 & 1497.156 & 1496.811 & 1493.806 & 1489.076 & 1493.642 & 1491.129 & 1491.867 & 1487.595 & 1488.584 & 1494.049 & 1494.526 & 1493.809 & 1495.705 & 1493.788 & 1485.041 & 1479.082 \\
\hline
\end{tabular} $\begin{array}{llllllllllllllllllllll}1494.475 & 1495.024 & 1495.731 & 1496.051 & 1497.131 & 1496.768 & 1493.792 & 1489.064 & 1493.625 & 1491.126 & 1491.863 & 1487.598 & 1488.595 & 1494.036 & 1494.493 & 1493.793 & 1495.662 & 1493.771 & 1485.038 & 1479.077 \\ 1494.472 & 1495.029 & 1495.741 & 1496.037 & 1497.119 & 1496.746 & 1493.781 & 1489.055 & 1493.613 & 1491.122 & 1491.86 & 1487.605 & 1488.586 & 1494.024 & 1494.477 & 1493.781 & 1495.646 & 1493.76 & 1485.038 & 1479.073\end{array}$ 
Well

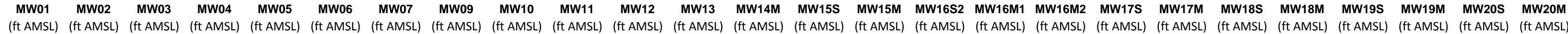

$12 / 30 / 112: 00$ 12/30/11 3:00 $12 / 30 / 115 \cdot 00$ 12/30/11 5:00 12/30/11 6:00 $12 / 30 / 118: 00$ 12/30/11 9:00 12/30/11 10:00 2/30/11 11:00 12/30/11 12:00 12/30/111 14:00 $12 / 30 / 1115: 00$ $12 / 30 / 11116: 00$ $12 / 30 / 11$ 17:00 $12 / 30 / 1118: 00$ 12/30/11 19:00 2/30/11 20:00 2/30/11 21:00 12/30/11 22:00 12/31/11 0:00 12/31/11 1:00 $12 / 31 / 112: 00$ 12/31/11 3:00 12/31/111 4:00 $12 / 31 / 115: 00$ $12 / 31 / 116: 00$ $12 / 31 / 118: 00$ 12/31/11 9:00 12/31/11 10:00 $12 / 31 / 1111: 00$ $12 / 31 / 1112: 00$ 12/31/1113:00 $12 / 31 / 1114: 00$ 12/31/11 15:00 $12 / 31 / 1117: 00$ $12 / 31 / 1118: 00$ 12/31/11 19:00 12/31/11 20:00 $12 / 31 / 1121: 00$ 12/31/11 22:00 1/1/12 0:00

$1 / 1 / 123: 00$

1/1/12 4:00

1/1/12 5:00

1/1/12 6:00 $\begin{array}{llll}1494.831 & 1495.261 & 1496.778 & 1495.576\end{array}$ $\begin{array}{rrrr}1494.829 & 1495.25 & 1496.766 & 1495.56 \\ 1494.826 & 1495.224 & 1496735 & 1495.534\end{array}$ $\begin{array}{lrrr}1494.826 & 1495.224 & 1496.735 & 1495.534 \\ 1494.836 & 1495.229 & 1496752 & 1495.53\end{array}$ $\begin{array}{lllll}1494.829 & 1495.217 & 1496.738 & 1495.518\end{array}$ $\begin{array}{lllll}1494.833 & 1495.219 & 1496.745 & 1455.516\end{array}$ $\begin{array}{llllll}1494.831 & 1495.201 & 1496.721 & 1495.502\end{array}$ $\begin{array}{llllll}1494.826 & 1495.187 & 1496.705 & 1495.488\end{array}$ $\begin{array}{llll}1494.826 & 1495.175 & 1496.689 & 1495.469\end{array}$ $\begin{array}{llll}1494.831 & 1495.175 & 1496.698 & 1495.467\end{array}$ $\begin{array}{rrrr}1494.836 & 1495.177 & 1496.707 & 1495.469 \\ 149484 & 1455187 & 1496.728 & 1955.481\end{array}$ $\begin{array}{rrrrr}1494.84 & 1495.187 & 1496.728 & 1495.481 \\ 1494.845 & 1495.198 & 1496.749 & 1495.492\end{array}$ $\begin{array}{lllll}1494.842 & 1495.196 & 1496.738 & 1495.497\end{array}$ $\begin{array}{lllll}1494.842 & 1495.191 & 1496.728 & 1495.49\end{array}$ $\begin{array}{lllll}1494.847 & 1495.196 & 1496.731 & 1495.492\end{array}$ $\begin{array}{lllll}1494.849 & 1495.201 & 1496.738 & 1495.497\end{array}$ $\begin{array}{rrrr}1494.849 & 1495.205 & 1496.74 & 1495.497\end{array}$ $\begin{array}{llll}1494.852 & 1495.205 & 1496.745 & 1495.502\end{array}$ $\begin{array}{llll}1494.863 & 1495.217 & 1496.766 & 1495.513 \\ 1494.868 & 1495231 & 1496.789 & 1495.532\end{array}$ $\begin{array}{llll}1494.866 & 1495.236 & 1496.796 & 1495.541\end{array}$ $1494.873 \quad 1495.25 \quad 1496.813 \quad 1495.553$ $\begin{array}{lllll}1494.875 & 1495.259 & 1496.829 & 1495.569\end{array}$ $\begin{array}{lllll}1494.877 & 1495.271 & 1496.848 & 1495.586\end{array}$ $\begin{array}{lllll}1494.882 & 1495.282 & 1496.857 & 1495.598\end{array}$ $\begin{array}{lllll}1494.891 & 1495.299 & 1496.89 & 1495.621\end{array}$ $\begin{array}{lllll}1494.894 & 1495.32 & 1496.915 & 1495.644\end{array}$ $\begin{array}{lllll}1494.901 & 1495.336 & 1496.939 & 1495.668 \\ 1494.903 & 1495352 & 1496.955 & 1495.688\end{array}$ $\begin{array}{lllll}1494.898 & 1495.357 & 1496.955 & 1495.698\end{array}$ $\begin{array}{llllll}1494.908 & 1495.371 & 1496.967 & 1495.709\end{array}$ $1494.905 \quad 1495.378 \quad 1496.976 \quad 1495.719$ $\begin{array}{lllll}1494.915 & 1495.394 & 1496.997 & 1495.737\end{array}$ $1494.917 \quad 1495.406 \quad 1497.011 \quad 1495.749$ $\begin{array}{lllll}1494.912 & 1495.397 & 1496.976 & 1495.738 \\ 1499.908 & 1495397 & 1496965 & 14957\end{array}$ $\begin{array}{llll}1494.908 & 1495.397 & 1496.965 & 1495.73 \\ 1494.905 & 1495383 & 1496929 & 1495.707\end{array}$ $\begin{array}{llll}1494.894 & 1495.348 & 1496864 & 1495.707\end{array}$ $\begin{array}{lllll}1494.891 & 1495.31 & 1496.81 & 1495.618\end{array}$ $1494.887 \quad 1495.289 \quad 1496.787 \quad 1495.583$ $\begin{array}{llllll}1494.873 & 1495.25 & 1496.735 & 1495.541\end{array}$ $\begin{array}{lllll}1494.859 & 1495.203 & 1496.658 & 1495.481\end{array}$ $1494.851495 .1491496 .602 \quad 1495.422$ $\begin{array}{llll}1494.847 & 1495.129 & 1496.581 & 1495.383\end{array}$ $\begin{array}{lllll}1494.836 & 1495.096 & 1496.546 & 1495.338 \\ 1494826 & 1495.061 & 1496506 & 1495301\end{array}$ $\begin{array}{lllll}1494.824 & 1455.045 & 1496.495 & 1495.273\end{array}$ $\begin{array}{llll}1494.817 & 1495.033 & 1496.497 & 1495.259\end{array}$ $\begin{array}{lllll}1494.808 & 1495.005 & 1496.464 & 1495.236\end{array}$ $\begin{array}{lllll}1494.801 & 1494.984 & 1496.443 & 1495.208\end{array}$ $\begin{array}{lllll}1494.789 & 1494.963 & 1496.42 & 1495.184\end{array}$ $\begin{array}{llll}1494.794 & 1494.968 & 1496.439 & 1495.184 \\ 1494.789 & 1494.963 & 1496.439 & 1495.182\end{array}$

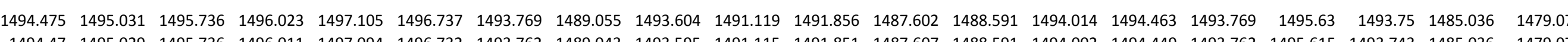

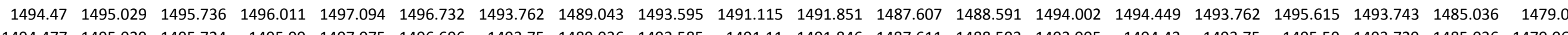

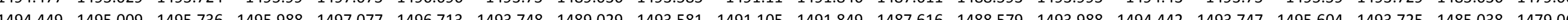
$\begin{array}{lllllllllllllllllllllll}1494.451 & 1495.021 & 1495.722 & 1495.977 & 1497.063 & 1496.705 & 1493.743 & 1489.026 & 1493.576 & 1491.098 & 1491.846 & 1487.607 & 1488.586 & 1493.986 & 1494.425 & 1493.743 & 1495.583 & 1493.72 & 1485.034 & 1479.066\end{array}$

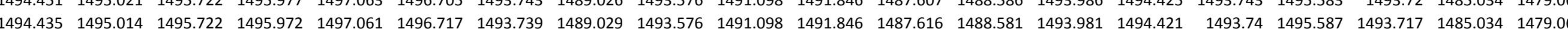
$\begin{array}{llllllllllllllllllll}1494.442 & 1495.024 & 1495.712 & 1495.956 & 1497.047 & 1496.691 & 1493.732 & 1489.024 & 1493.564 & 1491.091 & 1491.846 & 1487.621 & 1488.586 & 1493.977 & 1494.407 & 1493.731 & 1495.566 & 1493.71 & 1485.034 & 1499.068\end{array}$ $\begin{array}{lllllllllllllllllllll}1494.442 & 1495.021 & 1495.705 & 1495.944 & 1497.033 & 1496.679 & 1493.725 & 1489.015 & 1493.559 & 1491.087 & 1491.844 & 1487.616 & 1488.584 & 1493.967 & 1494.395 & 1493.724 & 1495.554 & 1493.706 & 1485.034 & 1479.066\end{array}$

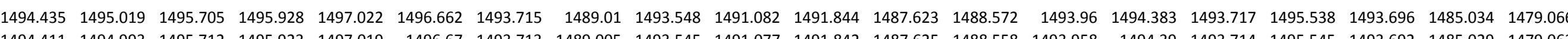

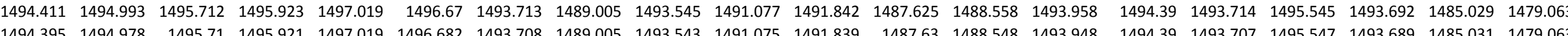

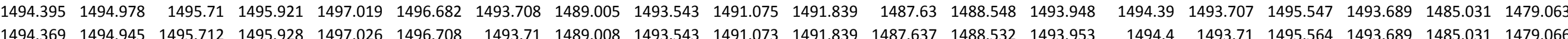
$\begin{array}{llllllllllllllllllllll}1494.35 & 1494.933 & 1495.707 & 1495.93 & 1497.033 & 1466.734 & 1493.715 & 1489.015 & 1493.545 & 1491.07 & 1491.839 & 1487.641 & 1488.525 & 1493.958 & 1494.411 & 1493.712 & 1495.58 & 1493.689 & 1485.029 & 1479.063\end{array}$ $\begin{array}{llllllllllllllllllll}1494.355 & 1494.933 & 1495698 & 1495925 & 1497.031 & 1496729 & 149371 & 1489012 & 1493545 & 149107 & 1491837 & 1487.646 & 1488.525 & 1493951 & 1494.404 & 1493.71 & 1495.573 & 1493.689 & 1485.029 & 1479.061\end{array}$ $\begin{array}{llllllllllllllllllllll}1494.362 & 1494.931 & 1495.691 & 1495.919 & 1497.024 & 1496.722 & 1493.703 & 1489.01 & 1493.541 & 1491.068 & 1491.832 & 1487.6 & 1488.523 & 1493.951 & 1494.397 & 1493.707 & 1495.568 & 1493.685 & 1485.027 & 1479.059\end{array}$

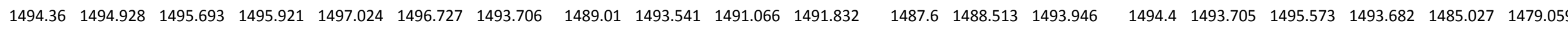
$\begin{array}{llllllllllllllllllllllll}1494.355 & 1494.926 & 1495.691 & 1495.919 & 1497.028 & 1496.737 & 1493.706 & 1489.012 & 1493.541 & 1491.063 & 1491.832 & 1487.605 & 1488.515 & 1493.946 & 1494.404 & 1493.707 & 1495.575 & 1493.689 & 1485.029 & 1479.061\end{array}$

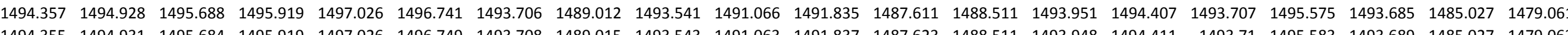

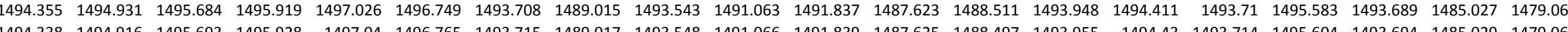

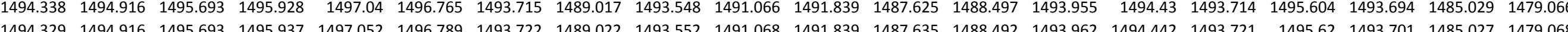
$\begin{array}{llllllllllllllllllllllll}1494.336 & 1494.928 & 1495.679 & 1495.939 & 1497.054 & 1496.796 & 1493.722 & 1489.027 & 1493.557 & 1491.068 & 1491.844 & 1487.644 & 1488.501 & 1493.967 & 1494.444 & 1493.726 & 1495.62 & 1493.706 & 1485.027 & 1479.073\end{array}$ $\begin{array}{llllllllllllllllllllll}1494.327 & 1494.928 & 1495686 & 1495949 & 1497.061 & 1496806 & 1493732 & 1489031 & 1493562 & 1491.073 & 1491844 & 1487.653 & 1488.492 & 1493969 & 1494.456 & 1493.731 & 1495.639 & 1493.71 & 1485.029 & 1479.073\end{array}$ $\begin{array}{lllllllllllllllllllllllll}1494.324 & 1494.931 & 1495.686 & 1495.958 & 1497.073 & 1496.825 & 1493.736 & 1489.036 & 1493.569 & 1491.075 & 1491.849 & 1487.658 & 1488.492 & 1493.974 & 1494.472 & 1493.738 & 1495.653 & 1493.717 & 1485.027 & 1479.075\end{array}$ $\begin{array}{lllllllllllllllllllllllll}1494.322 & 1494.933 & 1495.681 & 1495.967 & 1497.082 & 1496.842 & 1493.743 & 1489.041 & 1493.578 & 1491.077 & 1491.849 & 1487.669 & 1488.494 & 1493.984 & 1494.474 & 1493.743 & 1495.667 & 1493.725 & 1485.027 & 1479.077 \\ \end{array}$ $\begin{array}{lllllllllllllllllllll}1494.329 & 1494.935 & 1495.676 & 1495.977 & 1497.091 & 1496.849 & 1493.748 & 1489.05 & 1493.583 & 1491.082 & 1491.853 & 1487.674 & 1488.497 & 1493.988 & 1494.491 & 1493.75 & 1495.681 & 1493.732 & 1485.027 & 1479.077\end{array}$

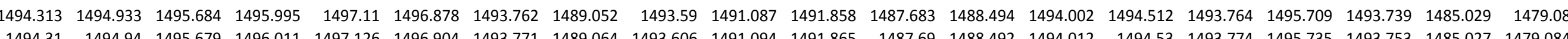

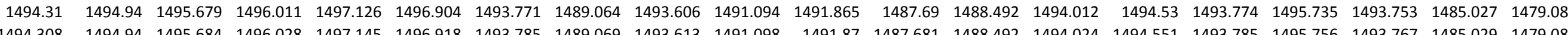
$\begin{array}{llllllllllllllllllll}1494.308 & 1494.94 & 1495.684 & 1496.028 & 1497.145 & 1496.918 & 1493.785 & 1489.069 & 1493.613 & 1491.098 & 1491.87 & 1487.681 & 1488.492 & 1494.024 & 1494.551 & 1493.785 & 1495.756 & 1493.767 & 1485.029 & 1479.089 \\ 1494.317 & 1494.95 & 1495.676 & 1496.042 & 1497.161 & 1496.935 & 1493.797 & 1489.078 & 1493.627 & 1491.105 & 1491.877 & 1487.699 & 1488.504 & 1494.035 & 1494.57 & 1493.797 & 1495.775 & 1493.778 & 1485.029 & 1479.094\end{array}$ $\begin{array}{llllllllllllllllllll}1494.338 & 1494.954 & 1495.665 & 1496.051 & 1497.166 & 1496.938 & 1493.806 & 1489.078 & 1493.637 & 1491.11 & 1491.879 & 1487.699 & 1488.52 & 1494.045 & 1494.568 & 1493.807 & 1495.77 & 1493.786 & 1485.027 & 1479.096\end{array}$

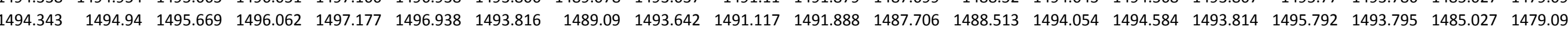

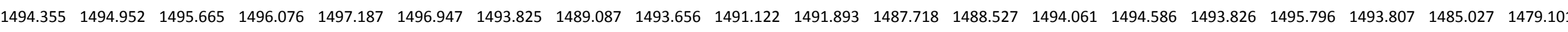
$\begin{array}{lllllllllllllllllllllll}1494.353 & 1494.947 & 1495.667 & 1496.093 & 1497.203 & 1496.959 & 1493.834 & 1489.097 & 1493.665 & 1491.129 & 1491.895 & 1487.718 & 1488.525 & 1494.071 & 1494.605 & 1493.835 & 1495.82 & 1493.816 & 1485.024 & 1479.105 & \end{array}$

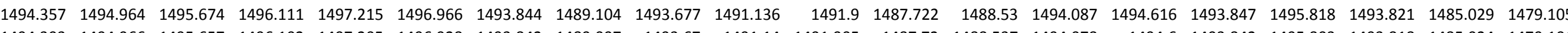

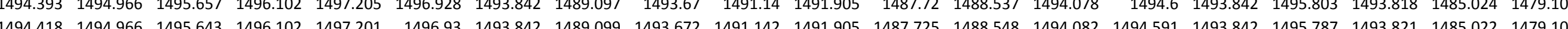

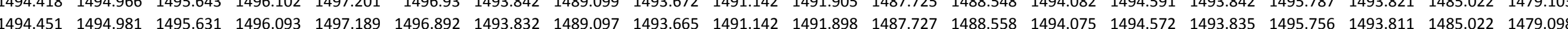

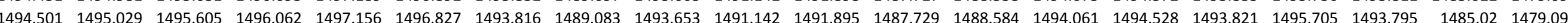
$\begin{array}{llllllllllllllllllllll}1494.527 & 1495.053 & 1495.61 & 1496.035 & 1497.131 & 1496.763 & 1493.72 & 1489.069 & 1493.55 & 1491.138 & 1491.872 & 1487.727 & 1488.577 & 1493.96 & 1494.463 & 1493.721 & 1495.655 & 1493.703 & 1485.022 & 1479.084\end{array}$ $\begin{array}{llllllllllllllllllll}1494.534 & 1495.057 & 1495.608 & 1496.021 & 1497.11 & 1496.746 & 1493.746 & 1489.055 & 1493.574 & 1491.136 & 1491.867 & 1487.704 & 1488.586 & 1493.984 & 1494.451 & 1493.747 & 1495.622 & 1493.725 & 1485.022 & 1479.082\end{array}$ \begin{tabular}{llllllllllllllllllllll}
1494.557 & 1495.057 & 1495.593 & 1495.988 & 1497.082 & 1496.71 & 1493.739 & 1489.041 & 1493.571 & 1491.131 & 1491.863 & 1487.704 & 1488.602 & 1493.979 & 1494.423 & 1493.738 & 1495.575 & 1493.72 & 1485.017 & 1479.077 \\
\hline
\end{tabular} $\begin{array}{lllllllllllllllllllll}1494.599 & 1495.06 & 1495.565 & 1495.942 & 1497.035 & 1496.643 & 1493.722 & 1489.027 & 1493.555 & 1491.117 & 1491.849 & 1487.699 & 1488.626 & 1493.962 & 1494.365 & 1493.721 & 1495.512 & 1493.703 & 1485.017 & 1479.075 \\ \end{array}$

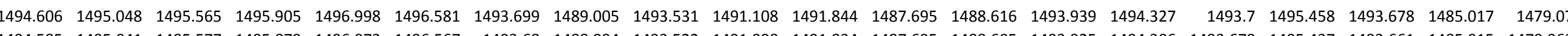

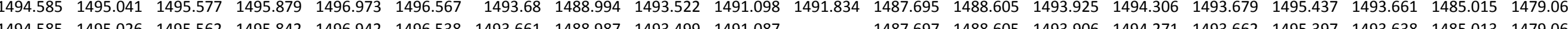

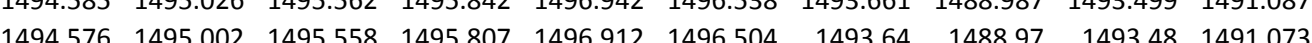
$1494.551494 .993 \quad 1495.565 \quad 149578414968941496.495 \quad 1493.6241488 .958 \quad 1493.4611491 .063$ $\begin{array}{llllllllllll}1494.512 & 1494.988 & 1495.565 & 1495.77 & 1496.88 & 1496.507 & 1493.612 & 1488.949 & 1493.452 & 1491.052\end{array}$ $\begin{array}{llllllllllll}1494.505 & 1494.971 & 1495.551 & 1495.74 & 1496.856 & 1496.488 & 1493.596 & 1488.944 & 1493.435 & 1491.04\end{array}$ $\begin{array}{lllllllllll}1494.491 & 1494.952 & 1495.548 & 1495.714 & 1496.831 & 1496.471 & 1493.577 & 1488.937 & 1493.416 & 1491.028\end{array}$ $\begin{array}{llllllllllll}1494.472 & 1494.94 & 1495.543 & 1495.691 & 1496.812 & 1496.459 & 1493.561 & 1488.933 & 1493.405 & 1491.014\end{array}$

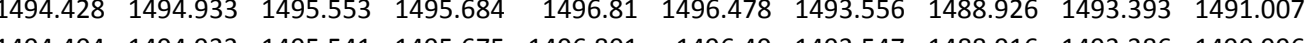
$\begin{array}{llllllllll}1487.697 & 1488.605 & 1493.906 & 1494.271 & 1493.662 & 1495.397 & 1493.638 & 1485.013 & 1479.06 \\ 1487.06 & 1488593 & 1493.887 & 1494.243 & 1493.641 & 1495.357 & 1493.619 & 148501 & 1479052\end{array}$ $\begin{array}{llllllllll}487.697 & 1488.577 & 1493.871 & 1494.225 & 1493.622 & 1495.34 & 1493.605 & 1485.013 & 1479.047\end{array}$ $\begin{array}{llllllllll}1487.699 & 1488.567 & 1493.859 & 1494.22 & 1493.612 & 1495.338 & 1493.593 & 1485.01 & 1479.045\end{array}$ $\begin{array}{llllllllll}1487.697 & 1488.567 & 1493.843 & 1494.197 & 1493.596 & 1495.308 & 1493.579 & 1485.008 & 1479.042\end{array}$ $\begin{array}{lllllllllll}1487.697 & 1488.56 & 1493.826 & 1494.173 & 1493.577 & 1495.282 & 1493.56 & 1485.008 & 1479.038\end{array}$ $\begin{array}{lllllllllll}1487.699 & 1488.551 & 1493.812 & 1494.155 & 1493.56 & 1495.261 & 1493.544 & 1485.008 & 1479.033\end{array}$ $\begin{array}{lrrrrrrrr}1487.702 & 1488.53 & 1493.803 & 1494.162 & 1493.556 & 1495.272 & 1493.534 & 1485.008 & 1479.033 \\ 1487.697 & 1488518 & 1493.793 & 1494162 & 1493.548 & 1495275 & 1493.53 & 1485.006 & 1479.033\end{array}$ 
Well

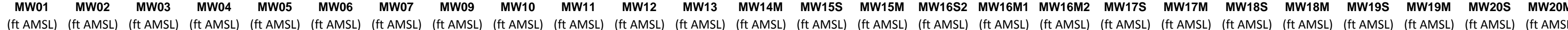

1/1/128:00 $1 / 1 / 129: 00$ 1/1/1211:00 1/1/12 12:00 1/1/12 13:00 1/1/12 14:00 1/1/12 15:00 $1 / 1 / 121600$ 1/1/12 17:00 1/1/12 18:00 $1 / 1 / 1220: 00$ 1/1/12 21:00 1/1/112 22:00 1/1/12 23:00 1/2/12 0:00 1/2/12 1:00 1/2/12 2:00 1/2/122 3:00 $1 / 2 / 124: 000$ 1/2/12 7:00 $1 / 2 / 128: 00$ 1/2/12 9:00 1/2/12 10:00 1/2/12 11:00 1/2/12 12:00 1/2/12 14:00 1/2/12 15:00 1/2/12 16:00 1/2/12 17:00 $1 / 2 / 1218: 00$ 1/2/12 19:00 //2/1220:00 1/2/12 21:00 $1 / 2 / 1222: 00$ 1/3/1220:00 1/3/12 1:00 1/3/12 2:00 1/3/12 3:00 1/3/12 4:00 $1 / 3 / 125.00$ $1 / 3 / 127: 00$ 1/3/12 8:00 1/3/12 9:00 1/3/12 10:00 1/3/12 11:00 $1 / 3 / 1212: 00$
$1 / 3 / 1213: 00$ $\begin{array}{llll}1494.775 & 1494.937 & 1496.399 & 1495.156 \\ 1494.763 & 1494.912 & 1496361 & 1495.128\end{array}$ $1494.763 \quad 1494.912 \quad 1496.361 \quad 1495.128$ $\begin{array}{rrrr}1494.756 & 1494.888 & 1496.333 & 1495.1 \\ 1494.756 & 1494.884 & 1496.333 & 1495091\end{array}$ $\begin{array}{llll}1494.759 & 1494.895 & 1496.364 & 1495.103\end{array}$ $\begin{array}{llll}1494.766 & 1494.907 & 1496.392 & 1495.121\end{array}$ $\begin{array}{lllll}1494.466 & 1494.907 & 1496.392 & 1495.121 \\ 1494.766 & 1494.916 & 1496.415 & 1495.14\end{array}$ $\begin{array}{lllll}1494.761 & 1494.921 & 1496.422 & 1495.154\end{array}$ $1494.759 \quad 1494.926 \quad 1496.42 \quad 1495.156$ $\begin{array}{llll}1494.752 & 1494.923 & 1496.413 & 1495.159\end{array}$ $\begin{array}{llll}1494.749 & 1494.923 & 1496.397 & 1495.154 \\ 1494.745 & 1494.916 & 1496382 & 1495.147\end{array}$ $\begin{array}{rrrrr}1494.745 & 1494.916 & 1496.382 & 1495.147 \\ 1494.74 & 1494.912 & 1496.371 & 1495.137\end{array}$

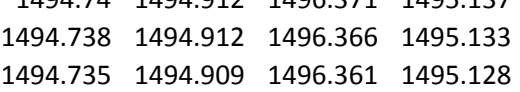
$\begin{array}{lllll}1494.731 & 1494.907 & 1496.357 & 1495.124\end{array}$ $\begin{array}{lllll}1494.731 & 1494.907 & 1496.354 & 1495.123\end{array}$ $\begin{array}{lllll}1494.728 & 1494.907 & 1496.354 & 1495.123\end{array}$ $\begin{array}{lllll}1494.731 & 1494.909 & 1496.361 & 1495.124\end{array}$ $\begin{array}{lllll}1494.728 & 1494.912 & 1496.361 & 1495.128 \\ 1494.726 & 1494914 & 1496366 & 1495.131\end{array}$ $\begin{array}{lllll}1494.721 & 1494.916 & 1496.359 & 1495.13\end{array}$ $\begin{array}{lllll}1494.721 & 1494.916 & 1496357 & 1495.131\end{array}$ $\begin{array}{llll}1494.717 & 1494.916 & 1496.354 & 1495.13\end{array}$ $\begin{array}{lllll}1494.719 & 1494.921 & 1496.361 & 1495.135\end{array}$ $\begin{array}{lllll}1494.71 & 1494.909 & 1496.333 & 1495.121\end{array}$ $\begin{array}{lllll}1494.707 & 1494.907 & 1496.324 & 1495.114\end{array}$ $\begin{array}{llllll}1494.717 & 1494.912 & 1496.345 & 1495.121\end{array}$ $\begin{array}{llll}1494.726 & 1494.933 & 1496.387 & 1495.145 \\ 1494.731 & 1494.954 & 1496.427 & 1495.177\end{array}$ $\begin{array}{lllll}1494.735 & 1494.975 & 1496.453 & 1495.203\end{array}$ $\begin{array}{lllll}1494.738 & 1494.989 & 1496.471 & 1495.203\end{array}$ $\begin{array}{lrrrr}1494.738 & 1495 & 1496.481 & 1495.24\end{array}$ $\begin{array}{llll}1494.74 & 1495.007 & 1496.485 & 1495.252\end{array}$ $\begin{array}{lllll}1494.745 & 1495.024 & 1496.504 & 1495.268\end{array}$ $1494.747 \quad 1495.031 \quad 1496.5091495 .275$ $\begin{array}{lllll}1494.749 & 1495.045 & 1496.523 & 1495.292\end{array}$ $\begin{array}{llll}1494.754 & 1455.059 & 1496.541 & 1495.306 \\ 1494.756 & 1495068 & 1496544 & 1495313\end{array}$ $\begin{array}{lllll}1494.761 & 1495.082 & 1496.56 & 1495.329\end{array}$ $1494.763 \quad 1495.089 \quad 1496.565 \quad 1495.336$ $\begin{array}{lllll}1494.766 & 1495.101 & 1496.581 & 1495.348\end{array}$ $\begin{array}{rrrr}1494.775 & 1495.117 & 1496.6 & 1495.364\end{array}$ $1494.777 \begin{array}{lll}1495.131 & 1496.612 & 1455.376\end{array}$ $\begin{array}{lllll}1494.784 & 1495.147 & 1496.635 & 1495.397 \\ 1494.791 & 1495.168 & 1496658 & 1495418\end{array}$ $\begin{array}{rrrrr}1494.791 & 1495.168 & 1496.658 & 1495.418 \\ 1494.791 & 1495.18 & 1496.663 & 1495.429\end{array}$ $1494.801 \quad 1495.194 \quad 1496.682 \quad 1495.446$ $\begin{array}{llll}1494.805 & 1495.203 & 1496.691 & 1495.457\end{array}$ $1494.808 \quad 1495.208 \quad 1496.6891495 .464$ $1494.812 \quad 1495.212 \quad 1496.6911495 .467$ $1494.826 \quad 1495.24 \quad 1496.728 \quad 1495.488$ $\begin{array}{rrrr}1494.84 & 1495.275 & 1496.78 & 1495.525 \\ 1494.847 & 1495.303 & 1496.82 & 1495.56\end{array}$ $\begin{array}{llllllllll}1494.409 & 1494.907 & 1495.527 & 1495.645 & 1496.775 & 1496.461 & 1493.533 & 1488.912 & 1493.372 & 1490.984\end{array}$ $\begin{array}{lllllllllll}1494.409 & 1494.897 & 1495.515 & 1495.612 & 1496.749 & 1496.428 & 1493.514 & 1488.905 & 1493.353 & 1490.973\end{array}$ $\begin{array}{llllllllllll}1494.397 & 1494.892 & 1495.51 & 1495.584 & 1496.726 & 1496.406 & 1493.498 & 4888.902 & 1493.337 & 1490.961 \\ 1494364 & 1494883 & 1495.52 & 1495573 & 1496717 & 1496.409 & 1493.486 & 1488895 & 149333 & 1490.952\end{array}$ $\begin{array}{lllllllllll}1494.313 & 1494.868 & 1495.527 & 1495.575 & 1496.724 & 1496.442 & 1493.483 & 1488.895 & 1493.323 & 1490.945\end{array}$ $\begin{array}{llllllllll}1494.268 & 1494.847 & 1495.534 & 1495.582 & 1496.733 & 1496.473 & 1493.483 & 1488.898 & 1493.32 & 1490.94\end{array}$ $\begin{array}{lllllllllll}1494.24 & 1494.835 & 1495.522 & 1495.587 & 1496.742 & 1496.512 & 1493.486 & 1488.905 & 1493.323 & 1490.935\end{array}$ $\begin{array}{lllllllllll}1494.228 & 1494.837 & 1495.515 & 1495.587 & 1496.742 & 1496.526 & 1493.486 & 1488.905 & 1493.325 & 1490.931\end{array}$ $\begin{array}{lllllllllll}1494.221 & 1494.844 & 1495.508 & 1495.587 & 1496.745 & 1496.536 & 1493.486 & 1488.905 & 1493.32 & 1490.926\end{array}$ $\begin{array}{llllllllll}1494.223 & 1494.859 & 1495.503 & 1495.582 & 1496.742 & 1496.536 & 1493.481 & 1488.9 & 1493.316 & 1490.924\end{array}$ $\begin{array}{rrrrrrrrrr}1494.228 & 1494.854 & 1495.498 & 1495.573 & 1496.735 & 1496.521 & 1493.474 & 1488.895 & 1493.309 & 1490.919 \\ 1494.23 & 1494.852 & 1495.489 & 1495.563 & 1496726 & 1496514 & 1493.467 & 1488893 & 1493304 & 1490.914\end{array}$ $\begin{array}{lllllllllll}1494.233 & 1449.847 & 1495.479 & 1495.552 & 1496.722 & 1496.507 & 1493.462 & 1488.883 & 1493.302 & 1490.912\end{array}$ $\begin{array}{lllllllllll}1494.226 & 1494844 & 1495.479 & 1495545 & 1496717 & 1496504 & 1493.46 & 1488.891 & 1493297 & 1490.91\end{array}$ $\begin{array}{lllllllllll}1494.219 & 1494.84 & 1495.472 & 1495.54 & 1496.712 & 1496.504 & 1493.453 & 1488.888 & 1493.295 & 1490.905\end{array}$ $\begin{array}{llllllllll}1494.212 & 1494.835 & 1495.47 & 1495.531 & 1496.708 & 1496.504 & 1493.451 & 1488.886 & 1493.288 & 1490.9\end{array}$ $\begin{array}{lllllllllll}1494.204 & 1494.828 & 1495.465 & 1495.526 & 1496.705 & 1496.504 & 1493.448 & 1488.886 & 1493.285 & 1490.898\end{array}$ $\begin{array}{llllllllllll}1494.2 & 1494.828 & 1495.463 & 1495.524 & 1496.703 & 1496.509 & 1493.448 & 1488.886 & 1493.283 & 1490.896\end{array}$ $\begin{array}{lllllllllllll}1494.183 & 1494.82 & 1495.46 & 1495.524 & 1496.70 & 1496.514 & 1433.446 & 1488.883 & 1493.283 & 1490.893\end{array}$ $\begin{array}{lllllllllll}1494.176 & 1494.816 & 1495.46 & 1495.522 & 1496.708 & 1496.519 & 1493.444 & 1488.886 & 1493.28 & 1490.893 \\ 1494.167 & 1494.813 & 1495.151 & 1495.524 & 1496708 & 1496524 & 1493.444 & 1488881 & 149328 & 1490891\end{array}$ $\begin{array}{llllllllllll}1494.169 & 1494.816 & 1495.439 & 1495.519 & 1496.705 & 1496.526 & 1493.441 & 1488.881 & 1493.278 & 1490.889\end{array}$ $\begin{array}{lllllllllll}1494.164 & 1494.808 & 1495.439 & 1495517 & 1496708 & 1496521 & 1493.439 & 1488.881 & 1493.278 & 1490.889\end{array}$ $\begin{array}{lllllllllll}1494.16 & 1494.811 & 1495.434 & 1495.515 & 1496.705 & 1496.526 & 1493.439 & 1488.883 & 1493.273 & 1490.886\end{array}$ $\begin{array}{llllllllll}1494.15 & 1494.808 & 1495.437 & 1495.517 & 1496.708 & 1496.536 & 1493.439 & 1488.886 & 1493.273 & 1490.886\end{array}$ $\begin{array}{lllllllllll}1494.164 & 1494.804 & 1495.418 & 1495.503 & 1496.694 & 1496.507 & 1493.432 & 1488.883 & 1493.269 & 1490.886\end{array}$ $\begin{array}{lllllllllll}1494.162 & 1494.799 & 1495.415 & 1495.494 & 1496.689 & 1496.495 & 1493.427 & 1488.876 & 1493.264 & 1490.884\end{array}$ $\begin{array}{llllllllll}1494.132 & 1494.785 & 1495.429 & 1495.501 & 1496.696 & 1496.507 & 1493.427 & 1488.879 & 1493.264 & 1490.884 \\ 1494.096 & 1494.749 & 1495.434 & 1495519 & 1496717 & 149655 & 1493.437 & 1488886 & 1493269 & 149086\end{array}$ $\begin{array}{rrrrrrrrrrr}1494.096 & 1494.749 & 1495.434 & 1495.519 & 1496.717 & 1496.55 & 1493.437 & 1488.886 & 1493.269 & 1490.886 \\ 1494.068 & 1494.73 & 1495.434 & 1495.54 & 1496.738 & 1496591 & 1493.448 & 1488.9 & 1493.283 & 1490.889\end{array}$ $\begin{array}{lllllllllll}1494.054 & 1494.72 & 1495.432 & 1495.556 & 1496.756 & 1496.624 & 1493.458 & 1488.907 & 1493.288 & 1490.891\end{array}$ $\begin{array}{llllllllllll}1494.052 & 1494.715 & 1495.422 & 1495.57 & 1496.77 & 1496.646 & 1493.467 & 1488.919 & 1493.297 & 1490.896\end{array}$ $\begin{array}{lllllllllll}1494.056 & 1494.72 & 1495.42 & 1495.582 & 1496.782 & 1496.655 & 1493.472 & 1488.921 & 1493.304 & 1490.9\end{array}$ $\begin{array}{lllllllllll}1494.066 & 1494.725 & 1495.41 & 1495.589 & 1496.789 & 1496.655 & 1493.476 & 1488.923 & 1493.309 & 1490.905\end{array}$ $\begin{array}{lllllllllll}1494.066 & 1494.725 & 1495.415 & 1495.603 & 1496.803 & 1496.674 & 1493.483 & 1488.933 & 1493.318 & 1490.91\end{array}$ $\begin{array}{rlllllllll}1494.078 & 1494.732 & 1495.406 & 1495.612 & 1496.81 & 1496.677 & 1493.488 & 1488.933 & 1493.32 & 1490.914 \\ 1494.08 & 1494.732 & 1495.403 & 1495.624 & 1496.824 & 1496686 & 1493.95 & 148894 & 1493327 & 149099\end{array}$ $\begin{array}{llllllllll}1494.08 & 1494.732 & 1455.403 & 1455.624 & 1496.824 & 1466.686 & 1493.495 & 1488.94 & 1493.327 & 1490.919 \\ 1494.08 & 1494.732 & 1495.406 & 1495638 & 1496838 & 1496703 & 1493.502 & 1488944 & 1493337 & 1490926\end{array}$ $\begin{array}{llllllllllll}1494.092 & 1494.741 & 1495.403 & 1495647 & 1496845 & 1496701 & 1493.509 & 1488.949 & 1493346 & 1490.933\end{array}$ $\begin{array}{llllllllllll}1494.092 & 1494.737 & 1495.399 & 1495.661 & 1496.856 & 1496.713 & 1493.516 & 1488.951 & 1493.351 & 1490.94\end{array}$ $\begin{array}{llllllllll}1494.099 & 1494.746 & 1495.396 & 1495.67 & 1496.863 & 1496.715 & 1493.526 & 1488.956 & 1493.36 & 1490.945\end{array}$ $\begin{array}{lllllllllll}1494.101 & 1494.741 & 1495.394 & 1495.684 & 1496.877 & 1496.727 & 1493.535 & 1488.961 & 1493.367 & 1490.949\end{array}$ $\begin{array}{lllllllllll}1494.099 & 1494.746 & 1495.394 & 1495.698 & 1496.891 & 1496.741 & 1493.544 & 1488.959 & 1493.379 & 1490.956\end{array}$ $\begin{array}{llllllllll}1494.103 & 1494.753 & 1495.396 & 1495.71 & 1496.903 & 1496.751 & 1493.556 & 1488.963 & 1493.391 & 1490.963\end{array}$ $\begin{array}{lllllllllll}1494.101 & 1494.751 & 1495.399 & 1495.726 & 1496.917 & 1496.765 & 1493.565 & 1488.97 & 1493.398 & 1490.973 \\ 1494.099 & 1494.768 & 1495399 & 1495.747 & 1496935 & 1496792 & 1493.577 & 1488.975 & 1493407 & 1490.98\end{array}$

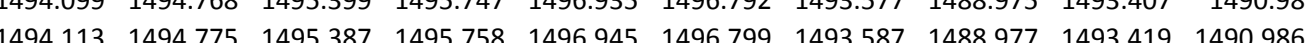
$\begin{array}{lllllllllllll}1494.113 & 1494.777 & 1495.391 & 1495.772 & 1496.961 & 1496.804 & 1493.596 & 1488.987 & 1493.428 & 1490.993\end{array}$ $\begin{array}{llllllllllll}1494.125 & 1494.773 & 1495.387 & 1495.786 & 1496.973 & 1496.811 & 1493.605 & 1488.991 & 1493.438 & 1491.003\end{array}$ $\begin{array}{lllllllllll}1494.141 & 1494.787 & 1495.382 & 1495.795 & 1496.98 & 1496.806 & 1493.61 & 1488.994 & 1493.445 & 1491.007\end{array}$ $\begin{array}{llllllllll}1494.15 & 1494.785 & 1495.384 & 1495.805 & 1496.987 & 1496.794 & 1493.615 & 1488.998 & 1493.447 & 1491.014\end{array}$ $\begin{array}{llllllllll}1494.132 & 1494.782 & 1495.389 & 1495.826 & 1497.008 & 1496.82 & 1493.631 & 1489.001 & 1493.461 & 1491.021\end{array}$ $\begin{array}{llllllllll}1494.108 & 1494.799 & 1495.399 & 1495.856 & 1497.04 & 1496.868 & 1493.647 & 1489.008 & 1493.477 & 1491.031\end{array}$ $\begin{array}{lllllllll}1487.695 & 1488.52 & 1493.781 & 1494.125 & 1493.532 & 1495.235 & 1493.516 & 1485.003 & 1479.028\end{array}$ $\begin{array}{llllllllll}1487.678 & 1488.513 & 1493.76 & 1494.099 & 1493.518 & 1495.218 & 1493.499 & 1485.006 & 1479.024 \\ 1487.674 & 1488.497 & 1493.746 & 1494.08 & 1493.499 & 1495.174 & 1493.478 & 1485003 & 1479009\end{array}$ $\begin{array}{lllllllll}1487.667 & 1488.4971 & 1493.746 & 1494.08 & 1493.499 & 1495.174 & 1493.478 & 1485.003 & 1499.019\end{array}$ $\begin{array}{llllllllll}1487.648 & 1488.44 & 1493.73 & 1494.092 & 1493.484 & 1495.202 & 1493.469 & 1485.003 & 1479.017\end{array}$ $\begin{array}{lllllllllll}1487.63 & 1488.41 & 1493.728 & 1494.115 & 1493.487 & 1495.225 & 1493.466 & 1485.003 & 1479.019\end{array}$ $\begin{array}{lllllllllll}1487.577 & 1488.389 & 1493.732 & 1494.125 & 1493.489 & 1495.246 & 1493.466 & 1485.003 & 1479.019\end{array}$ $\begin{array}{llllllllll}1487.584 & 1488.375 & 1493.73 & 1494.129 & 1493.487 & 1495.251 & 1493.469 & 1485.003 & 1479.019\end{array}$ $\begin{array}{llllllllll}1487.593 & 1488.365 & 1493.73 & 1494.132 & 1493.484 & 1495.258 & 1493.466 & 1485.001 & 1479.017\end{array}$ $\begin{array}{lllllllll}1487.6 & 4488.363 & 1493.725 & 1494.125 & 1493.482 & 1495.251 & 1493.466 & 1485.001 & 1479.012\end{array}$ $\begin{array}{llllllllll}1487.556 & 1488.358 & 1493.723 & 1494.118 & 1493.475 & 1495.242 & 1493.457 & 1485.001 & 1479.012\end{array}$ $\begin{array}{lllllllllll}1487.57 & 1488.354 & 1493.706 & 1494.104 & 1493.461 & 1495.225 & 1493.448 & 1484.999 & 1479.007\end{array}$ $\begin{array}{llllllllll}1487.574 & 1488.347 & 1493.704 & 1494.099 & 1493.461 & 1495.221 & 1493.443 & 1484.999 & 1479.005\end{array}$ $\begin{array}{llllllllll}1487.584 & 1488.335 & 1493.701 & 1494.094 & 1493.458 & 1495.221 & 1493.436 & 1484.996 & 1479.005\end{array}$ $\begin{array}{llllllllll}1487.586 & 1488.337 & 1493.692 & 1494.094 & 1493.451 & 1495.216 & 1493.436 & 1484.996 & 1479.005\end{array}$ \begin{tabular}{llllllllll}
1487.591 & 1488.325 & 1493.694 & 1494.089 & 1493.451 & 1495.214 & 1493.431 & 1484.996 & 1479.000 \\
\hline
\end{tabular} $\begin{array}{lllllllll}1487.591 & 1488.321 & 1493.692 & 1494.09 & 1493.447 & 1495.211 & 1493.429 & 1484.999 & 1479.005\end{array}$ $\begin{array}{llllllllllll}4487.595 & 1488.311 & 1493.694 & 1494.094 & 1493.449 & 1495.225 & 1493.429 & 1484.999 & 1479.005\end{array}$

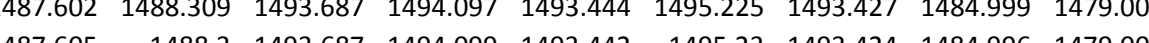

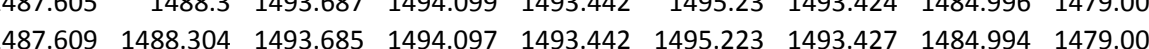
$\begin{array}{lllllllllll}1487.623 & 1488.295 & 1493.683 & 1494.094 & 1493.437 & 1495.225 & 1493.422 & 1484.994 & 1479.005\end{array}$ $\begin{array}{lllllllll}1487.628 & 1488.293 & 1493.683 & 1494.099 & 1493.439 & 1495.23 & 1493.422 & 1484.994 & 1479.005\end{array}$ $\begin{array}{lllllllll}1487.63 & 1488.295 & 1493.678 & 1494.083 & 1493.435 & 1495.207 & 1493.415 & 1484.994 & 1479.003\end{array}$ $\begin{array}{lllllllll}1487.623 & 14888.285 & 1493.673 & 1494.078 & 1493.428 & 1495.199 & 1493.408 & 1444.992 & 1479.000\end{array}$ $\begin{array}{lllllllll}1487.605 & 1488.26 & 4993.671 & 1494.085 & 1493.428 & 1495.218 & 1493.408 & 1484.994 & 1479.005\end{array}$ $\begin{array}{llllllllll}1487.609 & 1488.234 & 1493.68 & 1494.113 & 1493.439 & 1495.251 & 1493.412 & 1484.994 & 1479.005 \\ 1487.595 & 1488.217 & 1493.687 & 1494.138 & 1493.447 & 1495.282 & 1493.424 & 1484992 & 1479.01\end{array}$ $\begin{array}{llllllllll}1487.605 & 1488.208 & 1493.699 & 1494.157 & 1493.458 & 1495.308 & 1493.436 & 1484.992 & 1479.012\end{array}$ $\begin{array}{lllllllllll}1487.611 & 1488.206 & 1493.709 & 1494.173 & 1493.466 & 1495.329 & 1493.443 & 1484.992 & 1479.012\end{array}$ $\begin{array}{llllllllll}1487.565 & 1488.206 & 1493.713 & 1494.178 & 1493.473 & 1495.34 & 1493.455 & 1484.992 & 1479.012\end{array}$ $\begin{array}{llllllllll}4487.563 & 1488.208 & 1493.716 & 1494.19 & 1439.477 & 1495.348 & 1493.459 & 1484.992 & 1479.014\end{array}$ $\begin{array}{llllllllll}1487.572 & 1488.208 & 1493.723 & 1494.199 & 1493.484 & 1495.357 & 1493.466 & 1484.992 & 1479.014\end{array}$

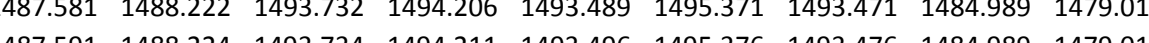

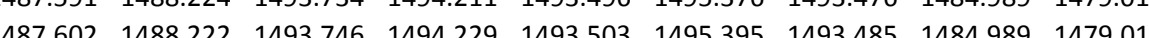
$\begin{array}{llllllllll}1487.611 & 1488232 & 1493.749 & 1494.236 & 1493.511 & 1495.397 & 1493.49 & 1484.992 & 1479019\end{array}$ $\begin{array}{lllllllllll}1487.618 & 1488.236 & 1493.758 & 1494.246 & 1493.52 & 1495.416 & 1493.502 & 1484.989 & 1479.024\end{array}$ $\begin{array}{lllllllll}1487.625 & 1488.241 & 1493.765 & 1494.257 & 1493.527 & 1495.425 & 1493.506 & 1484.989 & 1479.026\end{array}$ $\begin{array}{llllllllll}1487.635 & 1488.243 & 1493.774 & 1494.269 & 1493.537 & 1495.432 & 1493.516 & 1484.989 & 1479.031\end{array}$ $\begin{array}{lllllllll}4487.641 & 1488.241 & 1493.786 & 1494.283 & 1493.546 & 1495.458 & 1493.525 & 1484.992 & 1479.033\end{array}$ $\begin{array}{llllllllll}1487.653 & 1488.248 & 1493.793 & 1494.295 & 1493.556 & 1495.467 & 1493.534 & 1484.992 & 1479.035\end{array}$ $\begin{array}{lllllllll}148.66 & 1488.246 & 1493.805 & 1494.311 & 1493.567 & 1455.486 & 1493.544 & 1484.992 & 1479.04\end{array}$

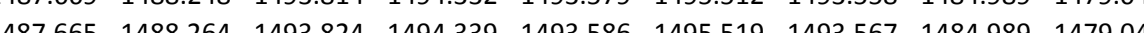
$\begin{array}{llllllllllll}1487.662 & 1488.262 & 1493.833 & 1494.351 & 1493.596 & 1495.531 & 1493.577 & 1484.989 & 1479.047\end{array}$ $\begin{array}{lllllllllll}1487.672 & 1488.269 & 1493.84 & 1494.36 & 1493.608 & 1495.545 & 1493.581 & 1484.989 & 1479.049\end{array}$ $\begin{array}{llllllllll}1487.678 & 1488.276 & 1493.847 & 1494.365 & 1493.612 & 1495.545 & 1493.591 & 1484.989 & 1479.052\end{array}$ $\begin{array}{llllllllll}4487.685 & 1488.278 & 1493.854 & 1494.367 & 1493.617 & 1495.552 & 1493.595 & 1484.992 & 1479.052\end{array}$ $\begin{array}{llllllllll}1487.692 & 1488.264 & 1493.866 & 1494.395 & 1493.629 & 1495.58 & 1493.605 & 1484.989 & 1479.056\end{array}$ $\begin{array}{llllllllll}1487.697 & 1488.25 & 1493.88 & 1494.435 & 1493.65 & 1495.625 & 1493.619 & 1484.989 & 1479.061 \\ 1487.706 & 1488.255 & 1493.904 & 1494.46 & 1493.669 & 1495.658 & 1493.64 & 1484.989 & 1479.066\end{array}$ 
Well

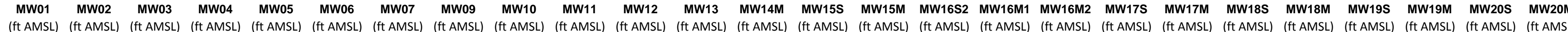

1/3/12 14:00 $1 / 3 / 1215: 00$ $1 / 3 / 1216: 00$ 1/3/12 17:00 $1 / 3 / 121218: 00$
$1 / 3 / 12$ 19:00 $1 / 3 / 1220: 00$ $1 / 3 / 1221: 00$ $1 / 3 / 1222: 00$ 1/3/12 23:00 1/4/120:00 1/4/12 1:00 1/4/12 2:00 1/4/123:00 1/4/12 5:00 1/4/12 6:00 1/4/12 7:00 1/4/12 8:00 $1 / 4 / 1210.00$ 1/4/12 10:00 1/4/12 12:00 1/4/1212 13:00 1/4/12 14:00 1/4/12 15:00 1/4/1212 16:00 1/4/12 17:00 1/4/12 18:00
1/4/12 19:00 1/4/12 19:00 1/4/12 21:00 1/4/12 22:00 1/4/12 23:00 1/5/12 0:00 1/5/121:00 $1 / 5 / 123.00$ 1/5/12 3:00 1/5/12 5:00 1/5/125:00 1/5/12 7:00 1/5/12 8:00 1/5/12 9:00 1/5/12 10:00 $1 / 5 / 1212.00$ 1/5/12 12:00 1/5/12 14:00 1/5/12 15:00 1/5/12 16:00 1/5/12 17:00 $1 / 5 / 1218: 00$
$1 / 5 / 1219: 00$ $\begin{array}{llll}1494.852 & 1495.324 & 1496.838 & 1495.588 \\ 1494.849 & 1495329 & 1496827 & 1495.595\end{array}$ $1494.849 \quad 1495.329 \quad 1496.8271495 .595$ $\begin{array}{rrrr}1494.859 & 1495.336 & 1496.829 & 1495.6 \\ 1494.859 & 1495.343 & 1496834 & 1495607\end{array}$ $\begin{array}{lllll}1494.852 & 1495.334 & 1496.799 & 1495.59\end{array}$ $\begin{array}{llll}1494.859 & 1495.334 & 1496.796 & 1495.583\end{array}$ $\begin{array}{lllll}1494.859 & 1495.334 & 1496.796 & 1495.583 \\ 1494.863 & 1495.336 & 1496.803 & 1495.583\end{array}$ $\begin{array}{lllll}1494.856 & 1495.322 & 1496.764 & 1495.56\end{array}$ $\begin{array}{lllll}1494.856 & 1495.31 & 1496.747 & 1495.537\end{array}$ $\begin{array}{llll}1494.854 & 1495.301 & 1496.726 & 1495.516 \\ 1499852 & 1495287 & 1496707 & 1459.497\end{array}$ $\begin{array}{llll}1494.852 & 1495.287 & 1496.707 & 1495.497 \\ 1494.852 & 1495282 & 1496703 & 1495483\end{array}$ $\begin{array}{llll}1494.852 & 1495.282 & 1496.703 & 1495.483 \\ 1494.852 & 1495.275 & 1496.689 & 1495.467\end{array}$ $\begin{array}{llllll}1494.845 & 1495.254 & 1496.663 & 1459.467\end{array}$ $\begin{array}{lllll}1494.847 & 1495.243 & 1496.644 & 1495.425\end{array}$ $\begin{array}{lllll}1494.847 & 1459.243 & 1496.644 & 1495.425 \\ 1495.224 & 1496.623 & 1495.404\end{array}$ $\begin{array}{lllll}1494.84 & 1495.217 & 1496.614 & 1495.39\end{array}$ $1494.833 \quad 1495.196 \quad 1496.588 \quad 1495.366$ $\begin{array}{llll}1494.826 & 1495.175 & 1496.56 & 1495.345\end{array}$ $\begin{array}{llll}1494.822 & 1495.161 & 1496.544 & 1495.322 \\ 1494.822 & 1495.152 & 1496539 & 1495308\end{array}$ $\begin{array}{lllll}1494.822 & 1495.152 & 1496.539 & 1495.308 \\ 1494.824 & 1495.154 & 1496.548 & 1495.308\end{array}$ $\begin{array}{lllll}1494.829 & 1495.163 & 1496579 & 1495315\end{array}$ $\begin{array}{lllll}1494.836 & 1495.177 & 1496.612 & 1495.336\end{array}$ $\begin{array}{lllll}1494.84 & 1495.194 & 1496.644 & 1495.359\end{array}$ $\begin{array}{llll}1494.842 & 1495.201 & 1496.651 & 1495.373\end{array}$ $\begin{array}{rrrr}1494.845 & 1495.212 & 1496.668 & 1495.39\end{array}$ $\begin{array}{rrrrr}1494.842 & 1495.208 & 1496.658 & 1495.392 \\ 1494.84 & 1495205 & 1496644 & 1495387\end{array}$ $\begin{array}{rrrrr}1494.84 & 1495.205 & 1496.644 & 1495.387 \\ 1494.845 & 1495208 & 1496.654 & 1495394\end{array}$ $\begin{array}{lllll}1494.845 & 1495.21 & 1496.661 & 1495.397\end{array}$ $\begin{array}{lllll}1494.845 & 1495.21 & 1496.658 & 1495.397\end{array}$ $\begin{array}{lllll}1494.852 & 1495.217 & 1496.665 & 1495.401\end{array}$ $\begin{array}{lllll}1494.854 & 1495.222 & 1496.677 & 1495.411\end{array}$ $\begin{array}{llll}1494.859 & 1495.233 & 1496.698 & 1495.425\end{array}$ $\begin{array}{llll}1494.861 & 1495.24 & 1496.705 & 1495.436 \\ 1499.863 & 1955.25 & 1469714 & 195446\end{array}$ $\begin{array}{lllll}1494.863 & 1495.25 & 1496.714 & 1495.446\end{array}$ $\begin{array}{lllll}1494.868 & 1495.254 & 1496.724 & 1495.455 \\ 1494.87 & 1495.264 & 1496.735 & 1495.467\end{array}$ $\begin{array}{lllll}1494.873 & 1495.268 & 1496.742 & 1495.474\end{array}$ $\begin{array}{llllll}1494.873 & 1495.273 & 1496.735 & 1495.476\end{array}$ $1494.877 \quad 1495.275 \quad 1496.745 \quad 1495.481$ $1494.877 \quad 1495.28 \quad 1496.752 \quad 1495.488$ $\begin{array}{llll}1494.875 & 1495.28 & 1496.747 & 1495.485\end{array}$ $\begin{array}{llll}1494.88 & 1495.282 & 1496.745 & 1495.485\end{array}$ $1494.884 \quad 1495.287 \quad 1496.756 \quad 1495.492$ $1494.903 \quad 1495.322 \quad 1496.822 \quad 1495.539$

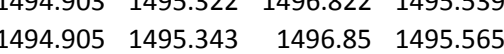
$1494.905 \quad 1495.35 \quad 1496.857 \quad 1495.579$ $\begin{array}{rrrr}1494.908 & 1495.357 & 1496.862 & 1495.588 \\ 1494.91 & 1495.364 & 1496.864 & 1495.597\end{array}$ $\begin{array}{lllll}494.915 & 1495.371 & 1496.871 & 1495.604\end{array}$ $\begin{array}{llllllllll}1494.113 & 1494.828 & 1495.399 & 1495.909 & 1497.087 & 1496.928 & 1493.685 & 1489.031 & 1493.515 & 1491.049\end{array}$ $\begin{array}{llllllllll}1494.146 & 1494.823 & 1495.391 & 1495.916 & 1497.091 & 1496.916 & 1493.689 & 1489.036 & 1493.522 & 1491.059 \\ 1494.164 & 194.837 & 1995391 & 1495928 & 1497.098 & 1496909 & 1493699 & 1489038 & 1493531 & 1491066\end{array}$ $\begin{array}{llllllllll}1494.186 & 1494.835 & 1495.382 & 1495.942 & 1497.108 & 1496.916 & 1493.706 & 1489.048 & 1493536 & 1491.067\end{array}$ $\begin{array}{lllllllllll}1494.23 & 1494.849 & 1495.37 & 1495.935 & 1497.098 & 1496.883 & 1493.701 & 1489.048 & 1493.536 & 1491.077\end{array}$ $\begin{array}{lllllllllll}1494.247 & 1494.842 & 1495.37 & 1495.939 & 1497.101 & 1496.873 & 1493.706 & 1489.045 & 1493.536 & 1491.084\end{array}$ $\begin{array}{llllllllllll}1494.254 & 1494.835 & 1495.37 & 1495.946 & 1497.108 & 1496.873 & 1493.706 & 1489.045 & 1493.538 & 1491.089\end{array}$ $\begin{array}{lllllllllll}1494.291 & 1494.866 & 1495.353 & 1495.932 & 1497.091 & 1496.823 & 1493.584 & 1489.043 & 1493.412 & 1491.089\end{array}$ $\begin{array}{lllllllllll}1494.308 & 1494.875 & 1495.356 & 1495.928 & 1497.08 & 1496.804 & 1493.626 & 1489.041 & 1493.456 & 1491.089\end{array}$

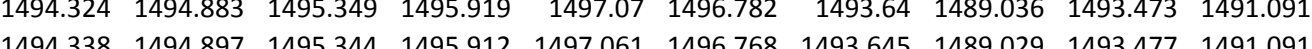
$\begin{array}{llllllllll}1494.338 & 1494.897 & 1495.344 & 1495.912 & 1497.061 & 1496.768 & 1493.645 & 1489.029 & 1493.477 & 1491.091 \\ 1494.338 & 1494899 & 1495344 & 1495907 & 1497.054 & 1496758 & 149365 & 1489024 & 1493.482 & 1491.091\end{array}$ $\begin{array}{llllllllllll}1494.343 & 1494.909 & 1495.342 & 1495.898 & 1497.045 & 1496.744 & 1493.65 & 1489.022 & 1493.487 & 1491.089\end{array}$ $\begin{array}{lllllllllllll}1494.357 & 1494.926 & 1495.332 & 1495.884 & 1497.028 & 1496.72 & 1493.647 & 1489.015 & 1493.482 & 1491.089\end{array}$ $\begin{array}{lllllllllll}1494.36 & 1494.931 & 1495.332 & 1495.872 & 1497.019 & 1496.701 & 1493.643 & 1489.01 & 1493.477 & 1491.084\end{array}$ $\begin{array}{lllllllllll}1494.362 & 1494.931 & 1495.33 & 1495.858 & 1497.003 & 1496.686 & 1493.636 & 1488.996 & 1493.47 & 1491.08\end{array}$ $\begin{array}{llllllllllll}1494.357 & 1494.921 & 1495.33 & 1495.849 & 1496.996 & 1496.677 & 1493.631 & 1488.994 & 1493.466 & 1491.077\end{array}$ $\begin{array}{lllllllllll}1494.362 & 1494.919 & 1495.318 & 1495.833 & 1496.977 & 1496.653 & 1493.622 & 1488.989 & 1493.459 & 1491.07\end{array}$ $\begin{array}{lllllllllllll}1494.367 & 1494.99 & 1455.313 & 1455.814 & 1496.959 & 1496.631 & 1493.61 & 1488.9779 & 1491.066\end{array}$ $\begin{array}{llllllllll}1494.362 & 1494.914 & 1495.308 & 1495.798 & 1496.945 & 1496.615 & 1493.598 & 1488.973 & 1493.438 & 1491.061 \\ 1494346 & 1494.909 & 1495.313 & 1495791 & 1496938 & 1496617 & 1493.427 & 1488963 & 1493255 & 1491.054\end{array}$ $\begin{array}{llllllllllll}1494.317 & 1494.89 & 1495.32 & 1495.786 & 1496.935 & 1496.627 & 1493.507 & 1488.963 & 1493.337 & 1491.052\end{array}$ $\begin{array}{llllllllll}1494.28 & 1494.854 & 1495.327 & 1495.793 & 1496.945 & 1496.66 & 1493.53 & 1488.959 & 1493.367 & 1491.047\end{array}$ $\begin{array}{llllllllll}1494.249 & 1494.825 & 1495.332 & 1495.807 & 1496.959 & 1496.696 & 1493.551 & 1488.97 & 1493.384 & 1491.047\end{array}$ $\begin{array}{lllllllllll}1494.226 & 1494.813 & 1495.33 & 1495.821 & 1496.973 & 1496.729 & 1493.57 & 1488.98 & 1493.4 & 1491.045\end{array}$ $\begin{array}{llllllllll}1494.219 & 1494.82 & 1495.327 & 1495.826 & 1496.98 & 1496.736 & 1493.579 & 1488.984 & 1493.414 & 1491.047\end{array}$

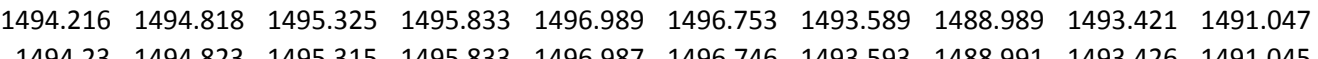
$\begin{array}{lllllllllll}1494.23 & 1494.823 & 1495.315 & 1495.833 & 1496.987 & 1496.746 & 1493.593 & 1488.991 & 1493.426 & 1491.045 \\ 1494224 & 1494.832 & 1495308 & 1495.83 & 1496984 & 1496734 & 1493593 & 1488991 & 1493.428 & 1491.047\end{array}$ $\begin{array}{rrrrrrrrrr}1494.244 & 1494.832 & 1495.308 & 1495.83 & 1496.984 & 1496.734 & 1493.593 & 1488.991 & 1493.428 & 1491.047 \\ 1494.244 & 1494.823 & 1495308 & 1495833 & 1496987 & 1496744 & 1493.598 & 1488.984 & 1493.433 & 1491.049\end{array}$ $\begin{array}{lllllllllll}1494.247 & 1494.827 & 1495.311 & 1495.837 & 1496.989 & 1496.748 & 1493.601 & 1488.991 & 1493.435 & 1491.047\end{array}$ $\begin{array}{llllllllllll}1494.256 & 1494.835 & 1495.306 & 1495.837 & 1496.989 & 1496.744 & 1493.603 & 1488.991 & 1493.435 & 1491.049\end{array}$ $\begin{array}{lllllllllll}1494.251 & 1494.825 & 1495.308 & 1495.84 & 1496.994 & 1496.751 & 1493.603 & 1488.991 & 1493.442 & 1491.049\end{array}$ $\begin{array}{lllllllllll}1494.249 & 1494.823 & 1495.308 & 1495.844 & 1497.001 & 1496.763 & 1493.61 & 1488.998 & 1493.447 & 1491.049\end{array}$ $\begin{array}{llllllllll}1494.24 & 1494.828 & 1495.311 & 1495.858 & 1497.012 & 1496.784 & 1493.619 & 1489.003 & 1493.454 & 1491.054\end{array}$ $\begin{array}{llllllllll}1494.242 & 1494.823 & 1495.306 & 1495.863 & 1497.022 & 1496.789 & 1493.626 & 1489.005 & 1493.461 & 1491.056\end{array}$ $\begin{array}{lllllllllll}1494244 & 1944.818 & 1995.308 & 1495.874 & 1497.026 & 1496.801 & 1493.638 & 1489.008 & 1493.47 & 1491.059\end{array}$ $\begin{array}{llllllllllll}1494.247 & 1494.83 & 1495.308 & 1495.886 & 1497.042 & 1496.816 & 1493.652 & 1489.012 & 1493.487 & 1491.066\end{array}$ $\begin{array}{llllllllllll}1494.251 & 1494.837 & 1495.306 & 1495.893 & 1497.047 & 1496.82 & 1493.659 & 1489.017 & 1493.491 & 1491.068\end{array}$ $\begin{array}{lllllllllll}1494.263 & 1494.847 & 1495.301 & 1495.895 & 1497.049 & 1496.813 & 1493.661 & 1489.022 & 1493.496 & 1491.073\end{array}$ $\begin{array}{lllllllllll}1494.263 & 1494.84 & 1495.304 & 1495.9 & 1497.054 & 1496.818 & 1493.666 & 1489.024 & 1493.498 & 1491.075\end{array}$ $\begin{array}{lllllllllll}1494.268 & 1494.84 & 1495.299 & 1495.907 & 1497.059 & 1496.823 & 1493.671 & 1489.022 & 1493.506 & 1491.077\end{array}$ $\begin{array}{llllllllll}1494.282 & 1494.854 & 1495.299 & 1495.905 & 1497.059 & 1496.816 & 1493.671 & 1489.024 & 1493.508 & 1491.082\end{array}$ $\begin{array}{llllllllllll}1494.284 & 1494.849 & 1495.296 & 1455.909 & 1497.061 & 1496.818 & 1493.673 & 1489.022 & 1493.508 & 1491.082\end{array}$ $\begin{array}{llllllllll}1494258 & 1494864 & 1495311 & 1495.935 & 1497.087 & 1496856 & 1493687 & 1498034 & 149352 & 1491.087\end{array}$ $\begin{array}{lllllllllll}1494.242 & 1494.861 & 1495.313 & 1495.953 & 1497.105 & 1496.887 & 1493.703 & 1489.036 & 1493.531 & 1491.096\end{array}$ $\begin{array}{llllllllll}1494.235 & 1494.861 & 1495.315 & 1495.972 & 1497.124 & 1496.911 & 1493.715 & 1489.045 & 1493.548 & 1491.101\end{array}$ $\begin{array}{lllllllllll}1494.247 & 1494.868 & 1495.311 & 1495.981 & 1497.133 & 1496.916 & 1493.725 & 1489.05 & 1493.557 & 1491.105\end{array}$ $\begin{array}{llllllllll}1494.261 & 1494.866 & 1495.308 & 1495.993 & 1497.14 & 1496.916 & 1493.732 & 1489.055 & 1493.564 & 1491.11\end{array}$ $\begin{array}{lllllllllll}1494.275 & 1494.878 & 1495.308 & 1496 & 1497.147 & 1496.916 & 1493.739 & 1489.057 & 1493.571 & 1491.115\end{array}$ $\begin{array}{llllllllll}1494.287 & 1494.868 & 1495.306 & 1496.009 & 1497.154 & 1496.918 & 1493.743 & 1489.059 & 1493.576 & 1491.119\end{array}$ $\begin{array}{lllllllll}1487.715 & 1488.264 & 1493.92 & 1494.477 & 1493.684 & 1495.679 & 1493.659 & 1484.987 & 1479.07\end{array}$ $\begin{array}{rrrrrrrrr}1487.72 & 1488.281 & 1493.927 & 1494.477 & 1493.693 & 1495.669 & 1493.666 & 1484.987 & 1479.07\end{array}$

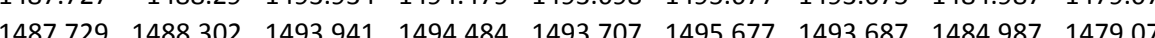
$\begin{array}{lllllllllll}1487.732 & 1488.33 & 1493.941 & 1494.467 & 1493.702 & 1495.653 & 1493.685 & 1484.985 & 1479.07\end{array}$ $\begin{array}{lllllllllll}1487.732 & 1488.337 & 1493.944 & 1494.463 & 1493.705 & 1495.648 & 1493.687 & 1484.984 & 1479.07\end{array}$ $\begin{array}{llllllllll}1487.732 & 1488.342 & 1493.946 & 1494.467 & 1493.705 & 1495.653 & 1493.685 & 1484.985 & 1479.073\end{array}$ $\begin{array}{llllllllll}1487.732 & 1488.368 & 1493.819 & 1494.39 & 1493.586 & 1495.613 & 1493.567 & 1484.984 & 1479.068\end{array}$ $\begin{array}{llllllllll}14887.736 & 1488.375 & 1493.864 & 1494.397 & 1493.627 & 1495.592 & 1493.607 & 1484.984 & 1479.066\end{array}$ $\begin{array}{llllllllll}1487.739 & 1488.391 & 1493.88 & 1494.388 & 1493.641 & 1495.568 & 1493.624 & 1484.984 & 1479.066\end{array}$ $\begin{array}{lllllllllll}1487.736 & 1488.408 & 1493.887 & 1494.376 & 1493.646 & 1495.552 & 1493.628 & 1484.984 & 1479.066 \\ 1487.741 & 1488.41 & 149389 & 1494376 & 1493.648 & 1495.55 & 1493.633 & 1484.984 & 1479.068\end{array}$ $\begin{array}{llllllllll}1487.741 & 1488.41 & 1493.892 & 4494.376 & 449.648 & 1495.55 & 1493.633 & 1444.984 & 1479.068\end{array}$ $\begin{array}{lllllllllll}1487.743 & 1488.429 & 1493.89 & 1494.351 & 1493.646 & 1495.503 & 1493.628 & 1484.982 & 1479.068\end{array}$ $\begin{array}{lllllllllll}1487.746 & 1488.426 & 1493.883 & 1494.337 & 1493.641 & 1495.486 & 1493.624 & 1484.985 & 1479.066\end{array}$ $\begin{array}{lllllllllll}1487.746 & 1488.44 & 1493.88 & 1494.32 & 1493.636 & 1495.47 & 1493.619 & 1484.98 & 1479.066\end{array}$ $\begin{array}{llllllllll}1487.732 & 1488.436 & 1493.873 & 1494.306 & 1493.629 & 1495.456 & 1493.614 & 1484.982 & 1479.063\end{array}$ $\begin{array}{llllllllll}1487.725 & 1488.438 & 1493.864 & 1494.297 & 1493.62 & 1495.432 & 1493.605 & 1484.982 & 1479.061\end{array}$ $\begin{array}{lllllllll}1487.727 & 1488.45 & 1493.852 & 4994.271 & 1493.61 & 1495.406 & 1493.593 & 1484.98 & 1479.059 \\ 1487.729 & 1488.43 & 1493845 & 1494257 & 1493598 & 1495395 & 1493.581 & 148988 & 1479056\end{array}$ $\begin{array}{lllllllll}1487.729 & 1488.443 & 1493.845 & 1494.257 & 1493.598 & 1495.395 & 1493.581 & 1484.98 & 1479.056\end{array}$ $\begin{array}{llllllllll}1487.741 & 1488.41 & 1493.744 & 1494.215 & 1493.506 & 1495.387 & 1493.478 & 1484.982 & 1479.052\end{array}$ $\begin{array}{lllllllllll}1487.746 & 1488389 & 1493.772 & 1494246 & 1493.532 & 1495.409 & 1493506 & 1484.98 & 1479.054\end{array}$ $\begin{array}{llllllllllll}1487.752 & 1488.37 & 1493.796 & 1494.271 & 1493.553 & 1495.43 & 1493.527 & 1484.98 & 1479.059\end{array}$ $\begin{array}{llllllllll}1487.759 & 1488.363 & 1493.81 & 1494.29 & 1493.57 & 1495.451 & 1493.546 & 1484.977 & 1479.061\end{array}$ $\begin{array}{lllllllllll}1487.764 & 1488.358 & 1493.821 & 1494.306 & 1493.582 & 1495.47 & 1493.556 & 1484.977 & 1479.061\end{array}$ $\begin{array}{llllllllll}1487.718 & 1488.356 & 1493.831 & 1494.313 & 1493.591 & 1495.479 & 1493.567 & 1484.977 & 1479.063\end{array}$ $\begin{array}{lllllllllll}148.715 & 4488.363 & 1493.835 & 1494.309 & 1493.596 & 1495.472 & 1493.5774 & 1484.977 & 1479.061\end{array}$

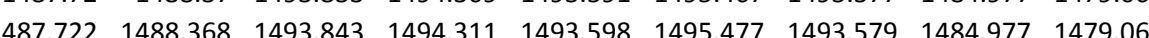
$\begin{array}{llllllllll}1487.727 & 1488.37 & 1493.838 & 1494.316 & 1493.598 & 1495.481 & 1493.581 & 1484.977 & 1479.059\end{array}$ $\begin{array}{llllllllll}1487.729 & 1488.377 & 1493.84 & 1494.32 & 1493.601 & 1495.474 & 1493.581 & 1484.977 & 1479.059\end{array}$ $\begin{array}{lllllllll}1487.734 & 1488.37 & 1493.843 & 1494.33 & 1493.603 & 1495.486 & 1493.584 & 1484.977 & 1479.059\end{array}$ $\begin{array}{llllllllll}1487.736 & 1488.375 & 1493.85 & 1494.332 & 1493.61 & 1495.493 & 1493.591 & 1484.975 & 1479.059\end{array}$ $\begin{array}{llllllllll}1487.739 & 1488.375 & 1493.861 & 1494.346 & 1493.62 & 1495.512 & 1493.598 & 1484.977 & 1479.063\end{array}$ $\begin{array}{llllllllll}1487.75 & 1488.379 & 1493.868 & 1494.36 & 1493.629 & 1495.521 & 1493.607 & 1484.975 & 1479.066\end{array}$

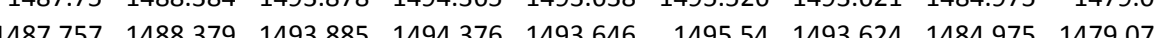
$\begin{array}{lllllllllll}1487.766 & 1488.386 & 1493.89 & 1494.39 & 1493.653 & 1495.552 & 1493.631 & 1484.975 & 1479.08\end{array}$ $\begin{array}{lllllllllll}1487.776 & 1488.391 & 1493.901 & 1494.39 & 1493.657 & 1495.557 & 1493.638 & 1484.975 & 1479.08\end{array}$ $\begin{array}{llllllllll}1487.766 & 1488.396 & 1493.901 & 1494.393 & 1493.662 & 1495.554 & 1493.642 & 1484.977 & 1479.08\end{array}$ $\begin{array}{llllllllll}1487.769 & 1488.398 & 1493.908 & 1494.397 & 1493.665 & 1495.564 & 1493.645 & 1484.975 & 1479.084\end{array}$ $\begin{array}{lllllllllll}1487.766 & 1488.4 & 1493.908 & 1494.404 & 1493.672 & 1495.571 & 1493.649 & 1484.975 & 1479.08\end{array}$ $\begin{array}{lllllllll}1487.766 & 1488.408 & 1493.913 & 1494.397 & 1493.672 & 1495.566 & 1493.652 & 1484.973 & 1479.084\end{array}$ $\begin{array}{llllllllll}1487.766 & 4488.408 & 1493.913 & 1494.402 & 1493.674 & 1495.564 & 1493.649 & 1484.973 & 1479.084\end{array}$

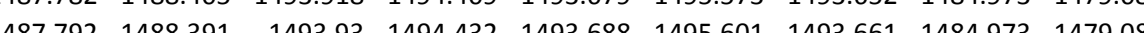
$\begin{array}{lllllllllll}1487.799 & 1488.382 & 1493.941 & 1494.458 & 1493.702 & 1495.63 & 1493.675 & 1484.973 & 1479.089\end{array}$ $\begin{array}{lllllllllll}1487.803 & 1488.377 & 1493.955 & 1494.477 & 1493.714 & 1495.655 & 1493.687 & 1484.97 & 1479.094\end{array}$ $\begin{array}{llllllllll}1487.81 & 1488.386 & 1493.965 & 1494.479 & 1493.726 & 1495.658 & 1493.701 & 1484.973 & 1479.098\end{array}$ $\begin{array}{lllllllllll}1487.815 & 1488.391 & 1493.972 & 1494.488 & 1493.733 & 1495.669 & 1493.71 & 1484.973 & 1479.100\end{array}$ $\begin{array}{llllllllllll}1487.82 & 1488.393 & 1493.979 & 1494.495 & 1493.738 & 1495.674 & 1493.717 & 1484.97 & 1479.103\end{array}$ $\begin{array}{rrrrrrrrr}1487.822 & 1488.398 & 1493.984 & 1494.5 & 1493.743 & 1495.681 & 1493.725 & 1484.973 & 1479.103\end{array}$ 
Well

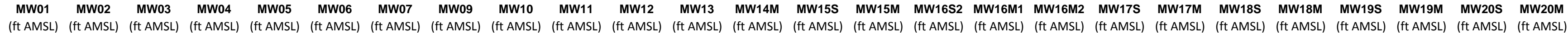

$1 / 5 / 1220: 00$ $1 / 5 / 1221: 00$ 1/5/1222:00 1/5/12 23:00 1/6/120:00 1/6/12 2:00 1/6/12 3:00 1/6/12 4:00 1/6/125:00 1/6/12 6:00 $1 / 6 / 128.00$ 1/6/12 9:00 1/6/12 9:00 1/6/12 11:00 1/6/12 12:00 1/6/12 13:00 $1 / 6 / 1214: 00$
$1 / 6 / 1215: 00$ 1/6/12 15:00 1/6/12 16:00 1/6/12 18:00 1/6/12 18:00 1/6/12 20:00 1/6/12 21:00 1/6/12 22:00 1/6/12 23:00 1/7/120:00 1/7/12 1:00 $1 / 7 / 123: 00$ 1/7//12 4:00 1/7/12 5:00 1/7/12 6:00 1/7/122 7:00 $1 / 7 / 128: 00$ 1/77/12 9:00 1/7/12 11:00 1/7/12 12:00 1/7/12 13:00 1/7/12 14:00 1/7/1215:00 1/7/12 16:00 1/7/12218.00 1/7/12 18:00 1/7/12 20:00 1/7/12 21:00 1/7/12 22:00 1/7/12 23:00 1/8/12 0:00 $\begin{array}{llll}1494.917 & 1495.378 & 1496.866 & 1495.607 \\ 1499.922 & 1495385 & 1496873 & 1495.612\end{array}$ $\begin{array}{llll}1494.922 & 1495.385 & 1496.873 & 1495.612 \\ 1494.929 & 1495392 & 1496887 & 1995.618\end{array}$ $\begin{array}{llll}1494.929 & 1495.392 & 1496.887 & 1495.618\end{array}$ $\begin{array}{lllll}1494.931 & 1495.408 & 1496.904 & 1495.635\end{array}$ $\begin{array}{lllll}1494.936 & 1495.415 & 1496.913 & 1495.635\end{array}$ $\begin{array}{rrrrr}1494.936 & 1495.415 & 1496.913 & 1495.642 \\ 1494.938 & 1495.42 & 1496.915 & 1495.646\end{array}$ $\begin{array}{lllll}1494.938 & 1495.42 & 1496.911 & 1495.649\end{array}$ $1494.94 \quad 1495.425 \quad 1496.915 \quad 1495.651$ $1494.943 \quad 1495.432 \quad 1496.918 \quad 1495.653$ $\begin{array}{llll}1494.945 & 1495.432 & 1496.922 & 1495.656 \\ 1494.943 & 1495.427 & 1496908 & 1495651\end{array}$ $\begin{array}{rrrrr}1494.943 & 1495.427 & 1496.908 & 1495.651 \\ 1494.947 & 1495.434 & 1496.92 & 1495.653\end{array}$ $\begin{array}{lllll}1494.933 & 1495.418 & 1496.876 & 1495.63\end{array}$ $\begin{array}{rrrr}1494.936 & 1495.404 & 1496.855 & 1495.611\end{array}$ $\begin{array}{lllll}1494.936 & 1459.404 & 1496.855 & 1495.611 \\ 1494.936 & 1495.392 & 1496.834 & 1495.588\end{array}$ $\begin{array}{lllll}1494.94 & 1495.392 & 1496.843 & 1495.586\end{array}$ $\begin{array}{lllll}1494.94 & 1495.387 & 1496.838 & 1495.576\end{array}$ $\begin{array}{lllll}1494.938 & 1495.38 & 1496.831 & 1495.567\end{array}$ $\begin{array}{rrrr}1494.936 & 1495.364 & 1496.806 & 1495.548 \\ 1494.931 & 1495348 & 149678 & 1995527\end{array}$ $\begin{array}{lllll}1494.924 & 1495.32 & 1496.742 & 1495.599\end{array}$ $\begin{array}{lllll}1494.919 & 1495.299 & 1496.714 & 1495.471\end{array}$ $\begin{array}{llllll}1494.915 & 1495.273 & 1496.686 & 1495.443\end{array}$ $\begin{array}{lllll}1494.91 & 1495.259 & 1496.672 & 1495.422\end{array}$ $\begin{array}{lllll}1494.91 & 1495.252 & 1496.665 & 1495.408\end{array}$ $\begin{array}{lllll}1494.905 & 1495.243 & 1496.658 & 1495.394\end{array}$ $\begin{array}{llll}1494.905 & 1495.236 & 1496.654 & 1495.385\end{array}$ $\begin{array}{llll}1494.903 & 1495.229 & 1496.654 & 1495.378 \\ 1494.903 & 1495231 & 1496.658 & 1495378\end{array}$ $\begin{array}{llllll}1494.901 & 1495.226 & 1496.654 & 1495.373\end{array}$ $\begin{array}{llllll}1494.901 & 1495.224 & 1496.654 & 1495.373\end{array}$ $1494.901 \quad 1495.226 \quad 1496.658 \quad 1495.376$ $\begin{array}{lllll}1494.901 & 1495.229 & 1496.663 & 1495.378\end{array}$ $\begin{array}{lllll}1494.894 & 1495.217 & 1496.642 & 1495.369\end{array}$ $\begin{array}{llll}1494.898 & 1459.219 & 1496.649 & 1495.371\end{array}$ $\begin{array}{lllll}1494.894 & 1495.212 & 1496.64 & 1495.364\end{array}$ $\begin{array}{lllll}1494.891 & 1495.205 & 1496.628 & 1495.357 \\ 1494.889 & 1495.203 & 1496.626 & 1495.352\end{array}$ $\begin{array}{lllll}1494.884 & 1495.194 & 1496.612 & 1495.343\end{array}$ $\begin{array}{lllll}1494.894 & 1495.205 & 1496.64 & 1495.355\end{array}$ $1494.901 \quad 1495.2261496 .6791495 .378$ $1494.901 \quad 1495.231 \quad 1496.6891495 .394$ $1494.898 \quad 1495.23314966 .6891495 .399$ $1494.896 \quad 1495.231 \quad 1496.6751495 .397$ $\begin{array}{llll}1494.894 & 1495.224 & 1496.663 & 1495.392 \\ 1494.898 & 1495229 & 149667 & 1495394\end{array}$ $\begin{array}{llll}1494.894 & 1495.224 & 1496.656 & 1495.385\end{array}$ $1494.891 \quad 1495.222 \quad 1496.649 \quad 1495.378$ $1494.889 \begin{array}{llll}1495.215 & 1496.64 & 1495.366\end{array}$ $1494.887 \quad 1495.212 \quad 1496.628 \quad 1495.359$ $1494.882 \quad 1495.208 \quad 1496.616 \quad 1495.348$ $\begin{array}{llll}1494.882 & 1495.203 & 1496.609 & 1495.341 \\ 1494.882 & 1495.203 & 1496.614 & 1495.338\end{array}$ $\begin{array}{llllllllll}1494.315 & 1494.863 & 1495.306 & 1496.021 & 1497.159 & 1496.911 & 1493.746 & 1489.059 & 1493.581 & 1491.129\end{array}$ $\begin{array}{lllllllllll}1494.324 & 1494.873 & 1495.306 & 1496.028 & 1497.168 & 1496.916 & 1493.748 & 1489.069 & 1493.588 & 1491.131\end{array}$ $\begin{array}{lllllllllll}1494.327 & 1494.885 & 1495.311 & 1466.037 & 1477.177 & 1496.923 & 1493.755 & 1489.069 & 1493.59 & 1491.136 \\ 1494.334 & 1494.88 & 1495308 & 1496046 & 1497.184 & 1496.93 & 1493.76 & 1489073 & 1493597 & 1491.14\end{array}$ $\begin{array}{lllllllllll}1494.338 & 1494.887 & 1495.311 & 1496.055 & 1497.191 & 1496.938 & 1493.764 & 1489.073 & 1493.606 & 1491.145\end{array}$ $\begin{array}{lllllllllllll}1494.346 & 1494.902 & 1495.311 & 1496.065 & 1497.201 & 1496.942 & 1493.774 & 1489.083 & 1493.611 & 1491.147\end{array}$ $\begin{array}{llllllllllll}1494.357 & 1494.887 & 1495.311 & 1496.072 & 1497.205 & 1496.945 & 1493.783 & 1489.08 & 1493.62 & 1491.152\end{array}$ $\begin{array}{lllllllllll}1494.371 & 1494.885 & 1495.306 & 1496.074 & 1497.205 & 1496.935 & 1493.788 & 1489.08 & 1493.627 & 1491.156\end{array}$ $\begin{array}{llllllllll}1494.378 & 1494.926 & 1495.306 & 1496.079 & 1497.21 & 1496.933 & 1493.795 & 1489.09 & 1493.632 & 1491.161\end{array}$ $\begin{array}{llllllllll}1494.388 & 1494.928 & 1495.311 & 1496.086 & 1497.215 & 1496.938 & 1493.799 & 1489.08 & 1493.637 & 1491.163 \\ 1494397 & 1499.935 & 1495308 & 1496093 & 1497.219 & 149692 & 1493804 & 1489087 & 149642 & 149.166\end{array}$ $\begin{array}{rrrrrrrrrr}1494.397 & 1494.935 & 1495.308 & 1496.093 & 1497.219 & 1496.942 & 1493.804 & 1489.087 & 1493.642 & 1491.166 \\ 1494.414 & 1494.94 & 1495306 & 1496088 & 1497.215 & 1496923 & 1493802 & 1489.092 & 1493642 & 1491.168\end{array}$ $\begin{array}{llllllllllll}1494.414 & 1494.933 & 1495.308 & 1496.097 & 1497.219 & 1496.933 & 1493.809 & 1489.092 & 1493.646 & 1491.17\end{array}$ $\begin{array}{lllllllllll}1494.451 & 1494.971 & 1495.294 & 1496.079 & 1497.201 & 1496.885 & 1493.799 & 1489.09 & 1493.639 & 1491.17\end{array}$ $\begin{array}{llllllllll}1494.463 & 1494.974 & 1495.289 & 1496.069 & 1497.189 & 1496.863 & 1493.792 & 1489.078 & 1493.632 & 1491.17\end{array}$ $\begin{array}{llllllllllll}1494.47 & 1494.969 & 1495.289 & 1496.06 & 1497.177 & 1496.842 & 1493.785 & 1489.073 & 1493.627 & 1491.168\end{array}$ $\begin{array}{lllllllllll}1494.458 & 1494.962 & 1495.296 & 1496.06 & 1497.177 & 1496.849 & 1493.783 & 1489.066 & 1493.623 & 1491.168\end{array}$ $\begin{array}{lllllllllll}1494.456 & 1494.95 & 1495.294 & 1496.053 & 1497.173 & 1496.847 & 1493.781 & 1489.069 & 1493.623 & 1491.166\end{array}$ $\begin{array}{llllllllll}1494.461 & 1494.942 & 1495.289 & 1496.051 & 1497.166 & 1496.842 & 1493.718 & 1489.064 & 1493.618 & 1491.166\end{array}$ $\begin{array}{llllllllll}1494.472 & 1494.954 & 1495.282 & 1496.037 & 1497.149 & 1496.818 & 1493.776 & 1489.066 & 1493.613 & 1491.161 \\ 1494.487 & 1494.964 & 1495273 & 1496021 & 1497.135 & 1496794 & 1493764 & 1489059 & 1493604 & 1491.159\end{array}$ $\begin{array}{lllllllllll}1494.505 & 1494.988 & 1495.268 & 1496.002 & 1497.115 & 1496.76 & 1493.75 & 1489.043 & 1493.597 & 1491.152\end{array}$ $\begin{array}{llllllllllll}1494.51 & 1495.002 & 1495.266 & 1495.981 & 1497.094 & 1496.736 & 1493.736 & 1489.034 & 1493.581 & 1491.147\end{array}$ $\begin{array}{lllllllllll}1494.517 & 1495 & 1495.263 & 1495.958 & 1497.075 & 1496.705 & 1493.72 & 1489.029 & 1493.566 & 1491.14\end{array}$ $\begin{array}{lllllllllll}1494.508 & 1494.993 & 1495.261 & 1495.944 & 1497.061 & 1496.696 & 1493.708 & 1489.017 & 1493.55 & 1491.133\end{array}$ $\begin{array}{llllllllll}1494.496 & 1494.986 & 1495.266 & 1495.935 & 1497.049 & 1496.696 & 1493.696 & 1489.015 & 1493.541 & 1491.126\end{array}$ $\begin{array}{llllllllll}1494.489 & 1494.983 & 1495.263 & 1495.923 & 1497.04 & 1496.694 & 1493.687 & 1489.008 & 1493.531 & 1491.122\end{array}$ $\begin{array}{llllllllll}1494.472 & 1494.974 & 1495.263 & 1495.912 & 1497.031 & 1496.691 & 1493.678 & 1488.998 & 1493.522 & 1491.115 \\ 1494.463 & 1494971 & 1495266 & 1495.905 & 1497.026 & 1496693 & 1493673 & 1488998 & 1493517 & 1491108\end{array}$ $\begin{array}{llllllllllll}1494.463 & 1494.971 & 1495.266 & 1495.905 & 1497.026 & 1496.693 & 1493.673 & 1488.998 & 1493.517 & 1491.108 \\ 1494.444 & 1494.964 & 1495.263 & 14959 & 1497.024 & 1496703 & 1493.668 & 1488.996 & 1493515 & 1491.103\end{array}$ $\begin{array}{llllllllllll}1494.44 & 1494.966 & 1495.261 & 1495.893 & 1497.019 & 1496.703 & 1493.668 & 1488.994 & 1493.513 & 1491.101\end{array}$ $\begin{array}{lllllllllll}1494.428 & 1494.962 & 1495.258 & 1495.886 & 1497.012 & 1496.705 & 1493.666 & 1488.991 & 1493.508 & 1491.096\end{array}$ $\begin{array}{lllllllllll}1494.414 & 1494.954 & 1495.261 & 1495.886 & 1497.012 & 1496.72 & 1493.668 & 1488.991 & 1493.51 & 1491.091\end{array}$ $\begin{array}{llllllllllll}1494.407 & 1494.954 & 1495.261 & 1495.881 & 1497.012 & 1496.725 & 1493.668 & 1488.994 & 1493.513 & 1491.089\end{array}$ $\begin{array}{lllllllllll}1494.416 & 1494.961 & 1495.254 & 1495.87 & 1497.001 & 1496.705 & 1493.664 & 1488.991 & 1493.508 & 1491.087\end{array}$

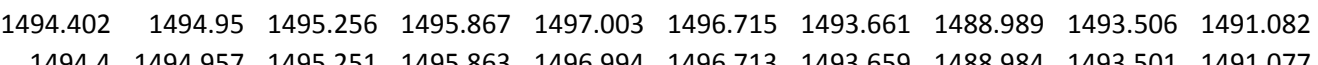

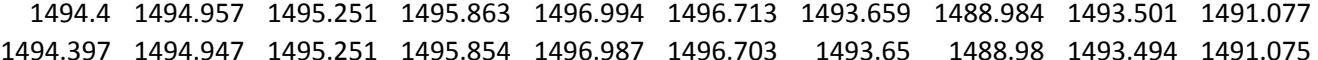
$\begin{array}{llllllllll}1449.393 & 1444.935 & 1495.249 & 1495.844 & 1496.984 & 1496.701 & 1493.645 & 1488.98 & 1493.487 & 1491.07\end{array}$ $\begin{array}{llllllllllll}1494.393 & 1494.933 & 1495.244 & 1495.837 & 1496.975 & 1496.694 & 1493.64 & 1488.982 & 1493.48 & 1491.068\end{array}$ $\begin{array}{lllllllllll}1494.362 & 1494.904 & 1495.256 & 1495.844 & 1496.984 & 1496.722 & 1493.64 & 1488.975 & 1493.484 & 1491.066\end{array}$ $\begin{array}{lllllllllll}1494.331 & 1494.873 & 1495.266 & 1495.858 & 1497.003 & 1496.76 & 1493.652 & 1488.987 & 1493.491 & 1491.066\end{array}$ $\begin{array}{lllllllllll}1494.327 & 1494.88 & 1495.258 & 1495.865 & 1497.008 & 1496.772 & 1493.657 & 1488.991 & 1493.496 & 1491.068\end{array}$ $\begin{array}{llllllllll}1494.331 & 1494.88 & 1495.256 & 1495.865 & 1497.01 & 1496.772 & 1493.659 & 1488.991 & 1493.501 & 1491.066\end{array}$ $\begin{array}{llllllllll}1494.343 & 1494.887 & 1495.249 & 1495.858 & 1497.005 & 1496.758 & 1493.659 & 1488.996 & 1493.501 & 1491.066 \\ 1494.355 & 1494.897 & 1495247 & 1495856 & 1497.001 & 1496751 & 1493657 & 1488994 & 1493499 & 1491066\end{array}$

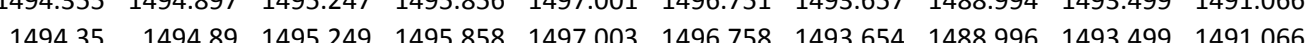
$\begin{array}{llllllllllll}1494.36 & 1494.906 & 1495.244 & 1495.851 & 1496.996 & 1496.748 & 1493.505 & 1488.989 & 1493.334 & 1491.066\end{array}$ $\begin{array}{lllllllllll}1494.367 & 1494.916 & 1495.239 & 1495.847 & 1496.994 & 1496.741 & 1493.558 & 1488.984 & 1493.395 & 1491.063\end{array}$ $\begin{array}{llllllllll}1494.371 & 1494.93 & 1495.235 & 1495.842 & 1496.984 & 1496.734 & 1493.575 & 1488.984 & 1493.414 & 1491.061\end{array}$ $\begin{array}{llllllllll}1494.376 & 1494.933 & 1495.235 & 1495.835 & 1496.982 & 1496.727 & 1493.582 & 1488.98 & 1493.423 & 1491.061\end{array}$ $\begin{array}{lllllllllll}1494.378 & 1494.931 & 1495.232 & 1495.828 & 1496.973 & 1496.717 & 1493.582 & 1488.977 & 1493.421 & 1491.061\end{array}$ $\begin{array}{llllllllllll}1494.376 & 1494.93 & 1495.23 & 1495.823 & 1496.97 & 1496.713 & 1493.584 & 1488.973 & 1493.428 & 1491.056\end{array}$ $\begin{array}{lllllllll}1487.824 & 1488.417 & 1493.986 & 1494.502 & 1493.747 & 1495.679 & 1493.729 & 1484.97 & 1479.101\end{array}$ $\begin{array}{llllllllll}1487.822 & 1488.424 & 1493.991 & 1494.505 & 1493.752 & 1495.684 & 1493.732 & 1484.97 & 1479.100\end{array}$

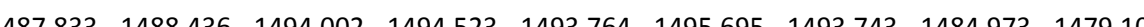
$\begin{array}{lllllllllll}1487.833 & 1488.445 & 1494.009 & 1494.533 & 1493.771 & 1495.709 & 1493.75 & 1484.97 & 1479.105\end{array}$ $\begin{array}{lllllllllll}1487.84 & 1488.45 & 1494.021 & 1494.542 & 1493.781 & 1495.719 & 1493.76 & 1484.97 & 1479.11\end{array}$ $\begin{array}{llllllllll}1487.843 & 1488.462 & 1494.028 & 1494.544 & 1493.788 & 1495.724 & 1493.767 & 1484.97 & 1479.112\end{array}$ $\begin{array}{llllllllll}1487.845 & 1488.473 & 1494.031 & 1494.542 & 1493.79 & 1495.716 & 1493.774 & 1484.97 & 1479.117\end{array}$ $\begin{array}{llllllllll}1487.852 & 1488.48 & 1494.038 & 1494.547 & 1493.8 & 1495.724 & 1493.776 & 1484.97 & 1479.12\end{array}$ $\begin{array}{llllllllll}1487.859 & 1488.49 & 1494.045 & 1494.551 & 1493.804 & 1495.724 & 1493.783 & 1484.973 & 1479.124\end{array}$ $\begin{array}{lllllllll}1487.852 & 1488.501 & 1494.049 & 1494.554 & 1493.809 & 1495.726 & 1493.79 & 1484.97 & 1479.127 \\ 1487.857 & 1488.518 & 1494.047 & 1494549 & 1493809 & 1495.714 & 1493.79 & 1484.97 & 1479.129\end{array}$ $\begin{array}{llllllllll}1487.85 & 1488.515 & 1494.049 & 1494.558 & 1493.814 & 1495.724 & 1493.795 & 1484.97 & 1479.129\end{array}$ $\begin{array}{lllllllll}1487854 & 1488537 & 1494.047 & 1494.526 & 1493.804 & 1495686 & 1493.783 & 1484.968 & 1479.124\end{array}$ $\begin{array}{llllllllll}1487.859 & 1488.544 & 1494.04 & 1494.509 & 1493.797 & 1495.669 & 1493.776 & 1484.968 & 1479.122\end{array}$ $\begin{array}{llllllllll}1487.863 & 1488.539 & 1494.031 & 1494.502 & 1493.79 & 1495.648 & 1493.767 & 1484.968 & 1479.12\end{array}$ $\begin{array}{lllllllllll}1487.866 & 1488.53 & 1494.033 & 1494.502 & 1493.788 & 1495.651 & 1493.764 & 1484.97 & 1479.12\end{array}$ $\begin{array}{lllllllll}1487.87 & 1488.53 & 1494.028 & 1494.493 & 1493.788 & 1495.646 & 1493.762 & 1484.968 & 1479.12\end{array}$ $\begin{array}{llllllllll}1487.873 & 1488.534 & 1494.026 & 1494.491 & 1493.781 & 1495.641 & 1493.764 & 1484.966 & 1479.12\end{array}$ $\begin{array}{llllllllll}1487.877 & 1488.539 & 1494.021 & 1494.472 & 1493.774 & 1495.611 & 1493.762 & 1484.966 & 1479.117\end{array}$ $\begin{array}{lllllllllll}1487.822 & 1488.548 & 1493.998 & 1494.425 & 1493.755 & 1495.561 & 1493.741 & 1484.966 & 1479.112\end{array}$ $\begin{array}{lllllllll}1487.82 & 1488548 & 1493.986 & 1494.404 & 1493.743 & 1495531 & 1493727 & 1484.963 & 1479.105\end{array}$ $\begin{array}{llllllllllll}1487.82 & 1488.541 & 1493.972 & 1494.381 & 1493.726 & 1495.505 & 1493.708 & 1484.963 & 1479.098\end{array}$ $\begin{array}{llllllllll}1487.82 & 1488.537 & 1493.962 & 1494.367 & 1493.714 & 1495.479 & 1493.696 & 1484.963 & 1479.094\end{array}$ $\begin{array}{llllllllll}1487.82 & 1488.534 & 1493.948 & 1494.358 & 1493.7 & 1495.47 & 1493.685 & 1484.963 & 1479.089\end{array}$

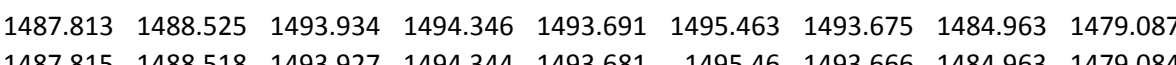

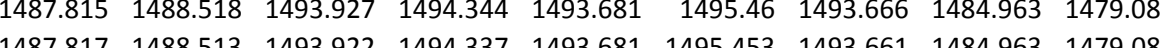
$\begin{array}{llllllllll}1487.817 & 1488.513 & 1493.922 & 1494.337 & 1493.681 & 1495.453 & 1493.661 & 1484.963 & 1479.082 \\ 1487.82 & 1488.504 & 1493.92 & 1494.332 & 1493.676 & 1495.456 & 1493.659 & 1484963 & 1479084\end{array}$ $\begin{array}{llllllllll}1487.824 & 1488.506 & 1493.92 & 1494.332 & 1493.672 & 1495.451 & 1493.656 & 1484.963 & 1479.089\end{array}$ $\begin{array}{llllllllll}1487.824 & 1488.501 & 1493.918 & 1494.334 & 1493.672 & 1495.456 & 1493.654 & 1484.963 & 1479.091\end{array}$ $\begin{array}{llllllllll}1487.831 & 1488.497 & 1493.918 & 1494.337 & 1493.674 & 1495.458 & 1493.654 & 1484.963 & 1479.096\end{array}$ $\begin{array}{llllllllll}1487.838 & 1488.494 & 1493.92 & 1494.344 & 1493.672 & 1495.465 & 1493.656 & 1484.963 & 1479.098\end{array}$ $\begin{array}{llllllllll}1487.838 & 1488.499 & 1493.913 & 1494.325 & 1493.667 & 1495.446 & 1493.649 & 1484.961 & 1479.098\end{array}$ $\begin{array}{llllllllll}1487.843 & 1488.49 & 1493.913 & 1494.334 & 1493.665 & 1495.451 & 1493.647 & 1484.961 & 1479.101\end{array}$

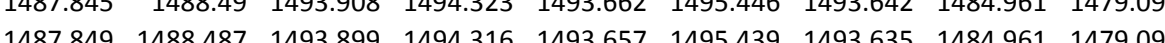
$\begin{array}{lllllllll}1487847 & 1488478 & 1493894 & 1494318 & 1493648 & 1495.434 & 1493628 & 1484959 & 1479091\end{array}$ $\begin{array}{lllllllllll}1487.843 & 1488.476 & 1493.894 & 1494.306 & 1493.646 & 1495.42 & 1493.624 & 1484.959 & 1479.091\end{array}$ $\begin{array}{llllllllll}1487.838 & 1488.447 & 1493.892 & 1494.318 & 1493.648 & 1495.446 & 1493.621 & 1484.959 & 1479.091\end{array}$ $\begin{array}{lllllllll}1487.838 & 1488.426 & 1493.897 & 1494.344 & 1493.655 & 1495.477 & 1493.631 & 1484.959 & 1479.096\end{array}$ $\begin{array}{lllllllllll}1487.801 & 1488.429 & 1493.901 & 1494.351 & 1493.66 & 1495.479 & 1493.638 & 1484.959 & 1479.098\end{array}$ $\begin{array}{llllllllll}1487.773 & 1488.426 & 1493.908 & 1494.353 & 1493.665 & 1495.481 & 1493.645 & 1484.959 & 1479.098\end{array}$ $\begin{array}{llllllllll}1487.75 & 1488.429 & 1493.904 & 1494.344 & 1433.662 & 1495.474 & 1493.642 & 1484.956 & 1479.101\end{array}$

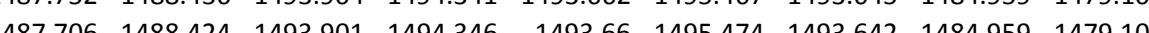
$\begin{array}{lllllllllll}4887.713 & 1488.436 & 1493.744 & 1494.271 & 1493.508 & 1495.458 & 1493.495 & 1484.959 & 1479.908\end{array}$ $\begin{array}{llllllllll}1487.725 & 1488.433 & 1493.8 & 1494.285 & 1493.563 & 1495.449 & 1493.549 & 1484.956 & 1479.096\end{array}$ $\begin{array}{lllllllll}1487.739 & 1488.438 & 1493.822 & 1494.29 & 1493.579 & 1495.434 & 1493.567 & 1484.956 & 1479.091\end{array}$ $\begin{array}{lllllllllll}1487.741 & 1488.438 & 1493.828 & 1494.285 & 1493.584 & 1495.43 & 1493.572 & 1484.954 & 1479.088\end{array}$ $\begin{array}{llllllllll}1487.752 & 1488.433 & 1493.828 & 1494.278 & 1493.586 & 1495.423 & 1493.567 & 1484.954 & 1479.084\end{array}$ $\begin{array}{llllllllll}1487.755 & 1488.431 & 1493.828 & 1494.281 & 1493.591 & 1495.416 & 1493.574 & 1484.954 & 1479.082 \\ 1487.762 & 1488.426 & 1493.838 & 1494281 & 1493593 & 1495.413 & 1493.577 & 1484.954 & 1479.082\end{array}$ 
Well

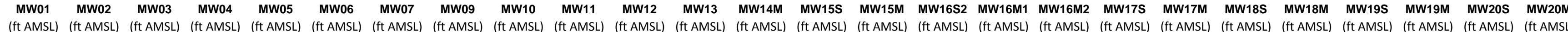

$1 / 8 / 122: 00$ $1 / 8 / 123: 00$ $1 / 8 / 124: 00$ $1 / 8 / 126: 00$ $1 / 8 / 127: 00$ 1/8/12 8:00 1/8/12 9:00 1/8/12 10:00 1/8/12 11:00 1/8/12 12:00 $1 / 8 / 1214: 00$ $1 / 8 / 1215: 00$ 1/8/12 15:00 1/8/12 17:00 $1 / 8 / 1218: 00$ 1/8/12 19:00 1/8/12 20:00 1/8/1221:00 1/8/12 22:00 1/8/12 23:00 1/9/120:12 1:00 1/9/12 2:00 1/9/12 3:00 1/9/12 4:00 $1 / 9 / 125.00$ $1 / 9 / 127.00$ $1 / 9 / 128: 00$ 1/9/12 9:00 1/9/12 10:00 1/9/12 11:00 $1 / 9 / 1212: 00$ 1/9/12 13:00 1/9/12 14:00 1/9/9/12 16:00 1/9/12 17:00 1/9/12 18:00 1/9/12 19:00 1/9/12 20:00 $1 / 9 / 1221: 00$ 1/9/1222:00 1/10/12000 1/10/120:00 1/10/12 1:00 1/10/12 3:00 1/10/12 4:00 1/10/12 5:00 $1 / 10 / 126: 00$ $\begin{array}{rrrr}1494.88 & 1495.198 & 1496.604 & 1495.331 \\ 1494.877 & 1495.196 & 1496.602 & 1495.329\end{array}$ $\begin{array}{rlll}1494.877 & 1495.196 & 1496.602 & 1495.329 \\ 1494.88 & 1495198 & 196650 & 1995331\end{array}$ $\begin{array}{lllll}1494.88 & 1495.198 & 1496.609 & 1495.331\end{array}$ $\begin{array}{rrrr}1494.877 & 1495.198 & 1496.607 & 1495.331\end{array}$ $\begin{array}{lllll}1494.873 & 1495.194 & 1496.598 & 1455.327\end{array}$ $\begin{array}{rrrrr}1494.873 & 1495.194 & 1496.598 & 1495.327 \\ 1494.87 & 1495.191 & 1496.595 & 1495.322\end{array}$ $\begin{array}{llllll}1494.866 & 1495.182 & 1496.577 & 1495.313\end{array}$ $\begin{array}{llll}1494.856 & 1495.168 & 1496.555 & 1495.294\end{array}$ $\begin{array}{lllll}1494.861 & 1495.166 & 1496.558 & 1495.292\end{array}$ $\begin{array}{rrrr}1494.863 & 1495.17 & 1496.572 & 1495.296 \\ 1494.873 & 1495.189 & 1496.612 & 1495317\end{array}$ $\begin{array}{rrrrr}1494.873 & 1495.189 & 1496.612 & 1495.317 \\ 1494.87 & 1495.194 & 1496.621 & 1495.327\end{array}$ $\begin{array}{lllll}1494.873 & 1495.201 & 1496.63 & 1495.341\end{array}$ $\begin{array}{rrrr}1494.866 & 1495.196 & 1496.616 & 1495.338\end{array}$ $\begin{array}{lllll}1494.866 & 1495.194 & 1496.602 & 1495.334\end{array}$ $\begin{array}{lllll}1494.861 & 1495.184 & 1496.586 & 1495.32\end{array}$ $\begin{array}{lllll}1494.861 & 1495.184 & 1496.586 & 1495.313\end{array}$ $\begin{array}{lllll}1494.861 & 1495.182 & 1496.581 & 1495.308\end{array}$ $\begin{array}{llll}1494.859 & 1495.184 & 1496.586 & 1495.308 \\ 1494.854 & 1495.177 & 1496.574 & 1495301\end{array}$ $\begin{array}{lllll}1494.854 & 1455.177 & 1466.567 & 1495.294\end{array}$ $\begin{array}{llllll}1494.856 & 1495.18 & 1496.579 & 1495.296\end{array}$ $\begin{array}{lllll}1494.856 & 1495.182 & 1496.584 & 1495.301\end{array}$ $\begin{array}{lllll}1494.859 & 1495.191 & 1496.598 & 1495.31\end{array}$ $\begin{array}{lllll}1494.861 & 1495.201 & 1496.616 & 1495.322\end{array}$ $\begin{array}{lllll}1494.856 & 1495.201 & 1496.614 & 1495.329\end{array}$ $\begin{array}{lllll}1494.859 & 1495.198 & 1496.609 & 1495.327\end{array}$ $\begin{array}{llll}1494.861 & 1495.201 & 1496.612 & 1495.331 \\ 1494.854 & 1495.196 & 1496.598 & 1495.327\end{array}$ $\begin{array}{lllll}1494.859 & 1495.198 & 1496.605 & 1495.327\end{array}$ $\begin{array}{llll}1494.856 & 1495.196 & 1496.6 & 1495.327\end{array}$ $\begin{array}{llll}1494.859 & 1495.205 & 1496.612 & 1495.334\end{array}$ $\begin{array}{lllll}1494.856 & 1495.203 & 1496.609 & 1495.331\end{array}$ $\begin{array}{llll}1494.875 & 1495.229 & 1496.665 & 1495.362\end{array}$ $\begin{array}{rrrr}1494.884 & 1495.252 & 1496.71 & 1495.397 \\ 1498.884 & 1495259 & 1496719 & 1955.413\end{array}$ $\begin{array}{rrrr}1494.884 & 1495.259 & 1496.719 & 1495.413 \\ 1494887 & 1495264 & 1496.717 & 1495.42\end{array}$ $1494.88 \quad 1495.259 \quad 1496703 \quad 1495.418$ $\begin{array}{lllll}1494.877 & 1495.257 & 1496.689 & 1495.413\end{array}$ $\begin{array}{llllll}1494.877 & 1495.254 & 1496.677 & 1495.406\end{array}$ $1494.882 \quad 1495.254 \quad 1496.682 \quad 1495.404$ $1494.887 \quad 1495.264 \quad 1496.696 \quad 1495.411$ $1494.894 \quad 1495.275 \quad 1496.721 \quad 1495.427$ $1494.9011495 .289 \quad 1496.747 \quad 1495.443$ $\begin{array}{lllll}1494.901 & 1495.296 & 1496.747 & 1495.453 \\ 1494.896 & 1495.289 & 1496733 & 1495.448\end{array}$ $1494.898 \quad 1495.292 \quad 1496.728 \quad 1495.446$ $\begin{array}{llll}1494.905 & 1495.299 & 1496.74 & 1495.453\end{array}$ $1494.905 \quad 1495.301 \quad 1496.742 \quad 1495.455$ $1494.908 \quad 1495.303 \quad 1496.747 \quad 1495.457$ 1494.9121495 .3131496 .7591495 .464 $\begin{array}{llll}1494.917 & 1495.315 & 1496.766 & 1495.471 \\ 1494.917 & 1495.317 & 1496.766 & 1495.476\end{array}$ $\begin{array}{llllllllll}1494.369 & 1494.93 & 1495.23 & 1495.816 & 1496.966 & 1496.708 & 1493.589 & 1488.973 & 1493.428 & 1491.054 \\ 1494362 & 1494.928 & 149523 & 1495812 & 1496961 & 149671 & 1493.591 & 1488.961 & 1493.435 & 1491.052\end{array}$ $\begin{array}{llllllllll}1494.362 & 1494.928 & 1495.23 & 1495.812 & 1496.961 & 1496.71 & 1493.591 & 1488.961 & 1493.435 & 1491.052 \\ 1494352 & 1494.916 & 1495.232 & 1495812 & 1496.963 & 1496715 & 4493598 & 1488.97 & 1493.44 & 1491052\end{array}$

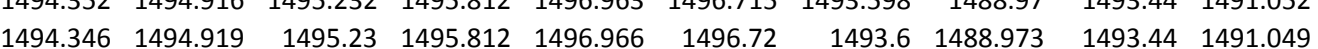
$\begin{array}{llllllllllll}1494.348 & 1494.916 & 1495.228 & 1495.807 & 1496.961 & 1496.715 & 1493.603 & 1488.966 & 1493.442 & 1491.049\end{array}$ $\begin{array}{llllllllllll}1494.355 & 1494.921 & 1495.223 & 1495.802 & 1496.954 & 1496.71 & 1493.603 & 1488.968 & 1493.442 & 1491.047\end{array}$ $\begin{array}{lllllllllll}1494.35 & 1494.919 & 1495.22 & 1495.8 & 1496.952 & 1496.708 & 1493.601 & 1488.968 & 1493.442 & 1491.045\end{array}$ $\begin{array}{lllllllllll}1494.36 & 1494.916 & 1495.211 & 1495.789 & 1496.94 & 1496.691 & 1493.591 & 1488.963 & 1493.435 & 1491.042\end{array}$ $\begin{array}{lllllllllll}1494.367 & 1494.911 & 1495.209 & 1495.775 & 1496.929 & 1496.674 & 1493.587 & 1488.961 & 1493.426 & 1491.04\end{array}$ $\begin{array}{llllllllll}1494.353 & 1494.907 & 1495.213 & 1495.772 & 1496.929 & 1496.677 & 1493.579 & 1488.956 & 1493.426 & 1491.04\end{array}$ $\begin{array}{llllllllll}1494.329 & 1494.885 & 1495.223 & 1495.775 & 1496.933 & 1496.689 & 1493.58 & 1488.954 & 1493.423 & 1491.035 \\ 1494.296 & 1494.854 & 1495232 & 1495789 & 1496952 & 1496729 & 1493589 & 1488961 & 1493.431 & 1491.038\end{array}$ $\begin{array}{lllllllllll}1494.291 & 1494.849 & 1495.228 & 1495.793 & 1496.954 & 1496.739 & 1493.593 & 1488.966 & 1493.433 & 1491.038\end{array}$ $\begin{array}{llllllllllll}1494.287 & 1494.844 & 1495.225 & 1495.802 & 1496.961 & 1496.753 & 1493.598 & 1488.97 & 1493.44 & 1491.04\end{array}$ $\begin{array}{llllllllll}1494.303 & 1494.861 & 1495.22 & 1495.795 & 1496.956 & 1496.739 & 1493.6 & 1488.968 & 1493.442 & 1491.04\end{array}$ $\begin{array}{lllllllllll}1494.313 & 1494.87 & 1495.216 & 1495.791 & 1496.952 & 1496.725 & 1493.345 & 1488.968 & 1493.168 & 1491.038\end{array}$ $\begin{array}{llllllllll}1494.324 & 1494.89 & 1495.209 & 1495.784 & 1496.945 & 1496.71 & 1493.47 & 1488.961 & 1493.304 & 1491.038\end{array}$ $\begin{array}{llllllllll}1494.322 & 1494.892 & 1495.211 & 1495.784 & 1496.942 & 1496.71 & 1493.505 & 1488.956 & 1493.341 & 1491.035\end{array}$

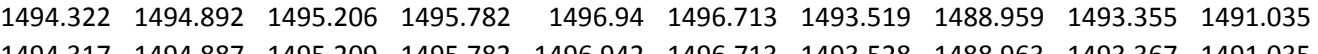
$\begin{array}{llllllllll}1494.317 & 1494.887 & 1495.209 & 1495.782 & 1496.942 & 1496.713 & 1493.528 & 1488.963 & 1493.367 & 1491.035 \\ 1494324 & 1494.892 & 1495204 & 1495.775 & 1496935 & 1496705 & 1493533 & 1488954 & 149337 & 1491.035\end{array}$ $\begin{array}{llllllllllll}1494.324 & 1494.887 & 1495.206 & 1495.77 & 1496.933 & 1496.701 & 1493.53 & 1488.954 & 1493.372 & 1491.031\end{array}$ $\begin{array}{lllllllllll}1494.315 & 149488 & 1495 & 211 & 1495772 & 1496935 & 1496.708 & 1493.537 & 1488.949 & 1493.377 & 1491.031\end{array}$ $\begin{array}{llllllllllll}1494.306 & 1494.88 & 1495.209 & 1495.775 & 1496.94 & 1496.717 & 1493.542 & 1488.954 & 1493.381 & 1491.033\end{array}$ $\begin{array}{lllllllllll}1494.296 & 1494.875 & 1495.211 & 1495.782 & 1496.947 & 1496.729 & 1493.549 & 1488.961 & 1493.391 & 1491.031\end{array}$ $\begin{array}{lllllllllll}1494.284 & 1494.866 & 1495.216 & 1495.791 & 1496.954 & 1496.746 & 1493.558 & 1488.961 & 1493.395 & 1491.033\end{array}$

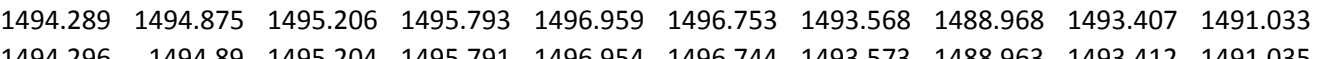
$\begin{array}{lllllllllll}1494.296 & 1494.89 & 1495.204 & 1495.791 & 1496.954 & 1496.744 & 1493.573 & 1488.963 & 1493.412 & 1491.035 \\ 1494296 & 1494887 & 1495204 & 1495.793 & 1496959 & 1496746 & 1493575 & 1488.963 & 1493.416 & 1491035\end{array}$ $\begin{array}{lllllllllll}1494.296 & 1494.887 & 1495.204 & 1495.793 & 1496.959 & 1496.746 & 1493.575 & 1488.963 & 1493.416 & 1491.035 \\ 1494.308 & 1494.892 & 1495.197 & 1495.789 & 1496.952 & 1496.734 & 1493.577 & 1488.966 & 1493.416 & 1491.038\end{array}$ $\begin{array}{lllllllllll}1494.298 & 1494.885 & 1495.201 & 1495.791 & 1496.956 & 1496.737 & 1493.58 & 1488.966 & 1493.419 & 1491.035\end{array}$ $\begin{array}{lllllllllll}1494.303 & 1494.89 & 1495.197 & 1495.789 & 1496.952 & 1496.734 & 1493.58 & 1488.963 & 1493.419 & 1491.038\end{array}$ $\begin{array}{lllllllllll}1494.289 & 1494.878 & 1495.199 & 1495.795 & 1496.961 & 1496.749 & 1493.582 & 1488.963 & 1493.421 & 1491.04\end{array}$ $\begin{array}{llllllllllll}1494.289 & 1494.88 & 1495.201 & 1495.795 & 1496.959 & 1496.744 & 1493.582 & 1488.965 & 1493.421 & 1491.038\end{array}$ $\begin{array}{lllllllllll}1494.247 & 1494.83 & 1495.225 & 1495.821 & 1496.984 & 1496.799 & 1493.591 & 1488.975 & 1493.43 & 1491.042\end{array}$ $\begin{array}{lllllllllll}1494.221 & 1494.815 & 1495.228 & 1495.842 & 1497.01 & 1496.839 & 1493.608 & 1488.984 & 1493.442 & 1491.047 \\ 1494228 & 1494815 & 1495225 & 1495851 & 1497.019 & 1496847 & 1493.615 & 1488998 & 1493454 & 1491052\end{array}$ $\begin{array}{lllllllllll}1494.228 & 1494.815 & 145.225 & 1455.851 & 1497.01 & 1496.847 & 1493.615 & 1488.998 & 1493.454 & 1491.052\end{array}$ $\begin{array}{lllllllllll}1494.266 & 1494.837 & 1495.211 & 1495.856 & 1497.022 & 1496.827 & 1493.626 & 1488.999 & 1493.466 & 1491.054\end{array}$ $\begin{array}{lllllllllll}1494.289 & 1494.851 & 1495.206 & 1495.854 & 1497.017 & 1496.811 & 1493.626 & 1488.998 & 1493.466 & 1491.061\end{array}$ $\begin{array}{llllllllll}1494.303 & 1494.859 & 1495.206 & 1495.851 & 1497.015 & 1496.799 & 1493.629 & 1488.998 & 1493.468 & 1491.061\end{array}$ $\begin{array}{llllllllll}1494.306 & 1494.858 & 1495.209 & 1495.854 & 1497.019 & 1496.801 & 1493.626 & 1488.994 & 1493.473 & 1491.063\end{array}$ $\begin{array}{llllllllllll}1494.301 & 1494.852 & 1495.209 & 1495.863 & 1497.026 & 1496.813 & 1493.631 & 1488.996 & 1493.47 & 1491.068\end{array}$ $\begin{array}{lllllllllll}1494.287 & 1494.832 & 1495.216 & 1495.874 & 1497.04 & 1496.835 & 1493.638 & 1489.001 & 1493.473 & 1491.07\end{array}$ $\begin{array}{llllllllllll}1494.277 & 1494.83 & 1495.22 & 1455.891 & 1497.054 & 1496.863 & 1493.643 & 1489.008 & 1493.482 & 1491.075\end{array}$

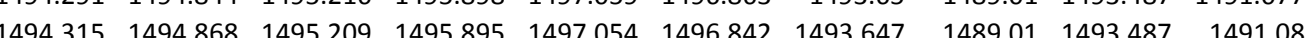
$\begin{array}{llllllllllll}1494.327 & 1494.878 & 1495.206 & 1495.898 & 1497.056 & 1496.839 & 1493.647 & 1489.012 & 1493.489 & 1491.082\end{array}$ $\begin{array}{lllllllllllll}1494.32 & 1494.87 & 1495.213 & 1495.905 & 1497.063 & 1496.847 & 1493.652 & 1489.01 & 1493.489 & 1491.084\end{array}$ $\begin{array}{lllllllllll}1494.327 & 1494.875 & 1495.213 & 1495.909 & 1497.066 & 1496.851 & 1493.659 & 1489.022 & 1493.498 & 1491.089\end{array}$ $\begin{array}{lllllllllll}1494.331 & 1494.883 & 1495.211 & 1495.912 & 1497.07 & 1496.849 & 1493.666 & 1489.015 & 1493.501 & 1491.089\end{array}$ $\begin{array}{llllllllllll}1494.324 & 1494.873 & 1495.216 & 1495.921 & 1497.077 & 1496.863 & 1493.671 & 1489.017 & 1493.51 & 1491.094\end{array}$ $\begin{array}{llllllllll}1494.329 & 1494.873 & 1495.216 & 1495.928 & 1497.084 & 1496.866 & 1493.678 & 1489.027 & 1493.517 & 1491.096\end{array}$ $\begin{array}{lllllllll}1487.769 & 1488.426 & 1493.838 & 1494.278 & 1493.596 & 1495.411 & 1493.574 & 1484.954 & 1479.084\end{array}$ $\begin{array}{llllllllll}1487.778 & 1488.424 & 1493.84 & 1494.276 & 1493.596 & 1495.409 & 1493.579 & 1484.954 & 1479.087 \\ 1487.78 & 1488.415 & 1493.845 & 1494.281 & 1493.601 & 1495.416 & 1493581 & 1484954 & 1470089\end{array}$ $\begin{array}{llllllllll}1484 & \end{array}$ $\begin{array}{llllllllll}1487.794 & 1488.412 & 1493.847 & 1494285 & 1493.608 & 1495.416 & 1493.586 & 1484.954 & 1479.096\end{array}$ $\begin{array}{llllllllll}1487.799 & 1488.415 & 1493.847 & 1494.278 & 1493.603 & 1495.399 & 1493.588 & 1484.952 & 1479.096\end{array}$ $\begin{array}{lllllllllll}1487.799 & 1488.415 & 1493.845 & 1494.274 & 1493.603 & 1495.402 & 1493.586 & 1484.952 & 1479.096\end{array}$ $\begin{array}{llllllllll}1487.787 & 1488.415 & 1493.843 & 1494.269 & 1493.596 & 1495.383 & 1493.581 & 1484.949 & 1479.094\end{array}$ $\begin{array}{lllllllllll}1487.787 & 1488.417 & 1493.833 & 1494.248 & 1493.589 & 1495.366 & 1493.57 & 1484.949 & 1479.089\end{array}$ $\begin{array}{llllllllll}1487.792 & 1488.398 & 1493.831 & 1494.25 & 1493.586 & 1495.369 & 1493.566 & 1484.949 & 1479.087\end{array}$ $\begin{array}{llllllllll}1487.801 & 1488.382 & 1493.829 & 1494.255 & 1493.586 & 1495.38 & 1493.56 & 1484.952 & 1479.087\end{array}$ $\begin{array}{llllllllll}1487.766 & 1488.358 & 1493.835 & 4494.281 & 1493.593 & 1495.416 & 1493.572 & 1484.952 & 1479.089 \\ 1487.75 & 1488.365 & 1493.843 & 1494.288 & 1493.598 & 1495.418 & 1493.577 & 1484.947 & 1479.099\end{array}$ $\begin{array}{llllllllll}1487.732 & 1488.358 & 1493.847 & 1494.297 & 1493.605 & 1495.43 & 1493.586 & 1484.947 & 1479.094\end{array}$ $\begin{array}{llllllllll}1487.672 & 1488.368 & 1493.847 & 1494.29 & 1493.605 & 1495.416 & 1493.588 & 1484.949 & 1479.096\end{array}$ $\begin{array}{llllllllll}1487.651 & 1488.365 & 1493.575 & 1494.213 & 1493.349 & 1495.406 & 1493.337 & 1484.949 & 1479.096\end{array}$ $\begin{array}{rrrrrrrrrr}1487.66 & 1488.37 & 1493.711 & 1494.215 & 1493.473 & 1495.395 & 1493.459 & 1484.947 & 1479.094 \\ 1487.676 & 1488368 & 1493.749 & 1494232 & 1493511 & 149539 & 1493.49 & 1484.947 & 1479.094\end{array}$ $\begin{array}{llllllllll}1487.676 & 1488.368 & 1493.749 & 1494.232 & 1493.511 & 1495.39 & 1493.49 & 1484.947 & 1479.094\end{array}$ $\begin{array}{llllllllll}1487.683 & 1488.365 & 1493.765 & 1494.234 & 1493.522 & 1495.385 & 1493.506 & 1484.947 & 1479.091\end{array}$

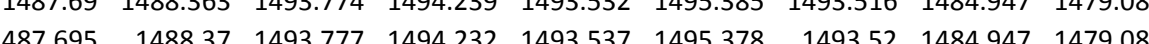
$\begin{array}{lllllllllll}487.697 & 1488.365 & 1493.777 & 1494.236 & 1493.537 & 1495.373 & 1493.518 & 1484.947 & 1479.082\end{array}$ $\begin{array}{llllllllllll}1487.704 & 1488361 & 1493.784 & 1494.239 & 1493.541 & 1495.378 & 1493.523 & 1484.947 & 1479.08\end{array}$ $\begin{array}{llllllllll}1487.709 & 1488.361 & 1493.786 & 1494.243 & 1493.546 & 1495.387 & 1493.527 & 1484.947 & 1479.082\end{array}$ $\begin{array}{llllllllll}1487.711 & 1488.358 & 1493.796 & 1494.255 & 1493.556 & 1495.397 & 1493.534 & 1484.945 & 1479.08\end{array}$ $\begin{array}{llllllllll}1487.72 & 1488.351 & 1493.805 & 1494.271 & 1493.563 & 1495.413 & 1493.544 & 1484.945 & 1479.087\end{array}$ $\begin{array}{lllllllll}1487.729 & 1488.361 & 1493.812 & 1494.276 & 1493.57 & 1495.416 & 1493.553 & 1484.945 & 1479.091\end{array}$ $\begin{array}{llllllllll}1487.743 & 1488.365 & 149.819 & 1494.271 & 1493.575 & 1455.411 & 1493.538 & 1484.945 & 1479.094\end{array}$ $\begin{array}{llllllllll}1487.729 & 1488.365 & 4493.821 & 1494.27 & 1493.579 & 1455.416 & 1493.563 & 1484.945 & 1479.096\end{array}$ $\begin{array}{llllllllll}1487.736 & 1488.365 & 1493.826 & 1494.274 & 1493.584 & 1495.411 & 1493.563 & 1484.942 & 1479.101\end{array}$ $\begin{array}{llllllllll}1487.734 & 1488.365 & 1493.824 & 1494.274 & 1493.584 & 1495.406 & 1493.563 & 1484.942 & 1479.098\end{array}$ $\begin{array}{llllllllll}1487.741 & 1488.354 & 1493.826 & 1494.285 & 1493.586 & 1495.416 & 1493.565 & 1484.942 & 1479.098\end{array}$ $\begin{array}{llllllllll}1487.748 & 1488.349 & 1493.826 & 1494.278 & 1493.584 & 1455.411 & 1493.56 & 1484.942 & 1479.094\end{array}$ $\begin{array}{llllllllll}1487.748 & 1488.314 & 1493.84 & 1494.316 & 1493.593 & 1495.46 & 1493.567 & 1484.942 & 1479.098\end{array}$ $\begin{array}{llllllllll}1487.752 & 1488.304 & 1493.854 & 1494.344 & 1493.61 & 1495.493 & 1493.586 & 1484.942 & 1479.101\end{array}$

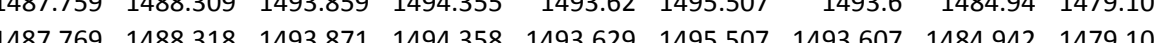
$\begin{array}{llllllllll}1487.72 & 1488.323 & 1493.871 & 1494.351 & 1493.629 & 1495.496 & 1493.61 & 1484.942 & 1479.1408\end{array}$ $\begin{array}{lllllllllll}1487.722 & 1488.335 & 1493.875 & 1494.344 & 1493.631 & 1495.484 & 1493.614 & 1484.94 & 1479.11\end{array}$ $\begin{array}{lllllllll}1487.734 & 1488.344 & 1493.875 & 1494.334 & 1493.631 & 1495.484 & 1493.617 & 1484.94 & 1479.11\end{array}$ $\begin{array}{lllllllll}1487.741 & 1488.339 & 1493.876 & 1494.344 & 1493.631 & 1495.477 & 1493.614 & 1484.94 & 1479.11\end{array}$ $\begin{array}{lllllllllll}1487.748 & 1488.344 & 1493.875 & 1494.351 & 1493.636 & 1495.489 & 1493.614 & 1484.94 & 1479.112\end{array}$ $\begin{array}{llllllllll}1487.757 & 1488.339 & 1493.883 & 1494.365 & 1493.643 & 1495.507 & 1493.621 & 1484.94 & 1479.112\end{array}$ $\begin{array}{llllllllll}1487.762 & 1488.34 & 1493.89 & 1494.379 & 1493.65 & 1455.533 & 1493.631 & 1484.94 & 1479.112\end{array}$

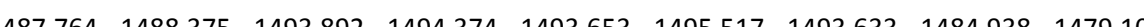
$\begin{array}{llllllllllll}1487.766 & 1488.38 & 1493.894 & 1494.369 & 1493.653 & 1495.519 & 1493.635 & 1484.938 & 1479.108\end{array}$ $\begin{array}{lllllllllll}1487.771 & 1488.377 & 1493.899 & 1494.383 & 1493.66 & 1495.528 & 1493.64 & 1484.938 & 1479.108\end{array}$ $\begin{array}{llllllllll}1487.78 & 1488.387 & 1493.904 & 1494.381 & 1493.662 & 1495.531 & 1493.645 & 1484.935 & 1479.11\end{array}$ $\begin{array}{llllllllll}1487.787 & 1488.389 & 1493.908 & 1494.388 & 1493.669 & 1495.533 & 1493.649 & 1484.94 & 1479.112\end{array}$ $\begin{array}{lllllllllll}1487.799 & 1488.391 & 1493.916 & 1494.4 & 1493.676 & 1495.547 & 1493.654 & 1484.94 & 1479.12\end{array}$ $\begin{array}{lrrrrrrrr}1487.792 & 1488.394 & 1493.927 & 1494.404 & 1493.686 & 1495.55 & 1493.664 & 1484.94 & 1479.124 \\ 1487.796 & 1488 . & 1493.932 & 1494.404 & 1493.686 & 1495.55 & 1493.668 & 1484.938 & 1479.127\end{array}$ 
Well

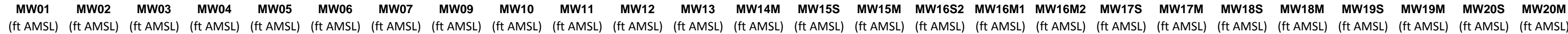

1/10/12 8:00 $1 / 10 / 129: 00$ $1 / 10 / 1210: 00$ 1/10/12 11:00 $12: 00$ $1 / 10 / 1212: 00$ 1/10/12 14:00 1/10/12 15:00 1/10/12 16:00 1/10/12 17:00 $1 / 10 / 12 ~ 18: 00$
$1 / 10 / 1219: 00$ 1/1/10/12 20:00 1/11/10/12 21:00 $1 / 10 / 1222: 00$ 1/10/12 23:00 1/11/12 0:00 1/11/121:00 $1 / 11 / 122: 00$ 1/11/123:00 1/111/12 5:00 1/11/12 6:00 1/11/122:00 1/11/12 8:00 1/11/12 9:00 1/11/12 10:00 1/11/12 11:00 $1 / 11 / 1212: 00$ 1/11/12 14:00 $1 / 11 / 1215: 00$ 1/11/12 16:00 1/11/12 17:00 1/11/12 18:00 1/11/12 19:00 1/11/12 21:00 $1 / 11 / 1222: 00$ 1/12/120:00 1/12/12 1:00 1/12/122:00 1/12/12 3:00 1/12/12 4:00

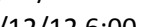
1/122/12 6:00 1/12/128:00 1/12/12 9:00 1/12/12 10:00 1/12/12 11:00 1/12/12 12:00 $\begin{array}{llll}1494.917 & 1495.32 & 1496.764 & 1495.476\end{array}$ $\begin{array}{llll}1494.929 & 1495.329 & 1496.784 & 1495.49 \\ 1494.924 & 1495331 & 1496787 & 1495.49\end{array}$ $\begin{array}{llll}1494.924 & 1495.331 & 1496.787 & 1495.49 \\ 1494.926 & 1495.329 & 1496773 & 1495.485\end{array}$ $\begin{array}{lllll}1494.938 & 1495.343 & 1496.808 & 1495.404\end{array}$ $\begin{array}{llll}1494.938 & 1455.343 & 1496.808 & 1495.504 \\ 1494.947 & 1495.364 & 1496.841 & 1495.527\end{array}$ $\begin{array}{lllll}1494.947 & 1495.364 & 1496.841 & 1455.527 \\ 1494.954 & 1495.383 & 1496.871 & 1495.551\end{array}$ $\begin{array}{lllll}1494.959 & 1495.397 & 1496.89 & 1495.572\end{array}$ $\begin{array}{llll}1494.964 & 1495.411 & 1496.904 & 1495.59\end{array}$ $\begin{array}{llll}1494.959 & 1495.413 & 1496.897 & 1495.597 \\ 1499.959 & 1495.413 & 1496885 & 1955595\end{array}$ $\begin{array}{rrrr}1494.959 & 1495.413 & 1496.885 & 1495.595 \\ 1499.966 & 1495.42 & 1466899 & 1455602\end{array}$ $\begin{array}{rrrr}1494.966 & 1495.42 & 1496.899 & 1495.602 \\ 1494.968 & 1495.429 & 1496.915 & 1495.614\end{array}$ $\begin{array}{llll}1494.968 & 1495.429 & 1466.915 & 1495.614 \\ 1494.971 & 1495.434 & 1496.913 & 1495.616\end{array}$ $\begin{array}{lllll}1494.973 & 1495.439 & 1496.911 & 1495.616\end{array}$ $\begin{array}{llllll}1494.973 & 1495.443 & 1496.915 & 1495.621\end{array}$ $\begin{array}{lllll}1494.978 & 1495.45 & 1496.927 & 1495.625\end{array}$ $\begin{array}{llll}1494.982 & 1495.457 & 1496.934 & 1495.635\end{array}$ $\begin{array}{llll}1494.982 & 1495.462 & 1496.941 & 1495.639\end{array}$ $\begin{array}{llll}1494.985 & 1495.469 & 1496.948 & 1495.644 \\ 1494.989 & 1495.474 & 1496.955 & 1495.651\end{array}$ $\begin{array}{lllll}1444.994 & 1455.481 & 1496.962 & 1495.658\end{array}$ $1494.9871495 .481 \quad 1496955 \quad 1495.658$ $\begin{array}{lllll}1494.982 & 1455.469 & 1496.929 & 1495.639\end{array}$ $\begin{array}{llllll}1494.978 & 1495.453 & 1496.894 & 1495.616\end{array}$ $\begin{array}{llll}1494.971 & 1495.432 & 1496.852 & 1495.586\end{array}$ $\begin{array}{lllll}1494.973 & 1495.418 & 1496.834 & 1495.562\end{array}$ $\begin{array}{lllll}1494.968 & 1495.401 & 1496.805 & 1495.539\end{array}$ $\begin{array}{llll}1494.971 & 1495.394 & 1496.801 & 1495.518 \\ 1494.968 & 1495.383 & 1496.794 & 1495.509\end{array}$ $\begin{array}{llll}1444.966 & 1455.371 & 1496.782 & 1495.492\end{array}$ $\begin{array}{lllll}1494.964 & 1495.362 & 1496.761 & 1455.474\end{array}$ $\begin{array}{llllll}1494.95 & 1495.327 & 1496.719 & 1495.441\end{array}$ $\begin{array}{lllll}1494.943 & 1495.301 & 1496.684 & 1495.411\end{array}$ $\begin{array}{lllll}1494.94 & 1495.285 & 1496.665 & 1495.383\end{array}$ $\begin{array}{rrrr}1494.936 & 1495.268 & 1496.649 & 1495.369 \\ 1499.926 & 149524 & 1496.626 & 1455345\end{array}$ $\begin{array}{llll}1494.926 & 1495.24 & 1496.626 & 1495.345 \\ 1494.926 & 1495.236 & 196618 & 195.329\end{array}$ $\begin{array}{lllll}1494.926 & 1495.236 & 1496.618 & 1495.329 \\ 1494.929 & 1495.238 & 1496.633 & 1495.327\end{array}$ $\begin{array}{lllll}1494.926 & 1495.236 & 1496.64 & 1495.327\end{array}$ $\begin{array}{llll}1494.922 & 1495.231 & 1496.63 & 1495.32\end{array}$ $\begin{array}{lllll}1494.917 & 1495.222 & 1496.628 & 1495.32\end{array}$ $1494.919 \quad 1495.224 \quad 1496.628 \quad 1495.315$ $1494.917 \quad 1495.229 \quad 1496.635 \quad 1495.32$ $\begin{array}{llll}1494.919 & 1495.233 & 1496.654 & 1495.329 \\ 1494.919 & 1495238 & 1496.658 & 1495336\end{array}$ $1494.9191495 .2381496 .658 \quad 1495.336$ $1494.919 \quad 1495.24 \quad 1496.663351495 .345$ $1494.9191495 .243 \quad 1496.665 \quad 1495.35$ $1494.917 \quad 1495.24 \quad 1496.654 \quad 1495.352$ $1494.915 \quad 1495.238 \quad 1496.64 \quad 1495.343$ $1494.912 \quad 1495.238 \quad 1496.647 \quad 1495.341$ $\begin{array}{llll}1494.919 & 1495.245 & 1496.665 & 1495.352 \\ 1494.924 & 1495.259 & 1496.689 & 1495.366\end{array}$ $\begin{array}{llllllllll}1494.341 & 1494.892 & 1495.213 & 1495.932 & 1497.089 & 1496.859 & 1493.687 & 1489.024 & 1493.522 & 1491.103\end{array}$ $\begin{array}{lllllllllll}1494.331 & 1494.878 & 1495.218 & 1495.946 & 1497.098 & 1496.88 & 1493.692 & 1489.027 & 1493.531 & 1491.105\end{array}$

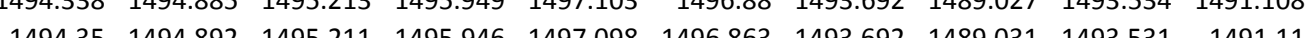
$\begin{array}{lllllllllll}1494.327 & 1494.859 & 1495.22 & 1495.963 & 1497.115 & 1496.894 & 1493.699 & 1489.031 & 1493.538 & 1491.115\end{array}$ $\begin{array}{lllllllllll}1494.31 & 1494.873 & 1495.23 & 1495.981 & 1497.133 & 1496.923 & 1493.71 & 1489.034 & 1493.548 & 1491.119\end{array}$ $\begin{array}{lllllllllll}1494.301 & 1494.919 & 1495.232 & 1496.002 & 1497.154 & 1496.954 & 1493.725 & 1489.045 & 1493.562 & 1491.122\end{array}$ $\begin{array}{lllllllllll}1494.301 & 1494.887 & 1495.237 & 1496.016 & 1497.168 & 1496.969 & 1493.734 & 1489.055 & 1493.571 & 1491.129\end{array}$ $\begin{array}{llllllllll}1494.308 & 1494.902 & 1495.239 & 1496.032 & 1497.182 & 1496.98 & 1493.746 & 1489.057 & 1493.585 & 1491.133\end{array}$ $\begin{array}{llllllllll}1494.331 & 1494.909 & 1495.232 & 1496.037 & 1497.184 & 1496.973 & 1493.753 & 1489.069 & 1493.59 & 1491.138\end{array}$ $\begin{array}{llllllllll}1494.357 & 1494.907 & 1495.242 & 1496.037 & 1497.182 & 1496.957 & 1493.76 & 1489.064 & 1493.595 & 1491.142 \\ 1494.364 & 1494.899 & 1495.242 & 1496.046 & 1497.191 & 1496.961 & 1493.764 & 1489.066 & 1493.604 & 1491.145\end{array}$ $\begin{array}{llllllllllll}1494.369 & 1494.909 & 1495.242 & 1496.06 & 1497.203 & 1496.973 & 1493.771 & 1489.071 & 1493.609 & 1491.149\end{array}$ $\begin{array}{llllllllllll}1494.381 & 1494.916 & 1495.242 & 1496.065 & 1497.208 & 1496.971 & 1493.776 & 1489.078 & 1493.613 & 1491.154\end{array}$ $\begin{array}{llllllllllll}1494.392 & 1494.916 & 1495.244 & 1496.069 & 1497.212 & 1496.969 & 1493.776 & 1489.078 & 1493.616 & 1491.159\end{array}$ $\begin{array}{lllllllllll}1494.4 & 1494.921 & 1495.247 & 1496.079 & 1497.217 & 1496.973 & 1493.778 & 1489.078 & 1493.616 & 1491.163\end{array}$ $\begin{array}{lllllllllll}1494.404 & 1494.918 & 1495.247 & 1496.088 & 1497.226 & 1496.978 & 1493.781 & 1489.083 & 1493.618 & 1491.168\end{array}$ $\begin{array}{llllllllll}1494.411 & 1494.926 & 1495.251 & 1496.097 & 1497.233 & 1496.983 & 1493.785 & 1489.085 & 1493.625 & 1491.168\end{array}$ $\begin{array}{lllllllllll}1494.418 & 1494.923 & 1495.251 & 1496.104 & 1497.24 & 196.985 & 1493.79 & 1489.085 & 1493.63 & 1491.173 \\ 1494.425 & 149493 & 1495.261 & 1496113 & 1497.245 & 1496988 & 1493.797 & 148909 & 1493637 & 1491.777\end{array}$ $\begin{array}{llllllllll}1494.425 & 1494.93 & 1495.261 & 1496.113 & 1497.245 & 1496.988 & 1493.997 & 1489.09 & 1493.637 & 1491.177 \\ 1494.43 & 1494.935 & 1495.261 & 1496123 & 1497.254 & 1496995 & 1493806 & 1489.094 & 1493646 & 1491.18\end{array}$ $\begin{array}{rlllllllllll}1494.435 & 1494.933 & 1495.258 & 1496.13 & 1497.259 & 1496.997 & 1493.816 & 1489.09 & 1493.656 & 1491.184\end{array}$ $\begin{array}{lllllllllll}1494.451 & 1494.945 & 1495.256 & 1496.132 & 1497.259 & 1496.988 & 1493.82 & 1489.094 & 1493.66 & 1491.187\end{array}$ $\begin{array}{llllllllllll}1494.479 & 1494.978 & 1495.251 & 1496.123 & 1497.247 & 1496.959 & 1493.818 & 1489.09 & 1493.658 & 1491.189\end{array}$ $\begin{array}{llllllllll}1494.508 & 1495.004 & 1495.242 & 1496.111 & 1497.231 & 1496.926 & 1493.811 & 1489.087 & 1493.656 & 1491.187\end{array}$ $\begin{array}{lllllllllll}1494.536 & 1495.028 & 1495.237 & 1496.093 & 1497.21 & 1496.882 & 1493.802 & 1489.078 & 1493.642 & 1491.184\end{array}$ $\begin{array}{lllllllllll}1494.538 & 1495.04 & 1495.237 & 1496.086 & 1497.201 & 1496.866 & 1493.792 & 1489.071 & 1493.637 & 1491.182\end{array}$

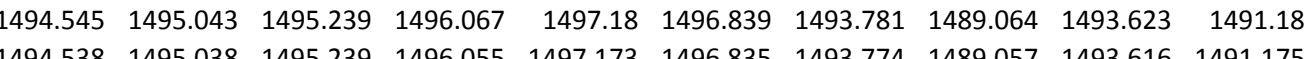
$\begin{array}{lllllllllll}1494.538 & 1495.038 & 1495.239 & 1496.055 & 1497.17 & 1496.835 & 1493.714 & 489.057 & 1493.616 & 1491.175 \\ 1494.536 & 1495.033 & 1495.235 & 1496.048 & 1497.166 & 1496.83 & 1493.762 & 1489.055 & 1493.606 & 1491.168\end{array}$ $\begin{array}{llllllllllll}1494.541 & 1495.038 & 1495.232 & 1496.037 & 1497.154 & 1496.815 & 1493.757 & 1489.043 & 1493.604 & 1491.168\end{array}$ $\begin{array}{lllllllllll}1494.536 & 1495.031 & 1495.225 & 1496.023 & 1497.14 & 1496.803 & 1493.746 & 1489.045 & 1493.592 & 1491.161\end{array}$ $\begin{array}{lllllllllll}1494.559 & 1495.028 & 1495.218 & 1495.997 & 1497.112 & 1496.76 & 1493.732 & 1489.036 & 1493.581 & 1491.156\end{array}$ $\begin{array}{llllllllllll}1494.569 & 1495.04 & 1495.211 & 1495.974 & 1497.089 & 1496.729 & 1493.72 & 1489.031 & 1493.566 & 1491.147\end{array}$ $\begin{array}{llllllllll}1494.557 & 1495.028 & 1495.211 & 1495.956 & 1497.075 & 1496.715 & 1493.708 & 1489.012 & 1493.557 & 1491.14\end{array}$

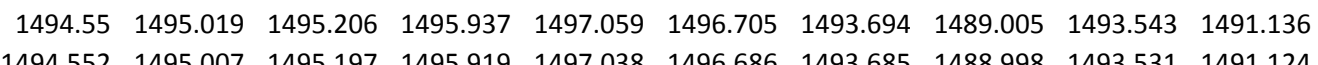
$\begin{array}{lllllllllll}1494.552 & 1495.007 & 1495.197 & 1495.919 & 1497.038 & 1496.686 & 1493.685 & 1488.998 & 1493.531 & 1491.124 \\ 1494536 & 1495 & 1495206 & 1495.902 & 1497.028 & 1496682 & 1493673 & 1488991 & 1493517 & 1491.117\end{array}$ $\begin{array}{lllllllllll}1494.505 & 1494.988 & 1495.211 & 1495.902 & 1497.028 & 1496.698 & 1943.664 & 1488.987 & 1493.51 & 1491.112\end{array}$ $\begin{array}{lllllllllll}1494.484 & 1494.988 & 1495.209 & 1495.898 & 1497.026 & 1496.713 & 1493.659 & 1488.984 & 1493.506 & 1491.105\end{array}$ $\begin{array}{llllllllll}1494.479 & 1494.976 & 1495.206 & 1495.886 & 1497.017 & 1496.705 & 1493.654 & 1488.982 & 1493.496 & 1491.101\end{array}$ $\begin{array}{llllllllll}1494.468 & 1494.964 & 1495.199 & 1495.879 & 1497.012 & 1496.708 & 1493.645 & 1488.982 & 1493.487 & 1491.089\end{array}$ $\begin{array}{lllllllllll}1494.454 & 1494.959 & 1495.201 & 1495.877 & 1497.008 & 1496.715 & 1493.64 & 1488.973 & 1493.484 & 1491.089\end{array}$ $\begin{array}{llllllllll}1494.439 & 1494.954 & 1495.201 & 1495.872 & 1497.008 & 1496.722 & 1493.64 & 1488.98 & 1493.482 & 1491.084\end{array}$

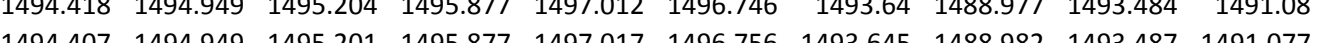

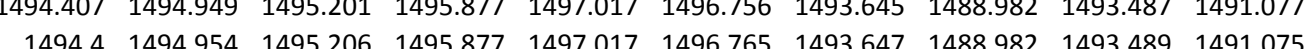
$\begin{array}{lllllllllll}1494.4 & 1494.954 & 1495.199 & 1495.874 & 1497.017 & 1496.763 & 1493.647 & 1488.984 & 1493.489 & 1491.073\end{array}$ $\begin{array}{lllllllllll}1494.395 & 1494.949 & 1495.201 & 1495.872 & 1497.017 & 1496.768 & 1493.65 & 1488.984 & 1493.494 & 1491.073\end{array}$ $\begin{array}{lllllllllll}1494.4 & 1494.952 & 1495.194 & 1495.87 & 1497.015 & 1496.763 & 1493.65 & 1488.98 & 1493.491 & 1491.073\end{array}$ $\begin{array}{lllllllllll}1494.402 & 1494.947 & 1495.192 & 1495.86 & 1497.005 & 1496.749 & 1493.645 & 1488.982 & 1493.487 & 1491.07\end{array}$ $\begin{array}{lllllllllll}1494.395 & 1494.94 & 1495.197 & 1495.86 & 1497.008 & 1496.756 & 1493.643 & 1488.977 & 1493.482 & 1491.068\end{array}$ $\begin{array}{lllllllllll}1494.374 & 1494.921 & 1495.204 & 1495.865 & 1497.017 & 1496.777 & 1493.643 & 1488.982 & 1493.487 & 1491.066\end{array}$ $\begin{array}{llllllllll}1487.783 & 1488.403 & 1493.934 & 1494.407 & 1493.688 & 1495.554 & 1493.668 & 1484.938 & 1479.129\end{array}$

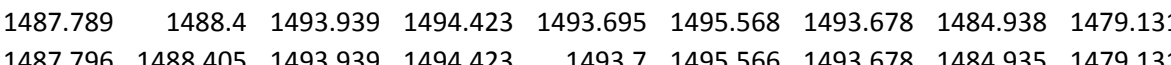

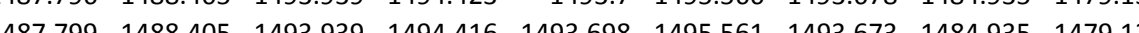
$\begin{array}{lllllllllll}1487.808 & 1488.384 & 1493944 & 1494.437 & 1493.705 & 1495.585 & 1493.678 & 1484.935 & 1479.129\end{array}$ $\begin{array}{lllllllllll}4878817 & 1488377 & 1493955 & 1494.458 & 1493.714 & 1495.615 & 1493687 & 1484.938 & 1479.131\end{array}$ $\begin{array}{llllllllll}1487.824 & 1488.368 & 1493.965 & 1494.484 & 1493.726 & 1495.639 & 1493.701 & 1484.935 & 1479.134\end{array}$ $\begin{array}{lllllllll}1487.836 & 1488.375 & 1493.979 & 1494.495 & 1493.738 & 1495.655 & 1493.715 & 1484.935 & 1479.138\end{array}$ $\begin{array}{lllllllll}1487.843 & 1488.379 & 1493.991 & 1494.509 & 1493.75 & 1495.672 & 1493.729 & 1484.935 & 1479.141\end{array}$ $\begin{array}{lllllllllll}1487.852 & 1488.393 & 1493.998 & 1494.512 & 1493.759 & 1495.672 & 1493.741 & 1484.935 & 1479.148\end{array}$ $\begin{array}{lllllllllll}1487.85 & 1488.403 & 1494.005 & 1494.509 & 1493.762 & 1495.667 & 1493.743 & 1484.935 & 1479.15 \\ 1487.857 & 1488.412 & 1494007 & 1494514 & 1493.771 & 1495674 & 1493.75 & 1484935 & 1479.152\end{array}$

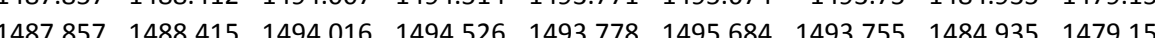
$\begin{array}{llllllllll}1487.859 & 1488.429 & 1494.019 & 1494.528 & 1493.778 & 1495.684 & 1493.757 & 1484.935 & 1479.155\end{array}$ $\begin{array}{llllllllll}487.861 & 1488.438 & 1494.019 & 1494.53 & 1493.778 & 1495.681 & 1493.762 & 1484.933 & 1479.152\end{array}$ $\begin{array}{lllllllllll}1487.863 & 1488.441 & 1494.026 & 1494.533 & 1493.783 & 1495.691 & 1493.762 & 1484.933 & 1479.152\end{array}$ $\begin{array}{llllllllll}1487.868 & 1488.45 & 1494.026 & 1494.54 & 1493.788 & 1495.7 & 1493.769 & 1484.935 & 1479.152\end{array}$ $\begin{array}{llllllllll}1487.868 & 1488.459 & 1494.031 & 1494.544 & 1493.793 & 1495.7 & 1493.771 & 1484.933 & 1479.152\end{array}$ $\begin{array}{llllllllll}1487.87 & 1488.466 & 1494.038 & 1494.549 & 1493.797 & 1455.709 & 1493.776 & 1484.933 & 1479.152\end{array}$ $\begin{array}{lllllllllll}1487.87 & 1488.466 & 1494.038 & 1494.549 & 1493.797 & 1495.709 & 1493.776 & 1484.933 & 1479.152 \\ 1487.884 & 1488.48 & 1494.042 & 1494.554 & 1493.804 & 1455.712 & 1493.783 & 1484.933 & 1479.155\end{array}$ $\begin{array}{llllllllll}1487.891 & 1488.483 & 1494.059 & 1494.57 & 1493821 & 1495.726 & 1493.797 & 1484.933 & 1479.1464\end{array}$ $\begin{array}{llllllllll}1487.882 & 1488.497 & 1494.066 & 1494.567 & 1493.826 & 1495.719 & 1493.804 & 1484.931 & 1479.169\end{array}$ $\begin{array}{lllllllllll}1487.873 & 1488.518 & 1494.066 & 1494.549 & 1493.823 & 1495.695 & 1493.802 & 1484.931 & 1479.169\end{array}$ $\begin{array}{llllllllll}1487.877 & 1488.532 & 1494.059 & 1494.528 & 1493.816 & 1495.672 & 1493.8 & 1484.928 & 1479.16\end{array}$ $\begin{array}{lllllllll}1487.877 & 1488.544 & 1494.047 & 1494.502 & 1493.804 & 1495.632 & 1493.79 & 1484.931 & 1479.166\end{array}$ $\begin{array}{llllllllll}1487.88 & 1488.541 & 1494.04 & 1494.491 & 1493.797 & 1495.615 & 1493.776 & 1484.931 & 1479.162\end{array}$ $\begin{array}{llllllllll}1487.887 & 1488.539 & 1494.026 & 1494.467 & 1493.783 & 1455.597 & 1493.767 & 1484.928 & 1479.159\end{array}$

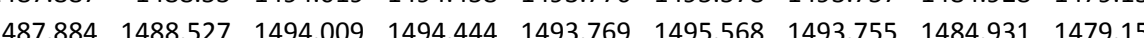
$\begin{array}{llllllllll}1487.889 & 1488.537 & 1494.002 & 1494.435 & 1493.759 & 1495.559 & 1493.743 & 1484.928 & 1479.155\end{array}$ $\begin{array}{llllllllll}1487.894 & 1488.537 & 1493.995 & 1494.421 & 1493.75 & 1495.538 & 1493.741 & 1484.928 & 1479.152\end{array}$ $\begin{array}{llllllllll}1487.838 & 1488.553 & 1493.979 & 1494.39 & 1493.738 & 1495.496 & 1493.729 & 1484.926 & 1479.15\end{array}$ $\begin{array}{lllllllll}1487.838 & 1488.556 & 1493.965 & 1494.365 & 1493.724 & 1495.463 & 1493.713 & 1484.928 & 1479.148\end{array}$ $\begin{array}{llllllllll}1487.838 & 1488.546 & 1493.955 & 1494.353 & 1493.714 & 1495.451 & 1493.699 & 1484.926 & 1479.145\end{array}$ $\begin{array}{lllllllll}1487.836 & 1488.544 & 1493.946 & 1494.334 & 1493.7 & 1495.434 & 1493.687 & 1484.926 & 1479.143\end{array}$

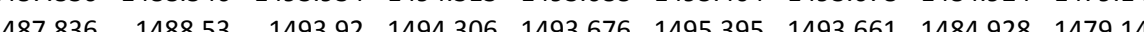
$\begin{array}{lllllllllll}1487836 & 1488509 & 1493.913 & 1494.309 & 1493.669 & 1495.406 & 1493.652 & 1444.928 & 1479.1436\end{array}$ $\begin{array}{llllllllll}1487.836 & 1488.513 & 1493.909 & 1494.306 & 1493.667 & 1495.409 & 1493.645 & 1484.928 & 1479.136\end{array}$ $\begin{array}{lllllllll}1487.838 & 1488.504 & 1493.897 & 1494.302 & 1493.657 & 1495.397 & 1493.635 & 1484.926 & 1479.131\end{array}$ $\begin{array}{lllllllll}1487.843 & 1488.509 & 1493.894 & 1494.297 & 1493.653 & 1495.397 & 1493.628 & 1484.926 & 1479.129\end{array}$ $\begin{array}{lllllllll}1487.836 & 1488.499 & 1493.887 & 1494.295 & 1493.643 & 1495.392 & 1493.628 & 1484.926 & 1479.129\end{array}$ $\begin{array}{llllllllll}1487.845 & 1488.492 & 1493.887 & 1494.302 & 1493.641 & 1495.399 & 1493.624 & 1484.926 & 1479.127\end{array}$

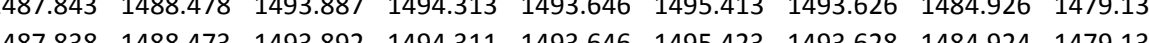

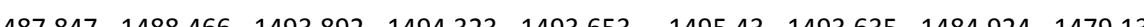
$\begin{array}{lllllllllll}1487.843 & 1488.464 & 1493.894 & 1494.32 & 1493.655 & 1495.425 & 1493.638 & 1484.924 & 1479.138\end{array}$ $\begin{array}{lllllllll}1487.847 & 1488.457 & 1493.897 & 1494.325 & 1493.655 & 1495.43 & 1493.635 & 1484.924 & 1479.141\end{array}$ $\begin{array}{lllllllll}1487.852 & 1488.459 & 1493.897 & 1494.323 & 1493.655 & 1495.425 & 1493.635 & 1484.924 & 1479.141\end{array}$ $\begin{array}{lllllllll}4487.852 & 1488.452 & 1493.89 & 1494.311 & 1493.65 & 1495.416 & 1493.626 & 1484.924 & 1479.141\end{array}$ $\begin{array}{lllllllllll}1487.857 & 1488.441 & 1493.887 & 1494.311 & 1493.648 & 1495.411 & 1493.624 & 1484.926 & 1479.136\end{array}$ $\begin{array}{llllllllll}1487.861 & 1488.422 & 1493.887 & 1494.323 & 1493.648 & 1495.442 & 1493.624 & 1484.926 & 1479.138 \\ 1487.861 & 1488.412 & 1493.894 & 1494.337 & 1493.653 & 1495.451 & 1493.626 & 1484.924 & 1479.141\end{array}$ 
Well

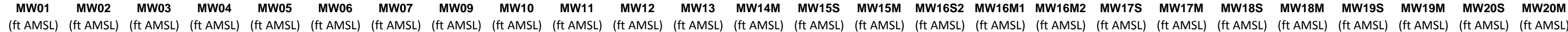

$1 / 12 / 1214: 00$ $1 / 12 / 1215: 00$ $1 / 12 / 1216: 00$ 1/12/12/12 18:00 $1 / 12 / 1219: 00$ 1/12/12 20:00 $1 / 12 / 1221: 00$ $1 / 12 / 1222: 00$ 1/122/1223:00 $1 / 13 / 120: 00$
$1 / 13 / 121: 00$
$1 / 13112200$ $1 / 13 / 121: 00$
$1 / 13 / 122: 00$ $1 / 13 / 123: 00$ 1/13/12 3:00 1/13/12 5:00 1/13/12 6:00 $1 / 13 / 127: 00$ 1/13/12 8:00 1/13/12 10:00 $-1 / 13 / 1211: 00$
$1 / 13 / 1212: 00$ 1/13/12 12:00 1/13/12 14:00 1/13/12 15:00 1/13/12 16:00 1/13/12 17:00 $1 / 13 / 1218: 00$ $1 / 13 / 1220: 00$ $1 / 13 / 1221: 00$ 1/13/12 22:00 1/13/12 23:00 1/14/12 0:00 $1 / 14 / 121: 00$
$1 / 14 / 122: 00$
$1 / 14 / 123: 00$ 1/141/12 3:00 1/14/12 4:00 $1 / 14 / 126: 00$ 1/14/12 7:00 $1 / 14 / 128: 00$ 1/14/12 9:00 1/14/12 10:00 $1 / 14 / 1211: 00$ 1/14/12 12:00 1/14/112 13:00 $1 / 14 / 1215: 00$ $1 / 14 / 1216: 00$
$1 / 14 / 1217: 00$ $1 / 14 / 1217: 00$
$1 / 14 / 1218: 00$ 1/14/12 18:00 $\begin{array}{lllll}1494.922 & 1495.264 & 1496.696 & 1495.378 \\ 1494.924 & 1495.268 & 14967 & 1495.383\end{array}$ $\begin{array}{rrrr}1494.924 & 1495.268 & 1496.7 & 1495.383 \\ 1494.919 & 1495264 & 1496.686 & 1455378\end{array}$ $\begin{array}{lllll}1494.919 & 1495.264 & 1496.686 & 1495.378\end{array}$ $\begin{array}{lllll}1494.919 & 1495.261 & 1496.677 & 1495.371\end{array}$ $\begin{array}{lllll}1494.917 & 1495.254 & 1496.663 & 1459.37\end{array}$ $\begin{array}{lllll}1494.917 & 1495.257 & 1496.663 & 14955.364\end{array}$ $\begin{array}{lllll}1494.915 & 1495.254 & 1496.661 & 1495.359\end{array}$ $\begin{array}{lllll}1494.91 & 1495.247 & 1496.644 & 1495.35\end{array}$ $\begin{array}{rrrr}1494.908 & 1495.243 & 1496.628 & 1495.338 \\ 1494.908 & 149524 & 1496.635 & 1455.327\end{array}$ $\begin{array}{llll}1494.908 & 1495.24 & 1496.635 & 1495.327 \\ 1499.912 & 149525 & 1966551 & 1495338\end{array}$ $\begin{array}{rrrr}1494.912 & 1495.25 & 1496.651 & 1495.338 \\ 1494.912 & 1495.252 & 1496.654 & 1495.343\end{array}$ $\begin{array}{lllll}1494.908 & 1495.247 & 1496.6467 & 1495.343 \\ 1495.341\end{array}$ $\begin{array}{lllll}1494.91 & 1455.252 & 1496.656 & 1495.348\end{array}$ $\begin{array}{lllll}1494.908 & 1495.252 & 1496.654 & 1495.345\end{array}$ $\begin{array}{lllll}1494.908 & 1495.25 & 1496.649 & 1495.343\end{array}$ $\begin{array}{lllll}1494.905 & 1495.247 & 1466.644 & 1495.343\end{array}$ $\begin{array}{llll}1494.908 & 1495.252 & 1496.651 & 1495.348\end{array}$ $\begin{array}{rrrr}1494.903 & 1495.243 & 1496.635 & 1495.338 \\ 1494.901 & 149524 & 1496.63 & 1495.331\end{array}$ $\begin{array}{rrrr}1494.901 & 1495.24 & 1496.63 & 1495.331 \\ 1494.898 & 1495.24 & 1496.628 & 1495.329\end{array}$ $\begin{array}{lllll}1494.91 & 1495.257 & 1496.665 & 1495.348\end{array}$ $\begin{array}{rrrrr}149.915 & 1495.257 & 1496.665 & 1495.348 \\ 1494.915 & 1495.271 & 1496.691 & 1495.366\end{array}$ $1494.917 \quad 1495.278 \quad 1496.703 \quad 1495.38$ $\begin{array}{llll}1494.917 & 1495.282 & 1496.705 & 1495.39\end{array}$ $\begin{array}{lllll}1494.917 & 1495.282 & 1496.707 & 1495.392\end{array}$ $\begin{array}{lllll}1494.915 & 1495.282 & 1496.693 & 1495.387\end{array}$ $\begin{array}{rrrrr}1494.917 & 1495.282 & 1496.693 & 1495.385 \\ 1494.919 & 1495285 & 1496.698 & 149539\end{array}$ $\begin{array}{lllll}1494.924 & 1495.292 & 1496.712 & 1495.394\end{array}$ $\begin{array}{lllll}1494.922 & 1495.294 & 1496.714 & 1495.399\end{array}$ $\begin{array}{llllll}1494.924 & 1495.296 & 1496.719 & 1495.404\end{array}$ $\begin{array}{llll}1494.929 & 1495.306 & 1496.738 & 1495.415\end{array}$ $\begin{array}{llll}1494.931 & 1495.31 & 1496.742 & 1495.42\end{array}$ $\begin{array}{rrrr}1494.931 & 1495.31 & 1496.738 & 1495.422 \\ 1499.926 & 1495303 & 1496719 & 1955.413\end{array}$ $\begin{array}{llll}1494.926 & 1495.303 & 1496.719 & 1495.413\end{array}$ $\begin{array}{rrrr}1494.924 & 1495.296 & 1496.7 & 1495.399 \\ 1494.919 & 1495.287 & 1496.679 & 1495.383\end{array}$ $\begin{array}{lllll}1494.915 & 1495.278 & 1496.658 & 1495.366\end{array}$ $\begin{array}{llllll}1494.908 & 1495.259 & 1496.626 & 1495.341\end{array}$ $\begin{array}{lllll}1494.901 & 1495.24 & 1496.595 & 1495.315\end{array}$ $\begin{array}{llll}1494.889 & 1495.217 & 1496.558 & 1495.282\end{array}$ $\begin{array}{llll}1494.88 & 1495.189 & 1496.527 & 1495.252\end{array}$ $1494.8751495 .173 \quad 1496.509 \quad 1495.231$ $\begin{array}{rlll}1494.877 & 1495.173 & 1496.523 & 1495.224 \\ 1494.88 & 1495.175 & 1496537 & 1495.226\end{array}$ $\begin{array}{llll}1494.882 & 1495.184 & 1496.558 & 1495.236\end{array}$ $1494.884 \quad 1495.194 \quad 1496.583 \quad 1495.252$ $1494.884 \quad 1495.198 \quad 1496.593 \quad 1495.261$ $\begin{array}{llll}1494.882 & 1495.203 & 1496.602 & 1495.273\end{array}$ $1494.88 \quad 1495.201 \quad 1496.593 \quad 1495.273$ $\begin{array}{rrrr}1494.88 & 1495.201 & 1496.588 & 1495.271 \\ 1494.877 & 1495.201 & 1496.588 & 1495.271\end{array}$ $\begin{array}{llllllllll}1494.357 & 1494.897 & 1495.206 & 1495.879 & 1497.031 & 1496.808 & 1493.65 & 1488.991 & 1493.487 & 1491.068 \\ 1494357 & 1494.902 & 1495206 & 1495884 & 1497.033 & 1496813 & 149365 & 1488996 & 1493.491 & 1491.068\end{array}$ $\begin{array}{llllllllll}1494.357 & 1494.902 & 1495.206 & 1495.884 & 1497.033 & 1496.813 & 1493.65 & 1488.996 & 1493.491 & 1491.068 \\ 1494374 & 1494.921 & 1495194 & 1495877 & 1497.028 & 1496801 & 1493.647 & 1488994 & 1493489 & 1491068\end{array}$ $\begin{array}{llllllllllll}1494.374 & 1494.921 & 1495.194 & 1495.877 & 1497.028 & 1496.801 & 1493.647 & 1488.994 & 1493.489 & 1491.068 \\ 1494.385 & 1494.935 & 1495.194 & 1495.874 & 1497.024 & 1496.787 & 1493.647 & 1488.994 & 1493.491 & 1491.068\end{array}$ $\begin{array}{llllllllllll}1494.385 & 1494.94 & 1495.197 & 1495.872 & 1497.024 & 1496.792 & 1493.65 & 1488.994 & 1493.489 & 1491.068\end{array}$ $\begin{array}{lllllllllll}1494.395 & 1494.947 & 1495.192 & 1495.867 & 1497.019 & 1496.78 & 1493.643 & 1488.989 & 1493.491 & 1491.068\end{array}$ $\begin{array}{lllllllllll}1494.395 & 1494.945 & 1495.192 & 1495.865 & 1497.019 & 1496.78 & 1493.647 & 1488.987 & 1493.489 & 1491.068\end{array}$ $\begin{array}{lllllllllll}1494.395 & 1494.942 & 1495.194 & 1495.865 & 1497.017 & 1496.78 & 1493.643 & 1488.987 & 1493.487 & 1491.068\end{array}$ $\begin{array}{lllllllllll}1494.407 & 1494.947 & 1495.185 & 1495.856 & 1497.01 & 1496.765 & 1493.638 & 1488.982 & 1493.482 & 1491.066\end{array}$ $\begin{array}{lllllllllll}1494.411 & 1494.94 & 1495.185 & 1495.849 & 1497.003 & 1496.751 & 1493.629 & 1488.977 & 1493.475 & 1491.066\end{array}$ $\begin{array}{rrrrrrrrrr}1494.4 & 1494.928 & 1495.192 & 1495.847 & 1497.001 & 1496.753 & 1493.629 & 1488.975 & 1493.47 & 1491.063 \\ 1494.383 & 1494.921 & 1495.192 & 1495.851 & 1497.008 & 1496.775 & 1493.626 & 1488.982 & 1493.47 & 1491.063\end{array}$ $\begin{array}{lllllllllll}1494.381 & 1494.925 & 1495.192 & 1495.854 & 1497.01 & 1496.777 & 1993.626 & 1488.977 & 1493.47 & 1491.061\end{array}$ $\begin{array}{llllllllll}1494.385 & 1494.935 & 1495.189 & 1495.849 & 1497.008 & 1496.772 & 1493.624 & 1488.991 & 1493.466 & 1491.063\end{array}$ $\begin{array}{llllllllllll}1494.374 & 1494.928 & 1495.189 & 1495.851 & 1497.01 & 1496.782 & 1493.626 & 1488.975 & 1493.468 & 1491.059\end{array}$ $\begin{array}{lllllllllll}1494.376 & 1494.935 & 1495.187 & 1495.851 & 1497.012 & 1496.78 & 1493.626 & 1488.982 & 1493.468 & 1491.059\end{array}$ $\begin{array}{llllllllll}1494.376 & 1494.935 & 1495.187 & 1495.851 & 1497.008 & 1496.777 & 1493.629 & 1488.98 & 1493.473 & 1491.061\end{array}$ $\begin{array}{llllllllll}1494.376 & 1494.935 & 1495.185 & 1495.847 & 1497.005 & 1496.777 & 1493.631 & 1488.977 & 1493.47 & 1491.059\end{array}$

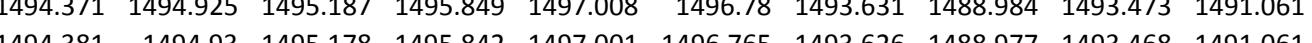
$\begin{array}{lrrrrrrrrr}1494.381 & 1494.93 & 1495.178 & 1495.842 & 1497.001 & 1496.765 & 1493.626 & 1488.977 & 1493.468 & 1491.061 \\ 1494.378 & 1494.925 & 1495.18 & 1495.84 & 1496996 & 1496763 & 1493.624 & 1488975 & 1493468 & 1491059\end{array}$ $\begin{array}{llllllllllll}1494.376 & 1494.925 & 1495.182 & 1495.837 & 1496.996 & 1496.76 & 1493.619 & 1488.973 & 1493.466 & 1491.056\end{array}$ $\begin{array}{lllllllllll}1494.341 & 1494.89 & 1495.197 & 1495.851 & 1497.01 & 1496.796 & 1493.624 & 1488.977 & 1493.468 & 1491.059\end{array}$ $\begin{array}{llllllllllll}1494.322 & 1494.873 & 1495.199 & 1495.863 & 1497.026 & 1496.827 & 1493.633 & 1488.994 & 1493.473 & 1491.061\end{array}$ $\begin{array}{lllllllllll}1494.32 & 1494.873 & 1495.197 & 1495.872 & 1497.033 & 1496.837 & 1493.636 & 1488.994 & 1493.477 & 1491.063\end{array}$ $\begin{array}{llllllllll}1494.327 & 1494.875 & 1495.194 & 1495.874 & 1497.038 & 1496.835 & 1493.638 & 1488.996 & 1493.477 & 1491.063\end{array}$ $\begin{array}{llllllllll}1494.334 & 1494.882 & 1495.194 & 1495.87 & 1497.04 & 1496.837 & 1493.643 & 1488.998 & 1493.482 & 1491.066\end{array}$

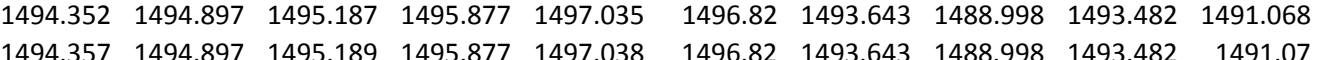

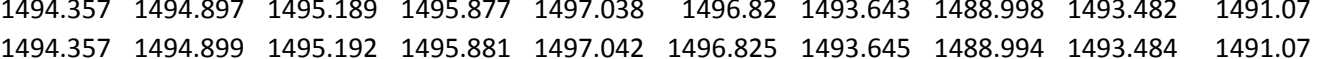
$\begin{array}{lllllllllll}1494.352 & 1494.894 & 1495.197 & 1495.888 & 1497.047 & 1496.837 & 1493.65 & 1489.005 & 1493.491 & 1491.073\end{array}$ $\begin{array}{lllllllllll}1494.36 & 1494.902 & 1495.192 & 1495.891 & 1497.052 & 1496.842 & 1493.652 & 1489.003 & 1493.496 & 1491.075\end{array}$ $\begin{array}{lllllllllll}1494.362 & 1494.909 & 1495.197 & 1495.895 & 1497.054 & 1496.842 & 1493.657 & 1489.005 & 1493.499 & 1491.077\end{array}$ $\begin{array}{lllllllllll}1494.352 & 1494.897 & 1495.199 & 1495.907 & 1497.063 & 1496.863 & 1493.659 & 1489.01 & 1493.499 & 1491.08\end{array}$ $\begin{array}{lllllllllll}1494.357 & 1494.899 & 1495.197 & 1495.909 & 1497.068 & 1496.863 & 1493.661 & 1489.01 & 1493.506 & 1491.082\end{array}$ $\begin{array}{llllllllll}1494.367 & 1494.911 & 1495.194 & 1495.914 & 1497.068 & 1496.861 & 1493.664 & 1489.015 & 1493.503 & 1491.084 \\ 1494385 & 1494.933 & 1495.187 & 1495907 & 1497.061 & 1496839 & 1493657 & 1489.012 & 1493501 & 1491.084\end{array}$ $\begin{array}{llllllllll}1494.385 & 1494.933 & 1455.187 & 145.907 & 147.061 & 1496.839 & 1493.657 & 1489.012 & 1493.501 & 1491.084 \\ 1494.397 & 1494.952 & 1495.187 & 1495.902 & 1497.052 & 1496825 & 1493.654 & 1489008 & 1493.496 & 1491.082\end{array}$ $\begin{array}{llllllllllll}1494.411 & 1494.961 & 1495.18 & 1495.891 & 1497.045 & 1496.801 & 1493.65 & 1489.001 & 1493.489 & 1491.082\end{array}$ $\begin{array}{llllllllllll}1494.421 & 1494.957 & 1495.178 & 1495.881 & 1497.033 & 1496.782 & 1493.643 & 1488.996 & 1493.484 & 1491.08\end{array}$ $\begin{array}{lllllllllll}1494.439 & 1494.952 & 1495.17 & 1495.865 & 1497.017 & 1496.751 & 1493.633 & 1488.984 & 1493.473 & 1491.077\end{array}$ $\begin{array}{llllllllll}1494.447 & 1494.945 & 1495.166 & 1495.844 & 1496.998 & 1496.724 & 1493.622 & 1488.977 & 1493.466 & 1491.073\end{array}$ $\begin{array}{lllllllllll}1494.463 & 1494.935 & 1495.159 & 1495.823 & 1496.977 & 1496.691 & 1493.612 & 1488.966 & 1493.452 & 1491.068\end{array}$ $\begin{array}{llllrlllll}1494.468 & 1494.926 & 1495.151 & 1495.8 & 1496.956 & 1496.662 & 1493.596 & 1488.961 & 1493.442 & 1491.061\end{array}$ $\begin{array}{lllllllllll}1494.456 & 1494.916 & 1495.151 & 1495.784 & 1496.94 & 1496.648 & 1493.584 & 1488.954 & 1493.43 & 1491.059 \\ 1494.421 & 1494.909 & 1495.163 & 1495782 & 149694 & 1496662 & 1493577 & 1488951 & 1493.419 & 1491.052\end{array}$ $\begin{array}{lllllllllll}1494.421 & 1494.909 & 495.163 & 1455.782 & 1496.94 & 1466.062 & 1493.577 & 1488.951 & 1493.419 & 1491.052 \\ 1494392 & 1494.892 & 1495.173 & 1495782 & 1496942 & 1496679 & 1493.572 & 1488947 & 1493.419 & 1491.047\end{array}$ $\begin{array}{llllllllllll}1494.362 & 1494.873 & 1495.17 & 1495.786 & 1496.945 & 1496.703 & 1493.572 & 1488.951 & 1493.416 & 1491.042\end{array}$ $\begin{array}{lllllllllll}1494.334 & 1494.846 & 1495.178 & 1495.793 & 1496.956 & 1496.729 & 1493.575 & 1488.947 & 1493.419 & 1491.042\end{array}$ $\begin{array}{lllllllllll}1494.324 & 1494.846 & 1495.175 & 1495.798 & 1496.961 & 1496.741 & 1493.575 & 1488.959 & 1493.419 & 1491.038\end{array}$ $\begin{array}{llllllllll}1494.317 & 1494.846 & 1495.173 & 1495.802 & 1496.966 & 1496.753 & 1493.575 & 1488.954 & 1493.419 & 1491.038\end{array}$

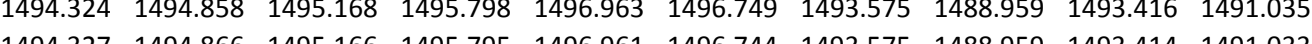

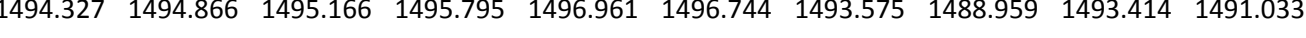

$\begin{array}{lllllllll}1487.863 & 1488.41 & 1493.894 & 1494.339 & 1493.653 & 1495.453 & 1493.631 & 1484.924 & 1479.138\end{array}$ $\begin{array}{llllllllll}1487.866 & 1488.408 & 1493.899 & 1494.348 & 1493.655 & 1495.463 & 1493.633 & 1484.921 & 1479.138 \\ 1487.822 & 1488.415 & 1493.897 & 1494.334 & 1493.653 & 1495.451 & 1493638 & 1484921 & 1479.138\end{array}$ $\begin{array}{lllllllll}1487824 & 1488.419 & 1493897 & 1494.327 & 1493.653 & 1495.449 & 1493.635 & 14844.921 & 1479.1436\end{array}$ $\begin{array}{lllllllllll}1487.826 & 1488.415 & 1493.894 & 1494.332 & 1493.653 & 1495.444 & 1493.638 & 1484.921 & 1479.138\end{array}$ $\begin{array}{llllllllll}1487.826 & 1488.419 & 1493.894 & 1494.325 & 1493.653 & 1495.437 & 1493.633 & 1484.919 & 1479.138\end{array}$ $\begin{array}{lllllllll}1487.836 & 1488.422 & 1493.894 & 1494.325 & 1493.65 & 1495.432 & 1493.633 & 1484.919 & 1479.138\end{array}$ $\begin{array}{llllllllll}1487.836 & 1488.424 & 1493.894 & 1494.327 & 1493.648 & 1495.432 & 1493.628 & 1484.919 & 1479.138\end{array}$ $\begin{array}{lllllllllll}1487.845 & 1488.431 & 1493.885 & 1494.306 & 1493.643 & 1495.418 & 1493.626 & 1484.919 & 1479.136\end{array}$ $\begin{array}{lllllllll}1487.836 & 1488.426 & 1493.878 & 1494.302 & 1493.636 & 1495.404 & 1493.619 & 1484.919 & 1479.134\end{array}$ $\begin{array}{llllllllll}1487.838 & 1488.415 & 1493.875 & 1494.299 & 1493.634 & 1495.411 & 1493.612 & 1484.919 & 1479.131 \\ & 1487843 & 1488.41 & 1493875 & 1494309 & 1493631 & 1495.42 & 1493612 & 1484919 & 1479.132\end{array}$ $\begin{array}{llllllllll}1487.845 & 1488.408 & 1493.875 & 1494.316 & 1493.631 & 1495.42 & 1493.61 & 1484.916 & 1479.129\end{array}$ $\begin{array}{llllllllll}1487.847 & 1488.408 & 1493.871 & 1494.306 & 1493.629 & 1495.416 & 1493.61 & 1484.916 & 1479.127\end{array}$ $\begin{array}{llllllllll}1487.847 & 1488.403 & 1493.875 & 1494.311 & 1493.631 & 1495.423 & 1493.612 & 1484.916 & 1479.122\end{array}$ $\begin{array}{lllllllllll}1487.854 & 1488.401 & 1493.875 & 1494.311 & 1493.634 & 1495.423 & 1493.612 & 1484.916 & 1479.129\end{array}$ $\begin{array}{llllllllll}1487.85 & 1488.41498 .405 & 1493.88 & 1494.311 & 1493.631 & 1495.413 & 1493.614 & 1484.914 & 1479.131\end{array}$ $\begin{array}{lllllllll}1487.861 & 1488.401 & 1493.878 & 1494.304 & 1493.636 & 1495.416 & 1493.617 & 1484.914 & 1479.134\end{array}$ $\begin{array}{lllllllll}1487.859 & 1488.394 & 1493.88 & 1494.311 & 1493.636 & 1455.423 & 1493.619 & 1484.914 & 1479.136\end{array}$ $\begin{array}{llllllllllll}1487.84 & 4888.398 & 1493.873 & 1494.304 & 1433.631 & 1495.409 & 1493.612 & 1484.914 & 1479.136\end{array}$ $\begin{array}{llllllllll}1487.836 & 1488.387 & 1493.871 & 1494.295 & 1493.624 & 1495.399 & 1493.605 & 1484.912 & 1499.136\end{array}$ $\begin{array}{llllllllll}1487.845 & 1488.358 & 1493.875 & 1494.316 & 1493.631 & 1495.432 & 1493.607 & 1484.914 & 1479.138\end{array}$ $\begin{array}{llllllllll}1487.838 & 1488.356 & 1493.88 & 1494.334 & 1493.638 & 1495.456 & 1493.614 & 1484.912 & 1479.138\end{array}$ $\begin{array}{llllllllll}4487.824 & 1488.351 & 1493.885 & 1494.341 & 1493.641 & 1495.463 & 1493.617 & 1484.912 & 1479.138\end{array}$ $\begin{array}{lllllllllll}1487.831 & 1488.356 & 1493.885 & 1494.344 & 1493.643 & 1495.465 & 1493.621 & 1484.909 & 1479.138\end{array}$ $\begin{array}{llllllllll}4487.783 & 1488.361 & 1493.89 & 1494.346 & 1493.648 & 1495.467 & 1493.626 & 1484.912 & 1479.141\end{array}$ $\begin{array}{llllllllll}1487.733 & 1488.37 & 1493.885 & 1494.334 & 1493.646 & 1495.456 & 1493.626 & 1484.909 & 1479.138\end{array}$ $\begin{array}{llllllllll}148.794 & 1488.372 & 1493.89 & 1494.339 & 1493.646 & 1495.46 & 1493.631 & 1484.912 & 1479.143 \\ 1487.813 & 1488372 & 1493894 & 1494.346 & 1493.65 & 1495.458 & 1493.635 & 1484.909 & 1479.145\end{array}$ $\begin{array}{llllllllll}1487.813 & 1488.368 & 1493.897 & 1494.351 & 1493.655 & 1495.472 & 1493.635 & 1484.912 & 1479.148\end{array}$ $\begin{array}{lllllllllll}1487.817 & 1488.38 & 1493.901 & 1494.355 & 1493.657 & 1495.474 & 1493.638 & 1484.912 & 1479.15\end{array}$ $\begin{array}{llllllllll}1487.826 & 1488.382 & 1493.904 & 1494.36 & 1493.66 & 1495.474 & 1493.642 & 1484.909 & 1479.152\end{array}$ $\begin{array}{llllllllll}4487.831 & 1488.375 & 1493.906 & 1494.367 & 1493.665 & 1495.491 & 1493.642 & 1484.909 & 1479.152\end{array}$ $\begin{array}{lllllllll}1487.838 & 1488.375 & 1493.908 & 1494.374 & 1493.669 & 1495.493 & 1493.647 & 1484.909 & 1479.152\end{array}$ $\begin{array}{lllllllllll}4487.843 & 1488.389 & 1493.908 & 1494.376 & 1493.669 & 1495.493 & 1493.647 & 1484.909 & 1479.15\end{array}$

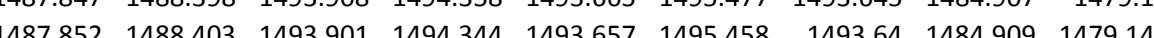
$\begin{array}{llllllllll}1487.854 & 1488.405 & 1493897 & 1494.334 & 1493.653 & 1495.437 & 1493.635 & 1484.907 & 1479.1443\end{array}$ $\begin{array}{lllllllll}1487.859 & 1488.415 & 1493.89 & 1494.316 & 1493.646 & 1495.423 & 1493.631 & 1484.907 & 1479.145\end{array}$ $\begin{array}{lllllllll}1487.857 & 1488.424 & 1493.88 & 1494.297 & 1493.638 & 1495.39 & 1493.621 & 1484.907 & 1479.143\end{array}$ $\begin{array}{lllllllll}1487.852 & 1488.424 & 1493.871 & 1494.278 & 1493.627 & 1495.371 & 1493.612 & 1484.905 & 1479.14\end{array}$ $\begin{array}{llllllllll}1487.857 & 1488.431 & 1493.859 & 1494.248 & 1493.615 & 1495.336 & 1493.595 & 1484.905 & 1479.141\end{array}$ $\begin{array}{lllllllll}1487.861 & 1488.429 & 1493.845 & 1494.229 & 1493.603 & 1495.308 & 1493.584 & 1484.905 & 1479.138\end{array}$ $\begin{array}{lllllllllll}4887.859 & 1488.417 & 1493.835 & 1494.21 & 1493.586 & 1455.286 & 1493.57 & 1484.905 & 1479.136\end{array}$

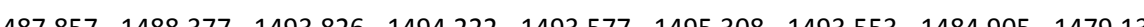
$\begin{array}{lllllllllll}1487.861 & 1488.361 & 1493.826 & 1494.227 & 1493.577 & 1495.315 & 1493.556 & 1484.905 & 1479.131\end{array}$ $\begin{array}{llllllllll}1487.863 & 1488.344 & 1493.824 & 1494.236 & 1493.579 & 1495.336 & 1493.556 & 1484.902 & 1479.134\end{array}$ $\begin{array}{llllllllll}1487.857 & 1488.342 & 1493.826 & 1494.25 & 1493.582 & 1495.348 & 1493.56 & 1484.902 & 1479.131\end{array}$ $\begin{array}{llllllllll}1487.845 & 1488.333 & 1493.826 & 1494.253 & 1493.582 & 1495.352 & 1493.563 & 1484.902 & 1479.12\end{array}$ $\begin{array}{lllllllllll}1487.826 & 1488.337 & 1493.826 & 1494.246 & 1493.579 & 1495.35 & 1493.563 & 1484.9 & 1479.131\end{array}$ $\begin{array}{lllllllll}1487.803 & 1488.34 & 1493.821 & 1494.246 & 1493.579 & 1495.345 & 1493.563 & 1484.902 & 1479.131 \\ 1487.78 & 1488.337 & 1493822 & 1494.246 & 1493.577 & 1495348 & 1493563 & 1484.902 & 1479.134\end{array}$ 
Well

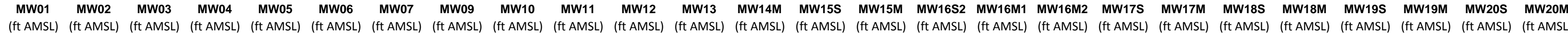

$1 / 14 / 1220: 00$ $1 / 14 / 1221: 00$ 1/14/11/12 22:00 $23: 00$ 1/14/15/12 $23: 00$ 1/15/120:00 1/15/122:00 $1 / 15 / 123: 00$ $1 / 15 / 124: 00$ 1/15/12 5:00 1/15/12 6:00 1/15/127:00 1/15/12 9:00 1/15/12 9:00 1/15/12 11:00 1/15/12 12:00 $1 / 15 / 1213: 00$ $1 / 15 / 1214: 00$
$1 / 15 / 1215: 00$
$1 / 15 / 1216: 00$ 1/15/12 17:00 1/15/12 18:00 $1 / 15 / 1219: 00$ 1/15/12 20:00 1/15/12 21:00 1/15/12 22:00 $1 / 15 / 1223: 00$ $1 / 16 / 122000$ 1/16/121:00 $1 / 16 / 123: 00$
$1 / 16 / 124: 00$ 1/16/12 5:00 $1 / 16 / 126: 00$ 1/16/12 7:00 $1 / 16 / 129: 00$ $1 / 16 / 1210: 00$ 1/16/12 12:00 1/16/12 13:00 1/16/12 14:00 $1 / 16 / 1215: 00$ 1/16/12 16:00 $1 / 16 / 1217.00$ 1/16/12 18:00 $1 / 16 / 1220: 00$ $1 / 16 / 1221: 00$ 1/16/12 22:00 $1 / 17 / 120: 00$
$1 / 17 / 121: 00$ $\begin{array}{rrrr}1494.877 & 1495.201 & 1496.593 & 1495.273 \\ 1494.88 & 1495.21 & 1496604 & 1495 \\ 1482\end{array}$ $\begin{array}{lllll}1494.88 & 1495.21 & 1496.604 & 1495.282 \\ 1494884 & 1495.219 & 1966.623 & 1995294\end{array}$ $\begin{array}{lllll}1494.884 & 1495.219 & 1496.623 & 1495.294 \\ 1494.884 & 1495226 & 1496.633 & 1495306\end{array}$ $\begin{array}{lllll}1494.891 & 1495.238 & 1496.656 & 1495.322\end{array}$ $\begin{array}{llll}1494.896 & 1495.25 & 1496.679 & 1495.322\end{array}$ $\begin{array}{lllll}1494.898 & 1495.261 & 1496.698 & 1495.355\end{array}$ $\begin{array}{lllll}1494.901 & 1495.268 & 1496.703 & 1495.369\end{array}$ $\begin{array}{lllll}1494.91 & 1495.285 & 1496.728 & 1495.387\end{array}$ $\begin{array}{llll}1494.915 & 1495.294 & 1496.742 & 1495.401\end{array}$ $\begin{array}{llll}1494.919 & 1495.308 & 1496.764 & 1495.42 \\ 1494.924 & 1495.322 & 1496777 & 1495.434\end{array}$ $\begin{array}{llll}1494.924 & 1495.322 & 1496.777 & 1495.434 \\ 1494.924 & 1495.324 & 1496.775 & 1495.439\end{array}$ $\begin{array}{lllll}1494.926 & 1495.327 & 1496.773 & 1455.439\end{array}$ $\begin{array}{lllll}1494.926 & 1455.327 & 1496.773 & 1495.441 \\ 1494.929 & 1495.327 & 1496.77 & 1495.441\end{array}$ $\begin{array}{lllll}1494.933 & 1495.331 & 1496.775 & 1495.441\end{array}$ $\begin{array}{lllll}1494.945 & 1495.352 & 1496.813 & 1495.462\end{array}$ $\begin{array}{lllll}1494.954 & 1495.371 & 1496.843 & 1495.483\end{array}$ $\begin{array}{llllll}1494.959 & 1495.387 & 1496.864 & 1495.504\end{array}$ $\begin{array}{lllll}1494.961 & 1495.399 & 1496.871 & 1495.516 \\ 1494.966 & 1495.404 & 1496873 & 1495.523\end{array}$ $\begin{array}{llll}1494.968 & 1495.408 & 1496.871 & 1495.527\end{array}$ $\begin{array}{lllll}1494.968 & 1495.406 & 1496.859 & 1495.52\end{array}$ $\begin{array}{lllll}1494.968 & 1495.401 & 1496.848 & 1495.511\end{array}$ $\begin{array}{llllll}1494.973 & 1495.406 & 1496.852 & 1495.506\end{array}$ $\begin{array}{lllll}1494.978 & 1495.415 & 1496.871 & 1495.511\end{array}$ $\begin{array}{llll}1494.985 & 1495.422 & 1496.887 & 1495.523\end{array}$ $\begin{array}{lllll}1494.992 & 1495.434 & 1496.906 & 1495.534\end{array}$ $\begin{array}{rrrrr}1494.996 & 1495.441 & 1496.915 & 1495.541 \\ 1494.996 & 1495.446 & 1496.92 & 1495548\end{array}$ $1494.999 \quad 1495.455 \quad 1496.927 \quad 1495.558$ $\begin{array}{lllll}1494.999 & 1495.455 & 1496.927 & 1495.56\end{array}$ $\begin{array}{lllll}1495.006 & 1495.467 & 1496.941 & 1495.567\end{array}$ $\begin{array}{lllll}1494.999 & 1495.462 & 1496.925 & 1495.565\end{array}$ $\begin{array}{lllll}1495.001 & 1495.462 & 1496.92 & 1495.558\end{array}$ $\begin{array}{llll}1494.994 & 1495.448 & 1496.885 & 1495.537 \\ 149987 & 1455.432 & 1469855 & 195513\end{array}$ $\begin{array}{lllll}1494.987 & 1455.432 & 1496.855 & 1495.513\end{array}$ $\begin{array}{rrrrr}1494.985 & 1495.418 & 1496.824 & 1495.488 \\ 1494.98 & 1495.399 & 1496.798 & 1495.457\end{array}$ $\begin{array}{lllll}1494.985 & 1495.399 & 1496.803 & 1495.45\end{array}$ $\begin{array}{lllll}1494.985 & 1495.392 & 1496.801 & 1495.441\end{array}$ $\begin{array}{llllll}1494.992 & 1495.404 & 1496.827 & 1495.448\end{array}$ $\begin{array}{lllll}1494.992 & 1495.406 & 1496.841 & 1495.455\end{array}$ $\begin{array}{lllll}1494.989 & 1495.404 & 1496.834 & 1495.455\end{array}$ $1494.994 \quad 1495.406 \quad 1496.8381495 .457$ $\begin{array}{lllll}1494.996 & 1495.404 & 1496.831 & 1495.45 \\ 1494.994 & 1495.401 & 1496.831 & 1495.45\end{array}$ $1494.99991495 .4041496 .834 \quad 1495.446$

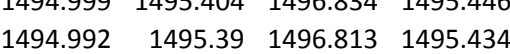
$1494.992 \quad 1495.387 \quad 1496.808 \quad 1495.427$ $1494.975 \quad 1495.362 \quad 1496.761 \quad 1495.401$ $1494.964 \quad 1495.315 \quad 1496.686 \quad 1495.35$ $\begin{array}{llll}1494.964 & 1495.299 & 1496.665 & 1495.322 \\ 1494.943 & 1495.254 & 1496.609 & 1495.271\end{array}$ $\begin{array}{llllllllll}1494.324 & 1494.875 & 1495.168 & 1495.798 & 1496.963 & 1496.751 & 1493.572 & 1488.959 & 1493.416 & 1491.031\end{array}$

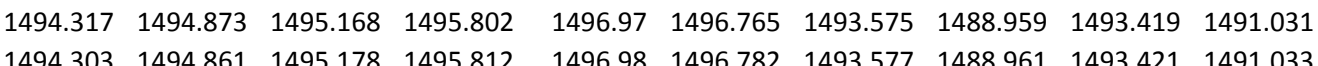
$\begin{array}{lllllllllll}1494.303 & 1494.861 & 1495.178 & 1495.812 & 1496.98 & 1496.782 & 1493.577 & 1488.961 & 1493.421 & 1491.033\end{array}$ $\begin{array}{llllllllllll}1494.289 & 1494.851 & 1495.18 & 1495.83 & 1496.998 & 1496.815 & 1493.589 & 1488.975 & 1493.431 & 1491.033\end{array}$ $\begin{array}{llllllllllll}1494.282 & 1494.851 & 1495.182 & 1495.842 & 1497.015 & 1496.835 & 1493.596 & 1488.977 & 1493.433 & 1491.035\end{array}$ $\begin{array}{lllllllllll}1494.282 & 1494.849 & 1495.18 & 1495.854 & 1497.024 & 1496.849 & 1493.601 & 1488.989 & 1493.44 & 1491.04\end{array}$ $\begin{array}{llllllllll}1494.287 & 1494.854 & 1495.18 & 1495.86 & 1497.033 & 1496.854 & 1493.605 & 1488.991 & 1493.442 & 1491.042\end{array}$

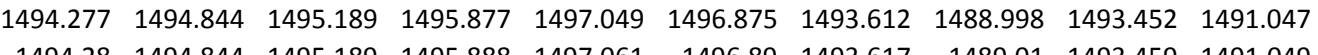

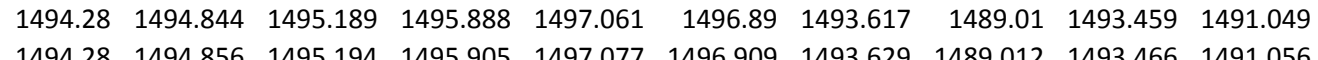
$\begin{array}{llllllllll}1494.28 & 1494.856 & 1495.194 & 1495.905 & 1497.077 & 1496.909 & 1493.629 & 1489.012 & 1493.466 & 1491.056 \\ 1494.287 & 1494856 & 1495.194 & 1495.916 & 1497.087 & 1496918 & 1493633 & 1489015 & 1493475 & 1491.061\end{array}$ $\begin{array}{llllllllll}1494.287 & 1494.856 & 1495.194 & 1495.916 & 1497.087 & 1496.918 & 1493.633 & 1489.015 & 1493.475 & 1491.061 \\ 1494.303 & 1494.866 & 1495.192 & 1495.923 & 1497.091 & 1496.916 & 1493.643 & 1489.017 & 1493.48 & 1491.066\end{array}$ $\begin{array}{llllllllllll}1494.315 & 1494.875 & 1495.189 & 1495.925 & 1497.094 & 1496.909 & 1493.643 & 1489.022 & 1493.487 & 1491.07\end{array}$ $\begin{array}{lllllllllll}1494.327 & 1494.878 & 1495.192 & 1495.93 & 1497.098 & 1496.904 & 1493.645 & 1489.024 & 1493.487 & 1491.073\end{array}$ $\begin{array}{lllllllllll}1494.331 & 1494.87 & 1495.197 & 1495.937 & 1497.103 & 1496.904 & 1493.647 & 1489.029 & 1493.489 & 1491.075\end{array}$ $\begin{array}{llllllllll}1494.31 & 1494.873 & 1495.206 & 1495.956 & 1497.122 & 1496.938 & 1493.654 & 1489.031 & 1493.496 & 1491.08\end{array}$ $\begin{array}{llllllllll}1494.301 & 1494.882 & 1495.213 & 1495.974 & 1497.14 & 1496.961 & 1493.668 & 1489.034 & 1493.506 & 1491.087\end{array}$

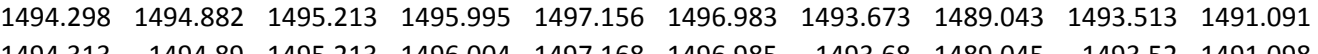
$\begin{array}{lllllllllll}1494.313 & 1494.89 & 1495.213 & 1496.004 & 1497.168 & 1496.985 & 1493.68 & 1489.045 & 1493.52 & 1491.098 \\ 1494.322 & 1494.885 & 1495218 & 1496014 & 1497.175 & 1496983 & 1493685 & 1489.05 & 1493524 & 1491.103\end{array}$ $\begin{array}{lllllllllllll}1494.341 & 1494.892 & 1495.213 & 1496.021 & 1497.18 & 1496.978 & 1493.687 & 1489.055 & 1493.527 & 1491.108\end{array}$ $\begin{array}{llllllllll}1494.367 & 1494.909 & 1495.213 & 1496.021 & 1497.177 & 1496964 & 1493.418 & 1489.057 & 1493234 & 1491.11\end{array}$ $\begin{array}{lllllllllll}1494.388 & 1494.911 & 1495.213 & 1496.021 & 1497.173 & 1496.949 & 1493.507 & 1489.057 & 1493.337 & 1491.11\end{array}$ $\begin{array}{lllllllllll}1494.392 & 1494.894 & 1495.216 & 1496.028 & 1497.18 & 1496.949 & 1493.563 & 1489.052 & 1493.395 & 1491.117\end{array}$ $\begin{array}{lllllllllll}1494.388 & 1494.921 & 1495.218 & 1496.037 & 1497.189 & 1496.961 & 1493.593 & 1489.064 & 1493.428 & 1491.119\end{array}$ $\begin{array}{lllllllllll}1494.39 & 1494.918 & 1495.223 & 1496.048 & 1497.198 & 1496.976 & 1493.619 & 1489.064 & 1493.454 & 1491.124\end{array}$ $\begin{array}{llllllllllll}1494.385 & 1494.913 & 1495.23 & 1496.062 & 1497.215 & 1496.992 & 1493.638 & 1489.073 & 1493.475 & 1491.129\end{array}$ $\begin{array}{rrrrrrrrrr}1494.392 & 1494.961 & 1495.232 & 1496.074 & 1497.222 & 1497 & 1493.652 & 1489.069 & 1493.489 & 1491.131 \\ 1494.402 & 1494.957 & 1495.23 & 1496.081 & 1497.231 & 1497.004 & 1493.661 & 1489.078 & 1493.501 & 1491.136\end{array}$ $\begin{array}{lllllllllll}1494.409 & 1494.959 & 1495.235 & 1496.09 & 1497.235 & 1497.007 & 1493.673 & 1489.076 & 1493.513 & 1491.138\end{array}$ $\begin{array}{llllllllll}1494.423 & 1494.959 & 1495.239 & 1496.097 & 1497.238 & 1497.002 & 1493.68 & 1489.083 & 1493.517 & 1491.142\end{array}$ $\begin{array}{lllllllllll}1494.421 & 1494.933 & 1495.239 & 1496.107 & 1497.252 & 1497.009 & 1493.687 & 1489.085 & 1493.527 & 1491.147\end{array}$ $\begin{array}{lllllllllll}1494.444 & 1494.978 & 1495.228 & 1496.109 & 1497.247 & 1496.995 & 1493.692 & 1489.083 & 1493.531 & 1491.145\end{array}$ $\begin{array}{lllllllllll}1494.458 & 1494.976 & 1495.235 & 1496.109 & 1497.247 & 1496.988 & 1493.694 & 1489.083 & 1493.534 & 1491.147\end{array}$

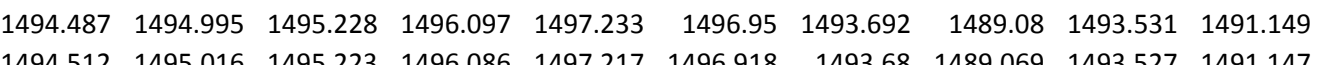
$\begin{array}{llllllllll}1494.512 & 1455.016 & 1455.223 & 1466.086 & 1497.217 & 1496.918 & 1493.68 & 1489.069 & 1493.527 & 1491.147 \\ 1494.531 & 1495.036 & 1495.218 & 1496.069 & 1497.201 & 1496.887 & 1493.673 & 1489.065 & 1493517 & 1491.145\end{array}$ $\begin{array}{lllllllllll}1494.541 & 1495.047 & 1495.213 & 1496.055 & 1497.187 & 1496.863 & 1493.666 & 1489.055 & 1493.508 & 1491.142\end{array}$ $\begin{array}{lllllllllll}1494.524 & 1495.028 & 1495.22 & 1496.051 & 1497.182 & 1496.866 & 1493.661 & 1489.052 & 1493.503 & 1491.138\end{array}$ $\begin{array}{llllllllllll}1494.515 & 1495.016 & 1495.223 & 1496.046 & 1497.177 & 1496.861 & 1493.657 & 1489.048 & 1493.498 & 1491.136\end{array}$ $\begin{array}{lllllllllll}1494.487 & 1494.971 & 1495.232 & 1496.053 & 1497.187 & 1496.892 & 1493.657 & 1489.05 & 1493.499 & 1491.138\end{array}$ $\begin{array}{lllllllllll}1494.477 & 1494.983 & 1495.23 & 1496.058 & 1497.189 & 1496.911 & 1493.659 & 1489.052 & 1493.501 & 1491.133\end{array}$ $\begin{array}{lllllllllll}1494.479 & 1494.983 & 1495.225 & 1496.051 & 1497.189 & 1496.894 & 1493.657 & 1489.057 & 1493.501 & 1491.131\end{array}$ $\begin{array}{llllllllllll}1494.475 & 1494.978 & 1495.225 & 1496.058 & 1497.187 & 1496.911 & 1493.657 & 1489.052 & 1493.499 & 1491.129\end{array}$ $\begin{array}{lllllllllll}1494.479 & 1494.969 & 1495.23 & 1496048 & 1497.187 & 1496894 & 1493.647 & 1489.05 & 1493.494 & 1491.129\end{array}$ $\begin{array}{lllllllllll}1494.477 & 1494.983 & 1495.232 & 1496.048 & 1497.187 & 1496.899 & 1493.65 & 1489.05 & 1493.491 & 1491.124\end{array}$ $\begin{array}{lllllllllll}1494.496 & 1494.988 & 1495.223 & 1496.037 & 1497.173 & 1496.885 & 1493.645 & 1489.05 & 1493.484 & 1491.119\end{array}$ $\begin{array}{llllllllll}1494.501 & 1494.988 & 1495.223 & 1496.035 & 1497.168 & 1496.88 & 1493.638 & 1489.048 & 1493.484 & 1491.117\end{array}$ $\begin{array}{llllllllll}1494.534 & 1495.028 & 1495.206 & 1496.014 & 1497.147 & 1496.839 & 1493.631 & 1489.041 & 1493.475 & 1491.115\end{array}$ $\begin{array}{llllllllllll}1494.573 & 1495.031 & 1495.197 & 1495.977 & 1497.108 & 1496.76 & 1493.61 & 1489.024 & 1493.454 & 1491.105\end{array}$ $\begin{array}{llllllllll}1494.569 & 1495.019 & 1495.197 & 1495.956 & 1497.094 & 1496.741 & 1493.596 & 1489.017 & 1493.442 & 1491.098\end{array}$ $\begin{array}{lllllllll}1487.757 & 1488.335 & 1493.821 & 1494.253 & 1493.577 & 1495.348 & 1493.56 & 1484.902 & 1479.134\end{array}$ $\begin{array}{llllllllll}1487.741 & 1488.328 & 1493.824 & 1494.255 & 1493.582 & 1495.362 & 1493.56 & 1484.902 & 1479.13\end{array}$

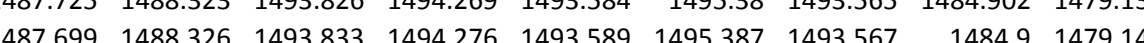
$\begin{array}{llllllllll}1487.678 & 1488.318 & 1493.835 & 1494.292 & 1493.593 & 1495.404 & 1493.574 & 1484.902 & 1479.143\end{array}$ $\begin{array}{llllllllll}1487.66 & 1488.318 & 1493.84 & 1494.306 & 1493.601 & 1495.425 & 1493.579 & 1484.902 & 1479.145\end{array}$ $\begin{array}{llllllllll}1487.635 & 1488.321 & 1493.845 & 1494.313 & 1493.605 & 1495.437 & 1493.584 & 1484.9 & 1479.148\end{array}$ $\begin{array}{lllllllll}1487.607 & 1488.323 & 1493.85 & 1494.32 & 1493.608 & 1495.449 & 1493.588 & 1484.9 & 1479.145\end{array}$ $\begin{array}{llllllllll}1487.588 & 1488.318 & 1493.862 & 1494.339 & 1493.615 & 1495.467 & 1493.593 & 1484.902 & 1479.15\end{array}$ $\begin{array}{lllllllll}1487.565 & 1488.323 & 1493.864 & 1494.346 & 1493.624 & 1495.477 & 1493.605 & 1484.9 & 1479.152\end{array}$ $\begin{array}{llllllllll}1487.531 & 1488.321 & 1493.873 & 1494.362 & 1493.634 & 1495.496 & 1493.61 & 1484.9 & 1479.157\end{array}$

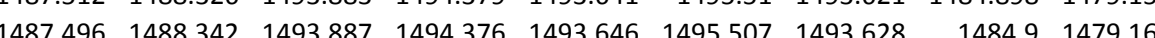
$\begin{array}{llllllllll}1487.477 & 1488.347 & 1493.892 & 1494.376 & 1493.646 & 1495.505 & 1493.628 & 1484.898 & 1479.164\end{array}$ $\begin{array}{llllllllll}1487.459 & 1488.351 & 1493.894 & 1494.376 & 1493.65 & 1495.507 & 1493.628 & 1484.898 & 1479.166\end{array}$ $\begin{array}{lllllllllll}1487.403 & 1488.351 & 1493.892 & 1494.379 & 1493.653 & 1495.505 & 1493.626 & 1484.898 & 1479.164\end{array}$ $\begin{array}{rrrrrrrrr}1487.38 & 1488.337 & 1493.904 & 1494.402 & 1493.66 & 1495.543 & 1493.633 & 1484.898 & 1479.169 \\ 1487.352 & 1488.33 & 1493.911 & 1494.418 & 1493.672 & 1495.561 & 1493.642 & 1484.898 & 1479.171\end{array}$ $\begin{array}{lrrrrrrrr}1487.352 & 1488.33 & 1493.911 & 1494.418 & 1493.672 & 1495.561 & 1493.642 & 1484.898 & 1479.171 \\ 1487.336 & 1488.333 & 1493.92 & 1494.437 & 1493.679 & 1495.583 & 1493.656 & 1484.898 & 1479.173\end{array}$ $\begin{array}{llllllllll}1487.336 & 1488.333 & 1493.92 & 1494.437 & 1493.679 & 1495.583 & 1493.656 & 1484.898 & 1479.173 \\ 1487.32 & 1488.34 & 1493.925 & 1494.435 & 1493.686 & 1495.587 & 1493.661 & 1484.895 & 1479.173\end{array}$ $\begin{array}{llllllllll}1487.253 & 1488.337 & 1493.927 & 1494.449 & 1493.688 & 1495.594 & 1493.666 & 1484.898 & 1479.1773\end{array}$ $\begin{array}{llllllllllll}4887.237 & 1488.354 & 1493.932 & 1494.444 & 1493.693 & 1495.597 & 1493675 & 1484895 & 147 & \end{array}$ $\begin{array}{llllllllll}1487.221 & 1488.365 & 1493.643 & 1494.36 & 1493.42 & 1495.58 & 1493.408 & 1484.895 & 1479.173 \\ 1487.2 & 1488 & 1493741 & 1494348 & 1493.513 & 1495.557 & 1493.495 & 1484895 & 1479.173\end{array}$ $\begin{array}{lllllllllll}1487.179 & 1488.38 & 1493.803 & 1494.374 & 1493.567 & 1495.561 & 1493.551 & 1484.895 & 1479.176\end{array}$ $\begin{array}{llllllllll}1487.165 & 1488.38 & 1493.835 & 1494.39 & 1493.601 & 1495.573 & 1493.581 & 1484.895 & 1479.18\end{array}$ $\begin{array}{llllllllll}1487.156 & 1488.382 & 1493.859 & 1494.409 & 1493.624 & 1495.583 & 1493.603 & 1484.895 & 1479.183\end{array}$ $\begin{array}{llllllllll} & 4487.147 & 1488.384 & 1493.88 & 1494.423 & 1493.643 & 1495.599 & 1493.621 & 1484.895 & 1479.187\end{array}$ $\begin{array}{llllllllll}1487.133 & 1488.394 & 1493.897 & 1494.437 & 1493.657 & 1455.611 & 1493.635 & 1484.895 & 1479.19\end{array}$ $\begin{array}{lllllllllll}1487.11 & 1488.405 & 1493.92 & 1494.453 & 1493.676 & 1495.62 & 1493.654 & 1484.895 & 1479.194\end{array}$ $\begin{array}{llllllllll}1487.096 & 1488.419 & 1493.925 & 1494.456 & 1493.688 & 1495.62 & 1493.664 & 1484.895 & 1479.194\end{array}$ $\begin{array}{lllllllll}1487.084 & 1488.417 & 1493.932 & 1494.467 & 1493.695 & 1495.637 & 1493.668 & 1484.895 & 1479.197\end{array}$ $\begin{array}{llllllllll}4487.073 & 1488.436 & 1493.939 & 1494.456 & 1493.698 & 1495.618 & 1493.678 & 1484.893 & 1479.197\end{array}$ $\begin{array}{llllllllll}1487.043 & 1488.441 & 1493.941 & 1494.458 & 1493.7 & 1495.613 & 1493.68 & 1484.893 & 1479.197\end{array}$ $\begin{array}{lllllllll}1487.026 & 1488.459 & 1493.939 & 1494.435 & 1493.698 & 1495.583 & 1493.675 & 1484.893 & 1479.194\end{array}$

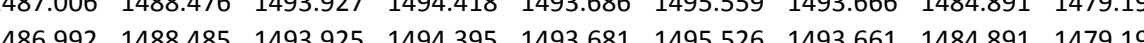

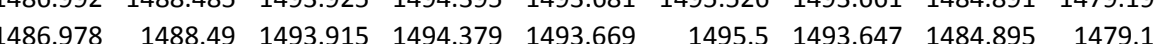
$\begin{array}{llllllllll}1486.964 & 1488.478 & 1493.913 & 1494.372 & 1493.667 & 1495.5 & 1493.642 & 1484.893 & 1479.19\end{array}$ $\begin{array}{llllllllll}1486.95 & 1488.473 & 1493.908 & 1494.369 & 1493.662 & 1495.496 & 1493.635 & 1484.891 & 1479.192\end{array}$ $\begin{array}{lllllllll}1486.936 & 1488.459 & 1493.908 & 1494.383 & 1493.662 & 1495.514 & 1493.638 & 1484.891 & 1479.19\end{array}$ $\begin{array}{lllllllllll}1486.927 & 1488.459 & 1493.911 & 1494.39 & 1493.667 & 1495.517 & 1493.645 & 1484.891 & 1479.192\end{array}$ $\begin{array}{llllllllll}1486.909 & 1488.459 & 1493.908 & 1494.383 & 1493.662 & 1495.514 & 1493.638 & 1484.891 & 1479.19\end{array}$ $\begin{array}{lllllllll}1486.842 & 1488.464 & 1493.904 & 1494.386 & 1493.662 & 1455.517 & 1493.642 & 1484.891 & 1479.19\end{array}$

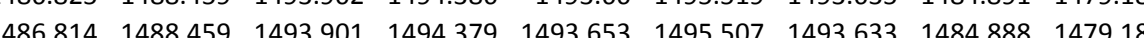
$\begin{array}{llllllllllll}1486.805 & 1488.455 & 1493.899 & 1494.383 & 1493.655 & 1495.512 & 1493.633 & 1484.891 & 1479.187\end{array}$ $\begin{array}{llllllllll}1486.791 & 1488.478 & 1493.89 & 1494.358 & 1493.65 & 1495.486 & 1493.628 & 1484.886 & 1479.185\end{array}$ $\begin{array}{lllllllll}1486.784 & 1488.478 & 1493.887 & 1494.355 & 1493.646 & 1495.489 & 1493.624 & 1484.888 & 1479.185\end{array}$ $\begin{array}{llllllllll}1486.772 & 1488.511 & 1493.88 & 1494.325 & 1493.636 & 1495.446 & 1493.624 & 1484.884 & 1479.185\end{array}$ $\begin{array}{lllllllllll}1486.747 & 1488.518 & 1493.857 & 1494.283 & 1493.617 & 1495.385 & 1493.595 & 1484.888 & 1479.18\end{array}$ $\begin{array}{llllllllll}1486.735 & 1488.511 & 1493.847 & 1494.267 & 1493.601 & 1495.366 & 1493.579 & 1484.886 & 1479.18 \\ 1486.721 & 1488.527 & 1493.824 & 1494215 & 1493577 & 1495.312 & 1493.563 & 1484.886 & 1479.176\end{array}$ 
Well

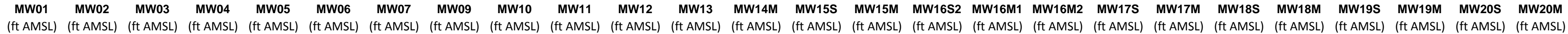

$1 / 17 / 122: 00$ $1 / 17 / 123: 00$ 1/17/122 4:00 1/17/12 5:00 $1 / 17 / 127: 00$ 1/17/12 8:00 1/17/12 9:00 1/17/12 10:00 1/17/12 11:00 1/17/12 12:00 1/177/12 13:00 $1 / 17 / 1215: 00$ 1/17/12 15:00 1/17/12 17:00 1/17/12 18:00 1/177/12 19:00 1/17/12 20:00 1/17/12 21:00 1/17/12 22:00 1/18/120:00 1/18/18 0:12 1:00 1/18/12 2:00 1/18/12 3:00 $1 / 18 / 124: 00$ $1 / 18 / 125: 00$ 1/18/12 6:00 $1 / 18 / 128: 00$ 1/18/12 9:00 1/18/12 10:00 1/18/12 11:00 1/18/12 12:00 1/18/12 13:00 $1 / 18 / 1214: 00$ 1/18/12 15:00 1/118/12 $16: 00$ 1/18/12 18:00 1/18/12 19:00 1/18/12 20:00 $1 / 18 / 1221: 00$ 1/18/12 22:00 1/19/120:00 1/19/12 1:00 1/19/12 3:00 1/19/12 4:00 1/19/12 5:00 $1 / 19 / 126: 00$
$1 / 19 / 127: 00$ $\begin{array}{llll}1494.94 & 1495.226 & 1496.581 & 1495.238\end{array}$ $\begin{array}{rrrr}1494.924 & 1495.194 & 1496.537 & 1495.196 \\ 1494.917 & 1495173 & 1496525 & 1495.17\end{array}$ $\begin{array}{llll}1494.917 & 1495.173 & 1496.525 & 1495.17 \\ 1494.908 & 1495.156 & 1496504 & 1495.142\end{array}$ $\begin{array}{lllll}1494.896 & 1495.129 & 1496.474 & 1495.117\end{array}$ $\begin{array}{lllll}1494.882 & 1495.098 & 1496.436 & 1495.081\end{array}$ $\begin{array}{lrrrr}1494.875 & 1495.08 & 1496.415 & 1995.058\end{array}$ $\begin{array}{llllll}1494.873 & 1495.063 & 1496.408 & 1495.037\end{array}$ $\begin{array}{lllll}1494.861 & 1495.049 & 1496.392 & 1495.018\end{array}$ $\begin{array}{llll}1494.856 & 1495.04 & 1496.389 & 1495.009\end{array}$ $\begin{array}{rrrr}1494.859 & 1495.04 & 1496.406 & 1495.016\end{array}$ $\begin{array}{llll}1494.866 & 1495.059 & 1496.448 & 1495.037 \\ 1494.863 & 1495.068 & 1496.464 & 1495.053\end{array}$ $\begin{array}{lllll}494.856 & 1495.066 & 1496.46 & 1455.053\end{array}$ $\begin{array}{rrrrr}1494.856 & 1495.066 & 1496.46 & 1495.058 \\ 1494.847 & 1495.059 & 1496.443 & 1495.054\end{array}$ $\begin{array}{lllll}1494.847 & 1459.059 & 1496.443 & 1495.054 \\ 1494.847 & 1495.061 & 1496.448 & 1495.058\end{array}$ $\begin{array}{llllll}1494.847 & 1495.063 & 1496.453 & 1495.063\end{array}$ $\begin{array}{lllll}1494.842 & 1495.066 & 1496.448 & 1495.063\end{array}$ $\begin{array}{llll}1494.838 & 1495.059 & 1496.441 & 1495.06\end{array}$ $\begin{array}{rrrr}1494.838 & 1495.066 & 1496.45 & 1495.067 \\ 1494.84 & 1495.07 & 1496.464 & 1495074\end{array}$ $\begin{array}{rrrrr}1494.84 & 1495.07 & 1496.464 & 1495.074 \\ 1494.838 & 1495.073 & 1496.469 & 1495.081\end{array}$ $\begin{array}{lllll}1494.842 & 1495.084 & 1496.49 & 1495098\end{array}$ $\begin{array}{llll}1494.847 & 1495.098 & 1496.518 & 1495.116\end{array}$ $\begin{array}{lllll}1494.849 & 1495.117 & 1496.544 & 1495.14\end{array}$ $\begin{array}{lllll}1494.856 & 1495.131 & 1496.569 & 1495.168\end{array}$ $\begin{array}{lllll}1494.852 & 1495.142 & 1496.584 & 1495.182\end{array}$ $\begin{array}{rrrr}1494.859 & 1495.159 & 1496.6 & 1495.201\end{array}$ $\begin{array}{lllll}1494.856 & 1495.163 & 1496.598 & 1495.208 \\ 1494.861 & 1495.177 & 1496.616 & 1495.222\end{array}$ $\begin{array}{lllll}1494.859 & 1455.182 & 1496.614 & 1495.229\end{array}$ $\begin{array}{lllll}1494.863 & 1495.187 & 1496.621 & 1495.231\end{array}$ $1494.861 \quad 1495.189 \quad 1496.618 \quad 1495.233$ $\begin{array}{lllll}1494.868 & 1495.205 & 1496.644 & 1495.25\end{array}$ $\begin{array}{lllll}1494.882 & 1495.243 & 1496.693 & 1495.285\end{array}$ $\begin{array}{lllll}1494.889 & 1495.268 & 1496.728 & 1495.31 \\ 1494896 & 1495294 & 1466.759 & 1495341\end{array}$ $\begin{array}{lllll}1494.896 & 1455.294 & 1496.759 & 1495.341\end{array}$ $\begin{array}{lllll}1494.901 & 1455.303 & 1496.766 & 1495.359 \\ 1494.903 & 1495315 & 1496775 & 1495371\end{array}$ $\begin{array}{lllll}1494.91 & 1495.329 & 1496.78 & 1495.383\end{array}$ $\begin{array}{llll}1494.905 & 1495.324 & 1496.768 & 1495.383\end{array}$ $\begin{array}{llll}1494.91 & 1495.331 & 1496.773 & 1495.385\end{array}$ $1494.9171495 .343 \quad 1496.791 \quad 1495.394$ $1494.9191495 .345 \quad 1496.789 \quad 1495.394$ $1494.924 \quad 1495.357 \quad 1496.8031495 .404$ $\begin{array}{llll}1494.929 & 1495.366 & 1496.815 & 1495.411\end{array}$ $1494.936 \quad 1495.373 \quad 1496822 \quad 1495.425$ $1494.938 \quad 1495.38 \quad 1496.831 \quad 14595.427$ $1494.933 \quad 1495.376 \quad 1496.81 \quad 1495.42$ $1494.931 \quad 1495.366 \quad 1496.794 \quad 1495.406$ $1494.926 \quad 1495.355 \quad 1496.766 \quad 1495.387$ $\begin{array}{rrrr}1494.922 & 1495.341 & 1496.74 & 1495.364 \\ 1494.91 & 1495.317 & 1496.691 & 1495.329\end{array}$ $\begin{array}{lrllllllll}1494.585 & 1495 & 1495.192 & 1495.895 & 1497.031 & 1496.669 & 1493.556 & 1488.991 & 1493.402 & 1491.08 \\ 1494.592 & 1494.99 & 1495.775 & 1495865 & 1497.001 & 1496.634 & 1493533 & 1488975 & 1493384 & 1491.068\end{array}$ $\begin{array}{llllllllll}1494.592 & 1494.99 & 1495.175 & 1495.865 & 1497.001 & 1496.634 & 1493.533 & 1488.975 & 1493.384 & 1491.068 \\ 1494571 & 1494.98 & 1495175 & 1495.844 & 1496.984 & 1496629 & 4493519 & 148897 & 1493365 & 1491059\end{array}$

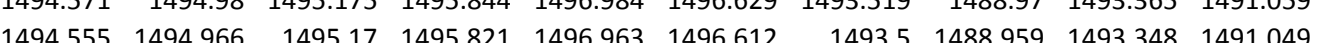
$\begin{array}{llllllllllll}1494.545 & 1494.954 & 1495.161 & 1495.793 & 1496.938 & 1496.593 & 1493.481 & 1488.949 & 1493.33 & 1491.035\end{array}$ \begin{tabular}{lllllllllll}
1494.536 & 1494.942 & 1495.149 & 1495.761 & 1496.912 & 1496.564 & 1493.46 & 1488.944 & 1493.311 & 1491.035 \\
\hline
\end{tabular} $\begin{array}{llllllllllll}1494.51 & 1494.93 & 1495.149 & 1495.74 & 1496.894 & 1496.55 & 1493.444 & 1488.933 & 1493.292 & 1491.012\end{array}$ $\begin{array}{lllllllllll}1494.489 & 1494.921 & 1495.147 & 1495.719 & 1496.877 & 1496.547 & 1493.427 & 1488.937 & 1493.278 & 1491\end{array}$ $\begin{array}{llllllllll}1494.47 & 1494.906 & 1495.14 & 1495.7 & 1496.859 & 1496.54 & 1493.413 & 1488.916 & 1493.262 & 1490.986\end{array}$

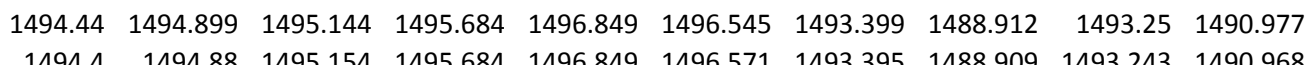
$\begin{array}{rrrrrrrrrr}1494.4 & 1494.88 & 1495.154 & 1495.684 & 1496.849 & 1496.571 & 1493.395 & 1488.909 & 1493.243 & 1490.968 \\ 1494.35 & 1494.846 & 1495.166 & 1495.691 & 1496861 & 1496.614 & 1493395 & 1488.909 & 1493243 & 1490961\end{array}$ $\begin{array}{rlllllllll}1494.35 & 1494.846 & 1495.166 & 1495.691 & 1496.861 & 1496.614 & 1493.395 & 1488.909 & 1493.243 & 1490.961 \\ 1494.322 & 1494.837 & 1495.161 & 1495.696 & 1496.868 & 1496.636 & 1493.397 & 1488.905 & 1493.241 & 1490.954\end{array}$ $\begin{array}{llllllllll}1494.32 & 1494.851 & 1495.151 & 1495.691 & 1496.863 & 1496.643 & 1493.39 & 1488.909 & 1493.236 & 1490.949\end{array}$ $\begin{array}{lllllllllll}1494.324 & 1494.866 & 1495.142 & 1495.679 & 1496.856 & 1496.626 & 1493.383 & 1488.902 & 1493.231 & 1490.942\end{array}$ $\begin{array}{lllllllllll}1494.313 & 1494.863 & 1495.149 & 1495.679 & 1496.856 & 1496.638 & 1493.378 & 1488.902 & 1493.224 & 1490.938\end{array}$ $\begin{array}{llllllllllll}1494.303 & 1494.861 & 1495.147 & 1495.679 & 1496.859 & 1496.648 & 1493.376 & 1488.902 & 1493.22 & 1490.933\end{array}$ $\begin{array}{llrrrrrrrr}1494.303 & 1494.861 & 1495.142 & 1495.675 & 1496.856 & 1496.648 & 1493.371 & 1488.905 & 1493.215 & 1490.931\end{array}$ $\begin{array}{llllllllll}1494.301 & 1494.856 & 1495.14 & 1495.67 & 1496.852 & 1496.646 & 1493.364 & 1488.902 & 1493.21 & 1490.926\end{array}$ $\begin{array}{rrrrrrrrrr}1494.291 & 1494.856 & 1495.144 & 1495.67 & 1496.854 & 1496.657 & 1493.366 & 1488.905 & 1493.21 & 1490.921 \\ 1494275 & 1494.846 & 1495.149 & 1495.672 & 1496863 & 1496669 & 1493.364 & 1488902 & 149321 & 1490.917\end{array}$ $\begin{array}{lllllllllllll}1494.27 & 1494.849 & 1495.147 & 1495.677 & 1496.868 & 1496.679 & 1493.366 & 1488.907 & 1493.212 & 1490.917\end{array}$

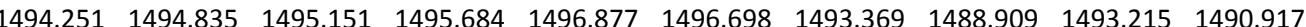
$\begin{array}{llllllllllll}1494.235 & 1494.82 & 1495.156 & 1495.698 & 1496.891 & 1496.729 & 1493.378 & 1488.919 & 1493.222 & 1490.917\end{array}$ $\begin{array}{lllllllllll}1494.221 & 1494.806 & 1495.159 & 1495.71 & 1496.905 & 1496.753 & 1493.388 & 1488.926 & 1493.229 & 1490.919\end{array}$ $\begin{array}{lllllllllll}1494.209 & 1494.799 & 1495.159 & 1495.726 & 1496.924 & 1496.777 & 1493.399 & 1488.935 & 1493.238 & 1490.924\end{array}$ $\begin{array}{lllllllllll}1494.219 & 1494.808 & 1495.154 & 1455.737 & 1496.933 & 1496.794 & 1493.404 & 1488.94 & 1493.248 & 1490.924\end{array}$ $\begin{array}{llllllllll}1494.211 & 1494.801 & 1495.161 & 1495.747 & 1496.945 & 1496.804 & 1493.411 & 1488.949 & 1493.252 & 1490.928 \\ 1494226 & 1494808 & 1995.154 & 1995.751 & 1969.949 & 1946801 & 1493.416 & 1488954 & 1493257 & 1490933\end{array}$ $\begin{array}{llllllllll}1494.226 & 1494.808 & 1495.154 & 1495.751 & 1496.949 & 1496.801 & 1493.416 & 1488.954 & 1493.257 & 1490.933 \\ 1494.223 & 1494.801 & 1495.159 & 1495.765 & 1496.963 & 1496815 & 1493.42 & 1488.956 & 1493262 & 1490.935\end{array}$ $\begin{array}{lllllllllll}1494.237 & 1494.811 & 1495.154 & 1495.768 & 1496.968 & 1496.816 & 1493.425 & 1488.959 & 1493.266 & 1490.938\end{array}$ $\begin{array}{llllllllllll}1494.242 & 1494.811 & 1495.156 & 1495.777 & 1496.973 & 1496.818 & 1493.425 & 1488.963 & 1493.271 & 1490.942\end{array}$ $\begin{array}{lllllllllll}1494.249 & 1494.813 & 1495.154 & 1495.779 & 1496.977 & 1496.811 & 1493.427 & 1488.966 & 1493.266 & 1490.945\end{array}$ $\begin{array}{llllllllll}1494.235 & 1494.796 & 1495.161 & 1495.793 & 1496.991 & 1496.837 & 1493.434 & 1488.968 & 1493.273 & 1490.949\end{array}$ $\begin{array}{lllllllllll}1494.207 & 1494.808 & 1495.173 & 1455.821 & 1497.022 & 1496.882 & 1493.448 & 1488.977 & 1493.29 & 1490.956\end{array}$ $\begin{array}{llrrrrrrrr}1494.2 & 1494.803 & 1495.175 & 1495.847 & 1497.045 & 1496.913 & 1493.46 & 1488.987 & 1493.302 & 1490.963\end{array}$

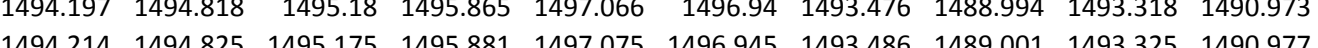
$\begin{array}{llllllllllll}1494.23 & 1494.837 & 1495.178 & 1495895 & 1497.089 & 1496.952 & 1493.493 & 1489003 & 1493332 & 1490994\end{array}$ $\begin{array}{llllllllllll}1494.24 & 1494.83 & 1495.18 & 1495.907 & 1497.101 & 1496.949 & 1493.502 & 1489.017 & 1493.341 & 1490.991\end{array}$ $\begin{array}{llllllllll}1494.27 & 1494.842 & 1495.173 & 1495.907 & 1497.101 & 1496.933 & 1493.5 & 1489.012 & 1493.339 & 1490.996\end{array}$ $\begin{array}{lllllllllll}1494.282 & 1494.839 & 1495.182 & 1495.916 & 1497.108 & 1496.935 & 1493.502 & 1489.019 & 1493.341 & 1491\end{array}$ $\begin{array}{lllllllllll}1494.282 & 1494.839 & 1495.187 & 1495.93 & 1497.122 & 1496.947 & 1493.507 & 1489.017 & 1493.346 & 1491.007\end{array}$ $\begin{array}{llllllllll}1494.298 & 1494.839 & 1495.182 & 1495.935 & 1497.124 & 1496.942 & 1493.509 & 1489.024 & 1493.351 & 1491.012\end{array}$ $\begin{array}{lllllllllll}1494.301 & 1494.854 & 1495.182 & 1495.946 & 1497.135 & 1496.95 & 1493.516 & 1489.027 & 1493.358 & 1491.014 \\ 1494.303 & 1494.849 & 1495.187 & 1495.96 & 1497.145 & 1496.957 & 1493.523 & 1489031 & 1493365 & 1491.019\end{array}$

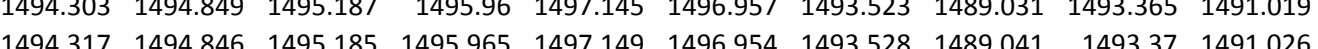
$\begin{array}{lllllllllllll}1494.324 & 1494.856 & 1495.187 & 1495.977 & 1497.156 & 1496.959 & 1493.537 & 1489.041 & 1493.379 & 1491.031\end{array}$ $\begin{array}{lllllllllll}1494.331 & 1494.861 & 1495.192 & 1495.986 & 1497.166 & 1496.959 & 1493.544 & 1489.043 & 1493.384 & 1491.035\end{array}$ $\begin{array}{llllllllll}1494.355 & 1494.906 & 1495.182 & 1495.984 & 1497.161 & 1496.945 & 1493.542 & 1489.045 & 1493.386 & 1491.04\end{array}$ $\begin{array}{llllllllll}1494.378 & 1494.923 & 1495.178 & 1495.979 & 1497.152 & 1496.918 & 1493.542 & 1489.041 & 1493.381 & 1491.04\end{array}$ $\begin{array}{lllllllllll}1494.404 & 1494.947 & 1495.175 & 1495.97 & 1497.14 & 1496.89 & 1493.537 & 1489.043 & 1493.381 & 1491.04\end{array}$ $\begin{array}{rrrrrrrrrr}1494.421 & 1494.966 & 1495.17 & 1495.96 & 1497.128 & 1496.87 & 1493.528 & 1489.036 & 1493.374 & 1491.04 \\ 1494.454 & 1494.971 & 1495.156 & 1495.937 & 1497.103 & 1496.818 & 1493.514 & 1489.022 & 1493.36 & 1491.035\end{array}$ $\begin{array}{lllllllll}1486.705 & 1488.513 & 1493.805 & 1494.204 & 1493.558 & 1495.286 & 1493.541 & 1484.886 & 1479.173\end{array}$ $\begin{array}{llllllllll}1486.684 & 1488.52 & 1493.786 & 1494.164 & 1493.539 & 1495.244 & 1493.518 & 1484.888 & 1479.169 \\ 1486673 & 1488509 & 1493767 & 1494143 & 1495522 & 1495223 & 1493504 & 148488 & 1479.166\end{array}$ $\begin{array}{llllllllll}1486.673 & 1488.509 & 1493.767 & 1494.143 & 149.522 & 1455.223 & 1439.504 & 1484.886 & 1479.166\end{array}$ $\begin{array}{llllllllll}1486.645 & 1488.511 & 1493.732 & 1494.101 & 1493.484 & 1495.169 & 1493.471 & 1484.884 & 1479.157\end{array}$ $\begin{array}{lllllllllll}1486.629 & 1488506 & 1493.713 & 1494.066 & 1493.466 & 1495136 & 1493.448 & 1484.884 & 1479.155\end{array}$ $\begin{array}{lllllllllll}1486.59 & 1488.495 & 1493.694 & 1494.052 & 1493.444 & 1495.115 & 1493.434 & 1484.884 & 1479.148\end{array}$ $\begin{array}{llllllllll}1486.559 & 1488.473 & 1493.68 & 1494.041 & 1493.432 & 1495.11 & 1493.415 & 1484.884 & 1479.145\end{array}$ $\begin{array}{lllllllll}1486.548 & 1488.464 & 1493.669 & 1494.024 & 1493.42 & 1495.094 & 1493.398 & 1484.884 & 1479.141\end{array}$ $\begin{array}{llllllllll}1486.509 & 1488.441 & 1493.652 & 1494.015 & 1493.406 & 1495.082 & 1493.384 & 1484.881 & 1479.144\end{array}$ $\begin{array}{lllllllllll}1486.495 & 1488.408 & 1493.647 & 1494.024 & 1493.397 & 1495.094 & 1493.38 & 1484.884 & 1479.14 \\ 1486.485 & 1488377 & 1493647 & 1494048 & 1493.402 & 1495.131 & 1493.377 & 1484886 & 1479.143\end{array}$ $\begin{array}{lllllllllll}148.485 & 1488.37 & 1493.647 & 1440.048 & 1493.00 & 4495.131 & 1493.377 & 1484.886 & 1479.143\end{array}$ $\begin{array}{llllllllll}1486.465 & 1488354 & 1493.643 & 1494.048 & 1493.397 & 1495.138 & 1493.377 & 1484.881 & 1479.141\end{array}$ $\begin{array}{llllllllll}1486.398 & 1488.351 & 1493.636 & 1494.04 & 1493.39 & 1495.129 & 1493.37 & 1484.879 & 1479.138\end{array}$ $\begin{array}{lllllllll}1486.388 & 1488.333 & 1493.629 & 1494.045 & 1493.385 & 1495.136 & 1493.363 & 1484.884 & 1479.136\end{array}$ $\begin{array}{llllllllll}1486.384 & 1488.33 & 1493.626 & 1494.045 & 1493.383 & 1495.141 & 1493.363 & 1484.881 & 1479.134\end{array}$ $\begin{array}{lllllllll}1486.379 & 1488.333 & 1493.622 & 1494.041 & 1493.375 & 1495.138 & 1493.356 & 1484.879 & 1479.131\end{array}$ $\begin{array}{lllllllll}1486.368 & 1488.328 & 1493.62 & 1494.038 & 1493.368 & 1455.134 & 1493.354 & 1484.879 & 1479.122\end{array}$ $\begin{array}{lllllllll}1486.363 & 1488.323 & 1493.617 & 1494.038 & 1493.371 & 1455.141 & 1439.349 & 1484.879 & 1479.131\end{array}$ $\begin{array}{llllllllll}1486.347 & 1488.304 & 1493.617 & 1494.057 & 1493.371 & 1495.164 & 1493.349 & 1484.881 & 1479.1334\end{array}$ $\begin{array}{llllllllll}148634 & 1488293 & 1493619 & 1494069 & 1493375 & 1495181 & 1493351 & 1484.881 & 1479.138\end{array}$ $\begin{array}{llllllllll}1486.333 & 1488.281 & 1493.627 & 1494.092 & 1493.383 & 1495.207 & 1493.363 & 1484.881 & 1479.141\end{array}$ $\begin{array}{lllllllll}1486.328 & 1488.276 & 1493.636 & 1494.108 & 1493.394 & 1495.23 & 1493.368 & 1484.877 & 1479.145\end{array}$ $\begin{array}{lllllllll}1486.326 & 1488.272 & 1493.645 & 1494.127 & 1493.402 & 1495.258 & 1493.38 & 1484.879 & 1479.15\end{array}$ $\begin{array}{llllllllll}1486.324 & 1488.274 & 1493.654 & 1494.134 & 1493.411 & 1495.261 & 1493.389 & 1484.877 & 1479.152\end{array}$ $\begin{array}{llllllllll}1486.321 & 1488.262 & 1493.657 & 1494.148 & 1493.418 & 1495.282 & 1493.391 & 1484.879 & 1479.157 & 1498\end{array}$

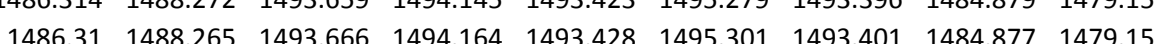
$\begin{array}{llllllllll}1486296 & 1488.272 & 1493.669 & 1494.162 & 1493.43 & 1495.298 & 1493.405 & 1484.877 & 1479.159\end{array}$ $\begin{array}{llllllllll}1486.289 & 1488.272 & 1493.673 & 1494.166 & 1493.432 & 1495.301 & 1493.405 & 1484.877 & 1479.159\end{array}$ $\begin{array}{llllllllll}1486.282 & 1488.269 & 1493.676 & 1494.164 & 1493.432 & 1495.303 & 1493.405 & 1484.877 & 1479.159\end{array}$ $\begin{array}{llllllllll}1486.275 & 1488.26 & 1493.683 & 1494.185 & 1493.442 & 1495.326 & 1493.412 & 1484.879 & 1479.162\end{array}$ $\begin{array}{llllllllll}1486.275 & 1488.25 & 1493.692 & 1494.22 & 1493.456 & 1495.362 & 1493.431 & 1484.877 & 1479.166\end{array}$ $\begin{array}{llllllllll}1486.277 & 1488.246 & 1493.709 & 1494.246 & 1493.468 & 1495.399 & 1493.438 & 1484.877 & 1479.171\end{array}$

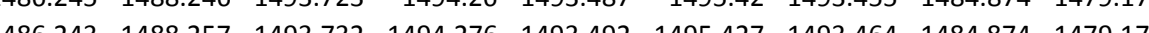
$\begin{array}{llllllllll}1486.238 & 1488267 & 1493.737 & 1494.278 & 1493.499 & 1495.439 & 1493.473 & 1484.874 & 1479.18\end{array}$ $\begin{array}{lllllllllll}1486.231 & 1488.265 & 1493.746 & 1494.288 & 1493.506 & 1495.449 & 1493.485 & 1484.874 & 1479.18\end{array}$ $\begin{array}{llllllllll}1486.169 & 1488.288 & 1493.746 & 1494.285 & 1493.506 & 1495.439 & 1493.485 & 1484.872 & 1479.178\end{array}$ $\begin{array}{llllllllll}1486.166 & 1488.288 & 1493.749 & 1494.29 & 1493.508 & 1495.442 & 1493.483 & 1484.872 & 1479.178\end{array}$ $\begin{array}{lllllllllll}4486.162 & 1488.293 & 1493.753 & 1494.297 & 1493.513 & 1495.453 & 1493.492 & 1484.874 & 1479.18\end{array}$ $\begin{array}{llllllllll}1486.157 & 1488.302 & 1493.756 & 1494.297 & 1493.515 & 1495.453 & 1493.49 & 1484.874 & 1479.178\end{array}$ $\begin{array}{llllllllll} & 1479.18\end{array}$

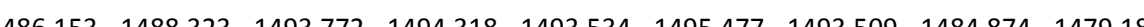
$\begin{array}{llllllllll}1486.148 & 1488.328 & 1493.784 & 1494.325 & 1493.544 & 1455.477 & 1493.52 & 1484.874 & 1479.19\end{array}$ $\begin{array}{llllllllll}1486.148 & 1488.335 & 1493.786 & 1494.332 & 1493.548 & 1495.484 & 1493.523 & 1484.874 & 1479.194\end{array}$ $\begin{array}{lllllllll}1486.148 & 1488.356 & 1493.793 & 1494.32 & 1493.553 & 1495.472 & 1493.53 & 1484.872 & 1479.197\end{array}$ $\begin{array}{llllllllll}1486.143 & 1488.372 & 1493.791 & 1494.304 & 1493.548 & 1495.451 & 1493.525 & 1484.87 & 1479.1997\end{array}$ $\begin{array}{lllllllllll}1486.143 & 1488.389 & 1493.784 & 1494.288 & 1493.541 & 1495.43 & 1493.523 & 1484.87 & 1479.197\end{array}$ $\begin{array}{rrrrrrrrr}1486.12 & 1488.391 & 1493.777 & 1494.271 & 1493.534 & 1495.406 & 1493.513 & 1484.867 & 1479.197 \\ 1486.102 & 1488.419 & 1493.763 & 1494.236 & 1493.52 & 1495.362 & 1493.502 & 1484.87 & 1479.192\end{array}$ 
Well

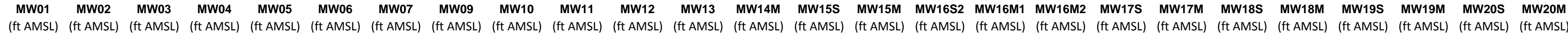

$1 / 19 / 128: 00$ $1 / 19 / 12$ 9:00 $1 / 19 / 1210: 00$ 1/19/12 11:00 1/19/12 12:00 $1 / 19 / 1214: 00$ 1/19/12 15:00 1/19/12 16:00 $1 / 19 / 1217: 00$ $1 / 19 / 1218: 00$
$1 / 19 / 1219: 00$ 1/19/12 19:00 1/19/12 20:00 1/19/12 22:00 $1 / 19 / 1223: 00$ $1 / 20 / 120: 00$ 1/20/12 1:00 $1 / 20 / 122: 00$ $1 / 20 / 123: 00$ 1/20/12 5:00 $1 / 20 / 126: 00$ $1 / 20 / 122: 00$ 1/20/12 8:00 1/20/12 9:00 1/20/12 10:00 $1 / 20 / 1211: 00$ $1 / 20 / 1212: 00$ 1/20/12 13:00 1/20/12 14:00 1/20/12 16:00 1/20/12 17:00 $1 / 20 / 1219: 00$
$1 / 20 / 1220: 00$
$1 / 20 / 1221: 00$ 1/20/12 21:00 1/20/1222:00 $1 / 21 / 120: 00$ 1/21/12 1:00 $1 / 21 / 122: 00$ $1 / 21 / 123: 00$ 1/21/12 4:00 $1 / 21 / 126.00$ 1/21/12 6:00 1/21/12 7:00 $1 / 21 / 129: 00$ $1 / 21 / 1210000$ $1 / 21 / 1211: 00$ 1/21/12 12:00 $\begin{array}{llll}1494.905 & 1495.296 & 1496.665 & 1495.299\end{array}$ $\begin{array}{lllll}1494.896 & 1495.271 & 1496.623 & 1495.264\end{array}$ $\begin{array}{llll}1494.896 & 1495.259 & 1496.614 & 1495.238\end{array}$ $\begin{array}{lllll}1494.901 & 1495.259 & 1496.633 & 1495.229\end{array}$ $\begin{array}{lllll}1494.901 & 1495.264 & 1496.656 & 1495.229\end{array}$ $\begin{array}{lllll}1494.898 & 1495.273 & 1496.675 & 1495.252\end{array}$ $\begin{array}{lllll}1494.901 & 1495.271 & 1496.672 & 1495.252\end{array}$ $\begin{array}{lllll}1494.898 & 1495.268 & 1496.668 & 1495.252\end{array}$ $\begin{array}{llll}1494.898 & 1495.264 & 1496.658 & 1495.245 \\ 149.903 & 1495273 & 196668 & 1495257\end{array}$ $\begin{array}{llll}1494.903 & 1495.273 & 1496.684 & 1495.257 \\ 1499891 & 1459264 & 1496651 & 195227\end{array}$ $\begin{array}{rrrr}1494.891 & 1495.264 & 1496.661 & 1495.247 \\ 1494.891 & 1495.259 & 1496.651 & 1495.24\end{array}$ $\begin{array}{llll}1494.891 & 1495.254 & 1496.649 & 1495.233\end{array}$ $\begin{array}{llll}1494.903 & 1495.268 & 1496.682 & 1495.247\end{array}$ $\begin{array}{lllll}1494.898 & 1495.275 & 1496.696 & 1495.261\end{array}$ $\begin{array}{lllll}1494.91 & 1495.292 & 1496.726 & 1495.28\end{array}$ $\begin{array}{lllll}1494.915 & 1495.308 & 1496.759 & 1495.308\end{array}$ $\begin{array}{lllll}1494.926 & 1495.331 & 1496.801 & 1495.338\end{array}$ $\begin{array}{lllll}1494.919 & 1495.324 & 1496.782 & 1495.338 \\ 1494.931 & 1495341 & 1496806 & 1955357\end{array}$ $\begin{array}{llll}1494.936 & 1495.35 & 1496.822 & 1495.371\end{array}$ $\begin{array}{lllll}1494.926 & 1495.338 & 1496.792 & 1495.362\end{array}$ $\begin{array}{lllll}1494.924 & 1455.331 & 1496.768 & 1495.348\end{array}$ $\begin{array}{lllll}1494.931 & 1495.334 & 1496.775 & 1495.345\end{array}$ $\begin{array}{lllll}1494.936 & 1495.343 & 1496.794 & 1495.355\end{array}$ $\begin{array}{llll}1494.929 & 1495.334 & 1496.775 & 1495.343\end{array}$ $\begin{array}{llll}1494.931 & 1495.331 & 1496.773 & 1495.338\end{array}$ $\begin{array}{llll}1494.931 & 1495.331 & 1496.768 & 1495.334 \\ 1494.933 & 1495331 & 1496.773 & 1495334\end{array}$ $\begin{array}{lllll}1494.931 & 1495.331 & 1496.768 & 1495.329\end{array}$ $\begin{array}{lllll}1494.922 & 1495.31 & 1496.728 & 1495.31\end{array}$ $\begin{array}{lllll}1494.915 & 1495.294 & 1496.693 & 1495.285\end{array}$ $\begin{array}{lllll}1494.903 & 1495.264 & 1496.647 & 1495.245\end{array}$ $\begin{array}{lllll}1494.894 & 1495.243 & 1496.607 & 1495.21\end{array}$ $\begin{array}{llll}1494.88 & 1495.21 & 1496.562 & 1495.166\end{array}$ $\begin{array}{lllll}1494.873 & 1495.182 & 1496.525 & 1495.128\end{array}$ $\begin{array}{rrrr}1494.866 & 1495.163 & 1496.509 & 1495.1 \\ 1494.859 & 1495.147 & 1496.492 & 1495.079\end{array}$ $\begin{array}{llll}1494.854 & 1455.138 & 1466.483 & 1495.063\end{array}$ $1494.859 \quad 1495.142 \quad 1496.506 \quad 1495.065$ $\begin{array}{lllll}1494.856 & 1495.142 & 1496.52 & 1495.067\end{array}$ $1494.845 \quad 1495.138 \quad 1496.509 \quad 1495.065$ $\begin{array}{lllll}1494.852 & 1495.142 & 1496.527 & 1495.074\end{array}$ $\begin{array}{llll}1494.856 & 1495.161 & 1496.572 & 1495.098 \\ 1494.845 & 1495147 & 1496537 & 1495091\end{array}$ $1494.8451495 .147 \quad 1496.53714955 .091$ $1449.845 \quad 1455.152 \quad 1496.546 \quad 1495.102$ $1494.845 \quad 1495.159 \quad 1496.56 \quad 1455.114$ $1494.847 \quad 1495.166 \quad 1496.579 \quad 1495.126$ $1494.845 \quad 1495.166 \quad 1496.5691495 .126$ $1494.849 \quad 1495.177 \quad 1496.595 \quad 1495.14$ $\begin{array}{rrrr}1494.856 & 1495.194 & 1496.628 & 1495.163 \\ 1494.87 & 1495.226 & 1496.689 & 1495.203\end{array}$ $\begin{array}{llllllllll}1494.465 & 1494.971 & 1495.159 & 1495.923 & 1497.087 & 1496.794 & 1493.502 & 1489.015 & 1493.346 & 1491.033 \\ 1494.482 & 1494.959 & 1495.151 & 1495895 & 1497.063 & 1496748 & 1493.483 & 1489.001 & 149333 & 1491.028\end{array}$ $\begin{array}{lllllllllll}1494.482 & 1494.959 & 1495.151 & 1495.895 & 1497.063 & 1496.748 & 1493.483 & 1489.001 & 1493.33 & 1491.028\end{array}$ $\begin{array}{lllllllllll}1494.465 & 1494.957 & 1495.151 & 1495.886 & 1497.049 & 1496.744 & 1493.472 & 1488.991 & 1493.316 & 1491.024 \\ 1494.442 & 1494.94 & 1495.156 & 1495881 & 1497.047 & 1496748 & 1493.465 & 1488991 & 1493313 & 1491.017\end{array}$ $\begin{array}{lllllllllll}1494.409 & 1494.916 & 1495.163 & 1495.881 & 1497.052 & 1496.765 & 1493.462 & 1488.989 & 1493.306 & 1491.014\end{array}$ $\begin{array}{lllllllllll}1494.383 & 1494.892 & 1495.163 & 1495.886 & 1497.056 & 1496.789 & 1493.462 & 1488.989 & 1493.311 & 1491.014\end{array}$ $\begin{array}{lllllllllll}1494.367 & 1494.875 & 1495.163 & 1495.893 & 1497.066 & 1496.815 & 1493.467 & 1488.994 & 1493.311 & 1491.007\end{array}$ $\begin{array}{lllllllllll}1494.369 & 1494.88 & 1495.156 & 1495.893 & 1497.063 & 1496.815 & 1493.467 & 1488.994 & 1493.309 & 1491.007\end{array}$ $\begin{array}{lllllllllll}1494.369 & 1494.885 & 1495.156 & 1495.891 & 1497.056 & 1496.815 & 1493.46 & 1488.989 & 1493.306 & 1491.005\end{array}$ $\begin{array}{llllllllll}1494.371 & 1494.892 & 1495.161 & 1495.886 & 1497.056 & 1496.803 & 1493.458 & 1488.991 & 1493.302 & 1491\end{array}$ $\begin{array}{llllllllll}1494.348 & 1494.873 & 1495.168 & 1495.893 & 1497.066 & 1496.83 & 1493.458 & 1488.996 & 1493.299 & 1490.998 \\ 1494.369 & 1494.897 & 1495.154 & 1495.884 & 1497.054 & 1496.808 & 1493.451 & 1488.991 & 1493295 & 1490996\end{array}$ $\begin{array}{lllllllllllll}1494.374 & 1494.909 & 1495.149 & 1495.877 & 1497.049 & 1496.799 & 1493.444 & 1488.991 & 1493.29 & 1490.993\end{array}$ $\begin{array}{lllllllllll}1494.371 & 1494.911 & 1495.154 & 1495.872 & 1497.049 & 1496.791 & 1493.439 & 1488.984 & 1493.285 & 1490.991\end{array}$ $\begin{array}{lllllllllll}1494.343 & 1494.88 & 1495.163 & 1495.884 & 1497.063 & 1496.825 & 1493.444 & 1488.989 & 1493.285 & 1490.991\end{array}$ $\begin{array}{lllllllllll}1494.336 & 1494.875 & 1495.166 & 1495.893 & 1497.07 & 1496.847 & 1493.446 & 1488.996 & 1493.292 & 1490.989\end{array}$ $\begin{array}{lllllllllll}1494.317 & 1494.863 & 1495.173 & 1495.905 & 1497.087 & 1496.873 & 1493.453 & 1489.003 & 1493.297 & 1490.991\end{array}$ $\begin{array}{llllllllll}1494.305 & 1494.856 & 1495.175 & 1495.925 & 1497.103 & 1496.906 & 1493.467 & 1489.012 & 1493.300 & 1490.991\end{array}$

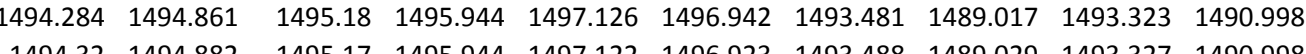
$\begin{array}{llllllllll}1494.32 & 1494.882 & 1495.17 & 1495.944 & 1497.122 & 1496.923 & 1493.488 & 1489.029 & 1493.327 & 1490.998\end{array}$ $\begin{array}{lllllllllll}1494.313 & 1494.88 & 1495.18 & 1495.972 & 1497.147 & 1496.957 & 1493.507 & 1489.036 & 1493.346 & 1491.007\end{array}$ $\begin{array}{lllllllllll}1494.348 & 1494.902 & 1495.17 & 1495.963 & 1497.14 & 1496.923 & 1493.5 & 1489.031 & 1493346 & 1491.007\end{array}$ $\begin{array}{llllllllllll}1494.374 & 1494.913 & 1495.166 & 1495.958 & 1497.131 & 1496.902 & 1493.5 & 1489.034 & 1493.341 & 1491.007\end{array}$ $\begin{array}{lllllllllll}1494.367 & 1494.897 & 1495.18 & 1495.958 & 1497.135 & 1496.897 & 1493.495 & 1489.031 & 1493.341 & 1491.012\end{array}$ $\begin{array}{llllllllll}1494.362 & 1494.882 & 1495.18 & 1495.97 & 1497.145 & 1496.921 & 1493.502 & 1489.034 & 1493.346 & 1491.012\end{array}$ $\begin{array}{llllllllll}1494.381 & 1494.897 & 1495.175 & 1495.965 & 1497.135 & 1496.902 & 1493.498 & 1489.038 & 1493.341 & 1491.014\end{array}$

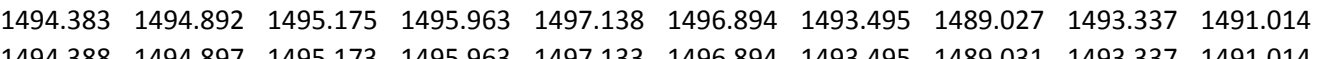
$\begin{array}{lllllllllll}1494.388 & 1494.897 & 1495.173 & 1495.963 & 1497.133 & 1496.894 & 1493.495 & 1489.031 & 1493.337 & 1491.014 \\ 1494.383 & 1494.887 & 1495.173 & 1495.963 & 1497.138 & 1496887 & 1493.495 & 1489.031 & 1493.337 & 1491.014\end{array}$ $\begin{array}{lllllllllll}1494.388 & 1494.89 & 1495.175 & 1495.965 & 1497.138 & 1496.887 & 1493.493 & 1489.031 & 1493.337 & 1491.014\end{array}$ $\begin{array}{llllllllllll}1494.418 & 1494.925 & 1495.166 & 1495.946 & 1497.119 & 1496.851 & 1493.483 & 1489.027 & 1493.332 & 1491.012\end{array}$ $\begin{array}{llllllllll}1494.439 & 1494.957 & 1495.159 & 1495.935 & 1497.098 & 1496.825 & 1493.477 & 1489.017 & 1493.318 & 1491.01\end{array}$ $\begin{array}{lllllllllll}1494.465 & 1494.961 & 1495.144 & 1495.907 & 1497.075 & 1496.77 & 1493.458 & 1489.005 & 1493.304 & 1491.003\end{array}$ $\begin{array}{lllllllllll}1494.479 & 1494.954 & 1495.142 & 1495.886 & 1497.049 & 1496.741 & 1493.441 & 1488.994 & 1493.287 & 1490.998\end{array}$ $\begin{array}{llllllllllll}1494.501 & 1494.949 & 1495.13 & 1495.86 & 1497.022 & 1496.70 & 1493.42 & 1488.977 & 1493.269 & 1490.989\end{array}$ $\begin{array}{llllllllllll}1494.496 & 1494.918 & 1455.13 & 1495.828 & 1496.998 & 1496.662 & 1493.397 & 1488.968 & 1493.245 & 1490.982 \\ 1494.484 & 1494.909 & 1495.125 & 1495812 & 1496.98 & 1496653 & 1493378 & 1488.956 & 1493227 & 149097\end{array}$ $\begin{array}{llllllllllll}14494.468 & 1494.897 & 1495.125 & 1495.791 & 1496.966 & 1496.638 & 1493.364 & 1488.954 & 1493.212 & 1490.963\end{array}$ $\begin{array}{llllllllllll}1494.451 & 1494.89 & 1495.123 & 1495.782 & 1496.949 & 1496.641 & 1493.35 & 1488.942 & 1493.198 & 1490.954\end{array}$ $\begin{array}{lllllllllll}1494.407 & 1494.878 & 1495.135 & 1495.779 & 1496.956 & 1496.66 & 1493.35 & 1488.94 & 1493.194 & 1490.947\end{array}$ $\begin{array}{lllllllllll}1494.381 & 1494.849 & 1495.14 & 1495.777 & 1496.954 & 1496.686 & 1493.346 & 1488.944 & 1493.189 & 1490.94\end{array}$ $\begin{array}{lllllllllll}1494.376 & 1494.873 & 1495.128 & 1495.768 & 1496.945 & 1496.681 & 1493.343 & 1488.937 & 1493.187 & 1490.933\end{array}$ $\begin{array}{llllllllll}1494.345 & 1494.842 & 1495.14 & 1495.768 & 1496.949 & 1496.693 & 1493.343 & 1488.935 & 1493.187 & 1490.928\end{array}$ $\begin{array}{lllllllllllll}1494.305 & 1494.818 & 1495.147 & 1495.789 & 1496.968 & 1496.756 & 1493.352 & 1488.944 & 1493.196 & 1490.926\end{array}$

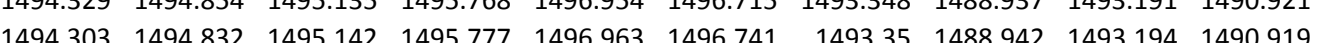
$\begin{array}{lllllllllll}1494.31 & 1494.856 & 1495.13 & 1495.772 & 1496.959 & 1496.729 & 1493.345 & 1488.94 & 1493.189 & 1490.917\end{array}$ $\begin{array}{lllllllllll}1494.298 & 1494.844 & 1495.135 & 1495.775 & 1496.963 & 1496.748 & 1493.345 & 1488.947 & 1493.189 & 1490.914\end{array}$ $\begin{array}{llllllllll}1494.284 & 1494.835 & 1495.137 & 1495.782 & 1496.973 & 1496.765 & 1493.348 & 1488.947 & 1493.191 & 1490.912\end{array}$ $\begin{array}{llllllllll}1494.287 & 1494.839 & 1495.13 & 1495.777 & 1496.97 & 1496.758 & 1493.346 & 1488.947 & 1493.189 & 1490.912\end{array}$ $\begin{array}{lllllllllll}1494.266 & 1494.813 & 1495.14 & 1495.789 & 1496.98 & 1496.784 & 1493.348 & 1488.954 & 1493.189 & 1490.912\end{array}$ $\begin{array}{lllllllllll}1494.242 & 1494.799 & 1495.147 & 1495.805 & 1496.998 & 1496.815 & 1493.357 & 1488.959 & 1493.201 & 1490.912\end{array}$ $\begin{array}{llllllllr}1486.097 & 1488.422 & 1493.751 & 1494.213 & 1493.508 & 1495.336 & 1493.49 & 1484.87 & 1479.19\end{array}$ $\begin{array}{rrrrrrrrr}1486.088 & 1488.424 & 1493.734 & 1494.183 & 1493.492 & 1495.301 & 1493.466 & 1484.865 & 1479.183 \\ 1486085 & 1488.415 & 1493.72 & 1494.169 & 1493.48 & 1495279 & 1493.45 & 1484867 & 1479.8\end{array}$ $\begin{array}{llllllllll}1486.079 & 1488.415 & 1493.72 & 1494.169 & 1493.48 & 1495.279 & 1493.45 & 1484.867 & 1479.18\end{array}$ $\begin{array}{llllllllll}1486.09 & 1488.375 & 1493.709 & 1494.176 & 1493.47 & 1495.296 & 1493.441 & 1484.872 & 1479.18\end{array}$ $\begin{array}{lllllllll}1486.076 & 1488.368 & 1493.711 & 1494.185 & 1493.468 & 1495.322 & 1493.441 & 1484.87 & 1479.183\end{array}$ $\begin{array}{llllllllll}1486.076 & 1488.368 & 1493.718 & 1494.197 & 1493.473 & 1495.322 & 1493.45 & 1484.865 & 1479.185\end{array}$ $\begin{array}{lllllllll}4886.072 & 1488.375 & 1493.713 & 1494.187 & 1493.475 & 1495.315 & 1493.452 & 1484.867 & 1479.187\end{array}$ $\begin{array}{llllllllll}1486.009 & 1488.377 & 1493.713 & 1494.19 & 1493.47 & 1495.31 & 1493.452 & 1484.867 & 1479.185\end{array}$ $\begin{array}{lllllllll}1486.009 & 1488.368 & 1493.709 & 1494.185 & 1493.461 & 1495.301 & 1493.443 & 1484.87 & 1479.18\end{array}$ $\begin{array}{lllllllll}1486.002 & 1488.358 & 1493.709 & 1494.199 & 1493.466 & 1495.322 & 1493.445 & 1484.867 & 1479.188 \\ 1486 & 1488377 & 1493704 & 1494.176 & 1493.458 & 1495303 & 1933.438 & 1484867 & 1479.18\end{array}$ $\begin{array}{lllllllllll}1485.993 & 1488.38 & 1493.694 & 1494.171 & 1493.456 & 1495296 & 1493.431 & 1484.863 & 1479.18\end{array}$ $\begin{array}{llllllllll}1485.988 & 1488368 & 1493.688 & 1494.166 & 1493.444 & 1495.293 & 1493.417 & 1484.867 & 1479.176\end{array}$ $\begin{array}{llllllllll}1485.988 & 1488.347 & 1493.692 & 1494.187 & 1493.449 & 1495.319 & 1493.419 & 1484.867 & 1479.178\end{array}$ $\begin{array}{llllllllll}1485.986 & 1488.356 & 1493.694 & 1494.197 & 1493.454 & 1495.333 & 1493.429 & 1484.867 & 1479.178\end{array}$ $\begin{array}{llllllllll}4485.984 & 1488.342 & 1493.701 & 1494.213 & 1493.458 & 1495.352 & 1493.434 & 1484.867 & 1479.18\end{array}$ $\begin{array}{lllllllll}1485.981 & 1488.349 & 1493.711 & 1494.239 & 1493.475 & 1495.383 & 1493.448 & 1484.865 & 1479.187\end{array}$ $\begin{array}{lllllllllll}4485.984 & 1488.335 & 1493.727 & 1494.27 & 1493.489 & 1495.423 & 1493.462 & 1484.867 & 1479.197\end{array}$ $\begin{array}{llllllllll}1485.984 & 1488.361 & 1493.732 & 1494.257 & 1493.494 & 1495.399 & 1493.47 & 1484.865 & 1479.197\end{array}$ $\begin{array}{lllllllllll}1485.986 & 1488.361 & 1493.751 & 1494.288 & 1493.511 & 1495.434 & 1493.492 & 1484.867 & 1479.208\end{array}$ $\begin{array}{llllllllll}1485.963 & 1488.38 & 1493.751 & 1494.267 & 1493.508 & 1495.416 & 1493.483 & 1484.865 & 1479.206\end{array}$ $\begin{array}{llllllllll}1485.951 & 1488.396 & 1493.749 & 1494.255 & 1493.503 & 1495.399 & 1493.485 & 1484.863 & 1479.206\end{array}$ $\begin{array}{llllllllll}1485.949 & 1488.377 & 1493.746 & 1494.264 & 1493.503 & 1495.406 & 1493.473 & 1484.87 & 1479.206\end{array}$ $\begin{array}{llllllllll}1485.949 & 1488.382 & 1493.746 & 1494.269 & 1493.508 & 1495.413 & 1493.483 & 1484.865 & 1479.206\end{array}$ $\begin{array}{llllllllll}1485.944 & 1488.396 & 1493.744 & 1494.26 & 1493.503 & 1495.399 & 1493.478 & 1484.863 & 1479.206\end{array}$ $\begin{array}{lllllllllll}1485.944 & 1488.394 & 1493.744 & 1494.257 & 1493.503 & 1455.392 & 1493.473 & 1484.865 & 1479.204\end{array}$ $\begin{array}{lllllllllll}14855.947 & 1488.398 & 1493.744 & 1494.25 & 1493.499 & 1495.39 & 1493.476 & 1484.863 & 1479.204\end{array}$ $\begin{array}{llllllllll}1485.944 & 1488.394 & 1493.741 & 1494.248 & 1493.501 & 1495.387 & 1493.476 & 1484.863 & 1479.206\end{array}$ $\begin{array}{llllllllll}1485.94 & 1488.412 & 1493.732 & 1494.222 & 1493.492 & 1495.357 & 1493.471 & 1484.863 & 1479.206\end{array}$ $\begin{array}{llllllllll}1485.935 & 1488.424 & 1493.727 & 1494.204 & 1493.482 & 1495.324 & 1493.457 & 1484.86 & 1479.206\end{array}$ $\begin{array}{llllllllll}1485.933 & 1488.246 & 1493.709 & 1494.169 & 1493.466 & 1495.282 & 1493.441 & 1484.86 & 1479.20\end{array}$ $\begin{array}{lllllllll}1485.926 & 1488.441 & 1493.692 & 1494.134 & 1493.447 & 1495.246 & 1493.427 & 1484.858 & 1479.197\end{array}$ $\begin{array}{lllllllll}1485.917 & 4488.452 & 1493.671 & 1494.097 & 1493.43 & 1495.199 & 1493.401 & 1484.856 & 1479.19\end{array}$ $\begin{array}{lllllllll}1485.903 & 1488.438 & 1493.652 & 1494.073 & 1493.404 & 1455.171 & 1493.382 & 1484.856 & 1479.18\end{array}$ $\begin{array}{lllllllllll}1485.887 & 1488.415 & 1493.612 & 1494.033 & 1493.366 & 1495.129 & 1493.347 & 1484.856 & 1479.169\end{array}$ $\begin{array}{llllllllllll}1485.88 & 1488.408 & 1493.603 & 1494.027 & 1493.359 & 1495.12 & 1493.333 & 1484.86 & 1479.166\end{array}$ $\begin{array}{lllllllll}1485.873 & 1488.38 & 1493.603 & 1494.038 & 1493.354 & 1495.136 & 1493.33 & 1484.858 & 1479.166\end{array}$ $\begin{array}{lllllllll}1485.866 & 1488.37 & 1493.596 & 1494.038 & 1493.352 & 1495.143 & 1493.328 & 1484.856 & 1479.169\end{array}$ $\begin{array}{llllllllll}1485.859 & 1488.372 & 1493.596 & 1494.031 & 1493.347 & 1495.131 & 1493.323 & 1484.856 & 1479.169\end{array}$ $\begin{array}{llllllllll}1485.859 & 1488.344 & 1493.594 & 1494.045 & 1493.349 & 1495.15 & 1493.326 & 1484.86 & 1499.173\end{array}$ $\begin{array}{lllllllllll}1485.864 & 1488.337 & 1493.603 & 1494.069 & 1493.359 & 1495.185 & 1493.335 & 1484.86 & 1479.18\end{array}$

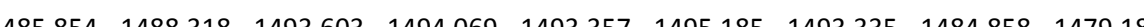
$\begin{array}{llllllllll}1485.857 & 1488.326 & 1493.596 & 1444.055 & 1493.352 & 1495.174 & 1493.328 & 1484.858 & 1479.183\end{array}$ $\begin{array}{lllllllll}1485.85 & 1488.316 & 1493.598 & 1494.066 & 1493.352 & 1495.183 & 1493.326 & 1484.856 & 1479.183\end{array}$ $\begin{array}{lllllllll}1485.833 & 1488.302 & 1493.596 & 1494.078 & 1493.352 & 1495.197 & 1493.33 & 1484.853 & 1479.18\end{array}$ $\begin{array}{llllllllll}1485.829 & 1488.302 & 1493.593 & 1494.071 & 1493.352 & 1495.195 & 1493.328 & 1484.853 & 1479.18\end{array}$ $\begin{array}{llllllllll}1485.827 & 1488.286 & 1493.596 & 1494.085 & 1493.354 & 1495.214 & 1493.333 & 1484.856 & 1479.183\end{array}$ $\begin{array}{rrrrrrrrr}1485.827 & 1488.274 & 1493.603 & 1494.113 & 1493.361 & 1495.239 & 1493.34 & 1484.858 & 1479.187 \\ 1485831 & 1488.25 & 1493619 & 1494.148 & 1493.378 & 1495289 & 1493.354 & 1484.858 & 1479.192\end{array}$ 
Well

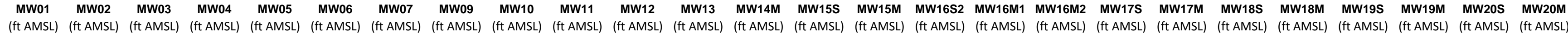

$1 / 21 / 1214: 00$ $1 / 21 / 1215: 00$ $1 / 21 / 1216: 00$ 1/21/121218:00 1/21/12 18:00 1/21/12 20:00 1/21/12 21:00 1/21/12 22:00 $1 / 21 / 1223: 00$
$1 / 22 / 120: 00$
$1 / 22 / 121: 00$ 1/22/12 1:00 $1 / 22 / 122: 00$ 1/22/123:00 $1 / 22 / 125: 00$ 1/22/12 6:00 $1 / 22 / 127: 00$ 1/22/12 8:00 $1 / 22 / 1210: 00$
$1 / 22 / 1211: 00$ 1/22/12 11:00 1/22/12 13:00 1/22/12 14:00 1/22/12 15:00 1/22/12 16:00 1/22/12 17:00 1/22/12 18:00 1/22/12 19:00 1/22/12 21:00 1/22/12 22:00 1/22/12 23:00 1/23/120:00 1/23/12 1:00 1/23/12 2:00 1/23/12 3:00 1/23/124:00 1/23/12 6:00 1/23/12 7:00 1/23/12 8:00 1/23/12 9:00 1/23/12 10:00 $1 / 23 / 1212.00$ 1/23/12 12:00 1/23/12 14:00 $1 / 23 / 1215: 00$ 1/23/12 16:00 1/23/12 18:00 $\begin{array}{lrrr}1494.882 & 1495.254 & 1496.735 & 1495.24\end{array}$ $\begin{array}{llll}1494.887 & 1495.28 & 1496.77 & 1495.28\end{array}$ $\begin{array}{rrrr}1494.894 & 1495.303 & 1496.794 & 1495.313 \\ 1494.903 & 1495.329 & 1496.82 & 1495341\end{array}$ $\begin{array}{lllll}1494.915 & 1495.352 & 1496.855 & 1495.371\end{array}$ $\begin{array}{lllll}1494.919 & 1495.376 & 1496.876 & 1495.371 \\ 1499 & 1496\end{array}$ $\begin{array}{llllll}1494.922 & 1495.383 & 1496.871 & 1495.408\end{array}$ $\begin{array}{lllll}1494.931 & 1495.397 & 1496.885 & 1495.418\end{array}$ $\begin{array}{lllll}1494.929 & 1495.401 & 1496.878 & 1495.422\end{array}$ $\begin{array}{lllll}1494.94 & 1495.415 & 1496.899 & 1495.436\end{array}$ $\begin{array}{llll}1494.95 & 1495.429 & 1496.918 & 1495.455 \\ 1499.961 & 1459.455 & 1496953 & 195 \\ 1485\end{array}$ $\begin{array}{rrrr}1494.961 & 1495.455 & 1496.953 & 1495.478 \\ 1494.966 & 1495.469 & 1496.96 & 1495.49\end{array}$ $\begin{array}{lllll}1494.966 & 1495.476 & 1496.964 & 1495.499\end{array}$ $\begin{array}{lllll}1444.973 & 1495.49 & 1496.976 & 1495.513\end{array}$ $\begin{array}{lllll}1494.985 & 1495.506 & 1497.002 & 1495.532\end{array}$ $\begin{array}{rrrr}1494.982 & 1495.511 & 1497 & 1495.541\end{array}$ $\begin{array}{llll}1494.98 & 1495.513 & 1496.993 & 1495.539\end{array}$ $\begin{array}{rrrr}1494.992 & 1455.523 & 1497.004 & 1495.546\end{array}$ $\begin{array}{rrrr}1494.987 & 1495.518 & 1496.99 & 1495.541 \\ 1494.987 & 1495.511 & 1496.974 & 1495.53\end{array}$ $\begin{array}{llll}1444.992 & 1495.509 & 1496.964 & 1495.518\end{array}$ $\begin{array}{llllll}1495.001 & 1495.516 & 1496.981 & 1495.525\end{array}$ $\begin{array}{lllll}1495.006 & 1495.527 & 1497.004 & 1495.539\end{array}$ $1494.999 \quad 1495.518 \quad 1496.978 \quad 1495.525$ $\begin{array}{lllll}1494.975 & 1495.506 & 1496.957 & 1495.513\end{array}$ $\begin{array}{lllll}1494.971 & 1495.474 & 1496.887 & 1495.471\end{array}$ $\begin{array}{rrrrr}1494.961 & 1495.434 & 1496.824 & 1495.418 \\ 1494.954 & 1495.404 & 149678 & 1955371\end{array}$ $\begin{array}{rrrrr}1494.954 & 1495.404 & 1496.78 & 1495.371 \\ 1494.945 & 1495364 & 1496.721 & 149531\end{array}$ $\begin{array}{llll}1494.933 & 1495.324 & 1496.677 & 1495.257\end{array}$ $\begin{array}{lllll}1494.929 & 1495.303 & 1496.658 & 1455.219\end{array}$ $\begin{array}{lllll}1494.924 & 1495.28 & 1496.642 & 1495.191\end{array}$ $\begin{array}{lllll}1494.95 & 1495.308 & 1496.707 & 1495.208\end{array}$ $\begin{array}{lllll}1494.917 & 1495.261 & 1496.633 & 1495.166\end{array}$ $\begin{array}{lllll}1494.91 & 1495.245 & 1496.616 & 1495.144\end{array}$ $\begin{array}{lllll}1494.901 & 1495.222 & 1496.591 & 1495.121 \\ 1494891 & 1495.196 & 1496562 & 1495.095\end{array}$ $\begin{array}{lllll}1494.891 & 1455.196 & 1496.562 & 1495.095 \\ 1494.889 & 1495189 & 1496562 & 1495084\end{array}$ $\begin{array}{lllll}1494.887 & 1495.177 & 1496.555 & 1495.072\end{array}$ $1494.882 \quad 1495.17 \quad 1496.553 \quad 1495.065$ $1494.8771495 .161 \quad 1496.548 \quad 1495.06$ $1494.87 \quad 1495.154 \quad 1496.537 \quad 1495.053$ $1494.866 \quad 1495.14 \quad 1496.525 \quad 1495.042$ $194.8561495 .124 \quad 1496.504 \quad 1495.028$ $1494.8611495 .122 \quad 1496.5091495 .021$ $1494.868 \quad 1495.142 \quad 1496.56 \quad 1495.049$ $\begin{array}{llll}1494.866 & 1495.145 & 1496.567 & 1495.06\end{array}$ $1494.863 \quad 1495.149 \quad 1496.579 \quad 1495.075$ $1494.863 \quad 1495.156 \quad 1496.59 \quad 1495.084$ $1494.8591495 .159 \quad 1496.588 \quad 1495.091$ $\begin{array}{llll}1494.856 & 1495.154 & 1496.576 & 1495.093 \\ 1494.849 & 1495.145 & 1496.555 & 1495.079\end{array}$ $\begin{array}{llllllllll}1494.188 & 1494.796 & 1495.161 & 1495.858 & 1497.056 & 1496.916 & 1493.39 & 1488.987 & 1493.229 & 1490.921\end{array}$ $\begin{array}{llllllllll}1494.188 & 1494.806 & 1495.166 & 1495.881 & 1497.08 & 1496.949 & 1493.406 & 1488.994 & 1493.245 & 1490.928 \\ 1494.193 & 1494.818 & 1495.168 & 1495.902 & 1497.103 & 1496969 & 193.423 & 1489005 & 1493262 & 1490935\end{array}$ $\begin{array}{lllllllllllll}144.193 & 1494.818 & 1495.168 & 1495.902 & 1497.103 & 1496.969 & 1493.423 & 4889.005 & 1493.262 & 1490.935 \\ 14942 & 1494811 & 1495.173 & 1495925 & 1497.126 & 1496992 & 1493.413 & 1489015 & 149325 & 1490942\end{array}$ $\begin{array}{lllllllllllll}1494.207 & 1494.835 & 1495.18 & 1495.951 & 1497.149 & 1497.019 & 1493.381 & 1489.027 & 1493215 & 1490.952\end{array}$ $\begin{array}{llllllllll}1494.221 & 1494.837 & 1495.18 & 1495.977 & 1497.17 & 1497.038 & 1493.42 & 1489.034 & 1493255 & 1490.952\end{array}$ $\begin{array}{lllllllllll}1494.249 & 1494.846 & 1495.178 & 1495.986 & 1497.18 & 1497.031 & 1493.437 & 1489.038 & 1493.273 & 1490.968\end{array}$ $\begin{array}{lllllllllll}1494.268 & 1494.849 & 1495.182 & 1496 & 1497.194 & 1497.031 & 1493.451 & 1489.043 & 1493.288 & 1490.975\end{array}$ $\begin{array}{llllllllll}1494.291 & 1494.868 & 1495.18 & 1496.007 & 1497.198 & 1497.019 & 1493.458 & 1489.05 & 1493.295 & 1490.98\end{array}$ $\begin{array}{llllllllll}1494.296 & 1494.868 & 1495.187 & 1496.025 & 1497.215 & 1497.038 & 1493.47 & 1489.055 & 1493.309 & 1490.986 \\ 1494301 & 1494.856 & 1995.192 & 1496046 & 197.233 & 1497.05 & 1493.484 & 1489064 & 149338 & 149096\end{array}$ $\begin{array}{llllllllll}1494.301 & 1494.856 & 1495.192 & 1496.046 & 1497.233 & 1497.05 & 1493.484 & 1489.064 & 1493.318 & 1490.996 \\ 1494.298 & 1494.913 & 1495.201 & 1496.074 & 1497.254 & 1497.081 & 1493.502 & 1489.069 & 1493.332 & 1491.003\end{array}$ $\begin{array}{lllllllllll}1494.313 & 1444.916 & 1495.206 & 1496.074 & 1497.254 & 1497.081 & 1493.502 & 1489.069 & 1493.332 & 1491.003 \\ 1497.268 & 1497.081 & 1493.514 & 1489.073 & 1493.351 & 1491.012\end{array}$ $\begin{array}{llllllllllll}1494.334 & 1494.911 & 1495.199 & 1496.1 & 1497.275 & 1497.076 & 1493.526 & 1489.078 & 1493.363 & 1491.019\end{array}$ $\begin{array}{llllllllll}1494.341 & 1494.925 & 1495.211 & 1496.116 & 1497.291 & 1497.086 & 1493.54 & 1489.083 & 1493.377 & 1491.024\end{array}$ $\begin{array}{lllllllllll}1494.343 & 1494.937 & 1495.218 & 1496.139 & 1497.315 & 1497.105 & 1493.556 & 1489.09 & 1493.391 & 1491.033\end{array}$ $\begin{array}{lllllllllll}1494.364 & 1494.937 & 1495.213 & 1496.146 & 1497.317 & 1497.098 & 1493.565 & 1489.095 & 1493.402 & 1491.042\end{array}$ $\begin{array}{llllllllll}1494.392 & 1494.937 & 1495.209 & 1496.153 & 1497.317 & 1497.091 & 1493.57 & 1489.099 & 1493.409 & 1491.047\end{array}$ $\begin{array}{llllllllllll}1494.397 & 1494.949 & 1455.216 & 1496.165 & 1497.331 & 1497.093 & 1493.48 & 1489.1491 .054\end{array}$ $\begin{array}{llllllllll}1494.425 & 1494.952 & 1495.213 & 1496.167 & 1497.328 & 1497.079 & 1493.579 & 1489.099 & 1493.419 & 1491.056 \\ 1494.447 & 1494.971 & 1495211 & 1496165 & 1497.322 & 1497.055 & 1493.577 & 1489.104 & 1493.416 & 1491.063\end{array}$ $\begin{array}{llllllllllll}1494.458 & 1494.969 & 1495.218 & 1496.16 & 1497.324 & 1497.036 & 1993.577 & 1489.109 & 1493.412 & 1491.063\end{array}$ $\begin{array}{llllllllll}1494.454 & 1494.973 & 1495.223 & 1496.172 & 1497.333 & 1497.052 & 1493.582 & 1489.106 & 1493.421 & 1491.068\end{array}$ $\begin{array}{lllllllllll}1494.444 & 1494.966 & 1495.23 & 1496.185 & 1497.34 & 1497.069 & 1493.589 & 1489.106 & 1493.428 & 1491.075\end{array}$ $\begin{array}{llllllllll}1494.472 & 1494.983 & 1495.216 & 1496.181 & 1497.335 & 1497.043 & 1493.587 & 1489.116 & 1493.428 & 1491.075\end{array}$ $\begin{array}{lllllllllll}1494.51 & 1495.036 & 1495.199 & 1496.181 & 1497.322 & 1497.043 & 1493.586 & 1489.109 & 1493.428 & 1491.075\end{array}$ $\begin{array}{llllllllll}1494.555 & 1495.059 & 1495.194 & 1496.151 & 1497.294 & 1496.954 & 1493.575 & 1489.099 & 1493.416 & 1491.073 \\ 1494592 & 149505 & 1495.187 & 149612 & 1497.261 & 149689 & 1493.558 & 1489083 & 1934 & 1491068\end{array}$ $\begin{array}{rrrrrrrrrr}1494.592 & 1495.05 & 1495.187 & 1496.12 & 1497.261 & 1496.89 & 1493.558 & 1489.083 & 1493.4 & 1491.068 \\ 1494.609 & 1495.045 & 1495.187 & 1496095 & 1497.231 & 1496847 & 1493147 & 1489071 & 1492.957 & 1491.063\end{array}$ $\begin{array}{llllllllll}1494.609 & 1495.045 & 1495.187 & 1496.095 & 1497.231 & 1496.847 & 1493.147 & 1489.071 & 1492.957 & 1491.063 \\ 1494.635 & 1495.043 & 1495.175 & 1496.055 & 1497.196 & 1496789 & 1493.292 & 1489.052 & 1493.126 & 1491.056\end{array}$ $\begin{array}{llllllllllll}1494.642 & 1495.028 & 1495.168 & 1496.023 & 1497.161 & 1496.744 & 1493.336 & 1489.036 & 1493.17 & 1491.045\end{array}$ $\begin{array}{lllllllllll}1494.623 & 1495.026 & 1495.173 & 1496.002 & 1497.14 & 1496.734 & 1493.348 & 1489.027 & 1493.187 & 1491.038\end{array}$ $\begin{array}{lllllllllll}1494.611 & 1494.997 & 1495.17 & 1495.979 & 1497.124 & 1496.72 & 1493.348 & 1489.012 & 1493.191 & 1491.028\end{array}$ $\begin{array}{llllllllllll}1494.522 & 1494.93 & 1495.204 & 1495.988 & 1497.145 & 1496.777 & 1493.362 & 1489.022 & 1493.205 & 1491.024\end{array}$ $\begin{array}{lllllllllll}1494.571 & 1494.98 & 1495.168 & 1495.953 & 1497.098 & 1496.72 & 1493.353 & 1489.003 & 1493.196 & 1491.012\end{array}$

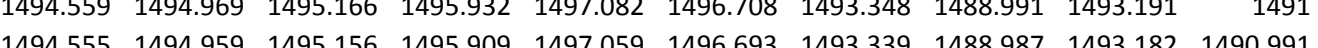

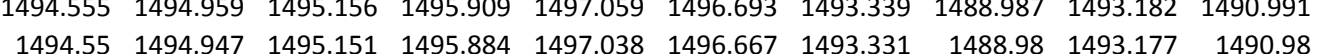
$\begin{array}{rrrrrrrrrr}1494.55 & 1494.947 & 1495.151 & 1495.884 & 1497.038 & 1496.667 & 1493.331 & 1488.98 & 1493.177 & 1490.98 \\ 1494.526 & 1494.937 & 1495.156 & 1495.87 & 1497.026 & 1496.667 & 1493.329 & 1488.975 & 1493.175 & 1490.968\end{array}$ $\begin{array}{lllllllllll}1494.505 & 1494.928 & 1495.151 & 1495.856 & 1497.015 & 1496.667 & 1493.324 & 1488.968 & 1493.168 & 1490.961\end{array}$ $\begin{array}{llllllllll}1494.486 & 1494.923 & 1495.151 & 1495.842 & 1497.005 & 1496.672 & 1493.322 & 1488.959 & 1493.166 & 1490.952\end{array}$ $\begin{array}{llllllllllll}1494.47 & 1494.918 & 1495.144 & 1495.833 & 1496.996 & 1496.677 & 1493.32 & 1488.961 & 1493.163 & 1490.942\end{array}$ $\begin{array}{lllllllllll}1494.463 & 1494.909 & 1495.144 & 1495.819 & 1496.984 & 1496.669 & 1493.313 & 1488.961 & 1493.156 & 1490.935\end{array}$ $\begin{array}{lllllllllll}1494.454 & 1494.904 & 1495.137 & 1495.805 & 1496.973 & 1496.662 & 1493.303 & 1488.954 & 1493.149 & 1490.926\end{array}$ $\begin{array}{lllllllllll}1494.449 & 1494.897 & 1495.132 & 1495.784 & 1496.956 & 1496.648 & 1493.289 & 1488.952 & 1493.135 & 1490.917\end{array}$

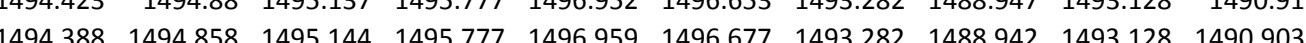
$\begin{array}{llllllllllll}1494.355 & 1494.835 & 1495.147 & 1495.786 & 1496.968 & 1496.713 & 1493.285 & 1488.949 & 1493.13 & 1490.898\end{array}$ $\begin{array}{lllllllllll}1494.341 & 1494.825 & 1495.144 & 1495.786 & 1496.97 & 1496.727 & 1493.289 & 1488.949 & 1493.13 & 1490.893\end{array}$ $\begin{array}{llllllllll}1494.331 & 1494.825 & 1495.137 & 1495.789 & 1496.975 & 1496.741 & 1493.292 & 1488.951 & 1493.135 & 1490.889\end{array}$ $\begin{array}{lllllllllll}1494.32 & 1494.825 & 1495.142 & 1495.791 & 1496.98 & 1496.753 & 1493.296 & 1488.954 & 1493.137 & 1490.889\end{array}$ $\begin{array}{llllllllllll}1494.324 & 1494.827 & 1495.137 & 1495.791 & 1496.977 & 1496.758 & 1493.299 & 1488.959 & 1493.142 & 1490.886\end{array}$ $\begin{array}{lllllllllll}1494.334 & 1494.842 & 1495.135 & 1495.784 & 1466.975 & 1496.748 & 1493.299 & 1488.959 & 1493.142 & 1490.882\end{array}$ $\begin{array}{llllllllll}1485.833 & 1488.241 & 1493.636 & 1494.183 & 1493.397 & 1495.34 & 1493.375 & 1484.858 & 1479.201\end{array}$ $\begin{array}{rrrrrrrrr}1485.831 & 1488.25 & 1493.652 & 1494.204 & 1493.413 & 1495.364 & 1493.391 & 1484.853 & 1479.204 \\ 1485776 & 1488.257 & 1493.666 & 14992.232 & 1493.43 & 1495392 & 1433.405 & 1488.858 & 1479.211\end{array}$ $\begin{array}{lllllllll}14885.773 & 1488.257 & 1493.666 & 1494.232 & 1493.43 & 1495.392 & 1433.405 & 1484.858 & 1479.211\end{array}$ $\begin{array}{llllllllll}148578 & 1488265 & 1493.619 & 1494.243 & 1493.39 & 1495.451 & 1493.366 & 1484.856 & 1479.22\end{array}$ $\begin{array}{llllllllll}1485.776 & 1488.281 & 1493.662 & 1494.269 & 1493.425 & 1495.463 & 1493.403 & 1484.853 & 1479.222\end{array}$ $\begin{array}{lllllllllll}1485.773 & 1488.302 & 1493.678 & 1494.278 & 1493.444 & 1495.47 & 1493.424 & 1484.855 & 1479.222\end{array}$ $\begin{array}{lllllllllll}1485.739 & 1488.302 & 1493.692 & 4494.292 & 1493.456 & 1495.481 & 1493.436 & 1484.856 & 1479.222\end{array}$ $\begin{array}{llllllllll}1485.743 & 1488.316 & 1493.701 & 1494.283 & 1493.466 & 1495.477 & 1493.441 & 1484.851 & 1479.222\end{array}$

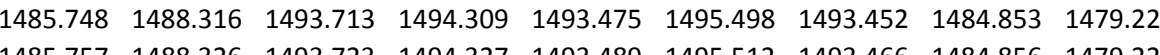
$\begin{array}{llllllllll}1485.757 & 1488.326 & 1493.723 & 1494.327 & 1493.489 & 1495.512 & 1493.466 & 1484.856 & 1479.225 \\ 1485762 & 1488326 & 1493739 & 1494348 & 1493508 & 1495543 & 1493.488 & 1484856 & 1479.232\end{array}$ $\begin{array}{llllllllll} & 1485.762 & 488.326 & 493.373 & 444.348 & 443.508 & 145.543 & 1439.488 & 1484.8568 & 149.232\end{array}$ $\begin{array}{llllllllll}1485.771 & 1488.347 & 1493.767 & 1494.367 & 1493.534 & 1495.554 & 1493.509 & 1484.853 & 1479.239\end{array}$ $\begin{array}{lllllllllll}1485.773 & 1488.342 & 1493.779 & 1494.381 & 1493.544 & 1495.564 & 1493.523 & 1484.856 & 1479.243\end{array}$ $\begin{array}{llllllllll}1485.783 & 1488.34 & 1493.796 & 1494.404 & 1493.563 & 1495.592 & 1493.539 & 1484.858 & 1479.253\end{array}$ $\begin{array}{llllllllll}1485.787 & 1488.358 & 1493.805 & 1494.404 & 1493.57 & 1495.59 & 1493.546 & 1484.853 & 1479.25\end{array}$ $\begin{array}{lllllllll}1485.79 & 1488.375 & 1493.817 & 1494.402 & 1493.579 & 1495.578 & 1493.549 & 1484.851 & 1479.26\end{array}$

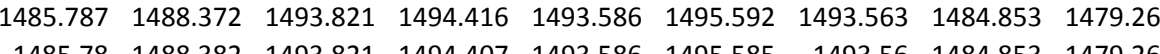
$\begin{array}{llllllllll}1485.78 & 1488.382 & 1493.821 & 1494.007 & 1493.586 & 1495.585 & 1493.56 & 1484.853 & 1499.262\end{array}$ $\begin{array}{llllllllll}1485.78 & 1488.398 & 1493.819 & 1494.39 & 1493.58 & 1482 & 14955.561 & 1493.558 & 1484.851 & 1479.257\end{array}$ $\begin{array}{lllllllll}1485.778 & 1488394 & 1493.826 & 1494.397 & 1493.586 & 1495.568 & 1493.56 & 1484.851 & 1499.257\end{array}$ $\begin{array}{lllllllllll}1485.783 & 1488.389 & 1493.833 & 1494.411 & 1493.596 & 1495.582 & 1493.567 & 1484.851 & 1479.26\end{array}$ $\begin{array}{lllllllllll}1485.776 & 1488.41 & 1493.833 & 1494.395 & 1493.593 & 1495.564 & 1493.565 & 1484.848 & 1479.26\end{array}$ $\begin{array}{llllllllll}1485.746 & 1488.434 & 1493.833 & 1494.374 & 1493.591 & 1495.533 & 1493.565 & 1484.837 & 1479.262\end{array}$ $\begin{array}{lllllllllll}1485.683 & 1488.459 & 1493.824 & 1494.339 & 1493.582 & 1495.484 & 1493.553 & 1484.846 & 1479.26\end{array}$ $\begin{array}{llllllllll}1485.688 & 1488.478 & 4938.805 & 1494.295 & 1493.565 & 1455.427 & 1493.537 & 1484.846 & 1479.257\end{array}$

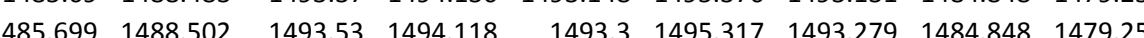
$\begin{array}{llllllllll}1485.699 & 1488.504 & 1493.577 & 1494.104 & 1493.34 & 1495.27 & 1493.316 & 1484.851 & 1479.25\end{array}$ $\begin{array}{llllllllll}1485.697 & 1488.502 & 1493.593 & 1494.097 & 1493.354 & 1495.242 & 1493.33 & 1484.846 & 1479.243\end{array}$ $\begin{array}{lllllllll}1485.688 & 1488.492 & 1493.596 & 1494.08 & 1493.357 & 1495.221 & 1493.33 & 1484.846 & 1479.236\end{array}$ $\begin{array}{llllllllll}1485.685 & 1488.431 & 1493.61 & 1494.129 & 1493.373 & 1495.289 & 1493.347 & 1484.856 & 1479.239\end{array}$ $\begin{array}{llllllllll}1485.681 & 1488.48 & 1493.598 & 1494.076 & 1493.359 & 1495.211 & 1493.333 & 1484.846 & 1479.229\end{array}$ $\begin{array}{llllllllll}4485.674 & 1488.473 & 1493.596 & 1494.064 & 1493.352 & 1495.188 & 1493.328 & 1484.846 & 1479.225\end{array}$ $\begin{array}{llllllllll}1485.674 & 1488.48 & 4953.591 & 1494.048 & 1493.347 & 1455.167 & 1493.323 & 1484.844 & 1479.225\end{array}$ $\begin{array}{llllllllll}1485.672 & 1488.455 & 1493.579 & 1494.024 & 1493.333 & 1495.141 & 1493.309 & 1484.844 & 1479.222\end{array}$ $\begin{array}{llllllllll}1485.676 & 1488.443 & 1493.575 & 1494.017 & 1493.328 & 1495.129 & 1493.307 & 1484.846 & 1479.225\end{array}$ $\begin{array}{llllllllll}1485.662 & 1488.434 & 1493.57 & 1494.017 & 1493.328 & 1495.129 & 1493.302 & 1484.846 & 1479.227\end{array}$ $\begin{array}{lllllllll}1485.655 & 1488.424 & 1493.568 & 1494.008 & 1493.323 & 1495.124 & 1493.297 & 1484.844 & 1479.227\end{array}$ $\begin{array}{lllllllll}1485.658 & 1488.419 & 1493.563 & 1494.001 & 1493.316 & 1495.115 & 1493.293 & 1484.841 & 1479.225\end{array}$ $\begin{array}{llllllllll}1485.653 & 1488.412 & 1493.556 & 1493.999 & 1493.309 & 1495.101 & 1493.283 & 1484.844 & 1479.222\end{array}$ $\begin{array}{llllllllll}1485.651 & 1488.405 & 1493.539 & 1493.975 & 1493.295 & 1495.08 & 1493.274 & 1484.841 & 1479.2218\end{array}$ $\begin{array}{llllllllll}1485.646 & 1488358 & 14935535 & 1493989 & 149329 & 1495.108 & 1493.265 & 148484 & 1479.21\end{array}$ $\begin{array}{lllllllllll}1485.648 & 1488.34 & 1493.537 & 1494.006 & 1493.293 & 1495.124 & 1493.267 & 1484.841 & 1479.213\end{array}$ $\begin{array}{llllllllll}1485.648 & 1488.333 & 1493.54 & 1494.01 & 1493.295 & 1495.134 & 1493.272 & 1484.841 & 1479.215\end{array}$ $\begin{array}{llllllllll}1485.651 & 1488.326 & 1493.544 & 1494.022 & 1493.3 & 1495.145 & 1493.274 & 1484.841 & 1479.218\end{array}$ $\begin{array}{llllllllll}1485.651 & 1488.318 & 1493.547 & 1494.027 & 1493.302 & 1495.16 & 1493.279 & 1484.841 & 1479.22\end{array}$ $\begin{array}{lllllllllll}1485.644 & 1488.316 & 1493.549 & 1494.029 & 1493.304 & 1495.16 & 1493.281 & 1484.839 & 1479.22\end{array}$ $\begin{array}{llllrrrrrr}1485.612 & 1488.316 & 1493.549 & 1494.029 & 1493.304 & 1495.155 & 1493.279 & 1484.839 & 1479.222 \\ 1485.607 & 1488321 & 1493.544 & 1494013 & 1493.3 & 1495.136 & 1493.274 & 1484.841 & 1479.222\end{array}$ 
Well

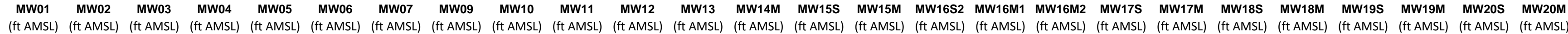

$1 / 23 / 1220: 00$ $1 / 23 / 1221: 00$ 1/23/12 22:00 1/23/12 23:00 1/24/120:00 $1 / 24 / 121: 00$ $1 / 24 / 123: 00$ $1 / 24 / 124: 00$ $1 / 24 / 125: 00$ $1 / 24 / 126: 00$ $1 / 24 / 127: 00$ $1 / 24 / 129: 00$ 1/24/12 9:00 1/24/12 11:00 $1 / 24 / 1212: 00$ 1/24/12 13:00 1/24/12 14:00 $1 / 24 / 12156: 00$
$1 / 24 / 1217: 00$ $1 / 24 / 1217: 00$
$1 / 24 / 1218: 00$ $1 / 24 / 1219: 00$ $1 / 241 / 121900$
$1 / 24 / 1220: 00$ 1/24/12 21:00 1/24/12 22:00 $1 / 24 / 1223: 00$ $1 / 25 / 120: 00$ 1/25/121:00 $1 / 25 / 123: 00$ $1 / 25 / 124: 00$ 1/25/12 5:00 $1 / 25 / 126: 00$ 1/25/12 7:00 1/25/12 9:00 $1 / 25 / 1210: 00$ 1/25/12 12:00 1/25/12 13:00 1/25/12 14:00 $1 / 25 / 1215: 00$ 1/25/12 16:00 $1 / 25 / 1217.00$ 1/25/12 18:00 $1 / 25 / 1220: 00$ $1 / 25 / 1221: 00$ 1/25/12 22:00 $1 / 25 / 1223: 00$ 1/26/12 0:00 $\begin{array}{rrrr}1494.849 & 1495.14 & 1496.546 & 1495.072\end{array}$ $\begin{array}{lllll}1494.849 & 1495.142 & 1496.558 & 1495.074\end{array}$ $\begin{array}{rrrr}1494.845 & 1495.138 & 1496.546 & 1495.07 \\ 1494.842 & 1495.136 & 1496.544 & 1495067\end{array}$ $\begin{array}{lllll}1494.842 & 1495.133 & 1496.541 & 1495.065\end{array}$ $\begin{array}{lllll}1494.842 & 1495.133 & 1496.541 & 1495.0653\end{array}$ $\begin{array}{lllll}1494.842 & 1495.133 & 1496.541 & 1495.063 \\ 1494.838 & 1495.133 & 1496.544 & 1495.063\end{array}$ $\begin{array}{llllll}1494.838 & 1495.135 & 1496.544 & 1495.063\end{array}$ $\begin{array}{lllll}1494.836 & 1495.133 & 1496.546 & 1495.065\end{array}$ $\begin{array}{llll}1494.836 & 1495.135 & 1496.551 & 1495.07\end{array}$

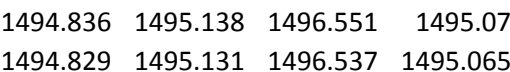
$\begin{array}{llll}1494.829 & 1495.131 & 1496.537 & 1495.065 \\ 1494.824 & 1495.124 & 1496.523 & 1495.058\end{array}$ $\begin{array}{lllll}494.826 & 1495122 & 1496523 & 1455.058\end{array}$ $\begin{array}{llll}1494.817 & 1495.11 & 1466.499 & 1495.04\end{array}$ $\begin{array}{lllll}1494.822 & 1495.108 & 1496.497 & 1495.03\end{array}$ $\begin{array}{lllll}1494.829 & 1495.119 & 1496.532 & 1495.046\end{array}$ $\begin{array}{llll}1494.838 & 1495.138 & 1496.572 & 1495.07\end{array}$ $\begin{array}{llll}1494.836 & 1495.149 & 1496.593 & 1495.088\end{array}$ $\begin{array}{lllll}1494.833 & 1495.149 & 1496.583 & 1495.093 \\ 1494.826 & 1495.147 & 1496569 & 1495088\end{array}$ $\begin{array}{llll}1494.824 & 1495.14 & 1496.558 & 1495.084\end{array}$ $\begin{array}{llll}1494.817 & 1495.126 & 1496.527 & 1495.065\end{array}$ $\begin{array}{llllll}1494.812 & 1495.119 & 1496.52 & 1495.056\end{array}$ $\begin{array}{lllll}1494.808 & 1495.11 & 1496.502 & 1495.042\end{array}$ $\begin{array}{lllll}1494.805 & 1495.105 & 1496.492 & 1495.032\end{array}$ $\begin{array}{lllll}1494.805 & 1495.101 & 1496.488 & 1495.023\end{array}$ 1494.7981495 .0891496 .4671495 .009 $\begin{array}{llllll}1494.801 & 1495.084 & 1496.457 & 1494.998 \\ 1494.801 & 1495.084 & 1496.464 & 1494.993\end{array}$ $\begin{array}{lllll}1494.803 & 1495.087 & 1496.481 & 1495\end{array}$ $\begin{array}{lllll}1494.801 & 1495.087 & 1496.483 & 1495.004\end{array}$ $\begin{array}{lllll}1494.808 & 1495.096 & 1496.504 & 1495.016\end{array}$ $\begin{array}{lllll}1494.803 & 1495.096 & 1496.504 & 1495.018\end{array}$ $1494.798 \quad 1495.094 \quad 1496.499 \quad 1495.021$ $1494.796 \quad 1495.098 \quad 1496.50614995 .025$ $\begin{array}{lllll}1494.787 & 1455.084 & 1496.474 & 1495.009\end{array}$ $\begin{array}{rrrr}1494.784 & 1495.077 & 1496.46 & 1495 \\ 1494.787 & 1495.075 & 1496.462 & 1494.995\end{array}$ $\begin{array}{lllll}1494.796 & 1495.08 & 1496.481 & 1495.002\end{array}$ $\begin{array}{lllll}1494.796 & 1495.091 & 1496.504 & 1495.016\end{array}$ $\begin{array}{lllll}1494.81 & 1495.124 & 1496.565 & 1495.051\end{array}$ $1494.8191495 .159 \quad 1496.618 \quad 1495.093$ $1494.815 \quad 1495.166 \quad 1496.623 \quad 1495.112$ $1494.8121495 .168 \quad 1496.6141495 .119$ $\begin{array}{llll}1494.812 & 1495.166 & 1496.609 & 1495.121\end{array}$ $1494.815 \quad 1495.177 \quad 1496.616 \quad 1495.13$ $\begin{array}{llll}1494.817 & 1495.184 & 1496.628 & 1495.14\end{array}$ $\begin{array}{lllll}1494.81 & 1495.173 & 1496.6 & 1495.123\end{array}$ $1494.812 \quad 1495.18 \quad 1496.609 \quad 1495.128$ $1494.817 \quad 1495.189 \quad 1496.633 \quad 1495.14$ $\begin{array}{rrrr}1494.819 & 1495.196 & 1496.64 & 1495.144 \\ 1494.817 & 1495.196 & 1496.637 & 1495.147\end{array}$ $\begin{array}{llllllllll}1494.348 & 1494.868 & 1495.13 & 1495.768 & 1496.959 & 1496.72 & 1493.287 & 1488.944 & 1493.13 & 1490.879\end{array}$ $\begin{array}{lrrrrrrrrr}1494.338 & 1494.863 & 1495.13 & 1495.77 & 1496.963 & 1496.736 & 1493.287 & 1488.947 & 1493.128 & 1490.877 \\ 1494.343 & 1494.87 & 1495.123 & 1495.763 & 1496956 & 1496727 & 1993.278 & 1488944 & 1493123 & 1490872\end{array}$ $\begin{array}{lllllllllll}1494.343 & 1494.87 & 1495.123 & 1495.763 & 1496.956 & 1496.727 & 1493.278 & 1488.944 & 1493.123 & 1490.872 \\ 1494.341 & 1494.868 & 1495.123 & 1495758 & 1496954 & 1496727 & 1493273 & 148894 & 1493116 & 149087\end{array}$ $\begin{array}{lllllllllll}1494.336 & 1494.866 & 1495.121 & 1495.756 & 1496952 & 1496.727 & 1493.271 & 1488.947 & 1493112 & 1490.865\end{array}$ $\begin{array}{lllllllllll}1494.331 & 1494.858 & 1495.121 & 1495.754 & 1496.952 & 1496.727 & 1493.268 & 1488.94 & 1493.112 & 1490.863\end{array}$ $\begin{array}{lllllllllll}1494.327 & 1494.858 & 1495.123 & 1495.751 & 1496.949 & 1496.729 & 1493.266 & 1488.94 & 1493.109 & 1490.861\end{array}$ $\begin{array}{lllllllllll}1494.32 & 1494.856 & 1495.121 & 1495.751 & 1496.952 & 1496.732 & 1493.266 & 1488.942 & 1493.109 & 1490.861\end{array}$ $\begin{array}{lllllllllll}1494.315 & 1494.851 & 1495.125 & 1495.749 & 1496.952 & 1496.736 & 1493.268 & 1488.94 & 1493.109 & 1490.861\end{array}$

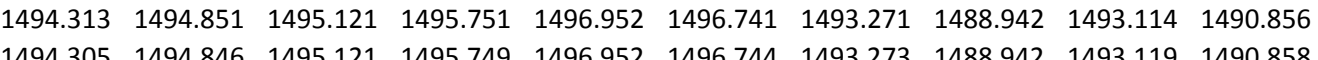
$\begin{array}{llllllllll}1494.305 & 1494.846 & 1495.121 & 1495.749 & 1496.952 & 1496.744 & 1493.273 & 1488.942 & 1493.119 & 1490.858 \\ 1494.315 & 1494849 & 1495.113 & 1495742 & 1496947 & 1496729 & 1493273 & 1488937 & 1493114 & 1490856\end{array}$ $\begin{array}{llllllllllll}1494.322 & 1494.851 & 1495.106 & 1495.737 & 1496.94 & 1496.724 & 1493.268 & 1488.935 & 1493.112 & 1490.854\end{array}$ $\begin{array}{llllllllllll}1494.317 & 1494846 & 1495113 & 1495735 & 1496938 & 1496.724 & 1493.264 & 1488.942 & 1493109 & 1490854\end{array}$ $\begin{array}{lllllllllll}1494.327 & 1494.849 & 1495.102 & 1495.719 & 1496.926 & 1496.698 & 1493.257 & 1488.935 & 1493.1 & 1490.849\end{array}$ $\begin{array}{lllllllllll}1494.317 & 1494.839 & 1495.104 & 1495.714 & 1496.922 & 1496.693 & 1493.25 & 1488.93 & 1493.095 & 1490.847\end{array}$ $\begin{array}{llllllllll}1494.282 & 1494.801 & 1495.121 & 1495.728 & 1496.938 & 1496.732 & 1493.252 & 1488.933 & 1493.095 & 1490.847\end{array}$ $\begin{array}{rlllllllll}1494.247 & 1494.782 & 1495.125 & 1495.747 & 1496.956 & 1496.772 & 1493.259 & 1488.94 & 1493.1 & 1490.847\end{array}$

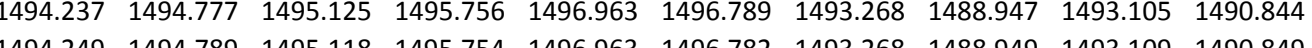
$\begin{array}{lllllllllll}1494.249 & 1494.789 & 1495.118 & 1495.754 & 1496.963 & 1496.782 & 1493.268 & 1488.949 & 1493.109 & 1490.849 \\ 1494263 & 1494.803 & 1495.113 & 1495.751 & 1496.959 & 1496.77 & 1493268 & 1488949 & 1493109 & 1490847\end{array}$ $\begin{array}{llllllllllll}1494.275 & 1494.82 & 1495.109 & 1495.749 & 1496.954 & 1496.76 & 1493.266 & 1488.952 & 1493.109 & 1490.847\end{array}$ $\begin{array}{llllllllll}1494.294 & 1494.842 & 1495.106 & 1495.733 & 1496.942 & 1496.729 & 1493.259 & 1488.94 & 1493.107 & 1490.847\end{array}$ $\begin{array}{llllllllllll}1494.298 & 1494.839 & 1495.099 & 1495.728 & 1496.935 & 1496.724 & 1493.254 & 1488.937 & 1493.1 & 1490.844\end{array}$ $\begin{array}{lllllllllll}1494.308 & 1494.842 & 1495.094 & 1495.719 & 1496.926 & 1496.71 & 1493.25 & 1488.933 & 1493.091 & 1490.84\end{array}$ $\begin{array}{lllllllllll}1494.305 & 1494.839 & 1495.094 & 1495.71 & 1496.919 & 1496.696 & 1493.242 & 1488.93 & 1493.086 & 1490.837\end{array}$ $\begin{array}{llllllllll}1494.301 & 1494.842 & 1495.094 & 1495.705 & 1496.915 & 1496.669 & 1493.233 & 1488.923 & 1493.076 & 1490.837\end{array}$ $\begin{array}{lllllllllll}1494.305 & 1494.83 & 1495.09 & 1455.693 & 1496.905 & 1496.679 & 1493.224 & 1488.928 & 1493.067 & 1490.833\end{array}$ $\begin{array}{lllllllllll}1494.298 & 1494.832 & 1495.092 & 1495.684 & 1496.898 & 1496.669 & 1493.217 & 1488.919 & 1493.06 & 1490.83 \\ 1494.287 & 1494.83 & 1495.094 & 1495.684 & 1496898 & 1496677 & 1493212 & 1488.916 & 1493.055 & 1490.826\end{array}$ $\begin{array}{lllllllllll}1494.27 & 1494.813 & 1495.099 & 1495.686 & 1496.901 & 1496.693 & 1493.212 & 1488.919 & 1493.055 & 1490.824\end{array}$ $\begin{array}{lllllllllll}1494.261 & 1494.811 & 1495.092 & 1495.689 & 1496.903 & 1496.703 & 1493.212 & 1488.919 & 1493.053 & 1490.823\end{array}$ $\begin{array}{llllllllll}1494.237 & 1494.787 & 1495.104 & 1495.693 & 1496.915 & 1496.72 & 1493.217 & 1488.928 & 1493.06 & 1490.823\end{array}$ $\begin{array}{lllllllllll}1494.24 & 1494.794 & 1495.099 & 1495.696 & 1496.915 & 1496.722 & 1493.217 & 1488.921 & 1493.067 & 1490.821\end{array}$ $\begin{array}{lllllllllll}1494.244 & 1494.801 & 1495.097 & 1495.693 & 1496.912 & 1496.72 & 1493.221 & 1488.923 & 1493.062 & 1490.819\end{array}$ $\begin{array}{llllllllllll}1494.242 & 1494.801 & 1495.094 & 1495.7 & 1496.915 & 1496.736 & 1493.224 & 1488.928 & 1493.067 & 1490.819\end{array}$ $\begin{array}{lllllllllll}1494.263 & 1494.832 & 1455.075 & 145.684 & 146.901 & 1496.701 & 1493.217 & 1488.919 & 1493.062 & 1490.817\end{array}$ $\begin{array}{lllllllllllll}1494.254 & 1494.82 & 1495.085 & 1495.672 & 1496.894 & 1496.691 & 1493.207 & 1488.914 & 1493.051 & 1490.814\end{array}$ $\begin{array}{lllllllllll}1494.228 & 1494.791 & 1495.097 & 1495.679 & 1496.903 & 1496.705 & 1493.207 & 1488.914 & 1493.048 & 1490.812\end{array}$ $\begin{array}{llllllllll}1494.209 & 1494.765 & 1495.097 & 1495.689 & 1496.915 & 1496.732 & 1493.207 & 1488.919 & 1493.051 & 1490.812\end{array}$ $\begin{array}{lllllllllll}1494.167 & 1494.715 & 1495.111 & 1495.712 & 1496.94 & 1496.784 & 1493.219 & 1488.93 & 1493.06 & 1490.814\end{array}$ $\begin{array}{lllllllllll}1494.134 & 1494.71 & 1495.116 & 1495.744 & 1496.968 & 1496.839 & 1493.233 & 1488.944 & 1493.076 & 1490.819\end{array}$ $\begin{array}{lllllllllll}1494.15 & 1494.727 & 1495.106 & 1495.754 & 1496.977 & 1496.847 & 1493.245 & 1488.956 & 1493.086 & 1490.821\end{array}$ $\begin{array}{lllllllllll}1494.171 & 1494.746 & 1495.102 & 1495.756 & 1496.98 & 1496.832 & 1493.25 & 1488.959 & 1493.093 & 1490.826 \\ 1494.188 & 1494.758 & 1495.104 & 1495758 & 1496982 & 1496827 & 1493252 & 1488959 & 1493098 & 149086\end{array}$

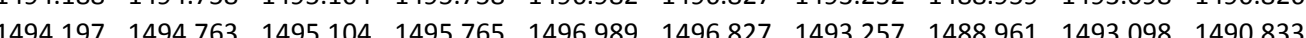
$\begin{array}{llllllllllll}1494.204 & 1494.77 & 1495.102 & 1495.77 & 1496.994 & 1496.83 & 1493.264 & 1488.966 & 1493.105 & 1490.833\end{array}$ $\begin{array}{lllllllllll}1494.207 & 1494.772 & 1495.104 & 1495.779 & 1497.003 & 1496.839 & 1493.268 & 1488.963 & 1493.107 & 1490.837\end{array}$ $\begin{array}{lllllllllll}1494.24 & 1494.811 & 1495.092 & 1495.772 & 1496.991 & 1496.806 & 1493.264 & 1488.966 & 1493.107 & 1490.84\end{array}$ $\begin{array}{llllllllllll}1494.235 & 1494.806 & 1495.097 & 1495.777 & 1496.998 & 1496.815 & 1493.264 & 1488.968 & 1493.105 & 1490.844\end{array}$ $\begin{array}{lllllllllll}1494.223 & 1494.794 & 1495.102 & 1495.789 & 1497.01 & 1496.837 & 1493.268 & 1488.982 & 1493.109 & 1490.844\end{array}$ $\begin{array}{llllllllll}1494.226 & 1494.791 & 1495.104 & 1495.795 & 1497.015 & 1496.839 & 1493.268 & 1488.973 & 1493.112 & 1490.847 \\ 1494235 & 1494.803 & 1495.104 & 1495798 & 1497.019 & 1496835 & 1493271 & 1488975 & 1493109 & 1490851\end{array}$ $\begin{array}{rrrrrrrrr}1485.602 & 1488.314 & 1493.54 & 1494.008 & 1493.293 & 1495.136 & 1493.267 & 1484.841 & 1479.22\end{array}$ $\begin{array}{lllllllll}1485.598 & 1488.302 & 1493.535 & 1494.008 & 1493.29 & 1495.138 & 1493.267 & 1484.839 & 1479.21 \\ 1485595 & 1488304 & 149353 & 1494008 & 1493285 & 145.129 & 149326 & 148483 & 1479.213\end{array}$ $\begin{array}{llllllllll}1485.595 & 1488.304 & 1493.53 & 1494.008 & 1493.285 & 1495.129 & 1493.26 & 1484.839 & 1499.213 \\ 1485.593 & 1488.304 & 1493.525 & 1494.001 & 1493281 & 1495.124 & 1493255 & 1488837 & 1479.211\end{array}$ $\begin{array}{llllllllll}1485.595 & 1488.304 & 1493.521 & 1494.001 & 1493.274 & 1495.127 & 1493.251 & 1484.839 & 1479.208\end{array}$ $\begin{array}{llllllllllll}1485.593 & 1488304 & 1493.516 & 1494.003 & 1493.276 & 1495.131 & 1493.253 & 1484.839 & 1479.206\end{array}$ $\begin{array}{llllllllll}1485.593 & 1488.3 & 1493.514 & 1494.003 & 1493.274 & 1495.127 & 1493.248 & 1484.839 & 1479.206\end{array}$ $\begin{array}{llllllllll}1485.598 & 1488.297 & 1493.516 & 1494.001 & 1493.271 & 1495.124 & 1493.248 & 1484.839 & 1479.208\end{array}$ $\begin{array}{lllllllll}1485.588 & 1488.297 & 1493.518 & 1494.003 & 1493.274 & 1495.131 & 1493.251 & 1484.839 & 1479.211\end{array}$ $\begin{array}{llllllllll}1485.588 & 1488.293 & 1493.521 & 1494.006 & 1493.276 & 1495.134 & 1493.253 & 1484.837 & 1479.21\end{array}$ $\begin{array}{lrrrrrrrrr}1485.593 & 1488.288 & 1493.523 & 1494.01 & 1493.281 & 1495.138 & 1493.255 & 1484.837 & 1479.218 \\ 1485593 & 148829 & 1493.518 & 1494003 & 1493276 & 1495.127 & 1493.253 & 1484837 & 1479.218\end{array}$ $\begin{array}{llllllllllll}1485.598 & 1488.295 & 1493.518 & 1493992 & 1493274 & 1495117 & 1493.251 & 1484.834 & 1479.218\end{array}$ $\begin{array}{lllllllllll}1485.593 & 1488.288 & 1493513 & 1493.992 & 1493.271 & 1495.117 & 1493.246 & 1484.834 & 1479.218\end{array}$ $\begin{array}{lllllllllll}1485.593 & 1488.286 & 1493.507 & 1493.978 & 1493.262 & 1495.089 & 1493.239 & 1484.834 & 1479.213\end{array}$ $\begin{array}{llllllllll}1485.586 & 1488.272 & 1493.499 & 1493.975 & 1493.257 & 1495.091 & 1493.232 & 1484.837 & 1479.211\end{array}$ $\begin{array}{llllllllll}1485.586 & 1488.255 & 1493.499 & 1493.994 & 1493.257 & 1495.124 & 1493.234 & 1484.834 & 1479.208\end{array}$ $\begin{array}{lllllllll}1485.586 & 1488.234 & 1493.506 & 1494.015 & 1493.266 & 1495.155 & 1493.241 & 1484.837 & 1479.213\end{array}$ $\begin{array}{llllllllll}1485.577 & 1488.232 & 1493.514 & 1494.027 & 493.271 & 1495.167 & 1493.251 & 1484.837 & 1479.213\end{array}$ $\begin{array}{lllllllll}1485.554 & 1488.239 & 143.516 & 1494.024 & 493.274 & 1495.162 & 1433.251 & 1484.834 & 1479.215\end{array}$ $\begin{array}{llllllllll}1485.507 & 148825 & 1493516 & 1494013 & 1493274 & 1495.138 & 1493248 & 1484832 & 1479215\end{array}$ $\begin{array}{llllllllll}1485.512 & 1488.255 & 1493.506 & 1493.987 & 1493.259 & 1495.108 & 1493.236 & 1484.83 & 1479.215\end{array}$ $\begin{array}{llllllllll}1485.514 & 1488.26 & 1493.499 & 1493.971 & 1493.255 & 1495.094 & 1493.229 & 1484.827 & 1479.213\end{array}$ $\begin{array}{llllllllll}1485.51 & 1488.253 & 1493.492 & 1493.964 & 1493.245 & 1495.082 & 1493.222 & 1484.83 & 1479.21\end{array}$

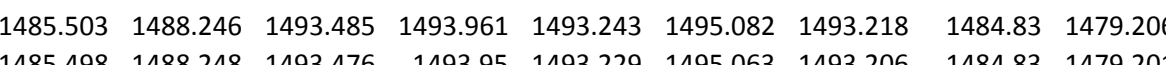
$\begin{array}{lllllllll}1485.498 & 1488.248 & 143.476 & 1493.95 & 1493.229 & 1495.063 & 1493.206 & 1484.83 & 1479.20\end{array}$ $\begin{array}{llllllllll}1485.496 & 1488.239 & 1493.464 & 1493.933 & 1493.219 & 1495.058 & 1493.199 & 1484.83 & 1479.199 \\ 1485.494 & 1488.234 & 1493.464 & 1493.938 & 1493214 & 1495.058 & 1493.194 & 1484.83 & 1479.197\end{array}$ $\begin{array}{lllllllllll}1485.498 & 1488.225 & 1493.464 & 1493.943 & 1493.217 & 1495.07 & 1493.19 & 1484.83 & 1479.197\end{array}$ $\begin{array}{llllllllll}1485.498 & 1488.232 & 1493.464 & 1493.945 & 1493.217 & 1495.068 & 1493.194 & 1484.827 & 1479.199\end{array}$ $\begin{array}{lllllllllll}1485.503 & 1488.204 & 1493.464 & 1493.961 & 1493.219 & 1495.091 & 1493.197 & 1484.83 & 1479.204\end{array}$ $\begin{array}{lllllllll}1485.501 & 1488.215 & 1493.469 & 1493.961 & 1493.226 & 1495.089 & 1493.199 & 1484.827 & 1479.206\end{array}$ $\begin{array}{lllllllll}1485.489 & 1488.218 & 1493.471 & 1493.961 & 1493.224 & 1495.094 & 1493.204 & 1484.827 & 1479.208\end{array}$ $\begin{array}{lllllllllll}1485.487 & 1488.225 & 1493.47 & 1493.959 & 1493.231 & 1495.087 & 1493.204 & 1484.827 & 1479.213\end{array}$

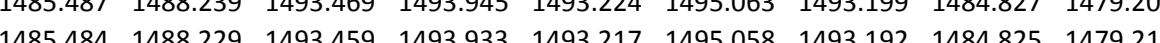
$\begin{array}{lllllllllll}1485.484 & 1488222 & 1493457 & 1493938 & 1493.212 & 1495.061 & 1493.19 & 1484.825 & 1479.2108\end{array}$ $\begin{array}{lllllllllll}1485.489 & 1488.199 & 1493.457 & 1493.947 & 1493.212 & 1495.075 & 1493.187 & 1484.825 & 1479.206\end{array}$ $\begin{array}{llllllllll}1485.503 & 1488.192 & 1493.457 & 1493.966 & 1493.214 & 1495.091 & 1493.187 & 1484.827 & 1479.206\end{array}$ $\begin{array}{llllllllll}1485.496 & 1488.168 & 1493.466 & 1493.994 & 1493.224 & 1495.138 & 1493.199 & 1484.827 & 1479.206\end{array}$ $\begin{array}{llllllllll}1485.498 & 1488.164 & 1493.483 & 1494.029 & 1493.24 & 1495.183 & 1493.218 & 1484.827 & 1479.211\end{array}$ $\begin{array}{llllllllll}1485.503 & 1488.183 & 1493.492 & 1494.036 & 1493.25 & 1495.188 & 1493.227 & 1484.825 & 1479.213\end{array}$ $\begin{array}{lllllllll}1485.503 & 1488.187 & 1493.499 & 1494.031 & 1493.255 & 1495.188 & 1493.232 & 1484.825 & 1479.215 \\ 148551 & 1488.194 & 1493.502 & 1494034 & 1493.259 & 1495.185 & 1493234 & 1484823 & 1479.218\end{array}$

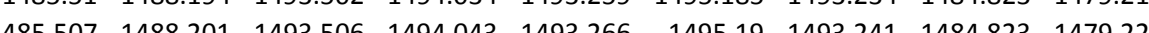
$\begin{array}{lllllllllll}1485.475 & 1488.203 & 1493.511 & 1494.045 & 1493.269 & 1495.195 & 1493.243 & 1484.825 & 1499.225\end{array}$ $\begin{array}{lllllllllll}1485.482 & 1488.208 & 1493.516 & 1494.055 & 1493.274 & 1495.202 & 1493.251 & 1484.825 & 1479.227\end{array}$ $\begin{array}{lllllllll}1485.484 & 1488.229 & 1493.513 & 1494.034 & 1493.269 & 1495.176 & 1493.243 & 1484.823 & 1479.225\end{array}$ $\begin{array}{llllllllll}1485.487 & 1488.227 & 1493.513 & 1494.038 & 1493.269 & 1495.185 & 1493.246 & 1484.823 & 1479.225\end{array}$ $\begin{array}{llllllllll}1485.487 & 1488.222 & 1493.516 & 1494.048 & 1493.274 & 1495.197 & 1493.248 & 1484.825 & 1479.225\end{array}$ $\begin{array}{lllllllll}1485.487 & 1488.229 & 1493.516 & 1494.057 & 1493.276 & 1495.204 & 1493.251 & 1484.823 & 1479.225 \\ 1485.484 & 1488.239 & 1493.518 & 1494.055 & 1493.276 & 1495.204 & 1493.253 & 1484.823 & 1479.222\end{array}$ 
Well

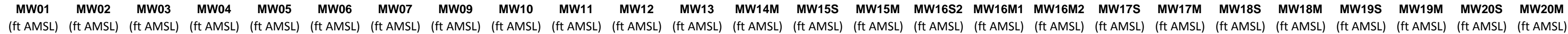

$1 / 26 / 122: 00$ $1 / 26 / 123: 00$ 1/26/12 4:00 1/26/12 5:00 1/26/126:00 $1 / 26 / 128: 00$ 1/26/12 9:00 1/26/12 10:00 1/26/12 11:00 1/26/12 12:00 1/26/12 13:00 $1 / 26 / 1215: 00$ 1/26/12 15:00 1/26/12 17:00 1/26/12 18:00 1/26/12 19:00 1/26/12 20:00 $1 / 26 / 1221: 00$ 1/26/12 22:00 1/27/120:00 1/277/120:00 1/27/12 2:00 1/27/12 3:00 $1 / 27 / 125: 00$ 1/27/122:00 $1 / 27 / 1227: 00$
$1 / 27 / 128: 00$ 1/27/12 9:00 1/27/12 10:00 1/27/12 11:00 1/27/12 12:00 $1 / 27 / 1213: 00$
$1 / 27 / 1214: 00$
$1 / 27 / 1215: 00$ 1/27/12 15:00 1/27/12 17:00 1/27/12 18:00 1/27/12 19:00 1/27/12 20:00 1/27/12 21:00 1/27/1222:00 $1 / 281120000$ 1/28/120:00 1/28/12 1:00 $1 / 28 / 123: 00$ 1/28/12 4:00 $1 / 28 / 125: 00$ $1 / 28 / 126: 00$
$1 / 28 / 127: 00$ $\begin{array}{rrrr}1494.817 & 1495.194 & 1496.63 & 1495.142 \\ 1494.822 & 1495.208 & 1496651 & 1495.154\end{array}$ $\begin{array}{llll}1494.822 & 1495.208 & 1496.651 & 1495.154\end{array}$ $\begin{array}{lllll}1494.822 & 1495.205 & 1496.649 & 1495.154\end{array}$ $\begin{array}{lllll}1494.808 & 1495.189 & 1496.604 & 1495.133\end{array}$ $\begin{array}{lllll}1494.81 & 1495.18 & 1496.586 & 1495.114\end{array}$ $\begin{array}{rrrr}1494.81 & 1495.18 & 1496.586 & 1495.114 \\ 1494.798 & 1495.166 & 1496.565 & 1495.1\end{array}$ $\begin{array}{lllll}1494.798 & 1495.156 & 1496.548 & 1495.081\end{array}$ $\begin{array}{lllll}1494.798 & 1495.159 & 1496.555 & 1495.077\end{array}$ $\begin{array}{llll}1494.798 & 1495.156 & 1496.558 & 1495.075\end{array}$ $\begin{array}{llll}1494.801 & 1495.161 & 1496.576 & 1495.081\end{array}$ $\begin{array}{llll}1494.805 & 1495.175 & 1496.604 & 1495.098 \\ 1494.805 & 1495.173 & 1496.609 & 1495.102\end{array}$ $\begin{array}{llllll}1494.805 & 1495.177 & 1496.616 & 1495.107\end{array}$ $\begin{array}{lllll}1494.803 & 1495.177 & 1496.609 & 1495.109\end{array}$ $\begin{array}{lllll}1494.798 & 1495.17 & 1496.595 & 1495.105\end{array}$ $\begin{array}{lllll}1494.798 & 1495.168 & 1496.588 & 1495.098\end{array}$ $\begin{array}{lllll}1494.798 & 1495.163 & 1496.586 & 1495.095\end{array}$ $1494.796 \quad 1495.163 \quad 1496.586 \quad 1495.093$ $\begin{array}{lllll}1494.803 & 1495.17 & 1496.607 & 1495.1 \\ 1494.796 & 1495.175 & 1496.607 & 1495.107\end{array}$ $\begin{array}{llrr}1494.796 & 1495.168 & 1496.6 & 1495.102\end{array}$ $\begin{array}{lllll}1494.805 & 1495.182 & 1496.626 & 1495.114\end{array}$ $\begin{array}{llll}1494.8051 & 1455.201 & 1496.626 & 1495.114 \\ 1495.13 & 1495.13\end{array}$ 1494.805 1495.201 $1496.644 \quad 1495.135$ $\begin{array}{lllll}1494.81 & 1495.203 & 1496.656 & 1495.142\end{array}$ $\begin{array}{llll}1494.815 & 1495.215 & 1496.668 & 1495.154\end{array}$ $\begin{array}{lllll}1494.819 & 1495.224 & 1496.686 & 1495.166\end{array}$ $\begin{array}{llll}1494.819 & 1495.229 & 1496.684 & 1495.173 \\ 1494.815 & 1495231 & 1496.686 & 1495.175\end{array}$ $\begin{array}{llll}1494.819 & 1495.229 & 1496.677 & 1495.173\end{array}$ $\begin{array}{llll}1494.826 & 1495.24 & 1496.705 & 1495.187\end{array}$ $\begin{array}{llll}1494.822 & 1495.236 & 1496.686 & 1495.18\end{array}$ $\begin{array}{lllll}1494.817 & 1495.229 & 1496.672 & 1495.17\end{array}$ $\begin{array}{llrr}1494.822 & 1495.226 & 1496.67 & 1495.166\end{array}$ $\begin{array}{llll}1494.822 & 1495.229 & 1496.677 & 1495.166 \\ 1498.805 & 1495208 & 1466628 & 149514\end{array}$ $\begin{array}{llll}1494.805 & 1495.208 & 1496.628 & 1495.14 \\ 1494.794 & 1495.175 & 1496558 & 1495.093\end{array}$ $\begin{array}{llll}1494.794 & 1495.175 & 1496.558 & 1495.093 \\ 1494.782 & 1495142 & 1496509 & 1495049\end{array}$ $\begin{array}{lllll}1494.775 & 1495.122 & 1496.481 & 1495.014\end{array}$ $\begin{array}{lllll}1494.763 & 1495.094 & 1496.438 & 1494.974\end{array}$ $1494.749 \begin{array}{lrl}1495.056 & 1496.38 & 1494.927\end{array}$ $1494.747 \quad 1495.028 \quad 1496.347 \quad 1494.888$ $\begin{array}{lllll}1494.749 & 1495.01 & 1496.336 & 1494.862\end{array}$ $1494.747 \quad 1495.003 \quad 1496.333 \quad 1494.85$

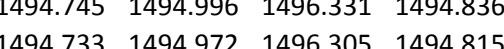
$1494.733 \quad 1494.961 \quad 1496296 \quad 1494.801$ $1494.7261494 .949 \quad 1496284 \quad 14494.792$ $1494.728 \quad 1494.947 \quad 1496.294 \quad 1494.79$ $\begin{array}{llll}1494.735 & 1494.954 & 1496.315 & 1494.799 \\ 1494.735 & 1494.968 & 1496.347 & 1494.818\end{array}$ $\begin{array}{lllll}1494.735 & 1494.968 & 1496.347 & 1494.818\end{array}$ $\begin{array}{rrrr}1494.728 & 1494.968 & 1496.347 & 1494.825 \\ 1494.719 & 1494.958 & 1496.326 & 1494.82\end{array}$ $\begin{array}{llllllllll}1494.244 & 1494.815 & 1495.092 & 1495.798 & 1497.015 & 1496.827 & 1493.271 & 1488.977 & 1493.112 & 1490.849\end{array}$ $\begin{array}{llllllllll}1494.235 & 1494.799 & 1495.099 & 1495.812 & 1497.028 & 1496.847 & 1493.275 & 1488.977 & 1493.116 & 1490.856 \\ 1492242 & 149.811 & 1495099 & 1495812 & 197.028 & 1496842 & 1493278 & 1489977 & 1493.119 & 1408566\end{array}$ $\begin{array}{llllllllllll}1494249 & 1494818 & 1495097 & 1495814 & 1497031 & 1496842 & 1493282 & 148898 & 1493121 & 1490.8568\end{array}$ $\begin{array}{lllllllllllll}1494.284 & 1494.863 & 1495.083 & 1495.802 & 1497.012 & 1496.799 & 1493.275 & 1488.975 & 1493.119 & 1490.856\end{array}$ $\begin{array}{llllllllllll}1494.294 & 1494.87 & 1495.09 & 1495.791 & 1497.005 & 1496.775 & 1493.271 & 1488.968 & 1493.112 & 14900.856\end{array}$ $\begin{array}{lllllllllll}1494.303 & 1494.861 & 1495.075 & 1495.782 & 1496.994 & 1496.758 & 1493.266 & 1488.961 & 1493.109 & 1490.854\end{array}$ $\begin{array}{llllllllllll}1494.308 & 1494.863 & 1495.075 & 1495.775 & 1496.984 & 1496.744 & 1493.259 & 1488.959 & 1493.102 & 1490.854\end{array}$ $\begin{array}{llllllllllll}1494.289 & 1494.849 & 1495.085 & 1495.772 & 1496.984 & 1496.751 & 1493.254 & 1488.949 & 1493.098 & 1490.851\end{array}$ $\begin{array}{lllllllllll}1494.277 & 1494.837 & 1495.078 & 4495.77 & 1496.982 & 1496.756 & 1493.247 & 1488.949 & 1493.095 & 1490.851\end{array}$ $\begin{array}{llllllllll}1494.254 & 1494.808 & 1495.083 & 1495.779 & 1496.991 & 1496.772 & 1493.247 & 1488.951 & 1493.091 & 1490.847 \\ 1494.228 & 1494775 & 1495092 & 1495.793 & 1497.005 & 1496801 & 1493257 & 1488.956 & 1493095 & 1490849\end{array}$

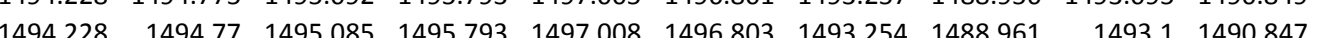
$\begin{array}{lllllllllll}1494.223 & 1494.77 & 1495.09 & 1495.798 & 1497.012 & 1496.813 & 1493.257 & 1488.956 & 1493.1 & 1490.847\end{array}$ $\begin{array}{llllllllllll}1494.233 & 1494.784 & 1495.085 & 1495.798 & 1497.01 & 1496.803 & 1493.259 & 1488.963 & 1493.1 & 1490.849\end{array}$ $\begin{array}{llllllllllll}1494.247 & 1494.803 & 1495.083 & 1495.793 & 1497.003 & 1496.791 & 1493.257 & 1488.963 & 1493.1 & 1490.847\end{array}$ $\begin{array}{lllllllllll}1494.254 & 1494.811 & 1495.08 & 1495.789 & 1497.001 & 1496.784 & 1493.257 & 1488.959 & 1493.1 & 1490.844\end{array}$ $\begin{array}{lllllllllll}1494.258 & 1494.818 & 1495.08 & 1495.789 & 1497.003 & 1496.784 & 1493.254 & 1488.956 & 1493.1 & 1490.844\end{array}$ $\begin{array}{lllllllllll}1494.258 & 1494.823 & 1495.075 & 1495.789 & 1497.001 & 1496.782 & 1493.254 & 1488.956 & 1493.098 & 1490.842 \\ 1492244 & 1498.806 & 1495087 & 1495.795 & 1497.01 & 1496801 & 1493257 & 148859 & 14931 & 1408844\end{array}$ $\begin{array}{rrrrrrrrrrr}1494.244 & 1494.806 & 1495.087 & 1495.795 & 1497.01 & 1496.801 & 1493.257 & 1488.959 & 1493.1 & 1490.844 \\ 1494.249 & 1494813 & 149508 & 14958 & 1497.01 & 1496811 & 1493259 & 1488.961 & 1493102 & 1490844\end{array}$ $\begin{array}{lllllllllll}1494.256 & 1494.823 & 1495.078 & 1495.795 & 1497.008 & 1496.794 & 1493.254 & 1488.961 & 1493.098 & 1490.844\end{array}$ $\begin{array}{llllllllllll}1494.235 & 1494.801 & 1495.087 & 1495.807 & 1497.019 & 1496.82 & 1493.257 & 1488.961 & 1493.1 & 1490.844\end{array}$ $\begin{array}{lllllllllll}1494.219 & 1494.779 & 1495.094 & 1495.819 & 1497.035 & 1496.844 & 1493.264 & 1488.968 & 1493.107 & 1490.847\end{array}$ $\begin{array}{lllllllllll}1494.235 & 1494.799 & 1495.083 & 1495.821 & 1497.033 & 1496.842 & 1493.266 & 1488.97 & 1493.107 & 1490.847\end{array}$ $\begin{array}{lllllllllll}1494.23 & 1494.791 & 1495.09 & 1495.828 & 1497.045 & 1496.849 & 1493.268 & 1488.973 & 1493.112 & 1490.849\end{array}$ $\begin{array}{llllllllll}1494.226 & 1494.784 & 1495.094 & 1495.835 & 1497.052 & 1496.856 & 1493.275 & 1488.973 & 1493.119 & 1490.849\end{array}$

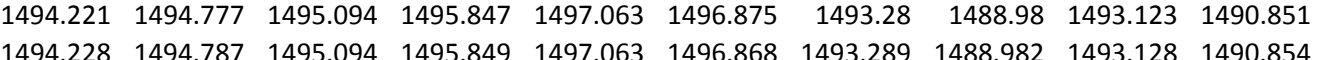

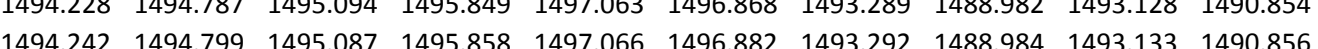
$\begin{array}{llllllllllll}1494.249 & 1494.808 & 1495.09 & 1495.854 & 1497.066 & 1496.856 & 1493.294 & 1488.984 & 1493.133 & 1490.858\end{array}$ $\begin{array}{llllllllll}1494.235 & 1494.789 & 1495.097 & 1495.872 & 1497.08 & 1496.894 & 1493.301 & 1488.998 & 1493.142 & 1490.861\end{array}$ $\begin{array}{llllllllll}1494.256 & 1494.808 & 1495.09 & 1495.863 & 1497.075 & 1496.863 & 1493.296 & 1488.994 & 1493.137 & 1490.863\end{array}$ $\begin{array}{llllllllllll}1494.27 & 1494.825 & 1495.087 & 1495.86 & 1497.068 & 1496.854 & 1493.294 & 1488.989 & 1493.135 & 1490.861\end{array}$ $\begin{array}{lllllllllll}1494.268 & 1494.825 & 1495.092 & 1495.858 & 1497.068 & 1496.847 & 1493.292 & 1488.984 & 1493.133 & 1490.863\end{array}$ $\begin{array}{llllllllll}1494.263 & 1494.815 & 1495.092 & 1495.863 & 1497.07 & 1496.851 & 1493.292 & 1488.982 & 1493.135 & 1490.865 \\ 1494301 & 1494866 & 1495071 & 1495844 & 1497.049 & 1496806 & 149328 & 1488984 & 1493123 & 149083\end{array}$

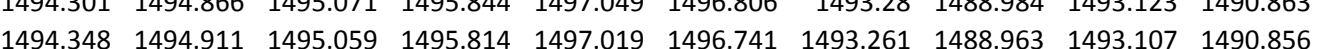

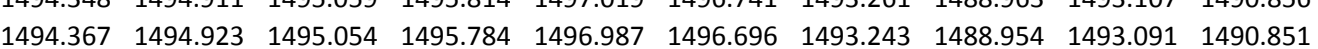
$\begin{array}{llllllllllll}1494.369 & 1494.887 & 1495.052 & 1495.763 & 1496.968 & 1496.667 & 1493.226 & 1488.935 & 1493.074 & 1490.842\end{array}$ $\begin{array}{lllllllllll}1494.378 & 1494.806 & 1495.042 & 1495.735 & 1496.94 & 1496.634 & 1493.207 & 1488.921 & 1493.055 & 1490.835\end{array}$ $\begin{array}{llllllllll}1494.392 & 1494.791 & 1495.033 & 1495.698 & 1496.905 & 1496.581 & 1493.189 & 1488.912 & 1493.032 & 1490.826\end{array}$ $\begin{array}{lllllllllll}1494.385 & 1494.787 & 1495.035 & 1495.668 & 1496.877 & 1496.552 & 1493.168 & 1488.902 & 1493.018 & 1490.817\end{array}$ $\begin{array}{llllllllll}1494.362 & 1494.779 & 1495.042 & 1495.647 & 1496.863 & 1496.545 & 1493.151 & 1488.895 & 1492.999 & 1490.807\end{array}$ $\begin{array}{lllllllllll}1494.336 & 1494.774 & 1495.042 & 1495.635 & 1496.852 & 1496.552 & 1493.14 & 1488.888 & 1492.985 & 1490.798 \\ 1494.313 & 1494.765 & 1495.035 & 1495624 & 149684 & 1496555 & 1493128 & 1488884 & 1492978 & 1490791\end{array}$ $\begin{array}{llllllllll}1494.313 & 1494.765 & 1455.035 & 1455.624 & 149684 & 1496.555 & 1493.128 & 1488.884 & 1492.978 & 1490.791 \\ 1494308 & 1494.76 & 1495.028 & 1495598 & 1496819 & 1496533 & 1493.114 & 1488879 & 1492962 & 1490.779\end{array}$ $\begin{array}{lllllllllll}1494.287 & 1494.756 & 1495.03 & 1495.584 & 1496.808 & 1496.531 & 1493.1 & 1488.874 & 1492.947 & 1490.77\end{array}$ $\begin{array}{lllllllllll}1494.268 & 1494.748 & 1495.028 & 1495.568 & 1496.796 & 1496.528 & 1493.088 & 1488.874 & 1492.936 & 1490.761\end{array}$ $\begin{array}{lllllllllll}1494.242 & 1494.744 & 1495.03 & 1495.563 & 1496.791 & 1496.54 & 1493.083 & 1488.87 & 1492.931 & 1490.754\end{array}$ $\begin{array}{lllllllllll}1494.202 & 1494.741 & 1495.04 & 1495.566 & 1496.798 & 1496.564 & 1493.079 & 1488.87 & 1492.924 & 1490.747\end{array}$ $\begin{array}{llrrrrrrrr}1494.169 & 1494.739 & 1495.042 & 1495.575 & 1496.808 & 1496.602 & 1493.083 & 1488.872 & 1492.929 & 1490.742\end{array}$ $\begin{array}{rrrrrrrrrr}1494.16 & 1494.734 & 1495.035 & 1495.573 & 1496.808 & 1496.61 & 1493.083 & 1488.877 & 1492.929 & 1490.737 \\ 1494.164 & 1494.729 & 1495.026 & 1495.563 & 1496.796 & 1496.595 & 1493.079 & 1488.87 & 1492.926 & 1490.73\end{array}$ $\begin{array}{llllllllll}1485.482 & 1488.248 & 1493.521 & 1494.05 & 1493.276 & 1495.197 & 1493.251 & 1484.823 & 1479.222\end{array}$ $\begin{array}{rlllllllll}1485.484 & 1488.239 & 1493.525 & 1494.069 & 1493.281 & 1495.214 & 1493.255 & 1484.82 & 1479.225 \\ 1485489 & 1488253 & 1493528 & 1494.059 & 1493283 & 1495216 & 1493.258 & 1484.823 & 1479227\end{array}$

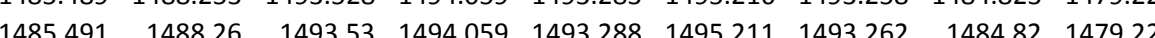
$\begin{array}{lllllllllll}1485.475 & 1488.286 & 1493.523 & 1494.041 & 1493.283 & 1495.178 & 1493.255 & 1484.82 & 1479.229\end{array}$ $\begin{array}{llllllllll}1485.466 & 1488.279 & 1493.518 & 1494.022 & 1493.278 & 1495.167 & 1493.255 & 1484.82 & 1479.229\end{array}$ $\begin{array}{llllllllllll}1485.466 & 1488.293 & 1493.518 & 1494.013 & 1493.271 & 1495.145 & 1493.246 & 1484.816 & 1479.229\end{array}$ $\begin{array}{llllllllll}1485.457 & 1488.29 & 1493.509 & 1494.001 & 1493.264 & 1495.127 & 1493.239 & 1484.818 & 1479.227\end{array}$ $\begin{array}{lllllllll}1485.454 & 1488.279 & 1493.504 & 1493.996 & 1493.262 & 1495.131 & 1493.236 & 1484.818 & 1479.227\end{array}$ $\begin{array}{llllllllll}1485.454 & 1488.272 & 1493.497 & 1493.999 & 1493.257 & 1495.127 & 1493.227 & 1484.818 & 1479.225\end{array}$ $\begin{array}{rrrrrrrrrr}1485.45 & 1488.26 & 1493.5 & 1494.008 & 1493.257 & 1495.152 & 1493.236 & 1484.82 & 1479.225 \\ 1485.452 & 1488.246 & 1493502 & 1494.024 & 1493262 & 1495.164 & 1493234 & 1484818 & 1479.222\end{array}$

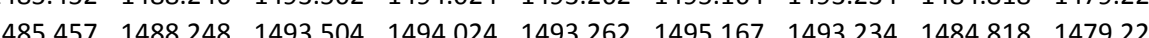
$\begin{array}{lllllllll}1485.457 & 1488.246 & 1493.506 & 1494.034 & 1493.264 & 1495.174 & 1493.236 & 1484.818 & 1479.225\end{array}$ $\begin{array}{llllllllll}1485.408 & 1488.253 & 1493.506 & 1494.029 & 1493.264 & 1495.167 & 1493.239 & 1484.818 & 1479.225\end{array}$ $\begin{array}{llllllllll}1485.413 & 1488.262 & 1493.506 & 1494.017 & 1493.264 & 1495.155 & 1493.239 & 1484.816 & 1479.225\end{array}$ $\begin{array}{llllllllll}4485.422 & 1488.257 & 1493.506 & 1494.015 & 1493.262 & 1495.15 & 1493.236 & 1484.818 & 1479.225\end{array}$ $\begin{array}{llllllllll}1485.429 & 1488.26 & 1493.504 & 1494.015 & 1493.262 & 1495.15 & 1493.236 & 1484.816 & 1479.229\end{array}$ $\begin{array}{lllllllll}1485.427 & 1488.262 & 1493.506 & 1494.01 & 1493.264 & 1495.152 & 1493.236 & 1484.816 & 1479.229\end{array}$ $\begin{array}{llllllllll}1485.433 & 1488.253 & 1493.511 & 1494.024 & 1493.264 & 1455.157 & 1493.239 & 1484.818 & 1479.232\end{array}$ $\begin{array}{llllllllll}1485.436 & 1488.265 & 1493.506 & 1494.017 & 1493.262 & 1495.152 & 1493.234 & 1484.813 & 1479.222\end{array}$ $\begin{array}{lllllllll}1485.436 & 1488255 & 1493.506 & 1494.036 & 1493264 & 1495178 & 1493239 & 1484.818 & 1479.229\end{array}$ $\begin{array}{lllllllllll}1485.436 & 1488.246 & 1493.514 & 1494.048 & 1493.271 & 1495.199 & 1493.246 & 1484.816 & 1479.232\end{array}$ $\begin{array}{llllllllll}1485.438 & 1488.265 & 1493.516 & 1494.041 & 1493.274 & 1495.19 & 1493.248 & 1484.811 & 1479.229\end{array}$ $\begin{array}{llllllllll}1485.443 & 1488.26 & 1493.516 & 1494.057 & 1493.274 & 1495.204 & 1493.251 & 1484.816 & 1479.232\end{array}$ $\begin{array}{lllllllll}4885.445 & 1488.262 & 1493.52 & 1494.066 & 1493.281 & 1495.211 & 1493.255 & 1484.816 & 1479.234\end{array}$ $\begin{array}{llllllllll}1485.44 & 1488.262 & 1493.528 & 1494.078 & 1493.288 & 1495.23 & 1493.265 & 1484.813 & 1479.236\end{array}$ $\begin{array}{llllllllll}1485.445 & 1488.269 & 1493.532 & 1494.08 & 1493.295 & 1495.237 & 1493.269 & 1484.816 & 1479.241 \\ 1485.447 & 1488.286 & 1493.539 & 1494.078 & 1493.297 & 1495.225 & 1493.274 & 1484.811 & 1479.246\end{array}$ $\begin{array}{lllllllllll}1485.45 & 1488.283 & 1493.539 & 1494.076 & 1493.302 & 1495.225 & 1493.272 & 1484.813 & 1479.24\end{array}$ $\begin{array}{llllllllll}1485.457 & 1488.281 & 1493.549 & 1494.092 & 1493.309 & 1495.244 & 1493.279 & 1484.816 & 1479.25\end{array}$ $\begin{array}{lllllllllll}1485.447 & 1488.283 & 1493.544 & 1494.082 & 1493.302 & 1495.23 & 1493.276 & 1484.813 & 1479.246\end{array}$ $\begin{array}{llllllllll}1485.447 & 1488.3 & 1493.544 & 1494.071 & 1493.302 & 1495.216 & 1493.274 & 1484.811 & 1479.243\end{array}$ $\begin{array}{lllllllll}1485.436 & 1488.29 & 1493.542 & 1494.071 & 1493.297 & 1495.214 & 1493.272 & 1484.811 & 1479.243\end{array}$ $\begin{array}{llllllllll}4485.445 & 1488.288 & 1493.542 & 1494.076 & 1493.3 & 1495.21 & 1493.272 & 1484.811 & 1479.241\end{array}$

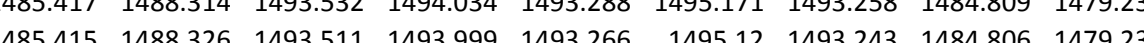
$\begin{array}{lllllllllll}1485.359 & 1488.335 & 1493.495 & 1493.964 & 1493.245 & 1495.073 & 1493.222 & 1484.806 & 1479.225\end{array}$ $\begin{array}{llllllllll}1485.355 & 1488.323 & 1493.481 & 1493.933 & 1493.231 & 1495.044 & 1493.208 & 1484.806 & 1479.222\end{array}$ $\begin{array}{llllllllll}1485.355 & 1488.337 & 1493.467 & 1493.905 & 1493.217 & 1495.004 & 1493.192 & 1484.806 & 1479.22\end{array}$ $\begin{array}{lllllllll}1485.355 & 1488.335 & 1493.438 & 1493.866 & 1493.193 & 1494.955 & 1493.168 & 1484.806 & 1479.215\end{array}$ $\begin{array}{lllllllll}4485.355 & 1488.326 & 1493.422 & 1493.838 & 1493.172 & 1494.92 & 1493.15 & 1484.806 & 1479.213\end{array}$ $\begin{array}{llllllllll}1485.357 & 1488.307 & 1493.403 & 1493.826 & 1493.16 & 1494.908 & 1493.133 & 1484.806 & 1479.211\end{array}$ $\begin{array}{lllllllllll}1485.366 & 1488.293 & 1493.394 & 1493.814 & 1493.143 & 1494.913 & 1493.121 & 1484.806 & 1479.208\end{array}$ $\begin{array}{lllllllll}1485357 & 1488281 & 1493365 & 1493784 & 1493.12 & 1494873 & 1493096 & 1484804 & 1479.1969\end{array}$ $\begin{array}{llllllllllll}1485.353 & 1488.262 & 1493.354 & 1493.775 & 1493.103 & 1494.863 & 1493.079 & 1484.804 & 1479.197\end{array}$ $\begin{array}{llllllllll}1485.353 & 1488.255 & 1493.339 & 1493.763 & 1493.098 & 1494.852 & 1493.072 & 1484.804 & 1479.194\end{array}$ $\begin{array}{llllllllll}1485.35 & 1488.239 & 1493.337 & 1493.763 & 1493.086 & 1494.859 & 1493.063 & 1484.804 & 1479.192\end{array}$ $\begin{array}{llllllllll}1485.353 & 1488.22 & 1493.333 & 1493.779 & 1493.084 & 1494.878 & 1493.063 & 1484.804 & 1479.194\end{array}$ $\begin{array}{llllllllll}1485.355 & 1488.213 & 1493.335 & 1493.796 & 1493.089 & 1494.903 & 1493.065 & 1484.804 & 1479.197\end{array}$ $\begin{array}{rrrrrrrrr}1485.346 & 1488.215 & 1493.335 & 1493.791 & 1493.091 & 1494.901 & 1493.065 & 1484.802 & 1479.199 \\ 148535 & 1488.22 & 1493.332 & 1493.784 & 1493.084 & 1494.889 & 1493.058 & 1484.802 & 1479.197\end{array}$ 
Well

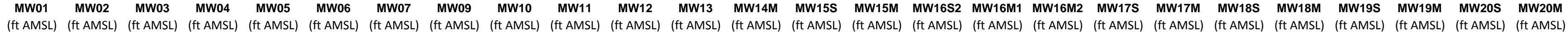

$1 / 28 / 128: 00$ $1 / 28 / 129: 00$ $1 / 28 / 1210: 00$ 1/28/12 11:00 1/28/12 12:00 1/28/12 13:00 1/28/12 15:00 1/28/12 16:00 $1 / 28 / 1217: 00$ $1 / 28 / 1218: 00$
$1 / 28 / 1219: 00$ 1/28/12 19:00 1/28/12 20:00 1/28/12 21:00 $1 / 28 / 1223: 00$ 1/29/120:00 1/29/12 1:00 1/29/122:00 1/29/122:00 1/29/12 4:00 1/29/12 6:00 $1 / 29 / 127: 00$ 1/29/12 8:00 1/29/12 9:00 1/29/12 10:00 1/29/12 11:00 $1 / 29 / 1212: 00$ 1/29/12 13:00 1/29/12 14:00 1/29/12 16:00 1/29/12 17:00 1/29/12 18:00 1/29/12 19:00 $1 / 29 / 1221: 00$ $1 / 29 / 12222000$ 1/30/12 0:00 1/30/12 1:00 1/30/122:00 1/30/12 3:00 $1 / 30 / 124: 00$ $1 / 30 / 125.00$ 1/30/12 6:00 1/30/12 7:00 1/30/12 9:00 1/30/12 $10: 00$ 1/30/12 11:00 1/30/12 12:00 $\begin{array}{llll}1494.712 & 1494.947 & 1496.305 & 1494.811 \\ 1494.707 & 1494.935 & 1496289 & 1494799\end{array}$ $\begin{array}{llll}1494.707 & 1494.935 & 1496.289 & 1494.799 \\ 1494705 & 1494926 & 1496275 & 1494.79\end{array}$ $\begin{array}{llll}1494.705 & 1494.926 & 1496.275 & 1494.79\end{array}$ $\begin{array}{lllll}1494.714 & 1494.928 & 1496.296 & 1494.79\end{array}$ $\begin{array}{lllll}1494.721 & 1494.947 & 1496.34 & 1494.818\end{array}$ $\begin{array}{llll}1494.721 & 1494.965 & 1496.375 & 1494.846\end{array}$ $\begin{array}{llllll}1494.721 & 1494.977 & 1496.394 & 1494.867\end{array}$ $\begin{array}{lllll}1494.721 & 1494.991 & 1496.413 & 1494.89\end{array}$ $1494.7191494 .998 \quad 1496.42 \quad 1494.904$ $\begin{array}{rrrr}1494.717 & 1494.996 & 1496.417 & 1494.911 \\ 1494.712 & 1494.998 & 1496.41 & 1494911\end{array}$ $\begin{array}{rrrr}1494.712 & 1494.998 & 1496.41 & 1494.911 \\ 1494.71 & 1494.998 & 1496.408 & 1494.913\end{array}$ $\begin{array}{lllll}1494.71 & 1495.003 & 1496.415 & 1494.918\end{array}$ $\begin{array}{rrrr}1494.705 & 1495.003 & 1496.415 & 1494.918\end{array}$ $\begin{array}{lllll}1494.705 & 1495.005 & 1496.413 & 1494.918\end{array}$ $\begin{array}{llll}1494.703 & 1495.003 & 1496.41 & 1494.918\end{array}$ $\begin{array}{llll}1494.707 & 1495.012 & 1496.424 & 1494.925\end{array}$ $\begin{array}{llll}1494.707 & 1495.024 & 1496.441 & 1494.937\end{array}$ $\begin{array}{llll}1494.705 & 1495.024 & 1496.434 & 1494.937 \\ 1494.703 & 1495.026 & 1496.436 & 1994.937\end{array}$ $\begin{array}{lllll}1494.705 & 1495.033 & 1496.443 & 1494.94 .944\end{array}$ $\begin{array}{lllll}1494.7 & 1495.031 & 1496.441 & 1494.944\end{array}$ $\begin{array}{llllll}1494.7 & 1495.033 & 1496.438 & 1494.944\end{array}$ $\begin{array}{lllll}1494.696 & 1495.028 & 1496.434 & 1494.941\end{array}$ $\begin{array}{lllll}1494.696 & 1495.024 & 1496.422 & 1494.934\end{array}$ $\begin{array}{llll}1494.698 & 1495.028 & 1496.431 & 1494.937\end{array}$ $\begin{array}{lllll}1494.698 & 1495.031 & 1496.436 & 1494.939\end{array}$ $\begin{array}{rrrrr}1494.705 & 1495.04 & 1496.45 & 1494.946 \\ 1494.707 & 1495.054 & 1496.474 & 1494.96\end{array}$ $\begin{array}{lrrrr}1494.717 & 1495.08 & 1496.509 & 1494.983\end{array}$ $\begin{array}{lllll}1494.714 & 1495.091 & 1496.525 & 14495.002\end{array}$ $\begin{array}{lllll}1494.714 & 1495.101 & 1496.527 & 1495.009\end{array}$ $\begin{array}{lllll}1494.714 & 1495.103 & 1496.53 & 1495.016\end{array}$ $\begin{array}{lllll}1494.712 & 1495.103 & 1496.523 & 1495.016\end{array}$ $\begin{array}{lllll}1494.712 & 1495.098 & 1496.518 & 1495.011 \\ 1494.712 & 1955.105 & 14965 & 1495009\end{array}$ $\begin{array}{rrrr}1494.712 & 1495.105 & 1496.52 & 1495.009\end{array}$ $1494.717 \quad 1495.112 \quad 146955391495901018$ $\begin{array}{lllll}1494.717 & 1495.119 & 1496.546 & 1495.025\end{array}$ $\begin{array}{lllll}1494.719 & 1495.126 & 1496.553 & 1495.03\end{array}$ $1494.728 \quad 1495.145 \quad 1496.584 \quad 1495.049$ $1494.724 \quad 1495.147 \quad 1496.583 \quad 1495.056$ $1494.728 \quad 1495.149 \quad 1496.59 \quad 1495.063$ $1494.731 \quad 1495.159 \quad 1496.597 \quad 1495.07$ $1494.73314331495 .168 \quad 1466.6021495 .077$ $1494.7351495 .168 \quad 1496.609 \quad 1495.084$ $1494.731 \quad 1495.166 \quad 1496.593 \quad 1495.079$ 1494.7311495 .1591496 .5861495 .067 $1494.735 \quad 1495.161 \quad 1496.595 \quad 1495.072$ $\begin{array}{llll}1494.735 & 1495.166 & 1496.602 & 1495.077\end{array}$ $\begin{array}{lrrr}1494.738 & 1495.17 & 1496.611 & 1495.081 \\ 1494.752 & 1495.194 & 1496.651 & 1495.105\end{array}$ $\begin{array}{llllllllll}1494.169 & 1494.724 & 1495.021 & 1495.549 & 1496.787 & 1496.579 & 1493.072 & 1488.865 & 1492.917 & 1490.726\end{array}$ \begin{tabular}{llllllllllll}
1494.171 & 1494.72 & 1495.014 & 1495.536 & 1496.773 & 1496.564 & 1493.062 & 1488.867 & 1492.908 & 1490.721 \\
\hline 1494.162 & 1494.715 & 1495014 & 1495524 & 1496766 & 1496557 & 1493055 & 14886 & 1492901 & 1490716
\end{tabular}

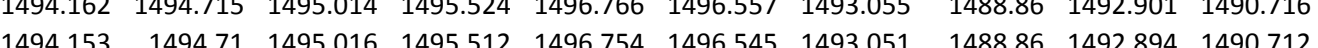
$\begin{array}{lllllllllll}1494.108 & 1494.708 & 1495.026 & 1495.519 & 1496.768 & 1496.581 & 1493.048 & 1488.862 & 1492.891 & 1490.707\end{array}$ $\begin{array}{llllllllllll}1494.068 & 1494.708 & 1495.035 & 1495.536 & 1496.789 & 1496.626 & 1493.051 & 1488.867 & 1492.896 & 1490.707\end{array}$ $\begin{array}{lllllllllll}1494.045 & 1494.705 & 1495.035 & 1495.554 & 1496.805 & 1496.665 & 1493.06 & 1488.879 & 1492.903 & 1490.707\end{array}$ $\begin{array}{llllllllllll}1494.033 & 1494.708 & 1495.033 & 1495.566 & 1496.817 & 1496.684 & 1493.067 & 1488.888 & 1492.908 & 1490.707\end{array}$ $\begin{array}{lllllllllll}1494.028 & 1494.71 & 1495.033 & 1495.575 & 1496.829 & 1496.705 & 1493.074 & 1488.893 & 1492.915 & 1490.709\end{array}$ $\begin{array}{lllllllllll}1494.033 & 1494.71 & 1495.03 & 1495.584 & 1496.838 & 1496.715 & 1493.079 & 1488.895 & 1492.922 & 1490.709 \\ 1494047 & 149471 & 1495.023 & 1495587 & 1496838 & 1496705 & 1493081 & 1488998 & 1492924 & 1490712\end{array}$ $\begin{array}{lllllllllll}1494.047 & 1494.71 & 1495.023 & 1495.587 & 1496.838 & 1496.705 & 1493.081 & 1488.898 & 1492.924 & 1490.712 \\ 1494.059 & 1494.71 & 1495018 & 1495587 & 1496842 & 1496703 & 1493083 & 1488905 & 1492929 & 1490714\end{array}$ $\begin{array}{lrrrrrrrrrr}14494.073 & 1494.708 & 1495.018 & 1495.589 & 1496.842 & 1496.698 & 1493.086 & 1488.905 & 1492.929 & 14900.714\end{array}$ $\begin{array}{lllllllllll}1494.068 & 1494.708 & 1495.021 & 1495.594 & 1496.847 & 1496.703 & 1493.088 & 1488.902 & 1492.933 & 1490.714\end{array}$ $\begin{array}{llllllllllll}1494.077 & 1494.705 & 1495.016 & 1495.596 & 1496.847 & 1496.703 & 1493.09 & 1488.9 & 1492.933 & 1490.719\end{array}$ $\begin{array}{lllllllllll}1494.08 & 1494.708 & 1495.014 & 1495.596 & 1496.849 & 1496.698 & 1493.09 & 1488.905 & 1492.931 & 1490.719\end{array}$ $\begin{array}{lllllllllll}1494.085 & 1494.705 & 1495.014 & 1495.596 & 1496.852 & 1496.698 & 1493.086 & 1488.905 & 1492.929 & 1490.719\end{array}$ $\begin{array}{llllllllll}1494.077 & 1494.705 & 1495.018 & 1495.603 & 1496.861 & 1496.708 & 1493.088 & 1488.9 & 1492.933 & 1490.721\end{array}$ $\begin{array}{lllllllllll}1494.066 & 1494.708 & 145.018 & 1455.612 & 4496.87 & 1496.722 & 1493.095 & 1488.912 & 1492.936 & 1490.723\end{array}$ $\begin{array}{rrrrrrrrrr}1494.077 & 1494.708 & 1495.014 & 1495.612 & 1496.868 & 1496.715 & 1493.095 & 1488.905 & 1492.936 & 1490.723 \\ 1494.08 & 1494.708 & 1495.011 & 1495.617 & 149687 & 1496717 & 1493095 & 1488905 & 149294 & 1490.726\end{array}$ $\begin{array}{lllllllllll}1494.075 & 1494.705 & 1495.014 & 1495.621 & 1496877 & 1496.722 & 1493.097 & 1488.909 & 1492.938 & 1490.728\end{array}$ $\begin{array}{llllllllll}1494.08 & 1494.708 & 1495.011 & 1495.621 & 1496877 & 1496.72 & 14931 & 1488.914 & 1492.943 & 1490.728\end{array}$ $\begin{array}{llllllllllll}1494.082 & 1494.708 & 1495.007 & 1495.626 & 1496.877 & 1496.72 & 1493.102 & 1488.907 & 1492.945 & 1490.73\end{array}$ $\begin{array}{llllllllllll}1494.092 & 1494.705 & 1495.009 & 1495.624 & 1496.877 & 1496.715 & 1493.1 & 1488.909 & 1492.943 & 1490.728\end{array}$ $\begin{array}{lllllllllll}1494.096 & 1494.703 & 1495.004 & 1495.621 & 1496.875 & 1496.698 & 1493.1 & 1488.907 & 1492.943 & 1490.73\end{array}$

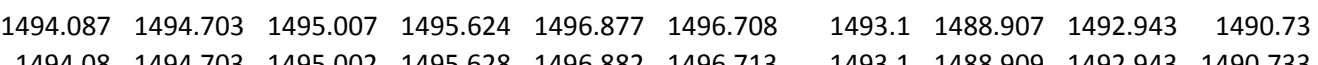
$\begin{array}{rlllllllll}1494.08 & 1494.703 & 1495.002 & 1495.628 & 1496.882 & 1496.713 & 1493.1 & 1488.909 & 1492.943 & 1490.733\end{array}$ $\begin{array}{lllllllllll}1494.063 & 1494.705 & 1495.014 & 1495.633 & 1496.891 & 1496.722 & 1493.104 & 1488.909 & 1492.945 & 1490.733 \\ 1494.049 & 1494.705 & 1495.016 & 1495.649 & 1496903 & 1496748 & 1493.107 & 1488.914 & 1492952 & 1490.735\end{array}$ $\begin{array}{llllllllllll}1494.028 & 1494.712 & 1495.021 & 1495.663 & 1496.919 & 1496.772 & 1493.114 & 1488.921 & 1492.957 & 1490.737\end{array}$ $\begin{array}{lllllllllll}1494.028 & 1494.717 & 1495.018 & 1495.677 & 1496.933 & 1496.791 & 1493.121 & 1488.923 & 1492.966 & 1490.742\end{array}$ $\begin{array}{llllllllllll}1494.037 & 1494.722 & 1495.016 & 1495.686 & 1496.938 & 1496.796 & 1493.126 & 1488.93 & 1492.969 & 1490.744\end{array}$ $\begin{array}{lllllllllll}1494.047 & 1494.724 & 1495.018 & 1495.691 & 1496.945 & 1496.791 & 1493.133 & 1488.93 & 1492.973 & 1490.747\end{array}$ $\begin{array}{llllllllll}1494.061 & 1494.727 & 1495.011 & 1495.693 & 1496.947 & 1496.787 & 1492.973 & 1488.935 & 1492.795 & 1490.749\end{array}$

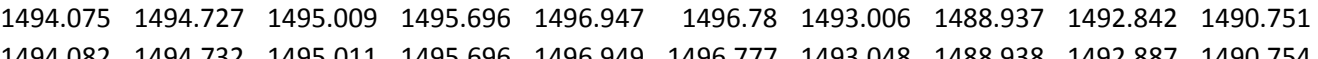

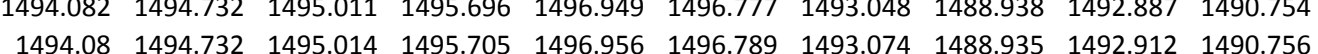
$\begin{array}{llllllllll}1494.082 & 1444.734 & 1495011 & 1495712 & 1496.963 & 1496791 & 14930 & 1488.942 & 1492.929 & 14900.758\end{array}$ $\begin{array}{lllllllllll}1494.082 & 1494.736 & 1495.011 & 1495.719 & 1496.97 & 1496.799 & 1493.102 & 1488.937 & 1492.94 & 1490.763\end{array}$ $\begin{array}{llllllllll}1494.087 & 1494.741 & 1495.016 & 1495.724 & 1496.973 & 1496.801 & 1493.114 & 1488.945 & 1492.95 & 1490.765\end{array}$ $\begin{array}{llllllllllll}1494.07 & 1494.746 & 1495.021 & 1495.742 & 1496.991 & 1496.827 & 1493.123 & 1488.954 & 1492.964 & 1490.768\end{array}$ $\begin{array}{lllllllllll}1494.085 & 1494.748 & 1495.014 & 1495.747 & 1496.994 & 1496.827 & 1493.133 & 1488.949 & 1492.973 & 1490.77\end{array}$ $\begin{array}{llllllllll}1494.087 & 1494.753 & 1495.021 & 1495.754 & 1497.003 & 1496.83 & 1493.137 & 1488.954 & 1492.98 & 1490.772\end{array}$ $\begin{array}{llllllllll}1494.092 & 1494.756 & 1495.018 & 1495.761 & 1497.008 & 1496.837 & 1433.147 & 1488.954 & 1492.987 & 1490.777 \\ 1494099 & 149476 & 1495018 & 1495768 & 1497.017 & 1496837 & 1493156 & 1488959 & 1492997 & 1490782\end{array}$

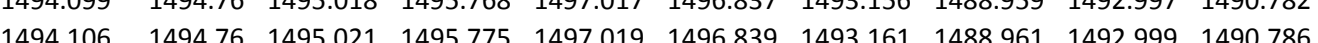
$\begin{array}{lllllllllll}1494.113 & 1494.765 & 1495.018 & 1495.779 & 1497.024 & 1496.842 & 1493.168 & 1488.959 & 1493.008 & 1490.786\end{array}$ $\begin{array}{llllllllllll}1494.132 & 1494.768 & 1495.016 & 1495.775 & 1497.022 & 1496.823 & 1493.165 & 1488.961 & 1493.008 & 1490.789\end{array}$ $\begin{array}{lllllllllll}1494.141 & 1494.768 & 1495.014 & 1495.777 & 1497.019 & 1496.813 & 1493.168 & 1488.959 & 1493.013 & 1490.791\end{array}$ $\begin{array}{lllllllllll}1494.132 & 1494.768 & 1495.018 & 1495.779 & 1497.024 & 1496.818 & 1493.17 & 1488.959 & 1493.011 & 1490.793\end{array}$ $\begin{array}{lllllllllllll}1494.129 & 1494.77 & 1495.018 & 1495.786 & 1497.026 & 1496.825 & 1493.172 & 1488.966 & 1493.013 & 1490.793\end{array}$ $\begin{array}{llllllllll}1494.124 & 1494.772 & 1495.018 & 1495.793 & 1497.033 & 1496.835 & 1493.179 & 1488.97 & 1493.02 & 1490.796\end{array}$ $\begin{array}{lllllllll}1485.346 & 1488.215 & 1493.323 & 1493.779 & 1493.077 & 1494.875 & 1493.053 & 1484.802 & 1479.197\end{array}$ $\begin{array}{lllllllllll}1485.346 & 1488.215 & 1493.314 & 1493.758 & 1493.07 & 1494.861 & 1493.046 & 1444.802 & 1479.194 \\ 1485348 & 1488204 & 1493312 & 1493.754 & 1493.063 & 1494.852 & 1493037 & 1484802 & 1479.92\end{array}$ $\begin{array}{lllllllll}1485346 & 1488.204 & 1493.312 & 1493.754 & 1493.063 & 1494.852 & 1493.037 & 14484.802 & 1499.1927\end{array}$ $\begin{array}{llllllllll}1485.348 & 1488.161 & 1493.297 & 1493.761 & 1493.056 & 1494.868 & 1493.028 & 1484.802 & 1497.187\end{array}$ $\begin{array}{llllllllllll}1485343 & 1488138 & 1493304 & 1493791 & 1493058 & 1494.901 & 1493.03 & 1484.802 & 1479.19\end{array}$ $\begin{array}{lllllllllll}1485.343 & 1488.121 & 1493.309 & 1493.807 & 1493.07 & 1494.927 & 1493.044 & 1484.799 & 1479.19\end{array}$ $\begin{array}{llllllllll}1485.343 & 1488.119 & 1493.316 & 1493.826 & 1493.072 & 1494.948 & 1493.049 & 1484.797 & 1479.19\end{array}$ $\begin{array}{llllllllll}1485.343 & 1488.112 & 1493.321 & 1493.835 & 1493.079 & 1494.974 & 1493.053 & 1484.799 & 1479.192\end{array}$ $\begin{array}{lllllllll}1485.346 & 1488.117 & 1493.328 & 1493.842 & 1493.089 & 1494.979 & 1493.06 & 1484.799 & 1479.194\end{array}$ $\begin{array}{llllllllll}1485.343 & 1488.117 & 1493.33 & 1493.845 & 1493.089 & 1494.976 & 1493.063 & 1484.799 & 1479.197\end{array}$

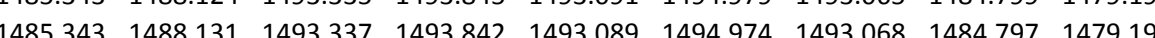
$\begin{array}{lllllllllll}1485.343 & 1488.128 & 1493.339 & 1493.847 & 1493.094 & 1494.986 & 1493.07 & 1484.797 & 1479.201\end{array}$ $\begin{array}{llllllllll}1485.341 & 1488.138 & 1493.342 & 1493.849 & 1493.096 & 1494.981 & 1493.07 & 1484.797 & 1479.201\end{array}$ $\begin{array}{llllllllll}1485.336 & 1488.136 & 1493.337 & 1493.849 & 1493.094 & 1494.979 & 1493.072 & 1484.797 & 1479.201\end{array}$ $\begin{array}{llllllllll}1485.341 & 1488.136 & 1493.34 & 1493.845 & 1493.096 & 1494.976 & 1493.068 & 1484.797 & 1479.201\end{array}$ $\begin{array}{lllllllll}1485.341 & 1488.135 & 1493.342 & 1493.861 & 1493.096 & 1494.99 & 1493.072 & 1484.797 & 1479.201\end{array}$ $\begin{array}{llllllllll}1485.343 & 1488.129 & 1493.344 & 1493.866 & 1493.101 & 1495.002 & 1493.075 & 1484.797 & 1479.204\end{array}$ $\begin{array}{lllllllllll}1485.339 & 1488.138 & 1493.344 & 1493.859 & 1493.098 & 1494.095 & 1433.075 & 1484.795 & 1479.204\end{array}$ $\begin{array}{llllllllllll}1485.346 & 1488.145 & 1493.349 & 1493.873 & 1493.105 & 1495.004 & 1493.079 & 1484.795 & 1479.206\end{array}$ $\begin{array}{llllllllll}1485.327 & 1488.152 & 1493.349 & 1493.866 & 1493.105 & 1494.997 & 1493.084 & 1484.795 & 1479.206 \\ 1485329 & 1488.152 & 1493349 & 1938.868 & 1493.11 & 1495 & 1493084 & 1484.795 & 1479.208\end{array}$ $\begin{array}{lllllllll}1485.329 & 1488.161 & 1493.354 & 1493.861 & 1493.108 & 1494.997 & 1493.082 & 1484.795 & 1479.208\end{array}$ $\begin{array}{llllllllll}1485.318 & 1488.159 & 1493.349 & 1493.859 & 1493.105 & 1494.986 & 1493.079 & 1484.795 & 1479.208\end{array}$ $\begin{array}{llllllllll}1485.322 & 1488.145 & 1493.331 & 1493.863 & 1493.105 & 1494.993 & 1493.084 & 1484.792 & 1479.208\end{array}$

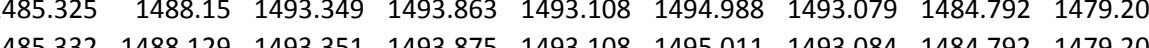
$\begin{array}{llllllllll}1485.332 & 1488.129 & 1493.351 & 1493.875 & 1493.108 & 1495.011 & 1493.084 & 1484.792 & 1479.206\end{array}$ $\begin{array}{llllllllll}1485.336 & 1488.114 & 1493.365 & 1493.915 & 1493.122 & 1495.054 & 1493.098 & 1484.792 & 1479.208\end{array}$ $\begin{array}{llllllllll}1485.343 & 1488.119 & 1493.37 & 1493.917 & 1493.129 & 1495.07 & 1493.103 & 1484.79 & 1479.208\end{array}$ $\begin{array}{lllllllll}1485.29 & 1488.126 & 1493.377 & 1493.924 & 1493.134 & 1495.073 & 1493.107 & 1484.79 & 1479.211\end{array}$

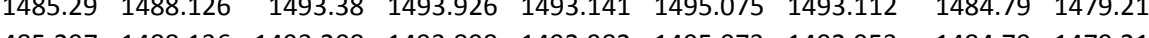
$\begin{array}{lllllllll}1485.297 & 1488.136 & 1493.208 & 1493.898 & 1492.982 & 1495.073 & 1492.953 & 1484.79 & 1479.213\end{array}$ $\begin{array}{lllllllll}1485.29 & 1488.15 & 1493.25 & 1493.863 & 1493.013 & 1495.058 & 1492.99 & 1484.787 & 1479.213 \\ 1485.29 & 1488.15 & 1493295 & 1493882 & 1493058 & 1495058 & 1493032 & 148479 & 1479.215\end{array}$ $\begin{array}{lllllllll}1485299 & 1488.147 & 1493321 & 1493898 & 1493082 & 1495068 & 1493056 & 1484788 & 1479.225\end{array}$ $\begin{array}{llllllllllll}1485.302 & 1488.157 & 1493.337 & 1493.91 & 1493098 & 1495.075 & 1493.072 & 148479 & 1479.22\end{array}$ $\begin{array}{llllllllll}1485.309 & 1488.157 & 1493.349 & 1493.915 & 1493.11 & 1495.084 & 1493.084 & 1484.79 & 1479.222\end{array}$ $\begin{array}{lllllllll}1485.313 & 1488.159 & 1493.359 & 1493.922 & 1493.117 & 1495.087 & 1493.093 & 1484.787 & 1479.225\end{array}$ $\begin{array}{lllllllll}1485.316 & 1488.152 & 1493.37 & 1493.947 & 1493.131 & 1495.113 & 1493.105 & 1484.79 & 1479.227\end{array}$ $\begin{array}{lllllllll}1485.32 & 1488.173 & 1493.377 & 1493.947 & 1493.139 & 1495.11 & 1493.112 & 1484.787 & 1479.227\end{array}$ $\begin{array}{llllllllll}1485.322 & 1488.171 & 1493.386 & 1493.954 & 1493.15 & 1495.122 & 1493.119 & 1484.79 & 1479.229\end{array}$ $\begin{array}{lllllllll}1485.325 & 1488.182 & 493.391 & 149.968 & 1493.153 & 1495.122 & 1493.126 & 1484.788 & 1479.229 \\ \end{array}$

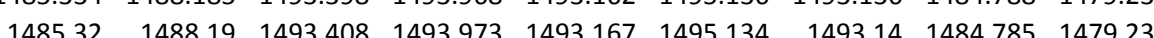
$\begin{array}{lllllllll}1485.313 & 1488.201 & 1493.415 & 1493.975 & 1493.174 & 1495.138 & 1493.145 & 1484.787 & 1479.23\end{array}$ $\begin{array}{lllllllllll}1485.318 & 1488.213 & 1493.415 & 1493.968 & 1493.174 & 1495.124 & 1493.147 & 1484.785 & 1479.236\end{array}$ $\begin{array}{llllllllll}1485.325 & 1488.22 & 1493.42 & 1493.971 & 1493.176 & 1495.115 & 1493.15 & 1484.785 & 1479.236\end{array}$ $\begin{array}{lllllllllll}1485.329 & 1488.213 & 1493.417 & 1493.971 & 1493.176 & 1495.124 & 1493.152 & 1484.785 & 1479.236\end{array}$ $\begin{array}{llllllllll}1485.329 & 1488.213 & 1493.419 & 1493.973 & 1493.181 & 1495.127 & 1493.154 & 1484.785 & 1479.236\end{array}$ $\begin{array}{lllllllll}1485.334 & 1488.208 & 1493.426 & 1493.982 & 1493.186 & 1495.131 & 1493.159 & 1484.783 & 1479.236 \\ 1485.339 & 1488.189 & 1493.436 & 1494.006 & 1493.195 & 1495.167 & 1493.166 & 1484.785 & 1479.239\end{array}$ 
Well

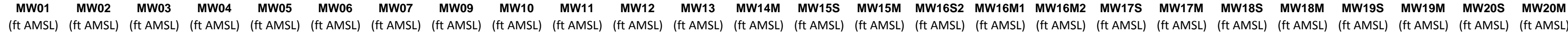

$1 / 30 / 1214: 00$ $1 / 30 / 1215: 00$ $1 / 30 / 1216: 00$ 1/30/12 17:00 1/30/12 18:00 1/30/12 20:00 1/30/12 21:00 1/30/12 22:00 $1 / 30 / 1223: 00$ 1/31/12 0:00 1/31/12 1:00 $1 / 31 / 122: 00$ 1/31/12 3:00 1/31/12 5:00 1/31/12 6:00 $1 / 31 / 127: 00$ 1/31/12 8:00 1/31/12 9:00 1/31/12 10:00 $1 / 31 / 1212: 00$ 1/31/12 13:00 1/31/12 14:00 1/31/12 15:00 1/31/12 16:00 $1 / 31 / 1227: 00$ 1/31/12 18:00 1/31/12 19:00 1/31/12 20:00 1/31/12 22:00 1/31/12 23:00 2/1/12 0:00 2/1/12 1:00 2/1/122 2:00 2/1/12 3:00 2/1/12 5:00 2/1/122 6:00 2/1/12 7:00 2/1/12 8:00 2/1/12 9:00 2/1/12 10:00 /21/12212.00 2/1/12 12:00 2/1/12 14:00 2/1/12 15:00 2/1/12 16:00 2/1/12 17:00 2/1/12 18:00 $\begin{array}{rrrr}1494.756 & 1495.215 & 1496.684 & 1495.13 \\ 1494.759 & 1495.226 & 1496.7 & 1495.147\end{array}$ $\begin{array}{llll}1494.759 & 1495.226 & 1496.7 & 1495.147\end{array}$ $\begin{array}{llll}1494.754 & 1495.224 & 1496.684 & 1495.144 \\ 1494.761 & 1495.226 & 1496684 & 1495.149\end{array}$ $\begin{array}{lllll}1494.759 & 1495.222 & 1496.668 & 1495.142\end{array}$ $\begin{array}{lllll}1494.754 & 1495.212 & 1496.649 & 1495.13\end{array}$ $\begin{array}{lllll}1494.756 & 1495.208 & 1496.642 & 1495.119\end{array}$ $\begin{array}{llll}1494.752 & 1495.201 & 1496.635 & 1495.112\end{array}$ $\begin{array}{lllll}1494.759 & 1495.205 & 1496.647 & 1495.105\end{array}$ $1494.761 \quad 1495.208 \quad 1496.6561495 .112$ $\begin{array}{llll}1494.763 & 1495.212 & 1496.665 & 1495.116 \\ 1494.768 & 1495217 & 1496675 & 1495.126\end{array}$ $\begin{array}{ccccc}1494.768 & 1495.217 & 1496.675 & 1495.126 \\ 1494.768 & 1495.215 & 1496.679 & 1495.13\end{array}$ $\begin{array}{llll}1494.77 & 1495.219 & 1496.682 & 1495.137\end{array}$ $\begin{array}{llll}1494.77 & 1495.219 & 1496.682 & 1495.137 \\ 1494.77 & 1495.217 & 1496.682 & 1495.137\end{array}$ $\begin{array}{rlll}1494.77 & 1459.217 & 1496.682 & 1495.137 \\ 1494.754 & 1495.194 & 1496.628 & 1495.109\end{array}$ $\begin{array}{lllll}1494.759 & 1495.189 & 1496.628 & 1495.1\end{array}$ $\begin{array}{rrrr}1494.754 & 1495.18 & 1496.609 & 1495.084\end{array}$ $\begin{array}{llll}1494.749 & 1495.163 & 1496.579 & 1495.06\end{array}$ $\begin{array}{rrrr}1494.745 & 1495.149 & 1496.562 & 1495.042 \\ 1494.74 & 1495.14 & 1496548 & 1495.023\end{array}$ $\begin{array}{rrrrr}1494.74 & 1495.14 & 1496.548 & 1495.023 \\ 1494.74 & 1495.129 & 1496.534 & 1495.009\end{array}$ $1494.735 \quad 1495.1191496 .527 \quad 1494.995$ $\begin{array}{lllll}1494.738 & 1495.119 & 1496.534 & 1494.99\end{array}$ $1494.738 \quad 1495.117 \quad 1496.541 \quad 1494.993$ $\begin{array}{llll}1494.731 & 1495.112 & 1496.534 & 1494.99\end{array}$ $\begin{array}{llll}1494.728 & 1495.105 & 1496.525 & 1494.981\end{array}$ $\begin{array}{llll}1494.721 & 1495.089 & 1496.495 & 1494.962 \\ 1494.714 & 1495075 & 1496.474 & 1994.944\end{array}$ $\begin{array}{rrrrr}1494.714 & 1495.075 & 1496.474 & 1494.944 \\ 1494.71 & 1495.068 & 1496.464 & 1494.93\end{array}$ $\begin{array}{rrrrr}1494.707 & 1495.056 & 1496.455 & 1494.918\end{array}$ $\begin{array}{llllll}1494.703 & 1495.047 & 1496.441 & 1494.906\end{array}$ $\begin{array}{lllll}1494.698 & 1495.035 & 1496.431 & 1494.895\end{array}$ $\begin{array}{lllll}1494.693 & 1495.031 & 1496.427 & 1494.888\end{array}$ $\begin{array}{llll}1494.691 & 1495.026 & 1496.42 & 1494.883\end{array}$ $\begin{array}{llll}1494.691 & 1495.024 & 1496.422 & 1494.881 \\ 1499.691 & 1495019 & 1469.424 & 194878\end{array}$ $\begin{array}{llll}194.691 & 1495.024 & 1496.422 & 1494881\end{array}$ $\begin{array}{lllll}1494.691 & 1495.024 & 1496.431 & 1494.883 \\ 1494.689 & 1495.021 & 1496.434 & 1494888\end{array}$ $\begin{array}{lllll}1494.684 & 1495.017 & 1496.424 & 1494.881\end{array}$ $\begin{array}{llllll}1494.686 & 1495.017 & 1496.427 & 1494.883\end{array}$ $\begin{array}{llllll}1494.682 & 1495.01 & 1496.422 & 1494.881\end{array}$ $\begin{array}{lllll}1494.679 & 1495.003 & 1496.406 & 1494.869\end{array}$ $1494.677 \quad \begin{array}{llll}1494.996 & 1496.396 & 1494.86\end{array}$ $1494.668 \quad 1494.984 \quad 1496.3751494 .848$ $\begin{array}{lllll}1494.673 & 1494.979 & 1496.373 & 1494.841 \\ 1494.679 & 1494.984 & 1496.392 & 1494846\end{array}$ $1494.684 \quad 1494.998 \quad 1496.422 \quad 1494.864$ $1494.686 \quad 1495.007 \quad 1496.446 \quad 1494.881$ $1494.684 \quad 1495.014 \quad 1496.455 \quad 1494.895$ $1494.682 \quad 1495.014 \quad 1496.453 \quad 1494.899$ $1494.675 \quad 1495.012 \quad 1496.443 \quad 1494.897$ $\begin{array}{rrrr}1494.673 & 1495.005 & 1496.434 & 1494.892 \\ 1494.666 & 1494.996 & 1496.41 & 1494.878\end{array}$ $\begin{array}{llllllllll}1494.085 & 1494.784 & 1495.035 & 1495.833 & 1497.073 & 1496.897 & 1493.198 & 1488.973 & 1493.037 & 1490.805\end{array}$

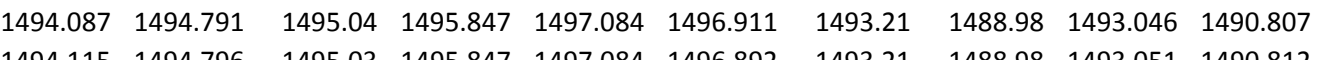
$\begin{array}{llllllllllll}1494.124 & 1494.796 & 1495.03 & 1495.847 & 1497.084 & 1496.892 & 1493.21 & 1488.98 & 1493.001 & 1490.812\end{array}$ $\begin{array}{lllllllllllll}1494.148 & 1494.796 & 1495.028 & 1495.851 & 1497.084 & 1496.875 & 1493214 & 1488.984 & 1493053 & 1490.816\end{array}$ $\begin{array}{lllllllllll}1494.167 & 1494.811 & 1495.026 & 1495.847 & 1497.075 & 1496.854 & 1493.212 & 1488.982 & 1493.053 & 1490.817\end{array}$ $\begin{array}{lllllllllll}1494.174 & 1494.813 & 1495.028 & 1495.844 & 1497.075 & 1496.842 & 1493.212 & 1488.977 & 1493.051 & 1490.819\end{array}$ $\begin{array}{lllllllllll}1494.186 & 1494.815 & 1495.026 & 1495.84 & 1497.07 & 1496.835 & 1493.21 & 1488.975 & 1493.051 & 1490.819\end{array}$ $\begin{array}{lllllllllll}1494.181 & 1494.803 & 1495.033 & 1495.844 & 1497.075 & 1496.844 & 1493.212 & 1488.977 & 1493.055 & 1490.819\end{array}$

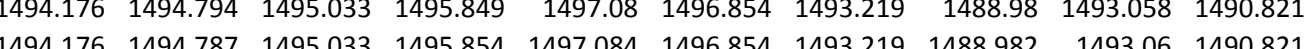
$\begin{array}{llrrrrrrrr}1494.176 & 1494.787 & 1495.033 & 1495.854 & 1497.084 & 1496.854 & 1493.219 & 1488.982 & 1493.06 & 1490.821 \\ 1494.171 & 1494.777 & 1495.035 & 1495.86 & 1497.091 & 1496.868 & 1493226 & 1488.982 & 1493065 & 1490824\end{array}$ $\begin{array}{lllllllllll}1494.174 & 1494.782 & 1495.035 & 1495.865 & 1497.096 & 1496.87 & 1493.226 & 1488.991 & 1493.065 & 1490.824\end{array}$ $\begin{array}{llllllllll}1494.179 & 1494.779 & 1495.035 & 1495.867 & 1497.098 & 1496.873 & 1493.229 & 1488.989 & 1493.069 & 1490.826\end{array}$ $\begin{array}{llllllllll}1494.183 & 1494.782 & 1495.037 & 1495.87 & 1497.098 & 1496.87 & 1493.231 & 1488.987 & 1493.072 & 1490.828\end{array}$ $\begin{array}{llllllllll}1494.23 & 1494.83 & 1495.018 & 1495.854 & 1497.073 & 1496.823 & 1493.222 & 1488.982 & 1493.067 & 1490.826\end{array}$ $\begin{array}{llllllllll}1494.228 & 1494.832 & 1495.026 & 1495.849 & 1497.07 & 1496.815 & 1493.219 & 1488.98 & 1493.06 & 1490.826\end{array}$ $\begin{array}{llllllllll}1494.24 & 1494.844 & 1495.023 & 1495.842 & 1497.061 & 1496.801 & 1493.214 & 1488.968 & 1493.058 & 1490.821\end{array}$ $\begin{array}{llllllllll}1494.258 & 1494.846 & 1495.016 & 1495.826 & 1497.042 & 1496.768 & 1493.205 & 1488.968 & 1493.051 & 1490.819\end{array}$ $\begin{array}{llllllllllll}1494.258 & 1494.794 & 1455.011 & 1455.814 & 1497.031 & 1496.753 & 1493.196 & 1488.959 & 1493.039 & 1490.819\end{array}$ $\begin{array}{llllllllllll}1494.249 & 1494.784 & 1495.011 & 1495.793 & 1497.012 & 1496.732 & 1493.179 & 1488.947 & 1493.025 & 1490.807\end{array}$

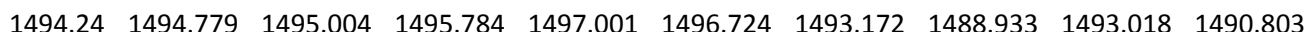
$\begin{array}{lllllllllll}1494.223 & 1494.775 & 1495.009 & 1495.779 & 1497.001 & 1496.734 & 1493.165 & 1488.94 & 1493.011 & 1490.8\end{array}$ $\begin{array}{lllllllllll}1494.204 & 1494.772 & 1495.014 & 1495.779 & 1497.003 & 1496.739 & 1493.165 & 1488.938 & 1493.008 & 1490.798\end{array}$ $\begin{array}{lllllllllll}1494.202 & 1494.77 & 1495.009 & 1495.775 & 1496.996 & 1496.741 & 1493.161 & 1488.942 & 1493.006 & 1490.793\end{array}$ $\begin{array}{lllllllllll}1494.202 & 1494.765 & 1455.004 & 1495.768 & 1496.989 & 1496.732 & 1493.156 & 1488.928 & 1492.999 & 1490.789\end{array}$

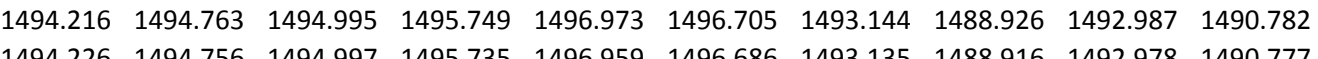
$\begin{array}{llllllllll}1494.226 & 1494.756 & 1494.997 & 1455.735 & 146.959 & 146.686 & 1493.135 & 1488.916 & 1492.978 & 1490.777 \\ 1494.221 & 1494.748 & 1494.992 & 1495.726 & 1496.952 & 14966679 & 1493.123 & 1488.916 & 1492.971 & 1490.772\end{array}$ $\begin{array}{lllllllllllll}1494.219 & 1494.744 & 1494.992 & 1495.717 & 1496.942 & 1496.672 & 1493.119 & 1488.909 & 1492.964 & 1490.765\end{array}$ $\begin{array}{lllllllllll}1494.214 & 1494.736 & 1494.988 & 1495.705 & 1496.933 & 1496.665 & 1493.112 & 1488.905 & 1492.955 & 1490.761\end{array}$ $\begin{array}{llllllllllll}1494.211 & 1494.732 & 1494.99 & 1495.693 & 1496.922 & 1496.657 & 1493.104 & 1488.898 & 1492.952 & 1490.756\end{array}$ $\begin{array}{llllllllllll}1494.202 & 1494.727 & 1494.985 & 1495.686 & 1496.915 & 1496.657 & 1493.1 & 1488.895 & 1492.943 & 1490.749\end{array}$ $\begin{array}{lllllllllll}1494.193 & 1494.72 & 1494.985 & 1495.677 & 1496.91 & 1496.655 & 1493.093 & 1488.895 & 1492.938 & 1490.744\end{array}$ $\begin{array}{llllllllll}1494.181 & 1494.717 & 1494.98 & 1495.672 & 1496.905 & 1496.657 & 1493.09 & 1488.893 & 1492.933 & 1490.74 \\ 1494.169 & 1494.712 & 1494.988 & 1495668 & 1496903 & 1496.66 & 1493.088 & 1488888 & 1492931 & 1490.737\end{array}$

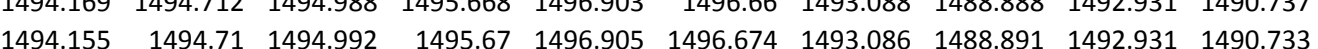
$\begin{array}{llllllllllll}1494.148 & 1494.71 & 1494.988 & 1495.666 & 1496903 & 1496.677 & 1493.086 & 1488.888 & 1492.929 & 1490.728\end{array}$ $\begin{array}{lllllllllll}1494.148 & 1494.705 & 1494.985 & 1495.659 & 1496.898 & 1496.672 & 1493.081 & 1488.886 & 1492.926 & 1490.726\end{array}$ $\begin{array}{llllllllll}1494.139 & 1494.705 & 1494.988 & 1495.656 & 1496.898 & 1496.677 & 1493.081 & 1488.881 & 1492.924 & 1490.723\end{array}$ $\begin{array}{lllllllllll}1494.141 & 1494.703 & 1494.98 & 1495.654 & 1496.894 & 1496.674 & 1493.079 & 1488.884 & 1492.919 & 1490.719\end{array}$ $\begin{array}{lllllllllll}1494.146 & 1494.701 & 1494.978 & 1495.642 & 1496.884 & 1496.66 & 1493.072 & 1488.884 & 1492.915 & 1490.716\end{array}$ $\begin{array}{lllllllllll}1494.143 & 1494.698 & 1494.976 & 1495.635 & 1496.88 & 1496.65 & 1493.067 & 1488.879 & 1492.91 & 1490.712\end{array}$ $\begin{array}{lllllllllllll}1494.146 & 1494.693 & 1494.969 & 1455.624 & 1496.866 & 1496.638 & 1493.058 & 4888.879 & 1492.903 & 1490.707\end{array}$

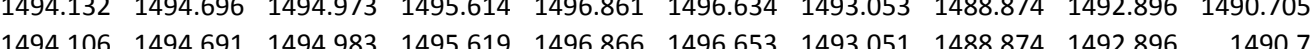
$\begin{array}{llllllllllll}1494.075 & 1494.689 & 1494.988 & 1495.628 & 1496.877 & 1496.684 & 1493.055 & 1488.881 & 1492.901 & 1490.702\end{array}$ $\begin{array}{llllllllllll}1494.056 & 1494.691 & 1494.988 & 1495.638 & 1496.891 & 1496.71 & 1493.065 & 1488.881 & 1492.905 & 1490.702\end{array}$ $\begin{array}{llllllllll}1494.054 & 1494.693 & 1494.988 & 1495.642 & 1496.898 & 1496.717 & 1493.065 & 1488.888 & 1492.908 & 1490.702\end{array}$ $\begin{array}{lllllllllll}1494.061 & 1494.693 & 1494.98 & 1495.645 & 1496.898 & 1496.717 & 1493.065 & 1488.891 & 1492.905 & 1490.7\end{array}$ $\begin{array}{llllllllll}1494.07 & 1494.691 & 1494.978 & 1495.642 & 1496.894 & 1496.71 & 1493.065 & 1488.891 & 1492.905 & 1490.7\end{array}$ $\begin{array}{rrrrrrrrrr}1494.08 & 1494.689 & 1494.976 & 1495.638 & 1496.891 & 1496.698 & 1493.058 & 1488.888 & 1492.903 & 1490.698 \\ 1494.099 & 1494.686 & 1494.966 & 1495.628 & 149688 & 1496.677 & 1493.053 & 1488.884 & 1492.894 & 1490.695\end{array}$ $\begin{array}{lllllllll}1485.346 & 1488.18 & 1493.443 & 1494.029 & 1493.207 & 1495.197 & 1493.18 & 1484.785 & 1479.241 \\ 1485346 & 1488.187 & 1493.455 & 1494.043 & 1493219 & 1495.207 & 1493.187 & 1484.785 & 1479.246\end{array}$ $\begin{array}{lllllllll}1485.346 & 1488.187 & 1493.455 & 1494.043 & 1493.219 & 1495.207 & 1493.187 & 1484.785 & 1479.246\end{array}$

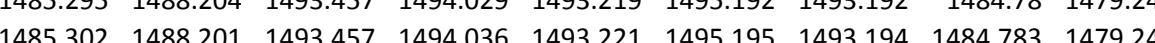
$\begin{array}{llllllllll}1485306 & 1488211 & 1493.462 & 1494.029 & 1493224 & 1495.183 & 1493.194 & 1484.785 & 1479.241\end{array}$ $\begin{array}{lllllllllllll}1485.306 & 1488.232 & 1493.459 & 1494.015 & 1493219 & 1495164 & 1493.192 & 1484.783 & 1479.241\end{array}$ $\begin{array}{lllllllllll}1485.313 & 1488.234 & 1493.459 & 1494.013 & 1493.217 & 1495.162 & 1493.192 & 1484.783 & 1479.241\end{array}$ $\begin{array}{llllllllll}4485.313 & 1488.241 & 1493.459 & 1494.006 & 1493.217 & 1495.155 & 1493.19 & 1484.783 & 1479.241\end{array}$ $\begin{array}{lllllllll}1485.32 & 1488.239 & 1493.457 & 1494.013 & 1493.219 & 1495.157 & 1493.192 & 1484.783 & 1479.243 \\ 148532 & 1488 & 1496\end{array}$ $\begin{array}{lllllllll}1485.32 & 1488.226 & 1493.466 & 1494.015 & 1493.224 & 1495.164 & 1493.197 & 1484.783 & 1479.246\end{array}$ $\begin{array}{llllllllll}1485.322 & 1488.251 & 1493.469 & 1494.024 & 1493.226 & 1495.174 & 1493.199 & 1484.783 & 1479.248 \\ 1485.327 & 1488.248 & 1493.474 & 1494.022 & 1493.231 & 1495.178 & 1493204 & 1484783 & 1479.25\end{array}$

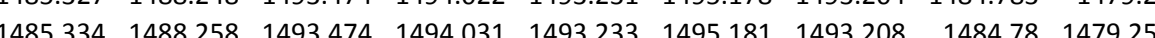
$\begin{array}{lllllllllll}1485.343 & 1488.262 & 1493.478 & 1494.038 & 1493.236 & 1495.181 & 1493.208 & 1484.78 & 1479.253\end{array}$ $\begin{array}{llllllllll}1485.339 & 1488.272 & 1493.478 & 1494.043 & 1493.238 & 1495.183 & 1493.211 & 1484.783 & 1479.255\end{array}$ $\begin{array}{llllllllll}1485.339 & 1488.3 & 1493.471 & 1493.999 & 1493.226 & 1495.138 & 1493.201 & 1484.776 & 1479.25\end{array}$ $\begin{array}{lllllllllll}1485.343 & 1488.293 & 1493.467 & 1493.996 & 1493.226 & 1495.134 & 1493.197 & 1484.78 & 1479.25\end{array}$ $\begin{array}{lllllllll}1485.302 & 1488.302 & 1493.464 & 1493.982 & 1493.219 & 1495.12 & 1493.192 & 1484.78 & 1479.248\end{array}$ $\begin{array}{llllllllll}1485.299 & 1488.312 & 1493.457 & 1493.961 & 1493.21 & 1495.091 & 1493.182 & 1484.778 & 1479.248\end{array}$ $\begin{array}{llllllllll}1485 & 300 & 1488.312 & 1493.441 & 1493.936 & 1493.203 & 1455.073 & 1493.173 & 1484.78 & 1479.246\end{array}$ $\begin{array}{llllllllll}1485.306 & 1488.305 & 1493.431 & 1493.929 & 1493.186 & 1495.049 & 1493.161 & 1484.776 & 1479.241\end{array}$ $\begin{array}{lllllllllll}1485.283 & 1488.281 & 1493.419 & 1493.917 & 1493.172 & 1495.042 & 1493.152 & 1484.776 & 1479.236\end{array}$ $\begin{array}{lllllllllll}1485.253 & 1488.269 & 1493.415 & 1493.924 & 1493.172 & 1495.047 & 1493.143 & 1484.776 & 1479.23\end{array}$ $\begin{array}{llllllllll}1485.23 & 1488.269 & 1493.412 & 1493.91 & 1493.167 & 1495.037 & 1493.14 & 1484.776 & 1479.234\end{array}$ $\begin{array}{lllllllll}1485.175 & 1488.272 & 1493.406 & 1493.908 & 1493.157 & 1495.028 & 1493.136 & 1484.773 & 1479.232\end{array}$

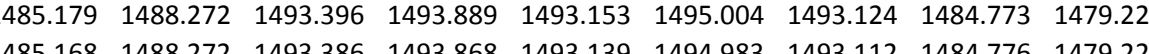
$\begin{array}{llllllllll}1485.168 & 1488.272 & 1493.386 & 1493.868 & 1493.139 & 1494.983 & 1493.112 & 1484.776 & 1479.227 \\ 1485.168 & 1488.269 & 1493.377 & 1493.863 & 1493.131 & 1494.974 & 1493.107 & 1484.773 & 1479.225\end{array}$ $\begin{array}{llllllllll}1485.177 & 1488.267 & 1493.373 & 1493.849 & 1493.124 & 1494.962 & 1493.098 & 1484.773 & 1479.222\end{array}$ $\begin{array}{llllllllll}1485.184 & 1488.262 & 1493.361 & 1493.842 & 1493.117 & 1494.955 & 1493.091 & 1484.773 & 1479222\end{array}$ $\begin{array}{llllllllll}1485.191 & 1488.255 & 1493.356 & 1493.838 & 1493.11 & 1494.943 & 1493.086 & 1484.773 & 1479.22\end{array}$ $\begin{array}{llllllllll}1485.193 & 1488.257 & 1493.356 & 1493.826 & 1493.103 & 1494.941 & 1493.079 & 1484.773 & 1479.22\end{array}$ $\begin{array}{llllllllll}1485.205 & 1488.251 & 1493.349 & 1493.824 & 1493.098 & 1494.934 & 1493.072 & 1484.771 & 1479.22\end{array}$ $\begin{array}{lllllllll}1485.207 & 1488.239 & 1493.344 & 1493.821 & 1493.096 & 1494.934 & 1493.072 & 1484.773 & 1479.222\end{array}$

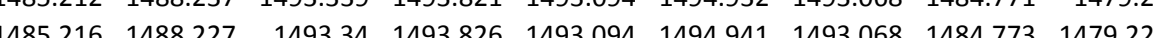

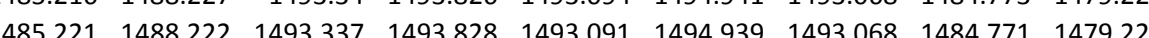
$\begin{array}{llllllllll}1485.225 & 1488.225 & 1493.335 & 1493.817 & 1493.086 & 1494.936 & 1493.063 & 1484.771 & 1479.222\end{array}$ $\begin{array}{lllllllll}1485.202 & 1488.218 & 1493.332 & 1493.824 & 1493.086 & 1494.936 & 1493.06 & 1484.771 & 1479.222\end{array}$ $\begin{array}{lllllllll}1485.202 & 1488.222 & 1493.328 & 1493.817 & 1493.084 & 1494.929 & 1493.058 & 1484.771 & 1479.222\end{array}$ $\begin{array}{lllllllllll}4485.212 & 1488.218 & 1493.321 & 1493.807 & 1493.075 & 1494.917 & 1493.051 & 1484.771 & 1479.22\end{array}$ $\begin{array}{llllllllll}1485.214 & 1488.215 & 1493.318 & 1493.798 & 1493.072 & 1494.913 & 1493.049 & 1484.771 & 1479.218\end{array}$

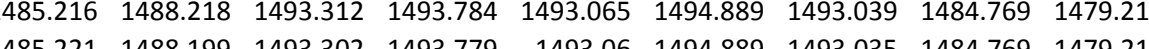

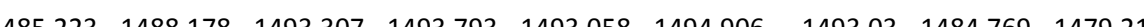
$\begin{array}{llllllllll}1485.228 & 1488.164 & 1493.309 & 1493.812 & 1493.065 & 1494.932 & 1493.037 & 1484.769 & 1479.213\end{array}$ $\begin{array}{llllllllll}1485.232 & 1488.152 & 1493.314 & 1493.835 & 1493.072 & 1494.95 & 1493.042 & 1484.766 & 1479.215\end{array}$ $\begin{array}{lllllllll}1485.237 & 1488.154 & 1493.314 & 1493.835 & 1493.072 & 1494.96 & 1493.046 & 1484.766 & 1479.213\end{array}$ $\begin{array}{rrrrrrrrr}1485.184 & 1488.152 & 1493.314 & 1493.831 & 1493.072 & 1494.962 & 1493.046 & 1484.766 & 1479.213 \\ 1485188 & 1488.159 & 1493.312 & 1493.824 & 1493.07 & 1494.953 & 1493.042 & 1484.766 & 1479211\end{array}$ $\begin{array}{llllllllll}1485.188 & 1488.159 & 1493.312 & 1493.824 & 1493.07 & 1494.953 & 1493.042 & 1484.766 & 1479.211\end{array}$ $\begin{array}{llllllllll}1485.191 & 1488.157 & 1493.311 & 1493.817 & 1493.063 & 1494.943 & 1493.037 & 1484.769 & 1479.211 \\ 1485.191 & 1488.166 & 1493.302 & 1493.807 & 1493.058 & 1494.922 & 1493.032 & 1484.766 & 1479.206\end{array}$ 
Well

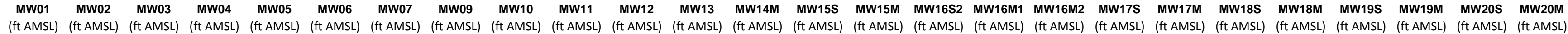

2/1/12 20:00 2/1/11221:00 2/1/12 22:00 2/1/122 23:00 2/2/120:00 2/2/122:00 2/2/12 3:00 2/2/12 4:00 2/2/122 5:00 2/2/122 6:00 2/2/12 7:00 2/2/12 9:00 2/2/12 10:00 2/2/12 11:00 2/2/12 12:00 2/2/12 13:00 2/2/12 14:00 2/2/122 15:00 2/2/12 16:00 2/2/12 18:00 2/2/2/12 18:00 2/2/12 20:00 2/2/12 21:00 2/2/122 22:00 2/2/1223:00 2/3/120:00 2/3/132 2:00 $2 / 3 / 123.00$ 2/3/12 4:00 2/3/12 5:00 2/3/12 6:00 2/3/12 7:00 2/3/128:00 2/3/12 9:00 2/3/12 11:00 2/3/12 12:00 2/3/12 13:00 2/3/12 14:00 2/3/12 15:00 2/3/12 16:00 $2 / 3 / 1218.00$ 2/3/12 18:00 2/3/12 20:00 2/3/12 21:00 2/3/12 22:00 2/3/12 23:00 2/4/12 0:00 $\begin{array}{llll}1494.666 & 1494.989 & 1496.396 & 1494.867 \\ 1494.668 & 1494.986 & 1496399 & 1494.862\end{array}$ $\begin{array}{rrrr}1494.668 & 1494.986 & 1496.399 & 1494.862 \\ 1494.67 & 1494.989 & 1496.408 & 1494.864\end{array}$ $\begin{array}{lllll}1494.67 & 1494.989 & 1496.408 & 1494.864 \\ 149.67 & 1494993 & 1496415 & 194.871\end{array}$ $\begin{array}{rrrr}1494.666 & 1494.989 & 1496.413 & 1494.871\end{array}$ $\begin{array}{lllll}1494.663 & 1494.986 & 1496.408 & 1449.871\end{array}$ $\begin{array}{llllll}1494.661 & 1494.979 & 1496.401 & 1494.867\end{array}$ $\begin{array}{llllll}1494.663 & 1494.982 & 1496.396 & 1494.862\end{array}$ $\begin{array}{lllll}1494.661 & 1494.979 & 1496.399 & 1494.862\end{array}$ $\begin{array}{lllll}1494.654 & 1494.972 & 1496.385 & 1494.855\end{array}$ $\begin{array}{llll}1494.654 & 1494.965 & 1496.371 & 1494.843 \\ 1494.654 & 1494965 & 1496371 & 19484\end{array}$ $\begin{array}{llll}1494.654 & 1494.965 & 1496.371 & 1494.841 \\ 1494.652 & 1494.961 & 1496.371 & 1494.839\end{array}$ $\begin{array}{lllll}1494.656 & 1494.963 & 1496.38 & 14494.841\end{array}$ $\begin{array}{llll}1494.652 & 1494.963 & 1496.385 & 1494.846\end{array}$ $\begin{array}{lllll}1494.654 & 1494.965 & 1496.392 & 1494.85\end{array}$ $\begin{array}{lllll}1494.661 & 1494.977 & 1496.41 & 1494.862\end{array}$ $1494.6661494 .996 \quad 1496.448 \quad 1494.885$ $\begin{array}{llll}1494.67 & 1495.019 & 1496.483 & 1494.913\end{array}$ $\begin{array}{lllll}1494.673 & 1495.031 & 1496.502 & 1494.932\end{array}$ $\begin{array}{llll}1494.675 & 1495.052 & 1496.523 & 1494.958\end{array}$ $\begin{array}{lllll}1494.673 & 1495.054 & 1496.52 & 1494.965\end{array}$ $\begin{array}{lllll}1494.67 & 1495.054 & 1496.513 & 1494.965\end{array}$ $\begin{array}{llrr}1494.666 & 1495.045 & 1496.49 & 1944.953\end{array}$ $\begin{array}{llll}1494.673 & 1495.054 & 1496.513 & 1494.963\end{array}$ $\begin{array}{lllll}1494.682 & 1495.07 & 1496.541 & 1494.976\end{array}$ 1494.6861495 .0891496 .5651494 .995 $\begin{array}{llll}1494.686 & 1495.098 & 1496.579 & 1495.009 \\ 1494.684 & 1495.089 & 1496.548 & 1494.997\end{array}$ $\begin{array}{llll}1444.684 & 1455.033 & 1496.509 & 1495.002\end{array}$ $\begin{array}{llll}1494.677 & 1494.996 & 1496.165 & 1494.997\end{array}$ $\begin{array}{lllll}1494.682 & 1494.989 & 1496.235 & 1495.009\end{array}$ $\begin{array}{lllll}1494.682 & 1494.991 & 1496.312 & 1495.004\end{array}$ $\begin{array}{lllll}1494.71 & 1495.01 & 1496.361 & 1495.028\end{array}$ $1494.6821494 .9861496 .347 \quad 1495.025$ $\begin{array}{lllll}1494.67 & 1494.961 & 1496.357 & 1495.018\end{array}$ $\begin{array}{lllll}1494.668 & 1496868 & 1496329 \quad 1495\end{array}$ $\begin{array}{llrr}1494.666 & 1498.828 & 1496.28 & 1494.995\end{array}$ $\begin{array}{lllll}1494.668 & 1500.094 & 1496.31 & 1495.004\end{array}$ $\begin{array}{lllll}1494.684 & 1501.036 & 1496.378 & 1495.025\end{array}$ $\begin{array}{lllll}1494.684 & 1502.027 & 1496.434 & 1495.046\end{array}$ $1494.6771502 .9411496 .471 \quad 1495.056$ $1494.684 \quad 1504.748 \quad 1496.546 \quad 1495.06$ $1494.677 \quad 1508.86 \quad 1496.5411495 .065$ $1494.67 \quad 1513.271 \quad 1496.2071495 .056$ $1494.675 \quad 1514.682 \quad 1496.261 \quad 1455.056$ $1494.808 \quad 1515.701 \quad 1496.256 \quad 1495.06$ $1495.166 \quad 1516.342 \quad 1496.242 \quad 1495.056$ $1495.492 \quad 1516.869 \quad 1496.247 \quad 1495.056$ $\begin{array}{llll}1495.755 & 1517.298 & 1496.289 & 1495.058 \\ 1495.972 & 1517.657 & 1496.301 & 1495.058\end{array}$ $\begin{array}{llllllllll}1494.106 & 1494.686 & 1494.966 & 1495.619 & 1496.873 & 1496.665 & 1493.046 & 1488.879 & 1492.891 & 1490.695 \\ 1494101 & 149468 & 1494969 & 1495617 & 149687 & 1496655 & 1493.044 & 1488.877 & 1492887 & 1490.693\end{array}$ $\begin{array}{llllllllll}1494.101 & 1494.684 & 1494.969 & 1495.617 & 1496.87 & 1496.665 & 1493.044 & 1488.877 & 1492.887 & 1490.693 \\ 1494.087 & 1494.681 & 1494971 & 1455621 & 1496875 & 1496679 & 1493044 & 1488874 & 1992889 & 1990691\end{array}$ $\begin{array}{lllllllllllll}1494.087 & 1494.681 & 1494.971 & 1455.621 & 1496.87 & 1496.679 & 1493.044 & 1488.874 & 1492.889 & 1490.691 \\ 1494 & 1494681 & 1494971 & 1495624 & 149688 & 1496686 & 1493046 & 1488881 & 1492889 & 1490.691\end{array}$ $\begin{array}{llllllllllll}1494.082 & 1494.677 & 1494.966 & 1495.621 & 1496.877 & 1496.689 & 1493.046 & 1488.879 & 1492.889 & 1490.691\end{array}$ $\begin{array}{llllllllllll}1494.085 & 1494.679 & 1494.966 & 1495.61 & 1496.877 & 1496.684 & 1493.046 & 1488.881 & 1492.889 & 1490.691\end{array}$ $\begin{array}{llllllllllll}1494.087 & 1494.677 & 1494.961 & 1495.614 & 1496.873 & 1496.674 & 1493.046 & 1488.872 & 1492.891 & 1490.688\end{array}$ $\begin{array}{lllllllllll}1494.087 & 1494.672 & 1494.961 & 1495.61 & 1496.87 & 1496.672 & 1493.041 & 1488.876 & 1492.884 & 1490.688\end{array}$ $\begin{array}{llllllllll}1494.08 & 1494.674 & 1494.964 & 1495.612 & 1496.868 & 1496.674 & 1493.041 & 1488.877 & 1492.887 & 1490.688\end{array}$ $\begin{array}{lllllllllll}1494.089 & 1494.669 & 1494.954 & 1495.605 & 1496.863 & 1496.665 & 1493.039 & 1488.874 & 1492.882 & 1490.686\end{array}$ $\begin{array}{llllllllll}1494.092 & 1494.667 & 1494.954 & 1495.598 & 1496.854 & 1496.65 & 1493.032 & 1488.872 & 1492.877 & 1490.684 \\ 1494087 & 1494665 & 1494959 & 1495596 & 1496854 & 1496.553 & 1493032 & 1488865 & 1492872 & 1490681\end{array}$ $\begin{array}{lllllllllllll}1494.08 & 1494.665 & 1494.954 & 1495591 & 1496852 & 1496653 & 1493027 & 1488867 & 149287 & 1490681\end{array}$ $\begin{array}{llllllllllll}1494.068 & 1494.665 & 1494.966 & 1495.596 & 1496.854 & 1496.665 & 1493.027 & 1488.87 & 1492872 & 1490.679\end{array}$ $\begin{array}{lllllllllll}1494.059 & 1494.662 & 1494.961 & 1495.596 & 1496.854 & 1496.674 & 1493.027 & 1488.867 & 1492.87 & 1490.677\end{array}$ $\begin{array}{lllllllllll}1494.049 & 1494.66 & 1494.959 & 1495.596 & 1496.859 & 1496.674 & 1493.027 & 1488.872 & 1492.87 & 1490.679\end{array}$ $\begin{array}{llllllllllll}1494.028 & 1494.662 & 1494.964 & 1495.605 & 1496.87 & 1496.691 & 1493.032 & 1488.877 & 1492.872 & 1490.677\end{array}$ $\begin{array}{lllllllllll}1494.005 & 1494.665 & 1494.973 & 1495.621 & 1496.884 & 1496.727 & 1493.039 & 1488.884 & 1492.884 & 1490.679\end{array}$

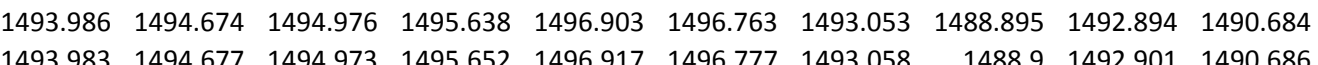
$\begin{array}{llllllllll}1493.983 & 1494.677 & 1494.973 & 1495.652 & 1496.917 & 1496.777 & 1493.058 & 1488.9 & 1492.901 & 1490.686 \\ 1493.988 & 1494.684 & 1494.971 & 1495661 & 1496.926 & 1496787 & 1493.065 & 1488907 & 1492905 & 1490691\end{array}$ $\begin{array}{llllllllllll}1493.993 & 1494.686 & 1494.973 & 1495.668 & 1496.935 & 1496.787 & 1493.069 & 1488.914 & 1492.91 & 1490.693\end{array}$ $\begin{array}{llllllllll}1494.009 & 1494.693 & 1494.976 & 1495.672 & 1496.938 & 1496.789 & 1493.072 & 1488.914 & 1492.912 & 1490.695\end{array}$ $\begin{array}{lllllllllllll}1494.026 & 1494.693 & 1494.969 & 1495.677 & 1496.938 & 1496.787 & 1493.072 & 1488.916 & 1492.912 & 1490.7\end{array}$ $\begin{array}{lllllllllll}1494.052 & 1494.693 & 1494.959 & 1495.668 & 1496.931 & 1496.756 & 1493.067 & 1488.914 & 1492.91 & 1490.7\end{array}$ $\begin{array}{llllllllll}1494.04 & 1494.696 & 1494.966 & 1495.682 & 1496.945 & 1496.777 & 1493.072 & 1488.919 & 1492.912 & 1490.702\end{array}$ $\begin{array}{lllllllllll}1494.028 & 1494.698 & 1494.976 & 1495.693 & 1496.956 & 1496.796 & 1493.079 & 1488.921 & 1492.922 & 1490.707\end{array}$ $\begin{array}{llllllllll}1494.019 & 1494.705 & 1494.978 & 1495.707 & 1496.975 & 1496.818 & 1493.09 & 1488.928 & 1492.933 & 1490.712 \\ 1494.026 & 1494.712 & 1494.973 & 1495721 & 1496.98 & 1496839 & 1493.107 & 1488.93 & 1492947 & 1490716\end{array}$ $\begin{array}{llllllllll}1494.026 & 1494.712 & 1494.973 & 1495.721 & 1496.98 & 1496.839 & 1493.107 & 1488.93 & 1492.947 & 1490.716 \\ 1494.052 & 1494.674 & 1494.976 & 1495712 & 1496973 & 1496803 & 1493128 & 1488.928 & 1492976 & 1490721\end{array}$ $\begin{array}{llllllllllll}1494.059 & 1494.655 & 1494.976 & 1495.71 & 1496.975 & 1496.791 & 1493.149 & 1488.933 & 1492.999 & 1490.728\end{array}$ $\begin{array}{llllllllllll}1494.085 & 1494.634 & 1494.964 & 1495.712 & 1496.97 & 1496.791 & 1493.175 & 1488.935 & 1493.023 & 1490.733\end{array}$ $\begin{array}{lllllllllll}1494.075 & 1494.626 & 1494.969 & 1495.672 & 1496.975 & 1496.803 & 1493.184 & 1488.938 & 1493.03 & 1490.74\end{array}$ $\begin{array}{lllllllllll}1494.08 & 1494.622 & 1494.971 & 1495.661 & 1496.973 & 1496.811 & 1493.184 & 1488.935 & 1493.027 & 1490.747\end{array}$ $\begin{array}{llllllllll}1494.056 & 1494.617 & 1495.011 & 1495.638 & 1496.998 & 1496.83 & 1493.182 & 1488.942 & 1493.02 & 1490.754\end{array}$ $\begin{array}{llllllllllll}1494.073 & 1494.636 & 1494.976 & 1495.619 & 1496.984 & 1496.83 & 1493.14 & 1488.945 & 1493.025 & 1490.754\end{array}$ $\begin{array}{llllllllllll}1494.099 & 1494.657 & 1494.961 & 1455.601 & 146.968 & 1496.787 & 1433.184 & 1488.945 & 1493.032 & 1490.756 \\ 1494113 & 1494.674 & 1494.966 & 1495582 & 1496966 & 1496753 & 1493.161 & 1488937 & 1493013 & 1490756\end{array}$ $\begin{array}{llllllllllll}1494.12 & 1494.689 & 1494.959 & 1495.575 & 1496.956 & 1496.736 & 1493.156 & 1488.935 & 1493.011 & 1490.758\end{array}$ $\begin{array}{lllllllllll}1494.129 & 1494.672 & 1494.954 & 1495.573 & 1496.949 & 1496.72 & 1493.154 & 1488.923 & 1493.006 & 1490.758\end{array}$ $\begin{array}{llllllllll}1494.11 & 1494.667 & 1494.966 & 1495.577 & 1496.952 & 1496.751 & 1493.165 & 1488.923 & 1493.018 & 1490.761\end{array}$ $\begin{array}{llllllllllll}1494.082 & 1494.693 & 1494.976 & 1495.582 & 1496.973 & 1496.789 & 1493.158 & 1488.928 & 1493.013 & 1490.768\end{array}$ $\begin{array}{lllllllllll}1494.073 & 1494.712 & 1494.985 & 1495.582 & 1496.977 & 1496.796 & 1493.177 & 1488.937 & 1493.018 & 1490.768\end{array}$ $\begin{array}{llllllllll}1494.08 & 1494.724 & 1494.973 & 1495.58 & 1496.977 & 1496.791 & 1493.182 & 1488.937 & 1493.023 & 1490.77\end{array}$ $\begin{array}{rrrrrrrrrr}1494.085 & 1494.734 & 1494.976 & 1495.591 & 1496.98 & 1496.78 & 1493.177 & 1488.94 & 1493.023 & 1490.775 \\ 1494.092 & 1494.741 & 1494.969 & 1495.649 & 1496.973 & 1496.78 & 1493182 & 1488.938 & 1493027 & 1490777\end{array}$

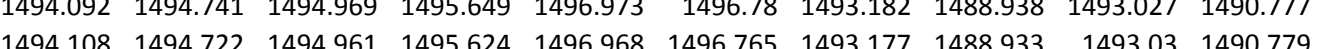
$\begin{array}{llllllllll}1444.122 & 1494.705 & 1494.954 & 1495.582 & 1496.956 & 1496.744 & 1493.175 & 1488.926 & 1493.03 & 1490.782\end{array}$ $\begin{array}{lllllllllll}1494.127 & 1494.684 & 1494.95 & 1495.556 & 1496.956 & 1496.732 & 1493.156 & 1488.921 & 1493.025 & 1490.786\end{array}$ $\begin{array}{llllllllll}1494.115 & 1494.684 & 1494.961 & 1495.556 & 1496.952 & 1496.746 & 1493.177 & 1488.921 & 1493.03 & 1490.789\end{array}$ $\begin{array}{lllllllllll}1494.12 & 1494.708 & 1494.999 & 1495.549 & 1496.947 & 1496.732 & 1493.168 & 1488.921 & 1493.025 & 1490.791\end{array}$ $\begin{array}{lllllllllll}1494.12 & 1494.71 & 1495.03 & 1495.542 & 1496.942 & 1496.724 & 1493.17 & 1488.914 & 1493.025 & 1490.798\end{array}$ $\begin{array}{lllllllllll}1494.117 & 1494.732 & 1495.075 & 1495.54 & 1496.942 & 1496.724 & 1493.175 & 1488.916 & 1493.025 & 1490.803 \\ 1494.117 & 1494.746 & 1495.116 & 1495.531 & 1496933 & 1496715 & 1493182 & 1488914 & 1493025 & 1490.805\end{array}$ $\begin{array}{rrrrrrrrr}1485.191 & 1488.166 & 1493.3 & 1493.793 & 1493.051 & 1494.91 & 1493.025 & 1484.766 & 1479.206 \\ 1485.177 & 1488.161 & 1493.293 & 1493.796 & 1493.048 & 1494.917 & 1493.023 & 1484.766 & 1479.204\end{array}$ $\begin{array}{llllllllll}1485.177 & 1488.161 & 1493.293 & 1493.796 & 1493.048 & 1494.917 & 1493.023 & 1484.766 & 1479.204 \\ 1485163 & 1488154 & 1493225 & 1493.8 & 1493.051 & 1494.92 & 1493.028 & 1484766 & 1479.204\end{array}$ $\begin{array}{llllllllll}1485.165 & 148815 & 1493225 & 1493805 & 1493053 & 1494.927 & 1493028 & 1484.766 & 1479.204\end{array}$ $\begin{array}{lllllllllll}1485.172 & 1488.154 & 1493.295 & 1493.803 & 1493.053 & 1494.925 & 1493.025 & 1484.764 & 1479.206\end{array}$ $\begin{array}{lllllllllll}1485.181 & 1488164 & 1493295 & 1493803 & 1493.053 & 1494.922 & 1493.025 & 1484.764 & 1479.208\end{array}$ $\begin{array}{lllllllllll}1485.186 & 1488.175 & 1493.295 & 1493.796 & 1493.048 & 1494.915 & 1493.023 & 1484.764 & 1479.208\end{array}$ $\begin{array}{llllllllll}1485.193 & 1488.168 & 1493.295 & 1493.793 & 1493.048 & 1494.92 & 1493.021 & 1484.764 & 1479.208\end{array}$ $\begin{array}{lllllllll}1485.205 & 1488.166 & 1493.295 & 1493.796 & 1493.051 & 1494.915 & 1493.023 & 1484.764 & 1479.211\end{array}$ $\begin{array}{llllllllll}1485.198 & 1488.168 & 1493.288 & 1493.789 & 1493.044 & 1494.906 & 1493.018 & 1484.764 & 1479.200\end{array}$ $\begin{array}{lllllllllll}1485.191 & 1488.171 & 1493.283 & 1493.775 & 1493.037 & 1494.894 & 1493.014 & 1484.762 & 1479.208\end{array}$ $\begin{array}{lllllllllll}1485.188 & 1488.166 & 1493.278 & 1493.777 & 1493.032 & 1494.894 & 1493.009 & 1484.762 & 1499.206\end{array}$ $\begin{array}{llllllllll}1485.191 & 1488.154 & 1493.278 & 1493.782 & 1493.032 & 1494.899 & 1493.006 & 1484.762 & 1479.206\end{array}$ $\begin{array}{llllllllll}1485.198 & 1488.152 & 1493.276 & 1493.782 & 1493.034 & 1494.899 & 1493.009 & 1484.762 & 1479.204\end{array}$ $\begin{array}{llllllllll}1485.202 & 1488.145 & 1493.279 & 1493.789 & 1493.037 & 1494.91 & 1493.004 & 1484.762 & 1479.206\end{array}$ $\begin{array}{llllllllll}1485.207 & 1488.124 & 1493.281 & 1493.8 & 1493.037 & 1494.925 & 1493.006 & 1484.764 & 1479.206\end{array}$ $\begin{array}{lllllllll}1485.216 & 1488.114 & 1493.29 & 1493.821 & 1493.046 & 1494.953 & 1493.016 & 1484.764 & 1479.208\end{array}$ $\begin{array}{llllllllll}1485.221 & 1488.107 & 1493.3 & 1493.847 & 1493.058 & 1494.983 & 1493.028 & 1484.762 & 1479.211\end{array}$ $\begin{array}{lllllllllll}1485.22 & 1488.11 & 1493.304 & 14938.859 & 1493.065 & 1495.002 & 1493.032 & 1484.759 & 1479.213\end{array}$ $\begin{array}{llllllllllll}1485.175 & 1488.11 & 1493.316 & 1493.875 & 1493.077 & 1495.019 & 1493.046 & 1484.759 & 1479.213\end{array}$ $\begin{array}{llllllll} & \end{array}$ $\begin{array}{llllllllll}1485.181 & 1488.136 & 1493.321 & 1493.866 & 1493.082 & 1495.014 & 1493.056 & 1484.757 & 1479.211\end{array}$ $\begin{array}{lllllllll}1485.184 & 1488.145 & 1493.319 & 1493.859 & 1493.077 & 1494.99 & 1493.046 & 1484.757 & 1479.206\end{array}$ $\begin{array}{lllllllllll}1485.191 & 1488.133 & 1493.321 & 1493.87 & 1493.079 & 1495.011 & 1493.046 & 1484.759 & 1479.208\end{array}$ $\begin{array}{lllllllllll}1485.198 & 1488.126 & 1493.325 & 1493.884 & 1493.086 & 1495.033 & 1493.053 & 1484.759 & 1479.208\end{array}$ $\begin{array}{lllllllllll}1485.205 & 1488.126 & 1493.342 & 1493.903 & 1493.101 & 1495.058 & 1493.065 & 1484.757 & 1479.215 \\ 1485.223 & 1488145 & 1493356 & 1493.922 & 1493.113 & 1495.063 & 1493091 & 1484.757 & 1479.225\end{array}$ $\begin{array}{lllllllll}1485.223 & 1488.145 & 143.356 & 1493.922 & 1493.113 & 1495.063 & 1493.091 & 1484.757 & 1479.225 \\ 1485246 & 1488.159 & 1493.38 & 1493.922 & 1493.134 & 1495.058 & 1493.105 & 1484.759 & 1479.248\end{array}$ $\begin{array}{llllllllll}1485.272 & 1488.168 & 1493.398 & 1493.929 & 1493.165 & 1495.058 & 1493.131 & 1484.757 & 1479.262\end{array}$ $\begin{array}{llllllllll}1485.295 & 1488.19 & 1493.422 & 1493.931 & 1493.188 & 1495.051 & 1493.161 & 1484.762 & 1479.281\end{array}$ $\begin{array}{llllllllll}1485.311 & 1488.187 & 1493.426 & 1493.947 & 1493.195 & 1495.061 & 1493.168 & 1484.759 & 1479.295\end{array}$ $\begin{array}{llllllllll}1485.325 & 1488.187 & 1493.427 & 1493.945 & 1493.193 & 1495.108 & 1493.175 & 1484.778 & 1479.302\end{array}$ $\begin{array}{lllllllll}1485.32 & 1488.143 & 1493.389 & 1493.982 & 1493.2 & 1495.176 & 1493.166 & 1484.787 & 1479.309\end{array}$

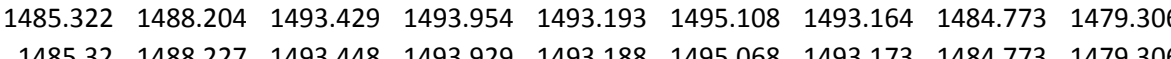
$\begin{array}{lllllllllll}1485.32 & 1488.227 & 1493.448 & 1493.929 & 1493.188 & 1495.068 & 1433.173 & 1484.773 & 1479.306\end{array}$ $\begin{array}{lllllllllll}1485.322 & 1488.222 & 1493.419 & 1493.912 & 1493.172 & 1495.061 & 1493.147 & 1484.776 & 1499.302\end{array}$ $\begin{array}{lllllllllll}1485.322 & 1488.232 & 1493.415 & 1493.91 & 1493.169 & 1495.065 & 1493.138 & 1484.778 & 1479.304\end{array}$ $\begin{array}{lllllllllll}1485.32 & 1488.229 & 1493.424 & 1493.917 & 1493.174 & 1495.073 & 1493.157 & 1484.785 & 1479.30\end{array}$ $\begin{array}{llllllllll}1485.327 & 1488.199 & 1493.401 & 1493.947 & 1493.176 & 1495.117 & 1493.152 & 1484.795 & 1479.31\end{array}$ $\begin{array}{llllllllll}1485.336 & 1488.206 & 1493.42 & 1493.964 & 1493.188 & 1495.134 & 1493.161 & 1484.802 & 1479.313\end{array}$ $\begin{array}{llllllllll}1485.346 & 1488.215 & 1493.429 & 1493.964 & 1493.188 & 1495.131 & 1493.164 & 1484.804 & 1479.313\end{array}$ $\begin{array}{lllllllll}1485.29 & 1488.211 & 1493.424 & 1493.964 & 1493.191 & 1455.136 & 1493.159 & 1484.809 & 1479.313\end{array}$

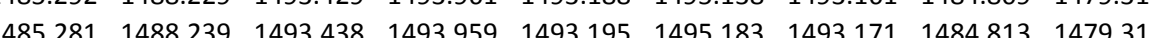
$\begin{array}{lllllllllll}1485.279 & 1488.244 & 1493.436 & 1493.95 & 1493.191 & 1495.15 & 1493.166 & 1484.823 & 1479.316\end{array}$ $\begin{array}{lllllllll}1485.292 & 1488.239 & 1493.422 & 1493.952 & 1493.193 & 1495.199 & 1493.161 & 1484.834 & 1479.318\end{array}$ $\begin{array}{llllllllll}1485.302 & 1488.244 & 1493.431 & 1493.957 & 1493.193 & 1495.166 & 1493.171 & 1484.844 & 1479.318\end{array}$ $\begin{array}{llllllllll}1485.309 & 1488.253 & 1493.426 & 1493.95 & 1493.191 & 1495.15 & 1493.164 & 1484.853 & 1479.31\end{array}$ $\begin{array}{llllllllll}1485.316 & 1488.255 & 1493.427 & 1493.95 & 1493.188 & 1495.148 & 1493.164 & 1484.858 & 1479.316\end{array}$ $\begin{array}{rrrrrrrrr}1485.325 & 1488.255 & 1493.429 & 1493.954 & 1493.188 & 1495.145 & 1493.164 & 1484.863 & 1479.316 \\ 1485.332 & 1488.265 & 1493.429 & 1493.95 & 1493.188 & 1495.138 & 1493.164 & 1484.865 & 1479.318\end{array}$ 
Well

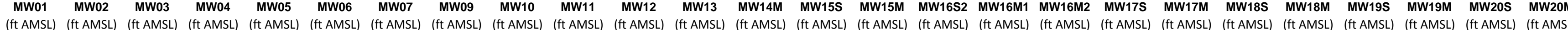

2/4/12 2:00 2/4/123:00

$2 / 4 / 124: 00$

2/4/12 6:00

2/4/12 7:00

2/4/12 8:00

2/4/12 9:00

2/4/12 10:00

2/4/12 11:00

2/4/1212 12:00

2/4/12 14:00

2/4/112 14:00

2/4/12 16:00

2/4/12 17:00

2/4/12 18:00

2/4/1219:00

2/4/1220:00

2/4/12 21:00

$2 / 4 / 1222300$

2/5/120:00

2/5/12 1:00

2/5/12 2:00

2/5/12 3:00

2/5/12 4:00

2/5/12 5:00

$2 / 5 / 126: 00$
$2 / 5 / 127.00$

2/5/12 8:00

2/5/12 9:00

2/5/12 10:00

2/5/12 11:00

2/5/1212:00

2/5/12 13:00

2/5/12 14:00

2/5/12 15:00

2/5/12 17:00

2/5/12 18:00

2/5/12 19:00

2/5/12 20:00

2/5/12 21:00

2/5/12222:00

2/5/12 23:00

2/6/120:00

2/6/12 1:00

2/6/12 3:00

2/6/12 4:00

2/6/12 5:00

2/6/12 6:00 $\begin{array}{llll}1496.165 & 1517.96 & 1496.315 & 1495.053 \\ 1496.354 & 1518.174 & 1496.329 & 1495.051\end{array}$ $\begin{array}{llll}1496.354 & 1518.174 & 1496.329 & 1495.051 \\ 1496547 & 1518.615 & 1996361 & 149509\end{array}$ $\begin{array}{lllll}1496.547 & 1518.615 & 1496.361 & 1495.049\end{array}$ $\begin{array}{lllll}1496.927 & 1521.168 & 1496.401 & 1495.04\end{array}$ $\begin{array}{rlll}1497.076 & 1523.287 & 1496389 & 1495.032\end{array}$ $\begin{array}{lllll}1497.076 & 1523.287 & 1496.389 & 1495.032 \\ 1497.201 & 1525.078 & 1496.392 & 1495.021\end{array}$ $\begin{array}{lllll}1497.309 & 1525.11 & 1496.413 & 1495.009\end{array}$ $\begin{array}{lllll}1497.404 & 1525.141 & 1496.406 & 1494.99\end{array}$ $\begin{array}{rllll}1497.499 & 1525.162 & 1496.401 & 1494.981 \\ 1497.59 & 1525176 & 1496396 & 1949\end{array}$ $\begin{array}{rrrr}1497.59 & 1525.176 & 1496.396 & 1494.981\end{array}$ $\begin{array}{rrrr}1497.688 & 1525.19 & 1496.403 & 1494.993 \\ 1497.777 & 1525.199 & 1496.338 & 1495.004\end{array}$ $\begin{array}{lllll}1497.865 & 1525.208 & 1496.324 & 1495.002\end{array}$ $\begin{array}{lllll}1497.937 & 1525.19 & 1496.31 & 1495.002\end{array}$ $\begin{array}{llll}1498.016 & 1525.197 & 1496.308 & 1495\end{array}$ $\begin{array}{lllll}1498.077 & 1525.213 & 1496.291 & 1494.99\end{array}$ $\begin{array}{llll}1498.131 & 1525.222 & 1496.287 & 1494.988\end{array}$ $\begin{array}{llll}1488.191 & 1525.232 & 1496.275 & 1494.983 \\ 148247 & 1525238 & 149627 & 144979\end{array}$ $\begin{array}{llll}1498.247 & 1525.238 & 1496.27 & 1494.979\end{array}$ $\begin{array}{llrr}1498.347 & 1525.218 & 1496289 & 1494.989\end{array}$ $\begin{array}{lllll}1498.387 & 1525.22 & 1496.287 & 1494.995\end{array}$ $\begin{array}{lllll}1498.424 & 1525.227 & 1496.277 & 1494.993\end{array}$ $\begin{array}{lllll}1498.466 & 1525.222 & 1496.284 & 1494.997\end{array}$ $\begin{array}{llll}1498.494 & 1525.229 & 1496.275 & 1494.995\end{array}$ $\begin{array}{rrrrr}1498.526 & 1525.222 & 1496.28 & 1494.997 \\ 1498.55 & 1525222 & 1496285 & 145000\end{array}$ $\begin{array}{llll}1498.55 & 1525.22 & 1496.284 & 1495.002\end{array}$ $\begin{array}{rrrr}1498.557 & 1525.213 & 1496.287 & 1495.007 \\ 1498.533 & 1525.224 & 149628 & 1495.007\end{array}$ $\begin{array}{lllll}1498.473 & 1525.22 & 1496.277 & 1495.007\end{array}$ $\begin{array}{lllll}1498.387 & 1525.231 & 1496.273 & 1495.002\end{array}$ $\begin{array}{lllll}1498.317 & 1525.234 & 1496.266 & 1495\end{array}$ $\begin{array}{lllll}1498.273 & 1525.234 & 1496.261 & 1494.997\end{array}$ $\begin{array}{llll}1498.294 & 1525.213 & 1496.28 & 1495.007\end{array}$ $\begin{array}{llll}1498.366 & 1525.166 & 1496.315 & 1495.025 \\ 1498.405 & 1525.127 & 149633 & 1995046\end{array}$ $\begin{array}{llll}1498.405 & 1525.127 & 1496.336 & 1495.046\end{array}$ $\begin{array}{rrrr}1498.396 & 1525.08 & 1496.357 & 1495.065 \\ 1498.352 & 1525.043 & 1496.373 & 1495.081\end{array}$ $\begin{array}{lllll}1498.277 & 1525.008 & 1496.387 & 1495.098\end{array}$ $1498.219 \quad 1524.991 \quad 1496.387 \quad 1495.107$ $1498.175 \quad 1524.984 \quad 1496.385 \quad 1495.105$ $1498.135 \quad 1524.98 \quad 1496.38 \quad 1495.105$ $1498.098 \quad 1524.973 \quad 1496.382 \quad 1495.105$ $1498.0581524 .966 \quad 1496.3891495 .109$ $1498.0161524 .8491496 .396 \quad 1495.112$ $1497.937 \quad 1524.4961496 .417 \quad 1495.121$ $\begin{array}{llll}1497.9 & 1523.726 & 1496.42 & 1495.123\end{array}$

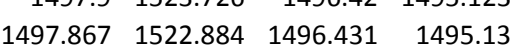
$1497.83 \quad 1521.949 \quad 1496.443 \quad 1495.142$ $1497.791 \quad 1521.0821496 .448 \quad 1495.147$ $\begin{array}{llll}1497.753 & 1520.259 & 1496.448 & 1495.149 \\ 1497.718 & 1519.478 & 1496.446 & 1495.151\end{array}$ $\begin{array}{llllllllll}1494.124 & 1494.758 & 1495.161 & 1495.526 & 1496.924 & 1496.703 & 1493.184 & 1488.907 & 1493.025 & 1490.807\end{array}$

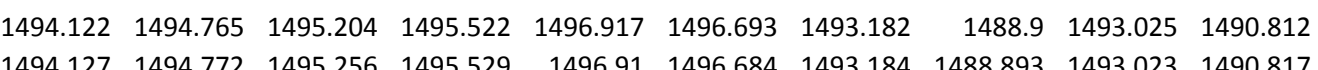
$\begin{array}{llllllllllll}1494.127 & 1494.772 & 1495.256 & 1495.529 & 1496.91 & 1496.684 & 1493.184 & 1488.893 & 149025 & 1490.817\end{array}$ $\begin{array}{lllllllllll}1494.132 & 1494.784 & 1495.353 & 1495.536 & 1496.889 & 1496.66 & 1493.186 & 1488.888 & 1493.018 & 1490.819\end{array}$ $\begin{array}{lllllllllll}1494.132 & 1494.789 & 1495.406 & 1495.542 & 1496.88 & 1496.65 & 1493.189 & 1488.877 & 1493.013 & 1490.819\end{array}$ $\begin{array}{llllllllllll}1494.139 & 1494.794 & 1495.458 & 1495.542 & 1496.863 & 1496.626 & 1493.184 & 1488.874 & 1493.006 & 1490.821\end{array}$ $\begin{array}{llllllllllll}1494.139 & 1494.794 & 1495.508 & 1495.555 & 1496.852 & 1496.612 & 1493.177 & 1488.865 & 1492.999 & 1490.824\end{array}$ $\begin{array}{lllllllllll}1494.141 & 1494.794 & 1495.558 & 1495.54 & 1496.833 & 1496.59 & 1493.168 & 1488.86 & 1492.99 & 1490.821\end{array}$ $\begin{array}{llllllllllll}1494.132 & 1494.796 & 1495.608 & 1495.542 & 1496.824 & 1496.581 & 1493.158 & 1488.855 & 1492.985 & 1490.821\end{array}$ $\begin{array}{lllllllllll}1494.113 & 1494.799 & 1495.653 & 1495.552 & 1496.817 & 1496.586 & 1493.147 & 1488.858 & 1492.976 & 1490.824 \\ 1494087 & 1494.806 & 1495.698 & 1495563 & 1496822 & 1496607 & 1493.149 & 1488851 & 1492.98 & 1490824\end{array}$ $\begin{array}{lllllllllllll}1494.07 & 1494.806 & 1495.731 & 1495.573 & 1496.822 & 1496.622 & 1493.154 & 1488.858 & 1492.983 & 1490.828\end{array}$ $\begin{array}{lllllllllll}1494.068 & 1494.811 & 1495.745 & 1495.573 & 1496.815 & 1496.61 & 1493.158 & 1488.855 & 1492.985 & 1490.828\end{array}$ $\begin{array}{llllllllll}1494.07 & 1494.813 & 1495.752 & 1495.573 & 1496.805 & 1496.602 & 1493.158 & 1488.851 & 1492.98 & 1490.828\end{array}$ $\begin{array}{lllllllllll}1494.063 & 1494.815 & 1495.762 & 1495.575 & 1496.803 & 1496.6 & 1493.156 & 1488.851 & 1492.978 & 1490.833\end{array}$ $\begin{array}{lllllllllll}1494.066 & 1494.815 & 1495.764 & 1495.573 & 1496.791 & 1496.588 & 1493.156 & 1488.844 & 1492.973 & 1490.833\end{array}$ $\begin{array}{lllllllllll}1494.061 & 1494.82 & 1495.762 & 1495.573 & 1496.784 & 1496.583 & 1493.154 & 1488.841 & 1492.964 & 1490.833\end{array}$ $\begin{array}{lllllllllll}1494.059 & 1494.825 & 1455.762 & 1495.57 & 1466.777 & 1496.581 & 1493.147 & 1488.844 & 1492.966 & 1490.835 \\ 1494.052 & 1494.823 & 1495.76 & 1495.568 & 1496.77 & 1496571 & 1493.13 & 1488837 & 1492955 & 1490837\end{array}$ $\begin{array}{rrrrrrrrrr}1494.052 & 1494.823 & 1495.76 & 1495.568 & 1496.77 & 1496.57 & 1493.13 & 1488.837 & 1492.955 & 1490.837 \\ 1494.033 & 1494.825 & 1495.764 & 1495577 & 1496.773 & 1496588 & 1493.128 & 1488837 & 1492.957 & 1490.837\end{array}$ $\begin{array}{lllllllllllll}1494.019 & 1494.832 & 1495.76 & 1495.587 & 1496.775 & 1496.598 & 1493.125 & 1488.839 & 1492.957 & 1490.84\end{array}$ $\begin{array}{lllllllllll}1494.016 & 1494.835 & 1495.745 & 1495.589 & 1496.773 & 1496.6 & 1493.123 & 1488.837 & 1492.957 & 1490.842\end{array}$ $\begin{array}{llllllllll}1494.016 & 1494.835 & 1495.729 & 1495.591 & 1496.766 & 1496.593 & 1493.123 & 1488.839 & 1492.959 & 1490.842\end{array}$ $\begin{array}{lllllllllll}1494.005 & 1494.839 & 1495.722 & 1495.601 & 1496.766 & 1496.6 & 1493.128 & 1488.837 & 1492.959 & 1490.847\end{array}$ $\begin{array}{lllllllllll}1494.009 & 1494.842 & 1495.698 & 1495.598 & 1496.761 & 1496.59 & 1493.128 & 1488.839 & 1492.964 & 1490.847\end{array}$ $\begin{array}{llllllllll}1493.998 & 1494.844 & 1495.684 & 1455.605 & 1496.761 & 1496.595 & 1493.128 & 1488.834 & 1492.964 & 1490.849\end{array}$ $\begin{array}{lllllllllll}1493.99 & 1494.849 & 1495.669 & 1495.614 & 1496.761 & 1496.602 & 1493.133 & 1488.834 & 1492.969 & 1490.854 \\ 1493.986 & 1494.854 & 1495.655 & 1495621 & 1496763 & 1496605 & 1493137 & 1488837 & 1492971 & 1490856\end{array}$ $\begin{array}{llllllllll}1493.986 & 1494.854 & 1455.655 & 1455.621 & 1496.763 & 1496.605 & 1493.137 & 1488.837 & 1492.971 & 1490.856 \\ 1493.988 & 1494.851 & 1495.636 & 1495.621 & 1496.756 & 1496.602 & 1493.137 & 1488.837 & 1492.966 & 1490.558\end{array}$ $\begin{array}{lllllllllll}1493.986 & 1494.856 & 1495.624 & 1495.626 & 1496.756 & 1496.602 & 1493.142 & 1488.837 & 1492.969 & 1490.863\end{array}$ $\begin{array}{lllllllllll}1493.986 & 1494.858 & 1495.605 & 1495.628 & 1496.752 & 1496.593 & 1493.14 & 1488.832 & 1492.966 & 1490.863\end{array}$ $\begin{array}{llllllllll}1493.983 & 1494.861 & 1495.591 & 1495.628 & 1496.749 & 1496.588 & 1493.133 & 1488.834 & 1492.964 & 1490.868\end{array}$ $\begin{array}{lllllllllll}1493.976 & 1494.863 & 1495.579 & 1495.633 & 1496.747 & 1496.59 & 1493.128 & 1488.83 & 1492.962 & 1490.87\end{array}$ $\begin{array}{llllllllllll}1493.96 & 1494.868 & 1495.574 & 1495.645 & 1496.752 & 1496.602 & 1493.128 & 1488.83 & 1492.964 & 1490.87\end{array}$ $\begin{array}{llllllllll}1493.929 & 1494.87 & 1495.591 & 1495.666 & 1496.77 & 1496.636 & 1493.135 & 1488.837 & 1492.971 & 1490.875 \\ 1493918 & 1494.875 & 1495.589 & 1495682 & 1496.78 & 1496.657 & 1493.142 & 1488848 & 149298 & 149082\end{array}$ $\begin{array}{lllllllllll}1439.918 & 1494.875 & 145.589 & 1495.682 & 1496.78 & 1496.657 & 1493.142 & 1488.848 & 1492.98 & 1490.882 \\ 1493 & 149488 & 1495.591 & 14957 & 1496794 & 1496677 & 1493.154 & 1488853 & 149299 & 1490886\end{array}$ $\begin{array}{lllllllllll}1493.904 & 1494.887 & 1495.586 & 1495.717 & 1496.803 & 1496.693 & 1493.163 & 1488.858 & 1492.997 & 1490.891\end{array}$ $\begin{array}{lllllllllll}1493.904 & 1494.892 & 1495.581 & 1495.733 & 1496.815 & 1496.701 & 1493.09 & 1488.86 & 1492.919 & 1490.898\end{array}$ $\begin{array}{llllllllll}1493.915 & 1494.897 & 1495.572 & 1495.744 & 1496.817 & 1496.705 & 1493.128 & 1488.872 & 1492.964 & 1490.903\end{array}$ $\begin{array}{lllllllllll}1493.932 & 1494.902 & 1495.553 & 1495.749 & 1496.819 & 1496.693 & 1493.14 & 1488.862 & 1492.976 & 1490.91\end{array}$ $\begin{array}{llllllllllll}1493.946 & 1494.904 & 1495.541 & 1495.754 & 1496.819 & 1496.689 & 1493.147 & 1488.865 & 1492.985 & 1490.914\end{array}$ $\begin{array}{lllllllllll}1493.951 & 1494.906 & 1495.541 & 1495.761 & 1496.822 & 1496.686 & 1493.151 & 1488.865 & 1492.99 & 1490.917\end{array}$ $\begin{array}{lllllllllll}1493.951 & 1494.911 & 1455.53 & 1495.77 & 1496.826 & 1496.691 & 1492.908 & 1488.865 & 1492.72 & 1490.921 \\ 1493.951 & 1494.913 & 1495.541 & 1495.779 & 1496833 & 1496.698 & 1492.964 & 1488.862 & 1492.788 & 1490.928\end{array}$

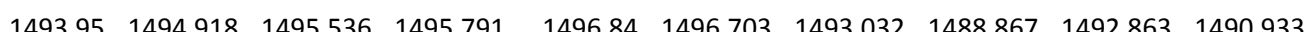
$\begin{array}{llllllllll}1493.95 & 1494.925 & 1495.534 & 1495.8 & 1496.847 & 1496.713 & 1493.069 & 1488.87 & 1492.901 & 1490.935\end{array}$ $\begin{array}{lllllllllll}1493.955 & 1494.925 & 1495.527 & 1495.807 & 1496.852 & 1496.713 & 1493.095 & 1488.872 & 1492.929 & 1490.942\end{array}$ $\begin{array}{llllllllll}1493.953 & 1494.93 & 1495.527 & 1495.819 & 1496.859 & 1496.72 & 1493.116 & 1488.872 & 1492.952 & 1490.945\end{array}$ $\begin{array}{lllllllllll}1493.951 & 1494.933 & 1495.527 & 1495.83 & 1496.868 & 1496.729 & 1493.135 & 1488.872 & 1492.971 & 1490.954\end{array}$ $\begin{array}{llllllllllll}1493.958 & 1494.935 & 1495.522 & 1495.835 & 1496.87 & 1496.729 & 1493.149 & 1488.874 & 1492.985 & 1490.956\end{array}$ $\begin{array}{llllllllll}1493.965 & 1494.94 & 1455.51 & 1455.84 & 1496.87 & 1496.727 & 1493.163 & 1488.877 & 1492.999 & 1490.963\end{array}$ $\begin{array}{lllllllll}1485.339 & 1488.272 & 1493.434 & 1493.943 & 1493.191 & 1495.141 & 1493.161 & 1484.872 & 1479.32\end{array}$ $\begin{array}{llllllllll}1485.341 & 1488.272 & 1493.426 & 1493.943 & 1493.188 & 1495.138 & 1493.159 & 1484.874 & 1479.32\end{array}$

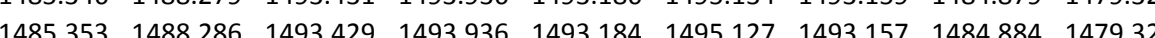
$\begin{array}{llllllllll}1485.357 & 1488.288 & 1493.431 & 1493.933 & 1493.181 & 1495.122 & 1493.154 & 1484.888 & 1479.32\end{array}$ $\begin{array}{lllllllll}1485.35 & 1488.293 & 1493.424 & 1493.919 & 1493.176 & 1495.115 & 1493.15 & 1484.891 & 1479.318\end{array}$ $\begin{array}{lllllllll}1485.35 & 1488.298 & 1493.422 & 1493.905 & 1493.169 & 1495.096 & 1493.143 & 1484.895 & 1479.313\end{array}$ $\begin{array}{llllllllll}1485.348 & 1488.298 & 1493.412 & 1493.896 & 1493.162 & 1495.087 & 1493.136 & 1484.9 & 1479.311\end{array}$ $\begin{array}{lllllllll}1485.339 & 1488.307 & 1493.403 & 1493.884 & 1493.153 & 1495.068 & 1493.129 & 1484.902 & 1479.309\end{array}$ $\begin{array}{lllllllll}1485.332 & 1488.298 & 1493.396 & 1493.873 & 1493.15 & 1495.061 & 1493.119 & 1444.907 & 1479.304\end{array}$ $\begin{array}{llllllllll}1485.329 & 1488.286 & 1493.382 & 1493.875 & 1493.143 & 1495.07 & 1493.114 & 1484.912 & 1479.304\end{array}$ $\begin{array}{lllllllll}1485.329 & 1488.272 & 1493.386 & 1493.903 & 1493.148 & 1495.101 & 1493.121 & 1484.923 & 1479.304\end{array}$ $\begin{array}{llllllllllll}1485304 & 1488.279 & 1493.391 & 1493.894 & 1493.148 & 1495.098 & 1493.124 & 1484.926 & 1479.304\end{array}$ $\begin{array}{llllllllll}1485.258 & 1488.283 & 1493.389 & 1493.891 & 1493.148 & 1495.084 & 1493.119 & 1484.928 & 1479.302\end{array}$ $\begin{array}{lllllllllll}1485.262 & 1488.279 & 1493.382 & 1493.894 & 1493.141 & 1495.089 & 1493.117 & 1484.931 & 1479.302\end{array}$ $\begin{array}{llllllllll}1485.262 & 1488.283 & 1493.382 & 1493.88 & 1493.139 & 1495.08 & 1493.112 & 1484.933 & 1479.295\end{array}$ $\begin{array}{lllllllll}1485.272 & 1488.286 & 1493.38 & 1493.873 & 1493.134 & 1495.073 & 1493.11 & 1484.933 & 1479.292\end{array}$ $\begin{array}{lllllllll}1488.279 & 1488.295 & 1493.37 & 1493.877 & 1493.129 & 1495.068 & 1493.1 & 1484.935 & 1479.288\end{array}$ $\begin{array}{lllllllll}1485.279 & 1488.283 & 1493.366 & 1493.868 & 1493.124 & 1495.063 & 1493.096 & 1484.938 & 1499.283 \\ 1485283 & 1488276 & 1493361 & 1493875 & 1493.122 & 1495075 & 1493096 & 148494 & 1479.281\end{array}$ $\begin{array}{llllllllll}1485.29 & 1488.276 & 1493.363 & 1493.882 & 1493.127 & 1495.084 & 1493.096 & 1484.94 & 1479.281\end{array}$ $\begin{array}{lllllllllll}1485.295 & 1488279 & 1493365 & 1493887 & 1493.127 & 1495.084 & 1493.098 & 1484.942 & 1479.278\end{array}$ $\begin{array}{lllllllllll}1485.297 & 1488.281 & 1493.368 & 1493.882 & 1493.124 & 1495.08 & 1493.098 & 1484.942 & 1479.276\end{array}$ $\begin{array}{lllllllll}1485.304 & 1488.281 & 1493.368 & 1493.884 & 1493.129 & 1495.087 & 1493.103 & 1484.942 & 1479.28\end{array}$ $\begin{array}{llllllllll}1485.313 & 1488.283 & 1493.37 & 1493.882 & 1493.129 & 1495.084 & 1493.103 & 1484.945 & 1479.28\end{array}$ $\begin{array}{lllllllll}1485.318 & 1488.279 & 1493.372 & 1493.889 & 1493.131 & 1495.089 & 1493.105 & 1484.945 & 1479.283\end{array}$

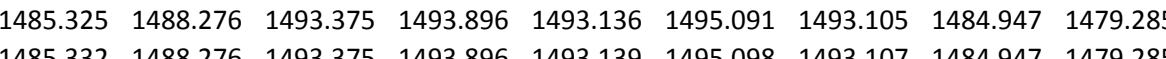
$\begin{array}{llllllllll}1485.332 & 1488.27 & 143.37 & 1493.896 & 1493.139 & 1495.098 & 1493.107 & 1484.947 & 1479.285 \\ 1485.336 & 1488.288 & 1493.377 & 1493.894 & 1493.136 & 1495.096 & 1493.11 & 1484947 & 1479.285\end{array}$ $\begin{array}{lllllllllll}1485.339 & 1488.286 & 1493.375 & 1493.896 & 1493.136 & 1495.091 & 1493.107 & 1484.949 & 1479.283\end{array}$ $\begin{array}{llllllllll}1485.325 & 1488.288 & 1493.37 & 1493.894 & 1493.134 & 1495.089 & 1493.105 & 1484.947 & 1479.281\end{array}$ $\begin{array}{llllllllll}1485.322 & 1488.279 & 1493.375 & 1493.887 & 1493.129 & 1495.082 & 1493.103 & 1484.949 & 1479.276\end{array}$ $\begin{array}{llllllllll}1485.327 & 1488.281 & 1493.37 & 1493.889 & 1493.131 & 1495.084 & 1493.1 & 1484.949 & 1479.27\end{array}$ $\begin{array}{llllllllll}1485.304 & 1488.265 & 1493.37 & 1493.901 & 1493.131 & 1495.098 & 1493.103 & 1484.952 & 1479.274\end{array}$

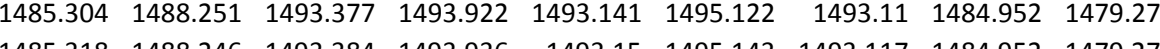
$\begin{array}{llllllllll}1485318 & 149823 & 1493394 & 1993954 & 1493.16 & 1495.162 & 1493.126 & 1484954 & 1479.276\end{array}$ $\begin{array}{lllllllllll}1485.274 & 1488.241 & 1493.403 & 1493.966 & 1493.167 & 1495.178 & 1493.136 & 1484.954 & 1479.281\end{array}$ $\begin{array}{llllllllll}1485.283 & 1488.241 & 1493.326 & 1493.938 & 1493.094 & 1495.192 & 1493.068 & 1484.954 & 1479.283\end{array}$ $\begin{array}{lllllllllll}1485.286 & 1488.251 & 1493.373 & 1493.959 & 1493.136 & 1495.192 & 1493.11 & 1484.954 & 1479.283\end{array}$ $\begin{array}{llllllllll}1485.295 & 1488.258 & 1493.384 & 1493.966 & 1493.148 & 1495.188 & 1493.124 & 1484.956 & 1479.28\end{array}$ $\begin{array}{llllllllll}1485.302 & 1488.269 & 1493.394 & 1493.971 & 1493.155 & 1495.181 & 1493.131 & 1484.956 & 1479.278\end{array}$ $\begin{array}{lllllllll}1485.306 & 1488.269 & 1493.396 & 1493.968 & 1493.16 & 1495.185 & 1493.133 & 1484.959 & 1479.276\end{array}$ $\begin{array}{llllllllll}1485.297 & 488.27 & 1493.13 & 1493.903 & 1492.913 & 1495.185 & 1492.887 & 1484.959 & 1479.271\end{array}$ $\begin{array}{lllllllll}1485295 & 1488279 & 1493272 & 1493922 & 1493039 & 1495188 & 1493016 & 1484961 & 1499.269\end{array}$ $\begin{array}{llllllllll}1485.302 & 1488.281 & 1493.309 & 1493.943 & 1493.079 & 1495.199 & 1493.049 & 1484.961 & 1479.271\end{array}$ $\begin{array}{lllllllllll}1485.311 & 1488.288 & 1493.335 & 1493.952 & 1493.101 & 1495.202 & 1493.077 & 1484.961 & 1479.274\end{array}$ $\begin{array}{lllllllll}1485.318 & 1488.298 & 1493.358 & 1493.968 & 1493.124 & 1495.209 & 1493.098 & 1484.961 & 1479.278\end{array}$ $\begin{array}{llllllllll}1485.325 & 1488.298 & 1493.377 & 1493.985 & 1493.146 & 1495.225 & 1493.117 & 1484.961 & 1479.283\end{array}$ $\begin{array}{lllllllllll}1485.332 & 1488.307 & 1493.394 & 1493.989 & 1493.158 & 1495.225 & 1493.129 & 1484.961 & 1479.285\end{array}$ $\begin{array}{llllllllll}1485.327 & 1488.314 & 1493.403 & 1493.996 & 1493.167 & 1495.23 & 1493.14 & 1484.961 & 1479.288 \\ 1485.332 & 1488316 & 1493.41 & 1493.999 & 1493.179 & 1495.228 & 1493.147 & 1484.963 & 1479.288\end{array}$ 
Well

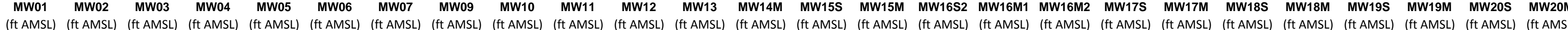

2/6/128:00 2/6/12 9:00 2/6/1211:00 2/6/12 12:00 2/6/12 13:00 2/6/12 14:00 2/6/12 15:00 2/6/12 16:00 2/6/12 17:00 2/6/12 18:00 2/6/12 20:00 2/6/12 21:00 2/6/12 22:00 2/6/12 23:00 2/7/12 0:00 2/7/121:00 2/7/12 2:00 2/7/7/123:00 2/7/12 5:00 2/7/12 6:00 2/7/12 7:00 2/7/12 8:00 2/7/12 9:00 2/7/12 10:00

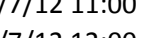
年/7/12 12:00 2/7/12 14:00 /7/1/12 15:00 2/7/12 16:00 2/7/12 17:00 2/7/1218:00 2/7/12 19:00 2/7/12 20:00 2/7/122 21:00 2/7/12 22:00 2/8/12 0:00 2/8/12 1:00 2/8/12 2:00 2/8/12 3:00 2/8/12 4:00 $2 / 8 / 126.00$ 2/8/12 7:00 2/8/12 8:00 2/8/12 9:00 2/8/12 10:00 2/8/12 11:00 2/8/12 12:00 $\begin{array}{llll}1497.686 & 1518.739 & 1496.443 & 1495.149\end{array}$ $\begin{array}{rrrr}1497.653 & 1518.03 & 1496.438 & 1495.149 \\ 1497.63 & 1517.328 & 196.455 & 195.156\end{array}$ $\begin{array}{lllll}1497.63 & 1517.328 & 1496.455 & 1495.156 \\ 1497.597 & 1516.65 & 1496.467 & 1495166\end{array}$ $\begin{array}{lllll}1497.567 & 1515.969 & 1496.492 & 1495.182\end{array}$ $\begin{array}{lllll}1497.541 & 1515.328 & 1496.52 & 1495.203\end{array}$ $\begin{array}{rrrr}1497.516 & 1514.708 & 1496.555 & 1495.226\end{array}$ $\begin{array}{lllll}1497.481 & 1514.132 & 1496.562 & 1495.245\end{array}$ $\begin{array}{llll}1497.451 & 1513.584 & 1496.565 & 1495.252\end{array}$ $\begin{array}{llll}1497.423 & 1513.064 & 1496.565 & 1495.259\end{array}$ $\begin{array}{llll}1497.392 & 1512.574 & 1496.562 & 1495.261 \\ 1493.364 & 15212108 & 1496555 & 1955257\end{array}$ $\begin{array}{llll}1497.364 & 1512.108 & 1496.555 & 1495.257 \\ 1497.336 & 1511.658 & 1496.551 & 1495.254\end{array}$ $\begin{array}{llll}1497.336 & 1511.658 & 1496.551 & 1495.254 \\ 1497.311 & 1511.229 & 1496.541 & 1495.245\end{array}$ $\begin{array}{llll}1497.283 & 1510.823 & 1496.52 & 1495.245\end{array}$ $\begin{array}{lllll}1497.262 & 1510.418 & 1496.52 & 1495.226\end{array}$ $\begin{array}{llrl}1497.232 & 1510.038 & 1496.511 & 1495.217\end{array}$ $\begin{array}{llll}1497.211 & 1509.662 & 1496.513 & 1495.212\end{array}$ $\begin{array}{llll}1497.18 & 1509.31 & 1496.509 & 1495.208\end{array}$ $\begin{array}{llll}1497.162 & 1508.951 & 1496.513 & 1495.205 \\ 1497.139 & 1508.609 & 1496523 & 1495.21\end{array}$ $\begin{array}{rrrrr}1497.118 & 1508.282 & 1496.53 & 1495.212\end{array}$ $\begin{array}{lllll}1497.09 & 1507.975 & 1496.523 & 1495.21\end{array}$ $\begin{array}{rrrr}1497.059 & 1507.681 & 1496.5066 & 1495.201\end{array}$ $\begin{array}{llllll}1497.034 & 1507.413 & 1496.469 & 1495.177\end{array}$ $\begin{array}{lllll}1497.013 & 1507.14 & 1496.446 & 1495.154\end{array}$ $\begin{array}{llll}1496.992 & 1506.869 & 1496.429 & 1495.137\end{array}$ $\begin{array}{llll}1496.969 & 1506.611 & 1496.417 & 1495.121 \\ 196955 & 1506343 & 1966.429 & 1955.123\end{array}$ $\begin{array}{llll}1496.955 & 1506.343 & 1496.429 & 1495.123 \\ 1496.929 & 1506.107 & 1496.436 & 1495.123\end{array}$ $\begin{array}{lllll}1496.906 & 1505.9 & 1496.441 & 1495.126\end{array}$ $\begin{array}{lllll}1496.885 & 1505.69 & 1496.436 & 1495.126\end{array}$ $1496.859 \begin{array}{llll}1505.464 & 1496.429 & 1495.121\end{array}$ $\begin{array}{lllll}1496.836 & 1505.219 & 1496.424 & 1495.116\end{array}$ $\begin{array}{lllll}1496.813 & 1504.974 & 1496.408 & 1495.109\end{array}$ $\begin{array}{llll}1496.789 & 1504.725 & 1496.396 & 1495.1\end{array}$ $\begin{array}{llll}1496.768 & 1504.498 & 1496.387 & 1495.093\end{array}$ $\begin{array}{rrrr}1496.743 & 1504.27 & 1496.382 & 1495.086 \\ 1496.717 & 1504.039 & 1496.373 & 1495.079\end{array}$ $\begin{array}{lllll}1496.701 & 1503.829 & 1496.366 & 1495.072\end{array}$ $\begin{array}{lllll}1496.68 & 1503.615 & 1496.373 & 1495.072\end{array}$ $\begin{array}{lllll}1496.659 & 1503.466 & 1496.38 & 1495.077\end{array}$ $1496.6381503 .288 \quad 1496.378 \quad 1495.077$ $1496.6191503 .109 \quad 1496.389 \quad 1495.084$ $\begin{array}{rrrrr}1496.601 & 1502.927 & 1496.401 & 1495.091 \\ 1496.58 & 1502.752 & 1496413 & 1495102\end{array}$ $1496.58 \quad 1502.7521496 .413 \quad 1495.102$ $\begin{array}{llll}1496.531 & 1502.449 & 1466.401 & 1495.102\end{array}$ $\begin{array}{llll}1496.514 & 1502.291 & 1496.399 & 1495.1\end{array}$ $1496.494 \quad 1502.132 \quad 1496.396 \quad 1495.1$ $1496.475 \quad 1501.985 \quad 1496.403 \quad 1495.105$ $1496.454 \quad 1501.822 \quad 1496.41 \quad 1495.112$ $\begin{array}{rrrr}1496.44 & 1501.738 & 1496.424 & 1495.119 \\ 1496.431 & 1501.612 & 1496.464 & 1495.144\end{array}$ $\begin{array}{llllllllll}1493.981 & 1494.945 & 1495.496 & 1495.849 & 1496.875 & 1496.717 & 1493.175 & 1488.877 & 1493.011 & 1490.97\end{array}$ $\begin{array}{llllllllll}1493.983 & 1494.945 & 1495.491 & 1495.849 & 1496.875 & 1496.71 & 1493.177 & 1488.877 & 1493.015 & 1490.975\end{array}$

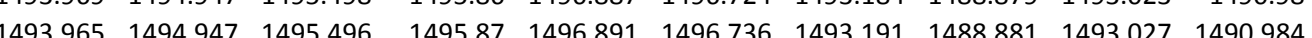
$\begin{array}{lllllllllll}1493.95 & 1494.954 & 1495.503 & 1495.884 & 1496.905 & 1496.756 & 1493.2 & 1488.879 & 1493.037 & 1490.986\end{array}$

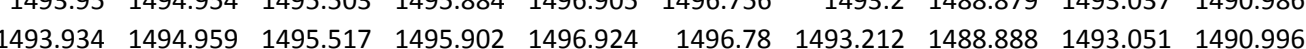
$\begin{array}{lllllllllll}1493.92 & 1494.966 & 1495.527 & 1495.923 & 1496.942 & 1496.808 & 1493.231 & 1488.891 & 1493.065 & 1491\end{array}$ $\begin{array}{lllllllllll}1493.929 & 1494.969 & 1495.515 & 1495.937 & 1496.954 & 1496.818 & 1493.243 & 1488.9 & 1493.079 & 1491.005\end{array}$ $\begin{array}{lllllllllll}1493.941 & 1494.971 & 1495.503 & 1495.944 & 1496.961 & 1496.811 & 1493.252 & 1488.905 & 1493.086 & 1491.012\end{array}$

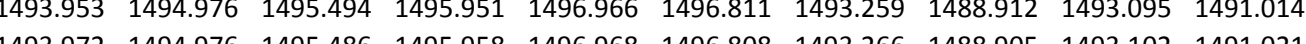
$\begin{array}{rrrrrrrrrr}1493.972 & 1494.976 & 1495.486 & 1495.958 & 1496.968 & 1496.808 & 1493.266 & 1488.905 & 1493.102 & 1491.021 \\ 1493.99 & 1494.978 & 1495.472 & 1495.958 & 1496.968 & 1496.791 & 1493.266 & 1488.909 & 1493.102 & 1491.026\end{array}$ $\begin{array}{llllllllllllll}1494.002 & 1494.983 & 1495.465 & 1495.96 & 1496.968 & 1496.787 & 1493.268 & 1488.907 & 1493.105 & 1491.028\end{array}$ $\begin{array}{llllllllll}1494.019 & 1494.983 & 1495.453 & 1495.958 & 1496966 & 1496.775 & 1493.264 & 1488.902 & 1493.105 & 1491.033\end{array}$ $\begin{array}{llllllllllll}1494.037 & 1494.983 & 1495.441 & 1495.951 & 1496.956 & 1496.751 & 1493.261 & 1488.895 & 1493.1 & 1491.033\end{array}$ $\begin{array}{llllllllllll}1494.04 & 1494.983 & 1495.439 & 1495.951 & 1496.959 & 1496.748 & 1493.259 & 1488.898 & 1493.1 & 1491.038\end{array}$ $\begin{array}{lllllllllll}1494.047 & 1494.98 & 1495.429 & 1495.946 & 1496.952 & 1496.739 & 1493.257 & 1488.898 & 1493.095 & 1491.04\end{array}$ $\begin{array}{llllllllll}1494.045 & 1494.983 & 1495.437 & 1495.946 & 1496.954 & 1496.741 & 1493.257 & 1488.888 & 1493.098 & 1491.04\end{array}$ $\begin{array}{lllllllllll}1494.049 & 1494.983 & 1495.427 & 1495.944 & 1496.947 & 1496.734 & 1493.264 & 1488.888 & 1493.1 & 1491.042\end{array}$ $\begin{array}{llllllllll}1494.047 & 1494.98 & 1495.429 & 1495.944 & 1496.952 & 1496.736 & 1493.264 & 1488.886 & 1493.105 & 1491.045 \\ 1494.037 & 1494.98 & 1495.434 & 1495949 & 1496.956 & 1496746 & 1493268 & 1488888 & 1493.109 & 1491.045\end{array}$ $\begin{array}{llllllllllll}14494.037 & 1494.985 & 1495.434 & 1495.949 & 1496.959 & 1496.746 & 1493.273 & 1488.888 & 1493.114 & 1491.049\end{array}$ $\begin{array}{lllllllllllll}1494.042 & 1494.983 & 1495.425 & 1495.946 & 1496.956 & 1496.741 & 1493.278 & 1488.888 & 1493.116 & 1491.052\end{array}$ $\begin{array}{llllllllllll}1494.059 & 1494.978 & 1495.41 & 1495.942 & 1496.947 & 1496.732 & 1493.278 & 1488.884 & 1493.116 & 1491.049\end{array}$ $\begin{array}{llllllllll}1494.08 & 1494.973 & 1495.389 & 1495.916 & 1496.929 & 1496.691 & 1493.268 & 1488.874 & 1493.107 & 1491.049\end{array}$ $\begin{array}{llllllllll}1494.087 & 1494.966 & 1495.377 & 1495.9 & 1496.917 & 1496.667 & 1493.257 & 1488.865 & 1493.1 & 1491.049\end{array}$ $\begin{array}{llllllllll}1494.087 & 1494.961 & 1495.375 & 1495.886 & 1496.905 & 1496.653 & 1493.247 & 1488.858 & 1493.088 & 1491.045\end{array}$ $\begin{array}{lllllllllll}1494.085 & 1494.952 & 1495.372 & 1495.874 & 1496.896 & 1496.641 & 1493.24 & 1488.851 & 1493.084 & 1491.042 \\ 1494.066 & 1494952 & 1495384 & 1495872 & 1496898 & 1496.65 & 149324 & 1488848 & 1493081 & 1491042\end{array}$ $\begin{array}{llllllllll}1494.066 & 1494.952 & 1495.384 & 145.872 & 146.898 & 1496.05 & 1493.24 & 1488.848 & 1493.081 & 1491.042 \\ 1494.049 & 1494.949 & 1495389 & 1495874 & 1496901 & 1496.665 & 1493.243 & 1488.844 & 1493.083 & 1491.04\end{array}$ $\begin{array}{lllllllllll}1494.04 & 1494.947 & 1495.394 & 1495.872 & 1496.901 & 1496.669 & 1493.245 & 1488.848 & 1493.083 & 1491.04\end{array}$ $\begin{array}{lllllllllll}1494.04 & 1494.942 & 1495.387 & 1495.867 & 1496.896 & 1496.665 & 1493.247 & 1488.846 & 1493.088 & 1491.04\end{array}$ $\begin{array}{lllllllllll}1494.04 & 1494.94 & 1495.382 & 1495.86 & 1496.891 & 1496.66 & 1493.245 & 1488.844 & 1493.086 & 1491.038\end{array}$ $\begin{array}{llllllllll}1494.04 & 1494.935 & 1495.377 & 1495.854 & 1496.887 & 1496.653 & 1493.247 & 1488.837 & 1493.086 & 1491.038\end{array}$ $\begin{array}{llllllllllll}1494.044 & 1494.93 & 1495.365 & 1495.844 & 1496.877 & 1496.643 & 1493.243 & 1488.839 & 1493.083 & 1491.035\end{array}$ $\begin{array}{llllllllll}1494.042 & 1494.925 & 1495.361 & 1495.83 & 1496.87 & 1496.631 & 1493.236 & 1488.832 & 1493.076 & 1491.035 \\ 1494042 & 1494.921 & 1495.356 & 1495.821 & 1496.866 & 1496.626 & 1493231 & 148833 & 1493074 & 149131\end{array}$

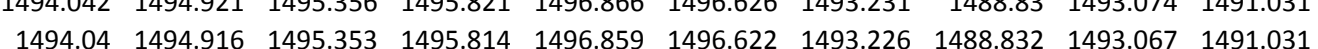
$\begin{array}{llllllllll}1494.037 & 1494.911 & 1495346 & 1495805 & 1496854 & 1496617 & 1493219 & 1488825 & 1493058 & 1491026\end{array}$ $\begin{array}{llllllllllll}1494.033 & 1494.906 & 1495.346 & 1495.793 & 1496.847 & 1496.605 & 1493.207 & 1488.83 & 1493.051 & 1491.024\end{array}$ $\begin{array}{llllllllll}1494.016 & 1494.904 & 1495.353 & 1495.795 & 1496.849 & 1496.617 & 1493.205 & 1488.827 & 1493.048 & 1491.021\end{array}$ $\begin{array}{lllllllllll}1494.007 & 1494.899 & 1495.356 & 1495.793 & 1496.849 & 1496.626 & 1493.207 & 1488.83 & 1493.048 & 1491.019\end{array}$ $\begin{array}{lllllllllll}1494 & 1494.894 & 1495.353 & 1495.791 & 1496.847 & 1496.626 & 1493.205 & 1488.83 & 1493.046 & 1491.019\end{array}$ $\begin{array}{lllllllllll}1493.988 & 1494.897 & 1495.356 & 1495.793 & 1496.852 & 1496.641 & 1493.21 & 1488.827 & 1493.048 & 1491.019\end{array}$ $\begin{array}{llllllllll}1493.976 & 1494.897 & 1495.361 & 1495.793 & 1496.859 & 1496.648 & 1493.212 & 1488.825 & 1493.053 & 1491.019 \\ 1493.969 & 1494.897 & 1495363 & 1495798 & 1496866 & 1496665 & 1493222 & 1488837 & 1493062 & 1491019\end{array}$ $\begin{array}{llllllllllll}1493972 & 1494894 & 1955.351 & 1995.795 & 1496863 & 1496.652 & 1493224 & 14888.834 & 1493065 & 1491.019\end{array}$ $\begin{array}{llllllllllll}1493.981 & 1494.892 & 1495.344 & 1495.791 & 1496.859 & 1496.655 & 1493.224 & 1488.83 & 1493.065 & 1491.021\end{array}$ $\begin{array}{lllllllllll}1493.979 & 1494.892 & 1495.339 & 1495.789 & 1496.859 & 1496.648 & 1493.224 & 1488.832 & 1493.062 & 1491.021\end{array}$ $\begin{array}{lllllllllll}1493.979 & 1494.885 & 1495.337 & 1495.784 & 1496.859 & 1496.648 & 1493.219 & 1488.83 & 1493.06 & 1491.021\end{array}$ $\begin{array}{lllllllllll}1493.972 & 1494.885 & 1495.337 & 1495.786 & 1496.861 & 1496.655 & 1493.221 & 1488.832 & 1493.06 & 1491.024\end{array}$ $\begin{array}{lllllllllll}1493.962 & 1494.885 & 1495.342 & 1495.791 & 1496.866 & 1496.665 & 1493.221 & 1488.83 & 1493.062 & 1491.024\end{array}$ $\begin{array}{llllllllll}1493.95 & 1494.887 & 1495.344 & 1495.793 & 1496.873 & 1496.677 & 1493.222 & 1488.832 & 1493.062 & 1491.026\end{array}$ $\begin{array}{lllllllll}1485.334 & 1488.321 & 1493.417 & 1493.999 & 1493.181 & 1495.223 & 1493.154 & 1484.963 & 1479.285\end{array}$ $\begin{array}{llllllllll}1485.341 & 1488.323 & 1493.42 & 1494.001 & 1493.186 & 1495.223 & 1493.159 & 1484.963 & 1479.28 \\ 1485348 & 1488.314 & 1493.429 & 1494.013 & 1493.191 & 1495.235 & 1493.161 & 1484.963 & 1479283\end{array}$ $\begin{array}{llllllllll}1485353 & 1488312 & 1493436 & 1494.02 & 14932 & 1495244 & 1493168 & 1484963 & 1479283\end{array}$ $\begin{array}{llllllllll}1485359 & 1488305 & 1493.445 & 1494.036 & 1493.21 & 1495.261 & 1493.175 & 1484.963 & 1479.281\end{array}$ $\begin{array}{lllllllllll}4485.369 & 1488.298 & 1493.457 & 1494.062 & 1493224 & 1495293 & 1493.185 & 1484.966 & 1479.283\end{array}$ $\begin{array}{lllllllllll}1485.38 & 1488.298 & 1493.471 & 1494.082 & 1493.24 & 1495.315 & 1493.204 & 1484.968 & 1479.288\end{array}$ $\begin{array}{lllllllllll}1485.383 & 1488.305 & 1493.485 & 1494.099 & 1493.25 & 1495.329 & 1493.218 & 1484.966 & 1479.29\end{array}$ $\begin{array}{llllllllll}1485.336 & 1488.309 & 1493.495 & 1494.104 & 1493.259 & 1495.331 & 1493.229 & 1484.963 & 1479.292\end{array}$ $\begin{array}{lllllllll}1485.341 & 1488.319 & 1493.504 & 1494.104 & 1493.269 & 1495.336 & 1493.236 & 1484.966 & 1479.297\end{array}$ $\begin{array}{llllllllll}1485.348 & 1488.328 & 1493.007 & 1494.111 & 1493.274 & 1495.331 & 1493.243 & 1484.966 & 1479.295\end{array}$ $\begin{array}{llllllllll}1485.357 & 1488.342 & 1493.513 & 1494.099 & 1493.276 & 1495.324 & 1493.248 & 1484.963 & 1479.29\end{array}$ $\begin{array}{llllllllll}1485357 & 1488.354 & 1493.511 & 1494.094 & 1493.276 & 149531 & 1493.246 & 1484.966 & 1479288\end{array}$ $\begin{array}{llllllllll}1485.362 & 1488.361 & 1493.504 & 1494.085 & 1493.269 & 1495.291 & 1493.241 & 1484.963 & 1479.28\end{array}$ $\begin{array}{llllllllll}1485.364 & 1488.363 & 1493.504 & 1494.08 & 1493.269 & 1495.291 & 1493.239 & 1484.963 & 1479.281\end{array}$ $\begin{array}{llllllllll}1485.369 & 1488.368 & 1493.502 & 1494.071 & 1493.267 & 1495.277 & 1493.236 & 1484.966 & 1479.276\end{array}$ $\begin{array}{lllllllll}1485.369 & 1488.368 & 1493.506 & 1494.071 & 1493.267 & 1495.282 & 1493.239 & 1484.963 & 1479.276\end{array}$

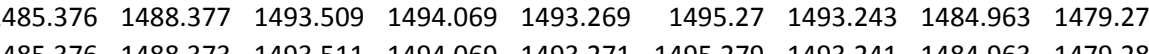
$\begin{array}{lllllllll}1485.376 & 1488.373 & 1493.511 & 1494.069 & 1493.27 & 1495.279 & 1493.241 & 1484.963 & 1479.28 \\ 1485383 & 1488373 & 1493.514 & 1494.083 & 1493281 & 1495284 & 1493251 & 1484963 & 1479.283\end{array}$ $\begin{array}{llllllllll}1485.392 & 1488.38 & 1493.523 & 1494.087 & 1493.281 & 1495.286 & 1493.253 & 1484.963 & 1479.285\end{array}$ $\begin{array}{lllllllllll}1485.38 & 1488382 & 1493525 & 149409 & 1493285 & 1495 & 279 & 1493258 & 1484.963 & 1479.288\end{array}$ $\begin{array}{lllllllllll}1485.376 & 1488.396 & 1493.525 & 1494.075 & 1493.283 & 1495.268 & 1493.26 & 1484.961 & 1479.288\end{array}$ $\begin{array}{llllllllll}1485.378 & 1488.408 & 1493.514 & 1494.05 & 1493.276 & 1495.237 & 1493.253 & 1484.959 & 1479.283\end{array}$ $\begin{array}{lllllllll}1485.385 & 1488.403 & 1493.506 & 1494.031 & 1493.266 & 1495.216 & 1493.239 & 1484.959 & 1479.278\end{array}$ $\begin{array}{lllllllll}1485.385 & 1488.403 & 1493.495 & 1494.02 & 1493.257 & 1495.199 & 1493.232 & 1484.959 & 1479.276\end{array}$ $\begin{array}{lllllllll}1485.387 & 1488.401 & 1493.49 & 1494.01 & 1493.25 & 1495.188 & 1493.225 & 1484.959 & 1479.274 \\ 1485396 & 1488389 & 1493.488 & 149401 & 149325 & 1495.195 & 1493218 & 1484.956 & 1479274\end{array}$

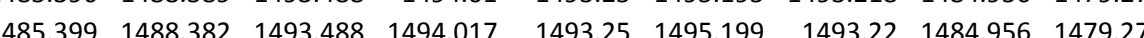
$\begin{array}{llllllllll}1485.408 & 1488.377 & 1493.49 & 1494.024 & 1493.255 & 1495.207 & 1493.227 & 1484.956 & 1479.278\end{array}$ $\begin{array}{llllllllll}1485.41 & 1488.38 & 1493.49 & 1494.022 & 1493.255 & 1495.199 & 1493.227 & 1484.956 & 1479.281\end{array}$ $\begin{array}{lllllllll}1485.415 & 1488.377 & 1493.492 & 1494.017 & 1493.255 & 1495.197 & 1493.229 & 1484.954 & 1479.283\end{array}$ $\begin{array}{lllllllll}4485.422 & 1488.377 & 1493.49 & 1494.017 & 1493.252 & 1455.192 & 1493.225 & 1484.954 & 1479.285\end{array}$ $\begin{array}{lllllllll}1485.417 & 1488.377 & 1493.49 & 1494.006 & 1493.252 & 1495.183 & 1493.229 & 1484.952 & 1479.283\end{array}$ $\begin{array}{lllllllll}4485.415 & 1488.38 & 1493.485 & 1493.999 & 1493.245 & 1495.176 & 1493.22 & 1484.952 & 1479.28 \\ 1485.413 & 1488375 & 1493.476 & 1493.992 & 1493238 & 1495.167 & 1493.213 & 1484952 & 1479228\end{array}$ $\begin{array}{lllllllll}1485 & 148838 & 1493.485 & 14939935 & 1493245 & 1495.176 & 149322 & 1484952 & 1479.281\end{array}$ $\begin{array}{llllllllll}1485.403 & 1488.37 & 1493.467 & 1493.975 & 1493.229 & 1495.145 & 1493206 & 1484.947 & 1479.271\end{array}$ $\begin{array}{llllllllll}1485.401 & 1488.361 & 1493.457 & 1493.971 & 1493.217 & 1495.148 & 1493.19 & 1484.949 & 1479.264\end{array}$ $\begin{array}{lllllllll}1485.403 & 1488.354 & 1493.455 & 1493.971 & 1493.214 & 1495.152 & 1493.19 & 1484.947 & 1479.262\end{array}$ $\begin{array}{lllllllll}1485.401 & 1488.347 & 1493.453 & 1493.978 & 1493.217 & 1495.157 & 1493.19 & 1484.947 & 1479.26\end{array}$ $\begin{array}{lllllllllll}1485.403 & 1488.344 & 1493.453 & 1493.975 & 1493.214 & 1495.152 & 1493.185 & 1484.945 & 1479.26\end{array}$ $\begin{array}{lllllllll}1485.401 & 1488.342 & 1493.457 & 1493.985 & 1493.219 & 1495.164 & 1493.19 & 1484.945 & 1479.26\end{array}$

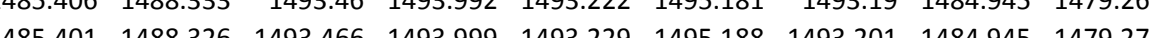

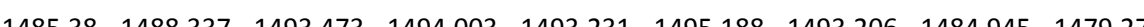
$\begin{array}{llllllllll}1485.385 & 1488.342 & 1493.474 & 1493.996 & 1493.231 & 1495.176 & 1493.208 & 1484.94 & 1479.274\end{array}$ $\begin{array}{lllllllll}1485.392 & 1488.335 & 1493.471 & 1493.996 & 1493.231 & 1495.178 & 1493.204 & 1484.942 & 1479.274\end{array}$ $\begin{array}{llllllllll}1485.396 & 1488.328 & 1493.466 & 1493.994 & 1493.226 & 1495.183 & 1493.199 & 1484.94 & 1479.26\end{array}$ $\begin{array}{llllllllll}1485.401 & 1488.316 & 1493.467 & 1493.999 & 1493.229 & 1495.178 & 1493.199 & 1484.942 & 1479.266\end{array}$ $\begin{array}{llllllllll}1485.406 & 1488.319 & 1493.469 & 1494.001 & 1493.229 & 1495.188 & 1493.199 & 1484.94 & 1479.267\end{array}$ $\begin{array}{llllllllll}1485.41 & 1488.309 & 1493.466 & 1494.01 & 1493.231 & 1495.199 & 1493.194 & 1484.94 & 1479.267 \\ 1485.42 & 1488.288 & 1493.474 & 1494.038 & 1493.238 & 1495.232 & 1493.197 & 1484.94 & 1479.269\end{array}$ 
Well

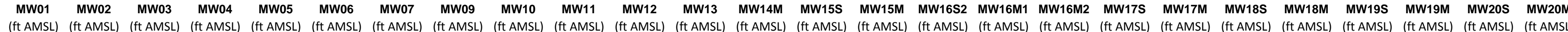

2/8/12 14:00 2/8/12 15:00 2/8/12 16:00 2/8/12 17:00 2/8/12 19:00 2/8/12 20:00 2/8/12 21:00 2/8/12 22:00 2/8/12 23:00 2/9/12 0:00 2/9/12 1:00 2/9/12 3:00 2/9/12 3:00 2/9/12 5:00 2/9/12 6:00 2/9/12 7:00 2/9/12 8:00 2/9/1210.00 2/9/12 11:00 /9/12 12:00 2/9/12 13:00 2/9/12 14:00 2/9/12 15:00 2/9/12 16:00 2/9/12 17:00 2/9/12 18:00 2/9/12 19:00 2/9/12 21:00 2/9/12 22:00 2/9/12 23:00 2/10/12 0:00 2/10/12 1:00 2/10/12 2:00 2/10/12 3:00 2/10/12 5:00 2/10/12 6:00 2/10/12 7:00 2/10/12 8:00 2/10/12 9:00 2/10/12 10:00 2/10/12 11:00 2/10/12 13:00 2/10/12 14:00 2/10/12 15:00 2/10/12 16:00 2/10/12 18:00

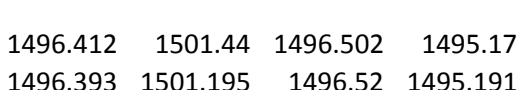
$\begin{array}{rrrr}1496.393 & 1501.195 & 1496.52 & 1495.191 \\ 1496372 & 1500.999 & 1496523 & 1995203\end{array}$ $\begin{array}{llll}1496.372 & 1500.999 & 1496.523 & 1495.203\end{array}$ $\begin{array}{lllll}1496.337 & 1500.782 & 1496.532 & 1495.222\end{array}$ $\begin{array}{llll}1496.321 & 1500.784 & 1496.52 & 1495.217\end{array}$ $\begin{array}{rrrrr}1496.305 & 1500.729 & 1496.523 & 1495.219\end{array}$ $\begin{array}{lllll}1496.286 & 1500.619 & 1496.53 & 1495.224\end{array}$ $\begin{array}{llll}1496.272 & 1500.509 & 1496.534 & 1495.226\end{array}$ $\begin{array}{llll}1496.256 & 1500.502 & 1496.53 & 1495.226\end{array}$ $\begin{array}{llll}1496.244 & 1500.407 & 1496.541 & 1495.229 \\ 1496233 & 1500314 & 1496558 & 149524\end{array}$ $\begin{array}{rrrr}1496.233 & 1500.314 & 1496.558 & 1495.24 \\ 1496.212 & 1500.223 & 1496.555 & 1495.243\end{array}$

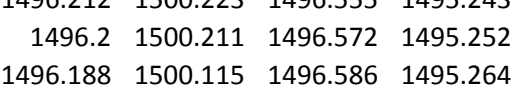
$\begin{array}{lllll}1496.174 & 1500.015 & 1496.59 & 1495.271\end{array}$ $\begin{array}{llll}1496.161 & 1499.927 & 1496.59 & 1495.275\end{array}$ $\begin{array}{llll}1496.147 & 1499.838 & 1496.59 & 1495.278\end{array}$ $\begin{array}{llll}1496.133 & 1499.761 & 1496.588 & 1495.275\end{array}$ $\begin{array}{llll}1496.116 & 1499.684 & 1496.588 & 1495.28 \\ 1496.109 & 1499.591 & 1496588 & 1495.278\end{array}$ $\begin{array}{lllll}1496.093 & 1499.616 & 1496593 & 149528\end{array}$ $\begin{array}{lllll}1496.091 & 1499.731 & 1496.623 & 1495296\end{array}$ $\begin{array}{lllll}1496.086 & 1499.717 & 1496.663 & 1495.327\end{array}$ $1496.074 \quad 1499.665 \quad 1496.689 \quad 1495.345$ $1496.058 \quad 1499.663 \quad 1496.691 \quad 1495.359$ $\begin{array}{lllll}1496.044 & 1499.614 & 1496.689 & 1495.364\end{array}$ $\begin{array}{llll}1496.035 & 1499.54 & 1496.689 & 1495.371\end{array}$ $\begin{array}{llll}1496.021 & 1499.533 & 1496.689 & 1495.373 \\ 1496.011 & 1499.633 & 1496.682 & 1495.371\end{array}$ $\begin{array}{llllll}1495.998 & 1499.605 & 1496.677 & 1495.366\end{array}$ $\begin{array}{llll}1495.986 & 1499.565 & 1496.672 & 1495.362\end{array}$ $\begin{array}{llllll}1495.979 & 1499.523 & 1496.672 & 1495.362\end{array}$ $\begin{array}{lllll}1495.97 & 1499.472 & 1496.679 & 1495.362\end{array}$ $\begin{array}{lllll}1495.96 & 1499.458 & 1496.684 & 1495.366\end{array}$ $\begin{array}{llll}1495.951 & 1499.505 & 1496.686 & 1495.369 \\ 1495937 & 1499.505 & 1466679 & 1495364\end{array}$ $\begin{array}{llll}1495.937 & 1499.505 & 1496.679 & 1495.364\end{array}$ $\begin{array}{llll}1495.93 & 1499.481 & 1496.675 & 1495.362 \\ 1495.918 & 1499.46 & 1496665 & 1495355\end{array}$ $\begin{array}{lllll}1495.904 & 1499.467 & 1496.642 & 1495.341\end{array}$ $\begin{array}{lllll}1495.89 & 1499.458 & 1496.604 & 1495.31\end{array}$ $\begin{array}{lllll}1495.883 & 1499.442 & 1496.59 & 1495.296\end{array}$ $\begin{array}{llll}1495.872 & 1499.418 & 1496.576 & 1495.28\end{array}$ $1495.858 \quad 1499.379 \quad 1496.555 \quad 1495.257$ $\begin{array}{llll}1495.848 & 1499.33 & 1496.539 & 1495.24 \\ 145832 & 1499316 & 1466504 & 1495215\end{array}$ 1495.832 1499.3161496 .5041495 .215 $\begin{array}{llll}1495.83 & 1499.167 & 1496.53 & 1495.208\end{array}$

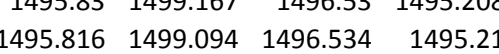
$1495.804 \quad 1499.031 \quad 1496.523 \quad 1495.205$ $1495.785 \quad 1499.003 \quad 1496.506 \quad 1495.194$ $\begin{array}{llll}1495.779 & 1498.982 & 1496.492 & 1495.184\end{array}$ $\begin{array}{llll}1495.769 & 1498.964 & 1496.476 & 1495.168 \\ 1495.751 & 1498.957 & 1496.441 & 1495.147\end{array}$ $\begin{array}{llllllllll}1493.906 & 1494.899 & 1495.377 & 1495.828 & 1496.912 & 1496.748 & 1493.243 & 1488.853 & 1493.081 & 1491.035 \\ 1493.904 & 1494906 & 1495377 & 1495.84 & 1496.926 & 1496765 & 1493257 & 14888 & 1493095 & 1491.04\end{array}$ $\begin{array}{lllllllllll}1493.904 & 1494.906 & 1495.377 & 1495.84 & 1496.926 & 1496.765 & 1493.257 & 1488.86 & 1493.095 & 1491.04\end{array}$ $\begin{array}{lllllllllll}143.915 & 1494.909 & 1495.368 & 1495.847 & 1496.933 & 1496.765 & 1493.264 & 1488.86 & 1493.1 & 1491.042 \\ 1493925 & 1494.916 & 1495.361 & 1495854 & 1496942 & 1496768 & 1493.273 & 1488.867 & 1493112 & 1491.049\end{array}$ $\begin{array}{llllllllllll}1493.943 & 1494.918 & 1495.351 & 1495.858 & 1496.945 & 1496.768 & 1493.278 & 1488.872 & 1493.116 & 1491.052\end{array}$ $\begin{array}{lllllllllll}1493.958 & 1494.918 & 1495.342 & 1495.854 & 1496.942 & 1496.756 & 1493.28 & 1488.872 & 1493.121 & 1491.052\end{array}$ $\begin{array}{lllllllllll}1493.962 & 1494.918 & 1495.334 & 1495.858 & 1496.949 & 1496.756 & 1493.285 & 1488.872 & 1493.121 & 1491.061\end{array}$ $\begin{array}{llllllllll}1493.969 & 1494.921 & 1495.332 & 1495.863 & 1496.954 & 1496.758 & 1493.285 & 1488.879 & 1493.126 & 1491.063\end{array}$ $\begin{array}{llllllllllll}1493.972 & 1494.923 & 1495.332 & 1495.867 & 1496.961 & 1496.765 & 1493.287 & 1488.877 & 1493.126 & 1491.07\end{array}$

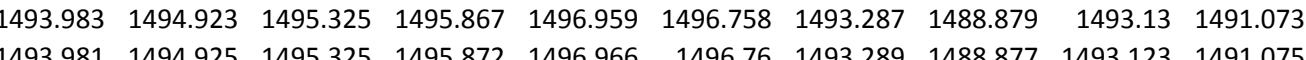
$\begin{array}{llllllllll}1493.981 & 1494.925 & 1495.325 & 1495.872 & 1496.966 & 1496.76 & 1493.289 & 1488.877 & 1493.123 & 1491.075 \\ 1493.974 & 1494.928 & 1495.334 & 1495881 & 1496975 & 1496775 & 1493.294 & 1488.879 & 1493.13 & 1491.077\end{array}$ $\begin{array}{lllllllllllll}1493.983 & 1494.928 & 1495.323 & 1495.881 & 1496.977 & 1496.772 & 1493.299 & 1488.884 & 1493.137 & 1491.082\end{array}$ $\begin{array}{lllllllllllll}1493.981 & 1494.933 & 1495.33 & 1495.893 & 1496.987 & 1496.787 & 1493.306 & 1488.884 & 1493.147 & 1491.087\end{array}$ $\begin{array}{lllllllllll}1493.979 & 1494.937 & 1495.337 & 1495.9 & 1496.996 & 1496.799 & 1493.315 & 1488.888 & 1493.156 & 1491.089\end{array}$ $\begin{array}{lllllllllll}1493.983 & 1494.942 & 1495.33 & 1495.907 & 1497.003 & 1496.801 & 1493.324 & 1488.884 & 1493.161 & 1491.094\end{array}$ $\begin{array}{lllllllllll}1493.993 & 1494.942 & 1495.327 & 1495.909 & 1497.005 & 1496.801 & 1493.331 & 1488.893 & 1493.17 & 1491.098\end{array}$ $\begin{array}{lllllllllll}1494.002 & 1494.947 & 1495.32 & 1495.914 & 1497.01 & 1496.796 & 1493.338 & 1488.893 & 1493.175 & 1491.103\end{array}$ $\begin{array}{llllllllll}1494.012 & 1494.947 & 1495.315 & 1495.914 & 1497.01 & 1466.794 & 1493.341 & 1488.895 & 1493.18 & 1491.105 \\ 1494016 & 149.945 & 1495311 & 1495.916 & 1497.012 & 1496789 & 1493346 & 1488895 & 1493.182 & 149108\end{array}$ $\begin{array}{lllllllllll}1494.016 & 1494.945 & 1495.311 & 1495.916 & 1497.012 & 1496.789 & 1493.346 & 1488.895 & 1493.182 & 1491.108 \\ 1494.019 & 1494.947 & 1495308 & 1495.919 & 1497.015 & 1496789 & 1493343 & 1488.955 & 1493184 & 1491112\end{array}$ $\begin{array}{lllllllllll}1494.021 & 1494.947 & 1495.306 & 1495.923 & 1497.019 & 1496.794 & 1493.345 & 1488.893 & 1493.184 & 1491.115\end{array}$ $\begin{array}{lllllllllll}1494.002 & 1494.952 & 1495.323 & 1495.935 & 1497.035 & 1496.82 & 1493.353 & 1488.9 & 1493.191 & 1491.119\end{array}$ $\begin{array}{lllllllllll}1493.981 & 1494.961 & 1495.342 & 1495.958 & 1497.056 & 1496.856 & 1493.366 & 1488.9 & 1493.203 & 1491.124\end{array}$ $\begin{array}{llllllllll}1493.976 & 1494.969 & 1495.349 & 1495.972 & 1497.073 & 1496.875 & 1493.381 & 1488.909 & 1493.215 & 1491.131\end{array}$ $\begin{array}{llllllllll}1493.99 & 1494.971 & 1495.339 & 1495.981 & 1497.08 & 1496.88 & 1493.388 & 1488.916 & 1493.227 & 1491.136\end{array}$ $\begin{array}{llllllllll}1494.005 & 1494.976 & 1495.334 & 1495.986 & 1497.084 & 1496.875 & 1493.395 & 1488.916 & 1493.236 & 1491.138 \\ 1494.016 & 1494978 & 149533 & 1495.99 & 1497.098 & 149683 & 1493404 & 1488923 & 1493241 & 149145\end{array}$ $\begin{array}{lllllllllll}1494.016 & 1494.978 & 1495.33 & 1495.99 & 1497.089 & 1496.87 & 1493.404 & 1488.923 & 1493.241 & 1491.145 \\ 1494.03 & 1494.98 & 1495323 & 1495.995 & 1497.094 & 149687 & 1493.413 & 1488923 & 14325 & 1491147\end{array}$ $\begin{array}{rrrrrrrrrr}1494.03 & 1494.98 & 1495.323 & 1495.995 & 1497.094 & 1496.87 & 1493.413 & 1488.923 & 1493.25 & 1491.147 \\ 1494.047 & 1494.983 & 1495.32 & 1495.997 & 1497.094 & 1496.858 & 1493.416 & 1488.923 & 1493255 & 1491.154\end{array}$ $\begin{array}{llllllllllll}1494.061 & 1494.983 & 1495.313 & 1495.997 & 1497.091 & 1496.854 & 1493.418 & 1488.926 & 1493.257 & 1491.156\end{array}$ $\begin{array}{lllllllllll}1494.075 & 1494.983 & 1495.301 & 1495.997 & 1497.091 & 1496.847 & 1493.42 & 1488.923 & 1493.257 & 1491.161\end{array}$ $\begin{array}{llllllllll}1494.08 & 1494.985 & 1495.301 & 1495.997 & 1497.094 & 1496.842 & 1493.42 & 1488.928 & 1493.257 & 1491.163\end{array}$ $\begin{array}{lllllllllll}1494.08 & 1494.985 & 1495.301 & 1496 & 1497.096 & 1496.847 & 1493.423 & 1488.926 & 1493.262 & 1491.166\end{array}$ $\begin{array}{lllllllllll}1494.087 & 1494.985 & 1495.301 & 1496.007 & 1497.101 & 1496.854 & 1493.425 & 1488.93 & 1493.264 & 1491.17\end{array}$ $\begin{array}{llllllllll}1494.089 & 1494.988 & 1495.296 & 1496.007 & 1497.103 & 1496.851 & 1493.427 & 1488.926 & 1493.266 & 1491.17 \\ 1494101 & 1494.99 & 1495292 & 1466007 & 1497.098 & 1496844 & 1493.43 & 1488928 & 1493269 & 1491.175\end{array}$ $\begin{array}{lllllllllll}1494.11 & 1494.99 & 1495287 & 1496007 & 1497098 & 1496837 & 1493.432 & 1488.928 & 1493.269 & 1491.175 \\ 1493 & 1493 & 1491.175\end{array}$ $\begin{array}{llllllllll}1494.12 & 1494.988 & 1495282 & 1496002 & 1497.096 & 149683 & 1493.434 & 1488928 & 1493273 & 1491.18\end{array}$ $\begin{array}{llllllllllll}1494.136 & 1494.988 & 1495.268 & 1495.995 & 1497.082 & 1496.806 & 1493.432 & 1488.921 & 1493.273 & 1491.18\end{array}$ $\begin{array}{lllllllllll}1494.162 & 1494.98 & 1495.247 & 1495.972 & 1497.059 & 1496.768 & 1493.425 & 1488.914 & 1493.264 & 1491.175\end{array}$ $\begin{array}{llllllllll}1494.162 & 1494.978 & 1495.249 & 1495.963 & 1497.054 & 1496.756 & 1493.423 & 1488.909 & 1493.262 & 1491.177\end{array}$ $\begin{array}{lllllllllll}1494.169 & 1494.971 & 1495.242 & 1495.951 & 1497.04 & 1496.744 & 1493.416 & 1488.898 & 1493.257 & 1491.173\end{array}$ $\begin{array}{llllllllll}1494.171 & 1494.966 & 1495.232 & 1495.939 & 1497.026 & 1496.727 & 1493.411 & 1488.893 & 1493.25 & 1491.17\end{array}$ $\begin{array}{lllllllllllll}1494.171 & 1494.959 & 1495.23 & 1455.925 & 1497.017 & 1496.71 & 1493.39 & 1488.879 & 149.243 & 1491.168\end{array}$

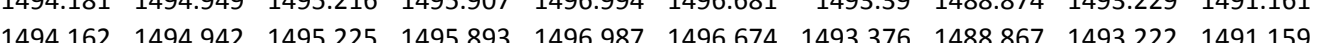
$\begin{array}{llllllllllll}1494.129 & 1494.942 & 1495.244 & 1495.898 & 1496.996 & 1496.705 & 1493.376 & 1488.87 & 1493.222 & 1491.156\end{array}$ $\begin{array}{lllllllllll}1494.115 & 1494.937 & 1495.249 & 1495.898 & 1496.996 & 1496.715 & 1493.376 & 1488.867 & 1493.217 & 1491.154\end{array}$ $\begin{array}{lllllllllll}1494.115 & 1494.937 & 1495.242 & 1495.888 & 1496.987 & 1496.708 & 1493.374 & 1488.862 & 1493.217 & 1491.149\end{array}$ $\begin{array}{lllllllllll}1494.122 & 1494.93 & 1495.23 & 1495.874 & 1496.975 & 1496.691 & 1493.371 & 1488.867 & 1493.212 & 1491.145\end{array}$ $\begin{array}{lllllllllll}1494.124 & 1494.925 & 1495.223 & 1495.865 & 1496.966 & 1496.679 & 1493.366 & 1488.858 & 1493.208 & 1491.142\end{array}$ $\begin{array}{llllllllll}1494.131 & 1494.918 & 1495.218 & 1495.854 & 1496.952 & 1496.669 & 1493.359 & 1488.851 & 1493.203 & 1491.138\end{array}$ $\begin{array}{lllllllll}1485.436 & 1488.283 & 1493.488 & 1494.059 & 1493.25 & 1495.263 & 1493.215 & 1484.938 & 1479.271\end{array}$ $\begin{array}{llllllllll}1485.459 & 1488.283 & 1493.502 & 1494.073 & 1493.264 & 1495.279 & 1493.234 & 1484.94 & 1479.276 \\ 1485.427 & 1488.29 & 1493506 & 1494.08 & 1493271 & 1495284 & 1493241 & 1484938 & 1479.278\end{array}$

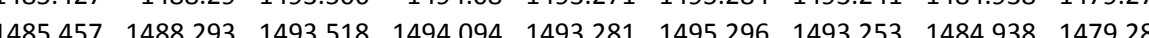
$\begin{array}{lllllllllll}1485.48 & 1488.302 & 1493.525 & 1494.094 & 1493.288 & 1495.289 & 1493.262 & 1484.935 & 1479.285\end{array}$ $\begin{array}{lllllllllll}1485.51 & 1488.307 & 1493.528 & 1494.092 & 1493.29 & 1495.289 & 1493.262 & 1484.935 & 1479.285\end{array}$ $\begin{array}{lllllllll}1485.542 & 1488.309 & 1493.528 & 1494.097 & 1493.293 & 1495.291 & 1493.262 & 1484.935 & 1479.285\end{array}$ $\begin{array}{llllllllll}1485.568 & 1488.307 & 1493.532 & 1494.094 & 1493.293 & 1495.293 & 1493.267 & 1484.935 & 1479.285\end{array}$ $\begin{array}{llllllllll}1485.598 & 1488.307 & 1493.532 & 1494.099 & 1493.297 & 1495.301 & 1493.265 & 1484.933 & 1479.283\end{array}$ $\begin{array}{llllllllll}1485.628 & 1488.314 & 4993.532 & 1494.101 & 1493.297 & 1495.293 & 1493.267 & 1484.933 & 1479.278\end{array}$ $\begin{array}{llllllllll}1485.658 & 1488.312 & 1493.532 & 1494.101 & 1493.297 & 1495.301 & 1493.265 & 1484.935 & 1479.276\end{array}$ $\begin{array}{lllllllllll}1485572 & 1488316 & 1493542 & 1494.115 & 1493.307 & 1495315 & 14932.27 & 1484.933 & 1479.278\end{array}$ $\begin{array}{llllllllll}1485.75 & 1488314 & 1493.551 & 1494.129 & 1493.316 & 1495.324 & 1493.283 & 1484.931 & 1479281\end{array}$ $\begin{array}{llllllllll}1485.785 & 1488.316 & 1493.563 & 1494.136 & 1493.326 & 1495.343 & 1493.293 & 1484.931 & 1479.28\end{array}$ $\begin{array}{llllllllll}1485.817 & 1488.321 & 1493.568 & 1494.148 & 1493.333 & 1495.345 & 1493.302 & 1484.931 & 1479.288\end{array}$ $\begin{array}{lllllllllll}1485.833 & 1488.33 & 1493.577 & 1494.15 & 1493.34 & 1495.35 & 1493.312 & 1484.931 & 1479.292\end{array}$ $\begin{array}{lllllllll}1485.854 & 1488.33 & 1493.582 & 1494.152 & 1493.347 & 1495.35 & 1493.316 & 1484.931 & 1479.292\end{array}$ $\begin{array}{lllllllllll}4885.887 & 1488.342 & 1493.587 & 1494.152 & 1493.349 & 1495.345 & 1493.321 & 4844.926 & 1479.295\end{array}$ $\begin{array}{llllllllll}1485.94 & 1488.342 & 1493.591 & 1494.15 & 1493.352 & 1495.35 & 143.321 & 1484.928 & 1479.295\end{array}$ $\begin{array}{lllllllllll}1485.974 & 1488.344 & 1493.591 & 1494.157 & 1493.354 & 1495.35 & 1493.319 & 1484.928 & 1479.292 \\ 1493.323 & 1484.926 & 1479.29\end{array}$ $\begin{array}{lllllllllll}1486.03 & 1488.328 & 1493.61 & 1494.201 & 1493.376 & 1495.409 & 1493.335 & 1484.931 & 1479.295\end{array}$ $\begin{array}{lllllllll}1486.06 & 1488.333 & 1493.615 & 1494.22 & 1493.39 & 1495.43 & 1493.347 & 1484.926 & 1479.295\end{array}$ $\begin{array}{llllllllll}1486.09 & 1488.34 & 1493.629 & 1494.227 & 1493.397 & 1495.439 & 1493.361 & 1484.926 & 1479.297\end{array}$ $\begin{array}{llllllllll}1486.118 & 1488.349 & 1493.641 & 1494.234 & 1493.404 & 1495.434 & 1493.375 & 1484.926 & 1479.299\end{array}$ $\begin{array}{lllllllll}1486.136 & 14888.352 & 1493.652 & 1494.33 & 1493.411 & 1455.444 & 4493.38 & 1484.926 & 1479.304\end{array}$ $\begin{array}{llllllllll}14866.145 & 1488.363 & 1493.654 & 1494.236 & 1493.423 & 1455.442 & 1493.389 & 1484.923 & 1479.306\end{array}$ $\begin{array}{llllllllll}1486.178 & 1488.387 & 1493.664 & 1494.234 & 1493.43 & 1495.43 & 1493.401 & 1484.923 & 1479.309\end{array}$ $\begin{array}{lllllllll}1486.203 & 1488.389 & 1493.662 & 1494.232 & 1493.428 & 1495.425 & 1493.401 & 1484.923 & 1479.309\end{array}$ $\begin{array}{llllllllll}1486.229 & 1488.387 & 1493.666 & 1494.236 & 1493.43 & 1495.425 & 1493.398 & 1484.923 & 1479.306\end{array}$ $\begin{array}{lllllllll}1486.25 & 1488.396 & 1493.666 & 1494.234 & 1493.432 & 1495.43 & 1493.403 & 1484.921 & 1479.306\end{array}$ $\begin{array}{llllllllll}1486.275 & 1488.396 & 1493.671 & 1494.234 & 1493.435 & 1495.432 & 1493.41 & 1484.921 & 1479.304\end{array}$ $\begin{array}{llllllllll}1486.296 & 1488.401 & 1493.669 & 1494.243 & 1493.435 & 1455.439 & 1493.403 & 1484.921 & 1479.302\end{array}$

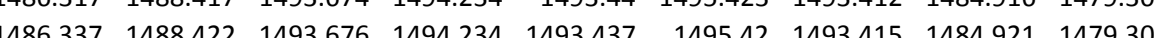
$\begin{array}{llllllllll}1486.363 & 1488.431 & 1493.678 & 1494.229 & 1493.44 & 1495411 & 1493.417 & 1484.919 & 1479304\end{array}$ $\begin{array}{lllllllllll}1486.386 & 1488.443 & 1493.678 & 1494.215 & 1493.444 & 1495.397 & 1493.419 & 1484.914 & 1479.304\end{array}$ $\begin{array}{llllllllll}1486.409 & 1488.455 & 1493.671 & 1494.194 & 1493.432 & 1495.364 & 1493.412 & 1484.916 & 1479.304\end{array}$ $\begin{array}{lllllllll}1486.423 & 1488.452 & 1493.666 & 1494.183 & 1493.428 & 1495.35 & 1493.405 & 1484.916 & 1479.304\end{array}$ $\begin{array}{llllllllll}1486.437 & 1488.457 & 1493.664 & 1494.176 & 1493.423 & 1495.338 & 1493.403 & 1484.914 & 1479.302\end{array}$ $\begin{array}{llllllllll}1486.437 & 1488.459 & 1493.652 & 1494.162 & 1493.418 & 1495.317 & 1493.396 & 1484.912 & 1479.302\end{array}$ $\begin{array}{lllllllllll}1486.423 & 1488.452 & 1493.65 & 1494.141 & 1493.406 & 1455.30 & 1493.387 & 1484.912 & 1479.297\end{array}$ $\begin{array}{llllllllll}14864 & 1488441 & 1493624 & 1494.115 & 1493385 & 14952.27 & 1493354 & 1484909 & 1479288\end{array}$ $\begin{array}{lllllllllll}1486.43 & 1488.417 & 1493.624 & 1494.127 & 1493.387 & 1495.284 & 1493.349 & 1484.909 & 1479.285\end{array}$ $\begin{array}{llllllllll}1486.448 & 1488.413 & 1493.624 & 1494.125 & 1493.385 & 1495.289 & 1493.354 & 1484.907 & 1479.283\end{array}$ $\begin{array}{llllllllll}4486.446 & 1488.415 & 1493.622 & 1494.125 & 1493.38 & 1495.277 & 1493.356 & 1484.907 & 1479.278\end{array}$ $\begin{array}{llllllllll}1486.411 & 1488.417 & 1493.619 & 1494.111 & 1493.378 & 1495.268 & 1493.358 & 1484.905 & 1479.278\end{array}$ $\begin{array}{lllllllllll}1486.439 & 1488.415 & 1493.615 & 1494.101 & 1493.375 & 1495.254 & 1493.356 & 1484.902 & 1479.278\end{array}$ $\begin{array}{rrrrrrrrr}1486.467 & 1488.413 & 1493.61 & 1494.087 & 1493.368 & 1495.237 & 1493.358 & 1484.902 & 1479.281 \\ 1486.49 & 1488.422 & 1493.601 & 1494.069 & 1493.361 & 1495214 & 1493349 & 1484.9 & 1479.278\end{array}$ 
Well

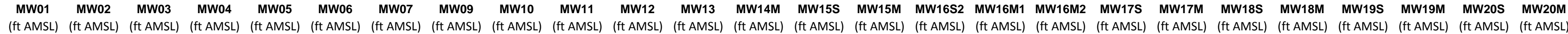

2/10/12 20:00 2/10/1221:00 2/10/12 22:00 2300 2/10/112 23:00 2/11/12 0:00 2/11/121:00 2/11/12 3:00 $2 / 11 / 124: 00$ 2/11/12 5:00

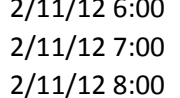
2/11/12 9:00 2/11/12 10:00 2/11/12 11:00 2/11/12 12:00 2/11/12 13:00 2/11/12 14:00 $2 / 11 / 1216: 00$

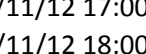
2/11/12 19:00 2/11/12 20:00 2/11/12 21:00 2/11/12 22:00 2/11/1223:00 2/12/120:00 2/12/122:00 2/12/12 3:00 2/12/12 5:00 2/12/12 6:00 2/12/12 7:00 2/12/12 9:00 2/12/12 10:00 2/12/12 12:00 2/12/12 13:00 2/12/12 14:00 2/12/12 15:00 2/12/12 16:00 $2 / 12 / 1218.00$ 2/12/12 19:00 2/12/12 20:00 2/12/12 21:00 2/12/12 22:00 2/12/122 23:00 $2 / 13 / 120: 00$
$2 / 13 / 121: 00$ $\begin{array}{rrrr}1495.744 & 1498.924 & 1496.429 & 1495.13\end{array}$ $\begin{array}{rrrr}1495.73 & 1498.891 & 1496.413 & 1495.116 \\ 1495.723 & 1498856 & 149641 & 1495.105\end{array}$ $\begin{array}{llll}1495.723 & 1498.856 & 1496.41 & 1495.105 \\ 1495.718 & 1498.807 & 1496.41 & 1495102\end{array}$ $\begin{array}{llrr}1495.699 & 1498.782 & 1496.399 & 1495.093\end{array}$ $\begin{array}{llll}1495.695 & 1498.749 & 1496.392 & 1459.093\end{array}$ $\begin{array}{llll}1495.681 & 1498.698 & 1496.387 & 1495.081\end{array}$ $\begin{array}{lllll}1495.667 & 1498.656 & 1496.357 & 1495.06\end{array}$ $\begin{array}{lllll}1495.657 & 1498.605 & 1496.347 & 1495.049\end{array}$ $\begin{array}{llll}1495.634 & 1498.584 & 1496.305 & 1495.023\end{array}$ $\begin{array}{llll}1495.632 & 1498.53 & 1496.296 & 1495.007 \\ 1495.623 & 1498.479 & 1966287 & 1494.997\end{array}$ $\begin{array}{llll}1495.623 & 1498.479 & 1496.287 & 1494.997 \\ 1495.613 & 1498.416 & 1496.291 & 1494.993\end{array}$ $\begin{array}{lllll}1495.599 & 1498.367 & 1496.277 & 1494.993\end{array}$ $\begin{array}{lllll}1495.597 & 1498.271 & 1496.294 & 1494.988\end{array}$ $\begin{array}{lllll}1495.585 & 1498.164 & 1496.294 & 1494.988\end{array}$ $\begin{array}{lllll}1495.583 & 1498.05 & 1496.312 & 1495.002\end{array}$ $\begin{array}{llll}1495.574 & 1497.961 & 1496.331 & 1495.016\end{array}$ $\begin{array}{llll}1495.569 & 1497.868 & 1496.352 & 1495.035\end{array}$ $\begin{array}{llll}1495.564 & 1497.765 & 1496.382 & 1495.06 \\ 1495.555 & 1497.702 & 1496.403 & 1495.084\end{array}$ $\begin{array}{llll}1455.546 & 1497.681 & 1496.422 & 1495.102\end{array}$ $\begin{array}{llll}1495.536 & 1497.679 & 1496.424 & 1495116\end{array}$ $\begin{array}{lllll}1495.525 & 1497.686 & 1496.413 & 1495.116\end{array}$ $\begin{array}{llllll}1495.511 & 1497.674 & 1496.399 & 1495.107\end{array}$ $\begin{array}{lllll}1495.506 & 1497.679 & 1496.385 & 1495.102\end{array}$ $\begin{array}{llll}1495.502 & 1497.656 & 1496.403 & 1495.112\end{array}$ $\begin{array}{llll}1495.497 & 1497.623 & 1496.42 & 1495.123\end{array}$ $\begin{array}{llll}1495.492 & 1497.586 & 1496.438 & 1495.135 \\ 1495.474 & 1497.569 & 1496.422 & 1495.133\end{array}$ $\begin{array}{lllll}1495.469 & 1497.546 & 1496.422 & 1495.135\end{array}$ $\begin{array}{llll}1495.474 & 1497.5 & 1496.45 & 1495.151\end{array}$ $\begin{array}{lllll}1495.469 & 1497.455 & 1496.488 & 1495.177\end{array}$ $1495.462 \quad 1497.423 \quad 1496.509 \quad 1495.198$ $\begin{array}{llll}1495.455 & 1497.392 & 1496.52 & 1495.215\end{array}$ $\begin{array}{llll}1495.446 & 1497.364 & 1496.53 & 1495.226\end{array}$ $\begin{array}{lllll}1495.441 & 1497.327 & 1496.534 & 1495.236\end{array}$ $\begin{array}{llll}1495.434 & 1497.27 & 1496.541 & 1495.243 \\ 1495.427 & 1497.21 & 1496541 & 1495245\end{array}$ $\begin{array}{rrrrr}1455.425 & 1497.152 & 1496.548 & 1495.254\end{array}$ $\begin{array}{lllll}1495.427 & 1497.096 & 1496.583 & 1495.275\end{array}$ $\begin{array}{llll}1495.427 & 1497.047 & 1496.63 & 1495.31\end{array}$ $\begin{array}{llll}1495.425 & 1497.005 & 1496.668 & 1495.343\end{array}$ $\begin{array}{llll}1495.422 & 1496.975 & 1496.698 & 1495.373\end{array}$ $\begin{array}{rrrr}1495.42 & 1496.952 & 1496.721 & 1495.401 \\ 1495.418 & 1496935 & 1496.742 & 1495.425\end{array}$ $1495.418 \quad 1496.935 \quad 1496.7421495 .425$ $1495.406 \quad 1496.931 \quad 1496756 \quad 1495.455$ $1495.404 \quad 1496.928 \quad 1496.766 \quad 1495.467$ $1495.401 \quad 1496.924 \quad 1496.77 \quad 1495.476$ $1495.406 \quad 1496.9191496 .798 \quad 1495.495$ $1495.399 \quad 1496.917 \quad 1496.815 \quad 1495.511$ $\begin{array}{llll}1495.397 & 1496.91 & 1496.824 & 1495.523 \\ 1495.399 & 1496.896 & 1496.838 & 1495.537\end{array}$ $\begin{array}{llllllllll}1494.143 & 1494.902 & 1495.206 & 1495.816 & 1496.924 & 1496.624 & 1493.343 & 1488.837 & 1493.189 & 1491.129\end{array}$ $\begin{array}{llllllllll}1494.139 & 1494.897 & 1495.199 & 1495.807 & 1496.908 & 1496.622 & 1493.338 & 1488.83 & 1493.182 & 1491.124 \\ 1494.127 & 1494.887 & 1495206 & 1495795 & 1496903 & 1496.624 & 1993331 & 14883 & 1493175 & 1491117\end{array}$

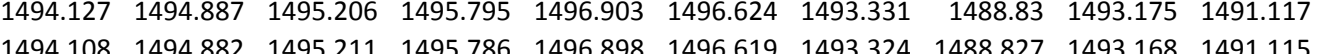
$\begin{array}{llllllllllll}1494.103 & 1494.878 & 1495.206 & 1495.775 & 1496.887 & 1496.622 & 1493.322 & 1488.827 & 1493.161 & 1491.11\end{array}$ $\begin{array}{lllllllllll}1494.099 & 1494.873 & 1495.199 & 1495.763 & 1496.88 & 1496.61 & 1493.313 & 1488.82 & 1493.156 & 1491.108\end{array}$ $\begin{array}{lllllllllll}1494.089 & 1494.868 & 1495.201 & 1495.756 & 1496.873 & 1496.617 & 1493.308 & 1488.823 & 1493.151 & 1491.101\end{array}$ $\begin{array}{lllllllllll}1494.099 & 1494.858 & 1495.189 & 1495.733 & 1496.856 & 1496.586 & 1493.299 & 1488.818 & 1493.14 & 1491.094\end{array}$ $\begin{array}{llllllllllll}1494.092 & 1494.853 & 1495.18 & 1495.719 & 1496.847 & 1496.581 & 1493.289 & 1488.813 & 1493.135 & 1491.091\end{array}$ $\begin{array}{lllllllllll}1494.106 & 1494.844 & 1495.168 & 1495.693 & 1496.822 & 1496.55 & 1493.282 & 1488.804 & 1493.126 & 1491.084\end{array}$ $\begin{array}{rrrrrrrrrrr}1494.094 & 1494.839 & 1495.163 & 1495.677 & 1496.81 & 1496.538 & 1493.271 & 1488.806 & 1493.119 & 1491.08 \\ 1494.08 & 149483 & 1495.168 & 1495661 & 1496798 & 1496531 & 1493266 & 1488804 & 1493114 & 1491.073\end{array}$

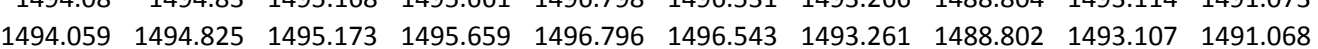
$\begin{array}{llllllllll}1494.052 & 1494.825 & 1495.17 & 1495.642 & 1496.784 & 1496.538 & 1493.257 & 1488.797 & 1493.1 & 1491.063\end{array}$ $\begin{array}{lllllllllll}1494.021 & 1494.82 & 1495.18 & 1495.645 & 1496.787 & 1496.555 & 1493.257 & 1488.799 & 1493.098 & 1491.059\end{array}$ $\begin{array}{lllllllllll}1494.007 & 1494.815 & 1495.178 & 1495.64 & 1496.784 & 1496.557 & 1493.252 & 1488.794 & 1493.095 & 1491.056\end{array}$ $\begin{array}{lllllllllll}1493.981 & 1494.811 & 1495.194 & 1495.642 & 1496.791 & 1496.574 & 1493.252 & 1488.799 & 1493.093 & 1491.054\end{array}$ $\begin{array}{llllllllll}1493.958 & 1494.813 & 1495.206 & 1495.652 & 1496.801 & 1496.6 & 1493.252 & 1488.799 & 1493.095 & 1491.052\end{array}$ $\begin{array}{lllllllllll}1493.939 & 1494.813 & 1495.211 & 1495.659 & 1496.808 & 1496.626 & 1493.254 & 1488.806 & 1493.098 & 1491.052 \\ 1493.915 & 1494818 & 1495.225 & 1495.675 & 1496829 & 1496655 & 1493266 & 1488818 & 149305 & 1491.054\end{array}$ $\begin{array}{lllllllllll}1493.915 & 1494.818 & 1495.225 & 1495.675 & 1496.829 & 1496.655 & 1493.266 & 1488.818 & 1493.105 & 1491.054 \\ 1493.906 & 149482 & 1495.225 & 1495686 & 1496838 & 1496679 & 1493273 & 1488825 & 1493116 & 1491.054\end{array}$ $\begin{array}{lllllllllll}1493.904 & 1494.825 & 1495.228 & 1495.696 & 1496.849 & 1496.693 & 1493.285 & 1488.827 & 1493.123 & 1491.056\end{array}$

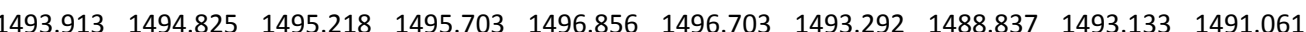
$\begin{array}{lllllllllll}1493.936 & 1494.827 & 1495.204 & 1495.698 & 1496.854 & 1496.681 & 1493.294 & 1488.83 & 1493.135 & 1491.063\end{array}$ $\begin{array}{llllllllll}1493.953 & 1494.825 & 1495.192 & 1495.693 & 1496.849 & 1496.672 & 1493.299 & 1488.827 & 1493.137 & 1491.068\end{array}$ $\begin{array}{lllllllllll}1493.967 & 1494.82 & 1495.182 & 1495.689 & 1496.845 & 1496.655 & 1493.294 & 1488.83 & 1493.137 & 1491.068\end{array}$ $\begin{array}{lllllllllll}1493.953 & 1494.82 & 1495.189 & 1455.696 & 1496.854 & 1496.674 & 1493.301 & 1488.83 & 1493.14 & 1491.07\end{array}$ $\begin{array}{lllllllllll}1493.946 & 1494.825 & 1495.194 & 1495.705 & 1496.863 & 1496.691 & 1493.308 & 1488.83 & 1493.144 & 1491.075 \\ 1493.936 & 1494827 & 1495201 & 1495.714 & 1496875 & 1496705 & 1493.31 & 1488834 & 1493.154 & 1491.08\end{array}$ $\begin{array}{llllllllll}143.936 & 1494.827 & 1455.201 & 1455.714 & 146.87 & 146.705 & 1493.31 & 1488.834 & 1493.154 & 1491.08 \\ 1493.955 & 1494.825 & 1495.187 & 1495.71 & 1496.87 & 1496.691 & 149331 & 1488.834 & 1493.152 & 1491.08\end{array}$ $\begin{array}{lllllllllll}1493.958 & 1494.825 & 1495.182 & 1495.712 & 1496.87 & 1496.689 & 1493.313 & 1488.837 & 1493.152 & 1491.082\end{array}$ $\begin{array}{llllllllll}1493.936 & 1494.827 & 1495.199 & 1495.724 & 1496.887 & 1496.713 & 1493.317 & 1488.839 & 1493.159 & 1491.087\end{array}$ $\begin{array}{llllllllll}1493.918 & 1494.835 & 1495.213 & 1495.742 & 1496.908 & 1496.748 & 1493.331 & 1488.841 & 1493.17 & 1491.094\end{array}$ $\begin{array}{lllllllllll}1493.913 & 1494.842 & 1495.216 & 1495.758 & 1496.922 & 1496.768 & 1493.345 & 1488.853 & 1493.182 & 1491.098\end{array}$ $\begin{array}{llllllllll}1493.918 & 1494.846 & 1495.216 & 1495.77 & 1496.933 & 1496.777 & 1493.355 & 1488.853 & 1493.194 & 1491.103\end{array}$

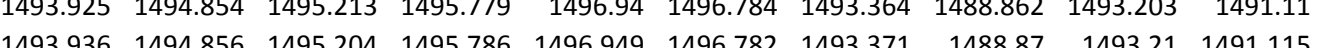
$\begin{array}{llllllllll}1493.936 & 1494.856 & 1495.204 & 1495.786 & 1496.949 & 1496.782 & 1493.37 & 1488.87 & 1493.21 & 1491.115 \\ 1493.939 & 1494.861 & 1495204 & 1495793 & 1496956 & 1496787 & 1493378 & 1488865 & 1493217 & 1491.119\end{array}$ $\begin{array}{lllllllllll}1493.948 & 1494.858 & 1495.194 & 1495798 & 1496.961 & 1496784 & 1493.383 & 1488.872 & 14932141 & 1491.124\end{array}$ $\begin{array}{lllllllllll}1493.95 & 1494.866 & 1495.199 & 1495.807 & 1496.966 & 1496.789 & 1493.388 & 1488.872 & 1493.224 & 1491.131\end{array}$ $\begin{array}{lllllllllll}1493.934 & 1494.875 & 1495.211 & 1495.823 & 1496.987 & 1496.815 & 1493.397 & 1488.881 & 1493.236 & 1491.135\end{array}$ $\begin{array}{lllllllllll}1493.915 & 1494.882 & 1495.23 & 1495.851 & 1497.015 & 1496.856 & 1493.413 & 1488.886 & 1493.252 & 1491.145\end{array}$ $\begin{array}{llllllllll}1493.906 & 1494.894 & 1495.239 & 1495.877 & 1497.04 & 1496.894 & 1493.427 & 1488.893 & 1493.264 & 1491.154\end{array}$ $\begin{array}{lllllllllll}1493.908 & 1494.904 & 1495.247 & 1495.905 & 1497.063 & 1496.921 & 1493.446 & 1488.902 & 1493.28 & 1491.161\end{array}$ $\begin{array}{llllllllll}1493.913 & 1494.913 & 1455.249 & 1455.923 & 1497.084 & 1466.935 & 1493.46 & 1488.914 & 1493.297 & 1491.17 \\ 1493.925 & 1494.923 & 1495251 & 1495944 & 1497.103 & 1496952 & 1493477 & 1488921 & 1493311 & 1491.18\end{array}$

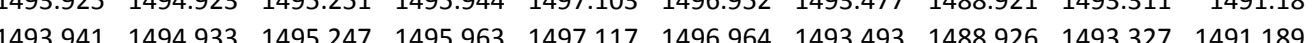
$\begin{array}{lllllllllll}1493.962 & 1494.942 & 1495.242 & 1495.977 & 1497.126 & 1496.961 & 1493.505 & 1488.933 & 1493.339 & 1491.196\end{array}$ $\begin{array}{lllllllllll}1493.981 & 1494.947 & 1495.242 & 1495.986 & 1497.138 & 1496.959 & 1493.516 & 1488.94 & 1493.351 & 1491.205\end{array}$ $\begin{array}{llllllllll}1493.998 & 1494.952 & 1495.239 & 1496 & 1497.147 & 1496.966 & 1493.528 & 1488.947 & 1493.36 & 1491.212\end{array}$ $\begin{array}{lllllllllll}1494 & 1494.957 & 1495.249 & 1496.023 & 1497.168 & 1496.98 & 1493.544 & 1488.949 & 1493.381 & 1491.224\end{array}$ $\begin{array}{lllllllllll}1494.012 & 1494.966 & 1495.251 & 1496.039 & 1497.182 & 1496.997 & 1493.556 & 1488.954 & 1493.393 & 1491.231\end{array}$ $\begin{array}{lllllllllll}1494.026 & 1494.971 & 1495.249 & 1496.055 & 1497.196 & 1497.002 & 1493.568 & 1488.961 & 1493.405 & 1491.238\end{array}$ $\begin{array}{lllllllll}1486.516 & 1488.415 & 1493.596 & 1494.059 & 1493.352 & 1495.204 & 1493.33 & 1484.898 & 1479.276\end{array}$ $\begin{array}{llllllllll}1486.541 & 1488.422 & 1493.586 & 1494.05 & 1493.345 & 1495.185 & 1493.328 & 1484.898 & 1479.276 \\ 1486562 & 1488.408 & 1493579 & 1494.038 & 149334 & 1495181 & 1493.319 & 1484898 & 1479.274\end{array}$ $\begin{array}{llllllllll}1486578 & 1488396 & 1493575 & 1494038 & 1493333 & 1495185 & 1493307 & 1484898 & 1479274\end{array}$ $\begin{array}{lllllllllll}1486.599 & 1488.401 & 1493.568 & 1494.033 & 1493.328 & 1495.169 & 1493.307 & 1484.891 & 1479.267\end{array}$ $\begin{array}{lllllllllll}1486.613 & 1488.384 & 1493.558 & 1494.029 & 1493.321 & 1495.167 & 1493.3 & 1484893 & 1479.26\end{array}$ $\begin{array}{lllllllllll}1486.626 & 1488.382 & 1493.554 & 1494.017 & 1493.314 & 1495.162 & 1493.297 & 1484.891 & 1479.26\end{array}$ $\begin{array}{llllllllll}1486.647 & 1488.382 & 1493.547 & 1494.003 & 1493.309 & 1495.134 & 1493.286 & 1484.888 & 1479.255\end{array}$ $\begin{array}{lllllllll}1486.661 & 1488.38 & 1493.537 & 1493.989 & 1493.3 & 1495.129 & 1493.276 & 1484.886 & 1479.25\end{array}$ $\begin{array}{llllllllll}1486.677 & 1488.389 & 1493.53 & 1493.968 & 1493.288 & 1495.091 & 1493.267 & 1444.886 & 1479.253\end{array}$ $\begin{array}{lllllllllll}1486.682 & 1488.368 & 1493.521 & 1493.959 & 1493.281 & 1495.087 & 1493.258 & 1484.886 & 1479.25 \\ 1486698 & 1488354 & 1493516 & 149.954 & 1492.276 & 1495077 & 1493248 & 1484884 & 1479.25\end{array}$ $\begin{array}{llllllllll}1486.719 & 1488.344 & 1493.511 & 1493.954 & 1493.269 & 1495.077 & 1493.246 & 1484.881 & 1479.253\end{array}$ $\begin{array}{lllllllllll}1486.733 & 1488344 & 1493.504 & 1493.94 & 1493.264 & 1495.066 & 1493.241 & 1484.879 & 1479.253\end{array}$ $\begin{array}{llllllllll}1486.744 & 1488.321 & 1493.502 & 1493.95 & 1493.267 & 1495.08 & 1493.239 & 1484.881 & 1479.248\end{array}$ $\begin{array}{llllllllll}1486.751 & 1488.309 & 1493.502 & 1493.954 & 1493.257 & 1495.077 & 1493.229 & 1484.879 & 1479.248\end{array}$ $\begin{array}{lllllllll}1486.763 & 1488.27 & 1493.497 & 1493.961 & 1493.259 & 1495.089 & 1493.222 & 1484.879 & 1479.243\end{array}$ $\begin{array}{lllllllll}1486.779 & 1488.262 & 1493.5 & 1493.975 & 1493.262 & 1495.11 & 1493.229 & 1484.879 & 1479.241\end{array}$ $\begin{array}{llllllllll}1486.79 & 1488.251 & 1493.502 & 1493.987 & 1493.264 & 1495.131 & 1493.227 & 1484.879 & 1479.24\end{array}$ $\begin{array}{llllllllll}4 & 1486.811 & 1488.232 & 149.509 & 1494.008 & 1493.274 & 1495.16 & 1493.241 & 1484.877 & 1479.241\end{array}$ $\begin{array}{llllllllll}1486.839 & 1488.234 & 1493.53 & 1494.043 & 1493.293 & 1495.197 & 1493.269 & 1484.874 & 1479.246\end{array}$ $\begin{array}{llllllllll}1486.855 & 1488241 & 1493539 & 1494048 & 1493302 & 1495202 & 1493283 & 1484.872 & 1479.25\end{array}$ $\begin{array}{llllllllllll}1486.869 & 1488.246 & 1493.539 & 1494.043 & 1493.304 & 1495.197 & 1493.283 & 1484.872 & 1479.25\end{array}$ $\begin{array}{llllllllll}1486.883 & 1488.255 & 1493.54 & 1494.038 & 1493.304 & 1495.188 & 1493.281 & 1484.872 & 1479.253\end{array}$ $\begin{array}{llllllllll}1486.895 & 1488.255 & 1493.542 & 1494.034 & 1493.304 & 1495.181 & 1493.276 & 1484.87 & 1479.253\end{array}$ $\begin{array}{llllllllll}1486.906 & 1488.251 & 1493.547 & 1494.043 & 1493.307 & 1495.192 & 1493.283 & 1484.87 & 1479.253\end{array}$ $\begin{array}{lllllllll}1486.92 & 1488.251 & 1493.553 & 1494.055 & 1493.314 & 1455.209 & 1493.29 & 1484.87 & 1479.255 \\ 1486941 & 1488244 & 1493558 & 1494.066 & 1493319 & 1495.223 & 1493295 & 1484.87 & 1479.2532\end{array}$ $\begin{array}{lllllllll}1486.952 & 1488.251 & 1493.554 & 1494.059 & 1493319 & 1495211 & 1493293 & 1484.877 & 149.253\end{array}$ $\begin{array}{llllllllll}1486.966 & 1488.255 & 1493.558 & 1494.059 & 1493.319 & 1495.211 & 1493.297 & 1484.867 & 1479.248\end{array}$ $\begin{array}{llllllllll}1486.98 & 1488.234 & 1493.563 & 1494.073 & 1493.328 & 1495.237 & 1493.297 & 1484.867 & 1479.255\end{array}$ $\begin{array}{lllllllll}1486.992 & 1488.234 & 1493.575 & 1494.104 & 1493.338 & 1495.268 & 1493.309 & 1484.867 & 1479.25\end{array}$ $\begin{array}{llllllllll}4487.006 & 1488.234 & 1493.587 & 1494.12 & 1493.352 & 1495.291 & 1493.323 & 1484.867 & 1479.26\end{array}$ $\begin{array}{lllllllll}1487.006 & 1488.234 & 1493.596 & 1494.132 & 1493.361 & 1495.305 & 1493.335 & 1484.865 & 1479.262\end{array}$ $\begin{array}{llllllllll}1487.022 & 1488.244 & 1493.607 & 1494.143 & 1493.373 & 1495.305 & 1493.347 & 1484.865 & 1479.264 \\ 1487.04 & 1488239 & 1493615 & 1494.15 & 1493.38 & 1495322 & 1493351 & 1484865 & 1479.269\end{array}$

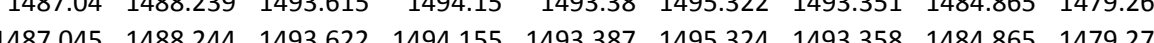

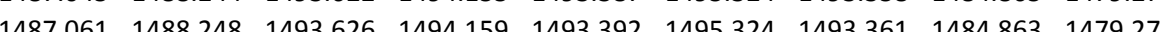
$\begin{array}{lllllllll}1487.077 & 1488.246 & 1493.631 & 1494.164 & 1493.397 & 1495.336 & 1493.366 & 1484.863 & 1479.271\end{array}$ $\begin{array}{lllllllll}1487.089 & 1488.234 & 1493.638 & 1494.187 & 1493.404 & 1495.364 & 1493.375 & 1484.863 & 1479.274\end{array}$ $\begin{array}{llllllllll}1487.105 & 1488.229 & 1493.654 & 1494.218 & 1493.421 & 1495.399 & 1493.387 & 1484.865 & 1479.276\end{array}$ $\begin{array}{llllllllll}1487.119 & 1488.229 & 1493.669 & 1494.246 & 1493.437 & 1495.437 & 1493.403 & 1484.865 & 1479.28\end{array}$ $\begin{array}{llllllllll}1487.133 & 1488.234 & 1493.685 & 1494.271 & 1493.456 & 1495.465 & 1493.419 & 1484.865 & 1479.281\end{array}$ $\begin{array}{llllllllll}1487.094 & 1488.239 & 1493.702 & 1494.29 & 1493.47 & 1495.489 & 1493.436 & 1484.865 & 1479.285\end{array}$

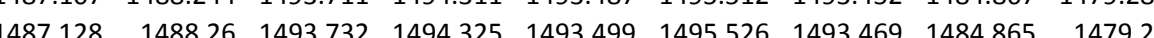
$\begin{array}{lllllllllll}1487.14 & 1488.272 & 1493.744 & 1494.334 & 1493.515 & 1495.531 & 1493.483 & 1484.865 & 1479.295\end{array}$ $\begin{array}{lllllllllll}1487.156 & 1488.279 & 1493.756 & 1494.346 & 1493.525 & 1495.54 & 1493.492 & 1484.865 & 1479.297\end{array}$ $\begin{array}{llllllllll}1487.179 & 1488.293 & 1493.767 & 1494.358 & 1493.537 & 1495.545 & 1493.509 & 1484.865 & 1479.302\end{array}$ $\begin{array}{lllllllllll}1487.193 & 1488.295 & 1493.784 & 1494.376 & 1493.551 & 1495.571 & 1493.52 & 1484.867 & 1479.30\end{array}$ $\begin{array}{lllllllllll}1487.211 & 1488.309 & 1493.796 & 1494.393 & 1493.565 & 1495.594 & 1493.539 & 1484.865 & 1479.316\end{array}$ $\begin{array}{rrrrrrrrr}1487.23 & 1488.316 & 1493.81 & 1494.402 & 1493.577 & 1495.597 & 1493.551 & 1484.865 & 1479.318 \\ 1487.246 & 1488.321 & 1493.817 & 1494.416 & 1493.589 & 1495.608 & 1493.556 & 1484.865 & 1479.32\end{array}$ 
Well

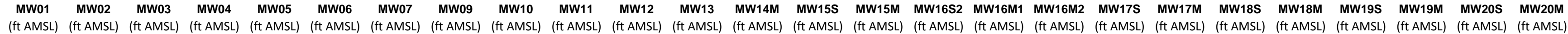

2/13/12 2:00 2/13/123:00 2/13/12 4:00 2/13/12 5:00 2/13/12 6:00 2/13/12 8:00 2/13/12 9:00 2/13/12 10:00 2/13/12 11:00 $12: 00$ 2/13/12 13:00 2/13/12 15:00 2/13/12 15:00 2/13/12 17:00 2/13/12 18:00 2/13/12 19:00 2/13/12 20:00 2/13/12 21:00 2/13/13/12 22:00 $23: 00$ 2/14/12 0:00 2/14/12 1:00 2/14/12 2:00 2/14/12 3:00 2/14/122 4:00 2/14/125:00 2/14/12 6:00 2/14/12 8:00 2/14/12 9:00 2/14/12 10:00 2/14/12 11:00 2/14/12 12:00 2/14/12 13:00 2/14/122 15:00 2/14/12 17:00 2/14/12 18:00 2/14/12 19:00 2/14/12 20:00 2/14/12 21:00 2/14/12 22:00 2/15/12 0:00 2/15/112 2:00 2/15/12 3:00 2/15/12 4:00 2/15/12 5:00 2/15/12 6:00 $\begin{array}{llll}1495.399 & 1496.877 & 1496.869 & 1495.562 \\ 1495.397 & 1496.863 & 1496.885 & 1495.579\end{array}$ $\begin{array}{lllll}1495.397 & 1496.863 & 1496.885 & 1495.579 \\ 1495.404 & 149684 & 1496915 & 1495.602\end{array}$ $\begin{array}{lllll}1495.404 & 1496.84 & 1496.915 & 1495.602\end{array}$ $\begin{array}{llll}1495.394 & 1496.837 & 1496.913 & 1495.609 \\ 1495.392 & 1496.835 & 1496.915 & 1495.618\end{array}$ $\begin{array}{lllll}1495.387 & 1496.835 & 1496.911 & 1455.618\end{array}$ $\begin{array}{lllll}1495.385 & 1496.833 & 1496.911 & 1495.618\end{array}$ $\begin{array}{lllll}1495.38 & 1496.835 & 1496.901 & 1495.616\end{array}$ $\begin{array}{lllll}1495.383 & 1496.823 & 1496.915 & 1495.623\end{array}$ $\begin{array}{lllll}1495.38 & 1496.816 & 1496.925 & 1495.628\end{array}$ $\begin{array}{rrrr}1495.378 & 1496.809 & 1496.936 & 1495.635 \\ 149538 & 1996805 & 149695 & 1955.644\end{array}$ $\begin{array}{rrrr}1495.38 & 1496.805 & 1496.95 & 1495.644 \\ 1495.38 & 1496.793 & 1496.964 & 1495.658\end{array}$ $\begin{array}{rrrrr}1495.371 & 1496.8 & 1496.953 & 1495.651\end{array}$ $\begin{array}{lllll}1495.369 & 1496.802 & 1496.932 & 1495.639\end{array}$ $\begin{array}{lllll}1495.376 & 1496.809 & 1496.906 & 1495.618\end{array}$ $\begin{array}{lllll}1495.392 & 1496.833 & 1496.866 & 1495.59\end{array}$ $\begin{array}{rrrrr}1495.406 & 1496.854 & 1496.843 & 1495.565\end{array}$ $\begin{array}{lllll}1495.42 & 1496.87 & 1496.831 & 1495.544\end{array}$ $\begin{array}{rrrr}1495.443 & 1496.898 & 1496.822 & 1495.53 \\ 1495.455 & 1496.924 & 1496813 & 1495.516\end{array}$ $\begin{array}{llll}1455.466 & 1466.933 & 1496.803 & 1495.502\end{array}$ $\begin{array}{lllll}1495.464 & 1496.954 & 1496.796 & 1495.492\end{array}$ $\begin{array}{lllll}1495.46 & 1496.963 & 1496.794 & 1495.485\end{array}$ $\begin{array}{lllll}1495.46 & 1496.97 & 1496.787 & 1995.476\end{array}$ $\begin{array}{lllll}1495.446 & 1496.97 & 1496.791 & 1495.471\end{array}$ $\begin{array}{lllll}1495.434 & 1496.968 & 1496.789 & 1495.469\end{array}$ $\begin{array}{lllll}1495.427 & 1496.97 & 1496.78 & 1495.462\end{array}$ $\begin{array}{rrrr}1495.406 & 1496.956 & 1496.791 & 1495.467 \\ 1495.401 & 1496.956 & 1496.775 & 1495.46\end{array}$ $\begin{array}{lllll}1495.392 & 1496.947 & 1496768 & 1495.453\end{array}$ $\begin{array}{lllll}1495.357 & 1496.91 & 1496.763 & 1495.443\end{array}$ $\begin{array}{lllll}1495.304 & 1496.856 & 1496.763 & 1495.439\end{array}$ $\begin{array}{lllll}1495.262 & 1496.816 & 1496.768 & 1495.439\end{array}$ $\begin{array}{llll}1495.22 & 1496.753 & 1496.773 & 1495.441\end{array}$ $\begin{array}{lllll}1495.143 & 1496.667 & 1496.805 & 1495.457\end{array}$ $\begin{array}{lllll}1455.157 & 1496.616 & 1496.827 & 1495.471\end{array}$ $\begin{array}{lllll}1495.166 & 1496572 & 1496834 & 145.45\end{array}$ $\begin{array}{llll}1495.185 & 1496.581 & 1496.841 & 1495.497\end{array}$ $1495.229 \quad 1496.609 \quad 1496.824 \quad 1495.49$ $1495.264 \quad 1496.6391496 .824 \quad 1495.49$ $1495.28 \quad 1496.66 \quad 1496.82 \quad 1495.485$ $1495.3041496 .6861496 .798 \quad 1495.474$ 1495.3291496 .7091496 .7891495 .464 $1495.3291496 .7191496 .798 \quad 1495.464$ $\begin{array}{llll}1495.29 & 1496.693 & 1496815 & 1495.471\end{array}$ $1495.264 \quad 1496.674 \quad 1496.838 \quad 1495.488$ $1495.266 \quad 1496.679 \quad 1496.82 \quad 1495.485$ $1495.255 \quad 1496.663 \quad 1496.831 \quad 1495.488$ $1495.245 \quad 1496.66 \quad 1496.843 \quad 1495.497$ $\begin{array}{rrrr}1495.241 & 1496.656 & 1496.836 & 1495.497 \\ 1495.25 & 1496.653 & 1496.82 & 1495.49\end{array}$ $\begin{array}{llllllllll}1494.037 & 1494.988 & 1495.258 & 1496.093 & 1497.228 & 1497.033 & 1493.596 & 1488.977 & 1493.428 & 1491.257 \\ 1494.049 & 1494.997 & 1495263 & 1496.111 & 1497.245 & 1497.038 & 1493608 & 1488.98 & 1493.442 & 1491266\end{array}$ $\begin{array}{llllllllll}1494.049 & 1494.997 & 1495.263 & 1496.111 & 1497.245 & 1497.038 & 1493.608 & 1488.98 & 1493.442 & 1491.266 \\ 1490.042 & 1495.002 & 1495273 & 1496139 & 1997.266 & 1497.069 & 1493624 & 1488.991 & 1493.56 & 1491273\end{array}$ $\begin{array}{llllllllll}1494.042 & 1495.002 & 1495.273 & 1496.139 & 1497.266 & 1497.069 & 1493.624 & 1488.991 & 1493.456 & 1491.273 \\ 1494.075 & 1495.007 & 1495263 & 1496148 & 1497273 & 1497.057 & 1493633 & 1488991 & 1493.47 & 1491.28\end{array}$ $\begin{array}{lllllllllll}1494.094 & 1495.014 & 1495.263 & 1496.158 & 1497.284 & 1497.052 & 1493.645 & 1489.001 & 1493.477 & 1491.291\end{array}$ $\begin{array}{lllllllllll}1494.115 & 1495.019 & 1495.258 & 1496.165 & 1497.284 & 1497.045 & 1493.654 & 1489.005 & 1493.489 & 1491.296\end{array}$ $\begin{array}{lllllllllllll}1494.136 & 1495.021 & 1495.251 & 1496.172 & 1497.289 & 1497.04 & 1493.659 & 1489.008 & 1493.498 & 1491.301\end{array}$ $\begin{array}{llllllllllll}1494.16 & 1495.024 & 1495.251 & 1496.174 & 1497.289 & 1497.026 & 1493.664 & 1489.005 & 1493.503 & 1491.308\end{array}$ $\begin{array}{lllllllllll}1494.162 & 1495.031 & 1495.254 & 1496.183 & 1497.298 & 1497.033 & 1493.673 & 1489.013 & 1493.51 & 1491.315\end{array}$ $\begin{array}{lllllllllll}1494.169 & 1495.033 & 1495.258 & 1496.195 & 1497.308 & 1497.038 & 1493.68 & 1489.013 & 1493.517 & 1491.319 \\ 1494178 & 149504 & 1495266 & 1496204 & 197.315 & 1497.045 & 1493.685 & 1489017 & 1993522 & 19134\end{array}$ $\begin{array}{llllllllll}1494.178 & 1495.04 & 1495.266 & 1496.204 & 1497.315 & 1497.045 & 1493.685 & 1489.017 & 1493.522 & 1491.324 \\ 1494.183 & 1495.045 & 1495.266 & 1496218 & 1497.324 & 1497.052 & 1493.694 & 1489.02 & 1493531 & 1491333\end{array}$ $\begin{array}{lrrrrrrrrrr}1494.183 & 1495.045 & 1495.266 & 1496.218 & 147.324 & 1497.052 & 1493.694 & 1489.02 & 1493.533 & 1491.333 \\ 1494.186 & 1495.05 & 1495.277 & 1496.232 & 1497.338 & 1497.064 & 1493.699 & 1489.024 & 1493.538 & 1491.338\end{array}$ $\begin{array}{lllllllllllll}1494.214 & 1495.054 & 1495.261 & 1496.234 & 1497.333 & 1497.05 & 1493.706 & 1489.031 & 1493.543 & 149134\end{array}$ $\begin{array}{lllllllllll}1494.242 & 1495.054 & 1495.247 & 1496.227 & 1497.326 & 1497.026 & 1493.704 & 1489.027 & 1493.543 & 1491.343\end{array}$ $\begin{array}{llllllllllll}1494.268 & 1495.057 & 1495.239 & 1496.22 & 1497.315 & 1496.992 & 1493.701 & 1489.031 & 1493.541 & 1491.345\end{array}$ $\begin{array}{llllllllll}1494.301 & 1495.057 & 1495.225 & 1496.206 & 1497.294 & 1496.957 & 1493.694 & 1489.027 & 1493.534 & 1491.345\end{array}$ $\begin{array}{llllllllll}1494.32 & 1495.055 & 1495.218 & 1496.19 & 1497.28 & 1496.928 & 1493.685 & 1489.008 & 1493.527 & 1491.343\end{array}$

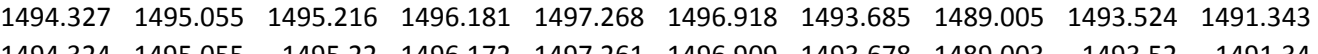
$\begin{array}{lllllllllll}1494.324 & 1495.055 & 1495.22 & 1496.172 & 1497.261 & 1496.909 & 1493.67 & 1489.003 & 1493.52 & 1491.34 \\ 1494.329 & 1495.055 & 1495.218 & 1496165 & 1497249 & 1496899 & 1493675 & 1488998 & 1493.517 & 1491338\end{array}$ $\begin{array}{llllllllllll}1449.331 & 1495.052 & 1495.22 & 1496.153 & 1497.242 & 1496.89 & 1493.671 & 1488.994 & 1493.513 & 1491.338\end{array}$ $\begin{array}{llllllllll}1494.334 & 149505 & 1495.22 & 1496148 & 1497.233 & 1496887 & 1493.666 & 1488991 & 1493.508 & 1491331\end{array}$ $\begin{array}{lllllllllll}1494.327 & 1495.05 & 1495.22 & 1496.141 & 1497.226 & 1496.88 & 1493.666 & 1488.991 & 1493.506 & 1491.331\end{array}$ $\begin{array}{lllllllllll}1494.324 & 1495.05 & 1495.22 & 1496.132 & 1497.219 & 1496.878 & 1493.659 & 1488.984 & 1493.503 & 1491.326\end{array}$ $\begin{array}{llllllllll}1494.315 & 1495.047 & 1495.223 & 1496.13 & 1497.217 & 1496.88 & 1493.659 & 1488.982 & 1493.501 & 1491.324\end{array}$ $\begin{array}{llllllllll}1494.312 & 1495.05 & 1495.228 & 1496.125 & 1497.212 & 1496.87 & 1493.659 & 1488.98 & 1493.501 & 1491.324\end{array}$ $\begin{array}{lllllllllll}1494.317 & 1495.047 & 1495.22 & 1496.118 & 1497.205 & 1496.873 & 1493.654 & 1488.977 & 1493.496 & 1491.322\end{array}$ $\begin{array}{lllllllllll}1494.301 & 1495.047 & 1495.232 & 1496.116 & 1497.208 & 1496.882 & 1493.654 & 1488.982 & 1493.494 & 1491.319 \\ 1494.315 & 1495.045 & 1495.223 & 1496109 & 1497.196 & 149687 & 1493.652 & 1488.982 & 1493.494 & 1491.317\end{array}$ $\begin{array}{llllllllllll}1494.312 & 1495.045 & 1495.22 & 1496.1 & 1497.191 & 1496.866 & 1493.65 & 1488.973 & 1493.494 & 1491.312\end{array}$ $\begin{array}{lllllllllll}1494.308 & 1495.04 & 1495.223 & 1496.093 & 1497.184 & 1496.856 & 1493.647 & 1488.975 & 1493.489 & 1491.31\end{array}$ $\begin{array}{llllllllll}1494.303 & 1495.042 & 1495.225 & 1496.09 & 1497.18 & 1496.861 & 1493.645 & 1488.973 & 1493.489 & 1491.308\end{array}$ $\begin{array}{lllllllllll}1494.294 & 1495.043 & 1495.23 & 1496.088 & 1497.18 & 1496.866 & 1493.645 & 1488.97 & 1493.484 & 1491.308\end{array}$ $\begin{array}{lllllllllll}1494.287 & 1495.04 & 1495.228 & 1496.088 & 1497.177 & 1496.873 & 1493.643 & 1488.973 & 1493.482 & 1491.303\end{array}$

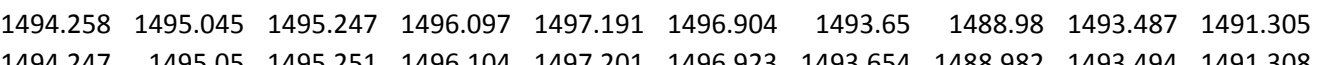
$\begin{array}{lllllllllllll}1494247 & 1495054 & 1495.256 & 1496109 & 1997205 & 149693 & 1493657 & 14888984 & 1493.494 & 1491.308\end{array}$

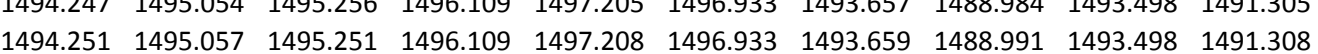
$\begin{array}{lllllllllll}1494.256 & 1495.059 & 1495.249 & 1496.113 & 1497.212 & 1496.942 & 1493.664 & 1488.991 & 1493.501 & 1491.308\end{array}$ $\begin{array}{lllllllllll}1494.28 & 1495.059 & 1495.242 & 1496.107 & 1497.205 & 1496.923 & 1493.661 & 1488.987 & 1493.498 & 1491.308\end{array}$ $\begin{array}{llllllllllll}1494.284 & 1495.059 & 1495.239 & 1496.107 & 1497.203 & 1496.923 & 1493.664 & 1488.987 & 1493.503 & 1491.31\end{array}$ $\begin{array}{lllllllllll}1494.291 & 1495.059 & 1495.242 & 1496.104 & 1497.201 & 1496.913 & 1493.661 & 1488.991 & 1493.506 & 1491.308\end{array}$ $\begin{array}{llllllllll}1494.312 & 1495.059 & 1495.23 & 1496.095 & 1497.191 & 1496.904 & 1493.659 & 1488.984 & 1493.498 & 1491.308\end{array}$ $\begin{array}{rrrrrrrrrr}1494.317 & 1495.057 & 1495.23 & 1496.09 & 1497.184 & 1496.89 & 1493.657 & 1488.982 & 1493.498 & 1491.308 \\ 149431 & 1495.062 & 1495.239 & 1496.09 & 1497.187 & 1496902 & 1493659 & 1488.982 & 1493501 & 1491.308\end{array}$ $\begin{array}{llllllllll}1494.31 & 1495.062 & 1495.239 & 1496.09 & 147.1487 & 1496.902 & 1493.659 & 1488.982 & 1493.501 & 1491.308\end{array}$ $\begin{array}{llllllllllll}1494.298 & 1495.062 & 1495.247 & 1496.093 & 1497.191 & 1496.918 & 1493.664 & 1488.987 & 1493.506 & 1491.308\end{array}$ $\begin{array}{lllllllllll}1494.284 & 1495.064 & 1495.256 & 1496.104 & 1497.203 & 1496.94 & 1493.673 & 1488.989 & 1493.51 & 1491.31\end{array}$ $\begin{array}{llllllllll}1494.303 & 1495.064 & 1495.244 & 1496.097 & 1497.196 & 1496.923 & 1493.671 & 1488.991 & 1493.515 & 1491.31\end{array}$ $\begin{array}{llllllllll}1494.296 & 1495.069 & 1495.251 & 1496.1 & 1497.201 & 1496.93 & 1493.673 & 1488.994 & 1493.517 & 1491.31\end{array}$ $\begin{array}{lllllllllll}1494.296 & 1495.069 & 1495.254 & 1496.109 & 1497.201 & 1496.947 & 1493.682 & 1488.996 & 1493.522 & 1491.312\end{array}$ $\begin{array}{lllllllllll}1494.303 & 1495.07 & 1455.251 & 1466.107 & 197.20 & 1496.947 & 1493.682 & 1488.996 & 1493.522 & 1491.312\end{array}$ $\begin{array}{lllllllll}1487.258 & 1488.328 & 1493.831 & 1494.437 & 1493.603 & 1495.632 & 1493.57 & 1484.867 & 1479.323\end{array}$ $\begin{array}{lllllllll}1487.274 & 1488.337 & 1493.845 & 1494.446 & 1493.617 & 1495.648 & 1493.584 & 1484.865 & 1479.32 \\ 1487292 & 188334 & 149359 & 1494.72 & 1493631 & 1495679 & 1493598 & 148487 & 1479332\end{array}$ $\begin{array}{llllllllll}1487.304 & 1488.34 & 1493.859 & 1494.472 & 1493.631 & 1495.679 & 1493.598 & 1484.87 & 149.332\end{array}$ $\begin{array}{lllllllllll}1487.299 & 1488.37 & 1493.883 & 1494.486 & 1493.653 & 1495.677 & 1493.621 & 1484.87 & 1479332\end{array}$ $\begin{array}{lllllllllll}1487306 & 1488387 & 1493895 & 1494.484 & 1493.66 & 1495.677 & 1493.631 & 1484.865 & 1479.337\end{array}$ $\begin{array}{lllllllllll}1487.325 & 1488.405 & 1493.901 & 1494.486 & 1493.667 & 1495.674 & 1493.642 & 1484.867 & 1479.341\end{array}$ $\begin{array}{lllllllllll}1487.341 & 1488.42 & 1493.909 & 1494.484 & 1493.674 & 1495.672 & 1493.645 & 1484.87 & 1479.341\end{array}$ $\begin{array}{lllllllll}1487.359 & 1488.42 & 1493.913 & 1494.493 & 1493.681 & 1495.681 & 1493.647 & 1484.87 & 1479.344\end{array}$ $\begin{array}{llllllllll}1487.369 & 1488.429 & 1493.923 & 1494.002 & 1493.688 & 1495.684 & 1493.659 & 1484.867 & 1479.346\end{array}$ $\begin{array}{lllllllllll}1487.383 & 1488.438 & 1493.932 & 1494.507 & 1493.695 & 1495.695 & 1493.666 & 1484.867 & 1479.346\end{array}$ $\begin{array}{lllllllllll}1487.406 & 1488.445 & 1493.944 & 1494.533 & 1493.712 & 1495.719 & 1493.678 & 1484.87 & 1479.348\end{array}$ $\begin{array}{lllllllllll}1487.42 & 1488.474 & 1493.949 & 1494.516 & 1493.714 & 1495.707 & 1493.687 & 1484.867 & 1479.348\end{array}$ $\begin{array}{llllllllll}1487.376 & 1488.49 & 1493.948 & 1494.507 & 1493.712 & 1495.684 & 1493.685 & 1484.867 & 1479.346\end{array}$ $\begin{array}{llllllllll}1487.383 & 1488.504 & 1493.949 & 1494.493 & 1493.712 & 1495.665 & 1493.682 & 1484.867 & 1479.344\end{array}$ $\begin{array}{lllllllllll}1487.383 & 1488.525 & 1493.939 & 1494.467 & 1493.703 & 1495.63 & 1493.678 & 1484.865 & 1479.339\end{array}$ $\begin{array}{lllllllll}1487.371 & 1488.532 & 1493.934 & 1494.449 & 1493.691 & 1495.608 & 1493.673 & 1484.865 & 1479.339\end{array}$

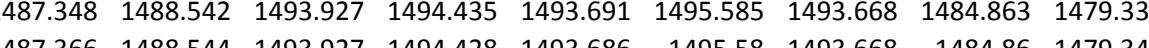

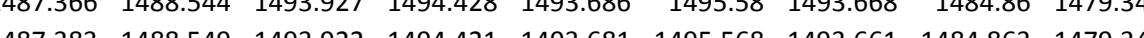
$\begin{array}{llllllllll}1487.399 & 1488.553 & 1493.92 & 1494.414 & 1493.679 & 1495.557 & 1493.654 & 1484.863 & 1479.339\end{array}$ $\begin{array}{lllllllllll}1487.424 & 1488.56 & 1493.915 & 1494.404 & 1493.672 & 1495.545 & 1493.654 & 1484.863 & 1479.339\end{array}$ $\begin{array}{llllllllll}1487.436 & 1488.563 & 1493.908 & 1494.393 & 1493.669 & 1495.538 & 1493.642 & 1484.86 & 1479.339\end{array}$ $\begin{array}{lllllllll}1487.447 & 1488.56 & 1493.906 & 1494.395 & 1493.667 & 1495.536 & 1493.642 & 1484.86 & 1479.339\end{array}$

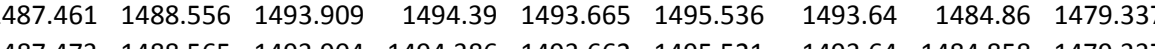
$\begin{array}{lllllllll}1487.473 & 1488.565 & 1493.904 & 1494.386 & 1493.662 & 1455.521 & 1493.64 & 1484.858 & 1479.337\end{array}$ $\begin{array}{llllllllll}1487.484 & 1488.553 & 1493.902 & 1494.395 & 1493.662 & 1495.538 & 1493.635 & 1484.86 & 1479.337 \\ 1487.496 & 1488.563 & 1493.902 & 1494.383 & 1493.66 & 1495.521 & 1493.635 & 1484.855 & 1479.337\end{array}$ $\begin{array}{llllllllll}1487.494 & 1488.56 & 1493.899 & 1494.372 & 1493.658 & 1495.514 & 1493.631 & 1484.855 & 1479.337\end{array}$ $\begin{array}{llllllllll}1487.505 & 1488.558 & 1493.897 & 1494.372 & 1493.653 & 1495.51 & 1493.626 & 1484.858 & 1479.339\end{array}$ $\begin{array}{llllllllll}1487.514 & 1488.551 & 1493.895 & 1494.367 & 1493.653 & 1495.503 & 1493.626 & 1484.855 & 1479.339\end{array}$ $\begin{array}{lllllllllll}4487.524 & 1488.542 & 1493.892 & 1494.374 & 1493.653 & 1495.505 & 1493.624 & 1484.855 & 1479.339\end{array}$ $\begin{array}{lllllllll}1487.528 & 1488.537 & 1493.89 & 1494.372 & 1493.65 & 1495.507 & 1493.619 & 1484.853 & 1479.339\end{array}$ $\begin{array}{llllllllll}1487.537 & 1488.513 & 1493.897 & 1494.39 & 1493.657 & 1495.54 & 1493.612 & 1484.855 & 1479.339\end{array}$

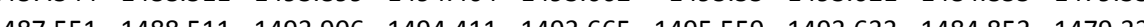
$\begin{array}{llllllllll}1487.51 & 1488.511 & 1493.906 & 1494.409 & 1493.669 & 1495.559 & 1493.638 & 148485 & 1479.337\end{array}$ $\begin{array}{lllllllll}1487.519 & 1488.516 & 1493.911 & 1494.416 & 1493.672 & 1495.566 & 1493.649 & 1484.853 & 1479.337\end{array}$ $\begin{array}{lllllllll}1487.526 & 1488.525 & 1493.906 & 1494.407 & 1493.669 & 1495.554 & 1493.645 & 1484.853 & 1479.334\end{array}$ $\begin{array}{lllllllll}1487.537 & 1488.525 & 1493.911 & 1494.409 & 1493.672 & 1495.552 & 1493.649 & 1484.853 & 1479.334\end{array}$ $\begin{array}{llllllllll}1487.547 & 1488.525 & 1493.909 & 1494.404 & 1493.672 & 1495.552 & 1493.645 & 1484.853 & 1479.33\end{array}$ $\begin{array}{llllllllll}1487.558 & 1488.542 & 1493.906 & 1494.395 & 1493.667 & 1495.526 & 1493.647 & 1484.851 & 1479.334\end{array}$ $\begin{array}{lllllllll}1487.563 & 1488.544 & 1493.904 & 1494.39 & 1493.665 & 1455.526 & 1493.642 & 1484.851 & 1479.334\end{array}$

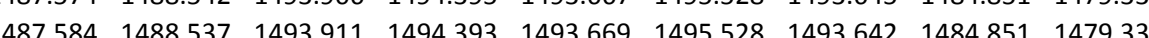
$\begin{array}{llllllllllll}1487.593 & 1488.528 & 1493.906 & 1494.402 & 1493.672 & 1495.543 & 1493.642 & 1484.851 & 1479.341\end{array}$ $\begin{array}{lllllllllll}1487.605 & 1488.53 & 1493.92 & 1494.418 & 1493.681 & 1495.564 & 1493.656 & 1484.851 & 1479.344\end{array}$ $\begin{array}{lllllllll}1487.614 & 1488.537 & 1493.918 & 1494.411 & 1493.679 & 1495.55 & 1493.654 & 1484.848 & 1479.344\end{array}$ $\begin{array}{llllllllll}1487.625 & 1488.532 & 1493.925 & 1494.418 & 1493.684 & 1495.559 & 1493.654 & 1484.851 & 1479.34\end{array}$ $\begin{array}{lllllllllll}1487.635 & 1488.537 & 1493.93 & 1494.421 & 1493.693 & 1495.564 & 1493.675 & 1484.851 & 1479.348\end{array}$ $\begin{array}{lllllllll}1487.623 & 1488.539 & 1493.93 & 1494.421 & 1493.688 & 1495.561 & 1493.661 & 1484.848 & 1479.348 \\ 1487.621 & 1488535 & 1493.93 & 1494.414 & 1493.686 & 1495.554 & 1493.661 & 1484.846 & 1479.348\end{array}$ 
Well

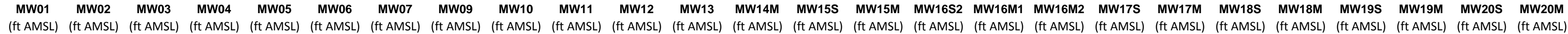

2/15/12 8:00 2/15/129:00 2/15/12 10:00 2/15/12 11:00 2/15/12 13:00 2/15/12 13:00 2/15/12 15:00 2/15/12 16:00 2/15/12 17:00 2/15/12 18:00 2/15/12 19:00 2/115/12 21:00 2/15/12 22:00 2/15/12 23:00 2/16/120:00 2/16/12 1:00 2/16/12 2:00 2/16/123:00 2/16/12 4:00 /216/12 6:00 2/16/12 6:00 2/16/12 8:00 2/16/12 9:00 2/16/12 10:00 2/16/12 11:00 2/16/12 12:00 2/16/12 14:00 2/16/12 15:00 2/16/12 16:00 2/16/12 17:00 2/16/12 18:00 2/16/12 19:00 2/16/12 20:00 2/16/122 21:00 2/16/12 23:00 2/117/1120:00 2/17/12 1:00 2/17/122:00 2/177/12 3:00 2/17/12 4:00 2/177/12 6:00 2/17/12 8:00 2/17/12 9:00 2/17/12 11:00 2/17/12 12:00 $\begin{array}{rrrr}1495.262 & 1496.66 & 1496.805 & 1495.478 \\ 1495.271 & 1496.658 & 1496.787 & 1495.464\end{array}$ $\begin{array}{rrrr}1495.271 & 1496.658 & 1496.787 & 1495.464 \\ 1495266 & 1496.646 & 1496.78 & 1955.457\end{array}$ $\begin{array}{llll}1495.266 & 1496.646 & 1496.78 & 1495.457\end{array}$ $\begin{array}{rrrr}1495.241 & 1496.618 & 1496.766 & 1495.448\end{array}$ $\begin{array}{lllll}1495.222 & 1496.593 & 1496.773 & 1455.443\end{array}$ $\begin{array}{rrrrr}1495.222 & 1496.593 & 1496.773 & 1495.439 \\ 1495.213 & 1496.579 & 1496.77 & 1495.439\end{array}$ $\begin{array}{llll}1495.229 & 1496.586 & 1496.754 & 1495.434\end{array}$ $\begin{array}{lllll}1495.248 & 1496.593 & 1496.738 & 1495.418\end{array}$ $\begin{array}{llllll}1495.259 & 1496.597 & 1496.721 & 1495.406\end{array}$ $\begin{array}{lllll}1495.306 & 1496.628 & 1496.661 & 1495.369\end{array}$ $\begin{array}{llll}1495.343 & 1496.644 & 1496.621 & 1495.331 \\ 1495.348 & 1496.644 & 1496.618 & 1495.313\end{array}$ $\begin{array}{lllll}1495355 & 1496.653 & 1496.597 & 1495.313\end{array}$ $\begin{array}{lllll}1495.355 & 1496.653 & 1496.597 & 1495.292 \\ 1495.369 & 1496.672 & 1496.581 & 1495.273\end{array}$ $\begin{array}{lllll}1495.411 & 1496.709 & 1496.551 & 1495.247\end{array}$ $\begin{array}{llll}1495.406 & 1496.716 & 1496.551 & 1495.236\end{array}$ $\begin{array}{llll}1495.415 & 1496.728 & 1496.534 & 1495.217\end{array}$ $\begin{array}{lllll}1495.394 & 1496.719 & 1496.56 & 1495.222\end{array}$ $\begin{array}{llll}1495.408 & 1496.739 & 1496.525 & 1495.203\end{array}$ $\begin{array}{llll}1495.39 & 1496.732 & 1496.523 & 1495.198\end{array}$ $\begin{array}{llll}1495.385 & 1496.732 & 1496.509 & 1495.187\end{array}$ $\begin{array}{lllll}1495.373 & 1496.735 & 1496.504 & 1495.18\end{array}$ $\begin{array}{lllll}1495.362 & 1496.728 & 1496.495 & 1495.173\end{array}$ $\begin{array}{llll}1495.322 & 1496.693 & 1496.483 & 1495.163\end{array}$ $\begin{array}{llll}1495.155 & 1496.632 & 1496.502 & 1495.173\end{array}$ $\begin{array}{llll}1495.145 & 1496.583 & 1496.511 & 1495.18\end{array}$ $\begin{array}{lllll}1495.143 & 1496.499 & 1496.506 & 1495.177 \\ 1495.152 & 1496.413 & 1496.548 & 1495201\end{array}$ $\begin{array}{lllll}1495.15 & 1496.355 & 1496.574 & 1495.224\end{array}$ $\begin{array}{llll}1495.147 & 1496.308 & 1496.597 & 1455.245\end{array}$ $\begin{array}{lllll}1495.138 & 1496.292 & 1496.593 & 1495.254\end{array}$ $\begin{array}{lllll}1495.141 & 1496.29 & 1496.6 & 1495.264\end{array}$ $\begin{array}{lllll}1495.138 & 1496.301 & 1496.618 & 1495.278\end{array}$ $\begin{array}{llll}1495.133 & 1496.336 & 1496.623 & 1495.289\end{array}$

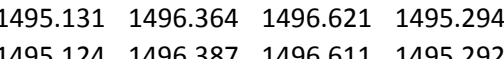

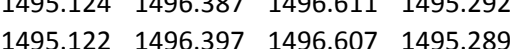
$\begin{array}{llllll}1495.126 & 1496.397 & 1496.614 & 1455.292\end{array}$ $1495.124 \quad 1496.394 \quad 1496.628 \quad 1495.303$ $1495.1261496 .399 \quad 1496.642 \quad 1495.313$ $1495.1271496 .404 \quad 1496.661 \quad 1495.327$ $1495.122 \quad 1496.413 \quad 1496.668 \quad 1495.338$ $\begin{array}{rrrr}1495.122 & 1496.411 & 1496.677 & 1495.348 \\ 1495.12 & 1496.411 & 1496686 & 1495357\end{array}$

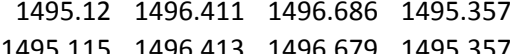
$1495.115 \quad 1496.399 \quad 1496.677 \quad 1495.362$ 1495.108 1496.411 1496.658 14955.352 $1495.106 \quad 1496.385 \quad 1496.647 \quad 1495.343$ $1495.108 \quad 1496.336 \quad 1496.654 \quad 1495.341$ $\begin{array}{llll}1495.106 & 1496.278 & 1496.656 & 1495.343\end{array}$ $\begin{array}{llll}1495.108 & 1496.215 & 1496.675 & 1495.352 \\ 1495.108 & 1496.171 & 1496.691 & 1495.362\end{array}$ $\begin{array}{llllllllll}1494.329 & 1495.069 & 1495.237 & 1496.088 & 1497.189 & 1496.904 & 1493.675 & 1488.987 & 1493.517 & 1491.312\end{array}$ $\begin{array}{lrrrrrrrrr}1494.343 & 1495.067 & 1495.232 & 1496.081 & 1497.18 & 1496.89 & 1493.668 & 1488.982 & 1493.513 & 1491.31 \\ 1494.338 & 1495.064 & 1495.23 & 1496076 & 1497.175 & 1496887 & 1933.666 & 1488.98 & 149351 & 1491312\end{array}$ $\begin{array}{rrrrrrrrrr}1494.338 & 1495.064 & 1495.23 & 1496.076 & 1497.175 & 1496.887 & 1493.666 & 1488.98 & 1493.51 & 1491.312 \\ 1494.341 & 1495.062 & 1495.235 & 1496069 & 1497.168 & 1496875 & 1493666 & 1488975 & 1493508 & 1491.308\end{array}$ $\begin{array}{llllllllllll}1494.341 & 1495.059 & 1495.232 & 1496.065 & 1497.163 & 1496.878 & 1493.661 & 1488.966 & 1493.503 & 1491305\end{array}$ $\begin{array}{lllllllllll}1494.329 & 1495.057 & 1495.239 & 1496.062 & 1497.166 & 1496.878 & 1493.659 & 1488.973 & 1493.506 & 1491.305\end{array}$ $\begin{array}{llllllllllll}1494.327 & 1495.059 & 1495.237 & 1496.058 & 1497.163 & 1496.878 & 1493.661 & 1488.97 & 1493.503 & 1491.305\end{array}$ $\begin{array}{lllllllllll}1494.338 & 1495.057 & 1495.23 & 1496.053 & 1497.149 & 1496.87 & 1493.657 & 1488.968 & 1493.498 & 1491.303\end{array}$ $\begin{array}{lllllllllll}1494.345 & 1495.055 & 1495.228 & 1496.042 & 1497.142 & 1496.851 & 1493.647 & 1488.968 & 1493.491 & 1491.301\end{array}$

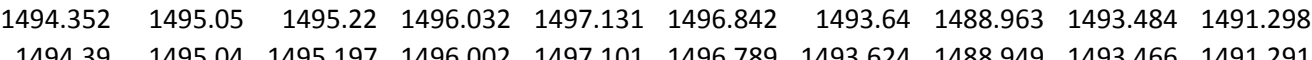
$\begin{array}{lllllllllll}1494.39 & 1495.04 & 1495.197 & 1496.002 & 1497.101 & 1496.789 & 1493.624 & 1488.949 & 1493.466 & 1491.291 \\ 1494.402 & 1495.028 & 1495.187 & 1495.977 & 1497.077 & 1496.748 & 1493.605 & 1488.945 & 1493.454 & 1491285\end{array}$ $\begin{array}{lllllllllll}1494.383 & 1495.021 & 1495.197 & 1495.965 & 1497.07 & 1496.746 & 1493.596 & 1488.933 & 1493.445 & 1491.282\end{array}$ $\begin{array}{lllllllllll}1494.383 & 1495.012 & 1495.192 & 1495.946 & 1497.052 & 1496.732 & 1493.586 & 1488.926 & 1493.433 & 1491.273\end{array}$ $\begin{array}{lllllllllll}1494.374 & 1495.007 & 1495.189 & 1495.935 & 1497.035 & 1496.722 & 1493.577 & 1488.921 & 1493.421 & 1491.266\end{array}$ $\begin{array}{llllllllllll}1494.381 & 1494.997 & 1495.182 & 1495.909 & 1497.015 & 1496.698 & 1493.413 & 1488.914 & 1493.252 & 1491.259\end{array}$ $\begin{array}{lllllllllll}1494.359 & 1494.99 & 1495.187 & 1495.9 & 1497.008 & 1496.701 & 1493.491 & 1488.909 & 1493.332 & 1491.252\end{array}$ $\begin{array}{lllrlllllll}1494.35 & 1494.98 & 1495.185 & 1495.881 & 1496.991 & 1496.686 & 1493.502 & 1488.9 & 1493.346 & 1491.243\end{array}$

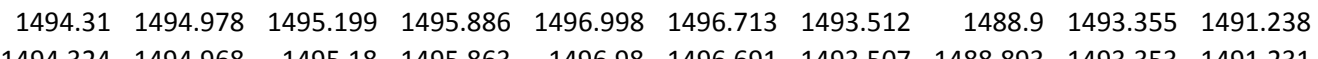
$\begin{array}{llllllllll}1494.324 & 1494.968 & 1495.18 & 1495.863 & 1496.98 & 1496.691 & 1493.507 & 1488.893 & 1493.353 & 1491.231 \\ 1494308 & 1494.963 & 1495.187 & 1495856 & 1496.973 & 1496698 & 1493.507 & 1488891 & 1493351 & 1491226\end{array}$ $\begin{array}{lllllllllll}1494.296 & 1494.959 & 1495.182 & 1495.849 & 1496.966 & 1496.696 & 1493.502 & 1488.888 & 1493.348 & 1491.219\end{array}$ $\begin{array}{llllllllll}1494.291 & 1494.952 & 1495.175 & 1495.835 & 1496.954 & 1496.689 & 1493.5 & 1488.891 & 1493.344 & 1491.215\end{array}$ $\begin{array}{llllllllllll}1494.282 & 1494.947 & 1495.175 & 1495.826 & 1496.947 & 1496.684 & 1493.495 & 1488.881 & 1493.339 & 1491.208\end{array}$ $\begin{array}{lllllllllll}1494.273 & 1494.942 & 1495.17 & 1495.814 & 1496.94 & 1496.681 & 1493.491 & 1488.879 & 1493.332 & 1491.201\end{array}$ $\begin{array}{lllllllllll}1494.265 & 1494.935 & 1495.17 & 1495.802 & 1496.929 & 1496.674 & 1493.484 & 1488.874 & 1493.327 & 1491.196\end{array}$ $\begin{array}{llllllllll}1494.233 & 1494.93 & 1495.18 & 1495.802 & 1496.935 & 1496.693 & 1493.484 & 1488.874 & 1493.327 & 1491.191\end{array}$ $\begin{array}{lllllllllll}1494.216 & 1494.928 & 1495.175 & 1495.807 & 1496.938 & 1496.715 & 1493.484 & 1488.872 & 1493.33 & 1491.189\end{array}$ $\begin{array}{llllllllll}1494.209 & 1494.925 & 1495.175 & 1495.798 & 1496.935 & 1496.708 & 1493.481 & 1488.87 & 1493.327 & 1491.184 \\ 1494.171 & 1494.928 & 1495.187 & 1495814 & 1496.952 & 1496748 & 1493.488 & 1488.872 & 1493337 & 1491.184\end{array}$ $\begin{array}{lllllllllll}1494.148 & 1494.932 & 1495.197 & 1495.826 & 1496.963 & 1496.772 & 1493.5 & 1488.879 & 1493.341 & 1491.184\end{array}$ $\begin{array}{lllllllllll}1494.136 & 1494.935 & 1495.199 & 1495.837 & 1496.977 & 1496.801 & 1493.507 & 1488.888 & 1493.351 & 1491.184\end{array}$ $\begin{array}{llllllllllll}1494.15 & 1494.937 & 1495.192 & 1495.84 & 1496.977 & 1496.806 & 1493.509 & 1488.891 & 1493.351 & 1491.184\end{array}$ $\begin{array}{lllllllllll}1494.148 & 1494.94 & 1495.192 & 1495.842 & 1496.984 & 1496.803 & 1493.512 & 1488.895 & 1493.353 & 1491.187\end{array}$ $\begin{array}{lllllllllll}1494.146 & 1494.942 & 1495.199 & 1495.851 & 1496.996 & 1496.818 & 1493.516 & 1488.9 & 1493.36 & 1491.189\end{array}$ $\begin{array}{rrrrrrrrrr}1494.148 & 1494.944 & 1495.194 & 1495.858 & 1497.001 & 1496.832 & 1493.523 & 1488.902 & 1493.362 & 1491.194 \\ 1494.16 & 199.949 & 1495.189 & 1495858 & 1497.003 & 149833 & 1493523 & 1488007 & 1493355 & 1991.94\end{array}$ $\begin{array}{rlllllllll}1494.16 & 1494.949 & 1495.189 & 1495.858 & 1497.003 & 1496.83 & 1493.523 & 1488.907 & 1493.365 & 1491.194 \\ 1494.176 & 1494.947 & 1495.182 & 1495858 & 1497.001 & 149682 & 1493.521 & 1488902 & 1493365 & 1491.194\end{array}$ $\begin{array}{rlrlrrrrrr}14494.186 & 1444.947 & 1495.178 & 1495.856 & 1497.001 & 1496.815 & 1493.523 & 1488.9 & 1493.367 & 1491.196\end{array}$ $\begin{array}{lllllllllll}1494.183 & 1494.947 & 1495.182 & 1495.858 & 1497.005 & 1496.82 & 1493.526 & 1488.909 & 1493.37 & 1491.198\end{array}$ $\begin{array}{llllllllll}1494.174 & 1494.949 & 1495.189 & 1495.867 & 1497.015 & 1496.832 & 1493.535 & 1488.902 & 1493.377 & 1491.203\end{array}$ $\begin{array}{lllllllllll}1494.169 & 1494.954 & 1495.192 & 1495.877 & 1497.024 & 1496.844 & 1493.542 & 1488.907 & 1493.381 & 1491.205\end{array}$ $\begin{array}{lllllllllll}1494.164 & 1494.959 & 1495.197 & 1495.888 & 1497.035 & 1496.863 & 1493.551 & 1488.914 & 1493.393 & 1491.208\end{array}$ $\begin{array}{lllllllllll}1494.169 & 1494.961 & 1495.192 & 1495.893 & 1497.04 & 1496.87 & 1493.556 & 1488.919 & 1493.398 & 1491.212\end{array}$ $\begin{array}{lllllllllll}1494.169 & 1494.963 & 1495.197 & 1495.9 & 1497.049 & 1496.875 & 1493.565 & 1488.921 & 1493.405 & 1491.217 \\ 1494.174 & 1494.966 & 1495.199 & 1495909 & 1497.056 & 1496885 & 1493575 & 1488923 & 149342 & 1491222\end{array}$ $\begin{array}{llllllllllll}1494.174 & 1494.966 & 1455.199 & 1495.909 & 1497.056 & 1466.885 & 1493.575 & 1488.923 & 1493.412 & 1491.222\end{array}$ $\begin{array}{lllllllllll}1494.193 & 1494.973 & 1495.189 & 1495.914 & 1497.061 & 1496.875 & 1493.575 & 1488.928 & 1493.414 & 1491.229\end{array}$ $\begin{array}{llllllllllll}1494.214 & 1494.971 & 1495.178 & 1495.905 & 1497.052 & 1496.854 & 1493.575 & 1488.926 & 1493.412 & 1491.229\end{array}$ $\begin{array}{llllllllll}1494.221 & 1494.971 & 1495.18 & 1495.902 & 1497.047 & 1496.842 & 1493.573 & 1488.921 & 1493.412 & 1491.229\end{array}$ $\begin{array}{lllllllllll}1494.214 & 1494.971 & 1495.182 & 1495.905 & 1497.052 & 1496.844 & 1493.57 & 1488.923 & 1493.412 & 1491.233\end{array}$ $\begin{array}{lllllllllll}1494.209 & 1494.971 & 1495.185 & 1495.909 & 1497.054 & 1496.851 & 1493.575 & 1488.919 & 1493.414 & 1491.236\end{array}$

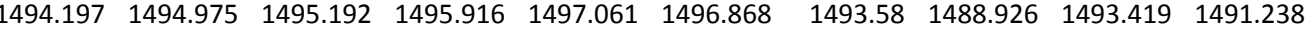

$\begin{array}{lllllllll}1487.635 & 1488.549 & 1493.925 & 1494.404 & 1493.684 & 1495.54 & 1493.659 & 1484.846 & 1479.346\end{array}$ $\begin{array}{llllllllll}1487.644 & 1488.556 & 1493.923 & 1494.393 & 1493.676 & 1495.524 & 1493.652 & 1484.846 & 1479.346\end{array}$

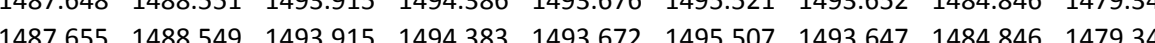
$\begin{array}{llllllllll}1487.662 & 1488.549 & 1493.913 & 1494.374 & 1493.672 & 1495.498 & 1493.649 & 1484.841 & 1479.346\end{array}$ $\begin{array}{llllllllllll}1487.672 & 1488535 & 1493.909 & 1494383 & 1493.667 & 1495.51 & 14936631 & 1484848 & 14793341\end{array}$ $\begin{array}{lllllllllll}1487.681 & 1488.53 & 1493.909 & 1494.379 & 1493.667 & 1495.507 & 1493.638 & 1484.841 & 1479.344\end{array}$ $\begin{array}{llllllllll}1487.688 & 1488.539 & 1493.904 & 1494.367 & 1493.665 & 1495.489 & 1493.645 & 1484.839 & 1479.341\end{array}$ $\begin{array}{lllllllllll}1487.637 & 1488.539 & 1493.895 & 1494.355 & 1493.655 & 1495.481 & 1493.633 & 1484.841 & 1479.337\end{array}$ $\begin{array}{lllllllll}1487.637 & 1488.546 & 1493.892 & 1494.348 & 1493.648 & 1459.465 & 1493.633 & 1484.837 & 1479.33\end{array}$ $\begin{array}{lllllllllll}1487.632 & 1488.563 & 1493.875 & 1494.299 & 1493.634 & 1495.409 & 1493.624 & 1484.834 & 1479.327 \\ 1487.641 & 1488.56 & 1493859 & 1494271 & 1493.612 & 1495.373 & 14936 & 1484.837 & 1479.32\end{array}$ $\begin{array}{llllllllllll}1487.641 & 1488.539 & 1493.852 & 1494.269 & 1493.605 & 1495.376 & 1493.586 & 1484.837 & 147932\end{array}$ $\begin{array}{lllllllllll}1487.646 & 1488.539 & 1493.838 & 1494.248 & 1493.593 & 1495.35 & 1493.579 & 1484.834 & 1479.316\end{array}$ $\begin{array}{llllllllll}1487.653 & 1488.535 & 1493.829 & 1494.236 & 1493.586 & 1495.333 & 1493.572 & 1484.834 & 1479.31\end{array}$ $\begin{array}{lllllllll}1487.658 & 1488.535 & 1493.659 & 1494.166 & 1493.423 & 1495.303 & 1493.422 & 1484.832 & 1479.311\end{array}$ $\begin{array}{llllllllll}1487.665 & 1488.52 & 1493.739 & 1494.18 & 1493.496 & 1495.298 & 1493.483 & 1484.832 & 1479.31\end{array}$ $\begin{array}{lllllllll}1487.672 & 1488.513 & 1493.753 & 1494.178 & 1493.508 & 1495.282 & 1493.497 & 1484.83 & 1479.31\end{array}$ $\begin{array}{lllllllll}1487.676 & 1488.488 & 1493.763 & 1494.19 & 1493.518 & 145.301 & 1493.492 & 1484.834 & 1479.31 \\ 1487.681 & 1488.49 & 1493758 & 1494.173 & 1493515 & 1495277 & 1493.497 & 1484 & 1493\end{array}$

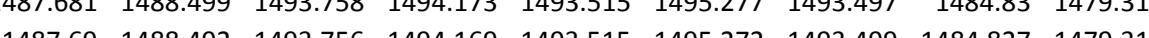
$\begin{array}{llllllllll}1487.697 & 1488.485 & 1493.758 & 1494.169 & 1493.513 & 1495.27 & 1493.499 & 1484.827 & 1479.311\end{array}$ $\begin{array}{llllllllll}1487.683 & 1488481 & 1493749 & 1494159 & 1493506 & 1495261 & 1493492 & 1484827 & 1479309\end{array}$ $\begin{array}{lllllllllll}1487.678 & 1488.462 & 1493.746 & 1494.157 & 1493.503 & 1495.256 & 1493.483 & 1484.827 & 1479.306\end{array}$ $\begin{array}{llllllllll}1487.685 & 1488.462 & 1493.737 & 1494.15 & 1493.499 & 1495.249 & 1493.476 & 1484.823 & 1479.304\end{array}$ $\begin{array}{llllllllll}1487.692 & 1488.45 & 1493.735 & 1494.136 & 1493.492 & 1495.239 & 1493.469 & 1484.823 & 1479.302\end{array}$ $\begin{array}{llllllllll}1487.699 & 1488.451 & 1493.735 & 1494.155 & 1493.492 & 1495.258 & 1493.466 & 1484.823 & 1479.29\end{array}$ $\begin{array}{llllllllll}1487.70 & 1488.427 & 1493.737 & 1494.155 & 1493.494 & 1495.258 & 1493.471 & 1484.82 & 1479.302\end{array}$ $\begin{array}{llllllllll}1487.715 & 1488.41 & 1493.734 & 1494.157 & 1493.494 & 1495.263 & 1493.459 & 1484.82 & 1479.299 \\ 1487.722 & 1488.382 & 1493.739 & 1494.183 & 1493.499 & 1495291 & 1493.459 & 1484.82 & 1479302\end{array}$ $\begin{array}{llllllllll}1487.729 & 1488.368 & 1493.751 & 1494.201 & 1493.508 & 1495.317 & 1493.466 & 1484.82 & 1479.304\end{array}$ $\begin{array}{lllllllllll}1487.736 & 1488.359 & 1493.756 & 1494.215 & 1493.518 & 1495.338 & 1493.485 & 1484.82 & 1479.300\end{array}$ $\begin{array}{lllllllll}1487.688 & 1488.366 & 1493.758 & 1494.218 & 1493.518 & 1495.338 & 1493.497 & 1484.818 & 1479.304\end{array}$ $\begin{array}{llllllllll}1487.692 & 1488.354 & 1493.761 & 1494.225 & 1493.522 & 1495.348 & 1493.497 & 1484.818 & 1479.304\end{array}$ $\begin{array}{lllllllll}1487.702 & 1488.349 & 1493.77 & 1494.236 & 1493.527 & 1495.362 & 1493.502 & 1484.818 & 1479.304\end{array}$ $\begin{array}{llllllllll}1487.706 & 1488.352 & 1493.768 & 1494.243 & 1493.529 & 1495.366 & 1493.511 & 1484.816 & 1479.304\end{array}$

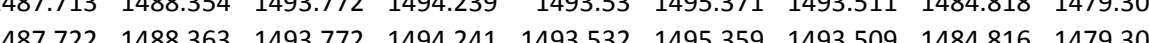

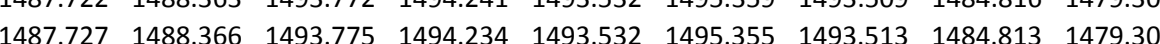
$\begin{array}{lllllllllll}1487.727 & 1488.361 & 1493.774 & 1494.243 & 1493.537 & 1495.366 & 1493.513 & 1484.816 & 1479.302\end{array}$ $\begin{array}{llllllllll}1487.736 & 1488.359 & 1493.784 & 1494.253 & 1493.544 & 1495.38 & 1493.518 & 1484.813 & 1479.304\end{array}$ $\begin{array}{llllllllll}1487.741 & 1488.354 & 1493.789 & 1494.267 & 1493.549 & 1495.392 & 1493.525 & 1484.816 & 1479.309\end{array}$ $\begin{array}{llllllllll}1487.755 & 1488.359 & 1493.796 & 1494.281 & 1493.56 & 1495.411 & 1493.537 & 1484.813 & 1479.313\end{array}$ $\begin{array}{llllllllll}1487.759 & 1488.366 & 1493.805 & 1494.295 & 1493.565 & 1495.413 & 1493.544 & 1484.813 & 1479.31\end{array}$ $\begin{array}{lllllllll}1487.77 & 1488.368 & 1493.815 & 1494.295 & 1493.575 & 1495.427 & 1493.549 & 1484.813 & 1479.32\end{array}$ $\begin{array}{llllllllll}1487 & 1479.32\end{array}$ $\begin{array}{lllllllllll}1487.766 & 1488.384 & 1493.824 & 1494.299 & 1493.582 & 1495.434 & 1493.563 & 1484.813 & 1479.323\end{array}$ $\begin{array}{llllllllll}1487.778 & 1488.391 & 1493.822 & 1494.295 & 1493.582 & 1495.413 & 1493.563 & 1484.811 & 1479.318\end{array}$ $\begin{array}{lllllllll}1487.771 & 1488.396 & 1493.819 & 1494.285 & 1493.579 & 1495.406 & 1493.556 & 1484.809 & 1479.316\end{array}$ $\begin{array}{llllllllll}1487.78 & 1488.385 & 1493.819 & 1494.29 & 1493.577 & 1495.411 & 1493.553 & 1484.809 & 1479.316\end{array}$ $\begin{array}{lllllllll}1487.792 & 1488.387 & 1493.822 & 1494.29 & 1493.582 & 1495.413 & 1493.551 & 1484.809 & 1479.316\end{array}$ $\begin{array}{lllllllll}1487.799 & 1488.377 & 1493.826 & 1494.299 & 1493.589 & 1495.423 & 1493.556 & 1484.809 & 1479.318 \\ 1487.808 & 1488.37 & 1493.831 & 1494.318 & 1493.591 & 1495.442 & 1493.558 & 1484.806 & 1479.318\end{array}$ 
Well

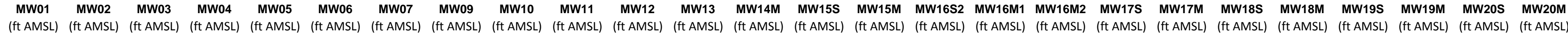

2/17/12 14:00 2/17/12 15:00 2/17/12 16:00 2/177/12 17:00 2/17/1212 19:00 2/17/12 20:00 2/17/12 21:00 2/17/12 22:00 2/17/122 23:00 2/18/12 0:00 2/18/12 1:00 2/18/12 3:00 //18/12 4:00 2/18/12 5:00 2/18/12 6:00 2/18/12 7:00 $2 / 18 / 128: 00$ 2/18/12 9:00 2/18/12 11:00 2/18/12 12:00 2/18/12 12:00 2/18/12 14:00 2/18/12 15:00 2/18/12 16:00 2/18/12 17:00 2/18/12 18:00 2/18/12 20:00 2/18/12 21:00 2/18/12 22:00 2/18/12 23:00 2/19/12 0:00 2/19/12 1:00 2/19/12 2:00 2/19/12 3:00 2/19/12 4:00 2/19/12 6:00 2/19/12 7:00 2/19/12 8:00 2/19/12 9:00 2/19/12 10:00 $2 / 19 / 1212.00$ 2/19/12 12:00 2/19/12 13:00 2/19/12 15:00 2/19/12 16:00 2/19/12 18:00 $\begin{array}{rrrr}1495.11 & 1496.131 & 1496.712 & 1495.38 \\ 1495.108 & 1496.108 & 1496.721 & 1495.39\end{array}$ $\begin{array}{llll}1495.108 & 1496.108 & 1496.721 & 1495.39 \\ 1495.106 & 1496.103 & 1496726 & 1495397\end{array}$ $\begin{array}{llll}1495.106 & 1496.103 & 1496.726 & 1495.397\end{array}$ $\begin{array}{rrrrr}1495.099 & 1496.119 & 1466.719 & 1495.397 \\ 1496.71 & 1495.394\end{array}$ $\begin{array}{rrrrr}1495.094 & 1496.143 & 1496.693 & 1495.385\end{array}$ $\begin{array}{llll}1495.094 & 1496.143 & 1496.693 & 1495.385 \\ 1495.092 & 1496.161 & 1496.684 & 1495.378\end{array}$ $\begin{array}{lllll}1495.089 & 1496.182 & 1496.668 & 1495.364\end{array}$ $\begin{array}{llll}1495.075 & 1496.213 & 1496.63 & 1495.338\end{array}$ $\begin{array}{llll}1495.078 & 1496.238 & 1496.614 & 1495.32\end{array}$ $\begin{array}{rrrr}1495.082 & 1496.25 & 1496.614 & 1495.31 \\ 1495078 & 1496264 & 1496.614 & 1495303\end{array}$ $\begin{array}{llll}1495.078 & 1496.264 & 1496.614 & 1495.303 \\ 1495.075 & 1496.278 & 1496.611 & 1495.301\end{array}$ $\begin{array}{lllll}1495.075 & 1466.278 & 1496.611 & 1495.301 \\ 1495.075 & 1496.299 & 1496.614 & 1495.301\end{array}$ $\begin{array}{lllll}1495.075 & 1496.301 & 1496.618 & 1495.301\end{array}$ $1495.073 \quad 1496.304 \quad 1496.6161495 .301$ $\begin{array}{llll}1495.066 & 1496.315 & 1496.597 & 1495.292\end{array}$ $\begin{array}{llll}1495.061 & 1496.329 & 1496.579 & 1495.28\end{array}$ $\begin{array}{llll}1495.066 & 1496.32 & 1496.586 & 1495.28\end{array}$ $\begin{array}{lrrrr}1495.061 & 1496.32 & 1496.579 & 1495.271 \\ 1495.064 & 1496.292 & 1496595 & 1495.28\end{array}$ $\begin{array}{rrrrr}1455.064 & 1466.234 & 1496.609 & 1495.287\end{array}$ $\begin{array}{lllll}1495.068 & 1496.171 & 1496.63 & 1495301\end{array}$ $\begin{array}{rrrrr}1495.068 & 1496.119 & 1496.651 & 1495.35 .32\end{array}$ $\begin{array}{lllll}1495.068 & 1496.07 & 1496.682 & 1495.341\end{array}$ $\begin{array}{lllll}1495.064 & 1496.056 & 1496.677 & 1495.35\end{array}$ $\begin{array}{lllll}1495.066 & 1496.045 & 1496.691 & 1495.362\end{array}$ $\begin{array}{lllll}1495.061 & 1496.056 & 1496.684 & 1495.364\end{array}$ $\begin{array}{rrrrr}1495.054 & 1496.08 & 1496.672 & 1495.359\end{array}$ $\begin{array}{lllll}1495.054 & 1496.14 & 1496.661 & 1455.35\end{array}$ $\begin{array}{llll}1495.05 & 1496.166 & 1496.663 & 1495.35\end{array}$ $\begin{array}{lllll}1495.052 & 1496.192 & 1496.665 & 1495.35\end{array}$ $\begin{array}{llll}1495.05 & 1496.215 & 1496.67 & 1495.35\end{array}$ $\begin{array}{llll}1495.05 & 1496.231 & 1496.668 & 1495.35\end{array}$ $\begin{array}{llll}1495.047 & 1496.25 & 1496.67 & 1495.352 \\ 1490.047 & 1496262 & 1496.672 & 195352\end{array}$ $\begin{array}{lllll}1495.047 & 1496.262 & 1496.672 & 1495.352\end{array}$ $\begin{array}{llll}1495.047 & 1496.264 & 1496.679 & 1495.359\end{array}$ $\begin{array}{lllll}1495.05 & 1496.25 & 1496.698 & 1495.376\end{array}$ $1495.045 \quad 1496.262 \quad 1496.691 \quad 1495.373$ $1495.043 \quad 1496.264 \quad 1496.684 \quad 1495.371$ $1495.038 \quad 1496.259 \quad 1496.672 \quad 1495.364$ $1495.043 \quad 1496.22 \quad 1496.679 \quad 1495.364$ $\begin{array}{llll}1495.043 & 1496.185 & 1496.684 & 1495.366 \\ 149504 & 1966.115 & 1966.691 & 195369\end{array}$ 1495.041496 .1151496 .6911495 .369 $1495.052 \quad 1495.984 \quad 1496.733 \quad 1495394$ $1495.052 \quad 1495.93 \quad 1496.761 \quad 1495.418$ $1495.061 \quad 1495.891 \quad 1496.7891495 .441$ $1495.0611495 .8771496 .798 \quad 1495.462$ $1495.059 \quad 1495.896 \quad 1496.801 \quad 1495.469$ $\begin{array}{llll}1495.059 & 1495.917 & 1496.805 & 1495.476 \\ 1495.057 & 1495.956 & 1496.803 & 1495.478\end{array}$ $\begin{array}{llllllllll}1494.174 & 1494.983 & 1495.204 & 1495.937 & 1497.084 & 1496.901 & 1493.594 & 1488.935 & 1493.433 & 1491.243\end{array}$ $\begin{array}{llllllllllll}1494.176 & 1494.985 & 1495.204 & 1495.946 & 1497.091 & 1496.909 & 1493.598 & 1488.938 & 1493.44 & 1491.247\end{array}$ \begin{tabular}{lllllllllll}
1494 & 1494.99 & 1455.199 & 1495.951 & 1497.096 & 1496.911 & 4993.603 & 4888.945 & 1493.442 & 1491.25 \\
\hline
\end{tabular} $\begin{array}{lllllllllll}1494.211 & 1494.99 & 1495.197 & 1495.953 & 1497.094 & 1496.894 & 1493.605 & 1488.94 & 1493.447 & 1491.257\end{array}$ $\begin{array}{lllllllllll}1494.228 & 1494.992 & 1495.187 & 1495.951 & 1497.089 & 1496.88 & 1493.6 & 1488.938 & 1493.44 & 1491.257\end{array}$ $\begin{array}{llllllllllll}1494.237 & 1494.99 & 1495.187 & 1495.946 & 1497.084 & 1496.87 & 1493.596 & 1488.94 & 1493.438 & 1491.259\end{array}$ $\begin{array}{lllllllllll}1494.251 & 1494.987 & 1495.178 & 1495.939 & 1497.077 & 1496.854 & 1493.594 & 1488.933 & 1493.433 & 1491.257\end{array}$ $\begin{array}{lllllllllll}1494.28 & 1494.983 & 1495.163 & 1495.923 & 1497.059 & 1496.818 & 1493.582 & 1488.923 & 1493.426 & 1491.254\end{array}$ $\begin{array}{llllllllll}1494.282 & 1494.975 & 1495.166 & 1455.912 & 1497.049 & 1496.803 & 1493.575 & 1488.916 & 1493.423 & 1491.252\end{array}$ $\begin{array}{lllllllllll}1494.273 & 1494.973 & 1495.17 & 1495.907 & 1497.047 & 1496.801 & 1493.573 & 1488.914 & 1493.419 & 1491.25 \\ 1492.265 & 1494971 & 1495.175 & 1495.907 & 1497.045 & 1496806 & 1493573 & 1488909 & 1493.416 & 149125\end{array}$ $\begin{array}{llllllllllll}1494.263 & 1494.968 & 1495.17 & 1495.902 & 1497.04 & 1496.803 & 1493.572 & 1488.905 & 1493.416 & 1491.25\end{array}$ $\begin{array}{llllllllllll}1494.256 & 1494.966 & 1495.17 & 1495.9 & 1497.04 & 1496.806 & 1493.577 & 1488.905 & 1493.419 & 1491.247\end{array}$ $\begin{array}{llllllllllll}1494.247 & 1494.963 & 1495.178 & 1495.902 & 1497.04 & 1496.818 & 1493.579 & 1488.905 & 1493.421 & 1491.245\end{array}$ $\begin{array}{lllllllllll}1494.244 & 1494.961 & 1495.173 & 1495.902 & 1497.04 & 1496.818 & 1493.577 & 1488.905 & 1493.421 & 1491.245\end{array}$ $\begin{array}{llllllllll}1494.254 & 1494.959 & 1495.166 & 1495.891 & 1497.029 & 1496.799 & 1493.573 & 1488.905 & 1493.414 & 1491.243\end{array}$ $\begin{array}{llllllllll}1494.258 & 1494.954 & 1495.159 & 1495.879 & 1497.022 & 1496.784 & 1493.565 & 1488.9 & 1493.412 & 1491.243\end{array}$ $\begin{array}{lllllllllll}1494.244 & 1494.952 & 1495.17 & 1495.879 & 1497.022 & 1496.749 & 1493.563 & 1488.895 & 1493.405 & 1491.238\end{array}$ $\begin{array}{lllllllllll}1494.242 & 1494.947 & 1495.163 & 1495.874 & 1497.015 & 1496.782 & 1493.558 & 1488.895 & 1493.405 & 1491.236 \\ 1494223 & 1494.947 & 1495.173 & 1495879 & 1497.022 & 1496806 & 1493558 & 1488.893 & 1493402 & 1491236\end{array}$ $\begin{array}{llllllllllll}1494.209 & 1494.947 & 1495.178 & 1495.884 & 1497.026 & 1496.813 & 1493.561 & 1488.895 & 1493.405 & 1491.233\end{array}$ $\begin{array}{lllllllllll}1494.186 & 1494.952 & 1495.187 & 1495.893 & 1497.038 & 1496.837 & 1493.565 & 1488.902 & 1493.409 & 1491.236\end{array}$ $\begin{array}{lllllllllll}1494.174 & 1494.956 & 1495.187 & 1495.905 & 1497.049 & 1496.861 & 1493.573 & 1488.905 & 1493.421 & 1491.238\end{array}$ $\begin{array}{llllllllll}1494.155 & 1494.963 & 1495.197 & 1495.919 & 1497.063 & 1496.887 & 1493.587 & 1488.912 & 1493.426 & 1491.24\end{array}$ $\begin{array}{llllllllll}1494.169 & 1494.966 & 1495.189 & 1495.921 & 1497.066 & 1496.885 & 1493.587 & 1488.916 & 1493.43 & 1491.24\end{array}$ $\begin{array}{llllllllll}1494.167 & 1494.968 & 1495.192 & 1455.928 & 1497.075 & 1496.894 & 1493.596 & 1488.921 & 1493.435 & 1491.245\end{array}$ $\begin{array}{llllllllll}1494.183 & 1494.971 & 1495.185 & 1495.93 & 1497.075 & 1496.89 & 1493.596 & 1488.926 & 1493.438 & 1491.247 \\ 1494.202 & 1494.973 & 1495.182 & 1495.928 & 1497.07 & 1496875 & 1493596 & 1488928 & 1493.438 & 1491247\end{array}$ $\begin{array}{lllllllllll}1494.202 & 1494.973 & 1495.182 & 1495.928 & 1497.07 & 1496.875 & 1493.596 & 1488.928 & 1493.438 & 1491.247 \\ 1494.214 & 1494.973 & 1495.175 & 1495.925 & 1497.068 & 1496.866 & 1493.594 & 1488.921 & 1493.433 & 1491.247\end{array}$ $\begin{array}{llllllllllll}1494.218 & 1494.973 & 1495.178 & 1495.925 & 1497.068 & 1496.863 & 1493.591 & 1488.923 & 1493.433 & 1491.25\end{array}$ $\begin{array}{llllllllll}1494.223 & 1494.976 & 1495.182 & 1495.925 & 1497.07 & 1496.866 & 1493.589 & 1488.921 & 1493.433 & 1491.25\end{array}$ $\begin{array}{llllllllll}1494.225 & 1494.973 & 1495.182 & 1495.928 & 1497.07 & 1496.863 & 1493.589 & 1488.921 & 1493.43 & 1491.25\end{array}$ $\begin{array}{llllllllll}1494.225 & 1494.978 & 1495.185 & 1495.93 & 1497.073 & 1496.868 & 1493.589 & 1488.919 & 1493.433 & 1491.254\end{array}$ $\begin{array}{lllllllllll}1494.228 & 1494.978 & 1495.182 & 1495.932 & 1497.073 & 1496.87 & 1493.591 & 1488.923 & 1493.435 & 1491.252\end{array}$ $\begin{array}{llllllllll}1494.23 & 1494.978 & 1495.182 & 1495.932 & 1497.075 & 1496.87 & 1493.596 & 1488.921 & 1493.438 & 1491.254 \\ 1494.23 & 1494.975 & 1495.182 & 1495.932 & 1497.077 & 149687 & 1493598 & 1488.93 & 149344 & 1491254\end{array}$ $\begin{array}{llllllllll}1494.23 & 1494.975 & 1455.182 & 1455.932 & 1497.077 & 1496.87 & 1493.598 & 1488.93 & 1493.44 & 1491.254 \\ 1494.228 & 1494.98 & 1495.189 & 1495937 & 1497.082 & 1496878 & 1493603 & 1488923 & 1493.455 & 1491.257\end{array}$ $\begin{array}{llllllllllll}1494.218 & 1494.983 & 1495.192 & 1495.946 & 1497.089 & 1496.892 & 1493.61 & 1488.928 & 1493.454 & 1491.259\end{array}$ $\begin{array}{lllllllllll}1494.218 & 1494.985 & 1495.187 & 1495.949 & 1497.094 & 1496.897 & 1493.617 & 1488.93 & 1493.454 & 1491.264\end{array}$ $\begin{array}{llllllllll}1494.23 & 1494.985 & 1495.187 & 1495.949 & 1497.091 & 1496.887 & 1493.617 & 1488.928 & 1493.461 & 1491.264\end{array}$ $\begin{array}{lllllllllll}1494.237 & 1494.985 & 1495.182 & 1495.949 & 1497.089 & 1496.88 & 1493.617 & 1488.93 & 1493.461 & 1491.266\end{array}$ $\begin{array}{lllllllllll}1494.249 & 1494.985 & 1495.18 & 1495.944 & 1497.082 & 1496.873 & 1493.615 & 1488.926 & 1493.456 & 1491.266\end{array}$ $\begin{array}{lllllllllll}1494.24 & 1494.987 & 1495.187 & 1495.946 & 1497.087 & 1496.878 & 1493.612 & 1488.928 & 1493.454 & 1491.266\end{array}$ $\begin{array}{llllllllll}1494.235 & 1494.987 & 1495.192 & 1495.946 & 1497.089 & 1496.878 & 1493.612 & 1488.93 & 1493.456 & 1491.266 \\ 1492.228 & 1494.987 & 1495189 & 1495.953 & 197.096 & 1496885 & 1493612 & 1488.923 & 149.454 & 141.268\end{array}$ $\begin{array}{llllllllll}1494.228 & 1494.987 & 1455.129 & 145.953 & 1497.096 & 1496.885 & 1493.612 & 1488.923 & 1493.454 & 1491.268 \\ 1494.221 & 1494.988 & 1495.192 & 1495.956 & 1497.098 & 1496.89 & 1493615 & 1488.93 & 1493.461 & 1491.271\end{array}$ $\begin{array}{llllllllll}1494.195 & 1494.992 & 1495.204 & 1495.97 & 1497.115 & 1496.916 & 1493.629 & 1488.93 & 1493.466 & 1491.273\end{array}$ $\begin{array}{lllllllllll}1494.186 & 1495.002 & 1495.211 & 1495.993 & 1497.131 & 1496.957 & 1493.64 & 1488.942 & 1493.48 & 1491.278\end{array}$ $\begin{array}{lllllllllll}1494.171 & 1495.011 & 1495.218 & 1496.007 & 1497.147 & 1496.973 & 1493.652 & 1488.947 & 1493.491 & 1491.28\end{array}$ $\begin{array}{lllllllllll}1494.178 & 1495.016 & 1495.218 & 1496.018 & 1497.161 & 1496.988 & 1493.661 & 1488.956 & 1493.498 & 1491.287\end{array}$ $\begin{array}{lllllllllll}1494.195 & 1495.018 & 1495.213 & 1496.025 & 1497.163 & 1496.985 & 1493.668 & 1488.961 & 1493.508 & 1491.289\end{array}$

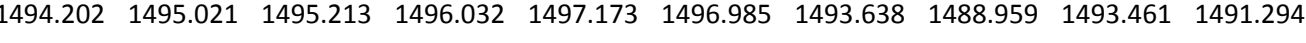

$\begin{array}{rrrrrrrrr}1487.824 & 1488.366 & 1493.84 & 1494.33 & 1493.601 & 1495.46 & 1493.57 & 1484.806 & 1479.323\end{array}$ $\begin{array}{lllllllll}1487.822 & 1488.37 & 1493.847 & 1494.337 & 1493.61 & 1495.47 & 1493.579 & 1484.806 & 1479.323\end{array}$ $\begin{array}{llllllllll}1487.776 & 1488.377 & 1493.855 & 1494.339 & 1493.612 & 1495.47 & 1493.579 & 1484806 & 1479.323\end{array}$ $\begin{array}{lllllllll}1487.785 & 1488389 & 1493852 & 1494.332 & 1493.612 & 1495.465 & 1493.588 & 1484804 & 1479.323\end{array}$ $\begin{array}{llllllllll}1487.787 & 1488.391 & 1493.85 & 1494.316 & 1493.608 & 1495.451 & 1493.586 & 1484.802 & 147932\end{array}$ $\begin{array}{llllllllll}1487.794 & 1488.394 & 1493.847 & 1494.318 & 1493.605 & 1495.446 & 1493.584 & 1484.802 & 1479.316\end{array}$ $\begin{array}{llllllllll}1487.796 & 1488.408 & 1493.838 & 1494.304 & 1493.603 & 1495.42 & 1493.579 & 1484.802 & 1479.313\end{array}$ $\begin{array}{llllllllll}1487.796 & 1488.422 & 1493.833 & 1494.278 & 1493.591 & 1495.39 & 1493.574 & 1484.799 & 1479.309\end{array}$ $\begin{array}{lllllllll}1487.801 & 1488.415 & 1493.829 & 1494.271 & 1493.582 & 1495.376 & 1493.567 & 1484.799 & 1479.30\end{array}$ $\begin{array}{llllllllll}1487.815 & 1488.41 & 1493.819 & 1494.269 & 1493.584 & 1495.378 & 1493.563 & 1484.799 & 1479.306\end{array}$ $\begin{array}{llllllllll}1487.831 & 1488.408 & 1493.824 & 1494.267 & 1493.582 & 1495.371 & 1493.563 & 1484.799 & 1479.311\end{array}$ $\begin{array}{llllllllll}1487.831 & 1488.405 & 1493.828 & 1494.267 & 1493.582 & 1495.371 & 1493.563 & 1484.799 & 1479.316\end{array}$ $\begin{array}{llllllllll}1487.838 & 1488.406 & 1493.826 & 1494.269 & 1493.584 & 1495.38 & 1493.565 & 1484.797 & 1479.32\end{array}$ $\begin{array}{lllllllll}1487.843 & 1488.408 & 1493.828 & 1494.269 & 1493.586 & 1495.373 & 1493.57 & 1484.797 & 1479.32\end{array}$ $\begin{array}{llllllllll}1487.843 & 1488.408 & 1493.824 & 1494.255 & 1493.582 & 1495.359 & 1493.563 & 1484.797 & 1479.31\end{array}$ $\begin{array}{lllllllllll}1487.845 & 1488.41 & 1493.814 & 1494.246 & 1493.572 & 1495.348 & 1493.549 & 1484.795 & 1479.316\end{array}$ $\begin{array}{llllllllll}1487.845 & 1488.396 & 1493.81 & 1494.25 & 1493.57 & 1495.357 & 1493.549 & 1484.797 & 1479.316\end{array}$ $\begin{array}{llllllllll}1487.847 & 1488.398 & 1493.808 & 1494.246 & 1493.567 & 1495.348 & 1493.541 & 1484.795 & 1479.313\end{array}$ $\begin{array}{lllllllllll}1487.822 & 1488.377 & 1493.812 & 1494.262 & 1493.57 & 1495.364 & 1493.541 & 1484.795 & 1479.311\end{array}$ $\begin{array}{llllllllll}1487831 & 1488366 & 1493817 & 1494274 & 1493.575 & 1495385 & 1493544 & 1484.792 & 1479.313\end{array}$ $\begin{array}{lllllllllll}1487.843 & 1488.361 & 1493.826 & 1494.295 & 1493.582 & 1495.409 & 1493.551 & 1484.792 & 1479.313\end{array}$ $\begin{array}{lllllllllll}1487.813 & 1488.352 & 1493.831 & 1494.304 & 1493.593 & 1495.427 & 1493.556 & 1484.795 & 1479.318\end{array}$ $\begin{array}{llllllllll}1487.824 & 1488.363 & 1493.84 & 1494.309 & 1493.596 & 1495.43 & 1493.57 & 1484.79 & 1479.32\end{array}$ $\begin{array}{llllllllll}1487.755 & 1488.359 & 1493.843 & 1494.316 & 1493.603 & 1495.444 & 1493.574 & 1484.79 & 1479.32\end{array}$ $\begin{array}{llllllllll}1487.764 & 1488.368 & 1493.845 & 1494.32 & 1493.603 & 1455.437 & 1433.584 & 1484.79 & 1479.323\end{array}$ $\begin{array}{llllllllll}1487785 & 1488.38 & 1493.845 & 1494.302 & 1493.603 & 1495.418 & 1493.581 & 1484.79 & 1479.32\end{array}$ $\begin{array}{llllllllll}1487.796 & 1488.382 & 1493.84 & 1494.304 & 1493.598 & 1495.418 & 1493.579 & 1484.787 & 1479.316\end{array}$ $\begin{array}{lllllllllll}1487.808 & 1488.384 & 1493.84 & 1494.302 & 1493.601 & 1495.418 & 1493.577 & 1484.787 & 1479.313\end{array}$ $\begin{array}{lllllllll}1487.817 & 1488.38 & 1493.838 & 1494.304 & 1493.596 & 1495.418 & 1493.577 & 1484.79 & 1479.31\end{array}$ $\begin{array}{llllllllll}1487.826 & 1488.385 & 1493.843 & 1494.309 & 1493.598 & 1495.423 & 1493.577 & 1484.787 & 1479.311\end{array}$ $\begin{array}{llllllllll}1487.831 & 1488.391 & 1493.84 & 1494.309 & 1493.603 & 1495.42 & 1493.581 & 1484.787 & 1479.31\end{array}$ $\begin{array}{llllllllll}1487.838 & 1488.391 & 1493.847 & 1494.306 & 1493.603 & 1495.42 & 1493.581 & 1484.787 & 1479.313\end{array}$ $\begin{array}{llllllllll}1487.85 & 1488.398 & 1493.85 & 1494.309 & 1493.605 & 1495.423 & 1493.584 & 1484.785 & 1479.316 \\ 1487.861 & 1488396 & 1493.852 & 1494.32 & 1493.612 & 1495.43 & 1493.586 & 1484.787 & 1479.32\end{array}$ $\begin{array}{llllllllll}1487.873 & 1488.399 & 1493.832 & 1494.32 & 1493.612 & 1495.43 & 1493.386 & 1484.787 & 149.327 \\ 1493.617 & 1495.446 & 1493.593 & 1484.785 & 1479.325\end{array}$ $\begin{array}{lllllllllll}1487.882 & 1488.398 & 1493.861 & 1494.33 & 1493.624 & 1495.451 & 1493.6 & 1484.785 & 1479.327\end{array}$ $\begin{array}{lllllllllll}1487.889 & 1488.405 & 1493.866 & 1494.332 & 1493.624 & 1495.446 & 1493.603 & 1484.785 & 1479.33\end{array}$ $\begin{array}{lllllllllll}1487.896 & 1488.41 & 1493.866 & 1494.325 & 1493.624 & 1495.444 & 1493.603 & 1484.783 & 1479.33\end{array}$ $\begin{array}{llllllllll}1487.898 & 1488.417 & 1493.862 & 1494.318 & 1493.622 & 1495.427 & 1493.6 & 1484.783 & 1479.327\end{array}$ $\begin{array}{llllllllll}1487.877 & 1488.405 & 1493.862 & 1494.323 & 1493.617 & 1495.434 & 1493.6 & 1484.785 & 1479.325\end{array}$ $\begin{array}{llllllllll}1487.877 & 1488.403 & 1493.85 & 1494.323 & 1493.622 & 1495.437 & 1493.598 & 1484.785 & 1479.323\end{array}$

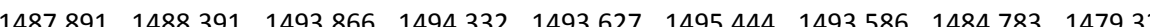
$\begin{array}{llllllllll}1487.866 & 1488.377 & 1493.873 & 1494.355 & 1493.639 & 1495.477 & 1493.593 & 1484.785 & 1479.325\end{array}$ $\begin{array}{llllllllll}1487.877 & 1488.377 & 1493.885 & 1494.376 & 1493.648 & 1495.503 & 1493.619 & 1484.783 & 1479.327\end{array}$ $\begin{array}{lllllllll}1487.889 & 1488.368 & 1493.897 & 1494.397 & 1493.66 & 1495.531 & 1493.631 & 1484.783 & 1479.334\end{array}$ $\begin{array}{lllllllllll}1487.85 & 1488.373 & 1493.904 & 1494.409 & 1493.669 & 1495.54 & 1493.645 & 1484.783 & 1479.339\end{array}$ $\begin{array}{llllllllll}1487.861 & 1488.387 & 1493.916 & 1494.414 & 1493.676 & 1495.547 & 1493.654 & 1484.783 & 1479.339\end{array}$ $\begin{array}{rrrrrrrrr}1487.873 & 1488.382 & 1493.878 & 1494.416 & 1493.65 & 1495.55 & 1493.614 & 1484.78 & 1479.341 \\ 1487.88 & 1488.396 & 1493.497 & 1494.285 & 1493.288 & 1495.536 & 1493.272 & 1484.78 & 1479.339\end{array}$ 
Well

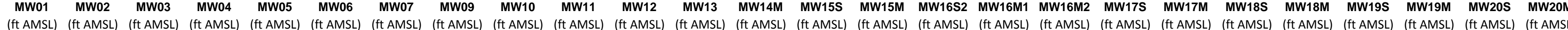

2/19/12 20:00 2/19/12 21:00 2/19/1222:00 2/120/12 20:00 2/20/120:00 2/20/12 2:00 2/20/12 3:00 2/20/12 4:00 2/20/12 5:00 2/20/12 6:00 2/20/12 7:00 2/20/129:00 2/20/12 9:00 2/20/12 11:00 2/20/12 12:00 2/20/12 14:00 2/20/12 16:00 2/20/12 17:00 2/20/12 19:00 2/20/12 20:00 2/20/12 21:00 2/20/12 22:00 2/20/12 23:00 2/21/120:00 2/21/121:00 2/21/12 3:00 2/21/12 4:00 2/21/12 5:00 2/21/12 6:00 2/21/12 7:00 $2 / 21 / 129000$ 2/21/1210:00 2/21/12 11:00 2/21/12 12:00 2/21/12 13:00 2/21/12 14:00 2/21/12 15:00 2/21/12 16:00 $2 / 21 / 1218.00$ 2/21/12 19:00 2/21/12 20:00 2/21/12 21:00 2/21/12 22:00 2/21/122 23:00 2/22/12 0:00 $\begin{array}{llll}1495.054 & 1495.982 & 1496.794 & 1495.471\end{array}$ $\begin{array}{lllll}1495.057 & 1495.989 & 1496.791 & 1495.457\end{array}$ $\begin{array}{lllll}1495.059 & 1495.998 & 1496.796 & 1495.455\end{array}$ $\begin{array}{lllll}1495.061 & 1496.033 & 1496.805 & 1495.453\end{array}$ $\begin{array}{llll}1495.057 & 1496.049 & 1496.798 & 1495.45\end{array}$ $\begin{array}{llll}1495.054 & 1496.063 & 1496.794 & 1495.448\end{array}$ $\begin{array}{llllll}1495.061 & 1496.042 & 1496.815 & 1495.455\end{array}$ $1495.068 \quad 1496.0191496 .845 \quad 1495.481$ $\begin{array}{lllll}1495.073 & 1496.01 & 1496.866 & 1495.497\end{array}$ $\begin{array}{rrrr}1495.075 & 1496.005 & 1496.88 & 1495.513 \\ 1495.08 & 1495.993 & 1496.894 & 149553\end{array}$ $\begin{array}{rrrr}1495.08 & 1495.993 & 1496.894 & 1495.53 \\ 1495.073 & 1496.003 & 1496.876 & 1495.525\end{array}$ $\begin{array}{lllll}1495.073 & 1496003 & 1496866 & 1455.525\end{array}$ $\begin{array}{lllll}1495.075 & 1496 & 1496.866 & 1495.525\end{array}$ $\begin{array}{lllll}1495.082 & 1495.984 & 1496.894 & 1495.537\end{array}$ $\begin{array}{lllll}1495.085 & 1495.963 & 1496.918 & 1495.555\end{array}$ $\begin{array}{llll}1495.089 & 1495.928 & 1496.939 & 1495.572\end{array}$ $\begin{array}{lllll}1495.103 & 1495.884 & 1496.985 & 1495.607\end{array}$ $\begin{array}{llll}1495.115 & 1495.854 & 1497.011 & 1495.635\end{array}$ $\begin{array}{llll}1495.103 & 1455.924 & 1496.974 & 1495.63\end{array}$ $1495.099 \quad 1495.961 \quad 14969391495.614$ $\begin{array}{llll}1495.092 & 1496.007 & 1496.899 & 1495.586\end{array}$ $\begin{array}{rrrr}1495.085 & 1496.047 & 1496.85 & 1495.551\end{array}$ $\begin{array}{llll}1495.075 & 1496.084 & 1496.808 & 1495.518\end{array}$ $\begin{array}{llll}1495.064 & 1496.129 & 1496.759 & 1495.474\end{array}$ $\begin{array}{lllll}1495.061 & 1496.157 & 1496.728 & 1495.439\end{array}$ $\begin{array}{rrrr}1495.057 & 1496.189 & 1496.712 & 1495.408 \\ 1495.043 & 1496.231 & 1496.67 & 1495371\end{array}$ $\begin{array}{lllll}1495.036 & 1496.259 & 1496.642 & 1495.341\end{array}$ $\begin{array}{lllll}1495.043 & 1496.252 & 1496.665 & 1495.338\end{array}$ $1495.033 \quad 1496.259 \quad 1496.658 \quad 1495.329$ $\begin{array}{lllll}1495.033 & 1496.252 & 1496.661 & 1495.327\end{array}$ $\begin{array}{lllll}1495.033 & 1496.248 & 1496.658 & 1495.32\end{array}$ $\begin{array}{lllll}1495.036 & 1496.229 & 1496.675 & 1495.327\end{array}$ $\begin{array}{llll}1495.029 & 1496.241 & 1496.656 & 1495.32\end{array}$ $\begin{array}{llll}1495.026 & 1496.208 & 1496.654 & 1495.317 \\ 1495.024 & 1496168 & 1496.649 & 1495315\end{array}$ $\begin{array}{lllll}1495.024 & 1496.115 & 1496.651 & 1495.315\end{array}$ $\begin{array}{lllll}1495.029 & 1496.061 & 1496.668 & 1495.322\end{array}$ $1495.038 \quad 1495.9791496 .712 \quad 1495.348$ $\begin{array}{lllll}1495.045 & 1495.903 & 1496.754 & 1495.378\end{array}$ $\begin{array}{llll}1495.045 & 1495.854 & 1496.782 & 1495.406\end{array}$ $1495.045 \quad 1495.837 \quad 1496.78 \quad 1495.418$ $\begin{array}{rrrr}1495.04 & 1495.844 & 1496.773 & 1495.422 \\ 1495.04 & 1495.865 & 1496.77 & 1495.425\end{array}$ $1495.04 \quad 1495.905 \quad 1496.763 \quad 1495.422$ $\begin{array}{llll}1495.043 & 1495.935 & 1496.768 & 1455.427\end{array}$ $1495.047 \quad 1495.951 \quad 1496.7911495 .441$ $1495.05 \quad 1495.963 \quad 1496.813 \quad 1495.455$ $\begin{array}{llll}1495.052 & 1495.979 & 1496.822 & 1495.462\end{array}$ $\begin{array}{llll}1495.054 & 1495.989 & 1496.836 & 1495.476 \\ 1495.057 & 1495.998 & 1496.845 & 1495.488\end{array}$ $\begin{array}{llllllllll}1494.242 & 1495.028 & 1495.206 & 1496.039 & 1497.175 & 1496.973 & 1493.322 & 1488.966 & 1493.144 & 1491.301\end{array}$

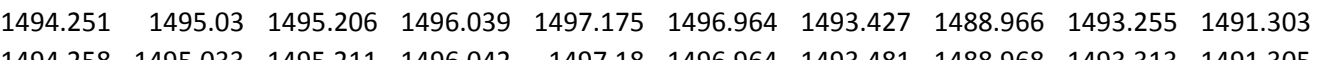
$\begin{array}{lllllllllllllll}1494.258 & 1495.033 & 1455.211 & 1496.042 & 1497.18 & 1496.964 & 1493.481 & 4888.968 & 1493.313 & 1491.305 \\ 1494268 & 1495036 & 1495211 & 1496048 & 1497.18 & 1496966 & 1493516 & 1488966 & 1493348 & 1491.308\end{array}$ $\begin{array}{lllllllllllll}1494.265 & 1495.04 & 1495.218 & 1496.055 & 1497.184 & 1496.976 & 1493.544 & 1488.973 & 1493.379 & 1491.31\end{array}$ $\begin{array}{llllllllllll}1494.28 & 1495.04 & 1495.216 & 1496.053 & 1497.184 & 1496.968 & 1493.561 & 1488.973 & 1493.395 & 1491.312\end{array}$ $\begin{array}{llllllllllll}1494.289 & 1495.04 & 1495.211 & 1496.051 & 1497.182 & 1496.954 & 1493.575 & 1488.97 & 1493.409 & 1491.312\end{array}$ $\begin{array}{lllllllllll}1494.275 & 1495.045 & 1495.223 & 1496.06 & 1497.194 & 1496.969 & 1493.594 & 1488.975 & 1493.433 & 1491.317\end{array}$ $\begin{array}{llllllllll}1494.258 & 1495.052 & 1495.237 & 1496.076 & 1497.21 & 1496.995 & 1493.617 & 1488.977 & 1493.452 & 1491.319\end{array}$

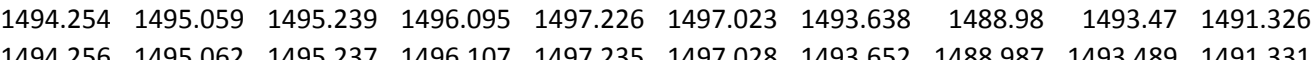

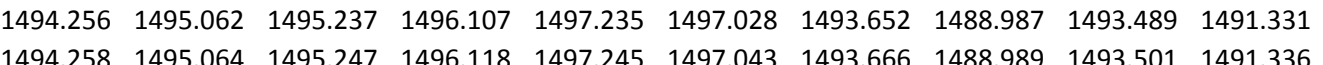
$\begin{array}{llllllllllll}1494.282 & 1495.066 & 1495.235 & 1496.113 & 1497.24 & 1497.021 & 1493.671 & 1488.994 & 1493.508 & 1491.336\end{array}$ $\begin{array}{llllllllll}1494.301 & 1495.069 & 1495.225 & 1496.116 & 1497.24 & 1497.014 & 1493.68 & 1488.994 & 1493.515 & 1491.34\end{array}$ $\begin{array}{llllllllll}1494.303 & 1495.071 & 1495.235 & 1496.116 & 1497.242 & 1497.004 & 1493.68 & 1488.994 & 1493.52 & 1491.34\end{array}$ $\begin{array}{llllllllllll}1494.291 & 1495.076 & 1495.247 & 1496.132 & 1497.256 & 1497.028 & 1493.689 & 1488.994 & 1493.529 & 1491.347\end{array}$ $\begin{array}{lllllllllll}1494.287 & 1495.076 & 1495.254 & 1496.153 & 1497.27 & 1497.064 & 1493.704 & 1489.008 & 1493.538 & 1491.347\end{array}$ $\begin{array}{llllllllll}1494.28 & 1495.081 & 1495.261 & 1496.167 & 1497.287 & 1497.067 & 1493.713 & 1489.003 & 1493.552 & 1491.352\end{array}$ $\begin{array}{lllllllllll}1494.258 & 1495.09 & 1495.277 & 1496.192 & 197.317 & 197.067 & 1493.713 & 1489.003 & 1493552 & 1491.3561\end{array}$

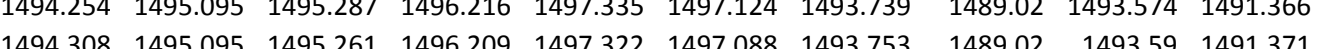
$\begin{array}{llllllllll}1494.308 & 1455.095 & 1495.261 & 1496.209 & 1497.322 & 1497.088 & 1493.753 & 1489.02 & 1493.59 & 1491.371 \\ 1494.317 & 1495.097 & 1495.266 & 1496.216 & 1497.328 & 1497.093 & 1493.762 & 1489.029 & 1493.602 & 1491.373\end{array}$ $\begin{array}{llllllllll}1494.357 & 1495.1 & 1495249 & 1496209 & 1497317 & 1497.062 & 1493.764 & 1489.024 & 1493.604 & 1491.378\end{array}$ $\begin{array}{llllllllllll}1494.397 & 1495.1 & 1495.23 & 1496.195 & 1497.301 & 1497.016 & 1493.76 & 1489.02 & 1493.599 & 1491.378\end{array}$ $\begin{array}{lllllllllll}1494.432 & 1495.095 & 1495.218 & 1496.172 & 1497.275 & 1496.969 & 1493.748 & 1489.013 & 1493.588 & 1491.375\end{array}$ $\begin{array}{llllllllll}1494.458 & 1495.088 & 1495.211 & 1496.153 & 1497.252 & 1496.928 & 1493.734 & 1488.996 & 1493.576 & 1491.371\end{array}$ $\begin{array}{rrrrrrrrrrr}1494.484 & 1495.081 & 1495.199 & 1496.12 & 1497.222 & 1496.878 & 1493.715 & 1488.991 & 1493.562 & 1491.366\end{array}$ $\begin{array}{llllllllll}1494.489 & 1495.073 & 1495.197 & 1496.102 & 1497.201 & 1496.854 & 1493.699 & 1488.977 & 1493.543 & 1491.359 \\ 1494.486 & 1495062 & 1495204 & 1496.083 & 1497.184 & 1496837 & 1493689 & 1488.963 & 1493531 & 1491354\end{array}$ $\begin{array}{lllllllllll}1494.486 & 1495.062 & 1495.204 & 1496.083 & 1497.184 & 1496.837 & 1493.689 & 1488.963 & 1493.531 & 1491.354 \\ 1494.498 & 1495.054 & 1495.189 & 1496053 & 1497.154 & 1496801 & 1493673 & 1488.954 & 1493515 & 1491.343\end{array}$ $\begin{array}{llllllllllll}1494.493 & 1495.045 & 1495.187 & 1496.03 & 1497.133 & 1496.777 & 1493.659 & 1488.945 & 1493.501 & 1491.336\end{array}$ $\begin{array}{lllllllllll}1494.454 & 1495.042 & 1495.209 & 1496.03 & 1497.133 & 1496.806 & 1493.657 & 1488.94 & 1493.501 & 1491.331\end{array}$ $\begin{array}{llllllllll}1494.444 & 1495.035 & 1495.206 & 1496.018 & 1497.124 & 1496.803 & 1493.652 & 1488.938 & 1493.496 & 1491.322\end{array}$ $\begin{array}{lllllllllll}1494.423 & 1495.03 & 1495.211 & 1496.009 & 1497.119 & 1496.803 & 1493.65 & 1488.935 & 1493.496 & 1491.317\end{array}$ $\begin{array}{llllllllll}1494.409 & 1495.026 & 1495.211 & 1496 & 1497.112 & 1496.806 & 1493.645 & 1488.928 & 1493.489 & 1491.31\end{array}$ $\begin{array}{lllrllllll}1494.383 & 1495.026 & 1495.22 & 1496 & 1497.112 & 1496.825 & 1493.647 & 1488.93 & 1493.491 & 1491.308 \\ 1493385 & 1495021 & 1495211 & 1495.988 & 1997.103 & 1496811 & 1493638 & 1488.928 & 1493487 & 141301\end{array}$ $\begin{array}{llllllllll}1494.385 & 1495.021 & 1495.211 & 1495.988 & 1497.103 & 1496.811 & 1493.638 & 1488.928 & 1493.487 & 1491.301 \\ 1494.376 & 1495.018 & 1495209 & 1495981 & 1497.096 & 1496818 & 1493.636 & 1488926 & 1493.48 & 1491294\end{array}$ $\begin{array}{llllllllll}1494.364 & 1495.011 & 1495211 & 1495974 & 1497.091 & 1496813 & 1493.631 & 1488.926 & 1493.475 & 1491.291\end{array}$ $\begin{array}{lllllllllll}1494.352 & 1495.009 & 1495.211 & 1495.972 & 1497.089 & 1496.823 & 1493.626 & 1488.923 & 1493.47 & 1491.287\end{array}$ $\begin{array}{lllllllllll}1494.331 & 1495.009 & 1495.22 & 1495.972 & 1497.091 & 1496.837 & 1493.626 & 1488.923 & 1493.473 & 1491.285\end{array}$ $\begin{array}{llllllllll}1494.291 & 1495.011 & 1495.237 & 1495.988 & 1497.115 & 1496.88 & 1493.636 & 1488.93 & 1493.477 & 1491.285\end{array}$ $\begin{array}{llllllllll}1494.261 & 1495.021 & 1495.249 & 1496.004 & 1497.133 & 1496.923 & 1493.647 & 1488.942 & 1493.491 & 1491.285\end{array}$ $\begin{array}{llllllllll}1494.251 & 1495.028 & 1495.249 & 1496.018 & 1497.147 & 1496.954 & 1493.659 & 1488.952 & 1493.503 & 1491.285\end{array}$ $\begin{array}{lllllllllll}1494.263 & 1495.033 & 1495.242 & 1496.023 & 1497.149 & 1496.949 & 1493.668 & 1488.956 & 1493.51 & 1491.289 \\ 1494.28 & 1495.038 & 1495235 & 1496023 & 1497.152 & 1496945 & 1493673 & 1488959 & 1493515 & 1491.289\end{array}$ $\begin{array}{llllllllllll}1494.28 & 1495.038 & 1455.235 & 1496.023 & 1497.152 & 1466.945 & 1493.67 & 1488.959 & 1493.515 & 1491.289\end{array}$ $\begin{array}{llllllllllll}1494.308 & 1495.04 & 1495.23 & 1496.021 & 1497.149 & 1496.933 & 1493.675 & 1488.956 & 1493.52 & 1491.291\end{array}$ $\begin{array}{lllllllllll}1494.31 & 1495.042 & 1495.237 & 1496.025 & 1497.154 & 1496.94 & 1493.678 & 1488.963 & 1493.52 & 1491.294\end{array}$ $\begin{array}{llllllllll}1494.303 & 1495.047 & 1495.242 & 1496.035 & 1497.163 & 1496.959 & 1493.682 & 1488.966 & 1493.524 & 1491.298\end{array}$ $\begin{array}{llllllllll}1494.296 & 1495.052 & 1495.249 & 1496.046 & 1497.177 & 1496.978 & 1493.685 & 1488.977 & 1493.527 & 1491.301\end{array}$ $\begin{array}{llllllllllll}1494.301 & 1495.059 & 1495.249 & 1496.053 & 1497.184 & 1496.99 & 1493.694 & 1488.97 & 1493.536 & 1491.303\end{array}$ $\begin{array}{llllllllll}1494.298 & 1495.062 & 1495.256 & 1496.065 & 1497.194 & 1497 & 1493.699 & 1488.984 & 1493.541 & 1491.308\end{array}$ $\begin{array}{llllllllll}1487.889 & 1488.408 & 1493.549 & 1494.253 & 1493.331 & 1495.517 & 1493.319 & 1484.78 & 1479.337\end{array}$ $\begin{array}{llllllllll}1487.896 & 1488.41 & 1493.664 & 1494.292 & 1493.439 & 1495.51 & 1493.419 & 1484.783 & 1479.334 \\ 1487.903 & 1488.41 & 1493.72 & 1492311 & 1493.489 & 1495.517 & 1493.469 & 188478 & 147932\end{array}$ $\begin{array}{llllllllll}1487.903 & 1488.41 & 1493.72 & 1494.311 & 1493.489 & 1495.517 & 1493.469 & 1484.78 & 1479.332 \\ 1487.91 & 1488.424 & 1493.756 & 1494332 & 1493.527 & 1495.51 & 1493.509 & 1484.78 & 1479.332\end{array}$ $\begin{array}{llllllllll}1487.914 & 1488.424 & 1493.781 & 1494.339 & 1493.553 & 1495.519 & 1493.53 & 1484.783 & 1479332\end{array}$ $\begin{array}{lllllllllll}1487.919 & 1488.434 & 1493.803 & 1494.341 & 1493.57 & 1495.514 & 1493.551 & 1484.778 & 1479.334\end{array}$ $\begin{array}{llllllllll}1487.926 & 1488.441 & 1493.817 & 1494.346 & 1493.584 & 1495.51 & 1493.563 & 1484.778 & 1479.334\end{array}$ $\begin{array}{llllllllll}1487.933 & 1488.431 & 1493.836 & 1494.372 & 1493.603 & 1495.533 & 1493.574 & 1484.783 & 1479.3396\end{array}$ $\begin{array}{llllllllll}1487.944 & 1488.427 & 1493.857 & 1494.393 & 1493.627 & 1495.559 & 1493.595 & 1484.783 & 1479.346\end{array}$ $\begin{array}{lllllllll}1487.956 & 1488.429 & 1493.878 & 1494.416 & 1493.648 & 1495.578 & 1493.619 & 1484.783 & 1479.353\end{array}$ $\begin{array}{llllllllll}1487.949 & 1488.436 & 1493.897 & 1494.43 & 1493.66 & 1495.594 & 1493.633 & 1484.78 & 1479.358 \\ 1487.956 & 1488.411 & 1493.908 & 1494.446 & 1493676 & 1495608 & 1493.649 & 1484783 & 1479358\end{array}$

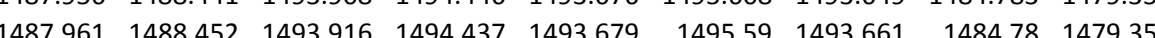
$\begin{array}{llllllllll}1487.963 & 1488.471 & 1493.918 & 1494.437 & 1493.686 & 1495.587 & 1493.666 & 1484.778 & 1479.358\end{array}$ $\begin{array}{llllllllll}1487.967 & 1488.464 & 1493.922 & 1494.439 & 1493.691 & 1495.583 & 1493.652 & 1484.783 & 1479.35\end{array}$ $\begin{array}{llllllllll}1487.977 & 1488.455 & 1493.934 & 1494.456 & 1493.698 & 1495.608 & 1493.664 & 1484.78 & 1479.358\end{array}$ $\begin{array}{llllllllll}1487.949 & 1488.462 & 1493.946 & 1494.472 & 1493.712 & 1495.627 & 1493.689 & 1484.776 & 1479.36\end{array}$ $\begin{array}{lllllllll}1487.951 & 1488.455 & 1493.958 & 1494.493 & 1493.724 & 1495.651 & 1493.682 & 1484.778 & 1479.362\end{array}$ $\begin{array}{llllllllll}1487.967 & 1488.441 & 1493.977 & 4994.526 & 1493.743 & 1495.686 & 1493.701 & 1484.78 & 1479.367\end{array}$

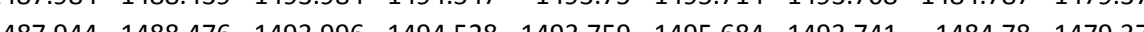
$\begin{array}{llllllllll}1487958 & 1488.485 & 1494.012 & 1494.535 & 1493.771 & 1495.693 & 1493.746 & 1484.778 & 1479.379\end{array}$ $\begin{array}{llllllllll}1487.97 & 1488.53 & 1494.005 & 1494.495 & 1493.769 & 1495.622 & 1493.743 & 1484.776 & 1479.376\end{array}$ $\begin{array}{llllllllll}1487.979 & 1488.544 & 1493.993 & 1494.465 & 1493.755 & 1495.585 & 1493.732 & 1484.776 & 1479.372\end{array}$ $\begin{array}{lllllllll}1487.986 & 1488.556 & 1493.979 & 1494.437 & 1493.745 & 1495.55 & 1493.72 & 1484.776 & 1479.365\end{array}$ $\begin{array}{llllllllll}1487.981 & 1488.565 & 1493.965 & 1494.402 & 1493.726 & 1495.503 & 1493.701 & 1484.773 & 1479.358\end{array}$ $\begin{array}{lllllllllll}1487.981 & 1488.56 & 4493.951 & 1494.374 & 1493.71 & 1455.474 & 1493.687 & 1484.773 & 1479.351\end{array}$

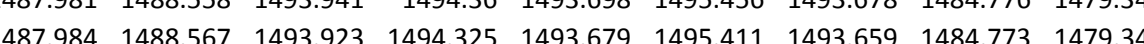
$\begin{array}{lllllllllll}1487.984 & 1488.57 & 1493.908 & 1494.306 & 1493.667 & 1495.385 & 1493.647 & 1484.771 & 1479.337\end{array}$ $\begin{array}{lllllllll}487.991 & 1488.544 & 1493.911 & 1494.316 & 1493.665 & 1495.404 & 1493.647 & 1484.773 & 1479.341\end{array}$ $\begin{array}{lllllllll}487.998 & 1488.546 & 1493.906 & 1494.309 & 1493.66 & 1495.395 & 1493.64 & 1484.769 & 1479.344\end{array}$ $\begin{array}{llllllllll}4488.002 & 1488.535 & 1493.904 & 1494.311 & 1493.662 & 1495.399 & 1493.635 & 1484.769 & 1479.344\end{array}$ $\begin{array}{lllllllllll}1487.995 & 1488.53 & 1493.895 & 1494.302 & 1493.658 & 1495.397 & 1493.633 & 1484.773 & 1479.346\end{array}$ $\begin{array}{lllllllllll}1487.991 & 1488.518 & 1493.899 & 1494.318 & 1493.655 & 1455.409 & 1493.633 & 1484.771 & 1479.346\end{array}$

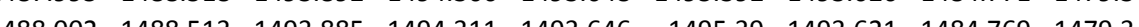
$\begin{array}{lllllllllll}1488.004 & 1488502 & 1493885 & 1494.297 & 1493.641 & 1495.385 & 1493.614 & 1484.766 & 1479.337\end{array}$ $\begin{array}{llllllllll}1488.009 & 1488.492 & 1493.878 & 1494.297 & 1493.634 & 1495.39 & 1493.61 & 1484.769 & 1479.337\end{array}$ $\begin{array}{llllllllll}1488.014 & 1488.476 & 1493.876 & 1494.304 & 1493.639 & 1495.402 & 1493.605 & 1484.766 & 1479.332\end{array}$ $\begin{array}{llllllllll}1488.021 & 1488.45 & 1493.885 & 1494.332 & 1493.643 & 1495.437 & 1493.621 & 1484.769 & 1479.334\end{array}$ $\begin{array}{lllllllllll}1488.028 & 1488.431 & 1493.899 & 1494.36 & 1493.655 & 1495.477 & 1493.635 & 1484.769 & 1479.339\end{array}$ $\begin{array}{llllllllll}1488.039 & 1488.434 & 1493.908 & 1494.381 & 1493.669 & 1495.496 & 1493.645 & 1484.766 & 1479.344\end{array}$ $\begin{array}{llllllllll}1488.044 & 1488.441 & 1493.918 & 1494.383 & 1493.67 & 1495.498 & 1493.655 & 1484.766 .766 & 1479.3446\end{array}$

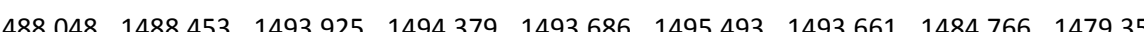
$\begin{array}{llllllllllll}4488.048 & 1488.452 & 1493.925 & 1494.381 & 1493.684 & 1495.496 & 1493.661 & 1484.766 & 1479.353\end{array}$ $\begin{array}{lllllllll}1488.053 & 1488.45 & 1493.927 & 1494.386 & 1493.684 & 1495.496 & 1493.664 & 1484.766 & 1479.353\end{array}$ $\begin{array}{llllllllll}1488.048 & 1488.443 & 1493.932 & 1494.4 & 1493.691 & 1495.519 & 1493.666 & 1484.766 & 1479.353\end{array}$ $\begin{array}{lllllllllll}1488.037 & 1488.439 & 1493.937 & 1494.414 & 1493.695 & 1495.536 & 1493.673 & 1484.766 & 1479.351\end{array}$ $\begin{array}{lllllllllll}1488.018 & 1488.448 & 1493.941 & 1494.423 & 1493.7 & 1495.543 & 1493.678 & 1484.766 & 1479.351\end{array}$

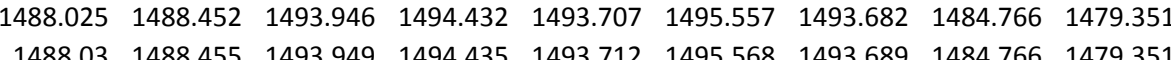


Well

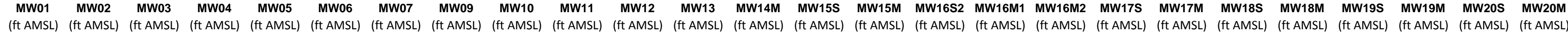

2/22/12 2:00 2/22/123:00 2/22/12 4:00 2/222/125:00 2/22/12 7:00 2/222/127:00 2/22/129:00 2/22/12 10:00 2/22/12 11:00 2/22/12 12:00 2/22/12 14:00 2/22/12 15:00 2/22/12 15:00 2/22/12 17:00 2/22/12 18:00 2/222/12 19:00 2/22/12 20:00 2/22/12 21:00 2/22/122 22:12 23:00 2/23/12 0:00 2/23/120:00 2/23/12 2:00 2/23/12 3:00 2/23/12 4:00 2/23/125:00 2/23/12 6:00 2/23/12 8:00 2/23/12 9:00 2/23/12 10:00 2/23/12 11:00 2/23/12 12:00 2/23/12 13:00 2/23/12 15:00 2/23/12 17:00 2/23/12 18:00 2/23/12 19:00 2/23/12 20:00 2/23/12 21:00 2/23/12 22:00 $2 / 24 / 120000$ 2/24/120:00 2/24/12 1:00 2/24/12 3:00 2/24/12 4:00 2/24/12 5:00 2/24/12 6:00 $\begin{array}{rrrr}1495.057 & 1496.007 & 1496.85 & 1495.495 \\ 1495.059 & 1496.012 & 1496854 & 1495.504\end{array}$ $\begin{array}{rrrr}1495.059 & 1496.012 & 1496.854 & 1495.504 \\ 1495066 & 1496 & 1968878 & 1995.518\end{array}$ $\begin{array}{rrrrr}1495.066 & 1496 & 1496.878 & 1495.518\end{array}$ $\begin{array}{llll}1495.066 & 1495.998 & 1496.893 & 1495.532 \\ 1496.883 & 1495.537\end{array}$ $\begin{array}{lllll}1495.071 & 1495.989 & 1496.892 & 14955.541\end{array}$ $\begin{array}{lllll}1495.071 & 1495.989 & 1496.892 & 1495.541 \\ 1495.071 & 1495.986 & 1496.887 & 1495.544\end{array}$ $\begin{array}{lllll}1495.073 & 1495.956 & 1496.894 & 1495.548\end{array}$ $\begin{array}{llll}1495.073 & 1495.917 & 1496.897 & 1495.553\end{array}$ $\begin{array}{rrrr}1495.075 & 1495.865 & 1496.894 & 1495.553 \\ 149508 & 1455798 & 196911 & 14955\end{array}$

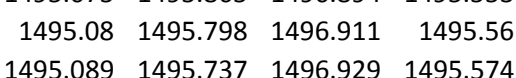
$\begin{array}{llll}1495.089 & 1495.737 & 1496.929 & 1495.574 \\ 1495.089 & 1495.683 & 1496.955 & 1495.595\end{array}$ $\begin{array}{lllll}1495.096 & 1495.651 & 1496.967 & 1495.604\end{array}$ $\begin{array}{llll}1495.096 & 1495.641 & 1496.971 & 1495.618\end{array}$ $\begin{array}{lllll}1495.0969 & 1459.641 & 1496.971 & 1495.618 \\ 1459.099 & 1495.653 & 1496.969 & 1495.621\end{array}$ $\begin{array}{llllll}1495.103 & 1495.676 & 1496.981 & 1495.628\end{array}$ $\begin{array}{llll}1495.106 & 1495.718 & 1496.981 & 1495.635\end{array}$ $\begin{array}{llll}1495.11 & 1495.756 & 1496.985 & 1495.639\end{array}$ $\begin{array}{llll}1495.12 & 1495.774 & 1497.011 & 1495.658 \\ 1495.122 & 1495.795 & 1497.028 & 1495.672\end{array}$ $\begin{array}{lllll}1455.131 & 1495.814 & 1497.056 & 1495.691\end{array}$ $\begin{array}{lllll}4495.138 & 1495.823 & 1497.081 & 1495.716\end{array}$ $\begin{array}{rrrrr}1495.129 & 1495.854 & 1497.081 .06 & 1495.716 \\ 1495.716\end{array}$ $\begin{array}{lllll}1495.14 & 1495.854 & 1497.072 & 1495.721\end{array}$ $\begin{array}{rrrr}1495.143 & 1495.877 & 1497.07 & 1495.723 \\ 1495.133 & 1499.917 & 1497.032 & 1495.705\end{array}$ $\begin{array}{llll}1495.133 & 1495.917 & 1497.032 & 1495.705 \\ 1495.145 & 1459.912 & 1497.065 & 1955721\end{array}$ $\begin{array}{llll}1495.145 & 1495.912 & 1497.065 & 1495.721 \\ 1495.143 & 1495.947 & 1497.051 & 1495714\end{array}$ $\begin{array}{lllll}1495.143 & 1495.947 & 1497.051 & 1495.714 \\ 1495.133 & 1495.986 & 1497.009 & 1495.693\end{array}$ $\begin{array}{llll}1495.12 & 1496.024 & 1496.929 & 1495.639\end{array}$ $\begin{array}{llll}1495.113 & 1496.024 & 1496.887 & 1495.639\end{array}$ $\begin{array}{llll}1495.108 & 1496.01 & 1496.85 & 1495.56\end{array}$ $\begin{array}{llll}1495.096 & 1496.01 & 1496.813 & 1495.52\end{array}$ $\begin{array}{lllll}1495.103 & 1495.968 & 1496.817 & 1495.502\end{array}$ $\begin{array}{llll}1495.103 & 1495.949 & 1496.801 & 1495.478 \\ 1495.096 & 1495.928 & 1496796 & 195.46\end{array}$ $\begin{array}{llll}1495.096 & 145.928 & 1496.796 & 1495.469\end{array}$ $\begin{array}{lllll}1495.082 & 1495.97 & 1496.742 & 1495.429 \\ 1495.075 & 1495.984 & 1496712 & 1495.399\end{array}$ $\begin{array}{llll}1495.071 & 1496.003 & 1496.689 & 1495.371\end{array}$ $\begin{array}{lllll}1495.057 & 1496.047 & 1496.647 & 1495.338\end{array}$ $\begin{array}{lllll}1495.047 & 1496.096 & 1496.616 & 1495.308\end{array}$ $\begin{array}{lllll}1495.043 & 1496.136 & 1496.586 & 1495.275\end{array}$ $1495.036 \quad 1496.159 \quad 1496.569 \quad 1495.252$

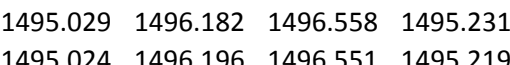
$\begin{array}{llll}1495.024 & 1496.196 & 1496.551 & 1495.219\end{array}$ $\begin{array}{llll}1495.012 & 1496.215 & 1496.53 & 1495.191\end{array}$ $\begin{array}{llll}1495.005 & 1496.224 & 1496.511 & 1455.175\end{array}$ $1495.008 \quad 1496.224 \quad 1496.504 \quad 1495.163$ $1495.003 \quad 1496.213 \quad 1496.506 \quad 1495.156$ $1494.998 \quad 1496.21 \quad 1496.499 \quad 1495.151$ $\begin{array}{llll}1494.996 & 1496.201 & 1496.492 & 1495.147 \\ 1494.984 & 1496.196 & 1496.471 & 1495.13\end{array}$ $\begin{array}{llllllllll}1494.315 & 1495.071 & 1495.256 & 1496.076 & 1497.205 & 1497.012 & 1493.713 & 1488.989 & 1493.55 & 1491.315\end{array}$ $\begin{array}{lllllllllll}1494.32 & 1495.073 & 1495.258 & 1496.083 & 1497.21 & 1497.014 & 1493.715 & 1488.994 & 1493.559 & 1491.317\end{array}$ $\begin{array}{llllllllll}1494.315 & 1495.081 & 1495.265 & 1496.097 & 1497.224 & 1497.033 & 1493.727 & 1488.991 & 1493.571 & 1491.324 \\ 1494.322 & 1495085 & 1495268 & 1496104 & 1497.233 & 1497.04 & 1493739 & 1489001 & 1493581 & 1491.326\end{array}$ $\begin{array}{lllllllllll}1494.336 & 1495.088 & 1495.258 & 1496.107 & 1497.233 & 1497.033 & 1493.743 & 1489.005 & 1493.585 & 1491.333\end{array}$ $\begin{array}{lllllllllll}1494.336 & 1495.093 & 1495.266 & 1496.118 & 1497.24 & 1497.043 & 1493.75 & 1489.003 & 1493.59 & 1491.338\end{array}$ $\begin{array}{llllllllllll}1494.35 & 1495.097 & 1495.263 & 1496.118 & 1497.242 & 1497.038 & 1493.755 & 1489.008 & 1493.592 & 1491.343\end{array}$ $\begin{array}{lllllllllll}1494.35 & 1495.1 & 1495.268 & 1496.125 & 1497.249 & 1497.043 & 1493.757 & 1489.005 & 1493.597 & 1491.345\end{array}$ $\begin{array}{llllllllllll}1494.357 & 1495.102 & 1495.266 & 1496.13 & 1497.252 & 1497.04 & 1493.76 & 1489.008 & 1493.599 & 1491.35\end{array}$ $\begin{array}{lllllllllll}1494.364 & 1495.102 & 1495.27 & 1496.132 & 1497.254 & 1497.031 & 1493.757 & 1489.008 & 1493.597 & 1491.352\end{array}$ $\begin{array}{llllllllll}1494.355 & 1495.105 & 1495.273 & 1496.141 & 1497.263 & 1497.047 & 1493.762 & 1489.013 & 1493.604 & 1491.354 \\ 1494.345 & 1495.109 & 1495.284 & 1496155 & 1497.277 & 1497.064 & 1493.769 & 1489017 & 1493609 & 1491359\end{array}$ $\begin{array}{llllllllllll}1494.338 & 1495.114 & 1495.294 & 1496.174 & 1497.291 & 1497.091 & 1493.781 & 1489.027 & 1493.62 & 1491.364\end{array}$ $\begin{array}{llllllllll}1494.341 & 1495.117 & 1495.289 & 1496.188 & 1497.303 & 1497.1 & 1493.79 & 1489.029 & 1493.632 & 1491368\end{array}$ $\begin{array}{lllllllllll}1494.35 & 1495.119 & 1495.292 & 1496.197 & 1497.312 & 1497.103 & 1493.799 & 1489.027 & 1493.641 & 1491.373\end{array}$ $\begin{array}{lllllllllll}1494.364 & 1495.121 & 1495.292 & 1496.199 & 1497.312 & 1497.098 & 1493.806 & 1489.029 & 1493.646 & 1491.378\end{array}$ $\begin{array}{lllllllllll}1494.371 & 1495.126 & 1495.294 & 1496.211 & 1497.324 & 1497.107 & 1493.811 & 1489.036 & 1493.653 & 1491.382\end{array}$ $\begin{array}{llllllllll}1494.383 & 1495.129 & 1495.296 & 1496.218 & 1497.328 & 1497.107 & 1493.81 & 1489.036 & 1493.663 & 1491.387\end{array}$ $\begin{array}{llllllllll}1494.397 & 1495.133 & 1495.294 & 1496.223 & 1497.333 & 1497.103 & 1493.823 & 1489.043 & 1493.665 & 1491.389\end{array}$ $\begin{array}{llllllllll}1494.388 & 1495.136 & 1495.311 & 1496.239 & 1497.352 & 1497.124 & 1493.83 & 1489.045 & 1493.672 & 1491.394 \\ 1494.392 & 1495.141 & 1495313 & 1496257 & 1497.361 & 1497141 & 1493.839 & 1489.043 & 1493.679 & 1491.399\end{array}$ $\begin{array}{llllllllllll}1494.388 & 1495.146 & 1495.322 & 1496.276 & 1497.38 & 1497.162 & 1493.846 & 1489.05 & 1493.686 & 1491.406\end{array}$ $\begin{array}{llllllllll}1494.388 & 1495.15 & 1495.33 & 1496.301 & 1497.398 & 1497.191 & 1493.86 & 1489.055 & 1493.7 & 1491.413\end{array}$ $\begin{array}{llllllllllll}1494.423 & 1495.155 & 1495.313 & 1496.299 & 1497.398 & 1497.17 & 1493.865 & 1489.062 & 1493.707 & 1491.415\end{array}$ $\begin{array}{lllllllllll}1494.425 & 1495.157 & 1495.32 & 1496.311 & 1497.408 & 1497.172 & 1493.87 & 1489.064 & 1493.712 & 1491.42\end{array}$ $\begin{array}{llllllllll}1494.439 & 1495.162 & 1495.318 & 1496.311 & 1497.41 & 1497.16 & 1493.877 & 1489.064 & 1493.717 & 1491.422\end{array}$ $\begin{array}{llllllllll}1494.484 & 1495.164 & 1495.301 & 1496.301 & 1497.398 & 1497.131 & 1493.874 & 1489.066 & 1493.719 & 1491.424 \\ 1494.47 & 1495.167 & 1495.32 & 149632 & 197.415 & 1497.16 & 149388 & 1489.073 & 1993.73 & 191.43\end{array}$

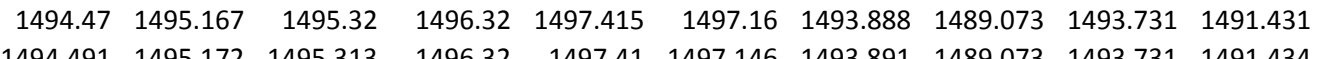
$\begin{array}{rrrrrrrrrr}1494.491 & 1495.172 & 1495.313 & 1496.32 & 1497.41 & 1497.146 & 1493.891 & 1489.073 & 1493.731 & 1491.434 \\ 1494.529 & 1495.172 & 1495289 & 1496306 & 1497.391 & 1497.11 & 1493.886 & 1489.069 & 1493.728 & 1491.431\end{array}$ $\begin{array}{lllllllllllll}1494.588 & 1495.164 & 1495.256 & 1496.267 & 1497.356 & 1497.019 & 1493.867 & 1489.05 & 1493.712 & 1491.429\end{array}$ $\begin{array}{llllllllll}1494.604 & 1495.16 & 1495.251 & 1496.248 & 1497.328 & 1496.983 & 1493.851 & 1489.038 & 1493.698 & 1491.424\end{array}$ $\begin{array}{llllllllll}1494.618 & 1495.155 & 1495.247 & 1496.223 & 1497.303 & 1496.942 & 1493.832 & 1489.031 & 1493.679 & 1491.42\end{array}$ $\begin{array}{lllllllllll}1494.625 & 1495.148 & 1495.244 & 1496.199 & 1497.275 & 1496.916 & 1493.816 & 1489.01 & 1493.66 & 1491.413\end{array}$ $\begin{array}{lllllllllll}1494.597 & 1495.141 & 1495.261 & 1496.188 & 1497.27 & 1496.909 & 1493.802 & 1489.015 & 1493.649 & 1491.406\end{array}$ $\begin{array}{llllllllll}1494.58 & 1495.136 & 1495.261 & 1496.172 & 1497.256 & 1496.892 & 1493.79 & 1489.001 & 1493.639 & 1491.401 \\ 1494569 & 1495.131 & 1495.261 & 149616 & 1497.242 & 1496899 & 1493.783 & 1488996 & 149363 & 1491394\end{array}$

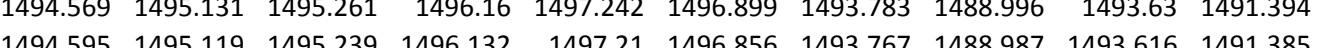
$\begin{array}{rrrrrrrrrrr}1444.595 & 1495.114 & 1495.232 & 1496.104 & 1497.187 & 1496.827 & 1493.755 & 1488.977 & 1493.599 & 1491.373\end{array}$ $\begin{array}{llllllllllll}1494.588 & 1495.1 & 1495.235 & 1496.079 & 1497.168 & 1496.808 & 1493.739 & 1488.966 & 1493.588 & 1491.364\end{array}$ $\begin{array}{llllllllll}1494.597 & 1495.093 & 1495.225 & 1496.051 & 1497.138 & 1496.777 & 1493.722 & 1488.959 & 1493.569 & 1491.354\end{array}$ $\begin{array}{lllllllllll}1494.595 & 1495.081 & 1495.22 & 1496.023 & 1497.11 & 1496.76 & 1493.706 & 1488.945 & 1493.555 & 1491.343\end{array}$ $\begin{array}{lllllllllll}1494.588 & 1495.069 & 1495.218 & 1495.993 & 1497.084 & 1496.727 & 1493.687 & 1488.94 & 1493.536 & 1491.331\end{array}$ $\begin{array}{lllllllllll}1494.571 & 1495.059 & 1495.218 & 1495.967 & 1497.068 & 1496.708 & 1493.666 & 1488.933 & 1493.517 & 1491.322\end{array}$ $\begin{array}{lllllllllll}1494.552 & 1495.05 & 1495.216 & 1495.951 & 1497.049 & 1496.715 & 1493.652 & 1488.928 & 1493.503 & 1491.31\end{array}$ $\begin{array}{lllllllllll}1494.529 & 1495.042 & 1495.22 & 145.935 & 147.038 & 1496714 & 1493.64 & 1488.923 & 1493.489 & 1491.298\end{array}$ $\begin{array}{llllllllllll}1494.496 & 1495.026 & 1495.218 & 1495.898 & 1497.01 & 1496.703 & 1493.615 & 1488.907 & 1493.461 & 1491.278\end{array}$ $\begin{array}{llllllllll}1494.484 & 1495.016 & 1495.211 & 1495.877 & 1496.991 & 1496.691 & 1493.598 & 1488.9 & 1493.449 & 1491.268\end{array}$ $\begin{array}{llllllllllll}1494.463 & 1495.009 & 1495.211 & 1495.863 & 1496.982 & 1496.691 & 1493.591 & 1488.893 & 1493.44 & 1491.259\end{array}$ $\begin{array}{llllllllll}1494.444 & 1495.004 & 1495.213 & 1495.854 & 1496.973 & 1496.698 & 1493.584 & 1488.898 & 1493.433 & 1491.25\end{array}$ $\begin{array}{llllllllllll}1494.428 & 1494.997 & 1495.216 & 1495.84 & 1496.966 & 1496.698 & 1493.577 & 1488.886 & 1493.43 & 1491.24\end{array}$ $\begin{array}{lllllllllllll}1494.414 & 1494.99 & 1455.211 & 1495.83 & 1466.954 & 1466.701 & 1493.57 & 1488.886 & 1493.419 & 1491.233\end{array}$ $\begin{array}{lllllllll}1488.039 & 1488.464 & 1493.958 & 1494.446 & 1493.721 & 1495.566 & 1493.694 & 1484.766 & 1479.353\end{array}$ $\begin{array}{lllllllll}1488.048 & 1488.469 & 1493.967 & 1494.449 & 1493.726 & 1495.575 & 1493.703 & 1484.766 & 1479.35\end{array}$

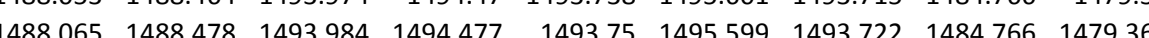
$\begin{array}{lllllllllll}1488.058 & 1488.483 & 1493.989 & 1494.479 & 1493.752 & 1495.604 & 1493.727 & 1484.766 & 1479.369\end{array}$ $\begin{array}{llllllllll}1488.06 & 1488.488 & 1493.998 & 1494.488 & 1493.757 & 1495.62 & 1493.732 & 1484.769 & 1479.369\end{array}$ $\begin{array}{llllllllll}1488.065 & 1488.497 & 1494.005 & 1494.484 & 1493.759 & 1495.608 & 1493.736 & 1484.764 & 1479.372\end{array}$ $\begin{array}{llllllllll}1488.072 & 1488.495 & 1494.005 & 1494.491 & 1493.764 & 1495.62 & 1493.741 & 1484.766 & 1479.372\end{array}$ $\begin{array}{lllllllll}1488.076 & 1488.502 & 1494.007 & 1494.493 & 1493.771 & 1495.613 & 1493.743 & 1484.764 & 1479.369\end{array}$ $\begin{array}{llllllllll}1488.083 & 1488.499 & 1494.007 & 1494.493 & 1493.766 & 1495.613 & 1493.743 & 1484.764 & 1479.367\end{array}$ $\begin{array}{llllllllll}1488.09 & 1488.492 & 1494.012 & 1494.5 & 1493.774 & 1495.627 & 1493.746 & 1484.764 & 1479.365\end{array}$ $\begin{array}{llllllllll}1488 & 106 & 1488.478 & 1494.024 & 1494.533 & 1493.788 & 1495.662 & 1493762 & 1484.764 & 1479.367\end{array}$

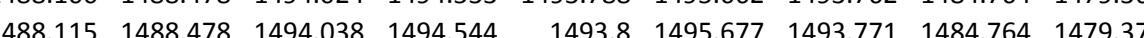
$\begin{array}{lllllllllll}1488.067 & 1488.483 & 1494.047 & 1494.551 & 1493.809 & 1495.688 & 1493.783 & 1484.764 & 1479.379\end{array}$ $\begin{array}{llllllllll}1488.062 & 1488.485 & 1494.054 & 1494.558 & 1493.814 & 1495.681 & 1493.79 & 1484.764 & 1479.379\end{array}$ $\begin{array}{lllllllllll}1488.067 & 1488.488 & 1494.064 & 1494.563 & 1493.819 & 1495.693 & 1493.8 & 1484.766 & 1479.388\end{array}$ $\begin{array}{llllllllll}1488.072 & 1488.497 & 1494.066 & 1494.577 & 1493.828 & 1495.7 & 1493.804 & 1484.764 & 1479.388\end{array}$

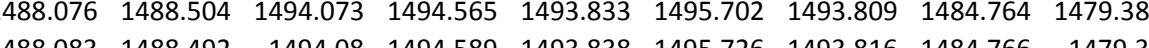
$\begin{array}{llllllllll}1488.083 & 4888.492 & 1494.08 & 1494.589 & 1493.838 & 1455.726 & 1493.816 & 1484.766 & 1479.39\end{array}$ $\begin{array}{llllllllll}1488.097 & 1488.502 & 1494.092 & 1494.617 & 1493.859 & 1495.761 & 1493.832 & 1484.766 & 1479.393\end{array}$ $\begin{array}{lllllll}1494.642 & 1493.868 & 1495.782 & 1493.847 & 1484.766 & 1479.395\end{array}$ $\begin{array}{llllllllll}1488.111 & 1488.544 & 1494.113 & 1494.628 & 1493.873 & 1495.768 & 1493.847 & 1484.764 & 1479.393\end{array}$ $\begin{array}{llllllllll}1488.118 & 1488.532 & 1494.123 & 1494.64 & 1493.88 & 1495.78 & 1493.856 & 1484.769 & 1479.395\end{array}$ $\begin{array}{lllllllllll}1488.125 & 1488.544 & 1494.125 & 1494.642 & 1493.885 & 1495.78 & 1493.861 & 1484.766 & 1479.397\end{array}$ $\begin{array}{llllllllll}1488.129 & 1488.574 & 1494.123 & 1494.617 & 1493.883 & 1495.756 & 1493.863 & 1484.766 & 1479.4\end{array}$ $\begin{array}{llllllllll}1488.141 & 1488.563 & 1494.137 & 1494.649 & 1493.897 & 1455.778 & 1493.872 & 1484.766 & 1479.404\end{array}$

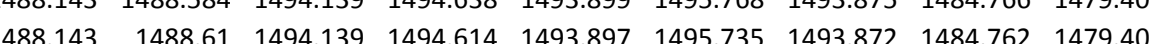
$\begin{array}{lllllllllll}1488.115 & 1488.631 & 1494.115 & 1494.563 & 1493.875 & 1495.669 & 1493.854 & 1484.764 & 1479.402\end{array}$ $\begin{array}{lllllllllll}1488.113 & 1488.636 & 1494.101 & 1494.535 & 1493.861 & 1495.625 & 1493.837 & 1484.759 & 1479.397\end{array}$ $\begin{array}{lllllllll}1488.09 & 1488.638 & 1494.085 & 1494.5 & 1493.845 & 1495.592 & 1493.818 & 1484.759 & 1479.388\end{array}$ $\begin{array}{lllllllllll}1488.092 & 1488.643 & 1494.066 & 1494.467 & 1493.828 & 1495.552 & 1493.797 & 1484.759 & 1479.386\end{array}$ $\begin{array}{llllllllll}1488.097 & 1488.615 & 1494.05 & 1494.47 & 1493.814 & 1495.545 & 1493.786 & 1484.762 & 1479.379\end{array}$ $\begin{array}{lllllllllll}1488.104 & 1488.61 & 1494.042 & 1494.453 & 1493.8 & 1495.543 & 1493.776 & 1484.762 & 1479.379\end{array}$

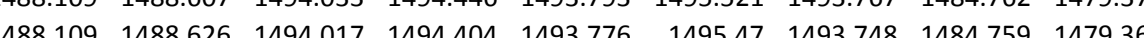
$\begin{array}{rrrrrrrrrrr}1488.06 & 1488.622 & 1494.007 & 1494.381 & 1493.762 & 1495.439 & 1493.739 & 1484.762 & 1479.367\end{array}$ $\begin{array}{llllllllll}1488.065 & 1488.612 & 1493.993 & 1494.365 & 1493.75 & 1495.427 & 1493.727 & 1484.759 & 1479.365\end{array}$ $\begin{array}{lllllllll}1488.067 & 1488.617 & 1493.974 & 1494.33 & 1493.731 & 1495.383 & 1493.706 & 1484.755 & 1479.362\end{array}$ $\begin{array}{llllllllll}1488.069 & 1488.614 & 1493.963 & 1494.306 & 1493.717 & 1495.355 & 1493.694 & 1484.755 & 1479.36\end{array}$ $\begin{array}{lllllllllll}4488.072 & 1488.603 & 1493.937 & 1494.283 & 1493.693 & 1495.324 & 1493.673 & 1484.759 & 1479.358\end{array}$ $\begin{array}{llllllllll}1488.072 & 1488.589 & 1493.923 & 1494.264 & 1493.679 & 1495.308 & 1493.656 & 1484.755 & 1479.353\end{array}$ $\begin{array}{llllllllll}1488.072 & 1488.584 & 1493.008 & 1494.248 & 1493.662 & 1455.289 & 1493.64 & 1484.755 & 1479.348\end{array}$

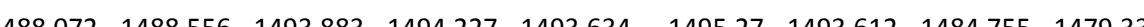
$\begin{array}{lllllllllll}1488.072 & 1488.544 & 1493.868 & 1494.22 & 1493.622 & 1495.258 & 1493.598 & 1484.755 & 1479.334\end{array}$ $\begin{array}{llllllllll}1488.069 & 1488.546 & 1493.857 & 1494.201 & 1493.61 & 1495.244 & 1493.586 & 1484.752 & 1479.332\end{array}$ $\begin{array}{llllllllll}1488.074 & 1488.53 & 1493.847 & 1494.197 & 1493.601 & 1495.239 & 1493.577 & 1484.752 & 1479.3\end{array}$ $\begin{array}{lllllllllll}1488.076 & 1488.521 & 1493.84 & 1494.185 & 1493.594 & 1495.232 & 1493.572 & 1484.755 & 1479.33\end{array}$ $\begin{array}{llllllllll}1488.078 & 1488.511 & 1493.833 & 1494.187 & 1493.589 & 1495.228 & 1493.565 & 1484.752 & 1479.33\end{array}$ $\begin{array}{llllllllll}1488.085 & 1488.504 & 1493.824 & 1494.176 & 1493.582 & 1495.228 & 1493.558 & 1484.752 & 1479.33 \\ 1488081 & 1488514 & 1493819 & 1494.162 & 1493.572 & 1495204 & 1493551 & 1484.75 & 1479.33\end{array}$ 
Well

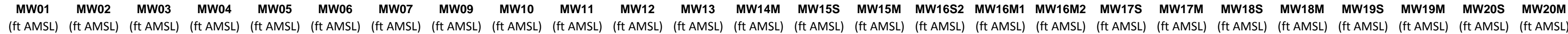

2/24/12 8:00 2/24/129:00 2/24/12 10:00 2/24/121212:00 2/24/12 12:00 2/24/12 14:00 2/24/12 15:00 2/24/12 16:00 2/24/12 17:00 2/24/12 18:00 2/24/12 19:00 2/24/12 20:00 2/24/122 21:00 2/24/12 23:00 2/25/12 0:00 2/25/12 1:00 2/25/122:00 2/25/12 3:00 2/25/12 4:00 /255/12 6:00 2/25/12 7:00 2/25/12 8:00 2/25/12 9:00 2/25/12 10:00 2/25/12 11:00 2/25/12 12:00 2/25/12 13:12 14:00 2/25/12 14:00 2/25/12 16:00 2/25/12 17:00 2/25/12 18:00 2/25/12 19:00 2/25/12 21:00

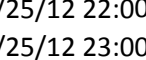
2/26/120:00 2/26/12 1:00 2/26/12 2:00 2/26/12 3:00 2/26/12 4:00 $2 / 26 / 126 \cdot 00$ 2/26/12 6:00 2/26/12 7:00 2/26/12 9:00 2/26/12 10:00 2/26/12 11:00 2/26/12 12:00 $\begin{array}{llll}1494.98 & 1496.182 & 1496.45 & 1495.114\end{array}$ $\begin{array}{llll}1444.98 & 1496.133 & 1496.424 & 1495.095 \\ 1494.977 & 1496.056 & 1496.424 & 1995088\end{array}$ $\begin{array}{rllll}1494.977 & 1496.056 & 1496.424 & 1495.088\end{array}$ $\begin{array}{rrrrr}1494.98 & 1495.97 & 1496.429 & 1495.086 \\ 1494.977 & 1495.898 & 1496.431 & 1495.088\end{array}$ $\begin{array}{lllll}1494.982 & 1495.823 & 1496.46 & 1455.088 \\ & 1495.1\end{array}$ $\begin{array}{rrrrr}1494.985 & 1495.758 & 1496.488 & 1495.123\end{array}$ $\begin{array}{lllll}1494.98 & 1495.711 & 1496.509 & 1495.142\end{array}$ $1494.977 \quad \begin{array}{llll}1495.695 & 1496.516 & 1495.154\end{array}$ $\begin{array}{rrrr}1494.978 & 1495.69 & 1496.53 & 1495.17 \\ 1494.97 & 1495.716 & 1496523 & 149.173\end{array}$ $\begin{array}{rrrr}1494.97 & 1495.716 & 1496.523 & 1495.173 \\ 1494.964 & 1495.772 & 1496.497 & 1495.163\end{array}$ $\begin{array}{llll}1494.964 & 1495.772 & 1496.497 & 1495.163 \\ 1494.961 & 1495.812 & 1496.469 & 1495.149\end{array}$ $\begin{array}{lllll}1494.957 & 1495.844 & 1496.457 & 1495.137\end{array}$ $\begin{array}{lllll}1494.954 & 1495.875 & 1496.445 & 1495.128\end{array}$ $\begin{array}{llll}1494.95 & 1495.903 & 1496.429 & 1495.116\end{array}$ $\begin{array}{lllll}1494.95 & 1495.912 & 1496.424 & 1495.107\end{array}$ $\begin{array}{lllll}1494.952 & 1495.912 & 1496.434 & 1495.109\end{array}$ $\begin{array}{lrrrr}1494.943 & 1495.919 & 1496.422 & 1495.102 \\ 1494.952 & 14959 & 1966488 & 1495\end{array}$ $\begin{array}{llll}1494.952 & 1495.9 & 1496.448 & 1495.114 \\ 1494.947 & 1495.9 & 1496.453 & 1495.121\end{array}$ $\begin{array}{rrrr}1494.947 & 1495.9 & 1496.453 & 1495.121 \\ 1494.943 & 1495.903 & 1496.448 & 1495.121\end{array}$ $\begin{array}{lllll}1494.94 & 1495898 & 1496453 & 1495.121\end{array}$ $\begin{array}{lllll}1494.94 & 1455.898 & 1496.453 & 1495.126 \\ 1494.94 & 1495.886 & 1496.467 & 1495.135\end{array}$ $\begin{array}{lllll}1494.938 & 1495.87 & 1496.469 & 1495.142\end{array}$ $\begin{array}{lllll}1494.936 & 1495.823 & 1496.469 & 1495.144\end{array}$ $\begin{array}{lllll}1494.94 & 1495.756 & 1496.492 & 1495.159\end{array}$ $\begin{array}{llll}1494.947 & 1495.662 & 1496.527 & 1495.187\end{array}$ $\begin{array}{rrrrr}1494.954 & 1495.576 & 1496.576 & 1495.219 \\ 1494.966 & 1495.49 & 1496.64 & 1495.266\end{array}$ $\begin{array}{lllll}1494.975 & 1495.425 & 1496.696 & 1495.315\end{array}$ $\begin{array}{lllll}1494.982 & 1495.38 & 1496.735 & 1495.357\end{array}$ $1494.987 \quad 1495.357 \quad 1496.761 \quad 1495.394$ $\begin{array}{lllll}1494.998 & 1495.352 & 1496.805 & 1495.429\end{array}$ $\begin{array}{llll}1495.001 & 1495.369 & 1496.82 & 1495.455\end{array}$ $\begin{array}{llll}1494.991 & 1495.422 & 1496.78 & 1495.448\end{array}$ $\begin{array}{lllll}1494.998 & 1495.453 & 1496.782 & 1495.457\end{array}$ $\begin{array}{rrrr}1495.003 & 1495.474 & 1496.789 & 1495.46 \\ 1495.001 & 1495.497 & 1496.789 & 1495.462\end{array}$ $\begin{array}{lllll}1495.003 & 1495.516 & 1496.794 & 1495.464\end{array}$ $\begin{array}{llllll}1495.005 & 1495.534 & 1496.801 & 1495.469\end{array}$ $\begin{array}{lllll}1495.01 & 1495.543 & 1496.815 & 1495.481\end{array}$ $1494.989 \quad 1495.606 \quad 1496.752 \quad 1495.45$ $1494.989 \quad 1495.655 \quad 1496.71 \quad 1495.418$ $\begin{array}{llll}1494.984 & 1495.704 & 1496.682 & 1495.387 \\ 1494.984 & 1495737 & 1996682 & 1995373\end{array}$ $1494.98 \quad 1495767 \quad 1466.6821495 .373$ $1494.975 \quad 1495.791 \quad 1496.658$ 1495.348 $1494.968 \quad 1495.809 \quad 1496.635 \quad 1495.329$ $1494.966 \quad 1495.788 \quad 1496.61814995 .31$ $1494.9661495 .7371496 .614 \quad 1495.299$ $1494.966 \quad 1495.676 \quad 1496.621 \quad 1495.296$ $\begin{array}{rrrr}1494.961 & 1495.616 & 1496.623 & 1495.294 \\ 1494.963 & 1495.555 & 1496.63 & 1495.296\end{array}$ $\begin{array}{llllllllll}1494.411 & 1494.975 & 1495.189 & 1495.791 & 1496.919 & 1496.672 & 1493.551 & 1488.872 & 1493.398 & 1491.217\end{array}$

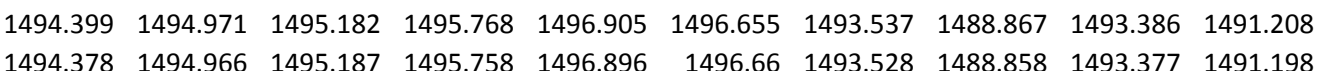
$\begin{array}{lllllllllll}1494.378 & 1494.966 & 1495.187 & 1495.758 & 1496.896 & 1496.66 & 1493.528 & 1488.858 & 1493.377 & 1491.198 \\ 1494.35 & 1494.956 & 1495.192 & 1495751 & 1496894 & 1496.667 & 1493521 & 1488855 & 1493367 & 1491.194\end{array}$ $\begin{array}{llllllllllll}1494.329 & 1494.951 & 1495.187 & 1495.742 & 1496891 & 1496.677 & 1493.512 & 1488.853 & 1493358 & 1491.184\end{array}$ $\begin{array}{llllllllllll}1494.294 & 1494.952 & 1495.208 & 1495.749 & 1496.896 & 1496.703 & 1493.512 & 1488.851 & 1493.36 & 1491.18\end{array}$ $\begin{array}{llllllllllll}1494.258 & 1494.949 & 1495.213 & 1495.761 & 1496.91 & 1496.741 & 1493.514 & 1488.855 & 1493.36 & 1491.177\end{array}$ $\begin{array}{llllllllll}1494.242 & 1494.949 & 1495.218 & 1495.768 & 1496.924 & 1496.765 & 1493.521 & 1488.86 & 1493.365 & 1491.173\end{array}$ $\begin{array}{llllllllllll}1494.235 & 1494.947 & 1495.216 & 1495.77 & 1496.926 & 1496.772 & 1493.523 & 1488.867 & 1493.367 & 1491.173\end{array}$ $\begin{array}{llllllllll}1494.225 & 1494.949 & 1495.216 & 1495.779 & 1496.935 & 1496.794 & 1493.35 & 1488.866 & 1493.374 & 1491.173\end{array}$ $\begin{array}{llllllllll}1494.237 & 1494.947 & 1495.206 & 1495.777 & 1496.933 & 1496.789 & 1493.53 & 1488.865 & 1493.377 & 1491.173 \\ 1494.261 & 1494947 & 1495.189 & 1495.765 & 1496922 & 1496768 & 1493528 & 1488865 & 1493372 & 1491.17\end{array}$ $\begin{array}{lllllllllllll}1494.277 & 1494.937 & 1495.182 & 1495.749 & 1496.91 & 1496.744 & 1493.521 & 1488.858 & 1493.365 & 1491.168\end{array}$ $\begin{array}{lllllllllllll}1494.282 & 1494.935 & 1495.178 & 1495.742 & 1496.903 & 1496.734 & 1493.514 & 1488.851 & 1493.362 & 1491.166\end{array}$ $\begin{array}{lllllllllll}1494.28 & 1494.93 & 1495.175 & 1495.733 & 1496.896 & 1496.724 & 1493.507 & 1488.848 & 1493.355 & 1491.166\end{array}$ $\begin{array}{llllllllll}1494.287 & 1494.925 & 1495.17 & 1495.721 & 1496.887 & 1496.713 & 1493.5 & 1488.848 & 1493.346 & 1491.161\end{array}$ $\begin{array}{rrrrrrrrrr}1494.28 & 1494.92 & 1495.168 & 1495.714 & 1496.882 & 1496.705 & 1493.493 & 1488.844 & 1493.339 & 1491.159 \\ 1494.261 & 1494.918 & 1495.178 & 1495.714 & 1946.884 & 1496.717 & 1493.488 & 1488.841 & 1493.337 & 1491.156 \\ 1494263 & 1494.916 & 149.168 & 1495705 & 1496877 & 149671 & 1493484 & 1488.837 & 1493.33 & 1491.154\end{array}$ $\begin{array}{lllllllllll}1494.263 & 1494.916 & 1455.168 & 1495.705 & 1496.877 & 1496.71 & 1493.484 & 1488.837 & 1493.33 & 1491.154\end{array}$

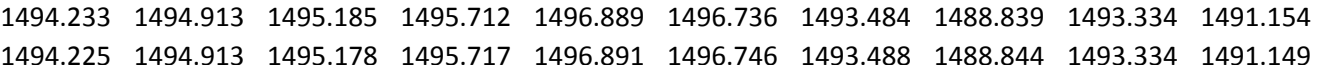
$\begin{array}{lllllllllll}1494.225 & 1494.911 & 1495.175 & 1495.712 & 1496.889 & 1496.741 & 1493.486 & 1488.844 & 1493.334 & 1491.149\end{array}$ $\begin{array}{llllllllllll}1494.218 & 1494.908 & 1495.173 & 1495.714 & 1496.889 & 1496.748 & 1493.488 & 1488.846 & 1493.337 & 1491.149\end{array}$ $\begin{array}{lllllllllll}1494.202 & 1494.906 & 1495.182 & 1495.721 & 1496.898 & 1496.765 & 1493.493 & 1488.846 & 1493.339 & 1491.149\end{array}$ $\begin{array}{lllllllllll}1494.2 & 1494.904 & 1495.175 & 1495.724 & 1496.903 & 1496.77 & 1493.498 & 1488.851 & 1493.341 & 1491.149\end{array}$ $\begin{array}{llllllllll}1494.195 & 1494.901 & 1495.17 & 1495.724 & 1496.903 & 1496.77 & 1493.495 & 1488.848 & 1493.339 & 1491.149\end{array}$

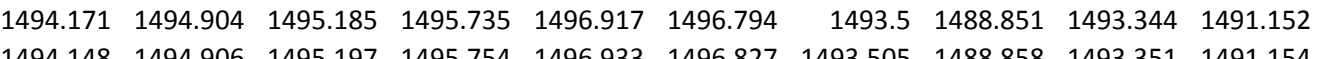

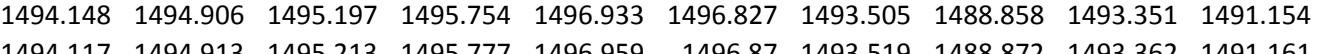
$\begin{array}{llllllllll}1494.117 & 1494.913 & 1495.213 & 1495.777 & 1496.959 & 1496.87 & 1493.519 & 1488.872 & 1493.362 & 1491.161 \\ 1494.082 & 1494.925 & 1495.235 & 1495.814 & 1496.996 & 1496.93 & 1493.54 & 1488.888 & 1493.379 & 1491.168\end{array}$ $\begin{array}{lllllllllll}1494.063 & 1494.942 & 1495.249 & 1495.847 & 1497.028 & 1496.983 & 1493.561 & 1488.905 & 1493.4 & 1491.177\end{array}$ $\begin{array}{lllllllllll}1494.061 & 1494.954 & 1495.251 & 1495.879 & 1497.059 & 1497.016 & 1493.579 & 1488.916 & 1493.419 & 1491.184\end{array}$ $\begin{array}{lllllllllll}1494.068 & 1494.968 & 1495.249 & 1495.902 & 1497.084 & 1497.04 & 1493.596 & 1488.926 & 1493.438 & 1491.196\end{array}$ $\begin{array}{llllllllll}1494.07 & 1494.98 & 1495.263 & 1495.935 & 1497.115 & 1497.079 & 1493.619 & 1488.938 & 1493.459 & 1491.21\end{array}$ $\begin{array}{llllllllll}1494.094 & 1494.992 & 1495.254 & 1495.956 & 1497.133 & 1497.083 & 1493.638 & 1488.949 & 1493.475 & 1491.219\end{array}$ $\begin{array}{llllllllll}1494.155 & 1494.995 & 1495.235 & 1455.951 & 1497.128 & 1497.045 & 1493.645 & 1488.954 & 1493.48 & 1491.229 \\ 1494.174 & 1495.002 & 1495232 & 1495.96 & 1497.138 & 1497.038 & 1493652 & 1488.952 & 1493.491 & 1491238\end{array}$ $\begin{array}{llllllllll}1494.174 & 1495.002 & 1495.232 & 1495.96 & 1497.138 & 1497.038 & 1493.652 & 1488.952 & 1493.491 & 1491.238 \\ 1494.193 & 1495.009 & 1495237 & 1495.972 & 1497.149 & 1497.04 & 1493.659 & 1488959 & 1493501 & 1491247\end{array}$ $\begin{array}{llllllllllll}1449.214 & 1495.011 & 1495.232 & 1495.979 & 1497.154 & 1497.038 & 1493.668 & 1488.963 & 1493.503 & 1491.254\end{array}$ $\begin{array}{lllllllllll}1494.228 & 1495.018 & 1495.239 & 1495.99 & 1497.161 & 1497.038 & 1493.671 & 1488.966 & 1493.51 & 1491.264\end{array}$ $\begin{array}{lllllllllll}1494.235 & 1495.023 & 1495.239 & 1495.995 & 1497.17 & 1497.033 & 1493.673 & 1488.968 & 1493.515 & 1491.271\end{array}$ $\begin{array}{lllllllllll}1494.242 & 1495.033 & 1495.251 & 1496.016 & 1497.182 & 1497.05 & 1493.682 & 1488.973 & 1493.524 & 1491.278\end{array}$ $\begin{array}{lllllllllll}1494.298 & 1495.028 & 1495.213 & 1496.002 & 1497.156 & 1497.004 & 1493.673 & 1488.973 & 1493.517 & 1491.282\end{array}$ $\begin{array}{llllllllll}1494.327 & 1495.023 & 1495.197 & 1495.979 & 1497.138 & 1496.937 & 1493.664 & 1488.959 & 1493.508 & 1491.282\end{array}$ $\begin{array}{lllllllllll}1494.348 & 1495.016 & 1495.189 & 1495.967 & 1497.122 & 1496.906 & 1493.654 & 1488.956 & 1493.498 & 1491.282\end{array}$ $\begin{array}{lllllllllll}1494.343 & 1495.011 & 1495201 & 1459.958 & 1497.112 & 1496899 & 1493647 & 1488947 & 1493.496 & 1491.285\end{array}$ $\begin{array}{lllllllllll}1494.345 & 1495.009 & 1495.201 & 1495.951 & 1497.103 & 1496.89 & 1493.643 & 1488.942 & 1493.487 & 1491.285\end{array}$ $\begin{array}{llllllllllll}1494.355 & 1495.004 & 1495.197 & 1495.942 & 1497.094 & 1496.87 & 1493.636 & 1488.935 & 1493.482 & 1491.282\end{array}$ $\begin{array}{llllllllll}1494.355 & 1494.999 & 1495.194 & 1495.93 & 1497.08 & 1496.851 & 1493.629 & 1488.933 & 1493.475 & 1491.28\end{array}$ $\begin{array}{llllllllll}1494.343 & 1494.992 & 1495.197 & 1495.921 & 1497.073 & 1496.844 & 1493.619 & 1488.923 & 1493.466 & 1491.278\end{array}$ $\begin{array}{lllllllllll}1494.324 & 1494.99 & 1495.201 & 1495.923 & 1497.075 & 1496.858 & 1493.617 & 1488.921 & 1493.463 & 1491.275\end{array}$ $\begin{array}{llllllllllll}1494.312 & 1494.987 & 1495.204 & 1495.921 & 1497.073 & 1496.856 & 1493.615 & 1488.923 & 1493.459 & 1491.273\end{array}$ $\begin{array}{lrlllllll}1488.095 & 1488.5 & 1493.805 & 1494.145 & 1493.558 & 1495.183 & 1493.537 & 1484.75 & 1479.325\end{array}$ $\begin{array}{llllllllll}1488.081 & 1488.483 & 1493.793 & 1494.132 & 1493.549 & 1495.169 & 1493.523 & 1484.75 & 1479.323\end{array}$ $\begin{array}{lllllllllll}1488085 & 1488.43 & 1493772 & 1494.125 & 149353 & 1495.164 & 1493.499 & 148475 & 1479313\end{array}$ $\begin{array}{lllllllllll}1488.085 & 1488.42 & 1493.768 & 1494.125 & 1493.52 & 1495.171 & 1493.492 & 1484.75 & 1479.300\end{array}$ $\begin{array}{llllllllll}1488.088 & 1488.389 & 1493.765 & 1494.134 & 1493.518 & 1495.192 & 1493.488 & 1484.75 & 1479.309\end{array}$ $\begin{array}{llllllllll}1488.092 & 1488.368 & 1493.768 & 1494.152 & 1493.525 & 1495.218 & 1493.497 & 1484.75 & 1479.309\end{array}$ $\begin{array}{llllllllll}1488.097 & 1488.354 & 1493.77 & 1494.171 & 1493.53 & 1495.232 & 1493.506 & 1484.75 & 1479.309\end{array}$ $\begin{array}{llllllllll}1488.099 & 1488.352 & 1493.775 & 1494.178 & 1493.532 & 1495.237 & 1493.509 & 1484.75 & 1479.311\end{array}$ $\begin{array}{llllllllll}1488.095 & 1488.345 & 1493.784 & 1494.185 & 1493.539 & 1495.265 & 1493.516 & 1484.748 & 1479.316\end{array}$ $\begin{array}{lllllllllll}1488.102 & 1488.345 & 1493.784 & 1494.183 & 1493.541 & 1495.254 & 1493.523 & 1484.748 & 1479.318\end{array}$

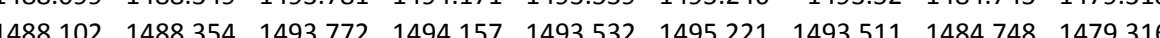
$\begin{array}{llllllllll}1488.102 & 1488349 & 1493.768 & 1494.15 & 1493.525 & 1495.209 & 1493.506 & 1484.745 & 1479.316\end{array}$ $\begin{array}{lllllllllll}1488.102 & 1488.349 & 1493.76 & 1494.143 & 1493.518 & 1495.199 & 1493.497 & 1484.745 & 1479.313\end{array}$ $\begin{array}{lllllllllll}1488.104 & 1488.349 & 1493.753 & 1494.127 & 1493.506 & 1495.188 & 1493.492 & 1484.745 & 1479.309\end{array}$ $\begin{array}{llllllllll}1488.104 & 1488.34 & 1493.746 & 1494.125 & 1493.501 & 1495.183 & 1493.48 & 1484.745 & 1479.304\end{array}$ $\begin{array}{llllllllll}1488.104 & 1488.326 & 1493.739 & 1494.127 & 1493.496 & 1495.19 & 1493.476 & 1484.745 & 1479.304\end{array}$ $\begin{array}{llllllllll}1888.104 & 1488.34 & 1493.734 & 1494.118 & 1493.492 & 1495.176 & 1493.476 & 1484.745 & 1479.304\end{array}$

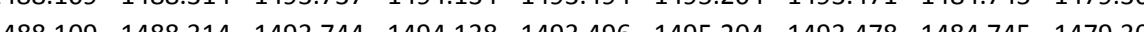
$\begin{array}{lllllllllll}1488.111 & 1488.314 & 1493.739 & 1494.136 & 1493.496 & 1495.204 & 1493.473 & 1484.745 & 1479.306\end{array}$ $\begin{array}{llllllllll}1488104 & 1488314 & 1493.739 & 1494.141 & 1493.499 & 1495204 & 1493.476 & 1484.745 & 1479.309\end{array}$ $\begin{array}{lllllllllll}1488.104 & 1488.307 & 1493.744 & 1494.148 & 1493.501 & 1495.223 & 1493.48 & 1484.745 & 1479.311\end{array}$ $\begin{array}{llllllllll}4488.109 & 1488.298 & 1493.749 & 1494.152 & 1493.503 & 1495.221 & 1493.483 & 1484.743 & 1479.31\end{array}$ $\begin{array}{llllllllll}1488.111 & 1488.293 & 1493.746 & 1494.152 & 1493.506 & 1495.228 & 1493.483 & 1484.743 & 1479.31\end{array}$ $\begin{array}{llllllllll}4888.111 & 1488.279 & 1493.751 & 1494.169 & 1493.511 & 1495.244 & 1493.483 & 1484.743 & 1479.311\end{array}$ $\begin{array}{llllllllll}1488.118 & 1488.262 & 1493.756 & 1494.197 & 1493.515 & 1495.275 & 1493.488 & 1484.745 & 1479.311\end{array}$ $\begin{array}{llllllllll}148.125 & 4488.237 & 1493.77 & 1494.22 & 1493.53 & 1455.324 & 1493.499 & 1484.743 & 1479.31\end{array}$ $\begin{array}{lllllllllll}1488.139 & 1488.222 & 1493.803 & 1494.302 & 1493.57 & 1495.425 & 1493.537 & 1484.748 & 1479.32\end{array}$ $\begin{array}{llllllllll}1488.141 & 1488.223 & 1493.824 & 1494.337 & 1493.591 & 1495.458 & 1493.567 & 1484.743 & 1479.327\end{array}$ $\begin{array}{llllllllll}1488.097 & 1488.23 & 1493.843 & 1494.36 & 1493.605 & 1495.489 & 1493.577 & 1484.745 & 1479.332\end{array}$ $\begin{array}{lllllllll}1488.104 & 1488.225 & 1493.864 & 1494.393 & 1493.631 & 1495.528 & 1493.605 & 1484.745 & 1479.339\end{array}$ $\begin{array}{llllllllll}1488.115 & 1488.244 & 1493.88 & 1494.407 & 1493.646 & 1495.538 & 1493.624 & 1484.748 & 1479.341\end{array}$ $\begin{array}{llllllllll} & 488.122 & 1488.274 & 1493.89 & 1494.395 & 493.653 & 1455.514 & 1493.631 & 1484.743 & 1479.344\end{array}$

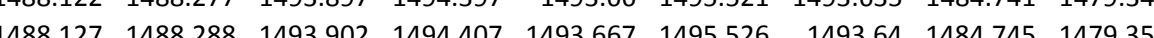
$\begin{array}{llllllllll}1488.136 & 1488.302 & 1493.911 & 1494.407 & 1493.672 & 1495.526 & 1493.652 & 1484.741 & 1479.318\end{array}$ $\begin{array}{llllllllll}1488.146 & 1488.309 & 1493.913 & 1494.409 & 1493.676 & 1495.528 & 1493.656 & 1484.743 & 1479.353\end{array}$ $\begin{array}{llllllllll}1488.152 & 1488.312 & 1493.92 & 1494.418 & 1493.684 & 1495.536 & 1493.659 & 1484.743 & 1479.35\end{array}$ $\begin{array}{lllllllll}1488.159 & 1488.323 & 1493.927 & 1494.428 & 1493.691 & 1495.545 & 1493.668 & 1484.741 & 1479.35\end{array}$ $\begin{array}{llllllllll}4488.164 & 1488.375 & 1493.925 & 1494.386 & 1493.684 & 1495.486 & 1493.673 & 1484.738 & 1479.346\end{array}$ $\begin{array}{llllllllll}1488.171 & 1488.375 & 1493.913 & 1494.362 & 1493.672 & 1495.463 & 1493.652 & 1484.738 & 1479.344\end{array}$ $\begin{array}{lllllllllll}1488.171 & 4888.385 & 1493.906 & 1494.344 & 1493.665 & 1455.434 & 1493.642 & 1484.741 & 1479.344\end{array}$

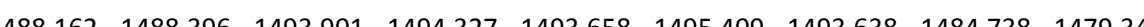
$\begin{array}{lllllllllll}1488.164 & 1488.396 & 1493.895 & 1494.318 & 1493.653 & 1495.404 & 1493.635 & 1484.738 & 1479.346\end{array}$ $\begin{array}{llllllllll}1488.171 & 1488.41 & 1493.89 & 1494.302 & 1493.648 & 1495.383 & 1493.628 & 1484.736 & 1479.344\end{array}$ $\begin{array}{llllllllll}1488.171 & 1488.408 & 1493.88 & 1494.292 & 1493.636 & 1495.364 & 1493.614 & 1484.738 & 1479.341\end{array}$ $\begin{array}{lllllllllll}1488.18 & 1488.399 & 1493.873 & 1494.283 & 1493.631 & 1495.359 & 1493.605 & 1484.736 & 1479.337\end{array}$ $\begin{array}{lllllllllll}1488.178 & 1488.389 & 1493.873 & 1494.283 & 1493.629 & 1495.357 & 1493.593 & 1484.738 & 1479.337\end{array}$ $\begin{array}{rllllllll}1488.18 & 1488.375 & 1493.869 & 1494.288 & 1493.62 & 1495.362 & 1493.591 & 1484.736 & 1479.334 \\ 1488.183 & 1488.366 & 1493.866 & 1494.288 & 1493.62 & 1495.364 & 1493.586 & 1484.736 & 1479.33\end{array}$ 
Well

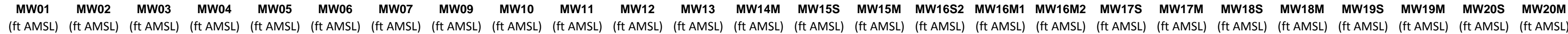

$2 / 26 / 1214: 00$ 2/26/12 15:00 2/26/12 16:00 2/26/12 17:00 18:00 2/26/12 18:00 2/26/12 20:00 2/26/12 21:00 2/26/12 22:00 2/26/122 23:00 2/27/1220:00 2/27/12 1:00 2/27/122:00 2/27/127/12 3:00 2/27/12 5:00 2/27/12 6:00 2/27/12 7:00 2/27/12 8:00 $2 / 27 / 1210: 00$ 2/27/12 11:00 2/27/12 13:00 2/27/12 14:00 2/27/12 15:00 2/27/12 16:00 2/27/12 17:00 2/27/12 18:00 2/27/12 20:00 2/27/12 21:00 2/27/12 22:00 2/27/12 23:00 2/28/120:00 2/28/12 1:00 2/28/122 2:00 2/28/12 3:00 2/28/12 5:00 2/28/12 6:00 2/28/12 7:00 2/28/12 8:00 2/28/129:00 2/28/12 10:00 $2 / 28 / 1212.00$ 2/28/12 13:00 2/2/28/12 13:00 2/28/12 15:00 2/28/12 16:00 2/28/12 18:00 $\begin{array}{llll}1494.959 & 1495.518 & 1496.633 & 1495.296\end{array}$ $\begin{array}{lllll}1494.961 & 1495.49 & 1496.635 & 1495.299\end{array}$ $\begin{array}{llll}1494.954 & 1495.492 & 1496.614 & 1495.287\end{array}$ $\begin{array}{rrrrr}1494.947 & 1495.52 & 1496.593 & 1495.273 \\ 1494.938 & 1495.574 & 1496.555 & 1495.247\end{array}$ $\begin{array}{lllll}1494.931 & 1495.641 & 1496.52 & 1495.247\end{array}$ $\begin{array}{rrrrr}1494.931 & 1495.641 & 1496.52 & 1495.219 \\ 1494.924 & 1495.693 & 1496.497 & 1495.191\end{array}$ $\begin{array}{llllll}1494.917 & 1495.737 & 1496.474 & 1495.163\end{array}$ $\begin{array}{lllll}1494.917 & 1495.767 & 1496.46 & 1495.137\end{array}$ $\begin{array}{llll}1494.912 & 1495.795 & 1496.446 & 1495.119 \\ 1499.915 & 1495802 & 196438 & 1495107\end{array}$ $\begin{array}{llll}1494.915 & 1495.802 & 1496.438 & 1495.107 \\ 1499.903 & 1459835 & 1466.413 & 1955086\end{array}$ $\begin{array}{llll}1494.903 & 1495.835 & 1496.413 & 1495.086 \\ 1494.901 & 1495.851 & 1496.389 & 1495.065\end{array}$ $\begin{array}{lllll}1494.903 & 1495.844 & 1496.396 & 1495.063\end{array}$ $\begin{array}{llll}1494.894 & 1495.863 & 1496.373 & 1495.044\end{array}$

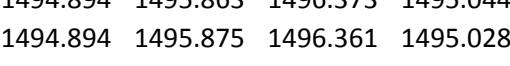
$\begin{array}{lllll}1494.891 & 1495.875 & 1496.359 & 1495.021\end{array}$ $\begin{array}{lllll}1494.891 & 1495.863 & 1496.361 & 1495.018\end{array}$ $\begin{array}{llll}1494.88 & 1495.863 & 1496.338 & 1495.009\end{array}$ $\begin{array}{llll}1494.88 & 1495.809 & 1496.336 & 1495.002 \\ 1494.882 & 1495.753 & 1496345 & 1495.004\end{array}$ $\begin{array}{llll}1494.882 & 1495.753 & 1496.345 & 1495.004 \\ 1495.697 & 1496.357 & 1495.011\end{array}$ $\begin{array}{llll}1494.884 & 1495.634 & 1496.38 & 1495.028\end{array}$ $\begin{array}{rrrr}1494.887 & 1495.578 & 1496.413 & 1495.051\end{array}$ $\begin{array}{llllll}1494.889 & 1495.546 & 1496.443 & 1495.077\end{array}$ $\begin{array}{lllll}1494.891 & 1495.513 & 1496.471 & 1495.102\end{array}$ $\begin{array}{lllll}1494.894 & 1495.504 & 1496.488 & 1495.126\end{array}$ $\begin{array}{rrrr}1494.891 & 1495.49 & 1496.506 & 1495.144\end{array}$ $\begin{array}{rrrr}1494.894 & 1495.49 & 1496.52 & 1495.163 \\ 1494.891 & 1495.509 & 1496.525 & 1495.175\end{array}$ $\begin{array}{llll}1494.894 & 1495.527 & 1496.537 & 1495.187\end{array}$ $\begin{array}{lllll}1494.891 & 1495.548 & 1496541 & 1495.197\end{array}$ $\begin{array}{lllll}1494.891 & 1495.555 & 1496.551 & 1495.208\end{array}$ $\begin{array}{lllll}1494.894 & 1495.555 & 1496.558 & 1495.217\end{array}$ $\begin{array}{lllll}1494.896 & 1495.534 & 1496.579 & 1495.233\end{array}$ $1494.901 \quad 1495.5131496 .6021495 .252$ $\begin{array}{llll}1494.903 & 1455.499 & 1496.621 & 1495.271\end{array}$ $\begin{array}{llll}1494.912 & 1455.478 & 1496.651 & 1495.292 \\ 1494.917 & 1495.467 & 1496675 & 1495315\end{array}$ $\begin{array}{lllll}1494.919 & 1495.457 & 1496.689 & 1495.334\end{array}$ $\begin{array}{llll}1494.922 & 1495.453 & 1496.7 & 1495.35\end{array}$ $1494.924 \quad 1495.453 \quad 1496.703 \quad 1495.359$ $1494.929 \quad 1495.45 \quad 1496.712 \quad 1495.369$ $1494.943 \quad 1495.42 \quad 1496.76171495 .399$

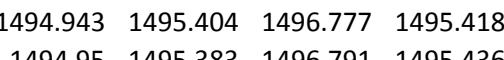

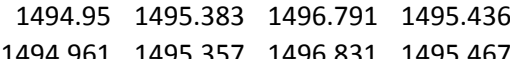
$1494.97 \quad 1495.329 \quad 1496.871 \quad 1495.499$ $1494.987 \quad 1495.301 \quad 1496.918 \quad 1495.541$ $1494.991 \quad 1495.282 \quad 1496.948 \quad 1495.574$ $1494.998 \quad 1495.254 \quad 1496.967 \quad 1495.6$ $1495.005 \quad 1495.247 \quad 1496.978 \quad 1495.623$ $\begin{array}{lrrr}1495.012 & 1495.25 & 1496.999 & 1495.644 \\ 1495.015 & 1495.259 & 1497.011 & 1495.658\end{array}$ $\begin{array}{llllllllll}1494.289 & 1494.985 & 1495.206 & 1495.923 & 1497.077 & 1496.873 & 1493.608 & 1488.928 & 1493.454 & 1491.268\end{array}$ $\begin{array}{rrrrrrrrrr}1494.28 & 1494.985 & 1495.204 & 1495.923 & 1497.075 & 1496.877 & 1493.612 & 1488.926 & 1493.454 & 1491.266 \\ 1494.294 & 1494.98 & 1495194 & 1495.914 & 197.066 & 1496858 & 4493603 & 1489921 & 149.449 & 1491264\end{array}$ $\begin{array}{rrrrrrrrrrr}1494.294 & 1494.98 & 1495.194 & 1495.914 & 1497.066 & 1496.858 & 1493.603 & 1488.921 & 1493.449 & 1491.264 \\ 1494.305 & 1494.978 & 1495.185 & 1495905 & 1497.052 & 1496844 & 1493.598 & 1488.914 & 1493.442 & 1491.261\end{array}$ $\begin{array}{lllllllllll}1494.329 & 1494.971 & 1495.175 & 1495.884 & 1497.033 & 1496.808 & 1493.589 & 1488.909 & 1493.433 & 1491.254\end{array}$ $\begin{array}{lllllllllll}1494.345 & 1494.961 & 1495.166 & 1495.86 & 1497.012 & 1496.772 & 1493.383 & 1488.9 & 1493.208 & 1491.252\end{array}$ $\begin{array}{llllllllllll}1494.348 & 1494.954 & 1495.161 & 1495.844 & 1496.996 & 1496.753 & 1493.308 & 1488.891 & 1493.142 & 1491.245\end{array}$ $\begin{array}{lllllllllll}1494.35 & 1494.942 & 1495.156 & 1495.828 & 1496.98 & 1496.736 & 1493.388 & 1488.886 & 1493.229 & 1491.238\end{array}$ $\begin{array}{lllllllllll}1494.341 & 1494.935 & 1495.159 & 1495.812 & 1496.966 & 1496.724 & 1493.416 & 1488.881 & 1493.259 & 1491.231\end{array}$

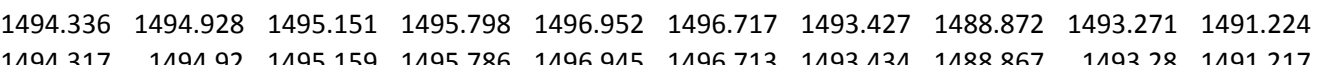
$\begin{array}{lllllllllll}1494.317 & 1494.92 & 1495.159 & 1495.786 & 1496.945 & 1496.713 & 1493.434 & 1488.867 & 1493.28 & 1491.217 \\ 1494.322 & 1494.913 & 1495.142 & 1495765 & 1496926 & 1496696 & 1493.432 & 148886 & 149328 & 1491212\end{array}$ $\begin{array}{lllllllllll}1494.317 & 1494.906 & 1495.137 & 1495.747 & 1496.908 & 1496.674 & 1493.4 & 1488.855 & 1493.273 & 1491.203\end{array}$ $\begin{array}{lllllllllllll}1494.289 & 1494.899 & 1495.144 & 1495.742 & 1496905 & 1496.691 & 1493.43 & 1488.855 & 1493276 & 1491.196\end{array}$ $\begin{array}{llllllllllll}1494.289 & 1494.894 & 1495.137 & 1495.721 & 1496.889 & 1496.669 & 1493.425 & 1488.846 & 1493.273 & 1491.189\end{array}$ $\begin{array}{lllllllllll}1494.282 & 1494.887 & 1495.132 & 1495.705 & 1496.875 & 1496.657 & 1493.42 & 1488.839 & 1493.269 & 1491.18\end{array}$ $\begin{array}{llllllllllll}1494.263 & 1494.884 & 1495.13 & 1495.698 & 1496.868 & 1496.665 & 1493.418 & 1488.834 & 1493.264 & 1491.175\end{array}$ $\begin{array}{lllllllllll}1494.242 & 1494.88 & 1495.135 & 1495.693 & 1496.866 & 1496.672 & 1493.416 & 1488.83 & 1493.262 & 1491.168\end{array}$ $\begin{array}{lllllllllll}1494.244 & 1494.875 & 1495.123 & 1495.67 & 1496.852 & 1496.657 & 1493.411 & 1488.83 & 1493.257 & 1491.159\end{array}$ $\begin{array}{lllllllllll}1494.223 & 1494.868 & 1495.123 & 1495.666 & 1496.845 & 1496.657 & 1493.406 & 1488.825 & 1493.255 & 1491.154 \\ 1494.197 & 1494.868 & 1495.135 & 1495.663 & 1496845 & 1496665 & 1493.404 & 1488825 & 149325 & 1491.147\end{array}$ $\begin{array}{llllllllllll}1494.171 & 1494.865 & 1495.137 & 1495.668 & 1496.847 & 1496.689 & 1493.404 & 1488.818 & 1493.248 & 1491.145\end{array}$ $\begin{array}{llllllllllll}1494.143 & 1494.863 & 1495.149 & 1495.675 & 1496.856 & 1496.71 & 1493.406 & 1488.825 & 1493.25 & 1491.142\end{array}$ $\begin{array}{lllllllllll}1494.115 & 1494.863 & 1495.161 & 1495.686 & 1496.87 & 1496.741 & 1493.413 & 1488.83 & 1493.257 & 1491.14\end{array}$ $\begin{array}{lllllllllll}1494.092 & 1494.865 & 1495.166 & 1495.703 & 1496.889 & 1496.772 & 1493.42 & 1488.839 & 1493.266 & 1491.14\end{array}$ $\begin{array}{llllllllll}1494.075 & 1494.87 & 1495.17 & 1495.717 & 1496.905 & 1496.803 & 1493.432 & 1488.846 & 1493.278 & 1491.142\end{array}$ $\begin{array}{llllllllll}1494.07 & 1494.872 & 1495.17 & 1495.728 & 1496.917 & 1496.82 & 1493.441 & 1488.855 & 1493.285 & 1491.142\end{array}$ $\begin{array}{llllllllllll}1494.068 & 1494.877 & 1495.17 & 1495.74 & 1496.931 & 1496.834 & 1493.451 & 1488.858 & 1493.294 & 1491.147 \\ 1494073 & 1494882 & 149517 & 1495.751 & 1496942 & 1496851 & 1493.46 & 1488865 & 1493304 & 1491.147\end{array}$

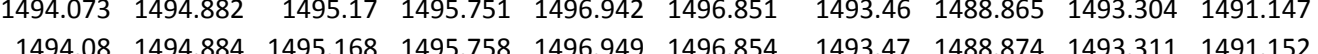
$\begin{array}{lllllllllll}1494.087 & 1494.889 & 1495.17 & 1495.765 & 1496.959 & 1496.861 & 1493.472 & 1488.87 & 1493.32 & 1491.156\end{array}$ $\begin{array}{llllllllllll}1494.094 & 1494.892 & 1495.166 & 1495.777 & 1496.966 & 1496.87 & 1493.483 & 1488.879 & 1493.325 & 1491.161\end{array}$ $\begin{array}{llllllllll}1494.099 & 1494.894 & 1495.168 & 1495.784 & 1496.975 & 1496.875 & 1493.493 & 1488.886 & 1493.334 & 1491.166\end{array}$ $\begin{array}{llllllllll}1494.103 & 1494.899 & 1495.166 & 1495.791 & 1496.982 & 1496.877 & 1493.495 & 1488.888 & 1493.339 & 1491.17\end{array}$ $\begin{array}{lllllllllll}1494.099 & 1494.906 & 1495.168 & 1495.807 & 1496.996 & 1496.897 & 1493.505 & 1488.893 & 1493.346 & 1491.175\end{array}$ $\begin{array}{llllllllll}1494.092 & 1494.911 & 1495.182 & 1495.821 & 1497.012 & 1496.921 & 1493.516 & 1488.895 & 1493.358 & 1491.182 \\ 1494092 & 1494.918 & 1495.182 & 1495837 & 1497.026 & 1496933 & 1493.526 & 1488902 & 149337 & 149189\end{array}$ $\begin{array}{llllllllll}1494.092 & 1494.918 & 1455.182 & 1455.837 & 147.026 & 1496.933 & 1493.526 & 1488.002 & 1493.37 & 1491.189 \\ 1494082 & 1494.923 & 1495.189 & 1495856 & 1497.047 & 1496959 & 1493.537 & 1488907 & 1493381 & 1491.194\end{array}$ $\begin{array}{llllllllllll}1494.08 & 1494.932 & 1495.192 & 1495.874 & 1497.066 & 1496.978 & 1493.549 & 1488.912 & 14933391 & 1491.203\end{array}$ $\begin{array}{lllllllllll}1494.089 & 1494.937 & 1495.192 & 1495.888 & 1497.08 & 1496.985 & 1493.563 & 1488.919 & 1493.402 & 1491.208\end{array}$ $\begin{array}{lllllllllll}1494.096 & 1494.944 & 1495.192 & 1495.907 & 1497.091 & 1496.997 & 1493.573 & 1488.93 & 1493.414 & 1491.217\end{array}$ $\begin{array}{lllllllllll}1494.113 & 1494.949 & 1495.187 & 1495.914 & 1497.103 & 1496.995 & 1493.582 & 1488.935 & 1493.421 & 1491.224\end{array}$ $\begin{array}{lllllllllll}1494.124 & 1494.956 & 1495.192 & 1495.925 & 1497.11 & 1497 & 1493.589 & 1488.933 & 1493.43 & 1491.231\end{array}$ $\begin{array}{llllllllll}1494.101 & 1494.963 & 1495.211 & 1495.96 & 1497.138 & 1497.04 & 1493.61 & 1488.942 & 1493.449 & 1491.243\end{array}$ $\begin{array}{rrrrrrrrrrr}1494.108 & 1494.973 & 1495.211 & 1495.974 & 1497.154 & 1497.055 & 1493.622 & 1488.952 & 1493.461 & 1491.247 \\ 1494.12 & 1494.98 & 1495211 & 1495988 & 1497.17 & 1497.062 & 1493633 & 1488954 & 1493.47 & 1491259\end{array}$ $\begin{array}{llllllllllll}1494.108 & 1494.987 & 1495.23 & 1496016 & 1497.196 & 1497.095 & 1493.65 & 1488.967 & 1493.482 & 1491.259\end{array}$ $\begin{array}{llllllllllll}1444.103 & 1494.999 & 1495.235 & 1496.048 & 1497.224 & 1497.131 & 1493.666 & 1488.975 & 1493.506 & 1491.275\end{array}$ $\begin{array}{lllllllllll}1494.096 & 1495.011 & 1495.254 & 1496.083 & 1497.259 & 1497.17 & 1493.687 & 1488.977 & 1493.522 & 1491.289\end{array}$ $\begin{array}{llllllllll}1494.101 & 1495.021 & 1495.256 & 1496.113 & 1497.282 & 1497.198 & 1493.708 & 1488.992 & 1493.545 & 1491.301\end{array}$ $\begin{array}{llllllllll}1494.122 & 1495.035 & 1495.258 & 1496.137 & 1497.303 & 1497.208 & 1493.722 & 1489.003 & 1493.562 & 1491.308\end{array}$ $\begin{array}{lllllllllll}1494.141 & 1495.042 & 1495.261 & 1496.165 & 1497.322 & 1497.213 & 1493.739 & 1489.01 & 1493.576 & 1491.324\end{array}$ $\begin{array}{llllllllll}1494.155 & 1495.05 & 1495.265 & 1496.188 & 1497.34 & 1497.229 & 1493.757 & 1489.02 & 1493.592 & 1491.333\end{array}$ $\begin{array}{lllllllll}1488.183 & 1488.366 & 1493.861 & 1494.285 & 1493.62 & 1495.362 & 1493.593 & 1484.736 & 1479.327\end{array}$ $\begin{array}{llllllllll}1488.18 & 1488.354 & 1493.862 & 1494.288 & 1493.617 & 1495.366 & 1493.593 & 1484.736 & 1479.327 \\ 1488.18 & 1488.361 & 1493.855 & 1494.274 & 1493.615 & 1495.352 & 1493593 & 1484.736 & 1473.9325\end{array}$ $\begin{array}{llllllllll}1488 & 183 & 488.361 & 493.855 & 1494.274 & 1493.615 & 1455.352 & 1493.593 & 1444.736 & 149.325\end{array}$ $\begin{array}{lllllllllll}1488.185 & 1488.375 & 1493.843 & 1494.236 & 1493.598 & 1495.301 & 1493.581 & 1484.734 & 1479.323\end{array}$ $\begin{array}{lllllllllll}1488.187 & 1488.382 & 1493.619 & 1494.162 & 1493.392 & 1495.27 & 1493.375 & 1484.734 & 147932\end{array}$ $\begin{array}{llllllllllll}1488.187 & 1488.377 & 1493.551 & 1494.083 & 1493.316 & 1495.235 & 1493.307 & 1484.734 & 1479.32\end{array}$ $\begin{array}{llllllllll}1488.185 & 1488.38 & 1493.638 & 1494.099 & 1493.399 & 1495.211 & 1493.384 & 1484.734 & 1479.318\end{array}$ $\begin{array}{llllllllll}1488.183 & 1488.37 & 1493.666 & 1494.092 & 1493.428 & 1495.195 & 1493.41 & 1484.734 & 1479.318\end{array}$ $\begin{array}{lllllllllll}1488.176 & 1488.37 & 1493.678 & 1494.097 & 1493.44 & 1495.181 & 1493.419 & 1484.734 & 1479.313\end{array}$ $\begin{array}{llllllllll}1488.176 & 1488.361 & 1493.687 & 1494.097 & 1493.444 & 1495.178 & 1493.427 & 1484.731 & 1479.311\end{array}$ $\begin{array}{lllllllllll}1488.166 & 1488363 & 1493.685 & 1494.064 & 1493.44 & 1495134 & 1493.424 & 1484.731 & 1479.304\end{array}$ $\begin{array}{lllllllllll}1488.166 & 1488.347 & 1493.683 & 1494.071 & 1493.437 & 1495.138 & 1493.422 & 1484.731 & 1479.304\end{array}$ $\begin{array}{lllllllllll}1488.18 & 1488.349 & 1493.676 & 1494.055 & 1493.435 & 1495.115 & 1493.419 & 1484.731 & 1479.299\end{array}$ $\begin{array}{lllllllll}1488.169 & 1488.34 & 1493.671 & 1494.05 & 1493.428 & 1495.11 & 1493.41 & 1484.731 & 1479.299\end{array}$ $\begin{array}{lllllllllll}1488.164 & 1488.333 & 1493.671 & 1494.045 & 1493.428 & 1495.101 & 1493.412 & 1484.729 & 1479.297\end{array}$ $\begin{array}{lllllllll}1488.155 & 1488.324 & 1493.666 & 1494.045 & 1493.425 & 1495.105 & 1493.403 & 1484.734 & 1479.297\end{array}$ $\begin{array}{lllllllll}1488.155 & 1488.34 & 1493.662 & 1494.027 & 1493.421 & 1455.089 & 1493.401 & 1484.726 & 1479.297\end{array}$ $\begin{array}{llllllllll}1488.159 & 1488.312 & 1493.659 & 1494.029 & 1493.416 & 1455.082 & 1493.396 & 1484.729 & 1479.295\end{array}$ $\begin{array}{lllllllll}1488.164 & 1488.281 & 1493.657 & 1494.038 & 1493.416 & 1455.101 & 1493.391 & 1484.729 & 1479.22\end{array}$ $\begin{array}{lllllllll}1488.132 & 1488262 & 1493.659 & 1494.052 & 1493.416 & 1495.122 & 1493.389 & 1484.734 & 1479.29\end{array}$ $\begin{array}{llllllllll}1488.129 & 1488.239 & 1493.666 & 1494.076 & 1493.423 & 1495.145 & 1493.396 & 1484.729 & 1479.29\end{array}$ $\begin{array}{lllllllll}1488.129 & 1488.225 & 1493.669 & 1494.094 & 1493.432 & 1495.174 & 1493.405 & 1484.729 & 1479.29\end{array}$ $\begin{array}{llllllllll}4488.125 & 1488.218 & 1493.683 & 1494.115 & 1493.442 & 1495.199 & 1493.417 & 1484.729 & 1479.292\end{array}$

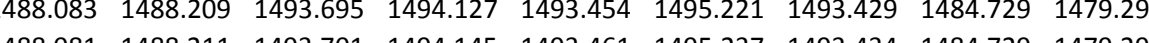

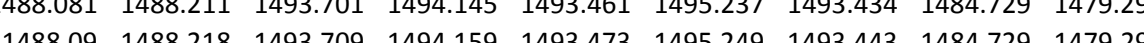

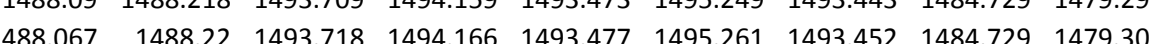
$\begin{array}{llllllllll}1488.072 & 1488.218 & 1493.723 & 1494.178 & 1493.485 & 1495.27 & 1493.459 & 1484.729 & 1479.304\end{array}$ $\begin{array}{llllllllll}1488.083 & 1488.227 & 1493.73 & 1494.183 & 1493.492 & 1495.275 & 1493.471 & 1484.726 & 1479.306\end{array}$ $\begin{array}{llllllllll}1488.092 & 1488.227 & 1493.739 & 1494.192 & 1493.501 & 1495.289 & 1493.48 & 1484.726 & 1479.311\end{array}$ $\begin{array}{llllllllll}4488.102 & 1488.227 & 1493.746 & 1494.201 & 1493.506 & 1495.291 & 1493.48 & 1484.727 & 1479.30\end{array}$ $\begin{array}{lllllllll}1488.115 & 1488.232 & 1493.753 & 1494.215 & 1493.513 & 1495.31 & 1493.492 & 1484.729 & 1479.313\end{array}$ $\begin{array}{lllllllll}4888.122 & 1488.225 & 1493.763 & 1494.234 & 1493.525 & 1495.336 & 1493.502 & 1484.727 & 1479.316\end{array}$

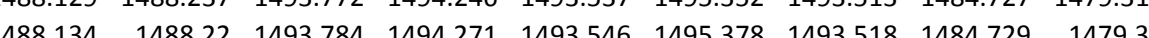
$\begin{array}{llllllllll}1488143 & 1488227 & 1493.796 & 1494.288 & 149356 & 1495399 & 149353 & 1444.726 & 147932323\end{array}$ $\begin{array}{llllllllll}1488.152 & 1488.232 & 1493.808 & 1494.302 & 1493.572 & 1495.416 & 1493.541 & 1484.726 & 1479.325\end{array}$ $\begin{array}{llllllllll}1488.146 & 1488.246 & 1493.822 & 1494.313 & 1493.584 & 1495.43 & 1493.56 & 1484.727 & 1479.33\end{array}$ $\begin{array}{lllllllll}1488.136 & 1488.251 & 1493.831 & 1494.318 & 1493.589 & 1495.432 & 1493.567 & 1484.726 & 1479.332\end{array}$ $\begin{array}{llllllllll}4488.146 & 1488.255 & 1493.838 & 1494.325 & 1493.601 & 1495.442 & 1493.57 & 1484.727 & 1479.334\end{array}$ $\begin{array}{lllllllll}1488.159 & 1488.248 & 1493.852 & 1494.367 & 1493.62 & 1495.479 & 1493.591 & 1484.736 & 1479.341\end{array}$

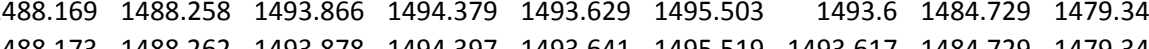

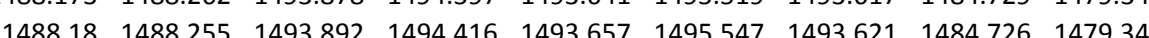
$\begin{array}{llllllllll}1488.189 & 1488.255 & 1493.909 & 1494.451 & 1493.676 & 1495.58 & 1493.64 & 1484.729 & 1479.353\end{array}$ $\begin{array}{llllllllll}1488.199 & 1488.251 & 1493.93 & 1494.486 & 1493.698 & 1495.627 & 1493.661 & 1484.731 & 1479.36\end{array}$ $\begin{array}{llllllllll}1488.21 & 1488.265 & 1493.946 & 1494.512 & 1493.714 & 1495.658 & 1493.68 & 1484.729 & 1479.362\end{array}$ $\begin{array}{llllllllll}1488.162 & 1488.274 & 1493.96 & 1494.53 & 1493.736 & 1495.672 & 1493.696 & 1484.729 & 1479.365\end{array}$ $\begin{array}{lllllllllll}1488.166 & 1488.286 & 1493.981 & 1494.542 & 1493.75 & 1495.688 & 1493.717 & 1484.729 & 1479.365\end{array}$ $\begin{array}{lllllllll}1488.176 & 1488.291 & 1493.996 & 1494.563 & 1493.766 & 1495.714 & 1493.739 & 1484.729 & 1479.369 \\ 1488.196 & 1488314 & 1494031 & 1494.579 & 1493.79 & 1495.733 & 1493769 & 1484.731 & 1479.39\end{array}$ 
Well

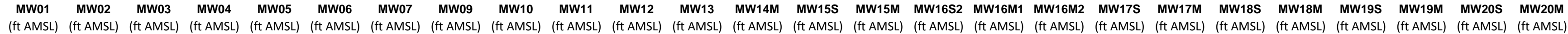

2/28/12 20:00 2/28/12 21:00 2/28/12 22:00 2/28/122 23:00 2/29/120:00 2/29/12 2:00 2/29/12 3:00 2/29/12 4:00 2/29/125:00 2/29/12 6:00 2/29/12 7:00 2/29/12 9:00 2/29/12 9:00 2/29/12 11:00 2/29/12 12:00 2/29/12 13:00 2/29/12 14:00 2/2/29/12 16:00 2/29/12 18:00 2/29/12 19:00 2/29/12 20:00 2/29/12 21:00 2/29/12 22:00 2/29/12 23:00 $3 / 1 / 120: 00$ 3/1/112 1:00 $2: 00$ 3/1/12 3:00 3/1/12 4:00 3/1/12 5:00 $3 / 1 / 126: 00$ 3/1/12 7:00 $3 / 1 / 128: 00$ 3/1/12 9:00 $3 / 1 / 1211: 00$ 3/11/12 12:00 3/1/12 13:00 3/1/12 14:00 3/1/12215:00 3/1/12 16:00 /1/11212.00 3/1/12 18:00 3/1/12 20:00 3/1/12 21:00 3/1/12 22:00 3/1/12 23:00 3/2/12 0:00 $\begin{array}{llll}1494.996 & 1495.387 & 1496.675 & 1495.649\end{array}$ $\begin{array}{llll}1494.994 & 1495.455 & 1496.703 & 1495.611 \\ 1494.985 & 1495.471 & 1496918 & 199555\end{array}$ $1494.985 \quad 1495.4711496 .9181495 .565$ $\begin{array}{llll}1494.977 & 1498.283 & 1497.011 & 1495.513 \\ 1494.975 & 1504.065 & 1496.915 & 1495.481\end{array}$ $\begin{array}{lllll}1494.973 & 1512.877 & 1496.878 & 1455.448\end{array}$ $\begin{array}{lllll}1494.973 & 1522.891 & 1496.808 & 1495.425\end{array}$ $\begin{array}{llllll}1494.97 & 1524.891 & 1496.707 & 1495.408\end{array}$ $\begin{array}{rrrrr}1494.966 & 1524.961 & 1496.7 & 1495.394\end{array}$ $\begin{array}{llll}1494.961 & 1525.019 & 1496.686 & 1495.378\end{array}$ $\begin{array}{rrrr}1494.961 & 1525.068 & 1496.667 & 1495.364 \\ 1494.957 & 1525.099 & 1496.658 & 1495.35\end{array}$ $\begin{array}{rrrr}1494.957 & 1525.099 & 1496.658 & 1495.35 \\ 1494.952 & 1525.136 & 1496.637 & 1495.336\end{array}$ $\begin{array}{lllll}1494.95 & 1525.157 & 1496.628 & 1495.324\end{array}$ $\begin{array}{rrrr}1494.952 & 1525.164 & 1496.623 & 1495.315\end{array}$ $\begin{array}{lllll}1494.945 & 1525.169 & 1496.621 & 1495.31\end{array}$ $\begin{array}{lllll}1494.947 & 1525.148 & 1496.628 & 1495.313\end{array}$ $\begin{array}{llll}1494.95 & 1525.103 & 1496.649 & 1495.322\end{array}$ $\begin{array}{rlll}1494.947 & 1525.068 & 1496.658 & 1495.331 \\ 1494.95 & 1525.024 & 14968 & 1995336\end{array}$ $\begin{array}{lllll}1494.95 & 1525.024 & 1496.66 & 1495.336 \\ 1494.945 & 1524.987 & 149666 & 14953343\end{array}$ $\begin{array}{rrrr}1494.947 & 1524.945 & 1496.665 & 1495.34345\end{array}$ $\begin{array}{lllll}1494.95 & 1524.903 & 1496677 & 1495355\end{array}$ $\begin{array}{rrrr}1494.947 & 1524.984 & 1496.672 & 1495.355 \\ 1495 & 1455\end{array}$ $1494.947 \quad 1524.875 \quad 1496.665 \quad 1495.355$ $\begin{array}{llll}1494.945 & 1524.868 & 1496.665 & 1495.357\end{array}$ $\begin{array}{rrrr}1494.95 & 1524.849 & 1496.677 & 1495.364 \\ 1494.95 & 152484 & 1469679 & 195336\end{array}$ $\begin{array}{rrrrr}1494.95 & 1524.84 & 1496.679 & 1495.369\end{array}$ $\begin{array}{rrrr}1494.954 & 1524.81 & 1496.71 & 1495.387 \\ 1494.959 & 1524.789 & 1496.726 & 1495.404\end{array}$ $\begin{array}{lllll}1494.963 & 1524.604 & 1496.752 & 1495.422\end{array}$ $\begin{array}{llll}1494.97 & 1524.369 & 1496.78 & 1495.446\end{array}$ $\begin{array}{lllll}1494.977 & 1523.583 & 1496.812 & 1495.474\end{array}$ $\begin{array}{lllll}1494.982 & 1522.693 & 1496.833 & 1495.497\end{array}$ $\begin{array}{lllll}1494.982 & 1521.867 & 1496.826 & 1495.506\end{array}$ $\begin{array}{llll}1494.984 & 1521.058 & 1496.843 & 1495.52 \\ 1494.991 & 1520284 & 196847 & 14953\end{array}$ $\begin{array}{lllll}1494.991 & 1520.284 & 1496.847 & 1495.53 \\ 1494.991 & 1519566 & 1466845 & 1495.532\end{array}$ $\begin{array}{rrrr}1494.991 & 1519.566 & 1496.845 & 1495.532 \\ 1494.996 & 1518.85 & 1496.859 & 1495.544\end{array}$ $\begin{array}{lllll}1494.998 & 1518.181 & 1496.857 & 1495.546\end{array}$ $\begin{array}{lllll}1495.001 & 1517.526 & 1496.864 & 1495.551\end{array}$ $1495.008 \quad 1516.843 \quad 1496.89 \quad 1495.567$ $1495.015 \quad 1516.214 \quad 1496.906 \quad 1495.581$

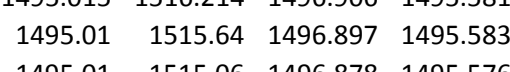
$\begin{array}{rrrr}1495.01 & 1515.06 & 1496.878 & 1495.576 \\ 1495.015 & 1514.526 & 1496883 & 1495.576\end{array}$ $\begin{array}{lrrrr}1495.015 & 1514.526 & 1496.883 & 1495.576\end{array}$ $\begin{array}{llll}1495.01 & 1513.593 & 1496.833 & 1495.541\end{array}$ $\begin{array}{llll}1495.01 & 1513.141 & 1496.834 & 1495.537\end{array}$ $1495.005 \quad 1512.7191496 .8151495 .523$ $1494.998 \quad 1512.311 \quad 1496.796 \quad 1495.511$ $1495.0191511 .863 \quad 1496.838 \quad 1495.523$ $\begin{array}{rrrr}1495.017 & 1511.413 & 1496.831 & 1495.52 \\ 1495.019 & 1511.033 & 1496.838 & 1495.523\end{array}$ $\begin{array}{rrrrrrrrrr}1494.24 & 1495.062 & 1495.239 & 1496.213 & 1497.349 & 1497.22 & 1493.849 & 1489.031 & 1493.665 & 1491.354\end{array}$ $\begin{array}{llllllllll}1494.315 & 1495.062 & 1495.209 & 1496.183 & 1497.324 & 1497.112 & 1493.821 & 1489.031 & 1493.658 & 1491.366\end{array}$ $\begin{array}{lllllllllll}1494.371 & 1495.057 & 1495.189 & 1496.16 & 1497.294 & 1497.05 & 1493.804 & 1489.027 & 1493.649 & 1491.371 \\ 1494.411 & 1495.052 & 1495.178 & 149613 & 1497.263 & 1496983 & 1493781 & 1489015 & 1493627 & 1491.373\end{array}$ $\begin{array}{llllllllllll}1494.423 & 1495.047 & 1495.18 & 1496.113 & 1497.247 & 1496.961 & 1493.769 & 1488.998 & 1493.616 & 1491.373\end{array}$ $\begin{array}{llllllllll}1494.428 & 1495.042 & 1495.18 & 1496.097 & 1497.228 & 1496.937 & 1493.757 & 1488.991 & 1493.602 & 1491.371\end{array}$ $\begin{array}{llllllllllll}1494.432 & 1495.035 & 1495.182 & 1496.083 & 1497.215 & 1496.918 & 1493.743 & 1488.984 & 1493.59 & 1491.368\end{array}$ $\begin{array}{llllllllllll}1494.421 & 1495.03 & 1495.185 & 1496.074 & 1497.203 & 1496.913 & 1493.734 & 1488.977 & 1493.583 & 1491.364\end{array}$ $\begin{array}{lllllllllll}1494.411 & 1495.026 & 1495.189 & 1496.065 & 1497.194 & 1496.909 & 1493.727 & 1488.975 & 1493.574 & 1491.361\end{array}$ $\begin{array}{llllllllll}1494.411 & 1495.026 & 1495.187 & 1496.053 & 1497.182 & 1496.897 & 1493.72 & 1488.97 & 1493.566 & 1491.357\end{array}$ $\begin{array}{rrrrrrrrrr}1494.409 & 1495.021 & 1495.185 & 1496.037 & 1497.168 & 1496.882 & 1493.711 & 1488.961 & 1493.557 & 1491.352 \\ 1494.404 & 1495.016 & 1495.18 & 1496025 & 1497.154 & 1496873 & 1493.704 & 1488.961 & 1493.55 & 1491345\end{array}$ $\begin{array}{lllllllllll}1494.404 & 1495.011 & 1495.178 & 1496.011 & 1497.14 & 1496.856 & 1493.694 & 1488.954 & 1493.541 & 1491.343\end{array}$ $\begin{array}{llllllllllll}1494.39 & 1495.009 & 1495.185 & 1496 & 1497.128 & 1496.847 & 1493.687 & 1488.949 & 1493.529 & 1491.338\end{array}$ $\begin{array}{lllllllllll}1494.376 & 1495.004 & 1495.18 & 1495.986 & 1497.124 & 1496.842 & 1493.678 & 1488.945 & 1493.527 & 1491.329\end{array}$ $\begin{array}{lllllllllll}1494.362 & 1495.004 & 1495.185 & 1495.981 & 1497.119 & 1496.846 & 1493.673 & 1488.94 & 1493.52 & 1491.326\end{array}$ $\begin{array}{lllllllllll}1494.345 & 1495.002 & 1495.189 & 1495.979 & 1497.11 & 1496.856 & 1493.668 & 1488.94 & 1493.513 & 1491.324\end{array}$ $\begin{array}{llllllllll}1494.312 & 1495.006 & 1495.204 & 1495.981 & 1497.119 & 1496.88 & 1493.668 & 1488.94 & 1493.515 & 1491.319\end{array}$ $\begin{array}{llllllllll}1494.303 & 1495.009 & 1495.201 & 1495.986 & 1497.119 & 1496.897 & 1493.671 & 1488.945 & 1493.515 & 1491.319 \\ 1494294 & 1495.011 & 1495.199 & 1495.984 & 1497.119 & 1496897 & 1493671 & 1488942 & 1493515 & 1991317\end{array}$ $\begin{array}{lllllllllll}1494.294 & 1495.011 & 1495.199 & 1495.984 & 1497.119 & 1496.897 & 1493.67 & 1488.942 & 1493.515 & 1491.317 \\ 1494289 & 1495.016 & 1495.199 & 1495981 & 1497.119 & 1496899 & 1493668 & 1488945 & 149351 & 1491317\end{array}$ $\begin{array}{llllllllll}1494.287 & 1495.018 & 1495.201 & 1495.981 & 1497.119 & 1496.904 & 1493.668 & 1488.947 & 1493.51 & 1491.317\end{array}$ $\begin{array}{lllllllllll}1494.28 & 1495.023 & 1495.204 & 1495.984 & 1497.124 & 1496.918 & 1493.668 & 1488.942 & 1493.515 & 1491.317\end{array}$ $\begin{array}{llllllllllll}1494.289 & 1495.026 & 1495.201 & 1495.981 & 1497.119 & 1496.916 & 1493.671 & 1488.949 & 1493.515 & 1491.319\end{array}$ $\begin{array}{llllllllllll}1494.296 & 1495.03 & 1495.201 & 1495.977 & 1497.117 & 1496.909 & 1493.673 & 1488.954 & 1493.517 & 1491.319\end{array}$ $\begin{array}{llllllllll}1494.298 & 1495.033 & 1495.199 & 1495.977 & 1497.117 & 1496.911 & 1493.671 & 1488.945 & 1493.517 & 1491.322\end{array}$ $\begin{array}{lllllllllll}1494.291 & 1495.038 & 1495.206 & 1495.979 & 1497.119 & 1496.923 & 1493.675 & 1488.949 & 1493.52 & 1491.322\end{array}$ $\begin{array}{lllllllllll}1494.289 & 1495.042 & 1495.206 & 1495.981 & 1497.124 & 1496.925 & 1493.678 & 1488.956 & 1493.524 & 1491.324 \\ 1494.273 & 1495.05 & 1495218 & 1495.993 & 1497.135 & 1496952 & 1493687 & 1488959 & 1493534 & 141331\end{array}$ $\begin{array}{lrrrrrrrrr}1494.273 & 1495.05 & 1455.218 & 1455.993 & 1477.135 & 1496.952 & 1493.687 & 1488.959 & 1493.534 & 1491.331 \\ 1494.265 & 1495.057 & 1495223 & 1496.002 & 1497.145 & 1496.969 & 1493.697 & 1488.966 & 1493.541 & 1491.333\end{array}$ $\begin{array}{llllllllllll}1494.254 & 1495.062 & 1495.23 & 1496.014 & 1497.159 & 1496.992 & 1493.704 & 1488.968 & 1493.548 & 1491.34\end{array}$ $\begin{array}{lllllllllll}1494.244 & 1495.071 & 1495.239 & 1496.03 & 1497.175 & 1497.019 & 1493.715 & 1488.977 & 1493.559 & 1491.345\end{array}$ $\begin{array}{llllllllll}1494.235 & 1495.078 & 1495.249 & 1496.046 & 1497.194 & 1497.047 & 1493.727 & 1488.984 & 1493.574 & 1491.354\end{array}$ $\begin{array}{lllllllllll}1494.237 & 1495.088 & 1495.249 & 1496.062 & 1497.208 & 1497.062 & 1493.741 & 1488.996 & 1493.583 & 1491.361\end{array}$ $\begin{array}{llllllllll}1494.258 & 1495.093 & 1495.239 & 1496.067 & 1497.21 & 1497.057 & 1493.748 & 1488.998 & 1493.59 & 1491.366\end{array}$ $\begin{array}{llllllllll}1494.263 & 1495.1 & 1495.246 & 1496.081 & 1497.222 & 1497.076 & 1493.757 & 1489.005 & 1493.597 & 1491.375 \\ 1494273 & 1495.107 & 1495247 & 1496086 & 1497.228 & 1497.069 & 1493.764 & 1489008 & 1493609 & 1491382\end{array}$ $\begin{array}{lllllllllll}1494.273 & 1495.107 & 1455.247 & 1466.086 & 1497.228 & 1497.065 & 1493.764 & 1489.008 & 1493.609 & 1491.382 \\ 1494.287 & 1495.114 & 1495244 & 1496093 & 1497.231 & 1497.069 & 1493.769 & 1489.01 & 1493609 & 1491.389\end{array}$ $\begin{array}{lllllllllll}1494.284 & 1495.119 & 1495.247 & 1496.102 & 1497.24 & 1497.078 & 1493.776 & 1489.008 & 1493.618 & 1491.396\end{array}$ $\begin{array}{lllllllllll}1494.296 & 1495.121 & 1495.246 & 1496.107 & 1497.242 & 1497.069 & 1493.781 & 1489.015 & 1493.62 & 1491.403\end{array}$ $\begin{array}{lllllllllllll}1494.301 & 1495.129 & 1495.249 & 1496.111 & 1497.247 & 1497.071 & 1493.785 & 1489.017 & 1493.627 & 1491.408\end{array}$ $\begin{array}{lllllllllll}1494.289 & 1495.136 & 1495.258 & 1496.127 & 1497.263 & 1497.095 & 1493.792 & 1489.02 & 1493.634 & 1491.417\end{array}$ $\begin{array}{lllllllllllll}1494.284 & 1495.141 & 1495.265 & 1496.139 & 1497.275 & 1497.107 & 1493.799 & 1489.024 & 1493.646 & 1491.42\end{array}$ $\begin{array}{llllllllll}1494.308 & 1495.148 & 1495.261 & 1496.141 & 1497.273 & 1497.1 & 1493.802 & 1489.024 & 1493.644 & 1491.429\end{array}$ $\begin{array}{lllllllllll}1494.331 & 1495.15 & 1495.246 & 1496.141 & 1497.268 & 1497.081 & 1493.804 & 1489.027 & 1493.646 & 1491.434 \\ 1494.336 & 1495.157 & 1495251 & 1496144 & 1497273 & 1497.076 & 1493806 & 1489034 & 1493651 & 1491.438\end{array}$

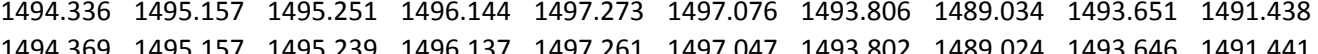
$\begin{array}{llllllllllll}1494.388 & 1495.157 & 1495.235 & 1496.127 & 1497.252 & 1497.021 & 1493.797 & 1489.022 & 1493.641 & 1491.443\end{array}$ $\begin{array}{lllllllllll}1494.392 & 1495.162 & 1495.242 & 1496.13 & 1497.249 & 1497.028 & 1493.797 & 1489.017 & 1493.639 & 1491.445\end{array}$ $\begin{array}{lllllllllll}1494.411 & 1495.162 & 1495.235 & 1496.12 & 1497.24 & 1497.007 & 1493.79 & 1489.013 & 1493.637 & 1491.445\end{array}$ $\begin{array}{llllllllll}1494.423 & 1495.162 & 1495.225 & 1496.113 & 1497.228 & 1496.997 & 1493.792 & 1489.01 & 1493.634 & 1491.445\end{array}$ $\begin{array}{lllllllllll}1494.388 & 1495.164 & 1495.258 & 1496.13 & 1497.245 & 1497.031 & 1493.797 & 1489.013 & 1493.641 & 1491.452\end{array}$ $\begin{array}{llllllllllll}1494.397 & 1495.169 & 1455.249 & 1496.123 & 1497.24 & 1497.016 & 1493.795 & 1489.013 & 1493.639 & 1491.452\end{array}$ $\begin{array}{lllllllll}1488.254 & 1488.38 & 1494.08 & 1494.586 & 1493.854 & 1495.695 & 1493.842 & 1484.731 & 1479.437\end{array}$ $\begin{array}{llllllllll}1488.254 & 1488.403 & 1494.064 & 1494.542 & 1493.83 & 1495.644 & 1493.807 & 1484.729 & 1479.432\end{array}$ $\begin{array}{lllllllllll}1488261 & 1488.436 & 149404 & 149446 & 14937788 & 1495531 & 1493771 & 1484.729 & 1479.425\end{array}$ $\begin{array}{llllllllll}1488268 & 1488.446 & 1494.031 & 1494.439 & 1493.778 & 1495.51 & 1493.76 & 1484.727 & 1479.423\end{array}$ $\begin{array}{lllllllllll}1488273 & 1488.453 & 1494.014 & 1494.421 & 1493.766 & 1495.486 & 1493.743 & 1484.727 & 1479.421\end{array}$ $\begin{array}{lllllllllll}1488.277 & 1488.457 & 1493.998 & 1494.402 & 1493.75 & 1495.467 & 1493.729 & 1484.727 & 1479.421\end{array}$ $\begin{array}{lllllllllll}1488.28 & 1488.462 & 1493.991 & 1494.397 & 1493.748 & 1495.465 & 1493.725 & 1484.724 & 1479.418\end{array}$ $\begin{array}{llllllllll}1488.284 & 1488.464 & 1493.984 & 1494.386 & 1493.736 & 1495.449 & 1493.715 & 1484.727 & 1479.418\end{array}$ $\begin{array}{llllllllll}1488.291 & 1488.471 & 1493.977 & 1494.372 & 1493.726 & 1495.442 & 1493.708 & 1484.726 & 1479.418\end{array}$ $\begin{array}{llllllllll}1488.277 & 1488.476 & 1493.965 & 1494.362 & 1493.719 & 1495.43 & 1493.696 & 1484.727 & 1479.416 \\ 1488275 & 1488.483 & 1493.958 & 1494351 & 1493714 & 1495.416 & 1493.692 & 1484.727 & 1479.414\end{array}$ $\begin{array}{lllllllll}1488.273 & 1488.483 & 1493.951 & 1494.332 & 1493.703 & 1495.402 & 1493.682 & 1484.726 & 1479.411\end{array}$ $\begin{array}{llllllllll}1488.277 & 1488.481 & 1493.939 & 1494.327 & 1493.698 & 1495387 & 1493.673 & 1484.731 & 1479.411\end{array}$ $\begin{array}{lllllllllll}1488.28 & 1488.471 & 1493.93 & 1494.32 & 1493.688 & 1495.378 & 1493.656 & 1484.729 & 1479.409\end{array}$ $\begin{array}{llllllllll}1488.284 & 1488.474 & 1493.925 & 1494.318 & 1493.681 & 1495.383 & 1493.654 & 1484.734 & 1479.407\end{array}$ $\begin{array}{llllllllll}1488.28 & 1488.467 & 1493.923 & 1494.32 & 1493.681 & 1495.387 & 1493.647 & 1484.736 & 1497.404\end{array}$ $\begin{array}{llllllllll}1488.282 & 1488.453 & 1493.918 & 1494.332 & 1493.681 & 1495.399 & 1493.645 & 1484.738 & 1479.4\end{array}$ $\begin{array}{llllllllll}1488.287 & 1488.455 & 1493.922 & 1494.337 & 1493.679 & 1495.409 & 1493.654 & 1484.741 & 1479.400\end{array}$

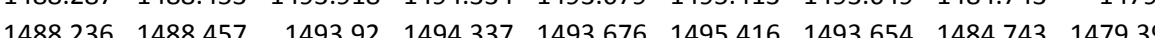
$\begin{array}{lllllllllll}1488.238 & 1488.457 & 1493.918 & 1494.341 & 1493.679 & 1455.423 & 1493.652 & 1484.745 & 1479.397\end{array}$ \begin{tabular}{llllllllll}
8.457 & 1493.918 & 1494.341 & 1493.679 & 1495.423 & 1493.652 & 1484745 & 1479397 \\
\hline
\end{tabular} $\begin{array}{llllllllll}1488.252 & 1488.467 & 1493.922 & 1494.348 & 1493.681 & 1495.432 & 1493.659 & 1484.75 & 1479.397\end{array}$ $\begin{array}{llllllllll}1488.259 & 1488.467 & 1493.923 & 1494.344 & 1493.679 & 1495.432 & 1493.656 & 1484.75 & 1479.397\end{array}$ $\begin{array}{lllllllllll}1488.266 & 1488.476 & 1493.923 & 1494.344 & 1493.681 & 1495.432 & 1493.659 & 1484.755 & 1479.397\end{array}$ $\begin{array}{lllllllll}1488.27 & 1488.476 & 1493.927 & 1494.351 & 1493.684 & 1495.439 & 1493.661 & 1484.757 & 1479.4\end{array}$ $\begin{array}{llllllllll}1488.275 & 1488.483 & 1493.927 & 1494.358 & 1493.688 & 1495.449 & 1493.664 & 1484.757 & 1479.402\end{array}$ $\begin{array}{llllllllll}1488.282 & 1488.481 & 1493.934 & 1494.37 & 1493.698 & 1495.474 & 143.671 & 1484.759 & 1479.404 \\ 1488.289 & 1488.483 & 1493.944 & 1494.393 & 1493.702 & 1495.489 & 1493.68 & 1484.762 & 1479.409\end{array}$ $\begin{array}{llllllllll}1488.293 & 1488.481 & 1493.955 & 1494.407 & 1493.714 & 1495.512 & 1493.687 & 1484.764 & 1479.411\end{array}$ $\begin{array}{lllllllll}1488.303 & 1488.485 & 1493.965 & 1494.428 & 1493.726 & 1495.543 & 1493.699 & 1484.769 & 1479.411\end{array}$ $\begin{array}{lllllllllll}1488.312 & 1488.488 & 1493.974 & 1494.456 & 1493.74 & 1495.568 & 1493.71 & 1484.769 & 1479.418\end{array}$ $\begin{array}{lllllllll}1488.321 & 1488.497 & 1493.984 & 1494.467 & 1493.752 & 1495.59 & 1493.722 & 1484.773 & 1479.421\end{array}$ $\begin{array}{llllllllll}1488.312 & 1488.518 & 1493.996 & 1494.467 & 1493.759 & 1495.585 & 1493.732 & 1484.771 & 1479.421\end{array}$ $\begin{array}{llllllllll}1488.31 & 1488.523 & 1494.002 & 1494.484 & 1493.766 & 1455.59 & 1493.743 & 1484.773 & 1479.423\end{array}$ $\begin{array}{llllllll}1489 & 149.423\end{array}$

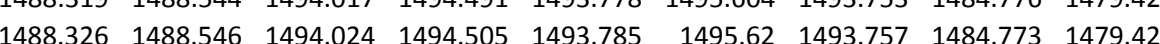
$\begin{array}{llllllllll}1488.333 & 1488.551 & 1494.031 & 1494.505 & 1493.79 & 1495.613 & 1493.757 & 1484.78 & 1479.425\end{array}$ $\begin{array}{llllllllll}1488.337 & 1488.558 & 1494.036 & 1494.509 & 1493.795 & 1495.627 & 1493.76 & 1484.78 & 1479.423\end{array}$ $\begin{array}{llllllllll}1488.347 & 1488.551 & 1494.04 & 1494.528 & 1493.802 & 1495.641 & 1493.767 & 1484.783 & 1479.425\end{array}$ $\begin{array}{llllllllll}1488.349 & 1488.549 & 1494.05 & 1494.537 & 1493.812 & 1495.658 & 1493.776 & 1484.783 & 1479.425\end{array}$ $\begin{array}{lllllllllll}1488.354 & 1488.565 & 1494.054 & 1494.537 & 1493.814 & 1495.648 & 1493.788 & 1484.783 & 1479.423\end{array}$ $\begin{array}{lllllllll}1488.351 & 1488.575 & 1494.054 & 494.528 & 1493.816 & 1495.639 & 1493.79 & 1484.783 & 1479.421\end{array}$ $\begin{array}{llllllllll}1488351 & 1488589 & 1494052 & 1494514 & 1493812 & 1495618 & 149379 & 1484783 & 1479.43\end{array}$ $\begin{array}{llllllllll}1488.354 & 1488.593 & 1494.047 & 1494.498 & 1493.804 & 1495.599 & 1493.781 & 1484.785 & 1479.418\end{array}$ $\begin{array}{lllllllllll}1488.356 & 1488.6 & 1494.05 & 1494.498 & 1493.804 & 1495.601 & 1493.786 & 1484.787 & 1479.416\end{array}$ $\begin{array}{llllllllll}1488.349 & 1488.612 & 1494.04 & 1494.484 & 1493.802 & 1495.585 & 1493.776 & 1484.785 & 1479.416\end{array}$ $\begin{array}{lllllllllll}1488.347 & 1488.631 & 1494.043 & 1494.477 & 1493.8 & 1495.566 & 1493.786 & 1484.785 & 1479.414\end{array}$ $\begin{array}{lllllllll}1488.351 & 1488.607 & 1494.045 & 1494.498 & 1493.804 & 1495.599 & 1493.783 & 1484.79 & 1479.418\end{array}$ $\begin{array}{lllllllll}1488.351 & 1488.617 & 1494.045 & 1494.498 & 1493.807 & 1495.597 & 1493.783 & 1484.79 & 1479.418 \\ 1488.358 & 1488619 & 1494.049 & 1494.502 & 1493.812 & 1495599 & 1493.786 & 1484.792 & 1479.421\end{array}$ 
Well

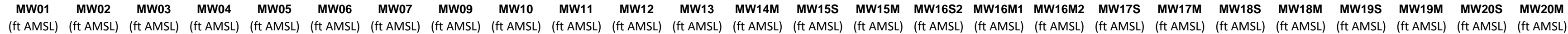

$3 / 2 / 122: 00$ $3 / 2 / 123: 00$ 3/2/12 4:00 3/2/12 5:00 $3 / 2 / 127: 00$ 3/2/12 8:00 3/2/12 9:00 3/2/12 10:00 3/2/12 11:00 3/2/12212:00 3/2/12 14:00 3/2/12 15:00 3/2/12 16:00 $3 / 2 / 1217: 00$ 3/2/12 18:00 3/2/12 19:00 3/2/122 20:00 3/2/12 21:00 3/2/12 22:00 $3 / 2 / 1223: 00$ 3/3/1/12:00 $3 / 3 / 122: 00$ $3 / 3 / 122: 00$ 3/3/122:00 3/3/12 5:00 $3 / 3 / 126: 00$ 3/3/12 8:00 3/3/12 9:00 3/3/12 10:00 3/3/12 11:00 $3 / 3 / 1212: 00$ 3/3/12 13:00 $3 / 3 / 1214: 00$ 3/3/1212 16:00 $3 / 3 / 1216: 00$
$3 / 3 / 1217: 00$ $3 / 3 / 1218: 00$ $3 / 3 / 1219: 00$ 3/3/12 20:00 $3 / 3 / 1221: 00$ $3 / 3 / 1222: 00$
$3 / 3 / 1223: 00$
$3 / 4 / 120$ 3/4/12 0:00 3/4/112 1:00 3/4/12 3:00 $3 / 4 / 124: 00$ 3/4/122:00 $3 / 4 / 126: 00$
$3 / 4 / 127: 00$ $\begin{array}{rrrr}1495.012 & 1510.688 & 1496.822 & 1495.518\end{array}$ $\begin{array}{rrrr}1495.01 & 1510.348 & 1496.81 & 1495.509 \\ 1494.998 & 1510.047 & 1496766 & 1995\end{array}$ $\begin{array}{llll}1494.998 & 1510.047 & 1496.766 & 1495.483\end{array}$ $\begin{array}{llll}1495.005 & 1509.114 & 1496.766 & 1495.476 \\ 1495.008 & 1509.399 & 1496.773 & 1495.471\end{array}$ $\begin{array}{llll}1494.987 & 1509.145 & 1496.707 & 1495.436\end{array}$ $\begin{array}{lllll}1494.984 & 1508.811 & 1496.668 & 1495.404\end{array}$ $\begin{array}{lllll}1494.982 & 1508.513 & 1496.66 & 1495.385\end{array}$ $\begin{array}{lllll}1494.977 & 1508.219 & 1496.637 & 1495.362\end{array}$ $\begin{array}{llll}1494.973 & 1507.93 & 1496.616 & 1495.341 \\ 1494.977 & 1507.604 & 149664 & 195533\end{array}$ $\begin{array}{rrrr}1494.977 & 1507.604 & 1496.64 & 1495.343 \\ 1494.98 & 1507.287 & 1496.658 & 149535\end{array}$ $\begin{array}{rrrr}1494.98 & 1507.287 & 1496.658 & 1495.35 \\ 1494.977 & 1506.993 & 1496.667 & 1495.357\end{array}$ $\begin{array}{lllll}1494.973 & 1506.727 & 1496.658 & 1459.357\end{array}$ $\begin{array}{llll}1494.973 & 1506.473 & 1496.653 & 1495.355\end{array}$ $\begin{array}{lllll}1494.97 & 1506.238 & 1496.647 & 1495.35\end{array}$ $\begin{array}{lllll}1494.966 & 1506.035 & 1496.632 & 1495.343\end{array}$ $\begin{array}{rrrr}1494.957 & 1505.86 & 1496.6 & 1495.322\end{array}$ $\begin{array}{llll}1494.95 & 1505.666 & 1496.574 & 1495.301 \\ 1494.95 & 1505.468 & 1996562 & 1495287\end{array}$ $\begin{array}{rrrr}1444.95 & 1505.468 & 1496.562 & 1495.287 \\ 1494.947 & 1505275 & 1496558 & 1495278\end{array}$ $\begin{array}{rrrr}1494.947 & 1505.275 & 1496.558 & 1495.278 \\ 1494.94 & 1505.095 & 1496.546 & 1495.266\end{array}$ $1494.94 \quad 1504.906 \quad 1496.551 \quad 14595.264$ $\begin{array}{rrrr}1494.94 & 1504.906 & 1496.551 & 1495.264 \\ 1494.936 & 1504.725 & 1496.546 & 1495.259\end{array}$ $\begin{array}{llllll}1494.931 & 1504.554 & 1496.534 & 1495.252\end{array}$ $\begin{array}{llll}1494.931 & 1504.37 & 1496.539 & 1495.252\end{array}$ $\begin{array}{llll}1494.931 & 1504.193 & 1496.546 & 1495.254\end{array}$ $\begin{array}{lllll}1494.929 & 1504.023 & 1496.546 & 1495.254\end{array}$ $\begin{array}{llll}1494.926 & 1503.981 & 1496.544 & 1495.257 \\ 1494.922 & 1503934 & 1496541 & 1495257\end{array}$ $\begin{array}{lllll}1494.922 & 1503.934 & 1496.541 & 1495.257 \\ 1494.917 & 1503.853 & 1496.532 & 1495.254\end{array}$ $\begin{array}{llll}1494.917 & 1503.727 & 1496.523 & 1495.25\end{array}$ $\begin{array}{lllll}1494.919 & 1503.559 & 1496.544 & 1495.257\end{array}$ $\begin{array}{lllll}1494.919 & 1503.389 & 1496.56 & 1495.271\end{array}$ $\begin{array}{lllll}1494.924 & 1503.221 & 1496.581 & 1495.285\end{array}$ $\begin{array}{llll}1494.926 & 1503.072 & 1496.602 & 1495.301 \\ 1494.933 & 1502.941 & 1496.632 & 1995.324\end{array}$ $\begin{array}{rrrr}1494.933 & 1502.941 & 1496.632 & 1495.324 \\ 1494.931 & 150285 & 1496.637 & 1995338\end{array}$ $\begin{array}{rrrr}1494.931 & 1502.85 & 1496.637 & 1495.338 \\ 1494.931 & 1502.789 & 1496.635 & 1495.348\end{array}$ $\begin{array}{llll}1494.926 & 1502.738 & 1496.626 & 1495.345\end{array}$ $\begin{array}{lllll}1494.924 & 1502.761 & 1496.609 & 1495.336\end{array}$ $\begin{array}{llll}1494.924 & 1502.764 & 1496.602 & 1495.331\end{array}$ $\begin{array}{lllll}1494.926 & 1502.734 & 1496.614 & 1495.338\end{array}$ $\begin{array}{lllll}1494.926 & 1502.715 & 1496.609 & 1455.338\end{array}$ $\begin{array}{llll}1494.924 & 1502.68 & 1496.616 & 1495.341 \\ 1494.926 & 1502.636 & 1496623 & 1495345\end{array}$ $\begin{array}{lllll}1494.926 & 1502.636 & 1496.623 & 1495.345 \\ 1494.929 & 1502587 & 1496.635 & 1495352\end{array}$ $\begin{array}{llll}1494.926 & 1502.54 & 1496.632 & 1495.355\end{array}$ $1494.929 \quad 1502.484 \quad 1496.637 \quad 1455.359$ $1494.931 \quad 1502.4 \quad 1496.651 \quad 1495.371$ $\begin{array}{rrrr}1494.94 & 1502.3 & 1496.677 & 1495.385 \\ 1494.936 & 1502.209 & 1496.677 & 1495.394\end{array}$ $\begin{array}{rrrr}1494.936 & 1502.209 & 1496.677 & 1495.394 \\ 1494.938 & 1502.12 & 1496.677 & 1495.399\end{array}$ $\begin{array}{rrrr}1494.938 & 1502.12 & 1496.677 & 1495.399 \\ 1494.936 & 1502.05 & 1496.67 & 1495.397\end{array}$ $\begin{array}{llllllllll}1494.407 & 1495.172 & 1495.249 & 1496.127 & 1497.238 & 1497.021 & 1493.799 & 1489.015 & 1493.646 & 1491.457 \\ 1494.414 & 1495.176 & 1495242 & 1496118 & 1497.231 & 1497.007 & 1493.797 & 1489.01 & 1493641 & 1491.457\end{array}$ $\begin{array}{llllllllll}1494.414 & 1495.172 & 1495.242 & 1496.118 & 1497.231 & 1497.007 & 1493.797 & 1489.01 & 1493.641 & 1491.457\end{array}$ $\begin{array}{llllllllllll}1494.43 & 1495.164 & 1495.22 & 1496.102 & 1496.093 & 1497.208 & 1496.956 & 1493.785 & 1489.003 & 1493.632 & 1491.454 \\ 1493 & 1491.454\end{array}$ $\begin{array}{llllllllllll}1494.418 & 1495.167 & 1495.239 & 1496.095 & 1497.205 & 1496.973 & 1493.783 & 1488.994 & 1493.63 & 1491.454\end{array}$ $\begin{array}{lllllllllll}1494.465 & 1495.157 & 1495.211 & 1496.065 & 1497.173 & 1496.916 & 1493.769 & 1488.98 & 1493.613 & 1491.45\end{array}$ $\begin{array}{llllllllllll}1494.475 & 1495.148 & 1495.201 & 1496.042 & 1497.149 & 1496.875 & 1493.753 & 1488.97 & 1493.599 & 1491.443\end{array}$ $\begin{array}{llllllllllll}1494.463 & 1495.143 & 1495.204 & 1496.03 & 1497.138 & 1496.866 & 1493.741 & 1488.968 & 1493.592 & 1491.441\end{array}$ $\begin{array}{lllllllllll}1494.456 & 1495.136 & 1495.208 & 1496.011 & 1497.124 & 1496.849 & 1493.732 & 1488.956 & 1493.581 & 1491.436\end{array}$ $\begin{array}{llllllllll}1494.451 & 1495.126 & 1495.197 & 1495.995 & 1497.105 & 1496.837 & 1493.72 & 1488.949 & 1493.569 & 1491.429\end{array}$ $\begin{array}{llllllllll}1494.411 & 1495.119 & 1495.22 & 1495.995 & 1497.11 & 1496.856 & 1493.718 & 1488.945 & 1493.564 & 1491.424 \\ 1494.378 & 1495.119 & 149523 & 1495.997 & 1497.112 & 1496877 & 1493718 & 1488947 & 1493564 & 1491.424\end{array}$ $\begin{array}{llllllllllll}1494.364 & 1495.119 & 1495.235 & 1495.997 & 1497.115 & 1496.892 & 1493.715 & 1488.949 & 1493.562 & 1491.422\end{array}$ $\begin{array}{lllllllllllll}1494.362 & 1495.117 & 1495.227 & 1495.993 & 1497108 & 1496.887 & 1493.713 & 1488.945 & 1493.557 & 1491.417\end{array}$ $\begin{array}{lllllllllll}1494.357 & 1495.114 & 1495.23 & 1495.986 & 1497.105 & 1496.885 & 1493.711 & 1488.945 & 1493.555 & 1491.417\end{array}$ $\begin{array}{lllllllllll}1494.355 & 1495.112 & 1495.223 & 1495.981 & 1497.098 & 1496.882 & 1493.703 & 1488.94 & 1493.552 & 1491.413\end{array}$ $\begin{array}{lllllllllll}1494.362 & 1495.105 & 1495.218 & 1495.972 & 1497.091 & 1496.875 & 1493.699 & 1488.942 & 1493.543 & 1491.408\end{array}$ $\begin{array}{lllllllllll}1494.383 & 1495.1 & 1495.208 & 1495.956 & 1497.073 & 1496.849 & 1493.692 & 1488.933 & 1493.538 & 1491.406\end{array}$ $\begin{array}{lrllllllll}1494.39 & 1495.09 & 1495.199 & 1495.935 & 1497.059 & 1496.825 & 1493.68 & 1488.928 & 1493.524 & 1491.401 \\ 1494388 & 1495.083 & 1495.201 & 1495.923 & 1497.047 & 1496813 & 1493.668 & 1488926 & 1493517 & 1491399\end{array}$ $\begin{array}{llllllllll}1494.388 & 1495.083 & 1495.201 & 1495.923 & 1497.047 & 1496.813 & 1493.668 & 1488.926 & 1493.517 & 1491.399 \\ 1494376 & 1495.078 & 1495206 & 1495.916 & 1497.04 & 1496813 & 1493664 & 1488928 & 1493513 & 1491394\end{array}$ $\begin{array}{lllllllllll}1494.376 & 1495.073 & 1495.201 & 1495.902 & 1497.031 & 1496.803 & 1493.657 & 1488.914 & 1493.506 & 1491.389\end{array}$ $\begin{array}{llllllllllll}1494.359 & 1495.069 & 1495.204 & 1495.898 & 1497.024 & 1496.81 & 1493.654 & 1488.912 & 1493.501 & 1491.387\end{array}$ $\begin{array}{llllllllllll}1494.35 & 1495.064 & 1495.204 & 1495.893 & 1497.019 & 1496.813 & 1493.652 & 1488.909 & 1493.498 & 1491.382\end{array}$ $\begin{array}{llllllllllll}1494.348 & 1495.059 & 1495.197 & 1495.884 & 1497.012 & 1496.803 & 1493.647 & 1488.907 & 1493.494 & 1491.38\end{array}$ $\begin{array}{llllllllll}1494.334 & 1495.054 & 1495.204 & 1495.877 & 1497.01 & 1496.81 & 1493.645 & 1488.905 & 1493.491 & 1491.378\end{array}$ $\begin{array}{llllllllll}1494.317 & 1495.05 & 1495.206 & 1495.877 & 1497.01 & 1496.82 & 1493.645 & 1488.9 & 1493.489 & 1491.375\end{array}$ $\begin{array}{lllllllllll}1494.312 & 1495.047 & 1495.206 & 1495.874 & 1497.008 & 1496.825 & 1493.645 & 1488.905 & 1493.491 & 1491.373 \\ 1494.303 & 1495.045 & 1495206 & 1495.87 & 1497.005 & 1496823 & 1493643 & 1488.905 & 1493.889 & 1491368\end{array}$ $\begin{array}{llllllllllll}1494.303 & 1495.045 & 1455.206 & 1495.87 & 1497.005 & 1496.823 & 1493.643 & 1488.905 & 1493.489 & 1491.368 \\ 1494.296 & 1495.04 & 1495.206 & 1495.867 & 1497.005 & 1496827 & 1493.638 & 1488.898 & 1493.484 & 1491368\end{array}$ $\begin{array}{llllllllllll}1494.296 & 1495.038 & 1495.197 & 1495.86 & 1496.998 & 1496.823 & 1493.636 & 1488.902 & 1493.482 & 1491.368\end{array}$ $\begin{array}{llllllllllll}1494.289 & 1495.033 & 1495.197 & 1495.851 & 1496.994 & 1496.815 & 1493.633 & 1488.893 & 1493.477 & 1491.364\end{array}$ $\begin{array}{lllllllllll}1494.265 & 1495.033 & 1495.204 & 1495.86 & 1497.001 & 1496.837 & 1493.633 & 1488.895 & 1493.477 & 1491.366\end{array}$ $\begin{array}{llllllllll}1494.244 & 1495.033 & 1495.216 & 1495.865 & 1497.008 & 1496.854 & 1493.636 & 1488.9 & 1493.482 & 1491.366\end{array}$ $\begin{array}{lllllllllll}1494.228 & 1495.035 & 1495.218 & 1495.874 & 1497.017 & 1496.87 & 1493.643 & 1488.909 & 1493.484 & 1491.366\end{array}$

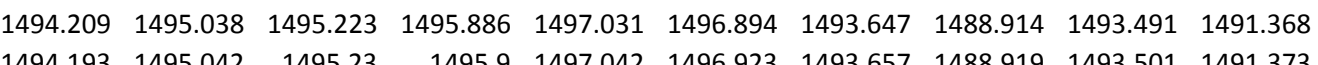
$\begin{array}{llllllllll}1494.193 & 1495.042 & 1495.23 & 1495.9 & 1497.042 & 146.923 & 1493.657 & 1488.919 & 1493.501 & 1491.373 \\ 1494.197 & 1495.047 & 1495.225 & 1495907 & 1497.052 & 1496.93 & 1493.661 & 1488923 & 1493506 & 1491.373\end{array}$ $\begin{array}{llllllllllll}1494.209 & 1495.052 & 1495.223 & 1495.907 & 1497.052 & 1496.925 & 1493.668 & 1488.928 & 1493.51 & 1491.378\end{array}$ $\begin{array}{llllllllllll}1494.225 & 1495.05 & 1495.213 & 1495.907 & 1497.049 & 1496.916 & 1493.664 & 1488.926 & 1493.506 & 1491.38\end{array}$ $\begin{array}{llllllllll}1494.242 & 1495.047 & 1495.209 & 1495.902 & 1497.045 & 1496.902 & 1493.661 & 1488.924 & 1493.506 & 1491.382\end{array}$ $\begin{array}{llllllllll}1494.251 & 1495.047 & 1495.208 & 1495.9 & 1497.042 & 1496.897 & 1493.659 & 1488.923 & 1493.506 & 1491.385\end{array}$ $\begin{array}{lllllllllll}1494.244 & 1495.045 & 1495.213 & 1495.907 & 1497.047 & 1496.906 & 1493.659 & 1488.919 & 1493.506 & 1491.387\end{array}$ $\begin{array}{llllllllll}1494.251 & 1495.045 & 1495.211 & 1495.905 & 1497.049 & 1496.904 & 1493.659 & 1488.923 & 1493.503 & 1491.389\end{array}$ $\begin{array}{lllllllllll}1494.251 & 1495.045 & 1495.213 & 1495.909 & 1497.052 & 1496.909 & 1493.664 & 1488.921 & 1493.506 & 1491.389\end{array}$ $\begin{array}{lllllllllllll}1494242 & 149505 & 1495.22 & 1495919 & 1497.063 & 1496921 & 1493.666 & 1488.928 & 19335515 & 1491.392\end{array}$ $\begin{array}{llllllllllll}1494.249 & 1495.05 & 1495.218 & 1495.921 & 1497.063 & 1496.925 & 1493.673 & 1488.931 & 1493.517 & 1491.396\end{array}$ $\begin{array}{llllllllllll}1494.249 & 1495.05 & 1495.218 & 1495.925 & 1497.068 & 1496.928 & 1493.678 & 1488.933 & 1493.522 & 1491.401\end{array}$ $\begin{array}{llllllllll}1494.242 & 1495.052 & 1495.22 & 1495.935 & 1497.075 & 1496.942 & 1493.687 & 1488.933 & 1493.529 & 1491.403\end{array}$ $\begin{array}{llllllllll}1494.228 & 1495.059 & 1495.23 & 1495.946 & 1497.091 & 1496.959 & 1493.694 & 1488.94 & 1493.536 & 1491.408\end{array}$ $\begin{array}{lllllllllll}1494.235 & 1495.062 & 1495.227 & 1495.951 & 1497.096 & 1496.966 & 1493.699 & 1488.938 & 1493.543 & 1491.413\end{array}$ $\begin{array}{lllllllllll}1494.24 & 1495.062 & 1495.225 & 1495.956 & 1497.096 & 1496.964 & 1493.701 & 1488.947 & 1493.545 & 1491.415\end{array}$ $\begin{array}{lllllllll}1488.358 & 1488.64 & 1494.052 & 1494.493 & 1493.812 & 1495.583 & 1493.793 & 1484.792 & 1479.421\end{array}$ $\begin{array}{llllllllllll}1488.363 & 1488.645 & 1494.047 & 1494.484 & 1493.807 & 1495.573 & 1493.788 & 1484.79 & 1479.423\end{array}$ $\begin{array}{lllllllllll}1488363 & 1488 & 1494035 & 1494458 & 1493795 & 149554 & 1493771 & 1484792 & 1479.48\end{array}$ $\begin{array}{llllllllll}1488.354 & 1488.652 & 1494.033 & 1494.458 & 1493.795 & 1495.543 & 1493.776 & 1484792 & 1479.418\end{array}$ $\begin{array}{llllllllllll}1488.342 & 1488.68 & 1494.007 & 1494.388 & 1493.762 & 1495.451 & 1493.746 & 1484.79 & 1479.409\end{array}$ $\begin{array}{llllllllll}1488.342 & 1488.669 & 1493.996 & 1494.379 & 1493.755 & 1495.444 & 1493.736 & 1484.79 & 1479.404\end{array}$ $\begin{array}{llllllllll}1488.342 & 1488.664 & 1493.986 & 1494.362 & 1493.743 & 1495.427 & 1493.715 & 1484.79 & 1479.402\end{array}$ $\begin{array}{lllllllll}1488.349 & 1488.664 & 1493.974 & 1494.346 & 1493.729 & 1495.404 & 1493.706 & 1484.787 & 1479.395\end{array}$ $\begin{array}{llllllllll}1488.351 & 1488.636 & 1493.97 & 1494.355 & 1493.726 & 1495.423 & 1493.696 & 1484.79 & 1479.395\end{array}$

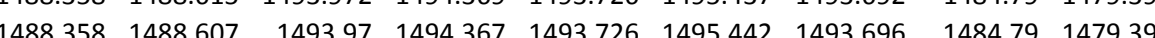
$\begin{array}{llllllllll}1488.361 & 1488605 & 1493.965 & 1494.36 & 1493.721 & 1495.437 & 1493.699 & 1484.79 & 1479.393\end{array}$ $\begin{array}{llllllllllll}1488.307 & 1488.598 & 1493.96 & 1494.362 & 1493.721 & 1495.437 & 1493.696 & 1484.79 & 1479.39\end{array}$ $\begin{array}{lllllllll}1488.31 & 1488.596 & 1493.958 & 1494.358 & 1493.714 & 1495.425 & 1493.694 & 1484.79 & 1479.388\end{array}$ $\begin{array}{lllllllllll}1488.312 & 1488.598 & 1493.951 & 1494.346 & 1493.71 & 1495.425 & 1493.692 & 1484.787 & 1479.386\end{array}$ $\begin{array}{llllllllll}1488.312 & 1488.607 & 1493.944 & 1494.323 & 1493.7 & 1495.385 & 1493.687 & 1484.787 & 1479.381\end{array}$

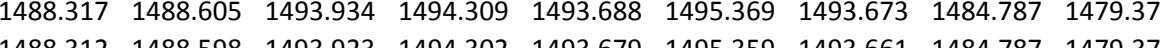
$\begin{array}{llllllllll}1488.312 & 1488.598 & 1493.923 & 1494.302 & 1493.679 & 1495.359 & 1493.661 & 1484.787 & 1479.374 \\ 148831 & 1488593 & 1493.92 & 149429 & 1493672 & 1495355 & 1493.654 & 1484.787 & 1479.374\end{array}$ $\begin{array}{llllllllll}1488307 & 1488.596 & 1493909 & 1494.281 & 1493.665 & 149534 & 1493.647 & 1484785 & 1479372\end{array}$ $\begin{array}{lllllllllll}1488312 & 1488589 & 1493.911 & 1494283 & 1493.665 & 1495343 & 1493.645 & 1484.785 & 1479372\end{array}$ $\begin{array}{lllllllllll}1488.314 & 1488.579 & 1493.904 & 1494.281 & 1493.662 & 1495.338 & 1493.642 & 1484.785 & 1479.374\end{array}$ $\begin{array}{lllllllllll}1488.314 & 1488.584 & 1493.899 & 1494.274 & 1493.658 & 1495.331 & 1493.638 & 1484.785 & 1479.374\end{array}$ $\begin{array}{lllllllllll}1488.319 & 1488.572 & 1493.899 & 1494.274 & 1493.655 & 1495.333 & 1493.635 & 1484.785 & 1479.37\end{array}$

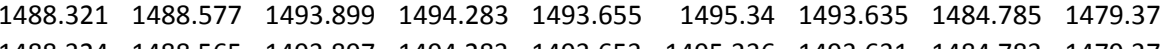
$\begin{array}{llllllllll}1488.324 & 1488.565 & 1493.897 & 1494.283 & 1493.653 & 1495.336 & 1493.631 & 1484.783 & 1479.376\end{array}$ $\begin{array}{llllllllll}1488.328 & 1488.558 & 1493.894 & 1494.278 & 1493.65 & 1495.34 & 1493.628 & 1484.783 & 1479.374 \\ 1488.33 & 1488.561 & 1493.892 & 1494.278 & 1493.648 & 149534 & 1493.626 & 1484.783 & 1479.374\end{array}$ $\begin{array}{llllllllll}1488.33 & 1488.568 & 1493.89 & 1494.274 & 1493.646 & 1495.333 & 1493.624 & 1484.783 & 1479.372\end{array}$ $\begin{array}{llllllllll}1488.324 & 1488.554 & 1493.883 & 1494.264 & 1493.643 & 1495.324 & 1493.614 & 1484.78 & 1479.36\end{array}$ $\begin{array}{llllllllll}1488.317 & 1488.535 & 1493.885 & 1494.281 & 1493.643 & 1495.343 & 1493.612 & 1484.785 & 1479.369\end{array}$ $\begin{array}{llllllllll}1488.303 & 1488.516 & 1493.887 & 1494.29 & 1493.646 & 1495.357 & 1493.605 & 1484.78 & 1479.369\end{array}$ $\begin{array}{lllllllll}1488.259 & 1488.502 & 1493.892 & 1494.306 & 1493.653 & 1495.373 & 1493.612 & 1484.78 & 1479.369\end{array}$ $\begin{array}{llllllllll}1488.25 & 1488.492 & 1493.899 & 1494.318 & 1493.66 & 1495.397 & 1493.619 & 1484.78 & 1479.372\end{array}$ $\begin{array}{lllllllll}1488252 & 1488485 & 1493913 & 149.334 & 1493672 & 1495.43 & 1493645 & 148478 & 1479374\end{array}$ $\begin{array}{llllllllll}1488.187 & 1488485 & 1493918 & 1494351 & 1493676 & 1495.43 & 1493652 & 148478 & 1479374\end{array}$ $\begin{array}{llllllllll}1488.187 & 1488.488 & 1493.916 & 1494.339 & 1493.674 & 1495.42 & 1493.649 & 1484.778 & 1479.372\end{array}$ $\begin{array}{llllllllll}1488.201 & 1488.492 & 1493.911 & 1494.33 & 1493.674 & 1495.411 & 1493.652 & 1484.778 & 1479.369\end{array}$ $\begin{array}{lllllllll}1488.208 & 1488.49 & 1493.911 & 1494.327 & 1493.669 & 1495.409 & 1493.649 & 1484.778 & 1479.367\end{array}$ $\begin{array}{llllllllll}1488.217 & 1488.483 & 1493.909 & 1494.232 & 1493.669 & 1495.416 & 1493.647 & 1484.778 & 1479.367\end{array}$ $\begin{array}{llllllllll}1488.226 & 1488.492 & 1493.911 & 1494.337 & 1493.669 & 1495.416 & 1493.647 & 1484.778 & 1479.367\end{array}$ $\begin{array}{lllllllll}1488.229 & 1488.495 & 1493.911 & 1494.337 & 1493.672 & 1495.42 & 1493.652 & 1484.776 & 1479.365 \\ 1488238 & 1488.995 & 1493918 & 1494344 & 1493674 & 1495.425 & 1493.654 & 1484778 & 1479366\end{array}$ $\begin{array}{lllllllll}1488.238 & 1488.495 & 1493.918 & 1494.374 & 1493.674 & 1495.425 & 1493.654 & 1484.778 & 1479.365\end{array}$ $\begin{array}{lllllllllll}1488.254 & 1488.502 & 1493.925 & 1494.351 & 1493.684 & 1495.432 & 1493.659 & 1484.778 & 1497.369\end{array}$ $\begin{array}{lllllllllll}1488.268 & 1488.502 & 1493.927 & 1494.358 & 1493.686 & 1495.442 & 1493.664 & 1484.776 & 1479.372\end{array}$ $\begin{array}{lllllllll}1488.273 & 1488.5 & 1493.937 & 1494.369 & 1493.693 & 1495.451 & 1493.675 & 1484.776 & 1479.376\end{array}$ $\begin{array}{lllllllllll}1488.282 & 1488.495 & 1493.942 & 1494.383 & 1493.7 & 1495.486 & 1493.673 & 1484.778 & 1479.379\end{array}$ $\begin{array}{lllllllllll}1488.289 & 1488.509 & 1493.949 & 1494.388 & 1493.71 & 1495.47 & 1493.685 & 1484.773 & 1479.381\end{array}$ $\begin{array}{lllllllll}1488.296 & 1488.511 & 1493.949 & 1494.39 & 1493.71 & 1495.479 & 1493.687 & 1484.776 & 1479.381 \\ 1488.296 & 1488518 & 1493953 & 1494.386 & 1493.712 & 1495.467 & 1493689 & 1484.776 & 1479.379\end{array}$ 
Well

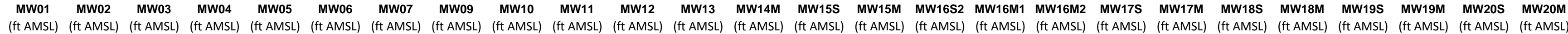

3/4/12 8:00 $3 / 4 / 129: 00$ $3 / 4 / 1211 \cdot 00$ $3 / 4 / 1212: 00$ $3 / 4 / 1213: 00$ 3/4/12 14:00 3/4/12 15:00 $3 / 4 / 1216: 00$ 3/4/12 17:00 $3 / 4 / 1218: 00$
$3 / 411219: 00$ 3/4/12 19:00 3/4/12 21:00 $3 / 4 / 4221: 00$ 3/4/12 23:00 3/5/12 0:00 3/5/121:00 3/5/12 2:00 $3 / 5 / 123: 00$ 3/5/12 5:00 $3 / 5 / 126.00$ 3/5/12 7:00 $3 / 5 / 128: 00$ 3/5/12 9:00 3/5/12 10:00 3/5/12 11:00 3/5/12 12:00 3/5/12 14:00 3/5/12 15:00 3/5/12 16:00 3/5/12 17:00 $3 / 5 / 1218: 00$ 3/5/12 19:00 3/5/12 20:00 3/5/12 21:00 3/5/12 22:00 3/5/6/12 23:00 3/6/12 1:00 3/6/12 2:00 3/6/12 3:00 $3 / 6 / 124: 00$
$3 / 6 / 125: 00$ $3 / 6 / 125: 00$ 3/6/12 6:00 3/6/12 8:00 3/6/12 9:00 3/6/12 10:00 $3 / 6 / 1211: 00$ $3 / 6 / 1212: 00$
$3 / 6 / 1213: 00$ $\begin{array}{lllll}1494.931 & 1501.966 & 1496.644 & 1495.383 \\ 1494.926 & 1501.85 & 1496.625 & 1495.369\end{array}$ $\begin{array}{llll}1494.926 & 1501.85 & 1496.625 & 1495.369 \\ 1494.926 & 1501.717 & 1496.611 & 1495.357\end{array}$ $\begin{array}{llll}1494.926 & 1501.717 & 1496.611 & 1495.357\end{array}$ $\begin{array}{rrrr}1494.924 & 1501.568 & 1496.602 & 1495.35 \\ 1494.929 & 1501.416 & 1496.607 & 1495.343\end{array}$ $\begin{array}{llll}1494.926 & 1501.267 & 1496.609 & 1495.345\end{array}$ $\begin{array}{llll}1494.926 & 1501.267 & 1496.609 & 1495.345 \\ 1494.929 & 1501.134 & 1496.625 & 1495.352\end{array}$ $1494.924 \quad 1501.013 \quad 1496.618 \quad 1495.35$ $\begin{array}{rrrr}1494.922 & 1500.924 & 1496.607 & 1495.343\end{array}$ $\begin{array}{llll}1494.919 & 1500.852 & 1496.595 & 1495.336\end{array}$ $\begin{array}{rrrr}1494.912 & 1500.794 & 1496.569 & 1495.32 \\ 1494.903 & 1500.749 & 1496.53 & 1495.287\end{array}$ $\begin{array}{rrrr}1494.903 & 1500.749 & 1496.53 & 1495.287 \\ 1494.898 & 1500.694 & 1496.504 & 1495.261\end{array}$ $\begin{array}{lllll}1494.896 & 1500.642 & 1466.504 & 1495.261 \\ 1495.243\end{array}$ $\begin{array}{rlrrr}1494.884 & 1500.612 & 1496.445 & 1495.243 \\ 1495.21\end{array}$ $\begin{array}{lllll}1494.88 & 1500.568 & 1496.429 & 1495.187\end{array}$ $\begin{array}{llll}1494.884 & 1500.495 & 1496.431 & 1495.177\end{array}$ $\begin{array}{llll}1494.877 & 1500.418 & 1496.429 & 1495.168\end{array}$ $\begin{array}{lllll}1494.875 & 1500.346 & 1496.424 & 1495.161\end{array}$ $\begin{array}{lllll}1494.87 & 1500.269 & 1496.431 & 1495.161 \\ 1494.875 & 1500.174 & 1496.453 & 1995.173\end{array}$ $\begin{array}{llll}1494.875 & 1500.092 & 1496.452 & 1495.18\end{array}$ $\begin{array}{lllll}1494.87 & 1500.008 & 1496.452 & 1495.182\end{array}$ $\begin{array}{rrrrr}1494.87 & 1509.008 & 1496.452 & 1495.182 \\ 1494.873 & 1499.924 & 1496.455 & 1495.182\end{array}$ $\begin{array}{llllll}1494.868 & 1499.833 & 1496.452 & 1495.182\end{array}$ $\begin{array}{lllll}1494.87 & 1499.7 & 1496.462 & 1495.189\end{array}$ $\begin{array}{rrrrr}1494.873 & 1499.551 & 1466.478 & 1495.198\end{array}$ $\begin{array}{rrrr}1494.875 & 1499.4 & 1496.502 & 1495.215 \\ 149488 & 1499.253 & 1965525 & 19523\end{array}$ $\begin{array}{rrrr}1494.88 & 1499.253 & 1496.525 & 1495.233 \\ 1494.891 & 1499.106 & 1496.574 & 1495.264\end{array}$ $\begin{array}{llll}1494.891 & 1499.106 & 1496.574 & 1495.264 \\ 1494.901 & 1498.964 & 1496.628 & 1495.301\end{array}$ $\begin{array}{lllll}1494.908 & 1498.847 & 1496.658 & 1495.331\end{array}$ $\begin{array}{lllll}1494.91 & 1498.758 & 1496.679 & 1495.357\end{array}$ $\begin{array}{lllll}1494.917 & 1498.693 & 1496.707 & 1495.385\end{array}$ $\begin{array}{lllll}1494.915 & 1498.658 & 1496.707 & 1495.399\end{array}$ $\begin{array}{llll}1494.922 & 1498.633 & 1496.707 & 1495.406 \\ 1499.924 & 1498.605 & 1496719 & 195 \\ 1498\end{array}$ $\begin{array}{lllll}1494.924 & 1498.605 & 1496.719 & 1495.418\end{array}$ $\begin{array}{lllll}1494.929 & 1498.563 & 1496.719 & 1495.422 \\ 1494.931 & 1498516 & 1496.728 & 1495.429\end{array}$ $\begin{array}{lllll}1494.936 & 1498.462 & 1496.733 & 1495.436\end{array}$ $\begin{array}{llllll}1494.94 & 1498.416 & 1496.749 & 1495.443\end{array}$ $\begin{array}{llllll}1494.943 & 1498.371 & 1496.759 & 1495.455\end{array}$ $\begin{array}{lllll}1494.95 & 1498.323 & 1496.777 & 1495.467\end{array}$ $\begin{array}{llll}1494.95 & 1498.281 & 1496.791 & 1495.481\end{array}$ $\begin{array}{rrrr}1494.954 & 1498.248 & 1496.798 & 1495.49\end{array}$ 1494.957 $1498.22 \quad 1496.796 \quad 1495.497$ $1494.9591498 .169 \quad 1496.784 \quad 1495.488$ $1494.957 \quad 1498.136 \quad 1496.773 \quad 1455.483$ $1494.959 \quad 1498.099 \quad 1496.763 \quad 1495.476$ $1494.963 \quad 1498.033 \quad 1496.777 \quad 1495.478$ $\begin{array}{llll}1494.966 & 1497.973 & 1496.78 & 1495.478\end{array}$ $\begin{array}{rrrr}1494.97 & 1497.891 & 1496.791 & 1495.483 \\ 1494.977 & 1497.798 & 1496.819 & 1495.502\end{array}$ $\begin{array}{llllllllll}1494.273 & 1495.062 & 1495.213 & 1495.946 & 1497.084 & 1496.928 & 1493.697 & 1488.954 & 1493.541 & 1491.417\end{array}$ $\begin{array}{llllrlllll}1494.284 & 1495.059 & 1495.204 & 1495.937 & 1497.077 & 1496.909 & 1493.692 & 1488.935 & 1493.536 & 1491.42 \\ 1494.287 & 1495.052 & 1495211 & 1955932 & 1497.07 & 1496897 & 1993.687 & 14893 & 1493531 & 149.42\end{array}$ $\begin{array}{rrrrrrrrrr}1494.287 & 1495.052 & 1495.211 & 1495.932 & 1497.07 & 1496.897 & 1493.687 & 1488.93 & 1493.531 & 1491.42 \\ 1494.282 & 1495.05 & 1495206 & 1495.928 & 1497.066 & 1496889 & 1493.682 & 1488.926 & 1493.524 & 1491.42\end{array}$ $\begin{array}{lllllllllll}1494.273 & 1495.047 & 1495.204 & 1495.928 & 1497.066 & 1496.892 & 1493.68 & 1488.921 & 1493.524 & 1491.42\end{array}$ $\begin{array}{lllllllllll}1494.261 & 1495.045 & 1495.211 & 1495.928 & 1497.07 & 1496.899 & 1493.682 & 1488.921 & 1493.527 & 1491.42\end{array}$ $\begin{array}{llllllllll}1494.247 & 1495.05 & 1495.216 & 1495.935 & 1497.077 & 1496.913 & 1493.685 & 1488.924 & 1493.529 & 1491.42\end{array}$ $\begin{array}{llllllllllll}1494.251 & 1495.047 & 1495.211 & 1495.935 & 1497.073 & 1496.911 & 1493.687 & 1488.926 & 1493.529 & 1491.42\end{array}$ $\begin{array}{lllllllllll}1494.258 & 1495.045 & 1495.206 & 1495.928 & 1497.066 & 1496.899 & 1493.685 & 1488.923 & 1493.527 & 1491.42\end{array}$ $\begin{array}{llllllllll}1494.268 & 1495.04 & 1495.199 & 1495.921 & 1497.061 & 1496.885 & 1493.67 & 1488.916 & 1493.515 & 1491.42\end{array}$

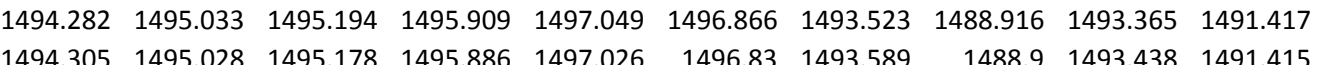
$\begin{array}{rlllllllll}1494.312 & 1455.021 & 1455.175 & 1495.87 & 1497.012 & 1496.803 & 1493.598 & 1488.895 & 1493.44 & 1491.41\end{array}$ $\begin{array}{llllllllllll}1494.31 & 1495.014 & 1495.173 & 1495.858 & 1497.001 & 1496.796 & 1493.596 & 1488.893 & 1493.442 & 1491.406\end{array}$ $\begin{array}{llllllllllll}1494.334 & 1495.004 & 1495.159 & 1495.826 & 1496.973 & 1496.753 & 1493.584 & 1488.884 & 1493.43 & 1491.399\end{array}$ $\begin{array}{lllllllllll}1494.324 & 1494.999 & 1495.159 & 1495.812 & 1496.959 & 1496.739 & 1493.577 & 1488.884 & 1493.426 & 1491.392\end{array}$ $\begin{array}{llllllllll}1494.303 & 1494.992 & 1495.166 & 1495.802 & 1496.954 & 1496.739 & 1493.57 & 1488.87 & 1493.419 & 1491.387\end{array}$ $\begin{array}{lllllllllll}1494.289 & 1494.985 & 1495.166 & 1495.793 & 1496.945 & 1496.743 & 1493.568 & 1488.87 & 1493.416 & 1491.382\end{array}$ $\begin{array}{llllllllll}1494.277 & 1494.983 & 1455.166 & 1495.786 & 1496.938 & 1496.739 & 1493.563 & 1488.87 & 1493.409 & 1491.375\end{array}$ $\begin{array}{rrrrrrrrrr}1494.258 & 1494.978 & 1495.17 & 1495.786 & 1496.94 & 1496.76 & 1493.563 & 1488.865 & 1493.412 & 1491.371 \\ 1494233 & 1494.975 & 1495.18 & 1495795 & 1496945 & 1496784 & 1493.565 & 1488.863 & 1493.414 & 1491.368\end{array}$ $\begin{array}{llllllllllll}1494.223 & 1494.975 & 1495.178 & 1495.789 & 1496.945 & 1496.782 & 1493.563 & 1488.865 & 1493.412 & 1491.364\end{array}$ $\begin{array}{lllllllllll}1494.218 & 1494.971 & 1495173 & 1495789 & 1496942 & 1496789 & 1493.565 & 1488.863 & 1493.409 & 1491361\end{array}$ $\begin{array}{llllllllllll}1494.204 & 1494.971 & 1495.173 & 1495.789 & 1496.945 & 1496.794 & 1493.561 & 1488.86 & 1493.407 & 1491.357\end{array}$ $\begin{array}{lllllllllll}1494.202 & 1494.963 & 1495.173 & 1495.784 & 1496.942 & 1496.789 & 1493.561 & 1488.86 & 1493.402 & 1491.354\end{array}$ $\begin{array}{lllllllllll}1494.186 & 1494.963 & 1495.173 & 1495.786 & 1496.947 & 1496.801 & 1493.556 & 1488.858 & 1493.405 & 1491.354\end{array}$ $\begin{array}{llllllllll}1494.167 & 1494.963 & 1495.18 & 1495.795 & 1496.952 & 1496.815 & 1493.558 & 1488.863 & 1493.402 & 1491.352\end{array}$ $\begin{array}{llllllllll}1494.146 & 1494.968 & 1495.187 & 1495.802 & 1496.963 & 1496.834 & 1493.423 & 1488.865 & 1493.243 & 1491.354 \\ 1494.127 & 1494.668 & 1495.192 & 1495816 & 1496.98 & 1496861 & 1493.46 & 1488872 & 1493297 & 1491354\end{array}$ $\begin{array}{llllllllllll}1494.127 & 1494.968 & 1495.192 & 1495.816 & 1496.98 & 1496.861 & 1493.46 & 1488.872 & 1493.297 & 1491.354 \\ 1494.094 & 1494.975 & 1495213 & 1495.84 & 1497.001 & 1496906 & 1493.437 & 1488.881 & 1493271 & 1491359\end{array}$ $\begin{array}{llllllllll}1494.063 & 1494.99 & 1495.22 & 1495.867 & 1497.033 & 1496.956 & 1493.5 & 1488.9 & 1493.339 & 1491.361\end{array}$ $\begin{array}{lllllllllll}1494.061 & 1495.002 & 1495.227 & 1495.891 & 1497.054 & 1496.985 & 1493.535 & 1488.907 & 1493.374 & 1491.368\end{array}$ $\begin{array}{lllllllllll}1494.066 & 1495.011 & 1495.227 & 1495.909 & 1497.073 & 1497.004 & 1493.563 & 1488.924 & 1493.402 & 1491.375\end{array}$ $\begin{array}{llllllllll}1494.07 & 1495.023 & 1495.23 & 1495.928 & 1497.094 & 1497.021 & 1493.584 & 1488.928 & 1493.426 & 1491.382\end{array}$ $\begin{array}{lllllllllll}1494.092 & 1495.03 & 1495.225 & 1495.939 & 1497.105 & 1497.028 & 1493.596 & 1488.945 & 1493.435 & 1491.389\end{array}$ $\begin{array}{lllllllllll}1494.117 & 1495.035 & 1495.22 & 1495.949 & 1497.11 & 1497.019 & 1493.608 & 1488.942 & 1493.447 & 1491.399 \\ 1494.127 & 1495.042 & 1495.225 & 1495.96 & 1497.122 & 1497.026 & 1493617 & 1488995 & 1493454 & 1491.403\end{array}$ $\begin{array}{lllllllllllll}1494.127 & 1455.042 & 1495.225 & 1495.96 & 1497.122 & 1497.026 & 1493.617 & 1488.945 & 1493.454 & 1491.403\end{array}$ $\begin{array}{lllllllllll}1449.157 & 1495.054 & 1495.22 & 1495.979 & 1497.135 & 1497.026 & 1493.629 & 1488.947 & 1493.466 & 1491.415\end{array}$ $\begin{array}{lllllllllll}1494.169 & 1495.059 & 1495.223 & 1495.988 & 1497.142 & 1497.033 & 1493.636 & 1488.956 & 1493.475 & 1491.42\end{array}$ $\begin{array}{llllllllll}1494.171 & 1495.064 & 1495.232 & 1496 & 1497.154 & 1497.04 & 1493.645 & 1488.956 & 1493.489 & 1491.427\end{array}$ $\begin{array}{llllllllll}1494.178 & 1495.069 & 1495.23 & 1496.011 & 1497.163 & 1497.05 & 1493.657 & 1488.963 & 1493.494 & 1491.431\end{array}$ $\begin{array}{llllllllll}1494.181 & 1495.073 & 1495.237 & 1496.023 & 1497.18 & 1497.055 & 1493.668 & 1488.961 & 1493.508 & 1491.441\end{array}$ $\begin{array}{llllllllll}1494.186 & 1495.078 & 1495.242 & 1496.037 & 1497.191 & 1497.066 & 1493.68 & 1488.968 & 1493.517 & 1491.443\end{array}$ $\begin{array}{rrrrrrrrrrr}1494.193 & 1495.088 & 1495.239 & 1496.053 & 1497.198 & 1497.076 & 1493.689 & 1488.973 & 1493.529 & 1491.45 \\ 1494.211 & 1495.09 & 1495237 & 1496058 & 1497203 & 1497.071 & 1493694 & 1488.98 & 1493538 & 1491.457\end{array}$

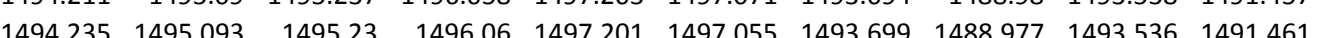
$\begin{array}{rrrrrrrrrrr}1494.235 & 1495.093 & 1495.23 & 1496.06 & 1497.201 & 1497.05 & 1493.699 & 1488.987 & 1493.536 & 1491.461 \\ 1494.244 & 1495.095 & 1495.232 & 1496.06 & 1497.203 & 1497.047 & 1493.701 & 1488.977 & 1493.543 & 1491.466\end{array}$ $\begin{array}{lllllllllll}1494.258 & 1495.1 & 1495.227 & 1496.065 & 1497.203 & 1497.038 & 1493.699 & 1488.98 & 1493.541 & 1491.468\end{array}$ $\begin{array}{llllllllll}1494.273 & 1495.097 & 1495.227 & 1496.06 & 1497.198 & 1497.026 & 1493.696 & 1488.98 & 1493.538 & 1491.468\end{array}$ $\begin{array}{llllllllll}1494.265 & 1495.1 & 1495.232 & 1496.069 & 1497.205 & 1497.036 & 1493.699 & 1488.977 & 1493.538 & 1491.473\end{array}$ $\begin{array}{llllllllll}1494.268 & 1495.102 & 1495.235 & 1496.072 & 1497.208 & 1497.035 & 1493.699 & 1488.984 & 1493.543 & 1491.475\end{array}$ $\begin{array}{llllllllll}1494.261 & 1495.107 & 1495.242 & 1496.081 & 1497.215 & 1497.043 & 1493.701 & 1488.984 & 1493.545 & 1491.478\end{array}$ $\begin{array}{lllllllll}1488.298 & 1488.525 & 1493.951 & 1494.367 & 1493.705 & 1495.449 & 1493.682 & 1484.771 & 1479.376\end{array}$ $\begin{array}{lllllllll}1488.291 & 1488.525 & 1493.942 & 1494.36 & 1493.703 & 1495.439 & 1493.673 & 1484.769 & 1479.377\end{array}$ $\begin{array}{lllllllll}1488298 & 1488521 & 1493939 & 149438 & 1493.695 & 1455.423 & 1493.666 & 1484.771 & 1479.372\end{array}$ $\begin{array}{llllllllll}1488.298 & 1488.514 & 1493.93 & 1494.346 & 1493.688 & 1495.416 & 1493.654 & 1484.769 & 1479.367\end{array}$ $\begin{array}{llllllllll}1488.305 & 1488.497 & 1493.932 & 1494348 & 1493.691 & 1495.423 & 1493.652 & 1484.771 & 1479.369\end{array}$ $\begin{array}{lllllllllll}1488.303 & 1488.488 & 1493.934 & 1494.355 & 1493.695 & 1495.43 & 1493.661 & 1484.769 & 1479.372\end{array}$ $\begin{array}{llllllllll}4488.307 & 1488.492 & 1493.934 & 1494.353 & 1493.695 & 1495.427 & 1493.671 & 1484.769 & 1479.369\end{array}$ $\begin{array}{lllllllll}1488.312 & 1488.488 & 1493.932 & 1494.344 & 1493.691 & 1495.418 & 1493.671 & 1484.766 & 1479.367\end{array}$ $\begin{array}{lllllllll}1488.312 & 1488.495 & 1493.923 & 1494.332 & 1493.679 & 1495.409 & 1493.659 & 1484.766 & 1479.365\end{array}$ $\begin{array}{lllllllllll}1488.298 & 1488.497 & 1493.772 & 1494.243 & 1493.529 & 1495.38 & 1493.52 & 1484.762 & 1479.362\end{array}$ $\begin{array}{llllllllll}1488.259 & 1488.502 & 1493.85 & 1494.248 & 1493.605 & 1495322 & 1493.593 & 1484762 & 1479351\end{array}$ $\begin{array}{lllllllll}1488.236 & 1488.5 & 1493.847 & 1494.241 & 1493.603 & 1495.315 & 1493.593 & 1484.759 & 1479.348\end{array}$ $\begin{array}{llllllllll}1488.203 & 1488.507 & 1493.838 & 1494.215 & 1493.594 & 1495.272 & 1493.579 & 1484.757 & 1479.341\end{array}$ $\begin{array}{lllllllll}1488.146 & 1488.5 & 1493.831 & 1494.199 & 1493.586 & 1495.256 & 1493.57 & 1484.757 & 1479.339\end{array}$ $\begin{array}{rrrrrrrrr}1488.12 & 1488.481 & 1493.829 & 1494.201 & 1493.579 & 1495.258 & 1493.558 & 1484.757 & 1479.339 \\ 1488.099 & 1488.478 & 1493.822 & 1494.197 & 1493.575 & 1495.254 & 1493.558 & 1484.755 & 1479.339\end{array}$ $\begin{array}{llllllllll}1488.099 & 1488.478 & 1493.822 & 1494.197 & 1493.575 & 1495.254 & 1493.558 & 1484.755 & 1479.339\end{array}$ $\begin{array}{lllllllllll}1488.078 & 1488.476 & 1493.817 & 1494.194 & 1493.572 & 1455.246 & 1493.551 & 1484.757 & 1479.339\end{array}$ $\begin{array}{lllllllllll}1488.055 & 488.474 & 1493.817 & 1494.192 & 1493.57 & 1455.249 & 1433.558 & 1484.752 & 1479.341\end{array}$ $\begin{array}{llllllllll}488.014 & 1488.455 & 1493.819 & 1494.204 & 1493.575 & 1495.265 & 1493.553 & 1484.752 & 1479.344\end{array}$ $\begin{array}{lllllllllll}1487.991 & 1488.453 & 1493.817 & 1494.208 & 1493.57 & 1495.265 & 1493.556 & 1484.752 & 1479346\end{array}$ $\begin{array}{llllllllll}1487.963 & 1488.448 & 1493.812 & 1494.211 & 1493.572 & 1495.268 & 1493.553 & 1484.752 & 1479.344\end{array}$ $\begin{array}{lllllllll}1487.937 & 1488.441 & 1493.808 & 1494.204 & 1493.565 & 1495.268 & 1493.541 & 1484.752 & 1479.341\end{array}$ $\begin{array}{lllllllll}1487.914 & 1488.431 & 1493.812 & 1494.208 & 1493.565 & 1495.277 & 1493.541 & 1484.75 & 1479.339\end{array}$ $\begin{array}{llllllllll}1487.882 & 1488.429 & 1493.808 & 1494.22 & 1493.567 & 1495.293 & 1493.537 & 1484.75 & 1479.337\end{array}$ $\begin{array}{llllllllll}1487.863 & 1488.413 & 1493.657 & 1494.211 & 1493.43 & 1495.31 & 1493.389 & 1484.75 & 1479.337\end{array}$ $\begin{array}{llllllllll}1487.847 & 1488.401 & 1493.704 & 1494.199 & 1493.47 & 1495.319 & 1493.436 & 1484.75 & 1479.339 \\ 1487.829 & 1488.382 & 1493.678 & 1494.213 & 1493.444 & 1495.357 & 1493.405 & 1484.752 & 1479.34\end{array}$ $\begin{array}{llllllllll}1487.806 & 1488.368 & 1493.742 & 1494.269 & 1493.513 & 1495.409 & 1493.476 & 1484.75 & 1479.35\end{array}$ $\begin{array}{lllllllllll}1487.789 & 1488.373 & 1493.777 & 1494.299 & 1493.546 & 1495.434 & 1493.516 & 1484.752 & 1479.35\end{array}$ $\begin{array}{lllllllllll}1487.718 & 1488.368 & 1493.803 & 1494.32 & 1493.575 & 1495.453 & 1493.541 & 1484.75 & 1479.358\end{array}$ $\begin{array}{lllllllllll}1487.697 & 1488.375 & 1493.829 & 1494.346 & 1493.594 & 1495.477 & 1493.565 & 1484.75 & 1479.362\end{array}$ $\begin{array}{llllllllll}1487.685 & 1488.387 & 1493.843 & 1494.353 & 1493.605 & 1495.484 & 1493.586 & 1484.75 & 1479.365\end{array}$ $\begin{array}{llllllllll}1487.667 & 1488.399 & 1493.855 & 1494.365 & 1493.615 & 1455.486 & 1493.593 & 1484.748 & 1479.362\end{array}$

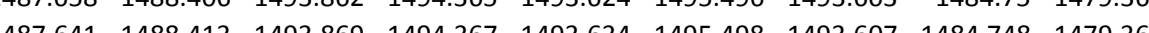
$\begin{array}{lllllllllll}1487.623 & 1488.417 & 1493.873 & 1494.379 & 1493.639 & 1495503 & 1493.612 & 1484.748 & 1479.36\end{array}$ $\begin{array}{lllllllllll}1487.611 & 1488.422 & 1493.885 & 1494.386 & 1493.646 & 1495.507 & 1493.621 & 1484.748 & 1479.36\end{array}$ $\begin{array}{llllllllll}1487.598 & 1488.424 & 1493.89 & 1494.397 & 1493.658 & 1495.521 & 1493.628 & 1484.75 & 1479.362\end{array}$ $\begin{array}{llllllllll}1487.584 & 1488.431 & 1493.899 & 1494.407 & 1493.667 & 1495.531 & 1493.64 & 1484.75 & 1479.367\end{array}$ $\begin{array}{llllllllll}4487.574 & 1488.436 & 1493.911 & 1494.418 & 1493.679 & 1495.543 & 1493.647 & 1484.75 & 1479.372\end{array}$ $\begin{array}{llllllllll}1487.561 & 1488.443 & 1493.925 & 1494.43 & 1493.686 & 1495.559 & 1493.661 & 1484.75 & 1479.374\end{array}$ $\begin{array}{lllllllll}1487.547 & 1488.453 & 1493.937 & 1494.444 & 1493.698 & 1495.568 & 1493.673 & 1484.75 & 1479.379\end{array}$ $\begin{array}{llllllllll}4877519 & 1488483 & 1493.944 & 1494.439 & 1493707 & 1495557 & 1493685 & 148475 & 1479.383\end{array}$ $\begin{array}{llllllllllll}1487.491 & 1488.485 & 1493.949 & 1494.437 & 1493.707 & 1495.557 & 1493.685 & 1484.75 & 1479.386\end{array}$ $\begin{array}{llllllllll}1487.473 & 1488.497 & 1493.946 & 1494.435 & 1493.707 & 1495.55 & 1493.685 & 1484.75 & 1479.381\end{array}$ $\begin{array}{llllllllll}1487.463 & 1488.507 & 1493.946 & 1494.428 & 1493.705 & 1495.538 & 1493.68 & 1484.748 & 1479.381\end{array}$ $\begin{array}{llllllllll}1487.443 & 1488.5 & 1493.946 & 1494.432 & 1493.71 & 1495.543 & 1493.678 & 1484.748 & 1479.376\end{array}$ $\begin{array}{llllllllll}1487.438 & 1488.5 & 1493.946 & 1494.435 & 1493.71 & 1495.545 & 1493.678 & 1484.745 & 1479.376\end{array}$ $\begin{array}{llllllllll}1487.403 & 1488.497 & 1493.951 & 1494.444 & 1493.712 & 1495.554 & 1493.675 & 1484.745 & 1479.372 \\ 1487.369 & 1488.488 & 1493.958 & 1494.456 & 1493.721 & 1495.578 & 1493.678 & 1484.748 & 1479.376\end{array}$ 
Well

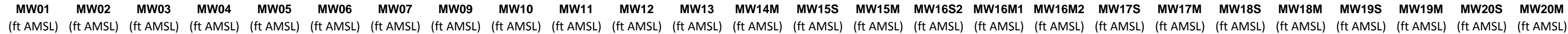

$3 / 6 / 1214: 00$ $3 / 6 / 1215: 00$ $3 / 6 / 1216: 00$ $3 / 6 / 1217: 00$ 3/6/12 19:00 3/6/12 20:00 3/6/12 21:00 3/6/12 22:00 3/6/12 23:00 $3 / 7 / 120: 00$ 3/7/12 1:00 3/7/122:00 3/7//12 3:00 3/7/125:00 3/7/12 6:00 3/7/12 7:00 3/7/12 8:00 (3/7/129.00 3/7//12 11:00 3/7/12 13:00 3/7/12 14:00 3/7/12 15:00 3/7/122 16:00 3/7/12 17:00 3/7/12 18:00 $3 / 7 / 1220: 00$ 3/7/1221:00 3/7//12 22:00 3/7/12 23:00 3/8/12 2:00 $3 / 8 / 121: 00$ 3/8/122 2:00 3/8/12 3:00 3/8/12 5:00 3/8/125:12 6:00 3/8/12 7:00 3/8/12 8:00 3/8/12 9:00 3/8/12 10:00 /8/12212.00 3/8/12 12:00 3/8/12 14:00 3/8/12 15:00 3/8/12 16:00 $3 / 8 / 1217: 00$ 3/8/12 18:00 $\begin{array}{rrrr}1494.984 & 1497.73 & 1496.843 & 1495.518\end{array}$ $\begin{array}{llll}1494.989 & 1497.674 & 1496.855 & 1495.53 \\ 1494.984 & 1497.651 & 1496843 & 1495532\end{array}$ $\begin{array}{lllll}1494.984 & 1497.651 & 1496.843 & 1495.532 \\ 1494.982 & 1497.658 & 1496.824 & 1495.523\end{array}$ $\begin{array}{lllll}1494.987 & 1497.656 & 1496.826 & 1495.523\end{array}$ $\begin{array}{lllll}1494.984 & 1497.667 & 1496812 & 1455.523\end{array}$ $\begin{array}{lllll}1494.984 & 1497.667 & 1496.812 & 1495.513 \\ 1494.987 & 1497.667 & 1496.815 & 1495.511\end{array}$ $\begin{array}{lllll}1494.989 & 1497.66 & 1496.808 & 1495.502\end{array}$ $1494.989 \quad 1497.658 \quad 1496.803 \quad 1495.495$ $\begin{array}{lllll}1494.991 & 1497.644 & 1496.803 & 1495.492\end{array}$ $\begin{array}{rrrr}1494.996 & 1497.623 & 1496.808 & 1495.49 \\ 1494.991 & 1497.616 & 1496.81 & 1495.49\end{array}$ $\begin{array}{rrrr}1494.991 & 1497.616 & 1496.81 & 1495.49 \\ 1494.994 & 1497.609 & 1496.801 & 1495.485\end{array}$ $\begin{array}{lllll}1494.998 & 1497.586 & 1496.822 & 1495.485\end{array}$ $\begin{array}{llll}1495.001 & 1497.574 & 1496.819 & 1495.495\end{array}$ $\begin{array}{lllll}1494.994 & 1497.576 & 1496.801 & 1495.483\end{array}$ $\begin{array}{lllll}1494.996 & 1497.567 & 1496.794 & 1495.478\end{array}$ $\begin{array}{lllll}1494.998 & 1497.551 & 1496.794 & 1495.474\end{array}$ $\begin{array}{llll}1494.998 & 1497.53 & 1496.789 & 1495.471\end{array}$ $\begin{array}{llll}1494.994 & 1497.511 & 1496.77 & 1495.455\end{array}$ $\begin{array}{llll}1494.987 & 1497.488 & 1496.738 & 1495.434 \\ 1494.987 & 1497.451 & 1496.726 & 1495.415\end{array}$ $\begin{array}{llll}1494.989 & 1497.416 & 1496.726 & 1495.411\end{array}$ $\begin{array}{lllll}1494.987 & 1497.383 & 1496.726 & 1495.404\end{array}$ $\begin{array}{llllll}1494.987 & 1497.364 & 1496.712 & 1495.392\end{array}$ $\begin{array}{lllll}1494.987 & 1497.334 & 1496.714 & 1495.387\end{array}$ $\begin{array}{llll}1494.977 & 1497.339 & 1496.682 & 1495.371\end{array}$ $\begin{array}{lllll}1494.97 & 1497.343 & 1496.663 & 1495.352\end{array}$ $\begin{array}{rrrrrr}1494.973 & 1497.364 & 1496.651 & 1495.338 \\ 1494.954 & 1497.42 & 1496.595 & 1495301\end{array}$ $\begin{array}{lllll}1494.957 & 1497.437 & 1496.579 & 1495.278\end{array}$ $\begin{array}{lllll}1494.952 & 1497.446 & 1496.565 & 1495.261\end{array}$ $\begin{array}{lllll}1494.94 & 1497.434 & 1496.537 & 1495.236\end{array}$ $\begin{array}{llll}1494.938 & 1497.427 & 1496.518 & 1495.215\end{array}$ $\begin{array}{llll}1494.938 & 1497.43 & 1496.52 & 1495.205\end{array}$ $\begin{array}{llll}1494.929 & 1497.467 & 1496.459 & 1495.163 \\ 1499.919 & 147.497 & 1466.445 & 195137\end{array}$ $\begin{array}{lllll}1494.919 & 1497.497 & 1496.445 & 1495.137\end{array}$ $\begin{array}{llll}1494.915 & 1497.469 & 1496.438 & 1495.126 \\ 1494.908 & 1497.462 & 1496.413 & 1495.102\end{array}$ $\begin{array}{llll}1494.891 & 1497.444 & 1496.389 & 1495.086\end{array}$ $\begin{array}{lllll}1494.88 & 1497.434 & 1496.361 & 1495.065\end{array}$ $\begin{array}{llll}1494.891 & 1497.392 & 1496.385 & 1495.067\end{array}$ $1494.871497 .397 \quad 1496.347 \quad 1495.044$ $1494.873 \quad 1497.353 \quad 1496.345 \quad 1495.037$ 1494.8591497 .3181496 .3221495 .021 $1494.863 \quad 1497.171 \quad 149633314995.009$ $1494.863 \quad 1497.113 \quad 1496354 \quad 1495.028$ $1494.866 \quad 1497.05 \quad 1496.385 \quad 1495.049$ $1494.8591496 .998 \quad 1496.375 \quad 1495.051$ $1494.8541496 .959 \quad 1496.373 \quad 1495.053$ $1494.854 \quad 1496.94 \quad 1496.375 \quad 1495.058$

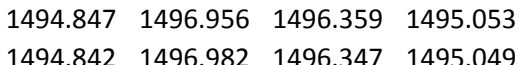

$\begin{array}{llllllllll}1494.237 & 1495.114 & 1495.261 & 1496.113 & 1497.245 & 1497.088 & 1493.722 & 1488.991 & 1493.562 & 1491.485\end{array}$

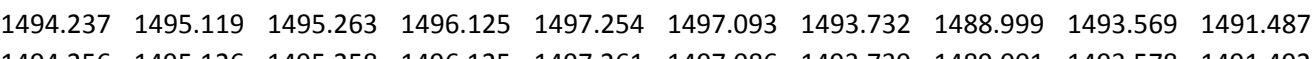
$\begin{array}{llllllllll}1494.256 & 1495.126 & 1495.258 & 1496.125 & 1497.261 & 1497.086 & 1493.739 & 1489.001 & 1493.578 & 1491.492 \\ 1494.284 & 1495.126 & 1495242 & 1496125 & 1497249 & 1497.066 & 1493734 & 1488996 & 1493576 & 1491.492\end{array}$ $\begin{array}{llllllllllll}1494.291 & 1495.126 & 1495.246 & 1496.127 & 1497.252 & 1497.066 & 1493.739 & 1489.003 & 1493.578 & 1491.496\end{array}$ $\begin{array}{llllllllll}1494.31 & 1495.126 & 1495.246 & 1496.12 & 1497.247 & 1497.047 & 1493.732 & 1488.999 & 1493.574 & 1491.496\end{array}$ $\begin{array}{llllllllllll}1494.317 & 1495.126 & 1495.247 & 1496.123 & 1497.247 & 1497.052 & 1493.732 & 1488.999 & 1493.574 & 1491.496\end{array}$ $\begin{array}{llllllllll}1494.324 & 1495.129 & 1495.254 & 1496.123 & 1497.245 & 1497.04 & 1493.727 & 1488.996 & 1493.569 & 1491.496\end{array}$ $\begin{array}{lllllllllll}1494.334 & 1495.131 & 1495.244 & 1496.123 & 1497.242 & 1497.035 & 1493.722 & 1488.996 & 1493.564 & 1491.499\end{array}$ $\begin{array}{llllllllll}1494.336 & 1495.131 & 1495.246 & 1496.123 & 1497.242 & 1497.031 & 1493.717 & 1488.994 & 1493.562 & 1491.496 \\ 1494334 & 1495.131 & 1495251 & 1966123 & 1997.245 & 1497.036 & 1493.72 & 1488999 & 149354 & 149196\end{array}$ $\begin{array}{llllllllll}1494.334 & 1495.131 & 1495.251 & 1496.123 & 1497.245 & 1497.036 & 1493.72 & 1488.999 & 1493.564 & 1491.496 \\ 1494.338 & 1495.136 & 1495.246 & 1496125 & 1497.242 & 1497.035 & 1493.72 & 1488.996 & 1493564 & 1491.496\end{array}$ $\begin{array}{llllllllll}1494.338 & 1495.136 & 445.246 & 446.125 & 147.242 & 1497.035 & 1493.72 & 1488.996 & 1493.564 & 1491.496 \\ 1494.345 & 1495.131 & 1495.246 & 1496.125 & 1497.24 & 1497.026 & 1493.72 & 1488.996 & 1493.562 & 1491.496\end{array}$ $\begin{array}{llllllllll}1494.331 & 1495.136 & 1495.265 & 1496.132 & 1497.252 & 1497.045 & 1493.727 & 1488.992 & 1493.569 & 1491.496\end{array}$ $\begin{array}{lllllllllll}1494.334 & 1495.141 & 1495.261 & 1496.137 & 1497.252 & 1497.047 & 1493.729 & 1489.005 & 1493.571 & 1491.499\end{array}$ $\begin{array}{lllllllllll}1494.352 & 1495.138 & 1495.249 & 1496.127 & 1497.242 & 1497.031 & 1493.727 & 1488.992 & 1493.574 & 1491.501\end{array}$ $\begin{array}{llllllllll}1494.359 & 1495.136 & 1495.249 & 1496.127 & 1497.24 & 1497.019 & 1493.722 & 1488.994 & 1493.569 & 1491.494\end{array}$ $\begin{array}{llllllllll}1494.362 & 1495.136 & 1495.249 & 1496.123 & 1497.238 & 1497.016 & 1493.725 & 1488.989 & 1493.569 & 1491.496\end{array}$ $\begin{array}{llllllllll}1494.359 & 1495.133 & 1495.251 & 1496.125 & 1497.235 & 197.019 & 1493.72 & 1488.994 & 1493.564 & 1491.494\end{array}$ $\begin{array}{rrrrrrrrrr}1494.374 & 1495.133 & 1495.244 & 1496.113 & 1497.222 & 1497 & 1493.713 & 1488.994 & 1493.557 & 1491.492 \\ 1494.39 & 1495.126 & 1495.23 & 1496097 & 1497.208 & 1496966 & 1493699 & 1488977 & 1493545 & 1491.487\end{array}$ $\begin{array}{lllllllllll}1494.388 & 1495.124 & 1495.23 & 1496.086 & 1497.201 & 1496.952 & 1493.692 & 1488.977 & 1493.534 & 1491.485\end{array}$ $\begin{array}{llllllllll}1494.374 & 1495.121 & 1495.235 & 1496086 & 1497196 & 1496.959 & 1493.685 & 1488.968 & 1493.529 & 1491.482\end{array}$ $\begin{array}{lllllllllll}1494.371 & 1495.119 & 1495.242 & 1496.086 & 1497.194 & 1496.966 & 1493.68 & 1488.968 & 1493.527 & 1491.48\end{array}$ $\begin{array}{llllllllllll}1494.371 & 1495.114 & 1495.239 & 1496.072 & 1497.184 & 1496.94 & 1493.673 & 1488.968 & 1493.524 & 1491.475\end{array}$ $\begin{array}{lllllllllll}1494.364 & 1495.114 & 1495.242 & 1496.069 & 1497.18 & 1496.957 & 1493.675 & 1488.968 & 1493.522 & 1491.473\end{array}$ $\begin{array}{llllllllll}1494.381 & 1495.107 & 1495.23 & 1496.053 & 1497.163 & 1496.923 & 1493.665 & 1488.956 & 1493.517 & 1491.468\end{array}$ $\begin{array}{llllllllll}1494.385 & 1495.102 & 1495.225 & 1496.042 & 1497.152 & 1496.909 & 1493.659 & 1488.952 & 1493.508 & 1491.464 \\ 1494.385 & 1495.095 & 1495225 & 1496028 & 1497.142 & 1496894 & 1493.65 & 1488.949 & 1493.96 & 1491.457\end{array}$ $\begin{array}{rrrrrrrrrrr}1494.385 & 1495.095 & 1495.225 & 1496.028 & 1497.142 & 1496.894 & 1493.65 & 1488.949 & 1493.496 & 1491.457 \\ 1494.418 & 1495.083 & 1495.201 & 1496 & 1497.11 & 1496.851 & 1493.633 & 1488.938 & 1493.48 & 1491.452\end{array}$ $\begin{array}{llllllllllll}1494.411 & 1495.073 & 1495.204 & 1495.981 & 1497.098 & 1496.83 & 1493.617 & 1488.928 & 1493.466 & 1491.445\end{array}$ $\begin{array}{llllllllll}1494.402 & 1455.066 & 1495.204 & 1495.967 & 1497.084 & 1496.825 & 1493.605 & 1488.923 & 1493.452 & 1491.436\end{array}$ $\begin{array}{lllllllllll}1494.404 & 1495.057 & 1495.199 & 1495.949 & 1497.063 & 1496.808 & 1493.586 & 1488.914 & 1493.435 & 1491.429\end{array}$ $\begin{array}{lllllllllll}1494.399 & 1495.045 & 1495.199 & 1495.93 & 1497.052 & 1496.789 & 1493.57 & 1488.905 & 1493.421 & 1491.42\end{array}$ $\begin{array}{llllllllll}1494.378 & 1495.038 & 1495.206 & 1495.919 & 1497.045 & 1496.789 & 1493.558 & 1488.902 & 1493.412 & 1491.415\end{array}$ $\begin{array}{llllllllll}1494.397 & 1495.026 & 1495.182 & 1495.87 & 1497.01 & 1496.705 & 1493.547 & 1488.895 & 1493.398 & 1491.401 \\ 1494388 & 1495.004 & 1495.182 & 1495856 & 1496.087 & 1496717 & 1493.537 & 1488884 & 1493384 & 1491394\end{array}$ $\begin{array}{llllllllll}1494.388 & 1495.004 & 1455.182 & 1495.856 & 1496.987 & 1496.717 & 1493.537 & 1488.884 & 1493.384 & 1491.394 \\ 1494.364 & 1495.002 & 1495.185 & 1495847 & 1496.98 & 1496732 & 1493.526 & 1488881 & 1493377 & 1491385\end{array}$ $\begin{array}{lllllllllllll}1494.359 & 1494.99 & 1495.182 & 1495.821 & 1496.963 & 1496.708 & 1493.509 & 1488.872 & 1493.365 & 1491.373\end{array}$ $\begin{array}{llllllllllll}1494.355 & 1494.985 & 1495.173 & 1495.805 & 1496.942 & 1496.7 & 1493.505 & 1488.867 & 1493.353 & 1491.366\end{array}$ $\begin{array}{llllllllll}1494.35 & 1494.975 & 1495.163 & 1495.784 & 1496.917 & 1496.681 & 1493.491 & 1488.856 & 1493.339 & 1491.357\end{array}$ $\begin{array}{llllllllllll}1494.301 & 1494.971 & 1495.18 & 1495.779 & 1496.924 & 1496.698 & 1493.484 & 1488.853 & 1493.33 & 1491.347\end{array}$ $\begin{array}{lllllllllll}1494.315 & 1494.963 & 1495.163 & 1495.758 & 1496.898 & 1496.689 & 1493.465 & 1488.846 & 1493.316 & 1491.338\end{array}$ $\begin{array}{llllllllll}1494.289 & 1494.954 & 1495.163 & 1495.74 & 1496.891 & 1496.674 & 1493.451 & 1488.844 & 1493.304 & 1491.329\end{array}$

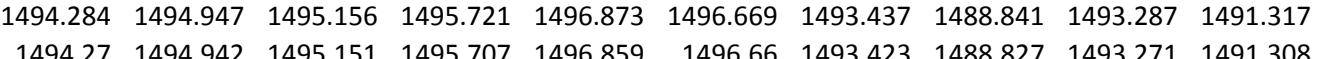
$\begin{array}{llllllllll}1494.221 & 1494.942 & 1495.151 & 1495.707 & 1466.859 & 1496.66 & 1433.423 & 1488.827 & 1493.271 & 1491.308\end{array}$ $\begin{array}{llllllllllll}1494.195 & 1494.935 & 1495.17 & 1495.71 & 1496.875 & 1496.708 & 1493.418 & 1488.827 & 1493.264 & 1491.294\end{array}$ $\begin{array}{lllllllllll}1494.16 & 1494.935 & 1495.182 & 1495.721 & 1496.88 & 1496.743 & 1493.42 & 1488.834 & 1493.266 & 1491.289\end{array}$ $\begin{array}{llllllllll}1494.16 & 1494.93 & 1495.175 & 1495.717 & 1496.88 & 1496.739 & 1493.418 & 1488.83 & 1493.266 & 1491.285\end{array}$ $\begin{array}{llllllllll}1494.15 & 1494.925 & 1495.168 & 1495.712 & 1496.877 & 1496.736 & 1493.418 & 1488.823 & 1493.264 & 1491.28\end{array}$ $\begin{array}{llllllllllll}1494.146 & 1494.928 & 1495.168 & 1495.712 & 1496.88 & 1496.741 & 1493.418 & 1488.825 & 1493.266 & 1491.278\end{array}$ $\begin{array}{llllllllll}1494.15 & 1494.92 & 1495.159 & 1495.703 & 1496.873 & 1496.734 & 1493.404 & 1488.827 & 1493.252 & 1491.273\end{array}$ $\begin{array}{rllllllll}1487.35 & 1488.49 & 1493.967 & 1494.474 & 1493.733 & 1495.599 & 1493.696 & 1484.752 & 1479.383\end{array}$ $\begin{array}{llllllllll}1487.343 & 1488.49 & 1493.979 & 1494.484 & 1493.738 & 1495.606 & 1493.708 & 1484.748 & 1479.38 \\ 1487.283 & 1488.504 & 1493979 & 1494484 & 1493745 & 1495.601 & 1493.717 & 1484748 & 147939\end{array}$

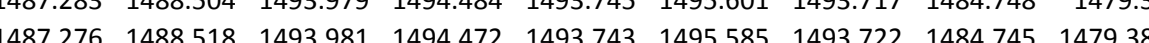
$\begin{array}{llllllllll}1487.248 & 1488.523 & 1493.981 & 1494.467 & 1493.745 & 1495.583 & 1493.727 & 1484.748 & 1479.39\end{array}$ $\begin{array}{lllllllllll}1487.246 & 1488.53 & 1493.981 & 1494.463 & 1493.738 & 1495.575 & 1493.72 & 1484.748 & 1479.39\end{array}$ $\begin{array}{lllllllll}1487.235 & 1488.537 & 1493.979 & 1494.463 & 1493.74 & 1495.575 & 1493.722 & 1484.748 & 1479.388\end{array}$ $\begin{array}{llllllllll}1487.225 & 1488.544 & 1493.974 & 1494.453 & 1493.733 & 1495.564 & 1493.71 & 1484.748 & 1479.381\end{array}$ $\begin{array}{lllllllllll}1487.216 & 1488.551 & 1493.967 & 1494.449 & 1493.731 & 1495.557 & 1493.708 & 1484.745 & 1479.381\end{array}$ $\begin{array}{lllllllllll}4487.207 & 1488.549 & 1493.963 & 1494.439 & 1493.729 & 1495.554 & 1493.701 & 1484.745 & 1479.379\end{array}$ $\begin{array}{lllllllllll}1487.198 & 1488.554 & 1493.967 & 1494.451 & 1493.729 & 1495.557 & 1493.701 & 1484.745 & 1479.379 \\ 1487.186 & 1488.563 & 1493.967 & 1494.444 & 1493.731 & 1495.552 & 1493703 & 1484745 & 1479.381\end{array}$ $\begin{array}{lllllllllll}1487.174 & 1488.57 & 1493.97 & 1494.442 & 1493.729 & 1495.543 & 1493.701 & 1484.745 & 1479.383\end{array}$ $\begin{array}{llllllllll}1487.168 & 1488.565 & 1493.972 & 1494.458 & 1493.733 & 1495.561 & 1493.701 & 1484.743 & 1479.386\end{array}$ $\begin{array}{lllllllllll}4487.158 & 1488.577 & 1493.979 & 1494.46 & 1493.738 & 1495.566 & 1493.717 & 1484.745 & 1479.39\end{array}$ $\begin{array}{lllllllllll}1487.149 & 1488.589 & 1493.977 & 1494.446 & 1493.736 & 1495.543 & 1493.717 & 1484.743 & 1479.39\end{array}$ $\begin{array}{lllllllllll}1487.128 & 1488.593 & 1493.974 & 1494.437 & 1493.733 & 1495.543 & 1493.708 & 1484.741 & 1479.39\end{array}$ $\begin{array}{lllllllll}1487.112 & 1488.596 & 1493.97 & 1494.435 & 1493.733 & 1495.543 & 1493.708 & 1484.743 & 1479.393\end{array}$

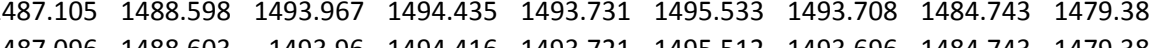
$\begin{array}{lllllllll}1487.006 & 1488.603 & 1493.96 & 1494.416 & 1493.721 & 1495.512 & 1493.696 & 1484.743 & 1479.386\end{array}$ $\begin{array}{llllllllll}1487.075 & 1488.598 & 1493.939 & 1494.383 & 1493.698 & 1459.474 & 1493.666 & 1484.738 & 1479.376\end{array}$ $\begin{array}{llllllllll}488.593 & 1493.937 & 1494.381 & 1493.693 & 1495.472 & 1493.661 & 1484.736 & 1499.372\end{array}$ $\begin{array}{lllllllllll}1487.059 & 1488.591 & 1493.934 & 1494.376 & 1493.688 & 1495.47 & 1493.666 & 1484.738 & 1479.372\end{array}$ $\begin{array}{lllllllll}1487.052 & 1488.589 & 1493.925 & 1494.372 & 1493.684 & 1495.46 & 1493.654 & 1484.741 & 1479.374\end{array}$ $\begin{array}{lllllllll}1487.045 & 1488.591 & 1493.927 & 1494.365 & 1493.684 & 1495.46 & 1493.661 & 1484.736 & 1479.374\end{array}$

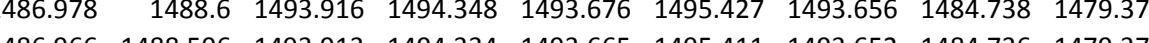

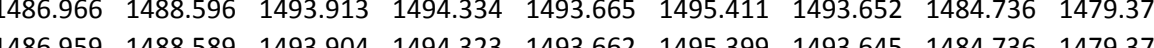
$\begin{array}{llllllllll}1486.959 & 1488.589 & 1493.904 & 1494.323 & 1493.662 & 1455.39 & 1493.645 & 1484.736 & 1479.374 \\ 1486.948 & 1488.615 & 1493.887 & 1494.285 & 1493.641 & 1495.35 & 1493633 & 1484.731 & 1479.372\end{array}$ $\begin{array}{lllllllll}1486.941 & 1488.596 & 1493.871 & 1494.271 & 1493.629 & 1495.338 & 1493.605 & 1484.736 & 1479.367\end{array}$ $\begin{array}{lllllllllll}1486.932 & 1488.593 & 1493.859 & 1494.253 & 1493.612 & 1495.317 & 1493.598 & 1484.734 & 1479.36\end{array}$ $\begin{array}{lllllllll}1486.909 & 1488.593 & 1493.845 & 1494.236 & 1493.596 & 1495.291 & 1493.581 & 1484.731 & 1479.353\end{array}$ $\begin{array}{llllllllll}4486.897 & 1488.584 & 1493.824 & 1494.211 & 1439.582 & 1495.279 & 1493.56 & 1484.729 & 1479.348\end{array}$ $\begin{array}{llllllllll}1486.888 & 1488.568 & 1493.817 & 1494.211 & 1493.567 & 1495.279 & 1493.553 & 1484.731 & 1479.346\end{array}$ $\begin{array}{llllllllll}4886.883 & 1488.579 & 1493.805 & 1494.176 & 1493.556 & 1495.232 & 1493.532 & 1484.738 & 1479.346\end{array}$

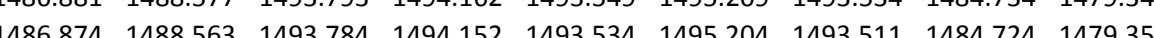
$\begin{array}{lllllllll}148686 & 1488.551 & 1493765 & 1494.132 & 1493.52 & 1495192 & 1493.483 & 1484.731 & 1479351\end{array}$

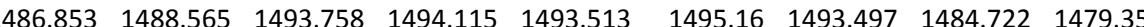
$\begin{array}{lllllllll}1486.825 & 1488.579 & 1493.749 & 1494.09 & 1493.501 & 1495.131 & 1493.497 & 1484.722 & 1479.35\end{array}$ $\begin{array}{llllllllll}1486.807 & 1488.521 & 1493.737 & 1494.101 & 1493.489 & 1495.155 & 1493.469 & 1484.726 & 1479.351\end{array}$ $\begin{array}{llllllllll}1486.795 & 1488.542 & 1493.721 & 1494.076 & 1493.475 & 1495.122 & 1493.457 & 1484.719 & 1479.346\end{array}$ $\begin{array}{llllllllll}1486.784 & 1488.514 & 1493.709 & 1494.071 & 1493.461 & 1495.124 & 1493.436 & 1484.726 & 1479.341\end{array}$ $\begin{array}{lllllllllll}1486.744 & 1488.14 & 1493.692 & 1494.05 & 1493.447 & 1455.096 & 1493.427 & 1484.722 & 1479.334\end{array}$ $\begin{array}{llllllllll}1486758 & 1488467 & 1493.671 & 1494055 & 1493.43 & 1495.113 & 1493401 & 1484.722 & 14793327\end{array}$ $\begin{array}{llllllllll}1486.749 & 1488.448 & 1493.671 & 1494.062 & 1493.425 & 1495.127 & 1493.394 & 1484.722 & 1479.323\end{array}$ $\begin{array}{llllllllll}1486.744 & 1488.434 & 1493.678 & 1494.073 & 1493.432 & 1495.148 & 1493.398 & 1484.724 & 1479.325\end{array}$ $\begin{array}{lllllllllll}1486.74 & 1488.427 & 1493.669 & 1494.073 & 1493.428 & 1495.148 & 1493.401 & 1484.719 & 1479.325\end{array}$ $\begin{array}{lllllllllll}1486.677 & 1488.413 & 1493.671 & 1494.071 & 1493.428 & 1495.145 & 1493.396 & 1484.722 & 1479.327 \\ \end{array}$ $\begin{array}{lllllllllll}1486.668 & 1488.403 & 1493.671 & 1494.076 & 1493.428 & 1495.148 & 1493.403 & 1484.722 & 1479.327\end{array}$ $\begin{array}{llllllllll}1486.666 & 1488.399 & 1493.659 & 1494.064 & 1493.416 & 1495.136 & 1493.401 & 1484.719 & 1479.33 \\ 1486.666 & 1488.403 & 1493.659 & 1494.057 & 1493.413 & 1495.127 & 1493.41 & 1484.719 & 1479.33\end{array}$ 
Well

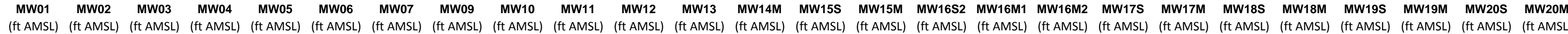

$3 / 8 / 1220: 00$ $3 / 8 / 1221: 00$ $3 / 8 / 1222: 00$
$3 / 8 / 1223: 00$ 3/8/12 23:00 3/9/120:00 $3 / 9 / 122: 00$ 3/9/12 3:00 3/9/12 2:00 3/9/12 5:00 3/9/12 6:00 3/9/12 7:00 3/9/12 9:00 3/9/12 9:00 3/9/12 11:00 3/9/12 12:00 3/9/12 13:00 $3 / 9 / 1214: 00$
$3 / 9 / 1215: 00$ $3 / 9 / 1216: 00$ 3/9/12 17:00 3/9/1218:00 3/9/12 20:00 $3 / 9 / 1221: 00$ $3 / 9 / 1222: 00$ 3/9/12 23:00 3/10/12 0:00 $3 / 10 / 121: 00$
$3 / 10 / 122: 00$ 3/10/12 3:00 3/10/12 4:00 3/10/12 5:00 3/10/12 6:00 $3 / 10 / 127: 00$
$3 / 10 / 128: 00$
$3 / 10 / 129: 00$ $3 / 10 / 1210: 00$ $3 / 10 / 1211: 00$ 3/10/12 12:00 3/10/12 13:00 3/10/12 14:00 $3 / 10 / 1215: 00$ 3/10/12 16:00 $3 / 10 / 1218.00$ 3/10/12 19:00 3/10/12 20:00 3/10/12 21:00 3/10/12 22:00 3/10/12 23:00 $3 / 11 / 120: 00$
$3 / 11 / 121: 00$ $\begin{array}{llll}1494.842 & 1496.989 & 1496.343 & 1495.044 \\ 1494.838 & 1497.001 & 1496329 & 1495.035\end{array}$ $\begin{array}{llll}1494.838 & 1497.001 & 1496.329 & 1495.035\end{array}$ $\begin{array}{lllll}4494.833 & 1497.017 & 1496.315 & 1495.023\end{array}$ $\begin{array}{llll}1494.826 & 1497.038 & 1496.294 & 1495.007 \\ 1494.831 & 1497.038 & 1496.293 & 1495.002\end{array}$ $\begin{array}{lllll}1494.824 & 1497.036 & 1496.293 & 1495.002 \\ 1495\end{array}$ $\begin{array}{rrrrr}1494.824 & 1497.036 & 1496.293 & 1495 \\ 1494.824 & 1497.029 & 1496.296 & 1494.997\end{array}$ $\begin{array}{lllll}1494.833 & 1497.008 & 1496.324 & 1495.014\end{array}$ $\begin{array}{lllll}1494.828 & 1496.989 & 1496.338 & 1495.023\end{array}$ $\begin{array}{rrrr}1494.824 & 1496.98 & 1496.331 & 1495.025 \\ 1494.824 & 1496.968 & 1496336 & 1455.032\end{array}$ $\begin{array}{llll}1494.824 & 1496.968 & 1496.336 & 1495.032 \\ 1498917 & 1469961 & 1466319 & 1455025\end{array}$ $\begin{array}{rrrr}1494.817 & 1496.961 & 1496.319 & 1495.025 \\ 1494.814 & 1496.938 & 1496.31 & 1495.021\end{array}$ $\begin{array}{rrrr}1494.814 & 1496.938 & 1496.31 & 1495.021 \\ 1494.812 & 1496.886 & 1496.303 & 1495.016\end{array}$ $\begin{array}{lllll}1494.812 & 446.886 & 1496.303 & 1495.016 \\ 149.817 & 1496.802 & 1496.317 & 1495.023\end{array}$ $\begin{array}{lllll}1494.817 & 1496.719 & 1496.331 & 1495.028\end{array}$ $\begin{array}{lllll}1494.814 & 1496.644 & 1496.343 & 1495.039\end{array}$ $\begin{array}{llll}1494.821 & 1496.565 & 1496.373 & 1495.058\end{array}$ $1494.8241496 .497 \quad 1496.4061495 .081$ $\begin{array}{llll}1494.826 & 1496.457 & 1496.431 & 1495.102 \\ 1494.826 & 1496.439 & 1496.441 & 1495.119\end{array}$ $\begin{array}{lllll}1494.826 & 1496.439 & 1496.441 & 1495.119 \\ 1494.828 & 1496.443 & 1496.455 & 1495.135\end{array}$ $\begin{array}{llllll}1494.826 & 1496.474 & 1496.464 & 1495.147\end{array}$ $\begin{array}{lllll}1494.828 & 1496.516 & 1496.471 & 1495.159\end{array}$ $\begin{array}{llllll}1494.826 & 1496.562 & 1496.464 & 1495.159\end{array}$ $\begin{array}{lllll}1494.826 & 1496.59 & 1496.462 & 1495.161\end{array}$ $\begin{array}{llll}1494.824 & 1496.621 & 1496.459 & 1495.161\end{array}$ $\begin{array}{llll}1494.821 & 1496.642 & 1496.462 & 1495.161\end{array}$ $\begin{array}{rrrrr}1494.821 & 1496.656 & 1496.462 & 1495.161 \\ 1494.824 & 1496.66 & 1496.478 & 1495.17\end{array}$ $\begin{array}{rrrrr}1494.824 & 1496.66 & 1496.478 & 1495.17 \\ 1494.828 & 1496.667 & 1496.495 & 1495.177\end{array}$ $\begin{array}{llllll}1494.831 & 1496.663 & 1496.516 & 1495.196\end{array}$ $\begin{array}{lllll}1494.833 & 1496.658 & 1496.532 & 1495.208\end{array}$ $\begin{array}{lllll}1494.833 & 1496.665 & 1496.53 & 1495.215\end{array}$ $\begin{array}{llll}1494.833 & 1496.672 & 1496.53 & 1495.217\end{array}$ $\begin{array}{llll}1494.831 & 1496.681 & 1496.527 & 1495.217 \\ 1499.831 & 1496.67 & 1496518 & 195212\end{array}$ $\begin{array}{llll}1494.831 & 1496.67 & 1496.518 & 1495.212\end{array}$ $\begin{array}{lllll}1494.828 & 1466562 & 14695511 & 1495.208\end{array}$ $\begin{array}{llll}1494.835 & 1496.497 & 1496.523 & 1495.208\end{array}$ $\begin{array}{lllll}1494.838 & 1496.427 & 1496.551 & 1495.219\end{array}$ $\begin{array}{lllll}1494.84 & 1496.373 & 1496.569 & 1495.236\end{array}$ $\begin{array}{lllll}1494.852 & 1496.329 & 1496.604 & 1495.261\end{array}$ $1494.8561496 .301 \quad 1496.63 \quad 1495.282$ $1494.859 \quad 1496.2914996 .644 \quad 1495.301$ $\begin{array}{llll}1494.861 & 1496.311 & 1496.642 & 1495.308 \\ 1494.859 & 1496345 & 1496.637 & 149531\end{array}$ $1494.861 \quad 1496.387 \quad 1496.63 \quad 1495.308$ $\begin{array}{llll}1494.866 & 1496.413 & 1496.644 & 1495.315\end{array}$ $1494.868 \quad 1496.434 \quad 1496.651 \quad 1495.32$ $1494.866 \quad 1496.45 \quad 1496.644 \quad 1495.32$ $1494.8751496 .448 \quad 1496.66 \quad 1495.329$ $\begin{array}{llll}1494.877 & 1496.455 & 1496.672 & 1495.338 \\ 1494.875 & 1496.464 & 1496.667 & 1495.336\end{array}$ $\begin{array}{llllllllll}1494.153 & 1494.916 & 1495.147 & 1495.689 & 1496.861 & 1496.722 & 1493.397 & 1488.82 & 1493.245 & 1491.266\end{array}$ $\begin{array}{llllllllll}1494.155 & 1494.911 & 1495.144 & 1495.679 & 1496.854 & 1496.712 & 1493.392 & 1488.818 & 1493.241 & 1491.261\end{array}$ $\begin{array}{rrrrrrrrrr}1494.162 & 1494.906 & 1495.135 & 1495.668 & 1496.845 & 1496.7 & 1493.381 & 1488.813 & 1493.231 & 1491.257 \\ 1494.167 & 1494.904 & 1495.125 & 1495.652 & 1496.831 & 1496681 & 1493371 & 1488811 & 1493217 & 1491.252\end{array}$ $\begin{array}{llllllllllll}1494.15 & 1494.899 & 1495.13 & 1495.645 & 1496.831 & 1496.684 & 1493.362 & 1488.809 & 1493.212 & 1491.247\end{array}$ $\begin{array}{lllllllllll}1494.141 & 1494.892 & 1495.13 & 1495.642 & 1496.824 & 1496.686 & 1493.357 & 1488.804 & 1493.208 & 1491.243\end{array}$ $\begin{array}{lllllllllll}1494.131 & 1494.892 & 1495.135 & 1495.638 & 1496.824 & 1496.691 & 1493.353 & 1488.804 & 1493.201 & 1491.24\end{array}$ $\begin{array}{lllllllllll}1494.099 & 1494.889 & 1495.147 & 1495.647 & 1496.838 & 1496.72 & 1493.355 & 1488.806 & 1493.205 & 1491.238\end{array}$ $\begin{array}{lllllllllll}1494.087 & 1494.889 & 1495.147 & 1495.654 & 1496.842 & 1496.739 & 1493.362 & 1488.813 & 1493.205 & 1491.236\end{array}$ $\begin{array}{llllllllllll}1494.087 & 1494.889 & 1495.14 & 1495.652 & 1496.838 & 1496.736 & 1493.362 & 4488.811 & 1493.21 & 1491.233\end{array}$ $\begin{array}{llllllllll}1494.08 & 1494.884 & 1495.137 & 1495.652 & 1496.845 & 1496.739 & 1493.364 & 1488.811 & 1493.21 & 1491.233 \\ 1494.087 & 1494.882 & 1495.125 & 1495.647 & 1496838 & 1496727 & 1493.36 & 1488809 & 1493208 & 1491233\end{array}$ $\begin{array}{llllllllllll}1494.089 & 1494.877 & 1495.125 & 1495.64 & 1496.835 & 1496.717 & 1493.353 & 1488.809 & 1493.203 & 1491.229\end{array}$ $\begin{array}{lllllllllll}1494.084 & 1494.875 & 1495.118 & 1495.635 & 1496.831 & 1496.71 & 1493.348 & 1488.804 & 1493.194 & 1491.229\end{array}$ $\begin{array}{lllllllllll}1494.063 & 1494.872 & 1495.128 & 1495.64 & 1496.838 & 1496.724 & 1493.343 & 1488.802 & 1493.194 & 1491.226\end{array}$ $\begin{array}{lllllllllll}1494.047 & 1494.872 & 1495.13 & 1495.647 & 1496.842 & 1496.739 & 1493.343 & 1488.809 & 1493.189 & 1491.226\end{array}$ $\begin{array}{llllllllll}1494.03 & 1494.872 & 1495.137 & 1495.652 & 1496.849 & 1496.753 & 1493.343 & 1488.811 & 1493.189 & 1491.224\end{array}$ $\begin{array}{llllllllll}1494.007 & 1494.872 & 1495.149 & 1495.666 & 1496.866 & 1496.782 & 1493.348 & 1488.82 & 1493.194 & 1491.226\end{array}$ $\begin{array}{lllllllllll}1493.986 & 1494.87 & 1495.159 & 1495.684 & 1496.884 & 1496.81 & 1493.357 & 1488.827 & 1493.201 & 1491.231 \\ 1493.976 & 1494.88 & 1495.163 & 14957 & 1496898 & 1496832 & 1493.366 & 1488834 & 149321 & 1491231\end{array}$ $\begin{array}{lllllllllll}1493.976 & 1494.88 & 1495.163 & 1495.7 & 1496.898 & 1496.832 & 1493.366 & 1488.834 & 1493.21 & 1491.231 \\ 1493.979 & 1494.882 & 1495.161 & 1495.71 & 1496.91 & 1496842 & 1493.374 & 1488839 & 1493219 & 1491.236\end{array}$

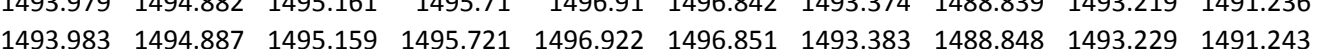
$\begin{array}{llllllllllll}1493.993 & 1494.892 & 1495.156 & 1495.728 & 1496.931 & 1496.856 & 1493.392 & 1488.851 & 1493.236 & 1491.245\end{array}$ $\begin{array}{lllllllllll}1494.005 & 1494.894 & 1495.156 & 1495.74 & 1496.938 & 1496.863 & 1493.399 & 1488.858 & 1493.243 & 1491.25\end{array}$ $\begin{array}{llllllllll}1494.023 & 1494.899 & 1495.149 & 1495.742 & 1496.94 & 1496.856 & 1493.402 & 1488.86 & 1493.248 & 1491.254\end{array}$ $\begin{array}{llllllllll}1494.035 & 1494.896 & 1495.144 & 1495.747 & 1496.945 & 1496.851 & 1493.399 & 1488.853 & 1493.245 & 1491.257\end{array}$

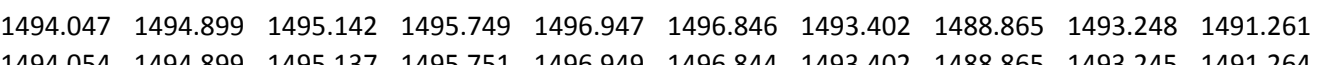
$\begin{array}{lllllllllll}1494.054 & 1494.899 & 1495.137 & 1495.751 & 1496.949 & 1496.844 & 1493.402 & 1488.865 & 1493.245 & 1491.264 \\ 1494056 & 1494899 & 1495.14 & 1495.756 & 1496952 & 1496842 & 1493399 & 1488872 & 1493243 & 1491266\end{array}$ $\begin{array}{llrlllllllll}1494.056 & 1494.899 & 1495.14 & 1495.756 & 1496.952 & 1496.842 & 1493.399 & 1488.872 & 1493.243 & 1491.266 \\ 1494.052 & 1494.904 & 1495.144 & 14957765 & 1496.963 & 1496.858 & 1493.404 & 1488.865 & 1493245 & 1491.271\end{array}$ $\begin{array}{lllllllllllll}1494.047 & 1494.906 & 1495.144 & 1495.777 & 1496.973 & 1496.87 & 1493.409 & 1488.867 & 1493.252 & 1491.273\end{array}$ $\begin{array}{lllllllllll}1494.037 & 1494.911 & 1495.154 & 1495.791 & 1496.987 & 1496.887 & 1493.416 & 1488.872 & 1493.257 & 1491.28\end{array}$ $\begin{array}{lllllllllll}1494.037 & 1494.916 & 1495.154 & 1495.802 & 1496.998 & 1496.899 & 1493.423 & 1488.877 & 1493.266 & 1491.282\end{array}$ $\begin{array}{lllllllllll}1494.047 & 1494.918 & 1495.149 & 1495.807 & 1497.003 & 1496.899 & 1493.43 & 1488.881 & 1493.271 & 1491.287\end{array}$ $\begin{array}{lllllllllll}1494.056 & 1494.92 & 1495.147 & 1495.814 & 1497.005 & 1496.892 & 1493.432 & 1488.881 & 1493.278 & 1491.289\end{array}$ $\begin{array}{rrrrrrrrrrr}1494.066 & 1494.923 & 1495.144 & 1495.816 & 1497.008 & 1496.885 & 1493.437 & 1488.881 & 1493.278 & 1491.294 \\ 1494.08 & 1494.923 & 1495.137 & 1495816 & 1497.005 & 1496875 & 1493.42 & 1488881 & 1493264 & 1491296\end{array}$

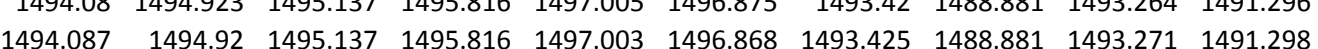
$\begin{array}{llllllllllll}1494.084 & 1494.923 & 1495.137 & 1495.819 & 1497.008 & 1496.866 & 1493.425 & 1488.881 & 1493.269 & 1491.301\end{array}$ $\begin{array}{lllllllllll}1494.073 & 1494.925 & 1495.144 & 1495.823 & 1497.012 & 1496.87 & 1493.427 & 1488.879 & 1493.269 & 1491.303\end{array}$ $\begin{array}{lllllllllll}1494.054 & 1494.928 & 1495.154 & 1495.84 & 1497.026 & 1496.899 & 1493.43 & 1488.888 & 1493.271 & 1491.305\end{array}$ $\begin{array}{llllllllll}1494.044 & 1494.935 & 1495.154 & 1495.854 & 1497.04 & 1496.913 & 1493.434 & 1488.888 & 1493.278 & 1491.305\end{array}$ $\begin{array}{lllllllllll}1494.026 & 1494.939 & 1495.168 & 1495.874 & 1497.061 & 1496.947 & 1493.448 & 1488.895 & 1493.29 & 1491.315\end{array}$ $\begin{array}{lllllllllll}1494.021 & 1494.947 & 1495.173 & 1495.891 & 1497.08 & 1496.971 & 1493.458 & 1488.898 & 1493.299 & 1491.317\end{array}$ $\begin{array}{llllllllll}1494.023 & 1494.954 & 1495.175 & 1495.907 & 1497.091 & 1496.983 & 1493.47 & 1488.907 & 1493.311 & 1491.322 \\ 1494.042 & 1494.959 & 1495.173 & 1495912 & 1497.096 & 1496976 & 1493.477 & 1488.912 & 1493318 & 1491329\end{array}$

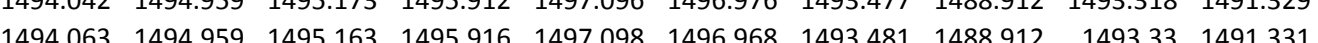
$\begin{array}{lllllllllll}1494.082 & 1494.963 & 1495.163 & 1495.921 & 1497.098 & 1496.959 & 1493.488 & 1488.916 & 1493.33 & 1491.336\end{array}$ $\begin{array}{llllllllllll}1494.087 & 1494.966 & 1495.17 & 1495.928 & 1497.108 & 1496.959 & 1493.493 & 1488.919 & 1493.337 & 1491.34\end{array}$ $\begin{array}{llllllllll}1494.094 & 1494.973 & 1495.168 & 1495.937 & 1497.117 & 1496.968 & 1493.498 & 1488.924 & 1493.339 & 1491.345\end{array}$ $\begin{array}{llllllllll}1494.108 & 1494.973 & 1495.166 & 1495.939 & 1497.115 & 1496.959 & 1493.5 & 1488.926 & 1493.344 & 1491.347\end{array}$ $\begin{array}{lllllllllllll}1494.103 & 1494.975 & 1495.175 & 1495.949 & 1497.128 & 1496.966 & 1493.502 & 1488.924 & 1493.346 & 1491.352\end{array}$ $\begin{array}{rrrrrrrrrr}1494.108 & 1494.98 & 1495.178 & 1495.958 & 1497.135 & 1496.978 & 1493.505 & 1488.928 & 1493.348 & 1491.357 \\ 1494.12 & 1494.983 & 1495.17 & 1495.96 & 1497.135 & 1496.971 & 1493.507 & 1488.931 & 1493.351 & 1491.359\end{array}$ $\begin{array}{lllllllll}1486.661 & 1488.392 & 1493.657 & 1494.057 & 1493.409 & 1495.122 & 1493.398 & 1484.722 & 1479.33\end{array}$ $\begin{array}{lllllllll}1486.626 & 1488.387 & 1493.648 & 1494.045 & 1493.402 & 1495.115 & 1493.382 & 1484.719 & 1479.325 \\ 1485585 & 1488387 & 1493638 & 1494031 & 1433.39 & 145.103 & 1493373 & 1484719 & 147932\end{array}$

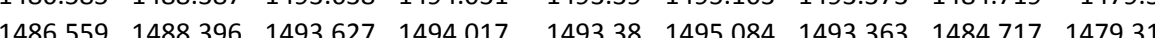
$\begin{array}{llllllllll}1486.562 & 1488.373 & 1493.617 & 1494.015 & 1493.371 & 1495.082 & 1493.347 & 1484.722 & 1479313\end{array}$ $\begin{array}{llllllllll}1486.553 & 1488.37 & 1493.61 & 1494.008 & 1493.366 & 1495.077 & 1493.344 & 1484.719 & 1479.311\end{array}$ $\begin{array}{llllllllll}1486.548 & 1488.361 & 1493.61 & 1494.01 & 1493.361 & 1495.08 & 1493.34 & 1484.719 & 1479.311\end{array}$ $\begin{array}{lllllllllll}1486.548 & 1488.349 & 1493.61 & 1494.027 & 1493.364 & 1495.108 & 1493.34 & 1484.722 & 1479.313\end{array}$ $\begin{array}{lllllllll}1486.518 & 1488.349 & 1493.612 & 1494.036 & 1493.371 & 1495.115 & 1493.349 & 1484.719 & 1479.318\end{array}$ $\begin{array}{lllllllll}1486.511 & 1488.34 & 1493.617 & 1494.031 & 1493.373 & 1459.115 & 1493.351 & 1484.717 & 1479.318\end{array}$ $\begin{array}{lllllllllll}1486.513 & 1488.345 & 1493.617 & 1494.038 & 1493.371 & 1495.115 & 1493.349 & 1484.719 & 1479.323\end{array}$

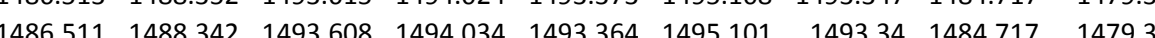
$\begin{array}{llllllllll}1486.504 & 1488.335 & 1493.603 & 1494.013 & 1493.357 & 1495.094 & 1493.333 & 1484.715 & 1479.316\end{array}$ $\begin{array}{llllllllll}1486.499 & 1488.314 & 1493.598 & 1494.024 & 1493.354 & 1495.103 & 1493.319 & 1484.717 & 1479.31\end{array}$ $\begin{array}{llllllllll}1486.495 & 1488.293 & 1493.596 & 1494.027 & 1493.352 & 1495.117 & 1493.3 & 1484.722 & 1479.306\end{array}$ $\begin{array}{llllllllll}1486.492 & 1488.281 & 1493.596 & 1494.034 & 1493.349 & 1495.129 & 1493.3 & 1484.717 & 1479.302\end{array}$ $\begin{array}{llllllllll}1486.49 & 1488.267 & 1493.601 & 1494.057 & 1493.357 & 1495.15 & 1493.297 & 1484.717 & 1479.302\end{array}$

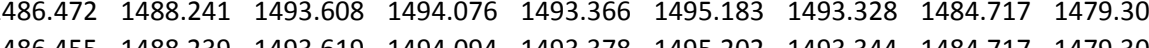

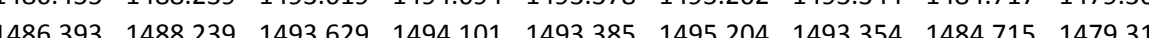
$\begin{array}{lllllllllll}1486.398 & 1488.241 & 1493.634 & 1494.115 & 1493.394 & 1495.195 & 1493.366 & 1484.715 & 1479.316\end{array}$ $\begin{array}{lllllllllll}1486.411 & 1488.253 & 1493.65 & 1494.134 & 1493.406 & 1495.225 & 1493.387 & 1484.715 & 1479.325\end{array}$ $\begin{array}{llllllllll}1486.416 & 1488.265 & 1493.652 & 1494.127 & 1493.411 & 1495.258 & 1493.389 & 1484.715 & 1479.32\end{array}$ $\begin{array}{lllllllll}1486.418 & 1488.27 & 1493.655 & 1494.127 & 1493.413 & 1495.286 & 1493.387 & 1484.715 & 1479.327\end{array}$ $\begin{array}{llllllllll}1486.414 & 1488.27 & 149.655 & 1494.129 & 1493.409 & 1495.305 & 1493.389 & 1484.715 & 1479.327\end{array}$ $\begin{array}{llllllllll}1486.414 & 1488.284 & 1493.65 & 1494.125 & 1493.409 & 1495.322 & 1493.387 & 1484.715 & 1479.325\end{array}$

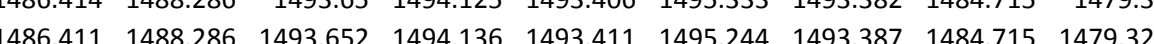
$\begin{array}{llllllllllll}1486.414 & 1488.288 & 1493.657 & 1494.143 & 1493.418 & 1495.254 & 1493.394 & 1484.715 & 1479.323\end{array}$ $\begin{array}{llllllllll}1486.411 & 1488.291 & 1493.664 & 1494.157 & 1493.425 & 1495.277 & 1493.396 & 1484.715 & 1479.325\end{array}$ $\begin{array}{lllllllllll}1486.409 & 1488.295 & 1493.674 & 1494.169 & 1493.432 & 1495.279 & 1493.403 & 1484.715 & 1479.33\end{array}$ $\begin{array}{llllllllll}1486.407 & 1488.307 & 1493.678 & 1494.173 & 1493.437 & 1495.289 & 1493.415 & 1484.715 & 1479.334\end{array}$ $\begin{array}{llllllllll}1486.405 & 1488.312 & 1493.683 & 1494.176 & 1493.442 & 1495.301 & 1493.417 & 1484.712 & 1479.33\end{array}$ $\begin{array}{llllllllll}1486.402 & 1488.328 & 1493.688 & 1494.176 & 1493.449 & 1495.31 & 1493.422 & 1484.715 & 1479.339\end{array}$

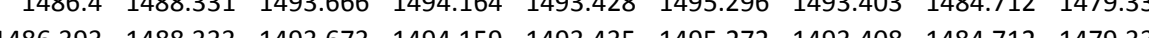
$\begin{array}{lllllllllll}1486.386 & 1488.328 & 1493678 & 1494.159 & 1493.435 & 1495.228 & 1493.394 & 1484.712 & 1479.332\end{array}$ $\begin{array}{lllllllllll}1486.384 & 1488.324 & 1493.674 & 1494.164 & 1493.435 & 1495.169 & 1493.382 & 1484.71 & 1479.327\end{array}$ $\begin{array}{llllllllll}1486.377 & 1488.312 & 1493.681 & 1494.178 & 1493.44 & 1495.298 & 1493.38 & 1484.712 & 1479.325\end{array}$ $\begin{array}{lllllllll}1486.37 & 1488.307 & 1493.685 & 1494.187 & 1493.444 & 1495.315 & 1493.394 & 1484.712 & 1479.325\end{array}$ $\begin{array}{llllllllll}1486.37 & 1488.295 & 1493.697 & 1494.218 & 1493.456 & 1495.345 & 1493.419 & 1484.712 & 1479.325\end{array}$ $\begin{array}{lllllllll}1486.37 & 1488.293 & 1493.706 & 1494.227 & 1493.468 & 1495.369 & 1493.434 & 1484.712 & 1479.33\end{array}$ $\begin{array}{llllllllll}1486.368 & 1488.302 & 493.718 & 1494.248 & 1493.48 & 1495.383 & 4993.45 & 1484.712 & 1479.332\end{array}$

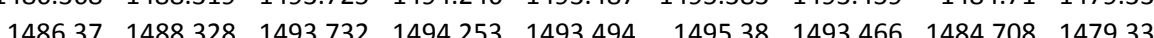
$\begin{array}{lllllllllll}1486.37 & 1488.345 & 1493.737 & 1494.243 & 1493.496 & 1495.376 & 1493.476 & 1484.712 & 1479.344\end{array}$ $\begin{array}{llllllllll}1486.368 & 1488.345 & 1493.744 & 1494.26 & 1493.503 & 1495.385 & 1493.473 & 1484.71 & 1479.346\end{array}$ $\begin{array}{lllllllll}1486.372 & 1488.349 & 1493.746 & 1494.26 & 1493.508 & 1495.39 & 1493.483 & 1484.71 & 1479.35\end{array}$ $\begin{array}{llllllllllll}1486.365 & 1488.356 & 1493.751 & 1494.262 & 1493.511 & 1495.385 & 1493.481 & 1484.71 & 1479.351\end{array}$ $\begin{array}{lllllllllll}1486.361 & 1488.347 & 1493.751 & 1494.274 & 1493.513 & 1495.404 & 1493.471 & 1484.71 & 1479.351\end{array}$ $\begin{array}{lllllllll}1486.361 & 1488.356 & 1493.756 & 1494.271 & 1493.515 & 1495.409 & 1493.483 & 1484.712 & 1479.351 \\ 1486.361 & 1488.37 & 1493.756 & 1494274 & 1493.518 & 1495.406 & 1493.49 & 1484.708 & 1479.348\end{array}$ 
Well

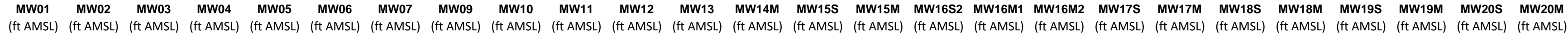

$3 / 11 / 122: 00$ 3/111/12 3:00 3/11/12 4:00 3/11/12 6:00 3/11/12 6:00 3/111/12 7:00 3/11/12 9:00 3/11/12 10:00 3/11/12 11:00 3/11/12 12:00 3/11/112 14:00 3/11/12 15:00 3/11/12 15:00 3/11/12 17:00 3/11/12 18:00 3/11/12 19:00 3/11/12 20:00 源/11/12 21:00 3/11/112 22:00 3/12/12 0:00 3/12/12 0:12 1:00 3/12/12 2:00 3/12/12 3:00 3/12/122 4:00 $3 / 12 / 125: 00$
$3 / 12 / 126: 00$
$3 / 12 / 127: 00$ 3/12/12 8:00 3/12/12 9:00 $3 / 12 / 1210: 00$ 3/12/12 11:00 3/12/12 12:00 3/12/12 13:00 3/12/12 15:00 3/12/12 16:00 3/12/12 17:00 3/12/12 19:00 3/12/12 20:00 $3 / 12 / 1221: 00$ 3/12/12 22:00 $3 / 13 / 120000$ 3/13/120:00 3/13/12 2:00 3/13/12 3:00 3/13/12 4:00 $3 / 13 / 125: 00$ 3/13/12 6:00 $\begin{array}{lllll}1494.877 & 1496.471 & 1496.667 & 1495.338 \\ 1494.882 & 1496.481 & 1496.677 & 1495.343\end{array}$ $\begin{array}{llll}1494.882 & 1496.481 & 1496.677 & 1495.343 \\ 1494.884 & 1496483 & 1496693 & 1495357\end{array}$ $\begin{array}{rrrr}1494.884 & 1496.483 & 1496.693 & 1495.357\end{array}$ $\begin{array}{lllll}1494.889 & 1496.49 & 1496.707 & 14953.369\end{array}$ $\begin{array}{lllll}1494.891 & 1496.492 & 1496.707 & 1459.373\end{array}$ $\begin{array}{llll}1494.894 & 1496.497 & 14966.71 & 1495.373\end{array}$ $\begin{array}{lllll}1494.898 & 1496.485 & 1496.719 & 1495.38\end{array}$ $\begin{array}{lllll}1494.901 & 1496.476 & 1496.733 & 1495.392\end{array}$ $1494.903 \quad 1496.469 \quad 1496.7541495 .408$ $\begin{array}{llll}1494.915 & 1496.443 & 1496.777 & 1495.425 \\ 1499.919 & 1469.434 & 1496.784 & 1955.436\end{array}$ $\begin{array}{llll}1494.919 & 1496.434 & 1496.784 & 1495.436 \\ 1494.929 & 1496.427 & 1496.805 & 1495.453\end{array}$ $\begin{array}{lllll}1494.931 & 1496.425 & 1496.822 & 1495.453 \\ 1495.467\end{array}$ $\begin{array}{lllll}1494.931 & 1496.425 & 1496.822 & 1495.467 \\ 1494.929 & 1496.429 & 1496.812 & 1495.471\end{array}$ $\begin{array}{lllll}1494.933 & 1496.427 & 1496.817 & 1495.476\end{array}$ $\begin{array}{lllll}1494.936 & 1496.427 & 1496.817 & 1495.476\end{array}$ $\begin{array}{lllll}1494.938 & 1496.439 & 1496.815 & 1995.476\end{array}$ $\begin{array}{lllll}1494.936 & 1496.453 & 1496.803 & 1495.467\end{array}$ $\begin{array}{rrrrr}1494.936 & 1496.46 & 1496.794 & 1495.455 \\ 1494.94 & 1496.471 & 1496.796 & 1495.448\end{array}$ $\begin{array}{rrrr}1494.94 & 1496.471 & 1496.796 & 1495.448 \\ 1494.943 & 1496.481 & 1496.798 & 1495.443\end{array}$ $\begin{array}{lllll}1494.945 & 1496.485 & 1496805 & 1495.446\end{array}$ $\begin{array}{lllll}1494.947 & 1496.478 & 1496.817 & 1495.453\end{array}$ $\begin{array}{lllll}1494.952 & 1496.471 & 1496.826 & 1995.457\end{array}$ $\begin{array}{lllll}1494.954 & 1496.46 & 1496.831 & 1495.462\end{array}$ $\begin{array}{rrrr}1494.95 & 1496.46 & 1496.819 & 1495.46\end{array}$ $\begin{array}{rrrr}1494.954 & 1496.455 & 1496.815 & 1495.457\end{array}$ $\begin{array}{rrrr}1494.95 & 1496.464 & 1496.791 & 1495.446 \\ 1494.945 & 1496.481 & 1496.768 & 1495.429\end{array}$ $\begin{array}{llll}1494.94 & 1496.495 & 1496.733 & 1495.404\end{array}$ $\begin{array}{llll}1494.94 & 1496.488 & 1496.712 & 1495.383\end{array}$ $\begin{array}{lllll}1494.938 & 1496.457 & 1496.703 & 1495.369\end{array}$ $\begin{array}{lllll}1494.936 & 1496.418 & 1496.689 & 1495.35\end{array}$ $1494.938 \quad 1496.366 \quad 1496.691 \quad 1495.345$ $\begin{array}{rrrr}1494.94 & 1496.315 & 1496.696 & 1495.345 \\ 1494.943 & 1496264 & 1496714 & 149535\end{array}$ $\begin{array}{llll}1494.943 & 1496.264 & 1496.714 & 1495.35 \\ 1494.94 & 1496.229 & 196.712 & 149535\end{array}$ $\begin{array}{rrrrr}1494.94 & 1496.229 & 1496.712 & 1495.35 \\ 1494.943 & 1496.208 & 1496.71 & 14953438\end{array}$ $\begin{array}{llll}1494.94 & 1496.208 & 1496.705 & 1495.345\end{array}$ $\begin{array}{lllll}1494.933 & 1496.238 & 1496.689 & 1495.334\end{array}$ $\begin{array}{lllll}1494.931 & 1496.28 & 1496.667 & 1495.32\end{array}$ $\begin{array}{lllll}1494.924 & 1496.325 & 1496.635 & 1495.299\end{array}$ $\begin{array}{lllll}1494.922 & 1496.357 & 1496.616 & 1495.28\end{array}$ $1494.919 \quad 1496.381496 .604 \quad 1495.264$ $1494.919 \quad 1496.399 \quad 1496.607 \quad 1495.257$ $\begin{array}{lllll}1494.922 & 1496.42 & 1496.625 & 1495.264\end{array}$ $1494.919 \quad 1496.432 \quad 1496.623 \quad 1495.266$ $1494.9191496 .434 \quad 1496.632 \quad 1495.268$ $1494.922 \quad 1496.434 \quad 1496.646 \quad 1495.278$ $1494.9191496 .436 \quad 1496.642 \quad 1495.28$ $\begin{array}{llll}1494.919 & 1496.425 & 1496.646 & 1495.285 \\ 1494.917 & 1496.427 & 1496.642 & 1495.282\end{array}$ $\begin{array}{llllllllll}1494.131 & 1494.985 & 1495.17 & 1495.967 & 1497.138 & 1496.971 & 1493.509 & 1488.931 & 1493.353 & 1491.361 \\ 1494.131 & 149499 & 1495.17 & 1495972 & 1497145 & 1496971 & 1493.514 & 1488.933 & 1493358 & 1491364\end{array}$ $\begin{array}{llrlllllllll}1494.131 & 1494.99 & 1495.17 & 1495.972 & 1497.145 & 1496.971 & 1493.514 & 1488.933 & 1493.358 & 1491.364\end{array}$

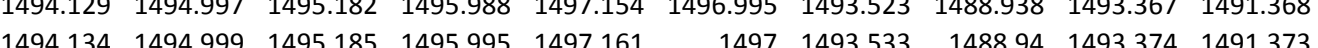
$\begin{array}{llllllllllll}1494.139 & 1495.002 & 1495.185 & 1496.002 & 1497.168 & 1496.997 & 1493.537 & 1488.945 & 1493.379 & 1491.378\end{array}$ $\begin{array}{lllllllllllll}1494.148 & 1495.004 & 1495.185 & 1496.007 & 1497.17 & 1496.995 & 1493.542 & 1488.945 & 1493.381 & 1491.378\end{array}$ $\begin{array}{lllllllllll}1494.157 & 1495.009 & 1495.185 & 1496.011 & 1497.175 & 1496.995 & 1493.547 & 1488.954 & 1493.388 & 1491.382\end{array}$ $\begin{array}{lllllllllll}1494.155 & 1495.011 & 1495.187 & 1496.023 & 1497.182 & 1497.007 & 1493.554 & 1488.949 & 1493.391 & 1491.387\end{array}$ $\begin{array}{rlllllllllll}1494.153 & 1494.999 & 1495.192 & 1496.03 & 1497.191 & 1497.004 & 1493.556 & 1488.961 & 1493.4 & 1491.389\end{array}$ $\begin{array}{llllllllll}1494.15 & 1495.011 & 1495.197 & 1496.051 & 1497.203 & 1497.04 & 1493.568 & 1488.952 & 1493.402 & 1491.396\end{array}$ $\begin{array}{rlllllllll}1494.143 & 1495.018 & 1495.208 & 1496.062 & 1497.219 & 1497.05 & 1493.573 & 1488.961 & 1493.412 & 1491.399 \\ 1494.15 & 1495.023 & 1495.208 & 1496.072 & 1497.228 & 1497.05 & 1493.573 & 1488.966 & 1493.416 & 1491.403\end{array}$ $\begin{array}{lrllllllllll}1494.15 & 1495.03 & 1495.216 & 1496.086 & 1497.242 & 1497.064 & 1993.582 & 1488.97 & 1493.421 & 1491.406\end{array}$ $\begin{array}{llllllllll}1494.153 & 1495.035 & 1495.22 & 1496.1 & 1497.254 & 1497.076 & 1493.594 & 1488.977 & 1493.433 & 1491.413\end{array}$ $\begin{array}{lllllllllll}1494.176 & 1495.04 & 1495.211 & 1496.104 & 1497.254 & 1497.069 & 1493.598 & 1488.975 & 1493.438 & 1491.417\end{array}$ $\begin{array}{lllllllllll}1494.188 & 1495.045 & 1495.213 & 1496.111 & 1497.261 & 1497.071 & 1493.605 & 1488.982 & 1493.447 & 1491.42\end{array}$ $\begin{array}{rrrrrrrrrr}1494.2 & 1495.05 & 1495.211 & 1496.116 & 1497.263 & 1497.064 & 1493.61 & 1488.984 & 1493.452 & 1491.424 \\ 1494.211 & 1495.054 & 1495.208 & 1496.12 & 1497.266 & 1497.059 & 1493.278 & 1488.984 & 1493.086 & 1491.429\end{array}$ $\begin{array}{llllllllll}1494.211 & 1495.054 & 1495.208 & 1496.12 & 1497.266 & 1497.059 & 1493.278 & 1488.984 & 1493.086 & 1491.429\end{array}$ $\begin{array}{lllllllllll}1494.232 & 1495.052 & 1455.208 & 1496.118 & 1497.261 & 1497.047 & 4993.203 & 1488.992 & 1493.008 & 1491.431\end{array}$

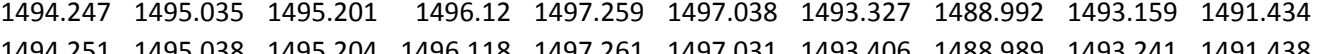
$\begin{array}{lllllllllll}1494.258 & 1495.047 & 1495.208 & 1496.123 & 1497.261 & 1497.033 & 1493.448 & 1488.992 & 1493.283 & 1491.438\end{array}$ $\begin{array}{llllllllll}1494.256 & 1495.056 & 1495211 & 1496125 & 1497.263 & 1497.035 & 1493.479 & 1488.992 & 1493.313 & 1491.438\end{array}$ $\begin{array}{lllllllllll}1494.256 & 1495.059 & 1495.216 & 1496.134 & 1497.27 & 1497.043 & 1493.498 & 1488.994 & 1493.337 & 1491.443\end{array}$ $\begin{array}{llllllllll}1494.258 & 1495.064 & 1495.22 & 1496.139 & 1497.28 & 1497.05 & 1493.519 & 1488.999 & 1493.355 & 1491.445\end{array}$ $\begin{array}{lllllllllll}1494.256 & 1495.069 & 1495.223 & 1496.148 & 1497.282 & 1497.055 & 1493.533 & 1488.999 & 1493.37 & 1491.447\end{array}$

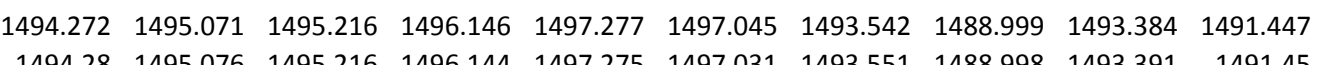
$\begin{array}{llllllllll}1494.28 & 1495.076 & 1495.216 & 1496.144 & 1497.275 & 1497.031 & 1493.551 & 1488.998 & 1493.391 & 1491.45 \\ 1494.305 & 1495076 & 1495204 & 1496137 & 1497.266 & 1497.011 & 1493556 & 1488996 & 1493398 & 1491.447\end{array}$ $\begin{array}{lllllllllll}1494.305 & 1495.076 & 1495.204 & 1496.137 & 1497.266 & 1497.011 & 1493.556 & 1488.996 & 1493.398 & 1491.447 \\ 1494.324 & 1495.076 & 1495.194 & 1496.127 & 1497.254 & 1496.99 & 1493.558 & 1488.989 & 1493395 & 1491.445\end{array}$ $\begin{array}{llllllllllll}1494.348 & 1495.071 & 1495.185 & 1496.109 & 1497.233 & 1496.956 & 1493.551 & 1488.982 & 1493.395 & 1491.445\end{array}$ $\begin{array}{lllllllllll}1494.352 & 1495.069 & 1495.187 & 1496.095 & 1497.222 & 1496.935 & 1493.547 & 1488.977 & 1493.391 & 1491.441\end{array}$ $\begin{array}{lllllllllll}1494.35 & 1495.064 & 1495.185 & 1496.088 & 1497.21 & 1496.923 & 1493.542 & 1488.97 & 1493.386 & 1491.436\end{array}$ $\begin{array}{lllllllllll}1494.348 & 1495.059 & 1495.194 & 1496.074 & 1497.201 & 1496.911 & 1493.535 & 1488.966 & 1493.379 & 1491.431\end{array}$ $\begin{array}{lllllllllll}1494.329 & 1495.059 & 1495.199 & 1496.072 & 1497.196 & 1496.913 & 1493.53 & 1488.961 & 1493.377 & 1491.427\end{array}$ $\begin{array}{llllllllll}1494.315 & 1495.057 & 1495.201 & 1496.069 & 1497.196 & 1496.923 & 1493.528 & 1488.959 & 1493.372 & 1491.424 \\ 1494296 & 1495.057 & 1495211 & 1496072 & 1497.198 & 149694 & 149353 & 1488959 & 1493372 & 1491.42\end{array}$

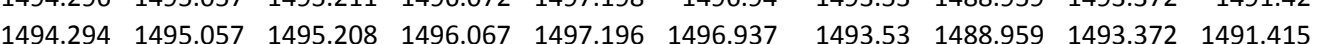
$\begin{array}{llllllllll}1494.291 & 1495.054 & 1495208 & 1496065 & 1497.194 & 1496937 & 1493.53 & 1488.959 & 1493.372 & 1491.413\end{array}$ $\begin{array}{llllllllll}1494.294 & 1495.057 & 1495.206 & 1496.06 & 1497.189 & 1496.935 & 1493.528 & 1488.959 & 1493.374 & 1491.41\end{array}$ $\begin{array}{llllllllll}1494.308 & 1495.052 & 1495.197 & 1496.053 & 1497.18 & 1496.921 & 1493.526 & 1488.959 & 1493.372 & 1491.406\end{array}$ $\begin{array}{lllllllllll}1494.317 & 1495.047 & 1495.194 & 1496.042 & 1497.168 & 1496.899 & 1493.523 & 1488.952 & 1493.37 & 1491.403\end{array}$ $\begin{array}{llllllllll}1494.336 & 1495.042 & 1495.178 & 1496.023 & 1497.149 & 1496.87 & 1493.514 & 1488.945 & 1493.362 & 1491.396\end{array}$ $\begin{array}{llllllllll}1494.343 & 1495.035 & 1495.18 & 1496.007 & 1497.133 & 1496.854 & 1493.509 & 1488.933 & 1493.353 & 1491.392\end{array}$ $\begin{array}{lllllllllll}1494.343 & 1495.03 & 1495.18 & 1495.995 & 1497.124 & 1496.844 & 1493.5 & 1488.93 & 1493.348 & 1491.387\end{array}$

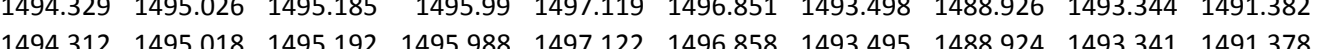
$\begin{array}{lllllllllll}1449.301 & 1455.018 & 1495.201 & 1495.988 & 1497.122 & 1496.873 & 1493.498 & 1488.931 & 1493.341 & 1491.373\end{array}$ $\begin{array}{llllllllllll}1494.294 & 1495.018 & 1495.197 & 1495.986 & 1497.117 & 1496.877 & 1493.493 & 1488.924 & 1493.341 & 1491.371\end{array}$ $\begin{array}{llllllllll}1494.284 & 1495.016 & 1495.201 & 1495.984 & 1497.122 & 1496.882 & 1493.493 & 1488.926 & 1493.339 & 1491.366\end{array}$ $\begin{array}{llllllllll}1494.27 & 1495.018 & 1495.208 & 1495.986 & 1497.124 & 1496.899 & 1493.498 & 1488.931 & 1493.341 & 1491.364\end{array}$ $\begin{array}{llllllllll}1494.27 & 1495.018 & 1495.204 & 1495.986 & 1497.124 & 1496.902 & 1493.495 & 1488.926 & 1493.344 & 1491.361\end{array}$ $\begin{array}{rrrrrrrrrr}1494.268 & 1495.018 & 1495.206 & 1495.983 & 1497.124 & 1496.897 & 1493.5 & 1488.933 & 1493.344 & 1491.359 \\ 1494.27 & 1495.018 & 1495.201 & 1495.983 & 1497.119 & 1496.901 & 1493.502 & 1488.931 & 1493.346 & 1491.357\end{array}$ $\begin{array}{lllllllll}1486.354 & 1488.382 & 1493.761 & 1494.278 & 1493.52 & 1495.399 & 1493.495 & 1484.71 & 1479.348\end{array}$ $\begin{array}{llllllllll}1486.354 & 1488.38 & 1493.763 & 1494.281 & 1493.525 & 1495.42 & 1493.492 & 1484.71 & 1479.35 \\ 1486356 & 1488392 & 1493772 & 149282 & 1493532 & 1495.418 & 1493504 & 148.71 & 147935\end{array}$ $\begin{array}{llllllllll}1486.356 & 1488.392 & 1493.772 & 1494.292 & 1493.532 & 1495.418 & 1493.504 & 1484.71 & 1479.355 \\ 1486.358 & 1488.394 & 1493.779 & 1494.299 & 1493.537 & 1495.43 & 1493.511 & 1484.71 & 1479.36\end{array}$ $\begin{array}{llllllllll}1486.358 & 1488.403 & 1493.789 & 1494.304 & 1493.548 & 1495.437 & 1493.516 & 1484.71 & 1479.365\end{array}$ 1486.3581488 .413 $\begin{array}{llllllllll}1486.356 & 1488.42 & 1493.796 & 1494.309 & 1493.553 & 1495.444 & 1493.527 & 1484.708 & 1479.369\end{array}$ $\begin{array}{llllllllll}1486.354 & 1488.42 & 1493.798 & 1494.318 & 1493.56 & 1495.446 & 1493.527 & 1484.712 & 1479.372\end{array}$ $\begin{array}{llllllllll}1486.358 & 1488.424 & 1493.808 & 1494.332 & 1493.565 & 1495.465 & 1493.523 & 1484.712 & 1479.374\end{array}$ $\begin{array}{llllllllll}1486.358 & 1488.434 & 193.812 & 1494.344 & 1493.575 & 1455.47 & 1493.546 & 1484.712 & 1479.346\end{array}$ $\begin{array}{llllllllll}1486.354 & 1488.422 & 1493.817 & 1494.355 & 1493.579 & 1495.496 & 1493.539 & 1484.708 & 1479.374\end{array}$ $\begin{array}{llllllllll}1486.349 & 1488.429 & 1493.828 & 1494.376 & 1493.594 & 1495.526 & 1493.546 & 1484.71 & 1479.374\end{array}$ $\begin{array}{lllllllll}1486.347 & 1488.436 & 1493.838 & 1494.39 & 1493.603 & 1495.538 & 1493.563 & 1484.71 & 1479376\end{array}$ $\begin{array}{llllllllll}1486.344 & 1488.457 & 1493.845 & 1494.39 & 1493.608 & 1495.533 & 1493.574 & 1484.71 & 1479.379\end{array}$ $\begin{array}{lllllllll}1486.344 & 1488.462 & 1493.855 & 1494.393 & 1493.612 & 1495.536 & 1493.579 & 1484.708 & 1479.381\end{array}$ $\begin{array}{lllllllll}1486.342 & 1488.467 & 1493.857 & 1494.393 & 1493.62 & 1495.538 & 1493.586 & 1484.708 & 1479.386\end{array}$ $\begin{array}{llllllllll}1486.344 & 1488.481 & 1493.5 & 1494.288 & 1493.29 & 1495.531 & 1493.262 & 1484.71 & 1479.38\end{array}$

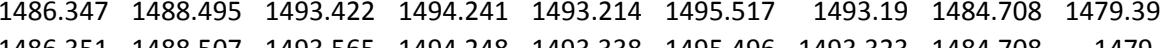
$\begin{array}{llllllllll}1486.351 & 1488.507 & 1493.565 & 1494.248 & 1493.338 & 1495.496 & 1493.323 & 1484.708 & 1479.4\end{array}$ $\begin{array}{rrrrrrrrrrr}1486.347 & 1488.514 & 1493.69 & 1494.297 & 1493.463 & 1495.498 & 1493.434 & 1484.708 & 1479.402\end{array}$ $\begin{array}{lllllllll}1486.34 & 1488.518 & 1493.744 & 1494.325 & 1493.508 & 1495.514 & 1493.478 & 1484.708 & 1479.402\end{array}$ $\begin{array}{lllllllll}1486.337 & 1488.525 & 1493.763 & 1494.339 & 1493.527 & 1495.524 & 1493.495 & 1484.708 & 1479.402\end{array}$ $\begin{array}{llllllllll}1486.342 & 1488.528 & 1493.779 & 1494.348 & 1493.546 & 1495.528 & 1493.506 & 1484.708 & 1479.402\end{array}$ $\begin{array}{llllllllll}1486.34 & 1488.549 & 1493.789 & 1494.351 & 1493.553 & 1495.519 & 1493.532 & 1484.708 & 1479.402\end{array}$ $\begin{array}{lllllllllll}1486.333 & 1488.542 & 493.798 & 1494.348 & 1493.563 & 1495.519 & 1493.532 & 1484.708 & 1479.404\end{array}$

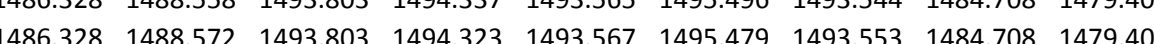
$\begin{array}{llllllllll}1486.326 & 1488.577 & 1493.803 & 1494.306 & 1493.56 & 1495.449 & 1493.546 & 1484.705 & 1479.402\end{array}$ $\begin{array}{llllllllll}486.321 & 1488.579 & 1493.796 & 1494.29 & 1493.558 & 1495.434 & 1493.539 & 1484.703 & 1479.397\end{array}$ $\begin{array}{lllllllll}1486.319 & 1488.577 & 1493.796 & 1494.281 & 1493.551 & 1495.42 & 1493.52 & 1484.705 & 1479.395\end{array}$ $\begin{array}{lllllllll}1486.114 & 1488.568 & 1493.779 & 1494.271 & 1493.544 & 1495.409 & 1493.502 & 1484.703 & 1479.388\end{array}$ $\begin{array}{llllllllll}1486.307 & 1488.554 & 1493.784 & 1494.271 & 1493.541 & 1495.404 & 1493.492 & 1484.705 & 1479.381\end{array}$

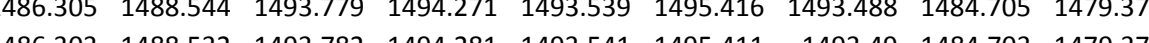

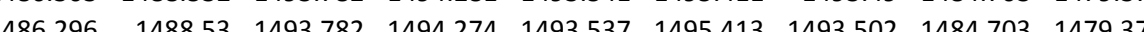
$\begin{array}{llllllllll}1486.275 & 1488.523 & 1493.777 & 1494.276 & 1493.537 & 1495.411 & 1493.502 & 1484.703 & 1479.372\end{array}$ $\begin{array}{lllllllll}1486.257 & 1488.521 & 1493.779 & 1494.274 & 1493.539 & 1495.411 & 1493.509 & 1484.703 & 1479.372\end{array}$ $\begin{array}{lllllllll}1486.243 & 1488.53 & 1493.779 & 1494.267 & 1493.537 & 1495.397 & 1493.516 & 1484.703 & 1479.372\end{array}$ $\begin{array}{lllllllll}1486.24 & 1488.537 & 1493.775 & 1494.25 & 1493.532 & 1495.376 & 1493.516 & 1484.703 & 1479.372\end{array}$ $\begin{array}{llllllllll}1486.245 & 1488.544 & 1493.77 & 1494.234 & 1493.525 & 1495.357 & 1493.511 & 1484.701 & 1479.32\end{array}$ $\begin{array}{lllllllll}1486.243 & 1488.546 & 1493.765 & 1494.218 & 1493.518 & 1495.331 & 1493.499 & 1484.703 & 1479.372\end{array}$ $\begin{array}{llllllllll}148.243 & 1488.539 & 1493.753 & 1494.208 & 1493.511 & 1455.322 & 1493.488 & 1484.703 & 1479.372\end{array}$

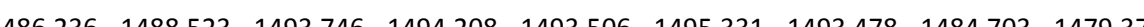
$\begin{array}{llllllllll}1486.236 & 1488.518 & 1493.746 & 1494.215 & 1493.506 & 1495.333 & 1493.48 & 1484.705 & 1479.374\end{array}$ $\begin{array}{lllllllllll}1486.231 & 1488.518 & 1493.746 & 1494.218 & 1493.503 & 1495.336 & 1493.478 & 1484.705 & 1479.372\end{array}$ $\begin{array}{llllllllll}1486.233 & 1488.516 & 1493.744 & 1494.22 & 1493.503 & 1495.352 & 1493.473 & 1484.703 & 1479.372\end{array}$ $\begin{array}{lllllllllll}1486.231 & 1488.509 & 1493.749 & 1494.229 & 1493.506 & 1495.362 & 1493.48 & 1484.703 & 1479.372\end{array}$ $\begin{array}{llllllllll}1486.213 & 1488.516 & 1493.749 & 1494.227 & 1493.508 & 1495.371 & 1493.483 & 1484.703 & 1479.372\end{array}$ $\begin{array}{llllllllll}1486.215 & 1488.514 & 1493.751 & 1494.229 & 1493.508 & 1495.385 & 1493.483 & 1484.703 & 1479.374 \\ 1486206 & 1488521 & 1493751 & 1494.229 & 1493511 & 1495395 & 1493485 & 1484.703 & 1479.374\end{array}$ 


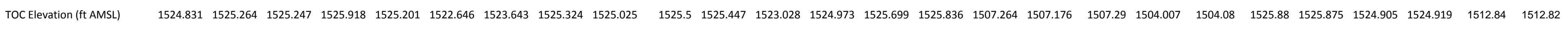

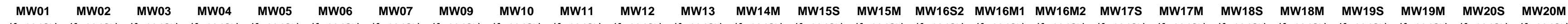
(ft AMSL) (ft AMSL) (ft AMSL) (ft AMSL) (ft AMSL) (ft AMSL) (ft AMSL) (ft AMSL) (ft AMSL) (ft AMSL) (ft AMSL) (ft AMSL) (ft AMSL) (ft AMSL) (ft AMSL) (ft AMSL) (ft AMSL) (ft AMSL) (ft AMSL) (ft AMSL) (ft AMSL) (ft AMSL) (ft AMSL) (ft AMSL) (ft AMSL) (ft AMSL)

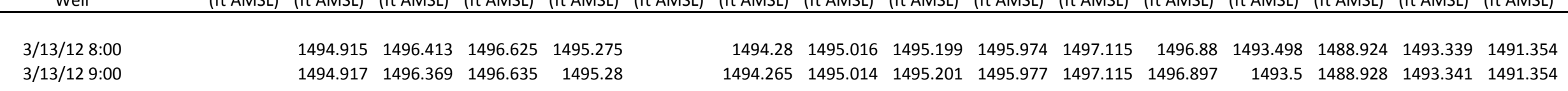

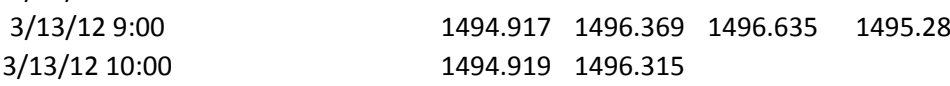
$\begin{array}{llllllllll}1494.254 & 1495.014 & 1495.206 & 1495.979 & 1497.122 & 1496.904 & 1493.495 & 1488.931 & 1493.341 & 1491.352\end{array}$ 1494.919

$\begin{array}{lllllllll}1486.203 & 1488.523 & 1493.749 & 1494.22 & 1493.503 & 1495.397 & 1493.478 & 1484.703 & 1479.372\end{array}$

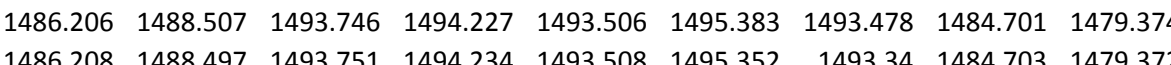
$\begin{array}{lllllllll}1486.208 & 1488.497 & 1493.751 & 1494.234 & 1493.508 & 1495.352 & 1493.34 & 1484.703 & 1479.372\end{array}$ $\begin{array}{lllllll}1486.213 & 1488.492 & 1493.751 & 1494.232 & 1493.508 & 1484.701 & 1479.369 \\ 1486.196 & 1488.488 & 1493.753 & 1494.25 & 1493.511 & 1484.703 & 1479.367\end{array}$ $\begin{array}{llllll}1486.194 & 1494.255 & 1493.513 & 1484.703 & 1479.367 \\ 14864 & 1484.701 & 1479.3656\end{array}$ 1486.194 $1484.701 \quad 1479.365$ 
TABLE S1.3 Automatically recorded groundwater levels in the Klassen wells, September 15, 2011, to March 13, 2012.

\begin{tabular}{|c|c|c|c|c|}
\hline \multicolumn{3}{|c|}{ Reference Elevation (ft AMSL) } & 1508.77 & \multirow{2}{*}{$\begin{array}{l}1509.12 \\
\text { Elevation in Klassen } \\
\text { Test Well (ft AMSL) }\end{array}$} \\
\hline Date and Time & $\begin{array}{l}\text { Depth in Klassen } \\
\text { Domestic Well } \\
(\mathrm{ft} \mathrm{BGL})\end{array}$ & $\begin{array}{c}\text { Depth in Klassen } \\
\text { Test Well } \\
\text { (ft BGL) }\end{array}$ & $\begin{array}{l}\text { Elevation in Klassen } \\
\text { Domestic Well } \\
\text { (ft AMSL) }\end{array}$ & \\
\hline 9/15/11 12:10 & 36.515 & 41.042 & 1472.25 & 1468.08 \\
\hline $9 / 15 / 1112: 20$ & 36.431 & 41.035 & 1472.34 & 1468.09 \\
\hline 9/15/11 12:30 & 36.367 & 41.03 & 1472.40 & 1468.09 \\
\hline 9/15/11 12:40 & 36.297 & 41.021 & 1472.47 & 1468.10 \\
\hline 9/15/11 12:50 & 36.26 & 41.016 & 1472.51 & 1468.11 \\
\hline 9/15/11 13:00 & 36.234 & 41.007 & 1472.54 & 1468.12 \\
\hline 9/15/11 13:10 & 36.177 & 41.002 & 1472.59 & 1468.12 \\
\hline 9/15/11 13:20 & 36.149 & 40.995 & 1472.62 & 1468.13 \\
\hline 9/15/11 13:30 & 36.095 & 40.986 & 1472.67 & 1468.14 \\
\hline 9/15/11 13:40 & 36.074 & 40.979 & 1472.70 & 1468.14 \\
\hline 9/15/11 13:50 & 36.027 & 40.972 & 1472.74 & 1468.15 \\
\hline 9/15/11 14:00 & 36.006 & 40.965 & 1472.76 & 1468.16 \\
\hline 9/15/11 14:10 & 35.964 & 40.955 & 1472.81 & 1468.17 \\
\hline $9 / 15 / 1114: 20$ & 37.791 & 40.948 & 1470.98 & 1468.17 \\
\hline 9/15/11 14:30 & 36.051 & 40.944 & 1472.72 & 1468.18 \\
\hline 9/15/11 14:40 & 35.964 & 40.934 & 1472.81 & 1468.19 \\
\hline 9/15/11 14:50 & 35.924 & 40.927 & 1472.85 & 1468.20 \\
\hline 9/15/11 15:00 & 35.875 & 40.92 & 1472.89 & 1468.20 \\
\hline 9/15/11 15:10 & 35.859 & 40.911 & 1472.91 & 1468.21 \\
\hline 9/15/11 15:20 & 35.821 & 40.901 & 1472.95 & 1468.22 \\
\hline $9 / 15 / 11$ 15:30 & 35.807 & 40.892 & 1472.96 & 1468.23 \\
\hline 9/15/11 15:40 & 35.795 & 40.885 & 1472.97 & 1468.24 \\
\hline $9 / 15 / 11$ 15:50 & 35.755 & 40.876 & 1473.01 & 1468.25 \\
\hline 9/15/11 16:00 & 35.72 & 40.864 & 1473.05 & 1468.26 \\
\hline 9/15/11 16:10 & 35.711 & 40.859 & 1473.06 & 1468.26 \\
\hline 9/15/11 16:20 & 35.678 & 40.847 & 1473.09 & 1468.28 \\
\hline 9/15/11 16:30 & 35.664 & 40.84 & 1473.11 & 1468.28 \\
\hline 9/15/11 16:40 & 35.638 & 40.831 & 1473.13 & 1468.29 \\
\hline 9/15/11 16:50 & 35.608 & 40.822 & 1473.16 & 1468.30 \\
\hline 9/15/11 17:00 & 35.603 & 40.812 & 1473.17 & 1468.31 \\
\hline 9/15/11 17:10 & 35.575 & 40.803 & 1473.19 & 1468.32 \\
\hline 9/15/11 17:20 & 35.544 & 40.793 & 1473.23 & 1468.33 \\
\hline 9/15/11 17:30 & 35.53 & 40.784 & 1473.24 & 1468.34 \\
\hline 9/15/11 17:40 & 35.523 & 40.777 & 1473.25 & 1468.35 \\
\hline 9/15/11 17:50 & 35.518 & 40.768 & 1473.25 & 1468.35 \\
\hline 9/15/11 18:00 & 35.49 & 40.758 & 1473.28 & 1468.36 \\
\hline 9/15/11 18:10 & 35.465 & 40.751 & 1473.30 & 1468.37 \\
\hline 9/15/11 18:20 & 35.821 & 40.744 & 1472.95 & 1468.38 \\
\hline
\end{tabular}


TABLE S1.3 Automatically recorded groundwater levels in the Klassen wells, September 15, 2011, to March 13, 2012.

\begin{tabular}{|c|c|c|c|c|}
\hline \multicolumn{3}{|c|}{ Reference Elevation (ft AMSL) } & 1508.77 & \multirow{2}{*}{$\begin{array}{l}1509.12 \\
\text { Elevation in Klassen } \\
\text { Test Well (ft AMSL) }\end{array}$} \\
\hline Date and Time & $\begin{array}{l}\text { Depth in Klassen } \\
\text { Domestic Well } \\
\text { (ft BGL) }\end{array}$ & $\begin{array}{c}\text { Depth in Klassen } \\
\text { Test Well } \\
\text { (ft BGL) }\end{array}$ & $\begin{array}{l}\text { Elevation in Klassen } \\
\text { Domestic Well } \\
\text { (ft AMSL) }\end{array}$ & \\
\hline 9/15/11 18:30 & 35.556 & 40.737 & 1473.21 & 1468.39 \\
\hline 9/15/11 18:40 & 35.526 & 40.73 & 1473.24 & 1468.39 \\
\hline 9/15/11 18:50 & 35.49 & 40.721 & 1473.28 & 1468.40 \\
\hline 9/15/11 19:00 & 35.474 & 40.714 & 1473.30 & 1468.41 \\
\hline 9/15/11 19:10 & 35.458 & 40.707 & 1473.31 & 1468.42 \\
\hline 9/15/11 19:20 & 35.434 & 40.7 & 1473.34 & 1468.42 \\
\hline 9/15/11 19:30 & 35.404 & 40.695 & 1473.37 & 1468.43 \\
\hline 9/15/11 19:40 & 35.399 & 40.693 & 1473.37 & 1468.43 \\
\hline 9/15/11 19:50 & 35.404 & 40.693 & 1473.37 & 1468.43 \\
\hline 9/15/11 20:00 & 35.404 & 40.695 & 1473.37 & 1468.43 \\
\hline 9/15/11 20:10 & 35.375 & 40.697 & 1473.39 & 1468.43 \\
\hline 9/15/11 20:20 & 35.408 & 40.707 & 1473.36 & 1468.42 \\
\hline 9/15/11 20:30 & 35.406 & 40.716 & 1473.36 & 1468.41 \\
\hline 9/15/11 20:40 & 35.397 & 40.721 & 1473.37 & 1468.40 \\
\hline 9/15/11 20:50 & 35.366 & 40.73 & 1473.40 & 1468.39 \\
\hline 9/15/11 21:00 & 35.382 & 40.739 & 1473.39 & 1468.38 \\
\hline 9/15/11 21:10 & 35.399 & 40.749 & 1473.37 & 1468.37 \\
\hline 9/15/11 21:20 & 35.401 & 40.761 & 1473.37 & 1468.36 \\
\hline 9/15/11 21:30 & 35.397 & 40.77 & 1473.37 & 1468.35 \\
\hline 9/15/11 21:40 & 35.476 & 40.784 & 1473.29 & 1468.34 \\
\hline 9/15/11 21:50 & 35.455 & 40.798 & 1473.31 & 1468.32 \\
\hline 9/15/11 22:00 & 35.783 & 40.812 & 1472.99 & 1468.31 \\
\hline 9/15/11 22:10 & 35.603 & 40.824 & 1473.17 & 1468.30 \\
\hline 9/15/11 22:20 & 35.528 & 40.831 & 1473.24 & 1468.29 \\
\hline $9 / 15 / 11$ 22:30 & 35.474 & 40.836 & 1473.30 & 1468.29 \\
\hline 9/15/11 22:40 & 35.45 & 40.836 & 1473.32 & 1468.29 \\
\hline $9 / 15 / 1122: 50$ & 39.658 & 40.838 & 1469.11 & 1468.28 \\
\hline 9/15/11 23:00 & 35.699 & 40.84 & 1473.07 & 1468.28 \\
\hline 9/15/11 23:10 & 41.935 & 40.845 & 1466.83 & 1468.28 \\
\hline 9/15/11 23:20 & 36.553 & 40.843 & 1472.22 & 1468.28 \\
\hline 9/15/11 23:30 & 35.711 & 40.843 & 1473.06 & 1468.28 \\
\hline 9/15/11 23:40 & 35.615 & 40.84 & 1473.15 & 1468.28 \\
\hline 9/15/11 23:50 & 35.537 & 40.84 & 1473.23 & 1468.28 \\
\hline 9/16/11 0:00 & 35.479 & 40.833 & 1473.29 & 1468.29 \\
\hline 9/16/11 0:10 & 35.432 & 40.829 & 1473.34 & 1468.29 \\
\hline 9/16/11 0:20 & 35.418 & 40.824 & 1473.35 & 1468.30 \\
\hline 9/16/11 0:30 & 35.38 & 40.819 & 1473.39 & 1468.30 \\
\hline 9/16/11 0:40 & 35.345 & 40.812 & 1473.42 & 1468.31 \\
\hline
\end{tabular}


TABLE S1.3 Automatically recorded groundwater levels in the Klassen wells, September 15, 2011, to March 13, 2012.

\begin{tabular}{|c|c|c|c|c|}
\hline \multicolumn{3}{|c|}{ Reference Elevation (ft AMSL) } & 1508.77 & \multirow{2}{*}{$\begin{array}{l}1509.12 \\
\text { Elevation in Klassen } \\
\text { Test Well (ft AMSL) }\end{array}$} \\
\hline Date and Time & $\begin{array}{l}\text { Depth in Klassen } \\
\text { Domestic Well } \\
(\mathrm{ft} \mathrm{BGL})\end{array}$ & $\begin{array}{c}\text { Depth in Klassen } \\
\text { Test Well } \\
\text { (ft BGL) }\end{array}$ & $\begin{array}{l}\text { Elevation in Klassen } \\
\text { Domestic Well } \\
\text { (ft AMSL) }\end{array}$ & \\
\hline $9 / 16 / 110: 50$ & 35.319 & 40.805 & 1473.45 & 1468.32 \\
\hline 9/16/11 1:00 & 35.31 & 40.8 & 1473.46 & 1468.32 \\
\hline 9/16/11 1:10 & 35.282 & 40.791 & 1473.49 & 1468.33 \\
\hline $9 / 16 / 111: 20$ & 35.253 & 40.786 & 1473.52 & 1468.34 \\
\hline $9 / 16 / 11$ 1:30 & 35.249 & 40.779 & 1473.52 & 1468.34 \\
\hline $9 / 16 / 11$ 1:40 & 35.265 & 40.777 & 1473.50 & 1468.35 \\
\hline $9 / 16 / 11$ 1:50 & 35.333 & 40.777 & 1473.44 & 1468.35 \\
\hline $9 / 16 / 112: 00$ & 35.312 & 40.775 & 1473.46 & 1468.35 \\
\hline $9 / 16 / 112: 10$ & 35.3 & 40.77 & 1473.47 & 1468.35 \\
\hline $9 / 16 / 112: 20$ & 35.277 & 40.765 & 1473.49 & 1468.36 \\
\hline $9 / 16 / 112: 30$ & 35.258 & 40.761 & 1473.51 & 1468.36 \\
\hline $9 / 16 / 112: 40$ & 35.253 & 40.754 & 1473.52 & 1468.37 \\
\hline $9 / 16 / 112: 50$ & 35.223 & 40.747 & 1473.55 & 1468.38 \\
\hline 9/16/11 3:00 & 35.218 & 40.739 & 1473.55 & 1468.38 \\
\hline 9/16/11 3:10 & 35.211 & 40.735 & 1473.56 & 1468.39 \\
\hline 9/16/11 3:20 & 35.214 & 40.728 & 1473.56 & 1468.39 \\
\hline 9/16/11 3:30 & 35.279 & 40.723 & 1473.49 & 1468.40 \\
\hline 9/16/11 3:40 & 35.258 & 40.718 & 1473.51 & 1468.40 \\
\hline 9/16/11 3:50 & 35.221 & 40.711 & 1473.55 & 1468.41 \\
\hline $9 / 16 / 114: 00$ & 35.188 & 40.704 & 1473.58 & 1468.42 \\
\hline $9 / 16 / 114: 10$ & 35.183 & 40.697 & 1473.59 & 1468.43 \\
\hline $9 / 16 / 114: 20$ & 35.155 & 40.688 & 1473.61 & 1468.43 \\
\hline 9/16/11 4:30 & 35.127 & 40.678 & 1473.64 & 1468.44 \\
\hline $9 / 16 / 114: 40$ & 35.103 & 40.671 & 1473.67 & 1468.45 \\
\hline 9/16/11 4:50 & 35.108 & 40.664 & 1473.66 & 1468.46 \\
\hline 9/16/11 5:00 & 35.296 & 40.66 & 1473.47 & 1468.46 \\
\hline 9/16/11 5:10 & 35.167 & 40.65 & 1473.60 & 1468.47 \\
\hline 9/16/11 5:20 & 35.132 & 40.643 & 1473.64 & 1468.48 \\
\hline 9/16/11 5:30 & 35.11 & 40.636 & 1473.66 & 1468.49 \\
\hline 9/16/11 5:40 & 35.08 & 40.627 & 1473.69 & 1468.50 \\
\hline 9/16/11 5:50 & 35.054 & 40.617 & 1473.72 & 1468.51 \\
\hline $9 / 16 / 116: 00$ & 35.096 & 40.61 & 1473.67 & 1468.51 \\
\hline 9/16/11 6:10 & 38.002 & 40.608 & 1470.77 & 1468.51 \\
\hline $9 / 16 / 116: 20$ & 35.277 & 40.606 & 1473.49 & 1468.52 \\
\hline $9 / 16 / 116: 30$ & 35.185 & 40.599 & 1473.58 & 1468.52 \\
\hline $9 / 16 / 116: 40$ & 35.162 & 40.589 & 1473.61 & 1468.53 \\
\hline $9 / 16 / 116: 50$ & 35.143 & 40.585 & 1473.63 & 1468.54 \\
\hline 9/16/11 7:00 & 35.106 & 40.575 & 1473.66 & 1468.55 \\
\hline
\end{tabular}


TABLE S1.3 Automatically recorded groundwater levels in the Klassen wells, September 15, 2011, to March 13, 2012.

\begin{tabular}{|c|c|c|c|c|}
\hline \multicolumn{3}{|c|}{ Reference Elevation (ft AMSL) } & 1508.77 & \multirow{2}{*}{$\begin{array}{l}1509.12 \\
\text { Elevation in Klassen } \\
\text { Test Well (ft AMSL) }\end{array}$} \\
\hline Date and Time & $\begin{array}{l}\text { Depth in Klassen } \\
\text { Domestic Well } \\
\text { (ft BGL) }\end{array}$ & $\begin{array}{c}\text { Depth in Klassen } \\
\text { Test Well } \\
\text { (ft BGL) }\end{array}$ & $\begin{array}{l}\text { Elevation in Klassen } \\
\text { Domestic Well } \\
\text { (ft AMSL) }\end{array}$ & \\
\hline 9/16/11 7:10 & 35.258 & 40.571 & 1473.51 & 1468.55 \\
\hline $9 / 16 / 117: 20$ & 35.171 & 40.564 & 1473.60 & 1468.56 \\
\hline 9/16/11 7:30 & 35.148 & 40.556 & 1473.62 & 1468.57 \\
\hline $9 / 16 / 117: 40$ & 35.448 & 40.552 & 1473.32 & 1468.57 \\
\hline $9 / 16 / 117: 50$ & 35.202 & 40.547 & 1473.57 & 1468.58 \\
\hline 9/16/11 8:00 & 35.509 & 40.542 & 1473.26 & 1468.58 \\
\hline $9 / 16 / 118: 10$ & 35.202 & 40.535 & 1473.57 & 1468.59 \\
\hline $9 / 16 / 118: 20$ & 38.71 & 40.528 & 1470.06 & 1468.59 \\
\hline $9 / 16 / 118: 30$ & 35.256 & 40.524 & 1473.51 & 1468.60 \\
\hline $9 / 16 / 118: 40$ & 35.162 & 40.517 & 1473.61 & 1468.61 \\
\hline $9 / 16 / 118: 50$ & 35.108 & 40.512 & 1473.66 & 1468.61 \\
\hline 9/16/11 9:00 & 35.094 & 40.502 & 1473.68 & 1468.62 \\
\hline 9/16/11 9:10 & 35.209 & 40.498 & 1473.56 & 1468.62 \\
\hline $9 / 16 / 119: 20$ & 47.378 & 40.498 & 1461.39 & 1468.62 \\
\hline 9/16/11 9:30 & 36.956 & 40.51 & 1471.81 & 1468.61 \\
\hline 9/16/11 9:40 & 36.025 & 40.514 & 1472.74 & 1468.61 \\
\hline 9/16/11 9:50 & 35.849 & 40.514 & 1472.92 & 1468.61 \\
\hline 9/16/11 10:00 & 35.833 & 40.519 & 1472.94 & 1468.60 \\
\hline 9/16/11 10:10 & 36.982 & 40.521 & 1471.79 & 1468.60 \\
\hline 9/16/11 10:20 & 36.013 & 40.533 & 1472.76 & 1468.59 \\
\hline $9 / 16 / 11$ 10:30 & 35.959 & 40.535 & 1472.81 & 1468.59 \\
\hline 9/16/11 10:40 & 35.938 & 40.538 & 1472.83 & 1468.58 \\
\hline 9/16/11 10:50 & 42.728 & 40.54 & 1466.04 & 1468.58 \\
\hline 9/16/11 11:00 & 40.509 & 40.545 & 1468.26 & 1468.58 \\
\hline 9/16/11 11:10 & 38.682 & 40.554 & 1470.09 & 1468.57 \\
\hline 9/16/11 11:20 & 38.223 & 40.571 & 1470.55 & 1468.55 \\
\hline 9/16/11 11:30 & 37.965 & 40.589 & 1470.80 & 1468.53 \\
\hline 9/16/11 11:40 & 42.16 & 40.61 & 1466.61 & 1468.51 \\
\hline 9/16/11 11:50 & 37.735 & 40.632 & 1471.03 & 1468.49 \\
\hline 9/16/11 12:00 & 37.348 & 40.648 & 1471.42 & 1468.47 \\
\hline 9/16/11 12:10 & 37.223 & 40.66 & 1471.55 & 1468.46 \\
\hline 9/16/11 12:20 & 37.167 & 40.669 & 1471.60 & 1468.45 \\
\hline 9/16/11 12:30 & 37.936 & 40.678 & 1470.83 & 1468.44 \\
\hline 9/16/11 12:40 & 37.252 & 40.69 & 1471.52 & 1468.43 \\
\hline 9/16/11 12:50 & 37.214 & 40.7 & 1471.56 & 1468.42 \\
\hline 9/16/11 13:00 & 37.202 & 40.709 & 1471.57 & 1468.41 \\
\hline 9/16/11 13:10 & 37.205 & 40.716 & 1471.56 & 1468.41 \\
\hline 9/16/11 13:20 & 37.219 & 40.725 & 1471.55 & 1468.40 \\
\hline
\end{tabular}


TABLE S1.3 Automatically recorded groundwater levels in the Klassen wells, September 15, 2011, to March 13, 2012.

\begin{tabular}{|c|c|c|c|c|}
\hline \multicolumn{3}{|c|}{ Reference Elevation (ft AMSL) } & 1508.77 & \multirow{2}{*}{$\begin{array}{l}1509.12 \\
\text { Elevation in Klassen } \\
\text { Test Well (ft AMSL) }\end{array}$} \\
\hline Date and Time & $\begin{array}{l}\text { Depth in Klassen } \\
\text { Domestic Well } \\
(\mathrm{ft} \mathrm{BGL})\end{array}$ & $\begin{array}{c}\text { Depth in Klassen } \\
\text { Test Well } \\
\text { (ft BGL) }\end{array}$ & $\begin{array}{l}\text { Elevation in Klassen } \\
\text { Domestic Well } \\
\text { (ft AMSL) }\end{array}$ & \\
\hline 9/16/11 13:30 & 37.228 & 40.732 & 1471.54 & 1468.39 \\
\hline $9 / 16 / 1113: 40$ & 37.245 & 40.739 & 1471.52 & 1468.38 \\
\hline 9/16/11 13:50 & 37.238 & 40.747 & 1471.53 & 1468.38 \\
\hline 9/16/11 14:00 & 37.155 & 40.747 & 1471.61 & 1468.38 \\
\hline 9/16/11 14:10 & 37.066 & 40.742 & 1471.70 & 1468.38 \\
\hline 9/16/11 14:20 & 36.98 & 40.737 & 1471.79 & 1468.39 \\
\hline 9/16/11 14:30 & 36.9 & 40.73 & 1471.87 & 1468.39 \\
\hline 9/16/11 14:40 & 36.834 & 40.723 & 1471.94 & 1468.40 \\
\hline 9/16/11 14:50 & 36.773 & 40.716 & 1472.00 & 1468.41 \\
\hline 9/16/11 15:00 & 36.738 & 40.711 & 1472.03 & 1468.41 \\
\hline 9/16/11 15:10 & 36.689 & 40.704 & 1472.08 & 1468.42 \\
\hline 9/16/11 15:20 & 36.654 & 40.697 & 1472.12 & 1468.43 \\
\hline 9/16/11 15:30 & 36.715 & 40.695 & 1472.05 & 1468.43 \\
\hline $9 / 16 / 1115: 40$ & 36.792 & 40.697 & 1471.98 & 1468.43 \\
\hline 9/16/11 15:50 & 36.862 & 40.7 & 1471.91 & 1468.42 \\
\hline 9/16/11 16:00 & 36.933 & 40.704 & 1471.84 & 1468.42 \\
\hline 9/16/11 16:10 & 36.998 & 40.711 & 1471.77 & 1468.41 \\
\hline $9 / 16 / 11$ 16:20 & 37.052 & 40.716 & 1471.72 & 1468.41 \\
\hline 9/16/11 16:30 & 37.099 & 40.721 & 1471.67 & 1468.40 \\
\hline 9/16/11 16:40 & 37.144 & 40.725 & 1471.63 & 1468.40 \\
\hline $9 / 16 / 1116: 50$ & 37.186 & 40.73 & 1471.58 & 1468.39 \\
\hline 9/16/11 17:00 & 37.226 & 40.732 & 1471.54 & 1468.39 \\
\hline 9/16/11 17:10 & 37.263 & 40.739 & 1471.51 & 1468.38 \\
\hline 9/16/11 17:20 & 37.299 & 40.742 & 1471.47 & 1468.38 \\
\hline 9/16/11 17:30 & 37.299 & 40.744 & 1471.47 & 1468.38 \\
\hline 9/16/11 17:40 & 37.24 & 40.744 & 1471.53 & 1468.38 \\
\hline 9/16/11 17:50 & 37.158 & 40.737 & 1471.61 & 1468.39 \\
\hline 9/16/11 18:00 & 37.092 & 40.73 & 1471.68 & 1468.39 \\
\hline 9/16/11 18:10 & 37.031 & 40.723 & 1471.74 & 1468.40 \\
\hline 9/16/11 18:20 & 36.963 & 40.714 & 1471.81 & 1468.41 \\
\hline 9/16/11 18:30 & 36.902 & 40.704 & 1471.87 & 1468.42 \\
\hline 9/16/11 18:40 & 36.848 & 40.695 & 1471.92 & 1468.43 \\
\hline $9 / 16 / 1118: 50$ & 36.792 & 40.688 & 1471.98 & 1468.43 \\
\hline 9/16/11 19:00 & 36.745 & 40.678 & 1472.02 & 1468.44 \\
\hline 9/16/11 19:10 & 36.703 & 40.667 & 1472.07 & 1468.46 \\
\hline 9/16/11 19:20 & 36.656 & 40.66 & 1472.11 & 1468.46 \\
\hline 9/16/11 19:30 & 36.623 & 40.65 & 1472.15 & 1468.47 \\
\hline 9/16/11 19:40 & 36.581 & 40.639 & 1472.19 & 1468.48 \\
\hline
\end{tabular}


TABLE S1.3 Automatically recorded groundwater levels in the Klassen wells, September 15, 2011, to March 13, 2012.

\begin{tabular}{|c|c|c|c|c|}
\hline \multicolumn{3}{|c|}{ Reference Elevation (ft AMSL) } & 1508.77 & \multirow{2}{*}{$\begin{array}{l}1509.12 \\
\text { Elevation in Klassen } \\
\text { Test Well (ft AMSL) }\end{array}$} \\
\hline Date and Time & $\begin{array}{l}\text { Depth in Klassen } \\
\text { Domestic Well } \\
(\mathrm{ft} \mathrm{BGL})\end{array}$ & $\begin{array}{c}\text { Depth in Klassen } \\
\text { Test Well } \\
\text { (ft BGL) }\end{array}$ & $\begin{array}{l}\text { Elevation in Klassen } \\
\text { Domestic Well } \\
\text { (ft AMSL) }\end{array}$ & \\
\hline 9/16/11 19:50 & 36.815 & 40.632 & 1471.95 & 1468.49 \\
\hline 9/16/11 20:00 & 36.654 & 40.625 & 1472.12 & 1468.50 \\
\hline 9/16/11 20:10 & 36.593 & 40.617 & 1472.18 & 1468.51 \\
\hline $9 / 16 / 1120: 20$ & 36.548 & 40.61 & 1472.22 & 1468.51 \\
\hline $9 / 16 / 1120: 30$ & 36.515 & 40.603 & 1472.25 & 1468.52 \\
\hline 9/16/11 20:40 & 36.485 & 40.594 & 1472.28 & 1468.53 \\
\hline $9 / 16 / 1120: 50$ & 38.809 & 40.587 & 1469.96 & 1468.54 \\
\hline 9/16/11 21:00 & 36.553 & 40.58 & 1472.22 & 1468.54 \\
\hline 9/16/11 21:10 & 36.518 & 40.578 & 1472.25 & 1468.54 \\
\hline $9 / 16 / 1121: 20$ & 36.496 & 40.571 & 1472.27 & 1468.55 \\
\hline 9/16/11 21:30 & 36.48 & 40.566 & 1472.29 & 1468.56 \\
\hline 9/16/11 21:40 & 36.485 & 40.561 & 1472.28 & 1468.56 \\
\hline 9/16/11 21:50 & 36.478 & 40.556 & 1472.29 & 1468.57 \\
\hline $9 / 16 / 1122: 00$ & 36.471 & 40.552 & 1472.30 & 1468.57 \\
\hline 9/16/11 22:10 & 36.461 & 40.545 & 1472.31 & 1468.58 \\
\hline 9/16/11 22:20 & 36.452 & 40.54 & 1472.32 & 1468.58 \\
\hline $9 / 16 / 1122: 30$ & 36.435 & 40.533 & 1472.33 & 1468.59 \\
\hline 9/16/11 22:40 & 38.234 & 40.528 & 1470.54 & 1468.59 \\
\hline 9/16/11 22:50 & 36.841 & 40.526 & 1471.93 & 1468.60 \\
\hline 9/16/11 23:00 & 36.797 & 40.526 & 1471.97 & 1468.60 \\
\hline 9/16/11 23:10 & 41.55 & 40.526 & 1467.22 & 1468.60 \\
\hline 9/16/11 23:20 & 36.879 & 40.528 & 1471.89 & 1468.59 \\
\hline $9 / 16 / 1123: 30$ & 38.929 & 40.526 & 1469.84 & 1468.60 \\
\hline 9/16/11 23:40 & 37.205 & 40.528 & 1471.56 & 1468.59 \\
\hline 9/16/11 23:50 & 36.94 & 40.528 & 1471.83 & 1468.59 \\
\hline 9/17/11 0:00 & 36.841 & 40.524 & 1471.93 & 1468.60 \\
\hline 9/17/11 0:10 & 36.766 & 40.519 & 1472.00 & 1468.60 \\
\hline 9/17/11 0:20 & 36.71 & 40.514 & 1472.06 & 1468.61 \\
\hline 9/17/11 0:30 & 36.668 & 40.51 & 1472.10 & 1468.61 \\
\hline 9/17/11 0:40 & 36.628 & 40.502 & 1472.14 & 1468.62 \\
\hline 9/17/11 0:50 & 36.6 & 40.498 & 1472.17 & 1468.62 \\
\hline 9/17/11 1:00 & 36.569 & 40.491 & 1472.20 & 1468.63 \\
\hline 9/17/11 1:10 & 36.546 & 40.486 & 1472.22 & 1468.64 \\
\hline 9/17/11 1:20 & 36.522 & 40.481 & 1472.25 & 1468.64 \\
\hline 9/17/11 1:30 & 36.503 & 40.474 & 1472.27 & 1468.65 \\
\hline 9/17/11 1:40 & 36.482 & 40.467 & 1472.29 & 1468.66 \\
\hline 9/17/11 1:50 & 36.464 & 40.463 & 1472.31 & 1468.66 \\
\hline $9 / 17 / 112: 00$ & 36.445 & 40.458 & 1472.32 & 1468.66 \\
\hline
\end{tabular}


TABLE S1.3 Automatically recorded groundwater levels in the Klassen wells, September 15, 2011, to March 13, 2012.

\begin{tabular}{|c|c|c|c|c|}
\hline \multicolumn{3}{|c|}{ Reference Elevation (ft AMSL) } & 1508.77 & \multirow{2}{*}{$\begin{array}{l}1509.12 \\
\text { Elevation in Klassen } \\
\text { Test Well (ft AMSL) }\end{array}$} \\
\hline Date and Time & $\begin{array}{l}\text { Depth in Klassen } \\
\text { Domestic Well } \\
(\mathrm{ft} \mathrm{BGL})\end{array}$ & $\begin{array}{c}\text { Depth in Klassen } \\
\text { Test Well } \\
\text { (ft BGL) }\end{array}$ & $\begin{array}{l}\text { Elevation in Klassen } \\
\text { Domestic Well } \\
\text { (ft AMSL) }\end{array}$ & \\
\hline 9/17/11 2:10 & 36.431 & 40.451 & 1472.34 & 1468.67 \\
\hline $9 / 17 / 112: 20$ & 36.414 & 40.444 & 1472.36 & 1468.68 \\
\hline 9/17/11 2:30 & 36.403 & 40.439 & 1472.37 & 1468.68 \\
\hline $9 / 17 / 112: 40$ & 36.384 & 40.432 & 1472.39 & 1468.69 \\
\hline $9 / 17 / 112: 50$ & 36.372 & 40.425 & 1472.40 & 1468.70 \\
\hline 9/17/11 3:00 & 41.421 & 40.42 & 1467.35 & 1468.70 \\
\hline 9/17/11 3:10 & 36.515 & 40.416 & 1472.25 & 1468.71 \\
\hline 9/17/11 3:20 & 36.433 & 40.411 & 1472.34 & 1468.71 \\
\hline 9/17/11 3:30 & 36.4 & 40.404 & 1472.37 & 1468.72 \\
\hline 9/17/11 3:40 & 36.374 & 40.399 & 1472.40 & 1468.72 \\
\hline $9 / 17 / 113: 50$ & 36.356 & 40.395 & 1472.41 & 1468.73 \\
\hline 9/17/11 4:00 & 36.339 & 40.385 & 1472.43 & 1468.74 \\
\hline $9 / 17 / 114: 10$ & 36.321 & 40.38 & 1472.45 & 1468.74 \\
\hline $9 / 17 / 114: 20$ & 36.309 & 40.376 & 1472.46 & 1468.75 \\
\hline 9/17/11 4:30 & 36.295 & 40.369 & 1472.47 & 1468.75 \\
\hline 9/17/11 4:40 & 36.281 & 40.362 & 1472.49 & 1468.76 \\
\hline $9 / 17 / 114: 50$ & 36.269 & 40.357 & 1472.50 & 1468.77 \\
\hline 9/17/11 5:00 & 36.257 & 40.35 & 1472.51 & 1468.77 \\
\hline 9/17/11 5:10 & 36.243 & 40.343 & 1472.53 & 1468.78 \\
\hline 9/17/11 5:20 & 36.231 & 40.336 & 1472.54 & 1468.79 \\
\hline 9/17/11 5:30 & 36.217 & 40.327 & 1472.55 & 1468.80 \\
\hline 9/17/11 5:40 & 36.206 & 40.322 & 1472.56 & 1468.80 \\
\hline 9/17/11 5:50 & 36.194 & 40.315 & 1472.58 & 1468.81 \\
\hline 9/17/11 6:00 & 36.182 & 40.312 & 1472.59 & 1468.81 \\
\hline $9 / 17 / 116: 10$ & 41.562 & 40.319 & 1467.21 & 1468.80 \\
\hline $9 / 17 / 116: 20$ & 36.607 & 40.322 & 1472.16 & 1468.80 \\
\hline $9 / 17 / 116: 30$ & 36.358 & 40.319 & 1472.41 & 1468.80 \\
\hline $9 / 17 / 116: 40$ & 36.292 & 40.315 & 1472.48 & 1468.81 \\
\hline 9/17/11 6:50 & 36.255 & 40.322 & 1472.51 & 1468.80 \\
\hline 9/17/11 7:00 & 36.224 & 40.322 & 1472.55 & 1468.80 \\
\hline 9/17/11 7:10 & 36.203 & 40.319 & 1472.57 & 1468.80 \\
\hline 9/17/11 7:20 & 36.185 & 40.315 & 1472.58 & 1468.81 \\
\hline 9/17/11 7:30 & 36.17 & 40.31 & 1472.60 & 1468.81 \\
\hline 9/17/11 7:40 & 36.154 & 40.301 & 1472.62 & 1468.82 \\
\hline 9/17/11 7:50 & 36.145 & 40.287 & 1472.62 & 1468.84 \\
\hline 9/17/11 8:00 & 36.133 & 40.28 & 1472.64 & 1468.84 \\
\hline 9/17/11 8:10 & 36.119 & 40.277 & 1472.65 & 1468.85 \\
\hline $9 / 17 / 118: 20$ & 36.105 & 40.273 & 1472.66 & 1468.85 \\
\hline
\end{tabular}


TABLE S1.3 Automatically recorded groundwater levels in the Klassen wells, September 15, 2011, to March 13, 2012.

\begin{tabular}{|c|c|c|c|c|}
\hline \multicolumn{3}{|c|}{ Reference Elevation (ft AMSL) } & 1508.77 & \multirow{2}{*}{$\begin{array}{l}1509.12 \\
\text { Elevation in Klassen } \\
\text { Test Well (ft AMSL) }\end{array}$} \\
\hline Date and Time & $\begin{array}{l}\text { Depth in Klassen } \\
\text { Domestic Well } \\
\text { (ft BGL) }\end{array}$ & $\begin{array}{c}\text { Depth in Klassen } \\
\text { Test Well } \\
\text { (ft BGL) }\end{array}$ & $\begin{array}{l}\text { Elevation in Klassen } \\
\text { Domestic Well } \\
\text { (ft AMSL) }\end{array}$ & \\
\hline $9 / 17 / 118: 30$ & 36.956 & 40.27 & 1471.81 & 1468.85 \\
\hline $9 / 17 / 118: 40$ & 36.321 & 40.273 & 1472.45 & 1468.85 \\
\hline $9 / 17 / 118: 50$ & 36.281 & 40.275 & 1472.49 & 1468.85 \\
\hline 9/17/11 9:00 & 40.359 & 40.277 & 1468.41 & 1468.85 \\
\hline 9/17/11 9:10 & 36.438 & 40.284 & 1472.33 & 1468.84 \\
\hline 9/17/11 9:20 & 36.372 & 40.282 & 1472.40 & 1468.84 \\
\hline 9/17/11 9:30 & 37.7 & 40.282 & 1471.07 & 1468.84 \\
\hline 9/17/11 9:40 & 36.604 & 40.284 & 1472.17 & 1468.84 \\
\hline 9/17/11 9:50 & 36.501 & 40.266 & 1472.27 & 1468.86 \\
\hline 9/17/11 10:00 & 36.499 & 40.266 & 1472.27 & 1468.86 \\
\hline 9/17/11 10:10 & 36.475 & 40.233 & 1472.29 & 1468.89 \\
\hline 9/17/11 10:20 & 36.464 & 40.216 & 1472.31 & 1468.91 \\
\hline 9/17/11 10:30 & 36.426 & 40.209 & 1472.34 & 1468.91 \\
\hline 9/17/11 10:40 & 36.391 & 40.205 & 1472.38 & 1468.92 \\
\hline 9/17/11 10:50 & 36.36 & 40.2 & 1472.41 & 1468.92 \\
\hline 9/17/11 11:00 & 36.332 & 40.195 & 1472.44 & 1468.93 \\
\hline 9/17/11 11:10 & 36.314 & 40.19 & 1472.46 & 1468.93 \\
\hline 9/17/11 11:20 & 36.292 & 40.186 & 1472.48 & 1468.94 \\
\hline 9/17/11 11:30 & 36.276 & 40.181 & 1472.49 & 1468.94 \\
\hline 9/17/11 11:40 & 36.248 & 40.176 & 1472.52 & 1468.95 \\
\hline 9/17/11 11:50 & 36.217 & 40.172 & 1472.55 & 1468.95 \\
\hline 9/17/11 12:00 & 36.196 & 40.165 & 1472.57 & 1468.96 \\
\hline 9/17/11 12:10 & 36.159 & 40.158 & 1472.61 & 1468.96 \\
\hline 9/17/11 12:20 & 36.131 & 40.153 & 1472.64 & 1468.97 \\
\hline 9/17/11 12:30 & 36.098 & 40.146 & 1472.67 & 1468.98 \\
\hline $9 / 17 / 11$ 12:40 & 36.067 & 40.139 & 1472.70 & 1468.98 \\
\hline $9 / 17 / 11$ 12:50 & 36.046 & 40.132 & 1472.72 & 1468.99 \\
\hline 9/17/11 13:00 & 36.016 & 40.127 & 1472.75 & 1469.00 \\
\hline 9/17/11 13:10 & 35.988 & 40.122 & 1472.78 & 1469.00 \\
\hline 9/17/11 13:20 & 35.971 & 40.113 & 1472.80 & 1469.01 \\
\hline 9/17/11 13:30 & 35.945 & 40.108 & 1472.82 & 1469.01 \\
\hline 9/17/11 13:40 & 35.924 & 40.104 & 1472.85 & 1469.02 \\
\hline $9 / 17 / 11$ 13:50 & 35.91 & 40.097 & 1472.86 & 1469.03 \\
\hline 9/17/11 14:00 & 35.887 & 40.09 & 1472.88 & 1469.03 \\
\hline 9/17/11 14:10 & 35.868 & 40.085 & 1472.90 & 1469.04 \\
\hline 9/17/11 14:20 & 35.854 & 40.078 & 1472.92 & 1469.04 \\
\hline 9/17/11 14:30 & 35.833 & 40.071 & 1472.94 & 1469.05 \\
\hline 9/17/11 14:40 & 35.816 & 40.066 & 1472.95 & 1469.06 \\
\hline
\end{tabular}


TABLE S1.3 Automatically recorded groundwater levels in the Klassen wells, September 15, 2011, to March 13, 2012.

\begin{tabular}{|c|c|c|c|c|}
\hline \multicolumn{3}{|c|}{ Reference Elevation (ft AMSL) } & 1508.77 & \multirow{2}{*}{$\begin{array}{l}1509.12 \\
\text { Elevation in Klassen } \\
\text { Test Well (ft AMSL) }\end{array}$} \\
\hline Date and Time & $\begin{array}{l}\text { Depth in Klassen } \\
\text { Domestic Well } \\
(\mathrm{ft} \mathrm{BGL})\end{array}$ & $\begin{array}{c}\text { Depth in Klassen } \\
\text { Test Well } \\
\text { (ft BGL) }\end{array}$ & $\begin{array}{l}\text { Elevation in Klassen } \\
\text { Domestic Well } \\
\text { (ft AMSL) }\end{array}$ & \\
\hline 9/17/11 14:50 & 35.805 & 40.059 & 1472.96 & 1469.06 \\
\hline 9/17/11 15:00 & 35.786 & 40.052 & 1472.98 & 1469.07 \\
\hline 9/17/11 15:10 & 35.769 & 40.045 & 1473.00 & 1469.08 \\
\hline $9 / 17 / 1115: 20$ & 35.76 & 40.04 & 1473.01 & 1469.08 \\
\hline 9/17/11 15:30 & 35.744 & 40.031 & 1473.03 & 1469.09 \\
\hline 9/17/11 15:40 & 35.736 & 40.026 & 1473.03 & 1469.10 \\
\hline $9 / 17 / 1115: 50$ & 35.751 & 40.022 & 1473.02 & 1469.10 \\
\hline 9/17/11 16:00 & 35.739 & 40.017 & 1473.03 & 1469.11 \\
\hline 9/17/11 16:10 & 35.727 & 40.01 & 1473.04 & 1469.11 \\
\hline $9 / 17 / 1116: 20$ & 35.706 & 40.003 & 1473.06 & 1469.12 \\
\hline 9/17/11 16:30 & 35.697 & 39.998 & 1473.07 & 1469.12 \\
\hline 9/17/11 16:40 & 35.683 & 39.991 & 1473.09 & 1469.13 \\
\hline 9/17/11 16:50 & 35.661 & 39.984 & 1473.11 & 1469.14 \\
\hline 9/17/11 17:00 & 35.655 & 39.977 & 1473.11 & 1469.15 \\
\hline 9/17/11 17:10 & 35.64 & 39.97 & 1473.13 & 1469.15 \\
\hline 9/17/11 17:20 & 35.624 & 39.965 & 1473.15 & 1469.16 \\
\hline 9/17/11 17:30 & 35.612 & 39.958 & 1473.16 & 1469.16 \\
\hline 9/17/11 17:40 & 35.605 & 39.951 & 1473.16 & 1469.17 \\
\hline 9/17/11 17:50 & 35.591 & 39.946 & 1473.18 & 1469.18 \\
\hline 9/17/11 18:00 & 35.575 & 39.939 & 1473.19 & 1469.18 \\
\hline 9/17/11 18:10 & 35.57 & 39.932 & 1473.20 & 1469.19 \\
\hline 9/17/11 18:20 & 35.558 & 39.928 & 1473.21 & 1469.19 \\
\hline $9 / 17 / 11$ 18:30 & 35.554 & 39.921 & 1473.22 & 1469.20 \\
\hline 9/17/11 18:40 & 35.551 & 39.911 & 1473.22 & 1469.21 \\
\hline 9/17/11 18:50 & 35.683 & 39.914 & 1473.09 & 1469.21 \\
\hline 9/17/11 19:00 & 35.821 & 39.918 & 1472.95 & 1469.20 \\
\hline 9/17/11 19:10 & 35.952 & 39.928 & 1472.82 & 1469.19 \\
\hline 9/17/11 19:20 & 36.032 & 39.939 & 1472.74 & 1469.18 \\
\hline 9/17/11 19:30 & 36.025 & 39.939 & 1472.74 & 1469.18 \\
\hline 9/17/11 19:40 & 38.417 & 39.942 & 1470.35 & 1469.18 \\
\hline 9/17/11 19:50 & 36.192 & 39.944 & 1472.58 & 1469.18 \\
\hline 9/17/11 20:00 & 36.079 & 39.944 & 1472.69 & 1469.18 \\
\hline 9/17/11 20:10 & 36.013 & 39.939 & 1472.76 & 1469.18 \\
\hline 9/17/11 20:20 & 38.635 & 39.935 & 1470.13 & 1469.19 \\
\hline 9/17/11 20:30 & 36.051 & 39.932 & 1472.72 & 1469.19 \\
\hline 9/17/11 20:40 & 35.962 & 39.928 & 1472.81 & 1469.19 \\
\hline 9/17/11 20:50 & 35.905 & 39.921 & 1472.86 & 1469.20 \\
\hline 9/17/11 21:00 & 35.861 & 39.916 & 1472.91 & 1469.21 \\
\hline
\end{tabular}


TABLE S1.3 Automatically recorded groundwater levels in the Klassen wells, September 15, 2011, to March 13, 2012.

\begin{tabular}{|c|c|c|c|c|}
\hline \multicolumn{3}{|c|}{ Reference Elevation (ft AMSL) } & 1508.77 & \multirow{2}{*}{$\begin{array}{l}1509.12 \\
\text { Elevation in Klassen } \\
\text { Test Well (ft AMSL) }\end{array}$} \\
\hline Date and Time & $\begin{array}{l}\text { Depth in Klassen } \\
\text { Domestic Well } \\
(\mathrm{ft} \mathrm{BGL})\end{array}$ & $\begin{array}{c}\text { Depth in Klassen } \\
\text { Test Well } \\
\text { (ft BGL) }\end{array}$ & $\begin{array}{l}\text { Elevation in Klassen } \\
\text { Domestic Well } \\
\text { (ft AMSL) }\end{array}$ & \\
\hline 9/17/11 21:10 & 35.833 & 39.909 & 1472.94 & 1469.21 \\
\hline $9 / 17 / 1121: 20$ & 35.802 & 39.904 & 1472.97 & 1469.22 \\
\hline 9/17/11 21:30 & 35.769 & 39.9 & 1473.00 & 1469.22 \\
\hline 9/17/11 21:40 & 35.744 & 39.895 & 1473.03 & 1469.23 \\
\hline 9/17/11 21:50 & 35.727 & 39.89 & 1473.04 & 1469.23 \\
\hline 9/17/11 22:00 & 35.718 & 39.883 & 1473.05 & 1469.24 \\
\hline 9/17/11 22:10 & 35.704 & 39.878 & 1473.07 & 1469.24 \\
\hline 9/17/11 22:20 & 35.68 & 39.876 & 1473.09 & 1469.25 \\
\hline $9 / 17 / 1122: 30$ & 35.662 & 39.874 & 1473.11 & 1469.25 \\
\hline $9 / 17 / 1122: 40$ & 39.688 & 39.867 & 1469.08 & 1469.26 \\
\hline $9 / 17 / 1122: 50$ & 35.929 & 39.869 & 1472.84 & 1469.25 \\
\hline 9/17/11 23:00 & 35.812 & 39.869 & 1472.96 & 1469.25 \\
\hline 9/17/11 23:10 & 39.423 & 39.864 & 1469.35 & 1469.26 \\
\hline $9 / 17 / 1123: 20$ & 35.98 & 39.864 & 1472.79 & 1469.26 \\
\hline 9/17/11 23:30 & 35.866 & 39.864 & 1472.90 & 1469.26 \\
\hline $9 / 17 / 1123: 40$ & 35.8 & 39.857 & 1472.97 & 1469.27 \\
\hline $9 / 17 / 1123: 50$ & 35.753 & 39.855 & 1473.02 & 1469.27 \\
\hline 9/18/11 0:00 & 35.734 & 39.85 & 1473.04 & 1469.27 \\
\hline 9/18/11 0:10 & 35.704 & 39.848 & 1473.07 & 1469.27 \\
\hline 9/18/11 0:20 & 35.676 & 39.843 & 1473.09 & 1469.28 \\
\hline 9/18/11 0:30 & 35.655 & 39.841 & 1473.11 & 1469.28 \\
\hline 9/18/11 0:40 & 35.636 & 39.836 & 1473.13 & 1469.29 \\
\hline $9 / 18 / 110: 50$ & 35.61 & 39.831 & 1473.16 & 1469.29 \\
\hline 9/18/11 1:00 & 35.629 & 39.829 & 1473.14 & 1469.29 \\
\hline 9/18/11 1:10 & 35.626 & 39.824 & 1473.14 & 1469.30 \\
\hline $9 / 18 / 11$ 1:20 & 35.608 & 39.82 & 1473.16 & 1469.30 \\
\hline 9/18/11 1:30 & 35.589 & 39.82 & 1473.18 & 1469.30 \\
\hline $9 / 18 / 11$ 1:40 & 35.608 & 39.815 & 1473.16 & 1469.31 \\
\hline 9/18/11 1:50 & 35.655 & 39.813 & 1473.11 & 1469.31 \\
\hline 9/18/11 2:00 & 35.647 & 39.81 & 1473.12 & 1469.31 \\
\hline 9/18/11 2:10 & 35.626 & 39.806 & 1473.14 & 1469.32 \\
\hline $9 / 18 / 112: 20$ & 35.612 & 39.801 & 1473.16 & 1469.32 \\
\hline 9/18/11 2:30 & 35.596 & 39.799 & 1473.17 & 1469.32 \\
\hline $9 / 18 / 112: 40$ & 35.582 & 39.792 & 1473.19 & 1469.33 \\
\hline $9 / 18 / 112: 50$ & 35.572 & 39.789 & 1473.20 & 1469.33 \\
\hline 9/18/11 3:00 & 35.558 & 39.787 & 1473.21 & 1469.34 \\
\hline 9/18/11 3:10 & 35.551 & 39.782 & 1473.22 & 1469.34 \\
\hline 9/18/11 3:20 & 35.561 & 39.785 & 1473.21 & 1469.34 \\
\hline
\end{tabular}


TABLE S1.3 Automatically recorded groundwater levels in the Klassen wells, September 15, 2011, to March 13, 2012.

\begin{tabular}{|c|c|c|c|c|}
\hline \multicolumn{3}{|c|}{ Reference Elevation (ft AMSL) } & 1508.77 & \multirow{2}{*}{$\begin{array}{l}1509.12 \\
\text { Elevation in Klassen } \\
\text { Test Well (ft AMSL) }\end{array}$} \\
\hline Date and Time & $\begin{array}{l}\text { Depth in Klassen } \\
\text { Domestic Well } \\
(\mathrm{ft} \mathrm{BGL})\end{array}$ & $\begin{array}{c}\text { Depth in Klassen } \\
\text { Test Well } \\
\text { (ft BGL) }\end{array}$ & $\begin{array}{l}\text { Elevation in Klassen } \\
\text { Domestic Well } \\
\text { (ft AMSL) }\end{array}$ & \\
\hline 9/18/11 3:30 & 35.61 & 39.785 & 1473.16 & 1469.34 \\
\hline 9/18/11 3:40 & 35.591 & 39.777 & 1473.18 & 1469.35 \\
\hline $9 / 18 / 113: 50$ & 35.568 & 39.777 & 1473.20 & 1469.35 \\
\hline $9 / 18 / 114: 00$ & 35.549 & 39.775 & 1473.22 & 1469.35 \\
\hline 9/18/11 4:10 & 35.53 & 39.768 & 1473.24 & 1469.35 \\
\hline $9 / 18 / 114: 20$ & 35.507 & 39.756 & 1473.26 & 1469.37 \\
\hline $9 / 18 / 114: 30$ & 35.483 & 39.728 & 1473.29 & 1469.39 \\
\hline $9 / 18 / 114: 40$ & 35.455 & 39.712 & 1473.31 & 1469.41 \\
\hline $9 / 18 / 114: 50$ & 35.434 & 39.707 & 1473.34 & 1469.42 \\
\hline 9/18/11 5:00 & 35.415 & 39.7 & 1473.35 & 1469.42 \\
\hline 9/18/11 5:10 & 35.396 & 39.705 & 1473.37 & 1469.42 \\
\hline 9/18/11 5:20 & 35.378 & 39.7 & 1473.39 & 1469.42 \\
\hline $9 / 18 / 115: 30$ & 36.077 & 39.695 & 1472.69 & 1469.43 \\
\hline $9 / 18 / 115: 40$ & 35.502 & 39.693 & 1473.27 & 1469.43 \\
\hline 9/18/11 5:50 & 40.092 & 39.688 & 1468.68 & 1469.43 \\
\hline 9/18/11 6:00 & 35.927 & 39.693 & 1472.84 & 1469.43 \\
\hline $9 / 18 / 116: 10$ & 35.582 & 39.7 & 1473.19 & 1469.42 \\
\hline 9/18/11 6:20 & 38.206 & 39.705 & 1470.56 & 1469.42 \\
\hline $9 / 18 / 116: 30$ & 35.65 & 39.716 & 1473.12 & 1469.41 \\
\hline $9 / 18 / 116: 40$ & 35.551 & 39.714 & 1473.22 & 1469.41 \\
\hline $9 / 18 / 116: 50$ & 35.497 & 39.707 & 1473.27 & 1469.42 \\
\hline 9/18/11 7:00 & 35.458 & 39.698 & 1473.31 & 1469.42 \\
\hline 9/18/11 7:10 & 35.427 & 39.691 & 1473.34 & 1469.43 \\
\hline 9/18/11 7:20 & 35.408 & 39.693 & 1473.36 & 1469.43 \\
\hline 9/18/11 7:30 & 35.389 & 39.695 & 1473.38 & 1469.43 \\
\hline 9/18/11 7:40 & 35.382 & 39.693 & 1473.39 & 1469.43 \\
\hline $9 / 18 / 117: 50$ & 35.373 & 39.691 & 1473.40 & 1469.43 \\
\hline 9/18/11 8:00 & 36.515 & 39.686 & 1472.25 & 1469.44 \\
\hline 9/18/11 8:10 & 35.46 & 39.674 & 1473.31 & 1469.45 \\
\hline 9/18/11 8:20 & 37.566 & 39.658 & 1471.20 & 1469.46 \\
\hline 9/18/11 8:30 & 35.533 & 39.651 & 1473.24 & 1469.47 \\
\hline 9/18/11 8:40 & 35.662 & 39.651 & 1473.11 & 1469.47 \\
\hline $9 / 18 / 118: 50$ & 35.561 & 39.651 & 1473.21 & 1469.47 \\
\hline 9/18/11 9:00 & 35.542 & 39.648 & 1473.23 & 1469.47 \\
\hline 9/18/11 9:10 & 35.535 & 39.653 & 1473.23 & 1469.47 \\
\hline 9/18/11 9:20 & 35.565 & 39.651 & 1473.20 & 1469.47 \\
\hline 9/18/11 9:30 & 35.575 & 39.651 & 1473.19 & 1469.47 \\
\hline 9/18/11 9:40 & 35.54 & 39.651 & 1473.23 & 1469.47 \\
\hline
\end{tabular}


TABLE S1.3 Automatically recorded groundwater levels in the Klassen wells, September 15, 2011, to March 13, 2012.

\begin{tabular}{|c|c|c|c|c|}
\hline \multicolumn{3}{|c|}{ Reference Elevation (ft AMSL) } & 1508.77 & \multirow{2}{*}{$\begin{array}{l}1509.12 \\
\text { Elevation in Klassen } \\
\text { Test Well (ft AMSL) }\end{array}$} \\
\hline Date and Time & $\begin{array}{l}\text { Depth in Klassen } \\
\text { Domestic Well } \\
(\mathrm{ft} \mathrm{BGL})\end{array}$ & $\begin{array}{c}\text { Depth in Klassen } \\
\text { Test Well } \\
\text { (ft BGL) }\end{array}$ & $\begin{array}{l}\text { Elevation in Klassen } \\
\text { Domestic Well } \\
\text { (ft AMSL) }\end{array}$ & \\
\hline 9/18/11 9:50 & 35.523 & 39.651 & 1473.25 & 1469.47 \\
\hline 9/18/11 10:00 & 35.54 & 39.651 & 1473.23 & 1469.47 \\
\hline 9/18/11 10:10 & 35.539 & 39.651 & 1473.23 & 1469.47 \\
\hline $9 / 18 / 1110: 20$ & 35.526 & 39.651 & 1473.24 & 1469.47 \\
\hline 9/18/11 10:30 & 35.509 & 39.655 & 1473.26 & 1469.47 \\
\hline 9/18/11 10:40 & 35.493 & 39.658 & 1473.28 & 1469.46 \\
\hline $9 / 18 / 1110: 50$ & 35.472 & 39.66 & 1473.30 & 1469.46 \\
\hline 9/18/11 11:00 & 35.45 & 39.665 & 1473.32 & 1469.46 \\
\hline 9/18/11 11:10 & 35.441 & 39.67 & 1473.33 & 1469.45 \\
\hline 9/18/11 11:20 & 35.415 & 39.67 & 1473.35 & 1469.45 \\
\hline 9/18/11 11:30 & 35.389 & 39.66 & 1473.38 & 1469.46 \\
\hline 9/18/11 11:40 & 35.373 & 39.66 & 1473.40 & 1469.46 \\
\hline 9/18/11 11:50 & 35.357 & 39.663 & 1473.41 & 1469.46 \\
\hline 9/18/11 12:00 & 35.345 & 39.665 & 1473.42 & 1469.46 \\
\hline 9/18/11 12:10 & 35.329 & 39.665 & 1473.44 & 1469.46 \\
\hline $9 / 18 / 11$ 12:20 & 35.331 & 39.665 & 1473.44 & 1469.46 \\
\hline $9 / 18 / 11$ 12:30 & 35.338 & 39.665 & 1473.43 & 1469.46 \\
\hline 9/18/11 12:40 & 35.406 & 39.667 & 1473.36 & 1469.46 \\
\hline 9/18/11 12:50 & 35.404 & 39.67 & 1473.37 & 1469.45 \\
\hline 9/18/11 13:00 & 35.443 & 39.674 & 1473.33 & 1469.45 \\
\hline 9/18/11 13:10 & 35.425 & 39.677 & 1473.34 & 1469.45 \\
\hline 9/18/11 13:20 & 35.406 & 39.681 & 1473.36 & 1469.44 \\
\hline $9 / 18 / 11$ 13:30 & 35.394 & 39.681 & 1473.38 & 1469.44 \\
\hline 9/18/11 13:40 & 35.371 & 39.679 & 1473.40 & 1469.44 \\
\hline 9/18/11 13:50 & 35.378 & 39.681 & 1473.39 & 1469.44 \\
\hline 9/18/11 14:00 & 36.989 & 39.681 & 1471.78 & 1469.44 \\
\hline 9/18/11 14:10 & 35.467 & 39.681 & 1473.30 & 1469.44 \\
\hline 9/18/11 14:20 & 35.425 & 39.681 & 1473.34 & 1469.44 \\
\hline 9/18/11 14:30 & 35.387 & 39.681 & 1473.38 & 1469.44 \\
\hline 9/18/11 14:40 & 35.352 & 39.684 & 1473.42 & 1469.44 \\
\hline 9/18/11 14:50 & 35.342 & 39.686 & 1473.43 & 1469.44 \\
\hline 9/18/11 15:00 & 35.345 & 39.686 & 1473.42 & 1469.44 \\
\hline 9/18/11 15:10 & 35.326 & 39.688 & 1473.44 & 1469.43 \\
\hline 9/18/11 15:20 & 35.303 & 39.686 & 1473.47 & 1469.44 \\
\hline 9/18/11 15:30 & 35.284 & 39.686 & 1473.49 & 1469.44 \\
\hline 9/18/11 15:40 & 35.265 & 39.684 & 1473.50 & 1469.44 \\
\hline 9/18/11 15:50 & 35.249 & 39.681 & 1473.52 & 1469.44 \\
\hline 9/18/11 16:00 & 35.232 & 39.679 & 1473.54 & 1469.44 \\
\hline
\end{tabular}


TABLE S1.3 Automatically recorded groundwater levels in the Klassen wells, September 15, 2011, to March 13, 2012.

\begin{tabular}{|c|c|c|c|c|}
\hline \multicolumn{3}{|c|}{ Reference Elevation (ft AMSL) } & 1508.77 & \multirow{2}{*}{$\begin{array}{l}1509.12 \\
\text { Elevation in Klassen } \\
\text { Test Well (ft AMSL) }\end{array}$} \\
\hline Date and Time & $\begin{array}{l}\text { Depth in Klassen } \\
\text { Domestic Well } \\
(\mathrm{ft} \mathrm{BGL})\end{array}$ & $\begin{array}{c}\text { Depth in Klassen } \\
\text { Test Well } \\
\text { (ft BGL) }\end{array}$ & $\begin{array}{l}\text { Elevation in Klassen } \\
\text { Domestic Well } \\
\text { (ft AMSL) }\end{array}$ & \\
\hline 9/18/11 16:10 & 35.228 & 39.677 & 1473.54 & 1469.45 \\
\hline $9 / 18 / 1116: 20$ & 35.23 & 39.674 & 1473.54 & 1469.45 \\
\hline 9/18/11 16:30 & 35.218 & 39.672 & 1473.55 & 1469.45 \\
\hline 9/18/11 16:40 & 36.848 & 39.667 & 1471.92 & 1469.46 \\
\hline 9/18/11 16:50 & 35.329 & 39.667 & 1473.44 & 1469.46 \\
\hline 9/18/11 17:00 & 35.27 & 39.665 & 1473.50 & 1469.46 \\
\hline 9/18/11 17:10 & 35.239 & 39.66 & 1473.53 & 1469.46 \\
\hline 9/18/11 17:20 & 35.216 & 39.658 & 1473.55 & 1469.46 \\
\hline 9/18/11 17:30 & 35.195 & 39.653 & 1473.57 & 1469.47 \\
\hline 9/18/11 17:40 & 35.174 & 39.651 & 1473.60 & 1469.47 \\
\hline 9/18/11 17:50 & 35.157 & 39.646 & 1473.61 & 1469.48 \\
\hline 9/18/11 18:00 & 35.493 & 39.644 & 1473.28 & 1469.48 \\
\hline 9/18/11 18:10 & 35.249 & 39.641 & 1473.52 & 1469.48 \\
\hline $9 / 18 / 1118: 20$ & 35.2 & 39.637 & 1473.57 & 1469.49 \\
\hline 9/18/11 18:30 & 35.169 & 39.634 & 1473.60 & 1469.49 \\
\hline 9/18/11 18:40 & 35.148 & 39.63 & 1473.62 & 1469.49 \\
\hline $9 / 18 / 11$ 18:50 & 35.129 & 39.625 & 1473.64 & 1469.50 \\
\hline 9/18/11 19:00 & 35.113 & 39.62 & 1473.66 & 1469.50 \\
\hline 9/18/11 19:10 & 35.643 & 39.62 & 1473.13 & 1469.50 \\
\hline 9/18/11 19:20 & 35.312 & 39.62 & 1473.46 & 1469.50 \\
\hline 9/18/11 19:30 & 35.253 & 39.618 & 1473.52 & 1469.50 \\
\hline 9/18/11 19:40 & 35.326 & 39.618 & 1473.44 & 1469.50 \\
\hline 9/18/11 19:50 & 35.298 & 39.618 & 1473.47 & 1469.50 \\
\hline 9/18/11 20:00 & 35.242 & 39.613 & 1473.53 & 1469.51 \\
\hline 9/18/11 20:10 & 35.188 & 39.609 & 1473.58 & 1469.51 \\
\hline 9/18/11 20:20 & 35.204 & 39.606 & 1473.57 & 1469.52 \\
\hline 9/18/11 20:30 & 35.207 & 39.602 & 1473.56 & 1469.52 \\
\hline 9/18/11 20:40 & 35.223 & 39.599 & 1473.55 & 1469.52 \\
\hline 9/18/11 20:50 & 35.237 & 39.599 & 1473.53 & 1469.52 \\
\hline 9/18/11 21:00 & 35.235 & 39.597 & 1473.53 & 1469.53 \\
\hline 9/18/11 21:10 & 35.195 & 39.592 & 1473.57 & 1469.53 \\
\hline 9/18/11 21:20 & 35.171 & 39.587 & 1473.60 & 1469.54 \\
\hline $9 / 18 / 1121: 30$ & 35.127 & 39.583 & 1473.64 & 1469.54 \\
\hline 9/18/11 21:40 & 35.089 & 39.576 & 1473.68 & 1469.55 \\
\hline 9/18/11 21:50 & 35.052 & 39.569 & 1473.72 & 1469.55 \\
\hline 9/18/11 22:00 & 35.019 & 39.564 & 1473.75 & 1469.56 \\
\hline 9/18/11 22:10 & 34.991 & 39.555 & 1473.78 & 1469.57 \\
\hline $9 / 18 / 1122: 20$ & 34.963 & 39.55 & 1473.81 & 1469.57 \\
\hline
\end{tabular}


TABLE S1.3 Automatically recorded groundwater levels in the Klassen wells, September 15, 2011, to March 13, 2012.

\begin{tabular}{|c|c|c|c|c|}
\hline \multicolumn{3}{|c|}{ Reference Elevation (ft AMSL) } & 1508.77 & \multirow{2}{*}{$\begin{array}{l}1509.12 \\
\text { Elevation in Klassen } \\
\text { Test Well (ft AMSL) }\end{array}$} \\
\hline Date and Time & $\begin{array}{l}\text { Depth in Klassen } \\
\text { Domestic Well } \\
\text { (ft BGL) }\end{array}$ & $\begin{array}{c}\text { Depth in Klassen } \\
\text { Test Well } \\
\text { (ft BGL) }\end{array}$ & $\begin{array}{l}\text { Elevation in Klassen } \\
\text { Domestic Well } \\
\text { (ft AMSL) }\end{array}$ & \\
\hline $9 / 18 / 1122: 30$ & 34.96 & 39.543 & 1473.81 & 1469.58 \\
\hline $9 / 18 / 1122: 40$ & 34.974 & 39.536 & 1473.80 & 1469.59 \\
\hline $9 / 18 / 1122: 50$ & 36.529 & 39.533 & 1472.24 & 1469.59 \\
\hline 9/18/11 23:00 & 35.516 & 39.533 & 1473.25 & 1469.59 \\
\hline 9/18/11 23:10 & 35.598 & 39.531 & 1473.17 & 1469.59 \\
\hline 9/18/11 23:20 & 35.439 & 39.529 & 1473.33 & 1469.59 \\
\hline $9 / 18 / 1123: 30$ & 35.715 & 39.526 & 1473.05 & 1469.60 \\
\hline 9/18/11 23:40 & 36.672 & 39.526 & 1472.10 & 1469.60 \\
\hline $9 / 18 / 1123: 50$ & 35.404 & 39.524 & 1473.37 & 1469.60 \\
\hline 9/19/11 0:00 & 35.256 & 39.519 & 1473.51 & 1469.60 \\
\hline 9/19/11 0:10 & 35.171 & 39.515 & 1473.60 & 1469.61 \\
\hline 9/19/11 0:20 & 35.108 & 39.51 & 1473.66 & 1469.61 \\
\hline 9/19/11 0:30 & 35.059 & 39.503 & 1473.71 & 1469.62 \\
\hline 9/19/11 0:40 & 35.019 & 39.498 & 1473.75 & 1469.62 \\
\hline 9/19/11 0:50 & 34.981 & 39.491 & 1473.79 & 1469.63 \\
\hline 9/19/11 1:00 & 34.951 & 39.487 & 1473.82 & 1469.64 \\
\hline 9/19/11 1:10 & 34.92 & 39.477 & 1473.85 & 1469.65 \\
\hline $9 / 19 / 11$ 1:20 & 34.895 & 39.472 & 1473.87 & 1469.65 \\
\hline 9/19/11 1:30 & 34.869 & 39.468 & 1473.90 & 1469.65 \\
\hline $9 / 19 / 11$ 1:40 & 34.848 & 39.461 & 1473.92 & 1469.66 \\
\hline 9/19/11 1:50 & 34.827 & 39.456 & 1473.94 & 1469.67 \\
\hline 9/19/11 2:00 & 34.806 & 39.449 & 1473.96 & 1469.67 \\
\hline 9/19/11 2:10 & 34.813 & 39.442 & 1473.96 & 1469.68 \\
\hline 9/19/11 2:20 & 34.796 & 39.44 & 1473.97 & 1469.68 \\
\hline 9/19/11 2:30 & 34.775 & 39.433 & 1473.99 & 1469.69 \\
\hline $9 / 19 / 112: 40$ & 34.754 & 39.426 & 1474.02 & 1469.70 \\
\hline $9 / 19 / 112: 50$ & 34.738 & 39.421 & 1474.03 & 1469.70 \\
\hline 9/19/11 3:00 & 34.719 & 39.414 & 1474.05 & 1469.71 \\
\hline 9/19/11 3:10 & 34.705 & 39.409 & 1474.06 & 1469.71 \\
\hline 9/19/11 3:20 & 35.422 & 39.404 & 1473.35 & 1469.72 \\
\hline 9/19/11 3:30 & 34.803 & 39.4 & 1473.97 & 1469.72 \\
\hline 9/19/11 3:40 & 34.747 & 39.393 & 1474.02 & 1469.73 \\
\hline 9/19/11 3:50 & 34.714 & 39.388 & 1474.06 & 1469.73 \\
\hline 9/19/11 4:00 & 34.688 & 39.383 & 1474.08 & 1469.74 \\
\hline 9/19/11 4:10 & 34.669 & 39.376 & 1474.10 & 1469.75 \\
\hline 9/19/11 4:20 & 34.677 & 39.372 & 1474.09 & 1469.75 \\
\hline 9/19/11 4:30 & 34.658 & 39.367 & 1474.11 & 1469.76 \\
\hline 9/19/11 4:40 & 34.639 & 39.36 & 1474.13 & 1469.76 \\
\hline
\end{tabular}


TABLE S1.3 Automatically recorded groundwater levels in the Klassen wells, September 15, 2011, to March 13, 2012.

\begin{tabular}{|c|c|c|c|c|}
\hline \multicolumn{3}{|c|}{ Reference Elevation (ft AMSL) } & 1508.77 & \multirow{2}{*}{$\begin{array}{l}1509.12 \\
\text { Elevation in Klassen } \\
\text { Test Well (ft AMSL) }\end{array}$} \\
\hline Date and Time & $\begin{array}{l}\text { Depth in Klassen } \\
\text { Domestic Well } \\
\text { (ft BGL) }\end{array}$ & $\begin{array}{c}\text { Depth in Klassen } \\
\text { Test Well } \\
\text { (ft BGL) }\end{array}$ & $\begin{array}{l}\text { Elevation in Klassen } \\
\text { Domestic Well } \\
\text { (ft AMSL) }\end{array}$ & \\
\hline 9/19/11 4:50 & 34.623 & 39.353 & 1474.15 & 1469.77 \\
\hline 9/19/11 5:00 & 34.606 & 39.348 & 1474.16 & 1469.77 \\
\hline 9/19/11 5:10 & 34.592 & 39.341 & 1474.18 & 1469.78 \\
\hline 9/19/11 5:20 & 34.576 & 39.336 & 1474.19 & 1469.79 \\
\hline 9/19/11 5:30 & 34.564 & 39.329 & 1474.21 & 1469.79 \\
\hline 9/19/11 5:40 & 34.552 & 39.325 & 1474.22 & 1469.80 \\
\hline 9/19/11 5:50 & 34.541 & 39.318 & 1474.23 & 1469.80 \\
\hline $9 / 19 / 116: 00$ & 34.529 & 39.311 & 1474.24 & 1469.81 \\
\hline 9/19/11 6:10 & 39.039 & 39.306 & 1469.73 & 1469.82 \\
\hline $9 / 19 / 116: 20$ & 34.754 & 39.304 & 1474.02 & 1469.82 \\
\hline $9 / 19 / 116: 30$ & 34.637 & 39.299 & 1474.13 & 1469.82 \\
\hline $9 / 19 / 116: 40$ & 34.592 & 39.292 & 1474.18 & 1469.83 \\
\hline $9 / 19 / 116: 50$ & 34.562 & 39.287 & 1474.21 & 1469.84 \\
\hline 9/19/11 7:00 & 34.541 & 39.282 & 1474.23 & 1469.84 \\
\hline 9/19/11 7:10 & 34.522 & 39.278 & 1474.25 & 1469.84 \\
\hline 9/19/11 7:20 & 34.519 & 39.271 & 1474.25 & 1469.85 \\
\hline 9/19/11 7:30 & 34.522 & 39.268 & 1474.25 & 1469.85 \\
\hline 9/19/11 7:40 & 34.505 & 39.261 & 1474.26 & 1469.86 \\
\hline 9/19/11 7:50 & 34.51 & 39.257 & 1474.26 & 1469.87 \\
\hline 9/19/11 8:00 & 36.285 & 39.252 & 1472.48 & 1469.87 \\
\hline 9/19/11 8:10 & 34.623 & 39.25 & 1474.15 & 1469.87 \\
\hline 9/19/11 8:20 & 34.557 & 39.245 & 1474.21 & 1469.88 \\
\hline 9/19/11 8:30 & 34.524 & 39.24 & 1474.25 & 1469.88 \\
\hline 9/19/11 8:40 & 34.991 & 39.236 & 1473.78 & 1469.89 \\
\hline 9/19/11 8:50 & 34.609 & 39.231 & 1474.16 & 1469.89 \\
\hline 9/19/11 9:00 & 34.55 & 39.226 & 1474.22 & 1469.90 \\
\hline 9/19/11 9:10 & 34.82 & 39.224 & 1473.95 & 1469.90 \\
\hline 9/19/11 9:20 & 34.665 & 39.219 & 1474.10 & 1469.90 \\
\hline 9/19/11 9:30 & 34.604 & 39.217 & 1474.17 & 1469.91 \\
\hline 9/19/11 9:40 & 34.564 & 39.212 & 1474.21 & 1469.91 \\
\hline 9/19/11 9:50 & 34.533 & 39.207 & 1474.24 & 1469.92 \\
\hline 9/19/11 10:00 & 34.726 & 39.203 & 1474.04 & 1469.92 \\
\hline 9/19/11 10:10 & 34.991 & 39.198 & 1473.78 & 1469.92 \\
\hline 9/19/11 10:20 & 34.655 & 39.196 & 1474.11 & 1469.93 \\
\hline 9/19/11 10:30 & 34.665 & 39.196 & 1474.10 & 1469.93 \\
\hline 9/19/11 10:40 & 34.644 & 39.193 & 1474.13 & 1469.93 \\
\hline 9/19/11 10:50 & 34.677 & 39.193 & 1474.09 & 1469.93 \\
\hline 9/19/11 11:00 & 34.641 & 39.191 & 1474.13 & 1469.93 \\
\hline
\end{tabular}


TABLE S1.3 Automatically recorded groundwater levels in the Klassen wells, September 15, 2011, to March 13, 2012.

\begin{tabular}{|c|c|c|c|c|}
\hline \multicolumn{3}{|c|}{ Reference Elevation (ft AMSL) } & 1508.77 & \multirow{2}{*}{$\begin{array}{l}1509.12 \\
\text { Elevation in Klassen } \\
\text { Test Well (ft AMSL) }\end{array}$} \\
\hline Date and Time & $\begin{array}{l}\text { Depth in Klassen } \\
\text { Domestic Well } \\
\text { (ft BGL) }\end{array}$ & $\begin{array}{c}\text { Depth in Klassen } \\
\text { Test Well } \\
\text { (ft BGL) }\end{array}$ & $\begin{array}{l}\text { Elevation in Klassen } \\
\text { Domestic Well } \\
\text { (ft AMSL) }\end{array}$ & \\
\hline 9/19/11 11:10 & 34.925 & 39.193 & 1473.84 & 1469.93 \\
\hline 9/19/11 11:20 & 34.798 & 39.191 & 1473.97 & 1469.93 \\
\hline 9/19/11 11:30 & 34.73 & 39.189 & 1474.04 & 1469.93 \\
\hline 9/19/11 11:40 & 34.677 & 39.182 & 1474.09 & 1469.94 \\
\hline 9/19/11 11:50 & 34.639 & 39.182 & 1474.13 & 1469.94 \\
\hline 9/19/11 12:00 & 34.709 & 39.177 & 1474.06 & 1469.95 \\
\hline 9/19/11 12:10 & 34.691 & 39.177 & 1474.08 & 1469.95 \\
\hline 9/19/11 12:20 & 34.738 & 39.177 & 1474.03 & 1469.95 \\
\hline 9/19/11 12:30 & 34.723 & 39.174 & 1474.05 & 1469.95 \\
\hline 9/19/11 12:40 & 35.005 & 39.174 & 1473.76 & 1469.95 \\
\hline $9 / 19 / 1112: 50$ & 34.883 & 39.174 & 1473.89 & 1469.95 \\
\hline 9/19/11 13:00 & 34.841 & 39.172 & 1473.93 & 1469.95 \\
\hline 9/19/11 13:10 & 34.855 & 39.172 & 1473.91 & 1469.95 \\
\hline 9/19/11 13:20 & 35.007 & 39.172 & 1473.76 & 1469.95 \\
\hline 9/19/11 13:30 & 34.949 & 39.172 & 1473.82 & 1469.95 \\
\hline 9/19/11 13:40 & 34.881 & 39.17 & 1473.89 & 1469.95 \\
\hline 9/19/11 13:50 & 34.913 & 39.17 & 1473.86 & 1469.95 \\
\hline 9/19/11 14:00 & 34.874 & 39.167 & 1473.90 & 1469.96 \\
\hline 9/19/11 14:10 & 34.848 & 39.16 & 1473.92 & 1469.96 \\
\hline 9/19/11 14:20 & 38.818 & 39.16 & 1469.95 & 1469.96 \\
\hline 9/19/11 14:30 & 35.035 & 39.163 & 1473.73 & 1469.96 \\
\hline 9/19/11 14:40 & 34.988 & 39.163 & 1473.78 & 1469.96 \\
\hline 9/19/11 14:50 & 34.958 & 39.16 & 1473.81 & 1469.96 \\
\hline 9/19/11 15:00 & 34.96 & 39.158 & 1473.81 & 1469.96 \\
\hline 9/19/11 15:10 & 34.909 & 39.156 & 1473.86 & 1469.97 \\
\hline 9/19/11 15:20 & 34.857 & 39.151 & 1473.91 & 1469.97 \\
\hline 9/19/11 15:30 & 34.827 & 39.146 & 1473.94 & 1469.98 \\
\hline 9/19/11 15:40 & 34.874 & 39.144 & 1473.90 & 1469.98 \\
\hline 9/19/11 15:50 & 34.841 & 39.142 & 1473.93 & 1469.98 \\
\hline 9/19/11 16:00 & 34.874 & 39.139 & 1473.90 & 1469.98 \\
\hline 9/19/11 16:10 & 34.848 & 39.137 & 1473.92 & 1469.99 \\
\hline 9/19/11 16:20 & 34.883 & 39.137 & 1473.89 & 1469.99 \\
\hline 9/19/11 16:30 & 35.031 & 39.135 & 1473.74 & 1469.99 \\
\hline 9/19/11 16:40 & 34.93 & 39.135 & 1473.84 & 1469.99 \\
\hline 9/19/11 16:50 & 34.864 & 39.13 & 1473.91 & 1469.99 \\
\hline 9/19/11 17:00 & 34.841 & 39.125 & 1473.93 & 1470.00 \\
\hline 9/19/11 17:10 & 34.822 & 39.123 & 1473.95 & 1470.00 \\
\hline 9/19/11 17:20 & 35.064 & 39.121 & 1473.71 & 1470.00 \\
\hline
\end{tabular}


TABLE S1.3 Automatically recorded groundwater levels in the Klassen wells, September 15, 2011, to March 13, 2012.

\begin{tabular}{|c|c|c|c|c|}
\hline \multicolumn{3}{|c|}{ Reference Elevation (ft AMSL) } & 1508.77 & \multirow{2}{*}{$\begin{array}{l}1509.12 \\
\text { Elevation in Klassen } \\
\text { Test Well (ft AMSL) }\end{array}$} \\
\hline Date and Time & $\begin{array}{l}\text { Depth in Klassen } \\
\text { Domestic Well } \\
\text { (ft BGL) }\end{array}$ & $\begin{array}{c}\text { Depth in Klassen } \\
\text { Test Well } \\
\text { (ft BGL) }\end{array}$ & $\begin{array}{l}\text { Elevation in Klassen } \\
\text { Domestic Well } \\
\text { (ft AMSL) }\end{array}$ & \\
\hline 9/19/11 17:30 & 34.89 & 39.118 & 1473.88 & 1470.00 \\
\hline 9/19/11 17:40 & 34.895 & 39.116 & 1473.87 & 1470.01 \\
\hline 9/19/11 17:50 & 34.871 & 39.116 & 1473.90 & 1470.01 \\
\hline 9/19/11 18:00 & 34.822 & 39.111 & 1473.95 & 1470.01 \\
\hline 9/19/11 18:10 & 34.787 & 39.106 & 1473.98 & 1470.02 \\
\hline 9/19/11 18:20 & 34.789 & 39.104 & 1473.98 & 1470.02 \\
\hline 9/19/11 18:30 & 34.801 & 39.099 & 1473.97 & 1470.02 \\
\hline 9/19/11 18:40 & 34.768 & 39.097 & 1474.00 & 1470.03 \\
\hline 9/19/11 18:50 & 34.73 & 39.092 & 1474.04 & 1470.03 \\
\hline 9/19/11 19:00 & 37.615 & 39.088 & 1471.15 & 1470.03 \\
\hline 9/19/11 19:10 & 34.829 & 39.083 & 1473.94 & 1470.04 \\
\hline 9/19/11 19:20 & 34.742 & 39.081 & 1474.03 & 1470.04 \\
\hline 9/19/11 19:30 & 34.693 & 39.076 & 1474.08 & 1470.05 \\
\hline 9/19/11 19:40 & 34.658 & 39.071 & 1474.11 & 1470.05 \\
\hline 9/19/11 19:50 & 35.056 & 39.067 & 1473.71 & 1470.06 \\
\hline 9/19/11 20:00 & 34.824 & 39.069 & 1473.95 & 1470.05 \\
\hline 9/19/11 20:10 & 34.763 & 39.067 & 1474.01 & 1470.06 \\
\hline 9/19/11 20:20 & 34.784 & 39.062 & 1473.99 & 1470.06 \\
\hline 9/19/11 20:30 & 34.782 & 39.064 & 1473.99 & 1470.06 \\
\hline 9/19/11 20:40 & 34.74 & 39.06 & 1474.03 & 1470.06 \\
\hline 9/19/11 20:50 & 34.698 & 39.057 & 1474.07 & 1470.07 \\
\hline 9/19/11 21:00 & 34.665 & 39.055 & 1474.10 & 1470.07 \\
\hline 9/19/11 21:10 & 34.634 & 39.05 & 1474.14 & 1470.07 \\
\hline 9/19/11 21:20 & 34.606 & 39.045 & 1474.16 & 1470.08 \\
\hline 9/19/11 21:30 & 34.641 & 39.045 & 1474.13 & 1470.08 \\
\hline 9/19/11 21:40 & 34.627 & 39.043 & 1474.14 & 1470.08 \\
\hline 9/19/11 21:50 & 34.667 & 39.041 & 1474.10 & 1470.08 \\
\hline 9/19/11 22:00 & 34.641 & 39.038 & 1474.13 & 1470.08 \\
\hline 9/19/11 22:10 & 34.62 & 39.036 & 1474.15 & 1470.09 \\
\hline 9/19/11 22:20 & 34.684 & 39.034 & 1474.09 & 1470.09 \\
\hline 9/19/11 22:30 & 35.244 & 39.034 & 1473.53 & 1470.09 \\
\hline 9/19/11 22:40 & 35.035 & 39.036 & 1473.73 & 1470.09 \\
\hline 9/19/11 22:50 & 38.506 & 39.038 & 1470.26 & 1470.08 \\
\hline 9/19/11 23:00 & 35.511 & 39.041 & 1473.26 & 1470.08 \\
\hline 9/19/11 23:10 & 35.003 & 39.043 & 1473.77 & 1470.08 \\
\hline 9/19/11 23:20 & 34.888 & 39.038 & 1473.88 & 1470.08 \\
\hline 9/19/11 23:30 & 34.815 & 39.036 & 1473.95 & 1470.09 \\
\hline 9/19/11 23:40 & 34.761 & 39.031 & 1474.01 & 1470.09 \\
\hline
\end{tabular}


TABLE S1.3 Automatically recorded groundwater levels in the Klassen wells, September 15, 2011, to March 13, 2012.

\begin{tabular}{|c|c|c|c|c|}
\hline \multicolumn{3}{|c|}{ Reference Elevation (ft AMSL) } & 1508.77 & \multirow{2}{*}{$\begin{array}{l}1509.12 \\
\text { Elevation in Klassen } \\
\text { Test Well (ft AMSL) }\end{array}$} \\
\hline Date and Time & $\begin{array}{l}\text { Depth in Klassen } \\
\text { Domestic Well } \\
(\mathrm{ft} \mathrm{BGL})\end{array}$ & $\begin{array}{c}\text { Depth in Klassen } \\
\text { Test Well } \\
\text { (ft BGL) }\end{array}$ & $\begin{array}{l}\text { Elevation in Klassen } \\
\text { Domestic Well } \\
\text { (ft AMSL) }\end{array}$ & \\
\hline 9/19/11 23:50 & 34.716 & 39.027 & 1474.05 & 1470.10 \\
\hline 9/20/11 0:00 & 34.679 & 39.022 & 1474.09 & 1470.10 \\
\hline 9/20/11 0:10 & 34.646 & 39.017 & 1474.12 & 1470.11 \\
\hline $9 / 20 / 110: 20$ & 34.616 & 39.013 & 1474.15 & 1470.11 \\
\hline 9/20/11 0:30 & 34.59 & 39.008 & 1474.18 & 1470.11 \\
\hline $9 / 20 / 110: 40$ & 34.566 & 39.003 & 1474.20 & 1470.12 \\
\hline 9/20/11 0:50 & 34.545 & 39.001 & 1474.22 & 1470.12 \\
\hline 9/20/11 1:00 & 34.524 & 38.994 & 1474.25 & 1470.13 \\
\hline 9/20/11 1:10 & 34.503 & 38.989 & 1474.27 & 1470.13 \\
\hline $9 / 20 / 111: 20$ & 34.487 & 38.984 & 1474.28 & 1470.14 \\
\hline $9 / 20 / 111: 30$ & 37.826 & 38.98 & 1470.94 & 1470.14 \\
\hline 9/20/11 1:40 & 34.613 & 38.977 & 1474.16 & 1470.15 \\
\hline $9 / 20 / 11$ 1:50 & 34.639 & 38.977 & 1474.13 & 1470.15 \\
\hline $9 / 20 / 112: 00$ & 34.609 & 38.98 & 1474.16 & 1470.14 \\
\hline $9 / 20 / 112: 10$ & 34.569 & 38.975 & 1474.20 & 1470.15 \\
\hline $9 / 20 / 112: 20$ & 34.562 & 38.973 & 1474.21 & 1470.15 \\
\hline $9 / 20 / 112: 30$ & 34.533 & 38.97 & 1474.24 & 1470.15 \\
\hline 9/20/11 2:40 & 34.505 & 38.966 & 1474.26 & 1470.16 \\
\hline $9 / 20 / 112: 50$ & 34.505 & 38.963 & 1474.26 & 1470.16 \\
\hline 9/20/11 3:00 & 34.484 & 38.959 & 1474.29 & 1470.16 \\
\hline 9/20/11 3:10 & 34.463 & 38.956 & 1474.31 & 1470.17 \\
\hline 9/20/11 3:20 & 34.482 & 38.954 & 1474.29 & 1470.17 \\
\hline 9/20/11 3:30 & 34.547 & 38.952 & 1474.22 & 1470.17 \\
\hline 9/20/11 3:40 & 34.536 & 38.952 & 1474.23 & 1470.17 \\
\hline 9/20/11 3:50 & 34.505 & 38.949 & 1474.26 & 1470.17 \\
\hline 9/20/11 4:00 & 34.48 & 38.945 & 1474.29 & 1470.18 \\
\hline 9/20/11 4:10 & 34.456 & 38.942 & 1474.31 & 1470.18 \\
\hline 9/20/11 4:20 & 34.594 & 38.94 & 1474.18 & 1470.18 \\
\hline 9/20/11 4:30 & 34.503 & 38.938 & 1474.27 & 1470.18 \\
\hline 9/20/11 4:40 & 34.461 & 38.933 & 1474.31 & 1470.19 \\
\hline 9/20/11 4:50 & 34.433 & 38.928 & 1474.34 & 1470.19 \\
\hline 9/20/11 5:00 & 34.409 & 38.926 & 1474.36 & 1470.20 \\
\hline 9/20/11 5:10 & 34.39 & 38.921 & 1474.38 & 1470.20 \\
\hline 9/20/11 5:20 & 34.372 & 38.919 & 1474.40 & 1470.20 \\
\hline 9/20/11 5:30 & 34.358 & 38.914 & 1474.41 & 1470.21 \\
\hline 9/20/11 5:40 & 34.341 & 38.909 & 1474.43 & 1470.21 \\
\hline $9 / 20 / 11$ 5:50 & 34.329 & 38.905 & 1474.44 & 1470.22 \\
\hline $9 / 20 / 116: 00$ & 34.315 & 38.9 & 1474.45 & 1470.22 \\
\hline
\end{tabular}


TABLE S1.3 Automatically recorded groundwater levels in the Klassen wells, September 15, 2011, to March 13, 2012.

\begin{tabular}{|c|c|c|c|c|}
\hline \multicolumn{3}{|c|}{ Reference Elevation (ft AMSL) } & 1508.77 & \multirow{2}{*}{$\begin{array}{l}1509.12 \\
\text { Elevation in Klassen } \\
\text { Test Well (ft AMSL) }\end{array}$} \\
\hline Date and Time & $\begin{array}{l}\text { Depth in Klassen } \\
\text { Domestic Well } \\
(\mathrm{ft} \mathrm{BGL})\end{array}$ & $\begin{array}{c}\text { Depth in Klassen } \\
\text { Test Well } \\
\text { (ft BGL) }\end{array}$ & $\begin{array}{l}\text { Elevation in Klassen } \\
\text { Domestic Well } \\
\text { (ft AMSL) }\end{array}$ & \\
\hline $9 / 20 / 116: 10$ & 35.31 & 38.898 & 1473.46 & 1470.22 \\
\hline $9 / 20 / 116: 20$ & 34.536 & 38.895 & 1474.23 & 1470.23 \\
\hline 9/20/11 6:30 & 34.435 & 38.895 & 1474.33 & 1470.23 \\
\hline $9 / 20 / 116: 40$ & 34.393 & 38.893 & 1474.38 & 1470.23 \\
\hline $9 / 20 / 116: 50$ & 34.362 & 38.888 & 1474.41 & 1470.23 \\
\hline $9 / 20 / 117: 00$ & 34.339 & 38.884 & 1474.43 & 1470.24 \\
\hline $9 / 20 / 117: 10$ & 34.318 & 38.879 & 1474.45 & 1470.24 \\
\hline 9/20/11 7:20 & 34.329 & 38.874 & 1474.44 & 1470.25 \\
\hline $9 / 20 / 117: 30$ & 34.315 & 38.872 & 1474.45 & 1470.25 \\
\hline $9 / 20 / 117: 40$ & 34.299 & 38.867 & 1474.47 & 1470.26 \\
\hline $9 / 20 / 117: 50$ & 34.283 & 38.865 & 1474.49 & 1470.26 \\
\hline 9/20/11 8:00 & 34.264 & 38.858 & 1474.51 & 1470.26 \\
\hline $9 / 20 / 118: 10$ & 34.254 & 38.855 & 1474.52 & 1470.27 \\
\hline $9 / 20 / 118: 20$ & 34.247 & 38.851 & 1474.52 & 1470.27 \\
\hline $9 / 20 / 118: 30$ & 41.145 & 38.848 & 1467.62 & 1470.27 \\
\hline $9 / 20 / 118: 40$ & 34.458 & 38.846 & 1474.31 & 1470.28 \\
\hline $9 / 20 / 118: 50$ & 37.036 & 38.844 & 1471.73 & 1470.28 \\
\hline 9/20/11 9:00 & 34.482 & 38.844 & 1474.29 & 1470.28 \\
\hline 9/20/11 9:10 & 35.312 & 38.841 & 1473.46 & 1470.28 \\
\hline 9/20/11 9:20 & 35.509 & 38.841 & 1473.26 & 1470.28 \\
\hline 9/20/11 9:30 & 34.761 & 38.844 & 1474.01 & 1470.28 \\
\hline 9/20/11 9:40 & 34.705 & 38.844 & 1474.06 & 1470.28 \\
\hline 9/20/11 9:50 & 34.555 & 38.841 & 1474.21 & 1470.28 \\
\hline 9/20/11 10:00 & 34.482 & 38.839 & 1474.29 & 1470.28 \\
\hline 9/20/11 10:10 & 34.433 & 38.837 & 1474.34 & 1470.29 \\
\hline 9/20/11 10:20 & 34.395 & 38.834 & 1474.37 & 1470.29 \\
\hline 9/20/11 10:30 & 34.362 & 38.827 & 1474.41 & 1470.30 \\
\hline $9 / 20 / 11$ 10:40 & 34.594 & 38.825 & 1474.18 & 1470.30 \\
\hline 9/20/11 10:50 & 34.421 & 38.823 & 1474.35 & 1470.30 \\
\hline 9/20/11 11:00 & 34.369 & 38.818 & 1474.40 & 1470.30 \\
\hline 9/20/11 11:10 & 37.026 & 38.813 & 1471.74 & 1470.31 \\
\hline 9/20/11 11:20 & 34.456 & 38.816 & 1474.31 & 1470.31 \\
\hline 9/20/11 11:30 & 34.381 & 38.811 & 1474.39 & 1470.31 \\
\hline 9/20/11 11:40 & 34.341 & 38.808 & 1474.43 & 1470.31 \\
\hline 9/20/11 11:50 & 34.311 & 38.804 & 1474.46 & 1470.32 \\
\hline 9/20/11 12:00 & 34.29 & 38.801 & 1474.48 & 1470.32 \\
\hline $9 / 20 / 1112: 10$ & 34.336 & 38.801 & 1474.43 & 1470.32 \\
\hline 9/20/11 12:20 & 34.325 & 38.799 & 1474.44 & 1470.32 \\
\hline
\end{tabular}


TABLE S1.3 Automatically recorded groundwater levels in the Klassen wells, September 15, 2011, to March 13, 2012.

\begin{tabular}{|c|c|c|c|c|}
\hline \multicolumn{3}{|c|}{ Reference Elevation (ft AMSL) } & 1508.77 & \multirow{2}{*}{$\begin{array}{l}1509.12 \\
\text { Elevation in Klassen } \\
\text { Test Well (ft AMSL) }\end{array}$} \\
\hline Date and Time & $\begin{array}{l}\text { Depth in Klassen } \\
\text { Domestic Well } \\
(\mathrm{ft} \mathrm{BGL})\end{array}$ & $\begin{array}{c}\text { Depth in Klassen } \\
\text { Test Well } \\
\text { (ft BGL) }\end{array}$ & $\begin{array}{l}\text { Elevation in Klassen } \\
\text { Domestic Well } \\
\text { (ft AMSL) }\end{array}$ & \\
\hline 9/20/11 12:30 & 37.362 & 38.799 & 1471.41 & 1470.32 \\
\hline 9/20/11 12:40 & 36.668 & 38.799 & 1472.10 & 1470.32 \\
\hline 9/20/11 12:50 & 34.512 & 38.799 & 1474.26 & 1470.32 \\
\hline 9/20/11 13:00 & 34.414 & 38.797 & 1474.36 & 1470.33 \\
\hline 9/20/11 13:10 & 34.367 & 38.794 & 1474.40 & 1470.33 \\
\hline 9/20/11 13:20 & 34.329 & 38.792 & 1474.44 & 1470.33 \\
\hline 9/20/11 13:30 & 34.301 & 38.79 & 1474.47 & 1470.33 \\
\hline 9/20/11 13:40 & 34.28 & 38.787 & 1474.49 & 1470.34 \\
\hline 9/20/11 13:50 & 34.261 & 38.787 & 1474.51 & 1470.34 \\
\hline 9/20/11 14:00 & 34.243 & 38.787 & 1474.53 & 1470.34 \\
\hline 9/20/11 14:10 & 34.226 & 38.787 & 1474.54 & 1470.34 \\
\hline 9/20/11 14:20 & 34.212 & 38.79 & 1474.56 & 1470.33 \\
\hline 9/20/11 14:30 & 34.2 & 38.787 & 1474.57 & 1470.34 \\
\hline 9/20/11 14:40 & 34.186 & 38.787 & 1474.58 & 1470.34 \\
\hline 9/20/11 14:50 & 34.177 & 38.785 & 1474.59 & 1470.34 \\
\hline 9/20/11 15:00 & 34.165 & 38.785 & 1474.60 & 1470.34 \\
\hline 9/20/11 15:10 & 34.154 & 38.783 & 1474.62 & 1470.34 \\
\hline 9/20/11 15:20 & 34.142 & 38.78 & 1474.63 & 1470.34 \\
\hline 9/20/11 15:30 & 34.135 & 38.78 & 1474.63 & 1470.34 \\
\hline $9 / 20 / 11$ 15:40 & 34.151 & 38.778 & 1474.62 & 1470.34 \\
\hline $9 / 20 / 11$ 15:50 & 34.149 & 38.778 & 1474.62 & 1470.34 \\
\hline 9/20/11 16:00 & 34.13 & 38.776 & 1474.64 & 1470.35 \\
\hline 9/20/11 16:10 & 34.121 & 38.773 & 1474.65 & 1470.35 \\
\hline 9/20/11 16:20 & 34.109 & 38.773 & 1474.66 & 1470.35 \\
\hline 9/20/11 16:30 & 34.097 & 38.769 & 1474.67 & 1470.35 \\
\hline 9/20/11 16:40 & 34.09 & 38.766 & 1474.68 & 1470.36 \\
\hline 9/20/11 16:50 & 34.081 & 38.764 & 1474.69 & 1470.36 \\
\hline 9/20/11 17:00 & 34.109 & 38.762 & 1474.66 & 1470.36 \\
\hline 9/20/11 17:10 & 34.161 & 38.762 & 1474.61 & 1470.36 \\
\hline 9/20/11 17:20 & 34.177 & 38.764 & 1474.59 & 1470.36 \\
\hline 9/20/11 17:30 & 34.154 & 38.762 & 1474.62 & 1470.36 \\
\hline 9/20/11 17:40 & 34.163 & 38.762 & 1474.61 & 1470.36 \\
\hline $9 / 20 / 11$ 17:50 & 34.142 & 38.759 & 1474.63 & 1470.36 \\
\hline 9/20/11 18:00 & 35.204 & 38.759 & 1473.57 & 1470.36 \\
\hline 9/20/11 18:10 & 34.43 & 38.769 & 1474.34 & 1470.35 \\
\hline 9/20/11 18:20 & 34.365 & 38.771 & 1474.40 & 1470.35 \\
\hline 9/20/11 18:30 & 34.315 & 38.769 & 1474.45 & 1470.35 \\
\hline 9/20/11 18:40 & 34.278 & 38.769 & 1474.49 & 1470.35 \\
\hline
\end{tabular}


TABLE S1.3 Automatically recorded groundwater levels in the Klassen wells, September 15, 2011, to March 13, 2012.

\begin{tabular}{|c|c|c|c|c|}
\hline \multicolumn{3}{|c|}{ Reference Elevation (ft AMSL) } & 1508.77 & \multirow{2}{*}{$\begin{array}{l}1509.12 \\
\text { Elevation in Klassen } \\
\text { Test Well (ft AMSL) }\end{array}$} \\
\hline Date and Time & $\begin{array}{l}\text { Depth in Klassen } \\
\text { Domestic Well } \\
(\mathrm{ft} \mathrm{BGL})\end{array}$ & $\begin{array}{c}\text { Depth in Klassen } \\
\text { Test Well } \\
\text { (ft BGL) }\end{array}$ & $\begin{array}{l}\text { Elevation in Klassen } \\
\text { Domestic Well } \\
\text { (ft AMSL) }\end{array}$ & \\
\hline 9/20/11 18:50 & 34.25 & 38.766 & 1474.52 & 1470.36 \\
\hline 9/20/11 19:00 & 39.034 & 38.764 & 1469.74 & 1470.36 \\
\hline 9/20/11 19:10 & 45.87 & 38.766 & 1462.90 & 1470.36 \\
\hline 9/20/11 19:20 & 34.822 & 38.771 & 1473.95 & 1470.35 \\
\hline 9/20/11 19:30 & 34.555 & 38.773 & 1474.21 & 1470.35 \\
\hline 9/20/11 19:40 & 34.451 & 38.771 & 1474.32 & 1470.35 \\
\hline 9/20/11 19:50 & 34.386 & 38.766 & 1474.38 & 1470.36 \\
\hline 9/20/11 20:00 & 34.339 & 38.764 & 1474.43 & 1470.36 \\
\hline 9/20/11 20:10 & 34.304 & 38.762 & 1474.47 & 1470.36 \\
\hline $9 / 20 / 1120: 20$ & 34.275 & 38.759 & 1474.49 & 1470.36 \\
\hline $9 / 20 / 1120: 30$ & 34.25 & 38.755 & 1474.52 & 1470.37 \\
\hline 9/20/11 20:40 & 34.226 & 38.752 & 1474.54 & 1470.37 \\
\hline 9/20/11 20:50 & 37.617 & 38.747 & 1471.15 & 1470.38 \\
\hline 9/20/11 21:00 & 34.346 & 38.75 & 1474.42 & 1470.37 \\
\hline 9/20/11 21:10 & 34.271 & 38.747 & 1474.50 & 1470.38 \\
\hline 9/20/11 21:20 & 34.264 & 38.745 & 1474.51 & 1470.38 \\
\hline 9/20/11 21:30 & 34.451 & 38.745 & 1474.32 & 1470.38 \\
\hline 9/20/11 21:40 & 34.308 & 38.743 & 1474.46 & 1470.38 \\
\hline 9/20/11 21:50 & 34.259 & 38.743 & 1474.51 & 1470.38 \\
\hline 9/20/11 22:00 & 34.278 & 38.74 & 1474.49 & 1470.38 \\
\hline 9/20/11 22:10 & 34.315 & 38.74 & 1474.45 & 1470.38 \\
\hline 9/20/11 22:20 & 34.348 & 38.74 & 1474.42 & 1470.38 \\
\hline $9 / 20 / 1122: 30$ & 38.933 & 38.743 & 1469.84 & 1470.38 \\
\hline 9/20/11 22:40 & 34.918 & 38.747 & 1473.85 & 1470.38 \\
\hline 9/20/11 22:50 & 34.632 & 38.752 & 1474.14 & 1470.37 \\
\hline 9/20/11 23:00 & 35.087 & 38.755 & 1473.68 & 1470.37 \\
\hline 9/20/11 23:10 & 34.953 & 38.762 & 1473.82 & 1470.36 \\
\hline 9/20/11 23:20 & 34.745 & 38.764 & 1474.02 & 1470.36 \\
\hline 9/20/11 23:30 & 34.641 & 38.764 & 1474.13 & 1470.36 \\
\hline 9/20/11 23:40 & 34.566 & 38.762 & 1474.20 & 1470.36 \\
\hline 9/20/11 23:50 & 34.51 & 38.759 & 1474.26 & 1470.36 \\
\hline 9/21/11 0:00 & 34.465 & 38.757 & 1474.30 & 1470.37 \\
\hline 9/21/11 0:10 & 34.428 & 38.752 & 1474.34 & 1470.37 \\
\hline 9/21/11 0:20 & 34.395 & 38.75 & 1474.37 & 1470.37 \\
\hline 9/21/11 0:30 & 34.367 & 38.75 & 1474.40 & 1470.37 \\
\hline 9/21/11 0:40 & 34.341 & 38.745 & 1474.43 & 1470.38 \\
\hline 9/21/11 0:50 & 34.318 & 38.743 & 1474.45 & 1470.38 \\
\hline 9/21/11 1:00 & 34.297 & 38.738 & 1474.47 & 1470.38 \\
\hline
\end{tabular}


TABLE S1.3 Automatically recorded groundwater levels in the Klassen wells, September 15, 2011, to March 13, 2012.

\begin{tabular}{|c|c|c|c|c|}
\hline \multicolumn{3}{|c|}{ Reference Elevation (ft AMSL) } & 1508.77 & \multirow{2}{*}{$\begin{array}{l}1509.12 \\
\text { Elevation in Klassen } \\
\text { Test Well (ft AMSL) }\end{array}$} \\
\hline Date and Time & $\begin{array}{l}\text { Depth in Klassen } \\
\text { Domestic Well } \\
(\mathrm{ft} \mathrm{BGL})\end{array}$ & $\begin{array}{c}\text { Depth in Klassen } \\
\text { Test Well } \\
\text { (ft BGL) }\end{array}$ & $\begin{array}{l}\text { Elevation in Klassen } \\
\text { Domestic Well } \\
\text { (ft AMSL) }\end{array}$ & \\
\hline $9 / 21 / 11$ 1:10 & 34.275 & 38.736 & 1474.49 & 1470.39 \\
\hline $9 / 21 / 111: 20$ & 34.796 & 38.733 & 1473.97 & 1470.39 \\
\hline 9/21/11 1:30 & 34.346 & 38.731 & 1474.42 & 1470.39 \\
\hline $9 / 21 / 11$ 1:40 & 34.294 & 38.729 & 1474.48 & 1470.39 \\
\hline $9 / 21 / 111: 50$ & 34.264 & 38.726 & 1474.51 & 1470.40 \\
\hline $9 / 21 / 112: 00$ & 34.24 & 38.724 & 1474.53 & 1470.40 \\
\hline $9 / 21 / 112: 10$ & 34.222 & 38.722 & 1474.55 & 1470.40 \\
\hline $9 / 21 / 112: 20$ & 34.203 & 38.717 & 1474.57 & 1470.41 \\
\hline $9 / 21 / 112: 30$ & 34.189 & 38.715 & 1474.58 & 1470.41 \\
\hline $9 / 21 / 112: 40$ & 34.175 & 38.71 & 1474.59 & 1470.41 \\
\hline $9 / 21 / 112: 50$ & 34.16 & 38.705 & 1474.61 & 1470.42 \\
\hline 9/21/11 3:00 & 34.147 & 38.703 & 1474.62 & 1470.42 \\
\hline $9 / 21 / 113: 10$ & 34.137 & 38.701 & 1474.63 & 1470.42 \\
\hline $9 / 21 / 113: 20$ & 34.125 & 38.698 & 1474.64 & 1470.42 \\
\hline $9 / 21 / 113: 30$ & 34.116 & 38.698 & 1474.65 & 1470.42 \\
\hline $9 / 21 / 113: 40$ & 34.107 & 38.696 & 1474.66 & 1470.43 \\
\hline $9 / 21 / 113: 50$ & 34.095 & 38.691 & 1474.67 & 1470.43 \\
\hline 9/21/11 4:00 & 34.086 & 38.686 & 1474.68 & 1470.44 \\
\hline $9 / 21 / 114: 10$ & 34.076 & 38.682 & 1474.69 & 1470.44 \\
\hline $9 / 21 / 114: 20$ & 34.069 & 38.684 & 1474.70 & 1470.44 \\
\hline $9 / 21 / 114: 30$ & 34.06 & 38.677 & 1474.71 & 1470.45 \\
\hline $9 / 21 / 114: 40$ & 34.05 & 38.677 & 1474.72 & 1470.45 \\
\hline $9 / 21 / 114: 50$ & 34.043 & 38.67 & 1474.73 & 1470.45 \\
\hline 9/21/11 5:00 & 34.034 & 38.67 & 1474.74 & 1470.45 \\
\hline 9/21/11 5:10 & 34.025 & 38.675 & 1474.74 & 1470.45 \\
\hline 9/21/11 5:20 & 34.018 & 38.675 & 1474.75 & 1470.45 \\
\hline 9/21/11 5:30 & 34.01 & 38.672 & 1474.76 & 1470.45 \\
\hline 9/21/11 5:40 & 34.003 & 38.668 & 1474.77 & 1470.45 \\
\hline 9/21/11 5:50 & 33.996 & 38.663 & 1474.77 & 1470.46 \\
\hline 9/21/11 6:00 & 33.989 & 38.658 & 1474.78 & 1470.46 \\
\hline 9/21/11 6:10 & 34.775 & 38.656 & 1473.99 & 1470.47 \\
\hline $9 / 21 / 116: 20$ & 34.487 & 38.654 & 1474.28 & 1470.47 \\
\hline $9 / 21 / 116: 30$ & 34.29 & 38.654 & 1474.48 & 1470.47 \\
\hline $9 / 21 / 116: 40$ & 34.215 & 38.661 & 1474.55 & 1470.46 \\
\hline $9 / 21 / 116: 50$ & 34.172 & 38.656 & 1474.60 & 1470.47 \\
\hline 9/21/11 7:00 & 34.142 & 38.658 & 1474.63 & 1470.46 \\
\hline $9 / 21 / 11$ 7:10 & 34.116 & 38.656 & 1474.65 & 1470.47 \\
\hline $9 / 21 / 11$ 7:20 & 34.097 & 38.651 & 1474.67 & 1470.47 \\
\hline
\end{tabular}


TABLE S1.3 Automatically recorded groundwater levels in the Klassen wells, September 15, 2011, to March 13, 2012.

\begin{tabular}{|c|c|c|c|c|}
\hline \multicolumn{3}{|c|}{ Reference Elevation (ft AMSL) } & 1508.77 & \multirow{2}{*}{$\begin{array}{l}1509.12 \\
\text { Elevation in Klassen } \\
\text { Test Well (ft AMSL) }\end{array}$} \\
\hline Date and Time & $\begin{array}{l}\text { Depth in Klassen } \\
\text { Domestic Well } \\
(\mathrm{ft} \mathrm{BGL})\end{array}$ & $\begin{array}{c}\text { Depth in Klassen } \\
\text { Test Well } \\
\text { (ft BGL) }\end{array}$ & $\begin{array}{l}\text { Elevation in Klassen } \\
\text { Domestic Well } \\
\text { (ft AMSL) }\end{array}$ & \\
\hline $9 / 21 / 117: 30$ & 34.109 & 38.651 & 1474.66 & 1470.47 \\
\hline $9 / 21 / 117: 40$ & 34.09 & 38.644 & 1474.68 & 1470.48 \\
\hline $9 / 21 / 117: 50$ & 34.074 & 38.64 & 1474.70 & 1470.48 \\
\hline $9 / 21 / 118: 00$ & 34.06 & 38.637 & 1474.71 & 1470.49 \\
\hline $9 / 21 / 118: 10$ & 34.046 & 38.633 & 1474.72 & 1470.49 \\
\hline $9 / 21 / 118: 20$ & 34.034 & 38.63 & 1474.74 & 1470.49 \\
\hline $9 / 21 / 118: 30$ & 34.022 & 38.625 & 1474.75 & 1470.50 \\
\hline $9 / 21 / 118: 40$ & 34.013 & 38.621 & 1474.76 & 1470.50 \\
\hline $9 / 21 / 118: 50$ & 34.165 & 38.623 & 1474.60 & 1470.50 \\
\hline 9/21/11 9:00 & 34.086 & 38.623 & 1474.68 & 1470.50 \\
\hline 9/21/11 9:10 & 34.053 & 38.625 & 1474.72 & 1470.50 \\
\hline $9 / 21 / 119: 20$ & 34.034 & 38.623 & 1474.74 & 1470.50 \\
\hline 9/21/11 9:30 & 34.015 & 38.623 & 1474.75 & 1470.50 \\
\hline 9/21/11 9:40 & 34.003 & 38.621 & 1474.77 & 1470.50 \\
\hline $9 / 21 / 119: 50$ & 36.944 & 38.621 & 1471.83 & 1470.50 \\
\hline $9 / 21 / 11$ 10:00 & 34.304 & 38.623 & 1474.47 & 1470.50 \\
\hline 9/21/11 10:10 & 34.182 & 38.621 & 1474.59 & 1470.50 \\
\hline $9 / 21 / 11$ 10:20 & 34.125 & 38.618 & 1474.64 & 1470.50 \\
\hline 9/21/11 10:30 & 34.093 & 38.611 & 1474.68 & 1470.51 \\
\hline $9 / 21 / 11$ 10:40 & 34.402 & 38.602 & 1474.37 & 1470.52 \\
\hline $9 / 21 / 11$ 10:50 & 36.611 & 38.602 & 1472.16 & 1470.52 \\
\hline 9/21/11 11:00 & 34.273 & 38.6 & 1474.50 & 1470.52 \\
\hline 9/21/11 11:10 & 34.17 & 38.597 & 1474.60 & 1470.53 \\
\hline $9 / 21 / 11$ 11:20 & 34.123 & 38.595 & 1474.65 & 1470.53 \\
\hline 9/21/11 11:30 & 34.093 & 38.595 & 1474.68 & 1470.53 \\
\hline 9/21/11 11:40 & 34.067 & 38.588 & 1474.70 & 1470.53 \\
\hline 9/21/11 11:50 & 34.048 & 38.586 & 1474.72 & 1470.54 \\
\hline $9 / 21 / 1112: 00$ & 34.029 & 38.583 & 1474.74 & 1470.54 \\
\hline 9/21/11 12:10 & 35.601 & 38.579 & 1473.17 & 1470.54 \\
\hline $9 / 21 / 11$ 12:20 & 34.123 & 38.579 & 1474.65 & 1470.54 \\
\hline $9 / 21 / 11$ 12:30 & 34.067 & 38.576 & 1474.70 & 1470.55 \\
\hline 9/21/11 12:40 & 34.039 & 38.574 & 1474.73 & 1470.55 \\
\hline $9 / 21 / 11$ 12:50 & 34.018 & 38.569 & 1474.75 & 1470.55 \\
\hline 9/21/11 13:00 & 34.001 & 38.569 & 1474.77 & 1470.55 \\
\hline 9/21/11 13:10 & 33.987 & 38.574 & 1474.78 & 1470.55 \\
\hline $9 / 21 / 11$ 13:20 & 33.975 & 38.572 & 1474.79 & 1470.55 \\
\hline 9/21/11 13:30 & 33.966 & 38.572 & 1474.80 & 1470.55 \\
\hline 9/21/11 13:40 & 33.954 & 38.572 & 1474.82 & 1470.55 \\
\hline
\end{tabular}


TABLE S1.3 Automatically recorded groundwater levels in the Klassen wells, September 15, 2011, to March 13, 2012.

\begin{tabular}{|c|c|c|c|c|}
\hline \multicolumn{3}{|c|}{ Reference Elevation (ft AMSL) } & 1508.77 & \multirow{2}{*}{$\begin{array}{l}1509.12 \\
\text { Elevation in Klassen } \\
\text { Test Well (ft AMSL) }\end{array}$} \\
\hline Date and Time & $\begin{array}{l}\text { Depth in Klassen } \\
\text { Domestic Well } \\
\text { (ft BGL) }\end{array}$ & $\begin{array}{c}\text { Depth in Klassen } \\
\text { Test Well } \\
\text { (ft BGL) }\end{array}$ & $\begin{array}{l}\text { Elevation in Klassen } \\
\text { Domestic Well } \\
\text { (ft AMSL) }\end{array}$ & \\
\hline $9 / 21 / 1113: 50$ & 33.945 & 38.569 & 1474.82 & 1470.55 \\
\hline 9/21/11 14:00 & 34.789 & 38.564 & 1473.98 & 1470.56 \\
\hline 9/21/11 14:10 & 34.086 & 38.567 & 1474.68 & 1470.56 \\
\hline $9 / 21 / 1114: 20$ & 34.025 & 38.564 & 1474.74 & 1470.56 \\
\hline 9/21/11 14:30 & 34.517 & 38.562 & 1474.25 & 1470.56 \\
\hline 9/21/11 14:40 & 34.121 & 38.567 & 1474.65 & 1470.56 \\
\hline $9 / 21 / 1114: 50$ & 34.057 & 38.564 & 1474.71 & 1470.56 \\
\hline 9/21/11 15:00 & 34.022 & 38.562 & 1474.75 & 1470.56 \\
\hline 9/21/11 15:10 & 33.996 & 38.557 & 1474.77 & 1470.57 \\
\hline $9 / 21 / 1115: 20$ & 33.978 & 38.557 & 1474.79 & 1470.57 \\
\hline $9 / 21 / 1115: 30$ & 33.961 & 38.56 & 1474.81 & 1470.56 \\
\hline $9 / 21 / 1115: 40$ & 33.947 & 38.564 & 1474.82 & 1470.56 \\
\hline $9 / 21 / 11$ 15:50 & 34.151 & 38.569 & 1474.62 & 1470.55 \\
\hline 9/21/11 16:00 & 34.039 & 38.572 & 1474.73 & 1470.55 \\
\hline 9/21/11 16:10 & 33.996 & 38.569 & 1474.77 & 1470.55 \\
\hline $9 / 21 / 1116: 20$ & 33.973 & 38.567 & 1474.80 & 1470.56 \\
\hline $9 / 21 / 11$ 16:30 & 33.954 & 38.562 & 1474.82 & 1470.56 \\
\hline 9/21/11 16:40 & 33.938 & 38.555 & 1474.83 & 1470.57 \\
\hline 9/21/11 16:50 & 33.926 & 38.553 & 1474.84 & 1470.57 \\
\hline 9/21/11 17:00 & 34.273 & 38.55 & 1474.50 & 1470.57 \\
\hline 9/21/11 17:10 & 34.022 & 38.548 & 1474.75 & 1470.57 \\
\hline 9/21/11 17:20 & 33.975 & 38.546 & 1474.79 & 1470.58 \\
\hline 9/21/11 17:30 & 33.95 & 38.543 & 1474.82 & 1470.58 \\
\hline 9/21/11 17:40 & 33.931 & 38.543 & 1474.84 & 1470.58 \\
\hline 9/21/11 17:50 & 33.942 & 38.541 & 1474.83 & 1470.58 \\
\hline 9/21/11 18:00 & 33.935 & 38.539 & 1474.83 & 1470.58 \\
\hline 9/21/11 18:10 & 34.147 & 38.541 & 1474.62 & 1470.58 \\
\hline 9/21/11 18:20 & 34.029 & 38.536 & 1474.74 & 1470.59 \\
\hline 9/21/11 18:30 & 33.987 & 38.536 & 1474.78 & 1470.59 \\
\hline 9/21/11 18:40 & 33.959 & 38.529 & 1474.81 & 1470.59 \\
\hline 9/21/11 18:50 & 33.94 & 38.527 & 1474.83 & 1470.60 \\
\hline 9/21/11 19:00 & 33.924 & 38.522 & 1474.85 & 1470.60 \\
\hline 9/21/11 19:10 & 33.91 & 38.52 & 1474.86 & 1470.60 \\
\hline 9/21/11 19:20 & 36.149 & 38.52 & 1472.62 & 1470.60 \\
\hline 9/21/11 19:30 & 34.151 & 38.522 & 1474.62 & 1470.60 \\
\hline 9/21/11 19:40 & 36.332 & 38.522 & 1472.44 & 1470.60 \\
\hline 9/21/11 19:50 & 34.186 & 38.522 & 1474.58 & 1470.60 \\
\hline 9/21/11 20:00 & 38.598 & 38.522 & 1470.17 & 1470.60 \\
\hline
\end{tabular}


TABLE S1.3 Automatically recorded groundwater levels in the Klassen wells, September 15, 2011, to March 13, 2012.

\begin{tabular}{|c|c|c|c|c|}
\hline \multicolumn{3}{|c|}{ Reference Elevation (ft AMSL) } & 1508.77 & \multirow{2}{*}{$\begin{array}{l}1509.12 \\
\text { Elevation in Klassen } \\
\text { Test Well (ft AMSL) }\end{array}$} \\
\hline Date and Time & $\begin{array}{l}\text { Depth in Klassen } \\
\text { Domestic Well } \\
\text { (ft BGL) }\end{array}$ & $\begin{array}{c}\text { Depth in Klassen } \\
\text { Test Well } \\
\text { (ft BGL) }\end{array}$ & $\begin{array}{l}\text { Elevation in Klassen } \\
\text { Domestic Well } \\
\text { (ft AMSL) }\end{array}$ & \\
\hline 9/21/11 20:10 & 37.31 & 38.522 & 1471.46 & 1470.60 \\
\hline $9 / 21 / 1120: 20$ & 36.895 & 38.525 & 1471.87 & 1470.60 \\
\hline 9/21/11 20:30 & 34.47 & 38.525 & 1474.30 & 1470.60 \\
\hline 9/21/11 20:40 & 34.315 & 38.525 & 1474.45 & 1470.60 \\
\hline 9/21/11 20:50 & 34.29 & 38.525 & 1474.48 & 1470.60 \\
\hline 9/21/11 21:00 & 34.238 & 38.525 & 1474.53 & 1470.60 \\
\hline 9/21/11 21:10 & 34.189 & 38.522 & 1474.58 & 1470.60 \\
\hline 9/21/11 21:20 & 34.151 & 38.52 & 1474.62 & 1470.60 \\
\hline 9/21/11 21:30 & 34.121 & 38.518 & 1474.65 & 1470.60 \\
\hline 9/21/11 21:40 & 34.203 & 38.518 & 1474.57 & 1470.60 \\
\hline $9 / 21 / 1121: 50$ & 34.205 & 38.515 & 1474.56 & 1470.61 \\
\hline 9/21/11 22:00 & 34.29 & 38.518 & 1474.48 & 1470.60 \\
\hline 9/21/11 22:10 & 34.304 & 38.52 & 1474.47 & 1470.60 \\
\hline 9/21/11 22:20 & 34.268 & 38.522 & 1474.50 & 1470.60 \\
\hline $9 / 21 / 1122: 30$ & 35.68 & 38.518 & 1473.09 & 1470.60 \\
\hline $9 / 21 / 1122: 40$ & 34.587 & 38.52 & 1474.18 & 1470.60 \\
\hline $9 / 21 / 1122: 50$ & 34.369 & 38.522 & 1474.40 & 1470.60 \\
\hline 9/21/11 23:00 & 37.791 & 38.525 & 1470.98 & 1470.60 \\
\hline 9/21/11 23:10 & 34.742 & 38.529 & 1474.03 & 1470.59 \\
\hline 9/21/11 23:20 & 34.402 & 38.532 & 1474.37 & 1470.59 \\
\hline 9/21/11 23:30 & 34.313 & 38.534 & 1474.46 & 1470.59 \\
\hline 9/21/11 23:40 & 34.261 & 38.529 & 1474.51 & 1470.59 \\
\hline $9 / 21 / 1123: 50$ & 34.222 & 38.529 & 1474.55 & 1470.59 \\
\hline 9/22/11 0:00 & 34.186 & 38.532 & 1474.58 & 1470.59 \\
\hline 9/22/11 0:10 & 34.161 & 38.532 & 1474.61 & 1470.59 \\
\hline $9 / 22 / 110: 20$ & 34.137 & 38.532 & 1474.63 & 1470.59 \\
\hline 9/22/11 0:30 & 34.114 & 38.532 & 1474.66 & 1470.59 \\
\hline $9 / 22 / 110: 40$ & 34.095 & 38.534 & 1474.67 & 1470.59 \\
\hline $9 / 22 / 110: 50$ & 34.076 & 38.539 & 1474.69 & 1470.58 \\
\hline 9/22/11 1:00 & 34.06 & 38.536 & 1474.71 & 1470.59 \\
\hline 9/22/11 1:10 & 34.046 & 38.536 & 1474.72 & 1470.59 \\
\hline $9 / 22 / 11$ 1:20 & 34.261 & 38.539 & 1474.51 & 1470.58 \\
\hline $9 / 22 / 11$ 1:30 & 34.125 & 38.539 & 1474.64 & 1470.58 \\
\hline $9 / 22 / 11$ 1:40 & 34.083 & 38.536 & 1474.69 & 1470.59 \\
\hline $9 / 22 / 11$ 1:50 & 34.069 & 38.532 & 1474.70 & 1470.59 \\
\hline $9 / 22 / 112: 00$ & 34.062 & 38.532 & 1474.71 & 1470.59 \\
\hline $9 / 22 / 112: 10$ & 34.041 & 38.532 & 1474.73 & 1470.59 \\
\hline $9 / 22 / 112: 20$ & 34.02 & 38.527 & 1474.75 & 1470.60 \\
\hline
\end{tabular}


TABLE S1.3 Automatically recorded groundwater levels in the Klassen wells, September 15, 2011, to March 13, 2012.

\begin{tabular}{|c|c|c|c|c|}
\hline \multicolumn{3}{|c|}{ Reference Elevation (ft AMSL) } & 1508.77 & \multirow{2}{*}{$\begin{array}{l}1509.12 \\
\text { Elevation in Klassen } \\
\text { Test Well (ft AMSL) }\end{array}$} \\
\hline Date and Time & $\begin{array}{l}\text { Depth in Klassen } \\
\text { Domestic Well } \\
(\mathrm{ft} \mathrm{BGL})\end{array}$ & $\begin{array}{c}\text { Depth in Klassen } \\
\text { Test Well } \\
\text { (ft BGL) }\end{array}$ & $\begin{array}{l}\text { Elevation in Klassen } \\
\text { Domestic Well } \\
\text { (ft AMSL) }\end{array}$ & \\
\hline $9 / 22 / 112: 30$ & 34.006 & 38.522 & 1474.76 & 1470.60 \\
\hline $9 / 22 / 112: 40$ & 33.989 & 38.518 & 1474.78 & 1470.60 \\
\hline $9 / 22 / 112: 50$ & 33.978 & 38.51 & 1474.79 & 1470.61 \\
\hline 9/22/11 3:00 & 33.964 & 38.51 & 1474.81 & 1470.61 \\
\hline 9/22/11 3:10 & 33.954 & 38.508 & 1474.82 & 1470.61 \\
\hline $9 / 22 / 113: 20$ & 33.942 & 38.506 & 1474.83 & 1470.62 \\
\hline 9/22/11 3:30 & 33.931 & 38.506 & 1474.84 & 1470.62 \\
\hline $9 / 22 / 113: 40$ & 33.921 & 38.506 & 1474.85 & 1470.62 \\
\hline $9 / 22 / 113: 50$ & 33.914 & 38.506 & 1474.86 & 1470.62 \\
\hline $9 / 22 / 114: 00$ & 33.905 & 38.508 & 1474.86 & 1470.61 \\
\hline $9 / 22 / 114: 10$ & 33.898 & 38.508 & 1474.87 & 1470.61 \\
\hline $9 / 22 / 114: 20$ & 39.182 & 38.503 & 1469.59 & 1470.62 \\
\hline 9/22/11 4:30 & 34.06 & 38.506 & 1474.71 & 1470.62 \\
\hline $9 / 22 / 114: 40$ & 33.982 & 38.506 & 1474.79 & 1470.62 \\
\hline $9 / 22 / 114: 50$ & 33.947 & 38.506 & 1474.82 & 1470.62 \\
\hline 9/22/11 5:00 & 33.954 & 38.503 & 1474.82 & 1470.62 \\
\hline $9 / 22 / 115: 10$ & 33.933 & 38.503 & 1474.84 & 1470.62 \\
\hline 9/22/11 5:20 & 33.917 & 38.501 & 1474.85 & 1470.62 \\
\hline $9 / 22 / 115: 30$ & 33.905 & 38.499 & 1474.86 & 1470.62 \\
\hline $9 / 22 / 115: 40$ & 33.893 & 38.499 & 1474.88 & 1470.62 \\
\hline $9 / 22 / 115: 50$ & 33.881 & 38.496 & 1474.89 & 1470.63 \\
\hline $9 / 22 / 116: 00$ & 33.872 & 38.494 & 1474.90 & 1470.63 \\
\hline 9/22/11 6:10 & 38.281 & 38.494 & 1470.49 & 1470.63 \\
\hline $9 / 22 / 116: 20$ & 34.076 & 38.496 & 1474.69 & 1470.63 \\
\hline $9 / 22 / 116: 30$ & 33.996 & 38.496 & 1474.77 & 1470.63 \\
\hline $9 / 22 / 116: 40$ & 33.959 & 38.496 & 1474.81 & 1470.63 \\
\hline $9 / 22 / 116: 50$ & 33.935 & 38.494 & 1474.83 & 1470.63 \\
\hline 9/22/11 7:00 & 33.917 & 38.494 & 1474.85 & 1470.63 \\
\hline 9/22/11 7:10 & 33.945 & 38.492 & 1474.82 & 1470.63 \\
\hline $9 / 22 / 11$ 7:20 & 33.989 & 38.494 & 1474.78 & 1470.63 \\
\hline 9/22/11 7:30 & 33.973 & 38.494 & 1474.80 & 1470.63 \\
\hline $9 / 22 / 11$ 7:40 & 33.952 & 38.494 & 1474.82 & 1470.63 \\
\hline $9 / 22 / 117: 50$ & 33.933 & 38.494 & 1474.84 & 1470.63 \\
\hline $9 / 22 / 118: 00$ & 33.919 & 38.492 & 1474.85 & 1470.63 \\
\hline $9 / 22 / 118: 10$ & 33.905 & 38.489 & 1474.86 & 1470.63 \\
\hline $9 / 22 / 118: 20$ & 33.891 & 38.487 & 1474.88 & 1470.64 \\
\hline $9 / 22 / 118: 30$ & 33.879 & 38.485 & 1474.89 & 1470.64 \\
\hline $9 / 22 / 118: 40$ & 34.238 & 38.485 & 1474.53 & 1470.64 \\
\hline
\end{tabular}


TABLE S1.3 Automatically recorded groundwater levels in the Klassen wells, September 15, 2011, to March 13, 2012.

\begin{tabular}{|c|c|c|c|c|}
\hline \multicolumn{3}{|c|}{ Reference Elevation (ft AMSL) } & 1508.77 & \multirow{2}{*}{$\begin{array}{l}1509.12 \\
\text { Elevation in Klassen } \\
\text { Test Well (ft AMSL) }\end{array}$} \\
\hline Date and Time & $\begin{array}{l}\text { Depth in Klassen } \\
\text { Domestic Well } \\
(\mathrm{ft} \mathrm{BGL})\end{array}$ & $\begin{array}{c}\text { Depth in Klassen } \\
\text { Test Well } \\
\text { (ft BGL) }\end{array}$ & $\begin{array}{l}\text { Elevation in Klassen } \\
\text { Domestic Well } \\
\text { (ft AMSL) }\end{array}$ & \\
\hline $9 / 22 / 118: 50$ & 33.98 & 38.482 & 1474.79 & 1470.64 \\
\hline 9/22/11 9:00 & 33.935 & 38.482 & 1474.83 & 1470.64 \\
\hline 9/22/11 9:10 & 33.912 & 38.48 & 1474.86 & 1470.64 \\
\hline $9 / 22 / 119: 20$ & 33.893 & 38.478 & 1474.88 & 1470.64 \\
\hline 9/22/11 9:30 & 33.877 & 38.473 & 1474.89 & 1470.65 \\
\hline 9/22/11 9:40 & 33.865 & 38.473 & 1474.90 & 1470.65 \\
\hline 9/22/11 9:50 & 33.853 & 38.471 & 1474.92 & 1470.65 \\
\hline 9/22/11 10:00 & 35.76 & 38.466 & 1473.01 & 1470.66 \\
\hline 9/22/11 10:10 & 34.008 & 38.468 & 1474.76 & 1470.65 \\
\hline $9 / 22 / 1110: 20$ & 33.94 & 38.466 & 1474.83 & 1470.66 \\
\hline $9 / 22 / 1110: 30$ & 33.907 & 38.464 & 1474.86 & 1470.66 \\
\hline 9/22/11 10:40 & 33.886 & 38.461 & 1474.88 & 1470.66 \\
\hline $9 / 22 / 11$ 10:50 & 33.87 & 38.459 & 1474.90 & 1470.66 \\
\hline 9/22/11 11:00 & 33.856 & 38.454 & 1474.91 & 1470.67 \\
\hline 9/22/11 11:10 & 33.844 & 38.452 & 1474.93 & 1470.67 \\
\hline $9 / 22 / 11$ 11:20 & 33.835 & 38.449 & 1474.93 & 1470.67 \\
\hline 9/22/11 11:30 & 33.825 & 38.447 & 1474.94 & 1470.68 \\
\hline 9/22/11 11:40 & 33.816 & 38.445 & 1474.95 & 1470.68 \\
\hline 9/22/11 11:50 & 33.806 & 38.442 & 1474.96 & 1470.68 \\
\hline $9 / 22 / 11$ 12:00 & 34.43 & 38.44 & 1474.34 & 1470.68 \\
\hline 9/22/11 12:10 & 33.928 & 38.438 & 1474.84 & 1470.68 \\
\hline $9 / 22 / 11$ 12:20 & 33.879 & 38.438 & 1474.89 & 1470.68 \\
\hline $9 / 22 / 11$ 12:30 & 33.853 & 38.435 & 1474.92 & 1470.69 \\
\hline $9 / 22 / 11$ 12:40 & 33.865 & 38.433 & 1474.90 & 1470.69 \\
\hline $9 / 22 / 11$ 12:50 & 33.849 & 38.431 & 1474.92 & 1470.69 \\
\hline 9/22/11 13:00 & 33.832 & 38.428 & 1474.94 & 1470.69 \\
\hline 9/22/11 13:10 & 33.818 & 38.426 & 1474.95 & 1470.70 \\
\hline $9 / 22 / 11$ 13:20 & 33.83 & 38.421 & 1474.94 & 1470.70 \\
\hline 9/22/11 13:30 & 33.823 & 38.421 & 1474.95 & 1470.70 \\
\hline 9/22/11 13:40 & 33.806 & 38.417 & 1474.96 & 1470.71 \\
\hline 9/22/11 13:50 & 33.799 & 38.412 & 1474.97 & 1470.71 \\
\hline 9/22/11 14:00 & 34.039 & 38.412 & 1474.73 & 1470.71 \\
\hline 9/22/11 14:10 & 33.891 & 38.412 & 1474.88 & 1470.71 \\
\hline $9 / 22 / 11$ 14:20 & 34.297 & 38.41 & 1474.47 & 1470.71 \\
\hline 9/22/11 14:30 & 33.982 & 38.41 & 1474.79 & 1470.71 \\
\hline 9/22/11 14:40 & 38.213 & 38.405 & 1470.56 & 1470.72 \\
\hline $9 / 22 / 11$ 14:50 & 34.067 & 38.407 & 1474.70 & 1470.72 \\
\hline 9/22/11 15:00 & 33.973 & 38.405 & 1474.80 & 1470.72 \\
\hline
\end{tabular}


TABLE S1.3 Automatically recorded groundwater levels in the Klassen wells, September 15, 2011, to March 13, 2012.

\begin{tabular}{|c|c|c|c|c|}
\hline \multicolumn{3}{|c|}{ Reference Elevation (ft AMSL) } & 1508.77 & \multirow{2}{*}{$\begin{array}{l}1509.12 \\
\text { Elevation in Klassen } \\
\text { Test Well (ft AMSL) }\end{array}$} \\
\hline Date and Time & $\begin{array}{l}\text { Depth in Klassen } \\
\text { Domestic Well } \\
\text { (ft BGL) }\end{array}$ & $\begin{array}{c}\text { Depth in Klassen } \\
\text { Test Well } \\
\text { (ft BGL) }\end{array}$ & $\begin{array}{l}\text { Elevation in Klassen } \\
\text { Domestic Well } \\
\text { (ft AMSL) }\end{array}$ & \\
\hline 9/22/11 15:10 & 33.926 & 38.403 & 1474.84 & 1470.72 \\
\hline $9 / 22 / 1115: 20$ & 33.896 & 38.4 & 1474.87 & 1470.72 \\
\hline $9 / 22 / 11$ 15:30 & 33.872 & 38.396 & 1474.90 & 1470.73 \\
\hline $9 / 22 / 1115: 40$ & 33.884 & 38.396 & 1474.89 & 1470.73 \\
\hline $9 / 22 / 1115: 50$ & 33.867 & 38.393 & 1474.90 & 1470.73 \\
\hline 9/22/11 16:00 & 33.849 & 38.391 & 1474.92 & 1470.73 \\
\hline 9/22/11 16:10 & 33.917 & 38.391 & 1474.85 & 1470.73 \\
\hline $9 / 22 / 1116: 20$ & 33.905 & 38.391 & 1474.86 & 1470.73 \\
\hline 9/22/11 16:30 & 33.881 & 38.391 & 1474.89 & 1470.73 \\
\hline $9 / 22 / 1116: 40$ & 33.863 & 38.388 & 1474.91 & 1470.73 \\
\hline $9 / 22 / 1116: 50$ & 33.87 & 38.386 & 1474.90 & 1470.74 \\
\hline 9/22/11 17:00 & 33.856 & 38.384 & 1474.91 & 1470.74 \\
\hline 9/22/11 17:10 & 34.191 & 38.384 & 1474.58 & 1470.74 \\
\hline $9 / 22 / 1117: 20$ & 33.95 & 38.384 & 1474.82 & 1470.74 \\
\hline 9/22/11 17:30 & 34.006 & 38.386 & 1474.76 & 1470.74 \\
\hline 9/22/11 17:40 & 34.791 & 38.388 & 1473.98 & 1470.73 \\
\hline 9/22/11 17:50 & 34.205 & 38.393 & 1474.56 & 1470.73 \\
\hline 9/22/11 18:00 & 34.147 & 38.398 & 1474.62 & 1470.72 \\
\hline 9/22/11 18:10 & 34.093 & 38.398 & 1474.68 & 1470.72 \\
\hline $9 / 22 / 11$ 18:20 & 34.048 & 38.396 & 1474.72 & 1470.73 \\
\hline $9 / 22 / 1118: 30$ & 34.013 & 38.396 & 1474.76 & 1470.73 \\
\hline 9/22/11 18:40 & 33.982 & 38.391 & 1474.79 & 1470.73 \\
\hline $9 / 22 / 1118: 50$ & 33.957 & 38.388 & 1474.81 & 1470.73 \\
\hline 9/22/11 19:00 & 33.935 & 38.386 & 1474.83 & 1470.74 \\
\hline 9/22/11 19:10 & 33.919 & 38.386 & 1474.85 & 1470.74 \\
\hline 9/22/11 19:20 & 33.921 & 38.381 & 1474.85 & 1470.74 \\
\hline 9/22/11 19:30 & 33.917 & 38.379 & 1474.85 & 1470.74 \\
\hline 9/22/11 19:40 & 33.9 & 38.377 & 1474.87 & 1470.75 \\
\hline 9/22/11 19:50 & 34.057 & 38.377 & 1474.71 & 1470.75 \\
\hline 9/22/11 20:00 & 33.989 & 38.374 & 1474.78 & 1470.75 \\
\hline 9/22/11 20:10 & 33.957 & 38.374 & 1474.81 & 1470.75 \\
\hline 9/22/11 20:20 & 33.952 & 38.37 & 1474.82 & 1470.75 \\
\hline $9 / 22 / 1120: 30$ & 33.931 & 38.367 & 1474.84 & 1470.76 \\
\hline $9 / 22 / 1120: 40$ & 33.907 & 38.365 & 1474.86 & 1470.76 \\
\hline $9 / 22 / 1120: 50$ & 33.891 & 38.363 & 1474.88 & 1470.76 \\
\hline 9/22/11 21:00 & 33.872 & 38.36 & 1474.90 & 1470.76 \\
\hline 9/22/11 21:10 & 33.914 & 38.36 & 1474.86 & 1470.76 \\
\hline $9 / 22 / 1121: 20$ & 33.947 & 38.36 & 1474.82 & 1470.76 \\
\hline
\end{tabular}


TABLE S1.3 Automatically recorded groundwater levels in the Klassen wells, September 15, 2011, to March 13, 2012.

\begin{tabular}{|c|c|c|c|c|}
\hline \multicolumn{3}{|c|}{ Reference Elevation (ft AMSL) } & 1508.77 & \multirow{2}{*}{$\begin{array}{l}1509.12 \\
\text { Elevation in Klassen } \\
\text { Test Well (ft AMSL) }\end{array}$} \\
\hline Date and Time & $\begin{array}{l}\text { Depth in Klassen } \\
\text { Domestic Well } \\
(\mathrm{ft} \mathrm{BGL})\end{array}$ & $\begin{array}{c}\text { Depth in Klassen } \\
\text { Test Well } \\
\text { (ft BGL) }\end{array}$ & $\begin{array}{l}\text { Elevation in Klassen } \\
\text { Domestic Well } \\
\text { (ft AMSL) }\end{array}$ & \\
\hline $9 / 22 / 1121: 30$ & 33.999 & 38.36 & 1474.77 & 1470.76 \\
\hline $9 / 22 / 1121: 40$ & 34.027 & 38.365 & 1474.74 & 1470.76 \\
\hline $9 / 22 / 1121: 50$ & 34.02 & 38.365 & 1474.75 & 1470.76 \\
\hline $9 / 22 / 1122: 00$ & 34.069 & 38.367 & 1474.70 & 1470.76 \\
\hline 9/22/11 22:10 & 34.046 & 38.365 & 1474.72 & 1470.76 \\
\hline $9 / 22 / 1122: 20$ & 34.015 & 38.365 & 1474.75 & 1470.76 \\
\hline $9 / 22 / 1122: 30$ & 33.987 & 38.363 & 1474.78 & 1470.76 \\
\hline $9 / 22 / 1122: 40$ & 34.667 & 38.36 & 1474.10 & 1470.76 \\
\hline $9 / 22 / 1122: 50$ & 34.292 & 38.363 & 1474.48 & 1470.76 \\
\hline $9 / 22 / 1123: 00$ & 34.175 & 38.365 & 1474.59 & 1470.76 \\
\hline 9/22/11 23:10 & 34.562 & 38.365 & 1474.21 & 1470.76 \\
\hline $9 / 22 / 1123: 20$ & 34.36 & 38.367 & 1474.41 & 1470.76 \\
\hline $9 / 22 / 1123: 30$ & 34.205 & 38.367 & 1474.56 & 1470.76 \\
\hline $9 / 22 / 1123: 40$ & 34.132 & 38.365 & 1474.64 & 1470.76 \\
\hline 9/22/11 23:50 & 34.086 & 38.363 & 1474.68 & 1470.76 \\
\hline 9/23/11 0:00 & 34.05 & 38.36 & 1474.72 & 1470.76 \\
\hline 9/23/11 0:10 & 34.02 & 38.358 & 1474.75 & 1470.76 \\
\hline 9/23/11 0:20 & 33.994 & 38.353 & 1474.78 & 1470.77 \\
\hline 9/23/11 0:30 & 33.975 & 38.351 & 1474.79 & 1470.77 \\
\hline $9 / 23 / 110: 40$ & 33.954 & 38.349 & 1474.82 & 1470.77 \\
\hline 9/23/11 0:50 & 33.938 & 38.344 & 1474.83 & 1470.78 \\
\hline 9/23/11 1:00 & 33.921 & 38.342 & 1474.85 & 1470.78 \\
\hline $9 / 23 / 11$ 1:10 & 33.905 & 38.337 & 1474.86 & 1470.79 \\
\hline $9 / 23 / 11$ 1:20 & 33.891 & 38.335 & 1474.88 & 1470.79 \\
\hline $9 / 23 / 11$ 1:30 & 33.879 & 38.33 & 1474.89 & 1470.79 \\
\hline $9 / 23 / 11$ 1:40 & 33.903 & 38.327 & 1474.87 & 1470.80 \\
\hline $9 / 23 / 11$ 1:50 & 33.964 & 38.33 & 1474.81 & 1470.79 \\
\hline 9/23/11 2:00 & 33.973 & 38.33 & 1474.80 & 1470.79 \\
\hline 9/23/11 2:10 & 33.95 & 38.325 & 1474.82 & 1470.80 \\
\hline $9 / 23 / 112: 20$ & 33.928 & 38.323 & 1474.84 & 1470.80 \\
\hline 9/23/11 2:30 & 33.935 & 38.32 & 1474.83 & 1470.80 \\
\hline $9 / 23 / 112: 40$ & 33.914 & 38.318 & 1474.86 & 1470.80 \\
\hline $9 / 23 / 112: 50$ & 33.896 & 38.316 & 1474.87 & 1470.81 \\
\hline 9/23/11 3:00 & 33.905 & 38.313 & 1474.86 & 1470.81 \\
\hline 9/23/11 3:10 & 33.889 & 38.311 & 1474.88 & 1470.81 \\
\hline 9/23/11 3:20 & 33.91 & 38.309 & 1474.86 & 1470.81 \\
\hline 9/23/11 3:30 & 33.98 & 38.311 & 1474.79 & 1470.81 \\
\hline 9/23/11 3:40 & 34.175 & 38.313 & 1474.59 & 1470.81 \\
\hline
\end{tabular}


TABLE S1.3 Automatically recorded groundwater levels in the Klassen wells, September 15, 2011, to March 13, 2012.

\begin{tabular}{|c|c|c|c|c|}
\hline \multicolumn{3}{|c|}{ Reference Elevation (ft AMSL) } & 1508.77 & \multirow{2}{*}{$\begin{array}{l}1509.12 \\
\text { Elevation in Klassen } \\
\text { Test Well (ft AMSL) }\end{array}$} \\
\hline Date and Time & $\begin{array}{l}\text { Depth in Klassen } \\
\text { Domestic Well } \\
\text { (ft BGL) }\end{array}$ & $\begin{array}{c}\text { Depth in Klassen } \\
\text { Test Well } \\
\text { (ft BGL) }\end{array}$ & $\begin{array}{l}\text { Elevation in Klassen } \\
\text { Domestic Well } \\
\text { (ft AMSL) }\end{array}$ & \\
\hline $9 / 23 / 113: 50$ & 34.057 & 38.313 & 1474.71 & 1470.81 \\
\hline $9 / 23 / 114: 00$ & 34.006 & 38.311 & 1474.76 & 1470.81 \\
\hline 9/23/11 4:10 & 33.971 & 38.309 & 1474.80 & 1470.81 \\
\hline $9 / 23 / 114: 20$ & 33.942 & 38.306 & 1474.83 & 1470.82 \\
\hline $9 / 23 / 114: 30$ & 33.921 & 38.304 & 1474.85 & 1470.82 \\
\hline $9 / 23 / 114: 40$ & 33.903 & 38.302 & 1474.87 & 1470.82 \\
\hline $9 / 23 / 114: 50$ & 33.886 & 38.299 & 1474.88 & 1470.82 \\
\hline $9 / 23 / 115: 00$ & 33.872 & 38.295 & 1474.90 & 1470.83 \\
\hline 9/23/11 5:10 & 33.858 & 38.292 & 1474.91 & 1470.83 \\
\hline $9 / 23 / 115: 20$ & 33.846 & 38.29 & 1474.92 & 1470.83 \\
\hline $9 / 23 / 115: 30$ & 33.832 & 38.288 & 1474.94 & 1470.83 \\
\hline 9/23/11 5:40 & 33.823 & 38.285 & 1474.95 & 1470.84 \\
\hline $9 / 23 / 115: 50$ & 33.813 & 38.283 & 1474.96 & 1470.84 \\
\hline $9 / 23 / 116: 00$ & 33.818 & 38.281 & 1474.95 & 1470.84 \\
\hline $9 / 23 / 116: 10$ & 35.382 & 38.281 & 1473.39 & 1470.84 \\
\hline $9 / 23 / 116: 20$ & 33.961 & 38.281 & 1474.81 & 1470.84 \\
\hline $9 / 23 / 116: 30$ & 33.903 & 38.281 & 1474.87 & 1470.84 \\
\hline $9 / 23 / 116: 40$ & 33.872 & 38.278 & 1474.90 & 1470.84 \\
\hline $9 / 23 / 116: 50$ & 33.851 & 38.276 & 1474.92 & 1470.85 \\
\hline $9 / 23 / 11$ 7:00 & 33.832 & 38.276 & 1474.94 & 1470.85 \\
\hline $9 / 23 / 11$ 7:10 & 33.818 & 38.271 & 1474.95 & 1470.85 \\
\hline $9 / 23 / 11$ 7:20 & 33.806 & 38.271 & 1474.96 & 1470.85 \\
\hline $9 / 23 / 117: 30$ & 33.825 & 38.269 & 1474.94 & 1470.85 \\
\hline 9/23/11 7:40 & 33.816 & 38.266 & 1474.95 & 1470.86 \\
\hline $9 / 23 / 117: 50$ & 33.802 & 38.266 & 1474.97 & 1470.86 \\
\hline 9/23/11 8:00 & 33.792 & 38.264 & 1474.98 & 1470.86 \\
\hline $9 / 23 / 118: 10$ & 33.806 & 38.264 & 1474.96 & 1470.86 \\
\hline $9 / 23 / 118: 20$ & 33.792 & 38.262 & 1474.98 & 1470.86 \\
\hline 9/23/11 8:30 & 39.611 & 38.262 & 1469.16 & 1470.86 \\
\hline $9 / 23 / 118: 40$ & 34.008 & 38.262 & 1474.76 & 1470.86 \\
\hline $9 / 23 / 118: 50$ & 35.997 & 38.262 & 1472.77 & 1470.86 \\
\hline 9/23/11 9:00 & 34.034 & 38.262 & 1474.74 & 1470.86 \\
\hline 9/23/11 9:10 & 34.048 & 38.266 & 1474.72 & 1470.86 \\
\hline 9/23/11 9:20 & 34.003 & 38.269 & 1474.77 & 1470.85 \\
\hline 9/23/11 9:30 & 33.964 & 38.269 & 1474.81 & 1470.85 \\
\hline 9/23/11 9:40 & 33.931 & 38.266 & 1474.84 & 1470.86 \\
\hline 9/23/11 9:50 & 33.907 & 38.264 & 1474.86 & 1470.86 \\
\hline 9/23/11 10:00 & 33.884 & 38.262 & 1474.89 & 1470.86 \\
\hline
\end{tabular}


TABLE S1.3 Automatically recorded groundwater levels in the Klassen wells, September 15, 2011, to March 13, 2012.

\begin{tabular}{|c|c|c|c|c|}
\hline \multicolumn{3}{|c|}{ Reference Elevation (ft AMSL) } & 1508.77 & \multirow{2}{*}{$\begin{array}{l}1509.12 \\
\text { Elevation in Klassen } \\
\text { Test Well (ft AMSL) }\end{array}$} \\
\hline Date and Time & $\begin{array}{l}\text { Depth in Klassen } \\
\text { Domestic Well } \\
(\mathrm{ft} \mathrm{BGL})\end{array}$ & $\begin{array}{c}\text { Depth in Klassen } \\
\text { Test Well } \\
\text { (ft BGL) }\end{array}$ & $\begin{array}{l}\text { Elevation in Klassen } \\
\text { Domestic Well } \\
\text { (ft AMSL) }\end{array}$ & \\
\hline 9/23/11 10:10 & 33.867 & 38.259 & 1474.90 & 1470.86 \\
\hline $9 / 23 / 1110: 20$ & 33.849 & 38.257 & 1474.92 & 1470.87 \\
\hline 9/23/11 10:30 & 33.835 & 38.255 & 1474.93 & 1470.87 \\
\hline $9 / 23 / 1110: 40$ & 33.821 & 38.252 & 1474.95 & 1470.87 \\
\hline 9/23/11 10:50 & 33.959 & 38.252 & 1474.81 & 1470.87 \\
\hline 9/23/11 11:00 & 33.884 & 38.25 & 1474.89 & 1470.87 \\
\hline 9/23/11 11:10 & 33.853 & 38.25 & 1474.92 & 1470.87 \\
\hline 9/23/11 11:20 & 34.336 & 38.248 & 1474.43 & 1470.87 \\
\hline 9/23/11 11:30 & 33.933 & 38.248 & 1474.84 & 1470.87 \\
\hline 9/23/11 11:40 & 33.879 & 38.245 & 1474.89 & 1470.88 \\
\hline 9/23/11 11:50 & 33.851 & 38.245 & 1474.92 & 1470.88 \\
\hline 9/23/11 12:00 & 34.17 & 38.245 & 1474.60 & 1470.88 \\
\hline 9/23/11 12:10 & 33.947 & 38.248 & 1474.82 & 1470.87 \\
\hline $9 / 23 / 1112: 20$ & 34.327 & 38.25 & 1474.44 & 1470.87 \\
\hline 9/23/11 12:30 & 34.001 & 38.252 & 1474.77 & 1470.87 \\
\hline 9/23/11 12:40 & 34.104 & 38.257 & 1474.67 & 1470.87 \\
\hline 9/23/11 12:50 & 34.813 & 38.259 & 1473.96 & 1470.86 \\
\hline 9/23/11 13:00 & 35.453 & 38.264 & 1473.32 & 1470.86 \\
\hline 9/23/11 13:10 & 34.278 & 38.271 & 1474.49 & 1470.85 \\
\hline $9 / 23 / 11$ 13:20 & 35.364 & 38.276 & 1473.41 & 1470.85 \\
\hline $9 / 23 / 11$ 13:30 & 34.292 & 38.281 & 1474.48 & 1470.84 \\
\hline 9/23/11 13:40 & 35.284 & 38.29 & 1473.49 & 1470.83 \\
\hline $9 / 23 / 11$ 13:50 & 38.502 & 38.297 & 1470.27 & 1470.83 \\
\hline 9/23/11 14:00 & 34.587 & 38.309 & 1474.18 & 1470.81 \\
\hline 9/23/11 14:10 & 34.909 & 38.318 & 1473.86 & 1470.80 \\
\hline 9/23/11 14:20 & 34.721 & 38.327 & 1474.05 & 1470.80 \\
\hline 9/23/11 14:30 & 34.662 & 38.332 & 1474.11 & 1470.79 \\
\hline 9/23/11 14:40 & 34.634 & 38.337 & 1474.14 & 1470.79 \\
\hline 9/23/11 14:50 & 36.698 & 38.344 & 1472.07 & 1470.78 \\
\hline 9/23/11 15:00 & 37.648 & 38.349 & 1471.12 & 1470.77 \\
\hline 9/23/11 15:10 & 34.841 & 38.356 & 1473.93 & 1470.77 \\
\hline 9/23/11 15:20 & 34.752 & 38.36 & 1474.02 & 1470.76 \\
\hline $9 / 23 / 11$ 15:30 & 34.726 & 38.365 & 1474.04 & 1470.76 \\
\hline 9/23/11 15:40 & 34.707 & 38.37 & 1474.06 & 1470.75 \\
\hline 9/23/11 15:50 & 34.705 & 38.374 & 1474.06 & 1470.75 \\
\hline 9/23/11 16:00 & 34.695 & 38.379 & 1474.07 & 1470.74 \\
\hline 9/23/11 16:10 & 35.031 & 38.381 & 1473.74 & 1470.74 \\
\hline 9/23/11 16:20 & 34.777 & 38.386 & 1473.99 & 1470.74 \\
\hline
\end{tabular}


TABLE S1.3 Automatically recorded groundwater levels in the Klassen wells, September 15, 2011, to March 13, 2012.

\begin{tabular}{|c|c|c|c|c|}
\hline \multicolumn{3}{|c|}{ Reference Elevation (ft AMSL) } & 1508.77 & \multirow{2}{*}{$\begin{array}{l}1509.12 \\
\text { Elevation in Klassen } \\
\text { Test Well (ft AMSL) }\end{array}$} \\
\hline Date and Time & $\begin{array}{l}\text { Depth in Klassen } \\
\text { Domestic Well } \\
\text { (ft BGL) }\end{array}$ & $\begin{array}{c}\text { Depth in Klassen } \\
\text { Test Well } \\
\text { (ft BGL) }\end{array}$ & $\begin{array}{l}\text { Elevation in Klassen } \\
\text { Domestic Well } \\
\text { (ft AMSL) }\end{array}$ & \\
\hline 9/23/11 16:30 & 34.735 & 38.391 & 1474.03 & 1470.73 \\
\hline 9/23/11 16:40 & 35.143 & 38.396 & 1473.63 & 1470.73 \\
\hline 9/23/11 16:50 & 34.82 & 38.4 & 1473.95 & 1470.72 \\
\hline 9/23/11 17:00 & 34.77 & 38.403 & 1474.00 & 1470.72 \\
\hline 9/23/11 17:10 & 34.747 & 38.407 & 1474.02 & 1470.72 \\
\hline 9/23/11 17:20 & 34.733 & 38.41 & 1474.04 & 1470.71 \\
\hline 9/23/11 17:30 & 34.723 & 38.412 & 1474.05 & 1470.71 \\
\hline 9/23/11 17:40 & 38.276 & 38.414 & 1470.49 & 1470.71 \\
\hline 9/23/11 17:50 & 36.963 & 38.421 & 1471.81 & 1470.70 \\
\hline 9/23/11 18:00 & 46.527 & 38.438 & 1462.24 & 1470.68 \\
\hline 9/23/11 18:10 & 48.549 & 38.464 & 1460.22 & 1470.66 \\
\hline 9/23/11 18:20 & 49.365 & 38.496 & 1459.40 & 1470.63 \\
\hline $9 / 23 / 11$ 18:30 & 49.173 & 38.532 & 1459.60 & 1470.59 \\
\hline 9/23/11 18:40 & 51.115 & 38.572 & 1457.65 & 1470.55 \\
\hline 9/23/11 18:50 & 53.976 & 38.616 & 1454.79 & 1470.51 \\
\hline 9/23/11 19:00 & 53.183 & 38.661 & 1455.59 & 1470.46 \\
\hline 9/23/11 19:10 & 38.86 & 38.696 & 1469.91 & 1470.43 \\
\hline 9/23/11 19:20 & 37.897 & 38.715 & 1470.87 & 1470.41 \\
\hline 9/23/11 19:30 & 37.428 & 38.722 & 1471.34 & 1470.40 \\
\hline 9/23/11 19:40 & 37.118 & 38.726 & 1471.65 & 1470.40 \\
\hline 9/23/11 19:50 & 36.888 & 38.731 & 1471.88 & 1470.39 \\
\hline 9/23/11 20:00 & 37.034 & 38.733 & 1471.74 & 1470.39 \\
\hline 9/23/11 20:10 & 37.385 & 38.736 & 1471.38 & 1470.39 \\
\hline 9/23/11 20:20 & 36.64 & 38.74 & 1472.13 & 1470.38 \\
\hline $9 / 23 / 1120: 30$ & 36.48 & 38.743 & 1472.29 & 1470.38 \\
\hline $9 / 23 / 1120: 40$ & 36.36 & 38.745 & 1472.41 & 1470.38 \\
\hline 9/23/11 20:50 & 36.745 & 38.745 & 1472.02 & 1470.38 \\
\hline 9/23/11 21:00 & 36.292 & 38.747 & 1472.48 & 1470.38 \\
\hline 9/23/11 21:10 & 36.182 & 38.747 & 1472.59 & 1470.38 \\
\hline 9/23/11 21:20 & 36.102 & 38.747 & 1472.67 & 1470.38 \\
\hline 9/23/11 21:30 & 36.034 & 38.747 & 1472.74 & 1470.38 \\
\hline 9/23/11 21:40 & 35.973 & 38.747 & 1472.80 & 1470.38 \\
\hline 9/23/11 21:50 & 35.92 & 38.745 & 1472.85 & 1470.38 \\
\hline 9/23/11 22:00 & 35.905 & 38.747 & 1472.86 & 1470.38 \\
\hline 9/23/11 22:10 & 35.91 & 38.747 & 1472.86 & 1470.38 \\
\hline 9/23/11 22:20 & 35.936 & 38.752 & 1472.83 & 1470.37 \\
\hline $9 / 23 / 1122: 30$ & 36.196 & 38.757 & 1472.57 & 1470.37 \\
\hline $9 / 23 / 11$ 22:40 & 36.274 & 38.764 & 1472.50 & 1470.36 \\
\hline
\end{tabular}


TABLE S1.3 Automatically recorded groundwater levels in the Klassen wells, September 15, 2011, to March 13, 2012.

\begin{tabular}{|c|c|c|c|c|}
\hline \multicolumn{3}{|c|}{ Reference Elevation (ft AMSL) } & 1508.77 & \multirow{2}{*}{$\begin{array}{l}1509.12 \\
\text { Elevation in Klassen } \\
\text { Test Well (ft AMSL) }\end{array}$} \\
\hline Date and Time & $\begin{array}{l}\text { Depth in Klassen } \\
\text { Domestic Well } \\
(\mathrm{ft} \mathrm{BGL})\end{array}$ & $\begin{array}{c}\text { Depth in Klassen } \\
\text { Test Well } \\
\text { (ft BGL) }\end{array}$ & $\begin{array}{l}\text { Elevation in Klassen } \\
\text { Domestic Well } \\
\text { (ft AMSL) }\end{array}$ & \\
\hline $9 / 23 / 1122: 50$ & 36.098 & 38.766 & 1472.67 & 1470.36 \\
\hline 9/23/11 23:00 & 36.328 & 38.771 & 1472.44 & 1470.35 \\
\hline 9/23/11 23:10 & 36.077 & 38.773 & 1472.69 & 1470.35 \\
\hline $9 / 23 / 1123: 20$ & 35.983 & 38.773 & 1472.79 & 1470.35 \\
\hline 9/23/11 23:30 & 35.924 & 38.773 & 1472.85 & 1470.35 \\
\hline 9/23/11 23:40 & 35.875 & 38.771 & 1472.89 & 1470.35 \\
\hline $9 / 23 / 1123: 50$ & 35.826 & 38.771 & 1472.94 & 1470.35 \\
\hline 9/24/11 0:00 & 35.781 & 38.769 & 1472.99 & 1470.35 \\
\hline 9/24/11 0:10 & 35.744 & 38.764 & 1473.03 & 1470.36 \\
\hline 9/24/11 0:20 & 35.718 & 38.764 & 1473.05 & 1470.36 \\
\hline 9/24/11 0:30 & 35.69 & 38.762 & 1473.08 & 1470.36 \\
\hline 9/24/11 0:40 & 35.659 & 38.759 & 1473.11 & 1470.36 \\
\hline 9/24/11 0:50 & 35.631 & 38.757 & 1473.14 & 1470.37 \\
\hline 9/24/11 1:00 & 35.603 & 38.755 & 1473.17 & 1470.37 \\
\hline 9/24/11 1:10 & 35.577 & 38.75 & 1473.19 & 1470.37 \\
\hline $9 / 24 / 11$ 1:20 & 36.668 & 38.747 & 1472.10 & 1470.38 \\
\hline $9 / 24 / 11$ 1:30 & 35.659 & 38.747 & 1473.11 & 1470.38 \\
\hline $9 / 24 / 11$ 1:40 & 35.594 & 38.745 & 1473.18 & 1470.38 \\
\hline $9 / 24 / 11$ 1:50 & 35.556 & 38.743 & 1473.21 & 1470.38 \\
\hline $9 / 24 / 112: 00$ & 35.523 & 38.74 & 1473.25 & 1470.38 \\
\hline $9 / 24 / 112: 10$ & 35.495 & 38.738 & 1473.27 & 1470.38 \\
\hline $9 / 24 / 112: 20$ & 35.469 & 38.733 & 1473.30 & 1470.39 \\
\hline 9/24/11 2:30 & 35.45 & 38.731 & 1473.32 & 1470.39 \\
\hline $9 / 24 / 112: 40$ & 35.429 & 38.726 & 1473.34 & 1470.40 \\
\hline $9 / 24 / 112: 50$ & 35.411 & 38.724 & 1473.36 & 1470.40 \\
\hline 9/24/11 3:00 & 35.389 & 38.719 & 1473.38 & 1470.40 \\
\hline 9/24/11 3:10 & 35.373 & 38.717 & 1473.40 & 1470.41 \\
\hline $9 / 24 / 113: 20$ & 35.359 & 38.715 & 1473.41 & 1470.41 \\
\hline 9/24/11 3:30 & 35.343 & 38.71 & 1473.43 & 1470.41 \\
\hline 9/24/11 3:40 & 35.326 & 38.71 & 1473.44 & 1470.41 \\
\hline 9/24/11 3:50 & 35.671 & 38.705 & 1473.10 & 1470.42 \\
\hline $9 / 24 / 114: 00$ & 35.432 & 38.705 & 1473.34 & 1470.42 \\
\hline 9/24/11 4:10 & 35.38 & 38.703 & 1473.39 & 1470.42 \\
\hline 9/24/11 4:20 & 35.345 & 38.701 & 1473.42 & 1470.42 \\
\hline $9 / 24 / 114: 30$ & 35.321 & 38.698 & 1473.45 & 1470.42 \\
\hline 9/24/11 4:40 & 35.305 & 38.696 & 1473.46 & 1470.43 \\
\hline 9/24/11 4:50 & 35.289 & 38.694 & 1473.48 & 1470.43 \\
\hline 9/24/11 5:00 & 35.27 & 38.691 & 1473.50 & 1470.43 \\
\hline
\end{tabular}


TABLE S1.3 Automatically recorded groundwater levels in the Klassen wells, September 15, 2011, to March 13, 2012.

\begin{tabular}{|c|c|c|c|c|}
\hline \multicolumn{3}{|c|}{ Reference Elevation (ft AMSL) } & 1508.77 & \multirow{2}{*}{$\begin{array}{l}1509.12 \\
\text { Elevation in Klassen } \\
\text { Test Well (ft AMSL) }\end{array}$} \\
\hline Date and Time & $\begin{array}{l}\text { Depth in Klassen } \\
\text { Domestic Well } \\
(\mathrm{ft} \mathrm{BGL})\end{array}$ & $\begin{array}{c}\text { Depth in Klassen } \\
\text { Test Well } \\
\text { (ft BGL) }\end{array}$ & $\begin{array}{l}\text { Elevation in Klassen } \\
\text { Domestic Well } \\
\text { (ft AMSL) }\end{array}$ & \\
\hline $9 / 24 / 115: 10$ & 35.258 & 38.689 & 1473.51 & 1470.43 \\
\hline $9 / 24 / 115: 20$ & 35.249 & 38.686 & 1473.52 & 1470.44 \\
\hline 9/24/11 5:30 & 35.235 & 38.684 & 1473.53 & 1470.44 \\
\hline 9/24/11 5:40 & 35.221 & 38.682 & 1473.55 & 1470.44 \\
\hline $9 / 24 / 115: 50$ & 35.211 & 38.679 & 1473.56 & 1470.44 \\
\hline 9/24/11 6:00 & 35.202 & 38.679 & 1473.57 & 1470.44 \\
\hline $9 / 24 / 116: 10$ & 36.916 & 38.677 & 1471.85 & 1470.45 \\
\hline $9 / 24 / 116: 20$ & 35.324 & 38.677 & 1473.45 & 1470.45 \\
\hline $9 / 24 / 116: 30$ & 35.268 & 38.677 & 1473.50 & 1470.45 \\
\hline $9 / 24 / 116: 40$ & 35.237 & 38.677 & 1473.53 & 1470.45 \\
\hline $9 / 24 / 116: 50$ & 35.216 & 38.675 & 1473.55 & 1470.45 \\
\hline 9/24/11 7:00 & 35.197 & 38.672 & 1473.57 & 1470.45 \\
\hline $9 / 24 / 117: 10$ & 35.185 & 38.672 & 1473.58 & 1470.45 \\
\hline $9 / 24 / 117: 20$ & 35.174 & 38.67 & 1473.60 & 1470.45 \\
\hline $9 / 24 / 117: 30$ & 35.16 & 38.668 & 1473.61 & 1470.45 \\
\hline $9 / 24 / 117: 40$ & 35.148 & 38.665 & 1473.62 & 1470.46 \\
\hline $9 / 24 / 117: 50$ & 35.141 & 38.663 & 1473.63 & 1470.46 \\
\hline 9/24/11 8:00 & 35.162 & 38.663 & 1473.61 & 1470.46 \\
\hline 9/24/11 8:10 & 38.098 & 38.665 & 1470.67 & 1470.46 \\
\hline $9 / 24 / 118: 20$ & 35.368 & 38.668 & 1473.40 & 1470.45 \\
\hline $9 / 24 / 118: 30$ & 35.286 & 38.668 & 1473.48 & 1470.45 \\
\hline $9 / 24 / 118: 40$ & 35.3 & 38.67 & 1473.47 & 1470.45 \\
\hline 9/24/11 8:50 & 35.443 & 38.675 & 1473.33 & 1470.45 \\
\hline 9/24/11 9:00 & 35.354 & 38.675 & 1473.42 & 1470.45 \\
\hline 9/24/11 9:10 & 35.385 & 38.675 & 1473.38 & 1470.45 \\
\hline 9/24/11 9:20 & 35.427 & 38.682 & 1473.34 & 1470.44 \\
\hline 9/24/11 9:30 & 35.404 & 38.684 & 1473.37 & 1470.44 \\
\hline 9/24/11 9:40 & 35.368 & 38.682 & 1473.40 & 1470.44 \\
\hline 9/24/11 9:50 & 35.338 & 38.682 & 1473.43 & 1470.44 \\
\hline 9/24/11 10:00 & 35.312 & 38.679 & 1473.46 & 1470.44 \\
\hline 9/24/11 10:10 & 35.286 & 38.677 & 1473.48 & 1470.45 \\
\hline 9/24/11 10:20 & 35.265 & 38.675 & 1473.50 & 1470.45 \\
\hline $9 / 24 / 11$ 10:30 & 35.251 & 38.672 & 1473.52 & 1470.45 \\
\hline 9/24/11 10:40 & 35.23 & 38.672 & 1473.54 & 1470.45 \\
\hline 9/24/11 10:50 & 39.843 & 38.668 & 1468.93 & 1470.45 \\
\hline 9/24/11 11:00 & 35.42 & 38.67 & 1473.35 & 1470.45 \\
\hline 9/24/11 11:10 & 39.004 & 38.668 & 1469.77 & 1470.45 \\
\hline 9/24/11 11:20 & 35.755 & 38.67 & 1473.01 & 1470.45 \\
\hline
\end{tabular}


TABLE S1.3 Automatically recorded groundwater levels in the Klassen wells, September 15, 2011, to March 13, 2012.

\begin{tabular}{|c|c|c|c|c|}
\hline \multicolumn{3}{|c|}{ Reference Elevation (ft AMSL) } & 1508.77 & \multirow{2}{*}{$\begin{array}{l}1509.12 \\
\text { Elevation in Klassen } \\
\text { Test Well (ft AMSL) }\end{array}$} \\
\hline Date and Time & $\begin{array}{l}\text { Depth in Klassen } \\
\text { Domestic Well } \\
\text { (ft BGL) }\end{array}$ & $\begin{array}{c}\text { Depth in Klassen } \\
\text { Test Well } \\
\text { (ft BGL) }\end{array}$ & $\begin{array}{l}\text { Elevation in Klassen } \\
\text { Domestic Well } \\
\text { (ft AMSL) }\end{array}$ & \\
\hline 9/24/11 11:30 & 39.545 & 38.675 & 1469.22 & 1470.45 \\
\hline 9/24/11 11:40 & 44.292 & 38.686 & 1464.48 & 1470.44 \\
\hline 9/24/11 11:50 & 46.243 & 38.703 & 1462.53 & 1470.42 \\
\hline $9 / 24 / 1112: 00$ & 46.654 & 38.726 & 1462.12 & 1470.40 \\
\hline 9/24/11 12:10 & 45.23 & 38.747 & 1463.54 & 1470.38 \\
\hline 9/24/11 12:20 & 43.274 & 38.769 & 1465.50 & 1470.35 \\
\hline 9/24/11 12:30 & 42.263 & 38.794 & 1466.51 & 1470.33 \\
\hline $9 / 24 / 1112: 40$ & 43.779 & 38.818 & 1464.99 & 1470.30 \\
\hline $9 / 24 / 1112: 50$ & 45.383 & 38.846 & 1463.39 & 1470.28 \\
\hline 9/24/11 13:00 & 47.798 & 38.872 & 1460.97 & 1470.25 \\
\hline 9/24/11 13:10 & 47.156 & 38.898 & 1461.61 & 1470.22 \\
\hline 9/24/11 13:20 & 48.607 & 38.919 & 1460.16 & 1470.20 \\
\hline 9/24/11 13:30 & 47.271 & 38.938 & 1461.50 & 1470.18 \\
\hline 9/24/11 13:40 & 45.521 & 38.961 & 1463.25 & 1470.16 \\
\hline 9/24/11 13:50 & 43.361 & 38.982 & 1465.41 & 1470.14 \\
\hline 9/24/11 14:00 & 45.652 & 39.001 & 1463.12 & 1470.12 \\
\hline 9/24/11 14:10 & 46.574 & 39.022 & 1462.20 & 1470.10 \\
\hline 9/24/11 14:20 & 49.058 & 39.048 & 1459.71 & 1470.07 \\
\hline 9/24/11 14:30 & 47.845 & 39.069 & 1460.92 & 1470.05 \\
\hline 9/24/11 14:40 & 46.529 & 39.097 & 1462.24 & 1470.03 \\
\hline $9 / 24 / 1114: 50$ & 44.869 & 39.121 & 1463.90 & 1470.00 \\
\hline 9/24/11 15:00 & 45.88 & 39.149 & 1462.89 & 1469.97 \\
\hline 9/24/11 15:10 & 48.579 & 39.189 & 1460.19 & 1469.93 \\
\hline 9/24/11 15:20 & 44.205 & 39.231 & 1464.56 & 1469.89 \\
\hline 9/24/11 15:30 & 44.867 & 39.264 & 1463.90 & 1469.86 \\
\hline 9/24/11 15:40 & 47.022 & 39.287 & 1461.75 & 1469.84 \\
\hline 9/24/11 15:50 & 43.45 & 39.311 & 1465.32 & 1469.81 \\
\hline 9/24/11 16:00 & 47.254 & 39.329 & 1461.52 & 1469.79 \\
\hline 9/24/11 16:10 & 42.505 & 39.348 & 1466.26 & 1469.77 \\
\hline 9/24/11 16:20 & 47.24 & 39.367 & 1461.53 & 1469.76 \\
\hline 9/24/11 16:30 & 45.859 & 39.383 & 1462.91 & 1469.74 \\
\hline 9/24/11 16:40 & 46.823 & 39.4 & 1461.95 & 1469.72 \\
\hline $9 / 24 / 1116: 50$ & 43.274 & 39.416 & 1465.50 & 1469.71 \\
\hline 9/24/11 17:00 & 46.37 & 39.433 & 1462.40 & 1469.69 \\
\hline 9/24/11 17:10 & 48.692 & 39.451 & 1460.08 & 1469.67 \\
\hline 9/24/11 17:20 & 40.831 & 39.47 & 1467.94 & 1469.65 \\
\hline 9/24/11 17:30 & 41.614 & 39.477 & 1467.16 & 1469.65 \\
\hline 9/24/11 17:40 & 39.695 & 39.48 & 1469.07 & 1469.64 \\
\hline
\end{tabular}


TABLE S1.3 Automatically recorded groundwater levels in the Klassen wells, September 15, 2011, to March 13, 2012.

\begin{tabular}{|c|c|c|c|c|}
\hline \multicolumn{3}{|c|}{ Reference Elevation (ft AMSL) } & 1508.77 & \multirow{2}{*}{$\begin{array}{l}1509.12 \\
\text { Elevation in Klassen } \\
\text { Test Well (ft AMSL) }\end{array}$} \\
\hline Date and Time & $\begin{array}{l}\text { Depth in Klassen } \\
\text { Domestic Well } \\
(\mathrm{ft} \mathrm{BGL})\end{array}$ & $\begin{array}{c}\text { Depth in Klassen } \\
\text { Test Well } \\
\text { (ft BGL) }\end{array}$ & $\begin{array}{l}\text { Elevation in Klassen } \\
\text { Domestic Well } \\
\text { (ft AMSL) }\end{array}$ & \\
\hline 9/24/11 17:50 & 42.493 & 39.477 & 1466.28 & 1469.65 \\
\hline 9/24/11 18:00 & 44.595 & 39.482 & 1464.17 & 1469.64 \\
\hline 9/24/11 18:10 & 39.367 & 39.484 & 1469.40 & 1469.64 \\
\hline $9 / 24 / 1118: 20$ & 39.072 & 39.482 & 1469.70 & 1469.64 \\
\hline 9/24/11 18:30 & 38.872 & 39.477 & 1469.90 & 1469.65 \\
\hline 9/24/11 18:40 & 38.715 & 39.472 & 1470.05 & 1469.65 \\
\hline $9 / 24 / 1118: 50$ & 40.91 & 39.468 & 1467.86 & 1469.65 \\
\hline 9/24/11 19:00 & 38.668 & 39.468 & 1470.10 & 1469.65 \\
\hline 9/24/11 19:10 & 38.488 & 39.465 & 1470.28 & 1469.66 \\
\hline 9/24/11 19:20 & 38.359 & 39.461 & 1470.41 & 1469.66 \\
\hline 9/24/11 19:30 & 38.251 & 39.456 & 1470.52 & 1469.67 \\
\hline 9/24/11 19:40 & 38.155 & 39.451 & 1470.61 & 1469.67 \\
\hline 9/24/11 19:50 & 38.068 & 39.447 & 1470.70 & 1469.68 \\
\hline 9/24/11 20:00 & 37.988 & 39.442 & 1470.78 & 1469.68 \\
\hline 9/24/11 20:10 & 38.075 & 39.44 & 1470.69 & 1469.68 \\
\hline $9 / 24 / 1120: 20$ & 37.936 & 39.437 & 1470.83 & 1469.69 \\
\hline 9/24/11 20:30 & 37.847 & 39.433 & 1470.92 & 1469.69 \\
\hline 9/24/11 20:40 & 37.775 & 39.428 & 1470.99 & 1469.69 \\
\hline 9/24/11 20:50 & 37.709 & 39.423 & 1471.06 & 1469.70 \\
\hline 9/24/11 21:00 & 37.648 & 39.419 & 1471.12 & 1469.70 \\
\hline 9/24/11 21:10 & 37.589 & 39.414 & 1471.18 & 1469.71 \\
\hline 9/24/11 21:20 & 37.535 & 39.409 & 1471.23 & 1469.71 \\
\hline 9/24/11 21:30 & 37.484 & 39.404 & 1471.29 & 1469.72 \\
\hline 9/24/11 21:40 & 37.435 & 39.4 & 1471.33 & 1469.72 \\
\hline 9/24/11 21:50 & 37.73 & 39.397 & 1471.04 & 1469.73 \\
\hline 9/24/11 22:00 & 37.507 & 39.395 & 1471.26 & 1469.73 \\
\hline 9/24/11 22:10 & 37.42 & 39.39 & 1471.35 & 1469.73 \\
\hline $9 / 24 / 1122: 20$ & 37.36 & 39.386 & 1471.41 & 1469.74 \\
\hline 9/24/11 22:30 & 37.306 & 39.379 & 1471.46 & 1469.74 \\
\hline 9/24/11 22:40 & 37.467 & 39.376 & 1471.30 & 1469.75 \\
\hline 9/24/11 22:50 & 37.524 & 39.374 & 1471.25 & 1469.75 \\
\hline 9/24/11 23:00 & 39.435 & 39.372 & 1469.33 & 1469.75 \\
\hline 9/24/11 23:10 & 37.446 & 39.369 & 1471.32 & 1469.75 \\
\hline 9/24/11 23:20 & 37.324 & 39.365 & 1471.45 & 1469.76 \\
\hline 9/24/11 23:30 & 37.247 & 39.36 & 1471.52 & 1469.76 \\
\hline 9/24/11 23:40 & 37.191 & 39.353 & 1471.58 & 1469.77 \\
\hline 9/24/11 23:50 & 37.172 & 39.348 & 1471.60 & 1469.77 \\
\hline 9/25/11 0:00 & 37.13 & 39.346 & 1471.64 & 1469.78 \\
\hline
\end{tabular}


TABLE S1.3 Automatically recorded groundwater levels in the Klassen wells, September 15, 2011, to March 13, 2012.

\begin{tabular}{|c|c|c|c|c|}
\hline \multicolumn{3}{|c|}{ Reference Elevation (ft AMSL) } & 1508.77 & \multirow{2}{*}{$\begin{array}{l}1509.12 \\
\text { Elevation in Klassen } \\
\text { Test Well (ft AMSL) }\end{array}$} \\
\hline Date and Time & $\begin{array}{l}\text { Depth in Klassen } \\
\text { Domestic Well } \\
(\mathrm{ft} \mathrm{BGL})\end{array}$ & $\begin{array}{c}\text { Depth in Klassen } \\
\text { Test Well } \\
\text { (ft BGL) }\end{array}$ & $\begin{array}{l}\text { Elevation in Klassen } \\
\text { Domestic Well } \\
\text { (ft AMSL) }\end{array}$ & \\
\hline 9/25/11 0:10 & 37.085 & 39.339 & 1471.68 & 1469.78 \\
\hline 9/25/11 0:20 & 37.041 & 39.332 & 1471.73 & 1469.79 \\
\hline $9 / 25 / 110: 30$ & 37.003 & 39.325 & 1471.77 & 1469.80 \\
\hline $9 / 25 / 110: 40$ & 36.968 & 39.32 & 1471.80 & 1469.80 \\
\hline 9/25/11 0:50 & 36.942 & 39.313 & 1471.83 & 1469.81 \\
\hline 9/25/11 1:00 & 36.93 & 39.308 & 1471.84 & 1469.81 \\
\hline 9/25/11 1:10 & 36.916 & 39.304 & 1471.85 & 1469.82 \\
\hline $9 / 25 / 111: 20$ & 36.883 & 39.297 & 1471.89 & 1469.83 \\
\hline 9/25/11 1:30 & 36.848 & 39.292 & 1471.92 & 1469.83 \\
\hline $9 / 25 / 111: 40$ & 36.848 & 39.285 & 1471.92 & 1469.84 \\
\hline $9 / 25 / 11$ 1:50 & 36.881 & 39.282 & 1471.89 & 1469.84 \\
\hline $9 / 25 / 112: 00$ & 36.862 & 39.278 & 1471.91 & 1469.84 \\
\hline $9 / 25 / 112: 10$ & 36.834 & 39.275 & 1471.94 & 1469.85 \\
\hline $9 / 25 / 112: 20$ & 36.804 & 39.271 & 1471.97 & 1469.85 \\
\hline $9 / 25 / 112: 30$ & 37.306 & 39.266 & 1471.46 & 1469.86 \\
\hline $9 / 25 / 112: 40$ & 36.895 & 39.261 & 1471.87 & 1469.86 \\
\hline $9 / 25 / 112: 50$ & 36.825 & 39.259 & 1471.94 & 1469.86 \\
\hline 9/25/11 3:00 & 36.78 & 39.252 & 1471.99 & 1469.87 \\
\hline 9/25/11 3:10 & 36.747 & 39.247 & 1472.02 & 1469.88 \\
\hline $9 / 25 / 113: 20$ & 36.738 & 39.243 & 1472.03 & 1469.88 \\
\hline $9 / 25 / 113: 30$ & 36.764 & 39.24 & 1472.01 & 1469.88 \\
\hline $9 / 25 / 113: 40$ & 36.74 & 39.236 & 1472.03 & 1469.89 \\
\hline 9/25/11 3:50 & 36.7 & 39.231 & 1472.07 & 1469.89 \\
\hline 9/25/11 4:00 & 36.665 & 39.226 & 1472.10 & 1469.90 \\
\hline 9/25/11 4:10 & 36.632 & 39.219 & 1472.14 & 1469.90 \\
\hline $9 / 25 / 114: 20$ & 36.604 & 39.212 & 1472.17 & 1469.91 \\
\hline $9 / 25 / 114: 30$ & 36.579 & 39.207 & 1472.19 & 1469.92 \\
\hline $9 / 25 / 114: 40$ & 36.553 & 39.2 & 1472.22 & 1469.92 \\
\hline $9 / 25 / 114: 50$ & 36.525 & 39.193 & 1472.24 & 1469.93 \\
\hline 9/25/11 5:00 & 36.501 & 39.189 & 1472.27 & 1469.93 \\
\hline 9/25/11 5:10 & 36.478 & 39.184 & 1472.29 & 1469.94 \\
\hline $9 / 25 / 115: 20$ & 36.459 & 39.177 & 1472.31 & 1469.95 \\
\hline $9 / 25 / 115: 30$ & 36.44 & 39.172 & 1472.33 & 1469.95 \\
\hline 9/25/11 5:40 & 36.419 & 39.167 & 1472.35 & 1469.96 \\
\hline 9/25/11 5:50 & 36.398 & 39.163 & 1472.37 & 1469.96 \\
\hline $9 / 25 / 116: 00$ & 36.799 & 39.16 & 1471.97 & 1469.96 \\
\hline 9/25/11 6:10 & 36.686 & 39.16 & 1472.08 & 1469.96 \\
\hline $9 / 25 / 116: 20$ & 41.152 & 39.156 & 1467.62 & 1469.97 \\
\hline
\end{tabular}


TABLE S1.3 Automatically recorded groundwater levels in the Klassen wells, September 15, 2011, to March 13, 2012.

\begin{tabular}{|c|c|c|c|c|}
\hline \multicolumn{3}{|c|}{ Reference Elevation (ft AMSL) } & 1508.77 & \multirow{2}{*}{$\begin{array}{l}1509.12 \\
\text { Elevation in Klassen } \\
\text { Test Well (ft AMSL) }\end{array}$} \\
\hline Date and Time & $\begin{array}{l}\text { Depth in Klassen } \\
\text { Domestic Well } \\
\text { (ft BGL) }\end{array}$ & $\begin{array}{c}\text { Depth in Klassen } \\
\text { Test Well } \\
\text { (ft BGL) }\end{array}$ & $\begin{array}{l}\text { Elevation in Klassen } \\
\text { Domestic Well } \\
\text { (ft AMSL) }\end{array}$ & \\
\hline $9 / 25 / 116: 30$ & 36.708 & 39.158 & 1472.06 & 1469.96 \\
\hline $9 / 25 / 116: 40$ & 36.579 & 39.156 & 1472.19 & 1469.97 \\
\hline $9 / 25 / 116: 50$ & 36.513 & 39.153 & 1472.26 & 1469.97 \\
\hline 9/25/11 7:00 & 36.464 & 39.149 & 1472.31 & 1469.97 \\
\hline $9 / 25 / 117: 10$ & 36.426 & 39.144 & 1472.34 & 1469.98 \\
\hline $9 / 25 / 117: 20$ & 36.414 & 39.142 & 1472.36 & 1469.98 \\
\hline $9 / 25 / 117: 30$ & 36.386 & 39.137 & 1472.38 & 1469.99 \\
\hline $9 / 25 / 117: 40$ & 36.36 & 39.135 & 1472.41 & 1469.99 \\
\hline $9 / 25 / 117: 50$ & 36.337 & 39.13 & 1472.43 & 1469.99 \\
\hline $9 / 25 / 118: 00$ & 36.581 & 39.128 & 1472.19 & 1469.99 \\
\hline 9/25/11 8:10 & 36.403 & 39.125 & 1472.37 & 1470.00 \\
\hline $9 / 25 / 118: 20$ & 37.634 & 39.123 & 1471.14 & 1470.00 \\
\hline $9 / 25 / 118: 30$ & 38.244 & 39.123 & 1470.53 & 1470.00 \\
\hline $9 / 25 / 118: 40$ & 36.511 & 39.123 & 1472.26 & 1470.00 \\
\hline $9 / 25 / 118: 50$ & 36.421 & 39.123 & 1472.35 & 1470.00 \\
\hline 9/25/11 9:00 & 36.37 & 39.121 & 1472.40 & 1470.00 \\
\hline 9/25/11 9:10 & 36.337 & 39.116 & 1472.43 & 1470.01 \\
\hline 9/25/11 9:20 & 36.318 & 39.113 & 1472.45 & 1470.01 \\
\hline 9/25/11 9:30 & 36.297 & 39.111 & 1472.47 & 1470.01 \\
\hline 9/25/11 9:40 & 36.321 & 39.109 & 1472.45 & 1470.01 \\
\hline 9/25/11 9:50 & 36.335 & 39.111 & 1472.43 & 1470.01 \\
\hline $9 / 25 / 11$ 10:00 & 36.323 & 39.111 & 1472.45 & 1470.01 \\
\hline 9/25/11 10:10 & 36.356 & 39.113 & 1472.41 & 1470.01 \\
\hline 9/25/11 10:20 & 36.356 & 39.116 & 1472.41 & 1470.01 \\
\hline $9 / 25 / 11$ 10:30 & 36.379 & 39.123 & 1472.39 & 1470.00 \\
\hline $9 / 25 / 11$ 10:40 & 36.372 & 39.123 & 1472.40 & 1470.00 \\
\hline $9 / 25 / 11$ 10:50 & 36.346 & 39.125 & 1472.42 & 1470.00 \\
\hline 9/25/11 11:00 & 36.314 & 39.125 & 1472.46 & 1470.00 \\
\hline 9/25/11 11:10 & 36.306 & 39.123 & 1472.46 & 1470.00 \\
\hline 9/25/11 11:20 & 36.339 & 39.125 & 1472.43 & 1470.00 \\
\hline 9/25/11 11:30 & 36.335 & 39.125 & 1472.43 & 1470.00 \\
\hline 9/25/11 11:40 & 36.365 & 39.128 & 1472.40 & 1469.99 \\
\hline $9 / 25 / 1111: 50$ & 36.344 & 39.125 & 1472.43 & 1470.00 \\
\hline 9/25/11 12:00 & 36.309 & 39.123 & 1472.46 & 1470.00 \\
\hline $9 / 25 / 11$ 12:10 & 36.281 & 39.121 & 1472.49 & 1470.00 \\
\hline $9 / 25 / 11$ 12:20 & 36.253 & 39.116 & 1472.52 & 1470.01 \\
\hline $9 / 25 / 1112: 30$ & 36.236 & 39.113 & 1472.53 & 1470.01 \\
\hline $9 / 25 / 11$ 12:40 & 36.241 & 39.111 & 1472.53 & 1470.01 \\
\hline
\end{tabular}


TABLE S1.3 Automatically recorded groundwater levels in the Klassen wells, September 15, 2011, to March 13, 2012.

\begin{tabular}{|c|c|c|c|c|}
\hline \multicolumn{3}{|c|}{ Reference Elevation (ft AMSL) } & 1508.77 & \multirow{2}{*}{$\begin{array}{l}1509.12 \\
\text { Elevation in Klassen } \\
\text { Test Well (ft AMSL) }\end{array}$} \\
\hline Date and Time & $\begin{array}{l}\text { Depth in Klassen } \\
\text { Domestic Well } \\
(\mathrm{ft} \mathrm{BGL})\end{array}$ & $\begin{array}{c}\text { Depth in Klassen } \\
\text { Test Well } \\
\text { (ft BGL) }\end{array}$ & $\begin{array}{l}\text { Elevation in Klassen } \\
\text { Domestic Well } \\
\text { (ft AMSL) }\end{array}$ & \\
\hline $9 / 25 / 1112: 50$ & 36.22 & 39.106 & 1472.55 & 1470.02 \\
\hline 9/25/11 13:00 & 36.201 & 39.104 & 1472.57 & 1470.02 \\
\hline 9/25/11 13:10 & 36.18 & 39.099 & 1472.59 & 1470.02 \\
\hline $9 / 25 / 1113: 20$ & 36.156 & 39.097 & 1472.61 & 1470.03 \\
\hline 9/25/11 13:30 & 36.135 & 39.09 & 1472.63 & 1470.03 \\
\hline 9/25/11 13:40 & 36.124 & 39.085 & 1472.65 & 1470.04 \\
\hline $9 / 25 / 1113: 50$ & 36.109 & 39.083 & 1472.66 & 1470.04 \\
\hline 9/25/11 14:00 & 36.088 & 39.076 & 1472.68 & 1470.05 \\
\hline 9/25/11 14:10 & 36.07 & 39.071 & 1472.70 & 1470.05 \\
\hline $9 / 25 / 1114: 20$ & 36.051 & 39.067 & 1472.72 & 1470.06 \\
\hline $9 / 25 / 1114: 30$ & 36.034 & 39.06 & 1472.74 & 1470.06 \\
\hline 9/25/11 14:40 & 36.032 & 39.055 & 1472.74 & 1470.07 \\
\hline 9/25/11 14:50 & 36.02 & 39.052 & 1472.75 & 1470.07 \\
\hline 9/25/11 15:00 & 36.006 & 39.048 & 1472.76 & 1470.07 \\
\hline 9/25/11 15:10 & 35.988 & 39.043 & 1472.78 & 1470.08 \\
\hline $9 / 25 / 11$ 15:20 & 35.971 & 39.038 & 1472.80 & 1470.08 \\
\hline 9/25/11 15:30 & 35.962 & 39.031 & 1472.81 & 1470.09 \\
\hline 9/25/11 15:40 & 35.955 & 39.029 & 1472.81 & 1470.09 \\
\hline 9/25/11 15:50 & 36.956 & 39.024 & 1471.81 & 1470.10 \\
\hline 9/25/11 16:00 & 36.088 & 39.024 & 1472.68 & 1470.10 \\
\hline 9/25/11 16:10 & 36.246 & 39.022 & 1472.52 & 1470.10 \\
\hline 9/25/11 16:20 & 36.182 & 39.022 & 1472.59 & 1470.10 \\
\hline $9 / 25 / 1116: 30$ & 36.246 & 39.029 & 1472.52 & 1470.09 \\
\hline 9/25/11 16:40 & 36.318 & 39.036 & 1472.45 & 1470.09 \\
\hline 9/25/11 16:50 & 36.386 & 39.043 & 1472.38 & 1470.08 \\
\hline 9/25/11 17:00 & 36.7 & 39.052 & 1472.07 & 1470.07 \\
\hline 9/25/11 17:10 & 36.621 & 39.064 & 1472.15 & 1470.06 \\
\hline 9/25/11 17:20 & 36.647 & 39.074 & 1472.12 & 1470.05 \\
\hline 9/25/11 17:30 & 36.691 & 39.081 & 1472.08 & 1470.04 \\
\hline 9/25/11 17:40 & 36.745 & 39.09 & 1472.02 & 1470.03 \\
\hline 9/25/11 17:50 & 36.797 & 39.102 & 1471.97 & 1470.02 \\
\hline 9/25/11 18:00 & 36.846 & 39.111 & 1471.92 & 1470.01 \\
\hline 9/25/11 18:10 & 36.89 & 39.121 & 1471.88 & 1470.00 \\
\hline 9/25/11 18:20 & 36.956 & 39.132 & 1471.81 & 1469.99 \\
\hline 9/25/11 18:30 & 36.998 & 39.144 & 1471.77 & 1469.98 \\
\hline 9/25/11 18:40 & 37.043 & 39.153 & 1471.73 & 1469.97 \\
\hline 9/25/11 18:50 & 37.094 & 39.165 & 1471.68 & 1469.96 \\
\hline 9/25/11 19:00 & 37.146 & 39.177 & 1471.62 & 1469.95 \\
\hline
\end{tabular}


TABLE S1.3 Automatically recorded groundwater levels in the Klassen wells, September 15, 2011, to March 13, 2012.

\begin{tabular}{|c|c|c|c|c|}
\hline \multicolumn{3}{|c|}{ Reference Elevation (ft AMSL) } & 1508.77 & \multirow{2}{*}{$\begin{array}{l}1509.12 \\
\text { Elevation in Klassen } \\
\text { Test Well (ft AMSL) }\end{array}$} \\
\hline Date and Time & $\begin{array}{l}\text { Depth in Klassen } \\
\text { Domestic Well } \\
\text { (ft BGL) }\end{array}$ & $\begin{array}{c}\text { Depth in Klassen } \\
\text { Test Well } \\
\text { (ft BGL) }\end{array}$ & $\begin{array}{l}\text { Elevation in Klassen } \\
\text { Domestic Well } \\
\text { (ft AMSL) }\end{array}$ & \\
\hline 9/25/11 19:10 & 37.573 & 39.191 & 1471.20 & 1469.93 \\
\hline 9/25/11 19:20 & 40.868 & 39.205 & 1467.90 & 1469.92 \\
\hline 9/25/11 19:30 & 44.384 & 39.228 & 1464.39 & 1469.89 \\
\hline 9/25/11 19:40 & 38.354 & 39.257 & 1470.42 & 1469.87 \\
\hline 9/25/11 19:50 & 38.068 & 39.273 & 1470.70 & 1469.85 \\
\hline 9/25/11 20:00 & 37.965 & 39.289 & 1470.80 & 1469.83 \\
\hline 9/25/11 20:10 & 37.911 & 39.301 & 1470.86 & 1469.82 \\
\hline 9/25/11 20:20 & 41.323 & 39.315 & 1467.45 & 1469.81 \\
\hline 9/25/11 20:30 & 38.009 & 39.329 & 1470.76 & 1469.79 \\
\hline 9/25/11 20:40 & 37.85 & 39.334 & 1470.92 & 1469.79 \\
\hline $9 / 25 / 1120: 50$ & 37.749 & 39.336 & 1471.02 & 1469.79 \\
\hline 9/25/11 21:00 & 37.65 & 39.339 & 1471.12 & 1469.78 \\
\hline 9/25/11 21:10 & 37.535 & 39.336 & 1471.23 & 1469.79 \\
\hline 9/25/11 21:20 & 37.43 & 39.332 & 1471.34 & 1469.79 \\
\hline $9 / 25 / 1121: 30$ & 37.36 & 39.329 & 1471.41 & 1469.79 \\
\hline 9/25/11 21:40 & 37.315 & 39.327 & 1471.45 & 1469.80 \\
\hline $9 / 25 / 1121: 50$ & 37.24 & 39.322 & 1471.53 & 1469.80 \\
\hline 9/25/11 22:00 & 37.162 & 39.318 & 1471.61 & 1469.80 \\
\hline 9/25/11 22:10 & 37.118 & 39.313 & 1471.65 & 1469.81 \\
\hline $9 / 25 / 1122: 20$ & 37.055 & 39.311 & 1471.71 & 1469.81 \\
\hline $9 / 25 / 1122: 30$ & 37.925 & 39.306 & 1470.84 & 1469.82 \\
\hline $9 / 25 / 1122: 40$ & 37.315 & 39.304 & 1471.45 & 1469.82 \\
\hline $9 / 25 / 1122: 50$ & 37.247 & 39.304 & 1471.52 & 1469.82 \\
\hline 9/25/11 23:00 & 37.059 & 39.299 & 1471.71 & 1469.82 \\
\hline 9/25/11 23:10 & 36.961 & 39.294 & 1471.81 & 1469.83 \\
\hline 9/25/11 23:20 & 36.886 & 39.289 & 1471.88 & 1469.83 \\
\hline 9/25/11 23:30 & 36.822 & 39.285 & 1471.95 & 1469.84 \\
\hline $9 / 25 / 11$ 23:40 & 36.759 & 39.278 & 1472.01 & 1469.84 \\
\hline $9 / 25 / 1123: 50$ & 36.703 & 39.271 & 1472.07 & 1469.85 \\
\hline 9/26/11 0:00 & 36.654 & 39.264 & 1472.12 & 1469.86 \\
\hline 9/26/11 0:10 & 36.607 & 39.257 & 1472.16 & 1469.87 \\
\hline 9/26/11 0:20 & 36.564 & 39.25 & 1472.21 & 1469.87 \\
\hline $9 / 26 / 110: 30$ & 36.522 & 39.243 & 1472.25 & 1469.88 \\
\hline $9 / 26 / 110: 40$ & 36.482 & 39.236 & 1472.29 & 1469.89 \\
\hline 9/26/11 0:50 & 38.023 & 39.228 & 1470.75 & 1469.89 \\
\hline 9/26/11 1:00 & 36.56 & 39.224 & 1472.21 & 1469.90 \\
\hline 9/26/11 1:10 & 36.466 & 39.219 & 1472.30 & 1469.90 \\
\hline $9 / 26 / 11$ 1:20 & 36.407 & 39.21 & 1472.36 & 1469.91 \\
\hline
\end{tabular}


TABLE S1.3 Automatically recorded groundwater levels in the Klassen wells, September 15, 2011, to March 13, 2012.

\begin{tabular}{|c|c|c|c|c|}
\hline \multicolumn{3}{|c|}{ Reference Elevation (ft AMSL) } & 1508.77 & \multirow{2}{*}{$\begin{array}{l}1509.12 \\
\text { Elevation in Klassen } \\
\text { Test Well (ft AMSL) }\end{array}$} \\
\hline Date and Time & $\begin{array}{l}\text { Depth in Klassen } \\
\text { Domestic Well } \\
(\mathrm{ft} \mathrm{BGL})\end{array}$ & $\begin{array}{c}\text { Depth in Klassen } \\
\text { Test Well } \\
\text { (ft BGL) }\end{array}$ & $\begin{array}{l}\text { Elevation in Klassen } \\
\text { Domestic Well } \\
\text { (ft AMSL) }\end{array}$ & \\
\hline $9 / 26 / 11$ 1:30 & 36.36 & 39.203 & 1472.41 & 1469.92 \\
\hline 9/26/11 1:40 & 36.318 & 39.196 & 1472.45 & 1469.93 \\
\hline $9 / 26 / 11$ 1:50 & 36.281 & 39.189 & 1472.49 & 1469.93 \\
\hline $9 / 26 / 112: 00$ & 36.243 & 39.182 & 1472.53 & 1469.94 \\
\hline 9/26/11 2:10 & 36.213 & 39.174 & 1472.56 & 1469.95 \\
\hline $9 / 26 / 112: 20$ & 36.182 & 39.167 & 1472.59 & 1469.96 \\
\hline $9 / 26 / 112: 30$ & 36.154 & 39.158 & 1472.62 & 1469.96 \\
\hline $9 / 26 / 112: 40$ & 36.128 & 39.151 & 1472.64 & 1469.97 \\
\hline $9 / 26 / 112: 50$ & 36.105 & 39.144 & 1472.66 & 1469.98 \\
\hline 9/26/11 3:00 & 36.079 & 39.137 & 1472.69 & 1469.99 \\
\hline 9/26/11 3:10 & 36.051 & 39.13 & 1472.72 & 1469.99 \\
\hline $9 / 26 / 113: 20$ & 36.025 & 39.121 & 1472.74 & 1470.00 \\
\hline $9 / 26 / 113: 30$ & 36.002 & 39.113 & 1472.77 & 1470.01 \\
\hline $9 / 26 / 113: 40$ & 35.978 & 39.106 & 1472.79 & 1470.02 \\
\hline 9/26/11 3:50 & 35.952 & 39.097 & 1472.82 & 1470.03 \\
\hline 9/26/11 4:00 & 35.929 & 39.092 & 1472.84 & 1470.03 \\
\hline 9/26/11 4:10 & 35.908 & 39.085 & 1472.86 & 1470.04 \\
\hline 9/26/11 4:20 & 35.884 & 39.078 & 1472.89 & 1470.04 \\
\hline $9 / 26 / 114: 30$ & 35.887 & 39.074 & 1472.88 & 1470.05 \\
\hline $9 / 26 / 114: 40$ & 37.231 & 39.069 & 1471.54 & 1470.05 \\
\hline $9 / 26 / 114: 50$ & 35.99 & 39.062 & 1472.78 & 1470.06 \\
\hline 9/26/11 5:00 & 35.919 & 39.057 & 1472.85 & 1470.07 \\
\hline 9/26/11 5:10 & 35.877 & 39.05 & 1472.89 & 1470.07 \\
\hline 9/26/11 5:20 & 35.844 & 39.045 & 1472.93 & 1470.08 \\
\hline 9/26/11 5:30 & 35.816 & 39.038 & 1472.95 & 1470.08 \\
\hline 9/26/11 5:40 & 35.791 & 39.034 & 1472.98 & 1470.09 \\
\hline $9 / 26 / 115: 50$ & 35.767 & 39.027 & 1473.00 & 1470.10 \\
\hline 9/26/11 6:00 & 35.748 & 39.017 & 1473.02 & 1470.11 \\
\hline 9/26/11 6:10 & 40.087 & 39.015 & 1468.68 & 1470.11 \\
\hline $9 / 26 / 116: 20$ & 35.92 & 39.013 & 1472.85 & 1470.11 \\
\hline 9/26/11 6:30 & 35.826 & 39.008 & 1472.94 & 1470.11 \\
\hline $9 / 26 / 116: 40$ & 35.79 & 39.003 & 1472.98 & 1470.12 \\
\hline $9 / 26 / 116: 50$ & 35.774 & 39.001 & 1473.00 & 1470.12 \\
\hline 9/26/11 7:00 & 35.746 & 38.996 & 1473.02 & 1470.13 \\
\hline 9/26/11 7:10 & 35.718 & 38.991 & 1473.05 & 1470.13 \\
\hline $9 / 26 / 117: 20$ & 35.697 & 38.987 & 1473.07 & 1470.14 \\
\hline $9 / 26 / 117: 30$ & 37.174 & 38.987 & 1471.60 & 1470.14 \\
\hline $9 / 26 / 117: 40$ & 41.285 & 38.994 & 1467.48 & 1470.13 \\
\hline
\end{tabular}


TABLE S1.3 Automatically recorded groundwater levels in the Klassen wells, September 15, 2011, to March 13, 2012.

\begin{tabular}{|c|c|c|c|c|}
\hline \multicolumn{3}{|c|}{ Reference Elevation (ft AMSL) } & 1508.77 & \multirow{2}{*}{$\begin{array}{l}1509.12 \\
\text { Elevation in Klassen } \\
\text { Test Well (ft AMSL) }\end{array}$} \\
\hline Date and Time & $\begin{array}{l}\text { Depth in Klassen } \\
\text { Domestic Well } \\
\text { (ft BGL) }\end{array}$ & $\begin{array}{c}\text { Depth in Klassen } \\
\text { Test Well } \\
\text { (ft BGL) }\end{array}$ & $\begin{array}{l}\text { Elevation in Klassen } \\
\text { Domestic Well } \\
\text { (ft AMSL) }\end{array}$ & \\
\hline 9/26/11 7:50 & 38.499 & 39.003 & 1470.27 & 1470.12 \\
\hline $9 / 26 / 118: 00$ & 42.993 & 39.013 & 1465.78 & 1470.11 \\
\hline 9/26/11 8:10 & 40.558 & 39.027 & 1468.21 & 1470.10 \\
\hline $9 / 26 / 118: 20$ & 46.548 & 39.041 & 1462.22 & 1470.08 \\
\hline $9 / 26 / 118: 30$ & 41.173 & 39.057 & 1467.60 & 1470.07 \\
\hline $9 / 26 / 118: 40$ & 43.272 & 39.071 & 1465.50 & 1470.05 \\
\hline $9 / 26 / 118: 50$ & 41.412 & 39.085 & 1467.36 & 1470.04 \\
\hline 9/26/11 9:00 & 41.138 & 39.099 & 1467.63 & 1470.02 \\
\hline 9/26/11 9:10 & 42.808 & 39.113 & 1465.96 & 1470.01 \\
\hline 9/26/11 9:20 & 41.71 & 39.125 & 1467.06 & 1470.00 \\
\hline 9/26/11 9:30 & 43.354 & 39.139 & 1465.42 & 1469.98 \\
\hline 9/26/11 9:40 & 40.924 & 39.149 & 1467.85 & 1469.97 \\
\hline 9/26/11 9:50 & 39.442 & 39.158 & 1469.33 & 1469.96 \\
\hline 9/26/11 10:00 & 43.699 & 39.17 & 1465.07 & 1469.95 \\
\hline 9/26/11 10:10 & 40.181 & 39.179 & 1468.59 & 1469.94 \\
\hline $9 / 26 / 11$ 10:20 & 42.146 & 39.189 & 1466.62 & 1469.93 \\
\hline 9/26/11 10:30 & 42.536 & 39.2 & 1466.23 & 1469.92 \\
\hline 9/26/11 10:40 & 40.627 & 39.21 & 1468.14 & 1469.91 \\
\hline 9/26/11 10:50 & 44.892 & 39.217 & 1463.88 & 1469.91 \\
\hline 9/26/11 11:00 & 41.562 & 39.226 & 1467.21 & 1469.90 \\
\hline 9/26/11 11:10 & 41.63 & 39.236 & 1467.14 & 1469.89 \\
\hline 9/26/11 11:20 & 44.642 & 39.247 & 1464.13 & 1469.88 \\
\hline 9/26/11 11:30 & 43.035 & 39.257 & 1465.73 & 1469.87 \\
\hline 9/26/11 11:40 & 41.086 & 39.266 & 1467.68 & 1469.86 \\
\hline 9/26/11 11:50 & 45.015 & 39.273 & 1463.75 & 1469.85 \\
\hline 9/26/11 12:00 & 42.329 & 39.282 & 1466.44 & 1469.84 \\
\hline 9/26/11 12:10 & 39.93 & 39.292 & 1468.84 & 1469.83 \\
\hline 9/26/11 12:20 & 43.734 & 39.301 & 1465.04 & 1469.82 \\
\hline 9/26/11 12:30 & 42.862 & 39.308 & 1465.91 & 1469.81 \\
\hline 9/26/11 12:40 & 40.845 & 39.315 & 1467.92 & 1469.81 \\
\hline 9/26/11 12:50 & 39.747 & 39.32 & 1469.02 & 1469.80 \\
\hline 9/26/11 13:00 & 42.299 & 39.327 & 1466.47 & 1469.80 \\
\hline 9/26/11 13:10 & 40.514 & 39.334 & 1468.26 & 1469.79 \\
\hline 9/26/11 13:20 & 40.917 & 39.341 & 1467.85 & 1469.78 \\
\hline 9/26/11 13:30 & 44.747 & 39.348 & 1464.02 & 1469.77 \\
\hline 9/26/11 13:40 & 41.34 & 39.353 & 1467.43 & 1469.77 \\
\hline 9/26/11 13:50 & 41.372 & 39.36 & 1467.40 & 1469.76 \\
\hline 9/26/11 14:00 & 44.794 & 39.367 & 1463.98 & 1469.76 \\
\hline
\end{tabular}


TABLE S1.3 Automatically recorded groundwater levels in the Klassen wells, September 15, 2011, to March 13, 2012.

\begin{tabular}{|c|c|c|c|c|}
\hline \multicolumn{3}{|c|}{ Reference Elevation (ft AMSL) } & 1508.77 & \multirow{2}{*}{$\begin{array}{l}1509.12 \\
\text { Elevation in Klassen } \\
\text { Test Well (ft AMSL) }\end{array}$} \\
\hline Date and Time & $\begin{array}{l}\text { Depth in Klassen } \\
\text { Domestic Well } \\
\text { (ft BGL) }\end{array}$ & $\begin{array}{c}\text { Depth in Klassen } \\
\text { Test Well } \\
\text { (ft BGL) }\end{array}$ & $\begin{array}{l}\text { Elevation in Klassen } \\
\text { Domestic Well } \\
\text { (ft AMSL) }\end{array}$ & \\
\hline 9/26/11 14:10 & 41.032 & 39.374 & 1467.74 & 1469.75 \\
\hline 9/26/11 14:20 & 40.094 & 39.381 & 1468.68 & 1469.74 \\
\hline 9/26/11 14:30 & 45.148 & 39.388 & 1463.62 & 1469.73 \\
\hline 9/26/11 14:40 & 42.038 & 39.395 & 1466.73 & 1469.73 \\
\hline 9/26/11 14:50 & 41.009 & 39.4 & 1467.76 & 1469.72 \\
\hline 9/26/11 15:00 & 47.259 & 39.407 & 1461.51 & 1469.72 \\
\hline 9/26/11 15:10 & 43.408 & 39.416 & 1465.36 & 1469.71 \\
\hline 9/26/11 15:20 & 40.727 & 39.428 & 1468.04 & 1469.69 \\
\hline 9/26/11 15:30 & 45.169 & 39.435 & 1463.60 & 1469.69 \\
\hline 9/26/11 15:40 & 41.311 & 39.444 & 1467.46 & 1469.68 \\
\hline $9 / 26 / 11$ 15:50 & 40.27 & 39.451 & 1468.50 & 1469.67 \\
\hline 9/26/11 16:00 & 42.439 & 39.458 & 1466.33 & 1469.66 \\
\hline 9/26/11 16:10 & 40.53 & 39.465 & 1468.24 & 1469.66 \\
\hline 9/26/11 16:20 & 43.849 & 39.472 & 1464.92 & 1469.65 \\
\hline $9 / 26 / 11$ 16:30 & 44.189 & 39.48 & 1464.58 & 1469.64 \\
\hline 9/26/11 16:40 & 42.212 & 39.487 & 1466.56 & 1469.64 \\
\hline $9 / 26 / 11$ 16:50 & 40.331 & 39.491 & 1468.44 & 1469.63 \\
\hline 9/26/11 17:00 & 41.185 & 39.501 & 1467.58 & 1469.62 \\
\hline 9/26/11 17:10 & 45.814 & 39.505 & 1462.96 & 1469.62 \\
\hline 9/26/11 17:20 & 42.948 & 39.524 & 1465.82 & 1469.60 \\
\hline 9/26/11 17:30 & 41.408 & 39.545 & 1467.36 & 1469.58 \\
\hline 9/26/11 17:40 & 41.06 & 39.569 & 1467.71 & 1469.55 \\
\hline $9 / 26 / 11$ 17:50 & 46.229 & 39.594 & 1462.54 & 1469.53 \\
\hline 9/26/11 18:00 & 43.64 & 39.62 & 1465.13 & 1469.50 \\
\hline 9/26/11 18:10 & 44.522 & 39.644 & 1464.25 & 1469.48 \\
\hline 9/26/11 18:20 & 41.902 & 39.67 & 1466.87 & 1469.45 \\
\hline $9 / 26 / 11$ 18:30 & 43.706 & 39.698 & 1465.06 & 1469.42 \\
\hline $9 / 26 / 11$ 18:40 & 45.624 & 39.726 & 1463.15 & 1469.40 \\
\hline $9 / 26 / 11$ 18:50 & 43.27 & 39.749 & 1465.50 & 1469.37 \\
\hline 9/26/11 19:00 & 42.022 & 39.775 & 1466.75 & 1469.35 \\
\hline 9/26/11 19:10 & 46.171 & 39.801 & 1462.60 & 1469.32 \\
\hline 9/26/11 19:20 & 43.403 & 39.829 & 1465.37 & 1469.29 \\
\hline 9/26/11 19:30 & 42.242 & 39.857 & 1466.53 & 1469.27 \\
\hline 9/26/11 19:40 & 45.908 & 39.883 & 1462.86 & 1469.24 \\
\hline 9/26/11 19:50 & 47.78 & 39.907 & 1460.99 & 1469.22 \\
\hline 9/26/11 20:00 & 41.002 & 39.928 & 1467.77 & 1469.19 \\
\hline $9 / 26 / 1120: 10$ & 42.261 & 39.942 & 1466.51 & 1469.18 \\
\hline $9 / 26 / 1120: 20$ & 40.577 & 39.958 & 1468.19 & 1469.16 \\
\hline
\end{tabular}


TABLE S1.3 Automatically recorded groundwater levels in the Klassen wells, September 15, 2011, to March 13, 2012.

\begin{tabular}{|c|c|c|c|c|}
\hline \multicolumn{3}{|c|}{ Reference Elevation (ft AMSL) } & 1508.77 & \multirow{2}{*}{$\begin{array}{l}1509.12 \\
\text { Elevation in Klassen } \\
\text { Test Well (ft AMSL) }\end{array}$} \\
\hline Date and Time & $\begin{array}{l}\text { Depth in Klassen } \\
\text { Domestic Well } \\
(\mathrm{ft} \mathrm{BGL})\end{array}$ & $\begin{array}{c}\text { Depth in Klassen } \\
\text { Test Well } \\
\text { (ft BGL) }\end{array}$ & $\begin{array}{l}\text { Elevation in Klassen } \\
\text { Domestic Well } \\
\text { (ft AMSL) }\end{array}$ & \\
\hline $9 / 26 / 1120: 30$ & 40.57 & 39.97 & 1468.20 & 1469.15 \\
\hline $9 / 26 / 1120: 40$ & 40.842 & 39.984 & 1467.93 & 1469.14 \\
\hline $9 / 26 / 1120: 50$ & 41.778 & 39.998 & 1466.99 & 1469.12 \\
\hline 9/26/11 21:00 & 40.479 & 40.014 & 1468.29 & 1469.11 \\
\hline 9/26/11 21:10 & 40.575 & 40.029 & 1468.19 & 1469.09 \\
\hline 9/26/11 21:20 & 41.846 & 40.043 & 1466.92 & 1469.08 \\
\hline $9 / 26 / 1121: 30$ & 40.31 & 40.05 & 1468.46 & 1469.07 \\
\hline 9/26/11 21:40 & 40.293 & 40.05 & 1468.48 & 1469.07 \\
\hline $9 / 26 / 1121: 50$ & 40.793 & 40.047 & 1467.98 & 1469.08 \\
\hline $9 / 26 / 1122: 00$ & 39.918 & 40.043 & 1468.85 & 1469.08 \\
\hline 9/26/11 22:10 & 39.953 & 40.043 & 1468.82 & 1469.08 \\
\hline $9 / 26 / 1122: 20$ & 42.636 & 40.04 & 1466.13 & 1469.08 \\
\hline 9/26/11 22:30 & 43.183 & 40.04 & 1465.59 & 1469.08 \\
\hline $9 / 26 / 1122: 40$ & 42.885 & 40.043 & 1465.88 & 1469.08 \\
\hline 9/26/11 22:50 & 40.622 & 40.038 & 1468.15 & 1469.08 \\
\hline 9/26/11 23:00 & 42.083 & 40.036 & 1466.69 & 1469.09 \\
\hline 9/26/11 23:10 & 39.599 & 40.031 & 1469.17 & 1469.09 \\
\hline 9/26/11 23:20 & 39.55 & 40.024 & 1469.22 & 1469.10 \\
\hline 9/26/11 23:30 & 40.075 & 40.014 & 1468.69 & 1469.11 \\
\hline $9 / 26 / 11$ 23:40 & 39.205 & 40.007 & 1469.56 & 1469.12 \\
\hline $9 / 26 / 1123: 50$ & 39.189 & 39.998 & 1469.58 & 1469.12 \\
\hline 9/27/11 0:00 & 39.33 & 39.989 & 1469.44 & 1469.13 \\
\hline 9/27/11 0:10 & 43.054 & 39.982 & 1465.72 & 1469.14 \\
\hline 9/27/11 0:20 & 38.917 & 39.972 & 1469.85 & 1469.15 \\
\hline 9/27/11 0:30 & 38.966 & 39.963 & 1469.80 & 1469.16 \\
\hline 9/27/11 0:40 & 40.132 & 39.953 & 1468.64 & 1469.17 \\
\hline 9/27/11 0:50 & 42.292 & 39.944 & 1466.48 & 1469.18 \\
\hline 9/27/11 1:00 & 38.703 & 39.937 & 1470.07 & 1469.19 \\
\hline 9/27/11 1:10 & 38.743 & 39.928 & 1470.03 & 1469.19 \\
\hline $9 / 27 / 11$ 1:20 & 39.557 & 39.918 & 1469.21 & 1469.20 \\
\hline 9/27/11 1:30 & 38.483 & 39.909 & 1470.29 & 1469.21 \\
\hline 9/27/11 1:40 & 38.506 & 39.9 & 1470.26 & 1469.22 \\
\hline 9/27/11 1:50 & 38.701 & 39.89 & 1470.07 & 1469.23 \\
\hline 9/27/11 2:00 & 38.565 & 39.883 & 1470.20 & 1469.24 \\
\hline 9/27/11 2:10 & 39.876 & 39.874 & 1468.89 & 1469.25 \\
\hline $9 / 27 / 112: 20$ & 38.284 & 39.864 & 1470.49 & 1469.26 \\
\hline $9 / 27 / 112: 30$ & 38.326 & 39.857 & 1470.44 & 1469.27 \\
\hline $9 / 27 / 112: 40$ & 38.682 & 39.848 & 1470.09 & 1469.27 \\
\hline
\end{tabular}


TABLE S1.3 Automatically recorded groundwater levels in the Klassen wells, September 15, 2011, to March 13, 2012.

\begin{tabular}{|c|c|c|c|c|}
\hline \multicolumn{3}{|c|}{ Reference Elevation (ft AMSL) } & 1508.77 & \multirow{2}{*}{$\begin{array}{l}1509.12 \\
\text { Elevation in Klassen } \\
\text { Test Well (ft AMSL) }\end{array}$} \\
\hline Date and Time & $\begin{array}{l}\text { Depth in Klassen } \\
\text { Domestic Well } \\
\text { (ft BGL) }\end{array}$ & $\begin{array}{c}\text { Depth in Klassen } \\
\text { Test Well } \\
\text { (ft BGL) }\end{array}$ & $\begin{array}{l}\text { Elevation in Klassen } \\
\text { Domestic Well } \\
\text { (ft AMSL) }\end{array}$ & \\
\hline $9 / 27 / 112: 50$ & 40.612 & 39.838 & 1468.16 & 1469.28 \\
\hline 9/27/11 3:00 & 38.162 & 39.829 & 1470.61 & 1469.29 \\
\hline 9/27/11 3:10 & 38.276 & 39.82 & 1470.49 & 1469.30 \\
\hline 9/27/11 3:20 & 38.199 & 39.81 & 1470.57 & 1469.31 \\
\hline 9/27/11 3:30 & 38.671 & 39.801 & 1470.10 & 1469.32 \\
\hline 9/27/11 3:40 & 42.484 & 39.792 & 1466.29 & 1469.33 \\
\hline $9 / 27 / 113: 50$ & 38.03 & 39.785 & 1470.74 & 1469.34 \\
\hline $9 / 27 / 114: 00$ & 38.098 & 39.775 & 1470.67 & 1469.35 \\
\hline 9/27/11 4:10 & 39.104 & 39.768 & 1469.67 & 1469.35 \\
\hline $9 / 27 / 114: 20$ & 37.878 & 39.759 & 1470.89 & 1469.36 \\
\hline $9 / 27 / 114: 30$ & 37.946 & 39.749 & 1470.82 & 1469.37 \\
\hline $9 / 27 / 114: 40$ & 38.178 & 39.742 & 1470.59 & 1469.38 \\
\hline $9 / 27 / 114: 50$ & 42.292 & 39.733 & 1466.48 & 1469.39 \\
\hline 9/27/11 5:00 & 37.81 & 39.726 & 1470.96 & 1469.40 \\
\hline 9/27/11 5:10 & 37.899 & 39.716 & 1470.87 & 1469.41 \\
\hline 9/27/11 5:20 & 38.919 & 39.707 & 1469.85 & 1469.42 \\
\hline 9/27/11 5:30 & 37.69 & 39.7 & 1471.08 & 1469.42 \\
\hline 9/27/11 5:40 & 37.737 & 39.693 & 1471.03 & 1469.43 \\
\hline 9/27/11 5:50 & 37.955 & 39.684 & 1470.81 & 1469.44 \\
\hline 9/27/11 6:00 & 37.789 & 39.677 & 1470.98 & 1469.45 \\
\hline 9/27/11 6:10 & 38.084 & 39.67 & 1470.69 & 1469.45 \\
\hline $9 / 27 / 116: 20$ & 37.775 & 39.665 & 1470.99 & 1469.46 \\
\hline 9/27/11 6:30 & 37.779 & 39.66 & 1470.99 & 1469.46 \\
\hline $9 / 27 / 116: 40$ & 38.401 & 39.653 & 1470.37 & 1469.47 \\
\hline $9 / 27 / 116: 50$ & 39.721 & 39.648 & 1469.05 & 1469.47 \\
\hline 9/27/11 7:00 & 37.643 & 39.644 & 1471.13 & 1469.48 \\
\hline 9/27/11 7:10 & 37.686 & 39.637 & 1471.08 & 1469.49 \\
\hline 9/27/11 7:20 & 38.171 & 39.63 & 1470.60 & 1469.49 \\
\hline 9/27/11 7:30 & 42.35 & 39.627 & 1466.42 & 1469.50 \\
\hline 9/27/11 7:40 & 37.549 & 39.62 & 1471.22 & 1469.50 \\
\hline 9/27/11 7:50 & 37.646 & 39.616 & 1471.12 & 1469.51 \\
\hline 9/27/11 8:00 & 39.332 & 39.611 & 1469.44 & 1469.51 \\
\hline 9/27/11 8:10 & 38.422 & 39.609 & 1470.35 & 1469.51 \\
\hline 9/27/11 8:20 & 39.414 & 39.602 & 1469.36 & 1469.52 \\
\hline 9/27/11 8:30 & 37.456 & 39.597 & 1471.31 & 1469.53 \\
\hline 9/27/11 8:40 & 37.606 & 39.592 & 1471.16 & 1469.53 \\
\hline 9/27/11 8:50 & 37.364 & 39.585 & 1471.41 & 1469.54 \\
\hline 9/27/11 9:00 & 38.159 & 39.58 & 1470.61 & 1469.54 \\
\hline
\end{tabular}


TABLE S1.3 Automatically recorded groundwater levels in the Klassen wells, September 15, 2011, to March 13, 2012.

\begin{tabular}{|c|c|c|c|c|}
\hline \multicolumn{3}{|c|}{ Reference Elevation (ft AMSL) } & 1508.77 & \multirow{2}{*}{$\begin{array}{l}1509.12 \\
\text { Elevation in Klassen } \\
\text { Test Well (ft AMSL) }\end{array}$} \\
\hline Date and Time & $\begin{array}{l}\text { Depth in Klassen } \\
\text { Domestic Well } \\
\text { (ft BGL) }\end{array}$ & $\begin{array}{c}\text { Depth in Klassen } \\
\text { Test Well } \\
\text { (ft BGL) }\end{array}$ & $\begin{array}{l}\text { Elevation in Klassen } \\
\text { Domestic Well } \\
\text { (ft AMSL) }\end{array}$ & \\
\hline 9/27/11 9:10 & 37.294 & 39.576 & 1471.48 & 1469.55 \\
\hline 9/27/11 9:20 & 37.186 & 39.566 & 1471.58 & 1469.56 \\
\hline 9/27/11 9:30 & 38.467 & 39.564 & 1470.30 & 1469.56 \\
\hline 9/27/11 9:40 & 37.526 & 39.562 & 1471.24 & 1469.56 \\
\hline 9/27/11 9:50 & 37.278 & 39.557 & 1471.49 & 1469.57 \\
\hline 9/27/11 10:00 & 37.16 & 39.55 & 1471.61 & 1469.57 \\
\hline 9/27/11 10:10 & 37.078 & 39.543 & 1471.69 & 1469.58 \\
\hline $9 / 27 / 11$ 10:20 & 42.454 & 39.541 & 1466.32 & 1469.58 \\
\hline 9/27/11 10:30 & 41.881 & 39.541 & 1466.89 & 1469.58 \\
\hline $9 / 27 / 1110: 40$ & 40.057 & 39.545 & 1468.71 & 1469.58 \\
\hline $9 / 27 / 1110: 50$ & 46.229 & 39.55 & 1462.54 & 1469.57 \\
\hline 9/27/11 11:00 & 46.63 & 39.559 & 1462.14 & 1469.56 \\
\hline 9/27/11 11:10 & 50.268 & 39.573 & 1458.50 & 1469.55 \\
\hline 9/27/11 11:20 & 51.609 & 39.592 & 1457.16 & 1469.53 \\
\hline 9/27/11 11:30 & 47.662 & 39.613 & 1461.11 & 1469.51 \\
\hline 9/27/11 11:40 & 45.788 & 39.634 & 1462.98 & 1469.49 \\
\hline 9/27/11 11:50 & 43.76 & 39.651 & 1465.01 & 1469.47 \\
\hline 9/27/11 12:00 & 44.935 & 39.665 & 1463.83 & 1469.46 \\
\hline 9/27/11 12:10 & 41.827 & 39.674 & 1466.94 & 1469.45 \\
\hline 9/27/11 12:20 & 40.643 & 39.684 & 1468.13 & 1469.44 \\
\hline $9 / 27 / 11$ 12:30 & 44.764 & 39.688 & 1464.01 & 1469.43 \\
\hline 9/27/11 12:40 & 46.03 & 39.695 & 1462.74 & 1469.43 \\
\hline 9/27/11 12:50 & 43.453 & 39.7 & 1465.32 & 1469.42 \\
\hline 9/27/11 13:00 & 41.161 & 39.705 & 1467.61 & 1469.42 \\
\hline 9/27/11 13:10 & 39.489 & 39.707 & 1469.28 & 1469.42 \\
\hline 9/27/11 13:20 & 38.814 & 39.705 & 1469.96 & 1469.42 \\
\hline 9/27/11 13:30 & 38.537 & 39.698 & 1470.23 & 1469.42 \\
\hline 9/27/11 13:40 & 38.335 & 39.691 & 1470.43 & 1469.43 \\
\hline 9/27/11 13:50 & 38.178 & 39.681 & 1470.59 & 1469.44 \\
\hline 9/27/11 14:00 & 40.936 & 39.672 & 1467.83 & 1469.45 \\
\hline 9/27/11 14:10 & 38.07 & 39.667 & 1470.70 & 1469.46 \\
\hline 9/27/11 14:20 & 37.911 & 39.66 & 1470.86 & 1469.46 \\
\hline 9/27/11 14:30 & 38.532 & 39.651 & 1470.24 & 1469.47 \\
\hline 9/27/11 14:40 & 39.423 & 39.648 & 1469.35 & 1469.47 \\
\hline 9/27/11 14:50 & 44.041 & 39.651 & 1464.73 & 1469.47 \\
\hline 9/27/11 15:00 & 41.279 & 39.653 & 1467.49 & 1469.47 \\
\hline 9/27/11 15:10 & 57.67 & 39.663 & 1451.10 & 1469.46 \\
\hline 9/27/11 15:20 & 60.929 & 39.686 & 1447.84 & 1469.44 \\
\hline
\end{tabular}


TABLE S1.3 Automatically recorded groundwater levels in the Klassen wells, September 15, 2011, to March 13, 2012.

\begin{tabular}{|c|c|c|c|c|}
\hline \multicolumn{3}{|c|}{ Reference Elevation (ft AMSL) } & 1508.77 & \multirow{2}{*}{$\begin{array}{l}1509.12 \\
\text { Elevation in Klassen } \\
\text { Test Well (ft AMSL) }\end{array}$} \\
\hline Date and Time & $\begin{array}{l}\text { Depth in Klassen } \\
\text { Domestic Well } \\
(\mathrm{ft} \mathrm{BGL})\end{array}$ & $\begin{array}{c}\text { Depth in Klassen } \\
\text { Test Well } \\
\text { (ft BGL) }\end{array}$ & $\begin{array}{l}\text { Elevation in Klassen } \\
\text { Domestic Well } \\
\text { (ft AMSL) }\end{array}$ & \\
\hline 9/27/11 15:30 & 62.308 & 39.716 & 1446.46 & 1469.41 \\
\hline $9 / 27 / 1115: 40$ & 63.493 & 39.752 & 1445.28 & 1469.37 \\
\hline 9/27/11 15:50 & 64.103 & 39.792 & 1444.67 & 1469.33 \\
\hline 9/27/11 16:00 & 64.391 & 39.831 & 1444.38 & 1469.29 \\
\hline 9/27/11 16:10 & 64.806 & 39.871 & 1443.96 & 1469.25 \\
\hline 9/27/11 16:20 & 65.238 & 39.914 & 1443.53 & 1469.21 \\
\hline $9 / 27 / 1116: 30$ & 58.303 & 39.956 & 1450.47 & 1469.17 \\
\hline 9/27/11 16:40 & 46.705 & 39.998 & 1462.06 & 1469.12 \\
\hline 9/27/11 16:50 & 45.228 & 40.036 & 1463.54 & 1469.09 \\
\hline 9/27/11 17:00 & 44.649 & 40.066 & 1464.12 & 1469.06 \\
\hline 9/27/11 17:10 & 43.786 & 40.094 & 1464.98 & 1469.03 \\
\hline 9/27/11 17:20 & 43.333 & 40.12 & 1465.44 & 1469.00 \\
\hline 9/27/11 17:30 & 43.159 & 40.146 & 1465.61 & 1468.98 \\
\hline 9/27/11 17:40 & 43.028 & 40.167 & 1465.74 & 1468.96 \\
\hline 9/27/11 17:50 & 42.916 & 40.188 & 1465.85 & 1468.93 \\
\hline 9/27/11 18:00 & 48.415 & 40.209 & 1460.35 & 1468.91 \\
\hline 9/27/11 18:10 & 46.633 & 40.23 & 1462.14 & 1468.89 \\
\hline 9/27/11 18:20 & 44.703 & 40.251 & 1464.07 & 1468.87 \\
\hline 9/27/11 18:30 & 43.464 & 40.275 & 1465.31 & 1468.85 \\
\hline $9 / 27 / 11$ 18:40 & 48.279 & 40.294 & 1460.49 & 1468.83 \\
\hline $9 / 27 / 1118: 50$ & 46.311 & 40.315 & 1462.46 & 1468.81 \\
\hline 9/27/11 19:00 & 44.529 & 40.334 & 1464.24 & 1468.79 \\
\hline 9/27/11 19:10 & 47.43 & 40.355 & 1461.34 & 1468.77 \\
\hline 9/27/11 19:20 & 42.125 & 40.376 & 1466.64 & 1468.75 \\
\hline 9/27/11 19:30 & 42.102 & 40.39 & 1466.67 & 1468.73 \\
\hline 9/27/11 19:40 & 41.555 & 40.402 & 1467.21 & 1468.72 \\
\hline 9/27/11 19:50 & 41.501 & 40.406 & 1467.27 & 1468.72 \\
\hline 9/27/11 20:00 & 41.82 & 40.406 & 1466.95 & 1468.72 \\
\hline 9/27/11 20:10 & 41.297 & 40.413 & 1467.47 & 1468.71 \\
\hline 9/27/11 20:20 & 41.232 & 40.423 & 1467.54 & 1468.70 \\
\hline 9/27/11 20:30 & 41.283 & 40.434 & 1467.49 & 1468.69 \\
\hline 9/27/11 20:40 & 41.4 & 40.453 & 1467.37 & 1468.67 \\
\hline $9 / 27 / 1120: 50$ & 41.555 & 40.465 & 1467.21 & 1468.66 \\
\hline 9/27/11 21:00 & 41.633 & 40.472 & 1467.14 & 1468.65 \\
\hline 9/27/11 21:10 & 42.54 & 40.481 & 1466.23 & 1468.64 \\
\hline 9/27/11 21:20 & 45.181 & 40.488 & 1463.59 & 1468.63 \\
\hline 9/27/11 21:30 & 41.06 & 40.495 & 1467.71 & 1468.63 \\
\hline 9/27/11 21:40 & 41.065 & 40.5 & 1467.70 & 1468.62 \\
\hline
\end{tabular}


TABLE S1.3 Automatically recorded groundwater levels in the Klassen wells, September 15, 2011, to March 13, 2012.

\begin{tabular}{|c|c|c|c|c|}
\hline \multicolumn{3}{|c|}{ Reference Elevation (ft AMSL) } & 1508.77 & \multirow{2}{*}{$\begin{array}{l}1509.12 \\
\text { Elevation in Klassen } \\
\text { Test Well (ft AMSL) }\end{array}$} \\
\hline Date and Time & $\begin{array}{l}\text { Depth in Klassen } \\
\text { Domestic Well } \\
(\mathrm{ft} \mathrm{BGL})\end{array}$ & $\begin{array}{c}\text { Depth in Klassen } \\
\text { Test Well } \\
\text { (ft BGL) }\end{array}$ & $\begin{array}{l}\text { Elevation in Klassen } \\
\text { Domestic Well } \\
\text { (ft AMSL) }\end{array}$ & \\
\hline $9 / 27 / 1121: 50$ & 41.086 & 40.507 & 1467.68 & 1468.62 \\
\hline $9 / 27 / 1122: 00$ & 41.152 & 40.514 & 1467.62 & 1468.61 \\
\hline 9/27/11 22:10 & 41.161 & 40.519 & 1467.61 & 1468.60 \\
\hline $9 / 27 / 1122: 20$ & 44.351 & 40.524 & 1464.42 & 1468.60 \\
\hline 9/27/11 22:30 & 41.614 & 40.538 & 1467.16 & 1468.58 \\
\hline 9/27/11 22:40 & 41.33 & 40.545 & 1467.44 & 1468.58 \\
\hline $9 / 27 / 1122: 50$ & 42.17 & 40.556 & 1466.60 & 1468.57 \\
\hline 9/27/11 23:00 & 43.877 & 40.564 & 1464.89 & 1468.56 \\
\hline 9/27/11 23:10 & 44.611 & 40.559 & 1464.16 & 1468.56 \\
\hline $9 / 27 / 1123: 20$ & 40.8 & 40.549 & 1467.97 & 1468.57 \\
\hline $9 / 27 / 1123: 30$ & 40.673 & 40.54 & 1468.10 & 1468.58 \\
\hline 9/27/11 23:40 & 40.577 & 40.531 & 1468.19 & 1468.59 \\
\hline $9 / 27 / 1123: 50$ & 40.542 & 40.517 & 1468.23 & 1468.61 \\
\hline 9/28/11 0:00 & 40.704 & 40.505 & 1468.07 & 1468.62 \\
\hline 9/28/11 0:10 & 41.508 & 40.493 & 1467.26 & 1468.63 \\
\hline $9 / 28 / 110: 20$ & 43.769 & 40.481 & 1465.00 & 1468.64 \\
\hline 9/28/11 0:30 & 39.93 & 40.472 & 1468.84 & 1468.65 \\
\hline $9 / 28 / 110: 40$ & 39.871 & 40.463 & 1468.90 & 1468.66 \\
\hline $9 / 28 / 11$ 0:50 & 39.848 & 40.451 & 1468.92 & 1468.67 \\
\hline 9/28/11 1:00 & 39.829 & 40.439 & 1468.94 & 1468.68 \\
\hline $9 / 28 / 11$ 1:10 & 39.885 & 40.425 & 1468.88 & 1468.70 \\
\hline $9 / 28 / 11$ 1:20 & 39.686 & 40.418 & 1469.08 & 1468.70 \\
\hline $9 / 28 / 11$ 1:30 & 39.653 & 40.406 & 1469.12 & 1468.72 \\
\hline 9/28/11 1:40 & 39.651 & 40.397 & 1469.12 & 1468.73 \\
\hline $9 / 28 / 11$ 1:50 & 39.782 & 40.392 & 1468.99 & 1468.73 \\
\hline $9 / 28 / 112: 00$ & 40.026 & 40.383 & 1468.74 & 1468.74 \\
\hline 9/28/11 2:10 & 40.828 & 40.373 & 1467.94 & 1468.75 \\
\hline $9 / 28 / 112: 20$ & 42.815 & 40.362 & 1465.95 & 1468.76 \\
\hline $9 / 28 / 112: 30$ & 40.387 & 40.35 & 1468.38 & 1468.77 \\
\hline $9 / 28 / 112: 40$ & 39.212 & 40.341 & 1469.56 & 1468.78 \\
\hline $9 / 28 / 112: 50$ & 39.208 & 40.329 & 1469.56 & 1468.79 \\
\hline 9/28/11 3:00 & 41.705 & 40.317 & 1467.06 & 1468.81 \\
\hline 9/28/11 3:10 & 39.112 & 40.305 & 1469.66 & 1468.82 \\
\hline $9 / 28 / 113: 20$ & 39.109 & 40.296 & 1469.66 & 1468.83 \\
\hline 9/28/11 3:30 & 39.175 & 40.289 & 1469.59 & 1468.83 \\
\hline 9/28/11 3:40 & 39.205 & 40.28 & 1469.56 & 1468.84 \\
\hline $9 / 28 / 113: 50$ & 39.318 & 40.27 & 1469.45 & 1468.85 \\
\hline 9/28/11 4:00 & 39.71 & 40.256 & 1469.06 & 1468.87 \\
\hline
\end{tabular}


TABLE S1.3 Automatically recorded groundwater levels in the Klassen wells, September 15, 2011, to March 13, 2012.

\begin{tabular}{|c|c|c|c|c|}
\hline \multicolumn{3}{|c|}{ Reference Elevation (ft AMSL) } & 1508.77 & \multirow{2}{*}{$\begin{array}{l}1509.12 \\
\text { Elevation in Klassen } \\
\text { Test Well (ft AMSL) }\end{array}$} \\
\hline Date and Time & $\begin{array}{l}\text { Depth in Klassen } \\
\text { Domestic Well } \\
\text { (ft BGL) }\end{array}$ & $\begin{array}{c}\text { Depth in Klassen } \\
\text { Test Well } \\
\text { (ft BGL) }\end{array}$ & $\begin{array}{l}\text { Elevation in Klassen } \\
\text { Domestic Well } \\
\text { (ft AMSL) }\end{array}$ & \\
\hline 9/28/11 4:10 & 40.821 & 40.244 & 1467.95 & 1468.88 \\
\hline $9 / 28 / 114: 20$ & 43.408 & 40.233 & 1465.36 & 1468.89 \\
\hline 9/28/11 4:30 & 38.727 & 40.219 & 1470.04 & 1468.90 \\
\hline $9 / 28 / 114: 40$ & 38.732 & 40.209 & 1470.04 & 1468.91 \\
\hline $9 / 28 / 114: 50$ & 38.727 & 40.197 & 1470.04 & 1468.93 \\
\hline 9/28/11 5:00 & 38.741 & 40.186 & 1470.03 & 1468.94 \\
\hline 9/28/11 5:10 & 38.809 & 40.172 & 1469.96 & 1468.95 \\
\hline $9 / 28 / 115: 20$ & 38.628 & 40.162 & 1470.14 & 1468.96 \\
\hline 9/28/11 5:30 & 38.633 & 40.151 & 1470.14 & 1468.97 \\
\hline $9 / 28 / 115: 40$ & 38.621 & 40.141 & 1470.15 & 1468.98 \\
\hline $9 / 28 / 115: 50$ & 38.645 & 40.129 & 1470.12 & 1468.99 \\
\hline $9 / 28 / 116: 00$ & 38.764 & 40.118 & 1470.01 & 1469.00 \\
\hline $9 / 28 / 116: 10$ & 39.688 & 40.108 & 1469.08 & 1469.01 \\
\hline $9 / 28 / 116: 20$ & 42.383 & 40.108 & 1466.39 & 1469.01 \\
\hline $9 / 28 / 116: 30$ & 38.567 & 40.101 & 1470.20 & 1469.02 \\
\hline $9 / 28 / 116: 40$ & 38.528 & 40.092 & 1470.24 & 1469.03 \\
\hline $9 / 28 / 116: 50$ & 38.511 & 40.083 & 1470.26 & 1469.04 \\
\hline 9/28/11 7:00 & 38.497 & 40.073 & 1470.27 & 1469.05 \\
\hline 9/28/11 7:10 & 38.523 & 40.064 & 1470.25 & 1469.06 \\
\hline $9 / 28 / 117: 20$ & 38.581 & 40.054 & 1470.19 & 1469.07 \\
\hline $9 / 28 / 11$ 7:30 & 38.837 & 40.047 & 1469.93 & 1469.08 \\
\hline $9 / 28 / 117: 40$ & 39.365 & 40.04 & 1469.40 & 1469.08 \\
\hline $9 / 28 / 117: 50$ & 39.144 & 40.031 & 1469.63 & 1469.09 \\
\hline 9/28/11 8:00 & 39.672 & 40.024 & 1469.10 & 1469.10 \\
\hline 9/28/11 8:10 & 41.309 & 40.017 & 1467.46 & 1469.11 \\
\hline $9 / 28 / 118: 20$ & 38.394 & 40.01 & 1470.38 & 1469.11 \\
\hline $9 / 28 / 118: 30$ & 38.302 & 40.003 & 1470.47 & 1469.12 \\
\hline $9 / 28 / 118: 40$ & 38.305 & 39.993 & 1470.46 & 1469.13 \\
\hline 9/28/11 8:50 & 40.047 & 39.986 & 1468.72 & 1469.14 \\
\hline 9/28/11 9:00 & 48.136 & 39.986 & 1460.63 & 1469.14 \\
\hline 9/28/11 9:10 & 50.474 & 39.993 & 1458.30 & 1469.13 \\
\hline 9/28/11 9:20 & 48.962 & 40.003 & 1459.81 & 1469.12 \\
\hline 9/28/11 9:30 & 46.274 & 40.017 & 1462.50 & 1469.11 \\
\hline 9/28/11 9:40 & 53.894 & 40.031 & 1454.88 & 1469.09 \\
\hline 9/28/11 9:50 & 50.08 & 40.047 & 1458.69 & 1469.08 \\
\hline 9/28/11 10:00 & 46.534 & 40.061 & 1462.24 & 1469.06 \\
\hline 9/28/11 10:10 & 48.833 & 40.078 & 1459.94 & 1469.04 \\
\hline 9/28/11 10:20 & 51.487 & 40.094 & 1457.28 & 1469.03 \\
\hline
\end{tabular}


TABLE S1.3 Automatically recorded groundwater levels in the Klassen wells, September 15, 2011, to March 13, 2012.

\begin{tabular}{|c|c|c|c|c|}
\hline \multicolumn{3}{|c|}{ Reference Elevation (ft AMSL) } & 1508.77 & \multirow{2}{*}{$\begin{array}{l}1509.12 \\
\text { Elevation in Klassen } \\
\text { Test Well (ft AMSL) }\end{array}$} \\
\hline Date and Time & $\begin{array}{l}\text { Depth in Klassen } \\
\text { Domestic Well } \\
(\mathrm{ft} \mathrm{BGL})\end{array}$ & $\begin{array}{c}\text { Depth in Klassen } \\
\text { Test Well } \\
\text { (ft BGL) }\end{array}$ & $\begin{array}{l}\text { Elevation in Klassen } \\
\text { Domestic Well } \\
\text { (ft AMSL) }\end{array}$ & \\
\hline 9/28/11 10:30 & 52.63 & 40.113 & 1456.14 & 1469.01 \\
\hline $9 / 28 / 1110: 40$ & 51.138 & 40.132 & 1457.63 & 1468.99 \\
\hline $9 / 28 / 1110: 50$ & 49.269 & 40.148 & 1459.50 & 1468.97 \\
\hline 9/28/11 11:00 & 50.472 & 40.167 & 1458.30 & 1468.96 \\
\hline 9/28/11 11:10 & 46.963 & 40.183 & 1461.81 & 1468.94 \\
\hline 9/28/11 11:20 & 45.125 & 40.195 & 1463.64 & 1468.93 \\
\hline 9/28/11 11:30 & 45.038 & 40.205 & 1463.73 & 1468.92 \\
\hline 9/28/11 11:40 & 46.809 & 40.214 & 1461.96 & 1468.91 \\
\hline 9/28/11 11:50 & 44.452 & 40.221 & 1464.32 & 1468.90 \\
\hline $9 / 28 / 1112: 00$ & 40.697 & 40.226 & 1468.07 & 1468.90 \\
\hline 9/28/11 12:10 & 40.24 & 40.228 & 1468.53 & 1468.89 \\
\hline $9 / 28 / 1112: 20$ & 40.331 & 40.226 & 1468.44 & 1468.90 \\
\hline 9/28/11 12:30 & 39.646 & 40.223 & 1469.12 & 1468.90 \\
\hline $9 / 28 / 1112: 40$ & 39.576 & 40.223 & 1469.19 & 1468.90 \\
\hline 9/28/11 12:50 & 40.643 & 40.221 & 1468.13 & 1468.90 \\
\hline 9/28/11 13:00 & 39.236 & 40.221 & 1469.53 & 1468.90 \\
\hline 9/28/11 13:10 & 39.231 & 40.219 & 1469.54 & 1468.90 \\
\hline 9/28/11 13:20 & 41.168 & 40.216 & 1467.60 & 1468.91 \\
\hline 9/28/11 13:30 & 38.992 & 40.216 & 1469.78 & 1468.91 \\
\hline 9/28/11 13:40 & 39.102 & 40.214 & 1469.67 & 1468.91 \\
\hline $9 / 28 / 11$ 13:50 & 39.212 & 40.212 & 1469.56 & 1468.91 \\
\hline 9/28/11 14:00 & 38.739 & 40.209 & 1470.03 & 1468.91 \\
\hline 9/28/11 14:10 & 38.743 & 40.209 & 1470.03 & 1468.91 \\
\hline 9/28/11 14:20 & 38.964 & 40.207 & 1469.81 & 1468.92 \\
\hline 9/28/11 14:30 & 39.813 & 40.205 & 1468.96 & 1468.92 \\
\hline 9/28/11 14:40 & 38.528 & 40.202 & 1470.24 & 1468.92 \\
\hline 9/28/11 14:50 & 38.595 & 40.2 & 1470.17 & 1468.92 \\
\hline 9/28/11 15:00 & 38.811 & 40.197 & 1469.96 & 1468.93 \\
\hline 9/28/11 15:10 & 38.558 & 40.2 & 1470.21 & 1468.92 \\
\hline 9/28/11 15:20 & 38.635 & 40.197 & 1470.13 & 1468.93 \\
\hline 9/28/11 15:30 & 44.782 & 40.197 & 1463.99 & 1468.93 \\
\hline 9/28/11 15:40 & 42.142 & 40.205 & 1466.63 & 1468.92 \\
\hline 9/28/11 15:50 & 40.645 & 40.214 & 1468.12 & 1468.91 \\
\hline 9/28/11 16:00 & 42.414 & 40.221 & 1466.36 & 1468.90 \\
\hline 9/28/11 16:10 & 39.635 & 40.228 & 1469.13 & 1468.89 \\
\hline 9/28/11 16:20 & 39.104 & 40.233 & 1469.67 & 1468.89 \\
\hline 9/28/11 16:30 & 40.561 & 40.233 & 1468.21 & 1468.89 \\
\hline 9/28/11 16:40 & 38.607 & 40.23 & 1470.16 & 1468.89 \\
\hline
\end{tabular}


TABLE S1.3 Automatically recorded groundwater levels in the Klassen wells, September 15, 2011, to March 13, 2012.

\begin{tabular}{|c|c|c|c|c|}
\hline \multicolumn{3}{|c|}{ Reference Elevation (ft AMSL) } & 1508.77 & \multirow{2}{*}{$\begin{array}{l}1509.12 \\
\text { Elevation in Klassen } \\
\text { Test Well (ft AMSL) }\end{array}$} \\
\hline Date and Time & $\begin{array}{l}\text { Depth in Klassen } \\
\text { Domestic Well } \\
(\mathrm{ft} \mathrm{BGL})\end{array}$ & $\begin{array}{c}\text { Depth in Klassen } \\
\text { Test Well } \\
\text { (ft BGL) }\end{array}$ & $\begin{array}{l}\text { Elevation in Klassen } \\
\text { Domestic Well } \\
\text { (ft AMSL) }\end{array}$ & \\
\hline 9/28/11 16:50 & 38.424 & 40.228 & 1470.35 & 1468.89 \\
\hline 9/28/11 17:00 & 38.265 & 40.223 & 1470.50 & 1468.90 \\
\hline 9/28/11 17:10 & 38.138 & 40.216 & 1470.63 & 1468.91 \\
\hline $9 / 28 / 1117: 20$ & 38.028 & 40.209 & 1470.74 & 1468.91 \\
\hline 9/28/11 17:30 & 37.932 & 40.2 & 1470.84 & 1468.92 \\
\hline 9/28/11 17:40 & 39.112 & 40.188 & 1469.66 & 1468.93 \\
\hline $9 / 28 / 1117: 50$ & 37.951 & 40.183 & 1470.82 & 1468.94 \\
\hline 9/28/11 18:00 & 37.866 & 40.179 & 1470.90 & 1468.94 \\
\hline 9/28/11 18:10 & 37.765 & 40.174 & 1471.00 & 1468.95 \\
\hline $9 / 28 / 1118: 20$ & 37.681 & 40.167 & 1471.09 & 1468.96 \\
\hline $9 / 28 / 1118: 30$ & 37.603 & 40.16 & 1471.17 & 1468.96 \\
\hline 9/28/11 18:40 & 41.862 & 40.153 & 1466.91 & 1468.97 \\
\hline 9/28/11 18:50 & 37.65 & 40.151 & 1471.12 & 1468.97 \\
\hline 9/28/11 19:00 & 37.709 & 40.148 & 1471.06 & 1468.97 \\
\hline 9/28/11 19:10 & 40.856 & 40.148 & 1467.91 & 1468.97 \\
\hline 9/28/11 19:20 & 42.714 & 40.148 & 1466.06 & 1468.97 \\
\hline 9/28/11 19:30 & 40.542 & 40.148 & 1468.23 & 1468.97 \\
\hline 9/28/11 19:40 & 38.633 & 40.148 & 1470.14 & 1468.97 \\
\hline 9/28/11 19:50 & 37.751 & 40.151 & 1471.02 & 1468.97 \\
\hline $9 / 28 / 1120: 00$ & 42.99 & 40.155 & 1465.78 & 1468.97 \\
\hline 9/28/11 20:10 & 38.34 & 40.162 & 1470.43 & 1468.96 \\
\hline 9/28/11 20:20 & 40.312 & 40.165 & 1468.46 & 1468.96 \\
\hline $9 / 28 / 1120: 30$ & 37.897 & 40.172 & 1470.87 & 1468.95 \\
\hline 9/28/11 20:40 & 37.972 & 40.174 & 1470.80 & 1468.95 \\
\hline 9/28/11 20:50 & 40.91 & 40.174 & 1467.86 & 1468.95 \\
\hline 9/28/11 21:00 & 37.735 & 40.174 & 1471.03 & 1468.95 \\
\hline 9/28/11 21:10 & 37.906 & 40.172 & 1470.86 & 1468.95 \\
\hline 9/28/11 21:20 & 37.606 & 40.167 & 1471.16 & 1468.96 \\
\hline 9/28/11 21:30 & 37.655 & 40.167 & 1471.11 & 1468.96 \\
\hline 9/28/11 21:40 & 38.232 & 40.162 & 1470.54 & 1468.96 \\
\hline 9/28/11 21:50 & 37.54 & 40.162 & 1471.23 & 1468.96 \\
\hline 9/28/11 22:00 & 37.62 & 40.16 & 1471.15 & 1468.96 \\
\hline 9/28/11 22:10 & 37.946 & 40.158 & 1470.82 & 1468.96 \\
\hline 9/28/11 22:20 & 37.716 & 40.16 & 1471.05 & 1468.96 \\
\hline $9 / 28 / 1122: 30$ & 39.238 & 40.158 & 1469.53 & 1468.96 \\
\hline 9/28/11 22:40 & 42.801 & 40.158 & 1465.97 & 1468.96 \\
\hline $9 / 28 / 1122: 50$ & 37.737 & 40.16 & 1471.03 & 1468.96 \\
\hline 9/28/11 23:00 & 37.801 & 40.158 & 1470.97 & 1468.96 \\
\hline
\end{tabular}


TABLE S1.3 Automatically recorded groundwater levels in the Klassen wells, September 15, 2011, to March 13, 2012.

\begin{tabular}{|c|c|c|c|c|}
\hline \multicolumn{3}{|c|}{ Reference Elevation (ft AMSL) } & 1508.77 & \multirow{2}{*}{$\begin{array}{l}1509.12 \\
\text { Elevation in Klassen } \\
\text { Test Well (ft AMSL) }\end{array}$} \\
\hline Date and Time & $\begin{array}{l}\text { Depth in Klassen } \\
\text { Domestic Well } \\
(\mathrm{ft} \mathrm{BGL})\end{array}$ & $\begin{array}{c}\text { Depth in Klassen } \\
\text { Test Well } \\
\text { (ft BGL) }\end{array}$ & $\begin{array}{l}\text { Elevation in Klassen } \\
\text { Domestic Well } \\
\text { (ft AMSL) }\end{array}$ & \\
\hline 9/28/11 23:10 & 38.347 & 40.158 & 1470.42 & 1468.96 \\
\hline $9 / 28 / 1123: 20$ & 37.629 & 40.158 & 1471.14 & 1468.96 \\
\hline 9/28/11 23:30 & 37.653 & 40.155 & 1471.12 & 1468.97 \\
\hline $9 / 28 / 1123: 40$ & 38.692 & 40.153 & 1470.08 & 1468.97 \\
\hline 9/28/11 23:50 & 37.477 & 40.148 & 1471.29 & 1468.97 \\
\hline 9/29/11 0:00 & 37.578 & 40.146 & 1471.19 & 1468.98 \\
\hline 9/29/11 0:10 & 41.827 & 40.141 & 1466.94 & 1468.98 \\
\hline 9/29/11 0:20 & 37.414 & 40.139 & 1471.36 & 1468.98 \\
\hline 9/29/11 0:30 & 37.718 & 40.136 & 1471.05 & 1468.99 \\
\hline 9/29/11 0:40 & 37.294 & 40.132 & 1471.48 & 1468.99 \\
\hline $9 / 29 / 110: 50$ & 37.367 & 40.127 & 1471.40 & 1469.00 \\
\hline 9/29/11 1:00 & 38.877 & 40.125 & 1469.89 & 1469.00 \\
\hline 9/29/11 1:10 & 37.259 & 40.12 & 1471.51 & 1469.00 \\
\hline $9 / 29 / 111: 20$ & 37.399 & 40.115 & 1471.37 & 1469.01 \\
\hline 9/29/11 1:30 & 37.395 & 40.111 & 1471.37 & 1469.01 \\
\hline 9/29/11 1:40 & 41.52 & 40.106 & 1467.25 & 1469.02 \\
\hline 9/29/11 1:50 & 37.233 & 40.104 & 1471.54 & 1469.02 \\
\hline $9 / 29 / 112: 00$ & 37.533 & 40.097 & 1471.24 & 1469.03 \\
\hline 9/29/11 2:10 & 37.123 & 40.092 & 1471.65 & 1469.03 \\
\hline 9/29/11 2:20 & 37.226 & 40.087 & 1471.54 & 1469.04 \\
\hline $9 / 29 / 112: 30$ & 38.556 & 40.083 & 1470.21 & 1469.04 \\
\hline 9/29/11 2:40 & 37.102 & 40.078 & 1471.67 & 1469.04 \\
\hline $9 / 29 / 112: 50$ & 37.231 & 40.071 & 1471.54 & 1469.05 \\
\hline 9/29/11 3:00 & 41.902 & 40.066 & 1466.87 & 1469.06 \\
\hline 9/29/11 3:10 & 37.088 & 40.061 & 1471.68 & 1469.06 \\
\hline 9/29/11 3:20 & 37.378 & 40.054 & 1471.39 & 1469.07 \\
\hline 9/29/11 3:30 & 36.984 & 40.05 & 1471.79 & 1469.07 \\
\hline 9/29/11 3:40 & 37.083 & 40.043 & 1471.69 & 1469.08 \\
\hline 9/29/11 3:50 & 38.664 & 40.038 & 1470.11 & 1469.08 \\
\hline 9/29/11 4:00 & 37.005 & 40.033 & 1471.76 & 1469.09 \\
\hline 9/29/11 4:10 & 37.064 & 40.026 & 1471.71 & 1469.10 \\
\hline 9/29/11 4:20 & 38.232 & 40.022 & 1470.54 & 1469.10 \\
\hline 9/29/11 4:30 & 36.959 & 40.017 & 1471.81 & 1469.11 \\
\hline 9/29/11 4:40 & 37.076 & 40.01 & 1471.69 & 1469.11 \\
\hline 9/29/11 4:50 & 40.826 & 40.005 & 1467.94 & 1469.12 \\
\hline 9/29/11 5:00 & 40 & 40 & 1468.77 & 1469.12 \\
\hline 9/29/11 5:10 & 36.975 & 39.996 & 1471.79 & 1469.13 \\
\hline 9/29/11 5:20 & 37.172 & 39.989 & 1471.60 & 1469.13 \\
\hline
\end{tabular}


TABLE S1.3 Automatically recorded groundwater levels in the Klassen wells, September 15, 2011, to March 13, 2012.

\begin{tabular}{|c|c|c|c|c|}
\hline \multicolumn{3}{|c|}{ Reference Elevation (ft AMSL) } & 1508.77 & \multirow{2}{*}{$\begin{array}{l}1509.12 \\
\text { Elevation in Klassen } \\
\text { Test Well (ft AMSL) }\end{array}$} \\
\hline Date and Time & $\begin{array}{l}\text { Depth in Klassen } \\
\text { Domestic Well } \\
(\mathrm{ft} \mathrm{BGL})\end{array}$ & $\begin{array}{c}\text { Depth in Klassen } \\
\text { Test Well } \\
\text { (ft BGL) }\end{array}$ & $\begin{array}{l}\text { Elevation in Klassen } \\
\text { Domestic Well } \\
\text { (ft AMSL) }\end{array}$ & \\
\hline $9 / 29 / 115: 30$ & 36.869 & 39.984 & 1471.90 & 1469.14 \\
\hline $9 / 29 / 115: 40$ & 36.963 & 39.977 & 1471.81 & 1469.15 \\
\hline 9/29/11 5:50 & 37.547 & 39.972 & 1471.22 & 1469.15 \\
\hline $9 / 29 / 116: 00$ & 36.879 & 39.968 & 1471.89 & 1469.15 \\
\hline 9/29/11 6:10 & 39.834 & 39.965 & 1468.94 & 1469.16 \\
\hline $9 / 29 / 116: 20$ & 41.691 & 39.968 & 1467.08 & 1469.15 \\
\hline $9 / 29 / 116: 30$ & 37.073 & 39.965 & 1471.70 & 1469.16 \\
\hline $9 / 29 / 116: 40$ & 37.266 & 39.961 & 1471.50 & 1469.16 \\
\hline $9 / 29 / 116: 50$ & 39.522 & 39.956 & 1469.25 & 1469.17 \\
\hline $9 / 29 / 117: 00$ & 36.982 & 39.951 & 1471.79 & 1469.17 \\
\hline 9/29/11 7:10 & 37.137 & 39.946 & 1471.63 & 1469.18 \\
\hline $9 / 29 / 117: 20$ & 38.75 & 39.942 & 1470.02 & 1469.18 \\
\hline $9 / 29 / 117: 30$ & 40.983 & 39.949 & 1467.79 & 1469.17 \\
\hline $9 / 29 / 117: 40$ & 38.558 & 39.946 & 1470.21 & 1469.18 \\
\hline 9/29/11 7:50 & 37.022 & 39.944 & 1471.75 & 1469.18 \\
\hline 9/29/11 8:00 & 37.081 & 39.939 & 1471.69 & 1469.18 \\
\hline 9/29/11 8:10 & 37.146 & 39.939 & 1471.62 & 1469.18 \\
\hline $9 / 29 / 118: 20$ & 37.151 & 39.937 & 1471.62 & 1469.19 \\
\hline 9/29/11 8:30 & 38.99 & 39.935 & 1469.78 & 1469.19 \\
\hline 9/29/11 8:40 & 36.945 & 39.928 & 1471.82 & 1469.19 \\
\hline 9/29/11 8:50 & 37.085 & 39.925 & 1471.68 & 1469.20 \\
\hline 9/29/11 9:00 & 41.128 & 39.923 & 1467.64 & 1469.20 \\
\hline 9/29/11 9:10 & 36.884 & 39.916 & 1471.89 & 1469.21 \\
\hline 9/29/11 9:20 & 37.202 & 39.909 & 1471.57 & 1469.21 \\
\hline 9/29/11 9:30 & 36.794 & 39.909 & 1471.98 & 1469.21 \\
\hline 9/29/11 9:40 & 36.867 & 39.902 & 1471.90 & 1469.22 \\
\hline 9/29/11 9:50 & 37.742 & 39.897 & 1471.03 & 1469.23 \\
\hline 9/29/11 10:00 & 36.745 & 39.895 & 1472.02 & 1469.23 \\
\hline 9/29/11 10:10 & 36.867 & 39.89 & 1471.90 & 1469.23 \\
\hline 9/29/11 10:20 & 38.987 & 39.888 & 1469.78 & 1469.23 \\
\hline 9/29/11 10:30 & 36.726 & 39.883 & 1472.04 & 1469.24 \\
\hline 9/29/11 10:40 & 36.816 & 39.878 & 1471.95 & 1469.24 \\
\hline 9/29/11 10:50 & 38.776 & 39.874 & 1469.99 & 1469.25 \\
\hline 9/29/11 11:00 & 36.705 & 39.871 & 1472.06 & 1469.25 \\
\hline 9/29/11 11:10 & 36.848 & 39.867 & 1471.92 & 1469.26 \\
\hline 9/29/11 11:20 & 41.103 & 39.862 & 1467.67 & 1469.26 \\
\hline 9/29/11 11:30 & 36.675 & 39.86 & 1472.09 & 1469.26 \\
\hline 9/29/11 11:40 & 36.982 & 39.855 & 1471.79 & 1469.27 \\
\hline
\end{tabular}


TABLE S1.3 Automatically recorded groundwater levels in the Klassen wells, September 15, 2011, to March 13, 2012.

\begin{tabular}{|c|c|c|c|c|}
\hline \multicolumn{3}{|c|}{ Reference Elevation (ft AMSL) } & 1508.77 & \multirow{2}{*}{$\begin{array}{l}1509.12 \\
\text { Elevation in Klassen } \\
\text { Test Well (ft AMSL) }\end{array}$} \\
\hline Date and Time & $\begin{array}{l}\text { Depth in Klassen } \\
\text { Domestic Well } \\
(\mathrm{ft} \mathrm{BGL})\end{array}$ & $\begin{array}{c}\text { Depth in Klassen } \\
\text { Test Well } \\
\text { (ft BGL) }\end{array}$ & $\begin{array}{l}\text { Elevation in Klassen } \\
\text { Domestic Well } \\
\text { (ft AMSL) }\end{array}$ & \\
\hline 9/29/11 11:50 & 36.602 & 39.853 & 1472.17 & 1469.27 \\
\hline 9/29/11 12:00 & 36.672 & 39.848 & 1472.10 & 1469.27 \\
\hline 9/29/11 12:10 & 36.755 & 39.843 & 1472.01 & 1469.28 \\
\hline $9 / 29 / 1112: 20$ & 36.98 & 39.841 & 1471.79 & 1469.28 \\
\hline 9/29/11 12:30 & 40.476 & 39.841 & 1468.29 & 1469.28 \\
\hline 9/29/11 12:40 & 36.682 & 39.838 & 1472.09 & 1469.28 \\
\hline $9 / 29 / 1112: 50$ & 36.844 & 39.834 & 1471.93 & 1469.29 \\
\hline 9/29/11 13:00 & 36.937 & 39.831 & 1471.83 & 1469.29 \\
\hline 9/29/11 13:10 & 37.282 & 39.827 & 1471.49 & 1469.30 \\
\hline $9 / 29 / 1113: 20$ & 36.623 & 39.822 & 1472.15 & 1469.30 \\
\hline 9/29/11 13:30 & 36.717 & 39.817 & 1472.05 & 1469.31 \\
\hline 9/29/11 13:40 & 38.04 & 39.817 & 1470.73 & 1469.31 \\
\hline 9/29/11 13:50 & 36.722 & 39.817 & 1472.05 & 1469.31 \\
\hline 9/29/11 14:00 & 36.787 & 39.813 & 1471.98 & 1469.31 \\
\hline 9/29/11 14:10 & 36.804 & 39.81 & 1471.97 & 1469.31 \\
\hline 9/29/11 14:20 & 37.871 & 39.806 & 1470.90 & 1469.32 \\
\hline 9/29/11 14:30 & 36.642 & 39.801 & 1472.13 & 1469.32 \\
\hline 9/29/11 14:40 & 36.733 & 39.799 & 1472.04 & 1469.32 \\
\hline 9/29/11 14:50 & 38.921 & 39.794 & 1469.85 & 1469.33 \\
\hline 9/29/11 15:00 & 36.597 & 39.789 & 1472.17 & 1469.33 \\
\hline 9/29/11 15:10 & 36.748 & 39.789 & 1472.02 & 1469.33 \\
\hline 9/29/11 15:20 & 41.03 & 39.785 & 1467.74 & 1469.34 \\
\hline $9 / 29 / 11$ 15:30 & 36.562 & 39.785 & 1472.21 & 1469.34 \\
\hline 9/29/11 15:40 & 36.804 & 39.785 & 1471.97 & 1469.34 \\
\hline 9/29/11 15:50 & 40.687 & 39.782 & 1468.08 & 1469.34 \\
\hline 9/29/11 16:00 & 36.539 & 39.782 & 1472.23 & 1469.34 \\
\hline 9/29/11 16:10 & 36.773 & 39.782 & 1472.00 & 1469.34 \\
\hline 9/29/11 16:20 & 39.702 & 39.785 & 1469.07 & 1469.34 \\
\hline 9/29/11 16:30 & 36.818 & 39.794 & 1471.95 & 1469.33 \\
\hline 9/29/11 16:40 & 37.186 & 39.801 & 1471.58 & 1469.32 \\
\hline 9/29/11 16:50 & 36.93 & 39.813 & 1471.84 & 1469.31 \\
\hline 9/29/11 17:00 & 37.116 & 39.822 & 1471.65 & 1469.30 \\
\hline 9/29/11 17:10 & 37.578 & 39.829 & 1471.19 & 1469.29 \\
\hline 9/29/11 17:20 & 37.536 & 39.841 & 1471.23 & 1469.28 \\
\hline 9/29/11 17:30 & 38.818 & 39.853 & 1469.95 & 1469.27 \\
\hline 9/29/11 17:40 & 37.449 & 39.867 & 1471.32 & 1469.26 \\
\hline 9/29/11 17:50 & 37.592 & 39.876 & 1471.18 & 1469.25 \\
\hline 9/29/11 18:00 & 38.347 & 39.883 & 1470.42 & 1469.24 \\
\hline
\end{tabular}


TABLE S1.3 Automatically recorded groundwater levels in the Klassen wells, September 15, 2011, to March 13, 2012.

\begin{tabular}{|c|c|c|c|c|}
\hline \multicolumn{3}{|c|}{ Reference Elevation (ft AMSL) } & 1508.77 & \multirow{2}{*}{$\begin{array}{l}1509.12 \\
\text { Elevation in Klassen } \\
\text { Test Well (ft AMSL) }\end{array}$} \\
\hline Date and Time & $\begin{array}{l}\text { Depth in Klassen } \\
\text { Domestic Well } \\
\text { (ft BGL) }\end{array}$ & $\begin{array}{c}\text { Depth in Klassen } \\
\text { Test Well } \\
\text { (ft BGL) }\end{array}$ & $\begin{array}{l}\text { Elevation in Klassen } \\
\text { Domestic Well } \\
\text { (ft AMSL) }\end{array}$ & \\
\hline 9/29/11 18:10 & 37.486 & 39.892 & 1471.28 & 1469.23 \\
\hline $9 / 29 / 1118: 20$ & 37.62 & 39.9 & 1471.15 & 1469.22 \\
\hline 9/29/11 18:30 & 38.077 & 39.907 & 1470.69 & 1469.22 \\
\hline 9/29/11 18:40 & 40.031 & 39.916 & 1468.74 & 1469.21 \\
\hline 9/29/11 18:50 & 38.952 & 39.923 & 1469.82 & 1469.20 \\
\hline 9/29/11 19:00 & 37.653 & 39.93 & 1471.12 & 1469.19 \\
\hline 9/29/11 19:10 & 37.894 & 39.939 & 1470.88 & 1469.18 \\
\hline 9/29/11 19:20 & 38.452 & 39.946 & 1470.32 & 1469.18 \\
\hline 9/29/11 19:30 & 40.263 & 39.956 & 1468.51 & 1469.17 \\
\hline 9/29/11 19:40 & 37.814 & 39.965 & 1470.96 & 1469.16 \\
\hline 9/29/11 19:50 & 37.892 & 39.972 & 1470.88 & 1469.15 \\
\hline 9/29/11 20:00 & 38.03 & 39.984 & 1470.74 & 1469.14 \\
\hline 9/29/11 20:10 & 38.105 & 39.996 & 1470.66 & 1469.13 \\
\hline $9 / 29 / 1120: 20$ & 38.199 & 40.007 & 1470.57 & 1469.12 \\
\hline 9/29/11 20:30 & 38.455 & 40.024 & 1470.31 & 1469.10 \\
\hline 9/29/11 20:40 & 38.87 & 40.036 & 1469.90 & 1469.09 \\
\hline 9/29/11 20:50 & 41.307 & 40.047 & 1467.46 & 1469.08 \\
\hline 9/29/11 21:00 & 38.263 & 40.061 & 1470.51 & 1469.06 \\
\hline 9/29/11 21:10 & 38.321 & 40.071 & 1470.45 & 1469.05 \\
\hline 9/29/11 21:20 & 38.309 & 40.075 & 1470.46 & 1469.05 \\
\hline 9/29/11 21:30 & 38.415 & 40.078 & 1470.35 & 1469.04 \\
\hline 9/29/11 21:40 & 39.044 & 40.08 & 1469.73 & 1469.04 \\
\hline 9/29/11 21:50 & 41.726 & 40.085 & 1467.04 & 1469.04 \\
\hline 9/29/11 22:00 & 38.124 & 40.09 & 1470.65 & 1469.03 \\
\hline 9/29/11 22:10 & 38.115 & 40.09 & 1470.65 & 1469.03 \\
\hline 9/29/11 22:20 & 38.103 & 40.09 & 1470.67 & 1469.03 \\
\hline 9/29/11 22:30 & 38.291 & 40.09 & 1470.48 & 1469.03 \\
\hline 9/29/11 22:40 & 41.527 & 40.094 & 1467.24 & 1469.03 \\
\hline 9/29/11 22:50 & 40.835 & 40.099 & 1467.93 & 1469.02 \\
\hline 9/29/11 23:00 & 39.229 & 40.106 & 1469.54 & 1469.02 \\
\hline 9/29/11 23:10 & 38.319 & 40.108 & 1470.45 & 1469.01 \\
\hline 9/29/11 23:20 & 38.232 & 40.106 & 1470.54 & 1469.02 \\
\hline $9 / 29 / 1123: 30$ & 38.349 & 40.104 & 1470.42 & 1469.02 \\
\hline 9/29/11 23:40 & 39.14 & 40.101 & 1469.63 & 1469.02 \\
\hline 9/29/11 23:50 & 42.139 & 40.094 & 1466.63 & 1469.03 \\
\hline 9/30/11 0:00 & 37.77 & 40.092 & 1471.00 & 1469.03 \\
\hline 9/30/11 0:10 & 37.765 & 40.087 & 1471.00 & 1469.04 \\
\hline 9/30/11 0:20 & 37.793 & 40.085 & 1470.98 & 1469.04 \\
\hline
\end{tabular}


TABLE S1.3 Automatically recorded groundwater levels in the Klassen wells, September 15, 2011, to March 13, 2012.

\begin{tabular}{|c|c|c|c|c|}
\hline \multicolumn{3}{|c|}{ Reference Elevation (ft AMSL) } & 1508.77 & \multirow{2}{*}{$\begin{array}{l}1509.12 \\
\text { Elevation in Klassen } \\
\text { Test Well (ft AMSL) }\end{array}$} \\
\hline Date and Time & $\begin{array}{l}\text { Depth in Klassen } \\
\text { Domestic Well } \\
(\mathrm{ft} \mathrm{BGL})\end{array}$ & $\begin{array}{c}\text { Depth in Klassen } \\
\text { Test Well } \\
\text { (ft BGL) }\end{array}$ & $\begin{array}{l}\text { Elevation in Klassen } \\
\text { Domestic Well } \\
\text { (ft AMSL) }\end{array}$ & \\
\hline 9/30/11 0:30 & 37.915 & 40.08 & 1470.85 & 1469.04 \\
\hline 9/30/11 0:40 & 38.513 & 40.075 & 1470.26 & 1469.05 \\
\hline 9/30/11 0:50 & 40.741 & 40.073 & 1468.03 & 1469.05 \\
\hline $9 / 30 / 11$ 1:00 & 37.533 & 40.068 & 1471.24 & 1469.05 \\
\hline 9/30/11 1:10 & 37.538 & 40.066 & 1471.23 & 1469.06 \\
\hline $9 / 30 / 11$ 1:20 & 37.564 & 40.061 & 1471.21 & 1469.06 \\
\hline 9/30/11 1:30 & 37.643 & 40.057 & 1471.13 & 1469.07 \\
\hline $9 / 30 / 11$ 1:40 & 37.981 & 40.054 & 1470.79 & 1469.07 \\
\hline $9 / 30 / 11$ 1:50 & 39.271 & 40.054 & 1469.50 & 1469.07 \\
\hline 9/30/11 2:00 & 40.533 & 40.052 & 1468.24 & 1469.07 \\
\hline $9 / 30 / 112: 10$ & 39.022 & 40.05 & 1469.75 & 1469.07 \\
\hline $9 / 30 / 112: 20$ & 37.465 & 40.047 & 1471.30 & 1469.08 \\
\hline $9 / 30 / 112: 30$ & 37.5 & 40.043 & 1471.27 & 1469.08 \\
\hline 9/30/11 2:40 & 37.552 & 40.038 & 1471.22 & 1469.08 \\
\hline $9 / 30 / 112: 50$ & 37.761 & 40.036 & 1471.01 & 1469.09 \\
\hline 9/30/11 3:00 & 38.635 & 40.031 & 1470.13 & 1469.09 \\
\hline 9/30/11 3:10 & 42.747 & 40.026 & 1466.02 & 1469.10 \\
\hline 9/30/11 3:20 & 42.172 & 40.022 & 1466.60 & 1469.10 \\
\hline 9/30/11 3:30 & 37.444 & 40.022 & 1471.33 & 1469.10 \\
\hline 9/30/11 3:40 & 37.437 & 40.019 & 1471.33 & 1469.10 \\
\hline 9/30/11 3:50 & 37.463 & 40.014 & 1471.31 & 1469.11 \\
\hline 9/30/11 4:00 & 37.568 & 40.007 & 1471.20 & 1469.12 \\
\hline 9/30/11 4:10 & 38.028 & 40.003 & 1470.74 & 1469.12 \\
\hline $9 / 30 / 114: 20$ & 39.728 & 39.996 & 1469.04 & 1469.13 \\
\hline 9/30/11 4:30 & 38.943 & 39.989 & 1469.83 & 1469.13 \\
\hline 9/30/11 4:40 & 37.221 & 39.984 & 1471.55 & 1469.14 \\
\hline $9 / 30 / 114: 50$ & 37.247 & 39.979 & 1471.52 & 1469.14 \\
\hline 9/30/11 5:00 & 37.306 & 39.972 & 1471.46 & 1469.15 \\
\hline 9/30/11 5:10 & 37.47 & 39.968 & 1471.30 & 1469.15 \\
\hline 9/30/11 5:20 & 38.073 & 39.961 & 1470.70 & 1469.16 \\
\hline 9/30/11 5:30 & 40 & 39.956 & 1468.77 & 1469.17 \\
\hline 9/30/11 5:40 & 37.866 & 39.949 & 1470.90 & 1469.17 \\
\hline 9/30/11 5:50 & 37.085 & 39.942 & 1471.68 & 1469.18 \\
\hline 9/30/11 6:00 & 39.442 & 39.935 & 1469.33 & 1469.19 \\
\hline 9/30/11 6:10 & 43.248 & 39.93 & 1465.52 & 1469.19 \\
\hline $9 / 30 / 116: 20$ & 38.539 & 39.93 & 1470.23 & 1469.19 \\
\hline $9 / 30 / 116: 30$ & 40.662 & 39.923 & 1468.11 & 1469.20 \\
\hline $9 / 30 / 116: 40$ & 37.116 & 39.918 & 1471.65 & 1469.20 \\
\hline
\end{tabular}


TABLE S1.3 Automatically recorded groundwater levels in the Klassen wells, September 15, 2011, to March 13, 2012.

\begin{tabular}{|c|c|c|c|c|}
\hline \multicolumn{3}{|c|}{ Reference Elevation (ft AMSL) } & 1508.77 & \multirow{2}{*}{$\begin{array}{l}1509.12 \\
\text { Elevation in Klassen } \\
\text { Test Well (ft AMSL) }\end{array}$} \\
\hline Date and Time & $\begin{array}{l}\text { Depth in Klassen } \\
\text { Domestic Well } \\
(\mathrm{ft} \mathrm{BGL})\end{array}$ & $\begin{array}{c}\text { Depth in Klassen } \\
\text { Test Well } \\
\text { (ft BGL) }\end{array}$ & $\begin{array}{l}\text { Elevation in Klassen } \\
\text { Domestic Well } \\
\text { (ft AMSL) }\end{array}$ & \\
\hline $9 / 30 / 116: 50$ & 37.142 & 39.916 & 1471.63 & 1469.21 \\
\hline $9 / 30 / 117: 00$ & 37.158 & 39.911 & 1471.61 & 1469.21 \\
\hline 9/30/11 7:10 & 37.224 & 39.907 & 1471.55 & 1469.22 \\
\hline $9 / 30 / 117: 20$ & 37.31 & 39.902 & 1471.46 & 1469.22 \\
\hline $9 / 30 / 117: 30$ & 37.634 & 39.897 & 1471.14 & 1469.23 \\
\hline $9 / 30 / 11$ 7:40 & 38.387 & 39.895 & 1470.38 & 1469.23 \\
\hline $9 / 30 / 117: 50$ & 40.831 & 39.89 & 1467.94 & 1469.23 \\
\hline $9 / 30 / 118: 00$ & 39.409 & 39.885 & 1469.36 & 1469.24 \\
\hline $9 / 30 / 118: 10$ & 41.194 & 39.881 & 1467.58 & 1469.24 \\
\hline $9 / 30 / 118: 20$ & 40.491 & 39.878 & 1468.28 & 1469.24 \\
\hline $9 / 30 / 118: 30$ & 37.303 & 39.876 & 1471.47 & 1469.25 \\
\hline $9 / 30 / 118: 40$ & 40.894 & 39.874 & 1467.88 & 1469.25 \\
\hline $9 / 30 / 118: 50$ & 39.707 & 39.869 & 1469.06 & 1469.25 \\
\hline 9/30/11 9:00 & 39.918 & 39.867 & 1468.85 & 1469.26 \\
\hline 9/30/11 9:10 & 38.626 & 39.864 & 1470.14 & 1469.26 \\
\hline 9/30/11 9:20 & 37.906 & 39.862 & 1470.86 & 1469.26 \\
\hline 9/30/11 9:30 & 37.28 & 39.857 & 1471.49 & 1469.27 \\
\hline 9/30/11 9:40 & 37.247 & 39.855 & 1471.52 & 1469.27 \\
\hline 9/30/11 9:50 & 37.697 & 39.855 & 1471.07 & 1469.27 \\
\hline 9/30/11 10:00 & 37.521 & 39.85 & 1471.25 & 1469.27 \\
\hline 9/30/11 10:10 & 37.327 & 39.85 & 1471.44 & 1469.27 \\
\hline 9/30/11 10:20 & 38.485 & 39.848 & 1470.28 & 1469.27 \\
\hline 9/30/11 10:30 & 37.676 & 39.846 & 1471.09 & 1469.28 \\
\hline 9/30/11 10:40 & 40.798 & 39.846 & 1467.97 & 1469.28 \\
\hline 9/30/11 10:50 & 39.799 & 39.848 & 1468.97 & 1469.27 \\
\hline 9/30/11 11:00 & 41.295 & 39.846 & 1467.47 & 1469.28 \\
\hline 9/30/11 11:10 & 38.082 & 39.843 & 1470.69 & 1469.28 \\
\hline 9/30/11 11:20 & 41.032 & 39.841 & 1467.74 & 1469.28 \\
\hline 9/30/11 11:30 & 40.68 & 39.843 & 1468.09 & 1469.28 \\
\hline 9/30/11 11:40 & 43.178 & 39.848 & 1465.59 & 1469.27 \\
\hline 9/30/11 11:50 & 42.008 & 39.85 & 1466.76 & 1469.27 \\
\hline 9/30/11 12:00 & 40.739 & 39.853 & 1468.03 & 1469.27 \\
\hline 9/30/11 12:10 & 39.808 & 39.857 & 1468.96 & 1469.27 \\
\hline 9/30/11 12:20 & 43.701 & 39.86 & 1465.07 & 1469.26 \\
\hline 9/30/11 12:30 & 41.452 & 39.864 & 1467.32 & 1469.26 \\
\hline 9/30/11 12:40 & 39.785 & 39.869 & 1468.98 & 1469.25 \\
\hline $9 / 30 / 1112: 50$ & 39.13 & 39.871 & 1469.64 & 1469.25 \\
\hline 9/30/11 13:00 & 43.312 & 39.876 & 1465.46 & 1469.25 \\
\hline
\end{tabular}


TABLE S1.3 Automatically recorded groundwater levels in the Klassen wells, September 15, 2011, to March 13, 2012.

\begin{tabular}{|c|c|c|c|c|}
\hline \multicolumn{3}{|c|}{ Reference Elevation (ft AMSL) } & 1508.77 & \multirow{2}{*}{$\begin{array}{l}1509.12 \\
\text { Elevation in Klassen } \\
\text { Test Well (ft AMSL) }\end{array}$} \\
\hline Date and Time & $\begin{array}{l}\text { Depth in Klassen } \\
\text { Domestic Well } \\
\text { (ft BGL) }\end{array}$ & $\begin{array}{c}\text { Depth in Klassen } \\
\text { Test Well } \\
\text { (ft BGL) }\end{array}$ & $\begin{array}{l}\text { Elevation in Klassen } \\
\text { Domestic Well } \\
\text { (ft AMSL) }\end{array}$ & \\
\hline 9/30/11 13:10 & 43.204 & 39.878 & 1465.57 & 1469.24 \\
\hline $9 / 30 / 1113: 20$ & 40.209 & 39.883 & 1468.56 & 1469.24 \\
\hline 9/30/11 13:30 & 41.145 & 39.888 & 1467.62 & 1469.23 \\
\hline 9/30/11 13:40 & 44.616 & 39.89 & 1464.15 & 1469.23 \\
\hline 9/30/11 13:50 & 41.982 & 39.892 & 1466.79 & 1469.23 \\
\hline 9/30/11 14:00 & 40.516 & 39.897 & 1468.25 & 1469.23 \\
\hline 9/30/11 14:10 & 45.162 & 39.9 & 1463.61 & 1469.22 \\
\hline $9 / 30 / 1114: 20$ & 43.544 & 39.902 & 1465.23 & 1469.22 \\
\hline 9/30/11 14:30 & 41.117 & 39.914 & 1467.65 & 1469.21 \\
\hline $9 / 30 / 1114: 40$ & 39.977 & 39.918 & 1468.79 & 1469.20 \\
\hline $9 / 30 / 1114: 50$ & 42.456 & 39.923 & 1466.31 & 1469.20 \\
\hline 9/30/11 15:00 & 44.276 & 39.925 & 1464.49 & 1469.20 \\
\hline 9/30/11 15:10 & 44.618 & 39.928 & 1464.15 & 1469.19 \\
\hline $9 / 30 / 1115: 20$ & 41.609 & 39.932 & 1467.16 & 1469.19 \\
\hline 9/30/11 15:30 & 41.07 & 39.935 & 1467.70 & 1469.19 \\
\hline 9/30/11 15:40 & 45.061 & 39.939 & 1463.71 & 1469.18 \\
\hline 9/30/11 15:50 & 42.721 & 39.944 & 1466.05 & 1469.18 \\
\hline 9/30/11 16:00 & 41.196 & 39.949 & 1467.57 & 1469.17 \\
\hline 9/30/11 16:10 & 40.423 & 39.953 & 1468.35 & 1469.17 \\
\hline $9 / 30 / 11$ 16:20 & 40.854 & 39.958 & 1467.92 & 1469.16 \\
\hline $9 / 30 / 1116: 30$ & 43.945 & 39.963 & 1464.82 & 1469.16 \\
\hline 9/30/11 16:40 & 44.815 & 39.968 & 1463.95 & 1469.15 \\
\hline $9 / 30 / 1116: 50$ & 43.345 & 39.968 & 1465.42 & 1469.15 \\
\hline 9/30/11 17:00 & 42.095 & 39.97 & 1466.67 & 1469.15 \\
\hline 9/30/11 17:10 & 40.047 & 39.975 & 1468.72 & 1469.15 \\
\hline $9 / 30 / 11$ 17:20 & 42.831 & 39.979 & 1465.94 & 1469.14 \\
\hline 9/30/11 17:30 & 45.04 & 39.979 & 1463.73 & 1469.14 \\
\hline 9/30/11 17:40 & 43.553 & 39.982 & 1465.22 & 1469.14 \\
\hline 9/30/11 17:50 & 41.255 & 39.984 & 1467.51 & 1469.14 \\
\hline 9/30/11 18:00 & 40.237 & 39.986 & 1468.53 & 1469.14 \\
\hline 9/30/11 18:10 & 39.827 & 39.986 & 1468.94 & 1469.14 \\
\hline 9/30/11 18:20 & 42.343 & 39.993 & 1466.43 & 1469.13 \\
\hline $9 / 30 / 11$ 18:30 & 44.895 & 40 & 1463.87 & 1469.12 \\
\hline 9/30/11 18:40 & 44.072 & 40.005 & 1464.70 & 1469.12 \\
\hline 9/30/11 18:50 & 44.06 & 40.007 & 1464.71 & 1469.12 \\
\hline 9/30/11 19:00 & 41.347 & 40.014 & 1467.42 & 1469.11 \\
\hline 9/30/11 19:10 & 40.615 & 40.017 & 1468.15 & 1469.11 \\
\hline 9/30/11 19:20 & 40.139 & 40.019 & 1468.63 & 1469.10 \\
\hline
\end{tabular}


TABLE S1.3 Automatically recorded groundwater levels in the Klassen wells, September 15, 2011, to March 13, 2012.

\begin{tabular}{|c|c|c|c|c|}
\hline \multicolumn{3}{|c|}{ Reference Elevation (ft AMSL) } & 1508.77 & \multirow{2}{*}{$\begin{array}{l}1509.12 \\
\text { Elevation in Klassen } \\
\text { Test Well (ft AMSL) }\end{array}$} \\
\hline Date and Time & $\begin{array}{l}\text { Depth in Klassen } \\
\text { Domestic Well } \\
\text { (ft BGL) }\end{array}$ & $\begin{array}{c}\text { Depth in Klassen } \\
\text { Test Well } \\
\text { (ft BGL) }\end{array}$ & $\begin{array}{l}\text { Elevation in Klassen } \\
\text { Domestic Well } \\
\text { (ft AMSL) }\end{array}$ & \\
\hline 9/30/11 19:30 & 41.424 & 40.022 & 1467.35 & 1469.10 \\
\hline 9/30/11 19:40 & 44.485 & 40.024 & 1464.28 & 1469.10 \\
\hline 9/30/11 19:50 & 43.012 & 40.022 & 1465.76 & 1469.10 \\
\hline $9 / 30 / 1120: 00$ & 44.004 & 40.019 & 1464.77 & 1469.10 \\
\hline 9/30/11 20:10 & 38.633 & 40.017 & 1470.14 & 1469.11 \\
\hline $9 / 30 / 1120: 20$ & 38.516 & 40.01 & 1470.25 & 1469.11 \\
\hline $9 / 30 / 1120: 30$ & 38.497 & 40.005 & 1470.27 & 1469.12 \\
\hline $9 / 30 / 1120: 40$ & 38.366 & 40.003 & 1470.40 & 1469.12 \\
\hline $9 / 30 / 1120: 50$ & 38.377 & 39.998 & 1470.39 & 1469.12 \\
\hline 9/30/11 21:00 & 39.581 & 39.989 & 1469.19 & 1469.13 \\
\hline 9/30/11 21:10 & 39.062 & 39.984 & 1469.71 & 1469.14 \\
\hline 9/30/11 21:20 & 37.979 & 39.977 & 1470.79 & 1469.15 \\
\hline 9/30/11 21:30 & 38.063 & 39.97 & 1470.71 & 1469.15 \\
\hline $9 / 30 / 1121: 40$ & 39.51 & 39.963 & 1469.26 & 1469.16 \\
\hline 9/30/11 21:50 & 37.803 & 39.956 & 1470.97 & 1469.17 \\
\hline 9/30/11 22:00 & 38.089 & 39.946 & 1470.68 & 1469.18 \\
\hline 9/30/11 22:10 & 42.257 & 39.939 & 1466.51 & 1469.18 \\
\hline 9/30/11 22:20 & 37.693 & 39.935 & 1471.08 & 1469.19 \\
\hline $9 / 30 / 1122: 30$ & 38.009 & 39.928 & 1470.76 & 1469.19 \\
\hline 9/30/11 22:40 & 37.512 & 39.918 & 1471.26 & 1469.20 \\
\hline $9 / 30 / 1122: 50$ & 37.589 & 39.914 & 1471.18 & 1469.21 \\
\hline 9/30/11 23:00 & 38.223 & 39.909 & 1470.55 & 1469.21 \\
\hline 9/30/11 23:10 & 37.793 & 39.907 & 1470.98 & 1469.22 \\
\hline 9/30/11 23:20 & 43.546 & 39.904 & 1465.22 & 1469.22 \\
\hline 9/30/11 23:30 & 42.329 & 39.904 & 1466.44 & 1469.22 \\
\hline $9 / 30 / 1123: 40$ & 37.74 & 39.902 & 1471.03 & 1469.22 \\
\hline 9/30/11 23:50 & 38.087 & 39.895 & 1470.68 & 1469.23 \\
\hline 10/1/11 0:00 & 37.482 & 39.89 & 1471.29 & 1469.23 \\
\hline 10/1/11 0:10 & 37.549 & 39.883 & 1471.22 & 1469.24 \\
\hline $10 / 1 / 110: 20$ & 41.977 & 39.876 & 1466.79 & 1469.25 \\
\hline 10/1/11 0:30 & 37.362 & 39.869 & 1471.41 & 1469.25 \\
\hline 10/1/11 0:40 & 37.93 & 39.862 & 1470.84 & 1469.26 \\
\hline 10/1/11 0:50 & 37.231 & 39.855 & 1471.54 & 1469.27 \\
\hline $10 / 1 / 11$ 1:00 & 37.341 & 39.85 & 1471.43 & 1469.27 \\
\hline $10 / 1 / 11$ 1:10 & 39.726 & 39.843 & 1469.04 & 1469.28 \\
\hline $10 / 1 / 11$ 1:20 & 37.179 & 39.834 & 1471.59 & 1469.29 \\
\hline $10 / 1 / 11$ 1:30 & 37.972 & 39.829 & 1470.80 & 1469.29 \\
\hline $10 / 1 / 11$ 1:40 & 37.066 & 39.822 & 1471.70 & 1469.30 \\
\hline
\end{tabular}


TABLE S1.3 Automatically recorded groundwater levels in the Klassen wells, September 15, 2011, to March 13, 2012.

\begin{tabular}{|c|c|c|c|c|}
\hline \multicolumn{3}{|c|}{ Reference Elevation (ft AMSL) } & 1508.77 & \multirow{2}{*}{$\begin{array}{l}1509.12 \\
\text { Elevation in Klassen } \\
\text { Test Well (ft AMSL) }\end{array}$} \\
\hline Date and Time & $\begin{array}{l}\text { Depth in Klassen } \\
\text { Domestic Well } \\
(\mathrm{ft} \mathrm{BGL})\end{array}$ & $\begin{array}{c}\text { Depth in Klassen } \\
\text { Test Well } \\
\text { (ft BGL) }\end{array}$ & $\begin{array}{l}\text { Elevation in Klassen } \\
\text { Domestic Well } \\
\text { (ft AMSL) }\end{array}$ & \\
\hline $10 / 1 / 111: 50$ & 37.207 & 39.815 & 1471.56 & 1469.31 \\
\hline $10 / 1 / 112: 00$ & 40.779 & 39.81 & 1467.99 & 1469.31 \\
\hline $10 / 1 / 112: 10$ & 37.043 & 39.803 & 1471.73 & 1469.32 \\
\hline $10 / 1 / 112: 20$ & 37.446 & 39.796 & 1471.32 & 1469.33 \\
\hline $10 / 1 / 112: 30$ & 36.937 & 39.792 & 1471.83 & 1469.33 \\
\hline $10 / 1 / 112: 40$ & 37.043 & 39.782 & 1471.73 & 1469.34 \\
\hline $10 / 1 / 112: 50$ & 41.572 & 39.775 & 1467.20 & 1469.35 \\
\hline $10 / 1 / 113: 00$ & 41.729 & 39.77 & 1467.04 & 1469.35 \\
\hline $10 / 1 / 113: 10$ & 36.933 & 39.763 & 1471.84 & 1469.36 \\
\hline $10 / 1 / 113: 20$ & 37.453 & 39.756 & 1471.32 & 1469.37 \\
\hline $10 / 1 / 113: 30$ & 36.825 & 39.752 & 1471.94 & 1469.37 \\
\hline $10 / 1 / 113: 40$ & 36.937 & 39.745 & 1471.83 & 1469.38 \\
\hline $10 / 1 / 113: 50$ & 40.845 & 39.735 & 1467.92 & 1469.39 \\
\hline $10 / 1 / 114: 00$ & 36.801 & 39.728 & 1471.97 & 1469.39 \\
\hline $10 / 1 / 114: 10$ & 37.388 & 39.721 & 1471.38 & 1469.40 \\
\hline $10 / 1 / 114: 20$ & 36.712 & 39.714 & 1472.06 & 1469.41 \\
\hline $10 / 1 / 114: 30$ & 36.839 & 39.707 & 1471.93 & 1469.42 \\
\hline 10/1/11 4:40 & 39.032 & 39.702 & 1469.74 & 1469.42 \\
\hline $10 / 1 / 114: 50$ & 36.708 & 39.695 & 1472.06 & 1469.43 \\
\hline $10 / 1 / 115: 00$ & 37.477 & 39.686 & 1471.29 & 1469.44 \\
\hline $10 / 1 / 115: 10$ & 36.623 & 39.681 & 1472.15 & 1469.44 \\
\hline $10 / 1 / 115: 20$ & 36.872 & 39.672 & 1471.90 & 1469.45 \\
\hline 10/1/11 5:30 & 36.785 & 39.667 & 1471.98 & 1469.46 \\
\hline $10 / 1 / 115: 40$ & 39.843 & 39.663 & 1468.93 & 1469.46 \\
\hline 10/1/11 5:50 & 36.637 & 39.655 & 1472.13 & 1469.47 \\
\hline $10 / 1 / 116: 00$ & 37.003 & 39.648 & 1471.77 & 1469.47 \\
\hline $10 / 1 / 116: 10$ & 37.672 & 39.641 & 1471.10 & 1469.48 \\
\hline $10 / 1 / 116: 20$ & 37.545 & 39.639 & 1471.22 & 1469.48 \\
\hline 10/1/11 6:30 & 36.748 & 39.634 & 1472.02 & 1469.49 \\
\hline 10/1/11 6:40 & 37.451 & 39.63 & 1471.32 & 1469.49 \\
\hline $10 / 1 / 116: 50$ & 36.6 & 39.623 & 1472.17 & 1469.50 \\
\hline $10 / 1 / 11$ 7:00 & 36.719 & 39.618 & 1472.05 & 1469.50 \\
\hline $10 / 1 / 117: 10$ & 40.158 & 39.609 & 1468.61 & 1469.51 \\
\hline $10 / 1 / 117: 20$ & 36.562 & 39.604 & 1472.21 & 1469.52 \\
\hline $10 / 1 / 117: 30$ & 36.771 & 39.597 & 1472.00 & 1469.53 \\
\hline $10 / 1 / 117: 40$ & 36.733 & 39.594 & 1472.04 & 1469.53 \\
\hline $10 / 1 / 117: 50$ & 39.1 & 39.587 & 1469.67 & 1469.54 \\
\hline $10 / 1 / 118: 00$ & 36.565 & 39.583 & 1472.20 & 1469.54 \\
\hline
\end{tabular}


TABLE S1.3 Automatically recorded groundwater levels in the Klassen wells, September 15, 2011, to March 13, 2012.

\begin{tabular}{|c|c|c|c|c|}
\hline \multicolumn{3}{|c|}{ Reference Elevation (ft AMSL) } & 1508.77 & \multirow{2}{*}{$\begin{array}{l}1509.12 \\
\text { Elevation in Klassen } \\
\text { Test Well (ft AMSL) }\end{array}$} \\
\hline Date and Time & $\begin{array}{l}\text { Depth in Klassen } \\
\text { Domestic Well } \\
(\mathrm{ft} \mathrm{BGL})\end{array}$ & $\begin{array}{c}\text { Depth in Klassen } \\
\text { Test Well } \\
\text { (ft BGL) }\end{array}$ & $\begin{array}{l}\text { Elevation in Klassen } \\
\text { Domestic Well } \\
\text { (ft AMSL) }\end{array}$ & \\
\hline $10 / 1 / 118: 10$ & 36.731 & 39.583 & 1472.04 & 1469.54 \\
\hline $10 / 1 / 118: 20$ & 39.339 & 39.58 & 1469.43 & 1469.54 \\
\hline $10 / 1 / 118: 30$ & 36.752 & 39.58 & 1472.02 & 1469.54 \\
\hline $10 / 1 / 118: 40$ & 36.935 & 39.58 & 1471.83 & 1469.54 \\
\hline $10 / 1 / 118: 50$ & 37.05 & 39.578 & 1471.72 & 1469.54 \\
\hline 10/1/11 9:00 & 37.681 & 39.573 & 1471.09 & 1469.55 \\
\hline 10/1/11 9:10 & 36.642 & 39.569 & 1472.13 & 1469.55 \\
\hline $10 / 1 / 119: 20$ & 36.75 & 39.564 & 1472.02 & 1469.56 \\
\hline 10/1/11 9:30 & 39.902 & 39.559 & 1468.87 & 1469.56 \\
\hline $10 / 1 / 119: 40$ & 36.59 & 39.555 & 1472.18 & 1469.57 \\
\hline 10/1/11 9:50 & 36.846 & 39.55 & 1471.92 & 1469.57 \\
\hline 10/1/11 10:00 & 36.485 & 39.543 & 1472.28 & 1469.58 \\
\hline 10/1/11 10:10 & 36.597 & 39.538 & 1472.17 & 1469.58 \\
\hline $10 / 1 / 1110: 20$ & 36.919 & 39.533 & 1471.85 & 1469.59 \\
\hline 10/1/11 10:30 & 38.14 & 39.531 & 1470.63 & 1469.59 \\
\hline 10/1/11 10:40 & 39.846 & 39.526 & 1468.92 & 1469.60 \\
\hline 10/1/11 10:50 & 37.09 & 39.526 & 1471.68 & 1469.60 \\
\hline 10/1/11 11:00 & 36.921 & 39.526 & 1471.85 & 1469.60 \\
\hline 10/1/11 11:10 & 37.292 & 39.524 & 1471.48 & 1469.60 \\
\hline 10/1/11 11:20 & 54.595 & 39.526 & 1454.17 & 1469.60 \\
\hline 10/1/11 11:30 & 40.202 & 39.543 & 1468.57 & 1469.58 \\
\hline 10/1/11 11:40 & 38.281 & 39.552 & 1470.49 & 1469.57 \\
\hline 10/1/11 11:50 & 39.163 & 39.552 & 1469.61 & 1469.57 \\
\hline 10/1/11 12:00 & 41.075 & 39.55 & 1467.69 & 1469.57 \\
\hline 10/1/11 12:10 & 37.127 & 39.55 & 1471.64 & 1469.57 \\
\hline $10 / 1 / 11$ 12:20 & 37.102 & 39.548 & 1471.67 & 1469.57 \\
\hline 10/1/11 12:30 & 37.207 & 39.543 & 1471.56 & 1469.58 \\
\hline 10/1/11 12:40 & 37.125 & 39.541 & 1471.64 & 1469.58 \\
\hline $10 / 1 / 11$ 12:50 & 37.151 & 39.538 & 1471.62 & 1469.58 \\
\hline 10/1/11 13:00 & 37.496 & 39.533 & 1471.27 & 1469.59 \\
\hline 10/1/11 13:10 & 38.983 & 39.529 & 1469.79 & 1469.59 \\
\hline 10/1/11 13:20 & 39.477 & 39.526 & 1469.29 & 1469.60 \\
\hline 10/1/11 13:30 & 37.224 & 39.524 & 1471.55 & 1469.60 \\
\hline 10/1/11 13:40 & 37.7 & 39.519 & 1471.07 & 1469.60 \\
\hline 10/1/11 13:50 & 37.791 & 39.517 & 1470.98 & 1469.61 \\
\hline 10/1/11 14:00 & 40.202 & 39.512 & 1468.57 & 1469.61 \\
\hline 10/1/11 14:10 & 36.754 & 39.508 & 1472.02 & 1469.61 \\
\hline 10/1/11 14:20 & 36.79 & 39.505 & 1471.98 & 1469.62 \\
\hline
\end{tabular}


TABLE S1.3 Automatically recorded groundwater levels in the Klassen wells, September 15, 2011, to March 13, 2012.

\begin{tabular}{|c|c|c|c|c|}
\hline \multicolumn{3}{|c|}{ Reference Elevation (ft AMSL) } & 1508.77 & \multirow{2}{*}{$\begin{array}{l}1509.12 \\
\text { Elevation in Klassen } \\
\text { Test Well (ft AMSL) }\end{array}$} \\
\hline Date and Time & $\begin{array}{l}\text { Depth in Klassen } \\
\text { Domestic Well } \\
\text { (ft BGL) }\end{array}$ & $\begin{array}{c}\text { Depth in Klassen } \\
\text { Test Well } \\
\text { (ft BGL) }\end{array}$ & $\begin{array}{l}\text { Elevation in Klassen } \\
\text { Domestic Well } \\
\text { (ft AMSL) }\end{array}$ & \\
\hline 10/1/11 14:30 & 36.82 & 39.503 & 1471.95 & 1469.62 \\
\hline 10/1/11 14:40 & 36.844 & 39.498 & 1471.93 & 1469.62 \\
\hline 10/1/11 14:50 & 36.905 & 39.494 & 1471.86 & 1469.63 \\
\hline 10/1/11 15:00 & 36.949 & 39.491 & 1471.82 & 1469.63 \\
\hline 10/1/11 15:10 & 37.134 & 39.489 & 1471.64 & 1469.63 \\
\hline $10 / 1 / 11$ 15:20 & 37.995 & 39.484 & 1470.77 & 1469.64 \\
\hline 10/1/11 15:30 & 42.629 & 39.482 & 1466.14 & 1469.64 \\
\hline 10/1/11 15:40 & 41.548 & 39.48 & 1467.22 & 1469.64 \\
\hline 10/1/11 15:50 & 38.309 & 39.477 & 1470.46 & 1469.65 \\
\hline 10/1/11 16:00 & 36.686 & 39.477 & 1472.08 & 1469.65 \\
\hline 10/1/11 16:10 & 36.858 & 39.475 & 1471.91 & 1469.65 \\
\hline 10/1/11 16:20 & 36.708 & 39.472 & 1472.06 & 1469.65 \\
\hline 10/1/11 16:30 & 36.748 & 39.47 & 1472.02 & 1469.65 \\
\hline 10/1/11 16:40 & 41.417 & 39.468 & 1467.35 & 1469.65 \\
\hline 10/1/11 16:50 & 41.143 & 39.465 & 1467.63 & 1469.66 \\
\hline 10/1/11 17:00 & 36.661 & 39.465 & 1472.11 & 1469.66 \\
\hline 10/1/11 17:10 & 36.682 & 39.463 & 1472.09 & 1469.66 \\
\hline 10/1/11 17:20 & 36.755 & 39.461 & 1472.01 & 1469.66 \\
\hline 10/1/11 17:30 & 37.083 & 39.458 & 1471.69 & 1469.66 \\
\hline 10/1/11 17:40 & 38.771 & 39.454 & 1470.00 & 1469.67 \\
\hline 10/1/11 17:50 & 36.82 & 39.454 & 1471.95 & 1469.67 \\
\hline 10/1/11 18:00 & 37.008 & 39.451 & 1471.76 & 1469.67 \\
\hline 10/1/11 18:10 & 37.585 & 39.449 & 1471.18 & 1469.67 \\
\hline 10/1/11 18:20 & 40.814 & 39.447 & 1467.96 & 1469.68 \\
\hline 10/1/11 18:30 & 36.527 & 39.447 & 1472.24 & 1469.68 \\
\hline 10/1/11 18:40 & 36.56 & 39.444 & 1472.21 & 1469.68 \\
\hline 10/1/11 18:50 & 38.138 & 39.442 & 1470.63 & 1469.68 \\
\hline 10/1/11 19:00 & 37.256 & 39.442 & 1471.51 & 1469.68 \\
\hline 10/1/11 19:10 & 36.919 & 39.442 & 1471.85 & 1469.68 \\
\hline 10/1/11 19:20 & 36.78 & 39.442 & 1471.99 & 1469.68 \\
\hline 10/1/11 19:30 & 37.782 & 39.444 & 1470.99 & 1469.68 \\
\hline 10/1/11 19:40 & 36.881 & 39.449 & 1471.89 & 1469.67 \\
\hline 10/1/11 19:50 & 36.6 & 39.449 & 1472.17 & 1469.67 \\
\hline 10/1/11 20:00 & 36.457 & 39.447 & 1472.31 & 1469.68 \\
\hline 10/1/11 20:10 & 36.353 & 39.442 & 1472.42 & 1469.68 \\
\hline 10/1/11 20:20 & 36.731 & 39.44 & 1472.04 & 1469.68 \\
\hline 10/1/11 20:30 & 36.323 & 39.437 & 1472.45 & 1469.69 \\
\hline 10/1/11 20:40 & 36.844 & 39.435 & 1471.93 & 1469.69 \\
\hline
\end{tabular}


TABLE S1.3 Automatically recorded groundwater levels in the Klassen wells, September 15, 2011, to March 13, 2012.

\begin{tabular}{|c|c|c|c|c|}
\hline \multicolumn{3}{|c|}{ Reference Elevation (ft AMSL) } & 1508.77 & \multirow{2}{*}{$\begin{array}{l}1509.12 \\
\text { Elevation in Klassen } \\
\text { Test Well (ft AMSL) }\end{array}$} \\
\hline Date and Time & $\begin{array}{l}\text { Depth in Klassen } \\
\text { Domestic Well } \\
\text { (ft BGL) }\end{array}$ & $\begin{array}{c}\text { Depth in Klassen } \\
\text { Test Well } \\
\text { (ft BGL) }\end{array}$ & $\begin{array}{l}\text { Elevation in Klassen } \\
\text { Domestic Well } \\
\text { (ft AMSL) }\end{array}$ & \\
\hline $10 / 1 / 1120: 50$ & 36.281 & 39.435 & 1472.49 & 1469.69 \\
\hline 10/1/11 21:00 & 36.576 & 39.433 & 1472.19 & 1469.69 \\
\hline 10/1/11 21:10 & 36.452 & 39.433 & 1472.32 & 1469.69 \\
\hline $10 / 1 / 1121: 20$ & 40.209 & 39.433 & 1468.56 & 1469.69 \\
\hline 10/1/11 21:30 & 36.304 & 39.43 & 1472.47 & 1469.69 \\
\hline 10/1/11 21:40 & 36.712 & 39.43 & 1472.06 & 1469.69 \\
\hline 10/1/11 21:50 & 36.257 & 39.433 & 1472.51 & 1469.69 \\
\hline 10/1/11 22:00 & 36.375 & 39.433 & 1472.39 & 1469.69 \\
\hline $10 / 1 / 1122: 10$ & 36.185 & 39.433 & 1472.58 & 1469.69 \\
\hline $10 / 1 / 1122: 20$ & 36.269 & 39.435 & 1472.50 & 1469.69 \\
\hline $10 / 1 / 1122: 30$ & 40.369 & 39.435 & 1468.40 & 1469.69 \\
\hline 10/1/11 22:40 & 39.297 & 39.44 & 1469.47 & 1469.68 \\
\hline $10 / 1 / 1122: 50$ & 36.839 & 39.449 & 1471.93 & 1469.67 \\
\hline 10/1/11 23:00 & 39.688 & 39.454 & 1469.08 & 1469.67 \\
\hline 10/1/11 23:10 & 36.581 & 39.456 & 1472.19 & 1469.67 \\
\hline $10 / 1 / 1123: 20$ & 36.715 & 39.461 & 1472.05 & 1469.66 \\
\hline $10 / 1 / 1123: 30$ & 36.384 & 39.461 & 1472.39 & 1469.66 \\
\hline $10 / 1 / 11$ 23:40 & 36.426 & 39.463 & 1472.34 & 1469.66 \\
\hline 10/1/11 23:50 & 38.732 & 39.463 & 1470.04 & 1469.66 \\
\hline $10 / 2 / 110: 00$ & 36.302 & 39.461 & 1472.47 & 1469.66 \\
\hline $10 / 2 / 110: 10$ & 36.712 & 39.461 & 1472.06 & 1469.66 \\
\hline $10 / 2 / 110: 20$ & 36.217 & 39.458 & 1472.55 & 1469.66 \\
\hline 10/2/11 0:30 & 36.377 & 39.458 & 1472.39 & 1469.66 \\
\hline $10 / 2 / 110: 40$ & 36.175 & 39.458 & 1472.59 & 1469.66 \\
\hline $10 / 2 / 110: 50$ & 36.253 & 39.456 & 1472.52 & 1469.67 \\
\hline $10 / 2 / 111: 00$ & 38.07 & 39.456 & 1470.70 & 1469.67 \\
\hline $10 / 2 / 111: 10$ & 36.171 & 39.454 & 1472.60 & 1469.67 \\
\hline $10 / 2 / 11$ 1:20 & 36.473 & 39.451 & 1472.30 & 1469.67 \\
\hline $10 / 2 / 11$ 1:30 & 36.107 & 39.451 & 1472.66 & 1469.67 \\
\hline $10 / 2 / 11$ 1:40 & 36.227 & 39.451 & 1472.54 & 1469.67 \\
\hline $10 / 2 / 11$ 1:50 & 39.168 & 39.447 & 1469.60 & 1469.68 \\
\hline $10 / 2 / 112: 00$ & 36.168 & 39.447 & 1472.60 & 1469.68 \\
\hline $10 / 2 / 112: 10$ & 37.163 & 39.449 & 1471.61 & 1469.67 \\
\hline $10 / 2 / 112: 20$ & 36.117 & 39.449 & 1472.65 & 1469.67 \\
\hline $10 / 2 / 112: 30$ & 36.306 & 39.447 & 1472.46 & 1469.68 \\
\hline $10 / 2 / 112: 40$ & 36.056 & 39.444 & 1472.71 & 1469.68 \\
\hline $10 / 2 / 112: 50$ & 36.152 & 39.442 & 1472.62 & 1469.68 \\
\hline $10 / 2 / 113: 00$ & 36.919 & 39.44 & 1471.85 & 1469.68 \\
\hline
\end{tabular}


TABLE S1.3 Automatically recorded groundwater levels in the Klassen wells, September 15, 2011, to March 13, 2012.

\begin{tabular}{|c|c|c|c|c|}
\hline \multicolumn{3}{|c|}{ Reference Elevation (ft AMSL) } & 1508.77 & \multirow{2}{*}{$\begin{array}{l}1509.12 \\
\text { Elevation in Klassen } \\
\text { Test Well (ft AMSL) }\end{array}$} \\
\hline Date and Time & $\begin{array}{l}\text { Depth in Klassen } \\
\text { Domestic Well } \\
(\mathrm{ft} \mathrm{BGL})\end{array}$ & $\begin{array}{c}\text { Depth in Klassen } \\
\text { Test Well } \\
\text { (ft BGL) }\end{array}$ & $\begin{array}{l}\text { Elevation in Klassen } \\
\text { Domestic Well } \\
\text { (ft AMSL) }\end{array}$ & \\
\hline $10 / 2 / 113: 10$ & 36.084 & 39.437 & 1472.69 & 1469.69 \\
\hline $10 / 2 / 113: 20$ & 36.236 & 39.435 & 1472.53 & 1469.69 \\
\hline $10 / 2 / 113: 30$ & 36.013 & 39.435 & 1472.76 & 1469.69 \\
\hline $10 / 2 / 113: 40$ & 36.103 & 39.43 & 1472.67 & 1469.69 \\
\hline $10 / 2 / 113: 50$ & 38.516 & 39.428 & 1470.25 & 1469.69 \\
\hline $10 / 2 / 114: 00$ & 36.027 & 39.426 & 1472.74 & 1469.70 \\
\hline $10 / 2 / 114: 10$ & 36.382 & 39.423 & 1472.39 & 1469.70 \\
\hline $10 / 2 / 114: 20$ & 35.974 & 39.421 & 1472.80 & 1469.70 \\
\hline $10 / 2 / 114: 30$ & 36.095 & 39.421 & 1472.67 & 1469.70 \\
\hline $10 / 2 / 114: 40$ & 39.212 & 39.416 & 1469.56 & 1469.71 \\
\hline $10 / 2 / 114: 50$ & 36.006 & 39.414 & 1472.76 & 1469.71 \\
\hline $10 / 2 / 115: 00$ & 37.029 & 39.411 & 1471.74 & 1469.71 \\
\hline $10 / 2 / 115: 10$ & 35.948 & 39.409 & 1472.82 & 1469.71 \\
\hline $10 / 2 / 115: 20$ & 36.145 & 39.407 & 1472.62 & 1469.72 \\
\hline $10 / 2 / 115: 30$ & 41.717 & 39.404 & 1467.05 & 1469.72 \\
\hline $10 / 2 / 115: 40$ & 36.016 & 39.402 & 1472.75 & 1469.72 \\
\hline $10 / 2 / 115: 50$ & 41.06 & 39.4 & 1467.71 & 1469.72 \\
\hline $10 / 2 / 116: 00$ & 36.412 & 39.402 & 1472.36 & 1469.72 \\
\hline $10 / 2 / 116: 10$ & 39.433 & 39.402 & 1469.34 & 1469.72 \\
\hline $10 / 2 / 116: 20$ & 36.44 & 39.4 & 1472.33 & 1469.72 \\
\hline $10 / 2 / 116: 30$ & 37.369 & 39.402 & 1471.40 & 1469.72 \\
\hline $10 / 2 / 116: 40$ & 36.091 & 39.4 & 1472.68 & 1469.72 \\
\hline $10 / 2 / 116: 50$ & 36.253 & 39.397 & 1472.52 & 1469.73 \\
\hline $10 / 2 / 117: 00$ & 40.648 & 39.395 & 1468.12 & 1469.73 \\
\hline 10/2/11 7:10 & 36.049 & 39.39 & 1472.72 & 1469.73 \\
\hline $10 / 2 / 117: 20$ & 36.74 & 39.39 & 1472.03 & 1469.73 \\
\hline $10 / 2 / 117: 30$ & 35.967 & 39.386 & 1472.80 & 1469.74 \\
\hline $10 / 2 / 117: 40$ & 36.114 & 39.383 & 1472.66 & 1469.74 \\
\hline $10 / 2 / 117: 50$ & 39.815 & 39.381 & 1468.95 & 1469.74 \\
\hline $10 / 2 / 118: 00$ & 39.949 & 39.379 & 1468.82 & 1469.74 \\
\hline 10/2/11 8:10 & 36.023 & 39.376 & 1472.75 & 1469.75 \\
\hline $10 / 2 / 118: 20$ & 37.376 & 39.374 & 1471.39 & 1469.75 \\
\hline $10 / 2 / 118: 30$ & 36.656 & 39.376 & 1472.11 & 1469.75 \\
\hline $10 / 2 / 118: 40$ & 37.045 & 39.379 & 1471.72 & 1469.74 \\
\hline $10 / 2 / 118: 50$ & 36.119 & 39.379 & 1472.65 & 1469.74 \\
\hline 10/2/11 9:00 & 36.222 & 39.379 & 1472.55 & 1469.74 \\
\hline 10/2/11 9:10 & 35.992 & 39.376 & 1472.78 & 1469.75 \\
\hline $10 / 2 / 119: 20$ & 36.114 & 39.379 & 1472.66 & 1469.74 \\
\hline
\end{tabular}


TABLE S1.3 Automatically recorded groundwater levels in the Klassen wells, September 15, 2011, to March 13, 2012.

\begin{tabular}{|c|c|c|c|c|}
\hline \multicolumn{3}{|c|}{ Reference Elevation (ft AMSL) } & 1508.77 & \multirow{2}{*}{$\begin{array}{l}1509.12 \\
\text { Elevation in Klassen } \\
\text { Test Well (ft AMSL) }\end{array}$} \\
\hline Date and Time & $\begin{array}{l}\text { Depth in Klassen } \\
\text { Domestic Well } \\
\text { (ft BGL) }\end{array}$ & $\begin{array}{c}\text { Depth in Klassen } \\
\text { Test Well } \\
\text { (ft BGL) }\end{array}$ & $\begin{array}{l}\text { Elevation in Klassen } \\
\text { Domestic Well } \\
\text { (ft AMSL) }\end{array}$ & \\
\hline 10/2/11 9:30 & 37.289 & 39.381 & 1471.48 & 1469.74 \\
\hline 10/2/11 9:40 & 36.056 & 39.383 & 1472.71 & 1469.74 \\
\hline 10/2/11 9:50 & 36.253 & 39.386 & 1472.52 & 1469.74 \\
\hline 10/2/11 10:00 & 36.049 & 39.39 & 1472.72 & 1469.73 \\
\hline 10/2/11 10:10 & 36.121 & 39.395 & 1472.65 & 1469.73 \\
\hline 10/2/11 10:20 & 38.091 & 39.397 & 1470.68 & 1469.73 \\
\hline 10/2/11 10:30 & 36.037 & 39.4 & 1472.73 & 1469.72 \\
\hline 10/2/11 10:40 & 36.283 & 39.402 & 1472.49 & 1469.72 \\
\hline 10/2/11 10:50 & 35.957 & 39.404 & 1472.81 & 1469.72 \\
\hline 10/2/11 11:00 & 36.051 & 39.414 & 1472.72 & 1469.71 \\
\hline 10/2/11 11:10 & 38.544 & 39.419 & 1470.23 & 1469.70 \\
\hline 10/2/11 11:20 & 35.955 & 39.426 & 1472.81 & 1469.70 \\
\hline 10/2/11 11:30 & 36.227 & 39.428 & 1472.54 & 1469.69 \\
\hline 10/2/11 11:40 & 35.889 & 39.433 & 1472.88 & 1469.69 \\
\hline 10/2/11 11:50 & 35.988 & 39.435 & 1472.78 & 1469.69 \\
\hline 10/2/11 12:00 & 38.209 & 39.437 & 1470.56 & 1469.69 \\
\hline 10/2/11 12:10 & 36.006 & 39.444 & 1472.76 & 1469.68 \\
\hline $10 / 2 / 11$ 12:20 & 36.253 & 39.451 & 1472.52 & 1469.67 \\
\hline 10/2/11 12:30 & 36.023 & 39.458 & 1472.75 & 1469.66 \\
\hline $10 / 2 / 11$ 12:40 & 36.133 & 39.465 & 1472.64 & 1469.66 \\
\hline $10 / 2 / 1112: 50$ & 37.207 & 39.47 & 1471.56 & 1469.65 \\
\hline 10/2/11 13:00 & 36.011 & 39.472 & 1472.76 & 1469.65 \\
\hline 10/2/11 13:10 & 36.14 & 39.472 & 1472.63 & 1469.65 \\
\hline $10 / 2 / 11$ 13:20 & 39.552 & 39.472 & 1469.22 & 1469.65 \\
\hline 10/2/11 13:30 & 35.981 & 39.472 & 1472.79 & 1469.65 \\
\hline 10/2/11 13:40 & 36.231 & 39.475 & 1472.54 & 1469.65 \\
\hline $10 / 2 / 11$ 13:50 & 36.21 & 39.475 & 1472.56 & 1469.65 \\
\hline 10/2/11 14:00 & 38.781 & 39.475 & 1469.99 & 1469.65 \\
\hline 10/2/11 14:10 & 35.988 & 39.475 & 1472.78 & 1469.65 \\
\hline 10/2/11 14:20 & 36.175 & 39.472 & 1472.59 & 1469.65 \\
\hline 10/2/11 14:30 & 35.896 & 39.472 & 1472.87 & 1469.65 \\
\hline 10/2/11 14:40 & 35.964 & 39.47 & 1472.81 & 1469.65 \\
\hline $10 / 2 / 1114: 50$ & 36.527 & 39.47 & 1472.24 & 1469.65 \\
\hline 10/2/11 15:00 & 36.128 & 39.47 & 1472.64 & 1469.65 \\
\hline 10/2/11 15:10 & 40.563 & 39.47 & 1468.21 & 1469.65 \\
\hline 10/2/11 15:20 & 35.971 & 39.472 & 1472.80 & 1469.65 \\
\hline 10/2/11 15:30 & 36.318 & 39.472 & 1472.45 & 1469.65 \\
\hline $10 / 2 / 11$ 15:40 & 35.901 & 39.472 & 1472.87 & 1469.65 \\
\hline
\end{tabular}


TABLE S1.3 Automatically recorded groundwater levels in the Klassen wells, September 15, 2011, to March 13, 2012.

\begin{tabular}{|c|c|c|c|c|}
\hline \multicolumn{3}{|c|}{ Reference Elevation (ft AMSL) } & 1508.77 & \multirow{2}{*}{$\begin{array}{l}1509.12 \\
\text { Elevation in Klassen } \\
\text { Test Well (ft AMSL) }\end{array}$} \\
\hline Date and Time & $\begin{array}{l}\text { Depth in Klassen } \\
\text { Domestic Well } \\
\text { (ft BGL) }\end{array}$ & $\begin{array}{c}\text { Depth in Klassen } \\
\text { Test Well } \\
\text { (ft BGL) }\end{array}$ & $\begin{array}{l}\text { Elevation in Klassen } \\
\text { Domestic Well } \\
\text { (ft AMSL) }\end{array}$ & \\
\hline $10 / 2 / 11$ 15:50 & 35.983 & 39.475 & 1472.79 & 1469.65 \\
\hline 10/2/11 16:00 & 36.093 & 39.475 & 1472.68 & 1469.65 \\
\hline 10/2/11 16:10 & 36.876 & 39.48 & 1471.89 & 1469.64 \\
\hline 10/2/11 16:20 & 36.21 & 39.494 & 1472.56 & 1469.63 \\
\hline 10/2/11 16:30 & 36.461 & 39.51 & 1472.31 & 1469.61 \\
\hline 10/2/11 16:40 & 36.536 & 39.524 & 1472.23 & 1469.60 \\
\hline $10 / 2 / 1116: 50$ & 37.733 & 39.529 & 1471.04 & 1469.59 \\
\hline 10/2/11 17:00 & 36.405 & 39.531 & 1472.36 & 1469.59 \\
\hline 10/2/11 17:10 & 36.52 & 39.536 & 1472.25 & 1469.59 \\
\hline 10/2/11 17:20 & 40.892 & 39.543 & 1467.88 & 1469.58 \\
\hline 10/2/11 17:30 & 36.417 & 39.55 & 1472.35 & 1469.57 \\
\hline 10/2/11 17:40 & 36.672 & 39.552 & 1472.10 & 1469.57 \\
\hline $10 / 2 / 1117: 50$ & 36.698 & 39.555 & 1472.07 & 1469.57 \\
\hline 10/2/11 18:00 & 36.677 & 39.562 & 1472.09 & 1469.56 \\
\hline 10/2/11 18:10 & 40.057 & 39.566 & 1468.71 & 1469.56 \\
\hline $10 / 2 / 11$ 18:20 & 36.419 & 39.569 & 1472.35 & 1469.55 \\
\hline $10 / 2 / 11$ 18:30 & 36.689 & 39.573 & 1472.08 & 1469.55 \\
\hline 10/2/11 18:40 & 36.609 & 39.578 & 1472.16 & 1469.54 \\
\hline $10 / 2 / 11$ 18:50 & 36.682 & 39.585 & 1472.09 & 1469.54 \\
\hline 10/2/11 19:00 & 37.193 & 39.587 & 1471.58 & 1469.54 \\
\hline 10/2/11 19:10 & 36.433 & 39.587 & 1472.34 & 1469.54 \\
\hline 10/2/11 19:20 & 36.632 & 39.587 & 1472.14 & 1469.54 \\
\hline 10/2/11 19:30 & 38.802 & 39.592 & 1469.97 & 1469.53 \\
\hline 10/2/11 19:40 & 36.651 & 39.599 & 1472.12 & 1469.52 \\
\hline 10/2/11 19:50 & 36.579 & 39.606 & 1472.19 & 1469.52 \\
\hline 10/2/11 20:00 & 36.569 & 39.611 & 1472.20 & 1469.51 \\
\hline 10/2/11 20:10 & 36.546 & 39.616 & 1472.22 & 1469.51 \\
\hline $10 / 2 / 1120: 20$ & 36.879 & 39.623 & 1471.89 & 1469.50 \\
\hline $10 / 2 / 1120: 30$ & 36.651 & 39.625 & 1472.12 & 1469.50 \\
\hline $10 / 2 / 1120: 40$ & 36.558 & 39.625 & 1472.21 & 1469.50 \\
\hline $10 / 2 / 1120: 50$ & 36.494 & 39.625 & 1472.28 & 1469.50 \\
\hline 10/2/11 21:00 & 36.499 & 39.625 & 1472.27 & 1469.50 \\
\hline 10/2/11 21:10 & 36.485 & 39.627 & 1472.28 & 1469.50 \\
\hline 10/2/11 21:20 & 36.48 & 39.627 & 1472.29 & 1469.50 \\
\hline 10/2/11 21:30 & 36.482 & 39.63 & 1472.29 & 1469.49 \\
\hline $10 / 2 / 1121: 40$ & 36.45 & 39.63 & 1472.32 & 1469.49 \\
\hline $10 / 2 / 1121: 50$ & 39.792 & 39.63 & 1468.98 & 1469.49 \\
\hline $10 / 2 / 1122: 00$ & 36.621 & 39.632 & 1472.15 & 1469.49 \\
\hline
\end{tabular}


TABLE S1.3 Automatically recorded groundwater levels in the Klassen wells, September 15, 2011, to March 13, 2012.

\begin{tabular}{|c|c|c|c|c|}
\hline \multicolumn{3}{|c|}{ Reference Elevation (ft AMSL) } & 1508.77 & \multirow{2}{*}{$\begin{array}{l}1509.12 \\
\text { Elevation in Klassen } \\
\text { Test Well (ft AMSL) }\end{array}$} \\
\hline Date and Time & $\begin{array}{l}\text { Depth in Klassen } \\
\text { Domestic Well } \\
(\mathrm{ft} \mathrm{BGL})\end{array}$ & $\begin{array}{c}\text { Depth in Klassen } \\
\text { Test Well } \\
\text { (ft BGL) }\end{array}$ & $\begin{array}{l}\text { Elevation in Klassen } \\
\text { Domestic Well } \\
\text { (ft AMSL) }\end{array}$ & \\
\hline 10/2/11 22:10 & 36.546 & 39.637 & 1472.22 & 1469.49 \\
\hline $10 / 2 / 1122: 20$ & 36.52 & 39.637 & 1472.25 & 1469.49 \\
\hline $10 / 2 / 1122: 30$ & 36.522 & 39.639 & 1472.25 & 1469.48 \\
\hline $10 / 2 / 1122: 40$ & 36.745 & 39.644 & 1472.02 & 1469.48 \\
\hline $10 / 2 / 1122: 50$ & 36.71 & 39.641 & 1472.06 & 1469.48 \\
\hline 10/2/11 23:00 & 38.07 & 39.646 & 1470.70 & 1469.48 \\
\hline $10 / 2 / 1123: 10$ & 36.663 & 39.646 & 1472.11 & 1469.48 \\
\hline $10 / 2 / 1123: 20$ & 36.548 & 39.646 & 1472.22 & 1469.48 \\
\hline $10 / 2 / 1123: 30$ & 36.473 & 39.644 & 1472.30 & 1469.48 \\
\hline $10 / 2 / 1123: 40$ & 36.421 & 39.641 & 1472.35 & 1469.48 \\
\hline $10 / 2 / 1123: 50$ & 36.374 & 39.639 & 1472.40 & 1469.48 \\
\hline 10/3/11 0:00 & 36.335 & 39.634 & 1472.43 & 1469.49 \\
\hline 10/3/11 0:10 & 36.306 & 39.632 & 1472.46 & 1469.49 \\
\hline $10 / 3 / 110: 20$ & 36.271 & 39.63 & 1472.50 & 1469.49 \\
\hline $10 / 3 / 110: 30$ & 36.246 & 39.627 & 1472.52 & 1469.50 \\
\hline $10 / 3 / 110: 40$ & 36.217 & 39.623 & 1472.55 & 1469.50 \\
\hline $10 / 3 / 110: 50$ & 36.196 & 39.62 & 1472.57 & 1469.50 \\
\hline 10/3/11 1:00 & 36.173 & 39.618 & 1472.60 & 1469.50 \\
\hline $10 / 3 / 11$ 1:10 & 36.152 & 39.616 & 1472.62 & 1469.51 \\
\hline $10 / 3 / 11$ 1:20 & 36.74 & 39.613 & 1472.03 & 1469.51 \\
\hline $10 / 3 / 11$ 1:30 & 36.253 & 39.611 & 1472.52 & 1469.51 \\
\hline $10 / 3 / 11$ 1:40 & 36.224 & 39.611 & 1472.55 & 1469.51 \\
\hline $10 / 3 / 11$ 1:50 & 36.257 & 39.611 & 1472.51 & 1469.51 \\
\hline $10 / 3 / 112: 00$ & 36.241 & 39.613 & 1472.53 & 1469.51 \\
\hline $10 / 3 / 112: 10$ & 36.227 & 39.613 & 1472.54 & 1469.51 \\
\hline $10 / 3 / 112: 20$ & 36.203 & 39.611 & 1472.57 & 1469.51 \\
\hline $10 / 3 / 112: 30$ & 36.185 & 39.609 & 1472.58 & 1469.51 \\
\hline $10 / 3 / 112: 40$ & 36.168 & 39.606 & 1472.60 & 1469.52 \\
\hline $10 / 3 / 112: 50$ & 36.154 & 39.604 & 1472.62 & 1469.52 \\
\hline 10/3/11 3:00 & 36.135 & 39.602 & 1472.63 & 1469.52 \\
\hline 10/3/11 3:10 & 36.121 & 39.602 & 1472.65 & 1469.52 \\
\hline $10 / 3 / 113: 20$ & 36.124 & 39.597 & 1472.65 & 1469.53 \\
\hline 10/3/11 3:30 & 36.17 & 39.599 & 1472.60 & 1469.52 \\
\hline $10 / 3 / 113: 40$ & 36.156 & 39.597 & 1472.61 & 1469.53 \\
\hline $10 / 3 / 113: 50$ & 36.124 & 39.597 & 1472.65 & 1469.53 \\
\hline $10 / 3 / 114: 00$ & 36.1 & 39.592 & 1472.67 & 1469.53 \\
\hline $10 / 3 / 114: 10$ & 36.074 & 39.59 & 1472.70 & 1469.53 \\
\hline $10 / 3 / 114: 20$ & 36.051 & 39.587 & 1472.72 & 1469.54 \\
\hline
\end{tabular}


TABLE S1.3 Automatically recorded groundwater levels in the Klassen wells, September 15, 2011, to March 13, 2012.

\begin{tabular}{|c|c|c|c|c|}
\hline \multicolumn{3}{|c|}{ Reference Elevation (ft AMSL) } & 1508.77 & \multirow{2}{*}{$\begin{array}{l}1509.12 \\
\text { Elevation in Klassen } \\
\text { Test Well (ft AMSL) }\end{array}$} \\
\hline Date and Time & $\begin{array}{l}\text { Depth in Klassen } \\
\text { Domestic Well } \\
(\mathrm{ft} \mathrm{BGL})\end{array}$ & $\begin{array}{c}\text { Depth in Klassen } \\
\text { Test Well } \\
\text { (ft BGL) }\end{array}$ & $\begin{array}{l}\text { Elevation in Klassen } \\
\text { Domestic Well } \\
\text { (ft AMSL) }\end{array}$ & \\
\hline $10 / 3 / 114: 30$ & 36.034 & 39.585 & 1472.74 & 1469.54 \\
\hline $10 / 3 / 114: 40$ & 39.515 & 39.58 & 1469.25 & 1469.54 \\
\hline $10 / 3 / 114: 50$ & 36.131 & 39.578 & 1472.64 & 1469.54 \\
\hline $10 / 3 / 115: 00$ & 36.058 & 39.576 & 1472.71 & 1469.55 \\
\hline 10/3/11 5:10 & 36.018 & 39.573 & 1472.75 & 1469.55 \\
\hline $10 / 3 / 115: 20$ & 35.995 & 39.571 & 1472.77 & 1469.55 \\
\hline $10 / 3 / 115: 30$ & 35.971 & 39.566 & 1472.80 & 1469.56 \\
\hline $10 / 3 / 115: 40$ & 35.948 & 39.562 & 1472.82 & 1469.56 \\
\hline $10 / 3 / 115: 50$ & 35.934 & 39.557 & 1472.84 & 1469.57 \\
\hline $10 / 3 / 116: 00$ & 35.912 & 39.555 & 1472.86 & 1469.57 \\
\hline 10/3/11 6:10 & 36.095 & 39.552 & 1472.67 & 1469.57 \\
\hline $10 / 3 / 116: 20$ & 36.034 & 39.55 & 1472.74 & 1469.57 \\
\hline $10 / 3 / 116: 30$ & 36.22 & 39.552 & 1472.55 & 1469.57 \\
\hline $10 / 3 / 116: 40$ & 40.659 & 39.557 & 1468.11 & 1469.57 \\
\hline $10 / 3 / 116: 50$ & 40.057 & 39.562 & 1468.71 & 1469.56 \\
\hline $10 / 3 / 117: 00$ & 46.724 & 39.576 & 1462.05 & 1469.55 \\
\hline $10 / 3 / 117: 10$ & 39.405 & 39.599 & 1469.36 & 1469.52 \\
\hline $10 / 3 / 11$ 7:20 & 41.769 & 39.613 & 1467.00 & 1469.51 \\
\hline $10 / 3 / 117: 30$ & 42.5 & 39.627 & 1466.27 & 1469.50 \\
\hline $10 / 3 / 117: 40$ & 39.278 & 39.639 & 1469.49 & 1469.48 \\
\hline $10 / 3 / 117: 50$ & 43.061 & 39.648 & 1465.71 & 1469.47 \\
\hline $10 / 3 / 118: 00$ & 44.588 & 39.658 & 1464.18 & 1469.46 \\
\hline 10/3/11 8:10 & 40.089 & 39.667 & 1468.68 & 1469.46 \\
\hline $10 / 3 / 118: 20$ & 37.545 & 39.672 & 1471.22 & 1469.45 \\
\hline $10 / 3 / 118: 30$ & 37.266 & 39.672 & 1471.50 & 1469.45 \\
\hline $10 / 3 / 118: 40$ & 37.097 & 39.67 & 1471.67 & 1469.45 \\
\hline $10 / 3 / 118: 50$ & 36.963 & 39.667 & 1471.81 & 1469.46 \\
\hline 10/3/11 9:00 & 36.855 & 39.663 & 1471.91 & 1469.46 \\
\hline 10/3/11 9:10 & 36.771 & 39.658 & 1472.00 & 1469.46 \\
\hline $10 / 3 / 119: 20$ & 36.703 & 39.653 & 1472.07 & 1469.47 \\
\hline 10/3/11 9:30 & 36.635 & 39.651 & 1472.13 & 1469.47 \\
\hline $10 / 3 / 119: 40$ & 36.571 & 39.646 & 1472.20 & 1469.48 \\
\hline $10 / 3 / 119: 50$ & 36.518 & 39.653 & 1472.25 & 1469.47 \\
\hline 10/3/11 10:00 & 36.475 & 39.653 & 1472.29 & 1469.47 \\
\hline 10/3/11 10:10 & 36.438 & 39.653 & 1472.33 & 1469.47 \\
\hline $10 / 3 / 11$ 10:20 & 36.393 & 39.651 & 1472.38 & 1469.47 \\
\hline 10/3/11 10:30 & 37.024 & 39.653 & 1471.75 & 1469.47 \\
\hline 10/3/11 10:40 & 36.45 & 39.663 & 1472.32 & 1469.46 \\
\hline
\end{tabular}


TABLE S1.3 Automatically recorded groundwater levels in the Klassen wells, September 15, 2011, to March 13, 2012.

\begin{tabular}{|c|c|c|c|c|}
\hline \multicolumn{3}{|c|}{ Reference Elevation (ft AMSL) } & 1508.77 & \multirow{2}{*}{$\begin{array}{l}1509.12 \\
\text { Elevation in Klassen } \\
\text { Test Well (ft AMSL) }\end{array}$} \\
\hline Date and Time & $\begin{array}{l}\text { Depth in Klassen } \\
\text { Domestic Well } \\
\text { (ft BGL) }\end{array}$ & $\begin{array}{c}\text { Depth in Klassen } \\
\text { Test Well } \\
\text { (ft BGL) }\end{array}$ & $\begin{array}{l}\text { Elevation in Klassen } \\
\text { Domestic Well } \\
\text { (ft AMSL) }\end{array}$ & \\
\hline 10/3/11 10:50 & 36.553 & 39.672 & 1472.22 & 1469.45 \\
\hline 10/3/11 11:00 & 36.412 & 39.686 & 1472.36 & 1469.44 \\
\hline 10/3/11 11:10 & 36.349 & 39.702 & 1472.42 & 1469.42 \\
\hline 10/3/11 11:20 & 41.987 & 39.724 & 1466.78 & 1469.40 \\
\hline 10/3/11 11:30 & 41.239 & 39.752 & 1467.53 & 1469.37 \\
\hline 10/3/11 11:40 & 40.913 & 39.785 & 1467.86 & 1469.34 \\
\hline 10/3/11 11:50 & 39.165 & 39.824 & 1469.60 & 1469.30 \\
\hline 10/3/11 12:00 & 42.353 & 39.864 & 1466.42 & 1469.26 \\
\hline $10 / 3 / 1112: 10$ & 42.55 & 39.907 & 1466.22 & 1469.22 \\
\hline $10 / 3 / 1112: 20$ & 40.359 & 39.951 & 1468.41 & 1469.17 \\
\hline $10 / 3 / 1112: 30$ & 39.194 & 39.993 & 1469.58 & 1469.13 \\
\hline $10 / 3 / 1112: 40$ & 40.774 & 40.04 & 1468.00 & 1469.08 \\
\hline $10 / 3 / 1112: 50$ & 41.708 & 40.087 & 1467.06 & 1469.04 \\
\hline 10/3/11 13:00 & 39.316 & 40.134 & 1469.45 & 1468.99 \\
\hline 10/3/11 13:10 & 44.738 & 40.186 & 1464.03 & 1468.94 \\
\hline $10 / 3 / 11$ 13:20 & 40.596 & 40.235 & 1468.17 & 1468.89 \\
\hline $10 / 3 / 11$ 13:30 & 39.294 & 40.287 & 1469.48 & 1468.84 \\
\hline 10/3/11 13:40 & 44.407 & 40.334 & 1464.36 & 1468.79 \\
\hline $10 / 3 / 11$ 13:50 & 42.085 & 40.383 & 1466.68 & 1468.74 \\
\hline $10 / 3 / 11$ 14:00 & 43.443 & 40.427 & 1465.33 & 1468.70 \\
\hline 10/3/11 14:10 & 39.752 & 40.467 & 1469.02 & 1468.66 \\
\hline $10 / 3 / 11$ 14:20 & 44.764 & 40.502 & 1464.01 & 1468.62 \\
\hline $10 / 3 / 11$ 14:30 & 43.225 & 40.535 & 1465.54 & 1468.59 \\
\hline 10/3/11 14:40 & 41.046 & 40.561 & 1467.72 & 1468.56 \\
\hline $10 / 3 / 11$ 14:50 & 43.286 & 40.587 & 1465.48 & 1468.54 \\
\hline 10/3/11 15:00 & 44.745 & 40.61 & 1464.02 & 1468.51 \\
\hline $10 / 3 / 11$ 15:10 & 43.15 & 40.632 & 1465.62 & 1468.49 \\
\hline $10 / 3 / 11$ 15:20 & 41.196 & 40.653 & 1467.57 & 1468.47 \\
\hline $10 / 3 / 11$ 15:30 & 40.059 & 40.678 & 1468.71 & 1468.44 \\
\hline 10/3/11 15:40 & 45.172 & 40.707 & 1463.60 & 1468.42 \\
\hline $10 / 3 / 11$ 15:50 & 42.634 & 40.744 & 1466.14 & 1468.38 \\
\hline 10/3/11 16:00 & 40.896 & 40.784 & 1467.87 & 1468.34 \\
\hline 10/3/11 16:10 & 46.349 & 40.822 & 1462.42 & 1468.30 \\
\hline 10/3/11 16:20 & 42.545 & 40.866 & 1466.22 & 1468.26 \\
\hline $10 / 3 / 11$ 16:30 & 40.887 & 40.913 & 1467.88 & 1468.21 \\
\hline $10 / 3 / 11$ 16:40 & 42.245 & 40.962 & 1466.52 & 1468.16 \\
\hline $10 / 3 / 11$ 16:50 & 41.063 & 41.014 & 1467.71 & 1468.11 \\
\hline 10/3/11 17:00 & 40.291 & 41.063 & 1468.48 & 1468.06 \\
\hline
\end{tabular}


TABLE S1.3 Automatically recorded groundwater levels in the Klassen wells, September 15, 2011, to March 13, 2012.

\begin{tabular}{|c|c|c|c|c|}
\hline \multicolumn{3}{|c|}{ Reference Elevation (ft AMSL) } & 1508.77 & \multirow{2}{*}{$\begin{array}{l}1509.12 \\
\text { Elevation in Klassen } \\
\text { Test Well (ft AMSL) }\end{array}$} \\
\hline Date and Time & $\begin{array}{l}\text { Depth in Klassen } \\
\text { Domestic Well } \\
\text { (ft BGL) }\end{array}$ & $\begin{array}{c}\text { Depth in Klassen } \\
\text { Test Well } \\
\text { (ft BGL) }\end{array}$ & $\begin{array}{l}\text { Elevation in Klassen } \\
\text { Domestic Well } \\
\text { (ft AMSL) }\end{array}$ & \\
\hline 10/3/11 17:10 & 42.341 & 41.117 & 1466.43 & 1468.01 \\
\hline $10 / 3 / 1117: 20$ & 44.7 & 41.171 & 1464.07 & 1467.95 \\
\hline 10/3/11 17:30 & 41.053 & 41.225 & 1467.72 & 1467.90 \\
\hline 10/3/11 17:40 & 42.193 & 41.274 & 1466.58 & 1467.85 \\
\hline 10/3/11 17:50 & 45.28 & 41.321 & 1463.49 & 1467.80 \\
\hline 10/3/11 18:00 & 42.784 & 41.361 & 1465.99 & 1467.76 \\
\hline 10/3/11 18:10 & 41.246 & 41.392 & 1467.52 & 1467.73 \\
\hline 10/3/11 18:20 & 45.472 & 41.422 & 1463.30 & 1467.70 \\
\hline $10 / 3 / 1118: 30$ & 41.867 & 41.45 & 1466.90 & 1467.67 \\
\hline $10 / 3 / 11$ 18:40 & 40.863 & 41.474 & 1467.91 & 1467.65 \\
\hline $10 / 3 / 1118: 50$ & 44.975 & 41.495 & 1463.79 & 1467.63 \\
\hline 10/3/11 19:00 & 43.145 & 41.511 & 1465.62 & 1467.61 \\
\hline 10/3/11 19:10 & 41.379 & 41.528 & 1467.39 & 1467.59 \\
\hline 10/3/11 19:20 & 40.448 & 41.542 & 1468.32 & 1467.58 \\
\hline 10/3/11 19:30 & 45.39 & 41.551 & 1463.38 & 1467.57 \\
\hline 10/3/11 19:40 & 39.217 & 41.561 & 1469.55 & 1467.56 \\
\hline 10/3/11 19:50 & 39.142 & 41.568 & 1469.63 & 1467.55 \\
\hline 10/3/11 20:00 & 38.788 & 41.57 & 1469.98 & 1467.55 \\
\hline 10/3/11 20:10 & 38.661 & 41.57 & 1470.11 & 1467.55 \\
\hline $10 / 3 / 1120: 20$ & 38.558 & 41.572 & 1470.21 & 1467.55 \\
\hline $10 / 3 / 1120: 30$ & 38.481 & 41.572 & 1470.29 & 1467.55 \\
\hline $10 / 3 / 11$ 20:40 & 38.359 & 41.57 & 1470.41 & 1467.55 \\
\hline $10 / 3 / 1120: 50$ & 38.23 & 41.563 & 1470.54 & 1467.56 \\
\hline 10/3/11 21:00 & 38.115 & 41.554 & 1470.65 & 1467.57 \\
\hline 10/3/11 21:10 & 38.037 & 41.547 & 1470.73 & 1467.58 \\
\hline $10 / 3 / 1121: 20$ & 37.946 & 41.535 & 1470.82 & 1467.59 \\
\hline $10 / 3 / 1121: 30$ & 37.854 & 41.523 & 1470.92 & 1467.60 \\
\hline $10 / 3 / 11$ 21:40 & 37.772 & 41.514 & 1471.00 & 1467.61 \\
\hline $10 / 3 / 1121: 50$ & 37.728 & 41.504 & 1471.04 & 1467.62 \\
\hline 10/3/11 22:00 & 37.721 & 41.495 & 1471.05 & 1467.63 \\
\hline $10 / 3 / 1122: 10$ & 37.65 & 41.483 & 1471.12 & 1467.64 \\
\hline $10 / 3 / 1122: 20$ & 37.667 & 41.474 & 1471.10 & 1467.65 \\
\hline $10 / 3 / 1122: 30$ & 37.66 & 41.467 & 1471.11 & 1467.66 \\
\hline $10 / 3 / 1122: 40$ & 40.406 & 41.46 & 1468.36 & 1467.66 \\
\hline $10 / 3 / 1122: 50$ & 37.901 & 41.455 & 1470.87 & 1467.67 \\
\hline 10/3/11 23:00 & 38.382 & 41.448 & 1470.39 & 1467.67 \\
\hline 10/3/11 23:10 & 37.986 & 41.441 & 1470.78 & 1467.68 \\
\hline $10 / 3 / 11$ 23:20 & 37.751 & 41.429 & 1471.02 & 1467.69 \\
\hline
\end{tabular}


TABLE S1.3 Automatically recorded groundwater levels in the Klassen wells, September 15, 2011, to March 13, 2012.

\begin{tabular}{|c|c|c|c|c|}
\hline \multicolumn{3}{|c|}{ Reference Elevation (ft AMSL) } & 1508.77 & \multirow{2}{*}{$\begin{array}{l}1509.12 \\
\text { Elevation in Klassen } \\
\text { Test Well (ft AMSL) }\end{array}$} \\
\hline Date and Time & $\begin{array}{l}\text { Depth in Klassen } \\
\text { Domestic Well } \\
\text { (ft BGL) }\end{array}$ & $\begin{array}{c}\text { Depth in Klassen } \\
\text { Test Well } \\
\text { (ft BGL) }\end{array}$ & $\begin{array}{l}\text { Elevation in Klassen } \\
\text { Domestic Well } \\
\text { (ft AMSL) }\end{array}$ & \\
\hline $10 / 3 / 1123: 30$ & 37.629 & 41.418 & 1471.14 & 1467.70 \\
\hline $10 / 3 / 1123: 40$ & 37.533 & 41.406 & 1471.24 & 1467.72 \\
\hline $10 / 3 / 1123: 50$ & 37.456 & 41.392 & 1471.31 & 1467.73 \\
\hline $10 / 4 / 110: 00$ & 37.383 & 41.38 & 1471.39 & 1467.74 \\
\hline 10/4/11 0:10 & 37.32 & 41.366 & 1471.45 & 1467.76 \\
\hline $10 / 4 / 110: 20$ & 37.268 & 41.35 & 1471.50 & 1467.77 \\
\hline $10 / 4 / 110: 30$ & 37.221 & 41.335 & 1471.55 & 1467.79 \\
\hline $10 / 4 / 110: 40$ & 37.174 & 41.324 & 1471.60 & 1467.80 \\
\hline 10/4/11 0:50 & 37.134 & 41.31 & 1471.64 & 1467.81 \\
\hline $10 / 4 / 111: 00$ & 37.092 & 41.296 & 1471.68 & 1467.83 \\
\hline 10/4/11 1:10 & 37.052 & 41.281 & 1471.72 & 1467.84 \\
\hline $10 / 4 / 111: 20$ & 37.012 & 41.267 & 1471.76 & 1467.86 \\
\hline $10 / 4 / 111: 30$ & 36.973 & 41.253 & 1471.80 & 1467.87 \\
\hline $10 / 4 / 111: 40$ & 36.93 & 41.242 & 1471.84 & 1467.88 \\
\hline $10 / 4 / 11$ 1:50 & 36.895 & 41.228 & 1471.87 & 1467.89 \\
\hline $10 / 4 / 112: 00$ & 36.867 & 41.211 & 1471.90 & 1467.91 \\
\hline $10 / 4 / 112: 10$ & 36.839 & 41.197 & 1471.93 & 1467.93 \\
\hline $10 / 4 / 112: 20$ & 36.813 & 41.185 & 1471.96 & 1467.94 \\
\hline $10 / 4 / 112: 30$ & 36.785 & 41.171 & 1471.98 & 1467.95 \\
\hline $10 / 4 / 112: 40$ & 36.759 & 41.159 & 1472.01 & 1467.96 \\
\hline $10 / 4 / 112: 50$ & 36.729 & 41.145 & 1472.04 & 1467.98 \\
\hline 10/4/11 3:00 & 36.698 & 41.134 & 1472.07 & 1467.99 \\
\hline 10/4/11 3:10 & 36.665 & 41.12 & 1472.10 & 1468.00 \\
\hline $10 / 4 / 113: 20$ & 36.642 & 41.105 & 1472.13 & 1468.02 \\
\hline $10 / 4 / 113: 30$ & 36.621 & 41.094 & 1472.15 & 1468.03 \\
\hline $10 / 4 / 113: 40$ & 36.6 & 41.08 & 1472.17 & 1468.04 \\
\hline $10 / 4 / 113: 50$ & 36.581 & 41.068 & 1472.19 & 1468.05 \\
\hline 10/4/11 4:00 & 36.56 & 41.054 & 1472.21 & 1468.07 \\
\hline $10 / 4 / 114: 10$ & 36.536 & 41.04 & 1472.23 & 1468.08 \\
\hline $10 / 4 / 114: 20$ & 36.508 & 41.028 & 1472.26 & 1468.09 \\
\hline 10/4/11 4:30 & 36.684 & 41.016 & 1472.09 & 1468.11 \\
\hline $10 / 4 / 114: 40$ & 36.557 & 41.005 & 1472.21 & 1468.12 \\
\hline $10 / 4 / 114: 50$ & 36.515 & 40.993 & 1472.25 & 1468.13 \\
\hline $10 / 4 / 115: 00$ & 36.487 & 40.981 & 1472.28 & 1468.14 \\
\hline $10 / 4 / 115: 10$ & 36.459 & 40.969 & 1472.31 & 1468.15 \\
\hline $10 / 4 / 115: 20$ & 36.433 & 40.958 & 1472.34 & 1468.16 \\
\hline $10 / 4 / 115: 30$ & 36.405 & 40.946 & 1472.36 & 1468.18 \\
\hline $10 / 4 / 115: 40$ & 36.382 & 40.937 & 1472.39 & 1468.19 \\
\hline
\end{tabular}


TABLE S1.3 Automatically recorded groundwater levels in the Klassen wells, September 15, 2011, to March 13, 2012.

\begin{tabular}{|c|c|c|c|c|}
\hline \multicolumn{3}{|c|}{ Reference Elevation (ft AMSL) } & 1508.77 & \multirow{2}{*}{$\begin{array}{l}1509.12 \\
\text { Elevation in Klassen } \\
\text { Test Well (ft AMSL) }\end{array}$} \\
\hline Date and Time & $\begin{array}{l}\text { Depth in Klassen } \\
\text { Domestic Well } \\
\text { (ft BGL) }\end{array}$ & $\begin{array}{c}\text { Depth in Klassen } \\
\text { Test Well } \\
\text { (ft BGL) }\end{array}$ & $\begin{array}{l}\text { Elevation in Klassen } \\
\text { Domestic Well } \\
\text { (ft AMSL) }\end{array}$ & \\
\hline $10 / 4 / 115: 50$ & 36.367 & 40.922 & 1472.40 & 1468.20 \\
\hline $10 / 4 / 116: 00$ & 36.349 & 40.913 & 1472.42 & 1468.21 \\
\hline $10 / 4 / 116: 10$ & 36.349 & 40.901 & 1472.42 & 1468.22 \\
\hline $10 / 4 / 116: 20$ & 36.656 & 40.892 & 1472.11 & 1468.23 \\
\hline $10 / 4 / 116: 30$ & 36.503 & 40.885 & 1472.27 & 1468.24 \\
\hline $10 / 4 / 116: 40$ & 36.532 & 40.878 & 1472.24 & 1468.24 \\
\hline $10 / 4 / 116: 50$ & 38.019 & 40.871 & 1470.75 & 1468.25 \\
\hline $10 / 4 / 117: 00$ & 39.294 & 40.866 & 1469.48 & 1468.26 \\
\hline $10 / 4 / 117: 10$ & 43.406 & 40.869 & 1465.36 & 1468.25 \\
\hline $10 / 4 / 117: 20$ & 40.866 & 40.866 & 1467.90 & 1468.26 \\
\hline $10 / 4 / 117: 30$ & 39.224 & 40.871 & 1469.55 & 1468.25 \\
\hline $10 / 4 / 117: 40$ & 42.308 & 40.873 & 1466.46 & 1468.25 \\
\hline $10 / 4 / 117: 50$ & 43.469 & 40.876 & 1465.30 & 1468.25 \\
\hline $10 / 4 / 118: 00$ & 40.892 & 40.878 & 1467.88 & 1468.24 \\
\hline $10 / 4 / 118: 10$ & 39.527 & 40.883 & 1469.24 & 1468.24 \\
\hline $10 / 4 / 118: 20$ & 44.705 & 40.887 & 1464.06 & 1468.24 \\
\hline $10 / 4 / 118: 30$ & 41.07 & 40.89 & 1467.70 & 1468.23 \\
\hline $10 / 4 / 118: 40$ & 41.485 & 40.892 & 1467.28 & 1468.23 \\
\hline $10 / 4 / 118: 50$ & 45.725 & 40.894 & 1463.04 & 1468.23 \\
\hline 10/4/11 9:00 & 42.414 & 40.897 & 1466.36 & 1468.23 \\
\hline 10/4/11 9:10 & 40.526 & 40.899 & 1468.24 & 1468.22 \\
\hline $10 / 4 / 119: 20$ & 39.559 & 40.901 & 1469.21 & 1468.22 \\
\hline 10/4/11 9:30 & 44.489 & 40.906 & 1464.28 & 1468.22 \\
\hline 10/4/11 9:40 & 42.658 & 40.906 & 1466.11 & 1468.22 \\
\hline 10/4/11 9:50 & 40.809 & 40.908 & 1467.96 & 1468.21 \\
\hline 10/4/11 10:00 & 39.775 & 40.911 & 1468.99 & 1468.21 \\
\hline 10/4/11 10:10 & 44.212 & 40.915 & 1464.56 & 1468.21 \\
\hline $10 / 4 / 11$ 10:20 & 44.759 & 40.915 & 1464.01 & 1468.21 \\
\hline 10/4/11 10:30 & 41.954 & 40.918 & 1466.82 & 1468.20 \\
\hline 10/4/11 10:40 & 40.512 & 40.92 & 1468.26 & 1468.20 \\
\hline 10/4/11 10:50 & 41.454 & 40.92 & 1467.32 & 1468.20 \\
\hline 10/4/11 11:00 & 48.345 & 40.922 & 1460.42 & 1468.20 \\
\hline 10/4/11 11:10 & 42.461 & 40.925 & 1466.31 & 1468.20 \\
\hline 10/4/11 11:20 & 40.655 & 40.927 & 1468.11 & 1468.20 \\
\hline 10/4/11 11:30 & 45.514 & 40.932 & 1463.26 & 1468.19 \\
\hline $10 / 4 / 11$ 11:40 & 42.594 & 40.937 & 1466.18 & 1468.19 \\
\hline 10/4/11 11:50 & 40.962 & 40.939 & 1467.81 & 1468.18 \\
\hline $10 / 4 / 1112: 00$ & 41.086 & 40.939 & 1467.68 & 1468.18 \\
\hline
\end{tabular}


TABLE S1.3 Automatically recorded groundwater levels in the Klassen wells, September 15, 2011, to March 13, 2012.

\begin{tabular}{|c|c|c|c|c|}
\hline \multicolumn{3}{|c|}{ Reference Elevation (ft AMSL) } & 1508.77 & \multirow{2}{*}{$\begin{array}{l}1509.12 \\
\text { Elevation in Klassen } \\
\text { Test Well (ft AMSL) }\end{array}$} \\
\hline Date and Time & $\begin{array}{l}\text { Depth in Klassen } \\
\text { Domestic Well } \\
\text { (ft BGL) }\end{array}$ & $\begin{array}{c}\text { Depth in Klassen } \\
\text { Test Well } \\
\text { (ft BGL) }\end{array}$ & $\begin{array}{l}\text { Elevation in Klassen } \\
\text { Domestic Well } \\
\text { (ft AMSL) }\end{array}$ & \\
\hline 10/4/11 12:10 & 45.448 & 40.941 & 1463.32 & 1468.18 \\
\hline 10/4/11 12:20 & 46.168 & 40.946 & 1462.60 & 1468.18 \\
\hline 10/4/11 12:30 & 42.181 & 40.946 & 1466.59 & 1468.18 \\
\hline 10/4/11 12:40 & 43.345 & 40.948 & 1465.42 & 1468.17 \\
\hline 10/4/11 12:50 & 44.316 & 40.948 & 1464.45 & 1468.17 \\
\hline 10/4/11 13:00 & 41.942 & 40.951 & 1466.83 & 1468.17 \\
\hline 10/4/11 13:10 & 41.323 & 40.951 & 1467.45 & 1468.17 \\
\hline 10/4/11 13:20 & 42.158 & 40.953 & 1466.61 & 1468.17 \\
\hline 10/4/11 13:30 & 45.204 & 40.955 & 1463.57 & 1468.17 \\
\hline 10/4/11 13:40 & 42.54 & 40.955 & 1466.23 & 1468.17 \\
\hline 10/4/11 13:50 & 41.018 & 40.958 & 1467.75 & 1468.16 \\
\hline 10/4/11 14:00 & 42.808 & 40.958 & 1465.96 & 1468.16 \\
\hline 10/4/11 14:10 & 44.893 & 40.958 & 1463.88 & 1468.16 \\
\hline 10/4/11 14:20 & 42.393 & 40.96 & 1466.38 & 1468.16 \\
\hline 10/4/11 14:30 & 41.279 & 40.96 & 1467.49 & 1468.16 \\
\hline 10/4/11 14:40 & 45.812 & 40.96 & 1462.96 & 1468.16 \\
\hline 10/4/11 14:50 & 43.523 & 40.96 & 1465.25 & 1468.16 \\
\hline 10/4/11 15:00 & 41.987 & 40.962 & 1466.78 & 1468.16 \\
\hline 10/4/11 15:10 & 40.807 & 40.965 & 1467.96 & 1468.16 \\
\hline $10 / 4 / 11$ 15:20 & 46.14 & 40.967 & 1462.63 & 1468.16 \\
\hline $10 / 4 / 11$ 15:30 & 43.307 & 40.967 & 1465.46 & 1468.16 \\
\hline 10/4/11 15:40 & 44.412 & 40.969 & 1464.36 & 1468.15 \\
\hline $10 / 4 / 11$ 15:50 & 44.48 & 40.974 & 1464.29 & 1468.15 \\
\hline 10/4/11 16:00 & 42.196 & 40.976 & 1466.57 & 1468.15 \\
\hline 10/4/11 16:10 & 40.957 & 40.981 & 1467.81 & 1468.14 \\
\hline 10/4/11 16:20 & 44.843 & 40.986 & 1463.93 & 1468.14 \\
\hline 10/4/11 16:30 & 49.248 & 40.991 & 1459.52 & 1468.13 \\
\hline 10/4/11 16:40 & 53.143 & 41.002 & 1455.63 & 1468.12 \\
\hline 10/4/11 16:50 & 54.703 & 41.016 & 1454.07 & 1468.11 \\
\hline 10/4/11 17:00 & 58.544 & 41.033 & 1450.23 & 1468.09 \\
\hline 10/4/11 17:10 & 55.908 & 41.054 & 1452.86 & 1468.07 \\
\hline 10/4/11 17:20 & 53.373 & 41.08 & 1455.40 & 1468.04 \\
\hline 10/4/11 17:30 & 54.883 & 41.108 & 1453.89 & 1468.01 \\
\hline 10/4/11 17:40 & 51.138 & 41.136 & 1457.63 & 1467.99 \\
\hline 10/4/11 17:50 & 56.216 & 41.162 & 1452.55 & 1467.96 \\
\hline 10/4/11 18:00 & 51.063 & 41.192 & 1457.71 & 1467.93 \\
\hline 10/4/11 18:10 & 56.659 & 41.218 & 1452.11 & 1467.90 \\
\hline 10/4/11 18:20 & 51.288 & 41.242 & 1457.48 & 1467.88 \\
\hline
\end{tabular}


TABLE S1.3 Automatically recorded groundwater levels in the Klassen wells, September 15, 2011, to March 13, 2012.

\begin{tabular}{|c|c|c|c|c|}
\hline \multicolumn{3}{|c|}{ Reference Elevation (ft AMSL) } & 1508.77 & \multirow{2}{*}{$\begin{array}{l}1509.12 \\
\text { Elevation in Klassen } \\
\text { Test Well (ft AMSL) }\end{array}$} \\
\hline Date and Time & $\begin{array}{l}\text { Depth in Klassen } \\
\text { Domestic Well } \\
\text { (ft BGL) }\end{array}$ & $\begin{array}{c}\text { Depth in Klassen } \\
\text { Test Well } \\
\text { (ft BGL) }\end{array}$ & $\begin{array}{l}\text { Elevation in Klassen } \\
\text { Domestic Well } \\
\text { (ft AMSL) }\end{array}$ & \\
\hline 10/4/11 18:30 & 56.985 & 41.265 & 1451.78 & 1467.86 \\
\hline 10/4/11 18:40 & 51.994 & 41.291 & 1456.78 & 1467.83 \\
\hline 10/4/11 18:50 & 53.488 & 41.314 & 1455.28 & 1467.81 \\
\hline 10/4/11 19:00 & 57.848 & 41.338 & 1450.92 & 1467.78 \\
\hline 10/4/11 19:10 & 60.315 & 41.371 & 1448.45 & 1467.75 \\
\hline 10/4/11 19:20 & 53.223 & 41.406 & 1455.55 & 1467.72 \\
\hline 10/4/11 19:30 & 58.237 & 41.443 & 1450.53 & 1467.68 \\
\hline 10/4/11 19:40 & 52.974 & 41.481 & 1455.80 & 1467.64 \\
\hline 10/4/11 19:50 & 52.669 & 41.521 & 1456.10 & 1467.60 \\
\hline 10/4/11 20:00 & 44.03 & 41.554 & 1464.74 & 1467.57 \\
\hline 10/4/11 20:10 & 43.288 & 41.575 & 1465.48 & 1467.55 \\
\hline 10/4/11 20:20 & 42.869 & 41.594 & 1465.90 & 1467.53 \\
\hline 10/4/11 20:30 & 42.585 & 41.608 & 1466.18 & 1467.51 \\
\hline 10/4/11 20:40 & 42.35 & 41.617 & 1466.42 & 1467.51 \\
\hline 10/4/11 20:50 & 42.153 & 41.624 & 1466.62 & 1467.50 \\
\hline 10/4/11 21:00 & 41.98 & 41.626 & 1466.79 & 1467.50 \\
\hline 10/4/11 21:10 & 41.895 & 41.629 & 1466.87 & 1467.49 \\
\hline 10/4/11 21:20 & 41.527 & 41.619 & 1467.24 & 1467.50 \\
\hline 10/4/11 21:30 & 41.239 & 41.608 & 1467.53 & 1467.51 \\
\hline 10/4/11 21:40 & 41.107 & 41.596 & 1467.66 & 1467.53 \\
\hline $10 / 4 / 1121: 50$ & 41.082 & 41.591 & 1467.69 & 1467.53 \\
\hline 10/4/11 22:00 & 41.06 & 41.591 & 1467.71 & 1467.53 \\
\hline $10 / 4 / 1122: 10$ & 41.023 & 41.591 & 1467.75 & 1467.53 \\
\hline $10 / 4 / 1122: 20$ & 40.976 & 41.589 & 1467.79 & 1467.53 \\
\hline $10 / 4 / 1122: 30$ & 40.936 & 41.586 & 1467.83 & 1467.54 \\
\hline $10 / 4 / 1122: 40$ & 40.917 & 41.589 & 1467.85 & 1467.53 \\
\hline $10 / 4 / 1122: 50$ & 41.907 & 41.589 & 1466.86 & 1467.53 \\
\hline 10/4/11 23:00 & 41.508 & 41.591 & 1467.26 & 1467.53 \\
\hline 10/4/11 23:10 & 41.018 & 41.596 & 1467.75 & 1467.53 \\
\hline 10/4/11 23:20 & 40.985 & 41.591 & 1467.78 & 1467.53 \\
\hline 10/4/11 23:30 & 40.664 & 41.579 & 1468.11 & 1467.54 \\
\hline 10/4/11 23:40 & 40.448 & 41.57 & 1468.32 & 1467.55 \\
\hline $10 / 4 / 1123: 50$ & 40.254 & 41.556 & 1468.52 & 1467.57 \\
\hline 10/5/11 0:00 & 40.11 & 41.542 & 1468.66 & 1467.58 \\
\hline 10/5/11 0:10 & 39.953 & 41.525 & 1468.82 & 1467.60 \\
\hline $10 / 5 / 110: 20$ & 39.836 & 41.511 & 1468.93 & 1467.61 \\
\hline 10/5/11 0:30 & 39.703 & 41.495 & 1469.07 & 1467.63 \\
\hline $10 / 5 / 110: 40$ & 39.59 & 41.479 & 1469.18 & 1467.64 \\
\hline
\end{tabular}


TABLE S1.3 Automatically recorded groundwater levels in the Klassen wells, September 15, 2011, to March 13, 2012.

\begin{tabular}{|c|c|c|c|c|}
\hline \multicolumn{3}{|c|}{ Reference Elevation (ft AMSL) } & 1508.77 & \multirow{2}{*}{$\begin{array}{l}1509.12 \\
\text { Elevation in Klassen } \\
\text { Test Well (ft AMSL) }\end{array}$} \\
\hline Date and Time & $\begin{array}{l}\text { Depth in Klassen } \\
\text { Domestic Well } \\
\text { (ft BGL) }\end{array}$ & $\begin{array}{c}\text { Depth in Klassen } \\
\text { Test Well } \\
\text { (ft BGL) }\end{array}$ & $\begin{array}{l}\text { Elevation in Klassen } \\
\text { Domestic Well } \\
\text { (ft AMSL) }\end{array}$ & \\
\hline $10 / 5 / 110: 50$ & 39.487 & 41.462 & 1469.28 & 1467.66 \\
\hline 10/5/11 1:00 & 39.369 & 41.448 & 1469.40 & 1467.67 \\
\hline 10/5/11 1:10 & 39.294 & 41.432 & 1469.48 & 1467.69 \\
\hline $10 / 5 / 111: 20$ & 39.217 & 41.415 & 1469.55 & 1467.71 \\
\hline $10 / 5 / 111: 30$ & 39.14 & 41.401 & 1469.63 & 1467.72 \\
\hline $10 / 5 / 111: 40$ & 39.112 & 41.387 & 1469.66 & 1467.74 \\
\hline 10/5/11 1:50 & 39.112 & 41.373 & 1469.66 & 1467.75 \\
\hline $10 / 5 / 112: 00$ & 39.032 & 41.361 & 1469.74 & 1467.76 \\
\hline $10 / 5 / 112: 10$ & 38.952 & 41.347 & 1469.82 & 1467.78 \\
\hline $10 / 5 / 112: 20$ & 38.877 & 41.335 & 1469.89 & 1467.79 \\
\hline $10 / 5 / 112: 30$ & 38.792 & 41.319 & 1469.98 & 1467.80 \\
\hline $10 / 5 / 112: 40$ & 38.72 & 41.305 & 1470.05 & 1467.82 \\
\hline $10 / 5 / 112: 50$ & 38.661 & 41.291 & 1470.11 & 1467.83 \\
\hline 10/5/11 3:00 & 38.602 & 41.274 & 1470.17 & 1467.85 \\
\hline 10/5/11 3:10 & 38.534 & 41.26 & 1470.24 & 1467.86 \\
\hline 10/5/11 3:20 & 38.516 & 41.246 & 1470.25 & 1467.88 \\
\hline $10 / 5 / 113: 30$ & 38.523 & 41.235 & 1470.25 & 1467.89 \\
\hline 10/5/11 3:40 & 38.455 & 41.223 & 1470.31 & 1467.90 \\
\hline $10 / 5 / 113: 50$ & 38.366 & 41.209 & 1470.40 & 1467.91 \\
\hline $10 / 5 / 114: 00$ & 38.319 & 41.195 & 1470.45 & 1467.93 \\
\hline $10 / 5 / 114: 10$ & 38.241 & 41.181 & 1470.53 & 1467.94 \\
\hline $10 / 5 / 114: 20$ & 38.199 & 41.167 & 1470.57 & 1467.96 \\
\hline 10/5/11 4:30 & 38.131 & 41.152 & 1470.64 & 1467.97 \\
\hline $10 / 5 / 114: 40$ & 38.072 & 41.136 & 1470.70 & 1467.99 \\
\hline $10 / 5 / 114: 50$ & 38.03 & 41.124 & 1470.74 & 1468.00 \\
\hline $10 / 5 / 11$ 5:00 & 37.967 & 41.11 & 1470.80 & 1468.01 \\
\hline $10 / 5 / 115: 10$ & 38.201 & 41.096 & 1470.57 & 1468.03 \\
\hline 10/5/11 5:20 & 38.007 & 41.087 & 1470.76 & 1468.04 \\
\hline 10/5/11 5:30 & 37.934 & 41.075 & 1470.84 & 1468.05 \\
\hline 10/5/11 5:40 & 37.868 & 41.063 & 1470.90 & 1468.06 \\
\hline 10/5/11 5:50 & 37.803 & 41.049 & 1470.97 & 1468.07 \\
\hline $10 / 5 / 116: 00$ & 37.77 & 41.037 & 1471.00 & 1468.09 \\
\hline $10 / 5 / 116: 10$ & 37.749 & 41.026 & 1471.02 & 1468.10 \\
\hline $10 / 5 / 116: 20$ & 37.739 & 41.014 & 1471.03 & 1468.11 \\
\hline $10 / 5 / 116: 30$ & 37.678 & 41.002 & 1471.09 & 1468.12 \\
\hline $10 / 5 / 116: 40$ & 37.643 & 40.991 & 1471.13 & 1468.13 \\
\hline $10 / 5 / 116: 50$ & 37.589 & 40.979 & 1471.18 & 1468.14 \\
\hline $10 / 5 / 11$ 7:00 & 37.775 & 40.969 & 1470.99 & 1468.15 \\
\hline
\end{tabular}


TABLE S1.3 Automatically recorded groundwater levels in the Klassen wells, September 15, 2011, to March 13, 2012.

\begin{tabular}{|c|c|c|c|c|}
\hline \multicolumn{3}{|c|}{ Reference Elevation (ft AMSL) } & 1508.77 & \multirow{2}{*}{$\begin{array}{l}1509.12 \\
\text { Elevation in Klassen } \\
\text { Test Well (ft AMSL) }\end{array}$} \\
\hline Date and Time & $\begin{array}{l}\text { Depth in Klassen } \\
\text { Domestic Well } \\
(\mathrm{ft} \mathrm{BGL})\end{array}$ & $\begin{array}{c}\text { Depth in Klassen } \\
\text { Test Well } \\
\text { (ft BGL) }\end{array}$ & $\begin{array}{l}\text { Elevation in Klassen } \\
\text { Domestic Well } \\
\text { (ft AMSL) }\end{array}$ & \\
\hline $10 / 5 / 117: 10$ & 37.617 & 40.958 & 1471.15 & 1468.16 \\
\hline $10 / 5 / 117: 20$ & 37.535 & 40.948 & 1471.23 & 1468.17 \\
\hline $10 / 5 / 117: 30$ & 37.503 & 40.939 & 1471.27 & 1468.18 \\
\hline $10 / 5 / 117: 40$ & 37.444 & 40.925 & 1471.33 & 1468.20 \\
\hline $10 / 5 / 117: 50$ & 40.828 & 40.918 & 1467.94 & 1468.20 \\
\hline $10 / 5 / 118: 00$ & 37.603 & 40.913 & 1471.17 & 1468.21 \\
\hline 10/5/11 8:10 & 37.477 & 40.908 & 1471.29 & 1468.21 \\
\hline $10 / 5 / 118: 20$ & 37.42 & 40.904 & 1471.35 & 1468.22 \\
\hline $10 / 5 / 118: 30$ & 37.362 & 40.897 & 1471.41 & 1468.23 \\
\hline $10 / 5 / 118: 40$ & 37.306 & 40.89 & 1471.46 & 1468.23 \\
\hline 10/5/11 8:50 & 37.282 & 40.88 & 1471.49 & 1468.24 \\
\hline 10/5/11 9:00 & 37.397 & 40.869 & 1471.37 & 1468.25 \\
\hline 10/5/11 9:10 & 37.301 & 40.859 & 1471.47 & 1468.26 \\
\hline $10 / 5 / 119: 20$ & 37.233 & 40.852 & 1471.54 & 1468.27 \\
\hline 10/5/11 9:30 & 37.177 & 40.845 & 1471.59 & 1468.28 \\
\hline 10/5/11 9:40 & 38.912 & 40.836 & 1469.86 & 1468.29 \\
\hline $10 / 5 / 119: 50$ & 37.226 & 40.829 & 1471.54 & 1468.29 \\
\hline 10/5/11 10:00 & 37.165 & 40.819 & 1471.60 & 1468.30 \\
\hline 10/5/11 10:10 & 37.106 & 40.812 & 1471.66 & 1468.31 \\
\hline $10 / 5 / 11$ 10:20 & 37.055 & 40.803 & 1471.71 & 1468.32 \\
\hline 10/5/11 10:30 & 37.036 & 40.793 & 1471.73 & 1468.33 \\
\hline 10/5/11 10:40 & 36.989 & 40.784 & 1471.78 & 1468.34 \\
\hline 10/5/11 10:50 & 36.975 & 40.772 & 1471.79 & 1468.35 \\
\hline 10/5/11 11:00 & 36.935 & 40.763 & 1471.83 & 1468.36 \\
\hline 10/5/11 11:10 & 36.893 & 40.754 & 1471.88 & 1468.37 \\
\hline 10/5/11 11:20 & 37.772 & 40.744 & 1471.00 & 1468.38 \\
\hline 10/5/11 11:30 & 36.973 & 40.735 & 1471.80 & 1468.39 \\
\hline 10/5/11 11:40 & 36.919 & 40.728 & 1471.85 & 1468.39 \\
\hline 10/5/11 11:50 & 36.867 & 40.721 & 1471.90 & 1468.40 \\
\hline 10/5/11 12:00 & 36.822 & 40.714 & 1471.95 & 1468.41 \\
\hline 10/5/11 12:10 & 36.811 & 40.707 & 1471.96 & 1468.42 \\
\hline $10 / 5 / 11$ 12:20 & 36.768 & 40.7 & 1472.00 & 1468.42 \\
\hline 10/5/11 12:30 & 36.757 & 40.693 & 1472.01 & 1468.43 \\
\hline 10/5/11 12:40 & 36.726 & 40.683 & 1472.04 & 1468.44 \\
\hline 10/5/11 12:50 & 36.689 & 40.676 & 1472.08 & 1468.45 \\
\hline 10/5/11 13:00 & 38.75 & 40.669 & 1470.02 & 1468.45 \\
\hline 10/5/11 13:10 & 36.801 & 40.662 & 1471.97 & 1468.46 \\
\hline 10/5/11 13:20 & 36.731 & 40.657 & 1472.04 & 1468.47 \\
\hline
\end{tabular}


TABLE S1.3 Automatically recorded groundwater levels in the Klassen wells, September 15, 2011, to March 13, 2012.

\begin{tabular}{|c|c|c|c|c|}
\hline \multicolumn{3}{|c|}{ Reference Elevation (ft AMSL) } & 1508.77 & \multirow{2}{*}{$\begin{array}{l}1509.12 \\
\text { Elevation in Klassen } \\
\text { Test Well (ft AMSL) }\end{array}$} \\
\hline Date and Time & $\begin{array}{l}\text { Depth in Klassen } \\
\text { Domestic Well } \\
\text { (ft BGL) }\end{array}$ & $\begin{array}{c}\text { Depth in Klassen } \\
\text { Test Well } \\
\text { (ft BGL) }\end{array}$ & $\begin{array}{l}\text { Elevation in Klassen } \\
\text { Domestic Well } \\
\text { (ft AMSL) }\end{array}$ & \\
\hline 10/5/11 13:30 & 36.686 & 40.65 & 1472.08 & 1468.47 \\
\hline 10/5/11 13:40 & 36.642 & 40.646 & 1472.13 & 1468.48 \\
\hline 10/5/11 13:50 & 36.632 & 40.639 & 1472.14 & 1468.48 \\
\hline 10/5/11 14:00 & 36.595 & 40.629 & 1472.17 & 1468.49 \\
\hline 10/5/11 14:10 & 36.569 & 40.625 & 1472.20 & 1468.50 \\
\hline 10/5/11 14:20 & 36.555 & 40.617 & 1472.21 & 1468.51 \\
\hline 10/5/11 14:30 & 36.525 & 40.61 & 1472.24 & 1468.51 \\
\hline 10/5/11 14:40 & 36.518 & 40.601 & 1472.25 & 1468.52 \\
\hline 10/5/11 14:50 & 36.492 & 40.596 & 1472.28 & 1468.53 \\
\hline 10/5/11 15:00 & 36.464 & 40.592 & 1472.31 & 1468.53 \\
\hline 10/5/11 15:10 & 36.461 & 40.587 & 1472.31 & 1468.54 \\
\hline 10/5/11 15:20 & 38.173 & 40.58 & 1470.60 & 1468.54 \\
\hline 10/5/11 15:30 & 36.553 & 40.575 & 1472.22 & 1468.55 \\
\hline 10/5/11 15:40 & 36.489 & 40.571 & 1472.28 & 1468.55 \\
\hline 10/5/11 15:50 & 36.443 & 40.568 & 1472.33 & 1468.55 \\
\hline 10/5/11 16:00 & 36.438 & 40.568 & 1472.33 & 1468.55 \\
\hline 10/5/11 16:10 & 36.487 & 40.564 & 1472.28 & 1468.56 \\
\hline 10/5/11 16:20 & 36.52 & 40.561 & 1472.25 & 1468.56 \\
\hline 10/5/11 16:30 & 36.478 & 40.561 & 1472.29 & 1468.56 \\
\hline 10/5/11 16:40 & 36.48 & 40.559 & 1472.29 & 1468.56 \\
\hline 10/5/11 16:50 & 36.445 & 40.554 & 1472.32 & 1468.57 \\
\hline 10/5/11 17:00 & 36.433 & 40.549 & 1472.34 & 1468.57 \\
\hline 10/5/11 17:10 & 36.396 & 40.547 & 1472.37 & 1468.58 \\
\hline 10/5/11 17:20 & 38.398 & 40.542 & 1470.37 & 1468.58 \\
\hline 10/5/11 17:30 & 36.496 & 40.54 & 1472.27 & 1468.58 \\
\hline 10/5/11 17:40 & 36.454 & 40.538 & 1472.32 & 1468.58 \\
\hline 10/5/11 17:50 & 36.447 & 40.535 & 1472.32 & 1468.59 \\
\hline 10/5/11 18:00 & 36.424 & 40.535 & 1472.35 & 1468.59 \\
\hline 10/5/11 18:10 & 36.382 & 40.535 & 1472.39 & 1468.59 \\
\hline 10/5/11 18:20 & 36.365 & 40.533 & 1472.40 & 1468.59 \\
\hline 10/5/11 18:30 & 36.356 & 40.533 & 1472.41 & 1468.59 \\
\hline 10/5/11 18:40 & 36.33 & 40.531 & 1472.44 & 1468.59 \\
\hline 10/5/11 18:50 & 36.316 & 40.531 & 1472.45 & 1468.59 \\
\hline 10/5/11 19:00 & 36.29 & 40.526 & 1472.48 & 1468.60 \\
\hline 10/5/11 19:10 & 37.561 & 40.524 & 1471.21 & 1468.60 \\
\hline 10/5/11 19:20 & 36.384 & 40.524 & 1472.39 & 1468.60 \\
\hline 10/5/11 19:30 & 36.335 & 40.524 & 1472.43 & 1468.60 \\
\hline 10/5/11 19:40 & 36.316 & 40.524 & 1472.45 & 1468.60 \\
\hline
\end{tabular}


TABLE S1.3 Automatically recorded groundwater levels in the Klassen wells, September 15, 2011, to March 13, 2012.

\begin{tabular}{|c|c|c|c|c|}
\hline \multicolumn{3}{|c|}{ Reference Elevation (ft AMSL) } & 1508.77 & \multirow{2}{*}{$\begin{array}{l}1509.12 \\
\text { Elevation in Klassen } \\
\text { Test Well (ft AMSL) }\end{array}$} \\
\hline Date and Time & $\begin{array}{l}\text { Depth in Klassen } \\
\text { Domestic Well } \\
\text { (ft BGL) }\end{array}$ & $\begin{array}{c}\text { Depth in Klassen } \\
\text { Test Well } \\
\text { (ft BGL) }\end{array}$ & $\begin{array}{l}\text { Elevation in Klassen } \\
\text { Domestic Well } \\
\text { (ft AMSL) }\end{array}$ & \\
\hline 10/5/11 19:50 & 36.398 & 40.526 & 1472.37 & 1468.60 \\
\hline 10/5/11 20:00 & 36.454 & 40.528 & 1472.32 & 1468.59 \\
\hline 10/5/11 20:10 & 36.7 & 40.535 & 1472.07 & 1468.59 \\
\hline 10/5/11 20:20 & 36.63 & 40.54 & 1472.14 & 1468.58 \\
\hline 10/5/11 20:30 & 36.628 & 40.545 & 1472.14 & 1468.58 \\
\hline 10/5/11 20:40 & 36.67 & 40.552 & 1472.10 & 1468.57 \\
\hline 10/5/11 20:50 & 36.677 & 40.559 & 1472.09 & 1468.56 \\
\hline 10/5/11 21:00 & 36.672 & 40.564 & 1472.10 & 1468.56 \\
\hline 10/5/11 21:10 & 36.668 & 40.564 & 1472.10 & 1468.56 \\
\hline 10/5/11 21:20 & 36.665 & 40.566 & 1472.10 & 1468.56 \\
\hline 10/5/11 21:30 & 36.665 & 40.568 & 1472.10 & 1468.55 \\
\hline 10/5/11 21:40 & 39.974 & 40.571 & 1468.80 & 1468.55 \\
\hline 10/5/11 21:50 & 36.902 & 40.575 & 1471.87 & 1468.55 \\
\hline 10/5/11 22:00 & 36.799 & 40.578 & 1471.97 & 1468.54 \\
\hline 10/5/11 22:10 & 36.764 & 40.582 & 1472.01 & 1468.54 \\
\hline $10 / 5 / 1122: 20$ & 36.766 & 40.585 & 1472.00 & 1468.54 \\
\hline $10 / 5 / 1122: 30$ & 36.754 & 40.587 & 1472.02 & 1468.54 \\
\hline 10/5/11 22:40 & 37.034 & 40.589 & 1471.74 & 1468.53 \\
\hline $10 / 5 / 1122: 50$ & 37.12 & 40.594 & 1471.65 & 1468.53 \\
\hline 10/5/11 23:00 & 41.452 & 40.594 & 1467.32 & 1468.53 \\
\hline 10/5/11 23:10 & 37.165 & 40.599 & 1471.60 & 1468.52 \\
\hline 10/5/11 23:20 & 37.024 & 40.601 & 1471.75 & 1468.52 \\
\hline 10/5/11 23:30 & 36.956 & 40.603 & 1471.81 & 1468.52 \\
\hline 10/5/11 23:40 & 36.914 & 40.603 & 1471.86 & 1468.52 \\
\hline 10/5/11 23:50 & 36.907 & 40.606 & 1471.86 & 1468.52 \\
\hline 10/6/11 0:00 & 36.905 & 40.603 & 1471.86 & 1468.52 \\
\hline 10/6/11 0:10 & 36.879 & 40.601 & 1471.89 & 1468.52 \\
\hline $10 / 6 / 110: 20$ & 36.858 & 40.599 & 1471.91 & 1468.52 \\
\hline $10 / 6 / 110: 30$ & 36.839 & 40.599 & 1471.93 & 1468.52 \\
\hline $10 / 6 / 110: 40$ & 36.822 & 40.596 & 1471.95 & 1468.53 \\
\hline $10 / 6 / 110: 50$ & 36.808 & 40.594 & 1471.96 & 1468.53 \\
\hline 10/6/11 1:00 & 36.792 & 40.594 & 1471.98 & 1468.53 \\
\hline 10/6/11 1:10 & 36.776 & 40.594 & 1471.99 & 1468.53 \\
\hline $10 / 6 / 111: 20$ & 36.761 & 40.592 & 1472.01 & 1468.53 \\
\hline $10 / 6 / 11$ 1:30 & 36.747 & 40.589 & 1472.02 & 1468.53 \\
\hline $10 / 6 / 11$ 1:40 & 36.736 & 40.585 & 1472.03 & 1468.54 \\
\hline $10 / 6 / 111: 50$ & 36.724 & 40.582 & 1472.05 & 1468.54 \\
\hline $10 / 6 / 112: 00$ & 36.883 & 40.582 & 1471.89 & 1468.54 \\
\hline
\end{tabular}


TABLE S1.3 Automatically recorded groundwater levels in the Klassen wells, September 15, 2011, to March 13, 2012.

\begin{tabular}{|c|c|c|c|c|}
\hline \multicolumn{3}{|c|}{ Reference Elevation (ft AMSL) } & 1508.77 & \multirow{2}{*}{$\begin{array}{l}1509.12 \\
\text { Elevation in Klassen } \\
\text { Test Well (ft AMSL) }\end{array}$} \\
\hline Date and Time & $\begin{array}{l}\text { Depth in Klassen } \\
\text { Domestic Well } \\
\text { (ft BGL) }\end{array}$ & $\begin{array}{c}\text { Depth in Klassen } \\
\text { Test Well } \\
\text { (ft BGL) }\end{array}$ & $\begin{array}{l}\text { Elevation in Klassen } \\
\text { Domestic Well } \\
\text { (ft AMSL) }\end{array}$ & \\
\hline $10 / 6 / 112: 10$ & 36.801 & 40.58 & 1471.97 & 1468.54 \\
\hline $10 / 6 / 112: 20$ & 36.768 & 40.578 & 1472.00 & 1468.54 \\
\hline $10 / 6 / 112: 30$ & 36.747 & 40.578 & 1472.02 & 1468.54 \\
\hline $10 / 6 / 112: 40$ & 36.731 & 40.575 & 1472.04 & 1468.55 \\
\hline $10 / 6 / 112: 50$ & 36.715 & 40.571 & 1472.05 & 1468.55 \\
\hline 10/6/11 3:00 & 36.703 & 40.568 & 1472.07 & 1468.55 \\
\hline 10/6/11 3:10 & 36.689 & 40.568 & 1472.08 & 1468.55 \\
\hline $10 / 6 / 113: 20$ & 36.679 & 40.564 & 1472.09 & 1468.56 \\
\hline 10/6/11 3:30 & 36.67 & 40.561 & 1472.10 & 1468.56 \\
\hline 10/6/11 3:40 & 36.661 & 40.559 & 1472.11 & 1468.56 \\
\hline 10/6/11 3:50 & 36.649 & 40.556 & 1472.12 & 1468.57 \\
\hline $10 / 6 / 114: 00$ & 36.642 & 40.554 & 1472.13 & 1468.57 \\
\hline $10 / 6 / 114: 10$ & 36.632 & 40.552 & 1472.14 & 1468.57 \\
\hline $10 / 6 / 114: 20$ & 36.623 & 40.547 & 1472.15 & 1468.58 \\
\hline $10 / 6 / 114: 30$ & 36.616 & 40.542 & 1472.15 & 1468.58 \\
\hline $10 / 6 / 114: 40$ & 36.614 & 40.538 & 1472.16 & 1468.58 \\
\hline $10 / 6 / 114: 50$ & 36.621 & 40.533 & 1472.15 & 1468.59 \\
\hline 10/6/11 5:00 & 36.616 & 40.533 & 1472.15 & 1468.59 \\
\hline $10 / 6 / 115: 10$ & 36.607 & 40.528 & 1472.16 & 1468.59 \\
\hline $10 / 6 / 115: 20$ & 36.597 & 40.526 & 1472.17 & 1468.60 \\
\hline $10 / 6 / 115: 30$ & 36.59 & 40.524 & 1472.18 & 1468.60 \\
\hline $10 / 6 / 115: 40$ & 36.586 & 40.521 & 1472.18 & 1468.60 \\
\hline 10/6/11 5:50 & 36.576 & 40.517 & 1472.19 & 1468.61 \\
\hline $10 / 6 / 116: 00$ & 36.571 & 40.512 & 1472.20 & 1468.61 \\
\hline $10 / 6 / 116: 10$ & 37.078 & 40.51 & 1471.69 & 1468.61 \\
\hline $10 / 6 / 116: 20$ & 36.937 & 40.512 & 1471.83 & 1468.61 \\
\hline $10 / 6 / 116: 30$ & 36.78 & 40.512 & 1471.99 & 1468.61 \\
\hline $10 / 6 / 116: 40$ & 36.722 & 40.512 & 1472.05 & 1468.61 \\
\hline $10 / 6 / 116: 50$ & 36.684 & 40.507 & 1472.09 & 1468.62 \\
\hline 10/6/11 7:00 & 36.656 & 40.505 & 1472.11 & 1468.62 \\
\hline 10/6/11 7:10 & 36.637 & 40.502 & 1472.13 & 1468.62 \\
\hline $10 / 6 / 117: 20$ & 36.621 & 40.502 & 1472.15 & 1468.62 \\
\hline $10 / 6 / 117: 30$ & 36.609 & 40.498 & 1472.16 & 1468.62 \\
\hline $10 / 6 / 117: 40$ & 36.616 & 40.493 & 1472.15 & 1468.63 \\
\hline $10 / 6 / 117: 50$ & 36.602 & 40.491 & 1472.17 & 1468.63 \\
\hline $10 / 6 / 118: 00$ & 38.187 & 40.488 & 1470.58 & 1468.63 \\
\hline $10 / 6 / 118: 10$ & 36.724 & 40.489 & 1472.05 & 1468.63 \\
\hline $10 / 6 / 118: 20$ & 36.661 & 40.488 & 1472.11 & 1468.63 \\
\hline
\end{tabular}


TABLE S1.3 Automatically recorded groundwater levels in the Klassen wells, September 15, 2011, to March 13, 2012.

\begin{tabular}{|c|c|c|c|c|}
\hline \multicolumn{3}{|c|}{ Reference Elevation (ft AMSL) } & 1508.77 & \multirow{2}{*}{$\begin{array}{l}1509.12 \\
\text { Elevation in Klassen } \\
\text { Test Well (ft AMSL) }\end{array}$} \\
\hline Date and Time & $\begin{array}{l}\text { Depth in Klassen } \\
\text { Domestic Well } \\
\text { (ft BGL) }\end{array}$ & $\begin{array}{c}\text { Depth in Klassen } \\
\text { Test Well } \\
\text { (ft BGL) }\end{array}$ & $\begin{array}{l}\text { Elevation in Klassen } \\
\text { Domestic Well } \\
\text { (ft AMSL) }\end{array}$ & \\
\hline $10 / 6 / 118: 30$ & 36.63 & 40.488 & 1472.14 & 1468.63 \\
\hline $10 / 6 / 118: 40$ & 44.086 & 40.484 & 1464.68 & 1468.64 \\
\hline $10 / 6 / 118: 50$ & 58.181 & 40.491 & 1450.59 & 1468.63 \\
\hline 10/6/11 9:00 & 62.414 & 40.512 & 1446.36 & 1468.61 \\
\hline 10/6/11 9:10 & 44.492 & 40.538 & 1464.28 & 1468.58 \\
\hline 10/6/11 9:20 & 44.56 & 40.559 & 1464.21 & 1468.56 \\
\hline $10 / 6 / 119: 30$ & 45.183 & 40.58 & 1463.59 & 1468.54 \\
\hline $10 / 6 / 119: 40$ & 42.592 & 40.599 & 1466.18 & 1468.52 \\
\hline 10/6/11 9:50 & 46.45 & 40.613 & 1462.32 & 1468.51 \\
\hline 10/6/11 10:00 & 48.659 & 40.629 & 1460.11 & 1468.49 \\
\hline 10/6/11 10:10 & 42.475 & 40.648 & 1466.29 & 1468.47 \\
\hline 10/6/11 10:20 & 46.75 & 40.664 & 1462.02 & 1468.46 \\
\hline 10/6/11 10:30 & 42.325 & 40.676 & 1466.44 & 1468.45 \\
\hline 10/6/11 10:40 & 42.113 & 40.69 & 1466.66 & 1468.43 \\
\hline 10/6/11 10:50 & 40.197 & 40.702 & 1468.57 & 1468.42 \\
\hline 10/6/11 11:00 & 39.794 & 40.709 & 1468.98 & 1468.41 \\
\hline 10/6/11 11:10 & 41.283 & 40.718 & 1467.49 & 1468.40 \\
\hline 10/6/11 11:20 & 44.454 & 40.728 & 1464.32 & 1468.39 \\
\hline 10/6/11 11:30 & 41.103 & 40.737 & 1467.67 & 1468.39 \\
\hline 10/6/11 11:40 & 45.336 & 40.744 & 1463.43 & 1468.38 \\
\hline $10 / 6 / 11$ 11:50 & 43.237 & 40.754 & 1465.53 & 1468.37 \\
\hline 10/6/11 12:00 & 39.51 & 40.763 & 1469.26 & 1468.36 \\
\hline 10/6/11 12:10 & 39.006 & 40.765 & 1469.76 & 1468.36 \\
\hline $10 / 6 / 11$ 12:20 & 38.757 & 40.765 & 1470.01 & 1468.36 \\
\hline 10/6/11 12:30 & 38.574 & 40.763 & 1470.20 & 1468.36 \\
\hline $10 / 6 / 11$ 12:40 & 38.431 & 40.763 & 1470.34 & 1468.36 \\
\hline $10 / 6 / 11$ 12:50 & 38.307 & 40.763 & 1470.46 & 1468.36 \\
\hline 10/6/11 13:00 & 38.199 & 40.763 & 1470.57 & 1468.36 \\
\hline 10/6/11 13:10 & 38.112 & 40.763 & 1470.66 & 1468.36 \\
\hline 10/6/11 13:20 & 38.04 & 40.761 & 1470.73 & 1468.36 \\
\hline 10/6/11 13:30 & 37.962 & 40.758 & 1470.81 & 1468.36 \\
\hline 10/6/11 13:40 & 37.897 & 40.756 & 1470.87 & 1468.37 \\
\hline 10/6/11 13:50 & 37.833 & 40.756 & 1470.94 & 1468.37 \\
\hline 10/6/11 14:00 & 37.777 & 40.754 & 1470.99 & 1468.37 \\
\hline 10/6/11 14:10 & 37.73 & 40.751 & 1471.04 & 1468.37 \\
\hline $10 / 6 / 11$ 14:20 & 37.683 & 40.749 & 1471.09 & 1468.37 \\
\hline 10/6/11 14:30 & 37.904 & 40.747 & 1470.87 & 1468.38 \\
\hline 10/6/11 14:40 & 37.721 & 40.747 & 1471.05 & 1468.38 \\
\hline
\end{tabular}


TABLE S1.3 Automatically recorded groundwater levels in the Klassen wells, September 15, 2011, to March 13, 2012.

\begin{tabular}{|c|c|c|c|c|}
\hline \multicolumn{3}{|c|}{ Reference Elevation (ft AMSL) } & 1508.77 & \multirow{2}{*}{$\begin{array}{l}1509.12 \\
\text { Elevation in Klassen } \\
\text { Test Well (ft AMSL) }\end{array}$} \\
\hline Date and Time & $\begin{array}{l}\text { Depth in Klassen } \\
\text { Domestic Well } \\
\text { (ft BGL) }\end{array}$ & $\begin{array}{c}\text { Depth in Klassen } \\
\text { Test Well } \\
\text { (ft BGL) }\end{array}$ & $\begin{array}{l}\text { Elevation in Klassen } \\
\text { Domestic Well } \\
\text { (ft AMSL) }\end{array}$ & \\
\hline 10/6/11 14:50 & 37.643 & 40.744 & 1471.13 & 1468.38 \\
\hline 10/6/11 15:00 & 37.589 & 40.744 & 1471.18 & 1468.38 \\
\hline 10/6/11 15:10 & 37.545 & 40.742 & 1471.22 & 1468.38 \\
\hline $10 / 6 / 1115: 20$ & 37.503 & 40.739 & 1471.27 & 1468.38 \\
\hline 10/6/11 15:30 & 37.463 & 40.737 & 1471.31 & 1468.39 \\
\hline 10/6/11 15:40 & 37.453 & 40.737 & 1471.32 & 1468.39 \\
\hline 10/6/11 15:50 & 41.996 & 40.737 & 1466.77 & 1468.39 \\
\hline 10/6/11 16:00 & 38 & 40.737 & 1470.77 & 1468.39 \\
\hline 10/6/11 16:10 & 39.175 & 40.742 & 1469.59 & 1468.38 \\
\hline 10/6/11 16:20 & 39.888 & 40.749 & 1468.88 & 1468.37 \\
\hline 10/6/11 16:30 & 40.404 & 40.754 & 1468.37 & 1468.37 \\
\hline 10/6/11 16:40 & 41.049 & 40.756 & 1467.72 & 1468.37 \\
\hline 10/6/11 16:50 & 39.677 & 40.763 & 1469.09 & 1468.36 \\
\hline 10/6/11 17:00 & 40.739 & 40.77 & 1468.03 & 1468.35 \\
\hline 10/6/11 17:10 & 44.461 & 40.777 & 1464.31 & 1468.35 \\
\hline 10/6/11 17:20 & 42.156 & 40.786 & 1466.61 & 1468.34 \\
\hline 10/6/11 17:30 & 40.711 & 40.798 & 1468.06 & 1468.32 \\
\hline 10/6/11 17:40 & 39.89 & 40.808 & 1468.88 & 1468.31 \\
\hline 10/6/11 17:50 & 44.705 & 40.819 & 1464.06 & 1468.30 \\
\hline 10/6/11 18:00 & 43.012 & 40.829 & 1465.76 & 1468.29 \\
\hline 10/6/11 18:10 & 41.22 & 40.84 & 1467.55 & 1468.28 \\
\hline 10/6/11 18:20 & 40.193 & 40.852 & 1468.58 & 1468.27 \\
\hline 10/6/11 18:30 & 45.807 & 40.864 & 1462.96 & 1468.26 \\
\hline 10/6/11 18:40 & 41.194 & 40.876 & 1467.58 & 1468.25 \\
\hline 10/6/11 18:50 & 45.582 & 40.887 & 1463.19 & 1468.24 \\
\hline 10/6/11 19:00 & 42.421 & 40.904 & 1466.35 & 1468.22 \\
\hline 10/6/11 19:10 & 43.091 & 40.915 & 1465.68 & 1468.21 \\
\hline 10/6/11 19:20 & 39.222 & 40.925 & 1469.55 & 1468.20 \\
\hline 10/6/11 19:30 & 41.694 & 40.934 & 1467.08 & 1468.19 \\
\hline 10/6/11 19:40 & 38.975 & 40.941 & 1469.79 & 1468.18 \\
\hline 10/6/11 19:50 & 40.612 & 40.944 & 1468.16 & 1468.18 \\
\hline 10/6/11 20:00 & 38.762 & 40.948 & 1470.01 & 1468.17 \\
\hline 10/6/11 20:10 & 39.025 & 40.948 & 1469.74 & 1468.17 \\
\hline 10/6/11 20:20 & 43.206 & 40.953 & 1465.56 & 1468.17 \\
\hline 10/6/11 20:30 & 38.657 & 40.955 & 1470.11 & 1468.17 \\
\hline 10/6/11 20:40 & 38.94 & 40.955 & 1469.83 & 1468.17 \\
\hline 10/6/11 20:50 & 38.729 & 40.955 & 1470.04 & 1468.17 \\
\hline 10/6/11 21:00 & 38.495 & 40.958 & 1470.27 & 1468.16 \\
\hline
\end{tabular}


TABLE S1.3 Automatically recorded groundwater levels in the Klassen wells, September 15, 2011, to March 13, 2012.

\begin{tabular}{|c|c|c|c|c|}
\hline \multicolumn{3}{|c|}{ Reference Elevation (ft AMSL) } & 1508.77 & \multirow{2}{*}{$\begin{array}{l}1509.12 \\
\text { Elevation in Klassen } \\
\text { Test Well (ft AMSL) }\end{array}$} \\
\hline Date and Time & $\begin{array}{l}\text { Depth in Klassen } \\
\text { Domestic Well } \\
\text { (ft BGL) }\end{array}$ & $\begin{array}{c}\text { Depth in Klassen } \\
\text { Test Well } \\
\text { (ft BGL) }\end{array}$ & $\begin{array}{l}\text { Elevation in Klassen } \\
\text { Domestic Well } \\
\text { (ft AMSL) }\end{array}$ & \\
\hline 10/6/11 21:10 & 38.558 & 40.955 & 1470.21 & 1468.17 \\
\hline $10 / 6 / 1121: 20$ & 41.344 & 40.958 & 1467.43 & 1468.16 \\
\hline 10/6/11 21:30 & 38.607 & 40.962 & 1470.16 & 1468.16 \\
\hline 10/6/11 21:40 & 41.283 & 40.969 & 1467.49 & 1468.15 \\
\hline 10/6/11 21:50 & 38.652 & 40.976 & 1470.12 & 1468.15 \\
\hline 10/6/11 22:00 & 39.663 & 40.979 & 1469.11 & 1468.14 \\
\hline 10/6/11 22:10 & 42.268 & 40.983 & 1466.50 & 1468.14 \\
\hline $10 / 6 / 1122: 20$ & 40.207 & 40.986 & 1468.56 & 1468.14 \\
\hline $10 / 6 / 1122: 30$ & 40.111 & 40.988 & 1468.66 & 1468.13 \\
\hline 10/6/11 22:40 & 39.972 & 40.993 & 1468.80 & 1468.13 \\
\hline $10 / 6 / 1122: 50$ & 39.058 & 41 & 1469.71 & 1468.12 \\
\hline 10/6/11 23:00 & 42.618 & 41.005 & 1466.15 & 1468.12 \\
\hline 10/6/11 23:10 & 38.8 & 41.005 & 1469.97 & 1468.12 \\
\hline $10 / 6 / 1123: 20$ & 40.688 & 41.007 & 1468.08 & 1468.12 \\
\hline 10/6/11 23:30 & 38.664 & 41.009 & 1470.11 & 1468.11 \\
\hline $10 / 6 / 1123: 40$ & 39.318 & 41.009 & 1469.45 & 1468.11 \\
\hline $10 / 6 / 1123: 50$ & 38.565 & 41.009 & 1470.20 & 1468.11 \\
\hline 10/7/11 0:00 & 38.818 & 41.009 & 1469.95 & 1468.11 \\
\hline 10/7/11 0:10 & 38.483 & 41.007 & 1470.29 & 1468.12 \\
\hline $10 / 7 / 110: 20$ & 38.617 & 41.005 & 1470.15 & 1468.12 \\
\hline $10 / 7 / 110: 30$ & 38.408 & 41.002 & 1470.36 & 1468.12 \\
\hline 10/7/11 0:40 & 38.504 & 40.998 & 1470.27 & 1468.12 \\
\hline 10/7/11 0:50 & 39.325 & 40.993 & 1469.44 & 1468.13 \\
\hline 10/7/11 1:00 & 38.422 & 40.991 & 1470.35 & 1468.13 \\
\hline $10 / 7 / 11$ 1:10 & 41.424 & 40.986 & 1467.35 & 1468.14 \\
\hline $10 / 7 / 11$ 1:20 & 38.354 & 40.986 & 1470.42 & 1468.14 \\
\hline $10 / 7 / 11$ 1:30 & 39.416 & 40.981 & 1469.35 & 1468.14 \\
\hline 10/7/11 1:40 & 38.293 & 40.979 & 1470.48 & 1468.14 \\
\hline $10 / 7 / 11$ 1:50 & 38.652 & 40.974 & 1470.12 & 1468.15 \\
\hline 10/7/11 2:00 & 38.241 & 40.972 & 1470.53 & 1468.15 \\
\hline 10/7/11 2:10 & 38.422 & 40.969 & 1470.35 & 1468.15 \\
\hline $10 / 7 / 112: 20$ & 38.192 & 40.965 & 1470.58 & 1468.16 \\
\hline $10 / 7 / 112: 30$ & 38.319 & 40.96 & 1470.45 & 1468.16 \\
\hline $10 / 7 / 112: 40$ & 38.15 & 40.958 & 1470.62 & 1468.16 \\
\hline $10 / 7 / 112: 50$ & 38.248 & 40.953 & 1470.52 & 1468.17 \\
\hline $10 / 7 / 113: 00$ & 42.28 & 40.951 & 1466.49 & 1468.17 \\
\hline $10 / 7 / 113: 10$ & 38.192 & 40.946 & 1470.58 & 1468.18 \\
\hline $10 / 7 / 113: 20$ & 40.207 & 40.941 & 1468.56 & 1468.18 \\
\hline
\end{tabular}


TABLE S1.3 Automatically recorded groundwater levels in the Klassen wells, September 15, 2011, to March 13, 2012.

\begin{tabular}{|c|c|c|c|c|}
\hline \multicolumn{3}{|c|}{ Reference Elevation (ft AMSL) } & 1508.77 & \multirow{2}{*}{$\begin{array}{l}1509.12 \\
\text { Elevation in Klassen } \\
\text { Test Well (ft AMSL) }\end{array}$} \\
\hline Date and Time & $\begin{array}{l}\text { Depth in Klassen } \\
\text { Domestic Well } \\
(\mathrm{ft} \mathrm{BGL})\end{array}$ & $\begin{array}{c}\text { Depth in Klassen } \\
\text { Test Well } \\
\text { (ft BGL) }\end{array}$ & $\begin{array}{l}\text { Elevation in Klassen } \\
\text { Domestic Well } \\
\text { (ft AMSL) }\end{array}$ & \\
\hline $10 / 7 / 113: 30$ & 38.138 & 40.937 & 1470.63 & 1468.19 \\
\hline $10 / 7 / 113: 40$ & 38.802 & 40.932 & 1469.97 & 1468.19 \\
\hline $10 / 7 / 113: 50$ & 38.096 & 40.93 & 1470.67 & 1468.19 \\
\hline $10 / 7 / 114: 00$ & 38.363 & 40.927 & 1470.41 & 1468.20 \\
\hline 10/7/11 4:10 & 38.059 & 40.922 & 1470.71 & 1468.20 \\
\hline $10 / 7 / 114: 20$ & 38.211 & 40.92 & 1470.56 & 1468.20 \\
\hline $10 / 7 / 114: 30$ & 38.019 & 40.918 & 1470.75 & 1468.20 \\
\hline $10 / 7 / 114: 40$ & 38.209 & 40.915 & 1470.56 & 1468.21 \\
\hline $10 / 7 / 114: 50$ & 39.97 & 40.911 & 1468.80 & 1468.21 \\
\hline $10 / 7 / 115: 00$ & 38.091 & 40.908 & 1470.68 & 1468.21 \\
\hline 10/7/11 5:10 & 40.784 & 40.904 & 1467.99 & 1468.22 \\
\hline 10/7/11 5:20 & 38.035 & 40.901 & 1470.73 & 1468.22 \\
\hline 10/7/11 5:30 & 38.537 & 40.899 & 1470.23 & 1468.22 \\
\hline $10 / 7 / 115: 40$ & 38.016 & 40.897 & 1470.75 & 1468.23 \\
\hline $10 / 7 / 115: 50$ & 38.211 & 40.894 & 1470.56 & 1468.23 \\
\hline $10 / 7 / 116: 00$ & 38.002 & 40.89 & 1470.77 & 1468.23 \\
\hline $10 / 7 / 116: 10$ & 42.334 & 40.89 & 1466.44 & 1468.23 \\
\hline $10 / 7 / 116: 20$ & 40.828 & 40.89 & 1467.94 & 1468.23 \\
\hline $10 / 7 / 116: 30$ & 38.202 & 40.89 & 1470.57 & 1468.23 \\
\hline $10 / 7 / 116: 40$ & 40.108 & 40.887 & 1468.66 & 1468.24 \\
\hline $10 / 7 / 116: 50$ & 38.108 & 40.885 & 1470.66 & 1468.24 \\
\hline 10/7/11 7:00 & 38.467 & 40.883 & 1470.30 & 1468.24 \\
\hline 10/7/11 7:10 & 38.049 & 40.883 & 1470.72 & 1468.24 \\
\hline $10 / 7 / 117: 20$ & 38.173 & 40.883 & 1470.60 & 1468.24 \\
\hline 10/7/11 7:30 & 37.981 & 40.878 & 1470.79 & 1468.24 \\
\hline 10/7/11 7:40 & 38.07 & 40.873 & 1470.70 & 1468.25 \\
\hline $10 / 7 / 117: 50$ & 39.386 & 40.871 & 1469.38 & 1468.25 \\
\hline $10 / 7 / 118: 00$ & 39.906 & 40.871 & 1468.86 & 1468.25 \\
\hline 10/7/11 8:10 & 38.598 & 40.871 & 1470.17 & 1468.25 \\
\hline 10/7/11 8:20 & 38.066 & 40.869 & 1470.70 & 1468.25 \\
\hline 10/7/11 8:30 & 37.969 & 40.866 & 1470.80 & 1468.26 \\
\hline $10 / 7 / 118: 40$ & 37.897 & 40.861 & 1470.87 & 1468.26 \\
\hline $10 / 7 / 118: 50$ & 38.45 & 40.859 & 1470.32 & 1468.26 \\
\hline 10/7/11 9:00 & 37.913 & 40.857 & 1470.86 & 1468.27 \\
\hline 10/7/11 9:10 & 37.829 & 40.854 & 1470.94 & 1468.27 \\
\hline 10/7/11 9:20 & 37.768 & 40.85 & 1471.00 & 1468.27 \\
\hline 10/7/11 9:30 & 37.718 & 40.845 & 1471.05 & 1468.28 \\
\hline 10/7/11 9:40 & 37.676 & 40.838 & 1471.09 & 1468.28 \\
\hline
\end{tabular}


TABLE S1.3 Automatically recorded groundwater levels in the Klassen wells, September 15, 2011, to March 13, 2012.

\begin{tabular}{|c|c|c|c|c|}
\hline \multicolumn{3}{|c|}{ Reference Elevation (ft AMSL) } & 1508.77 & \multirow{2}{*}{$\begin{array}{l}1509.12 \\
\text { Elevation in Klassen } \\
\text { Test Well (ft AMSL) }\end{array}$} \\
\hline Date and Time & $\begin{array}{l}\text { Depth in Klassen } \\
\text { Domestic Well } \\
(\mathrm{ft} \mathrm{BGL})\end{array}$ & $\begin{array}{c}\text { Depth in Klassen } \\
\text { Test Well } \\
\text { (ft BGL) }\end{array}$ & $\begin{array}{l}\text { Elevation in Klassen } \\
\text { Domestic Well } \\
\text { (ft AMSL) }\end{array}$ & \\
\hline $10 / 7 / 119: 50$ & 37.639 & 40.833 & 1471.13 & 1468.29 \\
\hline 10/7/11 10:00 & 37.603 & 40.829 & 1471.17 & 1468.29 \\
\hline 10/7/11 10:10 & 37.571 & 40.824 & 1471.20 & 1468.30 \\
\hline 10/7/11 10:20 & 37.54 & 40.817 & 1471.23 & 1468.31 \\
\hline 10/7/11 10:30 & 37.514 & 40.812 & 1471.26 & 1468.31 \\
\hline 10/7/11 10:40 & 37.709 & 40.81 & 1471.06 & 1468.31 \\
\hline 10/7/11 10:50 & 37.587 & 40.803 & 1471.18 & 1468.32 \\
\hline 10/7/11 11:00 & 38.087 & 40.798 & 1470.68 & 1468.32 \\
\hline 10/7/11 11:10 & 38.347 & 40.796 & 1470.42 & 1468.33 \\
\hline 10/7/11 11:20 & 37.66 & 40.793 & 1471.11 & 1468.33 \\
\hline 10/7/11 11:30 & 37.573 & 40.789 & 1471.20 & 1468.33 \\
\hline 10/7/11 11:40 & 39.961 & 40.786 & 1468.81 & 1468.34 \\
\hline 10/7/11 11:50 & 37.657 & 40.782 & 1471.11 & 1468.34 \\
\hline 10/7/11 12:00 & 37.566 & 40.779 & 1471.20 & 1468.34 \\
\hline 10/7/11 12:10 & 37.507 & 40.775 & 1471.26 & 1468.35 \\
\hline 10/7/11 12:20 & 37.467 & 40.77 & 1471.30 & 1468.35 \\
\hline 10/7/11 12:30 & 37.432 & 40.765 & 1471.34 & 1468.36 \\
\hline 10/7/11 12:40 & 37.402 & 40.763 & 1471.37 & 1468.36 \\
\hline 10/7/11 12:50 & 37.374 & 40.756 & 1471.40 & 1468.37 \\
\hline 10/7/11 13:00 & 37.35 & 40.749 & 1471.42 & 1468.37 \\
\hline 10/7/11 13:10 & 37.327 & 40.742 & 1471.44 & 1468.38 \\
\hline 10/7/11 13:20 & 37.299 & 40.735 & 1471.47 & 1468.39 \\
\hline 10/7/11 13:30 & 37.277 & 40.728 & 1471.49 & 1468.39 \\
\hline 10/7/11 13:40 & 37.585 & 40.723 & 1471.18 & 1468.40 \\
\hline 10/7/11 13:50 & 37.352 & 40.718 & 1471.42 & 1468.40 \\
\hline 10/7/11 14:00 & 37.299 & 40.711 & 1471.47 & 1468.41 \\
\hline 10/7/11 14:10 & 37.261 & 40.704 & 1471.51 & 1468.42 \\
\hline 10/7/11 14:20 & 37.233 & 40.702 & 1471.54 & 1468.42 \\
\hline 10/7/11 14:30 & 37.212 & 40.695 & 1471.56 & 1468.43 \\
\hline 10/7/11 14:40 & 37.188 & 40.686 & 1471.58 & 1468.44 \\
\hline 10/7/11 14:50 & 37.167 & 40.678 & 1471.60 & 1468.44 \\
\hline 10/7/11 15:00 & 37.148 & 40.671 & 1471.62 & 1468.45 \\
\hline 10/7/11 15:10 & 37.132 & 40.667 & 1471.64 & 1468.46 \\
\hline 10/7/11 15:20 & 37.116 & 40.662 & 1471.65 & 1468.46 \\
\hline 10/7/11 15:30 & 37.109 & 40.657 & 1471.66 & 1468.47 \\
\hline 10/7/11 15:40 & 37.123 & 40.65 & 1471.65 & 1468.47 \\
\hline 10/7/11 15:50 & 37.109 & 40.646 & 1471.66 & 1468.48 \\
\hline 10/7/11 16:00 & 37.28 & 40.639 & 1471.49 & 1468.48 \\
\hline
\end{tabular}


TABLE S1.3 Automatically recorded groundwater levels in the Klassen wells, September 15, 2011, to March 13, 2012.

\begin{tabular}{|c|c|c|c|c|}
\hline \multicolumn{3}{|c|}{ Reference Elevation (ft AMSL) } & 1508.77 & \multirow{2}{*}{$\begin{array}{l}1509.12 \\
\text { Elevation in Klassen } \\
\text { Test Well (ft AMSL) }\end{array}$} \\
\hline Date and Time & $\begin{array}{l}\text { Depth in Klassen } \\
\text { Domestic Well } \\
\text { (ft BGL) }\end{array}$ & $\begin{array}{c}\text { Depth in Klassen } \\
\text { Test Well } \\
\text { (ft BGL) }\end{array}$ & $\begin{array}{l}\text { Elevation in Klassen } \\
\text { Domestic Well } \\
\text { (ft AMSL) }\end{array}$ & \\
\hline 10/7/11 16:10 & 37.184 & 40.634 & 1471.59 & 1468.49 \\
\hline 10/7/11 16:20 & 37.141 & 40.629 & 1471.63 & 1468.49 \\
\hline 10/7/11 16:30 & 37.113 & 40.625 & 1471.66 & 1468.50 \\
\hline 10/7/11 16:40 & 37.085 & 40.62 & 1471.68 & 1468.50 \\
\hline 10/7/11 16:50 & 37.263 & 40.615 & 1471.51 & 1468.51 \\
\hline 10/7/11 17:00 & 37.221 & 40.613 & 1471.55 & 1468.51 \\
\hline 10/7/11 17:10 & 37.184 & 40.61 & 1471.59 & 1468.51 \\
\hline 10/7/11 17:20 & 37.202 & 40.608 & 1471.57 & 1468.51 \\
\hline 10/7/11 17:30 & 37.228 & 40.606 & 1471.54 & 1468.52 \\
\hline 10/7/11 17:40 & 37.198 & 40.603 & 1471.57 & 1468.52 \\
\hline 10/7/11 17:50 & 37.153 & 40.601 & 1471.62 & 1468.52 \\
\hline 10/7/11 18:00 & 37.104 & 40.594 & 1471.67 & 1468.53 \\
\hline 10/7/11 18:10 & 37.083 & 40.589 & 1471.69 & 1468.53 \\
\hline 10/7/11 18:20 & 37.097 & 40.587 & 1471.67 & 1468.54 \\
\hline 10/7/11 18:30 & 49.266 & 40.587 & 1459.50 & 1468.54 \\
\hline 10/7/11 18:40 & 49.621 & 40.596 & 1459.15 & 1468.53 \\
\hline 10/7/11 18:50 & 53.978 & 40.613 & 1454.79 & 1468.51 \\
\hline 10/7/11 19:00 & 51.246 & 40.634 & 1457.52 & 1468.49 \\
\hline 10/7/11 19:10 & 52.585 & 40.653 & 1456.18 & 1468.47 \\
\hline 10/7/11 19:20 & 54.534 & 40.678 & 1454.24 & 1468.44 \\
\hline 10/7/11 19:30 & 56.595 & 40.704 & 1452.17 & 1468.42 \\
\hline 10/7/11 19:40 & 56.197 & 40.732 & 1452.57 & 1468.39 \\
\hline 10/7/11 19:50 & 51.863 & 40.761 & 1456.91 & 1468.36 \\
\hline 10/7/11 20:00 & 55.402 & 40.789 & 1453.37 & 1468.33 \\
\hline 10/7/11 20:10 & 57.585 & 40.819 & 1451.18 & 1468.30 \\
\hline 10/7/11 20:20 & 54.058 & 40.852 & 1454.71 & 1468.27 \\
\hline 10/7/11 20:30 & 54.222 & 40.883 & 1454.55 & 1468.24 \\
\hline 10/7/11 20:40 & 55.289 & 40.913 & 1453.48 & 1468.21 \\
\hline $10 / 7 / 1120: 50$ & 41.947 & 40.939 & 1466.82 & 1468.18 \\
\hline 10/7/11 21:00 & 40.957 & 40.953 & 1467.81 & 1468.17 \\
\hline 10/7/11 21:10 & 40.43 & 40.962 & 1468.34 & 1468.16 \\
\hline 10/7/11 21:20 & 40.057 & 40.967 & 1468.71 & 1468.16 \\
\hline 10/7/11 21:30 & 40.179 & 40.972 & 1468.59 & 1468.15 \\
\hline 10/7/11 21:40 & 39.674 & 40.972 & 1469.10 & 1468.15 \\
\hline 10/7/11 21:50 & 39.442 & 40.972 & 1469.33 & 1468.15 \\
\hline 10/7/11 22:00 & 39.255 & 40.969 & 1469.51 & 1468.15 \\
\hline 10/7/11 22:10 & 39.142 & 40.969 & 1469.63 & 1468.15 \\
\hline $10 / 7 / 1122: 20$ & 38.994 & 40.967 & 1469.78 & 1468.16 \\
\hline
\end{tabular}


TABLE S1.3 Automatically recorded groundwater levels in the Klassen wells, September 15, 2011, to March 13, 2012.

\begin{tabular}{|c|c|c|c|c|}
\hline \multicolumn{3}{|c|}{ Reference Elevation (ft AMSL) } & 1508.77 & \multirow{2}{*}{$\begin{array}{l}1509.12 \\
\text { Elevation in Klassen } \\
\text { Test Well (ft AMSL) }\end{array}$} \\
\hline Date and Time & $\begin{array}{l}\text { Depth in Klassen } \\
\text { Domestic Well } \\
(\mathrm{ft} \mathrm{BGL})\end{array}$ & $\begin{array}{c}\text { Depth in Klassen } \\
\text { Test Well } \\
\text { (ft BGL) }\end{array}$ & $\begin{array}{l}\text { Elevation in Klassen } \\
\text { Domestic Well } \\
\text { (ft AMSL) }\end{array}$ & \\
\hline $10 / 7 / 1122: 30$ & 39.386 & 40.965 & 1469.38 & 1468.16 \\
\hline $10 / 7 / 1122: 40$ & 39.194 & 40.962 & 1469.58 & 1468.16 \\
\hline $10 / 7 / 1122: 50$ & 38.875 & 40.958 & 1469.89 & 1468.16 \\
\hline $10 / 7 / 1123: 00$ & 42.484 & 40.958 & 1466.29 & 1468.16 \\
\hline 10/7/11 23:10 & 40.556 & 40.958 & 1468.21 & 1468.16 \\
\hline 10/7/11 23:20 & 39.632 & 40.958 & 1469.14 & 1468.16 \\
\hline 10/7/11 23:30 & 38.861 & 40.958 & 1469.91 & 1468.16 \\
\hline 10/7/11 23:40 & 38.678 & 40.955 & 1470.09 & 1468.17 \\
\hline $10 / 7 / 1123: 50$ & 38.546 & 40.953 & 1470.22 & 1468.17 \\
\hline 10/8/11 0:00 & 38.436 & 40.948 & 1470.33 & 1468.17 \\
\hline 10/8/11 0:10 & 38.345 & 40.946 & 1470.42 & 1468.18 \\
\hline $10 / 8 / 110: 20$ & 38.258 & 40.941 & 1470.51 & 1468.18 \\
\hline 10/8/11 0:30 & 38.18 & 40.937 & 1470.59 & 1468.19 \\
\hline $10 / 8 / 110: 40$ & 38.155 & 40.932 & 1470.61 & 1468.19 \\
\hline 10/8/11 0:50 & 38.101 & 40.927 & 1470.67 & 1468.20 \\
\hline 10/8/11 1:00 & 38.035 & 40.92 & 1470.73 & 1468.20 \\
\hline $10 / 8 / 11$ 1:10 & 37.976 & 40.913 & 1470.79 & 1468.21 \\
\hline $10 / 8 / 11$ 1:20 & 37.915 & 40.906 & 1470.85 & 1468.22 \\
\hline $10 / 8 / 11$ 1:30 & 37.864 & 40.901 & 1470.91 & 1468.22 \\
\hline $10 / 8 / 11$ 1:40 & 37.857 & 40.894 & 1470.91 & 1468.23 \\
\hline $10 / 8 / 111: 50$ & 37.882 & 40.892 & 1470.89 & 1468.23 \\
\hline $10 / 8 / 112: 00$ & 37.838 & 40.887 & 1470.93 & 1468.24 \\
\hline $10 / 8 / 112: 10$ & 37.791 & 40.883 & 1470.98 & 1468.24 \\
\hline $10 / 8 / 112: 20$ & 37.742 & 40.876 & 1471.03 & 1468.25 \\
\hline 10/8/11 2:30 & 37.953 & 40.873 & 1470.82 & 1468.25 \\
\hline $10 / 8 / 112: 40$ & 37.765 & 40.869 & 1471.00 & 1468.25 \\
\hline $10 / 8 / 112: 50$ & 37.695 & 40.864 & 1471.07 & 1468.26 \\
\hline $10 / 8 / 113: 00$ & 37.639 & 40.859 & 1471.13 & 1468.26 \\
\hline 10/8/11 3:10 & 37.603 & 40.852 & 1471.17 & 1468.27 \\
\hline $10 / 8 / 113: 20$ & 37.592 & 40.845 & 1471.18 & 1468.28 \\
\hline 10/8/11 3:30 & 37.615 & 40.84 & 1471.15 & 1468.28 \\
\hline $10 / 8 / 113: 40$ & 37.568 & 40.836 & 1471.20 & 1468.29 \\
\hline $10 / 8 / 113: 50$ & 37.519 & 40.831 & 1471.25 & 1468.29 \\
\hline $10 / 8 / 114: 00$ & 37.47 & 40.824 & 1471.30 & 1468.30 \\
\hline $10 / 8 / 114: 10$ & 37.423 & 40.817 & 1471.35 & 1468.31 \\
\hline $10 / 8 / 114: 20$ & 37.385 & 40.81 & 1471.38 & 1468.31 \\
\hline $10 / 8 / 114: 30$ & 37.348 & 40.805 & 1471.42 & 1468.32 \\
\hline $10 / 8 / 114: 40$ & 37.313 & 40.796 & 1471.46 & 1468.33 \\
\hline
\end{tabular}


TABLE S1.3 Automatically recorded groundwater levels in the Klassen wells, September 15, 2011, to March 13, 2012.

\begin{tabular}{|c|c|c|c|c|}
\hline \multicolumn{3}{|c|}{ Reference Elevation (ft AMSL) } & 1508.77 & \multirow{2}{*}{$\begin{array}{l}1509.12 \\
\text { Elevation in Klassen } \\
\text { Test Well (ft AMSL) }\end{array}$} \\
\hline Date and Time & $\begin{array}{l}\text { Depth in Klassen } \\
\text { Domestic Well } \\
\text { (ft BGL) }\end{array}$ & $\begin{array}{c}\text { Depth in Klassen } \\
\text { Test Well } \\
\text { (ft BGL) }\end{array}$ & $\begin{array}{l}\text { Elevation in Klassen } \\
\text { Domestic Well } \\
\text { (ft AMSL) }\end{array}$ & \\
\hline 10/8/11 4:50 & 37.275 & 40.789 & 1471.49 & 1468.33 \\
\hline 10/8/11 5:00 & 37.245 & 40.782 & 1471.52 & 1468.34 \\
\hline 10/8/11 5:10 & 37.214 & 40.772 & 1471.56 & 1468.35 \\
\hline $10 / 8 / 115: 20$ & 37.188 & 40.765 & 1471.58 & 1468.36 \\
\hline $10 / 8 / 115: 30$ & 37.163 & 40.758 & 1471.61 & 1468.36 \\
\hline $10 / 8 / 115: 40$ & 37.134 & 40.749 & 1471.64 & 1468.37 \\
\hline $10 / 8 / 115: 50$ & 37.111 & 40.742 & 1471.66 & 1468.38 \\
\hline $10 / 8 / 116: 00$ & 37.085 & 40.737 & 1471.68 & 1468.39 \\
\hline $10 / 8 / 116: 10$ & 37.693 & 40.732 & 1471.08 & 1468.39 \\
\hline $10 / 8 / 116: 20$ & 37.188 & 40.728 & 1471.58 & 1468.39 \\
\hline 10/8/11 6:30 & 37.118 & 40.723 & 1471.65 & 1468.40 \\
\hline $10 / 8 / 116: 40$ & 37.073 & 40.718 & 1471.70 & 1468.40 \\
\hline $10 / 8 / 116: 50$ & 37.038 & 40.714 & 1471.73 & 1468.41 \\
\hline $10 / 8 / 117: 00$ & 37.01 & 40.707 & 1471.76 & 1468.42 \\
\hline 10/8/11 7:10 & 36.984 & 40.7 & 1471.79 & 1468.42 \\
\hline $10 / 8 / 117: 20$ & 36.958 & 40.695 & 1471.81 & 1468.43 \\
\hline $10 / 8 / 117: 30$ & 36.935 & 40.688 & 1471.83 & 1468.43 \\
\hline $10 / 8 / 117: 40$ & 36.912 & 40.683 & 1471.86 & 1468.44 \\
\hline $10 / 8 / 117: 50$ & 39.212 & 40.678 & 1469.56 & 1468.44 \\
\hline $10 / 8 / 118: 00$ & 37.104 & 40.671 & 1471.67 & 1468.45 \\
\hline 10/8/11 8:10 & 36.984 & 40.667 & 1471.79 & 1468.46 \\
\hline 10/8/11 8:20 & 36.93 & 40.662 & 1471.84 & 1468.46 \\
\hline 10/8/11 8:30 & 36.897 & 40.655 & 1471.87 & 1468.47 \\
\hline $10 / 8 / 118: 40$ & 36.958 & 40.648 & 1471.81 & 1468.47 \\
\hline $10 / 8 / 118: 50$ & 36.961 & 40.646 & 1471.81 & 1468.48 \\
\hline 10/8/11 9:00 & 37.155 & 40.646 & 1471.61 & 1468.48 \\
\hline 10/8/11 9:10 & 37.531 & 40.648 & 1471.24 & 1468.47 \\
\hline 10/8/11 9:20 & 37.376 & 40.66 & 1471.39 & 1468.46 \\
\hline 10/8/11 9:30 & 37.266 & 40.664 & 1471.50 & 1468.46 \\
\hline 10/8/11 9:40 & 39.053 & 40.664 & 1469.72 & 1468.46 \\
\hline 10/8/11 9:50 & 37.303 & 40.664 & 1471.47 & 1468.46 \\
\hline 10/8/11 10:00 & 37.226 & 40.664 & 1471.54 & 1468.46 \\
\hline 10/8/11 10:10 & 37.146 & 40.662 & 1471.62 & 1468.46 \\
\hline 10/8/11 10:20 & 37.059 & 40.653 & 1471.71 & 1468.47 \\
\hline 10/8/11 10:30 & 36.991 & 40.639 & 1471.78 & 1468.48 \\
\hline 10/8/11 10:40 & 36.93 & 40.629 & 1471.84 & 1468.49 \\
\hline 10/8/11 10:50 & 36.876 & 40.622 & 1471.89 & 1468.50 \\
\hline 10/8/11 11:00 & 36.825 & 40.62 & 1471.94 & 1468.50 \\
\hline
\end{tabular}


TABLE S1.3 Automatically recorded groundwater levels in the Klassen wells, September 15, 2011, to March 13, 2012.

\begin{tabular}{|c|c|c|c|c|}
\hline \multicolumn{3}{|c|}{ Reference Elevation (ft AMSL) } & 1508.77 & \multirow{2}{*}{$\begin{array}{l}1509.12 \\
\text { Elevation in Klassen } \\
\text { Test Well (ft AMSL) }\end{array}$} \\
\hline Date and Time & $\begin{array}{l}\text { Depth in Klassen } \\
\text { Domestic Well } \\
(\mathrm{ft} \mathrm{BGL})\end{array}$ & $\begin{array}{c}\text { Depth in Klassen } \\
\text { Test Well } \\
\text { (ft BGL) }\end{array}$ & $\begin{array}{l}\text { Elevation in Klassen } \\
\text { Domestic Well } \\
\text { (ft AMSL) }\end{array}$ & \\
\hline 10/8/11 11:10 & 36.783 & 40.615 & 1471.99 & 1468.51 \\
\hline 10/8/11 11:20 & 36.74 & 40.613 & 1472.03 & 1468.51 \\
\hline 10/8/11 11:30 & 36.703 & 40.61 & 1472.07 & 1468.51 \\
\hline 10/8/11 11:40 & 36.665 & 40.608 & 1472.10 & 1468.51 \\
\hline 10/8/11 11:50 & 36.881 & 40.608 & 1471.89 & 1468.51 \\
\hline 10/8/11 12:00 & 36.7 & 40.603 & 1472.07 & 1468.52 \\
\hline 10/8/11 12:10 & 36.64 & 40.603 & 1472.13 & 1468.52 \\
\hline 10/8/11 12:20 & 36.595 & 40.596 & 1472.17 & 1468.53 \\
\hline 10/8/11 12:30 & 36.557 & 40.599 & 1472.21 & 1468.52 \\
\hline 10/8/11 12:40 & 36.94 & 40.601 & 1471.83 & 1468.52 \\
\hline 10/8/11 12:50 & 36.64 & 40.599 & 1472.13 & 1468.52 \\
\hline 10/8/11 13:00 & 36.567 & 40.596 & 1472.20 & 1468.53 \\
\hline 10/8/11 13:10 & 36.518 & 40.596 & 1472.25 & 1468.53 \\
\hline $10 / 8 / 1113: 20$ & 36.722 & 40.599 & 1472.05 & 1468.52 \\
\hline 10/8/11 13:30 & 36.543 & 40.596 & 1472.23 & 1468.53 \\
\hline 10/8/11 13:40 & 36.482 & 40.596 & 1472.29 & 1468.53 \\
\hline 10/8/11 13:50 & 36.44 & 40.596 & 1472.33 & 1468.53 \\
\hline 10/8/11 14:00 & 36.405 & 40.594 & 1472.36 & 1468.53 \\
\hline 10/8/11 14:10 & 36.372 & 40.592 & 1472.40 & 1468.53 \\
\hline $10 / 8 / 1114: 20$ & 36.346 & 40.589 & 1472.42 & 1468.53 \\
\hline $10 / 8 / 1114: 30$ & 36.318 & 40.589 & 1472.45 & 1468.53 \\
\hline 10/8/11 14:40 & 36.292 & 40.585 & 1472.48 & 1468.54 \\
\hline 10/8/11 14:50 & 41.651 & 40.585 & 1467.12 & 1468.54 \\
\hline 10/8/11 15:00 & 36.445 & 40.58 & 1472.32 & 1468.54 \\
\hline 10/8/11 15:10 & 36.342 & 40.578 & 1472.43 & 1468.54 \\
\hline 10/8/11 15:20 & 36.292 & 40.578 & 1472.48 & 1468.54 \\
\hline 10/8/11 15:30 & 36.257 & 40.58 & 1472.51 & 1468.54 \\
\hline 10/8/11 15:40 & 36.224 & 40.58 & 1472.55 & 1468.54 \\
\hline 10/8/11 15:50 & 36.199 & 40.58 & 1472.57 & 1468.54 \\
\hline 10/8/11 16:00 & 40.84 & 40.58 & 1467.93 & 1468.54 \\
\hline 10/8/11 16:10 & 36.314 & 40.58 & 1472.46 & 1468.54 \\
\hline 10/8/11 16:20 & 36.267 & 40.575 & 1472.50 & 1468.55 \\
\hline 10/8/11 16:30 & 36.487 & 40.571 & 1472.28 & 1468.55 \\
\hline 10/8/11 16:40 & 36.318 & 40.573 & 1472.45 & 1468.55 \\
\hline 10/8/11 16:50 & 36.25 & 40.571 & 1472.52 & 1468.55 \\
\hline 10/8/11 17:00 & 36.973 & 40.571 & 1471.80 & 1468.55 \\
\hline 10/8/11 17:10 & 36.295 & 40.573 & 1472.47 & 1468.55 \\
\hline 10/8/11 17:20 & 36.222 & 40.573 & 1472.55 & 1468.55 \\
\hline
\end{tabular}


TABLE S1.3 Automatically recorded groundwater levels in the Klassen wells, September 15, 2011, to March 13, 2012.

\begin{tabular}{|c|c|c|c|c|}
\hline \multicolumn{3}{|c|}{ Reference Elevation (ft AMSL) } & 1508.77 & \multirow{2}{*}{$\begin{array}{l}1509.12 \\
\text { Elevation in Klassen } \\
\text { Test Well (ft AMSL) }\end{array}$} \\
\hline Date and Time & $\begin{array}{l}\text { Depth in Klassen } \\
\text { Domestic Well } \\
(\mathrm{ft} \mathrm{BGL})\end{array}$ & $\begin{array}{c}\text { Depth in Klassen } \\
\text { Test Well } \\
\text { (ft BGL) }\end{array}$ & $\begin{array}{l}\text { Elevation in Klassen } \\
\text { Domestic Well } \\
\text { (ft AMSL) }\end{array}$ & \\
\hline 10/8/11 17:30 & 36.18 & 40.566 & 1472.59 & 1468.56 \\
\hline 10/8/11 17:40 & 36.142 & 40.561 & 1472.63 & 1468.56 \\
\hline 10/8/11 17:50 & 36.112 & 40.554 & 1472.66 & 1468.57 \\
\hline 10/8/11 18:00 & 36.084 & 40.549 & 1472.69 & 1468.57 \\
\hline 10/8/11 18:10 & 36.06 & 40.547 & 1472.71 & 1468.58 \\
\hline 10/8/11 18:20 & 36.037 & 40.542 & 1472.73 & 1468.58 \\
\hline 10/8/11 18:30 & 36.016 & 40.538 & 1472.75 & 1468.58 \\
\hline 10/8/11 18:40 & 35.995 & 40.538 & 1472.77 & 1468.58 \\
\hline 10/8/11 18:50 & 35.976 & 40.535 & 1472.79 & 1468.59 \\
\hline 10/8/11 19:00 & 35.988 & 40.531 & 1472.78 & 1468.59 \\
\hline 10/8/11 19:10 & 36.675 & 40.524 & 1472.09 & 1468.60 \\
\hline 10/8/11 19:20 & 36.124 & 40.524 & 1472.65 & 1468.60 \\
\hline 10/8/11 19:30 & 36.056 & 40.524 & 1472.71 & 1468.60 \\
\hline 10/8/11 19:40 & 36.011 & 40.524 & 1472.76 & 1468.60 \\
\hline 10/8/11 19:50 & 35.98 & 40.517 & 1472.79 & 1468.61 \\
\hline 10/8/11 20:00 & 36.292 & 40.512 & 1472.48 & 1468.61 \\
\hline 10/8/11 20:10 & 36.044 & 40.512 & 1472.73 & 1468.61 \\
\hline 10/8/11 20:20 & 35.99 & 40.507 & 1472.78 & 1468.62 \\
\hline 10/8/11 20:30 & 35.952 & 40.507 & 1472.82 & 1468.62 \\
\hline $10 / 8 / 1120: 40$ & 35.927 & 40.5 & 1472.84 & 1468.62 \\
\hline $10 / 8 / 1120: 50$ & 36.163 & 40.502 & 1472.61 & 1468.62 \\
\hline 10/8/11 21:00 & 36.013 & 40.5 & 1472.76 & 1468.62 \\
\hline 10/8/11 21:10 & 35.959 & 40.498 & 1472.81 & 1468.62 \\
\hline $10 / 8 / 1121: 20$ & 35.924 & 40.493 & 1472.85 & 1468.63 \\
\hline 10/8/11 21:30 & 35.894 & 40.491 & 1472.88 & 1468.63 \\
\hline 10/8/11 21:40 & 35.873 & 40.491 & 1472.90 & 1468.63 \\
\hline $10 / 8 / 1121: 50$ & 35.849 & 40.484 & 1472.92 & 1468.64 \\
\hline 10/8/11 22:00 & 36.916 & 40.474 & 1471.85 & 1468.65 \\
\hline 10/8/11 22:10 & 37.873 & 40.472 & 1470.90 & 1468.65 \\
\hline 10/8/11 22:20 & 36.102 & 40.47 & 1472.67 & 1468.65 \\
\hline 10/8/11 22:30 & 35.997 & 40.465 & 1472.77 & 1468.66 \\
\hline 10/8/11 22:40 & 35.964 & 40.463 & 1472.81 & 1468.66 \\
\hline $10 / 8 / 1122: 50$ & 36.192 & 40.463 & 1472.58 & 1468.66 \\
\hline 10/8/11 23:00 & 36.698 & 40.463 & 1472.07 & 1468.66 \\
\hline 10/8/11 23:10 & 36.051 & 40.463 & 1472.72 & 1468.66 \\
\hline $10 / 8 / 1123: 20$ & 35.971 & 40.46 & 1472.80 & 1468.66 \\
\hline $10 / 8 / 1123: 30$ & 35.924 & 40.458 & 1472.85 & 1468.66 \\
\hline $10 / 8 / 1123: 40$ & 35.887 & 40.451 & 1472.88 & 1468.67 \\
\hline
\end{tabular}


TABLE S1.3 Automatically recorded groundwater levels in the Klassen wells, September 15, 2011, to March 13, 2012.

\begin{tabular}{|c|c|c|c|c|}
\hline \multicolumn{3}{|c|}{ Reference Elevation (ft AMSL) } & 1508.77 & \multirow{2}{*}{$\begin{array}{l}1509.12 \\
\text { Elevation in Klassen } \\
\text { Test Well (ft AMSL) }\end{array}$} \\
\hline Date and Time & $\begin{array}{l}\text { Depth in Klassen } \\
\text { Domestic Well } \\
\text { (ft BGL) }\end{array}$ & $\begin{array}{c}\text { Depth in Klassen } \\
\text { Test Well } \\
\text { (ft BGL) }\end{array}$ & $\begin{array}{l}\text { Elevation in Klassen } \\
\text { Domestic Well } \\
\text { (ft AMSL) }\end{array}$ & \\
\hline $10 / 8 / 1123: 50$ & 35.856 & 40.451 & 1472.91 & 1468.67 \\
\hline 10/9/11 0:00 & 35.828 & 40.449 & 1472.94 & 1468.67 \\
\hline 10/9/11 0:10 & 35.805 & 40.446 & 1472.96 & 1468.68 \\
\hline 10/9/11 0:20 & 35.783 & 40.444 & 1472.99 & 1468.68 \\
\hline 10/9/11 0:30 & 35.762 & 40.439 & 1473.01 & 1468.68 \\
\hline 10/9/11 0:40 & 35.744 & 40.441 & 1473.03 & 1468.68 \\
\hline $10 / 9 / 110: 50$ & 35.755 & 40.437 & 1473.01 & 1468.69 \\
\hline $10 / 9 / 111: 00$ & 35.767 & 40.432 & 1473.00 & 1468.69 \\
\hline 10/9/11 1:10 & 35.744 & 40.425 & 1473.03 & 1468.70 \\
\hline $10 / 9 / 111: 20$ & 35.723 & 40.42 & 1473.05 & 1468.70 \\
\hline 10/9/11 1:30 & 35.701 & 40.416 & 1473.07 & 1468.71 \\
\hline $10 / 9 / 111: 40$ & 35.685 & 40.409 & 1473.08 & 1468.71 \\
\hline $10 / 9 / 111: 50$ & 35.669 & 40.406 & 1473.10 & 1468.72 \\
\hline $10 / 9 / 112: 00$ & 35.652 & 40.402 & 1473.12 & 1468.72 \\
\hline $10 / 9 / 112: 10$ & 35.633 & 40.397 & 1473.14 & 1468.73 \\
\hline $10 / 9 / 112: 20$ & 35.622 & 40.395 & 1473.15 & 1468.73 \\
\hline $10 / 9 / 112: 30$ & 35.605 & 40.39 & 1473.16 & 1468.73 \\
\hline $10 / 9 / 112: 40$ & 35.591 & 40.388 & 1473.18 & 1468.73 \\
\hline $10 / 9 / 112: 50$ & 35.577 & 40.385 & 1473.19 & 1468.74 \\
\hline $10 / 9 / 113: 00$ & 35.565 & 40.383 & 1473.20 & 1468.74 \\
\hline 10/9/11 3:10 & 35.551 & 40.38 & 1473.22 & 1468.74 \\
\hline 10/9/11 3:20 & 38.375 & 40.378 & 1470.39 & 1468.74 \\
\hline 10/9/11 3:30 & 35.685 & 40.376 & 1473.08 & 1468.75 \\
\hline $10 / 9 / 113: 40$ & 35.615 & 40.371 & 1473.15 & 1468.75 \\
\hline $10 / 9 / 113: 50$ & 35.579 & 40.369 & 1473.19 & 1468.75 \\
\hline $10 / 9 / 114: 00$ & 35.556 & 40.366 & 1473.21 & 1468.76 \\
\hline $10 / 9 / 114: 10$ & 35.537 & 40.364 & 1473.23 & 1468.76 \\
\hline 10/9/11 4:20 & 35.518 & 40.357 & 1473.25 & 1468.77 \\
\hline $10 / 9 / 114: 30$ & 35.504 & 40.355 & 1473.27 & 1468.77 \\
\hline $10 / 9 / 114: 40$ & 35.49 & 40.352 & 1473.28 & 1468.77 \\
\hline 10/9/11 4:50 & 35.476 & 40.348 & 1473.29 & 1468.77 \\
\hline 10/9/11 5:00 & 35.462 & 40.345 & 1473.31 & 1468.78 \\
\hline 10/9/11 5:10 & 35.45 & 40.338 & 1473.32 & 1468.78 \\
\hline $10 / 9 / 115: 20$ & 35.439 & 40.334 & 1473.33 & 1468.79 \\
\hline $10 / 9 / 115: 30$ & 35.429 & 40.331 & 1473.34 & 1468.79 \\
\hline $10 / 9 / 115: 40$ & 36.914 & 40.324 & 1471.86 & 1468.80 \\
\hline $10 / 9 / 115: 50$ & 36.166 & 40.317 & 1472.60 & 1468.81 \\
\hline $10 / 9 / 116: 00$ & 36.353 & 40.31 & 1472.42 & 1468.81 \\
\hline
\end{tabular}


TABLE S1.3 Automatically recorded groundwater levels in the Klassen wells, September 15, 2011, to March 13, 2012.

\begin{tabular}{|c|c|c|c|c|}
\hline \multicolumn{3}{|c|}{ Reference Elevation (ft AMSL) } & 1508.77 & \multirow{2}{*}{$\begin{array}{l}1509.12 \\
\text { Elevation in Klassen } \\
\text { Test Well (ft AMSL) }\end{array}$} \\
\hline Date and Time & $\begin{array}{l}\text { Depth in Klassen } \\
\text { Domestic Well } \\
\text { (ft BGL) }\end{array}$ & $\begin{array}{c}\text { Depth in Klassen } \\
\text { Test Well } \\
\text { (ft BGL) }\end{array}$ & $\begin{array}{l}\text { Elevation in Klassen } \\
\text { Domestic Well } \\
\text { (ft AMSL) }\end{array}$ & \\
\hline $10 / 9 / 116: 10$ & 35.683 & 40.305 & 1473.09 & 1468.82 \\
\hline $10 / 9 / 116: 20$ & 39.468 & 40.296 & 1469.30 & 1468.83 \\
\hline $10 / 9 / 116: 30$ & 35.807 & 40.287 & 1472.96 & 1468.84 \\
\hline $10 / 9 / 116: 40$ & 35.678 & 40.282 & 1473.09 & 1468.84 \\
\hline $10 / 9 / 116: 50$ & 37.303 & 40.277 & 1471.47 & 1468.85 \\
\hline 10/9/11 7:00 & 36.042 & 40.27 & 1472.73 & 1468.85 \\
\hline $10 / 9 / 117: 10$ & 35.814 & 40.261 & 1472.96 & 1468.86 \\
\hline $10 / 9 / 117: 20$ & 35.723 & 40.256 & 1473.05 & 1468.87 \\
\hline $10 / 9 / 117: 30$ & 35.662 & 40.249 & 1473.11 & 1468.87 \\
\hline $10 / 9 / 117: 40$ & 35.617 & 40.242 & 1473.15 & 1468.88 \\
\hline $10 / 9 / 117: 50$ & 35.582 & 40.233 & 1473.19 & 1468.89 \\
\hline 10/9/11 8:00 & 40.42 & 40.223 & 1468.35 & 1468.90 \\
\hline 10/9/11 8:10 & 35.72 & 40.219 & 1473.05 & 1468.90 \\
\hline $10 / 9 / 118: 20$ & 39.426 & 40.216 & 1469.34 & 1468.91 \\
\hline $10 / 9 / 118: 30$ & 35.73 & 40.219 & 1473.04 & 1468.90 \\
\hline $10 / 9 / 118: 40$ & 35.96 & 40.221 & 1472.81 & 1468.90 \\
\hline $10 / 9 / 118: 50$ & 35.69 & 40.223 & 1473.08 & 1468.90 \\
\hline 10/9/11 9:00 & 35.619 & 40.223 & 1473.15 & 1468.90 \\
\hline 10/9/11 9:10 & 35.601 & 40.223 & 1473.17 & 1468.90 \\
\hline 10/9/11 9:20 & 35.563 & 40.221 & 1473.21 & 1468.90 \\
\hline 10/9/11 9:30 & 35.533 & 40.219 & 1473.24 & 1468.90 \\
\hline 10/9/11 9:40 & 35.504 & 40.216 & 1473.27 & 1468.91 \\
\hline 10/9/11 9:50 & 35.511 & 40.216 & 1473.26 & 1468.91 \\
\hline 10/9/11 10:00 & 35.486 & 40.216 & 1473.28 & 1468.91 \\
\hline 10/9/11 10:10 & 35.474 & 40.216 & 1473.30 & 1468.91 \\
\hline 10/9/11 10:20 & 35.469 & 40.216 & 1473.30 & 1468.91 \\
\hline 10/9/11 10:30 & 35.446 & 40.216 & 1473.32 & 1468.91 \\
\hline 10/9/11 10:40 & 35.467 & 40.216 & 1473.30 & 1468.91 \\
\hline 10/9/11 10:50 & 35.486 & 40.219 & 1473.28 & 1468.90 \\
\hline 10/9/11 11:00 & 35.46 & 40.223 & 1473.31 & 1468.90 \\
\hline 10/9/11 11:10 & 35.483 & 40.228 & 1473.29 & 1468.89 \\
\hline 10/9/11 11:20 & 35.511 & 40.233 & 1473.26 & 1468.89 \\
\hline 10/9/11 11:30 & 35.483 & 40.237 & 1473.29 & 1468.89 \\
\hline 10/9/11 11:40 & 35.453 & 40.235 & 1473.32 & 1468.89 \\
\hline 10/9/11 11:50 & 35.439 & 40.237 & 1473.33 & 1468.89 \\
\hline 10/9/11 12:00 & 35.481 & 40.235 & 1473.29 & 1468.89 \\
\hline 10/9/11 12:10 & 35.469 & 40.237 & 1473.30 & 1468.89 \\
\hline $10 / 9 / 11$ 12:20 & 35.544 & 40.24 & 1473.23 & 1468.88 \\
\hline
\end{tabular}


TABLE S1.3 Automatically recorded groundwater levels in the Klassen wells, September 15, 2011, to March 13, 2012.

\begin{tabular}{|c|c|c|c|c|}
\hline \multicolumn{3}{|c|}{ Reference Elevation (ft AMSL) } & 1508.77 & \multirow{2}{*}{$\begin{array}{l}1509.12 \\
\text { Elevation in Klassen } \\
\text { Test Well (ft AMSL) }\end{array}$} \\
\hline Date and Time & $\begin{array}{l}\text { Depth in Klassen } \\
\text { Domestic Well } \\
(\mathrm{ft} \mathrm{BGL})\end{array}$ & $\begin{array}{c}\text { Depth in Klassen } \\
\text { Test Well } \\
\text { (ft BGL) }\end{array}$ & $\begin{array}{l}\text { Elevation in Klassen } \\
\text { Domestic Well } \\
\text { (ft AMSL) }\end{array}$ & \\
\hline 10/9/11 12:30 & 35.547 & 40.242 & 1473.22 & 1468.88 \\
\hline 10/9/11 12:40 & 35.511 & 40.24 & 1473.26 & 1468.88 \\
\hline 10/9/11 12:50 & 35.479 & 40.24 & 1473.29 & 1468.88 \\
\hline 10/9/11 13:00 & 35.514 & 40.242 & 1473.26 & 1468.88 \\
\hline 10/9/11 13:10 & 35.528 & 40.237 & 1473.24 & 1468.89 \\
\hline 10/9/11 13:20 & 35.563 & 40.237 & 1473.21 & 1468.89 \\
\hline 10/9/11 13:30 & 35.575 & 40.242 & 1473.19 & 1468.88 \\
\hline 10/9/11 13:40 & 35.537 & 40.24 & 1473.23 & 1468.88 \\
\hline 10/9/11 13:50 & 35.5 & 40.24 & 1473.27 & 1468.88 \\
\hline 10/9/11 14:00 & 35.469 & 40.237 & 1473.30 & 1468.89 \\
\hline 10/9/11 14:10 & 35.443 & 40.228 & 1473.33 & 1468.89 \\
\hline 10/9/11 14:20 & 35.415 & 40.228 & 1473.35 & 1468.89 \\
\hline 10/9/11 14:30 & 35.392 & 40.228 & 1473.38 & 1468.89 \\
\hline 10/9/11 14:40 & 35.371 & 40.223 & 1473.40 & 1468.90 \\
\hline 10/9/11 14:50 & 35.352 & 40.214 & 1473.42 & 1468.91 \\
\hline 10/9/11 15:00 & 35.333 & 40.207 & 1473.44 & 1468.92 \\
\hline 10/9/11 15:10 & 35.314 & 40.207 & 1473.46 & 1468.92 \\
\hline 10/9/11 15:20 & 35.472 & 40.202 & 1473.30 & 1468.92 \\
\hline 10/9/11 15:30 & 35.458 & 40.195 & 1473.31 & 1468.93 \\
\hline 10/9/11 15:40 & 35.46 & 40.193 & 1473.31 & 1468.93 \\
\hline $10 / 9 / 11$ 15:50 & 35.42 & 40.188 & 1473.35 & 1468.93 \\
\hline 10/9/11 16:00 & 35.467 & 40.183 & 1473.30 & 1468.94 \\
\hline 10/9/11 16:10 & 35.465 & 40.179 & 1473.30 & 1468.94 \\
\hline $10 / 9 / 11$ 16:20 & 35.507 & 40.179 & 1473.26 & 1468.94 \\
\hline 10/9/11 16:30 & 35.502 & 40.176 & 1473.27 & 1468.95 \\
\hline 10/9/11 16:40 & 35.465 & 40.172 & 1473.30 & 1468.95 \\
\hline 10/9/11 16:50 & 35.432 & 40.169 & 1473.34 & 1468.95 \\
\hline 10/9/11 17:00 & 35.42 & 40.169 & 1473.35 & 1468.95 \\
\hline 10/9/11 17:10 & 35.387 & 40.165 & 1473.38 & 1468.96 \\
\hline 10/9/11 17:20 & 35.359 & 40.16 & 1473.41 & 1468.96 \\
\hline 10/9/11 17:30 & 35.336 & 40.158 & 1473.43 & 1468.96 \\
\hline 10/9/11 17:40 & 35.338 & 40.151 & 1473.43 & 1468.97 \\
\hline 10/9/11 17:50 & 35.465 & 40.148 & 1473.30 & 1468.97 \\
\hline 10/9/11 18:00 & 35.375 & 40.144 & 1473.39 & 1468.98 \\
\hline 10/9/11 18:10 & 35.359 & 40.139 & 1473.41 & 1468.98 \\
\hline 10/9/11 18:20 & 35.326 & 40.136 & 1473.44 & 1468.99 \\
\hline 10/9/11 18:30 & 35.385 & 40.132 & 1473.38 & 1468.99 \\
\hline 10/9/11 18:40 & 35.406 & 40.129 & 1473.36 & 1468.99 \\
\hline
\end{tabular}


TABLE S1.3 Automatically recorded groundwater levels in the Klassen wells, September 15, 2011, to March 13, 2012.

\begin{tabular}{|c|c|c|c|c|}
\hline \multicolumn{3}{|c|}{ Reference Elevation (ft AMSL) } & 1508.77 & \multirow{2}{*}{$\begin{array}{l}1509.12 \\
\text { Elevation in Klassen } \\
\text { Test Well (ft AMSL) }\end{array}$} \\
\hline Date and Time & $\begin{array}{l}\text { Depth in Klassen } \\
\text { Domestic Well } \\
\text { (ft BGL) }\end{array}$ & $\begin{array}{c}\text { Depth in Klassen } \\
\text { Test Well } \\
\text { (ft BGL) }\end{array}$ & $\begin{array}{l}\text { Elevation in Klassen } \\
\text { Domestic Well } \\
\text { (ft AMSL) }\end{array}$ & \\
\hline 10/9/11 18:50 & 35.397 & 40.122 & 1473.37 & 1469.00 \\
\hline 10/9/11 19:00 & 35.429 & 40.12 & 1473.34 & 1469.00 \\
\hline 10/9/11 19:10 & 35.415 & 40.12 & 1473.35 & 1469.00 \\
\hline 10/9/11 19:20 & 35.389 & 40.118 & 1473.38 & 1469.00 \\
\hline 10/9/11 19:30 & 35.415 & 40.115 & 1473.35 & 1469.01 \\
\hline 10/9/11 19:40 & 35.415 & 40.12 & 1473.35 & 1469.00 \\
\hline 10/9/11 19:50 & 35.472 & 40.12 & 1473.30 & 1469.00 \\
\hline 10/9/11 20:00 & 35.561 & 40.12 & 1473.21 & 1469.00 \\
\hline 10/9/11 20:10 & 35.556 & 40.118 & 1473.21 & 1469.00 \\
\hline 10/9/11 20:20 & 35.523 & 40.12 & 1473.25 & 1469.00 \\
\hline 10/9/11 20:30 & 36.032 & 40.115 & 1472.74 & 1469.01 \\
\hline 10/9/11 20:40 & 35.591 & 40.111 & 1473.18 & 1469.01 \\
\hline 10/9/11 20:50 & 35.523 & 40.108 & 1473.25 & 1469.01 \\
\hline 10/9/11 21:00 & 35.53 & 40.108 & 1473.24 & 1469.01 \\
\hline 10/9/11 21:10 & 35.516 & 40.108 & 1473.25 & 1469.01 \\
\hline 10/9/11 21:20 & 35.472 & 40.106 & 1473.30 & 1469.02 \\
\hline 10/9/11 21:30 & 35.429 & 40.104 & 1473.34 & 1469.02 \\
\hline 10/9/11 21:40 & 35.394 & 40.097 & 1473.38 & 1469.03 \\
\hline 10/9/11 21:50 & 35.394 & 40.099 & 1473.38 & 1469.02 \\
\hline 10/9/11 22:00 & 36.1 & 40.094 & 1472.67 & 1469.03 \\
\hline 10/9/11 22:10 & 35.589 & 40.094 & 1473.18 & 1469.03 \\
\hline 10/9/11 22:20 & 35.558 & 40.09 & 1473.21 & 1469.03 \\
\hline $10 / 9 / 1122: 30$ & 35.565 & 40.092 & 1473.20 & 1469.03 \\
\hline 10/9/11 22:40 & 36.046 & 40.083 & 1472.72 & 1469.04 \\
\hline $10 / 9 / 1122: 50$ & 35.945 & 40.08 & 1472.82 & 1469.04 \\
\hline 10/9/11 23:00 & 35.638 & 40.075 & 1473.13 & 1469.05 \\
\hline 10/9/11 23:10 & 36.016 & 40.071 & 1472.75 & 1469.05 \\
\hline 10/9/11 23:20 & 35.751 & 40.073 & 1473.02 & 1469.05 \\
\hline 10/9/11 23:30 & 35.603 & 40.073 & 1473.17 & 1469.05 \\
\hline 10/9/11 23:40 & 35.53 & 40.066 & 1473.24 & 1469.06 \\
\hline 10/9/11 23:50 & 35.476 & 40.064 & 1473.29 & 1469.06 \\
\hline 10/10/11 0:00 & 35.436 & 40.057 & 1473.33 & 1469.07 \\
\hline 10/10/11 0:10 & 35.399 & 40.059 & 1473.37 & 1469.06 \\
\hline 10/10/11 0:20 & 35.368 & 40.059 & 1473.40 & 1469.06 \\
\hline 10/10/11 0:30 & 35.34 & 40.057 & 1473.43 & 1469.07 \\
\hline 10/10/11 0:40 & 35.317 & 40.052 & 1473.45 & 1469.07 \\
\hline 10/10/11 0:50 & 35.293 & 40.045 & 1473.48 & 1469.08 \\
\hline 10/10/11 1:00 & 35.272 & 40.043 & 1473.50 & 1469.08 \\
\hline
\end{tabular}


TABLE S1.3 Automatically recorded groundwater levels in the Klassen wells, September 15, 2011, to March 13, 2012.

\begin{tabular}{|c|c|c|c|c|}
\hline \multicolumn{3}{|c|}{ Reference Elevation (ft AMSL) } & 1508.77 & \multirow{2}{*}{$\begin{array}{l}1509.12 \\
\text { Elevation in Klassen } \\
\text { Test Well (ft AMSL) }\end{array}$} \\
\hline Date and Time & $\begin{array}{l}\text { Depth in Klassen } \\
\text { Domestic Well } \\
(\mathrm{ft} \mathrm{BGL})\end{array}$ & $\begin{array}{c}\text { Depth in Klassen } \\
\text { Test Well } \\
\text { (ft BGL) }\end{array}$ & $\begin{array}{l}\text { Elevation in Klassen } \\
\text { Domestic Well } \\
\text { (ft AMSL) }\end{array}$ & \\
\hline 10/10/11 1:10 & 38.354 & 40.038 & 1470.42 & 1469.08 \\
\hline 10/10/11 1:20 & 35.394 & 40.036 & 1473.38 & 1469.09 \\
\hline 10/10/11 1:30 & 35.314 & 40.026 & 1473.46 & 1469.10 \\
\hline 10/10/11 1:40 & 35.331 & 40.019 & 1473.44 & 1469.10 \\
\hline 10/10/11 1:50 & 35.382 & 40.01 & 1473.39 & 1469.11 \\
\hline $10 / 10 / 112: 00$ & 35.35 & 40 & 1473.42 & 1469.12 \\
\hline $10 / 10 / 112: 10$ & 35.314 & 39.989 & 1473.46 & 1469.13 \\
\hline $10 / 10 / 112: 20$ & 35.31 & 39.977 & 1473.46 & 1469.15 \\
\hline $10 / 10 / 112: 30$ & 35.279 & 39.965 & 1473.49 & 1469.16 \\
\hline $10 / 10 / 112: 40$ & 35.251 & 39.953 & 1473.52 & 1469.17 \\
\hline $10 / 10 / 112: 50$ & 35.253 & 39.939 & 1473.52 & 1469.18 \\
\hline 10/10/11 3:00 & 35.228 & 39.93 & 1473.54 & 1469.19 \\
\hline 10/10/11 3:10 & 35.209 & 39.918 & 1473.56 & 1469.20 \\
\hline $10 / 10 / 113: 20$ & 35.242 & 39.909 & 1473.53 & 1469.21 \\
\hline 10/10/11 3:30 & 35.298 & 39.9 & 1473.47 & 1469.22 \\
\hline 10/10/11 3:40 & 35.275 & 39.89 & 1473.49 & 1469.23 \\
\hline $10 / 10 / 113: 50$ & 35.244 & 39.878 & 1473.53 & 1469.24 \\
\hline 10/10/11 4:00 & 35.214 & 39.867 & 1473.56 & 1469.26 \\
\hline 10/10/11 4:10 & 35.19 & 39.857 & 1473.58 & 1469.27 \\
\hline $10 / 10 / 114: 20$ & 40.033 & 39.848 & 1468.74 & 1469.27 \\
\hline $10 / 10 / 114: 30$ & 35.317 & 39.838 & 1473.45 & 1469.28 \\
\hline $10 / 10 / 114: 40$ & 35.232 & 39.829 & 1473.54 & 1469.29 \\
\hline $10 / 10 / 114: 50$ & 35.19 & 39.82 & 1473.58 & 1469.30 \\
\hline $10 / 10 / 115: 00$ & 35.16 & 39.81 & 1473.61 & 1469.31 \\
\hline 10/10/11 5:10 & 35.134 & 39.799 & 1473.64 & 1469.32 \\
\hline $10 / 10 / 115: 20$ & 35.115 & 39.787 & 1473.65 & 1469.34 \\
\hline 10/10/11 5:30 & 35.122 & 39.777 & 1473.65 & 1469.35 \\
\hline 10/10/11 5:40 & 35.101 & 39.768 & 1473.67 & 1469.35 \\
\hline $10 / 10 / 115: 50$ & 35.082 & 39.754 & 1473.69 & 1469.37 \\
\hline $10 / 10 / 116: 00$ & 35.066 & 39.742 & 1473.70 & 1469.38 \\
\hline 10/10/11 6:10 & 35.146 & 39.733 & 1473.62 & 1469.39 \\
\hline $10 / 10 / 116: 20$ & 35.42 & 39.724 & 1473.35 & 1469.40 \\
\hline $10 / 10 / 116: 30$ & 35.244 & 39.714 & 1473.53 & 1469.41 \\
\hline $10 / 10 / 116: 40$ & 35.188 & 39.707 & 1473.58 & 1469.42 \\
\hline $10 / 10 / 116: 50$ & 35.392 & 39.698 & 1473.38 & 1469.42 \\
\hline 10/10/11 7:00 & 35.242 & 39.691 & 1473.53 & 1469.43 \\
\hline 10/10/11 7:10 & 35.214 & 39.684 & 1473.56 & 1469.44 \\
\hline $10 / 10 / 117: 20$ & 35.174 & 39.677 & 1473.60 & 1469.45 \\
\hline
\end{tabular}


TABLE S1.3 Automatically recorded groundwater levels in the Klassen wells, September 15, 2011, to March 13, 2012.

\begin{tabular}{|c|c|c|c|c|}
\hline \multicolumn{3}{|c|}{ Reference Elevation (ft AMSL) } & 1508.77 & \multirow{2}{*}{$\begin{array}{l}1509.12 \\
\text { Elevation in Klassen } \\
\text { Test Well (ft AMSL) }\end{array}$} \\
\hline Date and Time & $\begin{array}{l}\text { Depth in Klassen } \\
\text { Domestic Well } \\
\text { (ft BGL) }\end{array}$ & $\begin{array}{c}\text { Depth in Klassen } \\
\text { Test Well } \\
\text { (ft BGL) }\end{array}$ & $\begin{array}{l}\text { Elevation in Klassen } \\
\text { Domestic Well } \\
\text { (ft AMSL) }\end{array}$ & \\
\hline 10/10/11 7:30 & 35.143 & 39.667 & 1473.63 & 1469.46 \\
\hline 10/10/11 7:40 & 35.169 & 39.66 & 1473.60 & 1469.46 \\
\hline 10/10/11 7:50 & 35.141 & 39.653 & 1473.63 & 1469.47 \\
\hline 10/10/11 8:00 & 35.115 & 39.644 & 1473.65 & 1469.48 \\
\hline 10/10/11 8:10 & 35.089 & 39.637 & 1473.68 & 1469.49 \\
\hline $10 / 10 / 118: 20$ & 35.071 & 39.63 & 1473.70 & 1469.49 \\
\hline 10/10/11 8:30 & 35.052 & 39.623 & 1473.72 & 1469.50 \\
\hline 10/10/11 8:40 & 35.033 & 39.616 & 1473.74 & 1469.51 \\
\hline 10/10/11 8:50 & 35.214 & 39.611 & 1473.56 & 1469.51 \\
\hline 10/10/11 9:00 & 35.258 & 39.606 & 1473.51 & 1469.52 \\
\hline 10/10/11 9:10 & 35.148 & 39.599 & 1473.62 & 1469.52 \\
\hline 10/10/11 9:20 & 36.82 & 39.594 & 1471.95 & 1469.53 \\
\hline 10/10/11 9:30 & 35.209 & 39.592 & 1473.56 & 1469.53 \\
\hline 10/10/11 9:40 & 35.134 & 39.585 & 1473.64 & 1469.54 \\
\hline 10/10/11 9:50 & 35.092 & 39.58 & 1473.68 & 1469.54 \\
\hline 10/10/11 10:00 & 35.061 & 39.578 & 1473.71 & 1469.54 \\
\hline 10/10/11 10:10 & 35.038 & 39.573 & 1473.73 & 1469.55 \\
\hline 10/10/11 10:20 & 35.404 & 39.569 & 1473.37 & 1469.55 \\
\hline 10/10/11 10:30 & 35.12 & 39.564 & 1473.65 & 1469.56 \\
\hline 10/10/11 10:40 & 39.154 & 39.562 & 1469.62 & 1469.56 \\
\hline $10 / 10 / 11$ 10:50 & 35.185 & 39.559 & 1473.58 & 1469.56 \\
\hline 10/10/11 11:00 & 35.103 & 39.555 & 1473.67 & 1469.57 \\
\hline 10/10/11 11:10 & 35.061 & 39.55 & 1473.71 & 1469.57 \\
\hline 10/10/11 11:20 & 35.223 & 39.548 & 1473.55 & 1469.57 \\
\hline 10/10/11 11:30 & 35.103 & 39.543 & 1473.67 & 1469.58 \\
\hline 10/10/11 11:40 & 35.056 & 39.541 & 1473.71 & 1469.58 \\
\hline 10/10/11 11:50 & 35.026 & 39.538 & 1473.74 & 1469.58 \\
\hline $10 / 10 / 11$ 12:00 & 35.214 & 39.536 & 1473.56 & 1469.59 \\
\hline 10/10/11 12:10 & 35.089 & 39.533 & 1473.68 & 1469.59 \\
\hline $10 / 10 / 11$ 12:20 & 35.042 & 39.533 & 1473.73 & 1469.59 \\
\hline $10 / 10 / 11$ 12:30 & 35.01 & 39.536 & 1473.76 & 1469.59 \\
\hline $10 / 10 / 11$ 12:40 & 34.986 & 39.536 & 1473.78 & 1469.59 \\
\hline $10 / 10 / 11$ 12:50 & 34.965 & 39.531 & 1473.80 & 1469.59 \\
\hline 10/10/11 13:00 & 34.946 & 39.531 & 1473.82 & 1469.59 \\
\hline 10/10/11 13:10 & 35.692 & 39.529 & 1473.08 & 1469.59 \\
\hline 10/10/11 13:20 & 35.117 & 39.531 & 1473.65 & 1469.59 \\
\hline 10/10/11 13:30 & 35.042 & 39.531 & 1473.73 & 1469.59 \\
\hline 10/10/11 13:40 & 35 & 39.524 & 1473.77 & 1469.60 \\
\hline
\end{tabular}


TABLE S1.3 Automatically recorded groundwater levels in the Klassen wells, September 15, 2011, to March 13, 2012.

\begin{tabular}{|c|c|c|c|c|}
\hline \multicolumn{3}{|c|}{ Reference Elevation (ft AMSL) } & 1508.77 & \multirow{2}{*}{$\begin{array}{l}1509.12 \\
\text { Elevation in Klassen } \\
\text { Test Well (ft AMSL) }\end{array}$} \\
\hline Date and Time & $\begin{array}{l}\text { Depth in Klassen } \\
\text { Domestic Well } \\
(\mathrm{ft} \mathrm{BGL})\end{array}$ & $\begin{array}{c}\text { Depth in Klassen } \\
\text { Test Well } \\
\text { (ft BGL) }\end{array}$ & $\begin{array}{l}\text { Elevation in Klassen } \\
\text { Domestic Well } \\
\text { (ft AMSL) }\end{array}$ & \\
\hline 10/10/11 13:50 & 35.474 & 39.517 & 1473.30 & 1469.61 \\
\hline 10/10/11 14:00 & 36.077 & 39.508 & 1472.69 & 1469.61 \\
\hline 10/10/11 14:10 & 35.132 & 39.498 & 1473.64 & 1469.62 \\
\hline $10 / 10 / 1114: 20$ & 35.054 & 39.482 & 1473.72 & 1469.64 \\
\hline 10/10/11 14:30 & 35.01 & 39.463 & 1473.76 & 1469.66 \\
\hline $10 / 10 / 1114: 40$ & 34.979 & 39.444 & 1473.79 & 1469.68 \\
\hline $10 / 10 / 1114: 50$ & 34.951 & 39.43 & 1473.82 & 1469.69 \\
\hline 10/10/11 15:00 & 34.93 & 39.419 & 1473.84 & 1469.70 \\
\hline $10 / 10 / 1115: 10$ & 34.92 & 39.411 & 1473.85 & 1469.71 \\
\hline $10 / 10 / 1115: 20$ & 34.923 & 39.4 & 1473.85 & 1469.72 \\
\hline 10/10/11 15:30 & 34.904 & 39.39 & 1473.87 & 1469.73 \\
\hline 10/10/11 15:40 & 34.885 & 39.381 & 1473.88 & 1469.74 \\
\hline 10/10/11 15:50 & 34.869 & 39.374 & 1473.90 & 1469.75 \\
\hline 10/10/11 16:00 & 34.855 & 39.367 & 1473.91 & 1469.76 \\
\hline 10/10/11 16:10 & 34.897 & 39.358 & 1473.87 & 1469.76 \\
\hline $10 / 10 / 11$ 16:20 & 35.071 & 39.36 & 1473.70 & 1469.76 \\
\hline 10/10/11 16:30 & 35.258 & 39.365 & 1473.51 & 1469.76 \\
\hline 10/10/11 16:40 & 35.427 & 39.374 & 1473.34 & 1469.75 \\
\hline 10/10/11 16:50 & 35.579 & 39.386 & 1473.19 & 1469.74 \\
\hline 10/10/11 17:00 & 36.1 & 39.4 & 1472.67 & 1469.72 \\
\hline $10 / 10 / 11$ 17:10 & 35.995 & 39.414 & 1472.77 & 1469.71 \\
\hline 10/10/11 17:20 & 36.091 & 39.43 & 1472.68 & 1469.69 \\
\hline 10/10/11 17:30 & 36.452 & 39.447 & 1472.32 & 1469.68 \\
\hline 10/10/11 17:40 & 36.414 & 39.463 & 1472.36 & 1469.66 \\
\hline 10/10/11 17:50 & 38.638 & 39.48 & 1470.13 & 1469.64 \\
\hline 10/10/11 18:00 & 37.955 & 39.501 & 1470.81 & 1469.62 \\
\hline 10/10/11 18:10 & 40.57 & 39.517 & 1468.20 & 1469.61 \\
\hline 10/10/11 18:20 & 37.146 & 39.538 & 1471.62 & 1469.58 \\
\hline 10/10/11 18:30 & 36.923 & 39.55 & 1471.85 & 1469.57 \\
\hline 10/10/11 18:40 & 36.75 & 39.552 & 1472.02 & 1469.57 \\
\hline 10/10/11 18:50 & 36.607 & 39.555 & 1472.16 & 1469.57 \\
\hline 10/10/11 19:00 & 36.485 & 39.552 & 1472.28 & 1469.57 \\
\hline 10/10/11 19:10 & 36.386 & 39.548 & 1472.38 & 1469.57 \\
\hline 10/10/11 19:20 & 36.604 & 39.545 & 1472.17 & 1469.58 \\
\hline 10/10/11 19:30 & 36.344 & 39.543 & 1472.43 & 1469.58 \\
\hline 10/10/11 19:40 & 36.234 & 39.538 & 1472.54 & 1469.58 \\
\hline 10/10/11 19:50 & 36.173 & 39.533 & 1472.60 & 1469.59 \\
\hline $10 / 10 / 1120: 00$ & 36.1 & 39.529 & 1472.67 & 1469.59 \\
\hline
\end{tabular}


TABLE S1.3 Automatically recorded groundwater levels in the Klassen wells, September 15, 2011, to March 13, 2012.

\begin{tabular}{|c|c|c|c|c|}
\hline \multicolumn{3}{|c|}{ Reference Elevation (ft AMSL) } & 1508.77 & \multirow{2}{*}{$\begin{array}{l}1509.12 \\
\text { Elevation in Klassen } \\
\text { Test Well (ft AMSL) }\end{array}$} \\
\hline Date and Time & $\begin{array}{l}\text { Depth in Klassen } \\
\text { Domestic Well } \\
(\mathrm{ft} \mathrm{BGL})\end{array}$ & $\begin{array}{c}\text { Depth in Klassen } \\
\text { Test Well } \\
\text { (ft BGL) }\end{array}$ & $\begin{array}{l}\text { Elevation in Klassen } \\
\text { Domestic Well } \\
\text { (ft AMSL) }\end{array}$ & \\
\hline 10/10/11 20:10 & 36.128 & 39.529 & 1472.64 & 1469.59 \\
\hline $10 / 10 / 1120: 20$ & 36.356 & 39.529 & 1472.41 & 1469.59 \\
\hline 10/10/11 20:30 & 36.342 & 39.533 & 1472.43 & 1469.59 \\
\hline $10 / 10 / 1120: 40$ & 36.445 & 39.541 & 1472.32 & 1469.58 \\
\hline $10 / 10 / 1120: 50$ & 36.557 & 39.552 & 1472.21 & 1469.57 \\
\hline 10/10/11 21:00 & 36.672 & 39.564 & 1472.10 & 1469.56 \\
\hline $10 / 10 / 1121: 10$ & 36.731 & 39.578 & 1472.04 & 1469.54 \\
\hline 10/10/11 21:20 & 36.907 & 39.587 & 1471.86 & 1469.54 \\
\hline 10/10/11 21:30 & 36.754 & 39.594 & 1472.02 & 1469.53 \\
\hline $10 / 10 / 1121: 40$ & 36.665 & 39.597 & 1472.10 & 1469.53 \\
\hline $10 / 10 / 1121: 50$ & 36.811 & 39.599 & 1471.96 & 1469.52 \\
\hline 10/10/11 22:00 & 36.837 & 39.602 & 1471.93 & 1469.52 \\
\hline $10 / 10 / 1122: 10$ & 36.663 & 39.602 & 1472.11 & 1469.52 \\
\hline $10 / 10 / 1122: 20$ & 40.678 & 39.602 & 1468.09 & 1469.52 \\
\hline $10 / 10 / 1122: 30$ & 36.703 & 39.604 & 1472.07 & 1469.52 \\
\hline $10 / 10 / 1122: 40$ & 36.522 & 39.599 & 1472.25 & 1469.52 \\
\hline $10 / 10 / 1122: 50$ & 36.407 & 39.594 & 1472.36 & 1469.53 \\
\hline 10/10/11 23:00 & 36.316 & 39.59 & 1472.45 & 1469.53 \\
\hline 10/10/11 23:10 & 36.238 & 39.585 & 1472.53 & 1469.54 \\
\hline $10 / 10 / 1123: 20$ & 36.17 & 39.583 & 1472.60 & 1469.54 \\
\hline $10 / 10 / 1123: 30$ & 36.109 & 39.576 & 1472.66 & 1469.55 \\
\hline 10/10/11 23:40 & 36.056 & 39.569 & 1472.71 & 1469.55 \\
\hline $10 / 10 / 1123: 50$ & 36.004 & 39.564 & 1472.77 & 1469.56 \\
\hline 10/11/11 0:00 & 35.957 & 39.557 & 1472.81 & 1469.57 \\
\hline 10/11/11 0:10 & 35.912 & 39.55 & 1472.86 & 1469.57 \\
\hline 10/11/11 0:20 & 35.873 & 39.545 & 1472.90 & 1469.58 \\
\hline 10/11/11 0:30 & 35.833 & 39.538 & 1472.94 & 1469.58 \\
\hline 10/11/11 0:40 & 35.795 & 39.529 & 1472.97 & 1469.59 \\
\hline 10/11/11 0:50 & 35.922 & 39.522 & 1472.85 & 1469.60 \\
\hline 10/11/11 1:00 & 35.812 & 39.515 & 1472.96 & 1469.61 \\
\hline 10/11/11 1:10 & 35.755 & 39.51 & 1473.01 & 1469.61 \\
\hline 10/11/11 1:20 & 35.734 & 39.501 & 1473.04 & 1469.62 \\
\hline 10/11/11 1:30 & 35.701 & 39.496 & 1473.07 & 1469.63 \\
\hline 10/11/11 1:40 & 35.664 & 39.487 & 1473.11 & 1469.64 \\
\hline 10/11/11 1:50 & 35.631 & 39.48 & 1473.14 & 1469.64 \\
\hline 10/11/11 2:00 & 35.598 & 39.47 & 1473.17 & 1469.65 \\
\hline 10/11/11 2:10 & 35.57 & 39.461 & 1473.20 & 1469.66 \\
\hline $10 / 11 / 112: 20$ & 35.542 & 39.454 & 1473.23 & 1469.67 \\
\hline
\end{tabular}


TABLE S1.3 Automatically recorded groundwater levels in the Klassen wells, September 15, 2011, to March 13, 2012.

\begin{tabular}{|c|c|c|c|c|}
\hline \multicolumn{3}{|c|}{ Reference Elevation (ft AMSL) } & 1508.77 & \multirow{2}{*}{$\begin{array}{l}1509.12 \\
\text { Elevation in Klassen } \\
\text { Test Well (ft AMSL) }\end{array}$} \\
\hline Date and Time & $\begin{array}{l}\text { Depth in Klassen } \\
\text { Domestic Well } \\
\text { (ft BGL) }\end{array}$ & $\begin{array}{c}\text { Depth in Klassen } \\
\text { Test Well } \\
\text { (ft BGL) }\end{array}$ & $\begin{array}{l}\text { Elevation in Klassen } \\
\text { Domestic Well } \\
\text { (ft AMSL) }\end{array}$ & \\
\hline $10 / 11 / 112: 30$ & 35.516 & 39.447 & 1473.25 & 1469.68 \\
\hline $10 / 11 / 112: 40$ & 35.488 & 39.44 & 1473.28 & 1469.68 \\
\hline $10 / 11 / 112: 50$ & 35.465 & 39.43 & 1473.30 & 1469.69 \\
\hline 10/11/11 3:00 & 35.441 & 39.423 & 1473.33 & 1469.70 \\
\hline 10/11/11 3:10 & 35.42 & 39.416 & 1473.35 & 1469.71 \\
\hline $10 / 11 / 113: 20$ & 35.394 & 39.409 & 1473.38 & 1469.71 \\
\hline 10/11/11 3:30 & 35.375 & 39.404 & 1473.39 & 1469.72 \\
\hline 10/11/11 3:40 & 35.352 & 39.395 & 1473.42 & 1469.73 \\
\hline $10 / 11 / 113: 50$ & 35.333 & 39.386 & 1473.44 & 1469.74 \\
\hline 10/11/11 4:00 & 35.312 & 39.376 & 1473.46 & 1469.75 \\
\hline 10/11/11 4:10 & 35.293 & 39.369 & 1473.48 & 1469.75 \\
\hline 10/11/11 4:20 & 35.275 & 39.36 & 1473.49 & 1469.76 \\
\hline 10/11/11 4:30 & 35.282 & 39.353 & 1473.49 & 1469.77 \\
\hline $10 / 11 / 114: 40$ & 35.268 & 39.343 & 1473.50 & 1469.78 \\
\hline $10 / 11 / 114: 50$ & 35.246 & 39.339 & 1473.52 & 1469.78 \\
\hline 10/11/11 5:00 & 35.228 & 39.334 & 1473.54 & 1469.79 \\
\hline 10/11/11 5:10 & 38.994 & 39.325 & 1469.78 & 1469.80 \\
\hline 10/11/11 5:20 & 35.354 & 39.32 & 1473.42 & 1469.80 \\
\hline 10/11/11 5:30 & 35.275 & 39.315 & 1473.49 & 1469.81 \\
\hline 10/11/11 5:40 & 35.261 & 39.308 & 1473.51 & 1469.81 \\
\hline 10/11/11 5:50 & 35.228 & 39.304 & 1473.54 & 1469.82 \\
\hline 10/11/11 6:00 & 35.202 & 39.299 & 1473.57 & 1469.82 \\
\hline 10/11/11 6:10 & 39.58 & 39.294 & 1469.19 & 1469.83 \\
\hline $10 / 11 / 116: 20$ & 35.366 & 39.292 & 1473.40 & 1469.83 \\
\hline 10/11/11 6:30 & 35.277 & 39.289 & 1473.49 & 1469.83 \\
\hline $10 / 11 / 116: 40$ & 35.258 & 39.287 & 1473.51 & 1469.84 \\
\hline $10 / 11 / 116: 50$ & 35.221 & 39.285 & 1473.55 & 1469.84 \\
\hline 10/11/11 7:00 & 35.19 & 39.28 & 1473.58 & 1469.84 \\
\hline 10/11/11 7:10 & 35.164 & 39.275 & 1473.61 & 1469.85 \\
\hline 10/11/11 7:20 & 35.141 & 39.271 & 1473.63 & 1469.85 \\
\hline 10/11/11 7:30 & 35.12 & 39.266 & 1473.65 & 1469.86 \\
\hline 10/11/11 7:40 & 35.099 & 39.261 & 1473.67 & 1469.86 \\
\hline 10/11/11 7:50 & 37.517 & 39.259 & 1471.25 & 1469.86 \\
\hline 10/11/11 8:00 & 35.239 & 39.257 & 1473.53 & 1469.87 \\
\hline 10/11/11 8:10 & 35.167 & 39.252 & 1473.60 & 1469.87 \\
\hline 10/11/11 8:20 & 35.127 & 39.25 & 1473.64 & 1469.87 \\
\hline 10/11/11 8:30 & 35.099 & 39.247 & 1473.67 & 1469.88 \\
\hline 10/11/11 8:40 & 35.075 & 39.245 & 1473.69 & 1469.88 \\
\hline
\end{tabular}


TABLE S1.3 Automatically recorded groundwater levels in the Klassen wells, September 15, 2011, to March 13, 2012.

\begin{tabular}{|c|c|c|c|c|}
\hline \multicolumn{3}{|c|}{ Reference Elevation (ft AMSL) } & 1508.77 & \multirow{2}{*}{$\begin{array}{l}1509.12 \\
\text { Elevation in Klassen } \\
\text { Test Well (ft AMSL) }\end{array}$} \\
\hline Date and Time & $\begin{array}{l}\text { Depth in Klassen } \\
\text { Domestic Well } \\
\text { (ft BGL) }\end{array}$ & $\begin{array}{c}\text { Depth in Klassen } \\
\text { Test Well } \\
\text { (ft BGL) }\end{array}$ & $\begin{array}{l}\text { Elevation in Klassen } \\
\text { Domestic Well } \\
\text { (ft AMSL) }\end{array}$ & \\
\hline 10/11/11 8:50 & 38.007 & 39.243 & 1470.76 & 1469.88 \\
\hline 10/11/11 9:00 & 35.197 & 39.243 & 1473.57 & 1469.88 \\
\hline 10/11/11 9:10 & 35.113 & 39.24 & 1473.66 & 1469.88 \\
\hline 10/11/11 9:20 & 35.075 & 39.238 & 1473.69 & 1469.88 \\
\hline 10/11/11 9:30 & 35.045 & 39.236 & 1473.72 & 1469.89 \\
\hline 10/11/11 9:40 & 37.84 & 39.231 & 1470.93 & 1469.89 \\
\hline 10/11/11 9:50 & 35.185 & 39.233 & 1473.58 & 1469.89 \\
\hline 10/11/11 10:00 & 35.101 & 39.231 & 1473.67 & 1469.89 \\
\hline 10/11/11 10:10 & 35.059 & 39.231 & 1473.71 & 1469.89 \\
\hline $10 / 11 / 1110: 20$ & 35.028 & 39.226 & 1473.74 & 1469.90 \\
\hline 10/11/11 10:30 & 35.005 & 39.224 & 1473.76 & 1469.90 \\
\hline $10 / 11 / 11$ 10:40 & 34.986 & 39.221 & 1473.78 & 1469.90 \\
\hline 10/11/11 10:50 & 34.967 & 39.219 & 1473.80 & 1469.90 \\
\hline 10/11/11 11:00 & 34.951 & 39.214 & 1473.82 & 1469.91 \\
\hline 10/11/11 11:10 & 34.937 & 39.21 & 1473.83 & 1469.91 \\
\hline $10 / 11 / 11$ 11:20 & 34.92 & 39.207 & 1473.85 & 1469.92 \\
\hline 10/11/11 11:30 & 35.188 & 39.205 & 1473.58 & 1469.92 \\
\hline 10/11/11 11:40 & 34.995 & 39.205 & 1473.77 & 1469.92 \\
\hline 10/11/11 11:50 & 34.979 & 39.203 & 1473.79 & 1469.92 \\
\hline $10 / 11 / 11$ 12:00 & 34.949 & 39.2 & 1473.82 & 1469.92 \\
\hline $10 / 11 / 11$ 12:10 & 34.925 & 39.198 & 1473.84 & 1469.92 \\
\hline $10 / 11 / 11$ 12:20 & 34.906 & 39.196 & 1473.86 & 1469.93 \\
\hline $10 / 11 / 11$ 12:30 & 34.89 & 39.191 & 1473.88 & 1469.93 \\
\hline $10 / 11 / 11$ 12:40 & 34.874 & 39.184 & 1473.90 & 1469.94 \\
\hline $10 / 11 / 11$ 12:50 & 34.859 & 39.177 & 1473.91 & 1469.95 \\
\hline 10/11/11 13:00 & 34.845 & 39.167 & 1473.92 & 1469.96 \\
\hline 10/11/11 13:10 & 35.024 & 39.153 & 1473.75 & 1469.97 \\
\hline $10 / 11 / 11$ 13:20 & 34.923 & 39.135 & 1473.85 & 1469.99 \\
\hline 10/11/11 13:30 & 34.888 & 39.123 & 1473.88 & 1470.00 \\
\hline 10/11/11 13:40 & 34.862 & 39.111 & 1473.91 & 1470.01 \\
\hline 10/11/11 13:50 & 34.841 & 39.102 & 1473.93 & 1470.02 \\
\hline 10/11/11 14:00 & 34.824 & 39.09 & 1473.95 & 1470.03 \\
\hline $10 / 11 / 11$ 14:10 & 34.81 & 39.081 & 1473.96 & 1470.04 \\
\hline $10 / 11 / 11$ 14:20 & 34.798 & 39.076 & 1473.97 & 1470.05 \\
\hline 10/11/11 14:30 & 35.024 & 39.069 & 1473.75 & 1470.05 \\
\hline $10 / 11 / 11$ 14:40 & 34.864 & 39.062 & 1473.91 & 1470.06 \\
\hline $10 / 11 / 11$ 14:50 & 34.824 & 39.055 & 1473.95 & 1470.07 \\
\hline 10/11/11 15:00 & 34.803 & 39.045 & 1473.97 & 1470.08 \\
\hline
\end{tabular}


TABLE S1.3 Automatically recorded groundwater levels in the Klassen wells, September 15, 2011, to March 13, 2012.

\begin{tabular}{|c|c|c|c|c|}
\hline \multicolumn{3}{|c|}{ Reference Elevation (ft AMSL) } & 1508.77 & \multirow{2}{*}{$\begin{array}{l}1509.12 \\
\text { Elevation in Klassen } \\
\text { Test Well (ft AMSL) }\end{array}$} \\
\hline Date and Time & $\begin{array}{l}\text { Depth in Klassen } \\
\text { Domestic Well } \\
\text { (ft BGL) }\end{array}$ & $\begin{array}{c}\text { Depth in Klassen } \\
\text { Test Well } \\
\text { (ft BGL) }\end{array}$ & $\begin{array}{l}\text { Elevation in Klassen } \\
\text { Domestic Well } \\
\text { (ft AMSL) }\end{array}$ & \\
\hline 10/11/11 15:10 & 34.784 & 39.043 & 1473.99 & 1470.08 \\
\hline $10 / 11 / 11$ 15:20 & 34.768 & 39.034 & 1474.00 & 1470.09 \\
\hline $10 / 11 / 1115: 30$ & 34.754 & 39.027 & 1474.02 & 1470.10 \\
\hline $10 / 11 / 11$ 15:40 & 34.742 & 39.022 & 1474.03 & 1470.10 \\
\hline $10 / 11 / 1115: 50$ & 34.73 & 39.015 & 1474.04 & 1470.11 \\
\hline 10/11/11 16:00 & 34.719 & 39.008 & 1474.05 & 1470.11 \\
\hline $10 / 11 / 1116: 10$ & 34.707 & 39.003 & 1474.06 & 1470.12 \\
\hline $10 / 11 / 1116: 20$ & 34.7 & 39.001 & 1474.07 & 1470.12 \\
\hline 10/11/11 16:30 & 34.719 & 38.996 & 1474.05 & 1470.13 \\
\hline 10/11/11 16:40 & 34.705 & 38.991 & 1474.06 & 1470.13 \\
\hline 10/11/11 16:50 & 34.702 & 38.987 & 1474.07 & 1470.14 \\
\hline 10/11/11 17:00 & 34.709 & 38.982 & 1474.06 & 1470.14 \\
\hline 10/11/11 17:10 & 34.749 & 38.98 & 1474.02 & 1470.14 \\
\hline 10/11/11 17:20 & 34.759 & 38.977 & 1474.01 & 1470.15 \\
\hline $10 / 11 / 11$ 17:30 & 34.777 & 38.975 & 1473.99 & 1470.15 \\
\hline 10/11/11 17:40 & 35.113 & 38.973 & 1473.66 & 1470.15 \\
\hline $10 / 11 / 11$ 17:50 & 34.897 & 38.968 & 1473.87 & 1470.15 \\
\hline 10/11/11 18:00 & 34.857 & 38.963 & 1473.91 & 1470.16 \\
\hline 10/11/11 18:10 & 34.822 & 38.961 & 1473.95 & 1470.16 \\
\hline $10 / 11 / 11$ 18:20 & 34.791 & 38.959 & 1473.98 & 1470.16 \\
\hline $10 / 11 / 11$ 18:30 & 34.763 & 38.954 & 1474.01 & 1470.17 \\
\hline 10/11/11 18:40 & 34.745 & 38.952 & 1474.02 & 1470.17 \\
\hline $10 / 11 / 11$ 18:50 & 34.78 & 38.945 & 1473.99 & 1470.18 \\
\hline 10/11/11 19:00 & 34.902 & 38.947 & 1473.87 & 1470.18 \\
\hline 10/11/11 19:10 & 35.019 & 38.952 & 1473.75 & 1470.17 \\
\hline 10/11/11 19:20 & 35.129 & 38.959 & 1473.64 & 1470.16 \\
\hline 10/11/11 19:30 & 35.415 & 38.966 & 1473.35 & 1470.16 \\
\hline 10/11/11 19:40 & 35.42 & 38.973 & 1473.35 & 1470.15 \\
\hline $10 / 11 / 11$ 19:50 & 35.488 & 38.984 & 1473.28 & 1470.14 \\
\hline 10/11/11 20:00 & 35.563 & 38.996 & 1473.21 & 1470.13 \\
\hline $10 / 11 / 1120: 10$ & 35.655 & 39.008 & 1473.11 & 1470.11 \\
\hline $10 / 11 / 1120: 20$ & 35.73 & 39.02 & 1473.04 & 1470.10 \\
\hline $10 / 11 / 1120: 30$ & 35.791 & 39.034 & 1472.98 & 1470.09 \\
\hline 10/11/11 20:40 & 35.854 & 39.045 & 1472.92 & 1470.08 \\
\hline $10 / 11 / 1120: 50$ & 35.88 & 39.057 & 1472.89 & 1470.07 \\
\hline 10/11/11 21:00 & 35.812 & 39.062 & 1472.96 & 1470.06 \\
\hline 10/11/11 21:10 & 36.318 & 39.067 & 1472.45 & 1470.06 \\
\hline 10/11/11 21:20 & 35.809 & 39.071 & 1472.96 & 1470.05 \\
\hline
\end{tabular}


TABLE S1.3 Automatically recorded groundwater levels in the Klassen wells, September 15, 2011, to March 13, 2012.

\begin{tabular}{|c|c|c|c|c|}
\hline \multicolumn{3}{|c|}{ Reference Elevation (ft AMSL) } & 1508.77 & \multirow{2}{*}{$\begin{array}{l}1509.12 \\
\text { Elevation in Klassen } \\
\text { Test Well (ft AMSL) }\end{array}$} \\
\hline Date and Time & $\begin{array}{l}\text { Depth in Klassen } \\
\text { Domestic Well } \\
\text { (ft BGL) }\end{array}$ & $\begin{array}{c}\text { Depth in Klassen } \\
\text { Test Well } \\
\text { (ft BGL) }\end{array}$ & $\begin{array}{l}\text { Elevation in Klassen } \\
\text { Domestic Well } \\
\text { (ft AMSL) }\end{array}$ & \\
\hline 10/11/11 21:30 & 35.699 & 39.071 & 1473.07 & 1470.05 \\
\hline $10 / 11 / 1121: 40$ & 35.676 & 39.071 & 1473.09 & 1470.05 \\
\hline $10 / 11 / 1121: 50$ & 35.748 & 39.076 & 1473.02 & 1470.05 \\
\hline $10 / 11 / 1122: 00$ & 35.835 & 39.081 & 1472.93 & 1470.04 \\
\hline $10 / 11 / 1122: 10$ & 35.908 & 39.09 & 1472.86 & 1470.03 \\
\hline $10 / 11 / 1122: 20$ & 35.973 & 39.099 & 1472.80 & 1470.02 \\
\hline $10 / 11 / 1122: 30$ & 36.039 & 39.111 & 1472.73 & 1470.01 \\
\hline $10 / 11 / 1122: 40$ & 36.405 & 39.121 & 1472.36 & 1470.00 \\
\hline $10 / 11 / 1122: 50$ & 36.482 & 39.135 & 1472.29 & 1469.99 \\
\hline $10 / 11 / 1123: 00$ & 38.804 & 39.146 & 1469.97 & 1469.98 \\
\hline $10 / 11 / 1123: 10$ & 36.799 & 39.163 & 1471.97 & 1469.96 \\
\hline $10 / 11 / 1123: 20$ & 36.64 & 39.174 & 1472.13 & 1469.95 \\
\hline $10 / 11 / 1123: 30$ & 36.614 & 39.189 & 1472.16 & 1469.93 \\
\hline $10 / 11 / 1123: 40$ & 36.525 & 39.196 & 1472.24 & 1469.93 \\
\hline $10 / 11 / 1123: 50$ & 36.405 & 39.2 & 1472.36 & 1469.92 \\
\hline $10 / 12 / 110: 00$ & 36.292 & 39.203 & 1472.48 & 1469.92 \\
\hline 10/12/11 0:10 & 36.194 & 39.2 & 1472.58 & 1469.92 \\
\hline 10/12/11 0:20 & 36.109 & 39.196 & 1472.66 & 1469.93 \\
\hline $10 / 12 / 110: 30$ & 36.034 & 39.191 & 1472.74 & 1469.93 \\
\hline $10 / 12 / 110: 40$ & 35.964 & 39.186 & 1472.81 & 1469.94 \\
\hline $10 / 12 / 110: 50$ & 35.901 & 39.179 & 1472.87 & 1469.94 \\
\hline 10/12/11 1:00 & 35.842 & 39.174 & 1472.93 & 1469.95 \\
\hline 10/12/11 1:10 & 35.788 & 39.167 & 1472.98 & 1469.96 \\
\hline 10/12/11 1:20 & 35.741 & 39.16 & 1473.03 & 1469.96 \\
\hline 10/12/11 1:30 & 37.601 & 39.153 & 1471.17 & 1469.97 \\
\hline 10/12/11 1:40 & 35.772 & 39.146 & 1473.00 & 1469.98 \\
\hline $10 / 12 / 11$ 1:50 & 35.687 & 39.142 & 1473.08 & 1469.98 \\
\hline $10 / 12 / 112: 00$ & 35.631 & 39.135 & 1473.14 & 1469.99 \\
\hline $10 / 12 / 112: 10$ & 35.586 & 39.13 & 1473.18 & 1469.99 \\
\hline $10 / 12 / 112: 20$ & 35.547 & 39.125 & 1473.22 & 1470.00 \\
\hline $10 / 12 / 112: 30$ & 35.509 & 39.118 & 1473.26 & 1470.00 \\
\hline $10 / 12 / 112: 40$ & 35.474 & 39.111 & 1473.30 & 1470.01 \\
\hline $10 / 12 / 112: 50$ & 35.441 & 39.106 & 1473.33 & 1470.02 \\
\hline 10/12/11 3:00 & 35.411 & 39.099 & 1473.36 & 1470.02 \\
\hline 10/12/11 3:10 & 35.382 & 39.09 & 1473.39 & 1470.03 \\
\hline 10/12/11 3:20 & 35.354 & 39.085 & 1473.42 & 1470.04 \\
\hline 10/12/11 3:30 & 35.329 & 39.078 & 1473.44 & 1470.04 \\
\hline $10 / 12 / 113: 40$ & 35.303 & 39.071 & 1473.47 & 1470.05 \\
\hline
\end{tabular}


TABLE S1.3 Automatically recorded groundwater levels in the Klassen wells, September 15, 2011, to March 13, 2012.

\begin{tabular}{|c|c|c|c|c|}
\hline \multicolumn{3}{|c|}{ Reference Elevation (ft AMSL) } & 1508.77 & \multirow{2}{*}{$\begin{array}{l}1509.12 \\
\text { Elevation in Klassen } \\
\text { Test Well (ft AMSL) }\end{array}$} \\
\hline Date and Time & $\begin{array}{l}\text { Depth in Klassen } \\
\text { Domestic Well } \\
(\mathrm{ft} \mathrm{BGL})\end{array}$ & $\begin{array}{c}\text { Depth in Klassen } \\
\text { Test Well } \\
\text { (ft BGL) }\end{array}$ & $\begin{array}{l}\text { Elevation in Klassen } \\
\text { Domestic Well } \\
\text { (ft AMSL) }\end{array}$ & \\
\hline $10 / 12 / 113: 50$ & 35.277 & 39.064 & 1473.49 & 1470.06 \\
\hline 10/12/11 4:00 & 35.253 & 39.06 & 1473.52 & 1470.06 \\
\hline 10/12/11 4:10 & 35.232 & 39.052 & 1473.54 & 1470.07 \\
\hline $10 / 12 / 114: 20$ & 35.209 & 39.045 & 1473.56 & 1470.08 \\
\hline 10/12/11 4:30 & 35.19 & 39.036 & 1473.58 & 1470.09 \\
\hline $10 / 12 / 114: 40$ & 35.169 & 39.031 & 1473.60 & 1470.09 \\
\hline $10 / 12 / 114: 50$ & 35.15 & 39.024 & 1473.62 & 1470.10 \\
\hline 10/12/11 5:00 & 35.129 & 39.02 & 1473.64 & 1470.10 \\
\hline 10/12/11 5:10 & 35.11 & 39.013 & 1473.66 & 1470.11 \\
\hline $10 / 12 / 115: 20$ & 35.092 & 39.006 & 1473.68 & 1470.12 \\
\hline $10 / 12 / 115: 30$ & 35.075 & 39.001 & 1473.69 & 1470.12 \\
\hline 10/12/11 5:40 & 35.056 & 38.994 & 1473.71 & 1470.13 \\
\hline $10 / 12 / 115: 50$ & 35.232 & 38.987 & 1473.54 & 1470.14 \\
\hline 10/12/11 6:00 & 35.131 & 38.982 & 1473.64 & 1470.14 \\
\hline 10/12/11 6:10 & 35.096 & 38.977 & 1473.67 & 1470.15 \\
\hline $10 / 12 / 116: 20$ & 35.307 & 38.977 & 1473.46 & 1470.15 \\
\hline $10 / 12 / 116: 30$ & 35.181 & 38.973 & 1473.59 & 1470.15 \\
\hline $10 / 12 / 116: 40$ & 38.051 & 38.968 & 1470.72 & 1470.15 \\
\hline $10 / 12 / 116: 50$ & 35.253 & 38.963 & 1473.52 & 1470.16 \\
\hline $10 / 12 / 117: 00$ & 37.538 & 38.961 & 1471.23 & 1470.16 \\
\hline 10/12/11 7:10 & 38.987 & 38.961 & 1469.78 & 1470.16 \\
\hline 10/12/11 7:20 & 35.53 & 38.963 & 1473.24 & 1470.16 \\
\hline $10 / 12 / 117: 30$ & 35.42 & 38.963 & 1473.35 & 1470.16 \\
\hline $10 / 12 / 117: 40$ & 35.49 & 38.966 & 1473.28 & 1470.16 \\
\hline $10 / 12 / 117: 50$ & 35.568 & 38.97 & 1473.20 & 1470.15 \\
\hline 10/12/11 8:00 & 36.06 & 38.977 & 1472.71 & 1470.15 \\
\hline 10/12/11 8:10 & 35.837 & 38.987 & 1472.93 & 1470.14 \\
\hline $10 / 12 / 118: 20$ & 35.877 & 38.996 & 1472.89 & 1470.13 \\
\hline $10 / 12 / 118: 30$ & 35.929 & 39.003 & 1472.84 & 1470.12 \\
\hline $10 / 12 / 118: 40$ & 36.267 & 39.01 & 1472.50 & 1470.11 \\
\hline $10 / 12 / 118: 50$ & 36.17 & 39.02 & 1472.60 & 1470.10 \\
\hline 10/12/11 9:00 & 36.189 & 39.029 & 1472.58 & 1470.09 \\
\hline 10/12/11 9:10 & 36.224 & 39.041 & 1472.55 & 1470.08 \\
\hline 10/12/11 9:20 & 36.203 & 39.048 & 1472.57 & 1470.07 \\
\hline 10/12/11 9:30 & 36.112 & 39.052 & 1472.66 & 1470.07 \\
\hline 10/12/11 9:40 & 36.018 & 39.052 & 1472.75 & 1470.07 \\
\hline $10 / 12 / 119: 50$ & 35.934 & 39.052 & 1472.84 & 1470.07 \\
\hline 10/12/11 10:00 & 35.859 & 39.052 & 1472.91 & 1470.07 \\
\hline
\end{tabular}


TABLE S1.3 Automatically recorded groundwater levels in the Klassen wells, September 15, 2011, to March 13, 2012.

\begin{tabular}{|c|c|c|c|c|}
\hline \multicolumn{3}{|c|}{ Reference Elevation (ft AMSL) } & 1508.77 & \multirow{2}{*}{$\begin{array}{l}1509.12 \\
\text { Elevation in Klassen } \\
\text { Test Well (ft AMSL) }\end{array}$} \\
\hline Date and Time & $\begin{array}{l}\text { Depth in Klassen } \\
\text { Domestic Well } \\
\text { (ft BGL) }\end{array}$ & $\begin{array}{c}\text { Depth in Klassen } \\
\text { Test Well } \\
\text { (ft BGL) }\end{array}$ & $\begin{array}{l}\text { Elevation in Klassen } \\
\text { Domestic Well } \\
\text { (ft AMSL) }\end{array}$ & \\
\hline 10/12/11 10:10 & 35.793 & 39.052 & 1472.98 & 1470.07 \\
\hline $10 / 12 / 1110: 20$ & 35.732 & 39.052 & 1473.04 & 1470.07 \\
\hline 10/12/11 10:30 & 35.68 & 39.052 & 1473.09 & 1470.07 \\
\hline 10/12/11 10:40 & 35.631 & 39.055 & 1473.14 & 1470.07 \\
\hline $10 / 12 / 1110: 50$ & 35.586 & 39.06 & 1473.18 & 1470.06 \\
\hline 10/12/11 11:00 & 35.542 & 39.06 & 1473.23 & 1470.06 \\
\hline 10/12/11 11:10 & 35.504 & 39.062 & 1473.27 & 1470.06 \\
\hline 10/12/11 11:20 & 35.467 & 39.064 & 1473.30 & 1470.06 \\
\hline 10/12/11 11:30 & 35.432 & 39.067 & 1473.34 & 1470.06 \\
\hline 10/12/11 11:40 & 38.617 & 39.067 & 1470.15 & 1470.06 \\
\hline 10/12/11 11:50 & 35.533 & 39.067 & 1473.24 & 1470.06 \\
\hline $10 / 12 / 1112: 00$ & 35.441 & 39.069 & 1473.33 & 1470.05 \\
\hline 10/12/11 12:10 & 35.389 & 39.069 & 1473.38 & 1470.05 \\
\hline $10 / 12 / 1112: 20$ & 35.352 & 39.069 & 1473.42 & 1470.05 \\
\hline $10 / 12 / 11$ 12:30 & 35.317 & 39.069 & 1473.45 & 1470.05 \\
\hline $10 / 12 / 11$ 12:40 & 35.289 & 39.067 & 1473.48 & 1470.06 \\
\hline $10 / 12 / 1112: 50$ & 35.261 & 39.062 & 1473.51 & 1470.06 \\
\hline 10/12/11 13:00 & 35.235 & 39.06 & 1473.53 & 1470.06 \\
\hline 10/12/11 13:10 & 39.655 & 39.057 & 1469.11 & 1470.07 \\
\hline $10 / 12 / 11$ 13:20 & 35.359 & 39.06 & 1473.41 & 1470.06 \\
\hline $10 / 12 / 11$ 13:30 & 35.268 & 39.06 & 1473.50 & 1470.06 \\
\hline 10/12/11 13:40 & 35.223 & 39.06 & 1473.55 & 1470.06 \\
\hline $10 / 12 / 11$ 13:50 & 35.183 & 39.06 & 1473.59 & 1470.06 \\
\hline $10 / 12 / 11$ 14:00 & 35.162 & 39.06 & 1473.61 & 1470.06 \\
\hline $10 / 12 / 11$ 14:10 & 35.134 & 39.052 & 1473.64 & 1470.07 \\
\hline $10 / 12 / 11$ 14:20 & 35.762 & 39.05 & 1473.01 & 1470.07 \\
\hline $10 / 12 / 11$ 14:30 & 35.23 & 39.05 & 1473.54 & 1470.07 \\
\hline $10 / 12 / 11$ 14:40 & 35.162 & 39.045 & 1473.61 & 1470.08 \\
\hline $10 / 12 / 1114: 50$ & 35.127 & 39.043 & 1473.64 & 1470.08 \\
\hline 10/12/11 15:00 & 35.094 & 39.038 & 1473.68 & 1470.08 \\
\hline $10 / 12 / 11$ 15:10 & 35.066 & 39.034 & 1473.70 & 1470.09 \\
\hline $10 / 12 / 11$ 15:20 & 35.336 & 39.031 & 1473.43 & 1470.09 \\
\hline $10 / 12 / 11$ 15:30 & 35.139 & 39.027 & 1473.63 & 1470.10 \\
\hline 10/12/11 15:40 & 35.117 & 39.022 & 1473.65 & 1470.10 \\
\hline $10 / 12 / 11$ 15:50 & 36.628 & 39.02 & 1472.14 & 1470.10 \\
\hline 10/12/11 16:00 & 35.188 & 39.02 & 1473.58 & 1470.10 \\
\hline 10/12/11 16:10 & 35.11 & 39.015 & 1473.66 & 1470.11 \\
\hline 10/12/11 16:20 & 35.068 & 39.015 & 1473.70 & 1470.11 \\
\hline
\end{tabular}


TABLE S1.3 Automatically recorded groundwater levels in the Klassen wells, September 15, 2011, to March 13, 2012.

\begin{tabular}{|c|c|c|c|c|}
\hline \multicolumn{3}{|c|}{ Reference Elevation (ft AMSL) } & 1508.77 & \multirow{2}{*}{$\begin{array}{l}1509.12 \\
\text { Elevation in Klassen } \\
\text { Test Well (ft AMSL) }\end{array}$} \\
\hline Date and Time & $\begin{array}{l}\text { Depth in Klassen } \\
\text { Domestic Well } \\
(\mathrm{ft} \mathrm{BGL})\end{array}$ & $\begin{array}{c}\text { Depth in Klassen } \\
\text { Test Well } \\
\text { (ft BGL) }\end{array}$ & $\begin{array}{l}\text { Elevation in Klassen } \\
\text { Domestic Well } \\
\text { (ft AMSL) }\end{array}$ & \\
\hline 10/12/11 16:30 & 35.153 & 39.017 & 1473.62 & 1470.11 \\
\hline $10 / 12 / 1116: 40$ & 35.314 & 39.024 & 1473.46 & 1470.10 \\
\hline 10/12/11 16:50 & 35.427 & 39.029 & 1473.34 & 1470.09 \\
\hline 10/12/11 17:00 & 35.528 & 39.038 & 1473.24 & 1470.08 \\
\hline 10/12/11 17:10 & 35.617 & 39.05 & 1473.15 & 1470.07 \\
\hline $10 / 12 / 1117: 20$ & 35.725 & 39.06 & 1473.04 & 1470.06 \\
\hline 10/12/11 17:30 & 35.835 & 39.071 & 1472.93 & 1470.05 \\
\hline 10/12/11 17:40 & 35.938 & 39.083 & 1472.83 & 1470.04 \\
\hline 10/12/11 17:50 & 36.034 & 39.099 & 1472.74 & 1470.02 \\
\hline 10/12/11 18:00 & 36.03 & 39.106 & 1472.74 & 1470.02 \\
\hline 10/12/11 18:10 & 36.213 & 39.113 & 1472.56 & 1470.01 \\
\hline 10/12/11 18:20 & 36.044 & 39.116 & 1472.73 & 1470.01 \\
\hline 10/12/11 18:30 & 35.999 & 39.121 & 1472.77 & 1470.00 \\
\hline $10 / 12 / 1118: 40$ & 35.938 & 39.123 & 1472.83 & 1470.00 \\
\hline 10/12/11 18:50 & 35.95 & 39.125 & 1472.82 & 1470.00 \\
\hline 10/12/11 19:00 & 35.898 & 39.125 & 1472.87 & 1470.00 \\
\hline 10/12/11 19:10 & 35.917 & 39.128 & 1472.85 & 1469.99 \\
\hline 10/12/11 19:20 & 37.859 & 39.137 & 1470.91 & 1469.99 \\
\hline 10/12/11 19:30 & 36.166 & 39.144 & 1472.60 & 1469.98 \\
\hline $10 / 12 / 11$ 19:40 & 36.227 & 39.158 & 1472.54 & 1469.96 \\
\hline $10 / 12 / 11$ 19:50 & 36.285 & 39.17 & 1472.48 & 1469.95 \\
\hline $10 / 12 / 1120: 00$ & 36.555 & 39.186 & 1472.21 & 1469.94 \\
\hline $10 / 12 / 1120: 10$ & 36.52 & 39.203 & 1472.25 & 1469.92 \\
\hline $10 / 12 / 1120: 20$ & 36.534 & 39.217 & 1472.24 & 1469.91 \\
\hline 10/12/11 20:30 & 36.564 & 39.233 & 1472.21 & 1469.89 \\
\hline $10 / 12 / 1120: 40$ & 36.686 & 39.25 & 1472.08 & 1469.87 \\
\hline $10 / 12 / 1120: 50$ & 36.834 & 39.275 & 1471.94 & 1469.85 \\
\hline $10 / 12 / 1121: 00$ & 36.867 & 39.297 & 1471.90 & 1469.83 \\
\hline $10 / 12 / 1121: 10$ & 36.818 & 39.308 & 1471.95 & 1469.81 \\
\hline 10/12/11 21:20 & 36.832 & 39.322 & 1471.94 & 1469.80 \\
\hline 10/12/11 21:30 & 36.855 & 39.334 & 1471.91 & 1469.79 \\
\hline $10 / 12 / 1121: 40$ & 36.874 & 39.346 & 1471.90 & 1469.78 \\
\hline $10 / 12 / 1121: 50$ & 36.89 & 39.36 & 1471.88 & 1469.76 \\
\hline $10 / 12 / 1122: 00$ & 36.837 & 39.369 & 1471.93 & 1469.75 \\
\hline $10 / 12 / 1122: 10$ & 38.148 & 39.376 & 1470.62 & 1469.75 \\
\hline $10 / 12 / 1122: 20$ & 36.844 & 39.381 & 1471.93 & 1469.74 \\
\hline $10 / 12 / 1122: 30$ & 38.389 & 39.383 & 1470.38 & 1469.74 \\
\hline $10 / 12 / 1122: 40$ & 37.491 & 39.388 & 1471.28 & 1469.73 \\
\hline
\end{tabular}


TABLE S1.3 Automatically recorded groundwater levels in the Klassen wells, September 15, 2011, to March 13, 2012.

\begin{tabular}{|c|c|c|c|c|}
\hline \multicolumn{3}{|c|}{ Reference Elevation (ft AMSL) } & 1508.77 & \multirow{2}{*}{$\begin{array}{l}1509.12 \\
\text { Elevation in Klassen } \\
\text { Test Well (ft AMSL) }\end{array}$} \\
\hline Date and Time & $\begin{array}{l}\text { Depth in Klassen } \\
\text { Domestic Well } \\
(\mathrm{ft} \mathrm{BGL})\end{array}$ & $\begin{array}{c}\text { Depth in Klassen } \\
\text { Test Well } \\
\text { (ft BGL) }\end{array}$ & $\begin{array}{l}\text { Elevation in Klassen } \\
\text { Domestic Well } \\
\text { (ft AMSL) }\end{array}$ & \\
\hline $10 / 12 / 1122: 50$ & 39.163 & 39.39 & 1469.61 & 1469.73 \\
\hline $10 / 12 / 1123: 00$ & 37.057 & 39.393 & 1471.71 & 1469.73 \\
\hline $10 / 12 / 1123: 10$ & 36.731 & 39.393 & 1472.04 & 1469.73 \\
\hline $10 / 12 / 1123: 20$ & 36.581 & 39.39 & 1472.19 & 1469.73 \\
\hline $10 / 12 / 1123: 30$ & 36.501 & 39.39 & 1472.27 & 1469.73 \\
\hline $10 / 12 / 1123: 40$ & 36.41 & 39.386 & 1472.36 & 1469.74 \\
\hline $10 / 12 / 1123: 50$ & 36.33 & 39.383 & 1472.44 & 1469.74 \\
\hline 10/13/11 0:00 & 36.269 & 39.379 & 1472.50 & 1469.74 \\
\hline 10/13/11 0:10 & 36.224 & 39.374 & 1472.55 & 1469.75 \\
\hline $10 / 13 / 110: 20$ & 36.161 & 39.369 & 1472.61 & 1469.75 \\
\hline $10 / 13 / 110: 30$ & 36.107 & 39.365 & 1472.66 & 1469.76 \\
\hline 10/13/11 0:40 & 36.053 & 39.36 & 1472.72 & 1469.76 \\
\hline 10/13/11 0:50 & 36.027 & 39.353 & 1472.74 & 1469.77 \\
\hline 10/13/11 1:00 & 35.983 & 39.348 & 1472.79 & 1469.77 \\
\hline 10/13/11 1:10 & 35.938 & 39.341 & 1472.83 & 1469.78 \\
\hline 10/13/11 1:20 & 35.894 & 39.336 & 1472.88 & 1469.79 \\
\hline 10/13/11 1:30 & 35.854 & 39.329 & 1472.92 & 1469.79 \\
\hline 10/13/11 1:40 & 35.877 & 39.325 & 1472.89 & 1469.80 \\
\hline 10/13/11 1:50 & 35.912 & 39.32 & 1472.86 & 1469.80 \\
\hline $10 / 13 / 112: 00$ & 35.891 & 39.315 & 1472.88 & 1469.81 \\
\hline $10 / 13 / 112: 10$ & 35.847 & 39.313 & 1472.92 & 1469.81 \\
\hline $10 / 13 / 112: 20$ & 35.826 & 39.308 & 1472.94 & 1469.81 \\
\hline $10 / 13 / 112: 30$ & 35.783 & 39.301 & 1472.99 & 1469.82 \\
\hline $10 / 13 / 112: 40$ & 35.765 & 39.294 & 1473.00 & 1469.83 \\
\hline $10 / 13 / 112: 50$ & 35.73 & 39.287 & 1473.04 & 1469.84 \\
\hline 10/13/11 3:00 & 35.704 & 39.28 & 1473.07 & 1469.84 \\
\hline 10/13/11 3:10 & 39.182 & 39.273 & 1469.59 & 1469.85 \\
\hline 10/13/11 3:20 & 35.859 & 39.271 & 1472.91 & 1469.85 \\
\hline 10/13/11 3:30 & 35.847 & 39.271 & 1472.92 & 1469.85 \\
\hline 10/13/11 3:40 & 35.786 & 39.266 & 1472.98 & 1469.86 \\
\hline $10 / 13 / 113: 50$ & 35.727 & 39.257 & 1473.04 & 1469.87 \\
\hline 10/13/11 4:00 & 35.68 & 39.252 & 1473.09 & 1469.87 \\
\hline 10/13/11 4:10 & 35.638 & 39.245 & 1473.13 & 1469.88 \\
\hline $10 / 13 / 114: 20$ & 35.601 & 39.238 & 1473.17 & 1469.88 \\
\hline $10 / 13 / 114: 30$ & 35.568 & 39.233 & 1473.20 & 1469.89 \\
\hline $10 / 13 / 114: 40$ & 35.544 & 39.224 & 1473.23 & 1469.90 \\
\hline $10 / 13 / 114: 50$ & 35.537 & 39.214 & 1473.23 & 1469.91 \\
\hline 10/13/11 5:00 & 35.504 & 39.207 & 1473.27 & 1469.92 \\
\hline
\end{tabular}


TABLE S1.3 Automatically recorded groundwater levels in the Klassen wells, September 15, 2011, to March 13, 2012.

\begin{tabular}{|c|c|c|c|c|}
\hline \multicolumn{3}{|c|}{ Reference Elevation (ft AMSL) } & 1508.77 & \multirow{2}{*}{$\begin{array}{l}1509.12 \\
\text { Elevation in Klassen } \\
\text { Test Well (ft AMSL) }\end{array}$} \\
\hline Date and Time & $\begin{array}{l}\text { Depth in Klassen } \\
\text { Domestic Well } \\
(\mathrm{ft} \mathrm{BGL})\end{array}$ & $\begin{array}{c}\text { Depth in Klassen } \\
\text { Test Well } \\
\text { (ft BGL) }\end{array}$ & $\begin{array}{l}\text { Elevation in Klassen } \\
\text { Domestic Well } \\
\text { (ft AMSL) }\end{array}$ & \\
\hline 10/13/11 5:10 & 35.476 & 39.2 & 1473.29 & 1469.92 \\
\hline $10 / 13 / 115: 20$ & 35.474 & 39.193 & 1473.30 & 1469.93 \\
\hline 10/13/11 5:30 & 35.446 & 39.189 & 1473.32 & 1469.93 \\
\hline $10 / 13 / 115: 40$ & 35.483 & 39.184 & 1473.29 & 1469.94 \\
\hline 10/13/11 5:50 & 35.589 & 39.182 & 1473.18 & 1469.94 \\
\hline 10/13/11 6:00 & 35.687 & 39.182 & 1473.08 & 1469.94 \\
\hline 10/13/11 6:10 & 35.774 & 39.184 & 1473.00 & 1469.94 \\
\hline $10 / 13 / 116: 20$ & 37.669 & 39.191 & 1471.10 & 1469.93 \\
\hline 10/13/11 6:30 & 36.241 & 39.2 & 1472.53 & 1469.92 \\
\hline 10/13/11 6:40 & 36.238 & 39.207 & 1472.53 & 1469.92 \\
\hline $10 / 13 / 116: 50$ & 36.274 & 39.217 & 1472.50 & 1469.91 \\
\hline 10/13/11 7:00 & 36.316 & 39.224 & 1472.45 & 1469.90 \\
\hline 10/13/11 7:10 & 36.356 & 39.231 & 1472.41 & 1469.89 \\
\hline $10 / 13 / 117: 20$ & 36.398 & 39.238 & 1472.37 & 1469.88 \\
\hline $10 / 13 / 117: 30$ & 36.44 & 39.245 & 1472.33 & 1469.88 \\
\hline $10 / 13 / 11$ 7:40 & 36.48 & 39.252 & 1472.29 & 1469.87 \\
\hline $10 / 13 / 117: 50$ & 36.518 & 39.257 & 1472.25 & 1469.87 \\
\hline 10/13/11 8:00 & 36.785 & 39.266 & 1471.98 & 1469.86 \\
\hline 10/13/11 8:10 & 36.696 & 39.28 & 1472.07 & 1469.84 \\
\hline $10 / 13 / 118: 20$ & 36.628 & 39.287 & 1472.14 & 1469.84 \\
\hline $10 / 13 / 118: 30$ & 37.052 & 39.282 & 1471.72 & 1469.84 \\
\hline $10 / 13 / 118: 40$ & 36.571 & 39.28 & 1472.20 & 1469.84 \\
\hline $10 / 13 / 118: 50$ & 36.827 & 39.278 & 1471.94 & 1469.84 \\
\hline 10/13/11 9:00 & 36.435 & 39.273 & 1472.33 & 1469.85 \\
\hline 10/13/11 9:10 & 36.318 & 39.268 & 1472.45 & 1469.85 \\
\hline 10/13/11 9:20 & 36.255 & 39.266 & 1472.51 & 1469.86 \\
\hline 10/13/11 9:30 & 36.18 & 39.259 & 1472.59 & 1469.86 \\
\hline 10/13/11 9:40 & 36.114 & 39.252 & 1472.66 & 1469.87 \\
\hline 10/13/11 9:50 & 36.053 & 39.243 & 1472.72 & 1469.88 \\
\hline 10/13/11 10:00 & 36.257 & 39.236 & 1472.51 & 1469.89 \\
\hline 10/13/11 10:10 & 36.06 & 39.226 & 1472.71 & 1469.90 \\
\hline $10 / 13 / 1110: 20$ & 36.009 & 39.219 & 1472.76 & 1469.90 \\
\hline $10 / 13 / 11$ 10:30 & 35.948 & 39.21 & 1472.82 & 1469.91 \\
\hline $10 / 13 / 11$ 10:40 & 36.034 & 39.203 & 1472.74 & 1469.92 \\
\hline $10 / 13 / 11$ 10:50 & 35.931 & 39.196 & 1472.84 & 1469.93 \\
\hline 10/13/11 11:00 & 35.87 & 39.186 & 1472.90 & 1469.94 \\
\hline 10/13/11 11:10 & 35.84 & 39.177 & 1472.93 & 1469.95 \\
\hline $10 / 13 / 1111: 20$ & 35.805 & 39.167 & 1472.96 & 1469.96 \\
\hline
\end{tabular}


TABLE S1.3 Automatically recorded groundwater levels in the Klassen wells, September 15, 2011, to March 13, 2012.

\begin{tabular}{|c|c|c|c|c|}
\hline \multicolumn{3}{|c|}{ Reference Elevation (ft AMSL) } & 1508.77 & \multirow{2}{*}{$\begin{array}{l}1509.12 \\
\text { Elevation in Klassen } \\
\text { Test Well (ft AMSL) }\end{array}$} \\
\hline Date and Time & $\begin{array}{l}\text { Depth in Klassen } \\
\text { Domestic Well } \\
(\mathrm{ft} \mathrm{BGL})\end{array}$ & $\begin{array}{c}\text { Depth in Klassen } \\
\text { Test Well } \\
\text { (ft BGL) }\end{array}$ & $\begin{array}{l}\text { Elevation in Klassen } \\
\text { Domestic Well } \\
\text { (ft AMSL) }\end{array}$ & \\
\hline 10/13/11 11:30 & 35.762 & 39.16 & 1473.01 & 1469.96 \\
\hline 10/13/11 11:40 & 36.661 & 39.151 & 1472.11 & 1469.97 \\
\hline 10/13/11 11:50 & 35.828 & 39.149 & 1472.94 & 1469.97 \\
\hline 10/13/11 12:00 & 35.748 & 39.146 & 1473.02 & 1469.98 \\
\hline $10 / 13 / 1112: 10$ & 35.727 & 39.149 & 1473.04 & 1469.97 \\
\hline $10 / 13 / 1112: 20$ & 35.683 & 39.16 & 1473.09 & 1469.96 \\
\hline $10 / 13 / 1112: 30$ & 35.645 & 39.17 & 1473.12 & 1469.95 \\
\hline $10 / 13 / 1112: 40$ & 35.61 & 39.179 & 1473.16 & 1469.94 \\
\hline $10 / 13 / 1112: 50$ & 35.579 & 39.189 & 1473.19 & 1469.93 \\
\hline 10/13/11 13:00 & 35.551 & 39.196 & 1473.22 & 1469.93 \\
\hline 10/13/11 13:10 & 35.551 & 39.2 & 1473.22 & 1469.92 \\
\hline 10/13/11 13:20 & 35.523 & 39.21 & 1473.25 & 1469.91 \\
\hline 10/13/11 13:30 & 35.495 & 39.217 & 1473.27 & 1469.91 \\
\hline $10 / 13 / 1113: 40$ & 35.469 & 39.226 & 1473.30 & 1469.90 \\
\hline $10 / 13 / 11$ 13:50 & 35.443 & 39.236 & 1473.33 & 1469.89 \\
\hline $10 / 13 / 11$ 14:00 & 35.479 & 39.25 & 1473.29 & 1469.87 \\
\hline $10 / 13 / 11$ 14:10 & 35.479 & 39.261 & 1473.29 & 1469.86 \\
\hline $10 / 13 / 11$ 14:20 & 35.875 & 39.278 & 1472.89 & 1469.84 \\
\hline $10 / 13 / 11$ 14:30 & 35.772 & 39.297 & 1473.00 & 1469.83 \\
\hline $10 / 13 / 11$ 14:40 & 35.828 & 39.32 & 1472.94 & 1469.80 \\
\hline $10 / 13 / 1114: 50$ & 35.901 & 39.35 & 1472.87 & 1469.77 \\
\hline 10/13/11 15:00 & 35.889 & 39.379 & 1472.88 & 1469.74 \\
\hline $10 / 13 / 11$ 15:10 & 35.98 & 39.409 & 1472.79 & 1469.71 \\
\hline $10 / 13 / 11$ 15:20 & 36.053 & 39.44 & 1472.72 & 1469.68 \\
\hline 10/13/11 15:30 & 36.121 & 39.468 & 1472.65 & 1469.65 \\
\hline $10 / 13 / 11$ 15:40 & 36.18 & 39.494 & 1472.59 & 1469.63 \\
\hline $10 / 13 / 11$ 15:50 & 36.243 & 39.524 & 1472.53 & 1469.60 \\
\hline 10/13/11 16:00 & 36.304 & 39.552 & 1472.47 & 1469.57 \\
\hline $10 / 13 / 11$ 16:10 & 36.356 & 39.58 & 1472.41 & 1469.54 \\
\hline 10/13/11 16:20 & 36.405 & 39.611 & 1472.36 & 1469.51 \\
\hline 10/13/11 16:30 & 36.454 & 39.646 & 1472.32 & 1469.48 \\
\hline 10/13/11 16:40 & 36.487 & 39.681 & 1472.28 & 1469.44 \\
\hline $10 / 13 / 1116: 50$ & 36.435 & 39.714 & 1472.33 & 1469.41 \\
\hline 10/13/11 17:00 & 38.872 & 39.745 & 1469.90 & 1469.38 \\
\hline 10/13/11 17:10 & 36.454 & 39.78 & 1472.32 & 1469.34 \\
\hline $10 / 13 / 11$ 17:20 & 36.332 & 39.813 & 1472.44 & 1469.31 \\
\hline 10/13/11 17:30 & 36.316 & 39.848 & 1472.45 & 1469.27 \\
\hline $10 / 13 / 11$ 17:40 & 36.295 & 39.883 & 1472.47 & 1469.24 \\
\hline
\end{tabular}


TABLE S1.3 Automatically recorded groundwater levels in the Klassen wells, September 15, 2011, to March 13, 2012.

\begin{tabular}{|c|c|c|c|c|}
\hline \multicolumn{3}{|c|}{ Reference Elevation (ft AMSL) } & 1508.77 & \multirow{2}{*}{$\begin{array}{l}1509.12 \\
\text { Elevation in Klassen } \\
\text { Test Well (ft AMSL) }\end{array}$} \\
\hline Date and Time & $\begin{array}{l}\text { Depth in Klassen } \\
\text { Domestic Well } \\
(\mathrm{ft} \mathrm{BGL})\end{array}$ & $\begin{array}{c}\text { Depth in Klassen } \\
\text { Test Well } \\
\text { (ft BGL) }\end{array}$ & $\begin{array}{l}\text { Elevation in Klassen } \\
\text { Domestic Well } \\
\text { (ft AMSL) }\end{array}$ & \\
\hline $10 / 13 / 1117: 50$ & 36.365 & 39.923 & 1472.40 & 1469.20 \\
\hline $10 / 13 / 1118: 00$ & 36.431 & 39.965 & 1472.34 & 1469.16 \\
\hline 10/13/11 18:10 & 38.537 & 40.007 & 1470.23 & 1469.12 \\
\hline $10 / 13 / 1118: 20$ & 36.738 & 40.052 & 1472.03 & 1469.07 \\
\hline 10/13/11 18:30 & 36.703 & 40.099 & 1472.07 & 1469.02 \\
\hline $10 / 13 / 1118: 40$ & 36.937 & 40.148 & 1471.83 & 1468.97 \\
\hline $10 / 13 / 1118: 50$ & 36.839 & 40.195 & 1471.93 & 1468.93 \\
\hline 10/13/11 19:00 & 37.641 & 40.242 & 1471.13 & 1468.88 \\
\hline 10/13/11 19:10 & 38.422 & 40.294 & 1470.35 & 1468.83 \\
\hline $10 / 13 / 1119: 20$ & 37.219 & 40.345 & 1471.55 & 1468.78 \\
\hline 10/13/11 19:30 & 37.155 & 40.397 & 1471.61 & 1468.73 \\
\hline 10/13/11 19:40 & 37.134 & 40.446 & 1471.64 & 1468.68 \\
\hline 10/13/11 19:50 & 37.125 & 40.498 & 1471.64 & 1468.62 \\
\hline $10 / 13 / 1120: 00$ & 37.315 & 40.547 & 1471.45 & 1468.58 \\
\hline 10/13/11 20:10 & 37.256 & 40.599 & 1471.51 & 1468.52 \\
\hline $10 / 13 / 1120: 20$ & 37.245 & 40.648 & 1471.52 & 1468.47 \\
\hline $10 / 13 / 1120: 30$ & 37.228 & 40.7 & 1471.54 & 1468.42 \\
\hline $10 / 13 / 1120: 40$ & 37.195 & 40.747 & 1471.57 & 1468.38 \\
\hline $10 / 13 / 1120: 50$ & 37.214 & 40.796 & 1471.56 & 1468.33 \\
\hline $10 / 13 / 1121: 00$ & 37.231 & 40.847 & 1471.54 & 1468.28 \\
\hline $10 / 13 / 1121: 10$ & 37.242 & 40.897 & 1471.53 & 1468.23 \\
\hline $10 / 13 / 1121: 20$ & 37.948 & 40.948 & 1470.82 & 1468.17 \\
\hline $10 / 13 / 1121: 30$ & 37.496 & 40.998 & 1471.27 & 1468.12 \\
\hline $10 / 13 / 1121: 40$ & 37.444 & 41.049 & 1471.33 & 1468.07 \\
\hline $10 / 13 / 1121: 50$ & 37.36 & 41.096 & 1471.41 & 1468.03 \\
\hline $10 / 13 / 1122: 00$ & 37.245 & 41.141 & 1471.52 & 1467.98 \\
\hline $10 / 13 / 1122: 10$ & 37.153 & 41.181 & 1471.62 & 1467.94 \\
\hline $10 / 13 / 1122: 20$ & 38.37 & 41.22 & 1470.40 & 1467.90 \\
\hline $10 / 13 / 1122: 30$ & 37.941 & 41.265 & 1470.83 & 1467.86 \\
\hline $10 / 13 / 1122: 40$ & 37.174 & 41.305 & 1471.60 & 1467.82 \\
\hline $10 / 13 / 1122: 50$ & 38.551 & 41.35 & 1470.22 & 1467.77 \\
\hline 10/13/11 23:00 & 37.456 & 41.394 & 1471.31 & 1467.73 \\
\hline 10/13/11 23:10 & 37.35 & 41.443 & 1471.42 & 1467.68 \\
\hline $10 / 13 / 1123: 20$ & 37.374 & 41.49 & 1471.40 & 1467.63 \\
\hline 10/13/11 23:30 & 37.397 & 41.542 & 1471.37 & 1467.58 \\
\hline $10 / 13 / 1123: 40$ & 37.423 & 41.591 & 1471.35 & 1467.53 \\
\hline $10 / 13 / 1123: 50$ & 37.465 & 41.638 & 1471.30 & 1467.48 \\
\hline 10/14/11 0:00 & 37.503 & 41.69 & 1471.27 & 1467.43 \\
\hline
\end{tabular}


TABLE S1.3 Automatically recorded groundwater levels in the Klassen wells, September 15, 2011, to March 13, 2012.

\begin{tabular}{|c|c|c|c|c|}
\hline \multicolumn{3}{|c|}{ Reference Elevation (ft AMSL) } & 1508.77 & \multirow{2}{*}{$\begin{array}{l}1509.12 \\
\text { Elevation in Klassen } \\
\text { Test Well (ft AMSL) }\end{array}$} \\
\hline Date and Time & $\begin{array}{l}\text { Depth in Klassen } \\
\text { Domestic Well } \\
(\mathrm{ft} \mathrm{BGL})\end{array}$ & $\begin{array}{c}\text { Depth in Klassen } \\
\text { Test Well } \\
\text { (ft BGL) }\end{array}$ & $\begin{array}{l}\text { Elevation in Klassen } \\
\text { Domestic Well } \\
\text { (ft AMSL) }\end{array}$ & \\
\hline 10/14/11 0:10 & 37.531 & 41.739 & 1471.24 & 1467.38 \\
\hline $10 / 14 / 110: 20$ & 37.559 & 41.786 & 1471.21 & 1467.34 \\
\hline 10/14/11 0:30 & 37.582 & 41.833 & 1471.19 & 1467.29 \\
\hline $10 / 14 / 110: 40$ & 37.554 & 41.88 & 1471.22 & 1467.24 \\
\hline 10/14/11 0:50 & 37.444 & 41.922 & 1471.33 & 1467.20 \\
\hline 10/14/11 1:00 & 37.329 & 41.957 & 1471.44 & 1467.17 \\
\hline 10/14/11 1:10 & 37.226 & 41.99 & 1471.54 & 1467.13 \\
\hline 10/14/11 1:20 & 37.13 & 42.025 & 1471.64 & 1467.10 \\
\hline 10/14/11 1:30 & 37.045 & 42.056 & 1471.72 & 1467.07 \\
\hline 10/14/11 1:40 & 36.965 & 42.086 & 1471.80 & 1467.04 \\
\hline $10 / 14 / 111: 50$ & 36.895 & 42.117 & 1471.87 & 1467.01 \\
\hline 10/14/11 2:00 & 36.827 & 42.145 & 1471.94 & 1466.98 \\
\hline 10/14/11 2:10 & 36.764 & 42.175 & 1472.01 & 1466.95 \\
\hline $10 / 14 / 112: 20$ & 36.708 & 42.208 & 1472.06 & 1466.91 \\
\hline $10 / 14 / 112: 30$ & 36.654 & 42.232 & 1472.12 & 1466.89 \\
\hline $10 / 14 / 112: 40$ & 36.6 & 42.26 & 1472.17 & 1466.86 \\
\hline $10 / 14 / 112: 50$ & 36.553 & 42.288 & 1472.22 & 1466.83 \\
\hline 10/14/11 3:00 & 36.508 & 42.307 & 1472.26 & 1466.82 \\
\hline 10/14/11 3:10 & 36.461 & 42.319 & 1472.31 & 1466.80 \\
\hline $10 / 14 / 113: 20$ & 36.421 & 42.326 & 1472.35 & 1466.80 \\
\hline $10 / 14 / 113: 30$ & 36.382 & 42.326 & 1472.39 & 1466.80 \\
\hline 10/14/11 3:40 & 36.342 & 42.326 & 1472.43 & 1466.80 \\
\hline $10 / 14 / 113: 50$ & 36.304 & 42.319 & 1472.47 & 1466.80 \\
\hline $10 / 14 / 114: 00$ & 36.267 & 42.307 & 1472.50 & 1466.82 \\
\hline 10/14/11 4:10 & 36.234 & 42.293 & 1472.54 & 1466.83 \\
\hline $10 / 14 / 114: 20$ & 36.199 & 42.276 & 1472.57 & 1466.85 \\
\hline 10/14/11 4:30 & 36.168 & 42.26 & 1472.60 & 1466.86 \\
\hline 10/14/11 4:40 & 36.138 & 42.236 & 1472.63 & 1466.89 \\
\hline $10 / 14 / 114: 50$ & 36.405 & 42.218 & 1472.36 & 1466.90 \\
\hline $10 / 14 / 115: 00$ & 36.201 & 42.197 & 1472.57 & 1466.93 \\
\hline 10/14/11 5:10 & 36.135 & 42.173 & 1472.63 & 1466.95 \\
\hline 10/14/11 5:20 & 36.091 & 42.15 & 1472.68 & 1466.97 \\
\hline $10 / 14 / 115: 30$ & 36.053 & 42.126 & 1472.72 & 1467.00 \\
\hline 10/14/11 5:40 & 36.02 & 42.1 & 1472.75 & 1467.02 \\
\hline 10/14/11 5:50 & 36.011 & 42.075 & 1472.76 & 1467.05 \\
\hline 10/14/11 6:00 & 36.051 & 42.051 & 1472.72 & 1467.07 \\
\hline 10/14/11 6:10 & 36.649 & 42.028 & 1472.12 & 1467.09 \\
\hline $10 / 14 / 116: 20$ & 36.175 & 42.004 & 1472.59 & 1467.12 \\
\hline
\end{tabular}


TABLE S1.3 Automatically recorded groundwater levels in the Klassen wells, September 15, 2011, to March 13, 2012.

\begin{tabular}{|c|c|c|c|c|}
\hline \multicolumn{3}{|c|}{ Reference Elevation (ft AMSL) } & 1508.77 & \multirow{2}{*}{$\begin{array}{l}1509.12 \\
\text { Elevation in Klassen } \\
\text { Test Well (ft AMSL) }\end{array}$} \\
\hline Date and Time & $\begin{array}{l}\text { Depth in Klassen } \\
\text { Domestic Well } \\
(\mathrm{ft} \mathrm{BGL})\end{array}$ & $\begin{array}{c}\text { Depth in Klassen } \\
\text { Test Well } \\
\text { (ft BGL) }\end{array}$ & $\begin{array}{l}\text { Elevation in Klassen } \\
\text { Domestic Well } \\
\text { (ft AMSL) }\end{array}$ & \\
\hline 10/14/11 6:30 & 36.095 & 41.981 & 1472.67 & 1467.14 \\
\hline 10/14/11 6:40 & 36.041 & 41.953 & 1472.73 & 1467.17 \\
\hline 10/14/11 6:50 & 35.997 & 41.924 & 1472.77 & 1467.20 \\
\hline 10/14/11 7:00 & 35.957 & 41.896 & 1472.81 & 1467.23 \\
\hline 10/14/11 7:10 & 35.936 & 41.87 & 1472.83 & 1467.25 \\
\hline $10 / 14 / 117: 20$ & 35.92 & 41.842 & 1472.85 & 1467.28 \\
\hline 10/14/11 7:30 & 35.884 & 41.821 & 1472.89 & 1467.30 \\
\hline 10/14/11 7:40 & 40.181 & 41.802 & 1468.59 & 1467.32 \\
\hline 10/14/11 7:50 & 36.02 & 41.786 & 1472.75 & 1467.34 \\
\hline 10/14/11 8:00 & 35.924 & 41.769 & 1472.85 & 1467.35 \\
\hline 10/14/11 8:10 & 35.873 & 41.751 & 1472.90 & 1467.37 \\
\hline $10 / 14 / 118: 20$ & 36.025 & 41.732 & 1472.74 & 1467.39 \\
\hline 10/14/11 8:30 & 35.938 & 41.713 & 1472.83 & 1467.41 \\
\hline 10/14/11 8:40 & 36.665 & 41.697 & 1472.10 & 1467.43 \\
\hline $10 / 14 / 118: 50$ & 37.857 & 41.68 & 1470.91 & 1467.44 \\
\hline 10/14/11 9:00 & 36.063 & 41.664 & 1472.71 & 1467.46 \\
\hline 10/14/11 9:10 & 35.985 & 41.643 & 1472.78 & 1467.48 \\
\hline 10/14/11 9:20 & 36.065 & 41.629 & 1472.70 & 1467.49 \\
\hline 10/14/11 9:30 & 36.156 & 41.617 & 1472.61 & 1467.51 \\
\hline 10/14/11 9:40 & 36.503 & 41.61 & 1472.27 & 1467.51 \\
\hline 10/14/11 9:50 & 36.435 & 41.601 & 1472.33 & 1467.52 \\
\hline $10 / 14 / 11$ 10:00 & 36.482 & 41.594 & 1472.29 & 1467.53 \\
\hline 10/14/11 10:10 & 36.539 & 41.584 & 1472.23 & 1467.54 \\
\hline $10 / 14 / 11$ 10:20 & 36.958 & 41.577 & 1471.81 & 1467.55 \\
\hline 10/14/11 10:30 & 36.829 & 41.572 & 1471.94 & 1467.55 \\
\hline 10/14/11 10:40 & 36.862 & 41.568 & 1471.91 & 1467.55 \\
\hline 10/14/11 10:50 & 36.97 & 41.565 & 1471.80 & 1467.56 \\
\hline 10/14/11 11:00 & 37.062 & 41.565 & 1471.71 & 1467.56 \\
\hline 10/14/11 11:10 & 37.453 & 41.563 & 1471.32 & 1467.56 \\
\hline $10 / 14 / 11$ 11:20 & 37.198 & 41.556 & 1471.57 & 1467.57 \\
\hline 10/14/11 11:30 & 37.055 & 41.54 & 1471.71 & 1467.58 \\
\hline 10/14/11 11:40 & 36.933 & 41.523 & 1471.84 & 1467.60 \\
\hline 10/14/11 11:50 & 36.827 & 41.504 & 1471.94 & 1467.62 \\
\hline $10 / 14 / 11$ 12:00 & 36.733 & 41.486 & 1472.04 & 1467.64 \\
\hline $10 / 14 / 11$ 12:10 & 36.651 & 41.464 & 1472.12 & 1467.66 \\
\hline $10 / 14 / 11$ 12:20 & 36.579 & 41.443 & 1472.19 & 1467.68 \\
\hline $10 / 14 / 11$ 12:30 & 36.511 & 41.422 & 1472.26 & 1467.70 \\
\hline $10 / 14 / 11$ 12:40 & 36.45 & 41.399 & 1472.32 & 1467.72 \\
\hline
\end{tabular}


TABLE S1.3 Automatically recorded groundwater levels in the Klassen wells, September 15, 2011, to March 13, 2012.

\begin{tabular}{|c|c|c|c|c|}
\hline \multicolumn{3}{|c|}{ Reference Elevation (ft AMSL) } & 1508.77 & \multirow{2}{*}{$\begin{array}{l}1509.12 \\
\text { Elevation in Klassen } \\
\text { Test Well (ft AMSL) }\end{array}$} \\
\hline Date and Time & $\begin{array}{l}\text { Depth in Klassen } \\
\text { Domestic Well } \\
\text { (ft BGL) }\end{array}$ & $\begin{array}{c}\text { Depth in Klassen } \\
\text { Test Well } \\
\text { (ft BGL) }\end{array}$ & $\begin{array}{l}\text { Elevation in Klassen } \\
\text { Domestic Well } \\
\text { (ft AMSL) }\end{array}$ & \\
\hline $10 / 14 / 1112: 50$ & 36.391 & 41.373 & 1472.38 & 1467.75 \\
\hline 10/14/11 13:00 & 36.365 & 41.35 & 1472.40 & 1467.77 \\
\hline $10 / 14 / 1113: 10$ & 36.318 & 41.326 & 1472.45 & 1467.80 \\
\hline $10 / 14 / 1113: 20$ & 37.61 & 41.303 & 1471.16 & 1467.82 \\
\hline $10 / 14 / 1113: 30$ & 36.379 & 41.281 & 1472.39 & 1467.84 \\
\hline $10 / 14 / 1113: 40$ & 36.283 & 41.258 & 1472.49 & 1467.86 \\
\hline $10 / 14 / 1113: 50$ & 36.403 & 41.235 & 1472.37 & 1467.89 \\
\hline 10/14/11 14:00 & 36.257 & 41.211 & 1472.51 & 1467.91 \\
\hline 10/14/11 14:10 & 36.189 & 41.19 & 1472.58 & 1467.93 \\
\hline $10 / 14 / 1114: 20$ & 36.138 & 41.167 & 1472.63 & 1467.96 \\
\hline $10 / 14 / 1114: 30$ & 36.1 & 41.145 & 1472.67 & 1467.98 \\
\hline $10 / 14 / 1114: 40$ & 36.117 & 41.124 & 1472.65 & 1468.00 \\
\hline $10 / 14 / 1114: 50$ & 37.195 & 41.105 & 1471.57 & 1468.02 \\
\hline $10 / 14 / 1115: 00$ & 36.487 & 41.091 & 1472.28 & 1468.03 \\
\hline $10 / 14 / 11$ 15:10 & 36.527 & 41.08 & 1472.24 & 1468.04 \\
\hline $10 / 14 / 11$ 15:20 & 36.597 & 41.068 & 1472.17 & 1468.05 \\
\hline $10 / 14 / 1115: 30$ & 36.658 & 41.059 & 1472.11 & 1468.06 \\
\hline $10 / 14 / 11$ 15:40 & 41.201 & 41.047 & 1467.57 & 1468.08 \\
\hline $10 / 14 / 1115: 50$ & 42.388 & 41.04 & 1466.38 & 1468.08 \\
\hline 10/14/11 16:00 & 42.205 & 41.037 & 1466.56 & 1468.09 \\
\hline $10 / 14 / 1116: 10$ & 39.951 & 41.044 & 1468.82 & 1468.08 \\
\hline $10 / 14 / 11$ 16:20 & 43.22 & 41.052 & 1465.55 & 1468.07 \\
\hline $10 / 14 / 1116: 30$ & 40.275 & 41.056 & 1468.49 & 1468.07 \\
\hline $10 / 14 / 11$ 16:40 & 43.856 & 41.07 & 1464.91 & 1468.05 \\
\hline $10 / 14 / 1116: 50$ & 40.523 & 41.08 & 1468.25 & 1468.04 \\
\hline 10/14/11 17:00 & 45.099 & 41.084 & 1463.67 & 1468.04 \\
\hline $10 / 14 / 11$ 17:10 & 41.398 & 41.087 & 1467.37 & 1468.04 \\
\hline $10 / 14 / 11$ 17:20 & 44.215 & 41.082 & 1464.55 & 1468.04 \\
\hline $10 / 14 / 11$ 17:30 & 42.486 & 41.077 & 1466.28 & 1468.05 \\
\hline $10 / 14 / 11$ 17:40 & 40.713 & 41.073 & 1468.06 & 1468.05 \\
\hline $10 / 14 / 11$ 17:50 & 43.919 & 41.066 & 1464.85 & 1468.06 \\
\hline 10/14/11 18:00 & 40.826 & 41.056 & 1467.94 & 1468.07 \\
\hline 10/14/11 18:10 & 44.208 & 41.047 & 1464.56 & 1468.08 \\
\hline $10 / 14 / 11$ 18:20 & 39.32 & 41.04 & 1469.45 & 1468.08 \\
\hline $10 / 14 / 11$ 18:30 & 38.694 & 41.033 & 1470.08 & 1468.09 \\
\hline 10/14/11 18:40 & 38.281 & 41.021 & 1470.49 & 1468.10 \\
\hline $10 / 14 / 11$ 18:50 & 38.033 & 41.005 & 1470.74 & 1468.12 \\
\hline 10/14/11 19:00 & 37.845 & 40.986 & 1470.92 & 1468.14 \\
\hline
\end{tabular}


TABLE S1.3 Automatically recorded groundwater levels in the Klassen wells, September 15, 2011, to March 13, 2012.

\begin{tabular}{|c|c|c|c|c|}
\hline \multicolumn{3}{|c|}{ Reference Elevation (ft AMSL) } & 1508.77 & \multirow{2}{*}{$\begin{array}{l}1509.12 \\
\text { Elevation in Klassen } \\
\text { Test Well (ft AMSL) }\end{array}$} \\
\hline Date and Time & $\begin{array}{l}\text { Depth in Klassen } \\
\text { Domestic Well } \\
\text { (ft BGL) }\end{array}$ & $\begin{array}{c}\text { Depth in Klassen } \\
\text { Test Well } \\
\text { (ft BGL) }\end{array}$ & $\begin{array}{l}\text { Elevation in Klassen } \\
\text { Domestic Well } \\
\text { (ft AMSL) }\end{array}$ & \\
\hline 10/14/11 19:10 & 37.688 & 40.965 & 1471.08 & 1468.16 \\
\hline $10 / 14 / 1119: 20$ & 37.557 & 40.946 & 1471.21 & 1468.18 \\
\hline 10/14/11 19:30 & 37.716 & 40.93 & 1471.05 & 1468.19 \\
\hline $10 / 14 / 11$ 19:40 & 37.683 & 40.915 & 1471.09 & 1468.21 \\
\hline 10/14/11 19:50 & 37.707 & 40.904 & 1471.06 & 1468.22 \\
\hline $10 / 14 / 1120: 00$ & 37.735 & 40.897 & 1471.03 & 1468.23 \\
\hline $10 / 14 / 1120: 10$ & 37.765 & 40.89 & 1471.00 & 1468.23 \\
\hline $10 / 14 / 1120: 20$ & 37.798 & 40.885 & 1470.97 & 1468.24 \\
\hline $10 / 14 / 1120: 30$ & 37.826 & 40.88 & 1470.94 & 1468.24 \\
\hline $10 / 14 / 1120: 40$ & 37.852 & 40.873 & 1470.92 & 1468.25 \\
\hline $10 / 14 / 1120: 50$ & 37.878 & 40.869 & 1470.89 & 1468.25 \\
\hline 10/14/11 21:00 & 37.932 & 40.864 & 1470.84 & 1468.26 \\
\hline $10 / 14 / 1121: 10$ & 37.988 & 40.864 & 1470.78 & 1468.26 \\
\hline $10 / 14 / 1121: 20$ & 38.019 & 40.861 & 1470.75 & 1468.26 \\
\hline 10/14/11 21:30 & 38.089 & 40.859 & 1470.68 & 1468.26 \\
\hline $10 / 14 / 1121: 40$ & 38.131 & 40.861 & 1470.64 & 1468.26 \\
\hline $10 / 14 / 1121: 50$ & 38.162 & 40.861 & 1470.61 & 1468.26 \\
\hline $10 / 14 / 1122: 00$ & 38.464 & 40.854 & 1470.31 & 1468.27 \\
\hline $10 / 14 / 1122: 10$ & 38.042 & 40.84 & 1470.73 & 1468.28 \\
\hline $10 / 14 / 1122: 20$ & 37.88 & 40.826 & 1470.89 & 1468.30 \\
\hline $10 / 14 / 1122: 30$ & 37.754 & 40.81 & 1471.02 & 1468.31 \\
\hline $10 / 14 / 1122: 40$ & 38.049 & 40.798 & 1470.72 & 1468.32 \\
\hline $10 / 14 / 1122: 50$ & 37.958 & 40.789 & 1470.81 & 1468.33 \\
\hline $10 / 14 / 1123: 00$ & 41.187 & 40.777 & 1467.58 & 1468.35 \\
\hline $10 / 14 / 1123: 10$ & 40.024 & 40.765 & 1468.75 & 1468.36 \\
\hline $10 / 14 / 1123: 20$ & 37.885 & 40.754 & 1470.88 & 1468.37 \\
\hline $10 / 14 / 1123: 30$ & 37.664 & 40.742 & 1471.11 & 1468.38 \\
\hline $10 / 14 / 1123: 40$ & 41.002 & 40.728 & 1467.77 & 1468.39 \\
\hline $10 / 14 / 1123: 50$ & 37.552 & 40.711 & 1471.22 & 1468.41 \\
\hline 10/15/11 0:00 & 38.544 & 40.695 & 1470.23 & 1468.43 \\
\hline 10/15/11 0:10 & 37.683 & 40.681 & 1471.09 & 1468.44 \\
\hline 10/15/11 0:20 & 37.425 & 40.667 & 1471.34 & 1468.46 \\
\hline 10/15/11 0:30 & 37.294 & 40.65 & 1471.48 & 1468.47 \\
\hline 10/15/11 0:40 & 37.198 & 40.634 & 1471.57 & 1468.49 \\
\hline 10/15/11 0:50 & 37.113 & 40.617 & 1471.66 & 1468.51 \\
\hline 10/15/11 1:00 & 37.043 & 40.599 & 1471.73 & 1468.52 \\
\hline 10/15/11 1:10 & 36.977 & 40.582 & 1471.79 & 1468.54 \\
\hline 10/15/11 1:20 & 36.916 & 40.561 & 1471.85 & 1468.56 \\
\hline
\end{tabular}


TABLE S1.3 Automatically recorded groundwater levels in the Klassen wells, September 15, 2011, to March 13, 2012.

\begin{tabular}{|c|c|c|c|c|}
\hline \multicolumn{3}{|c|}{ Reference Elevation (ft AMSL) } & 1508.77 & \multirow{2}{*}{$\begin{array}{l}1509.12 \\
\text { Elevation in Klassen } \\
\text { Test Well (ft AMSL) }\end{array}$} \\
\hline Date and Time & $\begin{array}{l}\text { Depth in Klassen } \\
\text { Domestic Well } \\
(\mathrm{ft} \mathrm{BGL})\end{array}$ & $\begin{array}{c}\text { Depth in Klassen } \\
\text { Test Well } \\
\text { (ft BGL) }\end{array}$ & $\begin{array}{l}\text { Elevation in Klassen } \\
\text { Domestic Well } \\
\text { (ft AMSL) }\end{array}$ & \\
\hline 10/15/11 1:30 & 36.881 & 40.542 & 1471.89 & 1468.58 \\
\hline 10/15/11 1:40 & 36.881 & 40.526 & 1471.89 & 1468.60 \\
\hline 10/15/11 1:50 & 36.89 & 40.51 & 1471.88 & 1468.61 \\
\hline $10 / 15 / 112: 00$ & 37.09 & 40.498 & 1471.68 & 1468.62 \\
\hline 10/15/11 2:10 & 36.912 & 40.484 & 1471.86 & 1468.64 \\
\hline $10 / 15 / 112: 20$ & 36.837 & 40.467 & 1471.93 & 1468.66 \\
\hline $10 / 15 / 112: 30$ & 36.778 & 40.451 & 1471.99 & 1468.67 \\
\hline $10 / 15 / 112: 40$ & 36.715 & 40.434 & 1472.05 & 1468.69 \\
\hline $10 / 15 / 112: 50$ & 36.684 & 40.42 & 1472.09 & 1468.70 \\
\hline 10/15/11 3:00 & 36.63 & 40.406 & 1472.14 & 1468.72 \\
\hline 10/15/11 3:10 & 36.583 & 40.388 & 1472.19 & 1468.73 \\
\hline 10/15/11 3:20 & 36.6 & 40.371 & 1472.17 & 1468.75 \\
\hline 10/15/11 3:30 & 36.632 & 40.357 & 1472.14 & 1468.77 \\
\hline $10 / 15 / 113: 40$ & 36.576 & 40.345 & 1472.19 & 1468.78 \\
\hline 10/15/11 3:50 & 36.522 & 40.331 & 1472.25 & 1468.79 \\
\hline 10/15/11 4:00 & 36.473 & 40.319 & 1472.30 & 1468.80 \\
\hline 10/15/11 4:10 & 36.431 & 40.308 & 1472.34 & 1468.81 \\
\hline 10/15/11 4:20 & 36.389 & 40.294 & 1472.38 & 1468.83 \\
\hline 10/15/11 4:30 & 36.351 & 40.275 & 1472.42 & 1468.85 \\
\hline $10 / 15 / 114: 40$ & 36.314 & 40.263 & 1472.46 & 1468.86 \\
\hline $10 / 15 / 114: 50$ & 36.278 & 40.244 & 1472.49 & 1468.88 \\
\hline 10/15/11 5:00 & 36.246 & 40.228 & 1472.52 & 1468.89 \\
\hline 10/15/11 5:10 & 36.21 & 40.216 & 1472.56 & 1468.91 \\
\hline 10/15/11 5:20 & 36.182 & 40.2 & 1472.59 & 1468.92 \\
\hline 10/15/11 5:30 & 36.149 & 40.186 & 1472.62 & 1468.94 \\
\hline $10 / 15 / 115: 40$ & 37.442 & 40.172 & 1471.33 & 1468.95 \\
\hline 10/15/11 5:50 & 36.236 & 40.158 & 1472.53 & 1468.96 \\
\hline 10/15/11 6:00 & 36.159 & 40.141 & 1472.61 & 1468.98 \\
\hline 10/15/11 6:10 & 36.109 & 40.127 & 1472.66 & 1469.00 \\
\hline $10 / 15 / 116: 20$ & 36.246 & 40.113 & 1472.52 & 1469.01 \\
\hline 10/15/11 6:30 & 36.131 & 40.101 & 1472.64 & 1469.02 \\
\hline $10 / 15 / 116: 40$ & 36.077 & 40.083 & 1472.69 & 1469.04 \\
\hline $10 / 15 / 116: 50$ & 36.037 & 40.071 & 1472.73 & 1469.05 \\
\hline 10/15/11 7:00 & 36.002 & 40.054 & 1472.77 & 1469.07 \\
\hline 10/15/11 7:10 & 35.969 & 40.04 & 1472.80 & 1469.08 \\
\hline $10 / 15 / 117: 20$ & 35.941 & 40.026 & 1472.83 & 1469.10 \\
\hline $10 / 15 / 117: 30$ & 35.912 & 40.012 & 1472.86 & 1469.11 \\
\hline $10 / 15 / 11$ 7:40 & 35.887 & 39.996 & 1472.88 & 1469.13 \\
\hline
\end{tabular}


TABLE S1.3 Automatically recorded groundwater levels in the Klassen wells, September 15, 2011, to March 13, 2012.

\begin{tabular}{|c|c|c|c|c|}
\hline \multicolumn{3}{|c|}{ Reference Elevation (ft AMSL) } & 1508.77 & \multirow{2}{*}{$\begin{array}{l}1509.12 \\
\text { Elevation in Klassen } \\
\text { Test Well (ft AMSL) }\end{array}$} \\
\hline Date and Time & $\begin{array}{l}\text { Depth in Klassen } \\
\text { Domestic Well } \\
(\mathrm{ft} \mathrm{BGL})\end{array}$ & $\begin{array}{c}\text { Depth in Klassen } \\
\text { Test Well } \\
\text { (ft BGL) }\end{array}$ & $\begin{array}{l}\text { Elevation in Klassen } \\
\text { Domestic Well } \\
\text { (ft AMSL) }\end{array}$ & \\
\hline 10/15/11 7:50 & 36.654 & 39.979 & 1472.12 & 1469.14 \\
\hline 10/15/11 8:00 & 35.957 & 39.965 & 1472.81 & 1469.16 \\
\hline 10/15/11 8:10 & 35.894 & 39.949 & 1472.88 & 1469.17 \\
\hline $10 / 15 / 118: 20$ & 35.884 & 39.932 & 1472.89 & 1469.19 \\
\hline 10/15/11 8:30 & 35.917 & 39.921 & 1472.85 & 1469.20 \\
\hline 10/15/11 8:40 & 36.002 & 39.914 & 1472.77 & 1469.21 \\
\hline 10/15/11 8:50 & 35.978 & 39.904 & 1472.79 & 1469.22 \\
\hline 10/15/11 9:00 & 36.032 & 39.897 & 1472.74 & 1469.23 \\
\hline 10/15/11 9:10 & 36.117 & 39.892 & 1472.65 & 1469.23 \\
\hline 10/15/11 9:20 & 36.215 & 39.892 & 1472.55 & 1469.23 \\
\hline 10/15/11 9:30 & 36.328 & 39.888 & 1472.44 & 1469.23 \\
\hline 10/15/11 9:40 & 36.459 & 39.892 & 1472.31 & 1469.23 \\
\hline 10/15/11 9:50 & 36.604 & 39.902 & 1472.17 & 1469.22 \\
\hline 10/15/11 10:00 & 36.909 & 39.909 & 1471.86 & 1469.21 \\
\hline 10/15/11 10:10 & 36.893 & 39.918 & 1471.88 & 1469.20 \\
\hline $10 / 15 / 11$ 10:20 & 36.923 & 39.925 & 1471.85 & 1469.20 \\
\hline 10/15/11 10:30 & 36.965 & 39.928 & 1471.80 & 1469.19 \\
\hline 10/15/11 10:40 & 37.005 & 39.932 & 1471.76 & 1469.19 \\
\hline 10/15/11 10:50 & 37.048 & 39.932 & 1471.72 & 1469.19 \\
\hline 10/15/11 11:00 & 37.09 & 39.937 & 1471.68 & 1469.19 \\
\hline 10/15/11 11:10 & 37.132 & 39.942 & 1471.64 & 1469.18 \\
\hline $10 / 15 / 11$ 11:20 & 37.172 & 39.946 & 1471.60 & 1469.18 \\
\hline 10/15/11 11:30 & 37.212 & 39.949 & 1471.56 & 1469.17 \\
\hline $10 / 15 / 11$ 11:40 & 37.252 & 39.953 & 1471.52 & 1469.17 \\
\hline 10/15/11 11:50 & 37.693 & 39.956 & 1471.08 & 1469.17 \\
\hline $10 / 15 / 11$ 12:00 & 37.474 & 39.958 & 1471.30 & 1469.16 \\
\hline $10 / 15 / 11$ 12:10 & 37.463 & 39.961 & 1471.31 & 1469.16 \\
\hline $10 / 15 / 11$ 12:20 & 37.395 & 39.963 & 1471.37 & 1469.16 \\
\hline $10 / 15 / 11$ 12:30 & 37.277 & 39.958 & 1471.49 & 1469.16 \\
\hline $10 / 15 / 11$ 12:40 & 37.162 & 39.951 & 1471.61 & 1469.17 \\
\hline $10 / 15 / 11$ 12:50 & 37.059 & 39.939 & 1471.71 & 1469.18 \\
\hline 10/15/11 13:00 & 36.968 & 39.93 & 1471.80 & 1469.19 \\
\hline 10/15/11 13:10 & 36.886 & 39.916 & 1471.88 & 1469.21 \\
\hline $10 / 15 / 11$ 13:20 & 36.806 & 39.904 & 1471.96 & 1469.22 \\
\hline 10/15/11 13:30 & 36.738 & 39.89 & 1472.03 & 1469.23 \\
\hline $10 / 15 / 11$ 13:40 & 36.672 & 39.878 & 1472.10 & 1469.24 \\
\hline $10 / 15 / 11$ 13:50 & 36.611 & 39.867 & 1472.16 & 1469.26 \\
\hline $10 / 15 / 11$ 14:00 & 37.683 & 39.853 & 1471.09 & 1469.27 \\
\hline
\end{tabular}


TABLE S1.3 Automatically recorded groundwater levels in the Klassen wells, September 15, 2011, to March 13, 2012.

\begin{tabular}{|c|c|c|c|c|}
\hline \multicolumn{3}{|c|}{ Reference Elevation (ft AMSL) } & 1508.77 & \multirow{2}{*}{$\begin{array}{l}1509.12 \\
\text { Elevation in Klassen } \\
\text { Test Well (ft AMSL) }\end{array}$} \\
\hline Date and Time & $\begin{array}{l}\text { Depth in Klassen } \\
\text { Domestic Well } \\
\text { (ft BGL) }\end{array}$ & $\begin{array}{c}\text { Depth in Klassen } \\
\text { Test Well } \\
\text { (ft BGL) }\end{array}$ & $\begin{array}{l}\text { Elevation in Klassen } \\
\text { Domestic Well } \\
\text { (ft AMSL) }\end{array}$ & \\
\hline $10 / 15 / 1114: 10$ & 36.654 & 39.841 & 1472.12 & 1469.28 \\
\hline $10 / 15 / 1114: 20$ & 36.553 & 39.827 & 1472.22 & 1469.30 \\
\hline 10/15/11 14:30 & 36.696 & 39.813 & 1472.07 & 1469.31 \\
\hline $10 / 15 / 1114: 40$ & 36.539 & 39.803 & 1472.23 & 1469.32 \\
\hline 10/15/11 14:50 & 36.457 & 39.787 & 1472.31 & 1469.34 \\
\hline 10/15/11 15:00 & 36.597 & 39.775 & 1472.17 & 1469.35 \\
\hline $10 / 15 / 1115: 10$ & 36.438 & 39.763 & 1472.33 & 1469.36 \\
\hline $10 / 15 / 11$ 15:20 & 36.363 & 39.752 & 1472.41 & 1469.37 \\
\hline $10 / 15 / 1115: 30$ & 37.069 & 39.738 & 1471.70 & 1469.38 \\
\hline $10 / 15 / 1115: 40$ & 36.44 & 39.731 & 1472.33 & 1469.39 \\
\hline $10 / 15 / 1115: 50$ & 36.372 & 39.721 & 1472.40 & 1469.40 \\
\hline 10/15/11 16:00 & 36.33 & 39.709 & 1472.44 & 1469.41 \\
\hline 10/15/11 16:10 & 36.299 & 39.7 & 1472.47 & 1469.42 \\
\hline $10 / 15 / 1116: 20$ & 36.267 & 39.688 & 1472.50 & 1469.43 \\
\hline 10/15/11 16:30 & 36.869 & 39.677 & 1471.90 & 1469.45 \\
\hline 10/15/11 16:40 & 38.875 & 39.672 & 1469.89 & 1469.45 \\
\hline $10 / 15 / 1116: 50$ & 47.31 & 39.67 & 1461.46 & 1469.45 \\
\hline 10/15/11 17:00 & 47.585 & 39.677 & 1461.18 & 1469.45 \\
\hline 10/15/11 17:10 & 51.774 & 39.686 & 1457.00 & 1469.44 \\
\hline $10 / 15 / 11$ 17:20 & 47.653 & 39.7 & 1461.12 & 1469.42 \\
\hline $10 / 15 / 11$ 17:30 & 53.49 & 39.712 & 1455.28 & 1469.41 \\
\hline $10 / 15 / 11$ 17:40 & 51.159 & 39.728 & 1457.61 & 1469.39 \\
\hline $10 / 15 / 11$ 17:50 & 50.664 & 39.752 & 1458.11 & 1469.37 \\
\hline 10/15/11 18:00 & 50.097 & 39.77 & 1458.67 & 1469.35 \\
\hline 10/15/11 18:10 & 54.74 & 39.796 & 1454.03 & 1469.33 \\
\hline $10 / 15 / 11$ 18:20 & 50.915 & 39.829 & 1457.85 & 1469.29 \\
\hline 10/15/11 18:30 & 55.477 & 39.86 & 1453.29 & 1469.26 \\
\hline 10/15/11 18:40 & 52.311 & 39.885 & 1456.46 & 1469.24 \\
\hline $10 / 15 / 11$ 18:50 & 54.867 & 39.911 & 1453.90 & 1469.21 \\
\hline 10/15/11 19:00 & 50.366 & 39.939 & 1458.40 & 1469.18 \\
\hline 10/15/11 19:10 & 55.833 & 39.963 & 1452.94 & 1469.16 \\
\hline 10/15/11 19:20 & 62.707 & 39.989 & 1446.06 & 1469.13 \\
\hline 10/15/11 19:30 & 45.08 & 40.017 & 1463.69 & 1469.11 \\
\hline 10/15/11 19:40 & 41.196 & 40.033 & 1467.57 & 1469.09 \\
\hline 10/15/11 19:50 & 40.415 & 40.036 & 1468.35 & 1469.09 \\
\hline $10 / 15 / 1120: 00$ & 39.932 & 40.033 & 1468.84 & 1469.09 \\
\hline $10 / 15 / 1120: 10$ & 39.585 & 40.029 & 1469.18 & 1469.09 \\
\hline $10 / 15 / 1120: 20$ & 39.315 & 40.022 & 1469.45 & 1469.10 \\
\hline
\end{tabular}


TABLE S1.3 Automatically recorded groundwater levels in the Klassen wells, September 15, 2011, to March 13, 2012.

\begin{tabular}{|c|c|c|c|c|}
\hline \multicolumn{3}{|c|}{ Reference Elevation (ft AMSL) } & 1508.77 & \multirow{2}{*}{$\begin{array}{l}1509.12 \\
\text { Elevation in Klassen } \\
\text { Test Well (ft AMSL) }\end{array}$} \\
\hline Date and Time & $\begin{array}{l}\text { Depth in Klassen } \\
\text { Domestic Well } \\
(\mathrm{ft} \mathrm{BGL})\end{array}$ & $\begin{array}{c}\text { Depth in Klassen } \\
\text { Test Well } \\
\text { (ft BGL) }\end{array}$ & $\begin{array}{l}\text { Elevation in Klassen } \\
\text { Domestic Well } \\
\text { (ft AMSL) }\end{array}$ & \\
\hline $10 / 15 / 1120: 30$ & 39.072 & 40.012 & 1469.70 & 1469.11 \\
\hline $10 / 15 / 1120: 40$ & 38.856 & 40.003 & 1469.91 & 1469.12 \\
\hline $10 / 15 / 1120: 50$ & 38.668 & 39.991 & 1470.10 & 1469.13 \\
\hline 10/15/11 21:00 & 38.577 & 39.977 & 1470.19 & 1469.15 \\
\hline 10/15/11 21:10 & 38.46 & 39.965 & 1470.31 & 1469.16 \\
\hline $10 / 15 / 1121: 20$ & 38.41 & 39.956 & 1470.36 & 1469.17 \\
\hline $10 / 15 / 1121: 30$ & 38.422 & 39.951 & 1470.35 & 1469.17 \\
\hline 10/15/11 21:40 & 38.403 & 39.946 & 1470.37 & 1469.18 \\
\hline $10 / 15 / 1121: 50$ & 39.294 & 39.939 & 1469.48 & 1469.18 \\
\hline $10 / 15 / 1122: 00$ & 39.041 & 39.939 & 1469.73 & 1469.18 \\
\hline $10 / 15 / 1122: 10$ & 38.755 & 39.937 & 1470.01 & 1469.19 \\
\hline $10 / 15 / 1122: 20$ & 38.521 & 39.935 & 1470.25 & 1469.19 \\
\hline $10 / 15 / 1122: 30$ & 38.326 & 39.932 & 1470.44 & 1469.19 \\
\hline $10 / 15 / 1122: 40$ & 39.269 & 39.928 & 1469.50 & 1469.19 \\
\hline $10 / 15 / 1122: 50$ & 38.467 & 39.921 & 1470.30 & 1469.20 \\
\hline $10 / 15 / 1123: 00$ & 38.185 & 39.914 & 1470.58 & 1469.21 \\
\hline $10 / 15 / 1123: 10$ & 38.138 & 39.902 & 1470.63 & 1469.22 \\
\hline $10 / 15 / 1123: 20$ & 38.18 & 39.897 & 1470.59 & 1469.23 \\
\hline 10/15/11 23:30 & 38.213 & 39.897 & 1470.56 & 1469.23 \\
\hline $10 / 15 / 1123: 40$ & 38.241 & 39.897 & 1470.53 & 1469.23 \\
\hline $10 / 15 / 1123: 50$ & 38.267 & 39.897 & 1470.50 & 1469.23 \\
\hline 10/16/11 0:00 & 38.291 & 39.9 & 1470.48 & 1469.22 \\
\hline 10/16/11 0:10 & 38.314 & 39.9 & 1470.46 & 1469.22 \\
\hline 10/16/11 0:20 & 38.335 & 39.902 & 1470.43 & 1469.22 \\
\hline 10/16/11 0:30 & 38.352 & 39.904 & 1470.42 & 1469.22 \\
\hline 10/16/11 0:40 & 38.37 & 39.904 & 1470.40 & 1469.22 \\
\hline 10/16/11 0:50 & 38.389 & 39.907 & 1470.38 & 1469.22 \\
\hline 10/16/11 1:00 & 38.312 & 39.907 & 1470.46 & 1469.22 \\
\hline 10/16/11 1:10 & 38.176 & 39.897 & 1470.59 & 1469.23 \\
\hline $10 / 16 / 111: 20$ & 38.047 & 39.888 & 1470.72 & 1469.23 \\
\hline 10/16/11 1:30 & 37.929 & 39.876 & 1470.84 & 1469.25 \\
\hline 10/16/11 1:40 & 37.882 & 39.864 & 1470.89 & 1469.26 \\
\hline 10/16/11 1:50 & 37.866 & 39.855 & 1470.90 & 1469.27 \\
\hline $10 / 16 / 112: 00$ & 37.765 & 39.843 & 1471.00 & 1469.28 \\
\hline $10 / 16 / 112: 10$ & 37.674 & 39.831 & 1471.10 & 1469.29 \\
\hline $10 / 16 / 112: 20$ & 37.603 & 39.822 & 1471.17 & 1469.30 \\
\hline $10 / 16 / 112: 30$ & 37.517 & 39.813 & 1471.25 & 1469.31 \\
\hline 10/16/11 2:40 & 37.465 & 39.803 & 1471.30 & 1469.32 \\
\hline
\end{tabular}


TABLE S1.3 Automatically recorded groundwater levels in the Klassen wells, September 15, 2011, to March 13, 2012.

\begin{tabular}{|c|c|c|c|c|}
\hline \multicolumn{3}{|c|}{ Reference Elevation (ft AMSL) } & 1508.77 & \multirow{2}{*}{$\begin{array}{l}1509.12 \\
\text { Elevation in Klassen } \\
\text { Test Well (ft AMSL) }\end{array}$} \\
\hline Date and Time & $\begin{array}{l}\text { Depth in Klassen } \\
\text { Domestic Well } \\
(\mathrm{ft} \mathrm{BGL})\end{array}$ & $\begin{array}{c}\text { Depth in Klassen } \\
\text { Test Well } \\
\text { (ft BGL) }\end{array}$ & $\begin{array}{l}\text { Elevation in Klassen } \\
\text { Domestic Well } \\
\text { (ft AMSL) }\end{array}$ & \\
\hline $10 / 16 / 112: 50$ & 38.272 & 39.792 & 1470.50 & 1469.33 \\
\hline 10/16/11 3:00 & 37.442 & 39.782 & 1471.33 & 1469.34 \\
\hline 10/16/11 3:10 & 37.352 & 39.77 & 1471.42 & 1469.35 \\
\hline $10 / 16 / 113: 20$ & 37.32 & 39.759 & 1471.45 & 1469.36 \\
\hline 10/16/11 3:30 & 37.315 & 39.752 & 1471.45 & 1469.37 \\
\hline 10/16/11 3:40 & 37.235 & 39.74 & 1471.53 & 1469.38 \\
\hline $10 / 16 / 113: 50$ & 37.165 & 39.726 & 1471.60 & 1469.40 \\
\hline 10/16/11 4:00 & 37.094 & 39.714 & 1471.68 & 1469.41 \\
\hline 10/16/11 4:10 & 37.036 & 39.7 & 1471.73 & 1469.42 \\
\hline $10 / 16 / 114: 20$ & 36.998 & 39.686 & 1471.77 & 1469.44 \\
\hline $10 / 16 / 114: 30$ & 36.954 & 39.674 & 1471.82 & 1469.45 \\
\hline 10/16/11 4:40 & 36.897 & 39.663 & 1471.87 & 1469.46 \\
\hline $10 / 16 / 114: 50$ & 36.848 & 39.648 & 1471.92 & 1469.47 \\
\hline 10/16/11 5:00 & 36.801 & 39.632 & 1471.97 & 1469.49 \\
\hline 10/16/11 5:10 & 36.754 & 39.62 & 1472.02 & 1469.50 \\
\hline $10 / 16 / 115: 20$ & 36.712 & 39.604 & 1472.06 & 1469.52 \\
\hline $10 / 16 / 115: 30$ & 36.67 & 39.592 & 1472.10 & 1469.53 \\
\hline 10/16/11 5:40 & 36.63 & 39.576 & 1472.14 & 1469.55 \\
\hline $10 / 16 / 115: 50$ & 40.645 & 39.562 & 1468.12 & 1469.56 \\
\hline $10 / 16 / 116: 00$ & 37.329 & 39.548 & 1471.44 & 1469.57 \\
\hline $10 / 16 / 116: 10$ & 36.827 & 39.526 & 1471.94 & 1469.60 \\
\hline $10 / 16 / 116: 20$ & 36.708 & 39.524 & 1472.06 & 1469.60 \\
\hline $10 / 16 / 116: 30$ & 37.064 & 39.526 & 1471.71 & 1469.60 \\
\hline $10 / 16 / 116: 40$ & 36.7 & 39.515 & 1472.07 & 1469.61 \\
\hline $10 / 16 / 116: 50$ & 36.609 & 39.496 & 1472.16 & 1469.63 \\
\hline 10/16/11 7:00 & 36.548 & 39.484 & 1472.22 & 1469.64 \\
\hline 10/16/11 7:10 & 36.518 & 39.484 & 1472.25 & 1469.64 \\
\hline 10/16/11 7:20 & 36.471 & 39.47 & 1472.30 & 1469.65 \\
\hline $10 / 16 / 117: 30$ & 36.426 & 39.451 & 1472.34 & 1469.67 \\
\hline $10 / 16 / 117: 40$ & 36.384 & 39.44 & 1472.39 & 1469.68 \\
\hline 10/16/11 7:50 & 36.346 & 39.426 & 1472.42 & 1469.70 \\
\hline 10/16/11 8:00 & 36.314 & 39.416 & 1472.46 & 1469.71 \\
\hline 10/16/11 8:10 & 41.281 & 39.404 & 1467.49 & 1469.72 \\
\hline 10/16/11 8:20 & 41.346 & 39.397 & 1467.42 & 1469.73 \\
\hline 10/16/11 8:30 & 36.52 & 39.39 & 1472.25 & 1469.73 \\
\hline $10 / 16 / 118: 40$ & 36.396 & 39.383 & 1472.37 & 1469.74 \\
\hline $10 / 16 / 118: 50$ & 36.323 & 39.379 & 1472.45 & 1469.74 \\
\hline 10/16/11 9:00 & 36.269 & 39.372 & 1472.50 & 1469.75 \\
\hline
\end{tabular}


TABLE S1.3 Automatically recorded groundwater levels in the Klassen wells, September 15, 2011, to March 13, 2012.

\begin{tabular}{|c|c|c|c|c|}
\hline \multicolumn{3}{|c|}{ Reference Elevation (ft AMSL) } & 1508.77 & \multirow{2}{*}{$\begin{array}{l}1509.12 \\
\text { Elevation in Klassen } \\
\text { Test Well (ft AMSL) }\end{array}$} \\
\hline Date and Time & $\begin{array}{l}\text { Depth in Klassen } \\
\text { Domestic Well } \\
\text { (ft BGL) }\end{array}$ & $\begin{array}{c}\text { Depth in Klassen } \\
\text { Test Well } \\
\text { (ft BGL) }\end{array}$ & $\begin{array}{l}\text { Elevation in Klassen } \\
\text { Domestic Well } \\
\text { (ft AMSL) }\end{array}$ & \\
\hline 10/16/11 9:10 & 36.257 & 39.367 & 1472.51 & 1469.76 \\
\hline 10/16/11 9:20 & 36.285 & 39.36 & 1472.48 & 1469.76 \\
\hline 10/16/11 9:30 & 36.323 & 39.358 & 1472.45 & 1469.76 \\
\hline 10/16/11 9:40 & 36.318 & 39.353 & 1472.45 & 1469.77 \\
\hline 10/16/11 9:50 & 36.264 & 39.348 & 1472.51 & 1469.77 \\
\hline 10/16/11 10:00 & 36.217 & 39.343 & 1472.55 & 1469.78 \\
\hline 10/16/11 10:10 & 36.203 & 39.341 & 1472.57 & 1469.78 \\
\hline 10/16/11 10:20 & 36.187 & 39.341 & 1472.58 & 1469.78 \\
\hline 10/16/11 10:30 & 36.149 & 39.336 & 1472.62 & 1469.79 \\
\hline 10/16/11 10:40 & 36.109 & 39.334 & 1472.66 & 1469.79 \\
\hline $10 / 16 / 1110: 50$ & 36.074 & 39.332 & 1472.70 & 1469.79 \\
\hline 10/16/11 11:00 & 36.067 & 39.332 & 1472.70 & 1469.79 \\
\hline 10/16/11 11:10 & 36.037 & 39.332 & 1472.73 & 1469.79 \\
\hline 10/16/11 11:20 & 35.999 & 39.334 & 1472.77 & 1469.79 \\
\hline 10/16/11 11:30 & 35.973 & 39.336 & 1472.80 & 1469.79 \\
\hline 10/16/11 11:40 & 35.945 & 39.334 & 1472.82 & 1469.79 \\
\hline 10/16/11 11:50 & 35.92 & 39.332 & 1472.85 & 1469.79 \\
\hline $10 / 16 / 11$ 12:00 & 35.896 & 39.327 & 1472.87 & 1469.80 \\
\hline $10 / 16 / 11$ 12:10 & 35.873 & 39.322 & 1472.90 & 1469.80 \\
\hline $10 / 16 / 11$ 12:20 & 35.877 & 39.325 & 1472.89 & 1469.80 \\
\hline $10 / 16 / 11$ 12:30 & 35.88 & 39.322 & 1472.89 & 1469.80 \\
\hline $10 / 16 / 11$ 12:40 & 35.866 & 39.322 & 1472.90 & 1469.80 \\
\hline $10 / 16 / 1112: 50$ & 35.852 & 39.32 & 1472.92 & 1469.80 \\
\hline 10/16/11 13:00 & 35.823 & 39.311 & 1472.95 & 1469.81 \\
\hline 10/16/11 13:10 & 36.112 & 39.308 & 1472.66 & 1469.81 \\
\hline $10 / 16 / 11$ 13:20 & 35.929 & 39.299 & 1472.84 & 1469.82 \\
\hline $10 / 16 / 11$ 13:30 & 35.861 & 39.289 & 1472.91 & 1469.83 \\
\hline 10/16/11 13:40 & 35.835 & 39.28 & 1472.93 & 1469.84 \\
\hline $10 / 16 / 11$ 13:50 & 35.812 & 39.271 & 1472.96 & 1469.85 \\
\hline 10/16/11 14:00 & 35.774 & 39.268 & 1473.00 & 1469.85 \\
\hline 10/16/11 14:10 & 35.746 & 39.261 & 1473.02 & 1469.86 \\
\hline $10 / 16 / 11$ 14:20 & 35.723 & 39.252 & 1473.05 & 1469.87 \\
\hline $10 / 16 / 11$ 14:30 & 35.697 & 39.247 & 1473.07 & 1469.88 \\
\hline $10 / 16 / 11$ 14:40 & 35.676 & 39.24 & 1473.09 & 1469.88 \\
\hline $10 / 16 / 11$ 14:50 & 35.652 & 39.231 & 1473.12 & 1469.89 \\
\hline $10 / 16 / 11$ 15:00 & 35.633 & 39.221 & 1473.14 & 1469.90 \\
\hline 10/16/11 15:10 & 39.51 & 39.214 & 1469.26 & 1469.91 \\
\hline $10 / 16 / 11$ 15:20 & 35.72 & 39.205 & 1473.05 & 1469.92 \\
\hline
\end{tabular}


TABLE S1.3 Automatically recorded groundwater levels in the Klassen wells, September 15, 2011, to March 13, 2012.

\begin{tabular}{|c|c|c|c|c|}
\hline \multicolumn{3}{|c|}{ Reference Elevation (ft AMSL) } & 1508.77 & \multirow{2}{*}{$\begin{array}{l}1509.12 \\
\text { Elevation in Klassen } \\
\text { Test Well (ft AMSL) }\end{array}$} \\
\hline Date and Time & $\begin{array}{l}\text { Depth in Klassen } \\
\text { Domestic Well } \\
(\mathrm{ft} \mathrm{BGL})\end{array}$ & $\begin{array}{c}\text { Depth in Klassen } \\
\text { Test Well } \\
\text { (ft BGL) }\end{array}$ & $\begin{array}{l}\text { Elevation in Klassen } \\
\text { Domestic Well } \\
\text { (ft AMSL) }\end{array}$ & \\
\hline $10 / 16 / 1115: 30$ & 39.761 & 39.2 & 1469.01 & 1469.92 \\
\hline $10 / 16 / 1115: 40$ & 39.907 & 39.2 & 1468.86 & 1469.92 \\
\hline 10/16/11 15:50 & 43.811 & 39.203 & 1464.96 & 1469.92 \\
\hline 10/16/11 16:00 & 39.74 & 39.212 & 1469.03 & 1469.91 \\
\hline 10/16/11 16:10 & 41.558 & 39.224 & 1467.21 & 1469.90 \\
\hline $10 / 16 / 1116: 20$ & 45.026 & 39.24 & 1463.74 & 1469.88 \\
\hline 10/16/11 16:30 & 45.505 & 39.261 & 1463.26 & 1469.86 \\
\hline 10/16/11 16:40 & 41.616 & 39.282 & 1467.15 & 1469.84 \\
\hline $10 / 16 / 1116: 50$ & 42.486 & 39.308 & 1466.28 & 1469.81 \\
\hline 10/16/11 17:00 & 44.78 & 39.329 & 1463.99 & 1469.79 \\
\hline 10/16/11 17:10 & 50.305 & 39.355 & 1458.46 & 1469.77 \\
\hline 10/16/11 17:20 & 47.339 & 39.386 & 1461.43 & 1469.74 \\
\hline 10/16/11 17:30 & 42.618 & 39.414 & 1466.15 & 1469.71 \\
\hline $10 / 16 / 1117: 40$ & 45.514 & 39.442 & 1463.26 & 1469.68 \\
\hline 10/16/11 17:50 & 44.679 & 39.468 & 1464.09 & 1469.65 \\
\hline 10/16/11 18:00 & 43.441 & 39.491 & 1465.33 & 1469.63 \\
\hline 10/16/11 18:10 & 47.634 & 39.515 & 1461.14 & 1469.61 \\
\hline 10/16/11 18:20 & 41.776 & 39.529 & 1466.99 & 1469.59 \\
\hline 10/16/11 18:30 & 39.212 & 39.533 & 1469.56 & 1469.59 \\
\hline $10 / 16 / 11$ 18:40 & 38.804 & 39.533 & 1469.97 & 1469.59 \\
\hline $10 / 16 / 11$ 18:50 & 38.53 & 39.531 & 1470.24 & 1469.59 \\
\hline 10/16/11 19:00 & 38.506 & 39.531 & 1470.26 & 1469.59 \\
\hline 10/16/11 19:10 & 38.495 & 39.533 & 1470.27 & 1469.59 \\
\hline $10 / 16 / 11$ 19:20 & 38.471 & 39.536 & 1470.30 & 1469.59 \\
\hline 10/16/11 19:30 & 38.556 & 39.543 & 1470.21 & 1469.58 \\
\hline 10/16/11 19:40 & 38.6 & 39.555 & 1470.17 & 1469.57 \\
\hline 10/16/11 19:50 & 39.111 & 39.569 & 1469.66 & 1469.55 \\
\hline $10 / 16 / 1120: 00$ & 38.851 & 39.58 & 1469.92 & 1469.54 \\
\hline $10 / 16 / 1120: 10$ & 38.743 & 39.592 & 1470.03 & 1469.53 \\
\hline 10/16/11 20:20 & 38.727 & 39.599 & 1470.04 & 1469.52 \\
\hline 10/16/11 20:30 & 38.724 & 39.611 & 1470.05 & 1469.51 \\
\hline 10/16/11 20:40 & 38.72 & 39.618 & 1470.05 & 1469.50 \\
\hline $10 / 16 / 1120: 50$ & 38.706 & 39.625 & 1470.06 & 1469.50 \\
\hline 10/16/11 21:00 & 38.696 & 39.632 & 1470.07 & 1469.49 \\
\hline 10/16/11 21:10 & 38.696 & 39.637 & 1470.07 & 1469.49 \\
\hline $10 / 16 / 1121: 20$ & 38.699 & 39.641 & 1470.07 & 1469.48 \\
\hline 10/16/11 21:30 & 38.928 & 39.648 & 1469.84 & 1469.47 \\
\hline $10 / 16 / 11$ 21:40 & 38.799 & 39.653 & 1469.97 & 1469.47 \\
\hline
\end{tabular}


TABLE S1.3 Automatically recorded groundwater levels in the Klassen wells, September 15, 2011, to March 13, 2012.

\begin{tabular}{|c|c|c|c|c|}
\hline \multicolumn{3}{|c|}{ Reference Elevation (ft AMSL) } & 1508.77 & \multirow{2}{*}{$\begin{array}{l}1509.12 \\
\text { Elevation in Klassen } \\
\text { Test Well (ft AMSL) }\end{array}$} \\
\hline Date and Time & $\begin{array}{l}\text { Depth in Klassen } \\
\text { Domestic Well } \\
(\mathrm{ft} \mathrm{BGL})\end{array}$ & $\begin{array}{c}\text { Depth in Klassen } \\
\text { Test Well } \\
\text { (ft BGL) }\end{array}$ & $\begin{array}{l}\text { Elevation in Klassen } \\
\text { Domestic Well } \\
\text { (ft AMSL) }\end{array}$ & \\
\hline $10 / 16 / 1121: 50$ & 38.762 & 39.655 & 1470.01 & 1469.47 \\
\hline $10 / 16 / 1122: 00$ & 38.741 & 39.66 & 1470.03 & 1469.46 \\
\hline $10 / 16 / 1122: 10$ & 38.729 & 39.665 & 1470.04 & 1469.46 \\
\hline $10 / 16 / 1122: 20$ & 38.72 & 39.665 & 1470.05 & 1469.46 \\
\hline $10 / 16 / 1122: 30$ & 38.715 & 39.67 & 1470.05 & 1469.45 \\
\hline $10 / 16 / 1122: 40$ & 38.71 & 39.674 & 1470.06 & 1469.45 \\
\hline $10 / 16 / 1122: 50$ & 42.653 & 39.679 & 1466.12 & 1469.44 \\
\hline 10/16/11 23:00 & 39.149 & 39.686 & 1469.62 & 1469.44 \\
\hline $10 / 16 / 1123: 10$ & 38.924 & 39.691 & 1469.85 & 1469.43 \\
\hline $10 / 16 / 1123: 20$ & 39.264 & 39.693 & 1469.51 & 1469.43 \\
\hline $10 / 16 / 1123: 30$ & 39.203 & 39.7 & 1469.57 & 1469.42 \\
\hline $10 / 16 / 1123: 40$ & 38.95 & 39.705 & 1469.82 & 1469.42 \\
\hline $10 / 16 / 1123: 50$ & 38.79 & 39.705 & 1469.98 & 1469.42 \\
\hline 10/17/11 0:00 & 38.661 & 39.702 & 1470.11 & 1469.42 \\
\hline 10/17/11 0:10 & 38.553 & 39.695 & 1470.22 & 1469.43 \\
\hline 10/17/11 0:20 & 38.455 & 39.688 & 1470.31 & 1469.43 \\
\hline 10/17/11 0:30 & 38.368 & 39.681 & 1470.40 & 1469.44 \\
\hline 10/17/11 0:40 & 38.288 & 39.679 & 1470.48 & 1469.44 \\
\hline 10/17/11 0:50 & 38.216 & 39.674 & 1470.55 & 1469.45 \\
\hline 10/17/11 1:00 & 38.15 & 39.672 & 1470.62 & 1469.45 \\
\hline 10/17/11 1:10 & 38.087 & 39.667 & 1470.68 & 1469.46 \\
\hline 10/17/11 1:20 & 38.03 & 39.663 & 1470.74 & 1469.46 \\
\hline 10/17/11 1:30 & 37.974 & 39.658 & 1470.80 & 1469.46 \\
\hline $10 / 17 / 111: 40$ & 37.89 & 39.648 & 1470.88 & 1469.47 \\
\hline 10/17/11 1:50 & 37.789 & 39.637 & 1470.98 & 1469.49 \\
\hline 10/17/11 2:00 & 37.695 & 39.625 & 1471.07 & 1469.50 \\
\hline 10/17/11 2:10 & 37.634 & 39.611 & 1471.14 & 1469.51 \\
\hline $10 / 17 / 112: 20$ & 37.564 & 39.599 & 1471.21 & 1469.52 \\
\hline $10 / 17 / 112: 30$ & 37.488 & 39.587 & 1471.28 & 1469.54 \\
\hline $10 / 17 / 112: 40$ & 37.416 & 39.576 & 1471.35 & 1469.55 \\
\hline $10 / 17 / 112: 50$ & 37.348 & 39.562 & 1471.42 & 1469.56 \\
\hline 10/17/11 3:00 & 37.284 & 39.55 & 1471.49 & 1469.57 \\
\hline 10/17/11 3:10 & 37.226 & 39.536 & 1471.54 & 1469.59 \\
\hline 10/17/11 3:20 & 37.17 & 39.524 & 1471.60 & 1469.60 \\
\hline 10/17/11 3:30 & 37.786 & 39.51 & 1470.98 & 1469.61 \\
\hline 10/17/11 3:40 & 37.177 & 39.501 & 1471.59 & 1469.62 \\
\hline 10/17/11 3:50 & 37.087 & 39.484 & 1471.68 & 1469.64 \\
\hline 10/17/11 4:00 & 37.022 & 39.475 & 1471.75 & 1469.65 \\
\hline
\end{tabular}


TABLE S1.3 Automatically recorded groundwater levels in the Klassen wells, September 15, 2011, to March 13, 2012.

\begin{tabular}{|c|c|c|c|c|}
\hline \multicolumn{3}{|c|}{ Reference Elevation (ft AMSL) } & 1508.77 & \multirow{2}{*}{$\begin{array}{l}1509.12 \\
\text { Elevation in Klassen } \\
\text { Test Well (ft AMSL) }\end{array}$} \\
\hline Date and Time & $\begin{array}{l}\text { Depth in Klassen } \\
\text { Domestic Well } \\
\text { (ft BGL) }\end{array}$ & $\begin{array}{c}\text { Depth in Klassen } \\
\text { Test Well } \\
\text { (ft BGL) }\end{array}$ & $\begin{array}{l}\text { Elevation in Klassen } \\
\text { Domestic Well } \\
\text { (ft AMSL) }\end{array}$ & \\
\hline 10/17/11 4:10 & 36.994 & 39.463 & 1471.78 & 1469.66 \\
\hline $10 / 17 / 114: 20$ & 36.937 & 39.447 & 1471.83 & 1469.68 \\
\hline 10/17/11 4:30 & 36.886 & 39.435 & 1471.88 & 1469.69 \\
\hline $10 / 17 / 114: 40$ & 36.837 & 39.423 & 1471.93 & 1469.70 \\
\hline $10 / 17 / 114: 50$ & 36.792 & 39.409 & 1471.98 & 1469.71 \\
\hline 10/17/11 5:00 & 36.747 & 39.4 & 1472.02 & 1469.72 \\
\hline 10/17/11 5:10 & 36.708 & 39.388 & 1472.06 & 1469.73 \\
\hline $10 / 17 / 115: 20$ & 36.665 & 39.376 & 1472.10 & 1469.75 \\
\hline 10/17/11 5:30 & 36.628 & 39.365 & 1472.14 & 1469.76 \\
\hline 10/17/11 5:40 & 36.588 & 39.355 & 1472.18 & 1469.77 \\
\hline $10 / 17 / 115: 50$ & 36.553 & 39.343 & 1472.22 & 1469.78 \\
\hline 10/17/11 6:00 & 36.518 & 39.325 & 1472.25 & 1469.80 \\
\hline 10/17/11 6:10 & 38.345 & 39.313 & 1470.42 & 1469.81 \\
\hline $10 / 17 / 116: 20$ & 36.766 & 39.304 & 1472.00 & 1469.82 \\
\hline $10 / 17 / 116: 30$ & 36.651 & 39.294 & 1472.12 & 1469.83 \\
\hline 10/17/11 6:40 & 36.579 & 39.285 & 1472.19 & 1469.84 \\
\hline $10 / 17 / 116: 50$ & 36.52 & 39.273 & 1472.25 & 1469.85 \\
\hline 10/17/11 7:00 & 36.909 & 39.264 & 1471.86 & 1469.86 \\
\hline 10/17/11 7:10 & 36.564 & 39.254 & 1472.21 & 1469.87 \\
\hline $10 / 17 / 117: 20$ & 36.485 & 39.243 & 1472.28 & 1469.88 \\
\hline 10/17/11 7:30 & 36.431 & 39.233 & 1472.34 & 1469.89 \\
\hline 10/17/11 7:40 & 36.414 & 39.221 & 1472.36 & 1469.90 \\
\hline 10/17/11 7:50 & 36.37 & 39.21 & 1472.40 & 1469.91 \\
\hline 10/17/11 8:00 & 38.982 & 39.2 & 1469.79 & 1469.92 \\
\hline 10/17/11 8:10 & 36.44 & 39.189 & 1472.33 & 1469.93 \\
\hline 10/17/11 8:20 & 40.814 & 39.177 & 1467.96 & 1469.95 \\
\hline 10/17/11 8:30 & 36.428 & 39.165 & 1472.34 & 1469.96 \\
\hline 10/17/11 8:40 & 36.33 & 39.153 & 1472.44 & 1469.97 \\
\hline 10/17/11 8:50 & 36.271 & 39.149 & 1472.50 & 1469.97 \\
\hline 10/17/11 9:00 & 36.227 & 39.135 & 1472.54 & 1469.99 \\
\hline 10/17/11 9:10 & 40.275 & 39.123 & 1468.49 & 1470.00 \\
\hline 10/17/11 9:20 & 36.431 & 39.113 & 1472.34 & 1470.01 \\
\hline 10/17/11 9:30 & 36.276 & 39.109 & 1472.49 & 1470.01 \\
\hline 10/17/11 9:40 & 36.208 & 39.102 & 1472.56 & 1470.02 \\
\hline 10/17/11 9:50 & 36.159 & 39.092 & 1472.61 & 1470.03 \\
\hline 10/17/11 10:00 & 36.117 & 39.083 & 1472.65 & 1470.04 \\
\hline 10/17/11 10:10 & 36.079 & 39.081 & 1472.69 & 1470.04 \\
\hline $10 / 17 / 11$ 10:20 & 36.614 & 39.078 & 1472.16 & 1470.04 \\
\hline
\end{tabular}


TABLE S1.3 Automatically recorded groundwater levels in the Klassen wells, September 15, 2011, to March 13, 2012.

\begin{tabular}{|c|c|c|c|c|}
\hline \multicolumn{3}{|c|}{ Reference Elevation (ft AMSL) } & 1508.77 & \multirow{2}{*}{$\begin{array}{l}1509.12 \\
\text { Elevation in Klassen } \\
\text { Test Well (ft AMSL) }\end{array}$} \\
\hline Date and Time & $\begin{array}{l}\text { Depth in Klassen } \\
\text { Domestic Well } \\
(\mathrm{ft} \mathrm{BGL})\end{array}$ & $\begin{array}{c}\text { Depth in Klassen } \\
\text { Test Well } \\
\text { (ft BGL) }\end{array}$ & $\begin{array}{l}\text { Elevation in Klassen } \\
\text { Domestic Well } \\
\text { (ft AMSL) }\end{array}$ & \\
\hline 10/17/11 10:30 & 36.163 & 39.067 & 1472.61 & 1470.06 \\
\hline $10 / 17 / 11$ 10:40 & 40.608 & 39.057 & 1468.16 & 1470.07 \\
\hline 10/17/11 10:50 & 43.781 & 39.06 & 1464.99 & 1470.06 \\
\hline 10/17/11 11:00 & 42.871 & 39.057 & 1465.90 & 1470.07 \\
\hline 10/17/11 11:10 & 40.458 & 39.06 & 1468.31 & 1470.06 \\
\hline $10 / 17 / 1111: 20$ & 44.086 & 39.067 & 1464.68 & 1470.06 \\
\hline 10/17/11 11:30 & 44.311 & 39.074 & 1464.46 & 1470.05 \\
\hline 10/17/11 11:40 & 37.625 & 39.085 & 1471.14 & 1470.04 \\
\hline 10/17/11 11:50 & 37.205 & 39.081 & 1471.56 & 1470.04 \\
\hline 10/17/11 12:00 & 36.975 & 39.078 & 1471.79 & 1470.04 \\
\hline 10/17/11 12:10 & 37.104 & 39.071 & 1471.67 & 1470.05 \\
\hline $10 / 17 / 1112: 20$ & 36.799 & 39.069 & 1471.97 & 1470.05 \\
\hline 10/17/11 12:30 & 36.67 & 39.067 & 1472.10 & 1470.06 \\
\hline $10 / 17 / 1112: 40$ & 36.572 & 39.057 & 1472.20 & 1470.07 \\
\hline $10 / 17 / 11$ 12:50 & 36.731 & 39.05 & 1472.04 & 1470.07 \\
\hline 10/17/11 13:00 & 36.811 & 39.043 & 1471.96 & 1470.08 \\
\hline 10/17/11 13:10 & 36.555 & 39.031 & 1472.21 & 1470.09 \\
\hline 10/17/11 13:20 & 36.452 & 39.022 & 1472.32 & 1470.10 \\
\hline 10/17/11 13:30 & 36.372 & 39.013 & 1472.40 & 1470.11 \\
\hline $10 / 17 / 11$ 13:40 & 36.309 & 39.001 & 1472.46 & 1470.12 \\
\hline $10 / 17 / 11$ 13:50 & 36.257 & 38.999 & 1472.51 & 1470.12 \\
\hline 10/17/11 14:00 & 36.487 & 38.991 & 1472.28 & 1470.13 \\
\hline $10 / 17 / 11$ 14:10 & 36.285 & 38.984 & 1472.48 & 1470.14 \\
\hline $10 / 17 / 11$ 14:20 & 36.213 & 38.98 & 1472.56 & 1470.14 \\
\hline 10/17/11 14:30 & 36.154 & 38.973 & 1472.62 & 1470.15 \\
\hline $10 / 17 / 11$ 14:40 & 36.109 & 38.963 & 1472.66 & 1470.16 \\
\hline $10 / 17 / 11$ 14:50 & 36.065 & 38.956 & 1472.70 & 1470.17 \\
\hline 10/17/11 15:00 & 36.03 & 38.947 & 1472.74 & 1470.18 \\
\hline $10 / 17 / 11$ 15:10 & 35.997 & 38.938 & 1472.77 & 1470.18 \\
\hline 10/17/11 15:20 & 36.267 & 38.933 & 1472.50 & 1470.19 \\
\hline 10/17/11 15:30 & 36.093 & 38.926 & 1472.68 & 1470.20 \\
\hline $10 / 17 / 11$ 15:40 & 36.02 & 38.919 & 1472.75 & 1470.20 \\
\hline 10/17/11 15:50 & 35.999 & 38.914 & 1472.77 & 1470.21 \\
\hline 10/17/11 16:00 & 35.957 & 38.907 & 1472.81 & 1470.22 \\
\hline 10/17/11 16:10 & 36.098 & 38.902 & 1472.67 & 1470.22 \\
\hline $10 / 17 / 11$ 16:20 & 35.98 & 38.895 & 1472.79 & 1470.23 \\
\hline 10/17/11 16:30 & 35.927 & 38.886 & 1472.84 & 1470.24 \\
\hline $10 / 17 / 11$ 16:40 & 35.882 & 38.879 & 1472.89 & 1470.24 \\
\hline
\end{tabular}


TABLE S1.3 Automatically recorded groundwater levels in the Klassen wells, September 15, 2011, to March 13, 2012.

\begin{tabular}{|c|c|c|c|c|}
\hline \multicolumn{3}{|c|}{ Reference Elevation (ft AMSL) } & 1508.77 & \multirow{2}{*}{$\begin{array}{l}1509.12 \\
\text { Elevation in Klassen } \\
\text { Test Well (ft AMSL) }\end{array}$} \\
\hline Date and Time & $\begin{array}{l}\text { Depth in Klassen } \\
\text { Domestic Well } \\
\text { (ft BGL) }\end{array}$ & $\begin{array}{c}\text { Depth in Klassen } \\
\text { Test Well } \\
\text { (ft BGL) }\end{array}$ & $\begin{array}{l}\text { Elevation in Klassen } \\
\text { Domestic Well } \\
\text { (ft AMSL) }\end{array}$ & \\
\hline $10 / 17 / 1116: 50$ & 36.168 & 38.872 & 1472.60 & 1470.25 \\
\hline 10/17/11 17:00 & 35.915 & 38.867 & 1472.85 & 1470.26 \\
\hline 10/17/11 17:10 & 35.868 & 38.86 & 1472.90 & 1470.26 \\
\hline $10 / 17 / 1117: 20$ & 35.844 & 38.855 & 1472.93 & 1470.27 \\
\hline 10/17/11 17:30 & 35.835 & 38.848 & 1472.93 & 1470.27 \\
\hline $10 / 17 / 1117: 40$ & 35.8 & 38.839 & 1472.97 & 1470.28 \\
\hline $10 / 17 / 1117: 50$ & 35.793 & 38.834 & 1472.98 & 1470.29 \\
\hline 10/17/11 18:00 & 35.819 & 38.83 & 1472.95 & 1470.29 \\
\hline 10/17/11 18:10 & 35.786 & 38.823 & 1472.98 & 1470.30 \\
\hline $10 / 17 / 1118: 20$ & 35.751 & 38.818 & 1473.02 & 1470.30 \\
\hline $10 / 17 / 1118: 30$ & 35.72 & 38.811 & 1473.05 & 1470.31 \\
\hline $10 / 17 / 1118: 40$ & 35.725 & 38.806 & 1473.04 & 1470.32 \\
\hline $10 / 17 / 1118: 50$ & 35.718 & 38.804 & 1473.05 & 1470.32 \\
\hline 10/17/11 19:00 & 36.628 & 38.797 & 1472.14 & 1470.33 \\
\hline 10/17/11 19:10 & 35.905 & 38.792 & 1472.86 & 1470.33 \\
\hline 10/17/11 19:20 & 35.779 & 38.787 & 1472.99 & 1470.34 \\
\hline $10 / 17 / 11$ 19:30 & 35.72 & 38.783 & 1473.05 & 1470.34 \\
\hline 10/17/11 19:40 & 35.678 & 38.78 & 1473.09 & 1470.34 \\
\hline 10/17/11 19:50 & 36.947 & 38.776 & 1471.82 & 1470.35 \\
\hline $10 / 17 / 1120: 00$ & 36.03 & 38.778 & 1472.74 & 1470.34 \\
\hline $10 / 17 / 1120: 10$ & 35.983 & 38.778 & 1472.79 & 1470.34 \\
\hline $10 / 17 / 1120: 20$ & 35.992 & 38.783 & 1472.78 & 1470.34 \\
\hline $10 / 17 / 1120: 30$ & 35.931 & 38.783 & 1472.84 & 1470.34 \\
\hline $10 / 17 / 1120: 40$ & 35.884 & 38.778 & 1472.89 & 1470.34 \\
\hline $10 / 17 / 1120: 50$ & 35.931 & 38.773 & 1472.84 & 1470.35 \\
\hline 10/17/11 21:00 & 35.882 & 38.771 & 1472.89 & 1470.35 \\
\hline 10/17/11 21:10 & 35.91 & 38.769 & 1472.86 & 1470.35 \\
\hline $10 / 17 / 1121: 20$ & 35.884 & 38.766 & 1472.89 & 1470.36 \\
\hline $10 / 17 / 1121: 30$ & 35.833 & 38.764 & 1472.94 & 1470.36 \\
\hline 10/17/11 21:40 & 35.788 & 38.755 & 1472.98 & 1470.37 \\
\hline $10 / 17 / 1121: 50$ & 35.746 & 38.747 & 1473.02 & 1470.38 \\
\hline $10 / 17 / 1122: 00$ & 35.774 & 38.743 & 1473.00 & 1470.38 \\
\hline $10 / 17 / 1122: 10$ & 41.037 & 38.733 & 1467.73 & 1470.39 \\
\hline $10 / 17 / 1122: 20$ & 35.927 & 38.731 & 1472.84 & 1470.39 \\
\hline $10 / 17 / 1122: 30$ & 35.908 & 38.731 & 1472.86 & 1470.39 \\
\hline $10 / 17 / 1122: 40$ & 36.081 & 38.729 & 1472.69 & 1470.39 \\
\hline $10 / 17 / 1122: 50$ & 36.091 & 38.726 & 1472.68 & 1470.40 \\
\hline $10 / 17 / 1123: 00$ & 38.544 & 38.726 & 1470.23 & 1470.40 \\
\hline
\end{tabular}


TABLE S1.3 Automatically recorded groundwater levels in the Klassen wells, September 15, 2011, to March 13, 2012.

\begin{tabular}{|c|c|c|c|c|}
\hline \multicolumn{3}{|c|}{ Reference Elevation (ft AMSL) } & 1508.77 & \multirow{2}{*}{$\begin{array}{l}1509.12 \\
\text { Elevation in Klassen } \\
\text { Test Well (ft AMSL) }\end{array}$} \\
\hline Date and Time & $\begin{array}{l}\text { Depth in Klassen } \\
\text { Domestic Well } \\
\text { (ft BGL) }\end{array}$ & $\begin{array}{c}\text { Depth in Klassen } \\
\text { Test Well } \\
\text { (ft BGL) }\end{array}$ & $\begin{array}{l}\text { Elevation in Klassen } \\
\text { Domestic Well } \\
\text { (ft AMSL) }\end{array}$ & \\
\hline $10 / 17 / 1123: 10$ & 36.095 & 38.724 & 1472.67 & 1470.40 \\
\hline $10 / 17 / 1123: 20$ & 36.109 & 38.722 & 1472.66 & 1470.40 \\
\hline $10 / 17 / 1123: 30$ & 37.636 & 38.717 & 1471.13 & 1470.41 \\
\hline $10 / 17 / 1123: 40$ & 36.126 & 38.717 & 1472.64 & 1470.41 \\
\hline $10 / 17 / 1123: 50$ & 35.985 & 38.712 & 1472.78 & 1470.41 \\
\hline 10/18/11 0:00 & 35.896 & 38.71 & 1472.87 & 1470.41 \\
\hline 10/18/11 0:10 & 35.835 & 38.705 & 1472.93 & 1470.42 \\
\hline $10 / 18 / 110: 20$ & 35.805 & 38.701 & 1472.96 & 1470.42 \\
\hline 10/18/11 0:30 & 35.758 & 38.698 & 1473.01 & 1470.42 \\
\hline $10 / 18 / 110: 40$ & 35.713 & 38.694 & 1473.06 & 1470.43 \\
\hline $10 / 18 / 110: 50$ & 35.678 & 38.689 & 1473.09 & 1470.43 \\
\hline 10/18/11 1:00 & 35.64 & 38.682 & 1473.13 & 1470.44 \\
\hline 10/18/11 1:10 & 35.61 & 38.677 & 1473.16 & 1470.45 \\
\hline $10 / 18 / 111: 20$ & 35.584 & 38.675 & 1473.19 & 1470.45 \\
\hline 10/18/11 1:30 & 35.723 & 38.672 & 1473.05 & 1470.45 \\
\hline 10/18/11 1:40 & 35.687 & 38.67 & 1473.08 & 1470.45 \\
\hline 10/18/11 1:50 & 35.713 & 38.668 & 1473.06 & 1470.45 \\
\hline $10 / 18 / 112: 00$ & 35.669 & 38.663 & 1473.10 & 1470.46 \\
\hline $10 / 18 / 112: 10$ & 35.643 & 38.661 & 1473.13 & 1470.46 \\
\hline $10 / 18 / 112: 20$ & 35.61 & 38.656 & 1473.16 & 1470.47 \\
\hline $10 / 18 / 112: 30$ & 35.572 & 38.654 & 1473.20 & 1470.47 \\
\hline $10 / 18 / 112: 40$ & 35.568 & 38.649 & 1473.20 & 1470.47 \\
\hline $10 / 18 / 112: 50$ & 35.535 & 38.644 & 1473.23 & 1470.48 \\
\hline 10/18/11 3:00 & 35.526 & 38.642 & 1473.24 & 1470.48 \\
\hline 10/18/11 3:10 & 35.502 & 38.64 & 1473.27 & 1470.48 \\
\hline $10 / 18 / 113: 20$ & 35.523 & 38.637 & 1473.25 & 1470.49 \\
\hline 10/18/11 3:30 & 35.556 & 38.637 & 1473.21 & 1470.49 \\
\hline 10/18/11 3:40 & 35.526 & 38.633 & 1473.24 & 1470.49 \\
\hline $10 / 18 / 113: 50$ & 35.49 & 38.628 & 1473.28 & 1470.49 \\
\hline 10/18/11 4:00 & 35.483 & 38.625 & 1473.29 & 1470.50 \\
\hline 10/18/11 4:10 & 35.455 & 38.618 & 1473.31 & 1470.50 \\
\hline 10/18/11 4:20 & 35.429 & 38.611 & 1473.34 & 1470.51 \\
\hline $10 / 18 / 114: 30$ & 35.401 & 38.607 & 1473.37 & 1470.52 \\
\hline 10/18/11 4:40 & 35.38 & 38.604 & 1473.39 & 1470.52 \\
\hline $10 / 18 / 114: 50$ & 35.357 & 38.6 & 1473.41 & 1470.52 \\
\hline 10/18/11 5:00 & 36.002 & 38.597 & 1472.77 & 1470.53 \\
\hline 10/18/11 5:10 & 35.453 & 38.593 & 1473.32 & 1470.53 \\
\hline 10/18/11 5:20 & 35.389 & 38.59 & 1473.38 & 1470.53 \\
\hline
\end{tabular}


TABLE S1.3 Automatically recorded groundwater levels in the Klassen wells, September 15, 2011, to March 13, 2012.

\begin{tabular}{|c|c|c|c|c|}
\hline \multicolumn{3}{|c|}{ Reference Elevation (ft AMSL) } & 1508.77 & \multirow{2}{*}{$\begin{array}{l}1509.12 \\
\text { Elevation in Klassen } \\
\text { Test Well (ft AMSL) }\end{array}$} \\
\hline Date and Time & $\begin{array}{l}\text { Depth in Klassen } \\
\text { Domestic Well } \\
\text { (ft BGL) }\end{array}$ & $\begin{array}{c}\text { Depth in Klassen } \\
\text { Test Well } \\
\text { (ft BGL) }\end{array}$ & $\begin{array}{l}\text { Elevation in Klassen } \\
\text { Domestic Well } \\
\text { (ft AMSL) }\end{array}$ & \\
\hline 10/18/11 5:30 & 35.352 & 38.586 & 1473.42 & 1470.54 \\
\hline 10/18/11 5:40 & 35.324 & 38.579 & 1473.45 & 1470.54 \\
\hline 10/18/11 5:50 & 35.3 & 38.569 & 1473.47 & 1470.55 \\
\hline 10/18/11 6:00 & 35.277 & 38.564 & 1473.49 & 1470.56 \\
\hline 10/18/11 6:10 & 38.567 & 38.557 & 1470.20 & 1470.57 \\
\hline $10 / 18 / 116: 20$ & 35.389 & 38.553 & 1473.38 & 1470.57 \\
\hline 10/18/11 6:30 & 35.314 & 38.548 & 1473.46 & 1470.57 \\
\hline 10/18/11 6:40 & 35.275 & 38.541 & 1473.49 & 1470.58 \\
\hline 10/18/11 6:50 & 35.265 & 38.534 & 1473.50 & 1470.59 \\
\hline 10/18/11 7:00 & 35.265 & 38.529 & 1473.50 & 1470.59 \\
\hline 10/18/11 7:10 & 35.296 & 38.525 & 1473.47 & 1470.60 \\
\hline 10/18/11 7:20 & 35.326 & 38.522 & 1473.44 & 1470.60 \\
\hline 10/18/11 7:30 & 35.303 & 38.515 & 1473.47 & 1470.61 \\
\hline 10/18/11 7:40 & 35.268 & 38.51 & 1473.50 & 1470.61 \\
\hline $10 / 18 / 117: 50$ & 35.554 & 38.506 & 1473.22 & 1470.62 \\
\hline 10/18/11 8:00 & 35.359 & 38.501 & 1473.41 & 1470.62 \\
\hline 10/18/11 8:10 & 35.3 & 38.499 & 1473.47 & 1470.62 \\
\hline $10 / 18 / 118: 20$ & 35.261 & 38.494 & 1473.51 & 1470.63 \\
\hline 10/18/11 8:30 & 35.23 & 38.485 & 1473.54 & 1470.64 \\
\hline 10/18/11 8:40 & 35.204 & 38.48 & 1473.57 & 1470.64 \\
\hline $10 / 18 / 118: 50$ & 35.181 & 38.473 & 1473.59 & 1470.65 \\
\hline 10/18/11 9:00 & 35.16 & 38.473 & 1473.61 & 1470.65 \\
\hline 10/18/11 9:10 & 35.141 & 38.471 & 1473.63 & 1470.65 \\
\hline 10/18/11 9:20 & 39.339 & 38.461 & 1469.43 & 1470.66 \\
\hline 10/18/11 9:30 & 35.244 & 38.459 & 1473.53 & 1470.66 \\
\hline 10/18/11 9:40 & 35.174 & 38.454 & 1473.60 & 1470.67 \\
\hline 10/18/11 9:50 & 35.139 & 38.452 & 1473.63 & 1470.67 \\
\hline 10/18/11 10:00 & 35.113 & 38.449 & 1473.66 & 1470.67 \\
\hline 10/18/11 10:10 & 35.094 & 38.452 & 1473.68 & 1470.67 \\
\hline 10/18/11 10:20 & 35.073 & 38.452 & 1473.70 & 1470.67 \\
\hline 10/18/11 10:30 & 35.056 & 38.449 & 1473.71 & 1470.67 \\
\hline 10/18/11 10:40 & 35.042 & 38.447 & 1473.73 & 1470.68 \\
\hline $10 / 18 / 11$ 10:50 & 35.028 & 38.445 & 1473.74 & 1470.68 \\
\hline 10/18/11 11:00 & 35.014 & 38.44 & 1473.76 & 1470.68 \\
\hline 10/18/11 11:10 & 40.791 & 38.435 & 1467.98 & 1470.69 \\
\hline $10 / 18 / 11$ 11:20 & 35.183 & 38.433 & 1473.59 & 1470.69 \\
\hline 10/18/11 11:30 & 35.094 & 38.428 & 1473.68 & 1470.69 \\
\hline $10 / 18 / 11$ 11:40 & 35.052 & 38.426 & 1473.72 & 1470.70 \\
\hline
\end{tabular}


TABLE S1.3 Automatically recorded groundwater levels in the Klassen wells, September 15, 2011, to March 13, 2012.

\begin{tabular}{|c|c|c|c|c|}
\hline \multicolumn{3}{|c|}{ Reference Elevation (ft AMSL) } & 1508.77 & \multirow{2}{*}{$\begin{array}{l}1509.12 \\
\text { Elevation in Klassen } \\
\text { Test Well (ft AMSL) }\end{array}$} \\
\hline Date and Time & $\begin{array}{l}\text { Depth in Klassen } \\
\text { Domestic Well } \\
\text { (ft BGL) }\end{array}$ & $\begin{array}{c}\text { Depth in Klassen } \\
\text { Test Well } \\
\text { (ft BGL) }\end{array}$ & $\begin{array}{l}\text { Elevation in Klassen } \\
\text { Domestic Well } \\
\text { (ft AMSL) }\end{array}$ & \\
\hline 10/18/11 11:50 & 35.024 & 38.424 & 1473.75 & 1470.70 \\
\hline 10/18/11 12:00 & 35.005 & 38.419 & 1473.76 & 1470.70 \\
\hline $10 / 18 / 1112: 10$ & 34.986 & 38.419 & 1473.78 & 1470.70 \\
\hline $10 / 18 / 1112: 20$ & 34.97 & 38.419 & 1473.80 & 1470.70 \\
\hline $10 / 18 / 1112: 30$ & 34.956 & 38.424 & 1473.81 & 1470.70 \\
\hline $10 / 18 / 1112: 40$ & 34.939 & 38.426 & 1473.83 & 1470.70 \\
\hline $10 / 18 / 1112: 50$ & 34.927 & 38.428 & 1473.84 & 1470.69 \\
\hline 10/18/11 13:00 & 34.913 & 38.426 & 1473.86 & 1470.70 \\
\hline 10/18/11 13:10 & 35.298 & 38.426 & 1473.47 & 1470.70 \\
\hline $10 / 18 / 11$ 13:20 & 35.028 & 38.421 & 1473.74 & 1470.70 \\
\hline 10/18/11 13:30 & 34.984 & 38.421 & 1473.79 & 1470.70 \\
\hline $10 / 18 / 1113: 40$ & 34.956 & 38.417 & 1473.81 & 1470.71 \\
\hline $10 / 18 / 1113: 50$ & 35.094 & 38.407 & 1473.68 & 1470.72 \\
\hline 10/18/11 14:00 & 35 & 38.398 & 1473.77 & 1470.72 \\
\hline 10/18/11 14:10 & 34.96 & 38.391 & 1473.81 & 1470.73 \\
\hline $10 / 18 / 11$ 14:20 & 34.937 & 38.379 & 1473.83 & 1470.74 \\
\hline $10 / 18 / 11$ 14:30 & 34.913 & 38.363 & 1473.86 & 1470.76 \\
\hline $10 / 18 / 11$ 14:40 & 34.897 & 38.349 & 1473.87 & 1470.77 \\
\hline $10 / 18 / 1114: 50$ & 34.883 & 38.339 & 1473.89 & 1470.78 \\
\hline $10 / 18 / 11$ 15:00 & 34.864 & 38.33 & 1473.91 & 1470.79 \\
\hline $10 / 18 / 11$ 15:10 & 34.855 & 38.32 & 1473.91 & 1470.80 \\
\hline $10 / 18 / 11$ 15:20 & 34.843 & 38.311 & 1473.93 & 1470.81 \\
\hline $10 / 18 / 11$ 15:30 & 36.595 & 38.304 & 1472.17 & 1470.82 \\
\hline $10 / 18 / 11$ 15:40 & 34.967 & 38.299 & 1473.80 & 1470.82 \\
\hline $10 / 18 / 1115: 50$ & 34.935 & 38.297 & 1473.83 & 1470.83 \\
\hline 10/18/11 16:00 & 34.895 & 38.295 & 1473.87 & 1470.83 \\
\hline 10/18/11 16:10 & 34.878 & 38.29 & 1473.89 & 1470.83 \\
\hline $10 / 18 / 11$ 16:20 & 34.852 & 38.283 & 1473.92 & 1470.84 \\
\hline 10/18/11 16:30 & 34.834 & 38.278 & 1473.94 & 1470.84 \\
\hline 10/18/11 16:40 & 34.817 & 38.274 & 1473.95 & 1470.85 \\
\hline $10 / 18 / 1116: 50$ & 34.806 & 38.266 & 1473.96 & 1470.86 \\
\hline 10/18/11 17:00 & 34.789 & 38.264 & 1473.98 & 1470.86 \\
\hline 10/18/11 17:10 & 34.78 & 38.257 & 1473.99 & 1470.87 \\
\hline 10/18/11 17:20 & 34.768 & 38.252 & 1474.00 & 1470.87 \\
\hline 10/18/11 17:30 & 35.12 & 38.248 & 1473.65 & 1470.87 \\
\hline $10 / 18 / 11$ 17:40 & 34.878 & 38.241 & 1473.89 & 1470.88 \\
\hline 10/18/11 17:50 & 34.829 & 38.238 & 1473.94 & 1470.88 \\
\hline 10/18/11 18:00 & 34.798 & 38.231 & 1473.97 & 1470.89 \\
\hline
\end{tabular}


TABLE S1.3 Automatically recorded groundwater levels in the Klassen wells, September 15, 2011, to March 13, 2012.

\begin{tabular}{|c|c|c|c|c|}
\hline \multicolumn{3}{|c|}{ Reference Elevation (ft AMSL) } & 1508.77 & \multirow{2}{*}{$\begin{array}{l}1509.12 \\
\text { Elevation in Klassen } \\
\text { Test Well (ft AMSL) }\end{array}$} \\
\hline Date and Time & $\begin{array}{l}\text { Depth in Klassen } \\
\text { Domestic Well } \\
\text { (ft BGL) }\end{array}$ & $\begin{array}{c}\text { Depth in Klassen } \\
\text { Test Well } \\
\text { (ft BGL) }\end{array}$ & $\begin{array}{l}\text { Elevation in Klassen } \\
\text { Domestic Well } \\
\text { (ft AMSL) }\end{array}$ & \\
\hline $10 / 18 / 1118: 10$ & 38.668 & 38.224 & 1470.10 & 1470.90 \\
\hline $10 / 18 / 1118: 20$ & 34.93 & 38.222 & 1473.84 & 1470.90 \\
\hline $10 / 18 / 1118: 30$ & 34.852 & 38.215 & 1473.92 & 1470.91 \\
\hline $10 / 18 / 1118: 40$ & 34.815 & 38.21 & 1473.95 & 1470.91 \\
\hline $10 / 18 / 1118: 50$ & 34.794 & 38.203 & 1473.98 & 1470.92 \\
\hline 10/18/11 19:00 & 34.773 & 38.198 & 1474.00 & 1470.92 \\
\hline 10/18/11 19:10 & 34.756 & 38.194 & 1474.01 & 1470.93 \\
\hline $10 / 18 / 1119: 20$ & 34.745 & 38.191 & 1474.02 & 1470.93 \\
\hline 10/18/11 19:30 & 35.354 & 38.187 & 1473.42 & 1470.94 \\
\hline $10 / 18 / 11$ 19:40 & 34.866 & 38.184 & 1473.90 & 1470.94 \\
\hline $10 / 18 / 1119: 50$ & 34.81 & 38.18 & 1473.96 & 1470.94 \\
\hline 10/18/11 20:00 & 34.777 & 38.177 & 1473.99 & 1470.95 \\
\hline $10 / 18 / 1120: 10$ & 34.787 & 38.173 & 1473.98 & 1470.95 \\
\hline $10 / 18 / 1120: 20$ & 34.763 & 38.17 & 1474.01 & 1470.95 \\
\hline $10 / 18 / 1120: 30$ & 34.747 & 38.168 & 1474.02 & 1470.95 \\
\hline $10 / 18 / 1120: 40$ & 34.73 & 38.166 & 1474.04 & 1470.96 \\
\hline $10 / 18 / 1120: 50$ & 34.714 & 38.163 & 1474.06 & 1470.96 \\
\hline $10 / 18 / 1121: 00$ & 34.74 & 38.161 & 1474.03 & 1470.96 \\
\hline $10 / 18 / 1121: 10$ & 36.67 & 38.163 & 1472.10 & 1470.96 \\
\hline $10 / 18 / 1121: 20$ & 34.956 & 38.166 & 1473.81 & 1470.96 \\
\hline $10 / 18 / 1121: 30$ & 34.883 & 38.163 & 1473.89 & 1470.96 \\
\hline $10 / 18 / 1121: 40$ & 34.852 & 38.159 & 1473.92 & 1470.96 \\
\hline $10 / 18 / 1121: 50$ & 34.838 & 38.159 & 1473.93 & 1470.96 \\
\hline $10 / 18 / 1122: 00$ & 34.831 & 38.156 & 1473.94 & 1470.97 \\
\hline $10 / 18 / 1122: 10$ & 34.813 & 38.156 & 1473.96 & 1470.97 \\
\hline $10 / 18 / 1122: 20$ & 34.789 & 38.154 & 1473.98 & 1470.97 \\
\hline $10 / 18 / 1122: 30$ & 34.768 & 38.154 & 1474.00 & 1470.97 \\
\hline $10 / 18 / 1122: 40$ & 35.059 & 38.152 & 1473.71 & 1470.97 \\
\hline $10 / 18 / 1122: 50$ & 35.019 & 38.152 & 1473.75 & 1470.97 \\
\hline $10 / 18 / 1123: 00$ & 36.046 & 38.149 & 1472.72 & 1470.97 \\
\hline $10 / 18 / 1123: 10$ & 35.237 & 38.152 & 1473.53 & 1470.97 \\
\hline 10/18/11 23:20 & 35.028 & 38.152 & 1473.74 & 1470.97 \\
\hline $10 / 18 / 1123: 30$ & 34.946 & 38.149 & 1473.82 & 1470.97 \\
\hline $10 / 18 / 1123: 40$ & 34.895 & 38.149 & 1473.87 & 1470.97 \\
\hline $10 / 18 / 1123: 50$ & 34.857 & 38.147 & 1473.91 & 1470.98 \\
\hline 10/19/11 0:00 & 34.827 & 38.144 & 1473.94 & 1470.98 \\
\hline 10/19/11 0:10 & 34.801 & 38.142 & 1473.97 & 1470.98 \\
\hline 10/19/11 0:20 & 34.777 & 38.14 & 1473.99 & 1470.98 \\
\hline
\end{tabular}


TABLE S1.3 Automatically recorded groundwater levels in the Klassen wells, September 15, 2011, to March 13, 2012.

\begin{tabular}{|c|c|c|c|c|}
\hline \multicolumn{3}{|c|}{ Reference Elevation (ft AMSL) } & 1508.77 & \multirow{2}{*}{$\begin{array}{l}1509.12 \\
\text { Elevation in Klassen } \\
\text { Test Well (ft AMSL) }\end{array}$} \\
\hline Date and Time & $\begin{array}{l}\text { Depth in Klassen } \\
\text { Domestic Well } \\
\text { (ft BGL) }\end{array}$ & $\begin{array}{c}\text { Depth in Klassen } \\
\text { Test Well } \\
\text { (ft BGL) }\end{array}$ & $\begin{array}{l}\text { Elevation in Klassen } \\
\text { Domestic Well } \\
\text { (ft AMSL) }\end{array}$ & \\
\hline 10/19/11 0:30 & 34.759 & 38.137 & 1474.01 & 1470.99 \\
\hline 10/19/11 0:40 & 34.74 & 38.133 & 1474.03 & 1470.99 \\
\hline 10/19/11 0:50 & 34.726 & 38.13 & 1474.04 & 1470.99 \\
\hline 10/19/11 1:00 & 34.709 & 38.128 & 1474.06 & 1470.99 \\
\hline 10/19/11 1:10 & 34.698 & 38.128 & 1474.07 & 1470.99 \\
\hline 10/19/11 1:20 & 34.684 & 38.126 & 1474.09 & 1471.00 \\
\hline 10/19/11 1:30 & 37.993 & 38.121 & 1470.78 & 1471.00 \\
\hline 10/19/11 1:40 & 34.829 & 38.119 & 1473.94 & 1471.00 \\
\hline 10/19/11 1:50 & 34.754 & 38.114 & 1474.02 & 1471.01 \\
\hline $10 / 19 / 112: 00$ & 34.719 & 38.109 & 1474.05 & 1471.01 \\
\hline $10 / 19 / 112: 10$ & 34.695 & 38.107 & 1474.07 & 1471.02 \\
\hline $10 / 19 / 112: 20$ & 34.677 & 38.102 & 1474.09 & 1471.02 \\
\hline $10 / 19 / 112: 30$ & 34.66 & 38.1 & 1474.11 & 1471.02 \\
\hline $10 / 19 / 112: 40$ & 34.646 & 38.095 & 1474.12 & 1471.03 \\
\hline $10 / 19 / 112: 50$ & 34.634 & 38.091 & 1474.14 & 1471.03 \\
\hline 10/19/11 3:00 & 34.623 & 38.088 & 1474.15 & 1471.03 \\
\hline 10/19/11 3:10 & 34.611 & 38.083 & 1474.16 & 1471.04 \\
\hline 10/19/11 3:20 & 34.599 & 38.081 & 1474.17 & 1471.04 \\
\hline 10/19/11 3:30 & 34.59 & 38.076 & 1474.18 & 1471.05 \\
\hline $10 / 19 / 113: 40$ & 34.58 & 38.076 & 1474.19 & 1471.05 \\
\hline $10 / 19 / 113: 50$ & 34.571 & 38.074 & 1474.20 & 1471.05 \\
\hline 10/19/11 4:00 & 34.564 & 38.069 & 1474.21 & 1471.05 \\
\hline 10/19/11 4:10 & 34.555 & 38.069 & 1474.21 & 1471.05 \\
\hline 10/19/11 4:20 & 34.545 & 38.067 & 1474.22 & 1471.06 \\
\hline 10/19/11 4:30 & 34.538 & 38.065 & 1474.23 & 1471.06 \\
\hline 10/19/11 4:40 & 34.531 & 38.062 & 1474.24 & 1471.06 \\
\hline 10/19/11 4:50 & 34.522 & 38.058 & 1474.25 & 1471.06 \\
\hline 10/19/11 5:00 & 34.515 & 38.053 & 1474.25 & 1471.07 \\
\hline 10/19/11 5:10 & 34.508 & 38.048 & 1474.26 & 1471.07 \\
\hline 10/19/11 5:20 & 34.501 & 38.041 & 1474.27 & 1471.08 \\
\hline 10/19/11 5:30 & 34.888 & 38.037 & 1473.88 & 1471.09 \\
\hline 10/19/11 5:40 & 34.616 & 38.032 & 1474.15 & 1471.09 \\
\hline $10 / 19 / 115: 50$ & 34.569 & 38.03 & 1474.20 & 1471.09 \\
\hline 10/19/11 6:00 & 34.604 & 38.027 & 1474.17 & 1471.10 \\
\hline 10/19/11 6:10 & 34.648 & 38.025 & 1474.12 & 1471.10 \\
\hline $10 / 19 / 116: 20$ & 34.857 & 38.025 & 1473.91 & 1471.10 \\
\hline 10/19/11 6:30 & 34.726 & 38.022 & 1474.04 & 1471.10 \\
\hline 10/19/11 6:40 & 34.674 & 38.018 & 1474.10 & 1471.10 \\
\hline
\end{tabular}


TABLE S1.3 Automatically recorded groundwater levels in the Klassen wells, September 15, 2011, to March 13, 2012.

\begin{tabular}{|c|c|c|c|c|}
\hline \multicolumn{3}{|c|}{ Reference Elevation (ft AMSL) } & 1508.77 & \multirow{2}{*}{$\begin{array}{l}1509.12 \\
\text { Elevation in Klassen } \\
\text { Test Well (ft AMSL) }\end{array}$} \\
\hline Date and Time & $\begin{array}{l}\text { Depth in Klassen } \\
\text { Domestic Well } \\
\text { (ft BGL) }\end{array}$ & $\begin{array}{c}\text { Depth in Klassen } \\
\text { Test Well } \\
\text { (ft BGL) }\end{array}$ & $\begin{array}{l}\text { Elevation in Klassen } \\
\text { Domestic Well } \\
\text { (ft AMSL) }\end{array}$ & \\
\hline 10/19/11 6:50 & 34.641 & 38.013 & 1474.13 & 1471.11 \\
\hline 10/19/11 7:00 & 34.613 & 38.013 & 1474.16 & 1471.11 \\
\hline 10/19/11 7:10 & 34.594 & 38.008 & 1474.18 & 1471.11 \\
\hline 10/19/11 7:20 & 34.604 & 38.006 & 1474.17 & 1471.12 \\
\hline 10/19/11 7:30 & 34.613 & 37.999 & 1474.16 & 1471.12 \\
\hline 10/19/11 7:40 & 34.594 & 37.999 & 1474.18 & 1471.12 \\
\hline 10/19/11 7:50 & 34.601 & 37.994 & 1474.17 & 1471.13 \\
\hline 10/19/11 8:00 & 34.587 & 37.992 & 1474.18 & 1471.13 \\
\hline 10/19/11 8:10 & 34.904 & 37.994 & 1473.87 & 1471.13 \\
\hline 10/19/11 8:20 & 34.653 & 37.99 & 1474.12 & 1471.13 \\
\hline 10/19/11 8:30 & 34.611 & 37.987 & 1474.16 & 1471.14 \\
\hline 10/19/11 8:40 & 34.583 & 37.985 & 1474.19 & 1471.14 \\
\hline 10/19/11 8:50 & 34.562 & 37.983 & 1474.21 & 1471.14 \\
\hline 10/19/11 9:00 & 34.545 & 37.98 & 1474.22 & 1471.14 \\
\hline 10/19/11 9:10 & 34.531 & 37.98 & 1474.24 & 1471.14 \\
\hline 10/19/11 9:20 & 34.515 & 37.976 & 1474.25 & 1471.15 \\
\hline 10/19/11 9:30 & 34.503 & 37.978 & 1474.27 & 1471.14 \\
\hline 10/19/11 9:40 & 35.028 & 37.976 & 1473.74 & 1471.15 \\
\hline 10/19/11 9:50 & 34.62 & 37.973 & 1474.15 & 1471.15 \\
\hline 10/19/11 10:00 & 34.566 & 37.971 & 1474.20 & 1471.15 \\
\hline 10/19/11 10:10 & 34.538 & 37.971 & 1474.23 & 1471.15 \\
\hline 10/19/11 10:20 & 34.517 & 37.971 & 1474.25 & 1471.15 \\
\hline 10/19/11 10:30 & 34.503 & 37.969 & 1474.27 & 1471.15 \\
\hline 10/19/11 10:40 & 34.489 & 37.966 & 1474.28 & 1471.16 \\
\hline 10/19/11 10:50 & 34.475 & 37.966 & 1474.29 & 1471.16 \\
\hline 10/19/11 11:00 & 34.465 & 37.964 & 1474.30 & 1471.16 \\
\hline 10/19/11 11:10 & 34.454 & 37.961 & 1474.32 & 1471.16 \\
\hline 10/19/11 11:20 & 34.444 & 37.957 & 1474.33 & 1471.17 \\
\hline 10/19/11 11:30 & 35.223 & 37.957 & 1473.55 & 1471.17 \\
\hline 10/19/11 11:40 & 34.59 & 37.959 & 1474.18 & 1471.16 \\
\hline 10/19/11 11:50 & 34.531 & 37.961 & 1474.24 & 1471.16 \\
\hline 10/19/11 12:00 & 34.501 & 37.959 & 1474.27 & 1471.16 \\
\hline 10/19/11 12:10 & 34.48 & 37.961 & 1474.29 & 1471.16 \\
\hline $10 / 19 / 11$ 12:20 & 34.461 & 37.959 & 1474.31 & 1471.16 \\
\hline 10/19/11 12:30 & 34.447 & 37.964 & 1474.32 & 1471.16 \\
\hline 10/19/11 12:40 & 34.435 & 37.966 & 1474.33 & 1471.16 \\
\hline $10 / 19 / 11$ 12:50 & 35.329 & 37.966 & 1473.44 & 1471.16 \\
\hline 10/19/11 13:00 & 34.576 & 37.971 & 1474.19 & 1471.15 \\
\hline
\end{tabular}


TABLE S1.3 Automatically recorded groundwater levels in the Klassen wells, September 15, 2011, to March 13, 2012.

\begin{tabular}{|c|c|c|c|c|}
\hline \multicolumn{3}{|c|}{ Reference Elevation (ft AMSL) } & 1508.77 & \multirow{2}{*}{$\begin{array}{l}1509.12 \\
\text { Elevation in Klassen } \\
\text { Test Well (ft AMSL) }\end{array}$} \\
\hline Date and Time & $\begin{array}{l}\text { Depth in Klassen } \\
\text { Domestic Well } \\
(\mathrm{ft} \mathrm{BGL})\end{array}$ & $\begin{array}{c}\text { Depth in Klassen } \\
\text { Test Well } \\
\text { (ft BGL) }\end{array}$ & $\begin{array}{l}\text { Elevation in Klassen } \\
\text { Domestic Well } \\
\text { (ft AMSL) }\end{array}$ & \\
\hline 10/19/11 13:10 & 34.51 & 37.971 & 1474.26 & 1471.15 \\
\hline $10 / 19 / 1113: 20$ & 34.482 & 37.971 & 1474.29 & 1471.15 \\
\hline 10/19/11 13:30 & 34.461 & 37.971 & 1474.31 & 1471.15 \\
\hline $10 / 19 / 11$ 13:40 & 34.442 & 37.969 & 1474.33 & 1471.15 \\
\hline 10/19/11 13:50 & 34.686 & 37.966 & 1474.08 & 1471.16 \\
\hline 10/19/11 14:00 & 35.073 & 37.961 & 1473.70 & 1471.16 \\
\hline 10/19/11 14:10 & 38.687 & 37.954 & 1470.08 & 1471.17 \\
\hline 10/19/11 14:20 & 38.769 & 37.947 & 1470.00 & 1471.18 \\
\hline 10/19/11 14:30 & 34.733 & 37.943 & 1474.04 & 1471.18 \\
\hline $10 / 19 / 1114: 40$ & 34.836 & 37.938 & 1473.93 & 1471.18 \\
\hline 10/19/11 14:50 & 35.195 & 37.929 & 1473.57 & 1471.19 \\
\hline 10/19/11 15:00 & 38.509 & 37.919 & 1470.26 & 1471.20 \\
\hline 10/19/11 15:10 & 34.794 & 37.912 & 1473.98 & 1471.21 \\
\hline $10 / 19 / 1115: 20$ & 34.888 & 37.905 & 1473.88 & 1471.22 \\
\hline 10/19/11 15:30 & 35.122 & 37.898 & 1473.65 & 1471.22 \\
\hline 10/19/11 15:40 & 35.075 & 37.891 & 1473.69 & 1471.23 \\
\hline 10/19/11 15:50 & 34.855 & 37.891 & 1473.91 & 1471.23 \\
\hline 10/19/11 16:00 & 34.77 & 37.886 & 1474.00 & 1471.24 \\
\hline 10/19/11 16:10 & 34.712 & 37.884 & 1474.06 & 1471.24 \\
\hline $10 / 19 / 11$ 16:20 & 34.669 & 37.879 & 1474.10 & 1471.24 \\
\hline 10/19/11 16:30 & 36.897 & 37.875 & 1471.87 & 1471.25 \\
\hline 10/19/11 16:40 & 34.735 & 37.872 & 1474.03 & 1471.25 \\
\hline 10/19/11 16:50 & 34.665 & 37.868 & 1474.10 & 1471.25 \\
\hline 10/19/11 17:00 & 35.026 & 37.863 & 1473.74 & 1471.26 \\
\hline 10/19/11 17:10 & 38.431 & 37.863 & 1470.34 & 1471.26 \\
\hline 10/19/11 17:20 & 35.064 & 37.863 & 1473.71 & 1471.26 \\
\hline 10/19/11 17:30 & 34.963 & 37.865 & 1473.81 & 1471.26 \\
\hline 10/19/11 17:40 & 36.03 & 37.865 & 1472.74 & 1471.26 \\
\hline 10/19/11 17:50 & 35.038 & 37.868 & 1473.73 & 1471.25 \\
\hline 10/19/11 18:00 & 34.988 & 37.868 & 1473.78 & 1471.25 \\
\hline 10/19/11 18:10 & 34.991 & 37.868 & 1473.78 & 1471.25 \\
\hline 10/19/11 18:20 & 34.932 & 37.87 & 1473.84 & 1471.25 \\
\hline 10/19/11 18:30 & 34.876 & 37.868 & 1473.89 & 1471.25 \\
\hline 10/19/11 18:40 & 34.834 & 37.868 & 1473.94 & 1471.25 \\
\hline 10/19/11 18:50 & 34.791 & 37.865 & 1473.98 & 1471.26 \\
\hline 10/19/11 19:00 & 34.759 & 37.861 & 1474.01 & 1471.26 \\
\hline 10/19/11 19:10 & 34.728 & 37.858 & 1474.04 & 1471.26 \\
\hline 10/19/11 19:20 & 35.113 & 37.856 & 1473.66 & 1471.27 \\
\hline
\end{tabular}


TABLE S1.3 Automatically recorded groundwater levels in the Klassen wells, September 15, 2011, to March 13, 2012.

\begin{tabular}{|c|c|c|c|c|}
\hline \multicolumn{3}{|c|}{ Reference Elevation (ft AMSL) } & 1508.77 & \multirow{2}{*}{$\begin{array}{l}1509.12 \\
\text { Elevation in Klassen } \\
\text { Test Well (ft AMSL) }\end{array}$} \\
\hline Date and Time & $\begin{array}{l}\text { Depth in Klassen } \\
\text { Domestic Well } \\
(\mathrm{ft} \mathrm{BGL})\end{array}$ & $\begin{array}{c}\text { Depth in Klassen } \\
\text { Test Well } \\
\text { (ft BGL) }\end{array}$ & $\begin{array}{l}\text { Elevation in Klassen } \\
\text { Domestic Well } \\
\text { (ft AMSL) }\end{array}$ & \\
\hline 10/19/11 19:30 & 34.953 & 37.856 & 1473.82 & 1471.27 \\
\hline 10/19/11 19:40 & 34.897 & 37.854 & 1473.87 & 1471.27 \\
\hline 10/19/11 19:50 & 34.838 & 37.854 & 1473.93 & 1471.27 \\
\hline $10 / 19 / 1120: 00$ & 34.791 & 37.851 & 1473.98 & 1471.27 \\
\hline 10/19/11 20:10 & 34.787 & 37.851 & 1473.98 & 1471.27 \\
\hline $10 / 19 / 1120: 20$ & 34.777 & 37.851 & 1473.99 & 1471.27 \\
\hline $10 / 19 / 1120: 30$ & 34.775 & 37.856 & 1473.99 & 1471.27 \\
\hline $10 / 19 / 1120: 40$ & 34.78 & 37.858 & 1473.99 & 1471.26 \\
\hline $10 / 19 / 1120: 50$ & 34.773 & 37.861 & 1474.00 & 1471.26 \\
\hline 10/19/11 21:00 & 34.754 & 37.865 & 1474.02 & 1471.26 \\
\hline $10 / 19 / 1121: 10$ & 34.723 & 37.868 & 1474.05 & 1471.25 \\
\hline 10/19/11 21:20 & 34.695 & 37.868 & 1474.07 & 1471.25 \\
\hline 10/19/11 21:30 & 35.427 & 37.87 & 1473.34 & 1471.25 \\
\hline 10/19/11 21:40 & 34.871 & 37.875 & 1473.90 & 1471.25 \\
\hline $10 / 19 / 1121: 50$ & 34.803 & 37.877 & 1473.97 & 1471.25 \\
\hline $10 / 19 / 1122: 00$ & 34.756 & 37.877 & 1474.01 & 1471.25 \\
\hline $10 / 19 / 1122: 10$ & 34.719 & 37.875 & 1474.05 & 1471.25 \\
\hline $10 / 19 / 1122: 20$ & 34.691 & 37.875 & 1474.08 & 1471.25 \\
\hline 10/19/11 22:30 & 34.662 & 37.872 & 1474.11 & 1471.25 \\
\hline $10 / 19 / 1122: 40$ & 34.681 & 37.872 & 1474.09 & 1471.25 \\
\hline $10 / 19 / 1122: 50$ & 34.911 & 37.872 & 1473.86 & 1471.25 \\
\hline 10/19/11 23:00 & 38.096 & 37.87 & 1470.67 & 1471.25 \\
\hline 10/19/11 23:10 & 36.443 & 37.872 & 1472.33 & 1471.25 \\
\hline $10 / 19 / 1123: 20$ & 35.139 & 37.877 & 1473.63 & 1471.25 \\
\hline 10/19/11 23:30 & 34.923 & 37.875 & 1473.85 & 1471.25 \\
\hline 10/19/11 23:40 & 34.857 & 37.872 & 1473.91 & 1471.25 \\
\hline 10/19/11 23:50 & 34.806 & 37.87 & 1473.96 & 1471.25 \\
\hline 10/20/11 0:00 & 34.761 & 37.87 & 1474.01 & 1471.25 \\
\hline 10/20/11 0:10 & 34.723 & 37.868 & 1474.05 & 1471.25 \\
\hline 10/20/11 0:20 & 34.693 & 37.868 & 1474.08 & 1471.25 \\
\hline 10/20/11 0:30 & 34.667 & 37.865 & 1474.10 & 1471.26 \\
\hline 10/20/11 0:40 & 34.644 & 37.863 & 1474.13 & 1471.26 \\
\hline $10 / 20 / 110: 50$ & 34.623 & 37.861 & 1474.15 & 1471.26 \\
\hline 10/20/11 1:00 & 34.601 & 37.856 & 1474.17 & 1471.27 \\
\hline 10/20/11 1:10 & 34.585 & 37.854 & 1474.18 & 1471.27 \\
\hline $10 / 20 / 111: 20$ & 34.569 & 37.849 & 1474.20 & 1471.27 \\
\hline 10/20/11 1:30 & 34.552 & 37.846 & 1474.22 & 1471.28 \\
\hline 10/20/11 1:40 & 34.538 & 37.842 & 1474.23 & 1471.28 \\
\hline
\end{tabular}


TABLE S1.3 Automatically recorded groundwater levels in the Klassen wells, September 15, 2011, to March 13, 2012.

\begin{tabular}{|c|c|c|c|c|}
\hline \multicolumn{3}{|c|}{ Reference Elevation (ft AMSL) } & 1508.77 & \multirow{2}{*}{$\begin{array}{l}1509.12 \\
\text { Elevation in Klassen } \\
\text { Test Well (ft AMSL) }\end{array}$} \\
\hline Date and Time & $\begin{array}{l}\text { Depth in Klassen } \\
\text { Domestic Well } \\
\text { (ft BGL) }\end{array}$ & $\begin{array}{c}\text { Depth in Klassen } \\
\text { Test Well } \\
\text { (ft BGL) }\end{array}$ & $\begin{array}{l}\text { Elevation in Klassen } \\
\text { Domestic Well } \\
\text { (ft AMSL) }\end{array}$ & \\
\hline $10 / 20 / 111: 50$ & 34.524 & 37.839 & 1474.25 & 1471.28 \\
\hline $10 / 20 / 112: 00$ & 34.512 & 37.835 & 1474.26 & 1471.29 \\
\hline 10/20/11 2:10 & 34.501 & 37.835 & 1474.27 & 1471.29 \\
\hline $10 / 20 / 112: 20$ & 34.487 & 37.83 & 1474.28 & 1471.29 \\
\hline $10 / 20 / 112: 30$ & 34.477 & 37.83 & 1474.29 & 1471.29 \\
\hline $10 / 20 / 112: 40$ & 34.465 & 37.825 & 1474.30 & 1471.30 \\
\hline $10 / 20 / 112: 50$ & 34.456 & 37.823 & 1474.31 & 1471.30 \\
\hline 10/20/11 3:00 & 34.444 & 37.821 & 1474.33 & 1471.30 \\
\hline 10/20/11 3:10 & 34.435 & 37.821 & 1474.33 & 1471.30 \\
\hline $10 / 20 / 113: 20$ & 34.426 & 37.816 & 1474.34 & 1471.31 \\
\hline $10 / 20 / 113: 30$ & 34.416 & 37.814 & 1474.35 & 1471.31 \\
\hline 10/20/11 3:40 & 34.407 & 37.811 & 1474.36 & 1471.31 \\
\hline $10 / 20 / 113: 50$ & 34.4 & 37.811 & 1474.37 & 1471.31 \\
\hline $10 / 20 / 114: 00$ & 34.39 & 37.809 & 1474.38 & 1471.31 \\
\hline 10/20/11 4:10 & 34.383 & 37.807 & 1474.39 & 1471.32 \\
\hline $10 / 20 / 114: 20$ & 34.374 & 37.804 & 1474.40 & 1471.32 \\
\hline $10 / 20 / 114: 30$ & 34.367 & 37.8 & 1474.40 & 1471.32 \\
\hline $10 / 20 / 114: 40$ & 34.365 & 37.795 & 1474.40 & 1471.33 \\
\hline $10 / 20 / 114: 50$ & 34.383 & 37.793 & 1474.39 & 1471.33 \\
\hline $10 / 20 / 115: 00$ & 34.372 & 37.79 & 1474.40 & 1471.33 \\
\hline 10/20/11 5:10 & 34.36 & 37.785 & 1474.41 & 1471.34 \\
\hline $10 / 20 / 115: 20$ & 34.351 & 37.781 & 1474.42 & 1471.34 \\
\hline $10 / 20 / 115: 30$ & 34.341 & 37.781 & 1474.43 & 1471.34 \\
\hline 10/20/11 5:40 & 34.334 & 37.776 & 1474.44 & 1471.35 \\
\hline $10 / 20 / 115: 50$ & 34.327 & 37.771 & 1474.44 & 1471.35 \\
\hline $10 / 20 / 116: 00$ & 34.32 & 37.771 & 1474.45 & 1471.35 \\
\hline $10 / 20 / 116: 10$ & 34.798 & 37.769 & 1473.97 & 1471.35 \\
\hline $10 / 20 / 116: 20$ & 34.62 & 37.771 & 1474.15 & 1471.35 \\
\hline $10 / 20 / 116: 30$ & 34.555 & 37.767 & 1474.21 & 1471.36 \\
\hline $10 / 20 / 116: 40$ & 34.51 & 37.764 & 1474.26 & 1471.36 \\
\hline $10 / 20 / 116: 50$ & 35.324 & 37.762 & 1473.45 & 1471.36 \\
\hline 10/20/11 7:00 & 34.592 & 37.762 & 1474.18 & 1471.36 \\
\hline 10/20/11 7:10 & 34.526 & 37.762 & 1474.24 & 1471.36 \\
\hline $10 / 20 / 117: 20$ & 34.494 & 37.76 & 1474.28 & 1471.36 \\
\hline 10/20/11 7:30 & 34.489 & 37.755 & 1474.28 & 1471.37 \\
\hline 10/20/11 7:40 & 34.463 & 37.755 & 1474.31 & 1471.37 \\
\hline $10 / 20 / 117: 50$ & 34.442 & 37.75 & 1474.33 & 1471.37 \\
\hline $10 / 20 / 118: 00$ & 34.449 & 37.746 & 1474.32 & 1471.38 \\
\hline
\end{tabular}


TABLE S1.3 Automatically recorded groundwater levels in the Klassen wells, September 15, 2011, to March 13, 2012.

\begin{tabular}{|c|c|c|c|c|}
\hline \multicolumn{3}{|c|}{ Reference Elevation (ft AMSL) } & 1508.77 & \multirow{2}{*}{$\begin{array}{l}1509.12 \\
\text { Elevation in Klassen } \\
\text { Test Well (ft AMSL) }\end{array}$} \\
\hline Date and Time & $\begin{array}{l}\text { Depth in Klassen } \\
\text { Domestic Well } \\
(\mathrm{ft} \mathrm{BGL})\end{array}$ & $\begin{array}{c}\text { Depth in Klassen } \\
\text { Test Well } \\
\text { (ft BGL) }\end{array}$ & $\begin{array}{l}\text { Elevation in Klassen } \\
\text { Domestic Well } \\
\text { (ft AMSL) }\end{array}$ & \\
\hline 10/20/11 8:10 & 34.43 & 37.746 & 1474.34 & 1471.38 \\
\hline $10 / 20 / 118: 20$ & 34.414 & 37.741 & 1474.36 & 1471.38 \\
\hline 10/20/11 8:30 & 35.821 & 37.739 & 1472.95 & 1471.38 \\
\hline 10/20/11 8:40 & 34.501 & 37.739 & 1474.27 & 1471.38 \\
\hline 10/20/11 8:50 & 34.449 & 37.739 & 1474.32 & 1471.38 \\
\hline 10/20/11 9:00 & 34.419 & 37.739 & 1474.35 & 1471.38 \\
\hline 10/20/11 9:10 & 34.397 & 37.736 & 1474.37 & 1471.39 \\
\hline 10/20/11 9:20 & 34.383 & 37.739 & 1474.39 & 1471.38 \\
\hline 10/20/11 9:30 & 34.367 & 37.736 & 1474.40 & 1471.39 \\
\hline 10/20/11 9:40 & 34.355 & 37.734 & 1474.41 & 1471.39 \\
\hline 10/20/11 9:50 & 34.536 & 37.734 & 1474.23 & 1471.39 \\
\hline 10/20/11 10:00 & 34.437 & 37.734 & 1474.33 & 1471.39 \\
\hline 10/20/11 10:10 & 34.397 & 37.734 & 1474.37 & 1471.39 \\
\hline $10 / 20 / 1110: 20$ & 34.376 & 37.734 & 1474.39 & 1471.39 \\
\hline 10/20/11 10:30 & 34.358 & 37.736 & 1474.41 & 1471.39 \\
\hline $10 / 20 / 11$ 10:40 & 34.344 & 37.734 & 1474.43 & 1471.39 \\
\hline $10 / 20 / 11$ 10:50 & 34.329 & 37.732 & 1474.44 & 1471.39 \\
\hline 10/20/11 11:00 & 34.32 & 37.732 & 1474.45 & 1471.39 \\
\hline 10/20/11 11:10 & 34.308 & 37.732 & 1474.46 & 1471.39 \\
\hline $10 / 20 / 11$ 11:20 & 34.299 & 37.736 & 1474.47 & 1471.39 \\
\hline 10/20/11 11:30 & 34.29 & 37.743 & 1474.48 & 1471.38 \\
\hline 10/20/11 11:40 & 34.283 & 37.748 & 1474.49 & 1471.37 \\
\hline 10/20/11 11:50 & 34.273 & 37.755 & 1474.50 & 1471.37 \\
\hline $10 / 20 / 11$ 12:00 & 34.266 & 37.76 & 1474.50 & 1471.36 \\
\hline 10/20/11 12:10 & 38.889 & 37.767 & 1469.88 & 1471.36 \\
\hline $10 / 20 / 11$ 12:20 & 34.451 & 37.783 & 1474.32 & 1471.34 \\
\hline $10 / 20 / 11$ 12:30 & 34.367 & 37.797 & 1474.40 & 1471.33 \\
\hline $10 / 20 / 11$ 12:40 & 34.332 & 37.807 & 1474.44 & 1471.32 \\
\hline $10 / 20 / 11$ 12:50 & 34.308 & 37.818 & 1474.46 & 1471.30 \\
\hline 10/20/11 13:00 & 34.292 & 37.83 & 1474.48 & 1471.29 \\
\hline 10/20/11 13:10 & 34.278 & 37.837 & 1474.49 & 1471.29 \\
\hline 10/20/11 13:20 & 34.266 & 37.846 & 1474.50 & 1471.28 \\
\hline 10/20/11 13:30 & 34.254 & 37.854 & 1474.52 & 1471.27 \\
\hline $10 / 20 / 11$ 13:40 & 35.64 & 37.863 & 1473.13 & 1471.26 \\
\hline 10/20/11 13:50 & 34.348 & 37.872 & 1474.42 & 1471.25 \\
\hline 10/20/11 14:00 & 34.299 & 37.882 & 1474.47 & 1471.24 \\
\hline 10/20/11 14:10 & 34.283 & 37.886 & 1474.49 & 1471.24 \\
\hline $10 / 20 / 11$ 14:20 & 34.461 & 37.893 & 1474.31 & 1471.23 \\
\hline
\end{tabular}


TABLE S1.3 Automatically recorded groundwater levels in the Klassen wells, September 15, 2011, to March 13, 2012.

\begin{tabular}{|c|c|c|c|c|}
\hline \multicolumn{3}{|c|}{ Reference Elevation (ft AMSL) } & 1508.77 & \multirow{2}{*}{$\begin{array}{l}1509.12 \\
\text { Elevation in Klassen } \\
\text { Test Well (ft AMSL) }\end{array}$} \\
\hline Date and Time & $\begin{array}{l}\text { Depth in Klassen } \\
\text { Domestic Well } \\
\text { (ft BGL) }\end{array}$ & $\begin{array}{c}\text { Depth in Klassen } \\
\text { Test Well } \\
\text { (ft BGL) }\end{array}$ & $\begin{array}{l}\text { Elevation in Klassen } \\
\text { Domestic Well } \\
\text { (ft AMSL) }\end{array}$ & \\
\hline $10 / 20 / 1114: 30$ & 34.358 & 37.9 & 1474.41 & 1471.22 \\
\hline $10 / 20 / 1114: 40$ & 34.32 & 37.908 & 1474.45 & 1471.21 \\
\hline $10 / 20 / 1114: 50$ & 34.32 & 37.915 & 1474.45 & 1471.21 \\
\hline $10 / 20 / 1115: 00$ & 34.379 & 37.919 & 1474.39 & 1471.20 \\
\hline $10 / 20 / 1115: 10$ & 34.503 & 37.929 & 1474.27 & 1471.19 \\
\hline $10 / 20 / 1115: 20$ & 34.796 & 37.94 & 1473.97 & 1471.18 \\
\hline $10 / 20 / 1115: 30$ & 34.836 & 37.954 & 1473.93 & 1471.17 \\
\hline $10 / 20 / 1115: 40$ & 34.918 & 37.966 & 1473.85 & 1471.16 \\
\hline $10 / 20 / 1115: 50$ & 35 & 37.978 & 1473.77 & 1471.14 \\
\hline 10/20/11 16:00 & 35.078 & 37.992 & 1473.69 & 1471.13 \\
\hline $10 / 20 / 1116: 10$ & 35.153 & 38.004 & 1473.62 & 1471.12 \\
\hline $10 / 20 / 1116: 20$ & 35.225 & 38.018 & 1473.54 & 1471.10 \\
\hline $10 / 20 / 1116: 30$ & 35.296 & 38.032 & 1473.47 & 1471.09 \\
\hline $10 / 20 / 1116: 40$ & 35.361 & 38.046 & 1473.41 & 1471.08 \\
\hline $10 / 20 / 1116: 50$ & 35.603 & 38.06 & 1473.17 & 1471.06 \\
\hline 10/20/11 17:00 & 35.584 & 38.076 & 1473.19 & 1471.05 \\
\hline $10 / 20 / 11$ 17:10 & 36.168 & 38.093 & 1472.60 & 1471.03 \\
\hline $10 / 20 / 11$ 17:20 & 37.064 & 38.109 & 1471.71 & 1471.01 \\
\hline $10 / 20 / 11$ 17:30 & 37.613 & 38.128 & 1471.16 & 1470.99 \\
\hline $10 / 20 / 11$ 17:40 & 42.407 & 38.152 & 1466.36 & 1470.97 \\
\hline $10 / 20 / 11$ 17:50 & 40.455 & 38.173 & 1468.31 & 1470.95 \\
\hline 10/20/11 18:00 & 38.481 & 38.191 & 1470.29 & 1470.93 \\
\hline $10 / 20 / 11$ 18:10 & 41.773 & 38.21 & 1467.00 & 1470.91 \\
\hline $10 / 20 / 11$ 18:20 & 38.607 & 38.229 & 1470.16 & 1470.89 \\
\hline $10 / 20 / 11$ 18:30 & 41.893 & 38.248 & 1466.88 & 1470.87 \\
\hline $10 / 20 / 11$ 18:40 & 38.757 & 38.269 & 1470.01 & 1470.85 \\
\hline $10 / 20 / 11$ 18:50 & 42.514 & 38.295 & 1466.26 & 1470.83 \\
\hline 10/20/11 19:00 & 39.33 & 38.325 & 1469.44 & 1470.80 \\
\hline 10/20/11 19:10 & 43.045 & 38.356 & 1465.72 & 1470.77 \\
\hline 10/20/11 19:20 & 37.7 & 38.379 & 1471.07 & 1470.74 \\
\hline 10/20/11 19:30 & 38.905 & 38.403 & 1469.86 & 1470.72 \\
\hline $10 / 20 / 11$ 19:40 & 37.418 & 38.424 & 1471.35 & 1470.70 \\
\hline 10/20/11 19:50 & 37.294 & 38.445 & 1471.48 & 1470.68 \\
\hline $10 / 20 / 1120: 00$ & 37.226 & 38.464 & 1471.54 & 1470.66 \\
\hline $10 / 20 / 1120: 10$ & 37.181 & 38.485 & 1471.59 & 1470.64 \\
\hline $10 / 20 / 1120: 20$ & 37.155 & 38.503 & 1471.61 & 1470.62 \\
\hline $10 / 20 / 1120: 30$ & 37.153 & 38.522 & 1471.62 & 1470.60 \\
\hline $10 / 20 / 1120: 40$ & 37.174 & 38.546 & 1471.60 & 1470.58 \\
\hline
\end{tabular}


TABLE S1.3 Automatically recorded groundwater levels in the Klassen wells, September 15, 2011, to March 13, 2012.

\begin{tabular}{|c|c|c|c|c|}
\hline \multicolumn{3}{|c|}{ Reference Elevation (ft AMSL) } & 1508.77 & \multirow{2}{*}{$\begin{array}{l}1509.12 \\
\text { Elevation in Klassen } \\
\text { Test Well (ft AMSL) }\end{array}$} \\
\hline Date and Time & $\begin{array}{l}\text { Depth in Klassen } \\
\text { Domestic Well } \\
\text { (ft BGL) }\end{array}$ & $\begin{array}{c}\text { Depth in Klassen } \\
\text { Test Well } \\
\text { (ft BGL) }\end{array}$ & $\begin{array}{l}\text { Elevation in Klassen } \\
\text { Domestic Well } \\
\text { (ft AMSL) }\end{array}$ & \\
\hline $10 / 20 / 1120: 50$ & 37.179 & 38.567 & 1471.59 & 1470.56 \\
\hline 10/20/11 21:00 & 37.214 & 38.59 & 1471.56 & 1470.53 \\
\hline $10 / 20 / 1121: 10$ & 37.174 & 38.611 & 1471.60 & 1470.51 \\
\hline $10 / 20 / 1121: 20$ & 37.043 & 38.623 & 1471.73 & 1470.50 \\
\hline 10/20/11 21:30 & 36.909 & 38.633 & 1471.86 & 1470.49 \\
\hline 10/20/11 21:40 & 36.78 & 38.64 & 1471.99 & 1470.48 \\
\hline $10 / 20 / 1121: 50$ & 36.693 & 38.647 & 1472.08 & 1470.48 \\
\hline $10 / 20 / 1122: 00$ & 36.614 & 38.654 & 1472.16 & 1470.47 \\
\hline $10 / 20 / 1122: 10$ & 36.529 & 38.661 & 1472.24 & 1470.46 \\
\hline $10 / 20 / 1122: 20$ & 36.492 & 38.67 & 1472.28 & 1470.45 \\
\hline $10 / 20 / 1122: 30$ & 36.557 & 38.682 & 1472.21 & 1470.44 \\
\hline $10 / 20 / 1122: 40$ & 37.782 & 38.698 & 1470.99 & 1470.42 \\
\hline $10 / 20 / 1122: 50$ & 37.066 & 38.712 & 1471.70 & 1470.41 \\
\hline $10 / 20 / 1123: 00$ & 39.421 & 38.731 & 1469.35 & 1470.39 \\
\hline $10 / 20 / 1123: 10$ & 38.323 & 38.747 & 1470.45 & 1470.38 \\
\hline $10 / 20 / 1123: 20$ & 37.226 & 38.764 & 1471.54 & 1470.36 \\
\hline $10 / 20 / 1123: 30$ & 37.174 & 38.783 & 1471.60 & 1470.34 \\
\hline $10 / 20 / 1123: 40$ & 37.158 & 38.797 & 1471.61 & 1470.33 \\
\hline $10 / 20 / 1123: 50$ & 37.158 & 38.813 & 1471.61 & 1470.31 \\
\hline $10 / 21 / 110: 00$ & 37.165 & 38.825 & 1471.60 & 1470.30 \\
\hline 10/21/11 0:10 & 37.179 & 38.839 & 1471.59 & 1470.28 \\
\hline 10/21/11 0:20 & 37.193 & 38.853 & 1471.58 & 1470.27 \\
\hline $10 / 21 / 110: 30$ & 37.207 & 38.867 & 1471.56 & 1470.26 \\
\hline 10/21/11 0:40 & 37.226 & 38.879 & 1471.54 & 1470.24 \\
\hline $10 / 21 / 11$ 0:50 & 37.242 & 38.891 & 1471.53 & 1470.23 \\
\hline 10/21/11 1:00 & 37.238 & 38.905 & 1471.53 & 1470.22 \\
\hline 10/21/11 1:10 & 37.132 & 38.912 & 1471.64 & 1470.21 \\
\hline 10/21/11 1:20 & 37.01 & 38.916 & 1471.76 & 1470.21 \\
\hline 10/21/11 1:30 & 36.897 & 38.919 & 1471.87 & 1470.20 \\
\hline 10/21/11 1:40 & 36.853 & 38.921 & 1471.92 & 1470.20 \\
\hline 10/21/11 1:50 & 36.827 & 38.928 & 1471.94 & 1470.19 \\
\hline $10 / 21 / 112: 00$ & 36.733 & 38.93 & 1472.04 & 1470.19 \\
\hline 10/21/11 2:10 & 36.651 & 38.933 & 1472.12 & 1470.19 \\
\hline $10 / 21 / 112: 20$ & 36.588 & 38.933 & 1472.18 & 1470.19 \\
\hline $10 / 21 / 112: 30$ & 36.532 & 38.938 & 1472.24 & 1470.18 \\
\hline $10 / 21 / 112: 40$ & 36.457 & 38.935 & 1472.31 & 1470.19 \\
\hline $10 / 21 / 112: 50$ & 36.396 & 38.935 & 1472.37 & 1470.19 \\
\hline 10/21/11 3:00 & 36.344 & 38.938 & 1472.43 & 1470.18 \\
\hline
\end{tabular}


TABLE S1.3 Automatically recorded groundwater levels in the Klassen wells, September 15, 2011, to March 13, 2012.

\begin{tabular}{|c|c|c|c|c|}
\hline \multicolumn{3}{|c|}{ Reference Elevation (ft AMSL) } & 1508.77 & \multirow{2}{*}{$\begin{array}{l}1509.12 \\
\text { Elevation in Klassen } \\
\text { Test Well (ft AMSL) }\end{array}$} \\
\hline Date and Time & $\begin{array}{l}\text { Depth in Klassen } \\
\text { Domestic Well } \\
(\mathrm{ft} \mathrm{BGL})\end{array}$ & $\begin{array}{c}\text { Depth in Klassen } \\
\text { Test Well } \\
\text { (ft BGL) }\end{array}$ & $\begin{array}{l}\text { Elevation in Klassen } \\
\text { Domestic Well } \\
\text { (ft AMSL) }\end{array}$ & \\
\hline 10/21/11 3:10 & 36.283 & 38.938 & 1472.49 & 1470.18 \\
\hline $10 / 21 / 113: 20$ & 36.281 & 38.94 & 1472.49 & 1470.18 \\
\hline 10/21/11 3:30 & 36.485 & 38.945 & 1472.28 & 1470.18 \\
\hline $10 / 21 / 113: 40$ & 36.33 & 38.945 & 1472.44 & 1470.18 \\
\hline 10/21/11 3:50 & 36.241 & 38.942 & 1472.53 & 1470.18 \\
\hline 10/21/11 4:00 & 36.17 & 38.94 & 1472.60 & 1470.18 \\
\hline 10/21/11 4:10 & 36.109 & 38.94 & 1472.66 & 1470.18 \\
\hline 10/21/11 4:20 & 36.056 & 38.938 & 1472.71 & 1470.18 \\
\hline 10/21/11 4:30 & 36.006 & 38.935 & 1472.76 & 1470.19 \\
\hline $10 / 21 / 114: 40$ & 35.959 & 38.933 & 1472.81 & 1470.19 \\
\hline $10 / 21 / 114: 50$ & 35.915 & 38.93 & 1472.85 & 1470.19 \\
\hline 10/21/11 5:00 & 35.875 & 38.928 & 1472.89 & 1470.19 \\
\hline 10/21/11 5:10 & 35.835 & 38.928 & 1472.93 & 1470.19 \\
\hline $10 / 21 / 115: 20$ & 35.798 & 38.921 & 1472.97 & 1470.20 \\
\hline 10/21/11 5:30 & 35.762 & 38.919 & 1473.01 & 1470.20 \\
\hline $10 / 21 / 115: 40$ & 35.727 & 38.916 & 1473.04 & 1470.21 \\
\hline $10 / 21 / 115: 50$ & 35.697 & 38.912 & 1473.07 & 1470.21 \\
\hline 10/21/11 6:00 & 35.666 & 38.909 & 1473.10 & 1470.21 \\
\hline 10/21/11 6:10 & 36.731 & 38.907 & 1472.04 & 1470.22 \\
\hline $10 / 21 / 116: 20$ & 35.821 & 38.902 & 1472.95 & 1470.22 \\
\hline $10 / 21 / 116: 30$ & 35.732 & 38.9 & 1473.04 & 1470.22 \\
\hline $10 / 21 / 116: 40$ & 35.673 & 38.895 & 1473.10 & 1470.23 \\
\hline $10 / 21 / 116: 50$ & 35.629 & 38.891 & 1473.14 & 1470.23 \\
\hline $10 / 21 / 117: 00$ & 35.594 & 38.884 & 1473.18 & 1470.24 \\
\hline 10/21/11 7:10 & 35.558 & 38.881 & 1473.21 & 1470.24 \\
\hline $10 / 21 / 117: 20$ & 35.528 & 38.879 & 1473.24 & 1470.24 \\
\hline 10/21/11 7:30 & 35.497 & 38.879 & 1473.27 & 1470.24 \\
\hline 10/21/11 7:40 & 35.469 & 38.877 & 1473.30 & 1470.25 \\
\hline $10 / 21 / 11$ 7:50 & 35.443 & 38.877 & 1473.33 & 1470.25 \\
\hline $10 / 21 / 118: 00$ & 35.418 & 38.874 & 1473.35 & 1470.25 \\
\hline 10/21/11 8:10 & 35.394 & 38.872 & 1473.38 & 1470.25 \\
\hline $10 / 21 / 118: 20$ & 37.399 & 38.872 & 1471.37 & 1470.25 \\
\hline $10 / 21 / 118: 30$ & 37.878 & 38.872 & 1470.89 & 1470.25 \\
\hline 10/21/11 8:40 & 37.231 & 38.877 & 1471.54 & 1470.25 \\
\hline $10 / 21 / 118: 50$ & 44.266 & 38.884 & 1464.50 & 1470.24 \\
\hline 10/21/11 9:00 & 39.222 & 38.891 & 1469.55 & 1470.23 \\
\hline 10/21/11 9:10 & 43.708 & 38.902 & 1465.06 & 1470.22 \\
\hline 10/21/11 9:20 & 39.925 & 38.912 & 1468.84 & 1470.21 \\
\hline
\end{tabular}


TABLE S1.3 Automatically recorded groundwater levels in the Klassen wells, September 15, 2011, to March 13, 2012.

\begin{tabular}{|c|c|c|c|c|}
\hline \multicolumn{3}{|c|}{ Reference Elevation (ft AMSL) } & 1508.77 & \multirow{2}{*}{$\begin{array}{l}1509.12 \\
\text { Elevation in Klassen } \\
\text { Test Well (ft AMSL) }\end{array}$} \\
\hline Date and Time & $\begin{array}{l}\text { Depth in Klassen } \\
\text { Domestic Well } \\
\text { (ft BGL) }\end{array}$ & $\begin{array}{c}\text { Depth in Klassen } \\
\text { Test Well } \\
\text { (ft BGL) }\end{array}$ & $\begin{array}{l}\text { Elevation in Klassen } \\
\text { Domestic Well } \\
\text { (ft AMSL) }\end{array}$ & \\
\hline 10/21/11 9:30 & 40.87 & 38.921 & 1467.90 & 1470.20 \\
\hline 10/21/11 9:40 & 42.43 & 38.933 & 1466.34 & 1470.19 \\
\hline 10/21/11 9:50 & 43.434 & 38.947 & 1465.34 & 1470.18 \\
\hline 10/21/11 10:00 & 38.992 & 38.961 & 1469.78 & 1470.16 \\
\hline 10/21/11 10:10 & 43.349 & 38.973 & 1465.42 & 1470.15 \\
\hline 10/21/11 10:20 & 37.803 & 38.987 & 1470.97 & 1470.14 \\
\hline $10 / 21 / 1110: 30$ & 37.273 & 38.996 & 1471.50 & 1470.13 \\
\hline $10 / 21 / 11$ 10:40 & 37.148 & 39.003 & 1471.62 & 1470.12 \\
\hline $10 / 21 / 1110: 50$ & 37.087 & 39.015 & 1471.68 & 1470.11 \\
\hline 10/21/11 11:00 & 37.064 & 39.027 & 1471.71 & 1470.10 \\
\hline 10/21/11 11:10 & 37.055 & 39.038 & 1471.71 & 1470.08 \\
\hline 10/21/11 11:20 & 37.05 & 39.05 & 1471.72 & 1470.07 \\
\hline 10/21/11 11:30 & 37.055 & 39.057 & 1471.71 & 1470.07 \\
\hline 10/21/11 11:40 & 44.763 & 39.067 & 1464.01 & 1470.06 \\
\hline $10 / 21 / 1111: 50$ & 40.561 & 39.083 & 1468.21 & 1470.04 \\
\hline $10 / 21 / 1112: 00$ & 39.29 & 39.102 & 1469.48 & 1470.02 \\
\hline $10 / 21 / 11$ 12:10 & 41.476 & 39.121 & 1467.29 & 1470.00 \\
\hline $10 / 21 / 1112: 20$ & 43.903 & 39.142 & 1464.87 & 1469.98 \\
\hline $10 / 21 / 11$ 12:30 & 41.593 & 39.16 & 1467.18 & 1469.96 \\
\hline $10 / 21 / 1112: 40$ & 40.061 & 39.174 & 1468.71 & 1469.95 \\
\hline $10 / 21 / 1112: 50$ & 39.236 & 39.186 & 1469.53 & 1469.94 \\
\hline 10/21/11 13:00 & 44.492 & 39.196 & 1464.28 & 1469.93 \\
\hline $10 / 21 / 11$ 13:10 & 44.827 & 39.207 & 1463.94 & 1469.92 \\
\hline $10 / 21 / 11$ 13:20 & 42.116 & 39.219 & 1466.65 & 1469.90 \\
\hline 10/21/11 13:30 & 40.458 & 39.228 & 1468.31 & 1469.89 \\
\hline $10 / 21 / 11$ 13:40 & 39.484 & 39.24 & 1469.29 & 1469.88 \\
\hline $10 / 21 / 11$ 13:50 & 42.822 & 39.25 & 1465.95 & 1469.87 \\
\hline 10/21/11 14:00 & 43.345 & 39.259 & 1465.42 & 1469.86 \\
\hline $10 / 21 / 11$ 14:10 & 41.227 & 39.271 & 1467.54 & 1469.85 \\
\hline $10 / 21 / 11$ 14:20 & 44.599 & 39.287 & 1464.17 & 1469.84 \\
\hline $10 / 21 / 11$ 14:30 & 44.853 & 39.308 & 1463.92 & 1469.81 \\
\hline $10 / 21 / 11$ 14:40 & 41.049 & 39.327 & 1467.72 & 1469.80 \\
\hline $10 / 21 / 1114: 50$ & 43.08 & 39.348 & 1465.69 & 1469.77 \\
\hline 10/21/11 15:00 & 44.665 & 39.372 & 1464.10 & 1469.75 \\
\hline $10 / 21 / 11$ 15:10 & 42.512 & 39.393 & 1466.26 & 1469.73 \\
\hline $10 / 21 / 11$ 15:20 & 41.236 & 39.414 & 1467.53 & 1469.71 \\
\hline $10 / 21 / 11$ 15:30 & 40.523 & 39.435 & 1468.25 & 1469.69 \\
\hline $10 / 21 / 11$ 15:40 & 43.65 & 39.456 & 1465.12 & 1469.67 \\
\hline
\end{tabular}


TABLE S1.3 Automatically recorded groundwater levels in the Klassen wells, September 15, 2011, to March 13, 2012.

\begin{tabular}{|c|c|c|c|c|}
\hline \multicolumn{3}{|c|}{ Reference Elevation (ft AMSL) } & 1508.77 & \multirow{2}{*}{$\begin{array}{l}1509.12 \\
\text { Elevation in Klassen } \\
\text { Test Well (ft AMSL) }\end{array}$} \\
\hline Date and Time & $\begin{array}{l}\text { Depth in Klassen } \\
\text { Domestic Well } \\
(\mathrm{ft} \mathrm{BGL})\end{array}$ & $\begin{array}{c}\text { Depth in Klassen } \\
\text { Test Well } \\
\text { (ft BGL) }\end{array}$ & $\begin{array}{l}\text { Elevation in Klassen } \\
\text { Domestic Well } \\
\text { (ft AMSL) }\end{array}$ & \\
\hline $10 / 21 / 1115: 50$ & 41.269 & 39.477 & 1467.50 & 1469.65 \\
\hline $10 / 21 / 1116: 00$ & 44.794 & 39.501 & 1463.98 & 1469.62 \\
\hline 10/21/11 16:10 & 42.686 & 39.519 & 1466.08 & 1469.60 \\
\hline $10 / 21 / 1116: 20$ & 41.398 & 39.527 & 1467.37 & 1469.60 \\
\hline 10/21/11 16:30 & 41.506 & 39.533 & 1467.26 & 1469.59 \\
\hline $10 / 21 / 1116: 40$ & 49.663 & 39.55 & 1459.11 & 1469.57 \\
\hline $10 / 21 / 1116: 50$ & 51.858 & 39.571 & 1456.91 & 1469.55 \\
\hline 10/21/11 17:00 & 52.576 & 39.594 & 1456.19 & 1469.53 \\
\hline 10/21/11 17:10 & 53.131 & 39.618 & 1455.64 & 1469.50 \\
\hline $10 / 21 / 1117: 20$ & 50.158 & 39.648 & 1458.61 & 1469.47 \\
\hline 10/21/11 17:30 & 51.457 & 39.677 & 1457.31 & 1469.45 \\
\hline 10/21/11 17:40 & 54.862 & 39.707 & 1453.91 & 1469.42 \\
\hline 10/21/11 17:50 & 51.884 & 39.738 & 1456.89 & 1469.38 \\
\hline 10/21/11 18:00 & 56.734 & 39.768 & 1452.04 & 1469.35 \\
\hline 10/21/11 18:10 & 52.768 & 39.799 & 1456.00 & 1469.32 \\
\hline $10 / 21 / 11$ 18:20 & 51.759 & 39.831 & 1457.01 & 1469.29 \\
\hline 10/21/11 18:30 & 56.337 & 39.864 & 1452.43 & 1469.26 \\
\hline 10/21/11 18:40 & 45.859 & 39.9 & 1462.91 & 1469.22 \\
\hline $10 / 21 / 11$ 18:50 & 48.661 & 39.93 & 1460.11 & 1469.19 \\
\hline 10/21/11 19:00 & 47.407 & 39.953 & 1461.36 & 1469.17 \\
\hline 10/21/11 19:10 & 45.451 & 39.975 & 1463.32 & 1469.15 \\
\hline 10/21/11 19:20 & 45.341 & 39.993 & 1463.43 & 1469.13 \\
\hline 10/21/11 19:30 & 49.318 & 40.012 & 1459.45 & 1469.11 \\
\hline $10 / 21 / 11$ 19:40 & 42.62 & 40.024 & 1466.15 & 1469.10 \\
\hline 10/21/11 19:50 & 41.863 & 40.029 & 1466.91 & 1469.09 \\
\hline $10 / 21 / 1120: 00$ & 41.393 & 40.024 & 1467.38 & 1469.10 \\
\hline 10/21/11 20:10 & 41.025 & 40.012 & 1467.74 & 1469.11 \\
\hline $10 / 21 / 1120: 20$ & 40.725 & 40.003 & 1468.04 & 1469.12 \\
\hline 10/21/11 20:30 & 40.507 & 39.989 & 1468.26 & 1469.13 \\
\hline $10 / 21 / 1120: 40$ & 40.352 & 39.975 & 1468.42 & 1469.15 \\
\hline $10 / 21 / 1120: 50$ & 40.176 & 39.961 & 1468.59 & 1469.16 \\
\hline 10/21/11 21:00 & 40.024 & 39.942 & 1468.75 & 1469.18 \\
\hline 10/21/11 21:10 & 39.885 & 39.928 & 1468.88 & 1469.19 \\
\hline $10 / 21 / 1121: 20$ & 39.698 & 39.909 & 1469.07 & 1469.21 \\
\hline 10/21/11 21:30 & 39.524 & 39.888 & 1469.25 & 1469.23 \\
\hline $10 / 21 / 1121: 40$ & 39.367 & 39.869 & 1469.40 & 1469.25 \\
\hline $10 / 21 / 1121: 50$ & 39.236 & 39.848 & 1469.53 & 1469.27 \\
\hline $10 / 21 / 1122: 00$ & 39.285 & 39.831 & 1469.48 & 1469.29 \\
\hline
\end{tabular}


TABLE S1.3 Automatically recorded groundwater levels in the Klassen wells, September 15, 2011, to March 13, 2012.

\begin{tabular}{|c|c|c|c|c|}
\hline \multicolumn{3}{|c|}{ Reference Elevation (ft AMSL) } & 1508.77 & \multirow{2}{*}{$\begin{array}{l}1509.12 \\
\text { Elevation in Klassen } \\
\text { Test Well (ft AMSL) }\end{array}$} \\
\hline Date and Time & $\begin{array}{l}\text { Depth in Klassen } \\
\text { Domestic Well } \\
(\mathrm{ft} \mathrm{BGL})\end{array}$ & $\begin{array}{c}\text { Depth in Klassen } \\
\text { Test Well } \\
\text { (ft BGL) }\end{array}$ & $\begin{array}{l}\text { Elevation in Klassen } \\
\text { Domestic Well } \\
\text { (ft AMSL) }\end{array}$ & \\
\hline $10 / 21 / 1122: 10$ & 39.076 & 39.813 & 1469.69 & 1469.31 \\
\hline $10 / 21 / 1122: 20$ & 38.931 & 39.792 & 1469.84 & 1469.33 \\
\hline $10 / 21 / 1122: 30$ & 43.464 & 39.773 & 1465.31 & 1469.35 \\
\hline $10 / 21 / 1122: 40$ & 38.978 & 39.756 & 1469.79 & 1469.37 \\
\hline $10 / 21 / 1122: 50$ & 38.785 & 39.74 & 1469.98 & 1469.38 \\
\hline $10 / 21 / 1123: 00$ & 38.642 & 39.719 & 1470.13 & 1469.40 \\
\hline $10 / 21 / 1123: 10$ & 41.541 & 39.702 & 1467.23 & 1469.42 \\
\hline $10 / 21 / 1123: 20$ & 38.788 & 39.688 & 1469.98 & 1469.43 \\
\hline $10 / 21 / 1123: 30$ & 38.633 & 39.674 & 1470.14 & 1469.45 \\
\hline $10 / 21 / 1123: 40$ & 38.523 & 39.66 & 1470.25 & 1469.46 \\
\hline $10 / 21 / 1123: 50$ & 38.42 & 39.646 & 1470.35 & 1469.48 \\
\hline 10/22/11 0:00 & 38.305 & 39.627 & 1470.46 & 1469.50 \\
\hline 10/22/11 0:10 & 38.199 & 39.611 & 1470.57 & 1469.51 \\
\hline $10 / 22 / 110: 20$ & 38.103 & 39.594 & 1470.67 & 1469.53 \\
\hline $10 / 22 / 110: 30$ & 38.044 & 39.578 & 1470.73 & 1469.54 \\
\hline $10 / 22 / 110: 40$ & 37.958 & 39.562 & 1470.81 & 1469.56 \\
\hline $10 / 22 / 110: 50$ & 37.875 & 39.545 & 1470.89 & 1469.58 \\
\hline 10/22/11 1:00 & 37.801 & 39.529 & 1470.97 & 1469.59 \\
\hline 10/22/11 1:10 & 37.725 & 39.515 & 1471.04 & 1469.61 \\
\hline $10 / 22 / 111: 20$ & 37.657 & 39.496 & 1471.11 & 1469.63 \\
\hline $10 / 22 / 111: 30$ & 37.592 & 39.48 & 1471.18 & 1469.64 \\
\hline 10/22/11 1:40 & 37.528 & 39.461 & 1471.24 & 1469.66 \\
\hline 10/22/11 1:50 & 37.467 & 39.444 & 1471.30 & 1469.68 \\
\hline $10 / 22 / 112: 00$ & 37.409 & 39.426 & 1471.36 & 1469.70 \\
\hline $10 / 22 / 112: 10$ & 37.352 & 39.409 & 1471.42 & 1469.71 \\
\hline $10 / 22 / 112: 20$ & 37.296 & 39.39 & 1471.47 & 1469.73 \\
\hline $10 / 22 / 112: 30$ & 37.245 & 39.374 & 1471.52 & 1469.75 \\
\hline $10 / 22 / 112: 40$ & 37.193 & 39.358 & 1471.58 & 1469.76 \\
\hline $10 / 22 / 112: 50$ & 37.17 & 39.339 & 1471.60 & 1469.78 \\
\hline 10/22/11 3:00 & 37.125 & 39.325 & 1471.64 & 1469.80 \\
\hline 10/22/11 3:10 & 37.073 & 39.308 & 1471.70 & 1469.81 \\
\hline $10 / 22 / 113: 20$ & 37.026 & 39.292 & 1471.74 & 1469.83 \\
\hline $10 / 22 / 113: 30$ & 36.98 & 39.275 & 1471.79 & 1469.85 \\
\hline 10/22/11 3:40 & 36.935 & 39.261 & 1471.83 & 1469.86 \\
\hline $10 / 22 / 113: 50$ & 36.89 & 39.245 & 1471.88 & 1469.88 \\
\hline 10/22/11 4:00 & 36.851 & 39.228 & 1471.92 & 1469.89 \\
\hline 10/22/11 4:10 & 36.811 & 39.214 & 1471.96 & 1469.91 \\
\hline $10 / 22 / 114: 20$ & 41.131 & 39.198 & 1467.64 & 1469.92 \\
\hline
\end{tabular}


TABLE S1.3 Automatically recorded groundwater levels in the Klassen wells, September 15, 2011, to March 13, 2012.

\begin{tabular}{|c|c|c|c|c|}
\hline \multicolumn{3}{|c|}{ Reference Elevation (ft AMSL) } & 1508.77 & \multirow{2}{*}{$\begin{array}{l}1509.12 \\
\text { Elevation in Klassen } \\
\text { Test Well (ft AMSL) }\end{array}$} \\
\hline Date and Time & $\begin{array}{l}\text { Depth in Klassen } \\
\text { Domestic Well } \\
\text { (ft BGL) }\end{array}$ & $\begin{array}{c}\text { Depth in Klassen } \\
\text { Test Well } \\
\text { (ft BGL) }\end{array}$ & $\begin{array}{l}\text { Elevation in Klassen } \\
\text { Domestic Well } \\
\text { (ft AMSL) }\end{array}$ & \\
\hline 10/22/11 4:30 & 36.9 & 39.184 & 1471.87 & 1469.94 \\
\hline 10/22/11 4:40 & 36.792 & 39.17 & 1471.98 & 1469.95 \\
\hline 10/22/11 4:50 & 36.733 & 39.153 & 1472.04 & 1469.97 \\
\hline 10/22/11 5:00 & 36.684 & 39.142 & 1472.09 & 1469.98 \\
\hline 10/22/11 5:10 & 36.642 & 39.125 & 1472.13 & 1470.00 \\
\hline 10/22/11 5:20 & 36.602 & 39.113 & 1472.17 & 1470.01 \\
\hline 10/22/11 5:30 & 36.564 & 39.104 & 1472.21 & 1470.02 \\
\hline 10/22/11 5:40 & 36.527 & 39.092 & 1472.24 & 1470.03 \\
\hline 10/22/11 5:50 & 36.494 & 39.081 & 1472.28 & 1470.04 \\
\hline 10/22/11 6:00 & 36.461 & 39.069 & 1472.31 & 1470.05 \\
\hline 10/22/11 6:10 & 36.426 & 39.06 & 1472.34 & 1470.06 \\
\hline 10/22/11 6:20 & 36.663 & 39.052 & 1472.11 & 1470.07 \\
\hline 10/22/11 6:30 & 36.511 & 39.043 & 1472.26 & 1470.08 \\
\hline 10/22/11 6:40 & 36.443 & 39.034 & 1472.33 & 1470.09 \\
\hline $10 / 22 / 116: 50$ & 36.393 & 39.027 & 1472.38 & 1470.10 \\
\hline 10/22/11 7:00 & 40.678 & 39.015 & 1468.09 & 1470.11 \\
\hline 10/22/11 7:10 & 39.32 & 39.008 & 1469.45 & 1470.11 \\
\hline 10/22/11 7:20 & 38.429 & 39.003 & 1470.34 & 1470.12 \\
\hline 10/22/11 7:30 & 42.109 & 39.001 & 1466.66 & 1470.12 \\
\hline $10 / 22 / 11$ 7:40 & 42.015 & 38.999 & 1466.75 & 1470.12 \\
\hline $10 / 22 / 11$ 7:50 & 39.691 & 38.996 & 1469.08 & 1470.13 \\
\hline $10 / 22 / 118: 00$ & 38.753 & 38.999 & 1470.02 & 1470.12 \\
\hline $10 / 22 / 118: 10$ & 42.188 & 38.996 & 1466.58 & 1470.13 \\
\hline $10 / 22 / 118: 20$ & 45.765 & 38.996 & 1463.00 & 1470.13 \\
\hline $10 / 22 / 118: 30$ & 40.261 & 39.001 & 1468.51 & 1470.12 \\
\hline $10 / 22 / 118: 40$ & 42.423 & 39.008 & 1466.35 & 1470.11 \\
\hline $10 / 22 / 118: 50$ & 40.469 & 39.015 & 1468.30 & 1470.11 \\
\hline 10/22/11 9:00 & 39.423 & 39.015 & 1469.35 & 1470.11 \\
\hline 10/22/11 9:10 & 43.019 & 39.017 & 1465.75 & 1470.11 \\
\hline 10/22/11 9:20 & 42.583 & 39.024 & 1466.19 & 1470.10 \\
\hline 10/22/11 9:30 & 40.537 & 39.029 & 1468.23 & 1470.09 \\
\hline 10/22/11 9:40 & 39.55 & 39.034 & 1469.22 & 1470.09 \\
\hline 10/22/11 9:50 & 44.332 & 39.041 & 1464.44 & 1470.08 \\
\hline 10/22/11 10:00 & 41.769 & 39.05 & 1467.00 & 1470.07 \\
\hline 10/22/11 10:10 & 42.076 & 39.052 & 1466.69 & 1470.07 \\
\hline 10/22/11 10:20 & 44.822 & 39.057 & 1463.95 & 1470.07 \\
\hline 10/22/11 10:30 & 43.321 & 39.057 & 1465.45 & 1470.07 \\
\hline 10/22/11 10:40 & 41.185 & 39.06 & 1467.58 & 1470.06 \\
\hline
\end{tabular}


TABLE S1.3 Automatically recorded groundwater levels in the Klassen wells, September 15, 2011, to March 13, 2012.

\begin{tabular}{|c|c|c|c|c|}
\hline \multicolumn{3}{|c|}{ Reference Elevation (ft AMSL) } & 1508.77 & \multirow{2}{*}{$\begin{array}{l}1509.12 \\
\text { Elevation in Klassen } \\
\text { Test Well (ft AMSL) }\end{array}$} \\
\hline Date and Time & $\begin{array}{l}\text { Depth in Klassen } \\
\text { Domestic Well } \\
\text { (ft BGL) }\end{array}$ & $\begin{array}{c}\text { Depth in Klassen } \\
\text { Test Well } \\
\text { (ft BGL) }\end{array}$ & $\begin{array}{l}\text { Elevation in Klassen } \\
\text { Domestic Well } \\
\text { (ft AMSL) }\end{array}$ & \\
\hline 10/22/11 10:50 & 39.902 & 39.064 & 1468.87 & 1470.06 \\
\hline 10/22/11 11:00 & 43.912 & 39.067 & 1464.86 & 1470.06 \\
\hline 10/22/11 11:10 & 43.382 & 39.069 & 1465.39 & 1470.05 \\
\hline 10/22/11 11:20 & 41.368 & 39.071 & 1467.40 & 1470.05 \\
\hline 10/22/11 11:30 & 41.248 & 39.074 & 1467.52 & 1470.05 \\
\hline 10/22/11 11:40 & 43.786 & 39.078 & 1464.98 & 1470.04 \\
\hline 10/22/11 11:50 & 43.389 & 39.081 & 1465.38 & 1470.04 \\
\hline $10 / 22 / 1112: 00$ & 41.26 & 39.085 & 1467.51 & 1470.04 \\
\hline $10 / 22 / 1112: 10$ & 40.223 & 39.092 & 1468.55 & 1470.03 \\
\hline $10 / 22 / 1112: 20$ & 42.479 & 39.099 & 1466.29 & 1470.02 \\
\hline $10 / 22 / 1112: 30$ & 44.473 & 39.104 & 1464.30 & 1470.02 \\
\hline $10 / 22 / 1112: 40$ & 42.188 & 39.109 & 1466.58 & 1470.01 \\
\hline $10 / 22 / 1112: 50$ & 42.629 & 39.111 & 1466.14 & 1470.01 \\
\hline 10/22/11 13:00 & 47.43 & 39.116 & 1461.34 & 1470.01 \\
\hline 10/22/11 13:10 & 43.335 & 39.118 & 1465.43 & 1470.00 \\
\hline $10 / 22 / 11$ 13:20 & 41.44 & 39.125 & 1467.33 & 1470.00 \\
\hline 10/22/11 13:30 & 40.35 & 39.128 & 1468.42 & 1469.99 \\
\hline $10 / 22 / 11$ 13:40 & 42.758 & 39.132 & 1466.01 & 1469.99 \\
\hline $10 / 22 / 11$ 13:50 & 44.562 & 39.135 & 1464.21 & 1469.99 \\
\hline $10 / 22 / 11$ 14:00 & 41.569 & 39.149 & 1467.20 & 1469.97 \\
\hline $10 / 22 / 11$ 14:10 & 45.345 & 39.165 & 1463.42 & 1469.96 \\
\hline $10 / 22 / 11$ 14:20 & 39.407 & 39.182 & 1469.36 & 1469.94 \\
\hline $10 / 22 / 11$ 14:30 & 41.117 & 39.198 & 1467.65 & 1469.92 \\
\hline $10 / 22 / 11$ 14:40 & 39.194 & 39.212 & 1469.58 & 1469.91 \\
\hline $10 / 22 / 11$ 14:50 & 41.229 & 39.228 & 1467.54 & 1469.89 \\
\hline 10/22/11 15:00 & 44.503 & 39.254 & 1464.27 & 1469.87 \\
\hline $10 / 22 / 11$ 15:10 & 45.887 & 39.278 & 1462.88 & 1469.84 \\
\hline $10 / 22 / 11$ 15:20 & 43.408 & 39.304 & 1465.36 & 1469.82 \\
\hline $10 / 22 / 11$ 15:30 & 47.383 & 39.329 & 1461.39 & 1469.79 \\
\hline $10 / 22 / 11$ 15:40 & 42.667 & 39.362 & 1466.10 & 1469.76 \\
\hline 10/22/11 15:50 & 45.61 & 39.386 & 1463.16 & 1469.74 \\
\hline 10/22/11 16:00 & 45.54 & 39.409 & 1463.23 & 1469.71 \\
\hline 10/22/11 16:10 & 44.789 & 39.437 & 1463.98 & 1469.69 \\
\hline $10 / 22 / 11$ 16:20 & 48.802 & 39.463 & 1459.97 & 1469.66 \\
\hline 10/22/11 16:30 & 43.739 & 39.494 & 1465.03 & 1469.63 \\
\hline $10 / 22 / 11$ 16:40 & 47.067 & 39.512 & 1461.70 & 1469.61 \\
\hline $10 / 22 / 11$ 16:50 & 44.639 & 39.526 & 1464.13 & 1469.60 \\
\hline 10/22/11 17:00 & 47.137 & 39.543 & 1461.63 & 1469.58 \\
\hline
\end{tabular}


TABLE S1.3 Automatically recorded groundwater levels in the Klassen wells, September 15, 2011, to March 13, 2012.

\begin{tabular}{|c|c|c|c|c|}
\hline \multicolumn{3}{|c|}{ Reference Elevation (ft AMSL) } & 1508.77 & \multirow{2}{*}{$\begin{array}{l}1509.12 \\
\text { Elevation in Klassen } \\
\text { Test Well (ft AMSL) }\end{array}$} \\
\hline Date and Time & $\begin{array}{l}\text { Depth in Klassen } \\
\text { Domestic Well } \\
\text { (ft BGL) }\end{array}$ & $\begin{array}{c}\text { Depth in Klassen } \\
\text { Test Well } \\
\text { (ft BGL) }\end{array}$ & $\begin{array}{l}\text { Elevation in Klassen } \\
\text { Domestic Well } \\
\text { (ft AMSL) }\end{array}$ & \\
\hline 10/22/11 17:10 & 43.964 & 39.562 & 1464.81 & 1469.56 \\
\hline $10 / 22 / 1117: 20$ & 54.752 & 39.58 & 1454.02 & 1469.54 \\
\hline 10/22/11 17:30 & 64.45 & 39.613 & 1444.32 & 1469.51 \\
\hline 10/22/11 17:40 & 53.795 & 39.653 & 1454.97 & 1469.47 \\
\hline 10/22/11 17:50 & 50.474 & 39.7 & 1458.30 & 1469.42 \\
\hline 10/22/11 18:00 & 50.955 & 39.733 & 1457.81 & 1469.39 \\
\hline $10 / 22 / 1118: 10$ & 52.465 & 39.768 & 1456.30 & 1469.35 \\
\hline 10/22/11 18:20 & 49.419 & 39.796 & 1459.35 & 1469.33 \\
\hline 10/22/11 18:30 & 47.135 & 39.824 & 1461.63 & 1469.30 \\
\hline $10 / 22 / 1118: 40$ & 49.419 & 39.857 & 1459.35 & 1469.27 \\
\hline 10/22/11 18:50 & 52.475 & 39.885 & 1456.29 & 1469.24 \\
\hline 10/22/11 19:00 & 50.774 & 39.916 & 1458.00 & 1469.21 \\
\hline 10/22/11 19:10 & 47.838 & 39.949 & 1460.93 & 1469.17 \\
\hline $10 / 22 / 11$ 19:20 & 52.841 & 39.972 & 1455.93 & 1469.15 \\
\hline 10/22/11 19:30 & 49.454 & 39.993 & 1459.32 & 1469.13 \\
\hline 10/22/11 19:40 & 48.009 & 40.012 & 1460.76 & 1469.11 \\
\hline 10/22/11 19:50 & 42.967 & 40.024 & 1465.80 & 1469.10 \\
\hline $10 / 22 / 1120: 00$ & 42.16 & 40.031 & 1466.61 & 1469.09 \\
\hline $10 / 22 / 1120: 10$ & 41.701 & 40.033 & 1467.07 & 1469.09 \\
\hline $10 / 22 / 1120: 20$ & 41.332 & 40.033 & 1467.44 & 1469.09 \\
\hline $10 / 22 / 1120: 30$ & 41.028 & 40.026 & 1467.74 & 1469.10 \\
\hline $10 / 22 / 1120: 40$ & 40.767 & 40.022 & 1468.00 & 1469.10 \\
\hline $10 / 22 / 1120: 50$ & 40.54 & 40.012 & 1468.23 & 1469.11 \\
\hline $10 / 22 / 1121: 00$ & 40.364 & 40.007 & 1468.41 & 1469.12 \\
\hline 10/22/11 21:10 & 40.174 & 39.998 & 1468.60 & 1469.12 \\
\hline $10 / 22 / 1121: 20$ & 40.003 & 39.986 & 1468.77 & 1469.14 \\
\hline 10/22/11 21:30 & 39.841 & 39.975 & 1468.93 & 1469.15 \\
\hline $10 / 22 / 1121: 40$ & 39.695 & 39.963 & 1469.07 & 1469.16 \\
\hline $10 / 22 / 1121: 50$ & 39.555 & 39.951 & 1469.21 & 1469.17 \\
\hline $10 / 22 / 1122: 00$ & 40.666 & 39.939 & 1468.10 & 1469.18 \\
\hline $10 / 22 / 1122: 10$ & 39.646 & 39.928 & 1469.12 & 1469.19 \\
\hline $10 / 22 / 1122: 20$ & 39.384 & 39.916 & 1469.39 & 1469.21 \\
\hline $10 / 22 / 1122: 30$ & 39.398 & 39.907 & 1469.37 & 1469.22 \\
\hline $10 / 22 / 1122: 40$ & 40.603 & 39.897 & 1468.17 & 1469.23 \\
\hline $10 / 22 / 1122: 50$ & 39.914 & 39.888 & 1468.86 & 1469.23 \\
\hline $10 / 22 / 1123: 00$ & 40.2 & 39.883 & 1468.57 & 1469.24 \\
\hline $10 / 22 / 1123: 10$ & 39.538 & 39.876 & 1469.23 & 1469.25 \\
\hline $10 / 22 / 1123: 20$ & 39.212 & 39.867 & 1469.56 & 1469.26 \\
\hline
\end{tabular}


TABLE S1.3 Automatically recorded groundwater levels in the Klassen wells, September 15, 2011, to March 13, 2012.

\begin{tabular}{|c|c|c|c|c|}
\hline \multicolumn{3}{|c|}{ Reference Elevation (ft AMSL) } & 1508.77 & \multirow{2}{*}{$\begin{array}{l}1509.12 \\
\text { Elevation in Klassen } \\
\text { Test Well (ft AMSL) }\end{array}$} \\
\hline Date and Time & $\begin{array}{l}\text { Depth in Klassen } \\
\text { Domestic Well } \\
\text { (ft BGL) }\end{array}$ & $\begin{array}{c}\text { Depth in Klassen } \\
\text { Test Well } \\
\text { (ft BGL) }\end{array}$ & $\begin{array}{l}\text { Elevation in Klassen } \\
\text { Domestic Well } \\
\text { (ft AMSL) }\end{array}$ & \\
\hline $10 / 22 / 1123: 30$ & 39.025 & 39.857 & 1469.74 & 1469.27 \\
\hline $10 / 22 / 1123: 40$ & 38.882 & 39.846 & 1469.89 & 1469.28 \\
\hline $10 / 22 / 1123: 50$ & 38.76 & 39.836 & 1470.01 & 1469.29 \\
\hline $10 / 23 / 110: 00$ & 38.652 & 39.824 & 1470.12 & 1469.30 \\
\hline 10/23/11 0:10 & 38.553 & 39.813 & 1470.22 & 1469.31 \\
\hline $10 / 23 / 110: 20$ & 38.46 & 39.799 & 1470.31 & 1469.32 \\
\hline $10 / 23 / 110: 30$ & 38.375 & 39.785 & 1470.39 & 1469.34 \\
\hline $10 / 23 / 110: 40$ & 38.293 & 39.773 & 1470.48 & 1469.35 \\
\hline $10 / 23 / 110: 50$ & 38.218 & 39.761 & 1470.55 & 1469.36 \\
\hline 10/23/11 1:00 & 38.143 & 39.749 & 1470.63 & 1469.37 \\
\hline 10/23/11 1:10 & 38.073 & 39.735 & 1470.70 & 1469.39 \\
\hline 10/23/11 1:20 & 38.007 & 39.724 & 1470.76 & 1469.40 \\
\hline 10/23/11 1:30 & 37.955 & 39.709 & 1470.81 & 1469.41 \\
\hline 10/23/11 1:40 & 37.96 & 39.7 & 1470.81 & 1469.42 \\
\hline 10/23/11 1:50 & 37.96 & 39.691 & 1470.81 & 1469.43 \\
\hline $10 / 23 / 112: 00$ & 37.908 & 39.681 & 1470.86 & 1469.44 \\
\hline $10 / 23 / 112: 10$ & 37.852 & 39.67 & 1470.92 & 1469.45 \\
\hline $10 / 23 / 112: 20$ & 37.826 & 39.66 & 1470.94 & 1469.46 \\
\hline $10 / 23 / 112: 30$ & 37.756 & 39.651 & 1471.01 & 1469.47 \\
\hline $10 / 23 / 112: 40$ & 37.711 & 39.639 & 1471.06 & 1469.48 \\
\hline $10 / 23 / 112: 50$ & 37.65 & 39.63 & 1471.12 & 1469.49 \\
\hline 10/23/11 3:00 & 37.589 & 39.618 & 1471.18 & 1469.50 \\
\hline 10/23/11 3:10 & 39.677 & 39.606 & 1469.09 & 1469.52 \\
\hline 10/23/11 3:20 & 37.695 & 39.599 & 1471.07 & 1469.52 \\
\hline $10 / 23 / 113: 30$ & 37.653 & 39.592 & 1471.12 & 1469.53 \\
\hline $10 / 23 / 113: 40$ & 37.564 & 39.585 & 1471.21 & 1469.54 \\
\hline $10 / 23 / 113: 50$ & 37.488 & 39.573 & 1471.28 & 1469.55 \\
\hline 10/23/11 4:00 & 37.423 & 39.564 & 1471.35 & 1469.56 \\
\hline 10/23/11 4:10 & 37.364 & 39.552 & 1471.41 & 1469.57 \\
\hline $10 / 23 / 114: 20$ & 37.31 & 39.543 & 1471.46 & 1469.58 \\
\hline $10 / 23 / 114: 30$ & 37.259 & 39.533 & 1471.51 & 1469.59 \\
\hline $10 / 23 / 114: 40$ & 37.212 & 39.524 & 1471.56 & 1469.60 \\
\hline $10 / 23 / 114: 50$ & 37.167 & 39.512 & 1471.60 & 1469.61 \\
\hline 10/23/11 5:00 & 37.123 & 39.503 & 1471.65 & 1469.62 \\
\hline 10/23/11 5:10 & 37.08 & 39.494 & 1471.69 & 1469.63 \\
\hline 10/23/11 5:20 & 37.041 & 39.484 & 1471.73 & 1469.64 \\
\hline 10/23/11 5:30 & 37.001 & 39.477 & 1471.77 & 1469.65 \\
\hline 10/23/11 5:40 & 36.963 & 39.47 & 1471.81 & 1469.65 \\
\hline
\end{tabular}


TABLE S1.3 Automatically recorded groundwater levels in the Klassen wells, September 15, 2011, to March 13, 2012.

\begin{tabular}{|c|c|c|c|c|}
\hline \multicolumn{3}{|c|}{ Reference Elevation (ft AMSL) } & 1508.77 & \multirow{2}{*}{$\begin{array}{l}1509.12 \\
\text { Elevation in Klassen } \\
\text { Test Well (ft AMSL) }\end{array}$} \\
\hline Date and Time & $\begin{array}{l}\text { Depth in Klassen } \\
\text { Domestic Well } \\
\text { (ft BGL) }\end{array}$ & $\begin{array}{c}\text { Depth in Klassen } \\
\text { Test Well } \\
\text { (ft BGL) }\end{array}$ & $\begin{array}{l}\text { Elevation in Klassen } \\
\text { Domestic Well } \\
\text { (ft AMSL) }\end{array}$ & \\
\hline 10/23/11 5:50 & 41.33 & 39.465 & 1467.44 & 1469.66 \\
\hline 10/23/11 6:00 & 37.463 & 39.463 & 1471.31 & 1469.66 \\
\hline 10/23/11 6:10 & 37.097 & 39.461 & 1471.67 & 1469.66 \\
\hline $10 / 23 / 116: 20$ & 37.207 & 39.456 & 1471.56 & 1469.67 \\
\hline 10/23/11 6:30 & 37.029 & 39.454 & 1471.74 & 1469.67 \\
\hline $10 / 23 / 116: 40$ & 36.947 & 39.449 & 1471.82 & 1469.67 \\
\hline $10 / 23 / 116: 50$ & 36.888 & 39.444 & 1471.88 & 1469.68 \\
\hline 10/23/11 7:00 & 36.837 & 39.437 & 1471.93 & 1469.69 \\
\hline 10/23/11 7:10 & 36.792 & 39.43 & 1471.98 & 1469.69 \\
\hline $10 / 23 / 117: 20$ & 36.752 & 39.423 & 1472.02 & 1469.70 \\
\hline 10/23/11 7:30 & 36.74 & 39.419 & 1472.03 & 1469.70 \\
\hline 10/23/11 7:40 & 36.703 & 39.409 & 1472.07 & 1469.71 \\
\hline 10/23/11 7:50 & 36.665 & 39.4 & 1472.10 & 1469.72 \\
\hline 10/23/11 8:00 & 37.979 & 39.393 & 1470.79 & 1469.73 \\
\hline $10 / 23 / 118: 10$ & 36.738 & 39.386 & 1472.03 & 1469.74 \\
\hline $10 / 23 / 118: 20$ & 39.749 & 39.379 & 1469.02 & 1469.74 \\
\hline $10 / 23 / 118: 30$ & 36.755 & 39.372 & 1472.01 & 1469.75 \\
\hline 10/23/11 8:40 & 36.879 & 39.367 & 1471.89 & 1469.76 \\
\hline $10 / 23 / 118: 50$ & 36.694 & 39.358 & 1472.08 & 1469.76 \\
\hline 10/23/11 9:00 & 36.619 & 39.353 & 1472.15 & 1469.77 \\
\hline 10/23/11 9:10 & 36.567 & 39.346 & 1472.20 & 1469.78 \\
\hline 10/23/11 9:20 & 36.522 & 39.341 & 1472.25 & 1469.78 \\
\hline 10/23/11 9:30 & 36.485 & 39.329 & 1472.28 & 1469.79 \\
\hline 10/23/11 9:40 & 36.45 & 39.32 & 1472.32 & 1469.80 \\
\hline 10/23/11 9:50 & 36.417 & 39.315 & 1472.35 & 1469.81 \\
\hline 10/23/11 10:00 & 36.386 & 39.311 & 1472.38 & 1469.81 \\
\hline 10/23/11 10:10 & 36.372 & 39.306 & 1472.40 & 1469.82 \\
\hline $10 / 23 / 11$ 10:20 & 36.356 & 39.301 & 1472.41 & 1469.82 \\
\hline 10/23/11 10:30 & 36.325 & 39.297 & 1472.44 & 1469.83 \\
\hline 10/23/11 10:40 & 36.295 & 39.292 & 1472.47 & 1469.83 \\
\hline 10/23/11 10:50 & 36.267 & 39.287 & 1472.50 & 1469.84 \\
\hline 10/23/11 11:00 & 36.241 & 39.282 & 1472.53 & 1469.84 \\
\hline 10/23/11 11:10 & 36.278 & 39.28 & 1472.49 & 1469.84 \\
\hline $10 / 23 / 11$ 11:20 & 36.295 & 39.28 & 1472.47 & 1469.84 \\
\hline 10/23/11 11:30 & 36.311 & 39.278 & 1472.46 & 1469.84 \\
\hline $10 / 23 / 11$ 11:40 & 36.271 & 39.275 & 1472.50 & 1469.85 \\
\hline $10 / 23 / 11$ 11:50 & 36.231 & 39.273 & 1472.54 & 1469.85 \\
\hline $10 / 23 / 11$ 12:00 & 36.201 & 39.271 & 1472.57 & 1469.85 \\
\hline
\end{tabular}


TABLE S1.3 Automatically recorded groundwater levels in the Klassen wells, September 15, 2011, to March 13, 2012.

\begin{tabular}{|c|c|c|c|c|}
\hline \multicolumn{3}{|c|}{ Reference Elevation (ft AMSL) } & 1508.77 & \multirow{2}{*}{$\begin{array}{l}1509.12 \\
\text { Elevation in Klassen } \\
\text { Test Well (ft AMSL) }\end{array}$} \\
\hline Date and Time & $\begin{array}{l}\text { Depth in Klassen } \\
\text { Domestic Well } \\
\text { (ft BGL) }\end{array}$ & $\begin{array}{c}\text { Depth in Klassen } \\
\text { Test Well } \\
\text { (ft BGL) }\end{array}$ & $\begin{array}{l}\text { Elevation in Klassen } \\
\text { Domestic Well } \\
\text { (ft AMSL) }\end{array}$ & \\
\hline $10 / 23 / 1112: 10$ & 36.309 & 39.271 & 1472.46 & 1469.85 \\
\hline $10 / 23 / 1112: 20$ & 36.363 & 39.268 & 1472.41 & 1469.85 \\
\hline $10 / 23 / 1112: 30$ & 36.503 & 39.273 & 1472.27 & 1469.85 \\
\hline $10 / 23 / 1112: 40$ & 36.665 & 39.275 & 1472.10 & 1469.85 \\
\hline $10 / 23 / 1112: 50$ & 36.696 & 39.273 & 1472.07 & 1469.85 \\
\hline 10/23/11 13:00 & 36.654 & 39.261 & 1472.12 & 1469.86 \\
\hline $10 / 23 / 1113: 10$ & 36.611 & 39.247 & 1472.16 & 1469.88 \\
\hline 10/23/11 13:20 & 36.604 & 39.231 & 1472.17 & 1469.89 \\
\hline $10 / 23 / 1113: 30$ & 36.621 & 39.219 & 1472.15 & 1469.90 \\
\hline $10 / 23 / 1113: 40$ & 36.604 & 39.207 & 1472.17 & 1469.92 \\
\hline 10/23/11 13:50 & 36.621 & 39.2 & 1472.15 & 1469.92 \\
\hline 10/23/11 14:00 & 36.618 & 39.189 & 1472.15 & 1469.93 \\
\hline $10 / 23 / 1114: 10$ & 36.628 & 39.182 & 1472.14 & 1469.94 \\
\hline $10 / 23 / 1114: 20$ & 36.609 & 39.172 & 1472.16 & 1469.95 \\
\hline 10/23/11 14:30 & 36.625 & 39.167 & 1472.14 & 1469.96 \\
\hline $10 / 23 / 11$ 14:40 & 36.583 & 39.16 & 1472.19 & 1469.96 \\
\hline $10 / 23 / 11$ 14:50 & 36.853 & 39.153 & 1471.92 & 1469.97 \\
\hline 10/23/11 15:00 & 36.677 & 39.149 & 1472.09 & 1469.97 \\
\hline $10 / 23 / 11$ 15:10 & 36.679 & 39.142 & 1472.09 & 1469.98 \\
\hline $10 / 23 / 11$ 15:20 & 36.644 & 39.135 & 1472.13 & 1469.99 \\
\hline $10 / 23 / 11$ 15:30 & 36.59 & 39.13 & 1472.18 & 1469.99 \\
\hline $10 / 23 / 11$ 15:40 & 36.647 & 39.123 & 1472.12 & 1470.00 \\
\hline $10 / 23 / 11$ 15:50 & 36.6 & 39.121 & 1472.17 & 1470.00 \\
\hline $10 / 23 / 11$ 16:00 & 36.611 & 39.114 & 1472.16 & 1470.01 \\
\hline 10/23/11 16:10 & 37.16 & 39.111 & 1471.61 & 1470.01 \\
\hline $10 / 23 / 11$ 16:20 & 36.677 & 39.106 & 1472.09 & 1470.02 \\
\hline 10/23/11 16:30 & 36.588 & 39.102 & 1472.18 & 1470.02 \\
\hline $10 / 23 / 11$ 16:40 & 36.574 & 39.095 & 1472.20 & 1470.03 \\
\hline $10 / 23 / 11$ 16:50 & 36.586 & 39.09 & 1472.18 & 1470.03 \\
\hline 10/23/11 17:00 & 36.569 & 39.085 & 1472.20 & 1470.04 \\
\hline 10/23/11 17:10 & 36.607 & 39.085 & 1472.16 & 1470.04 \\
\hline 10/23/11 17:20 & 40.73 & 39.081 & 1468.04 & 1470.04 \\
\hline 10/23/11 17:30 & 36.686 & 39.078 & 1472.08 & 1470.04 \\
\hline $10 / 23 / 11$ 17:40 & 36.569 & 39.074 & 1472.20 & 1470.05 \\
\hline 10/23/11 17:50 & 36.513 & 39.071 & 1472.26 & 1470.05 \\
\hline 10/23/11 18:00 & 36.447 & 39.067 & 1472.32 & 1470.06 \\
\hline 10/23/11 18:10 & 37.127 & 39.064 & 1471.64 & 1470.06 \\
\hline $10 / 23 / 11$ 18:20 & 36.595 & 39.062 & 1472.17 & 1470.06 \\
\hline
\end{tabular}


TABLE S1.3 Automatically recorded groundwater levels in the Klassen wells, September 15, 2011, to March 13, 2012.

\begin{tabular}{|c|c|c|c|c|}
\hline \multicolumn{3}{|c|}{ Reference Elevation (ft AMSL) } & 1508.77 & \multirow{2}{*}{$\begin{array}{l}1509.12 \\
\text { Elevation in Klassen } \\
\text { Test Well (ft AMSL) }\end{array}$} \\
\hline Date and Time & $\begin{array}{l}\text { Depth in Klassen } \\
\text { Domestic Well } \\
\text { (ft BGL) }\end{array}$ & $\begin{array}{c}\text { Depth in Klassen } \\
\text { Test Well } \\
\text { (ft BGL) }\end{array}$ & $\begin{array}{l}\text { Elevation in Klassen } \\
\text { Domestic Well } \\
\text { (ft AMSL) }\end{array}$ & \\
\hline 10/23/11 18:30 & 36.492 & 39.062 & 1472.28 & 1470.06 \\
\hline $10 / 23 / 11$ 18:40 & 36.438 & 39.06 & 1472.33 & 1470.06 \\
\hline $10 / 23 / 1118: 50$ & 36.377 & 39.052 & 1472.39 & 1470.07 \\
\hline 10/23/11 19:00 & 36.349 & 39.05 & 1472.42 & 1470.07 \\
\hline 10/23/11 19:10 & 38.525 & 39.045 & 1470.24 & 1470.08 \\
\hline 10/23/11 19:20 & 36.454 & 39.043 & 1472.32 & 1470.08 \\
\hline $10 / 23 / 1119: 30$ & 36.464 & 39.043 & 1472.31 & 1470.08 \\
\hline $10 / 23 / 11$ 19:40 & 36.405 & 39.038 & 1472.36 & 1470.08 \\
\hline $10 / 23 / 1119: 50$ & 36.4 & 39.036 & 1472.37 & 1470.09 \\
\hline $10 / 23 / 1120: 00$ & 36.433 & 39.036 & 1472.34 & 1470.09 \\
\hline $10 / 23 / 1120: 10$ & 36.438 & 39.034 & 1472.33 & 1470.09 \\
\hline $10 / 23 / 1120: 20$ & 36.457 & 39.031 & 1472.31 & 1470.09 \\
\hline $10 / 23 / 1120: 30$ & 36.398 & 39.027 & 1472.37 & 1470.10 \\
\hline $10 / 23 / 1120: 40$ & 36.358 & 39.024 & 1472.41 & 1470.10 \\
\hline $10 / 23 / 1120: 50$ & 36.353 & 39.022 & 1472.42 & 1470.10 \\
\hline 10/23/11 21:00 & 36.386 & 39.02 & 1472.38 & 1470.10 \\
\hline 10/23/11 21:10 & 36.344 & 39.015 & 1472.43 & 1470.11 \\
\hline 10/23/11 21:20 & 36.372 & 39.01 & 1472.40 & 1470.11 \\
\hline $10 / 23 / 1121: 30$ & 36.363 & 39.008 & 1472.41 & 1470.11 \\
\hline $10 / 23 / 1121: 40$ & 36.393 & 39.006 & 1472.38 & 1470.12 \\
\hline $10 / 23 / 1121: 50$ & 36.386 & 39.003 & 1472.38 & 1470.12 \\
\hline $10 / 23 / 1122: 00$ & 36.339 & 39.001 & 1472.43 & 1470.12 \\
\hline $10 / 23 / 1122: 10$ & 36.407 & 39.001 & 1472.36 & 1470.12 \\
\hline $10 / 23 / 1122: 20$ & 36.396 & 39.003 & 1472.37 & 1470.12 \\
\hline $10 / 23 / 1122: 30$ & 36.921 & 39.003 & 1471.85 & 1470.12 \\
\hline $10 / 23 / 1122: 40$ & 36.883 & 39.006 & 1471.89 & 1470.12 \\
\hline $10 / 23 / 1122: 50$ & 36.6 & 39.006 & 1472.17 & 1470.12 \\
\hline $10 / 23 / 1123: 00$ & 36.485 & 39.003 & 1472.28 & 1470.12 \\
\hline $10 / 23 / 1123: 10$ & 36.677 & 39.001 & 1472.09 & 1470.12 \\
\hline $10 / 23 / 1123: 20$ & 37.001 & 38.996 & 1471.77 & 1470.13 \\
\hline $10 / 23 / 1123: 30$ & 36.529 & 38.991 & 1472.24 & 1470.13 \\
\hline $10 / 23 / 1123: 40$ & 36.417 & 38.987 & 1472.35 & 1470.14 \\
\hline $10 / 23 / 1123: 50$ & 36.363 & 38.98 & 1472.41 & 1470.14 \\
\hline 10/24/11 0:00 & 36.299 & 38.975 & 1472.47 & 1470.15 \\
\hline 10/24/11 0:10 & 36.267 & 38.97 & 1472.50 & 1470.15 \\
\hline 10/24/11 0:20 & 36.22 & 38.963 & 1472.55 & 1470.16 \\
\hline 10/24/11 0:30 & 36.192 & 38.956 & 1472.58 & 1470.17 \\
\hline 10/24/11 0:40 & 36.161 & 38.947 & 1472.61 & 1470.18 \\
\hline
\end{tabular}


TABLE S1.3 Automatically recorded groundwater levels in the Klassen wells, September 15, 2011, to March 13, 2012.

\begin{tabular}{|c|c|c|c|c|}
\hline \multicolumn{3}{|c|}{ Reference Elevation (ft AMSL) } & 1508.77 & \multirow{2}{*}{$\begin{array}{l}1509.12 \\
\text { Elevation in Klassen } \\
\text { Test Well (ft AMSL) }\end{array}$} \\
\hline Date and Time & $\begin{array}{l}\text { Depth in Klassen } \\
\text { Domestic Well } \\
(\mathrm{ft} \mathrm{BGL})\end{array}$ & $\begin{array}{c}\text { Depth in Klassen } \\
\text { Test Well } \\
\text { (ft BGL) }\end{array}$ & $\begin{array}{l}\text { Elevation in Klassen } \\
\text { Domestic Well } \\
\text { (ft AMSL) }\end{array}$ & \\
\hline $10 / 24 / 110: 50$ & 36.131 & 38.942 & 1472.64 & 1470.18 \\
\hline 10/24/11 1:00 & 36.107 & 38.933 & 1472.66 & 1470.19 \\
\hline 10/24/11 1:10 & 36.077 & 38.923 & 1472.69 & 1470.20 \\
\hline $10 / 24 / 111: 20$ & 41.278 & 38.916 & 1467.49 & 1470.21 \\
\hline 10/24/11 1:30 & 36.196 & 38.914 & 1472.57 & 1470.21 \\
\hline 10/24/11 1:40 & 36.168 & 38.907 & 1472.60 & 1470.22 \\
\hline 10/24/11 1:50 & 36.189 & 38.902 & 1472.58 & 1470.22 \\
\hline $10 / 24 / 112: 00$ & 36.156 & 38.898 & 1472.61 & 1470.22 \\
\hline $10 / 24 / 112: 10$ & 36.124 & 38.891 & 1472.65 & 1470.23 \\
\hline $10 / 24 / 112: 20$ & 36.091 & 38.884 & 1472.68 & 1470.24 \\
\hline $10 / 24 / 112: 30$ & 36.06 & 38.879 & 1472.71 & 1470.24 \\
\hline $10 / 24 / 112: 40$ & 36.037 & 38.869 & 1472.73 & 1470.25 \\
\hline $10 / 24 / 112: 50$ & 36.02 & 38.86 & 1472.75 & 1470.26 \\
\hline 10/24/11 3:00 & 36.004 & 38.851 & 1472.77 & 1470.27 \\
\hline 10/24/11 3:10 & 35.988 & 38.841 & 1472.78 & 1470.28 \\
\hline 10/24/11 3:20 & 36.006 & 38.832 & 1472.76 & 1470.29 \\
\hline $10 / 24 / 113: 30$ & 36.046 & 38.83 & 1472.72 & 1470.29 \\
\hline 10/24/11 3:40 & 36.004 & 38.823 & 1472.77 & 1470.30 \\
\hline 10/24/11 3:50 & 35.985 & 38.816 & 1472.78 & 1470.31 \\
\hline $10 / 24 / 114: 00$ & 35.943 & 38.806 & 1472.83 & 1470.32 \\
\hline 10/24/11 4:10 & 35.927 & 38.799 & 1472.84 & 1470.32 \\
\hline $10 / 24 / 114: 20$ & 35.889 & 38.792 & 1472.88 & 1470.33 \\
\hline $10 / 24 / 114: 30$ & 35.887 & 38.785 & 1472.88 & 1470.34 \\
\hline $10 / 24 / 114: 40$ & 35.877 & 38.778 & 1472.89 & 1470.34 \\
\hline $10 / 24 / 114: 50$ & 35.842 & 38.771 & 1472.93 & 1470.35 \\
\hline 10/24/11 5:00 & 35.835 & 38.764 & 1472.93 & 1470.36 \\
\hline 10/24/11 5:10 & 35.812 & 38.757 & 1472.96 & 1470.37 \\
\hline 10/24/11 5:20 & 35.795 & 38.75 & 1472.97 & 1470.37 \\
\hline $10 / 24 / 115: 30$ & 35.783 & 38.743 & 1472.99 & 1470.38 \\
\hline 10/24/11 5:40 & 35.76 & 38.738 & 1473.01 & 1470.38 \\
\hline 10/24/11 5:50 & 35.753 & 38.726 & 1473.02 & 1470.40 \\
\hline 10/24/11 6:00 & 35.938 & 38.722 & 1472.83 & 1470.40 \\
\hline 10/24/11 6:10 & 39.639 & 38.715 & 1469.13 & 1470.41 \\
\hline $10 / 24 / 116: 20$ & 35.962 & 38.71 & 1472.81 & 1470.41 \\
\hline 10/24/11 6:30 & 35.887 & 38.708 & 1472.88 & 1470.41 \\
\hline $10 / 24 / 116: 40$ & 35.842 & 38.703 & 1472.93 & 1470.42 \\
\hline $10 / 24 / 116: 50$ & 35.8 & 38.696 & 1472.97 & 1470.43 \\
\hline 10/24/11 7:00 & 35.774 & 38.689 & 1473.00 & 1470.43 \\
\hline
\end{tabular}


TABLE S1.3 Automatically recorded groundwater levels in the Klassen wells, September 15, 2011, to March 13, 2012.

\begin{tabular}{|c|c|c|c|c|}
\hline \multicolumn{3}{|c|}{ Reference Elevation (ft AMSL) } & 1508.77 & \multirow{2}{*}{$\begin{array}{l}1509.12 \\
\text { Elevation in Klassen } \\
\text { Test Well (ft AMSL) }\end{array}$} \\
\hline Date and Time & $\begin{array}{l}\text { Depth in Klassen } \\
\text { Domestic Well } \\
\text { (ft BGL) }\end{array}$ & $\begin{array}{c}\text { Depth in Klassen } \\
\text { Test Well } \\
\text { (ft BGL) }\end{array}$ & $\begin{array}{l}\text { Elevation in Klassen } \\
\text { Domestic Well } \\
\text { (ft AMSL) }\end{array}$ & \\
\hline 10/24/11 7:10 & 35.746 & 38.684 & 1473.02 & 1470.44 \\
\hline $10 / 24 / 117: 20$ & 35.737 & 38.675 & 1473.03 & 1470.45 \\
\hline 10/24/11 7:30 & 35.706 & 38.668 & 1473.06 & 1470.45 \\
\hline 10/24/11 7:40 & 35.697 & 38.665 & 1473.07 & 1470.46 \\
\hline 10/24/11 7:50 & 35.664 & 38.658 & 1473.11 & 1470.46 \\
\hline 10/24/11 8:00 & 35.884 & 38.658 & 1472.89 & 1470.46 \\
\hline 10/24/11 8:10 & 35.727 & 38.658 & 1473.04 & 1470.46 \\
\hline $10 / 24 / 118: 20$ & 35.699 & 38.654 & 1473.07 & 1470.47 \\
\hline 10/24/11 8:30 & 35.657 & 38.649 & 1473.11 & 1470.47 \\
\hline $10 / 24 / 118: 40$ & 36.201 & 38.647 & 1472.57 & 1470.48 \\
\hline 10/24/11 8:50 & 36.102 & 38.644 & 1472.67 & 1470.48 \\
\hline 10/24/11 9:00 & 35.798 & 38.642 & 1472.97 & 1470.48 \\
\hline 10/24/11 9:10 & 35.72 & 38.64 & 1473.05 & 1470.48 \\
\hline 10/24/11 9:20 & 35.697 & 38.64 & 1473.07 & 1470.48 \\
\hline 10/24/11 9:30 & 35.655 & 38.64 & 1473.11 & 1470.48 \\
\hline 10/24/11 9:40 & 35.922 & 38.637 & 1472.85 & 1470.49 \\
\hline 10/24/11 9:50 & 35.727 & 38.637 & 1473.04 & 1470.49 \\
\hline 10/24/11 10:00 & 35.685 & 38.64 & 1473.08 & 1470.48 \\
\hline 10/24/11 10:10 & 36.299 & 38.637 & 1472.47 & 1470.49 \\
\hline $10 / 24 / 11$ 10:20 & 35.725 & 38.637 & 1473.04 & 1470.49 \\
\hline $10 / 24 / 11$ 10:30 & 40.101 & 38.637 & 1468.67 & 1470.49 \\
\hline 10/24/11 10:40 & 35.779 & 38.637 & 1472.99 & 1470.49 \\
\hline $10 / 24 / 11$ 10:50 & 35.905 & 38.637 & 1472.86 & 1470.49 \\
\hline 10/24/11 11:00 & 35.744 & 38.635 & 1473.03 & 1470.49 \\
\hline 10/24/11 11:10 & 35.896 & 38.635 & 1472.87 & 1470.49 \\
\hline $10 / 24 / 11$ 11:20 & 36.947 & 38.633 & 1471.82 & 1470.49 \\
\hline 10/24/11 11:30 & 40.251 & 38.637 & 1468.52 & 1470.49 \\
\hline 10/24/11 11:40 & 36.067 & 38.64 & 1472.70 & 1470.48 \\
\hline 10/24/11 11:50 & 40.376 & 38.642 & 1468.39 & 1470.48 \\
\hline $10 / 24 / 11$ 12:00 & 35.973 & 38.642 & 1472.80 & 1470.48 \\
\hline $10 / 24 / 11$ 12:10 & 35.866 & 38.644 & 1472.90 & 1470.48 \\
\hline $10 / 24 / 11$ 12:20 & 35.8 & 38.647 & 1472.97 & 1470.48 \\
\hline $10 / 24 / 11$ 12:30 & 35.744 & 38.647 & 1473.03 & 1470.48 \\
\hline $10 / 24 / 11$ 12:40 & 35.72 & 38.647 & 1473.05 & 1470.48 \\
\hline $10 / 24 / 11$ 12:50 & 35.676 & 38.642 & 1473.09 & 1470.48 \\
\hline $10 / 24 / 11$ 13:00 & 35.664 & 38.64 & 1473.11 & 1470.48 \\
\hline 10/24/11 13:10 & 35.626 & 38.635 & 1473.14 & 1470.49 \\
\hline $10 / 24 / 11$ 13:20 & 35.617 & 38.633 & 1473.15 & 1470.49 \\
\hline
\end{tabular}


TABLE S1.3 Automatically recorded groundwater levels in the Klassen wells, September 15, 2011, to March 13, 2012.

\begin{tabular}{|c|c|c|c|c|}
\hline \multicolumn{3}{|c|}{ Reference Elevation (ft AMSL) } & 1508.77 & \multirow{2}{*}{$\begin{array}{l}1509.12 \\
\text { Elevation in Klassen } \\
\text { Test Well (ft AMSL) }\end{array}$} \\
\hline Date and Time & $\begin{array}{l}\text { Depth in Klassen } \\
\text { Domestic Well } \\
(\mathrm{ft} \mathrm{BGL})\end{array}$ & $\begin{array}{c}\text { Depth in Klassen } \\
\text { Test Well } \\
\text { (ft BGL) }\end{array}$ & $\begin{array}{l}\text { Elevation in Klassen } \\
\text { Domestic Well } \\
\text { (ft AMSL) }\end{array}$ & \\
\hline 10/24/11 13:30 & 35.584 & 38.628 & 1473.19 & 1470.49 \\
\hline $10 / 24 / 1113: 40$ & 36.192 & 38.623 & 1472.58 & 1470.50 \\
\hline 10/24/11 13:50 & 36.004 & 38.621 & 1472.77 & 1470.50 \\
\hline 10/24/11 14:00 & 35.744 & 38.621 & 1473.03 & 1470.50 \\
\hline 10/24/11 14:10 & 35.666 & 38.618 & 1473.10 & 1470.50 \\
\hline $10 / 24 / 1114: 20$ & 36.55 & 38.611 & 1472.22 & 1470.51 \\
\hline $10 / 24 / 1114: 30$ & 35.713 & 38.609 & 1473.06 & 1470.51 \\
\hline $10 / 24 / 1114: 40$ & 36.532 & 38.604 & 1472.24 & 1470.52 \\
\hline $10 / 24 / 1114: 50$ & 38.635 & 38.602 & 1470.13 & 1470.52 \\
\hline $10 / 24 / 1115: 00$ & 36.063 & 38.597 & 1472.71 & 1470.53 \\
\hline $10 / 24 / 1115: 10$ & 36.281 & 38.595 & 1472.49 & 1470.53 \\
\hline $10 / 24 / 11$ 15:20 & 36.862 & 38.593 & 1471.91 & 1470.53 \\
\hline 10/24/11 15:30 & 38.739 & 38.59 & 1470.03 & 1470.53 \\
\hline $10 / 24 / 1115: 40$ & 37.557 & 38.588 & 1471.21 & 1470.53 \\
\hline $10 / 24 / 11$ 15:50 & 38.513 & 38.586 & 1470.26 & 1470.54 \\
\hline 10/24/11 16:00 & 37.235 & 38.586 & 1471.53 & 1470.54 \\
\hline 10/24/11 16:10 & 36.156 & 38.59 & 1472.61 & 1470.53 \\
\hline 10/24/11 16:20 & 36.187 & 38.59 & 1472.58 & 1470.53 \\
\hline 10/24/11 16:30 & 36.321 & 38.595 & 1472.45 & 1470.53 \\
\hline $10 / 24 / 11$ 16:40 & 36.389 & 38.595 & 1472.38 & 1470.53 \\
\hline $10 / 24 / 1116: 50$ & 37.829 & 38.597 & 1470.94 & 1470.53 \\
\hline 10/24/11 17:00 & 36.783 & 38.602 & 1471.99 & 1470.52 \\
\hline $10 / 24 / 11$ 17:10 & 36.633 & 38.604 & 1472.14 & 1470.52 \\
\hline $10 / 24 / 11$ 17:20 & 36.651 & 38.607 & 1472.12 & 1470.52 \\
\hline 10/24/11 17:30 & 36.771 & 38.609 & 1472.00 & 1470.51 \\
\hline 10/24/11 17:40 & 36.654 & 38.611 & 1472.12 & 1470.51 \\
\hline 10/24/11 17:50 & 36.715 & 38.618 & 1472.05 & 1470.50 \\
\hline 10/24/11 18:00 & 36.853 & 38.621 & 1471.92 & 1470.50 \\
\hline 10/24/11 18:10 & 37.111 & 38.625 & 1471.66 & 1470.50 \\
\hline 10/24/11 18:20 & 37.263 & 38.63 & 1471.51 & 1470.49 \\
\hline 10/24/11 18:30 & 37.866 & 38.635 & 1470.90 & 1470.49 \\
\hline $10 / 24 / 11$ 18:40 & 39.032 & 38.642 & 1469.74 & 1470.48 \\
\hline $10 / 24 / 11$ 18:50 & 40.61 & 38.649 & 1468.16 & 1470.47 \\
\hline 10/24/11 19:00 & 39.719 & 38.654 & 1469.05 & 1470.47 \\
\hline 10/24/11 19:10 & 36.797 & 38.658 & 1471.97 & 1470.46 \\
\hline $10 / 24 / 11$ 19:20 & 36.595 & 38.658 & 1472.17 & 1470.46 \\
\hline 10/24/11 19:30 & 36.478 & 38.658 & 1472.29 & 1470.46 \\
\hline $10 / 24 / 11$ 19:40 & 36.384 & 38.656 & 1472.39 & 1470.47 \\
\hline
\end{tabular}


TABLE S1.3 Automatically recorded groundwater levels in the Klassen wells, September 15, 2011, to March 13, 2012.

\begin{tabular}{|c|c|c|c|c|}
\hline \multicolumn{3}{|c|}{ Reference Elevation (ft AMSL) } & 1508.77 & \multirow{2}{*}{$\begin{array}{l}1509.12 \\
\text { Elevation in Klassen } \\
\text { Test Well (ft AMSL) }\end{array}$} \\
\hline Date and Time & $\begin{array}{l}\text { Depth in Klassen } \\
\text { Domestic Well } \\
\text { (ft BGL) }\end{array}$ & $\begin{array}{c}\text { Depth in Klassen } \\
\text { Test Well } \\
\text { (ft BGL) }\end{array}$ & $\begin{array}{l}\text { Elevation in Klassen } \\
\text { Domestic Well } \\
\text { (ft AMSL) }\end{array}$ & \\
\hline $10 / 24 / 1119: 50$ & 36.337 & 38.651 & 1472.43 & 1470.47 \\
\hline $10 / 24 / 1120: 00$ & 36.529 & 38.654 & 1472.24 & 1470.47 \\
\hline $10 / 24 / 1120: 10$ & 36.4 & 38.656 & 1472.37 & 1470.47 \\
\hline $10 / 24 / 1120: 20$ & 36.4 & 38.654 & 1472.37 & 1470.47 \\
\hline $10 / 24 / 1120: 30$ & 36.358 & 38.656 & 1472.41 & 1470.47 \\
\hline $10 / 24 / 1120: 40$ & 36.276 & 38.654 & 1472.49 & 1470.47 \\
\hline $10 / 24 / 1120: 50$ & 36.229 & 38.651 & 1472.54 & 1470.47 \\
\hline 10/24/11 21:00 & 36.192 & 38.647 & 1472.58 & 1470.48 \\
\hline 10/24/11 21:10 & 36.175 & 38.644 & 1472.59 & 1470.48 \\
\hline $10 / 24 / 1121: 20$ & 36.117 & 38.642 & 1472.65 & 1470.48 \\
\hline $10 / 24 / 1121: 30$ & 36.086 & 38.64 & 1472.68 & 1470.48 \\
\hline $10 / 24 / 1121: 40$ & 36.039 & 38.637 & 1472.73 & 1470.49 \\
\hline $10 / 24 / 1121: 50$ & 36.032 & 38.635 & 1472.74 & 1470.49 \\
\hline $10 / 24 / 1122: 00$ & 35.992 & 38.633 & 1472.78 & 1470.49 \\
\hline $10 / 24 / 1122: 10$ & 35.988 & 38.633 & 1472.78 & 1470.49 \\
\hline $10 / 24 / 1122: 20$ & 35.999 & 38.63 & 1472.77 & 1470.49 \\
\hline $10 / 24 / 1122: 30$ & 39.17 & 38.628 & 1469.60 & 1470.49 \\
\hline $10 / 24 / 1122: 40$ & 36.576 & 38.628 & 1472.19 & 1470.49 \\
\hline $10 / 24 / 1122: 50$ & 36.138 & 38.625 & 1472.63 & 1470.50 \\
\hline $10 / 24 / 1123: 00$ & 36.363 & 38.625 & 1472.41 & 1470.50 \\
\hline $10 / 24 / 1123: 10$ & 36.482 & 38.625 & 1472.29 & 1470.50 \\
\hline $10 / 24 / 1123: 20$ & 36.161 & 38.623 & 1472.61 & 1470.50 \\
\hline $10 / 24 / 1123: 30$ & 36.053 & 38.621 & 1472.72 & 1470.50 \\
\hline $10 / 24 / 1123: 40$ & 36.002 & 38.616 & 1472.77 & 1470.51 \\
\hline $10 / 24 / 1123: 50$ & 35.941 & 38.611 & 1472.83 & 1470.51 \\
\hline $10 / 25 / 110: 00$ & 35.889 & 38.607 & 1472.88 & 1470.52 \\
\hline 10/25/11 0:10 & 35.866 & 38.602 & 1472.90 & 1470.52 \\
\hline 10/25/11 0:20 & 35.823 & 38.595 & 1472.95 & 1470.53 \\
\hline $10 / 25 / 110: 30$ & 35.809 & 38.588 & 1472.96 & 1470.53 \\
\hline 10/25/11 0:40 & 35.772 & 38.581 & 1473.00 & 1470.54 \\
\hline 10/25/11 0:50 & 35.744 & 38.576 & 1473.03 & 1470.55 \\
\hline 10/25/11 1:00 & 35.723 & 38.572 & 1473.05 & 1470.55 \\
\hline 10/25/11 1:10 & 35.69 & 38.562 & 1473.08 & 1470.56 \\
\hline 10/25/11 1:20 & 35.685 & 38.555 & 1473.08 & 1470.57 \\
\hline 10/25/11 1:30 & 35.647 & 38.548 & 1473.12 & 1470.57 \\
\hline 10/25/11 1:40 & 35.645 & 38.543 & 1473.12 & 1470.58 \\
\hline 10/25/11 1:50 & 35.615 & 38.534 & 1473.15 & 1470.59 \\
\hline $10 / 25 / 112: 00$ & 35.596 & 38.527 & 1473.17 & 1470.60 \\
\hline
\end{tabular}


TABLE S1.3 Automatically recorded groundwater levels in the Klassen wells, September 15, 2011, to March 13, 2012.

\begin{tabular}{|c|c|c|c|c|}
\hline \multicolumn{3}{|c|}{ Reference Elevation (ft AMSL) } & 1508.77 & \multirow{2}{*}{$\begin{array}{l}1509.12 \\
\text { Elevation in Klassen } \\
\text { Test Well (ft AMSL) }\end{array}$} \\
\hline Date and Time & $\begin{array}{l}\text { Depth in Klassen } \\
\text { Domestic Well } \\
(\mathrm{ft} \mathrm{BGL})\end{array}$ & $\begin{array}{c}\text { Depth in Klassen } \\
\text { Test Well } \\
\text { (ft BGL) }\end{array}$ & $\begin{array}{l}\text { Elevation in Klassen } \\
\text { Domestic Well } \\
\text { (ft AMSL) }\end{array}$ & \\
\hline $10 / 25 / 112: 10$ & 35.579 & 38.522 & 1473.19 & 1470.60 \\
\hline $10 / 25 / 112: 20$ & 35.554 & 38.515 & 1473.22 & 1470.61 \\
\hline $10 / 25 / 112: 30$ & 35.551 & 38.506 & 1473.22 & 1470.62 \\
\hline $10 / 25 / 112: 40$ & 35.523 & 38.499 & 1473.25 & 1470.62 \\
\hline $10 / 25 / 112: 50$ & 35.514 & 38.492 & 1473.26 & 1470.63 \\
\hline $10 / 25 / 113: 00$ & 35.497 & 38.485 & 1473.27 & 1470.64 \\
\hline 10/25/11 3:10 & 35.469 & 38.478 & 1473.30 & 1470.64 \\
\hline $10 / 25 / 113: 20$ & 35.474 & 38.471 & 1473.30 & 1470.65 \\
\hline $10 / 25 / 113: 30$ & 35.446 & 38.466 & 1473.32 & 1470.66 \\
\hline $10 / 25 / 113: 40$ & 35.441 & 38.457 & 1473.33 & 1470.67 \\
\hline $10 / 25 / 113: 50$ & 35.425 & 38.449 & 1473.34 & 1470.67 \\
\hline 10/25/11 4:00 & 38.413 & 38.442 & 1470.36 & 1470.68 \\
\hline 10/25/11 4:10 & 35.544 & 38.438 & 1473.23 & 1470.68 \\
\hline $10 / 25 / 114: 20$ & 35.497 & 38.433 & 1473.27 & 1470.69 \\
\hline $10 / 25 / 114: 30$ & 35.453 & 38.426 & 1473.32 & 1470.70 \\
\hline $10 / 25 / 114: 40$ & 35.441 & 38.421 & 1473.33 & 1470.70 \\
\hline $10 / 25 / 114: 50$ & 35.408 & 38.414 & 1473.36 & 1470.71 \\
\hline 10/25/11 5:00 & 35.404 & 38.407 & 1473.37 & 1470.72 \\
\hline 10/25/11 5:10 & 35.378 & 38.405 & 1473.39 & 1470.72 \\
\hline $10 / 25 / 115: 20$ & 35.368 & 38.396 & 1473.40 & 1470.73 \\
\hline $10 / 25 / 115: 30$ & 35.35 & 38.391 & 1473.42 & 1470.73 \\
\hline $10 / 25 / 115: 40$ & 35.336 & 38.384 & 1473.43 & 1470.74 \\
\hline $10 / 25 / 115: 50$ & 35.326 & 38.377 & 1473.44 & 1470.75 \\
\hline $10 / 25 / 116: 00$ & 35.303 & 38.37 & 1473.47 & 1470.75 \\
\hline $10 / 25 / 116: 10$ & 39.984 & 38.367 & 1468.79 & 1470.76 \\
\hline $10 / 25 / 116: 20$ & 35.619 & 38.367 & 1473.15 & 1470.76 \\
\hline 10/25/11 6:30 & 35.526 & 38.365 & 1473.24 & 1470.76 \\
\hline $10 / 25 / 116: 40$ & 35.469 & 38.36 & 1473.30 & 1470.76 \\
\hline $10 / 25 / 116: 50$ & 35.467 & 38.356 & 1473.30 & 1470.77 \\
\hline $10 / 25 / 117: 00$ & 35.462 & 38.351 & 1473.31 & 1470.77 \\
\hline 10/25/11 7:10 & 35.488 & 38.349 & 1473.28 & 1470.77 \\
\hline $10 / 25 / 117: 20$ & 35.495 & 38.346 & 1473.27 & 1470.78 \\
\hline $10 / 25 / 117: 30$ & 35.453 & 38.344 & 1473.32 & 1470.78 \\
\hline $10 / 25 / 117: 40$ & 35.436 & 38.339 & 1473.33 & 1470.78 \\
\hline $10 / 25 / 117: 50$ & 35.401 & 38.335 & 1473.37 & 1470.79 \\
\hline $10 / 25 / 118: 00$ & 35.755 & 38.337 & 1473.01 & 1470.79 \\
\hline $10 / 25 / 118: 10$ & 35.488 & 38.332 & 1473.28 & 1470.79 \\
\hline $10 / 25 / 118: 20$ & 35.42 & 38.33 & 1473.35 & 1470.79 \\
\hline
\end{tabular}


TABLE S1.3 Automatically recorded groundwater levels in the Klassen wells, September 15, 2011, to March 13, 2012.

\begin{tabular}{|c|c|c|c|c|}
\hline \multicolumn{3}{|c|}{ Reference Elevation (ft AMSL) } & 1508.77 & \multirow{2}{*}{$\begin{array}{l}1509.12 \\
\text { Elevation in Klassen } \\
\text { Test Well (ft AMSL) }\end{array}$} \\
\hline Date and Time & $\begin{array}{l}\text { Depth in Klassen } \\
\text { Domestic Well } \\
\text { (ft BGL) }\end{array}$ & $\begin{array}{c}\text { Depth in Klassen } \\
\text { Test Well } \\
\text { (ft BGL) }\end{array}$ & $\begin{array}{l}\text { Elevation in Klassen } \\
\text { Domestic Well } \\
\text { (ft AMSL) }\end{array}$ & \\
\hline $10 / 25 / 118: 30$ & 35.397 & 38.325 & 1473.37 & 1470.80 \\
\hline $10 / 25 / 118: 40$ & 35.364 & 38.318 & 1473.41 & 1470.80 \\
\hline $10 / 25 / 118: 50$ & 35.345 & 38.318 & 1473.42 & 1470.80 \\
\hline 10/25/11 9:00 & 35.326 & 38.311 & 1473.44 & 1470.81 \\
\hline 10/25/11 9:10 & 35.298 & 38.309 & 1473.47 & 1470.81 \\
\hline 10/25/11 9:20 & 35.298 & 38.304 & 1473.47 & 1470.82 \\
\hline 10/25/11 9:30 & 35.272 & 38.302 & 1473.50 & 1470.82 \\
\hline 10/25/11 9:40 & 35.27 & 38.295 & 1473.50 & 1470.83 \\
\hline 10/25/11 9:50 & 35.244 & 38.292 & 1473.53 & 1470.83 \\
\hline 10/25/11 10:00 & 35.235 & 38.283 & 1473.53 & 1470.84 \\
\hline 10/25/11 10:10 & 35.221 & 38.283 & 1473.55 & 1470.84 \\
\hline $10 / 25 / 1110: 20$ & 35.202 & 38.278 & 1473.57 & 1470.84 \\
\hline 10/25/11 10:30 & 35.204 & 38.276 & 1473.57 & 1470.85 \\
\hline $10 / 25 / 11$ 10:40 & 35.183 & 38.271 & 1473.59 & 1470.85 \\
\hline $10 / 25 / 1110: 50$ & 35.162 & 38.266 & 1473.61 & 1470.86 \\
\hline 10/25/11 11:00 & 35.167 & 38.264 & 1473.60 & 1470.86 \\
\hline 10/25/11 11:10 & 35.481 & 38.259 & 1473.29 & 1470.86 \\
\hline 10/25/11 11:20 & 35.284 & 38.255 & 1473.49 & 1470.87 \\
\hline 10/25/11 11:30 & 35.235 & 38.257 & 1473.53 & 1470.87 \\
\hline $10 / 25 / 11$ 11:40 & 35.195 & 38.252 & 1473.57 & 1470.87 \\
\hline $10 / 25 / 1111: 50$ & 35.19 & 38.25 & 1473.58 & 1470.87 \\
\hline $10 / 25 / 1112: 00$ & 35.326 & 38.245 & 1473.44 & 1470.88 \\
\hline $10 / 25 / 11$ 12:10 & 35.469 & 38.245 & 1473.30 & 1470.88 \\
\hline $10 / 25 / 11$ 12:20 & 35.563 & 38.245 & 1473.21 & 1470.88 \\
\hline $10 / 25 / 1112: 30$ & 35.758 & 38.248 & 1473.01 & 1470.87 \\
\hline $10 / 25 / 11$ 12:40 & 36.571 & 38.248 & 1472.20 & 1470.87 \\
\hline $10 / 25 / 1112: 50$ & 39.517 & 38.248 & 1469.25 & 1470.87 \\
\hline 10/25/11 13:00 & 36.633 & 38.248 & 1472.14 & 1470.87 \\
\hline $10 / 25 / 11$ 13:10 & 35.701 & 38.248 & 1473.07 & 1470.87 \\
\hline $10 / 25 / 11$ 13:20 & 35.739 & 38.25 & 1473.03 & 1470.87 \\
\hline $10 / 25 / 11$ 13:30 & 36.1 & 38.248 & 1472.67 & 1470.87 \\
\hline $10 / 25 / 11$ 13:40 & 37.629 & 38.248 & 1471.14 & 1470.87 \\
\hline $10 / 25 / 11$ 13:50 & 35.645 & 38.245 & 1473.12 & 1470.88 \\
\hline $10 / 25 / 11$ 14:00 & 35.711 & 38.245 & 1473.06 & 1470.88 \\
\hline $10 / 25 / 11$ 14:10 & 35.786 & 38.241 & 1472.98 & 1470.88 \\
\hline $10 / 25 / 11$ 14:20 & 36.009 & 38.241 & 1472.76 & 1470.88 \\
\hline $10 / 25 / 11$ 14:30 & 39.043 & 38.238 & 1469.73 & 1470.88 \\
\hline $10 / 25 / 11$ 14:40 & 36.7 & 38.241 & 1472.07 & 1470.88 \\
\hline
\end{tabular}


TABLE S1.3 Automatically recorded groundwater levels in the Klassen wells, September 15, 2011, to March 13, 2012.

\begin{tabular}{|c|c|c|c|c|}
\hline \multicolumn{3}{|c|}{ Reference Elevation (ft AMSL) } & 1508.77 & \multirow{2}{*}{$\begin{array}{l}1509.12 \\
\text { Elevation in Klassen } \\
\text { Test Well (ft AMSL) }\end{array}$} \\
\hline Date and Time & $\begin{array}{l}\text { Depth in Klassen } \\
\text { Domestic Well } \\
\text { (ft BGL) }\end{array}$ & $\begin{array}{c}\text { Depth in Klassen } \\
\text { Test Well } \\
\text { (ft BGL) }\end{array}$ & $\begin{array}{l}\text { Elevation in Klassen } \\
\text { Domestic Well } \\
\text { (ft AMSL) }\end{array}$ & \\
\hline $10 / 25 / 1114: 50$ & 35.776 & 38.238 & 1472.99 & 1470.88 \\
\hline 10/25/11 15:00 & 35.831 & 38.238 & 1472.94 & 1470.88 \\
\hline $10 / 25 / 1115: 10$ & 35.962 & 38.238 & 1472.81 & 1470.88 \\
\hline $10 / 25 / 1115: 20$ & 36.22 & 38.238 & 1472.55 & 1470.88 \\
\hline 10/25/11 15:30 & 36.872 & 38.238 & 1471.90 & 1470.88 \\
\hline $10 / 25 / 1115: 40$ & 39.848 & 38.236 & 1468.92 & 1470.89 \\
\hline $10 / 25 / 1115: 50$ & 35.793 & 38.236 & 1472.98 & 1470.89 \\
\hline 10/25/11 16:00 & 35.859 & 38.234 & 1472.91 & 1470.89 \\
\hline $10 / 25 / 1116: 10$ & 35.927 & 38.234 & 1472.84 & 1470.89 \\
\hline $10 / 25 / 1116: 20$ & 36.157 & 38.236 & 1472.61 & 1470.89 \\
\hline 10/25/11 16:30 & 35.931 & 38.238 & 1472.84 & 1470.88 \\
\hline 10/25/11 16:40 & 36.009 & 38.243 & 1472.76 & 1470.88 \\
\hline $10 / 25 / 1116: 50$ & 36.295 & 38.243 & 1472.47 & 1470.88 \\
\hline 10/25/11 17:00 & 35.995 & 38.248 & 1472.77 & 1470.87 \\
\hline 10/25/11 17:10 & 36.035 & 38.25 & 1472.73 & 1470.87 \\
\hline $10 / 25 / 11$ 17:20 & 36.21 & 38.255 & 1472.56 & 1470.87 \\
\hline 10/25/11 17:30 & 36.83 & 38.255 & 1471.94 & 1470.87 \\
\hline $10 / 25 / 11$ 17:40 & 38.727 & 38.255 & 1470.04 & 1470.87 \\
\hline $10 / 25 / 11$ 17:50 & 42.461 & 38.257 & 1466.31 & 1470.87 \\
\hline 10/25/11 18:00 & 35.99 & 38.259 & 1472.78 & 1470.86 \\
\hline 10/25/11 18:10 & 35.999 & 38.259 & 1472.77 & 1470.86 \\
\hline $10 / 25 / 11$ 18:20 & 39.672 & 38.257 & 1469.10 & 1470.87 \\
\hline $10 / 25 / 11$ 18:30 & 36.614 & 38.259 & 1472.16 & 1470.86 \\
\hline 10/25/11 18:40 & 36.016 & 38.259 & 1472.75 & 1470.86 \\
\hline $10 / 25 / 11$ 18:50 & 36.065 & 38.262 & 1472.70 & 1470.86 \\
\hline 10/25/11 19:00 & 36.271 & 38.259 & 1472.50 & 1470.86 \\
\hline 10/25/11 19:10 & 37.43 & 38.259 & 1471.34 & 1470.86 \\
\hline 10/25/11 19:20 & 39.128 & 38.259 & 1469.64 & 1470.86 \\
\hline 10/25/11 19:30 & 35.922 & 38.262 & 1472.85 & 1470.86 \\
\hline 10/25/11 19:40 & 35.807 & 38.262 & 1472.96 & 1470.86 \\
\hline 10/25/11 19:50 & 35.917 & 38.262 & 1472.85 & 1470.86 \\
\hline $10 / 25 / 1120: 00$ & 35.8 & 38.262 & 1472.97 & 1470.86 \\
\hline $10 / 25 / 1120: 10$ & 35.741 & 38.264 & 1473.03 & 1470.86 \\
\hline $10 / 25 / 1120: 20$ & 35.706 & 38.264 & 1473.06 & 1470.86 \\
\hline $10 / 25 / 1120: 30$ & 35.645 & 38.262 & 1473.12 & 1470.86 \\
\hline $10 / 25 / 1120: 40$ & 35.594 & 38.259 & 1473.18 & 1470.86 \\
\hline $10 / 25 / 1120: 50$ & 35.544 & 38.259 & 1473.23 & 1470.86 \\
\hline $10 / 25 / 1121: 00$ & 35.504 & 38.257 & 1473.27 & 1470.87 \\
\hline
\end{tabular}


TABLE S1.3 Automatically recorded groundwater levels in the Klassen wells, September 15, 2011, to March 13, 2012.

\begin{tabular}{|c|c|c|c|c|}
\hline \multicolumn{3}{|c|}{ Reference Elevation (ft AMSL) } & 1508.77 & \multirow{2}{*}{$\begin{array}{l}1509.12 \\
\text { Elevation in Klassen } \\
\text { Test Well (ft AMSL) }\end{array}$} \\
\hline Date and Time & $\begin{array}{l}\text { Depth in Klassen } \\
\text { Domestic Well } \\
\text { (ft BGL) }\end{array}$ & $\begin{array}{c}\text { Depth in Klassen } \\
\text { Test Well } \\
\text { (ft BGL) }\end{array}$ & $\begin{array}{l}\text { Elevation in Klassen } \\
\text { Domestic Well } \\
\text { (ft AMSL) }\end{array}$ & \\
\hline $10 / 25 / 1121: 10$ & 35.467 & 38.255 & 1473.30 & 1470.87 \\
\hline $10 / 25 / 1121: 20$ & 35.516 & 38.255 & 1473.25 & 1470.87 \\
\hline $10 / 25 / 1121: 30$ & 35.584 & 38.257 & 1473.19 & 1470.87 \\
\hline $10 / 25 / 1121: 40$ & 35.551 & 38.255 & 1473.22 & 1470.87 \\
\hline $10 / 25 / 1121: 50$ & 35.504 & 38.257 & 1473.27 & 1470.87 \\
\hline $10 / 25 / 1122: 00$ & 35.46 & 38.257 & 1473.31 & 1470.87 \\
\hline $10 / 25 / 1122: 10$ & 35.425 & 38.252 & 1473.34 & 1470.87 \\
\hline $10 / 25 / 1122: 20$ & 35.389 & 38.25 & 1473.38 & 1470.87 \\
\hline $10 / 25 / 1122: 30$ & 35.359 & 38.243 & 1473.41 & 1470.88 \\
\hline $10 / 25 / 1122: 40$ & 35.57 & 38.241 & 1473.20 & 1470.88 \\
\hline $10 / 25 / 1122: 50$ & 35.624 & 38.238 & 1473.15 & 1470.88 \\
\hline $10 / 25 / 1123: 00$ & 37.409 & 38.234 & 1471.36 & 1470.89 \\
\hline $10 / 25 / 1123: 10$ & 35.57 & 38.234 & 1473.20 & 1470.89 \\
\hline $10 / 25 / 1123: 20$ & 35.469 & 38.229 & 1473.30 & 1470.89 \\
\hline $10 / 25 / 1123: 30$ & 35.411 & 38.222 & 1473.36 & 1470.90 \\
\hline $10 / 25 / 1123: 40$ & 35.366 & 38.217 & 1473.40 & 1470.91 \\
\hline $10 / 25 / 1123: 50$ & 35.329 & 38.215 & 1473.44 & 1470.91 \\
\hline $10 / 26 / 110: 00$ & 35.298 & 38.21 & 1473.47 & 1470.91 \\
\hline 10/26/11 0:10 & 35.268 & 38.21 & 1473.50 & 1470.91 \\
\hline $10 / 26 / 110: 20$ & 35.242 & 38.205 & 1473.53 & 1470.92 \\
\hline $10 / 26 / 110: 30$ & 35.221 & 38.194 & 1473.55 & 1470.93 \\
\hline 10/26/11 0:40 & 35.2 & 38.187 & 1473.57 & 1470.94 \\
\hline $10 / 26 / 110: 50$ & 35.176 & 38.187 & 1473.59 & 1470.94 \\
\hline 10/26/11 1:00 & 35.157 & 38.182 & 1473.61 & 1470.94 \\
\hline 10/26/11 1:10 & 35.136 & 38.18 & 1473.63 & 1470.94 \\
\hline 10/26/11 1:20 & 35.117 & 38.17 & 1473.65 & 1470.95 \\
\hline 10/26/11 1:30 & 35.099 & 38.168 & 1473.67 & 1470.95 \\
\hline 10/26/11 1:40 & 35.15 & 38.156 & 1473.62 & 1470.97 \\
\hline 10/26/11 1:50 & 35.2 & 38.149 & 1473.57 & 1470.97 \\
\hline $10 / 26 / 112: 00$ & 35.174 & 38.149 & 1473.60 & 1470.97 \\
\hline $10 / 26 / 112: 10$ & 35.143 & 38.149 & 1473.63 & 1470.97 \\
\hline $10 / 26 / 112: 20$ & 35.141 & 38.147 & 1473.63 & 1470.98 \\
\hline $10 / 26 / 112: 30$ & 35.12 & 38.14 & 1473.65 & 1470.98 \\
\hline $10 / 26 / 112: 40$ & 35.092 & 38.128 & 1473.68 & 1470.99 \\
\hline $10 / 26 / 112: 50$ & 35.099 & 38.121 & 1473.67 & 1471.00 \\
\hline 10/26/11 3:00 & 37.059 & 38.114 & 1471.71 & 1471.01 \\
\hline 10/26/11 3:10 & 35.218 & 38.112 & 1473.55 & 1471.01 \\
\hline 10/26/11 3:20 & 35.204 & 38.112 & 1473.57 & 1471.01 \\
\hline
\end{tabular}


TABLE S1.3 Automatically recorded groundwater levels in the Klassen wells, September 15, 2011, to March 13, 2012.

\begin{tabular}{|c|c|c|c|c|}
\hline \multicolumn{3}{|c|}{ Reference Elevation (ft AMSL) } & 1508.77 & \multirow{2}{*}{$\begin{array}{l}1509.12 \\
\text { Elevation in Klassen } \\
\text { Test Well (ft AMSL) }\end{array}$} \\
\hline Date and Time & $\begin{array}{l}\text { Depth in Klassen } \\
\text { Domestic Well } \\
\text { (ft BGL) }\end{array}$ & $\begin{array}{c}\text { Depth in Klassen } \\
\text { Test Well } \\
\text { (ft BGL) }\end{array}$ & $\begin{array}{l}\text { Elevation in Klassen } \\
\text { Domestic Well } \\
\text { (ft AMSL) }\end{array}$ & \\
\hline $10 / 26 / 113: 30$ & 35.232 & 38.105 & 1473.54 & 1471.02 \\
\hline $10 / 26 / 113: 40$ & 35.19 & 38.105 & 1473.58 & 1471.02 \\
\hline 10/26/11 3:50 & 35.153 & 38.098 & 1473.62 & 1471.02 \\
\hline $10 / 26 / 114: 00$ & 35.117 & 38.091 & 1473.65 & 1471.03 \\
\hline 10/26/11 4:10 & 35.087 & 38.095 & 1473.68 & 1471.03 \\
\hline $10 / 26 / 114: 20$ & 35.061 & 38.093 & 1473.71 & 1471.03 \\
\hline $10 / 26 / 114: 30$ & 35.04 & 38.081 & 1473.73 & 1471.04 \\
\hline $10 / 26 / 114: 40$ & 35.021 & 38.065 & 1473.75 & 1471.06 \\
\hline $10 / 26 / 114: 50$ & 35.01 & 38.027 & 1473.76 & 1471.10 \\
\hline $10 / 26 / 115: 00$ & 34.986 & 38.051 & 1473.78 & 1471.07 \\
\hline 10/26/11 5:10 & 34.967 & 38.046 & 1473.80 & 1471.08 \\
\hline 10/26/11 5:20 & 34.953 & 38.044 & 1473.82 & 1471.08 \\
\hline $10 / 26 / 115: 30$ & 34.937 & 38.03 & 1473.83 & 1471.09 \\
\hline $10 / 26 / 115: 40$ & 34.923 & 38.027 & 1473.85 & 1471.10 \\
\hline $10 / 26 / 115: 50$ & 35.265 & 38.022 & 1473.50 & 1471.10 \\
\hline 10/26/11 6:00 & 35.003 & 38.02 & 1473.77 & 1471.10 \\
\hline 10/26/11 6:10 & 35.673 & 38.02 & 1473.10 & 1471.10 \\
\hline $10 / 26 / 116: 20$ & 35.127 & 38.013 & 1473.64 & 1471.11 \\
\hline $10 / 26 / 116: 30$ & 37.763 & 38.008 & 1471.01 & 1471.11 \\
\hline $10 / 26 / 116: 40$ & 35.558 & 38.011 & 1473.21 & 1471.11 \\
\hline $10 / 26 / 116: 50$ & 35.261 & 38.013 & 1473.51 & 1471.11 \\
\hline 10/26/11 7:00 & 36.227 & 38.008 & 1472.54 & 1471.11 \\
\hline 10/26/11 7:10 & 35.223 & 38.006 & 1473.55 & 1471.12 \\
\hline $10 / 26 / 117: 20$ & 35.141 & 38.001 & 1473.63 & 1471.12 \\
\hline 10/26/11 7:30 & 35.089 & 37.999 & 1473.68 & 1471.12 \\
\hline $10 / 26 / 117: 40$ & 35.052 & 37.997 & 1473.72 & 1471.13 \\
\hline $10 / 26 / 117: 50$ & 35.045 & 37.992 & 1473.72 & 1471.13 \\
\hline 10/26/11 8:00 & 35.017 & 37.99 & 1473.75 & 1471.13 \\
\hline 10/26/11 8:10 & 34.986 & 37.985 & 1473.78 & 1471.14 \\
\hline $10 / 26 / 118: 20$ & 34.965 & 37.983 & 1473.80 & 1471.14 \\
\hline $10 / 26 / 118: 30$ & 34.946 & 37.98 & 1473.82 & 1471.14 \\
\hline 10/26/11 8:40 & 34.923 & 37.983 & 1473.85 & 1471.14 \\
\hline $10 / 26 / 118: 50$ & 40.383 & 37.98 & 1468.39 & 1471.14 \\
\hline 10/26/11 9:00 & 35.106 & 37.978 & 1473.66 & 1471.14 \\
\hline 10/26/11 9:10 & 35.005 & 37.978 & 1473.76 & 1471.14 \\
\hline 10/26/11 9:20 & 34.956 & 37.976 & 1473.81 & 1471.15 \\
\hline 10/26/11 9:30 & 34.927 & 37.973 & 1473.84 & 1471.15 \\
\hline 10/26/11 9:40 & 37.578 & 37.973 & 1471.19 & 1471.15 \\
\hline
\end{tabular}


TABLE S1.3 Automatically recorded groundwater levels in the Klassen wells, September 15, 2011, to March 13, 2012.

\begin{tabular}{|c|c|c|c|c|}
\hline \multicolumn{3}{|c|}{ Reference Elevation (ft AMSL) } & 1508.77 & \multirow{2}{*}{$\begin{array}{l}1509.12 \\
\text { Elevation in Klassen } \\
\text { Test Well (ft AMSL) }\end{array}$} \\
\hline Date and Time & $\begin{array}{l}\text { Depth in Klassen } \\
\text { Domestic Well } \\
\text { (ft BGL) }\end{array}$ & $\begin{array}{c}\text { Depth in Klassen } \\
\text { Test Well } \\
\text { (ft BGL) }\end{array}$ & $\begin{array}{l}\text { Elevation in Klassen } \\
\text { Domestic Well } \\
\text { (ft AMSL) }\end{array}$ & \\
\hline 10/26/11 9:50 & 35.061 & 37.971 & 1473.71 & 1471.15 \\
\hline 10/26/11 10:00 & 34.977 & 37.971 & 1473.79 & 1471.15 \\
\hline 10/26/11 10:10 & 34.937 & 37.971 & 1473.83 & 1471.15 \\
\hline $10 / 26 / 11$ 10:20 & 34.906 & 37.973 & 1473.86 & 1471.15 \\
\hline 10/26/11 10:30 & 34.883 & 37.973 & 1473.89 & 1471.15 \\
\hline $10 / 26 / 11$ 10:40 & 34.862 & 37.973 & 1473.91 & 1471.15 \\
\hline $10 / 26 / 1110: 50$ & 34.845 & 37.971 & 1473.92 & 1471.15 \\
\hline 10/26/11 11:00 & 34.827 & 37.969 & 1473.94 & 1471.15 \\
\hline 10/26/11 11:10 & 40.856 & 37.969 & 1467.91 & 1471.15 \\
\hline $10 / 26 / 1111: 20$ & 39.559 & 37.966 & 1469.21 & 1471.16 \\
\hline 10/26/11 11:30 & 37.671 & 37.964 & 1471.10 & 1471.16 \\
\hline 10/26/11 11:40 & 38.166 & 37.964 & 1470.60 & 1471.16 \\
\hline 10/26/11 11:50 & 35.162 & 37.964 & 1473.61 & 1471.16 \\
\hline $10 / 26 / 1112: 00$ & 35.218 & 37.966 & 1473.55 & 1471.16 \\
\hline 10/26/11 12:10 & 35.272 & 37.969 & 1473.50 & 1471.15 \\
\hline $10 / 26 / 11$ 12:20 & 35.39 & 37.969 & 1473.38 & 1471.15 \\
\hline $10 / 26 / 11$ 12:30 & 35.72 & 37.973 & 1473.05 & 1471.15 \\
\hline $10 / 26 / 11$ 12:40 & 36.83 & 37.978 & 1471.94 & 1471.14 \\
\hline $10 / 26 / 11$ 12:50 & 40.108 & 37.976 & 1468.66 & 1471.15 \\
\hline 10/26/11 13:00 & 35.359 & 37.973 & 1473.41 & 1471.15 \\
\hline 10/26/11 13:10 & 35.373 & 37.971 & 1473.40 & 1471.15 \\
\hline $10 / 26 / 11$ 13:20 & 35.434 & 37.971 & 1473.34 & 1471.15 \\
\hline 10/26/11 13:30 & 35.563 & 37.973 & 1473.21 & 1471.15 \\
\hline 10/26/11 13:40 & 39.344 & 37.971 & 1469.43 & 1471.15 \\
\hline 10/26/11 13:50 & 36.888 & 37.969 & 1471.88 & 1471.15 \\
\hline 10/26/11 14:00 & 35.631 & 37.969 & 1473.14 & 1471.15 \\
\hline $10 / 26 / 11$ 14:10 & 35.648 & 37.969 & 1473.12 & 1471.15 \\
\hline $10 / 26 / 11$ 14:20 & 35.598 & 37.966 & 1473.17 & 1471.16 \\
\hline 10/26/11 14:30 & 35.631 & 37.966 & 1473.14 & 1471.16 \\
\hline $10 / 26 / 11$ 14:40 & 35.744 & 37.959 & 1473.03 & 1471.16 \\
\hline 10/26/11 14:50 & 36.161 & 37.959 & 1472.61 & 1471.16 \\
\hline 10/26/11 15:00 & 39.217 & 37.959 & 1469.55 & 1471.16 \\
\hline $10 / 26 / 11$ 15:10 & 35.474 & 37.954 & 1473.30 & 1471.17 \\
\hline $10 / 26 / 11$ 15:20 & 36.94 & 37.952 & 1471.83 & 1471.17 \\
\hline 10/26/11 15:30 & 39.932 & 37.95 & 1468.84 & 1471.17 \\
\hline $10 / 26 / 11$ 15:40 & 38.319 & 37.947 & 1470.45 & 1471.18 \\
\hline $10 / 26 / 11$ 15:50 & 41.185 & 37.954 & 1467.58 & 1471.17 \\
\hline 10/26/11 16:00 & 44.712 & 37.959 & 1464.06 & 1471.16 \\
\hline
\end{tabular}


TABLE S1.3 Automatically recorded groundwater levels in the Klassen wells, September 15, 2011, to March 13, 2012.

\begin{tabular}{|c|c|c|c|c|}
\hline \multicolumn{3}{|c|}{ Reference Elevation (ft AMSL) } & 1508.77 & \multirow{2}{*}{$\begin{array}{l}1509.12 \\
\text { Elevation in Klassen } \\
\text { Test Well (ft AMSL) }\end{array}$} \\
\hline Date and Time & $\begin{array}{l}\text { Depth in Klassen } \\
\text { Domestic Well } \\
\text { (ft BGL) }\end{array}$ & $\begin{array}{c}\text { Depth in Klassen } \\
\text { Test Well } \\
\text { (ft BGL) }\end{array}$ & $\begin{array}{l}\text { Elevation in Klassen } \\
\text { Domestic Well } \\
\text { (ft AMSL) }\end{array}$ & \\
\hline 10/26/11 16:10 & 40.582 & 37.969 & 1468.19 & 1471.15 \\
\hline $10 / 26 / 1116: 20$ & 41.044 & 37.98 & 1467.73 & 1471.14 \\
\hline 10/26/11 16:30 & 55.331 & 37.99 & 1453.44 & 1471.13 \\
\hline $10 / 26 / 1116: 40$ & 41.834 & 38.011 & 1466.94 & 1471.11 \\
\hline 10/26/11 16:50 & 37.587 & 38.027 & 1471.18 & 1471.10 \\
\hline 10/26/11 17:00 & 37.336 & 38.041 & 1471.43 & 1471.08 \\
\hline $10 / 26 / 1117: 10$ & 36.884 & 38.048 & 1471.89 & 1471.07 \\
\hline 10/26/11 17:20 & 36.623 & 38.053 & 1472.15 & 1471.07 \\
\hline 10/26/11 17:30 & 36.64 & 38.053 & 1472.13 & 1471.07 \\
\hline $10 / 26 / 1117: 40$ & 37.674 & 38.053 & 1471.10 & 1471.07 \\
\hline 10/26/11 17:50 & 36.37 & 38.058 & 1472.40 & 1471.06 \\
\hline 10/26/11 18:00 & 36.206 & 38.055 & 1472.56 & 1471.07 \\
\hline 10/26/11 18:10 & 36.33 & 38.058 & 1472.44 & 1471.06 \\
\hline $10 / 26 / 1118: 20$ & 36.117 & 38.055 & 1472.65 & 1471.07 \\
\hline 10/26/11 18:30 & 36.379 & 38.053 & 1472.39 & 1471.07 \\
\hline 10/26/11 18:40 & 36.032 & 38.051 & 1472.74 & 1471.07 \\
\hline $10 / 26 / 11$ 18:50 & 41.232 & 38.046 & 1467.54 & 1471.08 \\
\hline 10/26/11 19:00 & 36.065 & 38.046 & 1472.70 & 1471.08 \\
\hline 10/26/11 19:10 & 35.91 & 38.044 & 1472.86 & 1471.08 \\
\hline 10/26/11 19:20 & 37.514 & 38.037 & 1471.26 & 1471.09 \\
\hline 10/26/11 19:30 & 35.875 & 38.034 & 1472.89 & 1471.09 \\
\hline 10/26/11 19:40 & 35.769 & 38.037 & 1473.00 & 1471.09 \\
\hline 10/26/11 19:50 & 36.227 & 38.039 & 1472.54 & 1471.08 \\
\hline $10 / 26 / 1120: 00$ & 35.755 & 38.044 & 1473.01 & 1471.08 \\
\hline 10/26/11 20:10 & 35.856 & 38.044 & 1472.91 & 1471.08 \\
\hline $10 / 26 / 1120: 20$ & 35.92 & 38.046 & 1472.85 & 1471.08 \\
\hline 10/26/11 20:30 & 35.76 & 38.048 & 1473.01 & 1471.07 \\
\hline $10 / 26 / 1120: 40$ & 39.109 & 38.048 & 1469.66 & 1471.07 \\
\hline $10 / 26 / 1120: 50$ & 35.774 & 38.053 & 1473.00 & 1471.07 \\
\hline 10/26/11 21:00 & 35.666 & 38.053 & 1473.10 & 1471.07 \\
\hline 10/26/11 21:10 & 36.189 & 38.053 & 1472.58 & 1471.07 \\
\hline 10/26/11 21:20 & 35.659 & 38.055 & 1473.11 & 1471.07 \\
\hline 10/26/11 21:30 & 35.629 & 38.055 & 1473.14 & 1471.07 \\
\hline $10 / 26 / 1121: 40$ & 35.84 & 38.06 & 1472.93 & 1471.06 \\
\hline 10/26/11 21:50 & 35.669 & 38.067 & 1473.10 & 1471.06 \\
\hline $10 / 26 / 1122: 00$ & 36.623 & 38.074 & 1472.15 & 1471.05 \\
\hline $10 / 26 / 1122: 10$ & 37.371 & 38.081 & 1471.40 & 1471.04 \\
\hline $10 / 26 / 1122: 20$ & 39.876 & 38.088 & 1468.89 & 1471.03 \\
\hline
\end{tabular}


TABLE S1.3 Automatically recorded groundwater levels in the Klassen wells, September 15, 2011, to March 13, 2012.

\begin{tabular}{|c|c|c|c|c|}
\hline \multicolumn{3}{|c|}{ Reference Elevation (ft AMSL) } & 1508.77 & \multirow{2}{*}{$\begin{array}{l}1509.12 \\
\text { Elevation in Klassen } \\
\text { Test Well (ft AMSL) }\end{array}$} \\
\hline Date and Time & $\begin{array}{l}\text { Depth in Klassen } \\
\text { Domestic Well } \\
\text { (ft BGL) }\end{array}$ & $\begin{array}{c}\text { Depth in Klassen } \\
\text { Test Well } \\
\text { (ft BGL) }\end{array}$ & $\begin{array}{l}\text { Elevation in Klassen } \\
\text { Domestic Well } \\
\text { (ft AMSL) }\end{array}$ & \\
\hline $10 / 26 / 1122: 30$ & 35.966 & 38.095 & 1472.80 & 1471.03 \\
\hline $10 / 26 / 1122: 40$ & 36.067 & 38.102 & 1472.70 & 1471.02 \\
\hline $10 / 26 / 1122: 50$ & 37.123 & 38.107 & 1471.65 & 1471.02 \\
\hline $10 / 26 / 1123: 00$ & 35.999 & 38.109 & 1472.77 & 1471.01 \\
\hline $10 / 26 / 1123: 10$ & 35.842 & 38.112 & 1472.93 & 1471.01 \\
\hline $10 / 26 / 1123: 20$ & 35.901 & 38.119 & 1472.87 & 1471.00 \\
\hline $10 / 26 / 1123: 30$ & 35.758 & 38.116 & 1473.01 & 1471.01 \\
\hline $10 / 26 / 1123: 40$ & 36.468 & 38.114 & 1472.30 & 1471.01 \\
\hline $10 / 26 / 1123: 50$ & 35.755 & 38.112 & 1473.01 & 1471.01 \\
\hline $10 / 27 / 110: 00$ & 35.659 & 38.112 & 1473.11 & 1471.01 \\
\hline 10/27/11 0:10 & 35.8 & 38.109 & 1472.97 & 1471.01 \\
\hline $10 / 27 / 110: 20$ & 35.638 & 38.109 & 1473.13 & 1471.01 \\
\hline $10 / 27 / 110: 30$ & 38.839 & 38.1 & 1469.93 & 1471.02 \\
\hline 10/27/11 0:40 & 35.648 & 38.1 & 1473.12 & 1471.02 \\
\hline 10/27/11 0:50 & 35.551 & 38.098 & 1473.22 & 1471.02 \\
\hline 10/27/11 1:00 & 35.847 & 38.1 & 1472.92 & 1471.02 \\
\hline 10/27/11 1:10 & 35.551 & 38.095 & 1473.22 & 1471.03 \\
\hline 10/27/11 1:20 & 35.633 & 38.093 & 1473.14 & 1471.03 \\
\hline 10/27/11 1:30 & 35.516 & 38.088 & 1473.25 & 1471.03 \\
\hline 10/27/11 1:40 & 36.321 & 38.088 & 1472.45 & 1471.03 \\
\hline 10/27/11 1:50 & 35.526 & 38.086 & 1473.24 & 1471.04 \\
\hline 10/27/11 2:00 & 35.443 & 38.079 & 1473.33 & 1471.04 \\
\hline $10 / 27 / 112: 10$ & 35.605 & 38.076 & 1473.16 & 1471.05 \\
\hline $10 / 27 / 112: 20$ & 35.448 & 38.074 & 1473.32 & 1471.05 \\
\hline $10 / 27 / 112: 30$ & 39.444 & 38.069 & 1469.33 & 1471.05 \\
\hline $10 / 27 / 112: 40$ & 35.486 & 38.074 & 1473.28 & 1471.05 \\
\hline $10 / 27 / 112: 50$ & 35.387 & 38.067 & 1473.38 & 1471.06 \\
\hline 10/27/11 3:00 & 35.959 & 38.065 & 1472.81 & 1471.06 \\
\hline 10/27/11 3:10 & 35.411 & 38.058 & 1473.36 & 1471.06 \\
\hline 10/27/11 3:20 & 35.533 & 38.051 & 1473.24 & 1471.07 \\
\hline 10/27/11 3:30 & 38 & 38.051 & 1470.77 & 1471.07 \\
\hline 10/27/11 3:40 & 35.49 & 38.048 & 1473.28 & 1471.07 \\
\hline 10/27/11 3:50 & 35.385 & 38.046 & 1473.38 & 1471.08 \\
\hline 10/27/11 4:00 & 35.514 & 38.041 & 1473.26 & 1471.08 \\
\hline 10/27/11 4:10 & 35.373 & 38.039 & 1473.40 & 1471.08 \\
\hline $10 / 27 / 114: 20$ & 35.927 & 38.027 & 1472.84 & 1471.10 \\
\hline 10/27/11 4:30 & 35.382 & 38.025 & 1473.39 & 1471.10 \\
\hline 10/27/11 4:40 & 37.756 & 38.02 & 1471.01 & 1471.10 \\
\hline
\end{tabular}


TABLE S1.3 Automatically recorded groundwater levels in the Klassen wells, September 15, 2011, to March 13, 2012.

\begin{tabular}{|c|c|c|c|c|}
\hline \multicolumn{3}{|c|}{ Reference Elevation (ft AMSL) } & 1508.77 & \multirow{2}{*}{$\begin{array}{l}1509.12 \\
\text { Elevation in Klassen } \\
\text { Test Well (ft AMSL) }\end{array}$} \\
\hline Date and Time & $\begin{array}{l}\text { Depth in Klassen } \\
\text { Domestic Well } \\
\text { (ft BGL) }\end{array}$ & $\begin{array}{c}\text { Depth in Klassen } \\
\text { Test Well } \\
\text { (ft BGL) }\end{array}$ & $\begin{array}{l}\text { Elevation in Klassen } \\
\text { Domestic Well } \\
\text { (ft AMSL) }\end{array}$ & \\
\hline $10 / 27 / 114: 50$ & 35.434 & 38.015 & 1473.34 & 1471.11 \\
\hline 10/27/11 5:00 & 35.331 & 38.013 & 1473.44 & 1471.11 \\
\hline 10/27/11 5:10 & 35.516 & 38.008 & 1473.25 & 1471.11 \\
\hline 10/27/11 5:20 & 35.333 & 38.004 & 1473.44 & 1471.12 \\
\hline 10/27/11 5:30 & 36.768 & 37.999 & 1472.00 & 1471.12 \\
\hline 10/27/11 5:40 & 35.35 & 37.992 & 1473.42 & 1471.13 \\
\hline 10/27/11 5:50 & 35.268 & 37.99 & 1473.50 & 1471.13 \\
\hline 10/27/11 6:00 & 35.392 & 37.987 & 1473.38 & 1471.14 \\
\hline 10/27/11 6:10 & 36.126 & 37.987 & 1472.64 & 1471.14 \\
\hline $10 / 27 / 116: 20$ & 36.907 & 37.99 & 1471.86 & 1471.13 \\
\hline 10/27/11 6:30 & 35.544 & 37.99 & 1473.23 & 1471.13 \\
\hline 10/27/11 6:40 & 36.421 & 37.987 & 1472.35 & 1471.14 \\
\hline 10/27/11 6:50 & 35.514 & 37.985 & 1473.26 & 1471.14 \\
\hline 10/27/11 7:00 & 35.394 & 37.971 & 1473.38 & 1471.15 \\
\hline $10 / 27 / 117: 10$ & 35.488 & 37.969 & 1473.28 & 1471.15 \\
\hline 10/27/11 7:20 & 35.364 & 37.966 & 1473.41 & 1471.16 \\
\hline 10/27/11 7:30 & 35.634 & 37.959 & 1473.14 & 1471.16 \\
\hline 10/27/11 7:40 & 35.387 & 37.954 & 1473.38 & 1471.17 \\
\hline 10/27/11 7:50 & 37.927 & 37.952 & 1470.84 & 1471.17 \\
\hline 10/27/11 8:00 & 37.224 & 37.947 & 1471.55 & 1471.18 \\
\hline 10/27/11 8:10 & 35.429 & 37.947 & 1473.34 & 1471.18 \\
\hline $10 / 27 / 118: 20$ & 37.144 & 37.947 & 1471.63 & 1471.18 \\
\hline $10 / 27 / 118: 30$ & 35.429 & 37.945 & 1473.34 & 1471.18 \\
\hline $10 / 27 / 118: 40$ & 39.06 & 37.945 & 1469.71 & 1471.18 \\
\hline 10/27/11 8:50 & 35.455 & 37.947 & 1473.31 & 1471.18 \\
\hline 10/27/11 9:00 & 35.652 & 37.947 & 1473.12 & 1471.18 \\
\hline 10/27/11 9:10 & 36.257 & 37.95 & 1472.51 & 1471.17 \\
\hline 10/27/11 9:20 & 35.413 & 37.952 & 1473.36 & 1471.17 \\
\hline 10/27/11 9:30 & 35.556 & 37.95 & 1473.21 & 1471.17 \\
\hline 10/27/11 9:40 & 35.856 & 37.952 & 1472.91 & 1471.17 \\
\hline 10/27/11 9:50 & 35.406 & 37.954 & 1473.36 & 1471.17 \\
\hline 10/27/11 10:00 & 35.493 & 37.957 & 1473.28 & 1471.17 \\
\hline 10/27/11 10:10 & 36.611 & 37.959 & 1472.16 & 1471.16 \\
\hline $10 / 27 / 11$ 10:20 & 35.434 & 37.959 & 1473.34 & 1471.16 \\
\hline 10/27/11 10:30 & 36.874 & 37.959 & 1471.90 & 1471.16 \\
\hline $10 / 27 / 11$ 10:40 & 35.394 & 37.959 & 1473.38 & 1471.16 \\
\hline $10 / 27 / 11$ 10:50 & 39.379 & 37.961 & 1469.39 & 1471.16 \\
\hline 10/27/11 11:00 & 35.387 & 37.964 & 1473.38 & 1471.16 \\
\hline
\end{tabular}


TABLE S1.3 Automatically recorded groundwater levels in the Klassen wells, September 15, 2011, to March 13, 2012.

\begin{tabular}{|c|c|c|c|c|}
\hline \multicolumn{3}{|c|}{ Reference Elevation (ft AMSL) } & 1508.77 & \multirow{2}{*}{$\begin{array}{l}1509.12 \\
\text { Elevation in Klassen } \\
\text { Test Well (ft AMSL) }\end{array}$} \\
\hline Date and Time & $\begin{array}{l}\text { Depth in Klassen } \\
\text { Domestic Well } \\
\text { (ft BGL) }\end{array}$ & $\begin{array}{c}\text { Depth in Klassen } \\
\text { Test Well } \\
\text { (ft BGL) }\end{array}$ & $\begin{array}{l}\text { Elevation in Klassen } \\
\text { Domestic Well } \\
\text { (ft AMSL) }\end{array}$ & \\
\hline 10/27/11 11:10 & 35.49 & 37.966 & 1473.28 & 1471.16 \\
\hline 10/27/11 11:20 & 39.543 & 37.961 & 1469.23 & 1471.16 \\
\hline 10/27/11 11:30 & 38.818 & 37.959 & 1469.95 & 1471.16 \\
\hline 10/27/11 11:40 & 35.448 & 37.957 & 1473.32 & 1471.17 \\
\hline 10/27/11 11:50 & 35.917 & 37.957 & 1472.85 & 1471.17 \\
\hline $10 / 27 / 1112: 00$ & 35.415 & 37.959 & 1473.35 & 1471.16 \\
\hline $10 / 27 / 1112: 10$ & 35.3 & 37.954 & 1473.47 & 1471.17 \\
\hline $10 / 27 / 1112: 20$ & 35.436 & 37.957 & 1473.33 & 1471.17 \\
\hline $10 / 27 / 1112: 30$ & 35.514 & 37.959 & 1473.26 & 1471.16 \\
\hline $10 / 27 / 1112: 40$ & 39.379 & 37.959 & 1469.39 & 1471.16 \\
\hline $10 / 27 / 1112: 50$ & 35.46 & 37.959 & 1473.31 & 1471.16 \\
\hline 10/27/11 13:00 & 35.333 & 37.961 & 1473.44 & 1471.16 \\
\hline 10/27/11 13:10 & 35.46 & 37.964 & 1473.31 & 1471.16 \\
\hline $10 / 27 / 1113: 20$ & 35.612 & 37.964 & 1473.16 & 1471.16 \\
\hline 10/27/11 13:30 & 35.378 & 37.966 & 1473.39 & 1471.16 \\
\hline $10 / 27 / 11$ 13:40 & 36.466 & 37.964 & 1472.30 & 1471.16 \\
\hline 10/27/11 13:50 & 35.359 & 37.969 & 1473.41 & 1471.15 \\
\hline 10/27/11 14:00 & 35.27 & 37.966 & 1473.50 & 1471.16 \\
\hline $10 / 27 / 11$ 14:10 & 35.371 & 37.969 & 1473.40 & 1471.15 \\
\hline $10 / 27 / 11$ 14:20 & 37.482 & 37.969 & 1471.29 & 1471.15 \\
\hline $10 / 27 / 11$ 14:30 & 35.455 & 37.971 & 1473.31 & 1471.15 \\
\hline $10 / 27 / 11$ 14:40 & 35.598 & 37.971 & 1473.17 & 1471.15 \\
\hline $10 / 27 / 11$ 14:50 & 36.543 & 37.966 & 1472.23 & 1471.16 \\
\hline 10/27/11 15:00 & 38.429 & 37.959 & 1470.34 & 1471.16 \\
\hline 10/27/11 15:10 & 36.56 & 37.954 & 1472.21 & 1471.17 \\
\hline $10 / 27 / 11$ 15:20 & 36.37 & 37.95 & 1472.40 & 1471.17 \\
\hline $10 / 27 / 11$ 15:30 & 35.636 & 37.95 & 1473.13 & 1471.17 \\
\hline $10 / 27 / 11$ 15:40 & 35.718 & 37.947 & 1473.05 & 1471.18 \\
\hline $10 / 27 / 11$ 15:50 & 35.514 & 37.947 & 1473.26 & 1471.18 \\
\hline 10/27/11 16:00 & 35.891 & 37.947 & 1472.88 & 1471.18 \\
\hline 10/27/11 16:10 & 35.483 & 37.943 & 1473.29 & 1471.18 \\
\hline $10 / 27 / 11$ 16:20 & 38.199 & 37.94 & 1470.57 & 1471.18 \\
\hline 10/27/11 16:30 & 35.46 & 37.94 & 1473.31 & 1471.18 \\
\hline $10 / 27 / 11$ 16:40 & 35.359 & 37.933 & 1473.41 & 1471.19 \\
\hline 10/27/11 16:50 & 35.451 & 37.933 & 1473.32 & 1471.19 \\
\hline 10/27/11 17:00 & 36.961 & 37.931 & 1471.81 & 1471.19 \\
\hline 10/27/11 17:10 & 35.399 & 37.924 & 1473.37 & 1471.20 \\
\hline $10 / 27 / 11$ 17:20 & 35.748 & 37.915 & 1473.02 & 1471.21 \\
\hline
\end{tabular}


TABLE S1.3 Automatically recorded groundwater levels in the Klassen wells, September 15, 2011, to March 13, 2012.

\begin{tabular}{|c|c|c|c|c|}
\hline \multicolumn{3}{|c|}{ Reference Elevation (ft AMSL) } & 1508.77 & \multirow{2}{*}{$\begin{array}{l}1509.12 \\
\text { Elevation in Klassen } \\
\text { Test Well (ft AMSL) }\end{array}$} \\
\hline Date and Time & $\begin{array}{l}\text { Depth in Klassen } \\
\text { Domestic Well } \\
\text { (ft BGL) }\end{array}$ & $\begin{array}{c}\text { Depth in Klassen } \\
\text { Test Well } \\
\text { (ft BGL) }\end{array}$ & $\begin{array}{l}\text { Elevation in Klassen } \\
\text { Domestic Well } \\
\text { (ft AMSL) }\end{array}$ & \\
\hline 10/27/11 17:30 & 38.384 & 37.912 & 1470.39 & 1471.21 \\
\hline $10 / 27 / 1117: 40$ & 35.455 & 37.91 & 1473.31 & 1471.21 \\
\hline 10/27/11 17:50 & 35.558 & 37.908 & 1473.21 & 1471.21 \\
\hline 10/27/11 18:00 & 36.213 & 37.901 & 1472.56 & 1471.22 \\
\hline 10/27/11 18:10 & 35.725 & 37.896 & 1473.04 & 1471.23 \\
\hline $10 / 27 / 1118: 20$ & 35.694 & 37.894 & 1473.08 & 1471.23 \\
\hline $10 / 27 / 1118: 30$ & 35.666 & 37.891 & 1473.10 & 1471.23 \\
\hline 10/27/11 18:40 & 35.671 & 37.889 & 1473.10 & 1471.23 \\
\hline 10/27/11 18:50 & 35.706 & 37.889 & 1473.06 & 1471.23 \\
\hline 10/27/11 19:00 & 35.711 & 37.889 & 1473.06 & 1471.23 \\
\hline 10/27/11 19:10 & 35.748 & 37.891 & 1473.02 & 1471.23 \\
\hline 10/27/11 19:20 & 35.816 & 37.891 & 1472.95 & 1471.23 \\
\hline 10/27/11 19:30 & 35.955 & 37.896 & 1472.81 & 1471.23 \\
\hline 10/27/11 19:40 & 36.3 & 37.9 & 1472.47 & 1471.22 \\
\hline 10/27/11 19:50 & 37.003 & 37.905 & 1471.77 & 1471.22 \\
\hline $10 / 27 / 1120: 00$ & 35.983 & 37.912 & 1472.79 & 1471.21 \\
\hline $10 / 27 / 1120: 10$ & 36.035 & 37.919 & 1472.73 & 1471.20 \\
\hline $10 / 27 / 1120: 20$ & 36.433 & 37.924 & 1472.34 & 1471.20 \\
\hline 10/27/11 20:30 & 37.449 & 37.931 & 1471.32 & 1471.19 \\
\hline $10 / 27 / 1120: 40$ & 36.407 & 37.936 & 1472.36 & 1471.19 \\
\hline $10 / 27 / 1120: 50$ & 36.604 & 37.943 & 1472.17 & 1471.18 \\
\hline $10 / 27 / 1121: 00$ & 36.063 & 37.947 & 1472.71 & 1471.18 \\
\hline $10 / 27 / 1121: 10$ & 36.323 & 37.954 & 1472.45 & 1471.17 \\
\hline $10 / 27 / 1121: 20$ & 37.181 & 37.964 & 1471.59 & 1471.16 \\
\hline 10/27/11 21:30 & 38.835 & 37.973 & 1469.93 & 1471.15 \\
\hline $10 / 27 / 1121: 40$ & 38.718 & 37.98 & 1470.05 & 1471.14 \\
\hline $10 / 27 / 1121: 50$ & 35.924 & 37.985 & 1472.85 & 1471.14 \\
\hline $10 / 27 / 1122: 00$ & 36.363 & 37.992 & 1472.41 & 1471.13 \\
\hline $10 / 27 / 1122: 10$ & 36.67 & 37.997 & 1472.10 & 1471.13 \\
\hline $10 / 27 / 1122: 20$ & 37.594 & 38.004 & 1471.18 & 1471.12 \\
\hline $10 / 27 / 1122: 30$ & 38.847 & 38.006 & 1469.92 & 1471.12 \\
\hline $10 / 27 / 1122: 40$ & 37.639 & 38.013 & 1471.13 & 1471.11 \\
\hline $10 / 27 / 1122: 50$ & 36.41 & 38.02 & 1472.36 & 1471.10 \\
\hline $10 / 27 / 1123: 00$ & 36.365 & 38.03 & 1472.40 & 1471.09 \\
\hline $10 / 27 / 1123: 10$ & 37.838 & 38.034 & 1470.93 & 1471.09 \\
\hline $10 / 27 / 1123: 20$ & 37.034 & 38.044 & 1471.74 & 1471.08 \\
\hline $10 / 27 / 1123: 30$ & 37.134 & 38.051 & 1471.64 & 1471.07 \\
\hline $10 / 27 / 1123: 40$ & 37.477 & 38.058 & 1471.29 & 1471.06 \\
\hline
\end{tabular}


TABLE S1.3 Automatically recorded groundwater levels in the Klassen wells, September 15, 2011, to March 13, 2012.

\begin{tabular}{|c|c|c|c|c|}
\hline \multicolumn{3}{|c|}{ Reference Elevation (ft AMSL) } & 1508.77 & \multirow{2}{*}{$\begin{array}{l}1509.12 \\
\text { Elevation in Klassen } \\
\text { Test Well (ft AMSL) }\end{array}$} \\
\hline Date and Time & $\begin{array}{l}\text { Depth in Klassen } \\
\text { Domestic Well } \\
\text { (ft BGL) }\end{array}$ & $\begin{array}{c}\text { Depth in Klassen } \\
\text { Test Well } \\
\text { (ft BGL) }\end{array}$ & $\begin{array}{l}\text { Elevation in Klassen } \\
\text { Domestic Well } \\
\text { (ft AMSL) }\end{array}$ & \\
\hline $10 / 27 / 1123: 50$ & 38.727 & 38.065 & 1470.04 & 1471.06 \\
\hline 10/28/11 0:00 & 40.732 & 38.069 & 1468.04 & 1471.05 \\
\hline 10/28/11 0:10 & 36.166 & 38.074 & 1472.60 & 1471.05 \\
\hline $10 / 28 / 110: 20$ & 36.166 & 38.081 & 1472.60 & 1471.04 \\
\hline 10/28/11 0:30 & 36.178 & 38.083 & 1472.59 & 1471.04 \\
\hline 10/28/11 0:40 & 36.208 & 38.091 & 1472.56 & 1471.03 \\
\hline $10 / 28 / 110: 50$ & 36.278 & 38.091 & 1472.49 & 1471.03 \\
\hline 10/28/11 1:00 & 36.466 & 38.093 & 1472.30 & 1471.03 \\
\hline 10/28/11 1:10 & 36.982 & 38.095 & 1471.79 & 1471.03 \\
\hline 10/28/11 1:20 & 38.213 & 38.098 & 1470.56 & 1471.02 \\
\hline 10/28/11 1:30 & 40.821 & 38.098 & 1467.95 & 1471.02 \\
\hline 10/28/11 1:40 & 36.719 & 38.098 & 1472.05 & 1471.02 \\
\hline 10/28/11 1:50 & 37.435 & 38.102 & 1471.33 & 1471.02 \\
\hline $10 / 28 / 112: 00$ & 39.168 & 38.102 & 1469.60 & 1471.02 \\
\hline 10/28/11 2:10 & 38.49 & 38.1 & 1470.28 & 1471.02 \\
\hline $10 / 28 / 112: 20$ & 36.011 & 38.102 & 1472.76 & 1471.02 \\
\hline $10 / 28 / 112: 30$ & 36.035 & 38.105 & 1472.73 & 1471.02 \\
\hline $10 / 28 / 112: 40$ & 36.067 & 38.105 & 1472.70 & 1471.02 \\
\hline $10 / 28 / 112: 50$ & 36.124 & 38.105 & 1472.65 & 1471.02 \\
\hline 10/28/11 3:00 & 36.067 & 38.105 & 1472.70 & 1471.02 \\
\hline 10/28/11 3:10 & 36.086 & 38.107 & 1472.68 & 1471.02 \\
\hline 10/28/11 3:20 & 36.138 & 38.107 & 1472.63 & 1471.02 \\
\hline 10/28/11 3:30 & 36.257 & 38.107 & 1472.51 & 1471.02 \\
\hline $10 / 28 / 113: 40$ & 36.6 & 38.109 & 1472.17 & 1471.01 \\
\hline 10/28/11 3:50 & 37.512 & 38.107 & 1471.26 & 1471.02 \\
\hline 10/28/11 4:00 & 39.625 & 38.109 & 1469.14 & 1471.01 \\
\hline $10 / 28 / 114: 10$ & 36.475 & 38.109 & 1472.29 & 1471.01 \\
\hline $10 / 28 / 114: 20$ & 35.967 & 38.109 & 1472.80 & 1471.01 \\
\hline $10 / 28 / 114: 30$ & 36.855 & 38.107 & 1471.91 & 1471.02 \\
\hline $10 / 28 / 114: 40$ & 36.023 & 38.107 & 1472.75 & 1471.02 \\
\hline $10 / 28 / 114: 50$ & 36.034 & 38.107 & 1472.74 & 1471.02 \\
\hline 10/28/11 5:00 & 36.074 & 38.105 & 1472.70 & 1471.02 \\
\hline 10/28/11 5:10 & 36.159 & 38.1 & 1472.61 & 1471.02 \\
\hline 10/28/11 5:20 & 36.361 & 38.1 & 1472.41 & 1471.02 \\
\hline 10/28/11 5:30 & 36.909 & 38.1 & 1471.86 & 1471.02 \\
\hline 10/28/11 5:40 & 39.817 & 38.098 & 1468.95 & 1471.02 \\
\hline 10/28/11 5:50 & 40.289 & 38.098 & 1468.48 & 1471.02 \\
\hline 10/28/11 6:00 & 35.96 & 38.098 & 1472.81 & 1471.02 \\
\hline
\end{tabular}


TABLE S1.3 Automatically recorded groundwater levels in the Klassen wells, September 15, 2011, to March 13, 2012.

\begin{tabular}{|c|c|c|c|c|}
\hline \multicolumn{3}{|c|}{ Reference Elevation (ft AMSL) } & 1508.77 & \multirow{2}{*}{$\begin{array}{l}1509.12 \\
\text { Elevation in Klassen } \\
\text { Test Well (ft AMSL) }\end{array}$} \\
\hline Date and Time & $\begin{array}{l}\text { Depth in Klassen } \\
\text { Domestic Well } \\
\text { (ft BGL) }\end{array}$ & $\begin{array}{c}\text { Depth in Klassen } \\
\text { Test Well } \\
\text { (ft BGL) }\end{array}$ & $\begin{array}{l}\text { Elevation in Klassen } \\
\text { Domestic Well } \\
\text { (ft AMSL) }\end{array}$ & \\
\hline 10/28/11 6:10 & 38.746 & 38.095 & 1470.02 & 1471.03 \\
\hline $10 / 28 / 116: 20$ & 36.358 & 38.095 & 1472.41 & 1471.03 \\
\hline 10/28/11 6:30 & 36.295 & 38.098 & 1472.47 & 1471.02 \\
\hline 10/28/11 6:40 & 36.384 & 38.095 & 1472.39 & 1471.03 \\
\hline $10 / 28 / 116: 50$ & 36.562 & 38.093 & 1472.21 & 1471.03 \\
\hline 10/28/11 7:00 & 36.82 & 38.093 & 1471.95 & 1471.03 \\
\hline 10/28/11 7:10 & 37.184 & 38.091 & 1471.59 & 1471.03 \\
\hline $10 / 28 / 117: 20$ & 38.638 & 38.088 & 1470.13 & 1471.03 \\
\hline 10/28/11 7:30 & 40.045 & 38.088 & 1468.72 & 1471.03 \\
\hline 10/28/11 7:40 & 35.997 & 38.088 & 1472.77 & 1471.03 \\
\hline 10/28/11 7:50 & 36.018 & 38.088 & 1472.75 & 1471.03 \\
\hline 10/28/11 8:00 & 36.175 & 38.088 & 1472.59 & 1471.03 \\
\hline 10/28/11 8:10 & 39.093 & 38.086 & 1469.68 & 1471.04 \\
\hline $10 / 28 / 118: 20$ & 39.513 & 38.086 & 1469.26 & 1471.04 \\
\hline $10 / 28 / 118: 30$ & 36.086 & 38.086 & 1472.68 & 1471.04 \\
\hline $10 / 28 / 118: 40$ & 36.677 & 38.088 & 1472.09 & 1471.03 \\
\hline $10 / 28 / 118: 50$ & 36.74 & 38.091 & 1472.03 & 1471.03 \\
\hline 10/28/11 9:00 & 36.482 & 38.093 & 1472.29 & 1471.03 \\
\hline 10/28/11 9:10 & 36.398 & 38.098 & 1472.37 & 1471.02 \\
\hline 10/28/11 9:20 & 36.288 & 38.1 & 1472.48 & 1471.02 \\
\hline 10/28/11 9:30 & 36.307 & 38.102 & 1472.46 & 1471.02 \\
\hline 10/28/11 9:40 & 36.328 & 38.102 & 1472.44 & 1471.02 \\
\hline 10/28/11 9:50 & 36.644 & 38.105 & 1472.13 & 1471.02 \\
\hline 10/28/11 10:00 & 39.517 & 38.109 & 1469.25 & 1471.01 \\
\hline 10/28/11 10:10 & 39.892 & 38.112 & 1468.88 & 1471.01 \\
\hline $10 / 28 / 11$ 10:20 & 36.684 & 38.114 & 1472.09 & 1471.01 \\
\hline $10 / 28 / 11$ 10:30 & 36.342 & 38.116 & 1472.43 & 1471.01 \\
\hline $10 / 28 / 11$ 10:40 & 36.293 & 38.119 & 1472.48 & 1471.00 \\
\hline $10 / 28 / 11$ 10:50 & 36.332 & 38.123 & 1472.44 & 1471.00 \\
\hline 10/28/11 11:00 & 36.377 & 38.13 & 1472.39 & 1470.99 \\
\hline 10/28/11 11:10 & 40.53 & 38.137 & 1468.24 & 1470.99 \\
\hline 10/28/11 11:20 & 37.899 & 38.144 & 1470.87 & 1470.98 \\
\hline $10 / 28 / 11$ 11:30 & 42.247 & 38.149 & 1466.52 & 1470.97 \\
\hline $10 / 28 / 11$ 11:40 & 39.881 & 38.159 & 1468.89 & 1470.96 \\
\hline 10/28/11 11:50 & 39.599 & 38.166 & 1469.17 & 1470.96 \\
\hline $10 / 28 / 11$ 12:00 & 39.433 & 38.17 & 1469.34 & 1470.95 \\
\hline $10 / 28 / 11$ 12:10 & 40.873 & 38.175 & 1467.90 & 1470.95 \\
\hline $10 / 28 / 11$ 12:20 & 36.37 & 38.18 & 1472.40 & 1470.94 \\
\hline
\end{tabular}


TABLE S1.3 Automatically recorded groundwater levels in the Klassen wells, September 15, 2011, to March 13, 2012.

\begin{tabular}{|c|c|c|c|c|}
\hline \multicolumn{3}{|c|}{ Reference Elevation (ft AMSL) } & 1508.77 & \multirow{2}{*}{$\begin{array}{l}1509.12 \\
\text { Elevation in Klassen } \\
\text { Test Well (ft AMSL) }\end{array}$} \\
\hline Date and Time & $\begin{array}{l}\text { Depth in Klassen } \\
\text { Domestic Well } \\
\text { (ft BGL) }\end{array}$ & $\begin{array}{c}\text { Depth in Klassen } \\
\text { Test Well } \\
\text { (ft BGL) }\end{array}$ & $\begin{array}{l}\text { Elevation in Klassen } \\
\text { Domestic Well } \\
\text { (ft AMSL) }\end{array}$ & \\
\hline $10 / 28 / 1112: 30$ & 36.377 & 38.187 & 1472.39 & 1470.94 \\
\hline $10 / 28 / 1112: 40$ & 36.391 & 38.191 & 1472.38 & 1470.93 \\
\hline $10 / 28 / 1112: 50$ & 36.499 & 38.198 & 1472.27 & 1470.92 \\
\hline 10/28/11 13:00 & 36.504 & 38.203 & 1472.27 & 1470.92 \\
\hline 10/28/11 13:10 & 36.635 & 38.208 & 1472.13 & 1470.91 \\
\hline $10 / 28 / 1113: 20$ & 36.623 & 38.213 & 1472.15 & 1470.91 \\
\hline 10/28/11 13:30 & 36.672 & 38.213 & 1472.10 & 1470.91 \\
\hline 10/28/11 13:40 & 36.543 & 38.213 & 1472.23 & 1470.91 \\
\hline $10 / 28 / 1113: 50$ & 41.032 & 38.21 & 1467.74 & 1470.91 \\
\hline $10 / 28 / 1114: 00$ & 36.771 & 38.21 & 1472.00 & 1470.91 \\
\hline 10/28/11 14:10 & 36.576 & 38.21 & 1472.19 & 1470.91 \\
\hline $10 / 28 / 1114: 20$ & 36.574 & 38.205 & 1472.20 & 1470.92 \\
\hline $10 / 28 / 1114: 30$ & 36.478 & 38.198 & 1472.29 & 1470.92 \\
\hline $10 / 28 / 1114: 40$ & 36.475 & 38.194 & 1472.29 & 1470.93 \\
\hline $10 / 28 / 11$ 14:50 & 36.527 & 38.189 & 1472.24 & 1470.93 \\
\hline $10 / 28 / 11$ 15:00 & 36.492 & 38.182 & 1472.28 & 1470.94 \\
\hline $10 / 28 / 11$ 15:10 & 36.468 & 38.177 & 1472.30 & 1470.95 \\
\hline $10 / 28 / 11$ 15:20 & 36.482 & 38.175 & 1472.29 & 1470.95 \\
\hline 10/28/11 15:30 & 36.551 & 38.173 & 1472.22 & 1470.95 \\
\hline $10 / 28 / 11$ 15:40 & 36.658 & 38.17 & 1472.11 & 1470.95 \\
\hline $10 / 28 / 1115: 50$ & 36.473 & 38.168 & 1472.30 & 1470.95 \\
\hline 10/28/11 16:00 & 39.501 & 38.163 & 1469.27 & 1470.96 \\
\hline 10/28/11 16:10 & 39.036 & 38.163 & 1469.73 & 1470.96 \\
\hline $10 / 28 / 11$ 16:20 & 36.391 & 38.161 & 1472.38 & 1470.96 \\
\hline 10/28/11 16:30 & 36.389 & 38.161 & 1472.38 & 1470.96 \\
\hline 10/28/11 16:40 & 38.237 & 38.159 & 1470.53 & 1470.96 \\
\hline $10 / 28 / 11$ 16:50 & 39.771 & 38.156 & 1469.00 & 1470.97 \\
\hline 10/28/11 17:00 & 40.474 & 38.152 & 1468.30 & 1470.97 \\
\hline 10/28/11 17:10 & 38.042 & 38.152 & 1470.73 & 1470.97 \\
\hline $10 / 28 / 11$ 17:20 & 37.134 & 38.152 & 1471.64 & 1470.97 \\
\hline 10/28/11 17:30 & 37.331 & 38.149 & 1471.44 & 1470.97 \\
\hline 10/28/11 17:40 & 37.353 & 38.149 & 1471.42 & 1470.97 \\
\hline $10 / 28 / 11$ 17:50 & 37.268 & 38.152 & 1471.50 & 1470.97 \\
\hline 10/28/11 18:00 & 37.367 & 38.154 & 1471.40 & 1470.97 \\
\hline 10/28/11 18:10 & 36.806 & 38.156 & 1471.96 & 1470.97 \\
\hline $10 / 28 / 11$ 18:20 & 36.651 & 38.156 & 1472.12 & 1470.97 \\
\hline 10/28/11 18:30 & 39.635 & 38.156 & 1469.13 & 1470.97 \\
\hline $10 / 28 / 11$ 18:40 & 39.449 & 38.159 & 1469.32 & 1470.96 \\
\hline
\end{tabular}


TABLE S1.3 Automatically recorded groundwater levels in the Klassen wells, September 15, 2011, to March 13, 2012.

\begin{tabular}{|c|c|c|c|c|}
\hline \multicolumn{3}{|c|}{ Reference Elevation (ft AMSL) } & 1508.77 & \multirow{2}{*}{$\begin{array}{l}1509.12 \\
\text { Elevation in Klassen } \\
\text { Test Well (ft AMSL) }\end{array}$} \\
\hline Date and Time & $\begin{array}{l}\text { Depth in Klassen } \\
\text { Domestic Well } \\
\text { (ft BGL) }\end{array}$ & $\begin{array}{c}\text { Depth in Klassen } \\
\text { Test Well } \\
\text { (ft BGL) }\end{array}$ & $\begin{array}{l}\text { Elevation in Klassen } \\
\text { Domestic Well } \\
\text { (ft AMSL) }\end{array}$ & \\
\hline 10/28/11 18:50 & 36.902 & 38.161 & 1471.87 & 1470.96 \\
\hline 10/28/11 19:00 & 36.743 & 38.166 & 1472.03 & 1470.96 \\
\hline 10/28/11 19:10 & 36.708 & 38.163 & 1472.06 & 1470.96 \\
\hline $10 / 28 / 11$ 19:20 & 36.731 & 38.166 & 1472.04 & 1470.96 \\
\hline 10/28/11 19:30 & 36.841 & 38.163 & 1471.93 & 1470.96 \\
\hline $10 / 28 / 11$ 19:40 & 37.151 & 38.163 & 1471.62 & 1470.96 \\
\hline 10/28/11 19:50 & 37.904 & 38.166 & 1470.87 & 1470.96 \\
\hline 10/28/11 20:00 & 39.405 & 38.163 & 1469.36 & 1470.96 \\
\hline $10 / 28 / 1120: 10$ & 39.97 & 38.166 & 1468.80 & 1470.96 \\
\hline $10 / 28 / 1120: 20$ & 36.363 & 38.166 & 1472.41 & 1470.96 \\
\hline $10 / 28 / 1120: 30$ & 36.377 & 38.166 & 1472.39 & 1470.96 \\
\hline $10 / 28 / 1120: 40$ & 36.393 & 38.166 & 1472.38 & 1470.96 \\
\hline $10 / 28 / 1120: 50$ & 36.426 & 38.163 & 1472.34 & 1470.96 \\
\hline $10 / 28 / 1121: 00$ & 36.478 & 38.163 & 1472.29 & 1470.96 \\
\hline 10/28/11 21:10 & 36.586 & 38.163 & 1472.18 & 1470.96 \\
\hline $10 / 28 / 1121: 20$ & 36.776 & 38.163 & 1471.99 & 1470.96 \\
\hline 10/28/11 21:30 & 36.722 & 38.161 & 1472.05 & 1470.96 \\
\hline $10 / 28 / 1121: 40$ & 36.996 & 38.163 & 1471.77 & 1470.96 \\
\hline $10 / 28 / 1121: 50$ & 37.608 & 38.163 & 1471.16 & 1470.96 \\
\hline $10 / 28 / 1122: 00$ & 38.9 & 38.166 & 1469.87 & 1470.96 \\
\hline $10 / 28 / 1122: 10$ & 41.201 & 38.163 & 1467.57 & 1470.96 \\
\hline $10 / 28 / 1122: 20$ & 36.264 & 38.166 & 1472.51 & 1470.96 \\
\hline $10 / 28 / 1122: 30$ & 36.278 & 38.163 & 1472.49 & 1470.96 \\
\hline $10 / 28 / 1122: 40$ & 36.3 & 38.161 & 1472.47 & 1470.96 \\
\hline $10 / 28 / 1122: 50$ & 37.829 & 38.161 & 1470.94 & 1470.96 \\
\hline $10 / 28 / 1123: 00$ & 36.776 & 38.163 & 1471.99 & 1470.96 \\
\hline $10 / 28 / 1123: 10$ & 36.621 & 38.166 & 1472.15 & 1470.96 \\
\hline $10 / 28 / 1123: 20$ & 36.736 & 38.168 & 1472.03 & 1470.95 \\
\hline $10 / 28 / 1123: 30$ & 36.982 & 38.168 & 1471.79 & 1470.95 \\
\hline $10 / 28 / 1123: 40$ & 37.542 & 38.166 & 1471.23 & 1470.96 \\
\hline $10 / 28 / 1123: 50$ & 38.718 & 38.166 & 1470.05 & 1470.96 \\
\hline 10/29/11 0:00 & 40.788 & 38.166 & 1467.98 & 1470.96 \\
\hline 10/29/11 0:10 & 36.412 & 38.163 & 1472.36 & 1470.96 \\
\hline 10/29/11 0:20 & 36.281 & 38.166 & 1472.49 & 1470.96 \\
\hline 10/29/11 0:30 & 36.3 & 38.161 & 1472.47 & 1470.96 \\
\hline 10/29/11 0:40 & 36.328 & 38.163 & 1472.44 & 1470.96 \\
\hline 10/29/11 0:50 & 36.379 & 38.159 & 1472.39 & 1470.96 \\
\hline 10/29/11 1:00 & 36.471 & 38.159 & 1472.30 & 1470.96 \\
\hline
\end{tabular}


TABLE S1.3 Automatically recorded groundwater levels in the Klassen wells, September 15, 2011, to March 13, 2012.

\begin{tabular}{|c|c|c|c|c|}
\hline \multicolumn{3}{|c|}{ Reference Elevation (ft AMSL) } & 1508.77 & \multirow{2}{*}{$\begin{array}{l}1509.12 \\
\text { Elevation in Klassen } \\
\text { Test Well (ft AMSL) }\end{array}$} \\
\hline Date and Time & $\begin{array}{l}\text { Depth in Klassen } \\
\text { Domestic Well } \\
\text { (ft BGL) }\end{array}$ & $\begin{array}{c}\text { Depth in Klassen } \\
\text { Test Well } \\
\text { (ft BGL) }\end{array}$ & $\begin{array}{l}\text { Elevation in Klassen } \\
\text { Domestic Well } \\
\text { (ft AMSL) }\end{array}$ & \\
\hline 10/29/11 1:10 & 36.668 & 38.154 & 1472.10 & 1470.97 \\
\hline 10/29/11 1:20 & 37.127 & 38.152 & 1471.64 & 1470.97 \\
\hline 10/29/11 1:30 & 38.035 & 38.149 & 1470.73 & 1470.97 \\
\hline 10/29/11 1:40 & 39.885 & 38.149 & 1468.88 & 1470.97 \\
\hline 10/29/11 1:50 & 38.586 & 38.152 & 1470.18 & 1470.97 \\
\hline $10 / 29 / 112: 00$ & 36.314 & 38.152 & 1472.46 & 1470.97 \\
\hline $10 / 29 / 112: 10$ & 36.321 & 38.152 & 1472.45 & 1470.97 \\
\hline $10 / 29 / 112: 20$ & 36.365 & 38.154 & 1472.40 & 1470.97 \\
\hline $10 / 29 / 112: 30$ & 36.396 & 38.154 & 1472.37 & 1470.97 \\
\hline $10 / 29 / 112: 40$ & 36.4 & 38.152 & 1472.37 & 1470.97 \\
\hline $10 / 29 / 112: 50$ & 36.426 & 38.156 & 1472.34 & 1470.97 \\
\hline 10/29/11 3:00 & 36.475 & 38.156 & 1472.29 & 1470.97 \\
\hline 10/29/11 3:10 & 36.614 & 38.159 & 1472.16 & 1470.96 \\
\hline $10 / 29 / 113: 20$ & 36.956 & 38.163 & 1471.81 & 1470.96 \\
\hline 10/29/11 3:30 & 37.69 & 38.168 & 1471.08 & 1470.95 \\
\hline 10/29/11 3:40 & 39.055 & 38.17 & 1469.71 & 1470.95 \\
\hline 10/29/11 3:50 & 40.62 & 38.17 & 1468.15 & 1470.95 \\
\hline 10/29/11 4:00 & 36.304 & 38.173 & 1472.47 & 1470.95 \\
\hline 10/29/11 4:10 & 36.314 & 38.175 & 1472.46 & 1470.95 \\
\hline 10/29/11 4:20 & 36.332 & 38.175 & 1472.44 & 1470.95 \\
\hline $10 / 29 / 114: 30$ & 36.365 & 38.175 & 1472.40 & 1470.95 \\
\hline 10/29/11 4:40 & 36.426 & 38.173 & 1472.34 & 1470.95 \\
\hline $10 / 29 / 114: 50$ & 36.553 & 38.17 & 1472.22 & 1470.95 \\
\hline 10/29/11 5:00 & 36.867 & 38.166 & 1471.90 & 1470.96 \\
\hline 10/29/11 5:10 & 37.634 & 38.166 & 1471.14 & 1470.96 \\
\hline 10/29/11 5:20 & 39.217 & 38.163 & 1469.55 & 1470.96 \\
\hline 10/29/11 5:30 & 39.782 & 38.161 & 1468.99 & 1470.96 \\
\hline 10/29/11 5:40 & 37.081 & 38.161 & 1471.69 & 1470.96 \\
\hline $10 / 29 / 115: 50$ & 36.433 & 38.161 & 1472.34 & 1470.96 \\
\hline 10/29/11 6:00 & 36.501 & 38.159 & 1472.27 & 1470.96 \\
\hline 10/29/11 6:10 & 36.661 & 38.156 & 1472.11 & 1470.97 \\
\hline 10/29/11 6:20 & 36.377 & 38.152 & 1472.39 & 1470.97 \\
\hline 10/29/11 6:30 & 36.342 & 38.152 & 1472.43 & 1470.97 \\
\hline 10/29/11 6:40 & 36.351 & 38.149 & 1472.42 & 1470.97 \\
\hline 10/29/11 6:50 & 36.375 & 38.149 & 1472.39 & 1470.97 \\
\hline 10/29/11 7:00 & 36.375 & 38.147 & 1472.39 & 1470.98 \\
\hline 10/29/11 7:10 & 36.464 & 38.142 & 1472.31 & 1470.98 \\
\hline 10/29/11 7:20 & 36.696 & 38.14 & 1472.07 & 1470.98 \\
\hline
\end{tabular}


TABLE S1.3 Automatically recorded groundwater levels in the Klassen wells, September 15, 2011, to March 13, 2012.

\begin{tabular}{|c|c|c|c|c|}
\hline \multicolumn{3}{|c|}{ Reference Elevation (ft AMSL) } & 1508.77 & \multirow{2}{*}{$\begin{array}{l}1509.12 \\
\text { Elevation in Klassen } \\
\text { Test Well (ft AMSL) }\end{array}$} \\
\hline Date and Time & $\begin{array}{l}\text { Depth in Klassen } \\
\text { Domestic Well } \\
(\mathrm{ft} \mathrm{BGL})\end{array}$ & $\begin{array}{c}\text { Depth in Klassen } \\
\text { Test Well } \\
\text { (ft BGL) }\end{array}$ & $\begin{array}{l}\text { Elevation in Klassen } \\
\text { Domestic Well } \\
\text { (ft AMSL) }\end{array}$ & \\
\hline 10/29/11 7:30 & 37.125 & 38.135 & 1471.64 & 1470.99 \\
\hline 10/29/11 7:40 & 38.164 & 38.13 & 1470.61 & 1470.99 \\
\hline 10/29/11 7:50 & 40.258 & 38.128 & 1468.51 & 1470.99 \\
\hline 10/29/11 8:00 & 36.935 & 38.123 & 1471.83 & 1471.00 \\
\hline 10/29/11 8:10 & 37.01 & 38.121 & 1471.76 & 1471.00 \\
\hline 10/29/11 8:20 & 37.439 & 38.121 & 1471.33 & 1471.00 \\
\hline 10/29/11 8:30 & 38.856 & 38.119 & 1469.91 & 1471.00 \\
\hline 10/29/11 8:40 & 37.716 & 38.123 & 1471.05 & 1471.00 \\
\hline 10/29/11 8:50 & 36.963 & 38.126 & 1471.81 & 1471.00 \\
\hline 10/29/11 9:00 & 39.827 & 38.133 & 1468.94 & 1470.99 \\
\hline 10/29/11 9:10 & 37.156 & 38.144 & 1471.61 & 1470.98 \\
\hline 10/29/11 9:20 & 39.679 & 38.152 & 1469.09 & 1470.97 \\
\hline 10/29/11 9:30 & 37.052 & 38.161 & 1471.72 & 1470.96 \\
\hline 10/29/11 9:40 & 40.878 & 38.17 & 1467.89 & 1470.95 \\
\hline 10/29/11 9:50 & 37.285 & 38.177 & 1471.48 & 1470.95 \\
\hline 10/29/11 10:00 & 40.76 & 38.187 & 1468.01 & 1470.94 \\
\hline 10/29/11 10:10 & 37.819 & 38.198 & 1470.95 & 1470.92 \\
\hline 10/29/11 10:20 & 42.207 & 38.205 & 1466.56 & 1470.92 \\
\hline 10/29/11 10:30 & 38.037 & 38.215 & 1470.73 & 1470.91 \\
\hline $10 / 29 / 11$ 10:40 & 37.003 & 38.22 & 1471.77 & 1470.90 \\
\hline $10 / 29 / 11$ 10:50 & 38.424 & 38.227 & 1470.35 & 1470.90 \\
\hline 10/29/11 11:00 & 37.031 & 38.231 & 1471.74 & 1470.89 \\
\hline 10/29/11 11:10 & 38.809 & 38.238 & 1469.96 & 1470.88 \\
\hline 10/29/11 11:20 & 37.906 & 38.245 & 1470.86 & 1470.88 \\
\hline 10/29/11 11:30 & 38.356 & 38.255 & 1470.41 & 1470.87 \\
\hline 10/29/11 11:40 & 37.132 & 38.269 & 1471.64 & 1470.85 \\
\hline 10/29/11 11:50 & 37.58 & 38.283 & 1471.19 & 1470.84 \\
\hline 10/29/11 12:00 & 41.145 & 38.285 & 1467.62 & 1470.84 \\
\hline 10/29/11 12:10 & 37.543 & 38.292 & 1471.23 & 1470.83 \\
\hline $10 / 29 / 11$ 12:20 & 42.421 & 38.302 & 1466.35 & 1470.82 \\
\hline $10 / 29 / 11$ 12:30 & 37.89 & 38.309 & 1470.88 & 1470.81 \\
\hline $10 / 29 / 11$ 12:40 & 38.389 & 38.313 & 1470.38 & 1470.81 \\
\hline $10 / 29 / 11$ 12:50 & 37.622 & 38.325 & 1471.15 & 1470.80 \\
\hline 10/29/11 13:00 & 39.623 & 38.33 & 1469.15 & 1470.79 \\
\hline $10 / 29 / 11$ 13:10 & 37.594 & 38.342 & 1471.18 & 1470.78 \\
\hline 10/29/11 13:20 & 41.337 & 38.346 & 1467.43 & 1470.78 \\
\hline 10/29/11 13:30 & 37.517 & 38.351 & 1471.25 & 1470.77 \\
\hline $10 / 29 / 11$ 13:40 & 37.697 & 38.36 & 1471.07 & 1470.76 \\
\hline
\end{tabular}


TABLE S1.3 Automatically recorded groundwater levels in the Klassen wells, September 15, 2011, to March 13, 2012.

\begin{tabular}{|c|c|c|c|c|}
\hline \multicolumn{3}{|c|}{ Reference Elevation (ft AMSL) } & 1508.77 & \multirow{2}{*}{$\begin{array}{l}1509.12 \\
\text { Elevation in Klassen } \\
\text { Test Well (ft AMSL) }\end{array}$} \\
\hline Date and Time & $\begin{array}{l}\text { Depth in Klassen } \\
\text { Domestic Well } \\
(\mathrm{ft} \mathrm{BGL})\end{array}$ & $\begin{array}{c}\text { Depth in Klassen } \\
\text { Test Well } \\
\text { (ft BGL) }\end{array}$ & $\begin{array}{l}\text { Elevation in Klassen } \\
\text { Domestic Well } \\
\text { (ft AMSL) }\end{array}$ & \\
\hline $10 / 29 / 1113: 50$ & 41.457 & 38.363 & 1467.31 & 1470.76 \\
\hline 10/29/11 14:00 & 38.002 & 38.372 & 1470.77 & 1470.75 \\
\hline 10/29/11 14:10 & 40.094 & 38.377 & 1468.68 & 1470.75 \\
\hline $10 / 29 / 1114: 20$ & 39.381 & 38.379 & 1469.39 & 1470.74 \\
\hline 10/29/11 14:30 & 37.876 & 38.372 & 1470.89 & 1470.75 \\
\hline $10 / 29 / 1114: 40$ & 39.327 & 38.372 & 1469.44 & 1470.75 \\
\hline 10/29/11 14:50 & 38.593 & 38.379 & 1470.18 & 1470.74 \\
\hline 10/29/11 15:00 & 40.425 & 38.381 & 1468.34 & 1470.74 \\
\hline 10/29/11 15:10 & 37.303 & 38.384 & 1471.47 & 1470.74 \\
\hline $10 / 29 / 1115: 20$ & 37.927 & 38.384 & 1470.84 & 1470.74 \\
\hline 10/29/11 15:30 & 37.05 & 38.381 & 1471.72 & 1470.74 \\
\hline 10/29/11 15:40 & 37.195 & 38.379 & 1471.57 & 1470.74 \\
\hline 10/29/11 15:50 & 36.865 & 38.374 & 1471.90 & 1470.75 \\
\hline 10/29/11 16:00 & 36.937 & 38.37 & 1471.83 & 1470.75 \\
\hline 10/29/11 16:10 & 39.567 & 38.363 & 1469.20 & 1470.76 \\
\hline 10/29/11 16:20 & 36.86 & 38.36 & 1471.91 & 1470.76 \\
\hline 10/29/11 16:30 & 38.642 & 38.356 & 1470.13 & 1470.77 \\
\hline 10/29/11 16:40 & 49.027 & 38.358 & 1459.74 & 1470.76 \\
\hline 10/29/11 16:50 & 49.921 & 38.365 & 1458.85 & 1470.76 \\
\hline 10/29/11 17:00 & 43.582 & 38.377 & 1465.19 & 1470.75 \\
\hline 10/29/11 17:10 & 46.633 & 38.386 & 1462.14 & 1470.74 \\
\hline 10/29/11 17:20 & 40.84 & 38.4 & 1467.93 & 1470.72 \\
\hline 10/29/11 17:30 & 44.482 & 38.412 & 1464.29 & 1470.71 \\
\hline 10/29/11 17:40 & 38.621 & 38.424 & 1470.15 & 1470.70 \\
\hline 10/29/11 17:50 & 41.424 & 38.431 & 1467.35 & 1470.69 \\
\hline 10/29/11 18:00 & 37.768 & 38.435 & 1471.00 & 1470.69 \\
\hline 10/29/11 18:10 & 37.779 & 38.435 & 1470.99 & 1470.69 \\
\hline 10/29/11 18:20 & 37.414 & 38.431 & 1471.36 & 1470.69 \\
\hline 10/29/11 18:30 & 37.418 & 38.428 & 1471.35 & 1470.69 \\
\hline 10/29/11 18:40 & 40.444 & 38.426 & 1468.33 & 1470.70 \\
\hline 10/29/11 18:50 & 37.186 & 38.421 & 1471.58 & 1470.70 \\
\hline 10/29/11 19:00 & 37.446 & 38.414 & 1471.32 & 1470.71 \\
\hline 10/29/11 19:10 & 37.05 & 38.412 & 1471.72 & 1470.71 \\
\hline 10/29/11 19:20 & 37.069 & 38.41 & 1471.70 & 1470.71 \\
\hline 10/29/11 19:30 & 37.09 & 38.403 & 1471.68 & 1470.72 \\
\hline 10/29/11 19:40 & 38.345 & 38.4 & 1470.42 & 1470.72 \\
\hline 10/29/11 19:50 & 36.9 & 38.398 & 1471.87 & 1470.72 \\
\hline 10/29/11 20:00 & 36.933 & 38.391 & 1471.84 & 1470.73 \\
\hline
\end{tabular}


TABLE S1.3 Automatically recorded groundwater levels in the Klassen wells, September 15, 2011, to March 13, 2012.

\begin{tabular}{|c|c|c|c|c|}
\hline \multicolumn{3}{|c|}{ Reference Elevation (ft AMSL) } & 1508.77 & \multirow{2}{*}{$\begin{array}{l}1509.12 \\
\text { Elevation in Klassen } \\
\text { Test Well (ft AMSL) }\end{array}$} \\
\hline Date and Time & $\begin{array}{l}\text { Depth in Klassen } \\
\text { Domestic Well } \\
(\mathrm{ft} \mathrm{BGL})\end{array}$ & $\begin{array}{c}\text { Depth in Klassen } \\
\text { Test Well } \\
\text { (ft BGL) }\end{array}$ & $\begin{array}{l}\text { Elevation in Klassen } \\
\text { Domestic Well } \\
\text { (ft AMSL) }\end{array}$ & \\
\hline 10/29/11 20:10 & 38.462 & 38.386 & 1470.31 & 1470.74 \\
\hline $10 / 29 / 1120: 20$ & 36.827 & 38.382 & 1471.94 & 1470.74 \\
\hline 10/29/11 20:30 & 37.045 & 38.377 & 1471.72 & 1470.75 \\
\hline $10 / 29 / 1120: 40$ & 36.691 & 38.37 & 1472.08 & 1470.75 \\
\hline $10 / 29 / 1120: 50$ & 36.78 & 38.367 & 1471.99 & 1470.76 \\
\hline 10/29/11 21:00 & 38.509 & 38.36 & 1470.26 & 1470.76 \\
\hline $10 / 29 / 1121: 10$ & 36.647 & 38.356 & 1472.12 & 1470.77 \\
\hline 10/29/11 21:20 & 36.813 & 38.353 & 1471.96 & 1470.77 \\
\hline $10 / 29 / 1121: 30$ & 38.206 & 38.349 & 1470.56 & 1470.77 \\
\hline $10 / 29 / 1121: 40$ & 37.102 & 38.346 & 1471.67 & 1470.78 \\
\hline $10 / 29 / 1121: 50$ & 38.506 & 38.339 & 1470.26 & 1470.78 \\
\hline 10/29/11 22:00 & 38.76 & 38.332 & 1470.01 & 1470.79 \\
\hline $10 / 29 / 1122: 10$ & 37.256 & 38.33 & 1471.51 & 1470.79 \\
\hline $10 / 29 / 1122: 20$ & 36.689 & 38.327 & 1472.08 & 1470.80 \\
\hline $10 / 29 / 1122: 30$ & 36.769 & 38.325 & 1472.00 & 1470.80 \\
\hline $10 / 29 / 1122: 40$ & 38.389 & 38.323 & 1470.38 & 1470.80 \\
\hline $10 / 29 / 1122: 50$ & 36.977 & 38.325 & 1471.79 & 1470.80 \\
\hline 10/29/11 23:00 & 36.633 & 38.32 & 1472.14 & 1470.80 \\
\hline 10/29/11 23:10 & 36.682 & 38.321 & 1472.09 & 1470.80 \\
\hline $10 / 29 / 1123: 20$ & 40.305 & 38.321 & 1468.46 & 1470.80 \\
\hline $10 / 29 / 1123: 30$ & 36.558 & 38.32 & 1472.21 & 1470.80 \\
\hline 10/29/11 23:40 & 37.268 & 38.32 & 1471.50 & 1470.80 \\
\hline $10 / 29 / 1123: 50$ & 36.499 & 38.318 & 1472.27 & 1470.80 \\
\hline 10/30/11 0:00 & 36.687 & 38.313 & 1472.08 & 1470.81 \\
\hline 10/30/11 0:10 & 36.431 & 38.311 & 1472.34 & 1470.81 \\
\hline 10/30/11 0:20 & 36.518 & 38.309 & 1472.25 & 1470.81 \\
\hline 10/30/11 0:30 & 40.343 & 38.304 & 1468.43 & 1470.82 \\
\hline 10/30/11 0:40 & 36.41 & 38.299 & 1472.36 & 1470.82 \\
\hline 10/30/11 0:50 & 37.65 & 38.292 & 1471.12 & 1470.83 \\
\hline 10/30/11 1:00 & 36.33 & 38.285 & 1472.44 & 1470.84 \\
\hline 10/30/11 1:10 & 36.609 & 38.281 & 1472.16 & 1470.84 \\
\hline $10 / 30 / 11$ 1:20 & 36.262 & 38.276 & 1472.51 & 1470.85 \\
\hline 10/30/11 1:30 & 36.384 & 38.269 & 1472.39 & 1470.85 \\
\hline $10 / 30 / 111: 40$ & 36.271 & 38.264 & 1472.50 & 1470.86 \\
\hline $10 / 30 / 111: 50$ & 36.417 & 38.267 & 1472.35 & 1470.86 \\
\hline $10 / 30 / 112: 00$ & 36.555 & 38.264 & 1472.21 & 1470.86 \\
\hline $10 / 30 / 112: 10$ & 36.3 & 38.259 & 1472.47 & 1470.86 \\
\hline $10 / 30 / 112: 20$ & 36.398 & 38.255 & 1472.37 & 1470.87 \\
\hline
\end{tabular}


TABLE S1.3 Automatically recorded groundwater levels in the Klassen wells, September 15, 2011, to March 13, 2012.

\begin{tabular}{|c|c|c|c|c|}
\hline \multicolumn{3}{|c|}{ Reference Elevation (ft AMSL) } & 1508.77 & \multirow{2}{*}{$\begin{array}{l}1509.12 \\
\text { Elevation in Klassen } \\
\text { Test Well (ft AMSL) }\end{array}$} \\
\hline Date and Time & $\begin{array}{l}\text { Depth in Klassen } \\
\text { Domestic Well } \\
(\mathrm{ft} \mathrm{BGL})\end{array}$ & $\begin{array}{c}\text { Depth in Klassen } \\
\text { Test Well } \\
\text { (ft BGL) }\end{array}$ & $\begin{array}{l}\text { Elevation in Klassen } \\
\text { Domestic Well } \\
\text { (ft AMSL) }\end{array}$ & \\
\hline $10 / 30 / 112: 30$ & 40.235 & 38.252 & 1468.53 & 1470.87 \\
\hline $10 / 30 / 112: 40$ & 36.293 & 38.25 & 1472.48 & 1470.87 \\
\hline $10 / 30 / 112: 50$ & 37.116 & 38.243 & 1471.65 & 1470.88 \\
\hline $10 / 30 / 113: 00$ & 36.236 & 38.238 & 1472.53 & 1470.88 \\
\hline 10/30/11 3:10 & 36.436 & 38.234 & 1472.33 & 1470.89 \\
\hline $10 / 30 / 113: 20$ & 38.457 & 38.229 & 1470.31 & 1470.89 \\
\hline $10 / 30 / 113: 30$ & 36.375 & 38.229 & 1472.39 & 1470.89 \\
\hline $10 / 30 / 113: 40$ & 36.696 & 38.227 & 1472.07 & 1470.90 \\
\hline $10 / 30 / 113: 50$ & 37.116 & 38.227 & 1471.65 & 1470.90 \\
\hline $10 / 30 / 114: 00$ & 36.274 & 38.224 & 1472.50 & 1470.90 \\
\hline 10/30/11 4:10 & 36.44 & 38.227 & 1472.33 & 1470.90 \\
\hline $10 / 30 / 114: 20$ & 36.185 & 38.222 & 1472.58 & 1470.90 \\
\hline $10 / 30 / 114: 30$ & 36.276 & 38.222 & 1472.49 & 1470.90 \\
\hline $10 / 30 / 114: 40$ & 40.878 & 38.217 & 1467.89 & 1470.91 \\
\hline $10 / 30 / 114: 50$ & 36.18 & 38.215 & 1472.59 & 1470.91 \\
\hline $10 / 30 / 115: 00$ & 37.095 & 38.21 & 1471.67 & 1470.91 \\
\hline 10/30/11 5:10 & 36.11 & 38.205 & 1472.66 & 1470.92 \\
\hline $10 / 30 / 115: 20$ & 36.323 & 38.203 & 1472.45 & 1470.92 \\
\hline 10/30/11 5:30 & 36.051 & 38.196 & 1472.72 & 1470.93 \\
\hline $10 / 30 / 115: 40$ & 36.159 & 38.194 & 1472.61 & 1470.93 \\
\hline $10 / 30 / 115: 50$ & 39.381 & 38.191 & 1469.39 & 1470.93 \\
\hline $10 / 30 / 116: 00$ & 41.658 & 38.182 & 1467.11 & 1470.94 \\
\hline 10/30/11 6:10 & 36.314 & 38.182 & 1472.46 & 1470.94 \\
\hline $10 / 30 / 116: 20$ & 38.171 & 38.18 & 1470.60 & 1470.94 \\
\hline $10 / 30 / 116: 30$ & 36.257 & 38.18 & 1472.51 & 1470.94 \\
\hline $10 / 30 / 116: 40$ & 36.278 & 38.177 & 1472.49 & 1470.95 \\
\hline $10 / 30 / 116: 50$ & 38.903 & 38.17 & 1469.87 & 1470.95 \\
\hline 10/30/11 7:00 & 36.14 & 38.163 & 1472.63 & 1470.96 \\
\hline 10/30/11 7:10 & 36.271 & 38.159 & 1472.50 & 1470.96 \\
\hline $10 / 30 / 117: 20$ & 36.044 & 38.154 & 1472.73 & 1470.97 \\
\hline 10/30/11 7:30 & 36.131 & 38.152 & 1472.64 & 1470.97 \\
\hline $10 / 30 / 117: 40$ & 39.665 & 38.149 & 1469.10 & 1470.97 \\
\hline $10 / 30 / 117: 50$ & 36.046 & 38.142 & 1472.72 & 1470.98 \\
\hline $10 / 30 / 118: 00$ & 36.208 & 38.14 & 1472.56 & 1470.98 \\
\hline 10/30/11 8:10 & 36.149 & 38.137 & 1472.62 & 1470.99 \\
\hline $10 / 30 / 118: 20$ & 36.217 & 38.135 & 1472.55 & 1470.99 \\
\hline $10 / 30 / 118: 30$ & 36.363 & 38.133 & 1472.41 & 1470.99 \\
\hline 10/30/11 8:40 & 36.316 & 38.13 & 1472.45 & 1470.99 \\
\hline
\end{tabular}


TABLE S1.3 Automatically recorded groundwater levels in the Klassen wells, September 15, 2011, to March 13, 2012.

\begin{tabular}{|c|c|c|c|c|}
\hline \multicolumn{3}{|c|}{ Reference Elevation (ft AMSL) } & 1508.77 & \multirow{2}{*}{$\begin{array}{l}1509.12 \\
\text { Elevation in Klassen } \\
\text { Test Well (ft AMSL) }\end{array}$} \\
\hline Date and Time & $\begin{array}{l}\text { Depth in Klassen } \\
\text { Domestic Well } \\
\text { (ft BGL) }\end{array}$ & $\begin{array}{c}\text { Depth in Klassen } \\
\text { Test Well } \\
\text { (ft BGL) }\end{array}$ & $\begin{array}{l}\text { Elevation in Klassen } \\
\text { Domestic Well } \\
\text { (ft AMSL) }\end{array}$ & \\
\hline $10 / 30 / 118: 50$ & 42.029 & 38.13 & 1466.74 & 1470.99 \\
\hline 10/30/11 9:00 & 41.056 & 38.137 & 1467.71 & 1470.99 \\
\hline 10/30/11 9:10 & 36.881 & 38.142 & 1471.89 & 1470.98 \\
\hline 10/30/11 9:20 & 36.834 & 38.147 & 1471.94 & 1470.98 \\
\hline 10/30/11 9:30 & 36.79 & 38.154 & 1471.98 & 1470.97 \\
\hline 10/30/11 9:40 & 36.354 & 38.156 & 1472.42 & 1470.97 \\
\hline 10/30/11 9:50 & 36.438 & 38.159 & 1472.33 & 1470.96 \\
\hline 10/30/11 10:00 & 37.824 & 38.163 & 1470.95 & 1470.96 \\
\hline 10/30/11 10:10 & 36.361 & 38.168 & 1472.41 & 1470.95 \\
\hline $10 / 30 / 1110: 20$ & 37.674 & 38.168 & 1471.10 & 1470.95 \\
\hline $10 / 30 / 1110: 30$ & 36.274 & 38.17 & 1472.50 & 1470.95 \\
\hline $10 / 30 / 1110: 40$ & 36.515 & 38.17 & 1472.25 & 1470.95 \\
\hline $10 / 30 / 1110: 50$ & 36.173 & 38.17 & 1472.60 & 1470.95 \\
\hline 10/30/11 11:00 & 36.271 & 38.173 & 1472.50 & 1470.95 \\
\hline $10 / 30 / 11$ 11:10 & 38.509 & 38.177 & 1470.26 & 1470.95 \\
\hline $10 / 30 / 11$ 11:20 & 36.154 & 38.184 & 1472.62 & 1470.94 \\
\hline $10 / 30 / 11$ 11:30 & 37.428 & 38.189 & 1471.34 & 1470.93 \\
\hline 10/30/11 11:40 & 36.074 & 38.196 & 1472.70 & 1470.93 \\
\hline $10 / 30 / 1111: 50$ & 36.314 & 38.201 & 1472.46 & 1470.92 \\
\hline $10 / 30 / 11$ 12:00 & 36.006 & 38.201 & 1472.76 & 1470.92 \\
\hline $10 / 30 / 11$ 12:10 & 36.117 & 38.203 & 1472.65 & 1470.92 \\
\hline $10 / 30 / 11$ 12:20 & 39.1 & 38.21 & 1469.67 & 1470.91 \\
\hline $10 / 30 / 1112: 30$ & 36.06 & 38.213 & 1472.71 & 1470.91 \\
\hline $10 / 30 / 1112: 40$ & 37.266 & 38.22 & 1471.50 & 1470.90 \\
\hline $10 / 30 / 1112: 50$ & 36.067 & 38.231 & 1472.70 & 1470.89 \\
\hline 10/30/11 13:00 & 36.323 & 38.241 & 1472.45 & 1470.88 \\
\hline $10 / 30 / 11$ 13:10 & 36.105 & 38.25 & 1472.66 & 1470.87 \\
\hline $10 / 30 / 11$ 13:20 & 36.239 & 38.257 & 1472.53 & 1470.87 \\
\hline $10 / 30 / 11$ 13:30 & 39.998 & 38.266 & 1468.77 & 1470.86 \\
\hline $10 / 30 / 11$ 13:40 & 36.755 & 38.274 & 1472.01 & 1470.85 \\
\hline $10 / 30 / 11$ 13:50 & 36.178 & 38.278 & 1472.59 & 1470.84 \\
\hline 10/30/11 14:00 & 36.344 & 38.285 & 1472.43 & 1470.84 \\
\hline 10/30/11 14:10 & 36.213 & 38.295 & 1472.56 & 1470.83 \\
\hline $10 / 30 / 11$ 14:20 & 36.264 & 38.299 & 1472.51 & 1470.82 \\
\hline $10 / 30 / 11$ 14:30 & 37.885 & 38.304 & 1470.88 & 1470.82 \\
\hline $10 / 30 / 11$ 14:40 & 36.253 & 38.309 & 1472.52 & 1470.81 \\
\hline $10 / 30 / 1114: 50$ & 36.574 & 38.316 & 1472.20 & 1470.81 \\
\hline $10 / 30 / 11$ 15:00 & 36.248 & 38.32 & 1472.52 & 1470.80 \\
\hline
\end{tabular}


TABLE S1.3 Automatically recorded groundwater levels in the Klassen wells, September 15, 2011, to March 13, 2012.

\begin{tabular}{|c|c|c|c|c|}
\hline \multicolumn{3}{|c|}{ Reference Elevation (ft AMSL) } & 1508.77 & \multirow{2}{*}{$\begin{array}{l}1509.12 \\
\text { Elevation in Klassen } \\
\text { Test Well (ft AMSL) }\end{array}$} \\
\hline Date and Time & $\begin{array}{l}\text { Depth in Klassen } \\
\text { Domestic Well } \\
\text { (ft BGL) }\end{array}$ & $\begin{array}{c}\text { Depth in Klassen } \\
\text { Test Well } \\
\text { (ft BGL) }\end{array}$ & $\begin{array}{l}\text { Elevation in Klassen } \\
\text { Domestic Well } \\
\text { (ft AMSL) }\end{array}$ & \\
\hline $10 / 30 / 1115: 10$ & 36.328 & 38.323 & 1472.44 & 1470.80 \\
\hline $10 / 30 / 1115: 20$ & 39.055 & 38.323 & 1469.71 & 1470.80 \\
\hline 10/30/11 15:30 & 36.192 & 38.325 & 1472.58 & 1470.80 \\
\hline $10 / 30 / 1115: 40$ & 37.268 & 38.323 & 1471.50 & 1470.80 \\
\hline 10/30/11 15:50 & 36.119 & 38.323 & 1472.65 & 1470.80 \\
\hline 10/30/11 16:00 & 36.536 & 38.325 & 1472.23 & 1470.80 \\
\hline 10/30/11 16:10 & 36.487 & 38.337 & 1472.28 & 1470.79 \\
\hline 10/30/11 16:20 & 36.684 & 38.346 & 1472.09 & 1470.78 \\
\hline 10/30/11 16:30 & 40.943 & 38.351 & 1467.83 & 1470.77 \\
\hline $10 / 30 / 1116: 40$ & 36.642 & 38.358 & 1472.13 & 1470.76 \\
\hline 10/30/11 16:50 & 37.254 & 38.358 & 1471.52 & 1470.76 \\
\hline 10/30/11 17:00 & 36.511 & 38.356 & 1472.26 & 1470.77 \\
\hline 10/30/11 17:10 & 36.64 & 38.356 & 1472.13 & 1470.77 \\
\hline $10 / 30 / 1117: 20$ & 36.694 & 38.351 & 1472.08 & 1470.77 \\
\hline 10/30/11 17:30 & 39.379 & 38.349 & 1469.39 & 1470.77 \\
\hline 10/30/11 17:40 & 36.475 & 38.349 & 1472.29 & 1470.77 \\
\hline 10/30/11 17:50 & 36.895 & 38.344 & 1471.87 & 1470.78 \\
\hline 10/30/11 18:00 & 36.37 & 38.339 & 1472.40 & 1470.78 \\
\hline 10/30/11 18:10 & 36.49 & 38.332 & 1472.28 & 1470.79 \\
\hline 10/30/11 18:20 & 38.736 & 38.327 & 1470.03 & 1470.80 \\
\hline $10 / 30 / 11$ 18:30 & 36.457 & 38.323 & 1472.31 & 1470.80 \\
\hline 10/30/11 18:40 & 36.815 & 38.318 & 1471.95 & 1470.80 \\
\hline 10/30/11 18:50 & 39.47 & 38.311 & 1469.30 & 1470.81 \\
\hline 10/30/11 19:00 & 36.424 & 38.309 & 1472.35 & 1470.81 \\
\hline 10/30/11 19:10 & 36.783 & 38.304 & 1471.99 & 1470.82 \\
\hline 10/30/11 19:20 & 36.332 & 38.297 & 1472.44 & 1470.83 \\
\hline 10/30/11 19:30 & 36.438 & 38.295 & 1472.33 & 1470.83 \\
\hline 10/30/11 19:40 & 36.278 & 38.292 & 1472.49 & 1470.83 \\
\hline 10/30/11 19:50 & 36.339 & 38.292 & 1472.43 & 1470.83 \\
\hline 10/30/11 20:00 & 36.201 & 38.29 & 1472.57 & 1470.83 \\
\hline 10/30/11 20:10 & 36.135 & 38.29 & 1472.63 & 1470.83 \\
\hline $10 / 30 / 1120: 20$ & 36.079 & 38.288 & 1472.69 & 1470.83 \\
\hline 10/30/11 20:30 & 36.025 & 38.285 & 1472.74 & 1470.84 \\
\hline $10 / 30 / 1120: 40$ & 36.02 & 38.283 & 1472.75 & 1470.84 \\
\hline 10/30/11 20:50 & 35.973 & 38.281 & 1472.80 & 1470.84 \\
\hline $10 / 30 / 1121: 00$ & 35.938 & 38.276 & 1472.83 & 1470.85 \\
\hline 10/30/11 21:10 & 36.091 & 38.271 & 1472.68 & 1470.85 \\
\hline $10 / 30 / 1121: 20$ & 35.962 & 38.271 & 1472.81 & 1470.85 \\
\hline
\end{tabular}


TABLE S1.3 Automatically recorded groundwater levels in the Klassen wells, September 15, 2011, to March 13, 2012.

\begin{tabular}{|c|c|c|c|c|}
\hline \multicolumn{3}{|c|}{ Reference Elevation (ft AMSL) } & 1508.77 & \multirow{2}{*}{$\begin{array}{l}1509.12 \\
\text { Elevation in Klassen } \\
\text { Test Well (ft AMSL) }\end{array}$} \\
\hline Date and Time & $\begin{array}{l}\text { Depth in Klassen } \\
\text { Domestic Well } \\
\text { (ft BGL) }\end{array}$ & $\begin{array}{c}\text { Depth in Klassen } \\
\text { Test Well } \\
\text { (ft BGL) }\end{array}$ & $\begin{array}{l}\text { Elevation in Klassen } \\
\text { Domestic Well } \\
\text { (ft AMSL) }\end{array}$ & \\
\hline 10/30/11 21:30 & 35.915 & 38.264 & 1472.85 & 1470.86 \\
\hline 10/30/11 21:40 & 35.877 & 38.257 & 1472.89 & 1470.87 \\
\hline $10 / 30 / 1121: 50$ & 35.835 & 38.252 & 1472.93 & 1470.87 \\
\hline $10 / 30 / 1122: 00$ & 35.814 & 38.245 & 1472.96 & 1470.88 \\
\hline $10 / 30 / 1122: 10$ & 35.793 & 38.238 & 1472.98 & 1470.88 \\
\hline $10 / 30 / 1122: 20$ & 35.788 & 38.234 & 1472.98 & 1470.89 \\
\hline $10 / 30 / 1122: 30$ & 35.772 & 38.227 & 1473.00 & 1470.90 \\
\hline $10 / 30 / 1122: 40$ & 35.751 & 38.222 & 1473.02 & 1470.90 \\
\hline $10 / 30 / 1122: 50$ & 38.692 & 38.22 & 1470.08 & 1470.90 \\
\hline 10/30/11 23:00 & 36.647 & 38.217 & 1472.12 & 1470.91 \\
\hline $10 / 30 / 1123: 10$ & 35.87 & 38.213 & 1472.90 & 1470.91 \\
\hline $10 / 30 / 1123: 20$ & 37.763 & 38.213 & 1471.01 & 1470.91 \\
\hline $10 / 30 / 1123: 30$ & 36.004 & 38.21 & 1472.77 & 1470.91 \\
\hline $10 / 30 / 1123: 40$ & 35.889 & 38.208 & 1472.88 & 1470.91 \\
\hline $10 / 30 / 1123: 50$ & 35.823 & 38.208 & 1472.95 & 1470.91 \\
\hline 10/31/11 0:00 & 35.769 & 38.203 & 1473.00 & 1470.92 \\
\hline 10/31/11 0:10 & 35.739 & 38.201 & 1473.03 & 1470.92 \\
\hline 10/31/11 0:20 & 35.692 & 38.196 & 1473.08 & 1470.93 \\
\hline 10/31/11 0:30 & 35.676 & 38.194 & 1473.09 & 1470.93 \\
\hline 10/31/11 0:40 & 35.636 & 38.191 & 1473.13 & 1470.93 \\
\hline $10 / 31 / 11$ 0:50 & 35.624 & 38.187 & 1473.15 & 1470.94 \\
\hline 10/31/11 1:00 & 35.587 & 38.18 & 1473.18 & 1470.94 \\
\hline 10/31/11 1:10 & 35.577 & 38.173 & 1473.19 & 1470.95 \\
\hline 10/31/11 1:20 & 35.542 & 38.168 & 1473.23 & 1470.95 \\
\hline 10/31/11 1:30 & 35.537 & 38.166 & 1473.23 & 1470.96 \\
\hline 10/31/11 1:40 & 35.504 & 38.156 & 1473.27 & 1470.97 \\
\hline 10/31/11 1:50 & 35.5 & 38.152 & 1473.27 & 1470.97 \\
\hline 10/31/11 2:00 & 35.469 & 38.149 & 1473.30 & 1470.97 \\
\hline 10/31/11 2:10 & 35.49 & 38.142 & 1473.28 & 1470.98 \\
\hline 10/31/11 2:20 & 35.465 & 38.14 & 1473.30 & 1470.98 \\
\hline $10 / 31 / 112: 30$ & 35.458 & 38.133 & 1473.31 & 1470.99 \\
\hline $10 / 31 / 112: 40$ & 35.429 & 38.13 & 1473.34 & 1470.99 \\
\hline $10 / 31 / 112: 50$ & 35.425 & 38.128 & 1473.34 & 1470.99 \\
\hline 10/31/11 3:00 & 35.408 & 38.121 & 1473.36 & 1471.00 \\
\hline 10/31/11 3:10 & 35.394 & 38.116 & 1473.38 & 1471.01 \\
\hline 10/31/11 3:20 & 35.387 & 38.112 & 1473.38 & 1471.01 \\
\hline 10/31/11 3:30 & 35.364 & 38.107 & 1473.41 & 1471.02 \\
\hline 10/31/11 3:40 & 35.361 & 38.102 & 1473.41 & 1471.02 \\
\hline
\end{tabular}


TABLE S1.3 Automatically recorded groundwater levels in the Klassen wells, September 15, 2011, to March 13, 2012.

\begin{tabular}{|c|c|c|c|c|}
\hline \multicolumn{3}{|c|}{ Reference Elevation (ft AMSL) } & 1508.77 & \multirow{2}{*}{$\begin{array}{l}1509.12 \\
\text { Elevation in Klassen } \\
\text { Test Well (ft AMSL) }\end{array}$} \\
\hline Date and Time & $\begin{array}{l}\text { Depth in Klassen } \\
\text { Domestic Well } \\
(\mathrm{ft} \mathrm{BGL})\end{array}$ & $\begin{array}{c}\text { Depth in Klassen } \\
\text { Test Well } \\
\text { (ft BGL) }\end{array}$ & $\begin{array}{l}\text { Elevation in Klassen } \\
\text { Domestic Well } \\
\text { (ft AMSL) }\end{array}$ & \\
\hline $10 / 31 / 113: 50$ & 35.558 & 38.098 & 1473.21 & 1471.02 \\
\hline 10/31/11 4:00 & 35.441 & 38.093 & 1473.33 & 1471.03 \\
\hline 10/31/11 4:10 & 35.385 & 38.088 & 1473.38 & 1471.03 \\
\hline $10 / 31 / 114: 20$ & 35.371 & 38.083 & 1473.40 & 1471.04 \\
\hline 10/31/11 4:30 & 35.34 & 38.077 & 1473.43 & 1471.05 \\
\hline $10 / 31 / 114: 40$ & 35.331 & 38.074 & 1473.44 & 1471.05 \\
\hline $10 / 31 / 114: 50$ & 35.303 & 38.065 & 1473.47 & 1471.06 \\
\hline 10/31/11 5:00 & 35.298 & 38.06 & 1473.47 & 1471.06 \\
\hline 10/31/11 5:10 & 35.272 & 38.051 & 1473.50 & 1471.07 \\
\hline $10 / 31 / 115: 20$ & 35.272 & 38.048 & 1473.50 & 1471.07 \\
\hline $10 / 31 / 115: 30$ & 35.246 & 38.039 & 1473.52 & 1471.08 \\
\hline 10/31/11 5:40 & 35.246 & 38.032 & 1473.52 & 1471.09 \\
\hline $10 / 31 / 115: 50$ & 35.221 & 38.025 & 1473.55 & 1471.10 \\
\hline 10/31/11 6:00 & 35.223 & 38.018 & 1473.55 & 1471.10 \\
\hline 10/31/11 6:10 & 37.707 & 38.013 & 1471.06 & 1471.11 \\
\hline $10 / 31 / 116: 20$ & 35.375 & 38.011 & 1473.39 & 1471.11 \\
\hline $10 / 31 / 116: 30$ & 35.303 & 38.004 & 1473.47 & 1471.12 \\
\hline $10 / 31 / 116: 40$ & 35.251 & 37.999 & 1473.52 & 1471.12 \\
\hline $10 / 31 / 116: 50$ & 35.242 & 37.992 & 1473.53 & 1471.13 \\
\hline 10/31/11 7:00 & 35.207 & 37.985 & 1473.56 & 1471.14 \\
\hline 10/31/11 7:10 & 35.204 & 37.978 & 1473.57 & 1471.14 \\
\hline 10/31/11 7:20 & 36.438 & 37.976 & 1472.33 & 1471.15 \\
\hline 10/31/11 7:30 & 35.3 & 37.971 & 1473.47 & 1471.15 \\
\hline 10/31/11 7:40 & 35.246 & 37.964 & 1473.52 & 1471.16 \\
\hline 10/31/11 7:50 & 35.235 & 37.957 & 1473.53 & 1471.17 \\
\hline 10/31/11 8:00 & 35.197 & 37.952 & 1473.57 & 1471.17 \\
\hline 10/31/11 8:10 & 40.418 & 37.947 & 1468.35 & 1471.18 \\
\hline 10/31/11 8:20 & 35.378 & 37.945 & 1473.39 & 1471.18 \\
\hline $10 / 31 / 118: 30$ & 37.383 & 37.943 & 1471.39 & 1471.18 \\
\hline $10 / 31 / 118: 40$ & 35.465 & 37.943 & 1473.30 & 1471.18 \\
\hline $10 / 31 / 118: 50$ & 35.387 & 37.943 & 1473.38 & 1471.18 \\
\hline 10/31/11 9:00 & 35.324 & 37.94 & 1473.45 & 1471.18 \\
\hline 10/31/11 9:10 & 35.296 & 37.94 & 1473.47 & 1471.18 \\
\hline 10/31/11 9:20 & 35.258 & 37.94 & 1473.51 & 1471.18 \\
\hline 10/31/11 9:30 & 35.286 & 37.936 & 1473.48 & 1471.19 \\
\hline 10/31/11 9:40 & 35.472 & 37.938 & 1473.30 & 1471.18 \\
\hline 10/31/11 9:50 & 35.385 & 37.936 & 1473.38 & 1471.19 \\
\hline 10/31/11 10:00 & 35.411 & 37.94 & 1473.36 & 1471.18 \\
\hline
\end{tabular}


TABLE S1.3 Automatically recorded groundwater levels in the Klassen wells, September 15, 2011, to March 13, 2012.

\begin{tabular}{|c|c|c|c|c|}
\hline \multicolumn{3}{|c|}{ Reference Elevation (ft AMSL) } & 1508.77 & \multirow{2}{*}{$\begin{array}{l}1509.12 \\
\text { Elevation in Klassen } \\
\text { Test Well (ft AMSL) }\end{array}$} \\
\hline Date and Time & $\begin{array}{l}\text { Depth in Klassen } \\
\text { Domestic Well } \\
\text { (ft BGL) }\end{array}$ & $\begin{array}{c}\text { Depth in Klassen } \\
\text { Test Well } \\
\text { (ft BGL) }\end{array}$ & $\begin{array}{l}\text { Elevation in Klassen } \\
\text { Domestic Well } \\
\text { (ft AMSL) }\end{array}$ & \\
\hline 10/31/11 10:10 & 36.614 & 37.938 & 1472.16 & 1471.18 \\
\hline $10 / 31 / 1110: 20$ & 35.594 & 37.943 & 1473.18 & 1471.18 \\
\hline 10/31/11 10:30 & 37.364 & 37.943 & 1471.41 & 1471.18 \\
\hline $10 / 31 / 11$ 10:40 & 35.753 & 37.947 & 1473.02 & 1471.18 \\
\hline 10/31/11 10:50 & 35.662 & 37.95 & 1473.11 & 1471.17 \\
\hline 10/31/11 11:00 & 40.214 & 37.947 & 1468.56 & 1471.18 \\
\hline 10/31/11 11:10 & 35.676 & 37.945 & 1473.09 & 1471.18 \\
\hline 10/31/11 11:20 & 36.921 & 37.945 & 1471.85 & 1471.18 \\
\hline 10/31/11 11:30 & 35.647 & 37.947 & 1473.12 & 1471.18 \\
\hline 10/31/11 11:40 & 35.561 & 37.943 & 1473.21 & 1471.18 \\
\hline $10 / 31 / 1111: 50$ & 35.488 & 37.94 & 1473.28 & 1471.18 \\
\hline 10/31/11 12:00 & 35.432 & 37.936 & 1473.34 & 1471.19 \\
\hline $10 / 31 / 1112: 10$ & 35.591 & 37.936 & 1473.18 & 1471.19 \\
\hline $10 / 31 / 1112: 20$ & 35.46 & 37.936 & 1473.31 & 1471.19 \\
\hline $10 / 31 / 1112: 30$ & 35.401 & 37.938 & 1473.37 & 1471.18 \\
\hline $10 / 31 / 11$ 12:40 & 35.371 & 37.936 & 1473.40 & 1471.19 \\
\hline $10 / 31 / 1112: 50$ & 35.329 & 37.933 & 1473.44 & 1471.19 \\
\hline 10/31/11 13:00 & 37.28 & 37.936 & 1471.49 & 1471.19 \\
\hline $10 / 31 / 11$ 13:10 & 35.406 & 37.94 & 1473.36 & 1471.18 \\
\hline $10 / 31 / 11$ 13:20 & 35.326 & 37.938 & 1473.44 & 1471.18 \\
\hline $10 / 31 / 11$ 13:30 & 35.303 & 37.938 & 1473.47 & 1471.18 \\
\hline $10 / 31 / 11$ 13:40 & 35.263 & 37.933 & 1473.51 & 1471.19 \\
\hline $10 / 31 / 11$ 13:50 & 35.228 & 37.931 & 1473.54 & 1471.19 \\
\hline $10 / 31 / 11$ 14:00 & 35.223 & 37.926 & 1473.55 & 1471.20 \\
\hline $10 / 31 / 11$ 14:10 & 35.366 & 37.924 & 1473.40 & 1471.20 \\
\hline $10 / 31 / 11$ 14:20 & 38.722 & 37.919 & 1470.05 & 1471.20 \\
\hline $10 / 31 / 11$ 14:30 & 40.866 & 37.919 & 1467.90 & 1471.20 \\
\hline $10 / 31 / 11$ 14:40 & 36.393 & 37.915 & 1472.38 & 1471.21 \\
\hline $10 / 31 / 1114: 50$ & 35.91 & 37.908 & 1472.86 & 1471.21 \\
\hline $10 / 31 / 11$ 15:00 & 35.678 & 37.9 & 1473.09 & 1471.22 \\
\hline $10 / 31 / 11$ 15:10 & 35.727 & 37.896 & 1473.04 & 1471.23 \\
\hline $10 / 31 / 11$ 15:20 & 35.577 & 37.891 & 1473.19 & 1471.23 \\
\hline $10 / 31 / 11$ 15:30 & 35.521 & 37.886 & 1473.25 & 1471.24 \\
\hline $10 / 31 / 11$ 15:40 & 35.694 & 37.884 & 1473.08 & 1471.24 \\
\hline $10 / 31 / 11$ 15:50 & 36.168 & 37.884 & 1472.60 & 1471.24 \\
\hline 10/31/11 16:00 & 35.694 & 37.886 & 1473.08 & 1471.24 \\
\hline $10 / 31 / 11$ 16:10 & 35.652 & 37.889 & 1473.12 & 1471.23 \\
\hline $10 / 31 / 11$ 16:20 & 35.648 & 37.891 & 1473.12 & 1471.23 \\
\hline
\end{tabular}


TABLE S1.3 Automatically recorded groundwater levels in the Klassen wells, September 15, 2011, to March 13, 2012.

\begin{tabular}{|c|c|c|c|c|}
\hline \multicolumn{3}{|c|}{ Reference Elevation (ft AMSL) } & 1508.77 & \multirow{2}{*}{$\begin{array}{l}1509.12 \\
\text { Elevation in Klassen } \\
\text { Test Well (ft AMSL) }\end{array}$} \\
\hline Date and Time & $\begin{array}{l}\text { Depth in Klassen } \\
\text { Domestic Well } \\
\text { (ft BGL) }\end{array}$ & $\begin{array}{c}\text { Depth in Klassen } \\
\text { Test Well } \\
\text { (ft BGL) }\end{array}$ & $\begin{array}{l}\text { Elevation in Klassen } \\
\text { Domestic Well } \\
\text { (ft AMSL) }\end{array}$ & \\
\hline 10/31/11 16:30 & 35.594 & 37.891 & 1473.18 & 1471.23 \\
\hline $10 / 31 / 1116: 40$ & 36.074 & 37.893 & 1472.70 & 1471.23 \\
\hline 10/31/11 16:50 & 35.617 & 37.893 & 1473.15 & 1471.23 \\
\hline 10/31/11 17:00 & 35.528 & 37.893 & 1473.24 & 1471.23 \\
\hline 10/31/11 17:10 & 39.827 & 37.896 & 1468.94 & 1471.23 \\
\hline $10 / 31 / 1117: 20$ & 36.375 & 37.9 & 1472.39 & 1471.22 \\
\hline 10/31/11 17:30 & 35.863 & 37.903 & 1472.91 & 1471.22 \\
\hline 10/31/11 17:40 & 35.812 & 37.908 & 1472.96 & 1471.21 \\
\hline 10/31/11 17:50 & 35.718 & 37.912 & 1473.05 & 1471.21 \\
\hline 10/31/11 18:00 & 35.657 & 37.912 & 1473.11 & 1471.21 \\
\hline 10/31/11 18:10 & 35.601 & 37.915 & 1473.17 & 1471.21 \\
\hline 10/31/11 18:20 & 35.561 & 37.917 & 1473.21 & 1471.21 \\
\hline 10/31/11 18:30 & 35.509 & 37.919 & 1473.26 & 1471.20 \\
\hline $10 / 31 / 1118: 40$ & 35.462 & 37.917 & 1473.31 & 1471.21 \\
\hline 10/31/11 18:50 & 35.446 & 37.917 & 1473.32 & 1471.21 \\
\hline 10/31/11 19:00 & 35.404 & 37.912 & 1473.37 & 1471.21 \\
\hline 10/31/11 19:10 & 35.68 & 37.912 & 1473.09 & 1471.21 \\
\hline 10/31/11 19:20 & 35.472 & 37.912 & 1473.30 & 1471.21 \\
\hline 10/31/11 19:30 & 35.427 & 37.912 & 1473.34 & 1471.21 \\
\hline 10/31/11 19:40 & 35.375 & 37.915 & 1473.39 & 1471.21 \\
\hline 10/31/11 19:50 & 35.333 & 37.915 & 1473.44 & 1471.21 \\
\hline 10/31/11 20:00 & 35.298 & 37.912 & 1473.47 & 1471.21 \\
\hline $10 / 31 / 1120: 10$ & 35.293 & 37.91 & 1473.48 & 1471.21 \\
\hline $10 / 31 / 1120: 20$ & 35.261 & 37.908 & 1473.51 & 1471.21 \\
\hline 10/31/11 20:30 & 35.23 & 37.905 & 1473.54 & 1471.22 \\
\hline $10 / 31 / 1120: 40$ & 35.207 & 37.9 & 1473.56 & 1471.22 \\
\hline $10 / 31 / 1120: 50$ & 35.207 & 37.898 & 1473.56 & 1471.22 \\
\hline $10 / 31 / 1121: 00$ & 35.209 & 37.896 & 1473.56 & 1471.23 \\
\hline $10 / 31 / 1121: 10$ & 35.272 & 37.896 & 1473.50 & 1471.23 \\
\hline 10/31/11 21:20 & 35.263 & 37.893 & 1473.51 & 1471.23 \\
\hline 10/31/11 21:30 & 35.277 & 37.893 & 1473.49 & 1471.23 \\
\hline 10/31/11 21:40 & 35.314 & 37.896 & 1473.46 & 1471.23 \\
\hline $10 / 31 / 1121: 50$ & 35.293 & 37.898 & 1473.48 & 1471.22 \\
\hline $10 / 31 / 1122: 00$ & 35.256 & 37.896 & 1473.51 & 1471.23 \\
\hline $10 / 31 / 1122: 10$ & 35.244 & 37.894 & 1473.53 & 1471.23 \\
\hline $10 / 31 / 1122: 20$ & 35.209 & 37.893 & 1473.56 & 1471.23 \\
\hline $10 / 31 / 1122: 30$ & 39.942 & 37.889 & 1468.83 & 1471.23 \\
\hline $10 / 31 / 1122: 40$ & 38.657 & 37.891 & 1470.11 & 1471.23 \\
\hline
\end{tabular}


TABLE S1.3 Automatically recorded groundwater levels in the Klassen wells, September 15, 2011, to March 13, 2012.

\begin{tabular}{|c|c|c|c|c|}
\hline \multicolumn{3}{|c|}{ Reference Elevation (ft AMSL) } & 1508.77 & \multirow{2}{*}{$\begin{array}{l}1509.12 \\
\text { Elevation in Klassen } \\
\text { Test Well (ft AMSL) }\end{array}$} \\
\hline Date and Time & $\begin{array}{l}\text { Depth in Klassen } \\
\text { Domestic Well } \\
\text { (ft BGL) }\end{array}$ & $\begin{array}{c}\text { Depth in Klassen } \\
\text { Test Well } \\
\text { (ft BGL) }\end{array}$ & $\begin{array}{l}\text { Elevation in Klassen } \\
\text { Domestic Well } \\
\text { (ft AMSL) }\end{array}$ & \\
\hline $10 / 31 / 1122: 50$ & 35.46 & 37.893 & 1473.31 & 1471.23 \\
\hline $10 / 31 / 1123: 00$ & 35.359 & 37.893 & 1473.41 & 1471.23 \\
\hline 10/31/11 23:10 & 35.291 & 37.891 & 1473.48 & 1471.23 \\
\hline $10 / 31 / 1123: 20$ & 35.242 & 37.891 & 1473.53 & 1471.23 \\
\hline $10 / 31 / 1123: 30$ & 36.921 & 37.889 & 1471.85 & 1471.23 \\
\hline 10/31/11 23:40 & 35.343 & 37.889 & 1473.43 & 1471.23 \\
\hline $10 / 31 / 1123: 50$ & 35.413 & 37.889 & 1473.36 & 1471.23 \\
\hline $11 / 1 / 110: 00$ & 35.293 & 37.889 & 1473.48 & 1471.23 \\
\hline $11 / 1 / 110: 10$ & 35.235 & 37.886 & 1473.53 & 1471.24 \\
\hline $11 / 1 / 110: 20$ & 35.218 & 37.886 & 1473.55 & 1471.24 \\
\hline $11 / 1 / 110: 30$ & 35.178 & 37.884 & 1473.59 & 1471.24 \\
\hline $11 / 1 / 110: 40$ & 35.148 & 37.882 & 1473.62 & 1471.24 \\
\hline $11 / 1 / 110: 50$ & 35.124 & 37.877 & 1473.65 & 1471.25 \\
\hline $11 / 1 / 11$ 1:00 & 35.117 & 37.877 & 1473.65 & 1471.25 \\
\hline $11 / 1 / 11$ 1:10 & 35.092 & 37.872 & 1473.68 & 1471.25 \\
\hline $11 / 1 / 111: 20$ & 35.068 & 37.87 & 1473.70 & 1471.25 \\
\hline $11 / 1 / 11$ 1:30 & 35.066 & 37.868 & 1473.70 & 1471.25 \\
\hline $11 / 1 / 11$ 1:40 & 35.103 & 37.865 & 1473.67 & 1471.26 \\
\hline $11 / 1 / 111: 50$ & 35.155 & 37.868 & 1473.61 & 1471.25 \\
\hline $11 / 1 / 112: 00$ & 35.132 & 37.865 & 1473.64 & 1471.26 \\
\hline $11 / 1 / 112: 10$ & 35.124 & 37.863 & 1473.65 & 1471.26 \\
\hline $11 / 1 / 112: 20$ & 35.103 & 37.863 & 1473.67 & 1471.26 \\
\hline $11 / 1 / 112: 30$ & 35.089 & 37.863 & 1473.68 & 1471.26 \\
\hline $11 / 1 / 112: 40$ & 35.08 & 37.863 & 1473.69 & 1471.26 \\
\hline $11 / 1 / 112: 50$ & 35.059 & 37.863 & 1473.71 & 1471.26 \\
\hline 11/1/11 3:00 & 35.059 & 37.861 & 1473.71 & 1471.26 \\
\hline $11 / 1 / 113: 10$ & 35.035 & 37.861 & 1473.73 & 1471.26 \\
\hline $11 / 1 / 113: 20$ & 35.071 & 37.861 & 1473.70 & 1471.26 \\
\hline $11 / 1 / 113: 30$ & 35.122 & 37.861 & 1473.65 & 1471.26 \\
\hline $11 / 1 / 113: 40$ & 35.099 & 37.863 & 1473.67 & 1471.26 \\
\hline $11 / 1 / 113: 50$ & 35.066 & 37.861 & 1473.70 & 1471.26 \\
\hline $11 / 1 / 114: 00$ & 35.035 & 37.858 & 1473.73 & 1471.26 \\
\hline $11 / 1 / 114: 10$ & 35.01 & 37.856 & 1473.76 & 1471.27 \\
\hline $11 / 1 / 114: 20$ & 35.014 & 37.856 & 1473.76 & 1471.27 \\
\hline $11 / 1 / 114: 30$ & 34.988 & 37.851 & 1473.78 & 1471.27 \\
\hline $11 / 1 / 114: 40$ & 34.967 & 37.846 & 1473.80 & 1471.28 \\
\hline $11 / 1 / 114: 50$ & 34.946 & 37.844 & 1473.82 & 1471.28 \\
\hline $11 / 1 / 11$ 5:00 & 34.927 & 37.842 & 1473.84 & 1471.28 \\
\hline
\end{tabular}


TABLE S1.3 Automatically recorded groundwater levels in the Klassen wells, September 15, 2011, to March 13, 2012.

\begin{tabular}{|c|c|c|c|c|}
\hline \multicolumn{3}{|c|}{ Reference Elevation (ft AMSL) } & 1508.77 & \multirow{2}{*}{$\begin{array}{l}1509.12 \\
\text { Elevation in Klassen } \\
\text { Test Well (ft AMSL) }\end{array}$} \\
\hline Date and Time & $\begin{array}{l}\text { Depth in Klassen } \\
\text { Domestic Well } \\
\text { (ft BGL) }\end{array}$ & $\begin{array}{c}\text { Depth in Klassen } \\
\text { Test Well } \\
\text { (ft BGL) }\end{array}$ & $\begin{array}{l}\text { Elevation in Klassen } \\
\text { Domestic Well } \\
\text { (ft AMSL) }\end{array}$ & \\
\hline 11/1/11 5:10 & 34.939 & 37.837 & 1473.83 & 1471.29 \\
\hline $11 / 1 / 115: 20$ & 34.918 & 37.833 & 1473.85 & 1471.29 \\
\hline $11 / 1 / 115: 30$ & 34.902 & 37.83 & 1473.87 & 1471.29 \\
\hline $11 / 1 / 115: 40$ & 39.367 & 37.825 & 1469.40 & 1471.30 \\
\hline $11 / 1 / 115: 50$ & 35.047 & 37.823 & 1473.72 & 1471.30 \\
\hline $11 / 1 / 116: 00$ & 34.986 & 37.821 & 1473.78 & 1471.30 \\
\hline $11 / 1 / 116: 10$ & 39.843 & 37.821 & 1468.93 & 1471.30 \\
\hline $11 / 1 / 116: 20$ & 35.129 & 37.823 & 1473.64 & 1471.30 \\
\hline $11 / 1 / 116: 30$ & 35.035 & 37.821 & 1473.73 & 1471.30 \\
\hline $11 / 1 / 116: 40$ & 34.986 & 37.818 & 1473.78 & 1471.30 \\
\hline $11 / 1 / 116: 50$ & 34.949 & 37.816 & 1473.82 & 1471.31 \\
\hline $11 / 1 / 117: 00$ & 34.977 & 37.816 & 1473.79 & 1471.31 \\
\hline $11 / 1 / 117: 10$ & 35.021 & 37.814 & 1473.75 & 1471.31 \\
\hline $11 / 1 / 117: 20$ & 35.021 & 37.814 & 1473.75 & 1471.31 \\
\hline $11 / 1 / 11$ 7:30 & 34.991 & 37.811 & 1473.78 & 1471.31 \\
\hline $11 / 1 / 117: 40$ & 34.958 & 37.807 & 1473.81 & 1471.32 \\
\hline $11 / 1 / 117: 50$ & 34.958 & 37.802 & 1473.81 & 1471.32 \\
\hline $11 / 1 / 118: 00$ & 34.932 & 37.802 & 1473.84 & 1471.32 \\
\hline $11 / 1 / 118: 10$ & 37.13 & 37.797 & 1471.64 & 1471.33 \\
\hline $11 / 1 / 118: 20$ & 35.049 & 37.797 & 1473.72 & 1471.33 \\
\hline $11 / 1 / 118: 30$ & 34.972 & 37.795 & 1473.80 & 1471.33 \\
\hline $11 / 1 / 118: 40$ & 34.953 & 37.795 & 1473.82 & 1471.33 \\
\hline 11/1/11 8:50 & 34.925 & 37.795 & 1473.84 & 1471.33 \\
\hline $11 / 1 / 11$ 9:00 & 34.897 & 37.795 & 1473.87 & 1471.33 \\
\hline $11 / 1 / 119: 10$ & 34.874 & 37.795 & 1473.90 & 1471.33 \\
\hline $11 / 1 / 119: 20$ & 34.855 & 37.795 & 1473.91 & 1471.33 \\
\hline 11/1/11 9:30 & 34.862 & 37.793 & 1473.91 & 1471.33 \\
\hline 11/1/11 9:40 & 34.845 & 37.795 & 1473.92 & 1471.33 \\
\hline $11 / 1 / 119: 50$ & 34.824 & 37.795 & 1473.95 & 1471.33 \\
\hline 11/1/11 10:00 & 34.808 & 37.79 & 1473.96 & 1471.33 \\
\hline 11/1/11 10:10 & 34.791 & 37.785 & 1473.98 & 1471.34 \\
\hline 11/1/11 10:20 & 34.794 & 37.785 & 1473.98 & 1471.34 \\
\hline 11/1/11 10:30 & 34.789 & 37.783 & 1473.98 & 1471.34 \\
\hline 11/1/11 10:40 & 34.77 & 37.783 & 1474.00 & 1471.34 \\
\hline 11/1/11 10:50 & 34.756 & 37.781 & 1474.01 & 1471.34 \\
\hline 11/1/11 11:00 & 34.742 & 37.778 & 1474.03 & 1471.34 \\
\hline 11/1/11 11:10 & 34.73 & 37.776 & 1474.04 & 1471.35 \\
\hline 11/1/11 11:20 & 34.745 & 37.774 & 1474.02 & 1471.35 \\
\hline
\end{tabular}


TABLE S1.3 Automatically recorded groundwater levels in the Klassen wells, September 15, 2011, to March 13, 2012.

\begin{tabular}{|c|c|c|c|c|}
\hline \multicolumn{3}{|c|}{ Reference Elevation (ft AMSL) } & 1508.77 & \multirow{2}{*}{$\begin{array}{l}1509.12 \\
\text { Elevation in Klassen } \\
\text { Test Well (ft AMSL) }\end{array}$} \\
\hline Date and Time & $\begin{array}{l}\text { Depth in Klassen } \\
\text { Domestic Well } \\
\text { (ft BGL) }\end{array}$ & $\begin{array}{c}\text { Depth in Klassen } \\
\text { Test Well } \\
\text { (ft BGL) }\end{array}$ & $\begin{array}{l}\text { Elevation in Klassen } \\
\text { Domestic Well } \\
\text { (ft AMSL) }\end{array}$ & \\
\hline 11/1/11 11:30 & 34.728 & 37.769 & 1474.04 & 1471.35 \\
\hline 11/1/11 11:40 & 34.716 & 37.769 & 1474.05 & 1471.35 \\
\hline 11/1/11 11:50 & 34.705 & 37.767 & 1474.06 & 1471.36 \\
\hline $11 / 1 / 1112: 00$ & 34.691 & 37.76 & 1474.08 & 1471.36 \\
\hline 11/1/11 12:10 & 34.698 & 37.757 & 1474.07 & 1471.37 \\
\hline $11 / 1 / 1112: 20$ & 34.695 & 37.755 & 1474.07 & 1471.37 \\
\hline $11 / 1 / 1112: 30$ & 34.681 & 37.753 & 1474.09 & 1471.37 \\
\hline $11 / 1 / 1112: 40$ & 34.667 & 37.748 & 1474.10 & 1471.37 \\
\hline $11 / 1 / 1112: 50$ & 34.658 & 37.746 & 1474.11 & 1471.38 \\
\hline $11 / 1 / 11$ 13:00 & 34.648 & 37.741 & 1474.12 & 1471.38 \\
\hline 11/1/11 13:10 & 34.876 & 37.741 & 1473.89 & 1471.38 \\
\hline 11/1/11 13:20 & 34.756 & 37.739 & 1474.01 & 1471.38 \\
\hline $11 / 1 / 11$ 13:30 & 34.716 & 37.734 & 1474.05 & 1471.39 \\
\hline $11 / 1 / 11$ 13:40 & 34.691 & 37.732 & 1474.08 & 1471.39 \\
\hline $11 / 1 / 1113: 50$ & 34.672 & 37.729 & 1474.10 & 1471.39 \\
\hline 11/1/11 14:00 & 34.66 & 37.724 & 1474.11 & 1471.40 \\
\hline 11/1/11 14:10 & 34.669 & 37.722 & 1474.10 & 1471.40 \\
\hline $11 / 1 / 11$ 14:20 & 34.653 & 37.717 & 1474.12 & 1471.41 \\
\hline 11/1/11 14:30 & 34.935 & 37.715 & 1473.83 & 1471.41 \\
\hline $11 / 1 / 11$ 14:40 & 34.726 & 37.715 & 1474.04 & 1471.41 \\
\hline $11 / 1 / 1114: 50$ & 34.684 & 37.713 & 1474.09 & 1471.41 \\
\hline 11/1/11 15:00 & 34.681 & 37.708 & 1474.09 & 1471.41 \\
\hline $11 / 1 / 11$ 15:10 & 34.667 & 37.706 & 1474.10 & 1471.42 \\
\hline $11 / 1 / 11$ 15:20 & 34.648 & 37.703 & 1474.12 & 1471.42 \\
\hline 11/1/11 15:30 & 34.653 & 37.703 & 1474.12 & 1471.42 \\
\hline $11 / 1 / 11$ 15:40 & 40.988 & 37.701 & 1467.78 & 1471.42 \\
\hline $11 / 1 / 11$ 15:50 & 49.553 & 37.706 & 1459.22 & 1471.42 \\
\hline 11/1/11 16:00 & 37.285 & 37.717 & 1471.48 & 1471.41 \\
\hline 11/1/11 16:10 & 35.819 & 37.727 & 1472.95 & 1471.40 \\
\hline 11/1/11 16:20 & 35.528 & 37.732 & 1473.24 & 1471.39 \\
\hline 11/1/11 16:30 & 35.359 & 37.734 & 1473.41 & 1471.39 \\
\hline 11/1/11 16:40 & 35.244 & 37.732 & 1473.53 & 1471.39 \\
\hline $11 / 1 / 11$ 16:50 & 35.162 & 37.732 & 1473.61 & 1471.39 \\
\hline 11/1/11 17:00 & 35.099 & 37.732 & 1473.67 & 1471.39 \\
\hline 11/1/11 17:10 & 35.075 & 37.732 & 1473.69 & 1471.39 \\
\hline 11/1/11 17:20 & 35.028 & 37.729 & 1473.74 & 1471.39 \\
\hline 11/1/11 17:30 & 34.986 & 37.727 & 1473.78 & 1471.40 \\
\hline 11/1/11 17:40 & 36.049 & 37.724 & 1472.72 & 1471.40 \\
\hline
\end{tabular}


TABLE S1.3 Automatically recorded groundwater levels in the Klassen wells, September 15, 2011, to March 13, 2012.

\begin{tabular}{|c|c|c|c|c|}
\hline \multicolumn{3}{|c|}{ Reference Elevation (ft AMSL) } & 1508.77 & \multirow{2}{*}{$\begin{array}{l}1509.12 \\
\text { Elevation in Klassen } \\
\text { Test Well (ft AMSL) }\end{array}$} \\
\hline Date and Time & $\begin{array}{l}\text { Depth in Klassen } \\
\text { Domestic Well } \\
\text { (ft BGL) }\end{array}$ & $\begin{array}{c}\text { Depth in Klassen } \\
\text { Test Well } \\
\text { (ft BGL) }\end{array}$ & $\begin{array}{l}\text { Elevation in Klassen } \\
\text { Domestic Well } \\
\text { (ft AMSL) }\end{array}$ & \\
\hline $11 / 1 / 1117: 50$ & 35.136 & 37.724 & 1473.63 & 1471.40 \\
\hline $11 / 1 / 1118: 00$ & 35.094 & 37.724 & 1473.68 & 1471.40 \\
\hline 11/1/11 18:10 & 35.124 & 37.727 & 1473.65 & 1471.40 \\
\hline $11 / 1 / 1118: 20$ & 35.106 & 37.727 & 1473.66 & 1471.40 \\
\hline 11/1/11 18:30 & 35.141 & 37.729 & 1473.63 & 1471.39 \\
\hline $11 / 1 / 11$ 18:40 & 35.099 & 37.732 & 1473.67 & 1471.39 \\
\hline $11 / 1 / 1118: 50$ & 35.078 & 37.729 & 1473.69 & 1471.39 \\
\hline 11/1/11 19:00 & 35.038 & 37.729 & 1473.73 & 1471.39 \\
\hline 11/1/11 19:10 & 35.146 & 37.729 & 1473.62 & 1471.39 \\
\hline $11 / 1 / 11$ 19:20 & 35.047 & 37.727 & 1473.72 & 1471.40 \\
\hline 11/1/11 19:30 & 35.024 & 37.727 & 1473.75 & 1471.40 \\
\hline 11/1/11 19:40 & 34.981 & 37.727 & 1473.79 & 1471.40 \\
\hline 11/1/11 19:50 & 34.946 & 37.722 & 1473.82 & 1471.40 \\
\hline $11 / 1 / 1120: 00$ & 34.974 & 37.722 & 1473.80 & 1471.40 \\
\hline 11/1/11 20:10 & 35.052 & 37.724 & 1473.72 & 1471.40 \\
\hline $11 / 1 / 1120: 20$ & 35.045 & 37.727 & 1473.72 & 1471.40 \\
\hline $11 / 1 / 1120: 30$ & 35.061 & 37.727 & 1473.71 & 1471.40 \\
\hline $11 / 1 / 1120: 40$ & 35.073 & 37.729 & 1473.70 & 1471.39 \\
\hline $11 / 1 / 1120: 50$ & 35.064 & 37.729 & 1473.71 & 1471.39 \\
\hline $11 / 1 / 1121: 00$ & 35.174 & 37.734 & 1473.60 & 1471.39 \\
\hline 11/1/11 21:10 & 35.178 & 37.741 & 1473.59 & 1471.38 \\
\hline $11 / 1 / 1121: 20$ & 35.204 & 37.743 & 1473.57 & 1471.38 \\
\hline $11 / 1 / 1121: 30$ & 35.162 & 37.746 & 1473.61 & 1471.38 \\
\hline 11/1/11 21:40 & 35.115 & 37.743 & 1473.65 & 1471.38 \\
\hline $11 / 1 / 1121: 50$ & 35.073 & 37.743 & 1473.70 & 1471.38 \\
\hline $11 / 1 / 1122: 00$ & 35.108 & 37.743 & 1473.66 & 1471.38 \\
\hline $11 / 1 / 1122: 10$ & 35.11 & 37.746 & 1473.66 & 1471.38 \\
\hline $11 / 1 / 1122: 20$ & 35.092 & 37.746 & 1473.68 & 1471.38 \\
\hline $11 / 1 / 1122: 30$ & 35.115 & 37.748 & 1473.65 & 1471.37 \\
\hline 11/1/11 22:40 & 37.925 & 37.748 & 1470.84 & 1471.37 \\
\hline $11 / 1 / 1122: 50$ & 35.624 & 37.755 & 1473.15 & 1471.37 \\
\hline 11/1/11 23:00 & 38.797 & 37.76 & 1469.97 & 1471.36 \\
\hline 11/1/11 23:10 & 36.121 & 37.762 & 1472.65 & 1471.36 \\
\hline $11 / 1 / 1123: 20$ & 35.406 & 37.762 & 1473.36 & 1471.36 \\
\hline 11/1/11 23:30 & 35.286 & 37.764 & 1473.48 & 1471.36 \\
\hline 11/1/11 23:40 & 35.211 & 37.769 & 1473.56 & 1471.35 \\
\hline $11 / 1 / 1123: 50$ & 35.155 & 37.767 & 1473.61 & 1471.36 \\
\hline 11/2/11 0:00 & 35.136 & 37.764 & 1473.63 & 1471.36 \\
\hline
\end{tabular}


TABLE S1.3 Automatically recorded groundwater levels in the Klassen wells, September 15, 2011, to March 13, 2012.

\begin{tabular}{|c|c|c|c|c|}
\hline \multicolumn{3}{|c|}{ Reference Elevation (ft AMSL) } & 1508.77 & \multirow{2}{*}{$\begin{array}{l}1509.12 \\
\text { Elevation in Klassen } \\
\text { Test Well (ft AMSL) }\end{array}$} \\
\hline Date and Time & $\begin{array}{l}\text { Depth in Klassen } \\
\text { Domestic Well } \\
\text { (ft BGL) }\end{array}$ & $\begin{array}{c}\text { Depth in Klassen } \\
\text { Test Well } \\
\text { (ft BGL) }\end{array}$ & $\begin{array}{l}\text { Elevation in Klassen } \\
\text { Domestic Well } \\
\text { (ft AMSL) }\end{array}$ & \\
\hline $11 / 2 / 110: 10$ & 35.096 & 37.767 & 1473.67 & 1471.36 \\
\hline $11 / 2 / 110: 20$ & 35.061 & 37.767 & 1473.71 & 1471.36 \\
\hline $11 / 2 / 110: 30$ & 35.054 & 37.767 & 1473.72 & 1471.36 \\
\hline $11 / 2 / 110: 40$ & 35.021 & 37.769 & 1473.75 & 1471.35 \\
\hline $11 / 2 / 110: 50$ & 35.021 & 37.771 & 1473.75 & 1471.35 \\
\hline $11 / 2 / 111: 00$ & 34.991 & 37.771 & 1473.78 & 1471.35 \\
\hline $11 / 2 / 111: 10$ & 34.963 & 37.771 & 1473.81 & 1471.35 \\
\hline $11 / 2 / 111: 20$ & 34.937 & 37.769 & 1473.83 & 1471.35 \\
\hline $11 / 2 / 111: 30$ & 34.916 & 37.771 & 1473.85 & 1471.35 \\
\hline $11 / 2 / 111: 40$ & 34.892 & 37.769 & 1473.88 & 1471.35 \\
\hline $11 / 2 / 111: 50$ & 34.874 & 37.767 & 1473.90 & 1471.36 \\
\hline $11 / 2 / 112: 00$ & 34.857 & 37.764 & 1473.91 & 1471.36 \\
\hline $11 / 2 / 112: 10$ & 34.838 & 37.764 & 1473.93 & 1471.36 \\
\hline $11 / 2 / 112: 20$ & 34.822 & 37.764 & 1473.95 & 1471.36 \\
\hline $11 / 2 / 112: 30$ & 34.806 & 37.762 & 1473.96 & 1471.36 \\
\hline $11 / 2 / 112: 40$ & 34.791 & 37.762 & 1473.98 & 1471.36 \\
\hline $11 / 2 / 112: 50$ & 34.777 & 37.76 & 1473.99 & 1471.36 \\
\hline $11 / 2 / 113: 00$ & 34.916 & 37.762 & 1473.85 & 1471.36 \\
\hline $11 / 2 / 113: 10$ & 34.836 & 37.757 & 1473.93 & 1471.37 \\
\hline $11 / 2 / 113: 20$ & 34.798 & 37.757 & 1473.97 & 1471.37 \\
\hline $11 / 2 / 113: 30$ & 34.777 & 37.753 & 1473.99 & 1471.37 \\
\hline $11 / 2 / 113: 40$ & 34.759 & 37.753 & 1474.01 & 1471.37 \\
\hline 11/2/11 3:50 & 34.742 & 37.746 & 1474.03 & 1471.38 \\
\hline $11 / 2 / 114: 00$ & 34.728 & 37.739 & 1474.04 & 1471.38 \\
\hline $11 / 2 / 114: 10$ & 34.714 & 37.739 & 1474.06 & 1471.38 \\
\hline $11 / 2 / 114: 20$ & 34.7 & 37.739 & 1474.07 & 1471.38 \\
\hline $11 / 2 / 114: 30$ & 34.688 & 37.739 & 1474.08 & 1471.38 \\
\hline $11 / 2 / 114: 40$ & 34.677 & 37.736 & 1474.09 & 1471.39 \\
\hline $11 / 2 / 114: 50$ & 34.667 & 37.732 & 1474.10 & 1471.39 \\
\hline 11/2/11 5:00 & 34.655 & 37.727 & 1474.11 & 1471.40 \\
\hline 11/2/11 5:10 & 34.646 & 37.724 & 1474.12 & 1471.40 \\
\hline $11 / 2 / 115: 20$ & 34.634 & 37.722 & 1474.14 & 1471.40 \\
\hline $11 / 2 / 115: 30$ & 34.625 & 37.72 & 1474.14 & 1471.40 \\
\hline $11 / 2 / 115: 40$ & 34.618 & 37.717 & 1474.15 & 1471.41 \\
\hline 11/2/11 5:50 & 34.606 & 37.713 & 1474.16 & 1471.41 \\
\hline $11 / 2 / 116: 00$ & 34.599 & 37.708 & 1474.17 & 1471.41 \\
\hline $11 / 2 / 116: 10$ & 39.44 & 37.71 & 1469.33 & 1471.41 \\
\hline $11 / 2 / 116: 20$ & 34.902 & 37.713 & 1473.87 & 1471.41 \\
\hline
\end{tabular}


TABLE S1.3 Automatically recorded groundwater levels in the Klassen wells, September 15, 2011, to March 13, 2012.

\begin{tabular}{|c|c|c|c|c|}
\hline \multicolumn{3}{|c|}{ Reference Elevation (ft AMSL) } & 1508.77 & \multirow{2}{*}{$\begin{array}{l}1509.12 \\
\text { Elevation in Klassen } \\
\text { Test Well (ft AMSL) }\end{array}$} \\
\hline Date and Time & $\begin{array}{l}\text { Depth in Klassen } \\
\text { Domestic Well } \\
\text { (ft BGL) }\end{array}$ & $\begin{array}{c}\text { Depth in Klassen } \\
\text { Test Well } \\
\text { (ft BGL) }\end{array}$ & $\begin{array}{l}\text { Elevation in Klassen } \\
\text { Domestic Well } \\
\text { (ft AMSL) }\end{array}$ & \\
\hline $11 / 2 / 116: 30$ & 34.808 & 37.713 & 1473.96 & 1471.41 \\
\hline $11 / 2 / 116: 40$ & 34.759 & 37.713 & 1474.01 & 1471.41 \\
\hline $11 / 2 / 116: 50$ & 34.723 & 37.713 & 1474.05 & 1471.41 \\
\hline 11/2/11 7:00 & 34.719 & 37.708 & 1474.05 & 1471.41 \\
\hline $11 / 2 / 117: 10$ & 34.759 & 37.706 & 1474.01 & 1471.42 \\
\hline $11 / 2 / 117: 20$ & 34.735 & 37.706 & 1474.03 & 1471.42 \\
\hline $11 / 2 / 117: 30$ & 34.74 & 37.701 & 1474.03 & 1471.42 \\
\hline $11 / 2 / 117: 40$ & 34.721 & 37.701 & 1474.05 & 1471.42 \\
\hline $11 / 2 / 117: 50$ & 34.698 & 37.699 & 1474.07 & 1471.42 \\
\hline 11/2/11 8:00 & 36.825 & 37.696 & 1471.94 & 1471.43 \\
\hline 11/2/11 8:10 & 34.808 & 37.694 & 1473.96 & 1471.43 \\
\hline $11 / 2 / 118: 20$ & 34.742 & 37.694 & 1474.03 & 1471.43 \\
\hline $11 / 2 / 118: 30$ & 34.705 & 37.689 & 1474.06 & 1471.43 \\
\hline $11 / 2 / 118: 40$ & 34.679 & 37.689 & 1474.09 & 1471.43 \\
\hline $11 / 2 / 118: 50$ & 34.66 & 37.687 & 1474.11 & 1471.44 \\
\hline $11 / 2 / 119: 00$ & 34.639 & 37.685 & 1474.13 & 1471.44 \\
\hline $11 / 2 / 119: 10$ & 34.625 & 37.685 & 1474.14 & 1471.44 \\
\hline $11 / 2 / 119: 20$ & 34.909 & 37.685 & 1473.86 & 1471.44 \\
\hline $11 / 2 / 119: 30$ & 34.726 & 37.687 & 1474.04 & 1471.44 \\
\hline $11 / 2 / 119: 40$ & 34.679 & 37.687 & 1474.09 & 1471.44 \\
\hline $11 / 2 / 119: 50$ & 34.651 & 37.685 & 1474.12 & 1471.44 \\
\hline 11/2/11 10:00 & 34.627 & 37.692 & 1474.14 & 1471.43 \\
\hline 11/2/11 10:10 & 34.611 & 37.692 & 1474.16 & 1471.43 \\
\hline $11 / 2 / 11$ 10:20 & 34.597 & 37.694 & 1474.17 & 1471.43 \\
\hline 11/2/11 10:30 & 34.585 & 37.696 & 1474.18 & 1471.43 \\
\hline $11 / 2 / 11$ 10:40 & 34.571 & 37.703 & 1474.20 & 1471.42 \\
\hline $11 / 2 / 11$ 10:50 & 34.557 & 37.708 & 1474.21 & 1471.41 \\
\hline 11/2/11 11:00 & 34.55 & 37.715 & 1474.22 & 1471.41 \\
\hline 11/2/11 11:10 & 34.541 & 37.72 & 1474.23 & 1471.40 \\
\hline 11/2/11 11:20 & 34.533 & 37.72 & 1474.24 & 1471.40 \\
\hline 11/2/11 11:30 & 34.967 & 37.724 & 1473.80 & 1471.40 \\
\hline 11/2/11 11:40 & 34.618 & 37.729 & 1474.15 & 1471.39 \\
\hline $11 / 2 / 11$ 11:50 & 34.576 & 37.732 & 1474.19 & 1471.39 \\
\hline $11 / 2 / 1112: 00$ & 34.555 & 37.734 & 1474.21 & 1471.39 \\
\hline 11/2/11 12:10 & 34.533 & 37.736 & 1474.24 & 1471.39 \\
\hline $11 / 2 / 11$ 12:20 & 34.524 & 37.736 & 1474.25 & 1471.39 \\
\hline $11 / 2 / 11$ 12:30 & 34.515 & 37.736 & 1474.25 & 1471.39 \\
\hline $11 / 2 / 11$ 12:40 & 34.503 & 37.739 & 1474.27 & 1471.38 \\
\hline
\end{tabular}


TABLE S1.3 Automatically recorded groundwater levels in the Klassen wells, September 15, 2011, to March 13, 2012.

\begin{tabular}{|c|c|c|c|c|}
\hline \multicolumn{3}{|c|}{ Reference Elevation (ft AMSL) } & 1508.77 & \multirow{2}{*}{$\begin{array}{l}1509.12 \\
\text { Elevation in Klassen } \\
\text { Test Well (ft AMSL) }\end{array}$} \\
\hline Date and Time & $\begin{array}{l}\text { Depth in Klassen } \\
\text { Domestic Well } \\
(\mathrm{ft} \mathrm{BGL})\end{array}$ & $\begin{array}{c}\text { Depth in Klassen } \\
\text { Test Well } \\
\text { (ft BGL) }\end{array}$ & $\begin{array}{l}\text { Elevation in Klassen } \\
\text { Domestic Well } \\
\text { (ft AMSL) }\end{array}$ & \\
\hline $11 / 2 / 1112: 50$ & 34.496 & 37.741 & 1474.27 & 1471.38 \\
\hline $11 / 2 / 1113: 00$ & 34.484 & 37.743 & 1474.29 & 1471.38 \\
\hline 11/2/11 13:10 & 34.723 & 37.755 & 1474.05 & 1471.37 \\
\hline $11 / 2 / 1113: 20$ & 34.606 & 37.757 & 1474.16 & 1471.37 \\
\hline 11/2/11 13:30 & 34.562 & 37.762 & 1474.21 & 1471.36 \\
\hline $11 / 2 / 1113: 40$ & 34.536 & 37.762 & 1474.23 & 1471.36 \\
\hline $11 / 2 / 1113: 50$ & 34.515 & 37.767 & 1474.25 & 1471.36 \\
\hline 11/2/11 14:00 & 34.503 & 37.774 & 1474.27 & 1471.35 \\
\hline 11/2/11 14:10 & 35.465 & 37.771 & 1473.30 & 1471.35 \\
\hline $11 / 2 / 1114: 20$ & 34.604 & 37.776 & 1474.17 & 1471.35 \\
\hline $11 / 2 / 1114: 30$ & 34.555 & 37.774 & 1474.21 & 1471.35 \\
\hline $11 / 2 / 1114: 40$ & 34.529 & 37.778 & 1474.24 & 1471.34 \\
\hline $11 / 2 / 1114: 50$ & 36.996 & 37.778 & 1471.77 & 1471.34 \\
\hline $11 / 2 / 1115: 00$ & 34.648 & 37.785 & 1474.12 & 1471.34 \\
\hline 11/2/11 15:10 & 34.58 & 37.785 & 1474.19 & 1471.34 \\
\hline $11 / 2 / 11$ 15:20 & 34.545 & 37.788 & 1474.22 & 1471.33 \\
\hline $11 / 2 / 11$ 15:30 & 34.557 & 37.783 & 1474.21 & 1471.34 \\
\hline $11 / 2 / 11$ 15:40 & 34.538 & 37.804 & 1474.23 & 1471.32 \\
\hline $11 / 2 / 11$ 15:50 & 34.845 & 37.816 & 1473.92 & 1471.31 \\
\hline $11 / 2 / 11$ 16:00 & 36.543 & 37.823 & 1472.23 & 1471.30 \\
\hline $11 / 2 / 1116: 10$ & 34.747 & 37.832 & 1474.02 & 1471.29 \\
\hline $11 / 2 / 11$ 16:20 & 34.66 & 37.839 & 1474.11 & 1471.28 \\
\hline $11 / 2 / 11$ 16:30 & 34.883 & 37.849 & 1473.89 & 1471.27 \\
\hline 11/2/11 16:40 & 37.5 & 37.854 & 1471.27 & 1471.27 \\
\hline $11 / 2 / 11$ 16:50 & 35.572 & 37.858 & 1473.20 & 1471.26 \\
\hline 11/2/11 17:00 & 34.984 & 37.868 & 1473.79 & 1471.25 \\
\hline 11/2/11 17:10 & 34.923 & 37.87 & 1473.85 & 1471.25 \\
\hline $11 / 2 / 11$ 17:20 & 34.852 & 37.879 & 1473.92 & 1471.24 \\
\hline $11 / 2 / 11$ 17:30 & 34.874 & 37.882 & 1473.90 & 1471.24 \\
\hline 11/2/11 17:40 & 35.087 & 37.879 & 1473.68 & 1471.24 \\
\hline $11 / 2 / 11$ 17:50 & 34.986 & 37.884 & 1473.78 & 1471.24 \\
\hline 11/2/11 18:00 & 34.953 & 37.889 & 1473.82 & 1471.23 \\
\hline $11 / 2 / 11$ 18:10 & 34.899 & 37.891 & 1473.87 & 1471.23 \\
\hline $11 / 2 / 11$ 18:20 & 34.852 & 37.896 & 1473.92 & 1471.23 \\
\hline 11/2/11 18:30 & 34.81 & 37.896 & 1473.96 & 1471.23 \\
\hline $11 / 2 / 11$ 18:40 & 34.78 & 37.891 & 1473.99 & 1471.23 \\
\hline $11 / 2 / 11$ 18:50 & 34.747 & 37.896 & 1474.02 & 1471.23 \\
\hline 11/2/11 19:00 & 35.108 & 37.891 & 1473.66 & 1471.23 \\
\hline
\end{tabular}


TABLE S1.3 Automatically recorded groundwater levels in the Klassen wells, September 15, 2011, to March 13, 2012.

\begin{tabular}{|c|c|c|c|c|}
\hline \multicolumn{3}{|c|}{ Reference Elevation (ft AMSL) } & 1508.77 & \multirow{2}{*}{$\begin{array}{l}1509.12 \\
\text { Elevation in Klassen } \\
\text { Test Well (ft AMSL) }\end{array}$} \\
\hline Date and Time & $\begin{array}{l}\text { Depth in Klassen } \\
\text { Domestic Well } \\
(\mathrm{ft} \mathrm{BGL})\end{array}$ & $\begin{array}{c}\text { Depth in Klassen } \\
\text { Test Well } \\
\text { (ft BGL) }\end{array}$ & $\begin{array}{l}\text { Elevation in Klassen } \\
\text { Domestic Well } \\
\text { (ft AMSL) }\end{array}$ & \\
\hline 11/2/11 19:10 & 34.813 & 37.891 & 1473.96 & 1471.23 \\
\hline $11 / 2 / 1119: 20$ & 35.01 & 37.893 & 1473.76 & 1471.23 \\
\hline 11/2/11 19:30 & 34.829 & 37.893 & 1473.94 & 1471.23 \\
\hline 11/2/11 19:40 & 34.773 & 37.898 & 1474.00 & 1471.22 \\
\hline 11/2/11 19:50 & 34.733 & 37.903 & 1474.04 & 1471.22 \\
\hline $11 / 2 / 1120: 00$ & 34.707 & 37.903 & 1474.06 & 1471.22 \\
\hline $11 / 2 / 1120: 10$ & 34.684 & 37.903 & 1474.09 & 1471.22 \\
\hline $11 / 2 / 1120: 20$ & 34.693 & 37.905 & 1474.08 & 1471.22 \\
\hline $11 / 2 / 1120: 30$ & 34.747 & 37.905 & 1474.02 & 1471.22 \\
\hline $11 / 2 / 1120: 40$ & 34.775 & 37.905 & 1473.99 & 1471.22 \\
\hline $11 / 2 / 1120: 50$ & 34.967 & 37.903 & 1473.80 & 1471.22 \\
\hline 11/2/11 21:00 & 34.916 & 37.903 & 1473.85 & 1471.22 \\
\hline 11/2/11 21:10 & 34.859 & 37.898 & 1473.91 & 1471.22 \\
\hline $11 / 2 / 1121: 20$ & 34.82 & 37.893 & 1473.95 & 1471.23 \\
\hline $11 / 2 / 1121: 30$ & 34.841 & 37.889 & 1473.93 & 1471.23 \\
\hline $11 / 2 / 1121: 40$ & 34.82 & 37.889 & 1473.95 & 1471.23 \\
\hline $11 / 2 / 1121: 50$ & 34.836 & 37.891 & 1473.93 & 1471.23 \\
\hline $11 / 2 / 1122: 00$ & 34.85 & 37.893 & 1473.92 & 1471.23 \\
\hline $11 / 2 / 1122: 10$ & 34.82 & 37.891 & 1473.95 & 1471.23 \\
\hline $11 / 2 / 1122: 20$ & 34.787 & 37.889 & 1473.98 & 1471.23 \\
\hline $11 / 2 / 1122: 30$ & 34.761 & 37.882 & 1474.01 & 1471.24 \\
\hline $11 / 2 / 1122: 40$ & 40.235 & 37.884 & 1468.53 & 1471.24 \\
\hline $11 / 2 / 1122: 50$ & 37.367 & 37.884 & 1471.40 & 1471.24 \\
\hline $11 / 2 / 1123: 00$ & 35.026 & 37.886 & 1473.74 & 1471.24 \\
\hline 11/2/11 23:10 & 35.589 & 37.886 & 1473.18 & 1471.24 \\
\hline $11 / 2 / 1123: 20$ & 35.197 & 37.889 & 1473.57 & 1471.23 \\
\hline $11 / 2 / 1123: 30$ & 35.038 & 37.886 & 1473.73 & 1471.24 \\
\hline $11 / 2 / 1123: 40$ & 34.956 & 37.886 & 1473.81 & 1471.24 \\
\hline $11 / 2 / 1123: 50$ & 34.899 & 37.884 & 1473.87 & 1471.24 \\
\hline 11/3/11 0:00 & 34.857 & 37.882 & 1473.91 & 1471.24 \\
\hline 11/3/11 0:10 & 34.822 & 37.877 & 1473.95 & 1471.25 \\
\hline $11 / 3 / 110: 20$ & 34.791 & 37.872 & 1473.98 & 1471.25 \\
\hline 11/3/11 0:30 & 34.768 & 37.87 & 1474.00 & 1471.25 \\
\hline 11/3/11 0:40 & 34.742 & 37.865 & 1474.03 & 1471.26 \\
\hline $11 / 3 / 110: 50$ & 34.721 & 37.865 & 1474.05 & 1471.26 \\
\hline $11 / 3 / 11$ 1:00 & 34.702 & 37.861 & 1474.07 & 1471.26 \\
\hline $11 / 3 / 11$ 1:10 & 34.686 & 37.861 & 1474.08 & 1471.26 \\
\hline $11 / 3 / 11$ 1:20 & 34.667 & 37.858 & 1474.10 & 1471.26 \\
\hline
\end{tabular}


TABLE S1.3 Automatically recorded groundwater levels in the Klassen wells, September 15, 2011, to March 13, 2012.

\begin{tabular}{|c|c|c|c|c|}
\hline \multicolumn{3}{|c|}{ Reference Elevation (ft AMSL) } & 1508.77 & \multirow{2}{*}{$\begin{array}{l}1509.12 \\
\text { Elevation in Klassen } \\
\text { Test Well (ft AMSL) }\end{array}$} \\
\hline Date and Time & $\begin{array}{l}\text { Depth in Klassen } \\
\text { Domestic Well } \\
\text { (ft BGL) }\end{array}$ & $\begin{array}{c}\text { Depth in Klassen } \\
\text { Test Well } \\
\text { (ft BGL) }\end{array}$ & $\begin{array}{l}\text { Elevation in Klassen } \\
\text { Domestic Well } \\
\text { (ft AMSL) }\end{array}$ & \\
\hline $11 / 3 / 111: 30$ & 34.655 & 37.854 & 1474.11 & 1471.27 \\
\hline $11 / 3 / 111: 40$ & 34.705 & 37.854 & 1474.06 & 1471.27 \\
\hline $11 / 3 / 111: 50$ & 34.756 & 37.851 & 1474.01 & 1471.27 \\
\hline $11 / 3 / 112: 00$ & 34.733 & 37.844 & 1474.04 & 1471.28 \\
\hline $11 / 3 / 112: 10$ & 34.709 & 37.842 & 1474.06 & 1471.28 \\
\hline $11 / 3 / 112: 20$ & 34.927 & 37.839 & 1473.84 & 1471.28 \\
\hline $11 / 3 / 112: 30$ & 34.787 & 37.837 & 1473.98 & 1471.29 \\
\hline $11 / 3 / 112: 40$ & 34.74 & 37.835 & 1474.03 & 1471.29 \\
\hline $11 / 3 / 112: 50$ & 34.733 & 37.83 & 1474.04 & 1471.29 \\
\hline $11 / 3 / 113: 00$ & 34.705 & 37.825 & 1474.06 & 1471.30 \\
\hline 11/3/11 3:10 & 34.693 & 37.821 & 1474.08 & 1471.30 \\
\hline $11 / 3 / 113: 20$ & 34.73 & 37.821 & 1474.04 & 1471.30 \\
\hline $11 / 3 / 113: 30$ & 34.78 & 37.818 & 1473.99 & 1471.30 \\
\hline $11 / 3 / 113: 40$ & 34.756 & 37.818 & 1474.01 & 1471.30 \\
\hline $11 / 3 / 113: 50$ & 34.73 & 37.814 & 1474.04 & 1471.31 \\
\hline $11 / 3 / 114: 00$ & 34.702 & 37.811 & 1474.07 & 1471.31 \\
\hline $11 / 3 / 114: 10$ & 34.684 & 37.809 & 1474.09 & 1471.31 \\
\hline $11 / 3 / 114: 20$ & 34.665 & 37.804 & 1474.10 & 1471.32 \\
\hline $11 / 3 / 114: 30$ & 34.646 & 37.802 & 1474.12 & 1471.32 \\
\hline $11 / 3 / 114: 40$ & 34.63 & 37.795 & 1474.14 & 1471.33 \\
\hline $11 / 3 / 114: 50$ & 34.618 & 37.79 & 1474.15 & 1471.33 \\
\hline $11 / 3 / 11$ 5:00 & 34.604 & 37.783 & 1474.17 & 1471.34 \\
\hline 11/3/11 5:10 & 34.604 & 37.778 & 1474.17 & 1471.34 \\
\hline $11 / 3 / 115: 20$ & 34.609 & 37.771 & 1474.16 & 1471.35 \\
\hline $11 / 3 / 115: 30$ & 34.592 & 37.764 & 1474.18 & 1471.36 \\
\hline $11 / 3 / 115: 40$ & 34.578 & 37.76 & 1474.19 & 1471.36 \\
\hline $11 / 3 / 115: 50$ & 34.566 & 37.755 & 1474.20 & 1471.37 \\
\hline $11 / 3 / 116: 00$ & 34.557 & 37.753 & 1474.21 & 1471.37 \\
\hline $11 / 3 / 116: 10$ & 35.047 & 37.748 & 1473.72 & 1471.37 \\
\hline $11 / 3 / 116: 20$ & 34.902 & 37.743 & 1473.87 & 1471.38 \\
\hline $11 / 3 / 116: 30$ & 34.763 & 37.746 & 1474.01 & 1471.38 \\
\hline $11 / 3 / 116: 40$ & 34.709 & 37.739 & 1474.06 & 1471.38 \\
\hline $11 / 3 / 116: 50$ & 34.669 & 37.736 & 1474.10 & 1471.39 \\
\hline $11 / 3 / 11$ 7:00 & 34.641 & 37.729 & 1474.13 & 1471.39 \\
\hline $11 / 3 / 117: 10$ & 34.651 & 37.727 & 1474.12 & 1471.40 \\
\hline $11 / 3 / 117: 20$ & 34.681 & 37.722 & 1474.09 & 1471.40 \\
\hline $11 / 3 / 117: 30$ & 34.66 & 37.72 & 1474.11 & 1471.40 \\
\hline $11 / 3 / 11$ 7:40 & 34.637 & 37.717 & 1474.13 & 1471.41 \\
\hline
\end{tabular}


TABLE S1.3 Automatically recorded groundwater levels in the Klassen wells, September 15, 2011, to March 13, 2012.

\begin{tabular}{|c|c|c|c|c|}
\hline \multicolumn{3}{|c|}{ Reference Elevation (ft AMSL) } & 1508.77 & \multirow{2}{*}{$\begin{array}{l}1509.12 \\
\text { Elevation in Klassen } \\
\text { Test Well (ft AMSL) }\end{array}$} \\
\hline Date and Time & $\begin{array}{l}\text { Depth in Klassen } \\
\text { Domestic Well } \\
\text { (ft BGL) }\end{array}$ & $\begin{array}{c}\text { Depth in Klassen } \\
\text { Test Well } \\
\text { (ft BGL) }\end{array}$ & $\begin{array}{l}\text { Elevation in Klassen } \\
\text { Domestic Well } \\
\text { (ft AMSL) }\end{array}$ & \\
\hline $11 / 3 / 117: 50$ & 34.616 & 37.713 & 1474.15 & 1471.41 \\
\hline $11 / 3 / 118: 00$ & 34.599 & 37.708 & 1474.17 & 1471.41 \\
\hline $11 / 3 / 118: 10$ & 34.585 & 37.701 & 1474.18 & 1471.42 \\
\hline $11 / 3 / 118: 20$ & 34.571 & 37.696 & 1474.20 & 1471.43 \\
\hline $11 / 3 / 118: 30$ & 35.465 & 37.689 & 1473.30 & 1471.43 \\
\hline $11 / 3 / 118: 40$ & 34.674 & 37.685 & 1474.10 & 1471.44 \\
\hline $11 / 3 / 118: 50$ & 34.648 & 37.682 & 1474.12 & 1471.44 \\
\hline $11 / 3 / 119: 00$ & 34.62 & 37.682 & 1474.15 & 1471.44 \\
\hline 11/3/11 9:10 & 34.594 & 37.678 & 1474.18 & 1471.44 \\
\hline $11 / 3 / 119: 20$ & 34.573 & 37.673 & 1474.20 & 1471.45 \\
\hline 11/3/11 9:30 & 36.459 & 37.671 & 1472.31 & 1471.45 \\
\hline $11 / 3 / 119: 40$ & 34.7 & 37.673 & 1474.07 & 1471.45 \\
\hline $11 / 3 / 119: 50$ & 34.632 & 37.671 & 1474.14 & 1471.45 \\
\hline $11 / 3 / 1110: 00$ & 34.599 & 37.671 & 1474.17 & 1471.45 \\
\hline 11/3/11 10:10 & 34.576 & 37.671 & 1474.19 & 1471.45 \\
\hline $11 / 3 / 11$ 10:20 & 34.831 & 37.671 & 1473.94 & 1471.45 \\
\hline $11 / 3 / 11$ 10:30 & 34.651 & 37.668 & 1474.12 & 1471.45 \\
\hline $11 / 3 / 11$ 10:40 & 34.606 & 37.671 & 1474.16 & 1471.45 \\
\hline $11 / 3 / 11$ 10:50 & 34.578 & 37.675 & 1474.19 & 1471.45 \\
\hline 11/3/11 11:00 & 37.282 & 37.673 & 1471.49 & 1471.45 \\
\hline 11/3/11 11:10 & 34.677 & 37.675 & 1474.09 & 1471.45 \\
\hline $11 / 3 / 11$ 11:20 & 34.611 & 37.678 & 1474.16 & 1471.44 \\
\hline $11 / 3 / 1111: 30$ & 34.58 & 37.68 & 1474.19 & 1471.44 \\
\hline 11/3/11 11:40 & 34.557 & 37.685 & 1474.21 & 1471.44 \\
\hline $11 / 3 / 11$ 11:50 & 34.538 & 37.692 & 1474.23 & 1471.43 \\
\hline 11/3/11 12:00 & 37.958 & 37.694 & 1470.81 & 1471.43 \\
\hline 11/3/11 12:10 & 34.651 & 37.699 & 1474.12 & 1471.42 \\
\hline $11 / 3 / 11$ 12:20 & 34.583 & 37.701 & 1474.19 & 1471.42 \\
\hline $11 / 3 / 11$ 12:30 & 34.552 & 37.706 & 1474.22 & 1471.42 \\
\hline 11/3/11 12:40 & 34.531 & 37.708 & 1474.24 & 1471.41 \\
\hline $11 / 3 / 11$ 12:50 & 34.515 & 37.71 & 1474.25 & 1471.41 \\
\hline 11/3/11 13:00 & 34.498 & 37.715 & 1474.27 & 1471.41 \\
\hline 11/3/11 13:10 & 34.639 & 37.717 & 1474.13 & 1471.41 \\
\hline $11 / 3 / 11$ 13:20 & 34.592 & 37.72 & 1474.18 & 1471.40 \\
\hline 11/3/11 13:30 & 34.557 & 37.722 & 1474.21 & 1471.40 \\
\hline 11/3/11 13:40 & 34.531 & 37.722 & 1474.24 & 1471.40 \\
\hline $11 / 3 / 11$ 13:50 & 34.512 & 37.724 & 1474.26 & 1471.40 \\
\hline 11/3/11 14:00 & 34.498 & 37.724 & 1474.27 & 1471.40 \\
\hline
\end{tabular}


TABLE S1.3 Automatically recorded groundwater levels in the Klassen wells, September 15, 2011, to March 13, 2012.

\begin{tabular}{|c|c|c|c|c|}
\hline \multicolumn{3}{|c|}{ Reference Elevation (ft AMSL) } & 1508.77 & \multirow{2}{*}{$\begin{array}{l}1509.12 \\
\text { Elevation in Klassen } \\
\text { Test Well (ft AMSL) }\end{array}$} \\
\hline Date and Time & $\begin{array}{l}\text { Depth in Klassen } \\
\text { Domestic Well } \\
(\mathrm{ft} \mathrm{BGL})\end{array}$ & $\begin{array}{c}\text { Depth in Klassen } \\
\text { Test Well } \\
\text { (ft BGL) }\end{array}$ & $\begin{array}{l}\text { Elevation in Klassen } \\
\text { Domestic Well } \\
\text { (ft AMSL) }\end{array}$ & \\
\hline $11 / 3 / 1114: 10$ & 39.693 & 37.727 & 1469.08 & 1471.40 \\
\hline $11 / 3 / 1114: 20$ & 34.63 & 37.729 & 1474.14 & 1471.39 \\
\hline $11 / 3 / 1114: 30$ & 34.557 & 37.732 & 1474.21 & 1471.39 \\
\hline $11 / 3 / 1114: 40$ & 34.545 & 37.734 & 1474.22 & 1471.39 \\
\hline $11 / 3 / 1114: 50$ & 34.531 & 37.736 & 1474.24 & 1471.39 \\
\hline $11 / 3 / 11$ 15:00 & 34.51 & 37.736 & 1474.26 & 1471.39 \\
\hline $11 / 3 / 11$ 15:10 & 34.496 & 37.739 & 1474.27 & 1471.38 \\
\hline $11 / 3 / 11$ 15:20 & 34.489 & 37.741 & 1474.28 & 1471.38 \\
\hline $11 / 3 / 1115: 30$ & 34.496 & 37.741 & 1474.27 & 1471.38 \\
\hline $11 / 3 / 1115: 40$ & 34.48 & 37.741 & 1474.29 & 1471.38 \\
\hline $11 / 3 / 1115: 50$ & 34.465 & 37.743 & 1474.30 & 1471.38 \\
\hline 11/3/11 16:00 & 34.902 & 37.746 & 1473.87 & 1471.38 \\
\hline $11 / 3 / 11$ 16:10 & 34.564 & 37.748 & 1474.21 & 1471.37 \\
\hline $11 / 3 / 1116: 20$ & 34.517 & 37.748 & 1474.25 & 1471.37 \\
\hline 11/3/11 16:30 & 34.524 & 37.748 & 1474.25 & 1471.37 \\
\hline $11 / 3 / 11$ 16:40 & 34.51 & 37.748 & 1474.26 & 1471.37 \\
\hline $11 / 3 / 11$ 16:50 & 34.512 & 37.75 & 1474.26 & 1471.37 \\
\hline 11/3/11 17:00 & 34.494 & 37.746 & 1474.28 & 1471.38 \\
\hline 11/3/11 17:10 & 34.475 & 37.746 & 1474.29 & 1471.38 \\
\hline $11 / 3 / 11$ 17:20 & 34.461 & 37.746 & 1474.31 & 1471.38 \\
\hline $11 / 3 / 11$ 17:30 & 37.09 & 37.741 & 1471.68 & 1471.38 \\
\hline $11 / 3 / 11$ 17:40 & 34.672 & 37.739 & 1474.10 & 1471.38 \\
\hline $11 / 3 / 11$ 17:50 & 34.62 & 37.734 & 1474.15 & 1471.39 \\
\hline 11/3/11 18:00 & 34.576 & 37.727 & 1474.19 & 1471.40 \\
\hline 11/3/11 18:10 & 34.721 & 37.724 & 1474.05 & 1471.40 \\
\hline $11 / 3 / 11$ 18:20 & 34.618 & 37.717 & 1474.15 & 1471.41 \\
\hline $11 / 3 / 11$ 18:30 & 34.573 & 37.717 & 1474.20 & 1471.41 \\
\hline 11/3/11 18:40 & 34.543 & 37.713 & 1474.23 & 1471.41 \\
\hline $11 / 3 / 11$ 18:50 & 34.519 & 37.71 & 1474.25 & 1471.41 \\
\hline 11/3/11 19:00 & 38.882 & 37.71 & 1469.89 & 1471.41 \\
\hline 11/3/11 19:10 & 34.655 & 37.71 & 1474.11 & 1471.41 \\
\hline $11 / 3 / 11$ 19:20 & 34.587 & 37.708 & 1474.18 & 1471.41 \\
\hline $11 / 3 / 11$ 19:30 & 34.552 & 37.703 & 1474.22 & 1471.42 \\
\hline 11/3/11 19:40 & 34.526 & 37.699 & 1474.24 & 1471.42 \\
\hline 11/3/11 19:50 & 34.505 & 37.692 & 1474.26 & 1471.43 \\
\hline $11 / 3 / 1120: 00$ & 34.526 & 37.687 & 1474.24 & 1471.44 \\
\hline $11 / 3 / 1120: 10$ & 34.58 & 37.687 & 1474.19 & 1471.44 \\
\hline $11 / 3 / 1120: 20$ & 34.822 & 37.689 & 1473.95 & 1471.43 \\
\hline
\end{tabular}


TABLE S1.3 Automatically recorded groundwater levels in the Klassen wells, September 15, 2011, to March 13, 2012.

\begin{tabular}{|c|c|c|c|c|}
\hline \multicolumn{3}{|c|}{ Reference Elevation (ft AMSL) } & 1508.77 & \multirow{2}{*}{$\begin{array}{l}1509.12 \\
\text { Elevation in Klassen } \\
\text { Test Well (ft AMSL) }\end{array}$} \\
\hline Date and Time & $\begin{array}{l}\text { Depth in Klassen } \\
\text { Domestic Well } \\
\text { (ft BGL) }\end{array}$ & $\begin{array}{c}\text { Depth in Klassen } \\
\text { Test Well } \\
\text { (ft BGL) }\end{array}$ & $\begin{array}{l}\text { Elevation in Klassen } \\
\text { Domestic Well } \\
\text { (ft AMSL) }\end{array}$ & \\
\hline $11 / 3 / 1120: 30$ & 34.709 & 37.687 & 1474.06 & 1471.44 \\
\hline $11 / 3 / 1120: 40$ & 34.684 & 37.685 & 1474.09 & 1471.44 \\
\hline $11 / 3 / 1120: 50$ & 34.672 & 37.682 & 1474.10 & 1471.44 \\
\hline $11 / 3 / 1121: 00$ & 34.637 & 37.678 & 1474.13 & 1471.44 \\
\hline $11 / 3 / 1121: 10$ & 36.257 & 37.673 & 1472.51 & 1471.45 \\
\hline $11 / 3 / 1121: 20$ & 34.766 & 37.673 & 1474.00 & 1471.45 \\
\hline $11 / 3 / 1121: 30$ & 34.667 & 37.675 & 1474.10 & 1471.45 \\
\hline $11 / 3 / 1121: 40$ & 34.623 & 37.668 & 1474.15 & 1471.45 \\
\hline $11 / 3 / 1121: 50$ & 34.592 & 37.666 & 1474.18 & 1471.46 \\
\hline $11 / 3 / 1122: 00$ & 34.871 & 37.661 & 1473.90 & 1471.46 \\
\hline $11 / 3 / 1122: 10$ & 34.7 & 37.661 & 1474.07 & 1471.46 \\
\hline $11 / 3 / 1122: 20$ & 34.7 & 37.661 & 1474.07 & 1471.46 \\
\hline $11 / 3 / 1122: 30$ & 34.669 & 37.654 & 1474.10 & 1471.47 \\
\hline $11 / 3 / 1122: 40$ & 35.052 & 37.652 & 1473.72 & 1471.47 \\
\hline $11 / 3 / 1122: 50$ & 35.04 & 37.654 & 1473.73 & 1471.47 \\
\hline 11/3/11 23:00 & 35.232 & 37.652 & 1473.54 & 1471.47 \\
\hline 11/3/11 23:10 & 38.162 & 37.652 & 1470.61 & 1471.47 \\
\hline $11 / 3 / 11$ 23:20 & 35.019 & 37.652 & 1473.75 & 1471.47 \\
\hline $11 / 3 / 11$ 23:30 & 37.038 & 37.652 & 1471.73 & 1471.47 \\
\hline $11 / 3 / 11$ 23:40 & 35.078 & 37.652 & 1473.69 & 1471.47 \\
\hline $11 / 3 / 1123: 50$ & 34.942 & 37.654 & 1473.83 & 1471.47 \\
\hline 11/4/11 0:00 & 34.866 & 37.656 & 1473.90 & 1471.47 \\
\hline 11/4/11 0:10 & 34.813 & 37.656 & 1473.96 & 1471.47 \\
\hline $11 / 4 / 110: 20$ & 34.77 & 37.659 & 1474.00 & 1471.46 \\
\hline 11/4/11 0:30 & 34.735 & 37.654 & 1474.03 & 1471.47 \\
\hline $11 / 4 / 110: 40$ & 34.707 & 37.654 & 1474.06 & 1471.47 \\
\hline $11 / 4 / 110: 50$ & 34.679 & 37.656 & 1474.09 & 1471.47 \\
\hline $11 / 4 / 111: 00$ & 34.658 & 37.654 & 1474.11 & 1471.47 \\
\hline $11 / 4 / 11$ 1:10 & 34.829 & 37.652 & 1473.94 & 1471.47 \\
\hline $11 / 4 / 111: 20$ & 34.716 & 37.649 & 1474.05 & 1471.47 \\
\hline $11 / 4 / 11$ 1:30 & 34.672 & 37.649 & 1474.10 & 1471.47 \\
\hline $11 / 4 / 11$ 1:40 & 34.644 & 37.649 & 1474.13 & 1471.47 \\
\hline $11 / 4 / 11$ 1:50 & 34.62 & 37.645 & 1474.15 & 1471.48 \\
\hline $11 / 4 / 112: 00$ & 34.599 & 37.642 & 1474.17 & 1471.48 \\
\hline $11 / 4 / 112: 10$ & 34.58 & 37.64 & 1474.19 & 1471.48 \\
\hline $11 / 4 / 112: 20$ & 34.566 & 37.64 & 1474.20 & 1471.48 \\
\hline $11 / 4 / 112: 30$ & 34.55 & 37.638 & 1474.22 & 1471.48 \\
\hline $11 / 4 / 112: 40$ & 34.536 & 37.633 & 1474.23 & 1471.49 \\
\hline
\end{tabular}


TABLE S1.3 Automatically recorded groundwater levels in the Klassen wells, September 15, 2011, to March 13, 2012.

\begin{tabular}{|c|c|c|c|c|}
\hline \multicolumn{3}{|c|}{ Reference Elevation (ft AMSL) } & 1508.77 & \multirow{2}{*}{$\begin{array}{l}1509.12 \\
\text { Elevation in Klassen } \\
\text { Test Well (ft AMSL) }\end{array}$} \\
\hline Date and Time & $\begin{array}{l}\text { Depth in Klassen } \\
\text { Domestic Well } \\
\text { (ft BGL) }\end{array}$ & $\begin{array}{c}\text { Depth in Klassen } \\
\text { Test Well } \\
\text { (ft BGL) }\end{array}$ & $\begin{array}{l}\text { Elevation in Klassen } \\
\text { Domestic Well } \\
\text { (ft AMSL) }\end{array}$ & \\
\hline $11 / 4 / 112: 50$ & 34.716 & 37.633 & 1474.05 & 1471.49 \\
\hline $11 / 4 / 113: 00$ & 34.604 & 37.635 & 1474.17 & 1471.49 \\
\hline $11 / 4 / 113: 10$ & 34.564 & 37.633 & 1474.21 & 1471.49 \\
\hline $11 / 4 / 113: 20$ & 34.543 & 37.635 & 1474.23 & 1471.49 \\
\hline $11 / 4 / 113: 30$ & 34.524 & 37.633 & 1474.25 & 1471.49 \\
\hline $11 / 4 / 113: 40$ & 34.51 & 37.631 & 1474.26 & 1471.49 \\
\hline $11 / 4 / 113: 50$ & 34.498 & 37.631 & 1474.27 & 1471.49 \\
\hline $11 / 4 / 114: 00$ & 34.487 & 37.631 & 1474.28 & 1471.49 \\
\hline $11 / 4 / 114: 10$ & 34.475 & 37.628 & 1474.29 & 1471.49 \\
\hline $11 / 4 / 114: 20$ & 34.463 & 37.628 & 1474.31 & 1471.49 \\
\hline $11 / 4 / 114: 30$ & 34.454 & 37.628 & 1474.32 & 1471.49 \\
\hline $11 / 4 / 114: 40$ & 34.444 & 37.628 & 1474.33 & 1471.49 \\
\hline $11 / 4 / 114: 50$ & 34.435 & 37.628 & 1474.33 & 1471.49 \\
\hline $11 / 4 / 115: 00$ & 34.426 & 37.626 & 1474.34 & 1471.50 \\
\hline $11 / 4 / 11$ 5:10 & 34.419 & 37.626 & 1474.35 & 1471.50 \\
\hline $11 / 4 / 115: 20$ & 34.412 & 37.626 & 1474.36 & 1471.50 \\
\hline $11 / 4 / 115: 30$ & 34.404 & 37.624 & 1474.37 & 1471.50 \\
\hline $11 / 4 / 115: 40$ & 34.395 & 37.621 & 1474.37 & 1471.50 \\
\hline $11 / 4 / 11$ 5:50 & 34.388 & 37.619 & 1474.38 & 1471.50 \\
\hline $11 / 4 / 116: 00$ & 34.381 & 37.617 & 1474.39 & 1471.51 \\
\hline $11 / 4 / 116: 10$ & 35.418 & 37.614 & 1473.35 & 1471.51 \\
\hline $11 / 4 / 116: 20$ & 34.627 & 37.619 & 1474.14 & 1471.50 \\
\hline $11 / 4 / 116: 30$ & 34.571 & 37.617 & 1474.20 & 1471.51 \\
\hline $11 / 4 / 116: 40$ & 34.552 & 37.617 & 1474.22 & 1471.51 \\
\hline $11 / 4 / 116: 50$ & 34.536 & 37.614 & 1474.23 & 1471.51 \\
\hline $11 / 4 / 11$ 7:00 & 34.512 & 37.617 & 1474.26 & 1471.51 \\
\hline $11 / 4 / 11$ 7:10 & 34.529 & 37.612 & 1474.24 & 1471.51 \\
\hline $11 / 4 / 117: 20$ & 34.566 & 37.607 & 1474.20 & 1471.52 \\
\hline $11 / 4 / 117: 30$ & 34.557 & 37.602 & 1474.21 & 1471.52 \\
\hline $11 / 4 / 117: 40$ & 34.543 & 37.598 & 1474.23 & 1471.52 \\
\hline $11 / 4 / 11$ 7:50 & 34.541 & 37.595 & 1474.23 & 1471.53 \\
\hline $11 / 4 / 118: 00$ & 34.768 & 37.595 & 1474.00 & 1471.53 \\
\hline $11 / 4 / 118: 10$ & 34.618 & 37.591 & 1474.15 & 1471.53 \\
\hline $11 / 4 / 118: 20$ & 34.571 & 37.588 & 1474.20 & 1471.53 \\
\hline $11 / 4 / 118: 30$ & 34.541 & 37.586 & 1474.23 & 1471.54 \\
\hline $11 / 4 / 118: 40$ & 34.517 & 37.586 & 1474.25 & 1471.54 \\
\hline $11 / 4 / 118: 50$ & 34.984 & 37.586 & 1473.79 & 1471.54 \\
\hline 11/4/11 9:00 & 34.838 & 37.593 & 1473.93 & 1471.53 \\
\hline
\end{tabular}


TABLE S1.3 Automatically recorded groundwater levels in the Klassen wells, September 15, 2011, to March 13, 2012.

\begin{tabular}{|c|c|c|c|c|}
\hline \multicolumn{3}{|c|}{ Reference Elevation (ft AMSL) } & 1508.77 & \multirow{2}{*}{$\begin{array}{l}1509.12 \\
\text { Elevation in Klassen } \\
\text { Test Well (ft AMSL) }\end{array}$} \\
\hline Date and Time & $\begin{array}{l}\text { Depth in Klassen } \\
\text { Domestic Well } \\
(\mathrm{ft} \mathrm{BGL})\end{array}$ & $\begin{array}{c}\text { Depth in Klassen } \\
\text { Test Well } \\
\text { (ft BGL) }\end{array}$ & $\begin{array}{l}\text { Elevation in Klassen } \\
\text { Domestic Well } \\
\text { (ft AMSL) }\end{array}$ & \\
\hline 11/4/11 9:10 & 35.481 & 37.593 & 1473.29 & 1471.53 \\
\hline $11 / 4 / 119: 20$ & 34.761 & 37.605 & 1474.01 & 1471.52 \\
\hline $11 / 4 / 119: 30$ & 34.665 & 37.612 & 1474.10 & 1471.51 \\
\hline $11 / 4 / 119: 40$ & 34.618 & 37.619 & 1474.15 & 1471.50 \\
\hline $11 / 4 / 119: 50$ & 34.583 & 37.628 & 1474.19 & 1471.49 \\
\hline 11/4/11 10:00 & 34.555 & 37.633 & 1474.21 & 1471.49 \\
\hline 11/4/11 10:10 & 35.042 & 37.64 & 1473.73 & 1471.48 \\
\hline 11/4/11 10:20 & 34.637 & 37.647 & 1474.13 & 1471.48 \\
\hline 11/4/11 10:30 & 34.58 & 37.654 & 1474.19 & 1471.47 \\
\hline $11 / 4 / 11$ 10:40 & 35.92 & 37.659 & 1472.85 & 1471.46 \\
\hline $11 / 4 / 1110: 50$ & 34.655 & 37.666 & 1474.11 & 1471.46 \\
\hline 11/4/11 11:00 & 34.59 & 37.673 & 1474.18 & 1471.45 \\
\hline 11/4/11 11:10 & 34.559 & 37.68 & 1474.21 & 1471.44 \\
\hline $11 / 4 / 1111: 20$ & 34.531 & 37.685 & 1474.24 & 1471.44 \\
\hline 11/4/11 11:30 & 35.038 & 37.689 & 1473.73 & 1471.43 \\
\hline 11/4/11 11:40 & 34.651 & 37.694 & 1474.12 & 1471.43 \\
\hline 11/4/11 11:50 & 34.592 & 37.701 & 1474.18 & 1471.42 \\
\hline $11 / 4 / 11$ 12:00 & 34.78 & 37.703 & 1473.99 & 1471.42 \\
\hline 11/4/11 12:10 & 34.63 & 37.706 & 1474.14 & 1471.42 \\
\hline $11 / 4 / 11$ 12:20 & 34.583 & 37.71 & 1474.19 & 1471.41 \\
\hline $11 / 4 / 1112: 30$ & 34.552 & 37.713 & 1474.22 & 1471.41 \\
\hline 11/4/11 12:40 & 34.529 & 37.717 & 1474.24 & 1471.41 \\
\hline $11 / 4 / 1112: 50$ & 34.51 & 37.72 & 1474.26 & 1471.40 \\
\hline 11/4/11 13:00 & 38.208 & 37.722 & 1470.56 & 1471.40 \\
\hline 11/4/11 13:10 & 34.674 & 37.727 & 1474.10 & 1471.40 \\
\hline 11/4/11 13:20 & 34.578 & 37.729 & 1474.19 & 1471.39 \\
\hline 11/4/11 13:30 & 34.538 & 37.729 & 1474.23 & 1471.39 \\
\hline 11/4/11 13:40 & 37.036 & 37.734 & 1471.73 & 1471.39 \\
\hline $11 / 4 / 11$ 13:50 & 34.665 & 37.739 & 1474.10 & 1471.38 \\
\hline 11/4/11 14:00 & 34.583 & 37.741 & 1474.19 & 1471.38 \\
\hline 11/4/11 14:10 & 34.545 & 37.743 & 1474.22 & 1471.38 \\
\hline $11 / 4 / 11$ 14:20 & 34.515 & 37.746 & 1474.25 & 1471.38 \\
\hline $11 / 4 / 11$ 14:30 & 34.494 & 37.748 & 1474.28 & 1471.37 \\
\hline 11/4/11 14:40 & 37.369 & 37.748 & 1471.40 & 1471.37 \\
\hline 11/4/11 14:50 & 34.632 & 37.75 & 1474.14 & 1471.37 \\
\hline $11 / 4 / 11$ 15:00 & 34.555 & 37.753 & 1474.21 & 1471.37 \\
\hline 11/4/11 15:10 & 34.517 & 37.753 & 1474.25 & 1471.37 \\
\hline $11 / 4 / 11$ 15:20 & 34.494 & 37.753 & 1474.28 & 1471.37 \\
\hline
\end{tabular}


TABLE S1.3 Automatically recorded groundwater levels in the Klassen wells, September 15, 2011, to March 13, 2012.

\begin{tabular}{|c|c|c|c|c|}
\hline \multicolumn{3}{|c|}{ Reference Elevation (ft AMSL) } & 1508.77 & \multirow{2}{*}{$\begin{array}{l}1509.12 \\
\text { Elevation in Klassen } \\
\text { Test Well (ft AMSL) }\end{array}$} \\
\hline Date and Time & $\begin{array}{l}\text { Depth in Klassen } \\
\text { Domestic Well } \\
\text { (ft BGL) }\end{array}$ & $\begin{array}{c}\text { Depth in Klassen } \\
\text { Test Well } \\
\text { (ft BGL) }\end{array}$ & $\begin{array}{l}\text { Elevation in Klassen } \\
\text { Domestic Well } \\
\text { (ft AMSL) }\end{array}$ & \\
\hline $11 / 4 / 1115: 30$ & 34.498 & 37.753 & 1474.27 & 1471.37 \\
\hline $11 / 4 / 11$ 15:40 & 38.11 & 37.76 & 1470.66 & 1471.36 \\
\hline $11 / 4 / 11$ 15:50 & 34.669 & 37.762 & 1474.10 & 1471.36 \\
\hline 11/4/11 16:00 & 34.578 & 37.764 & 1474.19 & 1471.36 \\
\hline 11/4/11 16:10 & 34.536 & 37.764 & 1474.23 & 1471.36 \\
\hline $11 / 4 / 1116: 20$ & 34.508 & 37.764 & 1474.26 & 1471.36 \\
\hline $11 / 4 / 1116: 30$ & 34.487 & 37.764 & 1474.28 & 1471.36 \\
\hline 11/4/11 16:40 & 34.468 & 37.764 & 1474.30 & 1471.36 \\
\hline $11 / 4 / 1116: 50$ & 34.451 & 37.764 & 1474.32 & 1471.36 \\
\hline 11/4/11 17:00 & 34.435 & 37.762 & 1474.33 & 1471.36 \\
\hline 11/4/11 17:10 & 34.822 & 37.764 & 1473.95 & 1471.36 \\
\hline $11 / 4 / 1117: 20$ & 34.529 & 37.762 & 1474.24 & 1471.36 \\
\hline 11/4/11 17:30 & 34.484 & 37.757 & 1474.29 & 1471.37 \\
\hline $11 / 4 / 1117: 40$ & 34.458 & 37.755 & 1474.31 & 1471.37 \\
\hline 11/4/11 17:50 & 34.437 & 37.753 & 1474.33 & 1471.37 \\
\hline 11/4/11 18:00 & 34.449 & 37.746 & 1474.32 & 1471.38 \\
\hline 11/4/11 18:10 & 34.435 & 37.743 & 1474.33 & 1471.38 \\
\hline $11 / 4 / 11$ 18:20 & 34.623 & 37.739 & 1474.15 & 1471.38 \\
\hline 11/4/11 18:30 & 34.515 & 37.739 & 1474.25 & 1471.38 \\
\hline 11/4/11 18:40 & 34.557 & 37.739 & 1474.21 & 1471.38 \\
\hline $11 / 4 / 1118: 50$ & 34.745 & 37.746 & 1474.02 & 1471.38 \\
\hline 11/4/11 19:00 & 34.864 & 37.75 & 1473.91 & 1471.37 \\
\hline 11/4/11 19:10 & 34.845 & 37.748 & 1473.92 & 1471.37 \\
\hline 11/4/11 19:20 & 34.822 & 37.748 & 1473.95 & 1471.37 \\
\hline 11/4/11 19:30 & 34.803 & 37.746 & 1473.97 & 1471.38 \\
\hline 11/4/11 19:40 & 34.949 & 37.746 & 1473.82 & 1471.38 \\
\hline 11/4/11 19:50 & 34.843 & 37.746 & 1473.93 & 1471.38 \\
\hline 11/4/11 20:00 & 34.784 & 37.743 & 1473.99 & 1471.38 \\
\hline 11/4/11 20:10 & 34.768 & 37.741 & 1474.00 & 1471.38 \\
\hline 11/4/11 20:20 & 34.756 & 37.741 & 1474.01 & 1471.38 \\
\hline 11/4/11 20:30 & 34.721 & 37.741 & 1474.05 & 1471.38 \\
\hline 11/4/11 20:40 & 34.691 & 37.739 & 1474.08 & 1471.38 \\
\hline $11 / 4 / 1120: 50$ & 34.66 & 37.736 & 1474.11 & 1471.39 \\
\hline 11/4/11 21:00 & 34.639 & 37.734 & 1474.13 & 1471.39 \\
\hline 11/4/11 21:10 & 34.616 & 37.732 & 1474.15 & 1471.39 \\
\hline 11/4/11 21:20 & 34.63 & 37.727 & 1474.14 & 1471.40 \\
\hline 11/4/11 21:30 & 35.16 & 37.727 & 1473.61 & 1471.40 \\
\hline 11/4/11 21:40 & 34.693 & 37.724 & 1474.08 & 1471.40 \\
\hline
\end{tabular}


TABLE S1.3 Automatically recorded groundwater levels in the Klassen wells, September 15, 2011, to March 13, 2012.

\begin{tabular}{|c|c|c|c|c|}
\hline \multicolumn{3}{|c|}{ Reference Elevation (ft AMSL) } & 1508.77 & \multirow{2}{*}{$\begin{array}{l}1509.12 \\
\text { Elevation in Klassen } \\
\text { Test Well (ft AMSL) }\end{array}$} \\
\hline Date and Time & $\begin{array}{l}\text { Depth in Klassen } \\
\text { Domestic Well } \\
\text { (ft BGL) }\end{array}$ & $\begin{array}{c}\text { Depth in Klassen } \\
\text { Test Well } \\
\text { (ft BGL) }\end{array}$ & $\begin{array}{l}\text { Elevation in Klassen } \\
\text { Domestic Well } \\
\text { (ft AMSL) }\end{array}$ & \\
\hline $11 / 4 / 1121: 50$ & 34.639 & 37.722 & 1474.13 & 1471.40 \\
\hline $11 / 4 / 1122: 00$ & 34.611 & 37.72 & 1474.16 & 1471.40 \\
\hline 11/4/11 22:10 & 34.613 & 37.717 & 1474.16 & 1471.41 \\
\hline $11 / 4 / 1122: 20$ & 34.59 & 37.715 & 1474.18 & 1471.41 \\
\hline $11 / 4 / 1122: 30$ & 36.919 & 37.715 & 1471.85 & 1471.41 \\
\hline $11 / 4 / 1122: 40$ & 36.405 & 37.713 & 1472.36 & 1471.41 \\
\hline $11 / 4 / 1122: 50$ & 34.775 & 37.717 & 1473.99 & 1471.41 \\
\hline $11 / 4 / 1123: 00$ & 34.967 & 37.715 & 1473.80 & 1471.41 \\
\hline $11 / 4 / 1123: 10$ & 34.857 & 37.717 & 1473.91 & 1471.41 \\
\hline $11 / 4 / 1123: 20$ & 34.806 & 37.717 & 1473.96 & 1471.41 \\
\hline $11 / 4 / 1123: 30$ & 34.763 & 37.717 & 1474.01 & 1471.41 \\
\hline $11 / 4 / 1123: 40$ & 34.719 & 37.72 & 1474.05 & 1471.40 \\
\hline $11 / 4 / 1123: 50$ & 34.681 & 37.72 & 1474.09 & 1471.40 \\
\hline 11/5/11 0:00 & 34.651 & 37.722 & 1474.12 & 1471.40 \\
\hline 11/5/11 0:10 & 34.625 & 37.72 & 1474.14 & 1471.40 \\
\hline $11 / 5 / 110: 20$ & 34.601 & 37.72 & 1474.17 & 1471.40 \\
\hline $11 / 5 / 110: 30$ & 34.583 & 37.717 & 1474.19 & 1471.41 \\
\hline 11/5/11 0:40 & 34.564 & 37.715 & 1474.21 & 1471.41 \\
\hline $11 / 5 / 110: 50$ & 34.548 & 37.715 & 1474.22 & 1471.41 \\
\hline $11 / 5 / 11$ 1:00 & 34.531 & 37.713 & 1474.24 & 1471.41 \\
\hline $11 / 5 / 11$ 1:10 & 34.517 & 37.71 & 1474.25 & 1471.41 \\
\hline $11 / 5 / 11$ 1:20 & 34.503 & 37.708 & 1474.27 & 1471.41 \\
\hline 11/5/11 1:30 & 34.773 & 37.708 & 1474.00 & 1471.41 \\
\hline $11 / 5 / 11$ 1:40 & 34.665 & 37.708 & 1474.10 & 1471.41 \\
\hline $11 / 5 / 11$ 1:50 & 34.677 & 37.713 & 1474.09 & 1471.41 \\
\hline $11 / 5 / 112: 00$ & 34.644 & 37.71 & 1474.13 & 1471.41 \\
\hline $11 / 5 / 112: 10$ & 34.609 & 37.71 & 1474.16 & 1471.41 \\
\hline $11 / 5 / 112: 20$ & 34.606 & 37.708 & 1474.16 & 1471.41 \\
\hline 11/5/11 2:30 & 34.58 & 37.708 & 1474.19 & 1471.41 \\
\hline $11 / 5 / 112: 40$ & 34.559 & 37.708 & 1474.21 & 1471.41 \\
\hline $11 / 5 / 112: 50$ & 34.562 & 37.71 & 1474.21 & 1471.41 \\
\hline $11 / 5 / 113: 00$ & 34.543 & 37.708 & 1474.23 & 1471.41 \\
\hline 11/5/11 3:10 & 34.524 & 37.703 & 1474.25 & 1471.42 \\
\hline $11 / 5 / 113: 20$ & 34.58 & 37.703 & 1474.19 & 1471.42 \\
\hline $11 / 5 / 113: 30$ & 34.623 & 37.706 & 1474.15 & 1471.42 \\
\hline 11/5/11 3:40 & 34.601 & 37.706 & 1474.17 & 1471.42 \\
\hline $11 / 5 / 113: 50$ & 34.573 & 37.708 & 1474.20 & 1471.41 \\
\hline $11 / 5 / 114: 00$ & 34.55 & 37.713 & 1474.22 & 1471.41 \\
\hline
\end{tabular}


TABLE S1.3 Automatically recorded groundwater levels in the Klassen wells, September 15, 2011, to March 13, 2012.

\begin{tabular}{|c|c|c|c|c|}
\hline \multicolumn{3}{|c|}{ Reference Elevation (ft AMSL) } & 1508.77 & \multirow{2}{*}{$\begin{array}{l}1509.12 \\
\text { Elevation in Klassen } \\
\text { Test Well (ft AMSL) }\end{array}$} \\
\hline Date and Time & $\begin{array}{l}\text { Depth in Klassen } \\
\text { Domestic Well } \\
\text { (ft BGL) }\end{array}$ & $\begin{array}{c}\text { Depth in Klassen } \\
\text { Test Well } \\
\text { (ft BGL) }\end{array}$ & $\begin{array}{l}\text { Elevation in Klassen } \\
\text { Domestic Well } \\
\text { (ft AMSL) }\end{array}$ & \\
\hline $11 / 5 / 114: 10$ & 34.531 & 37.715 & 1474.24 & 1471.41 \\
\hline $11 / 5 / 114: 20$ & 34.515 & 37.715 & 1474.25 & 1471.41 \\
\hline $11 / 5 / 114: 30$ & 34.496 & 37.717 & 1474.27 & 1471.41 \\
\hline $11 / 5 / 114: 40$ & 34.482 & 37.717 & 1474.29 & 1471.41 \\
\hline $11 / 5 / 114: 50$ & 34.468 & 37.717 & 1474.30 & 1471.41 \\
\hline $11 / 5 / 115: 00$ & 34.456 & 37.717 & 1474.31 & 1471.41 \\
\hline $11 / 5 / 115: 10$ & 34.444 & 37.715 & 1474.33 & 1471.41 \\
\hline $11 / 5 / 115: 20$ & 34.433 & 37.715 & 1474.34 & 1471.41 \\
\hline $11 / 5 / 115: 30$ & 34.451 & 37.713 & 1474.32 & 1471.41 \\
\hline $11 / 5 / 115: 40$ & 34.472 & 37.713 & 1474.30 & 1471.41 \\
\hline $11 / 5 / 115: 50$ & 39.709 & 37.715 & 1469.06 & 1471.41 \\
\hline $11 / 5 / 116: 00$ & 34.627 & 37.715 & 1474.14 & 1471.41 \\
\hline $11 / 5 / 116: 10$ & 34.548 & 37.715 & 1474.22 & 1471.41 \\
\hline $11 / 5 / 116: 20$ & 34.538 & 37.713 & 1474.23 & 1471.41 \\
\hline $11 / 5 / 116: 30$ & 34.508 & 37.715 & 1474.26 & 1471.41 \\
\hline $11 / 5 / 116: 40$ & 34.484 & 37.715 & 1474.29 & 1471.41 \\
\hline $11 / 5 / 116: 50$ & 34.465 & 37.715 & 1474.30 & 1471.41 \\
\hline $11 / 5 / 117: 00$ & 34.449 & 37.715 & 1474.32 & 1471.41 \\
\hline $11 / 5 / 117: 10$ & 34.435 & 37.717 & 1474.33 & 1471.41 \\
\hline $11 / 5 / 117: 20$ & 34.421 & 37.715 & 1474.35 & 1471.41 \\
\hline $11 / 5 / 117: 30$ & 34.409 & 37.713 & 1474.36 & 1471.41 \\
\hline $11 / 5 / 117: 40$ & 34.4 & 37.71 & 1474.37 & 1471.41 \\
\hline $11 / 5 / 117: 50$ & 34.39 & 37.71 & 1474.38 & 1471.41 \\
\hline $11 / 5 / 118: 00$ & 34.381 & 37.71 & 1474.39 & 1471.41 \\
\hline $11 / 5 / 118: 10$ & 35.167 & 37.713 & 1473.60 & 1471.41 \\
\hline $11 / 5 / 118: 20$ & 34.475 & 37.713 & 1474.29 & 1471.41 \\
\hline $11 / 5 / 118: 30$ & 34.428 & 37.713 & 1474.34 & 1471.41 \\
\hline $11 / 5 / 118: 40$ & 34.566 & 37.715 & 1474.20 & 1471.41 \\
\hline $11 / 5 / 118: 50$ & 36.318 & 37.72 & 1472.45 & 1471.40 \\
\hline 11/5/11 9:00 & 35.207 & 37.724 & 1473.56 & 1471.40 \\
\hline 11/5/11 9:10 & 35.211 & 37.729 & 1473.56 & 1471.39 \\
\hline $11 / 5 / 119: 20$ & 34.82 & 37.734 & 1473.95 & 1471.39 \\
\hline 11/5/11 9:30 & 34.768 & 37.741 & 1474.00 & 1471.38 \\
\hline 11/5/11 9:40 & 34.754 & 37.748 & 1474.02 & 1471.37 \\
\hline 11/5/11 9:50 & 34.754 & 37.753 & 1474.02 & 1471.37 \\
\hline 11/5/11 10:00 & 34.749 & 37.76 & 1474.02 & 1471.36 \\
\hline 11/5/11 10:10 & 34.745 & 37.767 & 1474.02 & 1471.36 \\
\hline $11 / 5 / 11$ 10:20 & 34.742 & 37.776 & 1474.03 & 1471.35 \\
\hline
\end{tabular}


TABLE S1.3 Automatically recorded groundwater levels in the Klassen wells, September 15, 2011, to March 13, 2012.

\begin{tabular}{|c|c|c|c|c|}
\hline \multicolumn{3}{|c|}{ Reference Elevation (ft AMSL) } & 1508.77 & \multirow{2}{*}{$\begin{array}{l}1509.12 \\
\text { Elevation in Klassen } \\
\text { Test Well (ft AMSL) }\end{array}$} \\
\hline Date and Time & $\begin{array}{l}\text { Depth in Klassen } \\
\text { Domestic Well } \\
\text { (ft BGL) }\end{array}$ & $\begin{array}{c}\text { Depth in Klassen } \\
\text { Test Well } \\
\text { (ft BGL) }\end{array}$ & $\begin{array}{l}\text { Elevation in Klassen } \\
\text { Domestic Well } \\
\text { (ft AMSL) }\end{array}$ & \\
\hline $11 / 5 / 1110: 30$ & 34.745 & 37.783 & 1474.02 & 1471.34 \\
\hline $11 / 5 / 11$ 10:40 & 34.74 & 37.79 & 1474.03 & 1471.33 \\
\hline $11 / 5 / 11$ 10:50 & 34.74 & 37.797 & 1474.03 & 1471.33 \\
\hline 11/5/11 11:00 & 34.74 & 37.807 & 1474.03 & 1471.32 \\
\hline 11/5/11 11:10 & 35.012 & 37.821 & 1473.76 & 1471.30 \\
\hline 11/5/11 11:20 & 34.864 & 37.83 & 1473.91 & 1471.29 \\
\hline 11/5/11 11:30 & 34.829 & 37.839 & 1473.94 & 1471.28 \\
\hline 11/5/11 11:40 & 35.127 & 37.854 & 1473.64 & 1471.27 \\
\hline 11/5/11 11:50 & 34.909 & 37.865 & 1473.86 & 1471.26 \\
\hline $11 / 5 / 1112: 00$ & 34.876 & 37.872 & 1473.89 & 1471.25 \\
\hline 11/5/11 12:10 & 34.852 & 37.877 & 1473.92 & 1471.25 \\
\hline $11 / 5 / 1112: 20$ & 34.841 & 37.889 & 1473.93 & 1471.23 \\
\hline $11 / 5 / 1112: 30$ & 34.834 & 37.9 & 1473.94 & 1471.22 \\
\hline $11 / 5 / 1112: 40$ & 34.824 & 37.915 & 1473.95 & 1471.21 \\
\hline $11 / 5 / 11$ 12:50 & 34.834 & 37.919 & 1473.94 & 1471.20 \\
\hline 11/5/11 13:00 & 35.2 & 37.924 & 1473.57 & 1471.20 \\
\hline 11/5/11 13:10 & 34.951 & 37.929 & 1473.82 & 1471.19 \\
\hline $11 / 5 / 11$ 13:20 & 34.904 & 37.933 & 1473.87 & 1471.19 \\
\hline 11/5/11 13:30 & 34.892 & 37.938 & 1473.88 & 1471.18 \\
\hline 11/5/11 13:40 & 34.871 & 37.947 & 1473.90 & 1471.18 \\
\hline $11 / 5 / 11$ 13:50 & 34.857 & 37.95 & 1473.91 & 1471.17 \\
\hline 11/5/11 14:00 & 34.909 & 37.961 & 1473.86 & 1471.16 \\
\hline 11/5/11 14:10 & 35 & 37.976 & 1473.77 & 1471.15 \\
\hline $11 / 5 / 11$ 14:20 & 37.829 & 37.985 & 1470.94 & 1471.14 \\
\hline 11/5/11 14:30 & 35.218 & 37.994 & 1473.55 & 1471.13 \\
\hline 11/5/11 14:40 & 35.129 & 37.999 & 1473.64 & 1471.12 \\
\hline $11 / 5 / 11$ 14:50 & 35.092 & 38.004 & 1473.68 & 1471.12 \\
\hline 11/5/11 15:00 & 35.059 & 38.011 & 1473.71 & 1471.11 \\
\hline 11/5/11 15:10 & 35.031 & 38.02 & 1473.74 & 1471.10 \\
\hline 11/5/11 15:20 & 37.05 & 38.03 & 1471.72 & 1471.09 \\
\hline 11/5/11 15:30 & 35.178 & 38.039 & 1473.59 & 1471.08 \\
\hline 11/5/11 15:40 & 35.11 & 38.044 & 1473.66 & 1471.08 \\
\hline $11 / 5 / 11$ 15:50 & 35.073 & 38.055 & 1473.70 & 1471.07 \\
\hline 11/5/11 16:00 & 35.038 & 38.062 & 1473.73 & 1471.06 \\
\hline 11/5/11 16:10 & 35.021 & 38.072 & 1473.75 & 1471.05 \\
\hline 11/5/11 16:20 & 35.01 & 38.079 & 1473.76 & 1471.04 \\
\hline 11/5/11 16:30 & 34.995 & 38.088 & 1473.77 & 1471.03 \\
\hline 11/5/11 16:40 & 34.977 & 38.095 & 1473.79 & 1471.03 \\
\hline
\end{tabular}


TABLE S1.3 Automatically recorded groundwater levels in the Klassen wells, September 15, 2011, to March 13, 2012.

\begin{tabular}{|c|c|c|c|c|}
\hline \multicolumn{3}{|c|}{ Reference Elevation (ft AMSL) } & 1508.77 & \multirow{2}{*}{$\begin{array}{l}1509.12 \\
\text { Elevation in Klassen } \\
\text { Test Well (ft AMSL) }\end{array}$} \\
\hline Date and Time & $\begin{array}{l}\text { Depth in Klassen } \\
\text { Domestic Well } \\
\text { (ft BGL) }\end{array}$ & $\begin{array}{c}\text { Depth in Klassen } \\
\text { Test Well } \\
\text { (ft BGL) }\end{array}$ & $\begin{array}{l}\text { Elevation in Klassen } \\
\text { Domestic Well } \\
\text { (ft AMSL) }\end{array}$ & \\
\hline $11 / 5 / 1116: 50$ & 34.967 & 38.098 & 1473.80 & 1471.02 \\
\hline 11/5/11 17:00 & 34.953 & 38.105 & 1473.82 & 1471.02 \\
\hline 11/5/11 17:10 & 34.949 & 38.102 & 1473.82 & 1471.02 \\
\hline $11 / 5 / 1117: 20$ & 35.176 & 38.107 & 1473.59 & 1471.02 \\
\hline 11/5/11 17:30 & 35.047 & 38.112 & 1473.72 & 1471.01 \\
\hline $11 / 5 / 1117: 40$ & 35.005 & 38.119 & 1473.76 & 1471.00 \\
\hline $11 / 5 / 1117: 50$ & 34.981 & 38.126 & 1473.79 & 1471.00 \\
\hline $11 / 5 / 1118: 00$ & 34.97 & 38.126 & 1473.80 & 1471.00 \\
\hline 11/5/11 18:10 & 34.963 & 38.133 & 1473.81 & 1470.99 \\
\hline $11 / 5 / 1118: 20$ & 34.963 & 38.14 & 1473.81 & 1470.98 \\
\hline $11 / 5 / 1118: 30$ & 34.965 & 38.154 & 1473.80 & 1470.97 \\
\hline 11/5/11 18:40 & 34.956 & 38.161 & 1473.81 & 1470.96 \\
\hline $11 / 5 / 1118: 50$ & 34.944 & 38.168 & 1473.83 & 1470.95 \\
\hline 11/5/11 19:00 & 34.935 & 38.173 & 1473.83 & 1470.95 \\
\hline 11/5/11 19:10 & 35.218 & 38.177 & 1473.55 & 1470.95 \\
\hline 11/5/11 19:20 & 35.017 & 38.187 & 1473.75 & 1470.94 \\
\hline 11/5/11 19:30 & 40.136 & 38.189 & 1468.63 & 1470.93 \\
\hline 11/5/11 19:40 & 35.136 & 38.201 & 1473.63 & 1470.92 \\
\hline 11/5/11 19:50 & 35.061 & 38.205 & 1473.71 & 1470.92 \\
\hline 11/5/11 20:00 & 35.021 & 38.217 & 1473.75 & 1470.91 \\
\hline 11/5/11 20:10 & 34.998 & 38.227 & 1473.77 & 1470.90 \\
\hline $11 / 5 / 1120: 20$ & 34.981 & 38.234 & 1473.79 & 1470.89 \\
\hline $11 / 5 / 1120: 30$ & 34.967 & 38.241 & 1473.80 & 1470.88 \\
\hline 11/5/11 20:40 & 34.956 & 38.248 & 1473.81 & 1470.87 \\
\hline $11 / 5 / 1120: 50$ & 34.946 & 38.257 & 1473.82 & 1470.87 \\
\hline 11/5/11 21:00 & 35.92 & 38.267 & 1472.85 & 1470.86 \\
\hline 11/5/11 21:10 & 35.089 & 38.276 & 1473.68 & 1470.85 \\
\hline 11/5/11 21:20 & 35.033 & 38.288 & 1473.74 & 1470.83 \\
\hline 11/5/11 21:30 & 35.017 & 38.297 & 1473.75 & 1470.83 \\
\hline 11/5/11 21:40 & 34.998 & 38.306 & 1473.77 & 1470.82 \\
\hline 11/5/11 21:50 & 34.979 & 38.311 & 1473.79 & 1470.81 \\
\hline 11/5/11 22:00 & 34.97 & 38.318 & 1473.80 & 1470.80 \\
\hline 11/5/11 22:10 & 35.033 & 38.328 & 1473.74 & 1470.79 \\
\hline $11 / 5 / 1122: 20$ & 35.101 & 38.342 & 1473.67 & 1470.78 \\
\hline 11/5/11 22:30 & 35.16 & 38.351 & 1473.61 & 1470.77 \\
\hline 11/5/11 22:40 & 40.092 & 38.36 & 1468.68 & 1470.76 \\
\hline $11 / 5 / 1122: 50$ & 35.547 & 38.374 & 1473.22 & 1470.75 \\
\hline 11/5/11 23:00 & 35.744 & 38.386 & 1473.03 & 1470.74 \\
\hline
\end{tabular}


TABLE S1.3 Automatically recorded groundwater levels in the Klassen wells, September 15, 2011, to March 13, 2012.

\begin{tabular}{|c|c|c|c|c|}
\hline \multicolumn{3}{|c|}{ Reference Elevation (ft AMSL) } & 1508.77 & \multirow{2}{*}{$\begin{array}{l}1509.12 \\
\text { Elevation in Klassen } \\
\text { Test Well (ft AMSL) }\end{array}$} \\
\hline Date and Time & $\begin{array}{l}\text { Depth in Klassen } \\
\text { Domestic Well } \\
\text { (ft BGL) }\end{array}$ & $\begin{array}{c}\text { Depth in Klassen } \\
\text { Test Well } \\
\text { (ft BGL) }\end{array}$ & $\begin{array}{l}\text { Elevation in Klassen } \\
\text { Domestic Well } \\
\text { (ft AMSL) }\end{array}$ & \\
\hline $11 / 5 / 1123: 10$ & 35.983 & 38.403 & 1472.79 & 1470.72 \\
\hline $11 / 5 / 1123: 20$ & 35.701 & 38.419 & 1473.07 & 1470.70 \\
\hline $11 / 5 / 1123: 30$ & 35.647 & 38.431 & 1473.12 & 1470.69 \\
\hline $11 / 5 / 1123: 40$ & 35.626 & 38.447 & 1473.14 & 1470.68 \\
\hline $11 / 5 / 1123: 50$ & 35.615 & 38.459 & 1473.15 & 1470.66 \\
\hline $11 / 6 / 110: 00$ & 35.615 & 38.471 & 1473.15 & 1470.65 \\
\hline 11/6/11 0:10 & 35.61 & 38.482 & 1473.16 & 1470.64 \\
\hline $11 / 6 / 110: 20$ & 35.615 & 38.496 & 1473.15 & 1470.63 \\
\hline 11/6/11 0:30 & 35.617 & 38.508 & 1473.15 & 1470.61 \\
\hline 11/6/11 0:40 & 35.619 & 38.518 & 1473.15 & 1470.60 \\
\hline $11 / 6 / 110: 50$ & 35.629 & 38.529 & 1473.14 & 1470.59 \\
\hline $11 / 6 / 111: 00$ & 35.657 & 38.539 & 1473.11 & 1470.58 \\
\hline $11 / 6 / 11$ 1:10 & 35.678 & 38.55 & 1473.09 & 1470.57 \\
\hline $11 / 6 / 111: 20$ & 35.683 & 38.56 & 1473.09 & 1470.56 \\
\hline $11 / 6 / 11$ 1:30 & 35.685 & 38.569 & 1473.08 & 1470.55 \\
\hline $11 / 6 / 11$ 1:40 & 35.685 & 38.581 & 1473.08 & 1470.54 \\
\hline $11 / 6 / 11$ 1:50 & 35.685 & 38.59 & 1473.08 & 1470.53 \\
\hline 11/6/11 2:00 & 35.683 & 38.602 & 1473.09 & 1470.52 \\
\hline $11 / 6 / 112: 10$ & 35.685 & 38.607 & 1473.08 & 1470.52 \\
\hline $11 / 6 / 112: 20$ & 35.685 & 38.616 & 1473.08 & 1470.51 \\
\hline $11 / 6 / 112: 30$ & 35.683 & 38.625 & 1473.09 & 1470.50 \\
\hline $11 / 6 / 112: 40$ & 35.683 & 38.633 & 1473.09 & 1470.49 \\
\hline $11 / 6 / 112: 50$ & 35.683 & 38.642 & 1473.09 & 1470.48 \\
\hline $11 / 6 / 113: 00$ & 35.68 & 38.649 & 1473.09 & 1470.47 \\
\hline $11 / 6 / 113: 10$ & 35.683 & 38.658 & 1473.09 & 1470.46 \\
\hline $11 / 6 / 113: 20$ & 37.697 & 38.663 & 1471.07 & 1470.46 \\
\hline $11 / 6 / 113: 30$ & 35.814 & 38.672 & 1472.96 & 1470.45 \\
\hline $11 / 6 / 113: 40$ & 35.767 & 38.675 & 1473.00 & 1470.45 \\
\hline $11 / 6 / 113: 50$ & 35.748 & 38.679 & 1473.02 & 1470.44 \\
\hline 11/6/11 4:00 & 35.739 & 38.686 & 1473.03 & 1470.44 \\
\hline 11/6/11 4:10 & 35.73 & 38.691 & 1473.04 & 1470.43 \\
\hline $11 / 6 / 114: 20$ & 35.725 & 38.703 & 1473.04 & 1470.42 \\
\hline $11 / 6 / 114: 30$ & 35.723 & 38.708 & 1473.05 & 1470.41 \\
\hline $11 / 6 / 114: 40$ & 35.723 & 38.715 & 1473.05 & 1470.41 \\
\hline $11 / 6 / 114: 50$ & 35.72 & 38.722 & 1473.05 & 1470.40 \\
\hline $11 / 6 / 115: 00$ & 35.72 & 38.726 & 1473.05 & 1470.40 \\
\hline $11 / 6 / 115: 10$ & 35.72 & 38.733 & 1473.05 & 1470.39 \\
\hline $11 / 6 / 115: 20$ & 35.723 & 38.738 & 1473.05 & 1470.38 \\
\hline
\end{tabular}


TABLE S1.3 Automatically recorded groundwater levels in the Klassen wells, September 15, 2011, to March 13, 2012.

\begin{tabular}{|c|c|c|c|c|}
\hline \multicolumn{3}{|c|}{ Reference Elevation (ft AMSL) } & 1508.77 & \multirow{2}{*}{$\begin{array}{l}1509.12 \\
\text { Elevation in Klassen } \\
\text { Test Well (ft AMSL) }\end{array}$} \\
\hline Date and Time & $\begin{array}{l}\text { Depth in Klassen } \\
\text { Domestic Well } \\
(\mathrm{ft} \mathrm{BGL})\end{array}$ & $\begin{array}{c}\text { Depth in Klassen } \\
\text { Test Well } \\
\text { (ft BGL) }\end{array}$ & $\begin{array}{l}\text { Elevation in Klassen } \\
\text { Domestic Well } \\
\text { (ft AMSL) }\end{array}$ & \\
\hline $11 / 6 / 115: 30$ & 35.72 & 38.743 & 1473.05 & 1470.38 \\
\hline $11 / 6 / 115: 40$ & 35.72 & 38.75 & 1473.05 & 1470.37 \\
\hline $11 / 6 / 115: 50$ & 35.723 & 38.752 & 1473.05 & 1470.37 \\
\hline $11 / 6 / 116: 00$ & 35.723 & 38.755 & 1473.05 & 1470.37 \\
\hline $11 / 6 / 116: 10$ & 35.723 & 38.762 & 1473.05 & 1470.36 \\
\hline $11 / 6 / 116: 20$ & 35.725 & 38.766 & 1473.04 & 1470.36 \\
\hline $11 / 6 / 116: 30$ & 35.725 & 38.769 & 1473.04 & 1470.35 \\
\hline $11 / 6 / 116: 40$ & 35.725 & 38.764 & 1473.04 & 1470.36 \\
\hline $11 / 6 / 116: 50$ & 41.635 & 38.764 & 1467.13 & 1470.36 \\
\hline $11 / 6 / 117: 00$ & 36.056 & 38.766 & 1472.71 & 1470.36 \\
\hline $11 / 6 / 117: 10$ & 40.842 & 38.769 & 1467.93 & 1470.35 \\
\hline $11 / 6 / 117: 20$ & 36.056 & 38.769 & 1472.71 & 1470.35 \\
\hline $11 / 6 / 117: 30$ & 35.964 & 38.769 & 1472.81 & 1470.35 \\
\hline $11 / 6 / 117: 40$ & 35.927 & 38.769 & 1472.84 & 1470.35 \\
\hline $11 / 6 / 117: 50$ & 35.901 & 38.769 & 1472.87 & 1470.35 \\
\hline $11 / 6 / 118: 00$ & 35.882 & 38.766 & 1472.89 & 1470.36 \\
\hline $11 / 6 / 118: 10$ & 35.873 & 38.766 & 1472.90 & 1470.36 \\
\hline $11 / 6 / 118: 20$ & 35.861 & 38.766 & 1472.91 & 1470.36 \\
\hline $11 / 6 / 118: 30$ & 35.854 & 38.762 & 1472.92 & 1470.36 \\
\hline $11 / 6 / 118: 40$ & 35.849 & 38.762 & 1472.92 & 1470.36 \\
\hline $11 / 6 / 118: 50$ & 35.842 & 38.762 & 1472.93 & 1470.36 \\
\hline $11 / 6 / 119: 00$ & 36.112 & 38.769 & 1472.66 & 1470.35 \\
\hline 11/6/11 9:10 & 35.999 & 38.778 & 1472.77 & 1470.34 \\
\hline $11 / 6 / 119: 20$ & 36.199 & 38.78 & 1472.57 & 1470.34 \\
\hline 11/6/11 9:30 & 36.081 & 38.79 & 1472.69 & 1470.33 \\
\hline $11 / 6 / 11$ 9:40 & 36.306 & 38.799 & 1472.46 & 1470.32 \\
\hline $11 / 6 / 119: 50$ & 36.177 & 38.806 & 1472.59 & 1470.32 \\
\hline $11 / 6 / 11$ 10:00 & 36.124 & 38.811 & 1472.65 & 1470.31 \\
\hline 11/6/11 10:10 & 36.109 & 38.816 & 1472.66 & 1470.31 \\
\hline $11 / 6 / 11$ 10:20 & 36.095 & 38.816 & 1472.67 & 1470.31 \\
\hline 11/6/11 10:30 & 36.088 & 38.818 & 1472.68 & 1470.30 \\
\hline 11/6/11 10:40 & 36.084 & 38.823 & 1472.69 & 1470.30 \\
\hline $11 / 6 / 11$ 10:50 & 36.079 & 38.827 & 1472.69 & 1470.30 \\
\hline 11/6/11 11:00 & 36.077 & 38.837 & 1472.69 & 1470.29 \\
\hline 11/6/11 11:10 & 36.074 & 38.848 & 1472.70 & 1470.27 \\
\hline 11/6/11 11:20 & 36.072 & 38.858 & 1472.70 & 1470.26 \\
\hline 11/6/11 11:30 & 36.07 & 38.867 & 1472.70 & 1470.26 \\
\hline 11/6/11 11:40 & 36.067 & 38.877 & 1472.70 & 1470.25 \\
\hline
\end{tabular}


TABLE S1.3 Automatically recorded groundwater levels in the Klassen wells, September 15, 2011, to March 13, 2012.

\begin{tabular}{|c|c|c|c|c|}
\hline \multicolumn{3}{|c|}{ Reference Elevation (ft AMSL) } & 1508.77 & \multirow{2}{*}{$\begin{array}{l}1509.12 \\
\text { Elevation in Klassen } \\
\text { Test Well (ft AMSL) }\end{array}$} \\
\hline Date and Time & $\begin{array}{l}\text { Depth in Klassen } \\
\text { Domestic Well } \\
(\mathrm{ft} \mathrm{BGL})\end{array}$ & $\begin{array}{c}\text { Depth in Klassen } \\
\text { Test Well } \\
\text { (ft BGL) }\end{array}$ & $\begin{array}{l}\text { Elevation in Klassen } \\
\text { Domestic Well } \\
\text { (ft AMSL) }\end{array}$ & \\
\hline $11 / 6 / 1111: 50$ & 36.07 & 38.888 & 1472.70 & 1470.23 \\
\hline $11 / 6 / 1112: 00$ & 36.065 & 38.893 & 1472.70 & 1470.23 \\
\hline 11/6/11 12:10 & 36.065 & 38.902 & 1472.70 & 1470.22 \\
\hline $11 / 6 / 1112: 20$ & 36.065 & 38.914 & 1472.70 & 1470.21 \\
\hline $11 / 6 / 1112: 30$ & 36.063 & 38.926 & 1472.71 & 1470.20 \\
\hline $11 / 6 / 1112: 40$ & 36.06 & 38.935 & 1472.71 & 1470.19 \\
\hline $11 / 6 / 1112: 50$ & 36.06 & 38.947 & 1472.71 & 1470.18 \\
\hline 11/6/11 13:00 & 36.058 & 38.952 & 1472.71 & 1470.17 \\
\hline 11/6/11 13:10 & 36.06 & 38.959 & 1472.71 & 1470.16 \\
\hline $11 / 6 / 1113: 20$ & 36.058 & 38.966 & 1472.71 & 1470.16 \\
\hline $11 / 6 / 1113: 30$ & 36.058 & 38.975 & 1472.71 & 1470.15 \\
\hline 11/6/11 13:40 & 36.056 & 38.985 & 1472.71 & 1470.14 \\
\hline $11 / 6 / 1113: 50$ & 36.056 & 38.991 & 1472.71 & 1470.13 \\
\hline $11 / 6 / 1114: 00$ & 36.053 & 39.001 & 1472.72 & 1470.12 \\
\hline 11/6/11 14:10 & 36.053 & 39.013 & 1472.72 & 1470.11 \\
\hline $11 / 6 / 11$ 14:20 & 36.051 & 39.024 & 1472.72 & 1470.10 \\
\hline $11 / 6 / 11$ 14:30 & 36.051 & 39.041 & 1472.72 & 1470.08 \\
\hline 11/6/11 14:40 & 36.049 & 39.055 & 1472.72 & 1470.07 \\
\hline $11 / 6 / 11$ 14:50 & 36.049 & 39.069 & 1472.72 & 1470.05 \\
\hline 11/6/11 15:00 & 36.241 & 39.09 & 1472.53 & 1470.03 \\
\hline $11 / 6 / 11$ 15:10 & 36.149 & 39.109 & 1472.62 & 1470.01 \\
\hline $11 / 6 / 11$ 15:20 & 36.128 & 39.125 & 1472.64 & 1470.00 \\
\hline $11 / 6 / 11$ 15:30 & 36.112 & 39.144 & 1472.66 & 1469.98 \\
\hline 11/6/11 15:40 & 36.098 & 39.163 & 1472.67 & 1469.96 \\
\hline $11 / 6 / 11$ 15:50 & 36.093 & 39.182 & 1472.68 & 1469.94 \\
\hline 11/6/11 16:00 & 36.086 & 39.21 & 1472.68 & 1469.91 \\
\hline 11/6/11 16:10 & 36.079 & 39.243 & 1472.69 & 1469.88 \\
\hline $11 / 6 / 11$ 16:20 & 36.086 & 39.273 & 1472.68 & 1469.85 \\
\hline 11/6/11 16:30 & 36.084 & 39.299 & 1472.69 & 1469.82 \\
\hline 11/6/11 16:40 & 36.086 & 39.32 & 1472.68 & 1469.80 \\
\hline 11/6/11 16:50 & 36.14 & 39.336 & 1472.63 & 1469.79 \\
\hline 11/6/11 17:00 & 36.206 & 39.355 & 1472.56 & 1469.77 \\
\hline 11/6/11 17:10 & 36.271 & 39.367 & 1472.50 & 1469.76 \\
\hline 11/6/11 17:20 & 36.382 & 39.381 & 1472.39 & 1469.74 \\
\hline 11/6/11 17:30 & 38.56 & 39.393 & 1470.21 & 1469.73 \\
\hline 11/6/11 17:40 & 36.661 & 39.407 & 1472.11 & 1469.72 \\
\hline $11 / 6 / 11$ 17:50 & 36.693 & 39.414 & 1472.08 & 1469.71 \\
\hline 11/6/11 18:00 & 36.722 & 39.421 & 1472.05 & 1469.70 \\
\hline
\end{tabular}


TABLE S1.3 Automatically recorded groundwater levels in the Klassen wells, September 15, 2011, to March 13, 2012.

\begin{tabular}{|c|c|c|c|c|}
\hline \multicolumn{3}{|c|}{ Reference Elevation (ft AMSL) } & 1508.77 & \multirow{2}{*}{$\begin{array}{l}1509.12 \\
\text { Elevation in Klassen } \\
\text { Test Well (ft AMSL) }\end{array}$} \\
\hline Date and Time & $\begin{array}{l}\text { Depth in Klassen } \\
\text { Domestic Well } \\
(\mathrm{ft} \mathrm{BGL})\end{array}$ & $\begin{array}{c}\text { Depth in Klassen } \\
\text { Test Well } \\
\text { (ft BGL) }\end{array}$ & $\begin{array}{l}\text { Elevation in Klassen } \\
\text { Domestic Well } \\
\text { (ft AMSL) }\end{array}$ & \\
\hline 11/6/11 18:10 & 36.794 & 39.428 & 1471.98 & 1469.69 \\
\hline $11 / 6 / 1118: 20$ & 36.848 & 39.437 & 1471.92 & 1469.69 \\
\hline 11/6/11 18:30 & 36.883 & 39.442 & 1471.89 & 1469.68 \\
\hline $11 / 6 / 1118: 40$ & 36.954 & 39.449 & 1471.82 & 1469.67 \\
\hline $11 / 6 / 11$ 18:50 & 36.984 & 39.456 & 1471.79 & 1469.67 \\
\hline 11/6/11 19:00 & 37.041 & 39.463 & 1471.73 & 1469.66 \\
\hline 11/6/11 19:10 & 37.099 & 39.47 & 1471.67 & 1469.65 \\
\hline 11/6/11 19:20 & 37.127 & 39.477 & 1471.64 & 1469.65 \\
\hline 11/6/11 19:30 & 37.193 & 39.482 & 1471.58 & 1469.64 \\
\hline 11/6/11 19:40 & 37.186 & 39.487 & 1471.58 & 1469.64 \\
\hline 11/6/11 19:50 & 37.111 & 39.489 & 1471.66 & 1469.63 \\
\hline 11/6/11 20:00 & 37.029 & 39.487 & 1471.74 & 1469.64 \\
\hline 11/6/11 20:10 & 36.951 & 39.482 & 1471.82 & 1469.64 \\
\hline $11 / 6 / 1120: 20$ & 37.348 & 39.477 & 1471.42 & 1469.65 \\
\hline 11/6/11 20:30 & 36.98 & 39.472 & 1471.79 & 1469.65 \\
\hline $11 / 6 / 1120: 40$ & 36.883 & 39.468 & 1471.89 & 1469.65 \\
\hline $11 / 6 / 1120: 50$ & 36.902 & 39.466 & 1471.87 & 1469.66 \\
\hline 11/6/11 21:00 & 36.86 & 39.461 & 1471.91 & 1469.66 \\
\hline 11/6/11 21:10 & 36.86 & 39.456 & 1471.91 & 1469.67 \\
\hline 11/6/11 21:20 & 36.827 & 39.451 & 1471.94 & 1469.67 \\
\hline $11 / 6 / 1121: 30$ & 36.768 & 39.442 & 1472.00 & 1469.68 \\
\hline 11/6/11 21:40 & 36.708 & 39.435 & 1472.06 & 1469.69 \\
\hline $11 / 6 / 1121: 50$ & 36.715 & 39.428 & 1472.05 & 1469.69 \\
\hline $11 / 6 / 1122: 00$ & 36.691 & 39.421 & 1472.08 & 1469.70 \\
\hline 11/6/11 22:10 & 36.64 & 39.414 & 1472.13 & 1469.71 \\
\hline $11 / 6 / 1122: 20$ & 36.668 & 39.409 & 1472.10 & 1469.71 \\
\hline $11 / 6 / 1122: 30$ & 36.628 & 39.404 & 1472.14 & 1469.72 \\
\hline $11 / 6 / 1122: 40$ & 36.611 & 39.397 & 1472.16 & 1469.73 \\
\hline $11 / 6 / 1122: 50$ & 36.604 & 39.393 & 1472.17 & 1469.73 \\
\hline 11/6/11 23:00 & 36.614 & 39.388 & 1472.16 & 1469.73 \\
\hline 11/6/11 23:10 & 36.579 & 39.381 & 1472.19 & 1469.74 \\
\hline $11 / 6 / 1123: 20$ & 36.607 & 39.374 & 1472.16 & 1469.75 \\
\hline $11 / 6 / 1123: 30$ & 36.586 & 39.372 & 1472.18 & 1469.75 \\
\hline 11/6/11 23:40 & 36.539 & 39.36 & 1472.23 & 1469.76 \\
\hline $11 / 6 / 1123: 50$ & 36.909 & 39.353 & 1471.86 & 1469.77 \\
\hline 11/7/11 0:00 & 36.766 & 39.343 & 1472.00 & 1469.78 \\
\hline 11/7/11 0:10 & 36.6 & 39.341 & 1472.17 & 1469.78 \\
\hline $11 / 7 / 11$ 0:20 & 37.226 & 39.334 & 1471.54 & 1469.79 \\
\hline
\end{tabular}


TABLE S1.3 Automatically recorded groundwater levels in the Klassen wells, September 15, 2011, to March 13, 2012.

\begin{tabular}{|c|c|c|c|c|}
\hline \multicolumn{3}{|c|}{ Reference Elevation (ft AMSL) } & 1508.77 & \multirow{2}{*}{$\begin{array}{l}1509.12 \\
\text { Elevation in Klassen } \\
\text { Test Well (ft AMSL) }\end{array}$} \\
\hline Date and Time & $\begin{array}{l}\text { Depth in Klassen } \\
\text { Domestic Well } \\
\text { (ft BGL) }\end{array}$ & $\begin{array}{c}\text { Depth in Klassen } \\
\text { Test Well } \\
\text { (ft BGL) }\end{array}$ & $\begin{array}{l}\text { Elevation in Klassen } \\
\text { Domestic Well } \\
\text { (ft AMSL) }\end{array}$ & \\
\hline $11 / 7 / 110: 30$ & 38.37 & 39.329 & 1470.40 & 1469.79 \\
\hline $11 / 7 / 110: 40$ & 36.912 & 39.325 & 1471.86 & 1469.80 \\
\hline $11 / 7 / 110: 50$ & 36.663 & 39.318 & 1472.11 & 1469.80 \\
\hline 11/7/11 1:00 & 36.872 & 39.313 & 1471.90 & 1469.81 \\
\hline 11/7/11 1:10 & 42.041 & 39.304 & 1466.73 & 1469.82 \\
\hline 11/7/11 1:20 & 37.104 & 39.299 & 1471.67 & 1469.82 \\
\hline $11 / 7 / 111: 30$ & 36.682 & 39.292 & 1472.09 & 1469.83 \\
\hline $11 / 7 / 111: 40$ & 36.623 & 39.285 & 1472.15 & 1469.84 \\
\hline $11 / 7 / 11$ 1:50 & 36.604 & 39.28 & 1472.17 & 1469.84 \\
\hline $11 / 7 / 112: 00$ & 36.55 & 39.273 & 1472.22 & 1469.85 \\
\hline 11/7/11 2:10 & 36.492 & 39.266 & 1472.28 & 1469.86 \\
\hline $11 / 7 / 112: 20$ & 36.454 & 39.257 & 1472.32 & 1469.87 \\
\hline $11 / 7 / 112: 30$ & 36.407 & 39.247 & 1472.36 & 1469.88 \\
\hline $11 / 7 / 112: 40$ & 36.379 & 39.24 & 1472.39 & 1469.88 \\
\hline $11 / 7 / 112: 50$ & 36.344 & 39.228 & 1472.43 & 1469.89 \\
\hline $11 / 7 / 113: 00$ & 36.318 & 39.221 & 1472.45 & 1469.90 \\
\hline $11 / 7 / 113: 10$ & 36.302 & 39.212 & 1472.47 & 1469.91 \\
\hline $11 / 7 / 113: 20$ & 36.323 & 39.205 & 1472.45 & 1469.92 \\
\hline $11 / 7 / 113: 30$ & 36.349 & 39.196 & 1472.42 & 1469.93 \\
\hline $11 / 7 / 113: 40$ & 36.309 & 39.189 & 1472.46 & 1469.93 \\
\hline $11 / 7 / 113: 50$ & 36.269 & 39.177 & 1472.50 & 1469.95 \\
\hline 11/7/11 4:00 & 36.231 & 39.17 & 1472.54 & 1469.95 \\
\hline 11/7/11 4:10 & 36.206 & 39.16 & 1472.56 & 1469.96 \\
\hline $11 / 7 / 114: 20$ & 36.18 & 39.153 & 1472.59 & 1469.97 \\
\hline $11 / 7 / 114: 30$ & 36.154 & 39.144 & 1472.62 & 1469.98 \\
\hline $11 / 7 / 114: 40$ & 36.128 & 39.135 & 1472.64 & 1469.99 \\
\hline $11 / 7 / 114: 50$ & 36.729 & 39.13 & 1472.04 & 1469.99 \\
\hline 11/7/11 5:00 & 36.201 & 39.123 & 1472.57 & 1470.00 \\
\hline 11/7/11 5:10 & 36.142 & 39.116 & 1472.63 & 1470.01 \\
\hline 11/7/11 5:20 & 36.109 & 39.107 & 1472.66 & 1470.02 \\
\hline 11/7/11 5:30 & 36.079 & 39.097 & 1472.69 & 1470.03 \\
\hline 11/7/11 5:40 & 36.053 & 39.088 & 1472.72 & 1470.03 \\
\hline 11/7/11 5:50 & 36.027 & 39.078 & 1472.74 & 1470.04 \\
\hline $11 / 7 / 116: 00$ & 36.004 & 39.069 & 1472.77 & 1470.05 \\
\hline $11 / 7 / 116: 10$ & 35.985 & 39.06 & 1472.78 & 1470.06 \\
\hline $11 / 7 / 116: 20$ & 35.971 & 39.052 & 1472.80 & 1470.07 \\
\hline $11 / 7 / 116: 30$ & 35.959 & 39.048 & 1472.81 & 1470.07 \\
\hline 11/7/11 6:40 & 35.957 & 39.038 & 1472.81 & 1470.08 \\
\hline
\end{tabular}


TABLE S1.3 Automatically recorded groundwater levels in the Klassen wells, September 15, 2011, to March 13, 2012.

\begin{tabular}{|c|c|c|c|c|}
\hline \multicolumn{3}{|c|}{ Reference Elevation (ft AMSL) } & 1508.77 & \multirow{2}{*}{$\begin{array}{l}1509.12 \\
\text { Elevation in Klassen } \\
\text { Test Well (ft AMSL) }\end{array}$} \\
\hline Date and Time & $\begin{array}{l}\text { Depth in Klassen } \\
\text { Domestic Well } \\
\text { (ft BGL) }\end{array}$ & $\begin{array}{c}\text { Depth in Klassen } \\
\text { Test Well } \\
\text { (ft BGL) }\end{array}$ & $\begin{array}{l}\text { Elevation in Klassen } \\
\text { Domestic Well } \\
\text { (ft AMSL) }\end{array}$ & \\
\hline $11 / 7 / 116: 50$ & 35.941 & 39.031 & 1472.83 & 1470.09 \\
\hline $11 / 7 / 117: 00$ & 35.966 & 39.024 & 1472.80 & 1470.10 \\
\hline $11 / 7 / 117: 10$ & 39.475 & 39.022 & 1469.29 & 1470.10 \\
\hline $11 / 7 / 117: 20$ & 36.152 & 39.015 & 1472.62 & 1470.11 \\
\hline $11 / 7 / 117: 30$ & 36.056 & 39.01 & 1472.71 & 1470.11 \\
\hline 11/7/11 7:40 & 36.011 & 39.003 & 1472.76 & 1470.12 \\
\hline $11 / 7 / 117: 50$ & 35.978 & 38.994 & 1472.79 & 1470.13 \\
\hline $11 / 7 / 118: 00$ & 35.962 & 38.991 & 1472.81 & 1470.13 \\
\hline $11 / 7 / 118: 10$ & 35.988 & 38.991 & 1472.78 & 1470.13 \\
\hline $11 / 7 / 118: 20$ & 39.433 & 38.989 & 1469.34 & 1470.13 \\
\hline $11 / 7 / 118: 30$ & 36.135 & 38.991 & 1472.63 & 1470.13 \\
\hline $11 / 7 / 118: 40$ & 36.051 & 38.985 & 1472.72 & 1470.14 \\
\hline $11 / 7 / 118: 50$ & 36.004 & 38.977 & 1472.77 & 1470.15 \\
\hline 11/7/11 9:00 & 35.966 & 38.968 & 1472.80 & 1470.15 \\
\hline 11/7/11 9:10 & 35.934 & 38.961 & 1472.84 & 1470.16 \\
\hline $11 / 7 / 11$ 9:20 & 36.518 & 38.956 & 1472.25 & 1470.17 \\
\hline $11 / 7 / 119: 30$ & 36.027 & 38.956 & 1472.74 & 1470.17 \\
\hline $11 / 7 / 11$ 9:40 & 35.964 & 38.952 & 1472.81 & 1470.17 \\
\hline 11/7/11 9:50 & 35.929 & 38.947 & 1472.84 & 1470.18 \\
\hline 11/7/11 10:00 & 36.128 & 38.942 & 1472.64 & 1470.18 \\
\hline 11/7/11 10:10 & 35.988 & 38.935 & 1472.78 & 1470.19 \\
\hline 11/7/11 10:20 & 35.934 & 38.935 & 1472.84 & 1470.19 \\
\hline 11/7/11 10:30 & 36.133 & 38.93 & 1472.64 & 1470.19 \\
\hline 11/7/11 10:40 & 35.997 & 38.923 & 1472.77 & 1470.20 \\
\hline 11/7/11 10:50 & 35.945 & 38.919 & 1472.82 & 1470.20 \\
\hline 11/7/11 11:00 & 35.908 & 38.916 & 1472.86 & 1470.21 \\
\hline 11/7/11 11:10 & 36.152 & 38.914 & 1472.62 & 1470.21 \\
\hline 11/7/11 11:20 & 35.976 & 38.909 & 1472.79 & 1470.21 \\
\hline 11/7/11 11:30 & 35.917 & 38.907 & 1472.85 & 1470.22 \\
\hline 11/7/11 11:40 & 35.875 & 38.898 & 1472.89 & 1470.22 \\
\hline 11/7/11 11:50 & 35.847 & 38.888 & 1472.92 & 1470.23 \\
\hline 11/7/11 12:00 & 35.826 & 38.879 & 1472.94 & 1470.24 \\
\hline 11/7/11 12:10 & 35.809 & 38.874 & 1472.96 & 1470.25 \\
\hline $11 / 7 / 11$ 12:20 & 35.791 & 38.867 & 1472.98 & 1470.26 \\
\hline 11/7/11 12:30 & 35.774 & 38.862 & 1473.00 & 1470.26 \\
\hline 11/7/11 12:40 & 37.657 & 38.858 & 1471.11 & 1470.26 \\
\hline $11 / 7 / 11$ 12:50 & 35.856 & 38.862 & 1472.91 & 1470.26 \\
\hline 11/7/11 13:00 & 35.791 & 38.867 & 1472.98 & 1470.26 \\
\hline
\end{tabular}


TABLE S1.3 Automatically recorded groundwater levels in the Klassen wells, September 15, 2011, to March 13, 2012.

\begin{tabular}{|c|c|c|c|c|}
\hline \multicolumn{3}{|c|}{ Reference Elevation (ft AMSL) } & 1508.77 & \multirow{2}{*}{$\begin{array}{l}1509.12 \\
\text { Elevation in Klassen } \\
\text { Test Well (ft AMSL) }\end{array}$} \\
\hline Date and Time & $\begin{array}{l}\text { Depth in Klassen } \\
\text { Domestic Well } \\
(\mathrm{ft} \mathrm{BGL})\end{array}$ & $\begin{array}{c}\text { Depth in Klassen } \\
\text { Test Well } \\
\text { (ft BGL) }\end{array}$ & $\begin{array}{l}\text { Elevation in Klassen } \\
\text { Domestic Well } \\
\text { (ft AMSL) }\end{array}$ & \\
\hline 11/7/11 13:10 & 35.755 & 38.867 & 1473.01 & 1470.26 \\
\hline $11 / 7 / 1113: 20$ & 35.725 & 38.855 & 1473.04 & 1470.27 \\
\hline 11/7/11 13:30 & 35.699 & 38.851 & 1473.07 & 1470.27 \\
\hline 11/7/11 13:40 & 35.68 & 38.841 & 1473.09 & 1470.28 \\
\hline 11/7/11 13:50 & 35.659 & 38.837 & 1473.11 & 1470.29 \\
\hline 11/7/11 14:00 & 36.25 & 38.827 & 1472.52 & 1470.30 \\
\hline 11/7/11 14:10 & 35.751 & 38.82 & 1473.02 & 1470.30 \\
\hline 11/7/11 14:20 & 35.692 & 38.816 & 1473.08 & 1470.31 \\
\hline 11/7/11 14:30 & 35.655 & 38.823 & 1473.11 & 1470.30 \\
\hline 11/7/11 14:40 & 35.631 & 38.823 & 1473.14 & 1470.30 \\
\hline $11 / 7 / 1114: 50$ & 35.819 & 38.83 & 1472.95 & 1470.29 \\
\hline 11/7/11 15:00 & 35.699 & 38.823 & 1473.07 & 1470.30 \\
\hline 11/7/11 15:10 & 35.65 & 38.82 & 1473.12 & 1470.30 \\
\hline $11 / 7 / 1115: 20$ & 36.527 & 38.818 & 1472.24 & 1470.30 \\
\hline 11/7/11 15:30 & 35.713 & 38.809 & 1473.06 & 1470.31 \\
\hline 11/7/11 15:40 & 35.655 & 38.769 & 1473.11 & 1470.35 \\
\hline $11 / 7 / 11$ 15:50 & 35.615 & 38.769 & 1473.15 & 1470.35 \\
\hline 11/7/11 16:00 & 35.586 & 38.78 & 1473.18 & 1470.34 \\
\hline 11/7/11 16:10 & 35.565 & 38.785 & 1473.20 & 1470.34 \\
\hline 11/7/11 16:20 & 35.551 & 38.787 & 1473.22 & 1470.34 \\
\hline $11 / 7 / 11$ 16:30 & 35.551 & 38.79 & 1473.22 & 1470.33 \\
\hline 11/7/11 16:40 & 35.537 & 38.79 & 1473.23 & 1470.33 \\
\hline 11/7/11 16:50 & 35.683 & 38.783 & 1473.09 & 1470.34 \\
\hline 11/7/11 17:00 & 35.603 & 38.783 & 1473.17 & 1470.34 \\
\hline 11/7/11 17:10 & 36.163 & 38.78 & 1472.61 & 1470.34 \\
\hline 11/7/11 17:20 & 35.659 & 38.783 & 1473.11 & 1470.34 \\
\hline 11/7/11 17:30 & 35.64 & 38.79 & 1473.13 & 1470.33 \\
\hline 11/7/11 17:40 & 35.662 & 38.792 & 1473.11 & 1470.33 \\
\hline 11/7/11 17:50 & 35.643 & 38.785 & 1473.13 & 1470.34 \\
\hline 11/7/11 18:00 & 35.687 & 38.792 & 1473.08 & 1470.33 \\
\hline 11/7/11 18:10 & 35.673 & 38.794 & 1473.10 & 1470.33 \\
\hline 11/7/11 18:20 & 35.713 & 38.799 & 1473.06 & 1470.32 \\
\hline 11/7/11 18:30 & 35.699 & 38.797 & 1473.07 & 1470.33 \\
\hline 11/7/11 18:40 & 35.715 & 38.792 & 1473.05 & 1470.33 \\
\hline 11/7/11 18:50 & 36.499 & 38.787 & 1472.27 & 1470.34 \\
\hline 11/7/11 19:00 & 35.884 & 38.787 & 1472.89 & 1470.34 \\
\hline 11/7/11 19:10 & 35.8 & 38.78 & 1472.97 & 1470.34 \\
\hline 11/7/11 19:20 & 35.751 & 38.776 & 1473.02 & 1470.35 \\
\hline
\end{tabular}


TABLE S1.3 Automatically recorded groundwater levels in the Klassen wells, September 15, 2011, to March 13, 2012.

\begin{tabular}{|c|c|c|c|c|}
\hline \multicolumn{3}{|c|}{ Reference Elevation (ft AMSL) } & 1508.77 & \multirow{2}{*}{$\begin{array}{l}1509.12 \\
\text { Elevation in Klassen } \\
\text { Test Well (ft AMSL) }\end{array}$} \\
\hline Date and Time & $\begin{array}{l}\text { Depth in Klassen } \\
\text { Domestic Well } \\
(\mathrm{ft} \mathrm{BGL})\end{array}$ & $\begin{array}{c}\text { Depth in Klassen } \\
\text { Test Well } \\
\text { (ft BGL) }\end{array}$ & $\begin{array}{l}\text { Elevation in Klassen } \\
\text { Domestic Well } \\
\text { (ft AMSL) }\end{array}$ & \\
\hline 11/7/11 19:30 & 35.723 & 38.773 & 1473.05 & 1470.35 \\
\hline 11/7/11 19:40 & 35.713 & 38.771 & 1473.06 & 1470.35 \\
\hline 11/7/11 19:50 & 35.678 & 38.769 & 1473.09 & 1470.35 \\
\hline 11/7/11 20:00 & 35.65 & 38.755 & 1473.12 & 1470.37 \\
\hline 11/7/11 20:10 & 35.629 & 38.755 & 1473.14 & 1470.37 \\
\hline $11 / 7 / 1120: 20$ & 35.608 & 38.757 & 1473.16 & 1470.37 \\
\hline $11 / 7 / 1120: 30$ & 38.194 & 38.757 & 1470.58 & 1470.37 \\
\hline $11 / 7 / 1120: 40$ & 35.741 & 38.759 & 1473.03 & 1470.36 \\
\hline $11 / 7 / 1120: 50$ & 35.683 & 38.759 & 1473.09 & 1470.36 \\
\hline 11/7/11 21:00 & 35.683 & 38.759 & 1473.09 & 1470.36 \\
\hline 11/7/11 21:10 & 35.655 & 38.757 & 1473.11 & 1470.37 \\
\hline $11 / 7 / 1121: 20$ & 35.626 & 38.755 & 1473.14 & 1470.37 \\
\hline 11/7/11 21:30 & 35.601 & 38.752 & 1473.17 & 1470.37 \\
\hline $11 / 7 / 1121: 40$ & 35.594 & 38.743 & 1473.18 & 1470.38 \\
\hline 11/7/11 21:50 & 35.601 & 38.733 & 1473.17 & 1470.39 \\
\hline $11 / 7 / 1122: 00$ & 35.657 & 38.729 & 1473.11 & 1470.39 \\
\hline 11/7/11 22:10 & 35.683 & 38.733 & 1473.09 & 1470.39 \\
\hline $11 / 7 / 1122: 20$ & 35.69 & 38.733 & 1473.08 & 1470.39 \\
\hline 11/7/11 22:30 & 35.685 & 38.733 & 1473.08 & 1470.39 \\
\hline $11 / 7 / 1122: 40$ & 35.659 & 38.733 & 1473.11 & 1470.39 \\
\hline $11 / 7 / 1122: 50$ & 36.056 & 38.736 & 1472.71 & 1470.39 \\
\hline 11/7/11 23:00 & 35.772 & 38.736 & 1473.00 & 1470.39 \\
\hline 11/7/11 23:10 & 35.685 & 38.729 & 1473.08 & 1470.39 \\
\hline 11/7/11 23:20 & 35.647 & 38.724 & 1473.12 & 1470.40 \\
\hline 11/7/11 23:30 & 35.591 & 38.724 & 1473.18 & 1470.40 \\
\hline 11/7/11 23:40 & 35.95 & 38.717 & 1472.82 & 1470.41 \\
\hline 11/7/11 23:50 & 35.8 & 38.719 & 1472.97 & 1470.40 \\
\hline 11/8/11 0:00 & 35.647 & 38.719 & 1473.12 & 1470.40 \\
\hline 11/8/11 0:10 & 36.135 & 38.719 & 1472.63 & 1470.40 \\
\hline 11/8/11 0:20 & 35.844 & 38.722 & 1472.93 & 1470.40 \\
\hline 11/8/11 0:30 & 35.662 & 38.724 & 1473.11 & 1470.40 \\
\hline $11 / 8 / 110: 40$ & 35.577 & 38.712 & 1473.19 & 1470.41 \\
\hline 11/8/11 0:50 & 35.516 & 38.712 & 1473.25 & 1470.41 \\
\hline 11/8/11 1:00 & 35.469 & 38.703 & 1473.30 & 1470.42 \\
\hline $11 / 8 / 11$ 1:10 & 35.429 & 38.698 & 1473.34 & 1470.42 \\
\hline $11 / 8 / 11$ 1:20 & 35.394 & 38.691 & 1473.38 & 1470.43 \\
\hline $11 / 8 / 11$ 1:30 & 35.361 & 38.684 & 1473.41 & 1470.44 \\
\hline $11 / 8 / 11$ 1:40 & 35.331 & 38.679 & 1473.44 & 1470.44 \\
\hline
\end{tabular}


TABLE S1.3 Automatically recorded groundwater levels in the Klassen wells, September 15, 2011, to March 13, 2012.

\begin{tabular}{|c|c|c|c|c|}
\hline \multicolumn{3}{|c|}{ Reference Elevation (ft AMSL) } & 1508.77 & \multirow{2}{*}{$\begin{array}{l}1509.12 \\
\text { Elevation in Klassen } \\
\text { Test Well (ft AMSL) }\end{array}$} \\
\hline Date and Time & $\begin{array}{l}\text { Depth in Klassen } \\
\text { Domestic Well } \\
(\mathrm{ft} \mathrm{BGL})\end{array}$ & $\begin{array}{c}\text { Depth in Klassen } \\
\text { Test Well } \\
\text { (ft BGL) }\end{array}$ & $\begin{array}{l}\text { Elevation in Klassen } \\
\text { Domestic Well } \\
\text { (ft AMSL) }\end{array}$ & \\
\hline $11 / 8 / 111: 50$ & 35.3 & 38.679 & 1473.47 & 1470.44 \\
\hline $11 / 8 / 112: 00$ & 35.275 & 38.679 & 1473.49 & 1470.44 \\
\hline $11 / 8 / 112: 10$ & 35.249 & 38.679 & 1473.52 & 1470.44 \\
\hline $11 / 8 / 112: 20$ & 35.225 & 38.677 & 1473.54 & 1470.45 \\
\hline $11 / 8 / 112: 30$ & 35.202 & 38.682 & 1473.57 & 1470.44 \\
\hline $11 / 8 / 112: 40$ & 35.178 & 38.682 & 1473.59 & 1470.44 \\
\hline $11 / 8 / 112: 50$ & 35.157 & 38.684 & 1473.61 & 1470.44 \\
\hline $11 / 8 / 113: 00$ & 35.139 & 38.682 & 1473.63 & 1470.44 \\
\hline $11 / 8 / 113: 10$ & 35.122 & 38.668 & 1473.65 & 1470.45 \\
\hline $11 / 8 / 113: 20$ & 35.106 & 38.654 & 1473.66 & 1470.47 \\
\hline $11 / 8 / 113: 30$ & 35.089 & 38.649 & 1473.68 & 1470.47 \\
\hline $11 / 8 / 113: 40$ & 35.073 & 38.637 & 1473.70 & 1470.49 \\
\hline $11 / 8 / 113: 50$ & 35.059 & 38.63 & 1473.71 & 1470.49 \\
\hline $11 / 8 / 114: 00$ & 35.235 & 38.625 & 1473.53 & 1470.50 \\
\hline $11 / 8 / 114: 10$ & 35.124 & 38.63 & 1473.65 & 1470.49 \\
\hline 11/8/11 4:20 & 35.082 & 38.623 & 1473.69 & 1470.50 \\
\hline $11 / 8 / 114: 30$ & 35.056 & 38.614 & 1473.71 & 1470.51 \\
\hline 11/8/11 4:40 & 35.033 & 38.619 & 1473.74 & 1470.50 \\
\hline $11 / 8 / 114: 50$ & 35.012 & 38.616 & 1473.76 & 1470.51 \\
\hline $11 / 8 / 11$ 5:00 & 34.998 & 38.609 & 1473.77 & 1470.51 \\
\hline $11 / 8 / 115: 10$ & 34.977 & 38.602 & 1473.79 & 1470.52 \\
\hline $11 / 8 / 11$ 5:20 & 34.995 & 38.6 & 1473.77 & 1470.52 \\
\hline 11/8/11 5:30 & 34.977 & 38.595 & 1473.79 & 1470.53 \\
\hline $11 / 8 / 115: 40$ & 34.958 & 38.597 & 1473.81 & 1470.53 \\
\hline $11 / 8 / 11$ 5:50 & 34.942 & 38.59 & 1473.83 & 1470.53 \\
\hline $11 / 8 / 116: 00$ & 34.927 & 38.583 & 1473.84 & 1470.54 \\
\hline $11 / 8 / 116: 10$ & 34.913 & 38.581 & 1473.86 & 1470.54 \\
\hline $11 / 8 / 116: 20$ & 34.899 & 38.576 & 1473.87 & 1470.55 \\
\hline 11/8/11 6:30 & 34.883 & 38.574 & 1473.89 & 1470.55 \\
\hline 11/8/11 6:40 & 34.874 & 38.569 & 1473.90 & 1470.55 \\
\hline $11 / 8 / 116: 50$ & 34.862 & 38.564 & 1473.91 & 1470.56 \\
\hline $11 / 8 / 11$ 7:00 & 34.876 & 38.557 & 1473.89 & 1470.57 \\
\hline $11 / 8 / 117: 10$ & 36.14 & 38.564 & 1472.63 & 1470.56 \\
\hline $11 / 8 / 117: 20$ & 35.141 & 38.564 & 1473.63 & 1470.56 \\
\hline $11 / 8 / 117: 30$ & 35.059 & 38.564 & 1473.71 & 1470.56 \\
\hline $11 / 8 / 11$ 7:40 & 35.028 & 38.56 & 1473.74 & 1470.56 \\
\hline $11 / 8 / 117: 50$ & 35 & 38.562 & 1473.77 & 1470.56 \\
\hline $11 / 8 / 118: 00$ & 34.967 & 38.557 & 1473.80 & 1470.57 \\
\hline
\end{tabular}


TABLE S1.3 Automatically recorded groundwater levels in the Klassen wells, September 15, 2011, to March 13, 2012.

\begin{tabular}{|c|c|c|c|c|}
\hline \multicolumn{3}{|c|}{ Reference Elevation (ft AMSL) } & 1508.77 & \multirow{2}{*}{$\begin{array}{l}1509.12 \\
\text { Elevation in Klassen } \\
\text { Test Well (ft AMSL) }\end{array}$} \\
\hline Date and Time & $\begin{array}{l}\text { Depth in Klassen } \\
\text { Domestic Well } \\
\text { (ft BGL) }\end{array}$ & $\begin{array}{c}\text { Depth in Klassen } \\
\text { Test Well } \\
\text { (ft BGL) }\end{array}$ & $\begin{array}{l}\text { Elevation in Klassen } \\
\text { Domestic Well } \\
\text { (ft AMSL) }\end{array}$ & \\
\hline 11/8/11 8:10 & 34.967 & 38.553 & 1473.80 & 1470.57 \\
\hline $11 / 8 / 118: 20$ & 34.998 & 38.555 & 1473.77 & 1470.57 \\
\hline 11/8/11 8:30 & 35.01 & 38.553 & 1473.76 & 1470.57 \\
\hline $11 / 8 / 118: 40$ & 34.993 & 38.548 & 1473.78 & 1470.57 \\
\hline $11 / 8 / 118: 50$ & 34.965 & 38.548 & 1473.80 & 1470.57 \\
\hline 11/8/11 9:00 & 34.939 & 38.546 & 1473.83 & 1470.58 \\
\hline 11/8/11 9:10 & 34.918 & 38.534 & 1473.85 & 1470.59 \\
\hline $11 / 8 / 119: 20$ & 34.897 & 38.529 & 1473.87 & 1470.59 \\
\hline 11/8/11 9:30 & 34.876 & 38.529 & 1473.89 & 1470.59 \\
\hline 11/8/11 9:40 & 34.857 & 38.529 & 1473.91 & 1470.59 \\
\hline 11/8/11 9:50 & 35.389 & 38.525 & 1473.38 & 1470.60 \\
\hline 11/8/11 10:00 & 34.935 & 38.525 & 1473.83 & 1470.60 \\
\hline 11/8/11 10:10 & 34.885 & 38.525 & 1473.88 & 1470.60 \\
\hline $11 / 8 / 1110: 20$ & 34.855 & 38.52 & 1473.91 & 1470.60 \\
\hline 11/8/11 10:30 & 34.834 & 38.515 & 1473.94 & 1470.61 \\
\hline 11/8/11 10:40 & 34.813 & 38.515 & 1473.96 & 1470.61 \\
\hline $11 / 8 / 11$ 10:50 & 35.373 & 38.508 & 1473.40 & 1470.61 \\
\hline 11/8/11 11:00 & 34.958 & 38.506 & 1473.81 & 1470.62 \\
\hline 11/8/11 11:10 & 34.885 & 38.518 & 1473.88 & 1470.60 \\
\hline $11 / 8 / 1111: 20$ & 35.028 & 38.508 & 1473.74 & 1470.61 \\
\hline 11/8/11 11:30 & 34.913 & 38.503 & 1473.86 & 1470.62 \\
\hline 11/8/11 11:40 & 36.316 & 38.506 & 1472.45 & 1470.62 \\
\hline 11/8/11 11:50 & 35.263 & 38.503 & 1473.51 & 1470.62 \\
\hline 11/8/11 12:00 & 35.087 & 38.501 & 1473.68 & 1470.62 \\
\hline 11/8/11 12:10 & 35.178 & 38.51 & 1473.59 & 1470.61 \\
\hline $11 / 8 / 11$ 12:20 & 35.042 & 38.506 & 1473.73 & 1470.62 \\
\hline 11/8/11 12:30 & 34.977 & 38.506 & 1473.79 & 1470.62 \\
\hline 11/8/11 12:40 & 34.932 & 38.501 & 1473.84 & 1470.62 \\
\hline $11 / 8 / 11$ 12:50 & 34.897 & 38.501 & 1473.87 & 1470.62 \\
\hline 11/8/11 13:00 & 34.866 & 38.506 & 1473.90 & 1470.62 \\
\hline 11/8/11 13:10 & 34.841 & 38.511 & 1473.93 & 1470.61 \\
\hline 11/8/11 13:20 & 34.817 & 38.501 & 1473.95 & 1470.62 \\
\hline 11/8/11 13:30 & 34.977 & 38.503 & 1473.79 & 1470.62 \\
\hline 11/8/11 13:40 & 34.869 & 38.496 & 1473.90 & 1470.63 \\
\hline 11/8/11 13:50 & 34.829 & 38.487 & 1473.94 & 1470.64 \\
\hline 11/8/11 14:00 & 34.801 & 38.473 & 1473.97 & 1470.65 \\
\hline 11/8/11 14:10 & 34.777 & 38.461 & 1473.99 & 1470.66 \\
\hline 11/8/11 14:20 & 34.761 & 38.435 & 1474.01 & 1470.69 \\
\hline
\end{tabular}


TABLE S1.3 Automatically recorded groundwater levels in the Klassen wells, September 15, 2011, to March 13, 2012.

\begin{tabular}{|c|c|c|c|c|}
\hline \multicolumn{3}{|c|}{ Reference Elevation (ft AMSL) } & 1508.77 & \multirow{2}{*}{$\begin{array}{l}1509.12 \\
\text { Elevation in Klassen } \\
\text { Test Well (ft AMSL) }\end{array}$} \\
\hline Date and Time & $\begin{array}{l}\text { Depth in Klassen } \\
\text { Domestic Well } \\
(\mathrm{ft} \mathrm{BGL})\end{array}$ & $\begin{array}{c}\text { Depth in Klassen } \\
\text { Test Well } \\
\text { (ft BGL) }\end{array}$ & $\begin{array}{l}\text { Elevation in Klassen } \\
\text { Domestic Well } \\
\text { (ft AMSL) }\end{array}$ & \\
\hline $11 / 8 / 1114: 30$ & 34.92 & 38.421 & 1473.85 & 1470.70 \\
\hline $11 / 8 / 1114: 40$ & 34.82 & 38.433 & 1473.95 & 1470.69 \\
\hline $11 / 8 / 1114: 50$ & 34.78 & 38.419 & 1473.99 & 1470.70 \\
\hline $11 / 8 / 1115: 00$ & 34.754 & 38.417 & 1474.02 & 1470.71 \\
\hline 11/8/11 15:10 & 34.984 & 38.412 & 1473.79 & 1470.71 \\
\hline $11 / 8 / 1115: 20$ & 34.852 & 38.4 & 1473.92 & 1470.72 \\
\hline $11 / 8 / 1115: 30$ & 34.796 & 38.4 & 1473.97 & 1470.72 \\
\hline $11 / 8 / 11$ 15:40 & 34.763 & 38.396 & 1474.01 & 1470.73 \\
\hline $11 / 8 / 11$ 15:50 & 34.738 & 38.391 & 1474.03 & 1470.73 \\
\hline $11 / 8 / 1116: 00$ & 34.714 & 38.393 & 1474.06 & 1470.73 \\
\hline 11/8/11 16:10 & 34.89 & 38.388 & 1473.88 & 1470.73 \\
\hline $11 / 8 / 11$ 16:20 & 34.775 & 38.388 & 1473.99 & 1470.73 \\
\hline $11 / 8 / 1116: 30$ & 34.733 & 38.382 & 1474.04 & 1470.74 \\
\hline $11 / 8 / 1116: 40$ & 34.705 & 38.379 & 1474.06 & 1470.74 \\
\hline 11/8/11 16:50 & 34.686 & 38.372 & 1474.08 & 1470.75 \\
\hline 11/8/11 17:00 & 34.902 & 38.367 & 1473.87 & 1470.76 \\
\hline 11/8/11 17:10 & 34.759 & 38.358 & 1474.01 & 1470.76 \\
\hline 11/8/11 17:20 & 34.714 & 38.346 & 1474.06 & 1470.78 \\
\hline 11/8/11 17:30 & 34.688 & 38.344 & 1474.08 & 1470.78 \\
\hline 11/8/11 17:40 & 34.665 & 38.342 & 1474.10 & 1470.78 \\
\hline $11 / 8 / 11$ 17:50 & 34.644 & 38.339 & 1474.13 & 1470.78 \\
\hline 11/8/11 18:00 & 34.63 & 38.327 & 1474.14 & 1470.80 \\
\hline 11/8/11 18:10 & 34.613 & 38.32 & 1474.16 & 1470.80 \\
\hline $11 / 8 / 11$ 18:20 & 34.601 & 38.313 & 1474.17 & 1470.81 \\
\hline 11/8/11 18:30 & 34.587 & 38.309 & 1474.18 & 1470.81 \\
\hline 11/8/11 18:40 & 34.576 & 38.306 & 1474.19 & 1470.82 \\
\hline 11/8/11 18:50 & 35.741 & 38.304 & 1473.03 & 1470.82 \\
\hline 11/8/11 19:00 & 34.684 & 38.299 & 1474.09 & 1470.82 \\
\hline 11/8/11 19:10 & 34.627 & 38.288 & 1474.14 & 1470.83 \\
\hline 11/8/11 19:20 & 34.601 & 38.278 & 1474.17 & 1470.84 \\
\hline 11/8/11 19:30 & 34.58 & 38.271 & 1474.19 & 1470.85 \\
\hline 11/8/11 19:40 & 34.564 & 38.264 & 1474.21 & 1470.86 \\
\hline 11/8/11 19:50 & 34.552 & 38.259 & 1474.22 & 1470.86 \\
\hline 11/8/11 20:00 & 34.541 & 38.252 & 1474.23 & 1470.87 \\
\hline 11/8/11 20:10 & 35.064 & 38.248 & 1473.71 & 1470.87 \\
\hline $11 / 8 / 1120: 20$ & 34.625 & 38.243 & 1474.14 & 1470.88 \\
\hline 11/8/11 20:30 & 38.117 & 38.236 & 1470.65 & 1470.89 \\
\hline 11/8/11 20:40 & 34.705 & 38.229 & 1474.06 & 1470.89 \\
\hline
\end{tabular}


TABLE S1.3 Automatically recorded groundwater levels in the Klassen wells, September 15, 2011, to March 13, 2012.

\begin{tabular}{|c|c|c|c|c|}
\hline \multicolumn{3}{|c|}{ Reference Elevation (ft AMSL) } & 1508.77 & \multirow{2}{*}{$\begin{array}{l}1509.12 \\
\text { Elevation in Klassen } \\
\text { Test Well (ft AMSL) }\end{array}$} \\
\hline Date and Time & $\begin{array}{l}\text { Depth in Klassen } \\
\text { Domestic Well } \\
\text { (ft BGL) }\end{array}$ & $\begin{array}{c}\text { Depth in Klassen } \\
\text { Test Well } \\
\text { (ft BGL) }\end{array}$ & $\begin{array}{l}\text { Elevation in Klassen } \\
\text { Domestic Well } \\
\text { (ft AMSL) }\end{array}$ & \\
\hline $11 / 8 / 1120: 50$ & 34.625 & 38.222 & 1474.14 & 1470.90 \\
\hline $11 / 8 / 1121: 00$ & 34.59 & 38.215 & 1474.18 & 1470.91 \\
\hline 11/8/11 21:10 & 34.927 & 38.208 & 1473.84 & 1470.91 \\
\hline $11 / 8 / 1121: 20$ & 34.658 & 38.201 & 1474.11 & 1470.92 \\
\hline 11/8/11 21:30 & 34.604 & 38.196 & 1474.17 & 1470.93 \\
\hline $11 / 8 / 1121: 40$ & 34.571 & 38.189 & 1474.20 & 1470.93 \\
\hline $11 / 8 / 1121: 50$ & 34.548 & 38.184 & 1474.22 & 1470.94 \\
\hline $11 / 8 / 1122: 00$ & 34.529 & 38.177 & 1474.24 & 1470.95 \\
\hline $11 / 8 / 1122: 10$ & 34.512 & 38.175 & 1474.26 & 1470.95 \\
\hline $11 / 8 / 1122: 20$ & 34.501 & 38.17 & 1474.27 & 1470.95 \\
\hline $11 / 8 / 1122: 30$ & 34.491 & 38.163 & 1474.28 & 1470.96 \\
\hline $11 / 8 / 1122: 40$ & 34.484 & 38.159 & 1474.29 & 1470.96 \\
\hline $11 / 8 / 1122: 50$ & 34.501 & 38.156 & 1474.27 & 1470.97 \\
\hline $11 / 8 / 1123: 00$ & 34.489 & 38.149 & 1474.28 & 1470.97 \\
\hline 11/8/11 23:10 & 36.965 & 38.147 & 1471.80 & 1470.98 \\
\hline $11 / 8 / 1123: 20$ & 34.606 & 38.144 & 1474.16 & 1470.98 \\
\hline $11 / 8 / 1123: 30$ & 34.543 & 38.142 & 1474.23 & 1470.98 \\
\hline 11/8/11 23:40 & 34.986 & 38.137 & 1473.78 & 1470.99 \\
\hline $11 / 8 / 1123: 50$ & 34.817 & 38.137 & 1473.95 & 1470.99 \\
\hline 11/9/11 0:00 & 37.097 & 38.137 & 1471.67 & 1470.99 \\
\hline 11/9/11 0:10 & 35.064 & 38.138 & 1473.71 & 1470.98 \\
\hline $11 / 9 / 11$ 0:20 & 34.796 & 38.138 & 1473.97 & 1470.98 \\
\hline 11/9/11 0:30 & 34.714 & 38.135 & 1474.06 & 1470.99 \\
\hline $11 / 9 / 110: 40$ & 34.663 & 38.13 & 1474.11 & 1470.99 \\
\hline $11 / 9 / 110: 50$ & 34.625 & 38.126 & 1474.14 & 1471.00 \\
\hline $11 / 9 / 11$ 1:00 & 34.597 & 38.121 & 1474.17 & 1471.00 \\
\hline $11 / 9 / 11$ 1:10 & 34.573 & 38.114 & 1474.20 & 1471.01 \\
\hline 11/9/11 1:20 & 35.35 & 38.112 & 1473.42 & 1471.01 \\
\hline $11 / 9 / 11$ 1:30 & 34.651 & 38.112 & 1474.12 & 1471.01 \\
\hline 11/9/11 1:40 & 34.597 & 38.109 & 1474.17 & 1471.01 \\
\hline 11/9/11 1:50 & 34.564 & 38.105 & 1474.21 & 1471.02 \\
\hline $11 / 9 / 112: 00$ & 34.541 & 38.1 & 1474.23 & 1471.02 \\
\hline $11 / 9 / 112: 10$ & 34.519 & 38.095 & 1474.25 & 1471.03 \\
\hline $11 / 9 / 112: 20$ & 34.505 & 38.093 & 1474.26 & 1471.03 \\
\hline $11 / 9 / 112: 30$ & 34.489 & 38.088 & 1474.28 & 1471.03 \\
\hline $11 / 9 / 112: 40$ & 34.475 & 38.083 & 1474.29 & 1471.04 \\
\hline $11 / 9 / 112: 50$ & 34.463 & 38.076 & 1474.31 & 1471.05 \\
\hline 11/9/11 3:00 & 34.451 & 38.069 & 1474.32 & 1471.05 \\
\hline
\end{tabular}


TABLE S1.3 Automatically recorded groundwater levels in the Klassen wells, September 15, 2011, to March 13, 2012.

\begin{tabular}{|c|c|c|c|c|}
\hline \multicolumn{3}{|c|}{ Reference Elevation (ft AMSL) } & 1508.77 & \multirow{2}{*}{$\begin{array}{l}1509.12 \\
\text { Elevation in Klassen } \\
\text { Test Well (ft AMSL) }\end{array}$} \\
\hline Date and Time & $\begin{array}{l}\text { Depth in Klassen } \\
\text { Domestic Well } \\
\text { (ft BGL) }\end{array}$ & $\begin{array}{c}\text { Depth in Klassen } \\
\text { Test Well } \\
\text { (ft BGL) }\end{array}$ & $\begin{array}{l}\text { Elevation in Klassen } \\
\text { Domestic Well } \\
\text { (ft AMSL) }\end{array}$ & \\
\hline $11 / 9 / 113: 10$ & 35.833 & 38.067 & 1472.94 & 1471.06 \\
\hline $11 / 9 / 113: 20$ & 34.578 & 38.062 & 1474.19 & 1471.06 \\
\hline $11 / 9 / 113: 30$ & 34.515 & 38.06 & 1474.25 & 1471.06 \\
\hline $11 / 9 / 113: 40$ & 34.487 & 38.051 & 1474.28 & 1471.07 \\
\hline $11 / 9 / 113: 50$ & 34.463 & 38.048 & 1474.31 & 1471.07 \\
\hline $11 / 9 / 114: 00$ & 34.447 & 38.048 & 1474.32 & 1471.07 \\
\hline $11 / 9 / 114: 10$ & 34.433 & 38.044 & 1474.34 & 1471.08 \\
\hline $11 / 9 / 114: 20$ & 34.421 & 38.037 & 1474.35 & 1471.09 \\
\hline $11 / 9 / 114: 30$ & 34.407 & 38.032 & 1474.36 & 1471.09 \\
\hline $11 / 9 / 114: 40$ & 34.397 & 38.03 & 1474.37 & 1471.09 \\
\hline $11 / 9 / 114: 50$ & 34.388 & 38.027 & 1474.38 & 1471.10 \\
\hline $11 / 9 / 115: 00$ & 34.379 & 38.02 & 1474.39 & 1471.10 \\
\hline $11 / 9 / 115: 10$ & 34.367 & 38.018 & 1474.40 & 1471.10 \\
\hline $11 / 9 / 115: 20$ & 34.36 & 38.011 & 1474.41 & 1471.11 \\
\hline $11 / 9 / 115: 30$ & 34.353 & 38.006 & 1474.42 & 1471.12 \\
\hline $11 / 9 / 115: 40$ & 34.344 & 38.001 & 1474.43 & 1471.12 \\
\hline $11 / 9 / 115: 50$ & 34.336 & 37.997 & 1474.43 & 1471.13 \\
\hline $11 / 9 / 116: 00$ & 34.332 & 37.994 & 1474.44 & 1471.13 \\
\hline $11 / 9 / 116: 10$ & 34.325 & 37.99 & 1474.44 & 1471.13 \\
\hline $11 / 9 / 116: 20$ & 34.315 & 37.987 & 1474.45 & 1471.14 \\
\hline $11 / 9 / 116: 30$ & 34.311 & 37.983 & 1474.46 & 1471.14 \\
\hline $11 / 9 / 116: 40$ & 34.304 & 37.98 & 1474.47 & 1471.14 \\
\hline 11/9/11 6:50 & 34.297 & 37.973 & 1474.47 & 1471.15 \\
\hline $11 / 9 / 11$ 7:00 & 34.292 & 37.971 & 1474.48 & 1471.15 \\
\hline 11/9/11 7:10 & 34.972 & 37.969 & 1473.80 & 1471.15 \\
\hline $11 / 9 / 117: 20$ & 34.775 & 37.969 & 1473.99 & 1471.15 \\
\hline $11 / 9 / 11$ 7:30 & 34.498 & 37.966 & 1474.27 & 1471.16 \\
\hline 11/9/11 7:40 & 34.44 & 37.964 & 1474.33 & 1471.16 \\
\hline 11/9/11 7:50 & 34.407 & 37.959 & 1474.36 & 1471.16 \\
\hline 11/9/11 8:00 & 34.383 & 37.954 & 1474.39 & 1471.17 \\
\hline 11/9/11 8:10 & 34.395 & 37.952 & 1474.37 & 1471.17 \\
\hline $11 / 9 / 118: 20$ & 34.428 & 37.952 & 1474.34 & 1471.17 \\
\hline $11 / 9 / 118: 30$ & 34.412 & 37.947 & 1474.36 & 1471.18 \\
\hline $11 / 9 / 118: 40$ & 34.393 & 37.943 & 1474.38 & 1471.18 \\
\hline $11 / 9 / 118: 50$ & 34.374 & 37.94 & 1474.40 & 1471.18 \\
\hline 11/9/11 9:00 & 34.36 & 37.938 & 1474.41 & 1471.18 \\
\hline 11/9/11 9:10 & 34.348 & 37.938 & 1474.42 & 1471.18 \\
\hline 11/9/11 9:20 & 34.336 & 37.933 & 1474.43 & 1471.19 \\
\hline
\end{tabular}


TABLE S1.3 Automatically recorded groundwater levels in the Klassen wells, September 15, 2011, to March 13, 2012.

\begin{tabular}{|c|c|c|c|c|}
\hline \multicolumn{3}{|c|}{ Reference Elevation (ft AMSL) } & 1508.77 & \multirow{2}{*}{$\begin{array}{l}1509.12 \\
\text { Elevation in Klassen } \\
\text { Test Well (ft AMSL) }\end{array}$} \\
\hline Date and Time & $\begin{array}{l}\text { Depth in Klassen } \\
\text { Domestic Well } \\
(\mathrm{ft} \mathrm{BGL})\end{array}$ & $\begin{array}{c}\text { Depth in Klassen } \\
\text { Test Well } \\
\text { (ft BGL) }\end{array}$ & $\begin{array}{l}\text { Elevation in Klassen } \\
\text { Domestic Well } \\
\text { (ft AMSL) }\end{array}$ & \\
\hline $11 / 9 / 119: 30$ & 34.325 & 37.933 & 1474.44 & 1471.19 \\
\hline $11 / 9 / 119: 40$ & 34.315 & 37.929 & 1474.45 & 1471.19 \\
\hline 11/9/11 9:50 & 34.48 & 37.926 & 1474.29 & 1471.20 \\
\hline 11/9/11 10:00 & 34.39 & 37.922 & 1474.38 & 1471.20 \\
\hline 11/9/11 10:10 & 34.358 & 37.917 & 1474.41 & 1471.21 \\
\hline $11 / 9 / 11$ 10:20 & 34.339 & 37.915 & 1474.43 & 1471.21 \\
\hline $11 / 9 / 11$ 10:30 & 34.322 & 37.912 & 1474.45 & 1471.21 \\
\hline 11/9/11 10:40 & 34.311 & 37.91 & 1474.46 & 1471.21 \\
\hline $11 / 9 / 1110: 50$ & 34.953 & 37.908 & 1473.82 & 1471.21 \\
\hline 11/9/11 11:00 & 34.407 & 37.91 & 1474.36 & 1471.21 \\
\hline 11/9/11 11:10 & 34.362 & 37.91 & 1474.41 & 1471.21 \\
\hline 11/9/11 11:20 & 34.336 & 37.912 & 1474.43 & 1471.21 \\
\hline 11/9/11 11:30 & 34.32 & 37.917 & 1474.45 & 1471.21 \\
\hline 11/9/11 11:40 & 34.306 & 37.917 & 1474.46 & 1471.21 \\
\hline 11/9/11 11:50 & 34.669 & 37.922 & 1474.10 & 1471.20 \\
\hline 11/9/11 12:00 & 34.433 & 37.926 & 1474.34 & 1471.20 \\
\hline 11/9/11 12:10 & 34.381 & 37.926 & 1474.39 & 1471.20 \\
\hline $11 / 9 / 11$ 12:20 & 34.351 & 37.929 & 1474.42 & 1471.19 \\
\hline 11/9/11 12:30 & 34.332 & 37.931 & 1474.44 & 1471.19 \\
\hline 11/9/11 12:40 & 34.315 & 37.936 & 1474.45 & 1471.19 \\
\hline $11 / 9 / 1112: 50$ & 34.301 & 37.938 & 1474.47 & 1471.18 \\
\hline 11/9/11 13:00 & 38.3 & 37.938 & 1470.47 & 1471.18 \\
\hline 11/9/11 13:10 & 34.458 & 37.938 & 1474.31 & 1471.18 \\
\hline 11/9/11 13:20 & 34.381 & 37.929 & 1474.39 & 1471.19 \\
\hline 11/9/11 13:30 & 34.346 & 37.915 & 1474.42 & 1471.21 \\
\hline 11/9/11 13:40 & 34.322 & 37.898 & 1474.45 & 1471.22 \\
\hline 11/9/11 13:50 & 34.306 & 37.877 & 1474.46 & 1471.25 \\
\hline 11/9/11 14:00 & 34.292 & 37.856 & 1474.48 & 1471.27 \\
\hline 11/9/11 14:10 & 34.484 & 37.828 & 1474.29 & 1471.29 \\
\hline 11/9/11 14:20 & 34.374 & 37.797 & 1474.40 & 1471.33 \\
\hline 11/9/11 14:30 & 34.334 & 37.767 & 1474.44 & 1471.36 \\
\hline 11/9/11 14:40 & 34.311 & 37.727 & 1474.46 & 1471.40 \\
\hline $11 / 9 / 11$ 14:50 & 34.294 & 37.694 & 1474.48 & 1471.43 \\
\hline 11/9/11 15:00 & 34.304 & 37.666 & 1474.47 & 1471.46 \\
\hline 11/9/11 15:10 & 34.463 & 37.642 & 1474.31 & 1471.48 \\
\hline $11 / 9 / 11$ 15:20 & 34.374 & 37.624 & 1474.40 & 1471.50 \\
\hline 11/9/11 15:30 & 34.332 & 37.61 & 1474.44 & 1471.51 \\
\hline 11/9/11 15:40 & 34.313 & 37.598 & 1474.46 & 1471.52 \\
\hline
\end{tabular}


TABLE S1.3 Automatically recorded groundwater levels in the Klassen wells, September 15, 2011, to March 13, 2012.

\begin{tabular}{|c|c|c|c|c|}
\hline \multicolumn{3}{|c|}{ Reference Elevation (ft AMSL) } & 1508.77 & \multirow{2}{*}{$\begin{array}{l}1509.12 \\
\text { Elevation in Klassen } \\
\text { Test Well (ft AMSL) }\end{array}$} \\
\hline Date and Time & $\begin{array}{l}\text { Depth in Klassen } \\
\text { Domestic Well } \\
\text { (ft BGL) }\end{array}$ & $\begin{array}{c}\text { Depth in Klassen } \\
\text { Test Well } \\
\text { (ft BGL) }\end{array}$ & $\begin{array}{l}\text { Elevation in Klassen } \\
\text { Domestic Well } \\
\text { (ft AMSL) }\end{array}$ & \\
\hline $11 / 9 / 1115: 50$ & 34.294 & 37.598 & 1474.48 & 1471.52 \\
\hline $11 / 9 / 1116: 00$ & 34.28 & 37.607 & 1474.49 & 1471.52 \\
\hline 11/9/11 16:10 & 34.268 & 37.631 & 1474.50 & 1471.49 \\
\hline $11 / 9 / 1116: 20$ & 34.259 & 37.666 & 1474.51 & 1471.46 \\
\hline 11/9/11 16:30 & 34.768 & 37.713 & 1474.00 & 1471.41 \\
\hline 11/9/11 16:40 & 34.372 & 37.769 & 1474.40 & 1471.35 \\
\hline $11 / 9 / 1116: 50$ & 34.322 & 37.835 & 1474.45 & 1471.29 \\
\hline 11/9/11 17:00 & 34.299 & 37.903 & 1474.47 & 1471.22 \\
\hline 11/9/11 17:10 & 34.278 & 37.98 & 1474.49 & 1471.14 \\
\hline $11 / 9 / 1117: 20$ & 38.164 & 38.065 & 1470.61 & 1471.06 \\
\hline $11 / 9 / 1117: 30$ & 34.402 & 38.154 & 1474.37 & 1470.97 \\
\hline 11/9/11 17:40 & 34.336 & 38.248 & 1474.43 & 1470.87 \\
\hline $11 / 9 / 1117: 50$ & 36.923 & 38.342 & 1471.85 & 1470.78 \\
\hline 11/9/11 18:00 & 34.555 & 38.433 & 1474.21 & 1470.69 \\
\hline 11/9/11 18:10 & 34.503 & 38.515 & 1474.27 & 1470.61 \\
\hline 11/9/11 18:20 & 35.237 & 38.588 & 1473.53 & 1470.53 \\
\hline 11/9/11 18:30 & 36.696 & 38.656 & 1472.07 & 1470.47 \\
\hline 11/9/11 18:40 & 35.122 & 38.715 & 1473.65 & 1470.41 \\
\hline 11/9/11 18:50 & 34.888 & 38.766 & 1473.88 & 1470.36 \\
\hline 11/9/11 19:00 & 35.927 & 38.806 & 1472.84 & 1470.32 \\
\hline 11/9/11 19:10 & 34.927 & 38.846 & 1473.84 & 1470.28 \\
\hline 11/9/11 19:20 & 34.824 & 38.874 & 1473.95 & 1470.25 \\
\hline 11/9/11 19:30 & 34.784 & 38.898 & 1473.99 & 1470.22 \\
\hline 11/9/11 19:40 & 34.777 & 38.916 & 1473.99 & 1470.21 \\
\hline 11/9/11 19:50 & 34.801 & 38.938 & 1473.97 & 1470.18 \\
\hline 11/9/11 20:00 & 34.761 & 38.952 & 1474.01 & 1470.17 \\
\hline 11/9/11 20:10 & 34.831 & 38.968 & 1473.94 & 1470.15 \\
\hline 11/9/11 20:20 & 34.998 & 38.982 & 1473.77 & 1470.14 \\
\hline 11/9/11 20:30 & 34.871 & 38.991 & 1473.90 & 1470.13 \\
\hline 11/9/11 20:40 & 34.806 & 38.996 & 1473.96 & 1470.13 \\
\hline 11/9/11 20:50 & 34.754 & 39.001 & 1474.02 & 1470.12 \\
\hline 11/9/11 21:00 & 34.714 & 39.003 & 1474.06 & 1470.12 \\
\hline 11/9/11 21:10 & 34.686 & 39.006 & 1474.08 & 1470.12 \\
\hline 11/9/11 21:20 & 34.763 & 39.01 & 1474.01 & 1470.11 \\
\hline 11/9/11 21:30 & 34.74 & 39.017 & 1474.03 & 1470.11 \\
\hline 11/9/11 21:40 & 34.791 & 39.022 & 1473.98 & 1470.10 \\
\hline 11/9/11 21:50 & 34.784 & 39.029 & 1473.99 & 1470.09 \\
\hline 11/9/11 22:00 & 34.745 & 39.036 & 1474.02 & 1470.09 \\
\hline
\end{tabular}


TABLE S1.3 Automatically recorded groundwater levels in the Klassen wells, September 15, 2011, to March 13, 2012.

\begin{tabular}{|c|c|c|c|c|}
\hline \multicolumn{3}{|c|}{ Reference Elevation (ft AMSL) } & 1508.77 & \multirow{2}{*}{$\begin{array}{l}1509.12 \\
\text { Elevation in Klassen } \\
\text { Test Well (ft AMSL) }\end{array}$} \\
\hline Date and Time & $\begin{array}{l}\text { Depth in Klassen } \\
\text { Domestic Well } \\
(\mathrm{ft} \mathrm{BGL})\end{array}$ & $\begin{array}{c}\text { Depth in Klassen } \\
\text { Test Well } \\
\text { (ft BGL) }\end{array}$ & $\begin{array}{l}\text { Elevation in Klassen } \\
\text { Domestic Well } \\
\text { (ft AMSL) }\end{array}$ & \\
\hline $11 / 9 / 1122: 10$ & 34.74 & 39.036 & 1474.03 & 1470.09 \\
\hline $11 / 9 / 1122: 20$ & 34.707 & 39.043 & 1474.06 & 1470.08 \\
\hline $11 / 9 / 1122: 30$ & 34.677 & 39.043 & 1474.09 & 1470.08 \\
\hline $11 / 9 / 1122: 40$ & 34.651 & 39.045 & 1474.12 & 1470.08 \\
\hline $11 / 9 / 1122: 50$ & 34.625 & 39.045 & 1474.14 & 1470.08 \\
\hline $11 / 9 / 1123: 00$ & 34.632 & 39.045 & 1474.14 & 1470.08 \\
\hline $11 / 9 / 1123: 10$ & 34.627 & 39.045 & 1474.14 & 1470.08 \\
\hline $11 / 9 / 1123: 20$ & 39.864 & 39.043 & 1468.91 & 1470.08 \\
\hline $11 / 9 / 1123: 30$ & 34.824 & 39.045 & 1473.95 & 1470.08 \\
\hline $11 / 9 / 1123: 40$ & 34.756 & 39.043 & 1474.01 & 1470.08 \\
\hline $11 / 9 / 1123: 50$ & 37.174 & 39.043 & 1471.60 & 1470.08 \\
\hline 11/10/11 0:00 & 34.906 & 39.043 & 1473.86 & 1470.08 \\
\hline 11/10/11 0:10 & 34.796 & 39.038 & 1473.97 & 1470.08 \\
\hline $11 / 10 / 110: 20$ & 34.738 & 39.036 & 1474.03 & 1470.09 \\
\hline $11 / 10 / 110: 30$ & 34.695 & 39.031 & 1474.07 & 1470.09 \\
\hline $11 / 10 / 110: 40$ & 34.658 & 39.024 & 1474.11 & 1470.10 \\
\hline $11 / 10 / 110: 50$ & 34.632 & 39.02 & 1474.14 & 1470.10 \\
\hline 11/10/11 1:00 & 34.606 & 39.013 & 1474.16 & 1470.11 \\
\hline 11/10/11 1:10 & 34.585 & 39.006 & 1474.18 & 1470.12 \\
\hline $11 / 10 / 111: 20$ & 34.566 & 38.999 & 1474.20 & 1470.12 \\
\hline $11 / 10 / 111: 30$ & 34.552 & 38.994 & 1474.22 & 1470.13 \\
\hline 11/10/11 1:40 & 34.606 & 38.989 & 1474.16 & 1470.13 \\
\hline $11 / 10 / 111: 50$ & 34.651 & 38.984 & 1474.12 & 1470.14 \\
\hline $11 / 10 / 112: 00$ & 34.627 & 38.98 & 1474.14 & 1470.14 \\
\hline $11 / 10 / 112: 10$ & 34.623 & 38.968 & 1474.15 & 1470.15 \\
\hline $11 / 10 / 112: 20$ & 34.599 & 38.959 & 1474.17 & 1470.16 \\
\hline $11 / 10 / 112: 30$ & 34.585 & 38.952 & 1474.18 & 1470.17 \\
\hline $11 / 10 / 112: 40$ & 34.583 & 38.942 & 1474.19 & 1470.18 \\
\hline $11 / 10 / 112: 50$ & 34.585 & 38.933 & 1474.18 & 1470.19 \\
\hline 11/10/11 3:00 & 34.564 & 38.921 & 1474.21 & 1470.20 \\
\hline 11/10/11 3:10 & 34.541 & 38.912 & 1474.23 & 1470.21 \\
\hline $11 / 10 / 113: 20$ & 34.587 & 38.905 & 1474.18 & 1470.22 \\
\hline $11 / 10 / 113: 30$ & 34.634 & 38.898 & 1474.14 & 1470.22 \\
\hline $11 / 10 / 113: 40$ & 34.611 & 38.891 & 1474.16 & 1470.23 \\
\hline $11 / 10 / 113: 50$ & 34.583 & 38.879 & 1474.19 & 1470.24 \\
\hline $11 / 10 / 114: 00$ & 34.557 & 38.869 & 1474.21 & 1470.25 \\
\hline $11 / 10 / 114: 10$ & 34.536 & 38.86 & 1474.23 & 1470.26 \\
\hline $11 / 10 / 114: 20$ & 34.515 & 38.851 & 1474.25 & 1470.27 \\
\hline
\end{tabular}


TABLE S1.3 Automatically recorded groundwater levels in the Klassen wells, September 15, 2011, to March 13, 2012.

\begin{tabular}{|c|c|c|c|c|}
\hline \multicolumn{3}{|c|}{ Reference Elevation (ft AMSL) } & 1508.77 & \multirow{2}{*}{$\begin{array}{l}1509.12 \\
\text { Elevation in Klassen } \\
\text { Test Well (ft AMSL) }\end{array}$} \\
\hline Date and Time & $\begin{array}{l}\text { Depth in Klassen } \\
\text { Domestic Well } \\
\text { (ft BGL) }\end{array}$ & $\begin{array}{c}\text { Depth in Klassen } \\
\text { Test Well } \\
\text { (ft BGL) }\end{array}$ & $\begin{array}{l}\text { Elevation in Klassen } \\
\text { Domestic Well } \\
\text { (ft AMSL) }\end{array}$ & \\
\hline $11 / 10 / 114: 30$ & 34.496 & 38.841 & 1474.27 & 1470.28 \\
\hline $11 / 10 / 114: 40$ & 37.148 & 38.832 & 1471.62 & 1470.29 \\
\hline $11 / 10 / 114: 50$ & 34.651 & 38.823 & 1474.12 & 1470.30 \\
\hline 11/10/11 5:00 & 34.562 & 38.811 & 1474.21 & 1470.31 \\
\hline 11/10/11 5:10 & 34.522 & 38.804 & 1474.25 & 1470.32 \\
\hline $11 / 10 / 115: 20$ & 34.494 & 38.792 & 1474.28 & 1470.33 \\
\hline $11 / 10 / 115: 30$ & 34.475 & 38.78 & 1474.29 & 1470.34 \\
\hline $11 / 10 / 11$ 5:40 & 34.456 & 38.773 & 1474.31 & 1470.35 \\
\hline $11 / 10 / 11$ 5:50 & 34.44 & 38.762 & 1474.33 & 1470.36 \\
\hline 11/10/11 6:00 & 34.426 & 38.752 & 1474.34 & 1470.37 \\
\hline 11/10/11 6:10 & 34.414 & 38.745 & 1474.36 & 1470.38 \\
\hline $11 / 10 / 116: 20$ & 34.4 & 38.736 & 1474.37 & 1470.39 \\
\hline $11 / 10 / 116: 30$ & 34.397 & 38.729 & 1474.37 & 1470.39 \\
\hline $11 / 10 / 116: 40$ & 34.407 & 38.722 & 1474.36 & 1470.40 \\
\hline $11 / 10 / 116: 50$ & 37.263 & 38.715 & 1471.51 & 1470.41 \\
\hline 11/10/11 7:00 & 34.871 & 38.71 & 1473.90 & 1470.41 \\
\hline 11/10/11 7:10 & 34.646 & 38.703 & 1474.12 & 1470.42 \\
\hline 11/10/11 7:20 & 35.08 & 38.703 & 1473.69 & 1470.42 \\
\hline 11/10/11 7:30 & 34.784 & 38.701 & 1473.99 & 1470.42 \\
\hline $11 / 10 / 11$ 7:40 & 34.695 & 38.696 & 1474.07 & 1470.43 \\
\hline $11 / 10 / 11$ 7:50 & 34.639 & 38.689 & 1474.13 & 1470.43 \\
\hline $11 / 10 / 118: 00$ & 34.597 & 38.679 & 1474.17 & 1470.44 \\
\hline 11/10/11 8:10 & 34.604 & 38.675 & 1474.17 & 1470.45 \\
\hline $11 / 10 / 118: 20$ & 34.613 & 38.67 & 1474.16 & 1470.45 \\
\hline $11 / 10 / 118: 30$ & 34.604 & 38.663 & 1474.17 & 1470.46 \\
\hline $11 / 10 / 118: 40$ & 34.585 & 38.656 & 1474.18 & 1470.47 \\
\hline $11 / 10 / 118: 50$ & 34.58 & 38.654 & 1474.19 & 1470.47 \\
\hline 11/10/11 9:00 & 34.794 & 38.651 & 1473.98 & 1470.47 \\
\hline 11/10/11 9:10 & 34.627 & 38.649 & 1474.14 & 1470.47 \\
\hline 11/10/11 9:20 & 34.578 & 38.642 & 1474.19 & 1470.48 \\
\hline 11/10/11 9:30 & 34.81 & 38.64 & 1473.96 & 1470.48 \\
\hline 11/10/11 9:40 & 34.653 & 38.633 & 1474.12 & 1470.49 \\
\hline $11 / 10 / 119: 50$ & 34.597 & 38.623 & 1474.17 & 1470.50 \\
\hline 11/10/11 10:00 & 34.559 & 38.618 & 1474.21 & 1470.50 \\
\hline 11/10/11 10:10 & 34.531 & 38.614 & 1474.24 & 1470.51 \\
\hline 11/10/11 10:20 & 34.508 & 38.609 & 1474.26 & 1470.51 \\
\hline 11/10/11 10:30 & 34.487 & 38.602 & 1474.28 & 1470.52 \\
\hline 11/10/11 10:40 & 34.47 & 38.597 & 1474.30 & 1470.53 \\
\hline
\end{tabular}


TABLE S1.3 Automatically recorded groundwater levels in the Klassen wells, September 15, 2011, to March 13, 2012.

\begin{tabular}{|c|c|c|c|c|}
\hline \multicolumn{3}{|c|}{ Reference Elevation (ft AMSL) } & 1508.77 & \multirow{2}{*}{$\begin{array}{l}1509.12 \\
\text { Elevation in Klassen } \\
\text { Test Well (ft AMSL) }\end{array}$} \\
\hline Date and Time & $\begin{array}{l}\text { Depth in Klassen } \\
\text { Domestic Well } \\
(\mathrm{ft} \mathrm{BGL})\end{array}$ & $\begin{array}{c}\text { Depth in Klassen } \\
\text { Test Well } \\
\text { (ft BGL) }\end{array}$ & $\begin{array}{l}\text { Elevation in Klassen } \\
\text { Domestic Well } \\
\text { (ft AMSL) }\end{array}$ & \\
\hline $11 / 10 / 11$ 10:50 & 34.454 & 38.595 & 1474.32 & 1470.53 \\
\hline 11/10/11 11:00 & 34.44 & 38.59 & 1474.33 & 1470.53 \\
\hline 11/10/11 11:10 & 34.426 & 38.59 & 1474.34 & 1470.53 \\
\hline $11 / 10 / 1111: 20$ & 34.414 & 38.59 & 1474.36 & 1470.53 \\
\hline 11/10/11 11:30 & 34.402 & 38.593 & 1474.37 & 1470.53 \\
\hline $11 / 10 / 1111: 40$ & 34.39 & 38.595 & 1474.38 & 1470.53 \\
\hline 11/10/11 11:50 & 34.379 & 38.595 & 1474.39 & 1470.53 \\
\hline $11 / 10 / 1112: 00$ & 34.369 & 38.593 & 1474.40 & 1470.53 \\
\hline $11 / 10 / 1112: 10$ & 34.358 & 38.59 & 1474.41 & 1470.53 \\
\hline $11 / 10 / 1112: 20$ & 34.351 & 38.588 & 1474.42 & 1470.53 \\
\hline $11 / 10 / 1112: 30$ & 34.341 & 38.586 & 1474.43 & 1470.54 \\
\hline $11 / 10 / 1112: 40$ & 34.332 & 38.588 & 1474.44 & 1470.53 \\
\hline $11 / 10 / 1112: 50$ & 34.325 & 38.586 & 1474.44 & 1470.54 \\
\hline $11 / 10 / 1113: 00$ & 34.315 & 38.581 & 1474.45 & 1470.54 \\
\hline 11/10/11 13:10 & 34.308 & 38.576 & 1474.46 & 1470.55 \\
\hline $11 / 10 / 11$ 13:20 & 34.299 & 38.567 & 1474.47 & 1470.56 \\
\hline 11/10/11 13:30 & 34.292 & 38.555 & 1474.48 & 1470.57 \\
\hline 11/10/11 13:40 & 34.285 & 38.546 & 1474.48 & 1470.58 \\
\hline $11 / 10 / 11$ 13:50 & 34.278 & 38.536 & 1474.49 & 1470.59 \\
\hline $11 / 10 / 11$ 14:00 & 39.632 & 38.527 & 1469.14 & 1470.60 \\
\hline $11 / 10 / 11$ 14:10 & 34.487 & 38.518 & 1474.28 & 1470.60 \\
\hline $11 / 10 / 11$ 14:20 & 34.402 & 38.508 & 1474.37 & 1470.61 \\
\hline $11 / 10 / 11$ 14:30 & 34.365 & 38.494 & 1474.40 & 1470.63 \\
\hline $11 / 10 / 11$ 14:40 & 34.341 & 38.489 & 1474.43 & 1470.63 \\
\hline $11 / 10 / 11$ 14:50 & 34.322 & 38.48 & 1474.45 & 1470.64 \\
\hline 11/10/11 15:00 & 34.308 & 38.471 & 1474.46 & 1470.65 \\
\hline $11 / 10 / 11$ 15:10 & 34.294 & 38.461 & 1474.48 & 1470.66 \\
\hline $11 / 10 / 11$ 15:20 & 34.285 & 38.452 & 1474.48 & 1470.67 \\
\hline 11/10/11 15:30 & 34.273 & 38.44 & 1474.50 & 1470.68 \\
\hline 11/10/11 15:40 & 34.266 & 38.426 & 1474.50 & 1470.70 \\
\hline 11/10/11 15:50 & 34.257 & 38.417 & 1474.51 & 1470.71 \\
\hline 11/10/11 16:00 & 34.28 & 38.405 & 1474.49 & 1470.72 \\
\hline $11 / 10 / 11$ 16:10 & 34.268 & 38.396 & 1474.50 & 1470.73 \\
\hline 11/10/11 16:20 & 34.257 & 38.384 & 1474.51 & 1470.74 \\
\hline 11/10/11 16:30 & 34.487 & 38.374 & 1474.28 & 1470.75 \\
\hline $11 / 10 / 11$ 16:40 & 34.346 & 38.365 & 1474.42 & 1470.76 \\
\hline $11 / 10 / 11$ 16:50 & 34.306 & 38.358 & 1474.46 & 1470.76 \\
\hline 11/10/11 17:00 & 34.308 & 38.349 & 1474.46 & 1470.77 \\
\hline
\end{tabular}


TABLE S1.3 Automatically recorded groundwater levels in the Klassen wells, September 15, 2011, to March 13, 2012.

\begin{tabular}{|c|c|c|c|c|}
\hline \multicolumn{3}{|c|}{ Reference Elevation (ft AMSL) } & 1508.77 & \multirow{2}{*}{$\begin{array}{l}1509.12 \\
\text { Elevation in Klassen } \\
\text { Test Well (ft AMSL) }\end{array}$} \\
\hline Date and Time & $\begin{array}{l}\text { Depth in Klassen } \\
\text { Domestic Well } \\
\text { (ft BGL) }\end{array}$ & $\begin{array}{c}\text { Depth in Klassen } \\
\text { Test Well } \\
\text { (ft BGL) }\end{array}$ & $\begin{array}{l}\text { Elevation in Klassen } \\
\text { Domestic Well } \\
\text { (ft AMSL) }\end{array}$ & \\
\hline $11 / 10 / 1117: 10$ & 34.301 & 38.339 & 1474.47 & 1470.78 \\
\hline $11 / 10 / 1117: 20$ & 34.283 & 38.33 & 1474.49 & 1470.79 \\
\hline 11/10/11 17:30 & 34.266 & 38.323 & 1474.50 & 1470.80 \\
\hline $11 / 10 / 11$ 17:40 & 34.271 & 38.313 & 1474.50 & 1470.81 \\
\hline 11/10/11 17:50 & 34.273 & 38.309 & 1474.50 & 1470.81 \\
\hline $11 / 10 / 1118: 00$ & 34.261 & 38.299 & 1474.51 & 1470.82 \\
\hline $11 / 10 / 1118: 10$ & 34.247 & 38.292 & 1474.52 & 1470.83 \\
\hline 11/10/11 18:20 & 34.318 & 38.285 & 1474.45 & 1470.84 \\
\hline $11 / 10 / 1118: 30$ & 34.381 & 38.285 & 1474.39 & 1470.84 \\
\hline $11 / 10 / 1118: 40$ & 34.367 & 38.283 & 1474.40 & 1470.84 \\
\hline $11 / 10 / 1118: 50$ & 34.344 & 38.278 & 1474.43 & 1470.84 \\
\hline 11/10/11 19:00 & 34.489 & 38.276 & 1474.28 & 1470.85 \\
\hline 11/10/11 19:10 & 34.39 & 38.271 & 1474.38 & 1470.85 \\
\hline $11 / 10 / 11$ 19:20 & 34.353 & 38.266 & 1474.42 & 1470.86 \\
\hline 11/10/11 19:30 & 34.327 & 38.264 & 1474.44 & 1470.86 \\
\hline 11/10/11 19:40 & 34.308 & 38.262 & 1474.46 & 1470.86 \\
\hline 11/10/11 19:50 & 34.292 & 38.257 & 1474.48 & 1470.87 \\
\hline 11/10/11 20:00 & 34.278 & 38.252 & 1474.49 & 1470.87 \\
\hline 11/10/11 20:10 & 34.266 & 38.245 & 1474.50 & 1470.88 \\
\hline $11 / 10 / 1120: 20$ & 34.465 & 38.241 & 1474.30 & 1470.88 \\
\hline $11 / 10 / 1120: 30$ & 34.447 & 38.241 & 1474.32 & 1470.88 \\
\hline $11 / 10 / 11$ 20:40 & 34.428 & 38.238 & 1474.34 & 1470.88 \\
\hline $11 / 10 / 1120: 50$ & 34.475 & 38.238 & 1474.29 & 1470.88 \\
\hline 11/10/11 21:00 & 34.472 & 38.236 & 1474.30 & 1470.89 \\
\hline 11/10/11 21:10 & 34.458 & 38.234 & 1474.31 & 1470.89 \\
\hline $11 / 10 / 1121: 20$ & 34.437 & 38.229 & 1474.33 & 1470.89 \\
\hline 11/10/11 21:30 & 34.409 & 38.227 & 1474.36 & 1470.90 \\
\hline $11 / 10 / 1121: 40$ & 34.395 & 38.22 & 1474.37 & 1470.90 \\
\hline $11 / 10 / 1121: 50$ & 34.461 & 38.217 & 1474.31 & 1470.91 \\
\hline $11 / 10 / 1122: 00$ & 34.442 & 38.213 & 1474.33 & 1470.91 \\
\hline $11 / 10 / 1122: 10$ & 34.501 & 38.208 & 1474.27 & 1470.91 \\
\hline $11 / 10 / 1122: 20$ & 34.501 & 38.205 & 1474.27 & 1470.92 \\
\hline $11 / 10 / 1122: 30$ & 34.562 & 38.205 & 1474.21 & 1470.92 \\
\hline $11 / 10 / 1122: 40$ & 34.555 & 38.201 & 1474.21 & 1470.92 \\
\hline $11 / 10 / 1122: 50$ & 34.562 & 38.199 & 1474.21 & 1470.92 \\
\hline $11 / 10 / 1123: 00$ & 34.606 & 38.196 & 1474.16 & 1470.93 \\
\hline 11/10/11 23:10 & 34.623 & 38.194 & 1474.15 & 1470.93 \\
\hline $11 / 10 / 1123: 20$ & 34.662 & 38.194 & 1474.11 & 1470.93 \\
\hline
\end{tabular}


TABLE S1.3 Automatically recorded groundwater levels in the Klassen wells, September 15, 2011, to March 13, 2012.

\begin{tabular}{|c|c|c|c|c|}
\hline \multicolumn{3}{|c|}{ Reference Elevation (ft AMSL) } & 1508.77 & \multirow{2}{*}{$\begin{array}{l}1509.12 \\
\text { Elevation in Klassen } \\
\text { Test Well (ft AMSL) }\end{array}$} \\
\hline Date and Time & $\begin{array}{l}\text { Depth in Klassen } \\
\text { Domestic Well } \\
\text { (ft BGL) }\end{array}$ & $\begin{array}{c}\text { Depth in Klassen } \\
\text { Test Well } \\
\text { (ft BGL) }\end{array}$ & $\begin{array}{l}\text { Elevation in Klassen } \\
\text { Domestic Well } \\
\text { (ft AMSL) }\end{array}$ & \\
\hline $11 / 10 / 1123: 30$ & 34.632 & 38.194 & 1474.14 & 1470.93 \\
\hline $11 / 10 / 1123: 40$ & 34.677 & 38.196 & 1474.09 & 1470.93 \\
\hline $11 / 10 / 1123: 50$ & 38.314 & 38.194 & 1470.46 & 1470.93 \\
\hline 11/11/11 0:00 & 34.998 & 38.198 & 1473.77 & 1470.92 \\
\hline 11/11/11 0:10 & 35.837 & 38.194 & 1472.93 & 1470.93 \\
\hline $11 / 11 / 110: 20$ & 35.279 & 38.196 & 1473.49 & 1470.93 \\
\hline $11 / 11 / 110: 30$ & 35.003 & 38.196 & 1473.77 & 1470.93 \\
\hline $11 / 11 / 110: 40$ & 34.904 & 38.194 & 1473.87 & 1470.93 \\
\hline $11 / 11 / 110: 50$ & 34.829 & 38.191 & 1473.94 & 1470.93 \\
\hline 11/11/11 1:00 & 34.77 & 38.184 & 1474.00 & 1470.94 \\
\hline 11/11/11 1:10 & 34.723 & 38.177 & 1474.05 & 1470.95 \\
\hline 11/11/11 1:20 & 34.684 & 38.175 & 1474.09 & 1470.95 \\
\hline 11/11/11 1:30 & 34.665 & 38.168 & 1474.10 & 1470.95 \\
\hline 11/11/11 1:40 & 34.705 & 38.166 & 1474.06 & 1470.96 \\
\hline $11 / 11 / 11$ 1:50 & 34.726 & 38.163 & 1474.04 & 1470.96 \\
\hline $11 / 11 / 112: 00$ & 34.688 & 38.159 & 1474.08 & 1470.96 \\
\hline $11 / 11 / 112: 10$ & 34.665 & 38.154 & 1474.10 & 1470.97 \\
\hline $11 / 11 / 112: 20$ & 34.644 & 38.152 & 1474.13 & 1470.97 \\
\hline $11 / 11 / 112: 30$ & 34.613 & 38.149 & 1474.16 & 1470.97 \\
\hline $11 / 11 / 112: 40$ & 34.601 & 38.142 & 1474.17 & 1470.98 \\
\hline $11 / 11 / 112: 50$ & 34.583 & 38.14 & 1474.19 & 1470.98 \\
\hline 11/11/11 3:00 & 34.555 & 38.135 & 1474.21 & 1470.99 \\
\hline 11/11/11 3:10 & 34.552 & 38.13 & 1474.22 & 1470.99 \\
\hline $11 / 11 / 113: 20$ & 34.592 & 38.128 & 1474.18 & 1470.99 \\
\hline 11/11/11 3:30 & 34.625 & 38.126 & 1474.14 & 1471.00 \\
\hline 11/11/11 3:40 & 34.594 & 38.119 & 1474.18 & 1471.00 \\
\hline $11 / 11 / 113: 50$ & 34.564 & 38.114 & 1474.21 & 1471.01 \\
\hline $11 / 11 / 114: 00$ & 34.536 & 38.107 & 1474.23 & 1471.02 \\
\hline $11 / 11 / 114: 10$ & 34.698 & 38.105 & 1474.07 & 1471.02 \\
\hline $11 / 11 / 114: 20$ & 34.594 & 38.1 & 1474.18 & 1471.02 \\
\hline 11/11/11 4:30 & 34.548 & 38.093 & 1474.22 & 1471.03 \\
\hline $11 / 11 / 114: 40$ & 34.517 & 38.088 & 1474.25 & 1471.03 \\
\hline $11 / 11 / 114: 50$ & 34.489 & 38.077 & 1474.28 & 1471.05 \\
\hline 11/11/11 5:00 & 34.505 & 38.069 & 1474.26 & 1471.05 \\
\hline 11/11/11 5:10 & 34.501 & 38.062 & 1474.27 & 1471.06 \\
\hline $11 / 11 / 115: 20$ & 34.477 & 38.053 & 1474.29 & 1471.07 \\
\hline 11/11/11 5:30 & 34.454 & 38.044 & 1474.32 & 1471.08 \\
\hline 11/11/11 5:40 & 34.433 & 38.037 & 1474.34 & 1471.09 \\
\hline
\end{tabular}


TABLE S1.3 Automatically recorded groundwater levels in the Klassen wells, September 15, 2011, to March 13, 2012.

\begin{tabular}{|c|c|c|c|c|}
\hline \multicolumn{3}{|c|}{ Reference Elevation (ft AMSL) } & 1508.77 & \multirow{2}{*}{$\begin{array}{l}1509.12 \\
\text { Elevation in Klassen } \\
\text { Test Well (ft AMSL) }\end{array}$} \\
\hline Date and Time & $\begin{array}{l}\text { Depth in Klassen } \\
\text { Domestic Well } \\
\text { (ft BGL) }\end{array}$ & $\begin{array}{c}\text { Depth in Klassen } \\
\text { Test Well } \\
\text { (ft BGL) }\end{array}$ & $\begin{array}{l}\text { Elevation in Klassen } \\
\text { Domestic Well } \\
\text { (ft AMSL) }\end{array}$ & \\
\hline $11 / 11 / 115: 50$ & 34.416 & 38.025 & 1474.35 & 1471.10 \\
\hline $11 / 11 / 116: 00$ & 34.426 & 38.02 & 1474.34 & 1471.10 \\
\hline $11 / 11 / 116: 10$ & 34.407 & 38.011 & 1474.36 & 1471.11 \\
\hline $11 / 11 / 116: 20$ & 34.416 & 38.006 & 1474.35 & 1471.12 \\
\hline $11 / 11 / 116: 30$ & 34.397 & 37.999 & 1474.37 & 1471.12 \\
\hline $11 / 11 / 116: 40$ & 34.379 & 37.994 & 1474.39 & 1471.13 \\
\hline $11 / 11 / 116: 50$ & 34.362 & 37.987 & 1474.41 & 1471.14 \\
\hline $11 / 11 / 11$ 7:00 & 34.346 & 37.98 & 1474.42 & 1471.14 \\
\hline $11 / 11 / 11$ 7:10 & 34.815 & 37.973 & 1473.95 & 1471.15 \\
\hline $11 / 11 / 117: 20$ & 34.48 & 37.966 & 1474.29 & 1471.16 \\
\hline $11 / 11 / 117: 30$ & 34.419 & 37.961 & 1474.35 & 1471.16 \\
\hline $11 / 11 / 117: 40$ & 34.386 & 37.952 & 1474.38 & 1471.17 \\
\hline $11 / 11 / 117: 50$ & 34.365 & 37.943 & 1474.40 & 1471.18 \\
\hline $11 / 11 / 118: 00$ & 34.39 & 37.936 & 1474.38 & 1471.19 \\
\hline $11 / 11 / 118: 10$ & 34.412 & 37.929 & 1474.36 & 1471.19 \\
\hline $11 / 11 / 118: 20$ & 34.419 & 37.926 & 1474.35 & 1471.20 \\
\hline $11 / 11 / 118: 30$ & 34.437 & 37.922 & 1474.33 & 1471.20 \\
\hline $11 / 11 / 118: 40$ & 34.414 & 37.917 & 1474.36 & 1471.21 \\
\hline $11 / 11 / 118: 50$ & 34.39 & 37.917 & 1474.38 & 1471.21 \\
\hline 11/11/11 9:00 & 34.367 & 37.917 & 1474.40 & 1471.21 \\
\hline 11/11/11 9:10 & 34.745 & 37.917 & 1474.02 & 1471.21 \\
\hline 11/11/11 9:20 & 34.573 & 37.912 & 1474.20 & 1471.21 \\
\hline 11/11/11 9:30 & 34.55 & 37.912 & 1474.22 & 1471.21 \\
\hline 11/11/11 9:40 & 35.633 & 37.91 & 1473.14 & 1471.21 \\
\hline 11/11/11 9:50 & 34.599 & 37.908 & 1474.17 & 1471.21 \\
\hline 11/11/11 10:00 & 34.524 & 37.905 & 1474.25 & 1471.22 \\
\hline 11/11/11 10:10 & 35.697 & 37.9 & 1473.07 & 1471.22 \\
\hline 11/11/11 10:20 & 40.861 & 37.896 & 1467.91 & 1471.23 \\
\hline 11/11/11 10:30 & 34.721 & 37.893 & 1474.05 & 1471.23 \\
\hline 11/11/11 10:40 & 34.594 & 37.893 & 1474.18 & 1471.23 \\
\hline 11/11/11 10:50 & 34.876 & 37.894 & 1473.89 & 1471.23 \\
\hline 11/11/11 11:00 & 39.597 & 37.893 & 1469.17 & 1471.23 \\
\hline 11/11/11 11:10 & 34.848 & 37.896 & 1473.92 & 1471.23 \\
\hline $11 / 11 / 11$ 11:20 & 42.449 & 37.9 & 1466.32 & 1471.22 \\
\hline 11/11/11 11:30 & 51.412 & 37.915 & 1457.36 & 1471.21 \\
\hline 11/11/11 11:40 & 52.601 & 37.931 & 1456.17 & 1471.19 \\
\hline 11/11/11 11:50 & 51.497 & 37.95 & 1457.27 & 1471.17 \\
\hline $11 / 11 / 11$ 12:00 & 37.2 & 37.961 & 1471.57 & 1471.16 \\
\hline
\end{tabular}


TABLE S1.3 Automatically recorded groundwater levels in the Klassen wells, September 15, 2011, to March 13, 2012.

\begin{tabular}{|c|c|c|c|c|}
\hline \multicolumn{3}{|c|}{ Reference Elevation (ft AMSL) } & 1508.77 & \multirow{2}{*}{$\begin{array}{l}1509.12 \\
\text { Elevation in Klassen } \\
\text { Test Well (ft AMSL) }\end{array}$} \\
\hline Date and Time & $\begin{array}{l}\text { Depth in Klassen } \\
\text { Domestic Well } \\
(\mathrm{ft} \mathrm{BGL})\end{array}$ & $\begin{array}{c}\text { Depth in Klassen } \\
\text { Test Well } \\
\text { (ft BGL) }\end{array}$ & $\begin{array}{l}\text { Elevation in Klassen } \\
\text { Domestic Well } \\
\text { (ft AMSL) }\end{array}$ & \\
\hline $11 / 11 / 1112: 10$ & 37.866 & 37.969 & 1470.90 & 1471.15 \\
\hline $11 / 11 / 1112: 20$ & 36.135 & 37.973 & 1472.63 & 1471.15 \\
\hline $11 / 11 / 1112: 30$ & 36.332 & 37.98 & 1472.44 & 1471.14 \\
\hline $11 / 11 / 1112: 40$ & 35.76 & 37.985 & 1473.01 & 1471.14 \\
\hline $11 / 11 / 1112: 50$ & 35.577 & 37.983 & 1473.19 & 1471.14 \\
\hline $11 / 11 / 11$ 13:00 & 35.462 & 37.976 & 1473.31 & 1471.15 \\
\hline $11 / 11 / 1113: 10$ & 35.619 & 37.983 & 1473.15 & 1471.14 \\
\hline $11 / 11 / 11$ 13:20 & 35.394 & 37.983 & 1473.38 & 1471.14 \\
\hline $11 / 11 / 1113: 30$ & 35.286 & 37.983 & 1473.48 & 1471.14 \\
\hline $11 / 11 / 1113: 40$ & 35.207 & 37.983 & 1473.56 & 1471.14 \\
\hline $11 / 11 / 1113: 50$ & 35.141 & 37.983 & 1473.63 & 1471.14 \\
\hline $11 / 11 / 1114: 00$ & 35.336 & 37.98 & 1473.43 & 1471.14 \\
\hline $11 / 11 / 1114: 10$ & 35.143 & 37.98 & 1473.63 & 1471.14 \\
\hline $11 / 11 / 1114: 20$ & 35.066 & 37.978 & 1473.70 & 1471.14 \\
\hline $11 / 11 / 11$ 14:30 & 35.005 & 37.973 & 1473.76 & 1471.15 \\
\hline $11 / 11 / 11$ 14:40 & 36.909 & 37.947 & 1471.86 & 1471.18 \\
\hline $11 / 11 / 11$ 14:50 & 35.059 & 37.9 & 1473.71 & 1471.22 \\
\hline 11/11/11 15:00 & 35.213 & 37.863 & 1473.56 & 1471.26 \\
\hline $11 / 11 / 11$ 15:30 & 34.817 & 37.844 & 1473.95 & 1471.28 \\
\hline 11/11/11 16:00 & 34.733 & 37.828 & 1474.04 & 1471.29 \\
\hline $11 / 11 / 11$ 16:30 & 34.653 & 37.807 & 1474.12 & 1471.32 \\
\hline 11/11/11 17:00 & 34.756 & 37.793 & 1474.01 & 1471.33 \\
\hline $11 / 11 / 11$ 17:30 & 34.644 & 37.776 & 1474.13 & 1471.35 \\
\hline 11/11/11 18:00 & 34.571 & 37.764 & 1474.20 & 1471.36 \\
\hline $11 / 11 / 11$ 18:30 & 34.515 & 37.755 & 1474.25 & 1471.37 \\
\hline 11/11/11 19:00 & 34.468 & 37.743 & 1474.30 & 1471.38 \\
\hline 11/11/11 19:30 & 34.543 & 37.734 & 1474.23 & 1471.39 \\
\hline $11 / 11 / 1120: 00$ & 34.526 & 37.724 & 1474.24 & 1471.40 \\
\hline $11 / 11 / 1120: 30$ & 34.477 & 37.71 & 1474.29 & 1471.41 \\
\hline 11/11/11 21:00 & 34.416 & 37.703 & 1474.35 & 1471.42 \\
\hline 11/11/11 21:30 & 34.47 & 37.706 & 1474.30 & 1471.42 \\
\hline $11 / 11 / 1122: 00$ & 34.393 & 37.703 & 1474.38 & 1471.42 \\
\hline $11 / 11 / 1122: 30$ & 34.346 & 37.692 & 1474.42 & 1471.43 \\
\hline $11 / 11 / 1123: 00$ & 34.583 & 37.682 & 1474.19 & 1471.44 \\
\hline $11 / 11 / 11$ 23:30 & 34.606 & 37.68 & 1474.16 & 1471.44 \\
\hline 11/12/11 0:00 & 34.454 & 37.671 & 1474.32 & 1471.45 \\
\hline 11/12/11 0:30 & 34.381 & 37.654 & 1474.39 & 1471.47 \\
\hline 11/12/11 1:00 & 34.578 & 37.649 & 1474.19 & 1471.47 \\
\hline
\end{tabular}


TABLE S1.3 Automatically recorded groundwater levels in the Klassen wells, September 15, 2011, to March 13, 2012.

\begin{tabular}{|c|c|c|c|c|}
\hline \multicolumn{3}{|c|}{ Reference Elevation (ft AMSL) } & 1508.77 & \multirow{2}{*}{$\begin{array}{l}1509.12 \\
\text { Elevation in Klassen } \\
\text { Test Well (ft AMSL) }\end{array}$} \\
\hline Date and Time & $\begin{array}{l}\text { Depth in Klassen } \\
\text { Domestic Well } \\
\text { (ft BGL) }\end{array}$ & $\begin{array}{c}\text { Depth in Klassen } \\
\text { Test Well } \\
\text { (ft BGL) }\end{array}$ & $\begin{array}{l}\text { Elevation in Klassen } \\
\text { Domestic Well } \\
\text { (ft AMSL) }\end{array}$ & \\
\hline $11 / 12 / 111: 30$ & 34.496 & 37.635 & 1474.27 & 1471.49 \\
\hline $11 / 12 / 112: 00$ & 34.437 & 37.619 & 1474.33 & 1471.50 \\
\hline $11 / 12 / 112: 30$ & 34.475 & 37.607 & 1474.29 & 1471.52 \\
\hline $11 / 12 / 113: 00$ & 38.516 & 37.591 & 1470.25 & 1471.53 \\
\hline $11 / 12 / 113: 30$ & 34.395 & 37.574 & 1474.37 & 1471.55 \\
\hline $11 / 12 / 114: 00$ & 34.325 & 37.556 & 1474.44 & 1471.57 \\
\hline $11 / 12 / 114: 30$ & 34.275 & 37.534 & 1474.49 & 1471.59 \\
\hline 11/12/11 5:00 & 34.236 & 37.516 & 1474.53 & 1471.61 \\
\hline $11 / 12 / 115: 30$ & 34.2 & 37.495 & 1474.57 & 1471.63 \\
\hline $11 / 12 / 116: 00$ & 34.17 & 37.476 & 1474.60 & 1471.65 \\
\hline $11 / 12 / 116: 30$ & 34.287 & 37.464 & 1474.48 & 1471.66 \\
\hline 11/12/11 7:00 & 34.193 & 37.452 & 1474.58 & 1471.67 \\
\hline 11/12/11 7:30 & 34.149 & 37.445 & 1474.62 & 1471.68 \\
\hline $11 / 12 / 118: 00$ & 34.144 & 37.436 & 1474.63 & 1471.69 \\
\hline $11 / 12 / 118: 30$ & 34.351 & 37.424 & 1474.42 & 1471.70 \\
\hline 11/12/11 9:00 & 34.285 & 37.427 & 1474.48 & 1471.70 \\
\hline 11/12/11 9:30 & 34.62 & 37.429 & 1474.15 & 1471.69 \\
\hline $11 / 12 / 11$ 10:00 & 34.428 & 37.427 & 1474.34 & 1471.70 \\
\hline $11 / 12 / 11$ 10:30 & 34.447 & 37.431 & 1474.32 & 1471.69 \\
\hline 11/12/11 11:00 & 34.578 & 37.441 & 1474.19 & 1471.68 \\
\hline 11/12/11 11:30 & 34.477 & 37.441 & 1474.29 & 1471.68 \\
\hline $11 / 12 / 11$ 12:00 & 37.65 & 37.441 & 1471.12 & 1471.68 \\
\hline $11 / 12 / 11$ 12:30 & 34.395 & 37.436 & 1474.37 & 1471.69 \\
\hline $11 / 12 / 11$ 13:00 & 34.482 & 37.431 & 1474.29 & 1471.69 \\
\hline $11 / 12 / 11$ 13:30 & 34.332 & 37.431 & 1474.44 & 1471.69 \\
\hline $11 / 12 / 11$ 14:00 & 34.365 & 37.443 & 1474.40 & 1471.68 \\
\hline $11 / 12 / 11$ 14:30 & 34.529 & 37.471 & 1474.24 & 1471.65 \\
\hline $11 / 12 / 11$ 15:00 & 34.313 & 37.492 & 1474.46 & 1471.63 \\
\hline $11 / 12 / 11$ 15:30 & 35.976 & 37.52 & 1472.79 & 1471.60 \\
\hline $11 / 12 / 11$ 16:00 & 39.393 & 37.544 & 1469.38 & 1471.58 \\
\hline $11 / 12 / 11$ 16:30 & 34.421 & 37.567 & 1474.35 & 1471.56 \\
\hline 11/12/11 17:00 & 34.426 & 37.591 & 1474.34 & 1471.53 \\
\hline $11 / 12 / 11$ 17:30 & 34.339 & 37.598 & 1474.43 & 1471.52 \\
\hline 11/12/11 18:00 & 34.268 & 37.598 & 1474.50 & 1471.52 \\
\hline $11 / 12 / 11$ 18:30 & 34.322 & 37.593 & 1474.45 & 1471.53 \\
\hline 11/12/11 19:00 & 35.242 & 37.584 & 1473.53 & 1471.54 \\
\hline 11/12/11 19:30 & 34.329 & 37.591 & 1474.44 & 1471.53 \\
\hline $11 / 12 / 1120: 00$ & 34.271 & 37.586 & 1474.50 & 1471.54 \\
\hline
\end{tabular}


TABLE S1.3 Automatically recorded groundwater levels in the Klassen wells, September 15, 2011, to March 13, 2012.

\begin{tabular}{|c|c|c|c|c|}
\hline \multicolumn{3}{|c|}{ Reference Elevation (ft AMSL) } & 1508.77 & \multirow{2}{*}{$\begin{array}{l}1509.12 \\
\text { Elevation in Klassen } \\
\text { Test Well (ft AMSL) }\end{array}$} \\
\hline Date and Time & $\begin{array}{l}\text { Depth in Klassen } \\
\text { Domestic Well } \\
(\mathrm{ft} \mathrm{BGL})\end{array}$ & $\begin{array}{c}\text { Depth in Klassen } \\
\text { Test Well } \\
\text { (ft BGL) }\end{array}$ & $\begin{array}{l}\text { Elevation in Klassen } \\
\text { Domestic Well } \\
\text { (ft AMSL) }\end{array}$ & \\
\hline $11 / 12 / 1120: 30$ & 34.21 & 37.572 & 1474.56 & 1471.55 \\
\hline $11 / 12 / 1121: 00$ & 34.168 & 37.563 & 1474.60 & 1471.56 \\
\hline 11/12/11 21:30 & 34.376 & 37.553 & 1474.39 & 1471.57 \\
\hline $11 / 12 / 1122: 00$ & 34.283 & 37.544 & 1474.49 & 1471.58 \\
\hline $11 / 12 / 1122: 30$ & 34.428 & 37.537 & 1474.34 & 1471.59 \\
\hline $11 / 12 / 1123: 00$ & 34.365 & 37.525 & 1474.40 & 1471.60 \\
\hline $11 / 12 / 1123: 30$ & 34.254 & 37.509 & 1474.52 & 1471.61 \\
\hline 11/13/11 0:00 & 34.233 & 37.492 & 1474.54 & 1471.63 \\
\hline $11 / 13 / 110: 30$ & 34.271 & 37.497 & 1474.50 & 1471.63 \\
\hline $11 / 13 / 111: 00$ & 34.58 & 37.506 & 1474.19 & 1471.62 \\
\hline $11 / 13 / 111: 30$ & 34.491 & 37.506 & 1474.28 & 1471.62 \\
\hline $11 / 13 / 112: 00$ & 34.4 & 37.49 & 1474.37 & 1471.63 \\
\hline $11 / 13 / 112: 30$ & 34.451 & 37.483 & 1474.32 & 1471.64 \\
\hline $11 / 13 / 113: 00$ & 34.355 & 37.466 & 1474.41 & 1471.66 \\
\hline 11/13/11 3:30 & 34.285 & 37.448 & 1474.48 & 1471.67 \\
\hline $11 / 13 / 114: 00$ & 34.231 & 37.438 & 1474.54 & 1471.68 \\
\hline $11 / 13 / 114: 30$ & 34.189 & 37.419 & 1474.58 & 1471.70 \\
\hline 11/13/11 5:00 & 34.151 & 37.403 & 1474.62 & 1471.72 \\
\hline $11 / 13 / 115: 30$ & 34.118 & 37.384 & 1474.65 & 1471.74 \\
\hline $11 / 13 / 116: 00$ & 34.43 & 37.373 & 1474.34 & 1471.75 \\
\hline $11 / 13 / 116: 30$ & 34.278 & 37.366 & 1474.49 & 1471.76 \\
\hline 11/13/11 7:00 & 34.175 & 37.351 & 1474.59 & 1471.77 \\
\hline 11/13/11 7:30 & 34.121 & 37.342 & 1474.65 & 1471.78 \\
\hline $11 / 13 / 118: 00$ & 34.083 & 37.34 & 1474.69 & 1471.78 \\
\hline $11 / 13 / 118: 30$ & 34.273 & 37.337 & 1474.50 & 1471.79 \\
\hline 11/13/11 9:00 & 34.236 & 37.347 & 1474.53 & 1471.78 \\
\hline 11/13/11 9:30 & 34.168 & 37.361 & 1474.60 & 1471.76 \\
\hline $11 / 13 / 11$ 10:00 & 34.111 & 37.377 & 1474.66 & 1471.75 \\
\hline $11 / 13 / 11$ 10:30 & 34.071 & 37.396 & 1474.70 & 1471.73 \\
\hline $11 / 13 / 11$ 11:00 & 34.095 & 37.405 & 1474.67 & 1471.72 \\
\hline $11 / 13 / 11$ 11:30 & 34.128 & 37.42 & 1474.64 & 1471.70 \\
\hline $11 / 13 / 1112: 00$ & 34.071 & 37.424 & 1474.70 & 1471.70 \\
\hline $11 / 13 / 11$ 12:30 & 34.036 & 37.429 & 1474.73 & 1471.69 \\
\hline $11 / 13 / 11$ 13:00 & 34.006 & 37.434 & 1474.76 & 1471.69 \\
\hline $11 / 13 / 11$ 13:30 & 33.982 & 37.438 & 1474.79 & 1471.68 \\
\hline $11 / 13 / 11$ 14:00 & 33.961 & 37.436 & 1474.81 & 1471.69 \\
\hline $11 / 13 / 11$ 14:30 & 34.339 & 37.424 & 1474.43 & 1471.70 \\
\hline $11 / 13 / 11$ 15:00 & 33.987 & 37.417 & 1474.78 & 1471.71 \\
\hline
\end{tabular}


TABLE S1.3 Automatically recorded groundwater levels in the Klassen wells, September 15, 2011, to March 13, 2012.

\begin{tabular}{|c|c|c|c|c|}
\hline \multicolumn{3}{|c|}{ Reference Elevation (ft AMSL) } & 1508.77 & \multirow{2}{*}{$\begin{array}{l}1509.12 \\
\text { Elevation in Klassen } \\
\text { Test Well (ft AMSL) }\end{array}$} \\
\hline Date and Time & $\begin{array}{l}\text { Depth in Klassen } \\
\text { Domestic Well } \\
\text { (ft BGL) }\end{array}$ & $\begin{array}{c}\text { Depth in Klassen } \\
\text { Test Well } \\
\text { (ft BGL) }\end{array}$ & $\begin{array}{l}\text { Elevation in Klassen } \\
\text { Domestic Well } \\
\text { (ft AMSL) }\end{array}$ & \\
\hline $11 / 13 / 1115: 30$ & 33.95 & 37.401 & 1474.82 & 1471.72 \\
\hline $11 / 13 / 1116: 00$ & 33.924 & 37.387 & 1474.85 & 1471.74 \\
\hline $11 / 13 / 1116: 30$ & 33.931 & 37.368 & 1474.84 & 1471.75 \\
\hline 11/13/11 17:00 & 33.931 & 37.356 & 1474.84 & 1471.77 \\
\hline $11 / 13 / 11$ 17:30 & 33.903 & 37.342 & 1474.87 & 1471.78 \\
\hline $11 / 13 / 11$ 18:00 & 34.01 & 37.326 & 1474.76 & 1471.80 \\
\hline $11 / 13 / 11$ 18:30 & 33.921 & 37.316 & 1474.85 & 1471.81 \\
\hline 11/13/11 19:00 & 34.022 & 37.312 & 1474.75 & 1471.81 \\
\hline 11/13/11 19:30 & 34.02 & 37.305 & 1474.75 & 1471.82 \\
\hline $11 / 13 / 1120: 00$ & 34.102 & 37.309 & 1474.67 & 1471.81 \\
\hline $11 / 13 / 1120: 30$ & 34.062 & 37.302 & 1474.71 & 1471.82 \\
\hline 11/13/11 21:00 & 34.24 & 37.305 & 1474.53 & 1471.82 \\
\hline $11 / 13 / 1121: 30$ & 34.2 & 37.302 & 1474.57 & 1471.82 \\
\hline $11 / 13 / 1122: 00$ & 34.111 & 37.288 & 1474.66 & 1471.83 \\
\hline $11 / 13 / 1122: 30$ & 34.053 & 37.279 & 1474.72 & 1471.84 \\
\hline $11 / 13 / 1123: 00$ & 34.217 & 37.276 & 1474.55 & 1471.85 \\
\hline $11 / 13 / 11$ 23:30 & 34.329 & 37.272 & 1474.44 & 1471.85 \\
\hline 11/14/11 0:00 & 34.163 & 37.262 & 1474.61 & 1471.86 \\
\hline $11 / 14 / 110: 30$ & 34.088 & 37.251 & 1474.68 & 1471.87 \\
\hline 11/14/11 1:00 & 34.039 & 37.236 & 1474.73 & 1471.89 \\
\hline $11 / 14 / 11$ 1:30 & 33.999 & 37.225 & 1474.77 & 1471.90 \\
\hline $11 / 14 / 112: 00$ & 33.968 & 37.213 & 1474.80 & 1471.91 \\
\hline $11 / 14 / 112: 30$ & 33.942 & 37.197 & 1474.83 & 1471.93 \\
\hline $11 / 14 / 113: 00$ & 33.919 & 37.185 & 1474.85 & 1471.94 \\
\hline $11 / 14 / 113: 30$ & 33.9 & 37.173 & 1474.87 & 1471.95 \\
\hline $11 / 14 / 114: 00$ & 33.884 & 37.164 & 1474.89 & 1471.96 \\
\hline $11 / 14 / 114: 30$ & 33.95 & 37.164 & 1474.82 & 1471.96 \\
\hline 11/14/11 5:00 & 33.898 & 37.159 & 1474.87 & 1471.96 \\
\hline $11 / 14 / 115: 30$ & 33.872 & 37.152 & 1474.90 & 1471.97 \\
\hline $11 / 14 / 116: 00$ & 33.849 & 37.143 & 1474.92 & 1471.98 \\
\hline $11 / 14 / 116: 30$ & 33.966 & 37.138 & 1474.80 & 1471.98 \\
\hline 11/14/11 7:00 & 33.898 & 37.126 & 1474.87 & 1472.00 \\
\hline $11 / 14 / 117: 30$ & 33.971 & 37.119 & 1474.80 & 1472.00 \\
\hline $11 / 14 / 118: 00$ & 33.961 & 37.114 & 1474.81 & 1472.01 \\
\hline $11 / 14 / 118: 30$ & 34.064 & 37.112 & 1474.71 & 1472.01 \\
\hline 11/14/11 9:00 & 34.339 & 37.114 & 1474.43 & 1472.01 \\
\hline 11/14/11 9:30 & 36.766 & 37.114 & 1472.00 & 1472.01 \\
\hline 11/14/11 10:00 & 34.386 & 37.126 & 1474.38 & 1472.00 \\
\hline
\end{tabular}


TABLE S1.3 Automatically recorded groundwater levels in the Klassen wells, September 15, 2011, to March 13, 2012.

\begin{tabular}{|c|c|c|c|c|}
\hline \multicolumn{3}{|c|}{ Reference Elevation (ft AMSL) } & 1508.77 & \multirow{2}{*}{$\begin{array}{l}1509.12 \\
\text { Elevation in Klassen } \\
\text { Test Well (ft AMSL) }\end{array}$} \\
\hline Date and Time & $\begin{array}{l}\text { Depth in Klassen } \\
\text { Domestic Well } \\
(\mathrm{ft} \mathrm{BGL})\end{array}$ & $\begin{array}{c}\text { Depth in Klassen } \\
\text { Test Well } \\
\text { (ft BGL) }\end{array}$ & $\begin{array}{l}\text { Elevation in Klassen } \\
\text { Domestic Well } \\
\text { (ft AMSL) }\end{array}$ & \\
\hline $11 / 14 / 1110: 30$ & 34.4 & 37.129 & 1474.37 & 1471.99 \\
\hline 11/14/11 11:00 & 41.869 & 37.131 & 1466.90 & 1471.99 \\
\hline 11/14/11 11:30 & 34.435 & 37.143 & 1474.33 & 1471.98 \\
\hline $11 / 14 / 1112: 00$ & 34.25 & 37.138 & 1474.52 & 1471.98 \\
\hline $11 / 14 / 1112: 30$ & 34.229 & 37.129 & 1474.54 & 1471.99 \\
\hline $11 / 14 / 1113: 00$ & 34.161 & 37.122 & 1474.61 & 1472.00 \\
\hline $11 / 14 / 1113: 30$ & 34.177 & 37.114 & 1474.59 & 1472.01 \\
\hline $11 / 14 / 1114: 00$ & 34.526 & 37.103 & 1474.24 & 1472.02 \\
\hline $11 / 14 / 1114: 30$ & 34.107 & 37.096 & 1474.66 & 1472.03 \\
\hline $11 / 14 / 1115: 00$ & 34.048 & 37.091 & 1474.72 & 1472.03 \\
\hline $11 / 14 / 1115: 30$ & 34.109 & 37.086 & 1474.66 & 1472.04 \\
\hline $11 / 14 / 1116: 00$ & 34.034 & 37.077 & 1474.74 & 1472.05 \\
\hline $11 / 14 / 1116: 30$ & 33.992 & 37.065 & 1474.78 & 1472.06 \\
\hline $11 / 14 / 1117: 00$ & 34.081 & 37.058 & 1474.69 & 1472.06 \\
\hline $11 / 14 / 11$ 17:30 & 34.177 & 37.06 & 1474.59 & 1472.06 \\
\hline $11 / 14 / 11$ 18:00 & 34.177 & 37.07 & 1474.59 & 1472.05 \\
\hline $11 / 14 / 11$ 18:30 & 34.09 & 37.068 & 1474.68 & 1472.05 \\
\hline 11/14/11 19:00 & 34.041 & 37.058 & 1474.73 & 1472.06 \\
\hline 11/14/11 19:30 & 34.252 & 37.061 & 1474.52 & 1472.06 \\
\hline $11 / 14 / 1120: 00$ & 34.13 & 37.056 & 1474.64 & 1472.07 \\
\hline $11 / 14 / 1120: 30$ & 34.067 & 37.044 & 1474.70 & 1472.08 \\
\hline $11 / 14 / 1121: 00$ & 37.102 & 37.037 & 1471.67 & 1472.09 \\
\hline $11 / 14 / 1121: 30$ & 34.064 & 37.035 & 1474.71 & 1472.09 \\
\hline $11 / 14 / 1122: 00$ & 34.006 & 37.028 & 1474.76 & 1472.09 \\
\hline $11 / 14 / 1122: 30$ & 34.001 & 37.021 & 1474.77 & 1472.10 \\
\hline $11 / 14 / 1123: 00$ & 34.196 & 37.023 & 1474.57 & 1472.10 \\
\hline 11/14/11 23:30 & 34.236 & 37.023 & 1474.53 & 1472.10 \\
\hline 11/15/11 0:00 & 34.095 & 37.018 & 1474.67 & 1472.10 \\
\hline 11/15/11 0:30 & 34.032 & 37.014 & 1474.74 & 1472.11 \\
\hline 11/15/11 1:00 & 33.987 & 37.002 & 1474.78 & 1472.12 \\
\hline 11/15/11 1:30 & 33.95 & 36.992 & 1474.82 & 1472.13 \\
\hline $11 / 15 / 112: 00$ & 33.919 & 36.983 & 1474.85 & 1472.14 \\
\hline $11 / 15 / 112: 30$ & 33.896 & 36.971 & 1474.87 & 1472.15 \\
\hline 11/15/11 3:00 & 33.872 & 36.962 & 1474.90 & 1472.16 \\
\hline 11/15/11 3:30 & 33.851 & 36.95 & 1474.92 & 1472.17 \\
\hline $11 / 15 / 114: 00$ & 33.832 & 36.941 & 1474.94 & 1472.18 \\
\hline $11 / 15 / 114: 30$ & 33.811 & 36.924 & 1474.96 & 1472.20 \\
\hline 11/15/11 5:00 & 33.905 & 36.922 & 1474.86 & 1472.20 \\
\hline
\end{tabular}


TABLE S1.3 Automatically recorded groundwater levels in the Klassen wells, September 15, 2011, to March 13, 2012.

\begin{tabular}{|c|c|c|c|c|}
\hline \multicolumn{3}{|c|}{ Reference Elevation (ft AMSL) } & 1508.77 & \multirow{2}{*}{$\begin{array}{l}1509.12 \\
\text { Elevation in Klassen } \\
\text { Test Well (ft AMSL) }\end{array}$} \\
\hline Date and Time & $\begin{array}{l}\text { Depth in Klassen } \\
\text { Domestic Well } \\
(\mathrm{ft} \mathrm{BGL})\end{array}$ & $\begin{array}{c}\text { Depth in Klassen } \\
\text { Test Well } \\
\text { (ft BGL) }\end{array}$ & $\begin{array}{l}\text { Elevation in Klassen } \\
\text { Domestic Well } \\
\text { (ft AMSL) }\end{array}$ & \\
\hline $11 / 15 / 115: 30$ & 33.839 & 36.917 & 1474.93 & 1472.21 \\
\hline $11 / 15 / 116: 00$ & 33.811 & 36.908 & 1474.96 & 1472.21 \\
\hline 11/15/11 6:30 & 33.95 & 36.908 & 1474.82 & 1472.21 \\
\hline $11 / 15 / 117: 00$ & 33.896 & 36.894 & 1474.87 & 1472.23 \\
\hline 11/15/11 7:30 & 34.015 & 36.899 & 1474.75 & 1472.22 \\
\hline $11 / 15 / 118: 00$ & 33.975 & 36.901 & 1474.79 & 1472.22 \\
\hline $11 / 15 / 118: 30$ & 33.917 & 36.894 & 1474.85 & 1472.23 \\
\hline 11/15/11 9:00 & 33.933 & 36.889 & 1474.84 & 1472.23 \\
\hline 11/15/11 9:30 & 33.877 & 36.887 & 1474.89 & 1472.24 \\
\hline $11 / 15 / 11$ 10:00 & 33.912 & 36.894 & 1474.86 & 1472.23 \\
\hline $11 / 15 / 11$ 10:30 & 33.858 & 36.894 & 1474.91 & 1472.23 \\
\hline 11/15/11 11:00 & 33.828 & 36.887 & 1474.94 & 1472.24 \\
\hline 11/15/11 11:30 & 34.034 & 36.887 & 1474.74 & 1472.24 \\
\hline $11 / 15 / 1112: 00$ & 33.893 & 36.889 & 1474.88 & 1472.23 \\
\hline $11 / 15 / 11$ 12:30 & 33.844 & 36.896 & 1474.93 & 1472.23 \\
\hline $11 / 15 / 11$ 13:00 & 34.156 & 36.901 & 1474.61 & 1472.22 \\
\hline $11 / 15 / 11$ 13:30 & 33.891 & 36.903 & 1474.88 & 1472.22 \\
\hline $11 / 15 / 11$ 14:00 & 33.839 & 36.896 & 1474.93 & 1472.23 \\
\hline $11 / 15 / 11$ 14:30 & 33.928 & 36.903 & 1474.84 & 1472.22 \\
\hline $11 / 15 / 11$ 15:00 & 34.097 & 36.908 & 1474.67 & 1472.21 \\
\hline $11 / 15 / 11$ 15:30 & 33.914 & 36.906 & 1474.86 & 1472.22 \\
\hline $11 / 15 / 11$ 16:00 & 33.961 & 36.903 & 1474.81 & 1472.22 \\
\hline $11 / 15 / 11$ 16:30 & 33.907 & 36.896 & 1474.86 & 1472.23 \\
\hline 11/15/11 17:00 & 33.856 & 36.889 & 1474.91 & 1472.23 \\
\hline $11 / 15 / 11$ 17:30 & 33.821 & 36.882 & 1474.95 & 1472.24 \\
\hline $11 / 15 / 11$ 18:00 & 37.805 & 36.877 & 1470.96 & 1472.25 \\
\hline 11/15/11 18:30 & 33.961 & 36.889 & 1474.81 & 1472.23 \\
\hline 11/15/11 19:00 & 33.884 & 36.892 & 1474.89 & 1472.23 \\
\hline 11/15/11 19:30 & 33.996 & 36.892 & 1474.77 & 1472.23 \\
\hline 11/15/11 20:00 & 34.161 & 36.906 & 1474.61 & 1472.22 \\
\hline $11 / 15 / 1120: 30$ & 34.027 & 36.903 & 1474.74 & 1472.22 \\
\hline $11 / 15 / 1121: 00$ & 33.959 & 36.899 & 1474.81 & 1472.22 \\
\hline $11 / 15 / 1121: 30$ & 33.912 & 36.894 & 1474.86 & 1472.23 \\
\hline $11 / 15 / 1122: 00$ & 33.9 & 36.892 & 1474.87 & 1472.23 \\
\hline $11 / 15 / 1122: 30$ & 33.889 & 36.885 & 1474.88 & 1472.24 \\
\hline $11 / 15 / 1123: 00$ & 36.182 & 36.885 & 1472.59 & 1472.24 \\
\hline $11 / 15 / 1123: 30$ & 34.287 & 36.889 & 1474.48 & 1472.23 \\
\hline 11/16/11 0:00 & 34.325 & 36.896 & 1474.44 & 1472.23 \\
\hline
\end{tabular}


TABLE S1.3 Automatically recorded groundwater levels in the Klassen wells, September 15, 2011, to March 13, 2012.

\begin{tabular}{|c|c|c|c|c|}
\hline \multicolumn{3}{|c|}{ Reference Elevation (ft AMSL) } & 1508.77 & \multirow{2}{*}{$\begin{array}{l}1509.12 \\
\text { Elevation in Klassen } \\
\text { Test Well (ft AMSL) }\end{array}$} \\
\hline Date and Time & $\begin{array}{l}\text { Depth in Klassen } \\
\text { Domestic Well } \\
(\mathrm{ft} \mathrm{BGL})\end{array}$ & $\begin{array}{c}\text { Depth in Klassen } \\
\text { Test Well } \\
\text { (ft BGL) }\end{array}$ & $\begin{array}{l}\text { Elevation in Klassen } \\
\text { Domestic Well } \\
\text { (ft AMSL) }\end{array}$ & \\
\hline $11 / 16 / 110: 30$ & 34.135 & 36.896 & 1474.63 & 1472.23 \\
\hline 11/16/11 1:00 & 34.172 & 36.906 & 1474.60 & 1472.22 \\
\hline 11/16/11 1:30 & 34.104 & 36.91 & 1474.67 & 1472.21 \\
\hline $11 / 16 / 112: 00$ & 34.06 & 36.908 & 1474.71 & 1472.21 \\
\hline $11 / 16 / 112: 30$ & 34.109 & 36.906 & 1474.66 & 1472.22 \\
\hline $11 / 16 / 113: 00$ & 34.036 & 36.899 & 1474.73 & 1472.22 \\
\hline $11 / 16 / 113: 30$ & 33.98 & 36.894 & 1474.79 & 1472.23 \\
\hline 11/16/11 4:00 & 33.94 & 36.889 & 1474.83 & 1472.23 \\
\hline $11 / 16 / 114: 30$ & 33.903 & 36.877 & 1474.87 & 1472.25 \\
\hline $11 / 16 / 115: 00$ & 33.874 & 36.868 & 1474.90 & 1472.25 \\
\hline $11 / 16 / 115: 30$ & 33.98 & 36.854 & 1474.79 & 1472.27 \\
\hline $11 / 16 / 116: 00$ & 33.903 & 36.847 & 1474.87 & 1472.28 \\
\hline $11 / 16 / 116: 30$ & 34.114 & 36.856 & 1474.66 & 1472.27 \\
\hline $11 / 16 / 117: 00$ & 33.994 & 36.852 & 1474.78 & 1472.27 \\
\hline 11/16/11 7:30 & 33.964 & 36.847 & 1474.81 & 1472.28 \\
\hline $11 / 16 / 118: 00$ & 33.928 & 36.842 & 1474.84 & 1472.28 \\
\hline $11 / 16 / 118: 30$ & 35.162 & 36.838 & 1473.61 & 1472.28 \\
\hline 11/16/11 9:00 & 34.036 & 36.84 & 1474.73 & 1472.28 \\
\hline 11/16/11 9:30 & 34.44 & 36.849 & 1474.33 & 1472.27 \\
\hline $11 / 16 / 11$ 10:00 & 34.344 & 36.868 & 1474.43 & 1472.25 \\
\hline $11 / 16 / 11$ 10:30 & 34.21 & 36.868 & 1474.56 & 1472.25 \\
\hline $11 / 16 / 11$ 11:00 & 34.21 & 36.863 & 1474.56 & 1472.26 \\
\hline $11 / 16 / 1111: 30$ & 34.111 & 36.866 & 1474.66 & 1472.26 \\
\hline $11 / 16 / 1112: 00$ & 34.21 & 36.863 & 1474.56 & 1472.26 \\
\hline $11 / 16 / 1112: 30$ & 34.09 & 36.859 & 1474.68 & 1472.26 \\
\hline 11/16/11 13:00 & 34.025 & 36.847 & 1474.74 & 1472.28 \\
\hline $11 / 16 / 1113: 30$ & 33.978 & 36.84 & 1474.79 & 1472.28 \\
\hline $11 / 16 / 11$ 14:00 & 34.048 & 36.833 & 1474.72 & 1472.29 \\
\hline $11 / 16 / 1114: 30$ & 33.966 & 36.826 & 1474.80 & 1472.30 \\
\hline $11 / 16 / 1115: 00$ & 33.926 & 36.819 & 1474.84 & 1472.30 \\
\hline $11 / 16 / 11$ 15:30 & 34.078 & 36.812 & 1474.69 & 1472.31 \\
\hline $11 / 16 / 11$ 16:00 & 33.942 & 36.805 & 1474.83 & 1472.32 \\
\hline $11 / 16 / 1116: 30$ & 33.919 & 36.8 & 1474.85 & 1472.32 \\
\hline 11/16/11 17:00 & 33.881 & 36.793 & 1474.89 & 1472.33 \\
\hline $11 / 16 / 11$ 17:30 & 33.945 & 36.795 & 1474.82 & 1472.33 \\
\hline 11/16/11 18:00 & 34.041 & 36.802 & 1474.73 & 1472.32 \\
\hline $11 / 16 / 1118: 30$ & 34.116 & 36.814 & 1474.65 & 1472.31 \\
\hline 11/16/11 19:00 & 34.039 & 36.81 & 1474.73 & 1472.31 \\
\hline
\end{tabular}


TABLE S1.3 Automatically recorded groundwater levels in the Klassen wells, September 15, 2011, to March 13, 2012.

\begin{tabular}{|c|c|c|c|c|}
\hline \multicolumn{3}{|c|}{ Reference Elevation (ft AMSL) } & 1508.77 & \multirow{2}{*}{$\begin{array}{l}1509.12 \\
\text { Elevation in Klassen } \\
\text { Test Well (ft AMSL) }\end{array}$} \\
\hline Date and Time & $\begin{array}{l}\text { Depth in Klassen } \\
\text { Domestic Well } \\
(\mathrm{ft} \mathrm{BGL})\end{array}$ & $\begin{array}{c}\text { Depth in Klassen } \\
\text { Test Well } \\
\text { (ft BGL) }\end{array}$ & $\begin{array}{l}\text { Elevation in Klassen } \\
\text { Domestic Well } \\
\text { (ft AMSL) }\end{array}$ & \\
\hline 11/16/11 19:30 & 34.003 & 36.805 & 1474.77 & 1472.32 \\
\hline $11 / 16 / 1120: 00$ & 33.982 & 36.802 & 1474.79 & 1472.32 \\
\hline $11 / 16 / 1120: 30$ & 33.961 & 36.807 & 1474.81 & 1472.32 \\
\hline $11 / 16 / 1121: 00$ & 33.945 & 36.812 & 1474.82 & 1472.31 \\
\hline 11/16/11 21:30 & 34.32 & 36.821 & 1474.45 & 1472.30 \\
\hline $11 / 16 / 1122: 00$ & 34.013 & 36.833 & 1474.76 & 1472.29 \\
\hline $11 / 16 / 1122: 30$ & 33.971 & 36.84 & 1474.80 & 1472.28 \\
\hline 11/16/11 23:00 & 34.217 & 36.852 & 1474.55 & 1472.27 \\
\hline $11 / 16 / 1123: 30$ & 34.29 & 36.856 & 1474.48 & 1472.27 \\
\hline 11/17/11 0:00 & 34.104 & 36.854 & 1474.67 & 1472.27 \\
\hline 11/17/11 0:30 & 34.027 & 36.849 & 1474.74 & 1472.27 \\
\hline 11/17/11 1:00 & 33.975 & 36.847 & 1474.79 & 1472.28 \\
\hline 11/17/11 1:30 & 33.935 & 36.842 & 1474.83 & 1472.28 \\
\hline $11 / 17 / 112: 00$ & 33.903 & 36.833 & 1474.87 & 1472.29 \\
\hline $11 / 17 / 112: 30$ & 33.874 & 36.828 & 1474.90 & 1472.29 \\
\hline 11/17/11 3:00 & 33.851 & 36.831 & 1474.92 & 1472.29 \\
\hline 11/17/11 3:30 & 33.954 & 36.831 & 1474.82 & 1472.29 \\
\hline $11 / 17 / 114: 00$ & 33.865 & 36.826 & 1474.90 & 1472.30 \\
\hline $11 / 17 / 114: 30$ & 33.83 & 36.819 & 1474.94 & 1472.30 \\
\hline 11/17/11 5:00 & 33.83 & 36.81 & 1474.94 & 1472.31 \\
\hline $11 / 17 / 11$ 5:30 & 33.802 & 36.802 & 1474.97 & 1472.32 \\
\hline $11 / 17 / 116: 00$ & 33.781 & 36.788 & 1474.99 & 1472.33 \\
\hline $11 / 17 / 116: 30$ & 33.9 & 36.786 & 1474.87 & 1472.34 \\
\hline 11/17/11 7:00 & 33.86 & 36.781 & 1474.91 & 1472.34 \\
\hline 11/17/11 7:30 & 33.964 & 36.779 & 1474.81 & 1472.34 \\
\hline 11/17/11 8:00 & 33.898 & 36.777 & 1474.87 & 1472.35 \\
\hline 11/17/11 8:30 & 33.851 & 36.774 & 1474.92 & 1472.35 \\
\hline 11/17/11 9:00 & 33.982 & 36.767 & 1474.79 & 1472.36 \\
\hline 11/17/11 9:30 & 33.86 & 36.767 & 1474.91 & 1472.36 \\
\hline 11/17/11 10:00 & 33.95 & 36.793 & 1474.82 & 1472.33 \\
\hline 11/17/11 10:30 & 34.025 & 36.807 & 1474.74 & 1472.32 \\
\hline 11/17/11 11:00 & 33.858 & 36.812 & 1474.91 & 1472.31 \\
\hline 11/17/11 11:30 & 33.809 & 36.816 & 1474.96 & 1472.31 \\
\hline $11 / 17 / 11$ 12:00 & 34.067 & 36.819 & 1474.70 & 1472.30 \\
\hline $11 / 17 / 11$ 12:30 & 33.853 & 36.828 & 1474.92 & 1472.29 \\
\hline 11/17/11 13:00 & 33.95 & 36.812 & 1474.82 & 1472.31 \\
\hline $11 / 17 / 11$ 13:30 & 33.846 & 36.741 & 1474.92 & 1472.38 \\
\hline $11 / 17 / 11$ 14:00 & 33.799 & 36.716 & 1474.97 & 1472.41 \\
\hline
\end{tabular}


TABLE S1.3 Automatically recorded groundwater levels in the Klassen wells, September 15, 2011, to March 13, 2012.

\begin{tabular}{|c|c|c|c|c|}
\hline \multicolumn{3}{|c|}{ Reference Elevation (ft AMSL) } & 1508.77 & \multirow{2}{*}{$\begin{array}{l}1509.12 \\
\text { Elevation in Klassen } \\
\text { Test Well (ft AMSL) }\end{array}$} \\
\hline Date and Time & $\begin{array}{l}\text { Depth in Klassen } \\
\text { Domestic Well } \\
(\mathrm{ft} \mathrm{BGL})\end{array}$ & $\begin{array}{c}\text { Depth in Klassen } \\
\text { Test Well } \\
\text { (ft BGL) }\end{array}$ & $\begin{array}{l}\text { Elevation in Klassen } \\
\text { Domestic Well } \\
\text { (ft AMSL) }\end{array}$ & \\
\hline $11 / 17 / 1114: 30$ & 33.86 & 36.709 & 1474.91 & 1472.41 \\
\hline $11 / 17 / 1115: 00$ & 35.357 & 36.706 & 1473.41 & 1472.42 \\
\hline $11 / 17 / 1115: 30$ & 34.074 & 36.709 & 1474.70 & 1472.41 \\
\hline $11 / 17 / 1116: 00$ & 33.928 & 36.702 & 1474.84 & 1472.42 \\
\hline $11 / 17 / 1116: 30$ & 33.966 & 36.699 & 1474.80 & 1472.42 \\
\hline 11/17/11 17:00 & 33.884 & 36.692 & 1474.89 & 1472.43 \\
\hline $11 / 17 / 1117: 30$ & 33.865 & 36.685 & 1474.90 & 1472.44 \\
\hline $11 / 17 / 11$ 18:00 & 33.928 & 36.683 & 1474.84 & 1472.44 \\
\hline $11 / 17 / 1118: 30$ & 33.881 & 36.676 & 1474.89 & 1472.45 \\
\hline $11 / 17 / 11$ 19:00 & 33.83 & 36.673 & 1474.94 & 1472.45 \\
\hline $11 / 17 / 1119: 30$ & 34.311 & 36.671 & 1474.46 & 1472.45 \\
\hline $11 / 17 / 1120: 00$ & 33.898 & 36.673 & 1474.87 & 1472.45 \\
\hline $11 / 17 / 1120: 30$ & 33.832 & 36.671 & 1474.94 & 1472.45 \\
\hline $11 / 17 / 1121: 00$ & 34.057 & 36.68 & 1474.71 & 1472.44 \\
\hline $11 / 17 / 1121: 30$ & 34.184 & 36.694 & 1474.59 & 1472.43 \\
\hline $11 / 17 / 1122: 00$ & 34.088 & 36.697 & 1474.68 & 1472.43 \\
\hline $11 / 17 / 1122: 30$ & 34.022 & 36.697 & 1474.75 & 1472.43 \\
\hline $11 / 17 / 1123: 00$ & 34.297 & 36.699 & 1474.47 & 1472.42 \\
\hline $11 / 17 / 1123: 30$ & 34.367 & 36.711 & 1474.40 & 1472.41 \\
\hline 11/18/11 0:00 & 34.158 & 36.706 & 1474.61 & 1472.42 \\
\hline 11/18/11 0:30 & 34.062 & 36.702 & 1474.71 & 1472.42 \\
\hline 11/18/11 1:00 & 33.996 & 36.694 & 1474.77 & 1472.43 \\
\hline 11/18/11 1:30 & 33.945 & 36.687 & 1474.82 & 1472.44 \\
\hline $11 / 18 / 112: 00$ & 33.905 & 36.678 & 1474.86 & 1472.44 \\
\hline $11 / 18 / 112: 30$ & 33.872 & 36.676 & 1474.90 & 1472.45 \\
\hline 11/18/11 3:00 & 33.928 & 36.676 & 1474.84 & 1472.45 \\
\hline 11/18/11 3:30 & 33.858 & 36.676 & 1474.91 & 1472.45 \\
\hline $11 / 18 / 114: 00$ & 33.823 & 36.673 & 1474.95 & 1472.45 \\
\hline $11 / 18 / 114: 30$ & 33.795 & 36.673 & 1474.97 & 1472.45 \\
\hline 11/18/11 5:00 & 33.774 & 36.669 & 1475.00 & 1472.45 \\
\hline 11/18/11 5:30 & 33.75 & 36.666 & 1475.02 & 1472.46 \\
\hline $11 / 18 / 116: 00$ & 33.729 & 36.662 & 1475.04 & 1472.46 \\
\hline $11 / 18 / 116: 30$ & 33.938 & 36.666 & 1474.83 & 1472.46 \\
\hline 11/18/11 7:00 & 33.842 & 36.652 & 1474.93 & 1472.47 \\
\hline 11/18/11 7:30 & 33.823 & 36.645 & 1474.95 & 1472.48 \\
\hline $11 / 18 / 118: 00$ & 38.687 & 36.641 & 1470.08 & 1472.48 \\
\hline 11/18/11 8:30 & 33.87 & 36.645 & 1474.90 & 1472.48 \\
\hline 11/18/11 9:00 & 34.018 & 36.65 & 1474.75 & 1472.47 \\
\hline
\end{tabular}


TABLE S1.3 Automatically recorded groundwater levels in the Klassen wells, September 15, 2011, to March 13, 2012.

\begin{tabular}{|c|c|c|c|c|}
\hline \multicolumn{3}{|c|}{ Reference Elevation (ft AMSL) } & 1508.77 & \multirow{2}{*}{$\begin{array}{l}1509.12 \\
\text { Elevation in Klassen } \\
\text { Test Well (ft AMSL) }\end{array}$} \\
\hline Date and Time & $\begin{array}{l}\text { Depth in Klassen } \\
\text { Domestic Well } \\
(\mathrm{ft} \mathrm{BGL})\end{array}$ & $\begin{array}{c}\text { Depth in Klassen } \\
\text { Test Well } \\
\text { (ft BGL) }\end{array}$ & $\begin{array}{l}\text { Elevation in Klassen } \\
\text { Domestic Well } \\
\text { (ft AMSL) }\end{array}$ & \\
\hline 11/18/11 9:30 & 33.9 & 36.65 & 1474.87 & 1472.47 \\
\hline $11 / 18 / 1110: 00$ & 33.931 & 36.643 & 1474.84 & 1472.48 \\
\hline $11 / 18 / 11$ 10:30 & 34.008 & 36.641 & 1474.76 & 1472.48 \\
\hline $11 / 18 / 1111: 00$ & 34.01 & 36.629 & 1474.76 & 1472.49 \\
\hline 11/18/11 11:30 & 33.879 & 36.603 & 1474.89 & 1472.52 \\
\hline $11 / 18 / 1112: 00$ & 34.043 & 36.584 & 1474.73 & 1472.54 \\
\hline $11 / 18 / 1112: 30$ & 33.889 & 36.575 & 1474.88 & 1472.55 \\
\hline $11 / 18 / 11$ 13:00 & 34.404 & 36.57 & 1474.37 & 1472.55 \\
\hline $11 / 18 / 1113: 30$ & 33.856 & 36.565 & 1474.91 & 1472.56 \\
\hline $11 / 18 / 1114: 00$ & 33.799 & 36.556 & 1474.97 & 1472.57 \\
\hline $11 / 18 / 1114: 30$ & 33.86 & 36.558 & 1474.91 & 1472.56 \\
\hline $11 / 18 / 1115: 00$ & 33.795 & 36.563 & 1474.97 & 1472.56 \\
\hline $11 / 18 / 1115: 30$ & 33.753 & 36.558 & 1475.02 & 1472.56 \\
\hline $11 / 18 / 1116: 00$ & 33.95 & 36.561 & 1474.82 & 1472.56 \\
\hline $11 / 18 / 11$ 16:30 & 33.799 & 36.558 & 1474.97 & 1472.56 \\
\hline $11 / 18 / 11$ 17:00 & 33.753 & 36.556 & 1475.02 & 1472.57 \\
\hline 11/18/11 17:30 & 33.947 & 36.554 & 1474.82 & 1472.57 \\
\hline 11/18/11 18:00 & 33.935 & 36.558 & 1474.83 & 1472.56 \\
\hline $11 / 18 / 11$ 18:30 & 33.865 & 36.558 & 1474.90 & 1472.56 \\
\hline 11/18/11 19:00 & 33.832 & 36.554 & 1474.94 & 1472.57 \\
\hline $11 / 18 / 11$ 19:30 & 33.931 & 36.554 & 1474.84 & 1472.57 \\
\hline $11 / 18 / 11$ 20:00 & 33.809 & 36.547 & 1474.96 & 1472.58 \\
\hline $11 / 18 / 1120: 30$ & 33.76 & 36.542 & 1475.01 & 1472.58 \\
\hline $11 / 18 / 1121: 00$ & 33.753 & 36.544 & 1475.02 & 1472.58 \\
\hline 11/18/11 21:30 & 33.72 & 36.551 & 1475.05 & 1472.57 \\
\hline $11 / 18 / 1122: 00$ & 33.874 & 36.558 & 1474.90 & 1472.56 \\
\hline $11 / 18 / 1122: 30$ & 33.783 & 36.558 & 1474.99 & 1472.56 \\
\hline $11 / 18 / 1123: 00$ & 33.966 & 36.563 & 1474.80 & 1472.56 \\
\hline $11 / 18 / 11$ 23:30 & 34.055 & 36.566 & 1474.71 & 1472.56 \\
\hline 11/19/11 0:00 & 35.068 & 36.565 & 1473.70 & 1472.56 \\
\hline 11/19/11 0:30 & 34.013 & 36.568 & 1474.76 & 1472.55 \\
\hline 11/19/11 1:00 & 34.046 & 36.568 & 1474.72 & 1472.55 \\
\hline 11/19/11 1:30 & 34.088 & 36.568 & 1474.68 & 1472.55 \\
\hline $11 / 19 / 112: 00$ & 33.973 & 36.565 & 1474.80 & 1472.56 \\
\hline $11 / 19 / 112: 30$ & 34.036 & 36.563 & 1474.73 & 1472.56 \\
\hline 11/19/11 3:00 & 33.947 & 36.561 & 1474.82 & 1472.56 \\
\hline 11/19/11 3:30 & 33.881 & 36.551 & 1474.89 & 1472.57 \\
\hline 11/19/11 4:00 & 33.835 & 36.544 & 1474.93 & 1472.58 \\
\hline
\end{tabular}


TABLE S1.3 Automatically recorded groundwater levels in the Klassen wells, September 15, 2011, to March 13, 2012.

\begin{tabular}{|c|c|c|c|c|}
\hline \multicolumn{3}{|c|}{ Reference Elevation (ft AMSL) } & 1508.77 & \multirow{2}{*}{$\begin{array}{l}1509.12 \\
\text { Elevation in Klassen } \\
\text { Test Well (ft AMSL) }\end{array}$} \\
\hline Date and Time & $\begin{array}{l}\text { Depth in Klassen } \\
\text { Domestic Well } \\
\text { (ft BGL) }\end{array}$ & $\begin{array}{c}\text { Depth in Klassen } \\
\text { Test Well } \\
\text { (ft BGL) }\end{array}$ & $\begin{array}{l}\text { Elevation in Klassen } \\
\text { Domestic Well } \\
\text { (ft AMSL) }\end{array}$ & \\
\hline $11 / 19 / 114: 30$ & 33.797 & 36.535 & 1474.97 & 1472.59 \\
\hline 11/19/11 5:00 & 33.764 & 36.53 & 1475.01 & 1472.59 \\
\hline 11/19/11 5:30 & 33.736 & 36.521 & 1475.03 & 1472.60 \\
\hline $11 / 19 / 116: 00$ & 33.713 & 36.511 & 1475.06 & 1472.61 \\
\hline $11 / 19 / 116: 30$ & 33.881 & 36.512 & 1474.89 & 1472.61 \\
\hline 11/19/11 7:00 & 33.774 & 36.511 & 1475.00 & 1472.61 \\
\hline $11 / 19 / 117: 30$ & 33.729 & 36.507 & 1475.04 & 1472.62 \\
\hline $11 / 19 / 118: 00$ & 33.699 & 36.507 & 1475.07 & 1472.62 \\
\hline $11 / 19 / 118: 30$ & 33.797 & 36.507 & 1474.97 & 1472.62 \\
\hline 11/19/11 9:00 & 33.886 & 36.514 & 1474.88 & 1472.61 \\
\hline 11/19/11 9:30 & 33.907 & 36.514 & 1474.86 & 1472.61 \\
\hline 11/19/11 10:00 & 33.821 & 36.507 & 1474.95 & 1472.62 \\
\hline $11 / 19 / 1110: 30$ & 38.284 & 36.507 & 1470.49 & 1472.62 \\
\hline 11/19/11 11:00 & 33.872 & 36.509 & 1474.90 & 1472.61 \\
\hline $11 / 19 / 1111: 30$ & 33.879 & 36.511 & 1474.89 & 1472.61 \\
\hline $11 / 19 / 11$ 12:00 & 33.832 & 36.512 & 1474.94 & 1472.61 \\
\hline $11 / 19 / 1112: 30$ & 33.896 & 36.518 & 1474.87 & 1472.60 \\
\hline 11/19/11 13:00 & 33.816 & 36.53 & 1474.95 & 1472.59 \\
\hline $11 / 19 / 11$ 13:30 & 33.9 & 36.535 & 1474.87 & 1472.59 \\
\hline $11 / 19 / 11$ 14:00 & 33.94 & 36.537 & 1474.83 & 1472.59 \\
\hline $11 / 19 / 11$ 14:30 & 33.982 & 36.549 & 1474.79 & 1472.57 \\
\hline $11 / 19 / 11$ 15:00 & 33.968 & 36.554 & 1474.80 & 1472.57 \\
\hline $11 / 19 / 1115: 30$ & 34.158 & 36.568 & 1474.61 & 1472.55 \\
\hline 11/19/11 16:00 & 34.625 & 36.575 & 1474.14 & 1472.55 \\
\hline $11 / 19 / 11$ 16:30 & 34.522 & 36.587 & 1474.25 & 1472.54 \\
\hline 11/19/11 17:00 & 34.273 & 36.605 & 1474.50 & 1472.52 \\
\hline $11 / 19 / 11$ 17:30 & 34.184 & 36.615 & 1474.59 & 1472.51 \\
\hline 11/19/11 18:00 & 34.334 & 36.622 & 1474.44 & 1472.50 \\
\hline $11 / 19 / 11$ 18:30 & 34.196 & 36.636 & 1474.57 & 1472.49 \\
\hline 11/19/11 19:00 & 39.62 & 36.643 & 1469.15 & 1472.48 \\
\hline 11/19/11 19:30 & 34.13 & 36.645 & 1474.64 & 1472.48 \\
\hline 11/19/11 20:00 & 34.078 & 36.645 & 1474.69 & 1472.48 \\
\hline $11 / 19 / 1120: 30$ & 34.118 & 36.652 & 1474.65 & 1472.47 \\
\hline 11/19/11 21:00 & 34.308 & 36.662 & 1474.46 & 1472.46 \\
\hline $11 / 19 / 1121: 30$ & 34.496 & 36.676 & 1474.27 & 1472.45 \\
\hline $11 / 19 / 1122: 00$ & 34.458 & 36.69 & 1474.31 & 1472.43 \\
\hline $11 / 19 / 1122: 30$ & 39.433 & 36.699 & 1469.34 & 1472.42 \\
\hline $11 / 19 / 1123: 00$ & 34.585 & 36.709 & 1474.18 & 1472.41 \\
\hline
\end{tabular}


TABLE S1.3 Automatically recorded groundwater levels in the Klassen wells, September 15, 2011, to March 13, 2012.

\begin{tabular}{|c|c|c|c|c|}
\hline \multicolumn{3}{|c|}{ Reference Elevation (ft AMSL) } & 1508.77 & \multirow{2}{*}{$\begin{array}{l}1509.12 \\
\text { Elevation in Klassen } \\
\text { Test Well (ft AMSL) }\end{array}$} \\
\hline Date and Time & $\begin{array}{l}\text { Depth in Klassen } \\
\text { Domestic Well } \\
(\mathrm{ft} \mathrm{BGL})\end{array}$ & $\begin{array}{c}\text { Depth in Klassen } \\
\text { Test Well } \\
\text { (ft BGL) }\end{array}$ & $\begin{array}{l}\text { Elevation in Klassen } \\
\text { Domestic Well } \\
\text { (ft AMSL) }\end{array}$ & \\
\hline $11 / 19 / 1123: 30$ & 35.88 & 36.723 & 1472.89 & 1472.40 \\
\hline 11/20/11 0:00 & 34.533 & 36.725 & 1474.24 & 1472.40 \\
\hline 11/20/11 0:30 & 34.409 & 36.727 & 1474.36 & 1472.40 \\
\hline 11/20/11 1:00 & 34.428 & 36.727 & 1474.34 & 1472.40 \\
\hline 11/20/11 1:30 & 34.336 & 36.718 & 1474.43 & 1472.40 \\
\hline $11 / 20 / 112: 00$ & 34.273 & 36.704 & 1474.50 & 1472.42 \\
\hline $11 / 20 / 112: 30$ & 34.332 & 36.706 & 1474.44 & 1472.42 \\
\hline 11/20/11 3:00 & 34.24 & 36.702 & 1474.53 & 1472.42 \\
\hline $11 / 20 / 113: 30$ & 34.168 & 36.697 & 1474.60 & 1472.43 \\
\hline $11 / 20 / 114: 00$ & 34.252 & 36.692 & 1474.52 & 1472.43 \\
\hline $11 / 20 / 114: 30$ & 34.118 & 36.687 & 1474.65 & 1472.44 \\
\hline 11/20/11 5:00 & 34.06 & 36.68 & 1474.71 & 1472.44 \\
\hline $11 / 20 / 115: 30$ & 34.022 & 36.664 & 1474.75 & 1472.46 \\
\hline $11 / 20 / 116: 00$ & 35.694 & 36.659 & 1473.08 & 1472.46 \\
\hline $11 / 20 / 116: 30$ & 34.259 & 36.659 & 1474.51 & 1472.46 \\
\hline 11/20/11 7:00 & 34.095 & 36.659 & 1474.67 & 1472.46 \\
\hline $11 / 20 / 11$ 7:30 & 34.027 & 36.655 & 1474.74 & 1472.47 \\
\hline 11/20/11 8:00 & 36.119 & 36.65 & 1472.65 & 1472.47 \\
\hline $11 / 20 / 118: 30$ & 36.494 & 36.645 & 1472.28 & 1472.48 \\
\hline 11/20/11 9:00 & 36.686 & 36.65 & 1472.08 & 1472.47 \\
\hline 11/20/11 9:30 & 34.562 & 36.659 & 1474.21 & 1472.46 \\
\hline $11 / 20 / 11$ 10:00 & 34.339 & 36.669 & 1474.43 & 1472.45 \\
\hline $11 / 20 / 11$ 10:30 & 34.339 & 36.683 & 1474.43 & 1472.44 \\
\hline $11 / 20 / 11$ 11:00 & 34.325 & 36.697 & 1474.44 & 1472.43 \\
\hline 11/20/11 11:30 & 34.212 & 36.723 & 1474.56 & 1472.40 \\
\hline $11 / 20 / 1112: 00$ & 34.135 & 36.732 & 1474.63 & 1472.39 \\
\hline $11 / 20 / 11$ 12:30 & 34.1 & 36.744 & 1474.67 & 1472.38 \\
\hline $11 / 20 / 11$ 13:00 & 34.046 & 36.746 & 1474.72 & 1472.38 \\
\hline 11/20/11 13:30 & 34.097 & 36.758 & 1474.67 & 1472.36 \\
\hline $11 / 20 / 11$ 14:00 & 34.142 & 36.786 & 1474.63 & 1472.34 \\
\hline $11 / 20 / 11$ 14:30 & 34.165 & 36.774 & 1474.60 & 1472.35 \\
\hline $11 / 20 / 11$ 15:00 & 34.207 & 36.77 & 1474.56 & 1472.35 \\
\hline $11 / 20 / 11$ 15:30 & 34.353 & 36.774 & 1474.42 & 1472.35 \\
\hline $11 / 20 / 11$ 16:00 & 34.268 & 36.774 & 1474.50 & 1472.35 \\
\hline 11/20/11 16:30 & 34.222 & 36.774 & 1474.55 & 1472.35 \\
\hline 11/20/11 17:00 & 34.147 & 36.767 & 1474.62 & 1472.36 \\
\hline 11/20/11 17:30 & 34.095 & 36.76 & 1474.67 & 1472.36 \\
\hline $11 / 20 / 11$ 18:00 & 34.47 & 36.751 & 1474.30 & 1472.37 \\
\hline
\end{tabular}


TABLE S1.3 Automatically recorded groundwater levels in the Klassen wells, September 15, 2011, to March 13, 2012.

\begin{tabular}{|c|c|c|c|c|}
\hline \multicolumn{3}{|c|}{ Reference Elevation (ft AMSL) } & 1508.77 & \multirow{2}{*}{$\begin{array}{l}1509.12 \\
\text { Elevation in Klassen } \\
\text { Test Well (ft AMSL) }\end{array}$} \\
\hline Date and Time & $\begin{array}{l}\text { Depth in Klassen } \\
\text { Domestic Well } \\
(\mathrm{ft} \mathrm{BGL})\end{array}$ & $\begin{array}{c}\text { Depth in Klassen } \\
\text { Test Well } \\
\text { (ft BGL) }\end{array}$ & $\begin{array}{l}\text { Elevation in Klassen } \\
\text { Domestic Well } \\
\text { (ft AMSL) }\end{array}$ & \\
\hline $11 / 20 / 1118: 30$ & 34.264 & 36.755 & 1474.51 & 1472.37 \\
\hline 11/20/11 19:00 & 34.275 & 36.751 & 1474.49 & 1472.37 \\
\hline 11/20/11 19:30 & 34.304 & 36.751 & 1474.47 & 1472.37 \\
\hline $11 / 20 / 1120: 00$ & 34.245 & 36.753 & 1474.52 & 1472.37 \\
\hline $11 / 20 / 1120: 30$ & 34.156 & 36.753 & 1474.61 & 1472.37 \\
\hline $11 / 20 / 1121: 00$ & 34.215 & 36.758 & 1474.55 & 1472.36 \\
\hline $11 / 20 / 1121: 30$ & 34.36 & 36.753 & 1474.41 & 1472.37 \\
\hline $11 / 20 / 1122: 00$ & 34.215 & 36.749 & 1474.55 & 1472.37 \\
\hline $11 / 20 / 1122: 30$ & 34.222 & 36.748 & 1474.55 & 1472.37 \\
\hline $11 / 20 / 1123: 00$ & 34.754 & 36.758 & 1474.02 & 1472.36 \\
\hline $11 / 20 / 1123: 30$ & 34.702 & 36.76 & 1474.07 & 1472.36 \\
\hline 11/21/11 0:00 & 34.332 & 36.763 & 1474.44 & 1472.36 \\
\hline $11 / 21 / 110: 30$ & 34.226 & 36.755 & 1474.54 & 1472.37 \\
\hline 11/21/11 1:00 & 34.273 & 36.753 & 1474.50 & 1472.37 \\
\hline 11/21/11 1:30 & 34.205 & 36.751 & 1474.56 & 1472.37 \\
\hline $11 / 21 / 112: 00$ & 34.156 & 36.741 & 1474.61 & 1472.38 \\
\hline $11 / 21 / 112: 30$ & 34.226 & 36.741 & 1474.54 & 1472.38 \\
\hline 11/21/11 3:00 & 34.144 & 36.732 & 1474.63 & 1472.39 \\
\hline 11/21/11 3:30 & 34.083 & 36.723 & 1474.69 & 1472.40 \\
\hline $11 / 21 / 114: 00$ & 34.034 & 36.716 & 1474.74 & 1472.41 \\
\hline $11 / 21 / 114: 30$ & 33.992 & 36.706 & 1474.78 & 1472.42 \\
\hline 11/21/11 5:00 & 33.964 & 36.699 & 1474.81 & 1472.42 \\
\hline $11 / 21 / 11$ 5:30 & 33.95 & 36.69 & 1474.82 & 1472.43 \\
\hline $11 / 21 / 116: 00$ & 34.043 & 36.683 & 1474.73 & 1472.44 \\
\hline $11 / 21 / 116: 30$ & 34.1 & 36.673 & 1474.67 & 1472.45 \\
\hline 11/21/11 7:00 & 34.095 & 36.666 & 1474.67 & 1472.46 \\
\hline 11/21/11 7:30 & 34.125 & 36.664 & 1474.64 & 1472.46 \\
\hline 11/21/11 8:00 & 35.833 & 36.666 & 1472.94 & 1472.46 \\
\hline $11 / 21 / 118: 30$ & 34.257 & 36.669 & 1474.51 & 1472.45 \\
\hline 11/21/11 9:00 & 34.076 & 36.662 & 1474.69 & 1472.46 \\
\hline 11/21/11 9:30 & 34.088 & 36.65 & 1474.68 & 1472.47 \\
\hline $11 / 21 / 11$ 10:00 & 34.21 & 36.664 & 1474.56 & 1472.46 \\
\hline $11 / 21 / 11$ 10:30 & 34.508 & 36.676 & 1474.26 & 1472.45 \\
\hline 11/21/11 11:00 & 34.756 & 36.678 & 1474.01 & 1472.44 \\
\hline 11/21/11 11:30 & 34.435 & 36.692 & 1474.33 & 1472.43 \\
\hline $11 / 21 / 11$ 12:00 & 34.297 & 36.697 & 1474.47 & 1472.43 \\
\hline $11 / 21 / 11$ 12:30 & 34.24 & 36.702 & 1474.53 & 1472.42 \\
\hline $11 / 21 / 11$ 13:00 & 34.137 & 36.704 & 1474.63 & 1472.42 \\
\hline
\end{tabular}


TABLE S1.3 Automatically recorded groundwater levels in the Klassen wells, September 15, 2011, to March 13, 2012.

Reference Elevation (ft AMSL)

1508.77

1509.12

\begin{tabular}{|c|c|c|c|c|}
\hline Date and Time & $\begin{array}{l}\text { Depth in Klassen } \\
\text { Domestic Well } \\
\text { (ft BGL) }\end{array}$ & $\begin{array}{c}\text { Depth in Klassen } \\
\text { Test Well } \\
\text { (ft BGL) }\end{array}$ & $\begin{array}{l}\text { Elevation in Klassen } \\
\text { Domestic Well } \\
\text { (ft AMSL) }\end{array}$ & $\begin{array}{l}\text { Elevation in Klassen } \\
\text { Test Well (ft AMSL) }\end{array}$ \\
\hline $11 / 21 / 11$ 13:30 & 37.458 & 36.702 & 1471.31 & 1472.42 \\
\hline $11 / 21 / 1114: 00$ & 34.095 & 36.702 & 1474.67 & 1472.42 \\
\hline $11 / 21 / 11$ 14:30 & 34.118 & 36.697 & 1474.65 & 1472.43 \\
\hline $11 / 21 / 11$ 15:00 & 34.299 & 36.671 & 1474.47 & 1472.45 \\
\hline $11 / 21 / 11$ 15:30 & 34.053 & 36.666 & 1474.72 & 1472.46 \\
\hline $11 / 21 / 11$ 16:00 & 34.025 & 36.655 & 1474.74 & 1472.47 \\
\hline $11 / 21 / 11$ 16:30 & 34.212 & 36.655 & 1474.56 & 1472.47 \\
\hline $11 / 21 / 11$ 17:00 & 34.2 & 36.652 & 1474.57 & 1472.47 \\
\hline $11 / 21 / 11$ 17:30 & 34.236 & 36.657 & 1474.53 & 1472.47 \\
\hline $11 / 21 / 11$ 18:00 & 34.09 & 36.659 & 1474.68 & 1472.46 \\
\hline $11 / 21 / 11$ 18:30 & 34.025 & 36.626 & 1474.74 & 1472.50 \\
\hline $11 / 21 / 11$ 19:00 & 34.043 & 36.641 & 1474.73 & 1472.48 \\
\hline $11 / 21 / 11$ 19:30 & 33.978 & 36.619 & 1474.79 & 1472.50 \\
\hline $11 / 21 / 1120: 00$ & 33.938 & 36.601 & 1474.83 & 1472.52 \\
\hline $11 / 21 / 1120: 30$ & 34.029 & 36.61 & 1474.74 & 1472.51 \\
\hline $11 / 21 / 1121: 00$ & 33.942 & 36.612 & 1474.83 & 1472.51 \\
\hline $11 / 21 / 1121: 30$ & 33.898 & 36.594 & 1474.87 & 1472.53 \\
\hline $11 / 21 / 1122: 00$ & 33.952 & 36.587 & 1474.82 & 1472.54 \\
\hline $11 / 21 / 1122: 30$ & 34.102 & 36.596 & 1474.67 & 1472.53 \\
\hline $11 / 21 / 1123: 00$ & 37.352 & 36.596 & 1471.42 & 1472.53 \\
\hline $11 / 21 / 1123: 30$ & 35.134 & 36.596 & 1473.64 & 1472.53 \\
\hline 11/22/11 0:00 & 34.36 & 36.596 & 1474.41 & 1472.53 \\
\hline $11 / 22 / 110: 30$ & 34.215 & 36.591 & 1474.55 & 1472.53 \\
\hline 11/22/11 1:00 & 34.121 & 36.591 & 1474.65 & 1472.53 \\
\hline $11 / 22 / 11$ 1:30 & 34.05 & 36.584 & 1474.72 & 1472.54 \\
\hline $11 / 22 / 112: 00$ & 33.996 & 36.587 & 1474.77 & 1472.54 \\
\hline $11 / 22 / 112: 30$ & 33.952 & 36.575 & 1474.82 & 1472.55 \\
\hline $11 / 22 / 113: 00$ & 34.114 & 36.57 & 1474.66 & 1472.55 \\
\hline $11 / 22 / 113: 30$ & 33.959 & 36.563 & 1474.81 & 1472.56 \\
\hline $11 / 22 / 114: 00$ & 33.903 & 36.556 & 1474.87 & 1472.57 \\
\hline $11 / 22 / 114: 30$ & 33.896 & 36.544 & 1474.87 & 1472.58 \\
\hline $11 / 22 / 115: 00$ & 33.856 & 36.542 & 1474.91 & 1472.58 \\
\hline $11 / 22 / 115: 30$ & 33.823 & 36.533 & 1474.95 & 1472.59 \\
\hline $11 / 22 / 116: 00$ & 33.799 & 36.521 & 1474.97 & 1472.60 \\
\hline $11 / 22 / 116: 30$ & 33.999 & 36.516 & 1474.77 & 1472.61 \\
\hline $11 / 22 / 117: 00$ & 33.91 & 36.514 & 1474.86 & 1472.61 \\
\hline $11 / 22 / 117: 30$ & 33.884 & 36.497 & 1474.89 & 1472.63 \\
\hline $11 / 22 / 118: 00$ & 33.839 & 36.493 & 1474.93 & 1472.63 \\
\hline
\end{tabular}

Page 230 of 372 
TABLE S1.3 Automatically recorded groundwater levels in the Klassen wells, September 15, 2011, to March 13, 2012.

\begin{tabular}{|c|c|c|c|c|}
\hline \multicolumn{3}{|c|}{ Reference Elevation (ft AMSL) } & 1508.77 & \multirow{2}{*}{$\begin{array}{l}1509.12 \\
\text { Elevation in Klassen } \\
\text { Test Well (ft AMSL) }\end{array}$} \\
\hline Date and Time & $\begin{array}{l}\text { Depth in Klassen } \\
\text { Domestic Well } \\
(\mathrm{ft} \mathrm{BGL})\end{array}$ & $\begin{array}{c}\text { Depth in Klassen } \\
\text { Test Well } \\
\text { (ft BGL) }\end{array}$ & $\begin{array}{l}\text { Elevation in Klassen } \\
\text { Domestic Well } \\
\text { (ft AMSL) }\end{array}$ & \\
\hline $11 / 22 / 118: 30$ & 39.369 & 36.495 & 1469.40 & 1472.63 \\
\hline 11/22/11 9:00 & 33.935 & 36.493 & 1474.83 & 1472.63 \\
\hline 11/22/11 9:30 & 33.863 & 36.486 & 1474.91 & 1472.64 \\
\hline $11 / 22 / 1110: 00$ & 33.821 & 36.474 & 1474.95 & 1472.65 \\
\hline $11 / 22 / 1110: 30$ & 33.79 & 36.479 & 1474.98 & 1472.64 \\
\hline $11 / 22 / 1111: 00$ & 33.79 & 36.493 & 1474.98 & 1472.63 \\
\hline $11 / 22 / 1111: 30$ & 33.762 & 36.497 & 1475.01 & 1472.63 \\
\hline $11 / 22 / 1112: 00$ & 33.816 & 36.497 & 1474.95 & 1472.63 \\
\hline $11 / 22 / 1112: 30$ & 33.76 & 36.502 & 1475.01 & 1472.62 \\
\hline $11 / 22 / 1113: 00$ & 34.667 & 36.5 & 1474.10 & 1472.62 \\
\hline $11 / 22 / 1113: 30$ & 33.764 & 36.469 & 1475.01 & 1472.65 \\
\hline $11 / 22 / 1114: 00$ & 33.86 & 36.436 & 1474.91 & 1472.69 \\
\hline $11 / 22 / 1114: 30$ & 33.774 & 36.425 & 1475.00 & 1472.70 \\
\hline $11 / 22 / 1115: 00$ & 33.94 & 36.425 & 1474.83 & 1472.70 \\
\hline $11 / 22 / 1115: 30$ & 33.959 & 36.425 & 1474.81 & 1472.70 \\
\hline $11 / 22 / 11$ 16:00 & 33.903 & 36.425 & 1474.87 & 1472.70 \\
\hline $11 / 22 / 11$ 16:30 & 33.867 & 36.425 & 1474.90 & 1472.70 \\
\hline $11 / 22 / 11$ 17:00 & 54.058 & 36.462 & 1454.71 & 1472.66 \\
\hline $11 / 22 / 11$ 17:30 & 36.088 & 36.535 & 1472.68 & 1472.59 \\
\hline $11 / 22 / 11$ 18:00 & 35.256 & 36.573 & 1473.51 & 1472.55 \\
\hline $11 / 22 / 11$ 18:30 & 35.11 & 36.591 & 1473.66 & 1472.53 \\
\hline $11 / 22 / 11$ 19:00 & 34.951 & 36.605 & 1473.82 & 1472.52 \\
\hline 11/22/11 19:30 & 34.829 & 36.631 & 1473.94 & 1472.49 \\
\hline $11 / 22 / 1120: 00$ & 34.651 & 36.648 & 1474.12 & 1472.47 \\
\hline $11 / 22 / 1120: 30$ & 34.7 & 36.662 & 1474.07 & 1472.46 \\
\hline $11 / 22 / 1121: 00$ & 34.63 & 36.68 & 1474.14 & 1472.44 \\
\hline 11/22/11 21:30 & 34.601 & 36.687 & 1474.17 & 1472.44 \\
\hline $11 / 22 / 1122: 00$ & 34.543 & 36.694 & 1474.23 & 1472.43 \\
\hline $11 / 22 / 1122: 30$ & 34.428 & 36.694 & 1474.34 & 1472.43 \\
\hline $11 / 22 / 1123: 00$ & 34.526 & 36.699 & 1474.24 & 1472.42 \\
\hline $11 / 22 / 1123: 30$ & 34.585 & 36.702 & 1474.18 & 1472.42 \\
\hline 11/23/11 0:00 & 34.552 & 36.709 & 1474.22 & 1472.41 \\
\hline $11 / 23 / 11$ 0:30 & 34.566 & 36.709 & 1474.20 & 1472.41 \\
\hline 11/23/11 1:00 & 34.555 & 36.709 & 1474.21 & 1472.41 \\
\hline $11 / 23 / 11$ 1:30 & 34.451 & 36.704 & 1474.32 & 1472.42 \\
\hline $11 / 23 / 112: 00$ & 34.449 & 36.699 & 1474.32 & 1472.42 \\
\hline $11 / 23 / 112: 30$ & 34.468 & 36.699 & 1474.30 & 1472.42 \\
\hline 11/23/11 3:00 & 34.36 & 36.697 & 1474.41 & 1472.43 \\
\hline
\end{tabular}


TABLE S1.3 Automatically recorded groundwater levels in the Klassen wells, September 15, 2011, to March 13, 2012.

\begin{tabular}{|c|c|c|c|c|}
\hline \multicolumn{3}{|c|}{ Reference Elevation (ft AMSL) } & 1508.77 & \multirow{2}{*}{$\begin{array}{l}1509.12 \\
\text { Elevation in Klassen } \\
\text { Test Well (ft AMSL) }\end{array}$} \\
\hline Date and Time & $\begin{array}{l}\text { Depth in Klassen } \\
\text { Domestic Well } \\
\text { (ft BGL) }\end{array}$ & $\begin{array}{c}\text { Depth in Klassen } \\
\text { Test Well } \\
\text { (ft BGL) }\end{array}$ & $\begin{array}{l}\text { Elevation in Klassen } \\
\text { Domestic Well } \\
\text { (ft AMSL) }\end{array}$ & \\
\hline $11 / 23 / 113: 30$ & 34.28 & 36.685 & 1474.49 & 1472.44 \\
\hline $11 / 23 / 114: 00$ & 34.217 & 36.687 & 1474.55 & 1472.44 \\
\hline $11 / 23 / 114: 30$ & 34.161 & 36.673 & 1474.61 & 1472.45 \\
\hline $11 / 23 / 115: 00$ & 34.116 & 36.664 & 1474.65 & 1472.46 \\
\hline 11/23/11 5:30 & 34.074 & 36.659 & 1474.70 & 1472.46 \\
\hline $11 / 23 / 116: 00$ & 34.039 & 36.645 & 1474.73 & 1472.48 \\
\hline $11 / 23 / 116: 30$ & 34.189 & 36.643 & 1474.58 & 1472.48 \\
\hline $11 / 23 / 11$ 7:00 & 34.078 & 36.638 & 1474.69 & 1472.48 \\
\hline $11 / 23 / 117: 30$ & 34.027 & 36.629 & 1474.74 & 1472.49 \\
\hline $11 / 23 / 118: 00$ & 34.139 & 36.629 & 1474.63 & 1472.49 \\
\hline $11 / 23 / 118: 30$ & 34.055 & 36.641 & 1474.71 & 1472.48 \\
\hline 11/23/11 9:00 & 34.027 & 36.641 & 1474.74 & 1472.48 \\
\hline 11/23/11 9:30 & 34.064 & 36.65 & 1474.71 & 1472.47 \\
\hline $11 / 23 / 11$ 10:00 & 34.257 & 36.664 & 1474.51 & 1472.46 \\
\hline $11 / 23 / 11$ 10:30 & 34.275 & 36.673 & 1474.49 & 1472.45 \\
\hline $11 / 23 / 11$ 11:00 & 34.39 & 36.694 & 1474.38 & 1472.43 \\
\hline 11/23/11 11:30 & 34.59 & 36.706 & 1474.18 & 1472.42 \\
\hline $11 / 23 / 11$ 12:00 & 34.766 & 36.697 & 1474.00 & 1472.43 \\
\hline $11 / 23 / 11$ 12:30 & 34.648 & 36.699 & 1474.12 & 1472.42 \\
\hline $11 / 23 / 11$ 13:00 & 34.677 & 36.711 & 1474.09 & 1472.41 \\
\hline $11 / 23 / 11$ 13:30 & 34.571 & 36.72 & 1474.20 & 1472.40 \\
\hline $11 / 23 / 11$ 14:00 & 34.531 & 36.718 & 1474.24 & 1472.40 \\
\hline $11 / 23 / 11$ 14:30 & 34.386 & 36.709 & 1474.38 & 1472.41 \\
\hline $11 / 23 / 11$ 15:00 & 34.358 & 36.699 & 1474.41 & 1472.42 \\
\hline $11 / 23 / 11$ 15:30 & 34.355 & 36.692 & 1474.41 & 1472.43 \\
\hline $11 / 23 / 11$ 16:00 & 34.254 & 36.683 & 1474.52 & 1472.44 \\
\hline $11 / 23 / 11$ 16:30 & 34.184 & 36.676 & 1474.59 & 1472.45 \\
\hline $11 / 23 / 11$ 17:00 & 34.299 & 36.676 & 1474.47 & 1472.45 \\
\hline $11 / 23 / 11$ 17:30 & 34.175 & 36.671 & 1474.59 & 1472.45 \\
\hline 11/23/11 18:00 & 34.233 & 36.673 & 1474.54 & 1472.45 \\
\hline $11 / 23 / 11$ 18:30 & 34.172 & 36.669 & 1474.60 & 1472.45 \\
\hline 11/23/11 19:00 & 36.759 & 36.664 & 1472.01 & 1472.46 \\
\hline 11/23/11 19:30 & 34.175 & 36.662 & 1474.59 & 1472.46 \\
\hline $11 / 23 / 1120: 00$ & 34.266 & 36.657 & 1474.50 & 1472.47 \\
\hline $11 / 23 / 1120: 30$ & 34.247 & 36.655 & 1474.52 & 1472.47 \\
\hline $11 / 23 / 1121: 00$ & 34.1 & 36.652 & 1474.67 & 1472.47 \\
\hline 11/23/11 21:30 & 34.043 & 36.643 & 1474.73 & 1472.48 \\
\hline $11 / 23 / 1122: 00$ & 34.327 & 36.636 & 1474.44 & 1472.49 \\
\hline
\end{tabular}


TABLE S1.3 Automatically recorded groundwater levels in the Klassen wells, September 15, 2011, to March 13, 2012.

\begin{tabular}{|c|c|c|c|c|}
\hline \multicolumn{3}{|c|}{ Reference Elevation (ft AMSL) } & 1508.77 & \multirow{2}{*}{$\begin{array}{l}1509.12 \\
\text { Elevation in Klassen } \\
\text { Test Well (ft AMSL) }\end{array}$} \\
\hline Date and Time & $\begin{array}{l}\text { Depth in Klassen } \\
\text { Domestic Well } \\
\text { (ft BGL) }\end{array}$ & $\begin{array}{c}\text { Depth in Klassen } \\
\text { Test Well } \\
\text { (ft BGL) }\end{array}$ & $\begin{array}{l}\text { Elevation in Klassen } \\
\text { Domestic Well } \\
\text { (ft AMSL) }\end{array}$ & \\
\hline $11 / 23 / 1122: 30$ & 38.026 & 36.633 & 1470.74 & 1472.49 \\
\hline $11 / 23 / 1123: 00$ & 34.339 & 36.641 & 1474.43 & 1472.48 \\
\hline $11 / 23 / 1123: 30$ & 34.362 & 36.648 & 1474.41 & 1472.47 \\
\hline $11 / 24 / 110: 00$ & 34.226 & 36.643 & 1474.54 & 1472.48 \\
\hline $11 / 24 / 110: 30$ & 34.177 & 36.634 & 1474.59 & 1472.49 \\
\hline 11/24/11 1:00 & 34.229 & 36.634 & 1474.54 & 1472.49 \\
\hline $11 / 24 / 111: 30$ & 34.17 & 36.626 & 1474.60 & 1472.50 \\
\hline $11 / 24 / 112: 00$ & 34.137 & 36.619 & 1474.63 & 1472.50 \\
\hline $11 / 24 / 112: 30$ & 34.271 & 36.615 & 1474.50 & 1472.51 \\
\hline $11 / 24 / 113: 00$ & 34.158 & 36.615 & 1474.61 & 1472.51 \\
\hline $11 / 24 / 113: 30$ & 34.083 & 36.612 & 1474.69 & 1472.51 \\
\hline 11/24/11 4:00 & 34.029 & 36.608 & 1474.74 & 1472.51 \\
\hline $11 / 24 / 114: 30$ & 33.987 & 36.601 & 1474.78 & 1472.52 \\
\hline 11/24/11 5:00 & 34.109 & 36.589 & 1474.66 & 1472.53 \\
\hline $11 / 24 / 11$ 5:30 & 33.98 & 36.579 & 1474.79 & 1472.54 \\
\hline $11 / 24 / 116: 00$ & 34.013 & 36.57 & 1474.76 & 1472.55 \\
\hline $11 / 24 / 116: 30$ & 34.039 & 36.565 & 1474.73 & 1472.56 \\
\hline 11/24/11 7:00 & 33.957 & 36.554 & 1474.81 & 1472.57 \\
\hline 11/24/11 7:30 & 33.912 & 36.544 & 1474.86 & 1472.58 \\
\hline $11 / 24 / 118: 00$ & 33.903 & 36.544 & 1474.87 & 1472.58 \\
\hline $11 / 24 / 118: 30$ & 34.083 & 36.554 & 1474.69 & 1472.57 \\
\hline 11/24/11 9:00 & 34.003 & 36.554 & 1474.77 & 1472.57 \\
\hline 11/24/11 9:30 & 34.034 & 36.558 & 1474.74 & 1472.56 \\
\hline $11 / 24 / 11$ 10:00 & 34.285 & 36.563 & 1474.48 & 1472.56 \\
\hline $11 / 24 / 11$ 10:30 & 36.672 & 36.565 & 1472.10 & 1472.56 \\
\hline $11 / 24 / 11$ 11:00 & 34.477 & 36.577 & 1474.29 & 1472.55 \\
\hline $11 / 24 / 11$ 11:30 & 34.43 & 36.584 & 1474.34 & 1472.54 \\
\hline $11 / 24 / 1112: 00$ & 34.416 & 36.582 & 1474.35 & 1472.54 \\
\hline $11 / 24 / 1112: 30$ & 34.376 & 36.582 & 1474.39 & 1472.54 \\
\hline $11 / 24 / 11$ 13:00 & 34.386 & 36.582 & 1474.38 & 1472.54 \\
\hline $11 / 24 / 11$ 13:30 & 34.848 & 36.587 & 1473.92 & 1472.54 \\
\hline $11 / 24 / 11$ 14:00 & 34.538 & 36.598 & 1474.23 & 1472.52 \\
\hline $11 / 24 / 11$ 14:30 & 34.487 & 36.598 & 1474.28 & 1472.52 \\
\hline $11 / 24 / 11$ 15:00 & 34.587 & 36.605 & 1474.18 & 1472.52 \\
\hline $11 / 24 / 11$ 15:30 & 34.538 & 36.61 & 1474.23 & 1472.51 \\
\hline $11 / 24 / 11$ 16:00 & 34.515 & 36.617 & 1474.25 & 1472.51 \\
\hline $11 / 24 / 11$ 16:30 & 34.789 & 36.624 & 1473.98 & 1472.50 \\
\hline $11 / 24 / 11$ 17:00 & 35.72 & 36.633 & 1473.05 & 1472.49 \\
\hline
\end{tabular}


TABLE S1.3 Automatically recorded groundwater levels in the Klassen wells, September 15, 2011, to March 13, 2012.

\begin{tabular}{|c|c|c|c|c|}
\hline \multicolumn{3}{|c|}{ Reference Elevation (ft AMSL) } & 1508.77 & \multirow{2}{*}{$\begin{array}{l}1509.12 \\
\text { Elevation in Klassen } \\
\text { Test Well (ft AMSL) }\end{array}$} \\
\hline Date and Time & $\begin{array}{l}\text { Depth in Klassen } \\
\text { Domestic Well } \\
\text { (ft BGL) }\end{array}$ & $\begin{array}{c}\text { Depth in Klassen } \\
\text { Test Well } \\
\text { (ft BGL) }\end{array}$ & $\begin{array}{l}\text { Elevation in Klassen } \\
\text { Domestic Well } \\
\text { (ft AMSL) }\end{array}$ & \\
\hline $11 / 24 / 1117: 30$ & 34.496 & 36.636 & 1474.27 & 1472.49 \\
\hline $11 / 24 / 1118: 00$ & 34.43 & 36.636 & 1474.34 & 1472.49 \\
\hline $11 / 24 / 1118: 30$ & 34.414 & 36.638 & 1474.36 & 1472.48 \\
\hline 11/24/11 19:00 & 36.344 & 36.648 & 1472.43 & 1472.47 \\
\hline 11/24/11 19:30 & 34.503 & 36.659 & 1474.27 & 1472.46 \\
\hline $11 / 24 / 1120: 00$ & 34.48 & 36.662 & 1474.29 & 1472.46 \\
\hline $11 / 24 / 1120: 30$ & 34.463 & 36.666 & 1474.31 & 1472.46 \\
\hline $11 / 24 / 1121: 00$ & 34.409 & 36.662 & 1474.36 & 1472.46 \\
\hline $11 / 24 / 1121: 30$ & 38.033 & 36.659 & 1470.74 & 1472.46 \\
\hline $11 / 24 / 1122: 00$ & 34.644 & 36.664 & 1474.13 & 1472.46 \\
\hline $11 / 24 / 1122: 30$ & 35.094 & 36.669 & 1473.68 & 1472.45 \\
\hline $11 / 24 / 1123: 00$ & 34.714 & 36.678 & 1474.06 & 1472.44 \\
\hline $11 / 24 / 1123: 30$ & 34.864 & 36.69 & 1473.91 & 1472.43 \\
\hline 11/25/11 0:00 & 34.719 & 36.692 & 1474.05 & 1472.43 \\
\hline $11 / 25 / 110: 30$ & 34.81 & 36.704 & 1473.96 & 1472.42 \\
\hline 11/25/11 1:00 & 34.869 & 36.718 & 1473.90 & 1472.40 \\
\hline $11 / 25 / 11$ 1:30 & 34.93 & 36.73 & 1473.84 & 1472.39 \\
\hline $11 / 25 / 112: 00$ & 34.951 & 36.734 & 1473.82 & 1472.39 \\
\hline $11 / 25 / 112: 30$ & 34.965 & 36.734 & 1473.80 & 1472.39 \\
\hline 11/25/11 3:00 & 34.981 & 36.741 & 1473.79 & 1472.38 \\
\hline $11 / 25 / 113: 30$ & 34.986 & 36.744 & 1473.78 & 1472.38 \\
\hline $11 / 25 / 114: 00$ & 34.998 & 36.751 & 1473.77 & 1472.37 \\
\hline $11 / 25 / 114: 30$ & 35 & 36.755 & 1473.77 & 1472.37 \\
\hline $11 / 25 / 11$ 5:00 & 35.005 & 36.753 & 1473.76 & 1472.37 \\
\hline 11/25/11 5:30 & 35.366 & 36.758 & 1473.40 & 1472.36 \\
\hline $11 / 25 / 116: 00$ & 35.333 & 36.76 & 1473.44 & 1472.36 \\
\hline $11 / 25 / 116: 30$ & 35.209 & 36.772 & 1473.56 & 1472.35 \\
\hline 11/25/11 7:00 & 35.15 & 36.774 & 1473.62 & 1472.35 \\
\hline $11 / 25 / 11$ 7:30 & 35.122 & 36.774 & 1473.65 & 1472.35 \\
\hline 11/25/11 8:00 & 35.106 & 36.777 & 1473.66 & 1472.35 \\
\hline $11 / 25 / 118: 30$ & 35.239 & 36.781 & 1473.53 & 1472.34 \\
\hline 11/25/11 9:00 & 35.12 & 36.784 & 1473.65 & 1472.34 \\
\hline 11/25/11 9:30 & 35.397 & 36.791 & 1473.37 & 1472.33 \\
\hline $11 / 25 / 11$ 10:00 & 35.385 & 36.8 & 1473.38 & 1472.32 \\
\hline $11 / 25 / 11$ 10:30 & 35.239 & 36.805 & 1473.53 & 1472.32 \\
\hline $11 / 25 / 11$ 11:00 & 35.19 & 36.807 & 1473.58 & 1472.32 \\
\hline $11 / 25 / 11$ 11:30 & 35.153 & 36.798 & 1473.62 & 1472.32 \\
\hline $11 / 25 / 11$ 12:00 & 35.239 & 36.8 & 1473.53 & 1472.32 \\
\hline
\end{tabular}


TABLE S1.3 Automatically recorded groundwater levels in the Klassen wells, September 15, 2011, to March 13, 2012.

\begin{tabular}{|c|c|c|c|c|}
\hline \multicolumn{3}{|c|}{ Reference Elevation (ft AMSL) } & 1508.77 & \multirow{2}{*}{$\begin{array}{l}1509.12 \\
\text { Elevation in Klassen } \\
\text { Test Well (ft AMSL) }\end{array}$} \\
\hline Date and Time & $\begin{array}{l}\text { Depth in Klassen } \\
\text { Domestic Well } \\
\text { (ft BGL) }\end{array}$ & $\begin{array}{c}\text { Depth in Klassen } \\
\text { Test Well } \\
\text { (ft BGL) }\end{array}$ & $\begin{array}{l}\text { Elevation in Klassen } \\
\text { Domestic Well } \\
\text { (ft AMSL) }\end{array}$ & \\
\hline $11 / 25 / 1112: 30$ & 35.188 & 36.802 & 1473.58 & 1472.32 \\
\hline $11 / 25 / 1113: 00$ & 40.05 & 36.81 & 1468.72 & 1472.31 \\
\hline $11 / 25 / 1113: 30$ & 35.277 & 36.805 & 1473.49 & 1472.32 \\
\hline $11 / 25 / 1114: 00$ & 35.256 & 36.812 & 1473.51 & 1472.31 \\
\hline $11 / 25 / 1114: 30$ & 35.45 & 36.81 & 1473.32 & 1472.31 \\
\hline $11 / 25 / 1115: 00$ & 35.249 & 36.807 & 1473.52 & 1472.32 \\
\hline $11 / 25 / 1115: 30$ & 35.636 & 36.812 & 1473.13 & 1472.31 \\
\hline $11 / 25 / 1116: 00$ & 35.277 & 36.812 & 1473.49 & 1472.31 \\
\hline $11 / 25 / 1116: 30$ & 35.237 & 36.809 & 1473.53 & 1472.31 \\
\hline $11 / 25 / 1117: 00$ & 35.211 & 36.807 & 1473.56 & 1472.32 \\
\hline $11 / 25 / 1117: 30$ & 35.176 & 36.802 & 1473.59 & 1472.32 \\
\hline 11/25/11 18:00 & 35.408 & 36.802 & 1473.36 & 1472.32 \\
\hline $11 / 25 / 1118: 30$ & 35.235 & 36.805 & 1473.53 & 1472.32 \\
\hline $11 / 25 / 11$ 19:00 & 35.235 & 36.802 & 1473.53 & 1472.32 \\
\hline 11/25/11 19:30 & 35.434 & 36.812 & 1473.34 & 1472.31 \\
\hline $11 / 25 / 1120: 00$ & 35.34 & 36.821 & 1473.43 & 1472.30 \\
\hline $11 / 25 / 1120: 30$ & 38.291 & 36.821 & 1470.48 & 1472.30 \\
\hline $11 / 25 / 1121: 00$ & 35.434 & 36.828 & 1473.34 & 1472.29 \\
\hline $11 / 25 / 1121: 30$ & 35.265 & 36.833 & 1473.50 & 1472.29 \\
\hline $11 / 25 / 1122: 00$ & 38.375 & 36.828 & 1470.39 & 1472.29 \\
\hline $11 / 25 / 1122: 30$ & 35.265 & 36.831 & 1473.50 & 1472.29 \\
\hline $11 / 25 / 1123: 00$ & 35.434 & 36.831 & 1473.34 & 1472.29 \\
\hline $11 / 25 / 1123: 30$ & 35.239 & 36.833 & 1473.53 & 1472.29 \\
\hline 11/26/11 0:00 & 35.101 & 36.821 & 1473.67 & 1472.30 \\
\hline 11/26/11 0:30 & 34.942 & 36.805 & 1473.83 & 1472.32 \\
\hline 11/26/11 1:00 & 34.953 & 36.793 & 1473.82 & 1472.33 \\
\hline 11/26/11 1:30 & 34.855 & 36.784 & 1473.91 & 1472.34 \\
\hline $11 / 26 / 112: 00$ & 34.773 & 36.779 & 1474.00 & 1472.34 \\
\hline $11 / 26 / 112: 30$ & 34.791 & 36.77 & 1473.98 & 1472.35 \\
\hline 11/26/11 3:00 & 34.686 & 36.758 & 1474.08 & 1472.36 \\
\hline 11/26/11 3:30 & 34.604 & 36.739 & 1474.17 & 1472.38 \\
\hline $11 / 26 / 114: 00$ & 34.533 & 36.727 & 1474.24 & 1472.40 \\
\hline $11 / 26 / 114: 30$ & 34.477 & 36.702 & 1474.29 & 1472.42 \\
\hline 11/26/11 5:00 & 34.421 & 36.671 & 1474.35 & 1472.45 \\
\hline 11/26/11 5:30 & 34.651 & 36.641 & 1474.12 & 1472.48 \\
\hline $11 / 26 / 116: 00$ & 34.4 & 36.624 & 1474.37 & 1472.50 \\
\hline $11 / 26 / 116: 30$ & 34.454 & 36.612 & 1474.32 & 1472.51 \\
\hline 11/26/11 7:00 & 34.351 & 36.594 & 1474.42 & 1472.53 \\
\hline
\end{tabular}


TABLE S1.3 Automatically recorded groundwater levels in the Klassen wells, September 15, 2011, to March 13, 2012.

\begin{tabular}{|c|c|c|c|c|}
\hline \multicolumn{3}{|c|}{ Reference Elevation (ft AMSL) } & 1508.77 & \multirow{2}{*}{$\begin{array}{l}1509.12 \\
\text { Elevation in Klassen } \\
\text { Test Well (ft AMSL) }\end{array}$} \\
\hline Date and Time & $\begin{array}{l}\text { Depth in Klassen } \\
\text { Domestic Well } \\
(\mathrm{ft} \mathrm{BGL})\end{array}$ & $\begin{array}{c}\text { Depth in Klassen } \\
\text { Test Well } \\
\text { (ft BGL) }\end{array}$ & $\begin{array}{l}\text { Elevation in Klassen } \\
\text { Domestic Well } \\
\text { (ft AMSL) }\end{array}$ & \\
\hline $11 / 26 / 117: 30$ & 34.297 & 36.57 & 1474.47 & 1472.55 \\
\hline $11 / 26 / 118: 00$ & 34.264 & 36.556 & 1474.51 & 1472.57 \\
\hline $11 / 26 / 118: 30$ & 34.388 & 36.54 & 1474.38 & 1472.58 \\
\hline 11/26/11 9:00 & 34.562 & 36.535 & 1474.21 & 1472.59 \\
\hline 11/26/11 9:30 & 39.501 & 36.556 & 1469.27 & 1472.57 \\
\hline $11 / 26 / 1110: 00$ & 35.146 & 36.589 & 1473.62 & 1472.53 \\
\hline $11 / 26 / 1110: 30$ & 34.803 & 36.615 & 1473.97 & 1472.51 \\
\hline 11/26/11 11:00 & 37.777 & 36.629 & 1470.99 & 1472.49 \\
\hline $11 / 26 / 1111: 30$ & 34.813 & 36.636 & 1473.96 & 1472.49 \\
\hline $11 / 26 / 1112: 00$ & 34.85 & 36.638 & 1473.92 & 1472.48 \\
\hline $11 / 26 / 1112: 30$ & 34.73 & 36.636 & 1474.04 & 1472.49 \\
\hline $11 / 26 / 11$ 13:00 & 36.956 & 36.636 & 1471.81 & 1472.49 \\
\hline $11 / 26 / 1113: 30$ & 34.728 & 36.636 & 1474.04 & 1472.49 \\
\hline $11 / 26 / 1114: 00$ & 34.676 & 36.636 & 1474.09 & 1472.49 \\
\hline $11 / 26 / 1114: 30$ & 34.735 & 36.634 & 1474.03 & 1472.49 \\
\hline $11 / 26 / 11$ 15:00 & 34.679 & 36.629 & 1474.09 & 1472.49 \\
\hline $11 / 26 / 11$ 15:30 & 34.646 & 36.626 & 1474.12 & 1472.50 \\
\hline 11/26/11 16:00 & 34.62 & 36.627 & 1474.15 & 1472.50 \\
\hline $11 / 26 / 11$ 16:30 & 34.597 & 36.619 & 1474.17 & 1472.50 \\
\hline $11 / 26 / 11$ 17:00 & 44.749 & 36.619 & 1464.02 & 1472.50 \\
\hline $11 / 26 / 11$ 17:30 & 35.404 & 36.652 & 1473.37 & 1472.47 \\
\hline $11 / 26 / 11$ 18:00 & 35.157 & 36.664 & 1473.61 & 1472.46 \\
\hline $11 / 26 / 11$ 18:30 & 35.005 & 36.669 & 1473.76 & 1472.45 \\
\hline $11 / 26 / 11$ 19:00 & 35.195 & 36.671 & 1473.57 & 1472.45 \\
\hline 11/26/11 19:30 & 35.038 & 36.678 & 1473.73 & 1472.44 \\
\hline $11 / 26 / 1120: 00$ & 35.108 & 36.683 & 1473.66 & 1472.44 \\
\hline $11 / 26 / 1120: 30$ & 34.942 & 36.69 & 1473.83 & 1472.43 \\
\hline $11 / 26 / 1121: 00$ & 35.511 & 36.683 & 1473.26 & 1472.44 \\
\hline $11 / 26 / 1121: 30$ & 34.749 & 36.678 & 1474.02 & 1472.44 \\
\hline $11 / 26 / 1122: 00$ & 35.293 & 36.671 & 1473.48 & 1472.45 \\
\hline $11 / 26 / 1122: 30$ & 35.19 & 36.673 & 1473.58 & 1472.45 \\
\hline $11 / 26 / 1123: 00$ & 34.738 & 36.671 & 1474.03 & 1472.45 \\
\hline $11 / 26 / 11$ 23:30 & 34.641 & 36.662 & 1474.13 & 1472.46 \\
\hline 11/27/11 0:00 & 34.573 & 36.659 & 1474.20 & 1472.46 \\
\hline 11/27/11 0:30 & 34.496 & 36.652 & 1474.27 & 1472.47 \\
\hline 11/27/11 1:00 & 34.496 & 36.645 & 1474.27 & 1472.48 \\
\hline $11 / 27 / 11$ 1:30 & 34.442 & 36.645 & 1474.33 & 1472.48 \\
\hline 11/27/11 2:00 & 34.456 & 36.633 & 1474.31 & 1472.49 \\
\hline
\end{tabular}


TABLE S1.3 Automatically recorded groundwater levels in the Klassen wells, September 15, 2011, to March 13, 2012.

\begin{tabular}{|c|c|c|c|c|}
\hline \multicolumn{3}{|c|}{ Reference Elevation (ft AMSL) } & 1508.77 & \multirow{2}{*}{$\begin{array}{l}1509.12 \\
\text { Elevation in Klassen } \\
\text { Test Well (ft AMSL) }\end{array}$} \\
\hline Date and Time & $\begin{array}{l}\text { Depth in Klassen } \\
\text { Domestic Well } \\
\text { (ft BGL) }\end{array}$ & $\begin{array}{c}\text { Depth in Klassen } \\
\text { Test Well } \\
\text { (ft BGL) }\end{array}$ & $\begin{array}{l}\text { Elevation in Klassen } \\
\text { Domestic Well } \\
\text { (ft AMSL) }\end{array}$ & \\
\hline $11 / 27 / 112: 30$ & 34.369 & 36.617 & 1474.40 & 1472.51 \\
\hline 11/27/11 3:00 & 34.313 & 36.605 & 1474.46 & 1472.52 \\
\hline 11/27/11 3:30 & 34.268 & 36.591 & 1474.50 & 1472.53 \\
\hline $11 / 27 / 114: 00$ & 34.226 & 36.584 & 1474.54 & 1472.54 \\
\hline $11 / 27 / 114: 30$ & 34.275 & 36.575 & 1474.49 & 1472.55 \\
\hline 11/27/11 5:00 & 34.322 & 36.566 & 1474.45 & 1472.56 \\
\hline $11 / 27 / 115: 30$ & 34.207 & 36.554 & 1474.56 & 1472.57 \\
\hline $11 / 27 / 116: 00$ & 34.156 & 36.542 & 1474.61 & 1472.58 \\
\hline $11 / 27 / 116: 30$ & 34.184 & 36.53 & 1474.59 & 1472.59 \\
\hline $11 / 27 / 11$ 7:00 & 34.128 & 36.514 & 1474.64 & 1472.61 \\
\hline $11 / 27 / 117: 30$ & 34.088 & 36.502 & 1474.68 & 1472.62 \\
\hline 11/27/11 8:00 & 34.055 & 36.497 & 1474.71 & 1472.63 \\
\hline $11 / 27 / 118: 30$ & 34.027 & 36.495 & 1474.74 & 1472.63 \\
\hline 11/27/11 9:00 & 34.083 & 36.486 & 1474.69 & 1472.64 \\
\hline 11/27/11 9:30 & 34.154 & 36.495 & 1474.62 & 1472.63 \\
\hline $11 / 27 / 11$ 10:00 & 34.116 & 36.502 & 1474.65 & 1472.62 \\
\hline $11 / 27 / 11$ 10:30 & 34.114 & 36.521 & 1474.66 & 1472.60 \\
\hline 11/27/11 11:00 & 34.107 & 36.563 & 1474.66 & 1472.56 \\
\hline 11/27/11 11:30 & 34.048 & 36.584 & 1474.72 & 1472.54 \\
\hline $11 / 27 / 1112: 00$ & 34.008 & 36.596 & 1474.76 & 1472.53 \\
\hline $11 / 27 / 11$ 12:30 & 33.975 & 36.605 & 1474.79 & 1472.52 \\
\hline $11 / 27 / 11$ 13:00 & 33.947 & 36.61 & 1474.82 & 1472.51 \\
\hline $11 / 27 / 11$ 13:30 & 33.924 & 36.612 & 1474.85 & 1472.51 \\
\hline $11 / 27 / 11$ 14:00 & 33.902 & 36.596 & 1474.87 & 1472.53 \\
\hline $11 / 27 / 11$ 14:30 & 33.903 & 36.577 & 1474.87 & 1472.55 \\
\hline $11 / 27 / 11$ 15:00 & 33.874 & 36.566 & 1474.90 & 1472.56 \\
\hline $11 / 27 / 11$ 15:30 & 33.856 & 36.565 & 1474.91 & 1472.56 \\
\hline $11 / 27 / 11$ 16:00 & 33.844 & 36.563 & 1474.93 & 1472.56 \\
\hline $11 / 27 / 11$ 16:30 & 33.987 & 36.565 & 1474.78 & 1472.56 \\
\hline 11/27/11 17:00 & 33.971 & 36.568 & 1474.80 & 1472.55 \\
\hline $11 / 27 / 11$ 17:30 & 33.919 & 36.561 & 1474.85 & 1472.56 \\
\hline $11 / 27 / 11$ 18:00 & 33.879 & 36.549 & 1474.89 & 1472.57 \\
\hline $11 / 27 / 11$ 18:30 & 33.851 & 36.535 & 1474.92 & 1472.59 \\
\hline 11/27/11 19:00 & 33.874 & 36.528 & 1474.90 & 1472.59 \\
\hline 11/27/11 19:30 & 33.889 & 36.528 & 1474.88 & 1472.59 \\
\hline $11 / 27 / 1120: 00$ & 33.9 & 36.518 & 1474.87 & 1472.60 \\
\hline $11 / 27 / 1120: 30$ & 33.98 & 36.523 & 1474.79 & 1472.60 \\
\hline $11 / 27 / 1121: 00$ & 34.02 & 36.523 & 1474.75 & 1472.60 \\
\hline
\end{tabular}


TABLE S1.3 Automatically recorded groundwater levels in the Klassen wells, September 15, 2011, to March 13, 2012.

\begin{tabular}{|c|c|c|c|c|}
\hline \multicolumn{3}{|c|}{ Reference Elevation (ft AMSL) } & 1508.77 & \multirow{2}{*}{$\begin{array}{l}1509.12 \\
\text { Elevation in Klassen } \\
\text { Test Well (ft AMSL) }\end{array}$} \\
\hline Date and Time & $\begin{array}{l}\text { Depth in Klassen } \\
\text { Domestic Well } \\
\text { (ft BGL) }\end{array}$ & $\begin{array}{c}\text { Depth in Klassen } \\
\text { Test Well } \\
\text { (ft BGL) }\end{array}$ & $\begin{array}{l}\text { Elevation in Klassen } \\
\text { Domestic Well } \\
\text { (ft AMSL) }\end{array}$ & \\
\hline $11 / 27 / 1121: 30$ & 33.968 & 36.523 & 1474.80 & 1472.60 \\
\hline $11 / 27 / 1122: 00$ & 33.912 & 36.521 & 1474.86 & 1472.60 \\
\hline $11 / 27 / 1122: 30$ & 33.872 & 36.516 & 1474.90 & 1472.61 \\
\hline $11 / 27 / 1123: 00$ & 33.844 & 36.514 & 1474.93 & 1472.61 \\
\hline $11 / 27 / 1123: 30$ & 33.816 & 36.509 & 1474.95 & 1472.61 \\
\hline $11 / 28 / 110: 00$ & 33.797 & 36.505 & 1474.97 & 1472.62 \\
\hline $11 / 28 / 110: 30$ & 33.778 & 36.495 & 1474.99 & 1472.63 \\
\hline 11/28/11 1:00 & 33.757 & 36.49 & 1475.01 & 1472.63 \\
\hline $11 / 28 / 111: 30$ & 33.741 & 36.488 & 1475.03 & 1472.63 \\
\hline $11 / 28 / 112: 00$ & 33.727 & 36.479 & 1475.04 & 1472.64 \\
\hline $11 / 28 / 112: 30$ & 33.734 & 36.476 & 1475.04 & 1472.65 \\
\hline 11/28/11 3:00 & 33.715 & 36.472 & 1475.05 & 1472.65 \\
\hline 11/28/11 3:30 & 33.699 & 36.46 & 1475.07 & 1472.66 \\
\hline $11 / 28 / 114: 00$ & 33.682 & 36.448 & 1475.09 & 1472.67 \\
\hline $11 / 28 / 114: 30$ & 33.666 & 36.443 & 1475.10 & 1472.68 \\
\hline 11/28/11 5:00 & 33.654 & 36.434 & 1475.12 & 1472.69 \\
\hline $11 / 28 / 11$ 5:30 & 33.64 & 36.42 & 1475.13 & 1472.70 \\
\hline $11 / 28 / 116: 00$ & 33.631 & 36.408 & 1475.14 & 1472.71 \\
\hline $11 / 28 / 116: 30$ & 33.663 & 36.404 & 1475.11 & 1472.72 \\
\hline $11 / 28 / 11$ 7:00 & 33.753 & 36.404 & 1475.02 & 1472.72 \\
\hline $11 / 28 / 117: 30$ & 33.764 & 36.401 & 1475.01 & 1472.72 \\
\hline 11/28/11 8:00 & 33.71 & 36.404 & 1475.06 & 1472.72 \\
\hline $11 / 28 / 118: 30$ & 33.682 & 36.406 & 1475.09 & 1472.72 \\
\hline 11/28/11 9:00 & 33.656 & 36.396 & 1475.11 & 1472.73 \\
\hline 11/28/11 9:30 & 33.635 & 36.399 & 1475.13 & 1472.72 \\
\hline $11 / 28 / 11$ 10:00 & 33.621 & 36.411 & 1475.15 & 1472.71 \\
\hline $11 / 28 / 11$ 10:30 & 33.605 & 36.413 & 1475.16 & 1472.71 \\
\hline $11 / 28 / 1111: 00$ & 33.593 & 36.418 & 1475.18 & 1472.70 \\
\hline 11/28/11 11:30 & 33.584 & 36.42 & 1475.19 & 1472.70 \\
\hline $11 / 28 / 11$ 12:00 & 33.572 & 36.422 & 1475.20 & 1472.70 \\
\hline $11 / 28 / 11$ 12:30 & 33.56 & 36.422 & 1475.21 & 1472.70 \\
\hline $11 / 28 / 11$ 13:00 & 33.551 & 36.361 & 1475.22 & 1472.76 \\
\hline $11 / 28 / 11$ 13:30 & 33.541 & 36.314 & 1475.23 & 1472.81 \\
\hline $11 / 28 / 11$ 14:00 & 33.534 & 36.298 & 1475.24 & 1472.82 \\
\hline $11 / 28 / 11$ 14:30 & 33.53 & 36.291 & 1475.24 & 1472.83 \\
\hline $11 / 28 / 11$ 15:00 & 33.523 & 36.284 & 1475.25 & 1472.84 \\
\hline $11 / 28 / 11$ 15:30 & 33.516 & 36.279 & 1475.25 & 1472.84 \\
\hline $11 / 28 / 11$ 16:00 & 33.558 & 36.27 & 1475.21 & 1472.85 \\
\hline
\end{tabular}


TABLE S1.3 Automatically recorded groundwater levels in the Klassen wells, September 15, 2011, to March 13, 2012.

\begin{tabular}{|c|c|c|c|c|}
\hline \multicolumn{3}{|c|}{ Reference Elevation (ft AMSL) } & 1508.77 & \multirow{2}{*}{$\begin{array}{l}1509.12 \\
\text { Elevation in Klassen } \\
\text { Test Well (ft AMSL) }\end{array}$} \\
\hline Date and Time & $\begin{array}{l}\text { Depth in Klassen } \\
\text { Domestic Well } \\
\text { (ft BGL) }\end{array}$ & $\begin{array}{c}\text { Depth in Klassen } \\
\text { Test Well } \\
\text { (ft BGL) }\end{array}$ & $\begin{array}{l}\text { Elevation in Klassen } \\
\text { Domestic Well } \\
\text { (ft AMSL) }\end{array}$ & \\
\hline $11 / 28 / 1116: 30$ & 33.56 & 36.265 & 1475.21 & 1472.86 \\
\hline 11/28/11 17:00 & 33.541 & 36.26 & 1475.23 & 1472.86 \\
\hline 11/28/11 17:30 & 33.551 & 36.256 & 1475.22 & 1472.87 \\
\hline $11 / 28 / 11$ 18:00 & 33.567 & 36.253 & 1475.20 & 1472.87 \\
\hline $11 / 28 / 1118: 30$ & 33.722 & 36.258 & 1475.05 & 1472.86 \\
\hline 11/28/11 19:00 & 33.706 & 36.263 & 1475.06 & 1472.86 \\
\hline 11/28/11 19:30 & 33.774 & 36.265 & 1475.00 & 1472.86 \\
\hline $11 / 28 / 1120: 00$ & 33.907 & 36.27 & 1474.86 & 1472.85 \\
\hline $11 / 28 / 1120: 30$ & 33.961 & 36.282 & 1474.81 & 1472.84 \\
\hline $11 / 28 / 1121: 00$ & 33.959 & 36.286 & 1474.81 & 1472.84 \\
\hline $11 / 28 / 1121: 30$ & 33.867 & 36.279 & 1474.90 & 1472.84 \\
\hline $11 / 28 / 1122: 00$ & 33.804 & 36.275 & 1474.97 & 1472.85 \\
\hline $11 / 28 / 1122: 30$ & 33.76 & 36.27 & 1475.01 & 1472.85 \\
\hline $11 / 28 / 1123: 00$ & 33.724 & 36.265 & 1475.05 & 1472.86 \\
\hline 11/28/11 23:30 & 33.694 & 36.265 & 1475.08 & 1472.86 \\
\hline 11/29/11 0:00 & 33.668 & 36.268 & 1475.10 & 1472.85 \\
\hline 11/29/11 0:30 & 33.652 & 36.27 & 1475.12 & 1472.85 \\
\hline $11 / 29 / 11$ 1:00 & 33.76 & 36.274 & 1475.01 & 1472.85 \\
\hline $11 / 29 / 11$ 1:30 & 33.729 & 36.27 & 1475.04 & 1472.85 \\
\hline $11 / 29 / 112: 00$ & 33.713 & 36.263 & 1475.06 & 1472.86 \\
\hline $11 / 29 / 112: 30$ & 33.811 & 36.258 & 1474.96 & 1472.86 \\
\hline 11/29/11 3:00 & 33.753 & 36.253 & 1475.02 & 1472.87 \\
\hline $11 / 29 / 113: 30$ & 33.706 & 36.258 & 1475.06 & 1472.86 \\
\hline $11 / 29 / 114: 00$ & 33.675 & 36.253 & 1475.09 & 1472.87 \\
\hline $11 / 29 / 114: 30$ & 33.649 & 36.249 & 1475.12 & 1472.87 \\
\hline 11/29/11 5:00 & 33.626 & 36.246 & 1475.14 & 1472.88 \\
\hline $11 / 29 / 115: 30$ & 33.607 & 36.237 & 1475.16 & 1472.89 \\
\hline $11 / 29 / 116: 00$ & 33.591 & 36.23 & 1475.18 & 1472.89 \\
\hline $11 / 29 / 116: 30$ & 33.652 & 36.225 & 1475.12 & 1472.90 \\
\hline 11/29/11 7:00 & 33.619 & 36.214 & 1475.15 & 1472.91 \\
\hline $11 / 29 / 117: 30$ & 33.694 & 36.209 & 1475.08 & 1472.91 \\
\hline $11 / 29 / 118: 00$ & 33.645 & 36.211 & 1475.12 & 1472.91 \\
\hline $11 / 29 / 118: 30$ & 33.612 & 36.209 & 1475.16 & 1472.91 \\
\hline 11/29/11 9:00 & 33.591 & 36.211 & 1475.18 & 1472.91 \\
\hline 11/29/11 9:30 & 33.57 & 36.213 & 1475.20 & 1472.91 \\
\hline 11/29/11 10:00 & 33.553 & 36.221 & 1475.22 & 1472.90 \\
\hline 11/29/11 10:30 & 33.541 & 36.221 & 1475.23 & 1472.90 \\
\hline 11/29/11 11:00 & 33.527 & 36.235 & 1475.24 & 1472.89 \\
\hline
\end{tabular}


TABLE S1.3 Automatically recorded groundwater levels in the Klassen wells, September 15, 2011, to March 13, 2012.

\begin{tabular}{|c|c|c|c|c|}
\hline \multicolumn{3}{|c|}{ Reference Elevation (ft AMSL) } & 1508.77 & \multirow{2}{*}{$\begin{array}{l}1509.12 \\
\text { Elevation in Klassen } \\
\text { Test Well (ft AMSL) }\end{array}$} \\
\hline Date and Time & $\begin{array}{l}\text { Depth in Klassen } \\
\text { Domestic Well } \\
\text { (ft BGL) }\end{array}$ & $\begin{array}{c}\text { Depth in Klassen } \\
\text { Test Well } \\
\text { (ft BGL) }\end{array}$ & $\begin{array}{l}\text { Elevation in Klassen } \\
\text { Domestic Well } \\
\text { (ft AMSL) }\end{array}$ & \\
\hline 11/29/11 11:30 & 33.516 & 36.235 & 1475.25 & 1472.89 \\
\hline $11 / 29 / 1112: 00$ & 33.506 & 36.237 & 1475.26 & 1472.89 \\
\hline $11 / 29 / 1112: 30$ & 33.495 & 36.242 & 1475.27 & 1472.88 \\
\hline $11 / 29 / 1113: 00$ & 33.485 & 36.249 & 1475.28 & 1472.87 \\
\hline $11 / 29 / 1113: 30$ & 33.476 & 36.244 & 1475.29 & 1472.88 \\
\hline $11 / 29 / 1114: 00$ & 33.466 & 36.228 & 1475.30 & 1472.89 \\
\hline $11 / 29 / 1114: 30$ & 33.459 & 36.192 & 1475.31 & 1472.93 \\
\hline $11 / 29 / 11$ 15:00 & 33.452 & 36.176 & 1475.32 & 1472.95 \\
\hline $11 / 29 / 1115: 30$ & 33.445 & 36.171 & 1475.32 & 1472.95 \\
\hline 11/29/11 16:00 & 33.464 & 36.169 & 1475.31 & 1472.95 \\
\hline 11/29/11 16:30 & 33.445 & 36.162 & 1475.32 & 1472.96 \\
\hline 11/29/11 17:00 & 33.436 & 36.155 & 1475.33 & 1472.97 \\
\hline $11 / 29 / 1117: 30$ & 33.689 & 36.16 & 1475.08 & 1472.96 \\
\hline 11/29/11 18:00 & 33.757 & 36.176 & 1475.01 & 1472.95 \\
\hline $11 / 29 / 11$ 18:30 & 33.682 & 36.181 & 1475.09 & 1472.94 \\
\hline 11/29/11 19:00 & 33.626 & 36.178 & 1475.14 & 1472.94 \\
\hline 11/29/11 19:30 & 33.586 & 36.174 & 1475.18 & 1472.95 \\
\hline 11/29/11 20:00 & 33.677 & 36.176 & 1475.09 & 1472.95 \\
\hline $11 / 29 / 1120: 30$ & 33.75 & 36.181 & 1475.02 & 1472.94 \\
\hline 11/29/11 21:00 & 33.764 & 36.183 & 1475.01 & 1472.94 \\
\hline 11/29/11 21:30 & 33.738 & 36.181 & 1475.03 & 1472.94 \\
\hline $11 / 29 / 1122: 00$ & 33.701 & 36.178 & 1475.07 & 1472.94 \\
\hline $11 / 29 / 1122: 30$ & 33.652 & 36.176 & 1475.12 & 1472.95 \\
\hline $11 / 29 / 1123: 00$ & 33.616 & 36.176 & 1475.15 & 1472.95 \\
\hline $11 / 29 / 1123: 30$ & 33.616 & 36.176 & 1475.15 & 1472.95 \\
\hline $11 / 30 / 110: 00$ & 33.609 & 36.174 & 1475.16 & 1472.95 \\
\hline $11 / 30 / 110: 30$ & 33.577 & 36.178 & 1475.19 & 1472.94 \\
\hline 11/30/11 1:00 & 33.551 & 36.181 & 1475.22 & 1472.94 \\
\hline 11/30/11 1:30 & 33.532 & 36.178 & 1475.24 & 1472.94 \\
\hline $11 / 30 / 112: 00$ & 33.516 & 36.174 & 1475.25 & 1472.95 \\
\hline $11 / 30 / 112: 30$ & 33.499 & 36.169 & 1475.27 & 1472.95 \\
\hline 11/30/11 3:00 & 33.487 & 36.171 & 1475.28 & 1472.95 \\
\hline $11 / 30 / 113: 30$ & 33.473 & 36.162 & 1475.30 & 1472.96 \\
\hline 11/30/11 4:00 & 33.462 & 36.164 & 1475.31 & 1472.96 \\
\hline $11 / 30 / 114: 30$ & 33.45 & 36.16 & 1475.32 & 1472.96 \\
\hline 11/30/11 5:00 & 33.438 & 36.152 & 1475.33 & 1472.97 \\
\hline 11/30/11 5:30 & 33.429 & 36.148 & 1475.34 & 1472.97 \\
\hline 11/30/11 6:00 & 33.417 & 36.141 & 1475.35 & 1472.98 \\
\hline
\end{tabular}


TABLE S1.3 Automatically recorded groundwater levels in the Klassen wells, September 15, 2011, to March 13, 2012.

\begin{tabular}{|c|c|c|c|c|}
\hline \multicolumn{3}{|c|}{ Reference Elevation (ft AMSL) } & 1508.77 & \multirow{2}{*}{$\begin{array}{l}1509.12 \\
\text { Elevation in Klassen } \\
\text { Test Well (ft AMSL) }\end{array}$} \\
\hline Date and Time & $\begin{array}{l}\text { Depth in Klassen } \\
\text { Domestic Well } \\
\text { (ft BGL) }\end{array}$ & $\begin{array}{c}\text { Depth in Klassen } \\
\text { Test Well } \\
\text { (ft BGL) }\end{array}$ & $\begin{array}{l}\text { Elevation in Klassen } \\
\text { Domestic Well } \\
\text { (ft AMSL) }\end{array}$ & \\
\hline $11 / 30 / 116: 30$ & 33.478 & 36.138 & 1475.29 & 1472.98 \\
\hline $11 / 30 / 117: 00$ & 33.448 & 36.134 & 1475.32 & 1472.99 \\
\hline 11/30/11 7:30 & 33.511 & 36.131 & 1475.26 & 1472.99 \\
\hline $11 / 30 / 118: 00$ & 33.469 & 36.134 & 1475.30 & 1472.99 \\
\hline 11/30/11 8:30 & 33.445 & 36.141 & 1475.32 & 1472.98 \\
\hline 11/30/11 9:00 & 33.616 & 36.138 & 1475.15 & 1472.98 \\
\hline 11/30/11 9:30 & 33.478 & 36.146 & 1475.29 & 1472.98 \\
\hline $11 / 30 / 11$ 10:00 & 33.443 & 36.152 & 1475.33 & 1472.97 \\
\hline $11 / 30 / 11$ 10:30 & 33.424 & 36.155 & 1475.35 & 1472.97 \\
\hline $11 / 30 / 1111: 00$ & 33.403 & 36.167 & 1475.37 & 1472.96 \\
\hline 11/30/11 11:30 & 33.389 & 36.174 & 1475.38 & 1472.95 \\
\hline $11 / 30 / 1112: 00$ & 33.375 & 36.134 & 1475.39 & 1472.99 \\
\hline $11 / 30 / 1112: 30$ & 33.368 & 36.089 & 1475.40 & 1473.03 \\
\hline $11 / 30 / 1113: 00$ & 33.356 & 36.073 & 1475.41 & 1473.05 \\
\hline 11/30/11 13:30 & 33.347 & 36.063 & 1475.42 & 1473.06 \\
\hline $11 / 30 / 11$ 14:00 & 33.337 & 36.059 & 1475.43 & 1473.06 \\
\hline $11 / 30 / 11$ 14:30 & 33.33 & 36.056 & 1475.44 & 1473.07 \\
\hline 11/30/11 15:00 & 33.323 & 36.056 & 1475.45 & 1473.07 \\
\hline 11/30/11 15:30 & 33.316 & 36.049 & 1475.45 & 1473.07 \\
\hline $11 / 30 / 11$ 16:00 & 33.34 & 36.052 & 1475.43 & 1473.07 \\
\hline 11/30/11 16:30 & 33.323 & 36.047 & 1475.45 & 1473.08 \\
\hline 11/30/11 17:00 & 33.335 & 36.042 & 1475.43 & 1473.08 \\
\hline 11/30/11 17:30 & 33.344 & 36.04 & 1475.43 & 1473.08 \\
\hline 11/30/11 18:00 & 33.328 & 36.033 & 1475.44 & 1473.09 \\
\hline 11/30/11 18:30 & 33.316 & 36.028 & 1475.45 & 1473.09 \\
\hline 11/30/11 19:00 & 33.307 & 36.028 & 1475.46 & 1473.09 \\
\hline 11/30/11 19:30 & 33.323 & 36.03 & 1475.45 & 1473.09 \\
\hline $11 / 30 / 1120: 00$ & 33.309 & 36.035 & 1475.46 & 1473.09 \\
\hline $11 / 30 / 1120: 30$ & 33.415 & 36.04 & 1475.35 & 1473.08 \\
\hline 11/30/11 21:00 & 33.48 & 36.056 & 1475.29 & 1473.07 \\
\hline 11/30/11 21:30 & 33.427 & 36.061 & 1475.34 & 1473.06 \\
\hline $11 / 30 / 1122: 00$ & 33.525 & 36.085 & 1475.24 & 1473.04 \\
\hline $11 / 30 / 1122: 30$ & 33.49 & 36.12 & 1475.28 & 1473.00 \\
\hline $11 / 30 / 1123: 00$ & 33.532 & 36.162 & 1475.24 & 1472.96 \\
\hline $11 / 30 / 1123: 30$ & 33.579 & 36.206 & 1475.19 & 1472.92 \\
\hline $12 / 1 / 110: 00$ & 33.518 & 36.242 & 1475.25 & 1472.88 \\
\hline $12 / 1 / 110: 30$ & 33.478 & 36.279 & 1475.29 & 1472.84 \\
\hline $12 / 1 / 11$ 1:00 & 33.452 & 36.317 & 1475.32 & 1472.81 \\
\hline
\end{tabular}


TABLE S1.3 Automatically recorded groundwater levels in the Klassen wells, September 15, 2011, to March 13, 2012.

\begin{tabular}{|c|c|c|c|c|}
\hline \multicolumn{3}{|c|}{ Reference Elevation (ft AMSL) } & 1508.77 & \multirow{2}{*}{$\begin{array}{l}1509.12 \\
\text { Elevation in Klassen } \\
\text { Test Well (ft AMSL) }\end{array}$} \\
\hline Date and Time & $\begin{array}{l}\text { Depth in Klassen } \\
\text { Domestic Well } \\
(\mathrm{ft} \mathrm{BGL})\end{array}$ & $\begin{array}{c}\text { Depth in Klassen } \\
\text { Test Well } \\
\text { (ft BGL) }\end{array}$ & $\begin{array}{l}\text { Elevation in Klassen } \\
\text { Domestic Well } \\
\text { (ft AMSL) }\end{array}$ & \\
\hline $12 / 1 / 111: 30$ & 33.473 & 36.354 & 1475.30 & 1472.77 \\
\hline $12 / 1 / 112: 00$ & 33.45 & 36.385 & 1475.32 & 1472.74 \\
\hline $12 / 1 / 112: 30$ & 33.429 & 36.411 & 1475.34 & 1472.71 \\
\hline 12/1/11 3:00 & 33.412 & 36.436 & 1475.36 & 1472.69 \\
\hline $12 / 1 / 113: 30$ & 33.398 & 36.462 & 1475.37 & 1472.66 \\
\hline $12 / 1 / 114: 00$ & 33.389 & 36.488 & 1475.38 & 1472.63 \\
\hline $12 / 1 / 114: 30$ & 33.377 & 36.511 & 1475.39 & 1472.61 \\
\hline $12 / 1 / 115: 00$ & 33.398 & 36.542 & 1475.37 & 1472.58 \\
\hline $12 / 1 / 115: 30$ & 33.382 & 36.566 & 1475.39 & 1472.56 \\
\hline $12 / 1 / 116: 00$ & 33.37 & 36.591 & 1475.40 & 1472.53 \\
\hline $12 / 1 / 116: 30$ & 33.499 & 36.619 & 1475.27 & 1472.50 \\
\hline $12 / 1 / 117: 00$ & 33.455 & 36.648 & 1475.31 & 1472.47 \\
\hline $12 / 1 / 117: 30$ & 33.506 & 36.676 & 1475.26 & 1472.45 \\
\hline $12 / 1 / 118: 00$ & 33.485 & 36.713 & 1475.28 & 1472.41 \\
\hline $12 / 1 / 118: 30$ & 33.455 & 36.739 & 1475.31 & 1472.38 \\
\hline $12 / 1 / 119: 00$ & 33.436 & 36.767 & 1475.33 & 1472.36 \\
\hline $12 / 1 / 119: 30$ & 33.419 & 36.793 & 1475.35 & 1472.33 \\
\hline $12 / 1 / 11$ 10:00 & 33.408 & 36.824 & 1475.36 & 1472.30 \\
\hline $12 / 1 / 11$ 10:30 & 33.398 & 36.842 & 1475.37 & 1472.28 \\
\hline 12/1/11 11:00 & 33.391 & 36.861 & 1475.38 & 1472.26 \\
\hline $12 / 1 / 1111: 30$ & 33.382 & 36.868 & 1475.39 & 1472.25 \\
\hline $12 / 1 / 11$ 12:00 & 33.375 & 36.887 & 1475.39 & 1472.24 \\
\hline $12 / 1 / 1112: 30$ & 33.37 & 36.896 & 1475.40 & 1472.23 \\
\hline $12 / 1 / 11$ 13:00 & 33.368 & 36.901 & 1475.40 & 1472.22 \\
\hline $12 / 1 / 11$ 13:30 & 33.359 & 36.91 & 1475.41 & 1472.21 \\
\hline 12/1/11 14:00 & 33.354 & 36.91 & 1475.42 & 1472.21 \\
\hline $12 / 1 / 11$ 14:30 & 33.351 & 36.903 & 1475.42 & 1472.22 \\
\hline 12/1/11 15:00 & 33.347 & 36.885 & 1475.42 & 1472.24 \\
\hline $12 / 1 / 11$ 15:30 & 33.37 & 36.873 & 1475.40 & 1472.25 \\
\hline $12 / 1 / 11$ 16:00 & 33.384 & 36.861 & 1475.39 & 1472.26 \\
\hline 12/1/11 16:30 & 33.598 & 36.849 & 1475.17 & 1472.27 \\
\hline 12/1/11 17:00 & 33.427 & 36.838 & 1475.34 & 1472.28 \\
\hline $12 / 1 / 11$ 17:30 & 33.652 & 36.835 & 1475.12 & 1472.29 \\
\hline 12/1/11 18:00 & 33.874 & 36.828 & 1474.90 & 1472.29 \\
\hline $12 / 1 / 11$ 18:30 & 35.497 & 36.826 & 1473.27 & 1472.30 \\
\hline 12/1/11 19:00 & 33.844 & 36.828 & 1474.93 & 1472.29 \\
\hline $12 / 1 / 11$ 19:30 & 33.722 & 36.828 & 1475.05 & 1472.29 \\
\hline $12 / 1 / 1120: 00$ & 33.649 & 36.828 & 1475.12 & 1472.29 \\
\hline
\end{tabular}


TABLE S1.3 Automatically recorded groundwater levels in the Klassen wells, September 15, 2011, to March 13, 2012.

\begin{tabular}{|c|c|c|c|c|}
\hline \multicolumn{3}{|c|}{ Reference Elevation (ft AMSL) } & 1508.77 & \multirow{2}{*}{$\begin{array}{l}1509.12 \\
\text { Elevation in Klassen } \\
\text { Test Well (ft AMSL) }\end{array}$} \\
\hline Date and Time & $\begin{array}{l}\text { Depth in Klassen } \\
\text { Domestic Well } \\
(\mathrm{ft} \mathrm{BGL})\end{array}$ & $\begin{array}{c}\text { Depth in Klassen } \\
\text { Test Well } \\
\text { (ft BGL) }\end{array}$ & $\begin{array}{l}\text { Elevation in Klassen } \\
\text { Domestic Well } \\
\text { (ft AMSL) }\end{array}$ & \\
\hline $12 / 1 / 1120: 30$ & 33.605 & 36.828 & 1475.16 & 1472.29 \\
\hline $12 / 1 / 1121: 00$ & 33.57 & 36.826 & 1475.20 & 1472.30 \\
\hline $12 / 1 / 1121: 30$ & 33.642 & 36.833 & 1475.13 & 1472.29 \\
\hline $12 / 1 / 1122: 00$ & 33.602 & 36.826 & 1475.17 & 1472.30 \\
\hline $12 / 1 / 1122: 30$ & 33.593 & 36.819 & 1475.18 & 1472.30 \\
\hline $12 / 1 / 1123: 00$ & 33.581 & 36.819 & 1475.19 & 1472.30 \\
\hline $12 / 1 / 1123: 30$ & 33.612 & 36.816 & 1475.16 & 1472.31 \\
\hline $12 / 2 / 110: 00$ & 33.565 & 36.814 & 1475.20 & 1472.31 \\
\hline $12 / 2 / 110: 30$ & 33.532 & 36.812 & 1475.24 & 1472.31 \\
\hline $12 / 2 / 111: 00$ & 33.64 & 36.81 & 1475.13 & 1472.31 \\
\hline $12 / 2 / 111: 30$ & 33.612 & 36.802 & 1475.16 & 1472.32 \\
\hline $12 / 2 / 112: 00$ & 33.593 & 36.798 & 1475.18 & 1472.32 \\
\hline $12 / 2 / 112: 30$ & 33.689 & 36.791 & 1475.08 & 1472.33 \\
\hline $12 / 2 / 113: 00$ & 33.633 & 36.784 & 1475.14 & 1472.34 \\
\hline $12 / 2 / 113: 30$ & 33.659 & 36.779 & 1475.11 & 1472.34 \\
\hline $12 / 2 / 114: 00$ & 33.6 & 36.779 & 1475.17 & 1472.34 \\
\hline $12 / 2 / 114: 30$ & 33.565 & 36.777 & 1475.20 & 1472.35 \\
\hline 12/2/11 5:00 & 33.539 & 36.767 & 1475.23 & 1472.36 \\
\hline $12 / 2 / 115: 30$ & 33.518 & 36.76 & 1475.25 & 1472.36 \\
\hline $12 / 2 / 116: 00$ & 33.534 & 36.753 & 1475.24 & 1472.37 \\
\hline $12 / 2 / 116: 30$ & 33.628 & 36.748 & 1475.14 & 1472.37 \\
\hline $12 / 2 / 117: 00$ & 33.588 & 36.737 & 1475.18 & 1472.39 \\
\hline $12 / 2 / 117: 30$ & 33.588 & 36.73 & 1475.18 & 1472.39 \\
\hline $12 / 2 / 118: 00$ & 33.717 & 36.732 & 1475.05 & 1472.39 \\
\hline $12 / 2 / 118: 30$ & 37.997 & 36.727 & 1470.77 & 1472.40 \\
\hline $12 / 2 / 119: 00$ & 33.905 & 36.727 & 1474.86 & 1472.40 \\
\hline $12 / 2 / 119: 30$ & 33.898 & 36.73 & 1474.87 & 1472.39 \\
\hline 12/2/11 10:00 & 33.846 & 36.727 & 1474.92 & 1472.40 \\
\hline $12 / 2 / 11$ 10:30 & 33.818 & 36.73 & 1474.95 & 1472.39 \\
\hline 12/2/11 11:00 & 37.256 & 36.737 & 1471.51 & 1472.39 \\
\hline 12/2/11 11:30 & 33.729 & 36.749 & 1475.04 & 1472.37 \\
\hline $12 / 2 / 1112: 00$ & 33.776 & 36.758 & 1474.99 & 1472.36 \\
\hline $12 / 2 / 1112: 30$ & 33.802 & 36.756 & 1474.97 & 1472.37 \\
\hline $12 / 2 / 11$ 13:00 & 33.692 & 36.744 & 1475.08 & 1472.38 \\
\hline $12 / 2 / 11$ 13:30 & 33.738 & 36.734 & 1475.03 & 1472.39 \\
\hline $12 / 2 / 1114: 00$ & 33.858 & 36.732 & 1474.91 & 1472.39 \\
\hline $12 / 2 / 1114: 30$ & 33.717 & 36.718 & 1475.05 & 1472.40 \\
\hline $12 / 2 / 11$ 15:00 & 33.806 & 36.706 & 1474.96 & 1472.42 \\
\hline
\end{tabular}


TABLE S1.3 Automatically recorded groundwater levels in the Klassen wells, September 15, 2011, to March 13, 2012.

\begin{tabular}{|c|c|c|c|c|}
\hline \multicolumn{3}{|c|}{ Reference Elevation (ft AMSL) } & 1508.77 & \multirow{2}{*}{$\begin{array}{l}1509.12 \\
\text { Elevation in Klassen } \\
\text { Test Well (ft AMSL) }\end{array}$} \\
\hline Date and Time & $\begin{array}{l}\text { Depth in Klassen } \\
\text { Domestic Well } \\
(\mathrm{ft} \mathrm{BGL})\end{array}$ & $\begin{array}{c}\text { Depth in Klassen } \\
\text { Test Well } \\
\text { (ft BGL) }\end{array}$ & $\begin{array}{l}\text { Elevation in Klassen } \\
\text { Domestic Well } \\
\text { (ft AMSL) }\end{array}$ & \\
\hline $12 / 2 / 1115: 30$ & 33.682 & 36.695 & 1475.09 & 1472.43 \\
\hline $12 / 2 / 1116: 00$ & 33.663 & 36.685 & 1475.11 & 1472.44 \\
\hline $12 / 2 / 1116: 30$ & 33.619 & 36.673 & 1475.15 & 1472.45 \\
\hline $12 / 2 / 1117: 00$ & 33.696 & 36.664 & 1475.07 & 1472.46 \\
\hline 12/2/11 17:30 & 33.616 & 36.65 & 1475.15 & 1472.47 \\
\hline $12 / 2 / 1118: 00$ & 33.581 & 36.631 & 1475.19 & 1472.49 \\
\hline $12 / 2 / 1118: 30$ & 33.574 & 36.622 & 1475.20 & 1472.50 \\
\hline 12/2/11 19:00 & 33.755 & 36.608 & 1475.01 & 1472.51 \\
\hline $12 / 2 / 1119: 30$ & 33.722 & 36.598 & 1475.05 & 1472.52 \\
\hline $12 / 2 / 1120: 00$ & 33.647 & 36.584 & 1475.12 & 1472.54 \\
\hline $12 / 2 / 1120: 30$ & 33.607 & 36.577 & 1475.16 & 1472.55 \\
\hline $12 / 2 / 1121: 00$ & 33.706 & 36.579 & 1475.06 & 1472.54 \\
\hline $12 / 2 / 1121: 30$ & 33.6 & 36.577 & 1475.17 & 1472.55 \\
\hline $12 / 2 / 1122: 00$ & 33.588 & 36.572 & 1475.18 & 1472.55 \\
\hline $12 / 2 / 1122: 30$ & 33.558 & 36.563 & 1475.21 & 1472.56 \\
\hline $12 / 2 / 1123: 00$ & 33.813 & 36.551 & 1474.96 & 1472.57 \\
\hline $12 / 2 / 1123: 30$ & 33.842 & 36.544 & 1474.93 & 1472.58 \\
\hline $12 / 3 / 110: 00$ & 33.783 & 36.535 & 1474.99 & 1472.59 \\
\hline $12 / 3 / 110: 30$ & 33.692 & 36.528 & 1475.08 & 1472.59 \\
\hline $12 / 3 / 11$ 1:00 & 33.635 & 36.523 & 1475.13 & 1472.60 \\
\hline $12 / 3 / 11$ 1:30 & 33.595 & 36.516 & 1475.17 & 1472.61 \\
\hline $12 / 3 / 112: 00$ & 33.558 & 36.5 & 1475.21 & 1472.62 \\
\hline $12 / 3 / 112: 30$ & 33.53 & 36.49 & 1475.24 & 1472.63 \\
\hline $12 / 3 / 113: 00$ & 33.509 & 36.481 & 1475.26 & 1472.64 \\
\hline 12/3/11 3:30 & 33.49 & 36.476 & 1475.28 & 1472.65 \\
\hline $12 / 3 / 114: 00$ & 33.591 & 36.481 & 1475.18 & 1472.64 \\
\hline $12 / 3 / 114: 30$ & 36.339 & 36.483 & 1472.43 & 1472.64 \\
\hline 12/3/11 5:00 & 33.696 & 36.488 & 1475.07 & 1472.63 \\
\hline 12/3/11 5:30 & 33.593 & 36.493 & 1475.18 & 1472.63 \\
\hline $12 / 3 / 116: 00$ & 33.633 & 36.5 & 1475.14 & 1472.62 \\
\hline $12 / 3 / 116: 30$ & 33.666 & 36.502 & 1475.10 & 1472.62 \\
\hline $12 / 3 / 117: 00$ & 33.577 & 36.507 & 1475.19 & 1472.62 \\
\hline $12 / 3 / 117: 30$ & 33.53 & 36.509 & 1475.24 & 1472.61 \\
\hline $12 / 3 / 118: 00$ & 33.502 & 36.507 & 1475.27 & 1472.62 \\
\hline $12 / 3 / 118: 30$ & 33.584 & 36.504 & 1475.19 & 1472.62 \\
\hline $12 / 3 / 11$ 9:00 & 33.938 & 36.493 & 1474.83 & 1472.63 \\
\hline 12/3/11 9:30 & 37.317 & 36.486 & 1471.45 & 1472.64 \\
\hline $12 / 3 / 11$ 10:00 & 33.764 & 36.502 & 1475.01 & 1472.62 \\
\hline
\end{tabular}


TABLE S1.3 Automatically recorded groundwater levels in the Klassen wells, September 15, 2011, to March 13, 2012.

\begin{tabular}{|c|c|c|c|c|}
\hline \multicolumn{3}{|c|}{ Reference Elevation (ft AMSL) } & 1508.77 & \multirow{2}{*}{$\begin{array}{l}1509.12 \\
\text { Elevation in Klassen } \\
\text { Test Well (ft AMSL) }\end{array}$} \\
\hline Date and Time & $\begin{array}{l}\text { Depth in Klassen } \\
\text { Domestic Well } \\
(\mathrm{ft} \mathrm{BGL})\end{array}$ & $\begin{array}{c}\text { Depth in Klassen } \\
\text { Test Well } \\
\text { (ft BGL) }\end{array}$ & $\begin{array}{l}\text { Elevation in Klassen } \\
\text { Domestic Well } \\
\text { (ft AMSL) }\end{array}$ & \\
\hline $12 / 3 / 1110: 30$ & 36.79 & 36.535 & 1471.98 & 1472.59 \\
\hline $12 / 3 / 1111: 00$ & 33.914 & 36.558 & 1474.86 & 1472.56 \\
\hline $12 / 3 / 1111: 30$ & 33.837 & 36.568 & 1474.93 & 1472.55 \\
\hline $12 / 3 / 1112: 00$ & 33.858 & 36.584 & 1474.91 & 1472.54 \\
\hline $12 / 3 / 1112: 30$ & 34.076 & 36.608 & 1474.69 & 1472.51 \\
\hline $12 / 3 / 1113: 00$ & 34.01 & 36.622 & 1474.76 & 1472.50 \\
\hline $12 / 3 / 1113: 30$ & 34.088 & 36.645 & 1474.68 & 1472.48 \\
\hline 12/3/11 14:00 & 34.149 & 36.662 & 1474.62 & 1472.46 \\
\hline $12 / 3 / 1114: 30$ & 33.961 & 36.669 & 1474.81 & 1472.45 \\
\hline $12 / 3 / 1115: 00$ & 34.566 & 36.666 & 1474.20 & 1472.46 \\
\hline $12 / 3 / 1115: 30$ & 34.069 & 36.662 & 1474.70 & 1472.46 \\
\hline $12 / 3 / 11$ 16:00 & 34.144 & 36.664 & 1474.63 & 1472.46 \\
\hline $12 / 3 / 1116: 30$ & 34.121 & 36.662 & 1474.65 & 1472.46 \\
\hline $12 / 3 / 1117: 00$ & 33.999 & 36.655 & 1474.77 & 1472.47 \\
\hline $12 / 3 / 11$ 17:30 & 34.062 & 36.643 & 1474.71 & 1472.48 \\
\hline $12 / 3 / 11$ 18:00 & 33.924 & 36.624 & 1474.85 & 1472.50 \\
\hline $12 / 3 / 1118: 30$ & 33.856 & 36.61 & 1474.91 & 1472.51 \\
\hline 12/3/11 19:00 & 34.088 & 36.601 & 1474.68 & 1472.52 \\
\hline 12/3/11 19:30 & 34.003 & 36.587 & 1474.77 & 1472.54 \\
\hline $12 / 3 / 1120: 00$ & 33.891 & 36.57 & 1474.88 & 1472.55 \\
\hline $12 / 3 / 1120: 30$ & 33.818 & 36.558 & 1474.95 & 1472.56 \\
\hline $12 / 3 / 1121: 00$ & 34.006 & 36.547 & 1474.76 & 1472.58 \\
\hline $12 / 3 / 1121: 30$ & 33.799 & 36.542 & 1474.97 & 1472.58 \\
\hline $12 / 3 / 1122: 00$ & 33.738 & 36.542 & 1475.03 & 1472.58 \\
\hline $12 / 3 / 1122: 30$ & 34.233 & 36.53 & 1474.54 & 1472.59 \\
\hline $12 / 3 / 1123: 00$ & 33.804 & 36.516 & 1474.97 & 1472.61 \\
\hline $12 / 3 / 1123: 30$ & 33.924 & 36.516 & 1474.85 & 1472.61 \\
\hline $12 / 4 / 11$ 0:00 & 33.795 & 36.505 & 1474.97 & 1472.62 \\
\hline 12/4/11 0:30 & 33.821 & 36.502 & 1474.95 & 1472.62 \\
\hline 12/4/11 1:00 & 33.893 & 36.495 & 1474.88 & 1472.63 \\
\hline 12/4/11 1:30 & 33.832 & 36.488 & 1474.94 & 1472.63 \\
\hline $12 / 4 / 112: 00$ & 33.816 & 36.486 & 1474.95 & 1472.64 \\
\hline $12 / 4 / 112: 30$ & 33.863 & 36.479 & 1474.91 & 1472.64 \\
\hline 12/4/11 3:00 & 33.785 & 36.472 & 1474.98 & 1472.65 \\
\hline $12 / 4 / 113: 30$ & 33.729 & 36.467 & 1475.04 & 1472.66 \\
\hline $12 / 4 / 114: 00$ & 33.689 & 36.46 & 1475.08 & 1472.66 \\
\hline $12 / 4 / 114: 30$ & 33.687 & 36.453 & 1475.08 & 1472.67 \\
\hline $12 / 4 / 11$ 5:00 & 33.649 & 36.448 & 1475.12 & 1472.67 \\
\hline
\end{tabular}


TABLE S1.3 Automatically recorded groundwater levels in the Klassen wells, September 15, 2011, to March 13, 2012.

\begin{tabular}{|c|c|c|c|c|}
\hline \multicolumn{3}{|c|}{ Reference Elevation (ft AMSL) } & 1508.77 & \multirow{2}{*}{$\begin{array}{l}1509.12 \\
\text { Elevation in Klassen } \\
\text { Test Well (ft AMSL) }\end{array}$} \\
\hline Date and Time & $\begin{array}{l}\text { Depth in Klassen } \\
\text { Domestic Well } \\
(\mathrm{ft} \mathrm{BGL})\end{array}$ & $\begin{array}{c}\text { Depth in Klassen } \\
\text { Test Well } \\
\text { (ft BGL) }\end{array}$ & $\begin{array}{l}\text { Elevation in Klassen } \\
\text { Domestic Well } \\
\text { (ft AMSL) }\end{array}$ & \\
\hline $12 / 4 / 115: 30$ & 33.619 & 36.436 & 1475.15 & 1472.69 \\
\hline $12 / 4 / 116: 00$ & 34.407 & 36.432 & 1474.36 & 1472.69 \\
\hline $12 / 4 / 116: 30$ & 33.877 & 36.427 & 1474.89 & 1472.70 \\
\hline $12 / 4 / 117: 00$ & 35.275 & 36.42 & 1473.49 & 1472.70 \\
\hline $12 / 4 / 117: 30$ & 33.959 & 36.415 & 1474.81 & 1472.71 \\
\hline $12 / 4 / 118: 00$ & 33.813 & 36.411 & 1474.96 & 1472.71 \\
\hline $12 / 4 / 118: 30$ & 34.043 & 36.415 & 1474.73 & 1472.71 \\
\hline $12 / 4 / 119: 00$ & 33.823 & 36.415 & 1474.95 & 1472.71 \\
\hline $12 / 4 / 119: 30$ & 33.821 & 36.42 & 1474.95 & 1472.70 \\
\hline $12 / 4 / 1110: 00$ & 33.973 & 36.434 & 1474.80 & 1472.69 \\
\hline $12 / 4 / 1110: 30$ & 34.015 & 36.467 & 1474.75 & 1472.66 \\
\hline 12/4/11 11:00 & 33.905 & 36.497 & 1474.86 & 1472.63 \\
\hline 12/4/11 11:30 & 33.825 & 36.514 & 1474.94 & 1472.61 \\
\hline $12 / 4 / 1112: 00$ & 33.769 & 36.516 & 1475.00 & 1472.61 \\
\hline $12 / 4 / 1112: 30$ & 33.755 & 36.518 & 1475.01 & 1472.60 \\
\hline $12 / 4 / 11$ 13:00 & 33.706 & 36.521 & 1475.06 & 1472.60 \\
\hline $12 / 4 / 11$ 13:30 & 33.668 & 36.514 & 1475.10 & 1472.61 \\
\hline $12 / 4 / 11$ 14:00 & 33.638 & 36.511 & 1475.13 & 1472.61 \\
\hline $12 / 4 / 11$ 14:30 & 33.734 & 36.507 & 1475.04 & 1472.62 \\
\hline $12 / 4 / 11$ 15:00 & 33.659 & 36.486 & 1475.11 & 1472.64 \\
\hline $12 / 4 / 11$ 15:30 & 33.619 & 36.46 & 1475.15 & 1472.66 \\
\hline 12/4/11 16:00 & 33.663 & 36.446 & 1475.11 & 1472.68 \\
\hline $12 / 4 / 11$ 16:30 & 33.612 & 36.434 & 1475.16 & 1472.69 \\
\hline $12 / 4 / 11$ 17:00 & 33.581 & 36.42 & 1475.19 & 1472.70 \\
\hline 12/4/11 17:30 & 34.637 & 36.415 & 1474.13 & 1472.71 \\
\hline 12/4/11 18:00 & 33.605 & 36.406 & 1475.16 & 1472.72 \\
\hline $12 / 4 / 11$ 18:30 & 33.563 & 36.394 & 1475.21 & 1472.73 \\
\hline 12/4/11 19:00 & 34.745 & 36.387 & 1474.02 & 1472.74 \\
\hline 12/4/11 19:30 & 33.811 & 36.385 & 1474.96 & 1472.74 \\
\hline $12 / 4 / 1120: 00$ & 33.832 & 36.385 & 1474.94 & 1472.74 \\
\hline $12 / 4 / 1120: 30$ & 33.835 & 36.387 & 1474.93 & 1472.74 \\
\hline 12/4/11 21:00 & 33.851 & 36.385 & 1474.92 & 1472.74 \\
\hline $12 / 4 / 1121: 30$ & 33.776 & 36.382 & 1474.99 & 1472.74 \\
\hline $12 / 4 / 1122: 00$ & 33.872 & 36.382 & 1474.90 & 1472.74 \\
\hline $12 / 4 / 1122: 30$ & 33.865 & 36.385 & 1474.90 & 1472.74 \\
\hline $12 / 4 / 1123: 00$ & 33.996 & 36.387 & 1474.77 & 1472.74 \\
\hline $12 / 4 / 1123: 30$ & 34.217 & 36.394 & 1474.55 & 1472.73 \\
\hline 12/5/11 0:00 & 33.935 & 36.392 & 1474.83 & 1472.73 \\
\hline
\end{tabular}


TABLE S1.3 Automatically recorded groundwater levels in the Klassen wells, September 15, 2011, to March 13, 2012.

\begin{tabular}{|c|c|c|c|c|}
\hline \multicolumn{3}{|c|}{ Reference Elevation (ft AMSL) } & 1508.77 & \multirow{2}{*}{$\begin{array}{l}1509.12 \\
\text { Elevation in Klassen } \\
\text { Test Well (ft AMSL) }\end{array}$} \\
\hline Date and Time & $\begin{array}{l}\text { Depth in Klassen } \\
\text { Domestic Well } \\
\text { (ft BGL) }\end{array}$ & $\begin{array}{c}\text { Depth in Klassen } \\
\text { Test Well } \\
\text { (ft BGL) }\end{array}$ & $\begin{array}{l}\text { Elevation in Klassen } \\
\text { Domestic Well } \\
\text { (ft AMSL) }\end{array}$ & \\
\hline $12 / 5 / 110: 30$ & 33.837 & 36.385 & 1474.93 & 1472.74 \\
\hline $12 / 5 / 111: 00$ & 33.776 & 36.382 & 1474.99 & 1472.74 \\
\hline 12/5/11 1:30 & 33.727 & 36.382 & 1475.04 & 1472.74 \\
\hline $12 / 5 / 112: 00$ & 33.687 & 36.373 & 1475.08 & 1472.75 \\
\hline $12 / 5 / 112: 30$ & 33.656 & 36.366 & 1475.11 & 1472.76 \\
\hline 12/5/11 3:00 & 33.626 & 36.357 & 1475.14 & 1472.77 \\
\hline $12 / 5 / 113: 30$ & 33.6 & 36.357 & 1475.17 & 1472.77 \\
\hline $12 / 5 / 114: 00$ & 33.577 & 36.364 & 1475.19 & 1472.76 \\
\hline $12 / 5 / 114: 30$ & 33.556 & 36.333 & 1475.21 & 1472.79 \\
\hline 12/5/11 5:00 & 33.539 & 36.319 & 1475.23 & 1472.80 \\
\hline $12 / 5 / 115: 30$ & 33.525 & 36.31 & 1475.24 & 1472.81 \\
\hline $12 / 5 / 116: 00$ & 33.556 & 36.317 & 1475.21 & 1472.81 \\
\hline $12 / 5 / 116: 30$ & 33.849 & 36.312 & 1474.92 & 1472.81 \\
\hline $12 / 5 / 117: 00$ & 33.816 & 36.307 & 1474.95 & 1472.82 \\
\hline $12 / 5 / 117: 30$ & 33.722 & 36.296 & 1475.05 & 1472.83 \\
\hline 12/5/11 8:00 & 33.785 & 36.303 & 1474.98 & 1472.82 \\
\hline $12 / 5 / 118: 30$ & 33.891 & 36.303 & 1474.88 & 1472.82 \\
\hline 12/5/11 9:00 & 36.7 & 36.317 & 1472.07 & 1472.81 \\
\hline $12 / 5 / 119: 30$ & 33.903 & 36.317 & 1474.87 & 1472.81 \\
\hline 12/5/11 10:00 & 33.867 & 36.324 & 1474.90 & 1472.80 \\
\hline $12 / 5 / 11$ 10:30 & 33.881 & 36.319 & 1474.89 & 1472.80 \\
\hline 12/5/11 11:00 & 33.917 & 36.321 & 1474.85 & 1472.80 \\
\hline 12/5/11 11:30 & 33.776 & 36.324 & 1474.99 & 1472.80 \\
\hline 12/5/11 12:00 & 33.968 & 36.331 & 1474.80 & 1472.79 \\
\hline $12 / 5 / 11$ 12:30 & 33.76 & 36.338 & 1475.01 & 1472.78 \\
\hline 12/5/11 13:00 & 33.912 & 36.352 & 1474.86 & 1472.77 \\
\hline 12/5/11 13:30 & 33.884 & 36.354 & 1474.89 & 1472.77 \\
\hline 12/5/11 14:00 & 33.863 & 36.361 & 1474.91 & 1472.76 \\
\hline $12 / 5 / 11$ 14:30 & 33.778 & 36.359 & 1474.99 & 1472.76 \\
\hline 12/5/11 15:00 & 33.748 & 36.352 & 1475.02 & 1472.77 \\
\hline 12/5/11 15:30 & 33.696 & 36.335 & 1475.07 & 1472.79 \\
\hline 12/5/11 16:00 & 33.659 & 36.321 & 1475.11 & 1472.80 \\
\hline 12/5/11 16:30 & 33.628 & 36.303 & 1475.14 & 1472.82 \\
\hline 12/5/11 17:00 & 33.624 & 36.296 & 1475.15 & 1472.83 \\
\hline 12/5/11 17:30 & 33.729 & 36.293 & 1475.04 & 1472.83 \\
\hline 12/5/11 18:00 & 33.811 & 36.293 & 1474.96 & 1472.83 \\
\hline 12/5/11 18:30 & 33.708 & 36.291 & 1475.06 & 1472.83 \\
\hline 12/5/11 19:00 & 37.625 & 36.284 & 1471.14 & 1472.84 \\
\hline
\end{tabular}


TABLE S1.3 Automatically recorded groundwater levels in the Klassen wells, September 15, 2011, to March 13, 2012.

\begin{tabular}{|c|c|c|c|c|}
\hline \multicolumn{3}{|c|}{ Reference Elevation (ft AMSL) } & 1508.77 & \multirow{2}{*}{$\begin{array}{l}1509.12 \\
\text { Elevation in Klassen } \\
\text { Test Well (ft AMSL) }\end{array}$} \\
\hline Date and Time & $\begin{array}{l}\text { Depth in Klassen } \\
\text { Domestic Well } \\
\text { (ft BGL) }\end{array}$ & $\begin{array}{c}\text { Depth in Klassen } \\
\text { Test Well } \\
\text { (ft BGL) }\end{array}$ & $\begin{array}{l}\text { Elevation in Klassen } \\
\text { Domestic Well } \\
\text { (ft AMSL) }\end{array}$ & \\
\hline 12/5/11 19:30 & 33.703 & 36.291 & 1475.07 & 1472.83 \\
\hline $12 / 5 / 1120: 00$ & 33.968 & 36.3 & 1474.80 & 1472.82 \\
\hline $12 / 5 / 1120: 30$ & 33.856 & 36.317 & 1474.91 & 1472.81 \\
\hline $12 / 5 / 1121: 00$ & 34.107 & 36.336 & 1474.66 & 1472.79 \\
\hline $12 / 5 / 1121: 30$ & 33.846 & 36.347 & 1474.92 & 1472.78 \\
\hline $12 / 5 / 1122: 00$ & 33.781 & 36.357 & 1474.99 & 1472.77 \\
\hline $12 / 5 / 1122: 30$ & 33.72 & 36.361 & 1475.05 & 1472.76 \\
\hline 12/5/11 23:00 & 33.881 & 36.373 & 1474.89 & 1472.75 \\
\hline $12 / 5 / 1123: 30$ & 34.102 & 36.387 & 1474.67 & 1472.74 \\
\hline $12 / 6 / 110: 00$ & 33.905 & 36.396 & 1474.86 & 1472.73 \\
\hline $12 / 6 / 110: 30$ & 33.804 & 36.397 & 1474.97 & 1472.73 \\
\hline $12 / 6 / 111: 00$ & 33.87 & 36.408 & 1474.90 & 1472.71 \\
\hline $12 / 6 / 111: 30$ & 33.809 & 36.413 & 1474.96 & 1472.71 \\
\hline $12 / 6 / 112: 00$ & 33.769 & 36.413 & 1475.00 & 1472.71 \\
\hline $12 / 6 / 112: 30$ & 33.851 & 36.413 & 1474.92 & 1472.71 \\
\hline $12 / 6 / 113: 00$ & 33.776 & 36.411 & 1474.99 & 1472.71 \\
\hline $12 / 6 / 113: 30$ & 33.72 & 36.406 & 1475.05 & 1472.72 \\
\hline $12 / 6 / 114: 00$ & 33.677 & 36.399 & 1475.09 & 1472.72 \\
\hline $12 / 6 / 114: 30$ & 33.645 & 36.396 & 1475.12 & 1472.73 \\
\hline $12 / 6 / 115: 00$ & 33.616 & 36.397 & 1475.15 & 1472.73 \\
\hline $12 / 6 / 115: 30$ & 33.684 & 36.387 & 1475.09 & 1472.74 \\
\hline $12 / 6 / 116: 00$ & 33.663 & 36.382 & 1475.11 & 1472.74 \\
\hline $12 / 6 / 116: 30$ & 33.731 & 36.378 & 1475.04 & 1472.74 \\
\hline $12 / 6 / 11$ 7:00 & 33.682 & 36.371 & 1475.09 & 1472.75 \\
\hline $12 / 6 / 117: 30$ & 33.724 & 36.364 & 1475.05 & 1472.76 \\
\hline $12 / 6 / 118: 00$ & 33.71 & 36.361 & 1475.06 & 1472.76 \\
\hline $12 / 6 / 118: 30$ & 33.813 & 36.368 & 1474.96 & 1472.75 \\
\hline 12/6/11 9:00 & 33.699 & 36.361 & 1475.07 & 1472.76 \\
\hline $12 / 6 / 119: 30$ & 33.774 & 36.361 & 1475.00 & 1472.76 \\
\hline 12/6/11 10:00 & 33.677 & 36.361 & 1475.09 & 1472.76 \\
\hline 12/6/11 10:30 & 33.703 & 36.366 & 1475.07 & 1472.76 \\
\hline 12/6/11 11:00 & 33.642 & 36.373 & 1475.13 & 1472.75 \\
\hline $12 / 6 / 11$ 11:30 & 33.602 & 36.394 & 1475.17 & 1472.73 \\
\hline $12 / 6 / 11$ 12:00 & 33.76 & 36.422 & 1475.01 & 1472.70 \\
\hline $12 / 6 / 1112: 30$ & 33.609 & 36.448 & 1475.16 & 1472.67 \\
\hline 12/6/11 13:00 & 33.567 & 36.45 & 1475.20 & 1472.67 \\
\hline $12 / 6 / 11$ 13:30 & 33.537 & 36.441 & 1475.23 & 1472.68 \\
\hline $12 / 6 / 11$ 14:00 & 33.513 & 36.42 & 1475.26 & 1472.70 \\
\hline
\end{tabular}


TABLE S1.3 Automatically recorded groundwater levels in the Klassen wells, September 15, 2011, to March 13, 2012.

\begin{tabular}{|c|c|c|c|c|}
\hline \multicolumn{3}{|c|}{ Reference Elevation (ft AMSL) } & 1508.77 & \multirow{2}{*}{$\begin{array}{l}1509.12 \\
\text { Elevation in Klassen } \\
\text { Test Well (ft AMSL) }\end{array}$} \\
\hline Date and Time & $\begin{array}{l}\text { Depth in Klassen } \\
\text { Domestic Well } \\
\text { (ft BGL) }\end{array}$ & $\begin{array}{c}\text { Depth in Klassen } \\
\text { Test Well } \\
\text { (ft BGL) }\end{array}$ & $\begin{array}{l}\text { Elevation in Klassen } \\
\text { Domestic Well } \\
\text { (ft AMSL) }\end{array}$ & \\
\hline $12 / 6 / 1114: 30$ & 33.595 & 36.404 & 1475.17 & 1472.72 \\
\hline $12 / 6 / 11$ 15:00 & 33.539 & 36.387 & 1475.23 & 1472.74 \\
\hline $12 / 6 / 11$ 15:30 & 33.506 & 36.368 & 1475.26 & 1472.75 \\
\hline $12 / 6 / 11$ 16:00 & 33.835 & 36.354 & 1474.93 & 1472.77 \\
\hline $12 / 6 / 1116: 30$ & 33.741 & 36.338 & 1475.03 & 1472.78 \\
\hline 12/6/11 17:00 & 33.577 & 36.317 & 1475.19 & 1472.81 \\
\hline $12 / 6 / 1117: 30$ & 33.541 & 36.289 & 1475.23 & 1472.83 \\
\hline $12 / 6 / 11$ 18:00 & 33.907 & 36.27 & 1474.86 & 1472.85 \\
\hline $12 / 6 / 1118: 30$ & 33.63 & 36.258 & 1475.14 & 1472.86 \\
\hline 12/6/11 19:00 & 33.565 & 36.249 & 1475.20 & 1472.87 \\
\hline 12/6/11 19:30 & 33.56 & 36.242 & 1475.21 & 1472.88 \\
\hline 12/6/11 20:00 & 33.795 & 36.242 & 1474.97 & 1472.88 \\
\hline $12 / 6 / 1120: 30$ & 33.659 & 36.237 & 1475.11 & 1472.89 \\
\hline $12 / 6 / 1121: 00$ & 33.692 & 36.239 & 1475.08 & 1472.88 \\
\hline $12 / 6 / 1121: 30$ & 33.624 & 36.239 & 1475.15 & 1472.88 \\
\hline $12 / 6 / 1122: 00$ & 33.659 & 36.235 & 1475.11 & 1472.89 \\
\hline $12 / 6 / 1122: 30$ & 33.701 & 36.239 & 1475.07 & 1472.88 \\
\hline 12/6/11 23:00 & 33.842 & 36.244 & 1474.93 & 1472.88 \\
\hline $12 / 6 / 1123: 30$ & 34.062 & 36.251 & 1474.71 & 1472.87 \\
\hline 12/7/11 0:00 & 33.818 & 36.249 & 1474.95 & 1472.87 \\
\hline $12 / 7 / 110: 30$ & 33.727 & 36.242 & 1475.04 & 1472.88 \\
\hline $12 / 7 / 11$ 1:00 & 33.666 & 36.239 & 1475.10 & 1472.88 \\
\hline $12 / 7 / 11$ 1:30 & 33.619 & 36.239 & 1475.15 & 1472.88 \\
\hline $12 / 7 / 112: 00$ & 33.581 & 36.237 & 1475.19 & 1472.89 \\
\hline $12 / 7 / 112: 30$ & 33.553 & 36.225 & 1475.22 & 1472.90 \\
\hline 12/7/11 3:00 & 33.525 & 36.221 & 1475.24 & 1472.90 \\
\hline $12 / 7 / 113: 30$ & 33.499 & 36.221 & 1475.27 & 1472.90 \\
\hline $12 / 7 / 114: 00$ & 33.478 & 36.211 & 1475.29 & 1472.91 \\
\hline $12 / 7 / 114: 30$ & 33.485 & 36.204 & 1475.28 & 1472.92 \\
\hline 12/7/11 5:00 & 33.459 & 36.197 & 1475.31 & 1472.93 \\
\hline $12 / 7 / 115: 30$ & 33.441 & 36.192 & 1475.33 & 1472.93 \\
\hline $12 / 7 / 116: 00$ & 33.434 & 36.185 & 1475.34 & 1472.94 \\
\hline $12 / 7 / 116: 30$ & 33.553 & 36.183 & 1475.22 & 1472.94 \\
\hline $12 / 7 / 117: 00$ & 33.485 & 36.176 & 1475.28 & 1472.95 \\
\hline $12 / 7 / 117: 30$ & 33.492 & 36.176 & 1475.28 & 1472.95 \\
\hline $12 / 7 / 118: 00$ & 33.469 & 36.174 & 1475.30 & 1472.95 \\
\hline $12 / 7 / 118: 30$ & 33.506 & 36.174 & 1475.26 & 1472.95 \\
\hline 12/7/11 9:00 & 33.532 & 36.167 & 1475.24 & 1472.96 \\
\hline
\end{tabular}


TABLE S1.3 Automatically recorded groundwater levels in the Klassen wells, September 15, 2011, to March 13, 2012.

\begin{tabular}{|c|c|c|c|c|}
\hline \multicolumn{3}{|c|}{ Reference Elevation (ft AMSL) } & 1508.77 & \multirow{2}{*}{$\begin{array}{l}1509.12 \\
\text { Elevation in Klassen } \\
\text { Test Well (ft AMSL) }\end{array}$} \\
\hline Date and Time & $\begin{array}{l}\text { Depth in Klassen } \\
\text { Domestic Well } \\
\text { (ft BGL) }\end{array}$ & $\begin{array}{c}\text { Depth in Klassen } \\
\text { Test Well } \\
\text { (ft BGL) }\end{array}$ & $\begin{array}{l}\text { Elevation in Klassen } \\
\text { Domestic Well } \\
\text { (ft AMSL) }\end{array}$ & \\
\hline $12 / 7 / 119: 30$ & 33.473 & 36.167 & 1475.30 & 1472.96 \\
\hline 12/7/11 10:00 & 33.448 & 36.162 & 1475.32 & 1472.96 \\
\hline 12/7/11 10:30 & 33.734 & 36.167 & 1475.04 & 1472.96 \\
\hline 12/7/11 11:00 & 33.48 & 36.195 & 1475.29 & 1472.93 \\
\hline 12/7/11 11:30 & 33.586 & 36.207 & 1475.18 & 1472.92 \\
\hline $12 / 7 / 1112: 00$ & 33.722 & 36.209 & 1475.05 & 1472.91 \\
\hline $12 / 7 / 1112: 30$ & 33.811 & 36.218 & 1474.96 & 1472.90 \\
\hline 12/7/11 13:00 & 33.635 & 36.211 & 1475.13 & 1472.91 \\
\hline $12 / 7 / 1113: 30$ & 33.649 & 36.204 & 1475.12 & 1472.92 \\
\hline 12/7/11 14:00 & 33.563 & 36.178 & 1475.21 & 1472.94 \\
\hline $12 / 7 / 1114: 30$ & 33.835 & 36.134 & 1474.93 & 1472.99 \\
\hline 12/7/11 15:00 & 33.565 & 36.117 & 1475.20 & 1473.01 \\
\hline $12 / 7 / 11$ 15:30 & 37.918 & 36.11 & 1470.85 & 1473.01 \\
\hline 12/7/11 16:00 & 33.626 & 36.108 & 1475.14 & 1473.01 \\
\hline $12 / 7 / 11$ 16:30 & 33.959 & 36.108 & 1474.81 & 1473.01 \\
\hline 12/7/11 17:00 & 33.68 & 36.108 & 1475.09 & 1473.01 \\
\hline 12/7/11 17:30 & 33.694 & 36.12 & 1475.08 & 1473.00 \\
\hline 12/7/11 18:00 & 33.692 & 36.127 & 1475.08 & 1473.00 \\
\hline $12 / 7 / 11$ 18:30 & 33.802 & 36.124 & 1474.97 & 1473.00 \\
\hline 12/7/11 19:00 & 33.642 & 36.127 & 1475.13 & 1473.00 \\
\hline 12/7/11 19:30 & 35.898 & 36.129 & 1472.87 & 1472.99 \\
\hline 12/7/11 20:00 & 33.626 & 36.129 & 1475.14 & 1472.99 \\
\hline $12 / 7 / 1120: 30$ & 33.673 & 36.136 & 1475.10 & 1472.99 \\
\hline 12/7/11 21:00 & 33.696 & 36.141 & 1475.07 & 1472.98 \\
\hline 12/7/11 21:30 & 33.654 & 36.131 & 1475.12 & 1472.99 \\
\hline $12 / 7 / 1122: 00$ & 33.682 & 36.131 & 1475.09 & 1472.99 \\
\hline $12 / 7 / 1122: 30$ & 35.052 & 36.129 & 1473.72 & 1472.99 \\
\hline 12/7/11 23:00 & 34.015 & 36.136 & 1474.75 & 1472.99 \\
\hline 12/7/11 23:30 & 33.736 & 36.131 & 1475.03 & 1472.99 \\
\hline 12/8/11 0:00 & 33.654 & 36.124 & 1475.12 & 1473.00 \\
\hline $12 / 8 / 110: 30$ & 33.6 & 36.122 & 1475.17 & 1473.00 \\
\hline $12 / 8 / 11$ 1:00 & 33.56 & 36.115 & 1475.21 & 1473.01 \\
\hline $12 / 8 / 11$ 1:30 & 33.527 & 36.113 & 1475.24 & 1473.01 \\
\hline $12 / 8 / 112: 00$ & 33.499 & 36.106 & 1475.27 & 1473.02 \\
\hline $12 / 8 / 112: 30$ & 33.476 & 36.103 & 1475.29 & 1473.02 \\
\hline $12 / 8 / 113: 00$ & 33.455 & 36.101 & 1475.31 & 1473.02 \\
\hline $12 / 8 / 113: 30$ & 33.438 & 36.094 & 1475.33 & 1473.03 \\
\hline $12 / 8 / 114: 00$ & 33.419 & 36.091 & 1475.35 & 1473.03 \\
\hline
\end{tabular}


TABLE S1.3 Automatically recorded groundwater levels in the Klassen wells, September 15, 2011, to March 13, 2012.

\begin{tabular}{|c|c|c|c|c|}
\hline \multicolumn{3}{|c|}{ Reference Elevation (ft AMSL) } & 1508.77 & \multirow{2}{*}{$\begin{array}{l}1509.12 \\
\text { Elevation in Klassen } \\
\text { Test Well (ft AMSL) }\end{array}$} \\
\hline Date and Time & $\begin{array}{l}\text { Depth in Klassen } \\
\text { Domestic Well } \\
\text { (ft BGL) }\end{array}$ & $\begin{array}{c}\text { Depth in Klassen } \\
\text { Test Well } \\
\text { (ft BGL) }\end{array}$ & $\begin{array}{l}\text { Elevation in Klassen } \\
\text { Domestic Well } \\
\text { (ft AMSL) }\end{array}$ & \\
\hline $12 / 8 / 114: 30$ & 33.403 & 36.085 & 1475.37 & 1473.04 \\
\hline 12/8/11 5:00 & 33.389 & 36.077 & 1475.38 & 1473.05 \\
\hline 12/8/11 5:30 & 33.375 & 36.07 & 1475.39 & 1473.05 \\
\hline $12 / 8 / 116: 00$ & 33.363 & 36.063 & 1475.41 & 1473.06 \\
\hline $12 / 8 / 116: 30$ & 33.64 & 36.066 & 1475.13 & 1473.06 \\
\hline 12/8/11 7:00 & 33.518 & 36.063 & 1475.25 & 1473.06 \\
\hline $12 / 8 / 117: 30$ & 33.602 & 36.068 & 1475.17 & 1473.05 \\
\hline $12 / 8 / 118: 00$ & 33.586 & 36.068 & 1475.18 & 1473.05 \\
\hline $12 / 8 / 118: 30$ & 33.605 & 36.073 & 1475.16 & 1473.05 \\
\hline 12/8/11 9:00 & 36.208 & 36.07 & 1472.56 & 1473.05 \\
\hline 12/8/11 9:30 & 33.567 & 36.075 & 1475.20 & 1473.05 \\
\hline 12/8/11 10:00 & 33.513 & 36.082 & 1475.26 & 1473.04 \\
\hline $12 / 8 / 1110: 30$ & 33.478 & 36.106 & 1475.29 & 1473.02 \\
\hline 12/8/11 11:00 & 36.621 & 36.117 & 1472.15 & 1473.01 \\
\hline $12 / 8 / 1111: 30$ & 33.499 & 36.117 & 1475.27 & 1473.01 \\
\hline $12 / 8 / 1112: 00$ & 33.455 & 36.12 & 1475.31 & 1473.00 \\
\hline $12 / 8 / 1112: 30$ & 33.424 & 36.12 & 1475.35 & 1473.00 \\
\hline 12/8/11 13:00 & 33.504 & 36.12 & 1475.27 & 1473.00 \\
\hline 12/8/11 13:30 & 33.464 & 36.12 & 1475.31 & 1473.00 \\
\hline $12 / 8 / 1114: 00$ & 33.588 & 36.12 & 1475.18 & 1473.00 \\
\hline $12 / 8 / 1114: 30$ & 33.459 & 36.108 & 1475.31 & 1473.01 \\
\hline 12/8/11 15:00 & 33.422 & 36.077 & 1475.35 & 1473.05 \\
\hline $12 / 8 / 11$ 15:30 & 33.396 & 36.042 & 1475.37 & 1473.08 \\
\hline 12/8/11 16:00 & 33.375 & 36.033 & 1475.39 & 1473.09 \\
\hline 12/8/11 16:30 & 33.452 & 36.033 & 1475.32 & 1473.09 \\
\hline 12/8/11 17:00 & 33.506 & 36.035 & 1475.26 & 1473.09 \\
\hline 12/8/11 17:30 & 33.574 & 36.047 & 1475.20 & 1473.08 \\
\hline 12/8/11 18:00 & 33.743 & 36.056 & 1475.03 & 1473.07 \\
\hline $12 / 8 / 11$ 18:30 & 33.598 & 36.059 & 1475.17 & 1473.06 \\
\hline 12/8/11 19:00 & 33.593 & 36.056 & 1475.18 & 1473.07 \\
\hline 12/8/11 19:30 & 33.532 & 36.042 & 1475.24 & 1473.08 \\
\hline 12/8/11 20:00 & 33.492 & 36.035 & 1475.28 & 1473.09 \\
\hline $12 / 8 / 1120: 30$ & 33.49 & 36.03 & 1475.28 & 1473.09 \\
\hline 12/8/11 21:00 & 33.455 & 36.028 & 1475.31 & 1473.09 \\
\hline 12/8/11 21:30 & 33.551 & 36.028 & 1475.22 & 1473.09 \\
\hline $12 / 8 / 1122: 00$ & 33.619 & 36.03 & 1475.15 & 1473.09 \\
\hline $12 / 8 / 1122: 30$ & 33.696 & 36.035 & 1475.07 & 1473.09 \\
\hline 12/8/11 23:00 & 33.877 & 36.04 & 1474.89 & 1473.08 \\
\hline
\end{tabular}


TABLE S1.3 Automatically recorded groundwater levels in the Klassen wells, September 15, 2011, to March 13, 2012.

\begin{tabular}{|c|c|c|c|c|}
\hline \multicolumn{3}{|c|}{ Reference Elevation (ft AMSL) } & 1508.77 & \multirow{2}{*}{$\begin{array}{l}1509.12 \\
\text { Elevation in Klassen } \\
\text { Test Well (ft AMSL) }\end{array}$} \\
\hline Date and Time & $\begin{array}{l}\text { Depth in Klassen } \\
\text { Domestic Well } \\
\text { (ft BGL) }\end{array}$ & $\begin{array}{c}\text { Depth in Klassen } \\
\text { Test Well } \\
\text { (ft BGL) }\end{array}$ & $\begin{array}{l}\text { Elevation in Klassen } \\
\text { Domestic Well } \\
\text { (ft AMSL) }\end{array}$ & \\
\hline $12 / 8 / 1123: 30$ & 33.692 & 36.035 & 1475.08 & 1473.09 \\
\hline 12/9/11 0:00 & 33.614 & 36.038 & 1475.16 & 1473.08 \\
\hline $12 / 9 / 110: 30$ & 33.689 & 36.038 & 1475.08 & 1473.08 \\
\hline 12/9/11 1:00 & 34.339 & 36.042 & 1474.43 & 1473.08 \\
\hline $12 / 9 / 111: 30$ & 33.858 & 36.047 & 1474.91 & 1473.08 \\
\hline $12 / 9 / 112: 00$ & 33.757 & 36.059 & 1475.01 & 1473.06 \\
\hline $12 / 9 / 112: 30$ & 33.896 & 36.061 & 1474.87 & 1473.06 \\
\hline 12/9/11 3:00 & 33.771 & 36.054 & 1475.00 & 1473.07 \\
\hline 12/9/11 3:30 & 33.692 & 36.054 & 1475.08 & 1473.07 \\
\hline 12/9/11 4:00 & 33.635 & 36.054 & 1475.13 & 1473.07 \\
\hline $12 / 9 / 114: 30$ & 33.593 & 36.049 & 1475.18 & 1473.07 \\
\hline $12 / 9 / 115: 00$ & 33.556 & 36.042 & 1475.21 & 1473.08 \\
\hline $12 / 9 / 115: 30$ & 33.525 & 36.038 & 1475.24 & 1473.08 \\
\hline $12 / 9 / 116: 00$ & 34.036 & 36.033 & 1474.73 & 1473.09 \\
\hline $12 / 9 / 116: 30$ & 33.593 & 36.028 & 1475.18 & 1473.09 \\
\hline 12/9/11 7:00 & 33.699 & 36.021 & 1475.07 & 1473.10 \\
\hline $12 / 9 / 117: 30$ & 33.708 & 36.016 & 1475.06 & 1473.11 \\
\hline 12/9/11 8:00 & 33.666 & 36.014 & 1475.10 & 1473.11 \\
\hline $12 / 9 / 118: 30$ & 33.602 & 36.014 & 1475.17 & 1473.11 \\
\hline 12/9/11 9:00 & 33.694 & 36.014 & 1475.08 & 1473.11 \\
\hline $12 / 9 / 119: 30$ & 38.281 & 36.014 & 1470.49 & 1473.11 \\
\hline 12/9/11 10:00 & 33.647 & 36.019 & 1475.12 & 1473.10 \\
\hline $12 / 9 / 11$ 10:30 & 37.036 & 36.033 & 1471.73 & 1473.09 \\
\hline 12/9/11 11:00 & 34.13 & 36.049 & 1474.64 & 1473.07 \\
\hline 12/9/11 11:30 & 33.689 & 36.056 & 1475.08 & 1473.07 \\
\hline 12/9/11 12:00 & 33.602 & 36.059 & 1475.17 & 1473.06 \\
\hline 12/9/11 12:30 & 33.556 & 36.054 & 1475.21 & 1473.07 \\
\hline 12/9/11 13:00 & 36.37 & 36.049 & 1472.40 & 1473.07 \\
\hline 12/9/11 13:30 & 33.556 & 36.054 & 1475.21 & 1473.07 \\
\hline 12/9/11 14:00 & 33.645 & 36.056 & 1475.12 & 1473.07 \\
\hline 12/9/11 14:30 & 33.541 & 36.059 & 1475.23 & 1473.06 \\
\hline 12/9/11 15:00 & 33.588 & 36.056 & 1475.18 & 1473.07 \\
\hline 12/9/11 15:30 & 33.628 & 36.049 & 1475.14 & 1473.07 \\
\hline 12/9/11 16:00 & 33.558 & 36.04 & 1475.21 & 1473.08 \\
\hline 12/9/11 16:30 & 33.539 & 36.038 & 1475.23 & 1473.08 \\
\hline 12/9/11 17:00 & 33.499 & 36.019 & 1475.27 & 1473.10 \\
\hline 12/9/11 17:30 & 33.593 & 36.012 & 1475.18 & 1473.11 \\
\hline 12/9/11 18:00 & 33.802 & 36.012 & 1474.97 & 1473.11 \\
\hline
\end{tabular}


TABLE S1.3 Automatically recorded groundwater levels in the Klassen wells, September 15, 2011, to March 13, 2012.

\begin{tabular}{|c|c|c|c|c|}
\hline \multicolumn{3}{|c|}{ Reference Elevation (ft AMSL) } & 1508.77 & \multirow{2}{*}{$\begin{array}{l}1509.12 \\
\text { Elevation in Klassen } \\
\text { Test Well (ft AMSL) }\end{array}$} \\
\hline Date and Time & $\begin{array}{l}\text { Depth in Klassen } \\
\text { Domestic Well } \\
(\mathrm{ft} \mathrm{BGL})\end{array}$ & $\begin{array}{c}\text { Depth in Klassen } \\
\text { Test Well } \\
\text { (ft BGL) }\end{array}$ & $\begin{array}{l}\text { Elevation in Klassen } \\
\text { Domestic Well } \\
\text { (ft AMSL) }\end{array}$ & \\
\hline $12 / 9 / 1118: 30$ & 33.607 & 36.007 & 1475.16 & 1473.12 \\
\hline 12/9/11 19:00 & 33.572 & 36.002 & 1475.20 & 1473.12 \\
\hline 12/9/11 19:30 & 33.652 & 36 & 1475.12 & 1473.12 \\
\hline $12 / 9 / 1120: 00$ & 33.553 & 35.995 & 1475.22 & 1473.13 \\
\hline 12/9/11 20:30 & 33.565 & 35.993 & 1475.20 & 1473.13 \\
\hline 12/9/11 21:00 & 33.896 & 35.991 & 1474.87 & 1473.13 \\
\hline $12 / 9 / 1121: 30$ & 33.581 & 35.993 & 1475.19 & 1473.13 \\
\hline $12 / 9 / 1122: 00$ & 33.558 & 35.991 & 1475.21 & 1473.13 \\
\hline $12 / 9 / 1122: 30$ & 33.994 & 35.993 & 1474.78 & 1473.13 \\
\hline $12 / 9 / 1123: 00$ & 34.487 & 36.009 & 1474.28 & 1473.11 \\
\hline $12 / 9 / 1123: 30$ & 33.86 & 36.016 & 1474.91 & 1473.11 \\
\hline $12 / 10 / 110: 00$ & 33.797 & 36.019 & 1474.97 & 1473.10 \\
\hline $12 / 10 / 110: 30$ & 33.731 & 36.021 & 1475.04 & 1473.10 \\
\hline $12 / 10 / 111: 00$ & 33.666 & 36.021 & 1475.10 & 1473.10 \\
\hline $12 / 10 / 11$ 1:30 & 33.614 & 36.026 & 1475.16 & 1473.10 \\
\hline $12 / 10 / 112: 00$ & 33.663 & 36.023 & 1475.11 & 1473.10 \\
\hline $12 / 10 / 112: 30$ & 33.595 & 36.019 & 1475.17 & 1473.10 \\
\hline $12 / 10 / 113: 00$ & 33.556 & 36.019 & 1475.21 & 1473.10 \\
\hline $12 / 10 / 113: 30$ & 33.523 & 36.014 & 1475.25 & 1473.11 \\
\hline $12 / 10 / 114: 00$ & 33.495 & 36.012 & 1475.27 & 1473.11 \\
\hline $12 / 10 / 114: 30$ & 33.473 & 36.012 & 1475.30 & 1473.11 \\
\hline $12 / 10 / 115: 00$ & 33.45 & 36.007 & 1475.32 & 1473.12 \\
\hline $12 / 10 / 115: 30$ & 33.462 & 36 & 1475.31 & 1473.12 \\
\hline $12 / 10 / 116: 00$ & 33.579 & 35.998 & 1475.19 & 1473.12 \\
\hline $12 / 10 / 116: 30$ & 33.57 & 35.993 & 1475.20 & 1473.13 \\
\hline $12 / 10 / 11$ 7:00 & 33.49 & 35.984 & 1475.28 & 1473.14 \\
\hline $12 / 10 / 117: 30$ & 33.452 & 35.979 & 1475.32 & 1473.14 \\
\hline $12 / 10 / 118: 00$ & 33.427 & 35.977 & 1475.34 & 1473.15 \\
\hline $12 / 10 / 118: 30$ & 33.502 & 35.981 & 1475.27 & 1473.14 \\
\hline 12/10/11 9:00 & 33.438 & 35.981 & 1475.33 & 1473.14 \\
\hline 12/10/11 9:30 & 33.548 & 35.988 & 1475.22 & 1473.13 \\
\hline 12/10/11 10:00 & 35.359 & 36 & 1473.41 & 1473.12 \\
\hline $12 / 10 / 11$ 10:30 & 33.556 & 36.03 & 1475.21 & 1473.09 \\
\hline 12/10/11 11:00 & 33.516 & 36.04 & 1475.25 & 1473.08 \\
\hline $12 / 10 / 1111: 30$ & 33.471 & 36.035 & 1475.30 & 1473.09 \\
\hline $12 / 10 / 1112: 00$ & 33.668 & 36.033 & 1475.10 & 1473.09 \\
\hline $12 / 10 / 1112: 30$ & 34.207 & 36.038 & 1474.56 & 1473.08 \\
\hline $12 / 10 / 11$ 13:00 & 33.86 & 36.042 & 1474.91 & 1473.08 \\
\hline
\end{tabular}


TABLE S1.3 Automatically recorded groundwater levels in the Klassen wells, September 15, 2011, to March 13, 2012.

\begin{tabular}{|c|c|c|c|c|}
\hline \multicolumn{3}{|c|}{ Reference Elevation (ft AMSL) } & 1508.77 & \multirow{2}{*}{$\begin{array}{l}1509.12 \\
\text { Elevation in Klassen } \\
\text { Test Well (ft AMSL) }\end{array}$} \\
\hline Date and Time & $\begin{array}{l}\text { Depth in Klassen } \\
\text { Domestic Well } \\
\text { (ft BGL) }\end{array}$ & $\begin{array}{c}\text { Depth in Klassen } \\
\text { Test Well } \\
\text { (ft BGL) }\end{array}$ & $\begin{array}{l}\text { Elevation in Klassen } \\
\text { Domestic Well } \\
\text { (ft AMSL) }\end{array}$ & \\
\hline $12 / 10 / 1113: 30$ & 33.677 & 36.047 & 1475.09 & 1473.08 \\
\hline $12 / 10 / 1114: 00$ & 33.6 & 36.047 & 1475.17 & 1473.08 \\
\hline $12 / 10 / 1114: 30$ & 33.677 & 36.052 & 1475.09 & 1473.07 \\
\hline $12 / 10 / 1115: 00$ & 33.586 & 36.054 & 1475.18 & 1473.07 \\
\hline $12 / 10 / 1115: 30$ & 33.666 & 36.052 & 1475.10 & 1473.07 \\
\hline $12 / 10 / 1116: 00$ & 33.565 & 36.047 & 1475.20 & 1473.08 \\
\hline $12 / 10 / 1116: 30$ & 33.577 & 36.042 & 1475.19 & 1473.08 \\
\hline 12/10/11 17:00 & 33.727 & 36.04 & 1475.04 & 1473.08 \\
\hline $12 / 10 / 1117: 30$ & 33.586 & 36.04 & 1475.18 & 1473.08 \\
\hline $12 / 10 / 1118: 00$ & 35.169 & 36.054 & 1473.60 & 1473.07 \\
\hline $12 / 10 / 1118: 30$ & 34.189 & 36.066 & 1474.58 & 1473.06 \\
\hline 12/10/11 19:00 & 33.954 & 36.066 & 1474.82 & 1473.06 \\
\hline $12 / 10 / 1119: 30$ & 33.832 & 36.068 & 1474.94 & 1473.05 \\
\hline $12 / 10 / 1120: 00$ & 33.757 & 36.068 & 1475.01 & 1473.05 \\
\hline $12 / 10 / 1120: 30$ & 33.72 & 36.066 & 1475.05 & 1473.06 \\
\hline $12 / 10 / 1121: 00$ & 33.694 & 36.07 & 1475.08 & 1473.05 \\
\hline $12 / 10 / 1121: 30$ & 33.663 & 36.077 & 1475.11 & 1473.05 \\
\hline $12 / 10 / 1122: 00$ & 36.102 & 36.075 & 1472.67 & 1473.05 \\
\hline $12 / 10 / 1122: 30$ & 33.828 & 36.077 & 1474.94 & 1473.05 \\
\hline $12 / 10 / 1123: 00$ & 34.426 & 36.092 & 1474.34 & 1473.03 \\
\hline $12 / 10 / 1123: 30$ & 33.961 & 36.096 & 1474.81 & 1473.03 \\
\hline $12 / 11 / 110: 00$ & 33.884 & 36.099 & 1474.89 & 1473.02 \\
\hline $12 / 11 / 110: 30$ & 33.966 & 36.103 & 1474.80 & 1473.02 \\
\hline 12/11/11 1:00 & 34.09 & 36.117 & 1474.68 & 1473.01 \\
\hline $12 / 11 / 11$ 1:30 & 34.083 & 36.124 & 1474.69 & 1473.00 \\
\hline $12 / 11 / 112: 00$ & 33.985 & 36.124 & 1474.78 & 1473.00 \\
\hline $12 / 11 / 112: 30$ & 34.018 & 36.131 & 1474.75 & 1472.99 \\
\hline 12/11/11 3:00 & 33.917 & 36.134 & 1474.85 & 1472.99 \\
\hline $12 / 11 / 113: 30$ & 33.839 & 36.143 & 1474.93 & 1472.98 \\
\hline 12/11/11 4:00 & 33.781 & 36.148 & 1474.99 & 1472.97 \\
\hline $12 / 11 / 114: 30$ & 33.731 & 36.148 & 1475.04 & 1472.97 \\
\hline 12/11/11 5:00 & 33.691 & 36.146 & 1475.08 & 1472.98 \\
\hline $12 / 11 / 115: 30$ & 33.654 & 36.141 & 1475.12 & 1472.98 \\
\hline $12 / 11 / 116: 00$ & 34.069 & 36.138 & 1474.70 & 1472.98 \\
\hline $12 / 11 / 116: 30$ & 33.771 & 36.136 & 1475.00 & 1472.99 \\
\hline 12/11/11 7:00 & 33.797 & 36.134 & 1474.97 & 1472.99 \\
\hline 12/11/11 7:30 & 33.694 & 36.122 & 1475.08 & 1473.00 \\
\hline $12 / 11 / 118: 00$ & 37.111 & 36.117 & 1471.66 & 1473.01 \\
\hline
\end{tabular}


TABLE S1.3 Automatically recorded groundwater levels in the Klassen wells, September 15, 2011, to March 13, 2012.

\begin{tabular}{|c|c|c|c|c|}
\hline \multicolumn{3}{|c|}{ Reference Elevation (ft AMSL) } & 1508.77 & \multirow{2}{*}{$\begin{array}{l}1509.12 \\
\text { Elevation in Klassen } \\
\text { Test Well (ft AMSL) }\end{array}$} \\
\hline Date and Time & $\begin{array}{l}\text { Depth in Klassen } \\
\text { Domestic Well } \\
\text { (ft BGL) }\end{array}$ & $\begin{array}{c}\text { Depth in Klassen } \\
\text { Test Well } \\
\text { (ft BGL) }\end{array}$ & $\begin{array}{l}\text { Elevation in Klassen } \\
\text { Domestic Well } \\
\text { (ft AMSL) }\end{array}$ & \\
\hline $12 / 11 / 118: 30$ & 33.865 & 36.115 & 1474.90 & 1473.01 \\
\hline 12/11/11 9:00 & 33.804 & 36.122 & 1474.97 & 1473.00 \\
\hline 12/11/11 9:30 & 33.706 & 36.12 & 1475.06 & 1473.00 \\
\hline $12 / 11 / 11$ 10:00 & 33.652 & 36.122 & 1475.12 & 1473.00 \\
\hline 12/11/11 10:30 & 33.663 & 36.131 & 1475.11 & 1472.99 \\
\hline $12 / 11 / 1111: 00$ & 33.741 & 36.155 & 1475.03 & 1472.97 \\
\hline $12 / 11 / 1111: 30$ & 33.804 & 36.181 & 1474.97 & 1472.94 \\
\hline $12 / 11 / 1112: 00$ & 33.874 & 36.197 & 1474.90 & 1472.93 \\
\hline $12 / 11 / 1112: 30$ & 34.003 & 36.221 & 1474.77 & 1472.90 \\
\hline $12 / 11 / 1113: 00$ & 33.9 & 36.235 & 1474.87 & 1472.89 \\
\hline $12 / 11 / 11$ 13:30 & 33.811 & 36.237 & 1474.96 & 1472.89 \\
\hline $12 / 11 / 11$ 14:00 & 33.959 & 36.244 & 1474.81 & 1472.88 \\
\hline $12 / 11 / 1114: 30$ & 33.945 & 36.253 & 1474.82 & 1472.87 \\
\hline $12 / 11 / 1115: 00$ & 33.874 & 36.253 & 1474.90 & 1472.87 \\
\hline $12 / 11 / 11$ 15:30 & 33.825 & 36.249 & 1474.94 & 1472.87 \\
\hline $12 / 11 / 11$ 16:00 & 33.757 & 36.242 & 1475.01 & 1472.88 \\
\hline $12 / 11 / 11$ 16:30 & 33.708 & 36.232 & 1475.06 & 1472.89 \\
\hline 12/11/11 17:00 & 34.365 & 36.23 & 1474.40 & 1472.89 \\
\hline $12 / 11 / 11$ 17:30 & 33.942 & 36.23 & 1474.83 & 1472.89 \\
\hline 12/11/11 18:00 & 33.842 & 36.218 & 1474.93 & 1472.90 \\
\hline $12 / 11 / 11$ 18:30 & 33.924 & 36.213 & 1474.85 & 1472.91 \\
\hline 12/11/11 19:00 & 34.128 & 36.214 & 1474.64 & 1472.91 \\
\hline 12/11/11 19:30 & 33.971 & 36.216 & 1474.80 & 1472.91 \\
\hline $12 / 11 / 1120: 00$ & 33.959 & 36.223 & 1474.81 & 1472.90 \\
\hline $12 / 11 / 11$ 20:30 & 34.01 & 36.23 & 1474.76 & 1472.89 \\
\hline $12 / 11 / 1121: 00$ & 33.957 & 36.239 & 1474.81 & 1472.88 \\
\hline 12/11/11 21:30 & 33.957 & 36.242 & 1474.81 & 1472.88 \\
\hline $12 / 11 / 1122: 00$ & 33.884 & 36.242 & 1474.89 & 1472.88 \\
\hline $12 / 11 / 1122: 30$ & 36.496 & 36.235 & 1472.27 & 1472.89 \\
\hline $12 / 11 / 1123: 00$ & 33.935 & 36.235 & 1474.83 & 1472.89 \\
\hline $12 / 11 / 1123: 30$ & 34.039 & 36.237 & 1474.73 & 1472.89 \\
\hline $12 / 12 / 110: 00$ & 37.043 & 36.239 & 1471.73 & 1472.88 \\
\hline $12 / 12 / 110: 30$ & 34.027 & 36.246 & 1474.74 & 1472.88 \\
\hline $12 / 12 / 11$ 1:00 & 34.039 & 36.256 & 1474.73 & 1472.87 \\
\hline $12 / 12 / 11$ 1:30 & 33.947 & 36.253 & 1474.82 & 1472.87 \\
\hline $12 / 12 / 112: 00$ & 33.889 & 36.242 & 1474.88 & 1472.88 \\
\hline $12 / 12 / 112: 30$ & 34.109 & 36.239 & 1474.66 & 1472.88 \\
\hline $12 / 12 / 113: 00$ & 33.942 & 36.237 & 1474.83 & 1472.89 \\
\hline
\end{tabular}


TABLE S1.3 Automatically recorded groundwater levels in the Klassen wells, September 15, 2011, to March 13, 2012.

\begin{tabular}{|c|c|c|c|c|}
\hline \multicolumn{3}{|c|}{ Reference Elevation (ft AMSL) } & 1508.77 & \multirow{2}{*}{$\begin{array}{l}1509.12 \\
\text { Elevation in Klassen } \\
\text { Test Well (ft AMSL) }\end{array}$} \\
\hline Date and Time & $\begin{array}{c}\text { Depth in Klassen } \\
\text { Domestic Well } \\
\text { (ft BGL) }\end{array}$ & $\begin{array}{c}\text { Depth in Klassen } \\
\text { Test Well } \\
\text { (ft BGL) }\end{array}$ & $\begin{array}{l}\text { Elevation in Klassen } \\
\text { Domestic Well } \\
\text { (ft AMSL) }\end{array}$ & \\
\hline $12 / 12 / 113: 30$ & 33.86 & 36.23 & 1474.91 & 1472.89 \\
\hline $12 / 12 / 114: 00$ & 33.797 & 36.218 & 1474.97 & 1472.90 \\
\hline $12 / 12 / 114: 30$ & 33.75 & 36.207 & 1475.02 & 1472.92 \\
\hline $12 / 12 / 115: 00$ & 33.708 & 36.197 & 1475.06 & 1472.93 \\
\hline $12 / 12 / 115: 30$ & 33.675 & 36.188 & 1475.09 & 1472.93 \\
\hline $12 / 12 / 116: 00$ & 33.729 & 36.176 & 1475.04 & 1472.95 \\
\hline $12 / 12 / 116: 30$ & 33.91 & 36.171 & 1474.86 & 1472.95 \\
\hline $12 / 12 / 117: 00$ & 33.804 & 36.169 & 1474.97 & 1472.95 \\
\hline $12 / 12 / 117: 30$ & 33.734 & 36.157 & 1475.04 & 1472.97 \\
\hline $12 / 12 / 118: 00$ & 33.914 & 36.15 & 1474.86 & 1472.97 \\
\hline $12 / 12 / 118: 30$ & 33.71 & 36.143 & 1475.06 & 1472.98 \\
\hline 12/12/11 9:00 & 34.179 & 36.138 & 1474.59 & 1472.98 \\
\hline 12/12/11 9:30 & 34.587 & 36.136 & 1474.18 & 1472.99 \\
\hline $12 / 12 / 11$ 10:00 & 33.753 & 36.131 & 1475.02 & 1472.99 \\
\hline $12 / 12 / 11$ 10:30 & 33.79 & 36.127 & 1474.98 & 1473.00 \\
\hline 12/12/11 11:00 & 37.005 & 36.122 & 1471.76 & 1473.00 \\
\hline 12/12/11 11:30 & 33.933 & 36.122 & 1474.84 & 1473.00 \\
\hline $12 / 12 / 11$ 12:00 & 33.76 & 36.127 & 1475.01 & 1473.00 \\
\hline $12 / 12 / 1112: 30$ & 33.945 & 36.131 & 1474.82 & 1472.99 \\
\hline $12 / 12 / 11$ 13:00 & 34.219 & 36.138 & 1474.55 & 1472.98 \\
\hline $12 / 12 / 11$ 13:30 & 33.727 & 36.138 & 1475.04 & 1472.98 \\
\hline $12 / 12 / 11$ 14:00 & 33.663 & 36.134 & 1475.11 & 1472.99 \\
\hline $12 / 12 / 11$ 14:30 & 33.818 & 36.129 & 1474.95 & 1472.99 \\
\hline $12 / 12 / 11$ 15:00 & 33.666 & 36.12 & 1475.10 & 1473.00 \\
\hline $12 / 12 / 11$ 15:30 & 33.612 & 36.11 & 1475.16 & 1473.01 \\
\hline $12 / 12 / 11$ 16:00 & 33.912 & 36.103 & 1474.86 & 1473.02 \\
\hline $12 / 12 / 11$ 16:30 & 33.649 & 36.094 & 1475.12 & 1473.03 \\
\hline 12/12/11 17:00 & 33.821 & 36.094 & 1474.95 & 1473.03 \\
\hline $12 / 12 / 11$ 17:30 & 33.893 & 36.099 & 1474.88 & 1473.02 \\
\hline 12/12/11 18:00 & 33.811 & 36.091 & 1474.96 & 1473.03 \\
\hline $12 / 12 / 11$ 18:30 & 33.961 & 36.089 & 1474.81 & 1473.03 \\
\hline 12/12/11 19:00 & 33.879 & 36.092 & 1474.89 & 1473.03 \\
\hline $12 / 12 / 11$ 19:30 & 33.79 & 36.084 & 1474.98 & 1473.04 \\
\hline $12 / 12 / 1120: 00$ & 33.825 & 36.082 & 1474.94 & 1473.04 \\
\hline $12 / 12 / 1120: 30$ & 33.856 & 36.085 & 1474.91 & 1473.04 \\
\hline $12 / 12 / 1121: 00$ & 33.928 & 36.092 & 1474.84 & 1473.03 \\
\hline $12 / 12 / 1121: 30$ & 33.884 & 36.096 & 1474.89 & 1473.03 \\
\hline $12 / 12 / 1122: 00$ & 33.802 & 36.094 & 1474.97 & 1473.03 \\
\hline
\end{tabular}


TABLE S1.3 Automatically recorded groundwater levels in the Klassen wells, September 15, 2011, to March 13, 2012.

\begin{tabular}{|c|c|c|c|c|}
\hline \multicolumn{3}{|c|}{ Reference Elevation (ft AMSL) } & 1508.77 & \multirow{2}{*}{$\begin{array}{l}1509.12 \\
\text { Elevation in Klassen } \\
\text { Test Well (ft AMSL) }\end{array}$} \\
\hline Date and Time & $\begin{array}{l}\text { Depth in Klassen } \\
\text { Domestic Well } \\
\text { (ft BGL) }\end{array}$ & $\begin{array}{c}\text { Depth in Klassen } \\
\text { Test Well } \\
\text { (ft BGL) }\end{array}$ & $\begin{array}{l}\text { Elevation in Klassen } \\
\text { Domestic Well } \\
\text { (ft AMSL) }\end{array}$ & \\
\hline $12 / 12 / 1122: 30$ & 33.865 & 36.103 & 1474.90 & 1473.02 \\
\hline $12 / 12 / 1123: 00$ & 33.999 & 36.094 & 1474.77 & 1473.03 \\
\hline $12 / 12 / 1123: 30$ & 34.034 & 36.099 & 1474.74 & 1473.02 \\
\hline $12 / 13 / 110: 00$ & 33.856 & 36.096 & 1474.91 & 1473.03 \\
\hline 12/13/11 0:30 & 33.778 & 36.101 & 1474.99 & 1473.02 \\
\hline $12 / 13 / 111: 00$ & 33.816 & 36.103 & 1474.95 & 1473.02 \\
\hline $12 / 13 / 111: 30$ & 33.734 & 36.099 & 1475.04 & 1473.02 \\
\hline $12 / 13 / 112: 00$ & 33.684 & 36.094 & 1475.09 & 1473.03 \\
\hline $12 / 13 / 112: 30$ & 33.647 & 36.084 & 1475.12 & 1473.04 \\
\hline $12 / 13 / 113: 00$ & 33.614 & 36.084 & 1475.16 & 1473.04 \\
\hline $12 / 13 / 113: 30$ & 33.586 & 36.075 & 1475.18 & 1473.05 \\
\hline 12/13/11 4:00 & 33.56 & 36.066 & 1475.21 & 1473.06 \\
\hline $12 / 13 / 114: 30$ & 33.537 & 36.061 & 1475.23 & 1473.06 \\
\hline $12 / 13 / 115: 00$ & 33.516 & 36.056 & 1475.25 & 1473.07 \\
\hline $12 / 13 / 115: 30$ & 33.495 & 36.052 & 1475.27 & 1473.07 \\
\hline $12 / 13 / 116: 00$ & 33.476 & 36.045 & 1475.29 & 1473.08 \\
\hline $12 / 13 / 116: 30$ & 33.724 & 36.054 & 1475.05 & 1473.07 \\
\hline 12/13/11 7:00 & 33.598 & 36.045 & 1475.17 & 1473.08 \\
\hline $12 / 13 / 11$ 7:30 & 33.628 & 36.038 & 1475.14 & 1473.08 \\
\hline $12 / 13 / 118: 00$ & 33.572 & 36.033 & 1475.20 & 1473.09 \\
\hline $12 / 13 / 118: 30$ & 33.53 & 36.035 & 1475.24 & 1473.09 \\
\hline 12/13/11 9:00 & 33.619 & 36.03 & 1475.15 & 1473.09 \\
\hline 12/13/11 9:30 & 33.544 & 36.023 & 1475.23 & 1473.10 \\
\hline $12 / 13 / 11$ 10:00 & 33.802 & 36.024 & 1474.97 & 1473.10 \\
\hline $12 / 13 / 11$ 10:30 & 33.537 & 36.023 & 1475.23 & 1473.10 \\
\hline $12 / 13 / 11$ 11:00 & 33.839 & 36.031 & 1474.93 & 1473.09 \\
\hline 12/13/11 11:30 & 33.797 & 36.035 & 1474.97 & 1473.09 \\
\hline $12 / 13 / 1112: 00$ & 33.91 & 36.04 & 1474.86 & 1473.08 \\
\hline $12 / 13 / 11$ 12:30 & 33.675 & 36.047 & 1475.09 & 1473.08 \\
\hline 12/13/11 13:00 & 33.694 & 36.052 & 1475.08 & 1473.07 \\
\hline $12 / 13 / 11$ 13:30 & 33.624 & 36.059 & 1475.15 & 1473.06 \\
\hline $12 / 13 / 11$ 14:00 & 33.565 & 36.054 & 1475.20 & 1473.07 \\
\hline $12 / 13 / 11$ 14:30 & 33.591 & 36.052 & 1475.18 & 1473.07 \\
\hline $12 / 13 / 11$ 15:00 & 33.534 & 36.042 & 1475.24 & 1473.08 \\
\hline $12 / 13 / 11$ 15:30 & 33.499 & 36.033 & 1475.27 & 1473.09 \\
\hline $12 / 13 / 11$ 16:00 & 33.546 & 36.028 & 1475.22 & 1473.09 \\
\hline $12 / 13 / 11$ 16:30 & 33.487 & 36.026 & 1475.28 & 1473.10 \\
\hline $12 / 13 / 11$ 17:00 & 33.455 & 36.007 & 1475.31 & 1473.12 \\
\hline
\end{tabular}


TABLE S1.3 Automatically recorded groundwater levels in the Klassen wells, September 15, 2011, to March 13, 2012.

\begin{tabular}{|c|c|c|c|c|}
\hline \multicolumn{3}{|c|}{ Reference Elevation (ft AMSL) } & 1508.77 & \multirow{2}{*}{$\begin{array}{l}1509.12 \\
\text { Elevation in Klassen } \\
\text { Test Well (ft AMSL) }\end{array}$} \\
\hline Date and Time & $\begin{array}{l}\text { Depth in Klassen } \\
\text { Domestic Well } \\
\text { (ft BGL) }\end{array}$ & $\begin{array}{c}\text { Depth in Klassen } \\
\text { Test Well } \\
\text { (ft BGL) }\end{array}$ & $\begin{array}{l}\text { Elevation in Klassen } \\
\text { Domestic Well } \\
\text { (ft AMSL) }\end{array}$ & \\
\hline $12 / 13 / 11$ 17:30 & 33.556 & 36.002 & 1475.21 & 1473.12 \\
\hline $12 / 13 / 11$ 18:00 & 33.806 & 36.002 & 1474.96 & 1473.12 \\
\hline $12 / 13 / 1118: 30$ & 33.76 & 36.005 & 1475.01 & 1473.12 \\
\hline $12 / 13 / 11$ 19:00 & 33.795 & 36.014 & 1474.97 & 1473.11 \\
\hline $12 / 13 / 11$ 19:30 & 33.701 & 36.012 & 1475.07 & 1473.11 \\
\hline $12 / 13 / 1120: 00$ & 33.68 & 36.007 & 1475.09 & 1473.12 \\
\hline $12 / 13 / 1120: 30$ & 33.806 & 36.012 & 1474.96 & 1473.11 \\
\hline $12 / 13 / 1121: 00$ & 33.717 & 36.014 & 1475.05 & 1473.11 \\
\hline $12 / 13 / 1121: 30$ & 33.67 & 36.016 & 1475.10 & 1473.11 \\
\hline $12 / 13 / 1122: 00$ & 33.853 & 36.021 & 1474.92 & 1473.10 \\
\hline $12 / 13 / 1122: 30$ & 33.722 & 36.026 & 1475.05 & 1473.10 \\
\hline $12 / 13 / 1123: 00$ & 36.464 & 36.038 & 1472.31 & 1473.08 \\
\hline $12 / 13 / 1123: 30$ & 33.928 & 36.045 & 1474.84 & 1473.08 \\
\hline $12 / 14 / 110: 00$ & 33.788 & 36.049 & 1474.98 & 1473.07 \\
\hline $12 / 14 / 110: 30$ & 33.713 & 36.052 & 1475.06 & 1473.07 \\
\hline 12/14/11 1:00 & 33.776 & 36.061 & 1474.99 & 1473.06 \\
\hline $12 / 14 / 11$ 1:30 & 33.717 & 36.063 & 1475.05 & 1473.06 \\
\hline $12 / 14 / 112: 00$ & 33.682 & 36.066 & 1475.09 & 1473.06 \\
\hline $12 / 14 / 112: 30$ & 33.75 & 36.07 & 1475.02 & 1473.05 \\
\hline 12/14/11 3:00 & 33.673 & 36.07 & 1475.10 & 1473.05 \\
\hline $12 / 14 / 113: 30$ & 33.614 & 36.068 & 1475.16 & 1473.05 \\
\hline $12 / 14 / 114: 00$ & 33.57 & 36.075 & 1475.20 & 1473.05 \\
\hline $12 / 14 / 114: 30$ & 33.558 & 36.075 & 1475.21 & 1473.05 \\
\hline 12/14/11 5:00 & 33.52 & 36.08 & 1475.25 & 1473.04 \\
\hline $12 / 14 / 115: 30$ & 33.581 & 36.089 & 1475.19 & 1473.03 \\
\hline $12 / 14 / 116: 00$ & 33.516 & 36.087 & 1475.25 & 1473.04 \\
\hline $12 / 14 / 116: 30$ & 33.811 & 36.089 & 1474.96 & 1473.03 \\
\hline 12/14/11 7:00 & 33.633 & 36.091 & 1475.14 & 1473.03 \\
\hline $12 / 14 / 11$ 7:30 & 33.628 & 36.096 & 1475.14 & 1473.03 \\
\hline $12 / 14 / 118: 00$ & 33.645 & 36.099 & 1475.12 & 1473.02 \\
\hline $12 / 14 / 118: 30$ & 33.574 & 36.099 & 1475.20 & 1473.02 \\
\hline 12/14/11 9:00 & 33.525 & 36.103 & 1475.24 & 1473.02 \\
\hline 12/14/11 9:30 & 33.558 & 36.106 & 1475.21 & 1473.02 \\
\hline 12/14/11 10:00 & 33.968 & 36.113 & 1474.80 & 1473.01 \\
\hline $12 / 14 / 11$ 10:30 & 33.546 & 36.117 & 1475.22 & 1473.01 \\
\hline 12/14/11 11:00 & 33.49 & 36.12 & 1475.28 & 1473.00 \\
\hline 12/14/11 11:30 & 33.527 & 36.122 & 1475.24 & 1473.00 \\
\hline $12 / 14 / 1112: 00$ & 33.602 & 36.124 & 1475.17 & 1473.00 \\
\hline
\end{tabular}


TABLE S1.3 Automatically recorded groundwater levels in the Klassen wells, September 15, 2011, to March 13, 2012.

\begin{tabular}{|c|c|c|c|c|}
\hline \multicolumn{3}{|c|}{ Reference Elevation (ft AMSL) } & 1508.77 & \multirow{2}{*}{$\begin{array}{l}1509.12 \\
\text { Elevation in Klassen } \\
\text { Test Well (ft AMSL) }\end{array}$} \\
\hline Date and Time & $\begin{array}{l}\text { Depth in Klassen } \\
\text { Domestic Well } \\
(\mathrm{ft} \mathrm{BGL})\end{array}$ & $\begin{array}{c}\text { Depth in Klassen } \\
\text { Test Well } \\
\text { (ft BGL) }\end{array}$ & $\begin{array}{l}\text { Elevation in Klassen } \\
\text { Domestic Well } \\
\text { (ft AMSL) }\end{array}$ & \\
\hline $12 / 14 / 1112: 30$ & 33.49 & 36.117 & 1475.28 & 1473.01 \\
\hline $12 / 14 / 1113: 00$ & 33.445 & 36.094 & 1475.32 & 1473.03 \\
\hline $12 / 14 / 1113: 30$ & 33.525 & 36.061 & 1475.24 & 1473.06 \\
\hline $12 / 14 / 1114: 00$ & 33.457 & 35.979 & 1475.31 & 1473.14 \\
\hline $12 / 14 / 1114: 30$ & 33.422 & 35.873 & 1475.35 & 1473.25 \\
\hline $12 / 14 / 1115: 00$ & 33.459 & 35.826 & 1475.31 & 1473.30 \\
\hline $12 / 14 / 1115: 30$ & 33.412 & 35.81 & 1475.36 & 1473.31 \\
\hline $12 / 14 / 11$ 16:00 & 37.685 & 35.817 & 1471.08 & 1473.31 \\
\hline $12 / 14 / 1116: 30$ & 33.565 & 35.822 & 1475.20 & 1473.30 \\
\hline $12 / 14 / 1117: 00$ & 33.495 & 35.819 & 1475.27 & 1473.30 \\
\hline $12 / 14 / 1117: 30$ & 33.471 & 35.817 & 1475.30 & 1473.31 \\
\hline $12 / 14 / 11$ 18:00 & 33.452 & 35.81 & 1475.32 & 1473.31 \\
\hline $12 / 14 / 1118: 30$ & 33.473 & 35.815 & 1475.30 & 1473.31 \\
\hline $12 / 14 / 11$ 19:00 & 33.633 & 35.815 & 1475.14 & 1473.31 \\
\hline $12 / 14 / 11$ 19:30 & 34.043 & 35.819 & 1474.73 & 1473.30 \\
\hline $12 / 14 / 1120: 00$ & 33.792 & 35.84 & 1474.98 & 1473.28 \\
\hline $12 / 14 / 1120: 30$ & 33.931 & 35.869 & 1474.84 & 1473.25 \\
\hline $12 / 14 / 1121: 00$ & 33.809 & 35.894 & 1474.96 & 1473.23 \\
\hline $12 / 14 / 1121: 30$ & 33.825 & 35.904 & 1474.94 & 1473.22 \\
\hline $12 / 14 / 1122: 00$ & 34.13 & 35.92 & 1474.64 & 1473.20 \\
\hline $12 / 14 / 1122: 30$ & 33.914 & 35.927 & 1474.86 & 1473.20 \\
\hline $12 / 14 / 1123: 00$ & 34.003 & 35.941 & 1474.77 & 1473.18 \\
\hline $12 / 14 / 1123: 30$ & 34.107 & 35.953 & 1474.66 & 1473.17 \\
\hline $12 / 15 / 110: 00$ & 38.614 & 35.96 & 1470.16 & 1473.16 \\
\hline 12/15/11 0:30 & 33.992 & 35.97 & 1474.78 & 1473.15 \\
\hline 12/15/11 1:00 & 33.863 & 35.963 & 1474.91 & 1473.16 \\
\hline $12 / 15 / 11$ 1:30 & 33.785 & 35.953 & 1474.98 & 1473.17 \\
\hline $12 / 15 / 112: 00$ & 33.724 & 35.951 & 1475.05 & 1473.17 \\
\hline $12 / 15 / 112: 30$ & 33.677 & 35.939 & 1475.09 & 1473.18 \\
\hline 12/15/11 3:00 & 33.757 & 35.925 & 1475.01 & 1473.20 \\
\hline 12/15/11 3:30 & 33.661 & 35.92 & 1475.11 & 1473.20 \\
\hline $12 / 15 / 114: 00$ & 33.614 & 35.911 & 1475.16 & 1473.21 \\
\hline $12 / 15 / 114: 30$ & 33.579 & 35.901 & 1475.19 & 1473.22 \\
\hline 12/15/11 5:00 & 33.574 & 35.899 & 1475.20 & 1473.22 \\
\hline 12/15/11 5:30 & 33.539 & 35.89 & 1475.23 & 1473.23 \\
\hline $12 / 15 / 116: 00$ & 33.513 & 35.883 & 1475.26 & 1473.24 \\
\hline $12 / 15 / 116: 30$ & 33.694 & 35.88 & 1475.08 & 1473.24 \\
\hline 12/15/11 7:00 & 33.593 & 35.876 & 1475.18 & 1473.25 \\
\hline
\end{tabular}


TABLE S1.3 Automatically recorded groundwater levels in the Klassen wells, September 15, 2011, to March 13, 2012.

\begin{tabular}{|c|c|c|c|c|}
\hline \multicolumn{3}{|c|}{ Reference Elevation (ft AMSL) } & 1508.77 & \multirow{2}{*}{$\begin{array}{l}1509.12 \\
\text { Elevation in Klassen } \\
\text { Test Well (ft AMSL) }\end{array}$} \\
\hline Date and Time & $\begin{array}{l}\text { Depth in Klassen } \\
\text { Domestic Well } \\
(\mathrm{ft} \mathrm{BGL})\end{array}$ & $\begin{array}{c}\text { Depth in Klassen } \\
\text { Test Well } \\
\text { (ft BGL) }\end{array}$ & $\begin{array}{l}\text { Elevation in Klassen } \\
\text { Domestic Well } \\
\text { (ft AMSL) }\end{array}$ & \\
\hline $12 / 15 / 117: 30$ & 33.563 & 35.871 & 1475.21 & 1473.25 \\
\hline $12 / 15 / 118: 00$ & 33.551 & 35.866 & 1475.22 & 1473.26 \\
\hline $12 / 15 / 118: 30$ & 33.513 & 35.876 & 1475.26 & 1473.25 \\
\hline 12/15/11 9:00 & 33.71 & 35.862 & 1475.06 & 1473.26 \\
\hline 12/15/11 9:30 & 40.913 & 35.866 & 1467.86 & 1473.26 \\
\hline $12 / 15 / 11$ 10:00 & 33.624 & 35.871 & 1475.15 & 1473.25 \\
\hline $12 / 15 / 1110: 30$ & 33.548 & 35.876 & 1475.22 & 1473.25 \\
\hline 12/15/11 11:00 & 33.506 & 35.88 & 1475.26 & 1473.24 \\
\hline $12 / 15 / 1111: 30$ & 33.478 & 35.883 & 1475.29 & 1473.24 \\
\hline $12 / 15 / 1112: 00$ & 33.553 & 35.887 & 1475.22 & 1473.24 \\
\hline $12 / 15 / 1112: 30$ & 33.495 & 35.899 & 1475.27 & 1473.22 \\
\hline $12 / 15 / 11$ 13:00 & 33.462 & 35.909 & 1475.31 & 1473.21 \\
\hline $12 / 15 / 1113: 30$ & 33.509 & 35.915 & 1475.26 & 1473.21 \\
\hline $12 / 15 / 1114: 00$ & 33.462 & 35.913 & 1475.31 & 1473.21 \\
\hline $12 / 15 / 11$ 14:30 & 33.464 & 35.918 & 1475.31 & 1473.20 \\
\hline $12 / 15 / 11$ 15:00 & 33.64 & 35.918 & 1475.13 & 1473.20 \\
\hline $12 / 15 / 11$ 15:30 & 33.591 & 35.911 & 1475.18 & 1473.21 \\
\hline $12 / 15 / 11$ 16:00 & 33.541 & 35.904 & 1475.23 & 1473.22 \\
\hline $12 / 15 / 11$ 16:30 & 33.508 & 35.897 & 1475.26 & 1473.23 \\
\hline $12 / 15 / 11$ 17:00 & 33.485 & 35.892 & 1475.28 & 1473.23 \\
\hline $12 / 15 / 11$ 17:30 & 33.452 & 35.885 & 1475.32 & 1473.24 \\
\hline $12 / 15 / 11$ 18:00 & 33.539 & 35.88 & 1475.23 & 1473.24 \\
\hline $12 / 15 / 11$ 18:30 & 33.473 & 35.873 & 1475.30 & 1473.25 \\
\hline $12 / 15 / 11$ 19:00 & 33.466 & 35.864 & 1475.30 & 1473.26 \\
\hline 12/15/11 19:30 & 33.431 & 35.855 & 1475.34 & 1473.27 \\
\hline $12 / 15 / 1120: 00$ & 33.534 & 35.847 & 1475.24 & 1473.28 \\
\hline $12 / 15 / 1120: 30$ & 33.687 & 35.854 & 1475.08 & 1473.27 \\
\hline $12 / 15 / 1121: 00$ & 33.628 & 35.85 & 1475.14 & 1473.27 \\
\hline $12 / 15 / 1121: 30$ & 33.631 & 35.848 & 1475.14 & 1473.27 \\
\hline $12 / 15 / 1122: 00$ & 33.579 & 35.843 & 1475.19 & 1473.28 \\
\hline $12 / 15 / 1122: 30$ & 33.534 & 35.838 & 1475.24 & 1473.28 \\
\hline $12 / 15 / 1123: 00$ & 33.513 & 35.836 & 1475.26 & 1473.29 \\
\hline $12 / 15 / 1123: 30$ & 34.264 & 35.838 & 1474.51 & 1473.28 \\
\hline 12/16/11 0:00 & 33.741 & 35.843 & 1475.03 & 1473.28 \\
\hline $12 / 16 / 110: 30$ & 33.628 & 35.843 & 1475.14 & 1473.28 \\
\hline 12/16/11 1:00 & 33.57 & 35.843 & 1475.20 & 1473.28 \\
\hline $12 / 16 / 11$ 1:30 & 33.525 & 35.843 & 1475.24 & 1473.28 \\
\hline $12 / 16 / 112: 00$ & 33.492 & 35.838 & 1475.28 & 1473.28 \\
\hline
\end{tabular}


TABLE S1.3 Automatically recorded groundwater levels in the Klassen wells, September 15, 2011, to March 13, 2012.

\begin{tabular}{|c|c|c|c|c|}
\hline \multicolumn{3}{|c|}{ Reference Elevation (ft AMSL) } & 1508.77 & \multirow{2}{*}{$\begin{array}{l}1509.12 \\
\text { Elevation in Klassen } \\
\text { Test Well (ft AMSL) }\end{array}$} \\
\hline Date and Time & $\begin{array}{l}\text { Depth in Klassen } \\
\text { Domestic Well } \\
\text { (ft BGL) }\end{array}$ & $\begin{array}{c}\text { Depth in Klassen } \\
\text { Test Well } \\
\text { (ft BGL) }\end{array}$ & $\begin{array}{l}\text { Elevation in Klassen } \\
\text { Domestic Well } \\
\text { (ft AMSL) }\end{array}$ & \\
\hline $12 / 16 / 112: 30$ & 33.464 & 35.838 & 1475.31 & 1473.28 \\
\hline 12/16/11 3:00 & 33.443 & 35.838 & 1475.33 & 1473.28 \\
\hline 12/16/11 3:30 & 33.546 & 35.838 & 1475.22 & 1473.28 \\
\hline $12 / 16 / 114: 00$ & 33.464 & 35.836 & 1475.31 & 1473.29 \\
\hline $12 / 16 / 114: 30$ & 33.431 & 35.833 & 1475.34 & 1473.29 \\
\hline $12 / 16 / 115: 00$ & 33.405 & 35.838 & 1475.36 & 1473.28 \\
\hline $12 / 16 / 115: 30$ & 33.387 & 35.831 & 1475.38 & 1473.29 \\
\hline $12 / 16 / 116: 00$ & 33.368 & 35.824 & 1475.40 & 1473.30 \\
\hline $12 / 16 / 116: 30$ & 33.586 & 35.822 & 1475.18 & 1473.30 \\
\hline $12 / 16 / 117: 00$ & 33.485 & 35.819 & 1475.28 & 1473.30 \\
\hline $12 / 16 / 117: 30$ & 33.471 & 35.822 & 1475.30 & 1473.30 \\
\hline 12/16/11 8:00 & 33.457 & 35.824 & 1475.31 & 1473.30 \\
\hline $12 / 16 / 118: 30$ & 33.757 & 35.84 & 1475.01 & 1473.28 \\
\hline 12/16/11 9:00 & 33.663 & 35.85 & 1475.11 & 1473.27 \\
\hline $12 / 16 / 119: 30$ & 33.68 & 35.855 & 1475.09 & 1473.27 \\
\hline $12 / 16 / 11$ 10:00 & 33.558 & 35.859 & 1475.21 & 1473.26 \\
\hline $12 / 16 / 11$ 10:30 & 33.502 & 35.871 & 1475.27 & 1473.25 \\
\hline 12/16/11 11:00 & 33.462 & 35.883 & 1475.31 & 1473.24 \\
\hline 12/16/11 11:30 & 33.431 & 35.892 & 1475.34 & 1473.23 \\
\hline $12 / 16 / 11$ 12:00 & 33.403 & 35.892 & 1475.37 & 1473.23 \\
\hline $12 / 16 / 11$ 12:30 & 33.382 & 35.892 & 1475.39 & 1473.23 \\
\hline $12 / 16 / 11$ 13:00 & 33.361 & 35.894 & 1475.41 & 1473.23 \\
\hline $12 / 16 / 11$ 13:30 & 33.344 & 35.89 & 1475.43 & 1473.23 \\
\hline $12 / 16 / 11$ 14:00 & 33.328 & 35.887 & 1475.44 & 1473.24 \\
\hline $12 / 16 / 11$ 14:30 & 33.314 & 35.871 & 1475.46 & 1473.25 \\
\hline $12 / 16 / 11$ 15:00 & 33.302 & 35.838 & 1475.47 & 1473.28 \\
\hline $12 / 16 / 11$ 15:30 & 33.434 & 35.817 & 1475.34 & 1473.31 \\
\hline 12/16/11 16:00 & 33.354 & 35.801 & 1475.42 & 1473.32 \\
\hline $12 / 16 / 11$ 16:30 & 33.321 & 35.796 & 1475.45 & 1473.33 \\
\hline 12/16/11 17:00 & 33.375 & 35.791 & 1475.39 & 1473.33 \\
\hline 12/16/11 17:30 & 33.485 & 35.791 & 1475.28 & 1473.33 \\
\hline 12/16/11 18:00 & 33.67 & 35.793 & 1475.10 & 1473.33 \\
\hline $12 / 16 / 11$ 18:30 & 33.595 & 35.798 & 1475.17 & 1473.32 \\
\hline 12/16/11 19:00 & 33.516 & 35.798 & 1475.25 & 1473.32 \\
\hline 12/16/11 19:30 & 33.884 & 35.791 & 1474.89 & 1473.33 \\
\hline $12 / 16 / 1120: 00$ & 33.696 & 35.794 & 1475.07 & 1473.33 \\
\hline $12 / 16 / 1120: 30$ & 33.687 & 35.784 & 1475.08 & 1473.34 \\
\hline $12 / 16 / 1121: 00$ & 33.624 & 35.787 & 1475.15 & 1473.34 \\
\hline
\end{tabular}


TABLE S1.3 Automatically recorded groundwater levels in the Klassen wells, September 15, 2011, to March 13, 2012.

\begin{tabular}{|c|c|c|c|c|}
\hline \multicolumn{3}{|c|}{ Reference Elevation (ft AMSL) } & 1508.77 & \multirow{2}{*}{$\begin{array}{l}1509.12 \\
\text { Elevation in Klassen } \\
\text { Test Well (ft AMSL) }\end{array}$} \\
\hline Date and Time & $\begin{array}{l}\text { Depth in Klassen } \\
\text { Domestic Well } \\
(\mathrm{ft} \mathrm{BGL})\end{array}$ & $\begin{array}{c}\text { Depth in Klassen } \\
\text { Test Well } \\
\text { (ft BGL) }\end{array}$ & $\begin{array}{l}\text { Elevation in Klassen } \\
\text { Domestic Well } \\
\text { (ft AMSL) }\end{array}$ & \\
\hline $12 / 16 / 1121: 30$ & 33.53 & 35.782 & 1475.24 & 1473.34 \\
\hline $12 / 16 / 1122: 00$ & 33.476 & 35.779 & 1475.29 & 1473.34 \\
\hline $12 / 16 / 1122: 30$ & 33.441 & 35.77 & 1475.33 & 1473.35 \\
\hline $12 / 16 / 1123: 00$ & 33.654 & 35.775 & 1475.12 & 1473.35 \\
\hline $12 / 16 / 1123: 30$ & 33.741 & 35.777 & 1475.03 & 1473.35 \\
\hline $12 / 17 / 110: 00$ & 33.642 & 35.775 & 1475.13 & 1473.35 \\
\hline $12 / 17 / 110: 30$ & 33.591 & 35.779 & 1475.18 & 1473.34 \\
\hline 12/17/11 1:00 & 33.652 & 35.791 & 1475.12 & 1473.33 \\
\hline $12 / 17 / 111: 30$ & 33.595 & 35.793 & 1475.17 & 1473.33 \\
\hline $12 / 17 / 112: 00$ & 33.56 & 35.794 & 1475.21 & 1473.33 \\
\hline $12 / 17 / 112: 30$ & 33.635 & 35.801 & 1475.13 & 1473.32 \\
\hline 12/17/11 3:00 & 33.567 & 35.801 & 1475.20 & 1473.32 \\
\hline 12/17/11 3:30 & 33.513 & 35.803 & 1475.26 & 1473.32 \\
\hline $12 / 17 / 114: 00$ & 33.476 & 35.803 & 1475.29 & 1473.32 \\
\hline $12 / 17 / 114: 30$ & 33.445 & 35.803 & 1475.32 & 1473.32 \\
\hline 12/17/11 5:00 & 33.419 & 35.796 & 1475.35 & 1473.33 \\
\hline $12 / 17 / 115: 30$ & 33.504 & 35.801 & 1475.27 & 1473.32 \\
\hline $12 / 17 / 116: 00$ & 33.433 & 35.794 & 1475.34 & 1473.33 \\
\hline $12 / 17 / 116: 30$ & 33.509 & 35.789 & 1475.26 & 1473.33 \\
\hline $12 / 17 / 11$ 7:00 & 33.434 & 35.784 & 1475.34 & 1473.34 \\
\hline $12 / 17 / 117: 30$ & 33.398 & 35.777 & 1475.37 & 1473.35 \\
\hline 12/17/11 8:00 & 33.45 & 35.779 & 1475.32 & 1473.34 \\
\hline $12 / 17 / 118: 30$ & 33.431 & 35.784 & 1475.34 & 1473.34 \\
\hline 12/17/11 9:00 & 33.556 & 35.789 & 1475.21 & 1473.33 \\
\hline 12/17/11 9:30 & 33.544 & 35.796 & 1475.23 & 1473.33 \\
\hline $12 / 17 / 11$ 10:00 & 33.577 & 35.81 & 1475.19 & 1473.31 \\
\hline $12 / 17 / 11$ 10:30 & 33.605 & 35.819 & 1475.16 & 1473.30 \\
\hline 12/17/11 11:00 & 33.502 & 35.829 & 1475.27 & 1473.29 \\
\hline 12/17/11 11:30 & 33.607 & 35.838 & 1475.16 & 1473.28 \\
\hline $12 / 17 / 11$ 12:00 & 33.478 & 35.845 & 1475.29 & 1473.28 \\
\hline $12 / 17 / 11$ 12:30 & 33.431 & 35.852 & 1475.34 & 1473.27 \\
\hline $12 / 17 / 11$ 13:00 & 33.398 & 35.845 & 1475.37 & 1473.28 \\
\hline $12 / 17 / 11$ 13:30 & 33.506 & 35.808 & 1475.26 & 1473.31 \\
\hline $12 / 17 / 11$ 14:00 & 33.412 & 35.793 & 1475.36 & 1473.33 \\
\hline $12 / 17 / 11$ 14:30 & 34.531 & 35.796 & 1474.24 & 1473.33 \\
\hline $12 / 17 / 11$ 15:00 & 33.469 & 35.798 & 1475.30 & 1473.32 \\
\hline $12 / 17 / 11$ 15:30 & 33.412 & 35.794 & 1475.36 & 1473.33 \\
\hline $12 / 17 / 11$ 16:00 & 33.38 & 35.793 & 1475.39 & 1473.33 \\
\hline
\end{tabular}


TABLE S1.3 Automatically recorded groundwater levels in the Klassen wells, September 15, 2011, to March 13, 2012.

\begin{tabular}{|c|c|c|c|c|}
\hline \multicolumn{3}{|c|}{ Reference Elevation (ft AMSL) } & 1508.77 & \multirow{2}{*}{$\begin{array}{l}1509.12 \\
\text { Elevation in Klassen } \\
\text { Test Well (ft AMSL) }\end{array}$} \\
\hline Date and Time & $\begin{array}{l}\text { Depth in Klassen } \\
\text { Domestic Well } \\
(\mathrm{ft} \mathrm{BGL})\end{array}$ & $\begin{array}{c}\text { Depth in Klassen } \\
\text { Test Well } \\
\text { (ft BGL) }\end{array}$ & $\begin{array}{l}\text { Elevation in Klassen } \\
\text { Domestic Well } \\
\text { (ft AMSL) }\end{array}$ & \\
\hline $12 / 17 / 1116: 30$ & 33.351 & 35.789 & 1475.42 & 1473.33 \\
\hline $12 / 17 / 1117: 00$ & 33.471 & 35.784 & 1475.30 & 1473.34 \\
\hline 12/17/11 17:30 & 33.384 & 35.786 & 1475.39 & 1473.34 \\
\hline $12 / 17 / 1118: 00$ & 44.559 & 35.824 & 1464.21 & 1473.30 \\
\hline 12/17/11 18:30 & 34.498 & 35.862 & 1474.27 & 1473.26 \\
\hline $12 / 17 / 11$ 19:00 & 34.118 & 35.864 & 1474.65 & 1473.26 \\
\hline $12 / 17 / 11$ 19:30 & 34.257 & 35.862 & 1474.51 & 1473.26 \\
\hline $12 / 17 / 1120: 00$ & 33.884 & 35.864 & 1474.89 & 1473.26 \\
\hline $12 / 17 / 1120: 30$ & 33.811 & 35.864 & 1474.96 & 1473.26 \\
\hline $12 / 17 / 1121: 00$ & 33.729 & 35.862 & 1475.04 & 1473.26 \\
\hline $12 / 17 / 1121: 30$ & 33.792 & 35.857 & 1474.98 & 1473.27 \\
\hline $12 / 17 / 1122: 00$ & 33.682 & 35.852 & 1475.09 & 1473.27 \\
\hline $12 / 17 / 1122: 30$ & 33.654 & 35.85 & 1475.12 & 1473.27 \\
\hline $12 / 17 / 1123: 00$ & 33.762 & 35.85 & 1475.01 & 1473.27 \\
\hline $12 / 17 / 1123: 30$ & 33.649 & 35.845 & 1475.12 & 1473.28 \\
\hline $12 / 18 / 110: 00$ & 33.781 & 35.843 & 1474.99 & 1473.28 \\
\hline $12 / 18 / 110: 30$ & 33.647 & 35.838 & 1475.12 & 1473.28 \\
\hline 12/18/11 1:00 & 33.584 & 35.836 & 1475.19 & 1473.29 \\
\hline 12/18/11 1:30 & 33.539 & 35.836 & 1475.23 & 1473.29 \\
\hline $12 / 18 / 112: 00$ & 33.502 & 35.831 & 1475.27 & 1473.29 \\
\hline $12 / 18 / 112: 30$ & 33.473 & 35.829 & 1475.30 & 1473.29 \\
\hline 12/18/11 3:00 & 33.445 & 35.824 & 1475.32 & 1473.30 \\
\hline $12 / 18 / 113: 30$ & 33.422 & 35.819 & 1475.35 & 1473.30 \\
\hline $12 / 18 / 114: 00$ & 33.401 & 35.819 & 1475.37 & 1473.30 \\
\hline $12 / 18 / 114: 30$ & 33.485 & 35.819 & 1475.28 & 1473.30 \\
\hline 12/18/11 5:00 & 33.412 & 35.819 & 1475.36 & 1473.30 \\
\hline 12/18/11 5:30 & 33.38 & 35.815 & 1475.39 & 1473.31 \\
\hline $12 / 18 / 116: 00$ & 33.912 & 35.805 & 1474.86 & 1473.32 \\
\hline $12 / 18 / 116: 30$ & 33.614 & 35.808 & 1475.16 & 1473.31 \\
\hline 12/18/11 7:00 & 33.476 & 35.805 & 1475.29 & 1473.32 \\
\hline 12/18/11 7:30 & 33.424 & 35.805 & 1475.35 & 1473.32 \\
\hline $12 / 18 / 118: 00$ & 33.422 & 35.801 & 1475.35 & 1473.32 \\
\hline $12 / 18 / 118: 30$ & 33.675 & 35.81 & 1475.09 & 1473.31 \\
\hline 12/18/11 9:00 & 33.584 & 35.812 & 1475.19 & 1473.31 \\
\hline 12/18/11 9:30 & 33.865 & 35.826 & 1474.90 & 1473.30 \\
\hline $12 / 18 / 11$ 10:00 & 33.713 & 35.836 & 1475.06 & 1473.29 \\
\hline $12 / 18 / 11$ 10:30 & 33.605 & 35.847 & 1475.16 & 1473.28 \\
\hline $12 / 18 / 11$ 11:00 & 33.661 & 35.871 & 1475.11 & 1473.25 \\
\hline
\end{tabular}


TABLE S1.3 Automatically recorded groundwater levels in the Klassen wells, September 15, 2011, to March 13, 2012.

\begin{tabular}{|c|c|c|c|c|}
\hline \multicolumn{3}{|c|}{ Reference Elevation (ft AMSL) } & 1508.77 & \multirow{2}{*}{$\begin{array}{l}1509.12 \\
\text { Elevation in Klassen } \\
\text { Test Well (ft AMSL) }\end{array}$} \\
\hline Date and Time & $\begin{array}{l}\text { Depth in Klassen } \\
\text { Domestic Well } \\
(\mathrm{ft} \mathrm{BGL})\end{array}$ & $\begin{array}{c}\text { Depth in Klassen } \\
\text { Test Well } \\
\text { (ft BGL) }\end{array}$ & $\begin{array}{l}\text { Elevation in Klassen } \\
\text { Domestic Well } \\
\text { (ft AMSL) }\end{array}$ & \\
\hline $12 / 18 / 1111: 30$ & 33.682 & 35.894 & 1475.09 & 1473.23 \\
\hline $12 / 18 / 1112: 00$ & 33.717 & 35.913 & 1475.05 & 1473.21 \\
\hline $12 / 18 / 1112: 30$ & 33.755 & 35.904 & 1475.01 & 1473.22 \\
\hline $12 / 18 / 1113: 00$ & 33.799 & 35.897 & 1474.97 & 1473.23 \\
\hline $12 / 18 / 1113: 30$ & 33.788 & 35.894 & 1474.98 & 1473.23 \\
\hline $12 / 18 / 1114: 00$ & 33.788 & 35.894 & 1474.98 & 1473.23 \\
\hline $12 / 18 / 1114: 30$ & 33.919 & 35.897 & 1474.85 & 1473.23 \\
\hline $12 / 18 / 1115: 00$ & 33.825 & 35.908 & 1474.94 & 1473.21 \\
\hline $12 / 18 / 1115: 30$ & 33.858 & 35.916 & 1474.91 & 1473.21 \\
\hline $12 / 18 / 1116: 00$ & 33.781 & 35.918 & 1474.99 & 1473.20 \\
\hline $12 / 18 / 1116: 30$ & 33.952 & 35.918 & 1474.82 & 1473.20 \\
\hline 12/18/11 17:00 & 33.91 & 35.925 & 1474.86 & 1473.20 \\
\hline $12 / 18 / 1117: 30$ & 34.243 & 35.93 & 1474.53 & 1473.19 \\
\hline $12 / 18 / 1118: 00$ & 33.823 & 35.927 & 1474.95 & 1473.20 \\
\hline $12 / 18 / 11$ 18:30 & 33.755 & 35.923 & 1475.01 & 1473.20 \\
\hline $12 / 18 / 11$ 19:00 & 33.68 & 35.92 & 1475.09 & 1473.20 \\
\hline $12 / 18 / 11$ 19:30 & 33.736 & 35.923 & 1475.03 & 1473.20 \\
\hline $12 / 18 / 1120: 00$ & 33.999 & 35.93 & 1474.77 & 1473.19 \\
\hline $12 / 18 / 1120: 30$ & 33.95 & 35.946 & 1474.82 & 1473.18 \\
\hline $12 / 18 / 1121: 00$ & 33.832 & 35.955 & 1474.94 & 1473.17 \\
\hline $12 / 18 / 1121: 30$ & 33.743 & 35.955 & 1475.03 & 1473.17 \\
\hline $12 / 18 / 1122: 00$ & 33.799 & 35.955 & 1474.97 & 1473.17 \\
\hline $12 / 18 / 1122: 30$ & 33.881 & 35.967 & 1474.89 & 1473.16 \\
\hline $12 / 18 / 1123: 00$ & 34.039 & 35.972 & 1474.73 & 1473.15 \\
\hline $12 / 18 / 1123: 30$ & 34.175 & 35.977 & 1474.59 & 1473.15 \\
\hline $12 / 19 / 110: 00$ & 33.87 & 35.972 & 1474.90 & 1473.15 \\
\hline 12/19/11 0:30 & 33.788 & 35.967 & 1474.98 & 1473.16 \\
\hline 12/19/11 1:00 & 33.828 & 35.967 & 1474.94 & 1473.16 \\
\hline 12/19/11 1:30 & 33.764 & 35.958 & 1475.01 & 1473.16 \\
\hline $12 / 19 / 112: 00$ & 33.715 & 35.951 & 1475.05 & 1473.17 \\
\hline $12 / 19 / 112: 30$ & 33.795 & 35.951 & 1474.97 & 1473.17 \\
\hline 12/19/11 3:00 & 33.713 & 35.953 & 1475.06 & 1473.17 \\
\hline 12/19/11 3:30 & 33.652 & 35.946 & 1475.12 & 1473.18 \\
\hline 12/19/11 4:00 & 33.692 & 35.946 & 1475.08 & 1473.18 \\
\hline $12 / 19 / 114: 30$ & 33.614 & 35.937 & 1475.16 & 1473.19 \\
\hline 12/19/11 5:00 & 33.593 & 35.932 & 1475.18 & 1473.19 \\
\hline 12/19/11 5:30 & 33.551 & 35.93 & 1475.22 & 1473.19 \\
\hline $12 / 19 / 116: 00$ & 33.518 & 35.923 & 1475.25 & 1473.20 \\
\hline
\end{tabular}


TABLE S1.3 Automatically recorded groundwater levels in the Klassen wells, September 15, 2011, to March 13, 2012.

\begin{tabular}{|c|c|c|c|c|}
\hline \multicolumn{3}{|c|}{ Reference Elevation (ft AMSL) } & 1508.77 & \multirow{2}{*}{$\begin{array}{l}1509.12 \\
\text { Elevation in Klassen } \\
\text { Test Well (ft AMSL) }\end{array}$} \\
\hline Date and Time & $\begin{array}{l}\text { Depth in Klassen } \\
\text { Domestic Well } \\
(\mathrm{ft} \mathrm{BGL})\end{array}$ & $\begin{array}{c}\text { Depth in Klassen } \\
\text { Test Well } \\
\text { (ft BGL) }\end{array}$ & $\begin{array}{l}\text { Elevation in Klassen } \\
\text { Domestic Well } \\
\text { (ft AMSL) }\end{array}$ & \\
\hline $12 / 19 / 116: 30$ & 33.708 & 35.918 & 1475.06 & 1473.20 \\
\hline $12 / 19 / 117: 00$ & 33.624 & 35.908 & 1475.15 & 1473.21 \\
\hline 12/19/11 7:30 & 33.656 & 35.906 & 1475.11 & 1473.22 \\
\hline $12 / 19 / 118: 00$ & 33.612 & 35.897 & 1475.16 & 1473.23 \\
\hline $12 / 19 / 118: 30$ & 33.692 & 35.894 & 1475.08 & 1473.23 \\
\hline 12/19/11 9:00 & 33.661 & 35.899 & 1475.11 & 1473.22 \\
\hline 12/19/11 9:30 & 33.666 & 35.904 & 1475.10 & 1473.22 \\
\hline 12/19/11 10:00 & 33.774 & 35.899 & 1475.00 & 1473.22 \\
\hline $12 / 19 / 1110: 30$ & 33.731 & 35.887 & 1475.04 & 1473.24 \\
\hline 12/19/11 11:00 & 33.731 & 35.892 & 1475.04 & 1473.23 \\
\hline $12 / 19 / 1111: 30$ & 33.609 & 35.887 & 1475.16 & 1473.24 \\
\hline $12 / 19 / 1112: 00$ & 33.98 & 35.885 & 1474.79 & 1473.24 \\
\hline $12 / 19 / 1112: 30$ & 33.584 & 35.892 & 1475.19 & 1473.23 \\
\hline $12 / 19 / 1113: 00$ & 33.518 & 35.911 & 1475.25 & 1473.21 \\
\hline $12 / 19 / 11$ 13:30 & 37.352 & 35.93 & 1471.42 & 1473.19 \\
\hline $12 / 19 / 11$ 14:00 & 33.598 & 35.955 & 1475.17 & 1473.17 \\
\hline $12 / 19 / 11$ 14:30 & 33.523 & 35.977 & 1475.25 & 1473.15 \\
\hline 12/19/11 15:00 & 33.659 & 36 & 1475.11 & 1473.12 \\
\hline 12/19/11 15:30 & 33.598 & 36.028 & 1475.17 & 1473.09 \\
\hline $12 / 19 / 11$ 16:00 & 33.509 & 36.021 & 1475.26 & 1473.10 \\
\hline $12 / 19 / 11$ 16:30 & 33.556 & 36.03 & 1475.21 & 1473.09 \\
\hline 12/19/11 17:00 & 33.502 & 36.068 & 1475.27 & 1473.05 \\
\hline 12/19/11 17:30 & 33.614 & 36.059 & 1475.16 & 1473.06 \\
\hline 12/19/11 18:00 & 33.753 & 36.059 & 1475.02 & 1473.06 \\
\hline 12/19/11 18:30 & 33.621 & 36.063 & 1475.15 & 1473.06 \\
\hline 12/19/11 19:00 & 33.602 & 36.066 & 1475.17 & 1473.06 \\
\hline 12/19/11 19:30 & 33.724 & 36.059 & 1475.05 & 1473.06 \\
\hline $12 / 19 / 1120: 00$ & 33.713 & 36.033 & 1475.06 & 1473.09 \\
\hline $12 / 19 / 1120: 30$ & 34.311 & 36.033 & 1474.46 & 1473.09 \\
\hline 12/19/11 21:00 & 34.28 & 36.035 & 1474.49 & 1473.09 \\
\hline 12/19/11 21:30 & 33.966 & 36.045 & 1474.80 & 1473.08 \\
\hline $12 / 19 / 1122: 00$ & 33.964 & 36.052 & 1474.81 & 1473.07 \\
\hline $12 / 19 / 1122: 30$ & 33.942 & 36.054 & 1474.83 & 1473.07 \\
\hline $12 / 19 / 1123: 00$ & 38.321 & 36.049 & 1470.45 & 1473.07 \\
\hline $12 / 19 / 11$ 23:30 & 34.008 & 36.08 & 1474.76 & 1473.04 \\
\hline $12 / 20 / 110: 00$ & 33.856 & 36.054 & 1474.91 & 1473.07 \\
\hline $12 / 20 / 110: 30$ & 33.76 & 36.054 & 1475.01 & 1473.07 \\
\hline $12 / 20 / 11$ 1:00 & 33.692 & 36.049 & 1475.08 & 1473.07 \\
\hline
\end{tabular}


TABLE S1.3 Automatically recorded groundwater levels in the Klassen wells, September 15, 2011, to March 13, 2012.

\begin{tabular}{|c|c|c|c|c|}
\hline \multicolumn{3}{|c|}{ Reference Elevation (ft AMSL) } & 1508.77 & \multirow{2}{*}{$\begin{array}{l}1509.12 \\
\text { Elevation in Klassen } \\
\text { Test Well (ft AMSL) }\end{array}$} \\
\hline Date and Time & $\begin{array}{l}\text { Depth in Klassen } \\
\text { Domestic Well } \\
(\mathrm{ft} \mathrm{BGL})\end{array}$ & $\begin{array}{c}\text { Depth in Klassen } \\
\text { Test Well } \\
\text { (ft BGL) }\end{array}$ & $\begin{array}{l}\text { Elevation in Klassen } \\
\text { Domestic Well } \\
\text { (ft AMSL) }\end{array}$ & \\
\hline $12 / 20 / 111: 30$ & 33.633 & 36.035 & 1475.14 & 1473.09 \\
\hline $12 / 20 / 112: 00$ & 33.586 & 36.03 & 1475.18 & 1473.09 \\
\hline $12 / 20 / 112: 30$ & 33.541 & 36.021 & 1475.23 & 1473.10 \\
\hline $12 / 20 / 113: 00$ & 37.13 & 36.016 & 1471.64 & 1473.11 \\
\hline $12 / 20 / 113: 30$ & 33.546 & 36.005 & 1475.22 & 1473.12 \\
\hline $12 / 20 / 114: 00$ & 33.492 & 36.002 & 1475.28 & 1473.12 \\
\hline $12 / 20 / 114: 30$ & 33.457 & 35.991 & 1475.31 & 1473.13 \\
\hline $12 / 20 / 115: 00$ & 33.427 & 35.979 & 1475.34 & 1473.14 \\
\hline $12 / 20 / 115: 30$ & 33.398 & 35.967 & 1475.37 & 1473.16 \\
\hline $12 / 20 / 116: 00$ & 33.373 & 35.955 & 1475.40 & 1473.17 \\
\hline $12 / 20 / 116: 30$ & 33.593 & 35.955 & 1475.18 & 1473.17 \\
\hline $12 / 20 / 117: 00$ & 33.485 & 35.946 & 1475.28 & 1473.18 \\
\hline $12 / 20 / 117: 30$ & 33.537 & 35.937 & 1475.23 & 1473.19 \\
\hline $12 / 20 / 118: 00$ & 33.49 & 35.937 & 1475.28 & 1473.19 \\
\hline $12 / 20 / 118: 30$ & 33.53 & 35.93 & 1475.24 & 1473.19 \\
\hline $12 / 20 / 119: 00$ & 33.457 & 35.923 & 1475.31 & 1473.20 \\
\hline $12 / 20 / 119: 30$ & 33.415 & 35.915 & 1475.35 & 1473.21 \\
\hline $12 / 20 / 11$ 10:00 & 33.448 & 35.904 & 1475.32 & 1473.22 \\
\hline $12 / 20 / 11$ 10:30 & 34.496 & 35.899 & 1474.27 & 1473.22 \\
\hline $12 / 20 / 1111: 00$ & 33.443 & 35.897 & 1475.33 & 1473.23 \\
\hline $12 / 20 / 1111: 30$ & 33.387 & 35.897 & 1475.38 & 1473.23 \\
\hline $12 / 20 / 1112: 00$ & 33.452 & 35.904 & 1475.32 & 1473.22 \\
\hline $12 / 20 / 1112: 30$ & 33.375 & 35.906 & 1475.39 & 1473.22 \\
\hline $12 / 20 / 1113: 00$ & 33.335 & 35.902 & 1475.43 & 1473.22 \\
\hline $12 / 20 / 1113: 30$ & 33.422 & 35.904 & 1475.35 & 1473.22 \\
\hline $12 / 20 / 1114: 00$ & 33.546 & 35.902 & 1475.22 & 1473.22 \\
\hline $12 / 20 / 1114: 30$ & 33.382 & 35.913 & 1475.39 & 1473.21 \\
\hline $12 / 20 / 11$ 15:00 & 33.335 & 35.923 & 1475.43 & 1473.20 \\
\hline $12 / 20 / 1115: 30$ & 33.305 & 35.927 & 1475.46 & 1473.20 \\
\hline $12 / 20 / 1116: 00$ & 33.816 & 35.923 & 1474.95 & 1473.20 \\
\hline $12 / 20 / 1116: 30$ & 33.321 & 35.927 & 1475.45 & 1473.20 \\
\hline $12 / 20 / 11$ 17:00 & 33.368 & 35.925 & 1475.40 & 1473.20 \\
\hline $12 / 20 / 1117: 30$ & 33.506 & 35.93 & 1475.26 & 1473.19 \\
\hline $12 / 20 / 11$ 18:00 & 33.459 & 35.93 & 1475.31 & 1473.19 \\
\hline $12 / 20 / 1118: 30$ & 33.452 & 35.923 & 1475.32 & 1473.20 \\
\hline 12/20/11 19:00 & 33.445 & 35.923 & 1475.32 & 1473.20 \\
\hline 12/20/11 19:30 & 33.405 & 35.92 & 1475.36 & 1473.20 \\
\hline $12 / 20 / 1120: 00$ & 33.49 & 35.913 & 1475.28 & 1473.21 \\
\hline
\end{tabular}


TABLE S1.3 Automatically recorded groundwater levels in the Klassen wells, September 15, 2011, to March 13, 2012.

\begin{tabular}{|c|c|c|c|c|}
\hline \multicolumn{3}{|c|}{ Reference Elevation (ft AMSL) } & 1508.77 & \multirow{2}{*}{$\begin{array}{l}1509.12 \\
\text { Elevation in Klassen } \\
\text { Test Well (ft AMSL) }\end{array}$} \\
\hline Date and Time & $\begin{array}{l}\text { Depth in Klassen } \\
\text { Domestic Well } \\
\text { (ft BGL) }\end{array}$ & $\begin{array}{c}\text { Depth in Klassen } \\
\text { Test Well } \\
\text { (ft BGL) }\end{array}$ & $\begin{array}{l}\text { Elevation in Klassen } \\
\text { Domestic Well } \\
\text { (ft AMSL) }\end{array}$ & \\
\hline $12 / 20 / 1120: 30$ & 33.375 & 35.911 & 1475.39 & 1473.21 \\
\hline $12 / 20 / 1121: 00$ & 33.619 & 35.915 & 1475.15 & 1473.21 \\
\hline $12 / 20 / 1121: 30$ & 33.767 & 35.92 & 1475.00 & 1473.20 \\
\hline $12 / 20 / 1122: 00$ & 33.68 & 35.92 & 1475.09 & 1473.20 \\
\hline $12 / 20 / 1122: 30$ & 33.823 & 35.913 & 1474.95 & 1473.21 \\
\hline $12 / 20 / 1123: 00$ & 35.483 & 35.916 & 1473.29 & 1473.21 \\
\hline $12 / 20 / 1123: 30$ & 33.708 & 35.913 & 1475.06 & 1473.21 \\
\hline $12 / 21 / 110: 00$ & 33.591 & 35.901 & 1475.18 & 1473.22 \\
\hline $12 / 21 / 110: 30$ & 33.52 & 35.892 & 1475.25 & 1473.23 \\
\hline $12 / 21 / 111: 00$ & 33.588 & 35.89 & 1475.18 & 1473.23 \\
\hline $12 / 21 / 111: 30$ & 33.534 & 35.89 & 1475.24 & 1473.23 \\
\hline $12 / 21 / 112: 00$ & 33.497 & 35.88 & 1475.27 & 1473.24 \\
\hline $12 / 21 / 112: 30$ & 33.574 & 35.876 & 1475.20 & 1473.25 \\
\hline $12 / 21 / 113: 00$ & 33.497 & 35.876 & 1475.27 & 1473.25 \\
\hline $12 / 21 / 113: 30$ & 35.889 & 35.871 & 1472.88 & 1473.25 \\
\hline $12 / 21 / 114: 00$ & 33.462 & 35.871 & 1475.31 & 1473.25 \\
\hline $12 / 21 / 114: 30$ & 33.401 & 35.864 & 1475.37 & 1473.26 \\
\hline 12/21/11 5:00 & 33.361 & 35.859 & 1475.41 & 1473.26 \\
\hline $12 / 21 / 115: 30$ & 33.328 & 35.857 & 1475.44 & 1473.27 \\
\hline $12 / 21 / 116: 00$ & 33.302 & 35.847 & 1475.47 & 1473.28 \\
\hline $12 / 21 / 116: 30$ & 33.487 & 35.85 & 1475.28 & 1473.27 \\
\hline 12/21/11 7:00 & 33.387 & 35.845 & 1475.38 & 1473.28 \\
\hline $12 / 21 / 11$ 7:30 & 33.366 & 35.838 & 1475.40 & 1473.28 \\
\hline $12 / 21 / 118: 00$ & 33.321 & 35.836 & 1475.45 & 1473.29 \\
\hline $12 / 21 / 118: 30$ & 33.29 & 35.84 & 1475.48 & 1473.28 \\
\hline 12/21/11 9:00 & 33.262 & 35.845 & 1475.51 & 1473.28 \\
\hline 12/21/11 9:30 & 33.359 & 35.85 & 1475.41 & 1473.27 \\
\hline $12 / 21 / 11$ 10:00 & 37.882 & 35.857 & 1470.89 & 1473.27 \\
\hline $12 / 21 / 11$ 10:30 & 33.445 & 35.88 & 1475.32 & 1473.24 \\
\hline 12/21/11 11:00 & 33.492 & 35.909 & 1475.28 & 1473.21 \\
\hline 12/21/11 11:30 & 33.485 & 35.916 & 1475.28 & 1473.21 \\
\hline $12 / 21 / 11$ 12:00 & 33.38 & 35.915 & 1475.39 & 1473.21 \\
\hline $12 / 21 / 11$ 12:30 & 33.323 & 35.916 & 1475.45 & 1473.21 \\
\hline $12 / 21 / 11$ 13:00 & 33.286 & 35.92 & 1475.48 & 1473.20 \\
\hline $12 / 21 / 11$ 13:30 & 34.599 & 35.923 & 1474.17 & 1473.20 \\
\hline $12 / 21 / 11$ 14:00 & 33.293 & 35.923 & 1475.48 & 1473.20 \\
\hline $12 / 21 / 11$ 14:30 & 33.314 & 35.923 & 1475.46 & 1473.20 \\
\hline $12 / 21 / 11$ 15:00 & 33.258 & 35.92 & 1475.51 & 1473.20 \\
\hline
\end{tabular}


TABLE S1.3 Automatically recorded groundwater levels in the Klassen wells, September 15, 2011, to March 13, 2012.

\begin{tabular}{|c|c|c|c|c|}
\hline \multicolumn{3}{|c|}{ Reference Elevation (ft AMSL) } & 1508.77 & \multirow{2}{*}{$\begin{array}{l}1509.12 \\
\text { Elevation in Klassen } \\
\text { Test Well (ft AMSL) }\end{array}$} \\
\hline Date and Time & $\begin{array}{l}\text { Depth in Klassen } \\
\text { Domestic Well } \\
\text { (ft BGL) }\end{array}$ & $\begin{array}{c}\text { Depth in Klassen } \\
\text { Test Well } \\
\text { (ft BGL) }\end{array}$ & $\begin{array}{l}\text { Elevation in Klassen } \\
\text { Domestic Well } \\
\text { (ft AMSL) }\end{array}$ & \\
\hline $12 / 21 / 1115: 30$ & 33.225 & 35.913 & 1475.54 & 1473.21 \\
\hline $12 / 21 / 1116: 00$ & 35.481 & 35.904 & 1473.29 & 1473.22 \\
\hline $12 / 21 / 1116: 30$ & 33.293 & 35.89 & 1475.48 & 1473.23 \\
\hline $12 / 21 / 1117: 00$ & 33.241 & 35.866 & 1475.53 & 1473.26 \\
\hline $12 / 21 / 1117: 30$ & 33.344 & 35.843 & 1475.43 & 1473.28 \\
\hline $12 / 21 / 1118: 00$ & 33.286 & 35.824 & 1475.48 & 1473.30 \\
\hline $12 / 21 / 1118: 30$ & 33.239 & 35.808 & 1475.53 & 1473.31 \\
\hline $12 / 21 / 11$ 19:00 & 33.23 & 35.798 & 1475.54 & 1473.32 \\
\hline $12 / 21 / 11$ 19:30 & 33.546 & 35.791 & 1475.22 & 1473.33 \\
\hline $12 / 21 / 1120: 00$ & 33.288 & 35.789 & 1475.48 & 1473.33 \\
\hline $12 / 21 / 1120: 30$ & 33.272 & 35.782 & 1475.50 & 1473.34 \\
\hline $12 / 21 / 1121: 00$ & 33.227 & 35.77 & 1475.54 & 1473.35 \\
\hline $12 / 21 / 1121: 30$ & 33.427 & 35.763 & 1475.34 & 1473.36 \\
\hline $12 / 21 / 1122: 00$ & 33.431 & 35.754 & 1475.34 & 1473.37 \\
\hline $12 / 21 / 1122: 30$ & 35.898 & 35.744 & 1472.87 & 1473.38 \\
\hline $12 / 21 / 1123: 00$ & 36.965 & 35.742 & 1471.80 & 1473.38 \\
\hline $12 / 21 / 1123: 30$ & 33.513 & 35.737 & 1475.26 & 1473.39 \\
\hline $12 / 22 / 110: 00$ & 33.41 & 35.735 & 1475.36 & 1473.39 \\
\hline $12 / 22 / 110: 30$ & 33.349 & 35.728 & 1475.42 & 1473.39 \\
\hline $12 / 22 / 11$ 1:00 & 36.815 & 35.728 & 1471.95 & 1473.39 \\
\hline $12 / 22 / 11$ 1:30 & 33.335 & 35.726 & 1475.43 & 1473.40 \\
\hline $12 / 22 / 112: 00$ & 33.281 & 35.711 & 1475.49 & 1473.41 \\
\hline $12 / 22 / 112: 30$ & 33.248 & 35.693 & 1475.52 & 1473.43 \\
\hline $12 / 22 / 113: 00$ & 33.22 & 35.676 & 1475.55 & 1473.45 \\
\hline $12 / 22 / 113: 30$ & 33.197 & 35.667 & 1475.57 & 1473.46 \\
\hline $12 / 22 / 114: 00$ & 33.173 & 35.653 & 1475.60 & 1473.47 \\
\hline $12 / 22 / 114: 30$ & 33.152 & 35.646 & 1475.62 & 1473.48 \\
\hline $12 / 22 / 115: 00$ & 33.162 & 35.641 & 1475.61 & 1473.48 \\
\hline $12 / 22 / 115: 30$ & 33.14 & 35.622 & 1475.63 & 1473.50 \\
\hline $12 / 22 / 116: 00$ & 33.169 & 35.613 & 1475.60 & 1473.51 \\
\hline $12 / 22 / 116: 30$ & 33.464 & 35.603 & 1475.31 & 1473.52 \\
\hline $12 / 22 / 11$ 7:00 & 33.321 & 35.589 & 1475.45 & 1473.53 \\
\hline $12 / 22 / 11$ 7:30 & 33.286 & 35.582 & 1475.48 & 1473.54 \\
\hline $12 / 22 / 118: 00$ & 33.237 & 35.573 & 1475.53 & 1473.55 \\
\hline $12 / 22 / 118: 30$ & 33.204 & 35.561 & 1475.57 & 1473.56 \\
\hline 12/22/11 9:00 & 33.293 & 35.552 & 1475.48 & 1473.57 \\
\hline 12/22/11 9:30 & 33.215 & 35.543 & 1475.55 & 1473.58 \\
\hline $12 / 22 / 11$ 10:00 & 37.099 & 35.526 & 1471.67 & 1473.60 \\
\hline
\end{tabular}


TABLE S1.3 Automatically recorded groundwater levels in the Klassen wells, September 15, 2011, to March 13, 2012.

\begin{tabular}{|c|c|c|c|c|}
\hline \multicolumn{3}{|c|}{ Reference Elevation (ft AMSL) } & 1508.77 & \multirow{2}{*}{$\begin{array}{l}1509.12 \\
\text { Elevation in Klassen } \\
\text { Test Well (ft AMSL) }\end{array}$} \\
\hline Date and Time & $\begin{array}{l}\text { Depth in Klassen } \\
\text { Domestic Well } \\
(\mathrm{ft} \mathrm{BGL})\end{array}$ & $\begin{array}{c}\text { Depth in Klassen } \\
\text { Test Well } \\
\text { (ft BGL) }\end{array}$ & $\begin{array}{l}\text { Elevation in Klassen } \\
\text { Domestic Well } \\
\text { (ft AMSL) }\end{array}$ & \\
\hline $12 / 22 / 1110: 30$ & 33.241 & 35.517 & 1475.53 & 1473.61 \\
\hline $12 / 22 / 1111: 00$ & 33.194 & 35.514 & 1475.58 & 1473.61 \\
\hline $12 / 22 / 1111: 30$ & 33.248 & 35.519 & 1475.52 & 1473.60 \\
\hline $12 / 22 / 1112: 00$ & 38.551 & 35.524 & 1470.22 & 1473.60 \\
\hline $12 / 22 / 1112: 30$ & 33.241 & 35.526 & 1475.53 & 1473.60 \\
\hline $12 / 22 / 1113: 00$ & 33.187 & 35.526 & 1475.58 & 1473.60 \\
\hline $12 / 22 / 1113: 30$ & 33.258 & 35.526 & 1475.51 & 1473.60 \\
\hline $12 / 22 / 1114: 00$ & 33.199 & 35.521 & 1475.57 & 1473.60 \\
\hline $12 / 22 / 1114: 30$ & 33.164 & 35.521 & 1475.61 & 1473.60 \\
\hline $12 / 22 / 1115: 00$ & 33.143 & 35.512 & 1475.63 & 1473.61 \\
\hline $12 / 22 / 1115: 30$ & 33.295 & 35.505 & 1475.47 & 1473.62 \\
\hline $12 / 22 / 1116: 00$ & 33.213 & 35.493 & 1475.56 & 1473.63 \\
\hline $12 / 22 / 1116: 30$ & 33.166 & 35.484 & 1475.60 & 1473.64 \\
\hline $12 / 22 / 1117: 00$ & 33.143 & 35.474 & 1475.63 & 1473.65 \\
\hline $12 / 22 / 11$ 17:30 & 33.211 & 35.467 & 1475.56 & 1473.66 \\
\hline $12 / 22 / 11$ 18:00 & 33.157 & 35.46 & 1475.61 & 1473.66 \\
\hline $12 / 22 / 1118: 30$ & 33.157 & 35.451 & 1475.61 & 1473.67 \\
\hline $12 / 22 / 11$ 19:00 & 35.542 & 35.442 & 1473.23 & 1473.68 \\
\hline $12 / 22 / 11$ 19:30 & 33.638 & 35.437 & 1475.13 & 1473.69 \\
\hline $12 / 22 / 1120: 00$ & 33.281 & 35.43 & 1475.49 & 1473.69 \\
\hline $12 / 22 / 1120: 30$ & 33.22 & 35.42 & 1475.55 & 1473.70 \\
\hline $12 / 22 / 1121: 00$ & 33.337 & 35.409 & 1475.43 & 1473.71 \\
\hline $12 / 22 / 1121: 30$ & 33.211 & 35.399 & 1475.56 & 1473.72 \\
\hline $12 / 22 / 1122: 00$ & 33.173 & 35.39 & 1475.60 & 1473.73 \\
\hline $12 / 22 / 1122: 30$ & 33.499 & 35.383 & 1475.27 & 1473.74 \\
\hline $12 / 22 / 1123: 00$ & 33.689 & 35.381 & 1475.08 & 1473.74 \\
\hline $12 / 22 / 1123: 30$ & 33.661 & 35.383 & 1475.11 & 1473.74 \\
\hline $12 / 23 / 110: 00$ & 33.459 & 35.378 & 1475.31 & 1473.74 \\
\hline $12 / 23 / 110: 30$ & 33.37 & 35.376 & 1475.40 & 1473.75 \\
\hline 12/23/11 1:00 & 33.314 & 35.369 & 1475.46 & 1473.75 \\
\hline $12 / 23 / 11$ 1:30 & 33.396 & 35.367 & 1475.37 & 1473.76 \\
\hline $12 / 23 / 112: 00$ & 33.298 & 35.362 & 1475.47 & 1473.76 \\
\hline $12 / 23 / 112: 30$ & 33.283 & 35.355 & 1475.49 & 1473.77 \\
\hline $12 / 23 / 113: 00$ & 33.237 & 35.345 & 1475.53 & 1473.78 \\
\hline $12 / 23 / 113: 30$ & 33.204 & 35.348 & 1475.57 & 1473.77 \\
\hline $12 / 23 / 114: 00$ & 33.178 & 35.338 & 1475.59 & 1473.78 \\
\hline $12 / 23 / 114: 30$ & 33.154 & 35.334 & 1475.62 & 1473.79 \\
\hline $12 / 23 / 11$ 5:00 & 33.133 & 35.324 & 1475.64 & 1473.80 \\
\hline
\end{tabular}


TABLE S1.3 Automatically recorded groundwater levels in the Klassen wells, September 15, 2011, to March 13, 2012.

\begin{tabular}{|c|c|c|c|c|}
\hline \multicolumn{3}{|c|}{ Reference Elevation (ft AMSL) } & 1508.77 & \multirow{2}{*}{$\begin{array}{l}1509.12 \\
\text { Elevation in Klassen } \\
\text { Test Well (ft AMSL) }\end{array}$} \\
\hline Date and Time & $\begin{array}{l}\text { Depth in Klassen } \\
\text { Domestic Well } \\
\text { (ft BGL) }\end{array}$ & $\begin{array}{c}\text { Depth in Klassen } \\
\text { Test Well } \\
\text { (ft BGL) }\end{array}$ & $\begin{array}{l}\text { Elevation in Klassen } \\
\text { Domestic Well } \\
\text { (ft AMSL) }\end{array}$ & \\
\hline $12 / 23 / 115: 30$ & 33.117 & 35.313 & 1475.65 & 1473.81 \\
\hline $12 / 23 / 116: 00$ & 33.103 & 35.306 & 1475.67 & 1473.82 \\
\hline $12 / 23 / 116: 30$ & 33.316 & 35.31 & 1475.45 & 1473.81 \\
\hline $12 / 23 / 117: 00$ & 33.215 & 35.306 & 1475.55 & 1473.82 \\
\hline $12 / 23 / 117: 30$ & 33.169 & 35.303 & 1475.60 & 1473.82 \\
\hline 12/23/11 8:00 & 33.169 & 35.303 & 1475.60 & 1473.82 \\
\hline $12 / 23 / 118: 30$ & 33.133 & 35.31 & 1475.64 & 1473.81 \\
\hline 12/23/11 9:00 & 33.112 & 35.306 & 1475.66 & 1473.82 \\
\hline 12/23/11 9:30 & 33.093 & 35.306 & 1475.68 & 1473.82 \\
\hline $12 / 23 / 11$ 10:00 & 33.077 & 35.313 & 1475.69 & 1473.81 \\
\hline $12 / 23 / 11$ 10:30 & 33.063 & 35.32 & 1475.71 & 1473.80 \\
\hline $12 / 23 / 1111: 00$ & 33.049 & 35.336 & 1475.72 & 1473.79 \\
\hline $12 / 23 / 1111: 30$ & 33.037 & 35.362 & 1475.73 & 1473.76 \\
\hline $12 / 23 / 1112: 00$ & 33.459 & 35.392 & 1475.31 & 1473.73 \\
\hline $12 / 23 / 11$ 12:30 & 33.356 & 35.423 & 1475.41 & 1473.70 \\
\hline $12 / 23 / 11$ 13:00 & 33.258 & 35.449 & 1475.51 & 1473.67 \\
\hline $12 / 23 / 11$ 13:30 & 35.286 & 35.47 & 1473.48 & 1473.65 \\
\hline $12 / 23 / 11$ 14:00 & 33.279 & 35.484 & 1475.49 & 1473.64 \\
\hline $12 / 23 / 11$ 14:30 & 33.516 & 35.498 & 1475.25 & 1473.62 \\
\hline $12 / 23 / 11$ 15:00 & 34.707 & 35.505 & 1474.06 & 1473.62 \\
\hline $12 / 23 / 11$ 15:30 & 33.49 & 35.505 & 1475.28 & 1473.62 \\
\hline $12 / 23 / 11$ 16:00 & 33.499 & 35.51 & 1475.27 & 1473.61 \\
\hline $12 / 23 / 11$ 16:30 & 33.548 & 35.512 & 1475.22 & 1473.61 \\
\hline $12 / 23 / 11$ 17:00 & 33.48 & 35.512 & 1475.29 & 1473.61 \\
\hline $12 / 23 / 11$ 17:30 & 33.504 & 35.507 & 1475.27 & 1473.62 \\
\hline $12 / 23 / 11$ 18:00 & 33.415 & 35.5 & 1475.35 & 1473.62 \\
\hline $12 / 23 / 11$ 18:30 & 33.377 & 35.496 & 1475.39 & 1473.63 \\
\hline $12 / 23 / 11$ 19:00 & 33.492 & 35.491 & 1475.28 & 1473.63 \\
\hline $12 / 23 / 11$ 19:30 & 33.328 & 35.486 & 1475.44 & 1473.64 \\
\hline $12 / 23 / 1120: 00$ & 33.424 & 35.484 & 1475.35 & 1473.64 \\
\hline $12 / 23 / 1120: 30$ & 33.307 & 35.479 & 1475.46 & 1473.64 \\
\hline $12 / 23 / 1121: 00$ & 33.26 & 35.477 & 1475.51 & 1473.65 \\
\hline $12 / 23 / 1121: 30$ & 33.227 & 35.472 & 1475.54 & 1473.65 \\
\hline $12 / 23 / 1122: 00$ & 33.342 & 35.47 & 1475.43 & 1473.65 \\
\hline $12 / 23 / 1122: 30$ & 34.721 & 35.472 & 1474.05 & 1473.65 \\
\hline $12 / 23 / 1123: 00$ & 37.043 & 35.477 & 1471.73 & 1473.65 \\
\hline $12 / 23 / 1123: 30$ & 33.795 & 35.481 & 1474.97 & 1473.64 \\
\hline $12 / 24 / 110: 00$ & 33.638 & 35.474 & 1475.13 & 1473.65 \\
\hline
\end{tabular}


TABLE S1.3 Automatically recorded groundwater levels in the Klassen wells, September 15, 2011, to March 13, 2012.

\begin{tabular}{|c|c|c|c|c|}
\hline \multicolumn{3}{|c|}{ Reference Elevation (ft AMSL) } & 1508.77 & \multirow{2}{*}{$\begin{array}{l}1509.12 \\
\text { Elevation in Klassen } \\
\text { Test Well (ft AMSL) }\end{array}$} \\
\hline Date and Time & $\begin{array}{l}\text { Depth in Klassen } \\
\text { Domestic Well } \\
\text { (ft BGL) }\end{array}$ & $\begin{array}{c}\text { Depth in Klassen } \\
\text { Test Well } \\
\text { (ft BGL) }\end{array}$ & $\begin{array}{l}\text { Elevation in Klassen } \\
\text { Domestic Well } \\
\text { (ft AMSL) }\end{array}$ & \\
\hline $12 / 24 / 110: 30$ & 33.527 & 35.474 & 1475.24 & 1473.65 \\
\hline 12/24/11 1:00 & 33.45 & 35.467 & 1475.32 & 1473.66 \\
\hline $12 / 24 / 111: 30$ & 33.391 & 35.463 & 1475.38 & 1473.66 \\
\hline $12 / 24 / 112: 00$ & 33.344 & 35.456 & 1475.43 & 1473.67 \\
\hline $12 / 24 / 112: 30$ & 33.666 & 35.449 & 1475.10 & 1473.67 \\
\hline $12 / 24 / 113: 00$ & 33.347 & 35.437 & 1475.42 & 1473.69 \\
\hline $12 / 24 / 113: 30$ & 33.293 & 35.423 & 1475.48 & 1473.70 \\
\hline $12 / 24 / 114: 00$ & 33.255 & 35.416 & 1475.51 & 1473.71 \\
\hline $12 / 24 / 114: 30$ & 33.225 & 35.411 & 1475.54 & 1473.71 \\
\hline $12 / 24 / 115: 00$ & 33.197 & 35.406 & 1475.57 & 1473.72 \\
\hline $12 / 24 / 115: 30$ & 33.173 & 35.397 & 1475.60 & 1473.73 \\
\hline 12/24/11 6:00 & 33.183 & 35.385 & 1475.59 & 1473.74 \\
\hline $12 / 24 / 116: 30$ & 33.241 & 35.376 & 1475.53 & 1473.75 \\
\hline $12 / 24 / 117: 00$ & 35.082 & 35.366 & 1473.69 & 1473.76 \\
\hline $12 / 24 / 117: 30$ & 33.215 & 35.364 & 1475.55 & 1473.76 \\
\hline $12 / 24 / 118: 00$ & 33.171 & 35.36 & 1475.60 & 1473.76 \\
\hline $12 / 24 / 118: 30$ & 33.173 & 35.362 & 1475.60 & 1473.76 \\
\hline 12/24/11 9:00 & 33.398 & 35.371 & 1475.37 & 1473.75 \\
\hline 12/24/11 9:30 & 33.344 & 35.369 & 1475.43 & 1473.75 \\
\hline $12 / 24 / 11$ 10:00 & 33.269 & 35.376 & 1475.50 & 1473.75 \\
\hline $12 / 24 / 11$ 10:30 & 33.251 & 35.388 & 1475.52 & 1473.73 \\
\hline $12 / 24 / 11$ 11:00 & 33.394 & 35.423 & 1475.38 & 1473.70 \\
\hline $12 / 24 / 11$ 11:30 & 33.333 & 35.442 & 1475.44 & 1473.68 \\
\hline $12 / 24 / 11$ 12:00 & 33.372 & 35.458 & 1475.40 & 1473.66 \\
\hline $12 / 24 / 11$ 12:30 & 33.349 & 35.465 & 1475.42 & 1473.66 \\
\hline $12 / 24 / 11$ 13:00 & 38.312 & 35.472 & 1470.46 & 1473.65 \\
\hline $12 / 24 / 11$ 13:30 & 33.307 & 35.481 & 1475.46 & 1473.64 \\
\hline $12 / 24 / 11$ 14:00 & 33.396 & 35.481 & 1475.37 & 1473.64 \\
\hline $12 / 24 / 11$ 14:30 & 33.412 & 35.493 & 1475.36 & 1473.63 \\
\hline $12 / 24 / 11$ 15:00 & 45.566 & 35.512 & 1463.20 & 1473.61 \\
\hline $12 / 24 / 11$ 15:30 & 38.234 & 35.543 & 1470.54 & 1473.58 \\
\hline $12 / 24 / 11$ 16:00 & 34.798 & 35.566 & 1473.97 & 1473.56 \\
\hline $12 / 24 / 11$ 16:30 & 34.332 & 35.608 & 1474.44 & 1473.51 \\
\hline $12 / 24 / 11$ 17:00 & 34.283 & 35.66 & 1474.49 & 1473.46 \\
\hline $12 / 24 / 11$ 17:30 & 34.292 & 35.69 & 1474.48 & 1473.43 \\
\hline $12 / 24 / 11$ 18:00 & 34.025 & 35.704 & 1474.74 & 1473.42 \\
\hline $12 / 24 / 11$ 18:30 & 34.261 & 35.709 & 1474.51 & 1473.41 \\
\hline $12 / 24 / 11$ 19:00 & 34.081 & 35.718 & 1474.69 & 1473.40 \\
\hline
\end{tabular}


TABLE S1.3 Automatically recorded groundwater levels in the Klassen wells, September 15, 2011, to March 13, 2012.

\begin{tabular}{|c|c|c|c|c|}
\hline \multicolumn{3}{|c|}{ Reference Elevation (ft AMSL) } & 1508.77 & \multirow{2}{*}{$\begin{array}{l}1509.12 \\
\text { Elevation in Klassen } \\
\text { Test Well (ft AMSL) }\end{array}$} \\
\hline Date and Time & $\begin{array}{l}\text { Depth in Klassen } \\
\text { Domestic Well } \\
\text { (ft BGL) }\end{array}$ & $\begin{array}{c}\text { Depth in Klassen } \\
\text { Test Well } \\
\text { (ft BGL) }\end{array}$ & $\begin{array}{l}\text { Elevation in Klassen } \\
\text { Domestic Well } \\
\text { (ft AMSL) }\end{array}$ & \\
\hline $12 / 24 / 1119: 30$ & 34.001 & 35.735 & 1474.77 & 1473.39 \\
\hline $12 / 24 / 1120: 00$ & 33.94 & 35.744 & 1474.83 & 1473.38 \\
\hline $12 / 24 / 1120: 30$ & 34.139 & 35.754 & 1474.63 & 1473.37 \\
\hline $12 / 24 / 1121: 00$ & 33.968 & 35.758 & 1474.80 & 1473.36 \\
\hline $12 / 24 / 1121: 30$ & 33.86 & 35.761 & 1474.91 & 1473.36 \\
\hline $12 / 24 / 1122: 00$ & 34.763 & 35.761 & 1474.01 & 1473.36 \\
\hline $12 / 24 / 1122: 30$ & 34.043 & 35.768 & 1474.73 & 1473.35 \\
\hline $12 / 24 / 1123: 00$ & 33.999 & 35.765 & 1474.77 & 1473.36 \\
\hline $12 / 24 / 1123: 30$ & 33.863 & 35.754 & 1474.91 & 1473.37 \\
\hline $12 / 25 / 110: 00$ & 33.797 & 35.747 & 1474.97 & 1473.38 \\
\hline $12 / 25 / 110: 30$ & 33.835 & 35.742 & 1474.93 & 1473.38 \\
\hline $12 / 25 / 111: 00$ & 33.872 & 35.749 & 1474.90 & 1473.37 \\
\hline $12 / 25 / 111: 30$ & 33.767 & 35.742 & 1475.00 & 1473.38 \\
\hline $12 / 25 / 112: 00$ & 33.689 & 35.73 & 1475.08 & 1473.39 \\
\hline $12 / 25 / 112: 30$ & 33.631 & 35.718 & 1475.14 & 1473.40 \\
\hline $12 / 25 / 113: 00$ & 33.579 & 35.707 & 1475.19 & 1473.42 \\
\hline $12 / 25 / 113: 30$ & 33.537 & 35.693 & 1475.23 & 1473.43 \\
\hline $12 / 25 / 114: 00$ & 33.525 & 35.688 & 1475.24 & 1473.43 \\
\hline $12 / 25 / 114: 30$ & 33.48 & 35.686 & 1475.29 & 1473.44 \\
\hline $12 / 25 / 11$ 5:00 & 33.441 & 35.676 & 1475.33 & 1473.45 \\
\hline $12 / 25 / 115: 30$ & 33.408 & 35.667 & 1475.36 & 1473.46 \\
\hline $12 / 25 / 116: 00$ & 33.741 & 35.66 & 1475.03 & 1473.46 \\
\hline $12 / 25 / 116: 30$ & 33.577 & 35.653 & 1475.19 & 1473.47 \\
\hline $12 / 25 / 11$ 7:00 & 33.476 & 35.639 & 1475.29 & 1473.48 \\
\hline $12 / 25 / 11$ 7:30 & 33.45 & 35.625 & 1475.32 & 1473.50 \\
\hline $12 / 25 / 118: 00$ & 33.668 & 35.618 & 1475.10 & 1473.50 \\
\hline $12 / 25 / 118: 30$ & 34.097 & 35.62 & 1474.67 & 1473.50 \\
\hline 12/25/11 9:00 & 33.654 & 35.632 & 1475.12 & 1473.49 \\
\hline 12/25/11 9:30 & 33.624 & 35.636 & 1475.15 & 1473.49 \\
\hline $12 / 25 / 11$ 10:00 & 33.556 & 35.655 & 1475.21 & 1473.47 \\
\hline $12 / 25 / 11$ 10:30 & 33.487 & 35.669 & 1475.28 & 1473.45 \\
\hline $12 / 25 / 11$ 11:00 & 33.577 & 35.676 & 1475.19 & 1473.45 \\
\hline $12 / 25 / 11$ 11:30 & 33.584 & 35.69 & 1475.19 & 1473.43 \\
\hline $12 / 25 / 1112: 00$ & 33.518 & 35.704 & 1475.25 & 1473.42 \\
\hline $12 / 25 / 1112: 30$ & 33.457 & 35.714 & 1475.31 & 1473.41 \\
\hline $12 / 25 / 11$ 13:00 & 33.408 & 35.723 & 1475.36 & 1473.40 \\
\hline $12 / 25 / 11$ 13:30 & 33.45 & 35.735 & 1475.32 & 1473.39 \\
\hline $12 / 25 / 1114: 00$ & 33.502 & 35.744 & 1475.27 & 1473.38 \\
\hline
\end{tabular}


TABLE S1.3 Automatically recorded groundwater levels in the Klassen wells, September 15, 2011, to March 13, 2012.

\begin{tabular}{|c|c|c|c|c|}
\hline \multicolumn{3}{|c|}{ Reference Elevation (ft AMSL) } & 1508.77 & \multirow{2}{*}{$\begin{array}{l}1509.12 \\
\text { Elevation in Klassen } \\
\text { Test Well (ft AMSL) }\end{array}$} \\
\hline Date and Time & $\begin{array}{l}\text { Depth in Klassen } \\
\text { Domestic Well } \\
\text { (ft BGL) }\end{array}$ & $\begin{array}{c}\text { Depth in Klassen } \\
\text { Test Well } \\
\text { (ft BGL) }\end{array}$ & $\begin{array}{l}\text { Elevation in Klassen } \\
\text { Domestic Well } \\
\text { (ft AMSL) }\end{array}$ & \\
\hline $12 / 25 / 1114: 30$ & 33.398 & 35.749 & 1475.37 & 1473.37 \\
\hline $12 / 25 / 1115: 00$ & 33.351 & 35.761 & 1475.42 & 1473.36 \\
\hline $12 / 25 / 1115: 30$ & 33.314 & 35.761 & 1475.46 & 1473.36 \\
\hline $12 / 25 / 1116: 00$ & 33.286 & 35.754 & 1475.48 & 1473.37 \\
\hline $12 / 25 / 1116: 30$ & 33.262 & 35.744 & 1475.51 & 1473.38 \\
\hline $12 / 25 / 1117: 00$ & 33.237 & 35.733 & 1475.53 & 1473.39 \\
\hline $12 / 25 / 1117: 30$ & 33.33 & 35.723 & 1475.44 & 1473.40 \\
\hline $12 / 25 / 11$ 18:00 & 33.258 & 35.716 & 1475.51 & 1473.41 \\
\hline $12 / 25 / 1118: 30$ & 33.225 & 35.714 & 1475.54 & 1473.41 \\
\hline $12 / 25 / 11$ 19:00 & 33.356 & 35.704 & 1475.41 & 1473.42 \\
\hline $12 / 25 / 11$ 19:30 & 33.232 & 35.707 & 1475.54 & 1473.42 \\
\hline $12 / 25 / 1120: 00$ & 33.45 & 35.704 & 1475.32 & 1473.42 \\
\hline $12 / 25 / 1120: 30$ & 33.295 & 35.704 & 1475.47 & 1473.42 \\
\hline $12 / 25 / 1121: 00$ & 33.267 & 35.7 & 1475.50 & 1473.42 \\
\hline $12 / 25 / 1121: 30$ & 33.225 & 35.695 & 1475.54 & 1473.43 \\
\hline $12 / 25 / 1122: 00$ & 33.197 & 35.697 & 1475.57 & 1473.43 \\
\hline $12 / 25 / 1122: 30$ & 33.553 & 35.704 & 1475.22 & 1473.42 \\
\hline $12 / 25 / 1123: 00$ & 33.361 & 35.707 & 1475.41 & 1473.42 \\
\hline $12 / 25 / 1123: 30$ & 35.113 & 35.707 & 1473.66 & 1473.42 \\
\hline $12 / 26 / 110: 00$ & 34.754 & 35.716 & 1474.02 & 1473.41 \\
\hline $12 / 26 / 110: 30$ & 33.635 & 35.721 & 1475.13 & 1473.40 \\
\hline 12/26/11 1:00 & 33.408 & 35.728 & 1475.36 & 1473.39 \\
\hline 12/26/11 1:30 & 33.328 & 35.73 & 1475.44 & 1473.39 \\
\hline $12 / 26 / 112: 00$ & 33.279 & 35.73 & 1475.49 & 1473.39 \\
\hline $12 / 26 / 112: 30$ & 33.239 & 35.723 & 1475.53 & 1473.40 \\
\hline 12/26/11 3:00 & 33.206 & 35.718 & 1475.56 & 1473.40 \\
\hline $12 / 26 / 113: 30$ & 33.272 & 35.726 & 1475.50 & 1473.40 \\
\hline $12 / 26 / 114: 00$ & 33.227 & 35.721 & 1475.54 & 1473.40 \\
\hline $12 / 26 / 114: 30$ & 33.197 & 35.723 & 1475.57 & 1473.40 \\
\hline $12 / 26 / 11$ 5:00 & 33.162 & 35.728 & 1475.61 & 1473.39 \\
\hline $12 / 26 / 115: 30$ & 33.136 & 35.721 & 1475.63 & 1473.40 \\
\hline $12 / 26 / 116: 00$ & 33.112 & 35.716 & 1475.66 & 1473.41 \\
\hline $12 / 26 / 116: 30$ & 33.246 & 35.721 & 1475.52 & 1473.40 \\
\hline $12 / 26 / 11$ 7:00 & 33.169 & 35.728 & 1475.60 & 1473.39 \\
\hline $12 / 26 / 11$ 7:30 & 33.129 & 35.73 & 1475.64 & 1473.39 \\
\hline $12 / 26 / 118: 00$ & 33.101 & 35.73 & 1475.67 & 1473.39 \\
\hline $12 / 26 / 118: 30$ & 33.244 & 35.735 & 1475.53 & 1473.39 \\
\hline 12/26/11 9:00 & 33.133 & 35.742 & 1475.64 & 1473.38 \\
\hline
\end{tabular}


TABLE S1.3 Automatically recorded groundwater levels in the Klassen wells, September 15, 2011, to March 13, 2012.

\begin{tabular}{|c|c|c|c|c|}
\hline \multicolumn{3}{|c|}{ Reference Elevation (ft AMSL) } & 1508.77 & \multirow{2}{*}{$\begin{array}{l}1509.12 \\
\text { Elevation in Klassen } \\
\text { Test Well (ft AMSL) }\end{array}$} \\
\hline Date and Time & $\begin{array}{l}\text { Depth in Klassen } \\
\text { Domestic Well } \\
\text { (ft BGL) }\end{array}$ & $\begin{array}{c}\text { Depth in Klassen } \\
\text { Test Well } \\
\text { (ft BGL) }\end{array}$ & $\begin{array}{l}\text { Elevation in Klassen } \\
\text { Domestic Well } \\
\text { (ft AMSL) }\end{array}$ & \\
\hline 12/26/11 9:30 & 33.211 & 35.758 & 1475.56 & 1473.36 \\
\hline $12 / 26 / 11$ 10:00 & 33.208 & 35.768 & 1475.56 & 1473.35 \\
\hline $12 / 26 / 11$ 10:30 & 33.213 & 35.777 & 1475.56 & 1473.35 \\
\hline $12 / 26 / 1111: 00$ & 33.743 & 35.794 & 1475.03 & 1473.33 \\
\hline $12 / 26 / 11$ 11:30 & 33.265 & 35.805 & 1475.50 & 1473.32 \\
\hline $12 / 26 / 1112: 00$ & 33.239 & 35.822 & 1475.53 & 1473.30 \\
\hline $12 / 26 / 1112: 30$ & 33.178 & 35.838 & 1475.59 & 1473.28 \\
\hline $12 / 26 / 11$ 13:00 & 37.327 & 35.847 & 1471.44 & 1473.28 \\
\hline $12 / 26 / 1113: 30$ & 33.565 & 35.859 & 1475.20 & 1473.26 \\
\hline $12 / 26 / 1114: 00$ & 33.356 & 35.864 & 1475.41 & 1473.26 \\
\hline $12 / 26 / 1114: 30$ & 33.45 & 35.866 & 1475.32 & 1473.26 \\
\hline $12 / 26 / 11$ 15:00 & 33.518 & 35.866 & 1475.25 & 1473.26 \\
\hline $12 / 26 / 1115: 30$ & 33.276 & 35.857 & 1475.49 & 1473.27 \\
\hline $12 / 26 / 1116: 00$ & 33.797 & 35.843 & 1474.97 & 1473.28 \\
\hline $12 / 26 / 11$ 16:30 & 33.473 & 35.826 & 1475.30 & 1473.30 \\
\hline $12 / 26 / 11$ 17:00 & 37.223 & 35.756 & 1471.55 & 1473.37 \\
\hline $12 / 26 / 11$ 17:30 & 33.741 & 35.697 & 1475.03 & 1473.43 \\
\hline 12/26/11 18:00 & 33.792 & 35.657 & 1474.98 & 1473.47 \\
\hline $12 / 26 / 11$ 18:30 & 33.67 & 35.643 & 1475.10 & 1473.48 \\
\hline 12/26/11 19:00 & 33.684 & 35.646 & 1475.09 & 1473.48 \\
\hline $12 / 26 / 11$ 19:30 & 33.584 & 35.636 & 1475.19 & 1473.49 \\
\hline $12 / 26 / 1120: 00$ & 33.588 & 35.643 & 1475.18 & 1473.48 \\
\hline $12 / 26 / 1120: 30$ & 33.539 & 35.657 & 1475.23 & 1473.47 \\
\hline $12 / 26 / 1121: 00$ & 33.506 & 35.655 & 1475.26 & 1473.47 \\
\hline $12 / 26 / 1121: 30$ & 33.492 & 35.66 & 1475.28 & 1473.46 \\
\hline $12 / 26 / 1122: 00$ & 33.415 & 35.66 & 1475.35 & 1473.46 \\
\hline $12 / 26 / 1122: 30$ & 37.409 & 35.657 & 1471.36 & 1473.47 \\
\hline $12 / 26 / 1123: 00$ & 36.747 & 35.655 & 1472.02 & 1473.47 \\
\hline $12 / 26 / 1123: 30$ & 33.647 & 35.655 & 1475.12 & 1473.47 \\
\hline 12/27/11 0:00 & 33.452 & 35.65 & 1475.32 & 1473.47 \\
\hline 12/27/11 0:30 & 33.377 & 35.641 & 1475.39 & 1473.48 \\
\hline 12/27/11 1:00 & 33.326 & 35.636 & 1475.44 & 1473.49 \\
\hline $12 / 27 / 11$ 1:30 & 33.283 & 35.636 & 1475.49 & 1473.49 \\
\hline $12 / 27 / 112: 00$ & 33.281 & 35.625 & 1475.49 & 1473.50 \\
\hline $12 / 27 / 112: 30$ & 33.356 & 35.618 & 1475.41 & 1473.50 \\
\hline $12 / 27 / 113: 00$ & 33.34 & 35.608 & 1475.43 & 1473.51 \\
\hline $12 / 27 / 113: 30$ & 33.448 & 35.599 & 1475.32 & 1473.52 \\
\hline $12 / 27 / 114: 00$ & 33.443 & 35.594 & 1475.33 & 1473.53 \\
\hline
\end{tabular}


TABLE S1.3 Automatically recorded groundwater levels in the Klassen wells, September 15, 2011, to March 13, 2012.

\begin{tabular}{|c|c|c|c|c|}
\hline \multicolumn{3}{|c|}{ Reference Elevation (ft AMSL) } & 1508.77 & \multirow{2}{*}{$\begin{array}{l}1509.12 \\
\text { Elevation in Klassen } \\
\text { Test Well (ft AMSL) }\end{array}$} \\
\hline Date and Time & $\begin{array}{l}\text { Depth in Klassen } \\
\text { Domestic Well } \\
\text { (ft BGL) }\end{array}$ & $\begin{array}{c}\text { Depth in Klassen } \\
\text { Test Well } \\
\text { (ft BGL) }\end{array}$ & $\begin{array}{l}\text { Elevation in Klassen } \\
\text { Domestic Well } \\
\text { (ft AMSL) }\end{array}$ & \\
\hline $12 / 27 / 114: 30$ & 33.354 & 35.587 & 1475.42 & 1473.54 \\
\hline 12/27/11 5:00 & 33.295 & 35.575 & 1475.47 & 1473.55 \\
\hline $12 / 27 / 115: 30$ & 33.251 & 35.568 & 1475.52 & 1473.55 \\
\hline $12 / 27 / 116: 00$ & 33.218 & 35.559 & 1475.55 & 1473.56 \\
\hline $12 / 27 / 116: 30$ & 33.323 & 35.55 & 1475.45 & 1473.57 \\
\hline $12 / 27 / 117: 00$ & 33.237 & 35.54 & 1475.53 & 1473.58 \\
\hline $12 / 27 / 117: 30$ & 33.194 & 35.533 & 1475.58 & 1473.59 \\
\hline $12 / 27 / 118: 00$ & 33.298 & 35.528 & 1475.47 & 1473.59 \\
\hline $12 / 27 / 118: 30$ & 33.302 & 35.533 & 1475.47 & 1473.59 \\
\hline 12/27/11 9:00 & 33.558 & 35.528 & 1475.21 & 1473.59 \\
\hline 12/27/11 9:30 & 34.548 & 35.531 & 1474.22 & 1473.59 \\
\hline $12 / 27 / 11$ 10:00 & 37.519 & 35.531 & 1471.25 & 1473.59 \\
\hline $12 / 27 / 11$ 10:30 & 33.417 & 35.531 & 1475.35 & 1473.59 \\
\hline $12 / 27 / 1111: 00$ & 33.607 & 35.54 & 1475.16 & 1473.58 \\
\hline $12 / 27 / 11$ 11:30 & 33.53 & 35.557 & 1475.24 & 1473.57 \\
\hline $12 / 27 / 1112: 00$ & 33.539 & 35.573 & 1475.23 & 1473.55 \\
\hline $12 / 27 / 11$ 12:30 & 33.492 & 35.587 & 1475.28 & 1473.54 \\
\hline $12 / 27 / 11$ 13:00 & 33.53 & 35.599 & 1475.24 & 1473.52 \\
\hline $12 / 27 / 11$ 13:30 & 33.579 & 35.606 & 1475.19 & 1473.52 \\
\hline $12 / 27 / 11$ 14:00 & 33.598 & 35.613 & 1475.17 & 1473.51 \\
\hline $12 / 27 / 11$ 14:30 & 33.563 & 35.61 & 1475.21 & 1473.51 \\
\hline $12 / 27 / 11$ 15:00 & 33.466 & 35.615 & 1475.30 & 1473.51 \\
\hline $12 / 27 / 11$ 15:30 & 36.473 & 35.625 & 1472.30 & 1473.50 \\
\hline $12 / 27 / 11$ 16:00 & 33.466 & 35.627 & 1475.30 & 1473.50 \\
\hline $12 / 27 / 11$ 16:30 & 33.434 & 35.622 & 1475.34 & 1473.50 \\
\hline $12 / 27 / 11$ 17:00 & 35.169 & 35.615 & 1473.60 & 1473.51 \\
\hline $12 / 27 / 11$ 17:30 & 33.415 & 35.606 & 1475.35 & 1473.52 \\
\hline $12 / 27 / 11$ 18:00 & 33.34 & 35.603 & 1475.43 & 1473.52 \\
\hline $12 / 27 / 11$ 18:30 & 33.293 & 35.601 & 1475.48 & 1473.52 \\
\hline 12/27/11 19:00 & 33.255 & 35.599 & 1475.51 & 1473.52 \\
\hline 12/27/11 19:30 & 33.225 & 35.594 & 1475.54 & 1473.53 \\
\hline $12 / 27 / 1120: 00$ & 33.527 & 35.594 & 1475.24 & 1473.53 \\
\hline $12 / 27 / 1120: 30$ & 33.452 & 35.596 & 1475.32 & 1473.53 \\
\hline $12 / 27 / 1121: 00$ & 33.38 & 35.596 & 1475.39 & 1473.53 \\
\hline $12 / 27 / 1121: 30$ & 33.312 & 35.594 & 1475.46 & 1473.53 \\
\hline $12 / 27 / 1122: 00$ & 33.269 & 35.587 & 1475.50 & 1473.54 \\
\hline $12 / 27 / 1122: 30$ & 38.443 & 35.585 & 1470.33 & 1473.54 \\
\hline $12 / 27 / 1123: 00$ & 34.541 & 35.585 & 1474.23 & 1473.54 \\
\hline
\end{tabular}


TABLE S1.3 Automatically recorded groundwater levels in the Klassen wells, September 15, 2011, to March 13, 2012.

\begin{tabular}{|c|c|c|c|c|}
\hline \multicolumn{3}{|c|}{ Reference Elevation (ft AMSL) } & 1508.77 & \multirow{2}{*}{$\begin{array}{l}1509.12 \\
\text { Elevation in Klassen } \\
\text { Test Well (ft AMSL) }\end{array}$} \\
\hline Date and Time & $\begin{array}{l}\text { Depth in Klassen } \\
\text { Domestic Well } \\
\text { (ft BGL) }\end{array}$ & $\begin{array}{c}\text { Depth in Klassen } \\
\text { Test Well } \\
\text { (ft BGL) }\end{array}$ & $\begin{array}{l}\text { Elevation in Klassen } \\
\text { Domestic Well } \\
\text { (ft AMSL) }\end{array}$ & \\
\hline $12 / 27 / 1123: 30$ & 33.565 & 35.592 & 1475.20 & 1473.53 \\
\hline $12 / 28 / 110: 00$ & 33.412 & 35.592 & 1475.36 & 1473.53 \\
\hline $12 / 28 / 110: 30$ & 33.337 & 35.596 & 1475.43 & 1473.53 \\
\hline 12/28/11 1:00 & 33.286 & 35.606 & 1475.48 & 1473.52 \\
\hline $12 / 28 / 111: 30$ & 33.244 & 35.604 & 1475.53 & 1473.52 \\
\hline $12 / 28 / 112: 00$ & 33.215 & 35.599 & 1475.55 & 1473.52 \\
\hline $12 / 28 / 112: 30$ & 33.185 & 35.608 & 1475.58 & 1473.51 \\
\hline $12 / 28 / 113: 00$ & 33.162 & 35.604 & 1475.61 & 1473.52 \\
\hline $12 / 28 / 113: 30$ & 33.143 & 35.596 & 1475.63 & 1473.53 \\
\hline $12 / 28 / 114: 00$ & 33.124 & 35.58 & 1475.65 & 1473.54 \\
\hline $12 / 28 / 114: 30$ & 33.103 & 35.582 & 1475.67 & 1473.54 \\
\hline $12 / 28 / 11$ 5:00 & 33.084 & 35.582 & 1475.69 & 1473.54 \\
\hline $12 / 28 / 115: 30$ & 33.068 & 35.573 & 1475.70 & 1473.55 \\
\hline $12 / 28 / 116: 00$ & 33.054 & 35.561 & 1475.72 & 1473.56 \\
\hline $12 / 28 / 116: 30$ & 33.272 & 35.564 & 1475.50 & 1473.56 \\
\hline $12 / 28 / 11$ 7:00 & 33.159 & 35.573 & 1475.61 & 1473.55 \\
\hline $12 / 28 / 11$ 7:30 & 33.112 & 35.571 & 1475.66 & 1473.55 \\
\hline $12 / 28 / 118: 00$ & 33.082 & 35.566 & 1475.69 & 1473.56 \\
\hline $12 / 28 / 118: 30$ & 33.154 & 35.568 & 1475.62 & 1473.55 \\
\hline 12/28/11 9:00 & 33.096 & 35.564 & 1475.67 & 1473.56 \\
\hline 12/28/11 9:30 & 33.595 & 35.564 & 1475.17 & 1473.56 \\
\hline $12 / 28 / 11$ 10:00 & 33.11 & 35.561 & 1475.66 & 1473.56 \\
\hline $12 / 28 / 11$ 10:30 & 33.07 & 35.568 & 1475.70 & 1473.55 \\
\hline $12 / 28 / 11$ 11:00 & 33.07 & 35.575 & 1475.70 & 1473.55 \\
\hline $12 / 28 / 11$ 11:30 & 33.152 & 35.589 & 1475.62 & 1473.53 \\
\hline $12 / 28 / 1112: 00$ & 33.105 & 35.604 & 1475.66 & 1473.52 \\
\hline $12 / 28 / 1112: 30$ & 33.061 & 35.62 & 1475.71 & 1473.50 \\
\hline $12 / 28 / 11$ 13:00 & 33.033 & 35.639 & 1475.74 & 1473.48 \\
\hline $12 / 28 / 11$ 13:30 & 33.187 & 35.65 & 1475.58 & 1473.47 \\
\hline $12 / 28 / 11$ 14:00 & 33.075 & 35.625 & 1475.69 & 1473.50 \\
\hline $12 / 28 / 11$ 14:30 & 33.04 & 35.538 & 1475.73 & 1473.58 \\
\hline $12 / 28 / 11$ 15:00 & 33.014 & 35.496 & 1475.76 & 1473.63 \\
\hline $12 / 28 / 1115: 30$ & 33.079 & 35.482 & 1475.69 & 1473.64 \\
\hline $12 / 28 / 11$ 16:00 & 33.03 & 35.477 & 1475.74 & 1473.65 \\
\hline $12 / 28 / 11$ 16:30 & 33.126 & 35.479 & 1475.64 & 1473.64 \\
\hline $12 / 28 / 11$ 17:00 & 33.049 & 35.481 & 1475.72 & 1473.64 \\
\hline $12 / 28 / 11$ 17:30 & 33.016 & 35.477 & 1475.75 & 1473.65 \\
\hline $12 / 28 / 11$ 18:00 & 33.049 & 35.474 & 1475.72 & 1473.65 \\
\hline
\end{tabular}


TABLE S1.3 Automatically recorded groundwater levels in the Klassen wells, September 15, 2011, to March 13, 2012.

\begin{tabular}{|c|c|c|c|c|}
\hline \multicolumn{3}{|c|}{ Reference Elevation (ft AMSL) } & 1508.77 & \multirow{2}{*}{$\begin{array}{l}1509.12 \\
\text { Elevation in Klassen } \\
\text { Test Well (ft AMSL) }\end{array}$} \\
\hline Date and Time & $\begin{array}{l}\text { Depth in Klassen } \\
\text { Domestic Well } \\
\text { (ft BGL) }\end{array}$ & $\begin{array}{c}\text { Depth in Klassen } \\
\text { Test Well } \\
\text { (ft BGL) }\end{array}$ & $\begin{array}{l}\text { Elevation in Klassen } \\
\text { Domestic Well } \\
\text { (ft AMSL) }\end{array}$ & \\
\hline $12 / 28 / 1118: 30$ & 33.152 & 35.474 & 1475.62 & 1473.65 \\
\hline $12 / 28 / 11$ 19:00 & 33.065 & 35.474 & 1475.70 & 1473.65 \\
\hline $12 / 28 / 11$ 19:30 & 33.025 & 35.467 & 1475.74 & 1473.66 \\
\hline $12 / 28 / 1120: 00$ & 33 & 35.465 & 1475.77 & 1473.66 \\
\hline $12 / 28 / 1120: 30$ & 32.979 & 35.458 & 1475.79 & 1473.66 \\
\hline $12 / 28 / 1121: 00$ & 32.988 & 35.456 & 1475.78 & 1473.67 \\
\hline $12 / 28 / 1121: 30$ & 33.115 & 35.46 & 1475.65 & 1473.66 \\
\hline $12 / 28 / 1122: 00$ & 33.049 & 35.458 & 1475.72 & 1473.66 \\
\hline $12 / 28 / 1122: 30$ & 33.035 & 35.456 & 1475.73 & 1473.67 \\
\hline $12 / 28 / 1123: 00$ & 33.394 & 35.46 & 1475.38 & 1473.66 \\
\hline $12 / 28 / 1123: 30$ & 38.063 & 35.479 & 1470.71 & 1473.64 \\
\hline 12/29/11 0:00 & 33.525 & 35.488 & 1475.24 & 1473.63 \\
\hline $12 / 29 / 110: 30$ & 33.391 & 35.498 & 1475.38 & 1473.62 \\
\hline 12/29/11 1:00 & 33.293 & 35.503 & 1475.48 & 1473.62 \\
\hline 12/29/11 1:30 & 33.227 & 35.503 & 1475.54 & 1473.62 \\
\hline $12 / 29 / 112: 00$ & 33.18 & 35.498 & 1475.59 & 1473.62 \\
\hline $12 / 29 / 112: 30$ & 33.14 & 35.503 & 1475.63 & 1473.62 \\
\hline 12/29/11 3:00 & 33.108 & 35.505 & 1475.66 & 1473.62 \\
\hline 12/29/11 3:30 & 33.079 & 35.507 & 1475.69 & 1473.62 \\
\hline $12 / 29 / 114: 00$ & 33.138 & 35.507 & 1475.63 & 1473.62 \\
\hline $12 / 29 / 114: 30$ & 33.084 & 35.505 & 1475.69 & 1473.62 \\
\hline 12/29/11 5:00 & 33.051 & 35.505 & 1475.72 & 1473.62 \\
\hline $12 / 29 / 115: 30$ & 33.025 & 35.5 & 1475.74 & 1473.62 \\
\hline $12 / 29 / 116: 00$ & 33.004 & 35.5 & 1475.77 & 1473.62 \\
\hline $12 / 29 / 116: 30$ & 33.117 & 35.498 & 1475.65 & 1473.62 \\
\hline 12/29/11 7:00 & 33.131 & 35.5 & 1475.64 & 1473.62 \\
\hline 12/29/11 7:30 & 33.119 & 35.5 & 1475.65 & 1473.62 \\
\hline 12/29/11 8:00 & 33.065 & 35.493 & 1475.70 & 1473.63 \\
\hline $12 / 29 / 118: 30$ & 33.232 & 35.5 & 1475.54 & 1473.62 \\
\hline 12/29/11 9:00 & 38.631 & 35.5 & 1470.14 & 1473.62 \\
\hline 12/29/11 9:30 & 33.133 & 35.505 & 1475.64 & 1473.62 \\
\hline $12 / 29 / 11$ 10:00 & 33.072 & 35.51 & 1475.70 & 1473.61 \\
\hline $12 / 29 / 11$ 10:30 & 33.086 & 35.514 & 1475.68 & 1473.61 \\
\hline 12/29/11 11:00 & 33.042 & 35.524 & 1475.73 & 1473.60 \\
\hline 12/29/11 11:30 & 33.009 & 35.535 & 1475.76 & 1473.59 \\
\hline $12 / 29 / 11$ 12:00 & 33.098 & 35.554 & 1475.67 & 1473.57 \\
\hline $12 / 29 / 11$ 12:30 & 33.018 & 35.568 & 1475.75 & 1473.55 \\
\hline $12 / 29 / 11$ 13:00 & 33.112 & 35.589 & 1475.66 & 1473.53 \\
\hline
\end{tabular}


TABLE S1.3 Automatically recorded groundwater levels in the Klassen wells, September 15, 2011, to March 13, 2012.

\begin{tabular}{|c|c|c|c|c|}
\hline \multicolumn{3}{|c|}{ Reference Elevation (ft AMSL) } & 1508.77 & \multirow{2}{*}{$\begin{array}{l}1509.12 \\
\text { Elevation in Klassen } \\
\text { Test Well (ft AMSL) }\end{array}$} \\
\hline Date and Time & $\begin{array}{l}\text { Depth in Klassen } \\
\text { Domestic Well } \\
\text { (ft BGL) }\end{array}$ & $\begin{array}{c}\text { Depth in Klassen } \\
\text { Test Well } \\
\text { (ft BGL) }\end{array}$ & $\begin{array}{l}\text { Elevation in Klassen } \\
\text { Domestic Well } \\
\text { (ft AMSL) }\end{array}$ & \\
\hline $12 / 29 / 1113: 30$ & 33.023 & 35.514 & 1475.75 & 1473.61 \\
\hline $12 / 29 / 1114: 00$ & 32.988 & 35.449 & 1475.78 & 1473.67 \\
\hline $12 / 29 / 1114: 30$ & 32.965 & 35.421 & 1475.80 & 1473.70 \\
\hline $12 / 29 / 1115: 00$ & 32.962 & 35.411 & 1475.81 & 1473.71 \\
\hline $12 / 29 / 1115: 30$ & 34.243 & 35.411 & 1474.53 & 1473.71 \\
\hline $12 / 29 / 1116: 00$ & 32.99 & 35.402 & 1475.78 & 1473.72 \\
\hline $12 / 29 / 1116: 30$ & 32.953 & 35.397 & 1475.82 & 1473.73 \\
\hline 12/29/11 17:00 & 33.068 & 35.397 & 1475.70 & 1473.73 \\
\hline $12 / 29 / 1117: 30$ & 33.004 & 35.392 & 1475.77 & 1473.73 \\
\hline $12 / 29 / 1118: 00$ & 32.96 & 35.383 & 1475.81 & 1473.74 \\
\hline $12 / 29 / 1118: 30$ & 32.936 & 35.388 & 1475.83 & 1473.73 \\
\hline 12/29/11 19:00 & 33.047 & 35.392 & 1475.72 & 1473.73 \\
\hline $12 / 29 / 1119: 30$ & 33.183 & 35.402 & 1475.59 & 1473.72 \\
\hline $12 / 29 / 1120: 00$ & 33.136 & 35.406 & 1475.63 & 1473.72 \\
\hline $12 / 29 / 1120: 30$ & 33.096 & 35.42 & 1475.67 & 1473.70 \\
\hline $12 / 29 / 1121: 00$ & 33.07 & 35.409 & 1475.70 & 1473.71 \\
\hline $12 / 29 / 1121: 30$ & 33.051 & 35.404 & 1475.72 & 1473.72 \\
\hline $12 / 29 / 1122: 00$ & 33.009 & 35.397 & 1475.76 & 1473.73 \\
\hline $12 / 29 / 1122: 30$ & 35.465 & 35.409 & 1473.30 & 1473.71 \\
\hline $12 / 29 / 1123: 00$ & 34.015 & 35.427 & 1474.75 & 1473.70 \\
\hline $12 / 29 / 1123: 30$ & 33.258 & 35.439 & 1475.51 & 1473.68 \\
\hline $12 / 30 / 110: 00$ & 33.164 & 35.439 & 1475.61 & 1473.68 \\
\hline $12 / 30 / 110: 30$ & 33.11 & 35.446 & 1475.66 & 1473.68 \\
\hline $12 / 30 / 11$ 1:00 & 33.07 & 35.444 & 1475.70 & 1473.68 \\
\hline $12 / 30 / 111: 30$ & 33.04 & 35.444 & 1475.73 & 1473.68 \\
\hline $12 / 30 / 112: 00$ & 33.058 & 35.442 & 1475.71 & 1473.68 \\
\hline $12 / 30 / 112: 30$ & 33.11 & 35.451 & 1475.66 & 1473.67 \\
\hline $12 / 30 / 113: 00$ & 33.084 & 35.446 & 1475.69 & 1473.68 \\
\hline $12 / 30 / 113: 30$ & 33.096 & 35.451 & 1475.67 & 1473.67 \\
\hline $12 / 30 / 114: 00$ & 33.161 & 35.456 & 1475.61 & 1473.67 \\
\hline $12 / 30 / 114: 30$ & 33.098 & 35.456 & 1475.67 & 1473.67 \\
\hline $12 / 30 / 11$ 5:00 & 33.058 & 35.453 & 1475.71 & 1473.67 \\
\hline $12 / 30 / 115: 30$ & 33.04 & 35.446 & 1475.73 & 1473.68 \\
\hline $12 / 30 / 116: 00$ & 33.023 & 35.437 & 1475.75 & 1473.69 \\
\hline $12 / 30 / 116: 30$ & 33.222 & 35.444 & 1475.55 & 1473.68 \\
\hline 12/30/11 7:00 & 33.138 & 35.442 & 1475.63 & 1473.68 \\
\hline $12 / 30 / 11$ 7:30 & 33.079 & 35.432 & 1475.69 & 1473.69 \\
\hline $12 / 30 / 118: 00$ & 33.042 & 35.423 & 1475.73 & 1473.70 \\
\hline
\end{tabular}


TABLE S1.3 Automatically recorded groundwater levels in the Klassen wells, September 15, 2011, to March 13, 2012.

\begin{tabular}{|c|c|c|c|c|}
\hline \multicolumn{3}{|c|}{ Reference Elevation (ft AMSL) } & 1508.77 & \multirow{2}{*}{$\begin{array}{l}1509.12 \\
\text { Elevation in Klassen } \\
\text { Test Well (ft AMSL) }\end{array}$} \\
\hline Date and Time & $\begin{array}{l}\text { Depth in Klassen } \\
\text { Domestic Well } \\
\text { (ft BGL) }\end{array}$ & $\begin{array}{c}\text { Depth in Klassen } \\
\text { Test Well } \\
\text { (ft BGL) }\end{array}$ & $\begin{array}{l}\text { Elevation in Klassen } \\
\text { Domestic Well } \\
\text { (ft AMSL) }\end{array}$ & \\
\hline $12 / 30 / 118: 30$ & 33.351 & 35.423 & 1475.42 & 1473.70 \\
\hline 12/30/11 9:00 & 33.15 & 35.416 & 1475.62 & 1473.71 \\
\hline $12 / 30 / 119: 30$ & 33.286 & 35.416 & 1475.48 & 1473.71 \\
\hline $12 / 30 / 1110: 00$ & 33.415 & 35.428 & 1475.35 & 1473.69 \\
\hline $12 / 30 / 1110: 30$ & 33.387 & 35.43 & 1475.38 & 1473.69 \\
\hline 12/30/11 11:00 & 33.326 & 35.442 & 1475.44 & 1473.68 \\
\hline $12 / 30 / 1111: 30$ & 34.001 & 35.458 & 1474.77 & 1473.66 \\
\hline $12 / 30 / 1112: 00$ & 35.631 & 35.489 & 1473.14 & 1473.63 \\
\hline $12 / 30 / 1112: 30$ & 33.769 & 35.528 & 1475.00 & 1473.59 \\
\hline $12 / 30 / 1113: 00$ & 33.56 & 35.542 & 1475.21 & 1473.58 \\
\hline $12 / 30 / 1113: 30$ & 33.509 & 35.547 & 1475.26 & 1473.58 \\
\hline $12 / 30 / 1114: 00$ & 33.523 & 35.545 & 1475.25 & 1473.58 \\
\hline $12 / 30 / 1114: 30$ & 33.415 & 35.545 & 1475.35 & 1473.58 \\
\hline $12 / 30 / 1115: 00$ & 34.433 & 35.543 & 1474.34 & 1473.58 \\
\hline $12 / 30 / 1115: 30$ & 33.344 & 35.54 & 1475.43 & 1473.58 \\
\hline $12 / 30 / 11$ 16:00 & 33.279 & 35.538 & 1475.49 & 1473.58 \\
\hline $12 / 30 / 1116: 30$ & 33.23 & 35.533 & 1475.54 & 1473.59 \\
\hline $12 / 30 / 11$ 17:00 & 33.194 & 35.526 & 1475.58 & 1473.60 \\
\hline $12 / 30 / 11$ 17:30 & 33.312 & 35.521 & 1475.46 & 1473.60 \\
\hline $12 / 30 / 11$ 18:00 & 33.295 & 35.521 & 1475.47 & 1473.60 \\
\hline $12 / 30 / 1118: 30$ & 33.215 & 35.517 & 1475.55 & 1473.61 \\
\hline 12/30/11 19:00 & 33.169 & 35.512 & 1475.60 & 1473.61 \\
\hline $12 / 30 / 11$ 19:30 & 33.274 & 35.507 & 1475.50 & 1473.62 \\
\hline $12 / 30 / 1120: 00$ & 33.175 & 35.5 & 1475.59 & 1473.62 \\
\hline $12 / 30 / 1120: 30$ & 33.3 & 35.5 & 1475.47 & 1473.62 \\
\hline $12 / 30 / 1121: 00$ & 33.162 & 35.498 & 1475.61 & 1473.62 \\
\hline $12 / 30 / 1121: 30$ & 33.176 & 35.496 & 1475.59 & 1473.63 \\
\hline $12 / 30 / 1122: 00$ & 33.18 & 35.493 & 1475.59 & 1473.63 \\
\hline $12 / 30 / 1122: 30$ & 33.255 & 35.491 & 1475.51 & 1473.63 \\
\hline $12 / 30 / 1123: 00$ & 37.148 & 35.505 & 1471.62 & 1473.62 \\
\hline $12 / 30 / 1123: 30$ & 33.49 & 35.512 & 1475.28 & 1473.61 \\
\hline $12 / 31 / 11$ 0:00 & 33.354 & 35.519 & 1475.42 & 1473.60 \\
\hline $12 / 31 / 110: 30$ & 33.279 & 35.519 & 1475.49 & 1473.60 \\
\hline 12/31/11 1:00 & 33.222 & 35.521 & 1475.55 & 1473.60 \\
\hline 12/31/11 1:30 & 33.18 & 35.519 & 1475.59 & 1473.60 \\
\hline $12 / 31 / 112: 00$ & 33.145 & 35.521 & 1475.62 & 1473.60 \\
\hline $12 / 31 / 112: 30$ & 33.115 & 35.521 & 1475.65 & 1473.60 \\
\hline 12/31/11 3:00 & 33.267 & 35.519 & 1475.50 & 1473.60 \\
\hline
\end{tabular}


TABLE S1.3 Automatically recorded groundwater levels in the Klassen wells, September 15, 2011, to March 13, 2012.

\begin{tabular}{|c|c|c|c|c|}
\hline \multicolumn{3}{|c|}{ Reference Elevation (ft AMSL) } & 1508.77 & \multirow{2}{*}{$\begin{array}{l}1509.12 \\
\text { Elevation in Klassen } \\
\text { Test Well (ft AMSL) }\end{array}$} \\
\hline Date and Time & $\begin{array}{l}\text { Depth in Klassen } \\
\text { Domestic Well } \\
(\mathrm{ft} \mathrm{BGL})\end{array}$ & $\begin{array}{c}\text { Depth in Klassen } \\
\text { Test Well } \\
\text { (ft BGL) }\end{array}$ & $\begin{array}{l}\text { Elevation in Klassen } \\
\text { Domestic Well } \\
\text { (ft AMSL) }\end{array}$ & \\
\hline $12 / 31 / 113: 30$ & 33.126 & 35.524 & 1475.64 & 1473.60 \\
\hline $12 / 31 / 114: 00$ & 33.084 & 35.528 & 1475.69 & 1473.59 \\
\hline $12 / 31 / 114: 30$ & 33.056 & 35.535 & 1475.71 & 1473.59 \\
\hline $12 / 31 / 115: 00$ & 33.033 & 35.545 & 1475.74 & 1473.58 \\
\hline 12/31/11 5:30 & 33.011 & 35.55 & 1475.76 & 1473.57 \\
\hline $12 / 31 / 116: 00$ & 32.993 & 35.554 & 1475.78 & 1473.57 \\
\hline $12 / 31 / 116: 30$ & 33.077 & 35.564 & 1475.69 & 1473.56 \\
\hline 12/31/11 7:00 & 33.016 & 35.566 & 1475.75 & 1473.56 \\
\hline $12 / 31 / 117: 30$ & 32.988 & 35.571 & 1475.78 & 1473.55 \\
\hline $12 / 31 / 118: 00$ & 32.995 & 35.571 & 1475.77 & 1473.55 \\
\hline $12 / 31 / 118: 30$ & 35.209 & 35.58 & 1473.56 & 1473.54 \\
\hline 12/31/11 9:00 & 33.159 & 35.592 & 1475.61 & 1473.53 \\
\hline 12/31/11 9:30 & 33.255 & 35.606 & 1475.51 & 1473.52 \\
\hline $12 / 31 / 11$ 10:00 & 33.384 & 35.615 & 1475.39 & 1473.51 \\
\hline 12/31/11 10:30 & 33.434 & 35.636 & 1475.34 & 1473.49 \\
\hline $12 / 31 / 11$ 11:00 & 34.137 & 35.648 & 1474.63 & 1473.47 \\
\hline $12 / 31 / 11$ 11:30 & 33.286 & 35.634 & 1475.48 & 1473.49 \\
\hline $12 / 31 / 11$ 12:00 & 33.309 & 35.535 & 1475.46 & 1473.59 \\
\hline $12 / 31 / 11$ 12:30 & 33.194 & 35.451 & 1475.58 & 1473.67 \\
\hline $12 / 31 / 11$ 13:00 & 33.138 & 35.425 & 1475.63 & 1473.70 \\
\hline $12 / 31 / 11$ 13:30 & 33.309 & 35.418 & 1475.46 & 1473.70 \\
\hline $12 / 31 / 11$ 14:00 & 33.152 & 35.409 & 1475.62 & 1473.71 \\
\hline $12 / 31 / 11$ 14:30 & 33.197 & 35.409 & 1475.57 & 1473.71 \\
\hline $12 / 31 / 11$ 15:00 & 33.115 & 35.409 & 1475.65 & 1473.71 \\
\hline $12 / 31 / 11$ 15:30 & 33.211 & 35.411 & 1475.56 & 1473.71 \\
\hline $12 / 31 / 11$ 16:00 & 33.237 & 35.413 & 1475.53 & 1473.71 \\
\hline 12/31/11 16:30 & 33.222 & 35.423 & 1475.55 & 1473.70 \\
\hline 12/31/11 17:00 & 33.171 & 35.435 & 1475.60 & 1473.69 \\
\hline 12/31/11 17:30 & 33.119 & 35.43 & 1475.65 & 1473.69 \\
\hline 12/31/11 18:00 & 33.19 & 35.439 & 1475.58 & 1473.68 \\
\hline 12/31/11 18:30 & 33.293 & 35.451 & 1475.48 & 1473.67 \\
\hline 12/31/11 19:00 & 33.211 & 35.46 & 1475.56 & 1473.66 \\
\hline 12/31/11 19:30 & 35.837 & 35.47 & 1472.93 & 1473.65 \\
\hline 12/31/11 20:00 & 33.246 & 35.481 & 1475.52 & 1473.64 \\
\hline 12/31/11 20:30 & 33.204 & 35.507 & 1475.57 & 1473.62 \\
\hline $12 / 31 / 1121: 00$ & 33.239 & 35.521 & 1475.53 & 1473.60 \\
\hline $12 / 31 / 1121: 30$ & 33.692 & 35.535 & 1475.08 & 1473.59 \\
\hline $12 / 31 / 1122: 00$ & 33.527 & 35.554 & 1475.24 & 1473.57 \\
\hline
\end{tabular}


TABLE S1.3 Automatically recorded groundwater levels in the Klassen wells, September 15, 2011, to March 13, 2012.

\begin{tabular}{|c|c|c|c|c|}
\hline \multicolumn{3}{|c|}{ Reference Elevation (ft AMSL) } & 1508.77 & \multirow{2}{*}{$\begin{array}{l}1509.12 \\
\text { Elevation in Klassen } \\
\text { Test Well (ft AMSL) }\end{array}$} \\
\hline Date and Time & $\begin{array}{l}\text { Depth in Klassen } \\
\text { Domestic Well } \\
(\mathrm{ft} \mathrm{BGL})\end{array}$ & $\begin{array}{c}\text { Depth in Klassen } \\
\text { Test Well } \\
\text { (ft BGL) }\end{array}$ & $\begin{array}{l}\text { Elevation in Klassen } \\
\text { Domestic Well } \\
\text { (ft AMSL) }\end{array}$ & \\
\hline $12 / 31 / 1122: 30$ & 33.567 & 35.566 & 1475.20 & 1473.56 \\
\hline $12 / 31 / 1123: 00$ & 33.419 & 35.575 & 1475.35 & 1473.55 \\
\hline $12 / 31 / 1123: 30$ & 33.356 & 35.585 & 1475.41 & 1473.54 \\
\hline 1/1/12 0:00 & 33.288 & 35.585 & 1475.48 & 1473.54 \\
\hline $1 / 1 / 120: 30$ & 33.239 & 35.582 & 1475.53 & 1473.54 \\
\hline $1 / 1 / 121: 00$ & 33.309 & 35.585 & 1475.46 & 1473.54 \\
\hline 1/1/12 1:30 & 33.251 & 35.585 & 1475.52 & 1473.54 \\
\hline $1 / 1 / 122: 00$ & 33.201 & 35.578 & 1475.57 & 1473.54 \\
\hline $1 / 1 / 122: 30$ & 33.192 & 35.568 & 1475.58 & 1473.55 \\
\hline 1/1/12 3:00 & 33.154 & 35.564 & 1475.62 & 1473.56 \\
\hline $1 / 1 / 123: 30$ & 33.126 & 35.559 & 1475.64 & 1473.56 \\
\hline $1 / 1 / 124: 00$ & 33.103 & 35.557 & 1475.67 & 1473.57 \\
\hline $1 / 1 / 124: 30$ & 33.084 & 35.54 & 1475.69 & 1473.58 \\
\hline $1 / 1 / 125: 00$ & 33.068 & 35.521 & 1475.70 & 1473.60 \\
\hline $1 / 1 / 125: 30$ & 33.178 & 35.517 & 1475.59 & 1473.61 \\
\hline $1 / 1 / 126: 00$ & 33.464 & 35.514 & 1475.31 & 1473.61 \\
\hline $1 / 1 / 126: 30$ & 33.295 & 35.51 & 1475.47 & 1473.61 \\
\hline $1 / 1 / 12$ 7:00 & 33.183 & 35.498 & 1475.59 & 1473.62 \\
\hline 1/1/12 7:30 & 33.136 & 35.477 & 1475.63 & 1473.65 \\
\hline 1/1/12 8:00 & 34.158 & 35.465 & 1474.61 & 1473.66 \\
\hline $1 / 1 / 128: 30$ & 33.342 & 35.456 & 1475.43 & 1473.67 \\
\hline 1/1/12 9:00 & 33.23 & 35.444 & 1475.54 & 1473.68 \\
\hline 1/1/12 9:30 & 33.408 & 35.437 & 1475.36 & 1473.69 \\
\hline $1 / 1 / 1210: 00$ & 33.337 & 35.442 & 1475.43 & 1473.68 \\
\hline $1 / 1 / 1210: 30$ & 33.675 & 35.446 & 1475.09 & 1473.68 \\
\hline $1 / 1 / 1211: 00$ & 33.302 & 35.46 & 1475.47 & 1473.66 \\
\hline 1/1/12 11:30 & 33.265 & 35.484 & 1475.50 & 1473.64 \\
\hline $1 / 1 / 1212: 00$ & 33.342 & 35.503 & 1475.43 & 1473.62 \\
\hline $1 / 1 / 1212: 30$ & 33.427 & 35.524 & 1475.34 & 1473.60 \\
\hline $1 / 1 / 1213: 00$ & 33.33 & 35.538 & 1475.44 & 1473.58 \\
\hline $1 / 1 / 1213: 30$ & 33.382 & 35.561 & 1475.39 & 1473.56 \\
\hline $1 / 1 / 1214: 00$ & 39.043 & 35.62 & 1469.73 & 1473.50 \\
\hline $1 / 1 / 1214: 30$ & 33.816 & 35.749 & 1474.95 & 1473.37 \\
\hline $1 / 1 / 1215: 00$ & 33.694 & 35.822 & 1475.08 & 1473.30 \\
\hline $1 / 1 / 1215: 30$ & 33.518 & 35.859 & 1475.25 & 1473.26 \\
\hline $1 / 1 / 1216: 00$ & 33.933 & 35.88 & 1474.84 & 1473.24 \\
\hline $1 / 1 / 1216: 30$ & 33.586 & 35.899 & 1475.18 & 1473.22 \\
\hline $1 / 1 / 1217: 00$ & 33.471 & 35.904 & 1475.30 & 1473.22 \\
\hline
\end{tabular}


TABLE S1.3 Automatically recorded groundwater levels in the Klassen wells, September 15, 2011, to March 13, 2012.

\begin{tabular}{|c|c|c|c|c|}
\hline \multicolumn{3}{|c|}{ Reference Elevation (ft AMSL) } & 1508.77 & \multirow{2}{*}{$\begin{array}{l}1509.12 \\
\text { Elevation in Klassen } \\
\text { Test Well (ft AMSL) }\end{array}$} \\
\hline Date and Time & $\begin{array}{l}\text { Depth in Klassen } \\
\text { Domestic Well } \\
(\mathrm{ft} \mathrm{BGL})\end{array}$ & $\begin{array}{c}\text { Depth in Klassen } \\
\text { Test Well } \\
\text { (ft BGL) }\end{array}$ & $\begin{array}{l}\text { Elevation in Klassen } \\
\text { Domestic Well } \\
\text { (ft AMSL) }\end{array}$ & \\
\hline $1 / 1 / 1217: 30$ & 33.401 & 35.902 & 1475.37 & 1473.22 \\
\hline $1 / 1 / 1218: 00$ & 33.696 & 35.906 & 1475.07 & 1473.22 \\
\hline $1 / 1 / 1218: 30$ & 33.417 & 35.904 & 1475.35 & 1473.22 \\
\hline $1 / 1 / 1219: 00$ & 33.443 & 35.901 & 1475.33 & 1473.22 \\
\hline $1 / 1 / 1219: 30$ & 33.527 & 35.899 & 1475.24 & 1473.22 \\
\hline $1 / 1 / 1220: 00$ & 33.647 & 35.897 & 1475.12 & 1473.23 \\
\hline $1 / 1 / 1220: 30$ & 33.769 & 35.902 & 1475.00 & 1473.22 \\
\hline $1 / 1 / 1221: 00$ & 33.525 & 35.892 & 1475.24 & 1473.23 \\
\hline $1 / 1 / 1221: 30$ & 33.509 & 35.883 & 1475.26 & 1473.24 \\
\hline $1 / 1 / 1222: 00$ & 33.419 & 35.876 & 1475.35 & 1473.25 \\
\hline $1 / 1 / 1222: 30$ & 38.178 & 35.869 & 1470.59 & 1473.25 \\
\hline $1 / 1 / 1223: 00$ & 33.992 & 35.866 & 1474.78 & 1473.26 \\
\hline $1 / 1 / 1223: 30$ & 33.792 & 35.864 & 1474.98 & 1473.26 \\
\hline $1 / 2 / 120: 00$ & 33.647 & 35.852 & 1475.12 & 1473.27 \\
\hline $1 / 2 / 120: 30$ & 33.546 & 35.838 & 1475.22 & 1473.28 \\
\hline 1/2/12 1:00 & 33.473 & 35.824 & 1475.30 & 1473.30 \\
\hline 1/2/12 1:30 & 33.419 & 35.815 & 1475.35 & 1473.31 \\
\hline $1 / 2 / 122: 00$ & 33.41 & 35.805 & 1475.36 & 1473.32 \\
\hline $1 / 2 / 122: 30$ & 33.452 & 35.801 & 1475.32 & 1473.32 \\
\hline 1/2/12 3:00 & 33.41 & 35.794 & 1475.36 & 1473.33 \\
\hline $1 / 2 / 123: 30$ & 33.38 & 35.782 & 1475.39 & 1473.34 \\
\hline $1 / 2 / 124: 00$ & 33.469 & 35.779 & 1475.30 & 1473.34 \\
\hline $1 / 2 / 124: 30$ & 33.396 & 35.768 & 1475.37 & 1473.35 \\
\hline $1 / 2 / 125: 00$ & 33.344 & 35.756 & 1475.43 & 1473.37 \\
\hline $1 / 2 / 125: 30$ & 33.912 & 35.749 & 1474.86 & 1473.37 \\
\hline $1 / 2 / 126: 00$ & 33.377 & 35.747 & 1475.39 & 1473.38 \\
\hline $1 / 2 / 126: 30$ & 33.541 & 35.747 & 1475.23 & 1473.38 \\
\hline 1/2/12 7:00 & 33.52 & 35.747 & 1475.25 & 1473.38 \\
\hline $1 / 2 / 12$ 7:30 & 33.532 & 35.747 & 1475.24 & 1473.38 \\
\hline $1 / 2 / 128: 00$ & 33.443 & 35.744 & 1475.33 & 1473.38 \\
\hline $1 / 2 / 128: 30$ & 33.483 & 35.749 & 1475.29 & 1473.37 \\
\hline 1/2/12 9:00 & 33.574 & 35.747 & 1475.20 & 1473.38 \\
\hline $1 / 2 / 129: 30$ & 33.785 & 35.744 & 1474.98 & 1473.38 \\
\hline 1/2/12 10:00 & 33.469 & 35.749 & 1475.30 & 1473.37 \\
\hline $1 / 2 / 1210: 30$ & 33.724 & 35.758 & 1475.05 & 1473.36 \\
\hline $1 / 2 / 1211: 00$ & 33.429 & 35.789 & 1475.34 & 1473.33 \\
\hline $1 / 2 / 1211: 30$ & 35.286 & 35.81 & 1473.48 & 1473.31 \\
\hline $1 / 2 / 1212: 00$ & 34.885 & 35.826 & 1473.88 & 1473.30 \\
\hline
\end{tabular}


TABLE S1.3 Automatically recorded groundwater levels in the Klassen wells, September 15, 2011, to March 13, 2012.

\begin{tabular}{|c|c|c|c|c|}
\hline \multicolumn{3}{|c|}{ Reference Elevation (ft AMSL) } & 1508.77 & \multirow{2}{*}{$\begin{array}{l}1509.12 \\
\text { Elevation in Klassen } \\
\text { Test Well (ft AMSL) }\end{array}$} \\
\hline Date and Time & $\begin{array}{l}\text { Depth in Klassen } \\
\text { Domestic Well } \\
(\mathrm{ft} \mathrm{BGL})\end{array}$ & $\begin{array}{c}\text { Depth in Klassen } \\
\text { Test Well } \\
\text { (ft BGL) }\end{array}$ & $\begin{array}{l}\text { Elevation in Klassen } \\
\text { Domestic Well } \\
\text { (ft AMSL) }\end{array}$ & \\
\hline $1 / 2 / 1212: 30$ & 33.438 & 35.84 & 1475.33 & 1473.28 \\
\hline $1 / 2 / 1213: 00$ & 33.757 & 35.848 & 1475.01 & 1473.27 \\
\hline $1 / 2 / 1213: 30$ & 33.448 & 35.852 & 1475.32 & 1473.27 \\
\hline $1 / 2 / 1214: 00$ & 33.373 & 35.859 & 1475.40 & 1473.26 \\
\hline $1 / 2 / 1214: 30$ & 33.347 & 35.843 & 1475.42 & 1473.28 \\
\hline $1 / 2 / 1215: 00$ & 33.434 & 35.81 & 1475.34 & 1473.31 \\
\hline $1 / 2 / 1215: 30$ & 33.344 & 35.794 & 1475.43 & 1473.33 \\
\hline $1 / 2 / 1216: 00$ & 33.366 & 35.784 & 1475.40 & 1473.34 \\
\hline $1 / 2 / 1216: 30$ & 33.309 & 35.777 & 1475.46 & 1473.35 \\
\hline $1 / 2 / 1217: 00$ & 33.269 & 35.768 & 1475.50 & 1473.35 \\
\hline $1 / 2 / 1217: 30$ & 33.368 & 35.761 & 1475.40 & 1473.36 \\
\hline $1 / 2 / 1218: 00$ & 33.298 & 35.756 & 1475.47 & 1473.37 \\
\hline $1 / 2 / 1218: 30$ & 33.471 & 35.751 & 1475.30 & 1473.37 \\
\hline $1 / 2 / 1219: 00$ & 33.373 & 35.747 & 1475.40 & 1473.38 \\
\hline $1 / 2 / 1219: 30$ & 33.631 & 35.756 & 1475.14 & 1473.37 \\
\hline $1 / 2 / 1220: 00$ & 33.518 & 35.754 & 1475.25 & 1473.37 \\
\hline $1 / 2 / 1220: 30$ & 33.506 & 35.751 & 1475.26 & 1473.37 \\
\hline $1 / 2 / 1221: 00$ & 33.52 & 35.751 & 1475.25 & 1473.37 \\
\hline $1 / 2 / 1221: 30$ & 33.537 & 35.747 & 1475.23 & 1473.38 \\
\hline $1 / 2 / 1222: 00$ & 33.748 & 35.747 & 1475.02 & 1473.38 \\
\hline $1 / 2 / 1222: 30$ & 37.381 & 35.747 & 1471.39 & 1473.38 \\
\hline $1 / 2 / 12$ 23:00 & 33.581 & 35.742 & 1475.19 & 1473.38 \\
\hline $1 / 2 / 12$ 23:30 & 33.457 & 35.73 & 1475.31 & 1473.39 \\
\hline $1 / 3 / 120: 00$ & 33.619 & 35.728 & 1475.15 & 1473.39 \\
\hline $1 / 3 / 120: 30$ & 33.457 & 35.721 & 1475.31 & 1473.40 \\
\hline 1/3/12 1:00 & 33.387 & 35.711 & 1475.38 & 1473.41 \\
\hline 1/3/12 1:30 & 33.335 & 35.704 & 1475.43 & 1473.42 \\
\hline $1 / 3 / 122: 00$ & 33.295 & 35.695 & 1475.47 & 1473.43 \\
\hline $1 / 3 / 122: 30$ & 33.26 & 35.686 & 1475.51 & 1473.44 \\
\hline 1/3/12 3:00 & 33.23 & 35.672 & 1475.54 & 1473.45 \\
\hline $1 / 3 / 123: 30$ & 33.204 & 35.664 & 1475.57 & 1473.46 \\
\hline $1 / 3 / 124: 00$ & 33.18 & 35.655 & 1475.59 & 1473.47 \\
\hline $1 / 3 / 124: 30$ & 33.159 & 35.646 & 1475.61 & 1473.48 \\
\hline $1 / 3 / 125: 00$ & 33.138 & 35.634 & 1475.63 & 1473.49 \\
\hline $1 / 3 / 125: 30$ & 33.119 & 35.627 & 1475.65 & 1473.50 \\
\hline $1 / 3 / 126: 00$ & 33.211 & 35.622 & 1475.56 & 1473.50 \\
\hline $1 / 3 / 126: 30$ & 33.356 & 35.632 & 1475.41 & 1473.49 \\
\hline $1 / 3 / 12$ 7:00 & 33.288 & 35.629 & 1475.48 & 1473.49 \\
\hline
\end{tabular}


TABLE S1.3 Automatically recorded groundwater levels in the Klassen wells, September 15, 2011, to March 13, 2012.

\begin{tabular}{|c|c|c|c|c|}
\hline \multicolumn{3}{|c|}{ Reference Elevation (ft AMSL) } & 1508.77 & \multirow{2}{*}{$\begin{array}{l}1509.12 \\
\text { Elevation in Klassen } \\
\text { Test Well (ft AMSL) }\end{array}$} \\
\hline Date and Time & $\begin{array}{l}\text { Depth in Klassen } \\
\text { Domestic Well } \\
(\mathrm{ft} \mathrm{BGL})\end{array}$ & $\begin{array}{c}\text { Depth in Klassen } \\
\text { Test Well } \\
\text { (ft BGL) }\end{array}$ & $\begin{array}{l}\text { Elevation in Klassen } \\
\text { Domestic Well } \\
\text { (ft AMSL) }\end{array}$ & \\
\hline $1 / 3 / 127: 30$ & 33.33 & 35.629 & 1475.44 & 1473.49 \\
\hline $1 / 3 / 128: 00$ & 33.703 & 35.632 & 1475.07 & 1473.49 \\
\hline $1 / 3 / 128: 30$ & 33.427 & 35.634 & 1475.34 & 1473.49 \\
\hline $1 / 3 / 129: 00$ & 33.781 & 35.632 & 1474.99 & 1473.49 \\
\hline $1 / 3 / 129: 30$ & 33.412 & 35.632 & 1475.36 & 1473.49 \\
\hline 1/3/12 10:00 & 33.319 & 35.648 & 1475.45 & 1473.47 \\
\hline 1/3/12 10:30 & 33.262 & 35.669 & 1475.51 & 1473.45 \\
\hline $1 / 3 / 1211: 00$ & 33.218 & 35.688 & 1475.55 & 1473.43 \\
\hline 1/3/12 11:30 & 33.183 & 35.704 & 1475.59 & 1473.42 \\
\hline $1 / 3 / 1212: 00$ & 33.154 & 35.718 & 1475.62 & 1473.40 \\
\hline $1 / 3 / 1212: 30$ & 33.126 & 35.735 & 1475.64 & 1473.39 \\
\hline $1 / 3 / 1213: 00$ & 33.105 & 35.648 & 1475.66 & 1473.47 \\
\hline 1/3/12 13:30 & 33.084 & 35.575 & 1475.69 & 1473.55 \\
\hline $1 / 3 / 1214: 00$ & 33.063 & 35.54 & 1475.71 & 1473.58 \\
\hline 1/3/12 14:30 & 33.044 & 35.524 & 1475.73 & 1473.60 \\
\hline $1 / 3 / 12$ 15:00 & 33.028 & 35.517 & 1475.74 & 1473.61 \\
\hline $1 / 3 / 12$ 15:30 & 33.267 & 35.51 & 1475.50 & 1473.61 \\
\hline 1/3/12 16:00 & 33.122 & 35.512 & 1475.65 & 1473.61 \\
\hline $1 / 3 / 1216: 30$ & 33.075 & 35.505 & 1475.69 & 1473.62 \\
\hline 1/3/12 17:00 & 34.212 & 35.512 & 1474.56 & 1473.61 \\
\hline $1 / 3 / 12$ 17:30 & 33.483 & 35.526 & 1475.29 & 1473.60 \\
\hline $1 / 3 / 1218: 00$ & 34.06 & 35.538 & 1474.71 & 1473.58 \\
\hline 1/3/12 18:30 & 33.492 & 35.552 & 1475.28 & 1473.57 \\
\hline 1/3/12 19:00 & 33.375 & 35.552 & 1475.39 & 1473.57 \\
\hline 1/3/12 19:30 & 33.434 & 35.559 & 1475.34 & 1473.56 \\
\hline $1 / 3 / 12$ 20:00 & 33.48 & 35.559 & 1475.29 & 1473.56 \\
\hline $1 / 3 / 12$ 20:30 & 33.509 & 35.564 & 1475.26 & 1473.56 \\
\hline $1 / 3 / 12$ 21:00 & 33.713 & 35.58 & 1475.06 & 1473.54 \\
\hline $1 / 3 / 12$ 21:30 & 33.713 & 35.592 & 1475.06 & 1473.53 \\
\hline $1 / 3 / 12$ 22:00 & 33.72 & 35.606 & 1475.05 & 1473.52 \\
\hline $1 / 3 / 1222: 30$ & 33.687 & 35.608 & 1475.08 & 1473.51 \\
\hline $1 / 3 / 12$ 23:00 & 33.753 & 35.613 & 1475.02 & 1473.51 \\
\hline $1 / 3 / 12$ 23:30 & 33.844 & 35.615 & 1474.93 & 1473.51 \\
\hline 1/4/12 0:00 & 33.633 & 35.61 & 1475.14 & 1473.51 \\
\hline $1 / 4 / 12$ 0:30 & 33.532 & 35.606 & 1475.24 & 1473.52 \\
\hline 1/4/12 1:00 & 33.459 & 35.596 & 1475.31 & 1473.53 \\
\hline 1/4/12 1:30 & 33.403 & 35.589 & 1475.37 & 1473.53 \\
\hline $1 / 4 / 122: 00$ & 33.391 & 35.587 & 1475.38 & 1473.54 \\
\hline
\end{tabular}


TABLE S1.3 Automatically recorded groundwater levels in the Klassen wells, September 15, 2011, to March 13, 2012.

\begin{tabular}{|c|c|c|c|c|}
\hline \multicolumn{3}{|c|}{ Reference Elevation (ft AMSL) } & 1508.77 & \multirow{2}{*}{$\begin{array}{l}1509.12 \\
\text { Elevation in Klassen } \\
\text { Test Well (ft AMSL) }\end{array}$} \\
\hline Date and Time & $\begin{array}{l}\text { Depth in Klassen } \\
\text { Domestic Well } \\
(\mathrm{ft} \mathrm{BGL})\end{array}$ & $\begin{array}{c}\text { Depth in Klassen } \\
\text { Test Well } \\
\text { (ft BGL) }\end{array}$ & $\begin{array}{l}\text { Elevation in Klassen } \\
\text { Domestic Well } \\
\text { (ft AMSL) }\end{array}$ & \\
\hline $1 / 4 / 122: 30$ & 33.431 & 35.587 & 1475.34 & 1473.54 \\
\hline $1 / 4 / 123: 00$ & 33.387 & 35.587 & 1475.38 & 1473.54 \\
\hline $1 / 4 / 123: 30$ & 33.354 & 35.58 & 1475.42 & 1473.54 \\
\hline $1 / 4 / 124: 00$ & 33.441 & 35.585 & 1475.33 & 1473.54 \\
\hline $1 / 4 / 124: 30$ & 33.365 & 35.58 & 1475.40 & 1473.54 \\
\hline $1 / 4 / 125: 00$ & 33.314 & 35.573 & 1475.46 & 1473.55 \\
\hline $1 / 4 / 125: 30$ & 33.387 & 35.571 & 1475.38 & 1473.55 \\
\hline $1 / 4 / 126: 00$ & 33.333 & 35.568 & 1475.44 & 1473.55 \\
\hline $1 / 4 / 126: 30$ & 33.417 & 35.561 & 1475.35 & 1473.56 \\
\hline 1/4/12 7:00 & 33.375 & 35.552 & 1475.39 & 1473.57 \\
\hline $1 / 4 / 127: 30$ & 33.429 & 35.547 & 1475.34 & 1473.58 \\
\hline $1 / 4 / 128: 00$ & 33.356 & 35.54 & 1475.41 & 1473.58 \\
\hline $1 / 4 / 128: 30$ & 33.377 & 35.54 & 1475.39 & 1473.58 \\
\hline 1/4/12 9:00 & 33.319 & 35.533 & 1475.45 & 1473.59 \\
\hline 1/4/12 9:30 & 33.288 & 35.528 & 1475.48 & 1473.59 \\
\hline 1/4/12 10:00 & 33.248 & 35.531 & 1475.52 & 1473.59 \\
\hline 1/4/12 10:30 & 33.342 & 35.535 & 1475.43 & 1473.59 \\
\hline 1/4/12 11:00 & 33.412 & 35.55 & 1475.36 & 1473.57 \\
\hline $1 / 4 / 1211: 30$ & 33.985 & 35.561 & 1474.78 & 1473.56 \\
\hline 1/4/12 12:00 & 33.337 & 35.568 & 1475.43 & 1473.55 \\
\hline $1 / 4 / 12$ 12:30 & 33.274 & 35.575 & 1475.50 & 1473.55 \\
\hline $1 / 4 / 1213: 00$ & 33.509 & 35.568 & 1475.26 & 1473.55 \\
\hline 1/4/12 13:30 & 33.295 & 35.564 & 1475.47 & 1473.56 \\
\hline 1/4/12 14:00 & 33.246 & 35.559 & 1475.52 & 1473.56 \\
\hline 1/4/12 14:30 & 33.204 & 35.554 & 1475.57 & 1473.57 \\
\hline 1/4/12 15:00 & 33.262 & 35.552 & 1475.51 & 1473.57 \\
\hline 1/4/12 15:30 & 33.199 & 35.543 & 1475.57 & 1473.58 \\
\hline 1/4/12 16:00 & 33.919 & 35.538 & 1474.85 & 1473.58 \\
\hline 1/4/12 16:30 & 33.201 & 35.533 & 1475.57 & 1473.59 \\
\hline 1/4/12 17:00 & 33.157 & 35.528 & 1475.61 & 1473.59 \\
\hline 1/4/12 17:30 & 33.126 & 35.524 & 1475.64 & 1473.60 \\
\hline 1/4/12 18:00 & 33.164 & 35.524 & 1475.61 & 1473.60 \\
\hline 1/4/12 18:30 & 33.126 & 35.521 & 1475.64 & 1473.60 \\
\hline 1/4/12 19:00 & 33.206 & 35.521 & 1475.56 & 1473.60 \\
\hline 1/4/12 19:30 & 33.283 & 35.526 & 1475.49 & 1473.60 \\
\hline $1 / 4 / 12$ 20:00 & 33.166 & 35.528 & 1475.60 & 1473.59 \\
\hline 1/4/12 20:30 & 33.124 & 35.533 & 1475.65 & 1473.59 \\
\hline $1 / 4 / 12$ 21:00 & 33.098 & 35.538 & 1475.67 & 1473.58 \\
\hline
\end{tabular}


TABLE S1.3 Automatically recorded groundwater levels in the Klassen wells, September 15, 2011, to March 13, 2012.

\begin{tabular}{|c|c|c|c|c|}
\hline \multicolumn{3}{|c|}{ Reference Elevation (ft AMSL) } & 1508.77 & \multirow{2}{*}{$\begin{array}{l}1509.12 \\
\text { Elevation in Klassen } \\
\text { Test Well (ft AMSL) }\end{array}$} \\
\hline Date and Time & $\begin{array}{l}\text { Depth in Klassen } \\
\text { Domestic Well } \\
(\mathrm{ft} \mathrm{BGL})\end{array}$ & $\begin{array}{c}\text { Depth in Klassen } \\
\text { Test Well } \\
\text { (ft BGL) }\end{array}$ & $\begin{array}{l}\text { Elevation in Klassen } \\
\text { Domestic Well } \\
\text { (ft AMSL) }\end{array}$ & \\
\hline $1 / 4 / 1221: 30$ & 33.075 & 35.54 & 1475.69 & 1473.58 \\
\hline $1 / 4 / 1222: 00$ & 33.054 & 35.545 & 1475.72 & 1473.58 \\
\hline $1 / 4 / 1222: 30$ & 33.152 & 35.55 & 1475.62 & 1473.57 \\
\hline $1 / 4 / 12$ 23:00 & 33.281 & 35.559 & 1475.49 & 1473.56 \\
\hline $1 / 4 / 1223: 30$ & 33.394 & 35.568 & 1475.38 & 1473.55 \\
\hline $1 / 5 / 120: 00$ & 34.435 & 35.571 & 1474.33 & 1473.55 \\
\hline $1 / 5 / 120: 30$ & 33.471 & 35.58 & 1475.30 & 1473.54 \\
\hline 1/5/12 1:00 & 33.321 & 35.58 & 1475.45 & 1473.54 \\
\hline $1 / 5 / 121: 30$ & 33.246 & 35.58 & 1475.52 & 1473.54 \\
\hline $1 / 5 / 122: 00$ & 33.22 & 35.573 & 1475.55 & 1473.55 \\
\hline $1 / 5 / 122: 30$ & 33.173 & 35.568 & 1475.60 & 1473.55 \\
\hline 1/5/12 3:00 & 33.138 & 35.568 & 1475.63 & 1473.55 \\
\hline $1 / 5 / 123: 30$ & 33.108 & 35.568 & 1475.66 & 1473.55 \\
\hline $1 / 5 / 124: 00$ & 33.082 & 35.566 & 1475.69 & 1473.56 \\
\hline $1 / 5 / 124: 30$ & 33.061 & 35.559 & 1475.71 & 1473.56 \\
\hline $1 / 5 / 12$ 5:00 & 33.04 & 35.557 & 1475.73 & 1473.57 \\
\hline $1 / 5 / 125: 30$ & 33.023 & 35.552 & 1475.75 & 1473.57 \\
\hline 1/5/12 6:00 & 33.084 & 35.549 & 1475.69 & 1473.57 \\
\hline $1 / 5 / 126: 30$ & 33.265 & 35.554 & 1475.50 & 1473.57 \\
\hline $1 / 5 / 127: 00$ & 33.154 & 35.557 & 1475.62 & 1473.57 \\
\hline $1 / 5 / 127: 30$ & 33.204 & 35.554 & 1475.57 & 1473.57 \\
\hline 1/5/12 8:00 & 33.138 & 35.552 & 1475.63 & 1473.57 \\
\hline $1 / 5 / 128: 30$ & 33.194 & 35.561 & 1475.58 & 1473.56 \\
\hline 1/5/12 9:00 & 33.436 & 35.564 & 1475.33 & 1473.56 \\
\hline 1/5/12 9:30 & 33.162 & 35.568 & 1475.61 & 1473.55 \\
\hline 1/5/12 10:00 & 33.133 & 35.578 & 1475.64 & 1473.54 \\
\hline 1/5/12 10:30 & 33.091 & 35.587 & 1475.68 & 1473.54 \\
\hline 1/5/12 11:00 & 33.061 & 35.599 & 1475.71 & 1473.52 \\
\hline 1/5/12 11:30 & 34.43 & 35.615 & 1474.34 & 1473.51 \\
\hline 1/5/12 12:00 & 33.19 & 35.636 & 1475.58 & 1473.49 \\
\hline 1/5/12 12:30 & 33.147 & 35.533 & 1475.62 & 1473.59 \\
\hline 1/5/12 13:00 & 33.361 & 35.493 & 1475.41 & 1473.63 \\
\hline 1/5/12 13:30 & 33.133 & 35.484 & 1475.64 & 1473.64 \\
\hline 1/5/12 14:00 & 33.082 & 35.474 & 1475.69 & 1473.65 \\
\hline 1/5/12 14:30 & 33.133 & 35.47 & 1475.64 & 1473.65 \\
\hline $1 / 5 / 1215: 00$ & 33.079 & 35.463 & 1475.69 & 1473.66 \\
\hline $1 / 5 / 1215: 30$ & 33.042 & 35.456 & 1475.73 & 1473.67 \\
\hline $1 / 5 / 1216: 00$ & 33.042 & 35.451 & 1475.73 & 1473.67 \\
\hline
\end{tabular}


TABLE S1.3 Automatically recorded groundwater levels in the Klassen wells, September 15, 2011, to March 13, 2012.

\begin{tabular}{|c|c|c|c|c|}
\hline \multicolumn{3}{|c|}{ Reference Elevation (ft AMSL) } & 1508.77 & \multirow{2}{*}{$\begin{array}{l}1509.12 \\
\text { Elevation in Klassen } \\
\text { Test Well (ft AMSL) }\end{array}$} \\
\hline Date and Time & $\begin{array}{l}\text { Depth in Klassen } \\
\text { Domestic Well } \\
\text { (ft BGL) }\end{array}$ & $\begin{array}{c}\text { Depth in Klassen } \\
\text { Test Well } \\
\text { (ft BGL) }\end{array}$ & $\begin{array}{l}\text { Elevation in Klassen } \\
\text { Domestic Well } \\
\text { (ft AMSL) }\end{array}$ & \\
\hline $1 / 5 / 1216: 30$ & 33.307 & 35.446 & 1475.46 & 1473.68 \\
\hline 1/5/12 17:00 & 33.07 & 35.442 & 1475.70 & 1473.68 \\
\hline $1 / 5 / 1217: 30$ & 42.843 & 35.442 & 1465.93 & 1473.68 \\
\hline $1 / 5 / 1218: 00$ & 33.415 & 35.458 & 1475.35 & 1473.66 \\
\hline $1 / 5 / 1218: 30$ & 33.312 & 35.46 & 1475.46 & 1473.66 \\
\hline $1 / 5 / 1219: 00$ & 33.267 & 35.458 & 1475.50 & 1473.66 \\
\hline $1 / 5 / 1219: 30$ & 33.443 & 35.47 & 1475.33 & 1473.65 \\
\hline $1 / 5 / 1220: 00$ & 33.445 & 35.479 & 1475.32 & 1473.64 \\
\hline $1 / 5 / 1220: 30$ & 33.419 & 35.489 & 1475.35 & 1473.63 \\
\hline $1 / 5 / 1221: 00$ & 33.483 & 35.5 & 1475.29 & 1473.62 \\
\hline $1 / 5 / 1221: 30$ & 33.375 & 35.503 & 1475.39 & 1473.62 \\
\hline $1 / 5 / 1222: 00$ & 33.558 & 35.507 & 1475.21 & 1473.62 \\
\hline $1 / 5 / 1222: 30$ & 36.056 & 35.514 & 1472.71 & 1473.61 \\
\hline $1 / 5 / 1223: 00$ & 39.074 & 35.521 & 1469.70 & 1473.60 \\
\hline $1 / 5 / 1223: 30$ & 33.656 & 35.526 & 1475.11 & 1473.60 \\
\hline 1/6/12 0:00 & 33.495 & 35.519 & 1475.27 & 1473.60 \\
\hline 1/6/12 0:30 & 33.403 & 35.51 & 1475.37 & 1473.61 \\
\hline 1/6/12 1:00 & 33.335 & 35.5 & 1475.43 & 1473.62 \\
\hline 1/6/12 1:30 & 33.283 & 35.491 & 1475.49 & 1473.63 \\
\hline $1 / 6 / 122: 00$ & 33.288 & 35.482 & 1475.48 & 1473.64 \\
\hline $1 / 6 / 122: 30$ & 33.321 & 35.479 & 1475.45 & 1473.64 \\
\hline 1/6/12 3:00 & 33.276 & 35.474 & 1475.49 & 1473.65 \\
\hline 1/6/12 3:30 & 33.251 & 35.47 & 1475.52 & 1473.65 \\
\hline 1/6/12 4:00 & 33.335 & 35.467 & 1475.43 & 1473.66 \\
\hline $1 / 6 / 124: 30$ & 33.26 & 35.46 & 1475.51 & 1473.66 \\
\hline 1/6/12 5:00 & 33.208 & 35.453 & 1475.56 & 1473.67 \\
\hline 1/6/12 5:30 & 33.169 & 35.446 & 1475.60 & 1473.68 \\
\hline 1/6/12 6:00 & 33.159 & 35.439 & 1475.61 & 1473.68 \\
\hline $1 / 6 / 126: 30$ & 33.37 & 35.437 & 1475.40 & 1473.69 \\
\hline 1/6/12 7:00 & 33.279 & 35.432 & 1475.49 & 1473.69 \\
\hline 1/6/12 7:30 & 33.22 & 35.437 & 1475.55 & 1473.69 \\
\hline 1/6/12 8:00 & 33.197 & 35.437 & 1475.57 & 1473.69 \\
\hline 1/6/12 8:30 & 33.152 & 35.43 & 1475.62 & 1473.69 \\
\hline 1/6/12 9:00 & 34.294 & 35.42 & 1474.48 & 1473.70 \\
\hline 1/6/12 9:30 & 33.375 & 35.418 & 1475.39 & 1473.70 \\
\hline 1/6/12 10:00 & 33.253 & 35.425 & 1475.52 & 1473.70 \\
\hline $1 / 6 / 12$ 10:30 & 33.363 & 35.428 & 1475.41 & 1473.69 \\
\hline $1 / 6 / 1211: 00$ & 33.323 & 35.432 & 1475.45 & 1473.69 \\
\hline
\end{tabular}


TABLE S1.3 Automatically recorded groundwater levels in the Klassen wells, September 15, 2011, to March 13, 2012.

\begin{tabular}{|c|c|c|c|c|}
\hline \multicolumn{3}{|c|}{ Reference Elevation (ft AMSL) } & 1508.77 & \multirow{2}{*}{$\begin{array}{l}1509.12 \\
\text { Elevation in Klassen } \\
\text { Test Well (ft AMSL) }\end{array}$} \\
\hline Date and Time & $\begin{array}{l}\text { Depth in Klassen } \\
\text { Domestic Well } \\
(\mathrm{ft} \mathrm{BGL})\end{array}$ & $\begin{array}{c}\text { Depth in Klassen } \\
\text { Test Well } \\
\text { (ft BGL) }\end{array}$ & $\begin{array}{l}\text { Elevation in Klassen } \\
\text { Domestic Well } \\
\text { (ft AMSL) }\end{array}$ & \\
\hline $1 / 6 / 1211: 30$ & 33.373 & 35.442 & 1475.40 & 1473.68 \\
\hline $1 / 6 / 1212: 00$ & 33.208 & 35.446 & 1475.56 & 1473.68 \\
\hline $1 / 6 / 1212: 30$ & 33.152 & 35.442 & 1475.62 & 1473.68 \\
\hline 1/6/12 13:00 & 33.215 & 35.437 & 1475.55 & 1473.69 \\
\hline $1 / 6 / 1213: 30$ & 33.147 & 35.43 & 1475.62 & 1473.69 \\
\hline $1 / 6 / 1214: 00$ & 33.213 & 35.425 & 1475.56 & 1473.70 \\
\hline 1/6/12 14:30 & 36.527 & 35.425 & 1472.24 & 1473.70 \\
\hline $1 / 6 / 1215: 00$ & 33.457 & 35.437 & 1475.31 & 1473.69 \\
\hline 1/6/12 15:30 & 33.429 & 35.444 & 1475.34 & 1473.68 \\
\hline 1/6/12 16:00 & 33.335 & 35.446 & 1475.43 & 1473.68 \\
\hline 1/6/12 16:30 & 33.262 & 35.449 & 1475.51 & 1473.67 \\
\hline $1 / 6 / 1217: 00$ & 33.359 & 35.451 & 1475.41 & 1473.67 \\
\hline $1 / 6 / 1217: 30$ & 33.241 & 35.453 & 1475.53 & 1473.67 \\
\hline $1 / 6 / 1218: 00$ & 33.19 & 35.453 & 1475.58 & 1473.67 \\
\hline $1 / 6 / 1218: 30$ & 33.18 & 35.453 & 1475.59 & 1473.67 \\
\hline $1 / 6 / 12$ 19:00 & 33.143 & 35.453 & 1475.63 & 1473.67 \\
\hline $1 / 6 / 1219: 30$ & 33.138 & 35.453 & 1475.63 & 1473.67 \\
\hline $1 / 6 / 12$ 20:00 & 33.11 & 35.451 & 1475.66 & 1473.67 \\
\hline $1 / 6 / 1220: 30$ & 33.084 & 35.451 & 1475.69 & 1473.67 \\
\hline $1 / 6 / 1221: 00$ & 33.065 & 35.449 & 1475.70 & 1473.67 \\
\hline $1 / 6 / 1221: 30$ & 33.206 & 35.446 & 1475.56 & 1473.68 \\
\hline $1 / 6 / 1222: 00$ & 33.14 & 35.444 & 1475.63 & 1473.68 \\
\hline $1 / 6 / 1222: 30$ & 33.408 & 35.451 & 1475.36 & 1473.67 \\
\hline $1 / 6 / 1223: 00$ & 33.23 & 35.456 & 1475.54 & 1473.67 \\
\hline $1 / 6 / 1223: 30$ & 33.926 & 35.453 & 1474.84 & 1473.67 \\
\hline 1/7/12 0:00 & 33.368 & 35.46 & 1475.40 & 1473.66 \\
\hline 1/7/12 0:30 & 33.262 & 35.458 & 1475.51 & 1473.66 \\
\hline 1/7/12 1:00 & 33.204 & 35.453 & 1475.57 & 1473.67 \\
\hline 1/7/12 1:30 & 33.162 & 35.453 & 1475.61 & 1473.67 \\
\hline $1 / 7 / 122: 00$ & 33.129 & 35.451 & 1475.64 & 1473.67 \\
\hline $1 / 7 / 12$ 2:30 & 33.103 & 35.449 & 1475.67 & 1473.67 \\
\hline 1/7/12 3:00 & 33.079 & 35.444 & 1475.69 & 1473.68 \\
\hline 1/7/12 3:30 & 33.058 & 35.432 & 1475.71 & 1473.69 \\
\hline 1/7/12 4:00 & 33.068 & 35.423 & 1475.70 & 1473.70 \\
\hline 1/7/12 4:30 & 33.042 & 35.413 & 1475.73 & 1473.71 \\
\hline $1 / 7 / 12$ 5:00 & 33.023 & 35.404 & 1475.75 & 1473.72 \\
\hline $1 / 7 / 125: 30$ & 33.007 & 35.392 & 1475.76 & 1473.73 \\
\hline $1 / 7 / 12$ 6:00 & 33.152 & 35.383 & 1475.62 & 1473.74 \\
\hline
\end{tabular}


TABLE S1.3 Automatically recorded groundwater levels in the Klassen wells, September 15, 2011, to March 13, 2012.

\begin{tabular}{|c|c|c|c|c|}
\hline \multicolumn{3}{|c|}{ Reference Elevation (ft AMSL) } & 1508.77 & \multirow{2}{*}{$\begin{array}{l}1509.12 \\
\text { Elevation in Klassen } \\
\text { Test Well (ft AMSL) }\end{array}$} \\
\hline Date and Time & $\begin{array}{l}\text { Depth in Klassen } \\
\text { Domestic Well } \\
(\mathrm{ft} \mathrm{BGL})\end{array}$ & $\begin{array}{c}\text { Depth in Klassen } \\
\text { Test Well } \\
\text { (ft BGL) }\end{array}$ & $\begin{array}{l}\text { Elevation in Klassen } \\
\text { Domestic Well } \\
\text { (ft AMSL) }\end{array}$ & \\
\hline $1 / 7 / 126: 30$ & 33.046 & 35.376 & 1475.72 & 1473.75 \\
\hline $1 / 7 / 127: 00$ & 33.016 & 35.371 & 1475.75 & 1473.75 \\
\hline 1/7/12 7:30 & 32.997 & 35.362 & 1475.77 & 1473.76 \\
\hline $1 / 7 / 128: 00$ & 32.981 & 35.357 & 1475.79 & 1473.77 \\
\hline $1 / 7 / 128: 30$ & 33.154 & 35.362 & 1475.62 & 1473.76 \\
\hline $1 / 7 / 129: 00$ & 33.272 & 35.378 & 1475.50 & 1473.74 \\
\hline 1/7/12 9:30 & 33.424 & 35.397 & 1475.35 & 1473.73 \\
\hline 1/7/12 10:00 & 33.546 & 35.416 & 1475.22 & 1473.71 \\
\hline 1/7/12 10:30 & 33.335 & 35.423 & 1475.43 & 1473.70 \\
\hline 1/7/12 11:00 & 33.464 & 35.437 & 1475.31 & 1473.69 \\
\hline 1/7/12 11:30 & 33.448 & 35.458 & 1475.32 & 1473.66 \\
\hline $1 / 7 / 1212: 00$ & 33.525 & 35.472 & 1475.24 & 1473.65 \\
\hline 1/7/12 12:30 & 33.382 & 35.481 & 1475.39 & 1473.64 \\
\hline 1/7/12 13:00 & 33.359 & 35.489 & 1475.41 & 1473.63 \\
\hline 1/7/12 13:30 & 33.504 & 35.498 & 1475.27 & 1473.62 \\
\hline 1/7/12 14:00 & 33.457 & 35.503 & 1475.31 & 1473.62 \\
\hline 1/7/12 14:30 & 33.663 & 35.51 & 1475.11 & 1473.61 \\
\hline 1/7/12 15:00 & 33.687 & 35.517 & 1475.08 & 1473.61 \\
\hline $1 / 7 / 1215: 30$ & 42.953 & 35.517 & 1465.82 & 1473.61 \\
\hline 1/7/12 16:00 & 33.853 & 35.531 & 1474.92 & 1473.59 \\
\hline 1/7/12 16:30 & 33.635 & 35.533 & 1475.13 & 1473.59 \\
\hline $1 / 7 / 1217: 00$ & 33.525 & 35.533 & 1475.24 & 1473.59 \\
\hline 1/7/12 17:30 & 33.445 & 35.533 & 1475.32 & 1473.59 \\
\hline 1/7/12 18:00 & 33.389 & 35.538 & 1475.38 & 1473.58 \\
\hline 1/7/12 18:30 & 33.34 & 35.535 & 1475.43 & 1473.59 \\
\hline 1/7/12 19:00 & 33.302 & 35.533 & 1475.47 & 1473.59 \\
\hline 1/7/12 19:30 & 33.29 & 35.526 & 1475.48 & 1473.60 \\
\hline 1/7/12 20:00 & 33.265 & 35.526 & 1475.50 & 1473.60 \\
\hline 1/7/12 20:30 & 33.398 & 35.531 & 1475.37 & 1473.59 \\
\hline 1/7/12 21:00 & 33.37 & 35.533 & 1475.40 & 1473.59 \\
\hline 1/7/12 21:30 & 33.342 & 35.533 & 1475.43 & 1473.59 \\
\hline 1/7/12 22:00 & 33.48 & 35.538 & 1475.29 & 1473.58 \\
\hline $1 / 7 / 12$ 22:30 & 33.509 & 35.538 & 1475.26 & 1473.58 \\
\hline 1/7/12 23:00 & 33.736 & 35.543 & 1475.03 & 1473.58 \\
\hline $1 / 7 / 1223: 30$ & 33.638 & 35.545 & 1475.13 & 1473.58 \\
\hline 1/8/12 0:00 & 33.527 & 35.543 & 1475.24 & 1473.58 \\
\hline $1 / 8 / 120: 30$ & 33.492 & 35.535 & 1475.28 & 1473.59 \\
\hline $1 / 8 / 121: 00$ & 33.436 & 35.535 & 1475.33 & 1473.59 \\
\hline
\end{tabular}


TABLE S1.3 Automatically recorded groundwater levels in the Klassen wells, September 15, 2011, to March 13, 2012.

\begin{tabular}{|c|c|c|c|c|}
\hline \multicolumn{3}{|c|}{ Reference Elevation (ft AMSL) } & 1508.77 & \multirow{2}{*}{$\begin{array}{l}1509.12 \\
\text { Elevation in Klassen } \\
\text { Test Well (ft AMSL) }\end{array}$} \\
\hline Date and Time & $\begin{array}{l}\text { Depth in Klassen } \\
\text { Domestic Well } \\
(\mathrm{ft} \mathrm{BGL})\end{array}$ & $\begin{array}{c}\text { Depth in Klassen } \\
\text { Test Well } \\
\text { (ft BGL) }\end{array}$ & $\begin{array}{l}\text { Elevation in Klassen } \\
\text { Domestic Well } \\
\text { (ft AMSL) }\end{array}$ & \\
\hline $1 / 8 / 121: 30$ & 33.403 & 35.528 & 1475.37 & 1473.59 \\
\hline $1 / 8 / 122: 00$ & 33.415 & 35.521 & 1475.35 & 1473.60 \\
\hline $1 / 8 / 122: 30$ & 33.471 & 35.526 & 1475.30 & 1473.60 \\
\hline $1 / 8 / 123: 00$ & 33.419 & 35.521 & 1475.35 & 1473.60 \\
\hline $1 / 8 / 123: 30$ & 33.506 & 35.519 & 1475.26 & 1473.60 \\
\hline $1 / 8 / 124: 00$ & 33.53 & 35.519 & 1475.24 & 1473.60 \\
\hline $1 / 8 / 124: 30$ & 33.436 & 35.514 & 1475.33 & 1473.61 \\
\hline $1 / 8 / 125: 00$ & 33.398 & 35.512 & 1475.37 & 1473.61 \\
\hline $1 / 8 / 125: 30$ & 33.342 & 35.5 & 1475.43 & 1473.62 \\
\hline $1 / 8 / 126: 00$ & 33.713 & 35.5 & 1475.06 & 1473.62 \\
\hline $1 / 8 / 126: 30$ & 33.544 & 35.503 & 1475.23 & 1473.62 \\
\hline $1 / 8 / 127: 00$ & 33.424 & 35.498 & 1475.35 & 1473.62 \\
\hline $1 / 8 / 127: 30$ & 33.384 & 35.491 & 1475.39 & 1473.63 \\
\hline $1 / 8 / 128: 00$ & 40.021 & 35.479 & 1468.75 & 1473.64 \\
\hline $1 / 8 / 128: 30$ & 34.529 & 35.479 & 1474.24 & 1473.64 \\
\hline 1/8/12 9:00 & 33.813 & 35.484 & 1474.96 & 1473.64 \\
\hline 1/8/12 9:30 & 33.781 & 35.498 & 1474.99 & 1473.62 \\
\hline 1/8/12 10:00 & 33.626 & 35.51 & 1475.14 & 1473.61 \\
\hline $1 / 8 / 1210: 30$ & 33.577 & 35.528 & 1475.19 & 1473.59 \\
\hline 1/8/12 11:00 & 33.492 & 35.538 & 1475.28 & 1473.58 \\
\hline $1 / 8 / 1211: 30$ & 33.45 & 35.55 & 1475.32 & 1473.57 \\
\hline $1 / 8 / 1212: 00$ & 33.422 & 35.564 & 1475.35 & 1473.56 \\
\hline 1/8/12 12:30 & 33.368 & 35.578 & 1475.40 & 1473.54 \\
\hline 1/8/12 13:00 & 33.429 & 35.596 & 1475.34 & 1473.53 \\
\hline 1/8/12 13:30 & 33.57 & 35.61 & 1475.20 & 1473.51 \\
\hline 1/8/12 14:00 & 33.647 & 35.62 & 1475.12 & 1473.50 \\
\hline 1/8/12 14:30 & 33.684 & 35.627 & 1475.09 & 1473.50 \\
\hline 1/8/12 15:00 & 33.642 & 35.634 & 1475.13 & 1473.49 \\
\hline 1/8/12 15:30 & 33.734 & 35.664 & 1475.04 & 1473.46 \\
\hline 1/8/12 16:00 & 33.645 & 35.737 & 1475.12 & 1473.39 \\
\hline 1/8/12 16:30 & 33.562 & 35.77 & 1475.21 & 1473.35 \\
\hline 1/8/12 17:00 & 33.722 & 35.782 & 1475.05 & 1473.34 \\
\hline 1/8/12 17:30 & 33.516 & 35.784 & 1475.25 & 1473.34 \\
\hline 1/8/12 18:00 & 33.495 & 35.779 & 1475.27 & 1473.34 \\
\hline $1 / 8 / 1218: 30$ & 33.572 & 35.784 & 1475.20 & 1473.34 \\
\hline $1 / 8 / 12$ 19:00 & 33.642 & 35.791 & 1475.13 & 1473.33 \\
\hline 1/8/12 19:30 & 33.699 & 35.791 & 1475.07 & 1473.33 \\
\hline $1 / 8 / 12$ 20:00 & 36.095 & 35.794 & 1472.67 & 1473.33 \\
\hline
\end{tabular}


TABLE S1.3 Automatically recorded groundwater levels in the Klassen wells, September 15, 2011, to March 13, 2012.

\begin{tabular}{|c|c|c|c|c|}
\hline \multicolumn{3}{|c|}{ Reference Elevation (ft AMSL) } & 1508.77 & \multirow{2}{*}{$\begin{array}{l}1509.12 \\
\text { Elevation in Klassen } \\
\text { Test Well (ft AMSL) }\end{array}$} \\
\hline Date and Time & $\begin{array}{l}\text { Depth in Klassen } \\
\text { Domestic Well } \\
(\mathrm{ft} \mathrm{BGL})\end{array}$ & $\begin{array}{c}\text { Depth in Klassen } \\
\text { Test Well } \\
\text { (ft BGL) }\end{array}$ & $\begin{array}{l}\text { Elevation in Klassen } \\
\text { Domestic Well } \\
\text { (ft AMSL) }\end{array}$ & \\
\hline $1 / 8 / 1220: 30$ & 33.799 & 35.793 & 1474.97 & 1473.33 \\
\hline $1 / 8 / 1221: 00$ & 33.713 & 35.791 & 1475.06 & 1473.33 \\
\hline $1 / 8 / 1221: 30$ & 33.621 & 35.782 & 1475.15 & 1473.34 \\
\hline $1 / 8 / 1222: 00$ & 34.238 & 35.779 & 1474.53 & 1473.34 \\
\hline $1 / 8 / 1222: 30$ & 33.788 & 35.779 & 1474.98 & 1473.34 \\
\hline $1 / 8 / 1223: 00$ & 34.069 & 35.782 & 1474.70 & 1473.34 \\
\hline $1 / 8 / 1223: 30$ & 34.266 & 35.787 & 1474.50 & 1473.34 \\
\hline $1 / 9 / 120: 00$ & 33.898 & 35.789 & 1474.87 & 1473.33 \\
\hline $1 / 9 / 120: 30$ & 33.764 & 35.779 & 1475.01 & 1473.34 \\
\hline $1 / 9 / 121: 00$ & 33.677 & 35.775 & 1475.09 & 1473.35 \\
\hline $1 / 9 / 121: 30$ & 33.609 & 35.772 & 1475.16 & 1473.35 \\
\hline $1 / 9 / 122: 00$ & 33.631 & 35.768 & 1475.14 & 1473.35 \\
\hline $1 / 9 / 122: 30$ & 33.668 & 35.768 & 1475.10 & 1473.35 \\
\hline $1 / 9 / 123: 00$ & 33.614 & 35.761 & 1475.16 & 1473.36 \\
\hline 1/9/12 3:30 & 33.584 & 35.756 & 1475.19 & 1473.37 \\
\hline 1/9/12 4:00 & 33.649 & 35.751 & 1475.12 & 1473.37 \\
\hline $1 / 9 / 124: 30$ & 33.563 & 35.74 & 1475.21 & 1473.38 \\
\hline 1/9/12 5:00 & 33.502 & 35.728 & 1475.27 & 1473.39 \\
\hline $1 / 9 / 12$ 5:30 & 33.452 & 35.721 & 1475.32 & 1473.40 \\
\hline $1 / 9 / 126: 00$ & 33.509 & 35.721 & 1475.26 & 1473.40 \\
\hline $1 / 9 / 126: 30$ & 33.684 & 35.716 & 1475.09 & 1473.41 \\
\hline 1/9/12 7:00 & 33.689 & 35.711 & 1475.08 & 1473.41 \\
\hline 1/9/12 7:30 & 33.687 & 35.711 & 1475.08 & 1473.41 \\
\hline $1 / 9 / 128: 00$ & 33.588 & 35.707 & 1475.18 & 1473.42 \\
\hline 1/9/12 8:30 & 33.645 & 35.709 & 1475.12 & 1473.41 \\
\hline 1/9/12 9:00 & 33.616 & 35.716 & 1475.15 & 1473.41 \\
\hline 1/9/12 9:30 & 33.621 & 35.726 & 1475.15 & 1473.40 \\
\hline 1/9/12 10:00 & 33.64 & 35.742 & 1475.13 & 1473.38 \\
\hline 1/9/12 10:30 & 33.595 & 35.756 & 1475.17 & 1473.37 \\
\hline 1/9/12 11:00 & 37.43 & 35.768 & 1471.34 & 1473.35 \\
\hline 1/9/12 11:30 & 33.598 & 35.789 & 1475.17 & 1473.33 \\
\hline 1/9/12 12:00 & 33.677 & 35.812 & 1475.09 & 1473.31 \\
\hline 1/9/12 12:30 & 33.495 & 35.829 & 1475.27 & 1473.29 \\
\hline 1/9/12 13:00 & 33.527 & 35.796 & 1475.24 & 1473.33 \\
\hline $1 / 9 / 1213: 30$ & 38.225 & 35.721 & 1470.54 & 1473.40 \\
\hline $1 / 9 / 1214: 00$ & 33.75 & 35.693 & 1475.02 & 1473.43 \\
\hline $1 / 9 / 1214: 30$ & 33.598 & 35.683 & 1475.17 & 1473.44 \\
\hline $1 / 9 / 12$ 15:00 & 33.457 & 35.676 & 1475.31 & 1473.45 \\
\hline
\end{tabular}


TABLE S1.3 Automatically recorded groundwater levels in the Klassen wells, September 15, 2011, to March 13, 2012.

\begin{tabular}{|c|c|c|c|c|}
\hline \multicolumn{3}{|c|}{ Reference Elevation (ft AMSL) } & 1508.77 & \multirow{2}{*}{$\begin{array}{l}1509.12 \\
\text { Elevation in Klassen } \\
\text { Test Well (ft AMSL) }\end{array}$} \\
\hline Date and Time & $\begin{array}{l}\text { Depth in Klassen } \\
\text { Domestic Well } \\
(\mathrm{ft} \mathrm{BGL})\end{array}$ & $\begin{array}{c}\text { Depth in Klassen } \\
\text { Test Well } \\
\text { (ft BGL) }\end{array}$ & $\begin{array}{l}\text { Elevation in Klassen } \\
\text { Domestic Well } \\
\text { (ft AMSL) }\end{array}$ & \\
\hline $1 / 9 / 1215: 30$ & 33.396 & 35.665 & 1475.37 & 1473.46 \\
\hline $1 / 9 / 1216: 00$ & 33.354 & 35.653 & 1475.42 & 1473.47 \\
\hline $1 / 9 / 1216: 30$ & 33.319 & 35.641 & 1475.45 & 1473.48 \\
\hline 1/9/12 17:00 & 33.495 & 35.634 & 1475.27 & 1473.49 \\
\hline $1 / 9 / 1217: 30$ & 33.398 & 35.627 & 1475.37 & 1473.50 \\
\hline $1 / 9 / 1218: 00$ & 34.034 & 35.662 & 1474.74 & 1473.46 \\
\hline 1/9/12 18:30 & 33.828 & 35.679 & 1474.94 & 1473.44 \\
\hline 1/9/12 19:00 & 33.687 & 35.676 & 1475.08 & 1473.45 \\
\hline 1/9/12 19:30 & 34.719 & 35.679 & 1474.05 & 1473.44 \\
\hline $1 / 9 / 1220: 00$ & 33.938 & 35.688 & 1474.83 & 1473.43 \\
\hline $1 / 9 / 1220: 30$ & 33.757 & 35.688 & 1475.01 & 1473.43 \\
\hline $1 / 9 / 1221: 00$ & 33.654 & 35.686 & 1475.12 & 1473.44 \\
\hline $1 / 9 / 1221: 30$ & 33.624 & 35.686 & 1475.15 & 1473.44 \\
\hline $1 / 9 / 1222: 00$ & 33.832 & 35.688 & 1474.94 & 1473.43 \\
\hline $1 / 9 / 1222: 30$ & 34.728 & 35.695 & 1474.04 & 1473.43 \\
\hline 1/9/12 23:00 & 35.708 & 35.709 & 1473.06 & 1473.41 \\
\hline $1 / 9 / 1223: 30$ & 34.334 & 35.728 & 1474.44 & 1473.39 \\
\hline 1/10/12 0:00 & 34.111 & 35.735 & 1474.66 & 1473.39 \\
\hline $1 / 10 / 12$ 0:30 & 33.931 & 35.737 & 1474.84 & 1473.39 \\
\hline $1 / 10 / 121: 00$ & 33.816 & 35.728 & 1474.95 & 1473.39 \\
\hline $1 / 10 / 121: 30$ & 33.727 & 35.723 & 1475.04 & 1473.40 \\
\hline $1 / 10 / 122: 00$ & 33.656 & 35.721 & 1475.11 & 1473.40 \\
\hline $1 / 10 / 122: 30$ & 33.598 & 35.718 & 1475.17 & 1473.40 \\
\hline 1/10/12 3:00 & 33.546 & 35.709 & 1475.22 & 1473.41 \\
\hline 1/10/12 3:30 & 33.504 & 35.7 & 1475.27 & 1473.42 \\
\hline 1/10/12 4:00 & 33.462 & 35.693 & 1475.31 & 1473.43 \\
\hline $1 / 10 / 124: 30$ & 33.424 & 35.686 & 1475.35 & 1473.44 \\
\hline 1/10/12 5:00 & 33.391 & 35.679 & 1475.38 & 1473.44 \\
\hline 1/10/12 5:30 & 33.581 & 35.679 & 1475.19 & 1473.44 \\
\hline 1/10/12 6:00 & 33.398 & 35.671 & 1475.37 & 1473.45 \\
\hline 1/10/12 6:30 & 33.49 & 35.669 & 1475.28 & 1473.45 \\
\hline $1 / 10 / 127: 00$ & 33.427 & 35.667 & 1475.34 & 1473.46 \\
\hline 1/10/12 7:30 & 33.391 & 35.657 & 1475.38 & 1473.47 \\
\hline $1 / 10 / 128: 00$ & 34.428 & 35.655 & 1474.34 & 1473.47 \\
\hline $1 / 10 / 128: 30$ & 33.41 & 35.669 & 1475.36 & 1473.45 \\
\hline 1/10/12 9:00 & 33.349 & 35.681 & 1475.42 & 1473.44 \\
\hline 1/10/12 9:30 & 33.312 & 35.683 & 1475.46 & 1473.44 \\
\hline 1/10/12 10:00 & 33.279 & 35.695 & 1475.49 & 1473.43 \\
\hline
\end{tabular}


TABLE S1.3 Automatically recorded groundwater levels in the Klassen wells, September 15, 2011, to March 13, 2012.

\begin{tabular}{|c|c|c|c|c|}
\hline \multicolumn{3}{|c|}{ Reference Elevation (ft AMSL) } & 1508.77 & \multirow{2}{*}{$\begin{array}{l}1509.12 \\
\text { Elevation in Klassen } \\
\text { Test Well (ft AMSL) }\end{array}$} \\
\hline Date and Time & $\begin{array}{l}\text { Depth in Klassen } \\
\text { Domestic Well } \\
(\mathrm{ft} \mathrm{BGL})\end{array}$ & $\begin{array}{c}\text { Depth in Klassen } \\
\text { Test Well } \\
\text { (ft BGL) }\end{array}$ & $\begin{array}{l}\text { Elevation in Klassen } \\
\text { Domestic Well } \\
\text { (ft AMSL) }\end{array}$ & \\
\hline $1 / 10 / 1210: 30$ & 33.253 & 35.709 & 1475.52 & 1473.41 \\
\hline $1 / 10 / 1211: 00$ & 33.262 & 35.723 & 1475.51 & 1473.40 \\
\hline 1/10/12 11:30 & 33.23 & 35.744 & 1475.54 & 1473.38 \\
\hline $1 / 10 / 1212: 00$ & 33.204 & 35.758 & 1475.57 & 1473.36 \\
\hline $1 / 10 / 1212: 30$ & 33.183 & 35.77 & 1475.59 & 1473.35 \\
\hline $1 / 10 / 1213: 00$ & 33.162 & 35.671 & 1475.61 & 1473.45 \\
\hline $1 / 10 / 1213: 30$ & 33.171 & 35.578 & 1475.60 & 1473.54 \\
\hline $1 / 10 / 1214: 00$ & 33.305 & 35.564 & 1475.46 & 1473.56 \\
\hline $1 / 10 / 1214: 30$ & 33.579 & 35.566 & 1475.19 & 1473.56 \\
\hline $1 / 10 / 1215: 00$ & 33.429 & 35.571 & 1475.34 & 1473.55 \\
\hline 1/10/12 15:30 & 33.342 & 35.564 & 1475.43 & 1473.56 \\
\hline 1/10/12 16:00 & 34.067 & 35.552 & 1474.70 & 1473.57 \\
\hline $1 / 10 / 1216: 30$ & 33.309 & 35.545 & 1475.46 & 1473.58 \\
\hline $1 / 10 / 1217: 00$ & 33.344 & 35.538 & 1475.43 & 1473.58 \\
\hline 1/10/12 17:30 & 33.363 & 35.538 & 1475.41 & 1473.58 \\
\hline $1 / 10 / 12$ 18:00 & 33.314 & 35.535 & 1475.46 & 1473.59 \\
\hline $1 / 10 / 12$ 18:30 & 33.321 & 35.531 & 1475.45 & 1473.59 \\
\hline 1/10/12 19:00 & 33.3 & 35.533 & 1475.47 & 1473.59 \\
\hline 1/10/12 19:30 & 33.316 & 35.533 & 1475.45 & 1473.59 \\
\hline 1/10/12 20:00 & 33.253 & 35.531 & 1475.52 & 1473.59 \\
\hline $1 / 10 / 1220: 30$ & 33.213 & 35.526 & 1475.56 & 1473.60 \\
\hline $1 / 10 / 12$ 21:00 & 33.41 & 35.528 & 1475.36 & 1473.59 \\
\hline $1 / 10 / 1221: 30$ & 33.581 & 35.547 & 1475.19 & 1473.58 \\
\hline $1 / 10 / 1222: 00$ & 33.609 & 35.559 & 1475.16 & 1473.56 \\
\hline $1 / 10 / 1222: 30$ & 35.469 & 35.564 & 1473.30 & 1473.56 \\
\hline $1 / 10 / 12$ 23:00 & 33.727 & 35.573 & 1475.04 & 1473.55 \\
\hline $1 / 10 / 12$ 23:30 & 33.856 & 35.587 & 1474.91 & 1473.54 \\
\hline 1/11/12 0:00 & 33.729 & 35.594 & 1475.04 & 1473.53 \\
\hline $1 / 11 / 12$ 0:30 & 33.598 & 35.594 & 1475.17 & 1473.53 \\
\hline 1/11/12 1:00 & 33.513 & 35.585 & 1475.26 & 1473.54 \\
\hline 1/11/12 1:30 & 33.445 & 35.578 & 1475.32 & 1473.54 \\
\hline $1 / 11 / 122: 00$ & 33.45 & 35.571 & 1475.32 & 1473.55 \\
\hline 1/11/12 2:30 & 33.492 & 35.571 & 1475.28 & 1473.55 \\
\hline 1/11/12 3:00 & 33.527 & 35.571 & 1475.24 & 1473.55 \\
\hline 1/11/12 3:30 & 33.457 & 35.566 & 1475.31 & 1473.56 \\
\hline 1/11/12 4:00 & 33.495 & 35.564 & 1475.27 & 1473.56 \\
\hline $1 / 11 / 124: 30$ & 33.412 & 35.557 & 1475.36 & 1473.57 \\
\hline $1 / 11 / 125: 00$ & 33.354 & 35.557 & 1475.42 & 1473.57 \\
\hline
\end{tabular}


TABLE S1.3 Automatically recorded groundwater levels in the Klassen wells, September 15, 2011, to March 13, 2012.

\begin{tabular}{|c|c|c|c|c|}
\hline \multicolumn{3}{|c|}{ Reference Elevation (ft AMSL) } & 1508.77 & \multirow{2}{*}{$\begin{array}{l}1509.12 \\
\text { Elevation in Klassen } \\
\text { Test Well (ft AMSL) }\end{array}$} \\
\hline Date and Time & $\begin{array}{l}\text { Depth in Klassen } \\
\text { Domestic Well } \\
(\mathrm{ft} \mathrm{BGL})\end{array}$ & $\begin{array}{c}\text { Depth in Klassen } \\
\text { Test Well } \\
\text { (ft BGL) }\end{array}$ & $\begin{array}{l}\text { Elevation in Klassen } \\
\text { Domestic Well } \\
\text { (ft AMSL) }\end{array}$ & \\
\hline $1 / 11 / 125: 30$ & 33.309 & 35.554 & 1475.46 & 1473.57 \\
\hline $1 / 11 / 126: 00$ & 33.272 & 35.543 & 1475.50 & 1473.58 \\
\hline 1/11/12 6:30 & 33.511 & 35.535 & 1475.26 & 1473.59 \\
\hline 1/11/12 7:00 & 33.403 & 35.531 & 1475.37 & 1473.59 \\
\hline $1 / 11 / 127: 30$ & 33.441 & 35.524 & 1475.33 & 1473.60 \\
\hline 1/11/12 8:00 & 33.347 & 35.514 & 1475.42 & 1473.61 \\
\hline 1/11/12 8:30 & 33.298 & 35.507 & 1475.47 & 1473.62 \\
\hline 1/11/12 9:00 & 33.347 & 35.503 & 1475.42 & 1473.62 \\
\hline 1/11/12 9:30 & 33.443 & 35.503 & 1475.33 & 1473.62 \\
\hline $1 / 11 / 1210: 00$ & 34.878 & 35.5 & 1473.89 & 1473.62 \\
\hline $1 / 11 / 1210: 30$ & 33.326 & 35.496 & 1475.44 & 1473.63 \\
\hline 1/11/12 11:00 & 33.265 & 35.491 & 1475.50 & 1473.63 \\
\hline 1/11/12 11:30 & 33.227 & 35.488 & 1475.54 & 1473.63 \\
\hline $1 / 11 / 1212: 00$ & 33.856 & 35.481 & 1474.91 & 1473.64 \\
\hline $1 / 11 / 12$ 12:30 & 33.227 & 35.479 & 1475.54 & 1473.64 \\
\hline 1/11/12 13:00 & 33.187 & 35.477 & 1475.58 & 1473.65 \\
\hline $1 / 11 / 12$ 13:30 & 33.248 & 35.479 & 1475.52 & 1473.64 \\
\hline 1/11/12 14:00 & 33.19 & 35.472 & 1475.58 & 1473.65 \\
\hline 1/11/12 14:30 & 33.342 & 35.477 & 1475.43 & 1473.65 \\
\hline $1 / 11 / 12$ 15:00 & 33.194 & 35.474 & 1475.58 & 1473.65 \\
\hline 1/11/12 15:30 & 33.23 & 35.467 & 1475.54 & 1473.66 \\
\hline 1/11/12 16:00 & 34.421 & 35.47 & 1474.35 & 1473.65 \\
\hline $1 / 11 / 12$ 16:30 & 33.556 & 35.474 & 1475.21 & 1473.65 \\
\hline 1/11/12 17:00 & 41.989 & 35.484 & 1466.78 & 1473.64 \\
\hline 1/11/12 17:30 & 33.724 & 35.503 & 1475.05 & 1473.62 \\
\hline 1/11/12 18:00 & 33.563 & 35.517 & 1475.21 & 1473.61 \\
\hline 1/11/12 18:30 & 33.45 & 35.517 & 1475.32 & 1473.61 \\
\hline 1/11/12 19:00 & 33.403 & 35.517 & 1475.37 & 1473.61 \\
\hline 1/11/12 19:30 & 33.624 & 35.528 & 1475.15 & 1473.59 \\
\hline 1/11/12 20:00 & 33.588 & 35.535 & 1475.18 & 1473.59 \\
\hline 1/11/12 20:30 & 33.56 & 35.545 & 1475.21 & 1473.58 \\
\hline 1/11/12 21:00 & 33.605 & 35.561 & 1475.16 & 1473.56 \\
\hline 1/11/12 21:30 & 33.703 & 35.585 & 1475.07 & 1473.54 \\
\hline $1 / 11 / 1222: 00$ & 33.839 & 35.608 & 1474.93 & 1473.51 \\
\hline $1 / 11 / 1222: 30$ & 33.846 & 35.625 & 1474.92 & 1473.50 \\
\hline 1/11/12 23:00 & 33.874 & 35.634 & 1474.90 & 1473.49 \\
\hline 1/11/12 23:30 & 33.677 & 35.634 & 1475.09 & 1473.49 \\
\hline 1/12/12 0:00 & 33.581 & 35.634 & 1475.19 & 1473.49 \\
\hline
\end{tabular}


TABLE S1.3 Automatically recorded groundwater levels in the Klassen wells, September 15, 2011, to March 13, 2012.

\begin{tabular}{|c|c|c|c|c|}
\hline \multicolumn{3}{|c|}{ Reference Elevation (ft AMSL) } & 1508.77 & \multirow{2}{*}{$\begin{array}{l}1509.12 \\
\text { Elevation in Klassen } \\
\text { Test Well (ft AMSL) }\end{array}$} \\
\hline Date and Time & $\begin{array}{l}\text { Depth in Klassen } \\
\text { Domestic Well } \\
(\mathrm{ft} \mathrm{BGL})\end{array}$ & $\begin{array}{c}\text { Depth in Klassen } \\
\text { Test Well } \\
\text { (ft BGL) }\end{array}$ & $\begin{array}{l}\text { Elevation in Klassen } \\
\text { Domestic Well } \\
\text { (ft AMSL) }\end{array}$ & \\
\hline $1 / 12 / 120: 30$ & 33.513 & 35.632 & 1475.26 & 1473.49 \\
\hline $1 / 12 / 121: 00$ & 33.457 & 35.627 & 1475.31 & 1473.50 \\
\hline $1 / 12 / 121: 30$ & 33.527 & 35.629 & 1475.24 & 1473.49 \\
\hline $1 / 12 / 122: 00$ & 33.427 & 35.629 & 1475.34 & 1473.49 \\
\hline $1 / 12 / 122: 30$ & 33.375 & 35.625 & 1475.39 & 1473.50 \\
\hline 1/12/12 3:00 & 33.337 & 35.618 & 1475.43 & 1473.50 \\
\hline 1/12/12 3:30 & 33.302 & 35.613 & 1475.47 & 1473.51 \\
\hline 1/12/12 4:00 & 33.274 & 35.601 & 1475.50 & 1473.52 \\
\hline $1 / 12 / 124: 30$ & 33.246 & 35.592 & 1475.52 & 1473.53 \\
\hline 1/12/12 5:00 & 33.251 & 35.58 & 1475.52 & 1473.54 \\
\hline $1 / 12 / 125: 30$ & 33.241 & 35.571 & 1475.53 & 1473.55 \\
\hline $1 / 12 / 126: 00$ & 33.208 & 35.557 & 1475.56 & 1473.57 \\
\hline $1 / 12 / 126: 30$ & 33.363 & 35.557 & 1475.41 & 1473.57 \\
\hline $1 / 12 / 127: 00$ & 33.3 & 35.549 & 1475.47 & 1473.57 \\
\hline 1/12/12 7:30 & 33.452 & 35.547 & 1475.32 & 1473.58 \\
\hline $1 / 12 / 128: 00$ & 33.359 & 35.547 & 1475.41 & 1473.58 \\
\hline $1 / 12 / 128: 30$ & 33.485 & 35.545 & 1475.28 & 1473.58 \\
\hline 1/12/12 9:00 & 33.417 & 35.54 & 1475.35 & 1473.58 \\
\hline 1/12/12 9:30 & 33.567 & 35.543 & 1475.20 & 1473.58 \\
\hline 1/12/12 10:00 & 33.459 & 35.552 & 1475.31 & 1473.57 \\
\hline $1 / 12 / 12$ 10:30 & 33.532 & 35.557 & 1475.24 & 1473.57 \\
\hline 1/12/12 11:00 & 33.38 & 35.559 & 1475.39 & 1473.56 \\
\hline $1 / 12 / 1211: 30$ & 33.316 & 35.568 & 1475.45 & 1473.55 \\
\hline $1 / 12 / 1212: 00$ & 33.438 & 35.592 & 1475.33 & 1473.53 \\
\hline $1 / 12 / 12$ 12:30 & 33.305 & 35.608 & 1475.46 & 1473.51 \\
\hline $1 / 12 / 12$ 13:00 & 33.255 & 35.61 & 1475.51 & 1473.51 \\
\hline $1 / 12 / 12$ 13:30 & 33.222 & 35.604 & 1475.55 & 1473.52 \\
\hline $1 / 12 / 1214: 00$ & 33.218 & 35.594 & 1475.55 & 1473.53 \\
\hline $1 / 12 / 12$ 14:30 & 33.305 & 35.589 & 1475.46 & 1473.53 \\
\hline $1 / 12 / 12$ 15:00 & 33.246 & 35.582 & 1475.52 & 1473.54 \\
\hline $1 / 12 / 12$ 15:30 & 33.337 & 35.571 & 1475.43 & 1473.55 \\
\hline 1/12/12 16:00 & 33.255 & 35.557 & 1475.51 & 1473.57 \\
\hline $1 / 12 / 12$ 16:30 & 33.23 & 35.545 & 1475.54 & 1473.58 \\
\hline $1 / 12 / 12$ 17:00 & 33.363 & 35.533 & 1475.41 & 1473.59 \\
\hline $1 / 12 / 12$ 17:30 & 33.234 & 35.521 & 1475.54 & 1473.60 \\
\hline $1 / 12 / 12$ 18:00 & 33.274 & 35.51 & 1475.50 & 1473.61 \\
\hline $1 / 12 / 12$ 18:30 & 33.37 & 35.498 & 1475.40 & 1473.62 \\
\hline $1 / 12 / 12$ 19:00 & 33.938 & 35.488 & 1474.83 & 1473.63 \\
\hline
\end{tabular}


TABLE S1.3 Automatically recorded groundwater levels in the Klassen wells, September 15, 2011, to March 13, 2012.

\begin{tabular}{|c|c|c|c|c|}
\hline \multicolumn{3}{|c|}{ Reference Elevation (ft AMSL) } & 1508.77 & \multirow{2}{*}{$\begin{array}{l}1509.12 \\
\text { Elevation in Klassen } \\
\text { Test Well (ft AMSL) }\end{array}$} \\
\hline Date and Time & $\begin{array}{l}\text { Depth in Klassen } \\
\text { Domestic Well } \\
\text { (ft BGL) }\end{array}$ & $\begin{array}{c}\text { Depth in Klassen } \\
\text { Test Well } \\
\text { (ft BGL) }\end{array}$ & $\begin{array}{l}\text { Elevation in Klassen } \\
\text { Domestic Well } \\
\text { (ft AMSL) }\end{array}$ & \\
\hline $1 / 12 / 12$ 19:30 & 33.49 & 35.486 & 1475.28 & 1473.64 \\
\hline $1 / 12 / 1220: 00$ & 33.384 & 35.479 & 1475.39 & 1473.64 \\
\hline $1 / 12 / 1220: 30$ & 33.438 & 35.482 & 1475.33 & 1473.64 \\
\hline $1 / 12 / 1221: 00$ & 33.591 & 35.491 & 1475.18 & 1473.63 \\
\hline $1 / 12 / 1221: 30$ & 33.441 & 35.484 & 1475.33 & 1473.64 \\
\hline $1 / 12 / 1222: 00$ & 33.391 & 35.482 & 1475.38 & 1473.64 \\
\hline $1 / 12 / 1222: 30$ & 34.496 & 35.486 & 1474.27 & 1473.64 \\
\hline $1 / 12 / 1223: 00$ & 33.654 & 35.498 & 1475.12 & 1473.62 \\
\hline $1 / 12 / 1223: 30$ & 34.353 & 35.51 & 1474.42 & 1473.61 \\
\hline 1/13/12 0:00 & 33.75 & 35.521 & 1475.02 & 1473.60 \\
\hline $1 / 13 / 120: 30$ & 33.605 & 35.531 & 1475.16 & 1473.59 \\
\hline $1 / 13 / 121: 00$ & 33.518 & 35.528 & 1475.25 & 1473.59 \\
\hline $1 / 13 / 121: 30$ & 33.452 & 35.531 & 1475.32 & 1473.59 \\
\hline $1 / 13 / 122: 00$ & 33.459 & 35.531 & 1475.31 & 1473.59 \\
\hline $1 / 13 / 122: 30$ & 33.478 & 35.531 & 1475.29 & 1473.59 \\
\hline 1/13/12 3:00 & 33.434 & 35.528 & 1475.34 & 1473.59 \\
\hline 1/13/12 3:30 & 33.609 & 35.535 & 1475.16 & 1473.59 \\
\hline 1/13/12 4:00 & 33.56 & 35.542 & 1475.21 & 1473.58 \\
\hline $1 / 13 / 124: 30$ & 33.464 & 35.538 & 1475.31 & 1473.58 \\
\hline $1 / 13 / 125: 00$ & 33.401 & 35.535 & 1475.37 & 1473.59 \\
\hline 1/13/12 5:30 & 33.354 & 35.533 & 1475.42 & 1473.59 \\
\hline 1/13/12 6:00 & 33.316 & 35.531 & 1475.45 & 1473.59 \\
\hline 1/13/12 6:30 & 34.658 & 35.526 & 1474.11 & 1473.60 \\
\hline $1 / 13 / 127: 00$ & 33.502 & 35.521 & 1475.27 & 1473.60 \\
\hline $1 / 13 / 12$ 7:30 & 33.495 & 35.531 & 1475.27 & 1473.59 \\
\hline $1 / 13 / 128: 00$ & 33.429 & 35.528 & 1475.34 & 1473.59 \\
\hline $1 / 13 / 128: 30$ & 33.368 & 35.519 & 1475.40 & 1473.60 \\
\hline 1/13/12 9:00 & 35.931 & 35.517 & 1472.84 & 1473.61 \\
\hline 1/13/12 9:30 & 33.546 & 35.519 & 1475.22 & 1473.60 \\
\hline 1/13/12 10:00 & 33.553 & 35.526 & 1475.22 & 1473.60 \\
\hline $1 / 13 / 12$ 10:30 & 33.591 & 35.531 & 1475.18 & 1473.59 \\
\hline 1/13/12 11:00 & 33.914 & 35.568 & 1474.86 & 1473.55 \\
\hline 1/13/12 11:30 & 33.762 & 35.603 & 1475.01 & 1473.52 \\
\hline $1 / 13 / 1212: 00$ & 33.717 & 35.622 & 1475.05 & 1473.50 \\
\hline $1 / 13 / 12$ 12:30 & 33.633 & 35.629 & 1475.14 & 1473.49 \\
\hline 1/13/12 13:00 & 33.612 & 35.632 & 1475.16 & 1473.49 \\
\hline 1/13/12 13:30 & 33.506 & 35.627 & 1475.26 & 1473.50 \\
\hline $1 / 13 / 12$ 14:00 & 33.577 & 35.578 & 1475.19 & 1473.54 \\
\hline
\end{tabular}


TABLE S1.3 Automatically recorded groundwater levels in the Klassen wells, September 15, 2011, to March 13, 2012.

\begin{tabular}{|c|c|c|c|c|}
\hline \multicolumn{3}{|c|}{ Reference Elevation (ft AMSL) } & 1508.77 & \multirow{2}{*}{$\begin{array}{l}1509.12 \\
\text { Elevation in Klassen } \\
\text { Test Well (ft AMSL) }\end{array}$} \\
\hline Date and Time & $\begin{array}{l}\text { Depth in Klassen } \\
\text { Domestic Well } \\
(\mathrm{ft} \mathrm{BGL})\end{array}$ & $\begin{array}{c}\text { Depth in Klassen } \\
\text { Test Well } \\
\text { (ft BGL) }\end{array}$ & $\begin{array}{l}\text { Elevation in Klassen } \\
\text { Domestic Well } \\
\text { (ft AMSL) }\end{array}$ & \\
\hline $1 / 13 / 1214: 30$ & 33.464 & 35.552 & 1475.31 & 1473.57 \\
\hline $1 / 13 / 1215: 00$ & 33.509 & 35.542 & 1475.26 & 1473.58 \\
\hline $1 / 13 / 12$ 15:30 & 33.417 & 35.535 & 1475.35 & 1473.59 \\
\hline $1 / 13 / 1216: 00$ & 33.401 & 35.531 & 1475.37 & 1473.59 \\
\hline $1 / 13 / 1216: 30$ & 33.405 & 35.526 & 1475.36 & 1473.60 \\
\hline $1 / 13 / 1217: 00$ & 33.443 & 35.524 & 1475.33 & 1473.60 \\
\hline $1 / 13 / 1217: 30$ & 33.509 & 35.519 & 1475.26 & 1473.60 \\
\hline $1 / 13 / 1218: 00$ & 34.512 & 35.542 & 1474.26 & 1473.58 \\
\hline $1 / 13 / 1218: 30$ & 33.957 & 35.557 & 1474.81 & 1473.57 \\
\hline $1 / 13 / 12$ 19:00 & 33.811 & 35.561 & 1474.96 & 1473.56 \\
\hline 1/13/12 19:30 & 33.86 & 35.559 & 1474.91 & 1473.56 \\
\hline 1/13/12 20:00 & 33.839 & 35.566 & 1474.93 & 1473.56 \\
\hline $1 / 13 / 1220: 30$ & 33.825 & 35.568 & 1474.94 & 1473.55 \\
\hline $1 / 13 / 1221: 00$ & 33.684 & 35.568 & 1475.09 & 1473.55 \\
\hline $1 / 13 / 12$ 21:30 & 33.602 & 35.564 & 1475.17 & 1473.56 \\
\hline $1 / 13 / 1222: 00$ & 34.109 & 35.575 & 1474.66 & 1473.55 \\
\hline $1 / 13 / 1222: 30$ & 34.149 & 35.599 & 1474.62 & 1473.52 \\
\hline $1 / 13 / 12$ 23:00 & 33.889 & 35.601 & 1474.88 & 1473.52 \\
\hline $1 / 13 / 12$ 23:30 & 33.781 & 35.599 & 1474.99 & 1473.52 \\
\hline 1/14/12 0:00 & 33.813 & 35.601 & 1474.96 & 1473.52 \\
\hline $1 / 14 / 12$ 0:30 & 33.708 & 35.599 & 1475.06 & 1473.52 \\
\hline $1 / 14 / 121: 00$ & 33.631 & 35.594 & 1475.14 & 1473.53 \\
\hline 1/14/12 1:30 & 33.57 & 35.585 & 1475.20 & 1473.54 \\
\hline $1 / 14 / 122: 00$ & 33.584 & 35.578 & 1475.19 & 1473.54 \\
\hline $1 / 14 / 122: 30$ & 33.621 & 35.573 & 1475.15 & 1473.55 \\
\hline 1/14/12 3:00 & 33.57 & 35.568 & 1475.20 & 1473.55 \\
\hline 1/14/12 3:30 & 33.534 & 35.559 & 1475.24 & 1473.56 \\
\hline 1/14/12 4:00 & 33.584 & 35.557 & 1475.19 & 1473.57 \\
\hline 1/14/12 4:30 & 33.511 & 35.547 & 1475.26 & 1473.58 \\
\hline 1/14/12 5:00 & 33.459 & 35.543 & 1475.31 & 1473.58 \\
\hline 1/14/12 5:30 & 33.419 & 35.528 & 1475.35 & 1473.59 \\
\hline $1 / 14 / 126: 00$ & 35.164 & 35.521 & 1473.61 & 1473.60 \\
\hline 1/14/12 6:30 & 37.768 & 35.514 & 1471.00 & 1473.61 \\
\hline 1/14/12 7:00 & 33.654 & 35.512 & 1475.12 & 1473.61 \\
\hline 1/14/12 7:30 & 33.715 & 35.507 & 1475.05 & 1473.62 \\
\hline 1/14/12 8:00 & 33.806 & 35.498 & 1474.96 & 1473.62 \\
\hline $1 / 14 / 128: 30$ & 33.588 & 35.498 & 1475.18 & 1473.62 \\
\hline $1 / 14 / 12$ 9:00 & 33.598 & 35.493 & 1475.17 & 1473.63 \\
\hline
\end{tabular}


TABLE S1.3 Automatically recorded groundwater levels in the Klassen wells, September 15, 2011, to March 13, 2012.

\begin{tabular}{|c|c|c|c|c|}
\hline \multicolumn{3}{|c|}{ Reference Elevation (ft AMSL) } & 1508.77 & \multirow{2}{*}{$\begin{array}{l}1509.12 \\
\text { Elevation in Klassen } \\
\text { Test Well (ft AMSL) }\end{array}$} \\
\hline Date and Time & $\begin{array}{l}\text { Depth in Klassen } \\
\text { Domestic Well } \\
\text { (ft BGL) }\end{array}$ & $\begin{array}{c}\text { Depth in Klassen } \\
\text { Test Well } \\
\text { (ft BGL) }\end{array}$ & $\begin{array}{l}\text { Elevation in Klassen } \\
\text { Domestic Well } \\
\text { (ft AMSL) }\end{array}$ & \\
\hline $1 / 14 / 129: 30$ & 33.626 & 35.503 & 1475.14 & 1473.62 \\
\hline $1 / 14 / 1210: 00$ & 33.713 & 35.507 & 1475.06 & 1473.62 \\
\hline $1 / 14 / 1210: 30$ & 33.638 & 35.521 & 1475.13 & 1473.60 \\
\hline 1/14/12 11:00 & 33.556 & 35.528 & 1475.21 & 1473.59 \\
\hline $1 / 14 / 1211: 30$ & 33.595 & 35.54 & 1475.17 & 1473.58 \\
\hline $1 / 14 / 1212: 00$ & 33.534 & 35.547 & 1475.24 & 1473.58 \\
\hline $1 / 14 / 1212: 30$ & 33.49 & 35.552 & 1475.28 & 1473.57 \\
\hline 1/14/12 13:00 & 33.438 & 35.557 & 1475.33 & 1473.57 \\
\hline $1 / 14 / 1213: 30$ & 33.398 & 35.552 & 1475.37 & 1473.57 \\
\hline 1/14/12 14:00 & 33.366 & 35.54 & 1475.40 & 1473.58 \\
\hline $1 / 14 / 1214: 30$ & 33.337 & 35.533 & 1475.43 & 1473.59 \\
\hline $1 / 14 / 12$ 15:00 & 33.312 & 35.528 & 1475.46 & 1473.59 \\
\hline $1 / 14 / 1215: 30$ & 33.288 & 35.524 & 1475.48 & 1473.60 \\
\hline 1/14/12 16:00 & 33.267 & 35.517 & 1475.50 & 1473.61 \\
\hline $1 / 14 / 1216: 30$ & 33.246 & 35.507 & 1475.52 & 1473.62 \\
\hline 1/14/12 17:00 & 33.227 & 35.5 & 1475.54 & 1473.62 \\
\hline $1 / 14 / 1217: 30$ & 33.211 & 35.493 & 1475.56 & 1473.63 \\
\hline $1 / 14 / 12$ 18:00 & 33.192 & 35.489 & 1475.58 & 1473.63 \\
\hline $1 / 14 / 1218: 30$ & 33.208 & 35.484 & 1475.56 & 1473.64 \\
\hline 1/14/12 19:00 & 33.213 & 35.479 & 1475.56 & 1473.64 \\
\hline 1/14/12 19:30 & 33.312 & 35.479 & 1475.46 & 1473.64 \\
\hline $1 / 14 / 12$ 20:00 & 33.262 & 35.474 & 1475.51 & 1473.65 \\
\hline $1 / 14 / 12$ 20:30 & 33.222 & 35.47 & 1475.55 & 1473.65 \\
\hline $1 / 14 / 12$ 21:00 & 33.19 & 35.465 & 1475.58 & 1473.66 \\
\hline $1 / 14 / 12$ 21:30 & 33.269 & 35.463 & 1475.50 & 1473.66 \\
\hline $1 / 14 / 12$ 22:00 & 38.877 & 35.467 & 1469.89 & 1473.66 \\
\hline $1 / 14 / 12$ 22:30 & 33.419 & 35.47 & 1475.35 & 1473.65 \\
\hline 1/14/12 23:00 & 33.309 & 35.463 & 1475.46 & 1473.66 \\
\hline $1 / 14 / 12$ 23:30 & 33.312 & 35.467 & 1475.46 & 1473.66 \\
\hline 1/15/12 0:00 & 33.255 & 35.472 & 1475.51 & 1473.65 \\
\hline $1 / 15 / 120: 30$ & 33.215 & 35.474 & 1475.55 & 1473.65 \\
\hline $1 / 15 / 121: 00$ & 33.187 & 35.472 & 1475.58 & 1473.65 \\
\hline 1/15/12 1:30 & 33.159 & 35.472 & 1475.61 & 1473.65 \\
\hline $1 / 15 / 122: 00$ & 33.14 & 35.467 & 1475.63 & 1473.66 \\
\hline $1 / 15 / 122: 30$ & 33.119 & 35.467 & 1475.65 & 1473.66 \\
\hline 1/15/12 3:00 & 33.105 & 35.463 & 1475.66 & 1473.66 \\
\hline $1 / 15 / 123: 30$ & 33.089 & 35.46 & 1475.68 & 1473.66 \\
\hline $1 / 15 / 124: 00$ & 33.072 & 35.458 & 1475.70 & 1473.66 \\
\hline
\end{tabular}


TABLE S1.3 Automatically recorded groundwater levels in the Klassen wells, September 15, 2011, to March 13, 2012.

\begin{tabular}{|c|c|c|c|c|}
\hline \multicolumn{3}{|c|}{ Reference Elevation (ft AMSL) } & 1508.77 & \multirow{2}{*}{$\begin{array}{l}1509.12 \\
\text { Elevation in Klassen } \\
\text { Test Well (ft AMSL) }\end{array}$} \\
\hline Date and Time & $\begin{array}{l}\text { Depth in Klassen } \\
\text { Domestic Well } \\
\text { (ft BGL) }\end{array}$ & $\begin{array}{c}\text { Depth in Klassen } \\
\text { Test Well } \\
\text { (ft BGL) }\end{array}$ & $\begin{array}{l}\text { Elevation in Klassen } \\
\text { Domestic Well } \\
\text { (ft AMSL) }\end{array}$ & \\
\hline $1 / 15 / 124: 30$ & 33.058 & 35.453 & 1475.71 & 1473.67 \\
\hline $1 / 15 / 125: 00$ & 33.044 & 35.449 & 1475.73 & 1473.67 \\
\hline 1/15/12 5:30 & 33.033 & 35.446 & 1475.74 & 1473.68 \\
\hline 1/15/12 6:00 & 33.598 & 35.449 & 1475.17 & 1473.67 \\
\hline $1 / 15 / 126: 30$ & 33.201 & 35.444 & 1475.57 & 1473.68 \\
\hline 1/15/12 7:00 & 33.272 & 35.437 & 1475.50 & 1473.69 \\
\hline 1/15/12 7:30 & 33.147 & 35.432 & 1475.62 & 1473.69 \\
\hline $1 / 15 / 128: 00$ & 33.405 & 35.425 & 1475.36 & 1473.70 \\
\hline $1 / 15 / 128: 30$ & 33.272 & 35.435 & 1475.50 & 1473.69 \\
\hline 1/15/12 9:00 & 33.164 & 35.437 & 1475.61 & 1473.69 \\
\hline 1/15/12 9:30 & 33.126 & 35.439 & 1475.64 & 1473.68 \\
\hline $1 / 15 / 1210: 00$ & 33.145 & 35.449 & 1475.62 & 1473.67 \\
\hline $1 / 15 / 1210: 30$ & 33.124 & 35.458 & 1475.65 & 1473.66 \\
\hline $1 / 15 / 1211: 00$ & 33.187 & 35.467 & 1475.58 & 1473.66 \\
\hline 1/15/12 11:30 & 33.267 & 35.489 & 1475.50 & 1473.63 \\
\hline $1 / 15 / 12$ 12:00 & 33.307 & 35.489 & 1475.46 & 1473.63 \\
\hline $1 / 15 / 12$ 12:30 & 33.342 & 35.484 & 1475.43 & 1473.64 \\
\hline $1 / 15 / 12$ 13:00 & 33.305 & 35.486 & 1475.46 & 1473.64 \\
\hline $1 / 15 / 12$ 13:30 & 35.045 & 35.484 & 1473.72 & 1473.64 \\
\hline 1/15/12 14:00 & 33.293 & 35.493 & 1475.48 & 1473.63 \\
\hline $1 / 15 / 12$ 14:30 & 33.222 & 35.496 & 1475.55 & 1473.63 \\
\hline $1 / 15 / 12$ 15:00 & 33.18 & 35.493 & 1475.59 & 1473.63 \\
\hline $1 / 15 / 12$ 15:30 & 35.321 & 35.493 & 1473.45 & 1473.63 \\
\hline 1/15/12 16:00 & 33.215 & 35.493 & 1475.55 & 1473.63 \\
\hline 1/15/12 16:30 & 33.187 & 35.493 & 1475.58 & 1473.63 \\
\hline 1/15/12 17:00 & 33.176 & 35.486 & 1475.59 & 1473.64 \\
\hline 1/15/12 17:30 & 41.243 & 35.486 & 1467.53 & 1473.64 \\
\hline $1 / 15 / 12$ 18:00 & 51.276 & 35.533 & 1457.49 & 1473.59 \\
\hline $1 / 15 / 12$ 18:30 & 34.749 & 35.603 & 1474.02 & 1473.52 \\
\hline 1/15/12 19:00 & 34.355 & 35.632 & 1474.41 & 1473.49 \\
\hline 1/15/12 19:30 & 34.067 & 35.636 & 1474.70 & 1473.49 \\
\hline 1/15/12 20:00 & 33.914 & 35.634 & 1474.86 & 1473.49 \\
\hline 1/15/12 20:30 & 34.918 & 35.636 & 1473.85 & 1473.49 \\
\hline $1 / 15 / 12$ 21:00 & 33.816 & 35.634 & 1474.95 & 1473.49 \\
\hline $1 / 15 / 12$ 21:30 & 33.696 & 35.625 & 1475.07 & 1473.50 \\
\hline $1 / 15 / 12$ 22:00 & 33.659 & 35.618 & 1475.11 & 1473.50 \\
\hline $1 / 15 / 12$ 22:30 & 35.603 & 35.611 & 1473.17 & 1473.51 \\
\hline $1 / 15 / 12$ 23:00 & 34.114 & 35.611 & 1474.66 & 1473.51 \\
\hline
\end{tabular}


TABLE S1.3 Automatically recorded groundwater levels in the Klassen wells, September 15, 2011, to March 13, 2012.

\begin{tabular}{|c|c|c|c|c|}
\hline \multicolumn{3}{|c|}{ Reference Elevation (ft AMSL) } & 1508.77 & \multirow{2}{*}{$\begin{array}{l}1509.12 \\
\text { Elevation in Klassen } \\
\text { Test Well (ft AMSL) }\end{array}$} \\
\hline Date and Time & $\begin{array}{l}\text { Depth in Klassen } \\
\text { Domestic Well } \\
\text { (ft BGL) }\end{array}$ & $\begin{array}{c}\text { Depth in Klassen } \\
\text { Test Well } \\
\text { (ft BGL) }\end{array}$ & $\begin{array}{l}\text { Elevation in Klassen } \\
\text { Domestic Well } \\
\text { (ft AMSL) }\end{array}$ & \\
\hline $1 / 15 / 1223: 30$ & 33.778 & 35.611 & 1474.99 & 1473.51 \\
\hline 1/16/12 0:00 & 33.642 & 35.606 & 1475.13 & 1473.52 \\
\hline $1 / 16 / 120: 30$ & 33.558 & 35.594 & 1475.21 & 1473.53 \\
\hline $1 / 16 / 121: 00$ & 33.492 & 35.587 & 1475.28 & 1473.54 \\
\hline $1 / 16 / 121: 30$ & 33.443 & 35.575 & 1475.33 & 1473.55 \\
\hline $1 / 16 / 122: 00$ & 33.452 & 35.568 & 1475.32 & 1473.55 \\
\hline $1 / 16 / 122: 30$ & 33.478 & 35.568 & 1475.29 & 1473.55 \\
\hline 1/16/12 3:00 & 33.436 & 35.566 & 1475.33 & 1473.56 \\
\hline $1 / 16 / 123: 30$ & 33.403 & 35.566 & 1475.37 & 1473.56 \\
\hline $1 / 16 / 124: 00$ & 33.487 & 35.568 & 1475.28 & 1473.55 \\
\hline $1 / 16 / 124: 30$ & 33.41 & 35.561 & 1475.36 & 1473.56 \\
\hline $1 / 16 / 125: 00$ & 33.361 & 35.552 & 1475.41 & 1473.57 \\
\hline $1 / 16 / 125: 30$ & 33.316 & 35.547 & 1475.45 & 1473.58 \\
\hline $1 / 16 / 126: 00$ & 33.312 & 35.54 & 1475.46 & 1473.58 \\
\hline $1 / 16 / 126: 30$ & 33.436 & 35.533 & 1475.33 & 1473.59 \\
\hline $1 / 16 / 127: 00$ & 33.394 & 35.54 & 1475.38 & 1473.58 \\
\hline 1/16/12 7:30 & 33.429 & 35.533 & 1475.34 & 1473.59 \\
\hline $1 / 16 / 128: 00$ & 33.537 & 35.528 & 1475.23 & 1473.59 \\
\hline $1 / 16 / 128: 30$ & 35.833 & 35.533 & 1472.94 & 1473.59 \\
\hline 1/16/12 9:00 & 33.684 & 35.538 & 1475.09 & 1473.58 \\
\hline 1/16/12 9:30 & 33.722 & 35.535 & 1475.05 & 1473.59 \\
\hline 1/16/12 10:00 & 33.631 & 35.54 & 1475.14 & 1473.58 \\
\hline 1/16/12 10:30 & 33.45 & 35.538 & 1475.32 & 1473.58 \\
\hline 1/16/12 11:00 & 33.464 & 35.54 & 1475.31 & 1473.58 \\
\hline 1/16/12 11:30 & 33.48 & 35.54 & 1475.29 & 1473.58 \\
\hline $1 / 16 / 1212: 00$ & 33.389 & 35.533 & 1475.38 & 1473.59 \\
\hline $1 / 16 / 1212: 30$ & 33.335 & 35.531 & 1475.43 & 1473.59 \\
\hline 1/16/12 13:00 & 33.319 & 35.519 & 1475.45 & 1473.60 \\
\hline $1 / 16 / 1213: 30$ & 33.452 & 35.521 & 1475.32 & 1473.60 \\
\hline 1/16/12 14:00 & 33.401 & 35.514 & 1475.37 & 1473.61 \\
\hline $1 / 16 / 1214: 30$ & 33.473 & 35.512 & 1475.30 & 1473.61 \\
\hline $1 / 16 / 12$ 15:00 & 34.074 & 35.503 & 1474.70 & 1473.62 \\
\hline $1 / 16 / 1215: 30$ & 33.462 & 35.505 & 1475.31 & 1473.62 \\
\hline 1/16/12 16:00 & 33.342 & 35.503 & 1475.43 & 1473.62 \\
\hline $1 / 16 / 1216: 30$ & 33.412 & 35.503 & 1475.36 & 1473.62 \\
\hline 1/16/12 17:00 & 33.302 & 35.503 & 1475.47 & 1473.62 \\
\hline 1/16/12 17:30 & 33.49 & 35.5 & 1475.28 & 1473.62 \\
\hline 1/16/12 18:00 & 33.539 & 35.51 & 1475.23 & 1473.61 \\
\hline
\end{tabular}


TABLE S1.3 Automatically recorded groundwater levels in the Klassen wells, September 15, 2011, to March 13, 2012.

\begin{tabular}{|c|c|c|c|c|}
\hline \multicolumn{3}{|c|}{ Reference Elevation (ft AMSL) } & 1508.77 & \multirow{2}{*}{$\begin{array}{l}1509.12 \\
\text { Elevation in Klassen } \\
\text { Test Well (ft AMSL) }\end{array}$} \\
\hline Date and Time & $\begin{array}{l}\text { Depth in Klassen } \\
\text { Domestic Well } \\
\text { (ft BGL) }\end{array}$ & $\begin{array}{c}\text { Depth in Klassen } \\
\text { Test Well } \\
\text { (ft BGL) }\end{array}$ & $\begin{array}{l}\text { Elevation in Klassen } \\
\text { Domestic Well } \\
\text { (ft AMSL) }\end{array}$ & \\
\hline $1 / 16 / 1218: 30$ & 33.511 & 35.517 & 1475.26 & 1473.61 \\
\hline $1 / 16 / 12$ 19:00 & 33.438 & 35.521 & 1475.33 & 1473.60 \\
\hline 1/16/12 19:30 & 33.45 & 35.514 & 1475.32 & 1473.61 \\
\hline $1 / 16 / 1220: 00$ & 33.443 & 35.512 & 1475.33 & 1473.61 \\
\hline $1 / 16 / 1220: 30$ & 33.363 & 35.505 & 1475.41 & 1473.62 \\
\hline $1 / 16 / 1221: 00$ & 33.34 & 35.503 & 1475.43 & 1473.62 \\
\hline $1 / 16 / 1221: 30$ & 33.412 & 35.498 & 1475.36 & 1473.62 \\
\hline $1 / 16 / 1222: 00$ & 33.436 & 35.493 & 1475.33 & 1473.63 \\
\hline $1 / 16 / 1222: 30$ & 33.851 & 35.486 & 1474.92 & 1473.64 \\
\hline $1 / 16 / 1223: 00$ & 33.9 & 35.477 & 1474.87 & 1473.65 \\
\hline 1/16/12 23:30 & 33.534 & 35.477 & 1475.24 & 1473.65 \\
\hline $1 / 17 / 12$ 0:00 & 33.434 & 35.465 & 1475.34 & 1473.66 \\
\hline 1/17/12 0:30 & 33.37 & 35.439 & 1475.40 & 1473.68 \\
\hline $1 / 17 / 121: 00$ & 33.326 & 35.425 & 1475.44 & 1473.70 \\
\hline 1/17/12 1:30 & 33.29 & 35.411 & 1475.48 & 1473.71 \\
\hline 1/17/12 2:00 & 33.262 & 35.402 & 1475.51 & 1473.72 \\
\hline 1/17/12 2:30 & 33.33 & 35.385 & 1475.44 & 1473.74 \\
\hline 1/17/12 3:00 & 33.267 & 35.381 & 1475.50 & 1473.74 \\
\hline $1 / 17 / 123: 30$ & 33.232 & 35.378 & 1475.54 & 1473.74 \\
\hline $1 / 17 / 124: 00$ & 33.204 & 35.378 & 1475.57 & 1473.74 \\
\hline 1/17/12 4:30 & 33.213 & 35.376 & 1475.56 & 1473.75 \\
\hline 1/17/12 5:00 & 33.183 & 35.374 & 1475.59 & 1473.75 \\
\hline 1/17/12 5:30 & 33.192 & 35.374 & 1475.58 & 1473.75 \\
\hline $1 / 17 / 126: 00$ & 33.164 & 35.366 & 1475.61 & 1473.76 \\
\hline $1 / 17 / 126: 30$ & 33.415 & 35.369 & 1475.35 & 1473.75 \\
\hline 1/17/12 7:00 & 33.316 & 35.367 & 1475.45 & 1473.76 \\
\hline $1 / 17 / 127: 30$ & 33.333 & 35.369 & 1475.44 & 1473.75 \\
\hline 1/17/12 8:00 & 33.49 & 35.371 & 1475.28 & 1473.75 \\
\hline 1/17/12 8:30 & 33.293 & 35.381 & 1475.48 & 1473.74 \\
\hline 1/17/12 9:00 & 33.241 & 35.388 & 1475.53 & 1473.73 \\
\hline 1/17/12 9:30 & 33.208 & 35.39 & 1475.56 & 1473.73 \\
\hline 1/17/12 10:00 & 33.349 & 35.399 & 1475.42 & 1473.72 \\
\hline 1/17/12 10:30 & 33.232 & 35.411 & 1475.54 & 1473.71 \\
\hline 1/17/12 11:00 & 33.394 & 35.425 & 1475.38 & 1473.70 \\
\hline 1/17/12 11:30 & 33.237 & 35.456 & 1475.53 & 1473.67 \\
\hline 1/17/12 12:00 & 33.194 & 35.481 & 1475.58 & 1473.64 \\
\hline $1 / 17 / 12$ 12:30 & 33.162 & 35.5 & 1475.61 & 1473.62 \\
\hline 1/17/12 13:00 & 33.609 & 35.505 & 1475.16 & 1473.62 \\
\hline
\end{tabular}


TABLE S1.3 Automatically recorded groundwater levels in the Klassen wells, September 15, 2011, to March 13, 2012.

\begin{tabular}{|c|c|c|c|c|}
\hline \multicolumn{3}{|c|}{ Reference Elevation (ft AMSL) } & 1508.77 & \multirow{2}{*}{$\begin{array}{l}1509.12 \\
\text { Elevation in Klassen } \\
\text { Test Well (ft AMSL) }\end{array}$} \\
\hline Date and Time & $\begin{array}{l}\text { Depth in Klassen } \\
\text { Domestic Well } \\
(\mathrm{ft} \mathrm{BGL})\end{array}$ & $\begin{array}{c}\text { Depth in Klassen } \\
\text { Test Well } \\
\text { (ft BGL) }\end{array}$ & $\begin{array}{l}\text { Elevation in Klassen } \\
\text { Domestic Well } \\
\text { (ft AMSL) }\end{array}$ & \\
\hline $1 / 17 / 1213: 30$ & 33.18 & 35.503 & 1475.59 & 1473.62 \\
\hline $1 / 17 / 1214: 00$ & 33.143 & 35.503 & 1475.63 & 1473.62 \\
\hline $1 / 17 / 1214: 30$ & 33.265 & 35.498 & 1475.50 & 1473.62 \\
\hline $1 / 17 / 1215: 00$ & 33.166 & 35.491 & 1475.60 & 1473.63 \\
\hline $1 / 17 / 1215: 30$ & 33.218 & 35.486 & 1475.55 & 1473.64 \\
\hline $1 / 17 / 1216: 00$ & 34.806 & 35.484 & 1473.96 & 1473.64 \\
\hline $1 / 17 / 1216: 30$ & 33.422 & 35.489 & 1475.35 & 1473.63 \\
\hline 1/17/12 17:00 & 33.356 & 35.486 & 1475.41 & 1473.64 \\
\hline $1 / 17 / 12$ 17:30 & 33.431 & 35.484 & 1475.34 & 1473.64 \\
\hline $1 / 17 / 1218: 00$ & 33.434 & 35.481 & 1475.34 & 1473.64 \\
\hline 1/17/12 18:30 & 33.38 & 35.479 & 1475.39 & 1473.64 \\
\hline 1/17/12 19:00 & 33.427 & 35.484 & 1475.34 & 1473.64 \\
\hline 1/17/12 19:30 & 33.558 & 35.489 & 1475.21 & 1473.63 \\
\hline $1 / 17 / 1220: 00$ & 33.509 & 35.486 & 1475.26 & 1473.64 \\
\hline $1 / 17 / 12$ 20:30 & 33.452 & 35.484 & 1475.32 & 1473.64 \\
\hline $1 / 17 / 12$ 21:00 & 33.525 & 35.484 & 1475.24 & 1473.64 \\
\hline $1 / 17 / 1221: 30$ & 33.422 & 35.484 & 1475.35 & 1473.64 \\
\hline $1 / 17 / 1222: 00$ & 33.448 & 35.489 & 1475.32 & 1473.63 \\
\hline $1 / 17 / 1222: 30$ & 35.382 & 35.493 & 1473.39 & 1473.63 \\
\hline $1 / 17 / 12$ 23:00 & 35.436 & 35.5 & 1473.33 & 1473.62 \\
\hline $1 / 17 / 12$ 23:30 & 33.602 & 35.505 & 1475.17 & 1473.62 \\
\hline 1/18/12 0:00 & 33.478 & 35.512 & 1475.29 & 1473.61 \\
\hline $1 / 18 / 120: 30$ & 33.403 & 35.512 & 1475.37 & 1473.61 \\
\hline 1/18/12 1:00 & 33.349 & 35.514 & 1475.42 & 1473.61 \\
\hline 1/18/12 1:30 & 33.307 & 35.514 & 1475.46 & 1473.61 \\
\hline $1 / 18 / 122: 00$ & 33.293 & 35.514 & 1475.48 & 1473.61 \\
\hline 1/18/12 2:30 & 33.253 & 35.517 & 1475.52 & 1473.61 \\
\hline 1/18/12 3:00 & 33.222 & 35.512 & 1475.55 & 1473.61 \\
\hline 1/18/12 3:30 & 33.197 & 35.51 & 1475.57 & 1473.61 \\
\hline 1/18/12 4:00 & 33.684 & 35.503 & 1475.09 & 1473.62 \\
\hline 1/18/12 4:30 & 33.225 & 35.5 & 1475.54 & 1473.62 \\
\hline 1/18/12 5:00 & 33.18 & 35.498 & 1475.59 & 1473.62 \\
\hline 1/18/12 5:30 & 33.152 & 35.493 & 1475.62 & 1473.63 \\
\hline 1/18/12 6:00 & 33.131 & 35.484 & 1475.64 & 1473.64 \\
\hline 1/18/12 6:30 & 33.314 & 35.491 & 1475.46 & 1473.63 \\
\hline 1/18/12 7:00 & 33.281 & 35.486 & 1475.49 & 1473.64 \\
\hline $1 / 18 / 127: 30$ & 33.373 & 35.486 & 1475.40 & 1473.64 \\
\hline $1 / 18 / 128: 00$ & 33.312 & 35.482 & 1475.46 & 1473.64 \\
\hline
\end{tabular}


TABLE S1.3 Automatically recorded groundwater levels in the Klassen wells, September 15, 2011, to March 13, 2012.

\begin{tabular}{|c|c|c|c|c|}
\hline \multicolumn{3}{|c|}{ Reference Elevation (ft AMSL) } & 1508.77 & \multirow{2}{*}{$\begin{array}{l}1509.12 \\
\text { Elevation in Klassen } \\
\text { Test Well (ft AMSL) }\end{array}$} \\
\hline Date and Time & $\begin{array}{l}\text { Depth in Klassen } \\
\text { Domestic Well } \\
(\mathrm{ft} \mathrm{BGL})\end{array}$ & $\begin{array}{c}\text { Depth in Klassen } \\
\text { Test Well } \\
\text { (ft BGL) }\end{array}$ & $\begin{array}{l}\text { Elevation in Klassen } \\
\text { Domestic Well } \\
\text { (ft AMSL) }\end{array}$ & \\
\hline $1 / 18 / 128: 30$ & 33.248 & 35.482 & 1475.52 & 1473.64 \\
\hline 1/18/12 9:00 & 33.382 & 35.484 & 1475.39 & 1473.64 \\
\hline 1/18/12 9:30 & 33.246 & 35.477 & 1475.52 & 1473.65 \\
\hline $1 / 18 / 1210: 00$ & 33.194 & 35.479 & 1475.58 & 1473.64 \\
\hline $1 / 18 / 1210: 30$ & 33.316 & 35.482 & 1475.45 & 1473.64 \\
\hline $1 / 18 / 1211: 00$ & 33.194 & 35.491 & 1475.58 & 1473.63 \\
\hline $1 / 18 / 1211: 30$ & 33.15 & 35.538 & 1475.62 & 1473.58 \\
\hline $1 / 18 / 1212: 00$ & 33.119 & 35.545 & 1475.65 & 1473.58 \\
\hline $1 / 18 / 1212: 30$ & 33.178 & 35.545 & 1475.59 & 1473.58 \\
\hline $1 / 18 / 1213: 00$ & 33.126 & 35.545 & 1475.64 & 1473.58 \\
\hline 1/18/12 13:30 & 33.171 & 35.545 & 1475.60 & 1473.58 \\
\hline $1 / 18 / 1214: 00$ & 33.213 & 35.543 & 1475.56 & 1473.58 \\
\hline $1 / 18 / 1214: 30$ & 33.147 & 35.524 & 1475.62 & 1473.60 \\
\hline $1 / 18 / 1215: 00$ & 33.377 & 35.456 & 1475.39 & 1473.67 \\
\hline $1 / 18 / 12$ 15:30 & 33.199 & 35.425 & 1475.57 & 1473.70 \\
\hline 1/18/12 16:00 & 33.143 & 35.406 & 1475.63 & 1473.72 \\
\hline $1 / 18 / 12$ 16:30 & 33.286 & 35.399 & 1475.48 & 1473.72 \\
\hline $1 / 18 / 12$ 17:00 & 33.143 & 35.397 & 1475.63 & 1473.73 \\
\hline $1 / 18 / 12$ 17:30 & 33.164 & 35.395 & 1475.61 & 1473.73 \\
\hline $1 / 18 / 1218: 00$ & 33.168 & 35.399 & 1475.60 & 1473.72 \\
\hline $1 / 18 / 1218: 30$ & 33.239 & 35.402 & 1475.53 & 1473.72 \\
\hline 1/18/12 19:00 & 33.166 & 35.406 & 1475.60 & 1473.72 \\
\hline $1 / 18 / 12$ 19:30 & 33.124 & 35.413 & 1475.65 & 1473.71 \\
\hline 1/18/12 20:00 & 33.211 & 35.416 & 1475.56 & 1473.71 \\
\hline $1 / 18 / 12$ 20:30 & 33.18 & 35.413 & 1475.59 & 1473.71 \\
\hline $1 / 18 / 12$ 21:00 & 33.124 & 35.421 & 1475.65 & 1473.70 \\
\hline 1/18/12 21:30 & 33.152 & 35.421 & 1475.62 & 1473.70 \\
\hline $1 / 18 / 1222: 00$ & 37.102 & 35.425 & 1471.67 & 1473.70 \\
\hline $1 / 18 / 1222: 30$ & 35.326 & 35.437 & 1473.44 & 1473.69 \\
\hline $1 / 18 / 12$ 23:00 & 33.412 & 35.444 & 1475.36 & 1473.68 \\
\hline $1 / 18 / 12$ 23:30 & 33.33 & 35.444 & 1475.44 & 1473.68 \\
\hline 1/19/12 0:00 & 33.253 & 35.439 & 1475.52 & 1473.68 \\
\hline 1/19/12 0:30 & 33.197 & 35.439 & 1475.57 & 1473.68 \\
\hline 1/19/12 1:00 & 33.154 & 35.43 & 1475.62 & 1473.69 \\
\hline 1/19/12 1:30 & 33.119 & 35.423 & 1475.65 & 1473.70 \\
\hline 1/19/12 2:00 & 33.138 & 35.416 & 1475.63 & 1473.71 \\
\hline $1 / 19 / 122: 30$ & 33.185 & 35.411 & 1475.58 & 1473.71 \\
\hline 1/19/12 3:00 & 33.157 & 35.399 & 1475.61 & 1473.72 \\
\hline
\end{tabular}


TABLE S1.3 Automatically recorded groundwater levels in the Klassen wells, September 15, 2011, to March 13, 2012.

\begin{tabular}{|c|c|c|c|c|}
\hline \multicolumn{3}{|c|}{ Reference Elevation (ft AMSL) } & 1508.77 & \multirow{2}{*}{$\begin{array}{l}1509.12 \\
\text { Elevation in Klassen } \\
\text { Test Well (ft AMSL) }\end{array}$} \\
\hline Date and Time & $\begin{array}{l}\text { Depth in Klassen } \\
\text { Domestic Well } \\
\text { (ft BGL) }\end{array}$ & $\begin{array}{c}\text { Depth in Klassen } \\
\text { Test Well } \\
\text { (ft BGL) }\end{array}$ & $\begin{array}{l}\text { Elevation in Klassen } \\
\text { Domestic Well } \\
\text { (ft AMSL) }\end{array}$ & \\
\hline 1/19/12 3:30 & 33.171 & 35.385 & 1475.60 & 1473.74 \\
\hline 1/19/12 4:00 & 33.23 & 35.378 & 1475.54 & 1473.74 \\
\hline 1/19/12 4:30 & 33.164 & 35.369 & 1475.61 & 1473.75 \\
\hline 1/19/12 5:00 & 33.122 & 35.357 & 1475.65 & 1473.77 \\
\hline $1 / 19 / 125: 30$ & 33.178 & 35.355 & 1475.59 & 1473.77 \\
\hline 1/19/12 6:00 & 33.49 & 35.343 & 1475.28 & 1473.78 \\
\hline 1/19/12 6:30 & 33.23 & 35.338 & 1475.54 & 1473.78 \\
\hline 1/19/12 7:00 & 33.201 & 35.322 & 1475.57 & 1473.80 \\
\hline 1/19/12 7:30 & 33.262 & 35.32 & 1475.51 & 1473.80 \\
\hline 1/19/12 8:00 & 33.649 & 35.317 & 1475.12 & 1473.81 \\
\hline 1/19/12 8:30 & 33.356 & 35.322 & 1475.41 & 1473.80 \\
\hline 1/19/12 9:00 & 33.403 & 35.322 & 1475.37 & 1473.80 \\
\hline 1/19/12 9:30 & 33.38 & 35.329 & 1475.39 & 1473.79 \\
\hline $1 / 19 / 1210: 00$ & 33.276 & 35.341 & 1475.49 & 1473.78 \\
\hline 1/19/12 10:30 & 33.349 & 35.357 & 1475.42 & 1473.77 \\
\hline 1/19/12 11:00 & 33.307 & 35.371 & 1475.46 & 1473.75 \\
\hline 1/19/12 11:30 & 35.762 & 35.388 & 1473.01 & 1473.73 \\
\hline 1/19/12 12:00 & 33.246 & 35.423 & 1475.52 & 1473.70 \\
\hline 1/19/12 12:30 & 33.19 & 35.439 & 1475.58 & 1473.68 \\
\hline 1/19/12 13:00 & 33.255 & 35.451 & 1475.51 & 1473.67 \\
\hline $1 / 19 / 12$ 13:30 & 33.176 & 35.463 & 1475.59 & 1473.66 \\
\hline 1/19/12 14:00 & 33.448 & 35.456 & 1475.32 & 1473.67 \\
\hline $1 / 19 / 12$ 14:30 & 33.206 & 35.46 & 1475.56 & 1473.66 \\
\hline 1/19/12 15:00 & 33.173 & 35.449 & 1475.60 & 1473.67 \\
\hline 1/19/12 15:30 & 33.133 & 35.442 & 1475.64 & 1473.68 \\
\hline 1/19/12 16:00 & 33.208 & 35.439 & 1475.56 & 1473.68 \\
\hline 1/19/12 16:30 & 33.14 & 35.427 & 1475.63 & 1473.70 \\
\hline 1/19/12 17:00 & 33.103 & 35.428 & 1475.67 & 1473.69 \\
\hline 1/19/12 17:30 & 33.108 & 35.418 & 1475.66 & 1473.70 \\
\hline 1/19/12 18:00 & 33.215 & 35.423 & 1475.55 & 1473.70 \\
\hline 1/19/12 18:30 & 33.131 & 35.416 & 1475.64 & 1473.71 \\
\hline 1/19/12 19:00 & 33.093 & 35.402 & 1475.68 & 1473.72 \\
\hline 1/19/12 19:30 & 33.241 & 35.402 & 1475.53 & 1473.72 \\
\hline 1/19/12 20:00 & 33.197 & 35.395 & 1475.57 & 1473.73 \\
\hline 1/19/12 20:30 & 33.143 & 35.383 & 1475.63 & 1473.74 \\
\hline 1/19/12 21:00 & 33.105 & 35.383 & 1475.66 & 1473.74 \\
\hline 1/19/12 21:30 & 33.434 & 35.39 & 1475.34 & 1473.73 \\
\hline $1 / 19 / 1222: 00$ & 33.45 & 35.411 & 1475.32 & 1473.71 \\
\hline
\end{tabular}


TABLE S1.3 Automatically recorded groundwater levels in the Klassen wells, September 15, 2011, to March 13, 2012.

\begin{tabular}{|c|c|c|c|c|}
\hline \multicolumn{3}{|c|}{ Reference Elevation (ft AMSL) } & 1508.77 & \multirow{2}{*}{$\begin{array}{l}1509.12 \\
\text { Elevation in Klassen } \\
\text { Test Well (ft AMSL) }\end{array}$} \\
\hline Date and Time & $\begin{array}{l}\text { Depth in Klassen } \\
\text { Domestic Well } \\
\text { (ft BGL) }\end{array}$ & $\begin{array}{c}\text { Depth in Klassen } \\
\text { Test Well } \\
\text { (ft BGL) }\end{array}$ & $\begin{array}{l}\text { Elevation in Klassen } \\
\text { Domestic Well } \\
\text { (ft AMSL) }\end{array}$ & \\
\hline $1 / 19 / 1222: 30$ & 33.544 & 35.427 & 1475.23 & 1473.70 \\
\hline $1 / 19 / 1223: 00$ & 33.755 & 35.435 & 1475.01 & 1473.69 \\
\hline $1 / 19 / 1223: 30$ & 33.45 & 35.435 & 1475.32 & 1473.69 \\
\hline $1 / 20 / 120: 00$ & 33.351 & 35.446 & 1475.42 & 1473.68 \\
\hline $1 / 20 / 12$ 0:30 & 33.283 & 35.456 & 1475.49 & 1473.67 \\
\hline 1/20/12 1:00 & 33.237 & 35.453 & 1475.53 & 1473.67 \\
\hline $1 / 20 / 121: 30$ & 33.197 & 35.456 & 1475.57 & 1473.67 \\
\hline $1 / 20 / 122: 00$ & 33.159 & 35.456 & 1475.61 & 1473.67 \\
\hline $1 / 20 / 122: 30$ & 33.131 & 35.442 & 1475.64 & 1473.68 \\
\hline 1/20/12 3:00 & 33.105 & 35.428 & 1475.66 & 1473.69 \\
\hline $1 / 20 / 123: 30$ & 33.082 & 35.425 & 1475.69 & 1473.70 \\
\hline $1 / 20 / 124: 00$ & 33.058 & 35.423 & 1475.71 & 1473.70 \\
\hline $1 / 20 / 124: 30$ & 33.218 & 35.413 & 1475.55 & 1473.71 \\
\hline $1 / 20 / 125: 00$ & 33.091 & 35.413 & 1475.68 & 1473.71 \\
\hline $1 / 20 / 125: 30$ & 33.086 & 35.392 & 1475.68 & 1473.73 \\
\hline 1/20/12 6:00 & 33.051 & 35.385 & 1475.72 & 1473.74 \\
\hline 1/20/12 6:30 & 33.171 & 35.381 & 1475.60 & 1473.74 \\
\hline 1/20/12 7:00 & 33.098 & 35.369 & 1475.67 & 1473.75 \\
\hline $1 / 20 / 12$ 7:30 & 33.143 & 35.362 & 1475.63 & 1473.76 \\
\hline $1 / 20 / 128: 00$ & 33.103 & 35.367 & 1475.67 & 1473.76 \\
\hline $1 / 20 / 128: 30$ & 38.478 & 35.376 & 1470.29 & 1473.75 \\
\hline 1/20/12 9:00 & 37.603 & 35.378 & 1471.17 & 1473.74 \\
\hline 1/20/12 9:30 & 33.366 & 35.376 & 1475.40 & 1473.75 \\
\hline 1/20/12 10:00 & 37.041 & 35.374 & 1471.73 & 1473.75 \\
\hline $1 / 20 / 12$ 10:30 & 33.359 & 35.371 & 1475.41 & 1473.75 \\
\hline 1/20/12 11:00 & 35.952 & 35.39 & 1472.82 & 1473.73 \\
\hline 1/20/12 11:30 & 33.354 & 35.402 & 1475.42 & 1473.72 \\
\hline $1 / 20 / 1212: 00$ & 33.26 & 35.402 & 1475.51 & 1473.72 \\
\hline $1 / 20 / 1212: 30$ & 35.912 & 35.406 & 1472.86 & 1473.72 \\
\hline $1 / 20 / 12$ 13:00 & 33.241 & 35.43 & 1475.53 & 1473.69 \\
\hline $1 / 20 / 12$ 13:30 & 33.18 & 35.423 & 1475.59 & 1473.70 \\
\hline $1 / 20 / 1214: 00$ & 33.305 & 35.42 & 1475.46 & 1473.70 \\
\hline $1 / 20 / 1214: 30$ & 33.162 & 35.404 & 1475.61 & 1473.72 \\
\hline $1 / 20 / 12$ 15:00 & 33.147 & 35.385 & 1475.62 & 1473.74 \\
\hline $1 / 20 / 12$ 15:30 & 33.112 & 35.374 & 1475.66 & 1473.75 \\
\hline 1/20/12 16:00 & 33.145 & 35.355 & 1475.62 & 1473.77 \\
\hline $1 / 20 / 12$ 16:30 & 33.218 & 35.338 & 1475.55 & 1473.78 \\
\hline $1 / 20 / 12$ 17:00 & 34.77 & 35.327 & 1474.00 & 1473.80 \\
\hline
\end{tabular}


TABLE S1.3 Automatically recorded groundwater levels in the Klassen wells, September 15, 2011, to March 13, 2012.

\begin{tabular}{|c|c|c|c|c|}
\hline \multicolumn{3}{|c|}{ Reference Elevation (ft AMSL) } & 1508.77 & \multirow{2}{*}{$\begin{array}{l}1509.12 \\
\text { Elevation in Klassen } \\
\text { Test Well (ft AMSL) }\end{array}$} \\
\hline Date and Time & $\begin{array}{l}\text { Depth in Klassen } \\
\text { Domestic Well } \\
\text { (ft BGL) }\end{array}$ & $\begin{array}{c}\text { Depth in Klassen } \\
\text { Test Well } \\
\text { (ft BGL) }\end{array}$ & $\begin{array}{l}\text { Elevation in Klassen } \\
\text { Domestic Well } \\
\text { (ft AMSL) }\end{array}$ & \\
\hline $1 / 20 / 1217: 30$ & 33.424 & 35.324 & 1475.35 & 1473.80 \\
\hline $1 / 20 / 1218: 00$ & 33.389 & 35.32 & 1475.38 & 1473.80 \\
\hline $1 / 20 / 1218: 30$ & 33.298 & 35.31 & 1475.47 & 1473.81 \\
\hline 1/20/12 19:00 & 33.248 & 35.299 & 1475.52 & 1473.82 \\
\hline $1 / 20 / 1219: 30$ & 33.471 & 35.294 & 1475.30 & 1473.83 \\
\hline $1 / 20 / 1220: 00$ & 33.272 & 35.294 & 1475.50 & 1473.83 \\
\hline $1 / 20 / 1220: 30$ & 33.239 & 35.294 & 1475.53 & 1473.83 \\
\hline $1 / 20 / 1221: 00$ & 33.19 & 35.289 & 1475.58 & 1473.83 \\
\hline $1 / 20 / 1221: 30$ & 33.19 & 35.289 & 1475.58 & 1473.83 \\
\hline $1 / 20 / 1222: 00$ & 33.236 & 35.289 & 1475.53 & 1473.83 \\
\hline $1 / 20 / 1222: 30$ & 36.187 & 35.294 & 1472.58 & 1473.83 \\
\hline $1 / 20 / 1223: 00$ & 38.511 & 35.305 & 1470.26 & 1473.82 \\
\hline $1 / 20 / 1223: 30$ & 33.492 & 35.32 & 1475.28 & 1473.80 \\
\hline $1 / 21 / 120: 00$ & 33.375 & 35.334 & 1475.39 & 1473.79 \\
\hline 1/21/12 0:30 & 33.356 & 35.338 & 1475.41 & 1473.78 \\
\hline 1/21/12 1:00 & 33.293 & 35.348 & 1475.48 & 1473.77 \\
\hline $1 / 21 / 121: 30$ & 33.244 & 35.348 & 1475.53 & 1473.77 \\
\hline $1 / 21 / 122: 00$ & 33.255 & 35.35 & 1475.51 & 1473.77 \\
\hline 1/21/12 2:30 & 33.29 & 35.352 & 1475.48 & 1473.77 \\
\hline 1/21/12 3:00 & 33.253 & 35.367 & 1475.52 & 1473.76 \\
\hline 1/21/12 3:30 & 33.23 & 35.376 & 1475.54 & 1473.75 \\
\hline $1 / 21 / 124: 00$ & 33.314 & 35.388 & 1475.46 & 1473.73 \\
\hline 1/21/12 4:30 & 33.412 & 35.374 & 1475.36 & 1473.75 \\
\hline 1/21/12 5:00 & 33.269 & 35.374 & 1475.50 & 1473.75 \\
\hline 1/21/12 5:30 & 33.215 & 35.378 & 1475.55 & 1473.74 \\
\hline 1/21/12 6:00 & 33.176 & 35.381 & 1475.59 & 1473.74 \\
\hline 1/21/12 6:30 & 33.274 & 35.371 & 1475.50 & 1473.75 \\
\hline 1/21/12 7:00 & 33.194 & 35.371 & 1475.58 & 1473.75 \\
\hline 1/21/12 7:30 & 33.154 & 35.371 & 1475.62 & 1473.75 \\
\hline $1 / 21 / 128: 00$ & 33.124 & 35.374 & 1475.65 & 1473.75 \\
\hline 1/21/12 8:30 & 33.103 & 35.383 & 1475.67 & 1473.74 \\
\hline 1/21/12 9:00 & 35.279 & 35.388 & 1473.49 & 1473.73 \\
\hline 1/21/12 9:30 & 33.424 & 35.392 & 1475.35 & 1473.73 \\
\hline 1/21/12 10:00 & 33.783 & 35.409 & 1474.99 & 1473.71 \\
\hline 1/21/12 10:30 & 33.551 & 35.425 & 1475.22 & 1473.70 \\
\hline 1/21/12 11:00 & 33.713 & 35.449 & 1475.06 & 1473.67 \\
\hline 1/21/12 11:30 & 33.624 & 35.472 & 1475.15 & 1473.65 \\
\hline $1 / 21 / 1212: 00$ & 33.673 & 35.507 & 1475.10 & 1473.62 \\
\hline
\end{tabular}


TABLE S1.3 Automatically recorded groundwater levels in the Klassen wells, September 15, 2011, to March 13, 2012.

\begin{tabular}{|c|c|c|c|c|}
\hline \multicolumn{3}{|c|}{ Reference Elevation (ft AMSL) } & 1508.77 & \multirow{2}{*}{$\begin{array}{l}1509.12 \\
\text { Elevation in Klassen } \\
\text { Test Well (ft AMSL) }\end{array}$} \\
\hline Date and Time & $\begin{array}{l}\text { Depth in Klassen } \\
\text { Domestic Well } \\
\text { (ft BGL) }\end{array}$ & $\begin{array}{c}\text { Depth in Klassen } \\
\text { Test Well } \\
\text { (ft BGL) }\end{array}$ & $\begin{array}{l}\text { Elevation in Klassen } \\
\text { Domestic Well } \\
\text { (ft AMSL) }\end{array}$ & \\
\hline $1 / 21 / 1212: 30$ & 33.713 & 35.54 & 1475.06 & 1473.58 \\
\hline $1 / 21 / 1213: 00$ & 33.666 & 35.554 & 1475.10 & 1473.57 \\
\hline $1 / 21 / 1213: 30$ & 33.548 & 35.557 & 1475.22 & 1473.57 \\
\hline $1 / 21 / 1214: 00$ & 33.638 & 35.554 & 1475.13 & 1473.57 \\
\hline $1 / 21 / 1214: 30$ & 33.473 & 35.549 & 1475.30 & 1473.57 \\
\hline $1 / 21 / 1215: 00$ & 33.398 & 35.542 & 1475.37 & 1473.58 \\
\hline $1 / 21 / 1215: 30$ & 33.344 & 35.528 & 1475.43 & 1473.59 \\
\hline $1 / 21 / 1216: 00$ & 34.761 & 35.491 & 1474.01 & 1473.63 \\
\hline $1 / 21 / 1216: 30$ & 33.469 & 35.451 & 1475.30 & 1473.67 \\
\hline $1 / 21 / 1217: 00$ & 33.405 & 35.428 & 1475.36 & 1473.69 \\
\hline $1 / 21 / 1217: 30$ & 33.34 & 35.413 & 1475.43 & 1473.71 \\
\hline $1 / 21 / 1218: 00$ & 33.396 & 35.413 & 1475.37 & 1473.71 \\
\hline $1 / 21 / 1218: 30$ & 33.492 & 35.42 & 1475.28 & 1473.70 \\
\hline 1/21/12 19:00 & 33.464 & 35.428 & 1475.31 & 1473.69 \\
\hline 1/21/12 19:30 & 33.502 & 35.435 & 1475.27 & 1473.69 \\
\hline $1 / 21 / 12$ 20:00 & 33.499 & 35.439 & 1475.27 & 1473.68 \\
\hline $1 / 21 / 1220: 30$ & 33.574 & 35.449 & 1475.20 & 1473.67 \\
\hline $1 / 21 / 1221: 00$ & 33.666 & 35.46 & 1475.10 & 1473.66 \\
\hline $1 / 21 / 1221: 30$ & 33.647 & 35.463 & 1475.12 & 1473.66 \\
\hline $1 / 21 / 1222: 00$ & 33.989 & 35.477 & 1474.78 & 1473.65 \\
\hline $1 / 21 / 1222: 30$ & 33.694 & 35.488 & 1475.08 & 1473.63 \\
\hline $1 / 21 / 12$ 23:00 & 33.755 & 35.503 & 1475.01 & 1473.62 \\
\hline $1 / 21 / 12$ 23:30 & 33.699 & 35.514 & 1475.07 & 1473.61 \\
\hline $1 / 22 / 120: 00$ & 33.724 & 35.524 & 1475.05 & 1473.60 \\
\hline $1 / 22 / 120: 30$ & 33.715 & 35.535 & 1475.05 & 1473.59 \\
\hline $1 / 22 / 121: 00$ & 33.605 & 35.54 & 1475.16 & 1473.58 \\
\hline $1 / 22 / 121: 30$ & 33.574 & 35.54 & 1475.20 & 1473.58 \\
\hline $1 / 22 / 122: 00$ & 33.551 & 35.54 & 1475.22 & 1473.58 \\
\hline $1 / 22 / 122: 30$ & 33.563 & 35.545 & 1475.21 & 1473.58 \\
\hline 1/22/12 3:00 & 33.511 & 35.535 & 1475.26 & 1473.59 \\
\hline 1/22/12 3:30 & 33.466 & 35.531 & 1475.30 & 1473.59 \\
\hline $1 / 22 / 124: 00$ & 33.541 & 35.533 & 1475.23 & 1473.59 \\
\hline $1 / 22 / 124: 30$ & 33.462 & 35.528 & 1475.31 & 1473.59 \\
\hline $1 / 22 / 125: 00$ & 33.396 & 35.531 & 1475.37 & 1473.59 \\
\hline 1/22/12 5:30 & 33.349 & 35.524 & 1475.42 & 1473.60 \\
\hline 1/22/12 6:00 & 33.57 & 35.514 & 1475.20 & 1473.61 \\
\hline $1 / 22 / 126: 30$ & 33.525 & 35.514 & 1475.24 & 1473.61 \\
\hline $1 / 22 / 127: 00$ & 33.408 & 35.5 & 1475.36 & 1473.62 \\
\hline
\end{tabular}


TABLE S1.3 Automatically recorded groundwater levels in the Klassen wells, September 15, 2011, to March 13, 2012.

\begin{tabular}{|c|c|c|c|c|}
\hline \multicolumn{3}{|c|}{ Reference Elevation (ft AMSL) } & 1508.77 & \multirow{2}{*}{$\begin{array}{l}1509.12 \\
\text { Elevation in Klassen } \\
\text { Test Well (ft AMSL) }\end{array}$} \\
\hline Date and Time & $\begin{array}{l}\text { Depth in Klassen } \\
\text { Domestic Well } \\
\text { (ft BGL) }\end{array}$ & $\begin{array}{c}\text { Depth in Klassen } \\
\text { Test Well } \\
\text { (ft BGL) }\end{array}$ & $\begin{array}{l}\text { Elevation in Klassen } \\
\text { Domestic Well } \\
\text { (ft AMSL) }\end{array}$ & \\
\hline $1 / 22 / 127: 30$ & 33.349 & 35.503 & 1475.42 & 1473.62 \\
\hline $1 / 22 / 128: 00$ & 33.506 & 35.498 & 1475.26 & 1473.62 \\
\hline $1 / 22 / 128: 30$ & 33.757 & 35.498 & 1475.01 & 1473.62 \\
\hline $1 / 22 / 129: 00$ & 33.675 & 35.5 & 1475.09 & 1473.62 \\
\hline $1 / 22 / 129: 30$ & 33.764 & 35.503 & 1475.01 & 1473.62 \\
\hline $1 / 22 / 1210: 00$ & 33.717 & 35.512 & 1475.05 & 1473.61 \\
\hline $1 / 22 / 1210: 30$ & 33.692 & 35.517 & 1475.08 & 1473.61 \\
\hline $1 / 22 / 1211: 00$ & 33.769 & 35.538 & 1475.00 & 1473.58 \\
\hline $1 / 22 / 1211: 30$ & 33.689 & 35.557 & 1475.08 & 1473.57 \\
\hline $1 / 22 / 1212: 00$ & 33.609 & 35.573 & 1475.16 & 1473.55 \\
\hline $1 / 22 / 1212: 30$ & 33.525 & 35.582 & 1475.24 & 1473.54 \\
\hline $1 / 22 / 1213: 00$ & 34.135 & 35.582 & 1474.63 & 1473.54 \\
\hline $1 / 22 / 1213: 30$ & 34.013 & 35.594 & 1474.76 & 1473.53 \\
\hline 1/22/12 14:00 & 34.071 & 35.62 & 1474.70 & 1473.50 \\
\hline $1 / 22 / 1214: 30$ & 34.139 & 35.639 & 1474.63 & 1473.48 \\
\hline $1 / 22 / 12$ 15:00 & 34.447 & 35.655 & 1474.32 & 1473.47 \\
\hline $1 / 22 / 1215: 30$ & 34.552 & 35.711 & 1474.22 & 1473.41 \\
\hline $1 / 22 / 1216: 00$ & 34.594 & 35.74 & 1474.18 & 1473.38 \\
\hline $1 / 22 / 1216: 30$ & 34.625 & 35.779 & 1474.14 & 1473.34 \\
\hline $1 / 22 / 12$ 17:00 & 49.921 & 35.808 & 1458.85 & 1473.31 \\
\hline $1 / 22 / 12$ 17:30 & 51.264 & 35.854 & 1457.51 & 1473.27 \\
\hline $1 / 22 / 12$ 18:00 & 35.737 & 35.883 & 1473.03 & 1473.24 \\
\hline $1 / 22 / 1218: 30$ & 35.425 & 35.878 & 1473.34 & 1473.24 \\
\hline 1/22/12 19:00 & 35.319 & 35.88 & 1473.45 & 1473.24 \\
\hline 1/22/12 19:30 & 35.103 & 35.866 & 1473.67 & 1473.26 \\
\hline $1 / 22 / 12$ 20:00 & 34.897 & 35.848 & 1473.87 & 1473.27 \\
\hline $1 / 22 / 12$ 20:30 & 34.719 & 35.843 & 1474.05 & 1473.28 \\
\hline $1 / 22 / 12$ 21:00 & 34.705 & 35.845 & 1474.06 & 1473.28 \\
\hline $1 / 22 / 12$ 21:30 & 34.529 & 35.84 & 1474.24 & 1473.28 \\
\hline $1 / 22 / 12$ 22:00 & 34.397 & 35.833 & 1474.37 & 1473.29 \\
\hline $1 / 22 / 1222: 30$ & 34.294 & 35.836 & 1474.48 & 1473.29 \\
\hline $1 / 22 / 12$ 23:00 & 36.874 & 35.864 & 1471.90 & 1473.26 \\
\hline $1 / 22 / 12$ 23:30 & 34.428 & 35.822 & 1474.34 & 1473.30 \\
\hline 1/23/12 0:00 & 34.254 & 35.81 & 1474.52 & 1473.31 \\
\hline $1 / 23 / 120: 30$ & 34.149 & 35.796 & 1474.62 & 1473.33 \\
\hline $1 / 23 / 121: 00$ & 34.069 & 35.779 & 1474.70 & 1473.34 \\
\hline $1 / 23 / 121: 30$ & 33.999 & 35.761 & 1474.77 & 1473.36 \\
\hline $1 / 23 / 122: 00$ & 33.994 & 35.74 & 1474.78 & 1473.38 \\
\hline
\end{tabular}


TABLE S1.3 Automatically recorded groundwater levels in the Klassen wells, September 15, 2011, to March 13, 2012.

\begin{tabular}{|c|c|c|c|c|}
\hline \multicolumn{3}{|c|}{ Reference Elevation (ft AMSL) } & 1508.77 & \multirow{2}{*}{$\begin{array}{l}1509.12 \\
\text { Elevation in Klassen } \\
\text { Test Well (ft AMSL) }\end{array}$} \\
\hline Date and Time & $\begin{array}{l}\text { Depth in Klassen } \\
\text { Domestic Well } \\
\text { (ft BGL) }\end{array}$ & $\begin{array}{c}\text { Depth in Klassen } \\
\text { Test Well } \\
\text { (ft BGL) }\end{array}$ & $\begin{array}{l}\text { Elevation in Klassen } \\
\text { Domestic Well } \\
\text { (ft AMSL) }\end{array}$ & \\
\hline $1 / 23 / 122: 30$ & 34.032 & 35.733 & 1474.74 & 1473.39 \\
\hline $1 / 23 / 123: 00$ & 33.968 & 35.73 & 1474.80 & 1473.39 \\
\hline $1 / 23 / 123: 30$ & 33.94 & 35.733 & 1474.83 & 1473.39 \\
\hline 1/23/12 4:00 & 33.996 & 35.733 & 1474.77 & 1473.39 \\
\hline $1 / 23 / 124: 30$ & 33.9 & 35.726 & 1474.87 & 1473.40 \\
\hline $1 / 23 / 125: 00$ & 33.83 & 35.723 & 1474.94 & 1473.40 \\
\hline $1 / 23 / 125: 30$ & 33.774 & 35.716 & 1475.00 & 1473.41 \\
\hline 1/23/12 6:00 & 33.727 & 35.714 & 1475.04 & 1473.41 \\
\hline 1/23/12 6:30 & 33.907 & 35.714 & 1474.86 & 1473.41 \\
\hline $1 / 23 / 127: 00$ & 33.804 & 35.711 & 1474.97 & 1473.41 \\
\hline $1 / 23 / 127: 30$ & 33.811 & 35.707 & 1474.96 & 1473.42 \\
\hline $1 / 23 / 128: 00$ & 33.755 & 35.702 & 1475.01 & 1473.42 \\
\hline $1 / 23 / 128: 30$ & 33.867 & 35.702 & 1474.90 & 1473.42 \\
\hline 1/23/12 9:00 & 33.71 & 35.697 & 1475.06 & 1473.43 \\
\hline 1/23/12 9:30 & 33.734 & 35.695 & 1475.04 & 1473.43 \\
\hline 1/23/12 10:00 & 33.811 & 35.688 & 1474.96 & 1473.43 \\
\hline $1 / 23 / 12$ 10:30 & 33.67 & 35.69 & 1475.10 & 1473.43 \\
\hline 1/23/12 11:00 & 33.696 & 35.702 & 1475.07 & 1473.42 \\
\hline 1/23/12 11:30 & 33.745 & 35.709 & 1475.02 & 1473.41 \\
\hline $1 / 23 / 1212: 00$ & 33.715 & 35.721 & 1475.05 & 1473.40 \\
\hline $1 / 23 / 1212: 30$ & 33.628 & 35.723 & 1475.14 & 1473.40 \\
\hline 1/23/12 13:00 & 33.673 & 35.726 & 1475.10 & 1473.40 \\
\hline $1 / 23 / 12$ 13:30 & 35.61 & 35.723 & 1473.16 & 1473.40 \\
\hline $1 / 23 / 1214: 00$ & 33.614 & 35.718 & 1475.16 & 1473.40 \\
\hline $1 / 23 / 1214: 30$ & 33.548 & 35.707 & 1475.22 & 1473.42 \\
\hline 1/23/12 15:00 & 33.506 & 35.688 & 1475.26 & 1473.43 \\
\hline $1 / 23 / 12$ 15:30 & 33.534 & 35.679 & 1475.24 & 1473.44 \\
\hline 1/23/12 16:00 & 33.624 & 35.671 & 1475.15 & 1473.45 \\
\hline $1 / 23 / 12$ 16:30 & 33.556 & 35.664 & 1475.21 & 1473.46 \\
\hline 1/23/12 17:00 & 33.49 & 35.65 & 1475.28 & 1473.47 \\
\hline $1 / 23 / 12$ 17:30 & 33.448 & 35.639 & 1475.32 & 1473.48 \\
\hline 1/23/12 18:00 & 33.415 & 35.629 & 1475.35 & 1473.49 \\
\hline $1 / 23 / 12$ 18:30 & 33.387 & 35.625 & 1475.38 & 1473.50 \\
\hline 1/23/12 19:00 & 33.363 & 35.615 & 1475.41 & 1473.51 \\
\hline 1/23/12 19:30 & 33.45 & 35.613 & 1475.32 & 1473.51 \\
\hline $1 / 23 / 12$ 20:00 & 33.415 & 35.613 & 1475.35 & 1473.51 \\
\hline $1 / 23 / 12$ 20:30 & 33.497 & 35.61 & 1475.27 & 1473.51 \\
\hline $1 / 23 / 12$ 21:00 & 33.419 & 35.606 & 1475.35 & 1473.52 \\
\hline
\end{tabular}


TABLE S1.3 Automatically recorded groundwater levels in the Klassen wells, September 15, 2011, to March 13, 2012.

\begin{tabular}{|c|c|c|c|c|}
\hline \multicolumn{3}{|c|}{ Reference Elevation (ft AMSL) } & 1508.77 & \multirow{2}{*}{$\begin{array}{l}1509.12 \\
\text { Elevation in Klassen } \\
\text { Test Well (ft AMSL) }\end{array}$} \\
\hline Date and Time & $\begin{array}{l}\text { Depth in Klassen } \\
\text { Domestic Well } \\
\text { (ft BGL) }\end{array}$ & $\begin{array}{c}\text { Depth in Klassen } \\
\text { Test Well } \\
\text { (ft BGL) }\end{array}$ & $\begin{array}{l}\text { Elevation in Klassen } \\
\text { Domestic Well } \\
\text { (ft AMSL) }\end{array}$ & \\
\hline $1 / 23 / 1221: 30$ & 33.37 & 35.599 & 1475.40 & 1473.52 \\
\hline $1 / 23 / 1222: 00$ & 33.34 & 35.594 & 1475.43 & 1473.53 \\
\hline $1 / 23 / 1222: 30$ & 37.425 & 35.596 & 1471.34 & 1473.53 \\
\hline $1 / 23 / 1223: 00$ & 33.849 & 35.604 & 1474.92 & 1473.52 \\
\hline $1 / 23 / 1223: 30$ & 33.666 & 35.606 & 1475.10 & 1473.52 \\
\hline $1 / 24 / 120: 00$ & 33.842 & 35.608 & 1474.93 & 1473.51 \\
\hline $1 / 24 / 120: 30$ & 33.624 & 35.606 & 1475.15 & 1473.52 \\
\hline $1 / 24 / 121: 00$ & 33.534 & 35.601 & 1475.24 & 1473.52 \\
\hline 1/24/12 1:30 & 33.473 & 35.592 & 1475.30 & 1473.53 \\
\hline $1 / 24 / 122: 00$ & 33.427 & 35.587 & 1475.34 & 1473.54 \\
\hline $1 / 24 / 122: 30$ & 33.391 & 35.578 & 1475.38 & 1473.54 \\
\hline $1 / 24 / 123: 00$ & 33.359 & 35.575 & 1475.41 & 1473.55 \\
\hline 1/24/12 3:30 & 33.328 & 35.571 & 1475.44 & 1473.55 \\
\hline $1 / 24 / 124: 00$ & 33.305 & 35.566 & 1475.46 & 1473.56 \\
\hline $1 / 24 / 124: 30$ & 33.281 & 35.559 & 1475.49 & 1473.56 \\
\hline 1/24/12 5:00 & 33.258 & 35.552 & 1475.51 & 1473.57 \\
\hline 1/24/12 5:30 & 33.239 & 35.545 & 1475.53 & 1473.58 \\
\hline 1/24/12 6:00 & 33.22 & 35.538 & 1475.55 & 1473.58 \\
\hline $1 / 24 / 126: 30$ & 33.408 & 35.531 & 1475.36 & 1473.59 \\
\hline $1 / 24 / 127: 00$ & 33.302 & 35.524 & 1475.47 & 1473.60 \\
\hline $1 / 24 / 127: 30$ & 33.258 & 35.519 & 1475.51 & 1473.60 \\
\hline 1/24/12 8:00 & 33.26 & 35.507 & 1475.51 & 1473.62 \\
\hline 1/24/12 8:30 & 33.298 & 35.507 & 1475.47 & 1473.62 \\
\hline $1 / 24 / 129: 00$ & 33.344 & 35.507 & 1475.43 & 1473.62 \\
\hline 1/24/12 9:30 & 33.272 & 35.503 & 1475.50 & 1473.62 \\
\hline 1/24/12 10:00 & 33.234 & 35.493 & 1475.54 & 1473.63 \\
\hline $1 / 24 / 12$ 10:30 & 33.208 & 35.496 & 1475.56 & 1473.63 \\
\hline 1/24/12 11:00 & 33.286 & 35.5 & 1475.48 & 1473.62 \\
\hline 1/24/12 11:30 & 33.225 & 35.507 & 1475.54 & 1473.62 \\
\hline $1 / 24 / 1212: 00$ & 33.192 & 35.519 & 1475.58 & 1473.60 \\
\hline $1 / 24 / 1212: 30$ & 33.171 & 35.519 & 1475.60 & 1473.60 \\
\hline $1 / 24 / 12$ 13:00 & 33.152 & 35.491 & 1475.62 & 1473.63 \\
\hline $1 / 24 / 12$ 13:30 & 33.136 & 35.463 & 1475.63 & 1473.66 \\
\hline $1 / 24 / 1214: 00$ & 33.122 & 35.453 & 1475.65 & 1473.67 \\
\hline $1 / 24 / 1214: 30$ & 33.187 & 35.449 & 1475.58 & 1473.67 \\
\hline $1 / 24 / 12$ 15:00 & 33.143 & 35.446 & 1475.63 & 1473.68 \\
\hline $1 / 24 / 12$ 15:30 & 33.117 & 35.442 & 1475.65 & 1473.68 \\
\hline $1 / 24 / 12$ 16:00 & 33.129 & 35.435 & 1475.64 & 1473.69 \\
\hline
\end{tabular}


TABLE S1.3 Automatically recorded groundwater levels in the Klassen wells, September 15, 2011, to March 13, 2012.

\begin{tabular}{|c|c|c|c|c|}
\hline \multicolumn{3}{|c|}{ Reference Elevation (ft AMSL) } & 1508.77 & \multirow{2}{*}{$\begin{array}{l}1509.12 \\
\text { Elevation in Klassen } \\
\text { Test Well (ft AMSL) }\end{array}$} \\
\hline Date and Time & $\begin{array}{l}\text { Depth in Klassen } \\
\text { Domestic Well } \\
(\mathrm{ft} \mathrm{BGL})\end{array}$ & $\begin{array}{c}\text { Depth in Klassen } \\
\text { Test Well } \\
\text { (ft BGL) }\end{array}$ & $\begin{array}{l}\text { Elevation in Klassen } \\
\text { Domestic Well } \\
\text { (ft AMSL) }\end{array}$ & \\
\hline $1 / 24 / 1216: 30$ & 33.194 & 35.432 & 1475.58 & 1473.69 \\
\hline $1 / 24 / 1217: 00$ & 33.14 & 35.428 & 1475.63 & 1473.69 \\
\hline $1 / 24 / 1217: 30$ & 33.112 & 35.42 & 1475.66 & 1473.70 \\
\hline $1 / 24 / 1218: 00$ & 33.201 & 35.421 & 1475.57 & 1473.70 \\
\hline $1 / 24 / 1218: 30$ & 33.22 & 35.423 & 1475.55 & 1473.70 \\
\hline $1 / 24 / 1219: 00$ & 33.162 & 35.416 & 1475.61 & 1473.71 \\
\hline $1 / 24 / 1219: 30$ & 33.129 & 35.413 & 1475.64 & 1473.71 \\
\hline $1 / 24 / 1220: 00$ & 33.218 & 35.413 & 1475.55 & 1473.71 \\
\hline $1 / 24 / 1220: 30$ & 33.15 & 35.416 & 1475.62 & 1473.71 \\
\hline $1 / 24 / 1221: 00$ & 33.119 & 35.411 & 1475.65 & 1473.71 \\
\hline $1 / 24 / 1221: 30$ & 33.098 & 35.413 & 1475.67 & 1473.71 \\
\hline $1 / 24 / 1222: 00$ & 33.082 & 35.409 & 1475.69 & 1473.71 \\
\hline $1 / 24 / 1222: 30$ & 33.811 & 35.406 & 1474.96 & 1473.72 \\
\hline $1 / 24 / 1223: 00$ & 33.558 & 35.418 & 1475.21 & 1473.70 \\
\hline $1 / 24 / 12$ 23:30 & 33.38 & 35.43 & 1475.39 & 1473.69 \\
\hline 1/25/12 0:00 & 33.267 & 35.432 & 1475.50 & 1473.69 \\
\hline 1/25/12 0:30 & 33.211 & 35.428 & 1475.56 & 1473.69 \\
\hline 1/25/12 1:00 & 33.173 & 35.432 & 1475.60 & 1473.69 \\
\hline $1 / 25 / 121: 30$ & 33.145 & 35.432 & 1475.62 & 1473.69 \\
\hline $1 / 25 / 122: 00$ & 33.122 & 35.435 & 1475.65 & 1473.69 \\
\hline $1 / 25 / 122: 30$ & 33.103 & 35.435 & 1475.67 & 1473.69 \\
\hline $1 / 25 / 123: 00$ & 33.086 & 35.432 & 1475.68 & 1473.69 \\
\hline 1/25/12 3:30 & 33.072 & 35.43 & 1475.70 & 1473.69 \\
\hline $1 / 25 / 124: 00$ & 33.056 & 35.425 & 1475.71 & 1473.70 \\
\hline $1 / 25 / 124: 30$ & 33.042 & 35.413 & 1475.73 & 1473.71 \\
\hline $1 / 25 / 125: 00$ & 33.028 & 35.406 & 1475.74 & 1473.72 \\
\hline $1 / 25 / 125: 30$ & 33.018 & 35.397 & 1475.75 & 1473.73 \\
\hline 1/25/12 6:00 & 33.007 & 35.39 & 1475.76 & 1473.73 \\
\hline $1 / 25 / 126: 30$ & 33.169 & 35.39 & 1475.60 & 1473.73 \\
\hline 1/25/12 7:00 & 33.084 & 35.383 & 1475.69 & 1473.74 \\
\hline 1/25/12 7:30 & 33.143 & 35.383 & 1475.63 & 1473.74 \\
\hline $1 / 25 / 128: 00$ & 33.133 & 35.378 & 1475.64 & 1473.74 \\
\hline 1/25/12 8:30 & 33.093 & 35.385 & 1475.68 & 1473.74 \\
\hline 1/25/12 9:00 & 33.227 & 35.381 & 1475.54 & 1473.74 \\
\hline 1/25/12 9:30 & 35.589 & 35.385 & 1473.18 & 1473.74 \\
\hline $1 / 25 / 12$ 10:00 & 33.162 & 35.399 & 1475.61 & 1473.72 \\
\hline $1 / 25 / 12$ 10:30 & 33.108 & 35.399 & 1475.66 & 1473.72 \\
\hline 1/25/12 11:00 & 33.18 & 35.418 & 1475.59 & 1473.70 \\
\hline
\end{tabular}


TABLE S1.3 Automatically recorded groundwater levels in the Klassen wells, September 15, 2011, to March 13, 2012.

\begin{tabular}{|c|c|c|c|c|}
\hline \multicolumn{3}{|c|}{ Reference Elevation (ft AMSL) } & 1508.77 & \multirow{2}{*}{$\begin{array}{l}1509.12 \\
\text { Elevation in Klassen } \\
\text { Test Well (ft AMSL) }\end{array}$} \\
\hline Date and Time & $\begin{array}{l}\text { Depth in Klassen } \\
\text { Domestic Well } \\
(\mathrm{ft} \mathrm{BGL})\end{array}$ & $\begin{array}{c}\text { Depth in Klassen } \\
\text { Test Well } \\
\text { (ft BGL) }\end{array}$ & $\begin{array}{l}\text { Elevation in Klassen } \\
\text { Domestic Well } \\
\text { (ft AMSL) }\end{array}$ & \\
\hline $1 / 25 / 1211: 30$ & 33.103 & 35.425 & 1475.67 & 1473.70 \\
\hline $1 / 25 / 1212: 00$ & 33.159 & 35.437 & 1475.61 & 1473.69 \\
\hline $1 / 25 / 1212: 30$ & 33.098 & 35.444 & 1475.67 & 1473.68 \\
\hline $1 / 25 / 1213: 00$ & 33.162 & 35.458 & 1475.61 & 1473.66 \\
\hline $1 / 25 / 1213: 30$ & 33.169 & 35.465 & 1475.60 & 1473.66 \\
\hline $1 / 25 / 1214: 00$ & 33.103 & 35.43 & 1475.67 & 1473.69 \\
\hline $1 / 25 / 1214: 30$ & 33.164 & 35.399 & 1475.61 & 1473.72 \\
\hline $1 / 25 / 12$ 15:00 & 33.105 & 35.381 & 1475.66 & 1473.74 \\
\hline $1 / 25 / 1215: 30$ & 33.072 & 35.374 & 1475.70 & 1473.75 \\
\hline $1 / 25 / 1216: 00$ & 33.049 & 35.371 & 1475.72 & 1473.75 \\
\hline $1 / 25 / 1216: 30$ & 33.706 & 35.369 & 1475.06 & 1473.75 \\
\hline $1 / 25 / 1217: 00$ & 33.101 & 35.369 & 1475.67 & 1473.75 \\
\hline $1 / 25 / 1217: 30$ & 33.054 & 35.364 & 1475.72 & 1473.76 \\
\hline $1 / 25 / 1218: 00$ & 37.085 & 35.378 & 1471.68 & 1473.74 \\
\hline $1 / 25 / 12$ 18:30 & 33.741 & 35.404 & 1475.03 & 1473.72 \\
\hline 1/25/12 19:00 & 33.506 & 35.409 & 1475.26 & 1473.71 \\
\hline $1 / 25 / 12$ 19:30 & 33.389 & 35.409 & 1475.38 & 1473.71 \\
\hline $1 / 25 / 1220: 00$ & 33.431 & 35.409 & 1475.34 & 1473.71 \\
\hline $1 / 25 / 1220: 30$ & 33.321 & 35.404 & 1475.45 & 1473.72 \\
\hline $1 / 25 / 1221: 00$ & 33.262 & 35.416 & 1475.51 & 1473.71 \\
\hline $1 / 25 / 1221: 30$ & 33.22 & 35.425 & 1475.55 & 1473.70 \\
\hline $1 / 25 / 1222: 00$ & 33.185 & 35.432 & 1475.58 & 1473.69 \\
\hline $1 / 25 / 1222: 30$ & 33.527 & 35.449 & 1475.24 & 1473.67 \\
\hline $1 / 25 / 12$ 23:00 & 33.886 & 35.46 & 1474.88 & 1473.66 \\
\hline $1 / 25 / 12$ 23:30 & 33.642 & 35.474 & 1475.13 & 1473.65 \\
\hline 1/26/12 0:00 & 33.464 & 35.482 & 1475.31 & 1473.64 \\
\hline $1 / 26 / 120: 30$ & 33.373 & 35.479 & 1475.40 & 1473.64 \\
\hline 1/26/12 1:00 & 33.312 & 35.479 & 1475.46 & 1473.64 \\
\hline 1/26/12 1:30 & 33.267 & 35.474 & 1475.50 & 1473.65 \\
\hline $1 / 26 / 122: 00$ & 33.354 & 35.472 & 1475.42 & 1473.65 \\
\hline $1 / 26 / 122: 30$ & 33.253 & 35.479 & 1475.52 & 1473.64 \\
\hline 1/26/12 3:00 & 33.208 & 35.482 & 1475.56 & 1473.64 \\
\hline 1/26/12 3:30 & 33.176 & 35.47 & 1475.59 & 1473.65 \\
\hline 1/26/12 4:00 & 33.15 & 35.467 & 1475.62 & 1473.66 \\
\hline 1/26/12 4:30 & 33.126 & 35.463 & 1475.64 & 1473.66 \\
\hline $1 / 26 / 125: 00$ & 33.131 & 35.453 & 1475.64 & 1473.67 \\
\hline $1 / 26 / 125: 30$ & 33.138 & 35.432 & 1475.63 & 1473.69 \\
\hline $1 / 26 / 126: 00$ & 33.105 & 35.423 & 1475.66 & 1473.70 \\
\hline
\end{tabular}


TABLE S1.3 Automatically recorded groundwater levels in the Klassen wells, September 15, 2011, to March 13, 2012.

\begin{tabular}{|c|c|c|c|c|}
\hline \multicolumn{3}{|c|}{ Reference Elevation (ft AMSL) } & 1508.77 & \multirow{2}{*}{$\begin{array}{l}1509.12 \\
\text { Elevation in Klassen } \\
\text { Test Well (ft AMSL) }\end{array}$} \\
\hline Date and Time & $\begin{array}{l}\text { Depth in Klassen } \\
\text { Domestic Well } \\
\text { (ft BGL) }\end{array}$ & $\begin{array}{c}\text { Depth in Klassen } \\
\text { Test Well } \\
\text { (ft BGL) }\end{array}$ & $\begin{array}{l}\text { Elevation in Klassen } \\
\text { Domestic Well } \\
\text { (ft AMSL) }\end{array}$ & \\
\hline $1 / 26 / 126: 30$ & 33.246 & 35.416 & 1475.52 & 1473.71 \\
\hline $1 / 26 / 127: 00$ & 33.157 & 35.413 & 1475.61 & 1473.71 \\
\hline 1/26/12 7:30 & 33.15 & 35.416 & 1475.62 & 1473.71 \\
\hline $1 / 26 / 128: 00$ & 33.112 & 35.402 & 1475.66 & 1473.72 \\
\hline $1 / 26 / 128: 30$ & 33.166 & 35.411 & 1475.60 & 1473.71 \\
\hline 1/26/12 9:00 & 33.307 & 35.425 & 1475.46 & 1473.70 \\
\hline $1 / 26 / 129: 30$ & 33.157 & 35.43 & 1475.61 & 1473.69 \\
\hline $1 / 26 / 1210: 00$ & 33.115 & 35.439 & 1475.65 & 1473.68 \\
\hline $1 / 26 / 1210: 30$ & 33.084 & 35.449 & 1475.69 & 1473.67 \\
\hline $1 / 26 / 1211: 00$ & 33.065 & 35.451 & 1475.70 & 1473.67 \\
\hline $1 / 26 / 1211: 30$ & 33.253 & 35.463 & 1475.52 & 1473.66 \\
\hline $1 / 26 / 1212: 00$ & 33.147 & 35.467 & 1475.62 & 1473.66 \\
\hline $1 / 26 / 1212: 30$ & 33.103 & 35.453 & 1475.67 & 1473.67 \\
\hline $1 / 26 / 1213: 00$ & 33.075 & 35.427 & 1475.69 & 1473.70 \\
\hline $1 / 26 / 12$ 13:30 & 33.206 & 35.418 & 1475.56 & 1473.70 \\
\hline $1 / 26 / 12$ 14:00 & 33.274 & 35.423 & 1475.50 & 1473.70 \\
\hline $1 / 26 / 1214: 30$ & 33.487 & 35.439 & 1475.28 & 1473.68 \\
\hline $1 / 26 / 12$ 15:00 & 33.556 & 35.458 & 1475.21 & 1473.66 \\
\hline $1 / 26 / 12$ 15:30 & 33.663 & 35.47 & 1475.11 & 1473.65 \\
\hline $1 / 26 / 12$ 16:00 & 33.659 & 35.479 & 1475.11 & 1473.64 \\
\hline $1 / 26 / 12$ 16:30 & 33.863 & 35.489 & 1474.91 & 1473.63 \\
\hline $1 / 26 / 12$ 17:00 & 33.776 & 35.5 & 1474.99 & 1473.62 \\
\hline $1 / 26 / 12$ 17:30 & 33.778 & 35.507 & 1474.99 & 1473.62 \\
\hline $1 / 26 / 1218: 00$ & 33.87 & 35.519 & 1474.90 & 1473.60 \\
\hline $1 / 26 / 12$ 18:30 & 33.884 & 35.531 & 1474.89 & 1473.59 \\
\hline 1/26/12 19:00 & 33.905 & 35.547 & 1474.86 & 1473.58 \\
\hline 1/26/12 19:30 & 37.017 & 35.559 & 1471.75 & 1473.56 \\
\hline $1 / 26 / 12$ 20:00 & 33.987 & 35.571 & 1474.78 & 1473.55 \\
\hline $1 / 26 / 1220: 30$ & 37.901 & 35.58 & 1470.87 & 1473.54 \\
\hline 1/26/12 21:00 & 34.076 & 35.592 & 1474.69 & 1473.53 \\
\hline $1 / 26 / 12$ 21:30 & 34.081 & 35.601 & 1474.69 & 1473.52 \\
\hline $1 / 26 / 1222: 00$ & 34.153 & 35.608 & 1474.62 & 1473.51 \\
\hline $1 / 26 / 1222: 30$ & 36.192 & 35.618 & 1472.58 & 1473.50 \\
\hline $1 / 26 / 12$ 23:00 & 34.226 & 35.625 & 1474.54 & 1473.50 \\
\hline $1 / 26 / 12$ 23:30 & 34.069 & 35.627 & 1474.70 & 1473.50 \\
\hline 1/27/12 0:00 & 33.921 & 35.625 & 1474.85 & 1473.50 \\
\hline $1 / 27 / 120: 30$ & 33.809 & 35.615 & 1474.96 & 1473.51 \\
\hline 1/27/12 1:00 & 33.724 & 35.608 & 1475.05 & 1473.51 \\
\hline
\end{tabular}


TABLE S1.3 Automatically recorded groundwater levels in the Klassen wells, September 15, 2011, to March 13, 2012.

\begin{tabular}{|c|c|c|c|c|}
\hline \multicolumn{3}{|c|}{ Reference Elevation (ft AMSL) } & 1508.77 & \multirow{2}{*}{$\begin{array}{l}1509.12 \\
\text { Elevation in Klassen } \\
\text { Test Well (ft AMSL) }\end{array}$} \\
\hline Date and Time & $\begin{array}{l}\text { Depth in Klassen } \\
\text { Domestic Well } \\
\text { (ft BGL) }\end{array}$ & $\begin{array}{c}\text { Depth in Klassen } \\
\text { Test Well } \\
\text { (ft BGL) }\end{array}$ & $\begin{array}{l}\text { Elevation in Klassen } \\
\text { Domestic Well } \\
\text { (ft AMSL) }\end{array}$ & \\
\hline $1 / 27 / 121: 30$ & 33.656 & 35.596 & 1475.11 & 1473.53 \\
\hline $1 / 27 / 122: 00$ & 33.6 & 35.58 & 1475.17 & 1473.54 \\
\hline $1 / 27 / 122: 30$ & 33.551 & 35.578 & 1475.22 & 1473.54 \\
\hline $1 / 27 / 123: 00$ & 34.672 & 35.573 & 1474.10 & 1473.55 \\
\hline 1/27/12 3:30 & 33.541 & 35.566 & 1475.23 & 1473.56 \\
\hline $1 / 27 / 124: 00$ & 33.478 & 35.564 & 1475.29 & 1473.56 \\
\hline $1 / 27 / 124: 30$ & 33.722 & 35.554 & 1475.05 & 1473.57 \\
\hline $1 / 27 / 125: 00$ & 33.466 & 35.55 & 1475.30 & 1473.57 \\
\hline $1 / 27 / 125: 30$ & 33.41 & 35.54 & 1475.36 & 1473.58 \\
\hline 1/27/12 6:00 & 33.37 & 35.524 & 1475.40 & 1473.60 \\
\hline $1 / 27 / 126: 30$ & 37.17 & 35.521 & 1471.60 & 1473.60 \\
\hline 1/27/12 7:00 & 37.106 & 35.507 & 1471.66 & 1473.62 \\
\hline 1/27/12 7:30 & 33.736 & 35.505 & 1475.03 & 1473.62 \\
\hline $1 / 27 / 128: 00$ & 33.6 & 35.507 & 1475.17 & 1473.62 \\
\hline 1/27/12 8:30 & 33.631 & 35.505 & 1475.14 & 1473.62 \\
\hline $1 / 27 / 129: 00$ & 33.502 & 35.505 & 1475.27 & 1473.62 \\
\hline $1 / 27 / 129: 30$ & 33.6 & 35.486 & 1475.17 & 1473.64 \\
\hline $1 / 27 / 12$ 10:00 & 35.852 & 35.493 & 1472.92 & 1473.63 \\
\hline $1 / 27 / 12$ 10:30 & 35.69 & 35.545 & 1473.08 & 1473.58 \\
\hline 1/27/12 11:00 & 34.505 & 35.571 & 1474.26 & 1473.55 \\
\hline $1 / 27 / 1211: 30$ & 34.404 & 35.58 & 1474.37 & 1473.54 \\
\hline $1 / 27 / 1212: 00$ & 34.226 & 35.594 & 1474.54 & 1473.53 \\
\hline $1 / 27 / 1212: 30$ & 34.13 & 35.592 & 1474.64 & 1473.53 \\
\hline $1 / 27 / 12$ 13:00 & 34.182 & 35.596 & 1474.59 & 1473.53 \\
\hline 1/27/12 13:30 & 39.508 & 35.589 & 1469.26 & 1473.53 \\
\hline 1/27/12 14:00 & 33.992 & 35.589 & 1474.78 & 1473.53 \\
\hline $1 / 27 / 1214: 30$ & 33.994 & 35.594 & 1474.78 & 1473.53 \\
\hline $1 / 27 / 12$ 15:00 & 34.067 & 35.608 & 1474.70 & 1473.51 \\
\hline $1 / 27 / 12$ 15:30 & 34.083 & 35.618 & 1474.69 & 1473.50 \\
\hline 1/27/12 16:00 & 34.093 & 35.629 & 1474.68 & 1473.49 \\
\hline $1 / 27 / 12$ 16:30 & 34.243 & 35.646 & 1474.53 & 1473.48 \\
\hline 1/27/12 17:00 & 34.182 & 35.653 & 1474.59 & 1473.47 \\
\hline $1 / 27 / 12$ 17:30 & 34.287 & 35.657 & 1474.48 & 1473.47 \\
\hline $1 / 27 / 12$ 18:00 & 34.257 & 35.667 & 1474.51 & 1473.46 \\
\hline $1 / 27 / 12$ 18:30 & 34.172 & 35.674 & 1474.60 & 1473.45 \\
\hline 1/27/12 19:00 & 34.212 & 35.674 & 1474.56 & 1473.45 \\
\hline 1/27/12 19:30 & 34.008 & 35.672 & 1474.76 & 1473.45 \\
\hline $1 / 27 / 12$ 20:00 & 33.91 & 35.667 & 1474.86 & 1473.46 \\
\hline
\end{tabular}


TABLE S1.3 Automatically recorded groundwater levels in the Klassen wells, September 15, 2011, to March 13, 2012.

\begin{tabular}{|c|c|c|c|c|}
\hline \multicolumn{3}{|c|}{ Reference Elevation (ft AMSL) } & 1508.77 & \multirow{2}{*}{$\begin{array}{l}1509.12 \\
\text { Elevation in Klassen } \\
\text { Test Well (ft AMSL) }\end{array}$} \\
\hline Date and Time & $\begin{array}{l}\text { Depth in Klassen } \\
\text { Domestic Well } \\
\text { (ft BGL) }\end{array}$ & $\begin{array}{c}\text { Depth in Klassen } \\
\text { Test Well } \\
\text { (ft BGL) }\end{array}$ & $\begin{array}{l}\text { Elevation in Klassen } \\
\text { Domestic Well } \\
\text { (ft AMSL) }\end{array}$ & \\
\hline $1 / 27 / 1220: 30$ & 33.889 & 35.66 & 1474.88 & 1473.46 \\
\hline $1 / 27 / 1221: 00$ & 33.978 & 35.657 & 1474.79 & 1473.47 \\
\hline $1 / 27 / 1221: 30$ & 33.802 & 35.655 & 1474.97 & 1473.47 \\
\hline $1 / 27 / 1222: 00$ & 36.874 & 35.657 & 1471.90 & 1473.47 \\
\hline $1 / 27 / 1222: 30$ & 33.957 & 35.674 & 1474.81 & 1473.45 \\
\hline $1 / 27 / 1223: 00$ & 34.121 & 35.676 & 1474.65 & 1473.45 \\
\hline $1 / 27 / 1223: 30$ & 33.947 & 35.676 & 1474.82 & 1473.45 \\
\hline 1/28/12 0:00 & 33.921 & 35.672 & 1474.85 & 1473.45 \\
\hline 1/28/12 0:30 & 33.811 & 35.667 & 1474.96 & 1473.46 \\
\hline 1/28/12 1:00 & 33.741 & 35.669 & 1475.03 & 1473.45 \\
\hline $1 / 28 / 121: 30$ & 33.682 & 35.665 & 1475.09 & 1473.46 \\
\hline $1 / 28 / 122: 00$ & 33.694 & 35.653 & 1475.08 & 1473.47 \\
\hline $1 / 28 / 122: 30$ & 33.734 & 35.657 & 1475.04 & 1473.47 \\
\hline $1 / 28 / 123: 00$ & 33.689 & 35.653 & 1475.08 & 1473.47 \\
\hline 1/28/12 3:30 & 33.654 & 35.65 & 1475.12 & 1473.47 \\
\hline $1 / 28 / 124: 00$ & 33.701 & 35.653 & 1475.07 & 1473.47 \\
\hline $1 / 28 / 124: 30$ & 33.626 & 35.65 & 1475.14 & 1473.47 \\
\hline 1/28/12 5:00 & 33.572 & 35.643 & 1475.20 & 1473.48 \\
\hline $1 / 28 / 125: 30$ & 33.532 & 35.627 & 1475.24 & 1473.50 \\
\hline $1 / 28 / 126: 00$ & 33.495 & 35.622 & 1475.27 & 1473.50 \\
\hline $1 / 28 / 126: 30$ & 33.659 & 35.618 & 1475.11 & 1473.50 \\
\hline 1/28/12 7:00 & 33.551 & 35.608 & 1475.22 & 1473.51 \\
\hline 1/28/12 7:30 & 33.497 & 35.599 & 1475.27 & 1473.52 \\
\hline $1 / 28 / 128: 00$ & 33.546 & 35.594 & 1475.22 & 1473.53 \\
\hline $1 / 28 / 128: 30$ & 36.438 & 35.599 & 1472.33 & 1473.52 \\
\hline 1/28/12 9:00 & 33.748 & 35.596 & 1475.02 & 1473.53 \\
\hline 1/28/12 9:30 & 33.914 & 35.596 & 1474.86 & 1473.53 \\
\hline 1/28/12 10:00 & 33.701 & 35.599 & 1475.07 & 1473.52 \\
\hline $1 / 28 / 12$ 10:30 & 33.985 & 35.613 & 1474.78 & 1473.51 \\
\hline 1/28/12 11:00 & 34.025 & 35.655 & 1474.74 & 1473.47 \\
\hline 1/28/12 11:30 & 33.797 & 35.672 & 1474.97 & 1473.45 \\
\hline $1 / 28 / 1212: 00$ & 33.708 & 35.681 & 1475.06 & 1473.44 \\
\hline $1 / 28 / 1212: 30$ & 33.701 & 35.681 & 1475.07 & 1473.44 \\
\hline $1 / 28 / 12$ 13:00 & 33.614 & 35.679 & 1475.16 & 1473.44 \\
\hline $1 / 28 / 12$ 13:30 & 33.572 & 35.625 & 1475.20 & 1473.50 \\
\hline $1 / 28 / 1214: 00$ & 33.668 & 35.603 & 1475.10 & 1473.52 \\
\hline $1 / 28 / 1214: 30$ & 38.605 & 35.622 & 1470.16 & 1473.50 \\
\hline $1 / 28 / 12$ 15:00 & 34.419 & 35.662 & 1474.35 & 1473.46 \\
\hline
\end{tabular}


TABLE S1.3 Automatically recorded groundwater levels in the Klassen wells, September 15, 2011, to March 13, 2012.

\begin{tabular}{|c|c|c|c|c|}
\hline \multicolumn{3}{|c|}{ Reference Elevation (ft AMSL) } & 1508.77 & \multirow{2}{*}{$\begin{array}{l}1509.12 \\
\text { Elevation in Klassen } \\
\text { Test Well (ft AMSL) }\end{array}$} \\
\hline Date and Time & $\begin{array}{l}\text { Depth in Klassen } \\
\text { Domestic Well } \\
(\mathrm{ft} \mathrm{BGL})\end{array}$ & $\begin{array}{c}\text { Depth in Klassen } \\
\text { Test Well } \\
\text { (ft BGL) }\end{array}$ & $\begin{array}{l}\text { Elevation in Klassen } \\
\text { Domestic Well } \\
\text { (ft AMSL) }\end{array}$ & \\
\hline $1 / 28 / 1215: 30$ & 34.519 & 35.695 & 1474.25 & 1473.43 \\
\hline $1 / 28 / 1216: 00$ & 34.587 & 35.718 & 1474.18 & 1473.40 \\
\hline $1 / 28 / 1216: 30$ & 34.639 & 35.74 & 1474.13 & 1473.38 \\
\hline $1 / 28 / 1217: 00$ & 34.688 & 35.758 & 1474.08 & 1473.36 \\
\hline $1 / 28 / 1217: 30$ & 34.831 & 35.779 & 1473.94 & 1473.34 \\
\hline $1 / 28 / 1218: 00$ & 34.812 & 35.801 & 1473.96 & 1473.32 \\
\hline $1 / 28 / 1218: 30$ & 34.827 & 35.817 & 1473.94 & 1473.31 \\
\hline $1 / 28 / 1219: 00$ & 34.965 & 35.838 & 1473.80 & 1473.28 \\
\hline $1 / 28 / 1219: 30$ & 36.593 & 35.862 & 1472.18 & 1473.26 \\
\hline $1 / 28 / 1220: 00$ & 35.19 & 35.887 & 1473.58 & 1473.24 \\
\hline $1 / 28 / 1220: 30$ & 35.251 & 35.908 & 1473.52 & 1473.21 \\
\hline $1 / 28 / 1221: 00$ & 35.293 & 35.937 & 1473.48 & 1473.19 \\
\hline $1 / 28 / 1221: 30$ & 35.504 & 35.981 & 1473.27 & 1473.14 \\
\hline $1 / 28 / 1222: 00$ & 35.699 & 36.028 & 1473.07 & 1473.09 \\
\hline $1 / 28 / 1222: 30$ & 36.145 & 36.082 & 1472.62 & 1473.04 \\
\hline $1 / 28 / 12$ 23:00 & 36.304 & 36.122 & 1472.47 & 1473.00 \\
\hline $1 / 28 / 12$ 23:30 & 35.833 & 36.127 & 1472.94 & 1473.00 \\
\hline 1/29/12 0:00 & 35.518 & 36.124 & 1473.25 & 1473.00 \\
\hline $1 / 29 / 12$ 0:30 & 35.282 & 36.117 & 1473.49 & 1473.01 \\
\hline $1 / 29 / 121: 00$ & 35.096 & 36.113 & 1473.67 & 1473.01 \\
\hline $1 / 29 / 121: 30$ & 34.944 & 36.108 & 1473.83 & 1473.01 \\
\hline $1 / 29 / 122: 00$ & 34.812 & 36.099 & 1473.96 & 1473.02 \\
\hline 1/29/12 2:30 & 34.7 & 36.082 & 1474.07 & 1473.04 \\
\hline 1/29/12 3:00 & 34.714 & 36.068 & 1474.06 & 1473.05 \\
\hline $1 / 29 / 123: 30$ & 34.564 & 36.052 & 1474.21 & 1473.07 \\
\hline 1/29/12 4:00 & 34.487 & 36.035 & 1474.28 & 1473.09 \\
\hline $1 / 29 / 124: 30$ & 34.4 & 36.019 & 1474.37 & 1473.10 \\
\hline 1/29/12 5:00 & 34.327 & 36.007 & 1474.44 & 1473.12 \\
\hline 1/29/12 5:30 & 34.259 & 35.991 & 1474.51 & 1473.13 \\
\hline 1/29/12 6:00 & 34.524 & 35.979 & 1474.25 & 1473.14 \\
\hline 1/29/12 6:30 & 34.358 & 35.97 & 1474.41 & 1473.15 \\
\hline $1 / 29 / 127: 00$ & 35.127 & 35.955 & 1473.64 & 1473.17 \\
\hline 1/29/12 7:30 & 34.236 & 35.944 & 1474.53 & 1473.18 \\
\hline $1 / 29 / 128: 00$ & 34.144 & 35.93 & 1474.63 & 1473.19 \\
\hline $1 / 29 / 128: 30$ & 34.109 & 35.92 & 1474.66 & 1473.20 \\
\hline 1/29/12 9:00 & 34.224 & 35.925 & 1474.55 & 1473.20 \\
\hline 1/29/12 9:30 & 34.097 & 35.925 & 1474.67 & 1473.20 \\
\hline 1/29/12 10:00 & 34.046 & 35.93 & 1474.72 & 1473.19 \\
\hline
\end{tabular}


TABLE S1.3 Automatically recorded groundwater levels in the Klassen wells, September 15, 2011, to March 13, 2012.

\begin{tabular}{|c|c|c|c|c|}
\hline \multicolumn{3}{|c|}{ Reference Elevation (ft AMSL) } & 1508.77 & \multirow{2}{*}{$\begin{array}{l}1509.12 \\
\text { Elevation in Klassen } \\
\text { Test Well (ft AMSL) }\end{array}$} \\
\hline Date and Time & $\begin{array}{l}\text { Depth in Klassen } \\
\text { Domestic Well } \\
(\mathrm{ft} \mathrm{BGL})\end{array}$ & $\begin{array}{c}\text { Depth in Klassen } \\
\text { Test Well } \\
\text { (ft BGL) }\end{array}$ & $\begin{array}{l}\text { Elevation in Klassen } \\
\text { Domestic Well } \\
\text { (ft AMSL) }\end{array}$ & \\
\hline $1 / 29 / 1210: 30$ & 33.982 & 35.937 & 1474.79 & 1473.19 \\
\hline $1 / 29 / 1211: 00$ & 34.142 & 35.951 & 1474.63 & 1473.17 \\
\hline 1/29/12 11:30 & 34.172 & 35.965 & 1474.60 & 1473.16 \\
\hline $1 / 29 / 1212: 00$ & 34.055 & 35.972 & 1474.71 & 1473.15 \\
\hline 1/29/12 12:30 & 34.071 & 35.934 & 1474.70 & 1473.19 \\
\hline $1 / 29 / 1213: 00$ & 34.118 & 35.93 & 1474.65 & 1473.19 \\
\hline $1 / 29 / 1213: 30$ & 34.123 & 35.941 & 1474.65 & 1473.18 \\
\hline 1/29/12 14:00 & 33.999 & 35.948 & 1474.77 & 1473.17 \\
\hline $1 / 29 / 1214: 30$ & 34.027 & 35.953 & 1474.74 & 1473.17 \\
\hline $1 / 29 / 1215: 00$ & 33.928 & 35.96 & 1474.84 & 1473.16 \\
\hline $1 / 29 / 1215: 30$ & 34.104 & 35.965 & 1474.67 & 1473.16 \\
\hline 1/29/12 16:00 & 33.945 & 35.965 & 1474.82 & 1473.16 \\
\hline $1 / 29 / 1216: 30$ & 33.914 & 35.965 & 1474.86 & 1473.16 \\
\hline $1 / 29 / 1217: 00$ & 47.589 & 35.958 & 1461.18 & 1473.16 \\
\hline 1/29/12 17:30 & 35.873 & 36 & 1472.90 & 1473.12 \\
\hline 1/29/12 18:00 & 34.836 & 36.019 & 1473.93 & 1473.10 \\
\hline $1 / 29 / 12$ 18:30 & 34.548 & 36.021 & 1474.22 & 1473.10 \\
\hline 1/29/12 19:00 & 34.531 & 36.028 & 1474.24 & 1473.09 \\
\hline 1/29/12 19:30 & 34.369 & 36.028 & 1474.40 & 1473.09 \\
\hline $1 / 29 / 1220: 00$ & 34.97 & 36.023 & 1473.80 & 1473.10 \\
\hline $1 / 29 / 12$ 20:30 & 34.334 & 36.019 & 1474.44 & 1473.10 \\
\hline 1/29/12 21:00 & 34.215 & 36.012 & 1474.55 & 1473.11 \\
\hline $1 / 29 / 12$ 21:30 & 34.111 & 36.002 & 1474.66 & 1473.12 \\
\hline $1 / 29 / 1222: 00$ & 34.06 & 35.993 & 1474.71 & 1473.13 \\
\hline $1 / 29 / 12$ 22:30 & 34.121 & 35.984 & 1474.65 & 1473.14 \\
\hline $1 / 29 / 12$ 23:00 & 34.564 & 35.974 & 1474.21 & 1473.15 \\
\hline $1 / 29 / 12$ 23:30 & 37.069 & 35.967 & 1471.70 & 1473.16 \\
\hline $1 / 30 / 12$ 0:00 & 34.114 & 35.965 & 1474.66 & 1473.16 \\
\hline $1 / 30 / 120: 30$ & 33.989 & 35.953 & 1474.78 & 1473.17 \\
\hline 1/30/12 1:00 & 33.912 & 35.941 & 1474.86 & 1473.18 \\
\hline 1/30/12 1:30 & 33.851 & 35.93 & 1474.92 & 1473.19 \\
\hline $1 / 30 / 122: 00$ & 33.802 & 35.92 & 1474.97 & 1473.20 \\
\hline $1 / 30 / 122: 30$ & 33.846 & 35.909 & 1474.92 & 1473.21 \\
\hline 1/30/12 3:00 & 37.592 & 35.901 & 1471.18 & 1473.22 \\
\hline 1/30/12 3:30 & 33.931 & 35.897 & 1474.84 & 1473.23 \\
\hline $1 / 30 / 124: 00$ & 33.813 & 35.885 & 1474.96 & 1473.24 \\
\hline 1/30/12 4:30 & 33.745 & 35.871 & 1475.02 & 1473.25 \\
\hline $1 / 30 / 125: 00$ & 33.788 & 35.862 & 1474.98 & 1473.26 \\
\hline
\end{tabular}


TABLE S1.3 Automatically recorded groundwater levels in the Klassen wells, September 15, 2011, to March 13, 2012.

\begin{tabular}{|c|c|c|c|c|}
\hline \multicolumn{3}{|c|}{ Reference Elevation (ft AMSL) } & 1508.77 & \multirow{2}{*}{$\begin{array}{l}1509.12 \\
\text { Elevation in Klassen } \\
\text { Test Well (ft AMSL) }\end{array}$} \\
\hline Date and Time & $\begin{array}{l}\text { Depth in Klassen } \\
\text { Domestic Well } \\
(\mathrm{ft} \mathrm{BGL})\end{array}$ & $\begin{array}{c}\text { Depth in Klassen } \\
\text { Test Well } \\
\text { (ft BGL) }\end{array}$ & $\begin{array}{l}\text { Elevation in Klassen } \\
\text { Domestic Well } \\
\text { (ft AMSL) }\end{array}$ & \\
\hline $1 / 30 / 125: 30$ & 33.71 & 35.85 & 1475.06 & 1473.27 \\
\hline $1 / 30 / 126: 00$ & 33.743 & 35.845 & 1475.03 & 1473.28 \\
\hline $1 / 30 / 126: 30$ & 33.844 & 35.84 & 1474.93 & 1473.28 \\
\hline $1 / 30 / 127: 00$ & 33.781 & 35.838 & 1474.99 & 1473.28 \\
\hline $1 / 30 / 127: 30$ & 33.757 & 35.829 & 1475.01 & 1473.29 \\
\hline $1 / 30 / 128: 00$ & 33.853 & 35.822 & 1474.92 & 1473.30 \\
\hline $1 / 30 / 128: 30$ & 36.616 & 35.819 & 1472.15 & 1473.30 \\
\hline 1/30/12 9:00 & 33.741 & 35.812 & 1475.03 & 1473.31 \\
\hline 1/30/12 9:30 & 33.79 & 35.808 & 1474.98 & 1473.31 \\
\hline $1 / 30 / 1210: 00$ & 34.111 & 35.801 & 1474.66 & 1473.32 \\
\hline 1/30/12 10:30 & 33.677 & 35.796 & 1475.09 & 1473.33 \\
\hline 1/30/12 11:00 & 33.619 & 35.791 & 1475.15 & 1473.33 \\
\hline $1 / 30 / 1211: 30$ & 33.577 & 35.782 & 1475.19 & 1473.34 \\
\hline $1 / 30 / 1212: 00$ & 33.654 & 35.77 & 1475.12 & 1473.35 \\
\hline $1 / 30 / 12$ 12:30 & 34.475 & 35.772 & 1474.29 & 1473.35 \\
\hline $1 / 30 / 12$ 13:00 & 33.598 & 35.765 & 1475.17 & 1473.36 \\
\hline $1 / 30 / 12$ 13:30 & 33.546 & 35.756 & 1475.22 & 1473.37 \\
\hline 1/30/12 14:00 & 34.927 & 35.751 & 1473.84 & 1473.37 \\
\hline $1 / 30 / 1214: 30$ & 34.625 & 35.754 & 1474.14 & 1473.37 \\
\hline $1 / 30 / 12$ 15:00 & 35.249 & 35.772 & 1473.52 & 1473.35 \\
\hline $1 / 30 / 12$ 15:30 & 40.952 & 35.803 & 1467.82 & 1473.32 \\
\hline 1/30/12 16:00 & 39.721 & 35.845 & 1469.05 & 1473.28 \\
\hline $1 / 30 / 12$ 16:30 & 37.65 & 35.887 & 1471.12 & 1473.24 \\
\hline 1/30/12 17:00 & 37.46 & 35.972 & 1471.31 & 1473.15 \\
\hline 1/30/12 17:30 & 38.551 & 36.08 & 1470.22 & 1473.04 \\
\hline 1/30/12 18:00 & 37.137 & 36.192 & 1471.63 & 1472.93 \\
\hline 1/30/12 18:30 & 36.916 & 36.286 & 1471.85 & 1472.84 \\
\hline 1/30/12 19:00 & 37.012 & 36.378 & 1471.76 & 1472.74 \\
\hline 1/30/12 19:30 & 36.942 & 36.457 & 1471.83 & 1472.67 \\
\hline $1 / 30 / 12$ 20:00 & 38.309 & 36.481 & 1470.46 & 1472.64 \\
\hline $1 / 30 / 12$ 20:30 & 36.25 & 36.488 & 1472.52 & 1472.63 \\
\hline $1 / 30 / 12$ 21:00 & 36.06 & 36.49 & 1472.71 & 1472.63 \\
\hline $1 / 30 / 1221: 30$ & 35.915 & 36.5 & 1472.85 & 1472.62 \\
\hline $1 / 30 / 1222: 00$ & 35.673 & 36.495 & 1473.10 & 1472.63 \\
\hline $1 / 30 / 1222: 30$ & 40.277 & 36.493 & 1468.49 & 1472.63 \\
\hline $1 / 30 / 12$ 23:00 & 35.678 & 36.493 & 1473.09 & 1472.63 \\
\hline $1 / 30 / 12$ 23:30 & 35.399 & 36.483 & 1473.37 & 1472.64 \\
\hline 1/31/12 0:00 & 35.223 & 36.472 & 1473.55 & 1472.65 \\
\hline
\end{tabular}


TABLE S1.3 Automatically recorded groundwater levels in the Klassen wells, September 15, 2011, to March 13, 2012.

\begin{tabular}{|c|c|c|c|c|}
\hline \multicolumn{3}{|c|}{ Reference Elevation (ft AMSL) } & 1508.77 & \multirow{2}{*}{$\begin{array}{l}1509.12 \\
\text { Elevation in Klassen } \\
\text { Test Well (ft AMSL) }\end{array}$} \\
\hline Date and Time & $\begin{array}{l}\text { Depth in Klassen } \\
\text { Domestic Well } \\
(\mathrm{ft} \mathrm{BGL})\end{array}$ & $\begin{array}{c}\text { Depth in Klassen } \\
\text { Test Well } \\
\text { (ft BGL) }\end{array}$ & $\begin{array}{l}\text { Elevation in Klassen } \\
\text { Domestic Well } \\
\text { (ft AMSL) }\end{array}$ & \\
\hline $1 / 31 / 120: 30$ & 35.085 & 36.455 & 1473.68 & 1472.67 \\
\hline $1 / 31 / 121: 00$ & 34.965 & 36.441 & 1473.80 & 1472.68 \\
\hline $1 / 31 / 121: 30$ & 34.862 & 36.425 & 1473.91 & 1472.70 \\
\hline $1 / 31 / 122: 00$ & 34.827 & 36.408 & 1473.94 & 1472.71 \\
\hline $1 / 31 / 122: 30$ & 34.801 & 36.401 & 1473.97 & 1472.72 \\
\hline 1/31/12 3:00 & 34.719 & 36.39 & 1474.05 & 1472.73 \\
\hline $1 / 31 / 123: 30$ & 34.651 & 36.373 & 1474.12 & 1472.75 \\
\hline 1/31/12 4:00 & 34.695 & 36.366 & 1474.07 & 1472.76 \\
\hline 1/31/12 4:30 & 34.613 & 36.35 & 1474.16 & 1472.77 \\
\hline 1/31/12 5:00 & 34.77 & 36.338 & 1474.00 & 1472.78 \\
\hline $1 / 31 / 125: 30$ & 34.545 & 36.333 & 1474.22 & 1472.79 \\
\hline $1 / 31 / 126: 00$ & 34.454 & 36.319 & 1474.32 & 1472.80 \\
\hline $1 / 31 / 126: 30$ & 34.658 & 36.314 & 1474.11 & 1472.81 \\
\hline $1 / 31 / 127: 00$ & 34.491 & 36.305 & 1474.28 & 1472.82 \\
\hline 1/31/12 7:30 & 34.472 & 36.3 & 1474.30 & 1472.82 \\
\hline 1/31/12 8:00 & 34.381 & 36.293 & 1474.39 & 1472.83 \\
\hline $1 / 31 / 128: 30$ & 34.311 & 36.286 & 1474.46 & 1472.84 \\
\hline 1/31/12 9:00 & 34.484 & 36.274 & 1474.29 & 1472.85 \\
\hline 1/31/12 9:30 & 34.287 & 36.268 & 1474.48 & 1472.85 \\
\hline 1/31/12 10:00 & 34.311 & 36.256 & 1474.46 & 1472.87 \\
\hline $1 / 31 / 12$ 10:30 & 34.215 & 36.249 & 1474.55 & 1472.87 \\
\hline 1/31/12 11:00 & 34.154 & 36.242 & 1474.62 & 1472.88 \\
\hline 1/31/12 11:30 & 34.104 & 36.225 & 1474.67 & 1472.90 \\
\hline $1 / 31 / 1212: 00$ & 34.064 & 36.211 & 1474.71 & 1472.91 \\
\hline 1/31/12 12:30 & 34.027 & 36.199 & 1474.74 & 1472.92 \\
\hline 1/31/12 13:00 & 34.081 & 36.195 & 1474.69 & 1472.93 \\
\hline 1/31/12 13:30 & 34.015 & 36.195 & 1474.75 & 1472.93 \\
\hline 1/31/12 14:00 & 34.095 & 36.199 & 1474.67 & 1472.92 \\
\hline 1/31/12 14:30 & 34.702 & 36.209 & 1474.07 & 1472.91 \\
\hline $1 / 31 / 12$ 15:00 & 34.222 & 36.214 & 1474.55 & 1472.91 \\
\hline $1 / 31 / 12$ 15:30 & 34.102 & 36.211 & 1474.67 & 1472.91 \\
\hline 1/31/12 16:00 & 34.036 & 36.207 & 1474.73 & 1472.92 \\
\hline $1 / 31 / 12$ 16:30 & 34.097 & 36.199 & 1474.67 & 1472.92 \\
\hline 1/31/12 17:00 & 34.027 & 36.199 & 1474.74 & 1472.92 \\
\hline 1/31/12 17:30 & 34.212 & 36.195 & 1474.56 & 1472.93 \\
\hline $1 / 31 / 12$ 18:00 & 34.095 & 36.195 & 1474.67 & 1472.93 \\
\hline 1/31/12 18:30 & 34.046 & 36.197 & 1474.72 & 1472.93 \\
\hline 1/31/12 19:00 & 34.02 & 36.195 & 1474.75 & 1472.93 \\
\hline
\end{tabular}


TABLE S1.3 Automatically recorded groundwater levels in the Klassen wells, September 15, 2011, to March 13, 2012.

\begin{tabular}{|c|c|c|c|c|}
\hline \multicolumn{3}{|c|}{ Reference Elevation (ft AMSL) } & 1508.77 & \multirow{2}{*}{$\begin{array}{l}1509.12 \\
\text { Elevation in Klassen } \\
\text { Test Well (ft AMSL) }\end{array}$} \\
\hline Date and Time & $\begin{array}{l}\text { Depth in Klassen } \\
\text { Domestic Well } \\
(\mathrm{ft} \mathrm{BGL})\end{array}$ & $\begin{array}{c}\text { Depth in Klassen } \\
\text { Test Well } \\
\text { (ft BGL) }\end{array}$ & $\begin{array}{l}\text { Elevation in Klassen } \\
\text { Domestic Well } \\
\text { (ft AMSL) }\end{array}$ & \\
\hline $1 / 31 / 12$ 19:30 & 34.139 & 36.195 & 1474.63 & 1472.93 \\
\hline $1 / 31 / 1220: 00$ & 34.003 & 36.188 & 1474.77 & 1472.93 \\
\hline $1 / 31 / 1220: 30$ & 34.123 & 36.188 & 1474.65 & 1472.93 \\
\hline $1 / 31 / 1221: 00$ & 34.081 & 36.188 & 1474.69 & 1472.93 \\
\hline $1 / 31 / 1221: 30$ & 34.022 & 36.183 & 1474.75 & 1472.94 \\
\hline $1 / 31 / 1222: 00$ & 34.071 & 36.176 & 1474.70 & 1472.95 \\
\hline $1 / 31 / 1222: 30$ & 38.558 & 36.167 & 1470.21 & 1472.96 \\
\hline $1 / 31 / 1223: 00$ & 35.612 & 36.167 & 1473.16 & 1472.96 \\
\hline $1 / 31 / 1223: 30$ & 34.158 & 36.164 & 1474.61 & 1472.96 \\
\hline $2 / 1 / 120: 00$ & 34.034 & 36.157 & 1474.74 & 1472.97 \\
\hline $2 / 1 / 120: 30$ & 33.964 & 36.148 & 1474.81 & 1472.97 \\
\hline 2/1/12 1:00 & 33.91 & 36.136 & 1474.86 & 1472.99 \\
\hline $2 / 1 / 121: 30$ & 33.865 & 36.124 & 1474.90 & 1473.00 \\
\hline $2 / 1 / 122: 00$ & 33.828 & 36.115 & 1474.94 & 1473.01 \\
\hline $2 / 1 / 122: 30$ & 33.795 & 36.101 & 1474.97 & 1473.02 \\
\hline 2/1/12 3:00 & 33.762 & 36.092 & 1475.01 & 1473.03 \\
\hline $2 / 1 / 123: 30$ & 33.736 & 36.085 & 1475.03 & 1473.04 \\
\hline $2 / 1 / 124: 00$ & 33.71 & 36.075 & 1475.06 & 1473.05 \\
\hline $2 / 1 / 124: 30$ & 33.687 & 36.066 & 1475.08 & 1473.06 \\
\hline $2 / 1 / 125: 00$ & 33.666 & 36.059 & 1475.10 & 1473.06 \\
\hline $2 / 1 / 125: 30$ & 33.647 & 36.049 & 1475.12 & 1473.07 \\
\hline $2 / 1 / 126: 00$ & 33.628 & 36.038 & 1475.14 & 1473.08 \\
\hline $2 / 1 / 126: 30$ & 33.83 & 36.038 & 1474.94 & 1473.08 \\
\hline $2 / 1 / 127: 00$ & 33.724 & 36.026 & 1475.05 & 1473.10 \\
\hline $2 / 1 / 12$ 7:30 & 33.734 & 36.019 & 1475.04 & 1473.10 \\
\hline $2 / 1 / 128: 00$ & 33.858 & 36.019 & 1474.91 & 1473.10 \\
\hline 2/1/12 8:30 & 33.701 & 36.014 & 1475.07 & 1473.11 \\
\hline 2/1/12 9:00 & 33.774 & 36.019 & 1475.00 & 1473.10 \\
\hline 2/1/12 9:30 & 33.684 & 36.009 & 1475.09 & 1473.11 \\
\hline 2/1/12 10:00 & 33.642 & 36.005 & 1475.13 & 1473.12 \\
\hline 2/1/12 10:30 & 33.612 & 35.995 & 1475.16 & 1473.13 \\
\hline 2/1/12 11:00 & 33.661 & 36.005 & 1475.11 & 1473.12 \\
\hline 2/1/12 11:30 & 33.677 & 36.014 & 1475.09 & 1473.11 \\
\hline 2/1/12 12:00 & 33.619 & 36.009 & 1475.15 & 1473.11 \\
\hline 2/1/12 12:30 & 33.584 & 35.995 & 1475.19 & 1473.13 \\
\hline 2/1/12 13:00 & 33.553 & 35.97 & 1475.22 & 1473.15 \\
\hline 2/1/12 13:30 & 33.53 & 35.951 & 1475.24 & 1473.17 \\
\hline $2 / 1 / 12$ 14:00 & 33.628 & 35.944 & 1475.14 & 1473.18 \\
\hline
\end{tabular}


TABLE S1.3 Automatically recorded groundwater levels in the Klassen wells, September 15, 2011, to March 13, 2012.

\begin{tabular}{|c|c|c|c|c|}
\hline \multicolumn{3}{|c|}{ Reference Elevation (ft AMSL) } & 1508.77 & \multirow{2}{*}{$\begin{array}{l}1509.12 \\
\text { Elevation in Klassen } \\
\text { Test Well (ft AMSL) }\end{array}$} \\
\hline Date and Time & $\begin{array}{l}\text { Depth in Klassen } \\
\text { Domestic Well } \\
\text { (ft BGL) }\end{array}$ & $\begin{array}{c}\text { Depth in Klassen } \\
\text { Test Well } \\
\text { (ft BGL) }\end{array}$ & $\begin{array}{l}\text { Elevation in Klassen } \\
\text { Domestic Well } \\
\text { (ft AMSL) }\end{array}$ & \\
\hline $2 / 1 / 1214: 30$ & 33.553 & 35.939 & 1475.22 & 1473.18 \\
\hline $2 / 1 / 1215: 00$ & 33.525 & 35.934 & 1475.24 & 1473.19 \\
\hline $2 / 1 / 1215: 30$ & 33.502 & 35.93 & 1475.27 & 1473.19 \\
\hline $2 / 1 / 1216: 00$ & 33.595 & 35.934 & 1475.17 & 1473.19 \\
\hline $2 / 1 / 1216: 30$ & 33.539 & 35.941 & 1475.23 & 1473.18 \\
\hline $2 / 1 / 1217: 00$ & 36.846 & 35.948 & 1471.92 & 1473.17 \\
\hline $2 / 1 / 1217: 30$ & 34.306 & 35.979 & 1474.46 & 1473.14 \\
\hline $2 / 1 / 1218: 00$ & 33.919 & 35.995 & 1474.85 & 1473.13 \\
\hline $2 / 1 / 1218: 30$ & 33.802 & 36.002 & 1474.97 & 1473.12 \\
\hline 2/1/12 19:00 & 33.727 & 36.009 & 1475.04 & 1473.11 \\
\hline 2/1/12 19:30 & 33.806 & 36.019 & 1474.96 & 1473.10 \\
\hline $2 / 1 / 1220: 00$ & 33.731 & 36.031 & 1475.04 & 1473.09 \\
\hline $2 / 1 / 1220: 30$ & 33.776 & 36.04 & 1474.99 & 1473.08 \\
\hline 2/1/12 21:00 & 33.896 & 36.07 & 1474.87 & 1473.05 \\
\hline $2 / 1 / 12$ 21:30 & 33.802 & 36.087 & 1474.97 & 1473.04 \\
\hline 2/1/12 22:00 & 33.736 & 36.094 & 1475.03 & 1473.03 \\
\hline $2 / 1 / 1222: 30$ & 38.72 & 36.096 & 1470.05 & 1473.03 \\
\hline 2/1/12 23:00 & 34.688 & 36.101 & 1474.08 & 1473.02 \\
\hline 2/1/12 23:30 & 33.945 & 36.106 & 1474.82 & 1473.02 \\
\hline $2 / 2 / 120: 00$ & 33.813 & 36.108 & 1474.96 & 1473.01 \\
\hline $2 / 2 / 12$ 0:30 & 33.769 & 36.108 & 1475.00 & 1473.01 \\
\hline 2/2/12 1:00 & 33.713 & 36.108 & 1475.06 & 1473.01 \\
\hline 2/2/12 1:30 & 33.668 & 36.101 & 1475.10 & 1473.02 \\
\hline $2 / 2 / 122: 00$ & 33.633 & 36.091 & 1475.14 & 1473.03 \\
\hline $2 / 2 / 122: 30$ & 33.605 & 36.087 & 1475.16 & 1473.04 \\
\hline 2/2/12 3:00 & 33.579 & 36.082 & 1475.19 & 1473.04 \\
\hline 2/2/12 3:30 & 33.558 & 36.075 & 1475.21 & 1473.05 \\
\hline $2 / 2 / 124: 00$ & 33.537 & 36.063 & 1475.23 & 1473.06 \\
\hline $2 / 2 / 124: 30$ & 33.518 & 36.056 & 1475.25 & 1473.07 \\
\hline 2/2/12 5:00 & 33.525 & 36.047 & 1475.24 & 1473.08 \\
\hline $2 / 2 / 125: 30$ & 33.502 & 36.038 & 1475.27 & 1473.08 \\
\hline 2/2/12 6:00 & 33.602 & 36.033 & 1475.17 & 1473.09 \\
\hline $2 / 2 / 126: 30$ & 36.858 & 36.035 & 1471.91 & 1473.09 \\
\hline 2/2/12 7:00 & 36.949 & 36.04 & 1471.82 & 1473.08 \\
\hline 2/2/12 7:30 & 34.015 & 36.049 & 1474.75 & 1473.07 \\
\hline 2/2/12 8:00 & 33.846 & 36.052 & 1474.92 & 1473.07 \\
\hline 2/2/12 8:30 & 33.83 & 36.059 & 1474.94 & 1473.06 \\
\hline 2/2/12 9:00 & 33.741 & 36.059 & 1475.03 & 1473.06 \\
\hline
\end{tabular}


TABLE S1.3 Automatically recorded groundwater levels in the Klassen wells, September 15, 2011, to March 13, 2012.

\begin{tabular}{|c|c|c|c|c|}
\hline \multicolumn{3}{|c|}{ Reference Elevation (ft AMSL) } & 1508.77 & \multirow{2}{*}{$\begin{array}{l}1509.12 \\
\text { Elevation in Klassen } \\
\text { Test Well (ft AMSL) }\end{array}$} \\
\hline Date and Time & $\begin{array}{l}\text { Depth in Klassen } \\
\text { Domestic Well } \\
(\mathrm{ft} \mathrm{BGL})\end{array}$ & $\begin{array}{c}\text { Depth in Klassen } \\
\text { Test Well } \\
\text { (ft BGL) }\end{array}$ & $\begin{array}{l}\text { Elevation in Klassen } \\
\text { Domestic Well } \\
\text { (ft AMSL) }\end{array}$ & \\
\hline $2 / 2 / 129: 30$ & 33.781 & 36.063 & 1474.99 & 1473.06 \\
\hline $2 / 2 / 12$ 10:00 & 33.689 & 36.061 & 1475.08 & 1473.06 \\
\hline $2 / 2 / 1210: 30$ & 33.806 & 36.063 & 1474.96 & 1473.06 \\
\hline 2/2/12 11:00 & 33.668 & 36.066 & 1475.10 & 1473.06 \\
\hline $2 / 2 / 1211: 30$ & 33.619 & 36.066 & 1475.15 & 1473.06 \\
\hline $2 / 2 / 12$ 12:00 & 33.872 & 36.07 & 1474.90 & 1473.05 \\
\hline 2/2/12 12:30 & 33.619 & 36.061 & 1475.15 & 1473.06 \\
\hline $2 / 2 / 12$ 13:00 & 33.57 & 36.035 & 1475.20 & 1473.09 \\
\hline 2/2/12 13:30 & 33.537 & 36.012 & 1475.23 & 1473.11 \\
\hline 2/2/12 14:00 & 33.724 & 36.005 & 1475.05 & 1473.12 \\
\hline $2 / 2 / 1214: 30$ & 33.588 & 36 & 1475.18 & 1473.12 \\
\hline $2 / 2 / 1215: 00$ & 33.692 & 35.995 & 1475.08 & 1473.13 \\
\hline $2 / 2 / 12$ 15:30 & 33.581 & 35.986 & 1475.19 & 1473.14 \\
\hline 2/2/12 16:00 & 33.537 & 35.979 & 1475.23 & 1473.14 \\
\hline 2/2/12 16:30 & 33.502 & 35.969 & 1475.27 & 1473.15 \\
\hline $2 / 2 / 12$ 17:00 & 33.473 & 35.962 & 1475.30 & 1473.16 \\
\hline 2/2/12 17:30 & 33.609 & 35.963 & 1475.16 & 1473.16 \\
\hline 2/2/12 18:00 & 33.553 & 35.96 & 1475.22 & 1473.16 \\
\hline $2 / 2 / 1218: 30$ & 33.509 & 35.96 & 1475.26 & 1473.16 \\
\hline 2/2/12 19:00 & 33.497 & 35.953 & 1475.27 & 1473.17 \\
\hline $2 / 2 / 1219: 30$ & 33.591 & 35.955 & 1475.18 & 1473.17 \\
\hline $2 / 2 / 12$ 20:00 & 33.6 & 35.951 & 1475.17 & 1473.17 \\
\hline $2 / 2 / 12$ 20:30 & 33.539 & 35.948 & 1475.23 & 1473.17 \\
\hline $2 / 2 / 1221: 00$ & 33.497 & 35.941 & 1475.27 & 1473.18 \\
\hline 2/2/12 21:30 & 33.677 & 35.939 & 1475.09 & 1473.18 \\
\hline $2 / 2 / 12$ 22:00 & 33.607 & 35.939 & 1475.16 & 1473.18 \\
\hline $2 / 2 / 12$ 22:30 & 33.567 & 35.93 & 1475.20 & 1473.19 \\
\hline 2/2/12 23:00 & 33.783 & 35.927 & 1474.99 & 1473.20 \\
\hline $2 / 2 / 12$ 23:30 & 33.811 & 35.932 & 1474.96 & 1473.19 \\
\hline $2 / 3 / 120: 00$ & 33.628 & 35.93 & 1475.14 & 1473.19 \\
\hline $2 / 3 / 120: 30$ & 33.539 & 35.883 & 1475.23 & 1473.24 \\
\hline $2 / 3 / 12$ 1:00 & 33.48 & 35.892 & 1475.29 & 1473.23 \\
\hline $2 / 3 / 121: 30$ & 33.438 & 35.878 & 1475.33 & 1473.24 \\
\hline $2 / 3 / 122: 00$ & 33.464 & 35.876 & 1475.31 & 1473.25 \\
\hline $2 / 3 / 122: 30$ & 33.476 & 35.873 & 1475.29 & 1473.25 \\
\hline 2/3/12 3:00 & 33.602 & 35.862 & 1475.17 & 1473.26 \\
\hline 2/3/12 3:30 & 33.504 & 35.859 & 1475.27 & 1473.26 \\
\hline $2 / 3 / 124: 00$ & 33.56 & 35.859 & 1475.21 & 1473.26 \\
\hline
\end{tabular}


TABLE S1.3 Automatically recorded groundwater levels in the Klassen wells, September 15, 2011, to March 13, 2012.

\begin{tabular}{|c|c|c|c|c|}
\hline \multicolumn{3}{|c|}{ Reference Elevation (ft AMSL) } & 1508.77 & \multirow{2}{*}{$\begin{array}{l}1509.12 \\
\text { Elevation in Klassen } \\
\text { Test Well (ft AMSL) }\end{array}$} \\
\hline Date and Time & $\begin{array}{l}\text { Depth in Klassen } \\
\text { Domestic Well } \\
\text { (ft BGL) }\end{array}$ & $\begin{array}{c}\text { Depth in Klassen } \\
\text { Test Well } \\
\text { (ft BGL) }\end{array}$ & $\begin{array}{l}\text { Elevation in Klassen } \\
\text { Domestic Well } \\
\text { (ft AMSL) }\end{array}$ & \\
\hline $2 / 3 / 124: 30$ & 33.485 & 35.84 & 1475.28 & 1473.28 \\
\hline $2 / 3 / 125: 00$ & 33.429 & 35.845 & 1475.34 & 1473.28 \\
\hline $2 / 3 / 125: 30$ & 33.391 & 35.817 & 1475.38 & 1473.31 \\
\hline $2 / 3 / 126: 00$ & 33.354 & 35.859 & 1475.42 & 1473.26 \\
\hline $2 / 3 / 126: 30$ & 33.48 & 35.857 & 1475.29 & 1473.27 \\
\hline $2 / 3 / 127: 00$ & 33.408 & 35.84 & 1475.36 & 1473.28 \\
\hline $2 / 3 / 127: 30$ & 33.495 & 35.852 & 1475.27 & 1473.27 \\
\hline $2 / 3 / 128: 00$ & 33.457 & 35.826 & 1475.31 & 1473.30 \\
\hline $2 / 3 / 128: 30$ & 33.504 & 35.826 & 1475.27 & 1473.30 \\
\hline 2/3/12 9:00 & 37.139 & 35.824 & 1471.63 & 1473.30 \\
\hline 2/3/12 9:30 & 33.495 & 35.831 & 1475.27 & 1473.29 \\
\hline $2 / 3 / 1210: 00$ & 33.743 & 35.81 & 1475.03 & 1473.31 \\
\hline 2/3/12 10:30 & 33.436 & 35.819 & 1475.33 & 1473.30 \\
\hline 2/3/12 11:00 & 33.48 & 35.801 & 1475.29 & 1473.32 \\
\hline 2/3/12 11:30 & 33.48 & 35.803 & 1475.29 & 1473.32 \\
\hline $2 / 3 / 12$ 12:00 & 33.71 & 35.798 & 1475.06 & 1473.32 \\
\hline 2/3/12 12:30 & 33.441 & 35.798 & 1475.33 & 1473.32 \\
\hline 2/3/12 13:00 & 33.727 & 35.824 & 1475.04 & 1473.30 \\
\hline 2/3/12 13:30 & 33.612 & 35.829 & 1475.16 & 1473.29 \\
\hline $2 / 3 / 12$ 14:00 & 34.794 & 35.836 & 1473.98 & 1473.29 \\
\hline $2 / 3 / 12$ 14:30 & 33.706 & 35.843 & 1475.06 & 1473.28 \\
\hline $2 / 3 / 12$ 15:00 & 33.79 & 35.843 & 1474.98 & 1473.28 \\
\hline 2/3/12 15:30 & 33.781 & 35.843 & 1474.99 & 1473.28 \\
\hline 2/3/12 16:00 & 33.975 & 35.847 & 1474.79 & 1473.28 \\
\hline 2/3/12 16:30 & 34.025 & 35.85 & 1474.74 & 1473.27 \\
\hline 2/3/12 17:00 & 34.484 & 35.852 & 1474.29 & 1473.27 \\
\hline 2/3/12 17:30 & 33.954 & 35.845 & 1474.82 & 1473.28 \\
\hline 2/3/12 18:00 & 33.797 & 35.836 & 1474.97 & 1473.29 \\
\hline 2/3/12 18:30 & 33.703 & 35.829 & 1475.07 & 1473.29 \\
\hline 2/3/12 19:00 & 33.633 & 35.819 & 1475.14 & 1473.30 \\
\hline 2/3/12 19:30 & 33.68 & 35.815 & 1475.09 & 1473.31 \\
\hline 2/3/12 20:00 & 33.586 & 35.81 & 1475.18 & 1473.31 \\
\hline $2 / 3 / 12$ 20:30 & 33.649 & 35.808 & 1475.12 & 1473.31 \\
\hline 2/3/12 21:00 & 33.539 & 35.803 & 1475.23 & 1473.32 \\
\hline 2/3/12 21:30 & 33.513 & 35.794 & 1475.26 & 1473.33 \\
\hline $2 / 3 / 12$ 22:00 & 35.277 & 35.791 & 1473.49 & 1473.33 \\
\hline $2 / 3 / 12$ 22:30 & 34.28 & 35.796 & 1474.49 & 1473.33 \\
\hline 2/3/12 23:00 & 34.292 & 35.791 & 1474.48 & 1473.33 \\
\hline
\end{tabular}


TABLE S1.3 Automatically recorded groundwater levels in the Klassen wells, September 15, 2011, to March 13, 2012.

\begin{tabular}{|c|c|c|c|c|}
\hline \multicolumn{3}{|c|}{ Reference Elevation (ft AMSL) } & 1508.77 & \multirow{2}{*}{$\begin{array}{l}1509.12 \\
\text { Elevation in Klassen } \\
\text { Test Well (ft AMSL) }\end{array}$} \\
\hline Date and Time & $\begin{array}{l}\text { Depth in Klassen } \\
\text { Domestic Well } \\
(\mathrm{ft} \mathrm{BGL})\end{array}$ & $\begin{array}{c}\text { Depth in Klassen } \\
\text { Test Well } \\
\text { (ft BGL) }\end{array}$ & $\begin{array}{l}\text { Elevation in Klassen } \\
\text { Domestic Well } \\
\text { (ft AMSL) }\end{array}$ & \\
\hline $2 / 3 / 1223: 30$ & 33.781 & 35.787 & 1474.99 & 1473.34 \\
\hline 2/4/12 0:00 & 33.677 & 35.784 & 1475.09 & 1473.34 \\
\hline 2/4/12 0:30 & 33.593 & 35.775 & 1475.18 & 1473.35 \\
\hline 2/4/12 1:00 & 33.53 & 35.765 & 1475.24 & 1473.36 \\
\hline 2/4/12 1:30 & 33.966 & 35.756 & 1474.80 & 1473.37 \\
\hline 2/4/12 2:00 & 33.577 & 35.747 & 1475.19 & 1473.38 \\
\hline $2 / 4 / 122: 30$ & 33.584 & 35.742 & 1475.19 & 1473.38 \\
\hline 2/4/12 3:00 & 33.534 & 35.735 & 1475.24 & 1473.39 \\
\hline 2/4/12 3:30 & 33.499 & 35.728 & 1475.27 & 1473.39 \\
\hline $2 / 4 / 124: 00$ & 33.544 & 35.728 & 1475.23 & 1473.39 \\
\hline $2 / 4 / 124: 30$ & 34.334 & 35.723 & 1474.44 & 1473.40 \\
\hline 2/4/12 5:00 & 33.497 & 35.711 & 1475.27 & 1473.41 \\
\hline $2 / 4 / 125: 30$ & 33.431 & 35.704 & 1475.34 & 1473.42 \\
\hline 2/4/12 6:00 & 33.441 & 35.693 & 1475.33 & 1473.43 \\
\hline $2 / 4 / 12$ 6:30 & 33.563 & 35.69 & 1475.21 & 1473.43 \\
\hline 2/4/12 7:00 & 33.476 & 35.679 & 1475.29 & 1473.44 \\
\hline $2 / 4 / 12$ 7:30 & 33.412 & 35.667 & 1475.36 & 1473.46 \\
\hline 2/4/12 8:00 & 33.534 & 35.653 & 1475.24 & 1473.47 \\
\hline 2/4/12 8:30 & 33.398 & 35.646 & 1475.37 & 1473.48 \\
\hline 2/4/12 9:00 & 33.734 & 35.648 & 1475.04 & 1473.47 \\
\hline 2/4/12 9:30 & 33.717 & 35.646 & 1475.05 & 1473.48 \\
\hline 2/4/12 10:00 & 33.689 & 35.632 & 1475.08 & 1473.49 \\
\hline 2/4/12 10:30 & 33.825 & 35.629 & 1474.94 & 1473.49 \\
\hline 2/4/12 11:00 & 33.659 & 35.62 & 1475.11 & 1473.50 \\
\hline 2/4/12 11:30 & 33.851 & 35.615 & 1474.92 & 1473.51 \\
\hline 2/4/12 12:00 & 33.689 & 35.618 & 1475.08 & 1473.50 \\
\hline 2/4/12 12:30 & 33.816 & 35.639 & 1474.95 & 1473.48 \\
\hline 2/4/12 13:00 & 36.471 & 35.641 & 1472.30 & 1473.48 \\
\hline 2/4/12 13:30 & 33.799 & 35.648 & 1474.97 & 1473.47 \\
\hline 2/4/12 14:00 & 33.912 & 35.655 & 1474.86 & 1473.47 \\
\hline 2/4/12 14:30 & 33.753 & 35.65 & 1475.02 & 1473.47 \\
\hline 2/4/12 15:00 & 33.736 & 35.646 & 1475.03 & 1473.48 \\
\hline 2/4/12 15:30 & 34.397 & 35.641 & 1474.37 & 1473.48 \\
\hline 2/4/12 16:00 & 34.243 & 35.641 & 1474.53 & 1473.48 \\
\hline 2/4/12 16:30 & 33.75 & 35.641 & 1475.02 & 1473.48 \\
\hline $2 / 4 / 12$ 17:00 & 33.654 & 35.641 & 1475.12 & 1473.48 \\
\hline 2/4/12 17:30 & 33.598 & 35.636 & 1475.17 & 1473.49 \\
\hline $2 / 4 / 1218: 00$ & 33.53 & 35.629 & 1475.24 & 1473.49 \\
\hline
\end{tabular}


TABLE S1.3 Automatically recorded groundwater levels in the Klassen wells, September 15, 2011, to March 13, 2012.

\begin{tabular}{|c|c|c|c|c|}
\hline \multicolumn{3}{|c|}{ Reference Elevation (ft AMSL) } & 1508.77 & \multirow{2}{*}{$\begin{array}{l}1509.12 \\
\text { Elevation in Klassen } \\
\text { Test Well (ft AMSL) }\end{array}$} \\
\hline Date and Time & $\begin{array}{l}\text { Depth in Klassen } \\
\text { Domestic Well } \\
(\mathrm{ft} \mathrm{BGL})\end{array}$ & $\begin{array}{c}\text { Depth in Klassen } \\
\text { Test Well } \\
\text { (ft BGL) }\end{array}$ & $\begin{array}{l}\text { Elevation in Klassen } \\
\text { Domestic Well } \\
\text { (ft AMSL) }\end{array}$ & \\
\hline $2 / 4 / 1218: 30$ & 33.509 & 35.629 & 1475.26 & 1473.49 \\
\hline 2/4/12 19:00 & 33.462 & 35.625 & 1475.31 & 1473.50 \\
\hline $2 / 4 / 1219: 30$ & 33.422 & 35.627 & 1475.35 & 1473.50 \\
\hline 2/4/12 20:00 & 33.387 & 35.622 & 1475.38 & 1473.50 \\
\hline $2 / 4 / 12$ 20:30 & 33.361 & 35.618 & 1475.41 & 1473.50 \\
\hline $2 / 4 / 1221: 00$ & 33.333 & 35.615 & 1475.44 & 1473.51 \\
\hline 2/4/12 21:30 & 33.309 & 35.613 & 1475.46 & 1473.51 \\
\hline $2 / 4 / 1222: 00$ & 33.286 & 35.613 & 1475.48 & 1473.51 \\
\hline 2/4/12 22:30 & 33.265 & 35.613 & 1475.50 & 1473.51 \\
\hline 2/4/12 23:00 & 33.424 & 35.613 & 1475.35 & 1473.51 \\
\hline 2/4/12 23:30 & 33.344 & 35.608 & 1475.43 & 1473.51 \\
\hline 2/5/12 0:00 & 33.293 & 35.606 & 1475.48 & 1473.52 \\
\hline $2 / 5 / 120: 30$ & 33.26 & 35.596 & 1475.51 & 1473.53 \\
\hline 2/5/12 1:00 & 33.232 & 35.589 & 1475.54 & 1473.53 \\
\hline 2/5/12 1:30 & 33.208 & 35.585 & 1475.56 & 1473.54 \\
\hline $2 / 5 / 122: 00$ & 33.272 & 35.58 & 1475.50 & 1473.54 \\
\hline $2 / 5 / 122: 30$ & 33.293 & 35.575 & 1475.48 & 1473.55 \\
\hline 2/5/12 3:00 & 33.272 & 35.566 & 1475.50 & 1473.56 \\
\hline 2/5/12 3:30 & 33.262 & 35.561 & 1475.51 & 1473.56 \\
\hline $2 / 5 / 124: 00$ & 33.335 & 35.561 & 1475.43 & 1473.56 \\
\hline $2 / 5 / 124: 30$ & 33.272 & 35.552 & 1475.50 & 1473.57 \\
\hline 2/5/12 5:00 & 33.23 & 35.547 & 1475.54 & 1473.58 \\
\hline $2 / 5 / 125: 30$ & 33.288 & 35.54 & 1475.48 & 1473.58 \\
\hline $2 / 5 / 126: 00$ & 34.374 & 35.545 & 1474.40 & 1473.58 \\
\hline $2 / 5 / 126: 30$ & 33.666 & 35.545 & 1475.10 & 1473.58 \\
\hline $2 / 5 / 12$ 7:00 & 33.471 & 35.54 & 1475.30 & 1473.58 \\
\hline 2/5/12 7:30 & 33.38 & 35.535 & 1475.39 & 1473.59 \\
\hline 2/5/12 8:00 & 33.429 & 35.538 & 1475.34 & 1473.58 \\
\hline $2 / 5 / 128: 30$ & 33.982 & 35.54 & 1474.79 & 1473.58 \\
\hline 2/5/12 9:00 & 33.427 & 35.542 & 1475.34 & 1473.58 \\
\hline 2/5/12 9:30 & 33.384 & 35.54 & 1475.39 & 1473.58 \\
\hline $2 / 5 / 12$ 10:00 & 33.41 & 35.545 & 1475.36 & 1473.58 \\
\hline 2/5/12 10:30 & 33.342 & 35.557 & 1475.43 & 1473.57 \\
\hline 2/5/12 11:00 & 33.314 & 35.571 & 1475.46 & 1473.55 \\
\hline 2/5/12 11:30 & 33.293 & 35.589 & 1475.48 & 1473.53 \\
\hline $2 / 5 / 1212: 00$ & 33.253 & 35.606 & 1475.52 & 1473.52 \\
\hline $2 / 5 / 1212: 30$ & 33.218 & 35.622 & 1475.55 & 1473.50 \\
\hline 2/5/12 13:00 & 33.19 & 35.629 & 1475.58 & 1473.49 \\
\hline
\end{tabular}


TABLE S1.3 Automatically recorded groundwater levels in the Klassen wells, September 15, 2011, to March 13, 2012.

\begin{tabular}{|c|c|c|c|c|}
\hline \multicolumn{3}{|c|}{ Reference Elevation (ft AMSL) } & 1508.77 & \multirow{2}{*}{$\begin{array}{l}1509.12 \\
\text { Elevation in Klassen } \\
\text { Test Well (ft AMSL) }\end{array}$} \\
\hline Date and Time & $\begin{array}{l}\text { Depth in Klassen } \\
\text { Domestic Well } \\
(\mathrm{ft} \mathrm{BGL})\end{array}$ & $\begin{array}{c}\text { Depth in Klassen } \\
\text { Test Well } \\
\text { (ft BGL) }\end{array}$ & $\begin{array}{l}\text { Elevation in Klassen } \\
\text { Domestic Well } \\
\text { (ft AMSL) }\end{array}$ & \\
\hline $2 / 5 / 1213: 30$ & 33.218 & 35.634 & 1475.55 & 1473.49 \\
\hline $2 / 5 / 1214: 00$ & 33.178 & 35.639 & 1475.59 & 1473.48 \\
\hline $2 / 5 / 1214: 30$ & 33.218 & 35.622 & 1475.55 & 1473.50 \\
\hline 2/5/12 15:00 & 33.22 & 35.608 & 1475.55 & 1473.51 \\
\hline $2 / 5 / 1215: 30$ & 33.206 & 35.596 & 1475.56 & 1473.53 \\
\hline $2 / 5 / 1216: 00$ & 33.394 & 35.589 & 1475.38 & 1473.53 \\
\hline $2 / 5 / 1216: 30$ & 33.19 & 35.582 & 1475.58 & 1473.54 \\
\hline 2/5/12 17:00 & 33.147 & 35.575 & 1475.62 & 1473.55 \\
\hline 2/5/12 17:30 & 33.363 & 35.566 & 1475.41 & 1473.56 \\
\hline $2 / 5 / 1218: 00$ & 33.178 & 35.559 & 1475.59 & 1473.56 \\
\hline 2/5/12 18:30 & 33.133 & 35.557 & 1475.64 & 1473.57 \\
\hline 2/5/12 19:00 & 33.19 & 35.557 & 1475.58 & 1473.57 \\
\hline 2/5/12 19:30 & 33.129 & 35.552 & 1475.64 & 1473.57 \\
\hline $2 / 5 / 1220: 00$ & 33.101 & 35.547 & 1475.67 & 1473.58 \\
\hline 2/5/12 20:30 & 33.122 & 35.542 & 1475.65 & 1473.58 \\
\hline $2 / 5 / 12$ 21:00 & 33.262 & 35.55 & 1475.51 & 1473.57 \\
\hline $2 / 5 / 1221: 30$ & 33.396 & 35.557 & 1475.37 & 1473.57 \\
\hline 2/5/12 22:00 & 33.419 & 35.564 & 1475.35 & 1473.56 \\
\hline $2 / 5 / 1222: 30$ & 39.184 & 35.575 & 1469.59 & 1473.55 \\
\hline 2/5/12 23:00 & 35.83 & 35.582 & 1472.94 & 1473.54 \\
\hline $2 / 5 / 12$ 23:30 & 33.612 & 35.587 & 1475.16 & 1473.54 \\
\hline $2 / 6 / 120: 00$ & 33.466 & 35.582 & 1475.30 & 1473.54 \\
\hline $2 / 6 / 120: 30$ & 33.382 & 35.582 & 1475.39 & 1473.54 \\
\hline $2 / 6 / 121: 00$ & 33.319 & 35.578 & 1475.45 & 1473.54 \\
\hline 2/6/12 1:30 & 33.272 & 35.571 & 1475.50 & 1473.55 \\
\hline $2 / 6 / 122: 00$ & 33.232 & 35.559 & 1475.54 & 1473.56 \\
\hline $2 / 6 / 12$ 2:30 & 33.218 & 35.55 & 1475.55 & 1473.57 \\
\hline 2/6/12 3:00 & 33.183 & 35.543 & 1475.59 & 1473.58 \\
\hline 2/6/12 3:30 & 33.213 & 35.535 & 1475.56 & 1473.59 \\
\hline $2 / 6 / 124: 00$ & 33.162 & 35.526 & 1475.61 & 1473.60 \\
\hline 2/6/12 4:30 & 33.129 & 35.514 & 1475.64 & 1473.61 \\
\hline $2 / 6 / 12$ 5:00 & 33.101 & 35.503 & 1475.67 & 1473.62 \\
\hline $2 / 6 / 125: 30$ & 33.077 & 35.493 & 1475.69 & 1473.63 \\
\hline $2 / 6 / 12$ 6:00 & 33.056 & 35.482 & 1475.71 & 1473.64 \\
\hline $2 / 6 / 12$ 6:30 & 33.187 & 35.477 & 1475.58 & 1473.65 \\
\hline 2/6/12 7:00 & 33.119 & 35.472 & 1475.65 & 1473.65 \\
\hline 2/6/12 7:30 & 33.159 & 35.47 & 1475.61 & 1473.65 \\
\hline 2/6/12 8:00 & 33.108 & 35.467 & 1475.66 & 1473.66 \\
\hline
\end{tabular}


TABLE S1.3 Automatically recorded groundwater levels in the Klassen wells, September 15, 2011, to March 13, 2012.

\begin{tabular}{|c|c|c|c|c|}
\hline \multicolumn{3}{|c|}{ Reference Elevation (ft AMSL) } & 1508.77 & \multirow{2}{*}{$\begin{array}{l}1509.12 \\
\text { Elevation in Klassen } \\
\text { Test Well (ft AMSL) }\end{array}$} \\
\hline Date and Time & $\begin{array}{l}\text { Depth in Klassen } \\
\text { Domestic Well } \\
(\mathrm{ft} \mathrm{BGL})\end{array}$ & $\begin{array}{c}\text { Depth in Klassen } \\
\text { Test Well } \\
\text { (ft BGL) }\end{array}$ & $\begin{array}{l}\text { Elevation in Klassen } \\
\text { Domestic Well } \\
\text { (ft AMSL) }\end{array}$ & \\
\hline $2 / 6 / 128: 30$ & 33.312 & 35.474 & 1475.46 & 1473.65 \\
\hline 2/6/12 9:00 & 33.23 & 35.484 & 1475.54 & 1473.64 \\
\hline 2/6/12 9:30 & 33.293 & 35.488 & 1475.48 & 1473.63 \\
\hline $2 / 6 / 12$ 10:00 & 37.96 & 35.5 & 1470.81 & 1473.62 \\
\hline 2/6/12 10:30 & 33.258 & 35.514 & 1475.51 & 1473.61 \\
\hline 2/6/12 11:00 & 33.251 & 35.519 & 1475.52 & 1473.60 \\
\hline 2/6/12 11:30 & 33.314 & 35.528 & 1475.46 & 1473.59 \\
\hline 2/6/12 12:00 & 38.122 & 35.531 & 1470.65 & 1473.59 \\
\hline 2/6/12 12:30 & 33.211 & 35.531 & 1475.56 & 1473.59 \\
\hline 2/6/12 13:00 & 33.347 & 35.519 & 1475.42 & 1473.60 \\
\hline 2/6/12 13:30 & 33.241 & 35.474 & 1475.53 & 1473.65 \\
\hline 2/6/12 14:00 & 33.354 & 35.451 & 1475.42 & 1473.67 \\
\hline 2/6/12 14:30 & 33.41 & 35.446 & 1475.36 & 1473.68 \\
\hline 2/6/12 15:00 & 33.656 & 35.446 & 1475.11 & 1473.68 \\
\hline 2/6/12 15:30 & 33.347 & 35.444 & 1475.42 & 1473.68 \\
\hline 2/6/12 16:00 & 33.281 & 35.437 & 1475.49 & 1473.69 \\
\hline 2/6/12 16:30 & 33.312 & 35.432 & 1475.46 & 1473.69 \\
\hline 2/6/12 17:00 & 33.323 & 35.43 & 1475.45 & 1473.69 \\
\hline $2 / 6 / 12$ 17:30 & 33.375 & 35.43 & 1475.39 & 1473.69 \\
\hline 2/6/12 18:00 & 33.539 & 35.432 & 1475.23 & 1473.69 \\
\hline 2/6/12 18:30 & 33.323 & 35.43 & 1475.45 & 1473.69 \\
\hline 2/6/12 19:00 & 33.274 & 35.428 & 1475.50 & 1473.69 \\
\hline 2/6/12 19:30 & 33.288 & 35.425 & 1475.48 & 1473.70 \\
\hline 2/6/12 20:00 & 33.3 & 35.428 & 1475.47 & 1473.69 \\
\hline 2/6/12 20:30 & 33.251 & 35.427 & 1475.52 & 1473.70 \\
\hline $2 / 6 / 12$ 21:00 & 33.3 & 35.428 & 1475.47 & 1473.69 \\
\hline 2/6/12 21:30 & 33.344 & 35.435 & 1475.43 & 1473.69 \\
\hline 2/6/12 22:00 & 36.009 & 35.446 & 1472.76 & 1473.68 \\
\hline 2/6/12 22:30 & 33.37 & 35.451 & 1475.40 & 1473.67 \\
\hline 2/6/12 23:00 & 33.441 & 35.458 & 1475.33 & 1473.66 \\
\hline 2/6/12 23:30 & 33.321 & 35.453 & 1475.45 & 1473.67 \\
\hline 2/7/12 0:00 & 33.255 & 35.449 & 1475.51 & 1473.67 \\
\hline $2 / 7 / 120: 30$ & 33.208 & 35.442 & 1475.56 & 1473.68 \\
\hline 2/7/12 1:00 & 33.169 & 35.432 & 1475.60 & 1473.69 \\
\hline 2/7/12 1:30 & 33.136 & 35.423 & 1475.63 & 1473.70 \\
\hline 2/7/12 2:00 & 33.105 & 35.411 & 1475.66 & 1473.71 \\
\hline $2 / 7 / 12$ 2:30 & 33.079 & 35.404 & 1475.69 & 1473.72 \\
\hline 2/7/12 3:00 & 35.387 & 35.392 & 1473.38 & 1473.73 \\
\hline
\end{tabular}


TABLE S1.3 Automatically recorded groundwater levels in the Klassen wells, September 15, 2011, to March 13, 2012.

\begin{tabular}{|c|c|c|c|c|}
\hline \multicolumn{3}{|c|}{ Reference Elevation (ft AMSL) } & 1508.77 & \multirow{2}{*}{$\begin{array}{l}1509.12 \\
\text { Elevation in Klassen } \\
\text { Test Well (ft AMSL) }\end{array}$} \\
\hline Date and Time & $\begin{array}{l}\text { Depth in Klassen } \\
\text { Domestic Well } \\
(\mathrm{ft} \mathrm{BGL})\end{array}$ & $\begin{array}{c}\text { Depth in Klassen } \\
\text { Test Well } \\
\text { (ft BGL) }\end{array}$ & $\begin{array}{l}\text { Elevation in Klassen } \\
\text { Domestic Well } \\
\text { (ft AMSL) }\end{array}$ & \\
\hline 2/7/12 3:30 & 33.101 & 35.385 & 1475.67 & 1473.74 \\
\hline $2 / 7 / 124: 00$ & 33.056 & 35.374 & 1475.71 & 1473.75 \\
\hline $2 / 7 / 124: 30$ & 33.025 & 35.364 & 1475.74 & 1473.76 \\
\hline 2/7/12 5:00 & 33.002 & 35.355 & 1475.77 & 1473.77 \\
\hline 2/7/12 5:30 & 32.981 & 35.341 & 1475.79 & 1473.78 \\
\hline $2 / 7 / 126: 00$ & 32.965 & 35.331 & 1475.80 & 1473.79 \\
\hline $2 / 7 / 126: 30$ & 33.065 & 35.329 & 1475.70 & 1473.79 \\
\hline 2/7/12 7:00 & 33.011 & 35.322 & 1475.76 & 1473.80 \\
\hline 2/7/12 7:30 & 33.096 & 35.315 & 1475.67 & 1473.81 \\
\hline 2/7/12 8:00 & 33.098 & 35.315 & 1475.67 & 1473.81 \\
\hline $2 / 7 / 128: 30$ & 33.14 & 35.313 & 1475.63 & 1473.81 \\
\hline 2/7/12 9:00 & 33.152 & 35.31 & 1475.62 & 1473.81 \\
\hline 2/7/12 9:30 & 49.079 & 35.313 & 1459.69 & 1473.81 \\
\hline $2 / 7 / 1210: 00$ & 36.89 & 35.357 & 1471.88 & 1473.77 \\
\hline 2/7/12 10:30 & 34.482 & 35.374 & 1474.29 & 1473.75 \\
\hline 2/7/12 11:00 & 34.06 & 35.381 & 1474.71 & 1473.74 \\
\hline 2/7/12 11:30 & 33.837 & 35.388 & 1474.93 & 1473.73 \\
\hline 2/7/12 12:00 & 33.689 & 35.395 & 1475.08 & 1473.73 \\
\hline $2 / 7 / 1212: 30$ & 33.584 & 35.397 & 1475.19 & 1473.73 \\
\hline 2/7/12 13:00 & 33.497 & 35.397 & 1475.27 & 1473.73 \\
\hline 2/7/12 13:30 & 33.427 & 35.395 & 1475.34 & 1473.73 \\
\hline 2/7/12 14:00 & 33.56 & 35.392 & 1475.21 & 1473.73 \\
\hline 2/7/12 14:30 & 33.387 & 35.388 & 1475.38 & 1473.73 \\
\hline 2/7/12 15:00 & 33.321 & 35.388 & 1475.45 & 1473.73 \\
\hline 2/7/12 15:30 & 33.295 & 35.383 & 1475.47 & 1473.74 \\
\hline 2/7/12 16:00 & 33.354 & 35.378 & 1475.42 & 1473.74 \\
\hline 2/7/12 16:30 & 33.281 & 35.374 & 1475.49 & 1473.75 \\
\hline 2/7/12 17:00 & 33.234 & 35.367 & 1475.54 & 1473.76 \\
\hline 2/7/12 17:30 & 33.192 & 35.359 & 1475.58 & 1473.76 \\
\hline 2/7/12 18:00 & 33.309 & 35.352 & 1475.46 & 1473.77 \\
\hline 2/7/12 18:30 & 33.208 & 35.348 & 1475.56 & 1473.77 \\
\hline 2/7/12 19:00 & 33.53 & 35.348 & 1475.24 & 1473.77 \\
\hline 2/7/12 19:30 & 33.419 & 35.348 & 1475.35 & 1473.77 \\
\hline 2/7/12 20:00 & 33.431 & 35.35 & 1475.34 & 1473.77 \\
\hline 2/7/12 20:30 & 33.511 & 35.357 & 1475.26 & 1473.77 \\
\hline 2/7/12 21:00 & 33.483 & 35.357 & 1475.29 & 1473.77 \\
\hline 2/7/12 21:30 & 33.38 & 35.362 & 1475.39 & 1473.76 \\
\hline 2/7/12 22:00 & 33.314 & 35.355 & 1475.46 & 1473.77 \\
\hline
\end{tabular}


TABLE S1.3 Automatically recorded groundwater levels in the Klassen wells, September 15, 2011, to March 13, 2012.

\begin{tabular}{|c|c|c|c|c|}
\hline \multicolumn{3}{|c|}{ Reference Elevation (ft AMSL) } & 1508.77 & \multirow{2}{*}{$\begin{array}{l}1509.12 \\
\text { Elevation in Klassen } \\
\text { Test Well (ft AMSL) }\end{array}$} \\
\hline Date and Time & $\begin{array}{l}\text { Depth in Klassen } \\
\text { Domestic Well } \\
(\mathrm{ft} \mathrm{BGL})\end{array}$ & $\begin{array}{c}\text { Depth in Klassen } \\
\text { Test Well } \\
\text { (ft BGL) }\end{array}$ & $\begin{array}{l}\text { Elevation in Klassen } \\
\text { Domestic Well } \\
\text { (ft AMSL) }\end{array}$ & \\
\hline $2 / 7 / 1222: 30$ & 33.743 & 35.35 & 1475.03 & 1473.77 \\
\hline $2 / 7 / 1223: 00$ & 34.609 & 35.36 & 1474.16 & 1473.76 \\
\hline 2/7/12 23:30 & 33.48 & 35.369 & 1475.29 & 1473.75 \\
\hline 2/8/12 0:00 & 33.368 & 35.366 & 1475.40 & 1473.76 \\
\hline $2 / 8 / 120: 30$ & 33.302 & 35.367 & 1475.47 & 1473.76 \\
\hline 2/8/12 1:00 & 33.251 & 35.369 & 1475.52 & 1473.75 \\
\hline 2/8/12 1:30 & 33.211 & 35.364 & 1475.56 & 1473.76 \\
\hline $2 / 8 / 122: 00$ & 33.251 & 35.364 & 1475.52 & 1473.76 \\
\hline $2 / 8 / 122: 30$ & 33.274 & 35.371 & 1475.50 & 1473.75 \\
\hline $2 / 8 / 123: 00$ & 33.232 & 35.369 & 1475.54 & 1473.75 \\
\hline $2 / 8 / 123: 30$ & 33.206 & 35.369 & 1475.56 & 1473.75 \\
\hline $2 / 8 / 124: 00$ & 33.288 & 35.371 & 1475.48 & 1473.75 \\
\hline $2 / 8 / 124: 30$ & 33.218 & 35.371 & 1475.55 & 1473.75 \\
\hline $2 / 8 / 125: 00$ & 33.169 & 35.364 & 1475.60 & 1473.76 \\
\hline 2/8/12 5:30 & 33.131 & 35.357 & 1475.64 & 1473.77 \\
\hline $2 / 8 / 126: 00$ & 33.101 & 35.348 & 1475.67 & 1473.77 \\
\hline $2 / 8 / 126: 30$ & 33.366 & 35.35 & 1475.40 & 1473.77 \\
\hline 2/8/12 7:00 & 33.234 & 35.343 & 1475.54 & 1473.78 \\
\hline 2/8/12 7:30 & 33.269 & 35.341 & 1475.50 & 1473.78 \\
\hline 2/8/12 8:00 & 33.204 & 35.338 & 1475.57 & 1473.78 \\
\hline $2 / 8 / 128: 30$ & 33.227 & 35.338 & 1475.54 & 1473.78 \\
\hline 2/8/12 9:00 & 33.159 & 35.336 & 1475.61 & 1473.79 \\
\hline 2/8/12 9:30 & 33.119 & 35.336 & 1475.65 & 1473.79 \\
\hline 2/8/12 10:00 & 33.086 & 35.336 & 1475.68 & 1473.79 \\
\hline 2/8/12 10:30 & 33.058 & 35.343 & 1475.71 & 1473.78 \\
\hline 2/8/12 11:00 & 33.274 & 35.348 & 1475.50 & 1473.77 \\
\hline 2/8/12 11:30 & 33.244 & 35.355 & 1475.53 & 1473.77 \\
\hline 2/8/12 12:00 & 33.851 & 35.364 & 1474.92 & 1473.76 \\
\hline 2/8/12 12:30 & 33.272 & 35.374 & 1475.50 & 1473.75 \\
\hline 2/8/12 13:00 & 33.274 & 35.388 & 1475.50 & 1473.73 \\
\hline 2/8/12 13:30 & 33.166 & 35.392 & 1475.60 & 1473.73 \\
\hline 2/8/12 14:00 & 33.356 & 35.392 & 1475.41 & 1473.73 \\
\hline 2/8/12 14:30 & 33.147 & 35.39 & 1475.62 & 1473.73 \\
\hline 2/8/12 15:00 & 33.091 & 35.381 & 1475.68 & 1473.74 \\
\hline 2/8/12 15:30 & 33.194 & 35.374 & 1475.58 & 1473.75 \\
\hline 2/8/12 16:00 & 33.145 & 35.371 & 1475.62 & 1473.75 \\
\hline 2/8/12 16:30 & 33.112 & 35.364 & 1475.66 & 1473.76 \\
\hline 2/8/12 17:00 & 33.204 & 35.355 & 1475.57 & 1473.77 \\
\hline
\end{tabular}


TABLE S1.3 Automatically recorded groundwater levels in the Klassen wells, September 15, 2011, to March 13, 2012.

\begin{tabular}{|c|c|c|c|c|}
\hline \multicolumn{3}{|c|}{ Reference Elevation (ft AMSL) } & 1508.77 & \multirow{2}{*}{$\begin{array}{l}1509.12 \\
\text { Elevation in Klassen } \\
\text { Test Well (ft AMSL) }\end{array}$} \\
\hline Date and Time & $\begin{array}{l}\text { Depth in Klassen } \\
\text { Domestic Well } \\
(\mathrm{ft} \mathrm{BGL})\end{array}$ & $\begin{array}{c}\text { Depth in Klassen } \\
\text { Test Well } \\
\text { (ft BGL) }\end{array}$ & $\begin{array}{l}\text { Elevation in Klassen } \\
\text { Domestic Well } \\
\text { (ft AMSL) }\end{array}$ & \\
\hline $2 / 8 / 1217: 30$ & 33.108 & 35.35 & 1475.66 & 1473.77 \\
\hline $2 / 8 / 1218: 00$ & 33.18 & 35.343 & 1475.59 & 1473.78 \\
\hline $2 / 8 / 1218: 30$ & 33.136 & 35.336 & 1475.63 & 1473.79 \\
\hline 2/8/12 19:00 & 33.321 & 35.336 & 1475.45 & 1473.79 \\
\hline 2/8/12 19:30 & 33.302 & 35.338 & 1475.47 & 1473.78 \\
\hline $2 / 8 / 1220: 00$ & 33.387 & 35.345 & 1475.38 & 1473.78 \\
\hline $2 / 8 / 1220: 30$ & 33.523 & 35.357 & 1475.25 & 1473.77 \\
\hline $2 / 8 / 1221: 00$ & 33.405 & 35.359 & 1475.36 & 1473.76 \\
\hline 2/8/12 21:30 & 33.335 & 35.369 & 1475.43 & 1473.75 \\
\hline $2 / 8 / 1222: 00$ & 33.253 & 35.374 & 1475.52 & 1473.75 \\
\hline $2 / 8 / 1222: 30$ & 35.007 & 35.374 & 1473.76 & 1473.75 \\
\hline 2/8/12 23:00 & 33.879 & 35.376 & 1474.89 & 1473.75 \\
\hline 2/8/12 23:30 & 33.427 & 35.385 & 1475.34 & 1473.74 \\
\hline 2/9/12 0:00 & 33.302 & 35.385 & 1475.47 & 1473.74 \\
\hline 2/9/12 0:30 & 33.232 & 35.388 & 1475.54 & 1473.73 \\
\hline 2/9/12 1:00 & 33.18 & 35.388 & 1475.59 & 1473.73 \\
\hline 2/9/12 1:30 & 33.262 & 35.385 & 1475.51 & 1473.74 \\
\hline 2/9/12 2:00 & 33.154 & 35.383 & 1475.62 & 1473.74 \\
\hline 2/9/12 2:30 & 33.108 & 35.383 & 1475.66 & 1473.74 \\
\hline 2/9/12 3:00 & 33.075 & 35.381 & 1475.69 & 1473.74 \\
\hline $2 / 9 / 123: 30$ & 33.044 & 35.376 & 1475.73 & 1473.75 \\
\hline 2/9/12 4:00 & 33.018 & 35.369 & 1475.75 & 1473.75 \\
\hline $2 / 9 / 124: 30$ & 33.079 & 35.364 & 1475.69 & 1473.76 \\
\hline $2 / 9 / 125: 00$ & 33.042 & 35.357 & 1475.73 & 1473.77 \\
\hline 2/9/12 5:30 & 33.002 & 35.348 & 1475.77 & 1473.77 \\
\hline $2 / 9 / 12$ 6:00 & 32.974 & 35.338 & 1475.80 & 1473.78 \\
\hline $2 / 9 / 126: 30$ & 33.131 & 35.336 & 1475.64 & 1473.79 \\
\hline 2/9/12 7:00 & 33.072 & 35.331 & 1475.70 & 1473.79 \\
\hline 2/9/12 7:30 & 33.101 & 35.324 & 1475.67 & 1473.80 \\
\hline 2/9/12 8:00 & 33.197 & 35.32 & 1475.57 & 1473.80 \\
\hline 2/9/12 8:30 & 33.065 & 35.315 & 1475.70 & 1473.81 \\
\hline 2/9/12 9:00 & 33.016 & 35.31 & 1475.75 & 1473.81 \\
\hline 2/9/12 9:30 & 32.983 & 35.306 & 1475.79 & 1473.82 \\
\hline 2/9/12 10:00 & 33.042 & 35.313 & 1475.73 & 1473.81 \\
\hline 2/9/12 10:30 & 32.988 & 35.315 & 1475.78 & 1473.81 \\
\hline 2/9/12 11:00 & 32.957 & 35.317 & 1475.81 & 1473.81 \\
\hline 2/9/12 11:30 & 33.009 & 35.329 & 1475.76 & 1473.79 \\
\hline 2/9/12 12:00 & 33.086 & 35.336 & 1475.68 & 1473.79 \\
\hline
\end{tabular}


TABLE S1.3 Automatically recorded groundwater levels in the Klassen wells, September 15, 2011, to March 13, 2012.

\begin{tabular}{|c|c|c|c|c|}
\hline \multicolumn{3}{|c|}{ Reference Elevation (ft AMSL) } & 1508.77 & \multirow{2}{*}{$\begin{array}{l}1509.12 \\
\text { Elevation in Klassen } \\
\text { Test Well (ft AMSL) }\end{array}$} \\
\hline Date and Time & $\begin{array}{l}\text { Depth in Klassen } \\
\text { Domestic Well } \\
(\mathrm{ft} \mathrm{BGL})\end{array}$ & $\begin{array}{c}\text { Depth in Klassen } \\
\text { Test Well } \\
\text { (ft BGL) }\end{array}$ & $\begin{array}{l}\text { Elevation in Klassen } \\
\text { Domestic Well } \\
\text { (ft AMSL) }\end{array}$ & \\
\hline 2/9/12 12:30 & 32.995 & 35.345 & 1475.77 & 1473.78 \\
\hline 2/9/12 13:00 & 32.948 & 35.352 & 1475.82 & 1473.77 \\
\hline 2/9/12 13:30 & 33.586 & 35.35 & 1475.18 & 1473.77 \\
\hline 2/9/12 14:00 & 32.979 & 35.36 & 1475.79 & 1473.76 \\
\hline 2/9/12 14:30 & 32.934 & 35.352 & 1475.84 & 1473.77 \\
\hline $2 / 9 / 1215: 00$ & 32.906 & 35.345 & 1475.86 & 1473.78 \\
\hline 2/9/12 15:30 & 32.913 & 35.336 & 1475.86 & 1473.79 \\
\hline 2/9/12 16:00 & 32.908 & 35.331 & 1475.86 & 1473.79 \\
\hline 2/9/12 16:30 & 32.934 & 35.324 & 1475.84 & 1473.80 \\
\hline $2 / 9 / 1217: 00$ & 33.694 & 35.32 & 1475.08 & 1473.80 \\
\hline 2/9/12 17:30 & 33.07 & 35.315 & 1475.70 & 1473.81 \\
\hline 2/9/12 18:00 & 33.126 & 35.317 & 1475.64 & 1473.81 \\
\hline 2/9/12 18:30 & 33.049 & 35.31 & 1475.72 & 1473.81 \\
\hline $2 / 9 / 1219: 00$ & 33.147 & 35.313 & 1475.62 & 1473.81 \\
\hline 2/9/12 19:30 & 33.244 & 35.315 & 1475.53 & 1473.81 \\
\hline 2/9/12 20:00 & 33.098 & 35.308 & 1475.67 & 1473.81 \\
\hline 2/9/12 20:30 & 33.033 & 35.303 & 1475.74 & 1473.82 \\
\hline 2/9/12 21:00 & 33.947 & 35.296 & 1474.82 & 1473.83 \\
\hline 2/9/12 21:30 & 33.225 & 35.301 & 1475.54 & 1473.82 \\
\hline 2/9/12 22:00 & 33.166 & 35.303 & 1475.60 & 1473.82 \\
\hline $2 / 9 / 12$ 22:30 & 33.105 & 35.301 & 1475.66 & 1473.82 \\
\hline 2/9/12 23:00 & 33.276 & 35.303 & 1475.49 & 1473.82 \\
\hline 2/9/12 23:30 & 33.483 & 35.308 & 1475.29 & 1473.81 \\
\hline 2/10/12 0:00 & 33.375 & 35.315 & 1475.39 & 1473.81 \\
\hline $2 / 10 / 12$ 0:30 & 33.391 & 35.32 & 1475.38 & 1473.80 \\
\hline $2 / 10 / 121: 00$ & 33.244 & 35.317 & 1475.53 & 1473.81 \\
\hline $2 / 10 / 121: 30$ & 33.312 & 35.317 & 1475.46 & 1473.81 \\
\hline 2/10/12 2:00 & 33.241 & 35.31 & 1475.53 & 1473.81 \\
\hline 2/10/12 2:30 & 33.225 & 35.31 & 1475.54 & 1473.81 \\
\hline 2/10/12 3:00 & 33.173 & 35.305 & 1475.60 & 1473.82 \\
\hline 2/10/12 3:30 & 33.143 & 35.299 & 1475.63 & 1473.82 \\
\hline $2 / 10 / 124: 00$ & 33.208 & 35.294 & 1475.56 & 1473.83 \\
\hline 2/10/12 4:30 & 33.133 & 35.287 & 1475.64 & 1473.84 \\
\hline $2 / 10 / 12$ 5:00 & 33.082 & 35.275 & 1475.69 & 1473.85 \\
\hline $2 / 10 / 125: 30$ & 34.034 & 35.254 & 1474.74 & 1473.87 \\
\hline $2 / 10 / 126: 00$ & 33.096 & 35.247 & 1475.67 & 1473.88 \\
\hline $2 / 10 / 126: 30$ & 33.248 & 35.252 & 1475.52 & 1473.87 \\
\hline $2 / 10 / 12$ 7:00 & 33.272 & 35.249 & 1475.50 & 1473.87 \\
\hline
\end{tabular}


TABLE S1.3 Automatically recorded groundwater levels in the Klassen wells, September 15, 2011, to March 13, 2012.

\begin{tabular}{|c|c|c|c|c|}
\hline \multicolumn{3}{|c|}{ Reference Elevation (ft AMSL) } & 1508.77 & \multirow{2}{*}{$\begin{array}{l}1509.12 \\
\text { Elevation in Klassen } \\
\text { Test Well (ft AMSL) }\end{array}$} \\
\hline Date and Time & $\begin{array}{l}\text { Depth in Klassen } \\
\text { Domestic Well } \\
(\mathrm{ft} \mathrm{BGL})\end{array}$ & $\begin{array}{c}\text { Depth in Klassen } \\
\text { Test Well } \\
\text { (ft BGL) }\end{array}$ & $\begin{array}{l}\text { Elevation in Klassen } \\
\text { Domestic Well } \\
\text { (ft AMSL) }\end{array}$ & \\
\hline $2 / 10 / 127: 30$ & 33.302 & 35.254 & 1475.47 & 1473.87 \\
\hline $2 / 10 / 128: 00$ & 37.17 & 35.251 & 1471.60 & 1473.87 \\
\hline $2 / 10 / 128: 30$ & 35.598 & 35.254 & 1473.17 & 1473.87 \\
\hline 2/10/12 9:00 & 33.415 & 35.256 & 1475.35 & 1473.87 \\
\hline 2/10/12 9:30 & 33.333 & 35.259 & 1475.44 & 1473.86 \\
\hline $2 / 10 / 12$ 10:00 & 33.328 & 35.261 & 1475.44 & 1473.86 \\
\hline $2 / 10 / 1210: 30$ & 35.319 & 35.261 & 1473.45 & 1473.86 \\
\hline 2/10/12 11:00 & 33.534 & 35.27 & 1475.24 & 1473.85 \\
\hline 2/10/12 11:30 & 33.546 & 35.287 & 1475.22 & 1473.84 \\
\hline $2 / 10 / 1212: 00$ & 33.614 & 35.32 & 1475.16 & 1473.80 \\
\hline $2 / 10 / 1212: 30$ & 33.546 & 35.345 & 1475.22 & 1473.78 \\
\hline $2 / 10 / 1213: 00$ & 33.673 & 35.369 & 1475.10 & 1473.75 \\
\hline $2 / 10 / 12$ 13:30 & 34.794 & 35.376 & 1473.98 & 1473.75 \\
\hline $2 / 10 / 1214: 00$ & 33.436 & 35.381 & 1475.33 & 1473.74 \\
\hline $2 / 10 / 12$ 14:30 & 33.431 & 35.383 & 1475.34 & 1473.74 \\
\hline $2 / 10 / 12$ 15:00 & 33.593 & 35.392 & 1475.18 & 1473.73 \\
\hline $2 / 10 / 12$ 15:30 & 33.572 & 35.395 & 1475.20 & 1473.73 \\
\hline $2 / 10 / 12$ 16:00 & 33.448 & 35.39 & 1475.32 & 1473.73 \\
\hline $2 / 10 / 12$ 16:30 & 33.523 & 35.388 & 1475.25 & 1473.73 \\
\hline 2/10/12 17:00 & 33.452 & 35.388 & 1475.32 & 1473.73 \\
\hline $2 / 10 / 12$ 17:30 & 33.436 & 35.381 & 1475.33 & 1473.74 \\
\hline $2 / 10 / 12$ 18:00 & 33.396 & 35.371 & 1475.37 & 1473.75 \\
\hline $2 / 10 / 12$ 18:30 & 33.398 & 35.366 & 1475.37 & 1473.76 \\
\hline 2/10/12 19:00 & 35.901 & 35.357 & 1472.87 & 1473.77 \\
\hline 2/10/12 19:30 & 33.638 & 35.355 & 1475.13 & 1473.77 \\
\hline $2 / 10 / 1220: 00$ & 33.361 & 35.35 & 1475.41 & 1473.77 \\
\hline $2 / 10 / 12$ 20:30 & 33.288 & 35.35 & 1475.48 & 1473.77 \\
\hline $2 / 10 / 12$ 21:00 & 33.239 & 35.352 & 1475.53 & 1473.77 \\
\hline $2 / 10 / 12$ 21:30 & 33.197 & 35.357 & 1475.57 & 1473.77 \\
\hline $2 / 10 / 1222: 00$ & 33.162 & 35.364 & 1475.61 & 1473.76 \\
\hline 2/10/12 22:30 & 34.482 & 35.369 & 1474.29 & 1473.75 \\
\hline 2/10/12 23:00 & 35.164 & 35.388 & 1473.61 & 1473.73 \\
\hline $2 / 10 / 12$ 23:30 & 33.431 & 35.397 & 1475.34 & 1473.73 \\
\hline 2/11/12 0:00 & 33.307 & 35.392 & 1475.46 & 1473.73 \\
\hline 2/11/12 0:30 & 33.265 & 35.397 & 1475.50 & 1473.73 \\
\hline 2/11/12 1:00 & 33.265 & 35.399 & 1475.50 & 1473.72 \\
\hline 2/11/12 1:30 & 33.208 & 35.404 & 1475.56 & 1473.72 \\
\hline $2 / 11 / 12$ 2:00 & 33.239 & 35.402 & 1475.53 & 1473.72 \\
\hline
\end{tabular}


TABLE S1.3 Automatically recorded groundwater levels in the Klassen wells, September 15, 2011, to March 13, 2012.

\begin{tabular}{|c|c|c|c|c|}
\hline \multicolumn{3}{|c|}{ Reference Elevation (ft AMSL) } & 1508.77 & \multirow{2}{*}{$\begin{array}{l}1509.12 \\
\text { Elevation in Klassen } \\
\text { Test Well (ft AMSL) }\end{array}$} \\
\hline Date and Time & $\begin{array}{l}\text { Depth in Klassen } \\
\text { Domestic Well } \\
\text { (ft BGL) }\end{array}$ & $\begin{array}{c}\text { Depth in Klassen } \\
\text { Test Well } \\
\text { (ft BGL) }\end{array}$ & $\begin{array}{l}\text { Elevation in Klassen } \\
\text { Domestic Well } \\
\text { (ft AMSL) }\end{array}$ & \\
\hline $2 / 11 / 122: 30$ & 33.253 & 35.399 & 1475.52 & 1473.72 \\
\hline $2 / 11 / 123: 00$ & 33.227 & 35.402 & 1475.54 & 1473.72 \\
\hline $2 / 11 / 123: 30$ & 33.204 & 35.402 & 1475.57 & 1473.72 \\
\hline 2/11/12 4:00 & 33.265 & 35.406 & 1475.50 & 1473.72 \\
\hline $2 / 11 / 124: 30$ & 33.307 & 35.406 & 1475.46 & 1473.72 \\
\hline $2 / 11 / 125: 00$ & 33.213 & 35.392 & 1475.56 & 1473.73 \\
\hline $2 / 11 / 125: 30$ & 33.164 & 35.392 & 1475.61 & 1473.73 \\
\hline $2 / 11 / 126: 00$ & 33.126 & 35.388 & 1475.64 & 1473.73 \\
\hline $2 / 11 / 126: 30$ & 33.185 & 35.385 & 1475.58 & 1473.74 \\
\hline $2 / 11 / 127: 00$ & 33.122 & 35.383 & 1475.65 & 1473.74 \\
\hline $2 / 11 / 127: 30$ & 33.084 & 35.383 & 1475.69 & 1473.74 \\
\hline 2/11/12 8:00 & 33.056 & 35.381 & 1475.71 & 1473.74 \\
\hline $2 / 11 / 128: 30$ & 33.112 & 35.376 & 1475.66 & 1473.75 \\
\hline 2/11/12 9:00 & 33.349 & 35.383 & 1475.42 & 1473.74 \\
\hline 2/11/12 9:30 & 33.312 & 35.397 & 1475.46 & 1473.73 \\
\hline 2/11/12 10:00 & 33.248 & 35.399 & 1475.52 & 1473.72 \\
\hline $2 / 11 / 12$ 10:30 & 33.302 & 35.406 & 1475.47 & 1473.72 \\
\hline 2/11/12 11:00 & 33.272 & 35.409 & 1475.50 & 1473.71 \\
\hline $2 / 11 / 12$ 11:30 & 33.361 & 35.428 & 1475.41 & 1473.69 \\
\hline 2/11/12 12:00 & 35.242 & 35.444 & 1473.53 & 1473.68 \\
\hline 2/11/12 12:30 & 33.459 & 35.463 & 1475.31 & 1473.66 \\
\hline 2/11/12 13:00 & 34.086 & 35.477 & 1474.68 & 1473.65 \\
\hline 2/11/12 13:30 & 33.337 & 35.489 & 1475.43 & 1473.63 \\
\hline $2 / 11 / 12$ 14:00 & 37.807 & 35.491 & 1470.96 & 1473.63 \\
\hline 2/11/12 14:30 & 33.391 & 35.5 & 1475.38 & 1473.62 \\
\hline 2/11/12 15:00 & 33.377 & 35.505 & 1475.39 & 1473.62 \\
\hline $2 / 11 / 12$ 15:30 & 33.64 & 35.507 & 1475.13 & 1473.62 \\
\hline $2 / 11 / 12$ 16:00 & 33.457 & 35.512 & 1475.31 & 1473.61 \\
\hline 2/11/12 16:30 & 33.347 & 35.514 & 1475.42 & 1473.61 \\
\hline 2/11/12 17:00 & 33.276 & 35.51 & 1475.49 & 1473.61 \\
\hline 2/11/12 17:30 & 33.377 & 35.505 & 1475.39 & 1473.62 \\
\hline 2/11/12 18:00 & 33.382 & 35.503 & 1475.39 & 1473.62 \\
\hline $2 / 11 / 12$ 18:30 & 33.387 & 35.503 & 1475.38 & 1473.62 \\
\hline 2/11/12 19:00 & 33.323 & 35.51 & 1475.45 & 1473.61 \\
\hline 2/11/12 19:30 & 33.609 & 35.528 & 1475.16 & 1473.59 \\
\hline 2/11/12 20:00 & 33.541 & 35.55 & 1475.23 & 1473.57 \\
\hline 2/11/12 20:30 & 33.509 & 35.573 & 1475.26 & 1473.55 \\
\hline 2/11/12 21:00 & 33.502 & 35.603 & 1475.27 & 1473.52 \\
\hline
\end{tabular}


TABLE S1.3 Automatically recorded groundwater levels in the Klassen wells, September 15, 2011, to March 13, 2012.

\begin{tabular}{|c|c|c|c|c|}
\hline \multicolumn{3}{|c|}{ Reference Elevation (ft AMSL) } & 1508.77 & \multirow{2}{*}{$\begin{array}{l}1509.12 \\
\text { Elevation in Klassen } \\
\text { Test Well (ft AMSL) }\end{array}$} \\
\hline Date and Time & $\begin{array}{l}\text { Depth in Klassen } \\
\text { Domestic Well } \\
(\mathrm{ft} \mathrm{BGL})\end{array}$ & $\begin{array}{c}\text { Depth in Klassen } \\
\text { Test Well } \\
\text { (ft BGL) }\end{array}$ & $\begin{array}{l}\text { Elevation in Klassen } \\
\text { Domestic Well } \\
\text { (ft AMSL) }\end{array}$ & \\
\hline $2 / 11 / 1221: 30$ & 33.701 & 35.634 & 1475.07 & 1473.49 \\
\hline $2 / 11 / 1222: 00$ & 33.412 & 35.66 & 1475.36 & 1473.46 \\
\hline $2 / 11 / 1222: 30$ & 34.243 & 35.686 & 1474.53 & 1473.44 \\
\hline $2 / 11 / 1223: 00$ & 33.72 & 35.707 & 1475.05 & 1473.42 \\
\hline $2 / 11 / 1223: 30$ & 33.499 & 35.718 & 1475.27 & 1473.40 \\
\hline $2 / 12 / 120: 00$ & 33.438 & 35.737 & 1475.33 & 1473.39 \\
\hline $2 / 12 / 120: 30$ & 33.359 & 35.74 & 1475.41 & 1473.38 \\
\hline $2 / 12 / 121: 00$ & 33.3 & 35.744 & 1475.47 & 1473.38 \\
\hline 2/12/12 1:30 & 33.255 & 35.749 & 1475.51 & 1473.37 \\
\hline $2 / 12 / 122: 00$ & 33.3 & 35.749 & 1475.47 & 1473.37 \\
\hline $2 / 12 / 122: 30$ & 33.396 & 35.758 & 1475.37 & 1473.36 \\
\hline $2 / 12 / 123: 00$ & 33.328 & 35.768 & 1475.44 & 1473.35 \\
\hline $2 / 12 / 123: 30$ & 33.286 & 35.779 & 1475.48 & 1473.34 \\
\hline $2 / 12 / 124: 00$ & 33.328 & 35.789 & 1475.44 & 1473.33 \\
\hline $2 / 12 / 124: 30$ & 33.258 & 35.791 & 1475.51 & 1473.33 \\
\hline $2 / 12 / 125: 00$ & 33.206 & 35.786 & 1475.56 & 1473.34 \\
\hline $2 / 12 / 125: 30$ & 33.169 & 35.779 & 1475.60 & 1473.34 \\
\hline 2/12/12 6:00 & 33.598 & 35.777 & 1475.17 & 1473.35 \\
\hline $2 / 12 / 126: 30$ & 33.366 & 35.775 & 1475.40 & 1473.35 \\
\hline $2 / 12 / 127: 00$ & 33.239 & 35.765 & 1475.53 & 1473.36 \\
\hline $2 / 12 / 127: 30$ & 33.18 & 35.756 & 1475.59 & 1473.37 \\
\hline $2 / 12 / 128: 00$ & 33.227 & 35.754 & 1475.54 & 1473.37 \\
\hline 2/12/12 8:30 & 33.403 & 35.754 & 1475.37 & 1473.37 \\
\hline 2/12/12 9:00 & 33.248 & 35.754 & 1475.52 & 1473.37 \\
\hline 2/12/12 9:30 & 33.178 & 35.749 & 1475.59 & 1473.37 \\
\hline $2 / 12 / 12$ 10:00 & 33.232 & 35.758 & 1475.54 & 1473.36 \\
\hline $2 / 12 / 12$ 10:30 & 33.225 & 35.768 & 1475.54 & 1473.35 \\
\hline 2/12/12 11:00 & 33.199 & 35.777 & 1475.57 & 1473.35 \\
\hline 2/12/12 11:30 & 33.143 & 35.791 & 1475.63 & 1473.33 \\
\hline 2/12/12 12:00 & 33.105 & 35.803 & 1475.66 & 1473.32 \\
\hline 2/12/12 12:30 & 33.072 & 35.815 & 1475.70 & 1473.31 \\
\hline $2 / 12 / 12$ 13:00 & 33.047 & 35.824 & 1475.72 & 1473.30 \\
\hline $2 / 12 / 12$ 13:30 & 33.326 & 35.833 & 1475.44 & 1473.29 \\
\hline $2 / 12 / 12$ 14:00 & 33.103 & 35.843 & 1475.67 & 1473.28 \\
\hline $2 / 12 / 12$ 14:30 & 33.072 & 35.845 & 1475.70 & 1473.28 \\
\hline $2 / 12 / 12$ 15:00 & 33.065 & 35.847 & 1475.70 & 1473.28 \\
\hline $2 / 12 / 12$ 15:30 & 33.077 & 35.845 & 1475.69 & 1473.28 \\
\hline $2 / 12 / 12$ 16:00 & 33.033 & 35.838 & 1475.74 & 1473.28 \\
\hline
\end{tabular}


TABLE S1.3 Automatically recorded groundwater levels in the Klassen wells, September 15, 2011, to March 13, 2012.

\begin{tabular}{|c|c|c|c|c|}
\hline \multicolumn{3}{|c|}{ Reference Elevation (ft AMSL) } & 1508.77 & \multirow{2}{*}{$\begin{array}{l}1509.12 \\
\text { Elevation in Klassen } \\
\text { Test Well (ft AMSL) }\end{array}$} \\
\hline Date and Time & $\begin{array}{l}\text { Depth in Klassen } \\
\text { Domestic Well } \\
(\mathrm{ft} \mathrm{BGL})\end{array}$ & $\begin{array}{c}\text { Depth in Klassen } \\
\text { Test Well } \\
\text { (ft BGL) }\end{array}$ & $\begin{array}{l}\text { Elevation in Klassen } \\
\text { Domestic Well } \\
\text { (ft AMSL) }\end{array}$ & \\
\hline $2 / 12 / 1216: 30$ & 35.443 & 35.829 & 1473.33 & 1473.29 \\
\hline $2 / 12 / 1217: 00$ & 33.077 & 35.826 & 1475.69 & 1473.30 \\
\hline $2 / 12 / 12$ 17:30 & 45.725 & 35.819 & 1463.04 & 1473.30 \\
\hline $2 / 12 / 1218: 00$ & 34.189 & 35.838 & 1474.58 & 1473.28 \\
\hline $2 / 12 / 1218: 30$ & 33.729 & 35.833 & 1475.04 & 1473.29 \\
\hline $2 / 12 / 12$ 19:00 & 33.516 & 35.824 & 1475.25 & 1473.30 \\
\hline $2 / 12 / 12$ 19:30 & 33.424 & 35.812 & 1475.35 & 1473.31 \\
\hline $2 / 12 / 1220: 00$ & 33.537 & 35.815 & 1475.23 & 1473.31 \\
\hline $2 / 12 / 1220: 30$ & 33.431 & 35.81 & 1475.34 & 1473.31 \\
\hline $2 / 12 / 1221: 00$ & 33.356 & 35.798 & 1475.41 & 1473.32 \\
\hline 2/12/12 21:30 & 33.422 & 35.791 & 1475.35 & 1473.33 \\
\hline $2 / 12 / 1222: 00$ & 33.427 & 35.789 & 1475.34 & 1473.33 \\
\hline $2 / 12 / 1222: 30$ & 33.328 & 35.784 & 1475.44 & 1473.34 \\
\hline $2 / 12 / 1223: 00$ & 35.021 & 35.777 & 1473.75 & 1473.35 \\
\hline $2 / 12 / 12$ 23:30 & 33.579 & 35.777 & 1475.19 & 1473.35 \\
\hline 2/13/12 0:00 & 37.226 & 35.775 & 1471.54 & 1473.35 \\
\hline $2 / 13 / 12$ 0:30 & 33.506 & 35.765 & 1475.26 & 1473.36 \\
\hline 2/13/12 1:00 & 33.382 & 35.765 & 1475.39 & 1473.36 \\
\hline $2 / 13 / 121: 30$ & 33.302 & 35.765 & 1475.47 & 1473.36 \\
\hline $2 / 13 / 12$ 2:00 & 33.244 & 35.754 & 1475.53 & 1473.37 \\
\hline $2 / 13 / 122: 30$ & 33.194 & 35.749 & 1475.58 & 1473.37 \\
\hline 2/13/12 3:00 & 33.176 & 35.737 & 1475.59 & 1473.39 \\
\hline 2/13/12 3:30 & 33.272 & 35.73 & 1475.50 & 1473.39 \\
\hline 2/13/12 4:00 & 33.166 & 35.725 & 1475.60 & 1473.40 \\
\hline $2 / 13 / 124: 30$ & 33.145 & 35.711 & 1475.62 & 1473.41 \\
\hline 2/13/12 5:00 & 33.098 & 35.695 & 1475.67 & 1473.43 \\
\hline $2 / 13 / 125: 30$ & 33.063 & 35.681 & 1475.71 & 1473.44 \\
\hline 2/13/12 6:00 & 33.056 & 35.669 & 1475.71 & 1473.45 \\
\hline 2/13/12 6:30 & 33.215 & 35.66 & 1475.55 & 1473.46 \\
\hline 2/13/12 7:00 & 33.136 & 35.646 & 1475.63 & 1473.48 \\
\hline 2/13/12 7:30 & 33.183 & 35.636 & 1475.59 & 1473.49 \\
\hline $2 / 13 / 128: 00$ & 33.115 & 35.625 & 1475.65 & 1473.50 \\
\hline 2/13/12 8:30 & 33.154 & 35.613 & 1475.62 & 1473.51 \\
\hline 2/13/12 9:00 & 33.173 & 35.601 & 1475.60 & 1473.52 \\
\hline 2/13/12 9:30 & 33.269 & 35.589 & 1475.50 & 1473.53 \\
\hline $2 / 13 / 12$ 10:00 & 33.22 & 35.585 & 1475.55 & 1473.54 \\
\hline 2/13/12 10:30 & 33.098 & 35.578 & 1475.67 & 1473.54 \\
\hline 2/13/12 11:00 & 33.154 & 35.568 & 1475.62 & 1473.55 \\
\hline
\end{tabular}


TABLE S1.3 Automatically recorded groundwater levels in the Klassen wells, September 15, 2011, to March 13, 2012.

\begin{tabular}{|c|c|c|c|c|}
\hline \multicolumn{3}{|c|}{ Reference Elevation (ft AMSL) } & 1508.77 & \multirow{2}{*}{$\begin{array}{l}1509.12 \\
\text { Elevation in Klassen } \\
\text { Test Well (ft AMSL) }\end{array}$} \\
\hline Date and Time & $\begin{array}{l}\text { Depth in Klassen } \\
\text { Domestic Well } \\
(\mathrm{ft} \mathrm{BGL})\end{array}$ & $\begin{array}{c}\text { Depth in Klassen } \\
\text { Test Well } \\
\text { (ft BGL) }\end{array}$ & $\begin{array}{l}\text { Elevation in Klassen } \\
\text { Domestic Well } \\
\text { (ft AMSL) }\end{array}$ & \\
\hline 2/13/12 11:30 & 33.232 & 35.564 & 1475.54 & 1473.56 \\
\hline $2 / 13 / 1212: 00$ & 33.19 & 35.559 & 1475.58 & 1473.56 \\
\hline 2/13/12 12:30 & 33.176 & 35.559 & 1475.59 & 1473.56 \\
\hline $2 / 13 / 1213: 00$ & 33.279 & 35.564 & 1475.49 & 1473.56 \\
\hline $2 / 13 / 1213: 30$ & 33.211 & 35.573 & 1475.56 & 1473.55 \\
\hline $2 / 13 / 12$ 14:00 & 33.818 & 35.582 & 1474.95 & 1473.54 \\
\hline $2 / 13 / 1214: 30$ & 33.187 & 35.58 & 1475.58 & 1473.54 \\
\hline $2 / 13 / 12$ 15:00 & 33.108 & 35.568 & 1475.66 & 1473.55 \\
\hline $2 / 13 / 12$ 15:30 & 33.061 & 35.552 & 1475.71 & 1473.57 \\
\hline $2 / 13 / 1216: 00$ & 33.133 & 35.535 & 1475.64 & 1473.59 \\
\hline 2/13/12 16:30 & 33.079 & 35.519 & 1475.69 & 1473.60 \\
\hline 2/13/12 17:00 & 33.865 & 35.5 & 1474.90 & 1473.62 \\
\hline $2 / 13 / 12$ 17:30 & 33.086 & 35.482 & 1475.68 & 1473.64 \\
\hline $2 / 13 / 1218: 00$ & 33.837 & 35.477 & 1474.93 & 1473.65 \\
\hline $2 / 13 / 12$ 18:30 & 33.448 & 35.463 & 1475.32 & 1473.66 \\
\hline 2/13/12 19:00 & 33.405 & 35.458 & 1475.36 & 1473.66 \\
\hline $2 / 13 / 12$ 19:30 & 33.328 & 35.449 & 1475.44 & 1473.67 \\
\hline $2 / 13 / 12$ 20:00 & 33.305 & 35.437 & 1475.46 & 1473.69 \\
\hline $2 / 13 / 12$ 20:30 & 33.22 & 35.427 & 1475.55 & 1473.70 \\
\hline 2/13/12 21:00 & 33.356 & 35.423 & 1475.41 & 1473.70 \\
\hline $2 / 13 / 12$ 21:30 & 33.201 & 35.416 & 1475.57 & 1473.71 \\
\hline $2 / 13 / 12$ 22:00 & 33.194 & 35.409 & 1475.58 & 1473.71 \\
\hline $2 / 13 / 1222: 30$ & 36.722 & 35.409 & 1472.05 & 1473.71 \\
\hline 2/13/12 23:00 & 33.448 & 35.411 & 1475.32 & 1473.71 \\
\hline 2/13/12 23:30 & 33.298 & 35.409 & 1475.47 & 1473.71 \\
\hline 2/14/12 0:00 & 33.22 & 35.395 & 1475.55 & 1473.73 \\
\hline 2/14/12 0:30 & 33.159 & 35.388 & 1475.61 & 1473.73 \\
\hline 2/14/12 1:00 & 33.117 & 35.376 & 1475.65 & 1473.75 \\
\hline 2/14/12 1:30 & 33.079 & 35.364 & 1475.69 & 1473.76 \\
\hline 2/14/12 2:00 & 33.049 & 35.364 & 1475.72 & 1473.76 \\
\hline 2/14/12 2:30 & 33.298 & 35.36 & 1475.47 & 1473.76 \\
\hline 2/14/12 3:00 & 33.061 & 35.36 & 1475.71 & 1473.76 \\
\hline 2/14/12 3:30 & 33.016 & 35.357 & 1475.75 & 1473.77 \\
\hline 2/14/12 4:00 & 32.986 & 35.348 & 1475.78 & 1473.77 \\
\hline 2/14/12 4:30 & 32.988 & 35.348 & 1475.78 & 1473.77 \\
\hline 2/14/12 5:00 & 32.96 & 35.336 & 1475.81 & 1473.79 \\
\hline 2/14/12 5:30 & 32.96 & 35.336 & 1475.81 & 1473.79 \\
\hline $2 / 14 / 126: 00$ & 32.929 & 35.334 & 1475.84 & 1473.79 \\
\hline
\end{tabular}


TABLE S1.3 Automatically recorded groundwater levels in the Klassen wells, September 15, 2011, to March 13, 2012.

\begin{tabular}{|c|c|c|c|c|}
\hline \multicolumn{3}{|c|}{ Reference Elevation (ft AMSL) } & 1508.77 & \multirow{2}{*}{$\begin{array}{l}1509.12 \\
\text { Elevation in Klassen } \\
\text { Test Well (ft AMSL) }\end{array}$} \\
\hline Date and Time & $\begin{array}{l}\text { Depth in Klassen } \\
\text { Domestic Well } \\
\text { (ft BGL) }\end{array}$ & $\begin{array}{c}\text { Depth in Klassen } \\
\text { Test Well } \\
\text { (ft BGL) }\end{array}$ & $\begin{array}{l}\text { Elevation in Klassen } \\
\text { Domestic Well } \\
\text { (ft AMSL) }\end{array}$ & \\
\hline 2/14/12 6:30 & 33.164 & 35.334 & 1475.61 & 1473.79 \\
\hline 2/14/12 7:00 & 33.162 & 35.324 & 1475.61 & 1473.80 \\
\hline 2/14/12 7:30 & 33.169 & 35.324 & 1475.60 & 1473.80 \\
\hline 2/14/12 8:00 & 33.086 & 35.322 & 1475.68 & 1473.80 \\
\hline $2 / 14 / 128: 30$ & 33.152 & 35.329 & 1475.62 & 1473.79 \\
\hline 2/14/12 9:00 & 36.156 & 35.336 & 1472.61 & 1473.79 \\
\hline 2/14/12 9:30 & 33.302 & 35.35 & 1475.47 & 1473.77 \\
\hline $2 / 14 / 12$ 10:00 & 33.171 & 35.383 & 1475.60 & 1473.74 \\
\hline $2 / 14 / 1210: 30$ & 33.101 & 35.411 & 1475.67 & 1473.71 \\
\hline 2/14/12 11:00 & 33.051 & 35.425 & 1475.72 & 1473.70 \\
\hline $2 / 14 / 1211: 30$ & 33.014 & 35.423 & 1475.76 & 1473.70 \\
\hline 2/14/12 12:00 & 32.983 & 35.432 & 1475.79 & 1473.69 \\
\hline $2 / 14 / 12$ 12:30 & 32.955 & 35.451 & 1475.81 & 1473.67 \\
\hline $2 / 14 / 12$ 13:00 & 33.124 & 35.465 & 1475.65 & 1473.66 \\
\hline $2 / 14 / 12$ 13:30 & 32.995 & 35.474 & 1475.77 & 1473.65 \\
\hline $2 / 14 / 12$ 14:00 & 32.953 & 35.47 & 1475.82 & 1473.65 \\
\hline $2 / 14 / 1214: 30$ & 32.925 & 35.47 & 1475.84 & 1473.65 \\
\hline $2 / 14 / 12$ 15:00 & 32.965 & 35.477 & 1475.80 & 1473.65 \\
\hline $2 / 14 / 12$ 15:30 & 32.922 & 35.477 & 1475.85 & 1473.65 \\
\hline $2 / 14 / 12$ 16:00 & 32.927 & 35.477 & 1475.84 & 1473.65 \\
\hline 2/14/12 16:30 & 32.894 & 35.474 & 1475.88 & 1473.65 \\
\hline $2 / 14 / 12$ 17:00 & 32.896 & 35.467 & 1475.87 & 1473.66 \\
\hline $2 / 14 / 12$ 17:30 & 33.485 & 35.463 & 1475.28 & 1473.66 \\
\hline $2 / 14 / 12$ 18:00 & 33.028 & 35.465 & 1475.74 & 1473.66 \\
\hline $2 / 14 / 12$ 18:30 & 32.983 & 35.465 & 1475.79 & 1473.66 \\
\hline 2/14/12 19:00 & 33.054 & 35.463 & 1475.72 & 1473.66 \\
\hline 2/14/12 19:30 & 33.058 & 35.463 & 1475.71 & 1473.66 \\
\hline $2 / 14 / 1220: 00$ & 33.103 & 35.463 & 1475.67 & 1473.66 \\
\hline 2/14/12 20:30 & 33.147 & 35.456 & 1475.62 & 1473.67 \\
\hline 2/14/12 21:00 & 33.628 & 35.467 & 1475.14 & 1473.66 \\
\hline 2/14/12 21:30 & 33.368 & 35.479 & 1475.40 & 1473.64 \\
\hline 2/14/12 22:00 & 33.248 & 35.472 & 1475.52 & 1473.65 \\
\hline $2 / 14 / 1222: 30$ & 33.171 & 35.465 & 1475.60 & 1473.66 \\
\hline 2/14/12 23:00 & 33.34 & 35.463 & 1475.43 & 1473.66 \\
\hline 2/14/12 23:30 & 33.389 & 35.467 & 1475.38 & 1473.66 \\
\hline 2/15/12 0:00 & 33.232 & 35.465 & 1475.54 & 1473.66 \\
\hline 2/15/12 0:30 & 33.152 & 35.458 & 1475.62 & 1473.66 \\
\hline 2/15/12 1:00 & 33.096 & 35.446 & 1475.67 & 1473.68 \\
\hline
\end{tabular}


TABLE S1.3 Automatically recorded groundwater levels in the Klassen wells, September 15, 2011, to March 13, 2012.

\begin{tabular}{|c|c|c|c|c|}
\hline \multicolumn{3}{|c|}{ Reference Elevation (ft AMSL) } & 1508.77 & \multirow{2}{*}{$\begin{array}{l}1509.12 \\
\text { Elevation in Klassen } \\
\text { Test Well (ft AMSL) }\end{array}$} \\
\hline Date and Time & $\begin{array}{l}\text { Depth in Klassen } \\
\text { Domestic Well } \\
\text { (ft BGL) }\end{array}$ & $\begin{array}{c}\text { Depth in Klassen } \\
\text { Test Well } \\
\text { (ft BGL) }\end{array}$ & $\begin{array}{l}\text { Elevation in Klassen } \\
\text { Domestic Well } \\
\text { (ft AMSL) }\end{array}$ & \\
\hline 2/15/12 1:30 & 33.054 & 35.435 & 1475.72 & 1473.69 \\
\hline 2/15/12 2:00 & 33.079 & 35.425 & 1475.69 & 1473.70 \\
\hline 2/15/12 2:30 & 33.096 & 35.423 & 1475.67 & 1473.70 \\
\hline 2/15/12 3:00 & 33.063 & 35.416 & 1475.71 & 1473.71 \\
\hline 2/15/12 3:30 & 33.047 & 35.406 & 1475.72 & 1473.72 \\
\hline 2/15/12 4:00 & 33.124 & 35.406 & 1475.65 & 1473.72 \\
\hline 2/15/12 4:30 & 33.054 & 35.399 & 1475.72 & 1473.72 \\
\hline $2 / 15 / 125: 00$ & 33.009 & 35.383 & 1475.76 & 1473.74 \\
\hline 2/15/12 5:30 & 32.974 & 35.378 & 1475.80 & 1473.74 \\
\hline 2/15/12 6:00 & 32.946 & 35.366 & 1475.82 & 1473.76 \\
\hline 2/15/12 6:30 & 33.126 & 35.362 & 1475.64 & 1473.76 \\
\hline 2/15/12 7:00 & 33.049 & 35.364 & 1475.72 & 1473.76 \\
\hline 2/15/12 7:30 & 33.021 & 35.352 & 1475.75 & 1473.77 \\
\hline $2 / 15 / 128: 00$ & 32.972 & 35.345 & 1475.80 & 1473.78 \\
\hline 2/15/12 8:30 & 33.004 & 35.338 & 1475.77 & 1473.78 \\
\hline 2/15/12 9:00 & 32.953 & 35.331 & 1475.82 & 1473.79 \\
\hline 2/15/12 9:30 & 33.044 & 35.327 & 1475.73 & 1473.80 \\
\hline 2/15/12 10:00 & 32.986 & 35.327 & 1475.78 & 1473.80 \\
\hline $2 / 15 / 12$ 10:30 & 32.936 & 35.317 & 1475.83 & 1473.81 \\
\hline 2/15/12 11:00 & 32.906 & 35.315 & 1475.86 & 1473.81 \\
\hline 2/15/12 11:30 & 32.967 & 35.315 & 1475.80 & 1473.81 \\
\hline $2 / 15 / 12$ 12:00 & 32.915 & 35.31 & 1475.85 & 1473.81 \\
\hline $2 / 15 / 12$ 12:30 & 32.882 & 35.313 & 1475.89 & 1473.81 \\
\hline 2/15/12 13:00 & 32.857 & 35.317 & 1475.91 & 1473.81 \\
\hline 2/15/12 13:30 & 32.922 & 35.317 & 1475.85 & 1473.81 \\
\hline $2 / 15 / 12$ 14:00 & 32.875 & 35.315 & 1475.89 & 1473.81 \\
\hline 2/15/12 14:30 & 33.044 & 35.308 & 1475.73 & 1473.81 \\
\hline $2 / 15 / 12$ 15:00 & 32.892 & 35.299 & 1475.88 & 1473.82 \\
\hline 2/15/12 15:30 & 32.857 & 35.296 & 1475.91 & 1473.83 \\
\hline 2/15/12 16:00 & 32.995 & 35.301 & 1475.77 & 1473.82 \\
\hline 2/15/12 16:30 & 32.887 & 35.308 & 1475.88 & 1473.81 \\
\hline 2/15/12 17:00 & 32.85 & 35.301 & 1475.92 & 1473.82 \\
\hline 2/15/12 17:30 & 32.941 & 35.301 & 1475.83 & 1473.82 \\
\hline 2/15/12 18:00 & 33.108 & 35.306 & 1475.66 & 1473.82 \\
\hline $2 / 15 / 12$ 18:30 & 32.965 & 35.313 & 1475.80 & 1473.81 \\
\hline 2/15/12 19:00 & 32.915 & 35.315 & 1475.85 & 1473.81 \\
\hline 2/15/12 19:30 & 32.913 & 35.322 & 1475.86 & 1473.80 \\
\hline 2/15/12 20:00 & 32.993 & 35.327 & 1475.78 & 1473.80 \\
\hline
\end{tabular}


TABLE S1.3 Automatically recorded groundwater levels in the Klassen wells, September 15, 2011, to March 13, 2012.

\begin{tabular}{|c|c|c|c|c|}
\hline \multicolumn{3}{|c|}{ Reference Elevation (ft AMSL) } & 1508.77 & \multirow{2}{*}{$\begin{array}{l}1509.12 \\
\text { Elevation in Klassen } \\
\text { Test Well (ft AMSL) }\end{array}$} \\
\hline Date and Time & $\begin{array}{l}\text { Depth in Klassen } \\
\text { Domestic Well } \\
(\mathrm{ft} \mathrm{BGL})\end{array}$ & $\begin{array}{c}\text { Depth in Klassen } \\
\text { Test Well } \\
\text { (ft BGL) }\end{array}$ & $\begin{array}{l}\text { Elevation in Klassen } \\
\text { Domestic Well } \\
\text { (ft AMSL) }\end{array}$ & \\
\hline $2 / 15 / 1220: 30$ & 32.906 & 35.329 & 1475.86 & 1473.79 \\
\hline $2 / 15 / 1221: 00$ & 32.904 & 35.329 & 1475.87 & 1473.79 \\
\hline $2 / 15 / 1221: 30$ & 33.002 & 35.338 & 1475.77 & 1473.78 \\
\hline $2 / 15 / 1222: 00$ & 33.004 & 35.343 & 1475.77 & 1473.78 \\
\hline $2 / 15 / 1222: 30$ & 33.351 & 35.35 & 1475.42 & 1473.77 \\
\hline $2 / 15 / 1223: 00$ & 33.274 & 35.364 & 1475.50 & 1473.76 \\
\hline $2 / 15 / 1223: 30$ & 33.122 & 35.369 & 1475.65 & 1473.75 \\
\hline 2/16/12 0:00 & 33.047 & 35.366 & 1475.72 & 1473.76 \\
\hline 2/16/12 0:30 & 33.025 & 35.362 & 1475.74 & 1473.76 \\
\hline $2 / 16 / 121: 00$ & 32.979 & 35.357 & 1475.79 & 1473.77 \\
\hline 2/16/12 1:30 & 33.03 & 35.362 & 1475.74 & 1473.76 \\
\hline $2 / 16 / 122: 00$ & 32.967 & 35.362 & 1475.80 & 1473.76 \\
\hline $2 / 16 / 122: 30$ & 32.932 & 35.345 & 1475.84 & 1473.78 \\
\hline $2 / 16 / 123: 00$ & 32.904 & 35.345 & 1475.87 & 1473.78 \\
\hline 2/16/12 3:30 & 32.882 & 35.341 & 1475.89 & 1473.78 \\
\hline 2/16/12 4:00 & 32.864 & 35.331 & 1475.91 & 1473.79 \\
\hline $2 / 16 / 124: 30$ & 32.845 & 35.327 & 1475.92 & 1473.80 \\
\hline 2/16/12 5:00 & 32.854 & 35.32 & 1475.92 & 1473.80 \\
\hline $2 / 16 / 125: 30$ & 32.833 & 35.313 & 1475.94 & 1473.81 \\
\hline $2 / 16 / 126: 00$ & 32.817 & 35.306 & 1475.95 & 1473.82 \\
\hline $2 / 16 / 126: 30$ & 33.035 & 35.31 & 1475.73 & 1473.81 \\
\hline $2 / 16 / 12$ 7:00 & 33.591 & 35.315 & 1475.18 & 1473.81 \\
\hline 2/16/12 7:30 & 33.199 & 35.324 & 1475.57 & 1473.80 \\
\hline $2 / 16 / 128: 00$ & 33.682 & 35.334 & 1475.09 & 1473.79 \\
\hline $2 / 16 / 128: 30$ & 33.103 & 35.338 & 1475.67 & 1473.78 \\
\hline 2/16/12 9:00 & 33.028 & 35.343 & 1475.74 & 1473.78 \\
\hline 2/16/12 9:30 & 32.983 & 35.343 & 1475.79 & 1473.78 \\
\hline 2/16/12 10:00 & 32.948 & 35.355 & 1475.82 & 1473.77 \\
\hline $2 / 16 / 12$ 10:30 & 34.81 & 35.362 & 1473.96 & 1473.76 \\
\hline 2/16/12 11:00 & 32.979 & 35.367 & 1475.79 & 1473.76 \\
\hline 2/16/12 11:30 & 32.932 & 35.367 & 1475.84 & 1473.76 \\
\hline $2 / 16 / 12$ 12:00 & 32.901 & 35.371 & 1475.87 & 1473.75 \\
\hline $2 / 16 / 12$ 12:30 & 32.946 & 35.383 & 1475.82 & 1473.74 \\
\hline $2 / 16 / 12$ 13:00 & 32.901 & 35.39 & 1475.87 & 1473.73 \\
\hline $2 / 16 / 12$ 13:30 & 32.953 & 35.397 & 1475.82 & 1473.73 \\
\hline $2 / 16 / 12$ 14:00 & 32.904 & 35.374 & 1475.87 & 1473.75 \\
\hline $2 / 16 / 12$ 14:30 & 32.875 & 35.341 & 1475.89 & 1473.78 \\
\hline $2 / 16 / 12$ 15:00 & 32.955 & 35.336 & 1475.81 & 1473.79 \\
\hline
\end{tabular}


TABLE S1.3 Automatically recorded groundwater levels in the Klassen wells, September 15, 2011, to March 13, 2012.

\begin{tabular}{|c|c|c|c|c|}
\hline \multicolumn{3}{|c|}{ Reference Elevation (ft AMSL) } & 1508.77 & \multirow{2}{*}{$\begin{array}{l}1509.12 \\
\text { Elevation in Klassen } \\
\text { Test Well (ft AMSL) }\end{array}$} \\
\hline Date and Time & $\begin{array}{l}\text { Depth in Klassen } \\
\text { Domestic Well } \\
(\mathrm{ft} \mathrm{BGL})\end{array}$ & $\begin{array}{c}\text { Depth in Klassen } \\
\text { Test Well } \\
\text { (ft BGL) }\end{array}$ & $\begin{array}{l}\text { Elevation in Klassen } \\
\text { Domestic Well } \\
\text { (ft AMSL) }\end{array}$ & \\
\hline $2 / 16 / 12$ 15:30 & 32.901 & 35.329 & 1475.87 & 1473.79 \\
\hline $2 / 16 / 1216: 00$ & 32.873 & 35.324 & 1475.90 & 1473.80 \\
\hline 2/16/12 16:30 & 32.904 & 35.334 & 1475.87 & 1473.79 \\
\hline $2 / 16 / 1217: 00$ & 33.227 & 35.341 & 1475.54 & 1473.78 \\
\hline $2 / 16 / 12$ 17:30 & 33.098 & 35.343 & 1475.67 & 1473.78 \\
\hline $2 / 16 / 12$ 18:00 & 33.002 & 35.343 & 1475.77 & 1473.78 \\
\hline $2 / 16 / 1218: 30$ & 32.95 & 35.341 & 1475.82 & 1473.78 \\
\hline 2/16/12 19:00 & 32.918 & 35.336 & 1475.85 & 1473.79 \\
\hline 2/16/12 19:30 & 32.889 & 35.331 & 1475.88 & 1473.79 \\
\hline $2 / 16 / 1220: 00$ & 32.868 & 35.331 & 1475.90 & 1473.79 \\
\hline $2 / 16 / 1220: 30$ & 32.85 & 35.331 & 1475.92 & 1473.79 \\
\hline $2 / 16 / 1221: 00$ & 32.836 & 35.329 & 1475.93 & 1473.79 \\
\hline $2 / 16 / 1221: 30$ & 32.821 & 35.329 & 1475.95 & 1473.79 \\
\hline $2 / 16 / 1222: 00$ & 32.807 & 35.32 & 1475.96 & 1473.80 \\
\hline $2 / 16 / 1222: 30$ & 32.796 & 35.315 & 1475.97 & 1473.81 \\
\hline $2 / 16 / 12$ 23:00 & 32.838 & 35.315 & 1475.93 & 1473.81 \\
\hline $2 / 16 / 1223: 30$ & 33.018 & 35.315 & 1475.75 & 1473.81 \\
\hline 2/17/12 0:00 & 32.925 & 35.313 & 1475.84 & 1473.81 \\
\hline $2 / 17 / 12$ 0:30 & 32.908 & 35.305 & 1475.86 & 1473.82 \\
\hline 2/17/12 1:00 & 32.866 & 35.301 & 1475.90 & 1473.82 \\
\hline $2 / 17 / 121: 30$ & 32.864 & 35.294 & 1475.91 & 1473.83 \\
\hline $2 / 17 / 12$ 2:00 & 32.833 & 35.284 & 1475.94 & 1473.84 \\
\hline 2/17/12 2:30 & 32.812 & 35.273 & 1475.96 & 1473.85 \\
\hline 2/17/12 3:00 & 32.793 & 35.266 & 1475.98 & 1473.86 \\
\hline 2/17/12 3:30 & 32.782 & 35.261 & 1475.99 & 1473.86 \\
\hline 2/17/12 4:00 & 32.765 & 35.254 & 1476.00 & 1473.87 \\
\hline 2/17/12 4:30 & 32.751 & 35.249 & 1476.02 & 1473.87 \\
\hline 2/17/12 5:00 & 32.866 & 35.245 & 1475.90 & 1473.88 \\
\hline 2/17/12 5:30 & 32.791 & 35.24 & 1475.98 & 1473.88 \\
\hline 2/17/12 6:00 & 32.763 & 35.235 & 1476.01 & 1473.89 \\
\hline 2/17/12 6:30 & 32.983 & 35.238 & 1475.79 & 1473.88 \\
\hline $2 / 17 / 127: 00$ & 32.889 & 35.238 & 1475.88 & 1473.88 \\
\hline 2/17/12 7:30 & 32.976 & 35.233 & 1475.79 & 1473.89 \\
\hline $2 / 17 / 128: 00$ & 32.939 & 35.23 & 1475.83 & 1473.89 \\
\hline $2 / 17 / 128: 30$ & 33.025 & 35.23 & 1475.74 & 1473.89 \\
\hline 2/17/12 9:00 & 33.056 & 35.238 & 1475.71 & 1473.88 \\
\hline 2/17/12 9:30 & 46.717 & 35.254 & 1462.05 & 1473.87 \\
\hline $2 / 17 / 12$ 10:00 & 50.097 & 35.317 & 1458.67 & 1473.81 \\
\hline
\end{tabular}


TABLE S1.3 Automatically recorded groundwater levels in the Klassen wells, September 15, 2011, to March 13, 2012.

\begin{tabular}{|c|c|c|c|c|}
\hline \multicolumn{3}{|c|}{ Reference Elevation (ft AMSL) } & 1508.77 & \multirow{2}{*}{$\begin{array}{l}1509.12 \\
\text { Elevation in Klassen } \\
\text { Test Well (ft AMSL) }\end{array}$} \\
\hline Date and Time & $\begin{array}{l}\text { Depth in Klassen } \\
\text { Domestic Well } \\
(\mathrm{ft} \mathrm{BGL})\end{array}$ & $\begin{array}{c}\text { Depth in Klassen } \\
\text { Test Well } \\
\text { (ft BGL) }\end{array}$ & $\begin{array}{l}\text { Elevation in Klassen } \\
\text { Domestic Well } \\
\text { (ft AMSL) }\end{array}$ & \\
\hline $2 / 17 / 1210: 30$ & 54.593 & 35.397 & 1454.18 & 1473.73 \\
\hline $2 / 17 / 1211: 00$ & 50.85 & 35.474 & 1457.92 & 1473.65 \\
\hline 2/17/12 11:30 & 52.925 & 35.557 & 1455.84 & 1473.57 \\
\hline $2 / 17 / 1212: 00$ & 56.849 & 35.646 & 1451.92 & 1473.48 \\
\hline $2 / 17 / 12$ 12:30 & 55.416 & 35.747 & 1453.35 & 1473.38 \\
\hline $2 / 17 / 12$ 13:00 & 43.776 & 35.876 & 1464.99 & 1473.25 \\
\hline $2 / 17 / 12$ 13:30 & 37.758 & 36.019 & 1471.01 & 1473.10 \\
\hline 2/17/12 14:00 & 36.975 & 36.075 & 1471.79 & 1473.05 \\
\hline $2 / 17 / 12$ 14:30 & 36.654 & 36.099 & 1472.12 & 1473.02 \\
\hline $2 / 17 / 12$ 15:00 & 36.253 & 36.106 & 1472.52 & 1473.02 \\
\hline 2/17/12 15:30 & 35.873 & 36.096 & 1472.90 & 1473.03 \\
\hline $2 / 17 / 12$ 16:00 & 35.586 & 36.08 & 1473.18 & 1473.04 \\
\hline $2 / 17 / 12$ 16:30 & 35.49 & 36.066 & 1473.28 & 1473.06 \\
\hline $2 / 17 / 1217: 00$ & 35.413 & 36.052 & 1473.36 & 1473.07 \\
\hline $2 / 17 / 12$ 17:30 & 35.598 & 36.04 & 1473.17 & 1473.08 \\
\hline $2 / 17 / 12$ 18:00 & 35.094 & 36.03 & 1473.68 & 1473.09 \\
\hline $2 / 17 / 12$ 18:30 & 35.019 & 36.026 & 1473.75 & 1473.10 \\
\hline 2/17/12 19:00 & 35.948 & 36.028 & 1472.82 & 1473.09 \\
\hline 2/17/12 19:30 & 35.024 & 36.021 & 1473.75 & 1473.10 \\
\hline $2 / 17 / 12$ 20:00 & 35.446 & 36.026 & 1473.32 & 1473.10 \\
\hline $2 / 17 / 1220: 30$ & 35.19 & 36.028 & 1473.58 & 1473.09 \\
\hline 2/17/12 21:00 & 34.923 & 36.021 & 1473.85 & 1473.10 \\
\hline $2 / 17 / 12$ 21:30 & 34.834 & 36.005 & 1473.94 & 1473.12 \\
\hline 2/17/12 22:00 & 34.782 & 35.991 & 1473.99 & 1473.13 \\
\hline 2/17/12 22:30 & 34.836 & 35.984 & 1473.93 & 1473.14 \\
\hline $2 / 17 / 12$ 23:00 & 35.336 & 35.981 & 1473.43 & 1473.14 \\
\hline 2/17/12 23:30 & 34.775 & 35.977 & 1473.99 & 1473.15 \\
\hline 2/18/12 0:00 & 34.59 & 35.962 & 1474.18 & 1473.16 \\
\hline 2/18/12 0:30 & 34.482 & 35.948 & 1474.29 & 1473.17 \\
\hline 2/18/12 1:00 & 34.369 & 35.937 & 1474.40 & 1473.19 \\
\hline 2/18/12 1:30 & 34.273 & 35.92 & 1474.50 & 1473.20 \\
\hline $2 / 18 / 122: 00$ & 34.254 & 35.904 & 1474.52 & 1473.22 \\
\hline 2/18/12 2:30 & 34.224 & 35.89 & 1474.55 & 1473.23 \\
\hline 2/18/12 3:00 & 34.147 & 35.878 & 1474.62 & 1473.24 \\
\hline 2/18/12 3:30 & 34.086 & 35.866 & 1474.68 & 1473.26 \\
\hline 2/18/12 4:00 & 34.137 & 35.854 & 1474.63 & 1473.27 \\
\hline $2 / 18 / 124: 30$ & 34.039 & 35.84 & 1474.73 & 1473.28 \\
\hline $2 / 18 / 12$ 5:00 & 34.093 & 35.824 & 1474.68 & 1473.30 \\
\hline
\end{tabular}


TABLE S1.3 Automatically recorded groundwater levels in the Klassen wells, September 15, 2011, to March 13, 2012.

\begin{tabular}{|c|c|c|c|c|}
\hline \multicolumn{3}{|c|}{ Reference Elevation (ft AMSL) } & 1508.77 & \multirow{2}{*}{$\begin{array}{l}1509.12 \\
\text { Elevation in Klassen } \\
\text { Test Well (ft AMSL) }\end{array}$} \\
\hline Date and Time & $\begin{array}{l}\text { Depth in Klassen } \\
\text { Domestic Well } \\
(\mathrm{ft} \mathrm{BGL})\end{array}$ & $\begin{array}{c}\text { Depth in Klassen } \\
\text { Test Well } \\
\text { (ft BGL) }\end{array}$ & $\begin{array}{l}\text { Elevation in Klassen } \\
\text { Domestic Well } \\
\text { (ft AMSL) }\end{array}$ & \\
\hline $2 / 18 / 125: 30$ & 33.961 & 35.805 & 1474.81 & 1473.32 \\
\hline $2 / 18 / 126: 00$ & 33.889 & 35.789 & 1474.88 & 1473.33 \\
\hline $2 / 18 / 126: 30$ & 33.935 & 35.77 & 1474.83 & 1473.35 \\
\hline $2 / 18 / 127: 00$ & 33.839 & 35.758 & 1474.93 & 1473.36 \\
\hline $2 / 18 / 127: 30$ & 33.813 & 35.747 & 1474.96 & 1473.38 \\
\hline $2 / 18 / 128: 00$ & 33.783 & 35.744 & 1474.99 & 1473.38 \\
\hline $2 / 18 / 128: 30$ & 33.821 & 35.733 & 1474.95 & 1473.39 \\
\hline 2/18/12 9:00 & 33.734 & 35.726 & 1475.04 & 1473.40 \\
\hline 2/18/12 9:30 & 33.713 & 35.718 & 1475.06 & 1473.40 \\
\hline $2 / 18 / 1210: 00$ & 33.825 & 35.721 & 1474.94 & 1473.40 \\
\hline $2 / 18 / 1210: 30$ & 33.743 & 35.714 & 1475.03 & 1473.41 \\
\hline 2/18/12 11:00 & 33.696 & 35.714 & 1475.07 & 1473.41 \\
\hline 2/18/12 11:30 & 34.036 & 35.718 & 1474.73 & 1473.40 \\
\hline $2 / 18 / 1212: 00$ & 33.806 & 35.723 & 1474.96 & 1473.40 \\
\hline 2/18/12 12:30 & 33.83 & 35.726 & 1474.94 & 1473.40 \\
\hline $2 / 18 / 12$ 13:00 & 34.128 & 35.725 & 1474.64 & 1473.40 \\
\hline $2 / 18 / 12$ 13:30 & 33.914 & 35.723 & 1474.86 & 1473.40 \\
\hline 2/18/12 14:00 & 33.877 & 35.707 & 1474.89 & 1473.42 \\
\hline $2 / 18 / 12$ 14:30 & 34.003 & 35.657 & 1474.77 & 1473.47 \\
\hline $2 / 18 / 12$ 15:00 & 34.149 & 35.66 & 1474.62 & 1473.46 \\
\hline $2 / 18 / 12$ 15:30 & 34.086 & 35.662 & 1474.68 & 1473.46 \\
\hline 2/18/12 16:00 & 33.926 & 35.657 & 1474.84 & 1473.47 \\
\hline $2 / 18 / 12$ 16:30 & 33.851 & 35.646 & 1474.92 & 1473.48 \\
\hline 2/18/12 17:00 & 33.771 & 35.636 & 1475.00 & 1473.49 \\
\hline 2/18/12 17:30 & 33.839 & 35.629 & 1474.93 & 1473.49 \\
\hline 2/18/12 18:00 & 33.849 & 35.632 & 1474.92 & 1473.49 \\
\hline 2/18/12 18:30 & 33.874 & 35.629 & 1474.90 & 1473.49 \\
\hline 2/18/12 19:00 & 33.926 & 35.632 & 1474.84 & 1473.49 \\
\hline 2/18/12 19:30 & 34.158 & 35.653 & 1474.61 & 1473.47 \\
\hline $2 / 18 / 1220: 00$ & 34.083 & 35.655 & 1474.69 & 1473.47 \\
\hline 2/18/12 20:30 & 34.057 & 35.66 & 1474.71 & 1473.46 \\
\hline $2 / 18 / 12$ 21:00 & 33.926 & 35.655 & 1474.84 & 1473.47 \\
\hline $2 / 18 / 1221: 30$ & 33.961 & 35.653 & 1474.81 & 1473.47 \\
\hline $2 / 18 / 1222: 00$ & 36.611 & 35.655 & 1472.16 & 1473.47 \\
\hline $2 / 18 / 1222: 30$ & 34.076 & 35.655 & 1474.69 & 1473.47 \\
\hline $2 / 18 / 12$ 23:00 & 34.144 & 35.655 & 1474.63 & 1473.47 \\
\hline 2/18/12 23:30 & 33.973 & 35.65 & 1474.80 & 1473.47 \\
\hline 2/19/12 0:00 & 33.851 & 35.643 & 1474.92 & 1473.48 \\
\hline
\end{tabular}


TABLE S1.3 Automatically recorded groundwater levels in the Klassen wells, September 15, 2011, to March 13, 2012.

\begin{tabular}{|c|c|c|c|c|}
\hline \multicolumn{3}{|c|}{ Reference Elevation (ft AMSL) } & 1508.77 & \multirow{2}{*}{$\begin{array}{l}1509.12 \\
\text { Elevation in Klassen } \\
\text { Test Well (ft AMSL) }\end{array}$} \\
\hline Date and Time & $\begin{array}{l}\text { Depth in Klassen } \\
\text { Domestic Well } \\
\text { (ft BGL) }\end{array}$ & $\begin{array}{c}\text { Depth in Klassen } \\
\text { Test Well } \\
\text { (ft BGL) }\end{array}$ & $\begin{array}{l}\text { Elevation in Klassen } \\
\text { Domestic Well } \\
\text { (ft AMSL) }\end{array}$ & \\
\hline 2/19/12 0:30 & 33.795 & 35.639 & 1474.97 & 1473.48 \\
\hline 2/19/12 1:00 & 33.717 & 35.632 & 1475.05 & 1473.49 \\
\hline 2/19/12 1:30 & 33.654 & 35.622 & 1475.12 & 1473.50 \\
\hline 2/19/12 2:00 & 33.682 & 35.61 & 1475.09 & 1473.51 \\
\hline 2/19/12 2:30 & 35.833 & 35.608 & 1472.94 & 1473.51 \\
\hline 2/19/12 3:00 & 33.696 & 35.601 & 1475.07 & 1473.52 \\
\hline 2/19/12 3:30 & 33.631 & 35.594 & 1475.14 & 1473.53 \\
\hline 2/19/12 4:00 & 33.692 & 35.585 & 1475.08 & 1473.54 \\
\hline 2/19/12 4:30 & 33.602 & 35.573 & 1475.17 & 1473.55 \\
\hline 2/19/12 5:00 & 33.541 & 35.559 & 1475.23 & 1473.56 \\
\hline 2/19/12 5:30 & 33.492 & 35.542 & 1475.28 & 1473.58 \\
\hline $2 / 19 / 126: 00$ & 34.463 & 35.531 & 1474.31 & 1473.59 \\
\hline 2/19/12 6:30 & 33.677 & 35.526 & 1475.09 & 1473.60 \\
\hline 2/19/12 7:00 & 33.537 & 35.519 & 1475.23 & 1473.60 \\
\hline 2/19/12 7:30 & 33.469 & 35.507 & 1475.30 & 1473.62 \\
\hline 2/19/12 8:00 & 33.57 & 35.505 & 1475.20 & 1473.62 \\
\hline $2 / 19 / 128: 30$ & 37.336 & 35.51 & 1471.43 & 1473.61 \\
\hline 2/19/12 9:00 & 33.724 & 35.521 & 1475.05 & 1473.60 \\
\hline 2/19/12 9:30 & 33.72 & 35.531 & 1475.05 & 1473.59 \\
\hline 2/19/12 10:00 & 33.626 & 35.55 & 1475.14 & 1473.57 \\
\hline $2 / 19 / 12$ 10:30 & 33.692 & 35.568 & 1475.08 & 1473.55 \\
\hline 2/19/12 11:00 & 33.588 & 35.585 & 1475.18 & 1473.54 \\
\hline 2/19/12 11:30 & 33.541 & 35.599 & 1475.23 & 1473.52 \\
\hline 2/19/12 12:00 & 33.476 & 35.611 & 1475.29 & 1473.51 \\
\hline 2/19/12 12:30 & 33.424 & 35.622 & 1475.35 & 1473.50 \\
\hline 2/19/12 13:00 & 33.38 & 35.62 & 1475.39 & 1473.50 \\
\hline 2/19/12 13:30 & 33.342 & 35.599 & 1475.43 & 1473.52 \\
\hline 2/19/12 14:00 & 34.669 & 35.578 & 1474.10 & 1473.54 \\
\hline 2/19/12 14:30 & 33.64 & 35.575 & 1475.13 & 1473.55 \\
\hline 2/19/12 15:00 & 33.755 & 35.589 & 1475.01 & 1473.53 \\
\hline 2/19/12 15:30 & 33.802 & 35.596 & 1474.97 & 1473.53 \\
\hline 2/19/12 16:00 & 35.8 & 35.594 & 1472.97 & 1473.53 \\
\hline 2/19/12 16:30 & 33.624 & 35.587 & 1475.15 & 1473.54 \\
\hline 2/19/12 17:00 & 33.541 & 35.58 & 1475.23 & 1473.54 \\
\hline 2/19/12 17:30 & 33.487 & 35.578 & 1475.28 & 1473.54 \\
\hline 2/19/12 18:00 & 34.132 & 35.575 & 1474.64 & 1473.55 \\
\hline 2/19/12 18:30 & 33.595 & 35.575 & 1475.17 & 1473.55 \\
\hline 2/19/12 19:00 & 33.607 & 35.578 & 1475.16 & 1473.54 \\
\hline
\end{tabular}


TABLE S1.3 Automatically recorded groundwater levels in the Klassen wells, September 15, 2011, to March 13, 2012.

\begin{tabular}{|c|c|c|c|c|}
\hline \multicolumn{3}{|c|}{ Reference Elevation (ft AMSL) } & 1508.77 & \multirow{2}{*}{$\begin{array}{l}1509.12 \\
\text { Elevation in Klassen } \\
\text { Test Well (ft AMSL) }\end{array}$} \\
\hline Date and Time & $\begin{array}{l}\text { Depth in Klassen } \\
\text { Domestic Well } \\
(\mathrm{ft} \mathrm{BGL})\end{array}$ & $\begin{array}{c}\text { Depth in Klassen } \\
\text { Test Well } \\
\text { (ft BGL) }\end{array}$ & $\begin{array}{l}\text { Elevation in Klassen } \\
\text { Domestic Well } \\
\text { (ft AMSL) }\end{array}$ & \\
\hline 2/19/12 19:30 & 33.506 & 35.578 & 1475.26 & 1473.54 \\
\hline $2 / 19 / 1220: 00$ & 33.553 & 35.573 & 1475.22 & 1473.55 \\
\hline 2/19/12 20:30 & 33.513 & 35.573 & 1475.26 & 1473.55 \\
\hline $2 / 19 / 1221: 00$ & 33.436 & 35.568 & 1475.33 & 1473.55 \\
\hline $2 / 19 / 1221: 30$ & 33.412 & 35.559 & 1475.36 & 1473.56 \\
\hline 2/19/12 22:00 & 33.478 & 35.559 & 1475.29 & 1473.56 \\
\hline $2 / 19 / 1222: 30$ & 33.389 & 35.55 & 1475.38 & 1473.57 \\
\hline 2/19/12 23:00 & 33.673 & 35.543 & 1475.10 & 1473.58 \\
\hline $2 / 19 / 1223: 30$ & 33.6 & 35.547 & 1475.17 & 1473.58 \\
\hline 2/20/12 0:00 & 33.448 & 35.54 & 1475.32 & 1473.58 \\
\hline $2 / 20 / 120: 30$ & 33.375 & 35.531 & 1475.39 & 1473.59 \\
\hline $2 / 20 / 121: 00$ & 33.323 & 35.524 & 1475.45 & 1473.60 \\
\hline $2 / 20 / 121: 30$ & 33.281 & 35.512 & 1475.49 & 1473.61 \\
\hline $2 / 20 / 122: 00$ & 33.244 & 35.505 & 1475.53 & 1473.62 \\
\hline $2 / 20 / 122: 30$ & 33.215 & 35.493 & 1475.55 & 1473.63 \\
\hline $2 / 20 / 12$ 3:00 & 33.298 & 35.486 & 1475.47 & 1473.64 \\
\hline $2 / 20 / 123: 30$ & 33.244 & 35.47 & 1475.53 & 1473.65 \\
\hline 2/20/12 4:00 & 33.194 & 35.46 & 1475.58 & 1473.66 \\
\hline $2 / 20 / 124: 30$ & 33.185 & 35.442 & 1475.58 & 1473.68 \\
\hline $2 / 20 / 12$ 5:00 & 33.145 & 35.442 & 1475.62 & 1473.68 \\
\hline $2 / 20 / 125: 30$ & 33.112 & 35.442 & 1475.66 & 1473.68 \\
\hline $2 / 20 / 126: 00$ & 33.086 & 35.437 & 1475.68 & 1473.69 \\
\hline 2/20/12 6:30 & 33.237 & 35.439 & 1475.53 & 1473.68 \\
\hline $2 / 20 / 12$ 7:00 & 33.14 & 35.435 & 1475.63 & 1473.69 \\
\hline $2 / 20 / 127: 30$ & 33.098 & 35.423 & 1475.67 & 1473.70 \\
\hline $2 / 20 / 128: 00$ & 33.072 & 35.411 & 1475.70 & 1473.71 \\
\hline $2 / 20 / 128: 30$ & 35.781 & 35.409 & 1472.99 & 1473.71 \\
\hline 2/20/12 9:00 & 33.14 & 35.404 & 1475.63 & 1473.72 \\
\hline 2/20/12 9:30 & 35.298 & 35.404 & 1473.47 & 1473.72 \\
\hline $2 / 20 / 12$ 10:00 & 33.26 & 35.402 & 1475.51 & 1473.72 \\
\hline 2/20/12 10:30 & 33.38 & 35.402 & 1475.39 & 1473.72 \\
\hline 2/20/12 11:00 & 33.351 & 35.399 & 1475.42 & 1473.72 \\
\hline $2 / 20 / 1211: 30$ & 33.462 & 35.397 & 1475.31 & 1473.73 \\
\hline $2 / 20 / 12$ 12:00 & 33.492 & 35.397 & 1475.28 & 1473.73 \\
\hline $2 / 20 / 12$ 12:30 & 33.771 & 35.406 & 1475.00 & 1473.72 \\
\hline $2 / 20 / 12$ 13:00 & 35.087 & 35.406 & 1473.68 & 1473.72 \\
\hline $2 / 20 / 12$ 13:30 & 33.687 & 35.416 & 1475.08 & 1473.71 \\
\hline $2 / 20 / 12$ 14:00 & 33.483 & 35.404 & 1475.29 & 1473.72 \\
\hline
\end{tabular}


TABLE S1.3 Automatically recorded groundwater levels in the Klassen wells, September 15, 2011, to March 13, 2012.

\begin{tabular}{|c|c|c|c|c|}
\hline \multicolumn{3}{|c|}{ Reference Elevation (ft AMSL) } & 1508.77 & \multirow{2}{*}{$\begin{array}{l}1509.12 \\
\text { Elevation in Klassen } \\
\text { Test Well (ft AMSL) }\end{array}$} \\
\hline Date and Time & $\begin{array}{l}\text { Depth in Klassen } \\
\text { Domestic Well } \\
(\mathrm{ft} \mathrm{BGL})\end{array}$ & $\begin{array}{c}\text { Depth in Klassen } \\
\text { Test Well } \\
\text { (ft BGL) }\end{array}$ & $\begin{array}{l}\text { Elevation in Klassen } \\
\text { Domestic Well } \\
\text { (ft AMSL) }\end{array}$ & \\
\hline $2 / 20 / 1214: 30$ & 33.487 & 35.39 & 1475.28 & 1473.73 \\
\hline $2 / 20 / 12$ 15:00 & 33.351 & 35.395 & 1475.42 & 1473.73 \\
\hline 2/20/12 15:30 & 33.279 & 35.352 & 1475.49 & 1473.77 \\
\hline $2 / 20 / 1216: 00$ & 36.977 & 35.341 & 1471.79 & 1473.78 \\
\hline $2 / 20 / 1216: 30$ & 33.328 & 35.343 & 1475.44 & 1473.78 \\
\hline $2 / 20 / 12$ 17:00 & 33.593 & 35.362 & 1475.18 & 1473.76 \\
\hline $2 / 20 / 1217: 30$ & 33.466 & 35.357 & 1475.30 & 1473.77 \\
\hline $2 / 20 / 12$ 18:00 & 33.417 & 35.343 & 1475.35 & 1473.78 \\
\hline $2 / 20 / 1218: 30$ & 33.415 & 35.334 & 1475.35 & 1473.79 \\
\hline $2 / 20 / 12$ 19:00 & 33.53 & 35.327 & 1475.24 & 1473.80 \\
\hline $2 / 20 / 12$ 19:30 & 33.504 & 35.322 & 1475.27 & 1473.80 \\
\hline $2 / 20 / 1220: 00$ & 33.659 & 35.32 & 1475.11 & 1473.80 \\
\hline $2 / 20 / 1220: 30$ & 33.633 & 35.322 & 1475.14 & 1473.80 \\
\hline $2 / 20 / 1221: 00$ & 33.98 & 35.36 & 1474.79 & 1473.76 \\
\hline $2 / 20 / 1221: 30$ & 33.675 & 35.381 & 1475.09 & 1473.74 \\
\hline $2 / 20 / 1222: 00$ & 33.534 & 35.392 & 1475.24 & 1473.73 \\
\hline $2 / 20 / 1222: 30$ & 34.541 & 35.404 & 1474.23 & 1473.72 \\
\hline $2 / 20 / 12$ 23:00 & 33.919 & 35.411 & 1474.85 & 1473.71 \\
\hline $2 / 20 / 12$ 23:30 & 33.577 & 35.418 & 1475.19 & 1473.70 \\
\hline 2/21/12 0:00 & 33.466 & 35.418 & 1475.30 & 1473.70 \\
\hline $2 / 21 / 12$ 0:30 & 33.398 & 35.418 & 1475.37 & 1473.70 \\
\hline $2 / 21 / 121: 00$ & 33.342 & 35.411 & 1475.43 & 1473.71 \\
\hline 2/21/12 1:30 & 33.3 & 35.406 & 1475.47 & 1473.72 \\
\hline 2/21/12 2:00 & 33.464 & 35.397 & 1475.31 & 1473.73 \\
\hline 2/21/12 2:30 & 33.41 & 35.409 & 1475.36 & 1473.71 \\
\hline 2/21/12 3:00 & 33.356 & 35.399 & 1475.41 & 1473.72 \\
\hline 2/21/12 3:30 & 33.319 & 35.39 & 1475.45 & 1473.73 \\
\hline 2/21/12 4:00 & 33.363 & 35.383 & 1475.41 & 1473.74 \\
\hline 2/21/12 4:30 & 33.288 & 35.381 & 1475.48 & 1473.74 \\
\hline 2/21/12 5:00 & 33.237 & 35.369 & 1475.53 & 1473.75 \\
\hline 2/21/12 5:30 & 33.197 & 35.357 & 1475.57 & 1473.77 \\
\hline $2 / 21 / 126: 00$ & 33.164 & 35.352 & 1475.61 & 1473.77 \\
\hline 2/21/12 6:30 & 33.302 & 35.352 & 1475.47 & 1473.77 \\
\hline 2/21/12 7:00 & 33.23 & 35.35 & 1475.54 & 1473.77 \\
\hline 2/21/12 7:30 & 33.274 & 35.338 & 1475.50 & 1473.78 \\
\hline $2 / 21 / 128: 00$ & 33.361 & 35.343 & 1475.41 & 1473.78 \\
\hline $2 / 21 / 128: 30$ & 33.232 & 35.345 & 1475.54 & 1473.78 \\
\hline 2/21/12 9:00 & 34.733 & 35.338 & 1474.04 & 1473.78 \\
\hline
\end{tabular}


TABLE S1.3 Automatically recorded groundwater levels in the Klassen wells, September 15, 2011, to March 13, 2012.

\begin{tabular}{|c|c|c|c|c|}
\hline \multicolumn{3}{|c|}{ Reference Elevation (ft AMSL) } & 1508.77 & \multirow{2}{*}{$\begin{array}{l}1509.12 \\
\text { Elevation in Klassen } \\
\text { Test Well (ft AMSL) }\end{array}$} \\
\hline Date and Time & $\begin{array}{l}\text { Depth in Klassen } \\
\text { Domestic Well } \\
(\mathrm{ft} \mathrm{BGL})\end{array}$ & $\begin{array}{c}\text { Depth in Klassen } \\
\text { Test Well } \\
\text { (ft BGL) }\end{array}$ & $\begin{array}{l}\text { Elevation in Klassen } \\
\text { Domestic Well } \\
\text { (ft AMSL) }\end{array}$ & \\
\hline $2 / 21 / 129: 30$ & 33.215 & 35.338 & 1475.55 & 1473.78 \\
\hline $2 / 21 / 1210: 00$ & 33.159 & 35.341 & 1475.61 & 1473.78 \\
\hline 2/21/12 10:30 & 33.124 & 35.338 & 1475.65 & 1473.78 \\
\hline $2 / 21 / 1211: 00$ & 33.194 & 35.338 & 1475.58 & 1473.78 \\
\hline 2/21/12 11:30 & 33.117 & 35.338 & 1475.65 & 1473.78 \\
\hline $2 / 21 / 12$ 12:00 & 33.192 & 35.348 & 1475.58 & 1473.77 \\
\hline $2 / 21 / 1212: 30$ & 33.103 & 35.357 & 1475.67 & 1473.77 \\
\hline 2/21/12 13:00 & 33.065 & 35.334 & 1475.70 & 1473.79 \\
\hline $2 / 21 / 12$ 13:30 & 33.328 & 35.317 & 1475.44 & 1473.81 \\
\hline $2 / 21 / 1214: 00$ & 33.096 & 35.308 & 1475.67 & 1473.81 \\
\hline $2 / 21 / 1214: 30$ & 33.15 & 35.301 & 1475.62 & 1473.82 \\
\hline $2 / 21 / 12$ 15:00 & 33.075 & 35.294 & 1475.69 & 1473.83 \\
\hline $2 / 21 / 12$ 15:30 & 33.035 & 35.294 & 1475.73 & 1473.83 \\
\hline $2 / 21 / 1216: 00$ & 33.007 & 35.299 & 1475.76 & 1473.82 \\
\hline $2 / 21 / 12$ 16:30 & 33.058 & 35.308 & 1475.71 & 1473.81 \\
\hline $2 / 21 / 12$ 17:00 & 33.049 & 35.32 & 1475.72 & 1473.80 \\
\hline $2 / 21 / 12$ 17:30 & 33.145 & 35.331 & 1475.62 & 1473.79 \\
\hline $2 / 21 / 12$ 18:00 & 33.093 & 35.338 & 1475.68 & 1473.78 \\
\hline $2 / 21 / 12$ 18:30 & 33.07 & 35.35 & 1475.70 & 1473.77 \\
\hline $2 / 21 / 12$ 19:00 & 36.049 & 35.364 & 1472.72 & 1473.76 \\
\hline $2 / 21 / 12$ 19:30 & 33.129 & 35.383 & 1475.64 & 1473.74 \\
\hline 2/21/12 20:00 & 33.065 & 35.395 & 1475.70 & 1473.73 \\
\hline $2 / 21 / 1220: 30$ & 33.025 & 35.397 & 1475.74 & 1473.73 \\
\hline 2/21/12 21:00 & 33.021 & 35.399 & 1475.75 & 1473.72 \\
\hline 2/21/12 21:30 & 33.215 & 35.409 & 1475.55 & 1473.71 \\
\hline 2/21/12 22:00 & 33.119 & 35.409 & 1475.65 & 1473.71 \\
\hline $2 / 21 / 1222: 30$ & 33.703 & 35.409 & 1475.07 & 1473.71 \\
\hline $2 / 21 / 12$ 23:00 & 33.408 & 35.416 & 1475.36 & 1473.71 \\
\hline $2 / 21 / 12$ 23:30 & 33.274 & 35.423 & 1475.50 & 1473.70 \\
\hline $2 / 22 / 12$ 0:00 & 33.171 & 35.421 & 1475.60 & 1473.70 \\
\hline 2/22/12 0:30 & 33.112 & 35.416 & 1475.66 & 1473.71 \\
\hline 2/22/12 1:00 & 33.068 & 35.409 & 1475.70 & 1473.71 \\
\hline 2/22/12 1:30 & 33.033 & 35.399 & 1475.74 & 1473.72 \\
\hline 2/22/12 2:00 & 33.004 & 35.39 & 1475.77 & 1473.73 \\
\hline 2/22/12 2:30 & 32.979 & 35.381 & 1475.79 & 1473.74 \\
\hline 2/22/12 3:00 & 32.955 & 35.369 & 1475.81 & 1473.75 \\
\hline 2/22/12 3:30 & 32.934 & 35.357 & 1475.84 & 1473.77 \\
\hline $2 / 22 / 124: 00$ & 32.913 & 35.35 & 1475.86 & 1473.77 \\
\hline
\end{tabular}


TABLE S1.3 Automatically recorded groundwater levels in the Klassen wells, September 15, 2011, to March 13, 2012.

\begin{tabular}{|c|c|c|c|c|}
\hline \multicolumn{3}{|c|}{ Reference Elevation (ft AMSL) } & 1508.77 & \multirow{2}{*}{$\begin{array}{l}1509.12 \\
\text { Elevation in Klassen } \\
\text { Test Well (ft AMSL) }\end{array}$} \\
\hline Date and Time & $\begin{array}{l}\text { Depth in Klassen } \\
\text { Domestic Well } \\
\text { (ft BGL) }\end{array}$ & $\begin{array}{c}\text { Depth in Klassen } \\
\text { Test Well } \\
\text { (ft BGL) }\end{array}$ & $\begin{array}{l}\text { Elevation in Klassen } \\
\text { Domestic Well } \\
\text { (ft AMSL) }\end{array}$ & \\
\hline $2 / 22 / 124: 30$ & 32.99 & 35.338 & 1475.78 & 1473.78 \\
\hline 2/22/12 5:00 & 32.936 & 35.324 & 1475.83 & 1473.80 \\
\hline 2/22/12 5:30 & 32.908 & 35.317 & 1475.86 & 1473.81 \\
\hline $2 / 22 / 126: 00$ & 32.885 & 35.313 & 1475.88 & 1473.81 \\
\hline $2 / 22 / 126: 30$ & 33.058 & 35.31 & 1475.71 & 1473.81 \\
\hline 2/22/12 7:00 & 33.025 & 35.313 & 1475.74 & 1473.81 \\
\hline 2/22/12 7:30 & 33.065 & 35.313 & 1475.70 & 1473.81 \\
\hline 2/22/12 8:00 & 33 & 35.306 & 1475.77 & 1473.82 \\
\hline $2 / 22 / 128: 30$ & 32.957 & 35.303 & 1475.81 & 1473.82 \\
\hline 2/22/12 9:00 & 33.103 & 35.303 & 1475.67 & 1473.82 \\
\hline 2/22/12 9:30 & 32.981 & 35.301 & 1475.79 & 1473.82 \\
\hline $2 / 22 / 12$ 10:00 & 32.939 & 35.294 & 1475.83 & 1473.83 \\
\hline $2 / 22 / 12$ 10:30 & 32.911 & 35.284 & 1475.86 & 1473.84 \\
\hline $2 / 22 / 1211: 00$ & 33.126 & 35.291 & 1475.64 & 1473.83 \\
\hline 2/22/12 11:30 & 33.966 & 35.291 & 1474.80 & 1473.83 \\
\hline $2 / 22 / 1212: 00$ & 33.244 & 35.296 & 1475.53 & 1473.83 \\
\hline $2 / 22 / 12$ 12:30 & 33.086 & 35.291 & 1475.68 & 1473.83 \\
\hline $2 / 22 / 12$ 13:00 & 33.143 & 35.291 & 1475.63 & 1473.83 \\
\hline $2 / 22 / 12$ 13:30 & 33.04 & 35.287 & 1475.73 & 1473.84 \\
\hline $2 / 22 / 1214: 00$ & 33.124 & 35.28 & 1475.65 & 1473.84 \\
\hline $2 / 22 / 12$ 14:30 & 33.021 & 35.27 & 1475.75 & 1473.85 \\
\hline $2 / 22 / 12$ 15:00 & 32.972 & 35.261 & 1475.80 & 1473.86 \\
\hline $2 / 22 / 12$ 15:30 & 32.934 & 35.252 & 1475.84 & 1473.87 \\
\hline $2 / 22 / 12$ 16:00 & 33.982 & 35.24 & 1474.79 & 1473.88 \\
\hline $2 / 22 / 12$ 16:30 & 32.974 & 35.235 & 1475.80 & 1473.89 \\
\hline $2 / 22 / 12$ 17:00 & 34.798 & 35.226 & 1473.97 & 1473.90 \\
\hline 2/22/12 17:30 & 33.15 & 35.226 & 1475.62 & 1473.90 \\
\hline $2 / 22 / 12$ 18:00 & 33.009 & 35.216 & 1475.76 & 1473.91 \\
\hline $2 / 22 / 12$ 18:30 & 32.948 & 35.212 & 1475.82 & 1473.91 \\
\hline 2/22/12 19:00 & 33.68 & 35.207 & 1475.09 & 1473.92 \\
\hline 2/22/12 19:30 & 33.204 & 35.221 & 1475.57 & 1473.90 \\
\hline $2 / 22 / 12$ 20:00 & 33.248 & 35.244 & 1475.52 & 1473.88 \\
\hline $2 / 22 / 1220: 30$ & 33.169 & 35.256 & 1475.60 & 1473.87 \\
\hline $2 / 22 / 12$ 21:00 & 33.244 & 35.268 & 1475.53 & 1473.85 \\
\hline $2 / 22 / 12$ 21:30 & 33.119 & 35.268 & 1475.65 & 1473.85 \\
\hline $2 / 22 / 1222: 00$ & 33.129 & 35.27 & 1475.64 & 1473.85 \\
\hline 2/22/12 22:30 & 34.463 & 35.28 & 1474.31 & 1473.84 \\
\hline $2 / 22 / 12$ 23:00 & 33.464 & 35.291 & 1475.31 & 1473.83 \\
\hline
\end{tabular}


TABLE S1.3 Automatically recorded groundwater levels in the Klassen wells, September 15, 2011, to March 13, 2012.

\begin{tabular}{|c|c|c|c|c|}
\hline \multicolumn{3}{|c|}{ Reference Elevation (ft AMSL) } & 1508.77 & \multirow{2}{*}{$\begin{array}{l}1509.12 \\
\text { Elevation in Klassen } \\
\text { Test Well (ft AMSL) }\end{array}$} \\
\hline Date and Time & $\begin{array}{l}\text { Depth in Klassen } \\
\text { Domestic Well } \\
(\mathrm{ft} \mathrm{BGL})\end{array}$ & $\begin{array}{c}\text { Depth in Klassen } \\
\text { Test Well } \\
\text { (ft BGL) }\end{array}$ & $\begin{array}{l}\text { Elevation in Klassen } \\
\text { Domestic Well } \\
\text { (ft AMSL) }\end{array}$ & \\
\hline $2 / 22 / 1223: 30$ & 33.248 & 35.291 & 1475.52 & 1473.83 \\
\hline 2/23/12 0:00 & 33.157 & 35.284 & 1475.61 & 1473.84 \\
\hline 2/23/12 0:30 & 33.096 & 35.277 & 1475.67 & 1473.85 \\
\hline 2/23/12 1:00 & 33.049 & 35.268 & 1475.72 & 1473.85 \\
\hline $2 / 23 / 121: 30$ & 33.011 & 35.263 & 1475.76 & 1473.86 \\
\hline 2/23/12 2:00 & 32.976 & 35.259 & 1475.79 & 1473.86 \\
\hline $2 / 23 / 122: 30$ & 32.948 & 35.247 & 1475.82 & 1473.88 \\
\hline $2 / 23 / 123: 00$ & 32.922 & 35.24 & 1475.85 & 1473.88 \\
\hline 2/23/12 3:30 & 33.002 & 35.223 & 1475.77 & 1473.90 \\
\hline 2/23/12 4:00 & 32.927 & 35.223 & 1475.84 & 1473.90 \\
\hline $2 / 23 / 124: 30$ & 32.892 & 35.221 & 1475.88 & 1473.90 \\
\hline $2 / 23 / 125: 00$ & 32.892 & 35.219 & 1475.88 & 1473.90 \\
\hline $2 / 23 / 125: 30$ & 32.864 & 35.214 & 1475.91 & 1473.91 \\
\hline $2 / 23 / 126: 00$ & 32.864 & 35.209 & 1475.91 & 1473.91 \\
\hline 2/23/12 6:30 & 33.082 & 35.212 & 1475.69 & 1473.91 \\
\hline 2/23/12 7:00 & 32.974 & 35.212 & 1475.80 & 1473.91 \\
\hline $2 / 23 / 127: 30$ & 33.047 & 35.209 & 1475.72 & 1473.91 \\
\hline 2/23/12 8:00 & 33.241 & 35.221 & 1475.53 & 1473.90 \\
\hline $2 / 23 / 128: 30$ & 34.149 & 35.226 & 1474.62 & 1473.90 \\
\hline 2/23/12 9:00 & 33.025 & 35.223 & 1475.74 & 1473.90 \\
\hline $2 / 23 / 12$ 9:30 & 32.967 & 35.228 & 1475.80 & 1473.89 \\
\hline $2 / 23 / 12$ 10:00 & 32.929 & 35.23 & 1475.84 & 1473.89 \\
\hline $2 / 23 / 12$ 10:30 & 32.901 & 35.235 & 1475.87 & 1473.89 \\
\hline 2/23/12 11:00 & 32.875 & 35.228 & 1475.89 & 1473.89 \\
\hline 2/23/12 11:30 & 32.861 & 35.235 & 1475.91 & 1473.89 \\
\hline $2 / 23 / 12$ 12:00 & 32.84 & 35.245 & 1475.93 & 1473.88 \\
\hline 2/23/12 12:30 & 32.828 & 35.244 & 1475.94 & 1473.88 \\
\hline 2/23/12 13:00 & 32.812 & 35.251 & 1475.96 & 1473.87 \\
\hline $2 / 23 / 12$ 13:30 & 32.828 & 35.249 & 1475.94 & 1473.87 \\
\hline 2/23/12 14:00 & 32.831 & 35.242 & 1475.94 & 1473.88 \\
\hline 2/23/12 14:30 & 32.807 & 35.235 & 1475.96 & 1473.89 \\
\hline 2/23/12 15:00 & 32.871 & 35.238 & 1475.90 & 1473.88 \\
\hline $2 / 23 / 12$ 15:30 & 32.854 & 35.242 & 1475.92 & 1473.88 \\
\hline 2/23/12 16:00 & 32.857 & 35.233 & 1475.91 & 1473.89 \\
\hline $2 / 23 / 12$ 16:30 & 32.854 & 35.235 & 1475.92 & 1473.89 \\
\hline 2/23/12 17:00 & 32.993 & 35.238 & 1475.78 & 1473.88 \\
\hline 2/23/12 17:30 & 32.875 & 35.238 & 1475.89 & 1473.88 \\
\hline $2 / 23 / 12$ 18:00 & 42.84 & 35.233 & 1465.93 & 1473.89 \\
\hline
\end{tabular}


TABLE S1.3 Automatically recorded groundwater levels in the Klassen wells, September 15, 2011, to March 13, 2012.

\begin{tabular}{|c|c|c|c|c|}
\hline \multicolumn{3}{|c|}{ Reference Elevation (ft AMSL) } & 1508.77 & \multirow{2}{*}{$\begin{array}{l}1509.12 \\
\text { Elevation in Klassen } \\
\text { Test Well (ft AMSL) }\end{array}$} \\
\hline Date and Time & $\begin{array}{l}\text { Depth in Klassen } \\
\text { Domestic Well } \\
(\mathrm{ft} \mathrm{BGL})\end{array}$ & $\begin{array}{c}\text { Depth in Klassen } \\
\text { Test Well } \\
\text { (ft BGL) }\end{array}$ & $\begin{array}{l}\text { Elevation in Klassen } \\
\text { Domestic Well } \\
\text { (ft AMSL) }\end{array}$ & \\
\hline $2 / 23 / 1218: 30$ & 33.253 & 35.254 & 1475.52 & 1473.87 \\
\hline $2 / 23 / 12$ 19:00 & 33.115 & 35.254 & 1475.65 & 1473.87 \\
\hline 2/23/12 19:30 & 36.691 & 35.259 & 1472.08 & 1473.86 \\
\hline $2 / 23 / 1220: 00$ & 33.169 & 35.263 & 1475.60 & 1473.86 \\
\hline $2 / 23 / 1220: 30$ & 33.185 & 35.273 & 1475.58 & 1473.85 \\
\hline $2 / 23 / 1221: 00$ & 33.204 & 35.28 & 1475.57 & 1473.84 \\
\hline $2 / 23 / 1221: 30$ & 33.122 & 35.277 & 1475.65 & 1473.85 \\
\hline $2 / 23 / 1222: 00$ & 33.119 & 35.277 & 1475.65 & 1473.85 \\
\hline $2 / 23 / 1222: 30$ & 33.194 & 35.282 & 1475.58 & 1473.84 \\
\hline $2 / 23 / 1223: 00$ & 33.283 & 35.287 & 1475.49 & 1473.84 \\
\hline 2/23/12 23:30 & 34.151 & 35.301 & 1474.62 & 1473.82 \\
\hline $2 / 24 / 12$ 0:00 & 33.631 & 35.31 & 1475.14 & 1473.81 \\
\hline $2 / 24 / 120: 30$ & 33.459 & 35.317 & 1475.31 & 1473.81 \\
\hline $2 / 24 / 121: 00$ & 33.298 & 35.315 & 1475.47 & 1473.81 \\
\hline $2 / 24 / 121: 30$ & 33.213 & 35.308 & 1475.56 & 1473.81 \\
\hline $2 / 24 / 122: 00$ & 33.241 & 35.305 & 1475.53 & 1473.82 \\
\hline $2 / 24 / 122: 30$ & 33.241 & 35.308 & 1475.53 & 1473.81 \\
\hline 2/24/12 3:00 & 33.194 & 35.308 & 1475.58 & 1473.81 \\
\hline $2 / 24 / 123: 30$ & 33.26 & 35.308 & 1475.51 & 1473.81 \\
\hline $2 / 24 / 124: 00$ & 33.265 & 35.315 & 1475.50 & 1473.81 \\
\hline $2 / 24 / 124: 30$ & 33.18 & 35.317 & 1475.59 & 1473.81 \\
\hline $2 / 24 / 125: 00$ & 33.126 & 35.315 & 1475.64 & 1473.81 \\
\hline 2/24/12 5:30 & 33.082 & 35.308 & 1475.69 & 1473.81 \\
\hline $2 / 24 / 126: 00$ & 33.063 & 35.301 & 1475.71 & 1473.82 \\
\hline $2 / 24 / 126: 30$ & 33.215 & 35.301 & 1475.55 & 1473.82 \\
\hline $2 / 24 / 127: 00$ & 33.162 & 35.296 & 1475.61 & 1473.83 \\
\hline $2 / 24 / 127: 30$ & 33.171 & 35.301 & 1475.60 & 1473.82 \\
\hline $2 / 24 / 128: 00$ & 33.145 & 35.301 & 1475.62 & 1473.82 \\
\hline $2 / 24 / 128: 30$ & 33.185 & 35.303 & 1475.58 & 1473.82 \\
\hline 2/24/12 9:00 & 33.323 & 35.303 & 1475.45 & 1473.82 \\
\hline 2/24/12 9:30 & 33.248 & 35.31 & 1475.52 & 1473.81 \\
\hline 2/24/12 10:00 & 33.363 & 35.32 & 1475.41 & 1473.80 \\
\hline $2 / 24 / 12$ 10:30 & 33.377 & 35.324 & 1475.39 & 1473.80 \\
\hline 2/24/12 11:00 & 33.185 & 35.331 & 1475.58 & 1473.79 \\
\hline 2/24/12 11:30 & 33.222 & 35.334 & 1475.55 & 1473.79 \\
\hline $2 / 24 / 12$ 12:00 & 33.52 & 35.343 & 1475.25 & 1473.78 \\
\hline $2 / 24 / 12$ 12:30 & 33.185 & 35.345 & 1475.58 & 1473.78 \\
\hline $2 / 24 / 12$ 13:00 & 33.117 & 35.348 & 1475.65 & 1473.77 \\
\hline
\end{tabular}


TABLE S1.3 Automatically recorded groundwater levels in the Klassen wells, September 15, 2011, to March 13, 2012.

\begin{tabular}{|c|c|c|c|c|}
\hline \multicolumn{3}{|c|}{ Reference Elevation (ft AMSL) } & 1508.77 & \multirow{2}{*}{$\begin{array}{l}1509.12 \\
\text { Elevation in Klassen } \\
\text { Test Well (ft AMSL) }\end{array}$} \\
\hline Date and Time & $\begin{array}{l}\text { Depth in Klassen } \\
\text { Domestic Well } \\
\text { (ft BGL) }\end{array}$ & $\begin{array}{c}\text { Depth in Klassen } \\
\text { Test Well } \\
\text { (ft BGL) }\end{array}$ & $\begin{array}{l}\text { Elevation in Klassen } \\
\text { Domestic Well } \\
\text { (ft AMSL) }\end{array}$ & \\
\hline $2 / 24 / 12$ 13:30 & 33.295 & 35.331 & 1475.47 & 1473.79 \\
\hline $2 / 24 / 12$ 14:00 & 33.126 & 35.315 & 1475.64 & 1473.81 \\
\hline $2 / 24 / 12$ 14:30 & 33.138 & 35.305 & 1475.63 & 1473.82 \\
\hline $2 / 24 / 12$ 15:00 & 33.239 & 35.308 & 1475.53 & 1473.81 \\
\hline $2 / 24 / 12$ 15:30 & 33.309 & 35.308 & 1475.46 & 1473.81 \\
\hline $2 / 24 / 1216: 00$ & 33.279 & 35.317 & 1475.49 & 1473.81 \\
\hline $2 / 24 / 1216: 30$ & 33.218 & 35.315 & 1475.55 & 1473.81 \\
\hline 2/24/12 17:00 & 33.152 & 35.305 & 1475.62 & 1473.82 \\
\hline 2/24/12 17:30 & 33.105 & 35.291 & 1475.66 & 1473.83 \\
\hline 2/24/12 18:00 & 35.146 & 35.308 & 1473.62 & 1473.81 \\
\hline $2 / 24 / 1218: 30$ & 33.858 & 35.331 & 1474.91 & 1473.79 \\
\hline 2/24/12 19:00 & 33.595 & 35.334 & 1475.17 & 1473.79 \\
\hline 2/24/12 19:30 & 33.551 & 35.334 & 1475.22 & 1473.79 \\
\hline $2 / 24 / 12$ 20:00 & 33.537 & 35.338 & 1475.23 & 1473.78 \\
\hline $2 / 24 / 12$ 20:30 & 33.427 & 35.336 & 1475.34 & 1473.79 \\
\hline 2/24/12 21:00 & 33.342 & 35.334 & 1475.43 & 1473.79 \\
\hline 2/24/12 21:30 & 33.375 & 35.336 & 1475.39 & 1473.79 \\
\hline $2 / 24 / 1222: 00$ & 33.394 & 35.343 & 1475.38 & 1473.78 \\
\hline $2 / 24 / 12$ 22:30 & 33.541 & 35.352 & 1475.23 & 1473.77 \\
\hline 2/24/12 23:00 & 33.708 & 35.359 & 1475.06 & 1473.76 \\
\hline 2/24/12 23:30 & 34.278 & 35.371 & 1474.49 & 1473.75 \\
\hline $2 / 25 / 120: 00$ & 33.572 & 35.378 & 1475.20 & 1473.74 \\
\hline 2/25/12 0:30 & 33.448 & 35.376 & 1475.32 & 1473.75 \\
\hline $2 / 25 / 121: 00$ & 33.37 & 35.374 & 1475.40 & 1473.75 \\
\hline $2 / 25 / 121: 30$ & 33.312 & 35.364 & 1475.46 & 1473.76 \\
\hline $2 / 25 / 122: 00$ & 33.347 & 35.36 & 1475.42 & 1473.76 \\
\hline $2 / 25 / 122: 30$ & 33.335 & 35.362 & 1475.43 & 1473.76 \\
\hline 2/25/12 3:00 & 33.448 & 35.364 & 1475.32 & 1473.76 \\
\hline 2/25/12 3:30 & 33.337 & 35.355 & 1475.43 & 1473.77 \\
\hline $2 / 25 / 124: 00$ & 33.389 & 35.352 & 1475.38 & 1473.77 \\
\hline $2 / 25 / 124: 30$ & 33.307 & 35.343 & 1475.46 & 1473.78 \\
\hline $2 / 25 / 125: 00$ & 33.248 & 35.334 & 1475.52 & 1473.79 \\
\hline $2 / 25 / 125: 30$ & 33.201 & 35.327 & 1475.57 & 1473.80 \\
\hline $2 / 25 / 126: 00$ & 33.185 & 35.32 & 1475.58 & 1473.80 \\
\hline $2 / 25 / 126: 30$ & 33.323 & 35.324 & 1475.45 & 1473.80 \\
\hline $2 / 25 / 127: 00$ & 33.215 & 35.32 & 1475.55 & 1473.80 \\
\hline $2 / 25 / 127: 30$ & 33.162 & 35.315 & 1475.61 & 1473.81 \\
\hline $2 / 25 / 128: 00$ & 33.302 & 35.31 & 1475.47 & 1473.81 \\
\hline
\end{tabular}


TABLE S1.3 Automatically recorded groundwater levels in the Klassen wells, September 15, 2011, to March 13, 2012.

\begin{tabular}{|c|c|c|c|c|}
\hline \multicolumn{3}{|c|}{ Reference Elevation (ft AMSL) } & 1508.77 & \multirow{2}{*}{$\begin{array}{l}1509.12 \\
\text { Elevation in Klassen } \\
\text { Test Well (ft AMSL) }\end{array}$} \\
\hline Date and Time & $\begin{array}{l}\text { Depth in Klassen } \\
\text { Domestic Well } \\
(\mathrm{ft} \mathrm{BGL})\end{array}$ & $\begin{array}{c}\text { Depth in Klassen } \\
\text { Test Well } \\
\text { (ft BGL) }\end{array}$ & $\begin{array}{l}\text { Elevation in Klassen } \\
\text { Domestic Well } \\
\text { (ft AMSL) }\end{array}$ & \\
\hline 2/25/12 8:30 & 33.281 & 35.313 & 1475.49 & 1473.81 \\
\hline 2/25/12 9:00 & 33.326 & 35.32 & 1475.44 & 1473.80 \\
\hline 2/25/12 9:30 & 33.401 & 35.329 & 1475.37 & 1473.79 \\
\hline $2 / 25 / 1210: 00$ & 33.621 & 35.36 & 1475.15 & 1473.76 \\
\hline $2 / 25 / 1210: 30$ & 33.579 & 35.388 & 1475.19 & 1473.73 \\
\hline $2 / 25 / 12$ 11:00 & 33.466 & 35.402 & 1475.30 & 1473.72 \\
\hline $2 / 25 / 1211: 30$ & 33.37 & 35.413 & 1475.40 & 1473.71 \\
\hline $2 / 25 / 12$ 12:00 & 33.429 & 35.423 & 1475.34 & 1473.70 \\
\hline $2 / 25 / 1212: 30$ & 39.977 & 35.435 & 1468.79 & 1473.69 \\
\hline $2 / 25 / 1213: 00$ & 33.541 & 35.383 & 1475.23 & 1473.74 \\
\hline 2/25/12 13:30 & 33.382 & 35.348 & 1475.39 & 1473.77 \\
\hline $2 / 25 / 12$ 14:00 & 33.359 & 35.327 & 1475.41 & 1473.80 \\
\hline $2 / 25 / 1214: 30$ & 33.38 & 35.315 & 1475.39 & 1473.81 \\
\hline $2 / 25 / 12$ 15:00 & 33.368 & 35.303 & 1475.40 & 1473.82 \\
\hline $2 / 25 / 12$ 15:30 & 33.314 & 35.296 & 1475.46 & 1473.83 \\
\hline $2 / 25 / 12$ 16:00 & 34.069 & 35.284 & 1474.70 & 1473.84 \\
\hline $2 / 25 / 12$ 16:30 & 33.276 & 35.277 & 1475.49 & 1473.85 \\
\hline $2 / 25 / 12$ 17:00 & 33.201 & 35.268 & 1475.57 & 1473.85 \\
\hline $2 / 25 / 12$ 17:30 & 38.319 & 35.256 & 1470.45 & 1473.87 \\
\hline $2 / 25 / 12$ 18:00 & 33.734 & 35.261 & 1475.04 & 1473.86 \\
\hline $2 / 25 / 12$ 18:30 & 33.347 & 35.256 & 1475.42 & 1473.87 \\
\hline 2/25/12 19:00 & 33.394 & 35.263 & 1475.38 & 1473.86 \\
\hline $2 / 25 / 12$ 19:30 & 33.645 & 35.27 & 1475.12 & 1473.85 \\
\hline $2 / 25 / 12$ 20:00 & 33.497 & 35.275 & 1475.27 & 1473.85 \\
\hline $2 / 25 / 12$ 20:30 & 33.619 & 35.28 & 1475.15 & 1473.84 \\
\hline $2 / 25 / 12$ 21:00 & 33.429 & 35.284 & 1475.34 & 1473.84 \\
\hline 2/25/12 21:30 & 33.361 & 35.275 & 1475.41 & 1473.85 \\
\hline $2 / 25 / 1222: 00$ & 33.286 & 35.268 & 1475.48 & 1473.85 \\
\hline 2/25/12 22:30 & 33.699 & 35.27 & 1475.07 & 1473.85 \\
\hline 2/25/12 23:00 & 34.463 & 35.27 & 1474.31 & 1473.85 \\
\hline $2 / 25 / 12$ 23:30 & 33.52 & 35.275 & 1475.25 & 1473.85 \\
\hline 2/26/12 0:00 & 33.431 & 35.28 & 1475.34 & 1473.84 \\
\hline 2/26/12 0:30 & 33.33 & 35.273 & 1475.44 & 1473.85 \\
\hline $2 / 26 / 121: 00$ & 33.262 & 35.263 & 1475.51 & 1473.86 \\
\hline 2/26/12 1:30 & 33.215 & 35.254 & 1475.55 & 1473.87 \\
\hline 2/26/12 2:00 & 33.175 & 35.242 & 1475.59 & 1473.88 \\
\hline $2 / 26 / 122: 30$ & 33.143 & 35.245 & 1475.63 & 1473.88 \\
\hline 2/26/12 3:00 & 33.112 & 35.245 & 1475.66 & 1473.88 \\
\hline
\end{tabular}


TABLE S1.3 Automatically recorded groundwater levels in the Klassen wells, September 15, 2011, to March 13, 2012.

\begin{tabular}{|c|c|c|c|c|}
\hline \multicolumn{3}{|c|}{ Reference Elevation (ft AMSL) } & 1508.77 & \multirow{2}{*}{$\begin{array}{l}1509.12 \\
\text { Elevation in Klassen } \\
\text { Test Well (ft AMSL) }\end{array}$} \\
\hline Date and Time & $\begin{array}{l}\text { Depth in Klassen } \\
\text { Domestic Well } \\
(\mathrm{ft} \mathrm{BGL})\end{array}$ & $\begin{array}{c}\text { Depth in Klassen } \\
\text { Test Well } \\
\text { (ft BGL) }\end{array}$ & $\begin{array}{l}\text { Elevation in Klassen } \\
\text { Domestic Well } \\
\text { (ft AMSL) }\end{array}$ & \\
\hline $2 / 26 / 123: 30$ & 33.086 & 35.235 & 1475.68 & 1473.89 \\
\hline $2 / 26 / 124: 00$ & 33.061 & 35.233 & 1475.71 & 1473.89 \\
\hline $2 / 26 / 124: 30$ & 33.037 & 35.228 & 1475.73 & 1473.89 \\
\hline 2/26/12 5:00 & 33.014 & 35.219 & 1475.76 & 1473.90 \\
\hline $2 / 26 / 125: 30$ & 32.993 & 35.212 & 1475.78 & 1473.91 \\
\hline $2 / 26 / 126: 00$ & 36.482 & 35.205 & 1472.29 & 1473.92 \\
\hline $2 / 26 / 126: 30$ & 33.185 & 35.209 & 1475.58 & 1473.91 \\
\hline $2 / 26 / 127: 00$ & 33.349 & 35.205 & 1475.42 & 1473.92 \\
\hline $2 / 26 / 127: 30$ & 33.103 & 35.202 & 1475.67 & 1473.92 \\
\hline $2 / 26 / 128: 00$ & 33.051 & 35.2 & 1475.72 & 1473.92 \\
\hline 2/26/12 8:30 & 33.68 & 35.207 & 1475.09 & 1473.92 \\
\hline 2/26/12 9:00 & 33.192 & 35.214 & 1475.58 & 1473.91 \\
\hline 2/26/12 9:30 & 33.211 & 35.223 & 1475.56 & 1473.90 \\
\hline $2 / 26 / 1210: 00$ & 33.246 & 35.235 & 1475.52 & 1473.89 \\
\hline $2 / 26 / 12$ 10:30 & 33.351 & 35.256 & 1475.42 & 1473.87 \\
\hline $2 / 26 / 12$ 11:00 & 33.382 & 35.282 & 1475.39 & 1473.84 \\
\hline 2/26/12 11:30 & 33.373 & 35.308 & 1475.40 & 1473.81 \\
\hline $2 / 26 / 12$ 12:00 & 33.415 & 35.334 & 1475.35 & 1473.79 \\
\hline $2 / 26 / 12$ 12:30 & 33.462 & 35.352 & 1475.31 & 1473.77 \\
\hline 2/26/12 13:00 & 33.455 & 35.374 & 1475.31 & 1473.75 \\
\hline $2 / 26 / 12$ 13:30 & 33.457 & 35.385 & 1475.31 & 1473.74 \\
\hline $2 / 26 / 12$ 14:00 & 33.469 & 35.395 & 1475.30 & 1473.73 \\
\hline $2 / 26 / 12$ 14:30 & 33.368 & 35.397 & 1475.40 & 1473.73 \\
\hline $2 / 26 / 12$ 15:00 & 33.295 & 35.392 & 1475.47 & 1473.73 \\
\hline $2 / 26 / 12$ 15:30 & 33.412 & 35.397 & 1475.36 & 1473.73 \\
\hline 2/26/12 16:00 & 33.485 & 35.411 & 1475.28 & 1473.71 \\
\hline $2 / 26 / 12$ 16:30 & 33.38 & 35.413 & 1475.39 & 1473.71 \\
\hline $2 / 26 / 12$ 17:00 & 34.533 & 35.416 & 1474.24 & 1473.71 \\
\hline $2 / 26 / 12$ 17:30 & 33.356 & 35.425 & 1475.41 & 1473.70 \\
\hline $2 / 26 / 12$ 18:00 & 33.279 & 35.43 & 1475.49 & 1473.69 \\
\hline $2 / 26 / 12$ 18:30 & 33.251 & 35.435 & 1475.52 & 1473.69 \\
\hline 2/26/12 19:00 & 33.206 & 35.435 & 1475.56 & 1473.69 \\
\hline $2 / 26 / 12$ 19:30 & 36.419 & 35.442 & 1472.35 & 1473.68 \\
\hline $2 / 26 / 1220: 00$ & 33.389 & 35.453 & 1475.38 & 1473.67 \\
\hline $2 / 26 / 1220: 30$ & 33.541 & 35.465 & 1475.23 & 1473.66 \\
\hline $2 / 26 / 12$ 21:00 & 33.52 & 35.479 & 1475.25 & 1473.64 \\
\hline $2 / 26 / 12$ 21:30 & 33.438 & 35.484 & 1475.33 & 1473.64 \\
\hline $2 / 26 / 12$ 22:00 & 33.438 & 35.491 & 1475.33 & 1473.63 \\
\hline
\end{tabular}


TABLE S1.3 Automatically recorded groundwater levels in the Klassen wells, September 15, 2011, to March 13, 2012.

\begin{tabular}{|c|c|c|c|c|}
\hline \multicolumn{3}{|c|}{ Reference Elevation (ft AMSL) } & 1508.77 & \multirow{2}{*}{$\begin{array}{l}1509.12 \\
\text { Elevation in Klassen } \\
\text { Test Well (ft AMSL) }\end{array}$} \\
\hline Date and Time & $\begin{array}{l}\text { Depth in Klassen } \\
\text { Domestic Well } \\
\text { (ft BGL) }\end{array}$ & $\begin{array}{c}\text { Depth in Klassen } \\
\text { Test Well } \\
\text { (ft BGL) }\end{array}$ & $\begin{array}{l}\text { Elevation in Klassen } \\
\text { Domestic Well } \\
\text { (ft AMSL) }\end{array}$ & \\
\hline $2 / 26 / 1222: 30$ & 33.401 & 35.493 & 1475.37 & 1473.63 \\
\hline 2/26/12 23:00 & 33.511 & 35.498 & 1475.26 & 1473.62 \\
\hline $2 / 26 / 12$ 23:30 & 33.598 & 35.505 & 1475.17 & 1473.62 \\
\hline 2/27/12 0:00 & 36.33 & 35.507 & 1472.44 & 1473.62 \\
\hline $2 / 27 / 120: 30$ & 33.638 & 35.512 & 1475.13 & 1473.61 \\
\hline $2 / 27 / 121: 00$ & 33.476 & 35.512 & 1475.29 & 1473.61 \\
\hline $2 / 27 / 121: 30$ & 33.394 & 35.51 & 1475.38 & 1473.61 \\
\hline $2 / 27 / 122: 00$ & 33.405 & 35.507 & 1475.36 & 1473.62 \\
\hline $2 / 27 / 122: 30$ & 33.427 & 35.517 & 1475.34 & 1473.61 \\
\hline $2 / 27 / 123: 00$ & 33.377 & 35.517 & 1475.39 & 1473.61 \\
\hline $2 / 27 / 123: 30$ & 33.342 & 35.514 & 1475.43 & 1473.61 \\
\hline $2 / 27 / 124: 00$ & 33.389 & 35.514 & 1475.38 & 1473.61 \\
\hline $2 / 27 / 124: 30$ & 33.321 & 35.512 & 1475.45 & 1473.61 \\
\hline $2 / 27 / 125: 00$ & 33.37 & 35.514 & 1475.40 & 1473.61 \\
\hline $2 / 27 / 125: 30$ & 33.293 & 35.51 & 1475.48 & 1473.61 \\
\hline $2 / 27 / 126: 00$ & 33.272 & 35.505 & 1475.50 & 1473.62 \\
\hline $2 / 27 / 126: 30$ & 33.431 & 35.505 & 1475.34 & 1473.62 \\
\hline $2 / 27 / 12$ 7:00 & 33.368 & 35.503 & 1475.40 & 1473.62 \\
\hline 2/27/12 7:30 & 33.427 & 35.503 & 1475.34 & 1473.62 \\
\hline 2/27/12 8:00 & 33.513 & 35.5 & 1475.26 & 1473.62 \\
\hline 2/27/12 8:30 & 33.605 & 35.5 & 1475.16 & 1473.62 \\
\hline 2/27/12 9:00 & 33.41 & 35.5 & 1475.36 & 1473.62 \\
\hline $2 / 27 / 129: 30$ & 33.34 & 35.498 & 1475.43 & 1473.62 \\
\hline $2 / 27 / 12$ 10:00 & 38.258 & 35.503 & 1470.51 & 1473.62 \\
\hline 2/27/12 10:30 & 33.499 & 35.51 & 1475.27 & 1473.61 \\
\hline 2/27/12 11:00 & 33.333 & 35.519 & 1475.44 & 1473.60 \\
\hline 2/27/12 11:30 & 33.462 & 35.526 & 1475.31 & 1473.60 \\
\hline 2/27/12 12:00 & 33.445 & 35.535 & 1475.32 & 1473.59 \\
\hline 2/27/12 12:30 & 33.305 & 35.542 & 1475.46 & 1473.58 \\
\hline $2 / 27 / 12$ 13:00 & 34.325 & 35.54 & 1474.44 & 1473.58 \\
\hline $2 / 27 / 12$ 13:30 & 33.307 & 35.528 & 1475.46 & 1473.59 \\
\hline 2/27/12 14:00 & 33.316 & 35.521 & 1475.45 & 1473.60 \\
\hline $2 / 27 / 1214: 30$ & 33.248 & 35.514 & 1475.52 & 1473.61 \\
\hline 2/27/12 15:00 & 33.305 & 35.505 & 1475.46 & 1473.62 \\
\hline $2 / 27 / 12$ 15:30 & 33.227 & 35.498 & 1475.54 & 1473.62 \\
\hline $2 / 27 / 12$ 16:00 & 33.187 & 35.498 & 1475.58 & 1473.62 \\
\hline $2 / 27 / 1216: 30$ & 33.246 & 35.5 & 1475.52 & 1473.62 \\
\hline 2/27/12 17:00 & 33.183 & 35.493 & 1475.59 & 1473.63 \\
\hline
\end{tabular}


TABLE S1.3 Automatically recorded groundwater levels in the Klassen wells, September 15, 2011, to March 13, 2012.

\begin{tabular}{|c|c|c|c|c|}
\hline \multicolumn{3}{|c|}{ Reference Elevation (ft AMSL) } & 1508.77 & \multirow{2}{*}{$\begin{array}{l}1509.12 \\
\text { Elevation in Klassen } \\
\text { Test Well (ft AMSL) }\end{array}$} \\
\hline Date and Time & $\begin{array}{l}\text { Depth in Klassen } \\
\text { Domestic Well } \\
(\mathrm{ft} \mathrm{BGL})\end{array}$ & $\begin{array}{c}\text { Depth in Klassen } \\
\text { Test Well } \\
\text { (ft BGL) }\end{array}$ & $\begin{array}{l}\text { Elevation in Klassen } \\
\text { Domestic Well } \\
\text { (ft AMSL) }\end{array}$ & \\
\hline $2 / 27 / 1217: 30$ & 33.169 & 35.486 & 1475.60 & 1473.64 \\
\hline $2 / 27 / 1218: 00$ & 33.251 & 35.482 & 1475.52 & 1473.64 \\
\hline 2/27/12 18:30 & 33.171 & 35.477 & 1475.60 & 1473.65 \\
\hline $2 / 27 / 12$ 19:00 & 39.053 & 35.477 & 1469.72 & 1473.65 \\
\hline 2/27/12 19:30 & 33.448 & 35.484 & 1475.32 & 1473.64 \\
\hline $2 / 27 / 1220: 00$ & 33.356 & 35.489 & 1475.41 & 1473.63 \\
\hline $2 / 27 / 1220: 30$ & 33.401 & 35.489 & 1475.37 & 1473.63 \\
\hline $2 / 27 / 1221: 00$ & 33.541 & 35.493 & 1475.23 & 1473.63 \\
\hline $2 / 27 / 1221: 30$ & 33.48 & 35.5 & 1475.29 & 1473.62 \\
\hline $2 / 27 / 1222: 00$ & 33.532 & 35.507 & 1475.24 & 1473.62 \\
\hline $2 / 27 / 1222: 30$ & 34.541 & 35.507 & 1474.23 & 1473.62 \\
\hline 2/27/12 23:00 & 36.018 & 35.51 & 1472.75 & 1473.61 \\
\hline $2 / 27 / 1223: 30$ & 33.52 & 35.514 & 1475.25 & 1473.61 \\
\hline 2/28/12 0:00 & 33.415 & 35.51 & 1475.35 & 1473.61 \\
\hline $2 / 28 / 12$ 0:30 & 33.347 & 35.507 & 1475.42 & 1473.62 \\
\hline $2 / 28 / 121: 00$ & 33.295 & 35.5 & 1475.47 & 1473.62 \\
\hline $2 / 28 / 121: 30$ & 33.253 & 35.498 & 1475.52 & 1473.62 \\
\hline 2/28/12 2:00 & 33.22 & 35.493 & 1475.55 & 1473.63 \\
\hline $2 / 28 / 122: 30$ & 33.192 & 35.493 & 1475.58 & 1473.63 \\
\hline $2 / 28 / 12$ 3:00 & 33.262 & 35.493 & 1475.51 & 1473.63 \\
\hline $2 / 28 / 123: 30$ & 33.19 & 35.484 & 1475.58 & 1473.64 \\
\hline $2 / 28 / 124: 00$ & 33.152 & 35.477 & 1475.62 & 1473.65 \\
\hline 2/28/12 4:30 & 33.157 & 35.47 & 1475.61 & 1473.65 \\
\hline $2 / 28 / 125: 00$ & 33.143 & 35.465 & 1475.63 & 1473.66 \\
\hline $2 / 28 / 125: 30$ & 33.114 & 35.458 & 1475.66 & 1473.66 \\
\hline $2 / 28 / 126: 00$ & 33.295 & 35.453 & 1475.47 & 1473.67 \\
\hline $2 / 28 / 126: 30$ & 33.239 & 35.453 & 1475.53 & 1473.67 \\
\hline 2/28/12 7:00 & 33.171 & 35.449 & 1475.60 & 1473.67 \\
\hline 2/28/12 7:30 & 33.347 & 35.449 & 1475.42 & 1473.67 \\
\hline 2/28/12 8:00 & 33.429 & 35.451 & 1475.34 & 1473.67 \\
\hline $2 / 28 / 128: 30$ & 33.232 & 35.456 & 1475.54 & 1473.67 \\
\hline 2/28/12 9:00 & 33.166 & 35.449 & 1475.60 & 1473.67 \\
\hline $2 / 28 / 129: 30$ & 33.131 & 35.437 & 1475.64 & 1473.69 \\
\hline $2 / 28 / 12$ 10:00 & 33.1 & 35.427 & 1475.67 & 1473.70 \\
\hline $2 / 28 / 12$ 10:30 & 33.072 & 35.435 & 1475.70 & 1473.69 \\
\hline 2/28/12 11:00 & 33.147 & 35.442 & 1475.62 & 1473.68 \\
\hline $2 / 28 / 12$ 11:30 & 33.086 & 35.451 & 1475.68 & 1473.67 \\
\hline $2 / 28 / 12$ 12:00 & 33.056 & 35.458 & 1475.71 & 1473.66 \\
\hline
\end{tabular}


TABLE S1.3 Automatically recorded groundwater levels in the Klassen wells, September 15, 2011, to March 13, 2012.

\begin{tabular}{|c|c|c|c|c|}
\hline \multicolumn{3}{|c|}{ Reference Elevation (ft AMSL) } & 1508.77 & \multirow{2}{*}{$\begin{array}{l}1509.12 \\
\text { Elevation in Klassen } \\
\text { Test Well (ft AMSL) }\end{array}$} \\
\hline Date and Time & $\begin{array}{l}\text { Depth in Klassen } \\
\text { Domestic Well } \\
\text { (ft BGL) }\end{array}$ & $\begin{array}{c}\text { Depth in Klassen } \\
\text { Test Well } \\
\text { (ft BGL) }\end{array}$ & $\begin{array}{l}\text { Elevation in Klassen } \\
\text { Domestic Well } \\
\text { (ft AMSL) }\end{array}$ & \\
\hline $2 / 28 / 1212: 30$ & 37.022 & 35.472 & 1471.75 & 1473.65 \\
\hline $2 / 28 / 1213: 00$ & 33.368 & 35.486 & 1475.40 & 1473.64 \\
\hline $2 / 28 / 12$ 13:30 & 33.208 & 35.491 & 1475.56 & 1473.63 \\
\hline $2 / 28 / 1214: 00$ & 33.14 & 35.503 & 1475.63 & 1473.62 \\
\hline $2 / 28 / 1214: 30$ & 33.171 & 35.505 & 1475.60 & 1473.62 \\
\hline $2 / 28 / 12$ 15:00 & 33.236 & 35.47 & 1475.53 & 1473.65 \\
\hline $2 / 28 / 1215: 30$ & 33.126 & 35.425 & 1475.64 & 1473.70 \\
\hline $2 / 28 / 12$ 16:00 & 38.38 & 35.404 & 1470.39 & 1473.72 \\
\hline $2 / 28 / 1216: 30$ & 33.22 & 35.395 & 1475.55 & 1473.73 \\
\hline $2 / 28 / 1217: 00$ & 33.138 & 35.392 & 1475.63 & 1473.73 \\
\hline $2 / 28 / 1217: 30$ & 33.204 & 35.39 & 1475.57 & 1473.73 \\
\hline $2 / 28 / 12$ 18:00 & 33.337 & 35.392 & 1475.43 & 1473.73 \\
\hline $2 / 28 / 1218: 30$ & 33.375 & 35.397 & 1475.39 & 1473.73 \\
\hline $2 / 28 / 1219: 00$ & 33.236 & 35.39 & 1475.53 & 1473.73 \\
\hline 2/28/12 19:30 & 33.356 & 35.362 & 1475.41 & 1473.76 \\
\hline $2 / 28 / 1220: 00$ & 33.192 & 35.35 & 1475.58 & 1473.77 \\
\hline $2 / 28 / 1220: 30$ & 33.326 & 35.338 & 1475.44 & 1473.78 \\
\hline 2/28/12 21:00 & 33.281 & 35.322 & 1475.49 & 1473.80 \\
\hline $2 / 28 / 12$ 21:30 & 33.199 & 35.322 & 1475.57 & 1473.80 \\
\hline $2 / 28 / 12$ 22:00 & 33.166 & 35.32 & 1475.60 & 1473.80 \\
\hline $2 / 28 / 1222: 30$ & 34.88 & 35.322 & 1473.89 & 1473.80 \\
\hline 2/28/12 23:00 & 33.884 & 35.341 & 1474.89 & 1473.78 \\
\hline $2 / 28 / 12$ 23:30 & 33.293 & 35.352 & 1475.48 & 1473.77 \\
\hline 2/29/12 0:00 & 33.201 & 35.352 & 1475.57 & 1473.77 \\
\hline 2/29/12 0:30 & 33.143 & 35.352 & 1475.63 & 1473.77 \\
\hline $2 / 29 / 12$ 1:00 & 33.1 & 35.35 & 1475.67 & 1473.77 \\
\hline 2/29/12 1:30 & 37.205 & 35.348 & 1471.56 & 1473.77 \\
\hline 2/29/12 2:00 & 33.107 & 35.35 & 1475.66 & 1473.77 \\
\hline 2/29/12 2:30 & 33.061 & 35.348 & 1475.71 & 1473.77 \\
\hline 2/29/12 3:00 & 33.028 & 35.343 & 1475.74 & 1473.78 \\
\hline 2/29/12 3:30 & 33.002 & 35.338 & 1475.77 & 1473.78 \\
\hline 2/29/12 4:00 & 32.981 & 35.334 & 1475.79 & 1473.79 \\
\hline 2/29/12 4:30 & 32.964 & 35.329 & 1475.81 & 1473.79 \\
\hline 2/29/12 5:00 & 32.946 & 35.324 & 1475.82 & 1473.80 \\
\hline 2/29/12 5:30 & 33.044 & 35.322 & 1475.73 & 1473.80 \\
\hline 2/29/12 6:00 & 32.981 & 35.322 & 1475.79 & 1473.80 \\
\hline $2 / 29 / 126: 30$ & 33.192 & 35.327 & 1475.58 & 1473.80 \\
\hline 2/29/12 7:00 & 33.091 & 35.327 & 1475.68 & 1473.80 \\
\hline
\end{tabular}


TABLE S1.3 Automatically recorded groundwater levels in the Klassen wells, September 15, 2011, to March 13, 2012.

\begin{tabular}{|c|c|c|c|c|}
\hline \multicolumn{3}{|c|}{ Reference Elevation (ft AMSL) } & 1508.77 & \multirow{2}{*}{$\begin{array}{l}1509.12 \\
\text { Elevation in Klassen } \\
\text { Test Well (ft AMSL) }\end{array}$} \\
\hline Date and Time & $\begin{array}{l}\text { Depth in Klassen } \\
\text { Domestic Well } \\
(\mathrm{ft} \mathrm{BGL})\end{array}$ & $\begin{array}{c}\text { Depth in Klassen } \\
\text { Test Well } \\
\text { (ft BGL) }\end{array}$ & $\begin{array}{l}\text { Elevation in Klassen } \\
\text { Domestic Well } \\
\text { (ft AMSL) }\end{array}$ & \\
\hline $2 / 29 / 127: 30$ & 33.133 & 35.334 & 1475.64 & 1473.79 \\
\hline $2 / 29 / 128: 00$ & 33.18 & 35.338 & 1475.59 & 1473.78 \\
\hline $2 / 29 / 128: 30$ & 33.126 & 35.338 & 1475.64 & 1473.78 \\
\hline 2/29/12 9:00 & 36.107 & 35.338 & 1472.66 & 1473.78 \\
\hline 2/29/12 9:30 & 33.15 & 35.341 & 1475.62 & 1473.78 \\
\hline $2 / 29 / 12$ 10:00 & 33.131 & 35.343 & 1475.64 & 1473.78 \\
\hline $2 / 29 / 1210: 30$ & 33.213 & 35.343 & 1475.56 & 1473.78 \\
\hline 2/29/12 11:00 & 33.164 & 35.345 & 1475.61 & 1473.78 \\
\hline 2/29/12 11:30 & 33.323 & 35.348 & 1475.45 & 1473.77 \\
\hline $2 / 29 / 1212: 00$ & 33.19 & 35.355 & 1475.58 & 1473.77 \\
\hline $2 / 29 / 1212: 30$ & 33.15 & 35.357 & 1475.62 & 1473.77 \\
\hline 2/29/12 13:00 & 33.281 & 35.355 & 1475.49 & 1473.77 \\
\hline 2/29/12 13:30 & 33.171 & 35.357 & 1475.60 & 1473.77 \\
\hline $2 / 29 / 1214: 00$ & 33.131 & 35.35 & 1475.64 & 1473.77 \\
\hline 2/29/12 14:30 & 33.227 & 35.352 & 1475.54 & 1473.77 \\
\hline $2 / 29 / 12$ 15:00 & 33.222 & 35.355 & 1475.55 & 1473.77 \\
\hline $2 / 29 / 12$ 15:30 & 33.187 & 35.352 & 1475.58 & 1473.77 \\
\hline 2/29/12 16:00 & 33.147 & 35.35 & 1475.62 & 1473.77 \\
\hline 2/29/12 16:30 & 33.361 & 35.35 & 1475.41 & 1473.77 \\
\hline $2 / 29 / 12$ 17:00 & 33.138 & 35.357 & 1475.63 & 1473.77 \\
\hline $2 / 29 / 12$ 17:30 & 35.317 & 35.369 & 1473.45 & 1473.75 \\
\hline 2/29/12 18:00 & 33.394 & 35.378 & 1475.38 & 1473.74 \\
\hline $2 / 29 / 12$ 18:30 & 33.258 & 35.376 & 1475.51 & 1473.75 \\
\hline 2/29/12 19:00 & 33.183 & 35.374 & 1475.59 & 1473.75 \\
\hline 2/29/12 19:30 & 33.541 & 35.378 & 1475.23 & 1473.74 \\
\hline 2/29/12 20:00 & 33.623 & 35.383 & 1475.15 & 1473.74 \\
\hline 2/29/12 20:30 & 33.487 & 35.39 & 1475.28 & 1473.73 \\
\hline 2/29/12 21:00 & 33.288 & 35.399 & 1475.48 & 1473.72 \\
\hline 2/29/12 21:30 & 33.628 & 35.402 & 1475.14 & 1473.72 \\
\hline 2/29/12 22:00 & 33.358 & 35.409 & 1475.41 & 1473.71 \\
\hline 2/29/12 22:30 & 33.274 & 35.406 & 1475.50 & 1473.72 \\
\hline 2/29/12 23:00 & 33.199 & 35.402 & 1475.57 & 1473.72 \\
\hline $2 / 29 / 12$ 23:30 & 33.171 & 35.402 & 1475.60 & 1473.72 \\
\hline $3 / 1 / 120: 00$ & 33.143 & 35.402 & 1475.63 & 1473.72 \\
\hline $3 / 1 / 12$ 0:30 & 33.098 & 35.395 & 1475.67 & 1473.73 \\
\hline $3 / 1 / 12$ 1:00 & 33.063 & 35.385 & 1475.71 & 1473.74 \\
\hline $3 / 1 / 12$ 1:30 & 33.032 & 35.381 & 1475.74 & 1473.74 \\
\hline $3 / 1 / 122: 00$ & 33.089 & 35.371 & 1475.68 & 1473.75 \\
\hline
\end{tabular}


TABLE S1.3 Automatically recorded groundwater levels in the Klassen wells, September 15, 2011, to March 13, 2012.

\begin{tabular}{|c|c|c|c|c|}
\hline \multicolumn{3}{|c|}{ Reference Elevation (ft AMSL) } & 1508.77 & \multirow{2}{*}{$\begin{array}{l}1509.12 \\
\text { Elevation in Klassen } \\
\text { Test Well (ft AMSL) }\end{array}$} \\
\hline Date and Time & $\begin{array}{l}\text { Depth in Klassen } \\
\text { Domestic Well } \\
(\mathrm{ft} \mathrm{BGL})\end{array}$ & $\begin{array}{c}\text { Depth in Klassen } \\
\text { Test Well } \\
\text { (ft BGL) }\end{array}$ & $\begin{array}{l}\text { Elevation in Klassen } \\
\text { Domestic Well } \\
\text { (ft AMSL) }\end{array}$ & \\
\hline $3 / 1 / 122: 30$ & 33.103 & 35.369 & 1475.67 & 1473.75 \\
\hline $3 / 1 / 123: 00$ & 33.082 & 35.366 & 1475.69 & 1473.76 \\
\hline $3 / 1 / 123: 30$ & 33.067 & 35.36 & 1475.70 & 1473.76 \\
\hline $3 / 1 / 124: 00$ & 33.347 & 35.359 & 1475.42 & 1473.76 \\
\hline $3 / 1 / 124: 30$ & 33.14 & 35.352 & 1475.63 & 1473.77 \\
\hline $3 / 1 / 125: 00$ & 33.075 & 35.348 & 1475.69 & 1473.77 \\
\hline $3 / 1 / 125: 30$ & 33.119 & 35.341 & 1475.65 & 1473.78 \\
\hline $3 / 1 / 126: 00$ & 33.091 & 35.336 & 1475.68 & 1473.79 \\
\hline $3 / 1 / 126: 30$ & 33.098 & 35.341 & 1475.67 & 1473.78 \\
\hline $3 / 1 / 127: 00$ & 33.126 & 35.338 & 1475.64 & 1473.78 \\
\hline $3 / 1 / 127: 30$ & 33.103 & 35.336 & 1475.67 & 1473.79 \\
\hline $3 / 1 / 128: 00$ & 33.075 & 35.343 & 1475.69 & 1473.78 \\
\hline $3 / 1 / 128: 30$ & 33.051 & 35.341 & 1475.72 & 1473.78 \\
\hline $3 / 1 / 129: 00$ & 33.004 & 35.341 & 1475.77 & 1473.78 \\
\hline $3 / 1 / 12$ 9:30 & 32.971 & 35.343 & 1475.80 & 1473.78 \\
\hline $3 / 1 / 12$ 10:00 & 38.18 & 35.343 & 1470.59 & 1473.78 \\
\hline $3 / 1 / 12$ 10:30 & 33.009 & 35.341 & 1475.76 & 1473.78 \\
\hline 3/1/12 11:00 & 32.96 & 35.338 & 1475.81 & 1473.78 \\
\hline $3 / 1 / 12$ 11:30 & 32.927 & 35.317 & 1475.84 & 1473.81 \\
\hline $3 / 1 / 12$ 12:00 & 32.999 & 35.291 & 1475.77 & 1473.83 \\
\hline $3 / 1 / 12$ 12:30 & 33.065 & 35.289 & 1475.70 & 1473.83 \\
\hline $3 / 1 / 12$ 13:00 & 32.983 & 35.284 & 1475.79 & 1473.84 \\
\hline $3 / 1 / 12$ 13:30 & 33.053 & 35.28 & 1475.72 & 1473.84 \\
\hline $3 / 1 / 12$ 14:00 & 32.969 & 35.275 & 1475.80 & 1473.85 \\
\hline $3 / 1 / 12$ 14:30 & 34.503 & 35.263 & 1474.27 & 1473.86 \\
\hline $3 / 1 / 12$ 15:00 & 32.988 & 35.261 & 1475.78 & 1473.86 \\
\hline $3 / 1 / 12$ 15:30 & 32.936 & 35.259 & 1475.83 & 1473.86 \\
\hline $3 / 1 / 12$ 16:00 & 37.854 & 35.263 & 1470.92 & 1473.86 \\
\hline $3 / 1 / 12$ 16:30 & 33.002 & 35.284 & 1475.77 & 1473.84 \\
\hline $3 / 1 / 12$ 17:00 & 32.938 & 35.31 & 1475.83 & 1473.81 \\
\hline $3 / 1 / 12$ 17:30 & 32.908 & 35.336 & 1475.86 & 1473.79 \\
\hline 3/1/12 18:00 & 32.99 & 35.371 & 1475.78 & 1473.75 \\
\hline $3 / 1 / 12$ 18:30 & 32.955 & 35.406 & 1475.81 & 1473.72 \\
\hline 3/1/12 19:00 & 32.971 & 35.451 & 1475.80 & 1473.67 \\
\hline 3/1/12 19:30 & 33.058 & 35.493 & 1475.71 & 1473.63 \\
\hline $3 / 1 / 12$ 20:00 & 32.995 & 35.533 & 1475.77 & 1473.59 \\
\hline $3 / 1 / 12$ 20:30 & 33.126 & 35.575 & 1475.64 & 1473.55 \\
\hline $3 / 1 / 12$ 21:00 & 33.023 & 35.622 & 1475.75 & 1473.50 \\
\hline
\end{tabular}


TABLE S1.3 Automatically recorded groundwater levels in the Klassen wells, September 15, 2011, to March 13, 2012.

\begin{tabular}{|c|c|c|c|c|}
\hline \multicolumn{3}{|c|}{ Reference Elevation (ft AMSL) } & 1508.77 & \multirow{2}{*}{$\begin{array}{l}1509.12 \\
\text { Elevation in Klassen } \\
\text { Test Well (ft AMSL) }\end{array}$} \\
\hline Date and Time & $\begin{array}{l}\text { Depth in Klassen } \\
\text { Domestic Well } \\
(\mathrm{ft} \mathrm{BGL})\end{array}$ & $\begin{array}{c}\text { Depth in Klassen } \\
\text { Test Well } \\
\text { (ft BGL) }\end{array}$ & $\begin{array}{l}\text { Elevation in Klassen } \\
\text { Domestic Well } \\
\text { (ft AMSL) }\end{array}$ & \\
\hline $3 / 1 / 1221: 30$ & 32.971 & 35.657 & 1475.80 & 1473.47 \\
\hline $3 / 1 / 1222: 00$ & 32.938 & 35.69 & 1475.83 & 1473.43 \\
\hline $3 / 1 / 1222: 30$ & 32.913 & 35.737 & 1475.86 & 1473.39 \\
\hline 3/1/12 23:00 & 34.153 & 35.777 & 1474.62 & 1473.35 \\
\hline $3 / 1 / 12$ 23:30 & 33.34 & 35.819 & 1475.43 & 1473.30 \\
\hline $3 / 2 / 120: 00$ & 33.185 & 35.869 & 1475.58 & 1473.25 \\
\hline $3 / 2 / 120: 30$ & 33.171 & 35.916 & 1475.60 & 1473.21 \\
\hline $3 / 2 / 121: 00$ & 33.084 & 35.953 & 1475.69 & 1473.17 \\
\hline $3 / 2 / 121: 30$ & 33.032 & 35.981 & 1475.74 & 1473.14 \\
\hline $3 / 2 / 122: 00$ & 32.988 & 36.016 & 1475.78 & 1473.11 \\
\hline $3 / 2 / 122: 30$ & 32.962 & 36.054 & 1475.81 & 1473.07 \\
\hline $3 / 2 / 123: 00$ & 33.049 & 36.089 & 1475.72 & 1473.03 \\
\hline $3 / 2 / 123: 30$ & 32.967 & 36.117 & 1475.80 & 1473.01 \\
\hline $3 / 2 / 124: 00$ & 32.936 & 36.155 & 1475.83 & 1472.97 \\
\hline $3 / 2 / 124: 30$ & 32.91 & 36.195 & 1475.86 & 1472.93 \\
\hline $3 / 2 / 125: 00$ & 32.889 & 36.228 & 1475.88 & 1472.89 \\
\hline $3 / 2 / 125: 30$ & 32.873 & 36.26 & 1475.90 & 1472.86 \\
\hline $3 / 2 / 126: 00$ & 32.901 & 36.286 & 1475.87 & 1472.84 \\
\hline $3 / 2 / 126: 30$ & 33.152 & 36.331 & 1475.62 & 1472.79 \\
\hline $3 / 2 / 127: 00$ & 33.037 & 36.366 & 1475.73 & 1472.76 \\
\hline $3 / 2 / 127: 30$ & 32.983 & 36.404 & 1475.79 & 1472.72 \\
\hline $3 / 2 / 128: 00$ & 33.089 & 36.439 & 1475.68 & 1472.68 \\
\hline $3 / 2 / 128: 30$ & 33.274 & 36.483 & 1475.50 & 1472.64 \\
\hline $3 / 2 / 129: 00$ & 50.014 & 36.521 & 1458.76 & 1472.60 \\
\hline $3 / 2 / 12$ 9:30 & 39.43 & 36.575 & 1469.34 & 1472.55 \\
\hline $3 / 2 / 12$ 10:00 & 33.72 & 36.612 & 1475.05 & 1472.51 \\
\hline $3 / 2 / 12$ 10:30 & 33.553 & 36.634 & 1475.22 & 1472.49 \\
\hline $3 / 2 / 12$ 11:00 & 36.452 & 36.652 & 1472.32 & 1472.47 \\
\hline $3 / 2 / 12$ 11:30 & 33.546 & 36.666 & 1475.22 & 1472.46 \\
\hline $3 / 2 / 12$ 12:00 & 33.405 & 36.662 & 1475.36 & 1472.46 \\
\hline $3 / 2 / 12$ 12:30 & 33.314 & 36.643 & 1475.46 & 1472.48 \\
\hline $3 / 2 / 12$ 13:00 & 33.382 & 36.631 & 1475.39 & 1472.49 \\
\hline $3 / 2 / 12$ 13:30 & 33.745 & 36.61 & 1475.02 & 1472.51 \\
\hline $3 / 2 / 12$ 14:00 & 33.433 & 36.591 & 1475.34 & 1472.53 \\
\hline $3 / 2 / 12$ 14:30 & 33.443 & 36.58 & 1475.33 & 1472.54 \\
\hline $3 / 2 / 1215: 00$ & 33.38 & 36.573 & 1475.39 & 1472.55 \\
\hline $3 / 2 / 1215: 30$ & 33.572 & 36.558 & 1475.20 & 1472.56 \\
\hline $3 / 2 / 12$ 16:00 & 33.372 & 36.547 & 1475.40 & 1472.58 \\
\hline
\end{tabular}


TABLE S1.3 Automatically recorded groundwater levels in the Klassen wells, September 15, 2011, to March 13, 2012.

\begin{tabular}{|c|c|c|c|c|}
\hline \multicolumn{3}{|c|}{ Reference Elevation (ft AMSL) } & 1508.77 & \multirow{2}{*}{$\begin{array}{l}1509.12 \\
\text { Elevation in Klassen } \\
\text { Test Well (ft AMSL) }\end{array}$} \\
\hline Date and Time & $\begin{array}{l}\text { Depth in Klassen } \\
\text { Domestic Well } \\
(\mathrm{ft} \mathrm{BGL})\end{array}$ & $\begin{array}{c}\text { Depth in Klassen } \\
\text { Test Well } \\
\text { (ft BGL) }\end{array}$ & $\begin{array}{l}\text { Elevation in Klassen } \\
\text { Domestic Well } \\
\text { (ft AMSL) }\end{array}$ & \\
\hline $3 / 2 / 1216: 30$ & 33.309 & 36.53 & 1475.46 & 1472.59 \\
\hline $3 / 2 / 1217: 00$ & 33.255 & 36.509 & 1475.51 & 1472.61 \\
\hline $3 / 2 / 1217: 30$ & 33.293 & 36.49 & 1475.48 & 1472.63 \\
\hline $3 / 2 / 12$ 18:00 & 33.222 & 36.469 & 1475.55 & 1472.65 \\
\hline $3 / 2 / 1218: 30$ & 33.255 & 36.455 & 1475.51 & 1472.67 \\
\hline $3 / 2 / 1219: 00$ & 35.575 & 36.434 & 1473.19 & 1472.69 \\
\hline 3/2/12 19:30 & 33.269 & 36.422 & 1475.50 & 1472.70 \\
\hline $3 / 2 / 1220: 00$ & 33.227 & 36.404 & 1475.54 & 1472.72 \\
\hline $3 / 2 / 12$ 20:30 & 33.173 & 36.387 & 1475.60 & 1472.74 \\
\hline $3 / 2 / 1221: 00$ & 33.136 & 36.368 & 1475.63 & 1472.75 \\
\hline $3 / 2 / 1221: 30$ & 36.585 & 36.35 & 1472.18 & 1472.77 \\
\hline $3 / 2 / 1222: 00$ & 33.196 & 36.34 & 1475.57 & 1472.78 \\
\hline $3 / 2 / 1222: 30$ & 33.135 & 36.321 & 1475.63 & 1472.80 \\
\hline $3 / 2 / 1223: 00$ & 33.394 & 36.312 & 1475.38 & 1472.81 \\
\hline $3 / 2 / 12$ 23:30 & 33.408 & 36.305 & 1475.36 & 1472.82 \\
\hline $3 / 3 / 120: 00$ & 33.265 & 36.293 & 1475.50 & 1472.83 \\
\hline $3 / 3 / 120: 30$ & 33.194 & 36.274 & 1475.58 & 1472.85 \\
\hline $3 / 3 / 121: 00$ & 33.15 & 36.253 & 1475.62 & 1472.87 \\
\hline $3 / 3 / 12$ 1:30 & 33.112 & 36.237 & 1475.66 & 1472.89 \\
\hline $3 / 3 / 122: 00$ & 33.168 & 36.221 & 1475.60 & 1472.90 \\
\hline $3 / 3 / 122: 30$ & 33.19 & 36.209 & 1475.58 & 1472.91 \\
\hline $3 / 3 / 123: 00$ & 33.159 & 36.197 & 1475.61 & 1472.93 \\
\hline $3 / 3 / 12$ 3:30 & 33.211 & 36.185 & 1475.56 & 1472.94 \\
\hline $3 / 3 / 124: 00$ & 33.239 & 36.176 & 1475.53 & 1472.95 \\
\hline $3 / 3 / 124: 30$ & 33.166 & 36.167 & 1475.60 & 1472.96 \\
\hline $3 / 3 / 12$ 5:00 & 33.119 & 36.15 & 1475.65 & 1472.97 \\
\hline $3 / 3 / 12$ 5:30 & 33.086 & 36.131 & 1475.68 & 1472.99 \\
\hline $3 / 3 / 126: 00$ & 34.796 & 36.122 & 1473.97 & 1473.00 \\
\hline $3 / 3 / 126: 30$ & 33.722 & 36.108 & 1475.05 & 1473.01 \\
\hline $3 / 3 / 12$ 7:00 & 33.114 & 36.094 & 1475.66 & 1473.03 \\
\hline $3 / 3 / 12$ 7:30 & 33.178 & 36.085 & 1475.59 & 1473.04 \\
\hline $3 / 3 / 128: 00$ & 33.117 & 36.08 & 1475.65 & 1473.04 \\
\hline $3 / 3 / 128: 30$ & 33.068 & 36.073 & 1475.70 & 1473.05 \\
\hline $3 / 3 / 12$ 9:00 & 33.701 & 36.063 & 1475.07 & 1473.06 \\
\hline $3 / 3 / 12$ 9:30 & 33.178 & 36.068 & 1475.59 & 1473.05 \\
\hline $3 / 3 / 12$ 10:00 & 33.255 & 36.075 & 1475.51 & 1473.05 \\
\hline $3 / 3 / 1210: 30$ & 33.138 & 36.077 & 1475.63 & 1473.05 \\
\hline $3 / 3 / 1211: 00$ & 51.269 & 36.089 & 1457.50 & 1473.03 \\
\hline
\end{tabular}


TABLE S1.3 Automatically recorded groundwater levels in the Klassen wells, September 15, 2011, to March 13, 2012.

\begin{tabular}{|c|c|c|c|c|}
\hline \multicolumn{3}{|c|}{ Reference Elevation (ft AMSL) } & 1508.77 & \multirow{2}{*}{$\begin{array}{l}1509.12 \\
\text { Elevation in Klassen } \\
\text { Test Well (ft AMSL) }\end{array}$} \\
\hline Date and Time & $\begin{array}{l}\text { Depth in Klassen } \\
\text { Domestic Well } \\
(\mathrm{ft} \mathrm{BGL})\end{array}$ & $\begin{array}{c}\text { Depth in Klassen } \\
\text { Test Well } \\
\text { (ft BGL) }\end{array}$ & $\begin{array}{l}\text { Elevation in Klassen } \\
\text { Domestic Well } \\
\text { (ft AMSL) }\end{array}$ & \\
\hline $3 / 3 / 1211: 30$ & 34.104 & 36.11 & 1474.67 & 1473.01 \\
\hline $3 / 3 / 1212: 00$ & 33.722 & 36.12 & 1475.05 & 1473.00 \\
\hline $3 / 3 / 1212: 30$ & 33.544 & 36.12 & 1475.23 & 1473.00 \\
\hline 3/3/12 13:00 & 33.438 & 36.077 & 1475.33 & 1473.05 \\
\hline $3 / 3 / 1213: 30$ & 33.558 & 36.056 & 1475.21 & 1473.07 \\
\hline $3 / 3 / 1214: 00$ & 33.37 & 36.042 & 1475.40 & 1473.08 \\
\hline $3 / 3 / 1214: 30$ & 33.422 & 36.033 & 1475.35 & 1473.09 \\
\hline $3 / 3 / 1215: 00$ & 33.323 & 36.021 & 1475.45 & 1473.10 \\
\hline $3 / 3 / 12$ 15:30 & 33.422 & 36.016 & 1475.35 & 1473.11 \\
\hline $3 / 3 / 12$ 16:00 & 33.342 & 36.012 & 1475.43 & 1473.11 \\
\hline $3 / 3 / 12$ 16:30 & 33.288 & 36.007 & 1475.48 & 1473.12 \\
\hline $3 / 3 / 1217: 00$ & 33.403 & 35.998 & 1475.37 & 1473.12 \\
\hline $3 / 3 / 1217: 30$ & 33.682 & 36.014 & 1475.09 & 1473.11 \\
\hline $3 / 3 / 1218: 00$ & 33.701 & 36.026 & 1475.07 & 1473.10 \\
\hline $3 / 3 / 1218: 30$ & 33.71 & 36.033 & 1475.06 & 1473.09 \\
\hline $3 / 3 / 12$ 19:00 & 33.621 & 36.033 & 1475.15 & 1473.09 \\
\hline $3 / 3 / 12$ 19:30 & 33.516 & 36.033 & 1475.25 & 1473.09 \\
\hline $3 / 3 / 12$ 20:00 & 33.461 & 36.03 & 1475.31 & 1473.09 \\
\hline $3 / 3 / 12$ 20:30 & 33.396 & 36.019 & 1475.37 & 1473.10 \\
\hline $3 / 3 / 12$ 21:00 & 33.342 & 36.012 & 1475.43 & 1473.11 \\
\hline $3 / 3 / 12$ 21:30 & 33.412 & 36.002 & 1475.36 & 1473.12 \\
\hline $3 / 3 / 12$ 22:00 & 33.347 & 35.993 & 1475.42 & 1473.13 \\
\hline $3 / 3 / 12$ 22:30 & 33.762 & 35.993 & 1475.01 & 1473.13 \\
\hline $3 / 3 / 12$ 23:00 & 39.449 & 36.002 & 1469.32 & 1473.12 \\
\hline $3 / 3 / 12$ 23:30 & 33.954 & 36.014 & 1474.82 & 1473.11 \\
\hline $3 / 4 / 120: 00$ & 33.776 & 36.009 & 1474.99 & 1473.11 \\
\hline $3 / 4 / 12$ 0:30 & 33.63 & 36 & 1475.14 & 1473.12 \\
\hline $3 / 4 / 12$ 1:00 & 33.532 & 35.988 & 1475.24 & 1473.13 \\
\hline $3 / 4 / 12$ 1:30 & 33.457 & 35.977 & 1475.31 & 1473.15 \\
\hline $3 / 4 / 122: 00$ & 33.398 & 35.965 & 1475.37 & 1473.16 \\
\hline $3 / 4 / 122: 30$ & 33.347 & 35.962 & 1475.42 & 1473.16 \\
\hline $3 / 4 / 123: 00$ & 33.302 & 35.948 & 1475.47 & 1473.17 \\
\hline $3 / 4 / 12$ 3:30 & 33.265 & 35.941 & 1475.50 & 1473.18 \\
\hline $3 / 4 / 12$ 4:00 & 33.323 & 35.941 & 1475.45 & 1473.18 \\
\hline $3 / 4 / 12$ 4:30 & 33.25 & 35.934 & 1475.52 & 1473.19 \\
\hline $3 / 4 / 12$ 5:00 & 33.208 & 35.92 & 1475.56 & 1473.20 \\
\hline $3 / 4 / 12$ 5:30 & 33.175 & 35.911 & 1475.59 & 1473.21 \\
\hline $3 / 4 / 12$ 6:00 & 33.452 & 35.899 & 1475.32 & 1473.22 \\
\hline
\end{tabular}


TABLE S1.3 Automatically recorded groundwater levels in the Klassen wells, September 15, 2011, to March 13, 2012.

\begin{tabular}{|c|c|c|c|c|}
\hline \multicolumn{3}{|c|}{ Reference Elevation (ft AMSL) } & 1508.77 & \multirow{2}{*}{$\begin{array}{l}1509.12 \\
\text { Elevation in Klassen } \\
\text { Test Well (ft AMSL) }\end{array}$} \\
\hline Date and Time & $\begin{array}{l}\text { Depth in Klassen } \\
\text { Domestic Well } \\
\text { (ft BGL) }\end{array}$ & $\begin{array}{c}\text { Depth in Klassen } \\
\text { Test Well } \\
\text { (ft BGL) }\end{array}$ & $\begin{array}{l}\text { Elevation in Klassen } \\
\text { Domestic Well } \\
\text { (ft AMSL) }\end{array}$ & \\
\hline $3 / 4 / 126: 30$ & 33.323 & 35.892 & 1475.45 & 1473.23 \\
\hline $3 / 4 / 127: 00$ & 33.232 & 35.88 & 1475.54 & 1473.24 \\
\hline $3 / 4 / 12$ 7:30 & 33.183 & 35.871 & 1475.59 & 1473.25 \\
\hline $3 / 4 / 128: 00$ & 33.293 & 35.862 & 1475.48 & 1473.26 \\
\hline $3 / 4 / 128: 30$ & 33.319 & 35.857 & 1475.45 & 1473.27 \\
\hline $3 / 4 / 129: 00$ & 33.368 & 35.859 & 1475.40 & 1473.26 \\
\hline $3 / 4 / 129: 30$ & 33.34 & 35.864 & 1475.43 & 1473.26 \\
\hline $3 / 4 / 12$ 10:00 & 33.288 & 35.869 & 1475.48 & 1473.25 \\
\hline $3 / 4 / 12$ 10:30 & 33.222 & 35.876 & 1475.55 & 1473.25 \\
\hline 3/4/12 11:00 & 33.178 & 35.885 & 1475.59 & 1473.24 \\
\hline 3/4/12 11:30 & 33.145 & 35.902 & 1475.62 & 1473.22 \\
\hline $3 / 4 / 12$ 12:00 & 33.243 & 35.923 & 1475.53 & 1473.20 \\
\hline $3 / 4 / 12$ 12:30 & 33.304 & 35.941 & 1475.47 & 1473.18 \\
\hline 3/4/12 13:00 & 33.302 & 35.958 & 1475.47 & 1473.16 \\
\hline $3 / 4 / 12$ 13:30 & 33.257 & 35.962 & 1475.51 & 1473.16 \\
\hline $3 / 4 / 12$ 14:00 & 33.199 & 35.96 & 1475.57 & 1473.16 \\
\hline $3 / 4 / 12$ 14:30 & 33.201 & 35.96 & 1475.57 & 1473.16 \\
\hline $3 / 4 / 12$ 15:00 & 33.49 & 35.97 & 1475.28 & 1473.15 \\
\hline $3 / 4 / 12$ 15:30 & 33.492 & 35.986 & 1475.28 & 1473.14 \\
\hline $3 / 4 / 12$ 16:00 & 33.483 & 35.991 & 1475.29 & 1473.13 \\
\hline $3 / 4 / 12$ 16:30 & 33.49 & 35.993 & 1475.28 & 1473.13 \\
\hline $3 / 4 / 12$ 17:00 & 33.358 & 35.986 & 1475.41 & 1473.14 \\
\hline $3 / 4 / 12$ 17:30 & 33.29 & 35.977 & 1475.48 & 1473.15 \\
\hline 3/4/12 18:00 & 33.241 & 35.967 & 1475.53 & 1473.16 \\
\hline $3 / 4 / 12$ 18:30 & 33.227 & 35.967 & 1475.54 & 1473.16 \\
\hline 3/4/12 19:00 & 33.236 & 35.974 & 1475.53 & 1473.15 \\
\hline 3/4/12 19:30 & 33.354 & 35.986 & 1475.42 & 1473.14 \\
\hline $3 / 4 / 12$ 20:00 & 33.293 & 35.995 & 1475.48 & 1473.13 \\
\hline $3 / 4 / 12$ 20:30 & 33.26 & 36.002 & 1475.51 & 1473.12 \\
\hline $3 / 4 / 12$ 21:00 & 33.241 & 36.005 & 1475.53 & 1473.12 \\
\hline $3 / 4 / 12$ 21:30 & 33.211 & 36.007 & 1475.56 & 1473.12 \\
\hline $3 / 4 / 12$ 22:00 & 33.171 & 36.007 & 1475.60 & 1473.12 \\
\hline $3 / 4 / 12$ 22:30 & 37.924 & 36 & 1470.85 & 1473.12 \\
\hline 3/4/12 23:00 & 33.269 & 35.998 & 1475.50 & 1473.12 \\
\hline 3/4/12 23:30 & 33.396 & 35.995 & 1475.37 & 1473.13 \\
\hline $3 / 5 / 12$ 0:00 & 33.253 & 35.991 & 1475.52 & 1473.13 \\
\hline $3 / 5 / 12$ 0:30 & 33.192 & 35.972 & 1475.58 & 1473.15 \\
\hline $3 / 5 / 12$ 1:00 & 33.15 & 35.963 & 1475.62 & 1473.16 \\
\hline
\end{tabular}


TABLE S1.3 Automatically recorded groundwater levels in the Klassen wells, September 15, 2011, to March 13, 2012.

\begin{tabular}{|c|c|c|c|c|}
\hline \multicolumn{3}{|c|}{ Reference Elevation (ft AMSL) } & 1508.77 & \multirow{2}{*}{$\begin{array}{l}1509.12 \\
\text { Elevation in Klassen } \\
\text { Test Well (ft AMSL) }\end{array}$} \\
\hline Date and Time & $\begin{array}{l}\text { Depth in Klassen } \\
\text { Domestic Well } \\
(\mathrm{ft} \mathrm{BGL})\end{array}$ & $\begin{array}{c}\text { Depth in Klassen } \\
\text { Test Well } \\
\text { (ft BGL) }\end{array}$ & $\begin{array}{l}\text { Elevation in Klassen } \\
\text { Domestic Well } \\
\text { (ft AMSL) }\end{array}$ & \\
\hline $3 / 5 / 121: 30$ & 33.117 & 35.951 & 1475.65 & 1473.17 \\
\hline $3 / 5 / 122: 00$ & 33.166 & 35.944 & 1475.60 & 1473.18 \\
\hline $3 / 5 / 122: 30$ & 33.213 & 35.941 & 1475.56 & 1473.18 \\
\hline $3 / 5 / 123: 00$ & 33.18 & 35.93 & 1475.59 & 1473.19 \\
\hline $3 / 5 / 123: 30$ & 33.175 & 35.92 & 1475.59 & 1473.20 \\
\hline $3 / 5 / 124: 00$ & 33.246 & 35.913 & 1475.52 & 1473.21 \\
\hline $3 / 5 / 124: 30$ & 33.175 & 35.901 & 1475.59 & 1473.22 \\
\hline $3 / 5 / 125: 00$ & 33.128 & 35.887 & 1475.64 & 1473.24 \\
\hline $3 / 5 / 125: 30$ & 33.096 & 35.876 & 1475.67 & 1473.25 \\
\hline $3 / 5 / 126: 00$ & 33.067 & 35.859 & 1475.70 & 1473.26 \\
\hline $3 / 5 / 126: 30$ & 33.293 & 35.859 & 1475.48 & 1473.26 \\
\hline $3 / 5 / 127: 00$ & 33.199 & 35.857 & 1475.57 & 1473.27 \\
\hline $3 / 5 / 127: 30$ & 33.239 & 35.852 & 1475.53 & 1473.27 \\
\hline $3 / 5 / 128: 00$ & 33.168 & 35.852 & 1475.60 & 1473.27 \\
\hline $3 / 5 / 128: 30$ & 33.241 & 35.85 & 1475.53 & 1473.27 \\
\hline $3 / 5 / 12$ 9:00 & 33.809 & 35.848 & 1474.96 & 1473.27 \\
\hline $3 / 5 / 12$ 9:30 & 33.452 & 35.85 & 1475.32 & 1473.27 \\
\hline 3/5/12 10:00 & 33.206 & 35.845 & 1475.56 & 1473.28 \\
\hline $3 / 5 / 12$ 10:30 & 33.279 & 35.838 & 1475.49 & 1473.28 \\
\hline $3 / 5 / 1211: 00$ & 35.547 & 35.819 & 1473.22 & 1473.30 \\
\hline $3 / 5 / 1211: 30$ & 33.272 & 35.805 & 1475.50 & 1473.32 \\
\hline $3 / 5 / 12$ 12:00 & 33.173 & 35.793 & 1475.60 & 1473.33 \\
\hline $3 / 5 / 12$ 12:30 & 33.119 & 35.777 & 1475.65 & 1473.35 \\
\hline 3/5/12 13:00 & 33.283 & 35.77 & 1475.49 & 1473.35 \\
\hline $3 / 5 / 12$ 13:30 & 33.124 & 35.758 & 1475.65 & 1473.36 \\
\hline $3 / 5 / 12$ 14:00 & 33.075 & 35.742 & 1475.69 & 1473.38 \\
\hline $3 / 5 / 12$ 14:30 & 33.135 & 35.73 & 1475.63 & 1473.39 \\
\hline 3/5/12 15:00 & 33.07 & 35.716 & 1475.70 & 1473.41 \\
\hline $3 / 5 / 12$ 15:30 & 33.054 & 35.702 & 1475.72 & 1473.42 \\
\hline 3/5/12 16:00 & 33.016 & 35.688 & 1475.75 & 1473.43 \\
\hline 3/5/12 16:30 & 33.1 & 35.679 & 1475.67 & 1473.44 \\
\hline $3 / 5 / 12$ 17:00 & 33.335 & 35.679 & 1475.43 & 1473.44 \\
\hline $3 / 5 / 12$ 17:30 & 33.539 & 35.681 & 1475.23 & 1473.44 \\
\hline 3/5/12 18:00 & 33.565 & 35.693 & 1475.20 & 1473.43 \\
\hline 3/5/12 18:30 & 33.372 & 35.69 & 1475.40 & 1473.43 \\
\hline $3 / 5 / 12$ 19:00 & 33.523 & 35.688 & 1475.25 & 1473.43 \\
\hline $3 / 5 / 12$ 19:30 & 33.44 & 35.688 & 1475.33 & 1473.43 \\
\hline $3 / 5 / 12$ 20:00 & 33.37 & 35.688 & 1475.40 & 1473.43 \\
\hline
\end{tabular}


TABLE S1.3 Automatically recorded groundwater levels in the Klassen wells, September 15, 2011, to March 13, 2012.

\begin{tabular}{|c|c|c|c|c|}
\hline \multicolumn{3}{|c|}{ Reference Elevation (ft AMSL) } & 1508.77 & \multirow{2}{*}{$\begin{array}{l}1509.12 \\
\text { Elevation in Klassen } \\
\text { Test Well (ft AMSL) }\end{array}$} \\
\hline Date and Time & $\begin{array}{l}\text { Depth in Klassen } \\
\text { Domestic Well } \\
(\mathrm{ft} \mathrm{BGL})\end{array}$ & $\begin{array}{c}\text { Depth in Klassen } \\
\text { Test Well } \\
\text { (ft BGL) }\end{array}$ & $\begin{array}{l}\text { Elevation in Klassen } \\
\text { Domestic Well } \\
\text { (ft AMSL) }\end{array}$ & \\
\hline $3 / 5 / 1220: 30$ & 33.281 & 35.681 & 1475.49 & 1473.44 \\
\hline $3 / 5 / 1221: 00$ & 33.461 & 35.681 & 1475.31 & 1473.44 \\
\hline $3 / 5 / 1221: 30$ & 33.45 & 35.688 & 1475.32 & 1473.43 \\
\hline $3 / 5 / 1222: 00$ & 33.487 & 35.69 & 1475.28 & 1473.43 \\
\hline $3 / 5 / 1222: 30$ & 33.973 & 35.693 & 1474.80 & 1473.43 \\
\hline $3 / 5 / 1223: 00$ & 37.183 & 35.693 & 1471.59 & 1473.43 \\
\hline 3/5/12 23:30 & 33.593 & 35.693 & 1475.18 & 1473.43 \\
\hline $3 / 6 / 120: 00$ & 33.448 & 35.683 & 1475.32 & 1473.44 \\
\hline $3 / 6 / 120: 30$ & 33.363 & 35.672 & 1475.41 & 1473.45 \\
\hline $3 / 6 / 121: 00$ & 33.29 & 35.657 & 1475.48 & 1473.47 \\
\hline $3 / 6 / 121: 30$ & 33.239 & 35.648 & 1475.53 & 1473.47 \\
\hline $3 / 6 / 122: 00$ & 33.192 & 35.634 & 1475.58 & 1473.49 \\
\hline $3 / 6 / 122: 30$ & 33.15 & 35.62 & 1475.62 & 1473.50 \\
\hline $3 / 6 / 123: 00$ & 33.117 & 35.61 & 1475.65 & 1473.51 \\
\hline $3 / 6 / 12$ 3:30 & 33.086 & 35.599 & 1475.68 & 1473.52 \\
\hline $3 / 6 / 12$ 4:00 & 33.056 & 35.587 & 1475.71 & 1473.54 \\
\hline $3 / 6 / 124: 30$ & 33.03 & 35.575 & 1475.74 & 1473.55 \\
\hline 3/6/12 5:00 & 33.1 & 35.568 & 1475.67 & 1473.55 \\
\hline $3 / 6 / 12$ 5:30 & 33.042 & 35.559 & 1475.73 & 1473.56 \\
\hline $3 / 6 / 126: 00$ & 33.011 & 35.55 & 1475.76 & 1473.57 \\
\hline $3 / 6 / 126: 30$ & 33.192 & 35.55 & 1475.58 & 1473.57 \\
\hline $3 / 6 / 12$ 7:00 & 33.107 & 35.547 & 1475.66 & 1473.58 \\
\hline $3 / 6 / 12$ 7:30 & 33.112 & 35.545 & 1475.66 & 1473.58 \\
\hline $3 / 6 / 128: 00$ & 33.182 & 35.54 & 1475.59 & 1473.58 \\
\hline $3 / 6 / 128: 30$ & 33.086 & 35.538 & 1475.68 & 1473.58 \\
\hline $3 / 6 / 12$ 9:00 & 33.523 & 35.535 & 1475.25 & 1473.59 \\
\hline $3 / 6 / 12$ 9:30 & 33.26 & 35.543 & 1475.51 & 1473.58 \\
\hline $3 / 6 / 12$ 10:00 & 33.255 & 35.545 & 1475.51 & 1473.58 \\
\hline $3 / 6 / 12$ 10:30 & 33.246 & 35.54 & 1475.52 & 1473.58 \\
\hline 3/6/12 11:00 & 33.154 & 35.533 & 1475.62 & 1473.59 \\
\hline $3 / 6 / 12$ 11:30 & 33.126 & 35.528 & 1475.64 & 1473.59 \\
\hline $3 / 6 / 12$ 12:00 & 33.206 & 35.521 & 1475.56 & 1473.60 \\
\hline $3 / 6 / 12$ 12:30 & 33.103 & 35.521 & 1475.67 & 1473.60 \\
\hline 3/6/12 13:00 & 33.051 & 35.514 & 1475.72 & 1473.61 \\
\hline 3/6/12 13:30 & 33.154 & 35.505 & 1475.62 & 1473.62 \\
\hline $3 / 6 / 12$ 14:00 & 33.053 & 35.507 & 1475.72 & 1473.62 \\
\hline $3 / 6 / 12$ 14:30 & 33.112 & 35.5 & 1475.66 & 1473.62 \\
\hline $3 / 6 / 12$ 15:00 & 33.028 & 35.493 & 1475.74 & 1473.63 \\
\hline
\end{tabular}


TABLE S1.3 Automatically recorded groundwater levels in the Klassen wells, September 15, 2011, to March 13, 2012.

\begin{tabular}{|c|c|c|c|c|}
\hline \multicolumn{3}{|c|}{ Reference Elevation (ft AMSL) } & 1508.77 & \multirow{2}{*}{$\begin{array}{l}1509.12 \\
\text { Elevation in Klassen } \\
\text { Test Well (ft AMSL) }\end{array}$} \\
\hline Date and Time & $\begin{array}{l}\text { Depth in Klassen } \\
\text { Domestic Well } \\
(\mathrm{ft} \mathrm{BGL})\end{array}$ & $\begin{array}{c}\text { Depth in Klassen } \\
\text { Test Well } \\
\text { (ft BGL) }\end{array}$ & $\begin{array}{l}\text { Elevation in Klassen } \\
\text { Domestic Well } \\
\text { (ft AMSL) }\end{array}$ & \\
\hline $3 / 6 / 1215: 30$ & 33.342 & 35.493 & 1475.43 & 1473.63 \\
\hline $3 / 6 / 1216: 00$ & 33.265 & 35.491 & 1475.50 & 1473.63 \\
\hline $3 / 6 / 1216: 30$ & 33.171 & 35.486 & 1475.60 & 1473.64 \\
\hline 3/6/12 17:00 & 33.201 & 35.486 & 1475.57 & 1473.64 \\
\hline $3 / 6 / 1217: 30$ & 33.236 & 35.493 & 1475.53 & 1473.63 \\
\hline $3 / 6 / 1218: 00$ & 33.253 & 35.5 & 1475.52 & 1473.62 \\
\hline $3 / 6 / 1218: 30$ & 33.272 & 35.503 & 1475.50 & 1473.62 \\
\hline $3 / 6 / 1219: 00$ & 33.394 & 35.51 & 1475.38 & 1473.61 \\
\hline 3/6/12 19:30 & 33.351 & 35.519 & 1475.42 & 1473.60 \\
\hline $3 / 6 / 1220: 00$ & 33.276 & 35.517 & 1475.49 & 1473.61 \\
\hline $3 / 6 / 1220: 30$ & 33.325 & 35.514 & 1475.44 & 1473.61 \\
\hline $3 / 6 / 1221: 00$ & 33.558 & 35.533 & 1475.21 & 1473.59 \\
\hline $3 / 6 / 1221: 30$ & 33.53 & 35.545 & 1475.24 & 1473.58 \\
\hline $3 / 6 / 1222: 00$ & 33.4 & 35.542 & 1475.37 & 1473.58 \\
\hline $3 / 6 / 1222: 30$ & 33.994 & 35.543 & 1474.78 & 1473.58 \\
\hline $3 / 6 / 12$ 23:00 & 33.642 & 35.547 & 1475.13 & 1473.58 \\
\hline $3 / 6 / 12$ 23:30 & 33.454 & 35.545 & 1475.32 & 1473.58 \\
\hline $3 / 7 / 12$ 0:00 & 33.347 & 35.543 & 1475.42 & 1473.58 \\
\hline $3 / 7 / 12$ 0:30 & 33.274 & 35.535 & 1475.50 & 1473.59 \\
\hline $3 / 7 / 12$ 1:00 & 33.22 & 35.526 & 1475.55 & 1473.60 \\
\hline $3 / 7 / 12$ 1:30 & 33.171 & 35.517 & 1475.60 & 1473.61 \\
\hline $3 / 7 / 12$ 2:00 & 33.211 & 35.512 & 1475.56 & 1473.61 \\
\hline $3 / 7 / 122: 30$ & 33.232 & 35.507 & 1475.54 & 1473.62 \\
\hline $3 / 7 / 123: 00$ & 33.194 & 35.5 & 1475.58 & 1473.62 \\
\hline $3 / 7 / 12$ 3:30 & 33.171 & 35.486 & 1475.60 & 1473.64 \\
\hline $3 / 7 / 12$ 4:00 & 33.208 & 35.481 & 1475.56 & 1473.64 \\
\hline $3 / 7 / 12$ 4:30 & 33.286 & 35.474 & 1475.48 & 1473.65 \\
\hline $3 / 7 / 12$ 5:00 & 33.152 & 35.465 & 1475.62 & 1473.66 \\
\hline $3 / 7 / 12$ 5:30 & 33.114 & 35.46 & 1475.66 & 1473.66 \\
\hline $3 / 7 / 126: 00$ & 33.075 & 35.451 & 1475.69 & 1473.67 \\
\hline $3 / 7 / 12$ 6:30 & 33.204 & 35.449 & 1475.57 & 1473.67 \\
\hline $3 / 7 / 12$ 7:00 & 33.107 & 35.442 & 1475.66 & 1473.68 \\
\hline $3 / 7 / 12$ 7:30 & 33.107 & 35.439 & 1475.66 & 1473.68 \\
\hline $3 / 7 / 128: 00$ & 36.923 & 35.435 & 1471.85 & 1473.69 \\
\hline $3 / 7 / 128: 30$ & 33.609 & 35.435 & 1475.16 & 1473.69 \\
\hline $3 / 7 / 12$ 9:00 & 33.607 & 35.439 & 1475.16 & 1473.68 \\
\hline 3/7/12 9:30 & 33.248 & 35.444 & 1475.52 & 1473.68 \\
\hline $3 / 7 / 12$ 10:00 & 33.154 & 35.451 & 1475.62 & 1473.67 \\
\hline
\end{tabular}


TABLE S1.3 Automatically recorded groundwater levels in the Klassen wells, September 15, 2011, to March 13, 2012.

\begin{tabular}{|c|c|c|c|c|}
\hline \multicolumn{3}{|c|}{ Reference Elevation (ft AMSL) } & 1508.77 & \multirow{2}{*}{$\begin{array}{l}1509.12 \\
\text { Elevation in Klassen } \\
\text { Test Well (ft AMSL) }\end{array}$} \\
\hline Date and Time & $\begin{array}{l}\text { Depth in Klassen } \\
\text { Domestic Well } \\
(\mathrm{ft} \mathrm{BGL})\end{array}$ & $\begin{array}{c}\text { Depth in Klassen } \\
\text { Test Well } \\
\text { (ft BGL) }\end{array}$ & $\begin{array}{l}\text { Elevation in Klassen } \\
\text { Domestic Well } \\
\text { (ft AMSL) }\end{array}$ & \\
\hline $3 / 7 / 1210: 30$ & 33.408 & 35.451 & 1475.36 & 1473.67 \\
\hline $3 / 7 / 1211: 00$ & 33.135 & 35.451 & 1475.63 & 1473.67 \\
\hline $3 / 7 / 1211: 30$ & 33.077 & 35.444 & 1475.69 & 1473.68 \\
\hline 3/7/12 12:00 & 33.673 & 35.439 & 1475.10 & 1473.68 \\
\hline $3 / 7 / 1212: 30$ & 33.093 & 35.435 & 1475.68 & 1473.69 \\
\hline $3 / 7 / 1213: 00$ & 33.037 & 35.423 & 1475.73 & 1473.70 \\
\hline $3 / 7 / 1213: 30$ & 32.995 & 35.416 & 1475.77 & 1473.71 \\
\hline $3 / 7 / 1214: 00$ & 33.075 & 35.413 & 1475.69 & 1473.71 \\
\hline $3 / 7 / 12$ 14:30 & 33.004 & 35.406 & 1475.77 & 1473.72 \\
\hline 3/7/12 15:00 & 33.056 & 35.395 & 1475.71 & 1473.73 \\
\hline 3/7/12 15:30 & 32.993 & 35.385 & 1475.78 & 1473.74 \\
\hline $3 / 7 / 1216: 00$ & 32.955 & 35.385 & 1475.81 & 1473.74 \\
\hline $3 / 7 / 1216: 30$ & 32.927 & 35.383 & 1475.84 & 1473.74 \\
\hline $3 / 7 / 1217: 00$ & 33.079 & 35.374 & 1475.69 & 1473.75 \\
\hline $3 / 7 / 12$ 17:30 & 32.999 & 35.371 & 1475.77 & 1473.75 \\
\hline $3 / 7 / 12$ 18:00 & 33.218 & 35.376 & 1475.55 & 1473.75 \\
\hline $3 / 7 / 12$ 18:30 & 33.227 & 35.383 & 1475.54 & 1473.74 \\
\hline $3 / 7 / 12$ 19:00 & 33.15 & 35.388 & 1475.62 & 1473.73 \\
\hline $3 / 7 / 12$ 19:30 & 33.171 & 35.399 & 1475.60 & 1473.72 \\
\hline $3 / 7 / 12$ 20:00 & 33.152 & 35.411 & 1475.62 & 1473.71 \\
\hline $3 / 7 / 12$ 20:30 & 33.105 & 35.423 & 1475.66 & 1473.70 \\
\hline $3 / 7 / 12$ 21:00 & 33.079 & 35.437 & 1475.69 & 1473.69 \\
\hline $3 / 7 / 12$ 21:30 & 33.199 & 35.449 & 1475.57 & 1473.67 \\
\hline $3 / 7 / 12$ 22:00 & 33.114 & 35.46 & 1475.66 & 1473.66 \\
\hline $3 / 7 / 12$ 22:30 & 33.1 & 35.467 & 1475.67 & 1473.66 \\
\hline $3 / 7 / 12$ 23:00 & 34.24 & 35.482 & 1474.53 & 1473.64 \\
\hline 3/7/12 23:30 & 33.417 & 35.5 & 1475.35 & 1473.62 \\
\hline $3 / 8 / 120: 00$ & 33.255 & 35.503 & 1475.51 & 1473.62 \\
\hline $3 / 8 / 120: 30$ & 33.178 & 35.491 & 1475.59 & 1473.63 \\
\hline $3 / 8 / 121: 00$ & 33.114 & 35.472 & 1475.66 & 1473.65 \\
\hline $3 / 8 / 12$ 1:30 & 33.075 & 35.444 & 1475.69 & 1473.68 \\
\hline $3 / 8 / 122: 00$ & 33.035 & 35.444 & 1475.73 & 1473.68 \\
\hline $3 / 8 / 122: 30$ & 33.002 & 35.43 & 1475.77 & 1473.69 \\
\hline $3 / 8 / 123: 00$ & 32.976 & 35.425 & 1475.79 & 1473.70 \\
\hline $3 / 8 / 123: 30$ & 32.955 & 35.397 & 1475.81 & 1473.73 \\
\hline $3 / 8 / 124: 00$ & 33.154 & 35.404 & 1475.62 & 1473.72 \\
\hline $3 / 8 / 12$ 4:30 & 32.983 & 35.402 & 1475.79 & 1473.72 \\
\hline $3 / 8 / 12$ 5:00 & 32.971 & 35.385 & 1475.80 & 1473.74 \\
\hline
\end{tabular}


TABLE S1.3 Automatically recorded groundwater levels in the Klassen wells, September 15, 2011, to March 13, 2012.

\begin{tabular}{|c|c|c|c|c|}
\hline \multicolumn{3}{|c|}{ Reference Elevation (ft AMSL) } & 1508.77 & \multirow{2}{*}{$\begin{array}{l}1509.12 \\
\text { Elevation in Klassen } \\
\text { Test Well (ft AMSL) }\end{array}$} \\
\hline Date and Time & $\begin{array}{l}\text { Depth in Klassen } \\
\text { Domestic Well } \\
(\mathrm{ft} \mathrm{BGL})\end{array}$ & $\begin{array}{c}\text { Depth in Klassen } \\
\text { Test Well } \\
\text { (ft BGL) }\end{array}$ & $\begin{array}{l}\text { Elevation in Klassen } \\
\text { Domestic Well } \\
\text { (ft AMSL) }\end{array}$ & \\
\hline $3 / 8 / 125: 30$ & 32.936 & 35.383 & 1475.83 & 1473.74 \\
\hline $3 / 8 / 126: 00$ & 32.938 & 35.367 & 1475.83 & 1473.76 \\
\hline $3 / 8 / 126: 30$ & 33.124 & 35.371 & 1475.65 & 1473.75 \\
\hline $3 / 8 / 127: 00$ & 33.053 & 35.385 & 1475.72 & 1473.74 \\
\hline $3 / 8 / 127: 30$ & 33.089 & 35.381 & 1475.68 & 1473.74 \\
\hline $3 / 8 / 128: 00$ & 33.258 & 35.374 & 1475.51 & 1473.75 \\
\hline $3 / 8 / 128: 30$ & 33.225 & 35.369 & 1475.54 & 1473.75 \\
\hline $3 / 8 / 129: 00$ & 33.077 & 35.383 & 1475.69 & 1473.74 \\
\hline $3 / 8 / 129: 30$ & 33.021 & 35.381 & 1475.75 & 1473.74 \\
\hline 3/8/12 10:00 & 32.99 & 35.378 & 1475.78 & 1473.74 \\
\hline $3 / 8 / 12$ 10:30 & 32.96 & 35.388 & 1475.81 & 1473.73 \\
\hline $3 / 8 / 1211: 00$ & 32.939 & 35.388 & 1475.83 & 1473.73 \\
\hline 3/8/12 11:30 & 32.915 & 35.411 & 1475.85 & 1473.71 \\
\hline $3 / 8 / 1212: 00$ & 32.899 & 35.413 & 1475.87 & 1473.71 \\
\hline $3 / 8 / 12$ 12:30 & 32.882 & 35.413 & 1475.89 & 1473.71 \\
\hline 3/8/12 13:00 & 32.866 & 35.418 & 1475.90 & 1473.70 \\
\hline 3/8/12 13:30 & 32.852 & 35.428 & 1475.92 & 1473.69 \\
\hline 3/8/12 14:00 & 33.138 & 35.435 & 1475.63 & 1473.69 \\
\hline $3 / 8 / 1214: 30$ & 33.281 & 35.435 & 1475.49 & 1473.69 \\
\hline $3 / 8 / 12$ 15:00 & 32.936 & 35.43 & 1475.83 & 1473.69 \\
\hline $3 / 8 / 12$ 15:30 & 32.946 & 35.421 & 1475.82 & 1473.70 \\
\hline $3 / 8 / 12$ 16:00 & 32.901 & 35.418 & 1475.87 & 1473.70 \\
\hline $3 / 8 / 12$ 16:30 & 32.87 & 35.413 & 1475.90 & 1473.71 \\
\hline 3/8/12 17:00 & 48.225 & 35.428 & 1460.54 & 1473.69 \\
\hline $3 / 8 / 12$ 17:30 & 34.444 & 35.47 & 1474.33 & 1473.65 \\
\hline 3/8/12 18:00 & 33.851 & 35.484 & 1474.92 & 1473.64 \\
\hline 3/8/12 18:30 & 33.759 & 35.5 & 1475.01 & 1473.62 \\
\hline 3/8/12 19:00 & 33.572 & 35.505 & 1475.20 & 1473.62 \\
\hline 3/8/12 19:30 & 33.448 & 35.51 & 1475.32 & 1473.61 \\
\hline $3 / 8 / 12$ 20:00 & 33.38 & 35.51 & 1475.39 & 1473.61 \\
\hline $3 / 8 / 12$ 20:30 & 33.351 & 35.505 & 1475.42 & 1473.62 \\
\hline $3 / 8 / 12$ 21:00 & 33.293 & 35.5 & 1475.48 & 1473.62 \\
\hline $3 / 8 / 12$ 21:30 & 33.466 & 35.503 & 1475.30 & 1473.62 \\
\hline $3 / 8 / 12$ 22:00 & 33.375 & 35.5 & 1475.39 & 1473.62 \\
\hline $3 / 8 / 12$ 22:30 & 33.297 & 35.496 & 1475.47 & 1473.63 \\
\hline $3 / 8 / 12$ 23:00 & 33.614 & 35.488 & 1475.16 & 1473.63 \\
\hline 3/8/12 23:30 & 33.623 & 35.493 & 1475.15 & 1473.63 \\
\hline $3 / 9 / 12$ 0:00 & 33.375 & 35.498 & 1475.39 & 1473.62 \\
\hline
\end{tabular}


TABLE S1.3 Automatically recorded groundwater levels in the Klassen wells, September 15, 2011, to March 13, 2012.

\begin{tabular}{|c|c|c|c|c|}
\hline \multicolumn{3}{|c|}{ Reference Elevation (ft AMSL) } & 1508.77 & \multirow{2}{*}{$\begin{array}{l}1509.12 \\
\text { Elevation in Klassen } \\
\text { Test Well (ft AMSL) }\end{array}$} \\
\hline Date and Time & $\begin{array}{l}\text { Depth in Klassen } \\
\text { Domestic Well } \\
(\mathrm{ft} \mathrm{BGL})\end{array}$ & $\begin{array}{c}\text { Depth in Klassen } \\
\text { Test Well } \\
\text { (ft BGL) }\end{array}$ & $\begin{array}{l}\text { Elevation in Klassen } \\
\text { Domestic Well } \\
\text { (ft AMSL) }\end{array}$ & \\
\hline $3 / 9 / 120: 30$ & 33.316 & 35.5 & 1475.45 & 1473.62 \\
\hline $3 / 9 / 121: 00$ & 33.251 & 35.5 & 1475.52 & 1473.62 \\
\hline $3 / 9 / 121: 30$ & 33.197 & 35.498 & 1475.57 & 1473.62 \\
\hline $3 / 9 / 122: 00$ & 33.157 & 35.498 & 1475.61 & 1473.62 \\
\hline $3 / 9 / 122: 30$ & 33.121 & 35.5 & 1475.65 & 1473.62 \\
\hline $3 / 9 / 123: 00$ & 33.091 & 35.503 & 1475.68 & 1473.62 \\
\hline $3 / 9 / 123: 30$ & 33.061 & 35.5 & 1475.71 & 1473.62 \\
\hline $3 / 9 / 124: 00$ & 33.389 & 35.498 & 1475.38 & 1473.62 \\
\hline $3 / 9 / 124: 30$ & 33.086 & 35.493 & 1475.68 & 1473.63 \\
\hline $3 / 9 / 125: 00$ & 33.042 & 35.484 & 1475.73 & 1473.64 \\
\hline $3 / 9 / 125: 30$ & 33.009 & 35.479 & 1475.76 & 1473.64 \\
\hline 3/9/12 6:00 & 32.986 & 35.474 & 1475.78 & 1473.65 \\
\hline $3 / 9 / 126: 30$ & 33.234 & 35.472 & 1475.54 & 1473.65 \\
\hline $3 / 9 / 127: 00$ & 33.389 & 35.472 & 1475.38 & 1473.65 \\
\hline $3 / 9 / 12$ 7:30 & 33.206 & 35.479 & 1475.56 & 1473.64 \\
\hline $3 / 9 / 128: 00$ & 33.161 & 35.481 & 1475.61 & 1473.64 \\
\hline $3 / 9 / 128: 30$ & 33.45 & 35.484 & 1475.32 & 1473.64 \\
\hline 3/9/12 9:00 & 44.205 & 35.486 & 1464.56 & 1473.64 \\
\hline $3 / 9 / 12$ 9:30 & 51.97 & 35.533 & 1456.80 & 1473.59 \\
\hline 3/9/12 10:00 & 51.998 & 35.601 & 1456.77 & 1473.52 \\
\hline $3 / 9 / 12$ 10:30 & 56.483 & 35.676 & 1452.29 & 1473.45 \\
\hline 3/9/12 11:00 & 50.526 & 35.765 & 1458.24 & 1473.36 \\
\hline 3/9/12 11:30 & 52.179 & 35.857 & 1456.59 & 1473.27 \\
\hline 3/9/12 12:00 & 39.29 & 35.939 & 1469.48 & 1473.18 \\
\hline $3 / 9 / 12$ 12:30 & 37.39 & 36.045 & 1471.38 & 1473.08 \\
\hline 3/9/12 13:00 & 36.71 & 36.124 & 1472.06 & 1473.00 \\
\hline 3/9/12 13:30 & 57.139 & 36.199 & 1451.63 & 1472.92 \\
\hline $3 / 9 / 12$ 14:00 & 52.674 & 36.3 & 1456.10 & 1472.82 \\
\hline $3 / 9 / 12$ 14:30 & 58.488 & 36.406 & 1450.28 & 1472.72 \\
\hline 3/9/12 15:00 & 41.323 & 36.507 & 1467.45 & 1472.62 \\
\hline $3 / 9 / 12$ 15:30 & 38.825 & 36.554 & 1469.94 & 1472.57 \\
\hline 3/9/12 16:00 & 38.009 & 36.561 & 1470.76 & 1472.56 \\
\hline 3/9/12 16:30 & 37.582 & 36.554 & 1471.19 & 1472.57 \\
\hline $3 / 9 / 12$ 17:00 & 36.989 & 36.535 & 1471.78 & 1472.59 \\
\hline $3 / 9 / 12$ 17:30 & 36.621 & 36.511 & 1472.15 & 1472.61 \\
\hline 3/9/12 18:00 & 36.459 & 36.49 & 1472.31 & 1472.63 \\
\hline 3/9/12 18:30 & 36.128 & 36.467 & 1472.64 & 1472.66 \\
\hline $3 / 9 / 12$ 19:00 & 48.314 & 36.448 & 1460.46 & 1472.67 \\
\hline
\end{tabular}


TABLE S1.3 Automatically recorded groundwater levels in the Klassen wells, September 15, 2011, to March 13, 2012.

\begin{tabular}{|c|c|c|c|c|}
\hline \multicolumn{3}{|c|}{ Reference Elevation (ft AMSL) } & 1508.77 & \multirow{2}{*}{$\begin{array}{l}1509.12 \\
\text { Elevation in Klassen } \\
\text { Test Well (ft AMSL) }\end{array}$} \\
\hline Date and Time & $\begin{array}{l}\text { Depth in Klassen } \\
\text { Domestic Well } \\
(\mathrm{ft} \mathrm{BGL})\end{array}$ & $\begin{array}{c}\text { Depth in Klassen } \\
\text { Test Well } \\
\text { (ft BGL) }\end{array}$ & $\begin{array}{l}\text { Elevation in Klassen } \\
\text { Domestic Well } \\
\text { (ft AMSL) }\end{array}$ & \\
\hline $3 / 9 / 1219: 30$ & 56.372 & 36.493 & 1452.40 & 1472.63 \\
\hline $3 / 9 / 12$ 20:00 & 56.07 & 36.565 & 1452.70 & 1472.56 \\
\hline $3 / 9 / 1220: 30$ & 39.074 & 36.638 & 1469.70 & 1472.48 \\
\hline $3 / 9 / 1221: 00$ & 37.711 & 36.652 & 1471.06 & 1472.47 \\
\hline $3 / 9 / 12$ 21:30 & 37.209 & 36.643 & 1471.56 & 1472.48 \\
\hline $3 / 9 / 1222: 00$ & 36.963 & 36.636 & 1471.81 & 1472.49 \\
\hline $3 / 9 / 1222: 30$ & 37.721 & 36.619 & 1471.05 & 1472.50 \\
\hline $3 / 9 / 1223: 00$ & 37.873 & 36.61 & 1470.90 & 1472.51 \\
\hline $3 / 9 / 12$ 23:30 & 36.327 & 36.594 & 1472.44 & 1472.53 \\
\hline $3 / 10 / 12$ 0:00 & 36.039 & 36.57 & 1472.73 & 1472.55 \\
\hline $3 / 10 / 12$ 0:30 & 35.821 & 36.544 & 1472.95 & 1472.58 \\
\hline $3 / 10 / 121: 00$ & 35.638 & 36.516 & 1473.13 & 1472.61 \\
\hline $3 / 10 / 121: 30$ & 35.481 & 36.488 & 1473.29 & 1472.63 \\
\hline $3 / 10 / 122: 00$ & 35.446 & 36.462 & 1473.32 & 1472.66 \\
\hline $3 / 10 / 12$ 2:30 & 35.333 & 36.443 & 1473.44 & 1472.68 \\
\hline $3 / 10 / 12$ 3:00 & 35.211 & 36.42 & 1473.56 & 1472.70 \\
\hline $3 / 10 / 123: 30$ & 35.108 & 36.396 & 1473.66 & 1472.73 \\
\hline 3/10/12 4:00 & 35.197 & 36.382 & 1473.57 & 1472.74 \\
\hline $3 / 10 / 124: 30$ & 35.024 & 36.361 & 1473.75 & 1472.76 \\
\hline $3 / 10 / 12$ 5:00 & 34.904 & 36.34 & 1473.87 & 1472.78 \\
\hline $3 / 10 / 125: 30$ & 34.801 & 36.319 & 1473.97 & 1472.80 \\
\hline $3 / 10 / 126: 00$ & 34.712 & 36.303 & 1474.06 & 1472.82 \\
\hline $3 / 10 / 126: 30$ & 34.838 & 36.286 & 1473.93 & 1472.84 \\
\hline $3 / 10 / 127: 00$ & 36.342 & 36.268 & 1472.43 & 1472.85 \\
\hline $3 / 10 / 12$ 7:30 & 35.274 & 36.265 & 1473.50 & 1472.86 \\
\hline $3 / 10 / 128: 00$ & 35.167 & 36.267 & 1473.60 & 1472.86 \\
\hline $3 / 10 / 128: 30$ & 34.89 & 36.26 & 1473.88 & 1472.86 \\
\hline 3/10/12 9:00 & 34.878 & 36.253 & 1473.89 & 1472.87 \\
\hline 3/10/12 9:30 & 34.754 & 36.253 & 1474.02 & 1472.87 \\
\hline $3 / 10 / 12$ 10:00 & 34.622 & 36.246 & 1474.15 & 1472.88 \\
\hline $3 / 10 / 12$ 10:30 & 34.524 & 36.232 & 1474.25 & 1472.89 \\
\hline $3 / 10 / 12$ 11:00 & 34.564 & 36.202 & 1474.21 & 1472.92 \\
\hline $3 / 10 / 12$ 11:30 & 34.423 & 36.183 & 1474.35 & 1472.94 \\
\hline $3 / 10 / 12$ 12:00 & 34.414 & 36.167 & 1474.36 & 1472.96 \\
\hline $3 / 10 / 12$ 12:30 & 34.322 & 36.15 & 1474.45 & 1472.97 \\
\hline $3 / 10 / 12$ 13:00 & 34.254 & 36.134 & 1474.52 & 1472.99 \\
\hline $3 / 10 / 12$ 13:30 & 34.226 & 36.124 & 1474.54 & 1473.00 \\
\hline $3 / 10 / 12$ 14:00 & 34.228 & 36.115 & 1474.54 & 1473.01 \\
\hline
\end{tabular}


TABLE S1.3 Automatically recorded groundwater levels in the Klassen wells, September 15, 2011, to March 13, 2012.

\begin{tabular}{|c|c|c|c|c|}
\hline \multicolumn{3}{|c|}{ Reference Elevation (ft AMSL) } & 1508.77 & \multirow{2}{*}{$\begin{array}{l}1509.12 \\
\text { Elevation in Klassen } \\
\text { Test Well (ft AMSL) }\end{array}$} \\
\hline Date and Time & $\begin{array}{l}\text { Depth in Klassen } \\
\text { Domestic Well } \\
\text { (ft BGL) }\end{array}$ & $\begin{array}{c}\text { Depth in Klassen } \\
\text { Test Well } \\
\text { (ft BGL) }\end{array}$ & $\begin{array}{l}\text { Elevation in Klassen } \\
\text { Domestic Well } \\
\text { (ft AMSL) }\end{array}$ & \\
\hline $3 / 10 / 1214: 30$ & 34.287 & 36.106 & 1474.48 & 1473.02 \\
\hline $3 / 10 / 12$ 15:00 & 34.221 & 36.094 & 1474.55 & 1473.03 \\
\hline $3 / 10 / 12$ 15:30 & 34.252 & 36.087 & 1474.52 & 1473.04 \\
\hline $3 / 10 / 1216: 00$ & 34.259 & 36.08 & 1474.51 & 1473.04 \\
\hline $3 / 10 / 1216: 30$ & 40.971 & 36.08 & 1467.80 & 1473.04 \\
\hline $3 / 10 / 12$ 17:00 & 34.423 & 36.08 & 1474.35 & 1473.04 \\
\hline $3 / 10 / 12$ 17:30 & 34.362 & 36.075 & 1474.41 & 1473.05 \\
\hline $3 / 10 / 12$ 18:00 & 34.25 & 36.063 & 1474.52 & 1473.06 \\
\hline $3 / 10 / 1218: 30$ & 34.153 & 36.052 & 1474.62 & 1473.07 \\
\hline $3 / 10 / 12$ 19:00 & 34.102 & 36.045 & 1474.67 & 1473.08 \\
\hline $3 / 10 / 12$ 19:30 & 34.114 & 36.04 & 1474.66 & 1473.08 \\
\hline $3 / 10 / 1220: 00$ & 34.097 & 36.038 & 1474.67 & 1473.08 \\
\hline $3 / 10 / 1220: 30$ & 34.008 & 36.031 & 1474.76 & 1473.09 \\
\hline $3 / 10 / 1221: 00$ & 34.153 & 36.028 & 1474.62 & 1473.09 \\
\hline $3 / 10 / 12$ 21:30 & 34.557 & 36.056 & 1474.21 & 1473.07 \\
\hline $3 / 10 / 12$ 22:00 & 36.135 & 36.08 & 1472.63 & 1473.04 \\
\hline $3 / 10 / 1222: 30$ & 34.569 & 36.089 & 1474.20 & 1473.03 \\
\hline $3 / 10 / 12$ 23:00 & 34.454 & 36.091 & 1474.32 & 1473.03 \\
\hline $3 / 10 / 12$ 23:30 & 34.404 & 36.089 & 1474.37 & 1473.03 \\
\hline $3 / 11 / 12$ 0:00 & 34.271 & 36.082 & 1474.50 & 1473.04 \\
\hline $3 / 11 / 12$ 0:30 & 34.172 & 36.073 & 1474.60 & 1473.05 \\
\hline $3 / 11 / 121: 00$ & 34.095 & 36.063 & 1474.67 & 1473.06 \\
\hline $3 / 11 / 12$ 1:30 & 34.029 & 36.054 & 1474.74 & 1473.07 \\
\hline $3 / 11 / 122: 00$ & 33.971 & 36.042 & 1474.80 & 1473.08 \\
\hline $3 / 11 / 12$ 2:30 & 34.107 & 36.033 & 1474.66 & 1473.09 \\
\hline 3/11/12 3:00 & 33.938 & 36.031 & 1474.83 & 1473.09 \\
\hline $3 / 11 / 12$ 3:30 & 33.872 & 36.031 & 1474.90 & 1473.09 \\
\hline 3/11/12 4:00 & 33.849 & 36.023 & 1474.92 & 1473.10 \\
\hline $3 / 11 / 124: 30$ & 33.799 & 36.014 & 1474.97 & 1473.11 \\
\hline 3/11/12 5:00 & 34.257 & 36.014 & 1474.51 & 1473.11 \\
\hline $3 / 11 / 12$ 5:30 & 33.863 & 36.012 & 1474.91 & 1473.11 \\
\hline 3/11/12 6:00 & 33.781 & 36.014 & 1474.99 & 1473.11 \\
\hline 3/11/12 6:30 & 33.727 & 36.014 & 1475.04 & 1473.11 \\
\hline $3 / 11 / 12$ 7:00 & 33.851 & 36.014 & 1474.92 & 1473.11 \\
\hline 3/11/12 7:30 & 33.844 & 36.024 & 1474.93 & 1473.10 \\
\hline 3/11/12 8:00 & 33.797 & 36.028 & 1474.97 & 1473.09 \\
\hline $3 / 11 / 128: 30$ & 33.713 & 36.033 & 1475.06 & 1473.09 \\
\hline 3/11/12 9:00 & 33.659 & 36.042 & 1475.11 & 1473.08 \\
\hline
\end{tabular}


TABLE S1.3 Automatically recorded groundwater levels in the Klassen wells, September 15, 2011, to March 13, 2012.

\begin{tabular}{|c|c|c|c|c|}
\hline \multicolumn{3}{|c|}{ Reference Elevation (ft AMSL) } & 1508.77 & \multirow{2}{*}{$\begin{array}{l}1509.12 \\
\text { Elevation in Klassen } \\
\text { Test Well (ft AMSL) }\end{array}$} \\
\hline Date and Time & $\begin{array}{l}\text { Depth in Klassen } \\
\text { Domestic Well } \\
(\mathrm{ft} \mathrm{BGL})\end{array}$ & $\begin{array}{c}\text { Depth in Klassen } \\
\text { Test Well } \\
\text { (ft BGL) }\end{array}$ & $\begin{array}{l}\text { Elevation in Klassen } \\
\text { Domestic Well } \\
\text { (ft AMSL) }\end{array}$ & \\
\hline $3 / 11 / 129: 30$ & 33.637 & 36.059 & 1475.13 & 1473.06 \\
\hline $3 / 11 / 12$ 10:00 & 33.722 & 36.08 & 1475.05 & 1473.04 \\
\hline $3 / 11 / 12$ 10:30 & 33.677 & 36.108 & 1475.09 & 1473.01 \\
\hline $3 / 11 / 1211: 00$ & 33.727 & 36.115 & 1475.04 & 1473.01 \\
\hline $3 / 11 / 12$ 11:30 & 33.809 & 36.143 & 1474.96 & 1472.98 \\
\hline $3 / 11 / 12$ 12:00 & 33.769 & 36.153 & 1475.00 & 1472.97 \\
\hline $3 / 11 / 12$ 12:30 & 33.839 & 36.162 & 1474.93 & 1472.96 \\
\hline $3 / 11 / 12$ 13:00 & 33.867 & 36.174 & 1474.90 & 1472.95 \\
\hline $3 / 11 / 12$ 13:30 & 33.752 & 36.169 & 1475.02 & 1472.95 \\
\hline $3 / 11 / 1214: 00$ & 33.851 & 36.178 & 1474.92 & 1472.94 \\
\hline $3 / 11 / 1214: 30$ & 33.848 & 36.181 & 1474.92 & 1472.94 \\
\hline $3 / 11 / 12$ 15:00 & 33.774 & 36.176 & 1475.00 & 1472.95 \\
\hline $3 / 11 / 12$ 15:30 & 33.724 & 36.164 & 1475.05 & 1472.96 \\
\hline $3 / 11 / 1216: 00$ & 33.684 & 36.15 & 1475.09 & 1472.97 \\
\hline $3 / 11 / 12$ 16:30 & 33.647 & 36.146 & 1475.12 & 1472.98 \\
\hline $3 / 11 / 12$ 17:00 & 33.825 & 36.141 & 1474.94 & 1472.98 \\
\hline $3 / 11 / 12$ 17:30 & 33.949 & 36.141 & 1474.82 & 1472.98 \\
\hline $3 / 11 / 12$ 18:00 & 33.849 & 36.136 & 1474.92 & 1472.99 \\
\hline $3 / 11 / 12$ 18:30 & 33.816 & 36.129 & 1474.95 & 1472.99 \\
\hline $3 / 11 / 12$ 19:00 & 34.205 & 36.143 & 1474.56 & 1472.98 \\
\hline $3 / 11 / 12$ 19:30 & 34.163 & 36.146 & 1474.61 & 1472.98 \\
\hline $3 / 11 / 12$ 20:00 & 34.003 & 36.136 & 1474.77 & 1472.99 \\
\hline $3 / 11 / 12$ 20:30 & 34.233 & 36.131 & 1474.54 & 1472.99 \\
\hline $3 / 11 / 12$ 21:00 & 34.071 & 36.131 & 1474.70 & 1472.99 \\
\hline $3 / 11 / 12$ 21:30 & 33.954 & 36.127 & 1474.82 & 1473.00 \\
\hline $3 / 11 / 12$ 22:00 & 34.067 & 36.124 & 1474.70 & 1473.00 \\
\hline $3 / 11 / 12$ 22:30 & 34.245 & 36.124 & 1474.52 & 1473.00 \\
\hline $3 / 11 / 12$ 23:00 & 34.812 & 36.127 & 1473.96 & 1473.00 \\
\hline $3 / 11 / 12$ 23:30 & 34.034 & 36.129 & 1474.74 & 1472.99 \\
\hline 3/12/12 0:00 & 33.884 & 36.127 & 1474.89 & 1473.00 \\
\hline 3/12/12 0:30 & 33.797 & 36.127 & 1474.97 & 1473.00 \\
\hline $3 / 12 / 12$ 1:00 & 33.729 & 36.122 & 1475.04 & 1473.00 \\
\hline $3 / 12 / 12$ 1:30 & 33.673 & 36.117 & 1475.10 & 1473.01 \\
\hline $3 / 12 / 12$ 2:00 & 33.832 & 36.122 & 1474.94 & 1473.00 \\
\hline $3 / 12 / 12$ 2:30 & 33.762 & 36.124 & 1475.01 & 1473.00 \\
\hline 3/12/12 3:00 & 33.71 & 36.127 & 1475.06 & 1473.00 \\
\hline 3/12/12 3:30 & 33.677 & 36.122 & 1475.09 & 1473.00 \\
\hline $3 / 12 / 124: 00$ & 33.738 & 36.115 & 1475.03 & 1473.01 \\
\hline
\end{tabular}


TABLE S1.3 Automatically recorded groundwater levels in the Klassen wells, September 15, 2011, to March 13, 2012.

\begin{tabular}{|c|c|c|c|c|}
\hline \multicolumn{3}{|c|}{ Reference Elevation (ft AMSL) } & 1508.77 & \multirow{2}{*}{$\begin{array}{l}1509.12 \\
\text { Elevation in Klassen } \\
\text { Test Well (ft AMSL) }\end{array}$} \\
\hline Date and Time & $\begin{array}{l}\text { Depth in Klassen } \\
\text { Domestic Well } \\
\text { (ft BGL) }\end{array}$ & $\begin{array}{c}\text { Depth in Klassen } \\
\text { Test Well } \\
\text { (ft BGL) }\end{array}$ & $\begin{array}{l}\text { Elevation in Klassen } \\
\text { Domestic Well } \\
\text { (ft AMSL) }\end{array}$ & \\
\hline $3 / 12 / 124: 30$ & 33.654 & 36.11 & 1475.12 & 1473.01 \\
\hline $3 / 12 / 125: 00$ & 33.616 & 36.106 & 1475.15 & 1473.02 \\
\hline $3 / 12 / 125: 30$ & 33.792 & 36.096 & 1474.98 & 1473.03 \\
\hline $3 / 12 / 126: 00$ & 33.682 & 36.085 & 1475.09 & 1473.04 \\
\hline $3 / 12 / 126: 30$ & 33.626 & 36.077 & 1475.14 & 1473.05 \\
\hline $3 / 12 / 127: 00$ & 33.572 & 36.061 & 1475.20 & 1473.06 \\
\hline $3 / 12 / 127: 30$ & 35.603 & 36.047 & 1473.17 & 1473.08 \\
\hline $3 / 12 / 128: 00$ & 33.715 & 36.035 & 1475.05 & 1473.09 \\
\hline $3 / 12 / 128: 30$ & 33.567 & 36.019 & 1475.20 & 1473.10 \\
\hline 3/12/12 9:00 & 33.595 & 36.005 & 1475.17 & 1473.12 \\
\hline 3/12/12 9:30 & 33.682 & 35.993 & 1475.09 & 1473.13 \\
\hline $3 / 12 / 1210: 00$ & 33.529 & 35.986 & 1475.24 & 1473.14 \\
\hline $3 / 12 / 12$ 10:30 & 33.473 & 35.977 & 1475.30 & 1473.15 \\
\hline $3 / 12 / 1211: 00$ & 33.431 & 35.967 & 1475.34 & 1473.16 \\
\hline $3 / 12 / 12$ 11:30 & 33.401 & 35.963 & 1475.37 & 1473.16 \\
\hline $3 / 12 / 12$ 12:00 & 33.464 & 35.927 & 1475.31 & 1473.20 \\
\hline $3 / 12 / 12$ 12:30 & 33.403 & 35.913 & 1475.37 & 1473.21 \\
\hline $3 / 12 / 12$ 13:00 & 33.445 & 35.909 & 1475.32 & 1473.21 \\
\hline $3 / 12 / 12$ 13:30 & 33.455 & 35.904 & 1475.31 & 1473.22 \\
\hline $3 / 12 / 12$ 14:00 & 33.506 & 35.897 & 1475.26 & 1473.23 \\
\hline $3 / 12 / 12$ 14:30 & 33.71 & 35.892 & 1475.06 & 1473.23 \\
\hline $3 / 12 / 12$ 15:00 & 33.508 & 35.894 & 1475.26 & 1473.23 \\
\hline $3 / 12 / 12$ 15:30 & 38.684 & 35.894 & 1470.09 & 1473.23 \\
\hline $3 / 12 / 12$ 16:00 & 33.59 & 35.897 & 1475.18 & 1473.23 \\
\hline $3 / 12 / 12$ 16:30 & 33.485 & 35.892 & 1475.28 & 1473.23 \\
\hline $3 / 12 / 12$ 17:00 & 33.567 & 35.885 & 1475.20 & 1473.24 \\
\hline $3 / 12 / 12$ 17:30 & 33.478 & 35.876 & 1475.29 & 1473.25 \\
\hline $3 / 12 / 12$ 18:00 & 34.36 & 35.876 & 1474.41 & 1473.25 \\
\hline $3 / 12 / 12$ 18:30 & 33.68 & 35.883 & 1475.09 & 1473.24 \\
\hline $3 / 12 / 12$ 19:00 & 33.67 & 35.89 & 1475.10 & 1473.23 \\
\hline $3 / 12 / 12$ 19:30 & 33.743 & 35.897 & 1475.03 & 1473.23 \\
\hline $3 / 12 / 12$ 20:00 & 33.978 & 35.918 & 1474.79 & 1473.20 \\
\hline $3 / 12 / 12$ 20:30 & 33.954 & 35.932 & 1474.82 & 1473.19 \\
\hline $3 / 12 / 12$ 21:00 & 33.842 & 35.934 & 1474.93 & 1473.19 \\
\hline $3 / 12 / 12$ 21:30 & 34.069 & 35.932 & 1474.70 & 1473.19 \\
\hline $3 / 12 / 12$ 22:00 & 34.282 & 35.937 & 1474.49 & 1473.19 \\
\hline $3 / 12 / 12$ 22:30 & 33.839 & 35.934 & 1474.93 & 1473.19 \\
\hline $3 / 12 / 12$ 23:00 & 33.722 & 35.93 & 1475.05 & 1473.19 \\
\hline
\end{tabular}


TABLE S1.3 Automatically recorded groundwater levels in the Klassen wells, September 15, 2011, to March 13, 2012.

\begin{tabular}{|c|c|c|c|c|}
\hline \multicolumn{3}{|c|}{ Reference Elevation (ft AMSL) } & 1508.77 & \multirow{2}{*}{$\begin{array}{l}1509.12 \\
\text { Elevation in Klassen } \\
\text { Test Well (ft AMSL) }\end{array}$} \\
\hline Date and Time & $\begin{array}{l}\text { Depth in Klassen } \\
\text { Domestic Well } \\
\text { (ft BGL) }\end{array}$ & $\begin{array}{c}\text { Depth in Klassen } \\
\text { Test Well } \\
\text { (ft BGL) }\end{array}$ & $\begin{array}{l}\text { Elevation in Klassen } \\
\text { Domestic Well } \\
\text { (ft AMSL) }\end{array}$ & \\
\hline $3 / 12 / 12$ 23:30 & 33.645 & 35.92 & 1475.12 & 1473.20 \\
\hline $3 / 13 / 120: 00$ & 33.584 & 35.913 & 1475.19 & 1473.21 \\
\hline $3 / 13 / 120: 30$ & 33.534 & 35.902 & 1475.24 & 1473.22 \\
\hline $3 / 13 / 121: 00$ & 33.492 & 35.892 & 1475.28 & 1473.23 \\
\hline $3 / 13 / 121: 30$ & 33.455 & 35.883 & 1475.31 & 1473.24 \\
\hline $3 / 13 / 122: 00$ & 33.419 & 35.873 & 1475.35 & 1473.25 \\
\hline $3 / 13 / 122: 30$ & 33.389 & 35.862 & 1475.38 & 1473.26 \\
\hline $3 / 13 / 123: 00$ & 33.358 & 35.855 & 1475.41 & 1473.27 \\
\hline $3 / 13 / 123: 30$ & 33.358 & 35.85 & 1475.41 & 1473.27 \\
\hline $3 / 13 / 124: 00$ & 33.325 & 35.847 & 1475.44 & 1473.28 \\
\hline $3 / 13 / 124: 30$ & 33.328 & 35.843 & 1475.44 & 1473.28 \\
\hline $3 / 13 / 125: 00$ & 33.293 & 35.836 & 1475.48 & 1473.29 \\
\hline $3 / 13 / 125: 30$ & 33.577 & 35.836 & 1475.19 & 1473.29 \\
\hline $3 / 13 / 126: 00$ & 33.476 & 35.831 & 1475.29 & 1473.29 \\
\hline $3 / 13 / 126: 30$ & 33.424 & 35.824 & 1475.35 & 1473.30 \\
\hline $3 / 13 / 12$ 7:00 & 34.311 & 35.817 & 1474.46 & 1473.31 \\
\hline $3 / 13 / 12$ 7:30 & 33.38 & 35.815 & 1475.39 & 1473.31 \\
\hline $3 / 13 / 128: 00$ & 33.326 & 35.812 & 1475.44 & 1473.31 \\
\hline $3 / 13 / 128: 30$ & 33.288 & 35.805 & 1475.48 & 1473.32 \\
\hline 3/13/12 9:00 & 34.695 & 35.798 & 1474.07 & 1473.32 \\
\hline $3 / 13 / 12$ 9:30 & 33.295 & 35.798 & 1475.47 & 1473.32 \\
\hline 3/13/12 10:00 & 33.248 & 35.798 & 1475.52 & 1473.32 \\
\hline $3 / 13 / 12$ 10:30 & 33.29 & 35.798 & 1475.48 & 1473.32 \\
\hline 3/13/12 11:00 & 33.321 & 35.798 & 1475.45 & 1473.32 \\
\hline $3 / 13 / 12$ 11:30 & 33.253 & 35.787 & 1475.52 & 1473.34 \\
\hline $3 / 13 / 12$ 12:00 & 33.218 & 35.754 & 1475.55 & 1473.37 \\
\hline $3 / 13 / 12$ 12:30 & 33.283 & 35.744 & 1475.49 & 1473.38 \\
\hline 3/13/12 13:00 & 33.48 & 35.74 & 1475.29 & 1473.38 \\
\hline $3 / 13 / 12$ 13:30 & 33.785 & 35.747 & 1474.98 & 1473.38 \\
\hline 3/13/12 14:00 & 34.128 & 35.758 & 1474.64 & 1473.36 \\
\hline $3 / 13 / 12$ 14:30 & 33.459 & 35.765 & 1475.31 & 1473.36 \\
\hline $3 / 13 / 12$ 15:00 & 33.384 & 35.761 & 1475.39 & 1473.36 \\
\hline $3 / 13 / 12$ 15:30 & 33.551 & 35.756 & 1475.22 & 1473.37 \\
\hline
\end{tabular}


TABLE S1.4 Daily rainfall data collected at the Hutchinson station, approximately 15 mi southwest of Inman, October 1, 2009, to March 31, 2010, from the Kansas Weather Data Library (http://wdl.agron.ksu.edu/).

\begin{tabular}{|c|c|}
\hline Date & $\begin{array}{c}\text { Total } \\
\text { Precipitation } \\
\text { (in.) }\end{array}$ \\
\hline 10/1/2009 & 0.02 \\
\hline $10 / 2 / 2009$ & 0.00 \\
\hline $10 / 3 / 2009$ & 0.00 \\
\hline $10 / 4 / 2009$ & 0.00 \\
\hline $10 / 5 / 2009$ & 0.01 \\
\hline 10/6/2009 & 0.06 \\
\hline 10/7/2009 & 0.02 \\
\hline 10/8/2009 & 0.72 \\
\hline 10/9/2009 & 0.00 \\
\hline 10/10/2009 & 0.00 \\
\hline 10/11/2009 & 0.00 \\
\hline 10/12/2009 & 0.02 \\
\hline 10/13/2009 & 0.43 \\
\hline 10/14/2009 & 0.02 \\
\hline 10/15/2009 & 0.00 \\
\hline 10/16/2009 & 0.00 \\
\hline 10/17/2009 & 0.00 \\
\hline 10/18/2009 & 0.00 \\
\hline 10/19/2009 & 0.00 \\
\hline 10/20/2009 & 0.00 \\
\hline 10/21/2009 & 0.25 \\
\hline 10/22/2009 & 0.00 \\
\hline 10/23/2009 & 0.00 \\
\hline 10/24/2009 & 0.00 \\
\hline 10/25/2009 & 0.12 \\
\hline 10/26/2009 & 0.00 \\
\hline 10/27/2009 & 0.00 \\
\hline 10/28/2009 & 0.14 \\
\hline 10/29/2009 & 0.87 \\
\hline 10/30/2009 & 0.01 \\
\hline 10/31/2009 & 0.00 \\
\hline $11 / 1 / 2009$ & 0.00 \\
\hline $11 / 2 / 2009$ & 0.00 \\
\hline $11 / 3 / 2009$ & 0.00 \\
\hline $11 / 4 / 2009$ & 0.00 \\
\hline $11 / 5 / 2009$ & 0.00 \\
\hline $11 / 6 / 2009$ & 0.00 \\
\hline 11/7/2009 & 0.00 \\
\hline $11 / 8 / 2009$ & 0.00 \\
\hline
\end{tabular}


TABLE S1.4 Daily rainfall data collected at the Hutchinson station, approximately $15 \mathrm{mi}$ southwest of Inman, October 1, 2009, to March 31, 2010, from the Kansas Weather Data Library (http://wdl.agron.ksu.edu/).

\begin{tabular}{|c|c|}
\hline Date & $\begin{array}{c}\text { Total } \\
\text { Precipitation } \\
\text { (in.) }\end{array}$ \\
\hline $11 / 9 / 2009$ & 0.12 \\
\hline 11/10/2009 & 0.00 \\
\hline 11/11/2009 & 0.00 \\
\hline $11 / 12 / 2009$ & 0.00 \\
\hline 11/13/2009 & 0.00 \\
\hline 11/14/2009 & 0.00 \\
\hline $11 / 15 / 2009$ & 0.17 \\
\hline 11/16/2009 & 0.06 \\
\hline 11/17/2009 & 0.00 \\
\hline 11/18/2009 & 0.00 \\
\hline 11/19/2009 & 0.00 \\
\hline 11/20/2009 & 0.00 \\
\hline $11 / 21 / 2009$ & 0.00 \\
\hline $11 / 22 / 2009$ & 0.00 \\
\hline 11/23/2009 & 0.02 \\
\hline 11/24/2009 & 0.00 \\
\hline $11 / 25 / 2009$ & 0.00 \\
\hline 11/26/2009 & 0.00 \\
\hline 11/27/2009 & 0.00 \\
\hline 11/28/2009 & 0.00 \\
\hline 11/29/2009 & 0.00 \\
\hline 11/30/2009 & 0.00 \\
\hline $12 / 1 / 2009$ & 0.00 \\
\hline $12 / 2 / 2009$ & 0.00 \\
\hline $12 / 3 / 2009$ & 0.00 \\
\hline $12 / 4 / 2009$ & 0.00 \\
\hline $12 / 5 / 2009$ & 0.00 \\
\hline 12/6/2009 & 0.00 \\
\hline 12/7/2009 & 0.00 \\
\hline $12 / 8 / 2009$ & 0.00 \\
\hline $12 / 9 / 2009$ & 0.00 \\
\hline 12/10/2009 & 0.05 \\
\hline $12 / 11 / 2009$ & 0.01 \\
\hline $12 / 12 / 2009$ & 0.00 \\
\hline 12/13/2009 & 0.00 \\
\hline $12 / 14 / 2009$ & 0.00 \\
\hline 12/15/2009 & 0.00 \\
\hline 12/16/2009 & 0.00 \\
\hline 12/17/2009 & 0.00 \\
\hline
\end{tabular}


TABLE S1.4 Daily rainfall data collected at the Hutchinson station, approximately 15 mi southwest of Inman, October 1, 2009, to March 31, 2010, from the Kansas Weather Data Library (http://wdl.agron.ksu.edu/).

\begin{tabular}{|c|c|}
\hline Date & $\begin{array}{c}\text { Total } \\
\text { Precipitation } \\
\text { (in.) }\end{array}$ \\
\hline $12 / 18 / 2009$ & 0.00 \\
\hline 12/19/2009 & 0.00 \\
\hline $12 / 20 / 2009$ & 0.00 \\
\hline $12 / 21 / 2009$ & 0.00 \\
\hline $12 / 22 / 2009$ & 0.05 \\
\hline $12 / 23 / 2009$ & 0.03 \\
\hline $12 / 24 / 2009$ & 0.00 \\
\hline $12 / 25 / 2009$ & 0.00 \\
\hline $12 / 26 / 2009$ & 0.00 \\
\hline 12/27/2009 & 0.00 \\
\hline $12 / 28 / 2009$ & 0.00 \\
\hline $12 / 29 / 2009$ & 0.00 \\
\hline $12 / 30 / 2009$ & 0.02 \\
\hline 12/31/2009 & 0.00 \\
\hline $1 / 1 / 2010$ & 0.00 \\
\hline $1 / 2 / 2010$ & 0.00 \\
\hline $1 / 3 / 2010$ & 0.00 \\
\hline $1 / 4 / 2010$ & 0.00 \\
\hline $1 / 5 / 2010$ & 0.00 \\
\hline $1 / 6 / 2010$ & 0.00 \\
\hline $1 / 7 / 2010$ & 0.00 \\
\hline $1 / 8 / 2010$ & 0.00 \\
\hline $1 / 9 / 2010$ & 0.00 \\
\hline $1 / 10 / 2010$ & 0.00 \\
\hline $1 / 11 / 2010$ & 0.00 \\
\hline $1 / 12 / 2010$ & 0.00 \\
\hline $1 / 13 / 2010$ & 0.00 \\
\hline $1 / 14 / 2010$ & 0.00 \\
\hline $1 / 15 / 2010$ & 0.00 \\
\hline $1 / 16 / 2010$ & 0.00 \\
\hline 1/17/2010 & 0.00 \\
\hline 1/18/2010 & 0.00 \\
\hline 1/19/2010 & 0.00 \\
\hline $1 / 20 / 2010$ & 0.00 \\
\hline $1 / 21 / 2010$ & 0.06 \\
\hline $1 / 22 / 2010$ & 0.02 \\
\hline $1 / 23 / 2010$ & 0.06 \\
\hline 1/24/2010 & 0.00 \\
\hline $1 / 25 / 2010$ & 0.00 \\
\hline
\end{tabular}


TABLE S1.4 Daily rainfall data collected at the Hutchinson station, approximately 15 mi southwest of Inman, October 1, 2009, to March 31, 2010, from the Kansas Weather Data Library (http://wdl.agron.ksu.edu/).

\begin{tabular}{|c|c|}
\hline Date & $\begin{array}{c}\text { Total } \\
\text { Precipitation } \\
\text { (in.) }\end{array}$ \\
\hline $1 / 26 / 2010$ & 0.00 \\
\hline $1 / 27 / 2010$ & 0.00 \\
\hline $1 / 28 / 2010$ & 0.00 \\
\hline $1 / 29 / 2010$ & 0.00 \\
\hline $1 / 30 / 2010$ & 0.06 \\
\hline $1 / 31 / 2010$ & 0.00 \\
\hline $2 / 1 / 2010$ & 0.01 \\
\hline $2 / 2 / 2010$ & 0.00 \\
\hline $2 / 3 / 2010$ & 0.00 \\
\hline 2/4/2010 & 0.14 \\
\hline $2 / 5 / 2010$ & 0.02 \\
\hline $2 / 6 / 2010$ & 0.00 \\
\hline $2 / 7 / 2010$ & 0.00 \\
\hline $2 / 8 / 2010$ & 0.11 \\
\hline $2 / 9 / 2010$ & 0.00 \\
\hline $2 / 10 / 2010$ & 0.00 \\
\hline $2 / 11 / 2010$ & 0.01 \\
\hline $2 / 12 / 2010$ & 0.00 \\
\hline $2 / 13 / 2010$ & 0.00 \\
\hline $2 / 14 / 2010$ & 0.00 \\
\hline $2 / 15 / 2010$ & 0.00 \\
\hline $2 / 16 / 2010$ & 0.00 \\
\hline $2 / 17 / 2010$ & 0.00 \\
\hline $2 / 18 / 2010$ & 0.00 \\
\hline 2/19/2010 & 0.01 \\
\hline $2 / 20 / 2010$ & 0.00 \\
\hline $2 / 21 / 2010$ & 0.25 \\
\hline $2 / 22 / 2010$ & 0.00 \\
\hline 2/23/2010 & 0.01 \\
\hline $2 / 24 / 2010$ & 0.02 \\
\hline $2 / 25 / 2010$ & 0.00 \\
\hline 2/26/2010 & 0.00 \\
\hline $2 / 27 / 2010$ & 0.00 \\
\hline 2/28/2010 & 0.00 \\
\hline $3 / 1 / 2010$ & 0.00 \\
\hline $3 / 2 / 2010$ & 0.00 \\
\hline $3 / 3 / 2010$ & 0.00 \\
\hline $3 / 4 / 2010$ & 0.00 \\
\hline $3 / 5 / 2010$ & 0.00 \\
\hline
\end{tabular}


TABLE S1.4 Daily rainfall data collected at the Hutchinson station, approximately 15 mi southwest of Inman, October 1, 2009, to March 31, 2010, from the Kansas Weather Data Library (http://wdl.agron.ksu.edu/).

\begin{tabular}{|c|c|}
\hline Date & $\begin{array}{c}\text { Total } \\
\text { Precipitation } \\
\text { (in.) }\end{array}$ \\
\hline $3 / 6 / 2010$ & 0.00 \\
\hline 3/7/2010 & 0.00 \\
\hline $3 / 8 / 2010$ & 0.89 \\
\hline $3 / 9 / 2010$ & 0.01 \\
\hline $3 / 10 / 2010$ & 0.18 \\
\hline $3 / 11 / 2010$ & 0.00 \\
\hline $3 / 12 / 2010$ & 0.00 \\
\hline $3 / 13 / 2010$ & 0.00 \\
\hline $3 / 14 / 2010$ & 0.00 \\
\hline $3 / 15 / 2010$ & 0.00 \\
\hline $3 / 16 / 2010$ & 0.00 \\
\hline $3 / 17 / 2010$ & 0.00 \\
\hline $3 / 18 / 2010$ & 0.00 \\
\hline $3 / 19 / 2010$ & 0.00 \\
\hline $3 / 20 / 2010$ & 0.00 \\
\hline $3 / 21 / 2010$ & 0.00 \\
\hline $3 / 22 / 2010$ & 0.00 \\
\hline $3 / 23 / 2010$ & 0.00 \\
\hline $3 / 24 / 2010$ & 0.05 \\
\hline $3 / 25 / 2010$ & 0.03 \\
\hline $3 / 26 / 2010$ & 0.00 \\
\hline $3 / 27 / 2010$ & 0.01 \\
\hline $3 / 28 / 2010$ & 0.00 \\
\hline $3 / 29 / 2010$ & 0.00 \\
\hline $3 / 30 / 2010$ & 0.00 \\
\hline $3 / 31 / 2010$ & 0.00 \\
\hline $4 / 1 / 2010$ & 0.00 \\
\hline $4 / 2 / 2010$ & 0.01 \\
\hline $4 / 3 / 2010$ & 0.00 \\
\hline $4 / 4 / 2010$ & 0.00 \\
\hline $4 / 5 / 2010$ & 0.00 \\
\hline $4 / 6 / 2010$ & 0.00 \\
\hline 4/7/2010 & 0.00 \\
\hline $4 / 8 / 2010$ & 0.00 \\
\hline $4 / 9 / 2010$ & 0.00 \\
\hline $4 / 10 / 2010$ & 0.00 \\
\hline $4 / 11 / 2010$ & 0.00 \\
\hline $4 / 12 / 2010$ & 0.00 \\
\hline $4 / 13 / 2010$ & 0.00 \\
\hline
\end{tabular}


TABLE S1.4 Daily rainfall data collected at the Hutchinson station, approximately 15 mi southwest of Inman, October 1, 2009, to March 31, 2010, from the Kansas Weather Data Library (http://wdl.agron.ksu.edu/).

\begin{tabular}{|c|c|}
\hline Date & $\begin{array}{c}\text { Total } \\
\text { Precipitation } \\
\text { (in.) }\end{array}$ \\
\hline $4 / 14 / 2010$ & 0.00 \\
\hline 4/15/2010 & 0.00 \\
\hline 4/16/2010 & 0.18 \\
\hline $4 / 17 / 2010$ & 0.00 \\
\hline 4/18/2010 & 0.00 \\
\hline 4/19/2010 & 0.00 \\
\hline $4 / 20 / 2010$ & 0.00 \\
\hline $4 / 21 / 2010$ & 0.00 \\
\hline $4 / 22 / 2010$ & 0.37 \\
\hline $4 / 23 / 2010$ & 0.30 \\
\hline $4 / 24 / 2010$ & 0.05 \\
\hline $4 / 25 / 2010$ & 0.00 \\
\hline $4 / 26 / 2010$ & 0.00 \\
\hline 4/27/2010 & 0.00 \\
\hline $4 / 28 / 2010$ & 0.00 \\
\hline 4/29/2010 & 0.00 \\
\hline $4 / 30 / 2010$ & 0.22 \\
\hline $5 / 1 / 2010$ & 0.00 \\
\hline $5 / 2 / 2010$ & 0.01 \\
\hline $5 / 3 / 2010$ & 0.00 \\
\hline $5 / 4 / 2010$ & 0.00 \\
\hline $5 / 5 / 2010$ & 0.00 \\
\hline $5 / 6 / 2010$ & 0.00 \\
\hline $5 / 7 / 2010$ & 0.00 \\
\hline $5 / 8 / 2010$ & 0.00 \\
\hline $5 / 9 / 2010$ & 0.00 \\
\hline $5 / 10 / 2010$ & 0.58 \\
\hline $5 / 11 / 2010$ & 0.00 \\
\hline $5 / 12 / 2010$ & 1.20 \\
\hline $5 / 13 / 2010$ & 0.01 \\
\hline $5 / 14 / 2010$ & 0.00 \\
\hline $5 / 15 / 2010$ & 0.03 \\
\hline $5 / 16 / 2010$ & 0.40 \\
\hline $5 / 17 / 2010$ & 0.01 \\
\hline $5 / 18 / 2010$ & 0.06 \\
\hline $5 / 19 / 2010$ & 0.70 \\
\hline $5 / 20 / 2010$ & 0.07 \\
\hline $5 / 21 / 2010$ & 0.00 \\
\hline $5 / 22 / 2010$ & 0.00 \\
\hline
\end{tabular}


TABLE S1.4 Daily rainfall data collected at the Hutchinson station, approximately 15 mi southwest of Inman, October 1, 2009, to March 31, 2010, from the Kansas Weather Data Library (http://wdl.agron.ksu.edu/).

\begin{tabular}{|c|c|}
\hline Date & $\begin{array}{c}\text { Total } \\
\text { Precipitation } \\
\text { (in.) }\end{array}$ \\
\hline $5 / 23 / 2010$ & 0.01 \\
\hline $5 / 24 / 2010$ & 0.21 \\
\hline $5 / 25 / 2010$ & 1.11 \\
\hline $5 / 26 / 2010$ & 0.51 \\
\hline $5 / 27 / 2010$ & 0.00 \\
\hline $5 / 28 / 2010$ & 0.00 \\
\hline 5/29/2010 & 0.00 \\
\hline $5 / 30 / 2010$ & 0.00 \\
\hline $5 / 31 / 2010$ & 0.00 \\
\hline $6 / 1 / 2010$ & 0.00 \\
\hline $6 / 2 / 2010$ & 0.00 \\
\hline $6 / 3 / 2010$ & 0.00 \\
\hline $6 / 4 / 2010$ & 0.00 \\
\hline $6 / 5 / 2010$ & 0.00 \\
\hline 6/6/2010 & 0.00 \\
\hline $6 / 7 / 2010$ & 0.64 \\
\hline 6/8/2010 & 0.00 \\
\hline $6 / 9 / 2010$ & 0.14 \\
\hline 6/10/2010 & 0.00 \\
\hline $6 / 11 / 2010$ & 0.00 \\
\hline $6 / 12 / 2010$ & 0.03 \\
\hline $6 / 13 / 2010$ & 3.16 \\
\hline $6 / 14 / 2010$ & 0.03 \\
\hline $6 / 15 / 2010$ & 0.16 \\
\hline 6/16/2010 & 0.00 \\
\hline 6/17/2010 & 0.00 \\
\hline 6/18/2010 & 0.00 \\
\hline 6/19/2010 & 0.00 \\
\hline 6/20/2010 & 0.00 \\
\hline $6 / 21 / 2010$ & 0.00 \\
\hline 6/22/2010 & 0.00 \\
\hline 6/23/2010 & 0.00 \\
\hline $6 / 24 / 2010$ & 0.00 \\
\hline $6 / 25 / 2010$ & 0.00 \\
\hline 6/26/2010 & 0.00 \\
\hline $6 / 27 / 2010$ & 0.00 \\
\hline 6/28/2010 & 0.00 \\
\hline 6/29/2010 & 0.00 \\
\hline 6/30/2010 & 0.00 \\
\hline
\end{tabular}


TABLE S1.4 Daily rainfall data collected at the Hutchinson station, approximately 15 mi southwest of Inman, October 1, 2009, to March 31, 2010, from the Kansas Weather Data Library (http://wdl.agron.ksu.edu/).

\begin{tabular}{|c|c|}
\hline Date & $\begin{array}{c}\text { Total } \\
\text { Precipitation } \\
\text { (in.) }\end{array}$ \\
\hline $7 / 1 / 2010$ & 0.00 \\
\hline $7 / 2 / 2010$ & 0.00 \\
\hline $7 / 3 / 2010$ & 0.83 \\
\hline $7 / 4 / 2010$ & 4.66 \\
\hline $7 / 5 / 2010$ & 0.99 \\
\hline $7 / 6 / 2010$ & 0.00 \\
\hline $12 / 23 / 2010$ & 0.00 \\
\hline $12 / 24 / 2010$ & 0.02 \\
\hline $12 / 25 / 2010$ & 0.00 \\
\hline $12 / 26 / 2010$ & 0.00 \\
\hline $12 / 27 / 2010$ & 0.00 \\
\hline $12 / 28 / 2010$ & 0.00 \\
\hline $12 / 29 / 2010$ & 0.00 \\
\hline 12/30/2010 & 0.00 \\
\hline $1 / 1 / 2011$ & 0.00 \\
\hline $1 / 2 / 2011$ & 0.00 \\
\hline $1 / 3 / 2011$ & 0.00 \\
\hline $1 / 4 / 2011$ & 0.00 \\
\hline $1 / 5 / 2011$ & 0.00 \\
\hline $1 / 6 / 2011$ & 0.00 \\
\hline $1 / 7 / 2011$ & 0.00 \\
\hline $1 / 8 / 2011$ & 0.00 \\
\hline $1 / 9 / 2011$ & 0.00 \\
\hline $1 / 10 / 2011$ & 0.00 \\
\hline $1 / 11 / 2011$ & 0.00 \\
\hline $1 / 12 / 2011$ & 0.00 \\
\hline $1 / 13 / 2011$ & 0.02 \\
\hline $1 / 14 / 2011$ & 0.00 \\
\hline $1 / 15 / 2011$ & 0.00 \\
\hline $1 / 16 / 2011$ & 0.00 \\
\hline $1 / 17 / 2011$ & 0.00 \\
\hline $1 / 18 / 2011$ & 0.00 \\
\hline $1 / 19 / 2011$ & 0.00 \\
\hline $1 / 20 / 2011$ & 0.00 \\
\hline $1 / 21 / 2011$ & 0.00 \\
\hline $1 / 22 / 2011$ & 0.00 \\
\hline $1 / 23 / 2011$ & 0.00 \\
\hline $1 / 24 / 2011$ & 0.00 \\
\hline $1 / 25 / 2011$ & 0.00 \\
\hline
\end{tabular}


TABLE S1.4 Daily rainfall data collected at the Hutchinson station, approximately 15 mi southwest of Inman, October 1, 2009, to March 31, 2010, from the Kansas Weather Data Library (http://wdl.agron.ksu.edu/).

\begin{tabular}{|c|c|}
\hline Date & $\begin{array}{c}\text { Total } \\
\text { Precipitation } \\
\text { (in.) }\end{array}$ \\
\hline $1 / 26 / 2011$ & 0.00 \\
\hline $1 / 27 / 2011$ & 0.00 \\
\hline $1 / 28 / 2011$ & 0.00 \\
\hline $1 / 29 / 2011$ & 0.00 \\
\hline $1 / 30 / 2011$ & 0.00 \\
\hline $1 / 31 / 2011$ & 0.00 \\
\hline $2 / 1 / 2011$ & 0.00 \\
\hline $2 / 2 / 2011$ & 0.00 \\
\hline $2 / 3 / 2011$ & 0.00 \\
\hline $2 / 4 / 2011$ & 0.00 \\
\hline $2 / 5 / 2011$ & 0.00 \\
\hline $2 / 6 / 2011$ & 0.00 \\
\hline $2 / 7 / 2011$ & 0.00 \\
\hline $2 / 8 / 2011$ & 0.00 \\
\hline $2 / 9 / 2011$ & 0.00 \\
\hline $2 / 10 / 2011$ & 0.00 \\
\hline $2 / 11 / 2011$ & 0.00 \\
\hline $2 / 12 / 2011$ & 0.00 \\
\hline $2 / 13 / 2011$ & 0.00 \\
\hline $2 / 14 / 2011$ & 0.00 \\
\hline $2 / 15 / 2011$ & 0.00 \\
\hline $2 / 16 / 2011$ & 0.00 \\
\hline $2 / 17 / 2011$ & 0.00 \\
\hline $2 / 18 / 2011$ & 0.00 \\
\hline $2 / 19 / 2011$ & 0.00 \\
\hline $2 / 20 / 2011$ & 0.00 \\
\hline $2 / 21 / 2011$ & 0.00 \\
\hline $2 / 22 / 2011$ & 0.00 \\
\hline $2 / 23 / 2011$ & 0.00 \\
\hline $2 / 24 / 2011$ & 0.22 \\
\hline $2 / 25 / 2011$ & 0.00 \\
\hline $2 / 26 / 2011$ & 0.00 \\
\hline $2 / 27 / 2011$ & 0.02 \\
\hline $2 / 28 / 2011$ & 0.00 \\
\hline $3 / 1 / 2011$ & 0.00 \\
\hline $3 / 2 / 2011$ & 0.00 \\
\hline $3 / 3 / 2011$ & 0.00 \\
\hline $3 / 4 / 2011$ & 0.00 \\
\hline $3 / 5 / 2011$ & 0.00 \\
\hline
\end{tabular}


TABLE S1.4 Daily rainfall data collected at the Hutchinson station, approximately 15 mi southwest of Inman, October 1, 2009, to March 31, 2010, from the Kansas Weather Data Library (http://wdl.agron.ksu.edu/).

\begin{tabular}{|c|c|}
\hline Date & $\begin{array}{c}\text { Total } \\
\text { Precipitation } \\
\text { (in.) }\end{array}$ \\
\hline $3 / 6 / 2011$ & 0.00 \\
\hline $3 / 7 / 2011$ & 0.00 \\
\hline $3 / 8 / 2011$ & 0.33 \\
\hline $3 / 9 / 2011$ & 0.00 \\
\hline $3 / 10 / 2011$ & 0.00 \\
\hline $3 / 11 / 2011$ & 0.00 \\
\hline $3 / 12 / 2011$ & 0.00 \\
\hline $3 / 13 / 2011$ & 0.11 \\
\hline $3 / 14 / 2011$ & 0.03 \\
\hline $3 / 15 / 2011$ & 0.00 \\
\hline $3 / 16 / 2011$ & 0.00 \\
\hline $3 / 17 / 2011$ & 0.00 \\
\hline $3 / 18 / 2011$ & 0.00 \\
\hline $3 / 19 / 2011$ & 0.18 \\
\hline $3 / 20 / 2011$ & 0.00 \\
\hline $3 / 21 / 2011$ & 0.00 \\
\hline $3 / 22 / 2011$ & 0.00 \\
\hline $3 / 23 / 2011$ & 0.00 \\
\hline $3 / 24 / 2011$ & 0.09 \\
\hline $3 / 25 / 2011$ & 0.00 \\
\hline $3 / 26 / 2011$ & 0.00 \\
\hline $3 / 27 / 2011$ & 0.00 \\
\hline $3 / 28 / 2011$ & 0.02 \\
\hline $3 / 29 / 2011$ & 0.00 \\
\hline $3 / 30 / 2011$ & 0.00 \\
\hline $3 / 31 / 2011$ & 0.01 \\
\hline $4 / 1 / 2011$ & 0.00 \\
\hline $4 / 2 / 2011$ & 0.00 \\
\hline $4 / 3 / 2011$ & 0.00 \\
\hline $4 / 4 / 2011$ & 0.00 \\
\hline $4 / 5 / 2011$ & 0.00 \\
\hline $4 / 6 / 2011$ & 0.00 \\
\hline $4 / 7 / 2011$ & 0.00 \\
\hline $4 / 8 / 2011$ & 0.00 \\
\hline $4 / 9 / 2011$ & 0.00 \\
\hline $4 / 10 / 2011$ & 0.00 \\
\hline $4 / 11 / 2011$ & 0.00 \\
\hline $4 / 12 / 2011$ & 0.00 \\
\hline $4 / 13 / 2011$ & 0.00 \\
\hline
\end{tabular}


TABLE S1.4 Daily rainfall data collected at the Hutchinson station, approximately 15 mi southwest of Inman, October 1, 2009, to March 31, 2010, from the Kansas Weather Data Library (http://wdl.agron.ksu.edu/).

\begin{tabular}{|c|c|}
\hline Date & $\begin{array}{c}\text { Total } \\
\text { Precipitation } \\
\text { (in.) }\end{array}$ \\
\hline $4 / 14 / 2011$ & 0.00 \\
\hline $4 / 15 / 2011$ & 0.13 \\
\hline $4 / 16 / 2011$ & 0.00 \\
\hline $4 / 17 / 2011$ & 0.00 \\
\hline $4 / 18 / 2011$ & 0.00 \\
\hline $4 / 19 / 2011$ & 0.00 \\
\hline $4 / 20 / 2011$ & 0.00 \\
\hline $4 / 21 / 2011$ & 0.00 \\
\hline $4 / 22 / 2011$ & 0.01 \\
\hline $4 / 23 / 2011$ & 0.00 \\
\hline $4 / 24 / 2011$ & 0.00 \\
\hline $4 / 25 / 2011$ & 0.15 \\
\hline $4 / 26 / 2011$ & 0.03 \\
\hline $4 / 27 / 2011$ & 0.02 \\
\hline $4 / 28 / 2011$ & 0.00 \\
\hline $4 / 29 / 2011$ & 0.00 \\
\hline $4 / 30 / 2011$ & 0.00 \\
\hline $5 / 1 / 2011$ & 0.00 \\
\hline $5 / 2 / 2011$ & 0.00 \\
\hline $5 / 3 / 2011$ & 0.00 \\
\hline $5 / 4 / 2011$ & 0.00 \\
\hline $5 / 5 / 2011$ & 0.00 \\
\hline $5 / 6 / 2011$ & 0.00 \\
\hline $5 / 7 / 2011$ & 0.00 \\
\hline $5 / 8 / 2011$ & 0.00 \\
\hline $5 / 9 / 2011$ & 0.00 \\
\hline $5 / 10 / 2011$ & 0.00 \\
\hline $5 / 11 / 2011$ & 0.00 \\
\hline $5 / 12 / 2011$ & 0.00 \\
\hline $5 / 13 / 2011$ & 0.01 \\
\hline $5 / 14 / 2011$ & 0.00 \\
\hline $5 / 15 / 2011$ & 0.00 \\
\hline $5 / 16 / 2011$ & 0.00 \\
\hline $5 / 17 / 2011$ & 0.00 \\
\hline $5 / 18 / 2011$ & 0.13 \\
\hline $5 / 19 / 2011$ & 0.01 \\
\hline $5 / 20 / 2011$ & 0.40 \\
\hline $5 / 21 / 2011$ & 0.00 \\
\hline $5 / 22 / 2011$ & 0.00 \\
\hline
\end{tabular}


TABLE S1.4 Daily rainfall data collected at the Hutchinson station, approximately 15 mi southwest of Inman, October 1, 2009, to March 31, 2010, from the Kansas Weather Data Library (http://wdl.agron.ksu.edu/).

\begin{tabular}{|c|c|}
\hline Date & $\begin{array}{c}\text { Total } \\
\text { Precipitation } \\
\text { (in.) }\end{array}$ \\
\hline $5 / 23 / 2011$ & 0.00 \\
\hline $5 / 24 / 2011$ & 0.70 \\
\hline $5 / 25 / 2011$ & 0.16 \\
\hline $5 / 26 / 2011$ & 0.00 \\
\hline $5 / 27 / 2011$ & 0.00 \\
\hline $5 / 28 / 2011$ & 0.00 \\
\hline $5 / 29 / 2011$ & 0.00 \\
\hline $5 / 30 / 2011$ & 0.00 \\
\hline $5 / 31 / 2011$ & 0.55 \\
\hline $6 / 1 / 2011$ & 0.00 \\
\hline $6 / 2 / 2011$ & 0.01 \\
\hline $6 / 3 / 2011$ & 0.00 \\
\hline $6 / 4 / 2011$ & 0.00 \\
\hline $6 / 5 / 2011$ & 0.00 \\
\hline $6 / 6 / 2011$ & 0.00 \\
\hline $6 / 7 / 2011$ & 0.00 \\
\hline $6 / 8 / 2011$ & 0.00 \\
\hline $6 / 9 / 2011$ & 0.03 \\
\hline $6 / 10 / 2011$ & 0.00 \\
\hline $6 / 11 / 2011$ & 0.66 \\
\hline $6 / 12 / 2011$ & 0.40 \\
\hline $6 / 13 / 2011$ & 0.00 \\
\hline $6 / 14 / 2011$ & 0.00 \\
\hline $6 / 15 / 2011$ & 0.00 \\
\hline $6 / 16 / 2011$ & 0.39 \\
\hline $6 / 17 / 2011$ & 0.03 \\
\hline $6 / 18 / 2011$ & 0.00 \\
\hline $6 / 19 / 2011$ & 0.00 \\
\hline $6 / 20 / 2011$ & 0.00 \\
\hline $6 / 21 / 2011$ & 0.00 \\
\hline $6 / 22 / 2011$ & 0.00 \\
\hline $6 / 23 / 2011$ & 0.20 \\
\hline $6 / 24 / 2011$ & 0.00 \\
\hline $6 / 25 / 2011$ & 0.00 \\
\hline $6 / 26 / 2011$ & 0.00 \\
\hline $6 / 27 / 2011$ & 0.00 \\
\hline $6 / 28 / 2011$ & 0.00 \\
\hline $6 / 29 / 2011$ & 0.00 \\
\hline $6 / 30 / 2011$ & 0.00 \\
\hline
\end{tabular}


TABLE S1.4 Daily rainfall data collected at the Hutchinson station, approximately 15 mi southwest of Inman, October 1, 2009, to March 31, 2010, from the Kansas Weather Data Library (http://wdl.agron.ksu.edu/).

\begin{tabular}{|c|c|}
\hline Date & $\begin{array}{c}\text { Total } \\
\text { Precipitation } \\
\text { (in.) }\end{array}$ \\
\hline $7 / 1 / 2011$ & 0.00 \\
\hline $7 / 2 / 2011$ & 0.00 \\
\hline $7 / 3 / 2011$ & 0.11 \\
\hline $7 / 4 / 2011$ & 0.00 \\
\hline $7 / 5 / 2011$ & 0.00 \\
\hline $7 / 6 / 2011$ & 0.00 \\
\hline $7 / 7 / 2011$ & 0.02 \\
\hline $7 / 8 / 2011$ & 0.00 \\
\hline $7 / 9 / 2011$ & 0.00 \\
\hline $7 / 10 / 2011$ & 0.08 \\
\hline $7 / 11 / 2011$ & 0.00 \\
\hline $7 / 12 / 2011$ & 0.00 \\
\hline $7 / 13 / 2011$ & 0.00 \\
\hline $7 / 14 / 2011$ & 0.00 \\
\hline $7 / 15 / 2011$ & 0.00 \\
\hline $7 / 16 / 2011$ & 0.00 \\
\hline $7 / 17 / 2011$ & 0.00 \\
\hline $7 / 18 / 2011$ & 0.00 \\
\hline $7 / 19 / 2011$ & 0.00 \\
\hline $7 / 20 / 2011$ & 0.00 \\
\hline $7 / 21 / 2011$ & 0.00 \\
\hline $7 / 22 / 2011$ & 0.00 \\
\hline $7 / 23 / 2011$ & 0.00 \\
\hline $7 / 24 / 2011$ & 0.14 \\
\hline $7 / 25 / 2011$ & 0.00 \\
\hline $7 / 26 / 2011$ & 0.00 \\
\hline $7 / 27 / 2011$ & 0.00 \\
\hline $7 / 28 / 2011$ & 0.00 \\
\hline $7 / 29 / 2011$ & 0.00 \\
\hline $7 / 30 / 2011$ & 0.06 \\
\hline $7 / 31 / 2011$ & 0.00 \\
\hline $8 / 1 / 2011$ & 0.00 \\
\hline $8 / 2 / 2011$ & 0.00 \\
\hline $8 / 3 / 2011$ & 0.14 \\
\hline $8 / 4 / 2011$ & 0.04 \\
\hline $8 / 5 / 2011$ & 0.00 \\
\hline $8 / 6 / 2011$ & 0.71 \\
\hline 8/7/2011 & 0.32 \\
\hline $8 / 8 / 2011$ & 0.04 \\
\hline
\end{tabular}


TABLE S1.4 Daily rainfall data collected at the Hutchinson station, approximately 15 mi southwest of Inman, October 1, 2009, to March 31, 2010, from the Kansas Weather Data Library (http://wdl.agron.ksu.edu/).

\begin{tabular}{|c|c|}
\hline Date & $\begin{array}{c}\text { Total } \\
\text { Precipitation } \\
\text { (in.) }\end{array}$ \\
\hline $8 / 9 / 2011$ & 0.06 \\
\hline $8 / 10 / 2011$ & 0.66 \\
\hline $8 / 11 / 2011$ & 0.00 \\
\hline $8 / 12 / 2011$ & 0.00 \\
\hline $8 / 13 / 2011$ & 0.00 \\
\hline $8 / 14 / 2011$ & 0.00 \\
\hline $8 / 15 / 2011$ & 0.00 \\
\hline $8 / 16 / 2011$ & 0.01 \\
\hline $8 / 17 / 2011$ & 0.00 \\
\hline $8 / 18 / 2011$ & 0.05 \\
\hline $8 / 19 / 2011$ & 0.05 \\
\hline $8 / 20 / 2011$ & 0.02 \\
\hline $8 / 21 / 2011$ & 0.00 \\
\hline $8 / 22 / 2011$ & 0.02 \\
\hline $8 / 23 / 2011$ & 0.00 \\
\hline $8 / 24 / 2011$ & 0.00 \\
\hline $8 / 25 / 2011$ & 0.00 \\
\hline $8 / 26 / 2011$ & 0.00 \\
\hline $8 / 27 / 2011$ & 0.00 \\
\hline $8 / 28 / 2011$ & 0.00 \\
\hline $8 / 29 / 2011$ & 0.00 \\
\hline $8 / 30 / 2011$ & 0.01 \\
\hline $8 / 31 / 2011$ & 0.00 \\
\hline $9 / 1 / 2011$ & 0.00 \\
\hline $9 / 2 / 2011$ & 0.00 \\
\hline $9 / 3 / 2011$ & 0.00 \\
\hline $9 / 4 / 2011$ & 0.00 \\
\hline $9 / 5 / 2011$ & 0.00 \\
\hline 9/6/2011 & 0.00 \\
\hline 9/7/2011 & 0.00 \\
\hline $9 / 8 / 2011$ & 0.00 \\
\hline 9/9/2011 & 0.00 \\
\hline $9 / 10 / 2011$ & 0.00 \\
\hline $9 / 11 / 2011$ & 0.00 \\
\hline $9 / 12 / 2011$ & 0.00 \\
\hline $9 / 13 / 2011$ & 0.00 \\
\hline $9 / 14 / 2011$ & 0.00 \\
\hline $9 / 15 / 2011$ & 0.00 \\
\hline $9 / 16 / 2011$ & 0.00 \\
\hline
\end{tabular}


TABLE S1.4 Daily rainfall data collected at the Hutchinson station, approximately 15 mi southwest of Inman, October 1, 2009, to March 31, 2010, from the Kansas Weather Data Library (http://wdl.agron.ksu.edu/).

\begin{tabular}{|c|c|}
\hline Date & $\begin{array}{c}\text { Total } \\
\text { Precipitation } \\
\text { (in.) }\end{array}$ \\
\hline $9 / 17 / 2011$ & 0.23 \\
\hline 9/18/2011 & 0.43 \\
\hline 9/19/2011 & 0.00 \\
\hline $9 / 20 / 2011$ & 0.00 \\
\hline $9 / 21 / 2011$ & 0.00 \\
\hline $9 / 22 / 2011$ & 0.00 \\
\hline $9 / 23 / 2011$ & 0.00 \\
\hline $9 / 24 / 2011$ & 0.00 \\
\hline $9 / 25 / 2011$ & 0.00 \\
\hline 9/26/2011 & 0.00 \\
\hline 9/27/2011 & 0.00 \\
\hline $9 / 28 / 2011$ & 0.00 \\
\hline 9/29/2011 & 0.00 \\
\hline 9/30/2011 & 0.00 \\
\hline $10 / 1 / 2011$ & 0.00 \\
\hline $10 / 2 / 2011$ & 0.00 \\
\hline $10 / 3 / 2011$ & 0.00 \\
\hline $10 / 4 / 2011$ & 0.00 \\
\hline $10 / 5 / 2011$ & 0.00 \\
\hline $10 / 6 / 2011$ & 0.00 \\
\hline $10 / 7 / 2011$ & 0.00 \\
\hline $10 / 8 / 2011$ & 0.48 \\
\hline $10 / 9 / 2011$ & 0.96 \\
\hline $10 / 10 / 2011$ & 0.00 \\
\hline $10 / 11 / 2011$ & 0.00 \\
\hline $10 / 12 / 2011$ & 0.00 \\
\hline $10 / 13 / 2011$ & 0.00 \\
\hline $10 / 14 / 2011$ & 0.00 \\
\hline $10 / 15 / 2011$ & 0.00 \\
\hline $10 / 16 / 2011$ & 0.00 \\
\hline 10/17/2011 & 0.02 \\
\hline 10/18/2011 & 0.00 \\
\hline $10 / 19 / 2011$ & 0.00 \\
\hline $10 / 20 / 2011$ & 0.00 \\
\hline 10/21/2011 & 0.00 \\
\hline $10 / 22 / 2011$ & 0.00 \\
\hline $10 / 23 / 2011$ & 0.00 \\
\hline $10 / 24 / 2011$ & 0.00 \\
\hline $10 / 25 / 2011$ & 0.00 \\
\hline
\end{tabular}


TABLE S1.4 Daily rainfall data collected at the Hutchinson station, approximately 15 mi southwest of Inman, October 1, 2009, to March 31, 2010, from the Kansas Weather Data Library (http://wdl.agron.ksu.edu/).

\begin{tabular}{|c|c|}
\hline Date & $\begin{array}{c}\text { Total } \\
\text { Precipitation } \\
\text { (in.) }\end{array}$ \\
\hline $10 / 26 / 2011$ & 0.00 \\
\hline 10/27/2011 & 0.00 \\
\hline $10 / 28 / 2011$ & 0.00 \\
\hline $10 / 29 / 2011$ & 0.00 \\
\hline $10 / 30 / 2011$ & 0.00 \\
\hline $10 / 31 / 2011$ & 0.00 \\
\hline $11 / 1 / 2011$ & 0.00 \\
\hline $11 / 2 / 2011$ & 0.23 \\
\hline $11 / 3 / 2011$ & 0.00 \\
\hline $11 / 4 / 2011$ & 0.00 \\
\hline $11 / 5 / 2011$ & 0.00 \\
\hline $11 / 6 / 2011$ & 0.00 \\
\hline $11 / 7 / 2011$ & 0.97 \\
\hline $11 / 8 / 2011$ & 0.44 \\
\hline $11 / 9 / 2011$ & 0.00 \\
\hline $11 / 10 / 2011$ & 0.00 \\
\hline $11 / 11 / 2011$ & 0.00 \\
\hline $11 / 12 / 2011$ & 0.00 \\
\hline $11 / 13 / 2011$ & 0.00 \\
\hline $11 / 14 / 2011$ & 0.00 \\
\hline $11 / 15 / 2011$ & 0.00 \\
\hline $11 / 16 / 2011$ & 0.00 \\
\hline $11 / 17 / 2011$ & 0.00 \\
\hline $11 / 18 / 2011$ & 0.00 \\
\hline $11 / 19 / 2011$ & 0.00 \\
\hline $11 / 20 / 2011$ & 0.00 \\
\hline $11 / 21 / 2011$ & 0.00 \\
\hline $11 / 22 / 2011$ & 0.01 \\
\hline $11 / 23 / 2011$ & 0.00 \\
\hline $11 / 24 / 2011$ & 0.00 \\
\hline $11 / 25 / 2011$ & 0.46 \\
\hline $11 / 26 / 2011$ & 0.35 \\
\hline $11 / 27 / 2011$ & 0.00 \\
\hline $11 / 28 / 2011$ & 0.00 \\
\hline $11 / 29 / 2011$ & 0.00 \\
\hline $11 / 30 / 2011$ & 0.00 \\
\hline $12 / 1 / 2011$ & 0.00 \\
\hline $12 / 2 / 2011$ & 0.04 \\
\hline $12 / 3 / 2011$ & 0.60 \\
\hline
\end{tabular}


TABLE S1.4 Daily rainfall data collected at the Hutchinson station, approximately 15 mi southwest of Inman, October 1, 2009, to March 31, 2010, from the Kansas Weather Data Library (http://wdl.agron.ksu.edu/).

\begin{tabular}{|c|c|}
\hline Date & $\begin{array}{c}\text { Total } \\
\text { Precipitation } \\
\text { (in.) }\end{array}$ \\
\hline $12 / 4 / 2011$ & 0.00 \\
\hline $12 / 5 / 2011$ & 0.00 \\
\hline $12 / 6 / 2011$ & 0.00 \\
\hline $12 / 7 / 2011$ & 0.00 \\
\hline $12 / 8 / 2011$ & 0.00 \\
\hline $12 / 9 / 2011$ & 0.00 \\
\hline $12 / 10 / 2011$ & 0.00 \\
\hline $12 / 11 / 2011$ & 0.00 \\
\hline $12 / 12 / 2011$ & 0.00 \\
\hline $12 / 13 / 2011$ & 0.19 \\
\hline $12 / 14 / 2011$ & 0.32 \\
\hline $12 / 15 / 2011$ & 0.00 \\
\hline $12 / 16 / 2011$ & 0.00 \\
\hline $12 / 17 / 2011$ & 0.00 \\
\hline $12 / 18 / 2011$ & 0.00 \\
\hline $12 / 19 / 2011$ & 1.27 \\
\hline $12 / 20 / 2011$ & 0.04 \\
\hline $12 / 21 / 2011$ & 0.01 \\
\hline $12 / 22 / 2011$ & 0.00 \\
\hline $12 / 23 / 2011$ & 0.00 \\
\hline $12 / 24 / 2011$ & 0.00 \\
\hline $12 / 25 / 2011$ & 0.00 \\
\hline $12 / 26 / 2011$ & 0.00 \\
\hline $12 / 27 / 2011$ & 0.00 \\
\hline $12 / 28 / 2011$ & 0.00 \\
\hline $12 / 29 / 2011$ & 0.00 \\
\hline $12 / 30 / 2011$ & 0.00 \\
\hline $12 / 31 / 2011$ & 0.00 \\
\hline $1 / 1 / 2012$ & 0.00 \\
\hline $1 / 2 / 2012$ & 0.00 \\
\hline $1 / 3 / 2012$ & 0.00 \\
\hline $1 / 4 / 2012$ & 0.00 \\
\hline $1 / 5 / 2012$ & 0.00 \\
\hline $1 / 6 / 2012$ & 0.00 \\
\hline $1 / 7 / 2012$ & 0.00 \\
\hline $1 / 8 / 2012$ & 0.00 \\
\hline 1/9/2012 & 0.00 \\
\hline $1 / 10 / 2012$ & 0.00 \\
\hline $1 / 11 / 2012$ & 0.00 \\
\hline
\end{tabular}


TABLE S1.4 Daily rainfall data collected at the Hutchinson station, approximately 15 mi southwest of Inman, October 1, 2009, to March 31, 2010, from the Kansas Weather Data Library (http://wdl.agron.ksu.edu/).

\begin{tabular}{|c|c|}
\hline Date & $\begin{array}{c}\text { Total } \\
\text { Precipitation } \\
\text { (in.) }\end{array}$ \\
\hline $1 / 12 / 2012$ & 0.00 \\
\hline $1 / 13 / 2012$ & 0.00 \\
\hline $1 / 14 / 2012$ & 0.00 \\
\hline $1 / 15 / 2012$ & 0.00 \\
\hline $1 / 16 / 2012$ & 0.00 \\
\hline $1 / 17 / 2012$ & 0.00 \\
\hline $1 / 18 / 2012$ & 0.00 \\
\hline $1 / 19 / 2012$ & 0.00 \\
\hline $1 / 20 / 2012$ & 0.00 \\
\hline $1 / 21 / 2012$ & 0.00 \\
\hline $1 / 22 / 2012$ & 0.07 \\
\hline $1 / 23 / 2012$ & 0.00 \\
\hline $1 / 24 / 2012$ & 0.00 \\
\hline $1 / 25 / 2012$ & 0.00 \\
\hline $1 / 26 / 2012$ & 0.00 \\
\hline $1 / 27 / 2012$ & 0.00 \\
\hline $1 / 28 / 2012$ & 0.00 \\
\hline $1 / 29 / 2012$ & 0.00 \\
\hline $1 / 30 / 2012$ & 0.00 \\
\hline $1 / 31 / 2012$ & 0.00 \\
\hline 2/1/2012 & 0.00 \\
\hline $2 / 2 / 2012$ & 0.68 \\
\hline 2/3/2012 & 1.44 \\
\hline $2 / 4 / 2012$ & 0.00 \\
\hline $2 / 5 / 2012$ & 0.00 \\
\hline 2/6/2012 & 0.01 \\
\hline 2/7/2012 & 0.05 \\
\hline 2/8/2012 & 0.00 \\
\hline 2/9/2012 & 0.00 \\
\hline 2/10/2012 & 0.00 \\
\hline $2 / 11 / 2012$ & 0.00 \\
\hline $2 / 12 / 2012$ & 0.00 \\
\hline $2 / 13 / 2012$ & 0.00 \\
\hline $2 / 14 / 2012$ & 0.00 \\
\hline $2 / 15 / 2012$ & 0.00 \\
\hline $2 / 16 / 2012$ & 0.00 \\
\hline $2 / 17 / 2012$ & 0.01 \\
\hline $2 / 18 / 2012$ & 0.02 \\
\hline 2/19/2012 & 0.00 \\
\hline
\end{tabular}


TABLE S1.4 Daily rainfall data collected at the Hutchinson station, approximately 15 mi southwest of Inman, October 1, 2009, to March 31, 2010, from the Kansas Weather Data Library (http://wdl.agron.ksu.edu/).

\begin{tabular}{|c|c|}
\hline Date & $\begin{array}{c}\text { Total } \\
\text { Precipitation } \\
\text { (in.) }\end{array}$ \\
\hline 2/20/2012 & 0.13 \\
\hline 2/21/2012 & 0.00 \\
\hline $2 / 22 / 2012$ & 0.00 \\
\hline 2/23/2012 & 0.00 \\
\hline $2 / 24 / 2012$ & 0.00 \\
\hline $2 / 25 / 2012$ & 0.00 \\
\hline 2/26/2012 & 0.00 \\
\hline 2/27/2012 & 0.00 \\
\hline 2/28/2012 & 0.47 \\
\hline 2/29/2012 & 0.00 \\
\hline $3 / 1 / 2012$ & 0.00 \\
\hline $3 / 2 / 2012$ & 0.00 \\
\hline $3 / 3 / 2012$ & 0.00 \\
\hline $3 / 4 / 2012$ & 0.00 \\
\hline $3 / 5 / 2012$ & 0.00 \\
\hline $3 / 6 / 2012$ & 0.00 \\
\hline $3 / 7 / 2012$ & 0.00 \\
\hline $3 / 8 / 2012$ & 0.07 \\
\hline $3 / 9 / 2012$ & 0.00 \\
\hline $3 / 10 / 2012$ & 0.00 \\
\hline $3 / 11 / 2012$ & 0.18 \\
\hline $3 / 12 / 2012$ & 0.00 \\
\hline $3 / 13 / 2012$ & 0.00 \\
\hline $3 / 14 / 2012$ & 0.00 \\
\hline $3 / 15 / 2012$ & 0.00 \\
\hline $3 / 16 / 2012$ & 0.00 \\
\hline $3 / 17 / 2012$ & 0.00 \\
\hline $3 / 18 / 2012$ & 0.00 \\
\hline 3/19/2012 & 0.76 \\
\hline $3 / 20 / 2012$ & 0.15 \\
\hline $3 / 21 / 2012$ & 0.63 \\
\hline $3 / 22 / 2012$ & 0.67 \\
\hline $3 / 23 / 2012$ & 0.02 \\
\hline $3 / 24 / 2012$ & 0.00 \\
\hline $3 / 25 / 2012$ & 0.00 \\
\hline $3 / 26 / 2012$ & 0.00 \\
\hline $3 / 27 / 2012$ & 0.00 \\
\hline $3 / 28 / 2012$ & 0.00 \\
\hline 3/29/2012 & 0.00 \\
\hline
\end{tabular}


TABLE S1.4 Daily rainfall data collected at the Hutchinson station, approximately 15 mi southwest of Inman, October 1, 2009, to March 31, 2010, from the Kansas Weather Data Library (http://wdl.agron.ksu.edu/).

\begin{tabular}{ll}
\hline & $\begin{array}{c}\text { Total } \\
\text { Precipitation } \\
\text { Date }\end{array}$ \\
\hline & (in.) \\
$3 / 30 / 2012$ & 0.00 \\
$3 / 31 / 2012$ & 0.00 \\
\hline
\end{tabular}




\section{Supplement 2:}

Quality Control for Sample Collection, Handling, and Analysis 


\section{Supplement 2:}

\section{Quality Control for Sample Collection, Handling, and Analysis}

This supplement documents the quality of the analytical data generated during the 20092011 investigations at Inman. The QA/QC procedures for sample collection, handling, and analysis during the investigation were described in detail in the Master Work Plan (Argonne 2002). The analytical results for quality control samples (replicates, duplicates, and verification samples) confirm that the data generated during the investigation reliably and accurately characterize the environmental conditions at the site. Field replicate samples verify the reproducibility of the sampling methodology, laboratory duplicate samples verify the reproducibility of the analytical results obtained at the AGEM Laboratory, and verification samples verify the accuracy of the analytical data. The AGEM QA/QC program (described in the Master Work Plan; Argonne 2002) is based on EPA (2008) guidelines.

\section{S2.1 Sampling to Monitor Sampling Collection, Handling, and Analysis Activities}

Sample collection and handling activities were monitored by the documentation of samples as they were collected and the use of chain-of-custody (COC) forms and custody seals to ensure sample integrity during handling and shipment. Field blanks, equipment rinsates, and trip blanks were collected to monitor sample collection and handling activities. Field replicate samples were collected, and samples were selected for duplicate analysis as a measure of analytical precision. The QA/QC samples are listed in Table S2.1. Analytical results for carbon tetrachloride and chloroform in QA/QC samples collected to monitor sample collection and handling activities are in Table S2.2.

\section{S2.1.1 Field Blanks}

To verify that contamination detected did not derive from water used during sampling activities, two field blanks of water were collected. The water in both field blanks came from the city water supply; one was from the field holding container storing water used for drilling, and the other was from the city water supply itself, which was the source of the water used for equipment decontamination (Table S2.1). Carbon tetrachloride was not detected in the field 
blanks. Chloroform was detected in both field blanks at low concentrations consistent with chlorination of the water supply $(<5 \mu \mathrm{g} / \mathrm{L}$; Table S2.2).

\section{S2.1.2 Equipment Rinsates}

To verify that equipment decontamination procedures were adequate during the field efforts, two equipment rinsates were collected (Table S2.1). Neither carbon tetrachloride nor chloroform was detected in the rinsate samples (Table S2.2).

\section{S2.1.3 Trip Blanks}

As an indicator of potential cross-contamination of samples during shipment, 45 trip blanks were prepared and included in shipments of soil or water samples to laboratories for organic analysis. Included in this total were 22 water trip blanks and 10 soil trip blanks sent to the AGEM Laboratory and 11 water trip blanks and 2 soil trip blanks sent to TestAmerica. The analytical results (Table S2.2) indicate that sample handling procedures were followed during the investigation and that cross-contamination of samples did not occur during shipment.

\section{S2.1.4 Replicate Samples and Duplicate Analyses}

As an indicator of the consistency of the sampling methodology followed and to provide a measure of analytical precision, replicate soil and groundwater samples were collected, and samples were also selected by the AGEM Laboratory for duplicate organic analysis. In addition, selected soil and groundwater samples were submitted for verification organic analysis at a secondary laboratory. Replicate samples, samples selected for duplicate analysis, and samples selected for verification organic analysis are listed in Table S2.1.

\section{S2.2 Quality Control for Organic Analysis of Soil and Water Samples at the AGEM Laboratory}

Soil samples collected during the investigation were quick-frozen on dry ice as they were collected and were shipped to the AGEM Laboratory for VOCs analysis by two analytical methods: (1) the headspace method to provide a rapid-turnaround qualitative measure of 
contaminant distribution for evaluation in the field, and (2) the purge-and-trap method to provide a quantitative measure of contaminant distribution.

The soil samples were first analyzed at the AGEM Laboratory (on a rapid-turnaround basis) by using a modification of the protocol in EPA Method 5021 (headspace analysis on a GC with electron capture detection). An 11-point calibration of the GC system was established on the basis of the mass of known quantities of carbon tetrachloride and chloroform ranging from 0.125 to 4.0 nanograms. Typical detection limits achieved were $0.1 \mu \mathrm{g} / \mathrm{kg}$ for carbon tetrachloride and $0.75 \mu \mathrm{g} / \mathrm{kg}$ for chloroform. The analytical data obtained with this method are acceptable for qualitative determination of contaminant distribution. The data were evaluated to select locations for deeper soil boring and sampling.

Aliquots of soil samples were subsequently analyzed for carbon tetrachloride, chloroform, and methylene chloride (contaminants of concern in the investigation) by a modification of EPA Method 8260B (purge-and-trap method), as referenced in the EPA's SW-846 (EPA 1998), to achieve a method detection limit of $10 \mu \mathrm{g} / \mathrm{kg}$. At the laboratory, the VOCs in each soil sample were extracted with methanol from the sample matrix. For the purgeand-trap soil analyses, an aliquot of the methanol extract was purged, and the volatile species were transferred to a sorbent tube. After purging, the sorbent tube was heated and backflushed with an inert gas to desorb the components into the GC-MS system.

Groundwater samples (and associated QC samples) were sent to the AGEM Laboratory for VOCs analysis by modified EPA Method 524.2 (EPA 1995) to achieve a method detection limit of $1 \mu \mathrm{g} / \mathrm{L}$. For the purge-and-trap analyses on a GC-MS system, the VOCs present in each groundwater sample were extracted (purged) from the sample matrix by bubbling an inert gas through the sample. The purged components were trapped in a sorbent tube, and the sorbent tube was heated and backflushed with an inert gas to desorb the components into the GC-MS system.

In total, 186 soil samples were analyzed for VOCs, including 13 replicate soil samples. A total of 105 groundwater samples were collected for VOCs analysis, including 5 replicate samples.

For both the soil and water analyses, the compounds eluting from the GC column were identified by retention time and by comparison with reference library spectra. The concentration of each component was calculated by comparison of the MS response for the quantitation ion to 
corresponding calibration curves, the response for internal standards, or both. The internal standard recovery limits were $80-120 \%$. Calibration checks with each sample delivery group (SDG) were required to be within $\pm 20 \%$ of the standard.

Every analytical laboratory statistically defines analyte-specific instrument detection limits and method detection limits. The instrument detection limit is less than the method detection limit. An instrument detection limit is the analyte concentration required to produce a signal three times the signal-to-noise ratio. Noise - a measure of the fluctuation in background signal that occurs even in the absence of an analyte - is dependent on instrument and laboratory conditions. The noise value is calculated from the standard deviation of a certain number of measurements of the background signal. The instrument detection limit is specific to each instrument and can vary by a small degree with each sample and analysis performed on that instrument.

A method detection limit is defined as "the minimum concentration of a substance that can be measured and reported with $99 \%$ confidence that the analyte concentration is greater than zero" (EPA 1997). The method detection limit is defined after the analyte in the sample has been extracted, isolated, and analyzed, thus taking into account matrix effects due to analysis of a lessthan-pure analyte. Given matrix effects and unavoidable analyte loss through all steps of the analytical procedure, the method detection limit can be 2 to 100 times higher than the instrument detection limit. For the AGEM Laboratory, the method detection limit is typically approximately 10 times the instrument detection limit.

A concentration in a sample below the method detection limit that does not produce a signal distinguishable from background noise is reported as non-detectable, with notation ND. For carbon tetrachloride and chloroform analyses at the AGEM Laboratory, such concentrations are $<1 \mu \mathrm{g} / \mathrm{L}$ in groundwater or $<10 \mu \mathrm{g} / \mathrm{kg}$ in soil.

A concentration in a sample above the instrument detection limit and below the method detection limit that produces a signal distinguishable from background noise is marked with a $\mathbf{J}$ qualifier. These results are associated with a relatively high degree of uncertainty, because they are below the $99 \%$ confidence level specified by the method detection limit. Because results with a J qualifier are below the method detection limit, they can also be reported with the notation ND. 
Samples submitted to the AGEM Laboratory for organic analysis by the purge-and-trap method were analyzed in 36 SDGs, as shown in Table S2.3. The QA/QC procedures followed included analysis of instrument calibration check standards, analysis of laboratory blanks, monitoring of surrogate spike recovery, and duplicate laboratory analyses. Significant results include the following:

- Samples shipped to the AGEM Laboratory were received with custody seals intact and at the appropriate temperature. Samples were analyzed within required holding times.

- Carbon tetrachloride and chloroform were not detected in laboratory method blanks analyzed with the samples.

- For each SDG, analytical instrument calibration was monitored by the analysis of calibration check standards. Table S2.3 shows the relative percent difference (RPD) between the known and calculated concentrations of the standards. The concentrations of calibration check standards measured in all SDGs were within the acceptable range of $\pm 20 \%$.

- Surrogate standard determinations were performed on samples and blanks by using surrogate spike compounds fluorobenzene, dichlorobenzene- $\mathrm{d} 4$, and bromofluorobenzene. Table S2.3 shows the percent recovery of these systemmonitoring compounds for each of the analyses. The surrogate recoveries were within the specified range of $80-120 \%$ for all samples in either the initial analysis or a successful reanalysis.

- Secondary QC analyses of soil and groundwater samples at the AGEM Laboratory were conducted throughout the investigation with the purge-andtrap method as a measure of the consistency in the sampling and analytical methodologies. This was accomplished through the analysis of replicate samples submitted to the laboratory or duplicate analysis of samples selected by the laboratory. Table S2.4 summarizes the analytical results for carbon tetrachloride and chloroform in the primary samples and their associated replicate or duplicate analyses. Consistency in both the sampling and analytical methodologies is indicated by the average RPD values of $17 \%$ for 
carbon tetrachloride and $10 \%$ for chloroform for dual analyses with the contaminants present above the method quantitation limit.

- Because the AGEM Laboratory was closed during a portion of December 2011, groundwater samples collected on December 22, 2011, were analyzed at TestAmerica, rather than the AGEM Laboratory. Sample identifier INTI25W-32701 incorrectly indicates location TI25 because of a typographical error in the sample identifier sent to TestAmerica. The groundwater result for this sample is for location TI24.

The analytical data from the AGEM Laboratory are acceptable for quantitative determination of contaminant distribution.

\section{S2.3 Quality Control for Verification Organic Analysis of Soil and Groundwater Samples by TestAmerica}

In accordance with the QA/QC procedures defined in the Master Work Plan (Argonne 2002), selected soil and groundwater samples analyzed at the AGEM Laboratory were subjected to verification analysis at a second laboratory with EPA Contract Laboratory Program methodology. Eighteen of the 186 vertical-profile soil samples (10\% of the soil samples) and 44 of the 105 groundwater samples ( $40 \%$ of the groundwater samples) analyzed at the AGEM Laboratory were also analyzed at TestAmerica. Table S2.5 compares the results from the two laboratories for the soil and groundwater samples submitted for verification organic analysis. The results from TestAmerica for the verification samples were reported in the 13 SDGs identified in Table S2.5. The data from TestAmerica are provided as Supplement 4 (on CD). An additional SDG from TestAmerica (also included in Supplement 4) provided the results for groundwater sampling on December 22, 2011, when the AGEM Laboratory was closed.

The QA/QC procedures followed at TestAmerica included initial and continuing calibration of instruments, analysis of laboratory blanks, monitoring of surrogate spike recovery, and analyses of laboratory control samples. Significant results included the following:

- Soil and groundwater samples shipped to TestAmerica were received with custody seals intact and at the appropriate preservation conditions. All samples were analyzed within required holding times. 
- Analytical instruments were properly tuned; initial and continuing calibration checks remained within the allowable range. Surrogate standard determinations were within allowable limits.

- Carbon tetrachloride and chloroform were not detected in the blanks analyzed with the samples.

Agreement between the two laboratories is good over the range of contaminant concentrations detected. The concentrations detected in analyses of soil and groundwater samples at the AGEM Laboratory are supported by the verification analyses at TestAmerica.

\section{S2.4 Quality Control for Inorganic Analysis of Groundwater Samples at TestAmerica}

Groundwater samples were collected for inorganic analysis to aid in geochemical characterization of the water-bearing zone. Groundwater samples collected for inorganic analysis were shipped immediately to TestAmerica Laboratories, Inc., University Park, Illinois, for filtration and analysis. The analyses included total alkalinity by EPA Method SM 2320B, dissolved anion concentrations (chloride, sulfate, nitrate, and phosphate) by EPA Method 300, and dissolved metals (aluminum, calcium, iron, magnesium, manganese, phosphorus, potassium, silicon, sodium, and zinc) by EPA Method 6010B.

Inorganic analyses of the groundwater samples were conducted in SDG 500-34569, included in Supplement 4 (on CD). The QA/QC procedures followed included instrument calibration through analysis of spiked calibration check standards, verification of inter-element and background correction factors through analysis of ICP (inductively coupled plasma) interference check samples, and analysis of laboratory control samples.

The inorganic results for groundwater samples from TestAmerica are acceptable for geochemical characterization on the basis of the recovery of known concentrations of the analytes of concern in laboratory control samples analyzed with the groundwater samples. 
TABLE S2.1 Quality control samples collected during the 2009-2011 investigation.

\begin{tabular}{|c|c|c|c|c|c|c|c|c|}
\hline $\begin{array}{l}\text { Sample } \\
\text { Date }\end{array}$ & Location & $\begin{array}{l}\text { Depth } \\
\text { (ft BGL) }\end{array}$ & Sample & $\begin{array}{l}\text { Sample } \\
\text { Medium }\end{array}$ & $\begin{array}{l}\text { Sample } \\
\text { Type }^{\mathrm{a}}\end{array}$ & $\begin{array}{l}\text { Chain of } \\
\text { Custody }\end{array}$ & $\begin{array}{l}\text { Shipping } \\
\text { Date }\end{array}$ & Sample Description \\
\hline \multicolumn{9}{|c|}{ Field blanks } \\
\hline $8 / 22 / 11$ & $\mathrm{QC}$ & - & INTRUCK-W-32731 & Water & FB & 1519 & $8 / 22 / 11$ & $\begin{array}{l}\text { Blank of water used during the Inman investigation, obtained } \\
\text { from the field holding container. }\end{array}$ \\
\hline $8 / 22 / 11$ & $\mathrm{QC}$ & - & INCITY-W-32732 & Water & FB & 1519 & $8 / 22 / 11$ & $\begin{array}{l}\text { Blank of water used during the Inman investigation, obtained } \\
\text { from the city water supply. }\end{array}$ \\
\hline \multicolumn{9}{|c|}{ Equipment rinsates } \\
\hline $11 / 9 / 09$ & $\mathrm{QC}$ & - & INQCTR-W-30753 & Water & $\mathrm{RI}$ & 3109 & $11 / 9 / 09$ & $\begin{array}{l}\text { Rinsate of decontaminated bailer after collection of sample } \\
\text { INTI08-W-30752. }\end{array}$ \\
\hline $11 / 11 / 09$ & $\mathrm{QC}$ & - & INQCIR-W-30759 & Water & $\mathrm{RI}$ & 3179 & $11 / 11 / 09$ & $\begin{array}{l}\text { Rinsate of decontaminated bailer after collection of sample } \\
\text { INTI02-W-30758. }\end{array}$ \\
\hline \multicolumn{9}{|c|}{ Trip blanks } \\
\hline $11 / 4 / 09$ & $\mathrm{QC}$ & - & INQCTB-S-30642 & Soil & TB & 3176 & $11 / 4 / 09$ & $\begin{array}{l}\text { Trip blank sent to the AGEM Laboratory for VOCs analysis } \\
\text { with soil samples listed on chain-of-custody forms } \\
\text { (COCs) } 3175 \text { and } 3176 \text {. }\end{array}$ \\
\hline $11 / 5 / 09$ & $\mathrm{QC}$ & - & INQCTB-S-30665 & Soil & TB & 3101 & $11 / 5 / 09$ & $\begin{array}{l}\text { Trip blank sent to the AGEM Laboratory for VOCs analysis } \\
\text { with soil samples listed on COCs } 3099 \text { and } 3101 .\end{array}$ \\
\hline $11 / 7 / 09$ & $\mathrm{QC}$ & - & INTB-S-30731 & Soil & TB & 3104 & $11 / 7 / 09$ & $\begin{array}{l}\text { Trip blank sent to the AGEM Laboratory for VOCs analysis } \\
\text { with soil samples listed on COCs } 3102,3103 \text {, and } 3104 \text {. }\end{array}$ \\
\hline $11 / 7 / 09$ & $\mathrm{QC}$ & - & INQCTB-S-30732 & Soil & TB & 3107 & $11 / 7 / 09$ & $\begin{array}{l}\text { Trip blank sent to the AGEM Laboratory for VOCs analysis } \\
\text { with soil samples listed on COCs } 3105,3106 \text {, and } 3107 \text {. }\end{array}$ \\
\hline $11 / 9 / 09$ & QC & - & INQCTB-S-30745 & Soil & TB & 3108 & $11 / 9 / 09$ & $\begin{array}{l}\text { Trip blank sent to the AGEM Laboratory for VOCs analysis } \\
\text { with soil samples listed on COC } 3108 \text {. }\end{array}$ \\
\hline $11 / 9 / 09$ & $\mathrm{QC}$ & - & INQCTB-W-30754 & Water & TB & 3109 & $11 / 9 / 09$ & $\begin{array}{l}\text { Trip blank sent to the AGEM Laboratory for VOCs analysis } \\
\text { with water samples listed on COC } 3109 \text {. }\end{array}$ \\
\hline $11 / 11 / 09$ & $\mathrm{QC}$ & - & INQCTB-W-30760 & Water & TB & 3179 & $11 / 11 / 09$ & $\begin{array}{l}\text { Trip blank sent to the AGEM Laboratory for VOCs analysis } \\
\text { with water samples listed on COC } 3179 \text {. }\end{array}$ \\
\hline $11 / 12 / 09$ & $\mathrm{QC}$ & - & IN-S-Meohblank-12Nov09 & Soil & TB & 3658 & $11 / 12 / 09$ & $\begin{array}{l}\text { Trip blank sent to TestAmerica for verification organic } \\
\text { analysis with soil samples listed on COC } 3658 \text {. }\end{array}$ \\
\hline $11 / 9 / 10$ & QC & - & INQCTB-W-32676 & Water & TB & 3020 & $11 / 9 / 10$ & $\begin{array}{l}\text { Trip blank sent to the AGEM Laboratory for VOCs analysis } \\
\text { with water samples listed on COC } 3020 \text {. }\end{array}$ \\
\hline $11 / 10 / 10$ & QC & - & INTI19-S-30776 & Soil & TB & 3022 & $11 / 10 / 10$ & $\begin{array}{l}\text { Trip blank sent to the AGEM Laboratory for VOCs analysis } \\
\text { with soil samples listed on COC } 3022 \text {. }\end{array}$ \\
\hline $11 / 10 / 10$ & $\mathrm{QC}$ & - & INTB-W-30764/AGEM & Water & TB & 3021 & $11 / 10 / 10$ & $\begin{array}{l}\text { Trip blank sent to the AGEM Laboratory for VOCs analysis } \\
\text { with water samples listed on COC } 3021 \text {. }\end{array}$ \\
\hline $11 / 10 / 10$ & QC & - & INTB-W-30764/TA & Water & TB & 3064 & $11 / 10 / 10$ & $\begin{array}{l}\text { Trip blank sent to TestAmerica for verification organic } \\
\text { analysis with water samples listed on COC } 3023 .\end{array}$ \\
\hline $11 / 11 / 10$ & QC & - & INQCTB-W-30787/AGEM & Water & TB & 3029 & $11 / 11 / 10$ & $\begin{array}{l}\text { Trip blank sent to the AGEM Laboratory for VOCs analysis } \\
\text { with water samples listed on COC } 3029 \text {. }\end{array}$ \\
\hline
\end{tabular}

Equipment rinsates

Trip blanks 
TABLE S2.1 (Cont.)

\begin{tabular}{|c|c|c|c|c|c|c|c|c|}
\hline $\begin{array}{l}\text { Sample } \\
\text { Date }\end{array}$ & Location & $\begin{array}{l}\text { Depth } \\
\text { (ft BGL) }\end{array}$ & Sample & $\begin{array}{l}\text { Sample } \\
\text { Medium }\end{array}$ & $\begin{array}{l}\text { Sample } \\
\text { Type }^{\mathrm{a}}\end{array}$ & $\begin{array}{l}\text { Chain of } \\
\text { Custody }\end{array}$ & $\begin{array}{l}\text { Shipping } \\
\text { Date }\end{array}$ & Sample Description \\
\hline \multicolumn{9}{|c|}{ Trip blanks (cont.) } \\
\hline $11 / 11 / 10$ & $\mathrm{QC}$ & - & INQCTB-W-30787/TA & Water & TB & 3030 & $11 / 11 / 10$ & $\begin{array}{l}\text { Trip blank sent to TestAmerica for verification organic } \\
\text { analysis with water samples listed on COC } 3030 .\end{array}$ \\
\hline $11 / 11 / 10$ & $\mathrm{QC}$ & - & INQCTB-S-30788 & Soil & TB & 3069 & $11 / 11 / 10$ & $\begin{array}{l}\text { Trip blank sent to the AGEM Laboratory for VOCs analysis } \\
\text { with soil samples listed on COC } 3069 \text {. }\end{array}$ \\
\hline $11 / 11 / 10$ & QC & - & INQCTB-S-30789 & Soil & TB & 3028 & $11 / 11 / 10$ & $\begin{array}{l}\text { Trip blank sent to the AGEM Laboratory for VOCs analysis } \\
\text { with soil samples listed on COC } 3028 \text {. }\end{array}$ \\
\hline $11 / 12 / 10$ & $\mathrm{QC}$ & - & INQCTB-S-30850 & Soil & TB & 3024 & $11 / 12 / 10$ & $\begin{array}{l}\text { Trip blank sent to the AGEM Laboratory for VOCs analysis } \\
\text { with soil samples listed on COC } 3024 \text {. }\end{array}$ \\
\hline $11 / 12 / 10$ & $\mathrm{QC}$ & - & INQCTB-S-30849 & Soil & TB & 3025 & $11 / 12 / 10$ & $\begin{array}{l}\text { Trip blank sent to the AGEM Laboratory for VOCs analysis } \\
\text { with soil samples listed on COC } 3025 \text {. }\end{array}$ \\
\hline $11 / 12 / 10$ & $\mathrm{QC}$ & - & INQCTB-W-30851 & Water & TB & 3031 & $11 / 12 / 10$ & $\begin{array}{l}\text { Trip blank sent to the AGEM Laboratory for VOCs analysis } \\
\text { with water samples listed on COC } 3031 \text {. }\end{array}$ \\
\hline $11 / 12 / 10$ & $\mathrm{QC}$ & - & INQCTB-W-30852 & Water & TB & 3031 & $11 / 12 / 10$ & $\begin{array}{l}\text { Trip blank sent to the AGEM Laboratory for VOCs analysis } \\
\text { with water samples listed on COC } 3031 \text {. }\end{array}$ \\
\hline $11 / 18 / 10$ & $\mathrm{QC}$ & - & INQCTB-W-32684 & Water & TB & 3032 & $11 / 18 / 10$ & $\begin{array}{l}\text { Trip blank sent to the AGEM Laboratory for VOCs analysis } \\
\text { with water samples listed on COC } 3032 \text {. }\end{array}$ \\
\hline $11 / 18 / 10$ & $\mathrm{QC}$ & - & IN-S-Meohblank-1118 & Meoh & TB & 4904 & $11 / 18 / 10$ & $\begin{array}{l}\text { Trip blank with soil samples for verification organic analysis } \\
\text { to TestAmerica listed on COC } 4904 \text {. }\end{array}$ \\
\hline $12 / 22 / 10$ & $\mathrm{QC}$ & - & INQCTB-W-32702 & Water & TB & 2705 & $12 / 22 / 10$ & $\begin{array}{l}\text { Trip blank with water samples for organic analysis to } \\
\text { TestAmerica, Inc. listed on COCs } 2704 \text { and } 2705 \text {. }\end{array}$ \\
\hline $5 / 24 / 11$ & $\mathrm{QC}$ & - & INQCTB-W-32692/AGEM & Water & TB & 2965 & $5 / 24 / 11$ & $\begin{array}{l}\text { Trip blank sent to the AGEM Laboratory for VOCs analysis } \\
\text { with water samples listed on COC } 2965 \text {. }\end{array}$ \\
\hline $5 / 24 / 11$ & $\mathrm{QC}$ & - & INQCTB-W-32692/TA & Water & TB & 2966 & $5 / 24 / 11$ & $\begin{array}{l}\text { Trip blank sent to TestAmerica for verification organic } \\
\text { analysis with water samples listed on COC } 2966 .\end{array}$ \\
\hline $6 / 23 / 11$ & $\mathrm{QC}$ & - & INQCTB-W-32696/AGEM & Water & TB & 2980 & $6 / 23 / 11$ & $\begin{array}{l}\text { Trip blank sent to the AGEM Laboratory for VOCs analysis } \\
\text { with water samples listed on COC } 2980 \text {. }\end{array}$ \\
\hline $6 / 23 / 11$ & $\mathrm{QC}$ & - & INQCTB-W-32696/TA & Water & TB & 2981 & $6 / 23 / 11$ & $\begin{array}{l}\text { Trip blank sent to TestAmerica for verification organic } \\
\text { analysis with water samples listed on COC } 2981 .\end{array}$ \\
\hline $8 / 22 / 11$ & $\mathrm{QC}$ & - & INQCTB-W-32733 & Water & TB & 1519 & $8 / 22 / 11$ & $\begin{array}{l}\text { Trip blank sent to the AGEM Laboratory for VOCs analysis } \\
\text { with water samples listed on COC } 1519 .\end{array}$ \\
\hline $8 / 24 / 11$ & $\mathrm{QC}$ & - & INQCTB-W-32738/AGEM & Water & TB & 2860 & $8 / 24 / 11$ & $\begin{array}{l}\text { Trip blank sent to the AGEM Laboratory for VOCs analysis } \\
\text { with water samples listed on COC } 2860 \text {. }\end{array}$ \\
\hline $8 / 24 / 11$ & $\mathrm{QC}$ & - & INQCTB-W-32738/TA & Water & TB & 2859 & $8 / 24 / 11$ & $\begin{array}{l}\text { Trip blank sent to TestAmerica for verification organic } \\
\text { analysis with water samples listed on COC } 2859 .\end{array}$ \\
\hline 8/25/11 & $\mathrm{QC}$ & - & INQCTB-W-32744/AGEM & Water & TB & 2850 & $8 / 25 / 11$ & $\begin{array}{l}\text { Trip blank sent to the AGEM Laboratory for VOCs analysis } \\
\text { with water samples listed on COC } 2850 \text {. }\end{array}$ \\
\hline 8/25/11 & $\mathrm{QC}$ & - & INQCTB-W-32744/TA & Water & TB & 2851 & $8 / 25 / 11$ & $\begin{array}{l}\text { Trip blank sent to TestAmerica for verification organic } \\
\text { analysis with water samples listed on COC } 2851 .\end{array}$ \\
\hline 8/27/11 & $\mathrm{QC}$ & - & INMW17QC-W-32746 & Water & TB & 2852 & $8 / 27 / 11$ & $\begin{array}{l}\text { Trip blank sent to the AGEM Laboratory for VOCs analysis } \\
\text { with water samples listed on COC } 2852 \text {. }\end{array}$ \\
\hline
\end{tabular}


TABLE S2.1 (Cont.)

\begin{tabular}{|c|c|c|c|c|c|c|c|c|}
\hline $\begin{array}{l}\text { Sample } \\
\text { Date }\end{array}$ & Location & $\begin{array}{l}\text { Depth } \\
\text { (ft BGL) }\end{array}$ & Sample & $\begin{array}{l}\text { Sample } \\
\text { Medium }\end{array}$ & $\begin{array}{l}\text { Sample } \\
\text { Type }^{a}\end{array}$ & $\begin{array}{l}\text { Chain of } \\
\text { Custody }\end{array}$ & $\begin{array}{l}\text { Shipping } \\
\text { Date }\end{array}$ & Sample Description \\
\hline \multicolumn{9}{|c|}{ Trip blanks (cont.) } \\
\hline 8/29/11 & QC & - & INQC-W-32763/AGEM & Water & TB & 3033 & $8 / 29 / 11$ & $\begin{array}{l}\text { Trip blank sent to the AGEM Laboratory for VOCs analysis } \\
\text { with water samples listed on COC } 3033 \text {. }\end{array}$ \\
\hline $8 / 29 / 11$ & $\mathrm{QC}$ & - & INQC-W-32763/TA & Water & TB & 2853 & $8 / 29 / 11$ & $\begin{array}{l}\text { Trip blank sent to TestAmerica for verification organic } \\
\text { analysis with water samples listed on COC } 2853 \text {. }\end{array}$ \\
\hline 8/29/11 & $\mathrm{QC}$ & - & INTB-W-32766 & Water & TB & 2854 & $8 / 29 / 11$ & $\begin{array}{l}\text { Trip blank sent to the AGEM Laboratory for VOCs analysis } \\
\text { with water samples listed on COC } 2854 \text {. }\end{array}$ \\
\hline 8/30/11 & QC & - & INQCTB-W-32769/AGEM & Water & TB & 2856 & $8 / 30 / 11$ & $\begin{array}{l}\text { Trip blank sent to the AGEM Laboratory for VOCs analysis } \\
\text { with water samples listed on COC } 2856 \text {. }\end{array}$ \\
\hline 8/30/11 & $\mathrm{QC}$ & - & INQCTB-W-32769/TA & Water & TB & 2855 & 8/30/11 & $\begin{array}{l}\text { Trip blank sent to TestAmerica for verification organic } \\
\text { analysis with water samples listed on COC } 2855 \text {. }\end{array}$ \\
\hline 8/31/11 & $\mathrm{QC}$ & - & INQCTB-W-32774 & Water & TB & 3035 & 8/31/11 & $\begin{array}{l}\text { Trip blank sent to the AGEM Laboratory for VOCs analysis } \\
\text { with water samples listed on COC } 3035 \text {. }\end{array}$ \\
\hline $9 / 1 / 11$ & $\mathrm{QC}$ & - & INQCTB-W-32778/AGEM & Water & TB & 3040 & $9 / 1 / 11$ & $\begin{array}{l}\text { Trip blank sent to the AGEM Laboratory for VOCs analysis } \\
\text { with water samples listed on COC } 3040 \text {. }\end{array}$ \\
\hline 9/1/11 & $\mathrm{QC}$ & - & INQCTB-W-32778/TA & Water & TB & 3039 & $9 / 1 / 11$ & $\begin{array}{l}\text { Trip blank sent to TestAmerica for verification organic } \\
\text { analysis with water samples listed on COC } 3039 .\end{array}$ \\
\hline $9 / 2 / 11$ & $\mathrm{QC}$ & - & INQCTB-W-32791 & Water & TB & 3037 & $9 / 2 / 11$ & $\begin{array}{l}\text { Trip blank sent to the AGEM Laboratory for VOCs analysis } \\
\text { with water samples listed on COC } 3037 \text {. }\end{array}$ \\
\hline 9/13/11 & $\mathrm{QC}$ & - & INQCTB-W-32777 & Water & TB & 3043 & $9 / 13 / 11$ & $\begin{array}{l}\text { Trip blank sent to the AGEM Laboratory for VOCs analysis } \\
\text { with water samples listed on COC } 3043 \text {. }\end{array}$ \\
\hline 9/15/11 & QC & - & INQCTB-W-32783/AGEM & Water & TB & 3044 & $9 / 15 / 11$ & $\begin{array}{l}\text { Trip blank sent to the AGEM Laboratory for VOCs analysis } \\
\text { with water samples listed on COC } 3044 \text {. }\end{array}$ \\
\hline 9/15/11 & QC & - & INQCTB-W-32783/TA & Water & TB & 3042 & $9 / 15 / 11$ & $\begin{array}{l}\text { Trip blank sent to TestAmerica for verification organic } \\
\text { analysis with water samples listed on COC } 3042 .\end{array}$ \\
\hline \multicolumn{9}{|c|}{ Replicate soil and groundwater samples } \\
\hline $11 / 4 / 09$ & TI01 & 34 & INTI01-S-30628 & Soil & CPT & 3175 & $11 / 4 / 09$ & Replicate of sample INTI01-S-30627. \\
\hline $11 / 4 / 09$ & TIO2 & 26 & INTI02-S-30638 & Soil & СРT & 3176 & $11 / 4 / 09$ & Replicate of sample INTI02-S-30637. \\
\hline $11 / 5 / 09$ & TIO4 & 38 & INTI04-S-30664 & Soil & CPT & 3101 & $11 / 5 / 09$ & Replicate of sample INTI04-S-30663. \\
\hline 11/6/09 & TI06 & 30 & INTI06-S-30684 & Soil & СРT & 3103 & 11/7/09 & Replicate of sample INTI06-S-30683. \\
\hline $11 / 6 / 09$ & TI07 & 38 & INTI07-S-30697 & Soil & CPT & 3104 & $11 / 7 / 09$ & Replicate of sample INTI07-S-30696. \\
\hline $11 / 7 / 09$ & T108 & 26 & INTI08-S-30705 & Soil & СРT & 3105 & 11/7/09 & Replicate of sample INTI08-S-30704. \\
\hline 11/7/09 & TI09 & 30 & INTI09-S-30717 & Soil & CPT & 3106 & $11 / 7 / 09$ & Replicate of sample INTI09-S-30716. \\
\hline $11 / 7 / 09$ & TI10 & 38 & INTI10-S-30730 & Soil & СРТ & 3107 & & Replicate of sample INTI10-S-30729. \\
\hline 11/8/09 & TI11 & 22 & INTI11-S-30740 & Soil & CPT & 3108 & $11 / 9 / 09$ & Replicate of sample INTI11-S-30739. \\
\hline 11/10/10 & TI19 & 24 & INTI19-S-30773 & Soil & СРТ & 3022 & 11/10/10 & Replicate of sample INTI19-S-30770. \\
\hline $11 / 11 / 10$ & TI20 & 36 & INTI20-S-30799 & Soil & CPT & 3069 & $11 / 11 / 10$ & Replicate of sample INTI20-S-30798. \\
\hline 11/11/10 & TI22 & 16 & INTI22-S-30818 & Soil & СРТ & 3028 & $11 / 11 / 10$ & Replicate of sample INTI22-S-30812. \\
\hline $11 / 12 / 10$ & TI24 & 32 & INTI24-S-30837 & Soil & СРТ & 3025 & $11 / 12 / 10$ & Replicate of sample INTI24-S-30835. \\
\hline
\end{tabular}


TABLE S2.1 (Cont.)

\begin{tabular}{|c|c|c|c|c|c|c|c|c|}
\hline $\begin{array}{l}\text { Sample } \\
\text { Date }\end{array}$ & Location & $\begin{array}{l}\text { Depth } \\
\text { (ft BGL) }\end{array}$ & Sample & $\begin{array}{l}\text { Sample } \\
\text { Medium }\end{array}$ & $\begin{array}{l}\text { Sample } \\
\text { Type }^{\mathrm{a}}\end{array}$ & $\begin{array}{l}\text { Chain of } \\
\text { Custody }\end{array}$ & $\begin{array}{l}\text { Shipping } \\
\text { Date }\end{array}$ & Sample Description \\
\hline
\end{tabular}

Replicate soil and groundwater samples (cont.)

$\begin{array}{cccllllll}\text { 12/22/10 } & \text { MW10 } & 31.7-41.7 & \text { INMW10DUP-W-32695 } & \text { Water } & \text { MW } & 2704 & 12 / 22 / 10 & \begin{array}{c}\text { Replicate of sample INMW10-W-32694. Analysis at } \\ \text { TestAmerica. }\end{array} \\ \text { 8/27/11 } & \text { MW17 } & 55-75 & \text { INMW17-W-32749 } & \text { Water } & \text { SB } & 2852 & 8 / 27 / 11 & \text { Replicate of sample INMW17-W-32748. } \\ \text { 8/28/11 } & \text { MW15M } & 86-96 & \text { INMW15-W-32751 } & \text { Water } & \text { MW } & 3033 & 8 / 29 / 11 & \text { Replicate of sample INMW15-W-32750. } \\ \text { 8/29/11 } & \text { MW18 } & 65-80 & \text { INMW18-W-32761 } & \text { Water } & \text { SB } & 3033 & 8 / 29 / 11 & \text { Replicate of sample INMW18-W-32760. } \\ \text { 8/29/11 } & \text { MW18 } & 85-90 & \text { INMW18-W-32755 } & \text { Water } & \text { MW } & 3033 & 8 / 29 / 11 & \text { Replicate of sample INMW18-W-32752. }\end{array}$

Samples selected by the AGEM Laboratory for duplicate organic analysis

\begin{tabular}{|c|c|c|c|c|c|c|c|c|}
\hline $11 / 4 / 09$ & TI02 & 10 & INTI02-S-30633 & Soil & CPT & 3175 & $11 / 4 / 09$ & Vertical-profile soil sample. \\
\hline $11 / 5 / 09$ & TIO3 & 30 & INTI03-S-30650 & Soil & СРT & 3099 & $11 / 5 / 09$ & Vertical-profile soil sample. \\
\hline 11/6/09 & TI07 & 30 & INTI07-S-30694 & Soil & СРT & 3103 & $11 / 7 / 09$ & Vertical-profile soil sample. \\
\hline 11/6/09 & TI07 & 34 & INTI07-S-30695 & Soil & СРT & 3103 & $11 / 7 / 09$ & Vertical-profile soil sample. \\
\hline 11/7/09 & TI09 & 38 & INTI09-S-30718 & Soil & CPT & 3106 & $11 / 7 / 09$ & Vertical-profile soil sample. \\
\hline $11 / 9 / 09$ & TI11 & $28-33$ & INTI11-W-30751 & Water & СРТ/P & 3109 & $11 / 9 / 09$ & $\begin{array}{l}\text { Temporary piezometer. Very little water available. One } \\
20-\mathrm{mL} \text { vial collected. }\end{array}$ \\
\hline $11 / 9 / 10$ & Bengston & - & INBENGSTEN-W-32675 & Water & DW & 3020 & $11 / 9 / 10$ & Private well sample. \\
\hline $11 / 10 / 10$ & MW04 & $30-40$ & INMW04-W-32677 & Water & MW & 3021 & $11 / 10 / 10$ & New monitoring well. \\
\hline $11 / 11 / 10$ & TI20 & 20 & INTI20-S-30794 & Soil & CPT & 3069 & $11 / 11 / 10$ & Vertical-profile soil sample. \\
\hline $11 / 11 / 10$ & TI20 & 24 & INTI20-S-30795 & Soil & CPT & 3069 & $11 / 11 / 10$ & Vertical-profile soil sample. \\
\hline $11 / 11 / 10$ & TI24 & 16 & INTI24-S-30831 & Soil & CPT & 3024 & $11 / 12 / 10$ & Vertical-profile soil sample. \\
\hline $11 / 12 / 10$ & TI25 & 16 & INTI25-S-30843 & Soil & CPT & 3025 & $11 / 12 / 10$ & Vertical-profile soil sample. \\
\hline $5 / 24 / 11$ & Hull & - & INHULL-W-32691 & Water & DW & 2965 & $5 / 24 / 11$ & Private well sample. \\
\hline 8/24/11 & MW14M & $85-95$ & INMW14S-W-32736 & Water & MW & 2860 & 8/24/11 & New monitoring well. \\
\hline $8 / 25 / 11$ & MW16 & $35-45$ & INMW16-W-32740 & Water & SB & 2850 & $8 / 25 / 11$ & New monitoring well. \\
\hline $8 / 27 / 11$ & MW17 & $15-31$ & INMW17-W-32745 & Water & SB & 2852 & $8 / 27 / 11$ & New monitoring well. \\
\hline $8 / 29 / 11$ & MW16D & $100-110$ & INMW16D-W-32756 & Water & MW & 3033 & $8 / 29 / 11$ & New monitoring well. \\
\hline 8/30/11 & MW19 & $15-50$ & INMW19-W-32768 & Water & SB & 2856 & 8/30/11 & New monitoring well. \\
\hline $8 / 31 / 11$ & MW17M & $72-82$ & INMW17M-W-32770 & Water & MW & 3035 & 8/31/11 & New monitoring well. \\
\hline 9/2/11 & MW19M & $85-95$ & INMW19D-W-32790 & Water & MW & 3037 & $9 / 2 / 11$ & New monitoring well. \\
\hline 9/15/11 & MW14D & $115-125$ & INMW14D-W-32782 & Water & $\mathrm{MW}$ & 3044 & 9/15/11 & New monitoring well. \\
\hline \multicolumn{9}{|c|}{ Samples submitted for verification organic analysis at TestAmerica } \\
\hline $11 / 4 / 09$ & TI01 & 34 & INTI01-S-30628 & Soil & CPT & 3175 & $11 / 4 / 09$ & Replicate of vertical profile sample INTI01-S-30627. \\
\hline $11 / 4 / 09$ & $\mathrm{TIO}$ & 10 & INTI02-S-30633 & Soil & СРT & 3175 & $11 / 4 / 09$ & Vertical soil profile. \\
\hline $11 / 5 / 09$ & TI04 & 6 & INTI04-S-30655 & Soil & CPT & 3099 & $11 / 5 / 09$ & Vertical soil profile. \\
\hline $11 / 5 / 09$ & TI04 & 34 & INTI04-S-30662 & Soil & CPT & 3101 & $11 / 5 / 09$ & Vertical soil profile. \\
\hline $11 / 6 / 09$ & TI05 & 6 & INTI05-S-30667 & Soil & CPT & 3102 & $11 / 7 / 09$ & Vertical soil profile. \\
\hline
\end{tabular}

11/7/09 Vertical soil profile. 
TABLE S2.1 (Cont.)

\begin{tabular}{|c|c|c|c|c|c|c|c|c|}
\hline $\begin{array}{l}\text { Sample } \\
\text { Date }\end{array}$ & Location & $\begin{array}{l}\text { Depth } \\
\text { (ft BGL) }\end{array}$ & Sample & $\begin{array}{l}\text { Sample } \\
\text { Medium }\end{array}$ & $\begin{array}{l}\text { Sample } \\
\text { Type }^{a}\end{array}$ & $\begin{array}{l}\text { Chain of } \\
\text { Custody }\end{array}$ & $\begin{array}{l}\text { Shipping } \\
\text { Date }\end{array}$ & Sample Description \\
\hline \multicolumn{9}{|c|}{ Samples submitted for verification organic analysis at TestAmerica (cont.) } \\
\hline 11/6/09 & TI05 & 18 & INTI05-S-30670 & Soil & СРT & 3102 & $11 / 7 / 09$ & Vertical soil profile. \\
\hline 11/6/09 & TI05 & 26 & INTI05-S-30672 & Soil & СРТ & 3102 & $11 / 7 / 09$ & Vertical soil profile. \\
\hline 11/6/09 & TI06 & 2 & INTI06-S-30676 & Soil & СРТ & 3102 & $11 / 7 / 09$ & Vertical soil profile. \\
\hline 11/6/09 & TIO7 & 34 & INTI07-S-30695 & Soil & СРT & 3103 & $11 / 7 / 09$ & Vertical soil profile. \\
\hline 11/6/09 & TIO7 & 38 & INTI07-S-30696 & Soil & СРT & 3104 & $11 / 7 / 09$ & Vertical soil profile. \\
\hline 11/8/09 & TI11 & 2 & INTI11-S-30734 & Soil & СРT & 3108 & $11 / 9 / 09$ & Vertical soil profile. \\
\hline $11 / 10 / 10$ & MW04 & $30-40$ & INMW04-W-32677 & Water & MW & 3021 & $11 / 10 / 10$ & Monitoring well. \\
\hline 11/10/10 & MW05 & $35-45$ & INMW05-W-32678 & Water & MW & 3021 & $11 / 10 / 10$ & Monitoring well. \\
\hline $11 / 10 / 10$ & TI14 & 29.5-39.5 & INTI14-W-30762 & Water & CPT/P & 3021 & $11 / 10 / 10$ & Grab sample. \\
\hline 11/10/10 & TI19 & 28 & INTI19-S-30771 & Soil & СРT & 3022 & $11 / 10 / 10$ & Vertical soil profile. \\
\hline $11 / 11 / 10$ & TI20 & 24 & INTI20-S-30795 & Soil & СРT & 3069 & $11 / 11 / 10$ & Vertical soil profile. \\
\hline 11/11/10 & TI21 & 36 & INTI21-S-30808 & Soil & СРТ & 3028 & $11 / 11 / 10$ & Vertical soil profile. \\
\hline $11 / 11 / 10$ & TI22 & 24 & INTI22-S-30814 & Soil & СРT & 3028 & $11 / 11 / 10$ & Vertical soil profile. \\
\hline 11/11/10 & TI18 & $30.6-40.6$ & INTI18-W-30786 & Water & СРТ/P & 3029 & 11/11/10 & Grab sample. \\
\hline 11/11/10 & TI16 & $31.2-41.2$ & INTI16-W-30785 & Water & CPT/P & 3029 & $11 / 11 / 10$ & Grab sample. \\
\hline 11/11/10 & TI23 & 8 & INTI23-S-30820 & Soil & CPT & 3024 & $11 / 12 / 10$ & Vertical soil profile. \\
\hline 11/11/10 & TI23 & 24 & INTI23-S-30824 & Soil & СРT & 3024 & $11 / 12 / 10$ & Vertical soil profile. \\
\hline 11/11/10 & TI15 & $31.7-41.7$ & INTI15-W-30784 & Water & СРТ/P & 3029 & 11/11/10 & Grab sample. \\
\hline 11/11/10 & TI13 & $31.7-41.7$ & INTI13-W-30783 & Water & CPT/P & 3029 & $11 / 11 / 10$ & Grab sample. \\
\hline $11 / 12 / 10$ & TI24 & 36 & INTI24-S-30836 & Soil & СРT & 3025 & $11 / 12 / 10$ & Vertical soil profile. \\
\hline $5 / 24 / 11$ & Hull & - & INHULL-W-32691 & Water & DW & 2965 & $5 / 24 / 11$ & $\begin{array}{l}\text { Windmill well. Also collected for cations and anions at } \\
\text { TestAmerica. }\end{array}$ \\
\hline $5 / 24 / 11$ & Brown & - & INBROWN-W-32686 & Water & DW & 2965 & $5 / 24 / 11$ & $\begin{array}{l}\text { Hydrant south of pond. Water has strong odor. Sulfur? Dead } \\
\text { fish? }\end{array}$ \\
\hline $5 / 24 / 11$ & Thiessen & - & INTHIESSEN-W-32685 & Water & DW & 2965 & $5 / 24 / 11$ & $\begin{array}{l}\text { Faucet on south side of house. Also collected for cations and } \\
\text { anions at TestAmerica. }\end{array}$ \\
\hline 6/23/11 & Klassen & - & INKLASSEN-W-32693 & Water & DW & 2980 & $6 / 23 / 11$ & $\begin{array}{l}\text { Second set of samples collected from the Klassen well. } \\
\text { Samples collected at the hydrant just west of the house. }\end{array}$ \\
\hline $6 / 23 / 11$ & Hull & - & INHULL-W-32694 & Water & DW & 2980 & $6 / 23 / 11$ & $\begin{array}{l}\text { Samples collected from the well supplying the Hull home. } \\
\text { Collected at faucet on the south side of house. }\end{array}$ \\
\hline 6/23/11 & Pauls & - & INPAULS-W-32695 & Water & DW & 2980 & $6 / 23 / 11$ & $\begin{array}{l}\text { Samples collected from the hydrant on the south end of the } \\
\text { place, near the garage. }\end{array}$ \\
\hline $8 / 22 / 11$ & MW14 & $75-90$ & INMW14-W-32730 & Water & SB & 1519 & $8 / 22 / 11$ & Monitoring well. \\
\hline 8/22/11 & MW14 & $95-120$ & INMW14-W-32734 & Water & SB & 1519 & $8 / 22 / 11$ & Monitoring well. \\
\hline 8/24/11 & MW14M & $85-95$ & INMW14S-W-32736 & Water & MW & 2860 & $8 / 24 / 11$ & Monitoring well. \\
\hline $8 / 24 / 11$ & MW15 & $55-75$ & INMW15-W-32735 & Water & SB & 2860 & $8 / 24 / 11$ & Monitoring well. \\
\hline $8 / 24 / 11$ & MW15 & $75-90$ & INMW15-W-32737 & Water & SB & 2860 & $8 / 24 / 11$ & Monitoring well. \\
\hline 8/25/11 & KTW & 135.2 & INKTW-W-32743 & Water & DW & 2850 & $8 / 25 / 11$ & Private well. \\
\hline 8/25/11 & MW16 & 75-91 & INMW16-W-32739 & Water & SB & 2850 & $8 / 25 / 11$ & \\
\hline 8/25/11 & MW16 & $35-45$ & INMW16-W-32740 & Water & SB & 2850 & 8/25/11 & \\
\hline 8/25/11 & MW16 & $15-30$ & INMW16-W-32741 & Water & SB & 2850 & $8 / 25 / 11$ & \\
\hline
\end{tabular}


TABLE S2.1 (Cont.)

\begin{tabular}{|c|c|c|c|c|c|c|c|c|}
\hline $\begin{array}{c}\text { Sample } \\
\text { Date }\end{array}$ & Location & $\begin{array}{l}\text { Depth } \\
\text { (ft BGL) }\end{array}$ & Sample & $\begin{array}{l}\text { Sample } \\
\text { Medium }\end{array}$ & $\begin{array}{l}\text { Sample } \\
\text { Type }^{a}\end{array}$ & $\begin{array}{l}\text { Chain of } \\
\text { Custody }\end{array}$ & $\begin{array}{l}\text { Shipping } \\
\text { Date }\end{array}$ & Sample Description \\
\hline \multicolumn{9}{|c|}{ Samples submitted for verification organic analysis at TestAmerica (cont.) } \\
\hline 8/25/11 & MW16 & $55-70$ & INMW16-W-32742 & Water & SB & 2850 & $8 / 25 / 11$ & \\
\hline $8 / 27 / 11$ & MW17 & $55-75$ & INMW17-W-32749 & Water & SB & 2852 & $8 / 27 / 11$ & Replicate of sample INMW17-W-32748. \\
\hline $8 / 27 / 11$ & MW15M & $86-96$ & INMW15-W-32747 & Water & MW & 2852 & $8 / 27 / 11$ & \\
\hline $8 / 27 / 11$ & MW17 & $15-31$ & INMW17-W-32745 & Water & SB & 2852 & $8 / 27 / 11$ & \\
\hline $8 / 27 / 11$ & MW17 & $55-75$ & INMW17-W-32748 & Water & SB & 2852 & 8/27/11 & \\
\hline $8 / 29 / 11$ & MW16M2 & $78-88$ & INMW16-W-32764 & Water & MW & 2854 & $8 / 29 / 11$ & \\
\hline $8 / 29 / 11$ & MW16S2 & $40-45$ & INMW16-W-32765 & Water & MW & 2854 & 8/29/11 & \\
\hline $8 / 29 / 11$ & MW18 & $65-80$ & INMW18-W-32761 & Water & SB & 3033 & $8 / 29 / 11$ & Replicate of sample INMW18-W-32760. \\
\hline 8/29/11 & MW18 & $85-90$ & INMW18-W-32752 & Water & SB & 3033 & 8/29/11 & \\
\hline $8 / 29 / 11$ & MW18 & $38-52$ & INMW18-W-32753 & Water & SB & 3033 & $8 / 29 / 11$ & \\
\hline 8/29/11 & MW18 & $95-102.5$ & INMW18-W-32754 & Water & SB & 3033 & 8/29/11 & \\
\hline $8 / 29 / 11$ & MW18 & $85-90$ & INMW18-W-32755 & Water & SB & 3033 & 8/29/11 & \\
\hline 8/29/11 & MW18 & $65-80$ & INMW18-W-32760 & Water & SB & 3033 & 8/29/11 & \\
\hline $8 / 29 / 11$ & MW16S1 & $25-35$ & INMW16-W-32762 & Water & MW & 3033 & 8/29/11 & \\
\hline 8/30/11 & MW16M1 & $60-70$ & INMW16-W-32767 & Water & MW & 2856 & 8/30/11 & \\
\hline $8 / 30 / 11$ & MW19 & $15-50$ & INMW19-W-32768 & Water & SB & 2856 & 8/30/11 & \\
\hline 8/31/11 & MW17D & $98-108$ & INMW17D-W-32773 & Water & MW & 3035 & $8 / 31 / 11$ & Monitoring well. \\
\hline 8/31/11 & MW17M & $72-82$ & INMW17M-W-32770 & Water & MW & 3035 & 8/31/11 & Monitoring well. \\
\hline $8 / 31 / 11$ & MW17S & $25-35$ & INMW17S-W-32772 & Water & MW & 3035 & $8 / 31 / 11$ & Monitoring well. \\
\hline 8/31/11 & MW19 & $75-90$ & INMW19-W-32771 & Water & SB & 3035 & 8/31/11 & \\
\hline 9/1/11 & MW18M & $85-95$ & INMW18M-W-32776 & Water & MW & 3040 & $9 / 1 / 11$ & Monitoring well. \\
\hline 9/1/11 & MW18S & $40-50$ & INMW18S-W-32775 & Water & MW & 3040 & $9 / 1 / 11$ & Monitoring well. \\
\hline $9 / 13 / 11$ & MW20 & $15-40$ & INMW20-W-32775 & Water & SB & 3043 & $9 / 13 / 11$ & \\
\hline 9/13/11 & MW20 & $65-89.5$ & INMW20-W-32776 & Water & SB & 3043 & 9/13/11 & \\
\hline $9 / 15 / 11$ & MW14D & $115-125$ & INMW14D-W-32782 & Water & MW & 3044 & $9 / 15 / 11$ & Monitoring well. \\
\hline 9/15/11 & MW20M & $72-82$ & INMW20M-W-32781 & Water & MW & 3044 & $9 / 15 / 11$ & Monitoring well. \\
\hline $9 / 15 / 11$ & MW20S & $30-40$ & INMW20S-W-32780 & Water & MW & 3044 & $9 / 15 / 11$ & Monitoring well. \\
\hline
\end{tabular}

a Sample types: CPT, cone penetrometer; CPT/P, piezometer; DW, domestic well; FB, field blank; MW, monitoring well; RI, rinsate; SB, soil boring; TB, trip blank. 
TABLE S2.2 Analytical results for quality control samples collected to monitor sample collection and handling.

\begin{tabular}{|c|c|c|c|c|c|c|c|c|c|c|}
\hline \multirow[b]{2}{*}{$\begin{array}{l}\text { Sample } \\
\text { Date }\end{array}$} & \multirow[b]{2}{*}{ Sample } & \multirow[b]{2}{*}{$\begin{array}{l}\text { Sample } \\
\text { Medium }\end{array}$} & \multirow[b]{2}{*}{$\begin{array}{l}\text { Analytical } \\
\text { Method }\end{array}$} & \multirow[b]{2}{*}{$\begin{array}{l}\text { Analytical } \\
\text { Laboratory }\end{array}$} & \multirow[b]{2}{*}{$\begin{array}{r}\text { Analysis } \\
\text { Date }\end{array}$} & \multicolumn{3}{|c|}{ Concentration } & \multirow{2}{*}{$\begin{array}{l}\text { Method } \\
\text { Detection } \\
\text { Limit }\end{array}$} & \multirow[b]{2}{*}{ Units } \\
\hline & & & & & & $\begin{array}{l}\text { Carbon } \\
\text { Tetrachloride }\end{array}$ & Chloroform & $\begin{array}{l}\text { Methylene } \\
\text { Chloride }\end{array}$ & & \\
\hline \multicolumn{11}{|c|}{ Field blanks } \\
\hline $8 / 22 / 11$ & INTRUCK-W-32731 & Water & E524.2 & AGEM & $8 / 23 / 11$ & $N D^{a}$ & 3.4 & ND & $<1$ & $\mu \mathrm{g} / \mathrm{L}$ \\
\hline 8/22/11 & INCITY-W-32732 & Water & E524.2 & AGEM & $8 / 23 / 11$ & ND & 1.9 & ND & $<1$ & $\mu \mathrm{g} / \mathrm{L}$ \\
\hline \multicolumn{11}{|c|}{ Equipment rinsates } \\
\hline $11 / 9 / 09$ & INQCTR-W-30753 & Water & E524.2 & AGEM & 11/10/09 & ND & ND & ND & $<1$ & $\mu \mathrm{g} / \mathrm{L}$ \\
\hline 11/11/09 & INQCIR-W-30759 & Water & E524.2 & AGEM & $11 / 16 / 09$ & ND & ND & ND & $<1$ & $\mu \mathrm{g} / \mathrm{L}$ \\
\hline \multicolumn{11}{|c|}{ Trip blanks } \\
\hline $11 / 4 / 09$ & INQCTB-S-30642 & Soil & SW8260B & AGEM & 11/10/09 & ND & ND & ND & $<10$ & $\mu \mathrm{g} / \mathrm{kg}$ \\
\hline $11 / 5 / 09$ & INQCTB-S-30665 & Soil & SW8260B & AGEM & $11 / 12 / 09$ & ND & ND & ND & $<10$ & $\mu \mathrm{g} / \mathrm{kg}$ \\
\hline $11 / 7 / 09$ & INTB-S-30731 & Soil & SW8260B & AGEM & 11/17/09 & ND & ND & ND & $<10$ & $\mu \mathrm{g} / \mathrm{kg}$ \\
\hline $11 / 7 / 09$ & INTB-S-30732 & Soil & SW8260B & AGEM & $11 / 17 / 09$ & ND & ND & ND & $<10$ & $\mu \mathrm{g} / \mathrm{kg}$ \\
\hline $11 / 9 / 09$ & INQCTB-S-30745 & Soil & SW8260B & AGEM & $11 / 18 / 09$ & ND & ND & ND & $<10$ & $\mu \mathrm{g} / \mathrm{kg}$ \\
\hline $11 / 9 / 09$ & INQCTB-W-30754 & Water & E524.2 & AGEM & $11 / 10 / 09$ & ND & ND & ND & $<1$ & $\mu \mathrm{g} / \mathrm{L}$ \\
\hline 11/11/09 & INQCTB-W-30760 & Water & E524.2 & AGEM & $11 / 16 / 09$ & ND & ND & ND & $<1$ & $\mu \mathrm{g} / \mathrm{L}$ \\
\hline $11 / 12 / 09$ & IN-S-MEOHBLANK-12NOV09 & Soil & SW8260B & $\mathrm{TA}^{\mathrm{b}}$ & $11 / 19 / 09$ & ND & ND & ND & $<10$ & $\mu \mathrm{g} / \mathrm{kg}$ \\
\hline $11 / 9 / 10$ & INQCTB-W-32676 & Water & E524.2 & AGEM & $11 / 11 / 10$ & ND & ND & ND & $<1$ & $\mu \mathrm{g} / \mathrm{L}$ \\
\hline $11 / 10 / 10$ & INTI19-S-30776 & Soil & SW8260B & AGEM & $11 / 16 / 10$ & ND & ND & ND & $<10$ & $\mu \mathrm{g} / \mathrm{kg}$ \\
\hline $11 / 10 / 10$ & INTB-W-30764/AGEM & Water & E524.2 & AGEM & $11 / 12 / 10$ & ND & ND & ND & $<1$ & $\mu \mathrm{g} / \mathrm{L}$ \\
\hline $11 / 10 / 10$ & INTB-W-30764/TA & Water & SW8260 & TA & $11 / 13 / 10$ & ND & ND & ND & $<0.5$ & $\mu \mathrm{g} / \mathrm{L}$ \\
\hline $11 / 11 / 10$ & INQCTB-W-30787/AGEM & Water & E524.2 & AGEM & $11 / 13 / 10$ & ND & ND & ND & $<1$ & $\mu \mathrm{g} / \mathrm{L}$ \\
\hline $11 / 11 / 10$ & INQCTB-W-30787/TA & Water & SW8260 & TA & $11 / 13 / 10$ & ND & ND & ND & 0.5 & $\mu \mathrm{g} / \mathrm{L}$ \\
\hline $11 / 11 / 10$ & INQCTB-S-30788 & Soil & SW8260B & AGEM & $11 / 18 / 10$ & ND & ND & ND & $<10$ & $\mu \mathrm{g} / \mathrm{kg}$ \\
\hline $11 / 11 / 10$ & INQCTB-S-30789 & Soil & SW8260B & AGEM & $11 / 18 / 10$ & ND & ND & ND & $<10$ & $\mu \mathrm{g} / \mathrm{kg}$ \\
\hline $11 / 12 / 10$ & INQCTB-S-30850 & Soil & SW8260B & AGEM & $11 / 23 / 10$ & ND & ND & ND & $<10$ & $\mu \mathrm{g} / \mathrm{kg}$ \\
\hline $11 / 12 / 10$ & INQCTB-S-30849 & Soil & SW8260B & AGEM & $11 / 23 / 10$ & ND & ND & ND & $<10$ & $\mu \mathrm{g} / \mathrm{kg}$ \\
\hline $11 / 12 / 10$ & INQCTB-W-30851 & Water & E524.2 & AGEM & $11 / 16 / 10$ & ND & ND & ND & $<1$ & $\mu \mathrm{g} / \mathrm{L}$ \\
\hline $11 / 12 / 10$ & INQCTB-W-30852 & Water & E524.2 & AGEM & $11 / 16 / 10$ & ND & ND & ND & $<1$ & $\mu \mathrm{g} / \mathrm{L}$ \\
\hline $11 / 18 / 10$ & INQCTB-W-32684 & Water & E524.2 & AGEM & $11 / 20 / 10$ & ND & ND & ND & $<1$ & $\mu \mathrm{g} / \mathrm{L}$ \\
\hline $11 / 18 / 10$ & IN-S-MEOHBLANK-1118 & Soil & SW8260B & TA & $11 / 24 / 10$ & ND & ND & ND & $<10$ & $\mu \mathrm{g} / \mathrm{kg}$ \\
\hline $12 / 22 / 10$ & INQCTB-W-32702 & Water & SW8260 & TA & $12 / 28 / 10$ & ND & ND & ND & $<0.5$ & $\mu \mathrm{g} / \mathrm{L}$ \\
\hline $5 / 24 / 11$ & INQCTB-W-32692/AGEM & Water & E524.2 & AGEM & $5 / 25 / 11$ & ND & ND & ND & $<1$ & $\mu \mathrm{g} / \mathrm{L}$ \\
\hline $5 / 24 / 11$ & INQCTB-W-32692/TA & Water & SW8260 & TA & $5 / 25 / 11$ & ND & $0.32 \mathrm{Jc}^{\mathrm{c}}$ & ND & 0.5 & $\mu g / L$ \\
\hline $6 / 23 / 11$ & INQCTB-W-32696/AGEM & Water & E524.2 & AGEM & $6 / 24 / 11$ & ND & ND & ND & $<1$ & $\mu \mathrm{g} / \mathrm{L}$ \\
\hline $6 / 23 / 11$ & INQCTB-W-32696/TA & Water & SW8260 & TA & $6 / 28 / 11$ & ND & ND & ND & 0.5 & $\mu \mathrm{g} / \mathrm{L}$ \\
\hline 8/22/11 & INQCTB-W-32733 & Water & E524.2 & AGEM & $8 / 23 / 11$ & ND & ND & ND & $<1$ & $\mu \mathrm{g} / \mathrm{L}$ \\
\hline
\end{tabular}




\begin{tabular}{|c|c|c|c|c|c|c|c|c|c|c|}
\hline \multirow[b]{2}{*}{$\begin{array}{l}\text { Sample } \\
\text { Date }\end{array}$} & \multirow[b]{2}{*}{ Sample } & \multirow[b]{2}{*}{$\begin{array}{l}\text { Sample } \\
\text { Medium }\end{array}$} & \multirow[b]{2}{*}{$\begin{array}{l}\text { Analytical } \\
\text { Method }\end{array}$} & \multirow[b]{2}{*}{$\begin{array}{l}\text { Analytical } \\
\text { Laboratory }\end{array}$} & \multirow[b]{2}{*}{$\begin{array}{r}\text { Analysis } \\
\text { Date }\end{array}$} & \multicolumn{3}{|c|}{ Concentration } & \multirow{2}{*}{$\begin{array}{l}\text { Method } \\
\text { Detection } \\
\text { Limit }\end{array}$} & \multirow[b]{2}{*}{ Units } \\
\hline & & & & & & $\begin{array}{l}\text { Carbon } \\
\text { Tetrachloride }\end{array}$ & Chloroform & $\begin{array}{l}\text { Methylene } \\
\text { Chloride }\end{array}$ & & \\
\hline \multicolumn{11}{|c|}{ Trip blanks (cont.) } \\
\hline 8/24/11 & INOCTB-W-32738/AGEM & Water & E524.2 & AGEM & $8 / 25 / 11$ & ND & ND & ND & $<1$ & $\mu \mathrm{g} / \mathrm{L}$ \\
\hline $8 / 24 / 11$ & INQCTB-W-32738/TA & Water & SW8260 & TA & $8 / 26 / 11$ & ND & $0.18 \mathrm{~J}$ & ND & $<0.5$ & $\mu \mathrm{g} / \mathrm{L}$ \\
\hline 8/25/11 & INQCTB-W-32744/AGEM & Water & E524.2 & AGEM & $8 / 26 / 11$ & ND & ND & ND & $<1$ & $\mu \mathrm{g} / \mathrm{L}$ \\
\hline 8/25/11 & INQCTB-W-32744/TA & Water & SW8260 & TA & $8 / 29 / 11$ & ND & ND & ND & $<0.5$ & $\mu \mathrm{g} / \mathrm{L}$ \\
\hline $8 / 27 / 11$ & INMW17QC-W-32746 & Water & E524.2 & AGEM & $8 / 29 / 11$ & ND & ND & ND & $<1$ & $\mu \mathrm{g} / \mathrm{L}$ \\
\hline 8/29/11 & INQC-W-32763/AGEM & Water & E524.2 & AGEM & $8 / 31 / 11$ & ND & ND & ND & $<1$ & $\mu \mathrm{g} / \mathrm{L}$ \\
\hline 8/29/11 & INQC-W-32763/TA & Water & SW8260 & TA & 9/1/11 & ND & $0.2 \mathrm{~J}$ & ND & $<0.5$ & $\mu \mathrm{g} / \mathrm{L}$ \\
\hline 8/29/11 & INTB-W-32766 & Water & E524.2 & AGEM & $8 / 30 / 11$ & ND & ND & ND & $<1$ & $\mu \mathrm{g} / \mathrm{L}$ \\
\hline 8/30/11 & INQCTB-W-32769/AGEM & Water & E524.2 & AGEM & $8 / 31 / 11$ & ND & ND & ND & $<1$ & $\mu \mathrm{g} / \mathrm{L}$ \\
\hline 8/30/11 & INQCTB-W-32769/TA & Water & SW8260 & TA & $9 / 1 / 11$ & ND & $0.13 \mathrm{~J}$ & ND & $<0.5$ & $\mu \mathrm{g} / \mathrm{L}$ \\
\hline 8/31/11 & INQCTB-W-32774 & Water & E524.2 & AGEM & $9 / 1 / 11$ & ND & ND & ND & $<1$ & $\mu \mathrm{g} / \mathrm{L}$ \\
\hline 9/1/11 & INQCTB-W-32778/AGEM & Water & E524.2 & AGEM & $9 / 2 / 11$ & ND & ND & ND & $<1$ & $\mu \mathrm{g} / \mathrm{L}$ \\
\hline 9/1/11 & INQCTB-W-32778/TA & Water & SW8260 & TA & 9/16/11 & ND & $0.17 \mathrm{~J}$ & ND & $<0.5$ & $\mu \mathrm{g} / \mathrm{L}$ \\
\hline 9/2/11 & INQCTB-W-32791 & Water & E524.2 & AGEM & 9/6/11 & ND & ND & ND & $<1$ & $\mu \mathrm{g} / \mathrm{L}$ \\
\hline 9/13/11 & INQCTB-W-32777 & Water & E524.2 & AGEM & 9/15/11 & ND & ND & ND & $<1$ & $\mu \mathrm{g} / \mathrm{L}$ \\
\hline $9 / 15 / 11$ & INQCTB-W-32783/AGEM & Water & E524.2 & AGEM & 9/16/11 & ND & ND & ND & $<\overline{1}$ & $\mu \mathrm{g} / \mathrm{L}$ \\
\hline $9 / 15 / 11$ & INQCTB-W-32783/TA & Water & SW8260 & TA & 9/20/11 & ND & $0.12 \mathrm{~J} \mathrm{~B}^{\mathrm{d}}$ & ND & $<0.5$ & $\mu \mathrm{g} / \mathrm{L}$ \\
\hline
\end{tabular}

a ND, compound analyzed for but not detected at a level greater than or equal to the indicated method detection limit.

b TA, TestAmerica

c J, compound identified with an estimated concentration between the instrument detection limit and the method detection limit.

d B, contaminant present in the associated analytical method blank. 
TABLE S2.3 Calibration and surrogate recovery during purge-and-trap organic analysis of water samples at the AGEM Laboratory.

\begin{tabular}{|c|c|c|c|c|c|c|c|c|c|}
\hline \multirow[b]{4}{*}{ Sample } & \multirow{2}{*}{\multicolumn{3}{|c|}{ Recovery of Surrogate Compounds ${ }^{a}(\%)$}} & \multicolumn{6}{|c|}{$\begin{array}{l}\text { Measured Concentration of } \\
\text { Calibration Check Standards }\end{array}$} \\
\hline & & & & \multirow{2}{*}{\multicolumn{2}{|c|}{$\begin{array}{c}\text { Carbon } \\
\text { Tetrachloride } \\
\end{array}$}} & \multirow{2}{*}{\multicolumn{2}{|c|}{ Chloroform }} & \multirow{2}{*}{\multicolumn{2}{|c|}{$\begin{array}{l}\text { Methylene } \\
\text { Chloride }\end{array}$}} \\
\hline & & & & & & & & & \\
\hline & Fluorobenzene & fluorobenzene & benzene- $\mathrm{d}_{4}$ & $\mathrm{ppb}^{\mathrm{b}}$ & $\mathrm{RPD}^{\mathrm{c}}$ & $\mathrm{ppb}^{\mathrm{b}}$ & $\mathrm{RPD}^{\mathrm{c}}$ & $\mathrm{ppb}^{\mathrm{b}}$ & $\mathrm{RPD}^{\mathrm{c}}$ \\
\hline \multicolumn{10}{|c|}{ SDG 09-11-6, analysis date November 6, 2009} \\
\hline 20-ppb standard & 106 & 114 & 100 & 22.68 & 12.6 & 23.93 & 17.9 & 20 & 0.0 \\
\hline Methanol blank & 91 & 100 & 98 & & & & & & \\
\hline INTI01-S-30621 & 90 & 107 & 104 & & & & & & \\
\hline INTI01-S-30626 & 118 & 90 & 94 & & & & & & \\
\hline INTI01-S-30627 & 82 & 95 & 94 & & & & & & \\
\hline INTI01-S-30630 & 81 & 99 & 96 & & & & & & \\
\hline INTI02-S-30632 & 81 & 89 & 92 & & & & & & \\
\hline INTI02-S-30634 & 82 & 95 & 90 & & & & & & \\
\hline INTI02-S-30640 & 117 & 89 & 86 & & & & & & \\
\hline INTI02-S-30635 & $77^{d}$ & 91 & 89 & \multirow{7}{*}{\multicolumn{6}{|c|}{ Reanalyzed in SDG 09-11-9 with recovery limits met. }} \\
\hline INTI01-S-30620 & 116 & 94 & 85 & & & & & & \\
\hline INTI01-S-30628 & 102 & 118 & 107 & & & & & & \\
\hline INTI02-S-30639 & 98 & 109 & 104 & & & & & & \\
\hline INTI02-S-30636 & 92 & 115 & 107 & & & & & & \\
\hline INTI01-S-30629 & 94 & 100 & 94 & & & & & & \\
\hline INTI01-S-30623 & 114 & 95 & 90 & & & & & & \\
\hline \multicolumn{10}{|c|}{ SDG 09-11-9, analysis date November 9, 2009} \\
\hline 20-ppb standard & 100 & 100 & 100 & 19.62 & 1.9 & 23.98 & 18.1 & 23.66 & 16.8 \\
\hline Methanol blank & 88 & 99 & 96 & & & & & & \\
\hline INTI02-S-30635 & 108 & 104 & 103 & & & & & & \\
\hline INTI01-S-30624 & 86 & 99 & 103 & & & & & & \\
\hline INTI01-S-30625 & 92 & 105 & 104 & & & & & & \\
\hline INTI02-S-30637 & 85 & 101 & 103 & & & & & & \\
\hline INTI02-S-30638 & 114 & 100 & 100 & & & & & & \\
\hline INTI02-S-30641 & 86 & 100 & 108 & & & & & & \\
\hline INTI02-S-30633 & 83 & 97 & 96 & & & & & & \\
\hline INTI02-S-30633DUP & 106 & 105 & 109 & & & & & & \\
\hline INTI01-S-30622 & $76^{\mathrm{d}}$ & 89 & 97 & Rea & alyzed in & G 09-11 & $1 \mathrm{~b}$ with & ery limits & met. \\
\hline
\end{tabular}

INTI01-S-30630

2-S-30632

INTI02-S-30635 


\begin{tabular}{|c|c|c|c|c|c|c|c|c|c|}
\hline \multirow[b]{3}{*}{ Sample } & \multirow{2}{*}{\multicolumn{3}{|c|}{ Recovery of Surrogate Compounds ${ }^{\mathrm{a}}(\%)$}} & \multicolumn{6}{|c|}{$\begin{array}{l}\text { Measured Concentration of } \\
\text { Calibration Check Standards }\end{array}$} \\
\hline & & & & \multicolumn{2}{|c|}{$\begin{array}{c}\text { Carbon } \\
\text { Tetrachloride }\end{array}$} & \multicolumn{2}{|c|}{ Chloroform } & \multicolumn{2}{|c|}{$\begin{array}{l}\text { Methylene } \\
\text { Chloride }\end{array}$} \\
\hline & Fluorobenzene & $\begin{array}{l}\text { Bromo- } \\
\text { fluorobenzene }\end{array}$ & $\begin{array}{l}\text { 1,4-Dichloro- } \\
\text { benzene- } \mathrm{d}_{4}\end{array}$ & $\mathrm{ppb}^{\mathrm{b}}$ & $\mathrm{RPD}^{\mathrm{c}}$ & $\mathrm{ppb}^{\mathrm{b}}$ & $\mathrm{RPD}^{\mathrm{c}}$ & $\mathrm{ppb}^{\mathrm{b}}$ & $\mathrm{RPD}^{\mathrm{c}}$ \\
\hline
\end{tabular}

SDG 09-11-9, analysis date November 9, 2009 (cont.)

INTI02-S-30631
INTI04-S-30656
INTI04-S-30663
INTI04-S-30657
INTI03-S-30651
Methanol blank 2
INTI03-S-30649
INTI03-S-30648
INTI03-S-30653
INTI04-S-30658
INTI04-S-30660
INQCTB-S-30642
Methanol blank 3

$75^{\mathrm{d}}$
82
93
95
84
105
89
108
96
87
86
85
100

SDG 09-11-10a, analysis date November 10, 2009

20-ppb standard
Methanol blank
INTI04-S-30662
INQCTB-S-30665
INTI04-S-30659
INTI03-S-30644
INTI03-S-30645
INTI03-S-30643
INTI03-S-30646
INTI04-S-30654
INTI04-S-30661
INTI03-S-30652
INTI03-S-30647
INTI04-S-30655

98
109
$140^{d}$
114
112
120
107
113
110
111
113
82
101
107

$\begin{array}{rr}90 & 89 \\ 95 & 101 \\ 113 & 117 \\ 105 & 106 \\ 99 & 101 \\ 82 & 82 \\ 85 & 90 \\ 95 & 93 \\ 99 & 93 \\ 89 & 90 \\ 84 & 85 \\ 98 & 95 \\ 100 & 100\end{array}$

$\begin{array}{rl}95 & 112 \\ 110 & 109 \\ 133^{d} & 124^{d} \\ 137^{d} & 137^{d} \\ 113 & 109 \\ 118 & 103 \\ 107 & 111 \\ 120 & 120 \\ 88 & 87 \\ 96 & 96 \\ 102 & 98 \\ 83 & 85 \\ 84 & 87 \\ 97 & 98\end{array}$

Reanalyzed in SDG 09-11-11b with recovery limits met.
20.28
1.4
23.2
14.8
21.54
7.4

Reanalyzed in SDG 09-11-12a with recovery limits met. Reanalyzed in SDG 09-11-12a with recovery limits met. 


\begin{tabular}{|c|c|c|c|c|c|c|c|c|c|}
\hline \multirow[b]{4}{*}{ Sample } & \multirow{2}{*}{\multicolumn{3}{|c|}{ Recovery of Surrogate Compounds ${ }^{a}(\%)$}} & \multicolumn{6}{|c|}{$\begin{array}{l}\text { Measured Concentration of } \\
\text { Calibration Check Standards }\end{array}$} \\
\hline & & & & \multirow{2}{*}{\multicolumn{2}{|c|}{$\begin{array}{c}\text { Carbon } \\
\text { Tetrachloride }\end{array}$}} & \multirow{2}{*}{\multicolumn{2}{|c|}{ Chloroform }} & \multirow{2}{*}{\multicolumn{2}{|c|}{$\begin{array}{l}\text { Methylene } \\
\text { Chloride }\end{array}$}} \\
\hline & \multirow[b]{2}{*}{ Fluorobenzene } & \multirow[b]{2}{*}{$\begin{array}{l}\text { Bromo- } \\
\text { fluorobenzene }\end{array}$} & \multirow[b]{2}{*}{$\begin{array}{l}\text { 1,4-Dichloro- } \\
\text { benzene- }_{4}\end{array}$} & & & & & & \\
\hline & & & & $\mathrm{ppb}^{\mathrm{b}}$ & $\mathrm{RPD}^{\mathrm{c}}$ & $\mathrm{ppb}^{\mathrm{b}}$ & $\mathrm{RPD}^{\mathrm{C}}$ & $\mathrm{ppb}^{\mathrm{b}}$ & $\mathrm{RPD}^{\mathrm{C}}$ \\
\hline \multicolumn{10}{|c|}{ SDG 09-11-10a, analysis date November 10, 2009 (cont.) } \\
\hline INTI03-S-30650 & 84 & 95 & 93 & & & & & & \\
\hline INTI03-S-30650DUP & 94 & 105 & 105 & & & & & & \\
\hline Methanol blank 2 & 88 & 90 & 91 & & & & & & \\
\hline \multicolumn{10}{|c|}{ SDG 09-11-10b, analysis date November 10, 2009} \\
\hline 20-ppb standard & 86 & 96 & 80 & 17.68 & 12.3 & 17.9 & 11.1 & 20.09 & 0.4 \\
\hline Laboratory blank & 100 & 100 & 100 & & & & & & \\
\hline INTI01-W-30733 & 100 & 88 & 85 & & & & & & \\
\hline INTI04-W-30747 & 115 & 117 & 117 & & & & & & \\
\hline INTI05-W-30748 & 100 & 98 & 100 & & & & & & \\
\hline INTI01-W-30749 & 97 & 97 & 101 & & & & & & \\
\hline INTI01-W-30750 & 99 & 96 & 108 & & & & & & \\
\hline INTI11-W-30751 & 101 & 117 & 118 & & & & & & \\
\hline INTI11-W-30751DUP & 105 & 119 & 113 & & & & & & \\
\hline INTI08-W-30752 & 95 & 101 & 99 & & & & & & \\
\hline INQCTR-W-30753 & 82 & 84 & 84 & & & & & & \\
\hline INQCCTB-W-30754 & 91 & 90 & 92 & & & & & & \\
\hline \multicolumn{10}{|c|}{ SDG 09-11-11a, analysis date November 11, 2009} \\
\hline 20-ppb standard & 100 & 100 & 100 & 19.69 & 1.6 & 20.53 & 2.6 & 23.26 & 15.1 \\
\hline Methanol blank & 100 & 94 & 94 & & & & & & \\
\hline INTI05-S-30672 & 100 & 106 & 106 & & & & & & \\
\hline INTI06-S-30681 & 106 & 110 & 106 & & & & & & \\
\hline INTI06-S-30682 & 100 & 109 & 106 & & & & & & \\
\hline INTI06-S-30685 & 115 & 96 & 96 & & & & & & \\
\hline INTI08-S-30703 & 114 & 102 & 99 & & & & & & \\
\hline INTI08-S-30706 & 112 & 115 & 99 & & & & & & \\
\hline INTI08-S-30708 & 87 & 94 & 93 & & & & & & \\
\hline
\end{tabular}

INTI03-S-30650

INTI03-S-30650DU 


\begin{tabular}{|c|c|c|c|c|c|c|c|c|c|}
\hline \multirow[b]{3}{*}{ Sample } & \multirow{2}{*}{\multicolumn{3}{|c|}{ Recovery of Surrogate Compounds ${ }^{a}(\%)$}} & \multicolumn{6}{|c|}{$\begin{array}{l}\text { Measured Concentration of } \\
\text { Calibration Check Standards }\end{array}$} \\
\hline & & & & \multicolumn{2}{|c|}{$\begin{array}{c}\text { Carbon } \\
\text { Tetrachloride } \\
\end{array}$} & \multicolumn{2}{|c|}{ Chloroform } & \multicolumn{2}{|c|}{$\begin{array}{l}\text { Methylene } \\
\text { Chloride }\end{array}$} \\
\hline & Fluorobenzene & $\begin{array}{l}\text { Bromo- } \\
\text { fluorobenzene }\end{array}$ & $\begin{array}{l}\text { 1,4-Dichloro- } \\
\text { benzene- } \mathrm{d}_{4}\end{array}$ & $\mathrm{ppb}^{\mathrm{b}}$ & $\mathrm{RPD}^{\mathrm{C}}$ & $\mathrm{ppb}^{\mathrm{b}}$ & $\mathrm{RPD}^{\mathrm{c}}$ & $\mathrm{ppb}^{\mathrm{b}}$ & $\mathrm{RPD}^{\mathrm{c}}$ \\
\hline
\end{tabular}

SDG 09-11-11a, analysis date November 11, 2009 (cont.)

$\begin{array}{lrrr}\text { INTI09-S-30718 } & 107 & 90 & 90 \\ \text { INTI09-S-30718DUP } & 85 & 91 & 89 \\ \text { INTI10-S-30729 } & 101 & 102 & 104 \\ \text { INTI05-S-30674 } & 104 & 93 & 92 \\ \text { INTI07-S-30696 } & 81 & 90 & 82 \\ \text { INTI06-S-30686 } & 85 & 93 & 91 \\ \text { INTI05-S-30675 } & 82 & 86 & 86 \\ \text { Methanol blank } 2 & 81 & 84 & 87\end{array}$

SDG 09-11-11b, analysis date November 11, 2009

20-ppb standard

Methanol blank

INTI01-S-30622

INTI02-S-30631

INTI04-S-30664

INTI07-S-30692

INTI09-S-30716

INTI07-S-30694

INTI07-S-30694DUP

Methanol blank 2

INTI09-S-30717

INTI10-S-30726

INTI09-S-30714

INTI10-S-30728

INTI09-S-30715

INTI10-S-30725

Methanol blank 3

Laboratory blank

INTI04B-W-30746

$\begin{array}{rrr}100 & 100 & 100 \\ 91 & 89 & 90 \\ 96 & 93 & 104 \\ 101 & 107 & 108 \\ 104 & 103 & 107 \\ 103 & 108 & 112 \\ 104 & 105 & 114 \\ 103 & 109 & 114 \\ 104 & 108 & 112 \\ 109 & 111 & 110 \\ 106 & 104 & 109 \\ 98 & 100 & 111 \\ 105 & 110 & 118 \\ 102 & 102 & 110 \\ 101 & 104 & 108 \\ 105 & 106 & 114 \\ 113 & 115 & 109 \\ 100 & 100 & 100 \\ 90 & 113 & 114\end{array}$

91

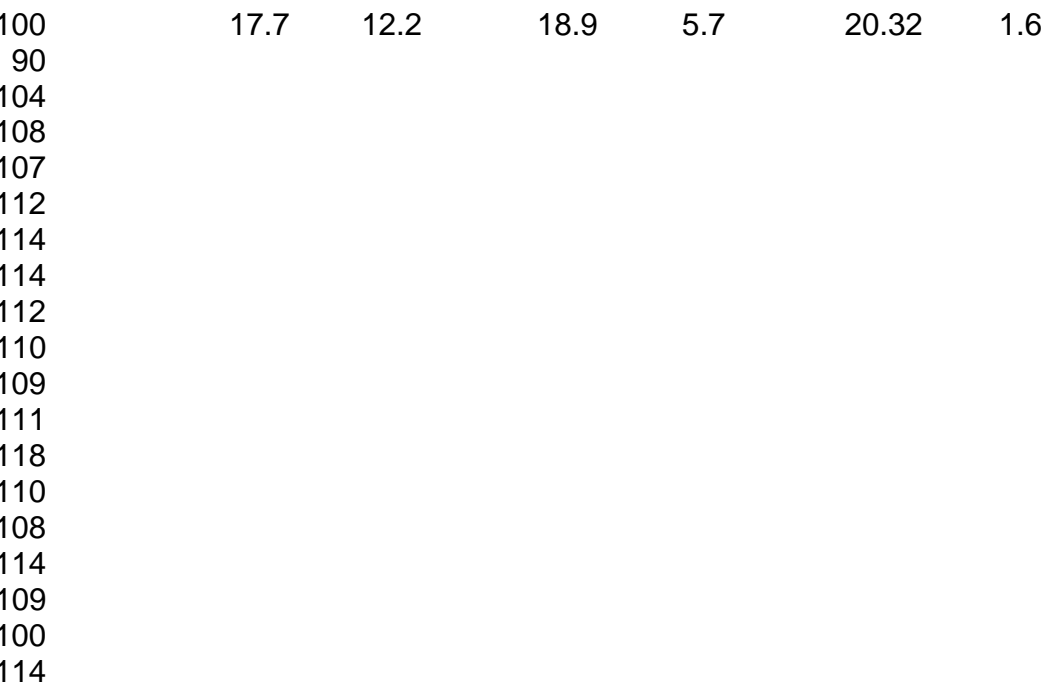




\begin{tabular}{|c|c|c|c|c|c|c|c|c|c|}
\hline \multirow[b]{4}{*}{ Sample } & \multirow{2}{*}{\multicolumn{3}{|c|}{ Recovery of Surrogate Compounds ${ }^{a}(\%)$}} & \multicolumn{6}{|c|}{$\begin{array}{l}\text { Measured Concentration of } \\
\text { Calibration Check Standards }\end{array}$} \\
\hline & & & & \multirow{2}{*}{\multicolumn{2}{|c|}{$\begin{array}{c}\text { Carbon } \\
\text { Tetrachloride }\end{array}$}} & \multirow{2}{*}{\multicolumn{2}{|c|}{ Chloroform }} & \multirow{2}{*}{\multicolumn{2}{|c|}{$\begin{array}{l}\text { Methylene } \\
\text { Chloride }\end{array}$}} \\
\hline & \multirow[b]{2}{*}{ Fluorobenzene } & \multirow[b]{2}{*}{$\begin{array}{l}\text { Bromo- } \\
\text { fluorobenzene }\end{array}$} & \multirow[b]{2}{*}{$\begin{array}{l}\text { 1,4-Dichloro- } \\
\text { benzene- } d_{4}\end{array}$} & & & & & & \\
\hline & & & & $\mathrm{ppb}^{\mathrm{b}}$ & $\mathrm{RPD}^{\mathrm{C}}$ & $\mathrm{ppb}^{\mathrm{b}}$ & $\mathrm{RPD}^{\mathrm{C}}$ & $\mathrm{ppb}^{\mathrm{b}}$ & $\mathrm{RPD}^{\mathrm{C}}$ \\
\hline \multicolumn{10}{|c|}{ SDG 09-11-12a, analysis date November 12, 2009} \\
\hline 20-ppb standard & 100 & 100 & 100 & 19.55 & 2.3 & 22.09 & 9.9 & 18.91 & 5.6 \\
\hline Methanol blank & 109 & 91 & 87 & & & & & & \\
\hline INTI04-S-30662 & 90 & 101 & 98 & & & & & & \\
\hline INQCTB-S-30665 & 88 & 107 & 98 & & & & & & \\
\hline INTI10-S-30727 & 103 & 110 & 113 & & & & & & \\
\hline INTI05-S-30671 & 88 & 99 & 100 & & & & & & \\
\hline INTI06-S-30683 & 81 & 93 & 93 & & & & & & \\
\hline INTI07-S-30693 & 82 & 89 & 92 & & & & & & \\
\hline Methanol blank 2 & 100 & 87 & 87 & & & & & & \\
\hline INTI08-S-30704 & 83 & 91 & 87 & & & & & & \\
\hline INTI05-S-30673 & 80 & 90 & 90 & & & & & & \\
\hline INTI08-S-30707 & 81 & 94 & 95 & & & & & & \\
\hline INTI06-S-30676 & 98 & 104 & 108 & & & & & & \\
\hline INTI07-S-30687 & $75^{d}$ & 89 & 86 & \multirow{2}{*}{\multicolumn{6}{|c|}{$\begin{array}{l}\text { Reanalyzed in SDG 09-11-16b with recovery limits met. } \\
\text { Reanalyzed in SDG 09-11-16b with recovery limits met. }\end{array}$}} \\
\hline INTI06-S-30678 & $78^{d}$ & 93 & 88 & & & & & & \\
\hline \multicolumn{10}{|c|}{ SDG 09-11-12b, analysis date November 12, 2009} \\
\hline 20-ppb standard & 98 & 107 & 94 & 20.67 & 3.3 & 23.32 & 15.3 & 20.62 & 3.1 \\
\hline Methanol blank & 95 & 91 & 86 & & & & & & \\
\hline INTI07-S-30697 & 93 & 95 & 94 & & & & & & \\
\hline INTI09-S-30712 & 96 & 97 & 101 & & & & & & \\
\hline INTI10-S-30723 & 95 & 99 & 98 & & & & & & \\
\hline INTI10-S-30722 & 94 & 91 & 98 & & & & & & \\
\hline INTI06-S-30677 & 96 & 95 & 100 & & & & & & \\
\hline INTI10-S-30720 & 99 & 96 & 103 & & & & & & \\
\hline INTI10-S-30720DUP & 97 & 95 & 101 & & & & & & \\
\hline Methanol blank 2 & 100 & 100 & 100 & & & & & & \\
\hline INTI10-S-30730 & 96 & 101 & 109 & & & & & & \\
\hline INTI06-S-30684 & 96 & 101 & 103 & & & & & & \\
\hline
\end{tabular}




\begin{tabular}{|c|c|c|c|c|c|c|c|c|c|}
\hline \multirow[b]{4}{*}{ Sample } & \multirow{2}{*}{\multicolumn{3}{|c|}{ Recovery of Surrogate Compounds ${ }^{\mathrm{a}}(\%)$}} & \multicolumn{6}{|c|}{$\begin{array}{l}\text { Measured Concentration of } \\
\text { Calibration Check Standards }\end{array}$} \\
\hline & & & & \multirow{2}{*}{\multicolumn{2}{|c|}{$\begin{array}{c}\text { Carbon } \\
\text { Tetrachloride } \\
\end{array}$}} & \multirow{2}{*}{\multicolumn{2}{|c|}{ Chloroform }} & \multirow{2}{*}{\multicolumn{2}{|c|}{$\begin{array}{l}\text { Methylene } \\
\text { Chloride }\end{array}$}} \\
\hline & & & & & & & & & \\
\hline & Fluorobenzene & fluorobenzene & benzene- $\mathrm{d}_{4}$ & $\mathrm{ppb}^{\mathrm{b}}$ & $\mathrm{RPD}^{\mathrm{c}}$ & $\mathrm{ppb}^{\mathrm{b}}$ & $\mathrm{RPD}^{\mathrm{c}}$ & $\mathrm{ppb}^{\mathrm{b}}$ & $\mathrm{RPD}^{\mathrm{c}}$ \\
\hline \multicolumn{10}{|c|}{ SDG 09-11-12b, analysis date November 12, 2009 (cont.) } \\
\hline INTI09-S-30713 & 96 & 94 & 101 & & & & & & \\
\hline INTI10-S-30724 & 97 & 102 & 103 & & & & & & \\
\hline INTI09-S-30711 & 97 & 95 & 103 & & & & & & \\
\hline \multicolumn{10}{|c|}{ SDG 09-11-16a, analysis date November 16, 2009} \\
\hline 20-ppb standard & 104 & 107 & 90 & 20.79 & 3.9 & 20.58 & 2.9 & 20.93 & 4.5 \\
\hline Laboratory blank & 109 & 100 & 96 & & & & & & \\
\hline INTI06-W-30757 & 87 & 96 & 93 & & & & & & \\
\hline INTI06-W-30756 & 98 & 97 & 99 & & & & & & \\
\hline INTI11-W-30755 & 92 & 99 & 92 & & & & & & \\
\hline INTI02-W-30758 & 107 & 89 & 86 & & & & & & \\
\hline INQCIR-W-30759 & 90 & 89 & 84 & & & & & & \\
\hline INQCTB-W-30760 & 87 & 87 & 83 & & & & & & \\
\hline \multicolumn{10}{|c|}{ SDG 09-11-16b, analysis date November 16, 2009} \\
\hline 20-ppb standard & 100 & 100 & 100 & 18.79 & 6.2 & 21.04 & 5.1 & 20.67 & 3.3 \\
\hline Methanol blank & 100 & 100 & 100 & & & & & & \\
\hline INTI07-S-30687 & 101 & 100 & 99 & & & & & & \\
\hline INTI06-S-30678 & 98 & 97 & 97 & & & & & & \\
\hline INTI08-S-30699 & 92 & 91 & 90 & & & & & & \\
\hline INTI05-S-30666 & 98 & 99 & 100 & & & & & & \\
\hline INTI10-S-30721 & 99 & 102 & 99 & & & & & & \\
\hline INTI07-S-30695 & 102 & 102 & 104 & & & & & & \\
\hline INTI07-S-30695DUP & 103 & 100 & 104 & & & & & & \\
\hline Methanol blank 2 & 103 & 95 & 94 & & & & & & \\
\hline INTI09-S-30719 & 100 & 93 & 99 & & & & & & \\
\hline INTI09-S-30710 & 101 & 96 & 97 & & & & & & \\
\hline INTI05-S-30670 & 103 & 97 & 98 & & & & & & \\
\hline
\end{tabular}

INTI09-S-30713

INTI10-S-3072

SDG 09-11-16a, analysis date November 16, 2009

ppb standard 


\begin{tabular}{|c|c|c|c|c|c|c|c|c|c|}
\hline \multirow[b]{3}{*}{ Sample } & \multirow{2}{*}{\multicolumn{3}{|c|}{ Recovery of Surrogate Compounds ${ }^{a}(\%)$}} & \multicolumn{6}{|c|}{$\begin{array}{l}\text { Measured Concentration of } \\
\text { Calibration Check Standards } \\
\end{array}$} \\
\hline & & & & \multicolumn{2}{|c|}{$\begin{array}{c}\text { Carbon } \\
\text { Tetrachloride } \\
\end{array}$} & \multicolumn{2}{|c|}{ Chloroform } & \multicolumn{2}{|c|}{$\begin{array}{l}\text { Methylene } \\
\text { Chloride }\end{array}$} \\
\hline & Fluorobenzene & $\begin{array}{l}\text { Bromo- } \\
\text { fluorobenzene }\end{array}$ & $\begin{array}{l}\text { 1,4-Dichloro- } \\
\text { benzene-d } 4\end{array}$ & $\mathrm{ppb}^{\mathrm{b}}$ & $\mathrm{RPD}^{\mathrm{c}}$ & $\mathrm{ppb}^{\mathrm{b}}$ & $\mathrm{RPD}^{\mathrm{c}}$ & $\mathrm{ppb}^{\mathrm{b}}$ & $\mathrm{RPD}^{\mathrm{c}}$ \\
\hline
\end{tabular}

SDG 09-11-16b, analysis date November 16, 2009 (cont.)

INTI08-S-30698

INTI08-S-30701

INTI07-S-30688

Methanol blank 3

101
102
100
93

SDG 09-11-17, analysis date November 17, 2009

20-ppb standard
Methanol blank
INTI07-S-30690
INTI05-S-30669
INTI06-S-30680
INTI07-S-30689
INTI05-S-30668
INTI09-S-30709
INTI07-S-30691
INTI05-S-30667
INTI06-S-30679
INTI11-S-30735
INTI11-S-30736
INTI08-S-30705
INTB-S-30731
Methanol blank 2

100
100
$127^{d}$
101
$141^{d}$
92
94
94
85
89
87
90
$74^{d}$
94
86
92

SDG 09-11-18, analysis date November 18, 2009

20-ppb standard

Methanol blank

INTI07-S-30690

100
105
103
114

91

92

95

88

97

$\begin{array}{cc}100 & 100 \\ 100 & 100 \\ 122^{\mathrm{d}} & 109 \\ 98 & 93 \\ 97 & 89 \\ 95 & 91 \\ 91 & 92 \\ 98 & 96 \\ 82 & 80 \\ 84 & 85 \\ 87 & 85 \\ 91 & 84 \\ 76^{\mathrm{d}} & 75^{\mathrm{d}} \\ 97 & 92 \\ 96 & 91 \\ 89 & 88\end{array}$

$22.76 \quad 12.9$

23.35

15.5

20.42

Reanalyzed in SDG 09-11-18 with recovery limits met.

Reanalyzed in SDG 09-11-18 with recovery limits met

Reanalyzed in SDG 09-11-18 with recovery limits met.

$\begin{array}{rr}100 & 100 \\ 98 & 99 \\ 104 & 111 \\ 118 & 116\end{array}$

$\begin{array}{lllllll}100 & 18.08 & 10.1 & 18.89 & 5.7 & 22.99 & 13.9\end{array}$




\begin{tabular}{|c|c|c|c|c|c|c|c|c|c|}
\hline \multirow[b]{4}{*}{ Sample } & \multirow{2}{*}{\multicolumn{3}{|c|}{ Recovery of Surrogate Compounds ${ }^{\mathrm{a}}(\%)$}} & \multicolumn{6}{|c|}{$\begin{array}{l}\text { Measured Concentration of } \\
\text { Calibration Check Standards }\end{array}$} \\
\hline & & & & \multirow{2}{*}{\multicolumn{2}{|c|}{$\begin{array}{c}\text { Carbon } \\
\text { Tetrachloride } \\
\end{array}$}} & \multirow{2}{*}{\multicolumn{2}{|c|}{ Chloroform }} & \multirow{2}{*}{\multicolumn{2}{|c|}{$\begin{array}{l}\text { Methylene } \\
\text { Chloride }\end{array}$}} \\
\hline & & & & & & & & & \\
\hline & Fluorobenzene & fluorobenzene & benzene- $\mathrm{d}_{4}$ & $\mathrm{ppb}^{\mathrm{b}}$ & $\mathrm{RPD}^{\mathrm{c}}$ & $\mathrm{ppb}^{\mathrm{b}}$ & $\mathrm{RPD}^{\mathrm{c}}$ & $\mathrm{ppb}^{\mathrm{b}}$ & $\mathrm{RPD}^{\mathrm{c}}$ \\
\hline \multicolumn{10}{|c|}{ SDG 09-11-18, analysis date November 18, 2009 (cont.) } \\
\hline INTI11-S-30736 & 101 & 107 & 113 & & & & & & \\
\hline INQCTB-S-30745 & 98 & 103 & 101 & & & & & & \\
\hline INTI11-S-30738 & 96 & 104 & 104 & & & & & & \\
\hline INTI11-S-30742 & 93 & 91 & 98 & & & & & & \\
\hline INTI11-S-30741 & 93 & 97 & 97 & & & & & & \\
\hline INTI11-S-30737 & 94 & 93 & 97 & & & & & & \\
\hline INTI11-S-30743 & 90 & 91 & 91 & & & & & & \\
\hline INTI11-S-30739 & 93 & 89 & 98 & & & & & & \\
\hline INTI11-S-30744 & 89 & 95 & 93 & & & & & & \\
\hline INTI11-S-30740 & 93 & 95 & 104 & & & & & & \\
\hline INTI11-S-30734 & 84 & 85 & 91 & & & & & & \\
\hline Methanol blank 2 & 91 & 86 & 91 & & & & & & \\
\hline INTI08-S-30702 & 93 & 100 & 101 & & & & & & \\
\hline \multicolumn{10}{|c|}{ SDG 09-11-19, analysis date November 19, 2009} \\
\hline 20-ppb standard & 100 & 100 & 100 & 19.51 & 2.5 & 20.66 & 3.2 & 20.7 & 3.4 \\
\hline Laboratory blank & 100 & 100 & 100 & & & & & & \\
\hline INTI08-S-30700 & 110 & 114 & 118 & & & & & & \\
\hline \multicolumn{10}{|c|}{ SDG 10-11-10, analysis date November 10, 2010} \\
\hline 20-ppb standard & 100 & 100 & 100 & 18.8 & 6.2 & 19.84 & 0.8 & 19.72 & 1.4 \\
\hline Laboratory blank & 104 & 107 & 102 & & & & & & \\
\hline INSISSON-W-30761 & 102 & 105 & 107 & & & & & & \\
\hline INMW01-W-32670 & 102 & 106 & 110 & & & & & & \\
\hline INMW02-W-32671 & 98 & 104 & 108 & & & & & & \\
\hline INMW03-W-32672 & 90 & 94 & 98 & & & & & & \\
\hline INWILLIAMS-W-32673 & 92 & 96 & 99 & & & & & & \\
\hline INPANKRATZ-W-32674 & 90 & 95 & 98 & & & & & & \\
\hline
\end{tabular}

INTI11-S-30736

INQCTB-S-30745

20-ppb standard

118

20-ppb standard

INMW02-W-32671

INWILLIAMS-W-32673 


\begin{tabular}{|c|c|c|c|c|c|c|c|c|c|}
\hline \multirow[b]{4}{*}{ Sample } & \multirow{2}{*}{\multicolumn{3}{|c|}{ Recovery of Surrogate Compounds ${ }^{a}(\%)$}} & \multicolumn{6}{|c|}{$\begin{array}{l}\text { Measured Concentration of } \\
\text { Calibration Check Standards }\end{array}$} \\
\hline & & & & \multirow{2}{*}{\multicolumn{2}{|c|}{$\begin{array}{c}\text { Carbon } \\
\text { Tetrachloride }\end{array}$}} & \multirow{2}{*}{\multicolumn{2}{|c|}{ Chloroform }} & \multirow{2}{*}{\multicolumn{2}{|c|}{$\begin{array}{l}\text { Methylene } \\
\text { Chloride }\end{array}$}} \\
\hline & & & & & & & & & \\
\hline & Fluorobenzene & $\begin{array}{l}\text { Bromo- } \\
\text { fluorobenzene }\end{array}$ & $\begin{array}{l}\text { 1,4-Dichloro- } \\
\text { benzene-d } 4\end{array}$ & $\mathrm{ppb}^{\mathrm{b}}$ & $\mathrm{RPD}^{\mathrm{C}}$ & $\mathrm{ppb}^{\mathrm{b}}$ & $\mathrm{RPD}^{\mathrm{C}}$ & $\mathrm{ppb}^{\mathrm{b}}$ & $\mathrm{RPD}^{\mathrm{C}}$ \\
\hline \multicolumn{10}{|c|}{ SDG 10-11-10, analysis date November 10, 2010 (cont.) } \\
\hline INBENGSTEN-W-32675 & 87 & 83 & 91 & & & & & & \\
\hline INBENGSTEN-W-32675DUP & 89 & 83 & 90 & & & & & & \\
\hline INQCTB-W-32676 & 91 & 88 & 92 & & & & & & \\
\hline \multicolumn{10}{|c|}{ SDG 10-11-11, analysis date November 11, 2010} \\
\hline 20-ppb standard & 104 & 105 & 108 & 18.66 & 6.9 & 20.14 & 0.7 & 18.9 & 5.7 \\
\hline Laboratory blank & 100 & 100 & 100 & & & & & & \\
\hline INTI14-W-30762 & 95 & 104 & 109 & & & & & & \\
\hline INTI12-W-32679 & 101 & 109 & 115 & & & & & & \\
\hline INTI14-W-30763 & 96 & 104 & 116 & & & & & & \\
\hline INMW05-W-32678 & 95 & 103 & 105 & & & & & & \\
\hline INMW04-W-32677 & 98 & 101 & 105 & & & & & & \\
\hline INMW04-W-32677DUP & 95 & 99 & 105 & & & & & & \\
\hline INTB-W-30764 & 92 & 92 & 93 & & & & & & \\
\hline \multicolumn{10}{|c|}{ SDG 10-11-12, analysis date November 12, 2010} \\
\hline 20-ppb standard & 91 & 98 & 99 & 19.33 & 3.4 & 20.56 & 2.8 & 20.42 & 2.1 \\
\hline Laboratory blank & 100 & 100 & 100 & & & & & & \\
\hline INTI16-W-30785 & 95 & 98 & 101 & & & & & & \\
\hline INTI15-W-30784 & 94 & 94 & 103 & & & & & & \\
\hline INTI13-W-30783 & 88 & 92 & 98 & & & & & & \\
\hline INTI18-W-30786 & 89 & 94 & 100 & & & & & & \\
\hline INQCTB-W-30787 & 87 & 88 & 94 & & & & & & \\
\hline Methanol blank & 102 & 101 & 101 & & & & & & \\
\hline INTI19-S-30772 & 105 & 109 & 115 & & & & & & \\
\hline INTI19-S-30773 & 99 & 103 & 104 & & & & & & \\
\hline INTI19-S-30769 & 99 & 105 & 103 & & & & & & \\
\hline INTI19-S-30767 & 102 & 107 & 111 & & & & & & \\
\hline
\end{tabular}

INBENGSTEN-W-32675

INBENGSTEN-W-32675DUP
105

INMW04-W-32677

INMW04-W-32677DUP

SDG 10-11-12, analysis date November 12, 2010

20-ppb standard

NTI15-W-30784

INTI19-S-30767 


\begin{tabular}{|c|c|c|c|c|c|c|c|c|c|}
\hline \multirow[b]{4}{*}{ Sample } & \multirow{2}{*}{\multicolumn{3}{|c|}{ Recovery of Surrogate Compounds ${ }^{\mathrm{a}}(\%)$}} & \multicolumn{6}{|c|}{$\begin{array}{l}\text { Measured Concentration of } \\
\text { Calibration Check Standards }\end{array}$} \\
\hline & & & & \multirow{2}{*}{\multicolumn{2}{|c|}{$\begin{array}{c}\text { Carbon } \\
\text { Tetrachloride }\end{array}$}} & \multirow{2}{*}{\multicolumn{2}{|c|}{ Chloroform }} & \multirow{2}{*}{\multicolumn{2}{|c|}{$\begin{array}{l}\text { Methylene } \\
\text { Chloride }\end{array}$}} \\
\hline & & & & & & & & & \\
\hline & Fluorobenzene & fluorobenzene & benzene- $\mathrm{d}_{4}$ & $\mathrm{ppb}^{\mathrm{b}}$ & $\mathrm{RPD}^{\mathrm{c}}$ & $\mathrm{ppb}^{\mathrm{b}}$ & $\mathrm{RPD}^{\mathrm{c}}$ & $\mathrm{ppb}^{\mathrm{b}}$ & $\mathrm{RPD}^{\mathrm{C}}$ \\
\hline \multicolumn{10}{|c|}{ SDG 10-11-12, analysis date November 12, 2010 (cont.) } \\
\hline INTI19-S-30765 & 98 & 110 & 110 & & & & & & \\
\hline INTI19-S-30770 & 98 & 109 & 113 & & & & & & \\
\hline INTI19-S-30766 & 96 & 104 & 108 & & & & & & \\
\hline INTI19-S-30768 & 93 & 108 & 105 & & & & & & \\
\hline \multicolumn{10}{|c|}{ SDG 10-11-15, analysis date November 15, 2010} \\
\hline 20-ppb standard & 85 & 98 & 90 & 23.8 & 17.4 & 23.87 & 17.6 & 20.36 & 1.8 \\
\hline Laboratory blank & 114 & 110 & 106 & & & & & & \\
\hline INTI17-W-30853 & 95 & 97 & 96 & & & & & & \\
\hline INQCTB-W-30852 & 87 & 91 & 97 & & & & & & \\
\hline INQCTB-W-30851 & 86 & 90 & 94 & & & & & & \\
\hline Methanol blank & 100 & 100 & 100 & & & & & & \\
\hline INTI19-S-30774 & 99 & 94 & 100 & & & & & & \\
\hline INTI19-S-30771 & 96 & 98 & 102 & & & & & & \\
\hline INTI19-S-30775 & 95 & 89 & 94 & & & & & & \\
\hline INTI19-S-30776 & 87 & 91 & 99 & & & & & & \\
\hline INTI21-S-30802 & 94 & 92 & 100 & & & & & & \\
\hline INTI21-S-30803 & 98 & 98 & 103 & & & & & & \\
\hline INTI21-S-30804 & 96 & 97 & 104 & & & & & & \\
\hline INTI20-S-30798 & 92 & 102 & 101 & & & & & & \\
\hline INTI20-S-30794 & 95 & 106 & 103 & & & & & & \\
\hline INTI20-S-30794DUP & 95 & 102 & 99 & & & & & & \\
\hline Methanol blank 2 & 94 & 93 & 95 & & & & & & \\
\hline INTI21-S-30801 & 114 & 97 & 117 & & & & & & \\
\hline INTI20-S-30793 & 101 & 106 & 111 & & & & & & \\
\hline INTI20-S-30790 & 107 & 113 & 118 & & & & & & \\
\hline INTI20-S-30792 & 94 & 98 & 99 & & & & & & \\
\hline INTI20-S-30799 & 104 & 114 & 114 & & & & & & \\
\hline INTI20-S-30795 & 90 & 103 & 110 & & & & & & \\
\hline
\end{tabular}

INTI19-S-30765
109

113

108 


\begin{tabular}{|c|c|c|c|c|c|c|c|c|c|}
\hline \multirow[b]{4}{*}{ Sample } & \multirow{2}{*}{\multicolumn{3}{|c|}{ Recovery of Surrogate Compounds ${ }^{\mathrm{a}}(\%)$}} & \multicolumn{6}{|c|}{$\begin{array}{l}\text { Measured Concentration of } \\
\text { Calibration Check Standards }\end{array}$} \\
\hline & & & & \multirow{2}{*}{\multicolumn{2}{|c|}{$\begin{array}{c}\text { Carbon } \\
\text { Tetrachloride }\end{array}$}} & \multirow{2}{*}{\multicolumn{2}{|c|}{ Chloroform }} & \multirow{2}{*}{\multicolumn{2}{|c|}{$\begin{array}{l}\text { Methylene } \\
\text { Chloride }\end{array}$}} \\
\hline & & Bromo & 14 Dicbloro & & & & & & \\
\hline & Fluorobenzene & fluorobenzene & benzene- $\mathrm{d}_{4}$ & $\mathrm{ppb}^{\mathrm{b}}$ & $\mathrm{RPD}^{\mathrm{c}}$ & $\mathrm{ppb}^{\mathrm{b}}$ & $\mathrm{RPD}^{\mathrm{c}}$ & $\mathrm{ppb}^{\mathrm{b}}$ & $\mathrm{RPD}^{\mathrm{c}}$ \\
\hline \multicolumn{10}{|c|}{ SDG 10-11-15, analysis date November 15, 2010 (cont.) } \\
\hline INTI20-S-30795DUP & 90 & 101 & 101 & & & & & & \\
\hline INTI20-S-30797 & 98 & 106 & 103 & & & & & & \\
\hline INTI20-S-30791 & 96 & 97 & 97 & & & & & & \\
\hline \multicolumn{10}{|c|}{ SDG 10-11-17, analysis date November 17, 2010} \\
\hline 20-ppb standard & 101 & 115 & 102 & 17.18 & 15.2 & 18.27 & 9.0 & 20.7 & 3.4 \\
\hline Methanol blank & 100 & 100 & 100 & & & & & & \\
\hline INTI22-S-30809 & 101 & 112 & 103 & & & & & & \\
\hline INTI21-S-30806 & 103 & 108 & 99 & & & & & & \\
\hline INTI22-S-30819 & 93 & 108 & 103 & & & & & & \\
\hline INTI21-S-30800 & 98 & 112 & 106 & & & & & & \\
\hline INTI20-S-30796 & 99 & 106 & 99 & & & & & & \\
\hline INTI22-S-30816 & 102 & 111 & 109 & & & & & & \\
\hline INTI22-S-30814 & 100 & 110 & 108 & & & & & & \\
\hline INTI22-S-30813 & 99 & 113 & 108 & & & & & & \\
\hline INTI21-S-30805 & 100 & 105 & 106 & & & & & & \\
\hline INTI21-S-30807 & 95 & 109 & 103 & & & & & & \\
\hline INTI22-S-30815 & 97 & 107 & 97 & & & & & & \\
\hline Methanol blank 2 & 95 & 102 & 98 & & & & & & \\
\hline INTI22-S-30817 & 95 & 102 & 95 & & & & & & \\
\hline INTI22-S-30812 & 98 & 108 & 98 & & & & & & \\
\hline INTI22-S-30810 & 101 & 112 & 110 & & & & & & \\
\hline INTI22-S-30811 & 105 & 111 & 110 & & & & & & \\
\hline INTI22-S-30818 & 101 & 114 & 105 & & & & & & \\
\hline INTI21-S-30808 & 102 & 107 & 109 & & & & & & \\
\hline INQCTB-S-30788 & 96 & 108 & 101 & & & & & & \\
\hline Methanol blank 3 & 95 & 101 & 100 & & & & & & \\
\hline INQCTB-S-30789 & 96 & 108 & 103 & & & & & & \\
\hline
\end{tabular}

INTI20-S-30795DUP

INTI20-S-30797

SDG 10-11-17, analysis date November 17, 2010

0-ppb standard

TI22-S-30813 


\begin{tabular}{|c|c|c|c|c|c|c|c|c|c|}
\hline \multirow[b]{4}{*}{ Sample } & \multirow{2}{*}{\multicolumn{3}{|c|}{ Recovery of Surrogate Compounds ${ }^{a}(\%)$}} & \multicolumn{6}{|c|}{$\begin{array}{l}\text { Measured Concentration of } \\
\text { Calibration Check Standards }\end{array}$} \\
\hline & & & & \multirow{2}{*}{\multicolumn{2}{|c|}{$\begin{array}{c}\text { Carbon } \\
\text { Tetrachloride }\end{array}$}} & \multirow{2}{*}{\multicolumn{2}{|c|}{ Chloroform }} & \multirow{2}{*}{\multicolumn{2}{|c|}{$\begin{array}{l}\text { Methylene } \\
\text { Chloride }\end{array}$}} \\
\hline & & & & & & & & & \\
\hline & Fluorobenzene & $\begin{array}{l}\text { Bromo- } \\
\text { fluorobenzene }\end{array}$ & $\begin{array}{l}\text { 1,4-Dichloro- } \\
\text { benzene-d }_{4}\end{array}$ & $\mathrm{ppb}^{\mathrm{b}}$ & $\mathrm{RPD}^{\mathrm{C}}$ & $\mathrm{ppb}^{\mathrm{b}}$ & $\mathrm{RPD}^{\mathrm{C}}$ & $\mathrm{ppb}^{\mathrm{b}}$ & $\mathrm{RPD}^{\mathrm{C}}$ \\
\hline \multicolumn{10}{|c|}{ SDG 10-11-18, analysis date November 18, 2010} \\
\hline 20-ppb standard & 100 & 100 & 100 & 22.84 & 13.3 & 23.31 & 15.3 & 21.84 & 8.8 \\
\hline Methanol blank & 100 & 100 & 100 & & & & & & \\
\hline INTI23-S-30839 & 89 & 102 & 104 & & & & & & \\
\hline INTI23-S-30824 & 89 & 103 & 106 & & & & & & \\
\hline INTI24-S-30830 & 88 & 98 & 100 & & & & & & \\
\hline INTI23-S-30826 & 90 & 96 & 105 & & & & & & \\
\hline INTI23-S-30823 & 85 & 96 & 103 & & & & & & \\
\hline INTI23-S-30827 & 88 & 95 & 106 & & & & & & \\
\hline INTI23-S-30822 & 87 & 99 & 102 & & & & & & \\
\hline INTI23-S-30821 & 91 & 100 & 101 & & & & & & \\
\hline INTI24-S-30831 & 88 & 98 & 102 & & & & & & \\
\hline INTI24-S-30831DUP & 87 & 95 & 97 & & & & & & \\
\hline INTI23-S-30825 & 97 & 102 & 101 & & & & & & \\
\hline INTI24-S-30829 & 93 & 102 & 107 & & & & & & \\
\hline INTI24-S-30828 & 89 & 98 & 99 & & & & & & \\
\hline INTI24-S-30832 & 85 & 95 & 98 & & & & & & \\
\hline \multicolumn{10}{|c|}{ SDG 10-11-19, analysis date November 19, 2010} \\
\hline 20-ppb standard & 89 & 95 & 85 & 21.33 & 6.4 & 23.31 & 15.3 & 20.8 & 3.9 \\
\hline Laboratory blank & 112 & 113 & 114 & & & & & & \\
\hline INTI22-W-32680 & 89 & 95 & 96 & & & & & & \\
\hline INTI23-W-32681 & 94 & 102 & 103 & & & & & & \\
\hline INTI24-W-32682 & 94 & 104 & 109 & & & & & & \\
\hline INTI26-W-32683 & 99 & 110 & 102 & & & & & & \\
\hline INQCTB-W-32684 & 88 & 87 & 86 & & & & & & \\
\hline Methanol blank & 100 & 100 & 100 & & & & & & \\
\hline INTI24-S-30833 & 91 & 101 & 101 & & & & & & \\
\hline INTI24-S-30835 & 88 & 92 & 103 & & & & & & \\
\hline INTI25-S-30840 & 88 & 98 & 102 & & & & & & \\
\hline
\end{tabular}

SDG 10-11-19, analysis date November 19, 2010

96

113

21.33

6.4 


\begin{tabular}{|c|c|c|c|c|c|c|c|c|c|}
\hline \multirow[b]{3}{*}{ Sample } & \multirow{2}{*}{\multicolumn{3}{|c|}{ Recovery of Surrogate Compounds ${ }^{\mathrm{a}}(\%)$}} & \multicolumn{6}{|c|}{$\begin{array}{l}\text { Measured Concentration of } \\
\text { Calibration Check Standards }\end{array}$} \\
\hline & & & & \multicolumn{2}{|c|}{$\begin{array}{c}\text { Carbon } \\
\text { Tetrachloride }\end{array}$} & \multicolumn{2}{|c|}{ Chloroform } & \multicolumn{2}{|c|}{$\begin{array}{l}\text { Methylene } \\
\text { Chloride }\end{array}$} \\
\hline & Fluorobenzene & $\begin{array}{l}\text { Bromo- } \\
\text { fluorobenzene }\end{array}$ & $\begin{array}{l}\text { 1,4-Dichloro- } \\
\text { benzene- } \mathrm{d}_{4}\end{array}$ & $\mathrm{ppb}^{\mathrm{b}}$ & $\mathrm{RPD}^{\mathrm{c}}$ & $\mathrm{ppb}^{\mathrm{b}}$ & $\mathrm{RPD}^{\mathrm{c}}$ & $\mathrm{ppb}^{\mathrm{b}}$ & $\mathrm{RPD}^{\mathrm{c}}$ \\
\hline
\end{tabular}

SDG 10-11-19, analysis date November 19, 2010 (cont.)

$\begin{array}{lrrr}\text { INTI24-S-30837 } & 87 & 95 & 106 \\ \text { INTI25-S-30845 } & 87 & 95 & 112 \\ \text { INTI24-S-30836 } & 86 & 97 & 105 \\ \text { INTI25-S-30843 } & 85 & 92 & 104 \\ \text { INTI25-S-30843DUP } & 81 & 84 & 90 \\ \text { Methanol blank 2 } & 85 & 88 & 95 \\ \text { INTI25-S-30848 } & 83 & 88 & 96 \\ \text { INTI25-S-30846 } & 92 & 101 & 112 \\ \text { INTI25-S-30842 } & 88 & 96 & 105 \\ \text { INTI24-S-30838 } & 90 & 93 & 108 \\ \text { INTI25-S-30844 } & 87 & 94 & 107\end{array}$

SDG 10-11-22, analysis date November 22, 2010

$\begin{array}{lrrr}\text { 20-ppb standard } & 100 & 100 & 100 \\ \text { Methanol blank } & 100 & 100 & 100 \\ \text { INTI25-S-30841 } & 90 & 100 & 102 \\ \text { INTI24-S-30834 } & 90 & 96 & 105 \\ \text { INQCTB-S-30849 } & 102 & 111 & 118 \\ \text { INQCTB-S-30850 } & 89 & 90 & 99\end{array}$

SDG 11-5-25, analysis date May 25, 2011

20-ppb standard

Laboratory blank

INTHIESSEN-W-32685

INBROWN-W-32686

INKLASSEN-W-32687

INGOERTZEN-W-32688

INISAAC-W-32689

\section{9}

100

102

$124^{\mathrm{d}}$

113

104

108
105

90

95

105

107

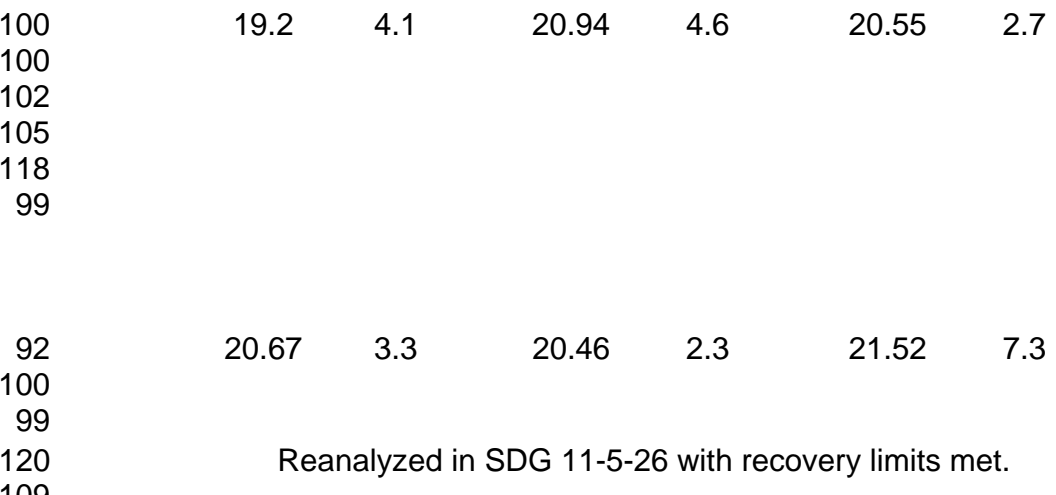




\begin{tabular}{|c|c|c|c|c|c|c|c|c|c|}
\hline \multirow[b]{4}{*}{ Sample } & \multirow{2}{*}{\multicolumn{3}{|c|}{ Recovery of Surrogate Compounds ${ }^{a}(\%)$}} & \multicolumn{6}{|c|}{$\begin{array}{l}\text { Measured Concentration of } \\
\text { Calibration Check Standards }\end{array}$} \\
\hline & & & & \multirow{2}{*}{\multicolumn{2}{|c|}{$\begin{array}{c}\text { Carbon } \\
\text { Tetrachloride }\end{array}$}} & \multirow{2}{*}{\multicolumn{2}{|c|}{ Chloroform }} & \multirow{2}{*}{\multicolumn{2}{|c|}{$\begin{array}{l}\text { Methylene } \\
\text { Chloride }\end{array}$}} \\
\hline & & & & & & & & & \\
\hline & Fluorobenzene & fluorobenzene & benzene- $\mathrm{d}_{4}$ & $\mathrm{ppb}^{\mathrm{b}}$ & $\mathrm{RPD}^{\mathrm{c}}$ & $\mathrm{ppb}^{\mathrm{b}}$ & $\mathrm{RPD}^{\mathrm{c}}$ & $\mathrm{ppb}^{\mathrm{b}}$ & $\mathrm{RPD}^{\mathrm{c}}$ \\
\hline \multicolumn{10}{|c|}{ SDG 11-5-25, analysis date May 25, 2011 (cont.) } \\
\hline INPANKRATZ-W-32690 & 106 & 99 & 96 & & & & & & \\
\hline INHULL-W-32691 & 106 & 104 & 102 & & & & & & \\
\hline INHULL-W-32691DUP & 106 & 98 & 98 & & & & & & \\
\hline INQCTB-W-32692 & 106 & 99 & 98 & & & & & & \\
\hline \multicolumn{10}{|c|}{ SDG 11-5-26, analysis date May 26, 2011} \\
\hline 20-ppb standard & 84 & 103 & 97 & 21.32 & 6.4 & 16.97 & 16.4 & 22.36 & 11.1 \\
\hline Laboratory blank & 100 & 100 & 100 & & & & & & \\
\hline INBROWN-W-32686 & 93 & 105 & 89 & & & & & & \\
\hline \multicolumn{10}{|c|}{ SDG 11-6-24, analysis date June 24, 2011} \\
\hline 20-ppb standard & 88 & 109 & 90 & 20.06 & 0.3 & 18.14 & 9.8 & 18.32 & 8.8 \\
\hline Laboratory blank & 100 & 100 & 100 & & & & & & \\
\hline INKLASSEN-W-32693 & 98 & 102 & 100 & & & & & & \\
\hline INHULL-W-32694 & 89 & 94 & 92 & & & & & & \\
\hline INPAULS-W-32695 & 91 & 92 & 92 & & & & & & \\
\hline INQCTB-W-32696 & 90 & 97 & 91 & & & & & & \\
\hline \multicolumn{10}{|c|}{ SDG 11-8-23, analysis date August 23, 2011} \\
\hline 20-ppb standard & 100 & 100 & 100 & 20.41 & 2.0 & 18.24 & 9.2 & 19.6 & 2.0 \\
\hline Laboratory blank & 97 & 111 & 99 & & & & & & \\
\hline INMW14-W-32730 & 98 & $70^{\mathrm{d}}$ & $72^{\mathrm{d}}$ & \multirow{4}{*}{\multicolumn{6}{|c|}{ Reanalyzed in SDG $11-8-25$ with recovery limits met. }} \\
\hline INTRUCK-W-32731 & 89 & 113 & 115 & & & & & & \\
\hline INCITY-W-32732 & 97 & 98 & 96 & & & & & & \\
\hline INQCTB-W-32733 & 95 & 91 & 96 & & & & & & \\
\hline
\end{tabular}

INPANKRATZ-W-32690

b standard

SDG 11-6-24, analysis date June 24, 201

pp standard

INHULL-W-32694

91 


\begin{tabular}{|c|c|c|c|c|c|c|c|c|c|}
\hline \multirow[b]{3}{*}{ Sample } & \multirow{2}{*}{\multicolumn{3}{|c|}{ Recovery of Surrogate Compounds ${ }^{\mathrm{a}}(\%)$}} & \multicolumn{6}{|c|}{$\begin{array}{l}\text { Measured Concentration of } \\
\text { Calibration Check Standards }\end{array}$} \\
\hline & & & & \multicolumn{2}{|c|}{$\begin{array}{c}\text { Carbon } \\
\text { Tetrachloride } \\
\end{array}$} & \multicolumn{2}{|c|}{ Chloroform } & \multicolumn{2}{|c|}{$\begin{array}{l}\text { Methylene } \\
\text { Chloride }\end{array}$} \\
\hline & Fluorobenzene & $\begin{array}{l}\text { Bromo- } \\
\text { fluorobenzene }\end{array}$ & $\begin{array}{l}\text { 1,4-Dichloro- } \\
\text { benzene- } d_{4}\end{array}$ & $\mathrm{ppb}^{\mathrm{b}}$ & $\mathrm{RPD}^{\mathrm{c}}$ & $\mathrm{ppb}^{\mathrm{b}}$ & $\mathrm{RPD}^{\mathrm{c}}$ & $\mathrm{ppb}^{\mathrm{b}}$ & $\mathrm{RPD}^{\mathrm{c}}$ \\
\hline
\end{tabular}

SDG 11-8-25, analysis date August 25, 2011

$\begin{array}{lrrr}\text { 20-ppb standard } & 99 & 114 & 119 \\ \text { Laboratory blank } & 100 & 100 & 100 \\ \text { INMW15-W-32735 } & 106 & 120 & 107 \\ \text { INMW15-W-32737 } & 100 & 103 & 103 \\ \text { INMW14S-W-32736 } & 88 & 116 & 105 \\ \text { INMW14S-W-32736DUP } & 98 & 120 & 118 \\ \text { INMW14-W-32734 } & 99 & 106 & 117 \\ \text { INMW14-W-32730 } & 96 & 108 & 104 \\ \text { INQCTB-W-32738 } & 94 & 99 & 95\end{array}$

INQCTB-W-32738

99

3.0

16.97

16.4

18.89

5.7

SDG 11-8-26, analysis date August 26, 2011

20-ppb standard

Laboratory blank

114

100

103

105

17

94

104
95

INMW16-W-32739

INMW16-W-32740

INMW16-W-32741

INMW16-W-32742

INKTW-W-32743

INQCTB-W-32744

INMW16-W-32740DUP

$\begin{array}{rrr}114 & 95 & 101 \\ 100 & 100 & 100 \\ 104 & 87 & 84 \\ 108 & 102 & 104 \\ 100 & 107 & 106 \\ 106 & 108 & 106 \\ 103 & 99 & 94 \\ 95 & 90 & 91 \\ 96 & 94 & 91\end{array}$

SDG 11-8-29, analysis date August 29, 2011

20-ppb standard

Laboratory blank

107

INMW17-W-32745

100

INMW15-W-32747

98

INMW17-W-32748

104

$\begin{array}{rr}96 & 106 \\ 100 & 100 \\ 100 & 90 \\ 111 & 109 \\ 110 & 111\end{array}$




\begin{tabular}{|c|c|c|c|c|c|c|c|c|c|}
\hline \multirow[b]{3}{*}{ Sample } & \multirow{2}{*}{\multicolumn{3}{|c|}{ Recovery of Surrogate Compounds ${ }^{\mathrm{a}}(\%)$}} & \multicolumn{6}{|c|}{$\begin{array}{l}\text { Measured Concentration of } \\
\text { Calibration Check Standards }\end{array}$} \\
\hline & & & & \multicolumn{2}{|c|}{$\begin{array}{c}\text { Carbon } \\
\text { Tetrachloride }\end{array}$} & \multicolumn{2}{|c|}{ Chloroform } & \multicolumn{2}{|c|}{$\begin{array}{l}\text { Methylene } \\
\text { Chloride }\end{array}$} \\
\hline & Fluorobenzene & $\begin{array}{l}\text { Bromo- } \\
\text { fluorobenzene }\end{array}$ & $\begin{array}{l}\text { 1,4-Dichloro- } \\
\text { benzene- } \mathrm{d}_{4}\end{array}$ & $\mathrm{ppb}^{\mathrm{b}}$ & $\mathrm{RPD}^{\mathrm{c}}$ & $\mathrm{ppb}^{\mathrm{b}}$ & $\mathrm{RPD}^{\mathrm{c}}$ & $\mathrm{ppb}^{\mathrm{b}}$ & $\mathrm{RPD}^{\mathrm{c}}$ \\
\hline
\end{tabular}

SDG 11-8-29, analysis date August 29, 2011 (cont.)

$\begin{array}{lllr}\text { INMW17-W-32745DUP } & 105 & 111 & 111 \\ \text { INMW17-W-32749 } & 100 & 103 & 100 \\ \text { INMW17QC-W-32746 } & 101 & 100 & 92\end{array}$

SDG 11-8-30a, analysis date August 30, 2011

20-ppb standard

Laboratory blank

INMW18-W-32752

INMW18-W-32753

INMW18-W-32754

INMW18-W-32755

INMW16-W-32764

INMW16D-W-32756

INMW16D-W-32756DUP

INMW16W-32765

INMW15-W-32750

INMW15-W-32751

INTB-W-32766

INQC-W-32763

100
97
114
106
103
98
104
97
95
93
93
90
90
$63^{\mathrm{d}}$

SDG 11-8-30b, analysis date August 30, 2011

20-ppb standard

Laboratory blank

INMW15S-W-32757

INMW17D-W-32758

INMW15D-W-32759

INMW18-W-32760

105
101
100
103
99
106

$\begin{array}{rr}94 & 93 \\ 91 & 91 \\ 109 & 110 \\ 112 & 114 \\ 109 & 109 \\ 94 & 101 \\ 104 & 102 \\ 102 & 108 \\ 101 & 101 \\ 87 & 100 \\ 93 & 94 \\ 95 & 96 \\ 88 & 85 \\ 64^{\mathrm{d}} & 70^{\mathrm{d}}\end{array}$

22.25

10.7

20.04

0.2

18.25

9.2

Reanalyzed in SDG 11-8-31 with recovery limits met.

$\begin{array}{rrrrrrrr}111 & 98 & 20 & 0.0 & 16.4 & 19.8 & 18.3 & 8.9 \\ 85 & 91 & & & & & & \\ 80 & 101 & & & & & & \\ 143^{\mathrm{d}} & 149^{\mathrm{d}} & & & \text { Reanalyzed in SDG 11-8-31 with recovery limits met. }\end{array}$




\begin{tabular}{|c|c|c|c|c|c|c|c|c|c|}
\hline \multirow[b]{4}{*}{ Sample } & \multirow{2}{*}{\multicolumn{3}{|c|}{ Recovery of Surrogate Compounds ${ }^{a}(\%)$}} & \multicolumn{6}{|c|}{$\begin{array}{l}\text { Measured Concentration of } \\
\text { Calibration Check Standards }\end{array}$} \\
\hline & & & & \multirow{2}{*}{\multicolumn{2}{|c|}{$\begin{array}{c}\text { Carbon } \\
\text { Tetrachloride }\end{array}$}} & \multirow{2}{*}{\multicolumn{2}{|c|}{ Chloroform }} & \multirow{2}{*}{\multicolumn{2}{|c|}{$\begin{array}{l}\text { Methylene } \\
\text { Chloride }\end{array}$}} \\
\hline & & & & & & & & & \\
\hline & Fluorobenzene & fluorobenzene & benzene- $\mathrm{d}_{4}$ & $\mathrm{ppb}^{\mathrm{b}}$ & $\mathrm{RPD}^{\mathrm{c}}$ & $\mathrm{ppb}^{\mathrm{b}}$ & $\mathrm{RPD}^{\mathrm{c}}$ & $\mathrm{ppb}^{\mathrm{b}}$ & $\mathrm{RPD}^{\mathrm{c}}$ \\
\hline \multicolumn{10}{|c|}{ SDG 11-8-30b, analysis date August 30, 2011 (cont.) } \\
\hline INMW18-W-32761 & 106 & $128^{d}$ & $121^{d}$ & \multirow{2}{*}{\multicolumn{6}{|c|}{ Reanalyzed in SDG 11-8-31 with recovery limits met. }} \\
\hline INMW16-W-32762 & 101 & 98 & 112 & & & & & & \\
\hline \multicolumn{10}{|c|}{ SDG 11-8-31, analysis date August 31, 2011} \\
\hline 20-ppb standard & 81 & 94 & 90 & 19.79 & 1.1 & 16.63 & 18.4 & 16.34 & 20.1 \\
\hline Laboratory blank & 100 & 100 & 100 & (1. & 1.1 & 10.00 & 10.4 & 10.04 & 20.1 \\
\hline INMW16-W-32767 & 99 & 91 & 100 & & & & & & \\
\hline INMW19-W-32768 & 96 & 119 & 115 & & & & & & \\
\hline INMW19-W-32768DUP & 96 & 116 & 109 & & & & & & \\
\hline INQCTB-W-32769 & 98 & 88 & 95 & & & & & & \\
\hline INQC-W-32763 & 99 & 81 & 92 & & & & & & \\
\hline INMW17D-W-32758 & 105 & 114 & 102 & & & & & & \\
\hline INMW18-W-32761 & 106 & 109 & 112 & & & & & & \\
\hline \multicolumn{10}{|c|}{ SDG 11-9-1, analysis date September 1, 2011} \\
\hline 20-ppb standard & 100 & 100 & 100 & 22.13 & 10.1 & 18.31 & 8.8 & 18.3 & 8.9 \\
\hline Laboratory blank & 100 & 100 & 100 & & & & & & \\
\hline INMW17D-W-32773 & 102 & 102 & 102 & & & & & & \\
\hline INMW17S-W-32772 & 99 & 118 & 114 & & & & & & \\
\hline INMW17M-W-32770 & 99 & 94 & 90 & & & & & & \\
\hline INMW17M-W-32770DUP & 94 & 92 & 88 & & & & & & \\
\hline INQCTB-W-32774 & 88 & 98 & 92 & & & & & & \\
\hline INMW19-W-32771 & 89 & 115 & 108 & & & & & & \\
\hline \multicolumn{10}{|c|}{ SDG 11-9-2, analysis date September 2, 2011} \\
\hline 20-ppb standard & 100 & 100 & 100 & 21.44 & 6.9 & 19.57 & 2.2 & 17.2 & 15.1 \\
\hline Laboratory blank & 100 & 100 & 100 & & & & & & \\
\hline
\end{tabular}

INMW18-W-32761
100 


\begin{tabular}{|c|c|c|c|c|c|c|c|c|c|}
\hline \multirow[b]{3}{*}{ Sample } & \multirow{2}{*}{\multicolumn{3}{|c|}{ Recovery of Surrogate Compounds ${ }^{\mathrm{a}}(\%)$}} & \multicolumn{6}{|c|}{$\begin{array}{l}\text { Measured Concentration of } \\
\text { Calibration Check Standards }\end{array}$} \\
\hline & & & & \multicolumn{2}{|c|}{$\begin{array}{c}\text { Carbon } \\
\text { Tetrachloride } \\
\end{array}$} & \multicolumn{2}{|c|}{ Chloroform } & \multicolumn{2}{|c|}{$\begin{array}{l}\text { Methylene } \\
\text { Chloride }\end{array}$} \\
\hline & Fluorobenzene & $\begin{array}{l}\text { Bromo- } \\
\text { fluorobenzene }\end{array}$ & $\begin{array}{l}\text { 1,4-Dichloro- } \\
\text { benzene-d } 4\end{array}$ & $\mathrm{ppb}^{\mathrm{b}}$ & $\mathrm{RPD}^{\mathrm{c}}$ & $\mathrm{ppb}^{\mathrm{b}}$ & $\mathrm{RPD}^{\mathrm{c}}$ & $\mathrm{ppb}^{\mathrm{b}}$ & $\mathrm{RPD}^{\mathrm{c}}$ \\
\hline
\end{tabular}

SDG 11-9-2, analysis date September 2, 2011 (cont.)

$\begin{array}{lrrr}\text { INMW18S-W-32775 } & 94 & 95 & 91 \\ \text { INMW18M-W-32776 } & 93 & 90 & 92 \\ \text { INMW18D-W-32777 } & 94 & 98 & 100 \\ \text { INQCTB-W-32778 } & 85 & 85 & 90 \\ & & & \\ \text { SDG 11-9-6, analysis date September 6, } & 2011 & & \\ & & 118 & 112 \\ \text { 20-ppb standard } & 101 & 100 & 88 \\ \text { Laboratory blank } & 100 & 86 & 93 \\ \text { INMW19S-W-32789 } & 88 & 98 & 92 \\ \text { INMW19D-W-32790 } & 92 & 98 & 92 \\ \text { INMW19D-W-32790DUP } & 91 & 95 & \end{array}$

SDG 11-9-15, analysis date September 15, 2011

$\begin{array}{lrrr}\text { 20-ppb standard } & 101 & 116 & 103 \\ \text { Laboratory blank } & 100 & 100 & 100 \\ \text { INMW20-W-32775 } & 87 & 85 & 89 \\ \text { INMW20-W-32776 } & 94 & 97 & 106 \\ \text { INMW20-W-32776DUP } & 92 & 97 & 98 \\ \text { INQCTB-W-32777 } & 92 & 91 & 90\end{array}$

SDG 11-9-16, analysis date September 16, 2011

$\begin{array}{lrrr}\text { 20-ppb standard } & 105 & 98 & 95 \\ \text { Laboratory blank } & 110 & 113 & 113 \\ \text { INMW20S-W-32780 } & 109 & 108 & 110 \\ \text { INMW20M-W-32781 } & 105 & 104 & 96 \\ \text { INMW14D-W-32782 } & 101 & 101 & 100\end{array}$

$\begin{array}{llllll}20.3 & 1.5 & 17.94 & 10.9 & 17.06 & 15.9\end{array}$




\begin{tabular}{|c|c|c|c|c|c|c|c|c|c|}
\hline \multirow[b]{4}{*}{ Sample } & \multirow{2}{*}{\multicolumn{3}{|c|}{ Recovery of Surrogate Compounds ${ }^{\mathrm{a}}(\%)$}} & \multicolumn{6}{|c|}{$\begin{array}{l}\text { Measured Concentration of } \\
\text { Calibration Check Standards }\end{array}$} \\
\hline & & & & \multirow{2}{*}{\multicolumn{2}{|c|}{$\begin{array}{c}\text { Carbon } \\
\text { Tetrachloride } \\
\end{array}$}} & \multirow{2}{*}{\multicolumn{2}{|c|}{ Chloroform }} & \multirow{2}{*}{\multicolumn{2}{|c|}{$\begin{array}{l}\text { Methylene } \\
\text { Chloride }\end{array}$}} \\
\hline & \multirow[b]{2}{*}{ Fluorobenzene } & \multirow[b]{2}{*}{$\begin{array}{l}\text { Bromo- } \\
\text { fluorobenzene }\end{array}$} & \multirow[b]{2}{*}{$\begin{array}{l}\text { 1,4-Dichloro- } \\
\text { benzene- } d_{4}\end{array}$} & & & & & & \\
\hline & & & & $\mathrm{ppb}^{\mathrm{b}}$ & $\mathrm{RPD}^{\mathrm{C}}$ & $\mathrm{ppb}^{\mathrm{b}}$ & $\mathrm{RPD}^{\mathrm{C}}$ & $\mathrm{ppb}^{\mathrm{b}}$ & $\mathrm{RPD}^{\mathrm{C}}$ \\
\hline SDG 11-9-16, analysis dat & 16, 2011 (cont.) & & & & & & & & \\
\hline INMW14D-W-32782DUP & 90 & 89 & 93 & & & & & & \\
\hline INQCTB-W-32783 & 90 & 87 & 87 & & & & & & \\
\hline
\end{tabular}

a Quality control range for recovery $=80-120 \%$.

b Concentration in parts per billion ( $\mu \mathrm{g} / \mathrm{L}$ in water or $\mu \mathrm{g} / \mathrm{kg}$ in soil).

c Quality control range for RPD $= \pm 20 \%$.

d Surrogate recovery outside quality control range. 
TABLE S2.4 Analytical results from the AGEM Laboratory for secondary quality control analyses.

\begin{tabular}{|c|c|c|c|c|c|c|c|c|c|c|c|c|}
\hline \multirow[b]{2}{*}{$\begin{array}{l}\text { Sample } \\
\text { Date }\end{array}$} & \multirow[b]{2}{*}{ Location } & \multirow[b]{2}{*}{$\begin{array}{l}\text { Depth } \\
\text { (ft BGL) }\end{array}$} & \multirow[b]{2}{*}{$\begin{array}{l}\text { Sample } \\
\text { Medium }\end{array}$} & \multirow[b]{2}{*}{ Sample } & \multirow[b]{2}{*}{ Analysis Type } & \multirow[b]{2}{*}{$\begin{array}{l}\text { Sample } \\
\text { Type }^{\mathrm{a}}\end{array}$} & \multirow[b]{2}{*}{$\begin{array}{l}\text { Analysis } \\
\text { Method }\end{array}$} & \multirow[b]{2}{*}{$\begin{array}{l}\text { Analysis } \\
\text { Date }\end{array}$} & \multicolumn{3}{|c|}{ Concentration } & \multirow[b]{2}{*}{ Units } \\
\hline & & & & & & & & & $\begin{array}{c}\text { Carbon } \\
\text { Tetrachloride }\end{array}$ & Chloroform & $\begin{array}{l}\text { Methylene } \\
\text { Chloride }\end{array}$ & \\
\hline $11 / 4 / 09$ & TI01 & 34 & Soil & INTI01-S-30627 & Primary sample & CPT & SW8260B & $11 / 6 / 09$ & $N D^{b}$ & ND & ND & $\mu \mathrm{g} / \mathrm{kg}$ \\
\hline $11 / 4 / 09$ & TI01 & 34 & Soil & INTI01-S-30628 & Replicate sample & CPT & SW8260B & $11 / 6 / 09$ & $3.5 \mathrm{~J}^{\mathrm{c}}$ & ND & ND & $\mu \mathrm{g} / \mathrm{kg}$ \\
\hline $11 / 4 / 09$ & T102 & 10 & Soil & INTI02-S-30633 & Primary sample & CPT & SW8260B & $11 / 9 / 09$ & ND & ND & ND & $\mu \mathrm{g} / \mathrm{kg}$ \\
\hline $11 / 4 / 09$ & TI02 & 10 & Soil & INTI02-S-30633DUP & Duplicate analysis & СРT & SW8260B & $11 / 9 / 09$ & ND & ND & ND & $\mu \mathrm{g} / \mathrm{kg}$ \\
\hline $11 / 4 / 09$ & T102 & 26 & Soil & INTI02-S-30637 & Primary sample & CPT & SW8260B & $11 / 9 / 09$ & $8.0 \mathrm{~J}$ & ND & ND & $\mu \mathrm{g} / \mathrm{kg}$ \\
\hline $11 / 4 / 09$ & TI02 & 26 & Soil & INTI02-S-30638 & Replicate sample & CPT & SW8260B & $11 / 9 / 09$ & ND & ND & ND & $\mu \mathrm{g} / \mathrm{kg}$ \\
\hline $11 / 5 / 09$ & T103 & 30 & Soil & INTI03-S-30650 & Primary sample & CPT & SW8260B & $11 / 10 / 09$ & ND & ND & ND & $\mu \mathrm{g} / \mathrm{kg}$ \\
\hline $11 / 5 / 09$ & TI03 & 30 & Soil & INTI03-S-30650DUP & Duplicate analysis & CPT & SW8260B & $11 / 10 / 09$ & ND & ND & ND & $\mu \mathrm{g} / \mathrm{kg}$ \\
\hline $11 / 5 / 09$ & T104 & 38 & Soil & INTI04-S-30663 & Primary sample & CPT & SW8260B & $11 / 10 / 09$ & ND & ND & ND & $\mu \mathrm{g} / \mathrm{kg}$ \\
\hline $11 / 5 / 09$ & T104 & 38 & Soil & INTI04-S-30664 & Replicate sample & CPT & SW8260B & $11 / 11 / 09$ & ND & ND & ND & $\mu \mathrm{g} / \mathrm{kg}$ \\
\hline $11 / 6 / 09$ & TI06 & 30 & Soil & INTI06-S-30683 & Primary sample & CPT & SW8260B & $11 / 12 / 09$ & ND & ND & ND & $\mu \mathrm{g} / \mathrm{kg}$ \\
\hline $11 / 6 / 09$ & TI06 & 30 & Soil & INTI06-S-30684 & Replicate sample & СРT & SW8260B & $11 / 12 / 09$ & ND & ND & ND & $\mu \mathrm{g} / \mathrm{kg}$ \\
\hline $11 / 6 / 09$ & TI07 & 30 & Soil & INTI07-S-30694DUP & Duplicate analysis & СРT & SW8260B & $11 / 11 / 09$ & ND & ND & ND & $\mu \mathrm{g} / \mathrm{kg}$ \\
\hline $11 / 6 / 09$ & T107 & 34 & Soil & INTI07-S-30695 & Primary sample & CPT & SW8260B & $11 / 16 / 09$ & ND & ND & ND & $\mu \mathrm{g} / \mathrm{kg}$ \\
\hline $11 / 6 / 09$ & T107 & 34 & Soil & INTI07-S-30695DUP & Duplicate analysis & CPT & SW8260B & $11 / 16 / 09$ & ND & ND & ND & $\mu \mathrm{g} / \mathrm{kg}$ \\
\hline $11 / 6 / 09$ & T107 & 38 & Soil & INTI07-S-30696 & Primary sample & CPT & SW8260B & $11 / 11 / 09$ & ND & ND & ND & $\mu \mathrm{g} / \mathrm{kg}$ \\
\hline $11 / 6 / 09$ & TI07 & 38 & Soil & INTI07-S-30697 & Replicate sample & СРT & SW8260B & $11 / 12 / 09$ & ND & ND & ND & $\mu \mathrm{g} / \mathrm{kg}$ \\
\hline $11 / 7 / 09$ & T108 & 26 & Soil & INTI08-S-30704 & Primary sample & CPT & SW8260B & $11 / 12 / 09$ & ND & ND & ND & $\mu \mathrm{g} / \mathrm{kg}$ \\
\hline $11 / 7 / 09$ & TI08 & 26 & Soil & INTI08-S-30705 & Replicate sample & CPT & SW8260B & $11 / 17 / 09$ & ND & ND & ND & $\mu \mathrm{g} / \mathrm{kg}$ \\
\hline $11 / 7 / 09$ & T109 & 30 & Soil & INTI09-S-30716 & Primary sample & CPT & SW8260B & $11 / 11 / 09$ & ND & ND & ND & $\mu \mathrm{g} / \mathrm{kg}$ \\
\hline $11 / 7 / 09$ & TI09 & 30 & Soil & INTI09-S-30717 & Replicate sample & CPT & SW8260B & $11 / 11 / 09$ & ND & ND & ND & $\mu \mathrm{g} / \mathrm{kg}$ \\
\hline $11 / 7 / 09$ & T109 & 38 & Soil & INTI09-S-30718 & Primary sample & CPT & SW8260B & $11 / 11 / 09$ & ND & ND & ND & $\mu \mathrm{g} / \mathrm{kg}$ \\
\hline $11 / 7 / 09$ & TI09 & 38 & Soil & INTI09-S-30718DUP & Duplicate analysis & CPT & SW8260B & $11 / 11 / 09$ & ND & ND & ND & $\mu \mathrm{g} / \mathrm{kg}$ \\
\hline $11 / 7 / 09$ & TI10 & 2 & Soil & INTI10-S-30720 & Primary sample & CPT & SW8260B & $11 / 12 / 09$ & ND & ND & ND & $\mu \mathrm{g} / \mathrm{kg}$ \\
\hline $11 / 7 / 09$ & TI10 & 2 & Soil & INTI10-S-30720DUP & Duplicate analysis & CPT & SW8260B & $11 / 12 / 09$ & ND & ND & ND & $\mu \mathrm{g} / \mathrm{kg}$ \\
\hline $11 / 7 / 09$ & TI10 & 38 & Soil & INTI10-S-30729 & Primary sample & CPT & SW8260B & 11/11/09 & $5.9 \mathrm{~J}$ & ND & ND & $\mu \mathrm{g} / \mathrm{kg}$ \\
\hline $11 / 7 / 09$ & TI10 & 38 & Soil & INTI10-S-30730 & Replicate sample & СPT & SW8260B & $11 / 12 / 09$ & ND & ND & ND & $\mu \mathrm{g} / \mathrm{kg}$ \\
\hline
\end{tabular}


TABLE S2.4 (Cont.)

\begin{tabular}{|c|c|c|c|c|c|c|c|c|c|c|c|c|}
\hline \multirow[b]{2}{*}{$\begin{array}{l}\text { Sample } \\
\text { Date }\end{array}$} & \multirow[b]{2}{*}{ Location } & \multirow[b]{2}{*}{$\begin{array}{c}\text { Depth } \\
\text { (ft BGL) }\end{array}$} & \multirow[b]{2}{*}{$\begin{array}{l}\text { Sample } \\
\text { Medium }\end{array}$} & \multirow[b]{2}{*}{ Sample } & \multirow[b]{2}{*}{ Analysis Type } & \multirow[b]{2}{*}{$\begin{array}{c}\text { Sample } \\
\text { Type }^{\text {a }}\end{array}$} & \multirow[b]{2}{*}{$\begin{array}{l}\text { Analysis } \\
\text { Method }\end{array}$} & \multirow[b]{2}{*}{$\begin{array}{l}\text { Analysis } \\
\text { Date }\end{array}$} & \multicolumn{3}{|c|}{ Concentration } & \multirow[b]{2}{*}{ Units } \\
\hline & & & & & & & & & $\begin{array}{c}\text { Carbon } \\
\text { Tetrachloride }\end{array}$ & Chloroform & $\begin{array}{l}\text { Methylene } \\
\text { Chloride }\end{array}$ & \\
\hline $11 / 9 / 09$ & TI11 & $28-33$ & Water & INTI11-W-30751 & Primary sample & CPT/P & E524.2 & $11 / 10 / 09$ & 12 & 4.5 & ND & $\mu \mathrm{g} / \mathrm{L}$ \\
\hline $11 / 9 / 09$ & TI11 & $28-33$ & Water & INTI11-W-30751DUP & Duplicate analysis & CPT/P & E524.2 & $11 / 10 / 09$ & 12 & 4.5 & ND & $\mu \mathrm{g} / \mathrm{L}$ \\
\hline $11 / 9 / 10$ & BENGSTON & - & Water & INBENGSTEN-W-32675 & Primary sample & DW & E524.2 & $11 / 11 / 10$ & 5.0 & 1.4 & ND & $\mu \mathrm{g} / \mathrm{L}$ \\
\hline $11 / 9 / 10$ & BENGSTON & - & Water & INBENGSTEN-W-32675DUP & Duplicate analysis & DW & E524.2 & $11 / 11 / 10$ & 5.1 & 1.4 & ND & $\mu \mathrm{g} / \mathrm{L}$ \\
\hline $11 / 10 / 10$ & MW04 & $30-40$ & Water & INMW04-W-32677 & Primary sample & MW & E524.2 & $11 / 12 / 10$ & ND & ND & ND & $\mu \mathrm{g} / \mathrm{L}$ \\
\hline $11 / 10 / 10$ & MW04 & $30-40$ & Water & INMW04-W-32677DUP & Duplicate analysis & MW & E524.2 & $11 / 12 / 10$ & ND & ND & ND & $\mu \mathrm{g} / \mathrm{L}$ \\
\hline $11 / 10 / 10$ & TI19 & 24 & Soil & INTI19-S-30770 & Primary sample & CPT & SW8260B & $11 / 13 / 10$ & ND & ND & ND & $\mu \mathrm{g} / \mathrm{kg}$ \\
\hline $11 / 10 / 10$ & TI19 & 24 & Soil & INTI19-S-30773 & Replicate sample & CPT & SW8260B & $11 / 13 / 10$ & ND & ND & ND & $\mu \mathrm{g} / \mathrm{kg}$ \\
\hline $11 / 11 / 10$ & TI20 & 20 & Soil & INTI20-S-30794 & Primary sample & CPT & SW8260B & $11 / 16 / 10$ & ND & ND & ND & $\mu \mathrm{g} / \mathrm{kg}$ \\
\hline $11 / 11 / 10$ & TI2O & 20 & Soil & INTI20-S-30794DUP & Duplicate analysis & CPT & SW8260B & $11 / 16 / 10$ & ND & ND & ND & $\mu \mathrm{g} / \mathrm{kg}$ \\
\hline $11 / 11 / 10$ & TI20 & 24 & Soil & INTI20-S-30795 & Primary sample & CPT & SW8260B & $11 / 16 / 10$ & ND & ND & ND & $\mu \mathrm{g} / \mathrm{kg}$ \\
\hline $11 / 11 / 10$ & $\mathrm{~T} 120$ & 24 & Soil & INTI20-S-30795DUP & Duplicate analysis & CPT & SW8260B & $11 / 16 / 10$ & ND & ND & ND & $\mu \mathrm{g} / \mathrm{kg}$ \\
\hline $11 / 11 / 10$ & TI20 & 36 & Soil & INTI20-S-30798 & Primary sample & CPT & SW8260B & $11 / 16 / 10$ & ND & ND & ND & $\mu \mathrm{g} / \mathrm{kg}$ \\
\hline $11 / 11 / 10$ & TI20 & 36 & Soil & INTI20-S-30799 & Replicate sample & CPT & SW8260B & $11 / 16 / 10$ & ND & ND & ND & $\mu \mathrm{g} / \mathrm{kg}$ \\
\hline $11 / 11 / 10$ & TI22 & 16 & Soil & INTI22-S-30812 & Primary sample & CPT & SW8260B & $11 / 18 / 10$ & ND & ND & ND & $\mu \mathrm{g} / \mathrm{kg}$ \\
\hline $11 / 11 / 10$ & TI22 & 16 & Soil & INTI22-S-30818 & Replicate sample & CPT & SW8260B & $11 / 18 / 10$ & ND & ND & ND & $\mu \mathrm{g} / \mathrm{kg}$ \\
\hline $11 / 11 / 10$ & TI24 & 16 & Soil & INTI24-S-30831 & Primary sample & CPT & SW8260B & $11 / 19 / 10$ & ND & ND & ND & $\mu \mathrm{g} / \mathrm{kg}$ \\
\hline $11 / 11 / 10$ & TI24 & 16 & Soil & INTI24-S-30831DUP & Duplicate analysis & CPT & SW8260B & 11/19/10 & ND & ND & ND & $\mu \mathrm{g} / \mathrm{kg}$ \\
\hline $11 / 12 / 10$ & TI24 & 32 & Soil & INTI24-S-30835 & Primary sample & CPT & SW8260B & $11 / 20 / 10$ & ND & ND & ND & $\mu \mathrm{g} / \mathrm{kg}$ \\
\hline $11 / 12 / 10$ & TI24 & 32 & Soil & INTI24-S-30837 & Replicate sample & CPT & SW8260B & $11 / 20 / 10$ & ND & ND & ND & $\mu \mathrm{g} / \mathrm{kg}$ \\
\hline $11 / 12 / 10$ & TI25 & 16 & Soil & INTI25-S-30843 & Primary sample & CPT & SW8260B & $11 / 20 / 10$ & ND & ND & ND & $\mu \mathrm{g} / \mathrm{kg}$ \\
\hline $11 / 12 / 10$ & TI25 & 16 & Soil & INTI25-S-30843DUP & Duplicate analysis & CPT & SW8260B & $11 / 20 / 10$ & ND & ND & ND & $\mu \mathrm{g} / \mathrm{kg}$ \\
\hline $5 / 24 / 11$ & HULL & - & Water & INHULL-W-32691 & Primary sample & DW & E524.2 & $5 / 25 / 11$ & ND & ND & ND & $\mu \mathrm{g} / \mathrm{L}$ \\
\hline $5 / 24 / 11$ & HULL & - & Water & INHULL-W-32691DUP & Duplicate analysis & DW & E524.2 & $5 / 25 / 11$ & ND & ND & ND & $\mu \mathrm{g} / \mathrm{L}$ \\
\hline $8 / 24 / 11$ & MW14M & $85-95$ & Water & INMW14S-W-32736 & Primary sample & MW & E524.2 & $8 / 25 / 11$ & 1.7 & 2.0 & ND & $\mu \mathrm{g} / \mathrm{L}$ \\
\hline $8 / 24 / 11$ & MW14M & $85-95$ & Water & INMW14S-W-32736DUP & Duplicate analysis & MW & E524.2 & $8 / 25 / 11$ & 1.5 & 1.8 & ND & $\mu \mathrm{g} / \mathrm{L}$ \\
\hline $8 / 25 / 11$ & MW16 & $35-45$ & Water & INMW16-W-32740 & Primary sample & SB & E524.2 & $8 / 26 / 11$ & 14 & 1.0 & ND & $\mu \mathrm{g} / \mathrm{L}$ \\
\hline $8 / 25 / 11$ & MW16 & $35-45$ & Water & INMW16-W-32740DUP & Duplicate analysis & SB & E524.2 & $8 / 26 / 11$ & 14 & 1.0 & ND & $\mu \mathrm{g} / \mathrm{L}$ \\
\hline $8 / 27 / 11$ & MW17 & $15-31$ & Water & INMW17-W-32745 & Primary sample & SB & E524.2 & $8 / 29 / 11$ & ND & ND & ND & $\mu \mathrm{g} / \mathrm{L}$ \\
\hline $8 / 27 / 11$ & MW17 & $15-31$ & Water & INMW17-W-32745DUP & Duplicate analysis & SB & E524.2 & $8 / 29 / 11$ & ND & ND & ND & $\mu \mathrm{g} / \mathrm{L}$ \\
\hline
\end{tabular}


TABLE S2.4 (Cont.)

\begin{tabular}{|c|c|c|c|c|c|c|c|c|c|c|c|c|}
\hline \multirow[b]{2}{*}{$\begin{array}{l}\text { Sample } \\
\text { Date }\end{array}$} & \multirow[b]{2}{*}{ Location } & \multirow[b]{2}{*}{$\begin{array}{l}\text { Depth } \\
\text { (ft BGL) }\end{array}$} & \multirow[b]{2}{*}{$\begin{array}{l}\text { Sample } \\
\text { Medium }\end{array}$} & \multirow[b]{2}{*}{ Sample } & \multirow[b]{2}{*}{ Analysis Type } & \multirow[b]{2}{*}{$\begin{array}{l}\text { Sample } \\
\text { Type }^{\mathrm{a}}\end{array}$} & \multirow[b]{2}{*}{$\begin{array}{l}\text { Analysis } \\
\text { Method }\end{array}$} & \multirow[b]{2}{*}{$\begin{array}{l}\text { Analysis } \\
\text { Date }\end{array}$} & \multicolumn{3}{|c|}{ Concentration } & \multirow[b]{2}{*}{ Units } \\
\hline & & & & & & & & & $\begin{array}{c}\text { Carbon } \\
\text { Tetrachloride }\end{array}$ & Chloroform & $\begin{array}{l}\text { Methylene } \\
\text { Chloride }\end{array}$ & \\
\hline $8 / 27 / 11$ & MW17 & $55-75$ & Water & INMW17-W-32748 & Primary sample & SB & E524.2 & $8 / 29 / 11$ & 1.0 & ND & ND & $\mu \mathrm{g} / \mathrm{L}$ \\
\hline 8/27/11 & MW17 & $55-75$ & Water & INMW17-W-32749 & Replicate sample & SB & E524.2 & $8 / 29 / 11$ & $0.9 \mathrm{~J}$ & ND & ND & $\mu \mathrm{g} / \mathrm{L}$ \\
\hline 8/28/11 & MW15M & $86-96$ & Water & INMW15-W-32750 & Primary sample & MW & E524.2 & 8/30/11 & 2.1 & 1.9 & ND & $\mu \mathrm{g} / \mathrm{L}$ \\
\hline 8/29/11 & MW16D & $100-110$ & Water & INMW16D-W-32756 & Primary sample & MW & E524.2 & 8/30/11 & ND & 1.8 & ND & $\mu \mathrm{g} / \mathrm{L}$ \\
\hline 8/29/11 & MW16D & $100-110$ & Water & INMW16D-W-32756DUP & Duplicate analysis & MW & E524.2 & $8 / 30 / 11$ & ND & 2.0 & ND & $\mu \mathrm{g} / \mathrm{L}$ \\
\hline 8/29/11 & MW18 & $65-80$ & Water & INMW18-W-32760 & Primary sample & SB & E524.2 & 8/30/11 & 2.2 & ND & ND & $\mu \mathrm{g} / \mathrm{L}$ \\
\hline 8/29/11 & MW18 & $65-80$ & Water & INMW18-W-32761 & Replicate sample & SB & E524.2 & $8 / 31 / 11$ & 1.6 & ND & ND & $\mu \mathrm{g} / \mathrm{L}$ \\
\hline 8/29/11 & MW18 & $85-90$ & Water & INMW18-W-32752 & Primary sample & SB & E524.2 & $8 / 30 / 11$ & 1.5 & $0.7 \mathrm{~J}$ & ND & $\mu \mathrm{g} / \mathrm{L}$ \\
\hline 8/29/11 & MW18 & $85-90$ & Water & INMW18-W-32755 & Replicate sample & SB & E524.2 & $8 / 30 / 11$ & 3.6 & 1.0 & ND & $\mu \mathrm{g} / \mathrm{L}$ \\
\hline 8/30/11 & MW19 & $15-50$ & Water & INMW19-W-32768 & Primary sample & SB & E524.2 & $8 / 31 / 11$ & ND & ND & ND & $\mu \mathrm{g} / \mathrm{L}$ \\
\hline 8/31/11 & MW17M & $72-82$ & Water & INMW17M-W-32770 & Primary sample & MW & E524.2 & 9/1/11 & $0.9 \mathrm{~J}$ & ND & ND & $\mu \mathrm{g} / \mathrm{L}$ \\
\hline 8/31/11 & MW17M & $72-82$ & Water & INMW17M-W-32770DUP & Duplicate analysis & MW & E524.2 & 9/1/11 & $0.9 \mathrm{~J}$ & ND & ND & $\mu \mathrm{g} / \mathrm{L}$ \\
\hline 9/2/11 & MW19M & $85-95$ & Water & INMW19D-W-32790 & Primary sample & MW & E524.2 & 9/6/11 & ND & ND & ND & $\mu \mathrm{g} / \mathrm{L}$ \\
\hline 9/2/11 & MW19M & 85-95 & Water & INMW19D-W-32790DUP & Duplicate analysis & MW & E524.2 & 9/6/11 & ND & ND & ND & $\mu \mathrm{g} / \mathrm{L}$ \\
\hline 9/13/11 & MW20 & $65-89.5$ & Water & INMW20-W-32776 & Primary sample & SB & E524.2 & 9/15/11 & ND & ND & ND & $\mu \mathrm{g} / \mathrm{L}$ \\
\hline 9/13/11 & MW20 & $65-89.5$ & Water & INMW20-W-32776DUP & Duplicate analysis & SB & E524.2 & 9/15/11 & ND & ND & ND & $\mu \mathrm{g} / \mathrm{L}$ \\
\hline 9/15/11 & MW14D & $115-125$ & Water & INMW14D-W-32782 & Primary sample & MW & E524.2 & 9/16/11 & ND & $0.6 \mathrm{~J}$ & ND & $\mu \mathrm{g} / \mathrm{L}$ \\
\hline 9/15/11 & MW14D & $115-125$ & Water & INMW14D-W-32782DUP & Duplicate analysis & MW & E524.2 & 9/16/11 & ND & $0.7 \mathrm{~J}$ & ND & $\mu \mathrm{g} / \mathrm{L}$ \\
\hline
\end{tabular}

a Sample types: CPT, cone penetrometer; CPT/P, piezometer; DW, domestic well; MW, monitoring well; SB, soil boring.

b ND, compound analyzed for but not detected at a level greater than or equal to the method detection limit $(<1 \mu \mathrm{g} / \mathrm{L}$ for water analyses or $<10 \mu \mathrm{g} / \mathrm{kg}$ for soil analyses).

c J, compound identified with an estimated concentration between the instrument detection limit and the method detection limit. 
TABLE S2.5 Analytical results from the AGEM Laboratory and TestAmerica for samples submitted for verification organic analysis.

\begin{tabular}{|c|c|c|c|c|c|c|c|c|c|c|c|c|c|c|}
\hline \multirow[b]{2}{*}{ Location } & \multirow[b]{2}{*}{ Sample } & \multirow[b]{2}{*}{$\begin{array}{l}\text { Sample } \\
\text { Date }\end{array}$} & \multirow[b]{2}{*}{$\begin{array}{l}\text { Sample } \\
\text { Type }^{a}\end{array}$} & \multirow[b]{2}{*}{$\begin{array}{c}\text { Depth } \\
\text { (ft BGL) }\end{array}$} & \multirow[b]{2}{*}{$\begin{array}{l}\text { Sample } \\
\text { Medium }\end{array}$} & \multirow[b]{2}{*}{$\begin{array}{l}\text { Analytical } \\
\text { Method }\end{array}$} & \multirow[b]{2}{*}{$\begin{array}{l}\text { Analytical } \\
\text { Laboratory }\end{array}$} & \multirow[b]{2}{*}{$\begin{array}{l}\text { Analysis } \\
\text { Date }\end{array}$} & \multirow[b]{2}{*}{$\begin{array}{c}\text { Sample } \\
\text { Delivery } \\
\text { Group }\end{array}$} & \multicolumn{3}{|c|}{ Concentration } & \multirow[b]{2}{*}{$\begin{array}{l}\text { Method } \\
\text { Detection } \\
\text { Limit }\end{array}$} & \multirow[b]{2}{*}{ Units } \\
\hline & & & & & & & & & & $\begin{array}{l}\text { Carbon } \\
\text { Tetrachloride }\end{array}$ & Chloroform & $\begin{array}{l}\text { Methylene } \\
\text { Chloride }\end{array}$ & & \\
\hline TI01 & INTI01-S-30628 & $11 / 4 / 09$ & CPT & 34 & Soil & SW8260B & AGEM & $11 / 6 / 09$ & $09-11-6$ & $3.5 \mathrm{~J}^{\mathrm{b}}$ & $N D^{c}$ & ND & $<10$ & $\mu \mathrm{g} / \mathrm{kg}$ \\
\hline TI01 & INTI01-S-30628 & $11 / 4 / 09$ & CPT & 34 & Soil & SW8260B & TA & $11 / 19 / 09$ & 134662 & 9.1 & ND & ND & $<8.9$ & $\mu \mathrm{g} / \mathrm{kg}$ \\
\hline T102 & INTIO2-S-30633 & $11 / 4 / 09$ & CPT & 10 & Soil & SW8260B & AGEM & $11 / 9 / 09$ & 09-11-9 & ND & ND & ND & $<10$ & $\mu \mathrm{g} / \mathrm{kg}$ \\
\hline T102 & INTIO2-S-30633 & $11 / 4 / 09$ & CPT & 10 & Soil & SW8260B & TA & $11 / 19 / 09$ & 134662 & ND & ND & ND & $<8.3$ & $\mu \mathrm{g} / \mathrm{kg}$ \\
\hline T104 & INTI04-S-30655 & $11 / 5 / 09$ & CPT & 6 & Soil & SW8260B & AGEM & $11 / 10 / 09$ & 09-11-10a & ND & ND & ND & $<10$ & $\mu \mathrm{g} / \mathrm{kg}$ \\
\hline T104 & INTI04-S-30655 & $11 / 5 / 09$ & CPT & 6 & Soil & SW8260B & TA & $11 / 19 / 09$ & 134662 & ND & ND & ND & $<8.4$ & $\mu \mathrm{g} / \mathrm{kg}$ \\
\hline T104 & INT104-S-30662 & $11 / 5 / 09$ & CPT & 34 & Soil & SW8260B & AGEM & $11 / 12 / 09$ & $09-11-12 a$ & ND & ND & ND & $<10$ & $\mu \mathrm{g} / \mathrm{kg}$ \\
\hline TI04 & INTI04-S-30662 & $11 / 5 / 09$ & CPT & 34 & Soil & SW8260B & TA & $11 / 19 / 09$ & 134662 & ND & ND & ND & $<9.6$ & $\mu \mathrm{g} / \mathrm{kg}$ \\
\hline T105 & INTI05-S-30667 & $11 / 6 / 09$ & CPT & 6 & Soil & SW8260B & AGEM & $11 / 17 / 09$ & $09-11-17$ & ND & ND & ND & $<10$ & $\mu \mathrm{g} / \mathrm{kg}$ \\
\hline T105 & INTI05-S-30667 & $11 / 6 / 09$ & CPT & 6 & Soil & SW8260B & TA & $11 / 19 / 09$ & 134662 & ND & ND & ND & $<9.3$ & $\mu \mathrm{g} / \mathrm{kg}$ \\
\hline T105 & INTI05-S-30670 & $11 / 6 / 09$ & CPT & 18 & Soil & SW8260B & AGEM & $11 / 16 / 09$ & 09-11-16b & ND & ND & ND & $<10$ & $\mu \mathrm{g} / \mathrm{kg}$ \\
\hline TI05 & INTIO5-S-30670 & $11 / 6 / 09$ & CPT & 18 & Soil & SW8260B & TA & $11 / 19 / 09$ & 134662 & ND & ND & ND & $<8.5$ & $\mu \mathrm{g} / \mathrm{kg}$ \\
\hline T105 & INTI05-S-30672 & $11 / 6 / 09$ & CPT & 26 & Soil & SW8260B & AGEM & $11 / 11 / 09$ & $09-11-11 a$ & ND & ND & ND & $<10$ & $\mu \mathrm{g} / \mathrm{kg}$ \\
\hline T105 & INTI05-S-30672 & $11 / 6 / 09$ & CPT & 26 & Soil & SW8260B & TA & $11 / 19 / 09$ & 134662 & ND & ND & ND & $<8.5$ & $\mu \mathrm{g} / \mathrm{kg}$ \\
\hline T106 & INT106-S-30676 & $11 / 6 / 09$ & CPT & 2 & Soil & SW8260B & AGEM & $11 / 12 / 09$ & $09-11-12 a$ & ND & ND & ND & $<10$ & $\mu \mathrm{g} / \mathrm{kg}$ \\
\hline T106 & INTI06-S-30676 & $11 / 6 / 09$ & CPT & 2 & Soil & SW8260B & TA & $11 / 19 / 09$ & 134662 & ND & ND & ND & $<9.9$ & $\mu \mathrm{g} / \mathrm{kg}$ \\
\hline T107 & INTI07-S-30695 & $11 / 6 / 09$ & CPT & 34 & Soil & SW8260B & AGEM & $11 / 16 / 09$ & 09-11-16b & ND & ND & ND & $<10$ & $\mu \mathrm{g} / \mathrm{kg}$ \\
\hline TI07 & INTI07-S-30695 & $11 / 6 / 09$ & CPT & 34 & Soil & SW8260B & TA & $11 / 19 / 09$ & 134662 & $2.0 \mathrm{~J}$ & ND & ND & $<8.0$ & $\mu \mathrm{g} / \mathrm{kg}$ \\
\hline T107 & INT107-S-30696 & $11 / 6 / 09$ & CPT & 38 & Soil & SW8260B & AGEM & $11 / 11 / 09$ & 09-11-11a & ND & ND & ND & $<10$ & $\mu \mathrm{g} / \mathrm{kg}$ \\
\hline TI07 & INTI07-S-30696 & $11 / 6 / 09$ & CPT & 38 & Soil & SW8260B & TA & $11 / 19 / 09$ & 134662 & ND & ND & ND & $<9.2$ & $\mu \mathrm{g} / \mathrm{kg}$ \\
\hline TI11 & INTI11-S-30734 & $11 / 8 / 09$ & СРT & 2 & Soil & SW8260B & AGEM & $11 / 18 / 09$ & 09-11-18 & ND & ND & ND & $<10$ & $\mu \mathrm{g} / \mathrm{kg}$ \\
\hline TI11 & INTI11-S-30734 & $11 / 8 / 09$ & CPT & 2 & Soil & SW8260B & TA & $11 / 19 / 09$ & 134662 & ND & 17 & ND & $<8.3$ & $\mu \mathrm{g} / \mathrm{kg}$ \\
\hline MW04 & INMW04-W-32677 & $11 / 10 / 10$ & MW & $30-40$ & Water & E524.2 & AGEM & $11 / 12 / 10$ & 10-11-11 & ND & ND & ND & $<1$ & $\mu \mathrm{g} / \mathrm{L}$ \\
\hline MW04 & INMW04-W-32677 & $11 / 10 / 10$ & MW & $30-40$ & Water & SW8260 & TA & $11 / 13 / 10$ & $200-2447$ & $0.12 \mathrm{~J}$ & ND & ND & $<0.5$ & $\mu \mathrm{g} / \mathrm{L}$ \\
\hline MW05 & INMW05-W-32678 & $11 / 10 / 10$ & MW & $35-45$ & Water & E524.2 & AGEM & $11 / 12 / 10$ & 10-11-11 & ND & ND & ND & $<1$ & $\mu \mathrm{g} / \mathrm{L}$ \\
\hline MW05 & INMW05-W-32678 & $11 / 10 / 10$ & MW & $35-45$ & Water & SW8260 & TA & $11 / 13 / 10$ & $200-2447$ & $0.081 \mathrm{~J}$ & ND & ND & $<0.5$ & $\mu \mathrm{g} / \mathrm{L}$ \\
\hline TI14 & INTI14-W-30762 & $11 / 10 / 10$ & CPT/P & $29.5-39.5$ & Water & E524.2 & AGEM & $11 / 11 / 10$ & 10-11-11 & ND & ND & ND & $<1$ & $\mu \mathrm{g} / \mathrm{L}$ \\
\hline TI14 & INTI14-W-30762 & $11 / 10 / 10$ & CPT/P & $29.5-39.5$ & Water & SW8260 & TA & $11 / 13 / 10$ & $200-2447$ & $0.044 \mathrm{~J}$ & ND & ND & $<0.5$ & $\mu \mathrm{g} / \mathrm{L}$ \\
\hline TI19 & INTI19-S-30771 & $11 / 10 / 10$ & CPT & 28 & Soil & SW8260B & AGEM & $11 / 16 / 10$ & 10-11-15 & ND & ND & ND & $<10$ & $\mu \mathrm{g} / \mathrm{kg}$ \\
\hline TI19 & INTI19-S-30771 & $11 / 10 / 10$ & CPT & 28 & Soil & SW8260B & TA & $11 / 24 / 10$ & $200-2608$ & ND & ND & ND & $<9.5$ & $\mu \mathrm{g} / \mathrm{kg}$ \\
\hline T120 & INTI20-S-30795 & $11 / 11 / 10$ & CPT & 24 & Soil & SW8260B & AGEM & $11 / 16 / 10$ & $10-11-15$ & ND & ND & ND & $<10$ & $\mu \mathrm{g} / \mathrm{kg}$ \\
\hline TI20 & INTI20-S-30795 & $11 / 11 / 10$ & CPT & 24 & Soil & SW8260B & TA & $11 / 24 / 10$ & $200-2608$ & ND & ND & ND & $<10$ & $\mu \mathrm{g} / \mathrm{kg}$ \\
\hline TI21 & INTI21-S-3080 & $11 / 11 / 10$ & CPT & 36 & Soil & SW8260B & AGEM & $11 / 18 / 10$ & $10-11-17$ & ND & ND & ND & $<10$ & $\mu \mathrm{g} / \mathrm{kg}$ \\
\hline TI21 & INTI21-S-30808 & $11 / 11 / 10$ & CPT & 36 & Soil & SW8260B & TA & $11 / 24 / 10$ & $200-2608$ & ND & ND & ND & $<9.5$ & $\mu \mathrm{g} / \mathrm{kg}$ \\
\hline TI22 & INTI22-S-30814 & $11 / 11 / 10$ & CPT & 24 & Soil & SW8260B & AGEM & $11 / 18 / 10$ & $10-11-17$ & ND & ND & ND & $<1$ & $\mu \mathrm{g} / \mathrm{kg}$ \\
\hline TI22 & INTI22-S-30814 & $11 / 11 / 10$ & CPT & 24 & Soil & SW8260B & TA & $11 / 24 / 10$ & $200-2608$ & ND & ND & ND & $<9.8$ & $\mu \mathrm{g} / \mathrm{kg}$ \\
\hline
\end{tabular}




\begin{tabular}{|c|c|c|c|c|c|c|c|c|c|c|c|c|c|c|}
\hline \multirow[b]{2}{*}{ Location } & \multirow[b]{2}{*}{ Sample } & \multirow[b]{2}{*}{$\begin{array}{l}\text { Sample } \\
\text { Date }\end{array}$} & \multirow[b]{2}{*}{$\begin{array}{l}\text { Sample } \\
\text { Type }^{a}\end{array}$} & \multirow[b]{2}{*}{$\begin{array}{c}\text { Depth } \\
\text { (ft BGL) }\end{array}$} & \multirow[b]{2}{*}{$\begin{array}{l}\text { Sample } \\
\text { Medium }\end{array}$} & \multirow[b]{2}{*}{$\begin{array}{l}\text { Analytical } \\
\text { Method }\end{array}$} & \multirow[b]{2}{*}{$\begin{array}{l}\text { Analytical } \\
\text { Laboratory }\end{array}$} & \multirow[b]{2}{*}{$\begin{array}{l}\text { Analysis } \\
\text { Date }\end{array}$} & \multirow[b]{2}{*}{$\begin{array}{l}\text { Sample } \\
\text { Delivery } \\
\text { Group }\end{array}$} & \multicolumn{3}{|c|}{ Concentration } & \multirow[b]{2}{*}{$\begin{array}{l}\text { Method } \\
\text { Detection } \\
\text { Limit }\end{array}$} & \multirow[b]{2}{*}{ Units } \\
\hline & & & & & & & & & & $\begin{array}{c}\text { Carbon } \\
\text { Tetrachloride }\end{array}$ & Chloroform & $\begin{array}{l}\text { Methylene } \\
\text { Chloride }\end{array}$ & & \\
\hline TI18 & INTI18-W-30786 & $11 / 11 / 10$ & CPT/P & $30.6-40.6$ & Water & E524.2 & AGEM & $11 / 12 / 10$ & 10-11-12 & ND & ND & ND & $<1$ & $\mu \mathrm{g} / \mathrm{L}$ \\
\hline TI18 & INTI18-W-30786 & $11 / 11 / 10$ & CPT/P & $30.6-40.6$ & Water & SW8260 & TA & $11 / 13 / 10$ & 200-2472 & ND & ND & ND & $<0.5$ & $\mu \mathrm{g} / \mathrm{L}$ \\
\hline TI16 & INTI16-W-30785 & $11 / 11 / 10$ & CPT/P & $31.2-41.2$ & Water & E524.2 & AGEM & $11 / 12 / 10$ & 10-11-12 & ND & ND & ND & $<1$ & $\mu \mathrm{g} / \mathrm{L}$ \\
\hline TI16 & INTI16-W-30785 & $11 / 11 / 10$ & CPT/P & $31.2-41.2$ & Water & SW8260 & TA & $11 / 13 / 10$ & $200-2472$ & ND & ND & ND & $<0.5$ & $\mu \mathrm{g} / \mathrm{L}$ \\
\hline TI23 & INTI23-S-30819 & $11 / 11 / 10$ & CPT & 4 & Soil & SW8260B & AGEM & $11 / 18 / 10$ & $10-11-17$ & ND & ND & ND & $<10$ & $\mu g / \mathrm{kg}$ \\
\hline TI23 & INTI23-S-30820 & $11 / 11 / 10$ & CPT & 8 & Soil & SW8260B & TA & $11 / 24 / 10$ & $200-2608$ & ND & ND & ND & $<9.8$ & $\mu \mathrm{g} / \mathrm{kg}$ \\
\hline TI23 & INTI23-S-30824 & $11 / 11 / 10$ & CPT & 24 & Soil & SW8260B & AGEM & $11 / 18 / 10$ & $10-11-18$ & ND & ND & ND & $<10$ & $\mu g / \mathrm{kg}$ \\
\hline TI23 & INTI23-S-30824 & $11 / 11 / 10$ & CPT & 24 & Soil & SW8260B & TA & $11 / 24 / 10$ & $200-2608$ & ND & ND & ND & $<9.5$ & $\mu \mathrm{g} / \mathrm{kg}$ \\
\hline TI15 & INTI15-W-30784 & $11 / 11 / 10$ & СРT/P & $31.7-41.7$ & Water & E524.2 & AGEM & $11 / 12 / 10$ & $10-11-12$ & ND & ND & ND & $<1$ & $\mu \mathrm{g} / \mathrm{L}$ \\
\hline TI15 & INTI15-W-30784 & $11 / 11 / 10$ & CPT/P & $31.7-41.7$ & Water & SW8260 & TA & $11 / 13 / 10$ & 200-2472 & ND & ND & ND & $<0.5$ & $\mu \mathrm{g} / \mathrm{L}$ \\
\hline TI13 & INTI13-W-30783 & $11 / 11 / 10$ & CPT/P & $31.7-41.7$ & Water & E524.2 & AGEM & $11 / 12 / 10$ & 10-11-12 & ND & ND & ND & $<1$ & $\mu \mathrm{g} / \mathrm{L}$ \\
\hline TI13 & INTI13-W-30783 & $11 / 11 / 10$ & CPT/P & $31.7-41.7$ & Water & SW8260 & TA & $11 / 13 / 10$ & $200-2472$ & ND & ND & ND & $<0.5$ & $\mu \mathrm{g} / \mathrm{L}$ \\
\hline TI24 & INTI24-S-30836 & $11 / 12 / 10$ & CPT & 36 & Soil & SW8260B & AGEM & $11 / 20 / 10$ & 10-11-19 & ND & ND & ND & $<10$ & $\mu \mathrm{g} / \mathrm{kg}$ \\
\hline TI24 & INTI24-S-30836 & $11 / 12 / 10$ & CPT & 36 & Soil & SW8260B & TA & $11 / 24 / 10$ & $200-2608$ & ND & ND & ND & $<8.0$ & $\mu \mathrm{g} / \mathrm{kg}$ \\
\hline BROWN & INBROWN-W-32686 & $5 / 24 / 11$ & DW & - & Water & E524.2 & AGEM & $5 / 27 / 11$ & $11-5-26$ & ND & ND & ND & $<1$ & $\mu \mathrm{g} / \mathrm{L}$ \\
\hline BROWN & INBROWN-W-32686 & $5 / 24 / 11$ & DW & - & Water & sW8260 & TA & $5 / 25 / 11$ & $200-5308$ & $0.072 \mathrm{~J} \mathrm{~B}^{\mathrm{d}}$ & ND & $0.071 \mathrm{~J}$ & $<0.5$ & $\mu \mathrm{g} / \mathrm{L}$ \\
\hline HULL & INHULL-W-32691 & $5 / 24 / 11$ & DW & - & Water & E524.2 & AGEM & $5 / 25 / 11$ & $11-5-25$ & ND & ND & ND & $<1$ & $\mu \mathrm{g} / \mathrm{L}$ \\
\hline HULL & INHULL-W-32691 & $5 / 24 / 11$ & DW & - & Water & sW8260 & TA & $5 / 25 / 11$ & $200-5308$ & $0.23 \mathrm{~J} \mathrm{~B}$ & ND & ND & $<0.5$ & $\mu \mathrm{g} / \mathrm{L}$ \\
\hline THIESSEN & INTHIESSEN-W-32685 & $5 / 24 / 11$ & DW & - & Water & E524.2 & AGEM & $5 / 25 / 11$ & $11-5-25$ & ND & ND & ND & $<1$ & $\mu \mathrm{g} / \mathrm{L}$ \\
\hline THIESSEN & INTHIESSEN-W-32685 & $5 / 24 / 11$ & DW & - & Water & sW8260 & TA & $5 / 25 / 11$ & $200-5308$ & ND & ND & ND & $<0.5$ & $\mu \mathrm{g} / \mathrm{L}$ \\
\hline HULL & INHULL-W-32694 & 6/23/11 & DW & - & Water & E524.2 & AGEM & 6/24/11 & $11-6-24$ & ND & ND & ND & $<1$ & $\mu \mathrm{g} / \mathrm{L}$ \\
\hline HULL & INHULL-W-32694 & 6/23/11 & DW & - & Water & SW8260 & TA & 6/28/11 & $200-5726$ & $0.25 \mathrm{~J}$ & ND & ND & $<0.5$ & $\mu \mathrm{g} / \mathrm{L}$ \\
\hline KLASSEN & INKLASSEN-W-32693 & $6 / 23 / 11$ & DW & - & Water & E524.2 & AGEM & $6 / 24 / 11$ & $11-6-24$ & 5.6 & ND & ND & $<1$ & $\mu \mathrm{g} / \mathrm{L}$ \\
\hline KLASSEN & INKLASSEN-W-32693 & 6/23/11 & DW & - & Water & SW8260 & TA & 6/28/11 & $200-5726$ & 6.9 & $0.25 \mathrm{~J}$ & ND & $<0.5$ & $\mu \mathrm{g} / \mathrm{L}$ \\
\hline PAULS & INPAULS-W-32695 & $6 / 23 / 11$ & DW & - & Water & E524.2 & AGEM & $6 / 24 / 11$ & $11-6-24$ & ND & ND & ND & $<1$ & $\mu \mathrm{g} / \mathrm{L}$ \\
\hline PAULS & INPAULS-W-32695 & 6/23/11 & DW & - & Water & SW8260 & TA & 6/28/11 & $200-5726$ & ND & ND & ND & $<0.5$ & $\mu \mathrm{g} / \mathrm{L}$ \\
\hline MW14M & INMW14S-W-32736 & 8/24/11 & MW & 85-95 & Water & E524.2 & AGEM & 8/25/11 & $11-8-25$ & 1.7 & 2.0 & ND & $<1$ & $\mu \mathrm{g} / \mathrm{L}$ \\
\hline MW14M & INMW14S-W-32736 & $8 / 24 / 11$ & MW & 85-95 & Water & sW8260 & TA & 8/26/11 & $200-6709$ & 1.1 & 1.7 & $0.05 \mathrm{~J}$ & $<0.5$ & $\mu \mathrm{g} / \mathrm{L}$ \\
\hline MW15 & INMW15-W-32735 & $8 / 24 / 11$ & SB & $55-75$ & Water & E524.2 & AGEM & 8/25/11 & $11-8-25$ & ND & ND & ND & $<1$ & $\mu \mathrm{g} / \mathrm{L}$ \\
\hline MW15 & INMW15-W-32735 & $8 / 24 / 11$ & SB & $55-75$ & Water & SW8260 & TA & 8/26/11 & $200-6709$ & $0.036 \mathrm{~J}$ & $0.031 \mathrm{~J}$ & ND & $<0.5$ & $\mu \mathrm{g} / \mathrm{L}$ \\
\hline MW15 & INMW15-W-32737 & $8 / 24 / 11$ & SB & $75-90$ & Water & E524.2 & AGEM & 8/25/11 & $11-8-25$ & 3.2 & $0.7 \mathrm{~J}$ & ND & $<1$ & $\mu \mathrm{g} / \mathrm{L}$ \\
\hline MW15 & INMW15-W-32737 & $8 / 24 / 11$ & SB & $75-90$ & Water & SW8260 & TA & 8/26/11 & $200-6709$ & 2.7 & 0.69 & ND & $<0.5$ & $\mu \mathrm{g} / \mathrm{L}$ \\
\hline KTW & INKTW-W-32743 & $8 / 25 / 11$ & DW & 135.2 & Water & E524.2 & AGEM & 8/26/11 & $11-8-26$ & 14 & $0.5 \mathrm{~J}$ & ND & $<1$ & $\mu \mathrm{g} / \mathrm{L}$ \\
\hline KTW & INKTW-W-32743 & 8/25/11 & DW & 135.2 & Water & SW8260 & TA & 8/29/11 & $200-6730$ & 12 & $0.48 \mathrm{~J}$ & ND & $<0.5$ & $\mu \mathrm{g} / \mathrm{L}$ \\
\hline MW16 & INMW16-W-32741 & 8/25/11 & SB & $15-$ & Water & E524 & AGEM & $8 / 26$ & 11 & 11 & $0.8 \mathrm{~J}$ & ND & $<1$ & $\mu \mathrm{g} / \mathrm{L}$ \\
\hline MW16 & INMW16-W-32741 & $8 / 25 / 11$ & SB & $15-30$ & Water & SW8260 & TA & 8/29/11 & $200-6730$ & 8.9 & 0.69 & ND & $<0.5$ & $\mu \mathrm{g} / \mathrm{L}$ \\
\hline
\end{tabular}


TABLE S2.5 (Cont.)

\begin{tabular}{|c|c|c|c|c|c|c|c|c|c|c|c|c|c|c|}
\hline \multirow[b]{2}{*}{ Location } & \multirow[b]{2}{*}{ Sample } & \multirow[b]{2}{*}{$\begin{array}{l}\text { Sample } \\
\text { Date }\end{array}$} & \multirow[b]{2}{*}{$\begin{array}{l}\text { Sample } \\
\text { Type }^{a}\end{array}$} & \multirow[b]{2}{*}{$\begin{array}{c}\text { Depth } \\
\text { (ft BGL) }\end{array}$} & \multirow[b]{2}{*}{$\begin{array}{l}\text { Sample } \\
\text { Medium }\end{array}$} & \multirow[b]{2}{*}{$\begin{array}{l}\text { Analytical } \\
\text { Method }\end{array}$} & \multirow[b]{2}{*}{$\begin{array}{l}\text { Analytical } \\
\text { Laboratory }\end{array}$} & \multirow[b]{2}{*}{$\begin{array}{c}\text { Analysis } \\
\text { Date }\end{array}$} & \multirow[b]{2}{*}{$\begin{array}{c}\text { Sample } \\
\text { Delivery } \\
\text { Group }\end{array}$} & \multicolumn{3}{|c|}{ Concentration } & \multirow[b]{2}{*}{$\begin{array}{l}\text { Method } \\
\text { Detection } \\
\text { Limit }\end{array}$} & \multirow[b]{2}{*}{ Units } \\
\hline & & & & & & & & & & $\begin{array}{c}\text { Carbon } \\
\text { Tetrachloride }\end{array}$ & Chloroform & $\begin{array}{l}\text { Methylene } \\
\text { Chloride }\end{array}$ & & \\
\hline MW16 & INMW16-W-32740 & $8 / 25 / 11$ & SB & $35-45$ & Water & E524.2 & AGEM & $8 / 26 / 11$ & $11-8-26$ & 14 & 1.0 & ND & $<1$ & $\mu \mathrm{g} / \mathrm{L}$ \\
\hline MW16 & INMW16-W-32740 & $8 / 25 / 11$ & SB & $35-45$ & Water & SW8260 & TA & $8 / 29 / 11$ & $200-6730$ & 13 & 0.94 & ND & $<0.5$ & $\mu \mathrm{g} / \mathrm{L}$ \\
\hline MW16 & INMW16-W-32742 & $8 / 25 / 11$ & $\mathrm{SB}$ & $55-70$ & Water & E524.2 & AGEM & $8 / 26 / 11$ & $11-8-26$ & ND & ND & ND & $<1$ & $\mu \mathrm{g} / \mathrm{L}$ \\
\hline MW16 & INMW16-W-32742 & $8 / 25 / 11$ & $\mathrm{SB}$ & $55-70$ & Water & SW8260 & TA & $8 / 29 / 11$ & $200-6730$ & $0.013 \mathrm{~J}$ & ND & ND & $<0.5$ & $\mu \mathrm{g} / \mathrm{L}$ \\
\hline MW16 & INMW16-W-32739 & $8 / 25 / 11$ & $\mathrm{SB}$ & $75-91$ & Water & E524.2 & AGEM & $8 / 26 / 11$ & $11-8-26$ & ND & ND & ND & $<1$ & $\mu \mathrm{g} / \mathrm{L}$ \\
\hline MW16 & INMW16-W-32739 & $8 / 25 / 11$ & SB & $75-91$ & Water & SW8260 & TA & $8 / 29 / 11$ & $200-6730$ & $0.1 \mathrm{~J}$ & $0.033 \mathrm{~J}$ & ND & $<0.5$ & $\mu \mathrm{g} / \mathrm{L}$ \\
\hline MW15M & INMW15-W-32747 & $8 / 27 / 11$ & MW & $86-96$ & Water & E524.2 & AGEM & 8/29/11 & $11-8-29$ & 1.2 & 2.5 & ND & $<1$ & $\mu g / L$ \\
\hline MW15M & INMW15-W-32747 & $8 / 27 / 11$ & MW & $86-96$ & Water & SW8260 & TA & 9/1/11 & $200-6762$ & 1.0 & 2.4 & $0.096 \mathrm{~J}$ & $<0.5$ & $\mu \mathrm{g} / \mathrm{L}$ \\
\hline MW17 & INMW17-W-32745 & $8 / 27 / 11$ & $\mathrm{SB}$ & $15-31$ & Water & E524.2 & AGEM & $8 / 29 / 11$ & $11-8-29$ & ND & ND & ND & $<1$ & $\mu \mathrm{g} / \mathrm{L}$ \\
\hline MW17 & INMW17-W-32745 & $8 / 27 / 11$ & SB & $15-31$ & Water & SW8260 & TA & 9/1/11 & $200-6762$ & $0.028 \mathrm{~J}$ & ND & ND & $<0.5$ & $\mu \mathrm{g} / \mathrm{L}$ \\
\hline MW17 & INMW17-W-32748 & $8 / 27 / 11$ & SB & $55-75$ & Water & E524.2 & AGEM & $8 / 29 / 11$ & $11-8-29$ & 1.0 & ND & ND & $<1$ & $\mu \mathrm{g} / \mathrm{L}$ \\
\hline MW17 & INMW17-W-32748 & $8 / 27 / 11$ & SB & $55-75$ & Water & SW8260 & TA & 9/1/11 & $200-6762$ & 0.76 & $0.26 \mathrm{~J}$ & $0.041 \mathrm{~J}$ & $<0.5$ & $\mu \mathrm{g} / \mathrm{L}$ \\
\hline MW17 & INMW17-W-32749 & $8 / 27 / 11$ & SB & $55-75$ & Water & E524.2 & AGEM & $8 / 29 / 11$ & $11-8-29$ & $0.9 \mathrm{~J}$ & ND & ND & $<1$ & $\mu \mathrm{g} / \mathrm{L}$ \\
\hline MW17 & INMW17-W-32749 & $8 / 27 / 11$ & $\mathrm{SB}$ & $55-75$ & Water & SW8260 & TA & $9 / 1 / 11$ & $200-6762$ & 0.62 & $0.23 \mathrm{~J}$ & $0.037 \mathrm{~J}$ & $<0.5$ & $\mu \mathrm{g} / \mathrm{L}$ \\
\hline MW16M2 & INMW16-W-32764 & 8/29/11 & MW & $78-88$ & Water & E524.2 & AGEM & $8 / 30 / 11$ & 11-8-30a & ND & $0.4 \mathrm{~J}$ & ND & $<1$ & $\mu \mathrm{g} / \mathrm{L}$ \\
\hline MW16M2 & INMW16-W-32764 & $8 / 29 / 11$ & MW & $78-88$ & Water & SW8260 & TA & 9/1/11 & $200-6783$ & $0.014 \mathrm{~J}$ & $0.44 \mathrm{~J}$ & ND & $<0.5$ & $\mu \mathrm{g} / \mathrm{L}$ \\
\hline MW16S1 & INMW16-W-32762 & 8/29/11 & MW & $25-35$ & Water & E524.2 & AGEM & 8/30/11 & 11-8-30b & 1.3 & $0.5 \mathrm{~J}$ & ND & $<1$ & $\mu g / L$ \\
\hline MW16S1 & INMW16-W-32762 & $8 / 29 / 11$ & MW & $25-35$ & Water & SW8260 & TA & 9/1/11 & $200-6762$ & 1.1 & $0.47 \mathrm{~J}$ & ND & $<0.5$ & $\mu \mathrm{g} / \mathrm{L}$ \\
\hline MW16S2 & INMW16-W-32765 & 8/29/11 & MW & $40-45$ & Water & E524.2 & AGEM & 8/30/11 & 11-8-30a & 2.3 & $0.4 \mathrm{~J}$ & ND & $<1$ & $\mu \mathrm{g} / \mathrm{L}$ \\
\hline MW16S2 & INMW16-W-32765 & 8/29/11 & MW & $40-45$ & Water & SW8260 & TA & 9/1/11 & $200-6783$ & 2.0 & $0.42 \mathrm{~J}$ & ND & $<0.5$ & $\mu \mathrm{g} / \mathrm{L}$ \\
\hline MW18 & INMW18-W-32753 & 8/29/11 & SB & $38-52$ & Water & E524.2 & AGEM & $8 / 30 / 11$ & 11-8-30a & ND & ND & ND & $<1$ & $\mu \mathrm{g} / \mathrm{L}$ \\
\hline MW18 & INMW18-W-32753 & $8 / 29 / 11$ & SB & $38-52$ & Water & SW8260 & TA & 9/1/11 & $200-6762$ & $0.14 \mathrm{~J}$ & $0.19 \mathrm{~J}$ & ND & $<0.5$ & $\mu g / L$ \\
\hline MW18 & INMW18-W-32760 & $8 / 29 / 11$ & $\mathrm{SB}$ & $65-80$ & Water & E524.2 & AGEM & 8/30/11 & $11-8-30 \mathrm{~b}$ & 2.2 & ND & ND & $<1$ & $\mu \mathrm{g} / \mathrm{L}$ \\
\hline MW18 & INMW18-W-32760 & $8 / 29 / 11$ & $\mathrm{SB}$ & $65-80$ & Water & SW8260 & TA & $9 / 1 / 11$ & $200-6762$ & 1.2 & $0.3 \mathrm{~J}$ & $\mathrm{ND}$ & $<0.5$ & $\mu \mathrm{g} / \mathrm{L}$ \\
\hline MW18 & INMW18-W-32761 & $8 / 29 / 11$ & $\mathrm{SB}$ & $65-80$ & Water & E524.2 & AGEM & $8 / 31 / 11$ & $11-8-31$ & 1.6 & ND & ND & $<1$ & $\mu \mathrm{g} / \mathrm{L}$ \\
\hline MW18 & INMW18-W-32761 & 8/29/11 & SB & $65-80$ & Water & SW8260 & TA & 9/1/11 & $200-6762$ & 1.3 & $0.33 \mathrm{~J}$ & ND & $<0.5$ & $\mu \mathrm{g} / \mathrm{L}$ \\
\hline MW18 & INMW18-W-32752 & $8 / 29 / 11$ & SB & $85-90$ & Water & E524.2 & AGEM & 8/30/11 & $11-8-30 a$ & 1.5 & $0.7 \mathrm{~J}$ & ND & $<1$ & $\mu g / L$ \\
\hline MW18 & INMW18-W-32752 & $8 / 29 / 11$ & $\mathrm{SB}$ & $85-90$ & Water & SW8260 & TA & 9/1/11 & $200-6762$ & 1.1 & 0.79 & ND & $<0.5$ & $\mu \mathrm{g} / \mathrm{L}$ \\
\hline MW18 & INMW18-W-32755 & 8/29/11 & SB & $85-90$ & Water & E524.2 & AGEM & 8/30/11 & 11-8-30a & 3.6 & 1.0 & ND & $<1$ & $\mu \mathrm{g} / \mathrm{L}$ \\
\hline MW18 & INMW18-W-32755 & $8 / 29 / 11$ & SB & $85-90$ & Water & SW8260 & TA & 9/1/11 & $200-6762$ & 3.0 & 0.96 & ND & $<0.5$ & $\mu \mathrm{g} / \mathrm{L}$ \\
\hline MW18 & INMW18-W-32754 & $8 / 29 / 11$ & SB & $95-102.5$ & Water & E524.2 & AGEM & $8 / 30 / 11$ & 11-8-30a & $0.6 \mathrm{~J}$ & $0.9 \mathrm{~J}$ & ND & $<1$ & $\mu \mathrm{g} / \mathrm{L}$ \\
\hline MW18 & INMW18-W-32754 & 8/29/11 & $\mathrm{SB}$ & $95-102.5$ & Water & SW8260 & TA & $9 / 1 / 11$ & $200-6762$ & $0.34 \mathrm{~J}$ & 0.98 & ND & $<0.5$ & $\mu \mathrm{g} / \mathrm{L}$ \\
\hline MW16M1 & INMW16-W-32767 & $8 / 30 / 11$ & MW & $60-70$ & Water & $\mathrm{E} 524.2$ & AGEM & $8 / 31 / 11$ & $11-8-31$ & ND & $0.4 \mathrm{~J}$ & ND & $<1$ & $\mu \mathrm{g} / \mathrm{L}$ \\
\hline MW16M1 & INMW16-W-32767 & 8/30/11 & MW & $60-70$ & Water & SW8260 & TA & $9 / 1 / 11$ & $200-6783$ & $0.012 \mathrm{~J}$ & $0.41 \mathrm{~J}$ & ND & $<0.5$ & $\mu \mathrm{g} / \mathrm{L}$ \\
\hline MW19 & INMW19-W-32768 & 8/30/11 & $\mathrm{SB}$ & $15-50$ & Water & E524.2 & AGEM & $8 / 31 / 11$ & $11-8-31$ & ND & ND & ND & $<1$ & $\mu \mathrm{g} / \mathrm{L}$ \\
\hline MW19 & INMW19-W-32768 & $8 / 30 / 11$ & SB & $15-50$ & Water & SW8260 & TA & 9/1/11 & $200-6783$ & $0.12 \mathrm{~J}$ & ND & ND & $<0.5$ & $\mu \mathrm{g} / \mathrm{L}$ \\
\hline MW17D & INMW17D-W-32773 & $8 / 31 / 11$ & MW & $98-108$ & Water & E524.2 & AGEM & 9/1/11 & $11-9-1$ & ND & ND & ND & $<1$ & $\mu g / L$ \\
\hline MW17D & INMW17D-W-32773 & $8 / 31 / 11$ & MW & $98-108$ & Water & SW8260 & TA & 9/6/11 & $200-6846$ & $\mathrm{ND}$ & $0.13 \mathrm{~J}$ & ND & $<0.5$ & $\mu g / L$ \\
\hline
\end{tabular}


TABLE S2.5 (Cont.)

\begin{tabular}{|c|c|c|c|c|c|c|c|c|c|c|c|c|c|c|}
\hline \multirow[b]{2}{*}{ Location } & \multirow[b]{2}{*}{ Sample } & \multirow[b]{2}{*}{$\begin{array}{l}\text { Sample } \\
\text { Date }\end{array}$} & \multirow[b]{2}{*}{$\begin{array}{l}\text { Sample } \\
\text { Type }^{a}\end{array}$} & \multirow[b]{2}{*}{$\begin{array}{c}\text { Depth } \\
\text { (ft BGL) }\end{array}$} & \multirow[b]{2}{*}{$\begin{array}{l}\text { Sample } \\
\text { Medium }\end{array}$} & \multirow[b]{2}{*}{$\begin{array}{l}\text { Analytical } \\
\text { Method }\end{array}$} & \multirow[b]{2}{*}{$\begin{array}{l}\text { Analytical } \\
\text { Laboratory }\end{array}$} & \multirow[b]{2}{*}{$\begin{array}{c}\text { Analysis } \\
\text { Date }\end{array}$} & \multirow[b]{2}{*}{$\begin{array}{c}\text { Sample } \\
\text { Delivery } \\
\text { Group }\end{array}$} & \multicolumn{3}{|c|}{ Concentration } & \multirow[b]{2}{*}{$\begin{array}{l}\text { Method } \\
\text { Detection } \\
\text { Limit }\end{array}$} & \multirow[b]{2}{*}{ Units } \\
\hline & & & & & & & & & & $\begin{array}{l}\text { Carbon } \\
\text { Tetrachloride }\end{array}$ & Chloroform & $\begin{array}{l}\text { Methylene } \\
\text { Chloride }\end{array}$ & & \\
\hline MW17M & INMW17M-W-32770 & $8 / 31 / 11$ & MW & $72-82$ & Water & E524.2 & AGEM & 9/1/11 & $11-9-1$ & $0.9 \mathrm{~J}$ & ND & ND & $<1$ & $\mu \mathrm{g} / \mathrm{L}$ \\
\hline MW17M & INMW17M-W-32770 & $8 / 31 / 11$ & MW & $72-82$ & Water & SW8260 & TA & 9/6/11 & $200-6846$ & 0.72 & $0.4 \mathrm{~J}$ & $0.049 \mathrm{~J}$ & $<0.5$ & $\mu \mathrm{g} / \mathrm{L}$ \\
\hline MW17S & INMW17S-W-32772 & $8 / 31 / 11$ & MW & $25-35$ & Water & E524.2 & AGEM & 9/1/11 & $11-9-1$ & ND & $0.8 \mathrm{~J}$ & ND & $<1$ & $\mu \mathrm{g} / \mathrm{L}$ \\
\hline MW17S & INMW17S-W-32772 & $8 / 31 / 11$ & MW & $25-35$ & Water & SW8260 & TA & 9/6/11 & $200-6846$ & $0.027 \mathrm{~J}$ & 0.63 & ND & $<0.5$ & $\mu \mathrm{g} / \mathrm{L}$ \\
\hline MW19 & INMW19-W-32771 & $8 / 31 / 11$ & SB & $75-90$ & Water & E524.2 & AGEM & 9/1/11 & $11-9-1$ & ND & ND & ND & $<1$ & $\mu \mathrm{g} / \mathrm{L}$ \\
\hline MW19 & INMW19-W-32771 & $8 / 31 / 11$ & SB & $75-90$ & Water & SW8260 & TA & $9 / 6 / 11$ & $200-6846$ & $0.19 \mathrm{~J}$ & $0.19 \mathrm{~J}$ & ND & $<0.5$ & $\mu \mathrm{g} / \mathrm{L}$ \\
\hline MW18M & INMW18M-W-32776 & 9/1/11 & MW & $85-95$ & Water & E524.2 & AGEM & 9/2/11 & $11-9-2$ & 3.3 & 1.0 & ND & $<1$ & $\mu \mathrm{g} / \mathrm{L}$ \\
\hline MW18M & INMW18M-W-32776 & 9/1/11 & MW & $85-95$ & Water & SW8260 & TA & 9/6/11 & $200-6846$ & ND & $0.15 \mathrm{~J}$ & ND & $<0.5$ & $\mu \mathrm{g} / \mathrm{L}$ \\
\hline MW18S & INMW18S-W-32775 & 9/1/11 & MW & $40-50$ & Water & E524.2 & AGEM & $9 / 2 / 11$ & $11-9-2$ & 4.5 & 1.0 & ND & $<1$ & $\mu \mathrm{g} / \mathrm{L}$ \\
\hline MW18S & INMW18S-W-32775 & 9/1/11 & MW & $40-50$ & Water & SW8260 & TA & 9/6/11 & $200-6846$ & 3.0 & 1.0 & ND & $<0.5$ & $\mu \mathrm{g} / \mathrm{L}$ \\
\hline MW20 & INMW20-W-32775 & $9 / 13 / 11$ & SB & $15-40$ & Water & E524.2 & AGEM & 9/15/11 & $11-9-2$ & ND & ND & ND & $<1$ & $\mu \mathrm{g} / \mathrm{L}$ \\
\hline MW20 & INMW20-W-32775 & 9/13/11 & SB & $15-40$ & Water & SW8260 & TA & 9/16/11 & $200-7066$ & ND & $0.04 \mathrm{~J}$ & ND & $<0.5$ & $\mu \mathrm{g} / \mathrm{L}$ \\
\hline MW20 & INMW20-W-32776 & 9/13/11 & SB & $65-89.5$ & Water & E524.2 & AGEM & 9/15/11 & $11-9-2$ & ND & ND & ND & $<1$ & $\mu \mathrm{g} / \mathrm{L}$ \\
\hline MW20 & INMW20-W-32776 & 9/13/11 & SB & $65-89.5$ & Water & SW8260 & TA & 9/16/11 & $200-7066$ & ND & $0.032 \mathrm{~J}$ & ND & $<0.5$ & $\mu \mathrm{g} / \mathrm{L}$ \\
\hline MW14D & INMW14D-W-32782 & 9/15/11 & MW & $115-125$ & Water & E524.2 & AGEM & 9/16/11 & $11-9-16$ & ND & $0.6 \mathrm{~J}$ & ND & $<1$ & $\mu \mathrm{g} / \mathrm{L}$ \\
\hline MW14D & INMW14D-W-32782 & 9/15/11 & MW & $115-125$ & Water & SW8260 & TA & 9/20/11 & $200-7045$ & ND & $0.56 \mathrm{~B}$ & $0.15 \mathrm{~J}$ & $<0.5$ & $\mu \mathrm{g} / \mathrm{L}$ \\
\hline MW20M & INMW20M-W-32781 & 9/15/11 & MW & $72-82$ & Water & E524.2 & AGEM & 9/16/11 & $11-9-16$ & ND & $0.4 \mathrm{~J}$ & ND & $<1$ & $\mu \mathrm{g} / \mathrm{L}$ \\
\hline MW20M & INMW20M-W-32781 & 9/15/11 & MW & $72-82$ & Water & sW8260 & TA & 9/20/11 & $200-7045$ & ND & $0.32 \mathrm{~J} \mathrm{~B}$ & ND & $<0.5$ & $\mu \mathrm{g} / \mathrm{L}$ \\
\hline MW20S & INMW20S-W-32780 & 9/15/11 & MW & $30-40$ & Water & E524.2 & AGEM & 9/16/11 & $11-9-16$ & ND & $0.7 \mathrm{~J}$ & ND & $<1$ & $\mu \mathrm{g} / \mathrm{L}$ \\
\hline MW20S & INMW20S-W-32780 & 9/15/11 & MW & $30-40$ & Water & SW8260 & TA & 9/20/11 & $200-7045$ & ND & $0.73 \mathrm{~B}$ & ND & $<0.5$ & $\mu \mathrm{g} / \mathrm{L}$ \\
\hline
\end{tabular}

a Sample types: CPT, cone penetrometer; CPT/P, piezometer; DW, domestic well; MW, monitoring well; SB, soil boring

b J, compound identified with an estimated concentration between the instrument detection limit and the method detection limit.

c ND, compound analyzed for but not detected at a level greater than or equal to the indicated method detection limit.

d B, contaminant present in associated laboratory blank. 


\section{Supplement 3:}

Data from Pace Analytical Services for Investigation-Derived Waste 
October 03, 2011

Mr. Travis Kamler

TCW Construction Inc

$141 \mathrm{M}$ Street

Lincoln, NE 68508

RE: Project: Inman KS/8A727-A5-167

Pace Project No.: 60106571

Dear Mr. Kamler:

Enclosed are the analytical results for sample(s) received by the laboratory on September 21, 2011. The results relate only to the samples included in this report. Results reported herein conform to the most current TNI standards, where applicable, unless otherwise narrated in the body of the report.

If you have any questions concerning this report, please feel free to contact me.

Sincerely,

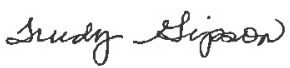

Trudy Gipson

trudy.gipson@pacelabs.com

Project Manager

Enclosures

cc: Mr. David Surgnier

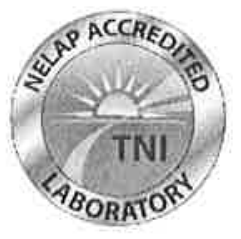




\title{
CERTIFICATIONS
}

\author{
Project: Inman KS/8A727-A5-167 \\ Pace Project No.: 60106571
}

\section{Kansas Certification IDs}

9608 Loiret Boulevard, Lenexa, KS 66219

A2LA Certification \#: 2456.01

Arkansas Certification \#: 05-008-0

Illinois Certification \#: 001191

lowa Certification \#: 118

Kansas/NELAP Certification \#: E-10116

Louisiana Certification \#: 03055

Nevada Certification \#: KS000212008A

Oklahoma Certification \#: 9205/9935

Texas Certification \#: T104704407-08-TX

Utah Certification \#: 9135995665 
SAMPLE SUMMARY

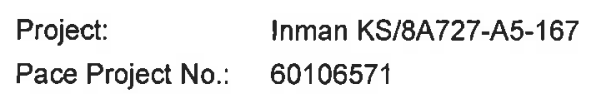

\begin{tabular}{|c|c|c|c|c|}
\hline Lab ID & Sample ID & Matrix & Date Collected & Date Received \\
\hline 60106571001 & INPURGE-W-30860 & Water & 09/20/11 16:00 & $09 / 21 / 1109: 00$ \\
\hline 60106571002 & INROLLF-S-30861 & Solid & 09/20/11 16:00 & 09/21/11 09:00 \\
\hline 60106571003 & INROLLR-S-30862 & Solid & 09/20/11 16:00 & 09/21/11 09:00 \\
\hline
\end{tabular}




\section{SAMPLE ANALYTE COUNT}

Project: Inman KS/8A727-A5-167

Pace Project No.: 60106571

\begin{tabular}{|c|c|c|c|c|}
\hline Lab ID & Sample ID & Method & Analysts & $\begin{array}{l}\text { Analytes } \\
\text { Reported }\end{array}$ \\
\hline \multirow[t]{3}{*}{60106571001} & INPURGE-W-30860 & EPA 504.1 & NAW & 1 \\
\hline & & EPA $5030 \mathrm{~B} / 8260$ & JDM & 70 \\
\hline & & EPA 353.2 & SRM1 & 1 \\
\hline \multirow[t]{2}{*}{60106571002} & INROLLF-S-30861 & EPA 8260 & RAB & 69 \\
\hline & & ASTM D2974-87 & DWC & 1 \\
\hline \multirow[t]{2}{*}{60106571003} & INROLLR-S-30862 & EPA 8260 & $\mathrm{RAB}$ & 69 \\
\hline & & ASTM D2974-87 & DWC & 1 \\
\hline
\end{tabular}




\section{ANALYTICAL RESULTS}

Project: Inman KS/8A727-A5-167

Pace Project No.: $\quad 60106571$

\begin{tabular}{|c|c|c|c|c|c|c|c|c|}
\hline \multirow{2}{*}{$\begin{array}{c}\text { Sample: INPURGE-W-30860 } \\
\text { Parameters }\end{array}$} & \multirow{2}{*}{\multicolumn{2}{|c|}{$\begin{array}{lr}\text { Lab ID: } & 60106571001 \\
\text { Results } & \text { Units }\end{array}$}} & \multicolumn{2}{|c|}{ Collected: $09 / 20 / 1116: 00$} & \multicolumn{2}{|c|}{ Received: 09/21/11 09:00 } & \multicolumn{2}{|l|}{ Matrix: Water } \\
\hline & & & Report Limit & DF & Prepared & Analyzed & CAS No. & Qual \\
\hline 504 GCS EDB and DBCP & \multicolumn{7}{|c|}{ Analytical Method: EPA 504.1 Preparation Method: EPA 504.1} & \\
\hline 1,2-Dibromoethane (EDB) & & ND ug/L & 0.030 & 1 & 09/26/11 00:00 & $09 / 27 / 1111: 41$ & $106-93-4$ & \\
\hline 8260 MSV & \multicolumn{7}{|c|}{ Analytical Method: EPA 5030B/8260 } & \\
\hline Acetone & & ND ug/L & 10.0 & 1 & & $09 / 27 / 1112: 42$ & $67-64-1$ & \\
\hline Benzene & & ND ug/L & 1.0 & 1 & & $09 / 27 / 1112: 42$ & $71-43-2$ & \\
\hline Bromobenzene & & ND ug/L & 1.0 & 1 & & $09 / 27 / 1112: 42$ & $108-86-1$ & \\
\hline Bromochloromethane & & ND ug/L & 1.0 & 1 & & $09 / 27 / 1112: 42$ & $74-97-5$ & \\
\hline Bromodichloromethane & & ND ug/L & 1.0 & 1 & & $09 / 27 / 1112: 42$ & $75-27-4$ & \\
\hline Bromoform & & ND ug/L & 1.0 & 1 & & $09 / 27 / 1112: 42$ & $75-25-2$ & \\
\hline Bromomethane & & ND ug/L & 1.0 & 1 & & $09 / 27 / 1112: 42$ & $74-83-9$ & \\
\hline 2-Butanone (MEK) & & JD ug/L & 10.0 & 1 & & $09 / 27 / 1112: 42$ & $78-93-3$ & \\
\hline n-Butylbenzene & & ND ug/L & 1.0 & 1 & & $09 / 27 / 1112: 42$ & 2 $104-51-8$ & \\
\hline sec-Butylbenzene & & ND ug/L & 1.0 & 1 & & $09 / 27 / 1112: 42$ & $135-98-8$ & \\
\hline tert-Butylbenzene & & JD ug/L & 1.0 & 1 & & $09 / 27 / 1112: 42$ & $98-06-6$ & \\
\hline Carbon disulfide & & ND ug/L & 5.0 & 1 & & $09 / 27 / 1112: 42$ & $75-15-0$ & \\
\hline Carbon tetrachloride & & ND ug/L & 1.0 & 1 & & $09 / 27 / 1112: 42$ & $56-23-5$ & \\
\hline Chlorobenzene & & JD ug/L & 1.0 & 1 & & $09 / 27 / 1112: 42$ & $108-90-7$ & \\
\hline Chloroethane & & JD ug/L & 1.0 & 1 & & $09 / 27 / 1112: 42$ & $75-00-3$ & \\
\hline Chloroform & & JD ug/L & 1.0 & 1 & & $09 / 27 / 1112: 42$ & $67-66-3$ & \\
\hline Chloromethane & & JD ug/L & 1.0 & 1 & & $09 / 27 / 1112: 42$ & $74-87-3$ & \\
\hline 2-Chlorotoluene & & JD ug/L & 1.0 & 1 & & $09 / 27 / 1112: 42$ & $95-49-8$ & \\
\hline 4-Chlorotoluene & & ID ug/L & 1.0 & 1 & & $09 / 27 / 1112: 42$ & $106-43-4$ & \\
\hline 1,2-Dibromo-3-chloropropane & & ID ug/L & 2.5 & 1 & & $09 / 27 / 1112: 42$ & $96-12-8$ & \\
\hline Dibromochloromethane & & ID ug/L & 1.0 & 1 & & $09 / 27 / 1112: 42$ & $124-48-1$ & \\
\hline 1,2-Dibromoethane (EDB) & & JD ug/L & 1.0 & 1 & & $09 / 27 / 1112: 42$ & $106-93-4$ & \\
\hline Dibromomethane & & ID ug/L & 1.0 & 1 & & $09 / 27 / 1112: 42$ & $74-95-3$ & \\
\hline 1,2-Dichlorobenzene & & JD ug/L & 1.0 & 1 & & $09 / 27 / 1112: 42$ & $95-50-1$ & \\
\hline 1,3-Dichlorobenzene & & ID ug/L & 1.0 & 1 & & $09 / 27 / 1112: 42$ & $541-73-1$ & \\
\hline 1,4-Dichlorobenzene & & ID ug/L & 1.0 & 1 & & $09 / 27 / 1112: 42$ & $106-46-7$ & \\
\hline Dichlorodifluoromethane & & ID ug/L & 1.0 & 1 & & $09 / 27 / 1112: 42$ & $75-71-8$ & \\
\hline 1,1-Dichloroethane & & ID ug/L & 1.0 & 1 & & $09 / 27 / 1112: 42$ & $75-34-3$ & \\
\hline 1,2-Dichloroethane & & ID ug/L & 1.0 & 1 & & $09 / 27 / 1112: 42$ & $107-06-2$ & \\
\hline 1,2-Dichloroethene (Total) & & ID ug/L & 1.0 & 1 & & $09 / 27 / 1112: 42$ & $540-59-0$ & \\
\hline 1,1-Dichloroethene & & JD ug/L & 1.0 & 1 & & $09 / 27 / 11 \quad 12: 42$ & $75-35-4$ & \\
\hline cis-1,2-Dichloroethene & & ID ug/L & 1.0 & 1 & & $09 / 27 / 11 \uparrow 2: 42$ & $156-59-2$ & \\
\hline trans-1,2-Dichloroethene & & ID ug/L & 1.0 & 1 & & $09 / 27 / 1112: 42$ & $156-60-5$ & \\
\hline 1,2-Dichloropropane & & ID ug/L & 1.0 & 1 & & $09 / 27 / 1112: 42$ & $78-87-5$ & \\
\hline 1,3-Dichloropropane & & ID ug/L & 1.0 & 1 & & $09 / 27 / 1112: 42$ & $142-28-9$ & \\
\hline 2,2-Dichloropropane & & ID ug/L & 1.0 & 1 & & $09 / 27 / 1112: 42$ & $594-20-7$ & \\
\hline 1,1-Dichloropropene & & ID ug/L & 1.0 & 1 & & $09 / 27 / 1112: 42$ & $563-58-6$ & \\
\hline cis-1,3-Dichloropropene & & ID ug/L & 1.0 & 1 & & $09 / 27 / 1112: 42$ & $10061-01-5$ & \\
\hline trans-1,3-Dichloropropene & & ID ug/L & 1.0 & 1 & & $09 / 27 / 1112: 42$ & $10061-02-6$ & \\
\hline Ethylbenzene & & ID ug/L & 1.0 & 1 & & $09 / 27 / 1112: 42$ & $100-41-4$ & \\
\hline Hexachloro-1,3-butadiene & & ID ug/L & 1.0 & 1 & & $09 / 27 / 1112: 42$ & $87-68-3$ & \\
\hline 2-Hexanone & & ID ug/L & 10.0 & 1 & & $09 / 27 / 1112: 42$ & $591-78-6$ & \\
\hline Isopropylbenzene (Cumene) & & ID ug/L & 1.0 & 1 & & $09 / 27 / 1112: 42$ & $98-82-8$ & \\
\hline p-Isopropyltoluene & & ID ug/L & 1.0 & 1 & & $09 / 27 / 1112: 42$ & $99-87-6$ & \\
\hline
\end{tabular}

\section{REPORT OF LABORATORY ANALYSIS}

This report shall not be reproduced, except in full, without the written consent of Pace Analytical Services, Inc.. 


\section{ANALYTICAL RESULTS}

Project: Inman KS/8A727-A5-167

Pace Project No.: 60106571

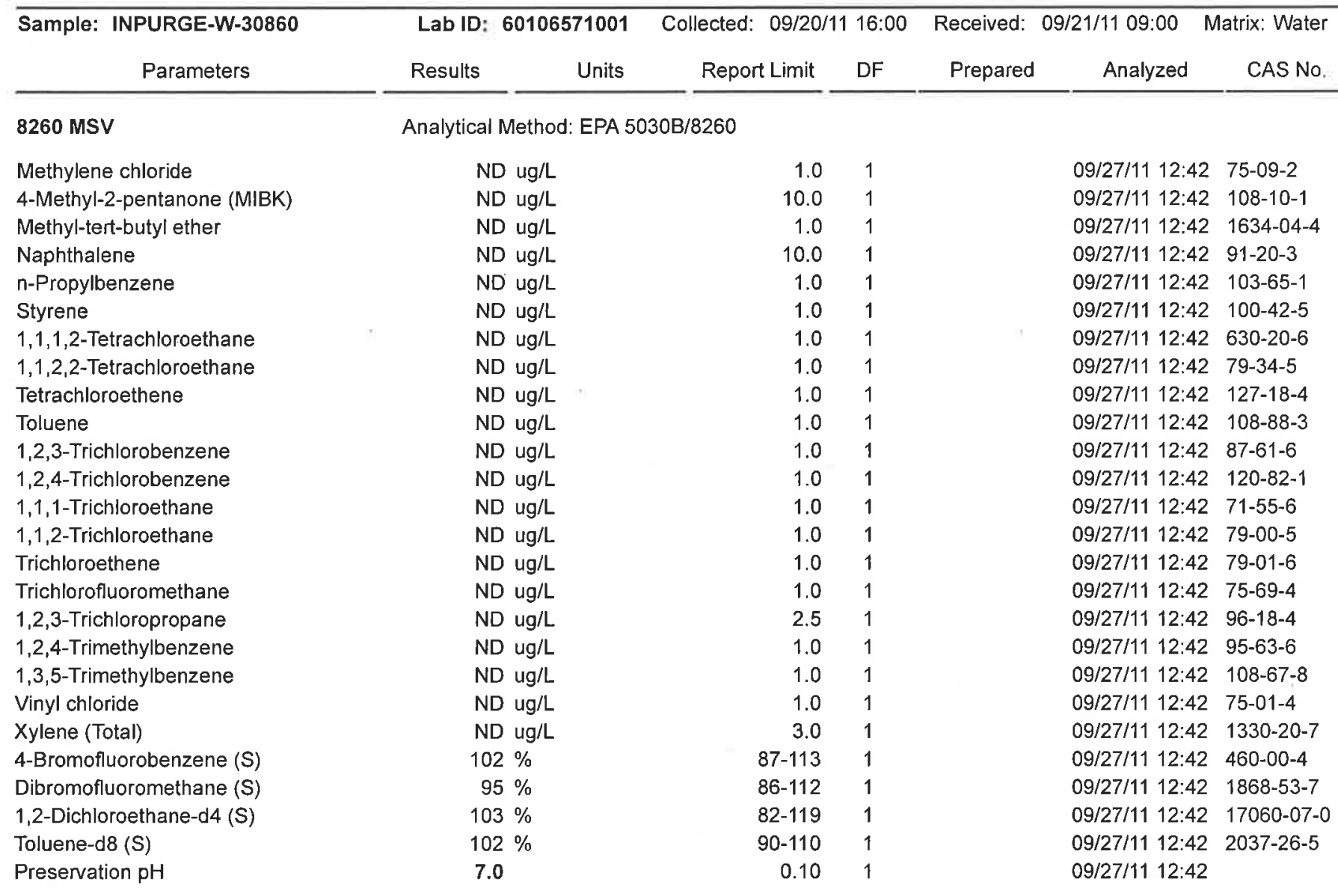

353.2 Nitrogen, NO2/NO3 unpres

Nitrogen, Nitrate
Analytical Method: EPA 353.2

$4.8 \mathrm{mg} / \mathrm{L}$
$0.10 \quad 1$

09/21/11 19:01 


\section{ANALYTICAL RESULTS}

Project:

Inman KS/8A727-A5-167

Pace Project No.: $\quad 60106571$

Sample: INROLLF-S-30861 $\quad$ Lab ID: $60106571002 \quad$ Collected: 09/20/11 16:00 Received: 09/21/11 09:00 Matrix: Solid

Results reported on a "dry-weight" basis

$\frac{\text { Parameters }}{8260 \text { MSV 5035A VOA }} \frac{\text { Results }}{\text { Analytical Method: EPA } 8260} \frac{\text { Units }}{\text { Report Limit }} \stackrel{\text { DF }}{-}$ Prepared

\section{Acetone}

Benzene

Bromobenzene

Bromochloromethane

Bromodichloromethane

Bromoform

Bromomethane

2-Butanone (MEK)

n-Butylbenzene

sec-Butylbenzene

tert-Butylbenzene

Carbon disulfide

Carbon tetrachloride

Chlorobenzene

Chloroethane

Chloroform

Chloromethane

2-Chlorotoluene

4-Chlorotoluene

1,2-Dibromo-3-chloropropane

Dibromochloromethane

1,2-Dibromoethane (EDB)

Dibromomethane

1,2-Dichlorobenzene

1,3-Dichlorobenzene

1,4-Dichlorobenzene

Dichlorodifluoromethane

1,1-Dichloroethane

1,2-Dichloroethane

1,2-Dichloroethene (Total)

1,1-Dichloroethene

cis-1,2-Dichloroethene

trans-1,2-Dichloroethene

1,2-Dichloropropane

1,3-Dichloropropane

2,2-Dichloropropane

1,1-Dichloropropene

cis-1,3-Dichloropropene

trans-1,3-Dichloropropene

Ethylbenzene

Hexachloro-1,3-butadiene

2-Hexanone

Isopropylbenzene (Cumene)

p-Isopropyltoluene

Methylene chloride

4-Methyl-2-pentanone (MIBK)

Date: 10/03/2011 03:27 PM

\begin{tabular}{|c|c|}
\hline ND ug $/ \mathrm{kg}$ & 21.4 \\
\hline ND ug $/ \mathrm{kg}$ & 5.4 \\
\hline ND ug/kg & 5.4 \\
\hline ND ug $/ \mathrm{kg}$ & 5.4 \\
\hline ND $u g / k g$ & 5.4 \\
\hline ND ug $/ \mathrm{kg}$ & 5.4 \\
\hline ND ug $/ \mathrm{kg}$ & 5.4 \\
\hline ND ug/kg & 10.7 \\
\hline ND ug $/ \mathrm{kg}$ & 5.4 \\
\hline ND ug/kg & 5.4 \\
\hline ND ug/kg & 5.4 \\
\hline ND ug/kg & 5.4 \\
\hline ND ug/kg & 5.4 \\
\hline ND ug $/ \mathrm{kg}$ & 5.4 \\
\hline ND ug/kg & 5.4 \\
\hline ND ug/kg & 5.4 \\
\hline ND ug/kg & 5.4 \\
\hline ND ug/kg & 5.4 \\
\hline ND ug/kg & 5.4 \\
\hline ND ug/kg & 10.7 \\
\hline ND ug/kg & 5.4 \\
\hline ND ug $/ \mathrm{kg}$ & 5.4 \\
\hline ND ug/kg & 5.4 \\
\hline $\mathrm{ND} u g / \mathrm{kg}$ & 5.4 \\
\hline $\mathrm{ND} u g / \mathrm{kg}$ & 5.4 \\
\hline ND ug $/ \mathrm{kg}$ & 5.4 \\
\hline ND ug/kg & 5.4 \\
\hline ND ug $/ \mathrm{kg}$ & 5.4 \\
\hline ND ug $/ \mathrm{kg}$ & 5.4 \\
\hline ND $u g / k g$ & 5.4 \\
\hline ND ug $/ \mathrm{kg}$ & 5.4 \\
\hline ND ug/kg & 5.4 \\
\hline ND ug $/ \mathrm{kg}$ & 5.4 \\
\hline ND ug/kg & 5.4 \\
\hline ND ug $/ \mathrm{kg}$ & 5.4 \\
\hline ND ug/kg & 5.4 \\
\hline ND ug/kg & 5.4 \\
\hline ND ug $/ \mathrm{kg}$ & 5.4 \\
\hline ND ug/kg & 5.4 \\
\hline ND ug $/ \mathrm{kg}$ & 5.4 \\
\hline ND ug/kg & 5.4 \\
\hline ND ug $/ \mathrm{kg}$ & 21.4 \\
\hline ND ug/kg & 5.4 \\
\hline ND ug $/ \mathrm{kg}$ & 5.4 \\
\hline ND ug/kg & 5.4 \\
\hline ND ug/kg & 10.7 \\
\hline
\end{tabular}

$09 / 29 / 11$ 12:22 67-64-1

$09 / 29 / 11$ 12:22 71-43-2

$09 / 29 / 11$ 12:22 108-86-1

09/29/11 12:22 74-97-5

$09 / 29 / 11 \quad 12: 22 \quad 75-27-4$

09/29/11 12:22 75-25-2

09/29/11 12:22 74-83-9

09/29/11 12:22 78-93-3

09/29/11 12:22 104-51-8

09/29/11 12:22 135-98-8

$09 / 29 / 11$ 12:22 98-06-6

$09 / 29 / 11$ 12:22 75-15-0

09/29/11 12:22 56-23-5

09/29/11 12:22 108-90-7

$09 / 29 / 11$ 12:22 75-00-3

09/29/11 12:22 67-66-3

09/29/11 12:22 74-87-3

$09 / 29 / 11 \quad 12: 22 \quad 95-49-8$

09/29/11 12:22 106-43-4

09/29/11 12:22 96-12-8

09/29/11 12:22 124-48-1

09/29/11 12:22 106-93-4

09/29/11 12:22 74-95-3

$09 / 29 / 11$ 12:22 95-50-1

09/29/11 12:22 541-73-1

09/29/11 12:22 106-46-7

09/29/11 12:22 75-71-8

$09 / 29 / 11$ 12:22 75-34-3

09/29/11 12:22 107-06-2

$09 / 29 / 11$ 12:22 540-59-0

$09 / 29 / 11 \quad 12: 22 \quad 75-35-4$

09/29/11 12:22 156-59-2

$09 / 29 / 11 \quad 12: 22$ 156-60-5

$09 / 29 / 11 \quad 12: 22 \quad 78-87-5$

$09 / 29 / 11 \quad 12: 22 \quad 142-28-9$

$09 / 29 / 11 \quad 12: 22 \quad 594-20-7$

09/29/11 12:22 563-58-6

$09 / 29 / 11$ 12:22 10061-01-5

09/29/11 12:22 10061-02-6

$09 / 29 / 11 \quad 12: 22 \quad 100-41-4$

$09 / 29 / 1112: 22 \quad 87-68-3$

$09 / 29 / 11 \quad 12: 22 \quad 591-78-6$

$09 / 29 / 11$ 12:22 98-82-8

$09 / 29 / 11 \quad 12: 22 \quad 99-87-6$

09/29/11 12:22 75-09-2

$09 / 29 / 11 \quad 12: 22 \quad 108-10-1$

L3

L3

L2

L3

\section{REPORT OF LABORATORY ANALYSIS}

Page 7 of 22
This report shall not be reproduced, except in full,

without the written consent of Pace Analytical Services, inc. 


\section{ANALYTICAL RESULTS}

Project: $\quad$ Inman KS/8A727-A5-167

Pace Project No.: 60106571

Sample: INROLLF-S-30861 Lab ID: 60106571002 Collected: 09/20/11 16:00 Received: 09/21/11 09:00 Matrix: Solid

Results reported on a "dry-weight" basis

Parameters

8260 MSV 5035A VOA

Methyl-tert-butyl ether

Naphthalene

n-Propylbenzene

Styrene

1,1,1,2-Tetrachloroethane

1,1,2,2-Tetrachloroethane

Tetrachloroethene

Toluene

1,2,3-Trichlorobenzene

1,2,4-Trichlorobenzene

1,1,1-Trichloroethane

1,1,2-Trichloroethane

Trichloroethene

Trichlorofluoromethane

1,2,3-Trichloropropane

1,2,4-Trimethylbenzene

1,3,5-Trimethylbenzene

Vinyl chloride

Xylene (Total)

Dibromofluoromethane (S)

Toluene-d8 (S)

4-Bromofluorobenzene (S)

1,2-Dichloroethane-d4 (S)

Percent Moisture

Percent Moisture

Results

Units Report Limit

DF

Analytical Method: EPA 8260

ND ug/kg
ND ug/kg
ND ug/kg
ND ug/kg
ND ug/kg
ND ug/kg
ND ug/kg
ND ug/kg
ND ug/kg
ND ug/kg
ND ug/kg
ND ug/kg
ND ug/kg
ND ug/kg
ND ug/kg
ND ug/kg
ND ug/kg
ND ug/kg
ND ug/kg
$107 \%$
$101 \%$
$102 \%$
$123 \%$

ND ug $/ \mathrm{kg}$

ND ug $/ \mathrm{kg}$

ND ug/kg

ND ug/kg

ND ug/kg

ND $u g / k g$

ND ug $/ \mathrm{kg}$

ND ug/kg

ND $u g / k g$

ND ug/kg

ND $u g / k g$

ND ug/kg

ND ug $/ \mathrm{kg}$

ND ug/kg

$107 \%$

$102 \%$

Analytical Method: ASTM D2974-87

$7.3 \%$

$\begin{array}{rr}5.4 & 1 \\ 10.7 & 1 \\ 5.4 & 1 \\ 5.4 & 1 \\ 5.4 & 1 \\ 5.4 & 1 \\ 5.4 & 1 \\ 5.4 & 1 \\ 5.4 & 1 \\ 5.4 & 1 \\ 5.4 & 1 \\ 5.4 & 1 \\ 5.4 & 1 \\ 5.4 & 1 \\ 5.4 & 1 \\ 5.4 & 1 \\ 5.4 & 1 \\ 5.4 & 1 \\ 5.4 & 1 \\ 68-129 & 1 \\ 81-121 & 1 \\ 75-131 & 1 \\ 77-131 & 1\end{array}$

$0.50 \quad 1$
09/29/11 12:22 1634-04-4

$09 / 29 / 11 \quad 12: 22 \quad 91-20-3$

$09 / 29 / 11$ 12:22 103-65-1

$09 / 29 / 11 \quad 12: 22 \quad 100-42-5$

$09 / 29 / 11 \quad 12: 22 \quad 630-20-6$

09/29/11 12:22 79-34-5

$09 / 29 / 11 \quad 12: 22$ 127-18-4

$09 / 29 / 11 \quad 12: 22 \quad 108-88-3$

$09 / 29 / 11$ 12:22 87-61-6

09/29/11 12:22 120-82-1

$09 / 29 / 11$ 12:22 71-55-6

09/29/11 12:22 79-00-5 "

09/29/11 12:22 79-01-6

$09 / 29 / 11 \quad 12: 22 \quad 75-69-4$

$09 / 29 / 11$ 12:22 96-18-4

$09 / 29 / 11$ 12:22 95-63-6

09/29/11 12:22 108-67-8

09/29/11 12:22 75-01-4

$09 / 29 / 11 \quad 12: 22 \quad 1330-20-7$

$09 / 29 / 11$ 12:22 1868-53-7

09/29/11 12:22 2037-26-5

09/29/11 12:22 460-00-4

09/29/11 12:22 17060-07-0

$09 / 26 / 1100: 00$ 


\section{ANALYTICAL RESULTS}

Project: Inman KS/8A727-A5-167

Pace Project No.: 60106571

Sample: INROLLR-S-30862 Lab ID: $60106571003 \quad$ Collected: 09/20/11 16:00 Received: 09/21/11 09:00 Matrix: Solid

Results reported on a "dry-weight" basis

$\frac{\text { Parameters }}{8260 \text { MSV 5035A VOA }} \frac{\text { Results }}{\text { Analytical Method: EPA } 8260} \frac{\text { Units }}{\text { Report Limit }} \stackrel{\text { DF }}{-} \frac{\text { Prepared }}{\text { Analyzed }}-$ CAS No.

\section{Acetone}

Benzerie

Bromobenzene

Bromochloromethane

Bromodichloromethane

Bromoform

Bromomethane

2-Butanone (MEK)

n-Butylbenzene

sec-Butylbenzene

tert-Butylbenzene

Carbon disulfide

Carbon tetrachloride

Chlorobenzene

Chloroethane

Chloroform

Chloromethane

2-Chlorotoluene

4-Chlorotoluene

1,2-Dibromo-3-chloropropane

Dibromochloromethane

1,2-Dibromoethane (EDB)

Dibromomethane

1,2-Dichlorobenzene

1,3-Dichlorobenzene

1,4-Dichlorobenzene

Dichlorodifluoromethane

1,1-Dichloroethane

1,2-Dichloroethane

1,2-Dichloroethene (Total)

1,1-Dichloroethene

cis-1,2-Dichloroethene

trans-1,2-Dichloroethene

1,2-Dichloropropane

1,3-Dichloropropane

2,2-Dichloropropane

1,1-Dichloropropene

cis-1,3-Dichloropropene

trans-1,3-Dichloropropene

Ethylbenzene

Hexachloro-1,3-butadiene

2-Hexanone

Isopropylbenzene (Cumene)

p-Isopropyltoluene

Methylene chloride

4-Methyl-2-pentanone (MIBK)

\begin{tabular}{|c|}
\hline ND ug/kg \\
\hline ND ug/kg \\
\hline ND ug/kg \\
\hline VD ug/kg \\
\hline ND ug $/ \mathrm{kg}$ \\
\hline ND ug $/ \mathrm{kg}$ \\
\hline ND ug $/ \mathrm{kg}$ \\
\hline ND ug $/ \mathrm{kg}$ \\
\hline ND ug/kg \\
\hline ND ug/kg \\
\hline ND ug $/ \mathrm{kg}$ \\
\hline ND ug/kg \\
\hline ND ug/kg \\
\hline ND ug $/ \mathrm{kg}$ \\
\hline ND ug/kg \\
\hline ND ug $/ \mathrm{kg}$ \\
\hline ND ug $/ \mathrm{kg}$ \\
\hline ND ug $/ \mathrm{kg}$ \\
\hline ND ug $/ \mathrm{kg}$ \\
\hline ND ug/kg \\
\hline ND ug/kg \\
\hline ND ug $/ \mathrm{kg}$ \\
\hline ND ug/kg \\
\hline ND ug/kg \\
\hline ND ug/kg \\
\hline ND ug/kg \\
\hline ND ug/kg \\
\hline ND ug $/ \mathrm{kg}$ \\
\hline ND ug/kg \\
\hline ND ug/kg \\
\hline ND ug/kg \\
\hline ND ug/kg \\
\hline ND ug $/ \mathrm{kg}$ \\
\hline ND ug $/ \mathrm{kg}$ \\
\hline ND ug/kg \\
\hline ND ug/kg \\
\hline ND ug/kg \\
\hline ND ug/kg \\
\hline ND ug/kg \\
\hline ND ug/kg \\
\hline ND ug $/ \mathrm{kg}$ \\
\hline ND ug/kg \\
\hline ND ug $/ \mathrm{kg}$ \\
\hline ND ug/kg \\
\hline $\begin{array}{l}\text { ND } \quad u g / k g \\
\text { ND } u g / k g\end{array}$ \\
\hline
\end{tabular}

\begin{tabular}{rl}
21.6 & 1 \\
5.4 & 1 \\
5.4 & 1 \\
5.4 & 1 \\
5.4 & 1 \\
5.4 & 1 \\
5.4 & 1 \\
10.8 & 1 \\
5.4 & 1 \\
5.4 & 1 \\
5.4 & 1 \\
5.4 & 1 \\
5.4 & 1 \\
5.4 & 1 \\
5.4 & 1 \\
5.4 & 1 \\
5.4 & 1 \\
5.4 & 1 \\
5.4 & 1 \\
10.8 & 1 \\
5.4 & 1 \\
5.4 & 1 \\
5.4 & 1 \\
5.4 & 1 \\
5.4 & 1 \\
5.4 & 1 \\
5.4 & 1 \\
5.4 & 1 \\
5.4 & 1 \\
5.4 & 1 \\
5.4 & 1 \\
5.4 & 1 \\
5.4 & 1 \\
5.4 & 1 \\
5.4 & 1 \\
5.4 & 1 \\
5.4 & 1 \\
5.4 & 1 \\
5.4 & 1 \\
5.4 & 1 \\
5.4 & 1 \\
21.6 & 1 \\
5.4 & 1 \\
5.4 & 1 \\
& 1 \\
& 1 \\
5.8 & 1 \\
\hline
\end{tabular}

09/29/11 12:37 67-64-1 $09 / 29 / 11 \quad 12: 37 \quad 71-43-2$

09/29/11 12:37 108-86-1 $09 / 29 / 11 \quad 12: 37 \quad 74-97-5$ $09 / 29 / 11 \quad 12: 37 \quad 75-27-4$ $09 / 29 / 11 \quad 12: 37 \quad 75-25-2$ 09/29/11 12:37 74-83-9 09/29/11 12:37 78-93-3 $09 / 29 / 11 \quad 12: 37 \quad 104-51-8$ $09 / 29 / 11 \quad 12: 37 \quad 135-98-8$ $09 / 29 / 11$ 12:37 98-06-6 $09 / 29 / 11 \quad 12: 37 \quad 75-15-0$ $09 / 29 / 11 \quad 12: 37 \quad 56-23-5$ 09/29/11 12:37 108-90-7 $09 / 29 / 11$ 12:37 75-00-3 $09 / 29 / 11$ 12:37 67-66-3 09/29/11 12:37 74-87-3 $09 / 29 / 11 \quad 12: 37 \quad 95-49-8$ 09/29/11 12:37 106-43-4 $09 / 29 / 11 \quad 12: 37 \quad 96-12-8$ $09 / 29 / 11 \quad 12: 37 \quad 124-48-1$ 09/29/11 12:37 106-93-4 $09 / 29 / 11 \quad 12: 37 \quad 74-95-3$ $09 / 29 / 11$ 12:37 95-50-1 09/29/11 12:37 541-73-1 09/29/11 12:37 106-46-7 $09 / 29 / 11 \quad 12: 37 \quad 75-71-8$ $09 / 29 / 11$ 12:37 75-34-3 09/29/11 12:37 107-06-2 09/29/11 12:37 540-59-0 $09 / 29 / 11$ 12:37 75-35-4 09/29/11 12:37 156-59-2 $09 / 29 / 11 \quad 12: 37 \quad 156-60-5$ 09/29/11 12:37 78-87-5 09/29/11 12:37 142-28-9 09/29/11 12:37 594-20-7 09/29/11 12:37 563-58-6 09/29/11 12:37 10061-01-5 09/29/11 12:37 10061-02-6 09/29/11 12:37 100-41-4 $09 / 29 / 11$ 12:37 87-68-3 $09 / 29 / 11 \quad 12: 37 \quad 591-78-6$ $09 / 29 / 11 \quad 12: 37 \quad 98-82-8$ $09 / 29 / 11 \quad 12: 37$ 99-87-6 $09 / 29 / 11 \quad 12: 37 \quad 75-09-2$ $09 / 29 / 11 \quad 12: 37 \quad 108-10-1$ 


\section{ANALYTICAL RESULTS}

Project: Inman KS/8A727-A5-167

Pace Project No.: 60106571

Sample: INROLLR-S-30862 Lab ID: 60106571003 Collected: 09/20/11 16:00 Received: 09/21/11 09:00 Matrix: Solid

Results reported on a "dry-weight" basis

\begin{tabular}{|c|c|c|c|c|c|c|c|c|}
\hline Parameters & Results & Units & Report Limit & $\mathrm{DF}$ & Prepared & Analyzed & CAS No. & Qual \\
\hline
\end{tabular}

\section{MSV 5035A VOA}

Methyl-tert-butyl ether

Naphthalene

n-Propylbenzene

Styrene

1,1,1,2-Tetrachloroethane

1,1,2,2-Tetrachloroethane

Tetrachloroethene

Toluene

1,2,3-Trichlorobenzene

1,2,4-Trichlorobenzene

1,1,1-Trichloroethane

1,1,2-Trichloroethane

Trichloroethene

Trichlorofluoromethane

1,2,3-Trichloropropane

1,2,4-Trimethylbenzene

1,3,5-Trimethylbenzene

Vinyl chloride

Xylene (Total)

Dibromofluoromethane (S)

Toluene-d8 (S)

4-Bromofluorobenzene (S)

1,2-Dichloroethane-d4 (S)

Percent Moisture

Percent Moisture
Analytical Method: EPA 8260

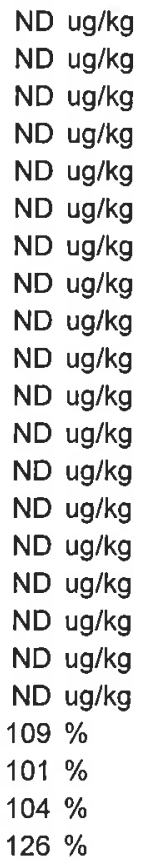

ND ug $/ \mathrm{kg}$

ND ug/kg

ND ug/kg

ND ug/kg

ND ug $/ \mathrm{kg}$

$\mathrm{g} / \mathrm{kg}$

ND ug $/ \mathrm{kg}$

ND ug $/ \mathrm{kg}$

ND ug $/ \mathrm{kg}$

ND $u g / \mathrm{kg}$

ND ug/kg

ND ug/kg

ND ug $/ \mathrm{kg}$

ND $u g / k g$

ND ug/kg

$101 \%$

$126 \%$

Analytical Method: ASTM D2974-87

$\begin{array}{rr}5.4 & 1 \\ 10.8 & 1 \\ 5.4 & 1 \\ 5.4 & 1 \\ 5.4 & 1 \\ 5.4 & 1 \\ 5.4 & 1 \\ 5.4 & 1 \\ 5.4 & 1 \\ 5.4 & 1 \\ 5.4 & 1 \\ 5.4 & 1 \\ 5.4 & 1 \\ 5.4 & 1 \\ 5.4 & 1 \\ 5.4 & 1 \\ 5.4 & 1 \\ 5.4 & 1 \\ 5.4 & 1 \\ 68-129 & 1 \\ 81-121 & 1 \\ 75-131 & 1 \\ 77-131 & 1\end{array}$

$0.50 \quad 1$
09/29/11 12:37 1634-04-4

09/29/11 12:37 91-20-3

$09 / 29 / 11$ 12:37 103-65-1

$09 / 29 / 11 \quad 12: 37 \quad 100-42-5$

09/29/11 12:37 630-20-6

$09 / 29 / 11 \quad 12: 37 \quad 79-34-5$

$09 / 29 / 11 \quad 12: 37 \quad 127-18-4$

09/29/11 12:37 108-88-3

$09 / 29 / 11 \quad 12: 37 \quad 87-61-6$

09/29/11 12:37 120-82-1

09/29/11 12:37 71-55-6

$09 / 29 / 11$ 12:37 79-00-5

$09 / 29 / 11$ 12:37 79-01-6

$09 / 29 / 11 \quad 12: 37 \quad 75-69-4$

09/29/11 12:37 96-18-4

$09 / 29 / 11$ 12:37 95-63-6

09/29/11 12:37 108-67-8

$09 / 29 / 11 \quad 12: 37 \quad 75-01-4$

$09 / 29 / 11 \quad 12: 37 \quad 1330-20-7$

09/29/11 12:37 1868-53-7

$09 / 29 / 11 \quad 12: 37 \quad 2037-26-5$

09/29/11 12:37 460-00-4

09/29/11 12:37 17060-07-0
$7.2 \%$
$09 / 26 / 1100: 00$ 


\section{QUALITY CONTROL DATA}

Project: Inman KS/8A727-A5-167

Pace Project No.: 60106571

\begin{tabular}{llll}
\hline QC Batch: & OEXT/30355 & Analysis Method: & EPA 504.1 \\
QC Batch Method: & EPA 504.1 & Analysis Description: & GCS 504 EDB DBCP
\end{tabular}

Associated Lab Samples: 60106571001

METHOD BLANK: $881162 \quad$ Matrix: Water

Associated Lab Samples: $\quad 60106571001$

$\frac{\text { Parameter }}{\text { 1,2-Dibromoethane (EDB) }} \frac{\text { Units }}{\mathrm{ug} / \mathrm{L}} \frac{\begin{array}{l}\text { Blank } \\ \text { Result }\end{array}}{\mathrm{ND}} \frac{\begin{array}{c}\text { Reporting } \\ \text { Limit }\end{array}}{0.030} \frac{\text { Analyzed }}{09 / 26 / 1117: 57} \frac{\text { Qualifiers }}{1}$

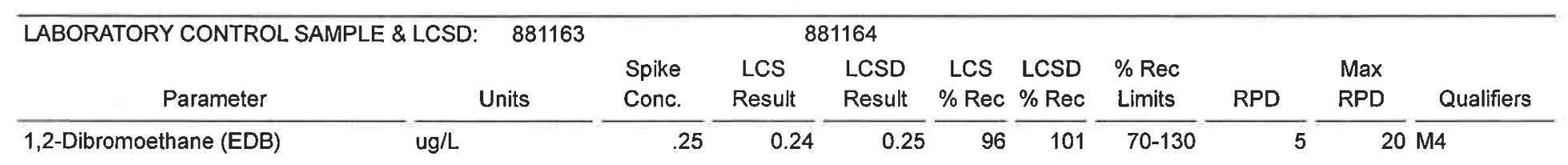




\title{
QUALITY CONTROL DATA
}

\author{
Project: Inman KS/8A727-A5-167
}

Pace Project No.: 60106571

\begin{tabular}{llll}
\hline QC Batch: & MSV/40401 & Analysis Method: & EPA 5030B/8260 \\
QC Batch Method: & EPA 5030B/8260 & Analysis Description: & 8260 MSV Water 7 day
\end{tabular}

Associated Lab Samples: $\quad 60106571001$

METHOD BLANK: $882014 \quad$ Matrix: Water

Associated Lab Samples: $\quad 60106571001$

\begin{tabular}{|c|c|c|c|c|c|}
\hline Parameter & Units & $\begin{array}{l}\text { Blank } \\
\text { Result }\end{array}$ & $\begin{array}{l}\text { Reporting } \\
\text { Limit }\end{array}$ & Analyzed & Qualifiers \\
\hline 1,1,1,2-Tetrachloroethane & $u g / L$ & ND & 1.0 & $09 / 27 / 1110: 53$ & \\
\hline 1,1,1-Trichloroethane & $u g / L$ & ND & 1.0 & $09 / 27 / 1110: 53$ & \\
\hline 1,1,2,2-Tetrachloroethane & ug/L & ND & 1.0 & $09 / 27 / 1110: 53$ & \\
\hline 1,1,2-Trichloroethane & ug/L & ND & 1.0 & $09 / 27 / 1110: 53$ & \\
\hline 1,1-Dichloroethane & ug/L & ND & 1.0 & $09 / 27 / 1110: 53$ & \\
\hline 1,1-Dichloroethene & ug/L & ND & 1.0 & $09 / 27 / 1110: 53$ & \\
\hline 1,1-Dichloropropene & ug/L & ND & 1.0 & $09 / 27 / 1110: 53$ & \\
\hline 1,2,3-Trichlorobenzene & $\mathrm{ug} / \mathrm{L}$ & ND & 1.0 & $09 / 27 / 1110: 53$ & \\
\hline 1,2,3-Trichloropropane & $\mathrm{ug} / \mathrm{L}$ & ND & 2.5 & $09 / 27 / 1110: 53$ & \\
\hline 1,2,4-Trichlorobenzene & ug/L & ND & 1.0 & $09 / 27 / 1110: 53$ & \\
\hline 1,2,4-Trimethylbenzene & $\mathrm{ug} / \mathrm{L}$ & ND & 1.0 & $09 / 27 / 1110: 53$ & \\
\hline 1,2-Dibromo-3-chloropropane & ug/L & ND & 2.5 & $09 / 27 / 1110: 53$ & \\
\hline 1,2-Dibromoethane (EDB) & $\mathrm{ug} / \mathrm{L}$ & ND & 1.0 & $09 / 27 / 1110: 53$ & \\
\hline 1,2-Dichlorobenzene & $\mathrm{ug} / \mathrm{L}$ & ND & 1.0 & $09 / 27 / 1110: 53$ & \\
\hline 1,2-Dichloroethane & $\mathrm{ug} / \mathrm{L}$ & ND & 1.0 & $09 / 27 / 1110: 53$ & \\
\hline 1,2-Dichloroethene (Total) & $\mathrm{ug} / \mathrm{L}$ & ND & 1.0 & $09 / 27 / 1110: 53$ & \\
\hline 1,2-Dichloropropane & $u g / L$ & ND & 1.0 & $09 / 27 / 1110: 53$ & \\
\hline 1,3,5-Trimethylbenzene & $\mathrm{ug} / \mathrm{L}$ & ND & 1.0 & $09 / 27 / 1110: 53$ & \\
\hline 1,3-Dichlorobenzene & $\mathrm{ug} / \mathrm{L}$ & ND & 1.0 & $09 / 27 / 1110: 53$ & \\
\hline 1,3-Dichloropropane & $u g / L$ & ND & 1.0 & $09 / 27 / 1110: 53$ & \\
\hline 1,4-Dichlorobenzene & $\mathrm{ug} / \mathrm{L}$ & ND & 1.0 & $09 / 27 / 1110: 53$ & \\
\hline 2,2-Dichloropropane & $\mathrm{ug} / \mathrm{L}$ & ND & 1.0 & $09 / 27 / 1110: 53$ & \\
\hline 2-Butanone (MEK) & $u g / L$ & ND & 10.0 & $09 / 27 / 1110: 53$ & \\
\hline 2-Chlorotoluene & $u g / L$ & ND & 1.0 & $09 / 27 / 1110: 53$ & \\
\hline 2-Hexanone & ug/L & ND & 10.0 & $09 / 27 / 1110: 53$ & \\
\hline 4-Chlorotoluene & $u g / L$ & ND & 1.0 & $09 / 27 / 1110: 53$ & \\
\hline 4-Methyl-2-pentanone (MIBK) & $\mathrm{ug} / \mathrm{L}$ & ND & 10.0 & $09 / 27 / 1110: 53$ & \\
\hline Acetone & $\mathrm{ug} / \mathrm{L}$ & ND & 10.0 & $09 / 27 / 1110: 53$ & \\
\hline Benzene & $\mathrm{ug} / \mathrm{L}$ & ND & 1.0 & $09 / 27 / 1110: 53$ & \\
\hline Bromobenzene & $u g / L$ & ND & 1.0 & $09 / 27 / 1110: 53$ & \\
\hline Bromochloromethane & ug/L & ND & 1.0 & $09 / 27 / 1110: 53$ & \\
\hline Bromodichloromethane & $u g / L$ & ND & 1.0 & $09 / 27 / 1110: 53$ & \\
\hline Bromoform & $\mathrm{ug} / \mathrm{L}$ & ND & 1.0 & $09 / 27 / 1110: 53$ & \\
\hline Bromomethane & $u g / L$ & ND & 1.0 & $09 / 27 / 1110: 53$ & \\
\hline Carbon disulfide & $u g / L$ & ND & 5.0 & $09 / 27 / 1110: 53$ & \\
\hline Carbon tetrachloride & $u g / L$ & ND & 1.0 & $09 / 27 / 1110: 53$ & \\
\hline Chlorobenzene & $\mathrm{ug} / \mathrm{L}$ & ND & 1.0 & $09 / 27 / 1110: 53$ & \\
\hline Chloroethane & $u g / L$ & ND & 1.0 & $09 / 27 / 1110: 53$ & \\
\hline Chloroform & $u g / L$ & ND & 1.0 & $09 / 27 / 1110: 53$ & \\
\hline Chloromethane & $\mathrm{ug} / \mathrm{L}$ & ND & 1.0 & $09 / 27 / 1110: 53$ & \\
\hline cis-1,2-Dichloroethene & $\mathrm{ug} / \mathrm{L}$ & ND & 1.0 & $09 / 27 / 1110: 53$ & \\
\hline cis-1,3-Dichloropropene & $u g / L$ & ND & 1.0 & $09 / 27 / 1110: 53$ & \\
\hline Dibromochloromethane & $u g / L$ & ND & 1.0 & $09 / 27 / 1110: 53$ & \\
\hline
\end{tabular}

Date: 10/03/2011 03:27 PM 


\section{QUALITY CONTROL DATA}

Project: Inman KS/8A727-A5-167

Pace Project No: $\quad 60106571$

METHOD BLANK: 882014

Matrix: Water

Associated Lab Samples: $\quad 60106571001$

\begin{tabular}{|c|c|c|c|c|c|}
\hline Parameter & Units & $\begin{array}{l}\text { Blank } \\
\text { Result }\end{array}$ & $\begin{array}{l}\text { Reporting } \\
\text { Limit }\end{array}$ & Analyzed & Qualifiers \\
\hline Dibromomethane & $\mathrm{ug} / \mathrm{L}$ & ND & 1.0 & $09 / 27 / 1110: 53$ & \\
\hline Dichlorodifluoromethane & ug/L & ND & 1.0 & $09 / 27 / 1110: 53$ & \\
\hline Ethylbenzene & $u g / L$ & ND & 1.0 & $09 / 27 / 1110: 53$ & \\
\hline Hexachloro-1,3-butadiene & $\mathrm{ug} / \mathrm{L}$ & ND & 1.0 & $09 / 27 / 11 \quad 10: 53$ & \\
\hline Isopropylbenzene (Cumene) & $u g / L$ & ND & 1.0 & $09 / 27 / 1110: 53$ & \\
\hline Methyl-tert-butyl ether & $\mathrm{ug} / \mathrm{L}$ & ND & 1.0 & $09 / 27 / 1110: 53$ & \\
\hline Methylene chloride & $\mathrm{ug} / \mathrm{L}$ & ND & 1.0 & $09 / 27 / 1110: 53$ & \\
\hline n-Butylbenzene & $u g / L$ & ND & 1.0 & $09 / 27 / 1110: 53$ & \\
\hline n-Propylbenzene & $\mathrm{ug} / \mathrm{L}$ & ND & 1.0 & $09 / 27 / 1110: 53$ & \\
\hline Naphthalene & $\mathrm{ug} / \mathrm{L}$ & ND & 10.0 & $09 / 27 / 1110: 53$ & \\
\hline p-Isopropyltoluene & $\mathrm{ug} / \mathrm{L}$ & ND & 1.0 & $09 / 27 / 1110: 53$ & \\
\hline sec-Butylbenzene & $\mathrm{ug} / \mathrm{L}$ & ND & 1.0 & $09 / 27 / 1110: 53$ & \\
\hline Styrene & $\mathrm{ug} / \mathrm{L}$ & ND & 1.0 & $09 / 27 / 1110: 53$ & \\
\hline tert-Butylbenzene & $\mathrm{ug} / \mathrm{L}$ & ND & 1.0 & $09 / 27 / 1110: 53$ & \\
\hline Tetrachloroethene & $\mathrm{ug} / \mathrm{L}$ & ND & 1.0 & $09 / 27 / 1110: 53$ & \\
\hline Toluene & $\mathrm{ug} / \mathrm{L}$ & ND & 1.0 & $09 / 27 / 1110: 53$ & \\
\hline trans-1,2-Dichloroethene & $\mathrm{ug} / \mathrm{L}$ & ND & 1.0 & $09 / 27 / 1110: 53$ & \\
\hline trans-1,3-Dichloropropene & $\mathrm{ug} / \mathrm{L}$ & ND & 1.0 & $09 / 27 / 1110: 53$ & \\
\hline Trichloroethene & ug/L & ND & 1.0 & $09 / 27 / 1110: 53$ & \\
\hline Trichlorofluoromethane & $\mathrm{ug} / \mathrm{L}$ & ND & 1.0 & $09 / 27 / 1110: 53$ & \\
\hline Vinyl chloride & $\mathrm{ug} / \mathrm{L}$ & ND & 1.0 & $09 / 27 / 1110: 53$ & \\
\hline Xylene (Total) & $\mathrm{ug} / \mathrm{L}$ & ND & 3.0 & $09 / 27 / 1110: 53$ & \\
\hline 1,2-Dichloroethane-d4 (S) & $\%$ & 102 & $82-119$ & $09 / 27 / 1110: 53$ & \\
\hline 4-Bromofluorobenzene (S) & $\%$ & 106 & $87-113$ & $09 / 27 / 11 \quad 10: 53$ & \\
\hline Dibromofluoromethane (S) & $\%$ & 96 & $86-112$ & $09 / 27 / 1110: 53$ & \\
\hline Toluene-d8 (S) & $\%$ & 99 & $90-110$ & $09 / 27 / 1110: 53$ & \\
\hline
\end{tabular}

\begin{tabular}{|c|c|c|c|c|c|c|}
\hline \multirow{2}{*}{$\begin{array}{c}\text { LABORATORY CONTROL SAMPLE: } \\
\text { Parameter }\end{array}$} & \multicolumn{6}{|l|}{882015} \\
\hline & Units & $\begin{array}{l}\text { Spike } \\
\text { Conc. }\end{array}$ & $\begin{array}{l}\text { LCS } \\
\text { Result }\end{array}$ & $\begin{array}{l}\text { LCS } \\
\% \operatorname{Rec}\end{array}$ & $\begin{array}{l}\% \text { Rec } \\
\text { Limits }\end{array}$ & Qualifiers \\
\hline $1,1,1,2$-Tetrachloroethane & $\mathrm{ug} / \mathrm{L}$ & 20 & 18.5 & 92 & $81-121$ & \\
\hline 1,1,1-Trichloroethane & $u g / L$ & 20 & 17.5 & 87 & $82-119$ & \\
\hline 1,1,2,2-Tetrachloroethane & $u g / L$ & 20 & 19.6 & 98 & $78-124$ & \\
\hline 1,1,2-Trichloroethane & $u g / L$ & 20 & 17.9 & 89 & $79-121$ & \\
\hline 1,1-Dichloroethane & $\mathrm{ug} / \mathrm{L}$ & 20 & 17.8 & 89 & $73-119$ & \\
\hline 1,1-Dichloroethene & $\mathrm{ug} / \mathrm{L}$ & 20 & 15.6 & 78 & $75-120$ & \\
\hline 1,1-Dichloropropene & $\mathrm{ug} / \mathrm{L}$ & 20 & 18.1 & 91 & $79-123$ & \\
\hline 1,2,3-Trichlorobenzene & $u g / L$ & 20 & 20.2 & 101 & $73-122$ & \\
\hline 1,2,3-Trichloropropane & $u g / L$ & 20 & 19.6 & 98 & $77-124$ & \\
\hline 1,2,4-Trichlorobenzene & $\mathrm{ug} / \mathrm{L}$ & 20 & 20.0 & 100 & $75-120$ & \\
\hline $1,2,4$-Trimethylbenzene & $\mathrm{ug} / \mathrm{L}$ & 20 & 20.4 & 102 & $77-120$ & \\
\hline 1,2-Dibromo-3-chloropropane & $\mathrm{ug} / \mathrm{L}$ & 20 & 19.4 & 97 & $69-125$ & \\
\hline 1,2-Dibromoethane (EDB) & $u g / L$ & 20 & 18.8 & 94 & $85-121$ & \\
\hline 1,2-Dichlorobenzene & $u g / L$ & 20 & 19.8 & 99 & $82-115$ & \\
\hline 1,2-Dichloroethane & $u g / L$ & 20 & 18.9 & 95 & $77-125$ & \\
\hline
\end{tabular}




\section{QUALITY CONTROL DATA}

Project: Inman KS/8A727-A5-167

Pace Project No.: 60106571

\begin{tabular}{|c|c|c|c|c|c|c|}
\hline \multirow{2}{*}{$\begin{array}{c}\text { LABORATORY CONTROL SAMPLE: } \\
\text { Parameter }\end{array}$} & \multicolumn{6}{|l|}{882015} \\
\hline & Units & $\begin{array}{l}\text { Spike } \\
\text { Conc. }\end{array}$ & $\begin{array}{l}\text { LCS } \\
\text { Result }\end{array}$ & $\begin{array}{c}\text { LCS } \\
\% \operatorname{Rec}\end{array}$ & $\begin{array}{l}\% \text { Rec } \\
\text { Limits }\end{array}$ & Qualifiers \\
\hline 1,2-Dichloroethene (Total) & $u g / L$ & 40 & 37.7 & 94 & $79-120$ & \\
\hline 1,2-Dichloropropane & $\mathrm{ug} / \mathrm{L}$ & 20 & 18.3 & 91 & $83-119$ & \\
\hline 1,3,5-Trimethylbenzene & $u g / L$ & 20 & 19.9 & 100 & $79-121$ & \\
\hline 1,3-Dichlorobenzene & $\mathrm{ug} / \mathrm{L}$ & 20 & 19.5 & 98 & $79-117$ & \\
\hline 1,3-Dichloropropane & $\mathrm{ug} / \mathrm{L}$ & 20 & 17.7 & 88 & $78-116$ & \\
\hline 1,4-Dichlorobenzene & ug/L & 20 & 19.8 & 99 & $83-115$ & \\
\hline 2,2-Dichloropropane & $\mathrm{ug} / \mathrm{L}$ & 20 & 16.8 & 84 & $66-123$ & \\
\hline 2-Butanone (MEK) & ug/L & 100 & 110 & 110 & $43-165$ & \\
\hline 2-Chlorotoluene & ug/L & 20 & 19.7 & 98 & $81-117$ & \\
\hline 2-Hexanone & ug/L & 100 & 115 & 115 & $47-159$ & \\
\hline 4-Chlorotoluene & ug/L & 20 & 19.9 & 99 & $84-116$ & \\
\hline 4-Methyl-2-pentanone (MIBK) & ug/L & 100 & 92.6 & 93 & $71-129$ & \\
\hline Acetone & ug/L & 100 & 97.2 & 97 & $18-192$ & \\
\hline Benzene & $\mathrm{ug} / \mathrm{L}$ & 20 & 18.8 & 94 & $82-117$ & \\
\hline Bromobenzene & $u g / L$ & 20 & 20.2 & 101 & $83-116$ & \\
\hline Bromochloromethane & $u g / L$ & 20 & 17.9 & 90 & $79-121$ & \\
\hline Bromodichloromethane & $u g / L$ & 20 & 17.6 & 88 & $79-114$ & \\
\hline Bromoform & $\mathrm{ug} / \mathrm{L}$ & 20 & 17.7 & 89 & $78-121$ & \\
\hline Bromomethane & $u g / L$ & 20 & 17.0 & 85 & $36-146$ & \\
\hline Carbon disulfide & $u g / L$ & 20 & 18.5 & 93 & $75-138$ & \\
\hline Carbon tetrachloride & $u g / L$ & 20 & 17.4 & 87 & $80-123$ & \\
\hline Chlorobenzene & $u g / L$ & 20 & 18.0 & 90 & $83-121$ & \\
\hline Chloroethane & ug/L & 20 & 18.4 & 92 & $42-166$ & \\
\hline Chloroform & $u g / L$ & 20 & 18.2 & 91 & $82-116$ & \\
\hline Chloromethane & $u g / L$ & 20 & 17.2 & 86 & $32-127$ & \\
\hline cis-1,2-Dichloroethene & $u g / L$ & 20 & 18.4 & 92 & $80-119$ & \\
\hline cis-1,3-Dichloropropene & $u g / L$ & 20 & 17.7 & 88 & $76-119$ & \\
\hline Dibromochloromethane & $u g / L$ & 20 & 18.1 & 90 & $81-123$ & \\
\hline Dibromomethane & ug/L & 20 & 17.7 & 89 & $79-123$ & \\
\hline Dichlorodifluoromethane & $\mathrm{ug} / \mathrm{L}$ & 20 & 16.8 & 84 & $10-163$ & \\
\hline Ethylbenzene & $\mathrm{ug} / \mathrm{L}$ & 20 & 18.3 & 91 & $79-121$ & \\
\hline Hexachloro-1,3-butadiene & $u g / L$ & 20 & 18.4 & 92 & $78-125$ & \\
\hline Isopropylbenzene (Cumene) & $u g / L$ & 20 & 19.9 & 100 & $80-120$ & \\
\hline Methyl-tert-butyl ether & $u g / L$ & 20 & 18.1 & 91 & $78-119$ & \\
\hline Methylene chloride & $u g / L$ & 20 & 18.2 & 91 & $75-118$ & \\
\hline n-Butylbenzene & $\mathrm{ug} / \mathrm{L}$ & 20 & 20.5 & 102 & $80-126$ & \\
\hline n-Propylbenzene & $\mathrm{ug} / \mathrm{L}$ & 20 & 18.6 & 93 & $83-116$ & \\
\hline Naphthalene & $u g / L$ & 20 & 19.5 & 98 & $66-133$ & \\
\hline p-lsopropyltoluene & $\mathrm{ug} / \mathrm{L}$ & 20 & 20.1 & 100 & $77-120$ & \\
\hline sec-Butylbenzene & $\mathrm{ug} / \mathrm{L}$ & 20 & 20.2 & 101 & $81-120$ & \\
\hline Styrene & $u g / L$ & 20 & 19.0 & 95 & $84-115$ & \\
\hline tert-Butylbenzene & $u g / L$ & 20 & 19.3 & 97 & $80-117$ & \\
\hline Tetrachloroethene & $u g / L$ & 20 & 18.6 & 93 & $80-124$ & \\
\hline Toluene & $u g / L$ & 20 & 18.3 & 92 & $80-120$ & \\
\hline trans-1,2-Dichloroethene & $u g / L$ & 20 & 19.3 & 97 & $79-120$ & \\
\hline trans-1,3-Dichloropropene & $u g / L$ & 20 & 18.7 & 93 & $76-118$ & \\
\hline Trichloroethene & $\mathrm{ug} / \mathrm{L}$ & 20 & 17.2 & 86 & $76-122$ & \\
\hline Trichlorofluoromethane & $\mathrm{ug} / \mathrm{L}$ & 20 & 17.0 & 85 & $72-120$ & \\
\hline
\end{tabular}




\section{QUALITY CONTROL DATA}

$\begin{array}{ll}\text { Project: } & \text { Inman KS/8A727-A5-167 } \\ \text { Pace Project No.: } & 60106571\end{array}$

\begin{tabular}{|c|c|c|c|c|c|c|}
\hline LABORATORY CONTROL SAMPLE: & 882015 & & & & & \\
\hline Parameter & Units & $\begin{array}{l}\text { Spike } \\
\text { Conc. }\end{array}$ & $\begin{array}{l}\text { LCS } \\
\text { Result }\end{array}$ & $\begin{array}{c}\text { LCS } \\
\% \operatorname{Rec}\end{array}$ & $\begin{array}{l}\% \text { Rec } \\
\text { Limits }\end{array}$ & Qualifiers \\
\hline Vinyl chloride & ug/L & 20 & 18.4 & 92 & $57-163$ & \\
\hline Xylene (Total) & $u g / L$ & 60 & 56.5 & 94 & $75-120$ & \\
\hline 1,2-Dichloroethane-d4 (S) & $\%$ & & & 96 & $82-119$ & \\
\hline 4-Bromofluorobenzene (S) & $\%$ & & & 99 & $87-113$ & \\
\hline Dibromofluoromethane (S) & $\%$ & & & 95 & $86-112$ & \\
\hline Toluene-d8 (S) & $\%$ & & & 99 & $90-110$ & \\
\hline
\end{tabular}




\section{QUALITY CONTROL DATA}

Project: Inman KS/8A727-A5-167

Pace Project No.: 60106571

\begin{tabular}{|c|c|c|c|}
\hline QC Batch: & MSV/40456 & Analysis Method: & EPA 8260 \\
\hline QC Batch Method: & EPA 8260 & Analysis Description: & 8260 MSV 5035A Volatile Organics \\
\hline
\end{tabular}

METHOD BLANK: $883230 \quad$ Matrix: Solid

Associated Lab Samples: $\quad 60106571002,60106571003$

\begin{tabular}{|c|c|c|c|c|c|}
\hline Parameter & Units & $\begin{array}{l}\text { Blank } \\
\text { Result }\end{array}$ & $\begin{array}{l}\text { Reporting } \\
\text { Limit }\end{array}$ & Analyzed & Qualifiers \\
\hline 1,1,1,2-Tetrachloroethane & $\mathrm{ug} / \mathrm{kg}$ & ND & 5.0 & 09/29/11 10:05 & \\
\hline $1,1,1$-Trichloroethane & $\mathrm{ug} / \mathrm{kg}$ & ND & 5.0 & $09 / 29 / 11 \quad 10: 05$ & \\
\hline 1,1,2,2-Tetrachloroethane & $\mathrm{ug} / \mathrm{kg}$ & ND & 5.0 & 09/29/11 10:05 & \\
\hline 1,1,2-Trichloroethane & $u g / k g$ & ND & 5.0 & 09/29/11 10:05 & \\
\hline 1,1-Dichloroethane & $\mathrm{ug} / \mathrm{kg}$ & ND & 5.0 & $09 / 29 / 11 \quad 10: 05$ & \\
\hline 1,1-Dichloroethene & $\mathrm{ug} / \mathrm{kg}$ & ND & 5.0 & 09/29/11 10:05 & \\
\hline 1,1-Dichloropropene & $\mathrm{ug} / \mathrm{kg}$ & ND & 5.0 & 09/29/11 10:05 & \\
\hline 1,2,3-Trichlorobenzene & $\mathrm{ug} / \mathrm{kg}$ & ND & 5.0 & 09/29/11 10:05 & \\
\hline 1,2,3-Trichloropropane & $\mathrm{ug} / \mathrm{kg}$ & ND & 5.0 & 09/29/11 10:05 & \\
\hline 1,2,4-Trichlorobenzene & $\mathrm{ug} / \mathrm{kg}$ & ND & 5.0 & 09/29/11 10:05 & \\
\hline 1,2,4-Trimethylbenzene & $\mathrm{ug} / \mathrm{kg}$ & ND & 5.0 & 09/29/11 10:05 & \\
\hline 1,2-Dibromo-3-chloropropane & $\mathrm{ug} / \mathrm{kg}$ & ND & 10.0 & 09/29/11 10:05 & \\
\hline 1,2-Dibromoethane (EDB) & $\mathrm{ug} / \mathrm{kg}$ & ND & 5.0 & 09/29/11 10:05 & \\
\hline 1,2-Dichlorobenzene & $\mathrm{ug} / \mathrm{kg}$ & ND & 5.0 & $09 / 29 / 1110: 05$ & \\
\hline 1,2-Dichloroethane & $\mathrm{ug} / \mathrm{kg}$ & ND & 5.0 & 09/29/11 10:05 & \\
\hline 1,2-Dichloroethene (Total) & $\mathrm{ug} / \mathrm{kg}$ & ND & 5.0 & $09 / 29 / 1110: 05$ & \\
\hline 1,2-Dichloropropane & $\mathrm{ug} / \mathrm{kg}$ & ND & 5.0 & 09/29/11 10:05 & \\
\hline 1,3,5-Trimethylbenzene & $\mathrm{ug} / \mathrm{kg}$ & ND & 5.0 & 09/29/11 10:05 & \\
\hline 1,3-Dichlorobenzene & $\mathrm{ug} / \mathrm{kg}$ & ND & 5.0 & 09/29/11 10:05 & \\
\hline 1,3-Dichloropropane & $\mathrm{ug} / \mathrm{kg}$ & ND & 5.0 & 09/29/11 10:05 & \\
\hline 1,4-Dichlorobenzene & $\mathrm{ug} / \mathrm{kg}$ & ND & 5.0 & 09/29/11 10:05 & \\
\hline 2,2-Dichloropropane & $\mathrm{ug} / \mathrm{kg}$ & ND & 5.0 & 09/29/11 10:05 & \\
\hline 2-Butanone (MEK) & $\mathrm{ug} / \mathrm{kg}$ & ND & 10.0 & 09/29/11 10:05 & \\
\hline 2-Chlorotoluene & $\mathrm{ug} / \mathrm{kg}$ & ND & 5.0 & 09/29/11 10:05 & \\
\hline 2-Hexanone & $\mathrm{ug} / \mathrm{kg}$ & ND & 20.0 & 09/29/11 10:05 & \\
\hline 4-Chlorotoluene & $\mathrm{ug} / \mathrm{kg}$ & ND & 5.0 & $09 / 29 / 1110: 05$ & \\
\hline 4-Methyl-2-pentanone (MIBK) & $\mathrm{ug} / \mathrm{kg}$ & ND & 10.0 & $09 / 29 / 11 \quad 10: 05$ & \\
\hline Acetone & $\mathrm{ug} / \mathrm{kg}$ & ND & 20.0 & $09 / 29 / 1110: 05$ & \\
\hline Benzene & $\mathrm{ug} / \mathrm{kg}$ & ND & 5.0 & 09/29/11 10:05 & \\
\hline Bromobenzene & $\mathrm{ug} / \mathrm{kg}$ & ND & 5.0 & 09/29/11 10:05 & \\
\hline Bromochloromethane & $\mathrm{ug} / \mathrm{kg}$ & ND & 5.0 & 09/29/11 10:05 & \\
\hline Bromodichloromethane & $\mathrm{ug} / \mathrm{kg}$ & ND & 5.0 & 09/29/11 10:05 & \\
\hline Bromoform & $\mathrm{ug} / \mathrm{kg}$ & ND & 5.0 & 09/29/11 10:05 & \\
\hline Bromomethane & $\mathrm{ug} / \mathrm{kg}$ & ND & 5.0 & $09 / 29 / 1110: 05$ & \\
\hline Carbon disulfide & $\mathrm{ug} / \mathrm{kg}$ & ND & 5.0 & $09 / 29 / 1110: 05$ & \\
\hline Carbon tetrachloride & $\mathrm{ug} / \mathrm{kg}$ & ND & 5.0 & 09/29/11 10:05 & \\
\hline Chlorobenzene & $\mathrm{ug} / \mathrm{kg}$ & ND & 5.0 & $09 / 29 / 1110: 05$ & \\
\hline Chloroethane & $\mathrm{ug} / \mathrm{kg}$ & ND & 5.0 & 09/29/11 10:05 & \\
\hline Chloroform & $\mathrm{ug} / \mathrm{kg}$ & ND & 5.0 & 09/29/11 10:05 & \\
\hline Chloromethane & $\mathrm{ug} / \mathrm{kg}$ & ND & 5.0 & 09/29/11 10:05 & \\
\hline cis-1,2-Dichloroethene & $\mathrm{ug} / \mathrm{kg}$ & ND & 5.0 & 09/29/11 10:05 & \\
\hline cis-1,3-Dichloropropene & $\mathrm{ug} / \mathrm{kg}$ & ND & 5.0 & 09/29/11 10:05 & \\
\hline Dibromochloromethane & $u g / \mathrm{kg}$ & ND & 5.0 & $09 / 29 / 11 \quad 10: 05$ & \\
\hline
\end{tabular}

Date: 10/03/2011 03:27 PM 


\section{QUALITY CONTROL DATA}

Project: Inman KS/8A727-A5-167

Pace Project No: $\quad 60106571$

METHOD BLANK: $883230 \quad$ Matrix: Solid

Associated Lab Samples: $\quad 60106571002,60106571003$

\begin{tabular}{|c|c|c|c|c|c|}
\hline Parameter & Units & $\begin{array}{l}\text { Blank } \\
\text { Result }\end{array}$ & $\begin{array}{l}\text { Reporting } \\
\text { Limit }\end{array}$ & Analyzed & Qualifiers \\
\hline Dibromomethane & $\mathrm{ug} / \mathrm{kg}$ & ND & 5.0 & 09/29/11 10:05 & \\
\hline Dichlorodifluoromethane & $\mathrm{ug} / \mathrm{kg}$ & ND & 5.0 & $09 / 29 / 1110: 05$ & \\
\hline Ethylbenzene & $\mathrm{ug} / \mathrm{kg}$ & ND & 5.0 & 09/29/11 10:05 & \\
\hline Hexachloro-1,3-butadiene & $\mathrm{ug} / \mathrm{kg}$ & ND & 5.0 & 09/29/11 10:05 & \\
\hline Isopropylbenzene (Cumene) & $\mathrm{ug} / \mathrm{kg}$ & ND & 5.0 & 09/29/11 10:05 & \\
\hline Methyl-tert-butyl ether & $\mathrm{ug} / \mathrm{kg}$ & ND & 5.0 & $09 / 29 / 1110: 05$ & \\
\hline Methylene chloride & $\mathrm{ug} / \mathrm{kg}$ & ND & 5.0 & 09/29/11 10:05 & \\
\hline n-Butylbenzene & $\mathrm{ug} / \mathrm{kg}$ & ND & 5.0 & 09/29/11 10:05 & \\
\hline n-Propylbenzene & $\mathrm{ug} / \mathrm{kg}$ & ND & 5.0 & 09/29/11 10:05 & \\
\hline Naphthalene & $\mathrm{ug} / \mathrm{kg}$ & ND & 10.0 & 09/29/11 10:05 & \\
\hline p-Isopropyltoluene & $\mathrm{ug} / \mathrm{kg}$ & ND & 5.0 & 09/29/11 10:05 & \\
\hline sec-Butylbenzene & $\mathrm{ug} / \mathrm{kg}$ & ND & 5.0 & 09/29/11 10:05 & \\
\hline Styrene & $\mathrm{ug} / \mathrm{kg}$ & ND & 5.0 & $09 / 29 / 1110: 05$ & \\
\hline tert-Butylbenzene & $\mathrm{ug} / \mathrm{kg}$ & ND & 5.0 & 09/29/11 10:05 & \\
\hline Tetrachloroethene & $\mathrm{ug} / \mathrm{kg}$ & ND & 5.0 & $09 / 29 / 11 \quad 10: 05$ & \\
\hline Toluene & $\mathrm{ug} / \mathrm{kg}$ & ND & 5.0 & $09 / 29 / 1110: 05$ & \\
\hline trans-1,2-Dichloroethene & $\mathrm{ug} / \mathrm{kg}$ & ND & 5.0 & 09/29/11 10:05 & \\
\hline trans-1,3-Dichloropropene & $u g / \mathrm{kg}$ & ND & 5.0 & $09 / 29 / 11 \quad 10: 05$ & \\
\hline Trichloroethene & $\mathrm{ug} / \mathrm{kg}$ & ND & 5.0 & $09 / 29 / 1110: 05$ & \\
\hline Trichlorofluoromethane & $\mathrm{ug} / \mathrm{kg}$ & ND & 5.0 & 09/29/11 10:05 & \\
\hline Vinyl chloride & $\mathrm{ug} / \mathrm{kg}$ & ND & 5.0 & $09 / 29 / 1110: 05$ & \\
\hline Xylene (Total) & $\mathrm{ug} / \mathrm{kg}$ & ND & 5.0 & 09/29/11 10:05 & \\
\hline 1,2-Dichloroethane-d4 (S) & $\%$ & 102 & $77-131$ & $09 / 29 / 1110: 05$ & \\
\hline 4-Bromofluorobenzene (S) & $\%$ & 97 & $75-131$ & 09/29/11 10:05 & \\
\hline Dibromofluoromethane (S) & $\%$ & 100 & $68-129$ & $09 / 29 / 1110: 05$ & \\
\hline Toluene-d8 (S) & $\%$ & 100 & $81-121$ & $09 / 29 / 1110: 05$ & \\
\hline
\end{tabular}

\begin{tabular}{|c|c|c|c|c|c|c|}
\hline \multirow{2}{*}{$\begin{array}{c}\text { LABORATORY CONTROL SAMPLE: } \\
\text { Parameter }\end{array}$} & \multicolumn{6}{|l|}{883231} \\
\hline & Units & $\begin{array}{l}\text { Spike } \\
\text { Conc. }\end{array}$ & $\begin{array}{l}\text { LCS } \\
\text { Result }\end{array}$ & $\begin{array}{l}\text { LCS } \\
\% \operatorname{Rec}\end{array}$ & $\begin{array}{l}\% \operatorname{Rec} \\
\text { Limits }\end{array}$ & Qualifiers \\
\hline 1,1,1,2-Tetrachloroethane & $\mathrm{ug} / \mathrm{kg}$ & 100 & 96.6 & 97 & $86-124$ & \\
\hline 1,1,1-Trichloroethane & $\mathrm{ug} / \mathrm{kg}$ & 100 & 102 & 102 & $83-119$ & \\
\hline 1,1,2,2-Tetrachloroethane & $\mathrm{ug} / \mathrm{kg}$ & 100 & 82.6 & 83 & $83-120$ & \\
\hline 1,1,2-Trichloroethane & $\mathrm{ug} / \mathrm{kg}$ & 100 & 95.9 & 96 & $85-120$ & \\
\hline 1,1-Dichloroethane & $\mathrm{ug} / \mathrm{kg}$ & 100 & 98.7 & 99 & $82-118$ & \\
\hline 1,1-Dichloroethene & $\mathrm{ug} / \mathrm{kg}$ & 100 & 87.7 & 88 & $78-125$ & \\
\hline 1,1-Dichloropropene & $\mathrm{ug} / \mathrm{kg}$ & 100 & 105 & 105 & $82-122$ & \\
\hline 1,2,3-Trichlorobenzene & $\mathrm{ug} / \mathrm{kg}$ & 100 & 86.1 & 86 & $81-126$ & \\
\hline 1,2,3-Trichloropropane & $\mathrm{ug} / \mathrm{kg}$ & 100 & 83.5 & 84 & $82-120$ & \\
\hline 1,2,4-Trichlorobenzene & $\mathrm{ug} / \mathrm{kg}$ & 100 & 86.4 & 86 & $74-122$ & \\
\hline 1,2,4-Trimethylbenzene & $\mathrm{ug} / \mathrm{kg}$ & 100 & 96.2 & 96 & $80-120$ & \\
\hline 1,2-Dibromo-3-chloropropane & $\mathrm{ug} / \mathrm{kg}$ & 100 & 88.8 & 89 & $73-120$ & \\
\hline 1,2-Dibromoethane (EDB) & $\mathrm{ug} / \mathrm{kg}$ & 100 & 97.8 & 98 & $85-121$ & \\
\hline 1,2-Dichlorobenzene & $\mathrm{ug} / \mathrm{kg}$ & 100 & 92.9 & 93 & $83-120$ & \\
\hline 1,2-Dichloroethane & $\mathrm{ug} / \mathrm{kg}$ & 100 & 101 & 101 & $80-120$ & \\
\hline
\end{tabular}




\section{QUALITY CONTROL DATA}

Project:

Inman KS/8A727-A5-167

Pace Project No.: 60106571

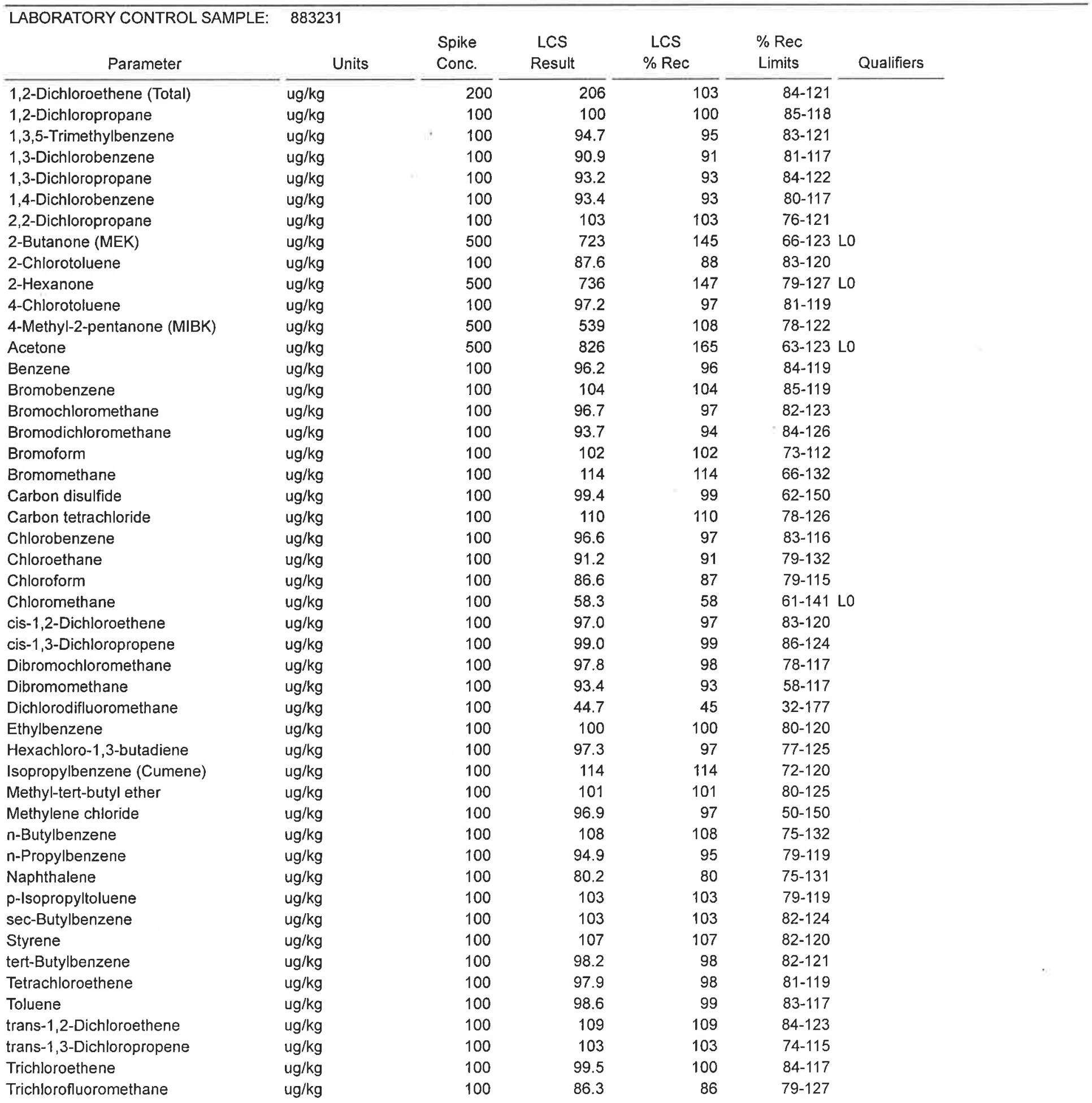




\section{QUALITY CONTROL DATA}

Project: Inman KS/8A727-A5-167

Pace Project No.: 60106571

\begin{tabular}{|c|c|c|c|c|c|c|}
\hline \multirow{2}{*}{$\begin{array}{c}\text { LABORATORY CONTROL SAMPLE: } \\
\text { Parameter }\end{array}$} & \multicolumn{6}{|l|}{883231} \\
\hline & Units & $\begin{array}{l}\text { Spike } \\
\text { Conc. }\end{array}$ & $\begin{array}{l}\text { LCS } \\
\text { Result }\end{array}$ & $\begin{array}{l}\text { LCS } \\
\% \operatorname{Rec}\end{array}$ & $\begin{array}{l}\% \text { Rec } \\
\text { Limits }\end{array}$ & Qualifiers \\
\hline Vinyl chloride & $\mathrm{ug} / \mathrm{kg}$ & 100 & 75.7 & 76 & $67-128$ & \\
\hline Xylene (Total) & $\mathrm{ug} / \mathrm{kg}$ & 300 & 311 & 104 & $80-120$ & \\
\hline 1,2-Dichloroethane-d4 (S) & $\%$ & & & 104 & $77-131$ & \\
\hline 4-Bromofluorobenzene (S) & $\%$ & & & 112 & $75-131$ & \\
\hline Dibromofluoromethane (S) & $\%$ & & & 98 & $68-129$ & \\
\hline Toluene-d8 (S) & $\%$ & & & 100 & $81-121$ & \\
\hline
\end{tabular}




\section{QUALITY CONTROL DATA}

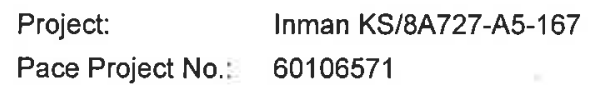

\begin{tabular}{llll}
\hline QC Batch: & PMST/6541 & Analysis Method: & ASTM D2974-87 \\
QC Batch Method: & ASTM D2974-87 & Analysis Description: & Dry Weight/Percent Moisture
\end{tabular}

Associated Lab Samples: $\quad 60106571002,60106571003$

METHOD BLANK: $881122 \quad$ Matrix: Solid

Associated Lab Samples: $\quad 60106571002,60106571003$

$\frac{\text { Parameter }}{\text { Percent Moisture }} \frac{\text { Units }}{\%} \frac{\text { Result }}{N D} \frac{\begin{array}{c}\text { Blank } \\ \text { Limit }\end{array}}{0.50} \frac{\text { Analyzed }}{09 / 26 / 1100: 00}-$ Qualifiers

\begin{tabular}{|c|c|c|c|c|c|c|c|}
\hline \multicolumn{8}{|c|}{ SAMPLE DUPLICATE: 881123} \\
\hline & & & 60106577004 & Dup & & $\operatorname{Max}$ & \\
\hline Parameter & & Units & Result & Result & RPD & RPD & Qualifiers \\
\hline Percent Moisture & & $\%$ & 19.8 & 19.6 & 1 & 20 & \\
\hline
\end{tabular}




\section{QUALITY CONTROL DATA}

Project: Inman KS/8A727-A5-167

Pace Project No.: 60106571

\begin{tabular}{llll}
\hline QC Batch: & WETA/17648 & Analysis Method: & EPA 353.2 \\
QC Batch Method: & EPA 353.2 & Analysis Description: & 353.2 Nitrate + Nitrite, Unpres.
\end{tabular}

Associated Lab Samples: 60106571001

METHOD BLANK: $878783 \quad$ Matrix: Water

Associated Lab Samples: 60106571001

$\frac{\text { Parameter }}{\text { Nitrogen, Nitrate }} \frac{\text { Units }}{\mathrm{mg} / \mathrm{L}} \frac{\text { Result }}{\mathrm{ND}} \frac{\begin{array}{c}\text { Blank } \\ \text { Limit }\end{array}}{0.10} \frac{\text { Analyzed }}{09 / 21 / 1118: 37} \frac{\text { Qualifiers }}{-}$

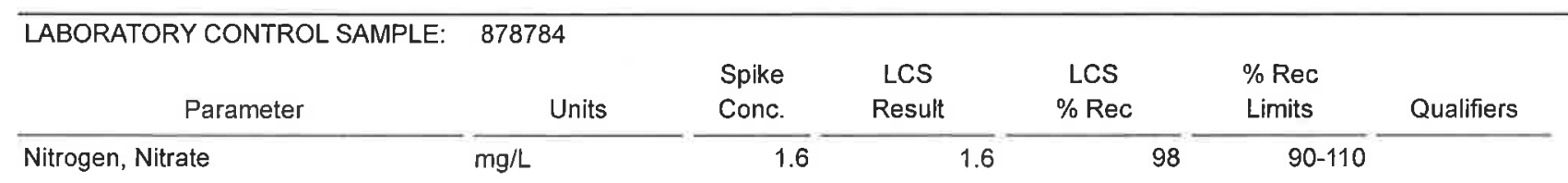

\begin{tabular}{|c|c|c|c|c|c|c|c|}
\hline MATRIX SPIKE SAMPLE: & 878785 & & & & & & \\
\hline Parameter & Units & $\begin{array}{c}60106549001 \\
\text { Result }\end{array}$ & $\begin{array}{l}\text { Spike } \\
\text { Conc. }\end{array}$ & $\begin{array}{c}\text { MS } \\
\text { Result }\end{array}$ & $\begin{array}{c}\text { MS } \\
\% \operatorname{Rec}\end{array}$ & $\begin{array}{l}\% \text { Rec } \\
\text { Limits }\end{array}$ & Qualifiers \\
\hline Nitrogen, Nitrate & $\mathrm{mg} / \mathrm{L}$ & 39.7 & 32 & 71.6 & 100 & $90-110$ & \\
\hline
\end{tabular}

\begin{tabular}{|c|c|c|c|c|c|c|c|}
\hline MATRIX SPIKE SAMPLE: & 878786 & & & & & & \\
\hline Parameter & Units & $\begin{array}{c}60106548001 \\
\text { Result }\end{array}$ & $\begin{array}{l}\text { Spike } \\
\text { Conc. }\end{array}$ & $\begin{array}{c}\text { MS } \\
\text { Result }\end{array}$ & $\begin{array}{c}\text { MS } \\
\% \operatorname{Rec}\end{array}$ & $\begin{array}{l}\% \text { Rec } \\
\text { Limits }\end{array}$ & Qualifiers \\
\hline Nitrogen, Nitrate & $\mathrm{mg} / \mathrm{L}$ & 33.9 & 32 & 62.8 & 90 & $90-110$ & \\
\hline
\end{tabular}

\section{SAMPLE DUPLICATE: $\quad 878787$}

\begin{tabular}{|c|c|c|c|c|c|c|}
\hline & & 60106546001 & Dup & & $\operatorname{Max}$ & \\
\hline Parameter & Units & Result & Result & RPD & RPD & Qualifiers \\
\hline Nitrogen, Nitrate & $\mathrm{mg} / \mathrm{L}$ & 2.5 & 2.5 & 1 & 15 & \\
\hline
\end{tabular}




\section{QUALIFIERS}

Project: $\quad$ Inman KS/8A727-A5-167

Pace Project No:: 60106571

\section{DEFINITIONS}

DF - Dilution Factor, if reported, represents the factor applied to the reported data due to changes in sample preparation, dilution of the sample aliquot, or moisture content.

ND - Not Detected at or above adjusted reporting limit.

$\mathrm{J}$ - Estimated concentration above the adjusted method detection limit and below the adjusted reporting limit.

MDL - Adjusted Method Detection Limit.

$S$ - Surrogate

1,2-Diphenylhydrazine ( 8270 listed analyte) decomposes to Azobenzene.

Consistent with EPA guidelines, unrounded data are displayed and have been used to calculate \% recovery and RPD values.

LCS(D) - Laboratory Control Sample (Duplicate)

MS(D) - Matrix Spike (Duplicate)

DUP - Sample Duplicate

RPD - Relative Percent Difference

NC - Not Calculable.

SG - Silica Gel - Clean-Up

$\mathrm{U}$ - Indicates the compound was analyzed for, but not detected.

$\mathrm{N}$-Nitrosodiphenylamine decomposes and cannot be separated from Diphenylamine using Method 8270 . The result reported for each analyte is a combined concentration.

Pace Analytical is TNI accredited. Contact your Pace PM for the current list of accredited analytes.

\section{BATCH QUALIFIERS}

Batch: MSV/40401

[M5] A matrix spike/matrix spike duplicate was not performed for this batch due to insufficient sample volume.

Batch: MSV/40456

[M5] A matrix spike/matrix spike duplicate was not performed for this batch due to insufficient sample volume.

\section{ANALYTE QUALIFIERS}

LO Analyte recovery in the laboratory control sample (LCS) was outside QC limits.

L2 Analyte recovery in the laboratory control sample (LCS) was below QC limits. Results for this analyte in associated samples may be biased low.

L3 Analyte recovery in the laboratory contral sample (LCS) exceeded QC limits. Analyte presence below reporting limits in associated samples. Results unaffected by high bias.

M4 A matrix spike/matrix spike duplicate was not performed for this batch due to sample dilution. 
Section A

Client Information:

Section Required Project Information:

Company. TCW Construction Adoress: 141 M Street Lincoln NE 68508

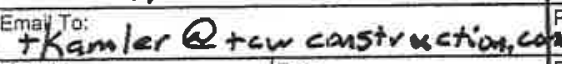
hone: $4167255^{\text {Fax }}$

$402416-255$

Requested Due DaterTAT:$$
\text { Section D }
$$

Required Cilient Information

SAMPLE ID

(A-Z, 0-9/.)

Sample IDS MUST BE UNIQUE

年

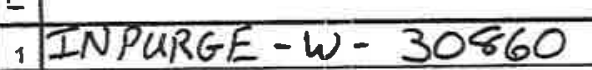

2 Well development water

3 Stored on site in a poly Tank

${ }_{5}$ INROLLF-S - 30861

6 INROLLR - S - 30862

7 Composite Soil samples of

8 monitoring well cuttings from

9 drill rig dumped into a

10 roll off containes

11

11

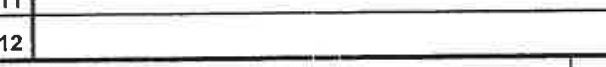

ADDITIONAL COMMENTS

ORIGINAL

$S L C 8 / 22$

SL $C 8 / 22$
tKamler@+cwconstruction.com surgnier@prodigy.net

urchase Order No:

Project Name: Inman $\mathrm{KS}$

$84727-45-167$
Section $C$

Involce Information:

Attention: Travis Kamler compareatei Construction Address: M Street Lincoln NE Pace Quote

Pace Projict Trady Gipson Manager: Trudy Gipson

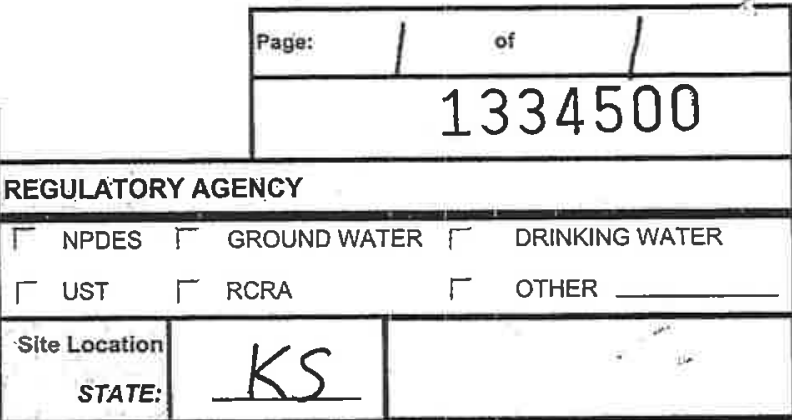

REGULATORY AGENCY

Requested Analysis Filtered (Y/N)

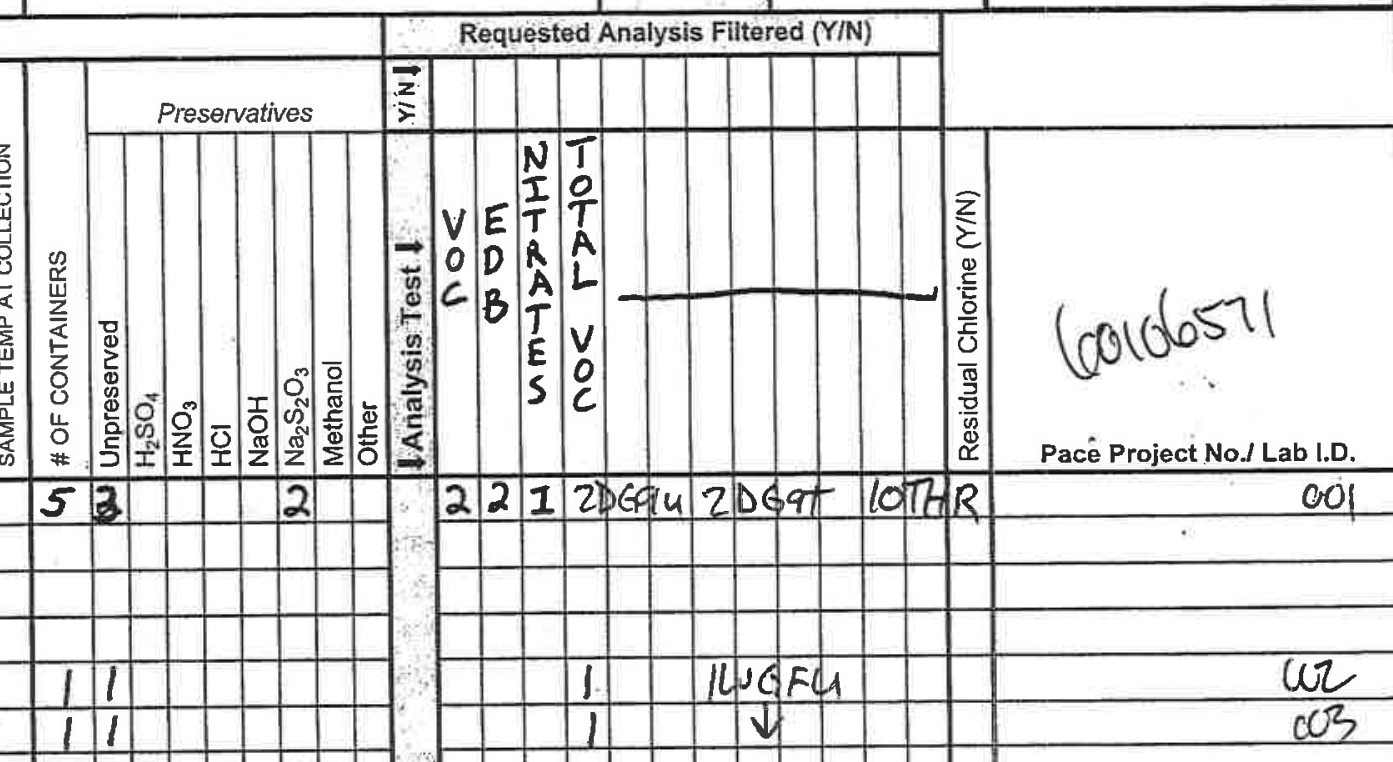

$9 / 20 / 16: 00$

$9 / 2016: 00$

11
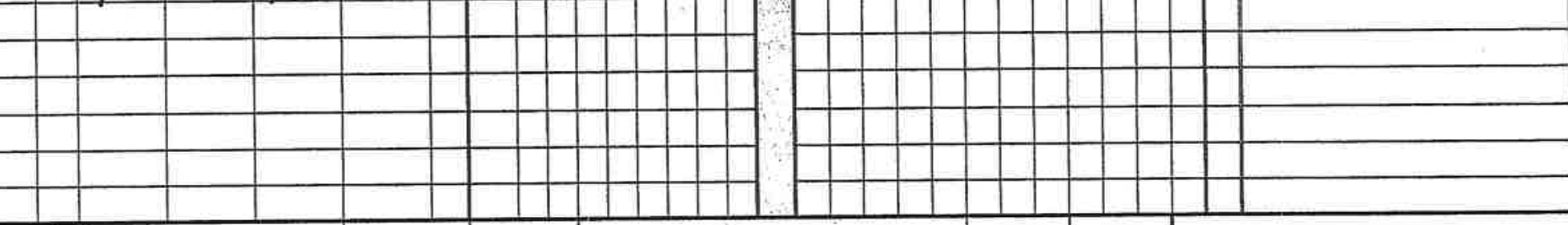

\begin{tabular}{c|c|c|c} 
RELINQUISHED BY/AFFLLATION & DATE & TIME & ACCEPTED BY IA \\
Travis Kamler /TCW & $9-20-11$ & $18: 00$ & pherquecey
\end{tabular}

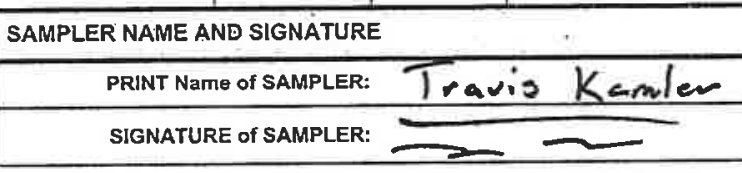

DATE SIgned

$\longrightarrow$ C 


\section{Sample Condition Upon Receipt}

Client Name: T $C_{L}$

Project \# 60106571

courier: fFed Ex $\square$ UPS $\square$ USPS $\square$ client $\square$ Commercial $\square$ Pace $\square$ other
Tracking \#: 875792181268
Pace Shipping Label Used?
Custody Seal on Cooler/Box Present:
Pace
$\square$ No
Seals intact: $\square$ Yes

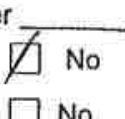

\begin{tabular}{l} 
Optional \\
\hline Proj. Due Date: $\quad 10 / 03$ \\
Proj. Name:
\end{tabular}

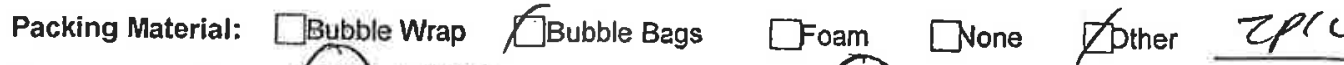

Thermometer Used: T-19) I T-194 Type of Ice: Wet Blue None

Cooler Temperature: 1.8

Temperature should be above freezing to $6^{\circ} \mathrm{C}$

Commențs:

$\square$ Samples on ice, cooling process has begun

Date and Initigls of person examining

Chain of Custody present:

Chain of Custody filled out:

Cres $\square$ No $\square$ N/A 1.

DYes $\square$ No $\square$ N/A 2

Chain of Custody relinquished: $\quad \forall$ Yes $\square$ No $\square$ N/A 3.

Sampler name \& signature on COC: $\quad$ VYes $\square$ No $\square$ N/A 4.

Samples arrived within holding time: $\quad$ f́res $\square$ No $\square$ N/A 5 .

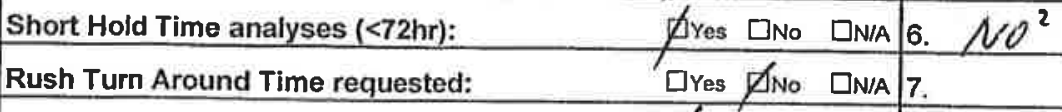

Sufficient volume:

Correct containers used:

-Pace containers used:

Containers intact:

Unpreserved 5035A soils frozen w/in 48hrs?

Filtered volume received for dissolved tests

Sample labels match COC:

-Includes date/time/ID/analyses Matrix:

All containers needing preservation have been checked.

All containers needing preservation are found to be in

compliance with EPA recommendation. Exceptions VOA). coliform, TOC. O\&G, MI-DRO (water),
Phenolics

Trip Blank present:

Pace Trip Blank lot \# (if purchased):

Headspace in VOA vials $(>6 \mathrm{~mm})$ :

Cyes $\square$ No $\square$ N/A 8.

Project sampled in USDA Regulated Area:

DYes $\square$ No $\square \mathrm{N} / \mathrm{A} 9$.

Project sampled in USDA Regulated Area:

Client Notification/ Resolution:

Person Contacted:

byes $\square$ No $\square \mathrm{N} / \mathrm{A}$

Gyes $\square$ No $\square \mathrm{N} / \mathrm{A} 10$.

$\square$ Yes $\square$ No Ø̋́N/A 11.

口Yes $\square$ NO DN/A 12.

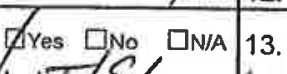

WT/SC

contents: $U,-21-11$

.

.
5.
1.
1.
12.
3.
14.

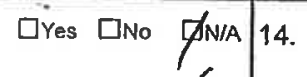

口Yes DNo $\not$ N/A

\begin{tabular}{l|l|l|}
\hline CYes $\square$ No & Lot \# of added when
\end{tabular}

口Yes Cino DN/A 15.

presenvative

Comments/ Resolution:

Project Manager Review:

Note: Whenever there is a discrepancy affecting North Carolina compliance samples, a copy of this form will be sent to the North Carolina DEHNR Certification Office ( i.e out of hold, incorrect preservative, out of temp, incorrect containers) 


\section{Special Waste Disposal Request \\ Kansas Department of Health and Environment \\ Bureau of Waste Management \\ Waste Reduction, Compliance and Enforcement Section \\ 1000 SW Jackson, Suite 320, Topeka, Kansas 66612-1366}

You may FAX this form to: $785-296-8909$ or $785-296-8721$

Please type or clearly print - See page 2 for instructions

I. REQUESTER INFORMATION (This is where the Disposal Authorization letter will be sent.)

Name: TCW Construction

Address: $141 \mathrm{M}$ Street

City: Lincoln

State: NE

Zip Code: 68508

Contact Person: Travis Kamler

Telephone Number: (402) 416-7255

E-Mail Address, if applicable. tkamler@tcwconstruction,com

Fax Number: $\quad$ (402) 475-5049

II. POINT OF GENERATION INFORMATION (only if different from the information in Section ( above)

Name: USDA

Address: 608 S Main Street

City: Inman __ State: KS Kip Code: 67546

Contact Person: Travis Kamler _ـ Telephone Number: (402) 416-7255

III. WASTE INFORMATION - Use back of form if additional space is required

Waste Description: Soil

Process Producing Waste: Monitoring Well Installations

Physical Characteristics of Waste: Solid

Quantity for Disposal: 20

(Please Select One) _Lbs. _Tons X Cubic Yards _Containers

Bags

Frequency (Select One): $\mathrm{X}$. One Time _ Week _..Month _ Year

Laboratory Analyses Attached: $X$ Yes _No Material Safety Data Sheets (MSDS) Attached: _. Yes $X$ No

Renewal of Previous Authorization: Previous Authorization No:

Date Issued:

\section{DISPOSAL INFORMATION}

Landfill Proposed for Disposal: Reno County

Solid Waste Transfer Station Proposed: McPherson Area Solid Waste Utility

\section{CERTIFICATION}

I hereby certify that I am a duly authorized representative of the generator identified above. I further certify that, to the best of my knowledge, the following items are true:

1. The waste identified for disposal is not a hazardous waste as defined by K.A.R. 28-31-3.

2. All analytical analyses provided are from a Kansas Department of Health and Environment (KDHE) certified laboratory and are representalive of the waste identified for disposal.

3. All information provided in any attached profile, re-certification, or other document completed by the authorized represenlative accurately characterizes the waste.

4. If this is a renewal, the materials and processes that generate the waste have not changed since the last disposal authorization indicated above, and the information previously provided to KDHE is still valid.

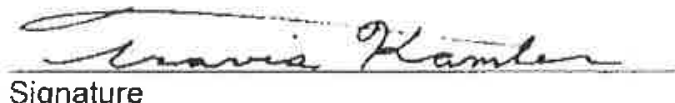

Signature

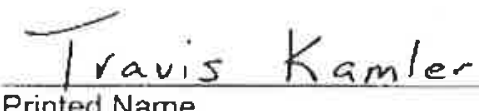

Printed Name $\frac{\text { Nov } 8,2011}{\text { Date }}$ 


\section{Instructions}

If you have any questions about information required to complete this form, please contact the Special Waste Coordinator at 785-296-1600 or send an e-mail to: swda@kdhe.state.ks.us

1. Requester Information - Requester information must be provided for the individual taking responsibility for the waste disposal request. This could be the actual generator of the waste, or a contractor or consultant managing the waste for a client. KDHE will e-mail you a copy of the special waste disposal authorization letter as a portable document file (pdf) if you provide your e-mail address. If you do not provide your e-mail address, we will mail or fax you a copy of the SWDA letter. Please note that you may complete this form on-line at our website; however, you must print the form and submit a signed copy via fax or regular mail.

II. Point of Generation Information - Point of generation information must be provided for the location where the waste is generated. If this information is identical to the information provided in Section I, this section may be left blank or marked "Same".

III. Waste Information - The following information must be provided concerning the waste:

Waste description - Provide a brief description of the waste. For example, "contaminated soil", "wastewater sludge", elc.

Process producing waste - Provide a brief description of the process that produced the waste. For example, "grinding operation", "wastewater treatment plant", "product spill", etc.

Physical Characteristics of Waste - Provide a brief description of the physical make-up of the waste. For example, "gray sludge", or "dark soils with petroleum odor", etc.

Quantity for Disposal - Estimate the quanlity of the waste for disposal in units of pounds, tons, cubic yards, containers, or bags. It is best to slightly overestimate.

Frequency - Indicate approximately how often the waste is to be disposed. If the request is for a one-lime-only disposal, indicate "One Time" even though you may need to make more than one trip to the landfill to complete the disposal.

Laboratory Analyses Attached - Indicate whether laboratory analyses performed by a KDHE certified laboratory are attached. If you have questions whether analyses are required or what analyses are required, please contact the Special Waste Coordinator at 785-296-1600 or send an e-mail to: swda@ kdhe.state.ks.us.

Material Safety Data Sheet (MSDS) Attached - Indicate whether an MSDS for the waste is attached. If you are using an MSDS to support your determination that the waste is not a hazardous waste, the MSDS must be attached.

Renewal of Previous Authorization - If you wish to renew a disposal authorization issued in the prior year, you must complete this section. Be sure to review the previous information (analyses, MSDS, etc.) provided to KDHE to make sure it is still valid.

IV. Disposal Information - The following information must be provided concerning the disposal site for the waste:

Landfill Proposed for Disposal - Indicate the landfill where you wish to dispose the waste. You should contact the landfill for tentative approval of acceptance prior to submitting this form.

Solid Waste Transfer Station Proposed - If the waste will be shipped through transfer station, indicate the name of that station, If the waste will be shipped directly to a landfill, leave this line blank or indicate "NA" for nol applicable.

v. Certification - The certification statement must be signed by an authorized representative of the generator/owner of the waste. This may be a consultant or contractor authorized to sign on behalf of the generator/owner of the waste. 
Mr. Travis Kamler

TCW Construction

$141 \mathrm{M}$ Street

Lincoln, NE 68508

\section{RE: Special Waste Disposal Authorization Number 11-1075}

THIS AUTHORIZATION EXPIRES May 9, 2012.

Dear Mr. Kamler:

We have considered your request for disposal of twenty (20) cubic yards of soil from monitor well intallation at USDA, $608 \mathrm{~S}$ Main St., Inman, KS. (Analysis provided)

Based solely on the analysis provided, the waste is not a characteristic hazardous waste with respect to the constituents tested. As stated in K.A.R.28-31-261, it is the responsibility of the generator to determine whether or not a waste is a hazardous waste by either knowledge of process or by proper testing by a K.D.H.E. certified lab. If there are questions as to the status of this waste, the department suggests the facility contact the Kansas Department of Health and Environment at telephone 316-337-6020. If TCW Construction is confident the material for disposal is not a hazardous waste for any characteristic or listed constituent not included in the testing, the following applies.

Approval is given to dispose of this waste at the Reno County landfill, operating under Kansas Permit 0723, via the McPherson Area Solid Waste Utility Transfer Station operating under Kansas Permit 0779 provided the following conditions are met:

1. Approval to deliver the waste must be obtained from both the transfer station operator and the landfill operator prior to transporting the waste to the transfer station. The final decision on whether to accept or reject the waste rests with the landfill operator. Please contact Steve Graves, Solid Waste Superintendent, telephone 620-694-2586, to obtain approval for landfill disposal, and contact John Hawk, telephone 620-241-6559 to obtain approval for the transfer station. If the transfer station or landfill operator refuses to accept this waste, you should contact us to determine alternate disposal options.

2. The waste must be transported separately to the landfill and be identified to the operator upon delivery.

3. Kansas Administrative Regulation 28-29-108(r) (12) and (13) requires solid waste disposal facilities to maintain a log of commercial or industrial wastes received such as sludges, barreled wastes, and special wastes. The log must indicate the source and quantity of waste and the disposal location thereof. The special waste authorization number should be used as identification when entering the shipment into the log. 
4. This approval is valid for disposal of the waste described and in the amount shown above. If additional shipments are required, you must contact us to receive another disposal authorization.

5. Operating standards as defined by K.A.R. 28-29-108(k) prohibit the disposal of liquid waste. "Liquid waste" means any waste material that is determined to contain "free liquids" as defined by method $9095 \mathrm{~A}$, revision 1, paint filter liquids test, as described in "Test Methods for Evaluating Solid Waste, Physical/Chemical Methods," EPA Pub. No. SW-846 dated December 1996. For purposes of this disposal authorization, all waste for disposal must be able to pass the "paint filter test".

6. Any change in the process producing this waste, any change in the materials used in producing this waste or any other change to this waste stream requires that a new Special Waste Disposal Authorization be obtained prior to disposal.

If you have any questions, feel free to contact me at 785-296-0681.

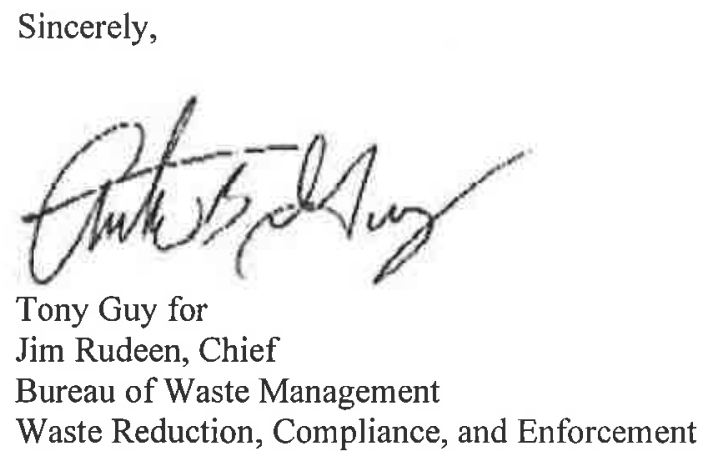

ABG

C Steve Graves
John Hawk
e-file

Requester phone: $402-416-7255$ 


\section{AGEM OI L}

CITY OF SABETHA Cash - Travis 805 MAIN

PO BOX 187

SABETHA KS 66534 785-284-2158

Receipt Na: 2.015320 Dec 19, '2011

TCW Const.

WASTEWATER FUND-MISC

Purged Water

50,00

502-00.000- 6632

MISCELLLANEOUS INCOME

Total:

50.00

Cash

50.00

Total Applied:

50.00

Change Tendered: 


\section{Supplement 4:}

\section{TestAmerica Data}




\section{Contents}

SDG 200-2447.

Page 3 of 866

SDG 200-2472

Page 49 of 866

SDG 200-2608

Page 98 of 866

SDG 200-3113

Page 177 of 866

SDG 200-5308

Page 292 of 866

SDG 200-5726 .

Page 342 of 866

SDG 200-6709

Page 388 of 866

SDG 200-6730

Page 435 of 866

SDG 200-6762

Page 488 of 866

SDG 200-6783

Page 559 of 866

SDG 200-6846

Page 621 of 866

SDG 200-7006

Page 677 of 866

SDG 200-7045

Page 721 of 866

SDG 500-34569

Page 768 of 866

SDG 134662.

Page 793 of 866 


\section{TestAmerica}

THE LEADER IN ENVIRONMENTAL TESTING

\section{ANALYTICAL REPORT}

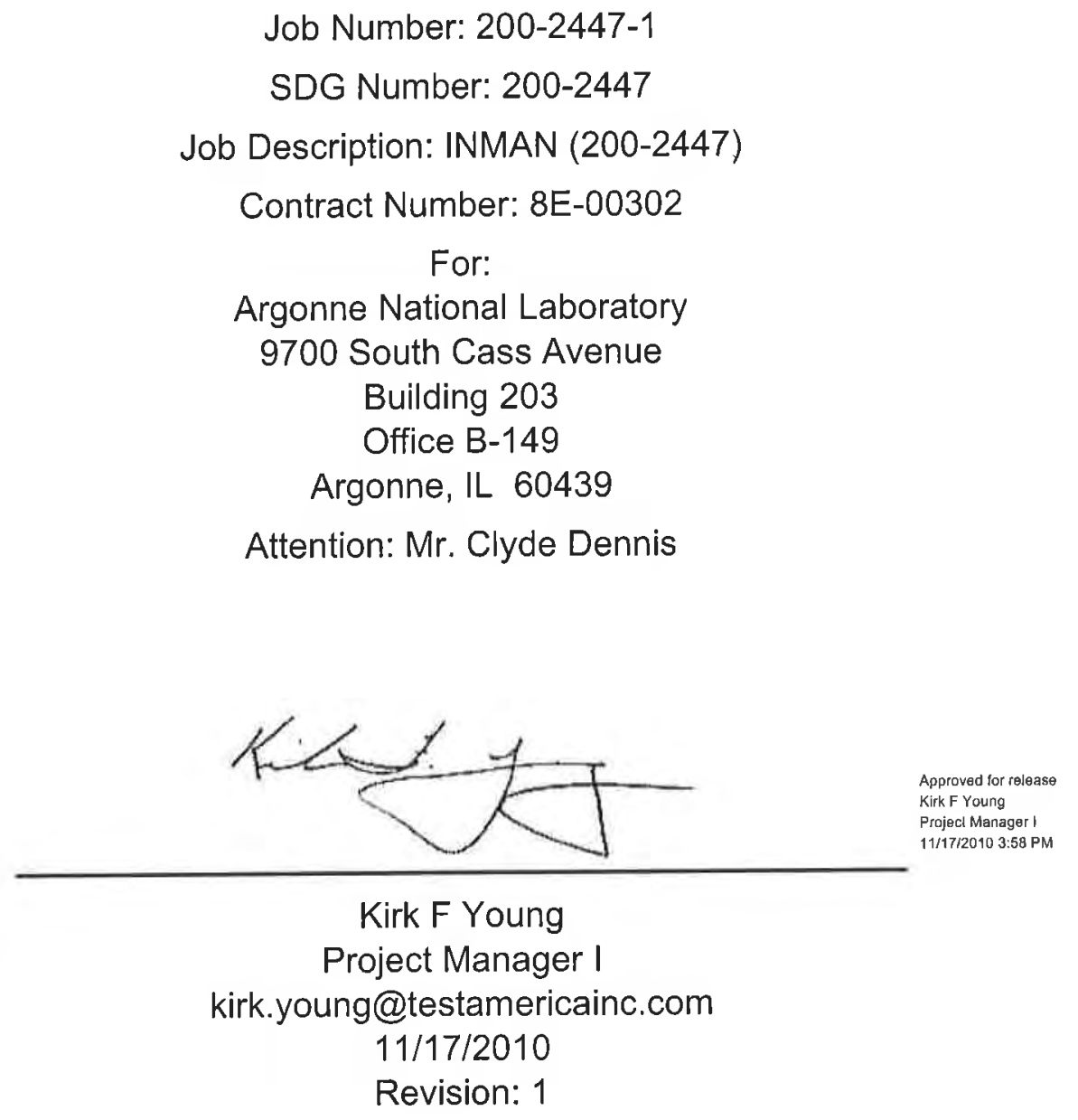

The test results in this report relate only to sample(s) as received by the laboratory. These test results were derived under a quality system that adheres to the requirements of NELAC. Pursuant to NELAC, this report may not be produced in full without written approval from the laboratory 


\section{Table of Contents}

Cover Title Page .......................... 1

Report Narrative . . . . . . . . . . . . . . . . . . 4

Case Narrative ................................ 4

Qualifier Definition ............................... 6

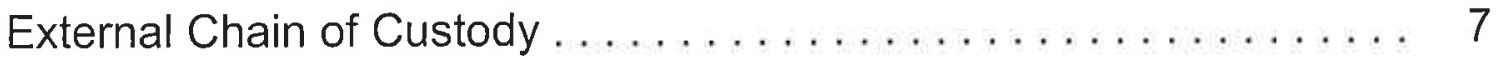

Internal Chain of Custody ..................... 8

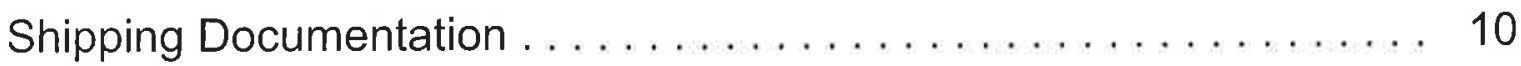

Sample Receipt and Log In Check List . . . . . . . . . . . . . . . . 11

Methodology Review ......................... 13

QC Summary - SOM01.2 Volatiles-Trace . . . . . . . . . . . . . . . . 14

QC Summary - SOM01.2 Volatiles-Trace . . . . . . . . . . . . . . . . 14

Deuterated Monitoring Compound Summary $\ldots \ldots \ldots \ldots \ldots \ldots \ldots \ldots \ldots \ldots \ldots \ldots$

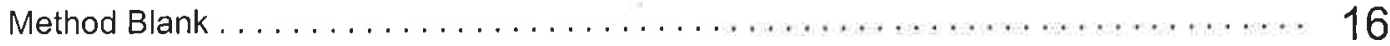

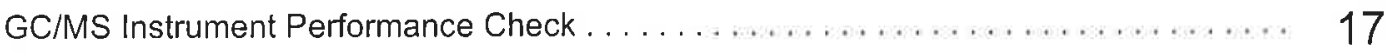

Internal Standard Area and $\mathrm{RT}$ Summary $\ldots \ldots \ldots \ldots \ldots \ldots \ldots \ldots \ldots \ldots \ldots \ldots \ldots \ldots$

Sample Data - SOM01.2 Volatiles-Trace . . . . . . . . . . . . . 20

Sample Data - SOM01.2 Volatiles-Trace ..................... 20

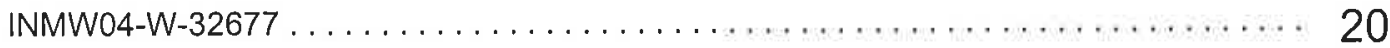

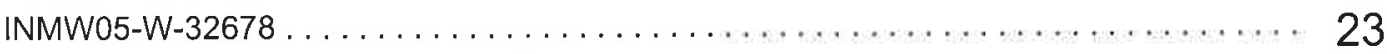

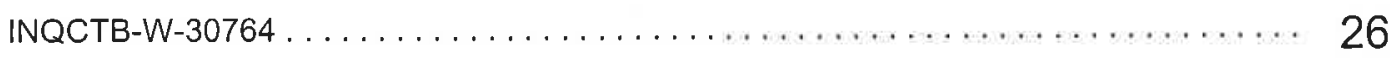

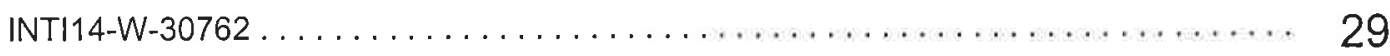

Standards - SOM01.2 Volatiles-Trace . . . . . . . . . . . . . . 32

Standards - SOM01.2 Volatiles-Trace ....................... 32

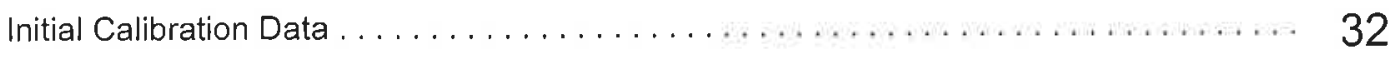

CCV Data, including closing CCV . . . . . . . . . . . . . . . . . . 35

Raw Qc Data - SOM01.2 Volatiles-Trace . . . . . . . . . . . . . . 41 


\section{Table of Contents}

Raw Qc Data - SOM01.2 Volatiles-Trace ...................... 41

Raw Qc Data - SOM01.2 Volatiles-Trace . ........................ 41

Blank Data . . . . . . . . . . . . . . . . . . 41 


\title{
CASE NARRATIVE
}

\author{
Client: Argonne National Laboratory \\ Project: Inman (200-2447)
}

Report Number: 200-2447-1

With the exceptions noted as flags or footnotes, standard analytical protocols were followed in the analysis of the samples and no problems were encountered or anomalies observed.

Calculations are performed before rounding to avoid round-off errors in calculated results.

All holding times were met and proper preservation noted for the methods performed on these samples, unless otherwise detailed in the individual sections below.

\section{Receipt}

The samples were received on 11/11/2010. Documentation of the condition of the samples at the time of their receipt and any exception to the laboratory's Sample Acceptance Policy is documented in the Sample Handling section of this submittal. The samples, as received, were not acid preserved. On that basis, the laboratory did provide for the analytical work to be performed within seven days of sample collection.

\section{SOM01.2 Volatile Organics (Trace Level Water)}

A storage blank was prepared for volatile organics analysis, and stored in association with the storage of the samples. That storage blank, identified as VHBLKO1, was carried through the holding period with the samples, and analyzed.

Each sample was analyzed without a dilution. Each of the analyses associated with the sample set exhibited an acceptable internal standard performance, and there was an acceptable recovery of each deuterated monitoring compound (DMC) in each analysis. Matrix spike and matrix spike duplicate analyses were not performed on samples in this sample set. Trace concentrations of acetone, carbon disulfide, trans-1,2-dichloroethene, 1,4-dichlorobenzene, 1,2,4-trichlorobenzene, and 1,2,3-trichlorobenzene were identified in the analysis of the method blank associated with the analytical work. The concentration of each compound in that analysis was below the established reporting limit, and the analysis did meet the technical acceptance criteria for a compliant method blank analysis. A trace concentration of acetone was identified in the analysis of the storage blank associated with the sample set. The concentration of acetone in that analysis was below the established reporting limit, and the analysis did meet the technical acceptance criteria for a compliant storage blank analysis. Present in the method blank and storage blank analyses was a non-target constituent that represents a compound that is related to the DMC formulation. The fact that the presence of this compound is not within the laboratory's control is at issue. The derived results for that compound have been qualified with an " $\mathrm{X}$ " qualifier to reflect the source of the contamination.

The responses for each of the target analytes met the relative standard deviation criterion in the initial calibration. The response for each target analyte met the percent difference criterion in the continuing calibration check acquisition. The response for each target analyte met the 50.0 percent difference criterion in the closing calibration check acquisition. 
The primary quantitation mass for methylcyclohexane that is specified in the Statement of Work is mass 83 . The laboratory did identify a contribution to mass 83 from 1,2-dichloropropane- $d_{6}$, one of the deuterated monitoring compounds (DMCs). The laboratory did change the primary quantitation mass assignment to mass 55 for the quantification of methylcyclohexane.

Manual integration was employed in deriving certain of the analytical results. The values that have been derived from manual integration are qualified on the quantitation reports. Extracted ion current profiles for each manual integration are included in the data package, and further documented in the Sample Preparation section of this submittal. 


\section{DATA REPORTING QUALIFIERS}

Client: Argonne National Laboratory

Job Number: $200-2447-1$

Sdg Number: $200-2447$

Lab Section

Description

GC/MS VOA

U

J

J

$\mathbf{X}$

B

N
Analyzed for but not detected.

Indicates an Estimated Value for TICs

Indicates an estimated value.

See case narrative notes for explanation of the ' $X$ ' flag

The analyte was found in an associated blank, as well as in the sample.

This flag indicates the presumptive evidence of a compound. 


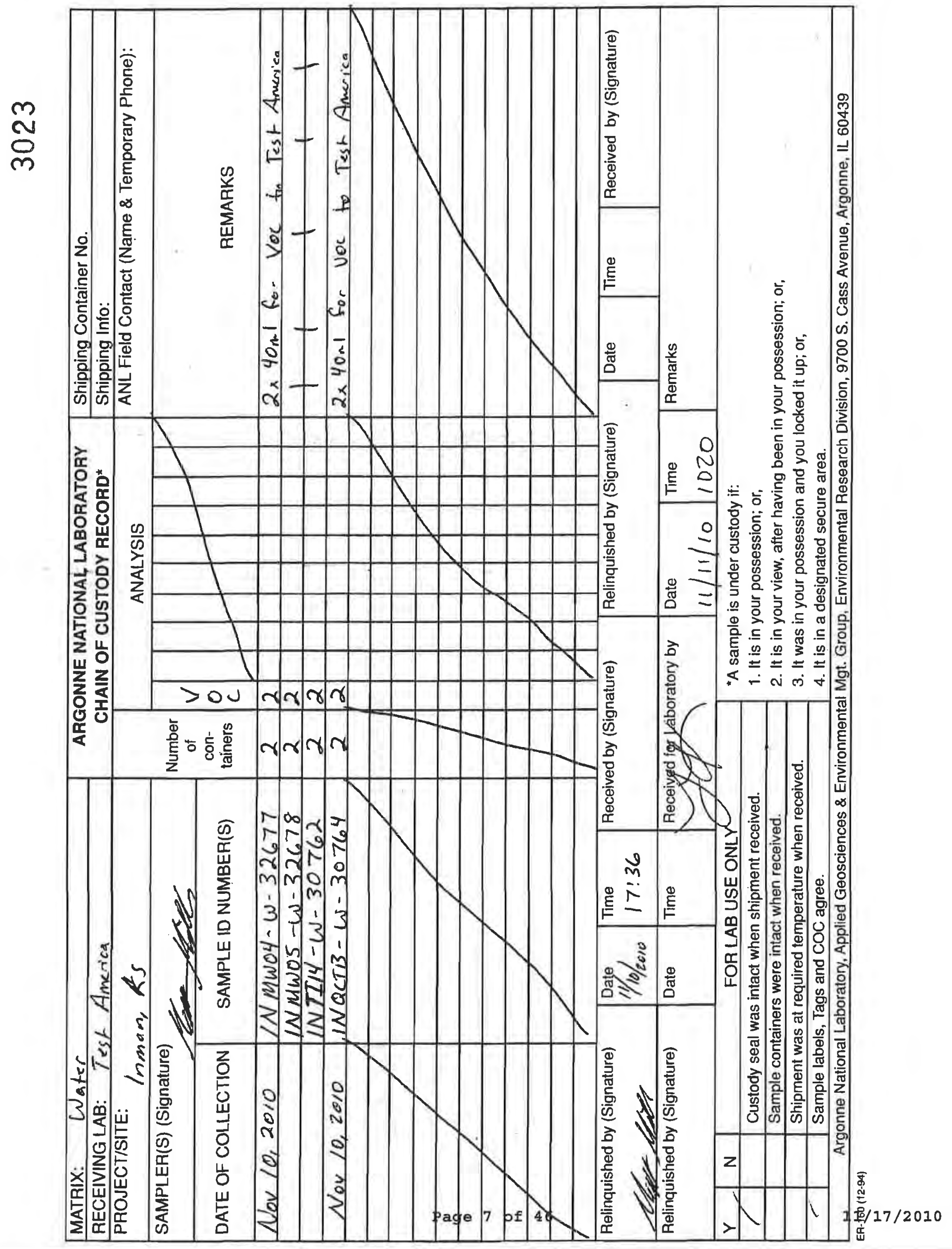




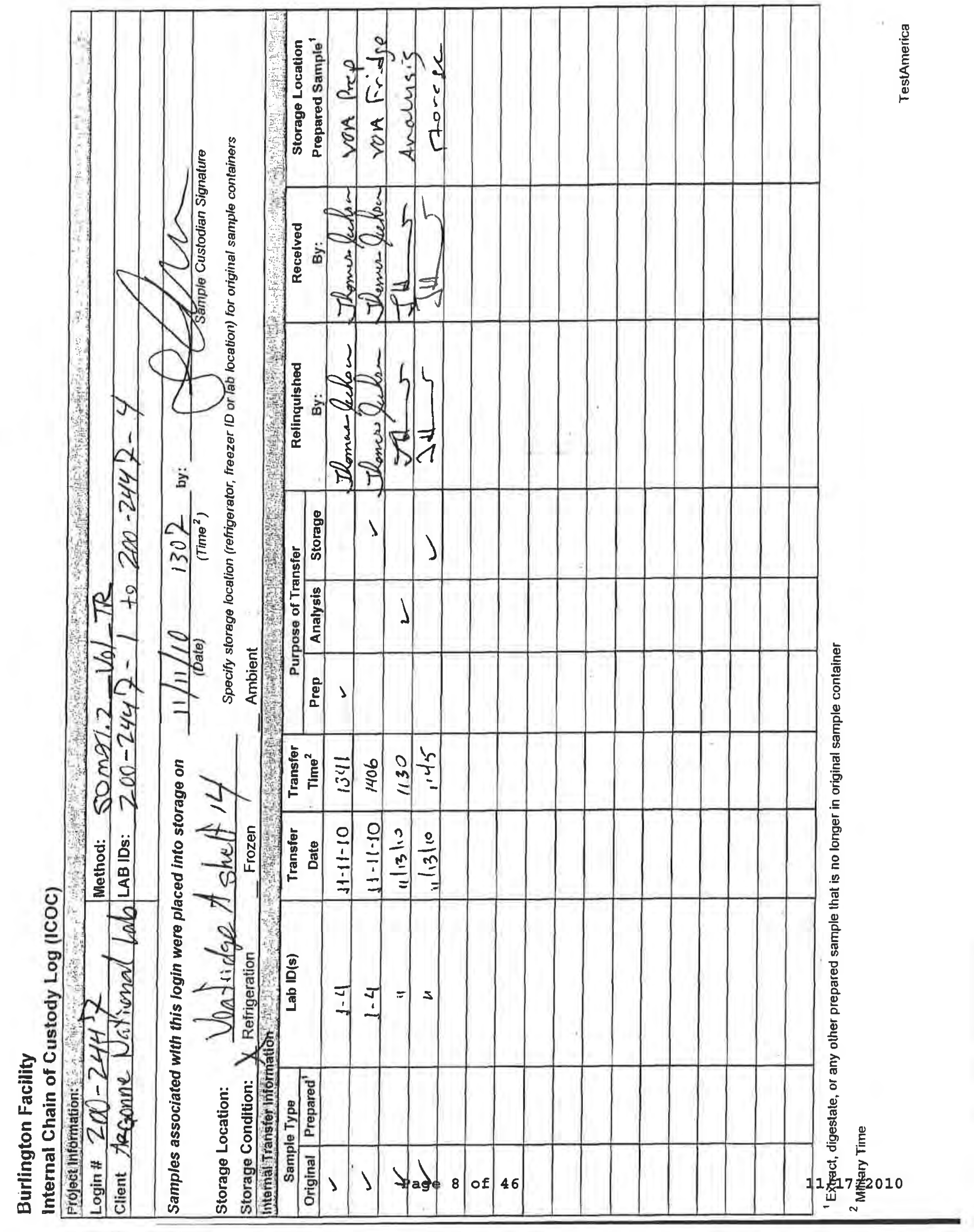




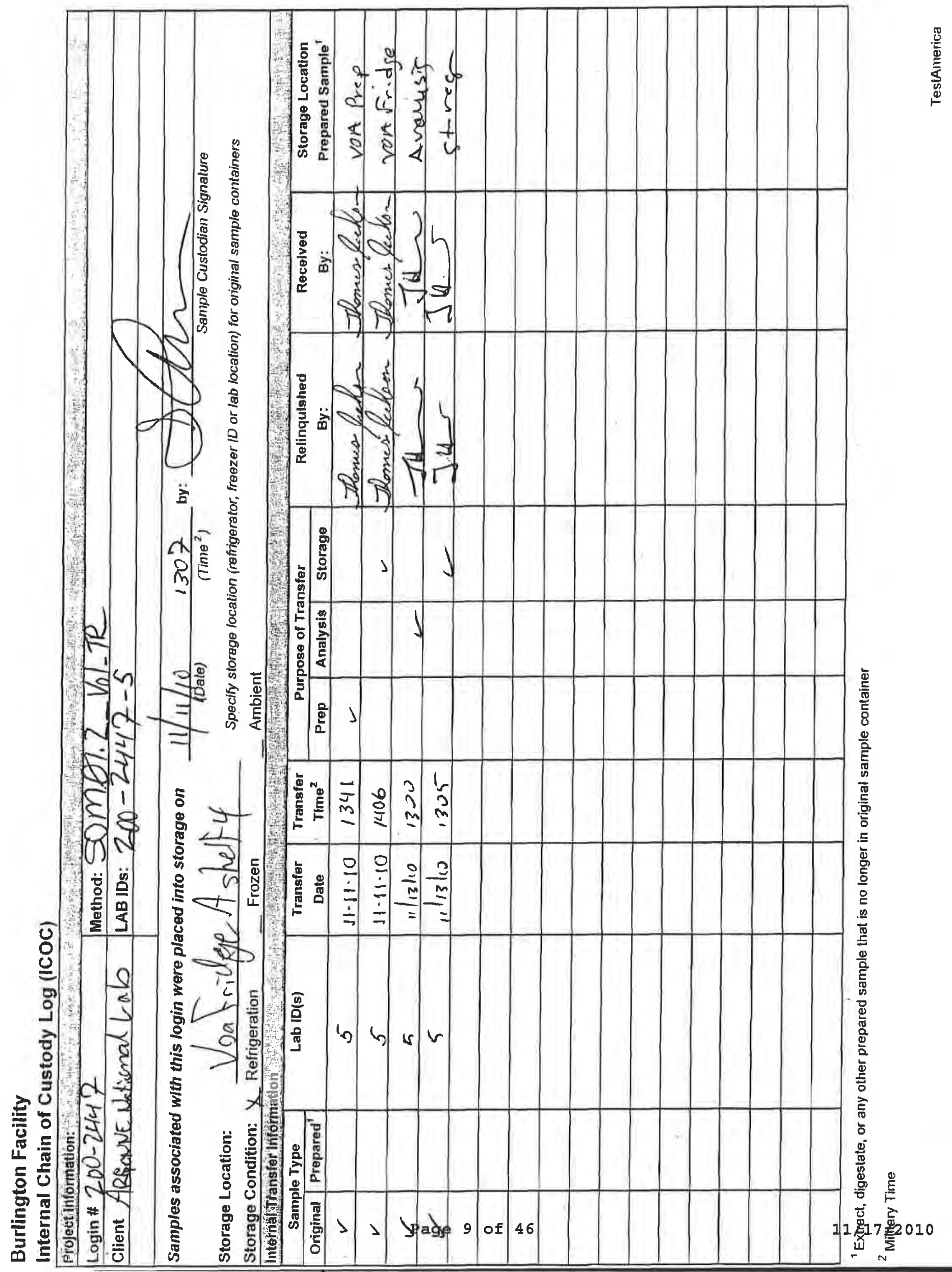




\section{Shipping and Receiving Documents}




\section{Login Sample Receipt Check List}

List Number: 1

\section{Question}

Radioactivity either was not measured or, if measured, is at or below background

The cooler's custody seal, if present, is intact.

The cooler or samples do not appear to have been compromised or tampered with.

Samples were received on ice.

Cooler Temperature is acceptable.

Cooler Temperature is recorded.

$\mathrm{COC}$ is present.

$\mathrm{COC}$ is filled out in ink and legible.

$\mathrm{COC}$ is filled out with all pertinent information.

Is the Fiètd Sampler's name present on COC?

There are no discrepancies between the sample IDs on the containers and the COC.

Samples are received within Holding Time.

Sample containers have legible labels.

Containers are not broken or leaking.

Sample collection date/times are provided.

Appropriate sample containers are used.

Sample bottles are completely filled.

Sample Preservation Verified

There is sufficient vol. for all requested analyses, incl. any requested MS/MSDs

VOA sample vials do not have headspace or bubble is $<6 \mathrm{~mm}(1 / 4 ")$ in diameter.

If necessary, staff have been informed of any short hold time or quick TAT needs

Multiphasic samples are not present.

Samples do not require splitting or compositing.

\section{T / F/NA Comment}

N/A

True

NO SEAL NUMBERS

True

True

True

True

True

True

True

True

True

True

True

True

True

False

True

N/A

True

True

True

N/A

N/A 
Job 200-2447-1

Client Job Description:

Purchase Order \#:

Work Order \#:

Project Manager:

Job Due Date:

Job TAT:

Max Deliverable Level: IV

Earliest Deliverable Due:

\section{Login 200-2447}

\section{Sample Receipt:}

Method of Delivery:

\section{Lab Sample \# Method}

200-2447-1 SOM01.2_Vol_Tr

200-2447-2 SOM01.2_Vol_T

200-2447-3

SOM 01.2_Vol_Tr SOM01.2 Trace Volatile Organics / In-Lab 200-2447-4 INQCTB-W-30764

SOM01.2_Vol_Tr SOM01.2 Trace Volatile Organics / In-Lab

200-2447-5 VHBLK01

SOM01.2_Vol_Tr SOM01.2 Trace Volatile Organics / In-Lab

\section{Client Sample ID Method Description / Work Location \\ INMW04-W-32677}

INMW05-W-32678

Tr SOM01.2 Trace Volatile Organics / In-Lab INTI14-W-30762

\section{Sample Login Acknowledgement}

INMAN (200-2447)

$8 \mathrm{E}-00302$

$8 \mathrm{E}-00302$

Kirk $F$ Young

$11 / 25 / 2010$

14 Days

IV
$11 / 25 / 2010$
Report To: Argonne National Laboratory

Jorge Alvarado

9700 South Cass Avenue

Building 203

Office B-149

Bill To: $\quad$ Argonne National Laboratory Accounts Payable Chief Financial Offices 9700 S. Cass Ave.

Building 201

Argonne, IL 60439
Argonne, IL 60439

Number of Coolers:

\section{1}

Cooler Temperature(s) (C $\left.\mathbf{C}^{\circ}\right): 1.9$ 


\section{METHODOLOGY SUMMARY}

\begin{tabular}{lll} 
Laboratory: & TestAmerica Laboratories & Project No: \\
Location: $\quad$ South Burlington, Vermont & SDG No: & 200-2447 \\
\hline VOA & \\
Volatile Organics Trace - USEPA CLP SOM01.2 &
\end{tabular}


$2 A$ - FORM II VOA-1

WATER VOLATILE DEUTERATED MONITORING COMPOUND RECOVERY

Lab Name: TESTAMERICA BURLINGTON

Contract: $8 \mathrm{E}-00302$

Lab Code: STLV Case No.: INMAN Mod. Ref No.:

SDG No.: 200-2447

Level: (TRACE or LOW) TRACE

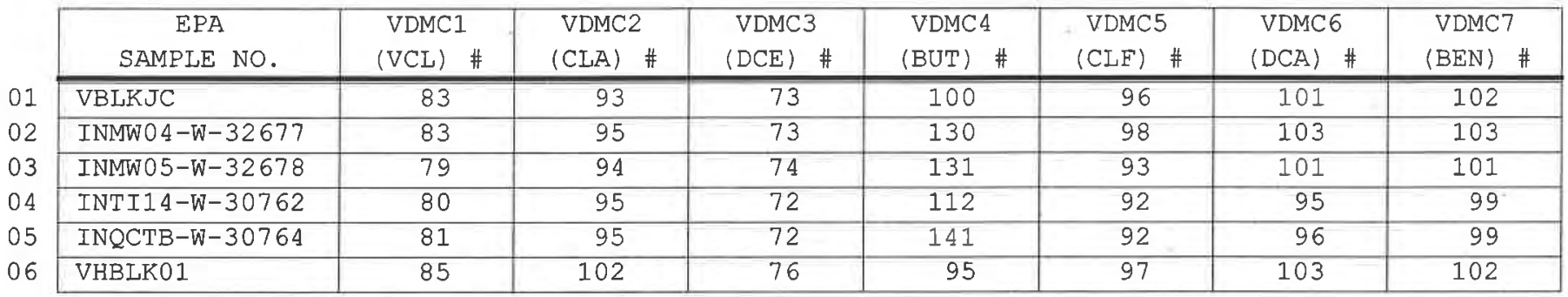

\footnotetext{
VDMC1 (VCL) = Vinyl Chloride-d3

VDMC2 $\quad(C L A)=$ Chloróethane-d5

VDMC3 $(D C E)=1,1-D i c h l o r o e t h e n e-d 2$

VDMC4 $(B U T)=2-B u t a n o n e-d 5$

VDMC5 $(\mathrm{CLF})=$ Chloroform-d

VDMC6 $(\mathrm{DCA})=1,2-\mathrm{Dichloroethane}-\mathrm{d} 4$

$\operatorname{VDMC7}(\mathrm{BEN})=$ Benzene-d6
}

QC LIMITS

(65-131)

$(71-131)$

$(55-104)$

(49-155)

$(78-121)$

$(78-129)$

$(77-124)$

\# Column to be used to flag recovery values

* Values outside of contract required QC limits

Page 1 of 1 
$2 B$ - FORM II VOA-2

WATER VOLATILE DEUTERATED MONITORING COMPOUND RECOVERY

Lab Name: TESTAMERICA BURLINGTON

Contract: $8 \mathrm{E}-00302$

Lab Code: STLV Case No.: INMAN Mod. Ref No.:

SDG No.: 200-2447

Level: (TRACE or LOW) TRACE

\begin{tabular}{|c|c|c|c|c|c|c|c|c|}
\hline $\begin{array}{c}\text { EPA } \\
\text { SAMPLE NO. }\end{array}$ & $\begin{array}{l}\text { VDMC8 } \\
(\mathrm{DPA}) \#\end{array}$ & $\begin{array}{l}\text { VDMC9 } \\
\text { (TOL) \# }\end{array}$ & $\begin{array}{l}\text { VDMC10 } \\
\text { (TDP) \# }\end{array}$ & $\begin{array}{l}\text { VDMC11 } \\
(\mathrm{HEX}) \quad \#\end{array}$ & $\begin{array}{l}\text { VDMC12 } \\
\text { (TCA) \# }\end{array}$ & $\begin{array}{l}\text { VDMC } 13 \\
\text { (DCZ) \# }\end{array}$ & OTHER & $\begin{array}{l}\text { TOT } \\
\text { OUT } \\
\end{array}$ \\
\hline VBLKJC & 94 & 101 & 103 & 101 & 98 & 102 & & 0 \\
\hline INMW0 4-W-32677 & 93 & 103 & 96 & 120 & 95 & 104 & & 0 \\
\hline INTI14-W-30762 & 89 & 97 & 93 & 110 & 91 & 100 & & 0 \\
\hline INQCTB-W-30764 & 90 & 99 & 94 & 131 & 89 & 100 & & 0 \\
\hline
\end{tabular}

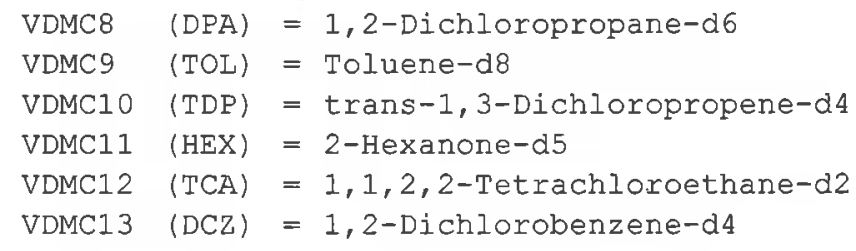

QC LIMITS

$(79-124)$
$(77-121)$
$(73-121)$
$(28-135)$
$(73-125)$
$(80-131)$


$4 A$ - FORM IV VOA

VOLATILE METHOD BLANK SUMMARY
EPA SAMPLE NO.

VBLKJC
Lab Name: TESTAMERICA BURLINGTON

Lab Code: STLV

Lab File ID: JBXA03.D

Case No.: INMAN Mod. Ref No.:

Contract: $\quad 8 \mathrm{E}-00302$

Instrument ID: J.i

Matrix: (SOIL/SED/WATER)

Level: (TRACE or LOW/MED)

GC Column: DB-624

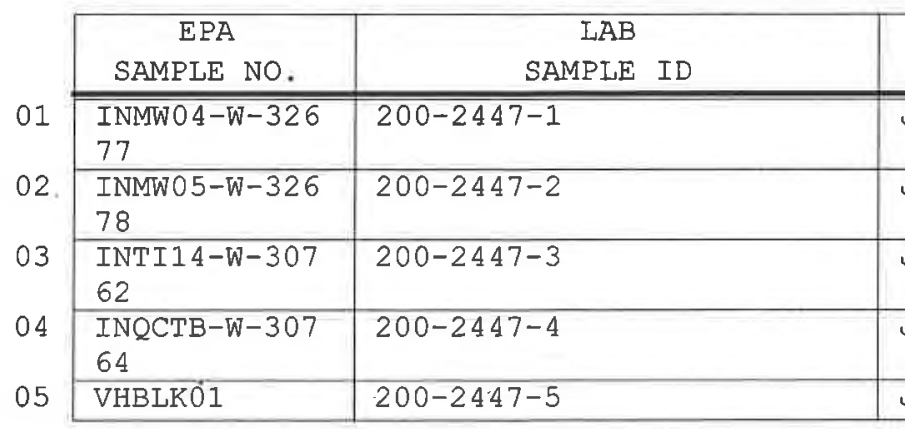

TRACE

ID: 0.20
SDG No.: 200-2447

Lab Sample ID: MB 200-9552/3

Date Analyzed: 11/13/2010

Time Analyzed: 1102

Heated Purge: ( $Y / N$ ) N

\begin{tabular}{|c|c|}
\hline $\begin{array}{c}\text { IAB } \\
\text { FILE ID }\end{array}$ & $\begin{array}{c}\text { TIME } \\
\text { ANALYZED }\end{array}$ \\
\hline JBXA14.D & 1605 \\
\hline JBXA15.D & 1630 \\
\hline JBXA16.D & 1656 \\
\hline JBXA17.D & 1722 \\
\hline JBXA24.D & 2022 \\
\hline
\end{tabular}

COMMENTS:

Page 1 of 1 
$5 A-F O R M V V O A$

VOLATILE ORGANICS INSTRUMENT

PERFORMANCE CHECK

BROMOFLUOROBENZENE (BFB)
EPA SAMPLE NO.

BFBJB
Lab Name: TESTAMERICA BURLINGTON

Lab code: STLV

Lab File Id: JBX01.D

Instrument Id: J.i

GC Column: DB-624

Contract: $8 \mathrm{E}-00302$

Case No.: INMAN Mod. Ref No.:

SDG No.: 200-2447

BFB Injection Date: 11/12/2010

BFB Injection Time: 1717 (mm)

\begin{tabular}{|r|l|r|}
\hline $\mathrm{m} / \mathrm{e}$ & ION ABUNDANCE CRITERIA & $\begin{array}{c}\text { RELATIVE } \\
\text { ABUNDANCE }\end{array}$ \\
\hline 50 & $15.0-40.0 \%$ of mass 95 & 19.0 \\
\hline 75 & $30.0-80.0 \%$ of mass 95 & 50.9 \\
\hline 95 & Base peak, 100\% relative abundance & 500 \\
\hline 96 & $5.0-9.0 \%$ of mass 95 & 0.7 \\
\hline 173 & Less than 2.0\% of mass 174 & $0.9) 1$ \\
\hline 174 & $50.0-120 \%$ of mass 95 & 80.0 \\
\hline 175 & $5.0-9.0 \%$ of mass 174 & $6.5(1)$ \\
\hline 176 & $95.0-101 \%$ of mass 174 & $(1)$ \\
\hline 177 & $5.0-9.0 \%$ of mass 176 & $5.3) 1$ \\
\hline
\end{tabular}

1 - Value is omass $174 \quad 2$ - Value is omass 176

\begin{tabular}{|c|c|c|c|c|}
\hline $\begin{array}{c}\text { EPA } \\
\text { SAMPLE NO. }\end{array}$ & $\begin{array}{r}\text { LAB } \\
\text { SAMPLE ID }\end{array}$ & $\begin{array}{l}\text { LAB } \\
\text { FILE ID }\end{array}$ & $\begin{array}{c}\text { DATE } \\
\text { ANALYZED }\end{array}$ & $\begin{array}{c}\text { TIME } \\
\text { ANALYZED }\end{array}$ \\
\hline VSTD0.5JB & IC 200-9510/3 & JBX03.D & $11 / 12 / 2010$ & 1810 \\
\hline VSTD001JB & IC $200-9510 / 4$ & JBX0 4.D & $11 / 12 / 2010$ & 1835 \\
\hline VSTD005JB & ICIS $200-9510 / 5$ & JBX05.D & $11 / 12 / 2010$ & 1901 \\
\hline VSTD010JB & IC $200-9510 / 6$ & JBX06.D & $11 / 12 / 2010$ & 1926 \\
\hline VSTD020JB & IC $200-9510 / 7$ & JBX07.D & $11 / 12 / 2010$ & 1951 \\
\hline
\end{tabular}


$5 A$ - FORM V VOA

VOLATILE ORGANICS INSTRUMENT

PERFORMANCE CHECK

BROMOFLUOROBENZENE

(BFB)
EPA SAMPLE NO.

BEBJC
Lab Name: TESTAMERICA BURLINGTON

Lab Code: STLV

Lab File Id: JBXA01.D

Instrument Id: J.i

GC Column: $\mathrm{DB}-624$

ID: 0.20
Contract: $8 E-00302$

SDG No.: 200-2447

BFB Injection Date: 11/13/2010

BFB Injection Time: 1017 (mm)

\begin{tabular}{|c|c|c|}
\hline m.te & ION ABUNDANCE CRITERIA & $\begin{array}{l}\text { 号 RELATIVE } \\
\text { ABUNDANCE }\end{array}$ \\
\hline 50 & $15.0-40.0 \%$ of mass 95 & 17.9 \\
\hline 75 & $30.0-80.0 \%$ of mass 95 & 50.1 \\
\hline 95 & Base peak, $100 \%$ relative abundance & 100 \\
\hline 96 & $5.0-9.0 \%$ of mass 95 & 6.7 \\
\hline 173 & Less than $2.0 \%$ of mass 174 & $1.0(1.2) 1$ \\
\hline 174 & $50.0-120 \%$ of mass 95 & 81.3 \\
\hline 175 & $5.0-9.0 \%$ of mass 174 & 6.31 \\
\hline 176 & $95.0-101 \%$ of mass 174 & $77.6(95.5) 1$ \\
\hline 177 & $5.0-9.0 \%$ of mass 176 & $5.5(7.0) 2$ \\
\hline
\end{tabular}

1 - Value is onass $174 \quad 2$ - Value is omass 176

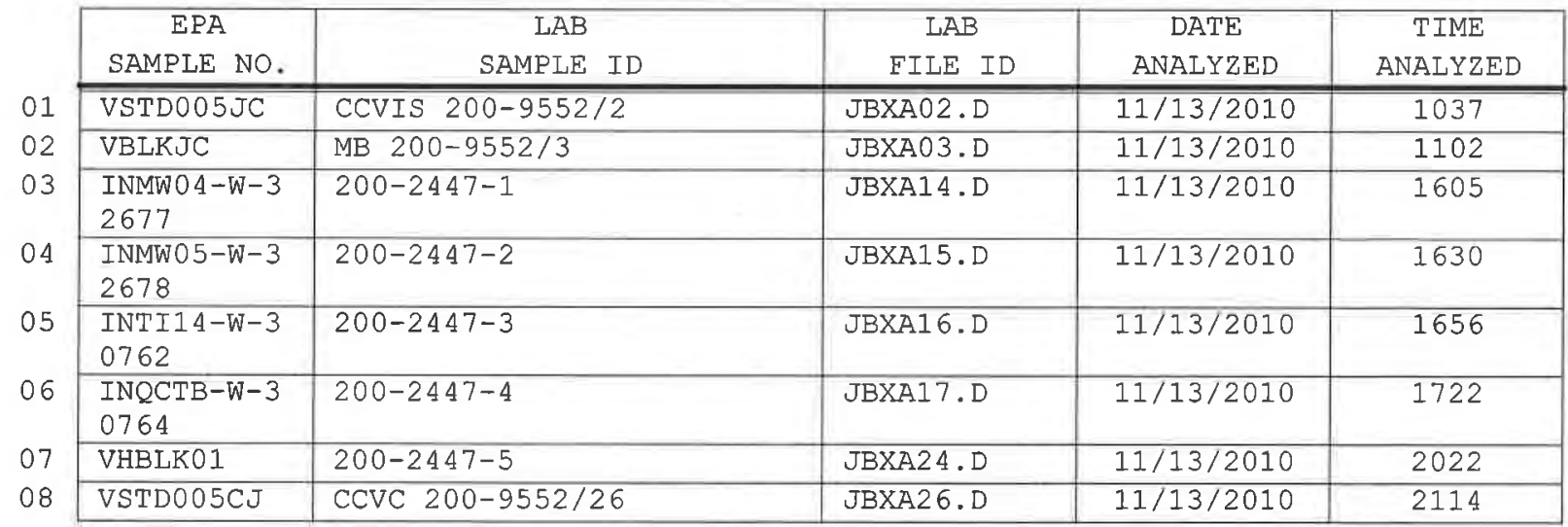


$8 A$ - FORM VIII VOA

VOLATILE INTERNAL STANDARD AREA AND RETENTION TIME SUMMARY

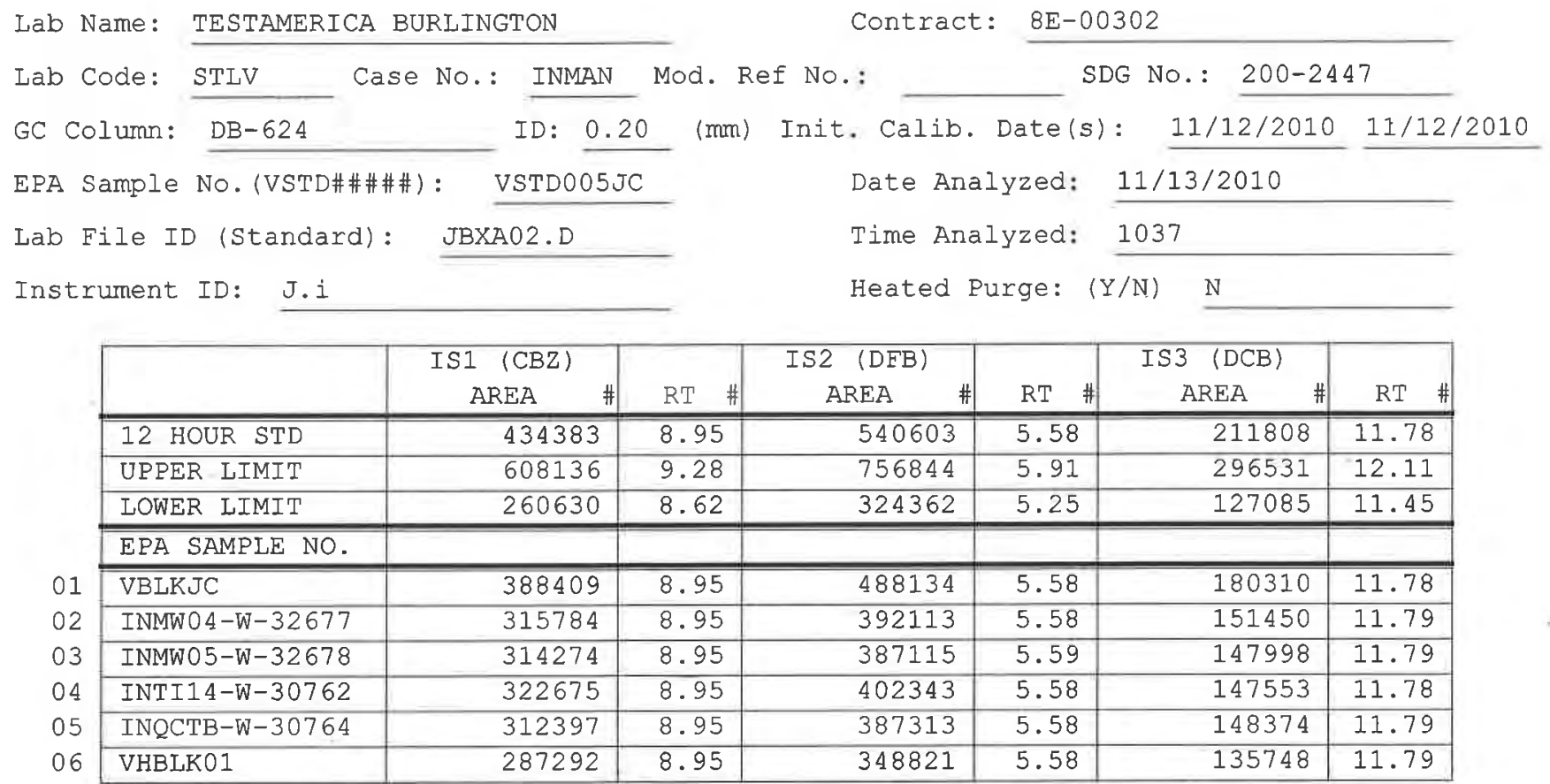

\footnotetext{
IS1 $(\mathrm{CBZ})=$ Chlorobenzene-d5

IS2 $(\mathrm{DFB})=1,4-\mathrm{Difluorobenzene}$

IS3 $(\mathrm{DCB})=1,4-\mathrm{Dichlorobenzene-d4}$
}

AREA UPPER LIMIT $=140 \%$ (Trace Volatiles) of internal standard area

AREA LOWER LIMIT $=60 \%$ (Trace Volatiles) of internal standard area

RT UPPER LIMIT $=+0.33$ (Trace Volatiles) minutes of internal standard RT

RT LOWER LIMIT $=-0.33$ (Trace Volatiles) minutes of internal standard RT

\# Column used to flag values outside contract required QC limits with an asterisk. 
Lab Name: TESTAMERICA BURLINGTON

Lab Code: STLV Case No.: INMAN

Matrix: (SOIL/SED/WATER)

Sample wt/vol: 25.0

\section{Water}

$(\mathrm{g} / \mathrm{mL}) \quad \mathrm{mL}$

Level: (TRACE/LOW/MED) TRACE

o Moisture: not dec.

GC Column: DB-624

Soil Extract Volume:

Purge Volume: 25.0
Contract: $\quad 8 \mathrm{E}-00302$

SDG No.: 200-2447

\begin{tabular}{|c|c|c|c|}
\hline CAS NO. & COMPOUND & $\begin{array}{l}\text { CONCENTRATION UNITS: } \\
(\mathrm{ug} / \mathrm{L} \text { or } \mathrm{ug} / \mathrm{kg}) \mathrm{ug} / \mathrm{L}\end{array}$ & 8 \\
\hline $75-71-8$ & Dichlorodifluoromethane & 0.50 & $\mathrm{U}$ \\
\hline $74-87-3$ & Chloromethane & 0.50 & $\mathrm{U}$ \\
\hline $75-01-4$ & Vinyl chloride & 0.50 & $\mathrm{U}$ \\
\hline $74-83-9$ & Bromomethane & 0.50 & U \\
\hline $75-00-3$ & Chloroethane & 0.50 & $\mathrm{U}$ \\
\hline $75-69-4$ & Trichlorofluoromethane & 0.50 & $\mathrm{U}$ \\
\hline $75-35-4$ & 1,1-Dichloroethene & 0.50 & $\mathrm{U}$ \\
\hline $76-13-1$ & 1,1,2-Trichloro-1,2,2-trifluoroethane & 0.50 & $\mathrm{U}$ \\
\hline $67-64-1$ & Acetone & 2.4 & $\mathrm{~J} \mathrm{~B}$ \\
\hline $75-15-0$ & Carbon disulfide & 0.088 & $\mathrm{~J} \mathrm{~B}$ \\
\hline $79-20-9$ & Methyl acetate & 0.50 & $\mathrm{U}$ \\
\hline $75-09-2$ & Methylene Chloride & 0.50 & $\mathrm{U}$ \\
\hline $156-60-5$ & trans-1,2-Dichloroethene & 0.50 & $\mathrm{U}$ \\
\hline $1634-04-4$ & Methyl tert-butyl ether & 0.50 & $\mathrm{U}$ \\
\hline $75-34-3$ & 1,1-Dichloroethane & 0.50 & $\mathrm{U}$ \\
\hline $156-59-2$ & cis-1,2-Dichloroethene & 0.50 & $\mathrm{U}$ \\
\hline $78-93-3$ & 2-Butanone & 5.0 & $\mathrm{U}$ \\
\hline $74-97-5$ & Bromochloromethane & 0.50 & $\mathrm{U}$ \\
\hline $67-66-3$ & Chloroform & 0.50 & $\mathrm{U}$ \\
\hline $71-55-6$ & 1,1,1-Trichloroethane & 0.50 & $\mathrm{U}$ \\
\hline $110-82-7$ & Cyclohexane & 0.50 & $\mathrm{U}$ \\
\hline $56-23-5$ & Carbon tetrachloride & 0.12 & $\mathrm{~J}$ \\
\hline $71-43-2$ & Benzene & 0.50 & $\mathrm{U}$ \\
\hline $107-06-2$ & 1,2-Dichloroethane & 0.50 & $\mathrm{U}$ \\
\hline
\end{tabular}

Report 1,4-Dioxane for Low-Medium VOA analysis only

Lab Sample ID: 200-2447-1

Lab File ID: JBXA14.D

Date Received: 11/11/2010

Date Analyzed: $11 / 13 / 2010$

Dilution Factor: 1.0

Soil Aliquot Volume:

(uL) (m山) 
$1 B$ - FORM I VOA-2

VOLATILE ORGANICS ANALYSIS DATA SHEET
EPA SAMPLE NO.

INMW0 $4-W-32677$

Lab Name: TESTAMERICA BURIINGTON

Lab Code: STLV

Case No.: INMAN

Matrix: (SOIL/SED/WATER)

Sample wt/vol: 25.0

Level: (TRACE/LOW/MED) TRACE

Water

$(g / m L) \quad m L$

응 Moisture: not dec.

GC Column: $\mathrm{DB}-624$

Soil Extract Volume:

ID: $0.20 \quad(\mathrm{~mm})$

(m工)

Purge Volume: 25.0
Contract: $8 E-00302$

SDG No.: 200-2447

Lab Sample ID: 200-2447-1

Lab File ID: JBXA14.D

Date Received: 11/11/2010

Date Analyzed: 11/13/2010

Dilution Factor: 1.0

Soil Aliquot Volume:

\begin{tabular}{|c|c|c|c|}
\hline CAS NO. & COMPOUND & $\begin{array}{l}\text { CONCENTRATION UNITS: } \\
(\mathrm{ug} / \mathrm{L} \text { or } \mathrm{ug} / \mathrm{kg}) \mathrm{ug} / \mathrm{L}\end{array}$ & $Q$ \\
\hline $79-01-6$ & Trichloroethene & 0.14 & $\bar{J}$ \\
\hline $108-87-2$ & Methylcyclohexane & 0.50 & $\mathrm{U}$ \\
\hline $78-87-5$ & 1,2-Dichloropropane & 0.50 & $\mathrm{U}$ \\
\hline $75-27-4$ & Bromodichloromethane & 0.50 & $\mathrm{U}$ \\
\hline $10061-01-5$ & cis-1,3-Dichloropropene & 0.50 & $\mathrm{U}$ \\
\hline $108-10-1$ & 4-Methyl-2-pentanone & 5.0 & $\mathrm{U}$ \\
\hline $108-88-3$ & Toluene & 0.13 & $\mathrm{~J}$ \\
\hline $10061-02-6$ & trans-1,3-Dichloropropene & 0.50 & $\mathrm{U}$ \\
\hline $79-00-5$ & $1,1,2$-Trichloroethane & 0.50 & $\mathrm{U}$ \\
\hline $127-18-4$ & Tetrachloroethene & 0.18 & $\mathrm{~J}$ \\
\hline $591-78-6$ & 2-Hexanone & 5.0 & $\mathrm{U}$ \\
\hline $124-48-1$ & Dibromochloromethane & 0.50 & $\mathrm{U}$ \\
\hline $106-93-4$ & 1,2-Dibromoethane & 0.50 & $\mathrm{U}$ \\
\hline $108-90-7$ & Chlorobenzene & 0.50 & $\mathrm{U}$ \\
\hline $100-41-4$ & Ethylbenzene & 0.50 & $\mathrm{U}$ \\
\hline $95-47-6$ & o-Xylene & 0.50 & $\mathrm{U}$ \\
\hline $179601-23-1$ & $m, p-x y l e n e$ & 0.061 & $\mathrm{~J}$ \\
\hline $100-42-5$ & Styrene & 0.14 & $\mathrm{~J}$ \\
\hline $75-25-2$ & Bromoform & 0.50 & $\mathrm{U}$ \\
\hline $98-82-8$ & Isopropylbenzene & 0.50 & $\mathrm{U}$ \\
\hline $79-34-5$ & $1,1,2,2$-Tetrachloroethane & 0.50 & $\mathrm{U}$ \\
\hline $541-73-1$ & 1,3-Dichlorobenzene & 0.50 & $\mathrm{U}$ \\
\hline $106-46-7$ & 1,4-Dichlorobenzene & 0.50 & $\mathrm{U}$ \\
\hline $95-50-1$ & 1,2-Dichlorobenzene & 0.50 & $\mathrm{U}$ \\
\hline $96-12-8$ & 1,2-Dibromo-3-Chloropropane & 0.50 & $\mathrm{U}$ \\
\hline $120-82-1$ & 1,2,4-Trichlorobenzene & 0.50 & $\mathrm{U}$ \\
\hline $87-61-6$ & 1,2,3-Trichlorobenzene & 0.50 & $\mathrm{U}$ \\
\hline
\end{tabular}


$1 \mathrm{~J}$ - FORM I VOA-TIC

VOLATILE ORGANICS ANALYSIS DATA SHEET

TENTATIVELY IDENTIEIED COMPOUNDS
EPA SAMPLE NO.

INMW04-W-32677

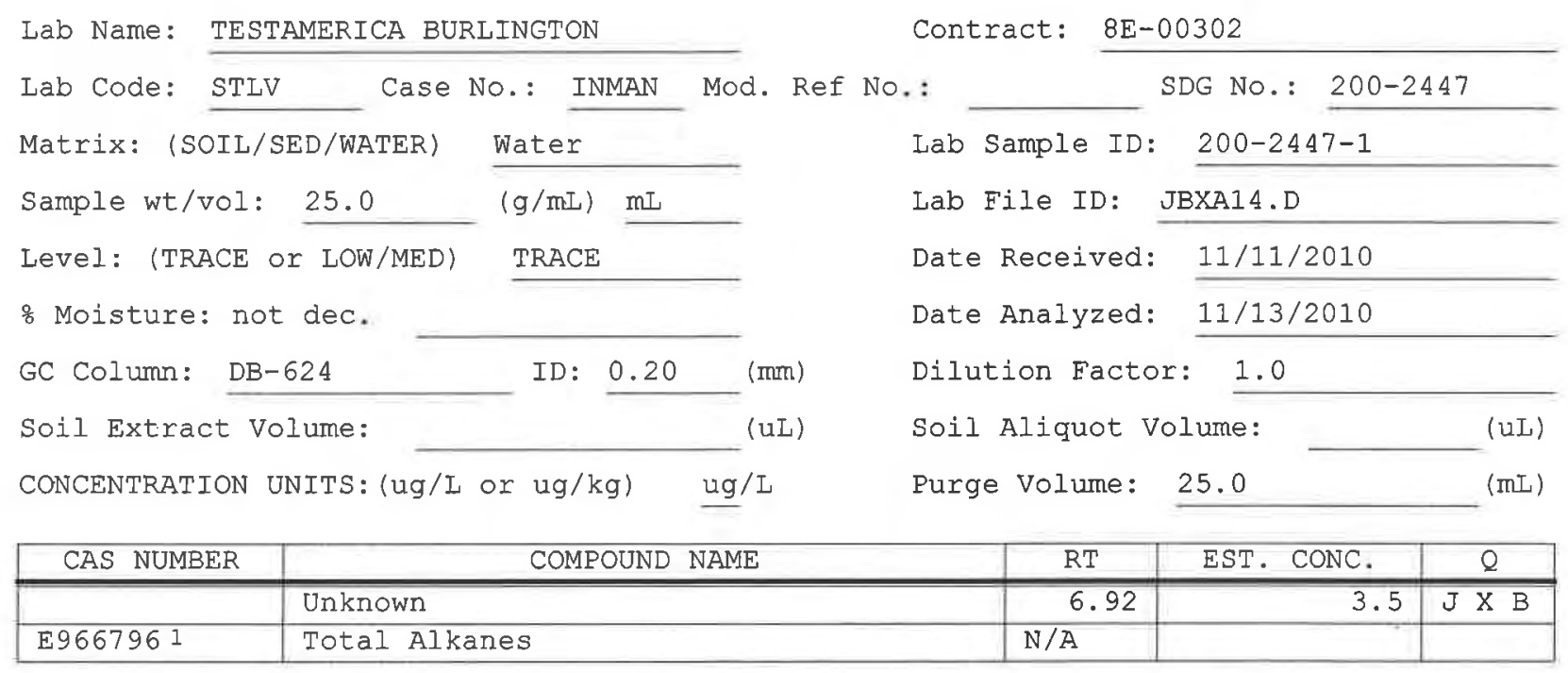

1 EPA-designated Registry Number. 
$1 A$ - FORM I VOA-1

VOLATILE ORGANICS ANALYSIS DATA SHEET
EPA SAMPLE NO.

INMW0 5-W-32678
Lab Name: TESTAMERICA BURLINGTON

Lab Code: STLV

Matrix: (SOIL/SED/WATER)

Sample wt/vol: 25.0

Level: (TRACE/LOW/MED) Case No.: INMAN Mod. Ref No.:

Contract: $\quad 8 E-00302$

o Moisture: not dec.

GC Column: $\mathrm{DB}-624$

ID: 0.20

Water

$(\mathrm{g} / \mathrm{mL}) \quad \mathrm{mL}$

Soil Extract Volume:

TRACE

Purge Volume: 25.0

\begin{tabular}{|c|c|c|c|}
\hline CAS NO. & COMPOUND & $\begin{array}{l}\text { CONCENTRATION UNITS: } \\
(\mathrm{ug} / \mathrm{L} \text { or } \mathrm{ug} / \mathrm{kg}) \mathrm{ug} / \mathrm{L}\end{array}$ & $Q$ \\
\hline $75-71-8$ & Dichlorodifluoromethane & 0.50 & $\overline{\mathrm{U}}$ \\
\hline $74-87-3$ & Chloromethane & 0.50 & $\mathrm{U}$ \\
\hline $75-01-4$ & Vinyl chloride & 0.50 & $\mathrm{U}$ \\
\hline $74-83-9$ & Bromomethane & 0.50 & $\mathrm{U}$ \\
\hline $75-00-3$ & Chloroethane & 0.50 & $\mathrm{U}$ \\
\hline $75-.69-4$ & Trichlorofluoromethane & 0.50 & $\mathrm{U}$ \\
\hline $75-35-4$ & 1,1-Dichloroethene & 0.50 & $\mathrm{U}$ \\
\hline $76-13-1$ & 1,1,2-Trichloro-1,2,2-trifluoroethane & 0.50 & $\mathrm{U}$ \\
\hline $67-64-1$ & Acetone & 2.1 & $\mathrm{~J} \mathrm{~B}$ \\
\hline $75-15-0$ & Carbon disulfide & 0.50 & $\mathrm{U}$ \\
\hline $79-20-9$ & Methyl acetate & 0.50 & $\mathrm{U}$ \\
\hline $75-09-2$ & Methylene Chloride & 0.50 & $\mathrm{U}$ \\
\hline $156-60-5$ & trans-1,2-Dichloroethene & 0.50 & $\mathrm{U}$ \\
\hline $1634-04-4$ & Methyl tert-butyl ether & 0.50 & $\mathrm{U}$ \\
\hline $75-34-3$ & 1,1-Dichloroethane & 0.50 & $\mathrm{U}$ \\
\hline $156-59-2$ & cis-1,2-Dichloroethene & 0.50 & $\mathrm{U}$ \\
\hline $78-93-3$ & 2-Butanone & 5.0 & $\mathrm{U}$ \\
\hline $74-97-5$ & Bromochloromethane & 0.50 & $\mathrm{U}$ \\
\hline $67-66-3$ & Chloroform & 0.50 & $\mathrm{U}$ \\
\hline $71-55-6$ & 1,1,1-Trichloroethane & 0.50 & $\mathrm{U}$ \\
\hline $110-82-7$ & Cyclohexane & 0.50 & $\mathrm{U}$ \\
\hline $56-23-5$ & Carbon tetrachloride & 0.081 & $\mathrm{~J}$ \\
\hline $71-43-2$ & Benzene & 0.50 & $\mathrm{U}$ \\
\hline $107-06-2$ & 1,2-Dichloroethane & 0.50 & $\mathrm{U}$ \\
\hline
\end{tabular}

Lab Sample ID: 200-2447-2

Lab File ID: JBXA15.D

Date Received: 11/11/2010

Date Analyzed: 11/13/2010

Dilution Factor: 1.0

Soil Aliquot Volume: (UL) (uL) (mL)

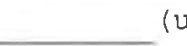


$1 B$ - FORM I VOA-2

VOLATILE ORGANICS ANALYSIS DATA SHEET
EPA SAMPLE NO.

INMW0 $5-W-32678$
Lab Name: TESTAMERICA BURLINGTON

Lab Code: STLV

Matrix: (SOIL/SED/WATER)

Sample wt/vol: 25.0

Water

$(\mathrm{g} / \mathrm{mL}) \mathrm{mL}$

Level: (TRACE/LOW/MED) TRACE

\% Moisture: not dec.

GC Column: DB-624

ID: 0.20

Soil Extract Volume:

Purge Volume: 25.0

$(\mathrm{mL})$
Contract: $8 E-00302$

SDG No.: 200-2447

Lab Sample ID: 200-2447-2

Lab File ID: JBXA15.D

Date Received: 11/11/2010

Date Analyzed: 11/13/2010

Dilution Factor: 1.0

Soil Aliquot Volume:

(uL)

\begin{tabular}{|c|c|c|c|}
\hline CAS NO. & COMPOUND & $\begin{array}{l}\text { CONCENTRATION UNITS: } \\
(\mathrm{ug} / \mathrm{L} \text { or } \mathrm{ug} / \mathrm{kg}) \mathrm{ug} / \mathrm{L}\end{array}$ & $Q$ \\
\hline $79-01-6$ & Trichloroethene & 0.13 & $\mathrm{~J}$ \\
\hline $108-87-2$ & Methylcyclohexane & 0.50 & $\mathrm{U}$ \\
\hline $78-87-5$ & 1,2-Dichloropropane & 0.50 & $\mathrm{U}$ \\
\hline $75-27-4$ & Bromodichloromethane & 0.50 & $\mathrm{U}$ \\
\hline $10061-01-5$ & cis-1,3-Dichloropropene & 0.50 & $\mathrm{U}$ \\
\hline $108-10-1$ & 4-Methyl-2-pentanone & 5.0 & $\mathrm{U}$ \\
\hline $108-88-3$ & Toluene & 0.11 & $\mathrm{~J}$ \\
\hline $10061-02-6$ & trans-1,3-Dichloropropene & 0.50 & $\mathrm{U}$ \\
\hline $79-00-5$ & 1,1,2-Trichloroethane & 0.50 & $\mathrm{U}$ \\
\hline $127-18-4$ & Tetrachloroethene & 0.17 & $\mathrm{~J}$ \\
\hline $591-78-6$ & 2-Hexanone & 4.3 & $\mathrm{~J}$ \\
\hline $124-48-1$ & Dibromochloromethane & 0.50 & $\mathrm{U}$ \\
\hline $106-93-4$ & 1,2-Dibromoethane & 0.50 & $\mathrm{U}$ \\
\hline $108-90-7$ & Chlorobenzene & 0.50 & $\mathrm{U}$ \\
\hline $100-41-4$ & Ethylbenzene & 0.50 & $\mathrm{U}$ \\
\hline $95-47-6$ & o-xylene & 0.50 & $\mathrm{U}$ \\
\hline $179601-23-1$ & $m, p-x y l e n e$ & 0.058 & $\mathrm{~J}$ \\
\hline $100-42-5$ & styrene & 0.14 & $\mathrm{~J}$ \\
\hline $75-25-2$ & Bromoform & 0.50 & $\mathrm{U}$ \\
\hline $98-82-8$ & Isopropylbenzene & 0.50 & $\mathrm{U}$ \\
\hline $79-34-5$ & $1,1,2,2$-Tetrachloroethane & 0.50 & $\mathrm{U}$ \\
\hline $541-73-1$ & 1,3-Dichlorobenzene & 0.50 & $\mathrm{U}$ \\
\hline $106-46-7$ & 1,4-Dichlorobenzene & 0.50 & $\mathrm{U}$ \\
\hline $95-50-1$ & 1,2-Dichlorobenzene & 0.50 & $\mathrm{U}$ \\
\hline $96-12-8$ & 1,2-Dibromo-3-Chloropropane & 0.50 & $\mathrm{U}$ \\
\hline $120-82-1$ & 1,2,4-Trichlorobenzene & 0.50 & $\mathrm{U}$ \\
\hline $87-61-6$ & 1,2,3-Trichlorobenzene & 0.50 & $\mathrm{U}$ \\
\hline
\end{tabular}


$1 \mathrm{~J}$ - FORM I VOA-TIC

VOLATILE ORGANICS ANALYSIS DATA SHEET

TENTATIVELY IDENTIFIED COMPOUNDS
EPA SAMPLE NO.

INMW05-W-32678

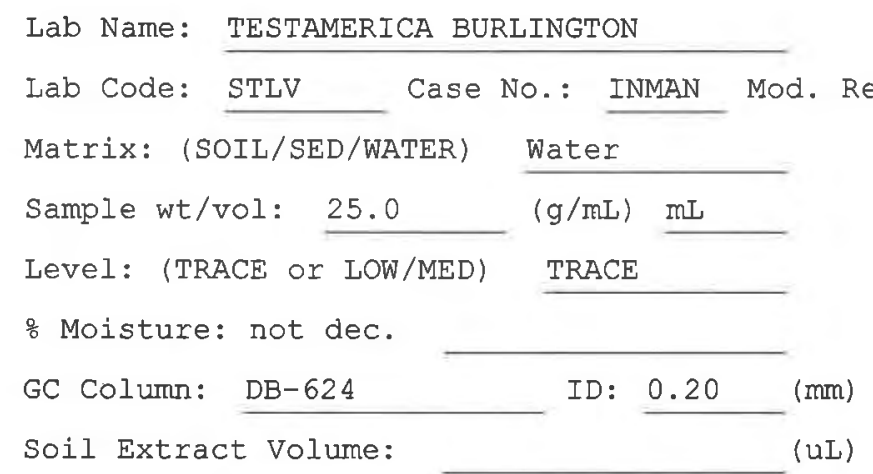

CONCENTRATION UNITS: (ug/L or $\mathrm{ug} / \mathrm{kg}$ ) ug/L
Contract: $8 \mathrm{E}-00302$

SDG No.: 200-2447

Lab Sample ID: 200-2447-2

Lab File ID: JBXA15.D

Date Received: 11/11/2010

Date Analyzed: 11/13/2010

Dilution Factor: 1.0

Soil Aliquot Volume:

(uL)

Purge Volume: 25.0

(mL)

01

02

\begin{tabular}{|l|l|c|c|c|}
\hline CAS NUMBER & \multicolumn{1}{|c|}{ COMPOUND NAME } & RT & EST. CONC. & $Q$ \\
\hline \hline & Unknown & 6.92 & 3.4 & $\mathrm{~J} X \mathrm{~B}$ \\
\hline E9667961 & Total Alkanes & N/A & & \\
\hline
\end{tabular}

I EPA-designated Registry Number. 
Lab Name: TESTAMERICA BURLINGTON

Lab Code: STLV

Matrix: (SOIL/SED/WATER)

Sample wt/vol: 25.0

Level: (TRACE/LOW/MED)

Case No.: INMAI
Water

$(\mathrm{g} / \mathrm{mL}) \quad \mathrm{mL}$
TRACE

응 Moisture: not dec.

GC Column: DB-624 ID $0.20 \quad(\mathrm{~mm})$

Soil Extract Volume: (uL) Mod. Ref No.:

Contract: $8 \mathrm{E}-00302$

Purge Volume: 25.0 $(\mathrm{mL})$
SDG No.: 200-2447

Lab Sample ID: $\quad 200-2447-4$

Lab File ID: JBXA17.D

Date Received: 11/11/2010

Date Analyzed: 11/13/2010

Dilution Factor: 1.0

Soil Aliquot volume: (uL)

\begin{tabular}{|c|c|c|c|}
\hline CAS NO. & COMPOUND & $\begin{array}{l}\text { CONCENTRATION UNITS: } \\
(\mathrm{ug} / \mathrm{L} \text { or } \mathrm{ug} / \mathrm{kg}) \mathrm{ug} / \mathrm{L}\end{array}$ & Q \\
\hline $75-71-8$ & Dichlorodifluoromethane & 0.50 & $\mathrm{U}$ \\
\hline $74-87-3$ & Chloromethane & 0.50 & $\mathrm{U}$ \\
\hline $75-01-4$ & Vinyl chloride & 0.50 & $\mathrm{U}$ \\
\hline $74-83-9$ & Bromomethane & 0.50 & $\mathrm{U}$ \\
\hline $75-00-3$ & Chloroethane & 0.50 & $\mathrm{U}$ \\
\hline $75-69-4$ & Trichlorofluoromethane & 0.50 & $\mathrm{U}$ \\
\hline $75-35-4$ & 1,1-Dichloroethene & 0.50 & U \\
\hline $76-13-1$ & 1,1,2-Trichloro-1,2,2-trifluoroethane & 0.50 & $\mathrm{U}$ \\
\hline $67-64-1$ & Acetone & 2.3 & $\mathrm{~J} \mathrm{~B}$ \\
\hline $75-15-0$ & Carbon disulfide & 0.50 & $\mathrm{U}$ \\
\hline $79-20-9$ & Methyl acetate & 0.50 & $\mathrm{U}$ \\
\hline $75-09-2$ & Methylene Chloride & 0.50 & $\mathrm{U}$ \\
\hline $156-60-5$ & trans-1,2-Dichloroethene & 0.50 & $\mathrm{U}$ \\
\hline $1634-04-4$ & Methyl tert-butyl ether & 0.50 & $\mathrm{U}$ \\
\hline $75-34-3$ & 1,1-Dichloroethane & 0.50 & $\mathrm{U}$ \\
\hline $156-59-2$ & cis-1,2-Dichloroethene & 0.50 & $\mathrm{U}$ \\
\hline $78-93-3$ & 2-Butanone & 2.1 & $\mathrm{~J}$ \\
\hline $74-97-5$ & Bromochloromethane & 0.50 & $\mathrm{U}$ \\
\hline $67-66-3$ & Chloroform & 0.50 & $\mathrm{U}$ \\
\hline $71-55-6$ & 1,1,1-Trichloroethane & 0.50 & $\mathrm{U}$ \\
\hline $110-82-7$ & Cyclohexane & 0.50 & $\mathrm{U}$ \\
\hline $56-23-5$ & Carbon tetrachloride & 0.50 & $\mathrm{U}$ \\
\hline $71-43-2$ & Benzene & 0.50 & $\mathrm{U}$ \\
\hline $107-06-2$ & 1,2-Dichloroethane & 0.50 & $\mathrm{U}$ \\
\hline
\end{tabular}

Report 1,4-Dioxane for Low-Medium VOA analysis only 
$1 B$ - FORM I VOA-2

VOLATILE ORGANICS ANALYSIS DATA SHEET
EPA SAMPLE NO.

INQCTB-W-30764
Lab Name: TESTAMERICA BURLINGTON

Lab Code: STIV

Level: (TRACE/LOW/MED) ID: $0.20 \quad(\mathrm{~mm})$ (UL)
Matrix: (SOIL/SED/WATER)

Sample wt/vol: 25.0

\% Moisture: not dec.

GC Column: DB-624

Soil Extract Volume:

Water

$(g / m L) \quad m L$

TRACE

Purge Volume: 25.0
Contract: $8 \mathrm{E}-00302$

SDG No.: 200-2447
Lab Sample ID: 200-2447-4

Lab File ID: JBXA17.D

Date Received: 11/11/2010

Date Analyzed: 11/13/2010

Dilution Factor: 1.0

Soil Aliquot volume: (uL)

\begin{tabular}{|c|c|c|c|}
\hline CAS NO. & COMPOUND & $\begin{array}{l}\text { CONCENTRATION UNITS: } \\
(\mathrm{ug} / \mathrm{L} \text { or } \mathrm{ug} / \mathrm{kg}) \mathrm{ug} / \mathrm{L}\end{array}$ & $\mathrm{Q}$ \\
\hline $79-01-6$ & Trichloroethene & 0.50 & $\overline{\mathrm{U}}$ \\
\hline $108-87-2$ & Methylcyclohexane & 0.50 & $\mathrm{U}$ \\
\hline $78-87-5$ & 1,2-Dichloropropane & 0.50 & $\mathrm{U}$ \\
\hline $75-27-4$ & Bromodichloromethane & 0.50 & $\mathrm{U}$ \\
\hline $10061-01-5$ & cis-1,3-Dichloropropene & 0.50 & $\mathrm{U}$ \\
\hline $108-10-1$ & 4-Methyl-2-pentanone & 5.0 & $\mathrm{U}$ \\
\hline $108-88-3$ & Toluene & 0.041 & $\mathrm{~J}$ \\
\hline $10061-02-6$ & trans-1,3-Dichloropropene & 0.50 & $\mathrm{U}$ \\
\hline $79-00-5$ & 1,1,2-Trichloroethane & 0.50 & $\mathrm{U}$ \\
\hline $127-18-4$ & Tetrachloroethene & 0.50 & $\mathrm{U}$ \\
\hline $591-78-6$ & 2-Hexanone & 5.0 & $\mathrm{U}$ \\
\hline $124-48-1$ & Dibromochloromethane & 0.50 & $\mathrm{U}$ \\
\hline $106-93-4$ & 1,2-Dibromoethane & 0.50 & $\mathrm{U}$ \\
\hline $108-90-7$ & Chlorobenzene & 0.50 & $\mathrm{U}$ \\
\hline $100-41-4$ & Ethylbenzene & 0.50 & $\mathrm{U}$ \\
\hline $95-47-6$ & o-Xylene & 0.044 & $\mathrm{~J}$ \\
\hline $179601-23-1$ & m,p-Xylene & 0.50 & $\mathrm{U}$ \\
\hline $100-42-5$ & Styrene & 0.50 & $\mathrm{U}$ \\
\hline $75-25-2$ & Bromoform & 0.50 & $\mathrm{U}$ \\
\hline $98-82-8$ & Isopropylbenzene & 0.50 & $\mathrm{U}$ \\
\hline $79-34-5$ & $1,1,2,2$-Tetrachloroethane & 0.50 & $\mathrm{U}$ \\
\hline $541-73-1$ & 1,3-Dichlorobenzene & 0.50 & $\mathrm{U}$ \\
\hline $106-46-7$ & 1,4-Dichlorobenzene & 0.50 & $\mathrm{U}$ \\
\hline $95-50-1$ & 1,2-Dichlorobenzene & 0.50 & $\mathrm{U}$ \\
\hline $96-12-8$ & 1,2-Dibromo-3-Chloropropane & 0.50 & $\mathrm{U}$ \\
\hline $120-82-1$ & 1,2,4-Trichlorobenzene & 0.50 & $\mathrm{U}$ \\
\hline $87-61-6$ & 1,2,3-Trichlorobenzene & 0.50 & $\mathrm{U}$ \\
\hline
\end{tabular}


1J - FORM I VOA-TIC

VOLATILE ORGANICS ANALYSIS DATA SHEET

TENTATIVELYY IDENTIFIED COMPOUNDS
EPA SAMPLE NO.

INQCTB-W-30764

Lab Name: TESTAMERICA BURLINGTON

Contract: $8 \mathrm{E}-00302$

Lab Code: STLV Case No.: INMAN Mod. Ref No.:

Matrix: (SOIL/SED/WATER)

Sample wt/vol: 25.0

Level: (TRACE or LOW/MED)

Water

$(\mathrm{g} / \mathrm{mL}) \mathrm{mL}$

TRACE

o Moisture: not dec.

GC Column: DB-624

Soil Extract Volume:

ID: $0.20 \quad(\mathrm{~mm})$

(UL)

CONCENTRATION UNITS: (ug/L or $\mathrm{ug} / \mathrm{kg}$ ) $\mathrm{ug} / \mathrm{L}$
Lab Sample ID: 200-2447-4

Lab File ID: JBXA17.D

Date Received: 11/11/2010

Date Analyzed: 11/13/2010

Dilution Factor: 1.0

Soil Aliquot Volume: (UL)

Purge Volume: 25.0 (mL)

01

\begin{tabular}{|c|l|c|c|c|}
\hline CAS NUMBER & \multicolumn{1}{|c|}{ COMPOUND NAME } & RT & EST. CONC. & Q \\
\hline \hline & Unknown & 6.92 & 3.3 & $\mathrm{~J} X \mathrm{~B}$ \\
\hline E9667961 & Total Alkanes & N/A & & \\
\hline
\end{tabular}

1 EPA-designated Registry Number. 
$1 A$ - FORM I VOA-1

VOLATILE ORGANICS ANALYSIS DATA SHEET
EPA SAMPLE NO.

INTI $14-W-30762$
Lab Name: TESTAMERICA BURLINGTON

Lab Code: STLV

Matrix: (SOIL/SED/WATER)

Sample wt/vol: 25.0 Case NO.: INMAN

Mod. Ref No.:
Contract: $8 \mathrm{E}-00302$

SDG No.: 200-2447
Level: (TRACE/LOW/MED) TRACE

음 Moisture: not dec.

GC Column: DB-624

Soil Extract Volume: ID: 0.20 (mm) (uL)

Purge Volume: 25.0 (mL)
Lab Sample ID: 200-2447-3

Lab File ID: JBXA16.D

Date Received: 11/11/2010

Date Analyzed: 11/13/2010

Dilution Factor: 1.0

Soil Aliquot Volume: (uL)

\begin{tabular}{|c|c|c|c|}
\hline CAS NO. & COMPOUND & $\begin{array}{l}\text { CONCENTRATION UNITS: } \\
(\mathrm{ug} / \mathrm{L} \text { or } \mathrm{ug} / \mathrm{kg}) \mathrm{ug} / \mathrm{L}\end{array}$ & $Q$ \\
\hline $75-71-8$ & Dichlorodifluoromethane & 0.50 & $\overline{\mathrm{U}}$ \\
\hline $74-87-3$ & Chloromethane & 0.50 & $\mathrm{U}$ \\
\hline $75-01-4$ & Vinyl chloride & 0.50 & $\mathrm{U}$ \\
\hline $74-83-9$ & Bromomethane & 0.50 & U \\
\hline $75-00-3$ & Chloroethane & 0.50 & $\mathrm{U}$ \\
\hline $75-69-4$ & Trichlorofluoromethane & 0.50 & U \\
\hline $75-35-4$ & 1,1-Dichloroethene & 0.50 & U \\
\hline $76-13-1$ & 1,1,2-Trichloro-1,2,2-trifluoroethane & 0.50 & $\mathrm{U}$ \\
\hline $67-64-1$ & Acetone & 2.4 & $J B$ \\
\hline $75-15-0$ & Carbon disulfide & 0.50 & U \\
\hline $79-20-9$ & Methyl acetate & 0.50 & $\mathrm{U}$ \\
\hline $75-09-2$ & Methylene Chloride & 0.50 & $\mathrm{U}$ \\
\hline $156-60-5$ & trans-1,2-Dichloroethene & 0.50 & $\mathrm{U}$ \\
\hline $1634-04-4$ & Methyl tert-butyl ether & 0.50 & $\mathrm{U}$ \\
\hline $75-34-3$ & 1,1-Dichloroethane & 0.50 & $\mathrm{U}$ \\
\hline $156-59-2$ & cis-1,2-Dichloroethene & 0.50 & $\mathrm{U}$ \\
\hline $78-93-3$ & 2-Butanone & 0.97 & $\mathrm{~J}$ \\
\hline $74-97-5$ & Bromochloromethane & 0.50 & $\mathrm{U}$ \\
\hline $67-66-3$ & Chloroform & 0.50 & $\mathrm{U}$ \\
\hline $71-55-6$ & 1,1,1-Trichloroethane & 0.50 & $\mathrm{U}$ \\
\hline $110-82-7$ & Cyclohexane & 0.50 & $\mathrm{U}$ \\
\hline $56-23-5$ & Carbon tetrachloride & 0.044 & $\mathrm{~J}$ \\
\hline $71-43-2$ & Benzene & 0.031 & $\mathrm{~J}$ \\
\hline $107-06-2$ & 1,2-Dichloroethane & 0.50 & $\mathrm{U}$ \\
\hline
\end{tabular}

Report 1,4-Dioxane for Low-Medium VOA analysis only 
Lab Name: TESTAMERICA BURLINGTON

Contract: $8 \mathrm{E}-00302$

Lab Code: STLV Case No.: INMAN Mod. Ref No.:

SDG No.: 200-2447

Matrix: (SOIL/SED/WATER)

Sample wt/vol: 25.0 Water

$(\mathrm{g} / \mathrm{mL}) \quad \mathrm{mL}$

Level: (TRACE/LOW/MED) TRACE

o Moisture: not dec.

GC Column: DB-624

Soil Extract Volume: ID: $0.20 \quad(\mathrm{~mm})$

Purge Volume: 25.0 (uL) (mL)
Lab Sample ID: 200-2447-3

Lab File ID: JBXA16.D

Date Received: 11/11/2010

Date Analyzed: 11/13/2010

Dilution Factor: 1.0

Soil Aliquot Volume:

(uL)

\begin{tabular}{|c|c|c|c|}
\hline CAS NO. & COMPOUND & $\begin{array}{l}\text { CONCENTRATION UNITS: } \\
(\mathrm{ug} / \mathrm{L} \text { or } u g / \mathrm{kg}) \mathrm{ug} / \mathrm{L}\end{array}$ & $Q$ \\
\hline $79-01-6$ & Trichloroethene & 0.057 & $\mathrm{~J}$ \\
\hline $108-87-2$ & Methylcyclohexane & 0.50 & $\mathrm{U}$ \\
\hline $78-87-5$ & 1,2-Dichloropropane & 0.50 & $\mathrm{U}$ \\
\hline $75-27-4$ & Bromodichloromethane & 0.50 & $\mathrm{U}$ \\
\hline $10061-01-5$ & cis-1,3-Dichloropropene & 0.50 & $\mathrm{U}$ \\
\hline $108-10-1$ & 4-Methyl-2-pentanone & 5.0 & $\mathrm{U}$ \\
\hline $108-88-3$ & Toluene & 0.036 & $\mathrm{~J}$ \\
\hline $10061-02-6$ & trans-1,3-Dichloropropene & 0.50 & $\mathrm{U}$ \\
\hline $79-00-5$ & 1,1,2-Trichloroethane & 0.50 & $\mathrm{U}$ \\
\hline $127-18-4$ & Tetrachloroethene & 0.50 & $\mathrm{U}$ \\
\hline $591-78-6$ & 2-Hexanone & 5.0 & $\mathrm{U}$ \\
\hline $124-48-1$ & Dibromochloromethane & 0.50 & $\mathrm{U}$ \\
\hline $106-93-4$ & 1,2-Dibromoethane & 0.50 & $\mathrm{U}$ \\
\hline $108-90-7$ & Chlorobenzene & 0.50 & $\mathrm{U}$ \\
\hline $100-41-4$ & Ethylbenzene & 0.50 & $\mathrm{U}$ \\
\hline $95-47-6$ & o-xylene & 0.50 & $\mathrm{U}$ \\
\hline $179601-23-1$ & m,p-Xylene & 0.50 & $\mathrm{U}$ \\
\hline $100-42-5$ & Styrene & 0.50 & $\mathrm{U}$ \\
\hline $75-25-2$ & Bromoform & 0.50 & $\mathrm{U}$ \\
\hline $98-82-8$ & Isopropylbenzene & 0.50 & $\mathrm{U}$ \\
\hline $79-34-5$ & $1,1,2,2$-Tetrachloroethane & 0.50 & $\mathrm{U}$ \\
\hline $541-73-1$ & 1,3-Dichlorobenzene & 0.50 & $\mathrm{U}$ \\
\hline $106-46-7$ & 1,4-Dichlorobenzene & 0.50 & $\mathrm{U}$ \\
\hline $95-50-1$ & 1,2-Dichlorobenzene & 0.50 & $\mathrm{U}$ \\
\hline $96-12-8$ & 1,2-Dibromo-3-Chloropropane & 0.50 & $\mathrm{U}$ \\
\hline $120-82-1$ & 1,2,4-Trichlorobenzene & 0.50 & $\mathrm{U}$ \\
\hline $87-61-6$ & 1,2,3-Trichlorobenzene & 0.50 & $\mathrm{U}$ \\
\hline
\end{tabular}




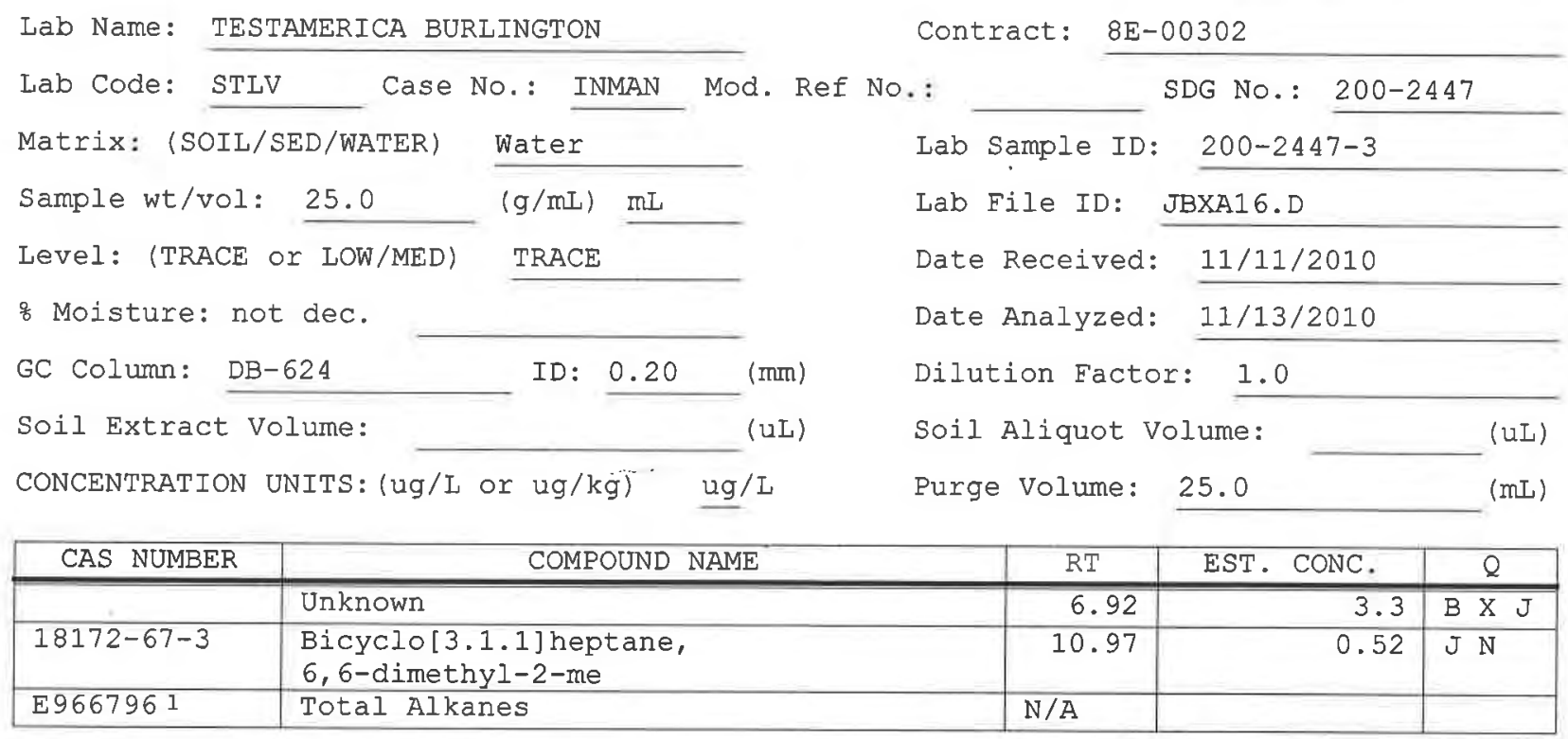


$6 A$ - FORM VI VOA-1

VOLATILE ORGANICS INITIAL CALIBRATION DATA

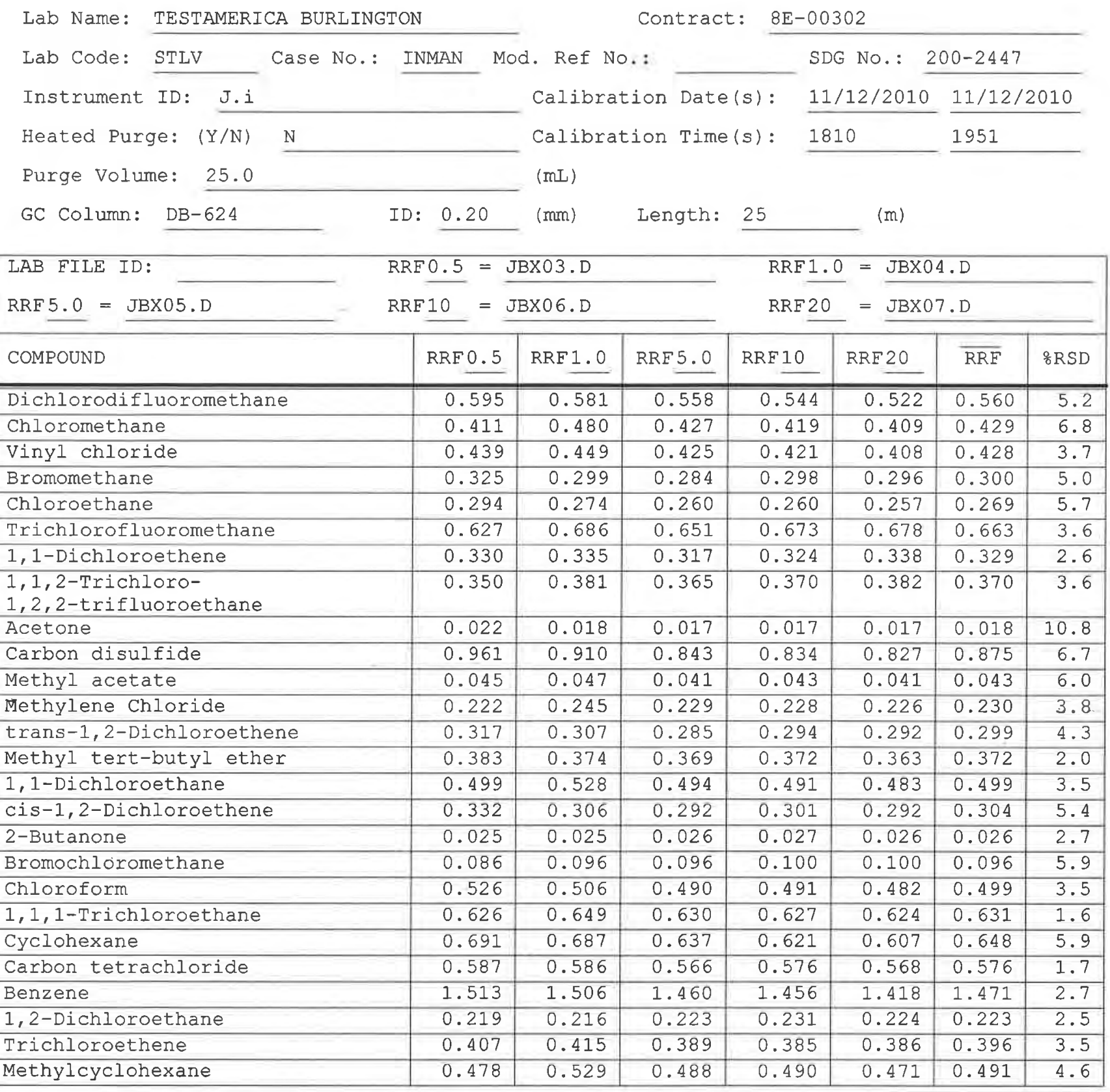

Report 1,4-Dioxane for Low-Medium VOA analysis only 
$6 \mathrm{~B}$ - FORM VI VOA-2

VOLATILE ORGANICS INITIAL CALIBRATION DATA

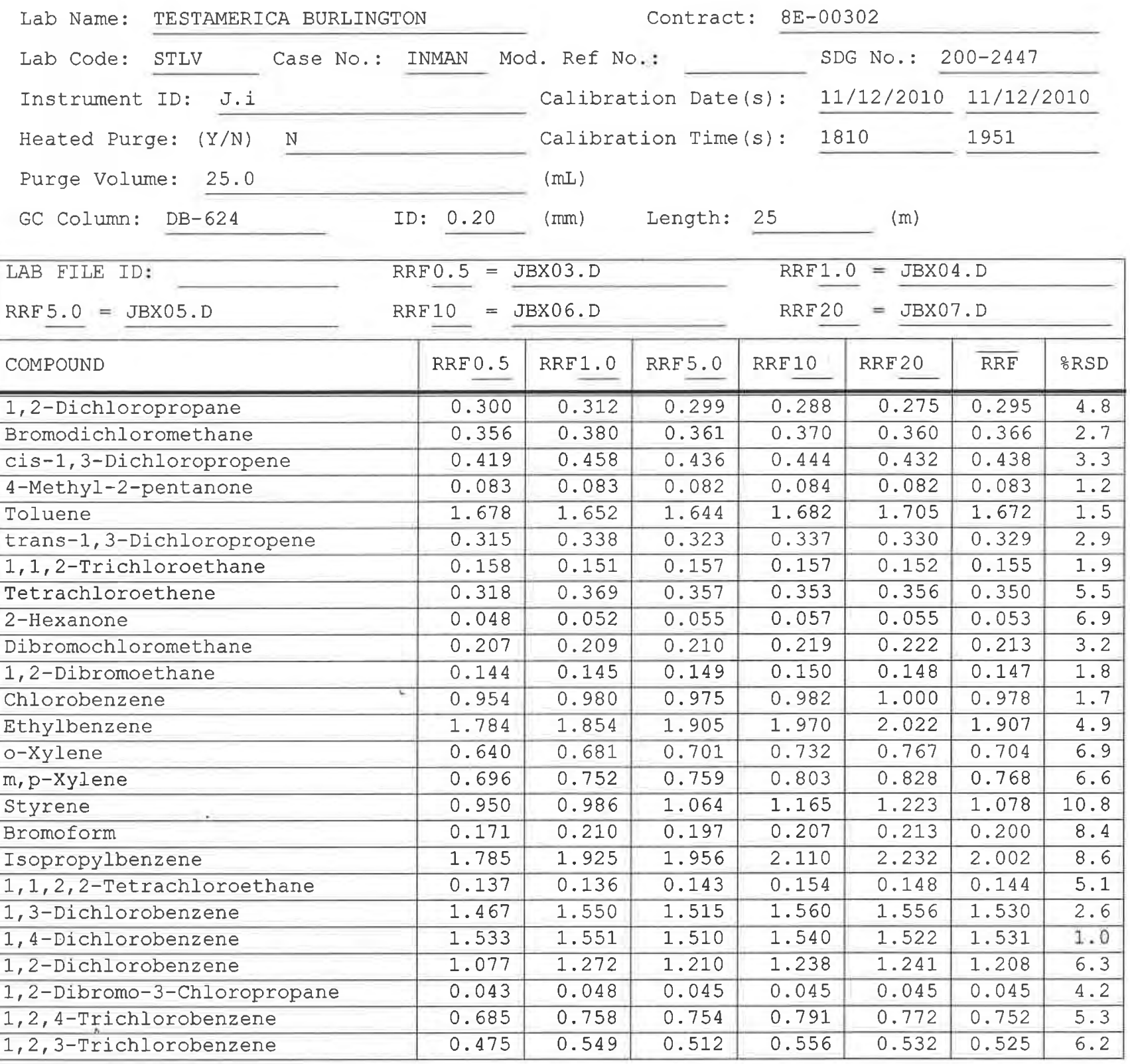


$6 C$ - FORM VI VOA-3

VOLATILE ORGANICS INITIAL CALIBRATION DATA

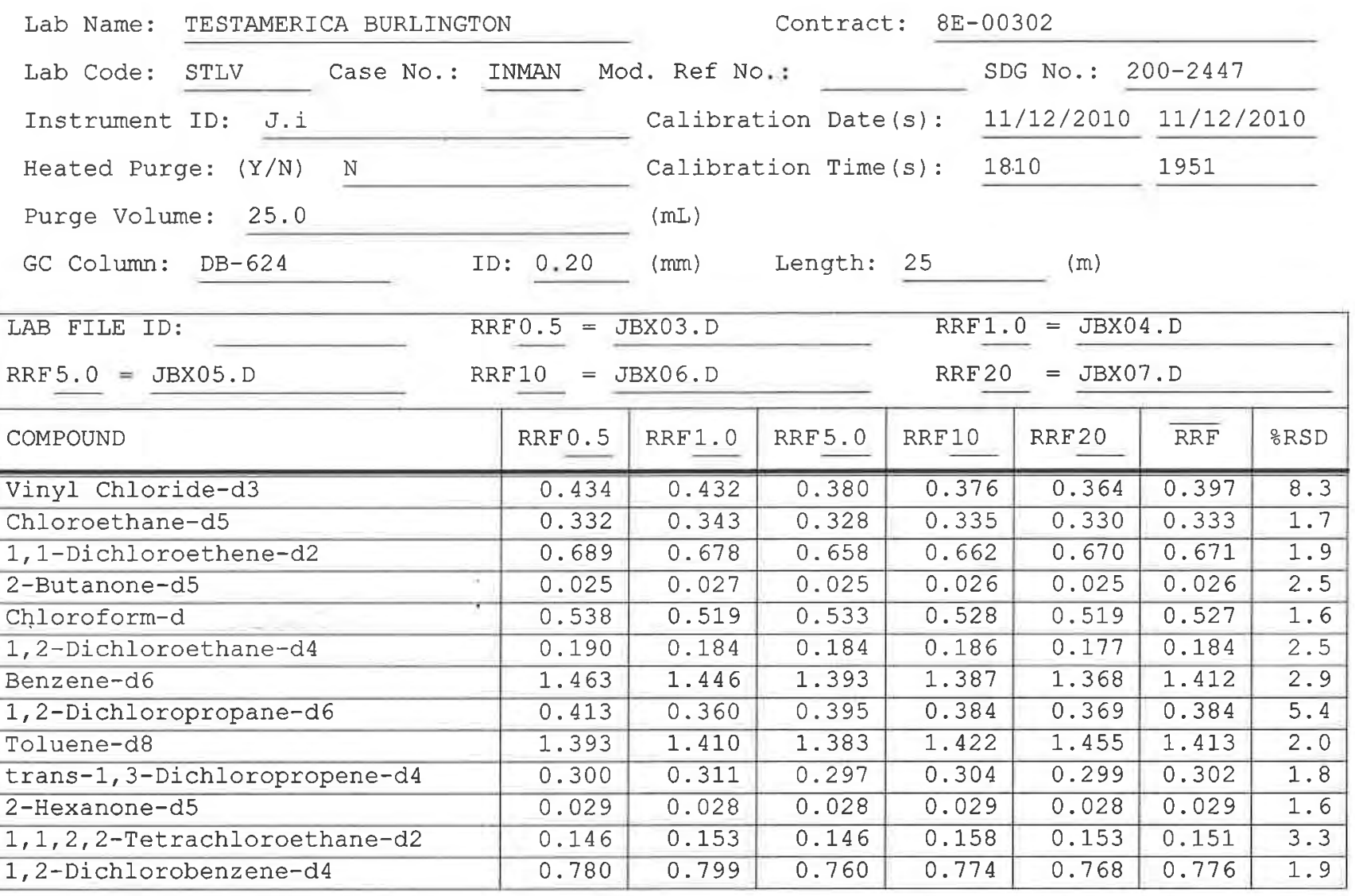

Report 1,4-Dioxane-d8 for Low-Medium VOA analysis only 
$7 A$ - FORM VII VOA-1

VOLATILE CONTINUING CALIBRATION DATA

Lab Name: TESTAMERICA BURLINGTON

Contract: $8 \mathrm{E}-00302$

Lab Code: STLV Case No.: INMAN Mod. Ref No.:

SDG No.: 200-2447

Instrument ID: J.i

Calibration Date: 11/13/2010 Time: 1037

Lab File Id: JBXA02.D

EPA Sample No. (VSTD\#\#\#\#): VSTD005JC

Init. Calib. Date(s): 11/12/2010 11/12/2010

Heated Purge: ( $\mathrm{Y} / \mathrm{N}) \underline{\mathrm{N}}$ GC Column: $\mathrm{DB}-624$ ID: 0.20 (mm) Length: 25 (m)

Purge Volume: 25.0

(mL)

\begin{tabular}{|c|c|c|c|c|c|}
\hline COMPOUND & $\overline{\mathrm{RRF}}$ & RRE5.0 & $\begin{array}{l}\text { MIN } \\
\text { RRF }\end{array}$ & $\because \mathrm{D}$ & MAX $\because D$ \\
\hline Dichlorodifluoromethane & 0.560 & 0.580 & 0.010 & 3.5 & 40.0 \\
\hline Chloromethane & 0.429 & 0.447 & 0.010 & 4.2 & 40.0 \\
\hline Vinyl chloride & 0.428 & 0.431 & 0.010 & 0.6 & 30.0 \\
\hline Bromomethane & 0.300 & 0.318 & 0.100 & 5.9 & 30.0 \\
\hline Chloroethane & 0.269 & 0.251 & 0.010 & -6.8 & 40.0 \\
\hline Trichlorofluoromethane & 0.663 & 0.655 & 0.010 & -1.3 & 40.0 \\
\hline 1,1-Dichloroethene & 0.329 & 0.329 & 0.100 & -0.1 & 30.0 \\
\hline 1,1,2-Trichloro-1,2,2-trifluoroethane & 0.370 & 0.367 & 0.010 & -0.6 & 40.0 \\
\hline Acetone & 0.018 & 0.017 & 0.010 & -7.9 & 40.0 \\
\hline Carbon disulfide & 0.875 & 0.858 & 0.010 & -1.9 & 40.0 \\
\hline Methyl acetate & 0.043 & 0.045 & 0.010 & 4.1 & 40.0 \\
\hline Methylene Chloride & 0.230 & 0.230 & 0.010 & 0.3 & 40.0 \\
\hline $\operatorname{trans}-1,2-$-Dichloroethene & 0.299 & 0.306 & 0.010 & 2.3 & 40.0 \\
\hline Methyl tert-butyl ether & 0.372 & 0.363 & 0.010 & -2.5 & 40.0 \\
\hline 1,1-Dichloroethane & 0.499 & 0.505 & 0.200 & 1.3 & 30.0 \\
\hline cis-1,2-Dichloroethene & 0.304 & 0.296 & 0.010 & -2.8 & 40.0 \\
\hline 2-Butanone & 0.026 & 0.025 & 0.010 & -5.8 & 40.0 \\
\hline Bromochloromethane & 0.096 & 0.097 & 0.050 & 1.0 & 30.0 \\
\hline Chloroform & 0.499 & 0.493 & 0.200 & -1.2 & 30.0 \\
\hline $1,1,1$-Trichloroethane & 0.631 & 0.662 & 0.100 & 4.8 & 30.0 \\
\hline Cyclohexane & 0.648 & 0.670 & 0.010 & 3.3 & 40.0 \\
\hline Carbon tetrachloride & 0.576 & 0.623 & 0.100 & 8.1 & 30.0 \\
\hline Benzene & 1.471 & 1.501 & 0.400 & 2.1 & 30.0 \\
\hline 1,2-Dichloroethane & 0.223 & 0.223 & 0.100 & 0.2 & 30.0 \\
\hline Trichloroethene & 0.396 & 0.411 & 0.300 & 3.7 & 30.0 \\
\hline Methylcyclohexane & 0.491 & 0.515 & 0.010 & 4.9 & 40.0 \\
\hline
\end{tabular}

Report 1,4-Dioxane for Low/Medium VOA analysis only 
$7 B$ - FORM VII VOA-2

VOLATILE CONTINUING CALIBRATION DATA

Lab Name: TESTAMERICA BURLINGTON

Contract: $8 \mathrm{E}-00302$

Lab Code: STLV Case No.: INMAN Mod. Ref No.:

SDG No.: 200-2447

Instrument ID: J.i

Calibration Date: 11/13/2010 Time: 1037

Lab File Id: JBXA02.D Init. Calib. Date(s): 11/12/2010 11/12/2010

EPA Sample No.(VSTD\#\#\#\#): VSTD005JC

Init. Calib. Time(s): $1810 \quad 1951$

Heated Purge: ( $\mathrm{Y} / \mathrm{N})$ GC Column: DB-624

ID: $0.20(\mathrm{~mm})$ Length: 25

(m)

Purge volume: 25.0 $(m L)$

\begin{tabular}{|c|c|c|c|c|c|}
\hline COMPOUND & $\overline{\mathrm{RRF}}$ & RRE5.0 & $\begin{array}{l}\text { MIN } \\
\text { RRF }\end{array}$ & $\% \mathrm{D}$ & $\mathrm{MAX} \div \mathrm{D}$ \\
\hline 1,2-Dichloropropane & 0.295 & 0.292 & 0.010 & -0.9 & 40.0 \\
\hline Bromodichloromethane & 0.366 & 0.375 & 0.200 & 2.6 & 30.0 \\
\hline cis-1,3-Dichloropropene & 0.438 & 0.445 & 0.200 & 1.6 & 30.0 \\
\hline 4-Methyl-2-pentanone & 0.083 & 0.080 & 0.010 & -3.3 & 40.0 \\
\hline Toluene & 1.672 & 1.695 & 0.400 & 1.4 & 30.0 \\
\hline trans-1,3-Dichloropropene & 0.329 & 0.335 & 0.100 & 1.8 & 30.0 \\
\hline 1,1,2-Trichloroethane & 0.155 & 0.153 & 0.100 & -1.3 & 30.0 \\
\hline Tetrachloroethene & 0.350 & 0.368 & 0.100 & 5.0 & 30.0 \\
\hline 2-Hexanone & 0.053 & 0.052 & 0.010 & -2.2 & 40.0 \\
\hline Dibromochloromethane & 0.213 & 0.216 & 0.100 & 1.2 & 30.0 \\
\hline 1,2-Dibromoethane & 0.147 & 0.150 & 0.010 & 1.5 & 40.0 \\
\hline Chlorobenzene & 0.978 & 1.005 & 0.500 & 2.8 & 30.0 \\
\hline Ethylbenzene & 1.907 & 1.978 & 0.100 & 3.7 & 30.0 \\
\hline o-xylene & 0.704 & 0.730 & 0.300 & 3.6 & 30.0 \\
\hline $\mathrm{m}, \mathrm{p}$-xylene & 0.768 & 0.789 & 0.300 & 2.8 & 30.0 \\
\hline Styrene & 1.078 & 1.074 & 0.300 & -0.3 & 30.0 \\
\hline Bromoform & 0.200 & 0.201 & 0.050 & 0.4 & 30.0 \\
\hline Isopropylbenzene & 2.002 & 2.087 & 0.010 & 4.3 & 40.0 \\
\hline $1,1,2,2$-Tetrachloroethane & 0.144 & 0.143 & 0.100 & -0.2 & 30.0 \\
\hline 1,3-Dichlorobenzene & 1.530 & 1.582 & 0.400 & 3.4 & 30.0 \\
\hline 1,4-Dichlorobenzene & 1.531 & 1.523 & 0.400 & -0.6 & 30.0 \\
\hline 1,2-Dichlorobenzene & 1.208 & 1.241 & 0.400 & 2.8 & 30.0 \\
\hline 1,2-Dibromo-3-Chloropropane & 0.045 & 0.043 & 0.010 & -5.7 & 40.0 \\
\hline 1,2,4-Trichlorobenzene & 0.752 & 0.760 & 0.200 & 1.1 & 30.0 \\
\hline 1,2,3-Trichlorobenzene & 0.525 & 0.515 & 0.200 & -1.9 & 30.0 \\
\hline
\end{tabular}


7C - FORM VII VOA-3

VOLATILE CONTINUING CALIBRATION DATA

Lab Name: TESTAMERICA BURLINGTON

Contract: $8 \mathrm{E}-00302$

Lab Code: STLV Case No.: INMAN Mod. Ref No.:

SDG NO.: 200-2447

Instrument ID: J.i

Calibration Date: 11/13/2010 Time: 1037

Lab File Id: JBXA02.D Init. Calib. Date(s): 11/12/2010 11/12/2010

EPA Sample No. (VSTD\#\#\#\#): VSTD005JC

Init. Calib. Time(s): $1810 \quad 1951$

Heated Purge: ( $\mathrm{Y} / \mathrm{N})$

GC Column:

ID: $0.20(\mathrm{~mm})$ Length: 25 (m)

Purge volume: 25.0

(mL)

\begin{tabular}{|c|c|c|c|c|c|}
\hline COMPOUND & $\overline{\mathrm{RRF}}$ & RRF5.0 & $\begin{array}{l}\text { MIN } \\
\text { RRF }\end{array}$ & $\because \mathrm{D}$ & MAX $\div D$ \\
\hline Vinyl Chloride-d3 & 0.397 & 0.386 & 0.010 & -2.8 & 30.0 \\
\hline Chloroethane-d5 & 0.333 & 0.325 & 0.010 & -2.6 & 40.0 \\
\hline 1,1-Dichloroethene-d2 & 0.671 & 0.667 & 0.010 & -0.7 & 30.0 \\
\hline 2-Butanone-d5 & 0.026 & 0.024 & 0.010 & -6.4 & 40.0 \\
\hline Chloroform-d & 0.527 & 0.527 & 0.010 & -0.1 & 30.0 \\
\hline 1,2-Dichloroethane-d4 & 0.184 & 0.178 & 0.010 & -3.6 & 30.0 \\
\hline Benzene-d6 & 1.412 & 1.457 & 0.010 & 3.2 & 30.0 \\
\hline 1,2-Dichloropropane-d6 & 0.384 & 0.401 & 0.010 & 4.3 & 40.0 \\
\hline Toluene-d8 & 1.413 & 1.469 & 0.010 & 4.0 & 30.0 \\
\hline trans-1,3-Dichloropropene-d4 & 0.302 & 0.303 & 0.010 & 0.4 & 30.0 \\
\hline 2-Hexanone-d5 & 0.029 & 0.027 & 0.010 & -6.5 & 40.0 \\
\hline $1,1,2,2$-Tetrachloroethane-d2 & 0.151 & 0.148 & 0.010 & -1.9 & 30.0 \\
\hline 1,2-Dichlorobenzene-d4 & 0.776 & 0.769 & 0.010 & -0.9 & 30.0 \\
\hline
\end{tabular}

Report 1,4-Dioxane-d8 for Low/Medium VOA analysis only 
Lab Name: TESTAMERICA BURLINGTON

Contract: $8 \mathrm{E}-00302$

Lab Code: STLV Case No.: INMAN Mod. Ref No.:

SDG No.: 200-2447

Instrument ID: J.i

Calibration Date: 11/13/2010 Time: 2114

Lab File Id: JBXA26.D

EPA Sample No. (VSTD\#\#\#\#)

VSTD005CJ

Init. Calib. Date(s): 11/12/2010 11/12/2010

Heated Purge: $(Y / N) \quad N$

GC Column:

Init. Calib. Time(s): 1810

1951

Purge volume: 25.0 ID: $0.20(\mathrm{~mm})$ Length: 25

(m)

\begin{tabular}{|c|c|c|c|c|c|}
\hline COMPOUND & $\overline{\mathrm{RRF}}$ & RRF5.0 & $\begin{array}{l}\text { MIN } \\
\text { RRF }\end{array}$ & $\frac{\circ}{8} \mathrm{D}$ & MAX 음 \\
\hline Dichlorodifluoromethane & 0.560 & 0.564 & 0.010 & 0.7 & 50.0 \\
\hline Chloromethane & 0.429 & 0.423 & 0.010 & -1.4 & 50.0 \\
\hline Vinyl chloride & 0.428 & 0.428 & 0.010 & 0.0 & 50.0 \\
\hline Bromomethane & 0.300 & 0.297 & 0.010 & -1.1 & 50.0 \\
\hline Chloroethane & 0.269 & 0.279 & 0.010 & 3.9 & 50.0 \\
\hline Trichlorofluoromethane & 0.663 & 0.685 & 0.010 & 3.3 & 50.0 \\
\hline 1,1-Dichloroethene & 0.329 & 0.328 & 0.010 & -0.2 & 50.0 \\
\hline 1,1,2-Trichloro-1,2,2-trifluoroethane & 0.370 & 0.362 & 0.010 & -2.1 & 50.0 \\
\hline Acetone & 0.018 & 0.017 & 0.010 & -6.8 & 50.0 \\
\hline Carbon disulfide & 0.875 & 0.817 & 0.010 & -6.7 & 50.0 \\
\hline Methyl acetate & 0.043 & 0.042 & 0.010 & -2.7 & 50.0 \\
\hline Methylene Chloride & 0.230 & 0.231 & 0.010 & 0.6 & 50.0 \\
\hline trans-1,2-Dichloroethene & 0.299 & 0.305 & 0.010 & 2.0 & 50.0 \\
\hline Methyl tert-butyl ether & 0.372 & 0.343 & 0.010 & -7.9 & 50.0 \\
\hline 1,1-Dichloroethane & 0.499 & 0.495 & 0.010 & -0.9 & 50.0 \\
\hline cis-1,2-Dichloroethene & 0.304 & 0.301 & 0.010 & -1.3 & 50.0 \\
\hline 2-Butanone & 0.026 & 0.025 & 0.010 & -4.3 & 50.0 \\
\hline Bromochloromethane & 0.096 & 0.098 & 0.010 & 2.2 & 50.0 \\
\hline Chloroform & 0.499 & 0.484 & 0.010 & -2.9 & 50.0 \\
\hline 1,1,1-Trichloroethane & 0.631 & 0.625 & 0.010 & -0.9 & 50.0 \\
\hline Cyclohexane & 0.648 & 0.625 & 0.010 & -3.7 & 50.0 \\
\hline Carbon tetrachloride & 0.576 & 0.582 & 0.010 & 1.0 & 50.0 \\
\hline Benzene & 1.471 & 1.474 & 0.010 & 0.2 & 50.0 \\
\hline 1,2-Dichloroethane & 0.223 & 0.220 & 0.010 & -1.2 & 50.0 \\
\hline Trichloroethene & 0.396 & 0.408 & 0.010 & 3.0 & 50.0 \\
\hline Methylcyclohexane & 0.491 & 0.502 & 0.010 & 2.4 & 50.0 \\
\hline
\end{tabular}

Report 1,4-Dioxane for Low/Medium VOA analysis only 
$7 \mathrm{~B}$ - FORM VII VOA-2

VOLATILE CONTINUING CALIBRATION DATA

Lab Name: TESTAMERICA BURLINGTON

Lab Code: STLV Case No.: INMAN Mod. Ref No.:

Contract: $8 E-00302$

Instrument ID: J.i

Calibration Date: 11/13/2010 Time: 2114

Lab File Id: JBXA26.D

EPA Sample No. (VSTD\#\#\#\#)

VSTD005CJ

Init. Calib. Date(s): 11/12/2010 11/12/2010

Heated Purge: ( $\mathrm{Y} / \mathrm{N})$

GC Column:

Init. Calib. Time(s): 1810

1951

Purge Volume: 25.0

ID: $0.20(\mathrm{~mm})$ Length: 25

(m)

(mL)

\begin{tabular}{|c|c|c|c|c|c|}
\hline COMPOUND & $\overline{\mathrm{RRF}}$ & RRF5.0 & $\begin{array}{l}\text { MIN } \\
\text { RRF }\end{array}$ & $\because \mathrm{D}$ & MAX :D \\
\hline 1,2-Dichloropropane & 0.295 & 0.279 & 0.010 & -5.4 & 50.0 \\
\hline Bromodichloromethane & 0.366 & 0.371 & 0.010 & 1.6 & 50.0 \\
\hline cis-1,3-Dichloropropene & 0.438 & 0.430 & 0.010 & -1.9 & 50.0 \\
\hline 4-Methyl-2-pentanone & 0.083 & 0.085 & 0.010 & 3.3 & 50.0 \\
\hline Toluene & 1.672 & 1.703 & 0.010 & 1.9 & 50.0 \\
\hline trans-1,3-Dichloropropene & 0.329 & 0.319 & 0.010 & -2.9 & 50.0 \\
\hline 1,1,2-Trichloroethane & 0.155 & 0.160 & 0.010 & 3.1 & 50.0 \\
\hline Tetrachloroethene & 0.350 & 0.366 & 0.010 & 4.6 & 50.0 \\
\hline 2-Hexanone & 0.053 & 0.058 & 0.010 & 7.9 & 50.0 \\
\hline Dibromochloromethane & 0.213 & 0.219 & 0.010 & 2.5 & 50.0 \\
\hline 1,2-Dibromoethane & 0.147 & 0.151 & 0.010 & 2.8 & 50.0 \\
\hline Chlorobenzene & 0.978 & 1.026 & 0.010 & 4.9 & 50.0 \\
\hline Ethylbenzene & 1.907 & 1.950 & 0.010 & 2.3 & 50.0 \\
\hline o-xylene & 0.704 & 0.745 & 0.010 & 5.7 & 50.0 \\
\hline $\mathrm{m}, \mathrm{p}$-Xylene & 0.768 & 0.800 & 0.010 & 4.2 & 50.0 \\
\hline Styrene & 1.078 & 1.112 & 0.010 & 3.2 & 50.0 \\
\hline Bromoform & 0.200 & 0.196 & 0.010 & -1.8 & 50.0 \\
\hline Isopropylbenzene & 2.002 & 2.092 & 0.010 & 4.5 & 50.0 \\
\hline $1,1,2,2$-Tetrachloroethane & 0.144 & 0.157 & 0.010 & 9.3 & 50.0 \\
\hline 1,3-Dichlorobenzene & 1.530 & 1.620 & 0.010 & 5.9 & 50.0 \\
\hline 1,4-Dichlorobenzene & 1.531 & 1.565 & 0.010 & 2.2 & 50.0 \\
\hline 1,2-Dichlorobenzene & 1.208 & 1.242 & 0.010 & 2.9 & 50.0 \\
\hline 1,2-Dibromo-3-Chloropropane & 0.045 & 0.043 & 0.010 & -3.9 & 50.0 \\
\hline 1,2,4-Trichlorobenzene & 0.752 & 0.795 & 0.010 & 5.7 & 50.0 \\
\hline $1,2,3$-Trichlorobenzene & 0.525 & 0.572 & 0.010 & 9.0 & 50.0 \\
\hline
\end{tabular}


Lab Name: TESTAMERICA BURLINGTON

Lab Code: STLV Case No.: INMAN Mod. Ref No.:

Contract: $8 \mathrm{E}-00302$

Calibration Date: $11 / 13 / 2010$ Time: 2114

Lab File Id: JBXA26.D Init. Calib. Date(s): 11/12/2010 11/12/2010

EPA Sample No. (VSTD\#\#\#\#): VSTD005CJ Init. Calib. Time(s): $1810 \quad 1951$

Heated Purge: ( $Y / N) \quad N$ GC Column: $\mathrm{DB}-624$ ID: $0.20(\mathrm{~mm})$ Length: 25 (m)

Purge Volume: 25.0 $(\mathrm{mL})$

\begin{tabular}{|c|c|c|c|c|c|}
\hline COMPOUND & $\overline{\mathrm{RRF}}$ & RRF5.0 & $\begin{array}{l}\text { MIN } \\
\text { RRE }\end{array}$ & $\frac{\circ}{0} \mathrm{D}$ & MAX $\because D$ \\
\hline Vinyl Chloride-d3 & 0.397 & 0.385 & 0.010 & -3.1 & 50.0 \\
\hline Chloroethane-d5 & 0.333 & 0.354 & 0.010 & 6.0 & 50.0 \\
\hline 1,1-Dichloroethene-d2 & 0.671 & 0.672 & 0.010 & 0.1 & 50.0 \\
\hline 2-Butanone-d5 & 0.026 & 0.024 & 0.010 & -4.9 & 50.0 \\
\hline Chloroform-d & 0.527 & 0.523 & 0.010 & -0.7 & 50.0 \\
\hline 1,2-Dichloroethane-d4 & 0.184 & 0.183 & 0.010 & -0.6 & 50.0 \\
\hline Benzene-d6 & 1.412 & 1.416 & 0.010 & 0.3 & 50.0 \\
\hline 1,2-Dichloropropane-d6 & 0.384 & 0.345 & 0.010 & -10.1 & 50.0 \\
\hline Toluene-d8 & 1.413 & 1.467 & 0.010 & 3.9 & 50.0 \\
\hline trans-1,3-Dichloropropene-d4 & 0.302 & 0.293 & 0.010 & -3.2 & 50.0 \\
\hline 2-Hexanone-d5 & 0.029 & 0.028 & 0.010 & -3.4 & 50.0 \\
\hline $1,1,2,2$-Tetrachloroethane-d2 & 0.151 & 0.151 & 0.010 & -0.1 & 50.0 \\
\hline 1,2-Dichlorobenzene-d4 & 0.776 & 0.794 & 0.010 & 2.2 & 50.0 \\
\hline
\end{tabular}

Report 1,4-Dioxane-d8 for Low/Medium VOA analysis only 
$1 A$ - FORM I VOA-1

VOLATILE ORGANICS ANALYSIS DATA SHEET
EPA SAMPLE NO.

VBLKJC

Lab Name: TESTAMERICA BURLINGTON

Lab Code: STLV Case No.: INMAN Mod. Ref No.:

Contract: $8 \mathrm{E}-00302$

Matrix: (SOIL/SED/WATER)

Sample wt/vol: 25.0

Level: (TRACE/LOW/MED) TRACE

\% Moisture: not dec.

GC Colurn: DB-624

Soil Extract Volume:

Water

$(\mathrm{g} / \mathrm{mL}) \mathrm{mL}$

Purge Volume: 25.0

\begin{tabular}{|c|c|c|c|}
\hline CAS NO. & COMPOUND & $\begin{array}{l}\text { CONCENTRATION UNITS: } \\
(\mathrm{ug} / \mathrm{L} \text { or } \mathrm{ug} / \mathrm{kg}) \mathrm{ug} / \mathrm{L} \\
\end{array}$ & $Q$ \\
\hline $75-71-8$ & Dichlorodifluoromethane & 0.50 & $\overline{\mathrm{U}}$ \\
\hline $74-87-3$ & Chloromethane & 0.50 & $\mathrm{U}$ \\
\hline $75-01-4$ & Vinyl chloride & 0.50 & $\mathrm{U}$ \\
\hline $74-83-9$ & Bromomethane & 0.50 & $\mathrm{U}$ \\
\hline $75-00-3$ & Chloroethane & 0.50 & $\mathrm{U}$ \\
\hline $75-69-4$ & Trichlorofluoromethane & 0.50 & $\mathrm{U}$ \\
\hline $75-35-4$ & 1,1-Dichloroethene & 0.50 & $\mathrm{U}$ \\
\hline $76-13-1$ & 1,1,2-Trichloro-1,2,2-trifluoroethane & 0.50 & $\mathrm{U}$ \\
\hline $67-64-1$ & Acetone & 1.8 & $\mathrm{~J}$ \\
\hline $75-15-0$ & Carbon disulfide & 0.16 & $\mathrm{~J}$ \\
\hline $79-20-9$ & Methyl acetate & 0.50 & $\mathrm{U}$ \\
\hline $75-09-2$ & Methylene Chloride & 0.50 & $\mathrm{U}$ \\
\hline $156-60-5$ & trans-1,2-Dichloroethene & 0.046 & $\mathrm{~J}$ \\
\hline $1634-04-4$ & Methyl tert-butyl ether & 0.50 & $\mathrm{U}$ \\
\hline $75-34-3$ & 1,1-Dichloroethane & 0.50 & $\mathrm{U}$ \\
\hline $156-59-2$ & cis-1,2-Dichloroethene & 0.50 & $\mathrm{U}$ \\
\hline $78-93-3$ & 2-Butanone & 5.0 & $\mathrm{U}$ \\
\hline $74-97-5$ & Bromochloromethane & 0.50 & $\mathrm{U}$ \\
\hline $67-66-3$ & Chloroform & 0.50 & $\mathrm{U}$ \\
\hline $71-55-6$ & 1,1,1-Trichloroethane & 0.50 & $\mathrm{U}$ \\
\hline $110-82-7$ & Cyclohexane & 0.50 & $\mathrm{U}$ \\
\hline $56-23-5$ & Carbon tetrachloride & 0.50 & $\mathrm{U}$ \\
\hline $71-43-2$ & Benzene & 0.50 & $\mathrm{U}$ \\
\hline $107-06-2$ & 1,2-Dichloroethane & 0.50 & $\mathrm{U}$ \\
\hline
\end{tabular}

Lab Sample ID: MB 200-9552/3

Lab File ID: JBXA03.D

Date Received:

Date Analyzed: 11/13/2010

Dilution Factor: 1.0

Soil Aliquot Volume: (uL) (uL) (mL) SDG No.: 200-2447 
Lab Name: TESTAMERICA BURLINGTON

Lab Code: STLV Case No.: INMAN

Matrix: (SOIL/SED/WATER)

Sample wt/vol: 25.0

Water

$(\mathrm{g} / \mathrm{mL}) \mathrm{mL}$

Level: (TRACE/LOW/MED) TRACE

음 Moisture: not dec.

GC Column: DB-624

Soil Extract Volume: ID: $0.20 \quad(\mathrm{~mm})$

Purge Volume: 25.0 (uL)

(mL)
Contract: $8 \mathrm{E}-00302$

SDG No.: 200-2447
Lab Sample ID: MB 200-9552/3

Lab File ID: JBXA03.D

Date Received:

Date Analyzed: 11/13/2010

Dilution Factor: 1.0

Soil Aliquot Volume:

\begin{tabular}{|c|c|c|c|}
\hline CAS NO. & COMPOUND & $\begin{array}{l}\text { CONCENTRATION UNITS: } \\
(\mathrm{ug} / \mathrm{L} \text { or } \mathrm{ug} / \mathrm{kg}) \mathrm{ug} / \mathrm{L}\end{array}$ & $Q$ \\
\hline $79-01-6$ & Trichloroethene & 0.50 & $\overline{\mathrm{U}}$ \\
\hline $108-87-2$ & Methylcyclohexane & 0.50 & $\mathrm{U}$ \\
\hline $78-87-5$ & 1,2-Dichloropropane & 0.50 & $\mathrm{U}$ \\
\hline $75-27-4$ & Bromodichloromethane & 0.50 & $\mathrm{U}$ \\
\hline $10061-01-5$ & cis-1,3-Dichloropropene & 0.50 & $\mathrm{U}$ \\
\hline $108-10-1$ & 4-Methyl-2-pentanone & 5.0 & $\mathrm{U}$ \\
\hline $108-88-3$ & Toluene & 0.50 & $\mathrm{U}$ \\
\hline $10061-02-6$ & trans-1,3-Dichloropropene & 0.50 & $\mathrm{U}$ \\
\hline $79-00-5$ & $1,1,2$-Trichloroethane & 0.50 & $\mathrm{U}$ \\
\hline $127-18-4$ & Tetrachloroethene & 0.50 & $\mathrm{U}$ \\
\hline $591-78-6$ & 2-Hexanone & 5.0 & $\mathrm{U}$ \\
\hline $124-48-1$ & Dibromochloromethane & 0.50 & $\mathrm{U}$ \\
\hline $106-93-4$ & 1,2-Dibromoethane & 0.50 & $\mathrm{U}$ \\
\hline $108-90-7$ & Chlorobenzene & 0.50 & $\mathrm{U}$ \\
\hline $100-41-4$ & Ethylbenzene & 0.50 & $\mathrm{U}$ \\
\hline $95-47-6$ & o-Xylene & 0.50 & $\mathrm{U}$ \\
\hline $179601-23-1$ & m,p-xylene & 0.50 & $\mathrm{U}$ \\
\hline $100-42-5$ & Styrene & 0.50 & $\mathrm{U}$ \\
\hline $75-25-2$ & Bromoform & 0.50 & $\mathrm{U}$ \\
\hline $98-82-8$ & Isopropylbenzene & 0.50 & $\mathrm{U}$ \\
\hline $79-34-5$ & $1,1,2,2$-Tetrachloroethane & 0.50 & $\mathrm{U}$ \\
\hline $541-73-1$ & 1,3-Dichlorobenzene & 0.50 & $\mathrm{U}$ \\
\hline $106-46-7$ & 1,4-Dichlorobenzene & 0.044 & $\mathrm{~J}$ \\
\hline $95-50-1$ & 1,2-Dichlorobenzene & 0.50 & $\mathrm{U}$ \\
\hline $96-12-8$ & 1,2-Dibromo-3-Chloropropane & 0.50 & $\mathrm{U}$ \\
\hline $120-82-1$ & 1,2,4-Trichlorobenzene & 0.14 & $\mathrm{~J}$ \\
\hline $87-61-6$ & 1,2,3-Trichlorobenzene & 0.19 & $\mathrm{~J}$ \\
\hline
\end{tabular}


1J - FORM I VOA-TIC

VOLATILE ORGANICS ANALYSIS DATA SHEET

TENTATIVELY IDENTIFIED COMPOUNDS
EPA SAMPLE NO.

VBLKJC

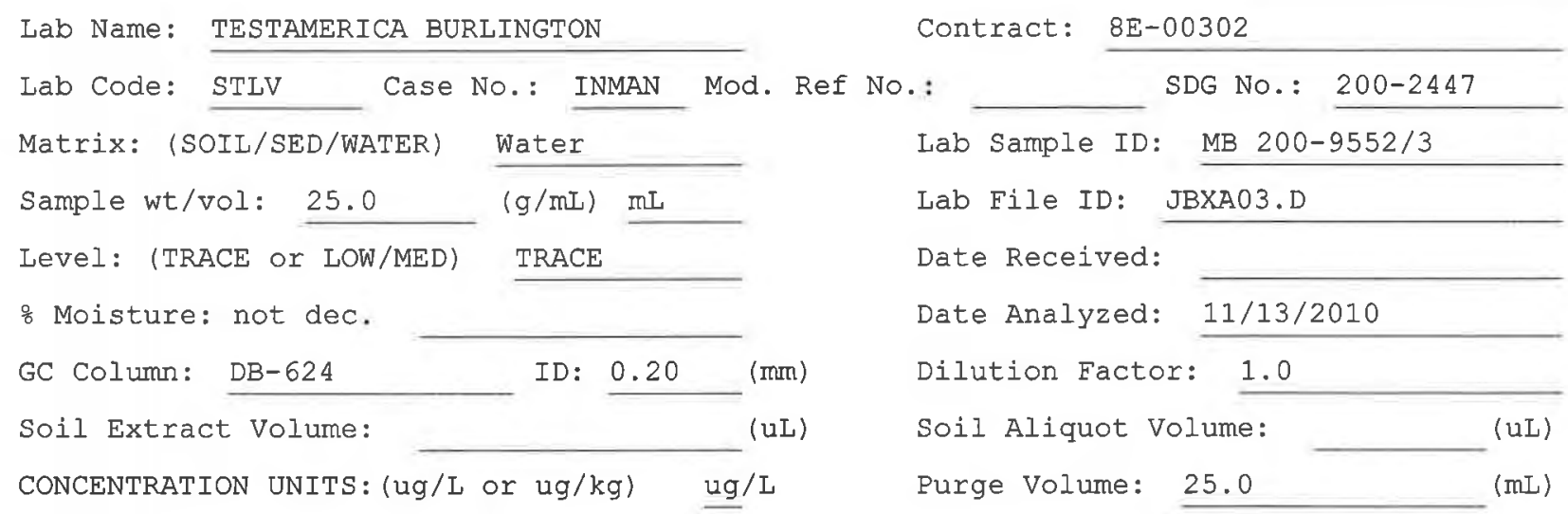

01

\begin{tabular}{|l|l|c|c|c|}
\hline CAS NUMBER & \multicolumn{1}{|c|}{ COMPOUND NAME } & RT & EST. CONC. & Q \\
\hline \hline & Unknown & 6.92 & 3.5 & X J \\
\hline E9667961 & Total Alkanes & N/A & & \\
\hline
\end{tabular}

1 EPA-designated Registry Number. 
$1 A$ - FORM I VOA-1

VOLATILE ORGANICS ANALYSIS DATA SHEET
EPA SAMPLE NO.

VHBLK01

Lab Name: TESTAMERICA BURLINGTON

Lab Code: STLV

Matrix: (SOIL/SED/WATER)

Sample wt/vol: 25.0

Level: (TRACE/LOW/MED)

응 Moisture: not dec.

GC Column: DB-624

Soil Extract Volume: ID: 0.20

Water

$(g / m L) \quad m L$

\section{RACE}

Purge Volume: 25.0
Contract: $\quad 8 \mathrm{E}-00302$

SDG No.: 200-2447
Lab Sample ID: 200-2447-5

Lab File ID: JBXA24.D

Date Received:

Date Analyzed: 11/13/2010

Dilution Factor: 1.0

Soil Aliquot Volume:

(UL)

\begin{tabular}{|c|c|c|c|}
\hline CAS NO. & COMPOUND & $\begin{array}{l}\text { CONCENTRATION UNITS: } \\
(\mathrm{ug} / \mathrm{L} \text { or } \mathrm{ug} / \mathrm{kg}) \mathrm{ug} / \mathrm{L}\end{array}$ & $Q$ \\
\hline $75-71-8$ & Dichlorodifluoromethane & 0.50 & $\mathrm{U}$ \\
\hline $74-87-3$ & Chloromethane & 0.50 & $\mathrm{U}$ \\
\hline $75-01-4$ & Vinyl chloride & 0.50 & $\mathrm{U}$ \\
\hline $74-83-9$ & Bromomethane & 0.50 & $\mathrm{U}$ \\
\hline $75-00-3$ & Chloroethane & 0.50 & $\mathrm{U}$ \\
\hline $75-69-4$ & Trichlorofluoromethane & 0.50 & $\mathrm{U}$ \\
\hline $75-35-4$ & 1,1-Dichloroethene & 0.50 & $\mathrm{U}$ \\
\hline $76-13-1$ & 1,1,2-Trichloro-1,2,2-trifluoroethane & 0.50 & $\mathrm{U}$ \\
\hline $67-64-1$ & Acetone & 2.7 & $\mathrm{~J} B$ \\
\hline $75-15-0$ & Carbon disulfide & 0.50 & $\mathrm{U}$ \\
\hline $79-20-9$ & Methyl acetate & 0.50 & $\mathrm{U}$ \\
\hline $75-09-2$ & Methylene Chloride & 0.50 & $\mathrm{U}$ \\
\hline $156-60-5$ & trans-1,2-Dichloroethene & 0.50 & $\mathrm{U}$ \\
\hline $1634-04-4$ & Methyl tert-butyl ether & 0.50 & $\mathrm{U}$ \\
\hline $75-34-3$ & 1,1-Dichloroethane & 0.50 & $\mathrm{U}$ \\
\hline $156-59-2$ & cis-1,2-Dichloroethene & 0.50 & $\mathrm{U}$ \\
\hline $78-93-3$ & 2-Butanone & 5.0 & $\mathrm{U}$ \\
\hline $74-97-5$ & Bromochloromethane & 0.50 & $\mathrm{U}$ \\
\hline $67-66-3$ & Chloroform & 0.50 & $\mathrm{U}$ \\
\hline $71-55-6$ & 1,1,1-Trichloroethane & 0.50 & $\mathrm{U}$ \\
\hline $110-82-7$ & Cyclohexane & 0.50 & $\mathrm{U}$ \\
\hline $56-23-5$ & Carbon tetrachloride & 0.50 & $\mathrm{U}$ \\
\hline $71-43-2$ & Benzene & 0.50 & $\mathrm{U}$ \\
\hline $107-06-2$ & 1,2-Dichloroethane & 0.50 & $\mathrm{U}$ \\
\hline
\end{tabular}

Report 1,4-Dioxane for Low-Medium VOA analysis only 
Lab Name: TESTAMERICA BURLINGTON

Lab code: STLV

Matrix: (SOIL/SED/WATER)

Sample wt/vol: 25.0

Water

$(g / m L) \quad m L$

Level: (TRACE/LOW/MED) TRACE

\% Moisture: not dec.

GC Column: DB-624 ID: $0.20 \quad$ (mim)

Soil Extract Volume: (uL)

Purge Volume: 25.0 (mL)
Contract: $8 \mathrm{E}-00302$

SDG No.: 200-2447
Lab Sample ID: 200-2447-5

Lab File ID: JBXA24.D

Date Received:

Date Analyzed: 11/13/2010

Dilution Factor: 1.0

Soil Aliquot Volume: (uL)

\begin{tabular}{|c|c|c|c|}
\hline CAS NO. & COMPOUND & $\begin{array}{l}\text { CONCENTRATION UNITS: } \\
(\mathrm{ug} / \mathrm{I} \text { or } \mathrm{ug} / \mathrm{kg}) \mathrm{ug} / \mathrm{L}\end{array}$ & $Q$ \\
\hline $79-01-6$ & Trichloroethene & 0.50 & $\overline{\mathrm{U}}$ \\
\hline $108-87-2$ & Methylcyclohexane & 0.50 & $\mathrm{U}$ \\
\hline $78-87-5$ & 1,2-Dichloropropane & 0.50 & $\mathrm{U}$ \\
\hline $75-27-4$ & Bromodichloromethane & 0.50 & U \\
\hline $10061-01-5$ & cis-1,3-Dichloropropene & 0.50 & $\mathrm{U}$ \\
\hline $108-10-1$ & 4-Methyl-2-pentanone & 5.0 & $\mathrm{U}$ \\
\hline $108-88-3$ & Toluene & 0.50 & U \\
\hline $10061-02-6$ & trans-1,3-Dichloropropene & 0.50 & $\mathrm{U}$ \\
\hline $79-00-5$ & 1,1,2-Trichloroethane & 0.50 & $\mathrm{U}$ \\
\hline $127-18-4$ & Tetrachloroethene & 0.50 & $\mathrm{U}$ \\
\hline $591-78-6$ & 2-Hexanone & 5.0 & $\mathrm{U}$ \\
\hline $124-48-1$ & Dibromochloromethane & 0.50 & U \\
\hline $106-93-4$ & 1,2-Dibromoethane & 0.50 & $\mathrm{U}$ \\
\hline $108-90-7$ & Chlorobenzene & 0.50 & $\mathrm{U}$ \\
\hline $100-41-4$ & Ethylbenzene & 0.50 & $\mathrm{U}$ \\
\hline $95-47-6$ & o-xylene & 0.50 & $\mathrm{U}$ \\
\hline $179601-23-1$ & m,p-Xylene & 0.50 & $\mathrm{U}$ \\
\hline $100-42-5$ & Styrene & 0.50 & $\mathrm{U}$ \\
\hline $75-25-2$ & Bromoform & 0.50 & $\mathrm{U}$ \\
\hline $98-82-8$ & Isopropylbenzene & 0.50 & $\mathrm{U}$ \\
\hline $79-34-5$ & $1,1,2,2$-Tetrachloroethane & 0.50 & $\mathrm{U}$ \\
\hline $541-73-1$ & 1,3-Dichlorobenzene & 0.50 & $\mathrm{U}$ \\
\hline $106-46-7$ & 1,4-Dichlorobenzene & 0.50 & $\mathrm{U}$ \\
\hline $95-50-1$ & 1,2-Dichlorobenzene & 0.50 & $\mathrm{U}$ \\
\hline $96-12-8$ & 1,2-Dibromo-3-Chloropropane & 0.50 & $\mathrm{U}$ \\
\hline $120-82-1$ & 1,2,4-Trichlorobenzene & 0.50 & $\mathrm{U}$ \\
\hline $87-61-6$ & 1,2,3-Trichlorobenzene & 0.50 & $\mathrm{U}$ \\
\hline
\end{tabular}


IJ - FORM I VOA-TIC

VOLATILE ORGANICS ANALYSIS DATA SHEET

TENTATIVELY IDENTIFIED COMPOUNDS
EPA SAMPLE NO.

VHBLKO1

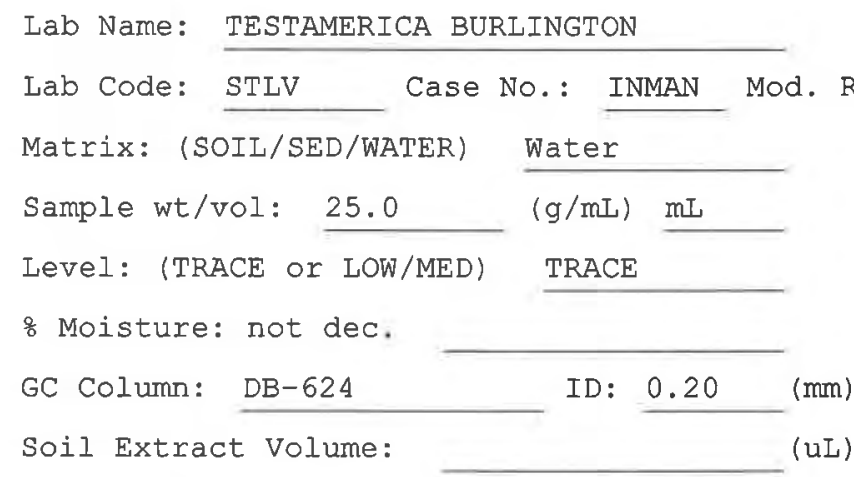

CONCENTRATION UNITS: (ug/L or $\mathrm{ug} / \mathrm{kg}$ ) ug/L
Contract: $8 \mathrm{E}-00302$

SDG No.: 200-2447

Lab Sample ID: 200-2447-5

Lab File ID: JBXA24.D

Date Received:

Date Analyzed: 11/13/2010

Dilution Factor: 1.0

Soil Aliquot Volume: (uL)

Purge Volume: 25.0 (mL)

01

\begin{tabular}{|l|l|c|r|r|}
\hline CAS NUMBER & \multicolumn{1}{|c|}{ COMPOUND NAME } & RT & EST. CONC. & Q \\
\hline \hline & Unknown & 6.92 & 3.4 & $\mathrm{~J} \mathrm{X} \mathrm{B}$ \\
\hline E9667961 & Total Alkanes & N/A & & \\
\hline
\end{tabular}

1 EPA-designated Registry Number. 


\section{TestAmerica}

THE LEADER IN ENVIRONMENTAL TESTING

\section{ANALYTICAL REPORT}

Job Number: 200-2472-1

SDG Number: 200-2472

Job Description: INMAN (200-2472)

Contract Number: 8E-00302

For:

Argonne National Laboratory

9700 South Cass Avenue

Building 203

Office B-149

Argonne, IL 60439

Attention: Mr. Clyde Dennis

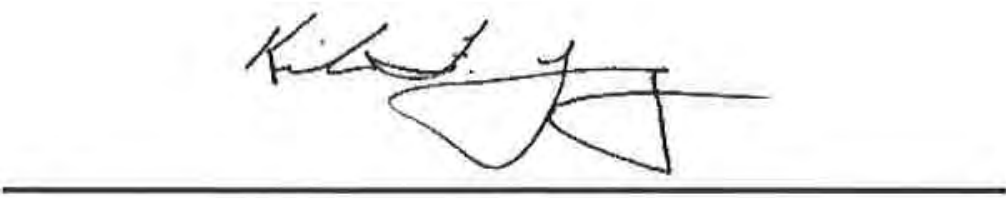

\section{Kirk F Young \\ Project Manager I \\ kirk.young@testamericainc.com}

$11 / 17 / 2010$

The test results in this report relate only to sample(s) as received by the laboratory. These test results were derived under a quality system that adheres to the requirements of NELAC. Pursuant to NELAC, this report may not be produced in full without written approval from the laboratory 


\section{Table of Contents}

Cover Title Page .......................... 1

Report Narrative $\ldots \ldots \ldots \ldots \ldots \ldots \ldots \ldots \ldots \ldots \ldots, 4$

Case Narrative .................................. 4

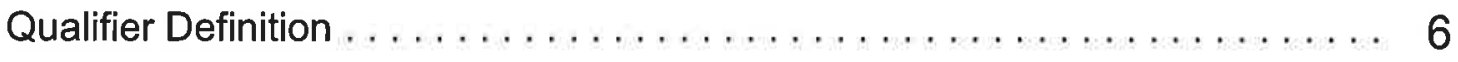

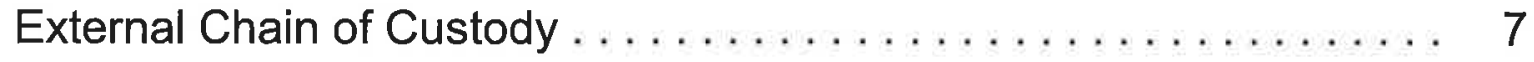

Internal Chain of Custody ........................ 8

Shipping Documentation .......................... 10

Sample Receipt and Log In Check List ..................... 11

Methodology Review............................ 13

QC Summary - SOM01.2 Volatiles-Trace .................. 14

QC Summary - SOM01.2 Volatiles-Trace $\ldots \ldots \ldots \ldots \ldots \ldots \ldots \ldots \ldots \ldots$

Deuterated Monitoring Compound Summary $\ldots \ldots \ldots \ldots \ldots \ldots \ldots \ldots \ldots \ldots \ldots \ldots \ldots \ldots \ldots$

Method Blank $\ldots \ldots \ldots \ldots \ldots \ldots \ldots \ldots \ldots \ldots \ldots \ldots \ldots \ldots \ldots \ldots \ldots, 16$

GC/MS Instrument Performance Check ............................... 17

Internal Standard Area and RT Summary $\ldots \ldots \ldots \ldots \ldots \ldots \ldots \ldots \ldots \ldots \ldots \ldots \ldots \ldots \ldots \ldots$

Sample Data - SOM01.2 Volatiles-Trace . . . . . . . . . . . . 20

Sample Data - SOM01.2 Volatiles-Trace . . . . . . . . . . . . . . . 20

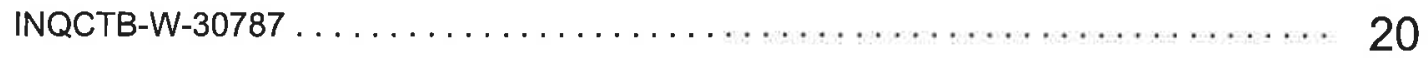

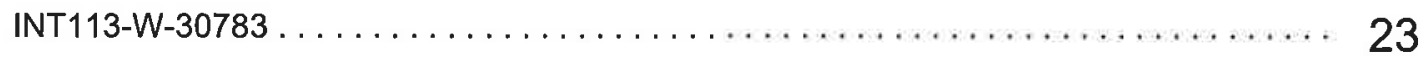

INT115-W-30784 ...................................... 26

INT116-W-30785 ....................................... 29

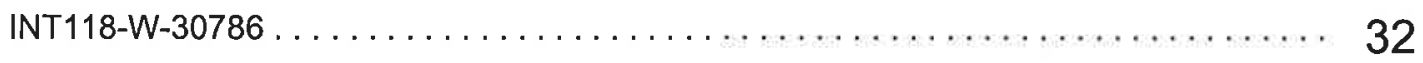

Standards - SOM01.2 Volatiles-Trace . . . . . . . . . . . . . 35

Standards - SOM01.2 Volatiles-Trace $\ldots \ldots \ldots \ldots \ldots \ldots \ldots \ldots \ldots \ldots, 35$

Initial Calibration Data $\ldots \ldots \ldots \ldots \ldots \ldots \ldots \ldots \ldots \ldots \ldots \ldots \ldots \ldots \ldots \ldots \ldots \ldots, \quad 35$

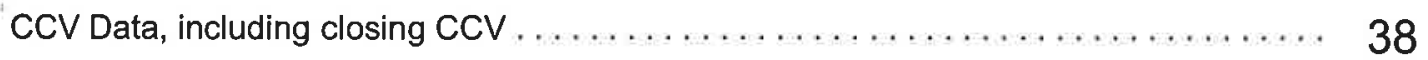

$\begin{array}{ll}\text { Page } 2 \text { of } 49 & 11 / 17 / 2010\end{array}$ 


\section{Table of Contents}

Raw Qc Data - SOM01.2 Volatiles-Trace . . . . . . . . . . . . . . . . 44

Raw Qc Data - SOM01.2 Volatiles-Trace .................... 44

Raw Qc Data - SOM01.2 Volatiles-Trace ........................ 44

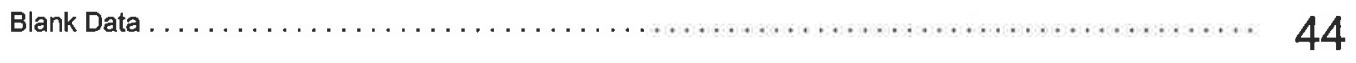




\title{
CASE NARRATIVE
}

\section{Client: Argonne National Laboratory}

\author{
Project: Inman (200-2472)
}

\author{
Report Number: 200-2472-1
}

With the exceptions noted as flags or footnotes, standard analytical protocols were followed in the analysis of the samples and no problems were encountered or anomalies observed.

Calculations are performed before rounding to avoid round-off errors in calculated results.

All holding times were met and proper preservation noted for the methods performed on these samples, unless otherwise detailed in the individual sections below.

\section{Recelpt}

The samples were received on 11/12/2010. Documentation of the condition of the samples at the time of their receipt and any exception to the laboratory's Sample Acceptance Policy is documented in the Sample Handling section of this submittal. The samples, as received, were not acid preserved. On that basis, the laboratory did provide for the analytical work to be performed within seven days of sample collection.

\section{SOM01.2 Volatile Organics (Trace Level Water)}

A storage blank was prepared for volatile organics analysis, and stored in association with the storage of the samples. That storage blank, identified as VHBLK01, was carried through the holding period with the samples, and analyzed.

Each sample was analyzed without a dilution. Each of the analyses associated with the sample set exhibited an acceptable internal standard performance. There was an acceptable recovery of each deuterated monitoring compound (DMC) in the analysis of the method blank associated with the analytical work, and in the analysis of the storage blank associated with the sample set. The analysis of the samples in this sample set did meet the technical acceptance criteria specific to DMC recoveries, although not all DMC recoveries were within the control range in each analysis. The technical acceptance criteria does provide for the recovery of up to three DMCs to fall outside of the control range in the analysis of field samples. Matrix spike and matrix spike duplicate analyses were not performed on samples in this sample set. Trace concentrations of acetone, carbon disulfide, trans-1,2-dichloroethene, 1,4-dichlorobenzene, 1,2,4-trichlorobenzene, and 1,2,3-trichlorobenzene were identified in the analysis of the method blank associated with the analytical work. The concentration of each compound in that analysis was below the established reporting limit, and the analysis did meet the technical acceptance criteria for a compliant method blank analysis. A trace concentration of acetone was identified in the analysis of the storage blank associated with the sample set. The concentration of acetone in that analysis was below the established reporting limit, and the analysis did meet the technical acceptance criteria for a compliant storage blank analysis. Present in the method blank and storage blank analyses was a non-target constituent that represents a compound that is related to the DMC formulation. The fact that the presence of this compound is not within the laboratory's control is at issue. The derived results for that compound have been qualified with an " $X$ " qualifier to reflect the source of the contamination. 
The responses for each of the target analytes met the relative standard deviation criterion in the initial calibration. The response for each target analyte met the percent difference criterion in the continuing calibration check acquisition. The response for each target analyte met the 50.0 percent difference criterion in the closing calibration check acquisition.

The primary quantitation mass for methylcyclohexane that is specified in the Statement of Work is mass 83 . The laboratory did identify a contribution to mass 83 from 1,2-dichloropropane- $d_{6}$, one of the deuterated monitoring compounds (DMCs). The laboratory did change the primary quantitation mass assignment to mass 55 for the quantification of methylcyclohexane.

Manual integration was employed in deriving certain of the analytical results. The values that have been derived from manual integration are qualified on the quantitation reports. Extracted ion current profiles for each manual integration are included in the data package, and further documented in the Sample Preparation section of this submittal. 


\section{DATA REPORTING QUALIFIERS}

Client: Argonne National Laboratory

Job Number: 200-2472-1

Sdg Number: $200-2472$

Description

GC/MS VOA

U

J

$J$

$\mathrm{X}$

*

B

N
Analyzed for but not detected.

Indicates an Estimated Value for TICs

Indicates an estimated value.

See case narrative notes for explanation of the ' $X$ ' flag

Surrogate exceeds the control limit

The analyte was found in an associated blank, as well as in the sample.

This flag indicates the presumptive evidence of a compound. 


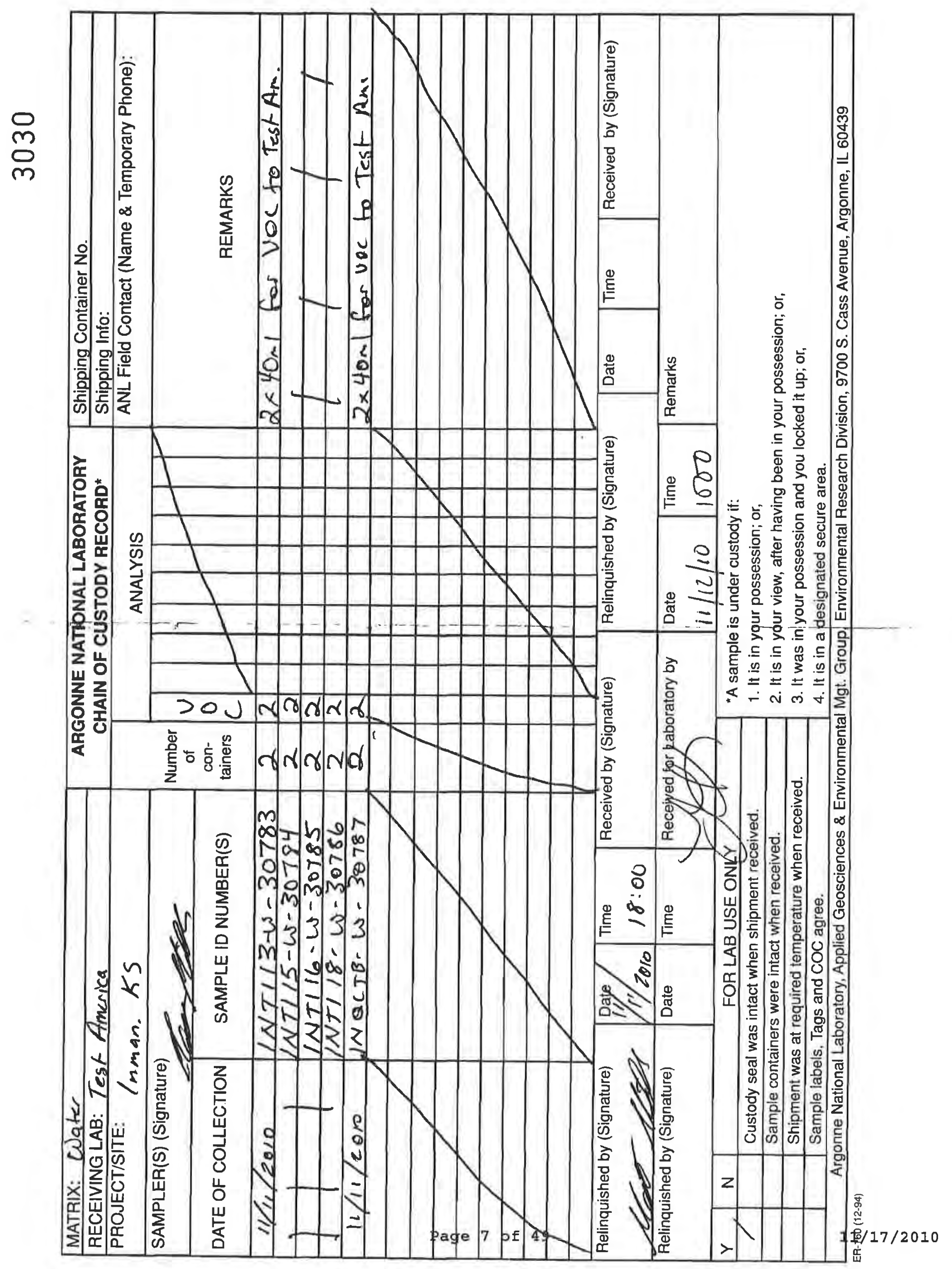




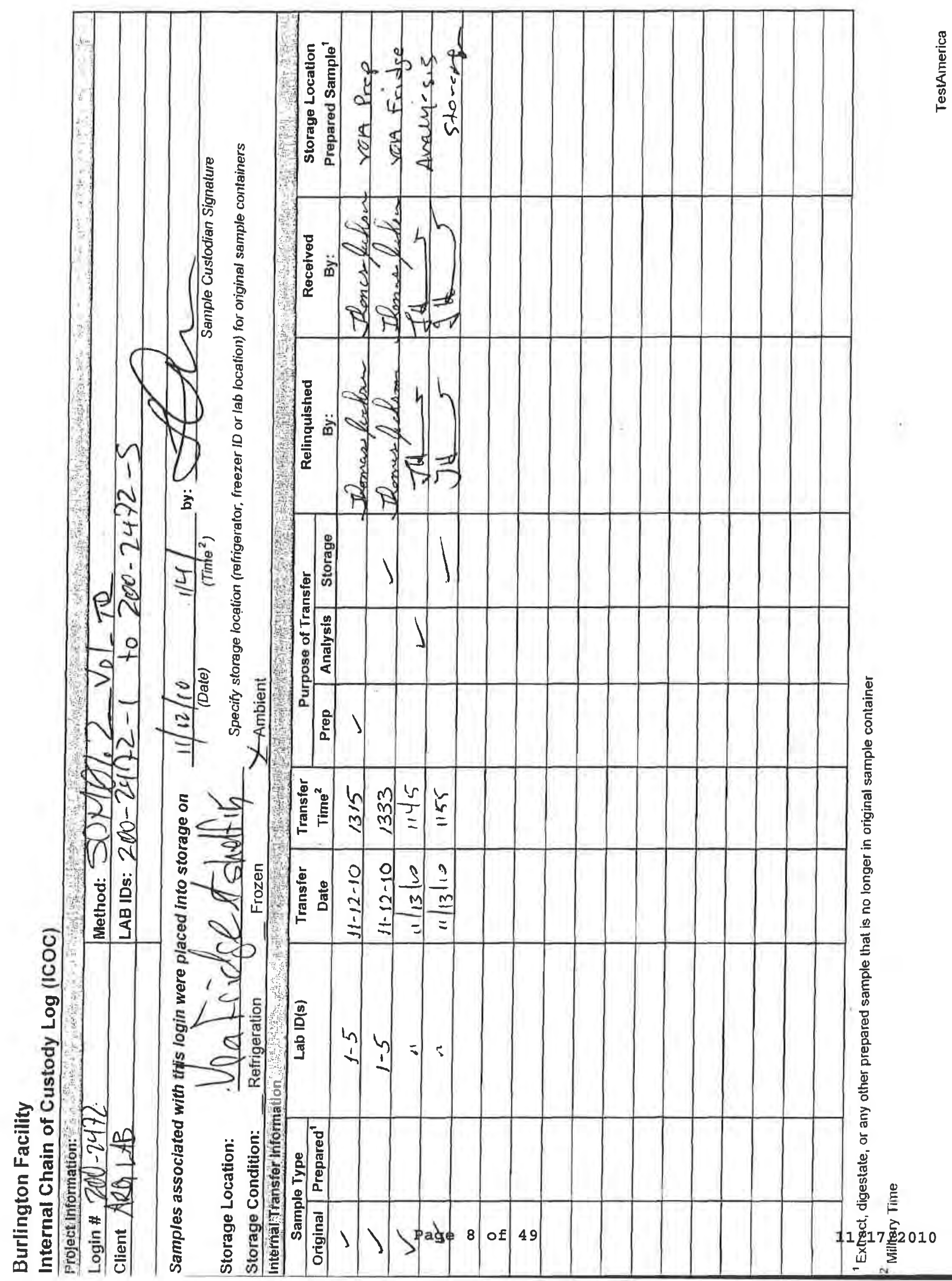




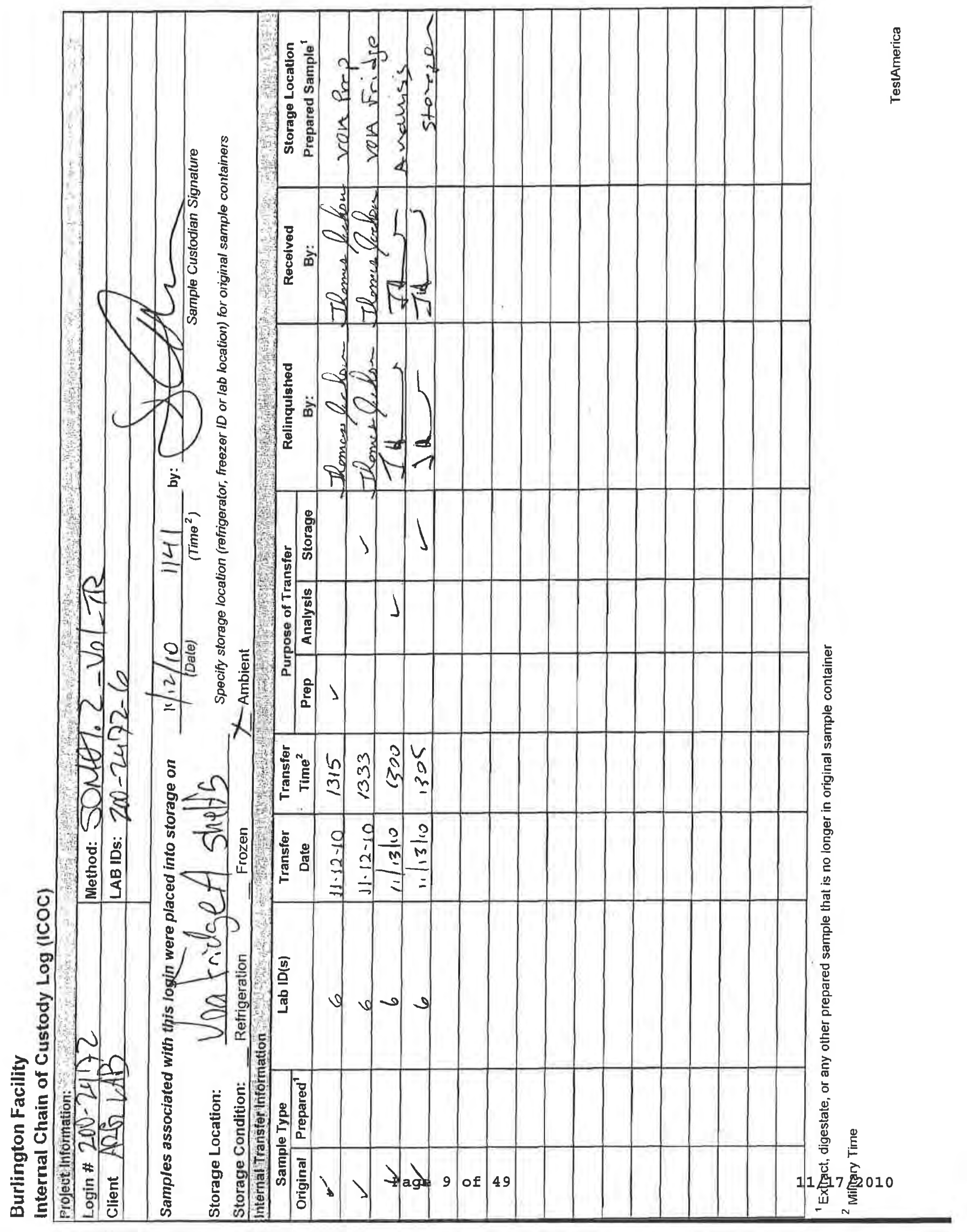




\section{Shipping and Receiving Documents}




\section{Login Sample Receipt Check List}

Login Number: 2472

List Source: TestAmerica Burlington Creator: Marion, Greg T

List Number: 1

Question

Radioactivity either was not measured or, if measured, is at or below background

The cooler's custody seal, if present, is intact.

The cooler or samples do not appear to have been compromised or tampered with.

Samples were received on ice.

Cooler Temperature is acceptable.

Cooler Temperature is recorded.

$\mathrm{COC}$ is present.

$\mathrm{COC}$ is filled out in ink and legible.

$\mathrm{COC}$ is filled out with all pertinent information.

Is the Field Sampler's name present on COC?

There are no discrepancies between the sample IDs on the containers and the COC.

Samples are received within Holding Time.

Sample containers have legible labels.

Containers are not broken or leaking.

Sample collection date/times are provided.

Appropriate sample containers are used.

Sample bottles are completely filled.

Sample Preservation Verified

There is sufficient vol. for all requested analyses, incl. any requested MS/MSDs

VOA sample vials do not have headspace or bubble is $<6 \mathrm{~mm}\left(1 / 4^{\prime \prime}\right)$ in diameter.

If necessary, staff have been informed of any short hold time or quick TAT needs

Multiphasic samples are not present.

Samples do not require splitting or compositing.
T/F/NA

Comment

N/A

True

True

True

True

True

True

True

True

True

True

True

True

True

True

False

True

N/A

True

N/A

True

N/A

N/A 


\section{Sample Login Acknowledgement}

Job 200-2472-1

\begin{tabular}{ll}
\hline Client Job Description: & INMAN (200-2472) \\
Purchase Order \#: & $8 \mathrm{E}-00302$ \\
Work Order \#: & $8 \mathrm{E}-00302$ \\
Project Manager: & Kirk F Young \\
Job Due Date: & $11 / 26 / 2010$ \\
Job TAT: & 14 Days \\
Max Deliverable Level: & IV
\end{tabular}

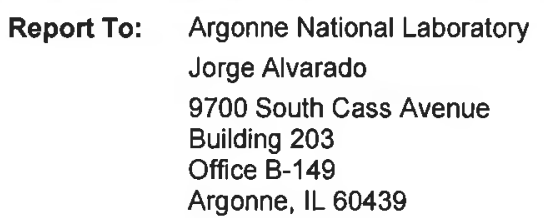

Bill To: $\quad$ Argonne National Laboratory Accounts Payable Chief Financial Offices 9700 S. Cass Ave. Building 201

Argonne, IL 60439

Login 200-2472

\section{Sample Receipt:}

Method of Delivery:

Lab Sample \#

Method

200-2472-1

SOM01.2_Vol_T

200-2472-2

SOM01.2_Vol_Tr SOM01.2 Trace Volatile Organics / In-Lab

200-2472-3

SOM01.2_Vol_Tr SOM01.2 Trace Volatile Organics / In-Lab

200-2472-4

SOM01.2_Vol_Tr SOM01.2 Trace Volatile Organics / In-Lab

200-2472-5

SOM01.2_Vol_Tr SOM01.2 Trace Volatile Organics / In-Lab

200-2472-6

SOM01.2_Vol_Tr SOM01.2 Trace Volatile Organics / In-Lab
Number of Coolers: $\quad 1$

Cooler Temperature(s) $\left(\mathrm{C}^{\circ}\right): 0.7$

Date Sampled Matríx

Rpt Basis Dry / Wet ${ }^{\text {** }}$

11/11/2010 12:00:00 AM Water

Total

Wet

11/11/2010 12:00:00 AM Water

Total Wet

11/11/2010 12:00:00 AM Water

Total Wet

11/11/2010 12:00:00 AM Water

Total Wet

11/11/2010 12:00:00 AM Water

Total Wet

11/12/2010 11:30:00 AM Water

Total Wet 


\section{METHODOLOGY SUMMARY}

Laboratory: TestAmerica Laboratories

Location: South Burlington, Vermont
Project No:

SDG No: $\quad 200-2472$

VOA

Volatile Organics Trace - USEPA CLP SOM01.2 
$2 A$ - FORM II VOA-1

WATER VOLATILE DEUTERATED MONITORING COMPOUND RECOVERY

Lab Name: TESTAMERICA BURLINGTON Contract: 8E-00302

Lab Code: STLV Case No.: INMAN Mod. Ref No.: SDG No.: 200-2472

Level: (TRACE or LOW) TRACE

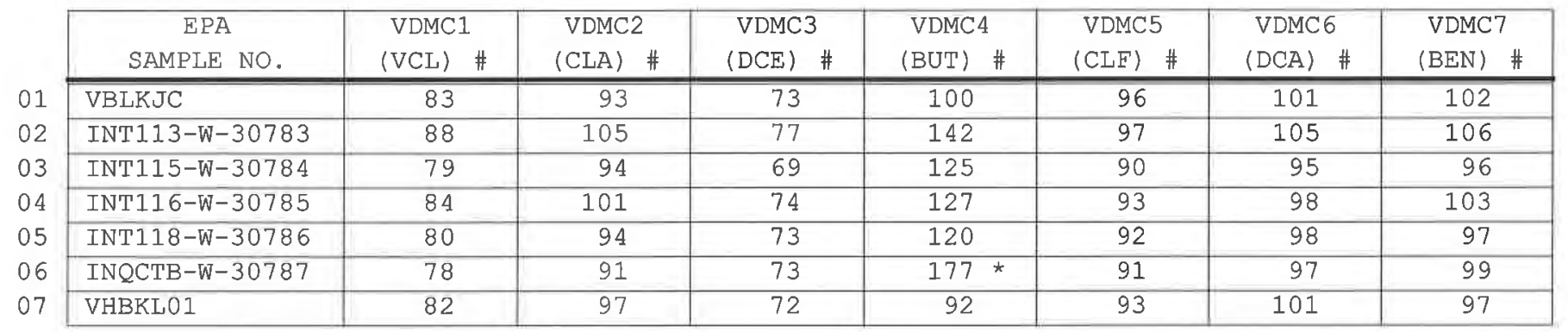

\footnotetext{
VDMC1 (VCL) = Vinyl Chloride-d3

VDMC2 $(\mathrm{CLA})=$ Chloroethane-d5

VDMC3 $(\mathrm{DCE})=1,1-\mathrm{Dich}$ loroethene-d2

VDMC4 (BUT) = 2-Butanone-d5

VDMC5 $($ CLF $)=$ Chloroform-d

VDMC6 $(\mathrm{DCA})=1,2-\mathrm{Dich}$ loroethane-d4

VDMC7 (BEN) = Benzene-d6
}

QC LIMITS

$(65-131)$

(71-131)

(55-104)

$(49-155)$

$(78-121)$

$(78-129)$

$(77-124)$

\# Column to be used to flag recovery values

* Values outside of contract required QC limits

Page 1 of 1 
$2 B$ - FORM II VOA-2

WATER VOLATILE DEUTERATED MONITORING COMPOUND RECOVERY

Lab Name: TESTAMERICA BURLINGTON

Contract: $8 \mathrm{E}-00302$

Lab Code: STLV Case No.: INMAN Mod. Ref No.:

SDG NO.: $200-2472$

Level: (TRACE or LOW) TRACE

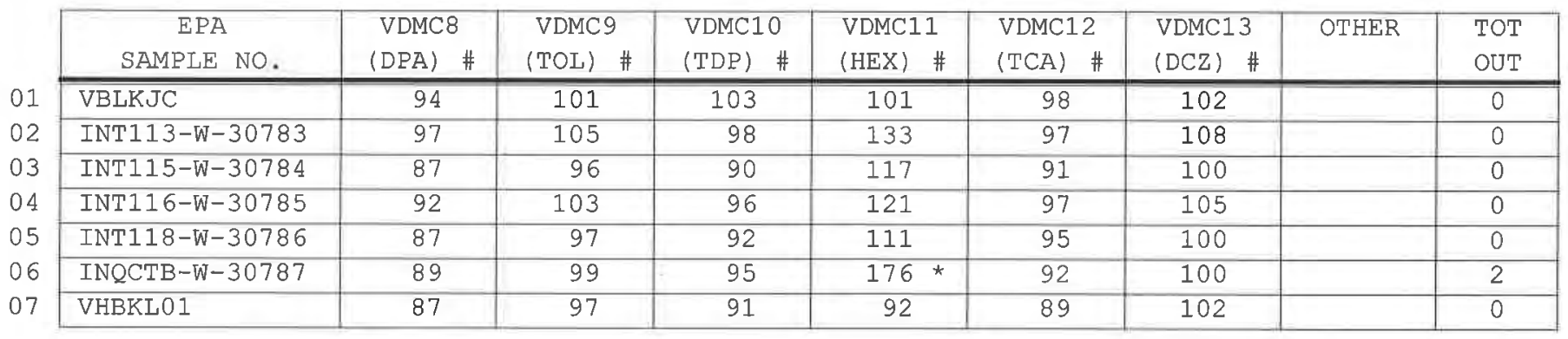

\footnotetext{
VDMC8 $(D P A)=1,2-$ Dichloropropane-d6

VDMC9 $($ TOL) = Toluene-d8

VDMC10 (TDP) = trans-1,3-Dichloropropene-d4

VDMC11 $(\mathrm{HEX})=2-$ Hexanone-d5

VDMC12 $(\mathrm{TCA})=1,1,2,2$-Tetrachloroethane-d2

VDMC13 $(\mathrm{DCZ})=1,2-\mathrm{Dichlorobenzene-d4}$
}

QC LIMITS

$(79-124)$
$(77-121)$
$(73-121)$
$(28-135)$
$(73-125)$
$(80-131)$

\# Column to be used to flag recovery values

* Values outside of contract required QC limits

Report 1,4-Dioxane-d8 for Low-Medium VOA analysis only

Page 1 of 1 
$4 A$ - FORM IV VOA

VOLATILE METHOD BLANK SUMMARY
EPA SAMPLE NO.

VBIKJC

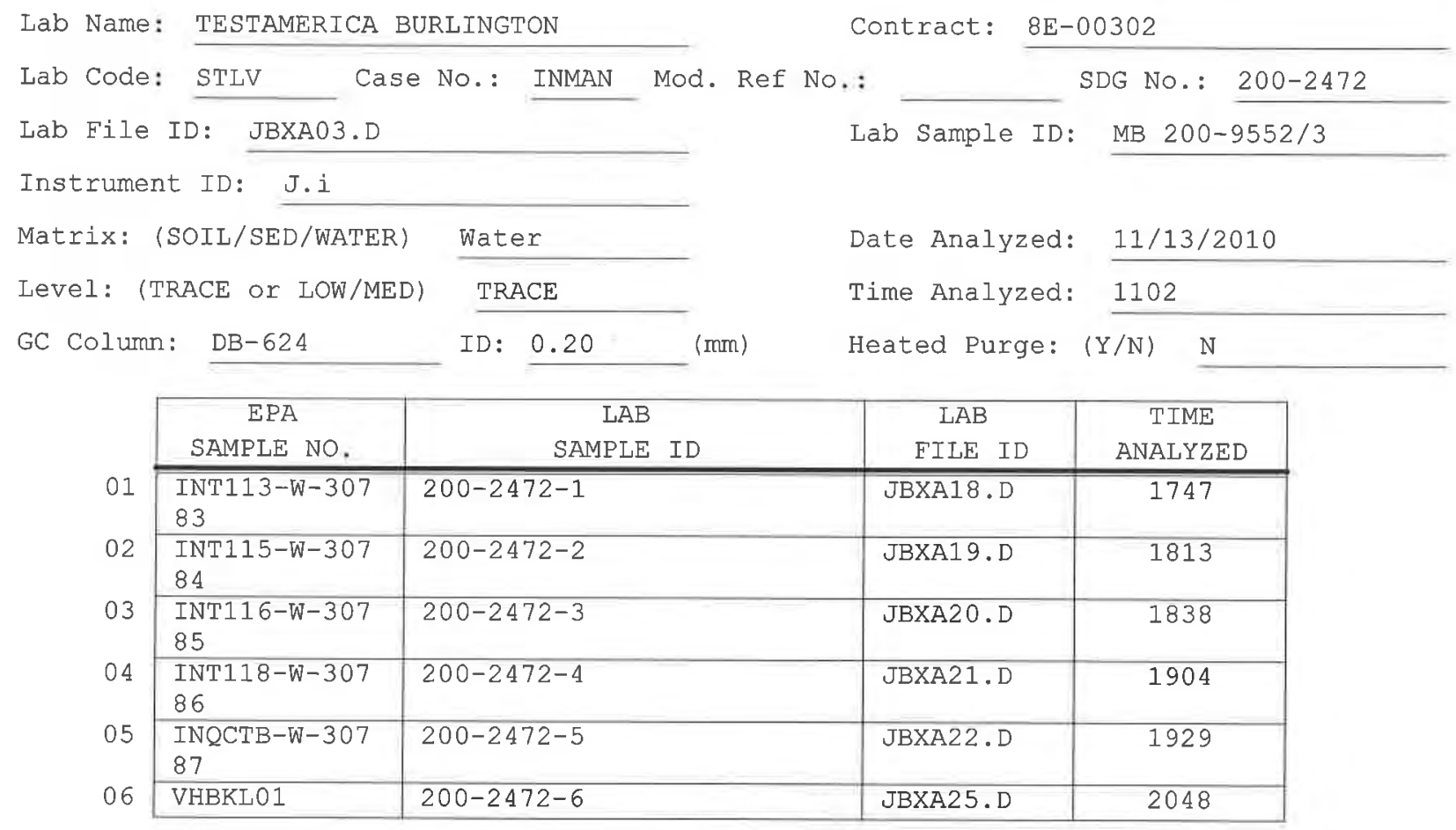

COMMENTS : 
$5 A-F O R M V V O A$

VOLATILE ORGANICS INSTRUMENT

PERFORMANCE CHECK

BROMOFLUOROBENZENE (BFB)
EPA SAMPLE NO.

BFBJB

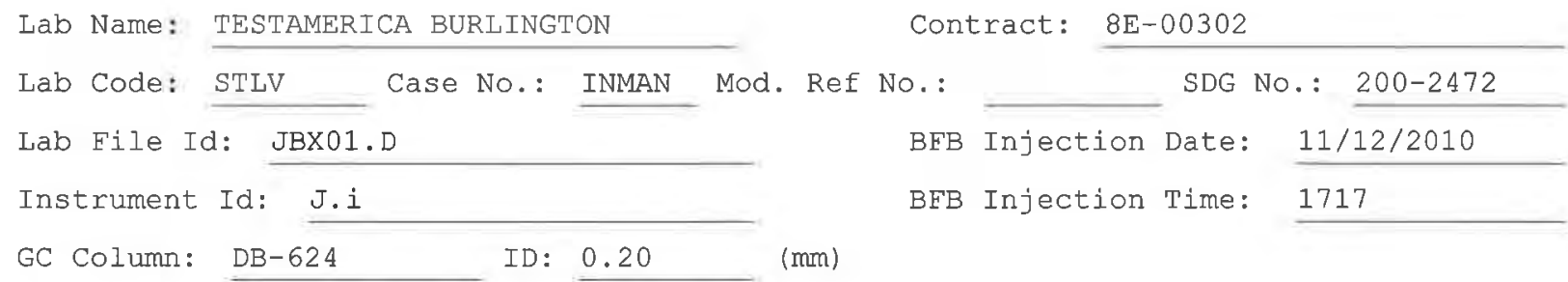

\begin{tabular}{|r|l|r|}
\hline $\mathrm{m} / \mathrm{e}$ & ION ABUNDANCE CRITERIA & $\begin{array}{c}\text { O RELATIVE } \\
\text { ABUNDANCE }\end{array}$ \\
\hline \hline 50 & $15.0-40.0 \%$ of mass 95 & 19.0 \\
\hline 75 & $30.0-80.0 \%$ of mass 95 & 50.9 \\
\hline 95 & Base peak, 100\% relative abundance & 500 \\
\hline 96 & $5.0-9.0 \%$ of mass 95 & 0.7 \\
\hline 173 & Less than 2.0\% of mass 174 & $0.9) 1$ \\
\hline 174 & $50.0-120 \%$ of mass 95 & 80.0 \\
\hline 175 & $5.0-9.0 \%$ of mass 174 & $6.5(16.2(1)$ \\
\hline 176 & $95.0-101 \%$ of mass 174 & $5.3) 1$ \\
\hline 177 & $5.0-9.0 \%$ of mass 176 & $6.8) 2$ \\
\hline
\end{tabular}

1 - Value is onass $174 \quad 2$ - Value is onmass 176

\begin{tabular}{|c|c|c|l|c|c|}
\hline \multicolumn{1}{c|}{\begin{tabular}{c} 
EPA \\
\cline { 2 - 7 }
\end{tabular}} & \multicolumn{1}{|c|}{ LAAB } & \multicolumn{1}{c|}{ LAB } & DATE & TIME \\
SAMPLE NO. & \multicolumn{1}{c|}{ SAMPLE ID } & FILE ID & ANALYZED & ANALYZED \\
\cline { 2 - 7 } 01 & VSTD0.5JB & IC 200-9510/3 & JBX03.D & $11 / 12 / 2010$ & 1810 \\
\cline { 2 - 7 } 03 & VSTD001JB & IC 200-9510/4 & JBX04.D & $11 / 12 / 2010$ & 1835 \\
\cline { 2 - 7 } 04 & VSTD005JB & ICIS 200-9510/5 & JBX05.D & $11 / 12 / 2010$ & 1901 \\
\cline { 2 - 7 } 05 & VSTD010JB & IC 200-9510/6 & JBX06.D & $11 / 12 / 2010$ & 1926 \\
\cline { 2 - 7 } & VSTD020JB & IC 200-9510/7 & JBX07.D & $11 / 12 / 2010$ & 1951 \\
\cline { 2 - 7 }
\end{tabular}


$5 A$ - FORM V VOA

VOLATILE ORGANICS INSTRUMENT

PERFORMANCE CHECK

BROMOFLUOROBENZENE (BEB)
EPA SAMPLE NO. $B F B J C$

Lab Name: TESTAMERICA BURLINGTON

Contract: $8 \mathrm{E}-00302$

Lab Code: STLV Case No.: INMAN Mod. Ref No.: SDG No.: 200-2472

Lab File Id: JBXAOI.D

Instrument Id: J.i

GC Column: DB-624

\begin{tabular}{|l|l}
\hline $\mathrm{m} / \mathrm{e}$ & ION ABUNDANCE CRITERIA \\
\hline
\end{tabular}

$50 \quad 15.0-40.0 \%$ of mass 95

$7530.0-80.0 \%$ of mass 95

95 Base peak, $100 \%$ relative abundance

$965.0-9.0 \%$ of mass 95

173 Less than $2.0 \%$ of mass 174

$17450.0-120 \%$ of mass 95

$175 \quad 5.0-9.0 \%$ of mass 174

$17695.0-101 \%$ of mass 174

$177 \quad 5.0-9.0 \%$ of mass 176
BFB Injection Date: 11/13/2010

BFB Injection Time: 1017 $(\mathrm{mm})$

1 - Value is omass 174

2 - Value is omass 176

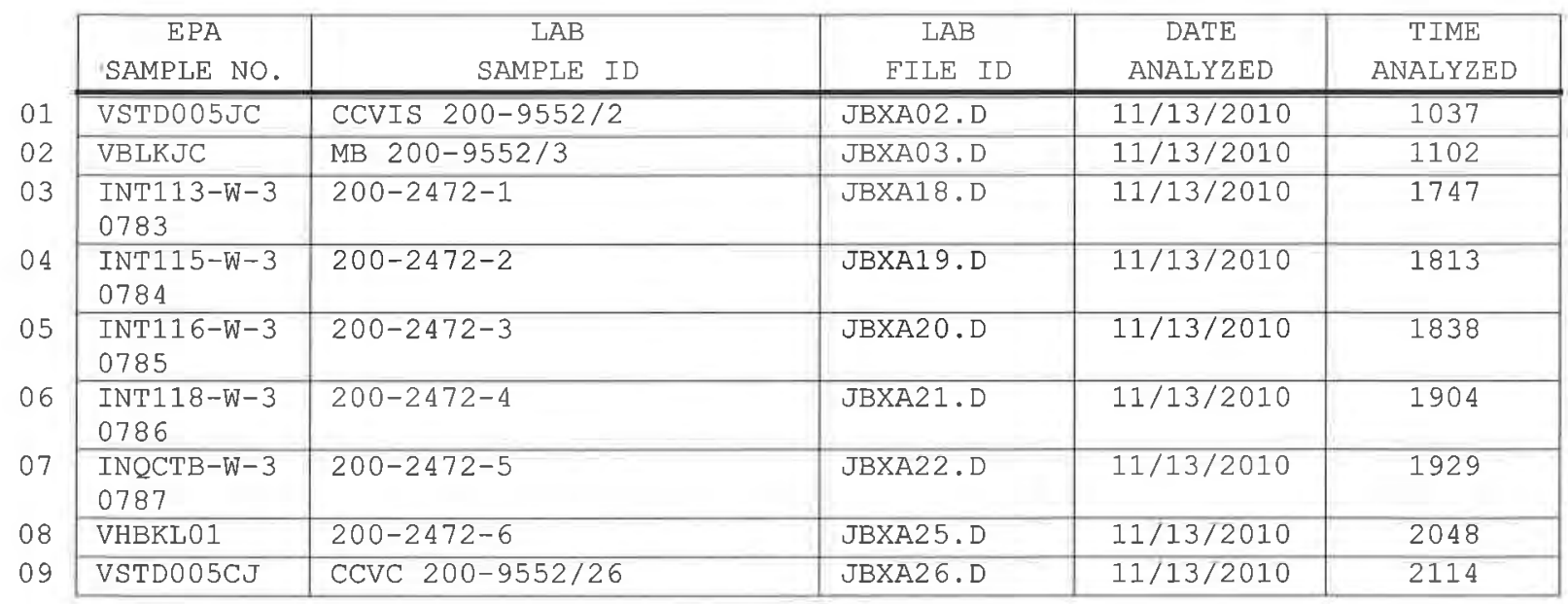


$8 \mathrm{~A}$ - FORM VIII VOA

VOLATILE INTERNAL STANDARD AREA AND RETENTION TIME SUMMARY

Lab Name: TESTAMERICA BURLINGTON Contract: 8E-00302

Lab Code: STLV Case No.: INMAN Mod. Ref No.:

SDG No.: 200-2472

GC Column: DB-624 ID: 0.20 (mm) Init.

EPA Sample No. (VSTD\#\#\#\#): VSTD005JC

Lab File ID (Standard): JBXA02.D

Instrument ID: J.i

Date Analyzed: 11/13/2010

Time Analyzed: 1037

Heated Purge: ( $\mathrm{Y} / \mathrm{N}) \mathrm{N}$

\begin{tabular}{|c|c|c|c|c|c|c|}
\hline & $\begin{array}{c}\text { IS } 1 \text { (CBZ) } \\
\text { AREA }\end{array}$ & RT \# & $\begin{array}{c}\text { IS2 (DFB) } \\
\text { AREA }\end{array}$ & RT \# & $\begin{array}{c}\text { IS3 (DCB) } \\
\text { AREA }\end{array}$ & $\mathrm{RT}$ \\
\hline 12 HOUR STD & 434383 & 8.95 & 540603 & 5.58 & 211808 & 11.78 \\
\hline UPPER IIMIT & 608136 & 9.28 & 756844 & 5.91 & 296531 & 12.11 \\
\hline LOWER IIMIT & 260630 & 8.62 & 324362 & 5.25 & 127085 & 11.45 \\
\hline EPA SAMPLE NO. & & & & & & \\
\hline VBLKJC & $\overline{388409}$ & 8.95 & $\overline{488134}$ & 5.58 & 180310 & 11.78 \\
\hline INT113-W-30783 & 300288 & 8.95 & 370544 & 5.59 & 145660 & 11.79 \\
\hline INT115-W-30784 & 321944 & 8.95 & 401741 & 5.58 & 151521 & 11.79 \\
\hline INT116-W-30785 & 296110 & 8.95 & 370136 & 5.58 & 141835 & 11.79 \\
\hline INT118-W-30786 & 308417 & 8.95 & 372249 & 5.58 & 148797 & 11.79 \\
\hline INQCTB-W-30787 & 307442 & 8.95 & 381166 & 5.59 & 148999 & 11.79 \\
\hline VHBKLO1 & 295186 & 8.95 & 356485 & 5.58 & 137385 & 11.79 \\
\hline
\end{tabular}

IS1 (CBZ) $=$ Chlorobenzene-d5

IS2 $(\mathrm{DFB})=1,4$-Difluorobenzene

IS3 $(\mathrm{DCB})=1,4$-Dichlorobenzene-d4

AREA UPPER LIMIT $=140 \%$ (Trace Volatiles) of internal standard area

AREA LOWER LIMIT $=60 \%$ (Trace Volatiles) of internal standard area

RT UPPER LIMIT $=+0.33$ (Trace Volatiles) minutes of internal standard RT

RT LOWER LIMIT $=-0.33$ (Trace Volatiles) minutes of internal standard RT

\# Column used to flag values outside contract required QC limits with an asterisk.

Page 1 of 1 
Lab Name: TESTAMERICA BURLINGTON

Lab Code: STLV

Matrix: (SOIL/SED/WATER)

Case No.: INMAN

Sample wt/vol: 25.0

Water

$(\mathrm{g} / \mathrm{mL}) \quad \mathrm{mL}$

Level: (TRACE/LOW/MED) TRACE

응 Moisture: not dec.

GC Column: DB-624

Soil Extract Volume:

Purge Volume: 25.0
Contract: 8E-00302

SDG No.: 200-2472

\begin{tabular}{|c|c|c|c|}
\hline CAS NO. & COMPOUND & $\begin{array}{l}\text { CONCENTRATION UNITS: } \\
(\mathrm{ug} / \mathrm{L} \text { or } \mathrm{ug} / \mathrm{kg} \text { ) ug/L }\end{array}$ & $Q$ \\
\hline $75-71-8$ & Dichlorodifluoromethane & 0.50 & $\mathrm{U}$ \\
\hline $74-87-3$ & Chloromethane & 0.50 & $\mathrm{U}$ \\
\hline $75-01-4$ & Vinyl chloride & 0.50 & $\mathrm{U}$ \\
\hline $74-83-9$ & Bromomethane & 0.50 & $\mathrm{U}$ \\
\hline $75-00-3$ & Chloroethane & 0.50 & $\mathrm{U}$ \\
\hline $75-69-4$ & Trichlorofluoromethane & 0.50 & $\mathrm{U}$ \\
\hline $75-35-4$ & 1,1-Dichloroethene & 0.50 & $\mathrm{U}$ \\
\hline $76-13-1$ & 1,1,2-Trichloro-1,2,2-trifluoroethane & 0.50 & $\mathrm{U}$ \\
\hline $67-64-1$ & Acetone & 3.7 & $\mathrm{~J} \mathrm{~B}$ \\
\hline $75-15-0$ & Carbon disulfide & 0.50 & $\mathrm{U}$ \\
\hline $79-20-9$ & Methyl acetate & 0.50 & $\mathrm{U}$ \\
\hline $75-09-2$ & Methylene Chloride & 0.50 & $\mathrm{U}$ \\
\hline $156-60-5$ & trans-1,2-Dichloroethene & 0.50 & $\mathrm{U}$ \\
\hline $1634-04-4$ & Methyl tert-butyl ether & 0.50 & $\mathrm{U}$ \\
\hline $75-34-3$ & 1,1-Dichloroethane & 0.50 & $\mathrm{U}$ \\
\hline $156-59-2$ & cis-1,2-Dichloroethene & 0.50 & $\mathrm{U}$ \\
\hline $78-93-3$ & 2-Butanone & 1.8 & $\mathrm{~J}$ \\
\hline $74-97-5$ & Bromochloromethane & 0.50 & $\mathrm{U}$ \\
\hline $67-66-3$ & Chloroform & 0.50 & $\mathrm{U}$ \\
\hline $71-55-6$ & 1,1,1-Trichloroethane & 0.50 & $\mathrm{U}$ \\
\hline $110-82-7$ & Cyclohexane & 0.50 & $\mathrm{U}$ \\
\hline $56-23-5$ & Carbon tetrachloride & 0.50 & $\mathrm{U}$ \\
\hline $71-43-2$ & Benzene & 0.50 & $\mathrm{U}$ \\
\hline $107-06-2$ & 1,2-Dichloroethane & 0.50 & $\mathrm{U}$ \\
\hline
\end{tabular}

Report 1,4-Dioxane for Low-Medium VOA analysis only

Lab Sample ID: 200-2472-5

Lab File ID: JBXA22.D

Date Received: 11/12/2010

Date Analyzed: 11/13/2010

Dilution Factor: 1.0

Soil Aliquot Volume: (uL) (mL) (ui) 
Lab Name: TESTAMERICA BURLINGTON

Contract: $8 \mathrm{E}-00302$

Lab Code: STLV Case No.: INMAN Mod. Ref No.:

SDG No.: 200-2472

Matrix: (SOIL/SED/WATER)

Sample wt/vol: 25.0

Level: (TRACE/LOW/MED)

TRACE

o Moisture: not dec.

GC Column: DB-624

Soil Extract Volume: ID: $0.20 \quad(\mathrm{~mm})$

Purge Volume: 25.0 (uL) (mL)
Lab Sample ID: 200-2472-5

Lab File ID: JBXA22.D

Date Received: 11/12/2010

Date Analyzed: 11/13/2010

Dilution Factor: 1.0

Soil Aliquot Volume:

(uL)

\begin{tabular}{|c|c|c|c|}
\hline CAS NO. & COMPOUND & $\begin{array}{l}\text { CONCENTRATION UNITS: } \\
(\mathrm{ug} / \mathrm{L} \text { or } u \mathrm{~g} / \mathrm{kg}) \mathrm{ug} / \mathrm{L}\end{array}$ & $Q$ \\
\hline $79-01-6$ & Trichloroethene & 0.50 & $\mathrm{U}$ \\
\hline $108-87-2$ & Methylcyclohexane & 0.50 & U \\
\hline $78-87-5$ & 1,2-Dichloropropane & 0.50 & $\mathrm{U}$ \\
\hline $75-27-4$ & Bromodichloromethane & 0.50 & $\mathrm{U}$ \\
\hline $10061-01-5$ & cis-1,3-Dichloropropene & 0.50 & $\mathrm{U}$ \\
\hline $108-10-1$ & 4-Methyl-2-pentanone & 5.0 & $\mathrm{U}$ \\
\hline $108-88-3$ & Toluene & 0.036 & $\mathrm{~J}$ \\
\hline $10061-02-6$ & trans-1,3-Dichloropropene & 0.50 & $\mathrm{U}$ \\
\hline $79-00-5$ & 1,1,2-Trichloroethane & 0.50 & $\mathrm{U}$ \\
\hline $127-18-4$ & Tetrachloroethene & 0.50 & $\mathrm{U}$ \\
\hline $591-78-6$ & 2-Hexanone & 5.0 & $\mathrm{U}$ \\
\hline $124-48-1$ & Dibromochloromethane & 0.50 & $\mathrm{U}$ \\
\hline $106-93-4$ & 1,2-Dibromoethane & 0.50 & $\mathrm{U}$ \\
\hline $108-90-7$ & Chlorobenzene & 0.50 & $\mathrm{U}$ \\
\hline $100-41-4$ & Ethylbenzene & 0.50 & $\mathrm{U}$ \\
\hline $95-47-6$ & o-Xylene & 0.50 & $\mathrm{U}$ \\
\hline $179601-23-1$ & m,p-xylene & 0.50 & $\mathrm{U}$ \\
\hline $100-42-5$ & Styrene & 0.50 & $\mathrm{U}$ \\
\hline $75-25-2$ & Bromoform & 0.50 & $\mathrm{U}$ \\
\hline $98-82-8$ & Isopropylbenzene & 0.50 & $\mathrm{U}$ \\
\hline $79-34-5$ & $1,1,2,2$-Tetrachloroethane & 0.50 & $\mathrm{U}$ \\
\hline $541-73-1$ & 1,3-Dichlorobenzene & 0.50 & $\mathrm{U}$ \\
\hline $106-46-7$ & 1,4-Dichlorobenzene & 0.50 & $\mathrm{U}$ \\
\hline $95-50-1$ & 1,2-Dichlorobenzene & 0.50 & $\mathrm{U}$ \\
\hline $96-12-8$ & 1,2-Dibromo-3-Chloropropane & 0.50 & $\mathrm{U}$ \\
\hline $120-82-1$ & $1,2,4$-Trichlorobenzene & 0.50 & $\mathrm{U}$ \\
\hline $87-61-6$ & 1,2,3-Trichlorobenzene & 0.50 & $\mathrm{U}$ \\
\hline
\end{tabular}


$1 \mathrm{~J}$ - FORM I VOA-TIC

VOLATILE ORGANICS ANALYSIS DATA SHEET

TENTATIVELY IDENTIFIED COMPOUNDS
EPA SAMPLE NO.

INQCTB-W-30787

Lab Name: TESTAMERICA BURLINGTON

Contract: $\quad 8 \mathrm{E}-00302$

Lab Code: STLV Case No.: INMAN Mod. Ref No.:

SDG No.: 200-2472

Matrix: (SOIL/SED/WATER)

water

Sample wt/vol: 25.0 $(\mathrm{g} / \mathrm{mL}) \quad \mathrm{mL}$

Level: (TRACE or LOW/MED)

TRACE

응 Moisture: not dec.

GC Column: DB-624

ID: $0.20 \quad(\mathrm{~mm})$

Soil Extract Volume:

(uL)

CONCENTRATION UNITS: (ug/L or $\mathrm{ug} / \mathrm{kg}$ ) $\underline{\mathrm{ug} / \mathrm{L}}$
Lab Sample ID: 200-2472-5

Lab File ID: JBXA22.D

Date Received: 11/12/2010

Date Analyzed: 11/13/2010

Dilution Factor: 1.0

Soil Aliquot Volume: (uL)

Purge Volume: 25.0 (mL)

\begin{tabular}{|c|c|c|c|c|c|}
\hline & CAS NUMBER & COMPOUND NAME & $\mathrm{RT}$ & EST. CONC. & $Q$ \\
\hline 01 & & Unknown & 6.92 & 3.4 & $\overline{J X B}$ \\
\hline 02 & & Unknown & 15.75 & 0.54 & $\mathrm{~J}$ \\
\hline 03 & & Unknown & 15.84 & 0.57 & $\mathrm{~J}$ \\
\hline 04 & E9667961 & Total Alkanes & $\mathrm{N} / \mathrm{A}$ & & \\
\hline
\end{tabular}

1 EPA-designated Registry Number. 
Lab Name: TESTAMERICA BURLINGTON

Contract: $8 \mathrm{E}-00302$

Lab Code: STLV Case No.: INMAN Mod. Ref No.: SDG No.: $\quad 200-2472$

Matrix: (SOIL/SED/WATER)

Sample wt/vol: 25.0

Level: (TRACE/LOW/MED)

Water

$(\mathrm{g} / \mathrm{mL}) \mathrm{mI}$

\% Moisture: not dec.

GC Column: DB-624

Soil Extract Volume:

TRACE

Purge Volume: 25.0
ID: $0.20 \quad(\mathrm{~mm})$

(uL)

(mL)
Lab Sample ID: 200-2472-1

Lab File ID: JBXA18.D

Date Received: 11/12/2010

Date Analyzed: 11/13/2010

Dilution Factor: 1.0

Soil Aliquot Volume:

(uL)

\begin{tabular}{|c|c|c|c|}
\hline CAS NO. & COMPOUND & $\begin{array}{l}\text { CONCENTRATION UNITS: } \\
(\mathrm{ug} / \mathrm{L} \text { or } \mathrm{ug} / \mathrm{kg}) \mathrm{ug} / \mathrm{L}\end{array}$ & 8 \\
\hline $75-71-8$ & Dichlorodifluoromethane & 0.50 & $\mathrm{U}$ \\
\hline $74-87-3$ & Chloromethane & 0.50 & $\mathrm{U}$ \\
\hline $75-01-4$ & Vinyl chloride & 0.50 & $\mathrm{U}$ \\
\hline $74-83-9$ & Bromomethane & 0.50 & $\mathrm{U}$ \\
\hline $75-00-3$ & Chloroethane & 0.50 & $\mathrm{U}$ \\
\hline $75-69-4$ & Trichlorofluoromethane & 0.50 & $\mathrm{U}$ \\
\hline $75-35-4$ & 1,1-Dichloroethene & 0.50 & $\mathrm{U}$ \\
\hline $76-13-1$ & 1,1,2-Trichloro-1,2,2-trifluoroethane & 0.50 & $\mathrm{U}$ \\
\hline $67-64-1$ & Acetone & 5.4 & $\mathrm{~B}$ \\
\hline $75-15-0$ & Carbon disulfide & 0.50 & $\mathrm{U}$ \\
\hline $79-20-9$ & Methyl acetate & 0.50 & $\mathrm{U}$ \\
\hline $75-09-2$ & Methylene Chloride & 0.50 & $\mathrm{U}$ \\
\hline $156-60-5$ & trans-1,2-Dichloroethene & 0.50 & $\mathrm{U}$ \\
\hline $1634-04-4$ & Methyl tert-butyl ether & 0.50 & $\mathrm{U}$ \\
\hline $75-34-3$ & 1,1-Dichloroethane & 0.50 & $\mathrm{U}$ \\
\hline $156-59-2$ & cis-1,2-Dichloroethene & 0.50 & $\mathrm{U}$ \\
\hline $78-93-3$ & 2-Butanone & 1.1 & $\mathrm{~J}$ \\
\hline $74-97-5$ & Bromochloromethane & 0.50 & $\mathrm{U}$ \\
\hline $67-66-3$ & Chloroform & 0.50 & $\mathrm{U}$ \\
\hline $71-55-6$ & 1,1,1-Trichloroethane & 0.50 & $\mathrm{U}$ \\
\hline $110-82-7$ & Cyclohexane & 0.50 & $\mathrm{U}$ \\
\hline $56-23-5$ & Carbon tetrachloride & 0.50 & $\mathrm{U}$ \\
\hline $71-43-2$ & Benzene & 0.11 & $\mathrm{~J}$ \\
\hline $107-06-2$ & 1,2-Dichloroethane & 0.50 & $\mathrm{U}$ \\
\hline
\end{tabular}

Report 1,4-Dioxane for Low-Medium VOA analysis only 
$1 B$ - FORM I VOA-2

VOLATILE ORGANICS ANALYSIS DATA SHEET
EPA SAMPLE NO. INT113-W-30783

Lab Name: TESTAMERICA BURLINGTON

Contract: $\quad 8 \mathrm{E}-00302$

Lab Code: STLV

Case No.: INMAN

Mod. Ref No.:

SDG No.: 200-2472

Matrix: (SOIL/SED/WATER)

Sample wt/vol: 25.0 Water $(\mathrm{g} / \mathrm{mL}) \mathrm{mL}$

Level: (TRACE/LOW/MED) TRACE

o Moisture: not dec.

GC Column: DB-624 ID: 0.20 $(\mathrm{mm})$

Soil Extract Volume: (uL) (mL)

Purge Volume: 25.0
Lab Sample ID: 200-2472-1

Lab File ID: JBXA18.D

Date Received: 11/12/2010

Date Analyzed: 11/13/2010

Dilution Factor: 1.0

Soil Aliquot Volume:

(uI)

\begin{tabular}{|c|c|c|c|}
\hline CAS NO. & COMPOUND & $\begin{array}{l}\text { CONCENTRATION UNITS: } \\
(\mathrm{ug} / \mathrm{L} \text { or } \mathrm{ug} / \mathrm{kg}) \mathrm{ug} / \mathrm{L}\end{array}$ & 8 \\
\hline $79-01-6$ & Trichloroethene & 0.50 & $\mathrm{U}$ \\
\hline $108-87-2$ & Methylcyclohexane & 0.50 & $\mathrm{U}$ \\
\hline $78-87-5$ & 1,2-Dichloropropane & 0.50 & $\mathrm{U}$ \\
\hline $75-27-4$ & Bromodichloromethane & 0.50 & $\mathrm{U}$ \\
\hline $10061-01-5$ & cis-1,3-Dichloropropene & 0.50 & $\mathrm{U}$ \\
\hline $108-10-1$ & 4-Methyl-2-pentanone & 5.0 & $\mathrm{U}$ \\
\hline $108-88-3$ & Toluene & 0.10 & $\mathrm{~J}$ \\
\hline $10061-02-6$ & trans-1,3-Dichloropropene & 0.50 & $\mathrm{U}$ \\
\hline $79-00-5$ & 1,1,2-Trichloroethane & 0.50 & $\mathrm{U}$ \\
\hline $127-18-4$ & Tetrachloroethene & 0.50 & $\mathrm{U}$ \\
\hline $591-78-6$ & 2-Hexanone & 5.0 & $\mathrm{U}$ \\
\hline $124-48-1$ & Dibromochloromethane & 0.50 & $\mathrm{U}$ \\
\hline $106-93-4$ & 1,2-Dibromoethane & 0.50 & $\mathrm{U}$ \\
\hline $108-90-7$ & Chlorobenzene & 0.50 & $\mathrm{U}$ \\
\hline $100-41-4$ & Ethylbenzene & 0.50 & $\mathrm{U}$ \\
\hline $95-47-6$ & o-Xylene & 0.50 & $\mathrm{U}$ \\
\hline $179601-23-1$ & $\mathrm{~m}, \mathrm{p}$-xylene & 0.049 & $\mathrm{~J}$ \\
\hline $100-42-5$ & Styrene & 0.50 & $\mathrm{U}$ \\
\hline $75-25-2$ & Bromoform & 0.50 & $\mathrm{U}$ \\
\hline $98-82-8$ & Isopropylbenzene & 0.50 & $\mathrm{U}$ \\
\hline $79-34-5$ & $1,1,2,2$-Tetrachloroethane & 0.50 & $\mathrm{U}$ \\
\hline $541-73-1$ & 1,3-Dichlorobenzene & 0.50 & $\mathrm{U}$ \\
\hline $106-46-7$ & 1,4-Dichlorobenzene & 0.50 & $\mathrm{U}$ \\
\hline $95-50-1$ & 1,2-Dichlorobenzene & 0.50 & $\mathrm{U}$ \\
\hline $96-12-8$ & 1,2-Dibromo-3-Chloropropane & 0.50 & $\mathrm{U}$ \\
\hline $120-82-1$ & 1,2,4-Trichlorobenzene & 0.50 & $\mathrm{U}$ \\
\hline $87-61-6$ & 1,2,3-Trichlorobenzene & 0.50 & $\mathrm{U}$ \\
\hline
\end{tabular}


$1 \mathrm{~J}$ - FORM I VOA-TIC

VOLATILE ORGANICS ANALYSIS DATA SHEET

TENTATIVELY IDENTIFIED COMPOUNDS
EPA SAMPLE NO.

INT113-W-30783
Lab Name: $\frac{\text { TESTAMERICA BURLINGTON }}{\text { Lab Code: STLV Case No.: INMAN Mod. Ref No. }}$

Matrix: (SOIL/SED/WATER)

Sample wt/vol: 25.0

Level: (TRACE or LOW/MED) TRACE

o Moisture: not dec.

GC Column: DB-624 ID: $0.20 \quad(\mathrm{~mm})$

Soil Extract Volume:

(uL)

CONCENTRATION UNITS: (ug/L or $\mathrm{ug} / \mathrm{kg}$ ) ug/L
Contract: $8 \mathrm{E}-00302$

SDG No.: 200-2472

Lab Sample ID: 200-2472-1

Lab File ID: JBXA18.D

Date Received: 11/12/2010

Date Analyzed: 11/13/2010

Dilution Factor: 1.0

Soil Aliquot Volume:

(UL)

Purge volume: 25.0 $(\mathrm{mL})$

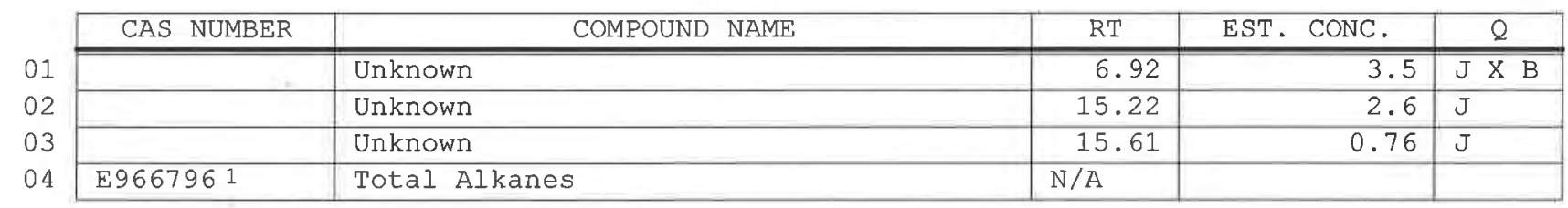

1 EPA-designated Registry Number. 
$1 A$ - FORM I VOA-1

VOLATILE ORGANICS ANALYSIS DATA SHEET
EPA SAMPLE NO.

INT115-W-30784

Lab Name: TESTAMERICA BURLINGTON

Contract: $8 \mathrm{E}-00302$

Lab Code: STLV Case No.: INMAN Mod. Ref No.: SDG NO.: 200-2472

Matrix: (SOIL/SED/WATER) Water

Sample wt/vol: 25.0 $(\mathrm{g} / \mathrm{mL}) \mathrm{mL}$

Level: (TRACE/LOW/MED) TRACE

\% Moisture: not dec.

GC Column: DB-624 ID: $0.20 \quad$ (mm)

Soil Extract Volume: (uL)

Purge Volume: 25.0 (mL)
Lab Sample ID: 200-2472-2

Lab File ID: JBXA19.D

Date Received: 11/12/2010

Date Analyzed: 11/13/2010

Dilution Factor: 1.0

Soil Aliquot Volume:

(uL)

\begin{tabular}{|c|c|c|c|}
\hline CAS NO. & COMPOUND & $\begin{array}{l}\text { CONCENTRATION UNITS: } \\
(\mathrm{ug} / \mathrm{L} \text { or } \mathrm{ug} / \mathrm{kg}) \mathrm{ug} / \mathrm{L}\end{array}$ & $Q$ \\
\hline $75-71-8$ & Dichlorodifluoromethane & 0.50 & $\overline{\mathrm{U}}$ \\
\hline $74-87-3$ & Chloromethane & 0.50 & $\mathrm{U}$ \\
\hline $75-01-4$ & Vinyl chloride & 0.50 & $\mathrm{U}$ \\
\hline $74-83-9$ & Bromomethane & 0.50 & $\mathrm{U}$ \\
\hline $75-00-3$ & Chloroethane & 0.50 & $\mathrm{U}$ \\
\hline $75-69-4$ & Trichlorofluoromethane & 0.50 & U \\
\hline $75-35-4$ & 1,1-Dichloroethene & 0.50 & $\mathrm{U}$ \\
\hline $76-13-1$ & 1,1,2-Trichloro-1,2,2-trifluoroethane & 0.50 & $\mathrm{U}$ \\
\hline $67-64-1$ & Acetone & 8.9 & $\mathrm{~B}$ \\
\hline $75-15-0$ & Carbon disulfide & 0.50 & $\mathrm{U}$ \\
\hline $79-20-9$ & Methyl acetate & 0.50 & $\mathrm{U}$ \\
\hline $75-09-2$ & Methylene Chloride & 0.50 & $\mathrm{U}$ \\
\hline $156-60-5$ & trans-1,2-Dichloroethene & 0.50 & $\mathrm{U}$ \\
\hline $1634-04-4$ & Methyl tert-butyl ether & 0.50 & $\mathrm{U}$ \\
\hline $75-34-3$ & 1,1-Dichloroethane & 0.50 & $\mathrm{U}$ \\
\hline $156-59-2$ & cis-1,2-Dichloroethene & 0.50 & $\mathrm{U}$ \\
\hline $78-93-3$ & 2-Butanone & 1.1 & $\mathrm{~J}$ \\
\hline $74-97-5$ & Bromochloromethane & 0.50 & $\mathrm{U}$ \\
\hline $67-66-3$ & Chloroform & 0.50 & $\mathrm{U}$ \\
\hline $71-55-6$ & 1,1,1-Trichloroethane & 0.50 & $\mathrm{U}$ \\
\hline $110-82-7$ & Cyclohexane & 0.50 & $\mathrm{U}$ \\
\hline $56-23-5$ & Carbon tetrachloride & 0.50 & $\mathrm{U}$ \\
\hline $71-43-2$ & Benzene & 0.078 & $\mathrm{~J}$ \\
\hline $107-06-2$ & 1,2-Dichloroethane & 0.50 & $\mathrm{U}$ \\
\hline
\end{tabular}

Report 1,4-Dioxane for Low-Medium VOA analysis only 
1B - FORM I VOA-2

VOLATILE ORGANICS ANALYSIS DATA SHEET
EPA SAMPLE NO.

INT115-W-30784

Lab Name: TESTAMERICA BURLINGTON

Lab Code: STLV

Matrix: (SOIL/SED/WATER)

Sample wt/vol: 25.0

Level: (TRACE/LOW/MED)

$\therefore$ Moisture: not dec.

GC Column: DB-624

Soil Extract Volume: ID: 0.20

Water

$(\mathrm{g} / \mathrm{mL}) \quad \mathrm{mI}$

TRACE

ID: $0.20 \quad(\mathrm{~mm})$

Purge Volume: 25.0
Contract: $\quad 8 \mathrm{E}-00302$

SDG No.: 200-2472
Lab Sample ID: 200-2472-2

Lab File ID: JBXA19.D

Date Received: 11/12/2010

Date Analyzed: 11/13/2010

Dilution Factor: 1.0

Soil Aliquot Volume:

(uL)

\begin{tabular}{|c|c|c|c|}
\hline CAS NO. & COMPOUND & $\begin{array}{l}\text { CONCENTRATION UNITS: } \\
(\mathrm{ug} / \mathrm{L} \text { or } \mathrm{ug} / \mathrm{kg}) \mathrm{ug} / \mathrm{L}\end{array}$ & $Q$ \\
\hline $79-01-6$ & Trichloroethene & 0.50 & $\overline{\mathrm{U}}$ \\
\hline $108-87-2$ & Methylcyclohexane & 0.50 & $\mathrm{U}$ \\
\hline $78-87-5$ & 1,2-Dichloropropane & 0.50 & $\mathrm{U}$ \\
\hline $75-27-4$ & Bromodichloromethane & 0.50 & $\mathrm{U}$ \\
\hline $10061-01-5$ & cis-1,3-Dichloropropene & 0.50 & $\mathrm{U}$ \\
\hline $108-10-1$ & 4-Methyl-2-pentanone & 5.0 & $\mathrm{U}$ \\
\hline $108-88-3$ & Toluene & 0.10 & $\mathrm{~J}$ \\
\hline $10061-02-6$ & trans-1,3-Dichloropropene & 0.50 & $\mathrm{U}$ \\
\hline $79-00-5$ & $1,1,2$-Trichloroethane & 0.50 & $\mathrm{U}$ \\
\hline $127-18-4$ & Tetrachloroethene & 0.50 & $\mathrm{U}$ \\
\hline $591-78-6$ & 2-Hexanone & 5.0 & $\mathrm{U}$ \\
\hline $124-48-1$ & Dibromochloromethane & 0.50 & $\mathrm{U}$ \\
\hline $106-93-4$ & 1,2-Dibromoethane & 0.50 & $\mathrm{U}$ \\
\hline $108-90-7$ & Chlorobenzene & 0.50 & $\mathrm{U}$ \\
\hline $100-41-4$ & Ethylbenzene & 0.50 & $\mathrm{U}$ \\
\hline $95-47-6$ & o-Xylene & 0.50 & $\mathrm{U}$ \\
\hline $179601-23-1$ & m, $\mathrm{p}$-Xylene & 0.060 & $\mathrm{~J}$ \\
\hline $100-42-5$ & Styrene & 0.50 & $\mathrm{U}$ \\
\hline $75-25-2$ & Bromoform & 0.50 & $\mathrm{U}$ \\
\hline $98-82-8$ & Isopropylbenzene & 0.50 & $\mathrm{U}$ \\
\hline $79-34-5$ & $1,1,2,2$-Tetrachloroethane & 0.50 & $\mathrm{U}$ \\
\hline $541-73-1$ & 1,3-Dichlorobenzene & 0.50 & $\mathrm{U}$ \\
\hline $106-46-7$ & 1,4-Dichlorobenzene & 0.50 & $\mathrm{U}$ \\
\hline $95-50-1$ & 1,2-Dichlorobenzene & 0.50 & $\mathrm{U}$ \\
\hline $96-12-8$ & 1,2-Dibromo-3-Chloropropane & 0.50 & $\mathrm{U}$ \\
\hline $120-82-1$ & 1,2,4-Trichlorobenzene & 0.50 & $\mathrm{U}$ \\
\hline $87-61-6$ & 1,2,3-Trichlorobenzene & 0.50 & $\mathrm{U}$ \\
\hline
\end{tabular}


$1 \mathrm{~J}$ - FORM I VOA-TIC

VOLATILE ORGANICS ANALYSIS DATA SHEET

TENTATIVELY IDENTIFIED COMPOUNDS
EPA SAMPLE NO. INT115-W-30784

\begin{abstract}
Lab Name: TESTAMERICA BURLINGTON
Lab Code: STLV Case No.: INMAN Mod. Ref No.:

Matrix: (SOIL/SED/WATER)

Sample wt/vol: 25.0

Level: (TRACE or LOW/MED)

Water

$(g / m L) \quad m L$

음 Moisture: not dec.

GC Column: DB-624

Soil Extract Volume:

ID: $0.20 \quad(\mathrm{~mm})$

CONCENTRATION UNITS: (ug/L or $u \mathrm{~g} / \mathrm{kg})$

(uL)

$\mathrm{ug} / \mathrm{L}$
\end{abstract}

Contract: $8 \mathrm{E}-00302$

SDG No.: 200-2472
Lab Sample ID: 200-2472-2

Lab File ID: JBXA19.D

Date Received: $11 / 12 / 2010$

Date Analyzed: 11/13/2010

Dilution Factor: 1.0

Soil Aliquot Volume: (uL)

Purge Volume: 25.0 $(\mathrm{mL})$

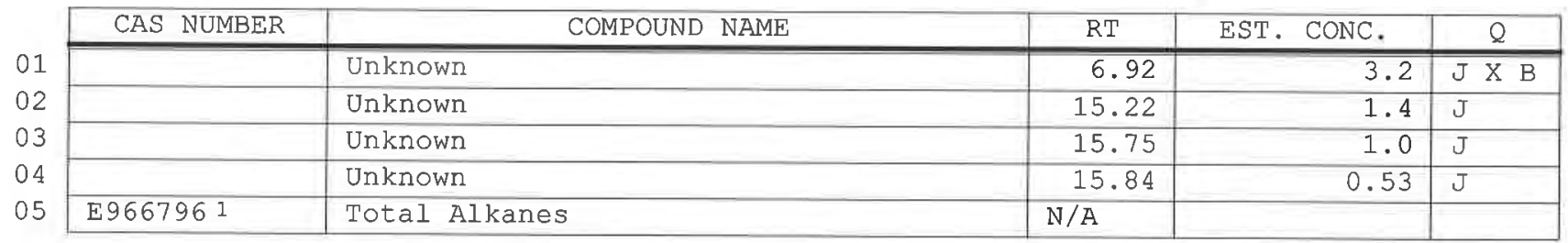

1 EPA-designated Registry Number. 
$1 A$ - FORM I VOA-1

VOLATILE ORGANICS ANALYSIS DATA SHEET
EPA SAMPLE NO.

INT116-W-30785

Lab Name: TESTAMERICA BURLINGTON

Contract: $\quad 8 \mathrm{E}-00302$

Lab Code: STLV Case No.: INMAN Mod. Ref No.: SDG No.: 200-2472

\section{Matrix: (SOIL/SED/WATER) Water}

Sample wt/vol: 25.0 $(\mathrm{g} / \mathrm{mL}) \quad \mathrm{mL}$

Level: (TRACE/LOW/MED) TRACE

응 Moisture: not dec.

GC Column: DB-624

Soil Extract Volume: ID: $0.20 \quad(\mathrm{~mm})$

Purge Volume: 25.0 (uL) $(m L)$
Lab Sample ID: 200-2472-3

Lab File ID: JBXA20.D

Date Received: 11/12/2010

Date Analyzed: 11/13/2010

Dilution Factor: 1.0

Soil Aliquot Volume:

(uL)

\begin{tabular}{|c|c|c|c|}
\hline CAS NO. & COMPOUND & $\begin{array}{l}\text { CONCENTRATION UNITS: } \\
(\mathrm{ug} / \mathrm{L} \text { or } \mathrm{ug} / \mathrm{kg}) \mathrm{ug} / \mathrm{L}\end{array}$ & $Q$ \\
\hline $75-71-8$ & Dichlorodifluoromethane & 0.50 & $\overline{\mathrm{U}}$ \\
\hline $74-87-3$ & Chloromethane & 0.50 & $\mathrm{U}$ \\
\hline $75-01-4$ & Vinyl chloride & 0.50 & $\mathrm{U}$ \\
\hline $74-83-9$ & Bromomethane & 0.50 & $\mathrm{U}$ \\
\hline $75-00-3$ & Chloroethane & 0.50 & $\mathrm{U}$ \\
\hline $75-69-4$ & Trichlorofluoromethane & 0.50 & $\mathrm{U}$ \\
\hline $75-35-4$ & 1,1-Dichloroethene & 0.50 & $\mathrm{U}$ \\
\hline $76-13-1$ & 1,1,2-Trichloro-1,2,2-trifluoroethane & 0.50 & $\mathrm{U}$ \\
\hline $67-64-1$ & Acetone & 3.8 & $\mathrm{~J} B$ \\
\hline $75-15-0$ & Carbon disulfide & 0.50 & $\mathrm{U}$ \\
\hline $79-20-9$ & Methyl acetate & 0.50 & $\mathrm{U}$ \\
\hline $75-09-2$ & Methylene Chloride & 0.50 & $\mathrm{U}$ \\
\hline $156-60-5$ & trans-1,2-Dichloroethene & 0.50 & $\mathrm{U}$ \\
\hline $1634-04-4$ & Methyl tert-butyl ether & 0.50 & $\mathrm{U}$ \\
\hline $75-34-3$ & 1,1-Dichloroethane & 0.50 & $\mathrm{U}$ \\
\hline $156-59-2$ & cis-1,2-Dichloroethene & 0.50 & $\mathrm{U}$ \\
\hline $78-93-3$ & 2-Butanone & 5.0 & $\mathrm{U}$ \\
\hline $74-97-5$ & Bromochloromethane & 0.50 & $\mathrm{U}$ \\
\hline $67-66-3$ & Chloroform & 0.50 & $\mathrm{U}$ \\
\hline $71-55-6$ & 1,1,1-Trichloroethane & 0.50 & $\mathrm{U}$ \\
\hline $110-82-7$ & Cyclohexane & 0.50 & U \\
\hline $56-23-5$ & Carbon tetrachloride & 0.50 & U \\
\hline $71-43-2$ & Benzene & 0.048 & $\mathrm{~J}$ \\
\hline $107-06-2$ & 1,2-Dichloroethane & 0.50 & $\mathrm{U}$ \\
\hline
\end{tabular}

Report 1,4-Dioxane for Low-Medium VOA analysis only 
Lab Name: TESTAMERICA BURLINGTON

Contract: 8E-00302

Lab Code: STLV Case No.: INMAN Mod. Ref No.: SDG No.: $\quad 200-2472$

Matrix: (SOIL/SED/WATER) Water

Sample wt/vol: $25.0 \quad(\mathrm{~g} / \mathrm{mL}) \mathrm{mL}$

Level: (TRACE/LOW/MED) TRACE

o Moisture: not dec.

GC Column: DB-624 ID: $0.20 \quad(\mathrm{~mm})$

Soil Extract Volume: (UL) $(\mathrm{mL})$

Purge Volume: 25.0
Lab Sample ID: 200-2472-3

Lab File ID: JBXA20.D

Date Received: 11/12/2010

Date Analyzed: 11/13/2010

Dilution Factor: 1.0

Soil Aliquot Volume:

(uL)

\begin{tabular}{|c|c|c|c|}
\hline CAS NO. & COMPOUND & $\begin{array}{l}\text { CONCENTRATION UNITS: } \\
(\mathrm{ug} / \mathrm{L} \text { or } \mathrm{ug} / \mathrm{kg}) \mathrm{ug} / \mathrm{L}\end{array}$ & c \\
\hline $79-01-6$ & Trichloroethene & 0.50 & $\mathrm{U}$ \\
\hline $108-87-2$ & Methylcyclohexane & 0.50 & $\mathrm{U}$ \\
\hline $78-87-5$ & 1,2-Dichloropropane & 0.50 & $\mathrm{U}$ \\
\hline $75-27-4$ & Bromodichloromethane & 0.50 & $\mathrm{U}$ \\
\hline $10061-01-5$ & cis-1,3-Dichloropropene & 0.50 & $\mathrm{U}$ \\
\hline $108-10-1$ & 4-Methyl-2-pentanone & 5.0 & $\mathrm{U}$ \\
\hline $108-88-3$ & Toluene & 0.068 & $\mathrm{~J}$ \\
\hline $10061-02-6$ & trans-1,3-Dichloropropene & 0.50 & $\mathrm{U}$ \\
\hline $79-00-5$ & 1,1,2-Trichloroethane & 0.50 & $\mathrm{U}$ \\
\hline $127-18-4$ & Tetrachloroethene & 0.50 & $\mathrm{U}$ \\
\hline $591-78-6$ & 2-Hexanone & 5.0 & $\mathrm{U}$ \\
\hline $124-48-1$ & Dibromochloromethane & 0.50 & $\mathrm{U}$ \\
\hline $106-93-4$ & 1,2-Dibromoethane & 0.50 & $\mathrm{U}$ \\
\hline $108-90-7$ & Chlorobenzene & 0.50 & $\mathrm{U}$ \\
\hline $100-41-4$ & Ethylbenzene & 0.50 & $\mathrm{U}$ \\
\hline $95-47-6$ & o-Xylene & 0.50 & $\mathrm{U}$ \\
\hline $179601-23-1$ & $\mathrm{~m}, \mathrm{p}$-Xylene & 0.50 & $\mathrm{U}$ \\
\hline $100-42-5$ & Styrene & 0.50 & $\mathrm{U}$ \\
\hline $75-25-2$ & Bromoform & 0.50 & $\mathrm{U}$ \\
\hline $98-82-8$ & Isopropylbenzene & 0.50 & $\mathrm{U}$ \\
\hline $79-34-5$ & $1,1,2,2$-Tetrachloroethane & 0.50 & $\mathrm{U}$ \\
\hline $541-73-1$ & 1,3-Dichlorobenzene & 0.50 & $\mathrm{U}$ \\
\hline $106-46-7$ & 1,4-Dichlorobenzene & 0.50 & $\mathrm{U}$ \\
\hline $95-50-1$ & 1,2-Dichlorobenzene & 0.50 & $\mathrm{U}$ \\
\hline $96-12-8$ & 1,2-Dibromo-3-Chloropropane & 0.50 & $\mathrm{U}$ \\
\hline $120-82-1$ & 1,2,4-Trichlorobenzene & 0.50 & $\mathrm{U}$ \\
\hline $87-61-6$ & 1,2,3-Trichlorobenzene & 0.50 & $\mathrm{U}$ \\
\hline
\end{tabular}


IJ - FORM I VOA-TIC

VOLATILE ORGANICS ANALYSIS DATA SHEET

TENTATIVELY IDENTIFIED COMPOUNDS
EPA SAMPLE NO.

INT116-W-30785
Lab Name: TESTAMERICA BURLINGTON

Lab Code: STLV

Matrix: (SOIL/SED/WATER)

Sample wt/vol: 25.0 Case No.: INMAN

Water

$(\mathrm{g} / \mathrm{mL}) \quad \mathrm{mL}$

Level: (TRACE or LOW/MED)

TRACE

응 Moisture: not dec.

GC Column: DB-624 ID: 0.20 $(\mathrm{mm})$

Soil Extract Volume: (UL)

CONCENTRATION UNITS: (ug/L or $\mathrm{ug} / \mathrm{kg}$ ) ug/L
Contract: $8 \mathrm{E}-00302$

Mod. Ref No.: SDG No.: 200-2472

Lab Sample ID: 200-2472-3

Lab File ID: JBXA20.D

Date Received: 11/12/2010

Date Analyzed: 11/13/2010

Dilution Factor: 1.0

Soil Aliquot volume: (uL)

Purge Volume: 25.0 (mL)

\begin{tabular}{|c|c|c|c|c|c|}
\hline & CAS NUMBER & COMPOUND NAME & RT & EST. CONC. & Q \\
\hline 01 & + & Unknown & $\overline{6.92}$ & 3.4 & $\overline{J X B}$ \\
\hline 02 & & Unknown & 15.00 & 1.0 & $\mathrm{~J}$ \\
\hline 03 & & Unknown & 15.75 & 1.5 & $\mathrm{~J}$ \\
\hline 04 & & Unknown & 15.84 & 1.5 & $\mathrm{~J}$ \\
\hline 05 & E9667961 & Total Alkanes & $\mathrm{N} / \mathrm{A}$ & & \\
\hline
\end{tabular}

1 EPA-designated Registry Number. 
$1 \mathrm{~A}$ - FORM I VOA-1

VOLATILE ORGANICS ANALYSIS DATA SHEET
EPA SAMPLE NO.

INT118-W-30786

Lab Name: TESTAMERICA BURLINGTON

Contract: $8 \mathrm{E}-00302$

Lab Code: STLV Case No.: INMAN Mod. Ref No.: SDG No.: $\quad 200-2472$

Matrix: (SOIL/SED/WATER)

Sample wt/vol: 25.0

water

$(\mathrm{g} / \mathrm{mL}) \mathrm{mL}$

Level: (TRACE/LOW/MED) TRACE

․ Moisture: not dec.

GC Column: DB-624 ID: 0.20 (mm)

Soil Extract Volume: (uL)

Purge Volume: 25.0 (mL)
Lab Sample ID: 200-2472-4

Lab File ID: JBXA21.D

Date Received: 11/12/2010

Date Analyzed: 11/13/2010

Dilution Factor: 1.0

Soil Aliquot Volume: (uL)

\begin{tabular}{|c|c|c|c|}
\hline CAS NO. & COMPOUND & $\begin{array}{l}\text { CONCENTRATION UNITS: } \\
(\mathrm{ug} / \mathrm{L} \text { or } \mathrm{ug} / \mathrm{kg}) \mathrm{ug} / \mathrm{L}\end{array}$ & Q \\
\hline $75-71-8$ & Dichlorodifluoromethane & 0.50 & $\overline{\mathrm{U}}$ \\
\hline $74-87-3$ & Chloromethane & 0.50 & $\mathrm{U}$ \\
\hline $75-01-4$ & Vinyl chloride & 0.50 & $\mathrm{U}$ \\
\hline $74-83-9$ & Bromomethane & 0.50 & $\mathrm{U}$ \\
\hline $75-00-3$ & Chloroethane & 0.50 & $\mathrm{U}$ \\
\hline $75-69-4$ & Trichlorofluoromethane & 0.50 & $\mathrm{U}$ \\
\hline $75-35-4$ & 1,1-Dichloroethene & 0.50 & $\mathrm{U}$ \\
\hline $76-13-1$ & 1,1,2-Trichloro-1,2,2-trifluoroethane & 0.50 & $\mathrm{U}$ \\
\hline $67-64-1$ & Acetone & 4.4 & $\mathrm{~J} B$ \\
\hline $75-15-0$ & Carbon disulfide & 0.50 & $\mathrm{U}$ \\
\hline $79-20-9$ & Methyl acetate & 0.50 & $\mathrm{U}$ \\
\hline $75-09-2$ & Methylene Chloride & 0.50 & $\mathrm{U}$ \\
\hline $156-60-5$ & trans-1,2-Dichloroethene & 0.50 & $\mathrm{U}$ \\
\hline $1634-04-4$ & Methyl tert-butyl ether & 0.50 & $\mathrm{U}$ \\
\hline $75-34-3$ & 1,1-Dichloroethane & 0.50 & $\mathrm{U}$ \\
\hline $156-59-2$ & cis-1,2-Dichloroethene & 0.50 & $\mathrm{U}$ \\
\hline $78-93-3$ & 2-Butanone & 5.0 & $\mathrm{U}$ \\
\hline $74-97-5$ & Bromochloromethane & 0.50 & $\mathrm{U}$ \\
\hline $67-66-3$ & Chloroform & 0.50 & $\mathrm{U}$ \\
\hline $71-55-6$ & 1,1,1-Trichloroethane & 0.50 & $\mathrm{U}$ \\
\hline $110-82-7$ & Cyclohexane & 0.50 & $\mathrm{U}$ \\
\hline $56-23-5$ & Carbon tetrachloride & 0.50 & $\mathrm{U}$ \\
\hline $71-43-2$ & Benzene & 0.050 & $\mathrm{~J}$ \\
\hline $107-06-2$ & 1,2-Dichloroethane & 0.50 & $\mathrm{U}$ \\
\hline
\end{tabular}

Report 1,4-Dioxane for Low-Medium VOA analysis only 
Lab Name: TESTAMERICA BURLINGTON

Contract: $8 \mathrm{E}-00302$

Lab Code: STLV Case No.: INMAN Mod. Ref No.:

SDG No.: 200-2472

Matrix: (SOIL/SED/WATER)

Sample wt/vol: 25.0 Water

Level: (TRACE/LOW/MED) TRACE

$\because$ Moisture: not dec.

GC Column: DB-624 ID: 0.20 (mm)

Soil Extract Volume: (uL) $(\mathrm{g} / \mathrm{mL}) \quad \mathrm{mL}$

Purge Volume: 25.0 $(\mathrm{mL})$
Lab Sample ID: 200-2472-4

Lab File ID: JBXA21.D

Date Received: 11/12/2010

Date Analyzed: 11/13/2010

Dilution Factor: 1.0

Soil Aliquot volume:

(uL)

\begin{tabular}{|c|c|c|c|}
\hline CAS NO. & COMPOUND & $\begin{array}{l}\text { CONCENTRATION UNITS: } \\
(\mathrm{ug} / \mathrm{L} \text { or } \mathrm{ug} / \mathrm{kg}) \mathrm{ug} / \mathrm{L}\end{array}$ & $Q$ \\
\hline $79-01-6$ & Trichloroethene & 0.50 & $\overline{\mathrm{U}}$ \\
\hline $108-87-2$ & Methylcyclohexane & 0.50 & $\mathrm{U}$ \\
\hline $78-87-5$ & 1,2-Dichloropropane & 0.50 & $\mathrm{U}$ \\
\hline $75-27-4$ & Bromodichloromethane & 0.50 & $\mathrm{U}$ \\
\hline $10061-01-5$ & cis-1,3-Dichloropropene & 0.50 & $\mathrm{U}$ \\
\hline $108-10-1$ & 4-Methyl-2-pentanone & 5.0 & $\mathrm{U}$ \\
\hline $108-88-3$ & Toluene & 0.048 & $\mathrm{~J}$ \\
\hline $10061-02-6$ & trans-1,3-Dichloropropene & 0.50 & $\mathrm{U}$ \\
\hline $79-00-5$ & 1,1,2-Trichloroethane & 0.50 & $\mathrm{U}$ \\
\hline $127-18-4$ & Tetrachloroethene & 0.50 & $\mathrm{U}$ \\
\hline $591-78-6$ & 2-Hexanone & 5.0 & $\mathrm{U}$ \\
\hline $124-48-1$ & Dibromochloromethane & 0.50 & $\mathrm{U}$ \\
\hline $106-93-4$ & 1,2-Dibromoethane & 0.50 & $\mathrm{U}$ \\
\hline $108-90-7$ & Chlorobenzene & 0.50 & $\mathrm{U}$ \\
\hline $100-41-4$ & Ethylbenzene & 0.50 & $\mathrm{U}$ \\
\hline $95-47-6$ & o-xylene & 0.50 & $\mathrm{U}$ \\
\hline $179601-23-1$ & m,p-xylene & 0.50 & $\mathrm{U}$ \\
\hline $100-42-5$ & styrene & 0.50 & $\mathrm{U}$ \\
\hline $75-25-2$ & Bromoform & 0.50 & $\mathrm{U}$ \\
\hline $98-82-8$ & Isopropylbenzene & 0.50 & $\mathrm{U}$ \\
\hline $79-34-5$ & $1,1,2,2$-Tetrachloroethane & 0.50 & $\mathrm{U}$ \\
\hline $541-73-1$ & 1,3-Dichlorobenzene & 0.50 & $\mathrm{U}$ \\
\hline $106-46-7$ & 1,4-Dichlorobenzene & 0.50 & $\mathrm{U}$ \\
\hline $95-50-1$ & 1,2-Dichlorobenzene & 0.50 & $\mathrm{U}$ \\
\hline $96-12-8$ & 1,2-Dibromo-3-Chloropropane & 0.50 & $\mathrm{U}$ \\
\hline $120-82-1$ & 1,2,4-Trichlorobenzene & 0.50 & $\mathrm{U}$ \\
\hline $87-61-6$ & 1,2,3-Trichlorobenzene & 0.50 & $\mathrm{U}$ \\
\hline
\end{tabular}


$1 \mathrm{~J}$ - FORM I VOA-TIC

VOLATILE ORGANICS ANALYSIS DATA SHEET

TENTATIVELY IDENTIFIED COMPOUNDS
EPA SAMPLE NO.

INT $118-W-30786$

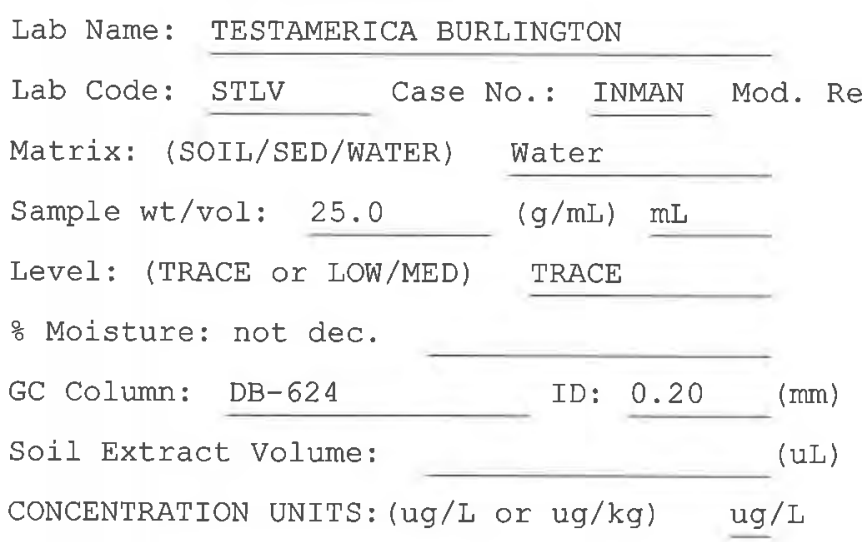

Contract: $8 \mathrm{E}-00302$

SDG No.: 200-2472

Lab Sample ID: 200-2472-4

Lab File ID: JBXA21.D

Date Received: 11/12/2010

Date Analyzed: 11/13/2010

Dilution Factor: 1.0

Soil Aliquot Volume:

$(u L)$

Purge Volume: 25.0 $(m L)$

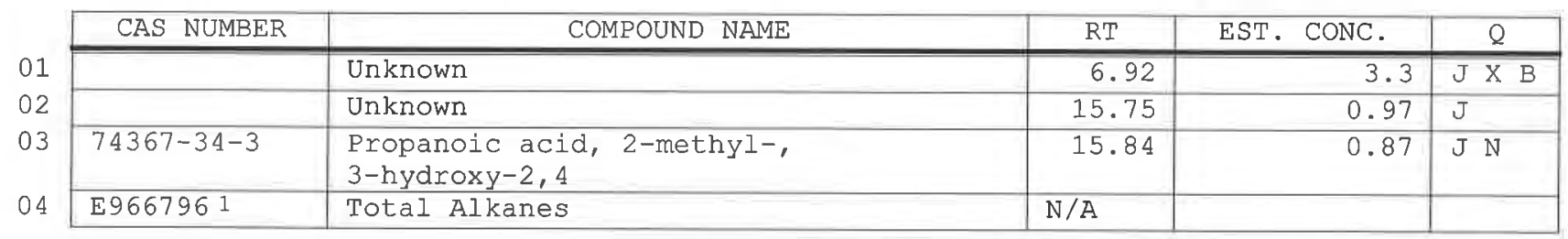

1 EPA-designated Registry Number. 
$6 A$ - FORM VI VOA-1

VOLATILE ORGANICS INITIAL CALIBRATION DATA

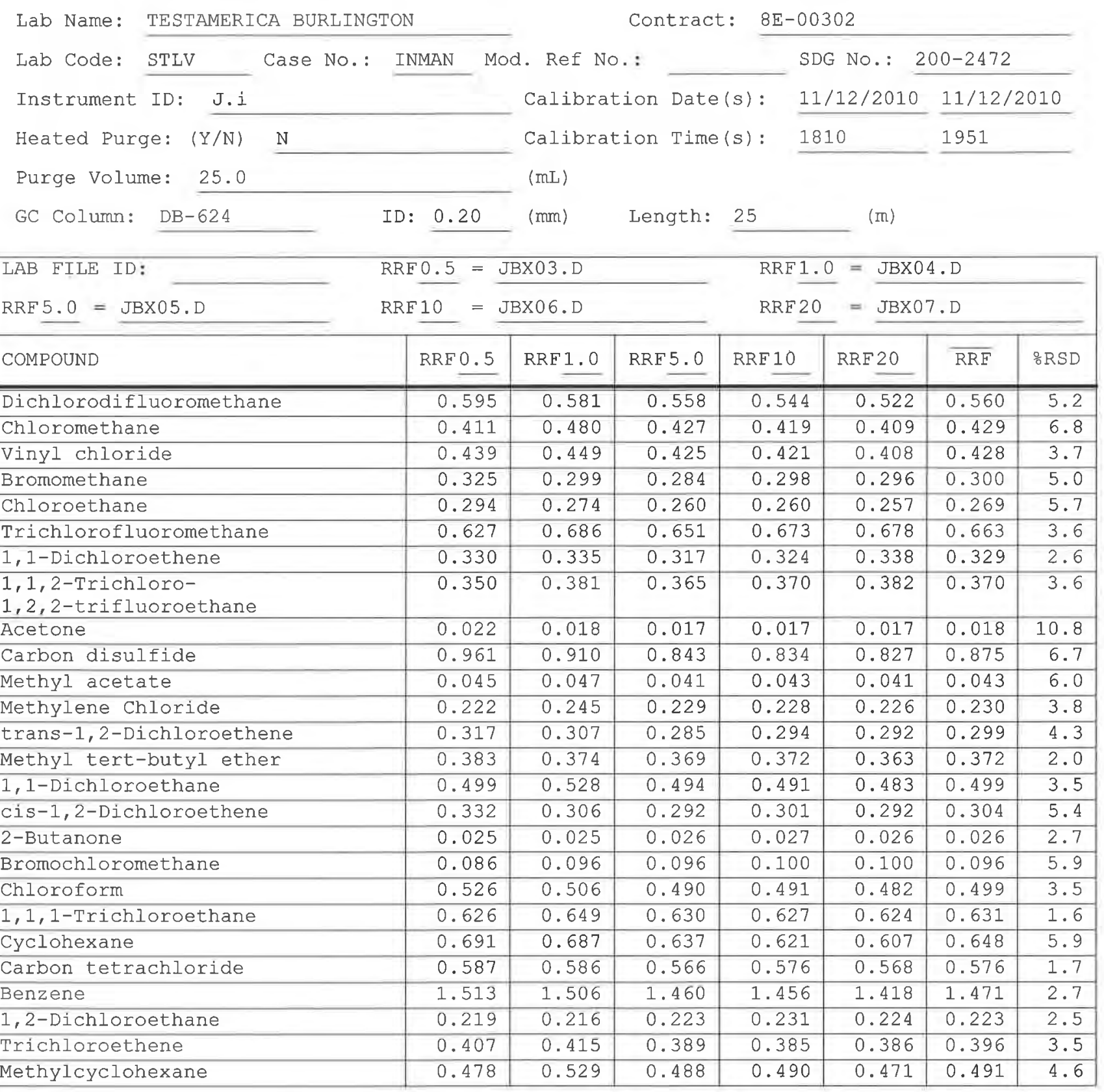

Report 1,4-Dioxane for Low-Medium VOA analysis only 
6B - FORM VI VOA-2

VOLATILE ORGANICS INITIAL CALIBRATION DATA

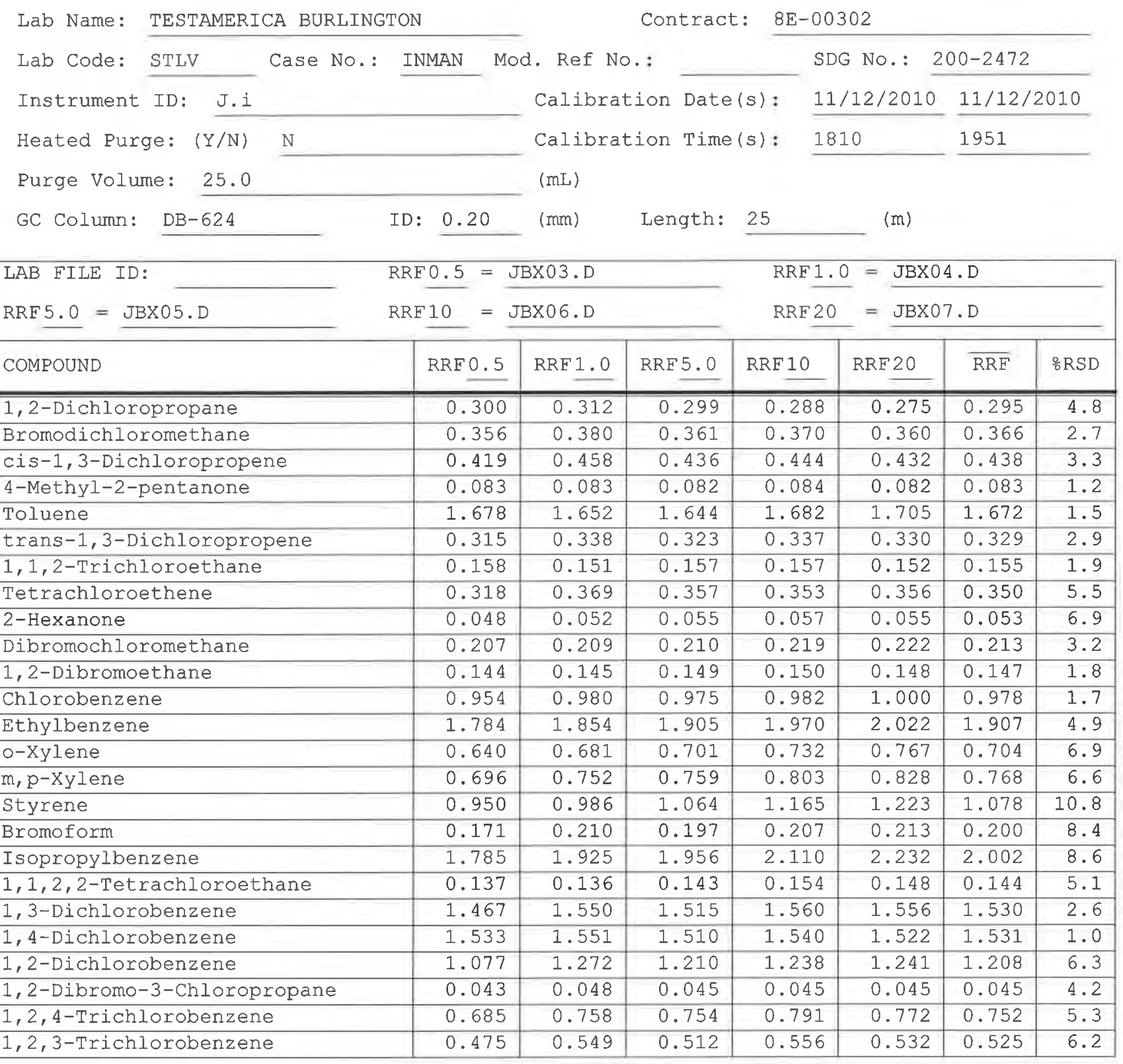


$6 C$ - FORM VI VOA-3

VOLATILE ORGANICS INITIAL CALIBRATION DATA

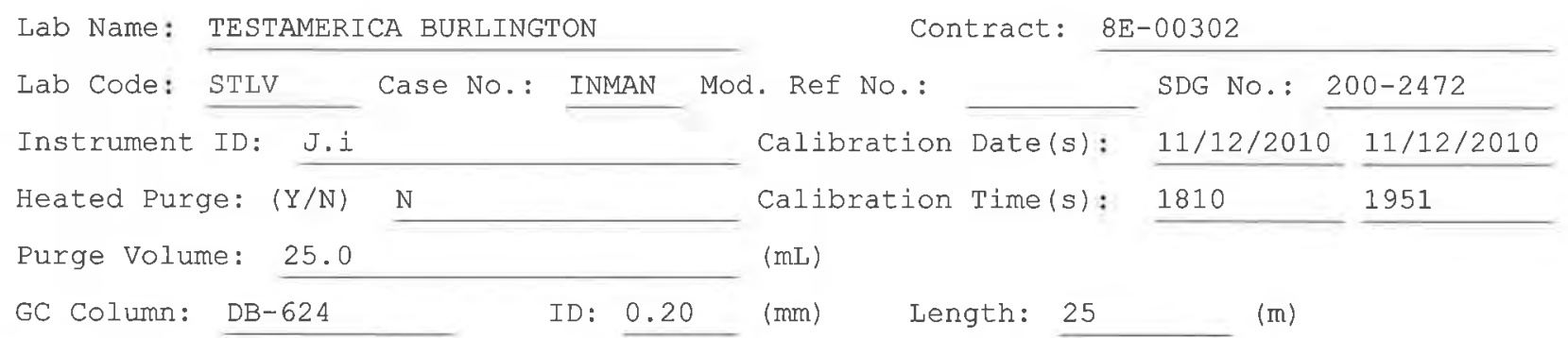

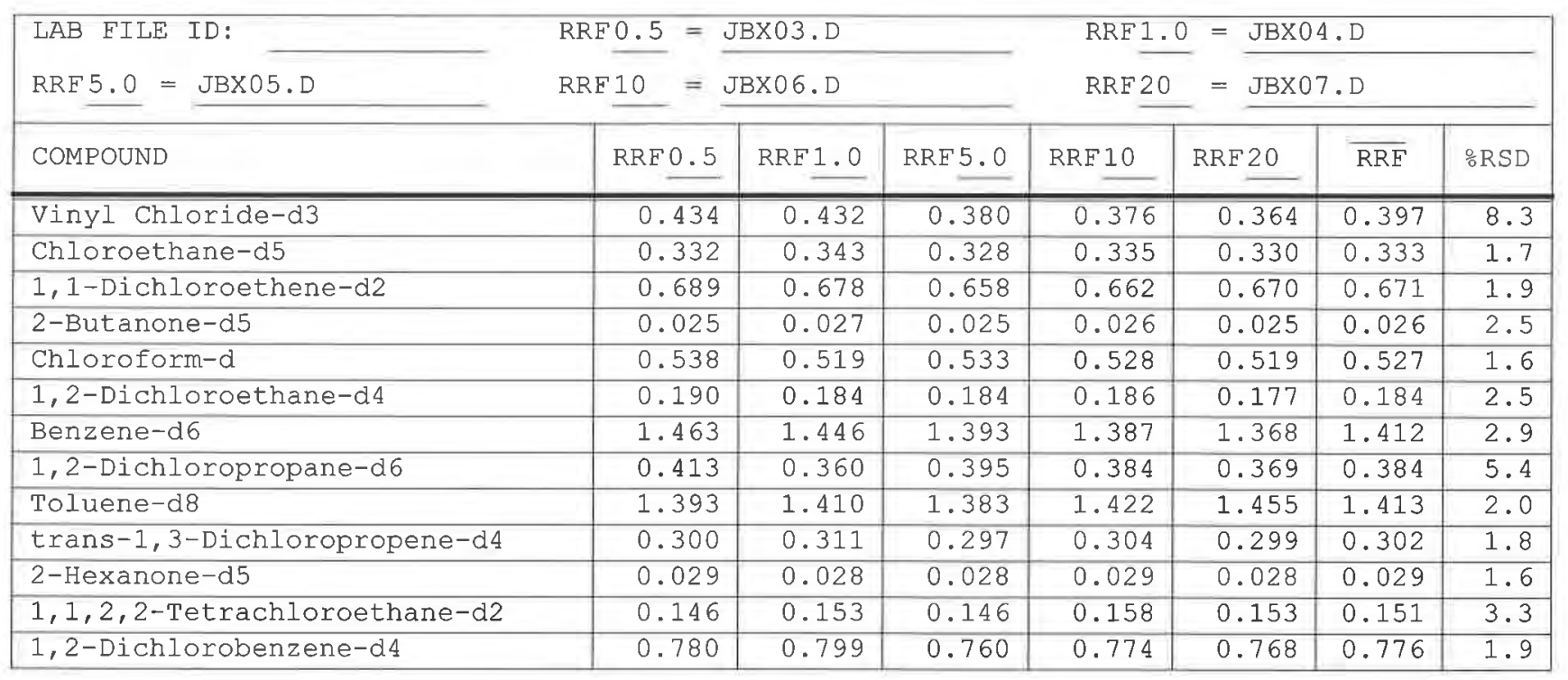

Report 1,4-Dioxane-d8 for Low-Medium VOA analysis only 
$7 A$ - FORM VII VOA-1

VOLATILE CONTINUING CALIBRATION DATA

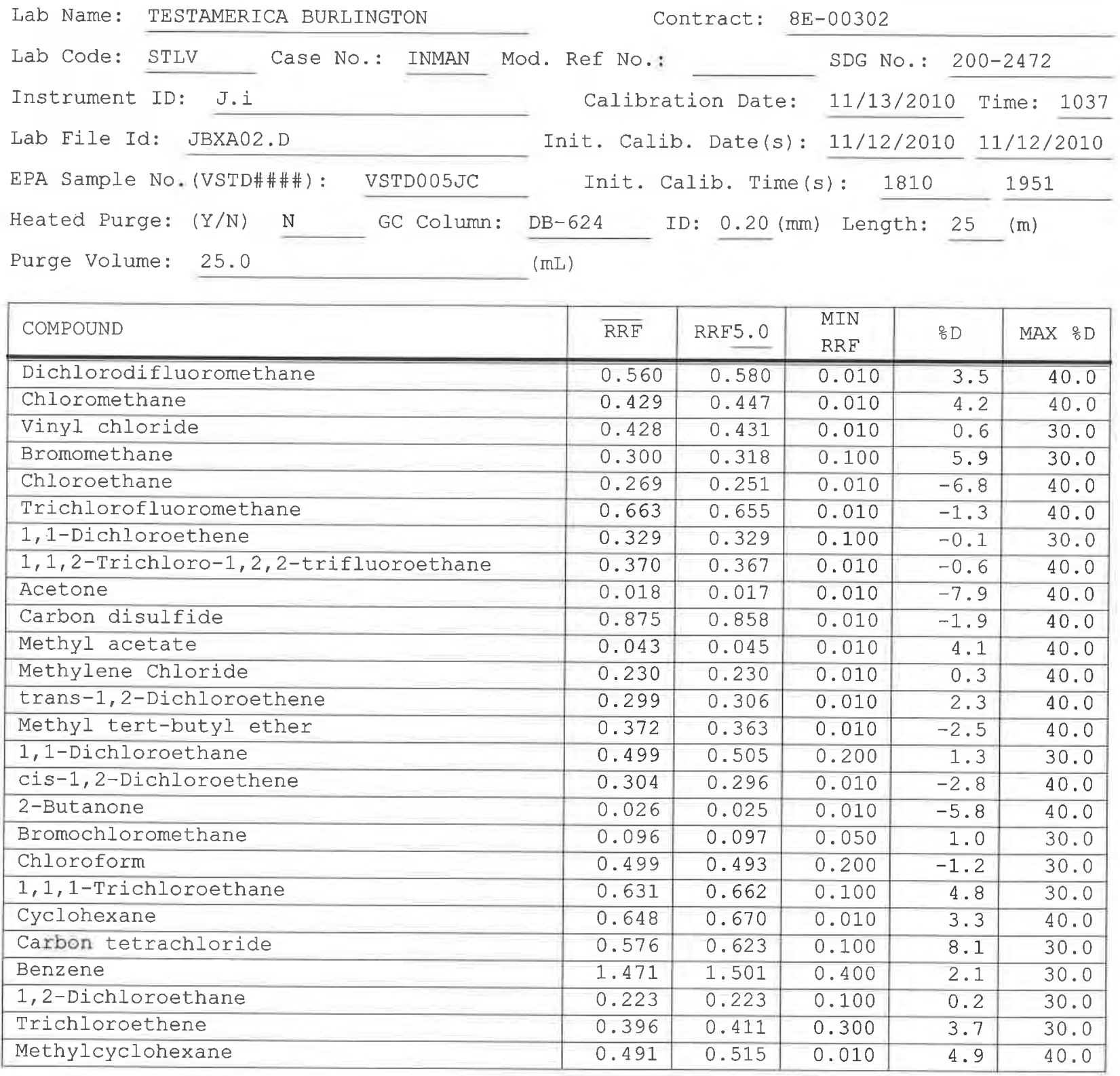

Report 1,4-Dioxane for Low/Medium VOA analysis only 
$7 \mathrm{~B}$ - FORM VII VOA-2

VOLATILE CONTINUING CALIBRATION DATA

Lab Name: TESTAMERICA BURLINGTON

Contract: $8 \mathrm{E}-00302$

Lab Code: STLV Case No.: INMAN Mod. Ref No.:

SDG NO.: 200-2472

Instrument ID: J.i

Calibration Date: 11/13/2010 Time: 1037

Lab File Id: JBXA02.D

EPA Sample No. (VSTD\#\#\#\#): VSTD005JC Init. Calib. Date(s): 11/12/2010 11/12/2010

Heated Purge: $(\mathrm{Y} / \mathrm{N}) \mathrm{N}$ GC Column: DB-624

Init. Calib. Time(s): 1810 1951

Purge Volume: 25.0 (mL)

\begin{tabular}{|c|c|c|c|c|c|}
\hline COMPOUND & $\overline{\mathrm{RRF}}$ & RRF5. 0 & $\begin{array}{l}\text { MIN } \\
\text { RRF }\end{array}$ & $\frac{\circ}{D}$ & $\mathrm{MAX} \div \mathrm{D}$ \\
\hline 1,2-Dichloropropane & 0.295 & 0.292 & 0.010 & -0.9 & 40.0 \\
\hline Bromodichloromethane & 0.366 & 0.375 & 0.200 & 2.6 & 30.0 \\
\hline cis-1,3-Dichloropropene & 0.438 & 0.445 & 0.200 & 1.6 & 30.0 \\
\hline 4-Methyl-2-pentanone & 0.083 & 0.080 & 0.010 & -3.3 & 40.0 \\
\hline Toluene & 1.672 & 1.695 & 0.400 & 1.4 & 30.0 \\
\hline trans-1,3-Dichloropropene & 0.329 & 0.335 & 0.100 & 1.8 & 30.0 \\
\hline 1,1,2-Trichloroethane & 0.155 & 0.153 & 0.100 & -1.3 & 30.0 \\
\hline Tetrachloroethene & 0.350 & 0.368 & 0.100 & 5.0 & 30.0 \\
\hline 2-Hexanone & 0.053 & 0.052 & 0.010 & -2.2 & 40.0 \\
\hline Dibromochloromethane & 0.213 & 0.216 & 0.100 & 1.2 & 30.0 \\
\hline 1,2-Dibromoethane & 0.147 & 0.150 & 0.010 & 1.5 & 40.0 \\
\hline Chlorobenzene & 0.978 & 1.005 & 0.500 & 2.8 & 30.0 \\
\hline Ethylbenzene & 1.907 & 1.978 & 0.100 & 3.7 & 30.0 \\
\hline o-xylene & 0.704 & 0.730 & 0.300 & 3.6 & 30.0 \\
\hline m,p-Xylene & 0.768 & 0.789 & 0.300 & 2.8 & 30.0 \\
\hline Styrene & 1.078 & 1.074 & 0.300 & -0.3 & 30.0 \\
\hline Bromoform & 0.200 & 0.201 & 0.050 & 0.4 & 30.0 \\
\hline Isopropylbenzene & 2.002 & 2.087 & 0.010 & 4.3 & 40.0 \\
\hline $1,1,2,2$-Tetrachloroethane & 0.144 & 0.143 & 0.100 & -0.2 & 30.0 \\
\hline 1,3-Dichlorobenzene & 1.530 & 1.582 & 0.400 & 3.4 & 30.0 \\
\hline 1,4-Dichlorobenzene & 1.531 & 1.523 & 0.400 & -0.6 & 30.0 \\
\hline 1,2-Dichlorobenzene & 1.208 & 1.241 & 0.400 & 2.8 & 30.0 \\
\hline 1,2-Dibromo-3-Chloropropane & 0.045 & 0.043 & 0.010 & -5.7 & 40.0 \\
\hline $1,2,4$-Trichlorobenzene & 0.752 & 0.760 & 0.200 & 1.1 & 30.0 \\
\hline 1,2,3-Trichlorobenzene & 0.525 & 0.515 & 0.200 & -1.9 & 30.0 \\
\hline
\end{tabular}


$7 C$ - FORM VII VOA-3

VOLATILE CONTINUING CALIBRATION DATA

Lab Name: TESTAMERICA BURLINGTON

Contract: $8 \mathrm{E}-00302$

Lab Code: STLV Case No.: INMAN Mod. R

Instrument ID: J.i

Calibration Date: 11/13/2010 Time: 1037

Lab File Id: JBXA02.D Init. Calib. Date(s): 11/12/2010 11/12/2010

EPA Sample No. (VSTD\#\#\#\#): VSTD005JC

Init. Calib. Time(s): $1810 \quad 1951$

Heated Purge: $(\mathrm{Y} / \mathrm{N}) \mathrm{N}$

GC Column: DB-624

ID: 0.20 (mm) Length: 25 (m)

Purge Volume: 25.0

$(\mathrm{mL})$

\begin{tabular}{|c|c|c|c|c|c|}
\hline COMPOUND & $\overline{\mathrm{RRF}}$ & RRF5.0 & $\begin{array}{l}\text { MIN } \\
\text { RRF }\end{array}$ & $\frac{\circ}{\partial}$ & $M A X \div D$ \\
\hline Vinyl Chloride-d3 & 0.397 & 0.386 & 0.010 & -2.8 & 30.0 \\
\hline Chloroethane-d5 & 0.333 & 0.325 & 0.010 & -2.6 & 40.0 \\
\hline 1,1-Dichloroethene-d2 & 0.671 & 0.667 & 0.010 & -0.7 & 30.0 \\
\hline 2-Butanone-d5 & 0.026 & 0.024 & 0.010 & -6.4 & 40.0 \\
\hline Chloroform-d & 0.527 & 0.527 & 0.010 & -0.1 & 30.0 \\
\hline 1,2-Dichloroethane-d4 & 0.184 & 0.178 & 0.010 & -3.6 & 30.0 \\
\hline Benzene-d6 & 1.412 & 1.457 & 0.010 & 3.2 & 30.0 \\
\hline 1,2-Dichloropropane-d6 & 0.384 & 0.401 & 0.010 & 4.3 & 40.0 \\
\hline Toluene-d8 & 1.413 & 1.469 & 0.010 & 4.0 & 30.0 \\
\hline trans-1,3-Dichloropropene-d4 & 0.302 & 0.303 & 0.010 & 0.4 & 30.0 \\
\hline 2-Hexanone-d5 & 0.029 & 0.027 & 0.010 & -6.5 & 40.0 \\
\hline $1,1,2,2-$ Tetrachloroethane-d2 & 0.151 & 0.148 & 0.010 & -1.9 & 30.0 \\
\hline 1,2-Dichlorobenzene-d4 & 0.776 & 0.769 & 0.010 & -0.9 & 30.0 \\
\hline
\end{tabular}

Report 1,4-Dioxane-d8 for Low/Medium VOA analysis only 
$7 A$ - FORM VII VOA-1

VOLATILE CONTINUING CALIBRATION DATA

Lab Name: TESTAMERICA BURLINGTON

Contract: $8 \mathrm{E}-00302$

Lab Code: STLV Case No.: INMAN Mod. Ref No.:

SDG No.: 200-2472

Instrument ID: J.i

Calibration Date: 11/13/2010 Time: 2114

Lab File Id: JBXA26.D Init. Calib. Date(s): 11/12/2010 11/12/2010

EPA Sample No.(VSTD\#\#\#\#): VSTD005CJ

Init. Calib. Time(s): $1810 \quad 1951$

Heated Purge: ( $Y / N) \quad N$

GC Column: DB-624

ID: $0.20(\mathrm{~mm})$ Length: 25

(m)

Purge Volume: 25.0

(mL)

\begin{tabular}{|c|c|c|c|c|c|}
\hline COMPOUND & $\overline{\mathrm{RRF}}$ & RRF5. 0 & $\begin{array}{l}\text { MIN } \\
\text { RRF }\end{array}$ & $\frac{\circ}{\circ} \mathrm{D}$ & MAX $\because D$ \\
\hline Dichlorodifluoromethane & 0.560 & 0.564 & 0.010 & 0.7 & $\overline{50.0}$ \\
\hline Chloromethane & 0.429 & 0.423 & 0.010 & -1.4 & 50.0 \\
\hline Vinyl chloride & 0.428 & 0.428 & 0.010 & 0.0 & 50.0 \\
\hline Bromomethane & 0.300 & 0.297 & 0.010 & -1.1 & 50.0 \\
\hline Chloroethane & 0.269 & 0.279 & 0.010 & 3.9 & 50.0 \\
\hline Trichlorofluoromethane & 0.663 & 0.685 & 0.010 & 3.3 & 50.0 \\
\hline 1,1-Dichloroethene & 0.329 & 0.328 & 0.010 & -0.2 & 50.0 \\
\hline 1,1,2-Trichloro-1,2,2-trifluoroethane & 0.370 & 0.362 & 0.010 & -2.1 & 50.0 \\
\hline Acetone & 0.018 & 0.017 & 0.010 & -6.8 & 50.0 \\
\hline Carbon disulfide & 0.875 & 0.817 & 0.010 & -6.7 & 50.0 \\
\hline Methyl acetate & 0.043 & 0.042 & 0.010 & -2.7 & 50.0 \\
\hline Methylene Chloride & 0.230 & 0.231 & 0.010 & 0.6 & 50.0 \\
\hline trans-1,2-Dichloroethene & 0.299 & 0.305 & 0.010 & 2.0 & 50.0 \\
\hline Methyl tert-butyl ether & 0.372 & 0.343 & 0.010 & -7.9 & 50.0 \\
\hline 1,1-Dichloroethane & 0.499 & 0.495 & 0.010 & -0.9 & 50.0 \\
\hline cis-1,2-Dichloroethene & 0.304 & 0.301 & 0.010 & -1.3 & 50.0 \\
\hline 2-Butanone & 0.026 & 0.025 & 0.010 & -4.3 & 50.0 \\
\hline Bromochloromethane & 0.096 & 0.098 & 0.010 & 2.2 & 50.0 \\
\hline Chloroform & 0.499 & 0.484 & 0.010 & -2.9 & 50.0 \\
\hline 1,1,1-Trichloroethane & 0.631 & 0.625 & 0.010 & -0.9 & 50.0 \\
\hline Cyclohexane & 0.648 & 0.625 & 0.010 & -3.7 & 50.0 \\
\hline Carbon tetrachloride & 0.576 & 0.582 & 0.010 & 1.0 & 50.0 \\
\hline Benzene & 1.471 & 1.474 & 0.010 & 0.2 & 50.0 \\
\hline 1,2-Dichloroethane & 0.223 & 0.220 & 0.010 & -1.2 & 50.0 \\
\hline Trichloroethene & 0.396 & 0.408 & 0.010 & 3.0 & 50.0 \\
\hline Methylcyclohexane & 0.491 & 0.502 & 0.010 & 2.4 & 50.0 \\
\hline
\end{tabular}

Report 1,4-Dioxane for Low/Medium VOA analysis only 
$7 B$ - FORM VII VOA-2

VOLATILE CONTINUING CALIBRATION DATA

Lab Name: TESTAMERICA BURLINGTON

Contract: $\quad 8 \mathrm{E}-00302$

Lab Code: STLV Case No.: INMAN Mod. Ref No.

SDG No.: 200-2472

Instrument ID: J.i

Calibration Date: 11/13/2010 Time: 2114

Lab File Id: JBXA26.D

EPA Sample No. (VSTD\#\#\#\#): VSTD005CJ Init. Calib. Date(s): 11/12/2010 11/12/2010

Heated Purge: ( $Y / N)$

GC Column: DB-624

Init. Calib. Time(s): $1810 \quad 1951$

Purge Volume: 25.0

(mL)

\begin{tabular}{|c|c|c|c|c|c|}
\hline COMPOUND & $\overline{\mathrm{RRF}}$ & RRF5.0 & $\begin{array}{l}\text { MIN } \\
\text { RRE }\end{array}$ & $\because \mathrm{D}$ & MAX $\because D$ \\
\hline 1,2-Dichloropropane & 0.295 & 0.279 & 0.010 & -5.4 & 50.0 \\
\hline Bromodichloromethane & 0.366 & 0.371 & 0.010 & 1.6 & 50.0 \\
\hline cis-1,3-Dichloropropene & 0.438 & 0.430 & 0.010 & -1.9 & 50.0 \\
\hline 4-Methyl-2-pentanone & 0.083 & 0.085 & 0.010 & 3.3 & 50.0 \\
\hline Toluene & 1.672 & 1.703 & 0.010 & 1.9 & 50.0 \\
\hline trans-1,3-Dichloropropene & 0.329 & 0.319 & 0.010 & -2.9 & 50.0 \\
\hline 1,1,2-Trichloroethane & 0.155 & 0.160 & 0.010 & 3.1 & 50.0 \\
\hline Tetrachloroethene & 0.350 & 0.366 & 0.010 & 4.6 & 50.0 \\
\hline 2-Hexanone & 0.053 & 0.058 & 0.010 & 7.9 & 50.0 \\
\hline Dibromochloromethane & 0.213 & 0.219 & 0.010 & 2.5 & 50.0 \\
\hline 1,2-Dibromoethane & 0.147 & 0.151 & 0.010 & 2.8 & 50.0 \\
\hline Chlorobenzene & 0.978 & 1.026 & 0.010 & 4.9 & 50.0 \\
\hline Ethylbenzene & 1.907 & 1.950 & 0.010 & 2.3 & 50.0 \\
\hline o-Xylene & 0.704 & 0.745 & 0.010 & 5.7 & 50.0 \\
\hline m,p-xylene & 0.768 & 0.800 & 0.010 & 4.2 & 50.0 \\
\hline Styrene & 1.078 & 1.112 & 0.010 & 3.2 & 50.0 \\
\hline Bromoform & 0.200 & 0.196 & 0.010 & -1.8 & 50.0 \\
\hline Isopropylbenzene & 2.002 & 2.092 & 0.010 & 4.5 & 50.0 \\
\hline $1,1,2,2$-Tetrachloroethane & 0.144 & 0.157 & 0.010 & 9.3 & 50.0 \\
\hline 1,3-Dichlorobenzene & 1.530 & 1.620 & 0.010 & 5.9 & 50.0 \\
\hline 1,4-Dichlorobenzene & 1.531 & 1.565 & 0.010 & 2.2 & 50.0 \\
\hline 1,2-Dichlorobenzene & 1.208 & 1.242 & 0.010 & 2.9 & 50.0 \\
\hline 1,2-Dibromo-3-Chloropropane & 0.045 & 0.043 & 0.010 & -3.9 & 50.0 \\
\hline 1,2,4-Trichlorobenzene & 0.752 & 0.795 & 0.010 & 5.7 & 50.0 \\
\hline 1,2,3-Trichlorobenzene & 0.525 & 0.572 & 0.010 & 9.0 & 50.0 \\
\hline
\end{tabular}


$7 C$ - FORM VII VOA-3

VOLATILE CONTINUING CALIBRATION DATA

Lab Name: TESTAMERICA BURLINGTON

Contract: $8 \mathrm{E}-00302$

Lab Code: STLV Case No.: INMAN Mod. Ref No.:

SDG No.: 200-2472

Instrument ID: J,i

Calibration Date: 11/13/2010 Time: 2114

Lab File Id: JBXA26. D Init. Calib. Date(s): 11/12/2010 11/12/2010

EPA Sample No. (VSTD\#\#\#\#): VSTD005CJ

Init. Calib. Time(s): $1810 \quad 1951$

Heated Purge: ( $\mathrm{Y} / \mathrm{N}) \quad \mathrm{N}$

GC Column: DB-624

ID: $0.20(\mathrm{~mm})$ Length: 25

(m)

Purge Volume: 25.0

$(\mathrm{mL})$

\begin{tabular}{|c|c|c|c|c|c|}
\hline COMPOUND & $\overline{\mathrm{RRF}}$ & RRF5.0 & $\begin{array}{l}\text { MIN } \\
\text { RRE }\end{array}$ & $\because \mathrm{D}$ & $\mathrm{MAX} \div \mathrm{D}$ \\
\hline Vinyl Chloride-d3 & 0.397 & 0.385 & 0.010 & -3.1 & 50.0 \\
\hline Chloroethane-d5 & 0.333 & 0.354 & 0.010 & 6.0 & 50.0 \\
\hline 1,1-Dichloroethene-d2 & 0.671 & 0.672 & 0.010 & 0.1 & 50.0 \\
\hline 2-Butanone-d5 & 0.026 & 0.024 & 0.010 & -4.9 & 50.0 \\
\hline Chloroform-d & 0.527 & 0.523 & 0.010 & -0.7 & 50.0 \\
\hline 1,2-Dichloroethane-d4 & 0.184 & 0.183 & 0.010 & -0.6 & 50.0 \\
\hline Benzene-d6 & 1.412 & 1.416 & 0.010 & 0.3 & 50.0 \\
\hline 1,2-Dichloropropane-d6 & 0.384 & 0.345 & 0.010 & -10.1 & 50.0 \\
\hline Toluene-d8 & 1.413 & 1.467 & 0.010 & 3.9 & 50.0 \\
\hline trans-1,3-Dichloropropene-d4 & 0.302 & 0.293 & 0.010 & -3.2 & 50.0 \\
\hline 2-Hexanone-d5 & 0.029 & 0.028 & 0.010 & -3.4 & 50.0 \\
\hline $1,1,2,2$-Tetrachloroethane-d2 & 0.151 & 0.151 & 0.010 & -0.1 & 50.0 \\
\hline 1,2-Dichlorobenzene-d4 & 0.776 & 0.794 & 0.010 & 2.2 & 50.0 \\
\hline
\end{tabular}

Report 1,4-Dioxane-d8 for Low/Medium VOA analysis only 
$1 A$ - FORM I VOA-1

VOLATILE ORGANICS ANALYSIS DATA SHEET
EPA SAMPLE NO.

VBLKJC

Lab Name: TESTAMERICA BURLINGTON

Contract: $\quad 8 \mathrm{E}-00302$

Lab Code: STLV

Case No.: INMAN Mod. Ref No.:

SDG No.: 200-2472

Matrix: (SOIL/SED/WATER) Water

Sample wt/vol: $25.0 \quad(\mathrm{~g} / \mathrm{mL}) \mathrm{mL}$

Level: (TRACE/LOW/MED) TRACE

\% Moisture: not dec.

GC Column: DB-624 ID $: 0.20 \quad$ (mm)

Soil Extract Volume: (uL) (mL)

Purge Volume: 25.0
Lab Sample ID: MB 200-9552/3

Lab File ID: JBXA03.D

Date Received:

Date Analyzed: 11/13/2010

Dilution Factor: 1.0

Soil Aliquot Volume:

(UL)

\begin{tabular}{|c|c|c|c|}
\hline CAS NO. & COMPOUND & $\begin{array}{l}\text { CONCENTRATION UNITS: } \\
\text { (ug/L or ug/kg) ug/L }\end{array}$ & $\mathrm{Q}$ \\
\hline $75-71-8$ & Dichlorodifluoromethane & 0.50 & $\bar{U}$ \\
\hline $74-87-3$ & Chloromethane & 0.50 & $\mathrm{U}$ \\
\hline $75-01-4$ & Vinyl chloride & 0.50 & $\mathrm{U}$ \\
\hline $74-83-9$ & Bromomethane & 0.50 & $\mathrm{U}$ \\
\hline $75-00-3$ & Chloroethane & 0.50 & $\mathrm{U}$ \\
\hline $75-69-4$ & Trichlorofluoromethane & 0.50 & $\mathrm{U}$ \\
\hline $75-35-4$ & 1,1-Dichloroethene & 0.50 & $\mathrm{U}$ \\
\hline $76-13-1$ & 1,1,2-Trichloro-1,2,2-trifluoroethane & 0.50 & $\mathrm{U}$ \\
\hline $67-64-1$ & Acetone & 1.8 & $\mathrm{~J}$ \\
\hline $75-15-0$ & Carbon disulfide & 0.16 & $\mathrm{~J}$ \\
\hline $79-20-9$ & Methyl acetate & 0.50 & $\mathrm{U}$ \\
\hline $75-09-2$ & Methylene Chloride & 0.50 & $\mathrm{U}$ \\
\hline $156-60-5$ & trans-1,2-Dichloroethene & 0.046 & $\mathrm{~J}$ \\
\hline $1634-04-4$ & Methyl tert-butyl ether & 0.50 & $\mathrm{U}$ \\
\hline $75-34-3$ & 1,1-Dichloroethane & 0.50 & $\mathrm{U}$ \\
\hline $156-59-2$ & cis-1,2-Dichloroethene & 0.50 & $\mathrm{U}$ \\
\hline $78-93-3$ & 2-Butanone & 5.0 & $\mathrm{U}$ \\
\hline $74-97-5$ & Bromochloromethane & 0.50 & $\mathrm{U}$ \\
\hline $67-66-3$ & Chloroform & 0.50 & $\mathrm{U}$ \\
\hline $71-55-6$ & 1,1,1-Trichloroethane & 0.50 & $\mathrm{U}$ \\
\hline $110-82-7$ & Cyclohexane & 0.50 & $\mathrm{U}$ \\
\hline $56-23-5$ & Carbon tetrachloride & 0.50 & $\mathrm{U}$ \\
\hline $71-43-2$ & Benzene & 0.50 & $\mathrm{U}$ \\
\hline $107-06-2$ & 1,2-Dichloroethane & 0.50 & U \\
\hline
\end{tabular}

Report 1,4-Dioxane for Low-Medium VOA analysis only 
$1 B$ - FORM I VOA-2

VOLATILE ORGANICS ANALYSIS DATA SHEET
EPA SAMPLE NO.

VBLKJC

Lab Name: TESTAMERICA BURLINGTON

Contract: $8 \mathrm{E}-00302$

Lab Code: STLV Case No.: INMAN Mod. Ref No.: SDG No.: 200-2472

Matrix: (SOIL/SED/WATER) Water

Sample wt/vol: 25.0 $(\mathrm{g} / \mathrm{mL}) \mathrm{mL}$

Level: (TRACE/LOW/MED) TRACE

응 Moisture: not dec.

GC Column: DB-624 ID : 0.20 (mm)

Soil Extract Volume: (uL) (mL)

Purge Volume: 25.0
Lab Sample ID: MB 200-9552/3

Lab File ID: JBXA03.D

Date Received:

Date Analyzed: $11 / 13 / 2010$

Dilution Factor: 1.0

Soil Aliquot volume:

(UL)

\begin{tabular}{|c|c|c|c|}
\hline CAS NO. & COMPOUND & $\begin{array}{l}\text { CONCENTRATION UNITS: } \\
(\mathrm{ug} / \mathrm{L} \text { or } u g / \mathrm{kg}) \mathrm{ug} / \mathrm{L}\end{array}$ & $Q$ \\
\hline $79-01-6$ & Trichloroethene & 0.50 & $\mathrm{U}$ \\
\hline $108-87-2$ & Methylcyclohexane & 0.50 & $\mathrm{U}$ \\
\hline $78-87-5$ & 1,2-Dichloropropane & 0.50 & $\mathrm{U}$ \\
\hline $75-27-4$ & Bromodichloromethane & 0.50 & $\mathrm{U}$ \\
\hline $10061-01-5$ & cis-1,3-Dichloropropene & 0.50 & $\mathrm{U}$ \\
\hline $108-10-1$ & 4-Methyl-2-pentanone & 5.0 & $\mathrm{U}$ \\
\hline $108-88-3$ & Toluene & 0.50 & $\mathrm{U}$ \\
\hline $10061-02-6$ & trans-1,3-Dichloropropene & 0.50 & $\mathrm{U}$ \\
\hline $79-00-5$ & $1,1,2$-Trichloroethane & 0.50 & $\mathrm{U}$ \\
\hline $127-18-4$ & Tetrachloroethene & 0.50 & $\mathrm{U}$ \\
\hline $591-78-6$ & 2-Hexanone & 5.0 & $\mathrm{U}$ \\
\hline $124-48-1$ & Dibromochloromethane & 0.50 & $\mathrm{U}$ \\
\hline $106-93-4$ & 1,2-Dibromoethane & 0.50 & $\mathrm{U}$ \\
\hline $108-90-7$ & Chlorobenzene & 0.50 & $\mathrm{U}$ \\
\hline $100-41-4$ & Ethylbenzene & 0.50 & $\mathrm{U}$ \\
\hline $95-47-6$ & o-Xylene & 0.50 & $\mathrm{U}$ \\
\hline $179601-23-1$ & m,p-xylene & 0.50 & $\mathrm{U}$ \\
\hline $100-42-5$ & Styrene & 0.50 & U \\
\hline $75-25-2$ & Bromoform & 0.50 & $\mathrm{U}$ \\
\hline $98-82-8$ & Isopropylbenzene & 0.50 & $\mathrm{U}$ \\
\hline $79-34-5$ & $1,1,2,2$-Tetrachloroethane & 0.50 & $\mathrm{U}$ \\
\hline $541-73-1$ & 1,3-Dichlorobenzene & 0.50 & $\mathrm{U}$ \\
\hline $106-46-7$ & 1,4-Dichlorobenzene & 0.044 & $\mathrm{~J}$ \\
\hline $95-50-1$ & 1,2-Dichlorobenzene & 0.50 & $\mathrm{U}$ \\
\hline $96-12-8$ & 1,2-Dibromo-3-Chloropropane & 0.50 & $\mathrm{U}$ \\
\hline $120-82-1$ & 1,2,4-Trichlorobenzene & 0.14 & $\mathrm{~J}$ \\
\hline $87-61-6$ & 1,2,3-Trichlorobenzene & 0.19 & $\mathrm{~J}$ \\
\hline
\end{tabular}


$1 \mathrm{~J}$ - FORM I VOA-TIC

VOLATILE ORGANICS ANALYSIS DATA SHEET TENTATIVELY IDENTIFIED COMPOUNDS
EPA SAMPLE NO.

VBLKJC
Lab Name: TESTAMERICA BURLINGTON

Lab Code: STLV Case No.: INMAN

Matrix: (SOIL/SED/WATER)

Sample wt/vol: 25.0

Level: (TRACE or LOW/MED)

Water

$(\mathrm{g} / \mathrm{mL}) \quad \mathrm{mL}$

\% Moisture: not dec.

GC Column: DB-624

ID: 0.20

$(\mathrm{mm})$

Soil Extract Volume:

(uL)

CONCENTRATION UNITS: $(\mathrm{ug} / \mathrm{L}$ or $\mathrm{ug} / \mathrm{kg}$ ) ug/L
Contract: $8 \mathrm{E}-00302$

SDG No.: 200-2472
Lab Sample ID: MB 200-9552/3

Lab File ID: JBXA03.D

Date Received:

Date Analyzed: 11/13/2010

Dilution Factor: 1.0

Soil Aliquot Volume: (uL)

Purge Volume: 25.0 (mL)

\begin{tabular}{|l|l|c|c|c|}
\hline CAS NUMBER & \multicolumn{1}{|c|}{ COMPOUND NAME } & RT & EST. CONC. & Q \\
\hline \hline E9667961 & Unknown & 6.92 & 3.5 & X J \\
\hline
\end{tabular}

1 EPA-designated Registry Number. 
Lab Name: TESTAMERICA BURLINGTON

Lab Code: STLV Case No.: INMA
Matrix: (SOIL/SED/WATER)

Sample wt/vol: 25.0

\section{Water}

$(\mathrm{g} / \mathrm{mL}) \quad \mathrm{mI}$

Level: (TRACE/LOW/MED) TRACE

o Moisture: not dec.

GC Column: DB-624

Purge Volume: 25.0
Soil Extract Volume:
Contract: $8 \mathrm{E}-00302$

SDG No.: 200-2472
Lab Sample ID: 200-2472-6

Lab File ID: JBXA25.D

Date Received:

Date Analyzed: 11/13/2010

Dilution Factor: 1.0

Soil Aliquot Volume:

(uL)

\begin{tabular}{|c|c|c|c|}
\hline CAS NO. & COMPOUND & $\begin{array}{l}\text { CONCENTRATION UNITS: } \\
(\mathrm{ug} / \mathrm{L} \text { or } \mathrm{ug} / \mathrm{kg}) \mathrm{ug} / \mathrm{L} \\
\end{array}$ & $Q$ \\
\hline $75-71-8$ & Dichlorodifluoromethane & 0.50 & $\overline{\mathrm{U}}$ \\
\hline $74-87-3$ & Chloromethane & 0.50 & $\mathrm{U}$ \\
\hline $75-01-4$ & Vinyl chloride & 0.50 & $\mathrm{U}$ \\
\hline $74-83-9$ & Bromomethane & 0.50 & $\mathrm{U}$ \\
\hline $75-00-3$ & Chloroethane & 0.50 & $\mathrm{U}$ \\
\hline $75-69-4$ & Trichlorofluoromethane & 0.50 & $\mathrm{U}$ \\
\hline $75-35-4$ & 1,1-Dichloroethene & 0.50 & $\mathrm{U}$ \\
\hline $76-13-1$ & 1,1,2-Trichloro-1,2,2-trifluoroethane & 0.50 & $\mathrm{U}$ \\
\hline $67-64-1$ & Acetone & 2.0 & $\mathrm{~J} \mathrm{~B}$ \\
\hline $75-15-0$ & Carbon disulfide & 0.50 & $\mathrm{U}$ \\
\hline $79-20-9$ & Methyl acetate & 0.50 & $\mathrm{U}$ \\
\hline $75-09-2$ & Methylene Chloride & 0.50 & $\mathrm{U}$ \\
\hline $156-60-5$ & trans-1,2-Dichloroethene & 0.50 & $\mathrm{U}$ \\
\hline $1634-04-4$ & Methyl tert-butyl ether & 0.50 & $\mathrm{U}$ \\
\hline $75-34-3$ & 1,1-Dichloroethane & 0.50 & $\mathrm{U}$ \\
\hline $156-59-2$ & cis-1,2-Dichloroethene & 0.50 & $\mathrm{U}$ \\
\hline $78-93-3$ & 2-Butanone & 5.0 & $\mathrm{U}$ \\
\hline $74-97-5$ & Bromochloromethane & 0.50 & $\mathrm{U}$ \\
\hline $67-66-3$ & Chloroform & 0.50 & $\mathrm{U}$ \\
\hline $71-55-6$ & 1,1,1-Trichloroethane & 0.50 & $\mathrm{U}$ \\
\hline $110-82-7$ & Cyclohexane & 0.50 & $\mathrm{U}$ \\
\hline $56-23-5$ & Carbon tetrachloride & 0.50 & $\mathrm{U}$ \\
\hline $71-43-2$ & Benzene & 0.50 & $\mathrm{U}$ \\
\hline $107-06-2$ & 1,2-Dichloroethane & 0.50 & $\mathrm{U}$ \\
\hline
\end{tabular}

Report 1,4-Dioxane for Low-Medium VOA analysis only 
$1 B$ - FORM I VOA-2

VOLATILE ORGANICS ANALYSIS DATA SHEET
EPA SAMPLE NO.

VHBKLO1
Lab Name: TESTAMERICA BURLINGTON

Lab Code: STLV

Matrix: (SOIL/SED/WATER)

Sample wt/vol: 25.0

Water

$(\mathrm{g} / \mathrm{mL}) \quad \mathrm{mL}$

Level: (TRACE/LOW/MED) TRACE

\% Moisture: not dec.

GC Column: DB-624

ID: 0.20

Soil Extract volume:

Purge Volume: 25.0
Contract: $8 E-00302$

SDG No.: 200-2472

Lab Sample ID: 200-2472-6

Lab File ID: JBXA25.D

Date Received:

Date Analyzed: 11/13/2010

Dilution Factor: 1.0

Soil Aliquot Volume:

(uL)

\begin{tabular}{|c|c|c|c|}
\hline CAS NO. & COMPOUND & $\begin{array}{l}\text { CONCENTRATION UNITS: } \\
(\mathrm{ug} / \mathrm{L} \text { or } \mathrm{ug} / \mathrm{kg}) \mathrm{ug} / \mathrm{L} \\
\end{array}$ & $Q$ \\
\hline $79-01-6$ & Trichloroethene & 0.50 & $\mathrm{U}$ \\
\hline $108-87-2$ & Methylcyclohexane & 0.50 & $\mathrm{U}$ \\
\hline $78-87-5$ & 1,2-Dichloropropane & 0.50 & $\mathrm{U}$ \\
\hline $75-27-4$ & Bromodichloromethane & 0.50 & $\mathrm{U}$ \\
\hline $10061-01-5$ & cis-1,3-Dichloropropene & 0.50 & $\mathrm{U}$ \\
\hline $108-10-1$ & 4-Methyl-2-pentanone & 5.0 & $\mathrm{U}$ \\
\hline $108-88-3$ & Toluene & 0.50 & $\mathrm{U}$ \\
\hline $10061-02-6$ & trans-1,3-Dichloropropene & 0.50 & $\mathrm{U}$ \\
\hline $79-00-5$ & 1,1,2-Trichloroethane & 0.50 & $\mathrm{U}$ \\
\hline $127-18-4$ & Tetrachloroethene & 0.50 & $\mathrm{U}$ \\
\hline $591-78-6$ & 2-Hexanone & 5.0 & $\mathrm{U}$ \\
\hline $124-48-1$ & Dibromochloromethane & 0.50 & $\mathrm{U}$ \\
\hline $106-93-4$ & 1,2-Dibromoethane & 0.50 & $\mathrm{U}$ \\
\hline $108-90-7$ & Chlorobenzene & 0.50 & $\mathrm{U}$ \\
\hline $100-41-4$ & Ethylbenzene & 0.50 & $\mathrm{U}$ \\
\hline $95-47-6$ & o-Xylene & 0.50 & $\mathrm{U}$ \\
\hline $179601-23-1$ & $\mathrm{~m}, \mathrm{p}$-Xylene & 0.50 & $\mathrm{U}$ \\
\hline $100-42-5$ & Styrene & 0.50 & $\mathrm{U}$ \\
\hline $75-25-2$ & Bromoform & 0.50 & $\mathrm{U}$ \\
\hline $98-82-8$ & Isopropylbenzene & 0.50 & $\mathrm{U}$ \\
\hline $79-34-5$ & $1,1,2,2$-Tetrachloroethane & 0.50 & $\mathrm{U}$ \\
\hline $541-73-1$ & 1,3-Dichlorobenzene & 0.50 & $\mathrm{U}$ \\
\hline $106-46-7$ & 1,4-Dichlorobenzene & 0.50 & $\mathrm{U}$ \\
\hline $95-50-1$ & 1,2-Dichlorobenzene & 0.50 & $\mathrm{U}$ \\
\hline $96-12-8$ & 1,2-Dibromo-3-Chloropropane & 0.50 & $\mathrm{U}$ \\
\hline $120-82-1$ & $1,2,4-$ Trichlorobenzene & 0.50 & $\mathrm{U}$ \\
\hline $87-61-6$ & 1,2,3-Trichlorobenzene & 0.50 & $\mathrm{U}$ \\
\hline
\end{tabular}


1J - FORM I VOA-TIC

VOLATILE ORGANICS ANALYSIS DATA SHEET

TENTATIVELY IDENTIFIED COMPOUNDS
EPA SAMPLE NO.

VHBKL01

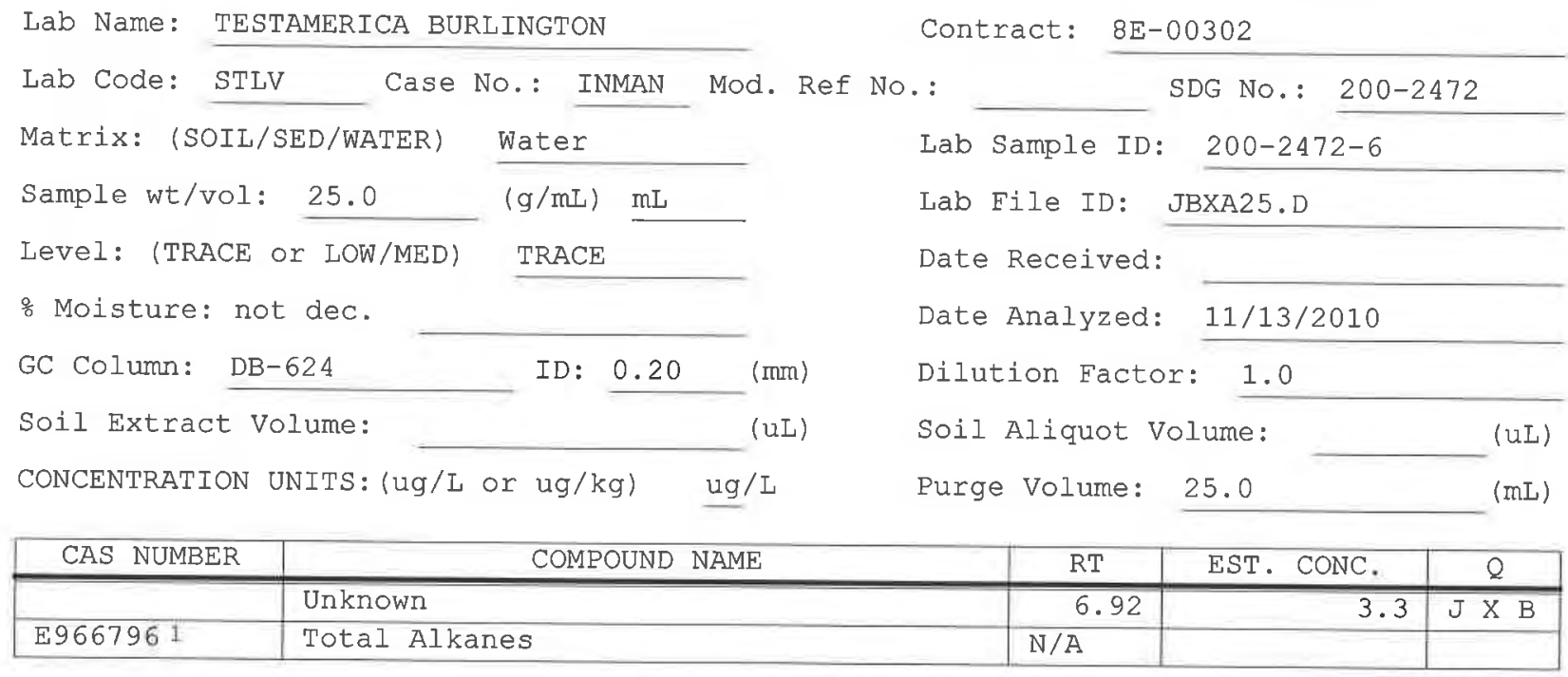

1 EPA-designated Registry Number. 


\section{TestAmerica}

THE LEADER IN ENVIRONMENTAL TESTING

\section{ANALYTICAL REPORT}

Job Number: 200-2608-1

SDG Number: INMAN (200-2608)

Job Description: INMAN (200-2608)

Contract Number: 8E-00302

For:

Argonne National Laboratory

9700 South Cass Avenue

Building 203

Office B-149

Argonne, IL 60439

Attention: Mr. Clyde Dennis

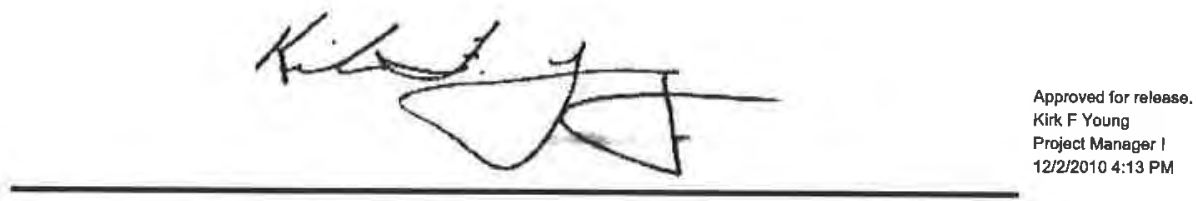

Kirk F Young

Project Manager I

kirk.young@testamericainc.com

$12 / 02 / 2010$

The test results in this report relate only to sample(s) as received by the laboratory. These test results were derived under a quality system that adheres to the requirements of NELAC. Pursuant to NELAC, this report may not be produced in full without written approval from the laboratory 


\section{Table of Contents}

Cover Title Page .......................... 1

Data Summaries ........................ 4

Report Narrative ............................. 4

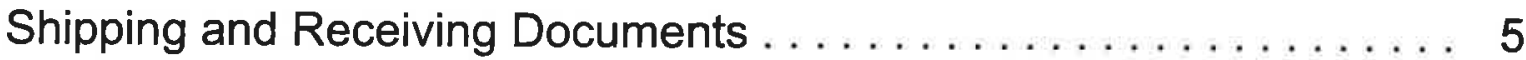

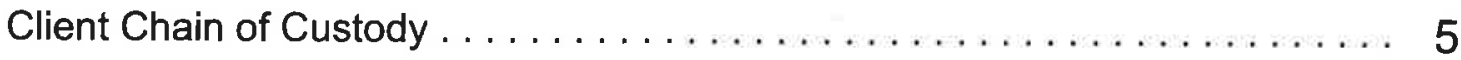

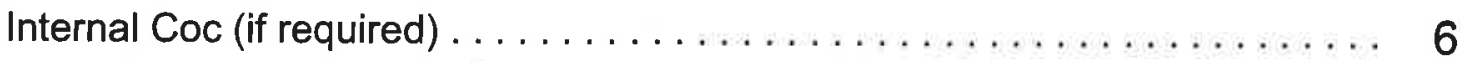

Data Summaries . . . . . . . . . . . . . . . . . . 7

Sample Summary ........................ 7

Method Summary $\ldots \ldots \ldots \ldots \ldots \ldots \ldots \ldots \ldots \ldots \ldots \ldots$

Method / Analyst Summary $\ldots \ldots \ldots \ldots \ldots \ldots \ldots \ldots \ldots \ldots \ldots . . \ldots \ldots$

QC Association Summary .......................... 10

Data Qualifiers ............................... 11

Organic Sample Data . . . . . . . . . . . . . . . . . . . . 12

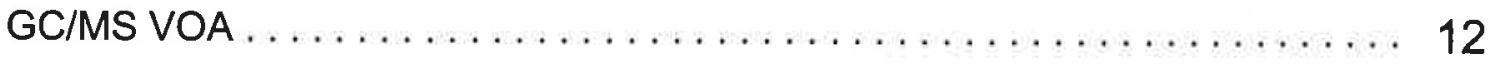

Method $8260 \mathrm{~B}, \ldots \ldots \ldots \ldots \ldots \ldots \ldots \ldots \ldots \ldots \ldots \ldots \ldots \ldots \ldots \ldots \ldots \ldots \ldots \ldots \ldots, 12$

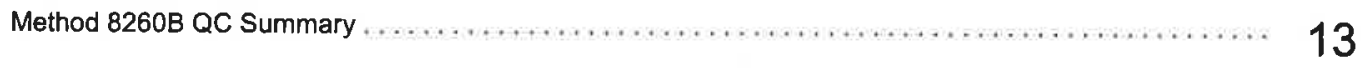

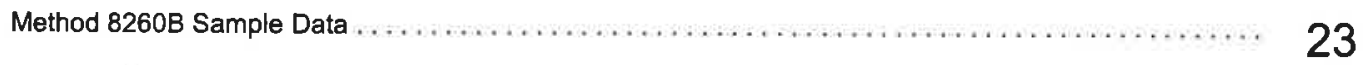

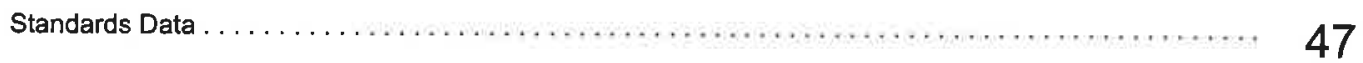

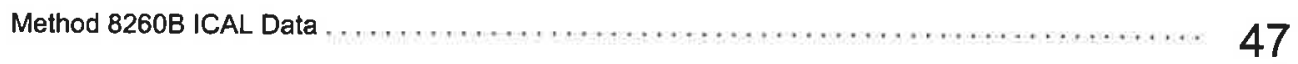

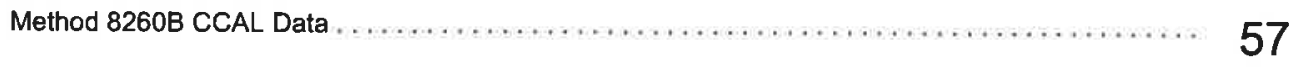

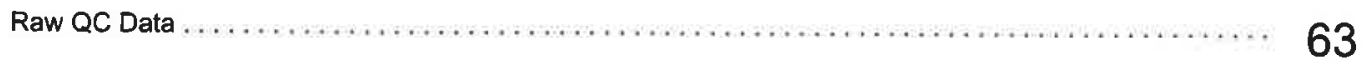

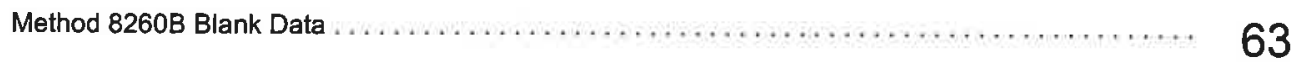

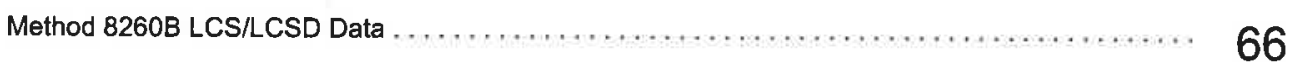

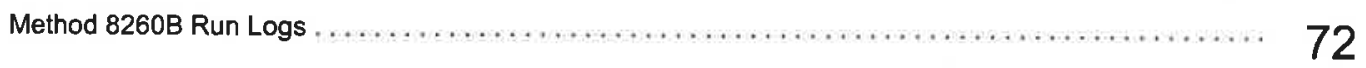

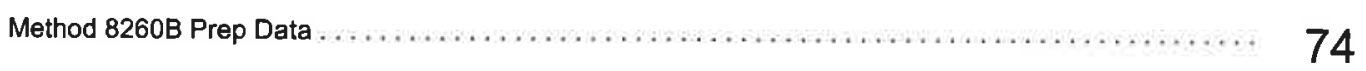

Shipping and Receiving Documents $\ldots \ldots \ldots \ldots \ldots \ldots \ldots$ 


\section{Table of Contents}

Sample Receipt Checklist . . . . . . . . . . . $\ldots \ldots \ldots \ldots \ldots \ldots$ 


\title{
CASE NARRATIVE
}

\section{Client: Argonne National Laboratory}

\author{
Project: Inman (200-2608)
}

Report Number: 200-2608-1

With the exceptions noted as flags or footnotes, standard analytical protocols were followed in the analysis of the samples and no problems were encountered or anomalies observed.

Calculations are performed before rounding to avoid round-off errors in calculated results.

All holding times were met and proper preservation noted for the methods performed on these samples, unless otherwise detailed in the individual sections below.

\section{Receipt}

The samples were received on 11/19/2010. Documentation of the condition of the samples at the time of their receipt and any exception to the laboratory's Sample Acceptance Policy is documented in the Sample Handling section of this submittal. The sample volumes were received without thermal preservation.

\section{SW846 Method(s) 5035/8260B Volatile Organics (Medium Level Soil)}

The samples were analyzed by the referenced method(s), using a low-level calibration. In performing the analytical work, 500 microliters of the methanol extract were added to the 5 milliliter purge volume. Each analysis associated with the samples in this sample set did exhibit an acceptable internal standard performance, and there was an acceptable recovery of the surrogate controls in each analysis. Two types of laboratory control sample analyses were performed in the analytical sequence. One was performed to evaluate method performance, and one was performed with 500 microliters of methanol added to the purge volume in order to characterize the affect on the analytical process. There was an acceptable recovery of each target analyte in the laboratory control sample analysis that defined method performance. With the exception of those for chloroethane, trichlorofluoromethane, methyl iodide, and 2-butanone, there was an acceptable recovery of each target analyte in the laboratory control sample analysis with methanol. In that analysis the recoveries of chloroethane (61 percent), trichlorofluoromethane (67 percent), methyl iodide (46 percent), and 2-butanone (66 percent) were low. Matrix spike and matrix spike duplicate analyses were not performed on samples in this sample set. The analysis of sample IN-S-MeOH BLANK was free of analyte contamination. Trace concentrations of chloromethane, bromomethane, and 1,2,4-trichlorobenzene were identified in the analysis of the instrument blank associated with the analytical work. The concentration of each compound in that analysis was below the established reporting limit of $10 \mathrm{ug} / \mathrm{Kg}$, and the analysis did meet the laboratory's technical acceptance criteria for a compliant method blank analysis.

Manual integration was employed in deriving certain of the analytical results. The values that have been derived from manual integration are qualified on the quantitation reports. Extracted ion current profiles for each manual integration are included in the data package, and further documented in the Sample Preparation section of this submittal. 


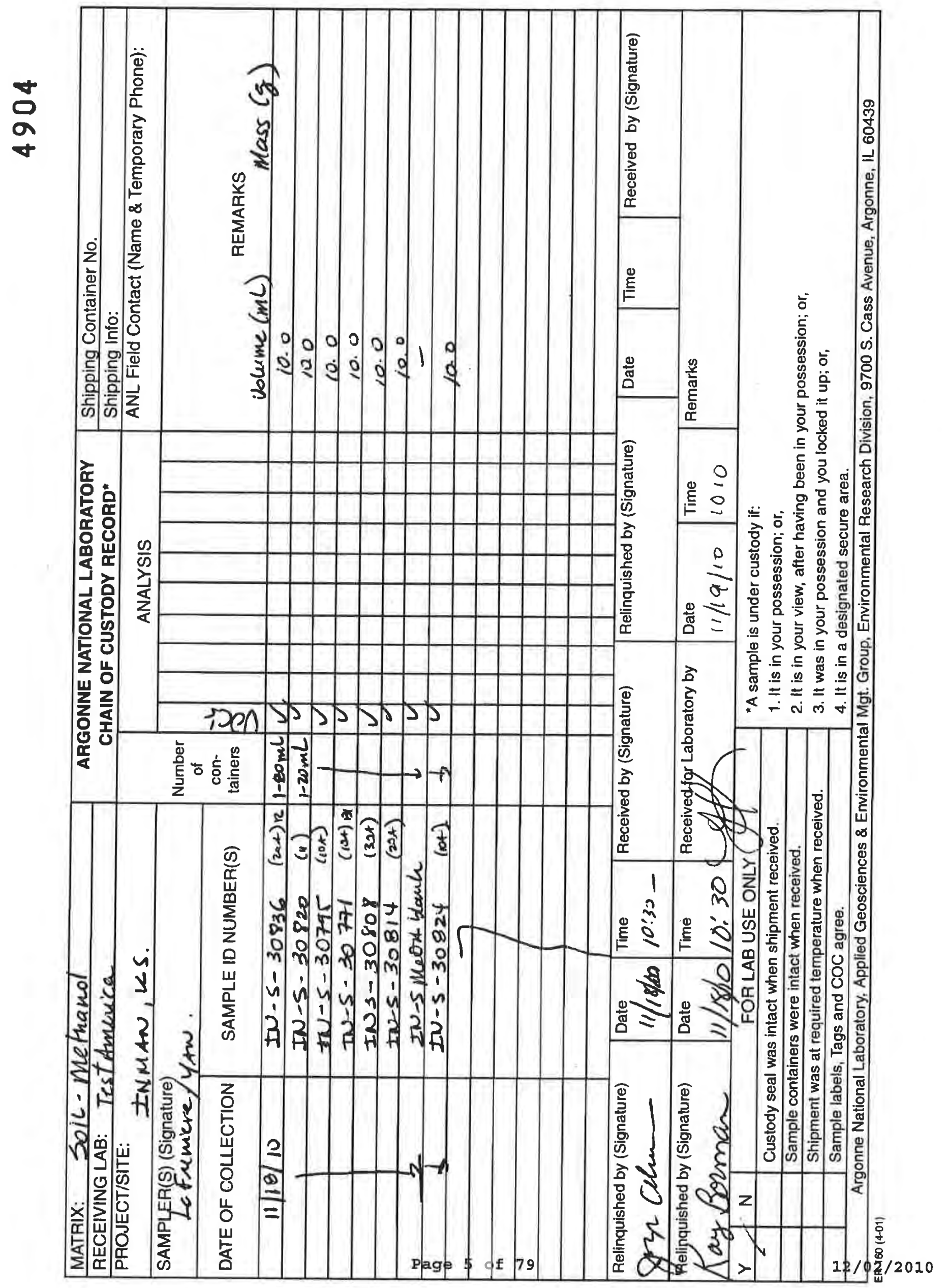




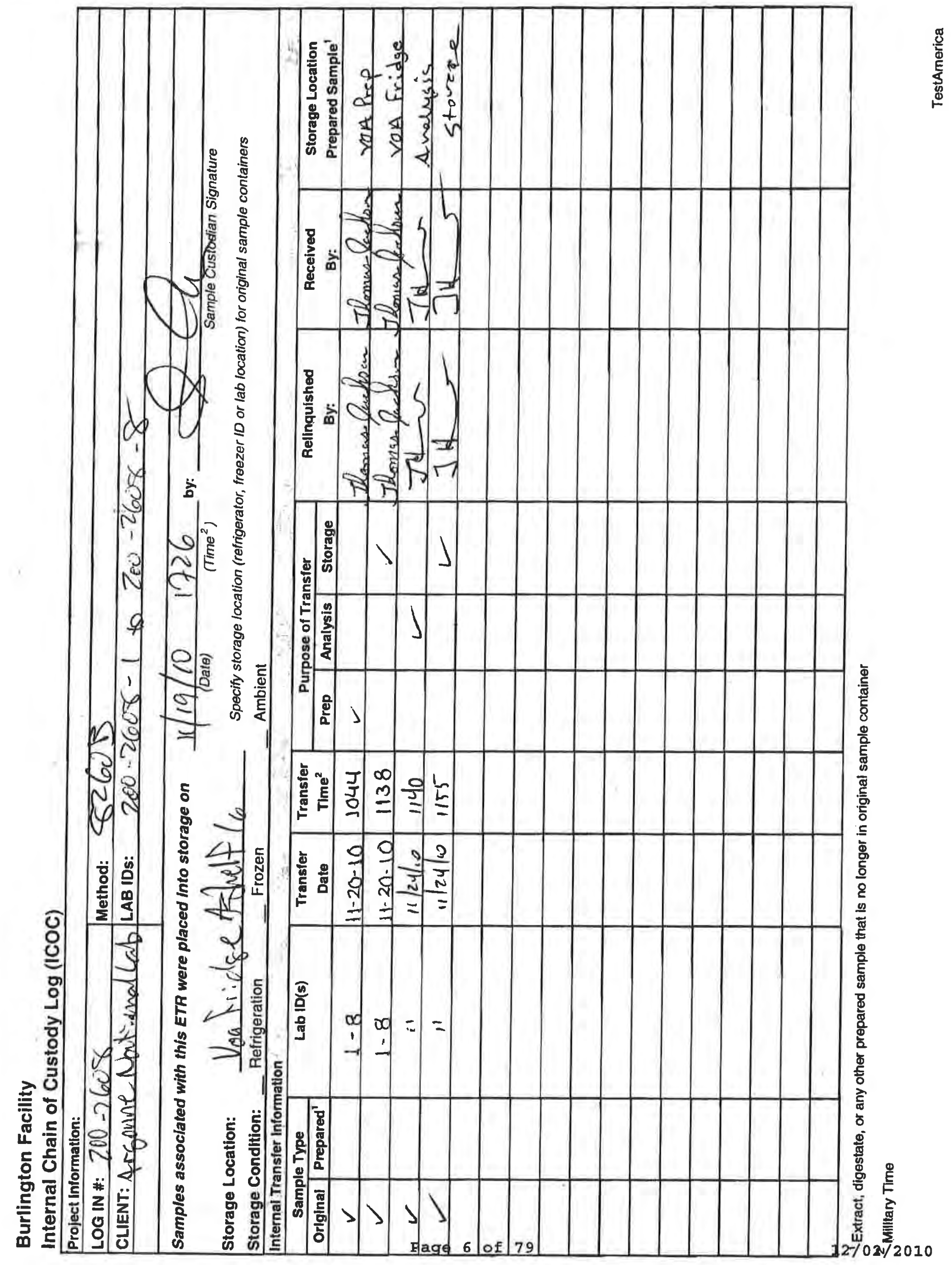




\section{SAMPLE SUMMARY}

Client: Argonne National Laboratory

Job Number: $200-2608-1$

Sdg Number: INMAN (200-2608)

\begin{tabular}{|c|c|c|c|c|}
\hline Lab Sample ID & Client Sample ID & Client Matrix & $\begin{array}{c}\text { Date/Time } \\
\text { Sampled }\end{array}$ & $\begin{array}{l}\text { Date/Time } \\
\text { Received }\end{array}$ \\
\hline $200-2608-1$ & IN-S-30836 & Solid & $11 / 18 / 20100000$ & $11 / 19 / 20101010$ \\
\hline $200-2608-2$ & IN-S-30820 & Solid & $11 / 18 / 20100000$ & $11 / 19 / 20101010$ \\
\hline $200-2608-3$ & IN-S-30795 & Solid & $11 / 18 / 20100000$ & $11 / 19 / 20101010$ \\
\hline $200-2608-4$ & IN-S-30771 & Solid & $11 / 18 / 20100000$ & $11 / 19 / 2010 \quad 1010$ \\
\hline $200-2608-5$ & IN-S-30808 & Solid & $11 / 18 / 20100000$ & $11 / 19 / 20101010$ \\
\hline $200-2608-6$ & IN-S-30814 & Solid & $11 / 18 / 20100000$ & $11 / 19 / 20101010$ \\
\hline $200-2608-7$ & IN-S-MEOH BLANK & Solid & $11 / 18 / 20100000$ & $11 / 19 / 20101010$ \\
\hline $200-2608-8$ & IN-S-30824 & Solid & $11 / 18 / 20100000$ & $11 / 19 / 20101010$ \\
\hline
\end{tabular}




\section{METHOD SUMMARY}

Client: Argonne National Laboratory

Description

Matrix: Solid

Volatile Organic Compounds (GC/MS)

Purge and Trap

Lab References:

TAL BUR = TestAmerica Burlington

Method References:

SW846 = "Test Methods For Evaluating Solid Waste, Physical/Chemical Methods", Third Edition, November 1986 And Its Updates.
Method

SW846 8260B

TAL BUR

TAL BUR
Preparation Method

SW846 5035
Job Number: 200-2608-1 Sdg Number: INMAN (200-2608) 


\section{METHOD / ANALYST SUMMARY}

Client: Argonne National Laboratory

Job Number: $200-2608-1$

Sdg Number: INMAN (200-2608)

Method

Analyst

Analyst ID

SW846 8260B

Heald, John

JRH 


\section{Quality Control Results}

Client: Argonne National Laboratory

Job Number: 200-2608-1

Sdg Number: INMAN (200-2608)

\section{QC Association Summary}

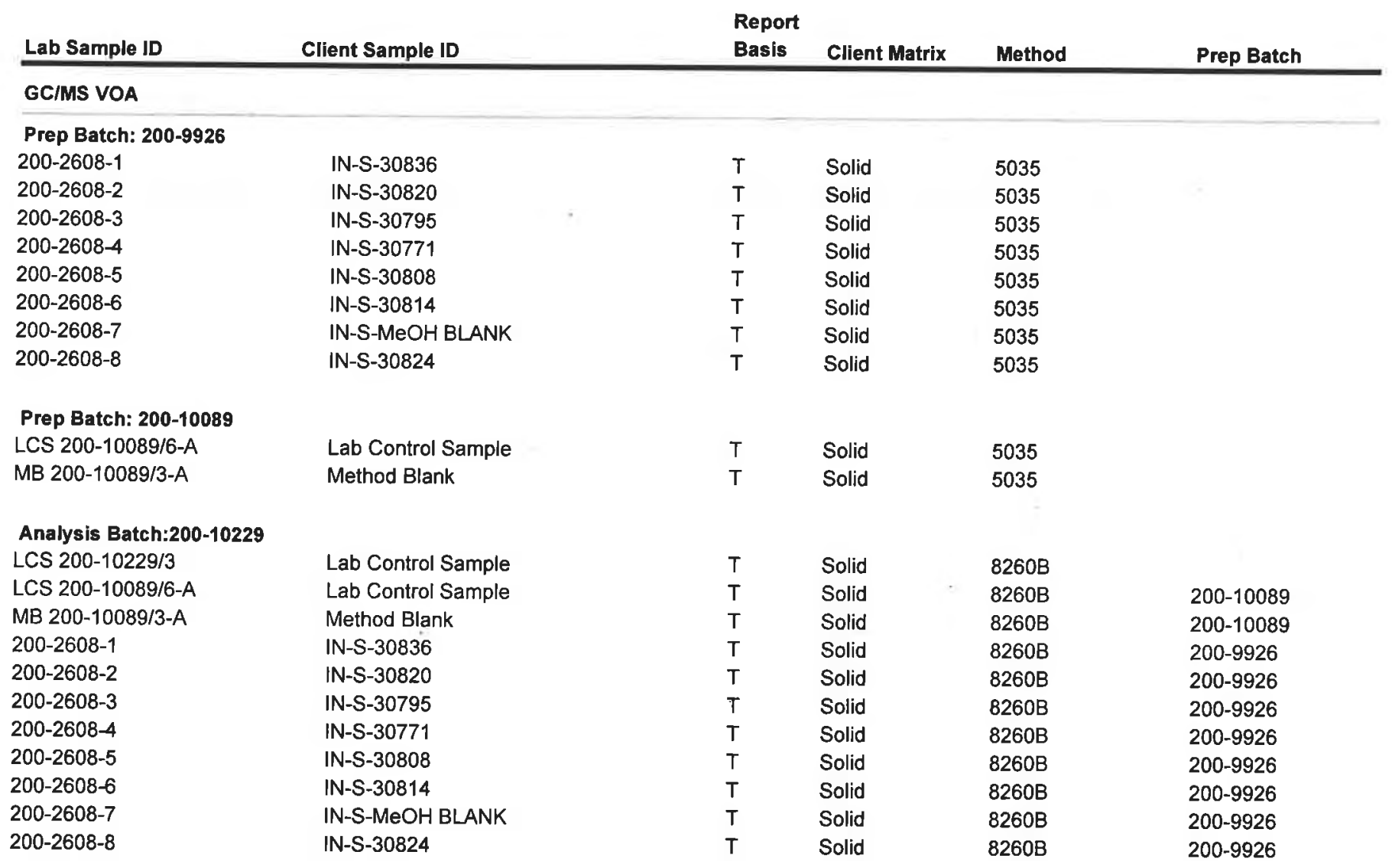

Report Basis

$T=$ Total 
DATA REPORTING QUALIFIERS

Client: Argonne National Laboratory

Job Number: 200-2608-1

Sdg Number: INMAN (200-2608)

Description

GC/MS VOA

B

U

J
Compound was found in the blank and sample.

Indicates the analyte was analyzed for but not detected.

Recovery or RPD exceeds control limits

Result is less than the RL but greater than or equal to the MDL and the concentration is an approximate value. 


\section{Method 8260B}

\section{Volatile Organic Compounds (GC/MS) by Method 8260B}


FORM II

GC/MS VOA SURROGATE RECOVERY

Lab Name: TestAmerica Burlington

Job No.: $200-2608-1$

SDG NO.: INMAN (200-2608)

Matrix: Solid

Level: Medium

GC Column (1): $\mathrm{DB}-624 \quad$ ID: 0.53 (mm)

\begin{tabular}{|l|l|r|r|r|r|}
\hline \multicolumn{1}{|c|}{ Client Sample ID } & Lab Sample ID & DCA \# & TOL \# & BFB \# & DCZ \# \\
\hline IN-S-30836 & $200-2608-1$ & 85 & 95 & 92 & 94 \\
\hline IN-S-30820 & $200-2608-2$ & 86 & 97 & 95 & 96 \\
\hline IN-S-30795 & $200-2608-3$ & 90 & 101 & 99 & 101 \\
\hline IN-S-30771 & $200-2608-4$ & 92 & 101 & 98 & 101 \\
\hline IN-S-30808 & $200-2608-5$ & 93 & 104 & 101 & 103 \\
\hline IN-S-30814 & $200-2608-6$ & 103 & 115 & 113 & 114 \\
\hline IN-S-MeOH BLANK & $200-2608-7$ & 92 & 104 & 101 & 102 \\
\hline IN-S-30824 & $200-2608-8$ & 99 & 110 & 107 & 108 \\
\hline & MB 200-10089/3-A & 90 & 102 & 100 & 101 \\
\hline & LCS $200-10089 / 6-A$ & 93 & 114 & 107 & 107 \\
\hline & LCS 200-10229/3 & 98 & 108 & 107 & 108 \\
\hline
\end{tabular}

$\mathrm{DCA}=1,2-$ Dichloroethane-d4

QC LIMITS

TOL = Toluene-d8

65-155

$80-115$

$80-115$

$\mathrm{BFB}=$ Bromofluorobenzene

$45-145$

\# Column to be used to flag recovery values

FORM II $8260 \mathrm{~B}$ 
FORM III

GC/MS VOA LAB CONTROL SAMPLE RECOVERY

Lab Name: TestAmerica Burlington

SDG No.: INMAN (200-2608)

Matrix: Solid

Lab ID: LCS 200-10089/6-A
Job No.: 200-2608-1

Lab File ID: Ifme04.d

Client ID:

\begin{tabular}{|c|c|c|c|c|c|}
\hline COMPOUND & $\begin{array}{c}\text { SPIKE } \\
\text { ADDED } \\
(\mathrm{ug} / \mathrm{Kg})\end{array}$ & $\begin{array}{c}\text { LCS } \\
\text { CONCENTRATION } \\
(\mathrm{ug} / \mathrm{Kg})\end{array}$ & $\begin{array}{l}\text { LCS } \\
\text { 응 } \\
\text { REC }\end{array}$ & $\begin{array}{l}\text { QC } \\
\text { LIMITS } \\
\text { REC }\end{array}$ & $\#$ \\
\hline Dichlorodifluoromethane & 250 & 203 & 81 & $30-180$ & \\
\hline Chloromethane & 250 & 263 & 105 & $55-150$ & \\
\hline Vinyl chloride & 250 & 229 & 91 & $65-145$ & \\
\hline Bromomethane & 250 & 162 & 65 & $65-145$ & \\
\hline Chloroethane & 250 & 152 & 61 & $70-135$ & $\star$ \\
\hline Trichlorofluoromethane & 250 & 167 & 67 & $70-140$ & * \\
\hline 1,1-Dichloroethene & 250 & 192 & 77 & $75-135$ & \\
\hline Freon TE & 250 & 211 & 84 & $75-140$ & \\
\hline Acetone & 1250 & 680 & 54 & $50-130$ & \\
\hline Methyl iodide & 250 & 115 & 46 & $70-150$ & * \\
\hline Carbon disulfide & 250 & 216 & 86 & $80-135$ & \\
\hline Methyl acetate & 250 & 257 & 103 & $60-140$ & \\
\hline Methylene Chloride & 250 & 257 & 103 & $75-140$ & \\
\hline trans-1,2-Dichloroethene & 250 & 260 & 104 & $80-130$ & \\
\hline Methyl t-butyl ether & 250 & 244 & 97 & $85-130$ & \\
\hline 1,1-Dichloroethane & 250 & 238 & 95 & $85-120$ & \\
\hline Vinyl acetate & 250 & 242 & 97 & $70-135$ & \\
\hline 2,2-Dichloropropane & 250 & 264 & 106 & $85-120$ & \\
\hline cis-1,2-Dichloroethene & 250 & 253 & 101 & $80-120$ & \\
\hline 2-Butanone & 1250 & 830 & 66 & $70-135$ & $\star$ \\
\hline Bromochloromethane & 250 & 211 & 84 & $75-125$ & \\
\hline Tetrahydrofuran & 3500 & 3050 & 87 & $75-125$ & \\
\hline Chloroform & 250 & 248 & 99 & $85-120$ & \\
\hline 1,1,1-Trichloroethane & 250 & 262 & 105 & $80-115$ & \\
\hline Cyclohexane & 250 & 281 & 112 & $60-140$ & \\
\hline 1,1-Dichloropropene & 250 & 275 & 110 & $85-120$ & \\
\hline Carbon tetrachloride & 250 & 263 & 105 & $80-115$ & \\
\hline Isobutyl alcohol & 12500 & 9310 & 75 & $70-135$ & \\
\hline Benzene & 250 & 278 & 111 & $85-120$ & \\
\hline 1,2-Dichloroethane & 250 & 244 & 98 & $75-120$ & \\
\hline Trichloroethene & 250 & 276 & 111 & $85-120$ & \\
\hline Methylcyclohexane & 250 & 297 & 119 & $60-140$ & \\
\hline 1,2-Dichloropropane & 250 & 265 & 106 & $85-120$ & \\
\hline Dibromomethane & 250 & 247 & 99 & $80-120$ & \\
\hline 1,4-Dioxane & 12500 & 10200 & 82 & $50-160$ & \\
\hline Bromodichloromethane & 250 & 250 & 100 & $80-115$ & \\
\hline 2-Chloroethyl vinyl ether & 250 & 209 & 83 & $65-145$ & \\
\hline cis-1,3-Dichloropropene & 250 & 265 & 106 & $85-120$ & \\
\hline 4-Methyl-2-pentanone & 1250 & 1080 & 86 & $65-135$ & \\
\hline Toluene & 250 & 290 & 116 & $75-125$ & \\
\hline trans-1,3-Dichloropropene & 250 & 267 & 107 & $85-120$ & \\
\hline 1,1,2-Trichloroethane & 250 & 269 & 108 & $75-125$ & \\
\hline
\end{tabular}

\# Column to be used to flag recovery and RPD values 
FORM III

GC/MS VOA LAB CONTROL SAMPLE RECOVERY

Lab Name: TestAmerica Burlington

SDG NO.: INMAN (200-2608)

Matrix: Solid

Lab ID: LCS 200-10089/6-A
Job No.: 200-2608-1
Lab File ID: Ifme04.d

Client ID:

\begin{tabular}{|c|c|c|c|c|c|}
\hline COMPOUND & $\begin{array}{c}\text { SPIKE } \\
\text { ADDED } \\
\text { (ug/Kg) }\end{array}$ & $\begin{array}{c}\text { LCS } \\
\text { CONCENTRATION } \\
(\mathrm{ug} / \mathrm{Kg})\end{array}$ & $\begin{array}{l}\text { LCS } \\
\frac{\circ}{6} \\
\operatorname{REC}\end{array}$ & $\begin{array}{l}\text { QC } \\
\text { LIMITS } \\
\text { REC }\end{array}$ & \# \\
\hline Tetrachloroethene & 250 & 299 & .120 & $85-120$ & \\
\hline 1,3-Dichloropropane & 250 & 273 & 109 & $85-120$ & \\
\hline 2-Hexanone & 1250 & 1110 & 89 & $70-135$ & \\
\hline Dibromochloromethane & 250 & 269 & 107 & $80-120$ & \\
\hline 1,2-Dibromoethane & 250 & 269 & 108 & $80-120$ & \\
\hline Chlorobenzene & 250 & 279 & 112 & $80-120$ & \\
\hline $1,1,1,2$-Tetrachloroethane & 250 & 272 & 109 & $80-115$ & \\
\hline Ethylbenzene & 250 & 281 & 113 & $80-120$ & \\
\hline$m \& p-x y l e n e$ & 500 & 571 & 114 & $80-120$ & \\
\hline o-xylene & 250 & 280 & 112 & $85-120$ & \\
\hline Styrene & 250 & 271 & 108 & $80-125$ & \\
\hline Bromoform & 250 & 247 & 99 & $75-130$ & \\
\hline Isopropylbenzene & 250 & 291 & 116 & $85-120$ & \\
\hline Bromobenzene & 250 & 278 & 111 & $85-120$ & \\
\hline $1,1,2,2$-Tetrachloroethane & 250 & 260 & 104 & $75-125$ & \\
\hline 1,2,3-Trichloropropane & 250 & 221 & 89 & $70-125$ & \\
\hline n-Propylbenzene & 250 & 291 & 117 & $85-120$ & \\
\hline 2-Chlorotoluene & 250 & 281 & 113 & $85-120$ & \\
\hline 4-Chlorotoluene & 250 & 286 & 114 & $85-120$ & \\
\hline $1,3,5$-Trimethylbenzene & 250 & 284 & 113 & $85-120$ & \\
\hline tert-Butylbenzene & 250 & 285 & 114 & $85-120$ & \\
\hline 1,2,4-Trimethylbenzene & 250 & 284 & 114 & $85-120$ & \\
\hline sec-Butylbenzene & 250 & 296 & 118 & $85-120$ & \\
\hline 1,3-Dichlorobenzene & 250 & 282 & 113 & $80-120$ & \\
\hline 4-Isopropyltoluene & 250 & 284 & 114 & $85-120$ & \\
\hline 1,4-Dichlorobenzene & 250 & 279 & 112 & $85-120$ & \\
\hline 1,2-Dichlorobenzene & 250 & 279 & 111 & $85-120$ & \\
\hline n-Butylbenzene & 250 & 312 & 125 & $85-125$ & \\
\hline 1,2-Dibromo-3-Chloropropane & 250 & 215 & 86 & $65-130$ & \\
\hline 1,2,4-Trichlorobenzene & 250 & 295 & 118 & $80-125$ & \\
\hline Hexachlorobutadiene & 250 & 331 & 132 & $65-150$ & \\
\hline Naphthalene & 250 & 255 & 102 & $80-125$ & \\
\hline 1,2,3-Trichlorobenzene & 250 & 279 & 112 & $70-125$ & \\
\hline
\end{tabular}

\# Column to be used to flag recovery and RPD values 
FORM III

GC/MS VOA LAB CONTROL SAMPLE RECOVERY

Lab Name: TestAmerica Burlington

SDG NO.: INMAN (200-2608)

Matrix: Solid

Lab ID: LCS 200-10229/3
Job No.: 200-2608-1

Level: Medium Lab File ID: lfme03.d

Client ID:

\begin{tabular}{|c|c|c|c|c|c|}
\hline COMPOUND & $\begin{array}{c}\text { SPIKE } \\
\text { ADDED } \\
\text { (ug/Kg) }\end{array}$ & $\begin{array}{c}\text { LCS } \\
\text { CONCENTRATION } \\
(\mathrm{ug} / \mathrm{Kg})\end{array}$ & $\begin{array}{c}\text { LCS } \\
\frac{\circ}{0} \\
\text { REC }\end{array}$ & $\begin{array}{c}Q C \\
\text { LIMITS } \\
\text { REC }\end{array}$ & \# \\
\hline Dichlorodifluoromethane & 25.0 & 24.4 & 97 & $30-180$ & \\
\hline Chloromethane & 25.0 & 24.6 & 98 & $55-150$ & \\
\hline Vinyl chloride & 25.0 & 27.4 & 110 & $65-145$ & \\
\hline Bromomethane & 25.0 & 23.7 & 95 & $65-145$ & \\
\hline Chloroethane & 25.0 & 29.1 & 116 & $70-135$ & \\
\hline Trichlorofluoromethane & 25.0 & 25.5 & 102 & $70-140$ & \\
\hline 1,1-Dichloroethene & 25.0 & 23.4 & 94 & $75-135$ & \\
\hline Freon TF & 25.0 & 24.6 & 98 & $75-140$ & \\
\hline Acetone & 125 & 117 & 94 & $50-130$ & \\
\hline Methyl iodide & 25.0 & 19.6 & 78 & $70-150$ & \\
\hline Carbon disulfide & 25.0 & 23.5 & 94 & $80-135$ & \\
\hline Methyl acetate & 25.0 & 27.4 & 110 & $60-140$ & \\
\hline Methylene Chloride & 25.0 & 26.0 & 104 & $75-140$ & \\
\hline trans-1,2-Dichloroethene & 25.0 & 24.9 & 100 & $80-130$ & \\
\hline Methyl t-butyl ether & 25.0 & 25.3 & 101 & $85-130$ & \\
\hline 1,1-Dichloroethane & 25.0 & 24.8 & 99 & $85-120$ & \\
\hline Vinyl acetate & 25.0 & 26.0 & 104 & $70-135$ & \\
\hline 2,2-Dichloropropane & 25.0 & 25.5 & 102 & $85-120$ & \\
\hline cis-1,2-Dichloroethene & 25.0 & 24.9 & 100 & $80-120$ & \\
\hline 2-Butanone & 125 & 128 & 102 & $70-135$ & \\
\hline Bromochloromethane & 25.0 & 25.7 & 103 & $75-125$ & \\
\hline Tetrahydrofuran & 350 & 348 & 99 & $75-125$ & \\
\hline Chloroform & 25.0 & 24.8 & 99 & $85-120$ & \\
\hline 1,1,1-Trichloroethane & 25.0 & 24.9 & 99 & $80-115$ & \\
\hline Cyclohexane & 25.0 & 26.2 & 105 & $60-140$ & \\
\hline 1,1-Dichloropropene & 25.0 & 25.6 & 102 & $85-120$ & \\
\hline Carbon tetrachloride & 25.0 & 25.3 & 101 & $80-115$ & \\
\hline Isobutyl alcohol & 1250 & 1300 & 104 & $70-135$ & \\
\hline Benzene & 25.0 & 26.2 & 105 & $85-120$ & \\
\hline 1,2-Dichloroethane & 25.0 & 24.6 & 98 & $75-120$ & \\
\hline Trichloroethene & 25.0 & 26.4 & 106 & $85-120$ & \\
\hline Methylcyclohexane & 25.0 & 27.2 & 109 & $60-140$ & \\
\hline 1,2-Dichloropropane & 25.0 & 26.0 & 104 & $85-120$ & \\
\hline Dibromomethane & 25.0 & 25.5 & 102 & $80-120$ & \\
\hline 1,4-Dioxane & 1250 & 1230 & 99 & $50-160$ & \\
\hline Bromodichloromethane & 25.0 & 25.6 & 102 & $80-115$ & \\
\hline 2-Chloroethyl vinyl ether & 25.0 & 26.9 & 108 & $65-145$ & \\
\hline cis-1,3-Dichloropropene & 25.0 & 25.8 & 103 & $85-120$ & \\
\hline 4-Methyl-2-pentanone & 125 & 133 & 107 & $65-135$ & \\
\hline Toluene & 25.0 & 26.5 & 106 & $75-125$ & \\
\hline trans-1,3-Dichloropropene & 25.0 & 25.1 & 100 & $85-120$ & \\
\hline 1,1,2-Trichloroethane & 25.0 & 26.7 & 107 & $75-125$ & \\
\hline
\end{tabular}

\# Colums to be used to flag recovery and RPD values 
FORM III

GC/MS VOA LAB CONTROL SAMPLE RECOVERY

Lab Name: TestAmerica Burlington

SDG No.: INMAN (200-2608)

Matrix: Solid

Lab ID: LCS 200-10229/3
Job No.: 200-2608-1

Lab File ID: Ifme03.d

Client ID:

\begin{tabular}{|c|c|c|c|c|c|c|}
\hline COMPOUND & $\begin{array}{c}\text { SPIKE } \\
\text { ADDED } \\
(\mathrm{ug} / \mathrm{Kg})\end{array}$ & \multicolumn{2}{|c|}{$\begin{array}{c}\text { LCS } \\
\text { CONCENTRATION } \\
(\mathrm{ug} / \mathrm{Kg})\end{array}$} & $\begin{array}{l}\text { LCS } \\
\frac{\circ}{6} \\
\text { REC }\end{array}$ & $\begin{array}{l}\text { QC } \\
\text { LIMITS } \\
\text { REC }\end{array}$ & $\#$ \\
\hline Tetrachloroethene & 25.0 & & 27.0 & 108 & $85-120$ & \\
\hline 1,3-Dichloropropane & 25.0 & & 26.3 & 105 & $85-120$ & \\
\hline 2-Hexanone & 125 & & 138 & 110 & $70-135$ & \\
\hline Dibromochloromethane & 25.0 & & 26.6 & 107 & $80-120$ & \\
\hline 1,2-Dibromoethane & 25.0 & & 26.5 & 106 & $80-120$ & \\
\hline Chlorobenzene & 25.0 & & 26.4 & 106 & $80-120$ & \\
\hline $1,1,1,2$-Tetrachloroethane & 25.0 & & 26.3 & 105 & $80-115$ & \\
\hline Ethylbenzene & 25.0 & & 26.3 & 105 & $80-120$ & \\
\hline $\mathrm{m} \& \mathrm{p}-\mathrm{xy}$ lene & 50.0 & + & 53.3 & 107 & $80-120$ & \\
\hline o-Xylene & 25.0 & & 26.7 & 107 & $85-120$ & \\
\hline Styrene & 25.0 & . & 26.3 & 105 & $80-125$ & \\
\hline Bromoform & 25.0 & & 26.1 & 104 & $75-130$ & \\
\hline Isopropylbenzene & 25.0 & & 26.8 & 107 & $85-120$ & \\
\hline Bromobenzene & 25.0 & & 26.6 & 106 & $85-120$ & \\
\hline $1,1,2,2$-Tetrachloroethane & 25.0 & & 27.4 & 110 & $75-125$ & \\
\hline 1,2,3-Trichloropropane & 25.0 & $\overline{-}$ & 23.4 & 93 & $70-125$ & \\
\hline n-Propylbenzene & 25.0 & & 26.7 & 107 & $85-120$ & \\
\hline 2-Chlorotoluene & 25.0 & & 26.5 & 106 & $85-120$ & \\
\hline 4-Chlorotoluene & 25.0 & & 26.7 & 107 & $85-120$ & \\
\hline 1,3,5-Trimethylbenzene & 25.0 & & 26.4 & 105 & $85-120$ & \\
\hline tert-Butylbenzene & 25.0 & & 26.4 & 106 & $85-120$ & \\
\hline 1,2,4-Trimethylbenzene & 25.0 & & 26.4 & 106 & $85-120$ & \\
\hline sec-Butylbenzene & 25.0 & & 26.8 & 107 & $85-120$ & \\
\hline 1,3-Dichlorobenzene & 25.0 & & 26.3 & 105 & $80-120$ & \\
\hline 4-Isopropyltoluene & 25.0 & & 25.9 & 104 & $85-120$ & \\
\hline 1,4-Dichlorobenzene & 25.0 & & 26.3 & 105 & $85-120$ & \\
\hline 1,2-Dichlorobenzene & 25.0 & & 26.7 & 107 & $85-120$ & \\
\hline n-Butylbenzene & 25.0 & & 27.4 & 110 & $85-125$ & \\
\hline 1,2-Dibromo-3-Chloropropane & 25.0 & & 25.0 & 100 & $65-130$ & \\
\hline $1,2,4$-Trichlorobenzene & 25.0 & & 26.9 & 108 & $80-125$ & \\
\hline Hexachlorobutadiene & 25.0 & & 28.3 & 113 & $65-150$ & \\
\hline Naphthalene & 25.0 & & 27.4 & 110 & $80-125$ & \\
\hline $1,2,3$-Trichlorobenzene & 25.0 & & 27.5 & 110 & $70-125$ & \\
\hline
\end{tabular}

\# Column to be used to flag recovery and RPD values 
FORM IV

GC/MS VOA METHOD BLANK SUMMARY

Lab Name: TestAmerica Burlington

SDG No.: INMAN (200-2608)

Lab File ID: lfme07.d

Matrix: Solid

Instrument ID: L.i

GC Colurnn: DB-624
Job No.: 200-2608-1

Lab Sample ID: MB 200-10089/3-A

Heated Purge: $(\mathrm{Y} / \mathrm{N}) \mathrm{N}$

Date Analyzed: 11/24/2010 11:03

THIS METHOD BLANK APPLIES TO THE FOLLOWING SAMPLES:

\begin{tabular}{|c|c|c|c|c|}
\hline & LCS 200-10229/3 & Ifme03.d & $11 / 24 / 2010$ & $08: 54$ \\
\hline IN-S-30836 & $200-2608-1$ & lfme.08.d & $11 / 24 / 2010$ & $12: 02$ \\
\hline$I N-S-30820$ & $200-2608-2$ & lfme09.d & $11 / 24 / 2010$ & $12: 34$ \\
\hline IN-S-30795 & $200-2608-3$ & Ifme10.d & $11 / 24 / 2010$ & $13: 06$ \\
\hline IN-S-30814 & $200-2608-6$ & Ifme13.d & $11 / 24 / 2010$ & $14: 43$ \\
\hline IN-S-MeOH BLANK & $200-2608-7$ & lfme14.d & $11 / 24 / 2010$ & $15: 15$ \\
\hline$I N-S-30824$ & $200-2608-8$ & lfme15.d & $11 / 24 / 2010$ & $15: 47$ \\
\hline
\end{tabular}


FORM V

GC/MS VOA INSTRUMENT PERFORMANCE CHECK

BROMOFLUOROBENZENE (BFB)

Lab Name: TestAmerica Burlington

SDG NO.: INMAN (200-2608)

Lab File ID: Ifm02.d

Instrument ID: I.i

Analysis Batch No.: 9968
Job No.: 200-2608-1

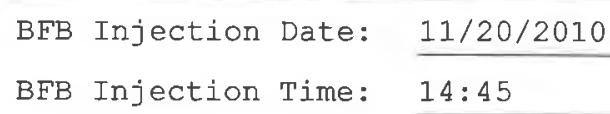

\begin{tabular}{|c|c|c|}
\hline$M / E$ & ION ABUNDANCE CRITERIA & $\begin{array}{l}\circ \text { RELATIVE } \\
\text { ABUNDANCE }\end{array}$ \\
\hline 50 & $15.0-40.0 \div$ of mass 95 & 23.8 \\
\hline 75 & $30.0-60.0 \%$ of mass 95 & 48.2 \\
\hline 95 & Base Peak, $100 \%$ relative abundance & 100.0 \\
\hline 96 & $5.0-9.0 \frac{\circ}{8}$ of mass 95 & 6.4 \\
\hline 173 & Less than 2.0 \% of mass 174 & $(0.0) 1$ \\
\hline 174 & $50.0-120.00$ of mass 95 & 75.5 \\
\hline 175 & $5.0-9.0$ of mass 174 & $(8.5) 1$ \\
\hline 176 & $95.0-101.0 \%$ of mass 174 & $(96.6) 1$ \\
\hline 177 & $5.0-9.0$ of mass 176 & $(7.1) 2$ \\
\hline
\end{tabular}

1-Value is $\frac{\circ}{6}$ mass 174

2-Value is 음 mass 176

THIS CHECK APPLIES TO THE FOLLOWING SAMPLES, MS, MSD, BLANKS AND STANDARDS:

\begin{tabular}{|c|c|c|c|c|}
\hline CLIENT SAMPLE ID & LAB SAMPLE ID & $\begin{array}{l}\text { LAB } \\
\text { FILE ID }\end{array}$ & $\begin{array}{c}\text { DATE } \\
\text { ANALYZED }\end{array}$ & $\begin{array}{c}\text { TIME } \\
\text { ANALYZED }\end{array}$ \\
\hline & IC $200-9968 / 4$ & $1 \mathrm{fm0} 4 . \mathrm{d}$ & $11 / 20 / 2010$ & $15: 36$ \\
\hline & IC $200-9968 / 5$ & lfm05.d & $11 / 20 / 2010$ & $16: 09$ \\
\hline & IC $200-9968 / 6$ & $\operatorname{lfm06.d}$ & $11 / 20 / 2010$ & $16: 41$ \\
\hline & ICIS $200-9968 / 7$ & $\operatorname{lfm} 07 . d$ & $11 / 20 / 2010$ & $17: 13$ \\
\hline & IC $200-9968 / 8$ & lfm08.d & $11 / 20 / 2010$ & $17: 45$ \\
\hline & IC $200-9968 / 9$ & lfm09.d & $11 / 20 / 2010$ & $18: 17$ \\
\hline & ICV $200-9968 / 12$ & $\operatorname{lfm} 12 \cdot d$ & $11 / 20 / 2010$ & $19: 53$ \\
\hline
\end{tabular}


FORM V

GC/MS VOA INSTRUMENT PERFORMANCE CHECK BROMOFLUOROBENZENE (BFB)

Lab Name: TestAmerica Burlington

SDG NO.: INMAN (200-2608)

Lab File ID: lfme01.d

Instrument ID: L.i

Analysis Batch No.: 10229

\begin{tabular}{|c|c|c|}
\hline$M / E$ & ION ABUNDANCE CRITERIA & $\begin{array}{l}\text { \% RELATIVE } \\
\text { ABUNDANCE }\end{array}$ \\
\hline 50 & $15.0-40.0$ of mass 95 & 22.3 \\
\hline 75 & $30.0-60.0 \div$ of mass 95 & 48.0 \\
\hline 95 & Base Peak, 100 응 relative abundance & 100.0 \\
\hline 96 & $5.0-9.0 \div$ of mass 95 & 6.9 \\
\hline 173 & Less than $2.0 \%$ of mass 174 & $(0.0) 1$ \\
\hline 174 & $50.0-120.00 \div$ of mass 95 & 74.8 \\
\hline 175 & $5.0-9.0 \div$ of mass 174 & $(5.3) 1$ \\
\hline 176 & $95.0-101.0 \div$ of mass 174 & $(96.9) 1$ \\
\hline 177 & $5.0-9.0$ of of mass 176 & $(7.1) 2$ \\
\hline
\end{tabular}

1-Value is $\frac{0}{0}$ mass 174

2-Value is \% mass 176
BFB Injection Date: 11/24/2010

BFB Injection Time: 08:02

THIS CHECK APPIIES TO THE FOLLOWING SAMPLES, MS, MSD, BLANKS AND STANDARDS:

\begin{tabular}{|c|c|c|c|c|}
\hline CLIENT SAMPLE ID & LAB SAMPLE ID & $\begin{array}{l}\text { LAB } \\
\text { FILE ID }\end{array}$ & $\begin{array}{c}\text { DATE } \\
\text { ANALYZED }\end{array}$ & $\begin{array}{c}\text { TIME } \\
\text { ANALYZED }\end{array}$ \\
\hline & CCVIS $200-10229 / 2$ & lfme02.d & $11 / 24 / 2010$ & $08: 22$ \\
\hline & LCS 200-10229/3 & lfme03.d & $11 / 24 / 2010$ & $08: 54$ \\
\hline & LCS 200-10089/6-A & Ifme04.d & $11 / 24 / 2010$ & $09: 26$ \\
\hline & MB 200-10089/3-A & lfme07.d & $11 / 24 / 2010$ & $11: 03$ \\
\hline$I N-S-30836$ & $200-2608-1$ & Ifme08.d & $11 / 24 / 2010$ & $12: 02$ \\
\hline$I N-S-30820$ & $200-2608-2$ & lfme09.d & $11 / 24 / 2010$ & $12: 34$ \\
\hline IN-S-30795 & $200-2608-3$ & Ifme10.d & $11 / 24 / 2010$ & $13: 06$ \\
\hline IN-S-30771 & $200-2608-4$ & lfme11.d & $11 / 24 / 2010$ & $13: 39$ \\
\hline$I N-S-30808$ & $200-2608-5$ & Ifme12.d & $11 / 24 / 2010$ & $14: 11$ \\
\hline$I N-S-30814$ & $200-2608-6$ & lfme13.d & $11 / 24 / 2010$ & $14: 43$ \\
\hline IN-S-MeOH BLANK & $200-2608-7$ & lfme14.d & $11 / 24 / 2010$ & $15: 15$ \\
\hline$I N-S-30824$ & $200-2608-8$ & lfme15.d & $11 / 24 / 2010$ & $15: 47$ \\
\hline
\end{tabular}


EORM VIII

GC/MS VOA INTERNAL STANDARD AREA AND RETENTION TIME SUMMARY

Lab Name: TestAmerica Burlington Job No.: 200-2608-1

SDG NO.: INMAN (200-2608)

Sample No.: ICIS 200-9968/7

Date Analyzed: 11/20/2010 17:13

Instrument ID: L.i

Lab File ID (Standard): lfm07.d

GC Column: DB-624

ID: $0.53(\mathrm{~mm})$

Calibration ID: 3377

\begin{tabular}{|c|c|c|c|c|c|c|c|}
\hline & \multicolumn{2}{|l|}{ FB } & \multicolumn{2}{|l|}{ CB2 } & \multicolumn{2}{|l|}{ DCB } \\
\hline & & AREA \# & RT \# & AREA \# & RT \# & AREA \# & RT \# \\
\hline INITIAL CALIBRATIO & INT & 1914600 & 9.84 & 1521264 & 15.66 & 888191 & 20.05 \\
\hline UPPER LIMIT & & 3829200 & $1,0.34$ & 3042528 & 16.16 & 1776382 & 20.55 \\
\hline LOWER LIMIT & & 957300 & 9.34 & 760632 & 15.16 & 444096 & 19.55 \\
\hline LAB SAMPLE ID & CLIENT SAMPLE ID & & & & & & \\
\hline ICV $200-9968 / 12$ & & 1990975 & 9.84 & 1581424 & 15.65 & 903144 & 20.04 \\
\hline
\end{tabular}

$\mathrm{FB}=$ Fluorobenzene

$\mathrm{CBZ}=\mathrm{Ch}$ lorobenzene-d5

$\mathrm{DCB}=1,4-\mathrm{Dichlorobenzene- \textrm {d } 4}$

Area Limit $=50 \%-200 \%$ of internal standard area

RT Limit $= \pm 0.50$ minutes of internal standard RT

\# Column used to flag values outside QC limits

FORM VIII $8260 \mathrm{~B}$ 
FORM VIII

GC/MS VOA INTERNAL STANDARD AREA AND RETENTION TIME SUMMARY

Lab Name: TestAmerica Burlington

SDG NO.: INMAN (200-2608)

Sample No.: CCVIS 200-10229/2

Instrument ID: L.i

Lab File ID (Standard): Ifme02.d

Calibration ID: 3377
Job No.: $200-2608-1$

Date Analyzed: 11/24/2010 08:22

GC Column: DB-624

ID : $0.53(\mathrm{~mm})$ Heated Purge: $(\mathrm{Y} / \mathrm{N}) \mathrm{N}$

\begin{tabular}{|c|c|c|c|c|c|c|c|}
\hline & & $\mathrm{FB}$ & & CBZ & & $\overline{D C B}$ & \\
\hline & & AREA \# & RT \# & AREA \# & RT \# & AREA \# & RT \# \\
\hline $12 / 24$ HOUR STD & & 2029415 & 9.84 & 1612349 & 15.65 & 960376 & 20.05 \\
\hline UPPER LIMIT & & 4058830 & 10.34 & 3224698 & 16.15 & 1920752 & 20.55 \\
\hline LOWER LIMIT & & 1014708 & 9.34 & 806175 & 15.15 & 480188 & 19.55 \\
\hline LAB SAMPLE ID & CLIENT SAMPLE ID & & & & & & \\
\hline LCS $200-10229 / 3$ & & 2098061 & 9.84 & 1650996 & 15.65 & 941946 & 20.06 \\
\hline LCS $200-10089 / 6-A$ & 7 & 2337525 & 9.81 & 1750468 & 15.64 & 967876 & 20.05 \\
\hline MB 200-10089/3-A & & 2122230 & 9.81 & 1669851 & 15.65 & 955060 & 20.05 \\
\hline $200-2608-1$ & IN-S-30836 & 2442608 & 9.81 & 1910379 & 15.65 & 1101171 & 20.06 \\
\hline $200-2608-2$ & IN-S-30820 & 2300243 & 9.80 & 1797882 & 15.65 & 1043099 & 20.06 \\
\hline $200-2608-3$ & IN-S-30795 & 2681071 & 9.81 & 2096860 & 15.65 & 1182644 & 20.06 \\
\hline $200-2608-4$ & IN-S-30771 & 1940167 & 9.82 & 1541896 & 15.65 & 890134 & 20.06 \\
\hline $200-2608-5$ & $\mathrm{IN}-\mathrm{S}-30808$ & 2713272 & 9.81 & 2163710 & 15.65 & 1246983 & 20.05 \\
\hline $200-2608-6$ & $I N-S-30814$ & 2389763 & 9.81 & 1881667 & 15.65 & 1098461 & 20.06 \\
\hline $200-2608-7$ & IN-S-MeOH BLANK & 2841197 & 9.81 & 2217152 & 15.65 & 1267596 & 20.06 \\
\hline $200-2608-8$ & IN-S-30824 & 2372847 & 9.80 & 1856493 & 15.65 & 1053508 & 20.07 \\
\hline
\end{tabular}

$\mathrm{FB}=$ Fluorobenzene

$\mathrm{CBZ}=$ Chlorobenzene $-\mathrm{d} 5$

$\mathrm{DCB}=1,4-\mathrm{Dich}$ lorobenzene-d4

Area limit $=50 \%-200 \%$ of internal standard area

$\mathrm{RT}$ Limit $= \pm 0.50$ minutes of internal standard RT

\# Column used to flag values outside QC limits

FORM VIII $8260 \mathrm{~B}$ 
FORM I

GC/MS VOA ORGANICS ANALYSIS DATA SHEET

Lab Name: TestAmerica Burlington

SDG No.: INMAN (200-2608)

Client Sample ID: IN-S-30836

Matrix: Solid

Analysis Method: $8260 \mathrm{~B}$

Sample wt/vol: $12.509(\mathrm{~g})$

Soil Aliquot Vol: 0.5 (mL)

Soil Extract Vol.: $10(\mathrm{~mL})$

\% Moisture:

Analysis Batch No.: 10229
Job No.: 200-2608-1

Lab Sample ID: 200-2608-1

Lab File ID: Ifme08.d

Date Collected: 11/18/2010 00:00

Date Analyzed: 11/24/2010 12:02

Dilution Factor: 1

GC Column: DB-624

ID : $0.53(\mathrm{~mm})$

Level: (low/med) Medium

Units: $u g / \mathrm{Kg}$

\begin{tabular}{|c|c|c|c|c|c|}
\hline CAS NO. & COMPOUND NAME & RESULT & $Q$ & RL & MDL \\
\hline $75-71-8$ & Dichlorodifluoromethane & 8.0 & U & 8.0 & 4.0 \\
\hline $74-87-3$ & Chloromethane & 8.0 & $\mathrm{U}$ & 8.0 & 3.0 \\
\hline $75-01-4$ & Vinyl chloride & 8.0 & $\mathrm{U}$ & 8.0 & 4.0 \\
\hline $74-83-9$ & Bromomethane & 5.1 & $\mathrm{JB} \bullet$ & 8.0 & 2.8 \\
\hline $75-00-3$ & Chloroethane & 8.0 & $\mathrm{U}$ * & 8.0 & 4.8 \\
\hline $75-69-4$ & Trichlorofluoromethane & 8.0 & $\mathrm{U} \star$ & 8.0 & 4.0 \\
\hline $75-35-4$ & 1,1-Dichloroethene & 8.0 & $\mathrm{U}$ & 8.0 & 1.7 \\
\hline $76-13-1$ & Freon $\mathrm{TF}$ & 8.0 & $\mathrm{U}$ & 8.0 & 2.2 \\
\hline $67-64-1$ & Acetone & 8.0 & $\mathrm{U}$ & 8.0 & 8.0 \\
\hline $74-88-4$ & Methyl iodide & 8.0 & $\mathrm{U}$ * & 8.0 & 4.0 \\
\hline $75-15-0$ & Carbon disulfide & 8.0 & $\mathrm{U}$ & 8.0 & 1.5 \\
\hline $79-20-9$ & Methyl acetate & 8.0 & $\mathrm{U}$ & 8.0 & 4.0 \\
\hline $75-09-2$ & Methylene Chloride & 8.0 & $\mathrm{U}$ & 8.0 & 4.0 \\
\hline $156-60-5$ & trans-1,2-Dichloroethene & 8.0 & $\mathrm{U}$ & 8.0 & 4.0 \\
\hline $1634-04-4$ & Methyl t-butyl ether & 8.0 & $\mathrm{U}$ & 8.0 & 4.0 \\
\hline $75-34-3$ & 1,1-Dichloroethane & 8.0 & $\mathrm{U}$ & 8.0 & 2.9 \\
\hline $108-05-4$ & Vinyl acetate & 8.0 & $\mathrm{U}$ & 8.0 & 4.0 \\
\hline $594-20-7$ & 2,2-Dichloropropane & 8.0 & $\mathrm{U}$ & 8.0 & 3.7 \\
\hline $156-59-2$ & cis-1,2-Dichloroethene & 8.0 & $\mathrm{U}$ & 8.0 & 1.7 \\
\hline $78-93-3$ & 2-Butanone & 8.0 & $\mathrm{U}$ * & 8.0 & 8.8 \\
\hline $74-97-5$ & Bromochloromethane & 8.0 & $\mathrm{U}$ & 8.0 & 4.2 \\
\hline $109-99-9$ & Tetrahydrofuran & 80 & $\mathrm{U}$ & 80 & 40 \\
\hline $67-66-3$ & Chloroform & 8.0 & $\mathrm{U}$ & 8.0 & 2.6 \\
\hline $71-55-6$ & 1,1,1-Trichloroethane & 8.0 & $\mathrm{U}$ & 8.0 & 2.9 \\
\hline $110-82-7$ & Cyclohexane & 8.0 & U & 8.0 & 2.8 \\
\hline $563-58-6$ & 1,1-Dichloropropene & 8.0 & 0 & 8.0 & 1.8 \\
\hline $56-23-5$ & Carbon tetrachloride & 8.0 & $\mathrm{U}$ & 8.0 & 2.6 \\
\hline $78-83-1$ & Isobutyl alcohol & 400 & $\mathrm{U}$ & 400 & 200 \\
\hline $71-43-2$ & Benzene & 8.0 & $\mathrm{U}$ & 8.0 & 2.5 \\
\hline $107-06-2$ & 1,2-Dichloroethane & 8.0 & $\mathrm{U}$ & 8.0 & 2.6 \\
\hline $79-01-6$ & Trichloroethene & 8.0 & $\mathrm{U}$ & 8.0 & 4.0 \\
\hline $108-87-2$ & Methylcyclohexane & 8.0 & $\mathrm{U}$ & 8.0 & 4.0 \\
\hline $78-87-5$ & 1,2-Dichloropropane & 8.0 & U & 8.0 & 3.0 \\
\hline $74-95-3$ & Dibromomethane & 8.0 & $\mathrm{U}$ & 8.0 & 2.0 \\
\hline $123-91-1$ & 1,4-Dioxane & 400 & $\mathrm{U}$ & 400 & 220 \\
\hline $75-27-4$ & Bromodichloromethane & 8.0 & $\mathrm{U}$ & 8.0 & 3.0 \\
\hline
\end{tabular}


FORM I

GC/MS VOA ORGANICS ANALYSIS DATA SHEET

Lab Name: TestAmerica Burlington

SDG NO.: INMAN (200-2608)

Client Sample ID: IN-S-30836

Matrix: Solid

Analysis Method: $8260 \mathrm{~B}$

Sample wt/vol: $12.509(\mathrm{~g})$

Soil Aliquot Vol: 0.5 (mL)

Soil Extract Vol.: $10(\mathrm{~mL})$

응 Moisture:

Analysis Batch No.: 10229
Job No.: $200-2608-1$

Lab Sample ID: 200-2608-1

Lab File ID: lfme08.d

Date Collected: 11/18/2010 00:00

Date Analyzed: 11/24/2010 12:02

Dilution Factor: 1

GC Column: DB-624

ID $: 0.53(\mathrm{~mm})$

Level: (low/med) Medium

Units: $u g / \mathrm{Kg}$

\begin{tabular}{|c|c|c|c|c|c|}
\hline CAS NO. & COMPOUND NAME & RESULT & $Q$ & RL & MDL \\
\hline $110-75-8$ & 2-Chloroethyl vinyl ether & 8.0 & $\mathrm{U}$ & 8.0 & 1.0 \\
\hline $10061-01-5$ & cis-1,3-Dichloropropene & 8.0 & $\mathrm{U}$ & 8.0 & 2.6 \\
\hline $108-10-1$ & 4-Methyl-2-pentanone & 8.0 & $\mathrm{U}$ & 8.0 & 1.7 \\
\hline $108-88-3$ & Toluene & 8.0 & $\mathrm{U}$ & 8.0 & 4.0 \\
\hline $10061-02-6$ & trans-1,3-Dichloropropene & 8.0 & $\mathrm{U}$ & 8.0 & 4.0 \\
\hline $79-00-5$ & 1,1,2-Trichloroethane & 8.0 & $\mathrm{U}$ & 8.0 & 3.4 \\
\hline $127-18-4$ & Tetrachloroethene & 8.0 & $\mathrm{U}$ & 8.0 & 4.0 \\
\hline $142-28-9$ & 1,3-Dichloropropane & 8.0 & U & 8.0 & 2.6 \\
\hline $591-78-6$ & 2-Hexanone & 8.0 & $\mathrm{U}$ & 8.0 & 8.0 \\
\hline $124-48-1$ & Dibromochlorome thane & 8.0 & $\mathrm{U}$ & 8.0 & 3.0 \\
\hline $106-93-4$ & 1,2-Dibromoethane & 8.0 & U & 8.0 & 4.0 \\
\hline $108-90-7$ & Chlorobenzene & 8.0 & $\mathrm{U}$ & 8.0 & 1.8 \\
\hline $630-20-6$ & $1,1,1,2$-Tetrachloroethane & 8.0 & $\mathrm{U}$ & 8.0 & 2.9 \\
\hline $100-41-4$ & Ethylbenzene & 8.0 & $\mathrm{U}$ & 8.0 & 4.0 \\
\hline $179601-23-1$ & $m \& p-x y l e n e$ & 8.0 & $\mathrm{U}$ & 8.0 & 4.0 \\
\hline $95-47-6$ & o-Xylene & 8.0 & $\mathrm{U}$ & 8.0 & 4.0 \\
\hline $100-42-5$ & Styrene & 8.0 & $\mathrm{U}$ & 8.0 & 4.0 \\
\hline $75-25-2$ & Bromo form & 8.0 & $\mathrm{U}$ & 8.0 & 3.1 \\
\hline $98-82-8$ & Isopropylbenzene & 8.0 & $\mathrm{U}$ & 8.0 & 4.0 \\
\hline $108-86-1$ & Bromobenzene & 8.0 & $\mathrm{U}$ & 8.0 & 3.0 \\
\hline $79-34-5$ & $1,1,2,2$-Tetrachloroethane & 8.0 & $\mathrm{U}$ & 8.0 & 3.0 \\
\hline $96-18-4$ & 1,2,3-Trichloropropane & 8.0 & $\mathrm{U}$ & 8.0 & 4.3 \\
\hline $103-65-1$ & n-Propylbenzene & 8.0 & $\mathrm{~J}$ & 8.0 & 4.0 \\
\hline $95-49-8$ & 2-Chlorotoluene & 8.0 & $\mathrm{~J}$ & 8.0 & 2.6 \\
\hline $106-43-4$ & 4-Chlorotoluene & 8.0 & U & 8.0 & 2.7 \\
\hline $108-67-8$ & 1,3,5-Trimethylbenzene & 8.0 & $\mathrm{U}$ & 8.0 & 2.6 \\
\hline $98-06-6$ & tert-Butylbenzene & 8.0 & $\mathrm{U}$ & 8.0 & 4.0 \\
\hline $95-63-6$ & 1,2,4-Trimethylbenzene & 8.0 & U & 8.0 & 2.7 \\
\hline $135-98-8$ & sec-Butylbenzene & 8.0 & $\mathrm{U}$ & 8.0 & 4.0 \\
\hline $541-73-1$ & 1,3-Dichlorobenzene & 8.0 & $\mathrm{U}$ & 8.0 & 2.0 \\
\hline $99-87-6$ & 4-Isopropyltoluene & 8.0 & $\mathrm{U}$ & 8.0 & 1.4 \\
\hline $106-46-7$ & 1,4-Dichlorobenzene & 8.0 & $\mathrm{U}$ & 8.0 & 1.8 \\
\hline $95-50-1$ & 1,2-Dichlorobenzene & 8.0 & $\mathrm{U}$ & 8.0 & 2.2 \\
\hline $104-51-8$ & n-Butylbenzene & 8.0 & $\mathrm{U}$ & 8.0 & 4.0 \\
\hline $96-12-8$ & 1,2-Dibromo-3-Chloropropane & 8.0 & $\mathrm{U}$ & 8.0 & 3.7 \\
\hline $120-82-1$ & 1,2,4-Trichlorobenzene & 1.6 & $\mathrm{~J} \mathrm{~B}$ & 8.0 & 1.3 \\
\hline
\end{tabular}


FORM I

GC/MS VOA ORGANICS ANALYSIS DATA SHEET

Lab Name: TestAmerica Burlington

SDG No.: INMAN (200-2608)

Client Sample ID: IN-S-30836

Matrix: Solid

Analysis Method: $8260 \mathrm{~B}$

Sample wt/vol: $12.509(\mathrm{~g})$

Soil Aliquot Vol: 0.5 (mL)

Soil Extract Vol.: $10(\mathrm{~mL})$

음 Moisture:

Analysis Batch No.: 10229
Job No.: 200-2608-1

Lab Sample ID: 200-2608-1

Lab File ID: lfme08.d

Date Collected: 11/18/2010 00:00

Date Analyzed: 11/24/2010 12:02

Dilution Factor: 1

GC Column: $\mathrm{DB}-624 \quad$ ID: 0.53 (mm)

Level: (low/med) Medium

Units: ug/Kg

\begin{tabular}{|l|l|r|r|r|r|}
\hline \multicolumn{1}{|c|}{ CAS NO. } & \multicolumn{1}{|c|}{ COMPOUND NAME } & RESULT & Q & RL & MDL \\
\hline \hline $87-68-3$ & Hexachlorobutadiene & 8.0 & U & 8.0 & 4.0 \\
\hline $91-20-3$ & Naphthalene & 8.0 & U & 8.0 & 4.0 \\
\hline $87-61-6$ & $1,2,3-$ Trichlorobenzene & 8.0 & U & 8.0 & 2.0 \\
\hline
\end{tabular}

\begin{tabular}{|l|l|r|r|r|}
\hline \multicolumn{1}{|c|}{ CAS NO. } & \multicolumn{1}{|c|}{ SURROGATE } & 을 & Q & LIMITS \\
\hline \hline $17060-07-0$ & 1,2 -Dichloroethane-d4 & 85 & & $65-155$ \\
\hline $2037-26-5$ & Toluene-d8 & 95 & & $80-115$ \\
\hline $460-00-4$ & Bromofluorobenzene & 92 & & $80-115$ \\
\hline $2199-69-1$ & 1,2-Dichlorobenzene-d4 & 94 & & $45-145$ \\
\hline
\end{tabular}


FORM I

GC/MS VOA ORGANICS ANALYSIS DATA SHEET

Lab Name: TestAmerica Burlington

SDG NO.: INMAN (200-2608)

Client Sample ID: IN-S-30820

Matrix: Solid

Analysis Method: $8260 B$

Sample wt/vol: $10.185(\mathrm{~g})$

Soil Aliquot Vol: 0.5 (mL)

Soil Extract Vol.: $10(\mathrm{~mL})$

응 Moisture:

Analysis Batch No.: 10229
Job No.: 200-2608-1

Lab Sample ID: 200-2608-2

Lab File ID: Ifme09.d

Date Collected: 11/18/2010 00:00

Date Analyzed: 11/24/2010 12:34

Dilution Factor: 1

GC Column: DB-624

ID : $0.53(\mathrm{~mm})$

Level: (low/med) Medium

Units: ug/Kg

\begin{tabular}{|c|c|c|c|c|c|}
\hline CAS NO. & COMPOUND NAME & RESULT & $Q$ & RL & MDL \\
\hline $75-71-8$ & Dichlorodifluoromethane & 9.8 & $\mathrm{U}$ & 9.8 & 4.9 \\
\hline $74-87-3$ & Chloromethane & 9.8 & $\mathrm{U}$ & 9.8 & 3.6 \\
\hline $75-01-4$ & Vinyl chloride & 9.8 & U & 9.8 & 4.9 \\
\hline $74-83-9$ & Bromomethane & 4.1 & $J$ B & 9.8 & 3.4 \\
\hline $75-00-3$ & Chloroethane & 9.8 & $\mathrm{U}$ * & 9.8 & 5.9 \\
\hline $75-69-4$ & Trichlorofluoromethane & 9.8 & $\mathrm{U} *$ & 9.8 & 4.9 \\
\hline $75-35-4$ & 1,1-Dichloroethene & 9.8 & U & 9.8 & 2.1 \\
\hline $76-13-1$ & Freon TF & 9.8 & $\mathrm{U}$ & 9.8 & 2.7 \\
\hline $67-64-1$ & Acetone & 9.8 & $\mathrm{U}$ & 9.8 & 9.8 \\
\hline $74-88-4$ & Methyl iodide & 9.8 & $\mathrm{U} *$ & 9.8 & 4.9 \\
\hline $75-15-0$ & Carbon disulfide & 9.8 & $\mathrm{U}$ & 9.8 & 1.9 \\
\hline $79-20-9$ & Methyl acetate & 9.8 & $\mathrm{U}$ & 9.8 & 4.9 \\
\hline $75-09-2$ & Methylene Chloride & 9.8 & $\mathrm{U}$ & 9.8 & 4.9 \\
\hline $156-60-5$ & trans-1,2-Dichloroethene & $9.8^{\circ}$ & $\mathrm{U}$ & 9.8 & 4.9 \\
\hline $1634-04-4$ & Methyl t-butyl ether & 9.8 & $\mathrm{U}$ & 9.8 & 4.9 \\
\hline $75-34-3$ & 1,1-Dichloroethane & 9.8 & $\mathrm{U}$ & 9.8 & 3.5 \\
\hline $108-05-4$ & Vinyl acetate & 9.8 & $\mathrm{U}$ & 9.8 & 4.9 \\
\hline $594-20-7$ & 2,2-Dichloropropane & 9.8 & $\mathrm{U}$ & 9.8 & 4.5 \\
\hline $156-59-2$ & cis-1,2-Dichloroethene & 9.8 & $\mathrm{U}$ & 9.8 & 2.1 \\
\hline $78-93-3$ & 2-Butanone & 9.8 & $\mathrm{U} *$ & 9.8 & 11 \\
\hline $74-97-5$ & Bromochloromethane & 9.8 & $\mathrm{U}$ & 9.8 & 5.2 \\
\hline $109-99-9$ & Tetrahydrofuran & 98 & $\mathrm{U}$ & 98 & 49 \\
\hline $67-66-3$ & Chloroform & 9.8 & $\mathrm{U}$ & 9.8 & 3.2 \\
\hline $71-55-6$ & 1,1,1-Trichloroethane & 9.8 & $\mathrm{U}$ & 9.8 & 3.5 \\
\hline $110-82-7$ & Cyclohexane & 9.8 & $\mathrm{U}$ & 9.8 & 3.4 \\
\hline $563-58-6$ & 1,1-Dichloropropene & 9.8 & $\mathrm{U}$ & 9.8 & 2.2 \\
\hline $56-23-5$ & Carbon tetrachloride & 9.8 & $\mathrm{U}$ & 9.8 & 3.1 \\
\hline $78-83-1$ & Isobutyl alcohol & 490 & $\mathrm{U}$ & 490 & 250 \\
\hline $71-43-2$ & Benzene & 9.8 & $\mathrm{U}$ & 9.8 & 3.0 \\
\hline $107-06-2$ & 1,2-Dichloroethane & 9.8 & $\mathrm{U}$ & 9.8 & 3.2 \\
\hline $79-01-6$ & Trichloroethene & 9.8 & $\mathrm{U}$ & 9.8 & 4.9 \\
\hline $108-87-2$ & Methylcyclohexane & 9.8 & $\mathrm{U}$ & 9.8 & 4.9 \\
\hline $78-87-5$ & 1,2-Dichloropropane & 9.8 & $\mathrm{U}$ & 9.8 & 3.7 \\
\hline $74-95-3$ & Dibromomethane & 9.8 & $\mathrm{U}$ & 9.8 & 2.5 \\
\hline $123-91-1$ & 1,4-Dioxane & 490 & $\mathrm{U}$ & 490 & 270 \\
\hline $75-27-4$ & Bromodichlorome thane & 9.8 & $\mathrm{U}$ & 9.8 & 3.6 \\
\hline
\end{tabular}


FORM I

GC/MS VOA ORGANICS ANALYSIS DATA SHEET

Lab Name: TestAmerica Burlington

SDG No.: INMAN (200-2608)

Client Sample ID: IN-S-30820

Matrix: Solid

Analysis Method: $8260 \mathrm{~B}$

Sample wt/vol: $10.185(\mathrm{~g})$

Soil Aliquot Vol: 0.5 (mL)

Soil Extract Vol.: $10(\mathrm{~mL})$

응 Moisture:

Analysis Batch No.: 10229
Job No.: 200-2608-1

Lab Sample ID: 200-2608-2

Lab File ID: lfme09.d

Date Collected: 11/18/2010 00:00

Date Analyzed: 11/24/2010 12:34

Dilution Factor: 1

GC Column: DB-624

ID : $0.53(\mathrm{~mm})$

Level: (Low/med) Medium

Units: $\underline{u g} / \mathrm{Kg}$

\begin{tabular}{|c|c|c|c|c|c|}
\hline CAS NO. & COMPOUND NAME & RESULT & $Q$ & RI & MDL \\
\hline $110-75-8$ & 2-Chloroethyl vinyl ether & 9.8 & $\mathrm{U}$ & 9.8 & 1.3 \\
\hline $10061-01-5$ & cis-1,3-Dichloropropene & 9.8 & $\mathrm{U}$ & 9.8 & 3.1 \\
\hline $108-10-1$ & 4-Methyl-2-pentanone & 9.8 & $\mathrm{U}$ & 9.8 & 2.1 \\
\hline $108-88-3$ & Toluene & 9.8 & $\mathrm{U}$ & 9.8 & 4.9 \\
\hline $10061-02-6$ & trans -1,3-Dichloropropene & 9.8 & $\mathrm{U}$ & 9.8 & 4.9 \\
\hline $79-00-5$ & 1,1,2-Trichloroethane & 9.8 & $\mathrm{U}$ & 9.8 & 4.1 \\
\hline $127-18-4$ & Tetrachloroethene & 9.8 & $\mathrm{U}$ & 9.8 & 4.9 \\
\hline $142-28-9$ & 1,3-Dichloropropane & 9.8 & $\mathrm{U}$ & 9.8 & 3.2 \\
\hline $591-78-6$ & 2-Hexanone & 9.8 & $\mathrm{u}$ & 9.8 & 9.8 \\
\hline $124-48-1$ & Dibromochloromethane & 9.8 & $\mathrm{U}$ & 9.8 & 3.6 \\
\hline $106-93-4$ & 1,2-Dibromoethane & 9.8 & $\mathrm{U}$ & 9.8 & 4.9 \\
\hline $108-90-7$ & Chlorobenzene & 9.8 & $\mathrm{U}$ & 9.8 & 2.3 \\
\hline $630-20-6$ & $1,1,1,2$-Tetrachloroethane & 9.8 & $\mathrm{U}$ & 9.8 & 3.5 \\
\hline $100-41-4$ & Ethylbenzene & 9.8 & $\mathrm{U}$ & 9.8 & 4.9 \\
\hline $179601-23-1$ & m\&p-Xylene & 9.8 & $\mathrm{U}$ & 9.8 & 4.9 \\
\hline $95-47-6$ & o-Xylene & 9.8 & $\mathrm{U}$ & 9.8 & 4.9 \\
\hline $100-42-5$ & Styrene & 9.8 & $\mathrm{U}$ & 9.8 & 4.9 \\
\hline $75-25-2$ & Bromoform & 9.8 & $\mathrm{U}$ & 9.8 & 3.8 \\
\hline $98-82-8$ & Isopropylbenzene & 9.8 & $\mathrm{U}$ & 9.8 & 4.9 \\
\hline $108-86-1$ & Bromobenzene & 9.8 & $\mathrm{U}$ & 9.8 & 3.6 \\
\hline $79-34-5$ & $1,1,2,2$-Tetrachloroethane & 9.8 & $\mathrm{U}$ & 9.8 & 3.6 \\
\hline $96-18-4$ & 1,2,3-Trichloropropane & 9.8 & $\mathrm{U}$ & 9.8 & 5.3 \\
\hline $103-65-1$ & n-Propylbenzene & 9.8 & $\mathrm{U}$ & 9.8 & 4.9 \\
\hline $95-49-8$ & 2-Chlorotoluene & 9.8 & $\mathrm{U}$ & 9.8 & 3.2 \\
\hline $106-43-4$ & 4-Chlorotoluene & 9.8 & $\mathrm{U}$ & 9.8 & 3.3 \\
\hline $108-67-8$ & 1,3,5-Trimethylbenzene & 9.8 & $\mathrm{U}$ & 9.8 & 3.1 \\
\hline $98-06-6$ & tert-Butylbenzene & 9.8 & $\mathrm{U}$ & 9.8 & 4.9 \\
\hline $95-63-6$ & 1,2,4-Trimethylbenzene & 9.8 & $\mathrm{U}$ & 9.8 & 3.3 \\
\hline $135-98-8$ & sec-Butylbenzene & 9.8 & $\mathrm{U}$ & 9.8 & 4.9 \\
\hline $541-73-1$ & 1,3-Dichlorobenzene & 9.8 & $\mathrm{U}$ & 9.8 & 2.5 \\
\hline $99-87-6$ & 4-Isopropyltoluene & 9.8 & $\mathrm{U}$ & 9.8 & 1.8 \\
\hline $106-46-7$ & 1,4-Dichlorobenzene & 9.8 & $\mathrm{U}$ & 9.8 & 2.3 \\
\hline $95-50-1$ & 1,2-Dichlorobenzene & 9.8 & $\mathrm{U}$ & 9.8 & 2.7 \\
\hline $104-51-8$ & n-Butylbenzene & 9.8 & $\mathrm{U}$ & 9.8 & 4.9 \\
\hline $96-12-8$ & 1,2-Dibromo-3-Chloropropane & 9.8 & $\mathrm{U}$ & 9.8 & 4.5 \\
\hline $120-82-1$ & 1,2,4-Trichlorobenzene & 9.8 & $\mathrm{U}$ & 9.8 & 1.6 \\
\hline
\end{tabular}


FORM I

GC/MS VOA ORGANICS ANALYSIS DATA SHEET

Lab Name: TestAmerica Burlington

SDG No.: INMAN (200-2608)

Client Sample ID: IN-S-30820

Matrix: Solid

Analysis Method: $8260 \mathrm{~B}$

Sample wt/vol: $10.185(\mathrm{~g})$

Soil Aliquot Vol: 0.5 (mL)

Soil Extract Vol.: 10 (mL)

음 Moisture:

Analysis Batch No.: 10229
Job No.: 200-2608-1

Lab Sample ID: 200-2608-2

Lab File ID: lfme09.d

Date Collected: 11/18/2010 00:00

Date Analyzed: 11/24/2010 12:34

Dilution Factor: 1

GC Column: DB-624

ID: $0.53(\mathrm{~mm})$

Level: (low/med) Medium

Units: ug/Kg

\begin{tabular}{|l|l|r|r|r|r|}
\hline \multicolumn{1}{|c|}{ CAS NO. } & \multicolumn{1}{|c|}{ COMPOUND NAME } & RESULT & Q & RL & MDI \\
\hline \hline $87-68-3$ & Hexachlorobutadiene & 9.8 & U & 9.8 & 9.9 \\
\hline $91-20-3$ & Naphthalene & 9.8 & U & 9.8 \\
\hline $87-61-6$ & $1,2,3-T r i c h l o r o b e n z e n e$ & 9.8 & U & 9.8 & \\
\hline
\end{tabular}

\begin{tabular}{|l|l|r|r|r|}
\hline \multicolumn{1}{|c|}{ CAS NO. } & \multicolumn{1}{|c|}{ SURROGATE } & oREC & Q & LIMITS \\
\hline \hline $17060-07-0$ & $1,2-$ Dichloroethane-d4 & 86 & & $65-155$ \\
\hline $2037-26-5$ & Toluene-d8 & 97 & $80-115$ \\
\hline $460-00-4$ & Bromofluorobenzene & 95 & \\
\hline $2199-69-1$ & 1,2-Dichlorobenzene-d4 & 96 & $80-115$ \\
\hline
\end{tabular}


FORM I

GC/MS VOA ORGANICS ANALYSIS DATA SHEET

Lab Name: TestAmerica Burlington

SDG No.: INMAN (200-2608)

Client Sample ID: IN-S-30795

Matrix: Solid

Analysis Method: $8260 \mathrm{~B}$

Sample wt/vol: $9.83(\mathrm{~g})$

Soil Aliquot Vol: 0.5 (mL)

Soil Extract Vol.: $10(\mathrm{~mL})$

응 Moisture:

Analysis Batch No.: 10229
Job No.: 200-2608-1

Lab Sample ID: 200-2608-3

Lab File ID: lfme10.d

Date Collected: 11/18/2010 00:00

Date Analyzed: 11/24/2010 13:06

Dilution Factor: 1

GC Column: DB-624

ID: $0.53(\mathrm{~mm})$

Level: (low/med) Medium

Units: ug/ $\mathrm{Kg}$

\begin{tabular}{|c|c|c|c|c|c|}
\hline CAS NO. & COMPOUND NAME & RESULT & $Q$ & RL & $\mathrm{MDL}$ \\
\hline $75-71-8$ & Dichlorodifluoromethane & 10 & $\mathrm{U}$ & 10 & 5.1 \\
\hline $74-87-3$ & Chloromethane & 10 & $\mathrm{U}$ & 10 & 3.8 \\
\hline $75-01-4$ & Vinyl chloride & 10 & U & 10 & 5.1 \\
\hline $74-83-9$ & Bromomethane & 10 & $\mathrm{U}$ & 10 & 3.6 \\
\hline $75-00-3$ & Chloroethane & 10 & $U \star$ & 10 & 6.1 \\
\hline $75-69-4$ & Trichlorofluoromethane & 10 & $U \star$ & 10 & 5.1 \\
\hline $75-35-4$ & 1,1-Dichloroethene & 10 & U & 10 & 2.1 \\
\hline $76-13-1$ & Freon TF & 10 & $\mathrm{U}$ & 10 & 2.8 \\
\hline $67-64-1$ & Acetone & 10 & $\mathrm{U}$ & 10 & 10 \\
\hline $74-88-4$ & Methyl iodide & 10 & $\mathrm{U} \star$ & 10 & 5.1 \\
\hline $75-15-0$ & Carbon disulfide & 10 & $\mathrm{U}$ & 10 & 1.9 \\
\hline $79-20-9$ & Methyl acetate & 10 & $\mathrm{U}$ & 10 & 5.1 \\
\hline $75-09-2$ & Methylene Chloride & 10 & $\mathrm{U}$ & 10 & 5.1 \\
\hline $156-60-5$ & trans-1,2-Dichloroethene & 10 & $\mathrm{U}$ & 10 & 5.1 \\
\hline $1634-04-4$ & Methyl t-butyl ether & 10 & $\mathrm{U}$ & 10 & 5.1 \\
\hline $75-34-3$ & 1,1-Dichloroethane & 10 & $\mathrm{U}$ & 10 & 3.7 \\
\hline $108-05-4$ & Vinyl acetate & 10 & $\mathrm{U}$ & 10 & 5.1 \\
\hline $594-20-7$ & 2,2-Dichloropropane & 10 & $\mathrm{U}$ & 10 & 4.7 \\
\hline $156-59-2$ & cis-1,2-Dichloroethene & 10 & $\mathrm{U}$ & 10 & 2.1 \\
\hline $78-93-3$ & 2-Butanone & 10 & $\mathrm{U} \star$ & 10 & 11 \\
\hline $74-97-5$ & Bromochloromethane & 10 & $\mathrm{U}$ & 10 & 5.4 \\
\hline $109-99-9$ & Tetrahydrofuran & 100 & $\mathrm{U}$ & 100 & 51 \\
\hline $67-66-3$ & Chloroform & 10 & $\mathrm{U}$ & 10 & 3.4 \\
\hline $71-55-6$ & 1,1,1-Trichloroethane & 10 & $\mathrm{U}$ & 10 & 3.7 \\
\hline $110-82-7$ & Cyclohexane & 10 & $\mathrm{U}$ & 10 & 3.6 \\
\hline $563-58-6$ & 1,1-Dichloropropene & 10 & $\mathrm{U}$ & 10 & 2.2 \\
\hline $56-23-5$ & Carbon tetrachloride & 10 & $\mathrm{~J}$ & 10 & 3.3 \\
\hline $78-83-1$ & Isobutyl alcohol & 510 & $\mathrm{U}$ & 510 & 250 \\
\hline $71-43-2$ & Benzene & 10 & $\mathrm{U}$ & 10 & 3.2 \\
\hline $107-06-2$ & 1,2-Dichloroethane & 10 & $\mathrm{U}$ & 10 & 3.4 \\
\hline $79-01-6$ & Trichloroethene & 10 & $\mathrm{U}$ & 10 & 5.1 \\
\hline $108-87-2$ & Methylcyclohexane & 10 & $\mathrm{U}$ & 10 & 5.1 \\
\hline $78-87-5$ & 1,2-Dichloropropane & 10 & $\mathrm{U}$ & 10 & 3.9 \\
\hline $74-95-3$ & Dibromomethane & 10 & $\mathrm{U}$ & 10 & 2.5 \\
\hline $123-91-1$ & 1,4-Dioxane & 510 & $\mathrm{O}$ & 510 & 270 \\
\hline $75-27-4$ & Bromodichloromethane & 10 & $\mathrm{U}$ & 10 & 3.8 \\
\hline
\end{tabular}


FORM I

GC/MS VOA ORGANICS ANALYSIS DATA SHEET

Lab Name: TestAmerica Burlington

SDG NO.: INMAN (200-2608)

Client Sample ID: IN-S-30795

Matrix: Solid

Analysis Method: $8260 \mathrm{~B}$

Sample wt/vol: $9.83(\mathrm{~g})$

Soil Aliquot Vol: 0.5 (mL)

Soil Extract Vol.: $10(\mathrm{~mL})$

응 Moisture:

Analysis Batch No.: 10229
Job No.: 200-2608-1

Lab Sample ID: 200-2608-3

Lab File ID: lfme10.d

Date Collected: 11/18/2010 00:00

Date Analyzed: 11/24/2010 13:06

Dilution Factor: 1

GC Column: DB-624

ID: $0.53(\mathrm{~mm})$

Level: (low/med) Medium

Units: $\mathrm{ug} / \mathrm{Kg}$

\begin{tabular}{|c|c|c|c|c|c|}
\hline CAS NO. & COMPOUND NAME & RESULT & $Q$ & RI & MDL \\
\hline $110-75-8$ & 2-Chloroethyl vinyl ether & 10 & $\mathrm{U}$ & 10 & 1.3 \\
\hline $10061-01-5$ & cis-1,3-Dichloropropene & 10 & $\mathrm{U}$ & 10 & 3.3 \\
\hline $108-10-1$ & 4-Methyl-2-pentanone & 10 & $\mathrm{U}$ & 10 & 2.1 \\
\hline $108-88-3$ & Toluene & 10 & $\mathrm{U}$ & 10 & 5.1 \\
\hline $10061-02-6$ & trans-1,3-Dichloropropene & 10 & $\mathrm{U}$ & 10 & 5.1 \\
\hline $79-00-5$ & 1,1,2-Trichloroethane & 10 & $\mathrm{U}$ & 10 & 4.3 \\
\hline $127-18-4$ & Tetrachloroethene & 10 & $\mathrm{U}$ & 10 & 5.1 \\
\hline $142-28-9$ & 1,3-Dichloropropane & 10 & $\mathrm{U}$ & 10 & 3.4 \\
\hline $591-78-6$ & 2-Hexanone & 10 & $\mathrm{U}$ & 10 & 10 \\
\hline $124-48-1$ & Dibromochloromethane & 10 & $\mathrm{U}$ & 10 & 3.8 \\
\hline $106-93-4$ & 1,2-Dibromoethane & 10 & $\mathrm{U}$ & 10 & 5.1 \\
\hline $108-90-7$ & Chlorobenzene & 10 & $\mathrm{U}$ & 10 & 2.3 \\
\hline $630-20-6$ & $1,1,1,2$-Tetrachloroethane & 10 & $\mathrm{U}$ & 10 & 3.7 \\
\hline $100-41-4$ & Ethylbenzene & 10 & $\mathrm{U}$ & 10 & 5.1 \\
\hline $179601-23-1$ & $m \& p-X y l e n e$ & 10 & $\mathrm{U}$ & 10 & 5.1 \\
\hline $95-47-6$ & o-Xylene & 10 & $\mathrm{U}$ & 10 & 5.1 \\
\hline $100-42-5$ & Styrene & 10 & $\mathrm{U}$ & 10 & 5.1 \\
\hline $75-25-2$ & Bromoform & 10 & $\mathrm{U}$ & 10 & 4.0 \\
\hline $98-82-8$ & Isopropylbenzene & 10 & $\mathrm{U}$ & 10 & 5.1 \\
\hline $108-86-1$ & Bromobenzene & 10 & $\mathrm{U}$ & 10 & 3.8 \\
\hline $79-34-5$ & $1,1,2,2$-Tetrachloroethane & 10 & $\mathrm{U}$ & 10 & 3.8 \\
\hline $96-18-4$ & 1,2,3-Trichloropropane & 10 & $\mathrm{U}$ & 10 & 5.5 \\
\hline $103-65-1$ & n-Propylbenzene & 10 & $\mathrm{U}$ & 10 & 5.1 \\
\hline $95-49-8$ & 2-Chlorotoluene & 10 & $\mathrm{U}$ & 10 & 3.4 \\
\hline $106-43-4$ & 4-Chlorotoluene & 10 & $\mathrm{U}$ & 10 & 3.5 \\
\hline $108-67-8$ & 1,3,5-Trimethylbenzene & 10 & $\mathrm{U}$ & 10 & 3.3 \\
\hline $98-06-6$ & tert-Butylbenzene & 10 & $\mathrm{U}$ & 10 & 5.1 \\
\hline $95-63-6$ & 1,2,4-Trimethylbenzene & 10 & $\mathrm{U}$ & 10 & 3.5 \\
\hline $135-98-8$ & sec-Butylbenzene & 10 & $\mathrm{U}$ & 10 & 5.1 \\
\hline $541-73-1$ & 1,3-Dichlorobenzene & 10 & $\mathrm{U}$ & 10 & 2.5 \\
\hline $99-87-6$ & 4-Isopropyltoluene & 10 & $\mathrm{U}$ & 10 & 1.8 \\
\hline $106-46-7$ & 1,4-Dichlorobenzene & 10 & $\mathrm{U}$ & 10 & 2.3 \\
\hline $95-50-1$ & 1,2-Dichlorobenzene & 10 & $\mathrm{U}$ & 10 & 2.8 \\
\hline $104-51-8$ & n-Butylbenzene & 10 & $\mathrm{U}$ & 10 & 5.1 \\
\hline $96-12-8$ & 1,2-Dibromo-3-Chloropropane & 10 & $\mathrm{U}$ & 10 & 4.7 \\
\hline $120-82-1$ & 1,2,4-Trichlorobenzene & 10 & $\mathrm{U}$ & 10 & 1.6 \\
\hline
\end{tabular}


FORM I

GC/MS VOA ORGANICS ANALYSIS DATA SHEET

Lab Name: TestAmerica Burlington

SDG NO.: INMAN (200-2608)

Client Sample ID: IN-S-30795

Matrix: Solid

Analysis Method: $8260 \mathrm{~B}$

Sample wt/vol: $9.83(\mathrm{~g})$

Soil Aliquot Vol: 0.5 (mL)

Soil Extract Vol.: $10(\mathrm{~mL})$

\% Moisture:

Analysis Batch No.: 10229
Job No.: 200-2608-1

Lab Sample ID: 200-2608-3

Lab File ID: lfme10.d

Date Collected: 11/18/2010 00:00

Date Analyzed: 11/24/2010 13:06

Dilution Factor: 1

GC Column: DB-624

ID: $0.53(\mathrm{~mm})$

Level: (low/med) Medium

Units: $\mathrm{ug} / \mathrm{Kg}$

\begin{tabular}{|l|l|r|r|r|r|}
\hline \multicolumn{1}{|c|}{ CAS NO. } & \multicolumn{1}{|c|}{ COMPOUND NAME } & RESULT & Q & RL & MDL \\
\hline \hline $87-68-3$ & Hexachlorobutadiene & 10 & U & 10 & 10 \\
\hline $91-20-3$ & Naphthalene & 10 & U & 5.1 \\
\hline $87-61-6$ & $1,2,3-$ Trichlorobenzene & 10 & U & 10 & 10 \\
\hline
\end{tabular}

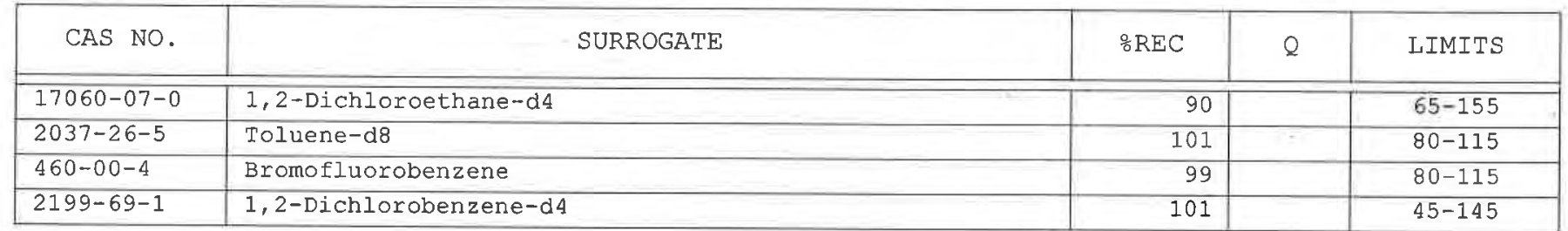


FORM I

GC/MS VOA ORGANICS ANALYSIS DATA SHEET

Lab Name: TestAmerica Burlington

SDG NO.: INMAN (200-2608)

Client Sample ID: IN-S-30771

Matrix: Solid

Analysis Method: $8260 \mathrm{~B}$

Sample wt/vol: $10.562(\mathrm{~g})$

Soil Aliquot Vol: 0.5 (mL)

Soil Extract Vol.: $10(\mathrm{~mL})$

응 Moisture:

Analysis Batch No.: 10229
Job No.: 200-2608-1

Lab Sample ID: 200-2608-4

Lab File ID: lfme11.d

Date Collected: 11/18/2010 00:00

Date Analyzed: 11/24/2010 13:39

Dilution Factor: 1

GC Column: DB-624

ID: $0.53(\mathrm{~mm})$

Level: (low/med) Medium

Units: ug/Kg

\begin{tabular}{|c|c|c|c|c|c|}
\hline CAS NO. & COMPOUND NAME & RESULT & Q & RL & MDL \\
\hline $75-71-8$ & Dichlorodifluoromethane & 9.5 & $\mathrm{U}$ & 9.5 & 4.7 \\
\hline $74-87-3$ & Chlorome thane & 9.5 & $\mathrm{U}$ & 9.5 & 3.5 \\
\hline $75-01-4$ & Vinyl. chloride & 9.5 & U & 9.5 & 4.7 \\
\hline $74-83-9$ & Bromomethane & 3.6 & $\mathrm{~J} \mathrm{~B}$ & 9.5 & 3.3 \\
\hline $75-00-3$ & Chloroethane & 9.5 & $\mathrm{U} \star$ & 9.5 & 5.7 \\
\hline $75-69-4$ & Trichlorofluoromethane & 9.5 & $\mathrm{U} *$ & 9.5 & 4.7 \\
\hline $75-35-4$ & 1,1-Dichloroethene & 9.5 & $\mathrm{U}$ & 9.5 & 2.0 \\
\hline $76-13-1$ & Ereon TF & 9.5 & $\mathrm{U}$ & 9.5 & 2.7 \\
\hline $67-64-1$ & Acetone & 9.5 & $\mathrm{U}$ & 9.5 & 9.5 \\
\hline $74-88-4$ & Methyl iodide & 9.5 & $U \star$ & 9.5 & 4.7 \\
\hline $75-15-0$ & Carbon disulfide & 9.5 & $\mathrm{U}$ & 9.5 & 1.8 \\
\hline $79-20-9$ & Methyl acetate & 9.5 & $\mathrm{U}$ & 9.5 & 4.7 \\
\hline $75-09-2$ & Methylene Chloride & 9.5 & $\mathrm{U}$ & 9.5 & 4.7 \\
\hline $156-60-5$ & trans-1,2-Dichloroethene & 9.5 & $\mathrm{U}$ & 9.5 & 4.7 \\
\hline $1634-04-4$ & Methyl t-butyl ether & 9.5 & $\mathrm{U}$ & 9.5 & 4.7 \\
\hline $75-34-3$ & 1,1-Dichloroethane & 9.5 & $\mathrm{U}$ & 9.5 & 3.4 \\
\hline $108-05-4$ & Vinyl acetate & 9.5 & $\mathrm{U}$ & 9.5 & 4.7 \\
\hline $594-20-7$ & 2,2-Dichloropropane & 9.5 & $\mathrm{U}$ & 9.5 & 4.4 \\
\hline $156-59-2$ & cis-1,2-Dichloroethene & 9.5 & $\mathrm{U}$ & 9.5 & 2.0 \\
\hline $78-93-3$ & 2-Butanone & 26 & * & 9.5 & 10 \\
\hline $74-97-5$ & Bromochloromethane & 9.5 & $\mathrm{U}$ & 9.5 & 5.0 \\
\hline $109-99-9$ & Tetrahydrofuran & 95 & $\mathrm{U}$ & 95 & 47 \\
\hline $67-66-3$ & Chloroform & 9.5 & $\mathrm{U}$ & 9.5 & 3.1 \\
\hline $71-55-6$ & 1,1,1-Trichloroethane & 9.5 & $\mathrm{U}$ & 9.5 & 3.4 \\
\hline $110-82-7$ & Cyclohexane & 9.5 & $\mathrm{U}$ & 9.5 & 3.3 \\
\hline $563-58-6$ & 1,1-Dichloropropene & 9.5 & $\mathrm{U}$ & 9.5 & 2.1 \\
\hline $56-23-5$ & Carbon tetrachloride & 9.5 & $\mathrm{U}$ & 9.5 & 3.0 \\
\hline $78-83-1$ & Isobutyl alcohol & 470 & $\mathrm{U}$ & 470 & 240 \\
\hline $71-43-2$ & Benzene & 9.5 & $\mathrm{U}$ & 9.5 & 2.9 \\
\hline $107-06-2$ & 1,2-Dichloroethane & 9.5 & $\mathrm{U}$ & 9.5 & 3.1 \\
\hline $79-01-6$ & Trichloroethene & 9.5 & U & 9.5 & 4.7 \\
\hline $108-87-2$ & Methylcyclohexane & 9.5 & $\mathrm{U}$ & 9.5 & 4.7 \\
\hline $78-87-5$ & 1,2-Dichloropropane & 9.5 & $\mathrm{U}$ & 9.5 & 3.6 \\
\hline $74-95-3$ & Dibromomethane & 9.5 & $\mathrm{U}$ & 9.5 & 2.4 \\
\hline $123-91-1$ & 1,4-Dioxane & 470 & $\mathrm{U}$ & 470 & 260 \\
\hline $75-27-4$ & Bromodichloromethane & 9.5 & $\mathrm{U}$ & 9.5 & 3.5 \\
\hline
\end{tabular}


FORM I

GC/MS VOA ORGANICS ANALYSIS DATA SHEET

Lab Name: TestAmerica Burlington

SDG No.: INMAN (200-2608)

Client Sample ID: IN-S-30771

Matrix: Solid

Analysis Method: $8260 \mathrm{~B}$

Sample wt/vol: $10.562(\mathrm{~g})$

Soil Aliquot Vol: 0.5 (mL)

Soil Extract Vol.: $10(\mathrm{~mL})$

응 Moisture:

Analysis Batch No.: 10229
Job No.: 200-2608-1

Lab Sample ID: 200-2608-4

Lab File ID: lfme11.d

Date Collected: 11/18/2010 00:00

Date Analyzed: 11/24/2010 13:39

Dilution Factor: 1

GC Column: DB-624

ID: $0.53(\mathrm{~mm})$

Level: (low/med) Medium

Units: ug/Kg

\begin{tabular}{|c|c|c|c|c|c|}
\hline CAS NO. & COMPOUND NAME & RESULT & $\mathrm{Q}$ & $\mathrm{RL}$ & MDL \\
\hline $110-75-8$ & 2-Chloroethyl vinyl ether & 9.5 & $\mathrm{U}$ & 9.5 & 1.2 \\
\hline $10061-01-5$ & cis-1,3-Dichloropropene & 9.5 & $\mathrm{U}$ & 9.5 & 3.0 \\
\hline $108-10-1$ & 4-Methyl-2-pentanone & 9.5 & $\mathrm{U}$ & 9.5 & 2.0 \\
\hline $108-88-3$ & Toluene & 9.5 & $\mathrm{U}$ & 9.5 & 4.7 \\
\hline $10061-02-6$ & trans-1,3-Dichloropropene & 9.5 & $\mathrm{U}$ & 9.5 & 4.7 \\
\hline $79-00-5$ & 1,1,2-Trichloroethane & 9.5 & $\mathrm{U}$ & 9.5 & 4.0 \\
\hline $127-18-4$ & Tetrachloroethene & 9.5 & $\mathrm{U}$ & 9.5 & 4.7 \\
\hline $142-28-9$ & 1,3-Dichloropropane & 9.5 & $\mathrm{U}$ & 9.5 & 3.1 \\
\hline $591-78-6$ & 2-Hexanone & 9.5 & $\mathrm{U}$ & 9.5 & 9.5 \\
\hline $124-48-1$ & Dibromochloromethane & 9.5 & $\mathrm{U}$ & 9.5 & 3.5 \\
\hline $106-93-4$ & 1,2-Dibromoethane & 9.5 & $\mathrm{U}$ & 9.5 & 4.7 \\
\hline $108-90-7$ & Chlorobenzene & 9.5 & $\mathrm{U}$ & 9.5 & 2.2 \\
\hline $630-20-6$ & $1,1,1,2$-Tetrachloroethane & 9.5 & $\mathrm{U}$ & 9.5 & 3.4 \\
\hline $100-41-4$ & Ethylbenzene & 9.5 & $\mathrm{U}$ & 9.5 & 4.7 \\
\hline $179601-23-1$ & m\&p-xylene & 9.5 & $\mathrm{U}$ & 9.5 & 4.7 \\
\hline $95-47-6$ & o-Xylene & 9.5 & $\mathrm{U}$ & 9.5 & 4.7 \\
\hline $100-42-5$ & Styrene & 9.5 & $\mathrm{U}$ & 9.5 & 4.7 \\
\hline $75-25-2$ & Bromoform & 9.5 & $\mathrm{U}$ & 9.5 & 3.7 \\
\hline $98-82-8$ & Isopropylbenzene & 9.5 & $\mathrm{U}$ & 9.5 & 4.7 \\
\hline $108-86-1$ & Bromobenzene & 9.5 & $\mathrm{U}$ & 9.5 & 3.5 \\
\hline $79-34-5$ & $1,1,2,2$-Tetrachloroethane & 9.5 & $\mathrm{U}$ & 9.5 & 3.5 \\
\hline $96-18-4$ & 1,2,3-Trichloropropane & 9.5 & $\mathrm{U}$ & 9.5 & 5.1 \\
\hline $103-65-1$ & n-Propylbenzene & 9.5 & $\mathrm{U}$ & 9.5 & 4.7 \\
\hline $95-49-8$ & 2-Chlorotoluene & 9.5 & $\mathrm{U}$ & 9.5 & 3.1 \\
\hline $106-43-4$ & 4-Chlorotoluene & 9.5 & $\mathrm{U}$ & 9.5 & 3.2 \\
\hline $108-67-8$ & 1,3,5-Trimethylbenzene & 9.5 & $\mathrm{U}$ & 9.5 & 3.0 \\
\hline $98-06-6$ & tert-Butylbenzene & 9.5 & $\mathrm{U}$ & 9.5 & 4.7 \\
\hline $95-63-6$ & 1,2,4-Trimethylbenzene & 9.5 & $\mathrm{U}$ & 9.5 & 3.2 \\
\hline $135-98-8$ & sec-Butylbenzene & 9.5 & $\mathrm{U}$ & 9.5 & 4.7 \\
\hline $541-73-1$ & 1,3-Dichlorobenzene & 9.5 & $\mathrm{U}$ & 9.5 & 2.4 \\
\hline $99-87-6$ & 4-Isopropyltoluene & 9.5 & $\mathrm{U}$ & 9.5 & 1.7 \\
\hline $106-46-7$ & 1,4-Dichlorobenzene & 9.5 & $\mathrm{U}$ & 9.5 & 2.2 \\
\hline $95-50-1$ & 1,2-Dichlorobenzene & 9.5 & $\mathrm{U}$ & 9.5 & 2.7 \\
\hline $104-51-8$ & n-Butylbenzene & 9.5 & $\mathrm{U}$ & 9.5 & 4.7 \\
\hline $96-12-8$ & 1,2-Dibromo-3-Chloropropane & 9.5 & $\mathrm{U}$ & 9.5 & 4.4 \\
\hline $120-82-1$ & 1,2,4-Trichlorobenzene & 9.5 & $\mathrm{U}$ & 9.5 & 1.5 \\
\hline
\end{tabular}


FORM I

GC/MS VOA ORGANICS ANALYSIS DATA SHEET

Lab Name: TestAmerica Burlington

SDG NO.: INMAN (200-2608)

Client Sample ID: IN-S-3077I

Matrix: Solid

Analysis Method: $8260 \mathrm{~B}$

Sample wt/vol: $10.562(\mathrm{~g})$

Soil Aliquot Vol: 0.5 (mL)

Soil Extract Vol.: $10(\mathrm{~mL})$

o Moisture:

Analysis Batch No.: 10229
Job No.: 200-2608-1

Lab Sample ID: 200-2608-4

Lab File ID: lfmell.d

Date Collected: 11/18/2010 00:00

Date Analyzed: 11/24/2010 13:39

Dilution Factor: 1

GC Column: $\mathrm{DB}-624 \quad$ ID: $0.53(\mathrm{~mm})$

Level: (low/med) Medium

Units: $u g / \mathrm{kg}$

\begin{tabular}{|l|l|r|r|r|r|}
\hline \multicolumn{1}{|c|}{ CAS NO. } & \multicolumn{1}{|c|}{ COMPOUND NAME } & RESULT & Q & RI & MDL \\
\hline \hline $87-68-3$ & Hexachlorobutadiene & 9.5 & U & 9.5 & 4.7 \\
\hline $91-20-3$ & Naphthalene & 9.5 & U & 9.5 & 9.7 \\
\hline $87-61-6$ & $1,2,3-T r i c h l o r o b e n z e n e$ & 9.5 & U & & 9.5 \\
\hline
\end{tabular}

\begin{tabular}{|l|l|r|r|r|}
\hline \multicolumn{1}{|c|}{ CAS NO. } & \multicolumn{1}{|c|}{ SURROGATE } & REC & Q & \multicolumn{1}{|c|}{ LIMITS } \\
\hline \hline $17060-07-0$ & 1,2-Dichloroethane-d4 & 92 & \\
\hline $2037-26-5$ & Toluene-d8 & 101 & $65-155$ \\
\hline $460-00-4$ & Bromofluorobenzene & 98 & $80-115$ \\
\hline $2199-69-1$ & $1,2-$ Dichlorobenzene-d4 & 101 & $80-115$ \\
\hline
\end{tabular}


FORM I

GC/MS VOA ORGANICS ANALYSIS DATA SHEET

Lab Name: TestAmerica Burlington

SDG No.: INMAN (200-2608)

Client Sample ID: IN-S-30808

Matrix: Solid

Analysis Method: $8260 \mathrm{~B}$

Sample wt/vol: $10.539(\mathrm{~g})$

Soil Aliquot Vol: 0.5 (m)

Soil Extract Vol.: $10(\mathrm{~mL})$

\% Moisture:

Analysis Batch No.: 10229
Job No.: 200-2608-1

Lab Sample ID: 200-2608-5

Lab File ID: lfme12.d

Date Collected: $\quad 11 / 18 / 2010 \quad 00: 00$

Date Analyzed: 11/24/2010 14:11

Dilution Factor: 1

GC Column: DB-624

ID: $0.53(\mathrm{~mm})$

Level: (low/med) Medium

Units: ug/ $\mathrm{kg}$

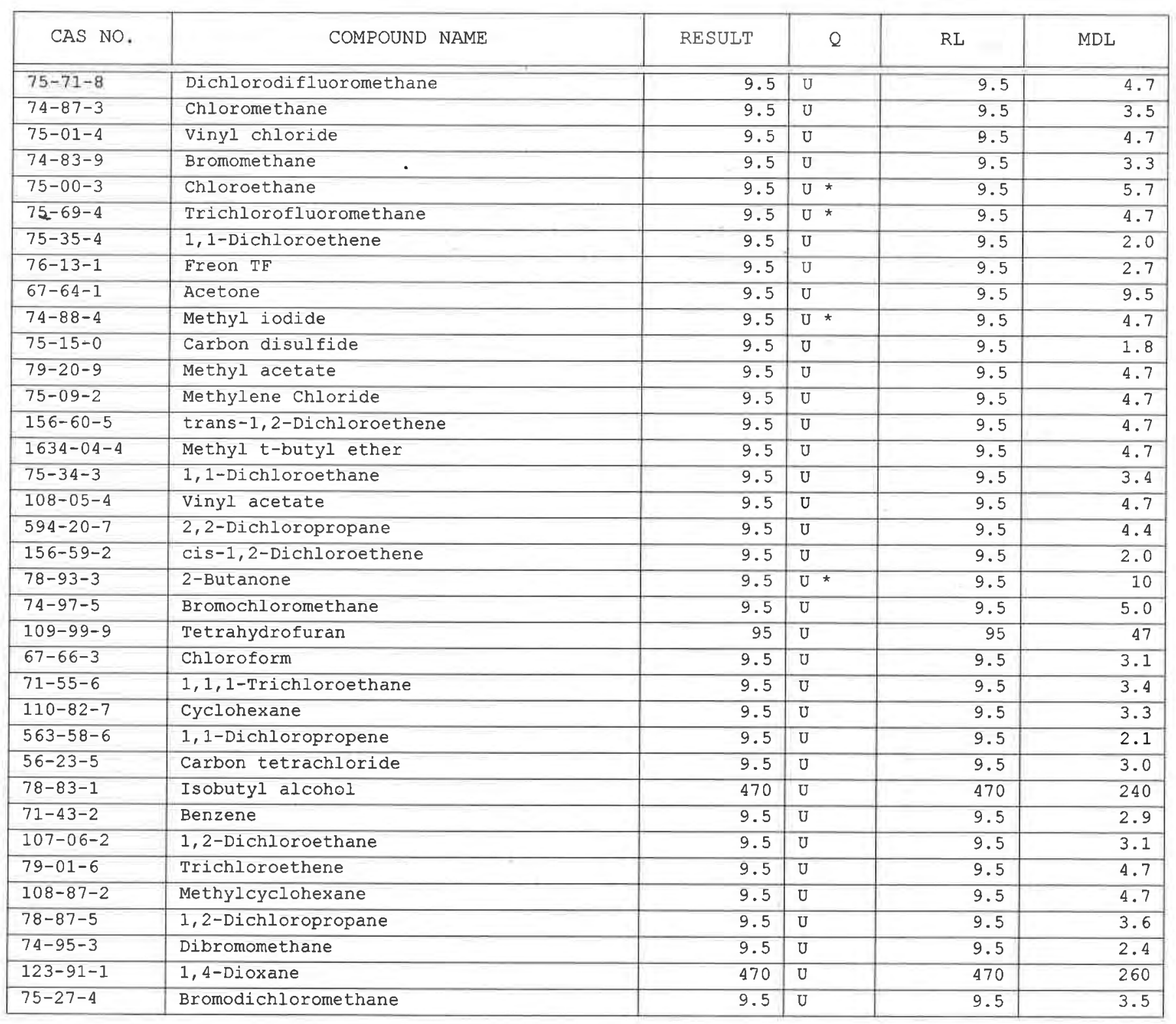


FORM I

GC/MS VOA ORGANICS ANALYSIS DATA SHEET

Lab Name: TestAmerica Burlington

SDG NO.: INMAN (200-2608)

Client Sample ID: IN-S-30808

Matrix: Solid

Analysis Method: $8260 \mathrm{~B}$

Sample wt/vol: $10.539(\mathrm{~g})$

Soil Aliquot Vol: 0.5 (mL)

Soil Extract Vol.: $10(\mathrm{~mL})$

음 Moisture:

Analysis Batch No.: 10229
Job No.: 200-2608-1

Lab Sample ID: 200-2608-5

Lab File ID: lfme12.d

Date Collected: 11/18/2010 00:00

Date Analyzed: 11/24/2010 14:11

Dilution Factor: 1

GC Column: DB-624

ID : $0.53(\mathrm{~mm})$

Level: (low/med) Medium

Units: $\mathrm{ug} / \mathrm{Kg}$

\begin{tabular}{|c|c|c|c|c|c|}
\hline CAS NO. & COMPOUND NAME & RESULT & $Q$ & RL & MDL \\
\hline $110-75-8$ & 2-Chloroethyl vinyl ether & 9.5 & U & 9.5 & 1.2 \\
\hline $10061-01-5$ & cis-1,3-Dichloropropene & 9.5 & $\mathrm{U}$ & 9.5 & 3.0 \\
\hline $108-10-1$ & 4-Methyl-2-pentanone & 9.5 & $\mathrm{U}$ & 9.5 & 2.0 \\
\hline $108-88-3$ & Toluene & 9.5 & $\mathrm{U}$ & 9.5 & 4.7 \\
\hline $10061-02-6$ & trans-1,3-Dichloropropene & 9.5 & $\mathrm{U}$ & 9.5 & 4.7 \\
\hline $79-00-5$ & $1,1,2$-Trichloroethane & 9.5 & $\mathrm{U}$ & 9.5 & 4.0 \\
\hline $127-18-4$ & Tetrachloroethene & 9.5 & $\mathrm{U}$ & 9.5 & 4.7 \\
\hline $142-28-9$ & 1,3-Dichloropropane & 9.5 & $\mathrm{U}$ & 9.5 & 3.1 \\
\hline $591-78-6$ & 2-Hexanone & 9.5 & $\mathrm{U}$ & 9.5 & 9.5 \\
\hline $124-48-1$ & Dibromochlorome thane & 9.5 & $\mathrm{U}$ & 9.5 & 3.5 \\
\hline $106-93-4$ & 1,2-Dibromoethane & 9.5 & $\mathrm{U}$ & 9.5 & 4.7 \\
\hline $108-90-7$ & Chlorobenzene & 9.5 & $\mathrm{U}$ & 9.5 & 2.2 \\
\hline $630-20-6$ & $1,1,1,2$-Tetrachloroethane & 9.5 & $\mathrm{U}$ & 9.5 & 3.4 \\
\hline $100-41-4$ & Ethylbenzene & 9.5 & $\mathrm{U}$ & 9.5 & 4.7 \\
\hline $179601-23-1$ & $\mathrm{m \& p}-\mathrm{xylene}$ & 9.5 & $\mathrm{U}$ & 9.5 & 4.7 \\
\hline $95-47-6$ & o-Xylene & 9.5 & $\mathrm{U}$ & 9.5 & 4.7 \\
\hline $100-42-5$ & Styrene & 9.5 & $\mathrm{U}$ & 9.5 & 4.7 \\
\hline $75-25-2$ & Bromoform & 9.5 & $\mathrm{U}$ & 9.5 & 3.7 \\
\hline $98-82-8$ & Isopropylbenzene & 9.5 & $\mathrm{U}$ & 9.5 & 4.7 \\
\hline $108-86-1$ & Bromobenzene & 9.5 & $\mathrm{U}$ & 9.5 & 3.5 \\
\hline $79-34-5$ & $1,1,2,2$-Tetrachloroethane & 9.5 & $\mathrm{U}$ & 9.5 & 3.5 \\
\hline $96-18-4$ & 1,2,3-Trichloropropane & 9.5 & $\mathrm{U}$ & 9.5 & 5.1 \\
\hline $103-65-1$ & n-Propylbenzene & 9.5 & $\mathrm{U}$ & 9.5 & 4.7 \\
\hline $95-49-8$ & 2-Chlorotoluene & 9.5 & $\mathrm{U}$ & 9.5 & 3.1 \\
\hline $106-43-4$ & 4-Chlorotoluene & 9.5 & $\mathrm{U}$ & 9.5 & 3.2 \\
\hline $108-67-8$ & 1,3,5-Trimethylbenzene & 9.5 & $\mathrm{U}$ & 9.5 & 3.0 \\
\hline $98-06-6$ & tert-Butylbenzene & 9.5 & $\mathrm{U}$ & 9.5 & 4.7 \\
\hline $95-63-6$ & $1,2,4$-Trimethylbenzene & 9.5 & $\mathrm{U}$ & 9.5 & 3.2 \\
\hline $135-98-8$ & sec-Butylbenzene & 9.5 & $\mathrm{U}$ & 9.5 & 4.7 \\
\hline $541-73-1$ & 1,3-Dichlorobenzene & 9.5 & $\mathrm{~J}$ & 9.5 & 2.4 \\
\hline $99-87-6$ & 4-Isopropyltoluene & 9.5 & $\mathrm{U}$ & 9.5 & 1.7 \\
\hline $106-46-7$ & 1,4-Dichlorobenzene & 9.5 & $\mathrm{U}$ & 9.5 & 2.2 \\
\hline $95-50-1$ & 1,2-Dichlorobenzene & 9.5 & $\mathrm{U}$ & 9.5 & 2.7 \\
\hline $104-51-8$ & n-Butylbenzene & 9.5 & $\mathrm{U}$ & 9.5 & 4.7 \\
\hline $96-12-8$ & 1,2-Dibromo-3-Chloropropane & 9.5 & $\mathrm{U}$ & 9.5 & 4.4 \\
\hline $120-82-1$ & 1,2,4-Trichlorobenzene & 9.5 & $\mathrm{U}$ & 9.5 & 1.5 \\
\hline
\end{tabular}

FORM I $8260 \mathrm{~B}$ 
FORM I

GC/MS VOA ORGANICS ANALYSIS DATA SHEET

Lab Name: TestAmerica Burlington

SDG NO.: INMAN (200-2608)

Client Sample ID: IN-S-30808

Matrix: Solid

Analysis Method: $8260 \mathrm{~B}$

Sample wt/vol: $10.539(\mathrm{~g})$

Soil Aliquot Vol: 0.5 (m山)

Soil Extract Vol.: 10 (mL)

\% Moisture:

Analysis Batch No.: 10229
Job No.: 200-2608-1

Lab Sample ID: 200-2608-5

Lab File ID: lfmel2.d

Date Collected: 11/18/2010 00:00

Date Analyzed: 11/24/2010 14:11

Dilution Factor: 1

GC Column: DB-624

ID: $0.53(\mathrm{~mm})$

Level: (low/med) Mediurn

Units: $u g / \mathrm{Kg}$

\begin{tabular}{|l|l|r|r|r|r|}
\hline \multicolumn{1}{|c|}{ CAS NO. } & \multicolumn{1}{|c|}{ COMPOUND NAME } & RESULT & Q & RL & MDL \\
\hline \hline $87-68-3$ & Hexachlorobutadiene & 9.5 & U & 9.5 & 9.7 \\
\hline $91-20-3$ & Naphthalene & 9.5 & U & 9.5 & 9.5 \\
\hline $87-61-6$ & $1,2,3-$ Trichlorobenzene & 9.5 & U & & 9.5 \\
\hline
\end{tabular}

\begin{tabular}{|c|c|c|c|c|}
\hline CAS NO. & SURROGATE & $\frac{\circ}{2} \mathrm{REC}$ & $Q$ & IIMITS \\
\hline $17060-07-0$ & 1,2-Dichloroethane-d4 & 93 & & $65-155$ \\
\hline $2037-26-5$ & Toluene-d8 & 104 & & $80-115$ \\
\hline $460-00-4$ & Bromofluorobenzene & 101 & & $80-115$ \\
\hline $2199-69-1$ & 1,2-Dichlorobenzene-d4 & 103 & & $45-145$ \\
\hline
\end{tabular}


FORM I

GC/MS VOA ORGANICS ANALYSIS DATA SHEET

Lab Name: TestAmerica Burlington

SDG NO.: INMAN (200-2608)

Client Sample ID: IN-S-30814

Matrix: Solid

Analysis Method: 8260B

Sample wt/vol: $10.245(\mathrm{~g})$

Soil Aliquot Vol: 0.5 (mL)

Soil Extract Vol.: $10(\mathrm{~mL})$

응 Moisture:

Analysis Batch No.: 10229
Job No.: 200-2608-1

Lab Sample ID: 200-2608-6

Lab File ID: lfme13.d

Date Collected: 11/18/2010 00:00

Date Analyzed: 11/24/2010 14:43

Dilution Factor: 1

GC Column: DB-624

ID $: 0.53(\mathrm{~mm})$

Level: (low/med) Medium

Units: ug/Kg

\begin{tabular}{|c|c|c|c|c|c|}
\hline CAS NO. & COMPOUND NAME & RESULT & $Q$ & RL & MDI \\
\hline $75-71-8$ & Dichlorodifluoromethane & 9.8 & $\mathrm{U}$ & 9.8 & 4.9 \\
\hline $74-87-3$ & Chlorome thane & 9.8 & $U$ & 9.8 & 3.6 \\
\hline $75-01-4$ & Vinyl chloride & 9.8 & $\mathrm{U}$ & 9.8 & 4.9 \\
\hline $74-83-9$ & Bromomethane & 9.8 & U & 9.8 & 3.4 \\
\hline $75-00-3$ & Chloroethane & 9.8 & $U *$ & 9.8 & 5.9 \\
\hline $75-69-4$ & Trichlorofluoromethane & 9.8 & $\mathrm{U} \star$ & 9.8 & 4.9 \\
\hline $75-35-4$ & 1,1-Dichloroethene & 9.8 & $\mathrm{U}$ & 9.8 & 2.0 \\
\hline $76-13-1$ & Freon TF & 9.8 & $\mathrm{U}$ & 9.8 & 2.7 \\
\hline $67-64-1$ & Acetone & 9.8 & $\mathrm{U}$ & 9.8 & 9.8 \\
\hline $74-88-4$ & Methyl iodide & 9.8 & $\mathrm{U} *$ & 9.8 & 4.9 \\
\hline $75-15-0$ & Carbon disulfide & 9.8 & $\mathrm{U}$ & 9.8 & 1.9 \\
\hline $79-20-9$ & Methyl acetate & 9.8 & $\mathrm{U}$ & 9.8 & 4.9 \\
\hline $75-09-2$ & Methylene Chloride & 9.8 & $\mathrm{U}$ & 9.8 & 4.9 \\
\hline $156-60-5$ & trans-1,2-Dichloroethene & 9.8 & $\mathrm{U}$ & 9.8 & 4.9 \\
\hline $1634-04-4$ & Methyl t-butyl ether & 9.8 & $\mathrm{U}$ & 9.8 & 4.9 \\
\hline $75-34-3$ & 1,1-Dichloroethane & 9.8 & $\mathrm{U}$ & 9.8 & 3.5 \\
\hline $108-05-4$ & Vinyl acetate & 9.8 & $\mathrm{U}$ & 9.8 & 4.9 \\
\hline $594-20-7$ & 2,2-Dichloropropane & 9.8 & $\mathrm{U}$ & 9.8 & 4.5 \\
\hline $156-59-2$ & cis-1,2-Dichloroethene & 9.8 & $\mathrm{U}$ & 9.8 & 2.0 \\
\hline $78-93-3$ & 2-Butanone & 9.8 & $\mathrm{U} *$ & 9.8 & 11 \\
\hline $74-97-5$ & Bromochloromethane & 9.8 & $\mathrm{U}$ & 9.8 & 5.2 \\
\hline $109-99-9$ & Tetrahydrofuran & 98 & U & 98 & 49 \\
\hline $67-66-3$ & Chloroform & 9.8 & $\mathrm{U}$ & 9.8 & 3.2 \\
\hline $71-55-6$ & 1,1,1-Trichloroethane & 9.8 & $\mathrm{~J}$ & 9.8 & 3.5 \\
\hline $110-82-7$ & Cyclohexane & 9.8 & $\mathrm{U}$ & 9.8 & 3.4 \\
\hline $563-58-6$ & 1,1-Dichloropropene & 9.8 & $\mathrm{U}$ & 9.8 & 2.1 \\
\hline $56-23-5$ & Carbon tetrachloride & 9.8 & $\mathrm{U}$ & 9.8 & 3.1 \\
\hline $78-83-1$ & Isobutyl alcohol & 490 & U & 490 & 240 \\
\hline $71-43-2$ & Benzene & 9.8 & $\mathrm{U}$ & 9.8 & 3.0 \\
\hline $107-06-2$ & 1,2-Dichloroethane & 9.8 & $\mathrm{U}$ & 9.8 & 3.2 \\
\hline $79-01-6$ & Trichloroethene & 9.8 & $\mathrm{U}$ & 9.8 & 4.9 \\
\hline $108-87-2$ & Methylcyclohexane & 9.8 & $\mathrm{U}$ & 9.8 & 4.9 \\
\hline $78-87-5$ & 1,2-Dichloropropane & 9.8 & $\mathrm{U}$ & 9.8 & 3.7 \\
\hline $74-95-3$ & Dibromome thane & 9.8 & $\mathrm{U}$ & 9.8 & 2.4 \\
\hline $123-91-1$ & 1,4-Dioxane & 490 & $\mathrm{U}$ & 490 & 260 \\
\hline $75-27-4$ & Bromodichloromethane & 9.8 & $\mathrm{U}$ & 9.8 & 3.6 \\
\hline
\end{tabular}


FORM I

GC/MS VOA ORGANICS ANALYSIS DATA SHEET

Lab Name: TestAmerica Burlington

SDG No.: INMAN (200-2608)

Client Sample ID: IN-S-30814

Matrix: Solid

Analysis Method: $8260 \mathrm{~B}$

Sample wt/vol: $10.245(\mathrm{~g})$

Soil Aliquot Vol: 0.5 (mL)

Soil Extract Vol.: $10(\mathrm{~mL})$

\% Moisture:

Analysis Batch No.: 10229
Job No.: 200-2608-1

Lab Sample ID: 200-2608-6

Lab File ID: lfme13.d

Date Collected: 11/18/2010 00:00

Date Analyzed: 11/24/2010 14:43

Dilution Factor: 1

GC Column: DB-624

ID: $0.53(\mathrm{~mm})$

Level: (low/med) Medium

Units: $\mathrm{ug} / \mathrm{Kg}$

\begin{tabular}{|c|c|c|c|c|c|}
\hline CAS NO. & COMPOUND NAME & RESULT & $Q$ & $\mathrm{RL}$ & MDL \\
\hline $110-75-8$ & 2-Chloroethyl vinyl ether & 9.8 & $\mathrm{U}$ & 9.8 & 1.3 \\
\hline $10061-01-5$ & cis-1,3-Dichloropropene & 9.8 & $\mathrm{U}$ & 9.8 & 3.1 \\
\hline $108-10-1$ & 4-Methyl-2-pentanone & 9.8 & $\mathrm{U}$ & 9.8 & 2.0 \\
\hline $108-88-3$ & Toluene & 9.8 & $\mathrm{U}$ & 9.8 & 4.9 \\
\hline $10061-02-6$ & trans-1,3-Dichloropropene & 9.8 & $\mathrm{U}$ & 9.8 & 4.9 \\
\hline $79-00-5$ & 1,1,2-Trichloroethane & 9.8 & $\mathrm{U}$ & 9.8 & 4.1 \\
\hline $127-18-4$ & Tetrachloroethene & 9.8 & $\mathrm{U}$ & 9.8 & 4.9 \\
\hline $142-28-9$ & 1,3-Dichloropropane & 9.8 & $\mathrm{U}$ & 9.8 & 3.2 \\
\hline $591-78-6$ & 2-Hexanone & 9.8 & $\mathrm{U}$ & 9.8 & 9.8 \\
\hline $124-48-1$ & Dibromochloromethane & 9.8 & $\mathrm{U}$ & 9.8 & 3.6 \\
\hline $106-93-4$ & 1,2-Dibromoethane & 9.8 & $\mathrm{U}$ & 9.8 & 4.9 \\
\hline $108-90-7$ & Chlorobenzene & 9.8 & $\mathrm{U}$ & 9.8 & 2.2 \\
\hline $630-20-6$ & $1,1,1,2$-Tetrachloroethane & 9.8 & $\mathrm{U}$ & 9.8 & 3.5 \\
\hline $100-41-4$ & Ethylbenzene & 9.8 & $\mathrm{U}$ & 9.8 & 4.9 \\
\hline $179601-23-1$ & m\&p-xylene & 9.8 & $\mathrm{U}$ & 9.8 & 4.9 \\
\hline $95-47-6$ & o-xylene & 9.8 & U & 9.8 & 4.9 \\
\hline $100-42-5$ & Styrene & 9.8 & $\mathrm{U}$ & 9.8 & 4.9 \\
\hline $75-25-2$ & Bromoform & 9.8 & $\mathrm{U}$ & 9.8 & 3.8 \\
\hline $98-82-8$ & Isopropylbenzene & 9.8 & $\mathrm{U}$ & 9.8 & 4.9 \\
\hline $108-86-1$ & Bromobenzene & 9.8 & 0 & 9.8 & 3.6 \\
\hline $79-34-5$ & $1,1,2,2$-Tetrachloroethane & 9.8 & $\mathrm{U}$ & 9.8 & 3.6 \\
\hline $96-18-4$ & 1,2,3-Trichloropropane & 9.8 & $\mathrm{U}$ & 9.8 & 5.3 \\
\hline $103-65-1$ & n-Propylbenzene & 9.8 & $\mathrm{U}$ & 9.8 & 4.9 \\
\hline $95-49-8$ & 2-Chlorotoluene & 9.8 & $\mathrm{U}$ & 9.8 & 3.2 \\
\hline $106-43-4$ & 4-Chlorotoluene & 9.8 & $\mathrm{U}$ & 9.8 & 3.3 \\
\hline $108-67-8$ & 1,3,5-Trimethylbenzene & 9.8 & $\mathrm{U}$ & 9.8 & 3.1 \\
\hline $98-06-6$ & tert-Butylbenzene & 9.8 & $\mathrm{U}$ & 9.8 & 4.9 \\
\hline $95-63-6$ & 1,2,4-Trimethylbenzene & 9.8 & $\mathrm{U}$ & 9.8 & 3.3 \\
\hline $135-98-8$ & sec-Butylbenzene & 9.8 & $\mathrm{U}$ & 9.8 & 4.9 \\
\hline $541-73-1$ & 1,3-Dichlorobenzene & 9.8 & $\mathrm{U}$ & 9.8 & 2.4 \\
\hline $99-87-6$ & 4-Isopropyltoluene & 9.8 & $\mathrm{U}$ & 9.8 & 1.8 \\
\hline $106-46-7$ & 1,4-Dichlorobenzene & 9.8 & $\mathrm{U}$ & 9.8 & 2.2 \\
\hline $95-50-1$ & 1,2-Dichlorobenzene & 9.8 & $\mathrm{U}$ & 9.8 & 2.7 \\
\hline $104-51-8$ & n-Butylbenzene & 9.8 & $\mathrm{U}$ & 9.8 & 4.9 \\
\hline $96-12-8$ & 1,2-Dibromo-3-Chloropropane & 9.8 & $\mathrm{U}$ & 9.8 & 4.5 \\
\hline $120-82-1$ & 1,2,4-Trichlorobenzene & 9.8 & $\mathrm{U}$ & 9.8 & 1.6 \\
\hline
\end{tabular}


FORM I

GC/MS VOA ORGANICS ANALYSIS DATA SHEET

Lab Name: TestAmerica Burlington

SDG NO.: INMAN (200-2608)

Client Sample ID: IN-S-30814

Matrix: Solid

Analysis Method: $8260 \mathrm{~B}$

Sample wt/vol: $10.245(\mathrm{~g})$

Soil Aliquot Vol: 0.5 (mL)

Soil Extract Vol.: $10(\mathrm{~mL})$

음 Moisture:

Analysis Batch No.: 10229
Job No.: 200-2608-1

Lab Sample ID: 200-2608-6

Lab File ID: lfme13.d

Date Collected: 11/18/2010 00:00

Date Analyzed: 11/24/2010 14:43

Dilution Factor: 1

GC Column: DB-624

ID: $0.53(\mathrm{~mm})$

Level: (low/med) Medium

Units: ug/ $\mathrm{kg}$

\begin{tabular}{|l|l|r|r|r|r|}
\hline \multicolumn{1}{|c|}{ CAS NO. } & \multicolumn{1}{|c|}{ COMPOUND NAME } & RESULT & Q & RL & MDL \\
\hline \hline $87-68-3$ & Hexachlorobutadiene & 9.8 & U & 9.8 & 4.9 \\
\hline $91-20-3$ & Naphthalene & 9.8 & U & 9.8 & 4.9 \\
\hline $87-61-6$ & $1,2,3-$ Trichlorobenzene & 9.8 & U & & 9.8 \\
\hline
\end{tabular}

\begin{tabular}{|c|c|c|c|c|}
\hline CAS NO. & SURROGATE & $\% \mathrm{REC}$ & $Q$ & LIMITS \\
\hline $17060-07-0$ & 1,2-Dichloroethane-d4 & 103 & & $65-155$ \\
\hline $2037-26-5$ & Toluene-d8 & 115 & & $80-115$ \\
\hline $460-00-4$ & Bromofluorobenzene & 113 & & $80-115$ \\
\hline $2199-69-1$ & 1,2-Dichlorobenzene-d4 & 114 & & $45-145$ \\
\hline
\end{tabular}


FORM I

GC/MS VOA ORGANICS ANALYSIS DATA SHEET

Lab Name: TestAmerica Burlington

SDG No.: INMAN (200-2608)

Client Sample ID: IN-S-MeOH BLANK

Matrix: Solid

Analysis Method: $8260 \mathrm{~B}$

Sample wt/vol: $10(\mathrm{~g})$

Soil Aliquot Vol: 0.5 (mL)

Soil Extract Vol.: $10(\mathrm{~mL})$

\% Moisture:

Analysis Batch No.: 10229
Job No.: $\quad 200-2608-1$

Lab Sample ID: $200-2608-7$

Lab File ID: Ifme14.d

Date Collected: 11/18/2010 00:00

Date Analyzed: 11/24/2010 15:15

Dilution Factor: 1

GC Column: DB-624

ID : $0.53(\mathrm{~mm})$

Level: (low/med) Medium

Units: ug/Kg

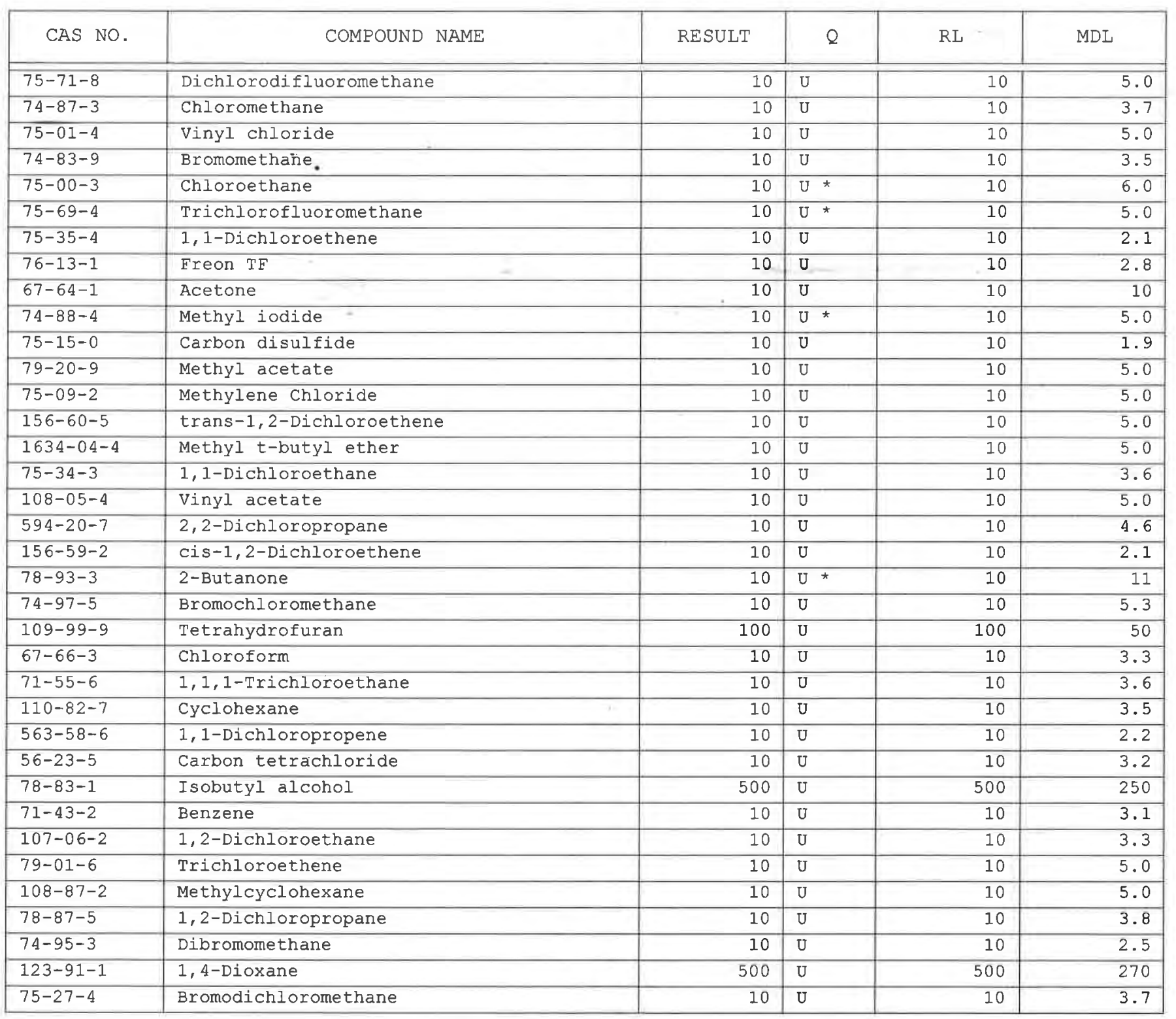


FORM I

GC/MS VOA ORGANICS ANALYSIS DATA SHEET

Lab Name: TestAmerica Burlington

SDG No.: INMAN (200-2608)

Client Sample ID: IN-S-MeOH BLANK

Matrix: Solid

Analysis Method: $8260 \mathrm{~B}$

Sample wt/vol: $10(\mathrm{~g})$

Soil Aliquot Vol: 0.5 (mL)

Soil Extract Vol.: $10(\mathrm{~mL})$

응 Moisture:

Analysis Batch No.: 10229
Job No.: 200-2608-1

Lab Sample ID: 200-2608-7

Lab File ID: lfme14.d

Date Collected: 11/18/2010 00:00

Date Analyzed: 11/24/2010 15:15

Dilution Eactor: 1

GC Column: DB-624

ID: $0.53(\mathrm{~mm})$

Level: (low/med) Medium

Units: ug/Kg

\begin{tabular}{|c|c|c|c|c|c|}
\hline CAS NO. & COMPOUND NAME & RESULT & Q & $\mathrm{RL}$ & MDL \\
\hline $110-75-8$ & 2-Chloroethyl vinyl ether & 10 & $\mathrm{U}$ & 10 & 1.3 \\
\hline $10061-01-5$ & cis-1,3-Dichloropropene & 10 & $\mathrm{U}$ & 10 & 3.2 \\
\hline $108-10-1$ & 4-Methy1-2-pentanone & 10 & $\mathrm{U}$ & 10 & 2.1 \\
\hline $108-88-3$ & Toluene & 10 & $\mathrm{U}$ & 10 & 5.0 \\
\hline $10061-02-6$ & trans-1,3-Dichloropropene & 10 & $\mathrm{U}$ & 10 & 5.0 \\
\hline $79-00-5$ & 1,1,2-Trichloroethane & 10 & $\mathrm{u}$ & 10 & 4.2 \\
\hline $127-18-4$ & Tetrachloroethene & 10 & $\mathrm{u}$ & 10 & 5.0 \\
\hline $142-28-9$ & 1,3-Dichloropropane & 10 & $\mathrm{U}$ & 10 & 3.3 \\
\hline $591-78-6$ & 2-Hexanone & 10 & $\mathrm{U}$ & 10 & 10 \\
\hline $124-48-1$ & Dibromochloromethane & 10 & o & 10 & 3.7 \\
\hline $106-93-4$ & 1,2-Dibromoethane & 10 & $\mathrm{U}$ & 10 & 5.0 \\
\hline $108-90-7$ & Chlorobenzene & 10 & $\mathrm{U}$ & 10 & 2.3 \\
\hline $630-20-6$ & $1,1,1,2$-Tetrachloroethane & 10 & $\mathrm{U}$ & 10 & 3.6 \\
\hline $100-41-4$ & Ethylbenzene & 10 & $\mathrm{U}$ & 10 & 5.0 \\
\hline $179601-23-1$ & $\mathrm{~m} \& \mathrm{p}$-Xylene & 10 & $\mathrm{U}$ & 10 & 5.0 \\
\hline $95-47-6$ & o-Xylene & 10 & $\mathrm{u}$ & 10 & 5.0 \\
\hline $100-42-5$ & Styrene & 10 & $\mathrm{U}$ & 10 & 5.0 \\
\hline $75-25-2$ & Bromoform & 10 & $\mathrm{U}$ & 10 & 3.9 \\
\hline $98-82-8$ & Isopropylbenzene & 10 & $\mathrm{U}$ & 10 & 5.0 \\
\hline $108-86-1$ & Bromobenzene & 10 & $\mathrm{U}$ & 10 & 3.7 \\
\hline $79-34-5$ & $1,1,2,2$-Tetrachloroethane & 10 & $\mathrm{U}$ & 10 & 3.7 \\
\hline $96-18-4$ & 1,2,3-Trichloropropane & 10 & $\mathrm{U}$ & 10 & 5.4 \\
\hline $103-65-1$ & n-Propylbenzene & 10 & $\mathrm{U}$ & 10 & 5.0 \\
\hline $95-49-8$ & 2-Chlorotoluene & 10 & $\mathrm{U}$ & 10 & 3.3 \\
\hline $106-43-4$ & 4-Chlorotoluene & 10 & U & 10 & 3.4 \\
\hline $108-67-8$ & 1,3,5-Trimethylbenzene & 10 & U & 10 & 3.2 \\
\hline $98-06-6$ & tert-Butylbenzene & 10 & U & 10 & 5.0 \\
\hline $95-63-6$ & 1,2,4-Trimethylbenzene & 10 & $\mathrm{U}$ & 10 & 3.4 \\
\hline $135-98-8$ & sec-Butylbenzene & 10 & $\mathrm{U}$ & 10 & 5.0 \\
\hline $541-73-1$ & 1,3-Dichlorobenzene & 10 & $\mathrm{u}$ & 10 & 2.5 \\
\hline $99-87-6$ & 4-Isopropyltoluene & 10 & $\mathrm{U}$ & 10 & 1.8 \\
\hline $106-46-7$ & 1,4-Dichlorobenzene & 10 & $\mathrm{U}$ & 10 & 2.3 \\
\hline $95-50-1$ & 1,2-Dichlorobenzene & 10 & $\mathrm{U}$ & 10 & 2.8 \\
\hline $104-51-8$ & n-Butylbenzene & 10 & U & 10 & 5.0 \\
\hline $96-12-8$ & 1,2-Dibromo-3-Chloropropane & 10 & $\mathrm{U}$ & 10 & 4.6 \\
\hline $120-82-1$ & 1,2,4-Trichlorobenzene & 10 & U & 10 & 1.6 \\
\hline
\end{tabular}


FORM I

GC/MS VOA ORGANICS ANALYSIS DATA SHEET

Lab Name: TestAmerica Burlington

SDG No.: INMAN (200-2608)

Client Sample ID: IN-S-MeOH BLANK

Matrix: Solid

Analysis Method: $8260 \mathrm{~B}$

Sample wt/vol: 10(g)

Soil Aliquot Vol: 0.5 (mL)

Soil Extract Vol.: 10 (mL)

8 Moisture:

Analysis Batch No.: 10229
Job No.: 200-2608-1

Lab Sample ID: 200-2608-7

Lab File ID: lfme14.d

Date Collected: 11/18/2010 00:00

Date Analyzed: 11/24/2010 15:15

Dilution Factor: 1

GC Column: $\mathrm{DB}-624$

ID: $0.53(\mathrm{~mm})$

Level: (low/med) Medium

Units: ug/Kg

\begin{tabular}{|c|c|c|c|c|c|}
\hline CAS NO. & COMPOUND NAME & RESULT & $Q$ & RL & MDL \\
\hline $87-68-3$ & Hexachlorobutadiene & 10 & $\mathrm{U}$ & 10 & 5.0 \\
\hline $91-20-3$ & Naphthalene & 10 & $\mathrm{U}$ & 10 & 5.0 \\
\hline $87-61-6$ & 1,2,3-Trichlorobenzene & 10 & $\mathrm{U}$ & 10 & 2.5 \\
\hline
\end{tabular}

\begin{tabular}{|l|l|r|r|r|}
\hline \multicolumn{1}{|c|}{ CAS NO. } & \multicolumn{1}{|c|}{ SURROGATE } & \%REC & Q & LIMITS \\
\hline \hline $17060-07-0$ & 1,2-Dichloroethane-d4 & 92 & & $65-155$ \\
\hline $2037-26-5$ & Toluene-d8 & 104 & $80-115$ \\
\hline $460-00-4$ & Bromofluorobenzene & 101 & & $80-115$ \\
\hline $2199-69-1$ & 1,2-Dichlorobenzene-d4 & 102 & & $45-145$ \\
\hline
\end{tabular}


FORM I

GC/MS VOA ORGANICS ANALYSIS DATA SHEET

Lab Name: TestAmerica Burlington

SDG NO.: INMAN (200-2608)

Client Sample ID: IN-S-30824

Matrix: Solid

Analysis Method: $8260 \mathrm{~B}$

Sample wt/vol: $10.542(\mathrm{~g})$

Soil Aliquot Vol: 0.5 (mL)

Soil Extract Vol.: $10(\mathrm{~mL})$

응 Moisture:

Analysis Batch No.: 10229
Job No.: 200-2608-1

Lab Sample ID: 200-2608-8

Lab File ID: Ifme15.d

Date Collected: 11/18/2010 00:00

Date Analyzed: 11/24/2010 15:47

Dilution Eactor: 1

GC Column: DB-624

ID: $0.53(\mathrm{~mm})$

Level: (low/med) Medium

Units: $u g / \mathrm{Kg}$

\begin{tabular}{|c|c|c|c|c|c|}
\hline CAS NO. & COMPOUND NAME & RESULT & $Q$ & RL & MDL \\
\hline $75-71-8$ & Dichlorodifluoromethane & 9.5 & $\mathrm{U}$ & 9.5 & 4.7 \\
\hline $74-87-3$ & Chloromethane & 9.5 & $\mathrm{U}$ & 9.5 & 3.5 \\
\hline $75-01-4$ & Vinyl chloride & 9.5 & $\mathrm{U}$ & 9.5 & 4.7 \\
\hline $74-83-9$ & Bromomethane & 3.4 & $\mathrm{~J} \mathrm{~B}$ & 9.5 & 3.3 \\
\hline $75-00-3$ & Chloroethane & 9.5 & $\mathrm{U} *$ & 9.5 & 5.7 \\
\hline $75-69-4$ & Trichlorofluoromethane & 9.5 & $\mathrm{U} *$ & 9.5 & 4.7 \\
\hline $75-35-4$ & 1,1-Dichloroethene & 9.5 & $\mathrm{U}$ & 9.5 & 2.0 \\
\hline $76-13-1$ & Freon TF & 9.5 & $\mathrm{U}$ & 9.5 & 2.7 \\
\hline $67-64-1$ & Acetone & 9.5 & $\mathrm{U}$ & 9.5 & 9.5 \\
\hline $74-88-4$ & Methyl iodide & 9.5 & $U$ * & 9.5 & 4.7 \\
\hline $75-15-0$ & Carbon disulfide & 9.5 & $\mathrm{U}$ & 9.5 & 1.8 \\
\hline $79-20-9$ & Methyl acetate & 9.5 & $\mathrm{U}$ & 9.5 & 4.7 \\
\hline $75-09-2$ & Methylene Chloride & 9.5 & $\mathrm{U}$ & 9.5 & 4.7 \\
\hline $156-60-5$ & trans-1,2-Dichloroethene & 9.5 & $\mathrm{U}$ & 9.5 & 4.7 \\
\hline $1634-04-4$ & Methyl t-butyl ether & 9.5 & $\mathrm{U}$ & 9.5 & 4.7 \\
\hline $75-34-3$ & 1,1-Dichloroethane & 9.5 & $\mathrm{U}$ & 9.5 & 3.4 \\
\hline $108-05-4$ & Vinyl acetate & 9.5 & U & 9.5 & 4.7 \\
\hline $594-20-7$ & 2,2-Dichloropropane & 9.5 & $\mathrm{U}$ & 9.5 & 4.4 \\
\hline $156-59-2$ & cis-1,2-Dichloroethene & 9.5 & $\mathrm{U}$ & 9.5 & 2.0 \\
\hline $78-93-3$ & 2-Butanone & 9.5 & $U \star$ & 9.5 & 10 \\
\hline $74-97-5$ & Bromochloromethane & 9.5 & $\mathrm{U}$ & 9.5 & 5.0 \\
\hline $109-99-9$ & Tetrahydrofuran & 95 & $\mathrm{U}$ & 95 & 47 \\
\hline $67-66-3$ & Chloroform & 9.5 & $\mathrm{U}$ & 9.5 & 3.1 \\
\hline $71-55-6$ & 1,1,1-Trichloroethane & 9.5 & $\mathrm{U}$ & 9.5 & 3.4 \\
\hline $110-82-7$ & Cyclohexane & 9.5 & $\mathrm{U}$ & 9.5 & 3.3 \\
\hline $563-58-6$ & 1,1-Dichloropropene & 9.5 & $\mathrm{U}$ & 9.5 & 2.1 \\
\hline $56-23-5$ & Carbon tetrachloride & 9.5 & $\mathrm{U}$ & 9.5 & 3.0 \\
\hline $78-83-1$ & Isobutyl alcohol & 470 & $\mathrm{U}$ & 470 & 240 \\
\hline $71-43-2$ & Benzene & 9.5 & $\mathrm{U}$ & 9.5 & 2.9 \\
\hline $107-06-2$ & 1.2-Dichloroethane & 9.5 & $\mathrm{U}$ & 9.5 & 3.1 \\
\hline $79-01-6$ & Trichloroethene & 9.5 & $\mathrm{U}$ & 9.5 & 4.7 \\
\hline $108-87-2$ & Methylcyclohexane & 9.5 & $\mathrm{U}$ & 9.5 & 4.7 \\
\hline $78-87-5$ & 1,2-Dichloropropane & 9.5 & $\mathrm{U}$ & 9.5 & 3.6 \\
\hline $74-95-3$ & Dibromomethane & 9.5 & $\mathrm{U}$ & 9.5 & 2.4 \\
\hline $123-91-1$ & 1,4-Dioxane & 470 & $\mathrm{U}$ & 470 & 260 \\
\hline $75-27-4$ & Bromodichloromethane & 9.5 & $\mathrm{U}$ & 9.5 & 3.5 \\
\hline
\end{tabular}


FORM I

GC/MS VOA ORGANICS ANALYSIS DATA SHEET

Lab Name: TestAmerica Burlington

SDG NO.: INMAN (200-2608)

Client Sample ID: IN-S-30824

Matrix: Solid

Analysis Method: $8260 \mathrm{~B}$

Sample wt/vol: $10.542(\mathrm{~g})$

Soil Aliquot Vol: 0.5 (mL)

Soil Extract Vol.: $10(\mathrm{~mL})$

응 Moisture:

Analysis Batch No.: 10229
Job No.: 200-2608-1

Lab Sample ID: 200-2608-8

Lab File ID: Ifme15.d

Date Collected: 11/18/2010 00:00

Date Analyzed: 11/24/2010 15:47

Dilution Factor: 1

GC Column: DB-624

ID: $0.53(\mathrm{~mm})$

Level: (low/med) Medium

Units: ug/ $\mathrm{Kg}$

\begin{tabular}{|c|c|c|c|c|c|}
\hline CAS NO. & COMPOUND NAME & RESULT & $Q$ & RI & MDL \\
\hline $1.1 .0-75-8$ & 2-Chloroethyl vinyl ether & 9.5 & $\mathrm{U}$ & 9.5 & 1.2 \\
\hline $10061-01-5$ & cis-1,3-Dichloropropene & 9.5 & $\mathrm{U}$ & 9.5 & 3.0 \\
\hline $108-10-1$ & 4-Methyl-2-pentanone & 9.5 & $\mathrm{U}^{-}$ & 9.5 & 2.0 \\
\hline $108-88-3$ & Toluene & 9.5 & $\mathrm{U}$ & 9.5 & 4.7 \\
\hline $10061-02-6$ & trans-1,3-Dichloropropene & 9.5 & $\mathrm{U}$ & 9.5 & 4.7 \\
\hline $79-00-5$ & 1,1,2-Trichloroethane & 9.5 & $\mathrm{U}$ & 9.5 & 4.0 \\
\hline $127-18-4$ & Tetrachloroethene & 9.5 & $\mathrm{U}$ & 9.5 & 4.7 \\
\hline $142-28-9$ & 1,3-Dichloropropane & 9.5 & $\mathrm{U}$ & 9.5 & 3.1 \\
\hline $591-78-6$ & 2-Hexanone & 9.5 & $\mathrm{U}$ & 9.5 & 9.5 \\
\hline $124-48-1$ & Dibromochlorome thane & 9.5 & $\mathrm{U}$ & 9.5 & 3.5 \\
\hline $106-93-4$ & 1,2-Dibromoethane & 9.5 & U & 9.5 & 4.7 \\
\hline $108-90-7$ & Chlorobenzene & 9.5 & $\mathrm{U}$ & 9.5 & 2.2 \\
\hline $630-20-6$ & $1,1,1,2-$ Tetrachloroethane & 9.5 & $\mathrm{U}$ & 9.5 & 3.4 \\
\hline $100-41-4$ & Ethylbenzene & 9.5 & $\mathrm{U}$ & 9.5 & 4.7 \\
\hline $179601-23-1$ & $m \& p-X y l$ ene & 9.5 & $\mathrm{U}$ & 9.5 & 4.7 \\
\hline $95-47-6$ & o-xylene & 9.5 & $\mathrm{U}$ & 9.5 & 4.7 \\
\hline $100-42-5$ & Styrene & 9.5 & $\mathrm{U}$ & 9.5 & 4.7 \\
\hline $75-25-2$ & Bromoform & 9.5 & $\mathrm{U}$ & 9.5 & 3.7 \\
\hline $98-82-8$ & Isopropylbenzene & 9.5 & $\mathrm{U}$ & 9.5 & 4.7 \\
\hline $108-86-1$ & Bromobenzene & 9.5 & $\mathrm{U}$ & 9.5 & 3.5 \\
\hline $79-34-5$ & $1,1,2,2$-Tetrachloroethane & 9.5 & $\mathrm{U}$ & 9.5 & 3.5 \\
\hline $96-18-4$ & 1,2,3-Trichloropropane & 9.5 & $\mathrm{U}$ & 9.5 & 5.1 \\
\hline $103-65-1$ & n-Propylbenzene & 9.5 & $\mathrm{U}$ & 9.5 & 4.7 \\
\hline $95-49-8$ & 2-Chlorotoluene & 9.5 & $\mathrm{U}$ & 9.5 & 3.1 \\
\hline $106-43-4$ & 4-Chlorotoluene & 9.5 & $\mathrm{U}$ & 9.5 & 3.2 \\
\hline $108-67-8$ & $1,3,5$-Trimethylbenzene & 9.5 & $\mathrm{U}$ & 9.5 & 3.0 \\
\hline $98-06-6:$ & tert-Butylbenzene & 9.5 & $\mathrm{U}$ & 9.5 & 4.7 \\
\hline $95-63-6$ & 1,2,4-Trimethylbenzene & 9.5 & $\mathrm{U}$ & 9.5 & 3.2 \\
\hline $135-98-8$ & sec-Butylbenzene & 9.5 & $\mathrm{U}$ & 9.5 & 4.7 \\
\hline $541-73-1$ & 1,3-Dichlorobenzene & 9.5 & $\mathrm{U}$ & 9.5 & 2.4 \\
\hline $99-87-6$ & 4-Isopropyltoluene & 9.5 & $\mathrm{U}$ & 9.5 & 1.7 \\
\hline $106-46-7$ & 1,4-Dichlorobenzene & 9.5 & $\mathrm{U}$ & 9.5 & 2.2 \\
\hline $95-50-1$ & 1,2-Dichlorobenzene & 9.5 & $\mathrm{U}$ & 9.5 & 2.7 \\
\hline $104-51-8$ & n-Butylbenzene & 9.5 & $\mathrm{U}$ & 9.5 & 4.7 \\
\hline $96-12-8$ & 1,2-Dibromo-3-Chloropropane & 9.5 & $\mathrm{U}$ & 9.5 & 4.4 \\
\hline $120-82-1$ & 1,2,4-Trichlorobenzene & 9.5 & $\mathrm{U}$ & 9.5 & 1.5 \\
\hline
\end{tabular}


FORM I

GC/MS VOA ORGANICS ANALYSIS DATA SHEET

Lab Name: TestAmerica Burlington

SDG NO.: INMAN (200-2608)

Client Sample ID: IN-S-30824

Matrix: Solid

Analysis Method: $8260 \mathrm{~B}$

Sample wt/vol: $10.542(\mathrm{~g})$

Soil Aliquot Vol: 0.5 (mL)

Soil Extract Vol.: 10 (mL)

음 Moisture:

Analysis Batch No.: 10229
Job No.: 200-2608-1

Lab Sample ID: 200-2608-8

Lab File ID: Ifmel5.d

Date Collected: 11/18/2010 00:00

Date Analyzed: 11/24/2010 15:47

Dilution Factor: 1

GC Column: DB-624 ID: 0.53 (mm)

Level: (low/med) Medium

Units: $u g / \mathrm{Kg}$

\begin{tabular}{|c|c|c|c|c|c|}
\hline CAS NO. & COMPOUND NAME & RESULT & Q & RL & MDL \\
\hline $87-68-3$ & Hexachlorobutadiene & 9.5 & U & 9.5 & 4.7 \\
\hline $91-20-3$ & Naphthalene & 9.5 & $\mathrm{U}$ & 9.5 & 4.7 \\
\hline $87-61-6$ & 1,2,3-Trichlorobenzene & 9.5 & $\mathrm{U}$ & 9.5 & 2.4 \\
\hline
\end{tabular}

\begin{tabular}{|l|l|r|r|r|}
\hline \multicolumn{1}{|c|}{ CAS NO. } & \multicolumn{1}{|c|}{ SURROGATE } & o.REC & Q & LIMITS \\
\hline \hline $17060-07-0$ & 1,2-Dichloroethane-d4 & 99 & \\
\hline $2037-26-5$ & Toluene-d8 & 110 & $65-155$ \\
\hline $460-00-4$ & Bromofluorobenzene & 107 & $80-115$ \\
\hline $2199-69-1$ & 1,2-Dichlorobenzene-d4 & 108 & $80-115$ \\
\hline
\end{tabular}




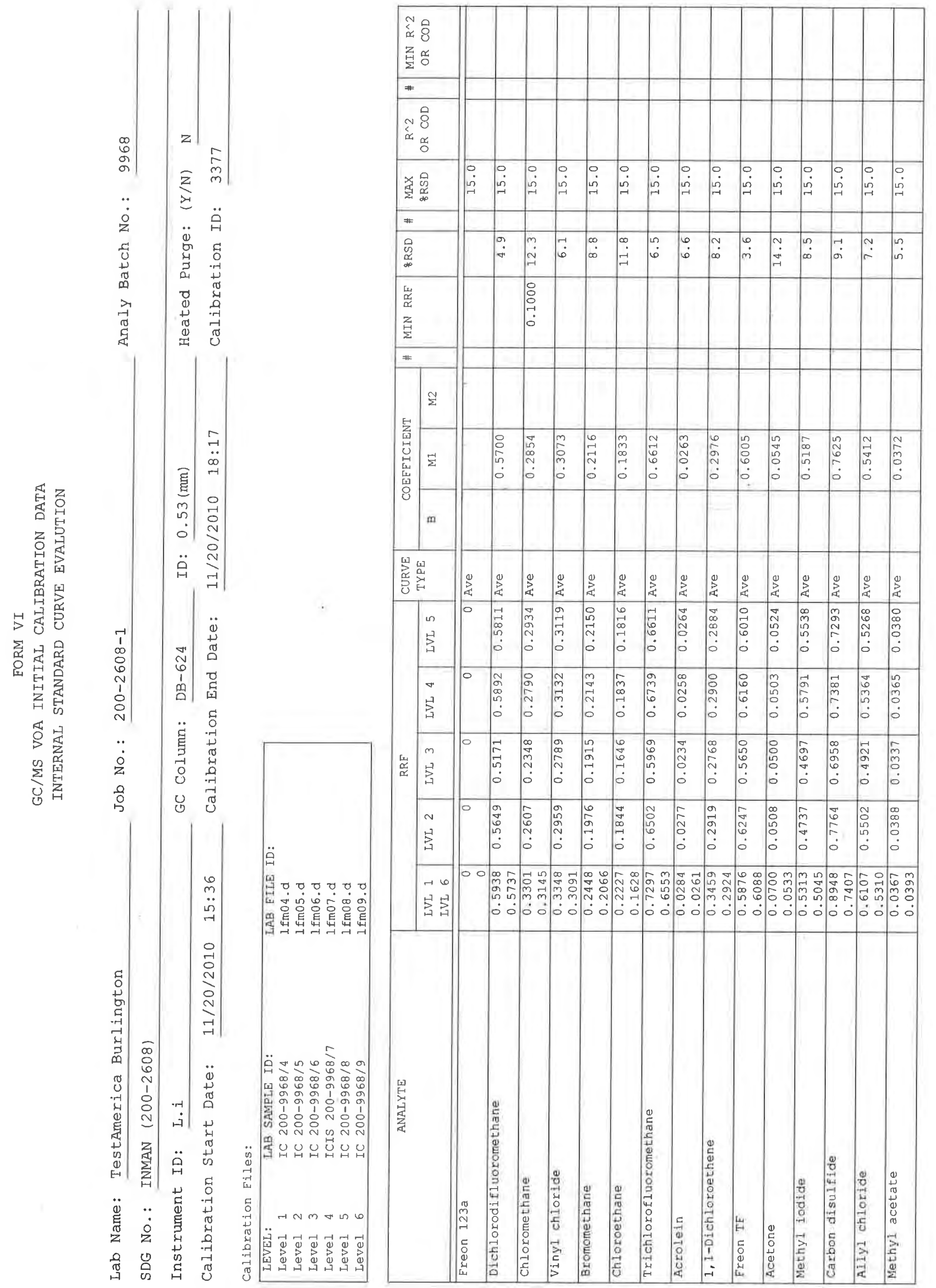

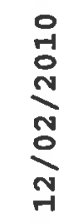

2
4
4
5
5
0
0
0

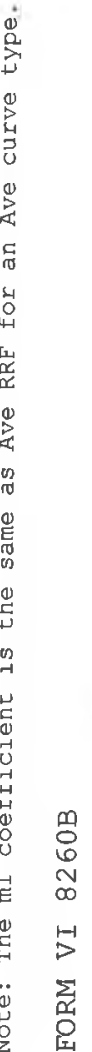




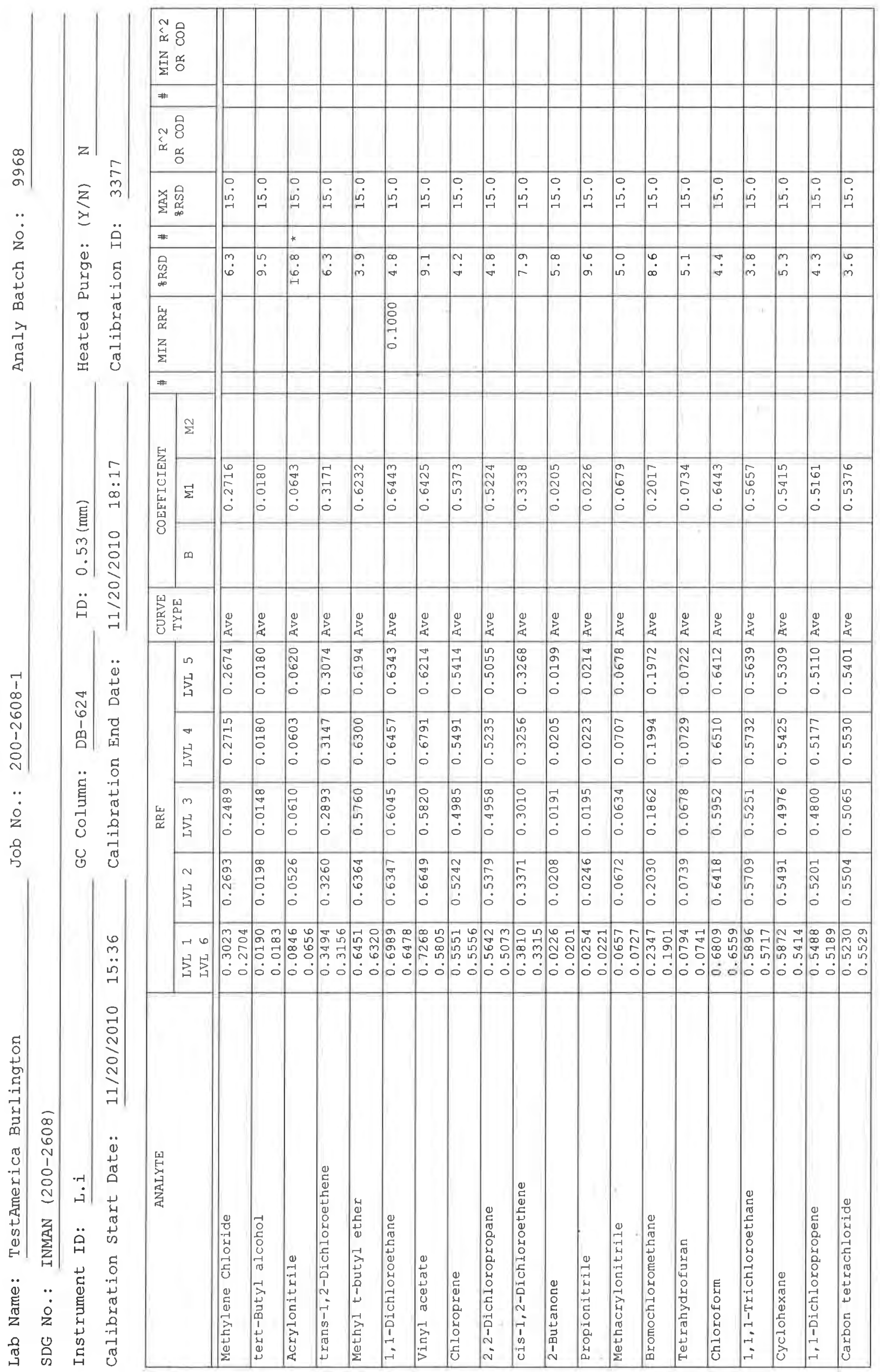

0
r-1
0
v
กิ
0
กิ

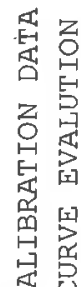

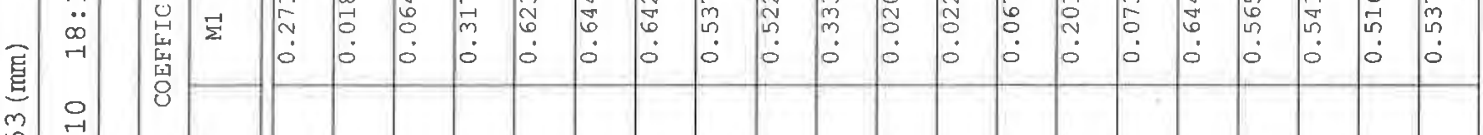

$>$ 芯

品晨品

是最

留 政

郘

c2

造

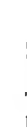




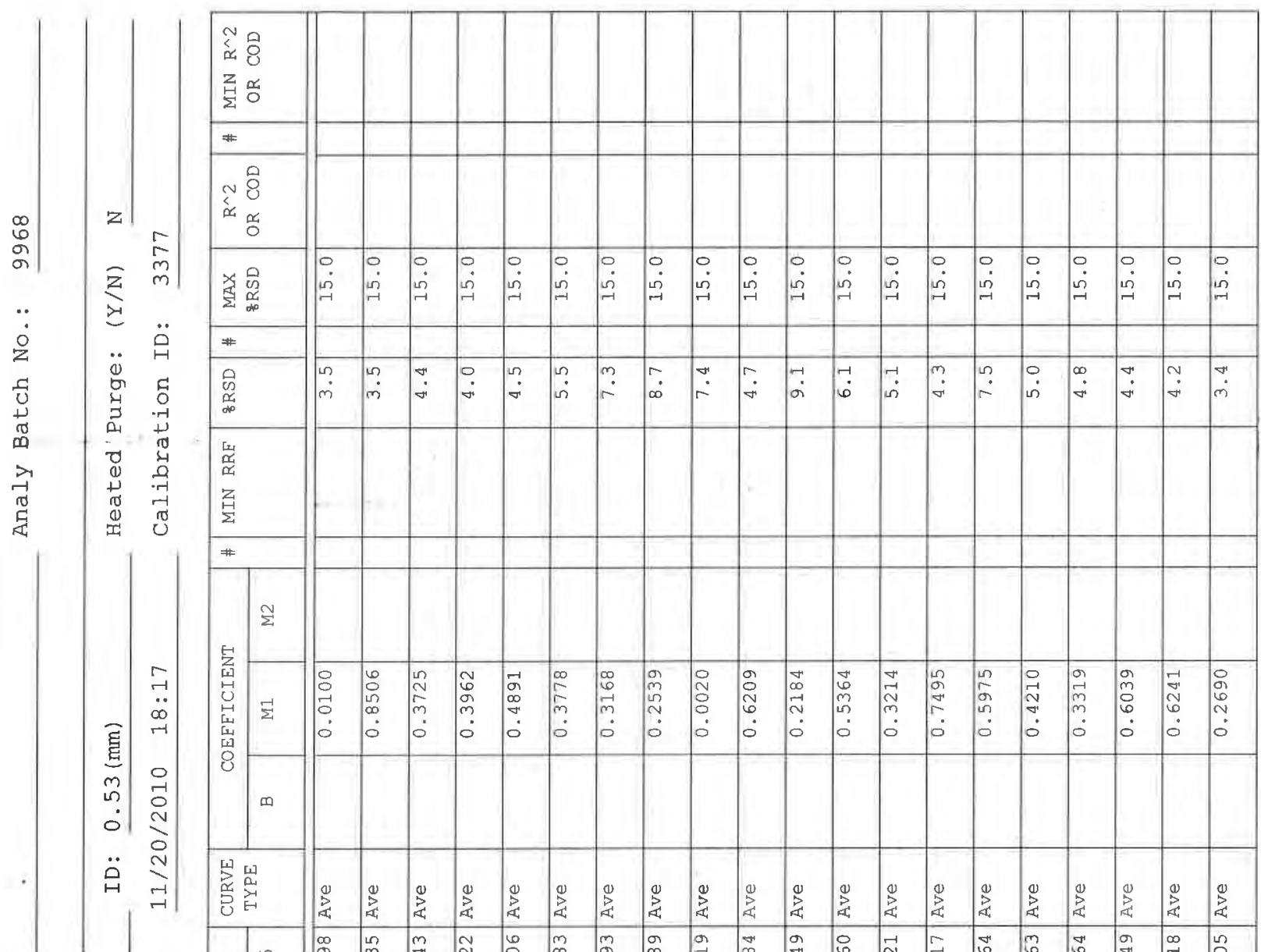

焉畐

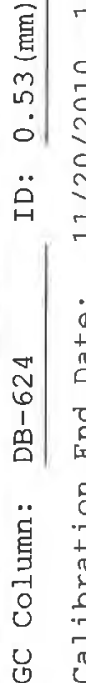

是焉

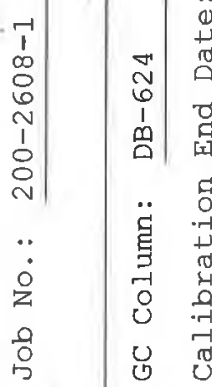

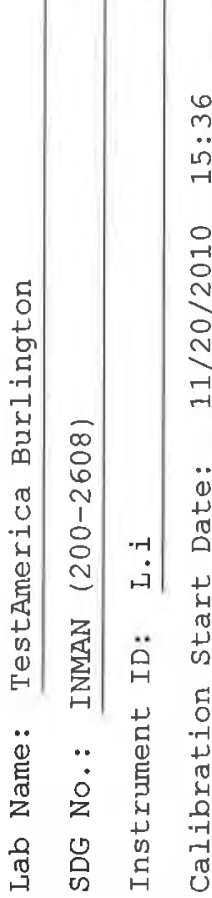

宸

岛

窝

㝠

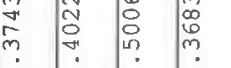

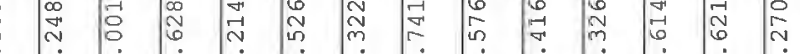

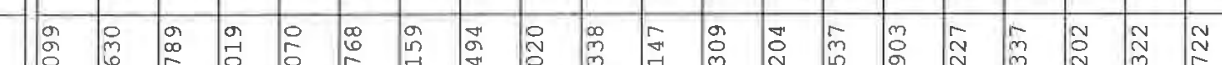

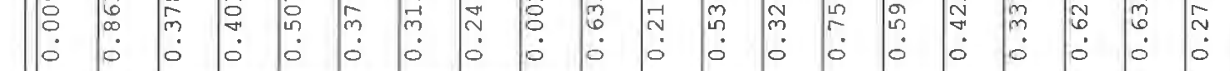

(1)

客

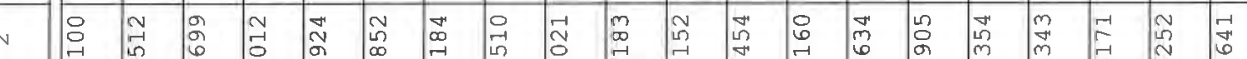

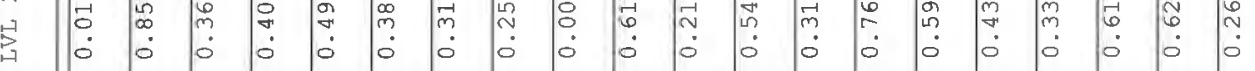

†

1

当

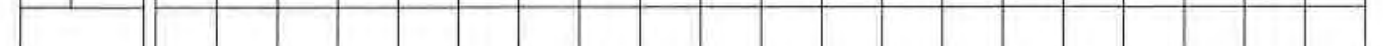




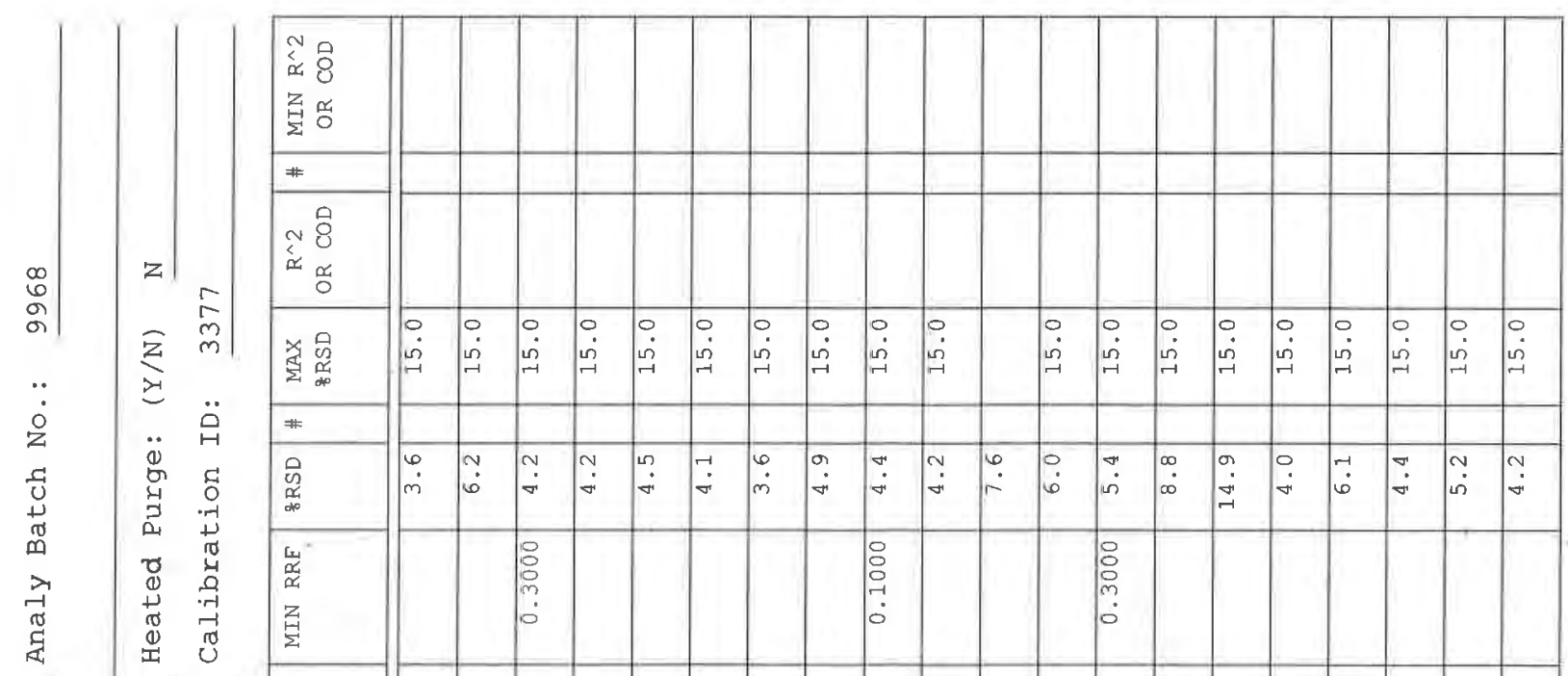

画㤎

号是

旨学

品

占苌虽

窟宾星

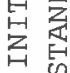

$1 \mid$

$\Sigma$

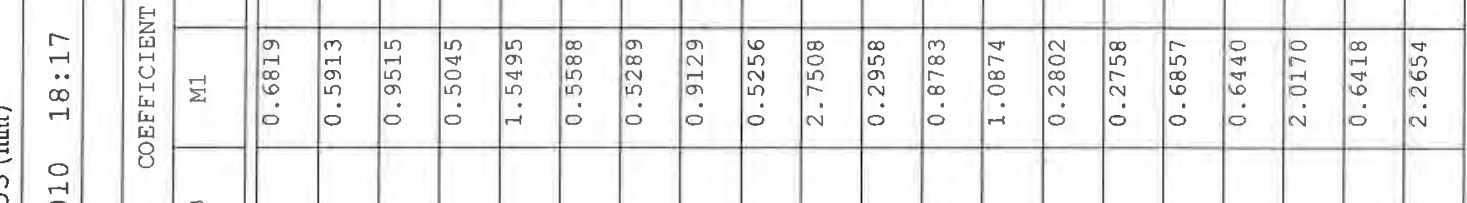

昏是

留

论舅

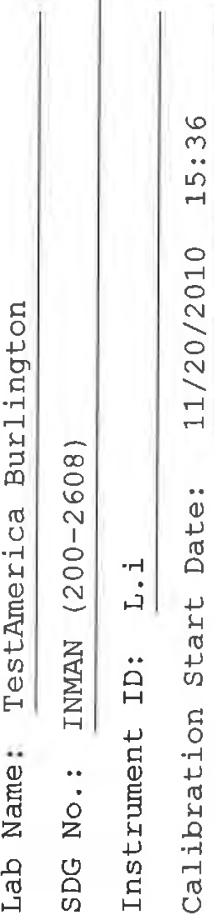

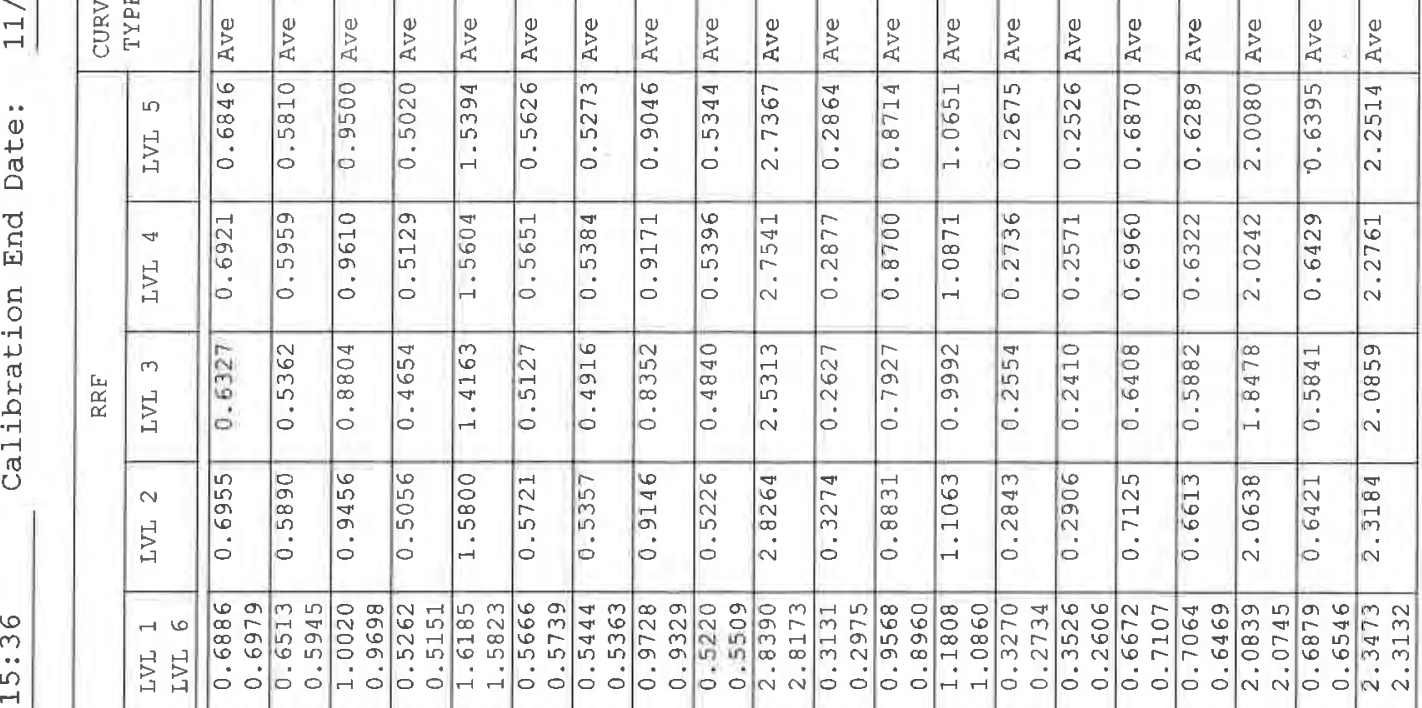

i.

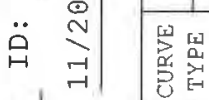
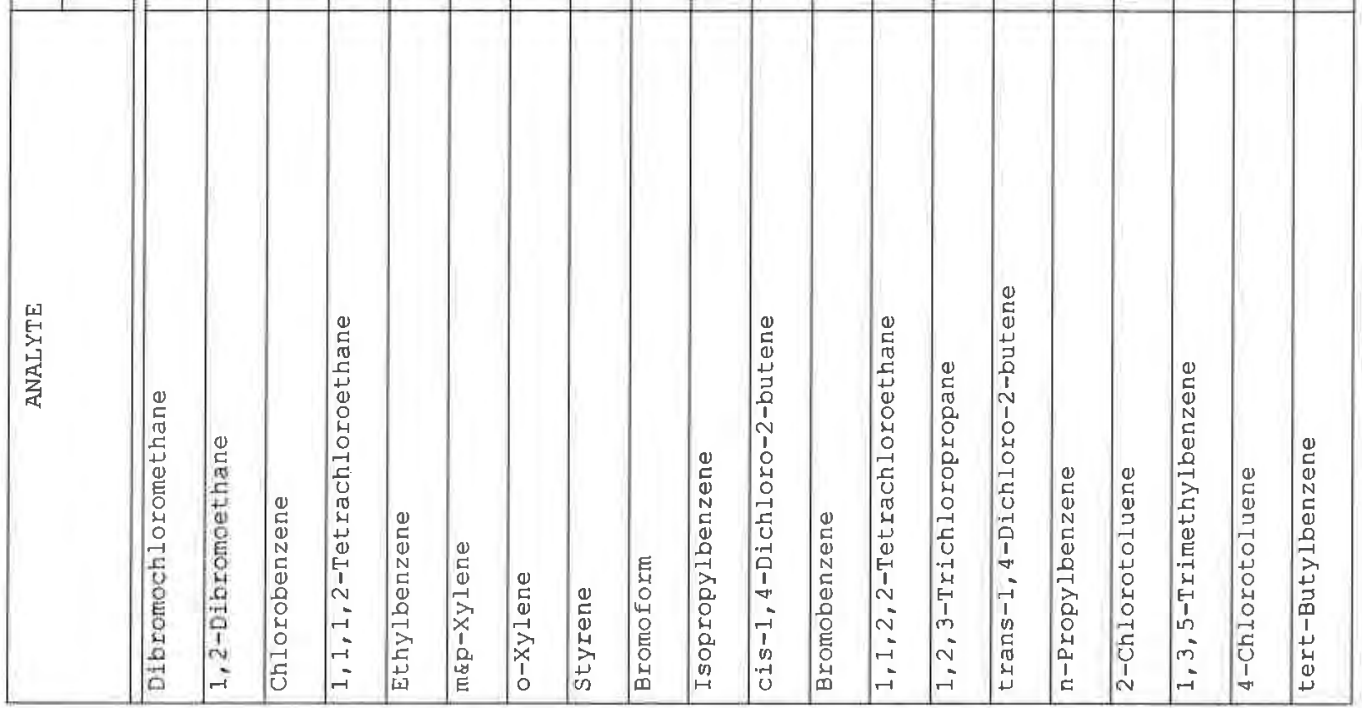

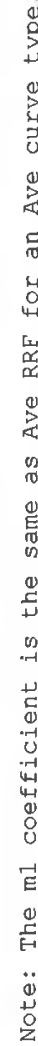




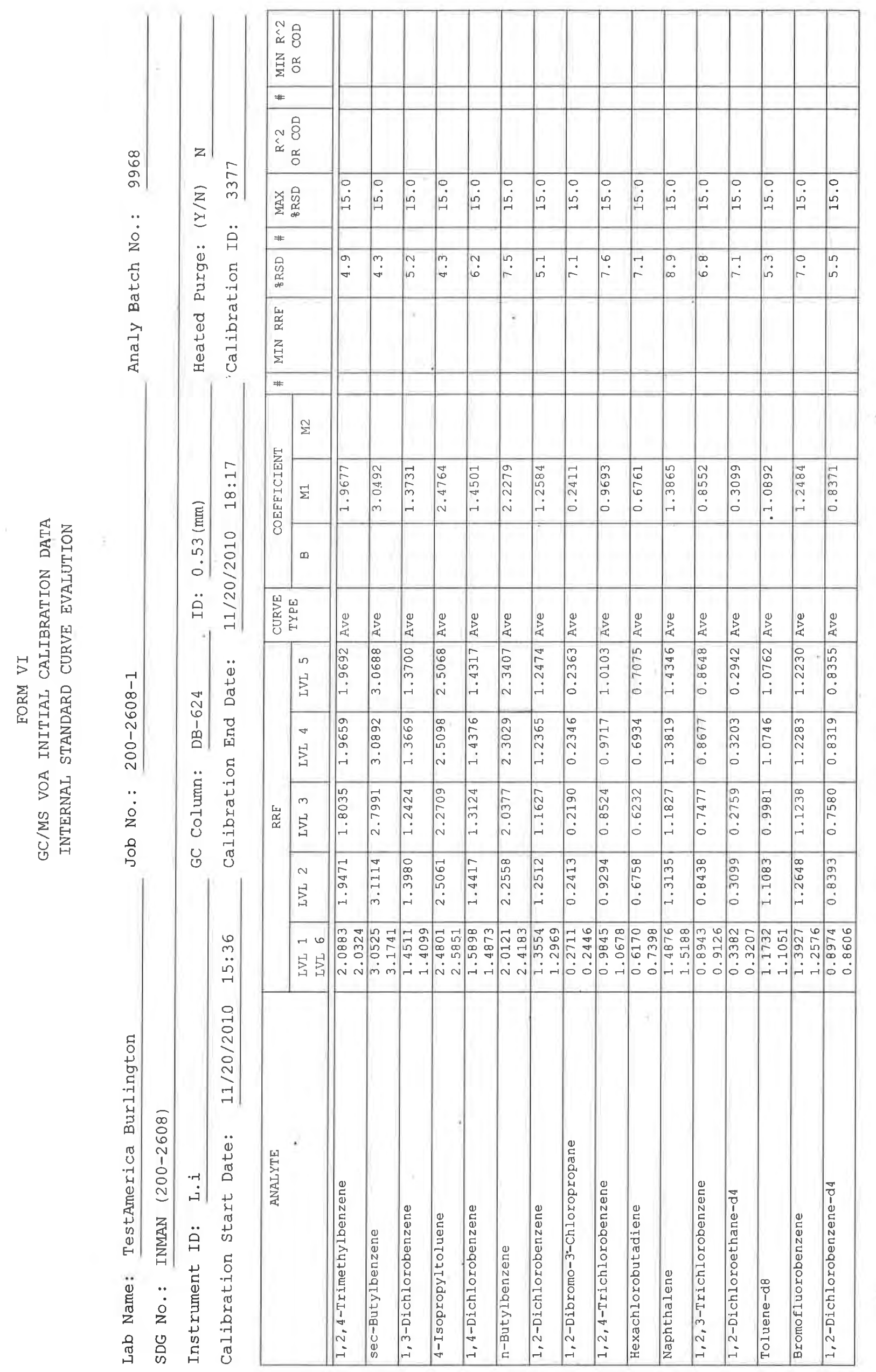

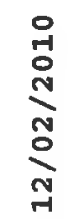

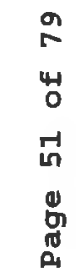

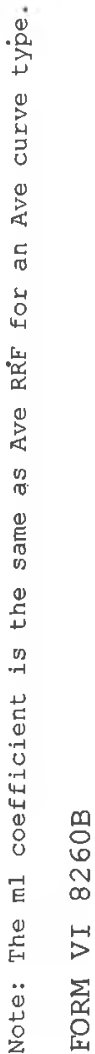



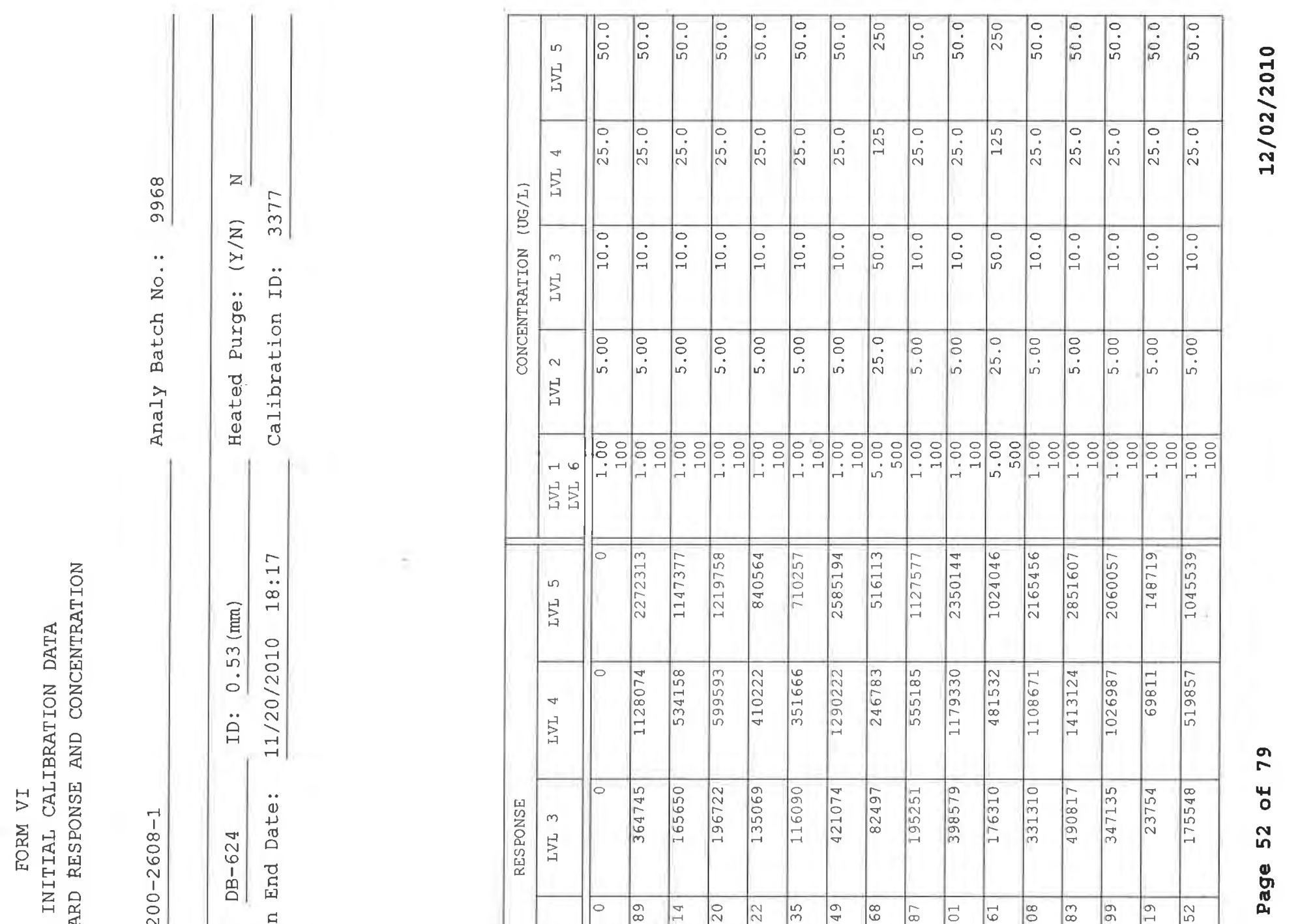

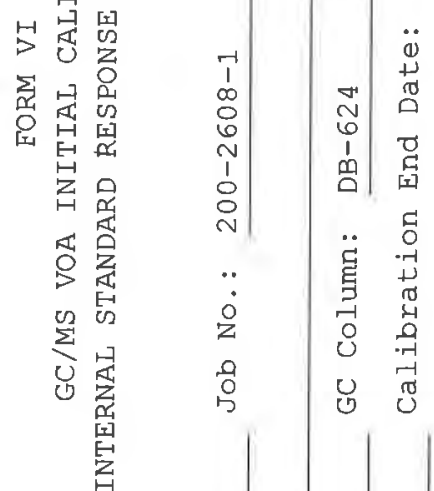

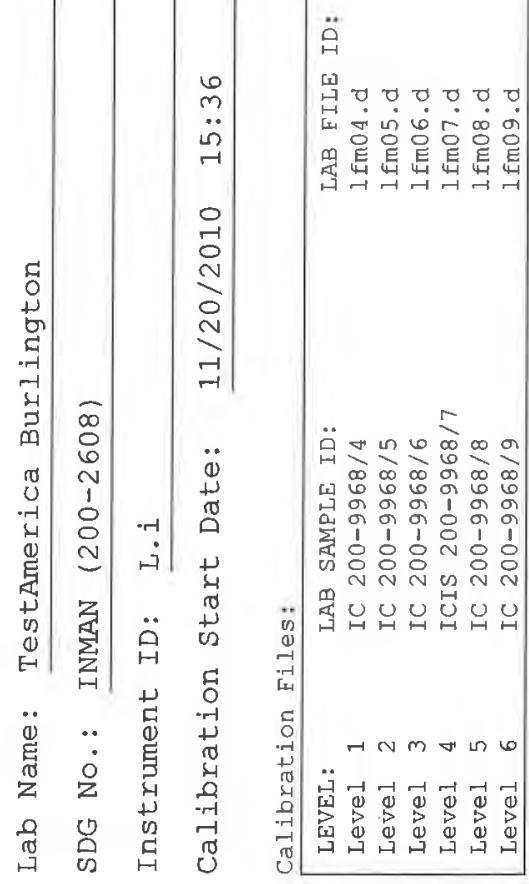




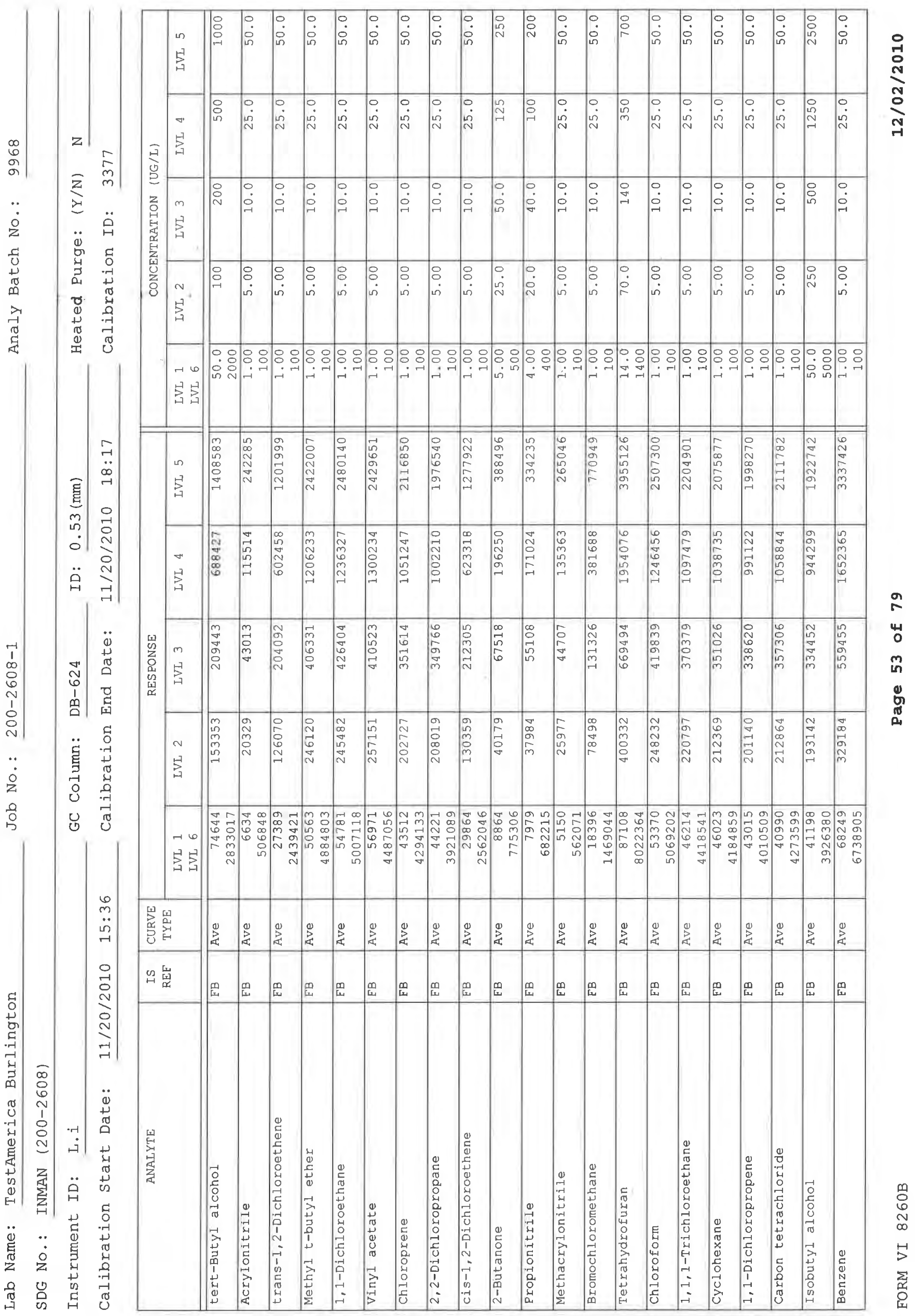




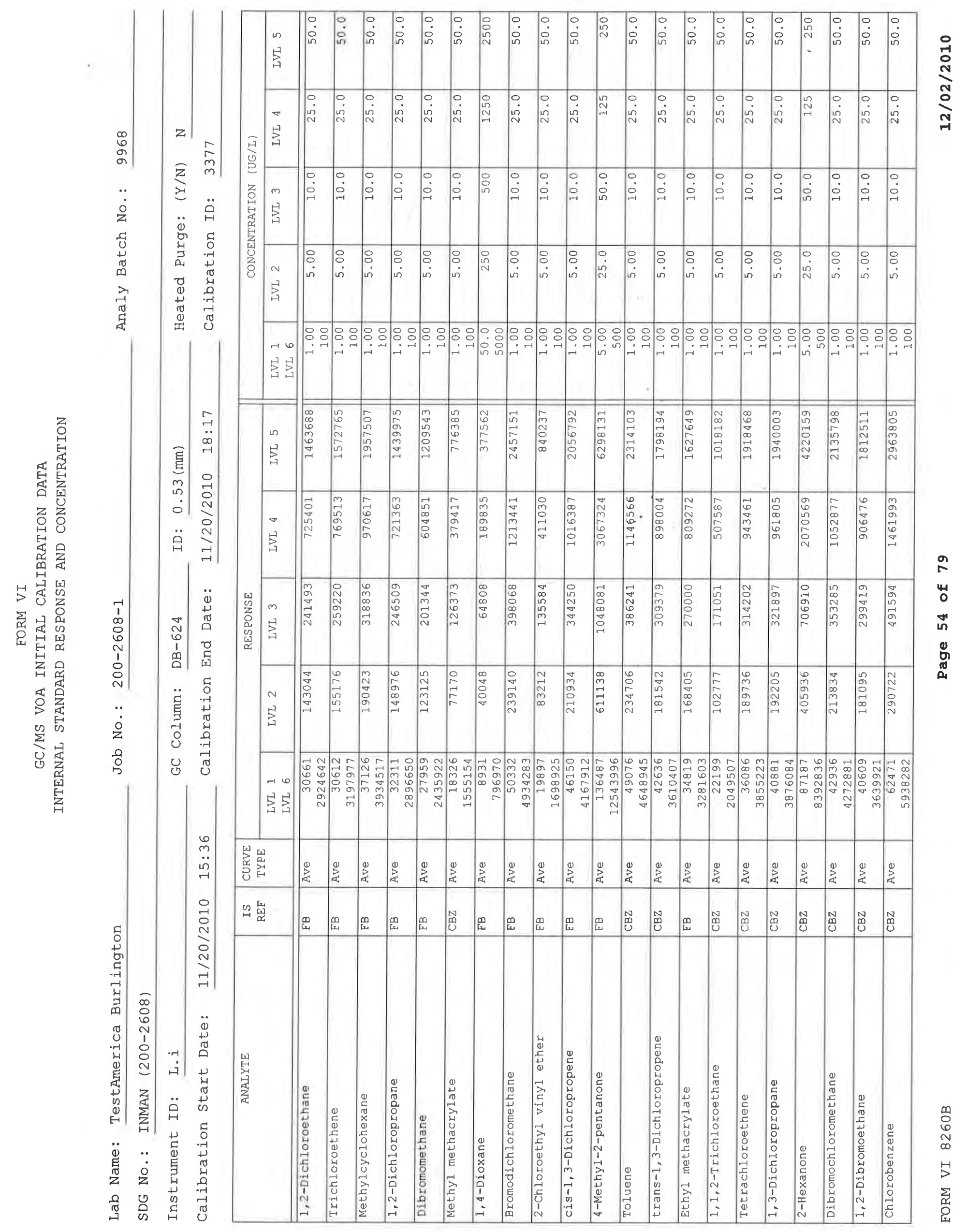




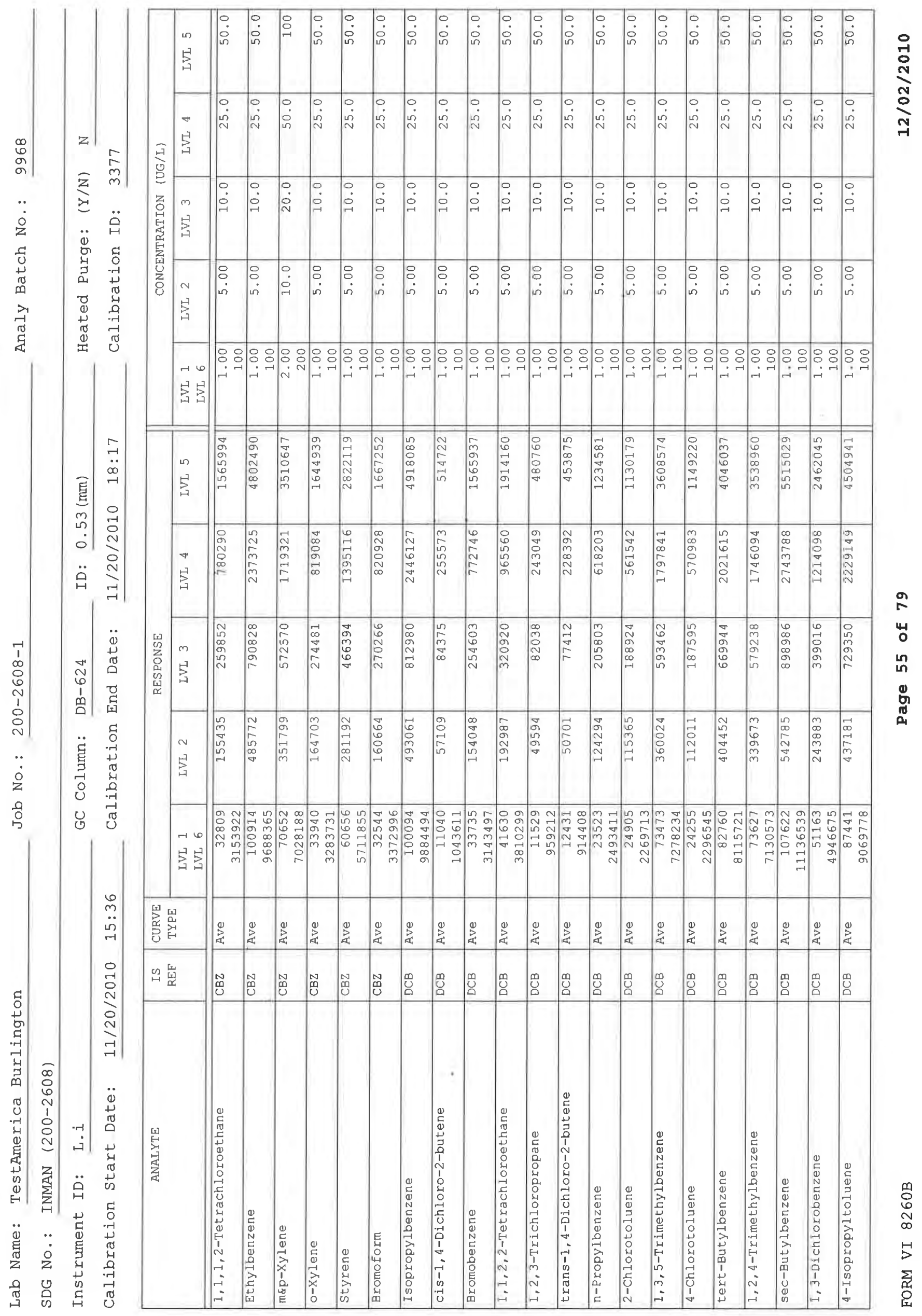




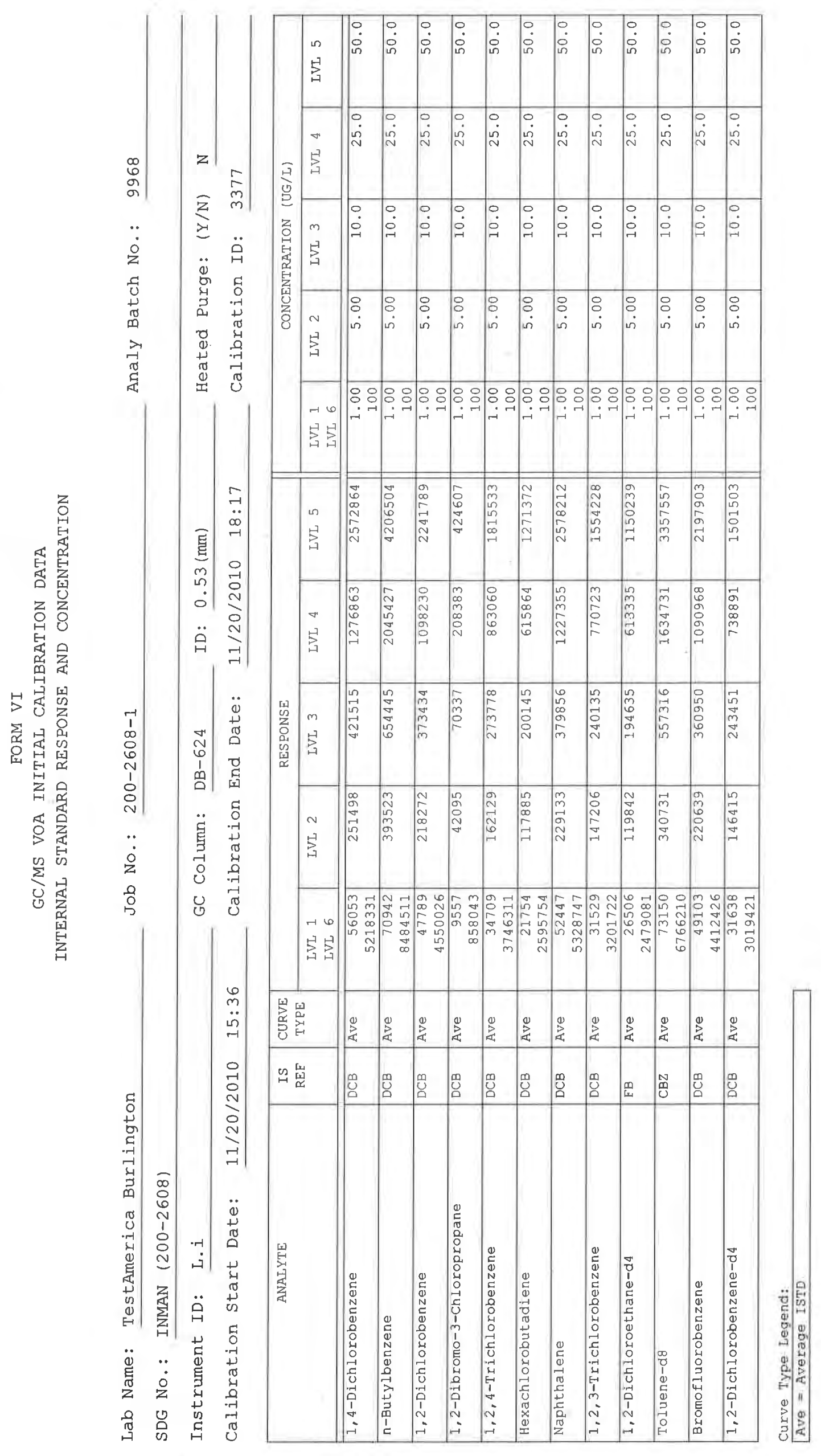

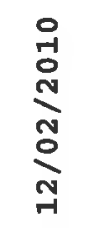

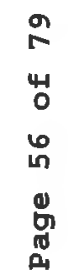


FORM VII

GC/MS VOA CONTINUING CALIBRATION DATA

Lab Name: TestAmerica Burlington

Job No.: 200-2608-1

SDG NO.: INMAN (200-2608)

Lab Sample ID: ICV 200-9968/12

Instrument ID: L.i

GC Column: DB-624

ID: $0.53(\mathrm{~mm})$

Calibration Date: 11/20/2010 19:53

Calib Start Date: 11/20/2010 15:36

Calib End Date: 11/20/2010 18:17

Lab File ID: Ifm12.d

Conc. Units: ug/Kg Heated Purge: (Y/N) N

EPA Sample No.: ICV

\begin{tabular}{|c|c|c|c|c|c|c|c|c|}
\hline ANAIYTE & $\begin{array}{l}\text { CURVE } \\
\text { TYPE }\end{array}$ & AVE RRE & RRF & MIN RRF & $\begin{array}{c}\text { CALC } \\
\text { AMOUNT }\end{array}$ & $\begin{array}{l}\text { SPIKE } \\
\text { AMOUNT }\end{array}$ & $\stackrel{\circ}{\circ}$ & $\begin{array}{l}\text { MAX } \\
\frac{\circ}{D}\end{array}$ \\
\hline Dichlorodifluoromethane & Ave & 0.5700 & 0.5713 & & 25.1 & 25.0 & 0.2 & 25.0 \\
\hline Chloromethane & Ave & 0.2854 & 0.2670 & 0.1000 & 23.4 & 25.0 & -6.4 & 25.0 \\
\hline Vinyl chloride & Ave & 0.3073 & 0.3142 & & 25.6 & 25.0 & 2.3 & 25.0 \\
\hline Bromomethane & Ave & 0.2116 & 0.2030 & & 24.0 & 25.0 & -4.1 & 25.0 \\
\hline Chloroethane & Ave & 0.1833 & 0.1846 & & 25.2 & 25.0 & 0.7 & 25.0 \\
\hline Trichlorofluoromethane & Ave & 0.6612 & 0.6329 & & 23.9 & 25.0 & -4.3 & 25.0 \\
\hline Acrolein & Ave & 0.0263 & 0.0213 & & 101 & 125 & -18.8 & 25.0 \\
\hline 1,1-Dichloroethene & Ave & 0.2976 & 0.2718 & & 22.8 & 25.0 & -8.7 & 25.0 \\
\hline Freon $T^{\prime} F$ & Ave & 0.6005 & 0.5718 & & 23.8 & 25.0 & -4.8 & 25.0 \\
\hline Acetone & Ave & 0.0545 & 0.0495 & & 114 & 125 & -9.1 & 25.0 \\
\hline Methyl iodide & Ave & 0.5187 & 0.5812 & & 28.0 & 25.0 & 12.0 & 25.0 \\
\hline Carbon disulfide & Ave & 0.7625 & 0.6845 & & 22.4 & 25.0 & -10.2 & 25.0 \\
\hline Allyl chloride & Ave & 0.5412 & 0.5153 & & 23.8 & 25.0 & -4.8 & 25.0 \\
\hline Methyl acetate & Ave & 0.0372 & 0.0407 & & 27.4 & 25.0 & 9.5 & 25.0 \\
\hline Methylene Chloride & Ave & 0.2716 & 0.2769 & & 25.5 & 25.0 & 1.9 & 25.0 \\
\hline tert-Butyl alcohol & Ave & 0.0180 & 0.0173 & & 482 & 500 & -3.7 & 25.0 \\
\hline Acrylonitrile & Ave & 0.0643 & 0.0612 & & 23.8 & 25.0 & -4.9 & 25.0 \\
\hline trans-1,2-Dichloroethene & Ave & 0.3171 & 0.3062 & & 24.1 & 25.0 & -3.4 & 25.0 \\
\hline Methyl t-butyl ether & Ave & 0.6232 & 0.6246 & & 25.1 & 25.0 & 0.2 & 25.0 \\
\hline 1,1-Dichloroethane & Ave & 0.6443 & 0.6297 & 0.1000 & 24.4 & 25.0 & -2.3 & 25.0 \\
\hline Vinyl acetate & Ave & 0.6425 & 0.5059 & & 19.7 & 25.0 & -21.3 & 25.0 \\
\hline Chloroprene & Ave & 0.5373 & 0.4746 & & 22.1 & 25.0 & -11.7 & 25.0 \\
\hline 2,2-Dichloropropane & Ave & 0.5224 & 0.4640 & & 22.2 & 25.0 & -11.2 & 25.0 \\
\hline cis-1,2-Dichloroethene & Ave & 0.3338 & 0.3236 & & 24.2 & 25.0 & -3.1 & 25.0 \\
\hline 2-Butanone & Ave & 0.0205 & 0.0207 & & 126 & 125 & 0.8 & 25.0 \\
\hline Propionitrile & Ave & 0.0226 & 0.0201 & & 89.0 & 100 & -11.0 & 25.0 \\
\hline Methacrylonitrile & Ave & 0.0679 & 0.0706 & & 26.0 & 25.0 & 4.0 & 25.0 \\
\hline Bromochloromethane & Ave & 0.2017 & 0.2013 & & 24.9 & 25.0 & -0.2 & 25.0 \\
\hline Tetrahydrofuran & Ave & 0.0734 & 0.0702 & & 335 & 350 & -4.4 & 25.0 \\
\hline Chloroform & Ave & 0.6443 & 0.6260 & & 24.3 & 25.0 & -2.9 & 25.0 \\
\hline 1,1,1-Trichloroethane & Ave & 0.5657 & 0.5617 & & 24.8 & 25.0 & -0.7 & 25.0 \\
\hline Cyclohexane & Ave & 0.5415 & 0.5403 & & 24.9 & 25.0 & -0.2 & 25.0 \\
\hline 1,1-Dichloropropene & Ave & 0.5161 & 0.5123 & & 24.8 & 25.0 & -0.7 & 25.0 \\
\hline Carbon tetrachloride & Ave & 0.5376 & 0.5359 & & 24.9 & 25.0 & -0.3 & 25.0 \\
\hline Isobutyl alcohol & Ave & 0.0100 & 0.0100 & & 1250 & 1250 & 0.3 & 25.0 \\
\hline Benzene & Ave & 0.8506 & 0.8650 & & 25.4 & 25.0 & 1.7 & 25.0 \\
\hline 1,2-Dichloroethane & Ave & 0.3725 & 0.3730 & & 25.0 & 25.0 & 0.1 & 25.0 \\
\hline Trichloroethene & Ave & 0.3962 & 0.4176 & & 26.3 & 25.0 & 5.4 & 25.0 \\
\hline Methylcyclohexane & Ave & 0.4891 & 0.5004 & & 25.6 & 25.0 & 2.3 & 25.0 \\
\hline
\end{tabular}


FORM VII

GC/MS VOA CONTINUING CALIBRATION DATA

Lab Name: TestAmerica Burlington

SDG NO.: INMAN (200-2608)

Lab Sample ID: ICV 200-9968/12

Instrument ID: L.i

GC Column: DB-624

ID: $0.53(\mathrm{~mm})$

Lab File ID: lfm12.d

EPA Sample No.: ICV
Job No.: 200-2608-1

Calibration Date: 11/20/2010 19:53

Calib Start Date: 11/20/2010 15:36

Calib End Date: 11/20/2010 18:17

Conc. Units: ug/Kg Heated Purge: ( $\mathrm{Y} / \mathrm{N}) \quad \mathrm{N}$

\begin{tabular}{|c|c|c|c|c|c|c|c|c|}
\hline ANALYTE & $\begin{array}{l}\text { CURVE } \\
\text { TYPE }\end{array}$ & AVE RRF & RRF & MIN RRF & $\begin{array}{c}\text { CALC } \\
\text { AMOUNT }\end{array}$ & $\begin{array}{l}\text { SPIKE } \\
\text { AMOUNT }\end{array}$ & 옹 & $\begin{array}{l}\text { MAX } \\
\text { 옹 D }\end{array}$ \\
\hline 1,2-Dichloropropane & Ave & 0.3778 & 0.3757 & & 24.9 & 25.0 & -0.6 & 25.0 \\
\hline Dibromomethane & Ave & 0.3168 & 0.3146 & & 24.8 & 25.0 & -0.7 & 25.0 \\
\hline Methyl methacrylate & Ave & 0.2539 & 0.2471 & & 24.3 & 25.0 & -2.7 & 25.0 \\
\hline 1,4-Dioxane & Ave & 0.0020 & 0.0019 & & 1150 & 1250 & -8.2 & 25.0 \\
\hline Bromodichloromethane & Ave & 0.6209 & 0.6287 & & 25.3 & 25.0 & 1.3 & 25.0 \\
\hline 2-Chloroethyl vinyl ether & Ave & 0.2184 & 0.2151 & & 24.6 & 25.0 & -1.5 & 25.0 \\
\hline cis-1,3-Dichloropropene & Ave & 0.5364 & 0.5274 & & 24.6 & 25.0 & -1.7 & 25.0 \\
\hline 4-Methyl-2-pentanone & Ave & 0.3214 & 0.3356 & & 131 & 125 & 4.4 & 25.0 \\
\hline Toluene & Ave & 0.7495 & 0.7536 & & 25.1 & 25.0 & 0.5 & 25.0 \\
\hline trans-1,3-Dichloropropene & Ave & 0.5975 & 0.5771 & & 24.1 & 25.0 & -3.4 & 25.0 \\
\hline Ethyl methacrylate & Ave & 0.4210 & 0.4268 & & 25.3 & 25.0 & 1.4 & 25.0 \\
\hline 1,1,2-Trichloroethane & Ave & 0.3319 & 0.3379 & & 25.5 & 25.0 & 1.8 & 25.0 \\
\hline Tetrachloroethene & Ave & 0.6039 & 0.6270 & & 26.0 & 25.0 & 3.8 & 25.0 \\
\hline 1,3-Dichloropropane & Ave & 0.6241 & 0.6372 & & 25.5 & 25.0 & 2.1 & 25.0 \\
\hline 2-Hexanone & Ave & 0.2690 & 0.2876 & & 134 & 125 & 6.9 & 25.0 \\
\hline Dibromochloromethane & Ave & 0.6819 & 0.7253 & & 26.6 & 25.0 & 6.4 & 25.0 \\
\hline 1,2-Dibromoethane & Ave & 0.5913 & 0.6033 & & 25.5 & 25.0 & 2.0 & 25.0 \\
\hline Chlorobenzene & Ave & 0.9515 & 0.9666 & 0.3000 & 25.4 & 25.0 & 1.6 & 25.0 \\
\hline 1, 1, 1,2-Tetrachloroethane & Ave & 0.5045 & 0.5171 & & 25.6 & 25.0 & 2.5 & 25.0 \\
\hline Ethylbenzene & Ave & 1.549 & 1.566 & & 25.3 & 25.0 & 1.1 & 25.0 \\
\hline $\mathrm{m \& p}-\mathrm{Xylene}$ & Ave & 0.5588 & 0.5689 & & 50.9 & 50.0 & 1.8 & 25.0 \\
\hline o-Xylene & Ave & 0.5289 & 0.5384 & & 25.4 & 25.0 & 1.8 & 25.0 \\
\hline Styrene & Ave & 0.9129 & 0.9227 & & 25.3 & 25.0 & 1.1 & 25.0 \\
\hline Bromoform & Ave & 0.5256 & 0.5371 & 0.1000 & 25.5 & 25.0 & 2.2 & 25.0 \\
\hline Isopropylbenzene & Ave & 2.751 & 2.808 & & 25.5 & 25.0 & 2.1 & 25.0 \\
\hline Bromobenzene & Ave & 0.8783 & 0.8911 & & 25.4 & 25.0 & 1.4 & 25.0 \\
\hline $1,1,2,2$-Tetrachloroethane & Åve & 1.087 & 1.086 & 0.3000 & 25.0 & 25.0 & -0.2 & 25.0 \\
\hline 1,2,3-Trichloropropane & Ave & 0.2802 & 0.2549 & & 22.7 & 25.0 & -9.0 & 25.0 \\
\hline trans-1,4-Dichloro-2-butene & Ave & 0.2758 & 0.2538 & & 23.0 & 25.0 & -8.0 & 25.0 \\
\hline n-Propylbenzene & Ave & 0.6857 & 0.6982 & & 25.5 & 25.0 & 1.8 & 25.0 \\
\hline 2-Chlorotoluene & Ave & 0.6440 & 0.6523 & & 25.3 & 25.0 & 1.3 & 25.0 \\
\hline $1,3,5$-Trimethylbenzene & Ave & 2.017 & 2.041 & & 25.3 & 25.0 & 1.2 & 25.0 \\
\hline 4-Chlorotoluene & Ave & 0.6418 & 0.6520 & & 25.4 & 25.0 & 1.6 & 25.0 \\
\hline tert-Butylbenzene & Ave & 2.265 & 2.316 & & 25.6 & 25.0 & 2.2 & 25.0 \\
\hline 1,2,4-Trimethylbenzene & Ave & 1.968 & 2.022 & & 25.7 & 25.0 & 2.8 & 25.0 \\
\hline sec-Butylbenzene & Ave & 3.049 & 3.145 & & 25.8 & 25.0 & 3.1 & 25.0 \\
\hline 1,3-Dichlorobenzene & Ave & 1.373 & 1.393 & & 25.4 & 25.0 & 1.5 & 25.0 \\
\hline 4-Isopropyltoluene & Ave & 2.476 & 2.481 & & 25.0 & 25.0 & 0.2 & 25.0 \\
\hline 1,4-Dichlorobenzene & Ave & 1.450 & 1.468 & & 25.3 & 25.0 & 1.2 & 25.0 \\
\hline
\end{tabular}


FORM VII

GC/MS VOA CONTINUING CALIBRATION DATA

Lab Name: TestAmerica Burlington

Job No.: 200-2608-1

SDG NO.: INMAN (200-2608)

Lab Sample ID: ICV 200-9968/12

Calibration Date: 11/20/2010 19:53

Instrument ID: L.i

Calib Start Date: 11/20/2010 15:36

GC Column: DB-624

ID: $0.53(\mathrm{~mm})$

Calib End Date: 11/20/2010 18:17

Lab File ID: $\ln 12 . \mathrm{d}$

Conc. Units: ug/Kg Heated Purge: ( $Y / N)$ N

EPA Sample No.: ICV

\begin{tabular}{|c|c|c|c|c|c|c|c|c|}
\hline ANALYTE & $\begin{array}{l}\text { CURVE } \\
\text { TYPE }\end{array}$ & AVE RRE & RRF & MIN RRF & $\begin{array}{c}\text { CALC } \\
\text { AMOUNT }\end{array}$ & $\begin{array}{l}\text { SPIKE } \\
\text { AMOUNT }\end{array}$ & $\because \mathrm{D}$ & $\begin{array}{l}\text { MAX } \\
\frac{\circ D}{\partial}\end{array}$ \\
\hline 1,2-Dichlorobenzene & Ave & 1.258 & 1.285 & & 25.5 & 25.0 & 2.1 & 25.0 \\
\hline n-Butylbenzene & Ave & 2.228 & 2.325 & & 26.1 & 25.0 & 4.4 & 25.0 \\
\hline 1,2-Dibromo-3-Chloropropane & Ave & 0.2411 & 0.2382 & & 24.7 & 25.0 & -1.2 & 25.0 \\
\hline 1,2,4-Trichlorobenzene & Ave & 0.9693 & 0.9933 & & 25.6 & 25.0 & 2.5 & 25.0 \\
\hline Hexachlorobutadiene & Ave & 0.6761 & 0.7074 & & 26.2 & 25.0 & 4.6 & 25.0 \\
\hline Naphthalene & Ave & 1.387 & 1.423 & & 25.7 & 25.0 & 2.6 & 25.0 \\
\hline 1,2,3-Trichlorobenzene & Ave & 0.8552 & 0.8729 & & 25.5 & 25.0 & 2.1 & 25.0 \\
\hline 1,2-Dichloroethane-d4 & Ave & 0.3099 & 0.3236 & & 26.1 & 25.0 & 4.4 & 25.0 \\
\hline Toluene-d8 & Ave & 1.089 & 1.116 & & 25.6 & 25.0 & 2.4 & 25.0 \\
\hline Bromofluorobenzene & Ave & 1.248 & 1.281 & & 25.6 & 25.0 & 2.6 & 25.0 \\
\hline 1,2-Dichlorobenzene-d 4 & Ave & 0.8371 & 0.8758 & & 26.2 & 25.0 & 4.6 & 25.0 \\
\hline
\end{tabular}


Lab Name: TestAmerica Burlington

SDG No.: INMAN (200-2608)

Lab Sample ID: CCVIS 200-10229/2

Instrument ID: L.i

GC Column: DB-624

ID: $0.53(\mathrm{~mm})$

Lab File ID: Ifme02.d

EPA Sample No.: CCVIS
Job No.: 200-2608-1

Calibration Date: 11/24/2010 08:22

Calib Start Date: 11/20/2010 15:36

Calib End Date: 11/20/2010 18:17

Conc. Units: ugg/Kg Heated Purge: ( $Y / N) \quad N$

\begin{tabular}{|c|c|c|c|c|c|c|c|c|}
\hline ANALYTE & $\begin{array}{l}\text { CURVE } \\
\text { TYPE }\end{array}$ & AVE RRF & RRF & MIN RRF & $\begin{array}{c}\text { CALC } \\
\text { AMOUNT }\end{array}$ & $\begin{array}{c}\text { SPIKE } \\
\text { AMOUNT }\end{array}$ & $\frac{\circ}{0} \mathrm{D}$ & $\begin{array}{l}\text { MAX } \\
\frac{O}{6} D\end{array}$ \\
\hline Dichlorodifluoromethane & Ave & 0.5700 & 0.5333 & & 23.4 & 25.0 & -6.4 & 20.0 \\
\hline Chloromethane & Ave & 0.2854 & 0.2377 & 0.1000 & 20.8 & 25.0 & -16.7 & 20.0 \\
\hline Vinyl chloride & Ave & 0.3073 & 0.3128 & & 25.4 & 25.0 & 1.8 & 20.0 \\
\hline Bromomethane & Ave & 0.2116 & 0.1798 & & 21.2 & 25.0 & -15.1 & 20.0 \\
\hline ChIoroethane & Ave & 0.1833 & 0.1959 & & 26.7 & 25.0 & 6.9 & 20.0 \\
\hline Trichlorofluoromethane & Ave & 0.6612 & $0 . \overline{6617}$ & & 25.0 & 25.0 & 0.0 & 20.0 \\
\hline Acrolein & Ave & 0.0263 & 0.0254 & & 121 & 125 & -3.6 & 20.0 \\
\hline 1,1-Dichloroethene & Ave & 0.2976 & 0.2830 & & 23.8 & 25.0 & -4.9 & 20.0 \\
\hline Freon TF & Ave & 0.6005 & 0.6025 & & 25.1 & 25.0 & 0.3 & 20.0 \\
\hline Acetone & Ave & 0.0545 & 0.0465 & & 107 & 125 & -14.6 & 20.0 \\
\hline Methyl iodide & Ave & 0.5187 & 0.3881 & & 18.7 & 25.0 & $-25.2 *$ & 20.0 \\
\hline Carbon disulfide & Ave & 0.7625 & 0.7253 & & 23.8 & 25.0 & -4.9 & 20.0 \\
\hline Aliyl chloride & Ave & 0.5412 & 0.5265 & & 24.3 & 25.0 & -2.7 & 20.0 \\
\hline Methyl acetate & Ave & 0.0372 & 0.0349 & & 23.5 & 25.0 & -6.1 & 20.0 \\
\hline Methylene Chloride & Ave & 0.2716 & 0.2654 & & 24.4 & 25.0 & -2.3 & 20.0 \\
\hline tert-Butyl alcohol & Ave & 0.0180 & 0.0152 & & 423 & 500 & -15.3 & 20.0 \\
\hline Acrylonitrile & Ave & 0.0643 & 0.0598 & & 23.3 & 25.0 & -7.0 & 20.0 \\
\hline trans-1,2-Dichloroethene & Ave & 0.3171 & 0.3029 & & 23.9 & 25.0 & -4.5 & 20.0 \\
\hline Methyl t-butyl ether & Ave & 0.6232 & 0.6029 & & 24.2 & 25.0 & -3.3 & 20.0 \\
\hline$\overline{1}, 1$-Dichloroethane & Ave & 0.6443 & 0.6152 & 0.1000 & 23.9 & 25.0 & -4.5 & 20.0 \\
\hline Vinyl acetate & Ave & 0.6425 & 0.6940 & & 27.0 & 25.0 & 8.0 & 20.0 \\
\hline Chloroprene & Ave & 0.5373 & 0.5303 & & 24.7 & 25.0 & -1.3 & 20.0 \\
\hline 2,2-Dichloropropane & Ave & 0.5224 & 0.5370 & & 25.7 & 25.0 & 2.8 & 20.0 \\
\hline cis-1,2-Dichloroethene & Ave & 0.3338 & 0.3195 & & 23.9 & 25.0 & -4.3 & 20.0 \\
\hline 2-Butanone & Ave & $0.020,5$ & 0.0181 & & 110 & 125 & -11.9 & 20.0 \\
\hline Propionitrile & Ave & 0.0226 & 0.0204 & & 90.3 & 100 & -9.7 & 20.0 \\
\hline Methacrylonitrile & Ave & 0.0679 & 0.0688 & & 25.3 & 25.0 & 1.4 & 20.0 \\
\hline Bromoch loromethane & Ave & 0.2017 & 0.1960 & & 24.3 & 25.0 & -2.9 & 20.0 \\
\hline Tetrahydrofuran & Ave & 0.0734 & 0.0680 & & 324 & 350 & -7.4 & 20.0 \\
\hline Chloroform & Ave & 0.6443 & 0.6291 & & 24.4 & 25.0 & -2.4 & 20.0 \\
\hline 1,1,1-Trichloroethane & Ave & 0.5657 & 0.5558 & & 24.6 & 25.0 & -1.8 & 20.0 \\
\hline Cyclohexane & Ave & 0.5415 & 0.5251 & & 24.2 & 25.0 & -3.0 & 20.0 \\
\hline 1,1-Dichloropropene & Ave & 0.5161 & 0.5038 & & 24.4 & 25.0 & -2.4 & $20 . \overline{0}$ \\
\hline Carbon tetrachloride & Ave & 0.5376 & 0.5317 & & 24.7 & 25.0 & -1.1 & 20.0 \\
\hline Isobutyl alcohol & Ave & 0.0100 & 0.0096 & & 1200 & 1250 & -3.6 & $2 \overline{0} . \overline{0}$ \\
\hline Benzene & Ave & 0.8506 & 0.8380 & & 24.6 & 25.0 & -1.5 & $2 \overline{0} .0$ \\
\hline I,2-Dichloroethane & Ave & 0.3725 & 0.3574 & & 24.0 & 25.0 & $-4 . \overline{0}$ & 20.0 \\
\hline Trichloroethene & Ave & 0.3962 & 0.3916 & & 24.7 & 25.0 & -1.2 & 20.0 \\
\hline Methylcyclohexane & Ave & 0.4891 & 0.4970 & & 25.4 & 25.0 & 1.6 & 20.0 \\
\hline
\end{tabular}


FORM VII

GC/MS VOA CONTINUING CALIBRATION DATA

Lab Name: TestAmerica Burlington

SDG No.: INMAN (200-2608)

Lab Sample ID: CCVIS 200-10229/2

Instrument ID: L.i

GC Column: $\mathrm{DB}-624$

ID: $0.53(\mathrm{~mm})$

Lab File ID: Ifme02.d

EPA Sample No.: CCVIS
Job No.: 200-2608-1

Calibration Date: 11/24/2010 08:22

Calib Start Date: 11/20/2010 15:36

Calib End Date: 11/20/2010 18:17

Conc. Units: ug/Kg Heated Purge: ( $\mathrm{Y} / \mathrm{N}) \quad \mathrm{N}$

\begin{tabular}{|c|c|c|c|c|c|c|c|c|}
\hline ANALYTE & $\begin{array}{l}\text { CURVE } \\
\text { TYPE }\end{array}$ & AVE RRF & RRF & MIN RRF & $\begin{array}{c}\text { CALC } \\
\text { AMOUNT }\end{array}$ & $\begin{array}{l}\text { SPIKE } \\
\text { AMOUNT }\end{array}$ & $\frac{\circ}{8} \mathrm{D}$ & $\begin{array}{l}\text { MAX } \\
\frac{\circ}{8} D\end{array}$ \\
\hline 1,2-Dichloropropane & Ave & 0.3778 & 0.3636 & & 24.1 & 25.0 & -3.7 & 20.0 \\
\hline Dibromomethane & Ave & 0.3168 & 0.3027 & & 23.9 & 25.0 & -4.4 & 20.0 \\
\hline Methyl methacrylate & Ave & 0.2539 & 0.2372 & & 23.4 & 25.0 & -6.6 & 20.0 \\
\hline 1,4-Dioxane & Ave & 0.0020 & 0.0021 & & 1270 & 1250 & 1.8 & 20.0 \\
\hline Bromodichloromethane & Ave & 0.6209 & 0.6127 & & 24.7 & 25.0 & -1.3 & 20.0 \\
\hline 2-Chloroethyl vinyl ether & Ave & 0.2184 & 0.2197 & & 25.1 & 25.0 & 0.6 & 20.0 \\
\hline cis-1,3-Dichloropropene & Ave & 0.5364 & 0.5264 & & 24.5 & 25.0 & -1.9 & 20.0 \\
\hline 4-Methyl-2-pentanone & Ave & 0.3214 & 0.2882 & & 112 & 125 & -10.3 & 20.0 \\
\hline Toluene & Ave & 0.7495 & 0.7248 & & 24.2 & 25.0 & -3.3 & 20.0 \\
\hline trans-1,3-Dichloropropene & Ave & 0.5975 & 0.5687 & & 23.8 & 25.0 & -4.8 & 20.0 \\
\hline Ethyl methacrylate & Ave & 0.4210 & 0.4046 & & 24.0 & 25.0 & -3.9 & 20.0 \\
\hline $1,1,2$-Trichloroethane & Ave & 0.3319 & 0.3214 & & 24.2 & 25.0 & -3.2 & 20.0 \\
\hline Tetrachloroethene - & Ave & 0.6039 & 0.6090 & & 25.2 & 25.0 & 0.9 & 20.0 \\
\hline 1,3-Dichloropropane & Ave & 0.6241 & 0.6151 & & 24.6 & 25.0 & -1.4 & 20.0 \\
\hline 2-Hexanone & Ave & 0.2690 & 0.2411 & & 112 & 125 & -10.3 & 20.0 \\
\hline Dibromochloromethane & Ave & 0.6819 & 0.6725 & & 24.7 & 25.0 & -1.4 & 20.0 \\
\hline 1,2-Dibromoethane & Ave & 0.5913 & 0.5711 & & 24.1 & 25.0 & -3.4 & 20.0 \\
\hline Chlorobenzene & Ave & 0.9515 & 0.9413 & 0.3000 & 24.7 & 25.0 & -1.1 & 20.0 \\
\hline $1,1,1,2$-Tetrachloroethane & Ave & 0.5045 & 0.4992 & & 24.7 & 25.0 & -1.1 & 20.0 \\
\hline Ethylbenzene & Ave & 1.549 & 1.527 & & 24.6 & 25.0 & -1.4 & 20.0 \\
\hline$m \& p-X y l e n e$ & Ave & 0.5588 & 0.5604 & & 50.1 & 50.0 & 0.3 & 20.0 \\
\hline o-Xylene & Ave & 0.5289 & 0.5330 & & 25.2 & 25.0 & 0.8 & 20.0 \\
\hline Styrene & Ave & 0.9129 & 0.9141 & & 25.0 & 25.0 & 0.1 & 20.0 \\
\hline Bromoform & Ave & 0.5256 & 0.5194 & 0.1000 & 24.7 & 25.0 & -1.2 & 20.0 \\
\hline Isopropylbenzene & Ave & 2.751 & 2.672 & & 24.3 & 25.0 & -2.9 & 20.0 \\
\hline Bromobenzene & Ave & 0.8783 & 0.8501 & & 24.2 & 25.0 & -3.2 & 20.0 \\
\hline $1,1,2,2$-Tetrachloroethane & Ave & 1.087 & 1.048 & 0.3000 & 24.1 & 25.0 & -3.6 & 20.0 \\
\hline 1,2,3-Trichloropropane & Ave & 0.2802 & 0.2558 & & 22.8 & 25.0 & -8.7 & 20.0 \\
\hline trans-1,4-Dichloro-2-butene & Ave & 0.2758 & 0.2448 & & 22.2 & 25.0 & -11.2 & 20.0 \\
\hline n-Propylbenzene & Ave & 0.6857 & 0.6717 & & 24.5 & 25.0 & -2.0 & 20.0 \\
\hline 2-Chlorotoluene & Ave & 0.6440 & 0.6146 & & 23.9 & 25.0 & -4.6 & 20.0 \\
\hline 1,3,5-Trimethylbenzene & Ave & 2.017 & 1.944 & & 24.1 & 25.0 & -3.6 & 20.0 \\
\hline 4-Chlorotoluene & Ave & 0.6418 & 0.6119 & & 23.8 & 25.0 & -4.7 & 20.0 \\
\hline tert-Butylbenzene & Ave & 2.265 & 2.211 & & 24.4 & 25.0 & -2.4 & 20.0 \\
\hline 1,2,4-Trimethylbenzene & Ave & 1.968 & 1.905 & & 24.2 & 25.0 & -3.2 & 20.0 \\
\hline sec-Butylbenzene & Ave & 3.049 & 3.004 & & 24.6 & 25.0 & -1.5 & 20.0 \\
\hline 1,3-Dichlorobenzene & Ave & 1.373 & 1.340 & & 24.4 & 25.0 & $-2,4$ & 20.0 \\
\hline 4-Isopropyltoluene & Ave & 2.476 & 2.429 & & 24.5 & 25.0 & -1.9 & 20.0 \\
\hline 1,4-Dichlorobenzene & Ave & 1.450 & 1.422 & & 24.5 & 25.0 & -1.9 & 20.0 \\
\hline
\end{tabular}


FORM VII

GC/MS VOA CONTINUING CALIBRATION DATA

Lab Name: TestAmerica Burlington

Job No.: 200-2608-1

SDG NO.: INMAN (200-2608)

Lab Sample ID: CCVIS 200-10229/2

Calibration Date: 11/24/2010 08:22

Instrument ID: I.i

GC Column: DB-624

ID : $0.53(\mathrm{~mm})$

Calib Start Date: 11/20/2010 15:36

Lab File ID: Ifme02.d

Calib End Date: 11/20/2010 18:17

Conc. Units: ug/Kg

Heated Purge: ( $\mathrm{Y} / \mathrm{N})$ N

EPA Sample No.: CCVIS

\begin{tabular}{|c|c|c|c|c|c|c|c|c|}
\hline ANALYTE & $\begin{array}{l}\text { CURVE } \\
\text { TYPE }\end{array}$ & AVE RRF & RRF & MIN RRF & $\begin{array}{c}\text { CALC } \\
\text { AMOUNT }\end{array}$ & $\begin{array}{l}\text { SPIKE } \\
\text { AMOUNT }\end{array}$ & $8 \mathrm{D}$ & $\begin{array}{l}\text { MAX } \\
8 D\end{array}$ \\
\hline 1,2-Dichlorobenzene & Ave & 1.258 & 1.228 & & 24.4 & 25.0 & -2.4 & 20.0 \\
\hline n-Butylbenzene & Ave & 2.228 & 2.228 & & 25.0 & 25.0 & -0.0 & 20.0 \\
\hline 1,2-Dibromo-3-Chloropropane & Ave & 0.2411 & 0.2248 & & 23.3 & 25.0 & -6.8 & 20.0 \\
\hline 1,2,4-Trichlorobenzene & Ave & 0.9693 & 0.9464 & & 24.4 & 25.0 & -2.4 & 20.0 \\
\hline Hexachlorobutadiene & Ave & 0.6761 & 0.6902 & & 25.5 & 25.0 & 2.1 & 20.0 \\
\hline Naphthalene & Ave & 1.387 & 1.289 & & 23.2 & 25.0 & -7.1 & 20.0 \\
\hline $1,2,3$-Trichlorobenzene & Ave & 0.8552 & 0.8130 & & 23.8 & 25.0 & -4.9 & 20.0 \\
\hline 1,2-Dichloroethane-d4 & Ave & 0.3099 & 0.3146 & & 25.4 & 25.0 & 1.5 & 20.0 \\
\hline Toluene-d8 & Ave & 1.089 & 1.061 & & 24.3 & 25.0 & -2.6 & 20.0 \\
\hline Bromofluorobenzene & Ave & 1.248 & 1.188 & & 23.8 & 25.0 & -4.8 & 20.0 \\
\hline 1,2-Dichlorobenzene-d4 & Ave & 0.8371 & 0.8225 & & 24.6 & 25.0 & -1.7 & 20.0 \\
\hline
\end{tabular}


FORM I

GC/MS VOA ORGANICS ANALYSIS DATA SHEET

Lab Name: TestAmerica Burlington

SDG No.: INMAN (200-2608)

Client Sample ID:

Matrix: Solid

Analysis Method: $8260 \mathrm{~B}$

Sample wt/vol: $10(\mathrm{~g})$

Soil Aliquot Vol: 0.5 (m工)

Soil Extract Vol.: $10(\mathrm{~mL})$

o Moisture:

Analysis Batch No.: 10229
Job No.: 200-2608-1

Lab Sample ID: MB 200-10089/3-A

Lab File ID: lfme07.d

Date Collected:

Date Analyzed: 11/24/2010 11:03

Dilution Factor: 1

GC Coliumn: DB-624

ID: $0.53(\mathrm{~mm})$

Level: (low/med) Medium

Units: $\mathrm{ug} / \mathrm{Kg}$

\begin{tabular}{|c|c|c|c|c|c|}
\hline CAS NO. & COMPOUND NAME & RESULT & $Q$ & RL & MDL \\
\hline $75-71-8$ & Dichlorodifluoromethane & 10 & $\mathrm{U}$ & 10 & 5.0 \\
\hline $74-87-3$ & Chloromethane & 5.14 & $\mathrm{~J}$ & 10 & 3.7 \\
\hline $75-01-4$ & Vinyl chloride & 10 & $\mathrm{U}$ & 10 & 5.0 \\
\hline $74-83-9$ & Bromomethane & 8.87 & $\mathrm{~J}$ & 10 & 3.5 \\
\hline $75-00-3$ & Chloroethane & 10 & $\mathrm{U}$ & 10 & 6.0 \\
\hline $75-69-4$ & Trichlorofluoromethane & 10 & $\mathrm{U}$ & 10 & 5.0 \\
\hline $75-35-4$ & 1,1-Dichloroethene & 10 & $\mathrm{U}$ & 10 & 2.1 \\
\hline $76-13-1$ & Freon TF & 10 & $\mathrm{U}$ & 10 & 2.8 \\
\hline $67-64-1$ & Acetone & 10 & $\mathrm{U}$ & 10 & 10 \\
\hline $74-88-4$ & Methyl iodide & 10 & $\mathrm{U}$ & 10 & 5.0 \\
\hline $75-15-0$ & Carbon disulfide & 10 & $\mathrm{U}$ & 10 & 1.9 \\
\hline $79-20-9$ & Methyl acetate & 10 & $\mathrm{U}$ & 10 & 5.0 \\
\hline $75-09-2$ & Methylene Chloride & 10 & $\mathrm{U}$ & 10 & 5.0 \\
\hline $156-60-5$ & trans-1,2-Dichloroethene & 10 & $\mathrm{U}$ & 10 & 5.0 \\
\hline $1634-04-4$ & Methyl t-butyl ether & 10 & $\mathrm{U}$ & 10 & 5.0 \\
\hline $75-34-3$ & 1,1-Dichloroethane & 10 & $\mathrm{U}$ & 10 & 3.6 \\
\hline $108-05-4$ & Vinyl acetate & 10 & $\mathrm{U}$ & 10 & 5.0 \\
\hline $594-20-7$ & 2,2-Dichloropropane & 10 & $\mathrm{U}$ & 10 & 4.6 \\
\hline $156-59-2$ & cis-1,2-Dichloroethene & 10 & $\mathrm{U}$ & 10 & 2.1 \\
\hline $78-93-3$ & 2-Butanone & 10 & $\mathrm{U}$ & 10 & 11 \\
\hline $74-97-5$ & Bromochloromethane & 10 & $\mathrm{U}$ & 10 & 5.3 \\
\hline $109-99-9$ & Tetrahydrofuran & 100 & $\mathrm{U}$ & 100 & 50 \\
\hline $67-66-3$ & Chloroform & 10 & $\mathrm{U}$ & 10 & 3.3 \\
\hline $71-55-6$ & 1,1,1-Trichloroethane & 10 & $\mathrm{U}$ & 10 & 3.6 \\
\hline $110-82-7$ & Cyclohexane & 10 & $\mathrm{U}$ & 10 & 3.5 \\
\hline $563-58-6$ & 1,1-Dichloropropene & 10 & $\mathrm{U}$ & 10 & 2.2 \\
\hline $56-23-5$ & Carbon tetrachloride & 10 & $\mathrm{U}$ & 10 & 3.2 \\
\hline $78-83-1$ & Isobutyl alcohol & 500 & $\mathrm{U}$ & 500 & 250 \\
\hline $71-43-2$ & Benzene & 10 & $\mathrm{U}$ & 10 & 3.1 \\
\hline $107-06-2$ & 1,2-Dichloroethane & 10 & $\mathrm{U}$ & 10 & 3.3 \\
\hline $79-01-6$ & Trichloroethene & 10 & U & 10 & 5.0 \\
\hline $108-87-2$ & Methylcyclohexane & 10 & $\mathrm{U}$ & 10 & 5.0 \\
\hline $78-87-5$ & 1,2-Dichloropropane & 10 & $\mathrm{U}$ & 10 & 3.8 \\
\hline $74-95-3$ & Dibromomethane & 10 & $\mathrm{U}$ & 10 & 2.5 \\
\hline $123-91-1$ & 1,4-Dioxane & 500 & $\mathrm{U}$ & 500 & 270 \\
\hline $75-27-4$ & Bromodichloromethane & 10 & $\mathrm{U}$ & 10 & 3.7 \\
\hline
\end{tabular}


FORM I

GC/MS VOA ORGANICS ANALYSIS DATA SHEET

Lab Name: TestAmerica Burlington

SDG No.: INMAN (200-2608)

Client Sample ID:

Matrix: Solid

Analysis Method: $8260 \mathrm{~B}$

Sample wt/vol: $10(\mathrm{~g})$

Soil Aliquot Vol: 0.5 (m山)

Soil Extract Vol.: $10(\mathrm{~mL})$

음 Moisture:

Analysis Batch No.: 10229
Job No.: 200-2608-1

Lab Sample ID: MB 200-10089/3-A

Lab File ID: lfme07.d

Date Collected:

Date Analyzed: 11/24/2010 11:03

Dilution Factor: 1

GC Column: DB-624

ID : $0.53(\mathrm{~mm})$

Level: (low/med) Medium

Units: ug/Kg

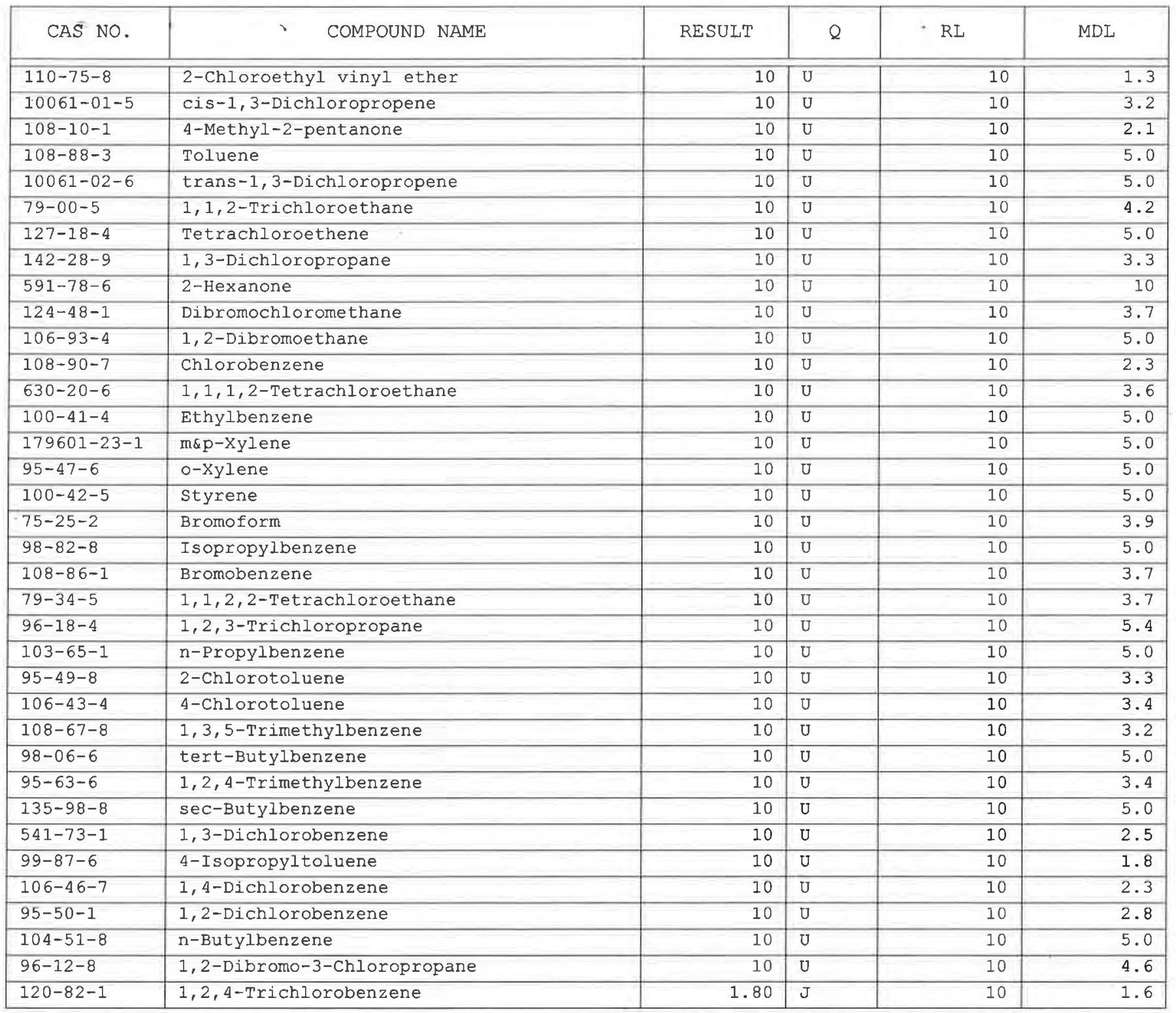


FORM I

GC/MS VOA ORGANICS ANALYSIS DATA SHEET

Lab Name: TestAmerica Burlington

SDG No.: INMAN (200-2608)

Client Sample ID:

Matrix: Solid

Analysis Method: $8260 \mathrm{~B}$

Sample wt/vol: $10(\mathrm{~g})$

Soil Aliquot Vol: 0.5 (mL)

Soil Extract Vol.: $10(\mathrm{~mL})$

응 Moisture:

Analysis Batch No.: 10229
Job No.: 200-2608-1

Lab Sample ID: MB 200-10089/3-A

Lab File ID: lfme07.d

Date collected:

Date Analyzed: 11/24/2010 11:03

Dilution Factor: 1

GC Column: DB-624

ID: $0.53(\mathrm{~mm})$

Level: (low/med) Medium

Units: ug/Kg

\begin{tabular}{|l|l|r|r|r|r|}
\hline \multicolumn{1}{|c|}{ CAS NO. } & \multicolumn{1}{|c|}{ COMPOUND NAME } & RESULT & Q & RL & MDI \\
\hline \hline $87-68-3$ & Hexachlorobutadiene & 10 & U & 10 & 5.0 \\
\hline $91-20-3$ & Naphthalene & 10 & U & 10 & 5.0 \\
\hline $87-61-6$ & $1,2,3-T r i c h l o r o b e n z e n e$ & 10 & U & & 10 \\
\hline
\end{tabular}

\begin{tabular}{|c|c|c|c|c|}
\hline CAS NO. & SURROGATE & 을 & $Q$ & LIMITS \\
\hline $17060-07-0$ & 1,2-Dichloroethane-d4 & 90 & & $65-155$ \\
\hline $2037-26-5$ & Toluene-d8 & 102 & & $80-115$ \\
\hline $460-00-4$ & Bromofluorobenzene & 100 & & $80-115$ \\
\hline $2199-69-1$ & 1,2-Dichlorobenzene-d4 & 101 & & $45-145$ \\
\hline
\end{tabular}


FORM I

GC/MS VOA ORGANICS ANALYSIS DATA SHEET

Lab Name: TestAmerica Burlington

Job No.: 200-2608-1

SDG NO.: INMAN (200-2608)

Client Sample ID:

Matrix: Solid

Analysis Method: $8260 \mathrm{~B}$

Sample wt/vol: $10(\mathrm{~g})$

Soil Aliquot Vol: 0.5 (m山)

Soil Extract Vol.: $10(\mathrm{~mL})$

\% Moisture:

Analysis Batch No.: 10229

Lab Sample ID: LCS 200-10089/6-A

Lab File ID: Ifme04.d

Date Collected:

Date Analyzed: 11/24/2010 09:26

Dilution Factor: 1

GC Column: DB-624

ID : 0.53 (Im)

Level: (low/med) Medium

Units: $u g / \mathrm{Kg}$

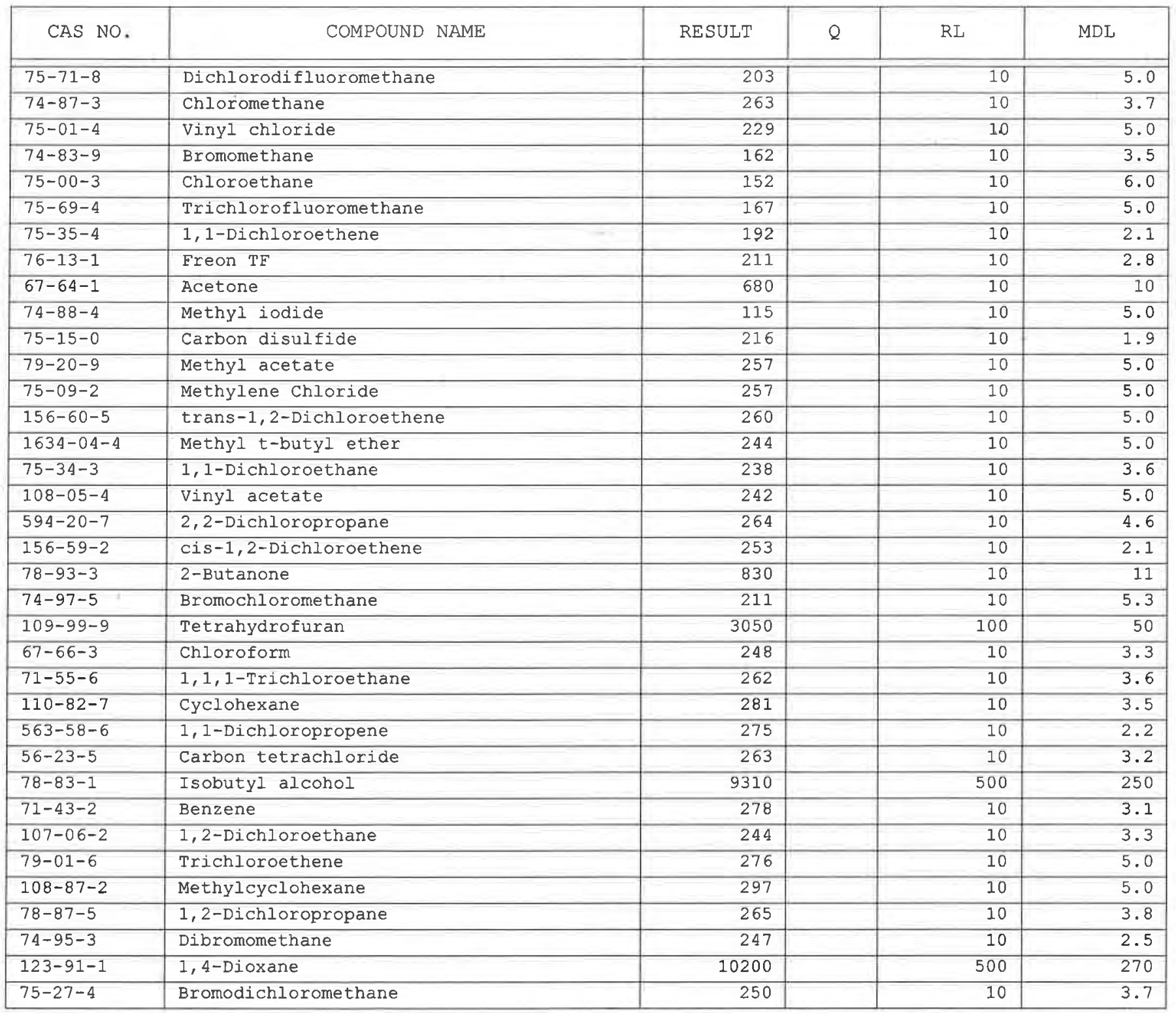

FORM I $8260 \mathrm{~B}$ 
Lab Name: TestAmerica Burlington

SDG NO.: INMAN (200-2608)

Client Sample ID:

Matrix: Solid

Analysis Method: $8260 \mathrm{~B}$

Sample wt/vol: $10(\mathrm{~g})$

Soil Aliquot Vol: 0.5 (mL)

Soil Extract Vol.: 10 (mL)

\% Moisture:

Analysis Batch No.: 10229
Job No.: 200-2608-1

Lab Sample ID: LCS 200-10089/6-A

Lab File ID: Ifme04.d

Date Collected:

Date Analyzed: 11/24/2010 09:26

Dilution Factor: 1

GC Column: $\mathrm{DB}-624$

ID: 0.53 (mm)

Level: (low/med) Medium

Units: ug/Kg

\begin{tabular}{|c|c|c|c|c|c|}
\hline CAS NO. & COMPOUND NAME & RESULT & $Q$ & RL & MDL \\
\hline $110-75-8$ & 2-Chloroethyl vinyl ether & 209 & & 10 & 1.3 \\
\hline $10061-01-5$ & cis-1,3-Dichloropropene & 265 & & 10 & 3.2 \\
\hline $108-10-1$ & 4-Methyl-2-pentanone & 1080 & & 10 & 2.1 \\
\hline $108-88-3$ & Toluene & 290 & & 10 & 5.0 \\
\hline $10061-02-6$ & trans-1,3-Dichloropropene & 267 & & 10 & 5.0 \\
\hline $79-00-5$ & 1,1,2-Trichloroethane & 269 & & 10 & 4.2 \\
\hline $127-18-4$ & Tetrachloroethene & 299 & & 10 & 5.0 \\
\hline $142-28-9$ & 1,3-Dichloropropane & 273 & & 10 & 3.3 \\
\hline $591-78-6$ & 2-Hexanone & 1110 & & 10 & 10 \\
\hline $124-48-1$ & Dibromochlorome thane & 269 & & 10 & 3.7 \\
\hline $106-93-4$ & 1,2-Dibromoethane & 269 & & 10 & 5.0 \\
\hline $108-90-7$ & Chlorobenzene & 279 & & 10 & 2.3 \\
\hline $630-20-6$ & $1,1,1,2$-Tetrachloroethane & 272 & & 10 & 3.6 \\
\hline $100-41-4$ & Ethylbenzene & 281 & & 10 & 5.0 \\
\hline $179601-23-1$ & m\&p-Xylene & 571 & & 10 & 5.0 \\
\hline $95-47-6$ & o-Xylene & 280 & & 10 & 5.0 \\
\hline $100-42-5$ & Styrene & 271 & & 10 & 5.0 \\
\hline $75-25-2$ & Bromoform & 247 & & 10 & 3.9 \\
\hline $98-82-8$ & Isopropylbenzene & 291 & & 10 & 5.0 \\
\hline $108-86-1$ & Bromobenzene & 278 & & 10 & 3.7 \\
\hline $79-34-5$ & $1,1,2,2$-Tetrachloroethane & 260 & & 10 & 3.7 \\
\hline $96-18-4$ & 1,2,3-Trichloropropane & 221 & & 10 & 5.4 \\
\hline $103-65-1$ & n-Propylbenzene & 291 & & 10 & 5.0 \\
\hline $95-49-8$ & 2-Chlorotoluene & 281 & & 10 & 3.3 \\
\hline $106-43-4$ & 4-Chlorotoluene & 286 & & 10 & 3.4 \\
\hline $108-67-8$ & 1,3,5-Trimethylbenzene & 284 & & 10 & 3.2 \\
\hline $98-06-6$ & tert-Butylbenzene & 285 & & 10 & 5.0 \\
\hline $95-63-6$ & $1,2,4$-Trimethylbenzene & 284 & & 10 & 3.4 \\
\hline $135-98-8$ & sec-Butylbenzene & 296 & & 10 & 5.0 \\
\hline $541-73-1$ & 1,3-Dichlorobenzene & 282 & & 10 & 2.5 \\
\hline $99-87-6$ & 4-Isopropyltoluene & 284 & & 10 & 1.8 \\
\hline $106-46-7$ & 1,4-Dichlorobenzene & 279 & & 10 & 2.3 \\
\hline $95-50-1$ & 1,2-Dichlorobenzene & 279 & & 10 & 2.8 \\
\hline $104-51-8$ & n-Butylbenzene & 312 & $=$ & 10 & 5.0 \\
\hline $96-12-8$ & 1,2-Dibromo-3-Chloropropane & 215 & & 10 & 4.6 \\
\hline $120-82-1$ & 1,2,4-Trichlorobenzene & 295 & & 10 & 1.6 \\
\hline
\end{tabular}

FORM I $8260 \mathrm{~B}$ 
FORM I

GC/MS VOA ORGANICS ANALYSIS DATA SHEET

Lab Name: TestAmerica Burlington

SDG No.: INMAN (200-2608)

Client Sample ID:

Matrix: Solid

Analysis Method: $8260 \mathrm{~B}$

Sample wt/vol: $10(\mathrm{~g})$

Soil Aliquot Vol: 0.5 (mL)

Soil Extract Vol.: $10(\mathrm{~mL})$

음 Moisture:

Analysis Batch No.: 10229
Job No.: 200-2608-1

Lab Sample ID: LCS 200-10089/6-A

Lab File ID: Ifme04.d

Date Collected:

Date Analyzed: 11/24/2010 09:26

Dilution Factor: 1

GC Column: DB-624

ID : $0.53(\mathrm{~mm})$

Level: (low/med) Medium

Units: ug/ $\mathrm{kg}$

\begin{tabular}{|c|c|c|c|c|c|}
\hline CAS NO. & COMPOUND NAME & RESULT & $Q$ & RL & MDL \\
\hline $87-68-3$ & Hexachlorobutadiene & 331 & & 10 & 5.0 \\
\hline $91-20-3$ & Naphthalene & 255 & & 10 & 5.0 \\
\hline $87-61-6$ & 1,2,3-Trichlorobenzene & 279 & & 10 & 2.5 \\
\hline
\end{tabular}

\begin{tabular}{|l|l|r|r|r|}
\hline \multicolumn{1}{|c|}{ CAS NO. } & \multicolumn{1}{|c|}{ SURROGATE } & 各REC & Q & \multicolumn{1}{c|}{ LIMITS } \\
\hline $17060-07-0$ & $1,2-$ Dichloroethane-d4 & 93 & \\
\hline $2037-26-5$ & Toluene-d8 & 114 & & $85-155$ \\
\hline $460-00-4$ & Bromofluorobenzene & 107 & & $80-115$ \\
\hline $2199-69-1$ & $1,2-$ Dichlorobenzene-d4 & 107 & & $45-145$ \\
\hline
\end{tabular}


FORM I

GC/MS VOA ORGANICS ANALYSIS DATA SHEET

Lab Name: TestAmerica Burlington

SDG NO.: INMAN (200-2608)

Client Sample ID:

Matrix: Solid

Analysis Method: $8260 \mathrm{~B}$

Sample wt/vol: $5(\mathrm{~mL})$

Soil Aliquot Vol: 5 (m山)

Soil Extract Vol.: $5(\mathrm{~mL})$

응 Moisture:

Analysis Batch No.: 10229
Job No.: 200-2608-1

Lab Sample ID: LCS 200-10229/3

Lab File ID: Ifme03.d

Date Collected:

Date Analyzed: 11/24/2010 08:54

Dilution Factor: 1

GC Column: DB-624

ID: $0.53(\mathrm{~mm})$

Level: (low/med) Medium

Units: $\underline{u g} / \mathrm{Kg}$

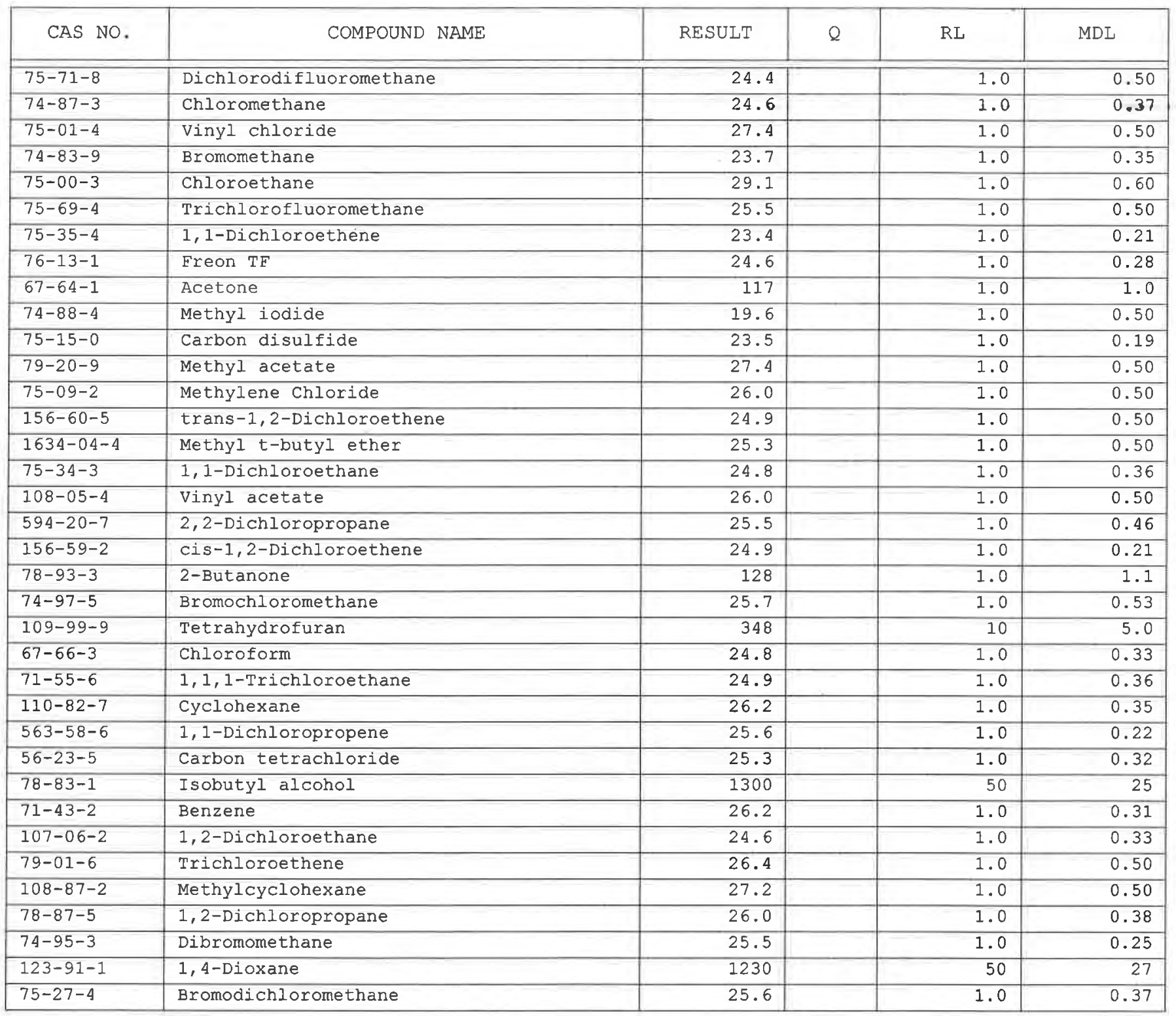


FORM I

GC/MS VOA ORGANICS ANALYSIS DATA SHEET

Lab Name: TestAmerica Burlington

SDG NO.: INMAN (200-2608)

Client Sample ID:

Matrix: Solid

Analysis Method: $8260 \mathrm{~B}$

Sample wt/vol: $5(\mathrm{~mL})$

Soil Aliquot Vol: $5(\mathrm{~mL})$

Soil Extract Vol.: 5 (mL)

\% Moisture:

Analysis Batch No.: 10229
Job No.: 200-2608-1

Lab Sample ID: LCS 200-10229/3

Lab File ID: lfme03.d

Date Collected:

Date Analyzed: 11/24/2010 08:54

Dilution Factor: 1

GC Column: DB-624

ID $: 0.53(\mathrm{~mm})$

Level: (low/med) Medium

Units: $\mathrm{ug} / \mathrm{Kg}$

\begin{tabular}{|c|c|c|c|c|c|}
\hline CAS NO. & COMPOUND NAME & RESULT & $Q$ & RL & MDL \\
\hline $110-75-8$ & 2-Chloroethyl vinyl ether & 26.9 & & 1.0 & 0.13 \\
\hline $10061-01-5$ & cis-1,3-Dichloropropene & 25.8 & & 1.0 & 0.32 \\
\hline $108-10-1$ & 4-Methyl-2-pentanone & $1 \overline{3}$ & & 1.0 & 0.21 \\
\hline $108-88-3$ & Toluene & 26.5 & & 1.0 & 0.50 \\
\hline $10061-02-6$ & trans-1,3-Dichloropropene & 25.1 & & 1.0 & 0.50 \\
\hline $79-00-5$ & $1,1,2$-Trichloroethane & 26.7 & & 1.0 & 0.42 \\
\hline $127-18-4$ & Tetrachloroethene & 27.0 & & 1.0 & 0.50 \\
\hline $142-28-9$ & 1,3-Dichloropropane & 26.3 & & 1.0 & 0.33 \\
\hline $591-78-6$ & 2-Hexanone & 138 & & 1.0 & 1.0 \\
\hline $124-48-1$ & Dibromochloromethane & 26.6 & & 1.0 & 0.37 \\
\hline $106-93-4$ & 1,2-Dibromoethane & 26.5 & & 1.0 & 0.50 \\
\hline $108-90-7$ & Chlorobenzene & 26.4 & & 1.0 & 0.23 \\
\hline $630-20-6$ & $1,1,1,2$-Tetrachloroethane & 26.3 & & 1.0 & 0.36 \\
\hline $100-41-4$ & Ethylbenzene & 26.3 & & 1.0 & 0.50 \\
\hline $179601-23-1$ & m\&p-xylene & 53.3 & & 1.0 & 0.50 \\
\hline $95-47-6$ & o-Xylene & 26.7 & & 1.0 & 0.50 \\
\hline $100-42-5$ & Styrene & 26.3 & & 1.0 & 0.50 \\
\hline $75-25-2$ & Bromoform & 26.1 & & 1.0 & 0.39 \\
\hline $98-82-8$ & Isopropylbenzene & 26.8 & & 1.0 & 0.50 \\
\hline $108-86-1$ & Bromobenzene & 26.6 & & 1.0 & 0.37 \\
\hline $79-34-5$ & $1,1,2,2$-Tetrachloroethane & 27.4 & & 1.0 & 0.37 \\
\hline $96-18-4$ & 1,2,3-Trichloropropane & 23.4 & & 1.0 & 0.54 \\
\hline $103-65-1$ & n-Propylbenzene & 26.7 & & 1.0 & 0.50 \\
\hline $95-49-8$ & 2-Chlorotoluene & 26.5 & & 1.0 & 0.33 \\
\hline $106-43-4$ & 4-Chlorotoluene & 26.7 & & 1.0 & 0.34 \\
\hline $108-67-8$ & 1,3,5-Trimethylbenzene & 26.4 & & 1.0 & 0.32 \\
\hline $98-06-6$ & tert-Butylbenzene & 26.4 & & 1.0 & 0.50 \\
\hline $95-63-6$ & 1,2,4-Trimethylbenzene & 26.4 & & 1.0 & 0.34 \\
\hline $135-98-8$ & sec-Butylbenzene & 26.8 & & 1.0 & 0.50 \\
\hline $541-73-1$ & 1,3-Dichlorobenzene & 26.3 & & 1.0 & 0.25 \\
\hline $99-87-6$ & A-Isopropyltoluene & 25.9 & & 1.0 & 0.18 \\
\hline $106-46-7$ & 1,4-Dichlorobenzene & 26.3 & & 1.0 & 0.23 \\
\hline $95-50-1$ & 1,2-Dichlorobenzene & 26.7 & & 1.0 & 0.28 \\
\hline $104-51-8$ & n-Butylbenzene & 27.4 & & 1.0 & 0.50 \\
\hline $96-12-8$ & 1,2-Dibromo-3-Chloropropane & 25.0 & & 1.0 & 0.46 \\
\hline $120-82-1$ & 1,2,4-Trichlorobenzene & 26.9 & & 1.0 & 0.16 \\
\hline
\end{tabular}


FORM I

GC/MS VOA ORGANICS ANALYSIS DATA SHEET

Lab Name: TestAmerica Burlington

SDG NO.: INMAN (200-2608)

Client Sample ID:

Matrix: Solid

Analysis Method: $8260 \mathrm{~B}$

Sample wt/vol: $5(\mathrm{~mL})$

Soil Aliquot Vol: 5 (mL)

Soil Extract Vol.: 5 (mL)

\% Moisture:

Analysis Batch No.: 10229
Job No.: 200-2608-1

Lab Sample ID: LCS 200-10229/3

Lab File ID: Ifme03.d

Date Collected:

Date Analyzed: 11/24/2010 08:54

Dilution Factor: 1

GC Column: DB-624 ID: $0.53(\mathrm{~mm})$

Level: (low/med) Medium

Units: ug/ $\mathrm{kg}$

\begin{tabular}{|c|c|c|c|c|c|}
\hline CAS NO. & COMPOUND NAME & RESULT & $Q$ & RL & MDL \\
\hline $87-68-3$ & Hexachlorobutadiene & 28.3 & & 1.0 & 0.50 \\
\hline $91-20-3$ & Naphthalene & 27.4 & & 1.0 & 0.50 \\
\hline $87-61-6$ & $1,2,3$-Trichlorobenzene & 27.5 & & 1.0 & 0.25 \\
\hline
\end{tabular}

\begin{tabular}{|l|l|r|r|r|}
\hline \multicolumn{1}{|c|}{ CAS NO. } & \multicolumn{1}{|c|}{ SURROGATE } & \%REC & Q & LIMITS \\
\hline \hline $17060-07-0$ & $1,2-$ Dichloroethane-d4 & 98 & \\
\hline $2037-26-5$ & Toluene-d8 & 108 & \\
\hline $460-00-4$ & Bromofluorobenzene & 107 & $80-1155$ \\
\hline $2199-69-1$ & $1,2-$ Dichlorobenzene-d4 & 108 & $80-115$ \\
\hline
\end{tabular}


GC/MS VOA ANALYSIS RUN LOG

Lab Name: TestAmerica Burlington

Job No.: 200-2608-1

SDG NO.: INMAN (200-2608)

Instrument ID: L.i

Analysis Batch Number: 9968

Start Date: 11/20/2010 14:38

End Date: 11/20/2010 20:58

\begin{tabular}{|c|c|c|c|c|c|}
\hline LAB SAMPLE ID & CLIENT SAMPLE ID & DATE ANALYZED & $\begin{array}{l}\text { DILUTION } \\
\text { FACTOR }\end{array}$ & IAB FILE ID & COLUMN ID \\
\hline BFB 200-9968/1 & & $11 / 20 / 2010 \quad 14: 38$ & 1 & & DB-624 $0.53(\mathrm{~mm})$ \\
\hline BFB $200-9 \overline{968 / 2}$ & & $11 / 20 / 2010 \quad 14: 45$ & 1 & $1 \mathrm{fm02} \cdot \mathrm{d}$ & DB-624 0.53 (murn) \\
\hline VIBLK $200-9968 / 3$ & & $11 / 20 / 2010 \quad 15: 04$ & 1 & & DB-624 0.53 (mu) \\
\hline IC $200-9968 / 4$ & & $11 / 20 / 2010 \quad 15: 36$ & 1 & $\operatorname{lfm} 04 . d$ & DB-624 $0.53(\mathrm{~mm})$ \\
\hline IC $200-9968 / 5$ & & $11 / 20 / 2010 \quad 16: 09$ & 1 & $1 \mathrm{fm} 05 . d$ & DB-624 $0.53(\mathrm{~mm})$ \\
\hline IC $200-9968 / 6$ & & $11 / 20 / 2010 \quad 16: 41$ & 1 & $1 \mathrm{fm} 06 . d$ & $\mathrm{DB}-6240.53(\mathrm{~mm})$ \\
\hline ICIS $200-9968 / 7$ & & $11 / 20 / 2010 \quad 17: 13$ & 1 & $1 f m 07 . d$ & $\mathrm{DB}-6240.53(\mathrm{~mm})$ \\
\hline IC $200-9968 / 8$ & & $11 / 20 / 2010 \quad 17: 45$ & 1 & lfm08.d & $\mathrm{DB}-6240.53(\mathrm{~mm})$ \\
\hline IC $200-9968 / 9$ & & $11 / 20 / 2010 \quad 18: 17$ & 1 & lfmog.d & $\mathrm{DB}-624+\theta .53(\mathrm{~mm})$ \\
\hline VIBLK $200-9968 / 10$ & - & $11 / 20 / 2010 \quad 18: 49$ & 1 & & $\mathrm{DB}-624 \quad 0.53(\mathrm{~mm})$ \\
\hline VIBLK $200-9968 / 11$ & & $11 / 20 / 2010 \quad 19: 21$ & 1 & & $\mathrm{DB}-624 \quad 0.53(\mathrm{~mm})$ \\
\hline ICV 200-9968/12 & & $11 / 20 / 2010 \quad 19: 53$ & 1 & lfm12.d & $\mathrm{DB}-6240.53(\mathrm{~mm})$ \\
\hline ICV $200-9968 / 13$ & & $11 / 20 / 2010 \quad 20: 26$ & 1 & - & $\mathrm{DB}-624 \quad 0.53(\mathrm{~mm})$ \\
\hline VIBLK $200-9968 / 14$ & & $11 / 20 / 2010 \quad 20: 58$ & 1 & & $\mathrm{DB}-624 \quad 0.53(\mathrm{~mm})$ \\
\hline
\end{tabular}


Lab Name: TestAmerica Burlington

SDG No.: INMAN (200-2608)

Instrument ID: I.i

Analysis Batch Number: 10229
Job No.: 200-2608-1

Start Date: 11/24/2010 08:02

End Date: 11/24/2010 15:47

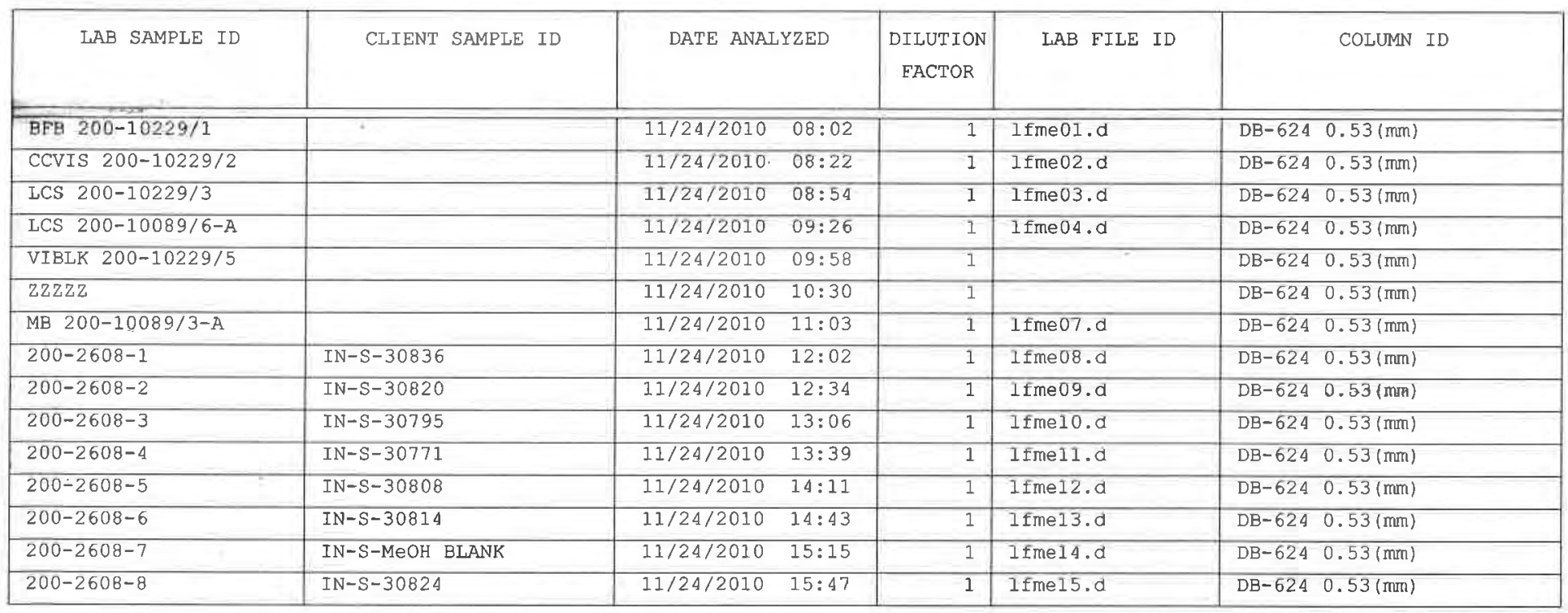




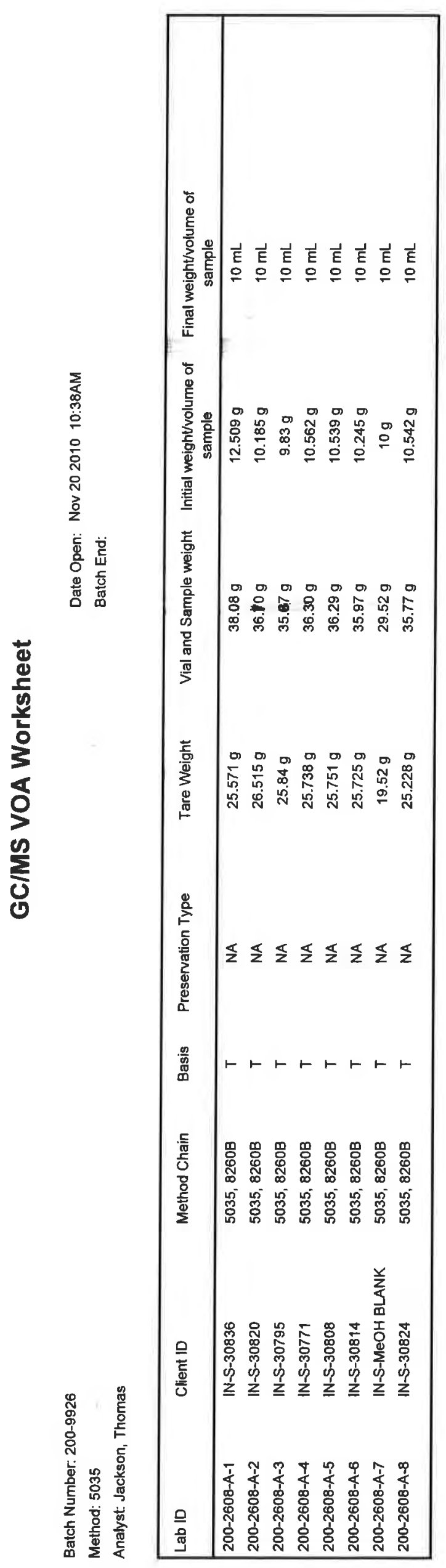

웅

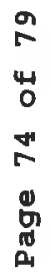




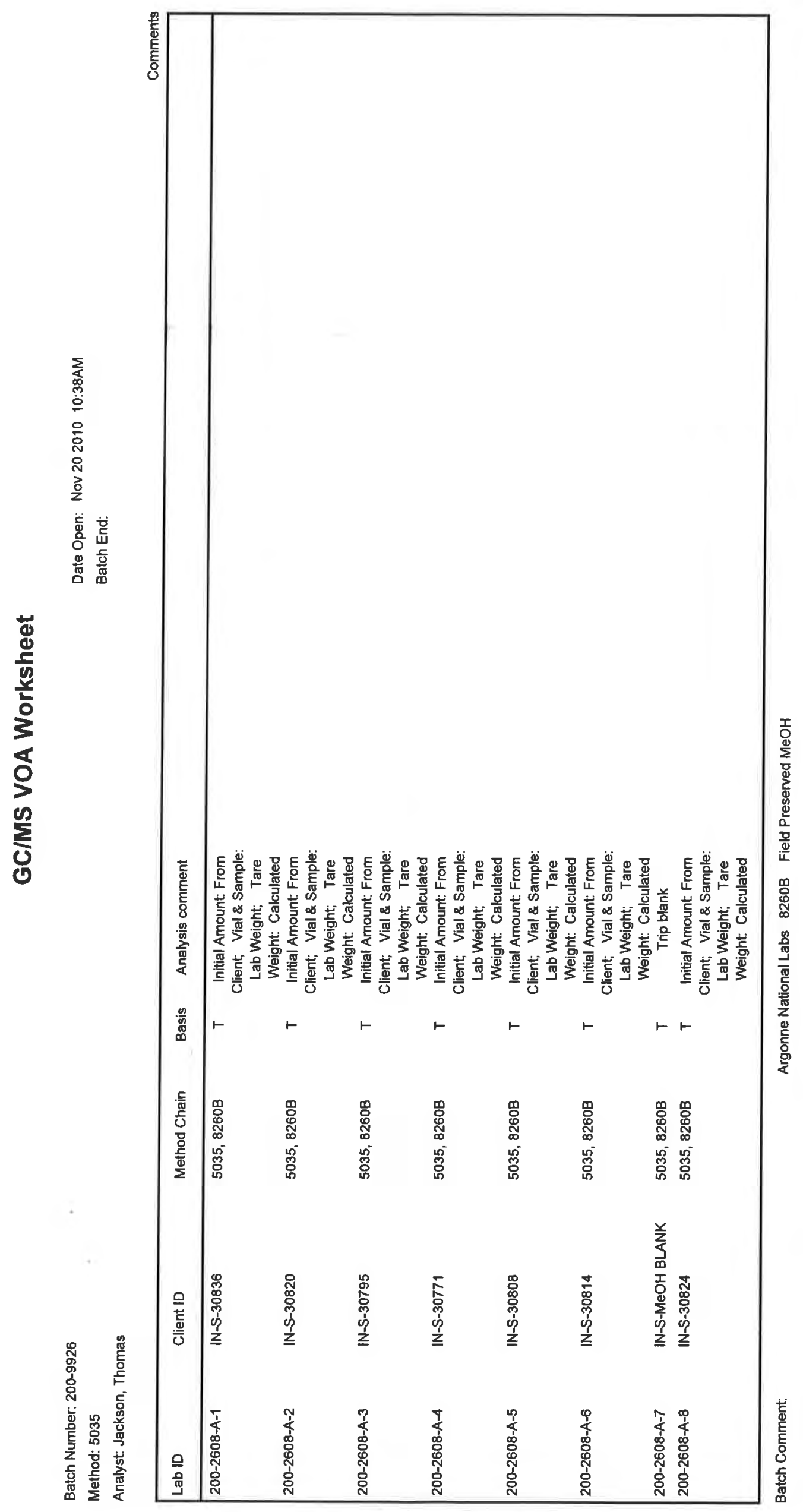

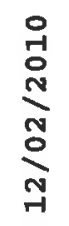

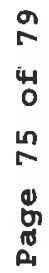




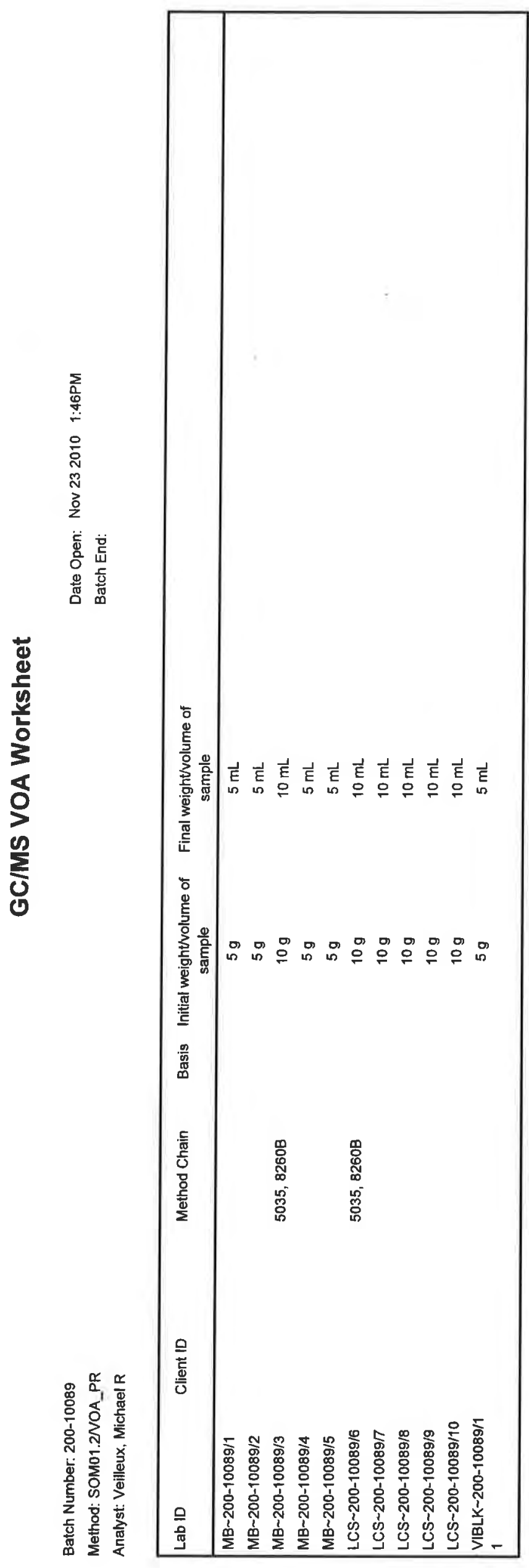

일

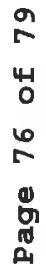




\section{Shipping and Receiving Documents}




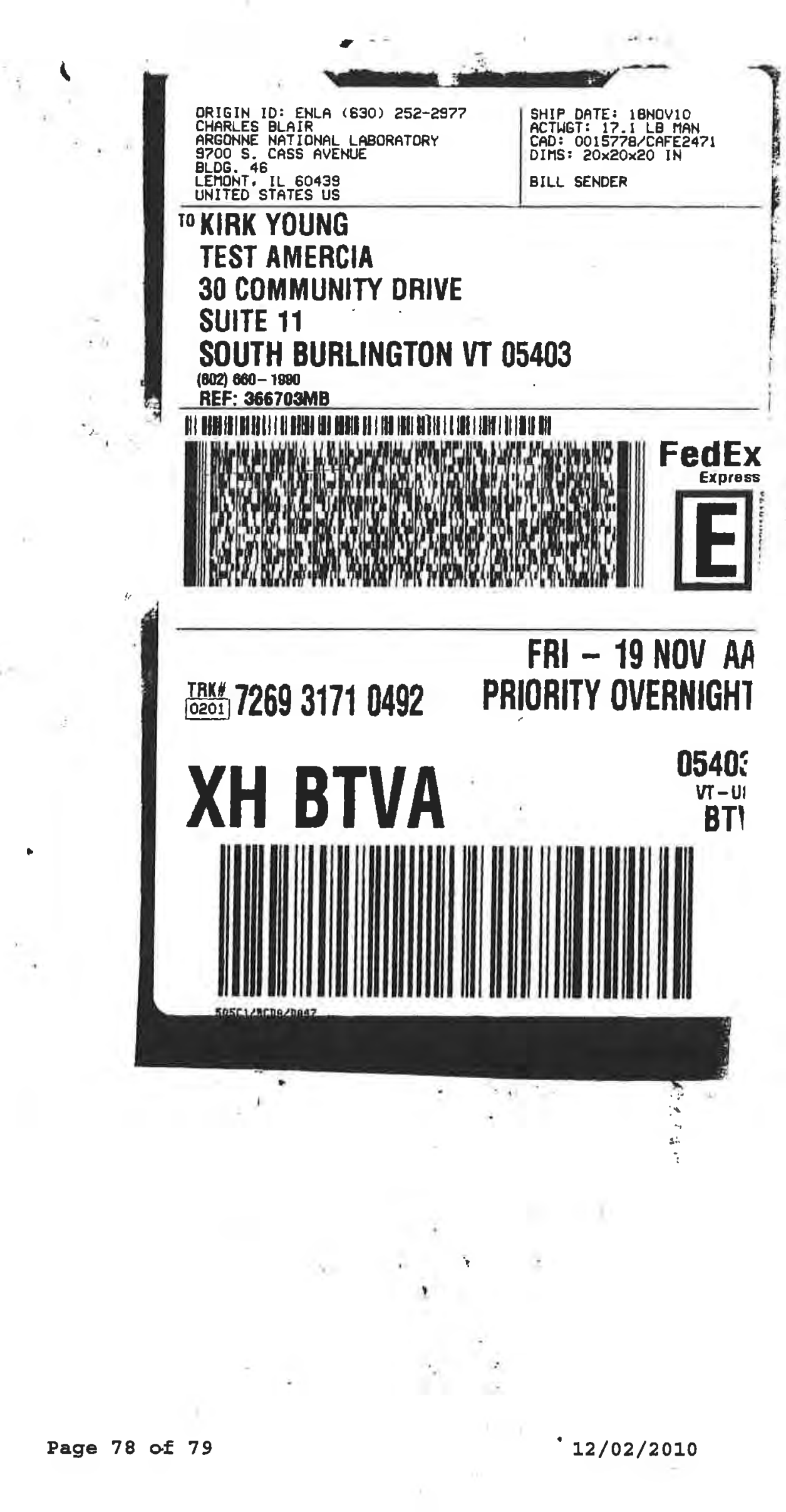




\section{Login Sample Receipt Check List}

Client: Argonne National Laboratory

Job Number: 200-2608-1

SDG Number: INMAN (200-2608)

Login Number: 2608

Creator: Marion, Greg T

List Number: 1

\section{Question}

Radioactivity either was not measured or, if measured, is at or below background

The cooler's custody seal, if present, is intact.

The cooler or samples do not appear to have been compromised or tampered with.

Samples were received on ice.

Cooler Temperature is acceptable.

Cooler Temperature is recorded.

$\mathrm{COC}$ is present.

$\mathrm{COC}$ is filled out in ink and legible.

$\mathrm{COC}$ is filled out with all pertinent information.

Is the Field Sampler's name present on COC?

There are no discrepancies between the sample IDs on the containers and the COC.

Samples are received within Holding Time.

Sample containers have legible labels.

Containers are not broken or leaking.

Sample collection date/times are provided.

Appropriate sample containers are used.

Sample bottles are completely filled.

Sample Preservation Verified

There is sufficient vol. for all requested analyses, incl. any requested MS/MSDs

VOA sample vials do not have headspace or bubble is $<6 \mathrm{~mm}(1 / 4 ")$ in diameter.

If necessary, staff have been informed of any short hold time or quick TAT needs

Multiphasic samples are not present.

Samples do not require splitting or compositing.
List Source: TestAmerica Burlington

T/FINA Comment
N/A
True
True
True
True
True
True
True
True
True
True
True
True
True
True
True
True
N/A
True
N/A
True
N/A
N/A




\section{TestAmerica}

THE LEADER IN ENVIRONMENTAL. TESTING

\section{ANALYTICAL REPORT}

Job Number: 200-3113-1

SDG Number: 200-3113

Job Description: Inman (200-3113)

Contract Number: 8E-00302

For:

Argonne National Laboratory

9700 South Cass Avenue

Building 203

Office B-149

Argonne, IL 60439

Attention: Mr. Clyde Dennis

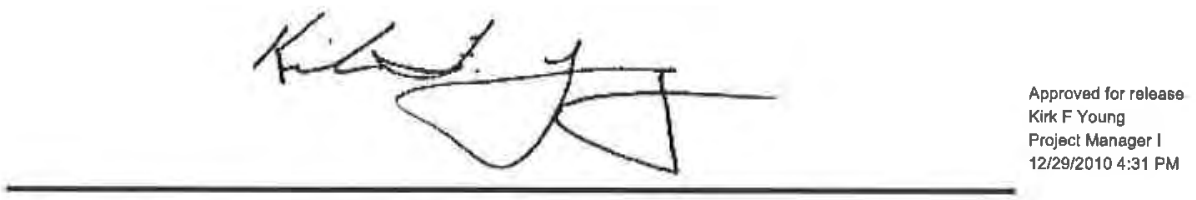

Kirk F Young

Project Manager I

kirk.young@testamericainc.com

$12 / 29 / 2010$

The test results in this report relate only to sample(s) as received by the laboratory. These test results were derived under a quality system that adheres to the requirements of NELAC. Pursuant to NELAC, this report may not be produced in full without written approval from the laboratory 


\section{Table of Contents}

Cover Title Page ........................ 1

Report Narrative . . . . . . . . . . . . . . . . . . 4

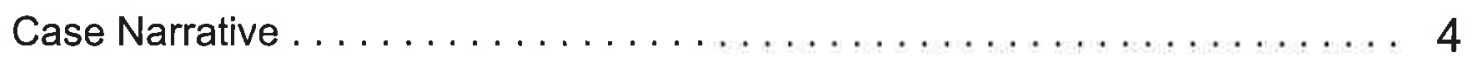

Qualifier Definition ............................ 6

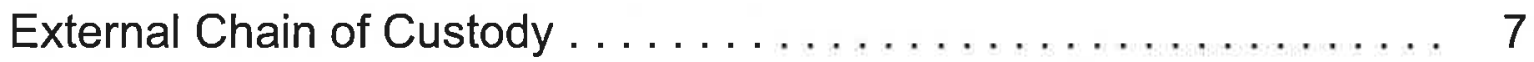

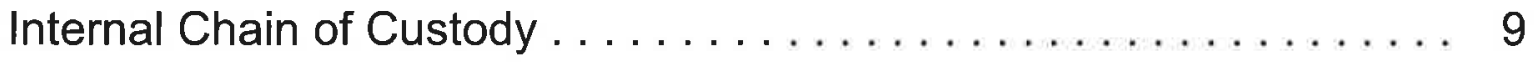

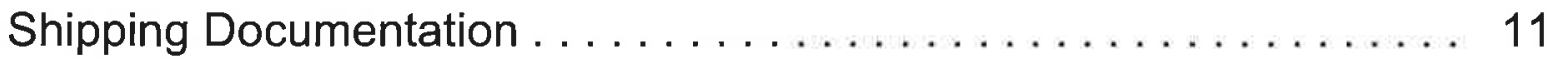

Airbills (if Applicable) . ............................ 12

Sample Receipt and Log In Check List ................... 13

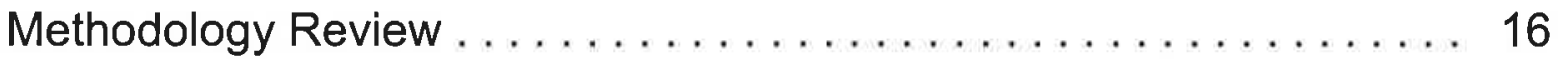

QC Summary - SOM01.2 Volatiles-Trace . . . . . . . . . . . . . . 17

QC Summary - SOM01.2 Volatiles-Trace . . . . . . . . . . . . . . 17

Deuterated Monitoring Compound Summary $\ldots \ldots \ldots \ldots \ldots \ldots \ldots \ldots \ldots \ldots \ldots \ldots \ldots \ldots$

Method Blank . . . . . . . . . . . . . . . . 19

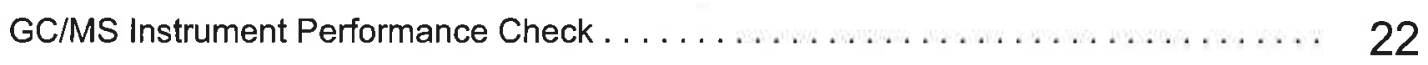

Internal Standard Area and RT Summary .......................... 26

Sample Data - SOM01.2 Volatiles-Trace . . . . . . . . . . . . . . 29

Sample Data - SOM01.2 Volatiles-Trace . . . . . . . . . . . . . . . . . 29

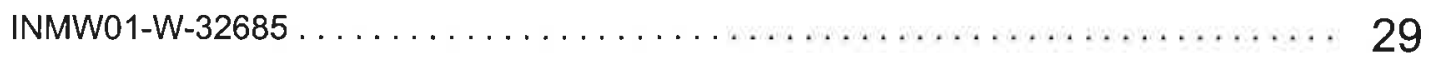

INMW02-W-32686 $\ldots \ldots \ldots \ldots \ldots \ldots \ldots \ldots \ldots \ldots \ldots \ldots \ldots \ldots \ldots \ldots \ldots \ldots \ldots \ldots \ldots \ldots, 32$

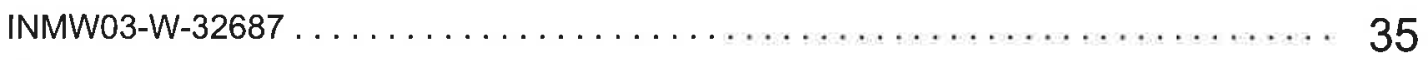

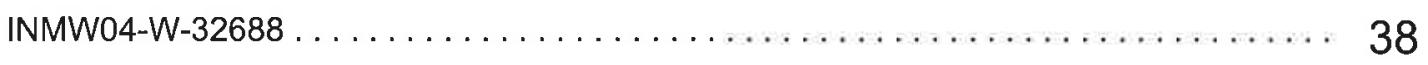

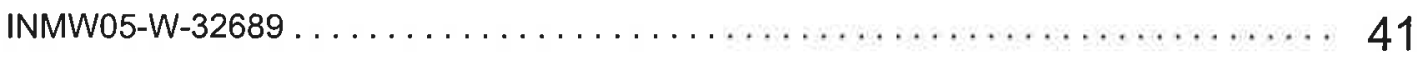

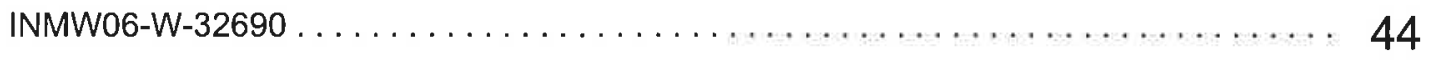

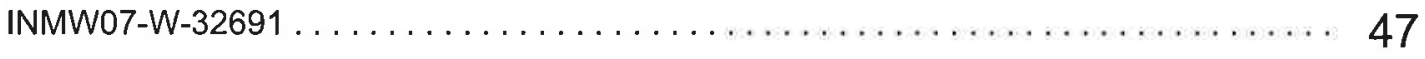

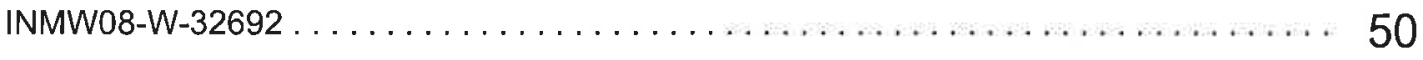




\section{Table of Contents}

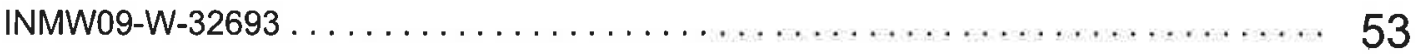

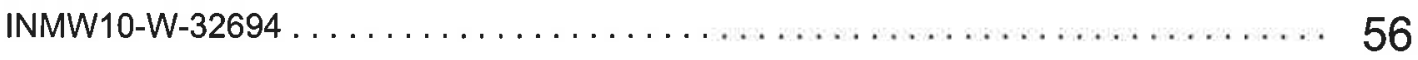

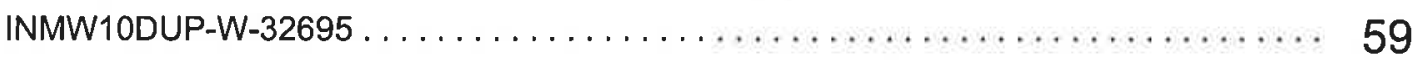

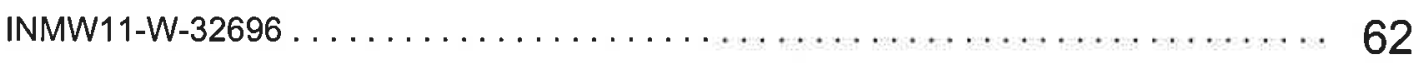

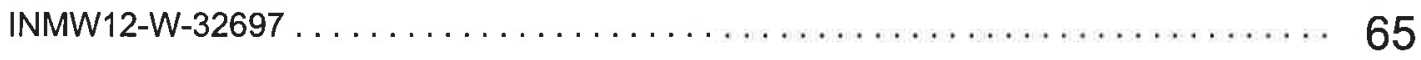

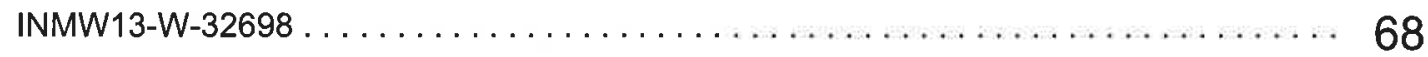

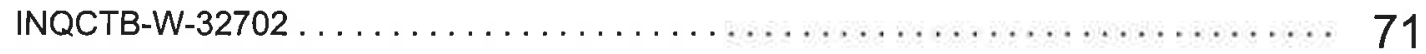

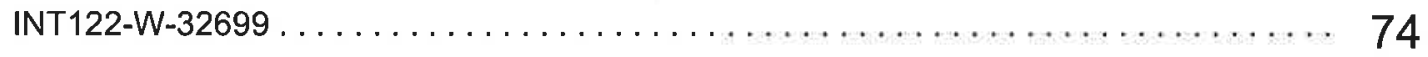

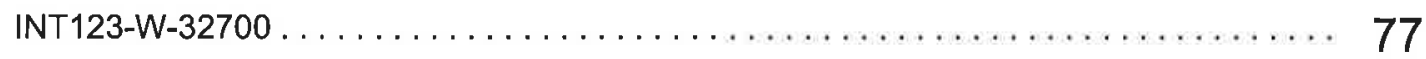

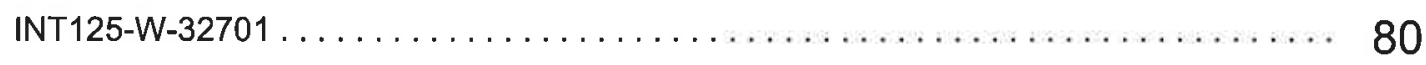

Standards - SOM01.2 Volatiles-Trace . . . . . . . . . . . . . . 83

Standards - SOM01.2 Volatiles-Trace . . . . . . . . . . . . . . 83

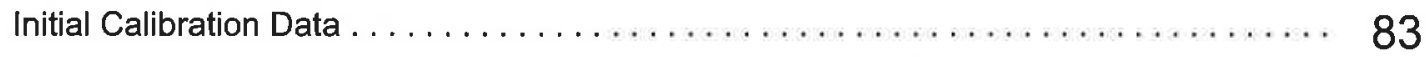

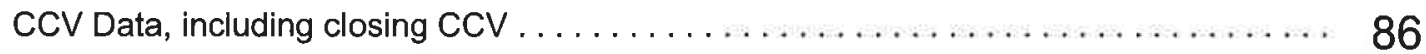

Raw Qc Data - SOM01.2 Volatiles-Trace ................... 104

Raw Qc Data - SOM01.2 Volatiles-Trace . . . . . . . . . . . . . . . . . . . 104

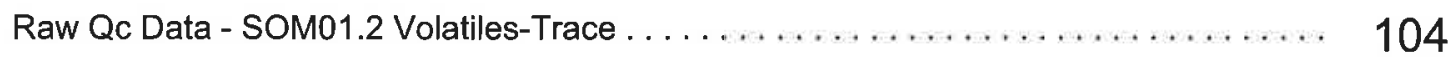

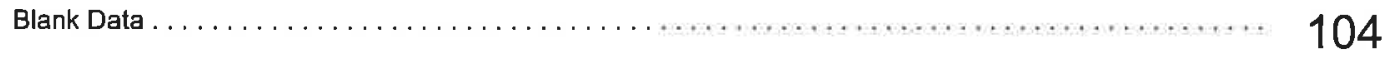




\title{
CASE NARRATIVE
}

\section{Client: Argonne National Laboratory \\ Project: Inman (200-3113)}

\author{
Report Number: 200-3113-1
}

With the exceptions noted as flags or footnotes, standard analytical protocols were followed in the analysis of the samples and no problems were encountered or anomalies observed.

Calculations were performed before rounding to avoid round-off errors in calculated results.

All holding times were met and proper preservation noted for the methods performed on these samples, unless otherwise detailed in the individual sections below.

\section{Receipt}

The samples were received on 12/23/2010. Documentation of the condition of the samples at the time of their receipt and any exception to the laboratory's Sample Acceptance Policy is documented in the Sample Handling section of this submittal. The samples, as received, were not acid preserved. On that basis, the laboratory did provide for the analytical work to be performed within seven days of sample collection.

\section{SOM01.2 Volatile Organics (Trace Level Water)}

A storage blank was prepared for volatile organics analysis, and stored in association with the storage of the samples. That storage blank, identified as VHBLK01, was carried through the holding period with the samples, and analyzed.

Samples INMW06-W-32690, INMW07-W-32691, INMW08-W-32692, INMW09-W-32693, INMW10-W-32694, INMW10DUP-W-32695, INMW11-W-32696, INMW12-W-32697, INMW13-W-32698, INT123-W-32700 had high constituent concentrations of acetone, methyl ethyl ketone, and tetrahydrofuran. Samples INMW10-W-32694 and INMW10DUP-W-32695 additionally had high constituent concentrations of methyl acetate. The presence of those compounds was suspected of being related to the construction of the sampling points. As such the laboratory did make a decision, based on past program experience, not to pursue a quantitative analysis of acetone, methyl acetate, and methyl ethyl ketone.

Samples INMW07-W-32691, INMW08-W-32692, INMW09-W-32693, INMW10-W-32694, INMW10DUP-W-32695, INMW11-W-32696, INMW12-W-32697, INMW13-W-32698 were analyzed at a dilution based on the constituent concentrations of acetone, methyl ethyl ketone, and tetrahydrofuran, which represented a constraint on the analytical process. The dilutions that were made represented a balance between maintaining the stability of the instrumental analysis and providing as low a reporting limit as possible for target analytes other than acetone, methyl acetate, and methyl ethyl ketone. The dilutions that were made ranged from a 2 -fold dilution up to a 7.5 -fold dilution.

Each of the analyses associated with the sample set exhibited an acceptable internal standard performance. There was an acceptable recovery of each deuterated monitoring compound (DMC) in the analysis of each method blank associated with the analytical work, and in the analysis of the storage blank associated with the sample set. The analysis of the samples in this 
sample set did meet the technical acceptance criteria specific to DMC recoveries, although not all DMC recoveries were within the control range in each analysis. The technical acceptance criteria does provide for the recovery of up to three DMCs to fall outside of the control range in the analysis of field samples. Matrix spike and matrix spike duplicate analyses were not performed on samples in this sample set. To varying extent, trace concentrations of chloromethane, acetone, carbon disulfide, trans-1,2-dichloroethene, methyl tert-butyl ether, 1,3-dichlorobenzene, 1,4-dichlorobenzene, 1,2,4-trichlorobenzene, and 1,2,3-trichlorobenzene were identified in the analysis of the method blanks associated with the analytical work. The concentration of each compound in each analysis was below the established reporting limit, and each analysis did meet the technical acceptance criteria for a compliant method blank analysis. A trace concentration of methyl tert-butyl ether was identified in the analysis of the storage blank associated with the sample set. The concentration of methyl tert-butyl ether in that analysis was below the established reporting limit, and the analysis did meet the technical acceptance criteria for a compliant storage blank analysis. Present in the method blank and storage blank analyses was a non-target constituent that represents a compound that is related to the DMC formulation. The fact that the presence of this compound is not within the laboratory's control is at issue. The derived results for that compound have been qualified with an " $\mathrm{X}$ " qualifier to reflect the source of the contamination.

The responses for each of the target analytes met the relative standard deviation criterion in the initial calibration. With the exception of that for bromomethane in the calibration check acquisition identified as VSTD005JF, the response for each target analyte met the percent difference criterion in each opening/continuing calibration check acquisition. In the referenced acquisition, the response for bromomethane was low and the change in response, relative to the average response in the initial calibration, represented a 51.5 percent difference. This did not meet the method allowance criterion of 40.0 percent, and the acquisition did not meet the technical acceptance criteria for a compliant opening/continuing calibration check. The issue was specific to bromomethane. The response for each target analyte met the 50.0 percent difference criterion in each closing calibration check acquisition.

The primary quantitation mass for methylcyclohexane that is specified in the Statement of Work is mass 83 . The laboratory did identify a contribution to mass 83 from 1,2-dichloropropane- $d_{6}$, one of the deuterated monitoring compounds (DMCs). The laboratory did change the primary quantitation mass assignment to mass 55 for the quantification of methylcyclohexane.

Manual integration was employed in deriving certain of the analytical results. The values that have been derived from manual integration are qualified on the quantitation reports. Extracted ion current profiles for each manual integration are included in the data package, and further documented in the Sample Preparation section of this submittal. 


\section{DATA REPORTING QUALIFIERS}

Client: Argonne National Laboratory

Job Number: $200-3113-1$

Sdg Number: 200-3113

Lab Section

Description

GC/MS VOA

U

E

$J$

$\mathrm{J}$

$\mathrm{X}$

*

B

N
Analyzed for but not detected.

Compound concentration exceeds the upper level of the calibration range of the instrument for that specific analysis.

Indicates an Estimated Value for TICs

Indicates an estimated value.

See case narrative notes for explanation of the ' $X$ ' flag

Surrogate exceeds the control limit

The analyte was found in an associated blank, as well as in the sample.

This flag indicates the presumptive evidence of a compound. 


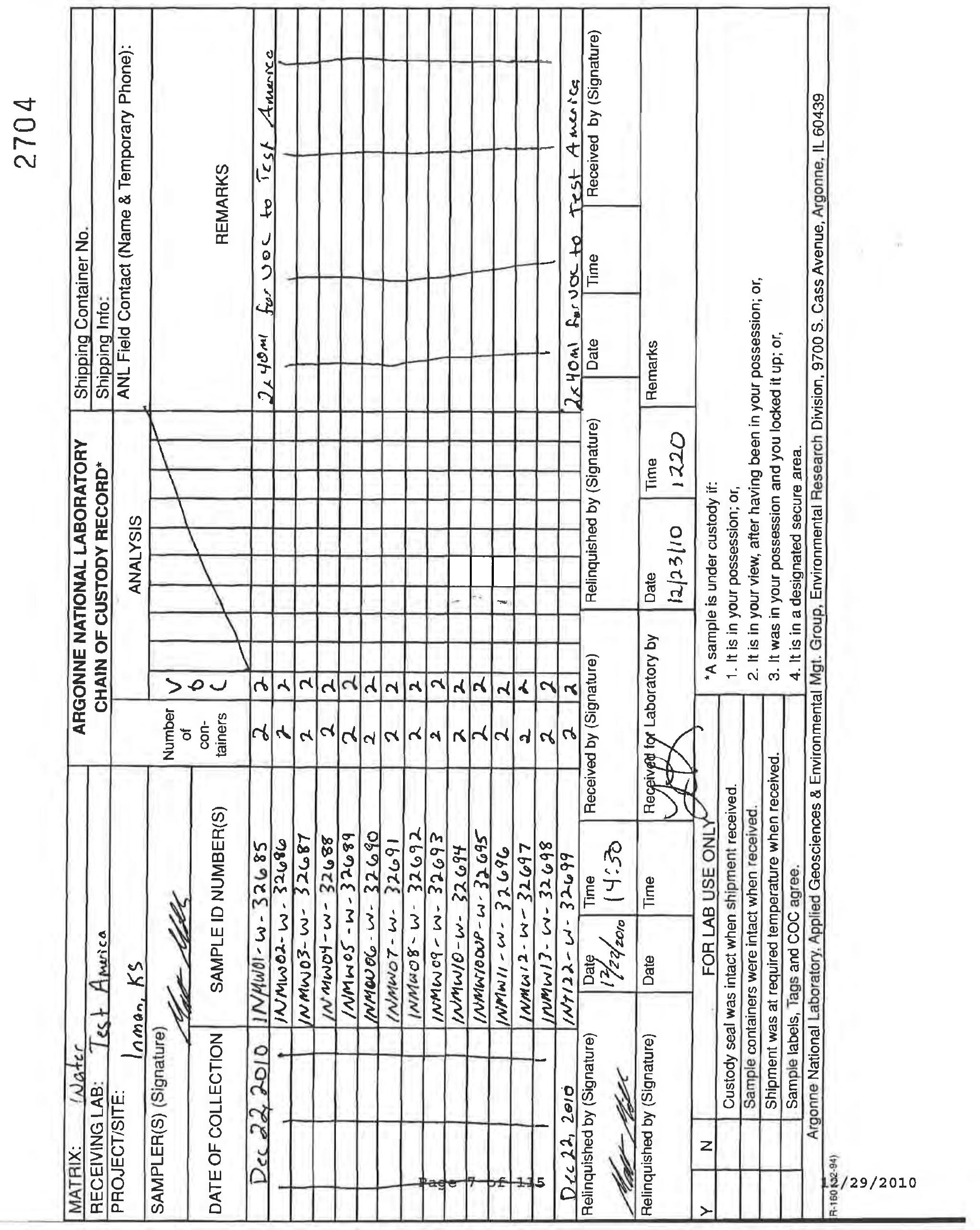




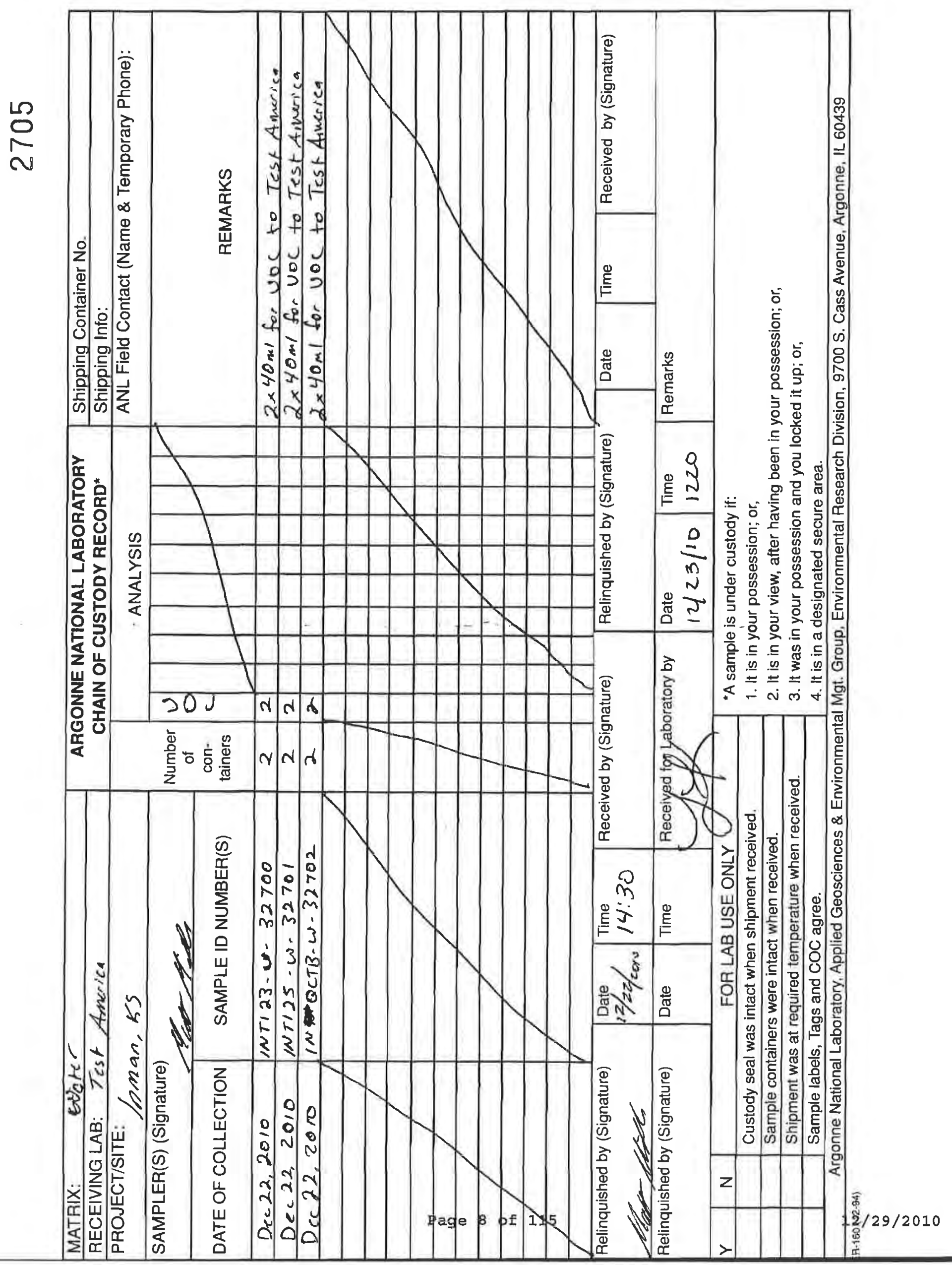




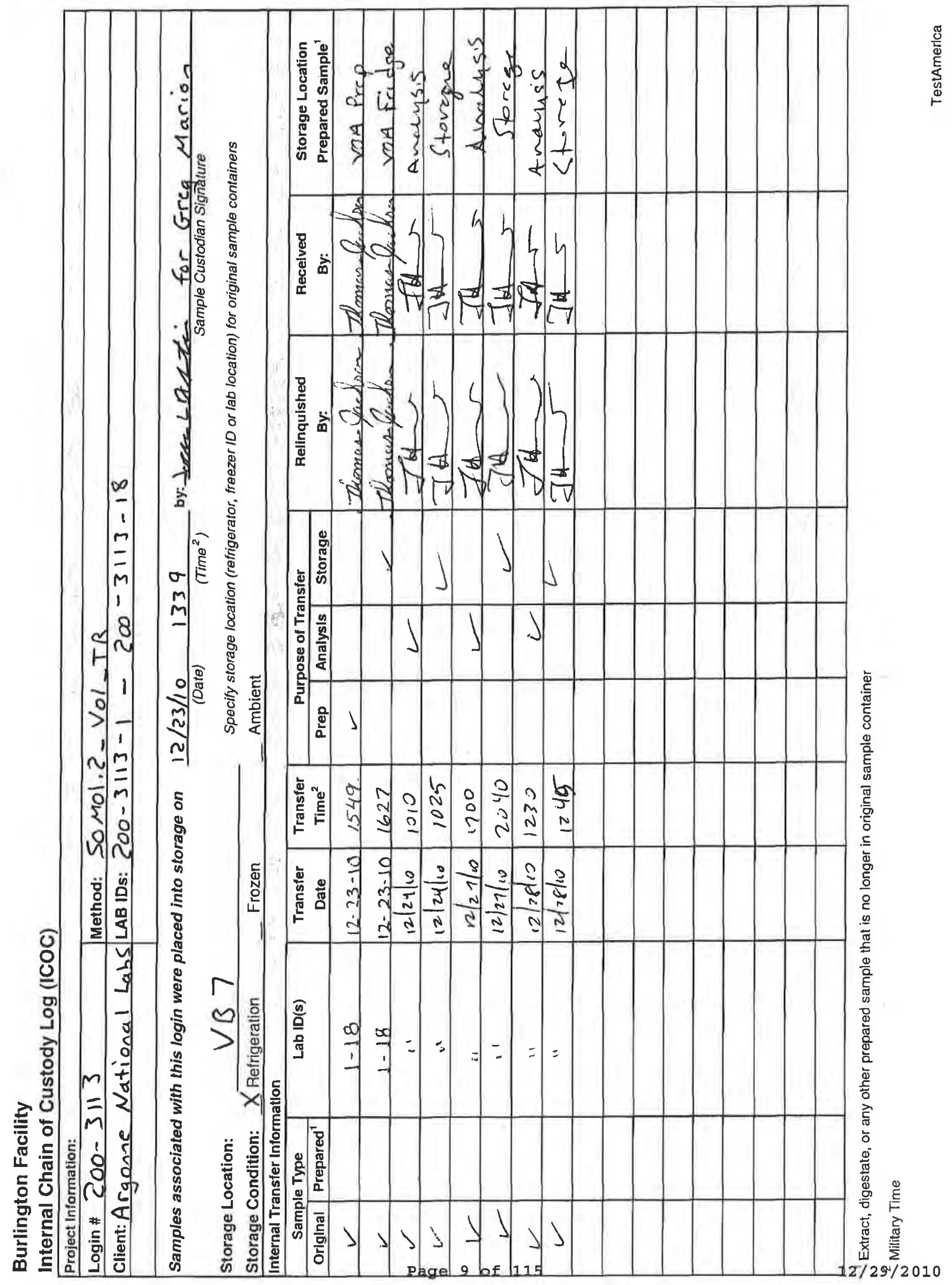




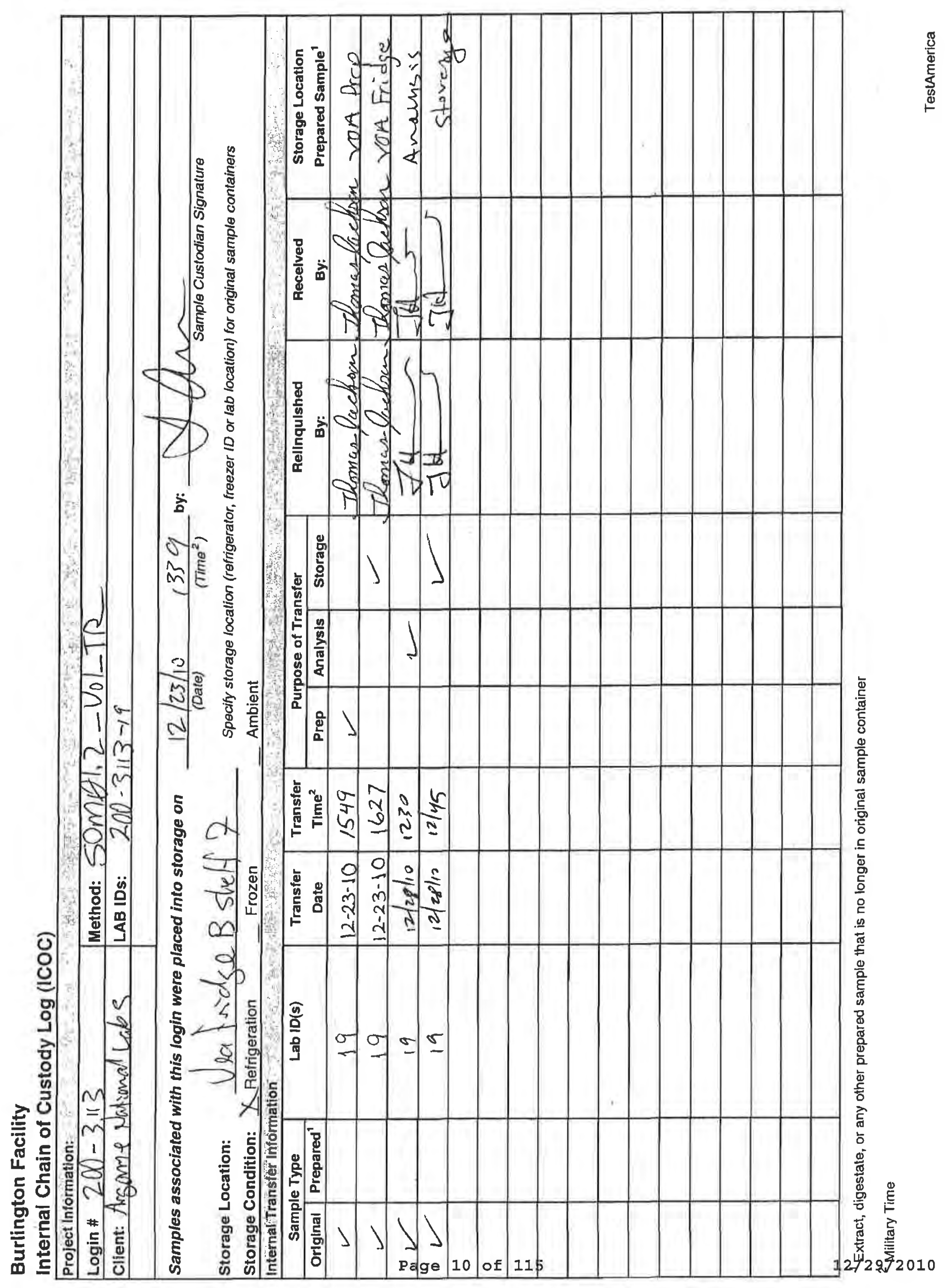




\section{Shipping and Receiving Documents}



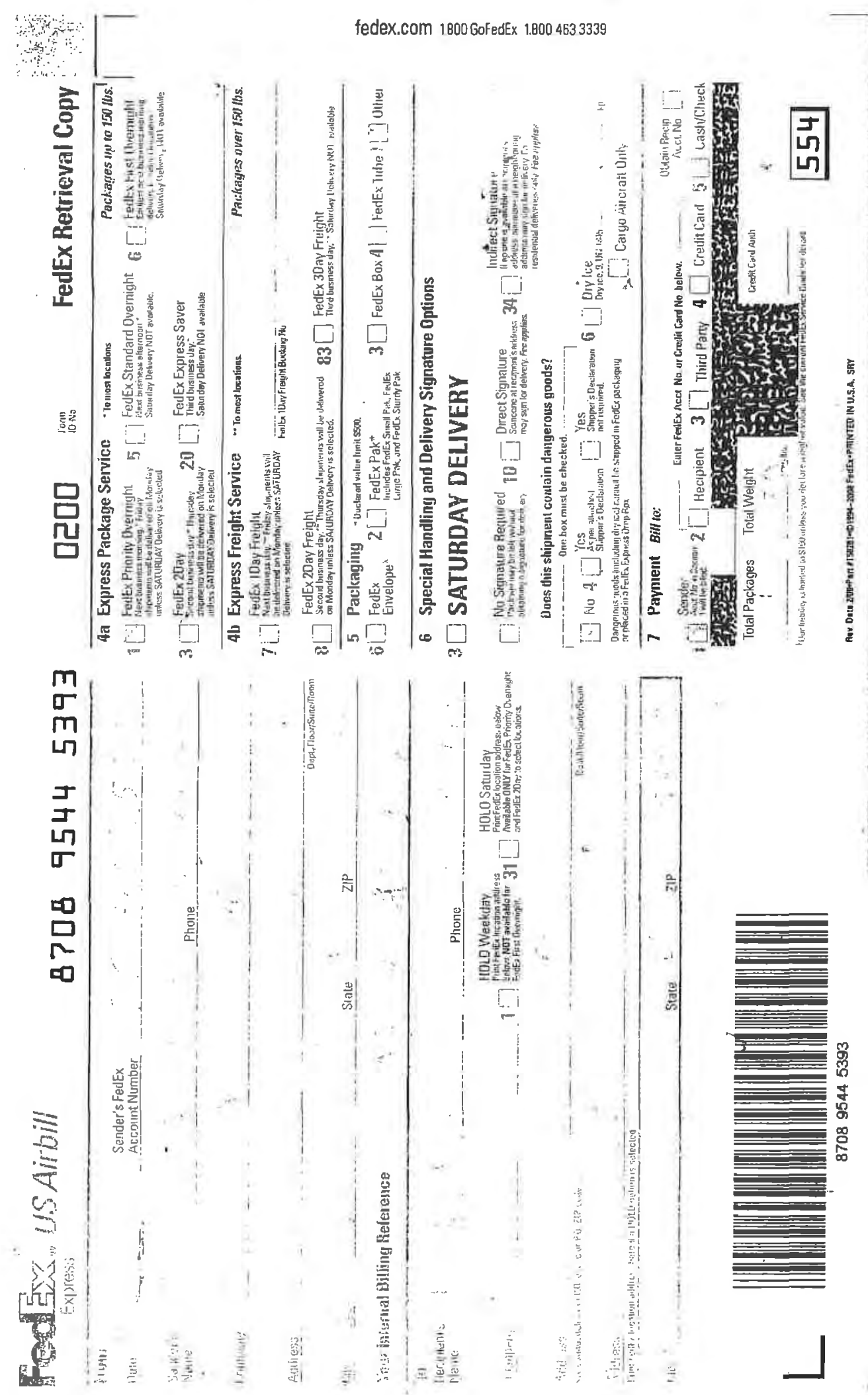


\section{Login Sample Receipt Check List}

Client: Argonne National Laboratory

Job Number: 200-3113-1

SDG Number: $200-3113$

Login Number: 3113

List Source: TestAmerica Burlington Creator: Marion, Greg T

List Number: 1

\section{Question}

Radioactivity either was not measured or, if measured, is at or below background

The cooler's custody seal, if present, is intact.

The cooler or samples do not appear to have been compromised or tampered with.

Samples were received on ice.

Cooler Temperature is acceptable.

Cooler Temperature is recorded.

$\mathrm{COC}$ is present.

$\mathrm{COC}$ is filled out in ink and legible.

$\mathrm{COC}$ is filled out with all pertinent information.

Is the Field Sampler's name present on COC?

There are no discrepancies between the sample IDs on the containers and the COC.

Samples are received within Holding Time.

Sample containers have legible labels.

Containers are not broken or leaking.

Sample collection date/times are provided.

Appropriate sample containers are used.

Sample bottles are completely filled.

Sample Preservation Verified

There is sufficient vol. for all requested analyses, incl. any requested MS/MSDs

VOA sample vials do not have headspace or bubble is $<6 \mathrm{~mm}\left(1 / 4^{\prime \prime}\right)$ in diameter.

If necessary, staff have been informed of any short hold time or quick TAT needs

Multiphasic samples are not present.

Samples do not require splitting or compositing.

$\begin{array}{ll}\text { T I F/NA } & \text { Comment } \\ \text { N/A } & \\ \text { True } & \text { NO SEAL NUMBERS } \\ \text { True } & \\ \text { True } & \\ \text { True } & \\ \text { True } & 2.5^{\circ} \mathrm{C} \text { IR GUN ID } 96 / \mathrm{CF}=-1 \\ \text { True } & \\ \text { True } & \\ \text { True } & \\ \text { True } & \\ \text { True } & \\ \text { True } & \\ \text { True } & \\ \text { True } & \\ \text { True } & \\ \text { False } & \\ \text { True } & \\ \text { N/A } & \\ \text { True } & \\ \text { True } & \\ \text { True } & \\ \text { N/A } & \\ \text { N/A } & \\ & \\ \end{array}$




\section{Sample Login Acknowledgement}

Job 200-3113-1

\begin{tabular}{|c|c|c|c|}
\hline Client Job Description: & Inman (200-3113) & Report To: & Argonne National Laboratory \\
\hline Purchase Order \#: & $8 E-00302$ & & Jorge Alvarado \\
\hline Work Order \#: & $8 E-00302$ & & 9700 South Cass Avenue \\
\hline Project Manager: & Kirk F Young & & Building 203 \\
\hline Job Due Date: & $1 / 6 / 2011$ & & $\begin{array}{l}\text { Office B-149 } \\
\text { Argonne, IL } 60439\end{array}$ \\
\hline Job TAT: & 14 Days & & \\
\hline Max Deliverable Level: & IV & Bill To: & $\begin{array}{l}\text { Argonne National Laboratory } \\
\text { Accounts Payable }\end{array}$ \\
\hline Earliest Deliverable Due: & $1 / 6 / 2011$ & & $\begin{array}{l}\text { Chief Financial Offices } \\
9700 \text { S. Cass Ave. } \\
\text { Building } 201 \\
\text { Argonne, IL } 60439\end{array}$ \\
\hline
\end{tabular}

Login 200-3113

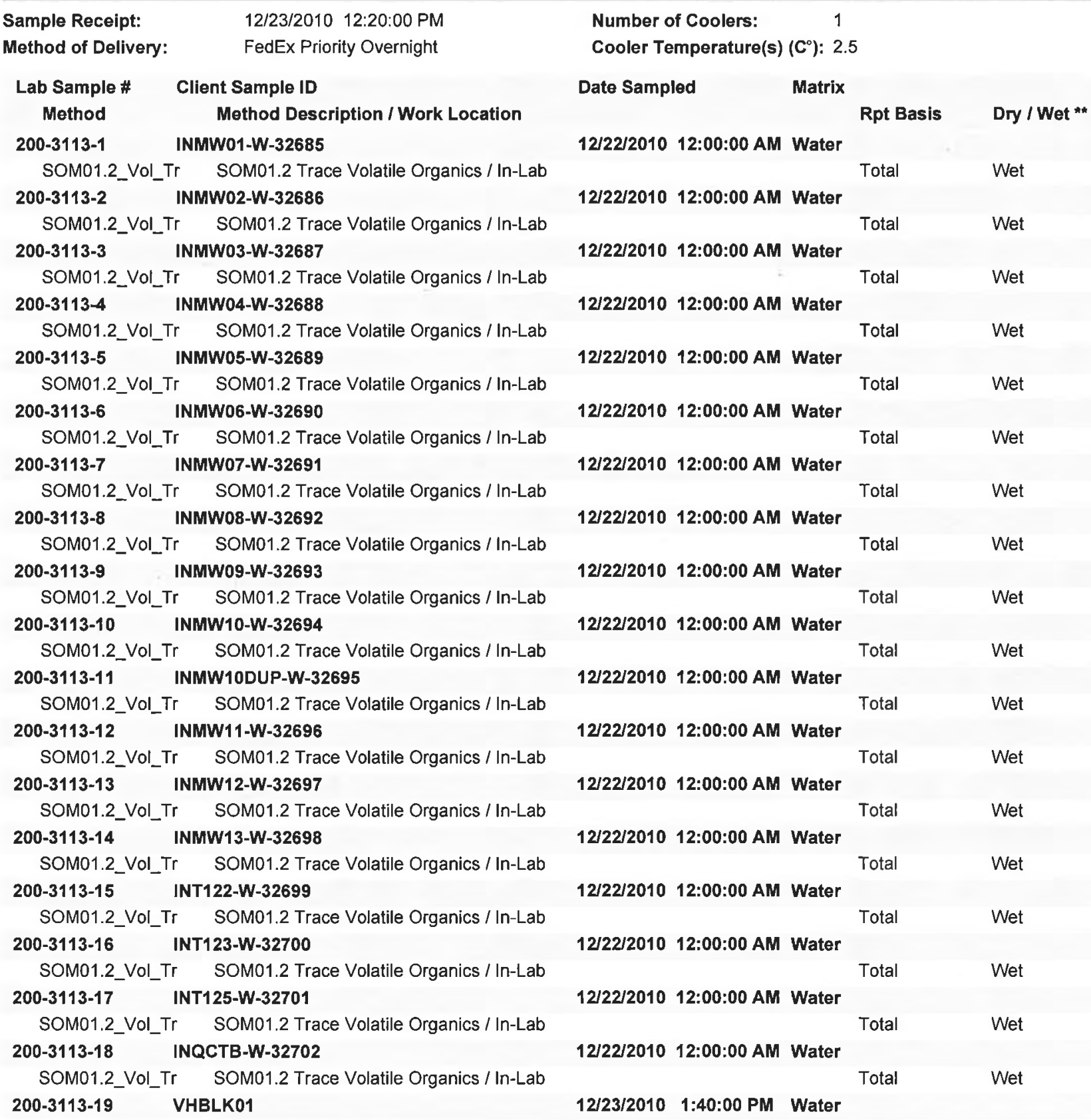

* Method on-hold

** WetDry indicates whether the reported results will be corrected fop agiatudeacoont1ang based on sample Wet weight or Dry 


\section{Sample Login Acknowledgement}

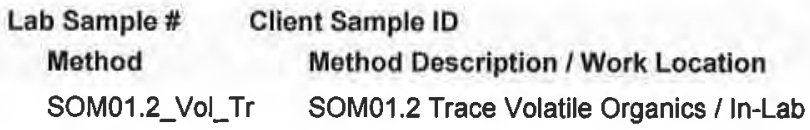

Date Sampled

Matrix

Rpt Basis

Total

Dry / Wet **

Wet 


\section{METHODOLOGY SUMMARY}

$\begin{array}{llll}\text { Laboratory: } & \text { TestAmerica Laboratories } & \text { Project No: } & \\ \text { Location: } & \text { South Burlington, Vermont } & \text { SDG No: } & 200-3113\end{array}$

VOA

Volatile Organics Trace - USEPA CLP SOM01.2 
$2 A$ - FORM II VOA-1

WATER VOLATILE DEUTERATED MONITORING COMPOUND RECOVERY

Lab Name: TESTAMERICA BURLINGTON

Contract: 8E-00302

Lab Code: STLV Case No.: INMAN Mod. Ref No.:

SDG No.: 200-3113

Level: (TRACE or LOW) TRACE

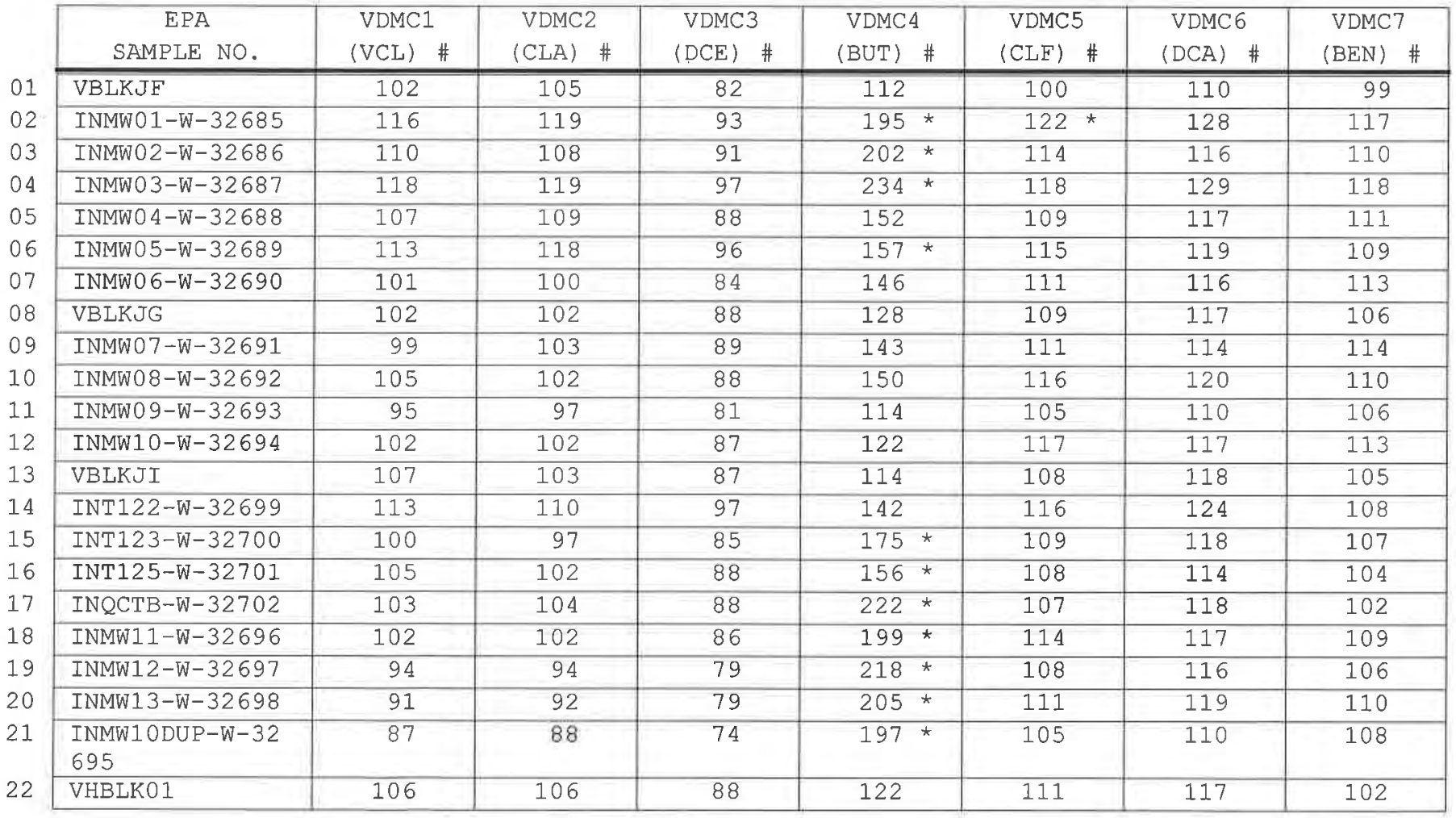

VDMC1 $(\mathrm{VCL})=$ Vinyl Chloride-d3

QC LIMITS

VDMC2 $($ CLA $)=$ Chloroethane-d5

(65-131)

VDMC3 $(\mathrm{DCE})=1,1-\mathrm{Dich}$ loroethene-d2

$(71-131)$

VDMC4 $\quad(B U T)=2$-Butanone-d5

(55-104)

VDMC5 $(C L F)=$ Chloroform $-\mathrm{d}$

$(49-155)$

VDMC $6 \quad(D C A)=1,2-$ Dichloroethane-d4

$(78-121)$

VDMC7 $(\mathrm{BEN})=$ Benzene-d6

$(78-129)$

$(77-124)$

\# Colum to be used to flag recovery values

* Values outside of contract required QC limits 
$2 B$ - FORM II VOA-2

WATER VOLATILE DEUTERATED MONITORING COMPOUND RECOVERY

Lab Name: TESTAMERICA BURLINGTON

Contract: $\quad 8 \mathrm{E}-00302$

Lab Code: STIV Case No.: INMAN Mod. Ref No.:

SDG No.: 200-3113

Level: (TRACE or LOW) TRACE

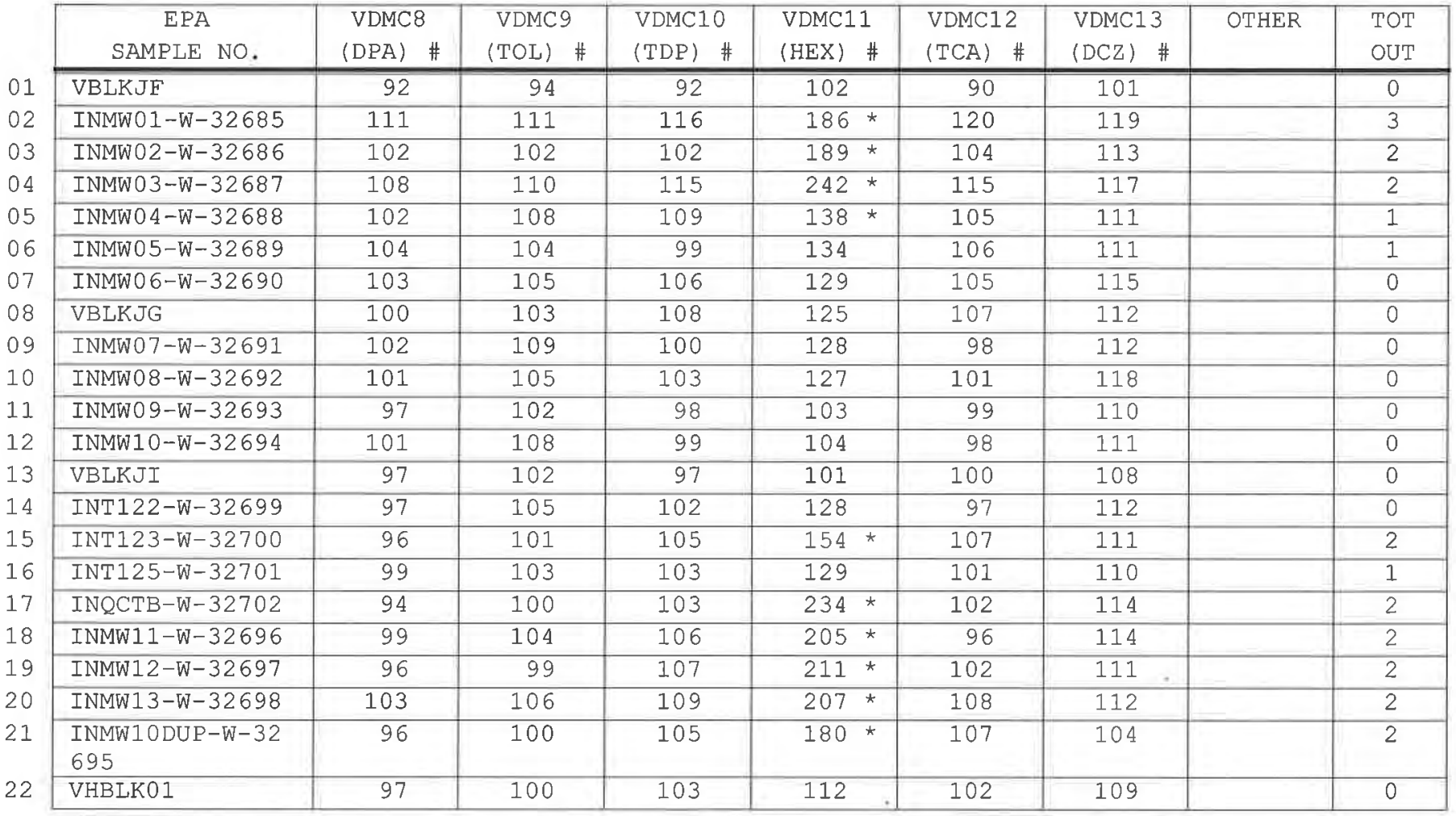

VDMC8 $(D P A)=1,2-$ Dichloropropane-d 6

QC LIMITS

VDMC9 $($ TOL $)=$ Toluene-d8

$(79-124)$

VDMC10 $(\mathrm{TDP})=$ trans-1,3-Dichloropropene-d4

$(77-121)$

VDMC11 $(\mathrm{HEX})=2$-Hexanone-d5

$(73-121)$

VDMC12 $(\mathrm{TCA})=1,1,2,2$-Tetrachloroethane-d2

$(28-135)$

VDMC13 $(\mathrm{DCZ})=1,2-\mathrm{Dich}$ lorobenzene-d4

$(73-125)$

$(80-131)$

\# Column to be used to flag recovery values

* Values outside of contract required QC limits

Report 1,4-Dioxane-d8 for Low-Medium VOA analysis only

Page 1 of 1 
$4 A$ - FORM IV VOA

VOLATILE METHOD BLANK SUMMARY
EPA SAMPLE NO.

VBLKJF

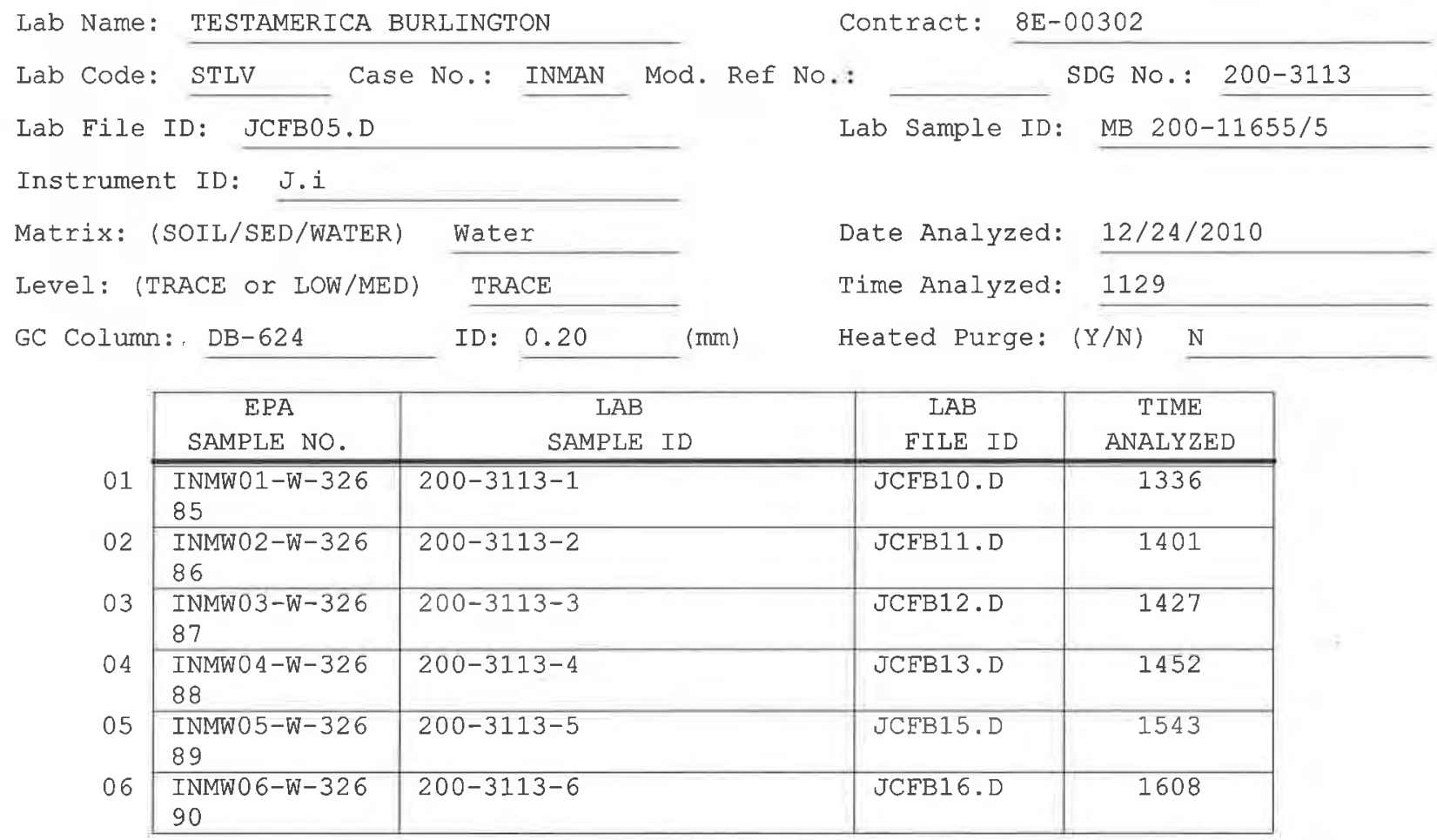

COMMENTS :

Page 1 of 1 
$4 \mathrm{~A}$ - FORM IV VOA

VOLATILE METHOD BLANK SUMMARY
EPA SAMPLE NO.

VBLKJG

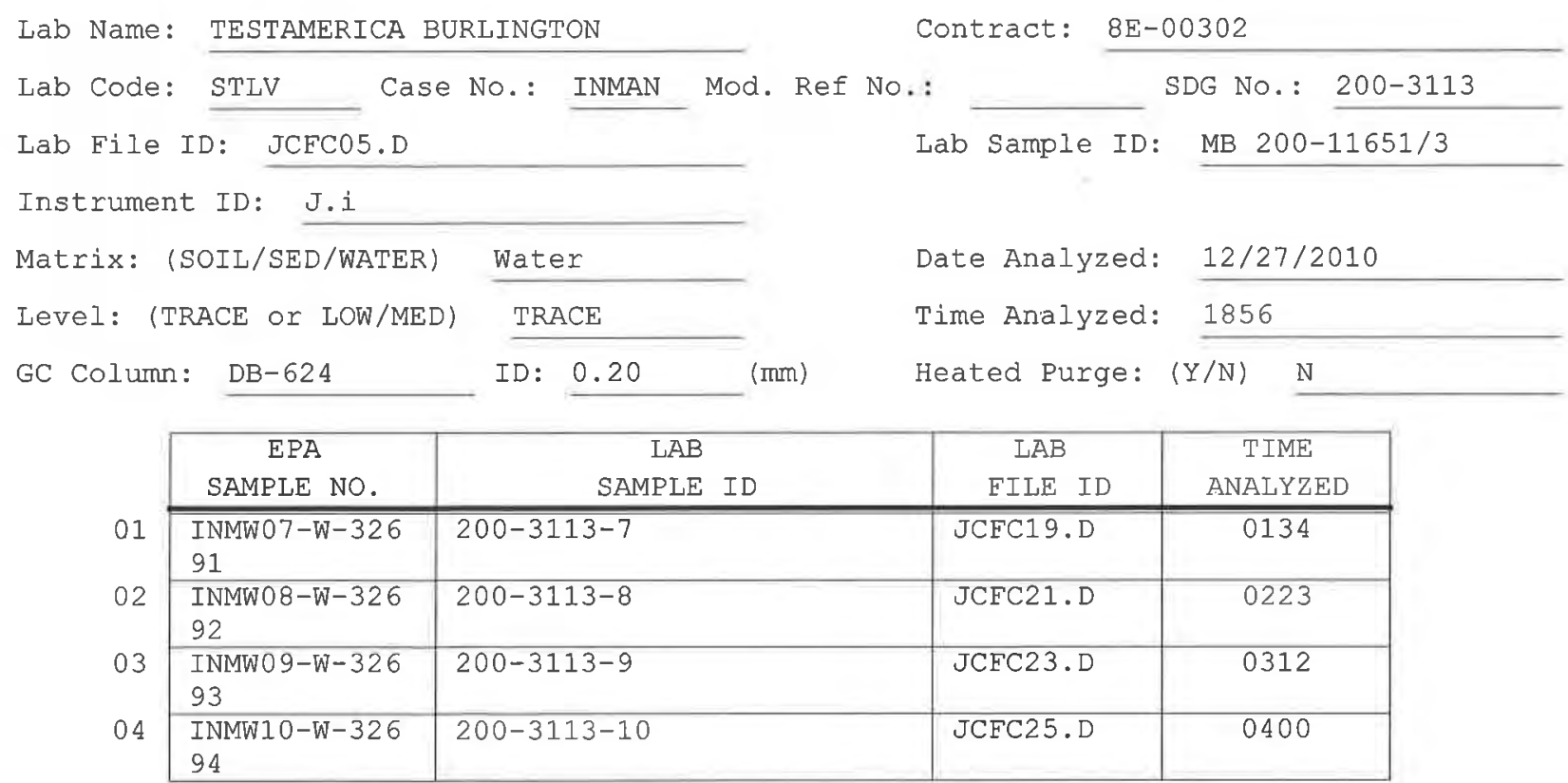

COMMENTS :

Page 1 of 1 
$4 A$ - FORM IV VOA

VOLATILE METHOD BLANK SUMMARY
EPA SAMPLE NO.

VBLKJI

Lab Name: TESTAMERICA BURLINGTON

Contract: $\quad 8 \mathrm{E}-00302$

Lab Code: STLV Case No.: INMAN Mod. Ref No.

Lab File ID: JCFE03.D

Instrument ID: J.i

Matrix: (SOIL/SED/WATER) Water

Level: (TRACE or LOW/MED) TRACE

GC Column: DB-624
: $\quad$ SDG No.: 200-3113
Date Analyzed: $12 / 28 / 2010$

Time Analyzed: 1404

Heated Purge: (Y/N) N

\begin{tabular}{|c|c|c|c|}
\hline $\begin{array}{c}\text { EPA } \\
\text { SAMPLE NO. }\end{array}$ & $\begin{array}{r}\text { LAB } \\
\text { SAMPLE ID }\end{array}$ & $\begin{array}{l}\text { LAB } \\
\text { FILE ID }\end{array}$ & $\begin{array}{c}\text { TIME } \\
\text { ANALYZED }\end{array}$ \\
\hline $\begin{array}{l}\text { INT122-W-326 } \\
99\end{array}$ & $200-3113-15$ & JCFE0 4.D & 1441 \\
\hline $\begin{array}{l}\text { INT123-W-327 } \\
00\end{array}$ & $200-3113-16$ & JCFE05.D & 1505 \\
\hline $\begin{array}{l}\operatorname{INT} 125-W-327 \\
01\end{array}$ & $200-3113-17$ & JCFE06.D & 1530 \\
\hline $\begin{array}{l}\text { INQCTB-W-327 } \\
02\end{array}$ & $200-3113-18$ & JCFE07.D & 1554 \\
\hline $\begin{array}{l}\text { INMW11-W-326 } \\
96\end{array}$ & $200-3113-12$ & JCFE09.D & 1721 \\
\hline $\begin{array}{l}\text { INMW12-W-326 } \\
97\end{array}$ & $200-3113-13$ & JCFE11.D & 1809 \\
\hline $\begin{array}{l}\text { INMW13-W-326 } \\
98\end{array}$ & $200-3113-14$ & JCFE13.D & 1858 \\
\hline $\begin{array}{l}\text { INMW1 ODUP-W- } \\
32695\end{array}$ & $200-3113-11$ & JCFE15.D & 1947 \\
\hline VHBLK01 & $200-3113-19$ & JCFE20.D & 2149 \\
\hline
\end{tabular}

COMMENTS :

Page 1 of 1 
$5 A$ - FORM V VOA

VOLATILE ORGANICS INSTRUMENT

PEREORMANCE CHECK

BROMOFLUOROBENZENE (BFB)
EPA SAMPLE NO.

BEBJD

Lab Name: TESTAMERICA BURLINGTON Contract: 8E-00302

Lab Code: STLV Case No.: INMAN Mod. Ref No.:

SDG No.: $200-3113$

Lab File Id: JCF01.D

Instrument Id: J.i

GC Column: DB-624

ID : 0.20 $(\mathrm{mm})$
BFB Injection Date: 12/23/2010

BFB Injection Time: 1152

\begin{tabular}{|r|l|r|}
\hline $\mathrm{m} / \mathrm{e}$ & ION ABUNDANCE CRITERIA & $\begin{array}{c}\text { ㅇ RELATIVE } \\
\text { ABUNDANCE }\end{array}$ \\
\hline \hline 50 & $15.0-40.0 \%$ of mass 95 & 18.7 \\
\hline 75 & $30.0-80.0 \%$ of mass 95 & 53.3 \\
\hline 95 & Base peak, 100\% relative abundance & 100 \\
\hline 96 & $5.0-9.0 \%$ of mass 95 & 6.5 \\
\hline 173 & Less than 2.0\% of mass 174 & 0.7( \\
\hline 174 & $50.0-120 \%$ of mass 95 & 77.7 \\
\hline 175 & $5.0-9.0 \%$ of mass 174 1 \\
\hline 176 & $95.0-101 \%$ of mass 174 & $6.2(1)$ \\
\hline 177 & $5.0-9.0 \%$ of mass 176 & $75.8(97.5) 1$ \\
\hline
\end{tabular}

1 - Value is omass 174

2 - Value is omass 176

\begin{tabular}{|c|c|c|c|c|}
\hline $\begin{array}{c}\text { EPA } \\
\text { SAMPLE NO. }\end{array}$ & $\begin{array}{c}\text { LAB } \\
\text { SAMPIE ID }\end{array}$ & $\begin{array}{l}\text { LAB } \\
\text { FILE ID }\end{array}$ & $\begin{array}{c}\text { DATE } \\
\text { ANALYZED }\end{array}$ & $\begin{array}{c}\text { TIME } \\
\text { ANALYZED }\end{array}$ \\
\hline VSTDO.5JD & IC $200-11592 / 3$ & JCF03.D & $12 / 23 / 2010$ & 1236 \\
\hline VSTD001JD & IC $200-11592 / 4$ & JCF04.D & $12 / 23 / 2010$ & 1301 \\
\hline VSTD005JD & ICIS $200-11592 / 5$ & JCF05.D & $12 / 23 / 2010$ & 1326 \\
\hline VSTD010JD & IC $200-11592 / 6$ & JCF06.D & $12 / 23 / 2010$ & 1352 \\
\hline VSTD020JD & IC $200-11592 / 7$ & JCF07.D & $12 / 23 / 2010$ & 1417 \\
\hline
\end{tabular}

Page 1 of 1 
$5 A$ - FORM V VOA

VOLATILE ORGANICS INSTRUMENT

PERFORMANCE CHECK

BROMOFLUOROBENZENE (BFB)
EPA SAMPLE NO.

$\mathrm{BFBJF}$

Lab Name: TESTAMERICA BURLINGTON

Contract: $8 \mathrm{E}-00302$

Lab Code: STLV Case No.: INMAN Mod. Ref No.:

SDG NO.: 200-3113

Lab File Id: JCFBO1.D

Instrument Id: J.i

GC Column: DB-624
ID: 0.20
BFB Injection Date: 12/24/2010

BFB Injection Time: 0941

\begin{tabular}{|r|l|r|}
\hline $\mathrm{m} / \mathrm{e}$ & ION ABUNDANCE CRITERIA & $\begin{array}{c}\text { \% RELATIVE } \\
\text { ABUNDANCE }\end{array}$ \\
\hline \hline 50 & $15.0-40.0 \%$ of mass 95 & 19.5 \\
\hline 75 & $30.0-80.0 \%$ of mass 95 & 54.9 \\
\hline 95 & Base peak, 100\% relative abundance & 100 \\
\hline 96 & $5.0-9.0 \%$ of mass 95 & 7.1 \\
\hline 173 & Less than 2.0\% of mass 174 & 0.8( \\
\hline 174 & $50.0-120 \%$ of mass 95 & 82.1 \\
\hline 175 & $5.0-9.0 \%$ of mass 174 & $6.9(9.4) 1$ \\
\hline 176 & $95.0-101 \%$ of mass 174 & $79.8(97.2) 1$ \\
\hline 177 & $5.0-9.0 \%$ of mass 176 & 5.5( \\
\hline
\end{tabular}

1 - Value is omass 174

2 - Value is omass 176

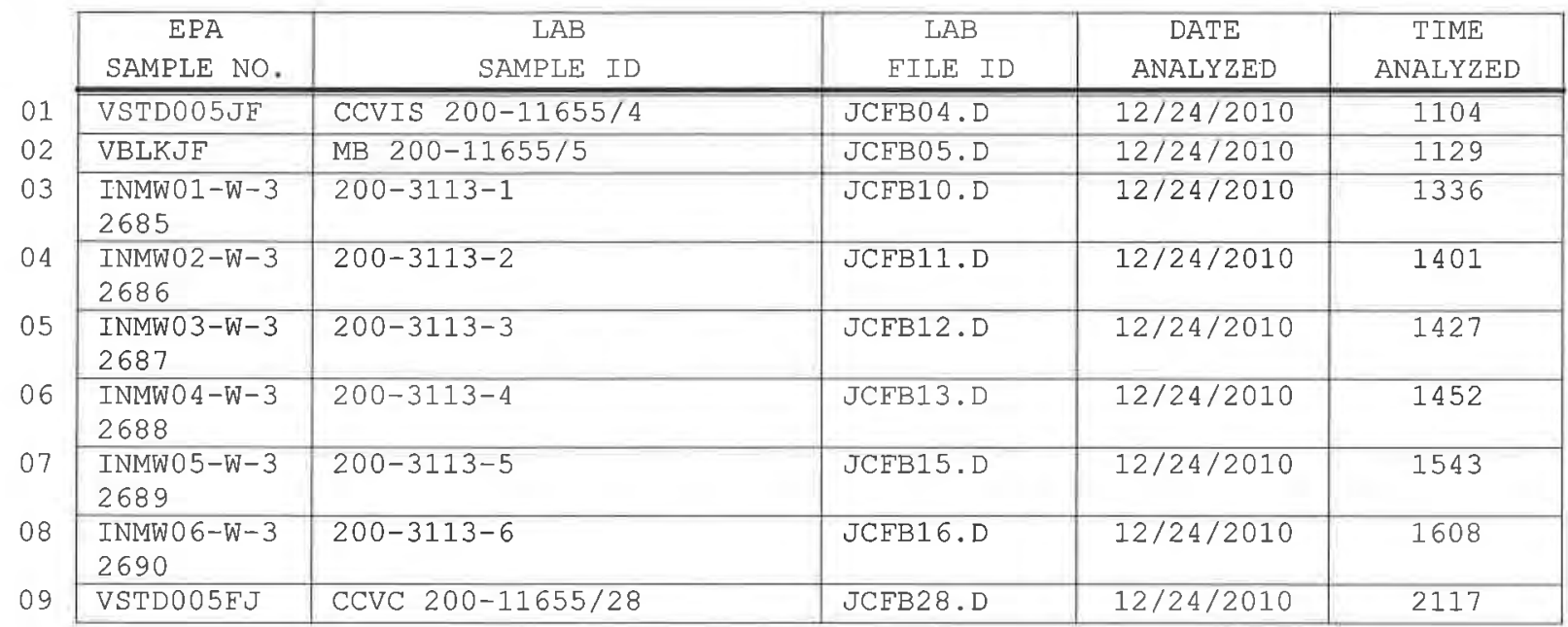

Page 1 of 1 
$5 A$ - FORM V VOA

VOLATILE ORGANICS INSTRUMENT

PERFORMANCE CHECK

BROMOFLUOROBENZENE (BFB)
EPA SAMPLE NO.

BFBJG
Lab Name: TESTAMERICA BURLINGTON
Contract: $8 \mathrm{E}-00302$

Lab Code: STLV Case No.: INMAN Mod. Ref No.:

SDG NO.: 200-3113

Lab File Id: JCFC01.D

Instrument Id: J.i

GC Column: DB-624
ID: 0.20 $(\mathrm{mm})$

\begin{tabular}{|r|l|r|}
\hline $\mathrm{m} / \mathrm{e}$ & ION ABUNDANCE CRITERIA & $\begin{array}{c}\text { \% } \\
\text { RELATIVE } \\
\text { ABUNDANCE }\end{array}$ \\
\hline \hline 50 & $15.0-40.0 \%$ of mass 95 & 16.3 \\
\hline 75 & $30.0-80.0 \%$ of mass 95 & 51.0 \\
\hline 95 & Base peak, 100\% relative abundance & 100 \\
\hline 96 & $5.0-9.0 \%$ of mass 95 & 6.0 \\
\hline 173 & Less than 2.0\% of mass 174 & $0.8(1$ \\
\hline 174 & $50.0-120 \%$ of mass 95 & 93.7 \\
\hline 175 & $5.0-9.0 \%$ of mass 174 1 \\
\hline 176 & $95.0-101 \%$ of mass 174 & $9.6(1) 1$ \\
\hline 177 & $5.0-9.0 \%$ of mass 176 & $5.9(9) 1$ \\
\hline
\end{tabular}

1 - Value is omass $174 \quad 2$ - Value is onass 176

\begin{tabular}{|c|c|c|c|c|}
\hline $\begin{array}{c}\text { EPA } \\
\text { SAMPLE NO. }\end{array}$ & $\begin{array}{r}\text { LAB } \\
\text { SAMPLE ID }\end{array}$ & $\begin{array}{l}\text { LAB } \\
\text { FILE ID }\end{array}$ & $\begin{array}{c}\text { DATE } \\
\text { ANALYZED }\end{array}$ & $\begin{array}{c}\text { TIME } \\
\text { ANALYZED }\end{array}$ \\
\hline VSTD005JG & CCVIS $200-11651 / 4$ & JCFC04.D & $12 / 27 / 2010$ & 1828 \\
\hline VBLKJG & MB 200-11651/3 & JCFC05.D & $12 / 27 / 2010$ & 1856 \\
\hline $\begin{array}{l}\text { INMW07-W-3 } \\
2691\end{array}$ & $200-3113-7$ & JCFC19.D & $12 / 28 / 2010$ & 0134 \\
\hline $\begin{array}{l}\text { INMW0 } 0-W-3 \\
2692\end{array}$ & $200-3113-8$ & JCFC21.D & $12 / 28 / 2010$ & 0223 \\
\hline $\begin{array}{l}\text { INMW09-W-3 } \\
2693\end{array}$ & $200-3113-9$ & JCFC23.D & $12 / 28 / 2010$ & 0312 \\
\hline $\begin{array}{l}\text { INMW10-W-3 } \\
2694\end{array}$ & $200-3113-10$ & JCFC25.D & $12 / 28 / 2010$ & 0400 \\
\hline VSTD005GJ & CCVC 200-11651/27 & JCFC27.D & $12 / 28 / 2010$ & 0449 \\
\hline
\end{tabular}


$5 A$ - FORM V VOA

VOLATILE ORGANICS INSTRUMENT

PERFORMANCE CHECK

BROMOFLUOROBENZENE (BFB)
EPA SAMPLE NO.

$\mathrm{BFBJI}$
Lab Name: $\frac{\text { TESTAMERICA BURLINGTON }}{\text { Lab Code: }}$ STLV No.: INMAN Mod. Ref No.
Lab File Id: JCFEO1.D

Instrument Id: J.i

GC Column: DB-624

ID: $0.20 \quad(\mathrm{~mm})$

Contract: $\quad 8 \mathrm{E}-00302$

BFB Injection Date: $\frac{12 / 28 / 2010}{1318}$

\begin{tabular}{|c|c|c|}
\hline $\mathrm{m} / \mathrm{e}$ & ION ABUNDANCE CRITERIA & $\begin{array}{l}\text { O RELATIVE } \\
\text { ABUNDANCE }\end{array}$ \\
\hline 50 & $15.0-40.0 \%$ of mass 95 & 18.0 \\
\hline 75 & $30.0-80.0 \%$ of mass 95 & 51.8 \\
\hline 95 & Base peak, $100 \%$ relative abundance & 100 \\
\hline 96 & $5.0-9.0 \mathrm{~g}$ of mass 95 & 6.2 \\
\hline 173 & Less than $2.0 \%$ of mass 174 & 0.81 \\
\hline 174 & $50.0-120 \%$ of mass 95 & 80.8 \\
\hline 175 & $5.0-9.0 \%$ of mass 174 & $5.7(7.1) 1$ \\
\hline 176 & $95.0-101 \%$ of mass 174 & $78.1(96.6) 1$ \\
\hline 177 & $5.0-9.0 \%$ of mass 176 & $4.9(6.2) 2$ \\
\hline
\end{tabular}

1 - Value is omass $174 \quad 2$ - Value is omass 176

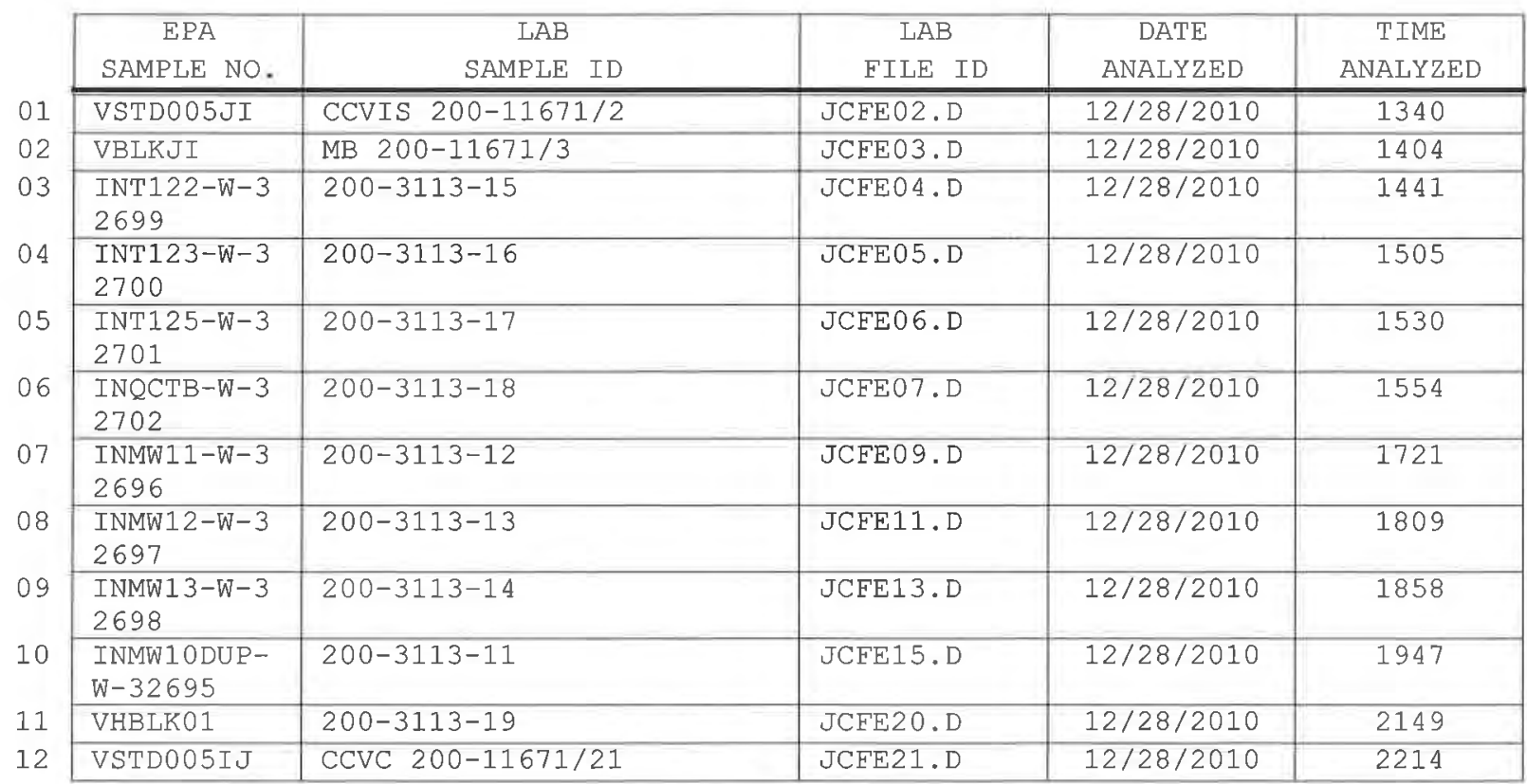

Page 1 of 1 
$8 A$ - FORM VIII VOA

VOLATILE INTERNAL STANDARD AREA AND RETENTION TIME SUMMARY

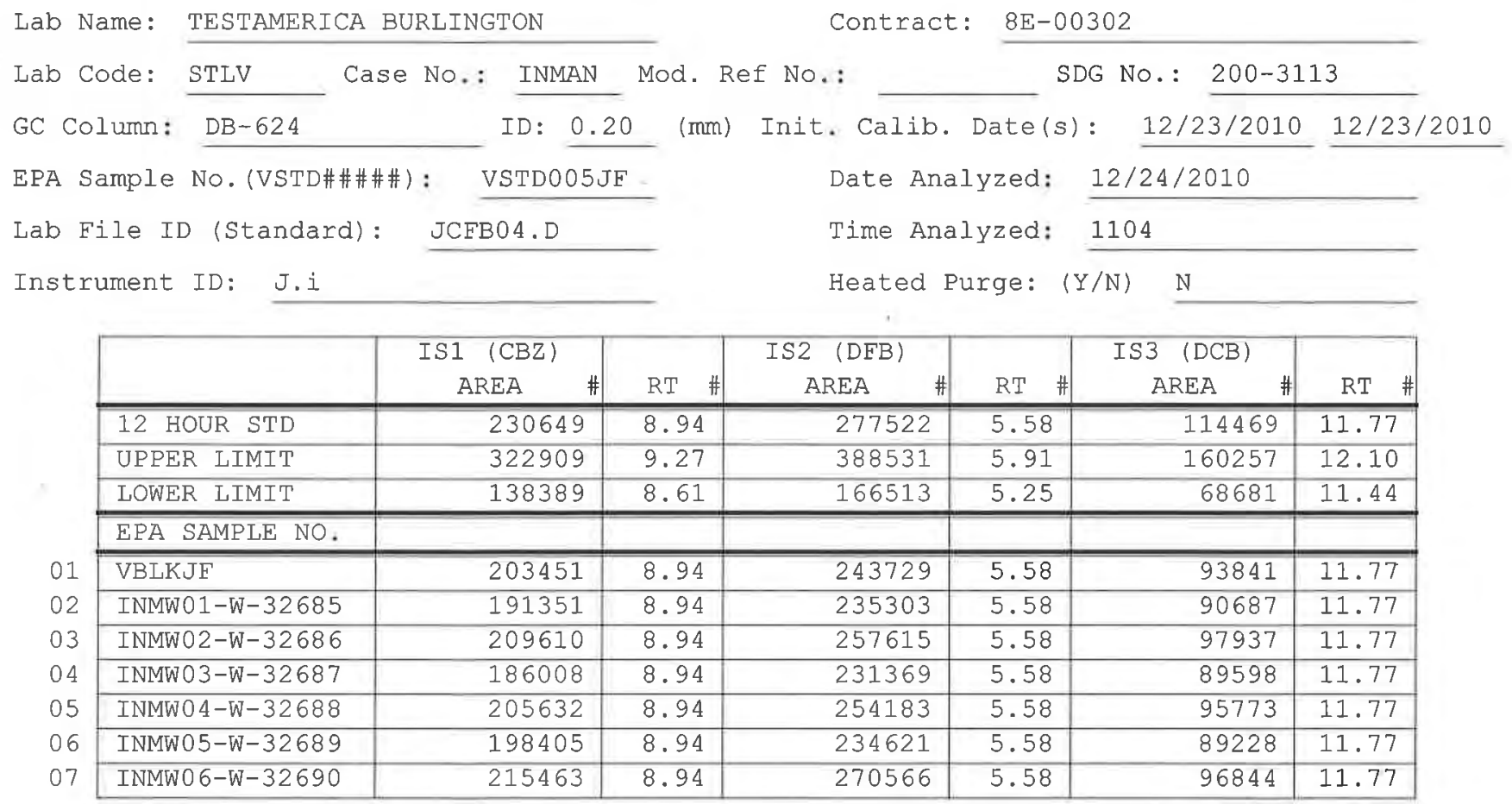

IS1 (CBZ) $=$ Chlorobenzene-d5

IS2 $(\mathrm{DFB})=1,4$-Difluorobenzene

IS3 $(\mathrm{DCB})=1,4-$ Dichlorobenzene-d4

AREA UPPER LIMIT $=140 \%$ (Trace Volatiles) of internal standard area

AREA LOWER LIMIT $=60 \%$ (Trace Volatiles) of internal standard area

RT UPPER LIMIT $=+0.33$ (Trace Volatiles) minutes of internal standard RT

RT LOWER LIMIT $=-0.33$ (Trace Volatiles) minutes of internal standard RT

\# Colum used to flag values outside contract required QC limits with an asterisk. 
$8 A$ - FORM VIII VOA

VOLATILE INTERNAL STANDARD AREA AND RETENTION TIME SUMMARY

Lab Name: TESTAMERICA BURLINGTON

Contract: $\quad 8 \mathrm{E}-00302$

Lab Code: STLV Case No.: INMAN Mod. Ref No.:

SDG No.: 200-3113

GC Column: DB-624 ID: 0.20 (mm) Init.

EPA Sample No. (VSTD\#\#\#\#\#): VSTD005JG

Lab File ID (Standard): JCFCO4.D

Instrument ID: J.i

Date Analyzed: 12/27/2010

Time Analyzed: 1828

Heated Purge: ( $\mathrm{Y} / \mathrm{N}) \mathrm{N}$

\begin{tabular}{|c|c|c|c|c|c|c|}
\hline & $\begin{array}{c}\text { IS1 (CBZ) } \\
\text { AREA }\end{array}$ & RT \# & $\begin{array}{c}\text { IS2 (DFB) } \\
\text { AREA }\end{array}$ & RT \# & $\begin{array}{c}\text { IS3 (DCB) } \\
\text { AREA }\end{array}$ & RT \# \\
\hline 12 HOUR STD & 235674 & 8.94 & 276913 & 5.58 & 121435 & 11.77 \\
\hline UPPER LIMIT & 329944 & 9.27 & 387678 & 5.91 & 170009 & 12.10 \\
\hline LOWER LIMIT & 141404 & 8.61 & 166148 & 5.25 & 72861 & 11.44 \\
\hline EPA SAMPLE NO. & & & & & & \\
\hline$\overline{\text { VBLKJG }}$ & 227266 & 8.94 & 275145 & 5.58 & 106227 & 11.77 \\
\hline INMW07-W-32691 & 204009 & 8.94 & 252171 & 5.58 & 92388 & 11.77 \\
\hline INMW08-W-32692 & 201454 & 8.94 & 241188 & 5.58 & 89805 & 11.77 \\
\hline INMW09-W-32693 & 200994 & 8.94 & 248913 & 5.58 & 89852 & 11.77 \\
\hline INMW10-W-32694 & 193747 & 8.94 & 237112 & 5.58 & 89324 & 11.77 \\
\hline
\end{tabular}

IS1 $(\mathrm{CBZ})=$ Chlorobenzene-d5

IS2 $(\mathrm{DFB})=1,4$-Difluorobenzene

IS3 $(\mathrm{DCB})=1,4$-Dichlorobenzene-d4

AREA UPPER LIMIT $=140 \%$ (Trace Volatiles) of internal standard area

AREA LOWER LIMIT $=60 \%$ (Trace Volatiles) of internal standard area

RT UPPER LIMIT $=+0.33$ (Trace Volatiles) minutes of internal standard RT

RT LOWER LIMIT $=-0.33$ (Trace Volatiles) minutes of internal standard RT

\# Column used to flag values outside contract required QC limits with an asterisk. 
$8 A$ - FORM VIII VOA

VOLATILE INTERNAL STANDARD AREA AND RETENTION TIME SUMMARY

Lab Name: TESTAMERICA BURLINGTON

Contract: $8 \mathrm{E}-00302$

Lab Code: STLV Case No.: INMAN Mod. Ref No.:

SDG No.: 200-3113

GC Column: DB-624

ID $: 0.20$

(mm) Init.

EPA Sample No. (VSTD\#\#\#\#\#): VSTD005JI

Calib. Date(s): 12/23/2010 12/23/2010

Lab File ID (Standard): JCFE02.D

Date Analyzed: 12/28/2010

Instrument ID: J.i

Time Analyzed: 1340

Heated Purge: $(\mathrm{Y} / \mathrm{N}) \mathrm{N}$

\begin{tabular}{|c|c|c|c|c|}
\hline & IS2 (DFB) & & IS3 (DCB) & \\
\hline RT \# & AREA & RT \# & AREA & RT \\
\hline 8.94 & 259749 & 5.57 & 110015 & 11.77 \\
\hline 9.27 & 363649 & 5.90 & 154021 & 12.10 \\
\hline 8.61 & 155849 & 5.24 & 66009 & 11.44 \\
\hline & & & & \\
\hline 8.94 & 239811 & 5.58 & 94372 & 11.77 \\
\hline 8.94 & 224442 & 5.57 & 90215 & 11.77 \\
\hline 8.94 & 250338 & 5.57 & 95628 & 11.77 \\
\hline 8.94 & 238434 & 5.58 & 93336 & 11.77 \\
\hline 8.94 & 242489 & 5.58 & 95328 & 11.77 \\
\hline 8.94 & 257027 & 5.57 & 96396 & 11.77 \\
\hline 8.94 & 260838 & 5.58 & 96780 & 11.77 \\
\hline 8.94 & 270302 & 5.58 & 99084 & 11.77 \\
\hline 8.94 & 279440 & 5.58 & 101123 & 11.77 \\
\hline 8.94 & 230131 & 5.58 & 91485 & 11.77 \\
\hline
\end{tabular}

IS1 (CBZ) = Chlorobenzene-d5

IS2 $(\mathrm{DFB})=1,4$-Difluorobenzene

IS3 $(\mathrm{DCB})=1,4$-Dichlorobenzene-d4

AREA UPPER IIMIT $=140 \%$ (Trace Volatiles) of internal standard area

AREA LOWER LIMIT $=60 \%$ (Trace Volatiles) of internal standard area

RT UPPER LIMIT $=+0.33$ (Trace Volatiles) minutes of internal standard RT

RT LOWER LIMIT $=-0.33$ (Trace Volatiles) minutes of internal standard RT

\# Column used to flag values outside contract required QC limits with an asterisk. 
$1 A$ - FORM I VOA-1

VOLATILE ORGANICS ANALYSIS DATA SHEET
EPA SAMPLE NO. INMW01-W-32685
Lab Name: TESTAMERICA BURLINGTON

Lab Code: STLV Case

Matrix: (SOIL/SED/WATER)

Water

Sample wt/vol: 25.0

$(\mathrm{g} / \mathrm{mL}) \mathrm{mL}$

Level: (TRACE/LOW/MED) TRACE

응 Moisture: not dec.

GC Column: DB-624 ID: 0.20 (mm)

Soil Extract Volume: (uL) (mL)

Purge Volume: 25.0
Contract: $\quad 8 \mathrm{E}-00302$

SDG No.: 200-3113

\begin{tabular}{|c|c|c|c|}
\hline CAS NO. & COMPOUND & $\begin{array}{l}\text { CONCENTRATION UNITS: } \\
(\mathrm{ug} / \mathrm{L} \text { or } \mathrm{ug} / \mathrm{kg}) \mathrm{ug} / \mathrm{L}\end{array}$ & Q \\
\hline $75-71-8$ & Dichlorodifluoromethane & 0.50 & U \\
\hline $74-87-3$ & Chloromethane & 0.50 & $\mathrm{U}$ \\
\hline $75-01-4$ & Vinyl chloride & 0.50 & $\mathrm{U}$ \\
\hline $74-83-9$ & Bromomethane & 0.50 & $\mathrm{U}$ \\
\hline $75-00-3$ & Chloroethane & 0.50 & $\mathrm{U}$ \\
\hline $75-69-4$ & Trichlorofluoromethane & 0.50 & $\mathrm{U}$ \\
\hline $75-35-4$ & 1,1-Dichloroethene & 0.50 & $\mathrm{U}$ \\
\hline $76-13-1$ & 1,1,2-Trichloro-1,2,2-trifluoroethane & 0.50 & $\mathrm{U}$ \\
\hline $67-64-1$ & Acetone & 2.5 & $\mathrm{~J} B$ \\
\hline $75-15-0$ & Carbon disulfide & 0.084 & $\mathrm{~J} B$ \\
\hline $79-20-9$ & Methyl acetate & 0.50 & $\mathrm{U}$ \\
\hline $75-09-2$ & Methylene Chloride & 0.50 & $\mathrm{U}$ \\
\hline $156-60-5$ & trans-1,2-Dichloroethene & 0.50 & $\mathrm{U}$ \\
\hline $1634-04-4$ & Methyl tert-butyl ether & 0.11 & $\mathrm{~J} \mathrm{~B}$ \\
\hline $75-34-3$ & 1,1-Dichloroethane & 0.50 & $\mathrm{U}$ \\
\hline $156-59-2$ & cis-1,2-Dichloroethene & 0.50 & $\mathrm{U}$ \\
\hline $78-93-3$ & 2-Butanone & 5.0 & $\mathrm{U}$ \\
\hline $74-97-5$ & Bromochloromethane & 0.50 & $\mathrm{U}$ \\
\hline $67-66-3$ & Chloroform & 3.2 & \\
\hline $71-55-6$ & 1,1,1-Trichloroethane & 0.50 & $\mathrm{U}$ \\
\hline $110-82-7$ & Cyclohexane & 0.50 & $\mathrm{U}$ \\
\hline $56-23-5$ & Carbon tetrachloride & 5.4 & \\
\hline $71-43-2$ & Benzene & 0.50 & $\mathrm{U}$ \\
\hline $107-06-2$ & 1,2-Dichloroethane & 0.50 & $\mathrm{U}$ \\
\hline
\end{tabular}

Report 1,4-Dioxane for Low-Medium VOA analysis only

Lab Sample ID: 200-3113-1

Lab File ID: JCFB10.D

Date Received: 12/23/2010

Date Analyzed: 12/24/2010

Dilution Factor: 1.0

Soil Aliquot Volume:

(uL) 
Lab Name: TESTAMERICA BURLINGTON

Lab Code: STLV Case No.: INMAN

Matrix: (SOIL/SED/WATER)

Water

Sample wt/vol: 25.0 $(\mathrm{g} / \mathrm{mL}) \mathrm{mL}$

Level: (TRACE/LOW/MED) TRACE

\% Moisture: not dec.

GC Column: DB-624 ID: 0.20 (nm)

Soil Extract Volume:

Purge Volume: 25.0

(uL)

( $\mathrm{mL})$
Contract: $\quad 8 E-00302$

SDG No.: 200-3113

\begin{tabular}{|c|c|c|c|}
\hline CAS NO. & COMPOUND & $\begin{array}{l}\text { CONCENTRATION UNITS: } \\
(\mathrm{ug} / \mathrm{L} \text { or } \mathrm{ug} / \mathrm{kg}) \mathrm{ug} / \mathrm{L}\end{array}$ & $Q$ \\
\hline $79-01-6$ & Trichloroethene & 0.50 & $\mathrm{U}$ \\
\hline $108-87-2$ & Methylcyclohexane & 0.50 & $\mathrm{U}$ \\
\hline $78-87-5$ & 1,2-Dichloropropane & 0.50 & $\mathrm{U}$ \\
\hline $75-27-4$ & Bromodichloromethane & 0.50 & $\mathrm{U}$ \\
\hline $10061-01-5$ & cis-1,3-Dichloropropene & 0.50 & $\mathrm{U}$ \\
\hline $108-10-1$ & 4-Methyl-2-pentanone & 5.0 & $\mathrm{U}$ \\
\hline $108-88-3$ & Toluene & 0.064 & $\mathrm{~J}$ \\
\hline $10061-02-6$ & trans-1,3-Dichloropropene & 0.50 & $\mathrm{U}$ \\
\hline $79-00-5$ & 1,1,2-Trichloroethane & 0.50 & $\mathrm{U}$ \\
\hline $127-18-4$ & Tetrachloroethene & 0.36 & $\mathrm{~J}$ \\
\hline $591-78-6$ & 2-Hexanone & 5.0 & $\mathrm{U}$ \\
\hline $124-48-1$ & Dibromochloromethane & 0.50 & $\mathrm{U}$ \\
\hline $106-93-4$ & 1,2-Dibromoethane & 0.50 & $\mathrm{U}$ \\
\hline $108-90-7$ & Chlorobenzene & 0.50 & $\mathrm{U}$ \\
\hline $100-41-4$ & Ethylbenzene & 0.50 & $\mathrm{U}$ \\
\hline $95-47-6$ & o-xylene & 0.50 & $\mathrm{U}$ \\
\hline 179601-23-1 & $\mathrm{m}, \mathrm{p}$-Xylene & 0.50 & $\mathrm{U}$ \\
\hline $100-42-5$ & Styrene & 0.50 & $\mathrm{U}$ \\
\hline $75-25-2$ & Bromoform & 0.50 & $\mathrm{U}$ \\
\hline $98-82-8$ & Isopropylbenzene & 0.50 & $\mathrm{U}$ \\
\hline $79-34-5$ & $1,1,2,2$-Tetrachloroethane & 0.50 & $\mathrm{U}$ \\
\hline $541-73-1$ & 1,3-Dichlorobenzene & 0.50 & U \\
\hline $106-46-7$ & 1,4-Dichlorobenzene & 0.50 & $\mathrm{U}$ \\
\hline $95-50-1$ & 1,2-Dichlorobenzene & 0.50 & $\mathrm{U}$ \\
\hline $96-12-8$ & 1,2-Dibromo-3-Chloropropane & 0.50 & $\mathrm{U}$ \\
\hline $120-82-1$ & 1,2,4-Trichlorobenzene & 0.50 & $\mathrm{U}$ \\
\hline $87-61-6$ & 1,2,3-Trichlorobenzene & 0.50 & $\mathrm{U}$ \\
\hline
\end{tabular}


IJ - FORM I VOA-TIC

VOLATILE ORGANICS ANALYSIS DATA SHEET

TENTATIVELY IDENTIEIED COMPOUNDS
EPA SAMPLE NO.

INMW01-W-32685

Lab Name: TESTAMERICA BURLINGTON

Contract: $8 \mathrm{E}-00302$

Lab Code: STLV Case No.: INMAN Mod. Ref No.: SDG No.: 200-3113

Matrix: (SOIL/SED/WATER)

Water

Sample wt/vol: 25.0

$(\mathrm{g} / \mathrm{mL}) \mathrm{mL}$

Level: (TRACE or LOW/MED) TRACE

응 Moisture: not dec.

GC Column: DB-624 ID $0.20 \quad(\mathrm{~mm})$

Soil Extract Volume: (uL)
Lab Sample ID: 200-3113-1

Lab File ID: JCFB10.D

Date Received: 12/23/2010

Date Analyzed: 12/24/2010

Dilution Factor: 1.0

Soil Aliquot Volume: (UL)

Purge Volume: 25.0 (mL)

\begin{tabular}{|c|c|c|c|c|}
\hline CAS NUMBER & COMPOUND NAME & $\mathrm{RT}$ & EST. CONC. & $Q$ \\
\hline $109-99-9$ & Furan, tetrahydro- & 4.50 & 14 & \\
\hline 02 & Unknown & 6.90 & 3.4 & $\mathrm{~B} \times \mathrm{J}$ \\
\hline E9667961 & Total Alkanes & $\mathrm{N} / \mathrm{A}$ & 20 & $\mathrm{~J}$ \\
\hline
\end{tabular}

1 EPA-designated Registry Number. 
$1 A$ - FORM I VOA-1

VOLATILE ORGANICS ANALYSIS DATA SHEET
EPA SAMPLE NO.

INMW02-W-32686

Lab Name: TESTAMERICA BURLINGTON

Contract: $8 \mathrm{E}-00302$

Lab Code: STLV Case No.: INMAN Mod. Ref No.:

SDG No.: $200-3113$

Matrix: (SOIL/SED/WATER) Water

Sample wt/vol: 25.0

$(\mathrm{g} / \mathrm{mL}) \mathrm{mL}$

Level: (TRACE/LOW/MED) TRACE

o Moisture: not dec.

GC Column: DB-624 ID $: 0.20 \quad(\mathrm{~mm})$

Soil Extract Volume:

Purge Volume: 25.0

(uL)

(mL)
Lab Sample ID: 200-3113-2

Lab Eile ID: JCFB11.D

Date Received: 12/23/2010

Date Analyzed: 12/24/2010

Dilution Factor: 1.0

Soil Aliquot Volume:

(uL)

\begin{tabular}{|c|c|c|c|}
\hline CAS NO. & COMPOUND & $\begin{array}{l}\text { CONCENTRATION UNITS: } \\
(\mathrm{ug} / \mathrm{L} \text { or } \mathrm{ug} / \mathrm{kg}) \mathrm{ug} / \mathrm{L}\end{array}$ & $Q$ \\
\hline $75-71-8$ & Dichlorodifluoromethane & 0.50 & $\overline{\mathrm{U}}$ \\
\hline $74-87-3$ & Chloromethane & 0.50 & $\mathrm{U}$ \\
\hline $75-01-4$ & Vinyl chloride & 0.50 & $\mathrm{U}$ \\
\hline $74-83-9$ & Bromomethane & 0.50 & $\mathrm{U}$ \\
\hline $75-00-3$ & Chloroethane & 0.50 & $\mathrm{U}$ \\
\hline $75-69-4$ & Trichlorofluoromethane & 0.50 & $\mathrm{U}$ \\
\hline $75-35-4$ & 1,1-Dichloroethene & 0.50 & $\mathrm{U}$ \\
\hline $76-13-1$ & 1,1,2-Trichloro-1,2,2-trifluoroethane & 0.50 & $\mathrm{U}$ \\
\hline $67-64-1$ & Acetone & 3.9 & $\mathrm{~J} B$ \\
\hline $75-15-0$ & Carbon disulfide & 0.10 & $\mathrm{~J} B$ \\
\hline $79-20-9$ & Methyl acetate & 0.50 & $\mathrm{U}$ \\
\hline $75-09-2$ & Methylene Chloride & 1.5 & \\
\hline $156-60-5$ & trans-1,2-Dichloroethene & 0.50 & $\mathrm{U}$ \\
\hline $1634-04-4$ & Methyl tert-butyl ether & 0.11 & $\mathrm{~J} B$ \\
\hline $75-34-3$ & 1,1-Dichloroethane & 0.50 & $\mathrm{U}$ \\
\hline $156-59-2$ & cis-1,2-Dichloroethene & 0.50 & $\mathrm{U}$ \\
\hline $78-93-3$ & 2-Butanone & 5.0 & $\mathrm{U}$ \\
\hline $74-97-5$ & Bromochloromethane & 0.50 & $\mathrm{U}$ \\
\hline $67-66-3$ & Chloroform & 4.8 & \\
\hline $71-55-6$ & 1,1,1-Trichloroethane & 0.50 & $\mathrm{U}$ \\
\hline $110-82-7$ & Cyclohexane & 0.50 & $\mathrm{U}$ \\
\hline $56-23-5$ & Carbon tetrachloride & 2.9 & \\
\hline $71-43-2$ & Benzene & 0.50 & $\mathrm{U}$ \\
\hline $107-06-2$ & 1,2-Dichloroethane & 0.50 & $\mathrm{U}$ \\
\hline
\end{tabular}

Report 1,4-Dioxane for Low-Medium VOA analysis only 
$1 B$ - FORM I VOA-2

VOLATILE ORGANICS ANALYSIS DATA SHEET
EPA SAMPLE NO.

INMW02-W-32686
Lab Name: TESTAMERICA BURLINGTON

Lab Code: STLV

Case No.: INMAN

Matrix: (SOIL/SED/WATER)

Sample wt/vol: 25.0

Water

$(\mathrm{g} / \mathrm{mL}) \quad \mathrm{mL}$

Level: (TRACE/LOW/MED)

TRACE

\% Moisture: not dec.

GC Column: DB-624

Soil Extract Volume: ID: $0.20 \quad$ (mm)

(uL)

$(\mathrm{mL})$

Purge Volume: 25.0
Contract: $\quad 8 E-00302$

SDG No.: 200-3113

\begin{tabular}{|c|c|c|c|}
\hline CAS NO. & COMPOUND & $\begin{array}{l}\text { CONCENTRATION UNITS: } \\
(\mathrm{ug} / \mathrm{L} \text { or } \mathrm{ug} / \mathrm{kg}) \mathrm{ug} / \mathrm{L}\end{array}$ & $Q$ \\
\hline $79-01-6$ & Trichloroethene & 0.078 & $\mathrm{~J}$ \\
\hline $108-87-2$ & Methylcyclohexane & 0.50 & $\mathrm{U}$ \\
\hline $78-87-5$ & 1,2-Dichloropropane & 0.50 & $\mathrm{U}$ \\
\hline $75-27-4$ & Bromodichloromethane & 0.50 & $\mathrm{U}$ \\
\hline $10061-01-5$ & cis-1,3-Dichloropropene & 0.50 & $\mathrm{U}$ \\
\hline $108-10-1$ & 4-Methyl-2-pentanone & 5.0 & $\mathrm{U}$ \\
\hline $108-88-3$ & Toluene & 0.15 & $\mathrm{~J}$ \\
\hline $10061-02-6$ & trans-1,3-Dichloropropene & 0.50 & $\mathrm{U}$ \\
\hline $79-00-5$ & 1,1,2-Trichloroethane & 0.50 & $\mathrm{U}$ \\
\hline $127-18-4$ & Tetrachloroethene & 0.35 & $\mathrm{~J}$ \\
\hline $591-78-6$ & 2-Hexanone & 5.0 & $\mathrm{U}$ \\
\hline $124-48-1$ & Dibromochloromethane & 0.50 & $\mathrm{U}$ \\
\hline $106-93-4$ & 1,2-Dibromoethane & 0.50 & $\mathrm{U}$ \\
\hline $108-90-7$ & Chlorobenzene & 0.50 & $\mathrm{U}$ \\
\hline $100-41-4$ & Ethylbenzene & 0.50 & $\mathrm{U}$ \\
\hline $95-47-6$ & o-Xylene & 0.50 & $\mathrm{U}$ \\
\hline $179601-23-1$ & $\mathrm{~m}, \mathrm{p}$-Xylene & 0.061 & $\mathrm{~J}$ \\
\hline $100-42-5$ & Styrene & 0.50 & $\mathrm{U}$ \\
\hline $75-25-2$ & Bromoform & 0.50 & $\mathrm{U}$ \\
\hline $98-82-8$ & Isopropylbenzene & 0.50 & $\mathrm{U}$ \\
\hline $79-34-5$ & $1,1,2,2$-Tetrachloroethane & 0.50 & $\mathrm{U}$ \\
\hline $541-73-1$ & 1,3-Dichlorobenzene & 0.50 & $\mathrm{U}$ \\
\hline $106-46-7$ & 1,4-Dichlorobenzene & 0.50 & $\mathrm{U}$ \\
\hline $95-50-1$ & 1,2-Dichlorobenzene & 0.50 & $\mathrm{U}$ \\
\hline $96-12-8$ & 1,2-Dibromo-3-Chloropropane & 0.50 & $\mathrm{U}$ \\
\hline $120-82-1$ & 1,2,4-Trichlorobenzene & 0.50 & $\mathrm{U}$ \\
\hline $87-61-6$ & 1,2,3-Trichlorobenzene & 0.50 & $\mathrm{U}$ \\
\hline
\end{tabular}


$1 \mathrm{~J}$ - FORM I VOA-TIC

VOLATILE ORGANICS ANALYSIS DATA SHEET

TENTATIVELY IDENTIEIED COMPOUNDS
EPA SAMPLE NO. INMW02-W-32686

Lab Name: TESTAMERICA BURLINGTON

Contract: $\quad 8 \mathrm{E}-00302$

Lab Code: STLV Case No.: INMAN Mod. Ref No.: SDG No.: 200-3113

Matrix: (SOIL/SED/WATER)

Sample wt/vol: 25.0

Level: (TRACE or LOW/MED)

Water

$(\mathrm{g} / \mathrm{mL}) \mathrm{mL}$

TRACE

\& Moisture: not dec.

GC Column: DB-624 ID: $0.20 \quad(\mathrm{~mm})$

Soil Extract volume: (uL)

CONCENTRATION UNITS: (ug/L or $u g / k g) ~ u g / L$
Lab Sample ID: 200-3113-2

Lab File ID: JCFB11.D

Date Received: 12/23/2010

Date Analyzed: 12/24/2010

Dilution Factor: 1.0

Soil Aliquot Volume: (uL)

Purge Volume: 25.0 (mL)

\begin{tabular}{|c|c|c|c|c|}
\hline CAS NUMBER & COMPOUND NAME & $\mathrm{RT}$ & EST. CONC. & $Q$ \\
\hline 01 & Unknown & 3.51 & 1.3 & $\bar{J}$ \\
\hline $109-99-9$ & Furan, tetrahydro- & 4.49 & 18 & $\mathrm{~J} \mathrm{~N}$ \\
\hline 03 & Unknown & 6.90 & 3.1 & $\mathrm{~B} \times \mathrm{J}$ \\
\hline E9667961 & Total Alkanes & $\mathrm{N} / \mathrm{A}$ & 17 & $\mathrm{~J}$ \\
\hline
\end{tabular}

1 EPA-designated Registry Number. 
$1 A$ - FORM I VOA-1

VOLATILE ORGANICS ANALYSIS DATA SHEET
EPA SAMPLE NO.

INMW03-W-32687

Lab Name: TESTAMERICA BURLINGTON

Contract: $8 \mathrm{E}-00302$

Lab Code: STLV Case No.: INMAN Mod. Ref No.:

SDG No.: 200-3113

Matrix: (SOIL/SED/WATER)

Sample wt/vol: 25.0 water

$(\mathrm{g} / \mathrm{mL}) \quad \mathrm{mL}$

Level: (TRACE/LOW/MED) TRACE

\% Moisture: not dec.

GC Column: DB-624 ID: $0.20 \quad(\mathrm{~mm})$

Soil Extract Volume: (uL)

Purge Volume: 25.0 (mL)
Lab Sample ID: 200-3113-3

Lab File ID: JCFB12.D

Date Received: 12/23/2010

Date Analyzed: 12/24/2010

Dilution Factor: 1.0

Soil Aliquot volume:

(uL)

\begin{tabular}{|c|c|c|c|}
\hline CAS NO. & COMPOUND & $\begin{array}{l}\text { CONCENTRATION UNITS: } \\
(\mathrm{ug} / \mathrm{L} \text { or } \mathrm{ug} / \mathrm{kg}) \mathrm{ug} / \mathrm{L}\end{array}$ & $Q$ \\
\hline $75-71-8$ & Dichlorodifluoromethane & 0.50 & $\overline{\mathrm{U}}$ \\
\hline $74-87-3$ & Chloromethane & 0.50 & $\mathrm{U}$ \\
\hline $75-01-4$ & Vinyl chloride & 0.50 & $\mathrm{U}$ \\
\hline $74-83-9$ & Bromomethane & 0.50 & $\mathrm{U}$ \\
\hline $75-00-3$ & Chloroethane & 0.50 & $\mathrm{U}$ \\
\hline $75-69-4$ & Trichlorofluoromethane & 0.50 & $\mathrm{U}$ \\
\hline $75-35-4$ & 1,1-Dichloroethene & 0.50 & $\mathrm{U}$ \\
\hline $76-13-1$ & 1,1,2-Trichloro-1,2,2-trifluoroethane & 0.50 & $\mathrm{U}$ \\
\hline $67-64-1$ & Acetone & 2.0 & $\mathrm{~J} B$ \\
\hline $75-15-0$ & Carbon disulfide & 0.19 & $\mathrm{~J} B$ \\
\hline $79-20-9$ & Methyl acetate & 0.50 & $\mathrm{U}$ \\
\hline $75-09-2$ & Methylene Chloride & 0.078 & $\mathrm{~J}$ \\
\hline $156-60-5$ & trans-1,2-Dichloroethene & 0.50 & $\mathrm{U}$ \\
\hline $1634-04-4$ & Methyl tert-butyl ether & 0.10 & $\mathrm{JB}$ \\
\hline $75-34-3$ & 1,1-Dichloroethane & 0.50 & $\mathrm{U}$ \\
\hline $156-59-2$ & cis-1,2-Dichloroethene & 0.50 & $\mathrm{U}$ \\
\hline $78-93-3$ & 2-Butanone & 6.2 & \\
\hline $74-97-5$ & Bromochloromethane & 0.50 & $\mathrm{U}$ \\
\hline $67-66-3$ & Chloroform & 1.5 & \\
\hline $71-55-6$ & 1,1,1-Trichloroethane & 0.50 & $\mathrm{U}$ \\
\hline $110-82-7$ & Cyclohexane & 0.50 & $\mathrm{U}$ \\
\hline $56-23-5$ & Carbon tetrachloride & 2.2 & \\
\hline $71-43-2$ & Benzene & 0.50 & $\mathrm{U}$ \\
\hline $107-06-2$ & 1,2-Dichloroethane & 0.50 & $\mathrm{U}$ \\
\hline
\end{tabular}

Report 1,4-Dioxane for Low-Medium VOA analysis only 
Lab Name: TESTAMERICA BURLINGTON

Lab Code: STLV Case No.: INMA Matrix: (SOIL/SED/WATER) Sample wt/vol: 25.0 water

Level: (TRACE/LOW/MED) TRACE

음 Moisture: not dec.

GC Column: DB-624 ID: $0.20 \quad$ (mm)

Soil Extract Volume:

Purge Volume: 25.0 (mL)

Contract: $8 \mathrm{E}-00302$

SDG NO.: 200-3113

Lab Sample ID: 200-3113-3

Lab File ID: JCFB12.D

Date Received: 12/23/2010

Date Analyzed: 12/24/2010

Dilution Factor: 1.0

Soil Aliquot Volume:

(uL)

\begin{tabular}{|c|c|c|c|}
\hline CAS NO. & COMPOUND & $\begin{array}{l}\text { CONCENTRATION UNITS: } \\
(\mathrm{ug} / \mathrm{L} \text { or } \mathrm{ug} / \mathrm{kg}) \mathrm{ug} / \mathrm{L}\end{array}$ & Q \\
\hline $79-01-6$ & Trichloroethene & 0.50 & $\overline{\mathrm{U}}$ \\
\hline $108-87-2$ & Methylcyclohexane & 0.50 & $\mathrm{U}$ \\
\hline $78-87-5$ & 1,2-Dichloropropane & 0.50 & $\mathrm{U}$ \\
\hline $75-27-4$ & Bromodichloromethane & 0.50 & $\mathrm{U}$ \\
\hline $10061-01-5$ & cis-1,3-Dichloropropene & 0.50 & $\mathrm{U}$ \\
\hline $108-10-1$ & 4-Methyl-2-pentanone & 5.0 & $\mathrm{U}$ \\
\hline $108-88-3$ & Toluene & 0.14 & $\mathrm{~J}$ \\
\hline $10061-02-6$ & trans-1,3-Dichloropropene & 0.50 & $\mathrm{U}$ \\
\hline $79-00-5$ & 1,1,2-Trichloroethane & 0.50 & $\mathrm{U}$ \\
\hline $127-18-4$ & Tetrachloroethene & 0.49 & $\mathrm{~J}$ \\
\hline $591-78-6$ & 2-Hexanone & 5.0 & $\mathrm{U}$ \\
\hline $124-48-1$ & Dibromochloromethane & 0.50 & $\mathrm{U}$ \\
\hline $106-93-4$ & 1,2-Dibromoethane & 0.50 & $\mathrm{U}$ \\
\hline $108-90-7$ & Chlorobenzene & 0.50 & $\mathrm{U}$ \\
\hline $100-41-4$ & Ethylbenzene & 0.50 & $\mathrm{U}$ \\
\hline $95-47-6$ & o-Xylene & 0.50 & $\mathrm{U}$ \\
\hline $179601-23-1$ & $\mathrm{~m}, \mathrm{p}$-Xylene & 0.070 & $\mathrm{~J}$ \\
\hline $100-42-5$ & Styrene & 0.50 & $\mathrm{U}$ \\
\hline $75-25-2$ & Bromoform & 0.50 & $\mathrm{U}$ \\
\hline $98-82-8$ & Isopropylbenzene & 0.50 & $\mathrm{U}$ \\
\hline $79-34-5$ & $1,1,2,2$-Tetrachloroethane & 0.50 & $\mathrm{U}$ \\
\hline $541-73-1$ & 1,3-Dichlorobenzene & 0.50 & $\mathrm{U}$ \\
\hline $106-46-7$ & 1,4-Dichlorobenzene & 0.50 & $\mathrm{U}$ \\
\hline $95-50-1$ & 1,2-Dichlorobenzene & 0.50 & $\mathrm{U}$ \\
\hline $96-12-8$ & 1,2-Dibromo-3-Chloropropane & 0.50 & $\mathrm{U}$ \\
\hline $120-82-1$ & 1,2,4-Trichlorobenzene & 0.50 & $\mathrm{U}$ \\
\hline $87-61-6$ & 1,2,3-Trichlorobenzene & 0.50 & $\mathrm{U}$ \\
\hline
\end{tabular}




\section{IJ - FORM I VOA-TIC \\ VOLATILE ORGANICS ANALYSIS DATA SHEET \\ TENTATIVELY IDENTIFIED COMPOUNDS}

EPA SAMPLE NO.

INMW03-W-32687

Lab Name: TESTAMERICA BURLINGTON

Contract: $8 \mathrm{E}-00302$

Lab Code: STLV Case No.: INMAN Mod. Ref No.:

SDG No.: 200-3113

Matrix: (SOIL/SED/WATER)

Sample wt/vol: 25.0

Level: (TRACE or LOW/MED)

Water

$(\mathrm{g} / \mathrm{mL}) \mathrm{mL}$

TRACE

o Moisture: not dec.

GC Column: $\mathrm{DB}-624$

ID: 0.20

(mm)

Soil Extract Volume:

(uL)
Lab Sample ID: 200-3113-3

Lab File ID: JCFB12.D

Date Received: 12/23/2010

Date Analyzed: 12/24/2010

Dilution Factor: 1.0

Soil Aliquot Volume: (uL)

Purge Volume: 25.0 $(\mathrm{mL})$

\begin{tabular}{l|l|l|r|r|r|}
\hline \multicolumn{1}{c|}{ CAS NUMBER } & \multicolumn{1}{|c|}{ COMPOUND NAME } & RT & EST. CONC. & Q \\
\cline { 2 - 6 } 01 & & Unknown & 6.90 & 3.3 & B X J \\
\cline { 2 - 6 } 02 & E9667961 & Total Alkanes & N/A & $\mathrm{J}$ \\
\cline { 2 - 6 }
\end{tabular}

1 EPA-designated Registry Number. 
$1 \mathrm{~A}$ - FORM I VOA-1

VOLATILE ORGANICS ANALYSIS DATA SHEET
EPA SAMPLE NO.

INMWO $4-W-32688$

Lab Name: TESTAMERICA BURLINGTON

Contract: $8 \mathrm{E}-00302$

Lab Code: STLV Case No.: INMAN Mod. Ref No.: SDG NO.: 200-3113

Matrix: (SOIL/SED/WATER)

Sample wt/vol: 25.0

Water

$(\mathrm{g} / \mathrm{mL}) \mathrm{mL}$

Level: (TRACE/LOW/MED) TRACE

응 Moisture: not dec.

GC Column: DB-624

Soil Extract Volume: ID $0.20 \quad(\mathrm{~mm})$

(uL)

(mL)

Purge Volume: 25.0

\section{: :}

Lab Sample ID: 200-3113-4

Lab File ID: JCFB13.D

Date Received: 12/23/2010

Date Analyzed: 12/24/2010

Dilution Factor: 1.0

Soil Aliquot Volume:

(uL)

\begin{tabular}{|c|c|c|c|}
\hline CAS NO. & COMPOUND & $\begin{array}{l}\text { CONCENTRATION UNITS: } \\
(\mathrm{ug} / \mathrm{L} \text { or } \mathrm{ug} / \mathrm{kg}) \mathrm{ug} / \mathrm{L}\end{array}$ & Q \\
\hline $75-71-8$ & Dichlorodifluoromethane & 0.50 & $\mathrm{U}$ \\
\hline $74-87-3$ & Chloromethane & 0.50 & $\mathrm{U}$ \\
\hline $75-01-4$ & Vinyl chloride & 0.50 & $\mathrm{U}$ \\
\hline $74-83-9$ & Bromomethane & 0.50 & $\mathrm{U}$ \\
\hline $75-00-3$ & Chloroethane & 0.50 & $\mathrm{U}$ \\
\hline $75-69-4$ & Trichlorofluoromethane & 0.50 & $\mathrm{U}$ \\
\hline $75-35-4$ & 1,1-Dichloroethene & 0.50 & $\mathrm{U}$ \\
\hline $76-13-1$ & 1,1,2-Trichloro-1,2,2-trifluoroethane & 0.50 & $\mathrm{U}$ \\
\hline $67-64-1$ & Acetone & 1.2 & $\mathrm{~J} \mathrm{~B}$ \\
\hline $75-15-0$ & Carbon disulfide & 0.11 & $\mathrm{~J} \mathrm{~B}$ \\
\hline $79-20-9$ & Methyl acetate & 0.50 & $\mathrm{U}$ \\
\hline $75-09-2$ & Methylene Chloride & 0.50 & $\mathrm{U}$ \\
\hline $156-60-5$ & trans-1,2-Dichloroethene & 0.50 & $\mathrm{U}$ \\
\hline $1634-04-4$ & Methyl tert-butyl ether & 0.098 & $\mathrm{~J} \mathrm{~B}$ \\
\hline $75-34-3$ & 1,1-Dichloroethane & 0.50 & $\mathrm{U}$ \\
\hline $156-59-2$ & cis-1,2-Dichloroethene & 0.50 & $\mathrm{U}$ \\
\hline $78-93-3$ & 2-Butanone & 5.0 & $\mathrm{U}$ \\
\hline $74-97-5$ & Bromochloromethane & 0.50 & $\mathrm{U}$ \\
\hline $67-66-3$ & Chloroform & 0.050 & $\mathrm{~J}$ \\
\hline $71-55-6$ & 1,1,1-Trichloroethane & 0.50 & $\mathrm{U}$ \\
\hline $110-82-7$ & Cyclohexane & 0.50 & $\mathrm{U}$ \\
\hline $56-23-5$ & Carbon tetrachloride & 0.22 & $\mathrm{~J}$ \\
\hline $71-43-2$ & Benzene & 0.50 & $\mathrm{U}$ \\
\hline $107-06-2$ & 1,2-Dichloroethane & 0.50 & $\mathrm{U}$ \\
\hline
\end{tabular}

Report 1,4-Dioxane for Low-Medium VOA analysis only 
Lab Name: TESTAMERICA BURLINGTON

Lab Code: STLV Case No.: INMA

Matrix: (SOIL/SED/WATER)

Sample wt/vol: 25.0 Water

Level: (TRACE/LOW/MED) TRACE

응 Moisture: not dec.

GC Column: DB-624 (mm) Soil Extract Volume: (uL) (mL)

Purge Volume: 25.0 ID: 0.20 $(\mathrm{g} / \mathrm{mL}) \mathrm{mL}$ (uI)
Contract: $8 \mathrm{E}-00302$

SDG No.: 200-3113

\begin{tabular}{|c|c|c|c|}
\hline CAS NO. & COMPOUND & $\begin{array}{l}\text { CONCENTRATION UNITS: } \\
(\mathrm{ug} / \mathrm{I} \text { or } \mathrm{ug} / \mathrm{kg}) \mathrm{ug} / \mathrm{L}\end{array}$ & $Q$ \\
\hline $79-01-6$ & Trichloroethene & 0.063 & $\overline{\mathrm{J}}$ \\
\hline $108-87-2$ & Methylcyclohexane & 0.50 & $\mathrm{U}$ \\
\hline $78-87-5$ & 1,2-Dichloropropane & 0.50 & $\mathrm{U}$ \\
\hline $75-27-4$ & Bromodichloromethane & 0.50 & $\mathrm{U}$ \\
\hline $10061-01-5$ & cis-1,3-Dichloropropene & 0.50 & $\mathrm{U}$ \\
\hline $108-10-1$ & 4-Methyl-2-pentanone & 5.0 & $\mathrm{U}$ \\
\hline $108-88-3$ & Toluene & 0.50 & $\mathrm{U}$ \\
\hline $10061-02-6$ & trans-1,3-Dichloropropene & 0.50 & $\mathrm{U}$ \\
\hline $79-00-5$ & $1,1,2$-Trichloroethane & 0.50 & $\mathrm{U}$ \\
\hline $127-18-4$ & Tetrachloroethene & 0.25 & $\mathrm{~J}$ \\
\hline $591-78-6$ & 2-Hexanone & 5.0 & $\mathrm{U}$ \\
\hline $124-48-1$ & Dibromochloromethane & 0.50 & $\mathrm{U}$ \\
\hline $106-93-4$ & 1,2-Dibromoethane & 0.50 & $\mathrm{U}$ \\
\hline $108-90-7$ & Chlorobenzene & 0.50 & $\mathrm{U}$ \\
\hline $100-41-4$ & Ethylbenzene & 0.50 & $\mathrm{U}$ \\
\hline $95-47-6$ & o-Xylene & 0.50 & $\mathrm{U}$ \\
\hline $179601-23-1$ & $m, p-X y l e n e$ & 0.50 & $\mathrm{U}$ \\
\hline $100-42-5$ & Styrene & 0.50 & $\mathrm{U}$ \\
\hline $75-25-2$ & Bromoform & 0.50 & $\mathrm{U}$ \\
\hline $98-82-8$ & Isopropylbenzene & 0.50 & $\mathrm{U}$ \\
\hline $79-34-5$ & $1,1,2,2$-Tetrachloroethane & 0.50 & $\mathrm{U}$ \\
\hline $541-73-1$ & 1,3-Dichlorobenzene & 0.50 & $\mathrm{U}$ \\
\hline $106-46-7$ & 1,4-Dichlorobenzene & 0.50 & $\mathrm{U}$ \\
\hline $95-50-1$ & 1,2-Dichlorobenzene & 0.50 & $\mathrm{U}$ \\
\hline $96-12-8$ & 1,2-Dibromo-3-Chloropropane & 0.50 & $\mathrm{U}$ \\
\hline $120-82-1$ & 1,2,4-Trichlorobenzene & 0.50 & $\mathrm{U}$ \\
\hline $87-61-6$ & 1,2,3-Trichlorobenzene & 0.50 & $\mathrm{U}$ \\
\hline
\end{tabular}

Lab Sample ID: 200-3113-4

Lab File ID: JCFB13.D

Date Received: 12/23/2010

Date Analyzed: 12/24/2010

Dilution Factor: 1.0

Soil Aliquot Volume:

(uL) 
$1 \mathrm{~J}$ - FORM I VOA-TIC

VOLATILE ORGANICS ANALYSIS DATA SHEET

TENTATIVELY IDENTIFIED COMPOUNDS
EPA SAMPLE NO.

INMWO $4-W-32688$

Lab Name: TESTAMERICA BURLINGTON

Contract: $8 \mathrm{E}-00302$

Lab Code: STLV Case No.: INMAN Mod. Ref No.: SDG No.: 200-3113

Matrix: (SOIL/SED/WATER) Water

Sample wt/vol: 25.0

$(\mathrm{g} / \mathrm{mL}) \quad \mathrm{mL}$

Level: (TRACE or LOW/MED) TRACE

\% Moisture: not dec.

GC Column: DB-624 ID: 0.20 (mm)

Soil Extract Volume: (uL)

CONCENTRATION UNITS: (ug/L or $\mathrm{ug} / \mathrm{kg}$ ) ug/L
Lab Sample ID: 200-3113-4

Lab File ID: JCFB13.D

Date Received: 12/23/2010

Date Analyzed: 12/24/2010

Dilution Factor: 1.0

Soil Aliquot Volume: (uI)

Purge Volume: 25.0 (mL)

\begin{tabular}{l|l|l|r|r|r|}
\hline \multicolumn{1}{|c|}{ CAS NUMBER } & \multicolumn{1}{|c|}{ COMPOUND NAME } & RT & EST. CONC. & $Q$ \\
\cline { 2 - 6 } 01 & $109-99-9$ & Furan, tetrahydro- & 4.49 & $\mathrm{~J}$ \\
02 & & Unknown & 6.90 & 18 & $\mathrm{~N}$ \\
\cline { 2 - 6 } 03 & E9667961 & Total Alkanes & N/A & $\mathrm{J}$ & $\mathrm{J}$ \\
\cline { 2 - 6 } & &
\end{tabular}

1 EPA-designated Registry Number. 
Lab Name: TESTAMERICA BURLINGTON

Contract: $\quad 8 \mathrm{E}-00302$

Lab Code: STLV Case No.: INMAN Mod. Ref No.:

SDG No.: $200-3113$

Matrix: (SOIL/SED/WATER) Water

Sample wt/vol: 25.0 $(g / m L) \quad m L$

Level: (TRACE/LOW/MED) TRACE

음 Moisture: not dec.

GC Column: DB-624 ID: $0.20 \quad$ (mm)

Soil Extract Volume:

Purge Volume: 25.0 (uL) (mL)
Lab Sample ID: 200-3113-5

Lab File ID: JCFB15.D

Date Received: 12/23/2010

Date Analyzed: 12/24/2010

Dilution Factor: 1.0

Soil Aliquot Volume:

(uL)

\begin{tabular}{|c|c|c|c|}
\hline CAS NO. & COMPOUND & $\begin{array}{l}\text { CONCENTRATION UNITS: } \\
(\mathrm{ug} / \mathrm{L} \text { or } \mathrm{ug} / \mathrm{kg}) \mathrm{ug} / \mathrm{L}\end{array}$ & $Q$ \\
\hline $75-71-8$ & Dichlorodifluoromethane & 0.50 & $\overline{\mathrm{U}}$ \\
\hline $74-87-3$ & Chloromethane & 0.50 & $\mathrm{U}$ \\
\hline $75-01-4$ & Vinyl chloride & 0.50 & $\mathrm{U}$ \\
\hline $74-83-9$ & Bromomethane & 0.50 & $\mathrm{U}$ \\
\hline $75-00-3$ & Chloroethane & 0.50 & $\mathrm{U}$ \\
\hline $75-69-4$ & Trichlorofluoromethane & 0.50 & $\mathrm{U}$ \\
\hline $75-35-4$ & 1,1-Dichloroethene & 0.50 & $\mathrm{U}$ \\
\hline $76-13-1$ & 1,1,2-Trichloro-1,2,2-trifluoroethane & 0.50 & $\mathrm{U}$ \\
\hline $67-64-1$ & Acetone & 0.98 & $\mathrm{~J} \mathrm{~B}$ \\
\hline $75-15-0$ & Carbon disulfide & 0.50 & $\mathrm{U}$ \\
\hline $79-20-9$ & Methyl acetate & 0.50 & $\mathrm{U}$ \\
\hline $75-09-2$ & Methylene Chloride & 0.50 & $\mathrm{U}$ \\
\hline $156-60-5$ & trans-1,2-Dichloroethene & 0.50 & $\mathrm{U}$ \\
\hline $1634-04-4$ & Methyl tert-butyl ether & 0.11 & $\mathrm{~J} \mathrm{~B}$ \\
\hline $75-34-3$ & 1,1-Dichloroethane & 0.50 & $\mathrm{U}$ \\
\hline $156-59-2$ & cis-1,2-Dichloroethene & 0.50 & $\mathrm{U}$ \\
\hline $78-93-3$ & 2-Butanone & 3.0 & $\mathrm{~J}$ \\
\hline $74-97-5$ & Bromochloromethane & 0.50 & $\mathrm{U}$ \\
\hline $67-66-3$ & Chloroform & 0.50 & $\mathrm{U}$ \\
\hline $71-55-6$ & 1,1,1-Trichloroethane & 0.50 & $\mathrm{U}$ \\
\hline $110-82-7$ & Cyclohexane & 0.50 & $\mathrm{U}$ \\
\hline $56-23-5$ & Carbon tetrachloride & 0.091 & $\mathrm{~J}$ \\
\hline $71-43-2$ & Benzene & 0.50 & $\mathrm{U}$ \\
\hline $107-06-2$ & 1,2-Dichloroethane & 0.50 & $\mathrm{U}$ \\
\hline
\end{tabular}

Report 1,4-Dioxane for Low-Medium VOA analysis only 
Lab Name: TESTAMERICA BURLINGTON

Lab Code: STLV Case No.: INMAN Mod. Ref No.:

Contract: $8 \mathrm{E}-00302$

Matrix: (SOIL/SED/WATER)

Sample wt/vol: 25.0

Level: (TRACE/LOW/MED)

o Moisture: not dec.

GC Column: DB-624

Soil Extract Volume:

Purge Volume: 25.0

\section{water}

$(\mathrm{g} / \mathrm{mL}) \mathrm{mL}$

\section{TRACE} ID: $0.20 \quad(\mathrm{~mm})$

(uL) (mL) SDG No.: 200-3113

Lab Sample ID: 200-3113-5

Lab File ID: JCFB15.D

Date Received: 12/23/2010

Date Analyzed: 12/24/2010

Dilution Factor: 1.0

Soil Aliquot Volume:

(UL)

(25.0

\begin{tabular}{|c|c|c|c|}
\hline CAS NO. & COMPOUND & $\begin{array}{l}\text { CONCENTRATION UNITS: } \\
(\mathrm{ug} / \mathrm{L} \text { or } \mathrm{ug} / \mathrm{kg}) \mathrm{ug} / \mathrm{L}\end{array}$ & $\mathrm{Q}$ \\
\hline $79-01-6$ & Trichloroethene & 0.50 & $\mathrm{U}$ \\
\hline $108-87-2$ & Methylcyclohexane & 0.50 & $\mathrm{U}$ \\
\hline $78-87-5$ & 1,2-Dichloropropane & 0.50 & $\mathrm{U}$ \\
\hline $75-27-4$ & Bromodichloromethane & 0.50 & $\mathrm{U}$ \\
\hline $10061-01-5$ & cis-1,3-Dichloropropene & 0.50 & $\mathrm{U}$ \\
\hline $108-10-1$ & 4-Methyl-2-pentanone & 5.0 & $\mathrm{U}$ \\
\hline $108-88-3$ & Toluene & 0.50 & $\mathrm{U}$ \\
\hline $10061-02-6$ & trans-1,3-Dichloropropene & 0.50 & $\mathrm{U}$ \\
\hline $79-00-5$ & 1,1,2-Trichloroethane & 0.50 & $\mathrm{U}$ \\
\hline $127-18-4$ & Tetrachloroethene & 0.20 & $\mathrm{~J}$ \\
\hline $591-78-6$ & 2-Hexanone & 5.0 & $\mathrm{U}$ \\
\hline $124-48-1$ & Dibromochloromethane & 0.50 & $\mathrm{U}$ \\
\hline $106-93-4$ & 1,2-Dibromoethane & 0.50 & $\mathrm{U}$ \\
\hline $108-90-7$ & Chlorobenzene & 0.50 & $\mathrm{U}$ \\
\hline $100-41-4$ & Ethylbenzene & 0.50 & $\mathrm{U}$ \\
\hline $95-47-6$ & o-Xylene & 0.50 & $\mathrm{U}$ \\
\hline $179601-23-1$ & m, p-xylene & 0.50 & $\mathrm{U}$ \\
\hline $100-42-5$ & Styrene & 0.50 & $\mathrm{U}$ \\
\hline $75-25-2$ & Bromoform & 0.50 & $\mathrm{U}$ \\
\hline $98-82-8$ & Isopropylbenzene & 0.50 & $\mathrm{U}$ \\
\hline $79-34-5$ & $1,1,2,2$-Tetrachloroethane & 0.50 & $\mathrm{U}$ \\
\hline $541-73-1$ & 1,3-Dichlorobenzene & 0.50 & $\mathrm{U}$ \\
\hline $106-46-7$ & 1,4-Dichlorobenzene & 0.50 & $\mathrm{U}$ \\
\hline $95-50-1$ & 1,2-Dichlorobenzene & 0.50 & $\mathrm{U}$ \\
\hline $96-12-8$ & 1,2-Dibromo-3-Chloropropane & 0.50 & $\mathrm{U}$ \\
\hline $120-82-1$ & 1,2,4-Trichlorobenzene & 0.50 & $\mathrm{U}$ \\
\hline $87-61-6$ & 1,2,3-Trichlorobenzene & 0.50 & $\mathrm{U}$ \\
\hline
\end{tabular}


$1 \mathrm{~J}$ - FORM I VOA-TIC

VOLATILE ORGANICS ANALYSIS DATA SHEET

TENTATIVELY IDENTIEIED COMPOUNDS
EPA SAMPLE NO.

Lab Name: TESTAMERICA BURLINGTON

Contract: $8 \mathrm{E}-00302$

Lab Code: STLV Case No.: INMAN Mod. Ref No.:

SDG No.: 200-3113

Matrix: (SOIL/SED/WATER) Water

Sample wt/vol: 25.0

$(\mathrm{g} / \mathrm{mL}) \mathrm{mL}$

Level: (TRACE or LOW/MED)

TRACE

o Moisture: not dec.

GC Column: DB-624

ID $: 0.20 \quad(\mathrm{~mm})$

Soil Extract Volume:

(uL)

CONCENTRATION UNITS: (ug/L or $\mathrm{ug} / \mathrm{kg}$ ) ug/L
Lab Sample ID: 200-3113-5

Lab File ID: JCFB15.D

Date Received: 12/23/2010

Date Analyzed: 12/24/2010

Dilution Factor: 1.0

Soil Aliquot Volume:

(uL)

Purge Volume: 25.0 (mL)

\begin{tabular}{l|l|l|r|r|r|}
\hline \multicolumn{1}{|c|}{ CAS NUMBER } & \multicolumn{1}{|c|}{ COMPOUND NAME } & RT & EST. CONC. & Q \\
\cline { 2 - 6 } 01 & & Unknown & 6.90 & 3.1 & B X J \\
\cline { 2 - 6 } 02 & E9667961 & Total Alkanes & N/A & $\mathrm{J}$ \\
\hline
\end{tabular}

1 EPA-designated Registry Number. 
$1 A$ - FORM I VOA-1

VOLATILE ORGANICS ANALYSIS DATA SHEET
EPA SAMPLE NO.

INMW06-W-32690
Lab Name: TESTAMERICA BURLINGTON

Lab Code: STLV Cas

Matrix: (SOIL/SED/WATER)

Sample wt/vol: 25.0

Water

Level: (TRACE/LOW/MED)

$(\mathrm{g} / \mathrm{mL}) \mathrm{mL}$

o Moisture: not dec.

GC Column: DB-624 ID: $0.20 \quad(\mathrm{~mm})$

Soil Extract Volume:

Purge Volume: 25.0

(uL)

$(\mathrm{mL})$
Contract: $8 \mathrm{E}-00302$

SDG No.: 200-3113

\begin{tabular}{|c|c|c|c|}
\hline CAS NO. & COMPOUND & $\begin{array}{l}\text { CONCENTRATION UNITS: } \\
(\mathrm{ug} / \mathrm{L} \text { or } \mathrm{ug} / \mathrm{kg}) \mathrm{ug} / \mathrm{L}\end{array}$ & $Q$ \\
\hline $75-71-8$ & Dichlorodifluoromethane & 0.50 & $\mathrm{U}$ \\
\hline $74-87-3$ & Chloromethane & 0.50 & $\mathrm{U}$ \\
\hline $75-01-4$ & Vinyl chloride & 0.50 & $\mathrm{U}$ \\
\hline $74-83-9$ & Bromomethane & 0.50 & $\mathrm{U}$ \\
\hline $75-00-3$ & Chloroethane & 0.50 & $\mathrm{U}$ \\
\hline $75-69-4$ & Trichlorofluoromethane & 0.50 & $\mathrm{U}$ \\
\hline $75-35-4$ & 1,1-Dichloroethene & 0.50 & $\mathrm{U}$ \\
\hline $76-13-1$ & 1,1,2-Trichloro-1,2,2-trifluoroethane & 0.50 & $\mathrm{U}$ \\
\hline $67-64-1$ & Acetone & 380 & E B \\
\hline $75-15-0$ & Carbon disulfide & 0.069 & $\mathrm{~J} \mathrm{~B}$ \\
\hline $79-20-9$ & Methyl acetate & 0.50 & $\mathrm{U}$ \\
\hline $75-09-2$ & Methylene Chloride & 0.50 & $\mathrm{U}$ \\
\hline $156-60-5$ & trans $-1,2-$ Dichloroethene & 0.50 & $\mathrm{U}$ \\
\hline $1634-04-4$ & Methyl tert-butyl ether & 0.098 & $\mathrm{~J} \mathrm{~B}$ \\
\hline $75-34-3$ & 1,1-Dichloroethane & 0.50 & $\mathrm{U}$ \\
\hline $156-59-2$ & cis-1,2-Dichloroethene & 0.50 & $\mathrm{U}$ \\
\hline $78-93-3$ & 2-Butanone & 11000 & $E$ \\
\hline $74-97-5$ & Bromochloromethane & 0.50 & $\mathrm{U}$ \\
\hline $67-66-3$ & Chloroform & 0.50 & $\mathrm{U}$ \\
\hline $71-55-6$ & 1,1,1-Trichloroethane & 0.50 & $\mathrm{U}$ \\
\hline $110-82-7$ & Cyclohexane & 0.50 & $\mathrm{U}$ \\
\hline $56-23-5$ & Carbon tetrachloride & 0.50 & $\mathrm{U}$ \\
\hline $71-43-2$ & Benzene & 0.50 & $\mathrm{U}$ \\
\hline $107-06-2$ & 1,2-Dichloroethane & 0.50 & $\mathrm{U}$ \\
\hline
\end{tabular}

Report 1,4-Dioxane for Low-Medium VOA analysis only 
$1 B$ - FORM I VOA-2

VOLATILE ORGANICS ANALYSIS DATA SHEET
EPA SAMPLE NO. INMW0 6-W-32690
Lab Name: TESTAMERICA BURLINGTON

Lab Code: STLV Case No.: INMAN

Matrix: (SOIL/SED/WATER)

Sample wt/vol: 25.0 Water

Level: (TRACE/LOW/MED) $(\mathrm{g} / \mathrm{mL}) \quad \mathrm{mL}$

응 Moisture: not dec.

GC Column: DB-624 ID: $0.20 \quad(\mathrm{~mm})$

Soil Extract Volume: (uI)

Purge Volume: 25.0 (mL)
Contract: $8 \mathrm{E}-00302$

SDG No.: $200-3113$

\begin{tabular}{|c|c|c|c|}
\hline CAS NO. & COMPOUND & $\begin{array}{l}\text { CONCENTRATION UNITS: } \\
(\mathrm{ug} / \mathrm{L} \text { or } \mathrm{ug} / \mathrm{kg}) \mathrm{ug} / \mathrm{L}\end{array}$ & $Q$ \\
\hline $79-01-6$ & Trichloroethene & 0.50 & $\overline{\mathrm{U}}$ \\
\hline $108-87-2$ & Methylcyclohexane & 0.50 & $\mathrm{U}$ \\
\hline $78-87-5$ & 1,2-Dichloropropane & 0.50 & $\mathrm{U}$ \\
\hline $75-27-4$ & Bromodichloromethane & 0.50 & $\mathrm{U}$ \\
\hline $10061-01-5$ & cis-1,3-Dichloropropene & 0.50 & $\mathrm{U}$ \\
\hline $108-10-1$ & 4-Methyl-2-pentanone & 5.0 & $\mathrm{U}$ \\
\hline $108-88-3$ & Toluene & 0.50 & $\mathrm{U}$ \\
\hline $10061-02-6$ & trans-1,3-Dichloropropene & 0.50 & $\mathrm{U}$ \\
\hline $79-00-5$ & 1,1,2-Trichloroethane & 0.50 & $\mathrm{U}$ \\
\hline $127-18-4$ & Tetrachloroethene & 0.50 & $\mathrm{U}$ \\
\hline $591-78-6$ & 2-Hexanone & 5.0 & $\mathrm{U}$ \\
\hline $124-48-1$ & Dibromochloromethane & 0.50 & $\mathrm{U}$ \\
\hline $106-93-4$ & 1,2-Dibromoethane & 0.50 & $\mathrm{U}$ \\
\hline $108-90-7$ & Chlorobenzene & 0.50 & $\mathrm{U}$ \\
\hline $100-41-4$ & Ethylbenzene & 0.50 & $\mathrm{U}$ \\
\hline $95-47-6$ & o-xylene & 0.50 & $\mathrm{U}$ \\
\hline $179601-23-1$ & m,p-xylene & 0.50 & $\mathrm{U}$ \\
\hline $100-42-5$ & Styrene & 0.50 & $\mathrm{U}$ \\
\hline $75-25-2$ & Bromoform & 0.50 & $\mathrm{U}$ \\
\hline $98-82-8$ & Isopropylbenzene & 0.50 & $\mathrm{U}$ \\
\hline $79-34-5$ & $1,1,2,2$-Tetrachloroethane & 0.50 & $\mathrm{U}$ \\
\hline $541-73-1$ & 1,3-Dichlorobenzene & 0.50 & $\mathrm{U}$ \\
\hline $106-46-7$ & 1,4-Dichlorobenzene & 0.50 & $\mathrm{U}$ \\
\hline $95-50-1$ & 1,2-Dichlorobenzene & 0.50 & $\mathrm{U}$ \\
\hline $96-12-8$ & 1,2-Dibromo-3-Chloropropane & 0.50 & $\mathrm{U}$ \\
\hline $120-82-1$ & 1,2,4-Trichlorobenzene & 0.50 & $\mathrm{U}$ \\
\hline $87-61-6$ & 1,2,3-Trichlorobenzene & 0.50 & $\mathrm{U}$ \\
\hline
\end{tabular}

Lab Sample ID: 200-3113-6

Lab File ID: JCFB16.D

Date Received: 12/23/2010

Date Analyzed: 12/24/2010

Dilution Factor: 1.0

Soil Aliquot volume:

(uL)

(n) 
$1 \mathrm{~J}$ - FORM I VOA-TIC

VOLATILE ORGANICS ANALYSIS DATA SHEET

TENTATIVELY IDENTIFIED COMPOUNDS
EPA SAMPLE NO.

INMW0 6-W-32690

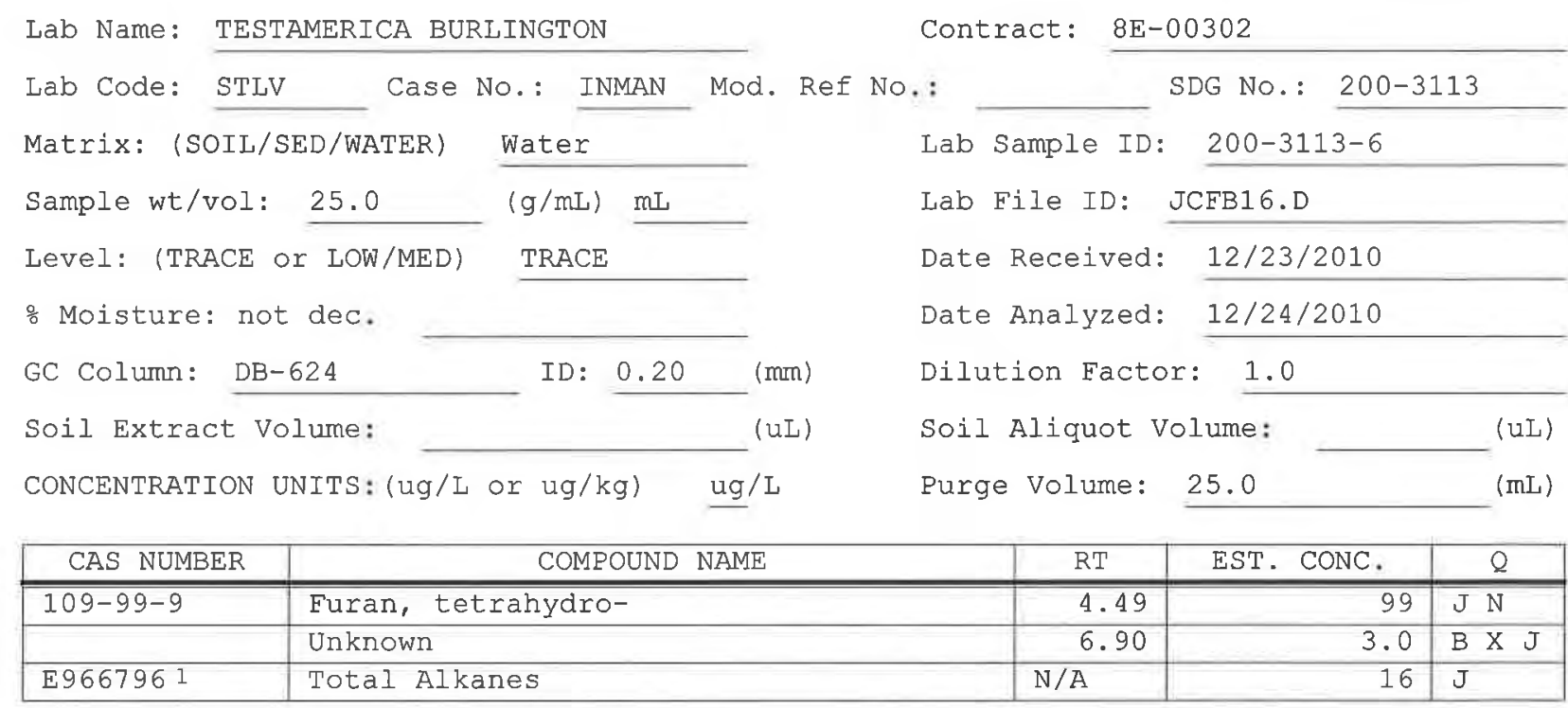

1 EPA-designated Registry Number. 
$1 \mathrm{~A}$ - FORM I VOA-1

VOLATILE ORGANICS ANALYSIS DATA SHEET
EPA SAMPLE NO.

INMW0 7-W-32691
Lab Name: TESTAMERICA BURLINGTON

Lab Code: STLV

Matrix: (SOIL/SED/WATER)

Case No.: INMA

\section{Water}

$(\mathrm{g} / \mathrm{mL}) \mathrm{mL}$

Sample wt/vol: 25.0

Level: (TRACE/LOW/MED) TRACE

o Moisture: not dec.

GC Column: DB-624 ID: $0.20 \quad$ (mm)

Soil Extract Volume:

Purge Volume: 25.0
Contract: $8 \mathrm{E}-00302$

SDG No.: 200-3113

\begin{tabular}{|c|c|c|c|}
\hline CAS NO. & COMPOUND & $\begin{array}{l}\text { CONCENTRATION UNITS: } \\
(\mathrm{ug} / \mathrm{L} \text { or } u g / \mathrm{kg}) \mathrm{ug} / \mathrm{L}\end{array}$ & Q \\
\hline $75-71-8$ & Dichlorodifluoromethane & 3.0 & $\mathrm{U}$ \\
\hline $74-87-3$ & Chloromethane & 3.0 & $\mathrm{U}$ \\
\hline $75-01-4$ & Vinyl chloride & 3.0 & $\mathrm{U}$ \\
\hline $74-83-9$ & Bromomethane & 3.0 & $\mathrm{U}$ \\
\hline $75-00-3$ & Chloroethane & 3.0 & $\mathrm{U}$ \\
\hline $75-69-4$ & Trichlorofluoromethane & 3.0 & $\mathrm{U}$ \\
\hline $75-35-4$ & 1,1-Dichloroethene & 3.0 & $\mathrm{U}$ \\
\hline $76-13-1$ & 1,1,2-Trichloro-1,2,2-trifluoroethane & 3.0 & $\mathrm{U}$ \\
\hline $67-64-1$ & Acetone & 1800 & $\mathrm{E} B$ \\
\hline $75-15-0$ & Carbon disulfide & 3.0 & $\mathrm{U}$ \\
\hline $79-20-9$ & Methyl acetate & 68 & \\
\hline $75-09-2$ & Methylene Chloride & 3.0 & $\mathrm{U}$ \\
\hline $156-60-5$ & trans-1,2-Dichloroethene & 3.0 & $\mathrm{U}$ \\
\hline $1634-04-4$ & Methyl tert-butyl ether & 0.66 & $\mathrm{~J} \mathrm{~B}$ \\
\hline $75-34-3$ & 1,1-Dichloroethane & 3.0 & $\mathrm{U}$ \\
\hline $156-59-2$ & cis-1,2-Dichloroethene & 3.0 & $\mathrm{U}$ \\
\hline $78-93-3$ & 2-Butanone & 35000 & $\mathrm{E}$ \\
\hline $74-97-5$ & Bromochloromethane & 3.0 & $\mathrm{U}$ \\
\hline $67-66-3$ & Chloroform & 3.0 & $\mathrm{U}$ \\
\hline $71-55-6$ & 1,1,1-Trichloroethane & 3.0 & $\mathrm{U}$ \\
\hline $110-82-7$ & Cyclohexane & 3.0 & $\mathrm{U}$ \\
\hline $56-23-5$ & Carbon tetrachloride & 3.0 & $\mathrm{U}$ \\
\hline $71-43-2$ & Benzene & 3.0 & $\mathrm{U}$ \\
\hline $107-06-2$ & 1,2-Dichloroethane & 3.0 & $\mathrm{U}$ \\
\hline
\end{tabular}

Report 1,4-Dioxane for Low-Medium VOA analysis only 
Lab Name: TESTAMERICA BURLINGTON

Lab Code: STLV Case No.: INMAN Mod. Ref No.:
Contract: $\quad 8 \mathrm{E}-00302$

SDG No.: 200-3113
Matrix: (SOIL/SED/WATER)

Sample wt/vol: 25.0

Water

$(\mathrm{g} / \mathrm{mL}) \quad \mathrm{mL}$

Level: (TRACE/LOW/MED) TRACE

o Moisture: not dec.

GC Column: DB-624

Soil Extract Volume: ID: $0.20 \quad$ (mm)

Purge Volume: 25.0 (uI) $(\mathrm{mL})$
Iab Sample ID: 200-3113-7

Lab File ID: JCFC19.D

Date Received: 12/23/2010

Date Analyzed: 12/28/2010

Dilution Factor: 6.1

Soil Aliquot Volume:

(uL)

\begin{tabular}{|c|c|c|c|}
\hline CAS NO. & COMPOUND & $\begin{array}{l}\text { CONCENTRATION UNITS: } \\
(\mathrm{ug} / \mathrm{L} \text { or } u \mathrm{~g} / \mathrm{kg}) \mathrm{ug} / \mathrm{L}\end{array}$ & $Q$ \\
\hline $79-01-6$ & Trichloroethene & 3.0 & $\overline{\mathrm{U}}$ \\
\hline $108-87-2$ & Methylcyclohexane & 3.0 & $\mathrm{U}$ \\
\hline $78-87-5$ & 1,2-Dichloropropane & 3.0 & $\mathrm{U}$ \\
\hline $75-27-4$ & Bromodichloromethane & 3.0 & $\mathrm{U}$ \\
\hline $10061-01-5$ & cis-1,3-Dichloropropene & 3.0 & $\mathrm{U}$ \\
\hline $108-10-1$ & 4-Methyl-2-pentanone & 30 & $\mathrm{U}$ \\
\hline $108-88-3$ & Toluene & 3.0 & $\mathrm{U}$ \\
\hline $10061-02-6$ & trans-1,3-Dichloropropene & 3.0 & $\mathrm{U}$ \\
\hline $79-00-5$ & 1,1,2-Trichloroethane & 3.0 & $\mathrm{U}$ \\
\hline $127-18-4$ & Tetrachloroethene & 3.0 & $\mathrm{U}$ \\
\hline $591-78-6$ & 2-Hexanone & 30 & $\mathrm{U}$ \\
\hline $124-48-1$ & Dibromochloromethane & 3.0 & $\mathrm{U}$ \\
\hline $106-93-4$ & 1,2-Dibromoethane & 3.0 & $\mathrm{U}$ \\
\hline $108-90-7$ & Chlorobenzene & 3.0 & $\mathrm{U}$ \\
\hline $100-41-4$ & Ethylbenzene & 3.0 & $\mathrm{U}$ \\
\hline $95-47-6$ & o-Xylene & 3.0 & $\mathrm{U}$ \\
\hline $179601-23-1$ & $\mathrm{~m}, \mathrm{p}$-Xylene & 3.0 & $\mathrm{U}$ \\
\hline $100-42-5$ & Styrene & 3.0 & $\mathrm{U}$ \\
\hline $75-25-2$ & Bromoform & 3.0 & $\mathrm{U}$ \\
\hline $98-82-8$ & Isopropylbenzene & 3.0 & $\mathrm{U}$ \\
\hline $79-34-5$ & $1,1,2,2$-Tetrachloroethane & 3.0 & $\mathrm{U}$ \\
\hline $541-73-1$ & 1,3-Dichlorobenzene & 3.0 & $\mathrm{U}$ \\
\hline $106-46-7$ & 1,4-Dichlorobenzene & 3.0 & $\mathrm{U}$ \\
\hline $95-50-1$ & 1,2-Dichlorobenzene & 3.0 & $\mathrm{U}$ \\
\hline $96-12-8$ & 1,2-Dibromo-3-Chloropropane & 3.0 & $\mathrm{U}$ \\
\hline $120-82-1$ & 1,2,4-Trichlorobenzene & 3.0 & $\mathrm{U}$ \\
\hline $87-61-6$ & 1,2,3-Trichlorobenzene & 3.0 & $\mathrm{U}$ \\
\hline
\end{tabular}


$1 \mathrm{~J}$ - FORM I VOA-TIC

VOLATILE ORGANICS ANALYSIS DATA SHEET

TENTATIVELY IDENTIFIED COMPOUNDS
EPA SAMPLE NO.

INMW07-W-32691

\begin{tabular}{|c|c|c|c|}
\hline Lab Name: TESTAMERICA BU & RLINGTON & & Contract: \\
\hline Lab Code: STLV & NO.: INMAN & Mod. Ref & SDG No.: $200-3113$ \\
\hline Matrix: (SOIL/SED/WATER) & Water & & Lab Sample ID: \\
\hline Sample wt/vol: 25.0 & $(\mathrm{~g} / \mathrm{mL}) \quad \mathrm{mL}$ & & Lab Eile ID: JCFC19.D \\
\hline Level: (TRACE or LOW/MED) & TRACE & & Date Received: \\
\hline$\frac{\circ}{6}$ Moisture: not dec. & & & Date Analyzed: $12 / 28 / 2010$ \\
\hline GC Column: DB-624 & ID: 0.20 & (mm) & Dilution Factor: \\
\hline Soil Extract Volume: & & (uL) & Soil Aliquot Volume: \\
\hline CONCENTRATION UNITS: $(\mathrm{ug} / \mathrm{I}$ & or ug/kg) & $\mathrm{ug} / \mathrm{L}$ & Purge Volume: 25.0 \\
\hline
\end{tabular}

01

\begin{tabular}{|l|l|r|r|r|}
\hline \multicolumn{1}{|c|}{ CAS NUMBER } & \multicolumn{1}{|c|}{ COMPOUND NAME } & RT & EST. CONC. & Q \\
\hline $109-99-9$ & Furan, tetrahydro- & 4.49 & 490 & $\mathrm{~J} \mathrm{~N}$ \\
\hline & Unknown & 6.90 & 7.86 & B X J \\
\hline 541-05-9 & Cyclotrisiloxane, hexamethyl- & N/A & 3.5 & B J N \\
\hline E9667961 & Total Alkanes & 100 & $\mathrm{~J}$ \\
\hline
\end{tabular}

1 EPA-designated Registry Number. 
Lab Name: TESTAMERICA BURLINGTON

Contract: $8 \mathrm{E}-00302$

Lab Code: STLV Case No.: INMAN Mod. Ref No.: SDG No.: 200-3113

Matrix: (SOIL/SED/WATER) Water

Sample wt/vol: 25.0 $(\mathrm{g} / \mathrm{mL}) \mathrm{mL}$

Level: (TRACE/LOW/MED) TRACE

응 Moisture: not dec.

GC Column: DB-624 ID: $0.20 \quad$ (mm)

Soil Extract Volume: (uL) (mL)

Purge Volume: 25.0
Lab Sample ID: 200-3113-8

Lab File ID: JCFC21.D

Date Received: 12/23/2010

Date Analyzed: 12/28/2010

Dilution Factor: 4.4

Soil Aliquot Volume:

(uL)

\begin{tabular}{|c|c|c|c|}
\hline CAS NO. & COMPOUND & $\begin{array}{l}\text { CONCENTRATION UNITS: } \\
(\mathrm{ug} / \mathrm{L} \text { or } \mathrm{ug} / \mathrm{kg}) \mathrm{ug} / \mathrm{L}\end{array}$ & $Q$ \\
\hline $75-71-8$ & Dichlorodifluoromethane & 2.2 & $\bar{U}$ \\
\hline $74-87-3$ & Chloromethane & 2.2 & $\mathrm{U}$ \\
\hline $75-01-4$ & Vinyl chloride & 2.2 & $\mathrm{U}$ \\
\hline $74-83-9$ & Bromomethane & 2.2 & $\mathrm{U}$ \\
\hline $75-00-3$ & Chloroethane & 2.2 & $\mathrm{U}$ \\
\hline $75-69-4$ & Trichlorofluoromethane & 2.2 & $\mathrm{U}$ \\
\hline $75-35-4$ & 1,1-Dichloroethene & 2.2 & $\mathrm{U}$ \\
\hline $76-13-1$ & 1,1,2-Trichloro-1,2,2-trifluoroethane & 2.2 & $\mathrm{U}$ \\
\hline $67-64-1$ & Acetone & 890 & E B \\
\hline $75-15-0$ & Carbon disulfide & 2.2 & $\mathrm{U}$ \\
\hline $79-20-9$ & Methyl acetate & 77 & \\
\hline $75-09-2$ & Methylene Chloride & 2.2 & $\mathrm{U}$ \\
\hline $156-60-5$ & trans-1,2-Dichloroethene & 2.2 & $\mathrm{U}$ \\
\hline $1634-04-4$ & Methyl tert-butyl ether & 0.36 & $\mathrm{~J} B$ \\
\hline $75-34-3$ & 1,1-Dichloroethane & 2.2 & $\mathrm{U}$ \\
\hline $156-59-2$ & cis-1,2-Dichloroethene & 2.2 & $\mathrm{U}$ \\
\hline $78-93-3$ & 2-Butanone & 22000 & $\mathrm{E}$ \\
\hline $74-97-5$ & Bromochloromethane & 2.2 & $\mathrm{U}$ \\
\hline $67-66-3$ & Chloroform & 2.2 & $\mathrm{U}$ \\
\hline $71-55-6$ & 1,1,1-Trichloroethane & 2.2 & $\mathrm{U}$ \\
\hline $110-82-7$ & Cyclohexane & 2.2 & $\mathrm{U}$ \\
\hline $56-23-5$ & Carbon tetrachloride & 2.2 & $\mathrm{U}$ \\
\hline $71-43-2$ & Benzene & 2.2 & U \\
\hline $107-06-2$ & 1,2-Dichloroethane & 2.2 & $\mathrm{U}$ \\
\hline
\end{tabular}

Report 1,4-Dioxane for Low-Medium VOA analysis only 
$1 B$ - FORM I VOA-2

VOLATILE ORGANICS ANALYSIS DATA SHEET
EPA SAMPIE NO.

INMW08-W-32692
Lab Name: TESTAMERICA BURLINGTON

Lab Code: STLV Case No.: INMAN Mod. Ref No.:
Matrix: (SOIL/SED/WATER)

Sample wt/vol: 25.0

Water

$(\mathrm{g} / \mathrm{mL}) \quad \mathrm{mL}$

Level: (TRACE/LOW/MED)

TRACE

응 Moisture: not dec.

GC Column: DB-624

Soil Extract Volume:

Purge Volume: 25.0

ID: $0.20 \quad$ (mm)

(uL)

$\left(m L^{\prime}\right)$
Contract: 8E-00302

SDG No.: 200-3113

\begin{tabular}{|c|c|c|c|}
\hline CAS NO. & COMPOUND & $\begin{array}{l}\text { CONCENTRATION UNITS: } \\
(\mathrm{ug} / \mathrm{L} \text { or } \mathrm{ug} / \mathrm{kg}) \mathrm{ug} / \mathrm{L}\end{array}$ & $Q$ \\
\hline $79-01-6$ & Trichloroethene & 2.2 & $\overline{\mathrm{U}}$ \\
\hline $108-87-2$ & Methylcyclohexane & 2.2 & $\mathrm{U}$ \\
\hline $78-87-5$ & 1,2-Dichloropropane & 2.2 & $\mathrm{U}$ \\
\hline $75-27-4$ & Bromodichloromethane & 2.2 & $\mathrm{U}$ \\
\hline $10061-01-5$ & cis-1,3-Dichloropropene & 2.2 & $\mathrm{U}$ \\
\hline $108-10-1$ & 4-Methyl-2-pentanone & 22 & $\mathrm{U}$ \\
\hline $108-88-3$ & Toluene & 2.2 & $\mathrm{U}$ \\
\hline $10061-02-6$ & trans-1,3-Dichloropropene & 2.2 & $\mathrm{U}$ \\
\hline $79-00-5$ & 1,1,2-Trichloroethane & 2.2 & $\mathrm{U}$ \\
\hline $127-18-4$ & Tetrachloroethene & 2.2 & $\mathrm{U}$ \\
\hline $591-78-6$ & 2-Hexanone & 22 & $\mathrm{U}$ \\
\hline $124-48-1$ & Dibromochloromethane & 2.2 & $\mathrm{U}$ \\
\hline $106-93-4$ & 1,2-Dibromoethane & 2.2 & $\mathrm{U}$ \\
\hline $108-90-7$ & Chlorobenzene & 2.2 & $\mathrm{U}$ \\
\hline $100-41-4$ & Ethylbenzene & 2.2 & $\mathrm{U}$ \\
\hline $95-47-6$ & o-Xylene & 2.2 & $\mathrm{U}$ \\
\hline $179601-23-1$ & m,p-xylene & 2.2 & $\mathrm{U}$ \\
\hline $100-42-5$ & Styrene & 2.2 & $\mathrm{U}$ \\
\hline $75-25-2$ & Bromoform & 2.2 & $\mathrm{U}$ \\
\hline $98-82-8$ & Isopropylbenzene & 2.2 & $\mathrm{U}$ \\
\hline $79-34-5$ & $1,1,2,2$-Tetrachloroethane & 2.2 & $\mathrm{U}$ \\
\hline $541-73-1$ & 1,3-Dichlorobenzene & 2.2 & $\mathrm{U}$ \\
\hline $106-46-7$ & 1,4-Dichlorobenzene & 2.2 & $\mathrm{U}$ \\
\hline $95-50-1$ & 1,2-Dichlorobenzene & 2.2 & $\mathrm{U}$ \\
\hline $96-12-8$ & 1,2-Dibromo-3-Chloropropane & 2.2 & $\mathrm{U}$ \\
\hline $120-82-1$ & 1,2,4-Trichlorobenzene & 2.2 & $\mathrm{U}$ \\
\hline $87-61-6$ & 1,2,3-Trichlorobenzene & 2.2 & $\mathrm{U}$ \\
\hline
\end{tabular}

Lab Sample ID: 200-3113-8

Lab File ID: JCFC21.D

Date Received: 12/23/2010

Date Analyzed: $12 / 28 / 2010$

Dilution Factor: 4.4

Soil Aliquot Volume:

(uL) 
$1 \mathrm{~J}$ - FORM I VOA-TIC

VOLATILE ORGANICS ANALYSIS DATA SHEET

TENTATIVELY IDENTIFIED COMPOUNDS
EPA SAMPLE NO.

INMW08-W-32692

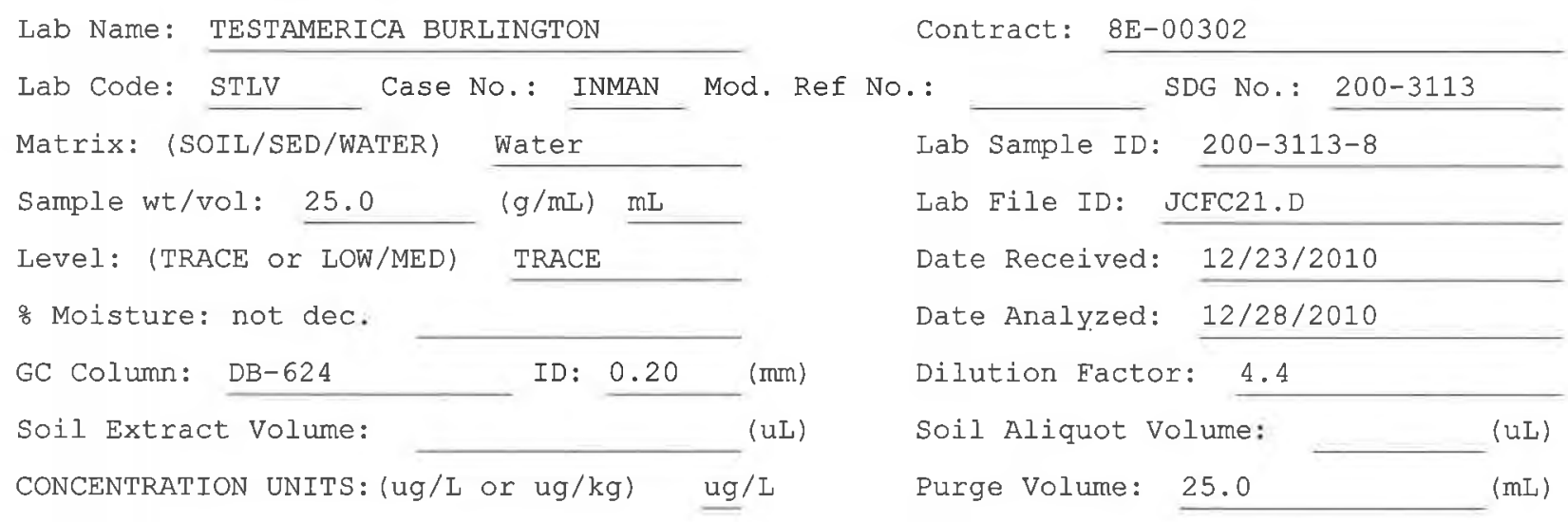

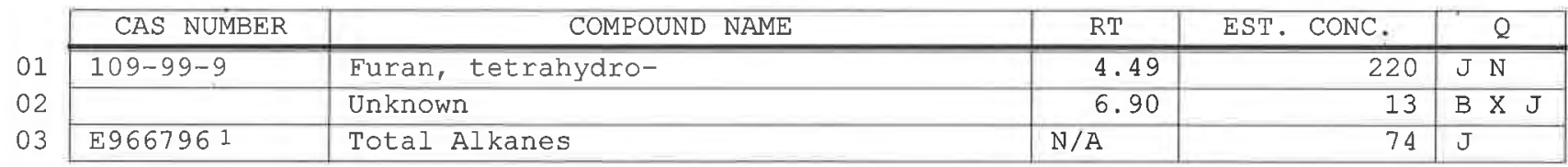

1 EPA-designated Registry Number. 
$1 A$ - FORM I VOA-1

VOLATILE ORGANICS ANALYSIS DATA SHEET
EPA SAMPLE NO. INMW09-W-32693
Lab Name: TESTAMERICA BURLINGTON

Lab Code: STLV Case No.: INMAN

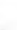

Mod. Ref No.:

Contract: $\quad 8 \mathrm{E}-00302$
Matrix: (SOIL/SED/WATER)

water

$(\mathrm{g} / \mathrm{mL}) \mathrm{mL}$

Sample wt/vol: 25.0

Level: (TRACE/LOW/MED) TRACE

음 Moisture: not dec.

GC Column: DB-624 ID: $0.20 \quad(\mathrm{~mm})$

Soil Extract Volume:

Purge Volume: 25.0

(mL)
SDG No.: 200-3113

Lab Sample ID: 200-3113-9

Lab File ID: JCFC23.D

Date Received: 12/23/2010

Date Analyzed: 12/28/2010

Dilution Factor: 7.5

Soil Aliquot Volume:

(uL)

\begin{tabular}{|c|c|c|c|}
\hline CAS NO. & COMPOUND & $\begin{array}{l}\text { CONCENTRATION UNITS: } \\
(\mathrm{ug} / \mathrm{L} \text { or } \mathrm{ug} / \mathrm{kg}) \mathrm{ug} / \mathrm{L}\end{array}$ & $Q$ \\
\hline $75-71-8$ & Dichlorodifluoromethane & 3.8 & $\overline{\mathrm{U}}$ \\
\hline $74-87-3$ & Chloromethane & 3.8 & $\mathrm{U}$ \\
\hline $75-01-4$ & Vinyl chloride & 3.8 & $\mathrm{U}$ \\
\hline $74-83-9$ & Bromomethane & 3.8 & $\mathrm{U}$ \\
\hline $75-00-3$ & Chloroethane & 3.8 & $\mathrm{U}$ \\
\hline $75-69-4$ & Trichlorofluoromethane & 3.8 & $\mathrm{U}$ \\
\hline $75-35-4$ & 1,1-Dichloroethene & 3.8 & $\mathrm{U}$ \\
\hline $76-13-1$ & 1,1,2-Trichloro-1,2,2-trifluoroethane & 3.8 & U \\
\hline $67-64-1$ & Acetone & 1700 & $E B$ \\
\hline $75-15-0$ & Carbon disulfide & 3.8 & $\mathrm{U}$ \\
\hline $79-20-9$ & Methyl acetate & 3.8 & $\mathrm{U}$ \\
\hline $75-09-2$ & Methylene Chloride & 3.8 & $\mathrm{U}$ \\
\hline $156-60-5$ & trans-1,2-Dichloroethene & 3.8 & $\mathrm{U}$ \\
\hline $1634-04-4$ & Methyl tert-butyl ether & 0.59 & $\mathrm{~J} B$ \\
\hline $75-34-3$ & 1,1-Dichloroethane & 3.8 & $\mathrm{U}$ \\
\hline $156-59-2$ & cis-1,2-Dichloroethene & 3.8 & $\mathrm{U}$ \\
\hline $78-93-3$ & 2-Butanone & 29000 & $\mathrm{E}$ \\
\hline $74-97-5$ & Bromochloromethane & 3.8 & $\mathrm{U}$ \\
\hline $67-66-3$ & Chloroform & 3.8 & $\mathrm{U}$ \\
\hline $71-55-6$ & 1,1,1-Trichloroethane & 3.8 & $\mathrm{U}$ \\
\hline $110-82-7$ & Cyclohexane & 3.8 & $\mathrm{U}$ \\
\hline $56-23-5$ & Carbon tetrachloride & 3.8 & $\mathrm{U}$ \\
\hline $71-43-2$ & Benzene & 3.8 & $\mathrm{U}$ \\
\hline $107-06-2$ & 1,2-Dichloroethane & 3.8 & $\mathrm{U}$ \\
\hline
\end{tabular}

Report 1,4-Dioxane for Low-Medium VOA analysis only 
Lab Name: TESTAMERICA BURLINGTON

Contract: $8 \mathrm{E}-00302$

Lab Code: STLV Case No.: INMAN Mod. Ref No.: SDG No.: $200-3113$

Matrix: (SOIL/SED/WATER)

Sample wt/vol: 25.0 Water

$(\mathrm{g} / \mathrm{mL}) \quad \mathrm{mL}$

Level: (TRACE/LOW/MED) TRACE

음 Moisture: not dec.

GC Column: DB-624

Soil Extract Volume: ID: $0.20 \quad(\mathrm{~mm})$

Purge Volume: 25.0 uL (mL)
Lab Sample ID: 200-3113-9

Lab File ID: JCFC23.D

Date Received: 12/23/2010

Date Analyzed: 12/28/2010

Dilution Factor: 7.5

Soil Aliquot Volume:

(uL)

\begin{tabular}{|c|c|c|c|}
\hline CAS NO. & COMPOUND & $\begin{array}{l}\text { CONCENTRATION UNITS: } \\
(\mathrm{ug} / \mathrm{L} \text { or } \mathrm{ug} / \mathrm{kg}) \mathrm{ug} / \mathrm{L}\end{array}$ & $Q$ \\
\hline $79-01-6$ & Trichloroethene & 3.8 & $\bar{U}$ \\
\hline $108-87-2$ & Methylcyclohexane & 3.8 & $\mathrm{U}$ \\
\hline $78-87-5$ & 1,2-Dichloropropane & 3.8 & $\mathrm{U}$ \\
\hline $75-27-4$ & Bromodichloromethane & 3.8 & $\mathrm{U}$ \\
\hline $10061-01-5$ & cis-1,3-Dichloropropene & 3.8 & $\mathrm{U}$ \\
\hline $108-10-1$ & 4-Methyl-2-pentanone & 38 & $\mathrm{U}$ \\
\hline $108-88-3$ & Toluene & 3.8 & $\mathrm{U}$ \\
\hline $10061-02-6$ & trans-1,3-Dichloropropene & 3.8 & $\mathrm{U}$ \\
\hline $79-00-5$ & $1,1,2$-Trichloroethane & 3.8 & $\mathrm{U}$ \\
\hline $127-18-4$ & Tetrachloroethene & 3.8 & $\mathrm{U}$ \\
\hline $591-78-6$ & 2-Hexanone & 38 & $\mathrm{U}$ \\
\hline $124-48-1$ & Dibromoch loromethane & 3.8 & U \\
\hline $106-93-4$ & 1,2-Dibromoethane & 3.8 & $\mathrm{U}$ \\
\hline $108-90-7$ & Chlorobenzene & 3.8 & $\mathrm{U}$ \\
\hline $100-41-4$ & Ethylbenzene & 3.8 & $\mathrm{U}$ \\
\hline $95-47-6$ & o-xylene & 3.8 & $\mathrm{U}$ \\
\hline $179601-23-1$ & m,p-xylene & 3.8 & $\mathrm{U}$ \\
\hline $100-42-5$ & Styrene & 3.8 & $\mathrm{U}$ \\
\hline $75-25-2$ & Bromoform & 3.8 & $\mathrm{U}$ \\
\hline $98-82-8$ & Isopropylbenzene & 3.8 & $\mathrm{U}$ \\
\hline $79-34-5$ & $1,1,2,2$-Tetrachloroethane & 3.8 & $\mathrm{U}$ \\
\hline $541-73-1$ & 1,3-Dichlorobenzene & 3.8 & $\mathrm{U}$ \\
\hline $106-46-7$ & 1,4-Dichlorobenzene & 3.8 & $\mathrm{U}$ \\
\hline $95-50-1$ & 1,2-Dichlorobenzene & 3.8 & $\mathrm{U}$ \\
\hline $96-12-8$ & 1,2-Dibromo-3-Chloropropane & 3.8 & $\mathrm{U}$ \\
\hline $120-82-1$ & 1,2,4-Trichlorobenzene & 3.8 & $\mathrm{U}$ \\
\hline $87-61-6$ & 1,2,3-Trichlorobenzene & 3.8 & $\mathrm{U}$ \\
\hline
\end{tabular}


$1 \mathrm{~J}$ - FORM I VOA-TIC

VOLATILE ORGANICS ANALYSIS DATA SHEET

TENTATIVELY IDENTIFIED COMPOUNDS
EPA SAMPLE NO.

INMW09-W-32693

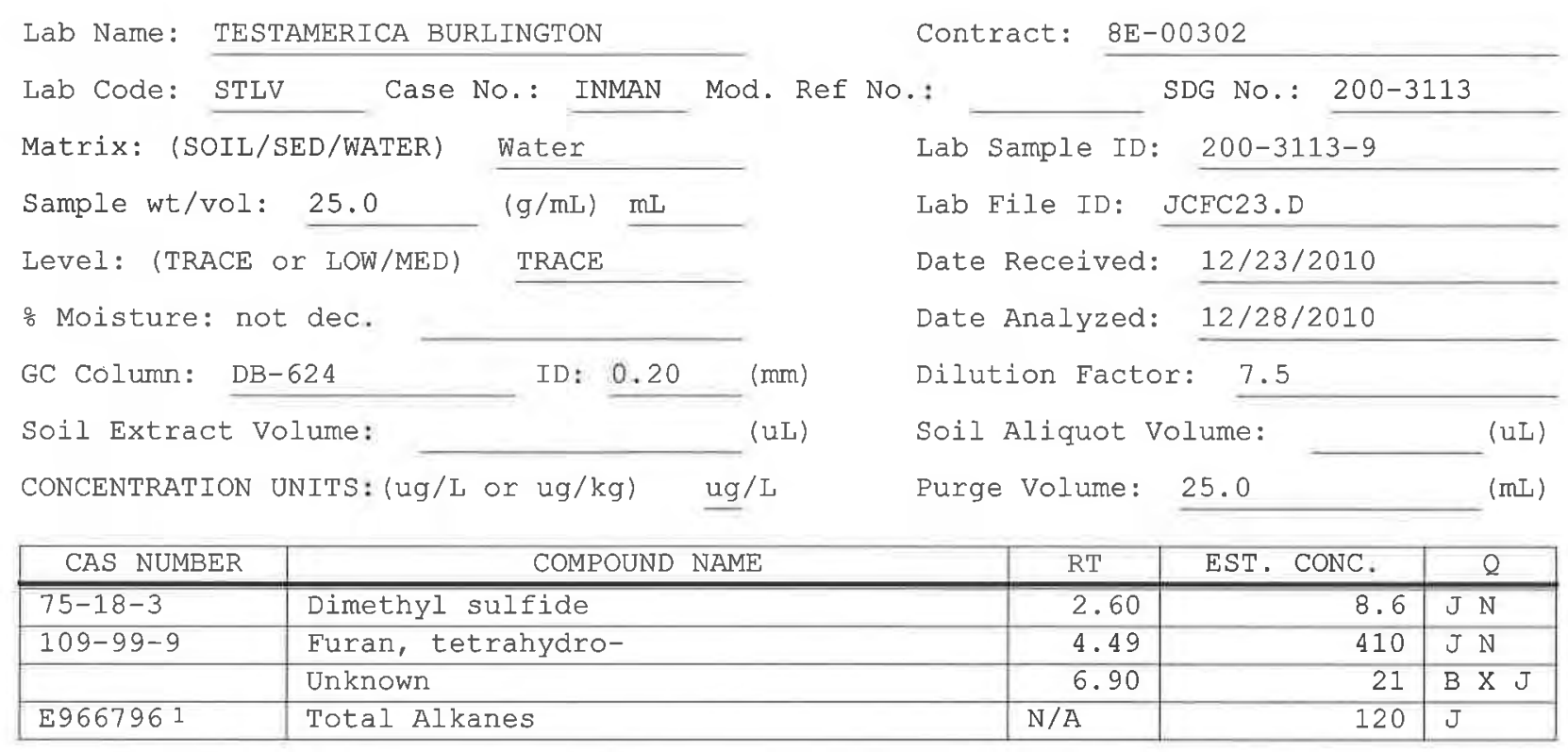

1 EPA-designated Registry Number. 
$1 A$ - FORM I VOA-1

VOLATILE ORGANICS ANALYSIS DATA SHEET
EPA SAMPLE NO.

INMW10-W-32694

Lab Name: TESTAMERICA BURLINGTON

Contract: $\quad 8 \mathrm{E}-00302$

Lab Code: STLV Case No.: INMAN Mod. Ref No.:

SDG No.: 200-3113

Matrix: (SOIL/SED/WATER)

Sample wt/vol: 25.0

Level: (TRACE/LOW/MED)

Water

$(\mathrm{g} / \mathrm{mL}) \quad \mathrm{mL}$

응 Moisture: not dec.

GC Column: DB-624

ID: 0.20 $(\mathrm{mm})$

Soil Extract Volume: (uL) (mL)

Purge Volume: 25.0
Lab Sample ID: 200-3113-10

Lab File ID: JCFC25.D

Date Received: 12/23/2010

Date Analyzed: 12/28/2010

Dilution Factor: 4.4

Soil Aliquot Volume:

(UL)

\begin{tabular}{|c|c|c|c|}
\hline CAS NO. & COMPOUND & $\begin{array}{l}\text { CONCENTRATION UNITS: } \\
(\mathrm{ug} / \mathrm{L} \text { or } \mathrm{ug} / \mathrm{kg}) \mathrm{ug} / \mathrm{I}\end{array}$ & $Q$ \\
\hline $75-71-8$ & Dichlorodifluoromethane & 2.2 & $\mathrm{U}$ \\
\hline $74-87-3$ & Chloromethane & 2.2 & $\mathrm{U}$ \\
\hline $75-01-4$ & Vinyl chloride & 2.2 & $\mathrm{U}$ \\
\hline $74-83-9$ & Bromomethane & 2.2 & $\mathrm{U}$ \\
\hline $75-00-3$ & Chloroethane & 2.2 & $\mathrm{U}$ \\
\hline $75-69-4$ & Trichlorofluoromethane & 2.2 & $\mathrm{U}$ \\
\hline $75-35-4$ & 1,1-Dichloroethene & 2.2 & $\mathrm{U}$ \\
\hline $76-13-1$ & 1,1,2-Trichloro-1,2,2-trifluoroethane & 2.2 & $\mathrm{U}$ \\
\hline $67-64-1$ & Acetone & 1600 & $\mathrm{E} B$ \\
\hline $75-15-0$ & Carbon disulfide & 2.2 & $\mathrm{U}$ \\
\hline $79-20-9$ & Methyl acetate & 150 & $E$ \\
\hline $75-09-2$ & Methylene Chloride & 2.2 & $\mathrm{U}$ \\
\hline $156-60-5$ & trans-1,2-Dichloroethene & 2.2 & $\mathrm{U}$ \\
\hline $1634-04-4$ & Methyl tert-butyl ether & 0.27 & $\mathrm{~J} B$ \\
\hline $75-34-3$ & 1,1-Dichloroethane & 2.2 & $\mathrm{U}$ \\
\hline $156-59-2$ & cis-1,2-Dichloroethene & 2.2 & $\mathrm{U}$ \\
\hline $78-93-3$ & 2-Butanone & 27000 & $\mathrm{E}$ \\
\hline $74-97-5$ & Bromochloromethane & 2.2 & $\mathrm{U}$ \\
\hline $67-66-3$ & Chloroform & 2.2 & $\mathrm{U}$ \\
\hline $71-55-6$ & 1,1,1-Trichloroethane & 2.2 & $\mathrm{U}$ \\
\hline $110-82-7$ & Cyclohexane & 2.2 & $\mathrm{U}$ \\
\hline $56-23-5$ & Carbon tetrachloride & 2.2 & $\mathrm{U}$ \\
\hline $71-43-2$ & Benzene & 2.2 & $\mathrm{U}$ \\
\hline $107-06-2$ & 1,2-Dichloroethane & 2.2 & $\mathrm{U}$ \\
\hline
\end{tabular}

Report 1,4-Dioxane for Low-Medium VOA analysis only 
$1 \mathrm{~B}$ - FORM I VOA-2

VOLATILE ORGANICS ANALYSIS DATA SHEET
EPA SAMPLE NO.

INMW10-W-32694
Lab Name: TESTAMERICA BURLINGTON

Lab Code: STLV Matrix: (SOIL/SED/WATER) Case No.: INMA IN

Sample wt/vol: 25.0

Water

$(\mathrm{g} / \mathrm{mL}) \mathrm{mL}$

Level: (TRACE/LOW/MED)

TRACE

\% Moisture: not dec.

GC Column: DB-624 ID: $0.20 \quad(\mathrm{~mm})$

Soil Extract Volume: (uL) (UL)

Purge Volume: 25.0 (mL)
Contract: $8 \mathrm{E}-00302$

SDG No.: 200-3113

\begin{tabular}{|c|c|c|c|}
\hline CAS NO. & COMPOUND & $\begin{array}{l}\text { CONCENTRATION UNITS: } \\
(\mathrm{ug} / \mathrm{L} \text { or } \mathrm{ug} / \mathrm{kg}) \mathrm{ug} / \mathrm{L}\end{array}$ & $\mathrm{Q}$ \\
\hline $79-01-6$ & Trichloroethene & 2.2 & $\mathrm{U}$ \\
\hline $108-87-2$ & Methylcyclohexane & 2.2 & $\mathrm{U}$ \\
\hline $78-87-5$ & 1,2-Dichloropropane & 2.2 & $\mathrm{U}$ \\
\hline $75-27-4$ & Bromodichloromethane & 2.2 & $\mathrm{U}$ \\
\hline $10061-01-5$ & cis-1,3-Dichloropropene & 2.2 & U \\
\hline $108-10-1$ & 4-Methyl-2-pentanone & 22 & $\mathrm{U}$ \\
\hline $108-88-3$ & Toluene & 2.2 & $\mathrm{U}$ \\
\hline $10061-02-6$ & trans-1,3-Dichloropropene & 2.2 & $\mathrm{U}$ \\
\hline $79-00-5$ & 1,1,2-Trichloroethane & 2.2 & $\mathrm{U}$ \\
\hline $127-18-4$ & Tetrachloroethene & 2.2 & $\mathrm{U}$ \\
\hline $591-78-6$ & 2-Hexanone & 22 & $\mathrm{U}$ \\
\hline $124-48-1$ & Dibromochloromethane & 2.2 & $\mathrm{U}$ \\
\hline $106-93-4$ & 1,2-Dibromoethane & 2.2 & $\mathrm{U}$ \\
\hline $108-90-7$ & Chlorobenzene & 2.2 & $\mathrm{U}$ \\
\hline $100-41-4$ & Ethylbenzene & 2.2 & $\mathrm{U}$ \\
\hline $95-47-6$ & o-xylene & 2.2 & $\mathrm{U}$ \\
\hline $179601-23-1$ & m, p-xylene & 2.2 & $\mathrm{U}$ \\
\hline $100-42-5$ & Styrene & 2.2 & $\mathrm{U}$ \\
\hline $75-25-2$ & Bromoform & 2.2 & $\mathrm{U}$ \\
\hline $98-82-8$ & Isopropylbenzene & 2.2 & $\mathrm{U}$ \\
\hline $79-34-5$ & $1,1,2,2$-Tetrachloroethane & 2.2 & $\mathrm{U}$ \\
\hline $541-73-1$ & 1,3-Dichlorobenzene & 2.2 & $\mathrm{U}$ \\
\hline $106-46-7$ & 1,4-Dichlorobenzene & 2.2 & $\mathrm{U}$ \\
\hline $95-50-1$ & 1,2-Dichlorobenzene & 2.2 & $\mathrm{U}$ \\
\hline $96-12-8$ & 1,2-Dibromo-3-Chloropropane & 2.2 & $\mathrm{U}$ \\
\hline $120-82-1$ & 1,2,4-Trichlorobenzene & 2.2 & $\mathrm{U}$ \\
\hline $87-61-6$ & 1,2,3-Trichlorobenzene & 2.2 & $\mathrm{U}$ \\
\hline
\end{tabular}


1J - FORM I VOA-TIC

VOLATILE ORGANICS ANALYSIS DATA SHEET

TENTATIVELY IDENTIFIED COMPOUNDS
EPA SAMPLE NO.

INMW10-W-326.94
Lab Name: TESTAMERICA BURLINGTON
Contract: $8 \mathrm{E}-00302$
Lab Code: STLV Case No.: INMAN Mod. Ref No.:
SDG No.: 200-3113
Matrix: (SOIL/SED/WATER)
Water
Sample wt/vol: 25.0
Level: (TRACE or LOW/MED)
$(\mathrm{g} / \mathrm{mL}) \mathrm{mI}$
\% Moisture: not dec.
GC Column: DB-624
ID: $0.20 \quad(\mathrm{~mm})$
Soil Extract Volume:
(uL)
CONCENTRATION UNITS: (ug/L or $\mathrm{ug} / \mathrm{kg}$ ) ug/L
Lab Sample ID: 200-3113-10
Lab File ID: JCFC25.D
Date Received: $12 / 23 / 2010$
Date Analyzed: 12/28/2010
Dilution Factor: 4.4
Soil Aliquot Volume: (uL)
Purge Volume: 25.0 (mL)

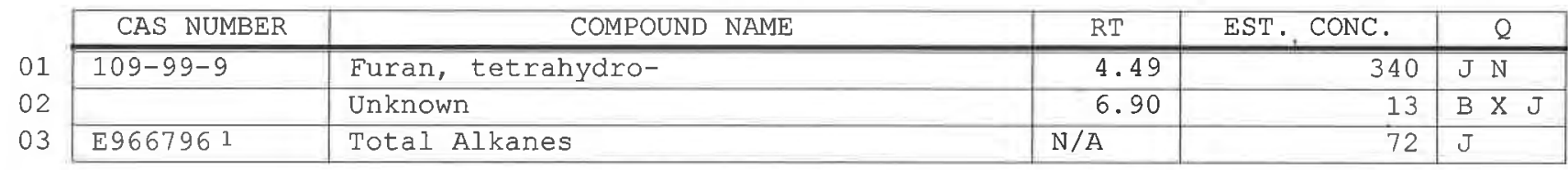

1 EPA-designated Registry Number. 
$1 A$ - FORM I VOA-1

VOLATILE ORGANICS ANALYSIS DATA SHEET
EPA SAMPLE NO.

INMW1 ODUP-W-32695
Lab Name: TESTAMERICA BURLINGTON

Lab Code: STLV Case No.: INMAN Mod. Ref No.:

Matrix: (SOIL/SED/WATER)

water

Sample wt/vol: 25.0

$(\mathrm{g} / \mathrm{mL}) \quad \mathrm{mL}$

Level: (TRACE/LOW/MED)

TRACE

음 Moisture: not dec.

GC Column: DB-624 ID $: 0.20 \quad(\mathrm{~mm})$

Soil Extract volume:

Purge Volume: 25.0

(UL)

$(\mathrm{mL})$
Contract: $8 \mathrm{E}-00302$

SDG No.: 200-3113
Lab Sample ID: 200-3113-11

Lab File ID: JCFE15.D

Date Received: 12/23/2010

Date Analyzed: 12/28/2010

Dilution Factor: 2.0

Soil Aliquot Volume:

(uL)

\begin{tabular}{|c|c|c|c|}
\hline CAS NO. & COMPOUND & $\begin{array}{l}\text { CONCENTRATION UNITS: } \\
(\mathrm{ug} / \mathrm{L} \text { or } \mathrm{ug} / \mathrm{kg}) \mathrm{ug} / \mathrm{L}\end{array}$ & 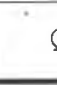 \\
\hline $75-71-8$ & Dichlorodifluoromethane & 1.0 & $\overline{\mathrm{U}}$ \\
\hline $74-87-3$ & Chloromethane & 1.0 & $\mathrm{U}$ \\
\hline $75-01-4$ & Vinyl chloride & 1.0 & $\mathrm{U}$ \\
\hline $74-83-9$ & Bromomethane & 1.0 & $\mathrm{U}$ \\
\hline $75-00-3$ & Chloroethane & 1.0 & $\mathrm{U}$ \\
\hline $75-69-4$ & Trichlorofluoromethane & 1.0 & $\mathrm{U}$ \\
\hline $75-35-4$ & 1,1-Dichloroethene & 1.0 & $\mathrm{U}$ \\
\hline $76-13-1$ & 1,1,2-Trichloro-1,2,2-trifluoroethane & 1.0 & $\mathrm{U}$ \\
\hline $67-64-1$ & Acetone & 3600 & $\mathrm{E} \mathrm{E}$ \\
\hline $75-15-0$ & Carbon disulfide & 1.0 & $\mathrm{U}$ \\
\hline $79-20-9$ & Methyl acetate & 130 & $\mathrm{E}$ \\
\hline $75-09-2$ & Methylene Chloride & 1.0 & $\mathrm{U}$ \\
\hline $156-60-5$ & trans-1,2-Dichloroethene & 1.0 & $\mathrm{U}$ \\
\hline $1634-04-4$ & Methyl tert-butyl ether & 0.18 & $\mathrm{JE}$ \\
\hline $75-34-3$ & 1,1-Dichloroethane & 1.0 & $\mathrm{U}$ \\
\hline $156-59-2$ & cis-1,2-Dichloroethene & 1.0 & $\mathrm{U}$ \\
\hline $78-93-3$ & 2-Butanone & 48000 & $\mathrm{E}$ \\
\hline $74-97-5$ & Bromochloromethane & 1.0 & $\mathrm{U}$ \\
\hline $67-66-3$ & Chloroform & 1.0 & $\mathrm{U}$ \\
\hline $71-55-6$ & 1,1,1-Trichloroethane & 1.0 & $\mathrm{U}$ \\
\hline $110-82-7$ & Cyclohexane & 1.0 & $\mathrm{U}$ \\
\hline $56-23-5$ & Carbon tetrachloride & 1.0 & $\mathrm{U}$ \\
\hline $71-43-2$ & Benzene & 1.0 & $\mathrm{U}$ \\
\hline $107-06-2$ & 1,2-Dichloroethane & 1.0 & $\mathrm{U}$ \\
\hline
\end{tabular}

Report 1,4-Dioxane for Low-Medium VOA analysis only 
$1 \mathrm{~B}$ - FORM I VOA-2

VOLATILE ORGANICS ANALYSIS DATA SHEET
EPA SAMPLE NO.

INMW1 ODUP-W-32695
Lab Name: TESTAMERICA BURLINGTON

Lab Code: STLV

Case No.: INMA

Matrix: (SOIL/SED/WATER)

Sample wt/vol: 25.0

Water

Level: (TRACE/LOW/MED) TRACE

응 Moisture: not dec.

GC Column: DB-624

ID: 0.20 (mm)

Soil Extract Volume: (uL) $(\mathrm{g} / \mathrm{mL}) \quad \mathrm{mL}$

Purge Volume: 25.0
Contract: $8 \mathrm{E}-00302$

SDG No.: 200-3113

\begin{tabular}{|c|c|c|c|}
\hline CAS NO. & COMPOUND & $\begin{array}{l}\text { CONCENTRATION UNITS: } \\
(\mathrm{ug} / \mathrm{L} \text { or } \mathrm{ug} / \mathrm{kg}) \mathrm{ug} / \mathrm{L}\end{array}$ & 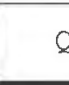 \\
\hline $79-01-6$ & Trichloroethene & 1.0 & $\overline{\mathrm{U}}$ \\
\hline $108-87-2$ & Methylcyclohexane & 1.0 & $\mathrm{U}$ \\
\hline $78-87-5$ & 1,2-Dichloropropane & 1.0 & $\mathrm{U}$ \\
\hline $75-27-4$ & Bromodichloromethane & 1.0 & $\mathrm{U}$ \\
\hline $10061-01-5$ & cis-1,3-Dichloropropene & 1.0 & $\mathrm{U}$ \\
\hline $108-10-1$ & 4-Methyl-2-pentanone & 10 & $\mathrm{U}$ \\
\hline $108-88-3$ & Toluene & 0.10 & $\mathrm{~J}$ \\
\hline $10061-02-6$ & trans-1,3-Dichloropropene & 1.0 & $\mathrm{U}$ \\
\hline $79-00-5$ & 1,1,2-Trichloroethane & 1.0 & $\mathrm{U}$ \\
\hline $127-18-4$ & Tetrachloroethene & 1.0 & $\mathrm{U}$ \\
\hline $591-78-6$ & 2-Hexanone & 10 & $\mathrm{U}$ \\
\hline $124-48-1$ & Dibromochloromethane & 1.0 & $\mathrm{U}$ \\
\hline $106-93-4$ & 1,2-Dibromoethane & 1.0 & $\mathrm{U}$ \\
\hline $108-90-7$ & Chlorobenzene & 1.0 & $\mathrm{U}$ \\
\hline $100-41-4$ & Ethylbenzene & 1.0 & $\mathrm{U}$ \\
\hline $95-47-6$ & o-xylene & 1.0 & $\mathrm{U}$ \\
\hline $179601-23-1$ & m, p-Xylene & 1.0 & U \\
\hline $100-42-5$ & Styrene & 1.0 & $\mathrm{U}$ \\
\hline $75-25-2$ & Bromoform & 1.0 & $\mathrm{U}$ \\
\hline $98-82-8$ & Isopropylbenzene & 1.0 & $\mathrm{U}$ \\
\hline $79-34-5$ & $1,1,2,2$-Tetrachloroethane & 1.0 & $\mathrm{U}$ \\
\hline $541-73-1$ & 1,3-Dichlorobenzene & 1.0 & $\mathrm{U}$ \\
\hline $106-46-7$ & 1,4-Dichlorobenzene & 1.0 & $\mathrm{U}$ \\
\hline $95-50-1$ & 1,2-Dichlorobenzene & 1.0 & $\mathrm{U}$ \\
\hline $96-12-8$ & 1,2-Dibromo-3-Chloropropane & 1.0 & $\mathrm{U}$ \\
\hline $120-82-1$ & 1,2,4-Trichlorobenzene & 1.0 & $\mathrm{U}$ \\
\hline $87-61-6$ & 1,2,3-Trichlorobenzene & 1.0 & $\mathrm{U}$ \\
\hline
\end{tabular}


$1 \mathrm{~J}$ - FORM I VOA-TIC

VOLATILE ORGANICS ANALYSIS DATA SHEET

TENTATIVELY IDENTIFIED COMPOUNDS
EPA SAMPLE NO.

INMW10DUP-W-32695

Lab Name: TESTAMERICA BURLINGTON Contract: 8E-00302

Lab Code: STLV Case No.: INMAN Mod. Ref No.:

SDG NO.: 200-3113

Matrix: (SOIL/SED/WATER)

Water

Sample wt/vol: 25.0

$(\mathrm{g} / \mathrm{mL}) \quad \mathrm{mL}$

Level: (TRACE or LOW/MED) TRACE

o Moisture: not dec.

GC Colurnn: DB-624

ID: $0.20 \quad(\mathrm{~mm})$

Soil Extract Volume: (uL)

CONCENTRATION UNITS: $(\mathrm{ug} / \mathrm{L}$ or $\mathrm{ug} / \mathrm{kg}) \mathrm{ug} / \mathrm{L}$
Lab Sample ID: 200-3113-11

Lab File ID: JCFE15.D

Date Received: 12/23/2010

Date Analyzed: $12 / 28 / 2010$

Dilution Factor: 2.0

Soil Aliquot Volume:

(uL)

Purge Volume: 25.0 (mL)

\begin{tabular}{|c|c|c|c|c|}
\hline CAS NUMBER & COMPOUND NAME & RT & EST. CONC. & Q \\
\hline $109-99-9$ & Furan, tetrahydro- & 4.50 & 1100 & \\
\hline 02 & Unknown & 6.90 & 6.2 & $\mathrm{~B} X \mathrm{~J}$ \\
\hline E9667961 & Total Alkanes & $\mathrm{N} / \mathrm{A}$ & 30 & $\mathrm{~J}$ \\
\hline
\end{tabular}

1 EPA-designated Registry Number. 
$1 A$ - FORM I VOA-1

VOLATILE ORGANICS ANALYSIS DATA SHEET
EPA SAMPLE NO.

INMW11-W-32696
Lab Name: TESTAMERICA BURLINGTON

Lab Code: STLV Case No.: INMAN

Matrix: (SOIL/SED/WATER)

Water

Sample wt/vol: 25.0

$(\mathrm{g} / \mathrm{mL}) \mathrm{mL}$

Level: (TRACE/LOW/MED) TRACE

\% Moisture: not dec.

GC Column: DB-624 ID $: 0.20 \quad(\mathrm{~mm})$

Soil Extract Volume:

Purge Volume: 25.0 (mL)
Contract: $8 \mathrm{E}-00302$

SDG No.: $200-3113$

Lab Sample ID: 200-3113-12

Lab File ID: JCFE09.D

Date Received: 12/23/2010

Date Analyzed: 12/28/2010

Dilution Factor: 2.0

Soil Aliquot volume:

(uL)

\begin{tabular}{|c|c|c|c|}
\hline CAS NO. & COMPOUND & $\begin{array}{l}\text { CONCENTRATION UNITS: } \\
(\mathrm{ug} / \mathrm{L} \text { or } \mathrm{ug} / \mathrm{kg}) \mathrm{ug} / \mathrm{L}\end{array}$ & 0 \\
\hline $75-71-8$ & Dichlorodifluoromethane & $\overline{1.0}$ & $\mathrm{U}$ \\
\hline $74-87-3$ & Chloromethane & 1.0 & $\mathrm{U}$ \\
\hline $75-01-4$ & Vinyl chloride & 1.0 & $\mathrm{U}$ \\
\hline $74-83-9$ & Bromomethane & 1.0 & $\mathrm{U}$ \\
\hline $75-00-3$ & Chloroethane & 1.0 & $\mathrm{U}$ \\
\hline $75-69-4$ & Trichlorofluoromethane & 1.0 & $\mathrm{U}$ \\
\hline $75-35-4$ & 1,1-Dichloroethene & 1.0 & $\mathrm{U}$ \\
\hline $76-13-1$ & $1,1,2$-Trichloro-1,2,2-trifluoroethane & 1.0 & $\mathrm{U}$ \\
\hline $67-64-1$ & Acetone & 640 & $\mathrm{E} B$ \\
\hline $75-15-0$ & Carbon disulfide & 1.0 & $\mathrm{U}$ \\
\hline $79-20-9$ & Methyl acetate & 1.0 & $\mathrm{U}$ \\
\hline $75-09-2$ & Methylene Chloride & 1.0 & $\mathrm{U}$ \\
\hline $156-60-5$ & trans-1,2-Dichloroethene & 1.0 & $\mathrm{U}$ \\
\hline $1634-04-4$ & Methyl tert-butyl ether & 0.12 & $\mathrm{~J} B$ \\
\hline $75-34-3$ & 1,1-Dichloroethane & 1.0 & $\mathrm{U}$ \\
\hline $156-59-2$ & cis-1,2-Dichloroethene & 1.0 & $\mathrm{U}$ \\
\hline $78-93-3$ & 2-Butanone & 10000 & $E$ \\
\hline $74-97-5$ & Bromochloromethane & 1.0 & $U$ \\
\hline $67-66-3$ & Chloroform & 1.0 & $\mathrm{U}$ \\
\hline $71-55-6$ & 1,1,1-Trichloroethane & 1.0 & $\mathrm{U}$ \\
\hline $110-82-7$ & Cyclohexane & 1.0 & $\mathrm{U}$ \\
\hline $56-23-5$ & Carbon tetrachloride & 1.0 & U \\
\hline $71-43-2$ & Benzene & 1.0 & U \\
\hline $107-06-2$ & 1,2-Dichloroethane & 1.0 & $\mathrm{U}$ \\
\hline
\end{tabular}

Report 1,4-Dioxane for Low-Medium VOA analysis only 
Lab Name: TESTAMERICA BURLINGTON

Lab Code: STLV Case No.: INMAN Mod. Ref No.:
Matrix: (SOIL/SED/WATER)

Sample wt/vol: 25.0

\section{Water}

$(\mathrm{g} / \mathrm{mL}) \quad \mathrm{mL}$

Level: (TRACE/LOW/MED) TRACE

응 Moisture: not dec.

GC Column: DB-624

Soil Extract Volume:

Purge Volume: 25.0
Contract: $8 \mathrm{E}-00302$ SDG No.: 200-3113

Lab Sample ID: 200-3113-12

Lab File ID: JCFE09.D

Date Received: 12/23/2010

Date Analyzed: 12/28/2010

Dilution Factor: 2.0

Soil Aliquot volume: (uL) ( (mI)

\begin{tabular}{|c|c|c|c|}
\hline CAS NO. & COMPOUND & $\begin{array}{l}\text { CONCENTRATION UNITS: } \\
(\mathrm{ug} / \mathrm{L} \text { or } \mathrm{ug} / \mathrm{kg}) \mathrm{ug} / \mathrm{L}\end{array}$ & $Q$ \\
\hline $79-01-6$ & Trichloroethene & 1.0 & $\mathrm{U}$ \\
\hline $108-87-2$ & Methylcyclohexane & 1.0 & $\mathrm{U}$ \\
\hline $78-87-5$ & 1,2-Dichloropropane & 1.0 & $\mathrm{U}$ \\
\hline $75-27-4$ & Bromodichloromethane & 1.0 & $\mathrm{U}$ \\
\hline $10061-01-5$ & cis-1,3-Dichloropropene & 1.0 & $\mathrm{U}$ \\
\hline $108-10-1$ & 4-Methyl-2-pentanone & 10 & $\mathrm{U}$ \\
\hline $108-88-3$ & Toluene & 1.0 & $\mathrm{U}$ \\
\hline $10061-02-6$ & trans-1,3-Dichloropropene & 1.0 & $\mathrm{U}$ \\
\hline $79-00-5$ & 1,1,2-Trichloroethane & 1.0 & $\mathrm{U}$ \\
\hline $127-18-4$ & Tetrachloroethene & 1.0 & $\mathrm{U}$ \\
\hline $591-78-6$ & 2-Hexanone & 10 & $\mathrm{U}$ \\
\hline $124-48-1$ & Dibromochloromethane & 1.0 & $\mathrm{U}$ \\
\hline $106-93-4$ & 1,2-Dibromoethane & 1.0 & $\mathrm{U}$ \\
\hline $108-90-7$ & Chlorobenzene & 1.0 & $\mathrm{U}$ \\
\hline $100-41-4$ & Ethylbenzene & 1.0 & $\mathrm{U}$ \\
\hline $95-47-6$ & o-Xylene & 1.0 & $\mathrm{U}$ \\
\hline $179601-23-1$ & $\mathrm{~m}, \mathrm{p}$-Xylene & 1.0 & $\mathrm{U}$ \\
\hline $100-42-5$ & Styrene & 1.0 & $\mathrm{U}$ \\
\hline $75-25-2$ & Bromoform & 1.0 & $\mathrm{U}$ \\
\hline $98-82-8$ & Isopropylbenzene & 1.0 & $\mathrm{U}$ \\
\hline $79-34-5$ & $1,1,2,2$-Tetrachloroethane & 1.0 & $\mathrm{U}$ \\
\hline $541-73-1$ & 1,3-Dichlorobenzene & 1.0 & $\mathrm{U}$ \\
\hline $106-46-7$ & 1,4-Dichlorobenzene & 1.0 & $\mathrm{U}$ \\
\hline $95-50-1$ & 1,2-Dichlorobenzene & 1.0 & $\mathrm{U}$ \\
\hline $96-12-8$ & 1,2-Dibromo-3-Chloropropane & 1.0 & $\mathrm{U}$ \\
\hline $120-82-1$ & $1,2,4$-Trichlorobenzene & 1.0 & $\mathrm{U}$ \\
\hline $87-61-6$ & 1,2,3-Trichlorobenzene & 1.0 & $\mathrm{U}$ \\
\hline
\end{tabular}


$1 \mathrm{~J}-$ EORM I VOA-TIC

VOLATILE ORGANICS ANALYSIS DATA SHEET

TENTATIVELY IDENTIFIED COMPOUNDS
EPA SAMPLE NO.

INMW11-W-32696
Lab Name: TESTAMERICA BURLINGTON
Contract: $8 E-00302$

Lab code: STLV Case No.: INMAN Mod. Ref No.:

SDG No.: 200-3113

Matrix: (SOIL/SED/WATER)

Water

Sample wt/vol: 25.0

$(\mathrm{g} / \mathrm{mL}) \mathrm{mL}$

Level: (TRACE or LOW/MED)

TRACE

o Moisture: not dec.

GC Column: DB-624

ID: $0.20 \quad$ (mm)

Soil Extract Volume:

(uL)
Lab Sample ID: 200-3113-12

Lab File ID: JCFE09.D

Date Received: 12/23/2010

Date Analyzed: 12/28/2010

Dilution Factor: 2.0

Soil Aliquot Volume:

(uL)

Purge Volume: 25.0

\begin{tabular}{|c|c|c|c|c|}
\hline CAS NUMBER & COMPOUND NAME & $\mathrm{RT}$ & EST. CONC. & $Q$ \\
\hline $109-99-9$ & Furan, tetrahydro- & 4.49 & 180 & $\overline{J N}$ \\
\hline 02 & Unknown & 6.90 & 6.2 & $\mathrm{~B} X \mathrm{~J}$ \\
\hline$E 9667961$ & Total Alkanes & $\mathrm{N} / \mathrm{A}$ & 36 & $\mathrm{~J}$ \\
\hline
\end{tabular}

1 EPA-designated Registry Number. 
$1 A-F O R M I V O A-1$

VOLATILE ORGANICS ANALYSIS DATA SHEET
EPA SAMPLE NO.

INMW12-W-32697
Lab Name: TESTAMERICA BURLINGTON

Lab Code: STLV

Matrix: (SOIL/SED/WATER)

Sample wt/vol: 25.0

$(\mathrm{g} / \mathrm{mL}) \quad \mathrm{mL}$

Level: (TRACE/LOW/MED) TRACE

\% Moisture: not dec.

GC Column: $\mathrm{DB}-624$

ID: $0.20 \quad(\mathrm{~mm})$

Soil Extract Volume:

Purge Volume: 25.0

$(\mathrm{mL})$
Contract: $8 E-00302$

SDG No.: 200-3113

\begin{tabular}{|c|c|c|c|}
\hline CAS NO. & COMPOUND & $\begin{array}{l}\text { CONCENTRATION UNITS: } \\
(\mathrm{ug} / \mathrm{L} \text { or } \mathrm{ug} / \mathrm{kg}) \mathrm{ug} / \mathrm{L}\end{array}$ & $Q$ \\
\hline $75-71-8$ & Dichlorodifluoromethane & 1.0 & $\overline{\mathrm{U}}$ \\
\hline $74-87-3$ & Chloromethane & 1.0 & $\mathrm{U}$ \\
\hline $75-01-4$ & Vinyl chloride & 1.0 & $\mathrm{U}$ \\
\hline $74-83-9$ & Bromomethane & 1.0 & $\mathrm{U}$ \\
\hline $75-00-3$ & Chloroethane & 1.0 & $\mathrm{U}$ \\
\hline $75-69-4$ & Trichlorofluoromethane & 1.0 & $\mathrm{U}$ \\
\hline $75-35-4$ & 1,1-Dichloroethene & 1.0 & $\mathrm{U}$ \\
\hline $76-13-1$ & 1,1,2-Trichloro-1,2,2-trifluoroethane & 1.0 & $\mathrm{U}$ \\
\hline $67-64-1$ & Acetone & 2200 & $\mathrm{E} B$ \\
\hline $75-15-0$ & Carbon disulfide & 1.0 & $\mathrm{U}$ \\
\hline $79-20-9$ & Methyl acetate & 1.0 & $\mathrm{U}$ \\
\hline $75-09-2$ & Methylene Chloride & 1.0 & $\mathrm{U}$ \\
\hline $156-60-5$ & trans-1,2-Dichloroethene & 1.0 & $\mathrm{U}$ \\
\hline $1634-04-4$ & Methyl tert-butyl ether & 0.22 & $\mathrm{~J} \mathrm{~B}$ \\
\hline $75-34-3$ & 1,1-Dichloroethane & 1.0 & $\mathrm{U}$ \\
\hline $156-59-2$ & cis-1,2-Dichloroethene & 1.0 & $\mathrm{U}$ \\
\hline $78-93-3$ & 2-Butanone & 19000 & $\mathrm{E}$ \\
\hline $74-97-5$ & Bromochloromethane & 1.0 & $\mathrm{U}$ \\
\hline $67-66-3$ & Chloroform & 1.0 & $\mathrm{U}$ \\
\hline $71-55-6$ & 1,1,1-Trichloroethane & 1.0 & $\mathrm{U}$ \\
\hline $110-82-7$ & Cyclohexane & 1.0 & $\mathrm{U}$ \\
\hline $56-23-5$ & Carbon tetrachloride & 1.0 & $\mathrm{U}$ \\
\hline $71-43-2$ & Benzene & 1.0 & $\mathrm{U}$ \\
\hline $107-06-2$ & 1,2-Dichloroethane & 1.0 & $\mathrm{U}$ \\
\hline
\end{tabular}

Report 1,4-Dioxane for Low-Medium VOA analysis only 
$1 B$ - FORM I VOA-2

VOLATILE ORGANICS ANALYSIS DATA SHEET
EPA SAMPLE NO.

INMW12-W-32697
Lab Name: TESTAMERICA BURLINGTON

Lab Code: STIV

Case No.: INMA

Matrix: (SOIL/SED/WATER)

Sample wt/vol: 25.0

Water

Level: (TRACE/LOW/MED)

$(\mathrm{g} / \mathrm{mL}) \mathrm{mL}$

o Moisture: not dec.

GC Column: DB-624

ID: $0.20^{\circ}(\mathrm{mm})$

Soil Extract Volume:

(uL)

Purge Volume: 25.0

(mL)
Contract: $8 \mathrm{E}-00302$

SDG No.: 200-3113

Lab Sample ID: 200-3113-13

Lab File ID: JCFEl1.D

Date Received: 12/23/2010

Date Analyzed: 12/28/2010

Dilution Factor: 2.0

Soil Aliquot volume:

(uL)

\begin{tabular}{|c|c|c|c|}
\hline CAS NO. & COMPOUND & $\begin{array}{l}\text { CONCENTRATION UNITS: } \\
(\mathrm{ug} / \mathrm{L} \text { or } \mathrm{ug} / \mathrm{kg}) \mathrm{ug} / \mathrm{L}\end{array}$ & $Q$ \\
\hline $79-01-6$ & Trichloroethene & 1.0 & $\overline{\mathrm{U}}$ \\
\hline $108-87-2$ & Methylcyclohexane & 1.0 & $\mathrm{U}$ \\
\hline $78-87-5$ & 1,2-Dichloropropane & 1.0 & $\mathrm{U}$ \\
\hline $75-27-4$ & Bromodichloromethane & 1.0 & $\mathrm{U}$ \\
\hline $10061-01-5$ & cis-1,3-Dichloropropene & 1.0 & $\mathrm{U}$ \\
\hline $108-10-1$ & 4-Methyl-2-pentanone & 10 & U \\
\hline $108-88-3$ & Toluene & 1.0 & $\mathrm{U}$ \\
\hline $10061-02-6$ & trans-1,3-Dichloropropene & 1.0 & $\mathrm{U}$ \\
\hline $79-00-5$ & 1,1,2-Trichloroethane & 1.0 & $\mathrm{U}$ \\
\hline $127-18-4$ & Tetrachloroethene & 1.0 & $\mathrm{U}$ \\
\hline $591-78-6$ & 2-Hexanone & 10 & $\mathrm{U}$ \\
\hline $124-48-1$ & Dibromochloromethane & 1.0 & $\mathrm{U}$ \\
\hline $106-93-4$ & 1,2-Dibromoethane & 1.0 & $\mathrm{U}$ \\
\hline $108-90-7$ & Chlorobenzene & 1.0 & $\mathrm{U}$ \\
\hline $100-41-4$ & Ethylbenzene & 1.0 & $\mathrm{U}$ \\
\hline $95-47-6$ & o-xylene & 1.0 & $\mathrm{U}$ \\
\hline $179601-23-1$ & $\mathrm{~m}, \mathrm{p}$-Xylene & 1.0 & $\mathrm{U}$ \\
\hline $100-42-5$ & Styrene & 1.0 & $\mathrm{U}$ \\
\hline $75-25-2$ & Bromoform & 1.0 & $\mathrm{U}$ \\
\hline $98-82-8$ & Isopropylbenzene & 1.0 & $\mathrm{U}$ \\
\hline $79-34-5$ & $1,1,2,2$-Tetrachloroethane & 1.0 & $\mathrm{U}$ \\
\hline $541-73-1$ & 1,3-Dichlorobenzene & 1.0 & $\mathrm{U}$ \\
\hline $106-46-7$ & 1,4-Dichlorobenzene & 1.0 & $\mathrm{U}$ \\
\hline $95-50-1$ & 1,2-Dichlorobenzene & 1.0 & $\mathrm{U}$ \\
\hline $96-12-8$ & 1,2-Dibromo-3-Chloropropane & 1.0 & $\mathrm{U}$ \\
\hline $120-82-1$ & 1,2,4-Trichlorobenzene & 1.0 & $\mathrm{U}$ \\
\hline $87-61-6$ & 1,2,3-Trichlorobenzene & 1.0 & $\mathrm{U}$ \\
\hline
\end{tabular}


$1 \mathrm{~J}$ - FORM I VOA-TIC

VOLATILE ORGANICS ANALYSIS DATA SHEET

TENTATIVELY IDENTIFIED COMPOUNDS
EPA SAMPLE NO.

INMW12-W-32697

Lab Name: TESTAMERICA BURLINGTON

Contract: $8 \mathrm{E}-00302$

Lab Code: STLV Case No.: INMAN Mod. Ref No.: SDG No.: 200-3113 Matrix: (SOIL/SED/WATER) Water

Sample wt/vol: 25.0 $(\mathrm{g} / \mathrm{mL}) \mathrm{mL}$ Level: (TRACE or LOW/MED) TRACE

\% Moisture: not dec.

GC Column: DB-624 ID: 0.20 ( $\mathrm{mm}$ )

Soil Extract Volume: (uL) CONCENTRATION UNITS: (ug/L or $\mathrm{ug} / \mathrm{kg}$ ) $\mathrm{ug} / \mathrm{L}$
Lab Sample ID: 200-3113-13

Lab File ID: JCFE11.D

Date Received: 12/23/2010

Date Analyzed: 12/28/2010

Dilution Factor: 2.0

Soil Aliquot Volume: (uL)

Purge Volume: 25.0 (mL)

\begin{tabular}{|c|c|c|c|c|c|}
\hline & CAS NUMBER & COMPOUND NAME & RT & EST. CONC. & Q \\
\hline 01 & $109-99-9$ & Furan, tetrahydro- & 4.49 & 120 & $\mathrm{~J} \mathrm{~N}$ \\
\hline 02 & & Unknown & 6.90 & 6.4 & $\mathrm{~B} \times \mathrm{J}$ \\
\hline 03 & E9667961 & Total Alkanes & $\mathrm{N} / \mathrm{A}$ & 32 & $\mathrm{~J}$ \\
\hline
\end{tabular}

1 EPA-designated Registry Number. 
$1 A$ - FORM I VOA-1

VOLATILE ORGANICS ANALYSIS DATA SHEET
EPA SAMPLE NO. INMW13-W-32698
Lab Name: TESTAMERICA BURLINGTON

Lab Code: STLV Matrix: (SOIL/SED/WATER)

Sample wt/vol: 25.0 Case No.: INMA water $(\mathrm{g} / \mathrm{mL}) \mathrm{mL}$

Leve1: (TRACE/LOW/MED) TRACE

o Moisture: not dec.

GC Column: $\mathrm{DB}-624$ ID: $0.20 \quad(\mathrm{~mm})$

Soil Extract Volume:

Purge Volume: 25.0 (mL)
Contract: $8 \mathrm{E}-00302$

SDG No.: 200-3113

\begin{tabular}{|c|c|c|c|}
\hline CAS NO. & COMPOUND & $\begin{array}{l}\text { CONCENTRATION UNITS: } \\
(\mathrm{ug} / \mathrm{L} \text { or } \mathrm{ug} / \mathrm{kg}) \mathrm{ug} / \mathrm{L}\end{array}$ & $Q$ \\
\hline $75-71-8$ & Dichlorodifluoromethane & 1.0 & $\mathrm{U}$ \\
\hline $74-87-3$ & Chloromethane & 1.0 & $\mathrm{U}$ \\
\hline $75-01-4$ & Vinyl chloride & 1.0 & $\mathrm{U}$ \\
\hline $74-83-9$ & Bromomethane & 1.0 & $\mathrm{U}$ \\
\hline $75-00-3$ & Chloroethane & 1.0 & $\mathrm{U}$ \\
\hline $75-69-4$ & Trichlorofluoromethane & 1.0 & $\mathrm{U}$ \\
\hline $75-35-4$ & 1,1-Dichloroethene & 1.0 & $\mathrm{U}$ \\
\hline $76-13-1$ & 1,1,2-Trichloro-1,2,2-trifluoroethane & 1.0 & $\mathrm{U}$ \\
\hline $67-64-1$ & Acetone & 6300 & E B \\
\hline $75-15-0$ & Carbon disulfide & 1.0 & $\mathrm{U}$ \\
\hline $79-20-9$ & Methyl acetate & 1.0 & $\mathrm{U}$ \\
\hline $75-09-2$ & Methylene Chloride & 1.0 & $\mathrm{U}$ \\
\hline $156-60-5$ & trans-1,2-Dichloroethene & 1.0 & $\mathrm{U}$ \\
\hline $1634-04-4$ & Methyl tert-butyl ether & 0.18 & $\mathrm{~J} \mathrm{~B}$ \\
\hline $75-34-3$ & 1,1-Dichloroethane & 1.0 & $\mathrm{U}$ \\
\hline $156-59-2$ & cis-1,2-Dichloroethene & 1.0 & $\mathrm{U}$ \\
\hline $78-93-3$ & 2-Butanone & 39000 & $\mathrm{E}$ \\
\hline $74-97-5$ & Bromochloromethane & 1.0 & $\mathrm{U}$ \\
\hline $67-66-3$ & Chloroform & 1.0 & $\mathrm{U}$ \\
\hline $71-55-6$ & $1,1,1$-Trichloroethane & 1.0 & $\mathrm{U}$ \\
\hline $110-82-7$ & Cyclohexane & 1.0 & $\mathrm{U}$ \\
\hline $56-23-5$ & Carbon tetrachloride & 1.0 & $\mathrm{U}$ \\
\hline $71-43-2$ & Benzene & 1.0 & $\mathrm{U}$ \\
\hline $107-06-2$ & 1,2-Dichloroethane & 1.0 & $\mathrm{U}$ \\
\hline
\end{tabular}

Report 1,4-Dioxane for Low-Medium VoA analysis only 
Lab Name: TESTAMERICA BURLINGTON

Contract: $8 \mathrm{E}-00302$

Lab Code: STLV Case No.: INMAN Mod. Ref No.: SDG No.: 200-3113

Matrix: (SOIL/SED/WATER)

Sample wt/vol: 25.0 Water

Level: (TRACE/LOW/MED) TRACE $(\mathrm{g} / \mathrm{mL}) \quad \mathrm{mL}$

o Moisture: not dec.

GC Column: DB-624

ID: 0.20 (mm)

Soil Extract Volume: (UL) (mL)

Purge Volume: 25.0
Lab Sample ID: 200-3113-14

Lab File ID: JCFE13.D

Date Received: 12/23/2010

Date Analyzed: 12/28/2010

Dilution Factor: 2.0

Soil Aliquot volume:

(uL)

\begin{tabular}{|c|c|c|c|}
\hline CAS NO. & COMPOUND & $\begin{array}{l}\text { CONCENTRATION UNITS: } \\
(\mathrm{ug} / \mathrm{L} \text { or } \mathrm{ug} / \mathrm{kg}) \mathrm{ug} / \mathrm{L}\end{array}$ & $Q$ \\
\hline $79-01-6$ & Trichloroethene & 1.0 & $\overline{\mathrm{U}}$ \\
\hline $108-87-2$ & Methylcyclohexane & 1.0 & $\mathrm{U}$ \\
\hline $78-87-5$ & 1,2-Dichloropropane & 1.0 & $\mathrm{U}$ \\
\hline $75-27-4$ & Bromodichloromethane & 1.0 & $\mathrm{U}$ \\
\hline $10061-01-5$ & cis-1,3-Dichloropropene & 1.0 & $\mathrm{U}$ \\
\hline $108-10-1$ & 4-Methyl-2-pentanone & 10 & $\mathrm{U}$ \\
\hline $108-88-3$ & Toluene & 1.0 & $\mathrm{U}$ \\
\hline $10061-02-6$ & trans-1,3-Dichloropropene & 1.0 & $\mathrm{U}$ \\
\hline $79-00-5$ & 1,1,2-Trichloroethane & 1.0 & $\mathrm{U}$ \\
\hline $127-18-4$ & Tetrachloroethene & 1.0 & $\mathrm{U}$ \\
\hline $591-78-6$ & 2-Hexanone & 10 & $\mathrm{U}$ \\
\hline $124-48-1$ & Dibromochloromethane & 1.0 & $\mathrm{U}$ \\
\hline $106-93-4$ & 1,2-Dibromoethane & 1.0 & $\mathrm{U}$ \\
\hline $108-90-7$ & Chlorobenzene & 1.0 & $\mathrm{U}$ \\
\hline $100-41-4$ & Ethylbenzene & 1.0 & $\mathrm{U}$ \\
\hline $95-47-6$ & o-xylene & 1.0 & $\mathrm{U}$ \\
\hline $179601-23-1$ & $\mathrm{~m}, \mathrm{p}$-Xylene & 1.0 & $\mathrm{U}$ \\
\hline $100-42-5$ & Styrene & 1.0 & $\mathrm{U}$ \\
\hline $75-25-2$ & Bromoform & 1.0 & $\mathrm{U}$ \\
\hline $98-82-8$ & Isopropylbenzene & 1.0 & $\mathrm{U}$ \\
\hline $79-34-5$ & $1,1,2,2$-Tetrachloroethane & 1.0 & $\mathrm{U}$ \\
\hline $541-73-1$ & 1,3-Dichlorobenzene & 1.0 & $\mathrm{U}$ \\
\hline $106-46-7$ & 1,4-Dichlorobenzene & 1.0 & $\mathrm{U}$ \\
\hline $95-50-1$ & 1,2-Dichlorobenzene & 1.0 & $\mathrm{U}$ \\
\hline $96-12-8$ & 1,2-Dibromo-3-Chloropropane & 1.0 & $\mathrm{U}$ \\
\hline $120-82-1$ & 1,2,4-Trichlorobenzene & 1.0 & $\mathrm{U}$ \\
\hline $87-61-6$ & 1,2,3-Trichlorobenzene & 1.0 & $\mathrm{U}$ \\
\hline
\end{tabular}


$1 \mathrm{~J}$ - FORM I VOA-TIC

VOLATILE ORGANICS ANALYSIS DATA SHEET

TENTATIVELY IDENTIFIED COMPOUNDS
EPA SAMPLE NO.

INMW13-W-32698

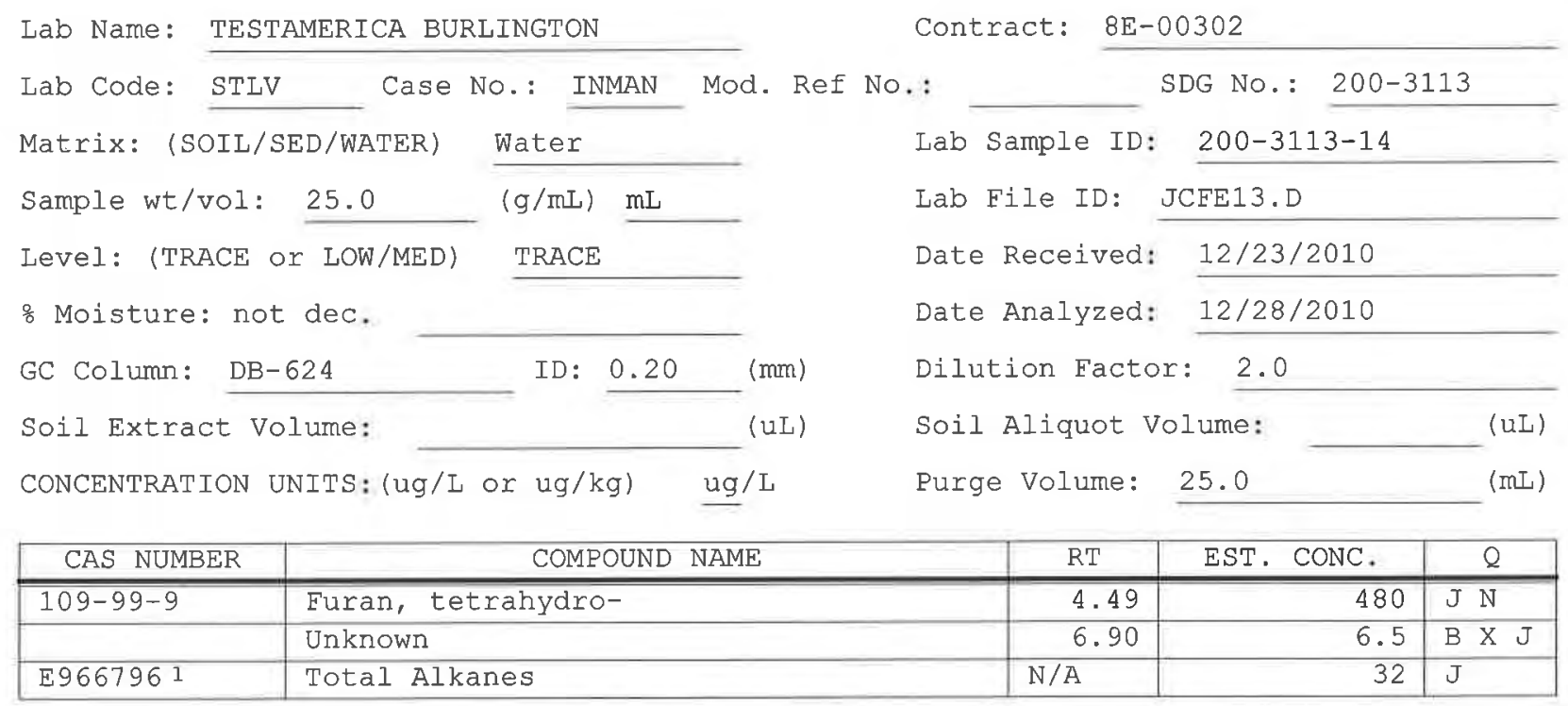

1 EPA-designated Registry Number. 
$1 A$ - FORM I VOA-1

VOLATILE ORGANICS ANALYSIS DATA SHEET
EPA SAMPLE NO.

INQCTB-W-32702

Lab Name: TESTAMERICA BURLINGTON

Contract: $8 \mathrm{E}-00302$

Lab Code: STLV

Case No.: INMAN Mod. Ref No.:

SDG No.: 200-3113

Matrix: (SOIL/SED/WATER) Water

Sample wt/vol: $25.0 \quad(\mathrm{~g} / \mathrm{mL}) \mathrm{mL}$

Level: (TRACE/LOW/MED) TRACE

\% Moisture: not dec.

GC Column: DB-624 ID: $0.20 \quad(\mathrm{~mm})$

Soil Extract Volume: (uL) (mL)

Purge Volume: 25.0
Lab Sample ID: 200-3113-18

Lab File ID: JCFE07.D

Date Received: 12/23/2010

Date Analyzed: 12/28/2010

Dilution Factor: 1.0

Soil Aliquot Volume:

(uL)

\begin{tabular}{|c|c|c|c|}
\hline CAS NO. & COMPOUND & $\begin{array}{l}\text { CONCENTRATION UNITS: } \\
(\mathrm{ug} / \mathrm{L} \text { or } \mathrm{ug} / \mathrm{kg}) \mathrm{ug} / \mathrm{L}\end{array}$ & $Q$ \\
\hline $75-71-8$ & Dichlorodifluoromethane & 0.50 & $\overline{\mathrm{U}}$ \\
\hline $74-87-3$ & Chloromethane & 0.50 & $\mathrm{U}$ \\
\hline $75-01-4$ & Vinyl chloride & 0.50 & $\mathrm{U}$ \\
\hline $74-83-9$ & Bromomethane & 0.50 & $\mathrm{U}$ \\
\hline $75-00-3$ & Chloroethane & 0.50 & $\mathrm{U}$ \\
\hline $75-69-4$ & Trichlorofluoromethane & 0.50 & $\mathrm{U}$ \\
\hline $75-35-4$ & 1,1-Dichloroethene & 0.50 & $\mathrm{U}$ \\
\hline $76-13-1$ & 1,1,2-Trichloro-1,2,2-trifluoroethane & 0.50 & $\mathrm{U}$ \\
\hline $67-64-1$ & Acetone & 2.8 & $\mathrm{~J} \mathrm{~B}$ \\
\hline $75-15-0$ & Carbon disulfide & 0.50 & $\mathrm{U}$ \\
\hline $79-20-9$ & Methyl acetate & 0.50 & $\mathrm{U}$ \\
\hline $75-09-2$ & Methylene Chloride & 0.50 & $\mathrm{U}$ \\
\hline $156-60-5$ & trans-1,2-Dichloroethene & 0.50 & $\mathrm{U}$ \\
\hline $1634-04-4$ & Methyl tert-butyl ether & 0.095 & $\mathrm{~J} B$ \\
\hline $75-34-3$ & 1,1-Dichloroethane & 0.50 & $\mathrm{U}$ \\
\hline $156-59-2$ & cis-1,2-Dichloroethene & 0.50 & $\mathrm{U}$ \\
\hline $78-93-3$ & 2-Butanone & 5.1 & \\
\hline $74-97-5$ & Bromochloromethane & 0.50 & $\mathrm{U}$ \\
\hline $67-66-3$ & Chloroform & 0.50 & $\mathrm{U}$ \\
\hline $71-55-6$ & 1,1,1-Trichloroethane & 0.50 & $\mathrm{U}$ \\
\hline $110-82-7$ & Cyclohexane & 0.50 & $\mathrm{U}$ \\
\hline $56-23-5$ & Carbon tetrachloride & 0.50 & $\mathrm{U}$ \\
\hline $71-43-2$ & Benzene & 0.50 & $\mathrm{U}$ \\
\hline $107-06-2$ & 1,2-Dichloroethane & 0.50 & $\mathrm{U}$ \\
\hline
\end{tabular}

Report 1,4-Dioxane for Low-Medium VOA analysis only 
Lab Name: TESTAMERICA BURLINGTON

Lab Code: STLV

Matrix: (SOIL/SED/WATER)

Sample wt/vol: 25.0

Level: (TRACE/LOW/MED)

TRACE

응 Moisture: not dec.

GC Column: DB-624

ID: 0.20 ( $\mathrm{mm})$

Soil Extract Volume:

Purge Volume: 25.0

(mL)
Contract: 8E-00302

SDG No.: 200-3113

Lab Sample ID: 200-3113-18

Lab File ID: JCFE07.D

Date Received: 12/23/2010

Date Analyzed: 12/28/2010

Dilution Factor: 1.0

Soil Aliquot Volume:

\begin{tabular}{|c|c|c|c|}
\hline CAS NO. & COMPOUND & $\begin{array}{l}\text { CONCENTRATION UNITS: } \\
(\mathrm{ug} / \mathrm{L} \text { or } \mathrm{ug} / \mathrm{kg}) \mathrm{ug} / \mathrm{L}\end{array}$ & $Q$ \\
\hline $79-01-6$ & Trichloroethene & 0.50 & $\mathrm{U}$ \\
\hline $108-87-2$ & Methylcyclohexane & 0.50 & $\mathrm{U}$ \\
\hline $78-87-5$ & 1,2-Dichloropropane & 0.50 & $\mathrm{U}$ \\
\hline $75-27-4$ & Bromodichloromethane & 0.50 & $\mathrm{U}$ \\
\hline $10061-01-5$ & cis-1,3-Dichloropropene & 0.50 & $\mathrm{U}$ \\
\hline $108-10-1$ & 4-Methyl-2-pentanone & 5.0 & $\mathrm{U}$ \\
\hline $108-88-3$ & Toluene & 0.13 & $\mathrm{~J}$ \\
\hline $10061-02-6$ & trans-1,3-Dichloropropene & 0.50 & $\mathrm{U}$ \\
\hline $79-00-5$ & 1,1,2-Trichloroethane & 0.50 & $\mathrm{U}$ \\
\hline $127-18-4$ & Tetrachloroethene & 0.50 & $\mathrm{U}$ \\
\hline $591-78-6$ & 2-Hexanone & 5.0 & $\mathrm{U}$ \\
\hline $124-48-1$ & Dibromochloromethane & 0.50 & $\mathrm{U}$ \\
\hline $106-93-4$ & 1,2-Dibromoethane & 0.50 & $\mathrm{U}$ \\
\hline $108-90-7$ & Chlorobenzene & 0.50 & $\mathrm{U}$ \\
\hline $100-41-4$ & Ethylbenzene & 0.50 & $\mathrm{U}$ \\
\hline $95-47-6$ & o-xylene & 0.052 & $\mathrm{~J}$ \\
\hline $179601-23-1$ & $\mathrm{~m}, \mathrm{p}$-Xylene & 0.052 & $\mathrm{~J}$ \\
\hline $100-42-5$ & styrene & 0.50 & $\mathrm{U}$ \\
\hline $75-25-2$ & Bromoform & 0.50 & $\mathrm{U}$ \\
\hline $98-82-8$ & Isopropylbenzene & 0.50 & $\mathrm{U}$ \\
\hline $79-34-5$ & $1,1,2,2$-Tetrachloroethane & 0.50 & $\mathrm{U}$ \\
\hline $541-73-1$ & 1,3-Dichlorobenzene & 0.50 & $\mathrm{U}$ \\
\hline $106-46-7$ & 1,4-Dichlorobenzene & 0.50 & $\mathrm{U}$ \\
\hline $95-50-1$ & 1,2-Dichlorobenzene & 0.50 & $\mathrm{U}$ \\
\hline $96-12-8$ & 1,2-Dibromo-3-Chloropropane & 0.50 & $\mathrm{U}$ \\
\hline $120-82-1$ & 1,2,4-Trichlorobenzene & 0.50 & $\mathrm{U}$ \\
\hline $87-61-6$ & 1,2,3-Trichlorobenzene & 0.50 & $\mathrm{U}$ \\
\hline
\end{tabular}


$1 \mathrm{~J}$ - FORM I VOA-TIC

VOLATILE ORGANICS ANALYSIS DATA SHEET

TENTATIVELY IDENTIFIED COMPOUNDS
EPA SAMPLE NO.

INQCTB-W-32702

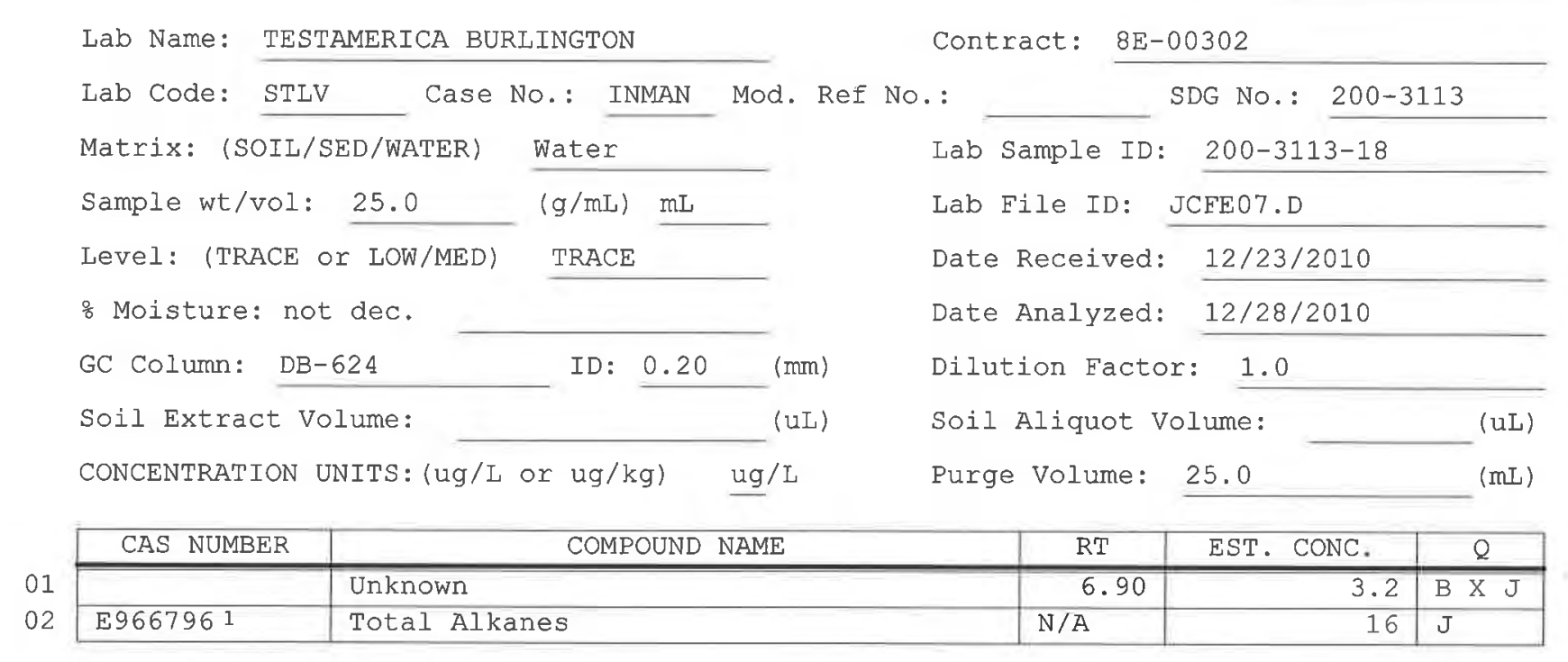

1 EPA-designated Registry Number. 
$1 A$ - FORM I VOA-1

VOLATILE ORGANICS ANALYSIS DATA SHEET
EPA SAMPLE NO.

INT122-W-32699
Lab Name: TESTAMERICA BURLINGTON

Lab Code: STLV Case

Matrix: (SOIL/SED/WATER)

Sample wt/vol: 25.0

Water

$(\mathrm{g} / \mathrm{mL}) \mathrm{mL}$

Level: (TRACE/LOW/MED) TRACE

o Moisture: not dec.

GC Column: DB-624

ID: 0.20

$(\mathrm{mm})$

Soil Extract Volume:

(uL)

(mL)
Contract: $8 \mathrm{E}-00302$

SDG No.: $200-3113$

Lab Sample ID: 200-3113-15

Lab File ID: JCFE04.D

Date Received: 12/23/2010

Date Analyzed: 12/28/2010

Dilution Factor: 1.0

Soil Aliquot Volume:

(uL)
CONCENTRATION UNITS: (ug/L or $u g / k g) ~ u g / L$
Q

\begin{tabular}{|c|c|c|c|}
\hline CAS NO. & COMPOUND & $\begin{array}{l}\text { CONCENTRATION UNITS: } \\
(\mathrm{ug} / \mathrm{L} \text { or } \mathrm{ug} / \mathrm{kg}) \mathrm{ug} / \mathrm{L}\end{array}$ & $Q$ \\
\hline $75-71-8$ & Dichlorodifluoromethane & 0.50 & $\mathrm{U}$ \\
\hline $74-87-3$ & Chloromethane & 0.50 & $\mathrm{U}$ \\
\hline $75-01-4$ & Vinyl chloride & 0.50 & $\mathrm{U}$ \\
\hline $74-83-9$ & Bromomethane & 0.50 & $\mathrm{U}$ \\
\hline $75-00-3$ & Chloroethane & 0.50 & $\mathrm{U}$ \\
\hline $75-69-4$ & Trichlorofluoromethane & 0.50 & $\mathrm{U}$ \\
\hline $75-35-4$ & 1,1-Dichloroethene & 0.50 & $\mathrm{U}$ \\
\hline $76-13-1$ & 1,1,2-Trichloro-1,2,2-trifluoroethane & 0.50 & $\mathrm{U}$ \\
\hline $67-64-1$ & Acetone & 9.9 & $\mathrm{~B}$ \\
\hline $75-15-0$ & Carbon disulfide & 0.13 & $\mathrm{~J} B$ \\
\hline $79-20-9$ & Methyl acetate & 0.50 & $\mathrm{U}$ \\
\hline $75-09-2$ & Methylene Chloride & 0.50 & $\mathrm{U}$ \\
\hline $156-60-5$ & trans-1,2-Dichloroethene & 0.50 & $\mathrm{U}$ \\
\hline $1634-04-4$ & Methyl tert-butyl ether & 0.50 & $\mathrm{U}$ \\
\hline $75-34-3$ & 1,1-Dichloroethane & 0.50 & $\mathrm{U}$ \\
\hline $156-59-2$ & cis-1,2-Dichloroethene & 0.50 & $\mathrm{U}$ \\
\hline $78-93-3$ & 2-Butanone & 76 & \\
\hline $74-97-5$ & Bromochloromethane & 0.50 & $\mathrm{U}$ \\
\hline $67-66-3$ & Chloroform & 0.50 & $\mathrm{U}$ \\
\hline $71-55-6$ & 1,1,1-Trichloroethane & 0.50 & $\mathrm{U}$ \\
\hline $110-82-7$ & Cyclohexane & 0.50 & $\mathrm{U}$ \\
\hline $56-23-5$ & Carbon tetrachloride & 0.050 & $\mathrm{~J}$ \\
\hline $71-43-2$ & Benzene & 0.50 & $\mathrm{U}$ \\
\hline $107-06-2$ & 1,2-Dichloroethane & 0.50 & $\mathrm{U}$ \\
\hline
\end{tabular}

Report 1,4-Dioxane for Low-Medium VOA analysis only 
Lab Name: TESTAMERICA BURLINGTON

Lab Code: STLV Case No.: INMAN Mod. Ref No.:

Contract: $8 \mathrm{E}-00302$

\section{Matrix: (SOIL/SED/WATER) Water}

Sample wt/vol: 25.0 $(\mathrm{g} / \mathrm{mL}) \quad \mathrm{mL}$

Level: (TRACE/LOW/MED) TRACE

응 Moisture: not dec.

GC Column: DB-624

Soil Extract Volume:

Purge Volume: 25.0
ID: $0.20 \quad(\mathrm{~mm})$

(uL)

(mL) SDG No.: 200-3113

Lab Sample ID: 200-3113-15

Lab File ID: JCFE04.D

Date Received: 12/23/2010

Date Analyzed: 12/28/2010

Dilution Factor: 1.0

Soil Aliquot volume:

(uL)

\begin{tabular}{|c|c|c|c|}
\hline CAS NO. & COMPOUND & $\begin{array}{l}\text { CONCENTRATION UNITS: } \\
(\mathrm{ug} / \mathrm{L} \text { or } \mathrm{ug} / \mathrm{kg}) \mathrm{ug} / \mathrm{L}\end{array}$ & $Q$ \\
\hline $79-01-6$ & Trichloroethene & 0.50 & $\mathrm{U}$ \\
\hline $108-87-2$ & Methylcyclohexane & 0.50 & $\mathrm{U}$ \\
\hline $78-87-5$ & 1,2-Dichloropropane & 0.50 & $\mathrm{U}$ \\
\hline $75-27-4$ & Bromodichloromethane & 0.50 & $\mathrm{U}$ \\
\hline $10061-01-5$ & cis-1,3-Dichloropropene & 0.50 & $\mathrm{U}$ \\
\hline $108-10-1$ & 4-Methyl-2-pentanone & 5.0 & $\mathrm{U}$ \\
\hline $108-88-3$ & Toluene & 0.50 & $\mathrm{U}$ \\
\hline $10061-02-6$ & trans-1,3-Dichloropropene & 0.50 & $\mathrm{U}$ \\
\hline $79-00-5$ & 1,1,2-Trichloroethane & 0.50 & $\mathrm{U}$ \\
\hline $127-18-4$ & Tetrachloroethene & 0.50 & $\mathrm{U}$ \\
\hline $591-78-6$ & 2-Hexanone & 5.0 & $\mathrm{U}$ \\
\hline $124-48-1$ & Dibromochloromethane & 0.50 & $\mathrm{U}$ \\
\hline $106-93-4$ & 1,2-Dibromoethane & 0.50 & $\mathrm{U}$ \\
\hline $108-90-7$ & Chlorobenzene & 0.50 & $\mathrm{U}$ \\
\hline $100-41-4$ & Ethylbenzene & 0.50 & $\mathrm{U}$ \\
\hline $95-47-6$ & o-Xylene & 0.50 & $\mathrm{U}$ \\
\hline $179601-23-1$ & m,p-Xylene & 0.50 & $\mathrm{U}$ \\
\hline $100-42-5$ & Styrene & 0.50 & $\mathrm{U}$ \\
\hline $75-25-2$ & Bromoform & 0.50 & $\mathrm{U}$ \\
\hline $98-82-8$ & Isopropylbenzene & 0.50 & $\mathrm{U}$ \\
\hline $79-34-5$ & $1,1,2,2$-Tetrachloroethane & 0.50 & $\mathrm{U}$ \\
\hline $541-73-1$ & 1,3-Dichlorobenzene & 0.50 & $\mathrm{U}$ \\
\hline $106-46-7$ & 1,4-Dichlorobenzene & 0.50 & $\mathrm{U}$ \\
\hline $95-50-1$ & 1,2-Dichlorobenzene & 0.50 & $\mathrm{U}$ \\
\hline $96-12-8$ & 1,2-Dibromo-3-Chloropropane & 0.50 & $\mathrm{U}$ \\
\hline $120-82-1$ & 1,2,4-Trichlorobenzene & 0.50 & $\mathrm{U}$ \\
\hline $87-61-6$ & 1,2,3-Trichlorobenzene & 0.50 & $\mathrm{U}$ \\
\hline
\end{tabular}


IJ - FORM I VOA-TIC

VOLATILE ORGANICS ANALYSIS DATA SHEET

TENTATIVELY IDENTIEIED COMPOUNDS
EPA SAMPLE NO.

INT122-W-32699

Lab Name: TESTAMERICA BURLINGTON

Contract: $8 \mathrm{E}-00302$

Lab Code: STLV Case No.: INMAN Mod. Ref No.: SDG No.: $200-3113$

Matrix: (SOIL/SED/WATER)

Water

Sample wt/vol: 25.0

Level: (TRACE or LOW/MED)

$(\mathrm{g} / \mathrm{mL}) \mathrm{mL}$

s Moisture: not dec.

GC Column: DB-624 ID: $0.20 \quad(\mathrm{~mm})$

Soil Extract volume: (uL)
Lab Sample ID: 200-3113-15

Lab File ID: JCFE04.D

Date Received: 12/23/2010

Date Analyzed: 12/28/2010

Dilution Factor: 1.0

Soil Aliquot Volume: (uL)

Purge Volume: 25.0 (mL)

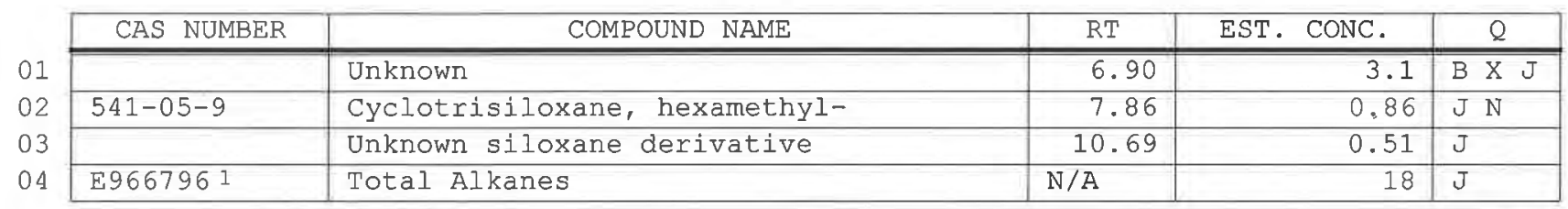

1 EPA-designated Registry Number. 
$1 A$ - FORM I VOA-1

VOLATILE ORGANICS ANALYSIS DATA SHEET
EPA SAMPLE NO.

INT123-W-32700

Lab Name: TESTAMERICA BURLINGTON

Contract: $8 \mathrm{E}-00302$

Lab Code: STLV Case No.: INMAN Mod. Ref No.:

SDG No.: 200-3113

Matrix: (SOIL/SED/WATER)

Water

Sample wt/vol: 25.0

$(\mathrm{g} / \mathrm{mL}) \mathrm{mL}$

Level: (TRACE/LOW/MED) TRACE

o Moisture: not dec.

GC Column: DB-624 ID: $0.20 \quad$ (mm)

Soil Extract Volume:

(uL)

Purge Volume: 25.0

(mL)
Lab Sample ID: 200-3113-16

Lab File ID: JCFE05.D

Date Received: 12/23/2010

Date Analyzed: 12/28/2010

Dilution Factor: 1.0

Soil Aliquot Volume:

(uL)

\begin{tabular}{|c|c|c|c|}
\hline CAS NO. & COMPOUND & $\begin{array}{l}\text { CONCENTRATION UNITS: } \\
(\mathrm{ug} / \mathrm{L} \text { or } \mathrm{ug} / \mathrm{kg}) \mathrm{ug} / \mathrm{L}\end{array}$ & s \\
\hline $75-71-8$ & Dichlorodifluoromethane & 0.50 & $\mathrm{U}$ \\
\hline $74-87-3$ & Chloromethane & 0.50 & $\mathrm{U}$ \\
\hline $75-01-4$ & Vinyl chloride & 0.50 & $\mathrm{U}$ \\
\hline $74-83-9$ & Bromomethane & 0.50 & $\mathrm{U}$ \\
\hline $75-00-3$ & Chloroethane & 0.50 & $\mathrm{U}$ \\
\hline $75-69-4$ & Trichlorofluoromethane & 0.50 & $\mathrm{U}$ \\
\hline $75-35-4$ & 1,1-Dichloroethene & 0.50 & $\mathrm{U}$ \\
\hline $76-13-1$ & 1,1,2-Trichloro-1,2,2-trifluoroethane & 0.50 & $\mathrm{U}$ \\
\hline $67-64-1$ & Acetone & 220 & $\mathrm{E} \mathrm{B}$ \\
\hline $75-15-0$ & Carbon disulfide & 0.11 & $\mathrm{~J} \mathrm{~B}$ \\
\hline $79-20-9$ & Methyl acetate & 0.50 & $\mathrm{U}$ \\
\hline $75-09-2$ & Methylene Chloride & 0.50 & $\mathrm{U}$ \\
\hline $156-60-5$ & trans-1,2-Dichloroethene & 0.50 & $\mathrm{U}$ \\
\hline $1634-04-4$ & Methyl tert-butyl ether & 0.12 & $\mathrm{JB}$ \\
\hline $75-34-3$ & 1,1-Dichloroethane & 0.50 & $\mathrm{U}$ \\
\hline $156-59-2$ & cis-1,2-Dichloroethene & 0.50 & $\mathrm{U}$ \\
\hline $78-93-3$ & 2-Butanone & 1600 & $\mathrm{E}$ \\
\hline $74-97-5$ & Bromochloromethane & 0.50 & $\mathrm{U}$ \\
\hline $67-66-3$ & Chloroform & 0.50 & $\mathrm{U}$ \\
\hline $71-55-6$ & 1,1,1-Trichloroethane & 0.50 & $\mathrm{U}$ \\
\hline $110-82-7$ & Cyclohexane & 0.50 & $\mathrm{U}$ \\
\hline $56-23-5$ & Carbon tetrachloride & 0.041 & $\mathrm{~J}$ \\
\hline $71-43-2$ & Benzene & 0.50 & $\mathrm{U}$ \\
\hline $107-06-2$ & 1,2-Dichloroethane & 0.50 & $\mathrm{U}$ \\
\hline
\end{tabular}

Report 1,4-Dioxane for Low-Medium VOA analysis only 
Lab Name: TESTAMERICA BURLINGTON

Lab Code: STLV Matrix: (SOIL/SED/WATER) Case No.: INMAN Mod. Ref No.:

Sample wt/vol: 25.0

Water

$(\mathrm{g} / \mathrm{mL}) \mathrm{mL}$

Level: (TRACE/LOW/MED) TRACE

응 Moisture: not dec.

GC Column: DB-624 ID: $0.20 \quad(\mathrm{~mm})$

Soil Extract Volume:

Purge Volume:

25.0
Contract: $8 \mathrm{E}-00302$

SDG NO.: 200-3113
Lab Sample ID: 200-3113-16

Lab File ID: JCFE05.D

Date Received: 12/23/2010

Date Analyzed: 12/28/2010

Dilution Factor: 1.0

Soil Aliquot volume: (uL)

\begin{tabular}{|c|c|c|c|}
\hline CAS NO. & COMPOUND & $\begin{array}{l}\text { CONCENTRATION UNITS: } \\
(\mathrm{ug} / \mathrm{L} \text { or } \mathrm{ug} / \mathrm{kg}) \mathrm{ug} / \mathrm{L}\end{array}$ & Q \\
\hline $79-01-6$ & Trichloroethene & 0.50 & $\overline{\mathrm{U}}$ \\
\hline $108-87-2$ & Methylcyclohexane & 0.50 & $\mathrm{U}$ \\
\hline $78-87-5$ & 1,2-Dichloropropane & 0.50 & $\mathrm{U}$ \\
\hline $75-27-4$ & Bromodichloromethane & 0.50 & $\mathrm{U}$ \\
\hline $10061-01-5$ & cis-1,3-Dichloropropene & 0.50 & $\mathrm{U}$ \\
\hline $108-10-1$ & 4-Methyl-2-pentanone & 5.0 & $\mathrm{U}$ \\
\hline $108-88-3$ & Toluene & 0.50 & $\mathrm{U}$ \\
\hline $10061-02-6$ & trans-1,3-Dichloropropene & 0.50 & $\mathrm{U}$ \\
\hline $79-00-5$ & 1,1,2-Trichloroethane & 0.50 & $\mathrm{U}$ \\
\hline $127-18-4$ & Tetrachloroethene & 0.50 & $\mathrm{U}$ \\
\hline $591-78-6$ & 2-Hexanone & 5.0 & $\mathrm{U}$ \\
\hline $124-48-1$ & Dibromochloromethane & 0.50 & $\mathrm{U}$ \\
\hline $106-93-4$ & 1,2-Dibromoethane & 0.50 & $\mathrm{U}$ \\
\hline $108-90-7$ & Chlorobenzene & 0.50 & $\mathrm{U}$ \\
\hline $100-41-4$ & Ethylbenzene & 0.50 & $\mathrm{U}$ \\
\hline $95-47-6$ & o-Xylene & 0.50 & $\mathrm{U}$ \\
\hline $179601-23-1$ & m,p-xylene & 0.50 & $\mathrm{U}$ \\
\hline $100-42-5$ & Styrene & 0.50 & $\mathrm{U}$ \\
\hline $75-25-2$ & Bromoform & 0.50 & $\mathrm{U}$ \\
\hline $98-82-8$ & Isopropylbenzene & 0.50 & $\mathrm{U}$ \\
\hline $79-34-5$ & $1,1,2,2$-Tetrachloroethane & 0.50 & $\mathrm{U}$ \\
\hline $541-73-1$ & 1,3-Dichlorobenzene & 0.50 & $\mathrm{U}$ \\
\hline $106-46-7$ & 1,4-Dichlorobenzene & 0.50 & $\mathrm{U}$ \\
\hline $95-50-1$ & 1,2-Dichlorobenzene & 0.50 & $\mathrm{U}$ \\
\hline $96-12-8$ & 1,2-Dibromo-3-Chloropropane & 0.50 & $\mathrm{U}$ \\
\hline $120-82-1$ & 1,2,4-Trichlorobenzene & 0.50 & $\mathrm{U}$ \\
\hline $87-61-6$ & 1,2,3-Trichlorobenzene & 0.50 & $\mathrm{U}$ \\
\hline
\end{tabular}


IJ - FORM I VOA-TIC

VOLATILE ORGANICS ANALYSIS DATA SHEET

TENTATIVELY IDENTIFIED COMPOUNDS
EPA SAMPLE NO.

INT $123-W-32700$

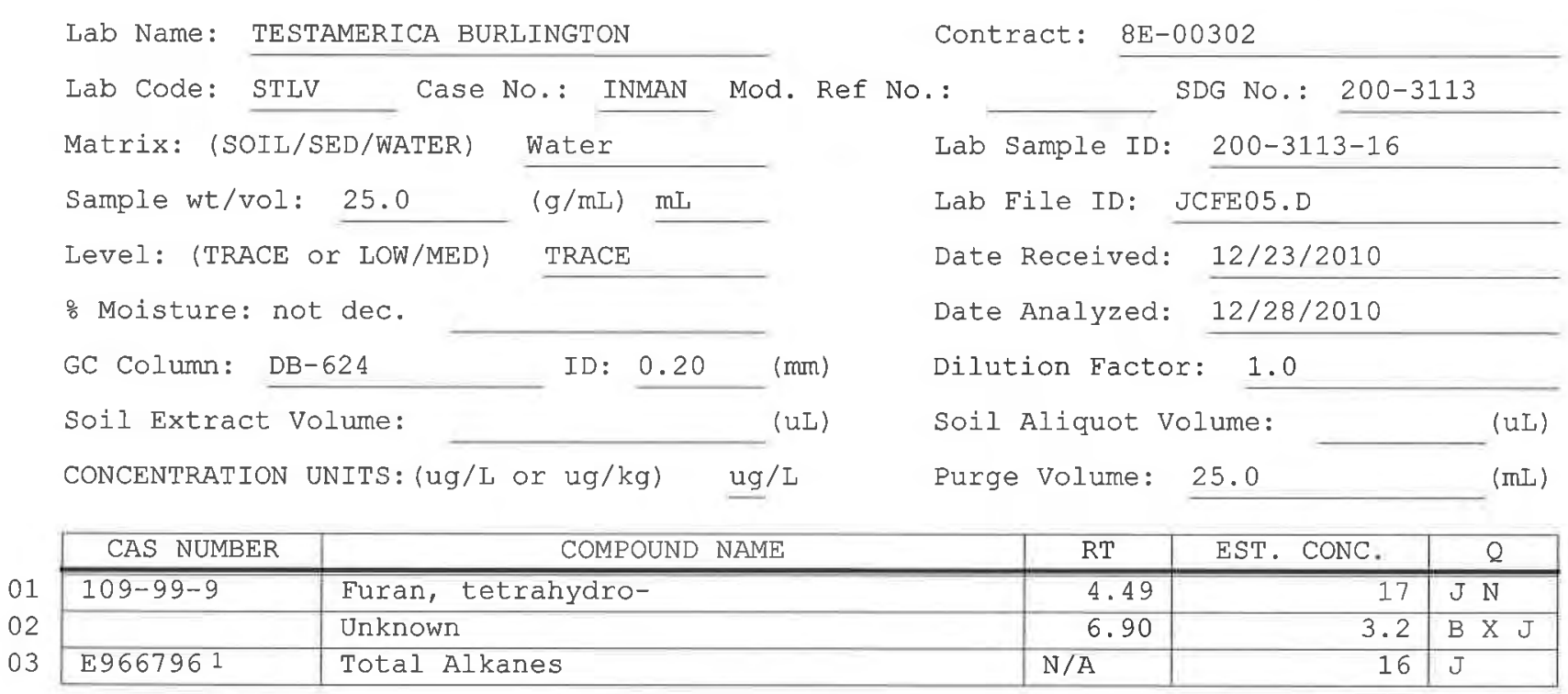

1 EPA-designated Registry Number. 
$1 A$ - FORM I VOA-1

VOLATILE ORGANICS ANALYSIS DATA SHEET
EPA SAMPLE NO. INT125-W-32701
Lab Name: TESTAMERICA BURLINGTON

Lab Code: STLV Case No.: INMAN Mod. Ref No.:

Matrix: (SOIL/SED/WATER) Water

Sample wt/vol: 25.0

$(\mathrm{g} / \mathrm{mL}) \mathrm{mL}$

Level: (TRACE/LOW/MED) TRACE

\% Moisture: not dec.

GC Column: DB-624

ID: 0.20 (mm)

Soil Extract Volume:

(uL) $(m L)$

Purge Volume: 25.0

(1)
Contract: $\quad 8 \mathrm{E}-00302$

SDG No.: $200-3113$

Lab Sample ID: 200-3113-17

Lab File ID: JCFEO6.D

Date Received: 12/23/2010

Date Analyzed: 12/28/2010

Dilution Factor: 1.0

Soil Aliquot Volume:

(uL)

\begin{tabular}{|c|c|c|c|}
\hline CAS NO. & COMPOUND & $\begin{array}{l}\text { CONCENTRATION UNITS: } \\
(\mathrm{ug} / \mathrm{L} \text { or } \mathrm{ug} / \mathrm{kg}) \mathrm{ug} / \mathrm{L}\end{array}$ & c \\
\hline $75-71-8$ & Dichlorodifluoromethane & 0.50 & $\mathrm{U}$ \\
\hline $74-87-3$ & Chloromethane & 0.50 & $\mathrm{U}$ \\
\hline $75-01-4$ & Vinyl chloride & 0.50 & $\mathrm{U}$ \\
\hline $74-83-9$ & Bromomethane & 0.50 & $\mathrm{U}$ \\
\hline $75-00-3$ & Chloroethane & 0.50 & $\mathrm{U}$ \\
\hline $75-69-4$ & Trichlorofluoromethane & 0.50 & $\mathrm{U}$ \\
\hline $75-35-4$ & 1,1-Dichloroethene & 0.50 & $\mathrm{U}$ \\
\hline $76-13-1$ & 1,1,2-Trichloro-1,2,2-trifluoroethane & 0.50 & $\mathrm{U}$ \\
\hline $67-64-1$ & Acetone & 7.6 & $\mathrm{~B}$ \\
\hline $75-15-0$ & Carbon disulfide & 0.50 & $\mathrm{U}$ \\
\hline $79-20-9$ & Methyl acetate & 0.50 & $\mathrm{U}$ \\
\hline $75-09-2$ & Methylene Chloride & 0.50 & $\mathrm{U}$ \\
\hline $156-60-5$ & trans-1,2-Dichloroethene & 0.50 & $\mathrm{U}$ \\
\hline $1634-04-4$ & Methyl tert-butyl ether & 0.093 & $\mathrm{~J} B$ \\
\hline $75-34-3$ & 1,1-Dichloroethane & 0.50 & $\mathrm{U}$ \\
\hline $156-59-2$ & cis-1,2-Dichloroethene & 0.50 & U \\
\hline $78-93-3$ & 2-Butanone & 61 & \\
\hline $74-97-5$ & Bromochloromethane & 0.50 & $\mathrm{U}$ \\
\hline $67-66-3$ & Chloroform & 0.50 & $\mathrm{U}$ \\
\hline $71-55-6$ & 1,1,1-Trichloroethane & 0.50 & $\mathrm{U}$ \\
\hline $110-82-7$ & Cyclohexane & 0.50 & $\mathrm{U}$ \\
\hline $56-23-5$ & Carbon tetrachloride & 0.27 & $\mathrm{~J}$ \\
\hline $71-43-2$ & Benzene & 0.50 & $\mathrm{U}$ \\
\hline $107-06-2$ & 1,2-Dichloroethane & 0.50 & $\mathrm{U}$ \\
\hline
\end{tabular}

Report 1,4-Dioxane for Low-Medium VOA analysis only 
Lab Name: TESTAMERICA BURLINGTON

Lab Code: STLV

Matrix: (SOIL/SED/WATER)

Sample wt/vol: 25.0

$(\mathrm{g} / \mathrm{mL}) \mathrm{mL}$

Level: (TRACE/LOW/MED) TRACE

g Moisture: not dec.

GC Column: DB-624 ID: $0.20 \quad$ (mm)

Soil Extract Volume:

(uL)

(mL)

Purge Volume: 25.0
Contract: $8 E-00302$ SDG No.: 200-3113

\section{. :}

Lab Sample ID: 200-3113-17

Lab File ID: JCFE06.D

Date Received: 12/23/2010

Date Analyzed: 12/28/2010

Dilution Factor: 1.0

Soil Aliquot Volume:

(uL)

\begin{tabular}{|c|c|c|c|}
\hline CAS NO. & COMPOUND & $\begin{array}{l}\text { CONCENTRATION UNITS: } \\
(\mathrm{ug} / \mathrm{L} \text { or } \mathrm{ug} / \mathrm{kg}) \mathrm{ug} / \mathrm{L}\end{array}$ & $Q$ \\
\hline $79-01-6$ & Trichloroethene & 0.50 & $\overline{\mathrm{U}}$ \\
\hline $108-87-2$ & Methylcyclohexane & 0.50 & $\mathrm{U}$ \\
\hline $78-87-5$ & 1,2-Dichloropropane & 0.50 & $\mathrm{U}$ \\
\hline $75-27-4$ & Bromodichloromethane & 0.50 & $\mathrm{U}$ \\
\hline $10061-01-5$ & cis-1,3-Dichloropropene & 0.50 & $\mathrm{U}$ \\
\hline $108-10-1$ & 4-Methyl-2-pentanone & 5.0 & $\mathrm{U}$ \\
\hline $108-88-3$ & Toluene & 0.50 & $\mathrm{U}$ \\
\hline $10061-02-6$ & trans-1,3-Dichloropropene & 0.50 & $\mathrm{U}$ \\
\hline $79-00-5$ & 1,1,2-Trichloroethane & 0.50 & $\mathrm{U}$ \\
\hline $127-18-4$ & Tetrachloroethene & 0.50 & $\mathrm{U}$ \\
\hline $591-78-6$ & 2-Hexanone & 5.0 & $\mathrm{U}$ \\
\hline $124-48-1$ & Dibromochloromethane & 0.50 & $\mathrm{U}$ \\
\hline $106-93-4$ & 1,2-Dibromoethane & 0.50 & $\mathrm{U}$ \\
\hline $108-90-7$ & Chlorobenzene & 0.50 & $\mathrm{U}$ \\
\hline $100-41-4$ & Ethylbenzene & 0.50 & $\mathrm{U}$ \\
\hline $95-47-6$ & o-Xylene & 0.50 & $\mathrm{U}$ \\
\hline $179601-23-1$ & $\mathrm{~m}, \mathrm{p}$-Xylene & 0.50 & $\mathrm{U}$ \\
\hline $100-42-5$ & Styrene & 0.50 & $\mathrm{U}$ \\
\hline $75-25-2$ & Bromoform & 0.50 & $\mathrm{U}$ \\
\hline $98-82-8$ & Isopropylbenzene & 0.50 & $\mathrm{U}$ \\
\hline $79-34-5$ & $1,1,2,2$-Tetrachloroethane & 0.50 & $\mathrm{U}$ \\
\hline $541-73-1$ & 1,3-Dichlorobenzene & 0.50 & $\mathrm{U}$ \\
\hline $106-46-7$ & 1,4-Dichlorobenzene & 0.50 & $\mathrm{U}$ \\
\hline $95-50-1$ & 1,2-Dichlorobenzene & 0.50 & $\mathrm{U}$ \\
\hline $96-12-8$ & 1,2-Dibromo-3-Chloropropane & 0.50 & $\mathrm{U}$ \\
\hline $120-82-1$ & 1,2,4-Trichlorobenzene & 0.50 & $\mathrm{U}$ \\
\hline $87-61-6$ & 1,2,3-Trichlorobenzene & 0.50 & $\mathrm{U}$ \\
\hline
\end{tabular}


$1 \mathrm{~J}$ - FORM I VOA-TIC

VOLATILE ORGANICS ANALYSIS DATA SHEET

TENTATIVELY IDENTIFIED COMPOUNDS
EPA SAMPLE NO.

INT125-W-32701

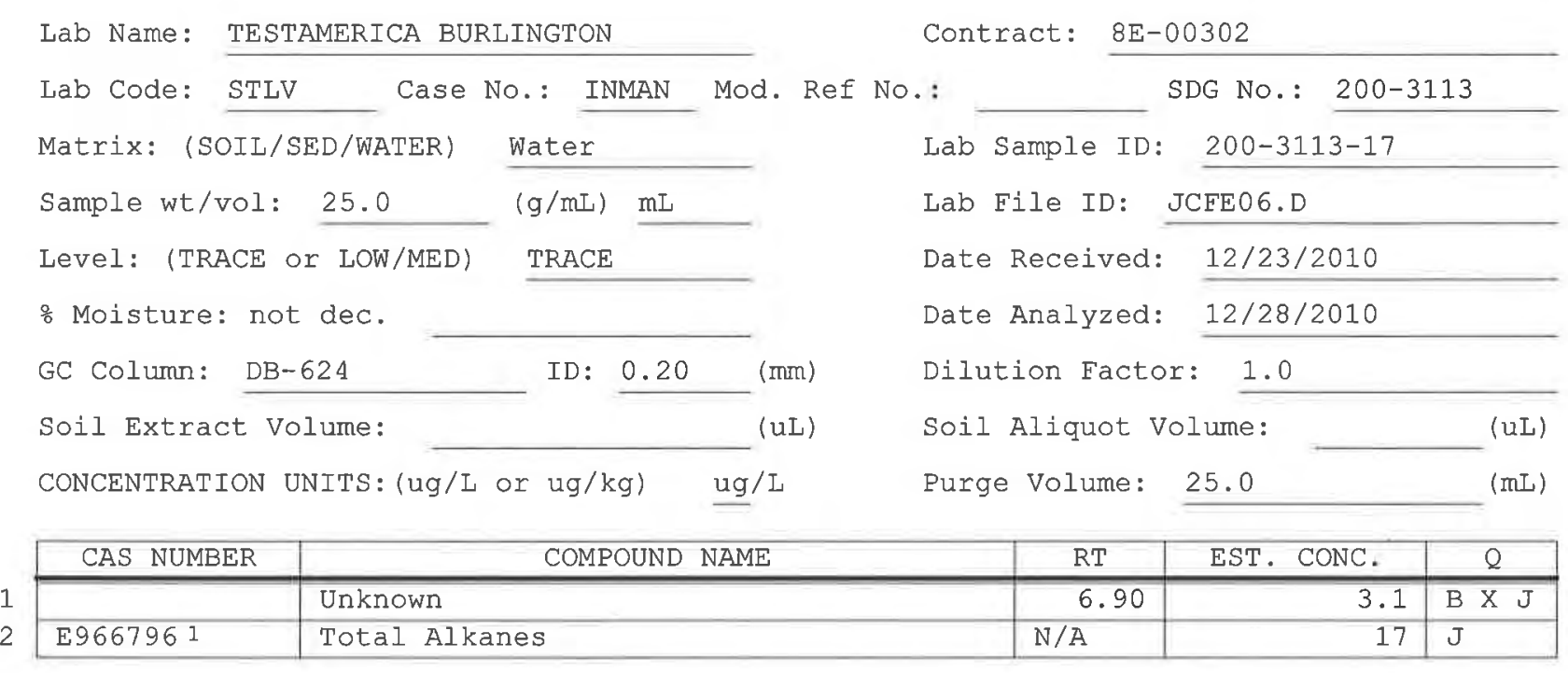

1EPA-designated Registry Number. 
$6 A$ - FORM VI VOA-1

VOLATILE ORGANICS INITIAL CALIBRATION DATA

Lab Name: TESTAMERICA BURLINGTON

Contract: $8 \mathrm{E}-00302$

Lab Code: STLV Case No.: INMAN Mod. Ref No.:

SDG No.: 200-3113

Instrument ID: J.i

Calibration Date(s): 12/23/2010 12/23/2010

Heated Purge: $(\mathrm{Y} / \mathrm{N}) \mathrm{N}$

Calibration Time(s): 1236

1417

Purge Volume: 25.0

(mL)

GC Column: DB-624

ID: $0.20 \quad$ (mm) Length: 25

(m)

\begin{tabular}{|c|c|c|c|c|c|c|c|}
\hline \multirow{3}{*}{$\begin{array}{l}\text { LAB FILE ID: } \\
\text { RRF } 5.0=\text { JCF05.D } \\
\text { COMPOUND }\end{array}$} & \multirow{2}{*}{\multicolumn{3}{|c|}{$\begin{array}{l}\text { RRF 0.5 }=\text { JCF03.D } \\
\operatorname{RRF10}=\text { JCF06.D }\end{array}$}} & \multirow{2}{*}{\multicolumn{4}{|c|}{$\begin{array}{l}\text { RRF 1.0 }=\text { JCF04.D } \\
\text { RRF 20 }=J C F 07 . D\end{array}$}} \\
\hline & & & & & & & \\
\hline & RRF 0.5 & RRE 1.0 & RRF 5.0 & RRF 10 & RRE 20 & $\overline{\mathrm{RRF}}$ & $\%$ RSD \\
\hline Dichlorodifluoromethane & 0.380 & 0.377 & 0.358 & 0.335 & 0.319 & 0.354 & $\overline{7.4}$ \\
\hline Chloromethane & 0.372 & 0.412 & 0.379 & 0.347 & 0.338 & 0.369 & 7.9 \\
\hline Vinyl chloride & 0.403 & 0.403 & 0.375 & 0.374 & 0.353 & 0.382 & 5.6 \\
\hline Bromomethane & 0.213 & 0.195 & 0.196 & 0.198 & 0.191 & 0.199 & 4.3 \\
\hline Chloroethane & 0.203 & 0.281 & 0.226 & 0.239 & 0.225 & 0.235 & 12.3 \\
\hline Trichlorofluoromethane & 0.561 & 0.570 & 0.562 & 0.555 & 0.554 & 0.561 & 1.2 \\
\hline 1,1-Dichloroethene & 0.300 & 0.306 & 0.277 & 0.277 & 0.271 & 0.286 & 5.4 \\
\hline $\begin{array}{l}1,1,2 \text {-Trichloro- } \\
1,2,2 \text {-trifluoroethane }\end{array}$ & 0.342 & 0.361 & 0.337 & 0.324 & 0.316 & 0.336 & 5.2 \\
\hline Acetone & 0.025 & 0.024 & 0.016 & 0.016 & 0.015 & 0.019 & 25.4 \\
\hline Carbon disulfide & 1.036 & 0.887 & 0.808 & 0.803 & 0.768 & 0.860 & 12.5 \\
\hline Methyl acetate & 0.060 & 0.044 & 0.045 & 0.039 & 0.039 & 0.045 & 19.2 \\
\hline Methylene Chloride & 0.248 & 0.245 & 0.238 & 0.234 & 0.221 & 0.237 & 4.5 \\
\hline trans-1,2-Dichloroethene & 0.353 & 0.322 & 0.298 & 0.297 & 0.280 & 0.310 & 9.1 \\
\hline Methyl tert-butyl ether & 0.333 & 0.364 & 0.345 & 0.334 & 0.334 & 0.342 & 3.8 \\
\hline 1,1-Dichloroethane & 0.520 & 0.517 & 0.503 & 0.479 & 0.465 & 0.497 & 4.8 \\
\hline cis-1,2-Dichloroethene & 0.307 & 0.279 & 0.284 & 0.282 & 0.278 & 0.286 & 4.3 \\
\hline 2-Butanone & 0.028 & 0.021 & 0.024 & 0.023 & 0.023 & 0.024 & 10.0 \\
\hline Bromochloromethane & 0.105 & 0.107 & 0.098 & 0.092 & 0.094 & 0.099 & 6.8 \\
\hline Chloroform & 0.495 & 0.498 & 0.491 & 0.471 & 0.464 & 0.484 & 3.2 \\
\hline 1,1,1-Trichloroethane & 0.617 & 0.643 & 0.622 & 0.597 & 0.590 & 0.614 & 3.4 \\
\hline Cyclohexane & 0.603 & 0.581 & 0.629 & 0.616 & 0.594 & 0.605 & 3.1 \\
\hline Carbon tetrachloride & 0.544 & 0.583 & 0.589 & 0.568 & 0.558 & 0.568 & 3.3 \\
\hline Benzene & 1.431 & 1.379 & 1.496 & 1.444 & 1.409 & 1.432 & 3.0 \\
\hline 1,2-Dichloroethane & 0.215 & 0.230 & 0.207 & 0.206 & 0.191 & 0.210 & 6.9 \\
\hline Trichloroethene & 0.416 & 0.374 & 0.389 & 0.373 & 0.371 & 0.385 & 4.9 \\
\hline Methylcyclohexane & 0.449 & 0.436 & 0.480 & 0.483 & 0.460 & 0.462 & 4.3 \\
\hline
\end{tabular}

Report 1,4-Dioxane for Low-Medium VOA analysis only 
$6 \mathrm{~B}$ - FORM VI VOA-2

VOLATILE ORGANICS INITIAL CALIBRATION DATA

Lab Name: TESTAMERICA BURLINGTON

Contract: $8 \mathrm{E}-00302$

Lab Code: STLV Case No.: INMAN Mod. Ref No.:

SDG NO.: 200-3113

Instrument ID: J.i

Calibration Date(s): 12/23/2010 12/23/2010

Heated Purge: $(\mathrm{Y} / \mathrm{N}) \mathrm{N}$

Calibration Time (s): 1236

1417

Purge Volume: 25.0

(mL)

GC Column: DB-624

ID: 0.20

(mm)

Length: 25

(m)

\begin{tabular}{|c|c|c|c|c|c|c|c|}
\hline \multirow{2}{*}{$\begin{array}{l}\text { LAB FILE ID: } \\
\text { RRF } 5.0=\text { JCF05.D }\end{array}$} & \multicolumn{3}{|c|}{$\mathrm{RRF} 0.5=\mathrm{JCF} 03 . \mathrm{D}$} & \multicolumn{4}{|c|}{$\mathrm{RRE} 1.0=\mathrm{JCF} 04 . \mathrm{D}$} \\
\hline & $\operatorname{RRF} 10=$ & CF06.D & & RRF 20 & $=\mathrm{JCE}$ &. $\mathrm{D}$ & \\
\hline COMPOUND & RRF 0.5 & $\mathrm{RRF} 1.0$ & RRE 5.0 & RRE 10 & RRE 20 & $\overline{R R F}$ & RSD \\
\hline 1,2-Dichloropropane & 0.228 & 0.295 & 0.300 & 0.276 & 0.275 & 0.275 & 10.3 \\
\hline Bromodichloromethane & 0.375 & 0.364 & 0.374 & 0.354 & 0.344 & 0.362 & 3.7 \\
\hline cis-1,3-Dichloropropene & 0.390 & 0.409 & 0.424 & 0.410 & 0.412 & 0.409 & 2.9 \\
\hline 4-Methyl-2-pentanone & 0.065 & 0.069 & 0.076 & 0.072 & 0.071 & 0.071 & 5.9 \\
\hline Toluene & 1.460 & 1.511 & 1.616 & 1.581 & 1.558 & 1.545 & 4.0 \\
\hline trans-1,3-Dichloropropene & 0.272 & 0.264 & 0.295 & 0.296 & 0.298 & 0.285 & 5.4 \\
\hline 1,1,2-Trichloroethane & 0.160 & 0.170 & 0.159 & 0.146 & 0.145 & 0.156 & 6.6 \\
\hline Tetrachloroethene & 0.345 & 0.334 & 0.341 & 0.351 & 0.357 & 0.345 & 2.5 \\
\hline 2-Hexanone & 0.045 & 0.046 & 0.050 & 0.047 & 0.047 & 0.047 & 4.1 \\
\hline Dibromochloromethane & 0.192 & 0.190 & 0.210 & 0.207 & 0.207 & 0.201 & 4.8 \\
\hline 1,2-Dibromoethane & 0.127 & 0.141 & 0.139 & 0.135 & 0.135 & 0.135 & 3.9 \\
\hline Chlorobenzene & 0.952 & 0.935 & 0.952 & 0.951 & 0.939 & 0.946 & 0.9 \\
\hline Ethylbenzene & 1.735 & 1.691 & 1.859 & 1.826 & 1.828 & 1.788 & 4.0 \\
\hline o-Xylene & 0.580 & 0.630 & 0.695 & 0.685 & 0.683 & 0.654 & 7.4 \\
\hline $\mathrm{m}, \mathrm{p}$-Xylene & 0.621 & 0.631 & 0.766 & 0.736 & 0.736 & 0.698 & 9.6 \\
\hline Styrene & 0.834 & 0.915 & 1.060 & 1.030 & 1.050 & 0.978 & 10.2 \\
\hline Bromoform & 0.121 & 0.176 & 0.193 & 0.196 & 0.193 & 0.176 & 18.0 \\
\hline Isopropylbenzene & 1.640 & 1.657 & 1.924 & 1.925 & 1.949 & 1.819 & 8.6 \\
\hline $1,1,2,2$-Tetrachloroethane & 0.128 & 0.149 & 0.147 & 0.132 & 0.136 & 0.139 & 6.6 \\
\hline 1,3-Dichlorobenzene & 1.371 & 1.539 & 1.485 & 1.481 & 1.467 & 1.469 & 4.2 \\
\hline 1,4-Dichlorobenzene & 1.519 & 1.556 & 1.488 & 1.465 & 1.415 & 1.488 & 3.6 \\
\hline 1,2-Dichlorobenzene & 1.204 & 1.229 & 1.226 & 1.179 & 1.154 & 1.198 & 2.7 \\
\hline 1,2-Dibromo-3-Chloropropane & 0.034 & 0.034 & 0.044 & 0.039 & 0.037 & 0.038 & 11.4 \\
\hline 1,2,4-Trichlorobenzene & 0.670 & 0.662 & 0.726 & 0.723 & 0.765 & 0.709 & 6.1 \\
\hline 1,2,3-Trichlorobenzene & 0.481 & 0.490 & 0.502 & 0.493 & 0.533 & 0.500 & 4.0 \\
\hline
\end{tabular}


$6 \mathrm{C}-$ FORM VI VOA-3

VOLATILE ORGANICS INITIAL CALIBRATION DATA

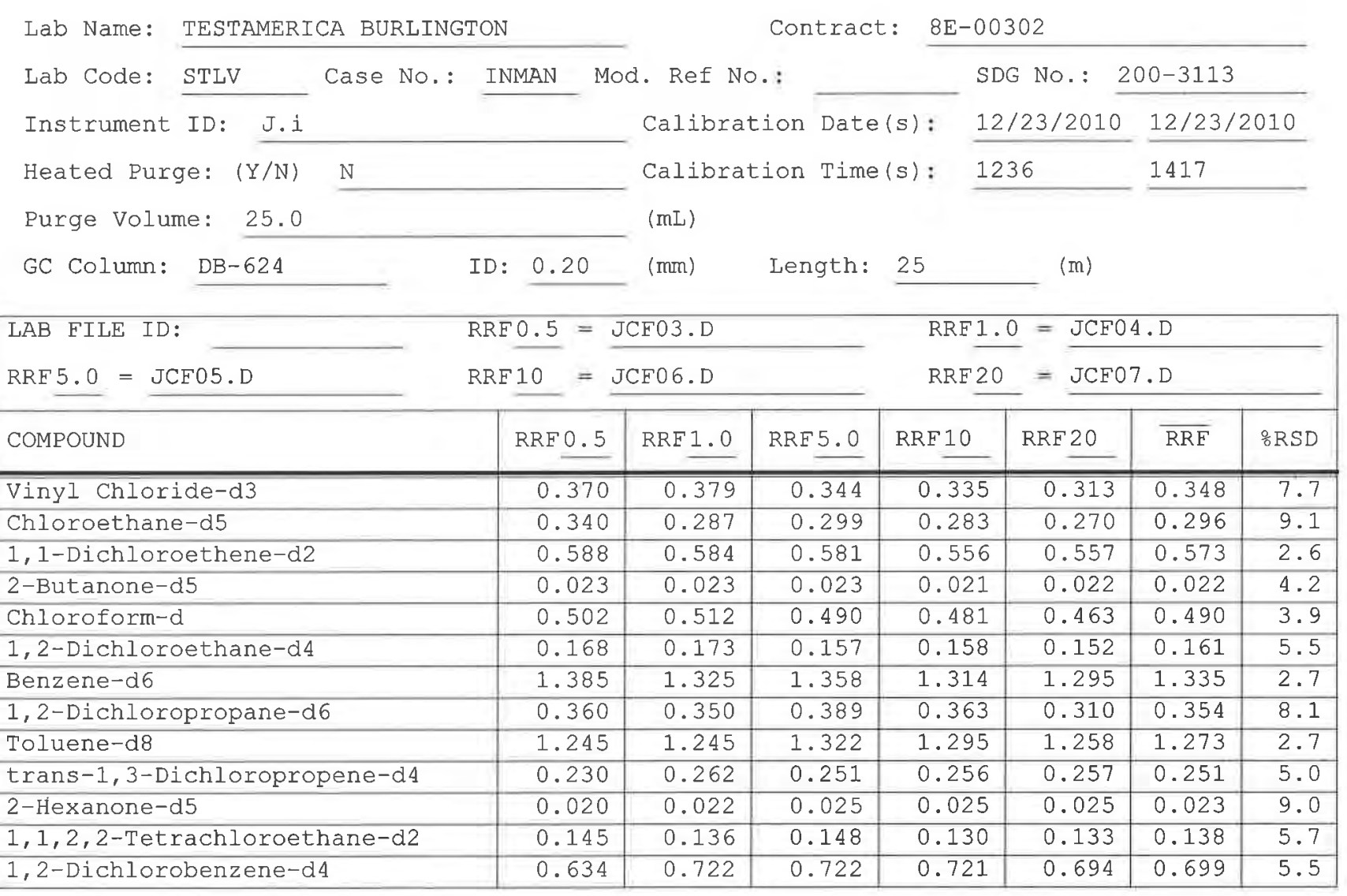

Report 1,4-Dioxane-d8 for Low-Medium VOA analysis only 
$7 A$ - FORM VII VOA-1

VOLATILE CONTINUING CALIBRATION DATA

Lab Name: TESTAMERICA BURLINGTON

Contract: $8 E-00302$

Lab Code: STLV Case No.: INMAN Mod. Ref No.:

SDG No.: $\quad 200-3113$

Instrument ID: J.i

Calibration Date: 12/24/2010 Time: 1104

Lab File Id: JCFBO4.D Init. Calib. Date(s): 12/23/2010 12/23/2010

EPA Sample No. (VSTD\#\#\#\#): VSTD005JF

Init. Calib. Time(s): $1236 \quad 1417$

Heated Purge: $(\mathrm{Y} / \mathrm{N}) \mathrm{N}$ GC Colunn: DB-624 ID: $0.20(\mathrm{~mm})$ Length: 25 $(\mathrm{m})$

Purge Volume: 25.0 ( $m L)$

\begin{tabular}{|c|c|c|c|c|c|}
\hline COMPOUND & $\overline{\mathrm{RRF}}$ & RRF5.0 & $\begin{array}{l}\text { MIN } \\
\text { RRE }\end{array}$ & $\stackrel{\circ}{\circ}$ & $\mathrm{MAX} \cong \mathrm{D}$ \\
\hline Dichlorodifluoromethane & 0.354 & 0.345 & 0.010 & -2.6 & 40.0 \\
\hline Chloromethane & 0.369 & 0.321 & 0.010 & -13.2 & 40.0 \\
\hline Vinyl chloride & 0.382 & 0.372 & 0.010 & -2.5 & 30.0 \\
\hline Bromomethane & 0.199 & 0.096 & 0.100 & -51.5 & 30.0 \\
\hline Chloroethane & 0.235 & 0.241 & 0.010 & 2.5 & 40.0 \\
\hline Trichlorofluoromethane & 0.561 & 0.581 & 0.010 & 3.6 & 40.0 \\
\hline 1,1-Dichloroethene & 0.286 & 0.287 & 0.100 & 0.5 & 30.0 \\
\hline 1,1,2-Trichloro-1,2,2-trifluoroethane & 0.336 & 0.340 & 0.010 & 1.3 & 40.0 \\
\hline Acetone & 0.019 & 0.017 & 0.010 & -10.0 & 40.0 \\
\hline Carbon disulfide & 0.860 & 0.821 & 0.010 & -4.6 & 40.0 \\
\hline Methyl acetate & 0.045 & 0.044 & 0.010 & -4.1 & 40.0 \\
\hline Methylene Chloride & 0.237 & 0.239 & 0.010 & 0.7 & 40.0 \\
\hline trans-1,2-Dichloroethene & 0.310 & 0.294 & 0.010 & -5.3 & 40.0 \\
\hline Methyl tert-butyl ether & 0.342 & 0.343 & 0.010 & 0.2 & 40.0 \\
\hline 1,1-Dichloroethane & 0.497 & 0.496 & 0.200 & -0.2 & 30.0 \\
\hline cis-1,2-Dichloroethene & 0.286 & 0.284 & 0.010 & -0.7 & 40.0 \\
\hline 2-Butanone & 0.024 & 0.025 & 0.010 & 6.9 & 40.0 \\
\hline Bromochloromethane & 0.099 & 0.099 & 0.050 & -0.6 & 30.0 \\
\hline Chloroform & 0.484 & 0.499 & 0.200 & 3.2 & 30.0 \\
\hline $1,1,1$-Trichloroethane & 0.614 & 0.595 & 0.100 & -3.0 & 30.0 \\
\hline Cyclohexane & 0.605 & 0.610 & 0.010 & 0.8 & 40.0 \\
\hline Carbon tetrachloride & 0.568 & $0.55 \overline{3}$ & 0.100 & -2.7 & 30.0 \\
\hline Benzene & 1.432 & 1.425 & 0.400 & -0.5 & 30.0 \\
\hline 1,2-Dichloroethane & 0.210 & 0.209 & 0.100 & -0.2 & 30.0 \\
\hline Trichloroethene & 0.385 & 0.370 & 0.300 & -3.7 & 30.0 \\
\hline Methylcyclohexane & 0.462 & 0.468 & 0.010 & 1.5 & 40.0 \\
\hline
\end{tabular}

Report 1,4-Dioxane for Low/Medium VOA analysis only 
Lab Name: TESTAMERICA BURLINGTON

Lab Code: STLV Case No.: INMAN Mod. Ref No.:
Contract: $8 \mathrm{E}-00302$

SDG No.: 200-3113

Calibration Date: 12/24/2010 Time: 1104 Init. Calib. Date(s): 12/23/2010 12/23/2010

Init. Calib. Time(s): $1236 \quad 1417$ ID: $0.20(\mathrm{~mm})$ Length: 25 (m)

Purge Volume: 25.0

$(\mathrm{mL})$

\begin{tabular}{|c|c|c|c|c|c|}
\hline COMPOUND & $\overline{\mathrm{RRF}}$ & RRF5.0 & $\begin{array}{l}\text { MIN } \\
\text { RRE }\end{array}$ & $\div \mathrm{D}$ & MAX $\% \mathrm{D}$ \\
\hline 1,2-Dichloropropane & 0.275 & 0.283 & 0.010 & 2.9 & 40.0 \\
\hline Bromodichloromethane & 0.362 & 0.359 & 0.200 & -0.9 & 30.0 \\
\hline cis-1,3-Dichloropropene & 0.409 & 0.405 & 0.200 & -1.1 & 30.0 \\
\hline 4-Methyl-2-pentanone & 0.071 & 0.074 & 0.010 & 4.1 & 40.0 \\
\hline Toluene & 1.545 & 1.569 & 0.400 & 1.5 & 30.0 \\
\hline trans-1,3-Dichloropropene & 0.285 & 0.284 & 0.100 & -0.4 & 30.0 \\
\hline 1,1,2-Trichloroethane & 0.156 & 0.153 & 0.100 & -1.9 & 30.0 \\
\hline Tetrachloroethene & 0.345 & 0.333 & 0.100 & -3.6 & 30.0 \\
\hline 2-Hexanone & 0.047 & 0.048 & 0.010 & 2.7 & 40.0 \\
\hline Dibromochloromethane & 0.201 & 0.205 & 0.100 & 2.0 & 30.0 \\
\hline 1,2-Dibromoethane & 0.135 & 0.141 & 0.010 & 4.0 & 40.0 \\
\hline Chlorobenzene & 0.946 & 0.934 & 0.500 & -1.2 & 30.0 \\
\hline Ethylbenzene & 1.788 & 1.785 & 0.100 & -0.2 & 30.0 \\
\hline o-xylene & 0.654 & 0.675 & 0.300 & 3.1 & 30.0 \\
\hline $\mathrm{m}, \mathrm{p}$-Xylene & 0.698 & 0.722 & 0.300 & 3.4 & 30.0 \\
\hline Styrene & 0.978 & 1.026 & 0.300 & 4.9 & 30.0 \\
\hline Bromoform & 0.176 & 0.209 & 0.050 & 18.8 & 30.0 \\
\hline Isopropylbenzene & 1.819 & 1.866 & 0.010 & 2.6 & 40.0 \\
\hline 1,1,2,2-Tetrachloroethane & 0.139 & 0.137 & 0.100 & -1.0 & 30.0 \\
\hline 1,3-Dichlorobenzene & 1.469 & 1.452 & 0.400 & -1.2 & 30.0 \\
\hline 1,4-Dichlorobenzene & 1.488 & 1.451 & 0.400 & -2.5 & 30.0 \\
\hline 1,2-Dichlorobenzene & 1.198 & 1.239 & 0.400 & 3.4 & 30.0 \\
\hline 1,2-Dibromo-3-Chloropropane & 0.038 & 0.038 & 0.010 & 1.1 & 40.0 \\
\hline 1,2,4-Trichlorobenzene & 0.709 & 0.684 & 0.200 & -3.5 & 30.0 \\
\hline 1,2,3-Trichlorobenzene & 0.500 & 0.477 & 0.200 & -4.6 & 30.0 \\
\hline
\end{tabular}


$7 C$ - FORM VII VOA-3

VOLATILE CONTINUING CALIBRATION DATA

Lab Name: TESTAMERICA BURLINGTON

Contract: $8 \mathrm{E}-00302$

Lab Code: STLV Case No.: INMAN Mod. Ref No.

SDG No.: 200-3113

Instrument ID: J.i

Calibration Date: 12/24/2010 Time: 1104

Lab File Id: JCFB04.D Init. Calib. Date(s): 12/23/2010 12/23/2010

EPA Sample No. (VSTD\#\#\#): VSTD005JF

Init. Calib. Time(s): 1236 1417

Heated Purge: ( $Y / N) \quad N$ GC Column: DB-624 ID: $0.20(\mathrm{~mm})$ Length: 25 (m)

Purge volume: 25.0

$(\mathrm{mL})$

\begin{tabular}{|c|c|c|c|c|c|}
\hline COMPOUND & $\overline{\mathrm{RRF}}$ & RRF5. 0 & $\begin{array}{l}\text { MIN } \\
\text { RRE }\end{array}$ & $\frac{\circ}{\partial} \mathrm{D}$ & MAX $\frac{\circ}{\partial}$ \\
\hline Vinyl Chloride-d3 & 0.348 & 0.338 & 0.010 & -3.0 & 30.0 \\
\hline Chloroethane-d5 & 0.296 & 0.301 & 0.010 & 1.7 & 40.0 \\
\hline 1,1-Dichloroethene-d2 & 0.573 & 0.580 & 0.010 & 1.2 & 30.0 \\
\hline 2-Butanone-d5 & 0.022 & 0.024 & 0.010 & 7.1 & 40.0 \\
\hline Chloroform-d & 0.490 & 0.498 & 0.010 & 1.6 & 30.0 \\
\hline 1,2-Dichloroethane-d4 & 0.161 & 0.167 & 0.010 & 3.6 & 30.0 \\
\hline Benzene-d6 & 1.335 & 1.310 & 0.010 & -1.9 & 30.0 \\
\hline 1,2-Dichloropropane-d6 & 0.354 & 0.363 & 0.010 & 2.6 & 40.0 \\
\hline Toluene-d8 & 1.273 & 1.273 & 0.010 & 0.0 & 30.0 \\
\hline trans-1,3-Dichloropropene-d4 & 0.251 & 0.250 & 0.010 & -0.6 & 30.0 \\
\hline 2-Hexanone-d5 & 0.023 & 0.025 & 0.010 & 8.1 & 40.0 \\
\hline $1,1,2,2$-Tetrachloroethane-d2 & 0.138 & 0.138 & 0.010 & -0.2 & 30.0 \\
\hline 1,2-Dichlorobenzene-d4 & 0.699 & 0.729 & 0.010 & 4.3 & 30.0 \\
\hline
\end{tabular}

Report 1,4-Dioxane-d8 for Low/Medium VOA analysis only 
$7 A$ - FORM VII VOA-1

VOLATILE CONTINUING CALIBRATION DATA

Lab Name: TESTAMERICA BURLINGTON

Contract: $8 \mathrm{E}-00302$

Lab Code: STLV Case No.: INMAN Mod. Ref No.: SDG No.: 200-3113

Instrument ID: J.i

Calibration Date: 12/24/2010 Time: 2117

Lab File Id: JCFB28.D Init. Calib. Date(s): 12/23/2010 12/23/2010

EPA Sample No. (VSTD\#\#\#\#)

VSTD005EJ

Init. Calib. Time(s): $1236 \quad 1417$

Heated Purge: ( $Y / N) \quad N$

GC Column: $\mathrm{DB}-624$

ID: $0.20(\mathrm{~mm})$ Length: 25

(II)

Purge Volume: 25.0

$(\mathrm{mL})$

\begin{tabular}{|c|c|c|c|c|c|}
\hline COMPOUND & $\overline{\mathrm{RRF}}$ & RRF5.0 & $\begin{array}{l}\text { MIN } \\
\text { RRF }\end{array}$ & $\because \mathrm{D}$ & $\operatorname{MAX} \div D$ \\
\hline Dichlorodifluoromethane & 0.354 & 0.346 & 0.010 & -2.3 & 50.0 \\
\hline Chloromethane & 0.369 & 0.337 & 0.010 & -8.9 & 50.0 \\
\hline Vinyl chloride & 0.382 & 0.371 & 0.010 & -2.7 & 50.0 \\
\hline Bromomethane & 0.199 & 0.133 & 0.010 & -33.2 & 50.0 \\
\hline Chloroethane & 0.235 & 0.254 & 0.010 & 8.3 & 50.0 \\
\hline Trichlorofluoromethane & 0.561 & 0.575 & 0.010 & 2.5 & 50.0 \\
\hline 1,1-Dichloroethene & 0.286 & 0.284 & 0.010 & -0.9 & 50.0 \\
\hline 1,1,2-Trichloro-1,2,2-trifluoroethane & 0.336 & 0.331 & 0.010 & -1.4 & 50.0 \\
\hline Acetone & 0.019 & 0.020 & 0.010 & 5.6 & 50.0 \\
\hline Carbon disulfide & 0.860 & 0.800 & 0.010 & -7.0 & 50.0 \\
\hline Methyl acetate & 0.045 & 0.040 & 0.010 & -11.1 & 50.0 \\
\hline Methylene Chloride & 0.237 & 0.244 & 0.010 & 2.8 & 50.0 \\
\hline trans-1,2-Dichloroethene & 0.310 & 0.297 & 0.010 & -4.2 & 50.0 \\
\hline Methyl tert-butyl ether & 0.342 & 0.363 & 0.010 & 6.1 & 50.0 \\
\hline 1,1-Dichloroethane & 0.497 & 0.497 & 0.010 & 0.1 & 50.0 \\
\hline cis-1,2-Dichloroethene & 0.286 & 0.288 & 0.010 & 0.7 & 50.0 \\
\hline 2-Butanone & 0.024 & 0.025 & 0.010 & 5.4 & 50.0 \\
\hline Bromochloromethane & 0.099 & 0.098 & 0.010 & -0.9 & 50.0 \\
\hline Chloroform & 0.484 & 0.486 & 0.010 & 0.5 & 50.0 \\
\hline 1,1,1-Trichloroethane & 0.614 & 0.595 & 0.010 & -3.0 & 50.0 \\
\hline Cyclohexane & 0.605 & 0.595 & 0.010 & -1.6 & 50.0 \\
\hline Carbon tetrachloride & 0.568 & 0.559 & 0.010 & -1.7 & 50.0 \\
\hline Benzene & 1.432 & 1.463 & 0.010 & 2.2 & 50.0 \\
\hline 1,2-Dichloroethane & 0.210 & 0.216 & 0.010 & 2.8 & 50.0 \\
\hline Trichloroethene & 0.385 & 0.367 & 0.010 & -4.5 & 50.0 \\
\hline Methylcyclohexane & 0.462 & 0.449 & 0.010 & -2.7 & 50.0 \\
\hline
\end{tabular}

Report 1,4-Dioxane for Low/Medium VOA analysis only 
$7 B$ - FORM VII VOA-2

VOLATILE CONTINUING CALIBRATION DATA

Lab Name: TESTAMERICA BURLINGTON

Contract: $8 \mathrm{E}-00302$

Lab Code: STLV Case No.: INMAN Mod.
Instrument ID: J.i
Lab File Id: JCFB28.D

EPA Sample No. (VSTD\#\#\#\#): VSTD005FJ

Init. Calib. Date(s): 12/23/2010 12/23/2010

Heated Purge: $(Y / N)$

$\mathrm{N} \quad \mathrm{GC}$ Column: $\mathrm{DB}-624$

Init. Calib. Time(s): 1236

1417

Purge Volume: 25.0

(mL)

\begin{tabular}{|c|c|c|c|c|c|}
\hline COMPOUND & $\overline{\mathrm{RRF}}$ & RRE5.0 & $\begin{array}{l}\text { MIN } \\
\text { RRE }\end{array}$ & $\frac{\circ}{\partial} \mathrm{D}$ & MAX $8 D$ \\
\hline 1,2-Dichloropropane & 0.275 & 0.291 & 0.010 & 5.9 & 50.0 \\
\hline Bromodichloromethane & 0.362 & 0.380 & 0.010 & 5.0 & 50.0 \\
\hline cis-1,3-Dichloropropene & 0.409 & 0.412 & 0.010 & 0.8 & 50.0 \\
\hline 4-Methyl-2-pentanone & 0.071 & 0.077 & 0.010 & 9.0 & 50.0 \\
\hline Toluene & 1.545 & 1.576 & 0.010 & 2.0 & 50.0 \\
\hline trans-1,3-Dichloropropene & 0.285 & 0.288 & 0.010 & 0.8 & 50.0 \\
\hline 1,1,2-Trichloroethane & 0.156 & 0.161 & 0.010 & 3.1 & 50.0 \\
\hline Tetrachloroethene & 0.345 & 0.332 & 0.010 & -3.8 & 50.0 \\
\hline 2-Hexanone & 0.047 & 0.050 & 0.010 & 6.3 & 50.0 \\
\hline Dibromochloromethane & 0.201 & 0.214 & 0.010 & 6.4 & 50.0 \\
\hline 1,2-Dibromoethane & 0.135 & 0.137 & 0.010 & 1.3 & 50.0 \\
\hline Chlorobenzene & 0.946 & 0.947 & 0.010 & 0.1 & 50.0 \\
\hline Ethylbenzene & 1.788 & 1.784 & 0.010 & -0.2 & 50.0 \\
\hline o-xylene & 0.654 & 0.678 & 0.010 & 3.6 & 50.0 \\
\hline m,p-xylene & 0.698 & 0.727 & 0.010 & 4.1 & 50.0 \\
\hline Styrene & 0.978 & 1.035 & 0.010 & 5.9 & 50.0 \\
\hline Bromoform & 0.176 & 0.208 & 0.010 & 18.4 & 50.0 \\
\hline Isopropylbenzene & 1.819 & 1.848 & 0.010 & 1.6 & 50.0 \\
\hline $1,1,2,2$-Tetrachloroethane & 0.139 & 0.143 & 0.010 & 3.5 & 50.0 \\
\hline 1,3-Dichlorobenzene & 1.469 & 1.411 & 0.010 & -3.9 & 50.0 \\
\hline 1,4-Dichlorobenzene & 1.488 & 1.424 & 0.010 & -4.3 & 50.0 \\
\hline 1,2-Dichlorobenzene & 1.198 & 1.187 & 0.010 & -0.9 & 50.0 \\
\hline 1,2-Dibromo-3-Chloropropane & 0.038 & 0.044 & 0.010 & 16.1 & 50.0 \\
\hline 1,2,4-Trichlorobenzene & 0.709 & 0.660 & 0.010 & -6.9 & 50.0 \\
\hline $1,2,3$-Trichlorobenzene & 0.500 & 0.464 & 0.010 & -7.3 & 50.0 \\
\hline
\end{tabular}


$7 \mathrm{C}$ - FORM VII VOA-3

VOLATILE CONTINUING CALIBRATION DATA

Lab Name: TESTAMERICA BURLINGTON

Contract: 8E-00302

Lab Code: STLV Case No.: INMAN Mod.
Instrument ID: J.i
Lab File Id: JCFB28.D

EPA Sample No. (VSTD\#\#\#\#): VSTD005EJ

Heated Purge: (Y/N) N GC Column: DB-624

$(\mathrm{mL})$

SDG No.: 200-3113

Purge Volume: 25.0

\begin{tabular}{|c|c|c|c|c|c|}
\hline COMPOUND & $\overline{\mathrm{RRF}}$ & RRF5. 0 & $\begin{array}{l}\text { MIN } \\
\text { RRF }\end{array}$ & $\frac{\circ}{\partial} \mathrm{D}$ & MAX $\% D$ \\
\hline Vinyl Chloride-d3 & 0.348 & 0.337 & 0.010 & -3.1 & 50.0 \\
\hline Chloroethane-d5 & 0.296 & 0.293 & 0.010 & -1.1 & 50.0 \\
\hline 1,1-Dichloroethene-d2 & 0.573 & 0.584 & 0.010 & 1.9 & 50.0 \\
\hline 2-Butanone-d5 & 0.022 & 0.026 & 0.010 & 13.5 & 50.0 \\
\hline Chloroform-d & 0.490 & 0.504 & 0.010 & 3.0 & 50.0 \\
\hline 1,2-Dichloroethane-d4 & 0.161 & 0.169 & 0.010 & 4.5 & 50.0 \\
\hline Benzene-d6 & 1.335 & 1.347 & 0.010 & 0.9 & 50.0 \\
\hline 1,2-Dichloropropane-d6 & 0.354 & 0.367 & 0.010 & 3.6 & 50.0 \\
\hline Toluene-d8 & 1.273 & 1.247 & 0.010 & -2.0 & 50.0 \\
\hline trans-1,3-Dichloropropene-d4 & 0.251 & 0.251 & 0.010 & -0.3 & 50.0 \\
\hline 2-Hexanone-d5 & 0.023 & 0.026 & 0.010 & 12.5 & 50.0 \\
\hline $1,1,2,2$-Tetrachloroethane-d2 & 0.138 & 0.152 & 0.010 & 10.1 & 50.0 \\
\hline 1,2-Dichlorobenzene-d4 & 0.699 & 0.701 & 0.010 & 0.4 & 50.0 \\
\hline
\end{tabular}

Report 1,4-Dioxane-d8 for Low/Medium VOA analysis only 
$7 \mathrm{~A}-$ FORM VII VOA-1

VOLATILE CONTINUING CALIBRATION DATA

Lab Name: TESTAMERICA BURLINGTON

Contract: $8 \mathrm{E}-00302$

Lab Code: STLV Case No.: INMAN Mod.
Instrument ID: J.i

Lab File Id: JCFCO4.D

EPA Sample No. (VSTD\#\#\#\#): VSTD005JG Init. Calib. Date(s): 12/23/2010 12/23/2010

Heated Purge: ( $\mathrm{Y} / \mathrm{N})$ $\mathrm{N}$ GC Column: DB-624 Init. Calib. Time(s): $1236 \quad 1417$ Purge Volume: 25.0 (mL) ID: $0.20(\mathrm{~mm})$ Length: 25 (m)

\begin{tabular}{|c|c|c|c|c|c|}
\hline COMPOUND & $\overline{\mathrm{RRF}}$ & RRF5.0 & $\begin{array}{l}\text { MIN } \\
\text { RRE }\end{array}$ & $\therefore \mathrm{D}$ & MAX $\% D$ \\
\hline Dichlorodifluoromethane & 0.354 & 0.306 & 0.010 & -13.4 & 40.0 \\
\hline Chloromethane & 0.369 & 0.341 & 0.010 & -7.8 & 40.0 \\
\hline Vinyl chloride & 0.382 & 0.363 & 0.010 & -4.8 & 30.0 \\
\hline Bromomethane & 0.199 & 0.149 & 0.100 & -25.2 & 30.0 \\
\hline Chloroethane & 0.235 & 0.229 & 0.010 & -2.3 & 40.0 \\
\hline Trichlorofluoromethane & 0.561 & 0.576 & 0.010 & 2.7 & 40.0 \\
\hline 1,1-Dichloroethene & 0.286 & 0.283 & 0.100 & -1.2 & 30.0 \\
\hline 1,1,2-Trichloro-1,2,2-trifluoroethane & 0.336 & 0.344 & 0.010 & 2.5 & 40.0 \\
\hline Acetone & 0.019 & 0.019 & 0.010 & -0.6 & 40.0 \\
\hline Carbon disulfide & 0.860 & 0.813 & 0.010 & -5.5 & 40.0 \\
\hline Methyl acetate & 0.045 & 0.049 & 0.010 & 8.5 & 40.0 \\
\hline Methylene Chloride & 0.237 & 0.253 & 0.010 & 6.6 & 40.0 \\
\hline trans-1,2-Dichloroethene & 0.310 & 0.297 & 0.010 & -4.1 & 40.0 \\
\hline Methyl tert-butyl ether & 0.342 & 0.374 & 0.010 & 9.4 & 40.0 \\
\hline 1,1-Dichloroethane & 0.497 & 0.495 & 0.200 & -0.4 & 30.0 \\
\hline cis-1,2-Dichloroethene & 0.286 & 0.300 & 0.010 & 4.9 & 40.0 \\
\hline 2-Butanone & 0.024 & 0.028 & 0.010 & 16.7 & 40.0 \\
\hline Bromochloromethane & 0.099 & 0.106 & 0.050 & 7.1 & 30.0 \\
\hline Chloroform & 0.484 & 0.507 & 0.200 & 4.8 & 30.0 \\
\hline 1,1,1-Trichloroethane & 0.614 & 0.583 & 0.100 & -5.0 & 30.0 \\
\hline Cyclohexane & 0.605 & 0.590 & 0.010 & -2.4 & 40.0 \\
\hline Carbon tetrachloride & 0.568 & 0.537 & 0.100 & -5.6 & 30.0 \\
\hline Benzene & 1.432 & 1.407 & 0.400 & -1.7 & 30.0 \\
\hline 1,2-Dichloroethane & 0.210 & 0.216 & 0.100 & 3.2 & 30.0 \\
\hline Trichloroethene & 0.385 & 0.365 & 0.300 & -5.2 & 30.0 \\
\hline Methylcyclohexane & 0.462 & 0.436 & 0.010 & -5.6 & 40.0 \\
\hline
\end{tabular}


$7 \mathrm{~B}$ - FORM VII VOA-2

VOLATILE CONTINUING CALIBRATION DATA

Lab Name: TESTAMERICA BURLINGTON

Contract: $8 \mathrm{E}-00302$

Lab Code: STLV Case No.: INMAN Mod.
Instrument ID: J.i
Lab File Id: JCFCO4.D

EPA Sample No. (VSTD\#\#\#\#): VSTD005JG

Heated Purge: ( $\mathrm{Y} / \mathrm{N}) \quad \underline{\mathrm{N}}$ GC Column:

-

- Ref No.

SDG No.: 200-3113

Calibration Date: 12/27/2010 Time: 1828 Init. Calib. Date(s): 12/23/2010 12/23/2010

Init. Calib. Time(s): $1236 \quad 1417$

Purge volume: 25.0 ID: $0.20(\mathrm{~mm})$ Length: 25

(m) (mL)

\begin{tabular}{|c|c|c|c|c|c|}
\hline COMPOUND & $\overline{\mathrm{RRF}}$ & RRF5.0 & $\begin{array}{l}\text { MIN } \\
\text { RRF }\end{array}$ & $\frac{\circ}{0} \mathrm{D}$ & MAX $\div D$ \\
\hline 1,2-Dichloropropane & 0.275 & 0.290 & 0.010 & 5.6 & 40.0 \\
\hline Bromodichloromethane & 0.362 & 0.372 & 0.200 & 2.9 & 30.0 \\
\hline cis-1,3-Dichloropropene & 0.409 & 0.422 & 0.200 & 3.2 & 30.0 \\
\hline 4-Methyl-2-pentanone & 0.071 & 0.082 & 0.010 & 15.6 & 40.0 \\
\hline Toluene & 1.545 & 1.531 & 0.400 & -0.9 & 30.0 \\
\hline trans-1,3-Dichloropropene & 0.285 & 0.307 & 0.100 & 7.6 & 30.0 \\
\hline 1,1,2-Trichloroethane & 0.156 & 0.157 & 0.100 & 0.4 & 30.0 \\
\hline Tetrachloroethene & 0.345 & 0.333 & 0.100 & -3.5 & 30.0 \\
\hline 2-Hexanone & 0.047 & 0.054 & 0.010 & 14.3 & 40.0 \\
\hline Dibromochloromethane & 0.201 & 0.215 & 0.100 & 6.7 & 30.0 \\
\hline 1,2-Dibromoethane & 0.135 & 0.143 & 0.010 & 5.6 & 40.0 \\
\hline Chlorobenzene & 0.946 & 0.937 & 0.500 & -1.0 & 30.0 \\
\hline Ethylbenzene & 1.788 & 1.762 & 0.100 & -1.5 & 30.0 \\
\hline o-Xylene & 0.654 & 0.672 & 0.300 & 2.7 & 30.0 \\
\hline $\mathrm{m}, \mathrm{p}$-Xylene & 0.698 & 0.715 & 0.300 & 2.4 & 30.0 \\
\hline Styrene & 0.978 & 1.030 & 0.300 & 5.3 & 30.0 \\
\hline Bromoform & 0.176 & 0.219 & 0.050 & 24.5 & 30.0 \\
\hline Isopropylbenzene & 1.819 & 1.805 & 0.010 & -0.8 & 40.0 \\
\hline $1,1,2,2$-Tetrachloroethane & 0.139 & 0.145 & 0.100 & 4.9 & 30.0 \\
\hline 1,3-Dichlorobenzene & 1.469 & 1.452 & 0.400 & -1.1 & 30.0 \\
\hline 1,4-Dichlorobenzene & 1.488 & 1.476 & 0.400 & -0.8 & 30.0 \\
\hline 1,2-Dichlorobenzene & 1.198 & 1.233 & 0.400 & 2.9 & 30.0 \\
\hline 1,2-Dibromo-3-Chloropropane & 0.038 & 0.041 & 0.010 & 8.3 & 40.0 \\
\hline 1,2,4-Trichlorobenzene & 0.709 & 0.734 & 0.200 & 3.6 & 30.0 \\
\hline $1,2,3$-Trichlorobenzene & 0.500 & 0.534 & 0.200 & 6.8 & 30.0 \\
\hline
\end{tabular}


$7 C$ - FORM VII VOA-3

VOLATILE CONTINUING CALIBRATION DATA

Lab Name: TESTAMERICA BURLINGTON

Contract: $8 \mathrm{E}-00302$

Lab Code: STLV Case No.: INMAN Mod.
Instrument ID: J.i
Lab File Id: JCFCO4.D

EPA Sample No. (VSTD\#\#\#\#): VSTD005JG

Init. Calib. Date(s): 12/23/2010 12/23/2010

Heated Purge: $(\mathrm{Y} / \mathrm{N})$

GC Column: DB-624

Init. Calib. Time(s): 1236 1417

Purge Volume: 25.0

$(\mathrm{mL})$

\begin{tabular}{|c|c|c|c|c|c|}
\hline COMPOUND & $\overline{\mathrm{RRF}}$ & RRF5.0 & $\begin{array}{l}\text { MIN } \\
\text { RRF }\end{array}$ & $\because \mathrm{D}$ & MAX $\because D$ \\
\hline Vinyl Chloride-d3 & 0.348 & 0.321 & 0.010 & -7.7 & 30.0 \\
\hline Chloroethane-d5 & 0.296 & 0.294 & 0.010 & -0.6 & 40.0 \\
\hline 1,1-Dichloroethene-d2 & 0.573 & 0.572 & 0.010 & -0.2 & 30.0 \\
\hline 2-Butanone-d5 & 0.022 & 0.027 & 0.010 & 20.1 & 40.0 \\
\hline Chloroform-d & 0.490 & 0.519 & 0.010 & 6.0 & 30.0 \\
\hline 1,2-Dichloroethane-d4 & 0.161 & 0.164 & 0.010 & 1.5 & 30.0 \\
\hline Benzene-d 6 & 1.335 & 1.292 & 0.010 & -3.3 & 30.0 \\
\hline 1,2-Dichloropropane-d6 & 0.354 & 0.317 & 0.010 & -10.5 & 40.0 \\
\hline Toluene-d8 & 1.273 & 1.224 & 0.010 & -3.9 & 30.0 \\
\hline trans-1,3-Dichloropropene-d4 & 0.251 & 0.267 & 0.010 & 6.1 & 30.0 \\
\hline 2-Hexanone-d5 & 0.023 & 0.029 & 0.010 & 23.8 & 40.0 \\
\hline $1,1,2,2$-Tetrachloroethane-d2 & 0.138 & 0.150 & 0.010 & 8.5 & 30.0 \\
\hline 1,2-Dichlorobenzene-d4 & 0.699 & 0.716 & 0.010 & 2.5 & 30.0 \\
\hline
\end{tabular}

Report 1,4-Dioxane-d8 for Low/Medium VOA analysis only 
$7 A$ - FORM VII VOA-1

VOLATILE CONTINUING CALIBRATION DATA

Lab Name: TESTAMERICA BURLINGTON

Contract: $\quad 8 \mathrm{E}-00302$

Lab Code: STLV Case No.: INMAN Mod, Ref No.: SDG No.: 200-3113

Instrument ID: J.i

Calibration Date: 12/28/2010 Time: 0449

Lab File Id: JCFC27.D

EPA Sample No. (VSTD\#\#\#\#):

VSTD005GJ Init. Calib. Date(s): 12/23/2010 12/23/2010

Heated Purge: $(\mathrm{Y} / \mathrm{N})$ $\mathrm{N} \quad$ GC Column: $\mathrm{DB}-624$

Init. Calib. Time(s): 1236 1417

Purge Volume: 25.0 $(\mathrm{mL})$

\begin{tabular}{|c|c|c|c|c|c|}
\hline COMPOUND & $\overline{\mathrm{RRF}}$ & RRF5.0 & $\begin{array}{l}\text { MIN } \\
\text { RRF }\end{array}$ & $\because \mathrm{D}$ & $\operatorname{MAX} \div D$ \\
\hline Dichlorodifluoromethane & 0.354 & 0.323 & 0.010 & -8.7 & 50.0 \\
\hline Chloromethane & 0.369 & 0.390 & 0.010 & 5.5 & 50.0 \\
\hline Vinyl chloride & 0.382 & 0.384 & 0.010 & 0.6 & 50.0 \\
\hline Bromomethane & 0.199 & 0.194 & 0.010 & -2.4 & 50.0 \\
\hline Chloroethane & 0.235 & 0.246 & 0.010 & 5.0 & 50.0 \\
\hline Trichlorofluoromethane & 0.561 & 0.630 & 0.010 & 12.4 & 50.0 \\
\hline 1,1-Dichloroethene & 0.286 & 0.313 & 0.010 & 9.4 & 50.0 \\
\hline 1,1,2-Trichloro-1,2,2-trifluoroethane & 0.336 & 0.356 & 0.010 & 6.0 & 50.0 \\
\hline Acetone & 0.019 & 0.019 & 0.010 & -1.5 & 50.0 \\
\hline Carbon disulfide & 0.860 & 0.876 & 0.010 & 1.8 & 50.0 \\
\hline Methyl acetate & 0.045 & 0.049 & 0.010 & 8.2 & 50.0 \\
\hline Methylene Chloride & 0.237 & 0.261 & 0.010 & 10.2 & 50.0 \\
\hline trans-1,2-Dichloroethene & 0.310 & 0.333 & 0.010 & 7.3 & 50.0 \\
\hline Methyl tert-butyl ether & 0.342 & 0.349 & 0.010 & 2.1 & 50.0 \\
\hline 1,1-Dichloroethane & 0.497 & 0.543 & 0.010 & 9.4 & 50.0 \\
\hline cis-1,2-Dichloroethene & 0.286 & 0.316 & 0.010 & 10.6 & 50.0 \\
\hline 2-Butanone & 0.024 & 0.025 & 0.010 & 5.6 & 50.0 \\
\hline Bromochloromethane & 0.099 & 0.109 & 0.010 & 9.6 & 50.0 \\
\hline Chloroform & 0.484 & 0.547 & 0.010 & 13.1 & 50.0 \\
\hline 1,1,1-Trichloroethane & 0.614 & 0.615 & 0.010 & 0.2 & 50.0 \\
\hline Cyclohexane & 0.605 & 0.626 & 0.010 & 3.6 & 50.0 \\
\hline Carbon tetrachloride & 0.568 & 0.581 & 0.010 & 2.3 & 50.0 \\
\hline Benzene & 1.432 & 1.482 & 0.010 & 3.5 & 50.0 \\
\hline 1,2-Dichloroethane & 0.210 & 0.228 & 0.010 & 8.9 & 50.0 \\
\hline Trichloroethene & 0.385 & 0.388 & 0.010 & 1.0 & 50.0 \\
\hline Methylcyclohexane & 0.462 & 0.447 & 0.010 & -3.2 & 50.0 \\
\hline
\end{tabular}

Report 1,4-Dioxane for Low/Medium VOA analysis only 
$7 B$ - FORM VII VOA-2

VOLATILE CONTINUING CALIBRATION DATA

Lab Name: TESTAMERICA BURLINGTON

Lab Code: STLV Case No.: INMAN Mod. Ref No.:

Contract: $8 \mathrm{E}-00302$

Instrument ID: J.i

Lab File Id: JCFC27.D

EPA Sample No. (VSTD\#\#\#\#):

VSTD005GJ

N GC Column: $\mathrm{DB}-624$

$(m L)$

Heated Purge: ( $\mathrm{Y} / \mathrm{N})$

Purge Volume: 25.0

Calibration Date: 12/28/2010 Time: 0449 Init. Calib. Date(s): 12/23/2010 12/23/2010

Init. Calib. Time(s): $1236 \quad 1417$

ID: $0.20(\mathrm{~mm})$ Length: 25 (m)

\begin{tabular}{|c|c|c|c|c|c|}
\hline COMPOUND & $\overline{\mathrm{RRF}}$ & RRF5.0 & $\begin{array}{l}\text { MIN } \\
\text { RRF }\end{array}$ & $\frac{\circ}{\partial} \mathrm{D}$ & MAX $\& D$ \\
\hline 1,2-Dichloropropane & 0.275 & 0.302 & 0.010 & 9.9 & 50.0 \\
\hline Bromodichloromethane & 0.362 & 0.365 & 0.010 & 0.8 & 50.0 \\
\hline cis-1,3-Dichloropropene & 0.409 & 0.411 & 0.010 & 0.4 & 50.0 \\
\hline 4-Methyl-2-pentanone & 0.071 & 0.073 & 0.010 & 3.6 & 50.0 \\
\hline Toluene & 1.545 & 1.655 & 0.010 & 7.1 & 50.0 \\
\hline trans-1,3-Dichloropropene & 0.285 & 0.284 & 0.010 & -0.5 & 50.0 \\
\hline 1,1,2-Trichloroethane & 0.156 & 0.159 & 0.010 & 2.1 & 50.0 \\
\hline Tetrachloroethene & 0.345 & 0.345 & 0.010 & -0.1 & 50.0 \\
\hline 2-Hexanone & 0.047 & 0.051 & 0.010 & 8.9 & 50.0 \\
\hline Dibromochloromethane & 0.201 & 0.216 & 0.010 & 7.3 & 50.0 \\
\hline 1,2-Dibromoethane & 0.135 & 0.139 & 0.010 & 2.9 & 50.0 \\
\hline Chlorobenzene & 0.946 & 0.987 & 0.010 & 4.3 & 50.0 \\
\hline Ethylbenzene & 1.788 & 1.857 & 0.010 & 3.9 & 50.0 \\
\hline o-xylene & 0.654 & 0.705 & 0.010 & 7.7 & 50.0 \\
\hline $\mathrm{m}, \mathrm{p}$-Xylene & 0.698 & 0.751 & 0.010 & 7.5 & 50.0 \\
\hline Styrene & 0.978 & 1.073 & 0.010 & 9.7 & 50.0 \\
\hline Bromoform & 0.176 & 0.218 & 0.010 & 23.8 & 50.0 \\
\hline Isopropylbenzene & 1.819 & 1.962 & 0.010 & 7.9 & 50.0 \\
\hline $1,1,2,2$-Tetrachloroethane & 0.139 & 0.152 & 0.010 & 9.8 & 50.0 \\
\hline 1,3-Dichlorobenzene & 1.469 & 1.556 & 0.010 & 5.9 & 50.0 \\
\hline 1,4-Dichlorobenzene & 1.488 & 1.560 & 0.010 & 4.8 & 50.0 \\
\hline 1,2-Dichlorobenzene & 1.198 & 1.259 & 0.010 & 5.1 & 50.0 \\
\hline 1,2-Dibromo-3-Chloropropane & 0.038 & 0.039 & 0.010 & 4.6 & 50.0 \\
\hline 1,2,4-Trichlorobenzene & 0.709 & 0.673 & 0.010 & -5.0 & 50.0 \\
\hline $1,2,3$-Trichlorobenzene & 0.500 & 0.462 & 0.010 & -7.6 & 50.0 \\
\hline
\end{tabular}


$7 C$ - FORM VII VOA-3

VOLATILE CONTINUING CALIBRATION DATA

Lab Name: TESTAMERICA BURLINGTON

Contract: $8 \mathrm{E}-00302$

Lab Code: STLV Case No.: INMAN Mod
Instrument ID: J.i
Lab File Id: JCFC27.D

Init. Calib. Date(s): 12/23/2010 12/23/2010

EPA Sample No. (VSTD\#\#\#\#): VSTD005GJ

Heated Purge: ( $\mathrm{Y} / \mathrm{N}) \quad \mathrm{N}$ GC Column: DB-624

Init. Calib. Time(s): $1236 \quad 1417$

Purge Volume: $25.0 \quad$ (mL) ID: $0.20(\mathrm{~mm})$ Length: $25(\mathrm{~m})$

\begin{tabular}{|c|c|c|c|c|c|}
\hline COMPOUND & $\overline{\mathrm{RRF}}$ & RRE5. 0 & $\begin{array}{l}\text { MIN } \\
\text { RRF }\end{array}$ & $\because \mathrm{D}$ & MAX $\because D$ \\
\hline Vinyl Chloride-d3 & 0.348 & 0.361 & 0.010 & 3.8 & 50.0 \\
\hline Chloroethane-d5 & 0.296 & 0.310 & 0.010 & 5.0 & 50.0 \\
\hline 1,1-Dichloroethene-d2 & 0.573 & 0.618 & 0.010 & 7.7 & 50.0 \\
\hline 2-Butanone-d5 & 0.022 & 0.024 & 0.010 & 5.0 & 50.0 \\
\hline Chloroform-d & 0.490 & 0.543 & 0.010 & 10.9 & 50.0 \\
\hline 1,2-Dichloroethane-d4 & 0.161 & 0.185 & 0.010 & 14.4 & 50.0 \\
\hline Benzene-d6 & 1.335 & 1.364 & 0.010 & 2.1 & 50.0 \\
\hline 1,2-Dichloropropane-d6 & 0.354 & 0.381 & 0.010 & 7.6 & 50.0 \\
\hline Toluene-d8 & 1.273 & 1.331 & 0.010 & 4.5 & 50.0 \\
\hline trans-1,3-Dichloropropene- $d 4$ & 0.251 & 0.249 & 0.010 & -0.8 & 50.0 \\
\hline 2-Hexanone-d5 & 0.023 & 0.025 & 0.010 & 5.5 & 50.0 \\
\hline $1,1,2,2$-Tetrachloroethane-d2 & 0.138 & 0.150 & 0.010 & 8.4 & 50.0 \\
\hline 1,2-Dichlorobenzene-d4 & 0.699 & 0.776 & 0.010 & 11.1 & 50.0 \\
\hline
\end{tabular}

Report 1,4-Dioxane-d8 for Low/Medium VOA analysis only 
$7 A$ - FORM VII VOA-1

VOLATILE CONTINUING CALIBRATION DATA

Lab Name: TESTAMERICA BURLINGTON

Contract: $\quad 8 \mathrm{E}-00302$

Lab Code: STLV Case No.: INMAN Mod. Ref No.:

SDG No.: 200-3113

Instrument ID: J.i

Calibration Date: 12/28/2010 Time: 1340

Lab File Id: JCFE02.D

Init. Calib. Date(s): 12/23/2010 12/23/2010

EPA Sample No. (VSTD\#\#\#\#): VSTD005JI

Init. Calib. Time(s): $1236 \quad 1417$

Heated Purge: ( $\mathrm{Y} / \mathrm{N}) \mathrm{N}$

GC Column: DB-624

ID: $0.20(\mathrm{~mm})$ Length: 25

(m)

Purge Volume: 25.0

(mL)

\begin{tabular}{|c|c|c|c|c|c|}
\hline COMPOUND & $\overline{\mathrm{RRE}}$ & RRF5.0 & $\begin{array}{l}\text { MIN } \\
\text { RRF }\end{array}$ & $\frac{8}{8} \mathrm{D}$ & MAX $\% \mathrm{D}$ \\
\hline Dichlorodifluoromethane & 0.354 & 0.341 & 0.010 & -3.6 & 40.0 \\
\hline Chloromethane & 0.369 & 0.403 & 0.010 & 9.1 & 40.0 \\
\hline Vinyl chloride & 0.382 & 0.393 & 0.010 & 3.1 & 30.0 \\
\hline Bromomethane & 0.199 & 0.214 & 0.100 & 7.6 & 30.0 \\
\hline Chloroethane & 0.235 & 0.246 & 0.010 & 4.8 & 40.0 \\
\hline Trichlorofluoromethane & 0.561 & 0.615 & 0.010 & 9.8 & 40.0 \\
\hline 1,1-Dichloroethene & 0.286 & 0.302 & 0.100 & 5.5 & 30.0 \\
\hline 1,1,2-Trichloro-1,2,2-trifluoroethane & 0.336 & 0.373 & 0.010 & 10.9 & 40.0 \\
\hline Acetone & 0.019 & 0.015 & 0.010 & -24.0 & 40.0 \\
\hline Carbon disulfide & 0.860 & 0.853 & 0.010 & -0.8 & 40.0 \\
\hline Methyl acetate & 0.045 & 0.046 & 0.010 & 0.7 & 40.0 \\
\hline Methylene Chloride & 0.237 & 0.242 & 0.010 & 2.1 & 40.0 \\
\hline trans-1,2-Dichloroethene & 0.310 & 0.324 & 0.010 & 4.5 & 40.0 \\
\hline Methyl tert-butyl ether & 0.342 & 0.298 & 0.010 & -13.0 & 40.0 \\
\hline 1,1-Dichloroethane & 0.497 & 0.518 & 0.200 & 4.2 & 30.0 \\
\hline cis-1,2-Dichloroethene & 0.286 & 0.304 & 0.010 & 6.3 & 40.0 \\
\hline 2-Butanone & 0.024 & 0.022 & 0.010 & -9.1 & 40.0 \\
\hline Bromochloromethane & 0.099 & 0.097 & 0.050 & -2.2 & 30.0 \\
\hline Chloroform & 0.484 & 0.515 & 0.200 & 6.5 & 30.0 \\
\hline 1,1,1-Trichloroethane & 0.614 & 0.637 & 0.100 & 3.8 & 30.0 \\
\hline Cyclohexane & 0.605 & 0.610 & 0.010 & 0.8 & 40.0 \\
\hline Carbon tetrachloride & 0.568 & 0.601 & 0.100 & 5.7 & 30.0 \\
\hline Benzene & 1.432 & 1.427 & 0.400 & -0.4 & 30.0 \\
\hline 1,2-Dichloroethane & 0.210 & 0.214 & 0.100 & 2.1 & 30.0 \\
\hline Trichloroethene & 0.385 & 0.390 & 0.300 & 1.4 & 30.0 \\
\hline Methylcyclohexane & 0.462 & 0.453 & 0.010 & -1.9 & 40.0 \\
\hline
\end{tabular}

Report 1,4-Dioxane for Low/Medium VOA analysis only 
Lab Name: TESTAMERICA BURLINGTON

Lab Code: STLV Case No.: INMAN Mod. Ref No.:
Contract: $8 E-00302$

SDG No.: $200-3113$

Calibration Date: 12/28/2010 Time: 1340 Init. Calib. Date(s): 12/23/2010 12/23/2010

Init. Calib. Time(s): $1236 \quad 1417$ ID: $0.20(\mathrm{~mm})$ Length: 25 (m)
Heated Purge: ( $Y / N)$

Purge volume: 25.0
VSTD005JI

GC Column: DB-624

(mL)

\begin{tabular}{|c|c|c|c|c|c|}
\hline COMPOUND & $\overline{\mathrm{RRF}}$ & RRF5.0 & $\begin{array}{l}\text { MIN } \\
\text { RRE }\end{array}$ & $8 D$ & MAX कD \\
\hline 1,2-Dichloropropane & 0.275 & 0.275 & 0.010 & -0.1 & 40.0 \\
\hline Bromodichloromethane & 0.362 & 0.352 & 0.200 & -2.8 & 30.0 \\
\hline cis-1,3-Dichloropropene & 0.409 & 0.390 & 0.200 & -4.6 & 30.0 \\
\hline 4-Methyl-2-pentanone & 0.071 & 0.064 & 0.010 & -10.0 & 40.0 \\
\hline Toluene & 1.545 & 1.612 & 0.400 & 4.3 & 30.0 \\
\hline trans-1,3-Dichloropropene & 0.285 & 0.277 & 0.100 & -2.7 & 30.0 \\
\hline 1,1,2-Trichloroethane & 0.156 & 0.142 & 0.100 & -8.8 & 30.0 \\
\hline Tetrachloroethene & 0.345 & 0.353 & 0.100 & 2.3 & 30.0 \\
\hline 2-Hexanone & 0.047 & 0.042 & 0.010 & -11.2 & 40.0 \\
\hline Dibromochloromethane & 0.201 & 0.201 & 0.100 & 0.0 & 30.0 \\
\hline 1,2-Dibromoethane & 0.135 & 0.132 & 0.010 & -2.5 & 40.0 \\
\hline Chlorobenzene & 0.946 & 0.949 & 0.500 & 0.3 & 30.0 \\
\hline Ethylbenzene & 1.788 & 1.873 & 0.100 & 4.8 & 30.0 \\
\hline o-xylene & 0.654 & 0.695 & 0.300 & 6.1 & 30.0 \\
\hline m,p-Xylene & 0.698 & 0.749 & 0.300 & 7.2 & 30.0 \\
\hline Styrene & 0.978 & 1.038 & 0.300 & 6.2 & 30.0 \\
\hline Bromoform & 0.176 & 0.189 & 0.050 & 7.8 & 30.0 \\
\hline Isopropylbenzene & 1.819 & 1.986 & 0.010 & 9.2 & 40.0 \\
\hline $1,1,2,2$-Tetrachloroethane & 0.139 & 0.127 & 0.100 & -8.7 & 30.0 \\
\hline 1,3-Dichlorobenzene & 1.469 & 1.499 & 0.400 & 2.0 & 30.0 \\
\hline 1,4-Dichlorobenzene & 1.488 & 1.472 & 0.400 & -1.1 & 30.0 \\
\hline 1,2-Dichlorobenzene & 1.198 & 1.185 & 0.400 & $-1 \cdot 1$ & 30.0 \\
\hline 1,2-Dibromo-3-Chloropropane & 0.038 & 0.036 & 0.010 & -5.1 & 40.0 \\
\hline 1,2,4-Trichlorobenzene & 0.709 & 0.670 & 0.200 & -5.5 & 30.0 \\
\hline $1,2,3$-Trichlorobenzene & 0.500 & 0.429 & 0.200 & $-14 \cdot 2$ & 30.0 \\
\hline
\end{tabular}


$7 C$ - FORM VII VOA-3

VOLATILE CONTINUING CALIBRATION DATA

Lab Name: TESTAMERICA BURLINGTON

Contract: $8 \mathrm{E}-00302$

Lab code: STIV Case No.: INMAN Mod. Ref No.: SDG No.: 200-3113

Instrument ID: J.i Calibration Date: 12/28/2010 Time: 1340

Lab File Id: JCFE02.D Init. Calib. Date(s): 12/23/2010 12/23/2010

EPA Sample No.(VSTD\#\#\#\#): VSTD005JI

Init. Calib. Time(s): $1236 \quad 1417$

Heated Purge: ( $\mathrm{Y} / \mathrm{N}) \quad \mathrm{N} \quad$ GC Column: DB-624 ID: 0.20 (mm) Length: 25 (m)

Purge Volume: 25.0 $(m L)$

\begin{tabular}{|c|c|c|c|c|c|}
\hline COMPOUND & $\overline{\mathrm{RRF}}$ & RRE5.0 & $\begin{array}{l}\text { MIN } \\
\text { RRE }\end{array}$ & $\frac{\circ}{\partial} \mathrm{D}$ & $\mathrm{MAX} \div \mathrm{D}$ \\
\hline Vinyl Chloride-d3 & 0.348 & 0.357 & 0.010 & 2.5 & 30.0 \\
\hline Chloroethane-d5 & 0.296 & 0.308 & 0.010 & 4.3 & 40.0 \\
\hline 1,1-Dichloroethene-d2 & 0.573 & 0.608 & 0.010 & 6.0 & 30.0 \\
\hline 2-Butanone-d5 & 0.022 & 0.020 & 0.010 & -9.1 & 40.0 \\
\hline Chloroform-d & 0.490 & 0.517 & 0.010 & 5.6 & 30.0 \\
\hline 1,2-Dichloroethane-d4 & 0.161 & 0.161 & 0.010 & -0.1 & 30.0 \\
\hline Benzene-d 6 & 1.335 & 1.323 & 0.010 & -0.9 & 30.0 \\
\hline 1,2-Dichloropropane-d6 & 0.354 & 0.365 & 0.010 & 3.0 & 40.0 \\
\hline Toluene-d8 & 1.273 & 1.290 & 0.010 & 1.3 & 30.0 \\
\hline trans-1,3-Dichloropropene-d4 & 0.251 & 0.237 & 0.010 & -5.9 & 30.0 \\
\hline 2-Hexanone-d5 & 0.023 & 0.021 & 0.010 & -9.4 & 40.0 \\
\hline $1,1,2,2$-Tetrachloroethane-d.2 & 0.138 & 0.134 & 0.010 & -3.0 & 30.0 \\
\hline 1,2-Dichlorobenzene-d4 & 0.699 & 0.698 & 0.010 & -0.1 & 30.0 \\
\hline
\end{tabular}


$7 A$ - FORM VII VOA-1

VOLATILE CONTINUING CALIBRATION DATA

Lab Name: TESTAMERICA BURLINGTON

Contract: $8 E-00302$

Lab Code: STLV Case No.: INMAN Mod. Ref No. SDG No.: 200-3113

Instrument ID: J.i

Calibration Date: 12/28/2010 Time: 2214

Lab File Id: JCFE21.D Init. Calib. Date(s): 12/23/2010 12/23/2010

EPA Sample No. (VSTD\#\#\#\#):

VSTD005IJ

Init. Calib. Time(s): 1236 1417

Heated Purge: $(\mathrm{Y} / \mathrm{N})$ GC Column: $\mathrm{DB}-624$ ID: $0.20(\mathrm{~mm})$ Length: 25

(m)

Purge Volume: 25.0 $(\mathrm{mL})$

\begin{tabular}{|c|c|c|c|c|c|}
\hline COMPOUND & $\overline{\mathrm{RRF}}$ & RRF5.0 & $\begin{array}{l}\text { MIN } \\
\text { RRF }\end{array}$ & $\frac{\circ}{\partial}$ & MAX $\stackrel{\circ}{8}$ \\
\hline Dichlorodifluoromethane & 0.354 & 0.310 & 0.010 & $-12 \cdot 3$ & 50.0 \\
\hline Chloromethane & 0.369 & 0.363 & 0.010 & -1.6 & 50.0 \\
\hline Vinyl chloride & 0.382 & 0.374 & 0.010 & -2.1 & 50.0 \\
\hline Bromomethane & 0.199 & 0.183 & 0.010 & -7.8 & 50.0 \\
\hline Chloroethane & 0.235 & 0.236 & 0.010 & 0.4 & 50.0 \\
\hline Trichlorofluoromethane & 0.561 & 0.581 & 0.010 & 3.6 & 50.0 \\
\hline 1,1-Dichloroethene & 0.286 & 0.289 & 0.010 & 1.0 & 50.0 \\
\hline 1,1,2-Trichloro-1,2,2-trifluoroethane & 0.336 & 0.346 & 0.010 & 2.9 & 50.0 \\
\hline Acetone & 0.019 & 0.018 & 0.010 & -5.0 & 50.0 \\
\hline Carbon disulfide & 0.860 & 0.797 & 0.010 & -7.3 & 50.0 \\
\hline Methyl acetate & 0.045 & 0.046 & 0.010 & 2.3 & 50.0 \\
\hline Methylene Chloride & 0.237 & 0.256 & 0.010 & 8.0 & 50.0 \\
\hline trans-1,2-Dichloroethene & 0.310 & 0.300 & 0.010 & -3.2 & 50.0 \\
\hline Methyl tert-butyl ether & 0.342 & 0.374 & 0.010 & 9.4 & 50.0 \\
\hline 1,1-Dichloroethane & 0.497 & 0.535 & 0.010 & 7.6 & 50.0 \\
\hline cis-1,2-Dichloroethene & 0.286 & 0.300 & 0.010 & 4.9 & 50.0 \\
\hline 2-Butanone & 0.024 & 0.027 & 0.010 & 14.9 & 50.0 \\
\hline Bromochloromethane & 0.099 & 0.105 & 0.010 & 5.6 & 50.0 \\
\hline Chloroform & 0.484 & 0.518 & 0.010 & 7.0 & 50.0 \\
\hline 1,1,1-Trichloroethane & 0.614 & 0.583 & 0.010 & -5.1 & 50.0 \\
\hline Cyclohexane & 0.605 & 0.578 & 0.010 & -4.5 & 50.0 \\
\hline Carbon tetrachloride & 0.568 & 0.537 & 0.010 & -5.5 & 50.0 \\
\hline Benzene & 1.432 & 1.390 & 0.010 & -2.9 & 50.0 \\
\hline 1,2-Dichloroethane & 0.210 & 0.222 & 0.010 & 5.6 & 50.0 \\
\hline Trichloroethene & 0.385 & 0.359 & 0.010 & -6.6 & 50.0 \\
\hline Methylcyclohexane & 0.462 & 0.429 & 0.010 & -7.1 & 50.0 \\
\hline
\end{tabular}

Report 1,4-Dioxane for Low/Medium VOA analysis only 
Lab Name: TESTAMERICA BURLINGTON

Lab Code: STLV Case No.: INMAN Mod. Ref No.:
Contract: $8 \mathrm{E}-00302$

Calibration Date: 12/28/2010 Time: 2214 Init. Calib. Date(s): 12/23/2010 12/23/2010

Init. Calib. Time(s): $1236 \quad 1417$ ID: $0.20(\mathrm{~mm})$ Length: 25 (m)

$(\mathrm{mL})$

\begin{tabular}{|c|c|c|c|c|c|}
\hline COMPOUND & $\overline{\mathrm{RRF}}$ & RRF5.0 & $\begin{array}{l}\text { MIN } \\
\text { RRE }\end{array}$ & $\div D$ & MAX $\div D$ \\
\hline 1,2-Dichloropropane & 0.275 & 0.281 & 0.010 & 2.3 & 50.0 \\
\hline Bromodichloromethane & 0.362 & 0.360 & 0.010 & -0.5 & 50.0 \\
\hline cis-1,3-Dichloropropene & 0.409 & 0.420 & 0.010 & 2.6 & 50.0 \\
\hline 4-Methyl-2-pentanone & 0.071 & 0.082 & 0.010 & 15.6 & 50.0 \\
\hline Toluene & 1.545 & 1.538 & 0.010 & -0.5 & 50.0 \\
\hline trans-1,3-Dichloropropene & 0.285 & 0.300 & 0.010 & 5.3 & 50.0 \\
\hline 1,1,2-Trichloroethane & 0.156 & 0.161 & 0.010 & 3.4 & 50.0 \\
\hline Tetrachloroethene & 0.345 & 0.330 & 0.010 & -4.6 & 50.0 \\
\hline 2-Hexanone & 0.047 & 0.054 & 0.010 & 15.1 & 50.0 \\
\hline Dibromochloromethane & 0.201 & 0.225 & 0.010 & 11.8 & 50.0 \\
\hline 1,2-Dibromoethane & 0.135 & 0.140 & 0.010 & 3.6 & 50.0 \\
\hline Chlorobenzene & 0.946 & 0.953 & 0.010 & 0.7 & 50.0 \\
\hline Ethylbenzene & 1.788 & 1.762 & 0.010 & -1.5 & 50.0 \\
\hline o-Xylene & 0.654 & 0.675 & 0.010 & 3.2 & 50.0 \\
\hline m,p-xylene & 0.698 & 0.726 & 0.010 & 4.0 & 50.0 \\
\hline Styrene & 0.978 & 1.049 & 0.010 & 7.3 & 50.0 \\
\hline Bromoform & 0.176 & 0.211 & 0.010 & 20.3 & 50.0 \\
\hline Isopropylbenzene & 1.819 & 1.820 & 0.010 & 0.1 & 50.0 \\
\hline $1,1,2,2$-Tetrachloroethane & 0.139 & 0.152 & 0.010 & 9.3 & 50.0 \\
\hline 1,3-Dichlorobenzene & 1.469 & 1.470 & 0.010 & 0.1 & 50.0 \\
\hline 1,4-Dichlorobenzene & 1.488 & 1.469 & 0.010 & -1.3 & 50.0 \\
\hline 1,2-Dichlorobenzene & 1.198 & 1.231 & 0.010 & 2.7 & 50.0 \\
\hline 1,2-Dibromo-3-Chloropropane & 0.038 & 0.043 & 0.010 & 15.9 & 50.0 \\
\hline 1,2,4-Trichlorobenzene & 0.709 & 0.703 & 0.010 & -0.9 & 50.0 \\
\hline 1,2,3-Trichlorobenzene & 0.500 & 0.495 & 0.010 & -0.9 & 50.0 \\
\hline
\end{tabular}


$7 \mathrm{C}$ - FORM VII VOA-3

VOLATILE CONTINUING CALIBRATION DATA

Lab Name: TESTAMERICA BURLINGTON

Contract: $\quad 8 \mathrm{E}-00302$

Lab code: STLV Case No.: INMAN Mod. Ref No.: SDG NO.: $200-3113$

Instrument ID: J.i

Lab File Id: JCEE21.D

Calibration Date: 12/28/2010 Time: 2214

EPA Sample No. (VSTD\#\#\#\#)

VSTD005IJ

$\frac{N}{\text { GC Column: }}$ (mL)

$\frac{N}{\text { GC Column: }} \frac{\mathrm{DB}-6}{(\mathrm{~mL})}$

Purge Volume: 25.0

\begin{tabular}{|c|c|c|c|c|c|}
\hline COMPOUND & $\overline{\mathrm{RRF}}$ & RRF5. 0 & $\begin{array}{l}\text { MIN } \\
\text { RRF }\end{array}$ & 응 & MAX 음 \\
\hline Vinyl Chloride-d3 & 0.348 & 0.330 & 0.010 & -5.3 & 50.0 \\
\hline Chloroethane-d5 & 0.296 & 0.288 & 0.010 & -2.5 & 50.0 \\
\hline 1,1-Dichloroethene-d2 & 0.573 & 0.585 & 0.010 & 2.0 & 50.0 \\
\hline 2-Butanone-d5 & 0.022 & 0.026 & 0.010 & 16.8 & 50.0 \\
\hline Chloroform-d & 0.490 & 0.526 & 0.010 & 7.3 & 50.0 \\
\hline 1,2-Dichloroethane-d4 & 0.161 & 0.179 & 0.010 & 10.7 & 50.0 \\
\hline Benzene-d6 & 1.335 & 1.274 & 0.010 & $-4 \cdot 6$ & 50.0 \\
\hline 1,2-Dichloropropane-d6 & 0.354 & 0.373 & 0.010 & 5.2 & 50.0 \\
\hline Toluene-d8 & 1.273 & 1.251 & 0.010 & -1.7 & 50.0 \\
\hline trans-1,3-Dichloropropene-d4 & 0.251 & 0.254 & 0.010 & 1.2 & 50.0 \\
\hline 2-Hexanone-d5 & 0.023 & 0.027 & 0.010 & 15.6 & 50.0 \\
\hline $1,1,2,2-$ Tetrachloroethane-d2 & 0.138 & 0.150 & 0.010 & 8.5 & 50.0 \\
\hline 1,2-Dichlorobenzene-d4 & 0.699 & 0.738 & 0.010 & 5.6 & 50.0 \\
\hline
\end{tabular}

Report 1,4-Dioxane-d8 for Low/Medium VOA analysis only 
$1 A$ - FORM I VOA-1

VOLATILE ORGANICS ANALYSIS DATA SHEET
EPA SAMPLE NO.

VBLKJF
Lab Name: TESTAMERICA BURLINGTON

Lab Code: STLV Case

Matrix: (SOIL/SED/WATER)

Sample wt/vol: 25.0 $(\mathrm{g} / \mathrm{mL}) \mathrm{mL}$

Level: (TRACE/LOW/MED) TRACE

\% Moisture: not dec.

GC Column: DB-624 ID $: 0.20 \quad(\mathrm{~mm})$

Soil Extract Volume:

Purge Volume: 25.0 (mL)
Contract: $8 \mathrm{E}-00302$

SDG No.: $\quad 200-3113$
Lab Sample ID: MB 200-11655/5

Lab File ID: JCFB05.D

Date Received:

Date Analyzed: 12/24/2010

Dilution Factor: 1.0

Soil Aliquot Volume:

(uL)

\begin{tabular}{|c|c|c|c|}
\hline CAS NO. & COMPOUND & $\begin{array}{l}\text { CONCENTRATION UNITS: } \\
(\mathrm{ug} / \mathrm{L} \text { or } \mathrm{ug} / \mathrm{kg}) \mathrm{ug} / \mathrm{L}\end{array}$ & c \\
\hline $75-71-8$ & Dichlorodifluoromethane & 0.50 & $\mathrm{U}$ \\
\hline $74-87-3$ & Chloromethane & 0.10 & $\mathrm{~J}$ \\
\hline $75-01-4$ & Vinyl chloride & 0.50 & $\mathrm{U}$ \\
\hline $74-83-9$ & Bromomethane & 0.50 & $\mathrm{U}$ \\
\hline $75-00-3$ & Chloroethane & 0.50 & $\mathrm{U}$ \\
\hline $75-69-4$ & Trichlorofluoromethane & 0.50 & $\mathrm{U}$ \\
\hline $75-35-4$ & 1,1-Dichloroethene & 0.50 & $\mathrm{U}$ \\
\hline $76-13-1$ & 1,1,2-Trichloro-1,2,2-trifluoroethane & 0.50 & $\mathrm{U}$ \\
\hline $67-64-1$ & Acetone & 1.9 & $\mathrm{~J}$ \\
\hline $75-15-0$ & Carbon disulfide & 0.17 & $\mathrm{~J}$ \\
\hline $79-20-9$ & Methyl acetate & 0.50 & $\mathrm{U}$ \\
\hline $75-09-2$ & Methylene Chloride & 0.50 & $\mathrm{U}$ \\
\hline $156-60-5$ & trans-1,2-Dichloroethene & 0.50 & $\mathrm{U}$ \\
\hline $1634-04-4$ & Methyl tert-butyl ether & 0.092 & $\mathrm{~J}$ \\
\hline $75-34-3$ & 1,1-Dichloroethane & 0.50 & $\mathrm{U}$ \\
\hline $156-59-2$ & cis-1,2-Dichloroethene & 0.50 & $\mathrm{U}$ \\
\hline $78-93-3$ & 2-Butanone & 5.0 & $\mathrm{U}$ \\
\hline $74-97-5$ & Bromochloromethane & 0.50 & $\mathrm{U}$ \\
\hline $67-66-3$ & Chloroform & 0.50 & $\mathrm{U}$ \\
\hline $71-55-6$ & 1,1,1-Trichloroethane & 0.50 & $\mathrm{U}$ \\
\hline $110-82-7$ & Cyclohexane & 0.50 & $\mathrm{U}$ \\
\hline $56-23-5$ & Carbon tetrachloride & 0.50 & $\mathrm{U}$ \\
\hline $71-43-2$ & Benzene & 0.50 & $\mathrm{U}$ \\
\hline $107-06-2$ & 1,2-Dichloroethane & 0.50 & $\mathrm{U}$ \\
\hline
\end{tabular}

Report 1,4-Dioxane for Low-Medium VOA analysis only 
Lab Name: TESTAMERICA BURLINGTON

Lab Code: STLV

Matrix: (SOIL/SED/WATER)

Sample wt/vol: 25.0

$(\mathrm{g} / \mathrm{mL}) \quad \mathrm{mL}$

Level: (TRACE/LOW/MED)

TRACE

응 Moisture: not dec.

GC Column: DB-624

ID: 0.20 (mm)

Soil Extract Volume:

Purge Volume: 25.0

(mL)
Contract: $8 \mathrm{E}-00302$

SDG No.: 200-3113

\begin{tabular}{|c|c|c|c|}
\hline CAS NO. & COMPOUND & $\begin{array}{l}\text { CONCENTRATION UNITS: } \\
(\mathrm{ug} / \mathrm{L} \text { or } \mathrm{ug} / \mathrm{kg}) \mathrm{ug} / \mathrm{L}\end{array}$ & $Q$ \\
\hline $79-01-6$ & Trichloroethene & 0.50 & $\mathrm{U}$ \\
\hline $108-87-2$ & Methylcyclohexane & 0.50 & $\mathrm{U}$ \\
\hline $78-87-5$ & 1,2-Dichloropropane & 0.50 & $\mathrm{U}$ \\
\hline $75-27-4$ & Bromodichloromethane & 0.50 & $\mathrm{U}$ \\
\hline $10061-01-5$ & cis-1,3-Dichloropropene & 0.50 & $\mathrm{U}$ \\
\hline $108-10-1$ & 4-Methyl-2-pentanone & 5.0 & $\mathrm{U}$ \\
\hline $108-88-3$ & Toluene & 0.50 & $\mathrm{U}$ \\
\hline $10061-02-6$ & trans-1,3-Dichloropropene & 0.50 & $\mathrm{U}$ \\
\hline $79-00-5$ & 1,1,2-Trichloroethane & 0.50 & $\mathrm{U}$ \\
\hline $127-18-4$ & Tetrachloroethene & 0.50 & $\mathrm{U}$ \\
\hline $591-78-6$ & 2-Hexanone & 5.0 & $\mathrm{U}$ \\
\hline $124-48-1$ & Dibromochloromethane & 0.50 & $\mathrm{U}$ \\
\hline $106-93-4$ & 1,2-Dibromoethane & 0.50 & $\mathrm{U}$ \\
\hline $108-90-7$ & Chlorobenzene & 0.50 & $\mathrm{U}$ \\
\hline $100-41-4$ & Ethylbenzene & 0.50 & $\mathrm{U}$ \\
\hline $95-47-6$ & o-Xylene & 0.50 & $\mathrm{U}$ \\
\hline $179601-23-1$ & m,p-Xylene & 0.50 & $\mathrm{U}$ \\
\hline $100-42-5$ & Styrene & 0.50 & $\mathrm{U}$ \\
\hline $75-25-2$ & Bromoform & 0.50 & $\mathrm{U}$ \\
\hline $98-82-8$ & Isopropylbenzene & 0.50 & $\mathrm{U}$ \\
\hline $79-34-5$ & $1,1,2,2$-Tetrachloroethane & 0.50 & $\mathrm{U}$ \\
\hline $541-73-1$ & 1,3-Dichlorobenzene & 0.50 & $\mathrm{U}$ \\
\hline $106-46-7$ & 1,4-Dichlorobenzene & 0.037 & $\mathrm{~J}$ \\
\hline $95-50-1$ & 1,2-Dichlorobenzene & 0.50 & $\mathrm{U}$ \\
\hline $96-12-8$ & 1,2-Dibromo-3-Chloropropane & 0.50 & $\mathrm{U}$ \\
\hline $120-82-1$ & 1,2,4-Trichlorobenzene & 0.11 & $\mathrm{~J}$ \\
\hline $87-61-6$ & 1,2,3-Trichlorobenzene & 0.50 & $\mathrm{U}$ \\
\hline
\end{tabular}


$1 \mathrm{~J}$ - FORM I VOA-TIC

VOLATILE ORGANICS ANALYSIS DATA SHEET

TENTATIVELY IDENTIFIED COMPOUNDS
EPA SAMPLE NO.

VBLKJF

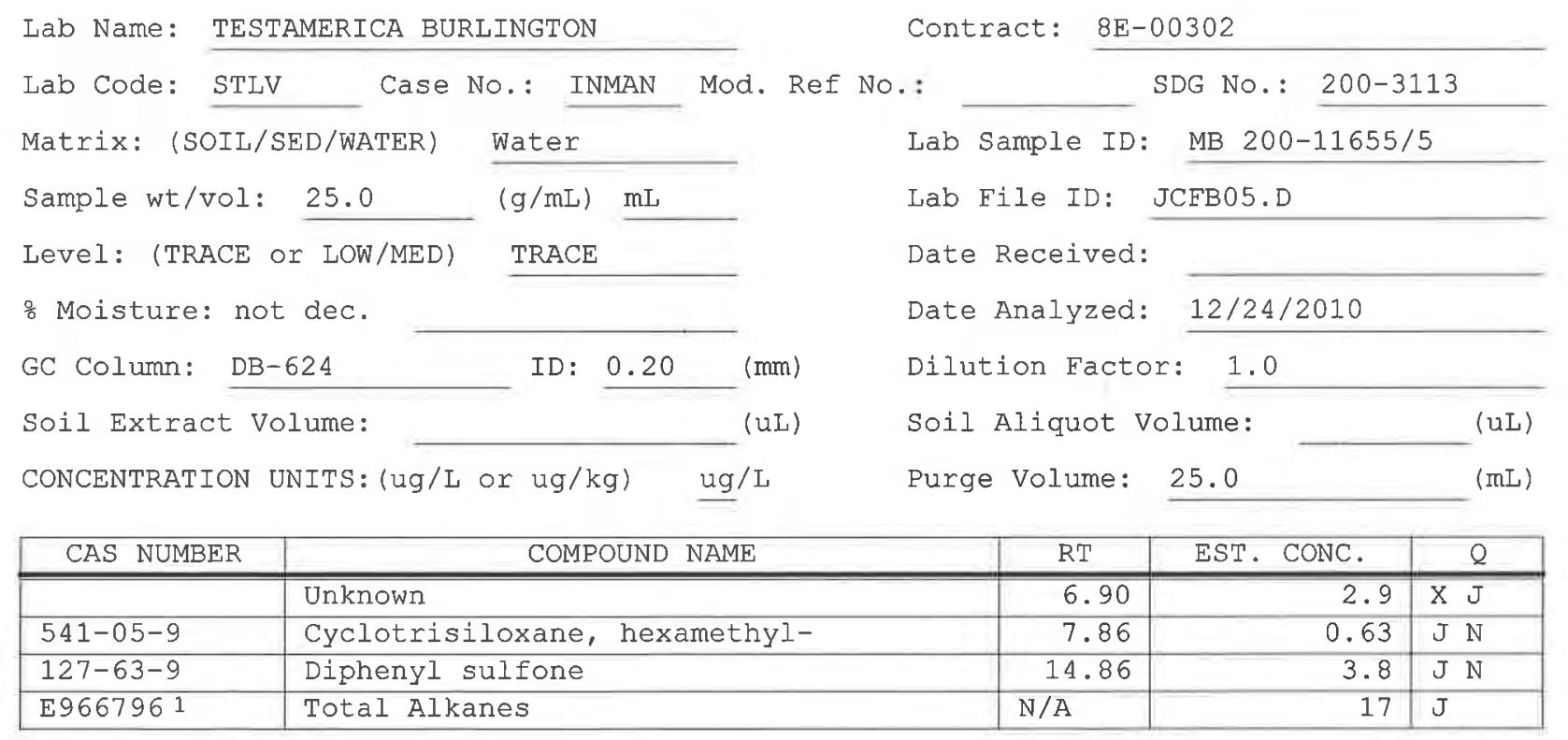

1 EPA-designated Registry Number. 
$1 A$ - FORM I VOA-1

VOLATILE ORGANICS ANALYSIS DATA SHEET
EPA SAMPLE NO.

VBLKJG

Lab Name: TESTAMERICA BURLINGTON

Lab Code: STLV ID: $0.20 \quad(\mathrm{~mm})$ Matrix: (SOIL/SED/WATER)

Sample wt/vol: 25.0

Level: (TRACE/LOW/MED)

TRACE

음 Moisture: not dec.

GC Column: DB-624

Soil Extract volume:

Water

$(\mathrm{g} / \mathrm{mL}) \mathrm{mL}$

Purge Volume: 25.0
Contract: $\quad 8 \mathrm{E}-00302$ SDG No.: 200-3113
Lab Sample ID: MB 200-11651/3

Lab File ID: JCFC05.D

Date Received:

Date Analyzed: $12 / 27 / 2010$

Dilution Factor: 1.0

Soil Aliquot Volume:

(uL) (mL)

\begin{tabular}{|c|c|c|c|}
\hline CAS NO. & COMPOUND & $\begin{array}{l}\text { CONCENTRATION UNITS: } \\
(\mathrm{ug} / \mathrm{L} \text { or } u g / k g) \mathrm{ug} / \mathrm{L}\end{array}$ & Q \\
\hline $75-71-8$ & Dichlorodifluoromethane & 0.50 & $\mathrm{U}$ \\
\hline $74-87-3$ & Chloromethane & 0.50 & $\mathrm{U}$ \\
\hline $75-01-4$ & Vinyl chloride & 0.50 & $\mathrm{U}$ \\
\hline $74-83-9$ & Bromomethane & 0.50 & $\mathrm{U}$ \\
\hline $75-00-3$ & Chloroethane & 0.50 & $\mathrm{U}$ \\
\hline $75-69-4$ & Trichlorofluoromethane & 0.50 & $\mathrm{U}$ \\
\hline $75-35-4$ & 1,1-Dichloroethene & 0.50 & $\mathrm{U}$ \\
\hline $76-13-1$ & 1,1,2-Trichloro-1,2,2-trifluoroethane & 0.50 & $\mathrm{U}$ \\
\hline $67-64-1$ & Acetone & 1.8 & $\mathrm{~J}$ \\
\hline $75-15-0$ & Carbon disulfide & 0.26 & $\mathrm{~J}$ \\
\hline $79-20-9$ & Methyl acetate & 0.50 & $\mathrm{U}$ \\
\hline $75-09-2$ & Methylene Chloride & 0.50 & $\mathrm{U}$ \\
\hline $156-60-5$ & trans-1,2-Dichloroethene & 0.093 & $\mathrm{~J}$ \\
\hline $1634-04-4$ & Methyl tert-butyl ether & 0.13 & $\mathrm{~J}$ \\
\hline $75-34-3$ & 1,1-Dichloroethane & 0.50 & $\mathrm{U}$ \\
\hline $156-59-2$ & cis-1,2-Dichloroethene & 0.50 & $\mathrm{U}$ \\
\hline $78-93-3$ & 2-Butanone & 5.0 & $\mathrm{U}$ \\
\hline $74-97-5$ & Bromochloromethane & 0.50 & $\mathrm{U}$ \\
\hline $67-66-3$ & Chloroform & 0.50 & $\mathrm{U}$ \\
\hline $71-55-6$ & 1,1,1-Trichloroethane & 0.50 & U \\
\hline $110-82-7$ & Cyclohexane & 0.50 & $\mathrm{U}$ \\
\hline $56-23-5$ & Carbon tetrachloride & 0.50 & $\mathrm{U}$ \\
\hline $71-43-2$ & Benzene & 0.50 & $\mathrm{U}$ \\
\hline $107-06-2$ & 1,2-Dichloroethane & 0.50 & $\mathrm{U}$ \\
\hline
\end{tabular}

Report 1,4-Dioxane for Low-Medium VOA analysis only 
$1 B$ - FORM I VOA-2

VOLATILE ORGANICS ANALYSIS DATA SHEET
EPA SAMPLE NO. VBLKJG
Lab Name: TESTAMERICA BURLINGTON

Lab Code: STLV Case No.: INMAN Mod. Ref No.:

Matrix: (SOIL/SED/WATER)

Sample wt/vol: 25.0

Water

$(\mathrm{g} / \mathrm{mL}) \quad \mathrm{mL}$

Level: (TRACE/LOW/MED)

TRACE

: Moisture: not dec.

GC Column: DB-624 ID: $0.20 \quad$ (mm)

Soil Extract Volume:

Purge Volume: 25.0

(uL)

(mL)
Contract: $8 \mathrm{E}-00302$

SDG No.: 200-3113

Lab Sample ID: MB 200-11651/3

Lab File ID: JCFC05.D

Date Received:

Date Analyzed: 12/27/2010

Dilution Factor: 1.0

Soil Aliquot Volume:

(uL)

\begin{tabular}{|c|c|c|c|}
\hline CAS NO. & COMPOUND & $\begin{array}{l}\text { CONCENTRATION UNITS: } \\
(\mathrm{ug} / \mathrm{L} \text { or } \mathrm{ug} / \mathrm{kg}) \mathrm{ug} / \mathrm{L}\end{array}$ & Q \\
\hline $79-01-6$ & Trichloroethene & 0.50 & $\overline{\mathrm{U}}$ \\
\hline $108-87-2$ & Methylcyclohexane & 0.50 & $\mathrm{U}$ \\
\hline $78-87-5$ & 1,2-Dichloropropane & 0.50 & $\mathrm{U}$ \\
\hline $75-27-4$ & Bromodichloromethane & 0.50 & $\mathrm{U}$ \\
\hline $10061-01-5$ & cis-1,3-Dichloropropene & 0.50 & $\mathrm{U}$ \\
\hline $108-10-1$ & 4-Methyl-2-pentanone & 5.0 & $\mathrm{U}$ \\
\hline $108-88-3$ & Toluene & 0.50 & $\mathrm{U}$ \\
\hline $10061-02-6$ & trans-1,3-Dichloropropene & 0.50 & $\mathrm{U}$ \\
\hline $79-00-5$ & 1,1,2-Trichloroethane & 0.50 & $\mathrm{U}$ \\
\hline $127-18-4$ & Tetrachloroethene & 0.50 & $\mathrm{U}$ \\
\hline $591-78-6$ & 2-Hexanone & 5.0 & $\mathrm{U}$ \\
\hline $124-48-1$ & Dibromochloromethane & 0.50 & $\mathrm{U}$ \\
\hline $106-93-4$ & 1,2-Dibromoethane & 0.50 & $\mathrm{U}$ \\
\hline $108-90-7$ & Chlorobenzene & 0.50 & $\mathrm{U}$ \\
\hline $100-41-4$ & Ethylbenzene & 0.50 & $\mathrm{U}$ \\
\hline $95-47-6$ & o-Xylene & 0.50 & $\mathrm{U}$ \\
\hline $179601-23-1$ & $m, p-x y l e n e$ & 0.50 & $\mathrm{U}$ \\
\hline $100-42-5$ & Styrene & 0.50 & $\mathrm{U}$ \\
\hline $75-25-2$ & Bromoform & 0.50 & $\mathrm{U}$ \\
\hline $98-82-8$ & Isopropylbenzene & 0.50 & $\mathrm{U}$ \\
\hline $79-34-5$ & $1,1,2,2-$ Tetrachloroethane & 0.50 & $\mathrm{U}$ \\
\hline $541-73-1$ & 1,3-Dichlorobenzene & 0.055 & $\mathrm{~J}$ \\
\hline $106-46-7$ & 1,4-Dichlorobenzene & 0.051 & $\mathrm{~J}$ \\
\hline $95-50-1$ & 1,2-Dichlorobenzene & 0.50 & $\mathrm{U}$ \\
\hline $96-12-8$ & 1,2-Dibromo-3-Chloropropane & 0.50 & $\mathrm{U}$ \\
\hline $120-82-1$ & 1,2,4-Trichlorobenzene & 0.092 & $\mathrm{~J}$ \\
\hline $87-61-6$ & 1,2,3-Trichlorobenzene & 0.12 & $\mathrm{~J}$ \\
\hline
\end{tabular}


$1 \mathrm{~J}$ - FORM I VOA-TIC

VOLATILE ORGANICS ANALYSIS DATA SHEET

TENTATIVELY IDENTIFIED COMPOUNDS
EPA SAMPLE NO.

VBLKJG
Lab Name: TESTAMERICA BURLINGTON Lab Code: STLV Case No.: INMAN Mod. Ref No.:

Matrix: (SOIL/SED/WATER) Water

Sample wt/vol: 25.0

Level: (TRACE or LOW/MED)

$(\mathrm{g} / \mathrm{mL}) \quad \mathrm{mL}$

o Moisture: not dec.

GC Column: DB-624

ID: $0.20 \quad$ (mm)

Soil Extract Volume: (uL)

CONCENTRATION UNITS: (ug/L or $\mathrm{ug} / \mathrm{kg}$ )
Contract: $\quad 8 \mathrm{E}-00302$

SDG NO.: 200-3113

Lab Sample ID: MB 200-11651/3

Lab File ID: JCFCO5.D

Date Received:

Date Analyzed: 12/27/2010

Dilution Factor: 1.0

Soil Aliquot Volume:

(uL)

Purge Volume: 25.0 (m山)

\begin{tabular}{l|l|l|r|r|r|}
\hline \multicolumn{1}{|c|}{ CAS NUMBER } & \multicolumn{1}{|c|}{ COMPOUND NAME } & RT & EST. CONC. & Q \\
\cline { 2 - 6 } 01 & & Unknown & 6.90 & 3.2 & X J \\
\hline
\end{tabular}

\footnotetext{
1 EPA-designated Registry Number.
} 
$1 A$ - FORM I VOA-1

VOLATILE ORGANICS ANALYSIS DATA SHEET
EPA SAMPLE NO.

VBLKJI

Lab Name: TESTAMERICA BURLINGTON

Lab Code: STLV Case No.: INMAN Mod.

Water

$(\mathrm{g} / \mathrm{mL}) \quad \mathrm{mL}$

Sample wt/vol: 25.0

Level: (TRACE/LOW/MED)

TRACE

o Moisture: not dec.

GC Column: DB-624

Soil Extract Volume: ID: $0.20 \quad$ (mm)

(uL)

(mL)

Purge Volume: 25.0
Contract: $8 E-00302$

SDG No.: 200-3113

. :

Lab Sample ID: MB 200-11671/3

Lab File ID: JCFE03.D

Date Received:

Date Analyzed: 12/28/2010

Dilution Factor: 1.0

Soil Aliquot Volume:

(uL)

\begin{tabular}{|c|c|c|c|}
\hline CAS NO. & COMPOUND & $\begin{array}{l}\text { CONCENTRATION UNITS: } \\
(\mathrm{ug} / \mathrm{L} \text { or } \mathrm{ug} / \mathrm{kg}) \mathrm{ug} / \mathrm{L}\end{array}$ & Q \\
\hline $75-71-8$ & Dichlorodifluoromethane & 0.50 & $\mathrm{U}$ \\
\hline $74-87-3$ & Chloromethane & 0.50 & $\mathrm{U}$ \\
\hline $75-01-4$ & Vinyl chloride & 0.50 & $\mathrm{U}$ \\
\hline $74-83-9$ & Bromomethane & 0.50 & $\mathrm{U}$ \\
\hline $75-00-3$ & Chloroethane & 0.50 & $\mathrm{U}$ \\
\hline $75-69-4$ & Trichlorofluoromethane & 0.50 & $\mathrm{U}$ \\
\hline $75-35-4$ & 1,1-Dichloroethene & 0.50 & $\mathrm{U}$ \\
\hline $76-13-1$ & 1,1,2-Trichloro-1,2,2-trifluoroethane & 0.50 & $\mathrm{U}$ \\
\hline $67-64-1$ & Acetone & 1.6 & $\mathrm{~J}$ \\
\hline $75-15-0$ & Carbon disulfide & 0.13 & $\mathrm{~J}$ \\
\hline $79-20-9$ & Methyl acetate & 0.50 & $\mathrm{U}$ \\
\hline $75-09-2$ & Methylene Chloride & 0.50 & $\mathrm{U}$ \\
\hline $156-60-5$ & trans-1,2-Dichloroethene & 0.50 & $\mathrm{U}$ \\
\hline $1634-04-4$ & Methyl tert-butyl ether & 0.096 & $\mathrm{~J}$ \\
\hline $75-34-3$ & 1,1-Dichloroethane & 0.50 & $\mathrm{U}$ \\
\hline $156-59-2$ & cis-1,2-Dichloroethene & 0.50 & $\mathrm{U}$ \\
\hline $78-93-3$ & 2-Butanone & 5.0 & $\mathrm{U}$ \\
\hline $74-97-5$ & Bromochloromethane & 0.50 & $\mathrm{U}$ \\
\hline $67-66-3$ & Chloroform & 0.50 & $\mathrm{U}$ \\
\hline $71-55-6$ & 1,1,1-Trichloroethane & 0.50 & $\mathrm{U}$ \\
\hline $110-82-7$ & Cyclohexane & 0.50 & $\mathrm{U}$ \\
\hline $56-23-5$ & Carbon tetrachloride & 0.50 & $\mathrm{U}$ \\
\hline $71-43-2$ & Benzene & 0.50 & $\mathrm{U}$ \\
\hline $107-06-2$ & 1,2-Dichloroethane & 0.50 & $\mathrm{U}$ \\
\hline
\end{tabular}

Report 1,4-Dioxane for Low-Medium VOA analysis only 
$1 B$ - FORM I VOA-2

VOLATILE ORGANICS ANALYSIS DATA SHEET
EPA SAMPLE NO.

VBLKJI
Lab Name: TESTAMERICA BURLINGTON

Lab Code: STLV

Matrix: (SOIL/SED/WATER)

Sample wt/vol: 25.0

Level: (TRACE/LOW/MED)

TRACE

o Moisture: not dec.

GC Column: DB-624

Soil Extract Volume:

Purge Volume: 25.0
Contract: $8 \mathrm{E}-00302$

SDG No.: 200-3113

Lab Sample ID: MB 200-11671/3

Lab File ID: JCFE03.D

Date Received:

Date Analyzed: 12/28/2010

Dilution Factor: 1.0

Soil Aliquot volume:

(mL)

\begin{tabular}{|c|c|c|c|}
\hline CAS NO. & COMPOUND & $\begin{array}{l}\text { CONCENTRATION UNITS: } \\
(\mathrm{ug} / \mathrm{L} \text { or } \mathrm{ug} / \mathrm{kg}) \mathrm{ug} / \mathrm{L}\end{array}$ & $Q$ \\
\hline $7.9-01-6$ & Trichloroethene & 0.50 & $\mathrm{U}$ \\
\hline $108-87-2$ & Methylcyclohexane & 0.50 & $\mathrm{U}$ \\
\hline $78-87-5$ & 1,2-Dichloropropane & 0.50 & $\mathrm{U}$ \\
\hline $75-27-4$ & Bromodichloromethane & 0.50 & $\mathrm{U}$ \\
\hline $10061-01-5$ & cis-1,3-Dichloropropene & 0.50 & $\mathrm{U}$ \\
\hline $108-10-1$ & 4-Methyl-2-pentanone & 5.0 & $\mathrm{U}$ \\
\hline $108-88-3$ & Toluene & 0.50 & $\mathrm{U}$ \\
\hline $10061-02-6$ & trans-1,3-Dichloropropene & 0.50 & $\mathrm{U}$ \\
\hline $79-00-5$ & $1,1,2$-Trichloroethane & 0.50 & $\mathrm{U}$ \\
\hline $127-18-4$ & Tetrachloroethene & 0.50 & $\mathrm{U}$ \\
\hline $591-78-6$ & 2-Hexanone & 5.0 & $\mathrm{U}$ \\
\hline $124-48-1$ & Dibromochloromethane & 0.50 & $\mathrm{U}$ \\
\hline $106-93-4$ & 1,2-Dibromoethane & 0.50 & $\mathrm{U}$ \\
\hline $108-90-7$ & Chlorobenzene & 0.50 & $\mathrm{U}$ \\
\hline $100-41-4$ & Ethylbenzene & 0.50 & $\mathrm{U}$ \\
\hline $95-47-6$ & o-xylene & 0.50 & $\mathrm{U}$ \\
\hline $179601-23-1$ & $\mathrm{~m}, \mathrm{p}$-xylene & 0.50 & $\mathrm{U}$ \\
\hline $100-42-5$ & Styrene & 0.50 & $\mathrm{U}$ \\
\hline $75-25-2$ & Bromoform & 0.50 & $\mathrm{U}$ \\
\hline $98-82-8$ & Isopropylbenzene & 0.50 & $\mathrm{U}$ \\
\hline $79-34-5$ & $1,1,2,2$-Tetrachloroethane & 0.50 & $\mathrm{U}$ \\
\hline $541-73-1$ & 1,3-Dichlorobenzene & 0.042 & $\mathrm{~J}$ \\
\hline $106-46-7$ & 1,4-Dichlorobenzene & 0.50 & $\mathrm{U}$ \\
\hline $95-50-1$ & 1,2-Dichlorobenzene & 0.50 & $\mathrm{U}$ \\
\hline $96-12-8$ & 1,2-Dibromo-3-Chloropropane & 0.50 & $\mathrm{U}$ \\
\hline $120-82-1$ & 1,2,4-Trichlorobenzene & 0.095 & $\mathrm{~J}$ \\
\hline $87-61-6$ & 1,2,3-Trichlorobenzene & 0.12 & $\mathrm{~J}$ \\
\hline
\end{tabular}


$1 \mathrm{~J}$ - FORM I VOA-TIC

VOLATILE ORGANICS ANALYSIS DATA SHEET

TENTATIVELY IDENTIFIED COMPOUNDS
EPA SAMPLE NO.

VBLKJI

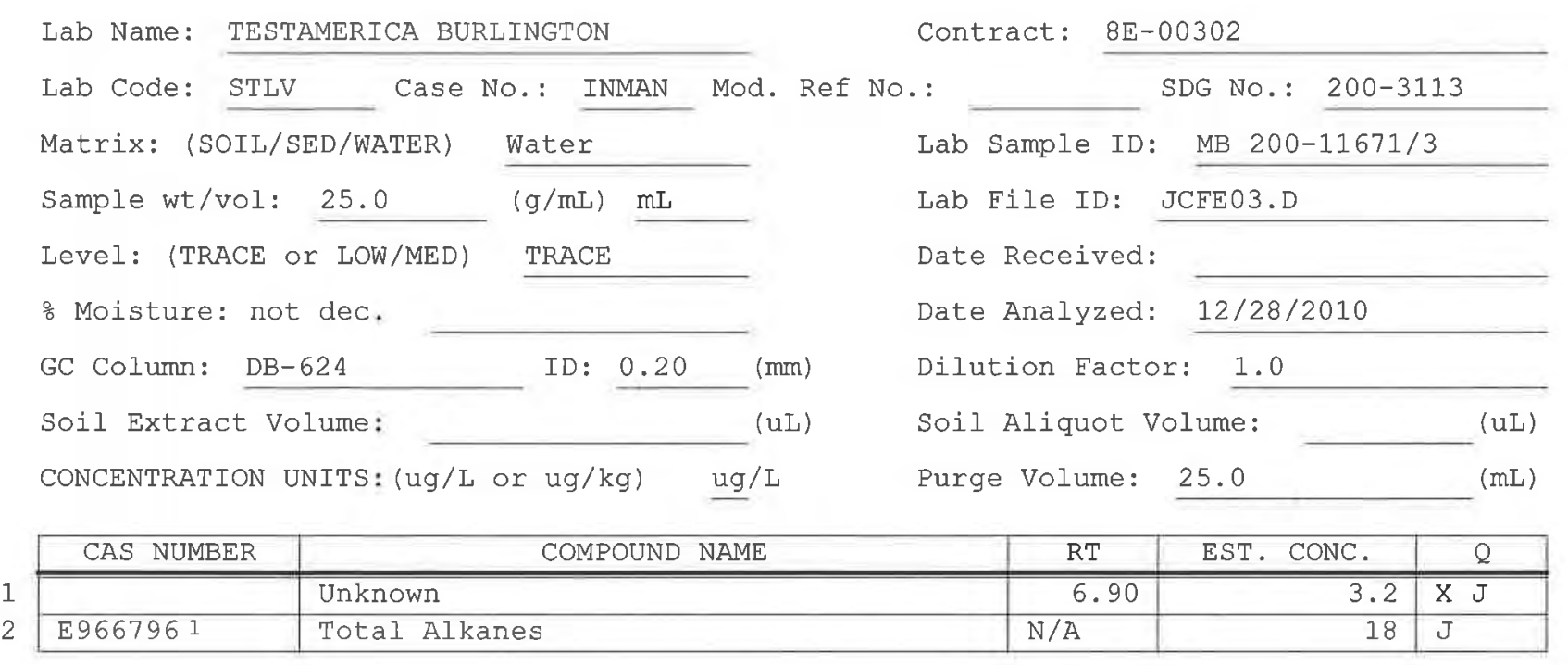

1 EPA-designated Registry Number. 
Lab Name: TESTAMERICA BURLINGTON

Contract: $8 \mathrm{E}-00302$

Lab Code: STLV Case No.: INMAN Mod. Ref No.: SDG No.: 200-3113

Matrix: (SOIL/SED/WATER) Water

Sample wt/vol: 25.0 $(g / m L) \quad m L$

Level: (TRACE/LOW/MED) TRACE

\% Moisture: not dec.

GC Column: DB-624 ID: $0.20 \quad$ (mm)

Soil Extract Volume: (uL) Purge Volume: 25.0 (mL)
Lab Sample ID: 200-3113-19

Lab File ID: JCFE20.D

Date Received:

Date Analyzed: 12/28/2010

Dilution Factor: 1.0

Soil Aliquot Volume:

(uL)

\begin{tabular}{|c|c|c|c|}
\hline CAS NO. & COMPOUND & $\begin{array}{l}\text { CONCENTRATION UNITS: } \\
(\mathrm{ug} / \mathrm{L} \text { or } \mathrm{ug} / \mathrm{kg}) \mathrm{ug} / \mathrm{L}\end{array}$ & 8 \\
\hline $75-71-8$ & Dichlorodifluoromethane & 0.50 & 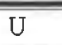 \\
\hline $74-87-3$ & Chloromethane & 0.50 & $\mathrm{U}$ \\
\hline $75-01-4$ & Vinyl chloride & 0.50 & $\mathrm{U}$ \\
\hline $74-83-9$ & Bromomethane & 0.50 & $\mathrm{U}$ \\
\hline $75-00-3$ & Chloroethane & 0.50 & $\mathrm{U}$ \\
\hline $75-69-4$ & Trichlorofluoromethane & 0.50 & $\mathrm{U}$ \\
\hline $75-35-4$ & 1,1-Dichloroethene & 0.50 & $\mathrm{U}$ \\
\hline $76-13-1$ & 1,1,2-Trichloro-1,2,2-trifluoroethane & 0.50 & $\mathrm{U}$ \\
\hline $67-64-1$ & Acetone & 5.0 & $\mathrm{U}$ \\
\hline $75-15-0$ & Carbon disulfide & 0.50 & $\mathrm{U}$ \\
\hline $79-20-9$ & Methyl acetate & 0.50 & $\mathrm{U}$ \\
\hline $75-09-2$ & Methylene Chloride & 0.50 & $\mathrm{U}$ \\
\hline $156-60-5$ & trans-1,2-Dichloroethene & 0.50 & $\mathrm{U}$ \\
\hline $1634-04-4$ & Methyl tert-butyl ether & 0.095 & $\mathrm{~J} \mathrm{~B}$ \\
\hline $75-34-3$ & 1,1-Dichloroethane & 0.50 & $\mathrm{U}$ \\
\hline $156-59-2$ & cis-1,2-Dichloroethene & 0.50 & $\mathrm{U}$ \\
\hline $78-93-3$ & 2-Butanone & 5.0 & $\mathrm{U}$ \\
\hline $74-97-5$ & Bromochloromethane & 0.50 & $\mathrm{U}$ \\
\hline $67-66-3$ & Chloroform & 0.50 & $\mathrm{U}$ \\
\hline $71-55-6$ & 1,1,1-Trichloroethane & 0.50 & $\mathrm{U}$ \\
\hline $110-82-7$ & Cyclohexane & 0.50 & $\mathrm{U}$ \\
\hline $56-23-5$ & Carbon tetrachloride & 0.50 & $\mathrm{U}$ \\
\hline $71-43-2$ & Benzene & 0.50 & $\mathrm{U}$ \\
\hline $107-06-2$ & 1,2-Dichloroethane & 0.50 & $\mathrm{U}$ \\
\hline
\end{tabular}

Report 1,4-Dioxane for Low-Medium VOA analysis only 
$1 B$ - FORM I VOA-2

VOLATILE ORGANICS ANALYSIS DATA SHEET
EPA SAMPLE NO. VHBLKO1
Lab Name: TESTAMERICA BURLINGTON

Lab Code: STLV Case No.: INMAN Mod. Ref No.:
Contract: $8 \mathrm{E}-00302$

SDG No.: 200-3113
Matrix: (SOIL/SED/WATER) Water

Sample wt/vol: 25.0

$(\mathrm{g} / \mathrm{mL}) \mathrm{mL}$

Level: (TRACE/LOW/MED) TRACE

음 Moisture: not dec.

GC Column: DB-624

Soil Extract Volume: ID: $0.20 \quad$ (mm)

Purge Volume: 25.0 (uL) (mL)
Lab Sample ID: 200-3113-19

Lab File ID: JCFE2O.D

Date Received:

Date Analyzed: 12/28/2010

Dilution Factor: 1.0

Soil Aliquot Volume:

(UL)

\begin{tabular}{|c|c|c|c|}
\hline CAS NO. & COMPOUND & $\begin{array}{l}\text { CONCENTRATION UNITS: } \\
(\mathrm{ug} / \mathrm{L} \text { or } \mathrm{ug} / \mathrm{kg}) \mathrm{ug} / \mathrm{L}\end{array}$ & $Q$ \\
\hline $79-01-6$ & Trichloroethene & 0.50 & $\overline{\mathrm{U}}$ \\
\hline $108-87-2$ & Methylcyclohexane & 0.50 & $\mathrm{U}$ \\
\hline $78-87-5$ & 1,2-Dichloropropane & 0.50 & $\mathrm{U}$ \\
\hline $75-27-4$ & Bromodichloromethane & 0.50 & $\mathrm{U}$ \\
\hline $10061-01-5$ & cis-1,3-Dichloropropene & 0.50 & $\mathrm{U}$ \\
\hline $108-10-1$ & 4-Methyl-2-pentanone & 5.0 & $\mathrm{U}$ \\
\hline $108-88-3$ & Toluene & 0.50 & $\mathrm{U}$ \\
\hline $10061-02-6$ & trans-1,3-Dichloropropene & 0.50 & $\mathrm{U}$ \\
\hline $79-00-5$ & 1,1,2-Trichloroethane & 0.50 & $\mathrm{U}$ \\
\hline $127-18-4$ & Tetrachloroethene & 0.50 & $\mathrm{U}$ \\
\hline $591-78-6$ & 2-Hexanone & 5.0 & $\mathrm{U}$ \\
\hline $124-48-1$ & Dibromochloromethane & 0.50 & $\mathrm{U}$ \\
\hline $106-93-4$ & 1,2-Dibromoethane & 0.50 & $\mathrm{U}$ \\
\hline $108-90-7$ & Chlorobenzene & 0.50 & $\mathrm{U}$ \\
\hline $100-41-4$ & Ethylbenzene & 0.50 & $\mathrm{U}$ \\
\hline $95-47-6$ & o-Xylene & 0.50 & $\mathrm{U}$ \\
\hline $179601-23-1$ & $\mathrm{~m}, \mathrm{p}$-xylene & 0.50 & $\mathrm{U}$ \\
\hline $100-42-5$ & Styrene & 0.50 & $\mathrm{U}$ \\
\hline $75-25-2$ & Bromoform & 0.50 & $\mathrm{U}$ \\
\hline $98-82-8$ & Isopropylbenzene & 0.50 & $\mathrm{U}$ \\
\hline $79-34-5$ & $1,1,2,2$-Tetrachloroethane & 0.50 & $\mathrm{U}$ \\
\hline $541-73-1$ & 1,3-Dichlorobenzene & 0.50 & $\mathrm{U}$ \\
\hline $106-46-7$ & 1,4-Dichlorobenzene & 0.50 & $\mathrm{U}$ \\
\hline $95-50-1$ & 1,2-Dichlorobenzene & 0.50 & $\mathrm{U}$ \\
\hline $96-12-8$ & 1,2-Dibromo-3-Chloropropane & 0.50 & $\mathrm{U}$ \\
\hline $120-82-1$ & 1,2,4-Trichlorobenzene & 0.50 & $\mathrm{U}$ \\
\hline $87-61-6$ & 1,2,3-Trichlorobenzene & 0.50 & $\mathrm{U}$ \\
\hline
\end{tabular}


$1 \mathrm{~J}$ - FORM I VOA-TIC

VOLATILE ORGANICS ANALYSIS DATA SHEET

TENTATIVELY IDENTIFIED COMPOUNDS
EPA SAMPLE NO.

VHBLKOI

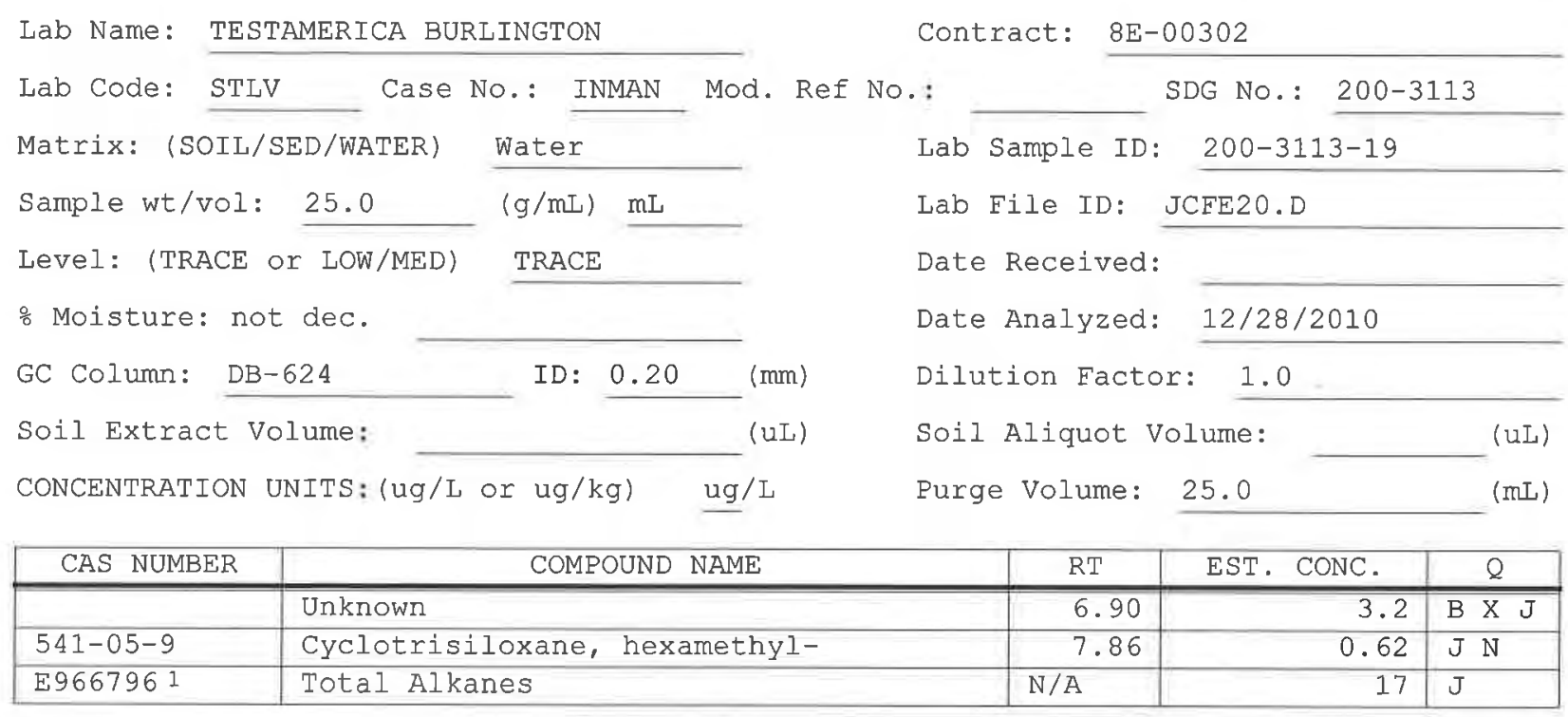

1 EPA-designated Registry Number. 


\section{ANALYTICAL REPORT}

Job Number: 200-5308-1

SDG Number: 200-5308

Job Description: Inman (200-5308)

Contract Number: EP-W-09-044

For:

Argonne National Laboratory

9700 South Cass Avenue

Building 203

Office B-149

Argonne, IL 60439

Attention: Mr. Clyde Dennis

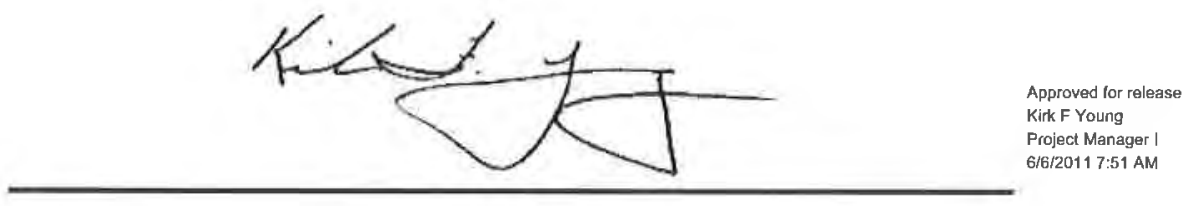

Kirk F Young

Project Manager I

kirk.young@testamericainc.com

06/06/2011

The test results in this report relate only to sample(s) as received by the laboratory. These test results were derived under a quality system that adheres to the requirements of NELAC. Pursuant to NELAC, this report may not be produced in full without written approval from the laboratory 


\section{Table of Contents}

Cover Title Page . . . . . . . . . . . . . . . . . . 1

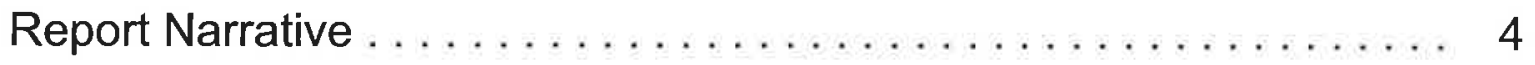

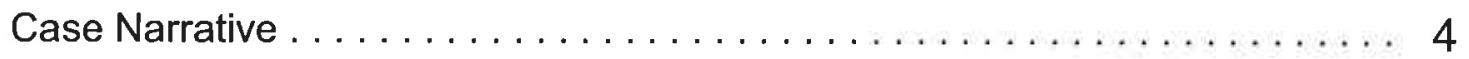

Qualifier Definition ............................ 6

External Chain of Custody . . . . . . . . . . . . . . . 7

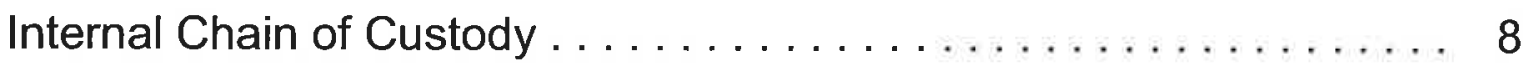

Shipping Documentation . . . . . . . . . . . . . . . . . 10

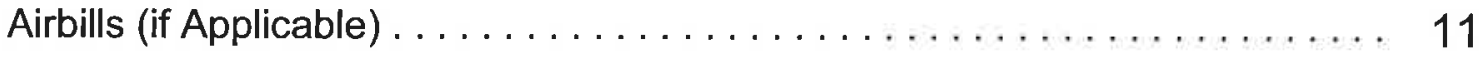

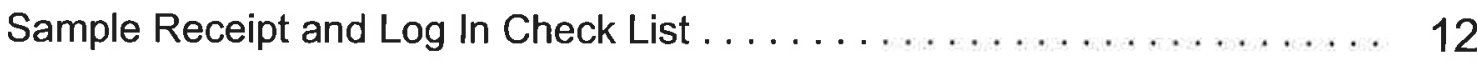

Methodology Review . . . . . . . . . . . . . . . . . . . . . . . . 14

QC Summary - SOM01.2 Volatiles-Trace . . . . . . . . . . . . . . . 15

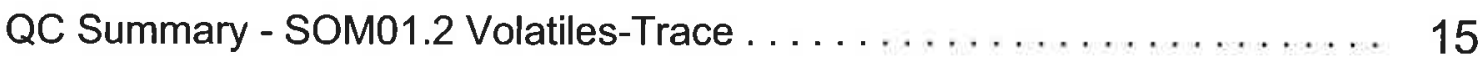

Deuterated Monitoring Compound Summary $\ldots \ldots \ldots \ldots \ldots \ldots \ldots \ldots \ldots \ldots \ldots \ldots \ldots \ldots$

Method Blank $\ldots \ldots \ldots \ldots \ldots \ldots \ldots \ldots \ldots \ldots \ldots \ldots \ldots \ldots \ldots \ldots \ldots \ldots, 17$

GC/MS Instrument Performance Check $\ldots \ldots \ldots \ldots \ldots \ldots \ldots \ldots \ldots \ldots \ldots \ldots \ldots \ldots \ldots \ldots, 18$

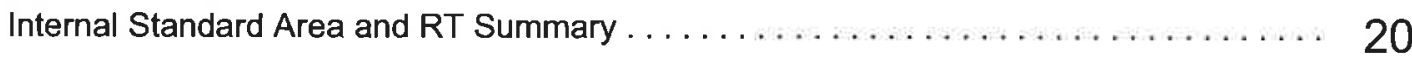

Sample Data - SOM01.2 Volatiles-Trace . . . . . . . . . . . . . 21

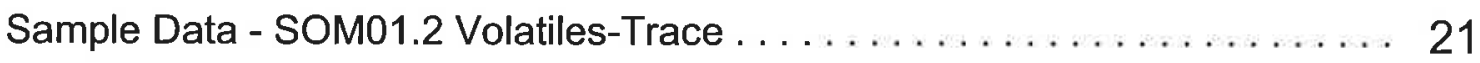

INBROWN-W-32686 $\ldots \ldots \ldots \ldots \ldots \ldots \ldots \ldots \ldots \ldots \ldots \ldots \ldots \ldots \ldots \ldots \ldots \ldots \ldots \ldots \ldots, 21$

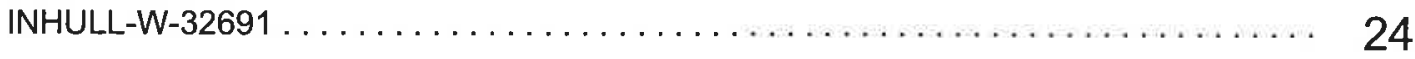

INQCTB-W-32692 $\ldots \ldots \ldots \ldots \ldots \ldots \ldots \ldots \ldots \ldots \ldots \ldots \ldots \ldots \ldots \ldots \ldots \ldots \ldots \ldots \ldots \ldots, 27$

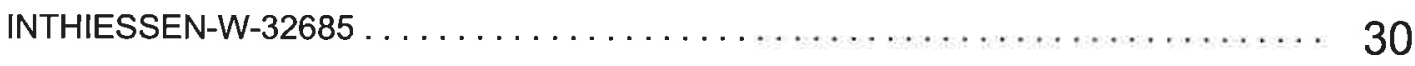

Standards - SOM01.2 Volatiles-Trace . . . . . . . . . . . . . . . 33

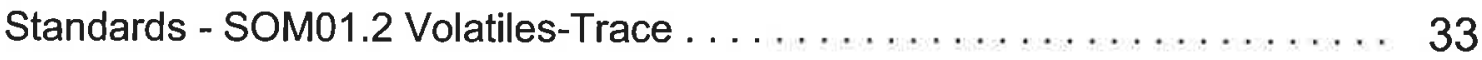

Initial Calibration Data $\ldots \ldots \ldots \ldots \ldots \ldots \ldots \ldots \ldots \ldots \ldots \ldots \ldots, 33$

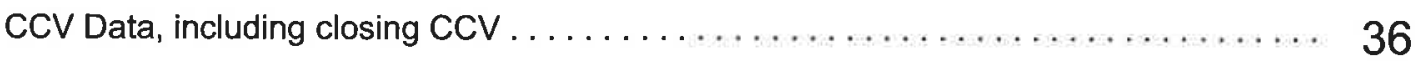




\section{Table of Contents}

Raw Qc Data - SOM01.2 Volatiles-Trace . . . . . . . . . . . . . . . . 42

Raw Qc Data - SOM01.2 Volatiles-Trace . . . . . . . . . . . . . . . . 42

Raw Qc Data - SOM01.2 Volatiles-Trace $\ldots \ldots \ldots \ldots \ldots \ldots \ldots \ldots \ldots \ldots \ldots, 42$

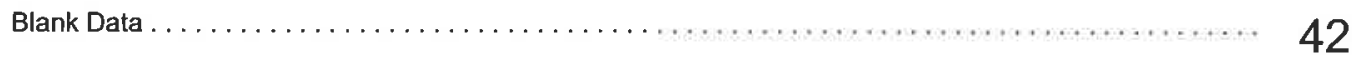




\title{
CASE NARRATIVE
}

\section{Client: Argonne National Laboratory}

\author{
Project: Inman (200-5308)
}

Report Number: 200-5308-1

\begin{abstract}
Enclosed is the data set for the referenced project work. With the exceptions noted as flags or footnotes, standard analytical protocols were followed in performing the analytical work and the applied control limits were met.
\end{abstract}

Calculations were performed before rounding to avoid round-off errors in calculated results.

All holding times were met and proper preservation noted for the methods performed on these samples, unless otherwise detailed in the individual sections below.

\section{Receipt}

The samples were received on 05/25/2011. Documentation of the condition of the samples at the time of their receipt and any exception to the laboratory's Sample Acceptance Policy is documented in the Sample Handling section of this submittal. The samples, as received, were not acid preserved. On that basis, the laboratory did provide for the analytical work to be performed within seven days of sample collection.

\section{SOM01.2 Volatile Organics (Trace Level Water)}

A storage blank was prepared for volatile organics analysis, and stored in association with the storage of the samples. That storage blank, identified as VHBLK01, was carried through the holding period with the samples, and analyzed.

With the exception of sample INBROWN-W-32686, each sample was analyzed without a dilution. Sample INBROWN-W-32686 was analyzed at a 2.2-fold dilution, and this represented the most concentrated analysis of that sample. Acetone, 2- butanone, and tetrahydrofuran were identified as constituents of sample INBROWN-W-32686. The presence of those compounds was suspected of being related to the construction of the sampling point. The laboratory did identify the issue to the project team, and a decision was made not to pursue a quantitative analysis of 2- butanone.

Each of the analyses associated with the sample set exhibited an acceptable internal standard performance. There was an acceptable recovery of each deuterated monitoring compound (DMC) in the analysis of the method blank and instrument blank associated with the analytical work, and in the analysis of the storage blank associated with the sample set. The analysis of the samples in this sample set did meet the technical acceptance criteria specific to DMC recoveries, although not all DMC recoveries were within the control range in each analysis. The technical acceptance criteria does provide for the recovery of up to three DMCs to fall outside of the control range in the analysis of field samples. Matrix spike and matrix spike duplicate analyses were not performed on samples in this sample set. Trace concentrations of acetone, carbon disulfide, trans-1,2-dichloroethene, carbon tetrachloride, toluene, and 1,2,3-trichlorobenzene were identified in the analysis of the method blank associated with the analytical work. The concentration of each compound in that analysis was below the established reporting limit, and the analysis did meet the technical acceptance criteria for a compliant method blank analysis. A 
trace concentration of carbon tetrachloride was identified in the analysis of the storage blank associated with the sample set. The concentration of carbon tetrachloride in that analysis was below the established reporting limit, and the analysis did meet the technical acceptance criteria for a compliant storage blank analysis. Trace concentrations of carbon disulfide and carbon tetrachloride were identified in the analysis of the instrument blank associated with the sample set. The concentration of each compound in that analysis was below the established reporting limit, and the analysis did meet the technical acceptance criteria for a compliant instrument blank analysis. Present in the method blank, storage blank, and instrument blank analyses was a nontarget constituent that represents a compound that is related to the DMC formulation. The fact that the presence of this compound is not within the laboratory's control is at issue. The derived results for that compound have been qualified with an "X" qualifier to reflect the source of the contamination.

The responses for each of the target analytes met the relative standard deviation criterion in the initial calibration. The response for each target analyte met the percent difference criterion in the opening/continuing calibration check acquisition. The response for each target analyte met the 50.0 percent difference criterion in the closing calibration check acquisition.

The primary quantitation mass for methylcyclohexane that is specified in the Statement of Work is mass 83 . The laboratory did identify a contribution to mass 83 from 1,2-dichloropropane- $d_{6}$, one of the deuterated monitoring compounds (DMCs). The laboratory did change the primary quantitation mass assignment to mass 55 for the quantification of methylcyclohexane.

Manual integration was employed in deriving certain of the analytical results. The values that have been derived from manual integration are qualified on the quantitation reports. Extracted ion current profiles for each manual integration are included in the data package, and further documented at the end of this submittal. 


\section{DATA REPORTING QUALIFIERS}

Client: Argonne National Laboratory

Job Number: 200-5308-1

Sdg Number: $200-5308$

Description

GC/MS VOA

U

E

J

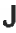

$x$

B

N
Analyzed for but not detected.

Compound concentration exceeds the upper level of the calibration range of the instrument for that specific analysis.

Indicates an Estimated Value for TICs

Indicates an estimated value.

See case narrative notes for explanation of the ' $X$ ' flag

Surrogate exceeds the control limit

The analyte was found in an associated blank, as well as in the sample.

This flag indicates the presumptive evidence of a compound. 

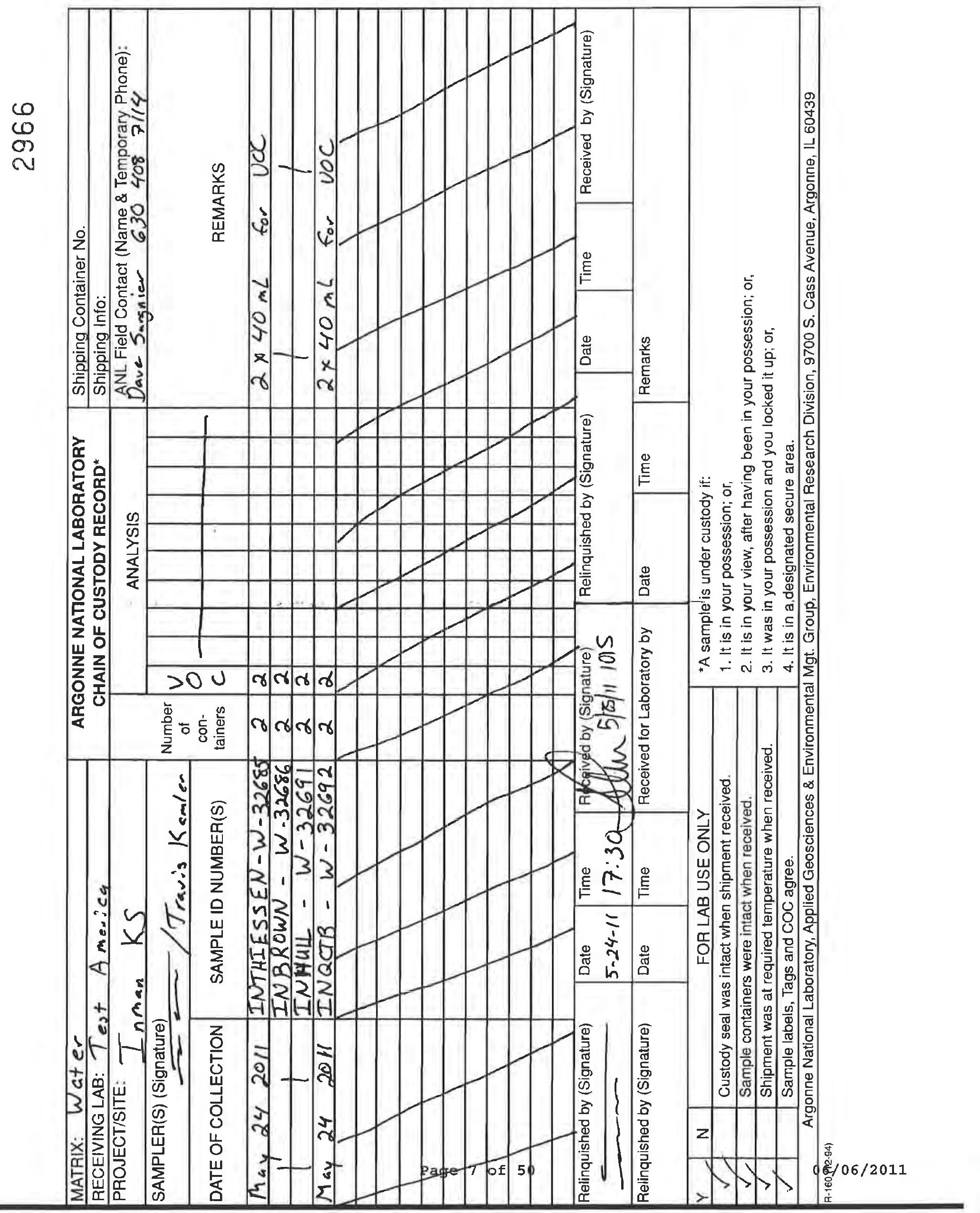


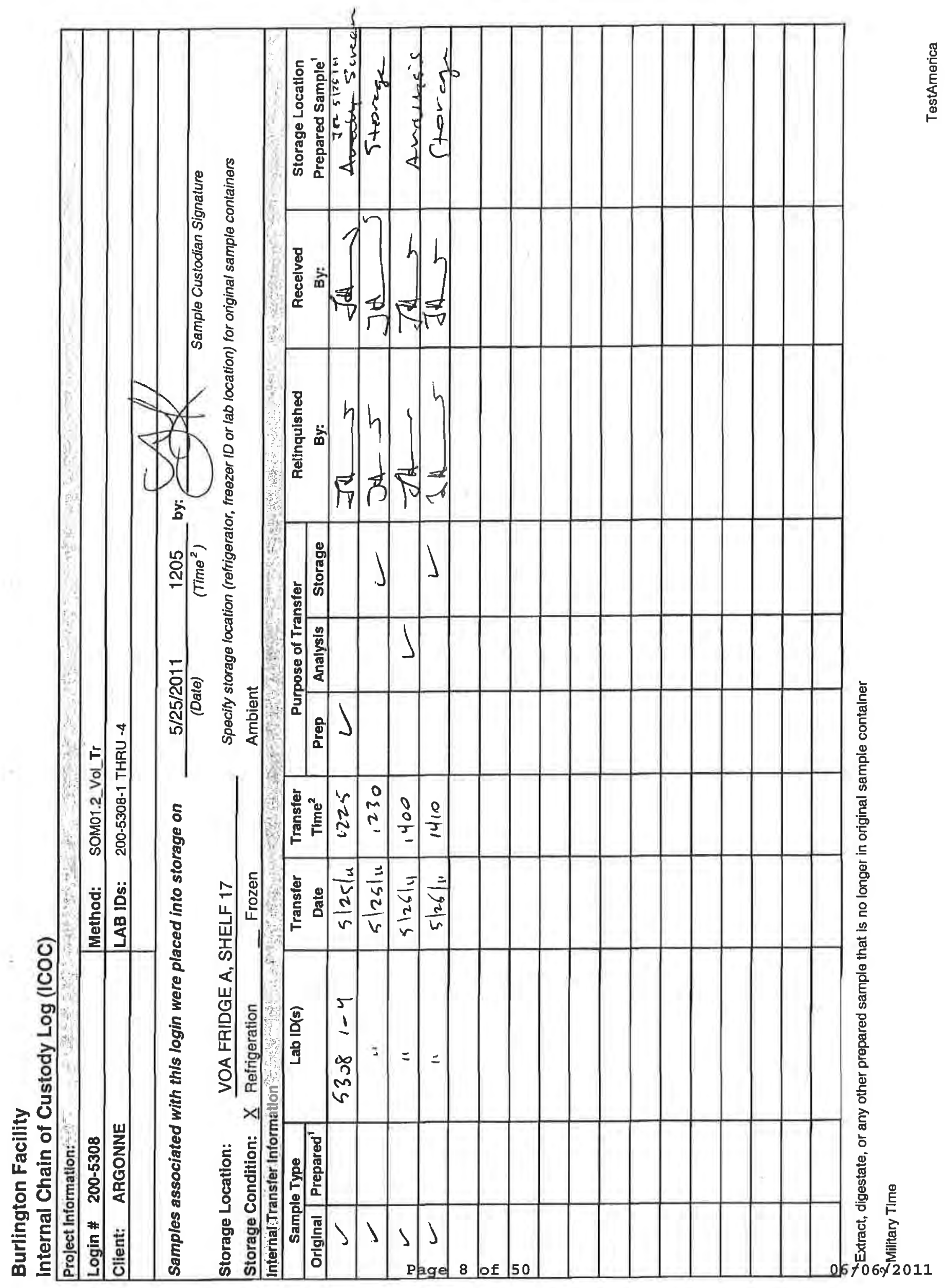




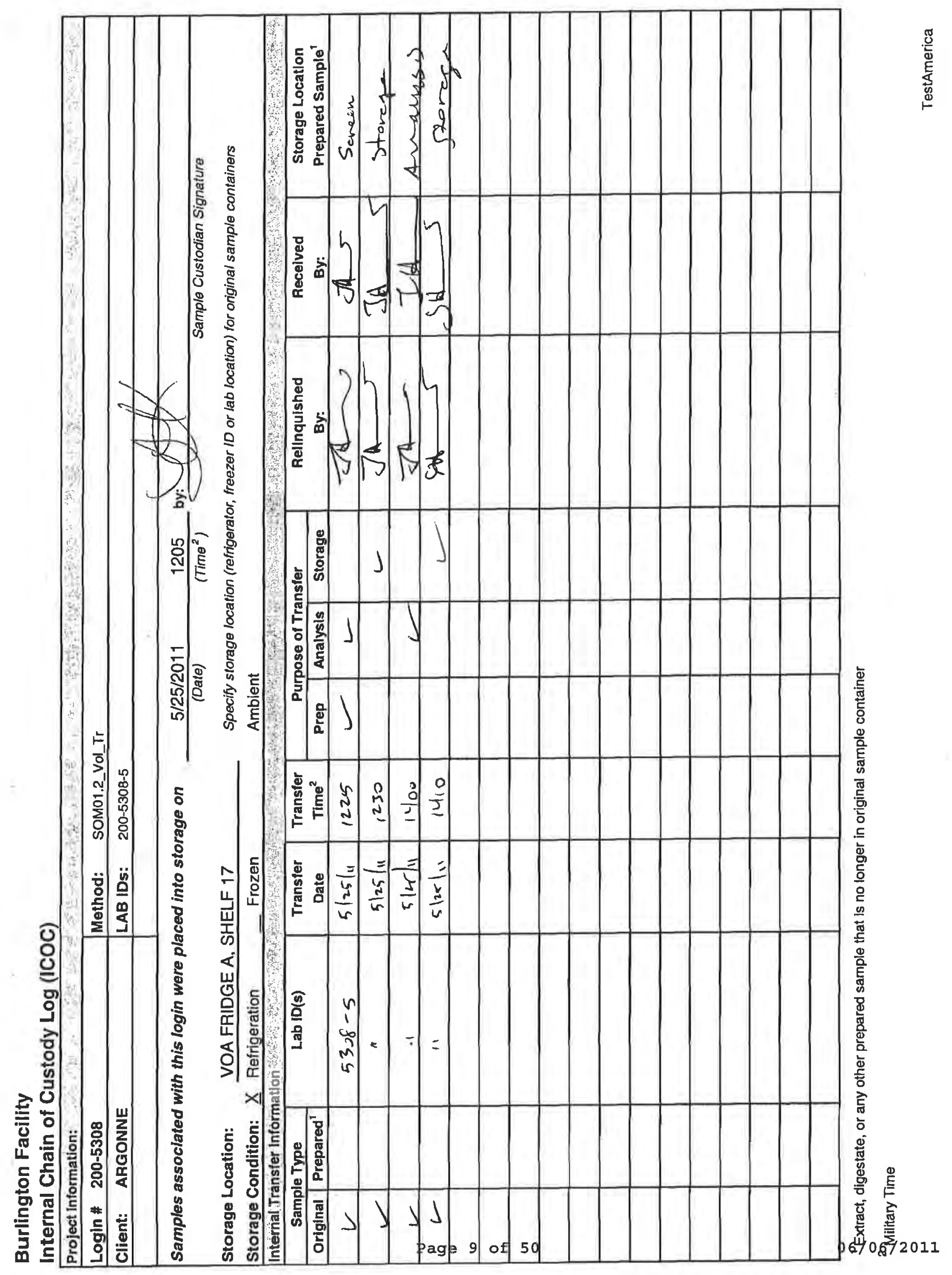




\section{Shipping and Receiving Documents}




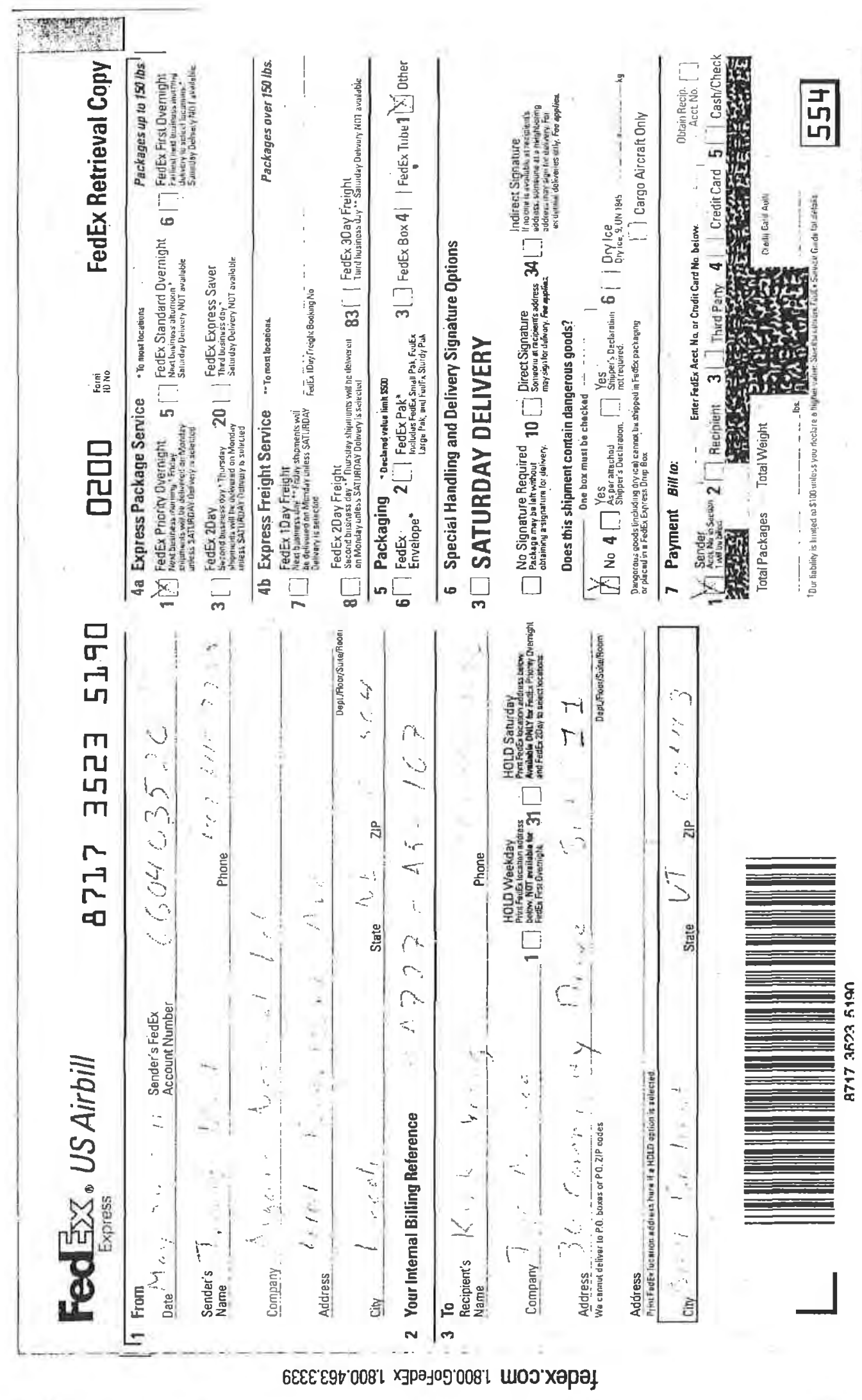




\section{Login Sample Receipt Checklist}

$\begin{array}{ll}\text { Answer } & \text { Comment } \\ \text { N/A } & \text { Lab does not accept radioactive samples. } \\ \text { True } & \text { NO CUSTODY SEAL NUMBERS } \\ \text { True } & \\ \text { True } & \\ \text { True } & \\ \text { True } & 0.1^{\circ} \mathrm{C}, \text { IR GUN ID 96, CF 0 } \\ \text { True } & \\ \text { True } & \\ \text { True } & \\ \text { True } & \\ \text { True } & \\ \text { True } & \\ \text { True } & \\ \text { True } & \\ \text { True } & \\ \text { True } & \\ \text { True } & \\ \text { N/A } & \\ \text { True } & \\ \text { True } & \\ \text { True } & \\ \text { True } & \\ \text { N/A } & \end{array}$

Radioactivity either was not measured or, if measured, is at or below background

The cooler's custody seal, if present, is intact.

The cooler or samples do not appear to have been compromised or tampered with.

Samples were received on ice.

Cooler Temperature is acceptable.

Cooler Temperature is recorded.

$\mathrm{COC}$ is present.

$\mathrm{COC}$ is filled out in ink and legible.

$\mathrm{COC}$ is filled out with all pertinent information.

Is the Field Sampler's name present on COC?

There are no discrepancies between the sample IDs on the containers and the $\mathrm{COC}$.

Samples are received within Holding Time.

Sample containers have legible labels.

Containers are not broken or leaking.

Sample collection date/times are provided.

Appropriate sample containers are used.

Sample bottles are completely filled.

Sample Preservation Verified.

There is sufficient vol. for all requested analyses, incl. any requested MS/MSDs

VOA sample vials do not have headspace or bubble is $<6 \mathrm{~mm}(1 / 4 ")$ in diameter.

Multiphasic samples are not present.

Samples do not require splitting or compositing.

Residual Chlorine Checked.
Check done at department level if required. 


\section{Sample Login Acknowledgement}

Job 200-5308-1

$\begin{array}{ll}\text { Client Job Description: } & \text { Inman (200-5308) } \\ \text { Purchase Order \#: } & 8 \mathrm{E}-00302 \\ \text { Work Order \#: } & 8 \mathrm{E}-00302 \\ \text { Project Manager: } & \text { Kirk F Young } \\ \text { Job Due Date: } & 6 / 8 / 2011 \\ \text { Job TAT: } & \text { 14 Days } \\ \text { Max Deliverable Level: } & \text { IV }\end{array}$

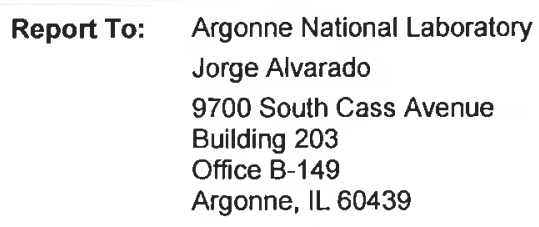

\section{Login 200-5308}

\begin{tabular}{|c|c|}
\hline Sample Receipt: & 5/25/2011 10:15:00 AM \\
\hline Method of Delivery: & FedEx Priority Overnight \\
\hline $\begin{array}{l}\text { Lab Sample \# } \\
\text { Method }\end{array}$ & $\begin{array}{l}\text { Client Sample ID } \\
\text { Method Description / Work Location }\end{array}$ \\
\hline 200-5308-1 & INTHIESSEN-W-32685 \\
\hline SOM01.2_Vol_Tr & SOM01.2 Trace Volatile Organics / In-Lab \\
\hline 200-5308-2 & INBROWN-W-32686 \\
\hline SOM01.2_Vol_Tr & SOM01.2 Trace Volatile Organics / In-Lab \\
\hline 200-5308-3 & INHULL-W-32691 \\
\hline SOM01.2_Vol_Tr & SOM01.2 Trace Volatile Organics / In-Lab \\
\hline 200-5308-4 & INQCTB-W-32692 \\
\hline SOM01.2_Vol_Tr & SOM01.2 Trace Volatile Organics / In-Lab \\
\hline 200-5308-5 & VHBLK01 \\
\hline SOM01.2_Vol_Tr & SOM01.2 \\
\hline
\end{tabular}

Number of Coolers: 1

Cooler Temperature(s) $\left(\mathbf{C}^{\circ}\right): 0.1$;

\begin{tabular}{|c|c|c|c|c|}
\hline \multicolumn{2}{|c|}{ Date Sampled } & \multirow[t]{2}{*}{ Matrix } & \multirow[b]{2}{*}{ Rpt Basis } & \multirow[b]{2}{*}{ Dry / Wet ** } \\
\hline & & & & \\
\hline \multirow[t]{2}{*}{$5 / 24 / 2011$} & 4:30:00 PM & Water & & \\
\hline & & & Total & Wet \\
\hline \multirow[t]{2}{*}{$5 / 24 / 2011$} & 4:05:00 PM & Water & & \\
\hline & & & Total & Wet \\
\hline \multirow[t]{2}{*}{$5 / 24 / 2011$} & 2:06:00 PM & Water & & \\
\hline & & & Total & Wet \\
\hline \multirow[t]{2}{*}{$5 / 24 / 2011$} & 2:32:00 PM & Water & & \\
\hline & & & Total & Wet \\
\hline
\end{tabular}

5/25/2011 11:45:00 AM Water 


\section{METHODOLOGY SUMMARY}

$\begin{array}{llll}\text { Laboratory: } & \text { TestAmerica Laboratories } & \text { Project No: } & \\ \text { Location: } & \text { South Burlington, Vermont } & \text { SDG No: } & \text { 200-5308 }\end{array}$

VOA

Volatile Organics Trace - USEPA CLP SOM01.2 
$2 A$ - FORM II VOA- 1

WATER VOLATILE DEUTERATED MONITORING COMPOUND RECOVERY

Lab Name: TESTAMERICA BURLINGTON

Contract: 8E-00302

Lab Code: STLV Case No.: INMAN Mod. Ref No.:

SDG No.: 200-5308

Level: (TRACE or LOW) TRACE

\begin{tabular}{|c|c|c|c|c|c|c|c|}
\hline $\begin{array}{c}\text { EPA } \\
\text { SAMPLE NO. }\end{array}$ & $\begin{array}{l}\text { VDMC1 } \\
\text { (VCL) \# }\end{array}$ & $\begin{array}{l}\text { VDMC2 } \\
\text { (CLA) \# }\end{array}$ & $\begin{array}{l}\text { VDMC3 } \\
\text { (DCE) \# }\end{array}$ & $\begin{array}{l}\text { VDMC4 } \\
\text { (BUT) \# }\end{array}$ & $\begin{array}{l}\text { VDMC5 } \\
(\mathrm{CLF}) \#\end{array}$ & $\begin{array}{l}\text { VDMC 6 } \\
\text { (DCA) \# }\end{array}$ & $\begin{array}{l}\text { VDMC7 } \\
(\mathrm{BEN}) \quad \#\end{array}$ \\
\hline VBLKJE & 106 & 103 & 76 & 103 & 100 & 110 & 97 \\
\hline $\begin{array}{l}\text { INBROWN-W-3268 } \\
6\end{array}$ & 102 & 97 & 72 & $183 \star$ & 101 & 110 & 107 \\
\hline INQCTB-W-32692 & 108 & 107 & 84 & 110 & 108 & 123 & 112 \\
\hline $\begin{array}{l}\text { INTHIESSEN-W-3 } \\
2685\end{array}$ & 106 & 114 & 85 & $237 \star$ & 115 & 129 & 109 \\
\hline
\end{tabular}

\footnotetext{
VDMC1 (VCL) = Vinyl Chloride-d3

VDMC2 $($ CLA $)=$ Chloroethane $-\mathrm{d} 5$

VDMC3 $(\mathrm{DCE})=1,1-\mathrm{Dich}$ loroethene-d2

VDMC4 $\quad($ BUT $)=2-$ Butanone-d5

VDMC5 $($ CLF $)=$ Chloroform-d

VDMC6 $(\mathrm{DCA})=1,2-\mathrm{Dich}$ loroethane-d4

VDMC7 (BEN) = Benzene-d6
}

$$
\begin{gathered}
\text { QC LIMITS } \\
\hline(65-131) \\
(71-131) \\
(55-104) \\
(49-155) \\
(78-121) \\
(78-129) \\
(77-124)
\end{gathered}
$$

\# Column to be used to flag recovery values

* Values outside of contract required QC limits

Page 1 of 1 
2B - FORM II VOA-2

WATER VOLATILE DEUTERATED MONITORING COMPOUND RECOVERY

Lab Name: TESTAMERICA BURLINGTON

Contract: $8 \mathrm{E}-00302$

Lab Code: STLV Case No.: INMAN Mod. Ref No.:

SDG NO.: 200-5308

Level: (TRACE or LOW) TRACE

\begin{tabular}{|c|c|c|c|c|c|c|c|c|}
\hline $\begin{array}{c}\text { EPA } \\
\text { SAMPLE NO. }\end{array}$ & $\begin{array}{l}\text { VDMC8 } \\
(\mathrm{DPA}) \#\end{array}$ & $\begin{array}{l}\text { VDMC9 } \\
\text { (TOL) \# }\end{array}$ & $\begin{array}{l}\text { VDMC10 } \\
(\mathrm{TDP}) \quad \#\end{array}$ & $\begin{array}{l}\text { VDMC11 } \\
\text { (HEX) \# }\end{array}$ & $\begin{array}{l}\text { VDMC12 } \\
\text { (TCA) \# }\end{array}$ & $\begin{array}{l}\text { VDMC13 } \\
\text { (DCZ) \# }\end{array}$ & OTHER & $\begin{array}{l}\text { TOT } \\
\text { OUT }\end{array}$ \\
\hline VBLKJE & 83 & 96 & 98 & 108 & 92 & 106 & & 0 \\
\hline $\begin{array}{l}\text { INBROWN-W-3268 } \\
6\end{array}$ & 83 & 105 & $\overline{94}$ & 182 * & 88 & 95 & & 2 \\
\hline INQCTB-W-32692 & 95 & 113 & 107 & 107 & 101 & i11 & & 0 \\
\hline $\begin{array}{l}\text { INTHIESSEN-W-3 } \\
2685\end{array}$ & 102 & 110 & 104 & $245 \star$ & 115 & 110 & & 2 \\
\hline
\end{tabular}

\footnotetext{
VDMC8 $(D P A)=1,2-D i c h l o r o p r o p a n e-d 6$

VDMC9 (TOL) = Toluene-d8

VDMC10 $(\mathrm{TDP})=$ trans $-1,3-\mathrm{Dichloropropene}-\mathrm{d} 4$

VDMC11 $($ HEX $)=2-$ Hexanone-d5

VDMC12 $(\mathrm{TCA})=1,1,2,2$-Tetrachloroethane-d2

VDMC13 $(\mathrm{DCZ})=1,2-\mathrm{Dich}$ lorobenzene-d4
}

QC LIMITS

$(79-124)$

$(77-121)$

$(73-121)$

$(28-135)$

$(73-125)$

$(80-131)$

\# Column to be used to flag recovery values

* Values outside of contract required QC limits

Report 1,4-Dioxane-d8 for Low-Medium VOA analysis only

Page 1 of 1 
$4 A$ - FORM IV VOA

VOLATILE METHOD BLANK SUMMARY
EPA SAMPLE NO.

VBLKJE
Lab Name: TESTAMERICA BURLINGTON

Contract: $8 \mathrm{E}-00302$

Lab Code: STLV Case No.: INMAN

Lab File ID: JCVB04.D

Instrument ID: J.i

Matrix: (SOIL/SED/WATER)

Water

Level: (TRACE or LOW/MED)

GC Column: DB-624

TRACE

ID: 0.20
SDG No.: 200-5308

\section{: :}

Lab Sample ID: MB 200-18645/4

Date Analyzed: 05/25/2011

Time Analyzed: 1329

Heated Purge: $(\mathrm{Y} / \mathrm{N}) \mathrm{N}$

\begin{tabular}{|l|l|l|l|c|}
\hline \multicolumn{1}{|c|}{$\begin{array}{c}\text { EPA } \\
\text { SAMPLE NO. }\end{array}$} & \multicolumn{1}{|c|}{ LAB } & \multicolumn{1}{c|}{$\begin{array}{c}\text { LAB } \\
\text { SAMPLE ID }\end{array}$} & $\begin{array}{c}\text { TIME } \\
\text { ANALYZED }\end{array}$ \\
\cline { 2 - 5 } 01 & $\begin{array}{l}\text { INBROWN-W-32 } \\
\text { 686 }\end{array}$ & $200-5308-2$ & JCVB05.D & 1420 \\
02 & VIBLKJH & VIBLK 200-18645/6 & JCVB06.D & 1444 \\
\cline { 2 - 5 } 03 & $\begin{array}{l}\text { INQCTB-W-326 } \\
\text { 92 }\end{array}$ & $200-5308-4$ & JCVB07.D & 1530 \\
\cline { 2 - 5 } 04 & $\begin{array}{l}\text { INTHIESSEN-W } \\
\text {-32685 }\end{array}$ & $200-5308-1$ & JCVB08.D & 1555 \\
\cline { 2 - 5 } 06 & $\begin{array}{l}\text { INHULL-W-326 } \\
\text { 91 }\end{array}$ & $200-5308-3$ & JCVB09.D & 1620 \\
\cline { 2 - 5 } & VHBLK01 & $200-5308-5$ & JCVB10.D & 1645 \\
\hline
\end{tabular}

COMMENTS :

Page 1 of 1 
$5 A$ - FORM V VOA

VOLATILE ORGANICS INSTRUMENT

PEREORMANCE CHECK

BROMOELUOROBENZENE
EPA SAMPLE NO

BEBJC
Lab Name: TESTAMERICA BURLINGTON

Lab Code: STLV

Case No.: INMA

Lab File Id: JCV03.D

Instrument Id: J.i

GC Column: DB-624

ID: 0.20
Contract: $\quad 8 \mathrm{E}-00302$

SDG No.: 200-5308

BFB Injection Date: 05/10/2011

BEB Injection Time: 0947 (mm)

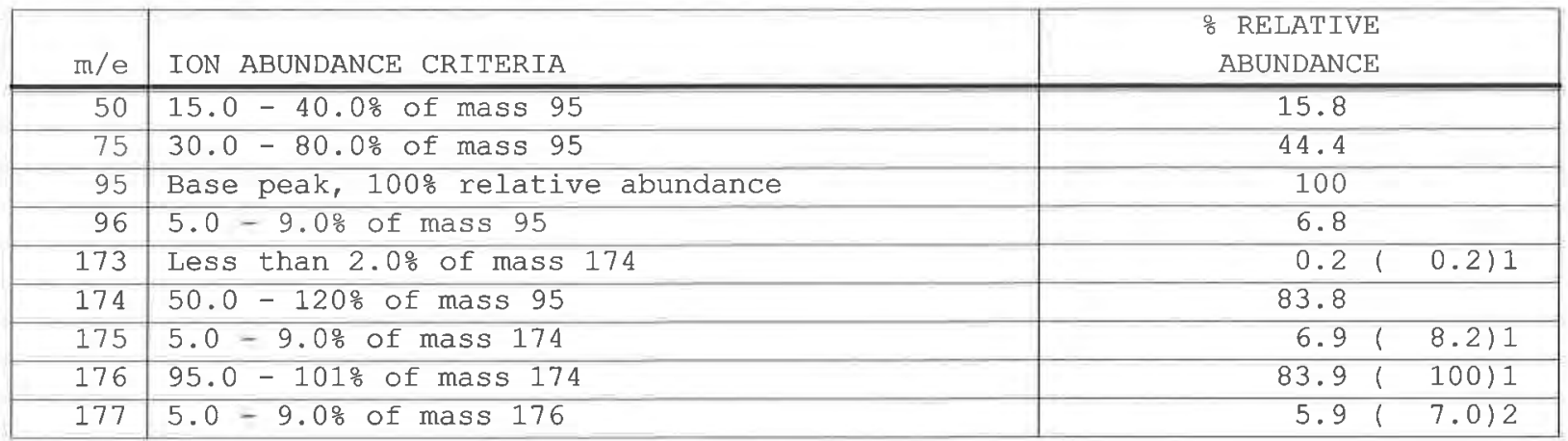

1 - Value is omass 174

2 - Value is omass 176

\begin{tabular}{|c|c|c|c|c|}
\hline $\begin{array}{c}\text { EPA } \\
\text { SAMPLE NO. }\end{array}$ & $\begin{array}{c}\text { LAB } \\
\text { SAMPLE ID }\end{array}$ & $\begin{array}{c}\text { LAB } \\
\text { EILE ID }\end{array}$ & $\begin{array}{c}\text { DATE } \\
\text { ANALYZED }\end{array}$ & $\begin{array}{c}\text { TIME } \\
\text { ANALYZED }\end{array}$ \\
\hline VSTDO.5JC & IC $200-17808 / 5$ & JCV05.D & $05 / 10 / 2011$ & 1032 \\
\hline VSTD001JC & IC $200-17808 / 6$ & JCV06.D & $05 / 10 / 2011$ & 1058 \\
\hline VSTD005JC & ICIS $200-17808 / 7$ & JCV07.D & $05 / 10 / 2011$ & 1123 \\
\hline VSTD010JC & IC $200-17808 / 8$ & JCV08.D & $05 / 10 / 2011$ & 1147 \\
\hline VSTD020JC & IC 200-17808/9 & JCV09.D & $05 / 10 / 2011$ & 1212 \\
\hline
\end{tabular}


$5 A$ - FORM V VOA

VOLATILE ORGANICS INSTRUMENT

PERFORMANCE CHECK

BROMOELUOROBENZENE (BEB)
EPA SAMPLE NO.

BFBJE
Lab Name: TESTAMERICA BURLINGTON Lab Code: STLV Case No.: INMAN Mod. Ref No.:
Contract: $8 \mathrm{E}-00302$

SDG No.: 200-5308

Lab File Id: JCVB01.D

BFB Injection Date: 05/25/2011

BFB Injection Time: 1221

Instrument Id: J.i

GC Column: $\mathrm{DB}-624$

ID: 0.20 $(\mathrm{Inm})$

\begin{tabular}{|c|c|c|}
\hline $\mathrm{m} / \mathrm{e}$ & ION ABUNDANCE CRITERIA & $\begin{array}{l}\text { O RELATIVE } \\
\text { ABUNDANCE }\end{array}$ \\
\hline 50 & $15.0-40.0 \%$ of mass 95 & 16.3 \\
\hline 75 & $30.0-80.0 \%$ of mass 95 & 49.4 \\
\hline 95 & Base peak, $100 \%$ relative abundance & 100 \\
\hline 96 & $5.0-9.0 \%$ of mass 95 & 6.3 \\
\hline 173 & Less than $2.0 \%$ of mass 174 & $0.7(0.7) 1$ \\
\hline 174 & $50.0-120 \%$ of mass 95 & 93.5 \\
\hline 175 & $5.0-9.0 \%$ of mass 174 & 6.21 \\
\hline 176 & $95.0-101 \%$ of mass 174 & $89.9(96.2) 1$ \\
\hline 177 & $5.0-9.0 \%$ of mass 176 & $6.1(6.8) 2$ \\
\hline
\end{tabular}

1 - Value is omass 174

2 - Value is omass 176

\begin{tabular}{|c|c|c|c|c|}
\hline $\begin{array}{c}\text { EPA } \\
\text { SAMPLE NO. }\end{array}$ & $\begin{array}{c}\text { LAB } \\
\text { SAMPLE ID }\end{array}$ & $\begin{array}{c}\text { LAB } \\
\text { FILE ID }\end{array}$ & $\begin{array}{c}\text { DATE } \\
\text { ANALYZED }\end{array}$ & $\begin{array}{c}\text { TIME } \\
\text { ANALYZED }\end{array}$ \\
\hline VSTD005JE & CCVIS $200-18645 / 3$ & JCVB03.D & $05 / 25 / 2011$ & 1305 \\
\hline VBLKJE & MB $200-18645 / 4$ & JCVB0 4.D & $05 / 25 / 2011$ & 1329 \\
\hline $\begin{array}{l}\text { INBROWN-W- } \\
32686\end{array}$ & $200-5308-2$ & JCVB05.D & $05 / 25 / 2011$ & 1420 \\
\hline VIBLKJH & VIBLK $200-18645 / 6$ & JCVB06.D & $05 / 25 / 2011$ & 1444 \\
\hline $\begin{array}{l}\text { INQCTB-W-3 } \\
2692\end{array}$ & $200-5308-4$ & JCVB07.D & $05 / 25 / 2011$ & 1530 \\
\hline $\begin{array}{l}\text { INTHIESSEN } \\
-W-32685\end{array}$ & $200-5308-1$ & JCVB08.D & $05 / 25 / 2011$ & 1555 \\
\hline $\begin{array}{l}\text { INHULL-W-3 } \\
2691\end{array}$ & $200-5308-3$ & JCVB09.D & $05 / 25 / 2011$ & 1620 \\
\hline VHBLK01 & $200-5308-5$ & JCVB10.D & $05 / 25 / 2011$ & 1645 \\
\hline VSTD005EJ & CCVC $200-18645 / 11$ & JCVB11.D & $05 / 25 / 2011$ & 1709 \\
\hline
\end{tabular}


$8 A$ - FORM VIII VOA

VOLATIJE INTERNAL STANDARD AREA AND RETENTION TIME SUMMARY

Lab Name: TESTAMERICA BURLINGTON

Lab Code: STLV

GC Column: DB-624 Case No.: INMAN Mod. Ref No.:

Contract: $\quad 8 E-00302$ ID: 0.20 (mm) Init.

EPA Sample No. (VSTD\#\#\#\#\#): VSTD005JE

Lab File ID (Standard): JCVB03.D

Instrument ID: J.i
SDG No.: 200-5308

\begin{tabular}{|c|c|c|c|c|c|c|c|}
\hline & & $\begin{array}{c}\text { IS1 (CBZ) } \\
\text { AREA }\end{array}$ & RT \# & $\begin{array}{c}\text { IS2 (DFB) } \\
\text { AREA }\end{array}$ & RT \# & $\begin{array}{c}\text { IS3 (DCB) } \\
\text { AREA }\end{array}$ & RT \\
\hline & 12 HOUR STD & 477654 & 8.93 & 568040 & 5.58 & 251717 & 11.76 \\
\hline & UPPER LIMIT & 668716 & 9.26 & 795256 & 5.91 & 352404 & 12.09 \\
\hline & LOWER IJMIT & 286592 & 8.60 & 340824 & 5.25 & 151030 & 11.43 \\
\hline & EPA SAMPLE NO. & & & & & & \\
\hline 01 & VBLKJE & 477385 & 8.93 & 575921 & 5.58 & 217900 & 11.76 \\
\hline 02 & $\begin{array}{l}\text { INBROWN-W-3268 } \\
6\end{array}$ & 461443 & 8.93 & 605142 & 5.58 & 217577 & 11.76 \\
\hline 03 & VIBLKJH & 462616 & 8.93 & 521056 & 5.58 & 220221 & 11.76 \\
\hline 04 & INQCTB-W-32692 & 403046 & 8.93 & 503671 & 5.58 & 196360 & 11.76 \\
\hline 05 & $\begin{array}{l}\text { INTHIESSEN-W-3 } \\
2685\end{array}$ & 381757 & 8.93 & 475745 & 5.58 & 195148 & 11.76 \\
\hline 06 & INHULL-W-32691 & 397126 & 8.93 & 496897 & 5.58 & 204493 & 11.76 \\
\hline 07 & VHBLKO1 & 410480 & 8.93 & 494409 & 5.58 & 194799 & 11.76 \\
\hline
\end{tabular}

IS1 $(\mathrm{CBZ})=$ Chlorobenzene-d5

IS2 $(\mathrm{DFB})=1,4-\mathrm{Diflu})=$

IS3 $(\mathrm{DCB})=1,4$-Dichlorobenzene-d 4

AREA UPPER LIMIT $=140 \%$ (Trace Volatiles) of internal standard area

AREA LOWER LIMIT $=60 \%$ (Trace Volatiles) of internal standard area

RT UPPER LIMIT $=+0.33$ (Trace Volatiles) minutes of internal standard RT

RT LOWER LIMIT $=-0.33$ (Trace Volatiles) minutes of internal standard RT

\# Column used to flag values outside contract required gC limits with an asterisk. 
1A - FORM I VOA-1

VOLATILE ORGANICS ANALYSIS DATA SHEET
EPA SAMPLE NO.

INBROWN-W-32686

Lab Name: TESTAMERICA BURLINGTON

Lab Code: STLV

Case No.: INMAN Mod. Ref No.:

Contract: $8 \mathrm{E}-00302$

Matrix: (SOII/SED/WATER)

Sample wt/vol: 25.0

Water

$(\mathrm{g} / \mathrm{mL}) \quad \mathrm{mL}$

Level: (TRACE/LOW/MED) TRACE

응 Moisture: not dec.

GC Column: DB-624

Soil Extract Volume: ID: 0.20 (mm) $(u L)$ $(\mathrm{mL})$

Purge Volume: 25.0
SDG No.: 200-5308

Lab Sample ID: 200-5308-2

Lab File ID: JCVB05.D

Date Received: 05/25/2011

Date Analyzed: 05/25/2011

Dilution Factor: 2.2

Soil Aliquot Volume:

(uL)

\begin{tabular}{|c|c|c|c|}
\hline CAS NO. & COMPOUND & $\begin{array}{l}\text { CONCENTRATION UNITS: } \\
(\mathrm{ug} / \mathrm{L} \text { or } \mathrm{ug} / \mathrm{kg}) \mathrm{ug} / \mathrm{L}\end{array}$ & $Q$ \\
\hline $75-71-8$ & Dichlorodifluoromethane & 1.1 & $\bar{U}$ \\
\hline $74-87-3$ & Chloromethane & 0.46 & $\mathrm{~J}$ \\
\hline $75-01-4$ & Vinyl chloride & 1.1 & $\mathrm{U}$ \\
\hline $74-83-9$ & Bromomethane & 1.1 & $\mathrm{U}$ \\
\hline $75-00-3$ & Chloroethane & 1.1 & $\mathrm{U}$ \\
\hline $75-69-4$ & Trichlorofluoromethane & 1.1 & $\mathrm{U}$ \\
\hline $75-35-4$ & 1,1-Dichloroethene & 1.1 & $\mathrm{U}$ \\
\hline $76-13-1$ & 1,1,2-Trichloro-1,2,2-trifluoroethane & 1.1 & $\mathrm{U}$ \\
\hline $67-64-1$ & Acetone & 33 & B \\
\hline $75-15-0$ & Carbon disulfide & 1.5 & B \\
\hline $79-20-9$ & Methyl acetate & 1.1 & $\mathrm{U}$ \\
\hline $75-09-2$ & Methylene Chloride & 0.071 & $\mathrm{~J}$ \\
\hline $156-60-5$ & trans-1,2-Dichloroethene & 1.1 & $\mathrm{U}$ \\
\hline $1634-04-4$ & Methyl tert-butyl ether & 1.1 & $\mathrm{U}$ \\
\hline $75-34-3$ & 1,1-Dichloroethane & 1.1 & $\mathrm{U}$ \\
\hline $156-59-2$ & cis-1,2-Dichloroethene & 1.1 & $\mathrm{U}$ \\
\hline $78-93-3$ & 2-Butanone & 640 & $\mathrm{E}$ \\
\hline $74-97-5$ & Bromochloromethane & 1.1 & $\mathrm{U}$ \\
\hline $67-66-3$ & Chloroform & 1.1 & $\mathrm{U}$ \\
\hline $71-55-6$ & 1,1,1-Trichloroethane & 1.1 & $\mathrm{U}$ \\
\hline $110-82-7$ & Cyclohexane & 1.1 & $\mathrm{U}$ \\
\hline $56-23-5$ & Carbon tetrachloride & 0.072 & $\mathrm{~J} \mathrm{~B}$ \\
\hline $71-43-2$ & Benzene & 0.16 & $\mathrm{~J}$ \\
\hline $107-06-2$ & 1,2-Dichloroethane & 1.1 & $\mathrm{U}$ \\
\hline
\end{tabular}

Report 1,4-Dioxane for Low-Medium VOA analysis only 
$1 B$ - FORM I VOA-2

VOLATILE ORGANICS ANALYSIS DATA SHEET
EPA SAMPLE NO.

INBROWN-W-32686
Lab Name: TESTAMERICA BURLINGTON

Lab Code: STLV Case No.: INMAN

Matrix: (SOIL/SED/WATER)

Water

Sample wt/vol: 25.0

$(\mathrm{g} / \mathrm{mL}) \quad \mathrm{mL}$

Level: (TRACE/LOW/MED) TRACE

o Moisture: not dec.

GC Column: DB-624

ID: 0.20

$(\mathrm{mm})$

Soil Extract Volume:

(mL)

Purge Volume: 25.0
Contract: 8E-00302

SDG No.: 200-5308

\begin{tabular}{|c|c|c|c|}
\hline CAS NO. & COMPOUND & $\begin{array}{l}\text { CONCENTRATION UNITS: } \\
(\mathrm{ug} / \mathrm{L} \text { or } \mathrm{ug} / \mathrm{kg}) \mathrm{ug} / \mathrm{L}\end{array}$ & 8 \\
\hline $79-01-6$ & Trichloroethene & 0.084 & $\mathrm{~J}$ \\
\hline $108-87-2$ & Methylcyclohexane & 1.1 & $\mathrm{U}$ \\
\hline $78-87-5$ & 1,2-Dichloropropane & 1.1 & $\mathrm{U}$ \\
\hline $75-27-4$ & Bromodichloromethane & 1.1 & $\mathrm{U}$ \\
\hline $10061-01-5$ & cis-1,3-Dichloropropene & 1.1 & $\mathrm{U}$ \\
\hline $108-10-1$ & 4-Methyl-2-pentanone & 11 & $\mathrm{U}$ \\
\hline $108-88-3$ & Toluene & 0.18 & $\mathrm{~J} \mathrm{~B}$ \\
\hline $10061-02-6$ & trans-1,3-Dichloropropene & 1.1 & $\mathrm{U}$ \\
\hline $79-00-5$ & 1,1,2-Trichloroethane & 1.1 & $\mathrm{U}$ \\
\hline $127-18-4$ & Tetrachloroethene & 1.1 & U \\
\hline $591-78-6$ & 2-Hexanone & 11 & $\mathrm{U}$ \\
\hline $124-48-1$ & Dibromochloromethane & 1.1 & $\mathrm{U}$ \\
\hline $106-93-4$ & 1,2-Dibromoethane & 1.1 & $\mathrm{U}$ \\
\hline $108-90-7$ & Chlorobenzene & 1.1 & $\mathrm{U}$ \\
\hline $100-41-4$ & Ethylbenzene & 1.1 & $\mathrm{U}$ \\
\hline $95-47-6$ & o-Xylene & 1.1 & $\mathrm{U}$ \\
\hline $179601-23-1$ & $\mathrm{~m}, \mathrm{p}$-Xylene & 1.1 & $\mathrm{U}$ \\
\hline $100-42-5$ & Styrene & 1.1 & $\mathrm{U}$ \\
\hline $75-25-2$ & Bromoform & 1.1 & $\mathrm{U}$ \\
\hline $98-82-8$ & Isopropylbenzene & 1.1 & $\mathrm{U}$ \\
\hline $79-34-5$ & $1,1,2,2$-Tetrachloroethane & 1.1 & $\mathrm{U}$ \\
\hline $541-73-1$ & 1,3-Dichlorobenzene & 1.1 & $\mathrm{U}$ \\
\hline $106-46-7$ & 1,4-Dichlorobenzene & 1.1 & $\mathrm{U}$ \\
\hline $95-50-1$ & 1,2-Dichlorobenzene & 1.1 & $\mathrm{U}$ \\
\hline $96-12-8$ & 1,2-Dibromo-3-Chloropropane & 1.1 & $\mathrm{U}$ \\
\hline $120-82-1$ & 1,2,4-Trichlorobenzene & 1.1 & $\mathrm{U}$ \\
\hline $87-61-6$ & 1,2,3-Trichlorobenzene & 1.1 & $\mathrm{U}$ \\
\hline
\end{tabular}

Lab Sample ID: 200-5308-2

Lab File ID: JCVB05.D

Date Received: 05/25/2011

Date Analyzed: 05/25/2011

Dilution Factor: 2.2

Soil Aliquot Volume:

(uL) 
$1 \mathrm{~J}$ - FORM I VOA-TIC

VOLATILE ORGANICS ANALYSIS DATA SHEET TENTATIVELY IDENTIFIED COMPOUNDS
EPA SAMPLE NO.

INBROWN-W-32686
Lab Name: TESTAMERICA BURLINGTON

Lab Code: STLV Case No.: INMAN

Matrix: (SOIL/SED/WATER)

Water

Sample wt/vol: 25.0 $(\mathrm{g} / \mathrm{mL}) \mathrm{mL}$

Level: (TRACE or LOW/MED) TRACE

\% Moisture: not dec.

GC Column: DB-624

ID: 0.20 (mm)

Soil Extract Volume: (uL)

CONCENTRATION UNITS: $(\mathrm{ug} / \mathrm{L}$ or $\mathrm{ug} / \mathrm{kg}) \mathrm{ug} / \mathrm{L}$
Contract: $8 \mathrm{E}-00302$ SDG No.: 200-5308

Lab Sample ID: 200-5308-2

Lab File ID: JCVB05.D

Date Received: 05/25/2011

Date Analyzed: 05/25/2011

Dilution Factor: 2.2

Soil Aliquot Volume:

(uL)

Purge Volume: 25.0 $(\mathrm{mL})$

\begin{tabular}{|c|c|c|c|c|}
\hline CAS NUMBER & COMPOUND NAME & RT & EST. CONC. & $Q$ \\
\hline $7446-09-5$ & Sulfur dioxide & 2.37 & 1.2 & $\mathrm{~J} \mathrm{~N}$ \\
\hline $109-99-9$ & Furan, tetrahydro- & 4.50 & 280 & J $N$ \\
\hline 03 & Unknown & 6.90 & 6.8 & $\mathrm{~B} \times \mathrm{X}$ \\
\hline $541-05-9$ & Cyclotrisiloxane, hexamethyl- & 7.85 & 4.3 & $\mathrm{~B} \mathrm{~J} \mathrm{~N}$ \\
\hline 05 & Unknown siloxane derivative & 10.69 & 5.4 & $\mathrm{~B} \mathrm{~J}$ \\
\hline 06 & Unknown & 12.88 & 1.5 & $\mathrm{~J}$ \\
\hline E9667961 & Total Alkanes & $\mathrm{N} / \mathrm{A}$ & & \\
\hline
\end{tabular}

1 EPA-designated Registry Number. 
Lab Name: TESTAMERICA BURIINGTON

Lab Code: STLV Cas

Matrix: (SOIL/SED/WATER)

water

Sample wt/vol: 25.0

$(\mathrm{g} / \mathrm{mL}) \mathrm{mL}$

Level: (TRACE/LOW/MED) TRACE

\% Moisture: not dec.

GC Column: DB-624

ID: $0.20 \quad$ (mm)

Soil Extract Volume:

(uL)

(mL)

Purge Volume: 25.0
Contract: $8 \mathrm{E}-00302$

SDG No.: 200-5308

. :

Lab Sample ID: 200-5308-3

Lab File ID: JCVB09.D

Date Received: 05/25/2011

Date Analyzed: 05/25/2011

Dilution Factor: 1.0

Soil Aliquot volume:

(uL)

\begin{tabular}{|c|c|c|c|}
\hline CAS NO. & COMPOUND & $\begin{array}{l}\text { CONCENTRATION UNITS: } \\
(\mathrm{ug} / \mathrm{L} \text { or } \mathrm{ug} / \mathrm{kg}) \mathrm{ug} / \mathrm{L}\end{array}$ & Q \\
\hline $75-71-8$ & Dichlorodifluoromethane & 0.50 & $\mathrm{U}$ \\
\hline $74-87-3$ & Chloromethane & 0.50 & $\mathrm{U}$ \\
\hline $75-01-4$ & Vinyl chloride & 0.50 & $\mathrm{U}$ \\
\hline $74-83-9$ & Bromomethane & 0.50 & $\mathrm{U}$ \\
\hline $75-00-3$ & Chloroethane & 0.50 & $\mathrm{U}$ \\
\hline $75-69-4$ & Trichlorofluoromethane & 0.50 & $\mathrm{U}$ \\
\hline $75-35-4$ & 1,1-Dichloroethene & 0.50 & $\mathrm{U}$ \\
\hline $76-13-1$ & 1,1,2-Trichloro-1,2,2-trifluoroethane & 0.50 & $\mathrm{U}$ \\
\hline $67-64-1$ & Acetone & 5.0 & $\mathrm{U}$ \\
\hline $75-15-0$ & Carbon disulfide & 0.50 & $\mathrm{U}$ \\
\hline $79-20-9$ & Methyl acetate & 0.50 & $\mathrm{U}$ \\
\hline $75-09-2$ & Methylene Chloride & 0.50 & $\mathrm{U}$ \\
\hline $156-60-5$ & trans-1,2-Dichloroethene & 0.50 & $\mathrm{U}$ \\
\hline $1634-04-4$ & Methyl tert-butyl ether & 0.50 & $\mathrm{U}$ \\
\hline $75-34-3$ & 1,1-Dichloroethane & 0.50 & $\mathrm{U}$ \\
\hline $156-59-2$ & cis-1,2-Dichloroethene & 0.50 & $\mathrm{U}$ \\
\hline $78-93-3$ & 2-Butanone & 5.0 & $\mathrm{U}$ \\
\hline $74-97-5$ & Bromochloromethane & 0.50 & $\mathrm{U}$ \\
\hline $67-66-3$ & Chloroform & 0.50 & $\mathrm{U}$ \\
\hline $71-55-6$ & 1,1,1-Trichloroethane & 0.50 & $\mathrm{U}$ \\
\hline $110-82-7$ & Cyclohexane & 0.50 & $\mathrm{U}$ \\
\hline $56-23-5$ & Carbon tetrachloride & 0.23 & $\mathrm{~J} \mathrm{~B}$ \\
\hline $71-43-2$ & Benzene & 0.50 & $\mathrm{U}$ \\
\hline $107-06-2$ & 1,2-Dichloroethane & 0.50 & $\mathrm{U}$ \\
\hline
\end{tabular}

Report 1,4-Dioxane for Low-Medium VOA analysis only 
$1 B$ - FORM I VOA-2

VOLATILE ORGANICS ANALYSIS DATA SHEET
EPA SAMPLE NO. INHULL-W-32691
Lab Name: TESTAMERICA BURLINGTON

Lab Code: STLV

Case No.: INMAN

Mod. Ref No.:
Matrix: (SOIL/SED/WATER)

Sample wt/vol: 25.0

Water

$(\mathrm{g} / \mathrm{mL}) \quad \mathrm{mL}$

Level: (TRACE/LOW/MED) TRACE

\% Moisture: not dec.

GC Column: DB-624

Soil Extract Volume: ID: $0.20 \quad$ (mm)

Purge Volume: 25.0

(mL)
Contract: $8 \mathrm{E}-00302$

SDG No.: 200-5308

Lab Sample ID: 200-5308-3

Lab File ID: JCVB09.D

Date Received: 05/25/2011

Date Analyzed: 05/25/2011

Dilution Factor: 1.0

Soil Aliquot Volume:

$(u L)$

\begin{tabular}{|c|c|c|c|}
\hline CAS NO. & COMPOUND & $\begin{array}{l}\text { CONCENTRATION UNITS: } \\
(\mathrm{ug} / \mathrm{L} \text { or } \mathrm{ug} / \mathrm{kg}) \mathrm{ug} / \mathrm{L}\end{array}$ & Q \\
\hline $79-01-6$ & Trichloroethene & 0.50 & $\mathrm{U}$ \\
\hline $108-87-2$ & Methylcyclohexane & 0.50 & $\mathrm{U}$ \\
\hline $78-87-5$ & 1,2-Dichloropropane & 0.50 & $\mathrm{U}$ \\
\hline $75-27-4$ & Bromodichloromethane & 0.50 & U \\
\hline $10061-01-5$ & cis-1,3-Dichloropropene & 0.50 & $\mathrm{U}$ \\
\hline $108-10-1$ & 4-Methyl-2-pentanone & 5.0 & $\mathrm{U}$ \\
\hline $108-88-3$ & Toluene & 0.50 & $\mathrm{U}$ \\
\hline $10061-02-6$ & trans-1,3-Dichloropropene & 0.50 & $\mathrm{U}$ \\
\hline $79-00-5$ & 1,1,2-Trichloroethane & 0.50 & $\mathrm{U}$ \\
\hline $127-18-4$ & Tetrachloroethene & 0.50 & $\mathrm{U}$ \\
\hline $591-78-6$ & 2-Hexanone & 5.0 & $\mathrm{U}$ \\
\hline $124-48-1$ & Dibromochloromethane & 0.50 & $\mathrm{U}$ \\
\hline $106-93-4$ & 1,2-Dibromoethane & 0.50 & $\mathrm{U}$ \\
\hline $108-90-7$ & Chlorobenzene & 0.50 & $\mathrm{U}$ \\
\hline $100-41-4$ & Ethylbenzene & 0.50 & $\mathrm{U}$ \\
\hline $95-47-6$ & o-xylene & 0.50 & $\mathrm{U}$ \\
\hline $179601-23-1$ & m,p-xylene & 0.50 & $\mathrm{U}$ \\
\hline $100-42-5$ & Styrene & 0.50 & U \\
\hline $75-25-2$ & Bromoform & 0.50 & $\mathrm{U}$ \\
\hline $98-82-8$ & Isopropylbenzene & 0.50 & $\mathrm{U}$ \\
\hline $79-34-5$ & 1,1,2,2-Tetrachloroethane & 0.50 & $\mathrm{U}$ \\
\hline $541-73-1$ & 1,3-Dichlorobenzene & 0.50 & $\mathrm{U}$ \\
\hline $106-46-7$ & 1,4-Dichlorobenzene & 0.50 & $\mathrm{U}$ \\
\hline $95-50-1$ & 1,2-Dichlorobenzene & 0.50 & $\mathrm{U}$ \\
\hline $96-12-8$ & 1,2-Dibromo-3-Chloropropane & 0.50 & $\mathrm{U}$ \\
\hline $120-82-1$ & 1,2,4-Trichlorobenzene & 0.50 & $\mathrm{U}$ \\
\hline $87-61-6$ & 1,2,3-Trichlorobenzene & 0.50 & $\mathrm{U}$ \\
\hline
\end{tabular}


IJ - FORM I VOA-TIC

VOLATILE ORGANICS ANALYSIS DATA SHEET TENTATIVELY IDENTIFIED COMPOUNDS
EPA SAMPLE NO. INHULL-W-32691

Lab Name: TESTAMERICA BURLINGTON Case No.: INMAN Mod. Ref No.: Contract: $8 \mathrm{E}-00302$

Lab Code: STLV

Water

Matrix: (SOIL/SED/WATER)

Sample wt/vol: 25.0 $(\mathrm{g} / \mathrm{mL}) \mathrm{mL}$

Level: (TRACE or LOW/MED) TRACE

응 Moisture: not dec.

GC Column: DB-624

ID: 0.20 (mm)

Soil Extract Volume: (uL)

CONCENTRATION UNITS: (ug/L or $\mathrm{ug} / \mathrm{kg}$ )
SDG No.: 200-5308

Lab Sample ID: 200-5308-3

Lab File ID: JCVB09.D

Date Received: 05/25/2011

Date Analyzed: 05/25/2011

Dilution Factor: 1.0

Soil Aliquot Volume:

(uL)

Purge Volume: 25.0 (mL)

\begin{tabular}{l|l|l|r|r|r|}
\hline \multicolumn{1}{|c|}{ CAS NUMBER } & \multicolumn{1}{|c|}{ COMPOUND NAME } & \multicolumn{1}{|c|}{ RT } & EST. CONC. & Q \\
\cline { 2 - 6 } 01 & & Unknown & 6.90 & 3.5 & B X J \\
\cline { 2 - 6 } 02 & E9667961 & Total Alkanes & N/A & & \\
\hline
\end{tabular}

1 EPA-designated Registry Number. 
$1 A$ - FORM I VOA-1

VOLATILE ORGANICS ANALYSIS DATA SHEET
EPA SAMPLE NO. INQCTB-W-32692

Lab Name: TESTAMERICA BURLINGTON

Lab Code: STLV Case No.:

Matrix: (SOIL/SED/WATER)

Sample wt/vol: 25.0

Water

$(\mathrm{g} / \mathrm{mL}) \mathrm{mL}$

Level: (TRACE/LOW/MED) TRACE

\% Moisture: not dec.

GC Column: DB-624

ID $: 0.20$ $(\mathrm{mm})$

Soil Extract Volume: (uL) (mL)

Purge Volume: 25.0
Contract: $\quad 8 \mathrm{E}-00302$

SDG No.: 200-5308 Mod. Ref No.:

Lab Sample ID: 200-5308-4

Lab File ID: JCVB07.D

Date Received: 05/25/2011

Date Analyzed: 05/25/2011

Dilution Factor: 1.0

Soil Aliquot Volume:

(uL)

\begin{tabular}{|c|c|c|c|}
\hline CAS NO. & COMPOUND & $\begin{array}{l}\text { CONCENTRATION UNITS: } \\
(\mathrm{ug} / \mathrm{L} \text { or } \mathrm{ug} / \mathrm{kg}) \mathrm{ug} / \mathrm{L}\end{array}$ & Q \\
\hline $75-71-8$ & Dichlorodifluoromethane & 0.50 & $\mathrm{U}$ \\
\hline $74-87-3$ & Chloromethane & 0.50 & $\mathrm{U}$ \\
\hline $75-01-4$ & Vinyl chloride & 0.50 & $\mathrm{U}$ \\
\hline $74-83-9$ & Bromomethane & 0.50 & $\mathrm{U}$ \\
\hline $75-00-3$ & Chloroethane & 0.50 & $\mathrm{U}$ \\
\hline $75-69-4$ & Trichlorofluoromethane & 0.50 & $\mathrm{U}$ \\
\hline $75-35-4$ & 1,1-Dichloroethene & 0.50 & $\mathrm{U}$ \\
\hline $76-13-1$ & 1,1,2-Trichloro-1,2,2-trifluoroethane & 0.50 & $\mathrm{U}$ \\
\hline $67-64-1$ & Acetone & 3.6 & $\mathrm{~J} \mathrm{~B}$ \\
\hline $75-15-0$ & Carbon disulfide & 0.21 & $\mathrm{~J} \mathrm{~B}$ \\
\hline $79-20-9$ & Methyl acetate & 0.50 & $\mathrm{U}$ \\
\hline $75-09-2$ & Methylene Chloride & 0.50 & $\mathrm{U}$ \\
\hline $156-60-5$ & trans-1,2-Dichloroethene & 0.50 & $\mathrm{U}$ \\
\hline $1634-04-4$ & Methyl tert-butyl ether & 0.50 & $\mathrm{U}$ \\
\hline $75-34-3$ & 1,1-Dichloroethane & 0.50 & $\mathrm{U}$ \\
\hline $156-59-2$ & cis-1,2-Dichloroethene & 0.50 & u \\
\hline $78-93-3$ & 2-Butanone & 5.0 & $\mathrm{U}$ \\
\hline $74-97-5$ & Bromochloromethane & 0.50 & $\mathrm{U}$ \\
\hline $67-66-3$ & Chloroform & 0.32 & $\mathrm{~J}$ \\
\hline $71-55-6$ & 1,1,1-Trichloroethane & 0.50 & $\mathrm{U}$ \\
\hline $110-82-7$ & Cyclohexane & 0.50 & $\mathrm{U}$ \\
\hline $56-23-5$ & Carbon tetrachloride & 0.50 & $\mathrm{U}$ \\
\hline $71-43-2$ & Benzene & 0.50 & $\mathrm{U}$ \\
\hline $107-06-2$ & 1,2-Dichloroethane & 0.50 & $\mathrm{U}$ \\
\hline
\end{tabular}

Report 1,4-Dioxane for Low-Medium VOA analysis only 
$1 B$ - FORM I VOA-2

VOLATILE ORGANICS ANALYSIS DATA SHEET
EPA SAMPLE NO. INQCTB-W-32692
Lab Name: TESTAMERICA BURLINGTON

Lab Code: STLV Case No.: INMAN

Matrix: (SOIL/SED/WATER)

Water

Sample wt/vol: 25.0

$(\mathrm{g} / \mathrm{mL}) \quad \mathrm{mL}$

Level: (TRACE/LOW/MED) TRACE

\% Moisture: not dec.

GC Column: DB-624 ID: 0.20 (mun)

Soil Extract Volume:

Purge Volume: 25.0

(mL)
Contract: $\quad 8 \mathrm{E}-00302$

SDG No.: 200-5308

\begin{tabular}{|c|c|c|c|}
\hline CAS NO. & COMPOUND & $\begin{array}{l}\text { CONCENTRATION UNITS: } \\
(\mathrm{ug} / \mathrm{L} \text { or } \mathrm{ug} / \mathrm{kg}) \mathrm{ug} / \mathrm{L}\end{array}$ & $Q$ \\
\hline $79-01-6$ & Trichloroethene & 0.50 & $\mathrm{U}$ \\
\hline $108-87-2$ & Methylcyclohexane & 0.50 & $\mathrm{U}$ \\
\hline $78-87-5$ & 1,2-Dichloropropane & 0.50 & $\mathrm{U}$ \\
\hline $75-27-4$ & Bromodichloromethane & 0.50 & $\mathrm{U}$ \\
\hline $10061-01-5$ & cis-1,3-Dichloropropene & 0.50 & $\mathrm{U}$ \\
\hline $108-10-1$ & 4-Methyl-2-pentanone & 5.0 & $\mathrm{U}$ \\
\hline $108-88-3$ & Toluene & 0.063 & $\mathrm{~J} B$ \\
\hline $10061-02-6$ & trans-1,3-Dichloropropene & 0.50 & $\mathrm{U}$ \\
\hline $79-00-5$ & 1,1,2-Trichloroethane & 0.50 & $\mathrm{U}$ \\
\hline $127-18-4$ & Tetrachloroethene & 0.50 & $\mathrm{U}$ \\
\hline $591-78-6$ & 2-Hexanone & 5.0 & $\mathrm{U}$ \\
\hline $124-48-1$ & Dibromochloromethane & 0.50 & $\mathrm{U}$ \\
\hline $106-93-4$ & 1,2-Dibromoethane & 0.50 & $\mathrm{U}$ \\
\hline $108-90-7$ & Chlorobenzene & 0.50 & $\mathrm{U}$ \\
\hline $100-41-4$ & Ethylbenzene & 0.50 & $\mathrm{U}$ \\
\hline $95-47-6$ & o-Xylene & 0.50 & $\mathrm{U}$ \\
\hline $179601-23-1$ & $\mathrm{~m}, \mathrm{p}$-xylene & 0.036 & $\mathrm{~J}$ \\
\hline $100-42-5$ & Styrene & 0.030 & $\mathrm{~J}$ \\
\hline $75-25-2$ & Bromoform & 0.50 & $\mathrm{U}$ \\
\hline $98-82-8$ & Isopropylbenzene & 0.50 & $\mathrm{U}$ \\
\hline $79-34-5$ & $1,1,2,2$-Tetrachloroethane & 0.50 & $\mathrm{U}$ \\
\hline $541-73-1$ & 1,3-Dichlorobenzene & 0.50 & $\mathrm{U}$ \\
\hline $106-46-7$ & 1,4-Dichlorobenzene & 0.49 & $\mathrm{~J}$ \\
\hline $95-50-1$ & 1,2-Dichlorobenzene & 0.50 & $\mathrm{U}$ \\
\hline $96-12-8$ & 1,2-Dibromo-3-Chloropropane & 0.50 & $\mathrm{U}$ \\
\hline $120-82-1$ & 1,2,4-Trichlorobenzene & 0.50 & U \\
\hline $87-61-6$ & 1,2,3-Trichlorobenzene & 0.50 & $\mathrm{U}$ \\
\hline
\end{tabular}

Lab Sample ID: 200-5308-4

Lab File ID: JCVB07.D

Date Received: 05/25/2011

Date Analyzed: 05/25/2011

Dilution Factor: 1.0

Soil Aliquot Volume:

(uL) 
$1 \mathrm{~J}$ - FORM I VOA-TIC

VOLATILE ORGANICS ANALYSIS DATA SHEET

TENTATIVELY IDENTIEIED COMPOUNDS
EPA SAMPLE NO,

INQCTB-W-32692

Lab Name: TESTAMERICA BURLINGTON

Lab Code: STLV Case No.: INMAN

Matrix: (SOIL/SED/WATER)

Water

Sample wt/vol: 25.0

Level: (TRACE or LOW/MED)

$(g / m L) \quad m L$

TRACE

응 Moisture: not dec.

GC Column: DB-624

Soil Extract Volume: ID: 0.20

(uI)

CONCENTRATION UNITS: (ug/L or ug/ $\mathrm{kg}$ ) ug/L
Contract: $8 E-00302$

SDG No.: 200-5308

Lab Sample ID: 200-5308-4

Lab File ID: JCVB07.D

Date Received: 05/25/2011

Date Analyzed: 05/25/2011

Dilution Factor: 1.0

Soil Aliquot Volume: (UL)

Purge Volume: 25.0 (mL)

\begin{tabular}{l|l|l|r|r|r|}
\hline \multicolumn{1}{l|}{ CAS NUMBER } & \multicolumn{1}{|c|}{ COMPOUND NAME } & \multicolumn{1}{|c|}{ RT } & EST. CONC. & Q \\
\cline { 2 - 6 } 01 & & Unknown & 6.90 & 3.4 & B X J \\
\cline { 2 - 6 } 02 & $541-05-9$ & Cyclotrisiloxane, hexamethyl- & 7.85 & 10.69 & B J N \\
\cline { 2 - 6 } 03 & & Unknown siloxane derivative & N/A & 1.4 & B J \\
\cline { 2 - 6 } 04 & E9667961 & Total Alkanes & &
\end{tabular}

1 EPA-designated Registry Number. 
$1 A$ - FORM I VOA-1

VOLATILE ORGANICS ANALYSIS DATA SHEET
EPA SAMPLE NO.
Lab Name: TESTAMERICA BURLINGTON

Lab Code: STLV Case No.: INMAN Mod. Ref No.:
Water

$(\mathrm{g} / \mathrm{mL}) \quad \mathrm{mL}$

Sample wt/vol: 25.0

Isevel: (TRACE/LOW/MED) TRACE

号 Moisture: not dec.

GC Column: DB-624 ID $: 0.20 \quad$ (mm)

Soil Extract Volume: (mL)
Purge Volume: 25.0

Contract: $8 \mathrm{E}-00302$

SDG No.: 200-5308

Lab Sample ID: 200-5308-1

Lab File ID: JCVB08.D

Date Received: 05/25/2011

Date Analyzed: 05/25/2011

Dilution Factor: 1.0

Soil Aliquot Volume:

\begin{tabular}{|c|c|c|c|}
\hline CAS NO. & COMPOUND & $\begin{array}{l}\text { CONCENTRATION UNITS: } \\
(\mathrm{ug} / \mathrm{L} \text { or } \mathrm{ug} / \mathrm{kg}) \mathrm{ug} / \mathrm{L}\end{array}$ & Q \\
\hline $75-71-8$ & Dichlorodifluoromethane & 0.50 & $\overline{0}$ \\
\hline $74-87-3$ & Chloromethane & 0.50 & $\mathrm{U}$ \\
\hline $75-01-4$ & Vinyl chloride & 0.50 & $\mathrm{U}$ \\
\hline $74-83-9$ & Bromomethane & 0.50 & $\mathrm{U}$ \\
\hline $75-00-3$ & Chloroethane & 0.50 & $\mathrm{U}$ \\
\hline $75-69-4$ & Trichlorofluoromethane & 0.50 & $\mathrm{U}$ \\
\hline $75-35-4$ & 1,1-Dichloroethene & 0.50 & $\mathrm{U}$ \\
\hline $76-13-1$ & 1,1,2-Trichloro-1,2,2-trifluoroethane & 0.50 & $\mathrm{U}$ \\
\hline $67-64-1$ & Acetone & 5.0 & U \\
\hline $75-15-0$ & Carbon disulfide & 0.17 & $\mathrm{~J} \mathrm{~B}$ \\
\hline $79-20-9$ & Methyl acetate & 0.50 & $\mathrm{U}$ \\
\hline $75-09-2$ & Methylene Chloride & 0.50 & $\mathrm{U}$ \\
\hline $156-60-5$ & trans-1,2-Dichloroethene & 0.50 & $\mathrm{U}$ \\
\hline $1634-04-4$ & Methyl tert-butyl ether & 0.50 & U \\
\hline $75-34-3$ & 1,1-Dichloroethane & 0.50 & $\mathrm{U}$ \\
\hline $156-59-2$ & cis-1,2-Dichloroethene & 0.50 & $\mathrm{U}$ \\
\hline $78-93-3$ & 2-Butanone & 5.0 & $\mathrm{U}$ \\
\hline $74-97-5$ & Bromochloromethane & 0.50 & $\mathrm{U}$ \\
\hline $67-66-3$ & Chloroform & 0.50 & $\mathrm{U}$ \\
\hline $71-55-6$ & 1,1,1-Trichloroethane & 0.50 & $\mathrm{U}$ \\
\hline $110-82-7$ & Cyclohexane & 0.50 & $\mathrm{U}$ \\
\hline $56-23-5$ & Carbon tetrachloride & 0.50 & $\mathrm{U}$ \\
\hline $71-43-2$ & Benzene & 0.50 & $\mathrm{U}$ \\
\hline $107-06-2$ & 1,2-Dichloroethane & 0.50 & $\mathrm{U}$ \\
\hline
\end{tabular}

Report 1,4-Dioxane for Low-Medium VOA analysis only 
$1 \mathrm{~B}$ - FORM I VOA-2

VOLATILE ORGANICS ANALYSIS DATA SHEET
EPA SAMPLE NO.

INTHIESSEN-W-3268
Lab Name: TESTAMERICA BURLINGTON Case No.: INMAN

Matrix: (SOIL/SED/WATER)

Water

Sample wt/vol: 25.0

$(\mathrm{g} / \mathrm{mL}) \quad \mathrm{mL}$

Level: (TRACE/LOW/MED) TRACE

\% Moisture: not dec.

GC Column: DB-624 ID: 0.20 $(\mathrm{mm})$

Soil Extract Volume:

Purge Volume: 25.0 $(\mathrm{mL})$
Lab Code: STLV

Contract: $8 \mathrm{E}-00302$

SDG No.: 200-5308

Lab Sample ID: 200-5308-1

Lab File ID: JCVB08.D

Date Received: 05/25/2011

Date Analyzed: 05/25/2011

Dilution Factor: 1.0

Soil Aliquot Volume:

(uL)

\begin{tabular}{|c|c|c|c|}
\hline CAS NO. & COMPOUND & $\begin{array}{l}\text { CONCENTRATION UNITS: } \\
(\mathrm{ug} / \mathrm{L} \text { or } \mathrm{ug} / \mathrm{kg}) \mathrm{ug} / \mathrm{L}\end{array}$ & $\varnothing$ \\
\hline $79-01-6$ & Trichloroethene & 0.50 & $\mathrm{U}$ \\
\hline $108-87-2$ & Methylcyclohexane & 0.50 & $\mathrm{U}$ \\
\hline $78-87-5$ & 1,2-Dichloropropane & 0.50 & $\mathrm{U}$ \\
\hline $75-27-4$ & Bromodichloromethane & 0.50 & $\mathrm{U}$ \\
\hline $10061-01-5$ & cis-1,3-Dichloropropene & 0.50 & $\mathrm{U}$ \\
\hline $108-10-1$ & 4-Methyl-2-pentanone & 5.0 & $\mathrm{U}$ \\
\hline $108-88-3$ & Toluene & 0.50 & $\mathrm{U}$ \\
\hline $10061-02-6$ & trans-1,3-Dichloropropene & 0.50 & $\mathrm{U}$ \\
\hline $79-00-5$ & 1,1,2-Trichloroethane & 0.50 & $\mathrm{U}$ \\
\hline $127-18-4$ & Tetrachloroethene & 0.50 & $\mathrm{U}$ \\
\hline $591-78-6$ & 2-Hexanone & 5.0 & $\mathrm{U}$ \\
\hline $124-48-1$ & Dibromochloromethane & 0.50 & $\mathrm{U}$ \\
\hline $106-93-4$ & 1,2-Dibromoethane & 0.50 & $\mathrm{U}$ \\
\hline $108-90-7$ & Chlorobenzene & 0.50 & U \\
\hline $100-41-4$ & Ethylbenzene & 0.50 & $\mathrm{U}$ \\
\hline $95-47-6$ & o-xylene & 0.50 & $\mathrm{U}$ \\
\hline $179601-23-1$ & m,p-xylene & 0.50 & $\mathrm{U}$ \\
\hline $100-42-5$ & Styrene & 0.50 & $\mathrm{U}$ \\
\hline $75-25-2$ & Bromoform & 0.50 & $\mathrm{U}$ \\
\hline $98-82-8$ & Isopropylbenzene & 0.50 & $\mathrm{U}$ \\
\hline $79-34-5$ & $1,1,2,2$-Tetrachloroethane & 0.50 & $\mathrm{U}$ \\
\hline $541-73-1$ & 1,3-Dichlorobenzene & 0.50 & $\mathrm{U}$ \\
\hline $106-46-7$ & 1,4-Dichlorobenzene & 0.50 & $\mathrm{U}$ \\
\hline $95-50-1$ & 1,2-Dichlorobenzene & 0.50 & $\mathrm{U}$ \\
\hline $96-12-8$ & 1,2-Dibromo-3-Chloropropane & 0.50 & $\mathrm{U}$ \\
\hline $120-82-1$ & 1,2,4-Trichlorobenzene & 0.50 & $\mathrm{U}$ \\
\hline $87-61-6$ & 1,2,3-Trichlorobenzene & 0.50 & $\mathrm{U}$ \\
\hline
\end{tabular}


$1 \mathrm{~J}$ - FORM I VOA-TIC

VOLATILE ORGANICS ANALYSIS DATA SHEET TENTATIVELY IDENTIFIED COMPOUNDS
EPA SAMPLE NO.

INTHIESSEN-W-3268
Lab Name: TESTAMERICA BURLINGTON

Lab Code: STLV Case No.: INMAN Mod. Ref No.:

Matrix: (SOIL/SED/WATER)

Sample wt/vol: 25.0

Water

Level: (TRACE or LOW/MED)

$(\mathrm{g} / \mathrm{mL}) \mathrm{mL}$

\% Moisture: not dec.

GC Column: DB-624

ID: 0.20

TRACE

Soil Extract Volume:

CONCENTRATION UNITS: (ug/L or $\mathrm{ug} / \mathrm{kg}$ )

$\mathrm{ug} / \mathrm{L}$

Contract: 8E-00302

SDG No.: 200-5308

Lab Sample ID: 200-5308-1

Lab File ID: JCVB08.D

Date Received: 05/25/2011

Date Analyzed: 05/25/2011

Dilution Factor: 1.0

Soil Aliquot Volume:

(uL)

Purge Volume: 25.0

(mL)

\begin{tabular}{|l|l|r|r|r|}
\hline \multicolumn{1}{|c|}{ CAS NUMBER } & \multicolumn{1}{|c|}{ COMPOUND NAME } & RT & EST. CONC. & Q \\
\hline & Unknown & 6.90 & 3.7 & $\mathrm{~B} X \mathrm{~J}$ \\
\hline $541-05-9$ & Cyclotrisiloxane, hexamethyl- & 7.85 & 0.64 & $\mathrm{~B} \mathrm{~J} \mathrm{~N}$ \\
\hline E9667961 & Total Alkanes & N/A & & \\
\hline
\end{tabular}

1 EPA-designated Registry Number. 
$6 \mathrm{~A}$ - FORM VI VOA-1

VOLATILE ORGANICS INITIAL CAIIBRATION DATA

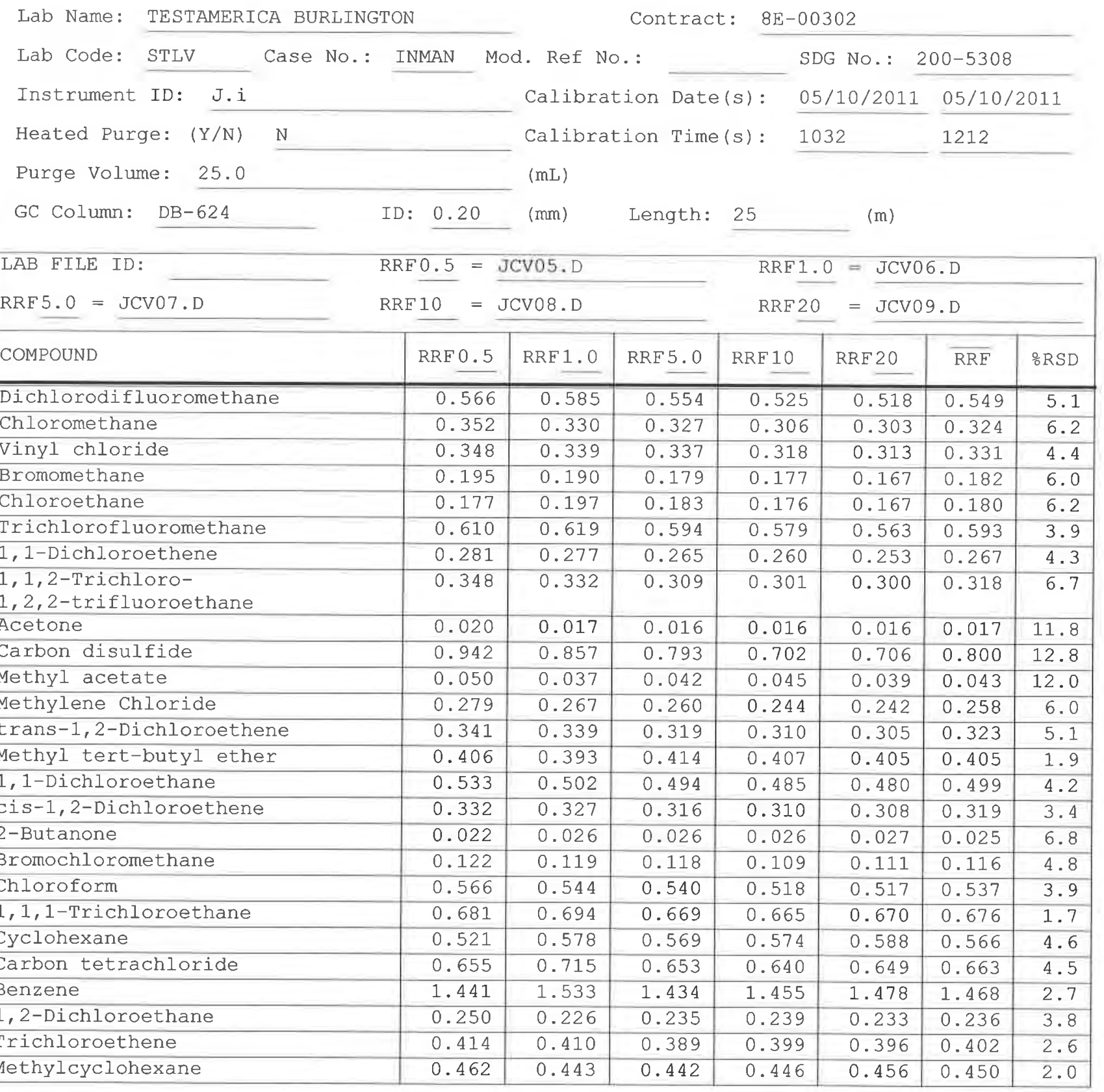

Report 1,4-Dioxane for Low-Medium VOA analysis only 
$6 B$ - EORM VI VOA-2

VOLATILE ORGANICS INITIAL CAIIBRATION DATA

Lab Name: TESTAMERICA BURLINGTON

Contract: $8 \mathrm{E}-00302$

Lab Code: STLV Case No.: INMAN Mod. Ref No.:

Instrument ID: J.i

Calibration Date $(s)$ :

SDG No.: 200-5308

$05 / 10 / 2011$

$05 / 10 / 2011$

Heated Purge: ( $Y / N) \quad N$

Purge Volume: 25.0

GC Column: DB-624

Calibration Time(s):

(mL)
(mm) Length: 25

ID: 0.20

(mL) Length: 25

(mm) Length: 25

1032 1212

\begin{tabular}{|c|c|c|c|c|c|c|c|}
\hline \multirow{3}{*}{$\begin{array}{l}\text { LAB EILE ID: } \\
\text { RRF } 5.0=\text { JCV07.D } \\
\text { COMPOUND }\end{array}$} & \multirow{2}{*}{$\begin{array}{l}\operatorname{RRF} \underline{0.5}= \\
\operatorname{RRF10}=\end{array}$} & \multirow{2}{*}{\multicolumn{2}{|c|}{$\begin{array}{l}\text { JCV0 5.D } \\
\text { JCV0 8.D }\end{array}$}} & \multirow{2}{*}{$\begin{array}{l}\text { RRF } 1.0 \\
\text { RRF 20 }\end{array}$} & \multirow{2}{*}{\multicolumn{3}{|c|}{$\begin{array}{l}=\text { JCV06.D } \\
=\text { JCV09.D }\end{array}$}} \\
\hline & & & & & & & \\
\hline & RRE 0.5 & RRE 1.0 & RRF 5.0 & RRF10 & RRF 20 & $\overline{\mathrm{R}} \overline{\mathrm{R} F}$ & $\stackrel{\circ}{\mathrm{RSD}}$ \\
\hline 1,2.Dichloropropane & 0.275 & 0.262 & 0.275 & 0.286 & 0.282 & 0.276 & 3.3 \\
\hline Brmodichlorome thane & 0.403 & 0.427 & 0.389 & 0.390 & 0.390 & 0.400 & 4.1 \\
\hline $\mathrm{c}^{\text {s-1,3-Dichloropropene }}$ & 0.409 & 0.447 & 0.445 & 0.451 & 0.464 & 0.443 & 4.7 \\
\hline -Methyl-2-pentanone & 0.069 & 0.072 & 0.075 & 0.076 & 0.078 & 0.074 & 4.8 \\
\hline Toluene & 1.562 & 1.652 & 1.632 & 1.646 & 1.665 & 1.631 & 2.5 \\
\hline trans-1,3-Dichloropropene & 0.311 & 0.316 & 0.335 & 0.346 & 0.355 & 0.333 & 5.6 \\
\hline 1,1,2-Trichloroethane & 0.176 & 0.212 & 0.165 & 0.172 & 0.168 & 0.179 & 10.6 \\
\hline Tetrachloroethene & 0.343 & 0.400 & 0.372 & 0.364 & 0.368 & 0.369 & 5.5 \\
\hline 2-Hexanone & 0.039 & 0.046 & 0.050 & 0.052 & 0.053 & 0.048 & 11.8 \\
\hline Dibromochloromethane & 0.229 & 0.253 & 0.250 & 0.245 & 0.250 & 0.245 & 3.9 \\
\hline 1,2-Dibromoethane & 0.159 & 0.170 & 0.157 & 0.158 & 0.161 & 0.161 & 3.2 \\
\hline Chilorobenzene & 0.977 & 1.043 & 1.005 & 1.015 & 1.019 & 1.012 & 2.4 \\
\hline Ethylbenzene & 1.709 & 1.787 & 1.808 & 1.870 & 1.911 & 1.817 & 4.3 \\
\hline o-Xylene & 0.626 & 0.676 & 0.684 & 0.693 & 0.709 & 0.678 & 4.6 \\
\hline m,p-Xylene & 0.602 & 0.726 & 0.739 & 0.748 & 0.759 & 0.715 & 9.0 \\
\hline Styrene & 0.806 & 0.949 & 1.063 & 1.087 & 1.097 & 1.001 & 12.4 \\
\hline Bromoform & 0.225 & 0.258 & 0.243 & 0.242 & 0.244 & 0.242 & 4.8 \\
\hline Isopropylbenzene & 1.595 & 1.737 & 1.886 & 1.943 & 1.987 & 1.830 & 8.8 \\
\hline $1,1,2,2$-Tetrachloroethane & 0.144 & 0.149 & 0.143 & 0.147 & 0.150 & 0.147 & 2.1 \\
\hline $1,3-$ Dichlorobenzene & 1.532 & 1.551 & 1.542 & 1.547 & 1.581 & 1.551 & 1.2 \\
\hline 1,4-Dichlorobenzene & 1.655 & 1.627 & 1.575 & 1.560 & 1.568 & 1.597 & 2.6 \\
\hline 1,2-Dichlorobenzene & 1.232 & 1.193 & 1.278 & 1.281 & 1.291 & 1.255 & 3.3 \\
\hline 1,2-Dibromo-3-Chloropropane & 0.066 & 0.038 & 0.044 & 0.043 & 0.044 & 0.047 & 22.8 \\
\hline 1,2,4-Trichlorobenzene & 0.699 & 0.760 & 0.765 & 0.791 & 0.809 & 0.765 & 5.5 \\
\hline 1,2,3-Trichlorobenzene & 0.522 & 0.527 & 0.573 & 0.556 & 0.579 & 0.552 & 4.7 \\
\hline
\end{tabular}


$6 \mathrm{C}-$ FORM VI VOA-3

VOLATILE ORGANICS INITIAL CALIBRATION DATA

Lab Name: TESTAMERICA BURLINGTON

Contract: $8 \mathrm{E}-00302$

Lab Code: STLV Case No.: INMAN Mod. Ref No.:

SDG NO.: 200-5308

Instrument ID: J.i

Calibration Date(s): 05/10/2011 05/10/2011

Heated Purge: ( $\mathrm{Y} / \mathrm{N}) \mathrm{N}$

Calibration Time(s): 1032

1212

Purge Volume: 25.0

$(\mathrm{mL})$

GC Column: DB-624

ID : 0.20

(mm) Length: 25

(m)

\begin{tabular}{|c|c|c|c|c|c|c|c|}
\hline \multirow{3}{*}{$\begin{array}{l}\text { LAB FILE ID: } \\
\text { RRF } 5.0=\text { JCV07.D } \\
\text { COMPOUND }\end{array}$} & \multirow{2}{*}{\multicolumn{3}{|c|}{$\begin{array}{l}\text { RRF 0.5 }=\text { JCV05.D } \\
\operatorname{RRF10}=\mathrm{JCV} 08 . \mathrm{D}\end{array}$}} & \multirow{2}{*}{\multicolumn{4}{|c|}{$\begin{array}{l}\text { RRE1.0 }=\text { JCV06.D } \\
\text { RRF20 }=\text { JCV09.D }\end{array}$}} \\
\hline & & & & & & & \\
\hline & RRE 0.5 & RRF 1.0 & RRE 5.0 & RRE 10 & RRE 20 & $\overline{\mathrm{RRF}}$ & 学 RSD \\
\hline Vinyl Chloride-d3 & 0.334 & 0.306 & 0.289 & 0.277 & 0.272 & 0.296 & 8.5 \\
\hline Chloroethane-d5 & 0.217 & 0.234 & 0.226 & 0.213 & 0.203 & 0.219 & 5.3 \\
\hline 1,1-Dichloroethene-d2 & 0.585 & 0.580 & 0.551 & 0.534 & 0.532 & 0.556 & 4.5 \\
\hline 2-Butanone-d5 & 0.020 & 0.024 & 0.025 & 0.025 & 0.025 & 0.023 & 9.4 \\
\hline Chloroform-d & 0.578 & 0.559 & 0.569 & 0.539 & 0.537 & 0.556 & 3.3 \\
\hline 1,2-Dichloroethane-d4 & 0.212 & 0.187 & 0.190 & 0.186 & 0.191 & 0.193 & 5.6 \\
\hline Benzene-d6 & 1.484 & 1.509 & 1.444 & 1.413 & 1.437 & 1.458 & 2.7 \\
\hline 1,2-Dichloropropane-d6 & 0.383 & 0.354 & 0.373 & 0.375 & 0.369 & 0.371 & 2.8 \\
\hline Toluene-d8 & 1.341 & 1.402 & 1.420 & 1.393 & 1.430 & 1.397 & 2.5 \\
\hline trans-1,3-Dichloropropene-d4 & 0.307 & 0.296 & 0.298 & 0.307 & 0.312 & 0.304 & 2.2 \\
\hline 2-Hexanone-d5 & 0.025 & 0.025 & 0.028 & 0.029 & 0.030 & 0.027 & 7.2 \\
\hline $1,1,2,2$-Tetrachloroethane-d2 & 0.145 & 0.158 & 0.152 & 0.156 & 0.151 & 0.152 & 3.3 \\
\hline 1,2-Dichlorobenzene-d4 & 0.895 & 0.875 & 0.806 & 0.771 & 0.788 & 0.827 & 6.7 \\
\hline
\end{tabular}

Report 1,4-Dioxane-d8 for Low-Medium VOA analysis only 
$7 \mathrm{~A}$ - FORM VII VOA-1

VOLATILE CONTINUING CALIBRATION DATA

Lab Name: TESTAMERICA BURLINGTON

Contract: $8 \mathrm{E}-00302$

Lab Code: STLV

Case No.: INMAN Mod. Ref No.:

SDG No.: 200-5308

Instrument ID: J.i

Calibration Date: 05/25/2011 Time: 1305

Lab File Id: JCVB03.D

EPA Sample No. (VSTD\#\#\#\#): VSTD005JE Init. Calib. Date(s) : 05/10/2011 05/10/2011

Heated Purge: $(\mathrm{Y} / \mathrm{N})$ GC Column: DB-624

Init. Calib. Time(s): 1032 1212

Purge Volume: 25.0 $(\mathrm{mL})$

\begin{tabular}{|c|c|c|c|c|c|}
\hline COMPOUND & $\overline{\text { RRE }}$ & RRE5.0 & $\begin{array}{l}\text { MIN } \\
\text { RRE }\end{array}$ & $\frac{5}{\partial}$ & MAX $\% \mathrm{D}$ \\
\hline Dichlorodifluoromethane & 0.549 & 0.633 & 0.010 & 15.2 & 40.0 \\
\hline Chloromethane & 0.324 & 0.379 & 0.010 & 17.1 & 40.0 \\
\hline Vinyl chloride & 0.331 & 0.358 & 0.010 & 8.1 & 30.0 \\
\hline Bromomethane & 0.182 & 0.195 & 0.100 & 7.2 & 30.0 \\
\hline Chloroethane & 0.180 & 0.191 & 0.010 & 6.2 & 40.0 \\
\hline Trichlorofluoromethane & 0.593 & 0.643 & 0.010 & 8.5 & 40.0 \\
\hline 1,1-Dichloroethene & 0.267 & 0.264 & 0.100 & -1.4 & 30.0 \\
\hline 1,1,2-Trichloro-1,2,2-trifluoroethane & 0.318 & 0.320 & 0.010 & 0.8 & 40.0 \\
\hline Acetone & 0.017 & 0.019 & 0.010 & 9.2 & 40.0 \\
\hline Carbon disulfide & 0.800 & 0.769 & 0.010 & -3.9 & 40.0 \\
\hline Methyl acetate & 0.043 & 0.049 & 0.010 & 13.8 & 40.0 \\
\hline Methylene Chloride & 0.258 & 0.287 & 0.010 & 11.0 & 40.0 \\
\hline trans-1,2-Dichloroethene & 0.323 & 0.359 & 0.010 & 11.2 & 40.0 \\
\hline Methyl tert-butyl ether & 0.405 & 0.500 & 0.010 & 23.5 & 40.0 \\
\hline 1,1-Dichloroethane & 0.499 & 0.544 & 0.200 & 9.2 & 30.0 \\
\hline cis-1,2-Dichloroethene & 0.319 & 0.336 & 0.010 & 5.4 & 40.0 \\
\hline 2-Butanone & 0.025 & 0.028 & 0.010 & 10.2 & 40.0 \\
\hline Bromochloromethane & 0.116 & 0.119 & 0.050 & 3.2 & 30.0 \\
\hline Chloroform & 0.537 & 0.562 & 0.200 & 4.7 & 30.0 \\
\hline 1,1,1-Trichloroethane & 0.676 & 0.721 & 0.100 & 6.7 & 30.0 \\
\hline Cyclohexane & 0.566 & 0.566 & 0.010 & 0.0 & 40.0 \\
\hline Carbon tetrachloride & 0.663 & 0.697 & 0.100 & 5.3 & 30.0 \\
\hline Benzene & 1.468 & 1.439 & 0.400 & -2.0 & 30.0 \\
\hline 1,2-Dichloroethane & 0.236 & 0.279 & 0.100 & 18.0 & 30.0 \\
\hline Trichloroethene & 0.402 & 0.395 & 0.300 & -1.6 & 30.0 \\
\hline Methylcyclohexane & 0.450 & 0.416 & 0.010 & -7.5 & 40.0 \\
\hline
\end{tabular}

Report 1,4-Dioxane for Low/Medium VOA analysis only 
$7 B$ - FORM VII VOA-2

VOLATILE CONTINUING CALIBRATION DATA

Lab Name: TESTAMERICA BURLINGTON

Contract: $8 \mathrm{E}-00302$

Lab Code: STLV Case No.: INMAN Mod. Ref No.:

SDG No.: 200-5308

Instrument ID: J.i

Calibration Date: 05/25/2011 Time: 1305

Lab File Id: JCVB03.D Init. Calib. Date(s): 05/10/2011 05/10/2011

EPA Sample No. (VSTD\#\#\#\#): VSTD005JE

Init. Calib. Time(s): $1032 \quad 1212$

Heated Purge: ( $\mathrm{Y} / \mathrm{N}) \mathrm{N}$

GC Column: DB-624

ID: $0.20(\mathrm{~mm})$ Length: 25

(II)

Purge Volume: 25.0

$(m L)$

\begin{tabular}{|c|c|c|c|c|c|}
\hline COMPOUND & $\overline{\mathrm{RRF}}$ & RRF5. 0 & $\begin{array}{l}\text { MIN } \\
\text { RRF }\end{array}$ & $\div \mathrm{D}$ & $M A X \div D$ \\
\hline 1,2-Dichloropropane & 0.276 & 0.274 & 0.010 & -0.6 & 40.0 \\
\hline Bromodichloromethane & 0.400 & 0.419 & 0.200 & 4.9 & 30.0 \\
\hline cis-1,3-Dichloropropene & 0.443 & 0.442 & 0.200 & -0.4 & 30.0 \\
\hline 4-Methyl-2-pentanone & 0.074 & 0.078 & 0.010 & 5.6 & 40.0 \\
\hline Toluene & 1.631 & 1.690 & 0.400 & 3.6 & 30.0 \\
\hline trans-1,3-Dichloropropene & 0.333 & 0.359 & 0.100 & 7.9 & 30.0 \\
\hline 1,1,2-Trichloroethane & 0.179 & 0.182 & 0.100 & 2.0 & 30.0 \\
\hline Tetrachloroethene & 0.369 & 0.389 & 0.100 & 5.3 & 30.0 \\
\hline 2-Hexanone & 0.048 & 0.058 & 0.010 & 19.8 & 40.0 \\
\hline Dibromochloromethane & 0.245 & 0.277 & 0.100 & 12.6 & 30.0 \\
\hline 1,2-Dibromoethane & 0.161 & 0.160 & 0.010 & -0.3 & 40.0 \\
\hline Chlorobenzene & 1.012 & 1.049 & 0.500 & 3.6 & 30.0 \\
\hline Ethylbenzene & 1.817 & 1.806 & 0.100 & -0.6 & 30.0 \\
\hline o-Xylene & 0.678 & 0.704 & 0.300 & 3.9 & 30.0 \\
\hline $\mathrm{m}, \mathrm{p}$-Xylene & 0.715 & 0.747 & 0.300 & 4.6 & 30.0 \\
\hline Styrene & 1.001 & 1.106 & 0.300 & 10.6 & 30.0 \\
\hline Bromoform & 0.242 & 0.259 & 0.050 & 6.6 & 30.0 \\
\hline Isopropylbenzene & 1.830 & 1.868 & 0.010 & 2.1 & 40.0 \\
\hline $1,1,2,2$-Tetrachloroethane & 0.147 & 0.155 & 0.100 & 5.6 & 30.0 \\
\hline 1,3-Dichlorobenzene & 1.551 & 1.478 & 0.400 & -4.7 & 30.0 \\
\hline 1,4-Dichlorobenzene & 1.597 & 1.550 & 0.400 & -3.0 & 30.0 \\
\hline 1,2-Dichlorobenzene & 1.255 & 1.337 & 0.400 & 6.5 & 30.0 \\
\hline 1,2-Dibromo-3-Chloropropane & 0.047 & 0.048 & 0.010 & 1.8 & 40.0 \\
\hline 1,2,4-Trichlorobenzene & 0.765 & 0.803 & 0.200 & 5.1 & 30.0 \\
\hline 1,2,3-Trichlorobenzene & 0.552 & 0.547 & 0.200 & -0.8 & 30.0 \\
\hline
\end{tabular}


$7 \mathrm{C}$ - FORM VII VOA-3

VOLATILE CONTINUING CALIBRATION DATA

Lab Name: TESTAMERICA BURLINGTON

Contract: $8 \mathrm{E}-00302$

Lab Code: STLV Case No.: INMAN Mod. Ref No.:

SDG No.: 200-5308

Instrument ID: J.i

Calibration Date: 05/25/2011 Time: 1305

Lab File Id: JCVB03.D Init. Calib. Date(s): 05/10/2011 05/10/2011

EPA Sample No. (VSTD\#\#\#\#): VSTD005JE

Init. Calib. Time(s): $1032 \quad 1212$

Heated Purge: ( $\mathrm{Y} / \mathrm{N})$

GC Column: DB-624

ID: $0.20(\mathrm{~mm})$ Length: 25

(m)

Purge Volume: 25.0

(mI)

\begin{tabular}{|c|c|c|c|c|c|}
\hline COMPOUND & $\overline{\mathrm{RRF}}$ & RRE5. 0 & $\begin{array}{l}\text { MIN } \\
\text { RRE }\end{array}$ & $\because \mathrm{D}$ & MAX $\because D$ \\
\hline Vinyl Chloride-d3 & 0.296 & 0.324 & 0.010 & 9.5 & 30.0 \\
\hline Chloroethane-d5 & 0.219 & 0.221 & 0.010 & 1.3 & 40.0 \\
\hline 1,1-Dichloroethene-d2 & 0.556 & 0.555 & 0.010 & -0.3 & 30.0 \\
\hline 2-Butanone-d5 & 0.023 & 0.028 & 0.010 & 20.3 & 40.0 \\
\hline Chloroform-d & 0.556 & 0.591 & 0.010 & 6.2 & 30.0 \\
\hline 1,2-Dichloroethane-d4 & 0.193 & 0.226 & 0.010 & 16.9 & 30.0 \\
\hline Benzene-d6 & 1.458 & 1.411 & 0.010 & -3.2 & 30.0 \\
\hline 1,2-Dichloropropane-d6 & 0.371 & 0.305 & 0.010 & -17.8 & 40.0 \\
\hline Toluene-d8 & 1.397 & 1.436 & 0.010 & 2.8 & 30.0 \\
\hline trans-1,3-Dichloropropene-d4 & 0.304 & 0.302 & 0.010 & -0.8 & 30.0 \\
\hline 2-Hexanone-d5 & 0.027 & 0.029 & 0.010 & 6.4 & 40.0 \\
\hline $1,1,2,2$-Tetrachloroethane-d2 & 0.152 & 0.158 & 0.010 & 4.0 & 30.0 \\
\hline 1,2-Dichlorobenzene-d4 & 0.827 & 0.835 & 0.010 & 1.0 & 30.0 \\
\hline
\end{tabular}

Report 1,4-Dioxane-d8 for Low/Medium VOA analysis only 
$7 A$ - FORM VII VOA-1

VOLATILE CONTINUING CALIBRATION DATA

Lab Name: TESTAMERICA BURLINGTON

Contract: $8 \mathrm{E}-00302$

Lab Code: STLV Case No.: INMAN Mod. Ref No.: SDG No.: 200-5308

Instrument ID: J.i

Calibration Date: 05/25/2011 Time: 1709

Lab File Id: JCVB11.D Init. Calib. Date(s): 05/10/2011 05/10/2011

EPA Sample No. (VSTD\#\#\#\#): VSTD005EJ

Init. Calib. Time(s): $1032 \quad 1212$

Heated Purge: ( $\mathrm{Y} / \mathrm{N}) \quad \mathrm{N}$ GC Column: DB-624 ID: $0.20(\mathrm{~mm})$ Length: 25 (m)

Purge Volume: 25.0 $(\mathrm{mL})$

\begin{tabular}{|c|c|c|c|c|c|}
\hline COMPOUND & $\overline{R R F}$ & RRF5.0 & $\begin{array}{l}\text { MIN } \\
\text { RRF }\end{array}$ & $\because \mathrm{D}$ & MAX $\because D$ \\
\hline Dichlorodifluoromethane & 0.549 & 0.633 & 0.010 & 15.3 & 50.0 \\
\hline Chloromethane & 0.324 & 0.338 & 0.010 & 4.4 & 50.0 \\
\hline Vinyl chloride & 0.331 & 0.316 & 0.010 & -4.4 & 50.0 \\
\hline Bromomethane & 0.182 & 0.157 & 0.010 & -13.7 & 50.0 \\
\hline Chloroethane & 0.180 & 0.207 & 0.010 & 14.9 & 50.0 \\
\hline Trichlorofluoromethane & 0.593 & 0.652 & 0.010 & 9.9 & 50.0 \\
\hline 1,1-Dichloroethene & 0.267 & 0.267 & 0.010 & -0.2 & 50.0 \\
\hline 1,1,2-Trichloro-1,2,2-trifluoroethane & 0.318 & 0.325 & 0.010 & 2.3 & 50.0 \\
\hline Acetone & 0.017 & 0.018 & 0.010 & 6.1 & 50.0 \\
\hline Carbon disulfide & 0.800 & 0.764 & 0.010 & -4.5 & 50.0 \\
\hline Methyl acetate & 0.043 & 0.049 & 0.010 & 15.6 & 50.0 \\
\hline Methylene Chloride & 0.258 & 0.277 & 0.010 & 7.1 & 50.0 \\
\hline trans-1,2-Dichloroethene & 0.323 & 0.360 & 0.010 & 11.4 & 50.0 \\
\hline Methyl tert-butyl ether & 0.405 & 0.490 & 0.010 & 21.0 & 50.0 \\
\hline 1,1-Dichloroethane & 0.499 & 0.514 & 0.010 & 3.0 & 50.0 \\
\hline cis-1,2-Dichloroethene & 0.319 & 0.330 & 0.010 & 3.7 & 50.0 \\
\hline 2-Butanone & 0.025 & 0.026 & 0.010 & 3.9 & 50.0 \\
\hline Bromochloromethane & 0.116 & 0.119 & 0.010 & 3.2 & 50.0 \\
\hline Chloroform & 0.537 & 0.565 & 0.010 & 5.2 & 50.0 \\
\hline 1,1,1-Trichloroethane & 0.676 & 0.782 & 0.010 & 15.7 & 50.0 \\
\hline Cyclohexane & 0.566 & 0.600 & 0.010 & 5.9 & 50.0 \\
\hline Carbon tetrachloride & 0.663 & 0.760 & 0.010 & 14.7 & 50.0 \\
\hline Benzene & 1.468 & 1.527 & 0.010 & 4.0 & 50.0 \\
\hline 1,2-Dichloroethane & 0.236 & 0.270 & 0.010 & 14.3 & 50.0 \\
\hline Trichloroethene & 0.402 & 0.419 & 0.010 & 4.4 & 50.0 \\
\hline Methylcyclohexane & 0.450 & 0.458 & 0.010 & 1.9 & 50.0 \\
\hline
\end{tabular}

Report 1,4-Dioxane for Low/Medium VOA analysis only 
7B - FORM VII VOA-2

VOLATILE CONTINUING CALIBRATION DATA

Lab Name: TESTAMERICA BURLINGTON

Contract: 8E-00302

Lab Code: STLV Case No.: INMAN

Mod. Ref No.:

SDG No.: 200-5308

Instrument ID: J.i

Calibration Date: 05/25/2011 Time: 1709

Lab File Id: JCVB11.D

Init. Calib. Date(s): 05/10/2011 05/10/2011

EPA Sample No, (VSTD\#\#\#\#): VSTDO05EJ

Init. Calib. Time(s): $1032 \quad 1212$

Heated Purge: ( $\mathrm{Y} / \mathrm{N}) \mathrm{N}$

GC Column: DB-624

ID: $0.20(\mathrm{~mm})$ Length: 25

(m)

Purge Volume: 25.0

(mL)

\begin{tabular}{|c|c|c|c|c|c|}
\hline COMPOUND & $\overline{\mathrm{RRF}}$ & RRF5. 0 & $\begin{array}{l}\text { MIN } \\
\text { RRF }\end{array}$ & $\frac{\circ}{\partial} \mathrm{D}$ & MAX $\because \mathrm{D}$ \\
\hline 1,2-Dichloropropane & 0.276 & 0.288 & 0.010 & 4.2 & 50.0 \\
\hline Bromodichloromethane & 0.400 & 0.442 & 0.010 & 10.6 & 50.0 \\
\hline cis-1,3-Dichloropropene & 0.443 & 0.439 & 0.010 & -1.0 & 50.0 \\
\hline 4-Methyl-2-pentanone & 0.074 & 0.081 & 0.010 & 10.4 & 50.0 \\
\hline Toluene & 1.631 & 1.759 & 0.010 & 7.8 & 50.0 \\
\hline trans-1,3-Dichloropropene & 0.333 & 0.372 & 0.010 & 11.8 & 50.0 \\
\hline 1,1,2-Trichloroethane & 0.179 & 0.194 & 0.010 & 8.4 & 50.0 \\
\hline Tetrachloroethene & 0.369 & 0.392 & 0.010 & 6.1 & 50.0 \\
\hline 2-Hexanone & 0.048 & 0.054 & 0.010 & 12.9 & 50.0 \\
\hline Dibromochloromethane & 0.245 & 0.276 & 0.010 & 12.6 & 50.0 \\
\hline 1,2-Dibromoethane & 0.161 & 0.170 & 0.010 & 5.4 & 50.0 \\
\hline Chlorobenzene & 1.012 & 1.052 & 0.010 & 4.0 & 50.0 \\
\hline Ethylbenzene & 1.817 & 1.971 & 0.010 & 8.5 & 50.0 \\
\hline o-Xylene & 0.678 & 0.704 & 0.010 & 3.9 & 50.0 \\
\hline $\mathrm{m}, \mathrm{p}$-xylene & 0.715 & 0.802 & 0.010 & 12.2 & 50.0 \\
\hline Styrene & 1.001 & 1.114 & 0.010 & 11.3 & 50.0 \\
\hline Bromoform & 0.242 & 0.250 & 0.010 & 3.1 & 50.0 \\
\hline Isopropylbenzene & 1.830 & 2.052 & 0.010 & 12.1 & 50.0 \\
\hline $1,1,2,2$-Tetrachloroethane & 0.147 & 0.163 & 0.010 & 11.0 & 50.0 \\
\hline 1,3-Dichlorobenzene & 1.551 & 1.587 & 0.010 & 2.4 & 50.0 \\
\hline 1,4-Dichlorobenzene & 1.597 & 1.617 & 0.010 & 1.2 & 50.0 \\
\hline 1,2-Dichlorobenzene & 1.255 & 1.257 & 0.010 & 0.2 & 50.0 \\
\hline 1,2-Dibromo-3-Chloropropane & 0.047 & 0.047 & 0.010 & 0.6 & 50.0 \\
\hline 1,2,4-Trichlorobenzene & 0.765 & 0.798 & 0.010 & 4.4 & 50.0 \\
\hline 1,2,3-Trichlorobenzene & 0.552 & 0.605 & 0.010 & 9.6 & 50.0 \\
\hline
\end{tabular}


7C - FORM VII VOA-3

VOLATILE CONTINUING CALIBRATION DATA

Lab Name: TESTAMERICA BURLINGTON

Contract: $8 \mathrm{E}-00302$

Lab Code: STLV Case No.: INMAN Mod. Ref No.:

SDG No.: 200-5308

Instrument ID: J.i

Calibration Date: 05/25/2011 Time: 1709

Lab File Id: JCVB11.D Init. Calib. Date(s): 05/10/2011 05/10/2011

EPA Sample No. (VSTD\#\#\#\#): VSTD005EJ

Init. Calib. Time(s): $1032 \quad 1212$

Heated Purge: ( $\mathrm{Y} / \mathrm{N}) \mathrm{N}$

GC Column: DB-624

ID : $0.20(\mathrm{~mm})$ Length: 25

(m)

Purge Volume: 25.0

(mL)

\begin{tabular}{|c|c|c|c|c|c|}
\hline COMPOUND & $\overline{\mathrm{RRE}}$ & RRE5.0 & $\begin{array}{l}\text { MIN } \\
\text { RRF }\end{array}$ & $\frac{\circ}{2} \mathrm{D}$ & $\operatorname{MAX} \div \mathrm{D}$ \\
\hline Vinyl Chloride-d3 & 0.296 & 0.289 & 0.010 & -2.4 & 50.0 \\
\hline Chloroethane-d5 & 0.219 & 0.216 & 0.010 & -1.1 & 50.0 \\
\hline 1,1-Dichloroethene-d2 & 0.556 & 0.554 & 0.010 & -0.5 & 50.0 \\
\hline 2-Butanone-d5 & 0.023 & 0.025 & 0.010 & 7.4 & 50.0 \\
\hline Chloroform-d & 0.556 & 0.580 & 0.010 & 4.3 & 50.0 \\
\hline 1,2-Dichloroethane-d4 & 0.193 & 0.223 & 0.010 & 15.2 & 50.0 \\
\hline Benzene-d6 & 1.458 & 1.528 & 0.010 & 4.9 & 50.0 \\
\hline 1,2-Dichloropropane-d6 & 0.371 & 0.384 & 0.010 & 3.5 & 50.0 \\
\hline Toluene-d8 & 1.397 & 1.520 & 0.010 & 8.7 & 50.0 \\
\hline trans-1,3-Dichloropropene-d4 & 0.304 & 0.327 & 0.010 & 7.7 & 50.0 \\
\hline 2-Hexanone-d5 & 0.027 & 0.030 & 0.010 & 8.6 & 50.0 \\
\hline $1,1,2,2$-Tetrachloroethane-d2 & 0.152 & 0.164 & 0.010 & 8.0 & 50.0 \\
\hline 1,2-Dichlorobenzene-d4 & 0.827 & 0.780 & 0.010 & -5.6 & 50.0 \\
\hline
\end{tabular}

Report 1,4-Dioxane-d8 for Low/Medium VOA analysis only 
$1 A$ - FORM I VOA-1

VOLATILE ORGANICS ANALYSIS DATA SHEET
EPA SAMPLE NO.

VBLKJE

Lab Name: TESTAMERICA BURLINGTON

Lab Code: STLV

Case No.: INMAN

Mod. Ref No.:

Water

$(\mathrm{g} / \mathrm{mL}) \quad \mathrm{mL}$

Sample wt/vol: 25.0

Level: (TRACE/LOW/MED)

TRACE

\% Moisture: not dec.

GC Column: DB-624

ID $: 0.20 \quad$ (mm)

Soil Extract Volume:

(uL)

(mL)

Purge Volume: 25.0

Contract: $8 \mathrm{E}-00302$

SDG No.: 200-5308

Lab Sample ID: MB 200-18645/4

Lab File ID: JCVBO4.D

Date Recej.ved:

Date Analyzed: 05/25/2011

Dilution Factor: 1.0

Soil Aliquot Volume:

(UL)

CONCENTRATION UNITS

\begin{tabular}{|c|c|c|c|}
\hline CAS NO. & COMPOUND & $\begin{array}{l}\text { CONCENTRATION UNITS: } \\
(\mathrm{ug} / \mathrm{L} \text { or } \mathrm{ug} / \mathrm{kg}) \mathrm{ug} / \mathrm{L}\end{array}$ & Q \\
\hline $75-71-8$ & Dichlorodifluoromethane & 0.50 & $\bar{U}$ \\
\hline $74-87-3$ & Chloromethane & 0.50 & $\mathrm{U}$ \\
\hline $75-01-4$ & Vinyl chloride & 0.50 & $\mathrm{U}$ \\
\hline $74-83-9$ & Bromomethane & 0.50 & $\mathrm{U}$ \\
\hline $75-00-3$ & Chloroethane & 0.50 & $\mathrm{U}$ \\
\hline $75-69-4$ & Trichlorofluoromethane & 0.50 & $\mathrm{U}$ \\
\hline $75-35-4$ & 1,1-Dichloroethene & 0.50 & $\mathrm{U}$ \\
\hline $76-13-1$ & 1,1,2-Trichloro-1,2,2-trifluoroethane & 0.50 & $\mathrm{U}$ \\
\hline $67-64-1$ & Acetone & 2.0 & $\mathrm{~J}$ \\
\hline $75-15-0$ & Carbon disulfide & 0.22 & $\mathrm{~J}$ \\
\hline $79-20-9$ & Methyl acetate & 0.50 & $\mathrm{U}$ \\
\hline $75-09-2$ & Methylene Chloride & 0.50 & $\mathrm{U}$ \\
\hline $156-60-5$ & trans-1,2-Dichloroethene & 0.055 & $\mathrm{~J}$ \\
\hline $1634-04-4$ & Methyl tert-butyl ether & 0.50 & $\mathrm{U}$ \\
\hline $75-34-3$ & 1,1-Dichloroethane & 0.50 & $\mathrm{U}$ \\
\hline $156-59-2$ & cis-1,2-Dichloroethene & 0.50 & $\mathrm{U}$ \\
\hline $78-93-3$ & 2-Butanone & 5.0 & $\mathrm{U}$ \\
\hline $74-97-5$ & Bromochloromethane & 0.50 & $\mathrm{U}$ \\
\hline $67-66-3$ & Chloroform & 0.50 & $\mathrm{U}$ \\
\hline $71-55-6$ & 1,1,1-Trichloroethane & 0.50 & $\mathrm{U}$ \\
\hline $110-82-7$ & Cyclohexane & 0.50 & $\mathrm{U}$ \\
\hline $56-23-5$ & Carbon tetrachloride & 0.024 & $\mathrm{~J}$ \\
\hline $71-43-2$ & Benzene & 0.50 & $\mathrm{U}$ \\
\hline $107-06-2$ & 1,2-Dichloroethane & 0.50 & $\mathrm{U}$ \\
\hline
\end{tabular}

Report 1,4-Dioxane for Low-Medium VOA analysis only

SOM01.2 (4/2007) 
Lab Name: TESTAMERICA BURLINGTON

Lab Code: STLV

Contract: $8 \mathrm{E}-00302$

Matrix: (SOIL/SED/WATER)

Sample wt/vol: 25.0

Water

$(\mathrm{g} / \mathrm{mL}) \quad \mathrm{mL}$

Level: (TRACE/LOW/MED) TRACE

응 Moisture: not dec.

GC Column: $\mathrm{DB}-624$

ID $: 0.20$ $(\mathrm{mm})$

Soil Extract Volume:

(mL)

Purge Volume: 25.0

SDG No.: 200-5308

Lab Sample ID: MB 200-18645/4

Lab File ID: JCVB04.D

Date Received:

Date Analyzed: 05/25/2011

Dilution Factor: 1.0

Soil Aliquot Volume:

\begin{tabular}{|c|c|c|c|}
\hline CAS NO. & COMPOUND & $\begin{array}{l}\text { CONCENTRATION UNITS: } \\
(\mathrm{ug} / \mathrm{L} \text { or } \mathrm{ug} / \mathrm{kg}) \mathrm{ug} / \mathrm{L}\end{array}$ & $Q$ \\
\hline $79-01-6$ & Trichloroethene & 0.50 & $\mathrm{U}$ \\
\hline $108-87-2$ & Methylcyclohexane & 0.50 & $\mathrm{U}$ \\
\hline $78-87-5$ & 1,2-Dichloropropane & 0.50 & $\mathrm{U}$ \\
\hline $75-27-4$ & Bromodichloromethane & 0.50 & $\mathrm{U}$ \\
\hline $10061-01-5$ & cis-1,3-Dichloropropene & 0.50 & $\mathrm{U}$ \\
\hline $108-10-1$ & 4-Methyl-2-pentanone & 5.0 & $\mathrm{U}$ \\
\hline $108-88-3$ & Toluene & 0.015 & $\mathrm{~J}$ \\
\hline $10061-02-6$ & trans-1,3-Dichloropropene & 0.50 & $\mathrm{U}$ \\
\hline $79-00-5$ & 1,1,2-Trichloroethane & 0.50 & $\mathrm{U}$ \\
\hline $127-18-4$ & Tetrachloroethene & 0.50 & $\mathrm{U}$ \\
\hline $591-78-6$ & 2-Hexanone & 5.0 & $\mathrm{U}$ \\
\hline $124-48-1$ & Dibromochloromethane & 0.50 & $\mathrm{U}$ \\
\hline $106-93-4$ & 1,2-Dibromoethane & 0.50 & $\mathrm{U}$ \\
\hline $108-90-7$ & Chlorobenzene & 0.50 & $\mathrm{U}$ \\
\hline $100-41-4$ & Ethylbenzene & 0.50 & $\mathrm{U}$ \\
\hline $95-47-6$ & o-xylene & 0.50 & $\mathrm{U}$ \\
\hline $179601-23-1$ & $\mathrm{~m}, \mathrm{p}$-Xylene & 0.50 & $\mathrm{U}$ \\
\hline $100-42-5$ & Styrene & 0.50 & $\mathrm{U}$ \\
\hline $75-25-2$ & Bromoform & 0.50 & $\mathrm{U}$ \\
\hline $98-82-8$ & Isopropylbenzene & 0.50 & $\mathrm{U}$ \\
\hline $79-34-5$ & $1,1,2,2$-Tetrachloroethane & 0.50 & $\mathrm{U}$ \\
\hline $541-73-1$ & 1,3-Dichlorobenzene & 0.50 & $\mathrm{U}$ \\
\hline $106-46-7$ & 1,4-Dichlorobenzene & 0.50 & $\mathrm{U}$ \\
\hline $95-50-1$ & 1,2-Dichlorobenzene & 0.50 & $\mathrm{U}$ \\
\hline $96-12-8$ & 1,2-Dibromo-3-Chloropropane & 0.50 & $\mathrm{U}$ \\
\hline $120-82-1$ & 1,2,4-Trichlorobenzene & 0.50 & $\mathrm{U}$ \\
\hline $87-61-6$ & 1,2,3-Trichlorobenzene & 0.11 & $\mathrm{~J}$ \\
\hline
\end{tabular}


1J - FORM I VOA-TIC

VOLATILE ORGANICS ANALYSIS DATA SHEET TENTATIVELY IDENTIFIED COMPOUNDS
EPA SAMPLE NO.

VBLKJE

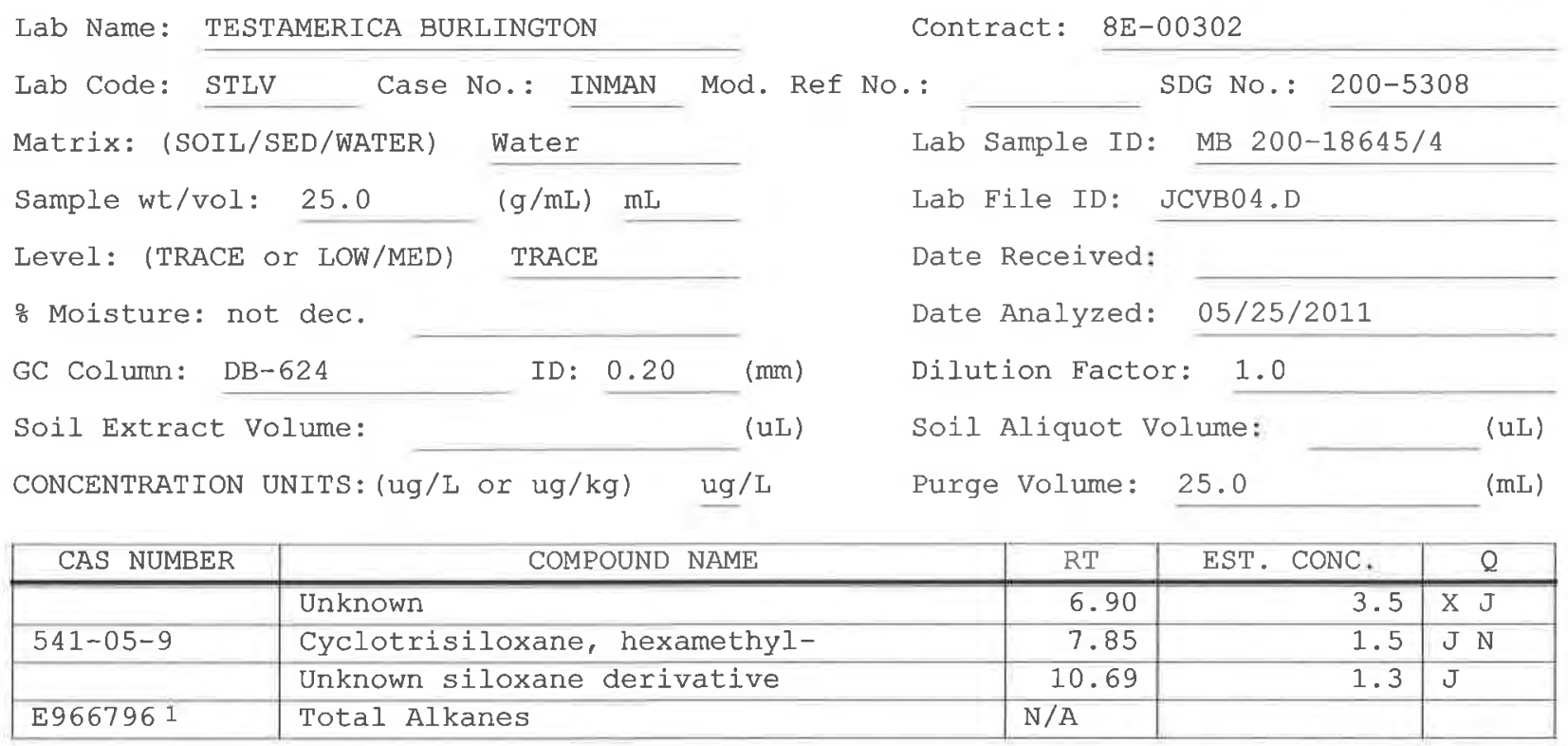

1 EPA-designated Registry Number. 
$1 \mathrm{~A}$ - FORM I VOA-1

VOLATILE ORGANICS ANALYSIS DATA SHEET
EPA SAMPLE NO.

VHBLK01
Lab Name: TESTAMERICA BURLINGTON

Lab Code: STLV Case No.

Matrix: (SOIL/SED/WATER)

Water

Sample wt/vol: 25.0

$(\mathrm{g} / \mathrm{mL}) \quad \mathrm{mL}$

Level: (TRACE/LOW/MED) TRACE

응 Moisture: not dec.

GC Column: DB-624 ID: 0.20 (mm)

Soil Extract Volume:

(mL)

Purge Volume: 25.0
Contract: $8 \mathrm{E}-00302$

SDG No.: 200-5308

\begin{tabular}{|c|c|c|c|}
\hline CAS NO. & COMPOUND & $\begin{array}{l}\text { CONCENTRATION UNITS: } \\
(\mathrm{ug} / \mathrm{L} \text { or } \mathrm{ug} / \mathrm{kg}) \mathrm{ug} / \mathrm{L}\end{array}$ & $Q$ \\
\hline $75-71-8$ & Dichlorodifluoromethane & 0.50 & $\mathrm{U}$ \\
\hline $74-87-3$ & Chloromethane & 0.50 & U \\
\hline $75-01-4$ & Vinyl chloride & 0.50 & $\mathrm{U}$ \\
\hline $74-83-9$ & Bromomethane & 0.50 & $\mathrm{U}$ \\
\hline $75-00-3$ & Chloroethane & 0.50 & $\mathrm{U}$ \\
\hline $75-69-4$ & Trichlorofluoromethane & 0.50 & $\mathrm{U}$ \\
\hline $75-35-4$ & 1,1-Dichloroethene & 0.50 & $\mathrm{U}$ \\
\hline $76-13-1$ & 1,1,2-Trichloro-1,2,2-trifluoroethane & 0.50 & $\mathrm{U}$ \\
\hline $67-64-1$ & Acetone & 5.0 & $\mathrm{U}$ \\
\hline $75-15-0$ & Carbon disulfide & 0.50 & $\mathrm{U}$ \\
\hline $79-20-9$ & Methyl acetate & 0.50 & $\mathrm{U}$ \\
\hline $75-09-2$ & Methylene Chloride & 0.50 & $\mathrm{U}$ \\
\hline $156-60-5$ & trans-1,2-Dichloroethene & 0.50 & $\mathrm{U}$ \\
\hline $1634-04-4$ & Methyl tert-butyl ether & 0.50 & $\mathrm{U}$ \\
\hline $75-34-3$ & 1,1-Dichloroethane & 0.50 & $\mathrm{U}$ \\
\hline $156-59-2$ & cis-1,2-Dichloroethene & 0.50 & $\mathrm{U}$ \\
\hline $78-93-3$ & 2-Butanone & 5.0 & $\mathrm{U}$ \\
\hline $74-97-5$ & Bromochloromethane & 0.50 & $\mathrm{U}$ \\
\hline $67-66-3$ & Chloroform & 0.50 & $\mathrm{U}$ \\
\hline $71-55-6$ & 1,1,1-Trichloroethane & 0.50 & $\mathrm{U}$ \\
\hline $110-82-7$ & Cyclohexane & 0.50 & $\mathrm{U}$ \\
\hline $56-23-5$ & Carbon tetrachloride & 0.021 & $\mathrm{~J} B$ \\
\hline $71-43-2$ & Benzene & 0.50 & $\mathrm{U}$ \\
\hline $107-06-2$ & 1,2-Dichloroethane & 0.50 & $\mathrm{U}$ \\
\hline
\end{tabular}

Report 1,4-Dioxane for Low-Medium VOA analysis only

Lab Sample ID: 200-5308-5

Lab File ID: JCVB10.D

Date Received:

Date Analyzed: 05/25/2011

Dilution Factor: 1.0

Soil Aliquot Volume: 
Lab Name: TESTAMERICA BURLINGTON

Lab Code: STLV Case No.: INMAN Mod. Ref No.:
Contract: $8 \mathrm{E}-00302$

SDG No.: 200-5308
Matrix: (SOIL/SED/WATER) Water

Sample wt/vol: 25.0

$(\mathrm{g} / \mathrm{mL}) \quad \mathrm{mL}$

Level: (TRACE/LOW/MED) TRACE

응 Moisture: not dec.

GC Column: DB-624

ID: 0.20 (mun)

Soil Extract Volume: (uI)

(mL)
Lab Sample ID: 200-5308-5

Lab File ID: JCVB10.D

Date Received:

Date Analyzed: 05/25/2011

Dilution Factor: 1.0

Soil Aliquot Volume:

\begin{tabular}{|c|c|c|c|}
\hline CAS NO. & COMPOUND & $\begin{array}{l}\text { CONCENTRATION UNITS: } \\
(\mathrm{ug} / \mathrm{L} \text { or } u g / \mathrm{kg}) \mathrm{ug} / \mathrm{L}\end{array}$ & ¿ \\
\hline $79-01-6$ & Trichloroethene & 0.50 & $\overline{\mathrm{U}}$ \\
\hline $108-87-2$ & Methylcyclohexane & 0.50 & $\mathrm{U}$ \\
\hline $78-87-5$ & 1,2-Dichloropropane & 0.50 & $\mathrm{U}$ \\
\hline $75-27-4$ & Bromodichloromethane & 0.50 & $\mathrm{U}$ \\
\hline $10061-01-5$ & cis-1,3-Dichloropropene & 0.50 & $\mathrm{U}$ \\
\hline $108-10-1$ & 4-Methyl-2-pentanone & 5.0 & $\mathrm{U}$ \\
\hline $108-88-3$ & Toluene & 0.50 & $\mathrm{U}$ \\
\hline $10061-02-6$ & trans-1,3-Dichloropropene & 0.50 & $\mathrm{U}$ \\
\hline $79-00-5$ & 1,1,2-Trichloroethane & 0.50 & $\mathrm{U}$ \\
\hline $127-18-4$ & Tetrachloroethene & 0.50 & $\mathrm{U}$ \\
\hline $591-78-6$ & 2-Hexanone & 5.0 & $\mathrm{U}$ \\
\hline $124-48-1$ & Dibromochloromethane & 0.50 & $\mathrm{U}$ \\
\hline $106-93-4$ & 1,2-Dibromoethane & 0.50 & $\mathrm{U}$ \\
\hline $108-90-7$ & Chlorobenzene & 0.50 & $\mathrm{U}$ \\
\hline $100-41-4$ & Ethylbenzene & 0.50 & $\mathrm{U}$ \\
\hline $95-47-6$ & o-Xylene & 0.50 & $\mathrm{U}$ \\
\hline $179601-23-1$ & $\mathrm{~m}, \mathrm{p}$-Xylene & 0.50 & $\mathrm{U}$ \\
\hline $100-42-5$ & Styrene & 0.50 & $\mathrm{U}$ \\
\hline $75-25-2$ & Bromoform & 0.50 & $\mathrm{U}$ \\
\hline $98-82-8$ & Isopropylbenzene & 0.50 & $\mathrm{U}$ \\
\hline $79-34-5$ & $1,1,2,2$-Tetrachloroethane & 0.50 & $\mathrm{U}$ \\
\hline $541-73-1$ & 1,3-Dichlorobenzene & 0.50 & $\mathrm{U}$ \\
\hline $106-46-7$ & 1,4-Dichlorobenzene & 0.50 & $\mathrm{U}$ \\
\hline $95-50-1$ & 1,2-Dichlorobenzene & 0.50 & $\mathrm{U}$ \\
\hline $96-12-8$ & 1,2-Dibromo-3-Chloropropane & 0.50 & $\mathrm{U}$ \\
\hline $120-82-1$ & 1,2,4-Trichlorobenzene & 0.50 & $\mathrm{U}$ \\
\hline $87-61-6$ & 1,2,3-Trichlorobenzene & 0.50 & $\mathrm{U}$ \\
\hline
\end{tabular}


$1 \mathrm{~J}$ - FORM I VOA-TIC

VOLATILE ORGANICS ANALYSIS DATA SHEET

TENTATIVELY IDENTIFIED COMPOUNDS
EPA SAMPLE NO.

VHBLKO1

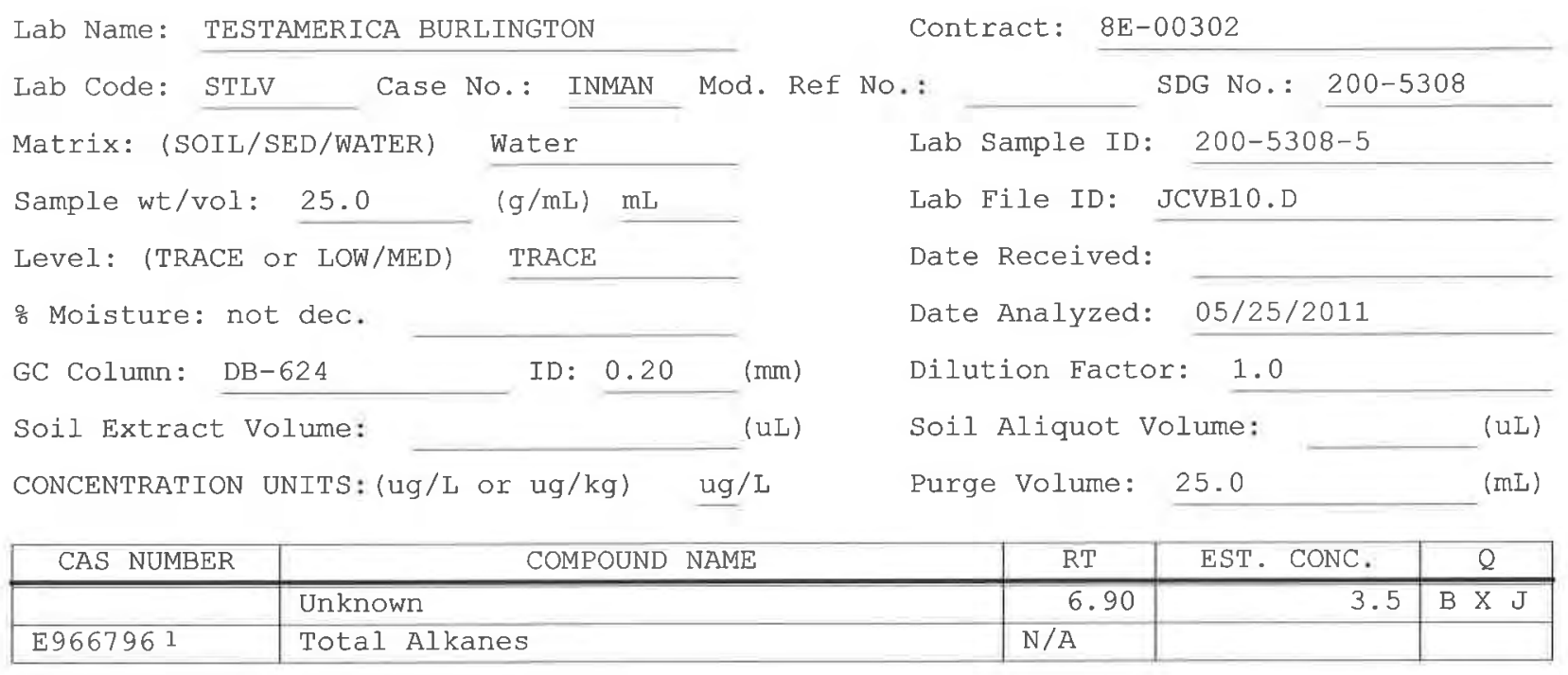

1 EPA-designated Registry Number. 
$1 \mathrm{~A}$ - FORM I VOA-1

VOLATILE ORGANICS ANALYSIS DATA SHEET
EPA SAMPLE NO.

VIBLKJH

Lab Name: TESTAMERICA BURLINGTON

Lab Code: STLV

Matrix: (SOIL/SED/WATER)

Sample wt/vol: 25.0

Water

$(\mathrm{g} / \mathrm{mI}) \quad \mathrm{mI}$

Level: (TRACE/LOW/MED) TRACE

․ㅡㅇ Moisture: not dec.

GC Column: DB-624

Soil Extract Volume: ID : $0.20 \quad(\mathrm{~mm})$

Purge Volume: 25.0

\begin{tabular}{|c|c|c|c|}
\hline CAS NO. & COMPOUND & $\begin{array}{l}\text { CONCENTRATION UNITS: } \\
(\mathrm{ug} / \mathrm{L} \text { or } \mathrm{ug} / \mathrm{kg} \text { ) ug/L }\end{array}$ & Q \\
\hline $75-71-8$ & Dichlorodifluoromethane & 0.50 & $\bar{U}$ \\
\hline $74-87-3$ & Chloromethane & 0.50 & $\mathrm{U}$ \\
\hline $75-01-4$ & Vinyl chloride & 0.50 & $\mathrm{U}$ \\
\hline $74-83-9$ & Bromomethane & 0.50 & $\mathrm{U}$ \\
\hline $75-00-3$ & Chloroethane & 0.50 & $\mathrm{U}$ \\
\hline $75-69-4$ & Trichlorofluoromethane & 0.50 & $\mathrm{U}$ \\
\hline $75-35-4$ & 1,1-Dichloroethene & 0.50 & $\mathrm{U}$ \\
\hline $76-13-1$ & 1,1,2-Trichloro-1,2,2-trifluoroethane & 0.50 & $\mathrm{U}$ \\
\hline $67-64-1$ & Acetone & 5.0 & $\mathrm{U}$ \\
\hline $75-15-0$ & Carbon disulfide & 0.16 & $\mathrm{~J} B$ \\
\hline $79-20-9$ & Methyl acetate & 0.50 & $\mathrm{U}$ \\
\hline $75-09-2$ & Methylene Chloride & 0.50 & $\mathrm{U}$ \\
\hline $156-60-5$ & trans-1,2-Dichloroethene & 0.50 & $\mathrm{U}$ \\
\hline $1634-04-4$ & Methyl tert-butyl ether & 0.50 & $\mathrm{U}$ \\
\hline $75-34-3$ & 1,1-Dichloroethane & 0.50 & $\mathrm{U}$ \\
\hline $156-59-2$ & cis-1,2-Dichloroethene & 0.50 & $\mathrm{U}$ \\
\hline $78-93-3$ & 2-Butanone & 5.0 & $\mathrm{U}$ \\
\hline $74-97-5$ & Bromochloromethane & 0.50 & $\mathrm{U}$ \\
\hline $67-66-3$ & Chloroform & 0.50 & $\mathrm{U}$ \\
\hline $71-55-6$ & 1,1,1-Trichloroethane & 0.50 & $\mathrm{U}$ \\
\hline $110-82-7$ & Cyclohexane & 0.50 & $\mathrm{U}$ \\
\hline $56-23-5$ & Carbon tetrachloride & 0.026 & $\mathrm{~J} B$ \\
\hline $71-43-2$ & Benzene & 0.50 & $\mathrm{U}$ \\
\hline $107-06-2$ & 1,2-Dichloroethane & 0.50 & $\mathrm{U}$ \\
\hline
\end{tabular}

Soil Aliquot Volume:

(mL)
(uL)

$(u L)$

Contract: $8 \mathrm{E}-00302$

SDG NO.: 200-5308

Lab Sample ID: VIBLK 200-18645/6

Lab File ID: JCVB06.D

Date Received:

Date Analyzed: 05/25/2011

Dilution Factor: 1.0 
Lab Name: TESTAMERICA BURLINGTON

Lab Code: STLV

Matrix: (SOIL/SED/WATER)

Sample wt/vol: 25.0

Water

$(\mathrm{g} / \mathrm{mL}) \quad \mathrm{mL}$

Level: (TRACE/LOW/MED) TRACE

\% Moisture: not dec.

GC Column: DB-624

ID: 0.20

Soil Extract Volume:

Purge Volume: 25.0

\begin{tabular}{|c|c|c|c|}
\hline CAS NO. & COMPOUND & $\begin{array}{l}\text { CONCENTRATION UNITS: } \\
(\mathrm{ug} / \mathrm{I} \text { or } \mathrm{ug} / \mathrm{kg}) \mathrm{ug} / \mathrm{L}\end{array}$ & $Q$ \\
\hline $79-01-6$ & Trichloroethene & 0.50 & $\bar{U}$ \\
\hline $108-87-2$ & Methylcyclohexane & 0.50 & $\mathrm{U}$ \\
\hline $78-87-5$ & 1,2-Dichloropropane & 0.50 & $\mathrm{U}$ \\
\hline $75-27-4$ & Bromodichloromethane & 0.50 & $\mathrm{U}$ \\
\hline $10061-01-5$ & cis-1,3-Dichloropropene & 0.50 & $\mathrm{U}$ \\
\hline $108-10-1$ & 4-Methyl-2-pentanone & 5.0 & $\mathrm{U}$ \\
\hline $108-88-3$ & Toluene & 0.50 & $\mathrm{U}$ \\
\hline $10061-02-6$ & trans $-1,3$-Dichloropropene & 0.50 & $\mathrm{U}$ \\
\hline $79-00-5$ & 1,1,2-Trichloroethane & 0.50 & $\mathrm{U}$ \\
\hline $127-18-4$ & Tetrachloroethene & 0.50 & $\mathrm{U}$ \\
\hline $591-78-6$ & 2-Hexanone & 5.0 & $\mathrm{U}$ \\
\hline $124-48-1$ & Dibromochloromethane & 0.50 & $\mathrm{U}$ \\
\hline $106-93-4$ & 1,2-Dibromoethane & 0.50 & $\mathrm{U}$ \\
\hline $108-90-7$ & Chlorobenzene & 0.50 & U \\
\hline $100-41-4$ & Ethylbenzene & 0.50 & $\mathrm{U}$ \\
\hline $95-47-6$ & o-Xylene & 0.50 & $\mathrm{U}$ \\
\hline $179601-23-1$ & m,p-xylene & 0.50 & $\mathrm{U}$ \\
\hline $100-42-5$ & Styrene & 0.50 & $\mathrm{U}$ \\
\hline $75-25-2$ & Bromoform & 0.50 & $\mathrm{U}$ \\
\hline $98-82-8$ & Isopropylbenzene & 0.50 & $\mathrm{U}$ \\
\hline $79-34-5$ & $1,1,2,2$-Tetrachloroethane & 0.50 & $\mathrm{U}$ \\
\hline $541-73-1$ & 1,3-Dichlorobenzene & 0.50 & $\mathrm{U}$ \\
\hline $106-46-7$ & 1,4-Dichlorobenzene & 0.50 & $\mathrm{U}$ \\
\hline $95-50-1$ & 1,2-Dichlorobenzene & 0.50 & $\mathrm{U}$ \\
\hline $96-12-8$ & 1,2-Dibromo-3-Chloropropane & 0.50 & $\mathrm{U}$ \\
\hline $120-82-1$ & 1,2,4-Trichlorobenzene & 0.50 & $\mathrm{U}$ \\
\hline $87-61-6$ & 1,2,3-Trichlorobenzene & 0.50 & $\mathrm{U}$ \\
\hline
\end{tabular}

Contract: $\quad 8 \mathrm{E}-00302$

SDG No.: 200-5308

Lab Sample ID: VIBLK 200-18645/6

Lab File ID: JCVB06.D

Date Received:

Date Analyzed: 05/25/2011

Dilution Factor: 1.0

Soil Aliquot Volume:

$(u L)$
(mL) 
IJ - FORM I VOA-TIC

VOLATILE ORGANICS ANALYSIS DATA SHEET TENTATIVELY IDENTIFIED COMPOUNDS
EPA SAMPLE NO.

VIBLKJH
Lab Name: TESTAMERICA BURLINGTON

Lab Code: STLV Case No.: INMAN

Matrix: (SOIL/SED/WATER)

Water

Sample wt/vol: 25.0

$(\mathrm{g} / \mathrm{mL}) \quad \mathrm{mL}$

Level: (TRACE or LOW/MED)

TRACE

응 Moisture: not dec.

GC Column: DB-624

ID: 0.20 (mm)

Soil Extract Volume: (uL)

CONCENTRATION UNITS: (ug/L or $\mathrm{ug} / \mathrm{kg}$ ) ug/L
Contract: $8 \mathrm{E}-00302$

SDG No.: 200-5308

Lab Sample ID: VIBLK 200-18645/6

Lab File ID: JCVB06.D

Date Received:

Date Analyzed: 05/25/2011

Dilution Factor: 1.0

Soil Aliquot Volume: (uL)

Purge Volume: 25.0 (mL)

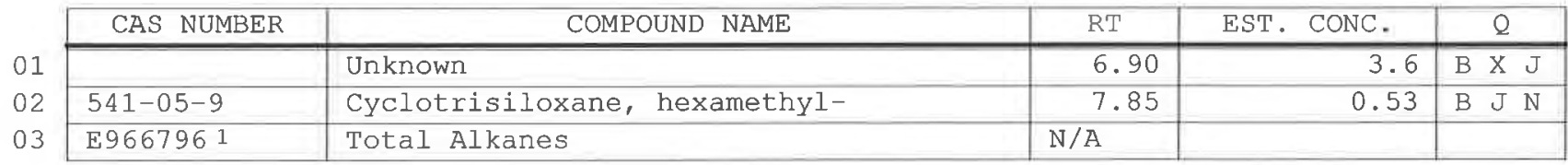

1EPA-designated Registry Number. 


\section{TestAmerica}

THE LEADER IN ENVIRONMENTAL TESTING

\section{ANALYTICAL REPORT}

Job Number: 200-5726-1

SDG Number: $200-5726$

Job Description: Inman (200-5726)

Contract Number: EP-W-09-044

For:

Argonne National Laboratory

9700 South Cass Avenue

Building 203

Office B-149

Argonne, IL 60439

Attention: Mr. Clyde Dennis

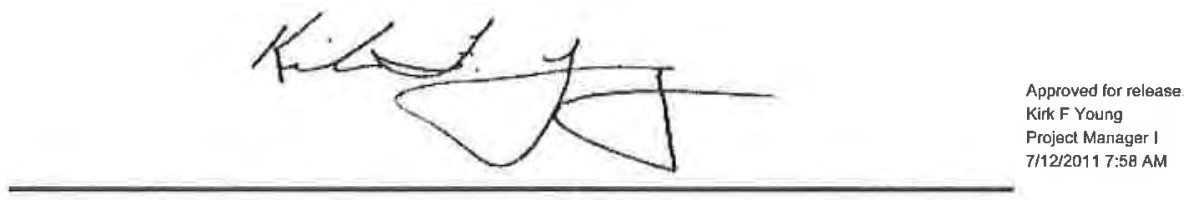

Kirk F Young

Project Manager I

kirk.young@testamericainc.com

07/12/2011

The test results in this report relate only to sample(s) as received by the laboratory. These test results were derived under a quality system that adheres to the requirements of NELAC. Pursuant to NELAC, this report may not be produced in full without written approval from the laboratory 


\section{Table of Contents}

Cover Title Page ........................ 1

Report Narrative ....................... 4

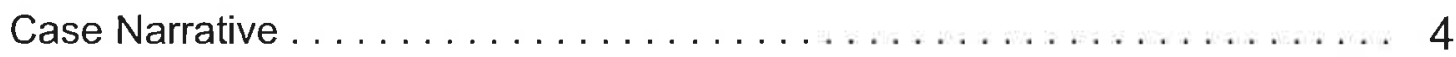

Qualifier Definition ............................. 6

External Chain of Custody . . . . . . . . . . . . . . 7

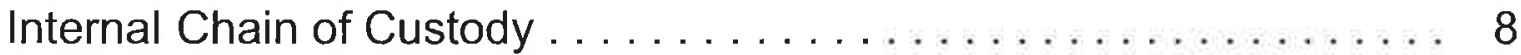

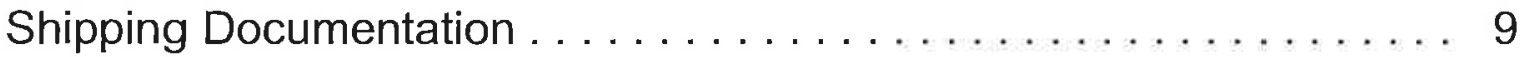

Airbills (if Applicable) . . . . . . . . . . . . . . . . . . . 10

Sample Receipt and Log In Check List.................. 11

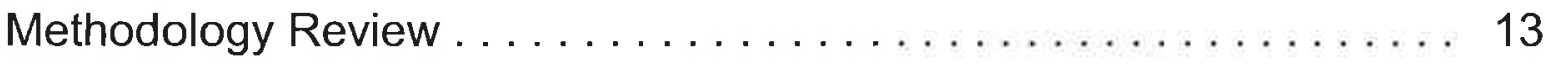

QC Summary - SOM01.2 Volatiles-Trace . . . . . . . . . . . . . 14

QC Summary - SOM01.2 Volatiles-Trace . . . . . . . . . . . . . . 14

Deuterated Monitoring Compound Summary $\ldots \ldots \ldots \ldots \ldots \ldots \ldots \ldots \ldots \ldots, 14$

Method Blank . . . . . . . . . . . . . . . . . . 16

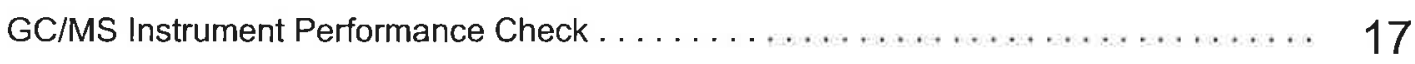

Internal Standard Area and RT Summary . . . . . . . . . . . . . . 19

Sample Data - SOM01.2 Volatiles-Trace . . . . . . . . . . . . . 20

Sample Data - SOM01.2 Volatiles-Trace . ................... 20

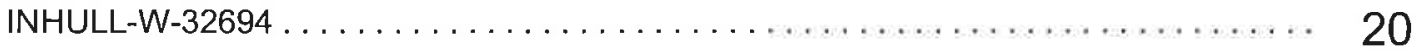

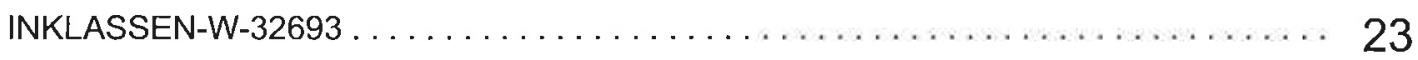

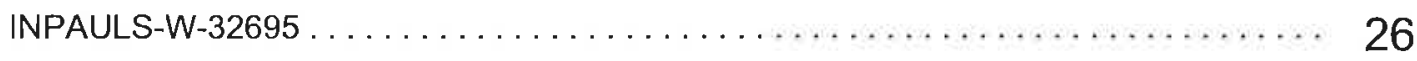

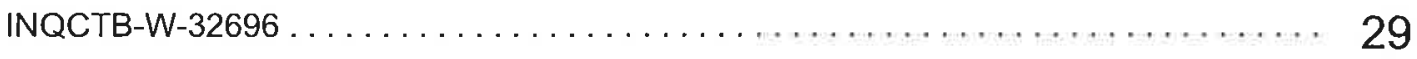

Standards - SOM01.2 Volatiles-Trace . . . . . . . . . . . . . 32

Standards - SOM01.2 Volatiles-Trace . . . . . . . . . . . . . . . . 32

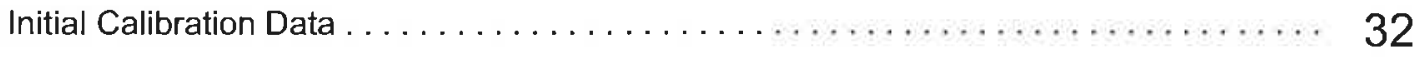

CCV Data, including closing CCV . . . . . . . . . . . 35 


\section{Table of Contents}

Raw Qc Data - SOM01.2 Volatiles-Trace . . . . . . . . . . . . . . . . 41

Raw Qc Data - SOM01.2 Volatiles-Trace . . . . . . . . . . . . . . . 41

Raw Qc Data - SOM01.2 Volatiles-Trace . . . . . . . . . . . . . . . . 41

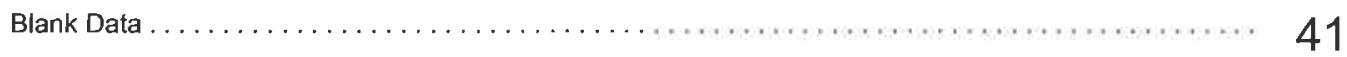




\title{
CASE NARRATIVE
}

\author{
Client: Argonne National Laboratory \\ Project: Inman (200-5726)
}

Report Number: 200-5726-1

Enclosed is the data set for the referenced project work. With the exceptions noted as flags or footnotes, standard analytical protocols were followed in performing the analytical work and the applied control limits were met.

Calculations were performed before rounding to avoid round-off errors in calculated results.

All holding times were met and proper preservation noted for the methods performed on these samples, unless otherwise detailed in the individual sections below.

\section{Receipt}

The samples were received on 06/25/2011. Documentation of the condition of the samples at the time of their receipt and any exception to the laboratory's Sample Acceptance Policy is documented in the Sample Handling section of this submittal. The samples, as received, were not acid preserved. On that basis, the laboratory did provide for the analytical work to be performed within seven days of sample collection.

\section{SOM01.2 Volatile Organics (Trace Level Water)}

A storage blank was prepared for volatile organics analysis, and stored in association with the storage of the samples. That storage blank, identified as VHBLK01, was carried through the holding period with the samples, and analyzed.

Each of the analyses associated with the sample set exhibited an acceptable internal standard performance. There was an acceptable recovery of each deuterated monitoring compound (DMC) in the method blank associated with the analytical work, and in the analysis of the storage blank associated with the sample set. The analysis of the samples in this sample set did meet the technical acceptance criteria specific to DMC recoveries, although not all DMC recoveries were within the control range in each analysis. The technical acceptance criteria does provide for the recovery of up to three DMCs to fall outside of the control range in the analysis of field samples. Matrix spike and matrix spike duplicate analyses were not performed on samples in this sample set. Trace concentrations of acetone, carbon disulfide, and toluene were identified in the analysis of the method blank associated with the analytical work. The concentration of each analyte in that analysis was below the established reporting limit, and the analysis did meet the technical acceptance criteria for a compliant method blank analysis. The analysis of the storage blank associated with the sample set was free of analyte contamination. Present in the method blank, storage blank, and instrument blank analyses was a non-target constituent that represents a compound that is related to the DMC formulation. The fact that the presence of this compound is not within the laboratory's control is at issue. The derived results for that compound have been qualified with an " $X$ " qualifier to reflect the source of the contamination.

The responses for each of the target analytes met the relative standard deviation criterion in the initial calibration. The response for each target analyte met the percent difference criterion in 
each opening/continuing calibration check acquisition. The response for each target analyte met the 50.0 percent difference criterion in each closing calibration check acquisition.

The primary quantitation mass for methylcyclohexane that is specified in the Statement of Work is mass 83 . The laboratory did identify a contribution to mass 83 from 1,2-dichloropropane- $d_{6}$, one of the deuterated monitoring compounds (DMCs). The laboratory did change the primary quantitation mass assignment to mass 55 for the quantification of methylcyclohexane.

Manual integration was employed in deriving certain of the analytical results. The values that have been derived from manual integration are qualified on the quantitation reports. Extracted ion current profiles for each manual integration are included in the data package, and further documented at the end of this submittal. 


\section{DATA REPORTING QUALIFIERS}

Client: Argonne National Laboratory

Job Number: 200-5726-1

Sdg Number: $200-5726$

Lab Section

Qualifier

Description

GC/MS VOA

U

$J$

$J$

$\mathrm{X}$

B
Analyzed for but not detected.

Indicates an Estimated Value for TICs

Indicates an estimated value.

See case narrative notes for explanation of the ' $X$ ' flag

Surrogate exceeds the control limit

The analyte was found in an associated blank, as well as in the sample. 


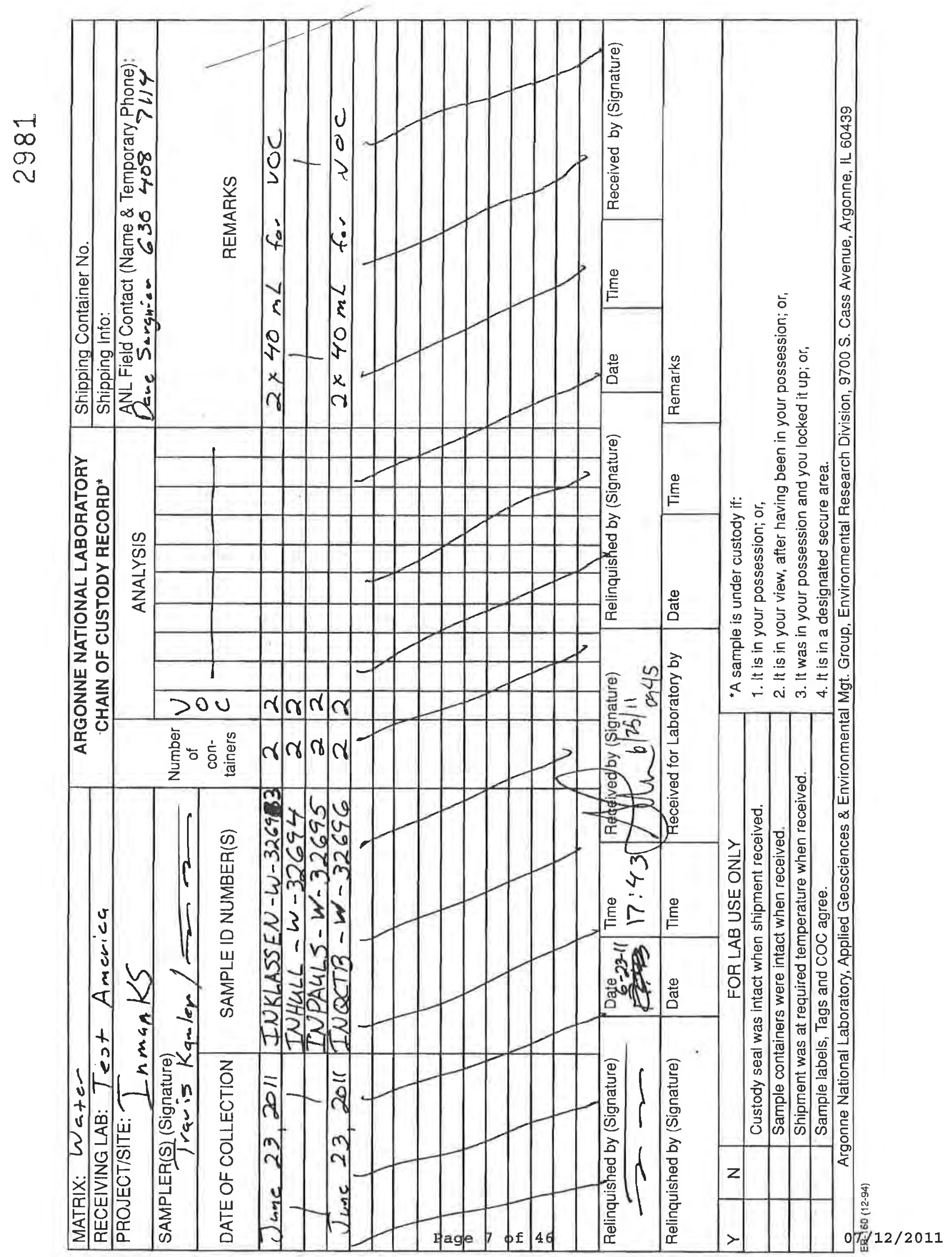




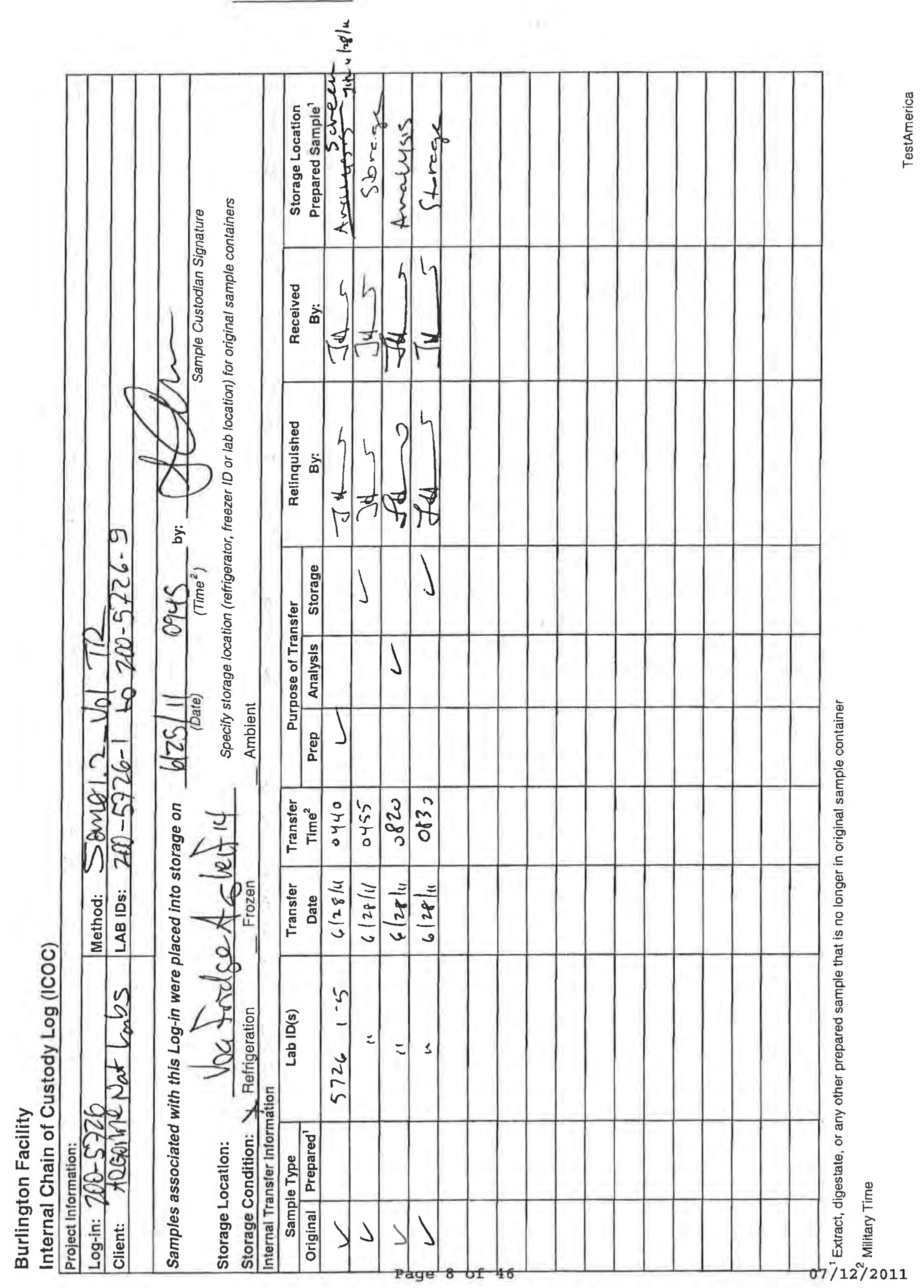




\section{Shipping and Receiving}

\section{Documents}




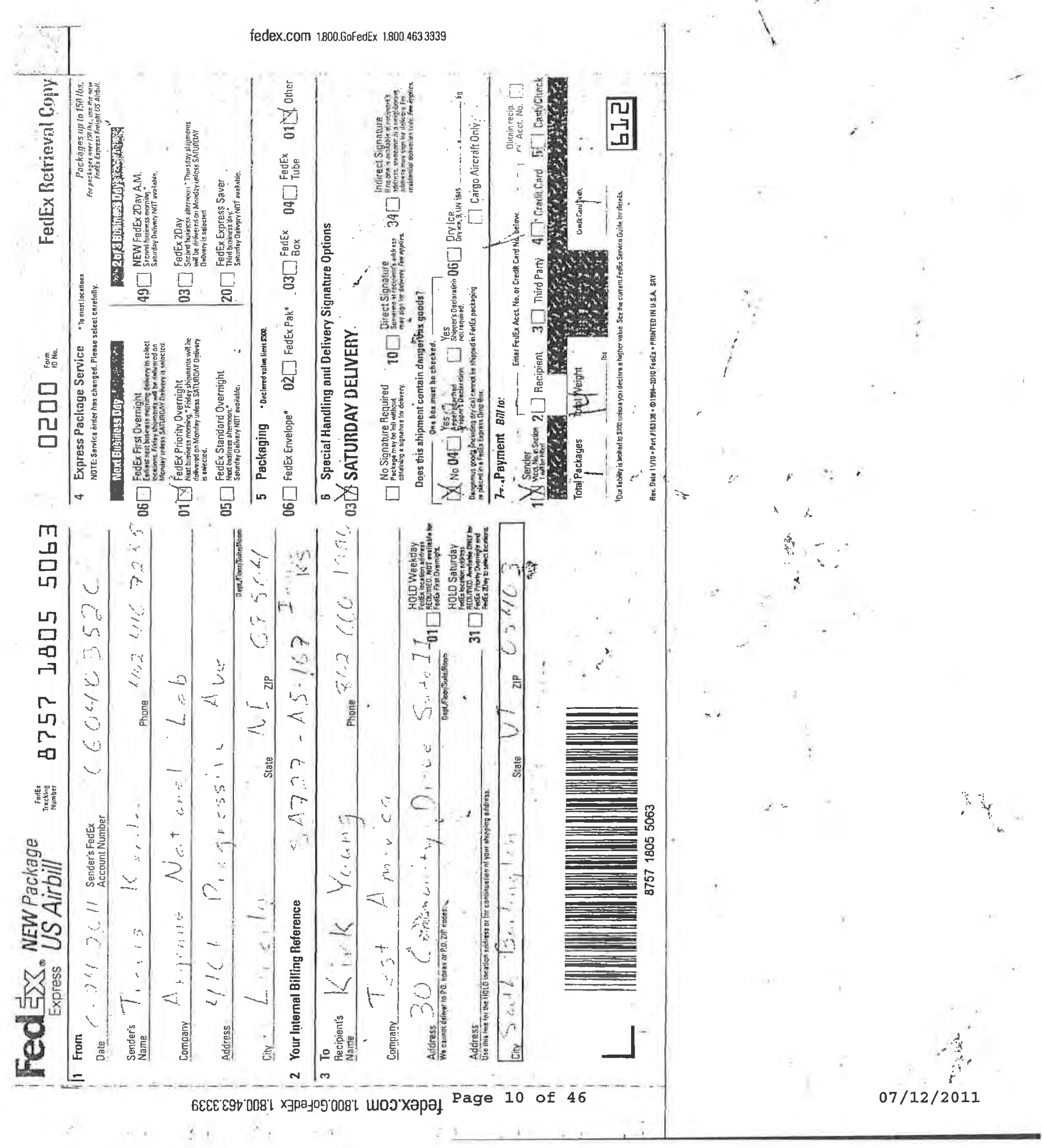




\section{Login Sample Receipt Checklist}

List Number: 1

Creator: Marion, Greg T

\begin{tabular}{|c|c|c|}
\hline Question & Answer & Comment \\
\hline $\begin{array}{l}\text { Radioactivity either was not measured or, if measured, is at or below } \\
\text { background }\end{array}$ & N/A & Lab does not accept radioactive samples. \\
\hline The cooler's custody seal, if present, is intact. & True & NO SEAL NUMBERS \\
\hline $\begin{array}{l}\text { The cooler or samples do not appear to have been compromised or } \\
\text { tampered with. }\end{array}$ & True & \\
\hline Samples were received on ice. & True & \\
\hline Cooler Temperature is acceptable. & True & \\
\hline Cooler Temperature is recorded. & True & $4.7^{\circ} \mathrm{C}$, IR GUN ID $96 / \mathrm{CF}=0$ \\
\hline COC is present. & True & \\
\hline COC is filled out in ink and legible. & True & \\
\hline COC is filled out with all pertinent information. & True & \\
\hline Is the Field Sampler's name present on COC? & True & \\
\hline $\begin{array}{l}\text { There are no discrepancies between the sample IDs on the containers and } \\
\text { the COC. }\end{array}$ & True & \\
\hline Samples are received within Holding Time. & True & \\
\hline Sample containers have legible labels. & True & \\
\hline Containers are not broken or leaking. & True & \\
\hline Sample collection date/times are provided. & True & \\
\hline Appropriate sample containers are used. & True & \\
\hline Sample bottles are completely filled. & True & \\
\hline Sample Preservation Verified. & $N / A$ & Sample volumes were received unpreserved. \\
\hline $\begin{array}{l}\text { There is sufficient vol. for all requested analyses, incl. any requested } \\
\text { MS/MSDs }\end{array}$ & True & \\
\hline $\begin{array}{l}\text { VOA sample vials do not have headspace or bubble is }<6 \mathrm{~mm}(1 / 4 ") \text { in } \\
\text { diameter. }\end{array}$ & True & \\
\hline Multiphasic samples are not present. & $N / A$ & \\
\hline Samples do not require splitting or compositing. & $N / A$ & \\
\hline Residual Chlorine Checked. & N/A & Check done at department level if required. \\
\hline
\end{tabular}




\section{Sample Login Acknowledgement}

Job 200-5726-1

Client Job Description:

Purchase Order \#:

Work Order \#:

Project Manager:

Job Due Date:

Job TAT:

Max Deliverable Level: IV

Earliest Deliverable Due:
Inman (200-5726)

$8 \mathrm{E}-00302$

8E-00302

Kirk F Young

7/9/2011

14 Days

IV

$7 / 9 / 2011$

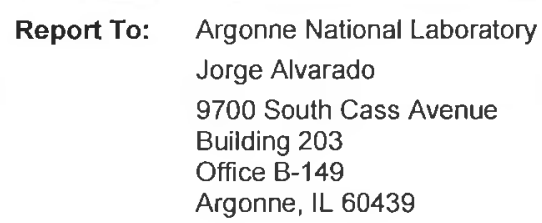

Office B-149

Argonne, IL 60439

Bill To: $\quad$ Argonne National Laboratory

Accounts Payable

Chief Financial Offices

9700 S. Cass Ave.

Building 201

Argonne, IL 60439

\section{Login 200-5726}

\begin{tabular}{|c|c|}
\hline Sample Receipt: & 6/25/2011 9:45:00 AM \\
\hline Method of Delivery: & FedEx Saturday Delivery \\
\hline Lab Sample \# & Client Sample ID \\
\hline Method & Method Description / Work Location \\
\hline $200-5726-1$ & INKLASSEN-W-32693 \\
\hline SOM01.2_Vol_Tr & SOM01.2 Trace Volatile Organics / In-Lab \\
\hline 200-5726-2 & INHULL-W-32694 \\
\hline SOM01.2_Vol_Tr & SOM01.2 Trace Volatile Organics / In-Lab \\
\hline $200-5726-3$ & INPAULS-W-32695 \\
\hline SOM01.2_Vol_Tr & SOM01.2 Trace Volatile Organics / In-Lab \\
\hline $200-5726-4$ & INQCTB-W-32696 \\
\hline SOM01.2_Vol_Tr & SOM01.2 Trace Volatile Organics / In-Lab \\
\hline 200-5726-5 & VHBLK01 \\
\hline SOM01.2_Vol_Tr & SOM01.2 Trace Volatile Or \\
\hline
\end{tabular}

$\begin{array}{lc}\text { Number of Coolers: } & 1 \\ \text { Cooler Temperature(s) }\left(\mathrm{C}^{\circ}\right): & 4.7 \text {; }\end{array}$

Date Sampled

Matrix

6/23/2011 12:00:00 AM Water

Rpt Basis Dry / Wet **

6/23/2011 12:00:00 AM Water

Total Wet

Total Wet

6/23/2011 12:00:00 AM Water

Total Wet

6/23/2011 12:00:00 AM Water

Total Wet

6/25/2011 4:50:00 PM Water

* Method on-hold

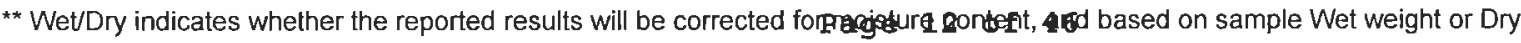




\section{METHODOLOGY SUMMARY}

Laboratory: TestAmerica Laboratories

Location: South Burlington, Vermont
Project No:

SDG No: $\quad 200-5726$

VOA

Volatile Organics Trace - USEPA CLP SOM01.2 
$2 A$ - FORM II VOA-1

WATER VOLATILE DEUTERATED MONITORING COMPOUND RECOVERY

Isab Name: TESTAMERICA BURLINGTON

Contract: $8 \mathrm{E}-00302$

Lab Code: STLV_Case No.: INMAN Mod. Ref No.: SDG No.: 200-5726

Level: (TRACE or LOW) TRACE

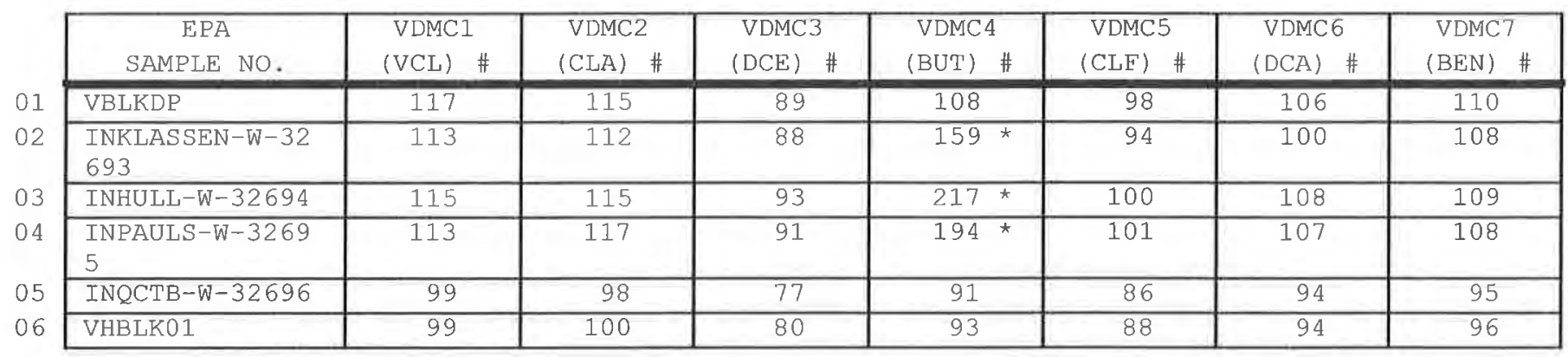

\footnotetext{
VDMC1 (VCL) = Vinyl Chloride-d3

VDMC2 $\quad($ CLA $)=$ Chloroethane $-\mathrm{d} 5$

VDMC3 $(\mathrm{DCE})=1,1-\mathrm{Dich}$ loroethene-d2

VDMC4 $\quad(B U T)=2-B u t a n o n e-d 5$

VDMC5 $(\mathrm{CLF})=$ Chloroform-d

VDMC6 $(\mathrm{DCA})=1,2-$ Dichloroethane-d4

VDMC7 $($ BEN $)=$ Benzene-d6
}

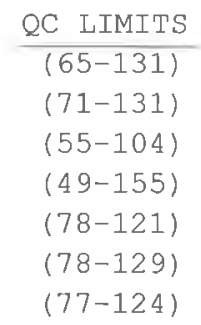

\# Column to be used to flag recovery values

* Values outside of contract required QC limits

Page 1 of 1
SOM01.2 (4/2007)

$07 / 12 / 2011$ 
$2 B$ - FORM II VOA-2

WATER VOLATILE DEUTERATED MONITORING COMPOUND RECOVERY

Lab Name: TESTAMERICA BURLINGTON

Contract: $8 \mathrm{E}-00302$

Lab Code: STLV Case No.: INMAN Mod. Ref No.:

SDG No.: $200-5726$

Level: (TRACE or LOW) TRACE

\begin{tabular}{|c|c|c|c|c|c|c|c|c|}
\hline $\begin{array}{c}\text { EPA } \\
\text { SAMPLE NO. }\end{array}$ & $\begin{array}{l}\text { VDMC8 } \\
\text { (DPA) \# }\end{array}$ & $\begin{array}{l}\text { VDMC9 } \\
\text { (TOL) \# }\end{array}$ & $\begin{array}{l}\text { VDMC10 } \\
\text { (TDP) \# }\end{array}$ & $\begin{array}{l}\text { VDMC11 } \\
(\mathrm{HEX}) \quad \#\end{array}$ & $\begin{array}{l}\text { VDMC12 } \\
\text { (TCA) \# }\end{array}$ & $\begin{array}{l}\text { VDMC13 } \\
\text { (DCZ) \# }\end{array}$ & OTHER & $\begin{array}{l}\text { TOT } \\
\text { OUT }\end{array}$ \\
\hline VBLKDP & 109 & 107 & 101 & 108 & 95 & 115 & & 0 \\
\hline $\begin{array}{l}\text { INKLASSEN-W-32 } \\
693\end{array}$ & 105 & 104 & 93 & 171 * & 87 & 108 & & 2 \\
\hline INHULL-W-32694 & 110 & 108 & 99 & $217 \star$ & 99 & 119 & & 2 \\
\hline $\begin{array}{l}\text { INPAULS-W-3269 } \\
5\end{array}$ & 107 & 106 & 99 & 197 * & 92 & 119 & & 2 \\
\hline VHBLK01 & 95 & 94 & 85 & 92 & 84 & 101 & & 0 \\
\hline
\end{tabular}

$\begin{array}{ll}\text { VDMC8 } & (\text { DPA })=1,2-\text { Dichloropropane-d6 } \\ \text { VDMC9 } & (\text { TOL })=\text { Toluene-d8 } \\ \text { VDMC10 } & \text { TDP }=\text { trans } 1,3-\text { Dichloropropene-d } 4 \\ \text { VDMC11 } & (\mathrm{HEX})=2-\text { Hexanone-d5 } \\ \text { VDMC12 } & (\mathrm{TCA})=1,1,2,2-\text { Tetrachloroethane-d2 } \\ \text { VDMC13 } & (\mathrm{DCZ})=1,2-\text { Dichlorobenzene-d4 }\end{array}$
QC LIMITS

$(79-124)$

$(77-121)$

$(73-121)$

$(28-135)$

$(73-125)$

$(80-131)$

\# Column to be used to flag recovery values

* Values outside of contract required QC limits

Report 1,4-Dioxane-d8 for Low-Medium VOA analysis only

Page 1 of 1 
Lab Name: TESTAMERICA BURLINGTON

Contract: $8 \mathrm{E}-00302$

Lab Code: STLV Case No.: INMAN Mod. Ref No.: SDG No.: 200-5726

Lab File ID: DHOI04.D

Lab Sample ID: MB 200-20420/4

Instrument ID: D.i

Matrix: (SOIL/SED/WATER)

Level: (TRACE or LOW/MED)

Water

TRACE

ID: 0.20

GC Column: DB-624
Date Analyzed: 06/28/2011

Time Analyzed: 0811

Heated Purge: (Y/N) N

\begin{tabular}{|c|c|c|c|}
\hline $\begin{array}{c}\text { EPA } \\
\text { SAMPLE NO. }\end{array}$ & $\begin{array}{r}\text { LAB } \\
\text { SAMPLE ID }\end{array}$ & $\begin{array}{l}\text { LAB } \\
\text { FILE ID }\end{array}$ & $\begin{array}{c}\text { TIME } \\
\text { ANALYZED }\end{array}$ \\
\hline $\begin{array}{l}\text { INKLASSEN-W- } \\
32693\end{array}$ & $200-5726-1$ & DHOI05.D & 0852 \\
\hline $\begin{array}{l}\text { INHULL-W-326 } \\
94\end{array}$ & $200-5726-2$ & DHOIO6.D & 0917 \\
\hline $\begin{array}{l}\text { INPAULS }-W-32 \\
695\end{array}$ & $200-5726-3$ & DHOI07.D & 0941 \\
\hline $\begin{array}{l}\text { INQCTB-W-326 } \\
96\end{array}$ & $200-5726-4$ & DHOI12.D & 1219 \\
\hline VHBLK01 & $200-5726-5$ & DHOI13.D & 1243 \\
\hline
\end{tabular}

COMMENTS :

Page 1 of 1 
$5 A-$ FORM V VOA

VOLATILE ORGANICS INSTRUMENT

PERFORMANCE CHECK BROMOFLUOROBENZENE

(BFB)
EPA SAMPLE NO.

BFBDE

Lab Name: TESTAMERICA BURLINGTON

Contract: $\quad$ 8E-00302

Lab Code: STLV Case No.: INMAN

Mod. Ref No.:

SDG No.: 200-5726

Lab File Id: DHO01.D

BFB Injection Date: 03/25/2011

Instrument Id: D.i.

BFB Injection Time: 1150

GC Column: DB-624

ID: 0.53 (mm)

\begin{tabular}{|c|c|c|}
\hline $\mathrm{m} / \mathrm{e}$ & ION ABUNDANCE CRITERIA & $\begin{array}{l}\text { REIATIVE } \\
\text { ABUNDANCE }\end{array}$ \\
\hline 50 & $15.0-40.0 \%$ of mass 95 & 22.3 \\
\hline 75 & $30.0-80.0$ o of mass 95 & 42.2 \\
\hline 95 & Base peak, $100 \%$ relative abundance & 100 \\
\hline 96 & $5.0-9.0 \%$ of mass 95 & 6.7 \\
\hline 173 & Less than $2.0 \%$ of mass 174 & 0.31 \\
\hline 174 & $50.0-120 \%$ of mass 95 & 74.2 \\
\hline 175 & $5.0-9.0 \%$ of mass 174 & $4.9(6.6) 1$ \\
\hline 176 & $95.0-101 \%$ of mass 174 & $72.2(97.4) 1$ \\
\hline 177 & $5.0-9.0 \%$ of mass 176 & $4.7(6.5) 2$ \\
\hline
\end{tabular}

1 - Value is onass 174

2 - Value is omass 176

\begin{tabular}{|c|c|c|c|c|}
\hline $\begin{array}{c}\text { EPA } \\
\text { SAMPLE NO. }\end{array}$ & $\begin{array}{c}\text { LAB } \\
\text { SAMPLE ID }\end{array}$ & $\begin{array}{c}\text { LAB } \\
\text { FILE ID }\end{array}$ & $\begin{array}{c}\text { DATE } \\
\text { ANALYZED }\end{array}$ & $\begin{array}{c}\text { TIME } \\
\text { ANALYZED }\end{array}$ \\
\hline VSTDO.5DE & IC $200-15665 / 3$ & DHO03.D & $03 / 25 / 2011$ & 1225 \\
\hline VSTD001DE & IC $200-15665 / 4$ & DHOO 4. D & $03 / 25 / 2011$ & 1250 \\
\hline VSTD005DE & ICIS $200-15665 / 5$ & DHO05.D & $03 / 25 / 2011$ & 1314 \\
\hline VSTD010DE & IC $200-15665 / 6$ & DHO06.D & $03 / 25 / 2011$ & 1339 \\
\hline VSTD020DE & IC $200-15665 / 7$ & DHO07.D & $03 / 25 / 2011$ & 1403 \\
\hline
\end{tabular}


5A - FORM V VOA

VOLATILE ORGANICS INSTRUMENT

PERFORMANCE CHECK

BROMOFLUOROBENZENE (BFB)
EPA SAMPLE NO.

BFBDP
Lab Name: TESTAMERICA BURLINGTON

Lab Code: STLV Case No.: INMAN

Lab File Id: DHOI01.D

Instrument Id: D.i

GC Column: DB-624

ID : 0.20
Contract: $8 \mathrm{E}-00302$

Mod. Ref No.:
SDG No.: 200-5726

BFB Injection Date: 06/28/2011

BFB Injection Time: 0703 $(\mathrm{mm})$

\begin{tabular}{|c|c|c|}
\hline $\mathrm{m} / \mathrm{e}$ & ION ABUNDANCE CRITERIA & $\begin{array}{l}\text { O RELATIVE } \\
\text { ABUNDANCE }\end{array}$ \\
\hline 50 & $15.0-40.0 \%$ of mass 95 & 24.1 \\
\hline 75 & $30.0-80.0 \%$ of mass 95 & 43.2 \\
\hline 95 & Base peak, $100 \%$ relative abundance & 100 \\
\hline 96 & $5.0-9.0 \%$ of mass 95 & 7.2 \\
\hline 173 & Less than $2.0 \%$ of mass 174 & $0.4(0.5) 1$ \\
\hline 174 & $50.0-120 \%$ of mass 95 & 75.8 \\
\hline 175 & $5.0-9.0 \%$ of mass 174 & 5.61 \\
\hline 176 & $95.0-101$ of of mass 174 & $74.8(98.7) 1$ \\
\hline 177 & $5.0-9.0 \%$ of mass 176 & $5.1(6.8) 2$ \\
\hline
\end{tabular}

1 - Value is omass 174

2 - Value is onass 176

\begin{tabular}{|c|c|c|c|c|}
\hline $\begin{array}{c}\text { EPA } \\
\text { SAMPLE NO. }\end{array}$ & $\begin{array}{c}\text { IAB } \\
\text { SAMPLE ID }\end{array}$ & $\begin{array}{c}\text { LAB } \\
\text { FILE ID }\end{array}$ & $\begin{array}{c}\text { DATE } \\
\text { ANALYZED }\end{array}$ & $\begin{array}{c}\text { TIME } \\
\text { ANALYZED }\end{array}$ \\
\hline VSTD005DP & CCVIS $200-20420 / 3$ & DHOI03.D & $06 / 28 / 2011$ & 0747 \\
\hline VBLKDP & MB $200-20420 / 4$ & DHOI04.D & $06 / 28 / 2011$ & 0811 \\
\hline $\begin{array}{l}\text { INKLASSEN- } \\
\text { W-32693 }\end{array}$ & $200-5726-1$ & DHOI05.D & $06 / 28 / 2011$ & 0852 \\
\hline $\begin{array}{l}\text { INHULL-W-3 } \\
2694\end{array}$ & $200-5726-2$ & DHOI06.D & $06 / 28 / 2011$ & 0917 \\
\hline $\begin{array}{l}\text { INPAULS-W- } \\
32695\end{array}$ & $200-5726-3$ & DHOI07.D & $06 / 28 / 2011$ & 0941 \\
\hline $\begin{array}{l}\text { INQCTB-W-3 } \\
2696\end{array}$ & $200-5726-4$ & DHOI 12.D & $06 / 28 / 2011$ & 1219 \\
\hline VHBLK01 & $200-5726-5$ & DHOI13.D & $06 / 28 / 2011$ & 1243 \\
\hline VSTD005PD & CCVC $200-20420 / 22$ & DHOI 22.D & $06 / 28 / 2011$ & 1636 \\
\hline
\end{tabular}


$8 A$ - FORM VIII VOA

VOLATILE INTERNAL STANDARD AREA AND RETENTION TIME SUMMARY

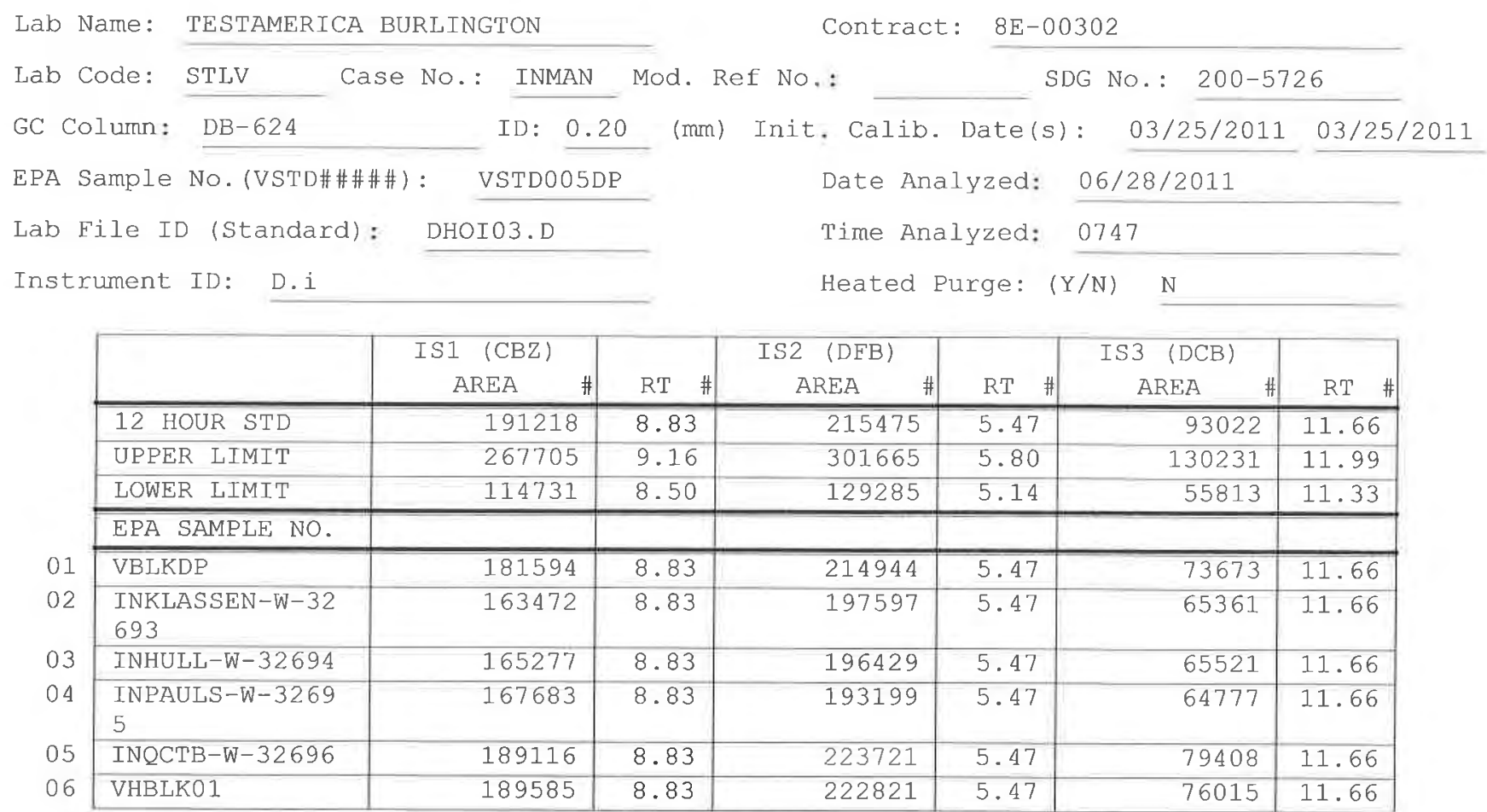

IS1 $(\mathrm{CBZ})=$ Chlorobenzene-d5

IS2 $(\mathrm{DFB})=1,4-\mathrm{Difluorobenzene}$

IS3 $($ DCB $)=1,4$-Dichlorobenzene-d4

AREA UPPER LIMIT $=140 \%$ (Trace Volatiles) of internal standard area

AREA LOWER LIMIT $=60 \%$ (Trace Volatiles) of internal standard area

RT UPPER LIMIT $=+0.33$ (Trace Volatiles) minutes of internal standard RT

RT LOWER LIMIT $=-0.33$ (Trace Volatiles) minutes of internal standard RT

\# Column used to flag values outside contract required QC limits with an asterisk. 
Lab Name: TESTAMERICA BURLINGTON

Contract: $8 \mathrm{E}-00302$

Lab Code: STLV Case No.: INMAN Mod. Ref No.:

SDG No.: 200-5726

Matrix: (SOIL/SED/WATER)

Sample wt/vol: 25.0

Water

$(\mathrm{g} / \mathrm{mL}) \quad \mathrm{mL}$

Level: (TRACE/LOW/MED) TRACE

음 Moisture: not dec.

GC Column: DB-624

Soil Extract Volume:

ID: $0.20 \quad(\mathrm{~mm})$

(uL)

(mL)

Purge Volume: 25.0
Lab Sample ID: 200-5726-2

Lab File ID: DHOI06.D

Date Received: 06/25/2011

Date Analyzed: 06/28/2011

Dilution Factor: 1.0

Soil Aliquot volume:

\begin{tabular}{|c|c|c|c|}
\hline CAS NO. & COMPOUND & $\begin{array}{l}\text { CONCENTRATION UNITS: } \\
(\mathrm{ug} / \mathrm{L} \text { or } u g / k g) \mathrm{ug} / \mathrm{L}\end{array}$ & Q \\
\hline $75-71-8$ & Dichlorodifluoromethane & 0.50 & $\mathrm{U}$ \\
\hline $74-87-3$ & Chloromethane & 0.50 & $\mathrm{U}$ \\
\hline $75-01-4$ & Vinyl chloride & 0.50 & $\mathrm{U}$ \\
\hline $74-83-9$ & Bromomethane & 0.50 & $\mathrm{U}$ \\
\hline $75-00-3$ & Chloroethane & 0.50 & $\mathrm{U}$ \\
\hline $75-69-4$ & Trichlorofluoromethane & 0.50 & $\mathrm{U}$ \\
\hline $75-35-4$ & 1,1-Dichloroethene & 0.50 & $\mathrm{U}$ \\
\hline $76-13-1$ & 1,1,2-Trichloro-1,2,2-trifluoroethane & 0.50 & $\mathrm{U}$ \\
\hline $67-64-1$ & Acetone & 5.0 & $\mathrm{U}$ \\
\hline $75-15-0$ & Carbon disulfide & 0.50 & $\mathrm{U}$ \\
\hline $79-20-9$ & Methyl acetate & 0.50 & $\mathrm{U}$ \\
\hline $75-09-2$ & Methylene Chloride & 0.50 & $\mathrm{U}$ \\
\hline $156-60-5$ & trans-1,2-Dichloroethene & 0.50 & $\mathrm{U}$ \\
\hline $1634-04-4$ & Methyl tert-butyl ether & 0.50 & $\mathrm{U}$ \\
\hline $75-34-3$ & 1,1-Dichloroethane & 0.50 & $\mathrm{U}$ \\
\hline $156-59-2$ & cis-1,2-Dichloroethene & 0.50 & $\mathrm{U}$ \\
\hline $78-93-3$ & 2-Butanone & 5.0 & $\mathrm{U}$ \\
\hline $74-97-5$ & Bromochloromethane & 0.50 & $\mathrm{U}$ \\
\hline $67-66-3$ & Chloroform & 0.50 & $\mathrm{U}$ \\
\hline $71-55-6$ & 1,1,1-Trichloroethane & 0.50 & $\mathrm{U}$ \\
\hline $110-82-7$ & Cyclohexane & 0.50 & $\mathrm{U}$ \\
\hline $56-23-5$ & Carbon tetrachloride & 0.25 & $\mathrm{~J}$ \\
\hline $71-43-2$ & Benzene & 0.50 & $\mathrm{U}$ \\
\hline $107-06-2$ & 1,2-Dichloroethane & 0.50 & $\mathrm{U}$ \\
\hline
\end{tabular}

Report 1,4-Dioxane for Low-Medium VOA analysis only 
$1 \mathrm{~B}$ - FORM I VOA-2

VOLATILE ORGANICS ANALYSIS DATA SHEET
EPA SAMPLE NO.

INHULL-W-32694
Lab Name: TESTAMERICA BURLINGTON

Lab Code: STLV Case No.: INMAN Mod. Ref No.:
Contract: $8 E-00302$

SDG No.: 200-5726
Matrix: (SOIL/SED/WATER)

Sample wt/vol: 25.0

water

$(\mathrm{g} / \mathrm{mL}) \mathrm{mL}$

Level: (TRACE/LOW/MED) TRACE

․ Moisture: not dec.

GC Column: DB-624 ID: $0.20 \quad$ (mm)

Soil Extract Volume: (uL) (mL)

Purge Volume: 25.0
Lab Sample ID: 200-5726-2

Lab File ID: DHOI06.D

Date Received: 06/25/2011

Date Analyzed: 06/28/2011

Dilution Factor: 1.0

Soil Aliquot Volume:

(uL)

\begin{tabular}{|c|c|c|c|}
\hline CAS NO. & COMPOUND & $\begin{array}{l}\text { CONCENTRATION UNITS: } \\
(\mathrm{ug} / \mathrm{L} \text { or } \mathrm{ug} / \mathrm{kg}) \mathrm{ug} / \mathrm{L}\end{array}$ & $Q$ \\
\hline $79-01-6$ & Trichloroethene & 0.50 & $\mathrm{U}$ \\
\hline $108-87-2$ & Methylcyclohexane & 0.50 & $\mathrm{U}$ \\
\hline $78-87-5$ & 1,2-Dichloropropane & 0.50 & $\mathrm{U}$ \\
\hline $75-27-4$ & Bromodichloromethane & 0.50 & $\mathrm{U}$ \\
\hline $10061-01-5$ & cis-1,3-Dichloropropene & 0.50 & $\mathrm{U}$ \\
\hline $108-10-1$ & 4-Methyl-2-pentanone & 5.0 & $\mathrm{U}$ \\
\hline $108-88-3$ & Toluene & 0.0098 & $\mathrm{~J} B$ \\
\hline $10061-02-6$ & trans-1,3-Dichloropropene & 0.50 & $\mathrm{U}$ \\
\hline $79-00-5$ & 1,1,2-Trichloroethane & 0.50 & $\mathrm{U}$ \\
\hline $127-18-4$ & Tetrachloroethene & 0.50 & $\mathrm{U}$ \\
\hline $591-78-6$ & 2-Hexanone & 5.0 & $\mathrm{U}$ \\
\hline $124-48-1$ & Dibromochloromethane & 0.50 & $\mathrm{U}$ \\
\hline $106-93-4$ & 1,2-Dibromoethane & 0.50 & $\mathrm{U}$ \\
\hline $108-90-7$ & Chlorobenzene & 0.50 & $\mathrm{U}$ \\
\hline $100-41-4$ & Ethylbenzene & 0.50 & $\mathrm{U}$ \\
\hline $95-47-6$ & o-xylene & 0.50 & $\mathrm{U}$ \\
\hline $179601-23-1$ & $\mathrm{~m}, \mathrm{p}$-Xylene & 0.50 & $\mathrm{U}$ \\
\hline $100-42-5$ & Styrene & 0.50 & $\mathrm{U}$ \\
\hline $75-25-2$ & Bromoform & 0.50 & $\mathrm{U}$ \\
\hline $98-82-8$ & Isopropylbenzene & 0.50 & $\mathrm{U}$ \\
\hline $79-34-5$ & $1,1,2,2$-Tetrachloroethane & 0.50 & $\mathrm{U}$ \\
\hline $541-73-1$ & 1,3-Dichlorobenzene & 0.50 & $\mathrm{U}$ \\
\hline $106-46-7$ & 1,4-Dichlorobenzene & 0.50 & $\mathrm{U}$ \\
\hline $95-50-1$ & 1,2-Dichlorobenzene & 0.50 & $\mathrm{U}$ \\
\hline $96-12-8$ & 1,2-Dibromo-3-Chloropropane & 0.50 & $\mathrm{U}$ \\
\hline $120-82-1$ & 1,2,4-Trichlorobenzene & 0.50 & $\mathrm{U}$ \\
\hline $87-61-6$ & 1,2,3-Trichlorobenzene & 0.50 & $\mathrm{U}$ \\
\hline
\end{tabular}


1J - FORM I VOA-TIC

VOLATILE ORGANICS ANALYSIS DATA SHEET

TENTATIVELY IDENTIFIED COMPOUNDS
EPA SAMPLE NO.

INHULL-W-32694
Lab Name: TESTAMERICA BURLINGTON

Lab Code: STLV Case No.: INMAN

Matrix: (SOIL/SED/WATER)

Water

Sample wt/vol: 25.0

$(\mathrm{g} / \mathrm{mL}) \quad \mathrm{mL}$

Level: (TRACE or LOW/MED)

TRACE

\% Moisture: not dec.

GC Column: DB-624

ID: 0.20 $(\mathrm{mm})$

Soil Extract Volume: (uL)

CONCENTRATION UNITS: (ug/L or $\mathrm{ug} / \mathrm{kg}$ ) ug/L
Contract: $8 \mathrm{E}-00302$

SDG No.: 200-5726

Lab Sample ID: 200-5726-2

Lab File ID: DHOI06.D

Date Received: 06/25/2011

Date Analyzed: 06/28/2011

Dilution Factor: 1.0

Soil Aliquot Volume: (uL)

Purge Volume: 25.0 (mL)

01

02

\begin{tabular}{|c|l|c|r|r|}
\hline CAS NUMBER & \multicolumn{1}{|c|}{ COMPOUND NAME } & RT & EST. CONC. & Q \\
\hline & Unknown & 6.79 & 3.1 & B X J \\
\hline E9667961 & Total Alkanes & N/A & & \\
\hline
\end{tabular}

1 EPA-designated Registry Number. 
Lab Name: TESTAMERICA BURLINGTON

Lab Code: STLV Case No.: INMAN

Matrix: (SOIL/SED/WATER)

Sample wt/vol: 25.0

Water

$(\mathrm{g} / \mathrm{mL}) \quad \mathrm{mL}$

Level: (TRACE/IOW/MED) TRACE

o Moisture: not dec.

GC Column: DB-624

Soil Extract Volume:

ID: $0.20 \quad$ (mm)

Purge Volume: 25.0

$(\mathrm{mL})$
Contract: $8 \mathrm{E}-00302$

SDG No.: 200-5726
Lab Sample ID: 200-5726-1

Lab File ID: DHOI05.D

Date Received: 06/25/2011

Date Analyzed: 06/28/2011

Dilution Factor: 1.0

Soil. Aliquot Volume: (uL)

\begin{tabular}{|c|c|c|c|}
\hline CAS NO. & COMPOUND & $\begin{array}{l}\text { CONCENTRATION UNITS: } \\
(\mathrm{ug} / \mathrm{L} \text { or } \mathrm{ug} / \mathrm{kg}) \mathrm{ug} / \mathrm{L}\end{array}$ & 0 \\
\hline $75-71-8$ & Dichlorodifluoromethane & 0.50 & $\mathrm{U}$ \\
\hline $74-87-3$ & Chloromethane & 0.50 & $\mathrm{U}$ \\
\hline $75-01-4$ & Vinyl chloride & 0.50 & $\mathrm{U}$ \\
\hline $74-83-9$ & Bromomethane & 0.50 & $\mathrm{U}$ \\
\hline $75-00-3$ & Chloroethane & 0.50 & $\mathrm{U}$ \\
\hline $75-69-4$ & Trichlorofluoromethane & 0.50 & $\mathrm{U}$ \\
\hline $75-35-4$ & 1,1-Dichloroethene & 0.50 & $\mathrm{U}$ \\
\hline $76-13-1$ & 1,1,2-Trichloro-1,2,2-trifluoroethane & 0.50 & $\mathrm{U}$ \\
\hline $67-64-1$ & Acetone & 5.0 & $\mathrm{U}$ \\
\hline $75-15-0$ & Carbon disulfide & 0.50 & $\mathrm{U}$ \\
\hline $79-20-9$ & Methyl acetate & 0.50 & $\mathrm{U}$ \\
\hline $75-09-2$ & Methylene Chloride & 0.50 & $\mathrm{U}$ \\
\hline $156-60-5$ & trans-1,2-Dichloroethene & 0.50 & $\mathrm{U}$ \\
\hline $1634-04-4$ & Methyl tert-butyl ether & 0.50 & $\mathrm{U}$ \\
\hline $75-34-3$ & 1,1-Dichloroethane & 0.50 & $\mathrm{U}$ \\
\hline $156-59-2$ & cis-1,2-Dichloroethene & 0.50 & $\mathrm{U}$ \\
\hline $78-93-3$ & 2-Butanone & 5.0 & $\mathrm{U}$ \\
\hline $74-97-5$ & Bromochloromethane & 0.50 & $\mathrm{U}$ \\
\hline $67-66-3$ & Chloroform & 0.25 & $\mathrm{~J}$ \\
\hline $71-55-6$ & 1,1,1-Trichloroethane & 0.50 & $\mathrm{U}$ \\
\hline $110-82-7$ & Cyclohexane & 0.50 & $\mathrm{U}$ \\
\hline $56-23-5$ & Carbon tetrachloride & 6.9 & \\
\hline $71-43-2$ & Benzene & 0.50 & $\mathrm{U}$ \\
\hline $107-06-2$ & 1,2-Dichloroethane & 0.50 & U \\
\hline
\end{tabular}

Report 1,4-Dioxane for Low-Medium VOA analysis only 
$1 B$ - FORM I VOA-2

VOLATILE; ORGANICS ANALYSIS DATA SHEET
EPA SAMPLE NO.

INKLASSEN-W-32693

Lab Name: TESTAMERICA BURLINGTON

Lab Code: STLV Case No.: INMAN Mod. Ref No.:

Contract: $\quad 8 \mathrm{E}-00302$

Matrix: (SOIL/SED/WATER)

Sample wt/vol: 25.0 Water

$(\mathrm{g} / \mathrm{mL}) \quad \mathrm{mL}$

Level: (TRACE/LOW/MED) TRACE

o Moisture: not dec.

GC Column: DB-624 ID: $0.20 \quad(\mathrm{~mm})$

Soil Extract Volume:

(uL)

Purge Volume: 25.0

SDG No.: 200-5726

Lab Sample ID: 200-5726-1

Lab File ID: DHOI05.D

Date Received: 06/25/2011

Date Analyzed: 06/28/2011

Dilution Factor: 1.0

Soil Aliquot Volume:

(uL)

\begin{tabular}{|c|c|c|c|}
\hline CAS NO. & COMPOUND & $\begin{array}{l}\text { CONCENTRATION UNITS: } \\
(\mathrm{ug} / \mathrm{L} \text { or } \mathrm{ug} / \mathrm{kg} \text { ) ug/L }\end{array}$ & $Q$ \\
\hline $79-01-6$ & Trichloroethene & 0.50 & $\mathrm{U}$ \\
\hline $108-87-2$ & Methylcyclohexane & 0.50 & $\mathrm{U}$ \\
\hline $78-87-5$ & 1,2-Dichloropropane & 0.50 & $\mathrm{U}$ \\
\hline $75-27-4$ & Bromodichloromethane & 0.50 & $\mathrm{U}$ \\
\hline $10061-01-5$ & cis-1,3-Dichloropropene & 0.50 & $\mathrm{U}$ \\
\hline $108-10-1$ & 4-Methyl-2-pentanone & 5.0 & $\mathrm{U}$ \\
\hline $108-88-3$ & Toluene & 0.012 & $J B$ \\
\hline $10061-02-6$ & trans-1,3-Dichloropropene & 0.50 & $\mathrm{U}$ \\
\hline $79-00-5$ & 1,1,2-Trichloroethane & 0.50 & $\mathrm{U}$ \\
\hline $127-18-4$ & Tetrachloroethene & 0.50 & $\mathrm{U}$ \\
\hline $591-78-6$ & 2-Hexanone & 5.0 & $\mathrm{U}$ \\
\hline $124-48-1$ & Dibromochloromethane & 0.50 & $\mathrm{U}$ \\
\hline $106-93-4$ & 1,2-Dibromoethane & 0.50 & $\mathrm{U}$ \\
\hline $108-90-7$ & Chlorobenzene & 0.50 & $\mathrm{U}$ \\
\hline $100-41-4$ & Ethylbenzene & 0.50 & $\mathrm{U}$ \\
\hline $95-47-6$ & o-xylene & 0.50 & $\mathrm{U}$ \\
\hline $179601-23-1$ & $\mathrm{~m}, \mathrm{p}$-Xylene & 0.50 & $\mathrm{U}$ \\
\hline $100-42-5$ & Styrene & 0.50 & $\mathrm{U}$ \\
\hline $75-25-2$ & Bromoform & 0.50 & $\mathrm{U}$ \\
\hline $98-82-8$ & Isopropylbenzene & 0.50 & $\mathrm{U}$ \\
\hline $79-34-5$ & $1,1,2,2$-Tetrachloroethane & 0.50 & $\mathrm{U}$ \\
\hline $541-73-1$ & 1,3-Dichlorobenzene & 0.50 & $\mathrm{U}$ \\
\hline $106-46-7$ & 1,4-Dichlorobenzene & 0.50 & $\mathrm{U}$ \\
\hline $95-50-1$ & 1,2-Dichlorobenzene & 0.50 & $\mathrm{U}$ \\
\hline $96-12-8$ & 1,2-Dibromo-3-Chloropropane & 0.50 & $\mathrm{U}$ \\
\hline $120-82-1$ & 1,2,4-Trichlorobenzene & 0.50 & $\mathrm{U}$ \\
\hline $87-61-6$ & 1,2,3-Trichlorobenzene & 0.50 & $\mathrm{U}$ \\
\hline
\end{tabular}


IJ - FORM I VOA-TIC

VOLATILE ORGANICS ANALYSIS DATA SHEET

TENTATIVELY IDENTIFIED COMPOUNDS
EPA SAMPLE NO.

INKLASSEN-W-32693

Lab Name: TESTAMERICA BURLINGTON

Contract: $8 E-00302$

Lab Code: STLV Case No.: INMAN Mod. Ref No.:

SDG No.: 200-5726

Matrix: (SOIL/SED/WATER)

Water

Sample wt/vol: 25.0

Level: (TRACE or LOW/MED)

$(\mathrm{g} / \mathrm{mL}) \mathrm{mL}$

TRACE

응 Moisture: not dec.

GC Column: DB-624 ID $: 0.20 \quad(\mathrm{~mm})$

Soil Extract Volume: (uL)

CONCENTRATION UNITS: (ug/L or $\mathrm{ug} / \mathrm{kg}$ ) ug/L
Lab Sample ID: 200-5726-1

Lab File ID: DHOI05.D

Date Received: 06/25/2011

Date Analyzed: 06/28/2011

Dilution Factor: 1.0

Soil Aliquot Volume: (uL)

Purge Volume: 25.0 (mL)

\begin{tabular}{|l|l|l|l|r|r|}
\hline \multicolumn{1}{|c|}{ CAS NUMBER } & \multicolumn{1}{|c|}{ COMPOUND NAME } & RT & EST. CONC. & Q \\
\cline { 2 - 6 } 01 & & Unknown & 6.79 & B X J \\
\cline { 2 - 6 } 02 & E9667961 & Total Alkanes & N/A & & \\
\hline
\end{tabular}

\footnotetext{
1 EPA-designated Registry Number.
} 
$1 \mathrm{~A}-$ FORM I VOA-1

VOLATILE ORGANICS ANALYSIS DATA SHEET
EPA SAMPLE NO.

INPAULS-W-32695

Iuab Name: TESTAMERICA BURLINGTON

Contract: $8 \mathrm{E}-00302$

Lab Code: STLV Case No.: INMAN Mod. Ref No.: SDG No.: $200-5726$

Matrix: (SOIL/SED/WATER)

Sample wt/vol: 25.0

Water

$(\mathrm{g} / \mathrm{mL}) \mathrm{mL}$

Level: (TRACE/LOW/MED) TRACE

o Moisture: not dec.

GC Column: DB-624

Soil Extract Volume: ID: $0.20 \quad(\mathrm{~mm})$ (uL) (mL)

Purge Volume: 25.0
Lab Sample ID: 200-5726-3

Lab File ID: DHOI07.D

Date Received: $06 / 25 / 2011$

Date Analyzed: 06/28/2011

Dilution Factor: 1.0

Soil Aliquot Volume:

\begin{tabular}{|c|c|c|c|}
\hline CAS NO. & COMPOUND & $\begin{array}{l}\text { CONCENTRATION UNITS: } \\
(u g / \mathrm{L} \text { or } u g / \mathrm{kg}) \mathrm{ug} / \mathrm{L}\end{array}$ & $Q$ \\
\hline $75-71-8$ & Dichlorodifluoromethane & 0.50 & $\mathrm{U}$ \\
\hline $74-87-3$ & Chloromethane & 0.50 & $\mathrm{U}$ \\
\hline $75-01-4$ & Vinyl chloride & 0.50 & $\mathrm{U}$ \\
\hline $74-83-9$ & Bromomethane & 0.50 & $\mathrm{U}$ \\
\hline $75-00-3$ & Chloroethane & 0.50 & $\mathrm{U}$ \\
\hline $75-69-4$ & Trichlorofluoromethane & 0.50 & $\mathrm{U}$ \\
\hline $75-35-4$ & 1,1-Dichloroethene & 0.50 & $\mathrm{U}$ \\
\hline $76-13-1$ & $1,1,2$-Trichloro-1,2,2-trifluoroethane & 0.50 & $\mathrm{U}$ \\
\hline $67-64-1$ & Acetone & 5.0 & $\mathrm{U}$ \\
\hline $75-15-0$ & Carbon disulfide & 0.50 & $\mathrm{U}$ \\
\hline $79-20-9$ & Methyl acetate & 0.50 & $\mathrm{U}$ \\
\hline $75-09-2$ & Methylene Chloride & 0.50 & $\mathrm{U}$ \\
\hline $156-60-5$ & trans $-1,2-$ Dichloroethene & 0.50 & $\mathrm{U}$ \\
\hline $1634-04-4$ & Methyl tert-butyl ether & 0.50 & $\mathrm{U}$ \\
\hline $75-34-3$ & 1,1-Dichloroethane & 0.50 & $\mathrm{U}$ \\
\hline $156-59-2$ & cis-1,2-Dichloroethene & 0.50 & $\mathrm{U}$ \\
\hline $78-93-3$ & 2-Butanone & 5.0 & $\mathrm{U}$ \\
\hline $74-97-5$ & Bromochloromethane & 0.50 & $\mathrm{U}$ \\
\hline $67-66-3$ & Chloroform & 0.50 & $\mathrm{U}$ \\
\hline $71-55-6$ & 1,1,1-Trichloroethane & 0.50 & $\mathrm{U}$ \\
\hline $110-82-7$ & Cyclohexane & 0.50 & $\mathrm{U}$ \\
\hline $56-23-5$ & Carbon tetrachloride & 0.50 & $\mathrm{U}$ \\
\hline $71-43-2$ & Benzene & 0.50 & $\mathrm{U}$ \\
\hline $107-06-2$ & 1,2-Dichloroethane & 0.50 & $\mathrm{U}$ \\
\hline
\end{tabular}

Report 1,4-Dioxane for Low-Medium VOA analysis only 
$1 \mathrm{~B}$ - FORM I VOA-2

VOLATILE ORGANICS ANALYSIS DATA SHEET
EPA SAMPLE NO.

INPAULS-W-32695
Lab Name: TESTAMERICA BURLINGTON

Lab Code: STLV Case No.: INMAN

Matrix: (SOIL/SED/WATER)

Water

Sample wt/vol: 25.0

$(\mathrm{g} / \mathrm{mL}) \quad \mathrm{mL}$

Level: (TRACE/LOW/MED) TRACE

o Moisture: not dec.

GC Column: DB-624 ID: $0.20 \quad$ (mm)

Soil Extract Volume: (uL) (mL)

Purge Volume: 25.0
Contract: $8 \mathrm{E}-00302$

SDG No.: 200-5726

Mod. Ref No.:

Lab Sample ID: 200-5726-3

Lab File ID: DHOI07.D

Date Received: 06/25/2011

Date Analyzed: 06/28/2011

Dilution Factor: 1.0

Soil Aliquot Volume:

(uL)

\begin{tabular}{|c|c|c|c|}
\hline CAS NO. & COMPOUND & $\begin{array}{l}\text { CONCENTRATION UNITS: } \\
(\mathrm{ug} / \mathrm{L} \text { or } u g / \mathrm{kg}) \mathrm{ug} / \mathrm{L}\end{array}$ & $\varnothing$ \\
\hline $79-01-6$ & Trichloroethene & 0.50 & $\mathrm{U}$ \\
\hline $108-87-2$ & Methylcyclohexane & 0.50 & $\mathrm{U}$ \\
\hline $78-87-5$ & 1,2-Dichloropropane & 0.50 & $\mathrm{U}$ \\
\hline $75-27-4$ & Bromodichloromethane & 0.50 & $\mathrm{U}$ \\
\hline $10061-01-5$ & cis-1,3-Dichloropropene & 0.50 & $\mathrm{U}$ \\
\hline $108-10-1$ & 4-Methyl-2-pentanone & 5.0 & $\mathrm{U}$ \\
\hline $108-88-3$ & Toluene & 0.50 & $\mathrm{U}$ \\
\hline $10061-02-6$ & trans-1,3-Dichloropropene & 0.50 & $\mathrm{U}$ \\
\hline $79-00-5$ & 1,1,2-Trichloroethane & 0.50 & $\mathrm{U}$ \\
\hline $127-18-4$ & Tetrachloroethene & 0.50 & $\mathrm{U}$ \\
\hline $591-78-6$ & 2-Hexanone & 5.0 & $\mathrm{U}$ \\
\hline $124-48-1$ & Dibromochloromethane & 0.50 & $\mathrm{U}$ \\
\hline $106-93-4$ & 1,2-Dibromoethane & 0.50 & $\mathrm{U}$ \\
\hline $108-90-7$ & Chlorobenzene & 0.50 & $\mathrm{U}$ \\
\hline $100-41-4$ & Ethylbenzene & 0.50 & $\mathrm{U}$ \\
\hline $95-47-6$ & o-Xylene & 0.50 & $\mathrm{U}$ \\
\hline $179601-23-1$ & $\mathrm{~m}, \mathrm{p}$-Xylene & 0.50 & $\mathrm{U}$ \\
\hline $100-42-5$ & Styrene & 0.50 & $\mathrm{U}$ \\
\hline $75-25-2$ & Bromoform & 0.50 & $\mathrm{U}$ \\
\hline $98-82-8$ & Isopropylbenzene & 0.50 & $\mathrm{U}$ \\
\hline $79-34-5$ & $1,1,2,2$-Tetrachloroethane & 0.50 & $\mathrm{U}$ \\
\hline $541-73-1$ & 1,3-Dichlorobenzene & 0.50 & $\mathrm{U}$ \\
\hline $106-46-7$ & 1,4-Dichlorobenzene & 0.50 & $\mathrm{U}$ \\
\hline $95-50-1$ & 1,2-Dichlorobenzene & 0.50 & $\mathrm{U}$ \\
\hline $96-12-8$ & 1,2-Dibromo-3-Chloropropane & 0.50 & $\mathrm{U}$ \\
\hline $120-82-1$ & $1,2,4$-Trichlorobenzene & 0.50 & $\mathrm{U}$ \\
\hline $87-61-6$ & $1,2,3$-Trichlorobenzene & 0.50 & $\mathrm{U}$ \\
\hline
\end{tabular}


IJ - FORM I VOA-TIC

VOLATILE ORGANICS ANALYSIS DATA SHEET

TENTATIVELY IDENTIFIED COMPOUNDS
EPA SAMPLE NO.

INPAULS-W-32695

Lab Name: TESTAMERICA BURLINGTON

Contract: $\quad 8 \mathrm{E}-00302$

Lab Code: STLV Case No.: INMAN Mod. Ref No.: Matrix: (SOIL/SED/WATER)

Water

Sample wt/vol: 25.0 $(\mathrm{g} / \mathrm{mL}) \quad \mathrm{mL}$

Level: (TRACE or LOW/MED)

TRACE

음 Moisture: not dec.

GC Column: DB-624

ID: $0.20 \quad(\mathrm{~mm})$

Soil Extract Volume: (uL)

CONCENTRATION UNITS: (ug/L or $\mathrm{ug} / \mathrm{kg}$ ) ug/L
SDG No.: 200-5726

Lab Sample ID: 200-5726-3

Lab File ID: DHOI07.D

Date Received: 06/25/2011

Date Analyzed: 06/28/2011

Dilution Factor: 1.0

Soil Aliquot Volume: (uL)

Purge Volume: 25.0 (mL)

\begin{tabular}{|l|l|l|r|r|r|}
\hline \multicolumn{1}{l|}{ CAS NUMBER } & \multicolumn{1}{|c|}{ COMPOUND NAME } & RT & EST. CONC. & Q \\
\cline { 2 - 6 } 01 & & Unknown & 6.79 & B X J \\
\cline { 2 - 6 } 02 & E9667961 & Total Alkanes & $\mathrm{N} / \mathrm{A}$ & & \\
\hline
\end{tabular}

1 EPA-designated Registry Number. 
$1 A$ - FORM I VOA-1

VOLATILE ORGANICS ANALYSIS DATA SHEET
EPA SAMPLE NO. INQCTB-W-32696

Lab Name: TESTAMERICA BURLINGTON

Contract: $\quad 8 \mathrm{E}-00302$

Lab Code: STLV Case No.: INMAN Mod. Ref No.:

SDG No.: 200-5726

Matrix: (SOIL/SED/WATER)

Sample wt/vol: 25.0 Water

$(\mathrm{g} / \mathrm{mL}) \quad \mathrm{mL}$

Level: (TRACE/LOW/MED) TRACE

o Moisture: not dec.

GC Column: DB-624

Soil Extract Volume: ID: $0.20 \quad(\mathrm{~mm})$

Purge Volume: 25.0
Lab Sample ID; 200-5726-4

Lab File ID: DHOI12.D

Date Received: 06/25/2011

Date Analyzed: 06/28/2011

Dilution Factor: 1.0

Soil Aliquot Volume:

(uL)

\begin{tabular}{|c|c|c|c|}
\hline CAS NO. & COMPOUND & $\begin{array}{l}\text { CONCENTRATION UNITS: } \\
(\mathrm{ug} / \mathrm{L} \text { or } \mathrm{ug} / \mathrm{kg}) \mathrm{ug} / \mathrm{L}\end{array}$ & 0 \\
\hline $75-71-8$ & Dichlorodifluoromethane & 0.50 & $\mathrm{U}$ \\
\hline $74-87-3$ & Chloromethane & 0.50 & $\mathrm{U}$ \\
\hline $75-01-4$ & Vinyl chloride & 0.50 & $\mathrm{U}$ \\
\hline $74-83-9$ & Bromomethane & 0.50 & $\mathrm{U}$ \\
\hline $75-00-3$ & Chloroethane & 0.50 & $\mathrm{U}$ \\
\hline $75-69-4$ & Trichlorofluoromethane & 0.50 & $\mathrm{U}$ \\
\hline $75-35-4$ & 1,1-Dichloroethene & 0.50 & $\mathrm{U}$ \\
\hline $76-13-1$ & 1,1,2-Trichloro-1,2,2-trifluoroethane & 0.50 & $\mathrm{U}$ \\
\hline $67-64-1$ & Acetone & 3.7 & $\mathrm{~J} \mathrm{~B}$ \\
\hline $75-15-0$ & Carbon disulfide & 0.50 & $\mathrm{U}$ \\
\hline $79-20-9$ & Methyl acetate & 0.50 & $\mathrm{U}$ \\
\hline $75-09-2$ & Methylene Chloride & 0.50 & $\mathrm{U}$ \\
\hline $156-60-5$ & trans-1,2-Dichloroethene & 0.50 & $\mathrm{U}$ \\
\hline $1634-04-4$ & Methyl tert-butyl ether & 0.50 & $\mathrm{U}$ \\
\hline $75-34-3$ & 1,1-Dichloroethane & 0.50 & $\mathrm{U}$ \\
\hline $156-59-2$ & cis-1,2-Dichloroethene & 0.50 & $\mathrm{U}$ \\
\hline $78-93-3$ & 2-Butanone & 5.0 & $\mathrm{U}$ \\
\hline $74-97-5$ & Bromochloromethane & 0.50 & $\mathrm{U}$ \\
\hline $67-66-3$ & Chloroform & 0.50 & $\mathrm{U}$ \\
\hline $71-55-6$ & 1,1,1-Trichloroethane & 0.50 & $\mathrm{u}$ \\
\hline $110-82-7$ & Cyclohexane & 0.50 & $\mathrm{U}$ \\
\hline $56-23-5$ & Carbon tetrachloride & 0.50 & $\mathrm{U}$ \\
\hline $71-43-2$ & Benzene & 0.50 & $\mathrm{U}$ \\
\hline $1.07-06-2$ & 1,2-Dichloroethane & 0.50 & $\mathrm{U}$ \\
\hline
\end{tabular}

Report 1,4-Dioxane for Low-Medium VOA analysis only 
$1 \mathrm{~B}$ - FORM I VOA-2

VOLATILE ORGANICS ANALYSIS DATA SHEET
EPA SAMPLE NO.

INQCTB-W-32696

Lab Name: TESTAMERICA BURLINGTON

Lab Code: STLV

Case NO.: INMAN

Matrix: (SOIL/SED/WATER)

Water

Sample wt/vol: 25.0

$(\mathrm{g} / \mathrm{mL}) \quad \mathrm{mL}$

Level: (TRACE/LOW/MED) TRACE

응 Moisture: not dec.

GC Column: DB-624

ID $: 0.20$ (mm)

Soil Extract Volume:

(uL) (mL)

Purge Volume: 25.0

Contract: $\quad 8 \mathrm{E}-00302$

Mod. Ref No.:

SDG No.: 200-5726

Lab Sample ID: 200-5726-4

Lab File ID: DHOI12.D

Date Received: 06/25/2011

Date Analyzed: 06/28/2011

Dilution Factor: 1.0

Soil Aliquot Volume:

(uL)

\begin{tabular}{|c|c|c|c|}
\hline CAS NO. & COMPOUND & $\begin{array}{l}\text { CONCENTRATION UNITS: } \\
(\mathrm{ug} / \mathrm{L} \text { or } \mathrm{ug} / \mathrm{kg}) \mathrm{ug} / \mathrm{L}\end{array}$ & Q \\
\hline $79-01-6$ & Trichloroethene & 0.50 & $\mathrm{U}$ \\
\hline $108-87-2$ & Methylcyclohexane & 0.50 & $\mathrm{U}$ \\
\hline $78-87-5$ & 1,2-Dichloropropane & 0.50 & $\mathrm{U}$ \\
\hline $75-27-4$ & Bromodichloromethane & 0.50 & $\mathrm{U}$ \\
\hline $10061-01-5$ & cis-1,3-Dichloropropene & 0.50 & U \\
\hline $108-10-1$ & 4-Methyl-2-pentanone & 5.0 & $\mathrm{U}$ \\
\hline $108-88-3$ & Toluene & 0.16 & $\mathrm{~J} B$ \\
\hline $10061-02-6$ & trans-1,3-Dichloropropene & 0.50 & $\mathrm{U}$ \\
\hline $79-00-5$ & 1,1,2-Trichloroethane & 0.50 & $\mathrm{U}$ \\
\hline $127-18-4$ & Tetrachloroethene & 0.50 & $\mathrm{U}$ \\
\hline $591-78-6$ & 2-Hexanone & 5.0 & $\mathrm{U}$ \\
\hline $124-48-1$ & Dibromochloromethane & 0.50 & $\mathrm{U}$ \\
\hline $106-93-4$ & 1,2-Dibromoethane & 0.50 & $\mathrm{U}$ \\
\hline $108-90-7$ & Chlorobenzene & 0.50 & $\mathrm{U}$ \\
\hline $100-41-4$ & Ethylbenzene & 0.018 & $\mathrm{~J}$ \\
\hline $95-47-6$ & o-Xylene & 0.039 & $\mathrm{~J}$ \\
\hline $179601-23-1$ & $\mathrm{~m}, \mathrm{p}$-Xylene & 0.042 & $\mathrm{~J}$ \\
\hline $100-42-5$ & Styrene & 0.025 & $\mathrm{~J}$ \\
\hline $75-25-2$ & Bromoform & 0.50 & $\mathrm{U}$ \\
\hline $98-82-8$ & Isopropylbenzene & 0.50 & $\mathrm{U}$ \\
\hline $79-34-5$ & $1,1,2,2$-Tetrachloroethane & 0.50 & $\mathrm{U}$ \\
\hline $541-73-1$ & 1,3-Dichlorobenzene & 0.50 & $\mathrm{U}$ \\
\hline $106-46-7$ & 1,4-Dichlorobenzene & 0.42 & $\mathrm{~J}$ \\
\hline $95-50-1$ & 1,2-Dichlorobenzene & 0.082 & $\mathrm{~J}$ \\
\hline $96-12-8$ & 1,2-Dibromo-3-Chloropropane & 0.50 & $\mathrm{U}$ \\
\hline $120-82-1$ & 1,2,4-Trichlorobenzene & 0.50 & $\mathrm{U}$ \\
\hline $87-61-6$ & 1,2,3-Trichlorobenzene & 0.50 & $\mathrm{U}$ \\
\hline
\end{tabular}


1J - FORM I VOA-TIC

VOLATILE ORGANICS ANALYSIS DATA SHEET

TENTATIVELY IDENTIEIED COMPOUNDS
EPA SAMPLE NO.

INQCTB-W-32696

Lab Name: TESTAMERICA BURLINGTON

Contract: $8 \mathrm{E}-00302$

Lab Code: STLV Case No.: INMAN Mod. Ref No.: SDG No.: 200-5726

Matrix: (SOIL/SED/WATER)

Sample wt/vol: 25.0 Water

$(\mathrm{g} / \mathrm{mL}) \quad \mathrm{mL}$

Level: (TRACE or LOW/MED)

TRACE

\% Moisture: not dec.

GC Column: DB-624

ID $: 0.20 \quad(\mathrm{~mm})$

Soil Extract Volume: (uL)

CONCENTRATION UNITS: (ug/L or $\mathrm{ug} / \mathrm{kg}) \mathrm{ug} / \mathrm{L}$
Lab Sample ID: 200-5726-4

Lab File ID: DHOI12.D

Date Received: 06/25/2011

Date Analyzed: 06/28/2011

Dilution Factor: 1.0

Soil Aliquot Volume: (UL)

Purge Volume: 25.0 (mL)

\begin{tabular}{|c|c|c|c|c|}
\hline CAS NUMBER & COMPOUND NAME & $\mathrm{RT}$ & EST. CONC. & $Q$ \\
\hline & Unknown & 1.50 & 2.9 & $\overline{\mathrm{J}}$ \\
\hline & Unknown & 6.79 & 2.6 & $\mathrm{~B} X \mathrm{~J}$ \\
\hline E9667961 & Total Alkanes & $\mathrm{N} / \mathrm{A}$ & & \\
\hline
\end{tabular}

1 EPA-designated Registry Number. 
$6 A-F O R M$ VI VOA-1

VOLATILE ORGANICS INITIAL CALIBRATION DATA

Lab Name: TESTAMERICA BURLINGTON

Contract: $8 \mathrm{E}-00302$

Lab Code: STLV Case No.: INMAN Mod. Ref No.:

SDG No.: $200-5726$

Instrument ID: D.i

Calibration Date(s):

$03 / 25 / 201103 / 25 / 2011$

Heated Purge: $(\mathrm{Y} / \mathrm{N}) \mathrm{N}$

Purge Volume: 25.0

Calibration Time(s): 1225 1403

GC Column: DB-624

ID: 0.20 $(\mathrm{mL})$

RRF $0.5=$ DHO03.D

LAB FILE ID:

RRF $10=\underline{D H O 06 . D}$

RRE $5.0=$ DHO05.D

Length: 25

(m)

\begin{tabular}{|c|c|c|c|c|c|c|c|}
\hline COMPOUND & RRF 0.5 & RRF 1.0 & RRF 5.0 & RRF 10 & RRF 20 & $\mathrm{RRF}$ & $\frac{\circ}{2} \mathrm{RSD}$ \\
\hline Dichlorodifluoromethane & 0.483 & 0.494 & 0.490 & 0.504 & 0.517 & 0.497 & 2.6 \\
\hline Chloromethane & 0.656 & 0.603 & 0.600 & 0.603 & 0.593 & 0.611 & 4.2 \\
\hline Vinyl chloride & 0.478 & 0.476 & 0.483 & 0.484 & 0.479 & 0.480 & 0.7 \\
\hline Bromomethane & 0.224 & 0.209 & 0.210 & 0.235 & 0.234 & 0.223 & 5.7 \\
\hline Chloroethane & 0.255 & 0.246 & 0.245 & 0.246 & 0.243 & 0.247 & 1.8 \\
\hline Trichlorofluoromethane & 0.493 & 0.507 & 0.517 & 0.512 & 0.535 & 0.513 & 3.0 \\
\hline 1,1-Dichloroethene & 0.263 & 0.269 & 0.277 & 0.276 & 0.270 & 0.271 & 2.2 \\
\hline $\begin{array}{l}\text { 1,1,2-Trichloro- } \\
1,2,2 \text {-trifluoroethane }\end{array}$ & 0.310 & 0.309 & 0.316 & 0.307 & 0.301 & 0.309 & 1.8 \\
\hline Acetone & 0.026 & 0.024 & 0.023 & 0.020 & 0.022 & 0.023 & 10.3 \\
\hline Carbon disulfide & 0.570 & 0.575 & 0.642 & 0.673 & 0.684 & 0.629 & 8.5 \\
\hline Methyl acetate & 0.086 & 0.081 & 0.070 & 0.062 & 0.066 & 0.073 & 13.7 \\
\hline Methylene Chloride & 0.237 & 0.236 & 0.242 & 0.233 & 0.239 & 0.238 & 1.4 \\
\hline trans $-1,2-$ Dichloroethene & 0.274 & 0.279 & 0.296 & 0.294 & 0.302 & 0.289 & 4.2 \\
\hline Methyl tert-butyl ether & 0.318 & 0.302 & 0.328 & 0.322 & 0.359 & 0.326 & 6.4 \\
\hline 1,1-Dichloroethane & 0.558 & 0.562 & 0.574 & 0.574 & 0.592 & 0.572 & 2.3 \\
\hline cis-1,2-Dichloroethene & 0.274 & 0.290 & 0.308 & 0.308 & 0.316 & 0.299 & 5.6 \\
\hline 2-Butanone & 0.036 & 0.038 & 0.040 & 0.037 & 0.042 & 0.039 & 5.9 \\
\hline Bromochloromethane & 0.100 & 0.096 & 0.103 & 0.100 & 0.108 & 0.101 & 4.5 \\
\hline Chloroform & 0.432 & 0.460 & 0.471 & 0.467 & 0.485 & 0.463 & 4.2 \\
\hline 1,1,1-Trichloroethane & 0.419 & 0.423 & 0.452 & 0.452 & 0.451 & 0.439 & 3.9 \\
\hline Cyclohexane & 0.700 & 0.753 & 0.821 & 0.818 & 0.799 & 0.778 & 6.6 \\
\hline Carbon tetrachloride & 0.383 & 0.396 & 0.409 & 0.416 & 0.417 & 0.404 & 3.6 \\
\hline Benzene & 1.409 & 1.468 & 1.441 & 1.415 & 1.390 & 1.425 & 2.1 \\
\hline 1,2-Dichloroethane & 0.246 & 0.229 & 0.269 & 0.257 & 0.283 & 0.257 & 8.0 \\
\hline Trichloroethene & 0.351 & 0.343 & 0.336 & 0.337 & 0.335 & 0.341 & 2.0 \\
\hline Methylcyclohexane & 0.606 & 0.646 & 0.688 & 0.682 & 0.672 & 0.659 & 5.1 \\
\hline
\end{tabular}

Report 1,4-Dioxane for Low-Medium VOA analysis only 
$6 \mathrm{~B}$ - FORM VI VOA-2

VOLATILE ORGANICS INITIAL CALIBRATION DATA

Lab Name: TESTAMERICA BURIJINGTON

Contract: $8 \mathrm{E}-00302$

Lab Code: STLV Case No.: INMAN Mod. Ref No.:

SDG No.: 200-5726

Instrument ID: D.i

Calibration Date(s):

$03 / 25 / 201103 / 25 / 2011$

Heated Purge: $(\mathrm{Y} / \mathrm{N}) \mathrm{N}$

Calibration Time(s): 12251403

Purge Volune: 25.0

(mL)

GC Column: DB-624

ID: 0.20

(mm) Length: 25

(m)

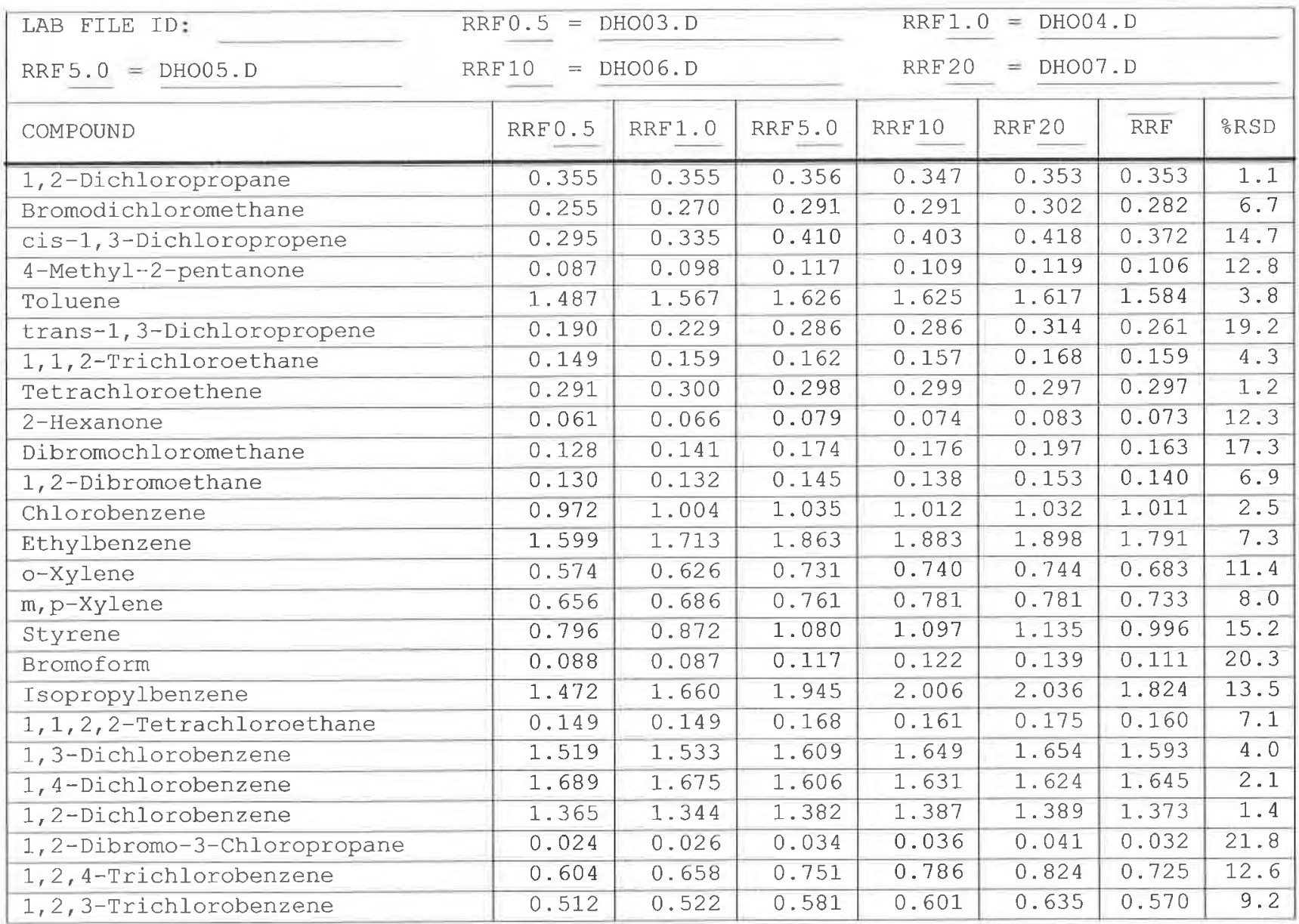


$6 C$ - FORM VI VOA-3

VOLATILE ORGANICS INITIAL CALIBRATION DATA

Lab Name: TESTAMERICA BURLINGTON

Contract: $\quad 8 \mathrm{E}-00302$

Lab Code: STLV Case No.: INMAN Mod. Ref No.:

SDG No.: 200-5726

Instrument ID: D.i Calibration Date(s):

$03 / 25 / 201103 / 25 / 2011$

Heated Purge: $(\mathrm{Y} / \mathrm{N}) \mathrm{N}$

Purge Volume: 25.0 Calibration Time(s) : 1225 1403

GC Column: DB-624 ID: 0.20 (mm) Length: 25

Column: DB-624 ID: 0.20 (mm) Length: 25 (mL)

(m)

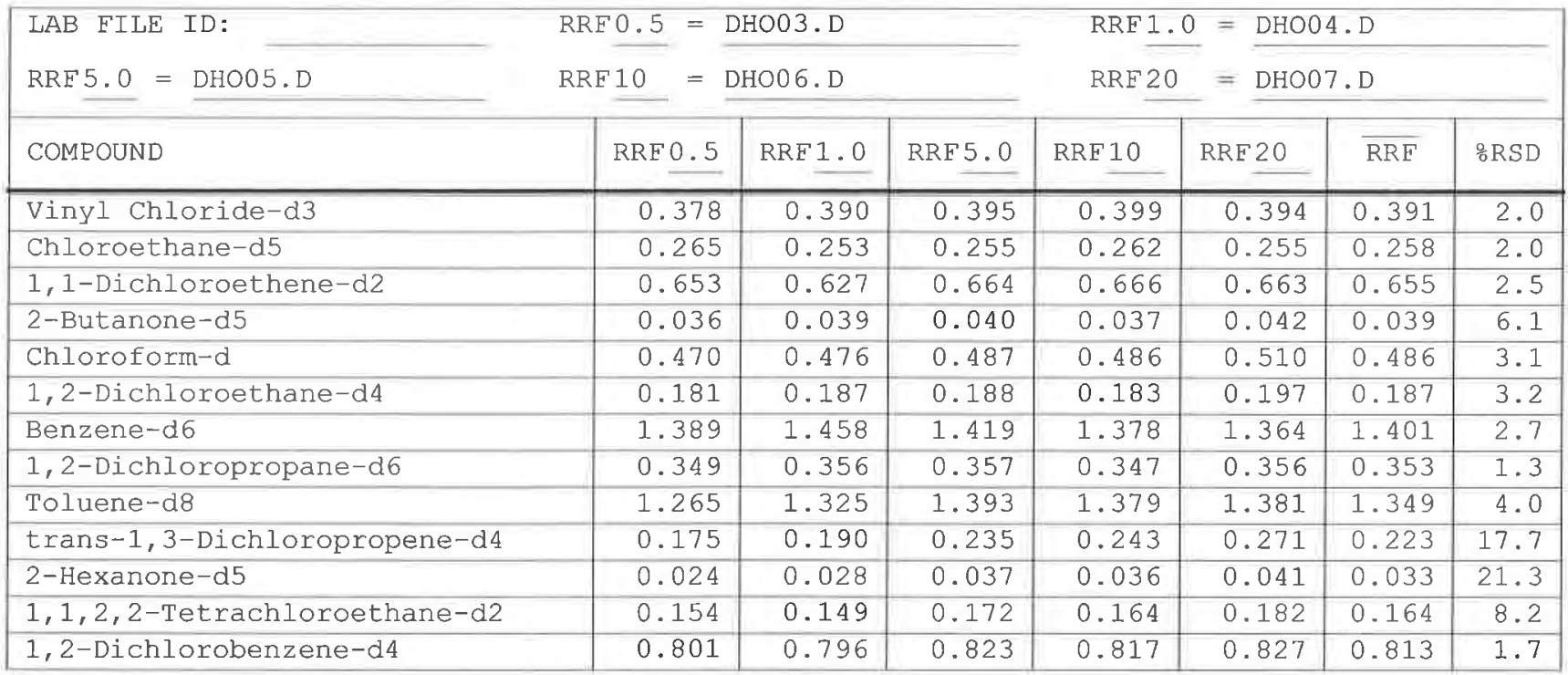

Report 1,4-Dioxane-d8 for Low-Medium VOA analysis only 
$7 A$ - FORM VII VOA-1

VOLATILE CONTINUING CALIBRATION DATA

Lab Name: TESTAMERICA BURLINGTON

Contract: $8 \mathrm{E}-00302$

Lab Code: STLV Case No.: INMAN Mod. Ref No.:

SDG No.: 200-5726

Instrument ID: D.i

Calibration Date: 06/28/2011 Time: 0747

Lab File Id: DHOI03.D Init. Calib. Date(s): 03/25/2011 03/25/2011

EPA Sample No. (VSTD\#\#\#\#): VSTD005DP

Init. Calib. Time(s): $1225 \quad 1403$

Heated Purge: ( $Y / N)$

GC Column: DB-624

ID: $0.20(\mathrm{~mm})$ Length: 25

(m)

Purge Volume: 25.0

$(m L)$

\begin{tabular}{|c|c|c|c|c|c|}
\hline COMPOUND & $\overline{\mathrm{RRF}}$ & RRE5.0 & $\begin{array}{l}\text { MIN } \\
\text { RRF }\end{array}$ & $\because \mathrm{D}$ & MAX $\% D$ \\
\hline Dichlorodifluoromethane & 0.497 & 0.498 & 0.010 & 0.1 & 40.0 \\
\hline Chloromethane & 0.611 & 0.706 & 0.010 & 15.6 & 40.0 \\
\hline Vinyl chloride & 0.480 & 0.536 & 0.010 & 11.7 & 30.0 \\
\hline Bromomethane & 0.223 & 0.223 & 0.100 & 0.1 & 30.0 \\
\hline Chloroethane & 0.247 & 0.281 & 0.010 & 14.0 & 40.0 \\
\hline Trichlorofluoromethane & 0.513 & 0.580 & 0.010 & 13.2 & 40.0 \\
\hline 1,1-Dichloroethene & 0.271 & 0.304 & 0.100 & 12.2 & 30.0 \\
\hline 1,1,2-Trichloro-1,2,2-trifluoroethane & 0.309 & 0.361 & 0.010 & 17.0 & 40.0 \\
\hline Acetone & 0.023 & 0.029 & 0.010 & 27.5 & 40.0 \\
\hline Carbon disulfide & 0.629 & 0.748 & 0.010 & 19.0 & 40.0 \\
\hline Methyl acetate & 0.073 & 0.080 & 0.010 & 9.8 & 40.0 \\
\hline Methylene Chloride & 0.238 & 0.253 & 0.010 & 6.6 & 40.0 \\
\hline trans-1,2-Dichloroethene & 0.289 & 0.316 & 0.010 & 9.2 & 40.0 \\
\hline Methyl tert-butyl ether & 0.326 & 0.301 & 0.010 & -7.6 & 40.0 \\
\hline 1,1-Dichloroethane & 0.572 & 0.613 & 0.200 & 7.2 & 30.0 \\
\hline cis-1,2-Dichloroethene & 0.299 & 0.309 & 0.010 & 3.3 & 40.0 \\
\hline 2-Butanone & 0.039 & 0.046 & 0.010 & 19.9 & 40.0 \\
\hline Bromochloromethane & 0.101 & 0.106 & 0.050 & 4.4 & 30.0 \\
\hline Chloroform & 0.463 & 0.476 & 0.200 & 2.8 & 30.0 \\
\hline 1,1,1-Trichloroethane & 0.439 & 0.454 & 0.100 & 3.4 & 30.0 \\
\hline Cyclohexane & 0.778 & 0.885 & 0.010 & 13.8 & 40.0 \\
\hline Carbon tetrachloride & 0.404 & 0.442 & 0.100 & 9.5 & 30.0 \\
\hline Benzene & 1.425 & 1.503 & 0.400 & 5.5 & 30.0 \\
\hline 1,2-Dichloroethane & 0.257 & 0.277 & 0.100 & 8.0 & 30.0 \\
\hline Trichloroethene & 0.341 & 0.354 & 0.300 & 4.0 & 30.0 \\
\hline Methylcyclohexane & 0.659 & 0.732 & 0.010 & 11.1 & 40.0 \\
\hline
\end{tabular}

Report 1,4-Dioxane for Low/Medium VOA analysis only 
$7 \mathrm{~B}$ - FORM VII VOA-2

VOLATILE CONTINUING CALIBRATION DATA

Lab Name: TESTAMERICA BURLINGTON

Contract: $\quad 8 \mathrm{E}-00302$

Lab Code: STLV Case No.: INMAN Mod. Ref No.:

SDG No.: 200-5726

Instrument ID: D.i

Calibration Date: 06/28/2011 Time: 0747

Lab File Id: DHOI03.D Init. Calib. Date(s): 03/25/2011 03/25/2011

EPA Sample No. (VSTD\#\#\#\#): VSTD005DP

Init. Calib. Time(s): $1225 \quad 1403$

Heated Purge: ( $\mathrm{Y} / \mathrm{N})$

GC Column: DB-624

ID: $0.20(\mathrm{~mm})$ Length: 25

(m)

Purge Volume: 25.0

(mL)

\begin{tabular}{|c|c|c|c|c|c|}
\hline COMPOUND & $\overline{\mathrm{RRF}}$ & RRF5. 0 & $\begin{array}{l}\text { MIN } \\
\text { RRE }\end{array}$ & $\frac{\circ}{\partial} \mathrm{D}$ & $\operatorname{MAX} \div \mathrm{D}$ \\
\hline 1,2-Dichloropropane & 0.353 & 0.364 & 0.010 & 2.9 & 40.0 \\
\hline Bromodichloromethane & 0.282 & 0.288 & 0.200 & 2.2 & 30.0 \\
\hline cis-1,3-Dichloropropene & 0.372 & 0.393 & 0.200 & 5.5 & 30.0 \\
\hline 4-Methyl-2-pentanone & 0.106 & 0.124 & 0.010 & 16.6 & 40.0 \\
\hline Toluene & 1.584 & 1.665 & 0.400 & 5.1 & 30.0 \\
\hline trans-1,3-Dichloropropene & 0.261 & 0.276 & 0.100 & 5.8 & 30.0 \\
\hline $1,1,2-$ Trichloroethane & 0.159 & 0.162 & 0.100 & 1.9 & 30.0 \\
\hline Tetrachloroethene & 0.297 & 0.319 & 0.100 & 7.4 & 30.0 \\
\hline 2-Hexanone & 0.073 & 0.088 & 0.010 & 21.5 & 40.0 \\
\hline Dibromochloromethane & 0.163 & 0.181 & 0.100 & 10.7 & 30.0 \\
\hline 1,2-Dibromoethane & 0.140 & 0.141 & 0.010 & 0.7 & 40.0 \\
\hline Chlorobenzene & 1.011 & 1.062 & 0.500 & 5.0 & 30.0 \\
\hline Ethylbenzene & 1.791 & 1.872 & 0.100 & 4.5 & 30.0 \\
\hline o-Xylene & 0.683 & 0.729 & 0.300 & 6.7 & 30.0 \\
\hline $\mathrm{m}, \mathrm{p}$-Xylene & 0.733 & 0.783 & 0.300 & 6.8 & 30.0 \\
\hline Styrene & 0.996 & 1.082 & 0.300 & 8.7 & 30.0 \\
\hline Bromoform & 0.111 & 0.135 & 0.050 & 22.0 & 30.0 \\
\hline Isopropylbenzene & 1.824 & 1.974 & 0.010 & 8.2 & 40.0 \\
\hline $1,1,2,2$-Tetrachloroethane & 0.160 & 0.163 & 0.100 & 1.6 & 30.0 \\
\hline 1,3-Dichlorobenzene & 1.593 & 1.626 & 0.400 & 2.1 & 30.0 \\
\hline 1,4-Dichlorobenzene & 1.645 & 1.668 & 0.400 & 1.4 & 30.0 \\
\hline 1,2-Dichlorobenzene & 1.373 & 1.433 & 0.400 & 4.4 & 30.0 \\
\hline 1,2-Dibromo-3-Chloropropane & 0.032 & 0.038 & 0.010 & 19.2 & 40.0 \\
\hline 1,2,4-Trichlorobenzene & 0.725 & 0.785 & 0.200 & 8.3 & 30.0 \\
\hline 1,2,3-Trichlorobenzene & 0.570 & 0.597 & 0.200 & 4.7 & 30.0 \\
\hline
\end{tabular}


7C - FORM VII VOA-3

VOLATILE CONTINUING CALIBRATION DATA

Lab Name: TESTAMERICA BURLINGTON

Contract: $\quad 8 \mathrm{E}-00302$

Lab Code: STLV Case No.: INMAN Mod. Ref No.: SDG No.: 200-5726

Instrument ID: D.i Calibration Date: 06/28/2011 Time: 0747

Lab File Id: DHOI03.D

EPA Sample No. (VSTD\#\#\#\#): VSTD005DP Init. Calib. Date(s): 03/25/2011 03/25/2011

Heated Purge: ( $\mathrm{Y} / \mathrm{N})$

N GC Column: DB-624

Init. Calib. Time(s): $1225 \quad 1403$

ID: $0.20(\mathrm{~mm})$ Length: 25

(m)

Purge Volume: 25.0

(mL)

\begin{tabular}{|c|c|c|c|c|c|}
\hline COMPOUND & RRF & RRF5. 0 & $\begin{array}{l}\text { MIN } \\
\text { RRF }\end{array}$ & $\frac{\circ}{8} \mathrm{D}$ & $\mathrm{MAX} \div \mathrm{D}$ \\
\hline Vinyl Chloride-d3 & 0.391 & 0.402 & 0.010 & 2.6 & 30.0 \\
\hline Chloroethane-d5 & 0.258 & 0.268 & 0.010 & 3.8 & 40.0 \\
\hline 1,1-Dichloroethene-d2 & 0.655 & 0.738 & 0.010 & 12.7 & 30.0 \\
\hline 2-Butanone-d5 & 0.039 & 0.041 & 0.010 & 5.7 & 40.0 \\
\hline Chloroform-d & 0.486 & 0.491 & 0.010 & 1.0 & 30.0 \\
\hline 1,2-Dichloroethane-d4 & 0.187 & 0.194 & 0.010 & 3.7 & 30.0 \\
\hline Benzene-d6 & 1.401 & 1.465 & 0.010 & 4.5 & 30.0 \\
\hline 1,2-Dichloropropane-d6 & 0.353 & 0.356 & 0.010 & 0.8 & 40.0 \\
\hline Toluene-d8 & 1.349 & 1.429 & 0.010 & 6.0 & 30.0 \\
\hline trans-1,3-Dichloropropene-d4 & 0.223 & 0.228 & 0.010 & 2.2 & 30.0 \\
\hline 2-Hexanone-d5 & 0.033 & 0.036 & 0.010 & 9.4 & 40.0 \\
\hline $1,1,2,2$-Tetrachloroethane-d2 & 0.164 & 0.163 & 0.010 & -0.8 & 30.0 \\
\hline 1,2-Dichlorobenzene-d4 & 0.813 & 0.840 & 0.010 & 3.4 & 30.0 \\
\hline
\end{tabular}

Report 1,4-Dioxane-d8 for Low/Medium VOA analysis only 
Lab Name: TESTAMERICA BURLINGTON

Contract: 8E-00302

Lab Code: STLV Case No.: INMAN Mod. Ref No.:

SDG NO.: 200-5726

Instrument ID: D.i

Calibration Date: 06/28/2011 Time: 1636

Lab File Id: DHOI22.D

EPA Sample No. (VSTD\#\#\#\#): VSTD005PD Init. Calib. Date(s): 03/25/2011 03/25/2011

Heated Purge: $(\mathrm{Y} / \mathrm{N})$ $\mathrm{N} \quad$ GC Column: $\mathrm{DB}-624$

Init. Calib. Time(s): 1225 1403

Purge Volume: 25.0 $(\mathrm{mL})$

\begin{tabular}{|c|c|c|c|c|c|}
\hline COMPOUND & $\overline{\mathrm{RRF}}$ & RRF5. 0 & $\begin{array}{l}\text { MIN } \\
\text { RRE }\end{array}$ & $\because \mathrm{D}$ & $\mathrm{MAX} \quad \frac{\circ}{8} \mathrm{D}$ \\
\hline Dichlorodifluoromethane & 0.497 & 0.448 & 0.010 & -9.9 & 50.0 \\
\hline Chloromethane & 0.611 & 0.653 & 0.010 & 7.0 & 50.0 \\
\hline Vinyl chloride & 0.480 & 0.480 & 0.010 & 0.1 & 50.0 \\
\hline Bromomethane & 0.223 & 0.197 & 0.010 & -11.7 & 50.0 \\
\hline Chloroethane & 0.247 & 0.249 & 0.010 & 0.8 & 50.0 \\
\hline Trichlorofluoromethane & 0.513 & 0.526 & 0.010 & 2.5 & 50.0 \\
\hline 1,1-Dichloroethene & 0.271 & 0.281 & 0.010 & 3.7 & 50.0 \\
\hline 1,1,2-Trichloro-1,2,2-trifluoroethane & 0.309 & 0.321 & 0.010 & 4.1 & 50.0 \\
\hline Acetone & 0.023 & 0.025 & 0.010 & 6.3 & 50.0 \\
\hline Carbon disulfide & 0.629 & 0.620 & 0.010 & -1.4 & 50.0 \\
\hline Methyl acetate & 0.073 & 0.071 & 0.010 & -3.2 & 50.0 \\
\hline Methylene Chloride & 0.238 & 0.226 & 0.010 & -5.1 & 50.0 \\
\hline trans-1,2-Dichloroethene & 0.289 & 0.277 & 0.010 & -4.2 & 50.0 \\
\hline Methyl tert-butyl ether & 0.326 & 0.234 & 0.010 & -28.2 & 50.0 \\
\hline 1,1-Dichloroethane & 0.572 & 0.536 & 0.010 & -6.3 & 50.0 \\
\hline cis-1,2-Dichloroethene & 0.299 & 0.266 & 0.010 & -11.0 & 50.0 \\
\hline 2-Butanone & 0.039 & 0.037 & 0.010 & -3.5 & 50.0 \\
\hline Bromochloromethane & 0.101 & 0.088 & 0.010 & -13.2 & 50.0 \\
\hline Chloroform & 0.463 & 0.413 & 0.010 & -10.9 & 50.0 \\
\hline 1,1,1-Trichloroethane & 0.439 & 0.421 & 0.010 & -4.1 & 50.0 \\
\hline Cyclohexane & 0.778 & 0.799 & 0.010 & 2.7 & 50.0 \\
\hline Carbon tetrachloride & 0.404 & 0.400 & 0.010 & -1.1 & 50.0 \\
\hline Benzene & 1.425 & 1.366 & 0.010 & -4.1 & 50.0 \\
\hline 1,2-Dichloroethane & 0.257 & 0.231 & 0.010 & -9.9 & 50.0 \\
\hline Trichloroethene & 0.341 & 0.322 & 0.010 & -5.5 & 50.0 \\
\hline Methylcyclohexane & 0.659 & 0.683 & 0.010 & 3.6 & 50.0 \\
\hline
\end{tabular}


$7 B$ - FORM VII VOA-2

VOLATILE CONTINUING CALIBRATION DATA

Lab Name: TESTAMERICA BURLINGTON

Contract: $8 \mathrm{E}-00302$

Lab Code: STIV Case No.: INMAN Mod. Ref No.:

SDG No.: $200-5726$

Instrument ID: D.i

Calibration Date: 06/28/2011 Time: 1636

Lab File Id: DHOI22. D

EPA Sample No. (VSTD\#\#\#\#): VSTD005PD Init. Calib. Date(s): 03/25/2011 03/25/2011

Heated Purge: $(Y / N)$

GC Column: DB-624

Init. Calib. Time(s): 1225 1403

Purge Volume: 25.0 $(\mathrm{mL})$

\begin{tabular}{|c|c|c|c|c|c|}
\hline COMPOUND & $\overline{\mathrm{RRF}}$ & RRE5. 0 & $\begin{array}{l}\text { MIN } \\
\text { RRF }\end{array}$ & $\frac{\circ}{\partial} \mathrm{D}$ & MAX $\because D$ \\
\hline 1,2-Dichloropropane & 0.353 & 0.325 & 0.010 & -8.0 & 50.0 \\
\hline Bromodichloromethane & 0.282 & 0.249 & 0.010 & -11.5 & 50.0 \\
\hline cis-1,3-Dichloropropene & 0.372 & 0.336 & 0.010 & -9.8 & 50.0 \\
\hline 4-Methyl-2-pentanone & 0.106 & 0.101 & 0.010 & -5.1 & 50.0 \\
\hline Toluene & 1.584 & 1.528 & 0.010 & -3.5 & 50.0 \\
\hline trans-1,3-Dichloropropene & 0.261 & 0.221 & 0.010 & -15.3 & 50.0 \\
\hline $1,1,2$-Trichloroethane & 0.159 & 0.134 & 0.010 & -15.6 & 50.0 \\
\hline Tetrachloroethene & 0.297 & 0.291 & 0.010 & -1.9 & 50.0 \\
\hline 2-Hexanone & 0.073 & 0.072 & 0.010 & -0.7 & 50.0 \\
\hline Dibromochloromethane & 0.163 & 0.145 & 0.010 & $-11 \cdot 1$ & 50.0 \\
\hline 1,2-Dibromoethane & 0.140 & 0.120 & 0.010 & -14.1 & 50.0 \\
\hline Chlorobenzene & 1.011 & 0.944 & 0.010 & -6.6 & 50.0 \\
\hline Ethylbenzene & 1.791 & 1.718 & 0.010 & -4.1 & 50.0 \\
\hline o-xylene & 0.683 & 0.657 & 0.010 & -3.8 & 50.0 \\
\hline $\mathrm{m}, \mathrm{p}$-Xylene & 0.733 & 0.710 & 0.010 & -3.2 & 50.0 \\
\hline Styrene & 0.996 & 0.970 & 0.010 & -2.6 & 50.0 \\
\hline Bromoform & 0.111 & 0.094 & 0.010 & -15.2 & 50.0 \\
\hline Isopropylbenzene & 1.824 & 1.809 & 0.010 & -0.8 & 50.0 \\
\hline 1,1,2,2-Tetrachloroethane & 0.160 & 0.134 & 0.010 & -16.3 & 50.0 \\
\hline 1,3-Dichlorobenzene & 1.593 & 1.428 & 0.010 & -10.4 & 50.0 \\
\hline 1,4-Dichlorobenzene & 1.645 & 1.494 & 0.010 & -9.2 & 50.0 \\
\hline 1,2-Dichlorobenzene & 1.373 & 1.243 & 0.010 & -9.5 & 50.0 \\
\hline 1,2-Dibromo-3-Chloropropane & 0.032 & 0.026 & 0.010 & -18.8 & 50.0 \\
\hline 1,2,4-Trichlorobenzene & 0.725 & 0.587 & 0.010 & $-18 \cdot 9$ & 50.0 \\
\hline 1,2,3-Trichlorobenzene & 0.570 & 0.476 & 0.010 & -16.5 & 50.0 \\
\hline
\end{tabular}


$7 C$ - FORM VII VOA-3

VOLATILE CONTINUING CALIBRATION DATA

Lab Name: TESTAMERICA BURLINGTON

Contract: $\quad 8 \mathrm{E}-00302$

Lab Code: STLV Case No.: INMAN Mod. Ref No.:

SDG No.: 200-5726

Instrument ID: D.i

Calibration Date: 06/28/2011 Time: 1636

Lab File Id: DHOI22.D

EPA Sample No. (VSTD\#\#\#\#): VSTD005PD

Init. Calib. Date(s): 03/25/2011 03/25/2011

Heated Purge: $(\mathrm{Y} / \mathrm{N}) \quad \mathrm{N}$

GC Column: DB-624

Init. Calib. Time(s): $1225 \quad 1403$

N

ID: $0.20(\mathrm{~mm})$ Length: 25

(m)

Purge Volume: 25.0

$(\mathrm{mL})$

\begin{tabular}{|c|c|c|c|c|c|}
\hline COMPOUND & $\overline{\mathrm{RRF}}$ & RRF5. 0 & $\begin{array}{l}\text { MIN } \\
\text { RRE }\end{array}$ & $\frac{\circ}{0} \mathrm{D}$ & MAX $\because D$ \\
\hline Vinyl Chloride-d3 & 0.391 & 0.356 & 0.010 & -9.0 & 50.0 \\
\hline Chloroethane-d5 & 0.258 & 0.254 & 0.010 & -1.7 & 50.0 \\
\hline 1,1-Dichloroethene-d2 & 0.655 & 0.671 & 0.010 & 2.5 & 50.0 \\
\hline 2-Butanone-d5 & 0.039 & 0.032 & 0.010 & -18.1 & 50.0 \\
\hline Chloroform-d & 0.486 & 0.426 & 0.010 & $-12 \cdot 3$ & 50.0 \\
\hline 1,2-Dichloroethane-d4 & 0.187 & 0.163 & 0.010 & -13.1 & 50.0 \\
\hline Benzene-d6 & 1.401 & 1.311 & 0.010 & -6.5 & 50.0 \\
\hline 1,2-Dichloropropane-d6 & 0.353 & 0.323 & 0.010 & -8.6 & 50.0 \\
\hline Toluene-d8 & 1.349 & 1.292 & 0.010 & -4.2 & 50.0 \\
\hline trans-1,3-Dichloropropene-d4 & 0.223 & 0.184 & 0.010 & -17.5 & 50.0 \\
\hline 2-Hexanone-d5 & 0.033 & 0.028 & 0.010 & -13.7 & 50.0 \\
\hline $1,1,2,2$-Tetrachloroethane-d2 & 0.164 & 0.139 & 0.010 & -15.4 & 50.0 \\
\hline 1,2-Dichlorobenzene-d4 & 0.813 & 0.721 & 0.010 & -11.3 & 50.0 \\
\hline
\end{tabular}

Report 1,4-Dioxane-d8 for Low/Medium VOA analysis only 
$1 A$ - FORM I VOA-1

VOLATILE ORGANICS ANALYSIS DATA SHEET
EPA SAMPLE NO.

VBLKDP

Lab Name: TESTAMERICA BURLINGTON

Contract: $8 \mathrm{E}-00302$

Lab Code: STLV Case No.: INMAN Mod. Ref No.: SDG No.: $200-5726$

Matrix: (SOIL/SED/WATER)

Sample wt/vol: 25.0 Water

$(\mathrm{g} / \mathrm{mL}) \quad \mathrm{mL}$

Level: (TRACE/LOW/MED) TRACE

응 Moisture: not dec.

GC Column: DB-624 ID: 0.20 $(\mathrm{mm})$

Soil Extract Volume: (uL) (mL)

Purge Volume: 25.0
Lab Sample ID: MB 200-20420/4

Lab File ID: DHOI04.D

Date Received:

Date Analyzed: 06/28/2011

Dilution Factor: 1.0

Soil Aliquot Volume:

(uL)

\begin{tabular}{|c|c|c|c|}
\hline CAS NO. & COMPOUND & $\begin{array}{l}\text { CONCENTRATION UNITS: } \\
(u g / L \text { or } u g / k g) u g / L\end{array}$ & $Q$ \\
\hline $75-71-8$ & Dichlorodifluoromethane & 0.50 & $\mathrm{U}$ \\
\hline $74-87-3$ & Chloromethane & 0.50 & $\mathrm{U}$ \\
\hline $75-01-4$ & Vinyl chloride & 0.50 & $\mathrm{U}$ \\
\hline $74-83-9$ & Bromomethane & 0.50 & $\mathrm{U}$ \\
\hline $75-00-3$ & Chloroethane & 0.50 & $\mathrm{U}$ \\
\hline $75-69-4$ & Trichlorofluoromethane & 0.50 & $\mathrm{U}$ \\
\hline $75-35-4$ & 1,1-Dichloroethene & 0.50 & $\mathrm{U}$ \\
\hline $76-13-1$ & 1,1,2-Trichloro-1,2,2-trifluoroethane & 0.50 & $\mathrm{U}$ \\
\hline $67-64-1$ & Acetone & 1.1 & $\mathrm{~J}$ \\
\hline $75-15-0$ & Carbon disulfide & 0.080 & $\mathrm{~J}$ \\
\hline $79-20-9$ & Methyl acetate & 0.50 & $\mathrm{~J}$ \\
\hline $75-09-2$ & Methylene Chloride & 0.50 & $\mathrm{U}$ \\
\hline $156-60-5$ & trans-1,2-Dichloroethene & 0.50 & $\mathrm{U}$ \\
\hline $1634-04-4$ & Methyl tert-butyl ether & 0.50 & $\mathrm{U}$ \\
\hline $75-34-3$ & 1,1-Dichloroethane & 0.50 & $\mathrm{U}$ \\
\hline $156-59-2$ & cis-1,2-Dichloroethene & 0.50 & $\mathrm{U}$ \\
\hline $78-93-3$ & 2-Butanone & 5.0 & $\mathrm{U}$ \\
\hline $74-97-5$ & Bromochloromethane & 0.50 & $\mathrm{U}$ \\
\hline $67-66-3$ & Chloroform & 0.50 & $\mathrm{U}$ \\
\hline $71-55-6$ & 1,1,1-Trichloroethane & 0.50 & $\mathrm{U}$ \\
\hline $110-82-7$ & Cyclohexane & 0.50 & $\mathrm{U}$ \\
\hline $56-23-5$ & Carbon tetrachloride & 0.50 & $\mathrm{U}$ \\
\hline $71-43-2$ & Benzene & 0.50 & $\mathrm{U}$ \\
\hline $107-06-2$ & 1,2-Dichloroethane & 0.50 & $\mathrm{U}$ \\
\hline
\end{tabular}

Report 1,4-Dioxane for Low-Medium VOA analysis only 
$1 B$ - FORM I VOA-2

VOLATILE ORGANICS ANALYSIS DATA SHEET
EPA SAMPLE NO.

VBLKDP
Lab Name: TESTAMERICA BURLINGTON

Lab Code: STLV Case No.: INMAN

Mod
Water

$(\mathrm{g} / \mathrm{mL}) \mathrm{mL}$

Sample wt/vol: 25.0

Level: (TRACE/LOW/MED)

TRACE

o Moisture: not dec.

GC Column: DB-624

ID: $0.20 \quad$ (mm)

Soil Extract volume:

Purge Volume: 25.0
Contract: $8 \mathrm{E}-00302$

SDG No.: 200-5726
Lab Sample ID: MB 200-20420/4

Lab File ID: DHOIO4.D

Date Received:

Date Analyzed: 06/28/2011

Dilution Factor: 1.0

Soil Aliquot Volume:

(uL)

\begin{tabular}{|c|c|c|c|}
\hline CAS NO. & COMPOUND & $\begin{array}{l}\text { CONCENTRATION UNITS: } \\
(\mathrm{ug} / \mathrm{L} \text { or } \mathrm{ug} / \mathrm{kg}) \mathrm{ug} / \mathrm{L}\end{array}$ & $Q$ \\
\hline $79-01-6$ & Trichloroethene & 0.50 & $\mathrm{U}$ \\
\hline $108-87-2$ & Methylcyclohexane & 0.50 & $\mathrm{U}$ \\
\hline $78-87-5$ & 1,2-Dichloropropane & 0.50 & $\mathrm{U}$ \\
\hline $75-27-4$ & Bromodichloromethane & 0.50 & $\mathrm{U}$ \\
\hline $10061-01-5$ & cis-1,3-Dichloropropene & 0.50 & $\mathrm{U}$ \\
\hline $108-10-1$ & 4-Methyl-2-pentanone & 5.0 & $\mathrm{U}$ \\
\hline $108-88-3$ & Toluene & 0.0072 & $J$ \\
\hline $10061-02-6$ & trans-1,3-Dichloropropene & 0.50 & $\mathrm{U}$ \\
\hline $79-00-5$ & $1,1,2$-Trichloroethane & 0.50 & $\mathrm{U}$ \\
\hline $127-18-4$ & Tetrachloroethene & 0.50 & $\mathrm{U}$ \\
\hline $591-78-6$ & 2-Hexanone & 5.0 & $\mathrm{U}$ \\
\hline $124-48-1$ & Dibromochloromethane & 0.50 & $\mathrm{U}$ \\
\hline $106-93-4$ & 1,2-Dibromoethane & 0.50 & $\mathrm{U}$ \\
\hline $108-90-7$ & Chlorobenzene & 0.50 & $\mathrm{U}$ \\
\hline $100-41-4$ & Ethylbenzene & 0.50 & $\mathrm{U}$ \\
\hline $95-47-6$ & o-Xylene & 0.50 & $\mathrm{U}$ \\
\hline $179601-23-1$ & $\mathrm{~m}, \mathrm{p}-\mathrm{xy}$ lene & 0.50 & $\mathrm{U}$ \\
\hline $100-42-5$ & Styrene & 0.50 & $\mathrm{U}$ \\
\hline $75-25-2$ & Bromoform & 0.50 & $\mathrm{U}$ \\
\hline $98-82-8$ & Isopropylbenzene & 0.50 & $\mathrm{U}$ \\
\hline $79-34-5$ & $1,1,2,2$-Tetrachloroethane & 0.50 & $\mathrm{U}$ \\
\hline $541-73-1$ & 1,3-Dichlorobenzene & 0.50 & $\mathrm{U}$ \\
\hline $106-46-7$ & 1,4-Dichlorobenzene & 0.50 & $\mathrm{U}$ \\
\hline $95-50-1$ & 1,2-Dichlorobenzene & 0.50 & $\mathrm{U}$ \\
\hline $96-12-8$ & 1,2-Dibromo-3-Chloropropane & 0.50 & $\mathrm{U}$ \\
\hline $120-82-1$ & $1,2,4$-Trichlorobenzene & 0.50 & $\mathrm{U}$ \\
\hline $87-61-6$ & 1,2,3-Trichlorobenzene & 0.50 & U \\
\hline
\end{tabular}


1J - FORM I VOA-TIC

VOLATILE ORGANICS ANALYSIS DATA SHEET

TENTATIVELY IDENTIFIED COMPOUNDS
EPA SAMPLE NO.

VBLKDP

\begin{abstract}
Lab Name: TESTAMERICA BURLINGTON Case No.: INMAN

Lab Code: STLV

Water

$(\mathrm{g} / \mathrm{mL}) \quad \mathrm{mL}$

Sample wt/vol: 25.0

Level: (TRACE or LOW/MED)

TRACE

음 Moisture: not dec.

GC Column: DB-624

Soil Extract Volume:

ID $: 0.20 \quad(\mathrm{~mm})$

CONCENTRATION UNITS: (ug/L or $\mathrm{ug} / \mathrm{kg}$ )

(uL)

$\mathrm{ug} / \mathrm{L}$
\end{abstract}

Mod. Ref No.:

Contract: $\quad 8 \mathrm{E}-00302$

SDG No.: 200-5726

Lab Sample ID: MB 200-20420/4

Lab File ID: DHOI04.D

Date Received:

Date Analyzed: 06/28/2011

Dilution Factor: 1.0

Soil Aliquot Volume:

Purge Volume: 25.0

01

02

\begin{tabular}{|l|l|c|c|c|}
\hline CAS NUMBER & \multicolumn{1}{|c|}{ COMPOUND NAME } & RT & EST. CONC. & Q \\
\hline & Unknown & 6.79 & 3.1 & X J \\
\hline E9667961 & Total Alkanes & N/A & & \\
\hline
\end{tabular}

1 EPA-designated Registry Number. 
$1 A$ - FORM I VOA-1

VOLATILE ORGANICS ANALYSIS DATA SHEET
EPA SAMPLE NO.

VHBLK01
Lab Name: TESTAMERICA BURLINGTON

Lab Code: STLV Case No.: INMA

Matrix: (SOIL/SED/WATER)

Sample wt/vol: 25.0

Water

$(\mathrm{g} / \mathrm{mL}) \quad \mathrm{mL}$

Level: (TRACE/LOW/MED) TRACE

o Moisture: not dec.

GC Column: DB-624 ID: 0.20 (nm)

Soil Extract Volume: (uL) (mL)

Purge Volume: 25.0
Contract: 8E-00302

SDG No.: 200-5726 Mod. Ref No.:

Lab Sample ID: 200-5726-5

Lab File ID: DHOI13.D

Date Received:

Date Analyzed: 06/28/2011

Dilution Factor: 1.0

Soil Aliquot Volume:

(UI)

\begin{tabular}{|c|c|c|c|}
\hline CAS NO. & COMPOUND & $\begin{array}{l}\text { CONCENTRATION UNITS: } \\
(\mathrm{ug} / \mathrm{L} \text { or } \mathrm{ug} / \mathrm{kg}) \mathrm{ug} / \mathrm{L}\end{array}$ & $Q$ \\
\hline $75-71-8$ & Dichlorodifluoromethane & 0.50 & $\mathrm{U}$ \\
\hline $74-87-3$ & Chloromethane & 0.50 & $\mathrm{U}$ \\
\hline $75-01-4$ & Vinyl chloride & 0.50 & $\mathrm{U}$ \\
\hline $74-83-9$ & Bromomethane & 0.50 & $\mathrm{U}$ \\
\hline $75-00-3$ & Chloroethane & 0.50 & $\mathrm{U}$ \\
\hline $75-69-4$ & Trichlorofluoromethane & 0.50 & $\mathrm{U}$ \\
\hline $75-35-4$ & 1,1-Dichloroethene & 0.50 & $\mathrm{U}$ \\
\hline $76-13-1$ & 1,1,2-Trichloro-1,2,2-trifluoroethane & 0.50 & U \\
\hline $67-64-1$ & Acetone & 5.0 & $\mathrm{U}$ \\
\hline $75-15-0$ & Carbon disulfide & 0.50 & $\mathrm{U}$ \\
\hline $79-20-9$ & Methyl acetate & 0.50 & U \\
\hline $75-09-2$ & Methylene Chloride & 0.50 & $\mathrm{U}$ \\
\hline $156-60-5$ & trans-1,2-Dichloroethene & 0.50 & $\mathrm{U}$ \\
\hline $1634-04-4$ & Methyl tert-butyl ether & 0.50 & $\mathrm{U}$ \\
\hline $75-34-3$ & 1,1-Dichloroethane & 0.50 & $\mathrm{U}$ \\
\hline $156-59-2$ & cis-1,2-Dichloroethene & 0.50 & $\mathrm{U}$ \\
\hline $78-93-3$ & 2-Butanone & 5.0 & $\mathrm{U}$ \\
\hline $74-97-5$ & Bromochloromethane & 0.50 & $\mathrm{U}$ \\
\hline $67-66-3$ & Chloroform & 0.50 & $\mathrm{U}$ \\
\hline $71-55-6$ & 1,1,1-Trichloroethane & 0.50 & $\mathrm{U}$ \\
\hline $110-82-7$ & Cyclohexane & 0.50 & $\mathrm{U}$ \\
\hline $56-23-5$ & Carbon tetrachloride & 0.50 & $\mathrm{U}$ \\
\hline $71-43-2$ & Benzene & 0.50 & $\mathrm{U}$ \\
\hline $107-06-2$ & 1,2-Dichloroethane & 0.50 & $\mathrm{U}$ \\
\hline
\end{tabular}

Report 1,4-Dioxane for Low-Medium VOA analysis only 
$1 B$ - FORM I VOA-2

VOLATILE ORGANICS ANALYSIS DATA SHEET
EPA SAMPLE NO.

VHBLKO1
Lab Name: TESTAMERICA BURLINGTON

Lab Code: STLV

Case No.: INMAN

Matrix: (SOIL/SED/WATER)

Water

Sample wt/vol: 25.0

$(\mathrm{g} / \mathrm{mL}) \mathrm{mL}$

Level: (TRACE/LOW/MED) TRACE

\% Moisture: not dec.

GC Column: DB-624

ID $: 0.20$

(mm)

Soil Extract Volume:

(uL)

(mL)

Purge Volume: 25.0

Contract: $8 \mathrm{E}-00302$

Mod. Ref No.:

SDG No.: 200-5726

Lab Sample ID: 200-5726-5

Lab File ID: DHOI13.D

Date Received:

Date Analyzed: 06/28/2011

Dilution Factor: 1.0

Soil Aliquot Volume:

(uL)

\begin{tabular}{|c|c|c|c|}
\hline CAS NO. & COMPOUND & $\begin{array}{l}\text { CONCENTRATION UNITS: } \\
(\mathrm{ug} / \mathrm{L} \text { or } u g / \mathrm{kg}) \mathrm{ug} / \mathrm{L}\end{array}$ & $Q$ \\
\hline $79-01-6$ & Trichloroethene & 0.50 & $\mathrm{U}$ \\
\hline $108-87-2$ & Methylcyclohexane & 0.50 & $\mathrm{U}$ \\
\hline $78-87-5$ & 1,2-Dichloropropane & 0.50 & $\mathrm{U}$ \\
\hline $75-27-4$ & Bromodichloromethane & 0.50 & $\mathrm{U}$ \\
\hline $10061-01-5$ & cis-1,3-Dichloropropene & 0.50 & $\mathrm{U}$ \\
\hline $108-10-1$ & 4-Methyl-2-pentanone & 5.0 & $\mathrm{U}$ \\
\hline $108-88-3$ & Toluene & 0.50 & $\mathrm{U}$ \\
\hline $10061-02-6$ & trans-1,3-Dichloropropene & 0.50 & $\mathrm{U}$ \\
\hline $79-00-5$ & 1,1,2-Trichloroethane & 0.50 & $\mathrm{U}$ \\
\hline $127-18-4$ & Tetrachloroethene & 0.50 & $\mathrm{U}$ \\
\hline $591-78-6$ & 2-Hexanone & 5.0 & $\mathrm{U}$ \\
\hline $124-48-1$ & Dibromochloromethane & 0.50 & $\mathrm{U}$ \\
\hline $106-93-4$ & 1,2-Dibromoethane & 0.50 & U \\
\hline $108-90-7$ & Chlorobenzene & 0.50 & $\mathrm{U}$ \\
\hline $100-41-4$ & Ethylbenzene & 0.50 & $\mathrm{U}$ \\
\hline $95-47-6$ & o-Xylene & 0.50 & $\mathrm{U}$ \\
\hline $179601-23-1$ & $\mathrm{~m}, \mathrm{p}$-Xylene & 0.50 & $\mathrm{U}$ \\
\hline $100-42-5$ & Styrene & 0.50 & U \\
\hline $75-25-2$ & Bromoform & 0.50 & U \\
\hline $98-82-8$ & Isopropylbenzene & 0.50 & $\mathrm{U}$ \\
\hline $79-34-5$ & $1,1,2,2$-Tetrachloroethane & 0.50 & U \\
\hline $541-73-1$ & 1,3-Dichlorobenzene & 0.50 & U \\
\hline $106-46-7$ & 1,4-Dichlorobenzene & 0.50 & $\mathrm{U}$ \\
\hline $95-50-1$ & 1,2-Dichlorobenzene & 0.50 & $\mathrm{U}$ \\
\hline $96-12-8$ & 1,2-Dibromo-3-Chloropropane & 0.50 & $\mathrm{U}$ \\
\hline $120-82-1$ & 1,2,4-Trichlorobenzene & 0.50 & $\mathrm{U}$ \\
\hline $87-61-6$ & 1,2,3-Trichlorobenzene & 0.50 & $\mathrm{U}$ \\
\hline
\end{tabular}


$1 \mathrm{~J}$ - FORM I VOA-TIC

VOLATILE ORGANICS ANALYSIS DATA SHEET TENTATIVELY IDENTIFIED COMPOUNDS
EPA SAMPLE NO.

VHBLKO1

Lab Name: TESTAMERICA BURLINGTON

Contract: $8 \mathrm{E}-00302$

Lab Code: STLV Case No.: INMAN Mod. Ref No.: SDG No.: 200-5726

Matrix: (SOIL/SED/WATER)

Water

Sample wt/vol: 25.0 $(\mathrm{g} / \mathrm{mL}) \quad \mathrm{mL}$

Level: (TRACE or LOW/MED) TRACE

\% Moisture: not dec.

GC Column: DB-624

Soil Extract Volume: ID : 0.20 (mm)

CONCENTRATION UNITS: (ug/L or $\mathrm{ug} / \mathrm{kg}$ ) (uI)
Lab Sample ID: 200-5726-5

Lab File ID: DHOI13.D

Date Received:

Date Analyzed: 06/28/2011

Dilution Factor: 1.0

Soil Aliquot Volume: (uL)

Purge Volume: 25.0 (mL)

01

\begin{tabular}{|l|l|c|c|c|}
\hline CAS NUMBER & \multicolumn{1}{|c|}{ COMPOUND NAME } & RT & EST. CONC. & $Q$ \\
\hline & Unknown & 6.79 & 2.7 & $\mathrm{~B} \times \mathrm{J}$ \\
\hline E9667961 & Total Alkanes & N/A & & \\
\hline
\end{tabular}

IEPA-designated Registry Number. 


\section{TestAmerica}

THE LEADER IN ENVIRONMENTAL TESTING

\section{ANALYTICAL REPORT}

Job Number: 200-6709-1

SDG Number: 200-6709

Job Description: Inman (200-6709)

Contract Number: EP-W-09-044

For:

Argonne National Laboratory

9700 South Cass Avenue

Building 203

Office B-149

Argonne, IL 60439

Attention: Mr. Clyde Dennis

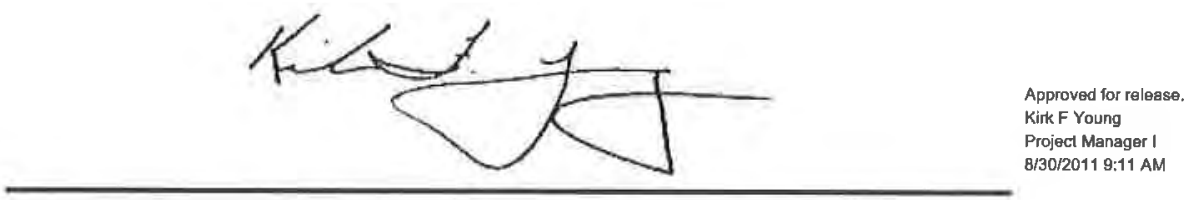

Kirk F Young

Project Manager I

kirk.young@testamericainc.com

08/30/2011

The test results in this report relate only to sample(s) as received by the laboratory. These test results were derived under a quality system that adheres to the requirements of NELAC. Pursuant to NELAC, this report may not be produced in full without written approval from the laboratory 


\section{Table of Contents}

Cover Title Page ......................... 1

Report Narrative . . . . . . . . . . . . . . . . . . 4

Case Narrative ............................... 4

Qualifier Definition ............................... 6

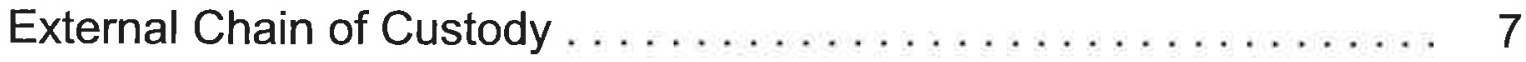

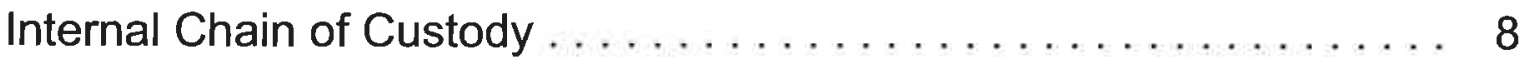

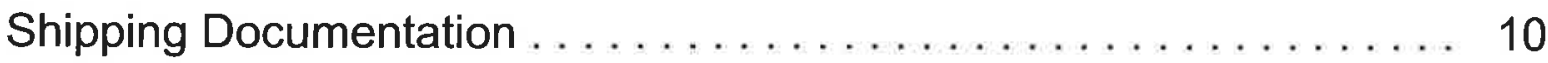

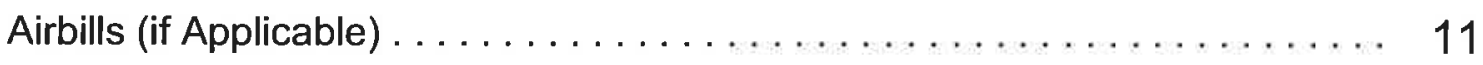

Sample Receipt and Log In Check List ................... 12

Methodology Review . . . . . . . . . . . . . . . . . . . 14

QC Summary - SOM01.2 Volatiles-Trace . . . . . . . . . . . . 15

QC Summary - SOM01.2 Volatiles-Trace . . . . . . . . . . . . . . 15

Deuterated Monitoring Compound Summary $\ldots \ldots \ldots \ldots \ldots \ldots \ldots \ldots \ldots \ldots, 15$

Method Blank . . . . . . . . . . . . . . . . 17

GC/MS Instrument Performance Check ...................... 18

Internal Standard Area and RT Summary ......................... 20

Sample Data - SOM01.2 Volatiles-Trace . . . . . . . . . . . . . . 21

Sample Data - SOM01.2 Volatiles-Trace . . . . . . . . . . . . . . 21

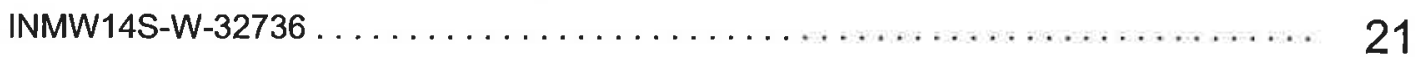

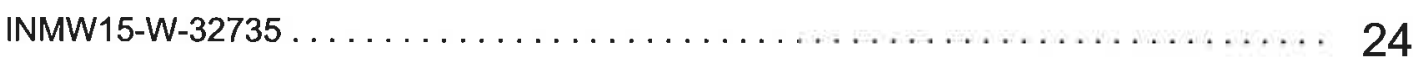

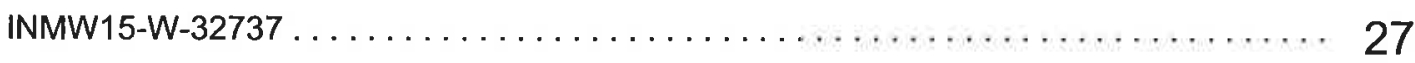

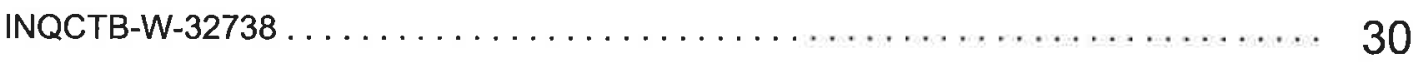

Standards - SOM01.2 Volatiles-Trace . . . . . . . . . . . . . 33

Standards - SOM01.2 Volatiles-Trace . . . . . . . . . . . . . . . 33

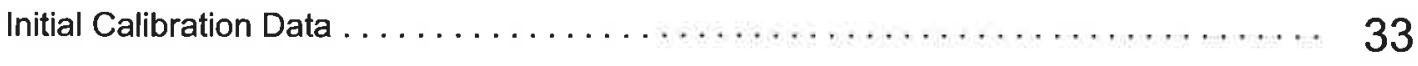

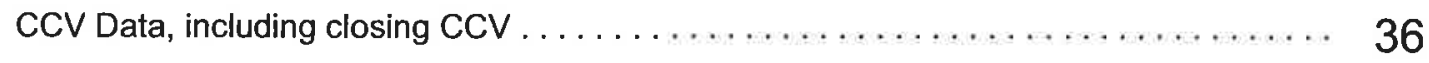




\section{Table of Contents}

Raw Qc Data - SOM01.2 Volatiles-Trace . . . . . . . . . . . . . . . 42

Raw Qc Data - SOM01.2 Volatiles-Trace . . . . . . . . . . . . . . 42

Raw Qc Data - SOM01.2 Volatiles-Trace . . . . . . . . . . . . . . . . 42

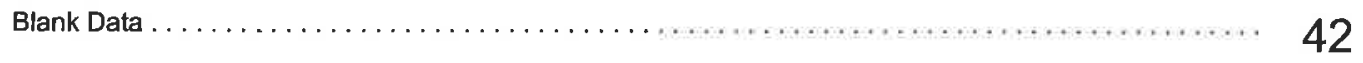




\section{CASE NARRATIVE}

\section{Client: Argonne National Laboratory}

Project: Inman (200-6709)

Report Number: 200-6709-1

Enclosed is the data set for the referenced project work. With the exceptions noted as flags or footnotes, standard analytical protocols were followed in performing the analytical work and the applied control limits were met.

Calculations were performed before rounding to avoid round-off errors in calculated results.

All holding times were met and proper preservation noted for the methods performed on these samples, unless otherwise detailed in the individual sections below.

\section{Receipt}

The samples were received on $08 / 25 / 2011$. Documentation of the condition of the samples at the time of their receipt and any exception to the laboratory's Sample Acceptance Policy is documented in the Shipping and Receiving section of this submittal. The samples, as received, were not acid preserved. On that basis, the laboratory did provide for the analytical work to be performed within seven days of sample collection.

Although listed on the chain-of-custody record, sample volumes were not received for samples INMW14-W-32730 and INMW14-W-32734. Additionally, for each of the other samples, the laboratory received four $20 \mathrm{~mL}$ vials and not the two $40 \mathrm{~mL}$ vials referenced on the chain-ofcustody record. The exception to this was sample INQCTB-W-32738, for which two $20 \mathrm{~mL}$ vials were received.

The container labels for sample INMW15-W-32735 did not match the information listed on the chain-of-custody record. The container labels referenced a sample date of 08/24/11. The chainof-custody record referenced a sample date of $8 / 22 / 11$. A sample date of $08 / 24 / 11$ was used for log-in.

\section{SOM01.2 Volatile Organics (Trace Level Water)}

A storage blank was prepared for volatile organics analysis, and stored in association with the storage of the samples. That storage blank, identified as VHBLK01, was carried through the holding period with the samples, and analyzed.

Each sample in the sample set was analyzed without a dilution. Each of the analyses associated with the sample set exhibited an acceptable internal standard performance. There was an acceptable recovery of each deuterated monitoring compound (DMC) in the analysis of the method blank associated with the analytical work, and in the analysis of the storage blank associated with the sample set. The analysis of the samples in this sample set did meet the technical acceptance criteria specific to DMC recoveries, although not all DMC recoveries were within the control range in each analysis. The technical acceptance criteria does provide for the recovery of up to three DMCs to fall outside of the control range in the analysis of field samples. Matrix spike and matrix spike duplicate analyses were not performed on samples in this sample set. A trace concentration of toluene was identified in the analysis of the method blank 
associated with the analytical work. The concentration of toluene in that analysis was below the established reporting limit, and the analysis did meet the technical acceptance criteria for a compliant method blank analysis. The analysis of the storage blank associated with the sample set was free of analyte contamination. Present in the method blank and storage blank analyses was a non-target constituent that represents a compound that is related to the DMC formulation. The fact that the presence of this compound is not within the laboratory's control is at issue. The derived results for that compound have been qualified with an " $X$ " qualifier to reflect the source of the contamination.

The responses for each of the target analytes met the relative standard deviation criterion in the initial calibration. The response for each target analyte met the percent difference criterion in the opening/continuing calibration check acquisition. The response for each target analyte met the 50.0 percent difference criterion in the closing calibration check acquisition.

The primary quantitation mass for methylcyclohexane that is specified in the Statement of Work is mass 83 . The laboratory did identify a contribution to mass 83 from 1,2-dichloropropane- $\mathrm{d}_{6}$, one of the deuterated monitoring compounds (DMCs). The laboratory did change the primary quantitation mass assignment to mass 55 for the quantification of methylcyclohexane.

Manual integration was employed in deriving certain of the analytical results. The values that have been derived from manual integration are qualified on the quantitation reports. Extracted ion current profiles for each manual integration are included in the data package, and further documented at the end of this submittal. 
DATA REPORTING QUALIFIERS

Client: Argonne National Laboratory

Job Number: 200-6709-1

Sdg Number: 200-6709

Lab Section

Qualifier

Description

GC/MS VOA

U

J

J

$\mathrm{X}$

*

B
Analyzed for but not detected.

Indicates an Estimated Value for TICs

Indicates an estimated value.

See case narrative notes for explanation of the ' $X$ ' flag

Surrogate exceeds the control limit

The analyte was found in an associated blank, as well as in the sample. 


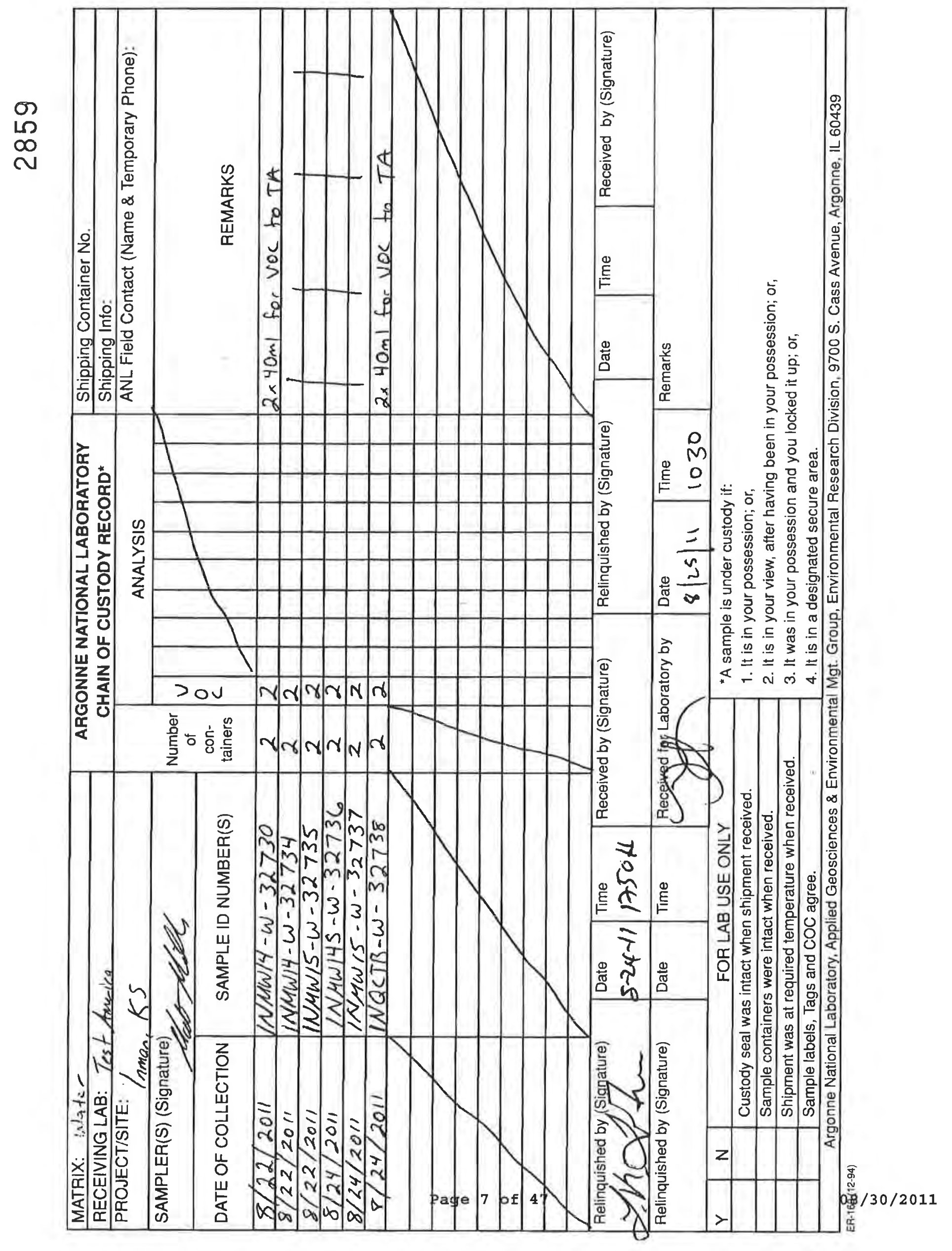




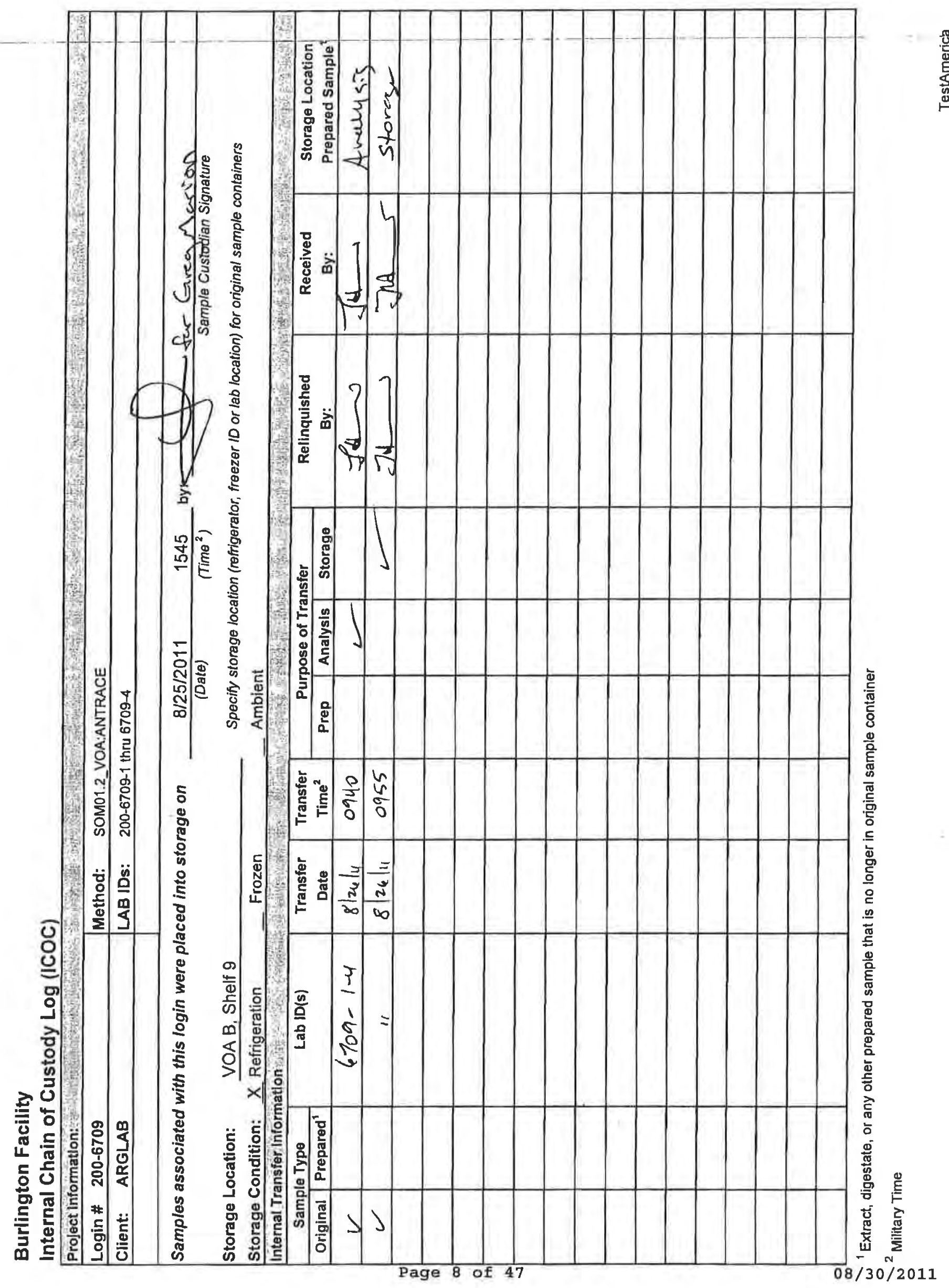




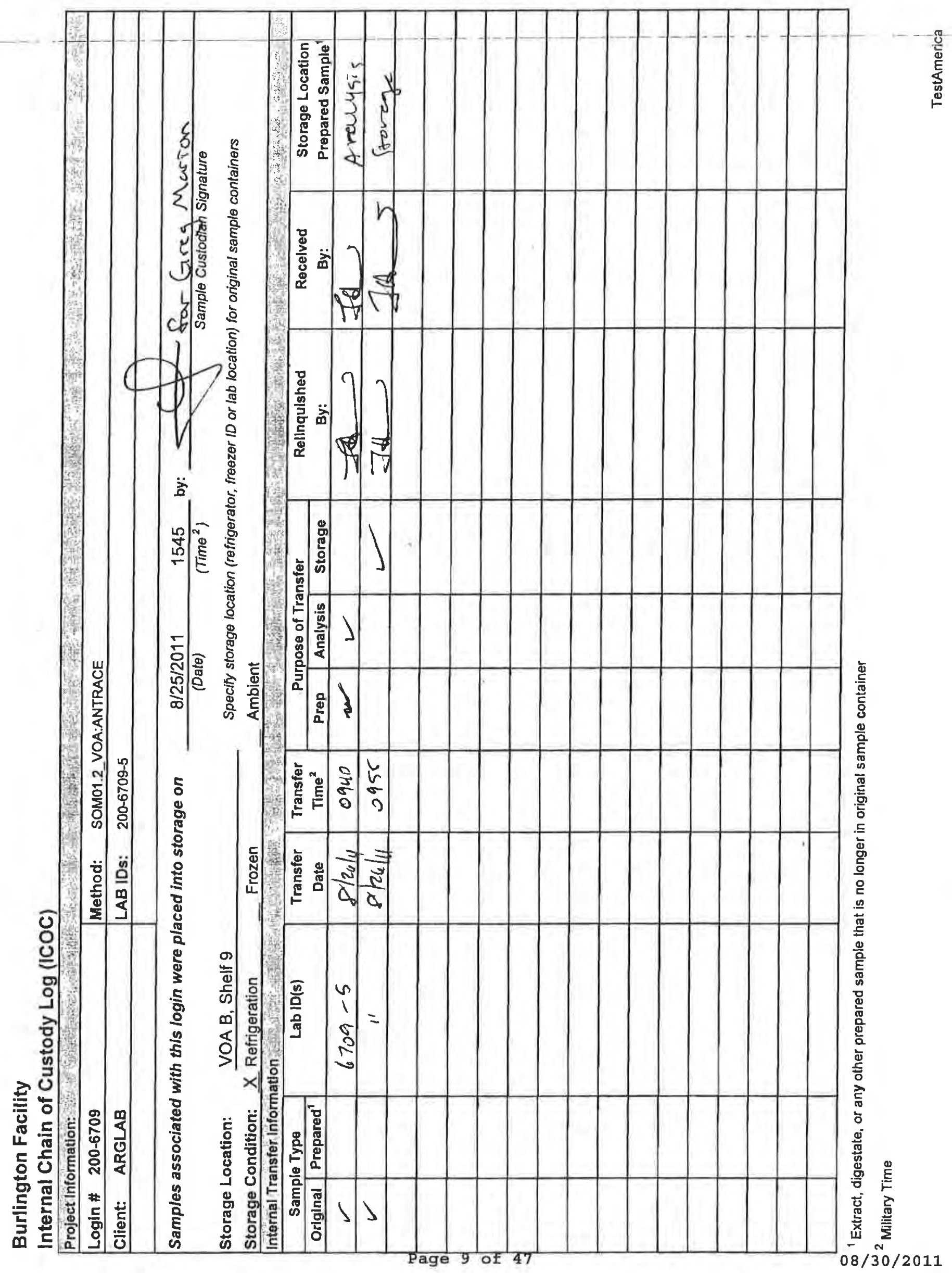




\section{Shipping and Receiving Documents}




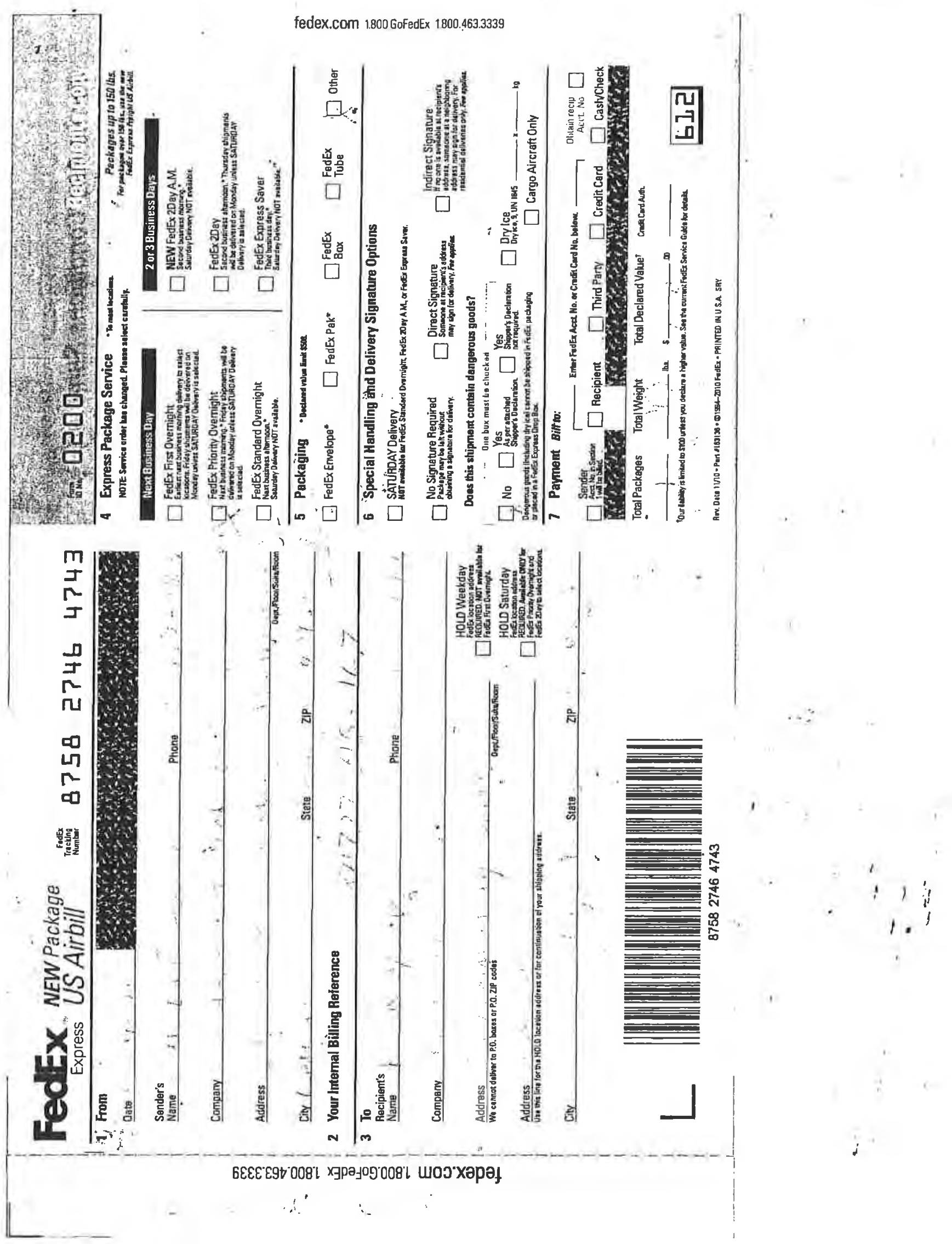




\section{Login Sample Receipt Checklist}

Login Number: 6709

List Number: 1

Creator: Marion, Greg T

Raestion background

The cooler's custody seal, if present, is intact.

The cooler or samples do not appear to have been compromised or tampered with.

Samples were received on ice.

Cooler Temperature is acceptable.

Cooler Temperature is recorded.

COC is present.

COC is filled out in ink and legible.

$\mathrm{COC}$ is filled out with all pertinent information.

Is the Field Sampler's name present on COC?

There are no discrepancies between the sample IDs on the containers and the COC.

Samples are received within Holding Time.

Sample containers have legible labels.

Containers are not broken or leaking.

Sample collection date/times are provided.

Appropriate sample containers are used.

Sample bottles are completely filled.

Sample Preservation Verified.

There is sufficient vol. for all requested analyses, incl. any requested MS/MSDs

VOA sample vials do not have headspace or bubble is $<6 \mathrm{~mm}\left(1 / 4^{\prime \prime}\right)$ in diameter.

Multiphasic samples are not present.

Samples do not require splitting or compositing.

Residual Chlorine Checked.
List Source: TestAmerica Burlington
Answer Comment

N/A

Lab does not accept radioactive samples.

True

NO SEAL NUMBERS

True

True

True

True

$5.1^{\circ} \mathrm{C}$, IR GUN ID $96 / \mathrm{CF}=0$

True

True

True

True

True

True

True

True

True

False

True

N/A

True

True

N/A

N/A

N/A 


\section{Sample Login Acknowledgement}

Job 200-6709-1

$\begin{array}{ll}\text { Client Job Description: } & \text { Inman (200-6709) } \\ \text { Purchase Order \#: } & 8 \mathrm{E}-00302 \\ \text { Work Order \#: } & 8 \mathrm{E}-00302 \\ \text { Project Manager: } & \text { Kirk F Young } \\ \text { Job Due Date: } & \text { 9/8/2011 } \\ \text { Job TAT: } & \text { 14 Days } \\ \text { Max Deliverable Level: } & \text { IV } \\ & \\ \text { Earliest Deliverable Due: } & 9 / 8 / 2011\end{array}$

\begin{tabular}{|c|c|}
\hline \multirow[t]{2}{*}{ Report To: } & $\begin{array}{l}\text { Argonne National Laboratory } \\
\text { Jorge Alvarado }\end{array}$ \\
\hline & $\begin{array}{l}9700 \text { South Cass Avenue } \\
\text { Building } 203 \\
\text { Office B-149 } \\
\text { Argonne, IL } 60439\end{array}$ \\
\hline Bill To: & $\begin{array}{l}\text { Argonne National Laboratory } \\
\text { Accounts Payable } \\
\text { Chief Financial Offices } \\
9700 \text { S. Cass Ave. } \\
\text { Building } 201 \\
\text { Argonne, IL } 60439\end{array}$ \\
\hline
\end{tabular}

Login 200-6709

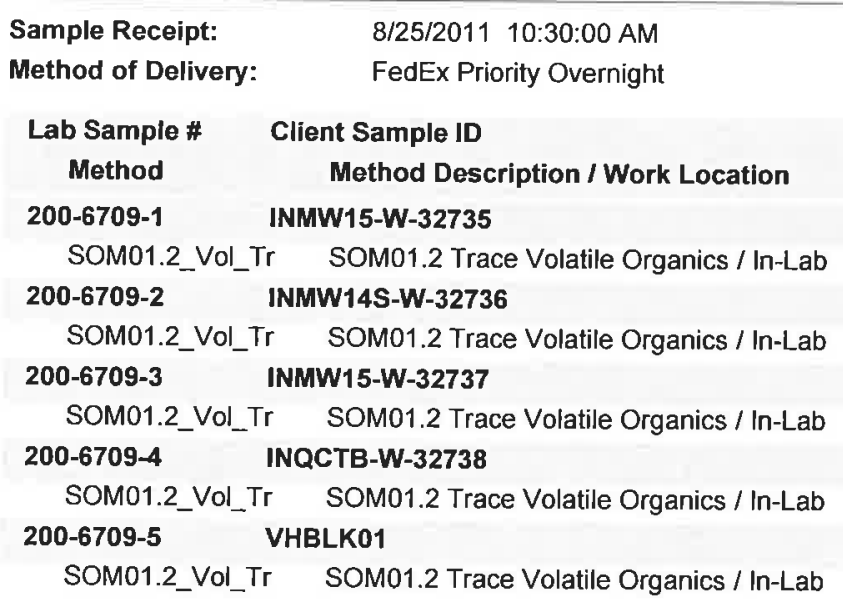

$\begin{array}{ll}\text { Number of Coolers: } & 1 \\ \text { Cooler Temperature(s) }\left(\mathbf{C}^{\circ}\right): & 5.1\end{array}$

Date Sampled

Matrix Rpt Basis Dry / Wet *

8/24/2011 12:00:00 AM Water Total Wet

8/24/2011 12:00:00 AM Water

8/24/2011 12:00:00 AM Water Total Wet

8/24/2011 12:00:00 AM Water Total Wet

8/25/2011 3:30:00 PM Water Total Wet

Total Wet




\section{METHODOLOGY SUMMARY}

\begin{tabular}{lll} 
Laboratory: & TestAmerica Laboratories & Project No: \\
Location: $\quad$ South Burlington, Vermont & SDG No: & 200-6709 \\
\hline VOA & \\
Volatile Organics Trace - USEPA CLP SOM01.2 &
\end{tabular}


Lab Name: TESTAMERICA BURLINGTON

Lab Code: STLV

Ievel: (TRACE Or LOW)
Contract: $8 \mathrm{E}-00302$

SDG No.: 200-6709

Level: (TRACE or LOW) TRACE

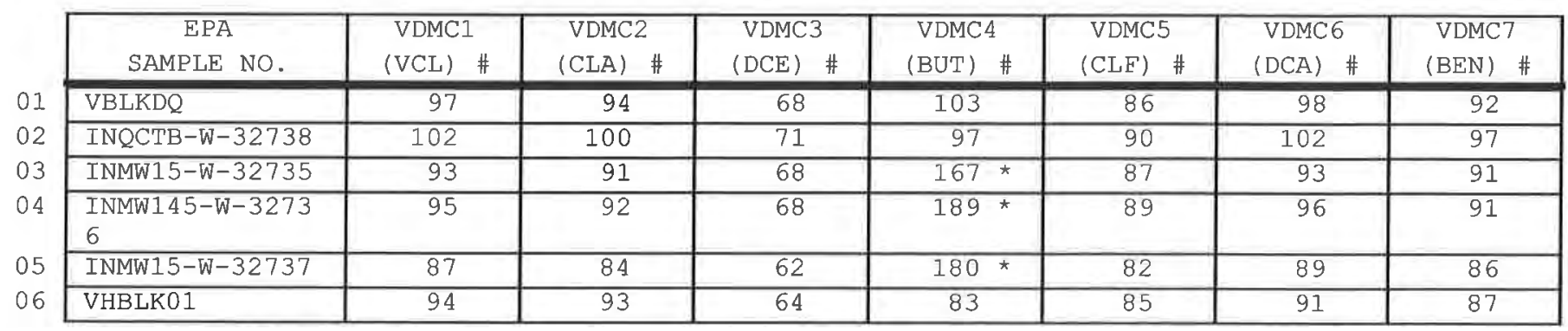

\footnotetext{
VDMC1 (VCL) = Vinyl Chloride-d3

VDMC2 (CLA) = Chloroethane-d5

VDMC3 $(D C E)=1,1-D i c h l o r o e t h e n e-d 2$

VDMC4 (BUT) = 2-Butanone-d5

VDMC5 (CLF) = Chloroform-d

VDMC6 $(\mathrm{DCA})=1,2-\mathrm{Dich}$ loroethane-d4

VDMC7
}

QC LIMITS

(65-131)

$(71-131)$

$(55-104)$

$(49-155)$

$(78-121)$

$(78-129)$

$(77-124)$

\# Column to be used to flag recovery values

* Values outside of contract required QC limits

Page 1 of 1 
Lab Name: TESTAMERICA BURIINGTON

Lab Code: STLV Case No.: INMAN Mod. Ref No.:

Level: (TRACE or LOW) TRACE
Contract: $8 \mathrm{E}-00302$

SDG No.: 200-6709

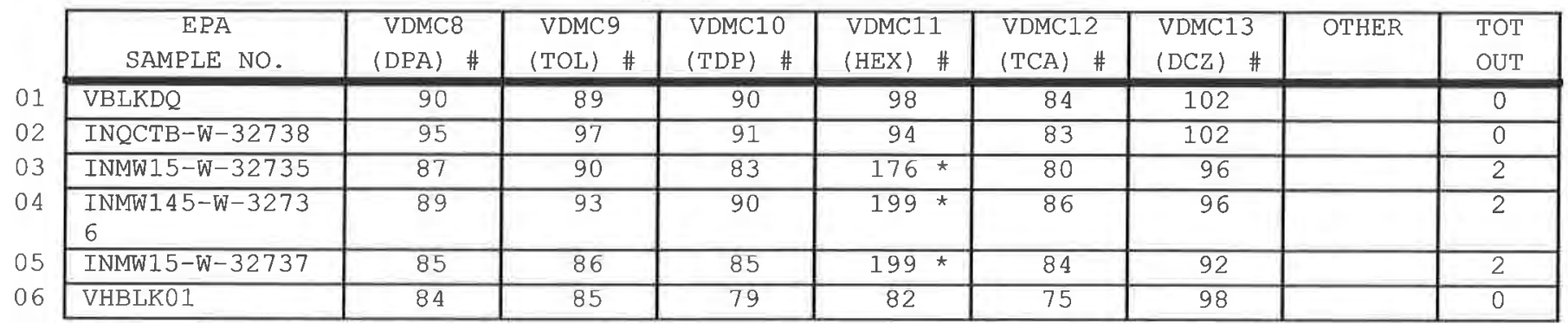

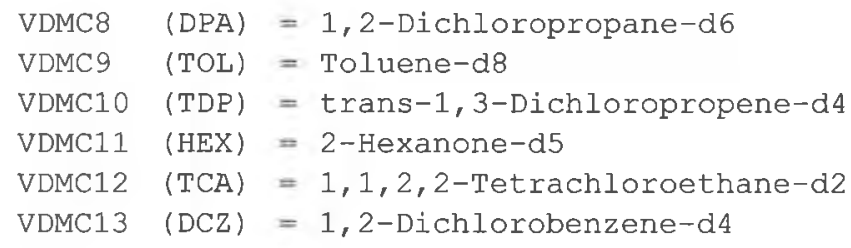

QC LIMITS

(79-124)

$(77-121)$

$(73-121)$

$(28-135)$

$(73-125)$

$(80-131)$

\# Column to be used to flag recovery values

* Values outside of contract required QC limits

Report 1,4-Dioxane-d8 for Low-Medium VOA analysis only

Page 1 of 1 
Lab Name: TESTAMERICA BURLINGTON

Lab Code: STLV

Lab File ID: DHPT03.D Case No.: INMAN Mod. Ref No.:

Contract: $8 \mathrm{E}-00302$

Instrument ID: D.i

Matrix: (SOIL/SED/WATER)

Level: (TRACE or LOW/MED)

GC Column: DB-624 water

TRACE

ID : 0.20 (mm)
SDG No.: 200-6709

Lab Sample ID: MB 200-24273/3

Date Analyzed: 08/26/2011

Time Analyzed: 0942

Heated Purge: ( $\mathrm{Y} / \mathrm{N}) \mathrm{N}$

\begin{tabular}{c|l|l|l|c|}
\cline { 2 - 5 } \multicolumn{1}{c|}{$\begin{array}{c}\text { EPA } \\
\text { SAMPLE NO. }\end{array}$} & \multicolumn{1}{|c|}{ LAB } & \multicolumn{1}{c|}{ LAB } & TIME \\
SAMPLE ID & \multicolumn{1}{|c|}{ SALE ID } & ANALYZED \\
\cline { 2 - 5 } 02 & $\begin{array}{l}\text { INQCTB-W-327 } \\
38\end{array}$ & $200-6709-4$ & DHPT04.D & 1018 \\
\cline { 2 - 5 } & $\begin{array}{l}\text { INMW15-W-327 } \\
35\end{array}$ & $200-6709-1$ & DHPT05.D & 1042 \\
\cline { 1 - 5 } 03 & $\begin{array}{l}\text { INMW145-W-32 } \\
736\end{array}$ & $200-6709-2$ & DHPT06.D & 1107 \\
\cline { 2 - 5 } 04 & $\begin{array}{l}\text { INMW15-W-327 } \\
37\end{array}$ & $200-6709-3$ & DHPT07.D & 1131 \\
\cline { 2 - 5 } 05 & VHBLK01 & $200-6709-5$ & DHPT08.D & 1209 \\
\hline
\end{tabular}

COMMENTS : 
$5 A$ - FORM V VOA

VOLATILE ORGANICS INSTRUMENT

PERFORMANCE CHECK

BROMOFLUOROBENZENE (BFB)
EPA SAMPLE NO.

BFBDV
Lab Name: TESTAMERICA BURLINGTON

Lab Code: STLV

Lab File Id: DHP01.D

Instrument Id: D.i

GC Column: DB-624
Case No.: INMAN Mod. Ref No.:

Contract: $8 \mathrm{E}-00302$

\begin{tabular}{|c|c|c|c|}
\hline $\mathrm{m} / \mathrm{e}$ & ION ABUNDANCE CRITERIA & $\begin{array}{l}\text { RELATIVE } \\
\text { ABUNDANCE }\end{array}$ & \\
\hline$\overline{50}$ & $15.0-40.0 \%$ of mass 95 & 26.1 & \\
\hline 75 & $30.0-80.0 \%$ of mass 95 & 46.4 & \\
\hline 95 & Base peak, $100 \%$ relative abundance & 100 & \\
\hline 96 & $5.0-9.0 \%$ of mass 95 & 6.3 & \\
\hline 173 & Less than $2.0 \%$ of mass 174 & 0 & 0) 1 \\
\hline 174 & $50.0-120 \%$ of mass 95 & 77.7 & \\
\hline 175 & $5.0-9.0 \%$ of mass 174 & 6.01 & $7.8) 1$ \\
\hline 176 & $95.0-101 \%$ of mass 174 & 78.21 & 101)1 \\
\hline 177 & $5.0-9.0 \%$ of mass 176 & 5.21 & $6 . 6 \longdiv { 2 }$ \\
\hline
\end{tabular}

1 - Value is omass $174 \quad 2$ - Value is omass 176

\begin{tabular}{|c|c|c|l|c|c|}
\hline \multicolumn{1}{c|}{\begin{tabular}{c} 
EPA \\
\cline { 2 - 6 }
\end{tabular}} & \multicolumn{1}{|c|}{ LAB } & \multicolumn{1}{c|}{ LAB } & DATE & TIME \\
SAMPLE NO. & \multicolumn{1}{c|}{ SAMPLE ID } & FILE ID & ANALYZED & ANALYZED \\
\cline { 2 - 7 } 02 & VSTD0.5DV & IC 200-21023/4 & DHP04.D & $07 / 07 / 2011$ & 1117 \\
\cline { 2 - 7 } 03 & VSTD001DV & IC 200-21023/5 & DHP05.D & $07 / 07 / 2011$ & 1141 \\
\cline { 2 - 7 } 04 & VSTD005DV & ICIS 200-21023/6 & DHP06.D & $07 / 07 / 2011$ & 1206 \\
\cline { 2 - 7 } 05 & VSTD010DV & IC 200-21023/7 & DHP07.D & $07 / 07 / 2011$ & 1231 \\
\cline { 2 - 7 } & VSTD020DV & IC 200-21023/8 & DHP08.D & $07 / 07 / 2011$ & 1256 \\
\hline
\end{tabular}


$5 A$ - FORM V VOA

VOLATILE ORGANICS INSTRUMENT

PERFORMANCE CHECK

BROMOFLUOROBENZENE (BEB)
EPA SAMPLE NO.

BFBDQ

Lab Name: TESTAMERICA BURLINGTON

Contract: $\quad 8 E-00302$

Lab Code: STLV Case No.: INMAN Mod. Ref No.:

SDG No.: 200-6709

Lab File Id: DHPT01.D

Instrument Id: D.i

BFB Injection Date: 08/26/2011

GC Column: DB-624

ID $: 0.20$

BFB Injection Time: 0859 (ImI)

\begin{tabular}{|c|c|c|}
\hline $\mathrm{m} / \mathrm{e}$ & ION ABUNDANCE CRITERIA & $\begin{array}{l}\text { RELATIVE } \\
\text { ABUNDANCE }\end{array}$ \\
\hline 50 & $15.0-40.0 \%$ of mass 95 & 26.5 \\
\hline 75 & $30.0-80.0 \%$ of mass 95 & 48.3 \\
\hline 95 & Base peak, $100 \%$ relative abundance & 100 \\
\hline 96 & $5.0-9.0 \div$ of mass 95 & 7.1 \\
\hline 173 & Less than $2.0 \%$ of mass 174 & 01 \\
\hline 174 & $50.0-120 \%$ of mass 95 & 74.6 \\
\hline 175 & $5.0-9.0 \%$ of mass 174 & 5.21 \\
\hline 176 & $95.0-101 \%$ of mass 174 & $72.4(97.1) 1$ \\
\hline 177 & $5.0-9.0 \%$ of mass 176 & $4.7(6.5) 2$ \\
\hline
\end{tabular}

1 - Value is omass $174 \quad 2$ - Value is omass 176

\begin{tabular}{|c|c|c|c|c|}
\hline $\begin{array}{c}\text { EPA } \\
\text { SAMPLE NO. }\end{array}$ & $\begin{array}{r}\text { LAB } \\
\text { SAMPLE ID } \\
\end{array}$ & \begin{tabular}{c}
\multicolumn{2}{c}{ LAB } \\
FILE ID
\end{tabular} & $\begin{array}{c}\text { DATE } \\
\text { ANALYZED }\end{array}$ & $\begin{array}{c}\text { TIME } \\
\text { ANALYZED }\end{array}$ \\
\hline VSTD005DQ & CCVIS $200-24273 / 2$ & $\overline{\mathrm{DHPTO2} . \mathrm{D}}$ & $08 / 26 / 2011$ & 0918 \\
\hline VBLKDQ & MB $200-24273 / 3$ & DHPT03.D & $08 / 26 / 2011$ & 0942 \\
\hline $\begin{array}{l}\text { INQCTB-W-3 } \\
2738\end{array}$ & $200-6709-4$ & DHPT04.D & $08 / 26 / 2011$ & 1018 \\
\hline $\begin{array}{l}\text { INMW15-W-3 } \\
2735\end{array}$ & $200-6709-1$ & DHPT05.D & $08 / 26 / 2011$ & 1042 \\
\hline $\begin{array}{l}\text { INMW145-W- } \\
32736\end{array}$ & $200-6709-2$ & DHPTO6.D & $08 / 26 / 2011$ & 1107 \\
\hline $\begin{array}{l}\text { INMW15-W-3 } \\
2737\end{array}$ & $200-6709-3$ & DHPT07.D & $08 / 26 / 2011$ & 1131 \\
\hline VHBLKO1 & $200-6709-5$ & DHPT08.D & $08 / 26 / 2011$ & 1209 \\
\hline VSTD005QD & CCVC $200-24273 / 9$ & DHPT09.D & $08 / 26 / 2011$ & 1244 \\
\hline
\end{tabular}


Lab Name: TESTAMERICA BURLINGTON

Contract: $8 \mathrm{E}-00302$

Lab Code: STLV Case No.: INMAN Mod. Ref No.: SDG No.: 200-6709

GC Column: DB-624 ID: 0.20 (mm) Init.

EPA Sample No. (VSTD\#\#\#\#\#): VSTD005DQ

Date Analyzed: 08/26/2011

Lab File ID (Standard): DHPT02.D

Time Analyzed: 0918

Instrument ID: D.i Heated Purge: ( $\mathrm{Y} / \mathrm{N}) \quad \mathrm{N}$

\begin{tabular}{|c|c|c|c|c|c|c|}
\hline & $\begin{array}{c}\text { IS1 (CBZ) } \\
\text { AREA }\end{array}$ & RT \# & $\begin{array}{c}\text { IS2 (DFB) } \\
\text { AREA }\end{array}$ & RT \# & $\begin{array}{c}\text { IS3 (DCB) } \\
\text { AREA }\end{array}$ & RT \\
\hline 12 HOUR STD & 144743 & 8.83 & 166850 & 5.47 & 69207 & 11.66 \\
\hline UPPER LIMIT & 202640 & 9.16 & 233590 & 5.80 & 96890 & 11.99 \\
\hline LOWER LIMIT & 86846 & 8.50 & 100110 & 5.14 & 41524 & 11.33 \\
\hline EPA SAMPLE NO. & & & & & & \\
\hline VBIJKDQ & 136944 & 8.83 & 158458 & 5.47 & 52791 & 11.66 \\
\hline INQCTB-W-32738 & 123188 & 8.83 & 147329 & 5.47 & 48348 & 11.66 \\
\hline INMW15-W-32735 & 132690 & 8.83 & 155334 & 5.47 & 57794 & 11.66 \\
\hline $\begin{array}{l}\text { INMW145-W-3273 } \\
6\end{array}$ & 134694 & 8.83 & 157571 & 5.47 & 58331 & 11.66 \\
\hline INMW15-W-32737 & 149779 & 8.83 & 178295 & 5.47 & 68723 & 11.66 \\
\hline VHBLK01 & 130857 & 8.83 & 152658 & 5.47 & 47421 & 11.66 \\
\hline
\end{tabular}

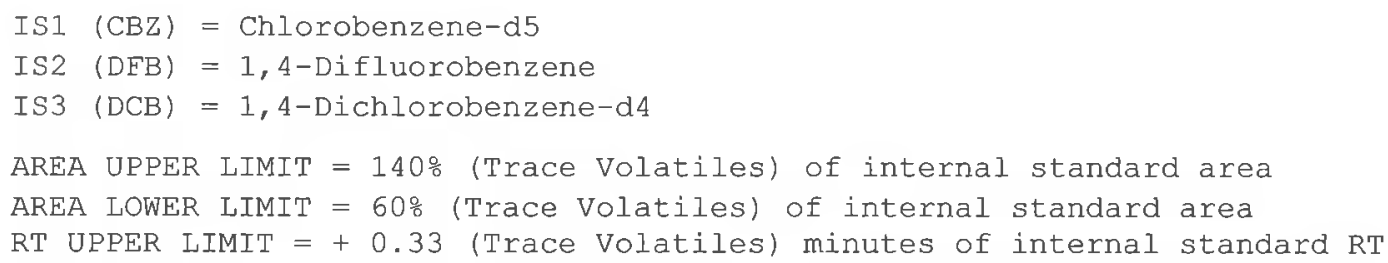


Lab Name: TESTAMERICA BURLINGTON

Lab Code: STLV Case No.: INMAN Mod. Ref No.:
Matrix: (SOIL/SED/WATER)

Sample wt/vol: 25.0

Water

$(\mathrm{g} / \mathrm{mL}) \quad \mathrm{mL}$

Level: (TRACE/LOW/MED) TRACE

\% Moisture: not dec.

GC Column: DB-624

Soil Extract Volume:

Purge Volume: 25.0
Contract: $8 \mathrm{E}-00302$
SDG No.: 200-6709

\begin{tabular}{|c|c|c|c|}
\hline CAS NO. & COMPOUND & $\begin{array}{l}\text { CONCENTRATION UNITS: } \\
(\mathrm{ug} / \mathrm{L} \text { or } \mathrm{ug} / \mathrm{kg}) \mathrm{ug} / \mathrm{L}\end{array}$ & 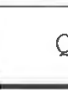 \\
\hline $75-71-8$ & Dichlorodifluoromethane & 0.50 & $\overline{\mathrm{U}}$ \\
\hline $74-87-3$ & Chloromethane & 0.50 & $\mathrm{U}$ \\
\hline $75-01-4$ & Vinyl chloride & 0.50 & $\mathrm{U}$ \\
\hline $74-83-9$ & Bromomethane & 0.50 & $\mathrm{U}$ \\
\hline $75-00-3$ & Chloroethane & 0.50 & $\mathrm{U}$ \\
\hline $75-69-4$ & Trichlorofluoromethane & 0.50 & $\mathrm{U}$ \\
\hline $75-35-4$ & 1,1-Dichloroethene & 0.50 & $\mathrm{U}$ \\
\hline $76-13-1$ & 1,1,2-Trichloro-1,2,2-trifluoroethane & 0.50 & $\mathrm{U}$ \\
\hline $67-64-1$ & Acetone & 1.9 & $\mathrm{~J}$ \\
\hline $75-15-0$ & Carbon disulfide & 0.50 & $\mathrm{U}$ \\
\hline $79-20-9$ & Methyl acetate & 0.50 & $\mathrm{U}$ \\
\hline $75-09-2$ & Methylene Chloride & 0.050 & $\mathrm{~J}$ \\
\hline $156-60-5$ & trans-1,2-Dichloroethene & 0.50 & $\mathrm{U}$ \\
\hline $1634-04-4$ & Methyl tert-butyl ether & 0.50 & $\mathrm{U}$ \\
\hline $75-34-3$ & 1,1-Dichloroethane & 0.50 & $\mathrm{U}$ \\
\hline $156-59-2$ & cis-1,2-Dichloroethene & 0.50 & $\mathrm{U}$ \\
\hline $78-93-3$ & 2-Butanone & 5.0 & $\mathrm{U}$ \\
\hline $74-97-5$ & Bromochloromethane & 0.50 & $\mathrm{U}$ \\
\hline $67-66-3$ & Chloroform & 1.7 & \\
\hline $71-55-6$ & 1,1,1-Trichloroethane & 0.50 & $\mathrm{U}$ \\
\hline $110-82-7$ & Cyclohexane & 0.50 & $\mathrm{U}$ \\
\hline $56-23-5$ & Carbon tetrachloride & 1.1 & \\
\hline $71-43-2$ & Benzene & 0.0074 & $\mathrm{~J}$ \\
\hline $107-06-2$ & 1,2-Dichloroethane & 0.50 & $\mathrm{U}$ \\
\hline
\end{tabular}

Lab Sample ID: 200-6709-2

Lab File ID: DHPT06.D

Date Received: 08/25/2011

Date Analyzed: 08/26/2011

Dilution Factor: 1.0

Soil Aliquot Volume: (uL) (mL) (uL)

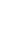


$1 B$ - FORM I VOA-2

VOLATILE ORGANICS ANALYSIS DATA SHEET
EPA SAMPLE NO.

INMW145-W-32736
Lab Name: TESTAMERICA BURLINGTON

Lab Code: STLV

Matrix: (SOIL/SED/WATER)

Sample wt/vol: 25.0

Water

$(g / m L) \quad m L$

Level: (TRACE/LOW/MED) TRACE

응 Moisture: not dec.

GC Column: DB-624

ID: $0.20 \quad(\mathrm{~mm})$

Soil Extract Volume: (uL)

(mI)

Purge Volume: 25.0
Contract: $\quad 8 E-00302$

SDG No.: 200-6709

Lab Sample ID: 200-6709-2

Lab File ID: DHPT06.D

Date Received: 08/25/2011

Date Analyzed: 08/26/2011

Dilution Factor: 1.0

Soil Aliquot Volume:

\begin{tabular}{|c|c|c|c|}
\hline CAS NO. & COMPOUND & $\begin{array}{l}\text { CONCENTRATION UNITS: } \\
(\mathrm{ug} / \mathrm{L} \text { or } \mathrm{ug} / \mathrm{kg}) \mathrm{ug} / \mathrm{L}\end{array}$ & $Q$ \\
\hline $79-01-6$ & Trichloroethene & 0.50 & $\mathrm{U}$ \\
\hline $108-87-2$ & Methylcyclohexane & 0.50 & $\mathrm{U}$ \\
\hline $78-87-5$ & 1,2-Dichloropropane & 0.50 & $\mathrm{U}$ \\
\hline $75-27-4$ & Bromodichloromethane & 1.7 & \\
\hline $10061-01-5$ & cis-1,3-Dichloropropene & 0.50 & $\mathrm{U}$ \\
\hline $108-10-1$ & 4-Methyl-2-pentanone & 5.0 & $\mathrm{U}$ \\
\hline $108-88-3$ & Toluene & 0.15 & $\mathrm{~J} \mathrm{~B}$ \\
\hline $10061-02-6$ & trans $-1,3-$ Dichloropropene & 0.50 & $\mathrm{U}$ \\
\hline $79-00-5$ & 1,1,2-Trichloroethane & 0.50 & $\mathrm{U}$ \\
\hline $127-18-4$ & Tetrachloroethene & 0.077 & $\mathrm{~J}$ \\
\hline $591-78-6$ & 2-Hexanone & 5.0 & $\mathrm{U}$ \\
\hline $124-48-1$ & Dibromochloromethane & 1.9 & \\
\hline $106-93-4$ & 1,2-Dibromoethane & 0.50 & $\mathrm{U}$ \\
\hline $108-90-7$ & Chlorobenzene & 0.50 & $\mathrm{U}$ \\
\hline $100-41-4$ & Ethylbenzene & 0.24 & $\mathrm{~J}$ \\
\hline $95-47-6$ & o-Xylene & 0.30 & $\mathrm{~J}$ \\
\hline $179601-23-1$ & m,p-Xylene & 0.72 & \\
\hline $100-42-5$ & Styrene & 0.50 & $\mathrm{U}$ \\
\hline $75-25-2$ & Bromoform & 0.50 & $\mathrm{U}$ \\
\hline $98-82-8$ & Isopropylbenzene & 0.50 & $\mathrm{U}$ \\
\hline $79-34-5$ & $1,1,2,2$-Tetrachloroethane & 0.50 & $\mathrm{U}$ \\
\hline $541-73-1$ & 1,3-Dichlorobenzene & 0.50 & $\mathrm{U}$ \\
\hline $106-46-7$ & 1,4-Dichlorobenzene & 0.50 & $\mathrm{U}$ \\
\hline $95-50-1$ & 1,2-Dichlorobenzene & 0.50 & $\mathrm{U}$ \\
\hline $96-12-8$ & 1,2-Dibromo-3-Chloropropane & 0.50 & $\mathrm{U}$ \\
\hline $120-82-1$ & 1,2,4-Trichlorobenzene & 0.50 & $\mathrm{U}$ \\
\hline $87-61-6$ & 1,2,3-Trichlorobenzene & 0.50 & $\mathrm{U}$ \\
\hline
\end{tabular}


1J - FORM I VOA-TIC

VOLATILE ORGANICS ANALYSIS DATA SHEET

TENTATIVELY IDENTIFIED COMPOUNDS
EPA SAMPLE NO.

INMW $145-W-32736$
Lab Name: TESTAMERICA BURLINGTON

Lab Code: STLV Case No.: INMAN

Matrix: (SOIL/SED/WATER)

Sample wt/vol: 25.0

Level: (TRACE or LOW/MED)

Water

$(\mathrm{g} / \mathrm{mL}) \mathrm{mL}$

TRACE

\& Moisture: not dec.

GC Column: DB-624

ID $: 0.20$

(mm)

Soil Extract Volume: (UL)

CONCENTRATION UNITS: (ug/L or $\mathrm{ug} / \mathrm{kg}) \mathrm{ug} / \mathrm{L}$
Contract: $8 E-00302$

SDG No.: 200-6709

Lab Sample ID: 200-6709-2

Lab File ID: DHPT06.D

Date Received: 08/25/2011

Date Analyzed: 08/26/2011

Dilution Factor: 1.0

Soil Aliquot Volume:

Purge Volume: 25.0

$(\mathrm{mL})$

01

02

\begin{tabular}{|c|l|c|c|c|}
\hline CAS NUMBER & \multicolumn{1}{|c|}{ COMPOUND NAME } & RT & EST. CONC. & Q \\
\hline & Unknown & 6.79 & 2.7 & B X J \\
\hline E9667961 & Total Alkanes & N/A & & \\
\hline
\end{tabular}

1 EPA-designated Registry Number. 
$1 A$ - FORM I VOA-1

VOLATILE ORGANICS ANALYSIS DATA SHEET
EPA SAMPLE NO.

INMW15-W-32735

Lab Name: TESTAMERICA BURLINGTON

Contract: $8 \mathrm{E}-00302$

Lab Code: STLV Case No.: INMAN Mod. Ref No.:

SDG No.: 200-6709

Matrix: (SOIL/SED/WATER)

Sample wt/vol: 25.0

Water

$(\mathrm{g} / \mathrm{mL}) \quad \mathrm{mL}$

Level: (TRACE/LOW/MED) TRACE

․ Moisture: not dec.

GC Column: DB-624

Soil Extract Volume:

ID: $0.20 \quad$ (mm)

(UL)

(mL)

Purge Volume: 25.0
Lab Sample ID: 200-6709-1

Lab File ID: DHPT05.D

Date Received: 08/25/2011

Date Analyzed: 08/26/2011

Dilution Factor: 1.0

Soil Aliquot Volume:

\begin{tabular}{|c|c|c|c|}
\hline CAS NO. & COMPOUND & $\begin{array}{l}\text { CONCENTRATION UNITS: } \\
(\mathrm{ug} / \mathrm{L} \text { or } \mathrm{ug} / \mathrm{kg}) \mathrm{ug} / \mathrm{L}\end{array}$ & Q \\
\hline $75-71-8$ & Dichlorodifluoromethane & 0.50 & $\mathrm{U}$ \\
\hline $74-87-3$ & Chloromethane & 0.50 & $\mathrm{U}$ \\
\hline $75-01-4$ & Vinyl chloride & 0.50 & $\mathrm{U}$ \\
\hline $74-83-9$ & Bromomethane & 0.50 & $\mathrm{U}$ \\
\hline $75-00-3$ & Chloroethane & 0.50 & $\mathrm{U}$ \\
\hline $75-69-4$ & Trichlorofluoromethane & 0.50 & $\mathrm{U}$ \\
\hline $75-35-4$ & 1,1-Dichloroethene & 0.50 & $\mathrm{U}$ \\
\hline $76-13-1$ & 1,1,2-Trichloro-1,2,2-trifluoroethane & 0.50 & $\mathrm{U}$ \\
\hline $67-64-1$ & Acetone & 2.1 & $\mathrm{~J}$ \\
\hline $75-15-0$ & Carbon disulfide & 0.50 & $\mathrm{U}$ \\
\hline $79-20-9$ & Methyl acetate & 0.50 & $\mathrm{U}$ \\
\hline $75-09-2$ & Methylene Chloride & 0.50 & $\mathrm{U}$ \\
\hline $156-60-5$ & trans $-1,2-$ Dichloroethene & 0.50 & $\mathrm{U}$ \\
\hline $1634-04-4$ & Methyl tert-butyl ether & 0.50 & $\mathrm{U}$ \\
\hline $75-34-3$ & 1,1-Dichloroethane & 0.50 & $\mathrm{U}$ \\
\hline $156-59-2$ & cis-1,2-Dichloroethene & 0.50 & $\mathrm{U}$ \\
\hline $78-93-3$ & 2-Butanone & 5.0 & $\mathrm{U}$ \\
\hline $74-97-5$ & Bromochloromethane & 0.50 & $\mathrm{U}$ \\
\hline $67-66-3$ & Chloroform & 0.031 & $\mathrm{~J}$ \\
\hline $71-55-6$ & 1,1,1-Trichloroethane & 0.50 & $\mathrm{U}$ \\
\hline $110-82-7$ & Cyclohexane & 0.50 & $\mathrm{U}$ \\
\hline $56-23-5$ & Carbon tetrachloride & 0.036 & $\mathrm{~J}$ \\
\hline $71-43-2$ & Benzene & 0.029 & $\mathrm{~J}$ \\
\hline $107-06-2$ & 1,2-Dichloroethane & 0.50 & $\mathrm{U}$ \\
\hline
\end{tabular}

Report 1,4-Dioxane for Low-Medium VOA analysis only 
Lab Name: TESTAMERICA BURLINGTON

Contract: $\quad 8 \mathrm{E}-00302$

Lab Code: STLV Case No.: INMAN Mod. Ref No.: SDG No.: 200-6709

Matrix: (SOIL/SED/WATER)

Sample wt/vol: 25.0

Water

$(\mathrm{g} / \mathrm{mL}) \quad \mathrm{mL}$

Level: (TRACE/LOW/MED) TRACE

\% Moisture: not dec.

GC Column: DB-624

Soil Extract Volume:

ID: $0.20 \quad$ (mm)

(uL)

(mL)

Purge Volume: 25.0
Lab Sample ID: 200-6709-1

Lab File ID: DHPT05.D

Date Received: 08/25/2011

Date Analyzed: 08/26/2011

Dilution Factor: 1.0

Soil Aliquot Volume:

(uL)

\begin{tabular}{|c|c|c|c|}
\hline CAS NO. & COMPOUND & $\begin{array}{l}\text { CONCENTRATION UNITS: } \\
(\mathrm{ug} / \mathrm{L} \text { or } \mathrm{ug} / \mathrm{kg}) \mathrm{ug} / \mathrm{L}\end{array}$ & 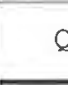 \\
\hline $79-01-6$ & Trichloroethene & 0.50 & $\mathrm{U}$ \\
\hline $108-87-2$ & Methylcyclohexane & 0.50 & $\mathrm{U}$ \\
\hline $78-87-5$ & 1,2-Dichloropropane & 0.50 & $\mathrm{U}$ \\
\hline $75-27-4$ & Bromodichloromethane & 0.50 & $\mathrm{U}$ \\
\hline $10061-01-5$ & cis-1,3-Dichloropropene & 0.50 & $\mathrm{U}$ \\
\hline $108-10-1$ & 4-Methyl-2-pentanone & 5.0 & $\mathrm{U}$ \\
\hline $108-88-3$ & Toluene & 0.047 & $\mathrm{~J} \mathrm{~B}$ \\
\hline $10061-02-6$ & trans-1,3-Dichloropropene & 0.50 & $\mathrm{U}$ \\
\hline $79-00-5$ & 1,1,2-Trichloroethane & 0.50 & $\mathrm{U}$ \\
\hline $127-18-4$ & Tetrachloroethene & 0.50 & $\mathrm{U}$ \\
\hline $591-78-6$ & 2-Hexanone & 5.0 & $\mathrm{U}$ \\
\hline $124-48-1$ & Dibromochloromethane & 0.50 & $\mathrm{U}$ \\
\hline $106-93-4$ & 1,2-Dibromoethane & 0.50 & $\mathrm{U}$ \\
\hline $108-90-7$ & Chlorobenzene & 0.50 & $\mathrm{U}$ \\
\hline $100-41-4$ & Ethylbenzene & 0.50 & $\mathrm{U}$ \\
\hline $95-47-6$ & o-Xylene & 0.50 & $\mathrm{U}$ \\
\hline $179601-23-1$ & $\mathrm{~m}, \mathrm{p}$-Xylene & 0.0059 & $\mathrm{~J}$ \\
\hline $100-42-5$ & Styrene & 0.50 & $\mathrm{U}$ \\
\hline $75-25-2$ & Bromoform & 0.50 & $\mathrm{U}$ \\
\hline $98-82-8$ & Isopropylbenzene & 0.50 & $\mathrm{U}$ \\
\hline $79-34-5$ & $1,1,2,2$-Tetrachloroethane & 0.50 & $\mathrm{U}$ \\
\hline $541-73-1$ & 1,3-Dichlorobenzene & 0.50 & $\mathrm{U}$ \\
\hline $106-46-7$ & 1,4-Dichlorobenzene & 0.50 & $\mathrm{U}$ \\
\hline $95-50-1$ & 1,2-Dichlorobenzene & 0.50 & $\mathrm{U}$ \\
\hline $96-12-8$ & 1,2-Dibromo-3-Chloropropane & 0.50 & $\mathrm{U}$ \\
\hline $120-82-1$ & 1,2,4-Trichlorobenzene & 0.50 & $\mathrm{U}$ \\
\hline $87-61-6$ & 1,2,3-Trichlorobenzene & 0.50 & $\mathrm{U}$ \\
\hline
\end{tabular}


IJ - FORM I VOA-TIC

VOLATILE ORGANICS ANALYSIS DATA SHEET

TENTATIVELY IDENTIEIED COMPOUNDS
EPA SAMPLE NO.

INMW15-W-32735

Lab Name: TESTAMERICA BURLINGTON

Contract: $8 \mathrm{E}-00302$

Lab Code: STLV Case No.: INMAN Mod. Ref No.: SDG NO.: 200-6709

Matrix: (SOIL/SED/WATER)

Water

Sample wt/vol: 25.0

Level: (TRACE or LOW/MED)

$(\mathrm{g} / \mathrm{mL}) \quad \mathrm{mL}$

TRACE

음 Moisture: not dec.

GC Column: DB-624 ID : $0.20 \quad(\mathrm{~mm})$

Soil Extract Volume: (uL)

CONCENTRATION UNITS: (ug/L or $\mathrm{ug} / \mathrm{kg}$ ) ug/L
Lab Sample ID: 200-6709-1

Lab File ID: DHPT05.D

Date Received: 08/25/2011

Date Analyzed: 08/26/2011

Dilution Factor: 1.0

Soil Aliquot Volume:

(uL)

Purge Volume: 25.0 (mL)

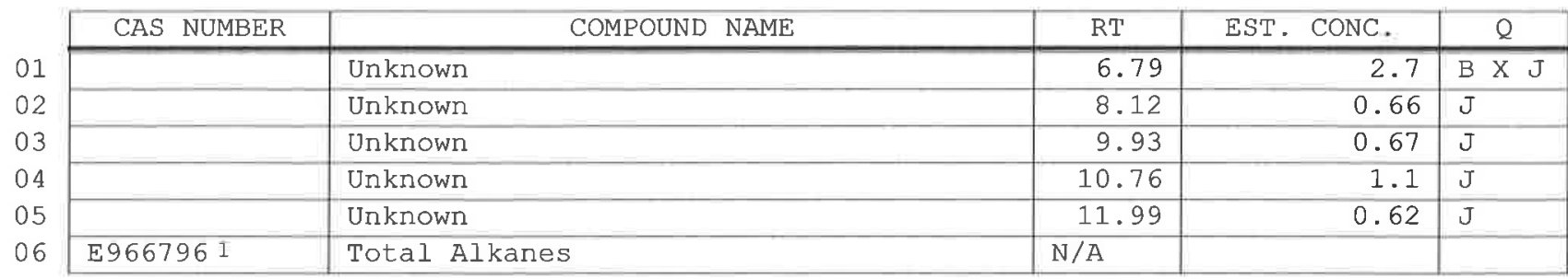

1 EPA-designated Registry Number. 
Lab Name: TESTAMERICA BURLINGTON

Lab Code: STLV

Matrix: (SOIL/SED/WATER)

Sample wt/vol: 25.0

$(\mathrm{g} / \mathrm{mL}) \quad \mathrm{mL}$

Level: (TRACE/LOW/MED) TRACE

응 Moisture: not dec.

GC Column: DB-624 ID : 0.20

Soil Extract Volume: (uL) (mL)

Purge Volume: 25.0
Contract: $8 \mathrm{E}-00302$

SDG No.: 200-6709

Lab Sample ID: 200-6709-3

Lab File ID: DHPT07.D

Date Received: 08/25/2011

Date Analyzed: 08/26/2011

Dilution Factor: 1.0

Soil Aliquot volume: (uL)

\begin{tabular}{|c|c|c|c|}
\hline CAS NO. & COMPOUND & $\begin{array}{l}\text { CONCENTRATION UNITS: } \\
(\mathrm{ug} / \mathrm{L} \text { or } \mathrm{ug} / \mathrm{kg}) \mathrm{ug} / \mathrm{L}\end{array}$ & $Q$ \\
\hline $75-71-8$ & Dichlorodifluoromethane & 0.50 & $\mathrm{U}$ \\
\hline $74-87-3$ & Chloromethane & 0.50 & $\mathrm{U}$ \\
\hline $75-01-4$ & Vinyl chloride & 0.50 & $\mathrm{U}$ \\
\hline $74-83-9$ & Bromomethane & 0.50 & $\mathrm{U}$ \\
\hline $75-00-3$ & Chloroethane & 0.50 & $\mathrm{U}$ \\
\hline $75-69-4$ & Trichlorofluoromethane & 0.50 & $\mathrm{U}$ \\
\hline $75-35-4$ & 1,1-Dichloroethene & 0.50 & $\mathrm{U}$ \\
\hline $76-13-1$ & 1,1,2-Trichloro-1,2,2-trifluoroethane & 0.50 & $\mathrm{U}$ \\
\hline $67-64-1$ & Acetone & 2.2 & $\mathrm{~J}$ \\
\hline $75-15-0$ & Carbon disulfide & 0.50 & $\mathrm{U}$ \\
\hline $79-20-9$ & Methyl acetate & 0.50 & $\mathrm{U}$ \\
\hline $75-09-2$ & Methylene Chloride & 0.50 & $\mathrm{U}$ \\
\hline $156-60-5$ & trans-1,2-Dichloroethene & 0.50 & $\mathrm{U}$ \\
\hline $1634-04-4$ & Methyl tert-butyl ether & 0.50 & U \\
\hline $75-34-3$ & 1,1-Dichloroethane & 0.50 & $\mathrm{U}$ \\
\hline $156-59-2$ & cis-1,2-Dichloroethene & 0.50 & $\mathrm{U}$ \\
\hline $78-93-3$ & 2-Butanone & 5.0 & $\mathrm{U}$ \\
\hline $74-97-5$ & Bromochloromethane & 0.50 & $\mathrm{U}$ \\
\hline $67-66-3$ & Chloroform & 0.69 & \\
\hline $71-55-6$ & 1,1,1-Trichloroethane & 0.50 & $\mathrm{U}$ \\
\hline $110-82-7$ & Cyclohexane & 0.50 & $\mathrm{U}$ \\
\hline $56-23-5$ & Carbon tetrachloride & 2.7 & \\
\hline $71-43-2$ & Benzene & 0.0091 & $\mathrm{~J}$ \\
\hline $107-06-2$ & 1,2-Dichloroethane & 0.50 & $\mathrm{U}$ \\
\hline
\end{tabular}

Report 1,4-Dioxane for Low-Medium VOA analysis only 
Lab Name: TESTAMERICA BURLINGTON

Contract: $8 \mathrm{E}-00302$

Lab Code: STLV Case No.: INMAN Mod. Ref No.: SDG No.: 200-6709

Matrix: (SOIL/SED/WATER)

Sample wt/vol: 25.0 Water

$(\mathrm{g} / \mathrm{mL}) \quad \mathrm{mL}$

Level: (TRACE/LOW/MED) TRACE

응 Moisture: not dec.

GC Column: DB-624 ID: $0.20 \quad$ (mm)

Soil Extract Volume: (uL) $(\mathrm{mL})$

Purge Volume: 25.0
Lab Sample ID: 200-6709-3

Lab File ID: DHPT07.D

Date Received: 08/25/2011

Date Analyzed: 08/26/2011

Dilution Factor: 1.0

Soil Aliquot Volume:

\begin{tabular}{|c|c|c|c|}
\hline CAS NO. & COMPOUND & $\begin{array}{l}\text { CONCENTRATION UNITS: } \\
(\mathrm{ug} / \mathrm{L} \text { or } \mathrm{ug} / \mathrm{kg}) \mathrm{ug} / \mathrm{L}\end{array}$ & 8 \\
\hline $79-01-6$ & Trichloroethene & 0.50 & $\mathrm{U}$ \\
\hline $108-87-2$ & Methylcyclohexane & 0.50 & $\mathrm{U}$ \\
\hline $78-87-5$ & 1,2-Dichloropropane & 0.50 & $\mathrm{U}$ \\
\hline $75-27-4$ & Bromodichloromethane & 0.50 & $\mathrm{U}$ \\
\hline $10061-01-5$ & cis-1,3-Dichloropropene & 0.50 & $\mathrm{U}$ \\
\hline $108-10-1$ & 4-Methyl-2-pentanone & 5.0 & $\mathrm{U}$ \\
\hline $108-88-3$ & Toluene & 0.019 & $\mathrm{~J} \mathrm{~B}$ \\
\hline $10061-02-6$ & trans-1,3-Dichloropropene & 0.50 & $\mathrm{U}$ \\
\hline $79-00-5$ & 1,1,2-Trichloroethane & 0.50 & $\mathrm{U}$ \\
\hline $127-18-4$ & Tetrachloroethene & 0.040 & $\mathrm{~J}$ \\
\hline $591-78-6$ & 2-Hexanone & 5.0 & $\mathrm{U}$ \\
\hline $124-48-1$ & Dibromochloromethane & 0.50 & $\mathrm{U}$ \\
\hline $106-93-4$ & 1,2-Dibromoethane & 0.50 & $\mathrm{U}$ \\
\hline $108-90-7$ & Chlorobenzene & 0.50 & $\mathrm{U}$ \\
\hline $100-41-4$ & Ethylbenzene & 0.50 & $\mathrm{U}$ \\
\hline $95-47-6$ & o-Xylene & 0.50 & $\mathrm{U}$ \\
\hline $179601-23-1$ & $\mathrm{~m}, \mathrm{p}-\mathrm{xy}$ lene & 0.50 & $\mathrm{U}$ \\
\hline $100-42-5$ & Styrene & 0.50 & $\mathrm{U}$ \\
\hline $75-25-2$ & Bromoform & 0.50 & $\mathrm{U}$ \\
\hline $98-82-8$ & Isopropylbenzene & 0.50 & $\mathrm{U}$ \\
\hline $79-34-5$ & $1,1,2,2$-Tetrachloroethane & 0.50 & $\mathrm{U}$ \\
\hline $541-73-1$ & 1,3-Dichlorobenzene & 0.50 & $\mathrm{U}$ \\
\hline $106-46-7$ & 1,4-Dichlorobenzene & 0.50 & $\mathrm{U}$ \\
\hline $95-50-1$ & 1,2-Dichlorobenzene & 0.50 & $\mathrm{U}$ \\
\hline $96-12-8$ & 1,2-Dibromo-3-Chloropropane & 0.50 & $\mathrm{U}$ \\
\hline $120-82-1$ & 1,2,4-Trichlorobenzene & 0.50 & $\mathrm{U}$ \\
\hline $87-61-6$ & 1,2,3-Trichlorobenzene & 0.50 & $\mathrm{U}$ \\
\hline
\end{tabular}


$1 \mathrm{~J}$ - FORM I VOA-TIC

VOLATILE ORGANICS ANALYSIS DATA SHEET

TENTATIVELY IDENTIFIED COMPOUNDS
EPA SAMPLE NO.

INMW 15-W-32737
Lab Name: TESTAMERICA BURLINGTON

Lab Code: STLV Case No.: INMAN

Matrix: (SOIL/SED/WATER)

Water

Sample wt/vol: 25.0

Level: (TRACE or LOW/MED)

$(\mathrm{g} / \mathrm{mL}) \quad \mathrm{mL}$

TRACE

응 Moisture: not dec.

GC Column: DB-624

ID : 0.20 (mm)

Soil Extract Volume: (uL)

CONCENTRATION UNITS: $(u g / L$ or $u g / k g) u g / L$

Contract: $\quad 8 \mathrm{E}-00302$ Mod. Ref No.: SDG No.: 200-6709

Lab Sample ID: 200-6709-3

Lab File ID: DHPT07.D

Date Received: 08/25/2011

Date Analyzed: 08/26/2011

Dilution Factor: 1.0

Soil Aliquot Volume: (uL)

Purge Volume: 25.0 (mI)

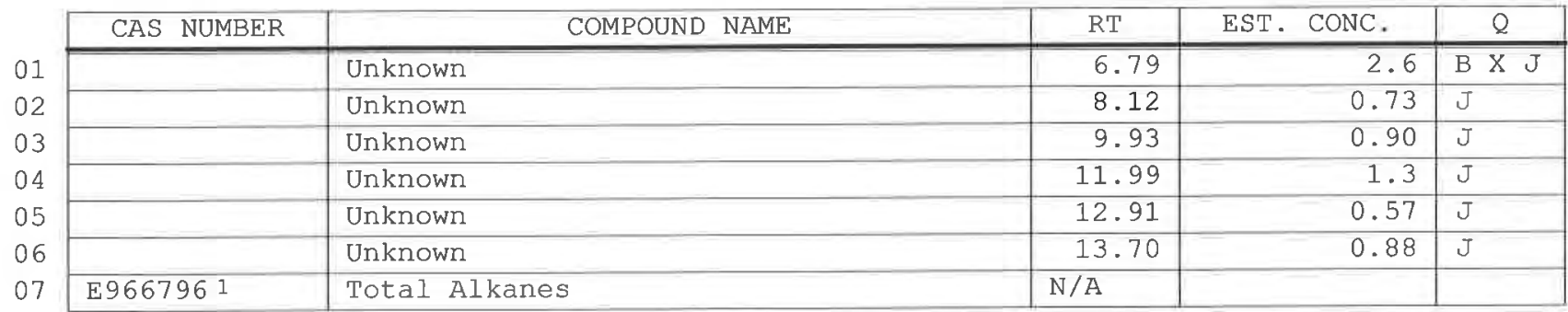

1 EPA-designated Registry Number. 
$1 A$ - FORM I VOA-1

VOLATILE ORGANICS ANALYSIS DATA SHEET
EPA SAMPLE NO.

INQCTB-W-32738
Lab Name: TESTAMERICA BURLINGTON

Lab Code: STLV Case No.: INMAN
Matrix: (SOIL/SED/WATER)

Sample wt/vol: 25.0

Water

$(\mathrm{g} / \mathrm{mL}) \quad \mathrm{mL}$

Level: (TRACE/IOW/MED) TRACE

응 Moisture: not dec.

GC Column: DB-624

Soil Extract Volume: ID $: 0.20 \quad(\mathrm{~mm})$

Purge Volume: 25.0
Contract: $8 \mathrm{E}-00302$

SDG No.: 200-6709

Lab Sample ID: 200-6709-4

Lab File ID: DHPT04.D

Date Received: 08/25/2011

Date Analyzed: 08/26/2011

Dilution Factor: 1.0

Soil Aliquot Volume:

\begin{tabular}{|c|c|c|c|}
\hline CAS NO. & COMPOUND & $\begin{array}{l}\text { CONCENTRATION UNITS: } \\
(\mathrm{ug} / \mathrm{L} \text { or } u g / \mathrm{kg}) \mathrm{ug} / \mathrm{L}\end{array}$ & $\varnothing$ \\
\hline $75-71-8$ & Dichlorodifluoromethane & 0.50 & $\mathrm{U}$ \\
\hline $74-87-3$ & Chloromethane & 0.50 & $\mathrm{U}$ \\
\hline $75-01-4$ & Vinyl chloride & 0.50 & $\mathrm{U}$ \\
\hline $74-83-9$ & Bromomethane & 0.50 & $\mathrm{U}$ \\
\hline $75-00-3$ & Chloroethane & 0.50 & $\mathrm{U}$ \\
\hline $75-69-4$ & Trichlorofluoromethane & 0.50 & $\mathrm{U}$ \\
\hline $75-35-4$ & 1,1-Dichloroethene & 0.50 & $\mathrm{U}$ \\
\hline $76-13-1$ & 1,1,2-Trichloro-1,2,2-trifluoroethane & 0.50 & $\mathrm{U}$ \\
\hline $67-64-1$ & Acetone & 2.5 & $\mathrm{~J}$ \\
\hline $75-15-0$ & Carbon disulfide & 0.50 & $\mathrm{U}$ \\
\hline $79-20-9$ & Methyl acetate & 0.50 & $\mathrm{U}$ \\
\hline $75-09-2$ & Methylene Chloride & 0.50 & $\mathrm{U}$ \\
\hline $156-60-5$ & trans-1,2-Dichloroethene & 0.50 & $\mathrm{U}$ \\
\hline $1634-04-4$ & Methyl tert-butyl ether & 0.50 & $\mathrm{U}$ \\
\hline $75-34-3$ & 1,1-Dichloroethane & 0.50 & $\mathrm{U}$ \\
\hline $156-59-2$ & cis-1,2-Dichloroethene & 0.50 & $\mathrm{U}$ \\
\hline $78-93-3$ & 2-Butanone & 5.0 & $\mathrm{U}$ \\
\hline $74-97-5$ & Bromochloromethane & 0.50 & $\mathrm{U}$ \\
\hline $67-66-3$ & Chloroform & 0.18 & $\mathrm{~J}$ \\
\hline $71-55-6$ & 1,1,1-Trichloroethane & 0.50 & $\mathrm{U}$ \\
\hline $110-82-7$ & Cyclohexane & 0.50 & $\mathrm{U}$ \\
\hline $56-23-5$ & Carbon tetrachloride & 0.50 & $\mathrm{U}$ \\
\hline $71-43-2$ & Benzene & 0.14 & $\mathrm{~J}$ \\
\hline $107-06-2$ & 1,2-Dichloroethane & 0.50 & $\mathrm{U}$ \\
\hline
\end{tabular}

Report 1,4-Dioxane for Low-Medium VOA analysis only 
$1 B$ - FORM I VOA-2

VOLATILE ORGANICS ANALYSIS DATA SHEET
EPA SAMPLE NO.

INQCTB-W-32738

Lab Name: TESTAMERICA BURLINGTON

Contract: 8E-00302

Lab Code: STLV Case No.: INMAN Mod. Ref No.: SDG No.: 200-6709

Matrix: (SOIL/SED/WATER)

Sample wt/vol: 25.0 Water

$(\mathrm{g} / \mathrm{mL}) \quad \mathrm{mL}$

Level: (TRACE/LOW/MED) TRACE

o Moisture: not dec.

GC Column: DB-624 ID: $0.20 \quad(\mathrm{~mm})$

Soil Extract volume: (uL)

Purge Volume: 25.0 (mL)
Lab Sample ID: 200-6709-4

Lab File ID: DHPT04.D

Date Received: $08 / 25 / 2011$

Date Analyzed: $08 / 26 / 2011$

Dilution Factor: 1.0

Soil Aliquot Volume:

(uL)

\begin{tabular}{|c|c|c|c|}
\hline CAS NO. & COMPOUND & $\begin{array}{l}\text { CONCENTRATION UNITS: } \\
(\mathrm{ug} / \mathrm{L} \text { or } \mathrm{ug} / \mathrm{kg}) \mathrm{ug} / \mathrm{L}\end{array}$ & $Q$ \\
\hline $79-01-6$ & Trichloroethene & 0.50 & $\mathrm{U}$ \\
\hline $108-87-2$ & Methylcyclohexane & 0.50 & $\mathrm{U}$ \\
\hline $78-87-5$ & 1,2-Dichloropropane & 0.50 & $\mathrm{U}$ \\
\hline $75-27-4$ & Bromodichloromethane & 0.50 & $\mathrm{U}$ \\
\hline $10061-01-5$ & cis-1,3-Dichloropropene & 0.50 & $\mathrm{U}$ \\
\hline $108-10-1$ & 4-Methyl-2-pentanone & 5.0 & $\mathrm{U}$ \\
\hline $108-88-3$ & Toluene & 0.42 & $\mathrm{~J} \mathrm{~B}$ \\
\hline $10061-02-6$ & trans-1,3-Dichloropropene & 0.50 & $\mathrm{U}$ \\
\hline $79-00-5$ & 1,1,2-Trichloroethane & 0.50 & $\mathrm{U}$ \\
\hline $127-18-4$ & Tetrachloroethene & 0.50 & $\mathrm{U}$ \\
\hline $591-78-6$ & 2-Hexanone & 5.0 & $\mathrm{U}$ \\
\hline $124-48-1$ & Dibromochloromethane & 0.50 & $\mathrm{U}$ \\
\hline $106-93-4$ & 1,2-Dibromoethane & 0.50 & $\mathrm{U}$ \\
\hline $108-90-7$ & Chlorobenzene & 0.50 & $\mathrm{U}$ \\
\hline $100-41-4$ & Ethylbenzene & 0.019 & $\mathrm{~J}$ \\
\hline $95-47-6$ & o-Xylene & 0.012 & $\mathrm{~J}$ \\
\hline $179601-23-1$ & $\mathrm{~m}, \mathrm{p}$-Xylene & 0.067 & $\mathrm{~J}$ \\
\hline $100-42-5$ & Styrene & 0.17 & $\mathrm{~J}$ \\
\hline $75-25-2$ & Bromoform & 0.50 & $\mathrm{U}$ \\
\hline $98-82-8$ & Isopropylbenzene & 0.50 & $\mathrm{U}$ \\
\hline $79-34-5$ & $1,1,2,2$-Tetrachloroethane & 0.50 & $\mathrm{U}$ \\
\hline $541-73-1$ & 1,3-Dichlorobenzene & 0.50 & $\mathrm{U}$ \\
\hline $106-46-7$ & 1,4-Dichlorobenzene & 0.50 & $\mathrm{U}$ \\
\hline $95-50-1$ & 1,2-Dichlorobenzene & 0.50 & $\mathrm{U}$ \\
\hline $96-12-8$ & 1,2-Dibromo-3-Chloropropane & 0.50 & $\mathrm{U}$ \\
\hline $120-82-1$ & 1,2,4-Trichlorobenzene & 0.50 & $\mathrm{U}$ \\
\hline $87-61-6$ & $1,2,3$-Trichlorobenzene & 0.50 & $\mathrm{U}$ \\
\hline
\end{tabular}


$1 \mathrm{~J}$ - FORM I VOA-TIC

VOLATILE ORGANICS ANALYSIS DATA SHEET

TENTATIVELY IDENTIFIED COMPOUNDS
EPA SAMPLE NO.

INQCTB-W-32738
Lab Name: TESTAMERICA BURLINGTON

Lab Code: STLV Case No.: INMAN

Matrix: (SOIL/SED/WATER)

Water

Sample wt/vol: 25.0

Level: (TRACE or LOW/MED)

$(\mathrm{g} / \mathrm{mL}) \quad \mathrm{mL}$

goisture: not dec.

GC Column: DB-624

ID: 0.20 $(\mathrm{mm})$

Soil Extract volume: (uL)

CONCENTRATION UNITS: (ug/L or $\mathrm{ug} / \mathrm{kg}$ ) $\mathrm{ug} / \mathrm{L}$
Contract: $8 \mathrm{E}-00302$

SDG No.: 200-6709

Lab Sample ID: 200-6709-4

Lab File ID: DHPT04.D

Date Received: 08/25/2011

Date Analyzed: 08/26/2011

Dilution Factor: 1.0

Soil Aliquot Volume:

Purge Volume: 25.0

01

02

\begin{tabular}{|c|l|c|c|c|}
\hline CAS NUMBER & \multicolumn{1}{|c|}{ COMPOUND NAME } & RT & EST. CONC. & Q \\
\hline \hline & Unknown & 6.79 & 2.8 & B X J \\
\hline E9667961 & Total Alkanes & N/A & & \\
\hline
\end{tabular}

1 EPA-designated Registry Number. 
$6 A-F O R M V I V O A-1$

VOLATILE ORGANICS INITIAL CALIBRATION DATA

Lab Name: TESTAMERICA BURLINGTON

Contract: $8 \mathrm{E}-00302$

Lab Code: STLV Case No.: INMAN Mod. Ref No.:

SDG No.: 200-6709

Instrument ID: D.i

Heated Purge: (Y/N) N

Calibration Date(s): 07/07/2011 07/07/2011

Purge Volume: 25.0

Calibration Time(s) : 1117

1256

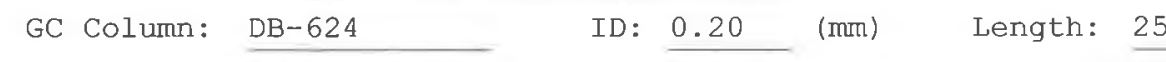

GC Column: DB-624 ID: 0.20 (mm) Length: 25

(mL)

\begin{tabular}{|c|c|c|c|c|c|c|c|}
\hline \multirow{3}{*}{$\begin{array}{l}\text { LAB FILE ID: } \\
\text { RRE } 5.0=\text { DHPO } 6 . \mathrm{D} \\
\text { COMPOUND }\end{array}$} & \multirow{2}{*}{\multicolumn{3}{|c|}{$\begin{array}{l}\text { RRE 0.5 }=\underline{\mathrm{DHP04.D}} \\
\operatorname{RRE10}=\mathrm{DHP07.D}\end{array}$}} & \multirow{2}{*}{\multicolumn{4}{|c|}{$\begin{array}{l}\text { RRF 1.0 }=\text { DHP05.D } \\
\text { RRF 20 }=\text { DHP08.D }\end{array}$}} \\
\hline & & & & & & & \\
\hline & $\operatorname{RRF} 0.5$ & RRF 1.0 & RRF 5.0 & RRF 10 & RRE 20 & $\overline{\mathrm{RRE}}$ & $\because \mathrm{RSD}$ \\
\hline Dichlorodifluoromethane & 0.418 & 0.398 & 0.390 & 0.409 & 0.398 & 0.403 & 2.7 \\
\hline Chloromethane & 0.769 & 0.713 & 0.655 & 0.659 & 0.639 & 0.687 & 7.8 \\
\hline Vinyl chloride & 0.524 & 0.491 & 0.475 & 0.499 & 0.491 & 0.496 & 3.6 \\
\hline Bromomethane & 0.180 & 0.203 & 0.205 & 0.219 & 0.239 & 0.209 & 10.3 \\
\hline Chloroethane & 0.292 & 0.280 & 0.259 & 0.269 & 0.259 & 0.272 & 5.2 \\
\hline Trichlorofluoromethane & 0.612 & 0.609 & 0.587 & 0.608 & 0.611 & 0.606 & 1.7 \\
\hline 1,1-Dichloroethene & 0.318 & 0.307 & 0.298 & 0.303 & 0.297 & 0.305 & 2.8 \\
\hline $\begin{array}{l}1,1,2 \text {-Trichloro- } \\
1,2,2 \text {-trifluoroethane }\end{array}$ & 0.377 & 0.358 & 0.336 & 0.358 & 0.347 & 0.355 & 4.3 \\
\hline Acetone & 0.047 & 0.038 & 0.032 & 0.032 & 0.030 & 0.036 & 19.3 \\
\hline Carbon disulfide & 0.758 & 0.707 & 0.719 & 0.760 & 0.766 & 0.742 & 3.6 \\
\hline Methyl acetate & 0.108 & 0.094 & 0.076 & 0.079 & 0.074 & 0.086 & 16.7 \\
\hline Methylene Chloride & 0.264 & 0.257 & 0.242 & 0.248 & 0.241 & 0.250 & 3.9 \\
\hline trans-1,2-Dichloroethene & 0.323 & 0.298 & 0.301 & 0.312 & 0.306 & 0.308 & 3.3 \\
\hline Methyl tert-butyl ether & 0.304 & 0.278 & 0.292 & 0.319 & 0.318 & 0.302 & 5.7 \\
\hline 1,1-Dichloroethane & 0.642 & 0.586 & 0.594 & 0.617 & 0.607 & 0.609 & 3.6 \\
\hline cis-1,2-Dichloroethene & 0.302 & 0.289 & 0.300 & 0.312 & 0.306 & 0.302 & 2.7 \\
\hline 2-Butanone & 0.045 & 0.046 & 0.047 & 0.049 & 0.048 & 0.047 & 3.4 \\
\hline Bromochloromethane & 0.104 & 0.103 & 0.100 & 0.099 & 0.101 & 0.101 & 2.0 \\
\hline Chloroform & 0.502 & 0.482 & 0.473 & 0.493 & 0.477 & 0.485 & 2.5 \\
\hline 1,1,1-Trichloroethane & 0.514 & 0.490 & 0.494 & 0.490 & 0.485 & 0.494 & 2.3 \\
\hline Cyclohexane & 0.761 & 0.795 & 0.901 & 0.909 & 0.883 & 0.850 & 7.9 \\
\hline Carbon tetrachloride & 0.487 & 0.459 & 0.477 & 0.474 & 0.464 & 0.472 & 2.4 \\
\hline Benzene & 1.617 & 1.501 & 1.518 & 1.495 & 1.447 & 1.515 & 4.1 \\
\hline 1,2-Dichloroethane & 0.291 & 0.271 & 0.277 & 0.284 & 0.285 & 0.282 & 2.7 \\
\hline Trichloroethene & 0.397 & 0.380 & 0.357 & 0.355 & 0.351 & 0.368 & 5.4 \\
\hline Methylcyclohexane & 0.703 & 0.708 & 0.769 & 0.757 & 0.751 & 0.737 & 4.1 \\
\hline
\end{tabular}

Report 1,4-Dioxane for Low-Medium VOA analysis only 
$6 B$ - FORM VI VOA-2

VOLATILE ORGANICS INITIAL CALIBRATION DATA

Lab Name: TESTAMERICA BURLINGTON

Contract: $8 \mathrm{E}-00302$

Lab Code: STLV Case No.: INMAN Mod. Ref No.:

SDG No.: 200-6709

Instrument ID: D.i

Calibration Date(s): 07/07/2011 07/07/2011

Heated Purge: (Y/N) N

Calibration Time(s): 1117

1256

Purge Volume: 25.0

(mL)

GC Column: DB-624

ID : 0.20

$(\mathrm{mm})$

Length: 25

(m)

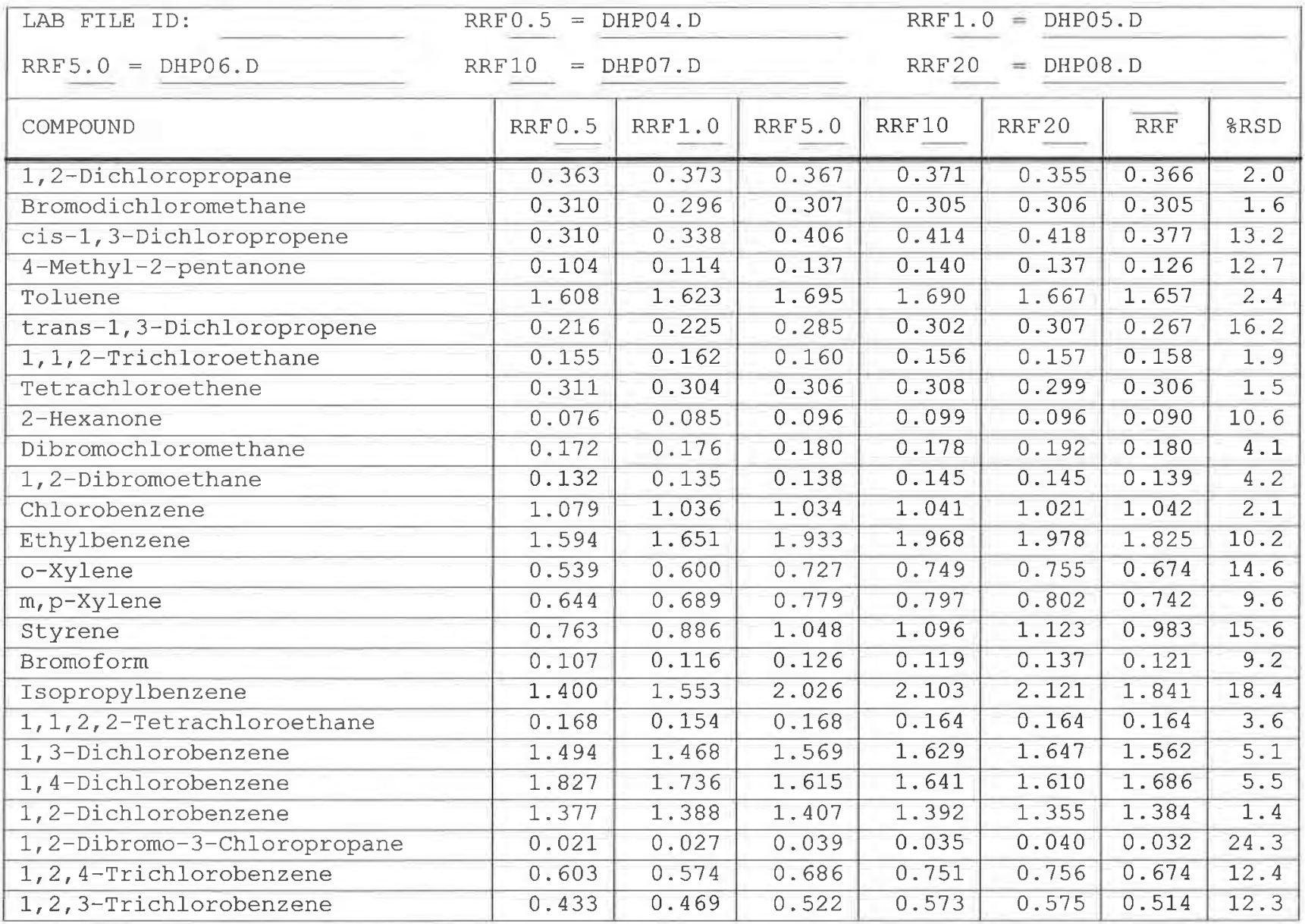


$6 C$ - FORM VI VOA-3

VOLATILE ORGANICS INITIAI CALIBRATION DATA

Lab Name: TESTAMERICA BURLINGTON

Contract: $\quad 8 \mathrm{E}-00302$

Lab Code: STLV Case No.: INMAN Mod. Ref No.:

SDG No.: 200-6709

Instrument ID: D.i

Calibration Date(s): 07/07/2011 07/07/2011

Heated Purge: $(\mathrm{Y} / \mathrm{N}) \mathrm{N}$

Calibration Time(s): 1117

1256

Purge Volume: 25.0

(mL)

GC Column: $\mathrm{DB}-624$

ID: 0.20

(mm) Length: 25

(II)

\begin{tabular}{|c|c|c|c|c|c|c|c|}
\hline \multirow{2}{*}{$\begin{array}{l}\text { LAB FILE ID: } \\
\text { RRE } 5.0=\text { DHP06.D }\end{array}$} & \multicolumn{3}{|c|}{$\mathrm{RRF} 0.5=\mathrm{DHPO} 4 . \mathrm{D}$} & \multicolumn{4}{|c|}{ RRF 1.0 = DHP05.D } \\
\hline & $\operatorname{RRF} 10=1$ & IP07.D & & RRF 20 & $=\underline{\mathrm{DHP}}$ & . D & \\
\hline COMPOUND & RRF 0.5 & RRF 1.0 & RRF 5.0 & RRF 10 & RRF 20 & $\overline{\mathrm{RRF}}$ & $\because \mathrm{RSD}$ \\
\hline Vinyl Chloride-d3 & 0.479 & 0.428 & 0.415 & 0.431 & 0.426 & 0.436 & 5.7 \\
\hline Chloroethane-d5 & 0.304 & 0.290 & 0.269 & 0.276 & 0.274 & 0.283 & 5.0 \\
\hline 1,1-Dichloroethene-d2 & 0.849 & 0.795 & 0.757 & 0.787 & 0.787 & 0.795 & 4.2 \\
\hline 2-Butanone-d5 & 0.041 & 0.038 & 0.040 & 0.044 & 0.043 & 0.041 & 5.5 \\
\hline Chloroform-d & 0.512 & 0.515 & 0.502 & 0.514 & 0.503 & 0.509 & 1.3 \\
\hline 1,2-Dichloroethane-d4 & 0.218 & 0.203 & 0.199 & 0.201 & 0.194 & 0.203 & 4.3 \\
\hline Benzene-d6 & 1.573 & 1.471 & 1.478 & 1.453 & 1.403 & 1.475 & 4.2 \\
\hline 1,2-Dichloropropane-d6 & 0.410 & 0.374 & 0.367 & 0.365 & 0.356 & 0.374 & 5.7 \\
\hline Toluene-d8 & 1.315 & 1.370 & 1.458 & 1.441 & 1.404 & 1.397 & 4.1 \\
\hline trans-1,3-Dichloropropene-d4 & 0.197 & 0.207 & 0.232 & 0.249 & 0.264 & 0.230 & 12.2 \\
\hline 2-Hexanone-d5 & 0.023 & 0.026 & 0.037 & 0.038 & 0.039 & 0.033 & 23.1 \\
\hline $1,1,2,2$-Tetrachloroethane-d2 & 0.151 & 0.161 & 0.167 & 0.171 & 0.169 & 0.164 & 4.8 \\
\hline 1,2-Dichlorobenzene-d4 & 0.885 & 0.843 & 0.815 & 0.807 & 0.799 & 0.830 & 4.2 \\
\hline
\end{tabular}

Report 1,4-Dioxane-d8 for Low-Medium VOA analysis only 
Lab Name: TESTAMERICA BURLINGTON

Lab Code: STLV Case No.: INMAN Mod. Ref No.:

Contract: $8 \mathrm{E}-00302$

Instrument ID: D.i

Calibration Date: 08/26/2011 Time: 0918

Lab File Id: DHPT02.D

EPA Sample No. (VSTD\#\#\#\#): VSTD005DQ Init. Calib. Date(s) : 07/07/2011 07/07/2011

Heated Purge: $(\mathrm{Y} / \mathrm{N})$

Purge Volume: 25.0
Init. Calib. Time(s): $1117 \quad \underline{1256}$

ID: $0.20(\mathrm{~mm})$ Length: 25 (m)
(mL)

\begin{tabular}{|c|c|c|c|c|c|}
\hline COMPOUND & $\overline{\mathrm{RRF}}$ & RRF5. 0 & $\begin{array}{l}\text { MIN } \\
\text { RRF }\end{array}$ & $\because \mathrm{D}$ & MAX $\because D$ \\
\hline Dichlorodifluoromethane & 0.403 & 0.465 & 0.010 & 15.4 & 40.0 \\
\hline Chloromethane & 0.687 & 0.712 & 0.010 & 3.6 & 40.0 \\
\hline Vinyl chloride & 0.496 & 0.499 & 0.010 & 0.6 & 30.0 \\
\hline Bromomethane & 0.209 & 0.207 & 0.010 & -1.1 & 30.0 \\
\hline Chloroethane & 0.272 & 0.258 & 0.010 & -5.0 & 40.0 \\
\hline Trichlorofluoromethane & 0.606 & 0.601 & 0.010 & -0.7 & 40.0 \\
\hline 1,1-Dichloroethene & 0.305 & 0.300 & 0.010 & -1.5 & 30.0 \\
\hline 1,1,2-Trichloro-1,2,2-trifluoroethane & 0.355 & 0.349 & 0.010 & -1.7 & 40.0 \\
\hline Acetone & 0.036 & 0.027 & 0.010 & -24.1 & 40.0 \\
\hline Carbon disulfide & 0.742 & 0.727 & 0.010 & -2.1 & 40.0 \\
\hline Methyl acetate & 0.086 & 0.077 & 0.010 & -9.9 & 40.0 \\
\hline Methylene Chloride & 0.250 & 0.230 & 0.010 & -8.2 & 40.0 \\
\hline trans-1,2-Dichloroethene & 0.308 & 0.289 & 0.010 & -6.2 & 40.0 \\
\hline Methyl tert-butyl ether & 0.302 & 0.248 & 0.010 & -17.8 & 40.0 \\
\hline 1,1-Dichloroethane & 0.609 & 0.590 & 0.010 & -3.1 & 30.0 \\
\hline cis-1,2-Dichloroethene & 0.302 & 0.284 & 0.010 & -5.9 & 40.0 \\
\hline 2-Butanone & 0.047 & 0.041 & 0.010 & -12.9 & 40.0 \\
\hline Bromochloromethane & 0.101 & 0.094 & 0.010 & -6.6 & 30.0 \\
\hline Chloroform & 0.485 & 0.461 & 0.010 & -5.0 & 30.0 \\
\hline 1,1,1-Trichloroethane & 0.494 & 0.467 & 0.010 & -5.6 & 30.0 \\
\hline Cyclohexane & 0.850 & 0.878 & 0.010 & 3.4 & 40.0 \\
\hline Carbon tetrachloride & 0.472 & 0.460 & 0.010 & -2.6 & 30.0 \\
\hline Benzene & 1.515 & 1.432 & 0.010 & -5.5 & 30.0 \\
\hline 1,2-Dichloroethane & 0.282 & 0.260 & 0.010 & -7.5 & 30.0 \\
\hline Trichloroethene & 0.368 & 0.339 & 0.010 & -7.8 & 30.0 \\
\hline Methylcyclohexane & 0.737 & 0.743 & 0.010 & 0.8 & 40.0 \\
\hline
\end{tabular}

Report 1,4-Dioxane for Low/Medium VOA analysis only 
$7 B$ - FORM VII VOA-2

VOLATILE CONTINUING CALIBRATION DATA

Lab Name: TESTAMERICA BURLINGTON

Contract: $8 \mathrm{E}-00302$

Lab Code: STLV Case No.: INMAN Mod. Ref No.:

SDG No.: 200-6709

Instrument ID: D.i

Calibration Date: 08/26/2011 Time: 0918

Lab File Id: DHPT02.D

EPA Sample No. (VSTD\#\#\#\#): VSTD005DQ Init. Calib. Date(s) : 07/07/2011 07/07/2011

Heated Purge: $(\mathrm{Y} / \mathrm{N})$

GC Column: DB-624

Init. Calib. Time(s): 111 1256

Purge Volume: 25.0 (mL) ID: $0.20(\mathrm{~mm})$ Length: 25 (m)

(a)

\begin{tabular}{|c|c|c|c|c|c|}
\hline COMPOUND & $\overline{\mathrm{RRF}}$ & RRF5.0 & $\begin{array}{l}\text { MIN } \\
\text { RRF }\end{array}$ & $\% \mathrm{D}$ & $\operatorname{MAX} \div \mathrm{D}$ \\
\hline 1,2-Dichloropropane & 0.366 & 0.351 & 0.010 & -4.0 & 40.0 \\
\hline Bromodichloromethane & 0.305 & 0.278 & 0.010 & -9.0 & 30.0 \\
\hline cis-1,3-Dichloropropene & 0.377 & 0.361 & 0.010 & -4.3 & 30.0 \\
\hline 4-Methyl-2-pentanone & 0.126 & 0.111 & 0.010 & -12.2 & 40.0 \\
\hline Toluene & 1.657 & 1.578 & 0.010 & -4.8 & 30.0 \\
\hline trans-1,3-Dichloropropene & 0.267 & 0.255 & 0.010 & -4.6 & 30.0 \\
\hline 1,1,2-Trichloroethane & 0.158 & 0.142 & 0.010 & -10.0 & 30.0 \\
\hline Tetrachloroethene & 0.306 & 0.292 & 0.010 & -4.7 & 30.0 \\
\hline 2-Hexanone & 0.090 & 0.078 & 0.010 & -13.6 & 40.0 \\
\hline Dibromochloromethane & 0.180 & 0.159 & 0.010 & -11.7 & 30.0 \\
\hline 1,2-Dibromoethane & 0.139 & 0.122 & 0.010 & -12.3 & 40.0 \\
\hline Chlorobenzene & 1.042 & 0.991 & 0.010 & -4.9 & 30.0 \\
\hline Ethylbenzene & 1.825 & 1.793 & 0.010 & -1.7 & 30.0 \\
\hline o-xylene & 0.674 & 0.679 & 0.010 & 0.6 & 30.0 \\
\hline $\mathrm{m}, \mathrm{p}$-Xylene & 0.742 & 0.736 & 0.010 & -0.8 & 30.0 \\
\hline styrene & 0.983 & 0.995 & 0.010 & 1.2 & 30.0 \\
\hline Bromoform & 0.121 & 0.105 & 0.010 & -13.1 & 30.0 \\
\hline Isopropylbenzene & 1.841 & 1.898 & 0.010 & 3.1 & 40.0 \\
\hline $1,1,2,2$-Tetrachloroethane & 0.164 & 0.134 & 0.010 & -17.9 & 30.0 \\
\hline 1,3-Dichlorobenzene & 1.562 & 1.423 & 0.010 & -8.9 & 30.0 \\
\hline 1,4-Dichlorobenzene & 1.686 & 1.530 & 0.010 & -9.2 & 30.0 \\
\hline 1,2-Dichlorobenzene & 1.384 & 1.279 & 0.010 & -7.6 & 30.0 \\
\hline 1,2-Dibromo-3-Chloropropane & 0.032 & 0.030 & 0.010 & -8.6 & 40.0 \\
\hline 1,2,4-Trichlorobenzene & 0.674 & 0.601 & 0.010 & -10.8 & 30.0 \\
\hline 1,2,3-Trichlorobenzene & 0.514 & 0.471 & 0.010 & -8.3 & 30.0 \\
\hline
\end{tabular}


Lab Name: TESTAMERICA BURLINGTON

Lab Code: STLV Case No.

Contract: $8 \mathrm{E}-00302$

Instrument ID: D.i

Lab File Id: DHPT02.D

EPA Sample No. (VSTD\#\#\#\#): VSTD005DQ GC Column: DB-624 Init Mod. Ref No.: SDG No.: 200-6709 Heated Purge: ( $\mathrm{Y} / \mathrm{N})$ $\mathrm{N}$ Purge Volume: 25.0 (mL)

\begin{tabular}{|c|c|c|c|c|c|}
\hline COMPOUND & $\overline{\mathrm{RRE}}$ & RRF5. 0 & $\begin{array}{l}\text { MIN } \\
\text { RRF }\end{array}$ & $\because \mathrm{D}$ & MAX $\% D$ \\
\hline Vinyl Chloride-d3 & 0.436 & 0.412 & 0.010 & -5.4 & 30.0 \\
\hline Chloroethane-d5 & 0.283 & 0.266 & 0.010 & -6.1 & 40.0 \\
\hline 1,1-Dichloroethene-d2 & 0.795 & 0.708 & 0.010 & -10.9 & 30.0 \\
\hline 2-Butanone-d5 & 0.041 & 0.035 & 0.010 & -16.2 & 40.0 \\
\hline Chloroform-d & 0.509 & 0.455 & 0.010 & -10.6 & 30.0 \\
\hline 1,2-Dichloroethane-d4 & 0.203 & 0.185 & 0.010 & -9.0 & 30.0 \\
\hline Benzene-d 6 & 1.475 & 1.329 & 0.010 & -9.9 & 30.0 \\
\hline 1,2-Dichloropropane-d6 & 0.374 & 0.325 & 0.010 & -13.1 & 40.0 \\
\hline Toluene-d8 & 1.397 & 1.298 & 0.010 & -7.1 & 30.0 \\
\hline trans-1,3-Dichloropropene-d4 & 0.230 & 0.199 & 0.010 & -13.5 & 30.0 \\
\hline 2-Hexanone-d5 & 0.033 & 0.028 & 0.010 & -15.1 & 40.0 \\
\hline $1,1,2,2$-Tetrachloroethane-d2 & 0.164 & 0.133 & 0.010 & -18.6 & 30.0 \\
\hline 1,2-Dichlorobenzene-d4 & 0.830 & 0.693 & 0.010 & -16.5 & 30.0 \\
\hline
\end{tabular}


Lab Name: TESTAMERICA BURLINGTON

Contract: $8 \mathrm{E}-00302$

Lab Code: STLV Case No.: INMAN Mod. Ref No.:

SDG No.: 200-6709

Instrument ID: D.i

Calibration Date: 08/26/2011 Time: 1244

Lab File Id: DHPT09.D

EPA Sample No. (VSTD\#\#\#\#): VSTD005QD Init. Calib. Date(s): 07/07/2011 07/07/2011

Heated Purge: ( $\mathrm{Y} / \mathrm{N}) \quad \mathrm{N}$ GC Column: DB-624

Init. Calib. Time(s): 1117 1256

Purge Volume: 25.0 ID: 0.20 (mm) Length: 25

(m) $(\mathrm{mL})$

\begin{tabular}{|c|c|c|c|c|c|}
\hline COMPOUND & $\overline{\mathrm{RRF}}$ & RRE5. 0 & $\begin{array}{l}\text { MIN } \\
\text { RRF }\end{array}$ & $8 \mathrm{D}$ & $\mathrm{MAX} \div \mathrm{D}$ \\
\hline Dichlorodifluoromethane & 0.403 & 0.452 & 0.010 & 12.2 & 50.0 \\
\hline Chloromethane & 0.687 & 0.693 & 0.010 & 0.9 & 50.0 \\
\hline Vinyl chloride & 0.496 & 0.490 & 0.100 & -1.4 & 50.0 \\
\hline Bromomethane & 0.209 & 0.190 & 0.100 & -9.3 & 50.0 \\
\hline Chloroethane & 0.272 & 0.262 & 0.010 & -3.5 & 50.0 \\
\hline Trichlorofluoromethane & 0.606 & 0.600 & 0.010 & -0.9 & 50.0 \\
\hline 1,1-Dichloroethene & 0.305 & 0.298 & 0.100 & -2.2 & 50.0 \\
\hline 1,1,2-Trichloro-1,2,2-trifluoroethane & 0.355 & 0.355 & 0.010 & 0.0 & 50.0 \\
\hline Acetone & 0.036 & 0.026 & 0.010 & -27.5 & 50.0 \\
\hline Carbon disulfide & 0.742 & 0.720 & 0.010 & -3.0 & 50.0 \\
\hline Methyl acetate & 0.086 & 0.072 & 0.010 & -16.2 & 50.0 \\
\hline Methylene Chloride & 0.250 & 0.232 & 0.010 & -7.2 & 50.0 \\
\hline trans-1,2-Dichloroethene & 0.308 & 0.287 & 0.010 & -6.9 & 50.0 \\
\hline Methyl tert-butyl ether & 0.302 & 0.251 & 0.010 & -16.9 & 50.0 \\
\hline 1,1-Dichloroethane & 0.609 & 0.576 & 0.200 & -5.4 & 50.0 \\
\hline cis-1,2-Dichloroethene & 0.302 & 0.282 & 0.010 & -6.5 & 50.0 \\
\hline 2-Butanone & 0.047 & 0.038 & 0.010 & -19.7 & 50.0 \\
\hline Bromochloromethane & 0.101 & 0.096 & 0.050 & -4.9 & 50.0 \\
\hline Chloroform & 0.485 & 0.468 & 0.200 & -3.6 & 50.0 \\
\hline $1,1,1$-Trichloroethane & 0.494 & 0.493 & 0.100 & -0.4 & 50.0 \\
\hline Cyclohexane & 0.850 & 0.923 & 0.010 & 8.6 & 50.0 \\
\hline Carbon tetrachloride & 0.472 & 0.478 & 0.100 & 1.2 & 50.0 \\
\hline Benzene & 1.515 & 1.471 & 0.400 & -2.9 & 50.0 \\
\hline 1,2-Dichloroethane & 0.282 & 0.272 & 0.100 & -3.5 & 50.0 \\
\hline Trichloroethene & 0.368 & 0.356 & 0.300 & -3.2 & 50.0 \\
\hline Methylcyclohexane & 0.737 & 0.775 & 0.010 & 5.1 & 50.0 \\
\hline
\end{tabular}


$7 B$ - FORM VII VOA-2

VOLATILE CONTINUING CALIBRATION DATA

Lab Name: TESTAMERICA BURLINGTON

Contract: $8 \mathrm{E}-00302$

Lab Code: STLV Case No.: INMAN Mod. Ref No.:

SDG No.: 200-6709

Instrument ID: D.i

Calibration Date: 08/26/2011 Time: 1244

Lab Eile Id: DHPT09.D

Init. Calib. Date(s): 07/07/2011 07/07/2011

EPA Sample No. (VSTD\#\#\#\#): VSTD005QD

Init. Calib. Time(s): $1117 \quad 1256$

Heated Purge: $(\mathrm{Y} / \mathrm{N}) \quad \mathrm{N}$

GC Column:

ID: $0.20(\mathrm{~mm})$ Length: 25

$(\mathrm{m})$

Purge Volume: 25.0

(mL)

\begin{tabular}{|c|c|c|c|c|c|}
\hline COMPOUND & $\overline{\mathrm{RRF}}$ & RRF5.0 & $\begin{array}{l}\text { MIN } \\
\text { RRE }\end{array}$ & $\div \mathrm{D}$ & MAX $\div D$ \\
\hline 1,2-Dichloropropane & 0.366 & 0.357 & 0.010 & -2.3 & 50.0 \\
\hline Bromodichloromethane & 0.305 & 0.287 & 0.200 & -5.9 & 50.0 \\
\hline cis-1,3-Dichloropropene & 0.377 & 0.384 & 0.200 & 1.7 & 50.0 \\
\hline 4-Methyl-2-pentanone & 0.126 & 0.116 & 0.010 & -8.6 & 50.0 \\
\hline Toluene & 1.657 & 1.647 & 0.400 & -0.6 & 50.0 \\
\hline trans-1,3-Dichloropropene & 0.267 & 0.261 & 0.100 & -2.3 & 50.0 \\
\hline 1,1,2-Trichloroethane & 0.158 & 0.151 & 0.100 & -4.2 & 50.0 \\
\hline Tetrachloroethene & 0.306 & 0.302 & 0.100 & $-1 \cdot 3$ & 50.0 \\
\hline 2-Hexanone & 0.090 & 0.081 & 0.010 & -10.2 & 50.0 \\
\hline Dibromochloromethane & 0.180 & 0.162 & 0.100 & -9.7 & 50.0 \\
\hline 1,2-Dibromoethane & 0.139 & 0.126 & 0.010 & -9.5 & 50.0 \\
\hline Chlorobenzene & 1.042 & 1.021 & 0.500 & -2.1 & 50.0 \\
\hline Ethylbenzene & 1.825 & 1.901 & 0.100 & 4.2 & 50.0 \\
\hline o-xylene & 0.674 & 0.714 & 0.300 & 5.9 & 50.0 \\
\hline m,p-Xylene & 0.742 & 0.782 & 0.300 & 5.3 & 50.0 \\
\hline Styrene & 0.983 & 1.085 & 0.300 & 10.3 & 50.0 \\
\hline Bromoform & 0.121 & 0.109 & 0.050 & -10.1 & 50.0 \\
\hline Isopropylbenzene & 1.841 & 1.984 & 0.010 & 7.8 & 50.0 \\
\hline $1,1,2,2$-Tetrachloroethane & 0.164 & 0.142 & 0.100 & -13.4 & 50.0 \\
\hline 1,3-Dichlorobenzene & 1.562 & 1.449 & 0.400 & -7.2 & 50.0 \\
\hline 1,4-Dichlorobenzene & 1.686 & 1.595 & 0.400 & -5.4 & 50.0 \\
\hline 1,2-Dichlorobenzene & 1.384 & 1.350 & 0.400 & -2.5 & 50.0 \\
\hline 1,2-Dibromo-3-Chloropropane & 0.032 & 0.026 & 0.010 & -18.9 & 50.0 \\
\hline 1,2,4-Trichlorobenzene & 0.674 & 0.611 & 0.200 & -9.4 & 50.0 \\
\hline 1,2,3-Trichlorobenzene & 0.514 & 0.485 & 0.200 & -5.7 & 50.0 \\
\hline
\end{tabular}


Lab Name: TESTAMERICA BURLINGTON

Contract: $8 \mathrm{E}-00302$

Lab Code: STLV Case No.: INMAN

Mod. Ref No.:

SDG No.: 200-6709

Instrument ID: D.i

Calibration Date: 08/26/2011 Time: 1244

Lab File Id: DHPT09.D Init. Calib. Date(s): 07/07/2011 07/07/2011

EPA Sample No. (VSTD\#\#\#\#): VSTD005QD

Init. Calib. Time(s): $1117 \quad 1256$

Heated Purge: $(\mathrm{Y} / \mathrm{N})$

GC Column:

ID: $0.20(\mathrm{~mm})$ Length: 25

(m)

Purge Volume: 25.0 $(\mathrm{mL})$

\begin{tabular}{|c|c|c|c|c|c|}
\hline COMPOUND & $\overline{\mathrm{RRF}}$ & RRF5. 0 & $\begin{array}{l}\text { MIN } \\
\text { RRF }\end{array}$ & $\stackrel{\circ}{0}$ & MAX $\because D$ \\
\hline Vinyl Chloride-d3 & 0.436 & 0.405 & 0.010 & -6.9 & 50.0 \\
\hline Chloroethane-d5 & 0.283 & 0.259 & 0.010 & -8.2 & 50.0 \\
\hline 1,1-Dichloroethene-d2 & 0.795 & 0.705 & 0.010 & -11.3 & 50.0 \\
\hline 2-Butanone-d5 & 0.041 & 0.034 & 0.010 & -18.8 & 50.0 \\
\hline Chloroform-d & 0.509 & 0.465 & 0.010 & -8.6 & 50.0 \\
\hline 1,2-Dichloroethane-d4 & 0.203 & 0.185 & 0.010 & -9.1 & 50.0 \\
\hline Benzene-d6 & 1.475 & 1.364 & 0.010 & -7.5 & 50.0 \\
\hline 1,2-Dichloropropane-d6 & 0.374 & 0.333 & 0.010 & -11.0 & 50.0 \\
\hline Toluene-d8 & 1.397 & 1.377 & 0.010 & -1.5 & 50.0 \\
\hline trans-1,3-Dichloropropene-d4 & 0.230 & 0.206 & 0.010 & -10.3 & 50.0 \\
\hline 2-Hexanone-d5 & 0.033 & 0.029 & 0.010 & $-11 \cdot 0$ & 50.0 \\
\hline $1,1,2,2$-Tetrachloroethane-d2 & 0.164 & 0.142 & 0.010 & -13.1 & 50.0 \\
\hline 1,2-Dichlorobenzene-d4 & 0.830 & 0.735 & 0.010 & -11.4 & 50.0 \\
\hline
\end{tabular}

Report 1,4-Dioxane-d8 for Low/Medium VOA analysis only 
Lab Name: TESTAMERICA BURLINGTON

Contract: $8 \mathrm{E}-00302$

Lab Code: STLV Case No.: INMAN Mod. Ref No.: SDG No.: 200-6709

Matrix: (SOIL/SED/WATER)

Sample wt/vol: 25.0 Water $(\mathrm{g} / \mathrm{mL}) \quad \mathrm{mL}$

Level: (TRACE/LOW/MED) TRACE

응 Moisture: not dec.

GC Column: DB-624 ID: $0.20 \quad(\mathrm{~mm})$

Soil Extract Volume: (uL) (mL)

Purge Volume: 25.0
Lab Sample ID: MB 200-24273/3

Lab File ID: DHPT03.D

Date Received:

Date Analyzed: 08/26/2011

Dilution Factor: 1.0

Soil Aliquot Volume: (uL)

\begin{tabular}{|c|c|c|c|}
\hline CAS NO. & COMPOUND & $\begin{array}{l}\text { CONCENTRATION UNITS: } \\
(\mathrm{ug} / \mathrm{L} \text { or } \mathrm{ug} / \mathrm{kg}) \mathrm{ug} / \mathrm{L}\end{array}$ & c \\
\hline $75-71-8$ & Dichlorodifluoromethane & 0.50 & $\overline{\mathrm{U}}$ \\
\hline $74-87-3$ & Chloromethane & 0.50 & $\mathrm{U}$ \\
\hline $75-01-4$ & Vinyl chloride & 0.50 & $\mathrm{U}$ \\
\hline $74-83-9$ & Bromomethane & 0.50 & $\mathrm{U}$ \\
\hline $75-00-3$ & Chloroethane & 0.50 & $\mathrm{U}$ \\
\hline $75-69-4$ & Trichlorofluoromethane & 0.50 & $\mathrm{U}$ \\
\hline $75-35-4$ & 1,1-Dichloroethene & 0.50 & $\mathrm{U}$ \\
\hline $76-13-1$ & 1,1,2-Trichloro-1,2,2-trifluoroethane & 0.50 & $\mathrm{U}$ \\
\hline $67-64-1$ & Acetone & 5.0 & $\mathrm{U}$ \\
\hline $75-15-0$ & Carbon disulfide & 0.50 & $\mathrm{U}$ \\
\hline $79-20-9$ & Methyl acetate & 0.50 & $\mathrm{U}$ \\
\hline $75-09-2$ & Methylene Chloride & 0.50 & $\mathrm{U}$ \\
\hline $156-60-5$ & trans-1,2-Dichloroethene & 0.50 & $\mathrm{U}$ \\
\hline $1634-04-4$ & Methyl tert-butyl ether & 0.50 & $\mathrm{U}$ \\
\hline $75-34-3$ & 1,1-Dichloroethane & 0.50 & $\mathrm{U}$ \\
\hline $156-59-2$ & cis-1,2-Dichloroethene & 0.50 & $\mathrm{U}$ \\
\hline $78-93-3$ & 2-Butanone & 5.0 & $\mathrm{U}$ \\
\hline $74-97-5$ & Bromochloromethane & 0.50 & $\mathrm{U}$ \\
\hline $67-66-3$ & Chloroform & 0.50 & $\mathrm{U}$ \\
\hline $71-55-6$ & 1,1,1-Trichloroethane & 0.50 & $\mathrm{U}$ \\
\hline $110-82-7$ & Cyclohexane & 0.50 & $\mathrm{U}$ \\
\hline $56-23-5$ & Carbon tetrachloride & 0.50 & U \\
\hline $71-43-2$ & Benzene & 0.50 & $\mathrm{U}$ \\
\hline $107-06-2$ & 1,2-Dichloroethane & 0.50 & $\mathrm{U}$ \\
\hline
\end{tabular}

Report 1,4-Dioxane for Low-Medium VOA analysis only 
$1 B$ - FORM I VOA-2

VOLATILE ORGANICS ANALYSIS DATA SHEET
EPA SAMPLE NO.

VBLKDQ
Lab Name: TESTAMERICA BURLINGTON

Lab Code: STLV

Matrix: (SOIL/SED/WATER)

Sample wt/vol: 25.0

Water

$(\mathrm{g} / \mathrm{mL}) \quad \mathrm{mL}$

Level: (TRACE/LOW/MED) TRACE

o Moisture: not dec.

GC Column: DB-624

ID: $0.20 \quad$ (mm)

Soil Extract Volume:

(mL)

Purge Volume: 25.0
Contract: $8 \mathrm{E}-00302$

SDG No.: 200-6709

Lab Sample ID: MB 200-24273/3

Lab File ID: DHPT03.D

Date Received:

Date Analyzed: 08/26/2011

Dilution Factor: 1.0

Soil Aliquot Volume:

\begin{tabular}{|c|c|c|c|}
\hline CAS NO. & COMPOUND & $\begin{array}{l}\text { CONCENTRATION UNITS: } \\
(\mathrm{ug} / \mathrm{L} \text { or } u g / \mathrm{kg}) \mathrm{ug} / \mathrm{L}\end{array}$ & $Q$ \\
\hline $79-01-6$ & Trichloroethene & 0.50 & $\mathrm{U}$ \\
\hline $108-87-2$ & Methylcyclohexane & 0.50 & $\mathrm{U}$ \\
\hline $78-87-5$ & 1,2-Dichloropropane & 0.50 & $\mathrm{U}$ \\
\hline $75-27-4$ & Bromodichloromethane & 0.50 & $\mathrm{U}$ \\
\hline $10061-01-5$ & cis-1,3-Dichloropropene & 0.50 & $\mathrm{U}$ \\
\hline $108-10-1$ & 4-Methyl-2-pentanone & 5.0 & $\mathrm{U}$ \\
\hline $108-88-3$ & Toluene & 0.0076 & $\mathrm{~J}$ \\
\hline $10061-02-6$ & trans-1,3-Dichloropropene & 0.50 & $\mathrm{U}$ \\
\hline $79-00-5$ & 1,1,2-Trichloroethane & 0.50 & $\mathrm{U}$ \\
\hline $127-18-4$ & Tetrachloroethene & 0.50 & $\mathrm{U}$ \\
\hline $591-78-6$ & 2-Hexanone & 5.0 & $\mathrm{U}$ \\
\hline $124-48-1$ & Dibromochloromethane & 0.50 & $\mathrm{U}$ \\
\hline $106-93-4$ & 1,2-Dibromoethane & 0.50 & $\mathrm{U}$ \\
\hline $108-90-7$ & Chlorobenzene & 0.50 & U \\
\hline $100-41-4$ & Ethylbenzene & 0.50 & U \\
\hline $95-47-6$ & o-xylene & 0.50 & $\mathrm{U}$ \\
\hline $179601-23-1$ & $\mathrm{~m}, \mathrm{p}-\mathrm{Xy}$ lene & 0.50 & $\mathrm{U}$ \\
\hline $100-42-5$ & Styrene & 0.50 & $\mathrm{U}$ \\
\hline $75-25-2$ & Bromoform & 0.50 & $\mathrm{U}$ \\
\hline $98-82-8$ & Isopropylbenzene & 0.50 & $\mathrm{U}$ \\
\hline $79-34-5$ & $1,1,2,2$-Tet rachloroethane & 0.50 & $\mathrm{U}$ \\
\hline $541-73-1$ & 1,3-Dichlorobenzene & 0.50 & $\mathrm{U}$ \\
\hline $106-46-7$ & 1,4-Dichlorobenzene & 0.50 & $\mathrm{U}$ \\
\hline $95-50-1$ & 1,2-Dichlorobenzene & 0.50 & $\mathrm{U}$ \\
\hline $96-12-8$ & 1,2-Dibromo-3-Chloropropane & 0.50 & $\mathrm{U}$ \\
\hline $120-82-1$ & 1,2,4-Trichlorobenzene & 0.50 & $\mathrm{U}$ \\
\hline $87-61-6$ & 1,2,3-Trichlorobenzene & 0.50 & $\mathrm{U}$ \\
\hline
\end{tabular}


1J - FORM I VOA-TIC

VOLATILE ORGANICS ANALYSIS DATA SHEET

TENTATIVELY IDENTIEIED COMPOUNDS
EPA SAMPLE NO.

VBLKDQ

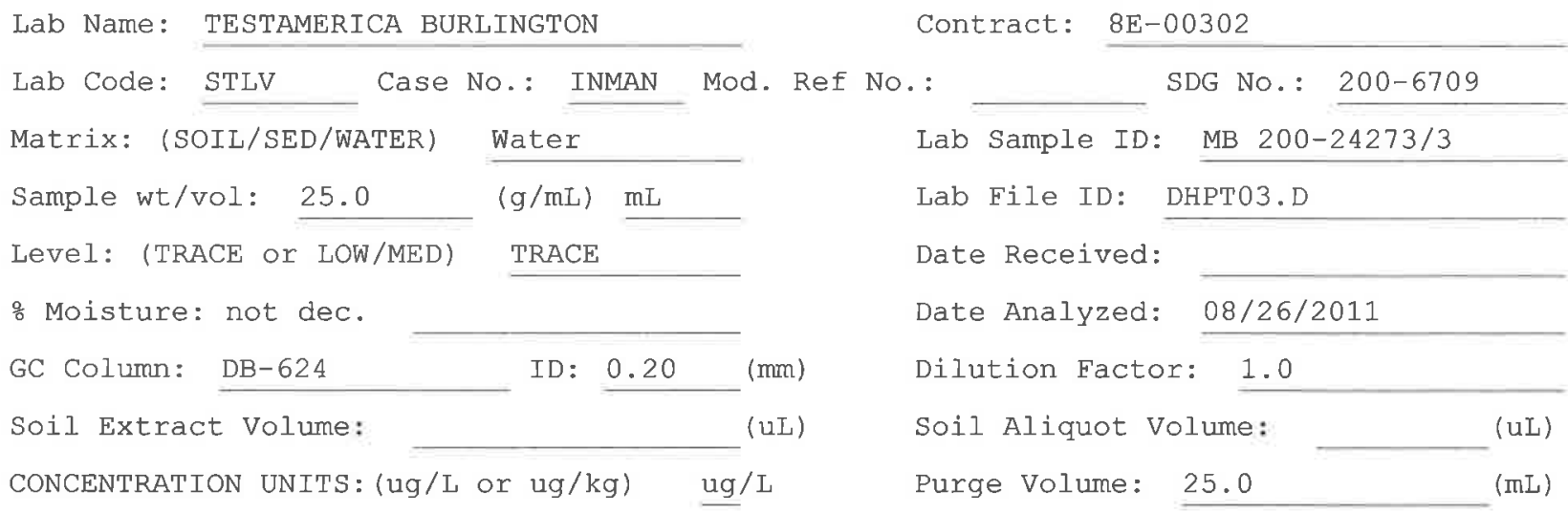

01

02

\begin{tabular}{|l|l|c|c|c|}
\hline CAS NUMBER & \multicolumn{1}{|c|}{ COMPOUND NAME } & RT & EST. CONC. & Q \\
\hline & Unknown & 6.79 & 2.8 & X J \\
\hline E9667961 & Total Alkanes & N/A & & \\
\hline
\end{tabular}

$1 \mathrm{EPA}$-designated Registry Number. 
$1 A$ - FORM I VOA-1

VOLATILE ORGANICS ANALYSIS DATA SHEET
EPA SAMPLE NO.

VHBLK01
Lab Name: TESTAMERICA BURLINGTON

Lab Code: STLV

Matrix: (SOIL/SED/WATER)

Sample wt/vol: 25.0

$(\mathrm{g} / \mathrm{mL}) \quad \mathrm{mL}$

Level: (TRACE/LOW/MED) TRACE

응 Moisture: not dec.

GC Column: DB-624

Soil Extract Volume: ID: 0.20 (mrn) (uL)

(mL)

Purge Volume: 25.0
Contract: $8 \mathrm{E}-00302$

SDG No.: $200-6709$

\begin{tabular}{|c|c|c|c|}
\hline CAS NO. & COMPOUND & $\begin{array}{l}\text { CONCENTRATION UNITS: } \\
(\mathrm{ug} / \mathrm{L} \text { or } \mathrm{ug} / \mathrm{kg}) \mathrm{ug} / \mathrm{L}\end{array}$ & $Q$ \\
\hline $75-71-8$ & Dichlorodifluoromethane & 0.50 & $\mathrm{U}$ \\
\hline $74-87-3$ & Chloromethane & 0.50 & $\mathrm{U}$ \\
\hline $75-01-4$ & Vinyl chloride & 0.50 & $\mathrm{U}$ \\
\hline $74-83-9$ & Bromomethane & 0.50 & $\mathrm{U}$ \\
\hline $75-00-3$ & Chloroethane & 0.50 & $\mathrm{U}$ \\
\hline $75-69-4$ & Trichlorofluoromethane & 0.50 & $\mathrm{U}$ \\
\hline $75-35-4$ & 1,1-Dichloroethene & 0.50 & $\mathrm{U}$ \\
\hline $76-13-1$ & 1,1,2-Trichloro-1,2,2-trifluoroethane & 0.50 & $\mathrm{U}$ \\
\hline $67-64-1$ & Acetone & 5.0 & $\mathrm{U}$ \\
\hline $75-15-0$ & Carbon disulfide & 0.50 & $\mathrm{U}$ \\
\hline $79-20-9$ & Methyl acetate & 0.50 & $\mathrm{U}$ \\
\hline $75-09-2$ & Methylene Chloride & 0.50 & $\mathrm{U}$ \\
\hline $156-60-5$ & trans-1,2-Dichloroethene & 0.50 & $\mathrm{U}$ \\
\hline $1634-04-4$ & Methyl tert-butyl ether & 0.50 & $\mathrm{U}$ \\
\hline $75-34-3$ & 1,1-Dichloroethane & 0.50 & $\mathrm{U}$ \\
\hline $156-59-2$ & cis-1,2-Dichloroethene & 0.50 & $\mathrm{U}$ \\
\hline $78-93-3$ & 2-Butanone & 5.0 & $\mathrm{U}$ \\
\hline $74-97-5$ & Bromochloromethane & 0.50 & $\mathrm{U}$ \\
\hline $67-66-3$ & Chloroform & 0.50 & $\mathrm{U}$ \\
\hline $71-55-6$ & 1,1,1-Trichloroethane & 0.50 & $\mathrm{U}$ \\
\hline $110-82-7$ & Cyclohexane & 0.50 & $\mathrm{U}$ \\
\hline $56-23-5$ & Carbon tetrachloride & 0.50 & $\mathrm{U}$ \\
\hline $71-43-2$ & Benzene & 0.50 & $\mathrm{U}$ \\
\hline $107-06-2$ & 1,2-Dichloroethane & 0.50 & $\mathrm{U}$ \\
\hline
\end{tabular}

Report 1,4-Dioxane for Low-Medium VOA analysis only

Lab Sample ID: 200-6709-5

Lab File ID: DHPT08.D

Date Received:

Date Analyzed: 08/26/2011

Dilution Factor: 1.0

Soil Aliquot Volume: 
Lab Name: TESTAMERICA BURLINGTON

Lab Code: STLV

Matrix: (SOIL/SED/WATER)

Sample wt/vol: 25.0

Case No.: INMAN

Water

$(\mathrm{g} / \mathrm{mL}) \quad \mathrm{mL}$

Level: (TRACE/LOW/MED) TRACE

\% Moisture: not dec.

GC Column: DB-624 ID $: 0.20$

Soil Extract volume:

Purge Volume: 25.0

(mL)
Contract: $8 \mathrm{E}-00302$

SDG No.: 200-6709

\begin{tabular}{|c|c|c|c|}
\hline CAS NO. & COMPOUND & $\begin{array}{l}\text { CONCENTRATION UNITS: } \\
(\mathrm{ug} / \mathrm{L} \text { or } \mathrm{ug} / \mathrm{kg}) \mathrm{ug} / \mathrm{L}\end{array}$ & $Q$ \\
\hline $79-01-6$ & Trichloroethene & 0.50 & $\bar{U}$ \\
\hline $108-87-2$ & Methylcyclohexane & 0.50 & $\mathrm{U}$ \\
\hline $78-87-5$ & 1,2-Dichloropropane & 0.50 & U \\
\hline $75-27-4$ & Bromodichloromethane & 0.50 & $\mathrm{U}$ \\
\hline $10061-01-5$ & cis-1,3-Dichloropropene & 0.50 & $\mathrm{U}$ \\
\hline $108-10-1$ & 4-Methyl-2-pentanone & 5.0 & $\mathrm{U}$ \\
\hline $108-88-3$ & Toluene & 0.50 & $\mathrm{U}$ \\
\hline $10061-02-6$ & trans-1,3-Dichloropropene & 0.50 & $\mathrm{U}$ \\
\hline $79-00-5$ & 1,1,2-Trichloroethane & 0.50 & $\mathrm{U}$ \\
\hline $127-18-4$ & Tetrachloroethene & 0.50 & $\mathrm{U}$ \\
\hline $591-78-6$ & 2-Hexanone & 5.0 & $\mathrm{U}$ \\
\hline $124-48-1$ & Dibromochloromethane & 0.50 & $\mathrm{U}$ \\
\hline $106-93-4$ & 1,2-Dibromoethane & 0.50 & $\mathrm{U}$ \\
\hline $108-90-7$ & Chlorobenzene & 0.50 & $\mathrm{U}$ \\
\hline $100-41-4$ & Ethylbenzene & 0.50 & $\mathrm{U}$ \\
\hline $95-47-6$ & o-Xylene & 0.50 & $\mathrm{U}$ \\
\hline $179601-23-1$ & $\mathrm{~m}, \mathrm{p}-\mathrm{xy}$ lene & 0.50 & $\mathrm{U}$ \\
\hline $100-42-5$ & Styrene & 0.50 & $\mathrm{U}$ \\
\hline $75-25-2$ & Bromoform & 0.50 & $\mathrm{U}$ \\
\hline $98-82-8$ & Isopropylbenzene & 0.50 & $\mathrm{U}$ \\
\hline $79-34-5$ & $1,1,2,2$-Tetrachloroethane & 0.50 & $\mathrm{U}$ \\
\hline $541-73-1$ & 1,3-Dichlorobenzene & 0.50 & $\mathrm{U}$ \\
\hline $106-46-7$ & 1,4-Dichlorobenzene & 0.50 & $\mathrm{U}$ \\
\hline $95-50-1$ & 1,2-Dichlorobenzene & 0.50 & $\mathrm{U}$ \\
\hline $96-12-8$ & 1,2-Dibromo-3-Chloropropane & 0.50 & $\mathrm{U}$ \\
\hline $120-82-1$ & 1,2,4-Trichlorobenzene & 0.50 & $\mathrm{U}$ \\
\hline $87-61-6$ & 1,2,3-Trichlorobenzene & 0.50 & $\mathrm{U}$ \\
\hline
\end{tabular}

Lab Sample ID: 200-6709-5

Lab File ID: DHPT08.D

Date Received:

Date Analyzed: 08/26/2011

Dilution Factor: 1.0

Soil Aliquot Volume:

(uL) 
$1 \mathrm{~J}$ - FORM I VOA-TIC

VOLATILE ORGANICS ANALYSIS DATA SHEET

TENTATIVELY IDENTIFIED COMPOUNDS
EPA SAMPLE NO.

VHBLK01
Lab Name: TESTAMERICA BURLINGTON

Lab Code: STLV Case No.: INMAN

Matrix: (SOIL/SED/WATER)

Sample wt/vol: 25.0

Water

$(\mathrm{g} / \mathrm{mL}) \quad \mathrm{mL}$

Level: (TRACE or LOW/MED)

TRACE

응 Moisture: not dec.

GC Column: DB-624

ID : 0.20 (mm)

Soil Extract Volume: (uL)

CONCENTRATION UNITS: (ug/L or $\mathrm{ug} / \mathrm{kg}$ )

$\mathrm{ug} / \mathrm{L}$
Contract: $\quad 8 \mathrm{E}-00302$

SDG No.: 200-6709

Lab Sample ID: 200-6709-5

Lab File ID: DHPT08.D

Date Received:

Date Analyzed: 08/26/2011

Dilution Factor: 1.0

Soil Aliquot Volume:

Purge Volume: 25.0 (mL)

\begin{tabular}{|l|l|l|r|r|r|}
\hline \multicolumn{1}{|c|}{ CAS NUMBER } & \multicolumn{1}{|c|}{ COMPOUND NAME } & RT & EST. CONC. & Q \\
\cline { 2 - 6 } 01 & & Unknown & 6.79 & B X J \\
\cline { 2 - 6 } & E9667961 & Total Alkanes & N/A & & \\
\hline
\end{tabular}

1 EPA-designated Registry Number. 


\section{TestAmerica}

THE LEADER IN ENVIRONMENTAL TESTING

\section{ANALYTICAL REPORT}

Job Number: 200-6730-1

SDG Number: $200-6730$

Job Description: Inman (200-6730)

Contract Number: EP-W-09-044

For:

Argonne National Laboratory

9700 South Cass Avenue

Building 203

Office B-149

Argonne, IL 60439

Attention: Mr. Clyde Dennis

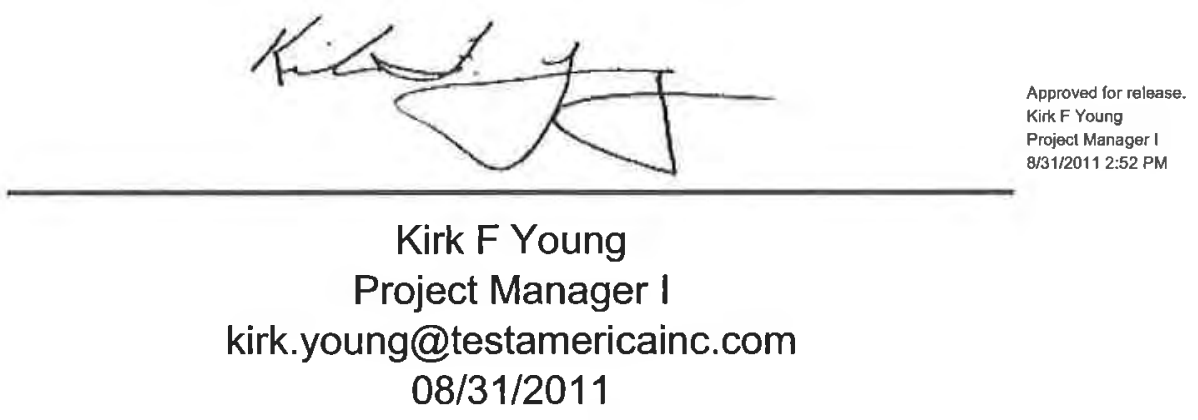

The test results in this report relate only to sample(s) as received by the laboratory. These test results were derived under a quality system that adheres to the requirements of NELAC. Pursuant to NELAC, this report may not be produced in full without written approval from the laboratory 


\section{Table of Contents}

Cover Title Page ........................ 1

Report Narrative . . . . . . . . . . . . . . . . . . 4

Case Narrative . . . . . . . . . . . . . . . . . . . . . 4

Qualifier Definition ............................ 6

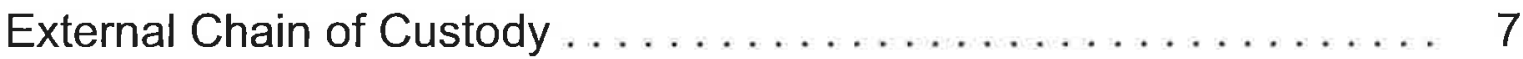

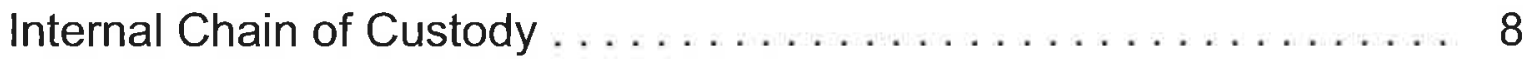

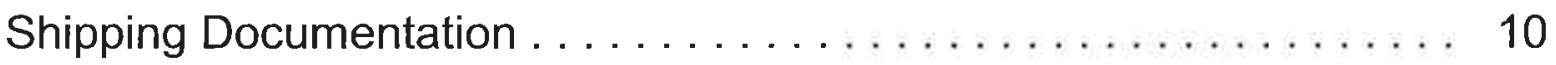

Airbills (if Applicable) . . . . . . . . . . . . . . . . . . . . 11

Sample Receipt and Log In Check List..................... 12

Methodology Review . . . . . . . . . . . . . . . . . . . . 14

QC Summary - SOM01.2 Volatiles-Trace . . . . . . . . . . . . . . 15

QC Summary - SOM01.2 Volatiles-Trace . . . . . . . . . . . . . . . 15

Deuterated Monitoring Compound Summary $\ldots \ldots \ldots \ldots \ldots \ldots \ldots \ldots \ldots \ldots \ldots \ldots \ldots \ldots \ldots \ldots$

Method Blank $\ldots \ldots \ldots \ldots \ldots \ldots \ldots \ldots \ldots \ldots \ldots \ldots \ldots \ldots \ldots \ldots, 17$

GC/MS Instrument Performance Check $\ldots \ldots \ldots \ldots \ldots \ldots \ldots \ldots \ldots \ldots \ldots \ldots \ldots \ldots \ldots \ldots \ldots, 18$

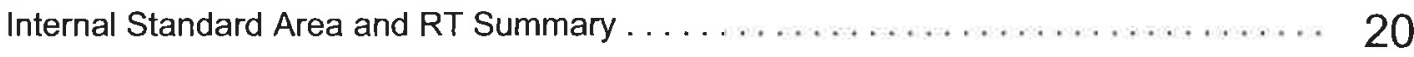

Sample Data - SOM01.2 Volatiles-Trace . . . . . . . . . . . . . 21

Sample Data - SOM01.2 Volatiles-Trace . . . . . . . . . . . . . . . 21

INKTW-W-32743 ............................................ 21

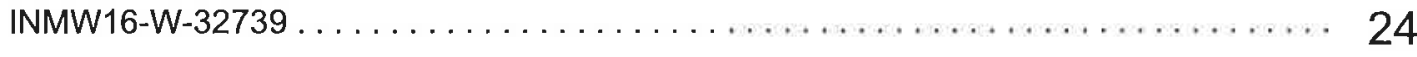

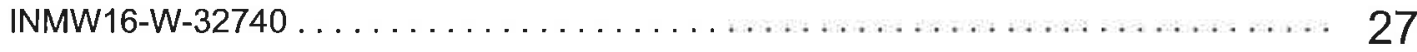

INMW16-W-32741 ........................................ 30

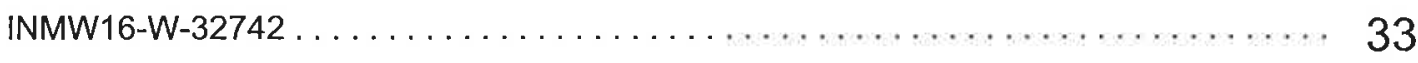

INQCTB-W-32744 .................................... 36

Standards - SOM01.2 Volatiles-Trace . . . . . . . . . . . . . 39

Standards - SOM01.2 Volatiles-Trace ...................... 39 


\section{Table of Contents}

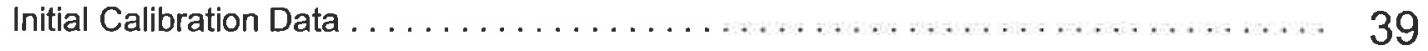

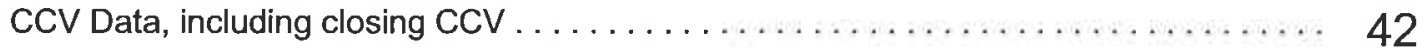

Raw Qc Data - SOM01.2 Volatiles-Trace . . . . . . . . . . . . . . . 48

Raw Qc Data - SOM01.2 Volatiles-Trace . . . . . . . . . . . . . . . . 48

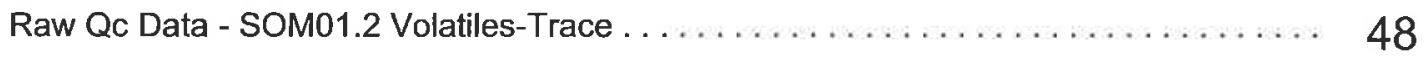

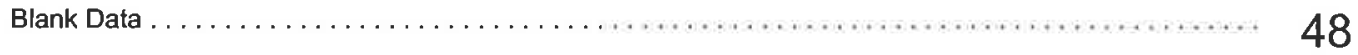




\title{
CASE NARRATIVE
}

\section{Client: Argonne National Laboratory}

\author{
Project: Inman (200-6730)
}

\author{
Report Number: 200-6730-1
}

Enclosed is the data set for the referenced project work. With the exceptions noted as flags or footnotes, standard analytical protocols were followed in performing the analytical work and the applied control limits were met.

Calculations were performed before rounding to avoid round-off errors in calculated results.

All holding times were met and proper preservation noted for the methods performed on these samples, unless otherwise detailed in the individual sections below.

\section{Receipt}

The samples were received on $08 / 26 / 2011$. Documentation of the condition of the samples at the time of their receipt and any exception to the laboratory's Sample Acceptance Policy is documented in the Shipping and Receiving section of this submittal. The samples, as received, were not acid preserved. On that basis, the laboratory did provide for the analytical work to be performed within seven days of sample collection.

\section{SOM01.2 Volatile Organics (Trace Level Water)}

A storage blank was prepared for volatile organics analysis, and stored in association with the storage of the samples. That storage blank, identified as VHBLK01, was carried through the holding period with the samples, and analyzed.

Each sample in the sample set was analyzed without a dilution. Each of the analyses associated with the sample set exhibited an acceptable internal standard performance. There was an acceptable recovery of each deuterated monitoring compound (DMC) in the analysis of the method blank associated with the analytical work, and in the analysis of the storage blank associated with the sample set. The analysis of the samples in this sample set did meet the technical acceptance criteria specific to DMC recoveries, although not all DMC recoveries were within the control range in each analysis. The technical acceptance criteria does provide for the recovery of up to three DMCs to fall outside of the control range in the analysis of field samples. Matrix spike and matrix spike duplicate analyses were not performed on samples in this sample set. The analysis of the method blank associated with the analytical work was free of analyte contamination. A trace concentration of chloroform was identified in the analysis of the method blank associated with the sample set. The concentration of chloroform in that analysis was below the established reporting limit, and the analysis did meet the technical acceptance criteria for a compliant method blank analysis. Present in the method blank and storage blank analyses was a non-target constituent that represents a compound that is related to the DMC formulation. The fact that the presence of this compound is not within the laboratory's control is at issue. The derived results for that compound have been qualified with an " $X$ " qualifier to reflect the source of the contamination.

The responses for each of the target analytes met the relative standard deviation criterion in the initial calibration. The response for each target analyte met the percent difference criterion in the 
opening/continuing calibration check acquisition. The response for each target analyte met the 50.0 percent difference criterion in the closing calibration check acquisition.

The primary quantitation mass for methylcyclohexane that is specified in the Statement of Work is mass 83 . The laboratory did identify a contribution to mass 83 from 1,2-dichloropropane- $\mathrm{d}_{6}$, one of the deuterated monitoring compounds (DMCs). The laboratory did change the primary quantitation mass assignment to mass 55 for the quantification of methylcyclohexane.

Manual integration was employed in deriving certain of the analytical results. The values that have been derived from manual integration are qualified on the quantitation reports. Extracted ion current profiles for each manual integration are included in the data package, and further documented at the end of this submittal. 


\section{DATA REPORTING QUALIFIERS}

Client: Argonne National Laboratory

Job Number: 200-6730-1

Sdg Number: $200-6730$

GC/MS VOA

U

J

J

X

B
Analyzed for but not detected.

Indicates an Estimated Value for TICs

Indicates an estimated value.

See case narrative notes for explanation of the ' $X$ ' flag

Surrogate exceeds the control limit

The analyte was found in an associated blank, as well as in the sample. 

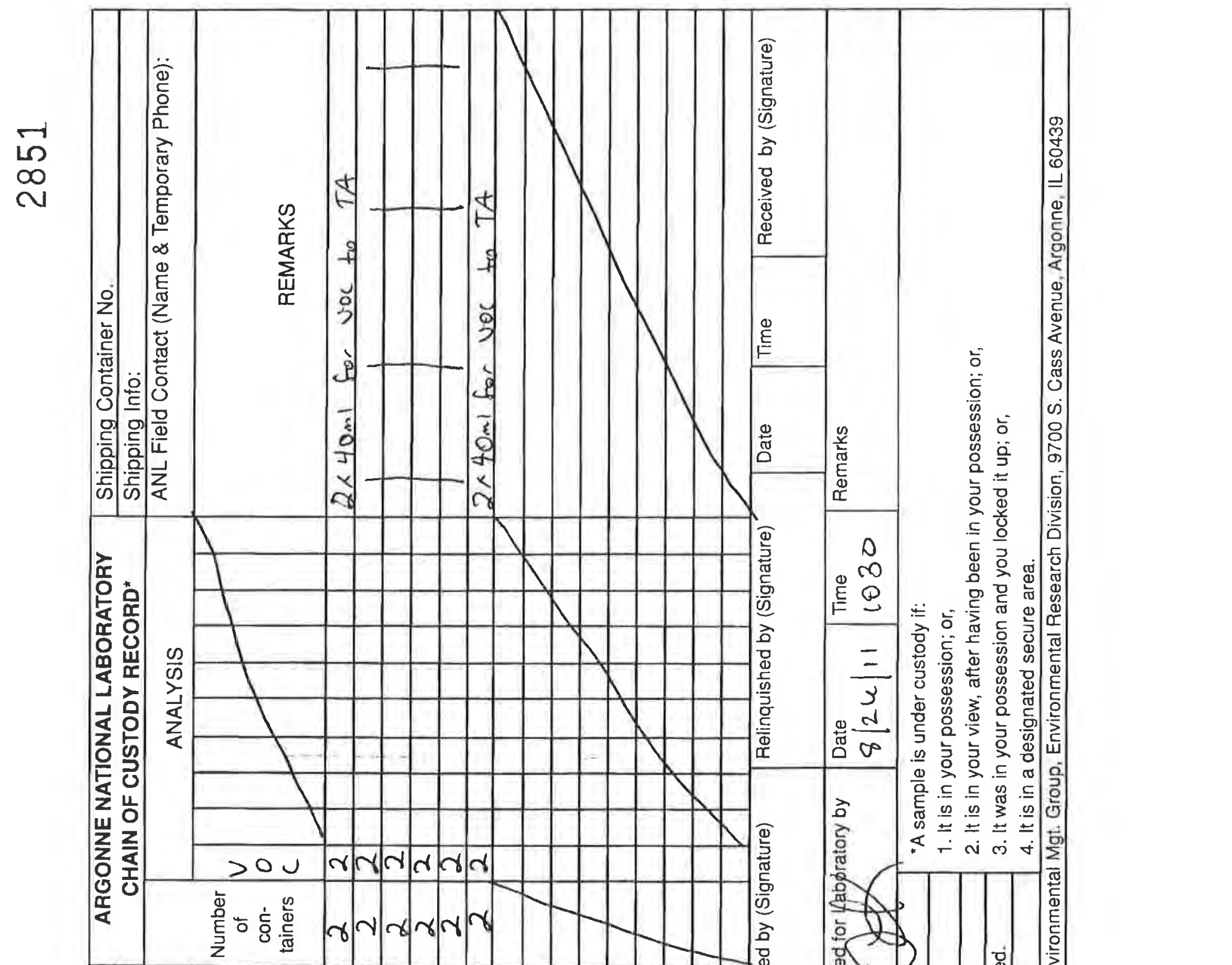


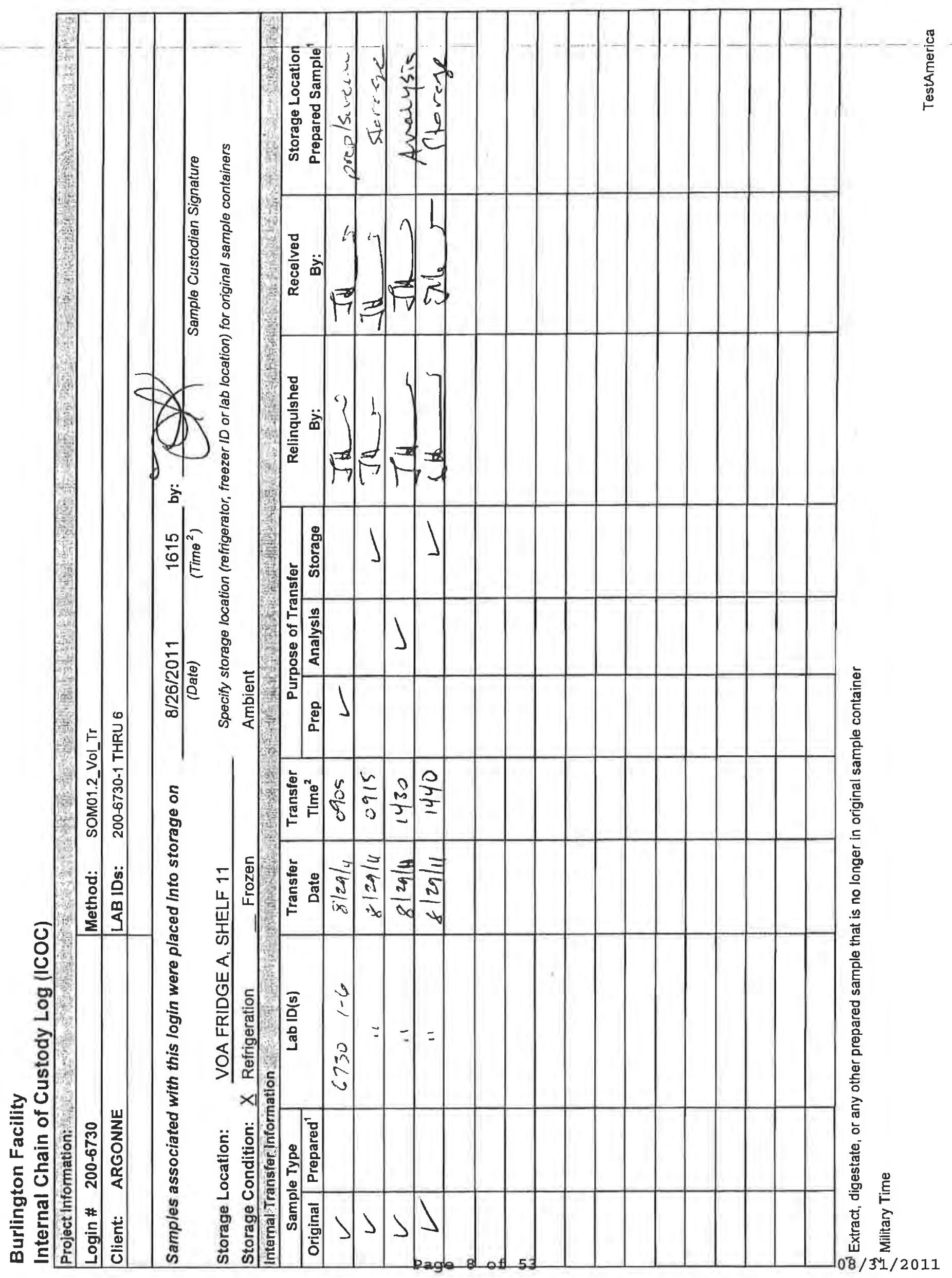




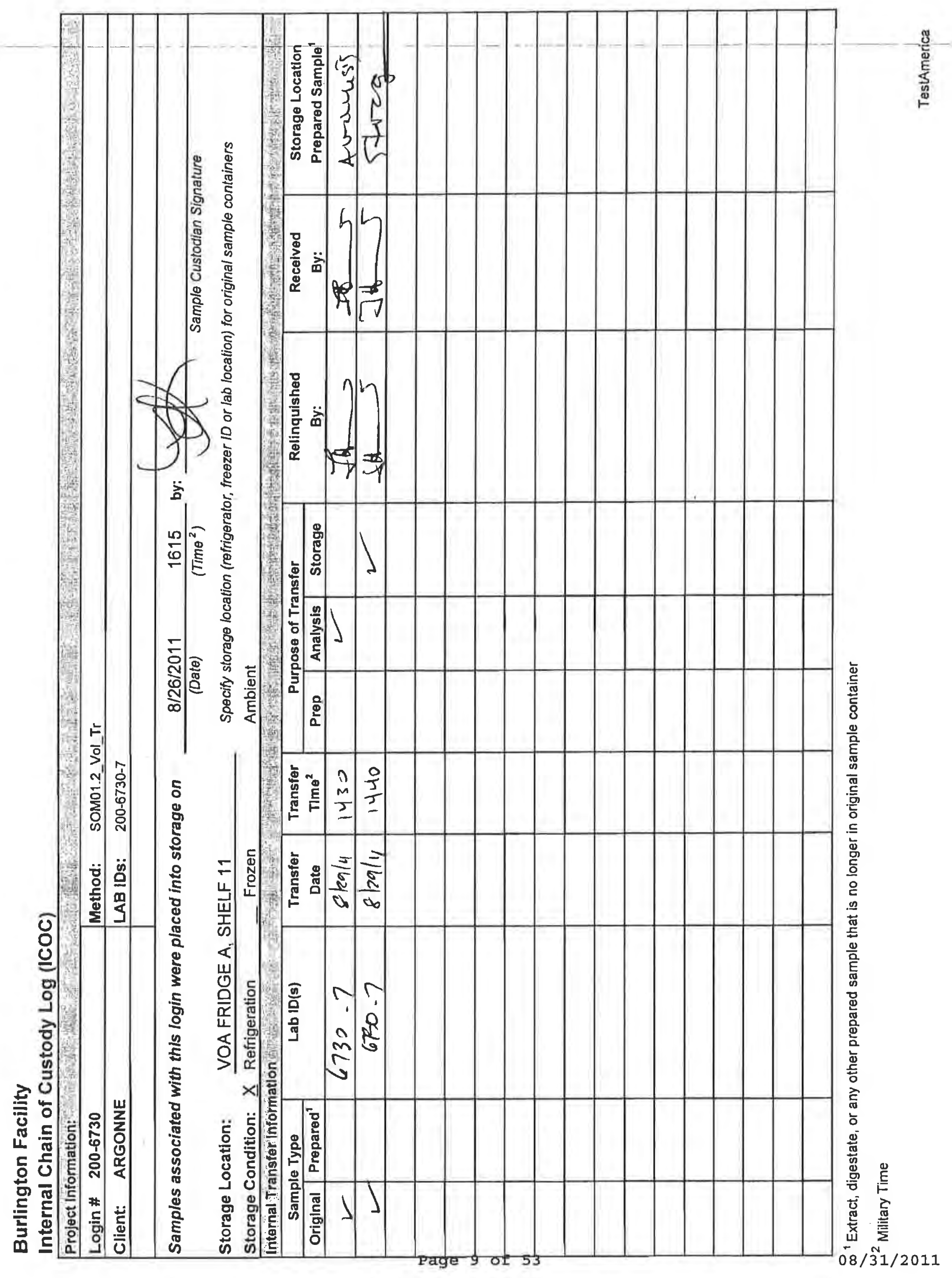




\section{Shipping and Receiving Documents}




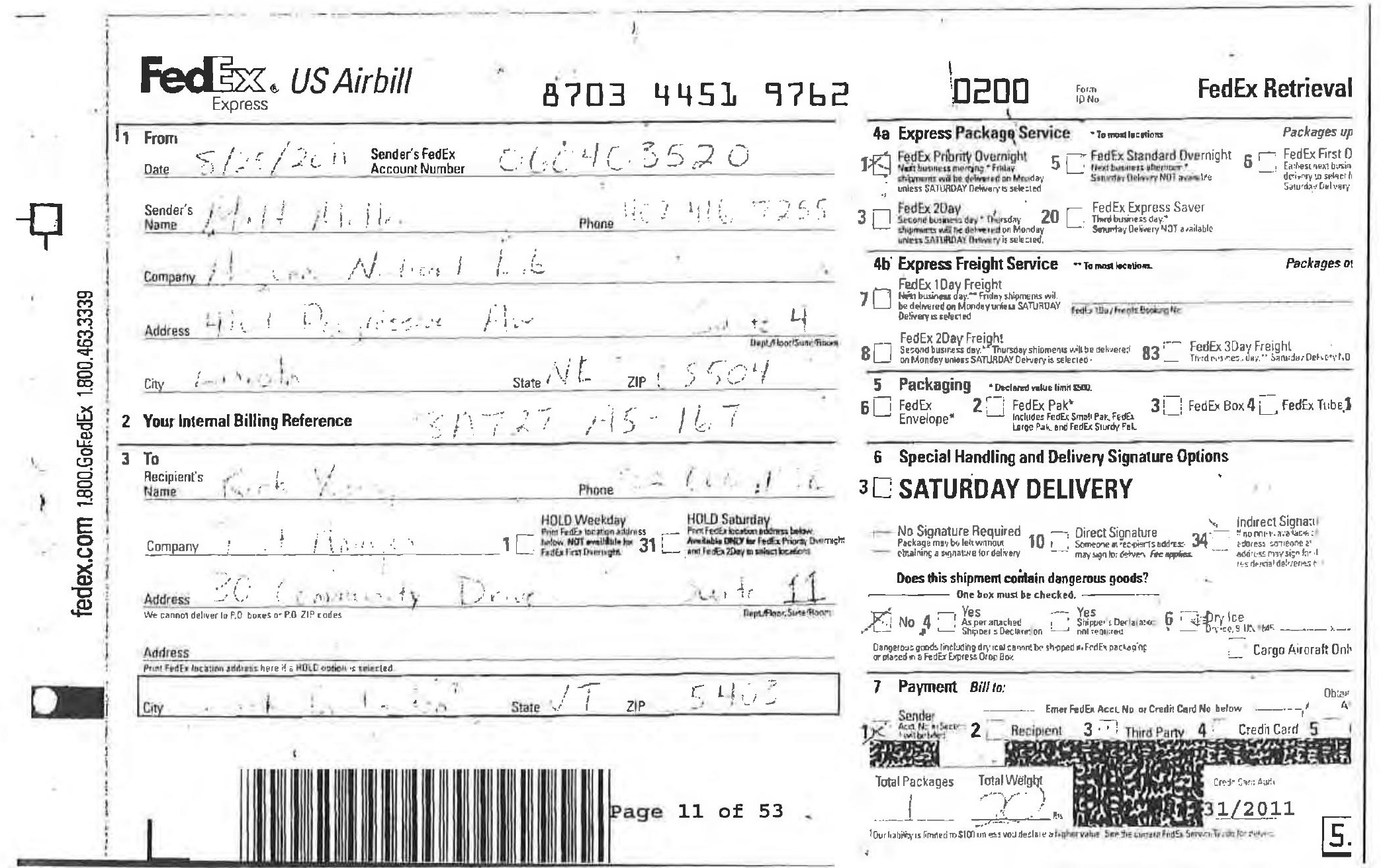




\section{Login Sample Receipt Checklist}

Client: Argonne National Laboratory

Job Number: 200-6730-1

SDG Number: $200-6730$

Login Number: 6730

List Source: TestAmerica Burlington

List Number: 1

Creator: Keeton, Jamie

Question

Radioactivity either was not measured or, if measured, is at or below background

The cooler's custody seal, if present, is intact.

The cooler or samples do not appear to have been compromised or tampered with.

Samples were received on ice.

Cooler Temperature is acceptable.

Cooler Temperature is recorded.

$\mathrm{COC}$ is present.

COC is filled out in ink and legible.

$\mathrm{COC}$ is filled out with all pertinent information.

Is the Field Sampler's name present on COC?

There are no discrepancies between the sample IDs on the containers and the COC.

Samples are received within Holding Time.

Sample containers have legible labels.

Containers are not broken or leaking.

Sample collection date/times are provided.

Appropriate sample containers are used.

Sample bottles are completely filled.

Sample Preservation Verified.

There is sufficient vol. for all requested analyses, incl. any requested

MS/MSDs

VOA sample vials do not have headspace or bubble is $<6 \mathrm{~mm}(1 / 4 ")$ in diameter.

Multiphasic samples are not present.

Samples do not require splitting or compositing.

Residual Chlorine Checked.

\begin{tabular}{l} 
Answ \\
N/A \\
True \\
True \\
True \\
True \\
True \\
True \\
True \\
False \\
True \\
True \\
\hline True \\
True \\
True \\
True \\
True \\
True \\
N/A \\
True \\
True \\
True \\
True \\
N/A
\end{tabular}

$5.1^{\circ} \mathrm{C}$, IR GUN ID 96, CF 0

Sampling times not listed on COC, logged in per container labels.

\section{(1)}

NO CUSTODY SEAL NUMBERS

True

True

True

True

False

True

True

True

True

True

True

True

True

N/A

True

True

True

N/A
Check done at department level as required. 


\section{Sample Login Acknowledgement}

Job 200-6730-1

$\begin{array}{ll}\text { Client Job Description: } & \text { Inman (200-6730) } \\ \text { Purchase Order \#: } & 8 \mathrm{E}-00302 \\ \text { Work Order \#: } & 8 \mathrm{E}-00302 \\ \text { Project Manager: } & \text { Kirk F Young } \\ \text { Job Due Date: } & 9 / 9 / 2011 \\ \text { Job TAT: } & \text { 14 Days } \\ \text { Max Deliverable Level: } & \text { IV } \\ & \\ \text { Earliest Deliverable Due: } & 9 / 9 / 2011\end{array}$

\begin{tabular}{|c|c|}
\hline Report To: & $\begin{array}{l}\text { Argonne National Laboratory } \\
\text { Jorge Alvarado }\end{array}$ \\
\hline & $\begin{array}{l}9700 \text { South Cass Avenue } \\
\text { Building } 203 \\
\text { Office B-149 } \\
\text { Argonne, IL } 60439\end{array}$ \\
\hline Bill To: & $\begin{array}{l}\text { Argonne National Laboratory } \\
\text { Accounts Payable } \\
\text { Chief Financial Offices } \\
9700 \text { S. Cass Ave. } \\
\text { Building } 201 \\
\text { Argonne, IL } 60439\end{array}$ \\
\hline
\end{tabular}

Login 200-6730

\begin{tabular}{|c|c|}
\hline Sample Receipt: & $8 / 26 / 2011 \quad 10: 30: 00$ AM \\
\hline Method of Delivery: & FedEx Priority Overnight \\
\hline $\begin{array}{l}\text { Lab Sample \# } \\
\text { Method }\end{array}$ & $\begin{array}{l}\text { Client Sample ID } \\
\text { Method Description / Work Location }\end{array}$ \\
\hline $200-6730-1$ & INMW16-W-32739 \\
\hline SOM01.2_Vol_Tr & SOM01.2 Trace Volatile Organics / In-La \\
\hline $200-6730-2$ & INMW16-W-32740 \\
\hline SOM01.2_Vol_Tr & SOM01.2 Trace Volatile Organics / In-Lab \\
\hline $200-6730-3$ & INMW16-W-32741 \\
\hline SOM01.2_Vol_Tr & SOM01.2 Trace Vo \\
\hline $200-6730-4$ & INMW16-W-32742 \\
\hline SOM01.2_Vol_Tr & SOM01.2 Trace Volatile Organics / In-La \\
\hline $200-6730-5$ & INKTW-W-32743 \\
\hline SOM01.2_Vol_Tr & SOM01.2 Trace Volatile Organics / In-Lab \\
\hline \multirow{2}{*}{$\begin{array}{l}\text { 200-6730-6 } \\
\text { SOM01.2_Vol_Tr }\end{array}$} & INQCTB-W-32744 \\
\hline & SOM01.2 Trace \\
\hline \multicolumn{2}{|c|}{ 200-6730-7 VHBLK01 } \\
\hline SOM01.2_Vol_Tr & SOM01.2 Tr \\
\hline
\end{tabular}

$\begin{array}{lc}\text { Number of Coolers: } & 1 \\ \text { Cooler Temperature(s) }\left(C^{\circ}\right): & 5.1 \text {; }\end{array}$

\begin{tabular}{|c|c|c|c|c|}
\hline \multirow{2}{*}{\multicolumn{2}{|c|}{ Date Sampled }} & \multicolumn{3}{|l|}{ Matrix } \\
\hline & & & Rpt Basis & Dry / Wet ** \\
\hline \multirow[t]{2}{*}{$8 / 25 / 2011$} & 4:15:00 PM & Water & & \\
\hline & & & Total & Wet \\
\hline \multirow[t]{2}{*}{$8 / 25 / 2011$} & 10:30:00 AM & Water & & \\
\hline & & & Total & Wet \\
\hline \multirow[t]{2}{*}{$8 / 25 / 2011$} & 10:00:00 AM & Water & & \\
\hline & & & Total & Wet \\
\hline \multirow[t]{2}{*}{$8 / 25 / 2011$} & 12:13:00 PM & Water & & \\
\hline & & & Total & Wet \\
\hline \multirow[t]{2}{*}{$8 / 25 / 2011$} & 11:18:00 AM & Water & & \\
\hline & & & Total & Wet \\
\hline \multirow[t]{2}{*}{$8 / 25 / 2011$} & 5:45:00 PM & Water & & \\
\hline & & & Total & Wet \\
\hline \multirow[t]{2}{*}{$8 / 26 / 2011$} & 6:12:00 PM & Water & & \\
\hline & & & Total & Wet \\
\hline
\end{tabular}




\section{METHODOLOGY SUMMARY}

\begin{tabular}{|c|c|c|}
\hline Laboratory: & TestAmerica Laboratories & Project No: \\
\hline Location: & South Burlington, Vermont & SDG No: \\
\hline
\end{tabular}

VOA

Volatile Organics Trace - USEPA CLP SOM01.2 
Lab Code: STLV Case No.: INMAN Mod. Ref No.: SDG No.: 200-6730 Level: (TRACE or LOW) TRACE

\begin{tabular}{|c|c|c|c|c|c|c|c|}
\hline $\begin{array}{c}\text { EPA } \\
\text { SAMPLE NO. }\end{array}$ & $\begin{array}{l}\text { VDMC1 } \\
\text { (VCL) \# }\end{array}$ & $\begin{array}{l}\text { VDMC2 } \\
\text { (CLA) \# }\end{array}$ & $\begin{array}{l}\text { VDMC3 } \\
\text { (DCE) \# }\end{array}$ & $\begin{array}{l}\text { VDMC4 } \\
\text { (BUT) \# }\end{array}$ & $\begin{array}{l}\text { VDMC5 } \\
\text { (CLF) \# }\end{array}$ & $\begin{array}{l}\text { VDMC6 } \\
\text { (DCA) \# }\end{array}$ & $\begin{array}{l}\text { VDMC7 } \\
\text { (BEN) \# }\end{array}$ \\
\hline VBLKDR & 90 & 89 & 64 & 96 & 83 & 93 & 91 \\
\hline INKTW-W-32743 & 97 & 96 & 70 & $189 *$ & 91 & 104 & 93 \\
\hline INQCTB-W-32744 & 94 & 93 & 67 & $171 *$ & 87 & 98 & 92 \\
\hline INMW1 6-W-32739 & 89 & 89 & 66 & $179 *$ & 84 & 94 & 94 \\
\hline INMW1 6-W-32740 & 102 & 100 & 73 & $193 \star$ & 94 & 103 & 101. \\
\hline INMW16-W-32741 & 94 & 93 & 68 & $176 *$ & 90 & 97 & 94 \\
\hline INMW1 $6-W-32742$ & 96 & 97 & 69 & $173 *$ & 91 & 96 & 97 \\
\hline VHBLK01 & 99 & 99 & 73 & 103 & 96 & 108 & 100 \\
\hline
\end{tabular}

\footnotetext{
VDMC1 (VCL) = Vinyl Chloride-d3

VDMC2 (CLA) = Chloroethane-d5

VDMC3 $(\mathrm{DCE})=1,1-\mathrm{Dich})$ loroethene-d2

VDMC4 (BUT) = 2-Butanone-d5

VDMC5 (CLF) = Chloroform-d

VDMC6 $(\mathrm{DCA})=1,2-\mathrm{Dichloroethane-d4}$

VDMC7 (BEN) = Benzene-d6
}

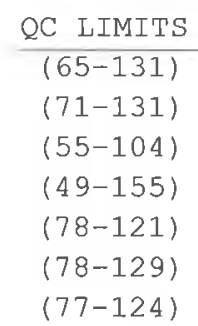

QC LIMITS

(65-131)

$(71-131)$

$(55-104)$

$(49-155)$

$(78-129)$

$(77-124)$

\# Column to be used to flag recovery values

* Values outside of contract required QC limits 
$2 \mathrm{~B}$ - FORM II VOA-2

WATER VOLATILE DEUTERATED MONITORING COMPOUND RECOVERY

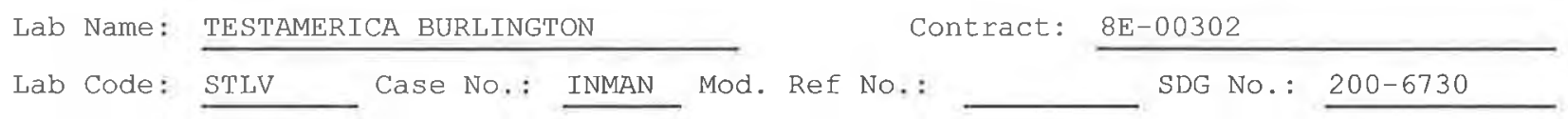

Level: (TRACE or LOW) TRACE

\begin{tabular}{|c|c|c|c|c|c|c|c|c|}
\hline $\begin{array}{c}\text { EPA } \\
\text { SAMPLE NO. }\end{array}$ & $\begin{array}{l}\text { VDMC8 } \\
\text { (DPA) \# }\end{array}$ & $\begin{array}{l}\text { VDMC9 } \\
\text { (TOL) \# }\end{array}$ & $\begin{array}{l}\text { VDMC } 10 \\
\text { (TDP) \# }\end{array}$ & $\begin{array}{l}\text { VDMC11 } \\
(\mathrm{HEX})\end{array}$ & $\begin{array}{l}\text { VDMC12 } \\
\text { (TCA) \# }\end{array}$ & $\begin{array}{l}\text { VDMC13 } \\
\text { (DCZ) \# }\end{array}$ & O'THER & $\begin{array}{l}\text { TOT } \\
\text { OUT }\end{array}$ \\
\hline VBLKDR & 89 & 90 & 88 & 98 & 82 & 99 & & 0 \\
\hline INKTW-W-32743 & 93 & 92 & 94 & 195 * & 87 & 103 & & 2 \\
\hline INMWI $6-W-32739$ & 90 & 93 & 88 & 208 * & 88 & 97 & & 2 \\
\hline INMW16-W-32740 & 100 & 101 & 97 & 212 * & 93 & 107 & & 2 \\
\hline VHBLKO1 & 100 & 101 & 97 & 108 & 92 & 117 & & 0 \\
\hline
\end{tabular}

$\begin{array}{ll}\text { VDMC8 } & (\text { DPA }=1,2-\text { Dichloropropane-d6 } \\ \text { VDMC9 } & (\text { TOL })=\text { Toluene-d8 } \\ \text { VDMC10 } & (\text { TDP }=\text { trans-1,3-Dichloropropene-d4 } \\ \text { VDMC11 } & (\mathrm{HEX})=2-\text { Hexanone-d5 } \\ \text { VDMC12 } & (\mathrm{TCA})=1,1,2,2-\text { Tetrachloroethane-d2 } \\ \text { VDMC13 } & (\mathrm{DCZ})=1,2-\mathrm{Dich} \text { lorobenzene-d4 }\end{array}$

\# Column to be used to flag recovery values

* Values outside of contract required QC limits

Report 1,4-Dioxane-d8 for Low-Medium VOA analysis only
QC LIMITS

$(79-124)$

$(77-121)$

$(73-121)$

$(28-135)$

$(73-125)$

$(80-131)$

Page 1 of 1 
$4 A$ - FORM IV VOA

VOLATILE METHOD BLANK SUMMARY
EPA SAMPLE NO.

VBLKDR
Lab Name: TESTAMERICA BURLINGTON

Lab Code: STLV Case No.: INMAN

Lab File ID: DHPU04.D

Instrument ID: D.i

Matrix: (SOIL/SED/WATER)

Water

Level: (TRACE or LOW/MED)

TRACE

GC Column: DB-624

\begin{tabular}{c|l|l|}
\hline \multicolumn{1}{|c|}{$\begin{array}{c}\text { EPA } \\
\text { SAMPLE NO. }\end{array}$} & \multicolumn{1}{c}{ LAB } \\
SAMPLE ID
\end{tabular}

Contract: $8 \mathrm{E}-00302$

SDG No.: 200-6730

Lab Sample ID: MB 200-24379/4

Date Analyzed: 08/29/2011

Time Analyzed: 1003

Heated Purge: (Y/N) N

\begin{tabular}{|c|c|}
\hline LAB & TIME \\
FILE ID & ANALYZED \\
\hline DHPU13.D & 1441 \\
\hline DHPU14.D & 1505 \\
\hline DHPU15.D & 1530 \\
\hline DHPU16.D & 1554 \\
\hline DHPU17.D & 1619 \\
\hline DHPU18.D & 1644 \\
\hline DHPU19.D & 1708 \\
\hline
\end{tabular}

COMMENTS :

Page 1 of 1 
$5 A$ - FORM V VOA

VOLATILE ORGANICS INSTRUMENT

PERFORMANCE CHECK

BROMOFLUOROBENZENE (BFB)
EPA SAMPLE NO.

BEBDV
Lab Name: TESTAMERICA BURLINGTON

Lab Code: STLV Case No.: INMA

Lab File Id: DHP01.D

Instrument Id: D.i

GC Column: DB-624

ID : 0.20
Contract: 8E-00302

SDG No.: 200-6730

BFB Injection Date: 07/07/2011

BFB Injection Time: 1005 (mrn)

\begin{tabular}{|c|c|c|c|}
\hline $\mathrm{m} / \mathrm{e}$ & ION ABUNDANCE CRITERIA & $\begin{array}{l}\text { : RELATIVE } \\
\text { ABUNDANCE } \\
\end{array}$ & \\
\hline$\overline{50}$ & $15.0-40.0$ o of mass 95 & 26.1 & \\
\hline 75 & $30.0-80.0 \%$ of mass 95 & 46.4 & \\
\hline 95 & Base peak, $100 \%$ relative abundance & 100 & \\
\hline 96 & $5.0-9.0 \%$ of mass 95 & 6.3 & \\
\hline 173 & Less than $2.0 \%$ of mass 174 & $0 \quad 1$ & 0) 1 \\
\hline 174 & $50.0-120 \%$ of mass 95 & 77.7 & \\
\hline 175 & $5.0-9.0 \%$ of mass 174 & 6.01 & $7.8) 1$ \\
\hline 176 & $95.0-101 \%$ of mass 174 & 78.21 & 101) 1 \\
\hline 177 & $5.0-9.0 \%$ of mass 176 & 5.21 & $6 . 6 \longdiv { 2 }$ \\
\hline
\end{tabular}

1 - Value is omass $174 \quad 2$ - Value is omass 176

\begin{tabular}{|c|c|l|l|c|c|}
\hline \multicolumn{1}{c|}{$\begin{array}{c}\text { EPA } \\
\text { SAMPLE NO. }\end{array}$} & \multicolumn{1}{|c|}{ LAB } & \multicolumn{1}{c|}{ LAB } & DATE & TIME \\
SAMPLE ID & \multicolumn{1}{c|}{ EILE ID } & ANALYZED & ANALYZED \\
\cline { 2 - 7 } 01 & VSTD0.5DV & IC 200-21023/4 & DHP04.D & $07 / 07 / 2011$ & 1117 \\
\cline { 2 - 7 } 02 & VSTD001DV & IC 200-21023/5 & DHP05.D & $07 / 07 / 2011$ & 1141 \\
\cline { 2 - 7 } 03 & VSTD005DV & ICIS 200-21023/6 & DHP06.D & $07 / 07 / 2011$ & 1206 \\
\cline { 2 - 7 } 05 & VSTD010DV & IC 200-21023/7 & DHP07.D & $07 / 07 / 2011$ & 1231 \\
\cline { 2 - 7 } & VSTD020DV & IC 200-21023/8 & DHP08.D & $07 / 07 / 2011$ & 1256 \\
\hline
\end{tabular}


$5 A$ - FORM V VOA

VOLATILE ORGANICS INSTRUMENT

PERFORMANCE CHECK

BROMOFLUOROBENZENE (BFB)
EPA SAMPLE NO.

BFBDR
Lab Name: TESTAMERICA BURLINGTON

Lab Code: STLV

Case No.: INMAN

Mod, Ref No.:
Contract: $8 \mathrm{E}-00302$

SDG No.: 200-6730

Lab File Id: DHPU01.D

BFB Injection Date: 08/29/2011

Instrument Id: D.i

BFB Injection Time: 0854

GC Column: DB-624

ID : 0.20

(mm)

\begin{tabular}{|c|c|c|}
\hline $\mathrm{m} / \mathrm{e}$ & ION ABUNDANCE CRITERIA & $\begin{array}{l}\text { \% RELATIVE } \\
\text { ABUNDANCE }\end{array}$ \\
\hline 50 & $15.0-40.0 \%$ of mass 95 & 27.2 \\
\hline 75 & $30.0-80.0 \%$ of mass 95 & 47.8 \\
\hline 95 & Base peak, $100 \%$ relative abundance & 100 \\
\hline 96 & $5.0-9.0 \%$ of mass 95 & 6.6 \\
\hline 173 & Less than $2.0 \%$ of mass 174 & 0.41 \\
\hline 174 & $50.0-120 \%$ of mass 95 & 75.9 \\
\hline 175 & $5.0-9.0 \%$ of mass 174 & $5.0(6.6) 1$ \\
\hline 176 & $95.0-101 \%$ of mass 174 & $72.1(95.0) 1$ \\
\hline 177 & $5.0-9.0 \%$ of mass 176 & $5.0(6.9) 2$ \\
\hline
\end{tabular}

1 - Value is omass $174 \quad 2$ - Value is omass 176

\begin{tabular}{|c|c|c|c|c|}
\hline $\begin{array}{c}\text { EPA } \\
\text { SAMPLE NO. }\end{array}$ & $\begin{array}{r}\text { LAB } \\
\text { SAMPLE ID }\end{array}$ & $\begin{array}{l}\text { LAB } \\
\text { FILE ID }\end{array}$ & $\begin{array}{c}\text { DATE } \\
\text { ANALYZED }\end{array}$ & $\begin{array}{c}\text { TIME } \\
\text { ANALYZED }\end{array}$ \\
\hline VSTD005DR & CCVIS $200-24379 / 3$ & DHPU03.D & $08 / 29 / 2011$ & 0939 \\
\hline VBLKDR & MB $200-24379 / 4$ & DHPU0 4.D & $08 / 29 / 2011$ & 1003 \\
\hline $\begin{array}{l}\text { INKTW-W-32 } \\
743\end{array}$ & $200-6730-5$ & DHPU13.D & $08 / 29 / 2011$ & 1441 \\
\hline $\begin{array}{l}\text { INQCTB-W-3 } \\
2744\end{array}$ & $200-6730-6$ & DHPU14.D & $08 / 29 / 2011$ & 1505 \\
\hline $\begin{array}{l}\text { INMW 16-W-3 } \\
2739\end{array}$ & $200-6730-1$ & DHPU15.D & $08 / 29 / 2011$ & 1530 \\
\hline $\begin{array}{l}\text { INMW16-W-3 } \\
2740\end{array}$ & $200-6730-2$ & DHPU16.D & $08 / 29 / 2011$ & 1554 \\
\hline $\begin{array}{l}\text { INMW16-W-3 } \\
2741\end{array}$ & $200-6730-3$ & DHPU17.D & $08 / 29 / 2011$ & 1619 \\
\hline $\begin{array}{l}\text { INMW 16-W-3 } \\
2742\end{array}$ & $200-6730-4$ & DHPU18.D & $08 / 29 / 2011$ & 1644 \\
\hline VHBLK01 & $200-6730-7$ & DHPU19.D & $08 / 29 / 2011$ & 1708 \\
\hline VSTD005RD & CCVC $200-24379 / 20$ & DHPU20.D & $08 / 29 / 2011$ & 1733 \\
\hline
\end{tabular}

Page 1 of 1 
Lab Code: STLV Case No.: INMAN Mod. Ref No.: SDG No.: $200-6730$

GC Column: DB-624

EPA Sample No. (VSTD\#\#\#\#\#):

Lab File ID (Standard): DHPU03.D

Instrument ID: D.i
ID: 0.20 (mm) Init. Calib. Date(s): 07/07/2011 07/07/2011

Date Analyzed: 08/29/2011

Time Analyzed: 0939

Heated Purge: $(\mathrm{Y} / \mathrm{N}) \mathrm{N}$

\begin{tabular}{|c|c|c|c|c|c|c|}
\hline & $\begin{array}{c}\text { IS1 (CBZ) } \\
\text { AREA }\end{array}$ & RT \# & $\begin{array}{c}\text { IS2 (DFB) } \\
\text { AREA }\end{array}$ & RT \# & $\begin{array}{c}\text { IS3 (DCB) } \\
\text { AREA }\end{array}$ & RT \# \\
\hline 12 HOUR STD & 157784 & 8.83 & 179248 & 5.47 & $\overline{72866}$ & 11.66 \\
\hline UPPER LIMIT & 220898 & 9.16 & 250947 & 5.80 & 102012 & 11.99 \\
\hline LOWER LIMIT & 94670 & 8.50 & 107549 & 5.14 & 43720 & 11.33 \\
\hline EPA SAMPLE NO. & & & & & & \\
\hline VBLKDR & 145940 & 8.83 & 177335 & 5.47 & 58919 & 11.66 \\
\hline INKTW-W-32743 & 138405 & 8.83 & 159283 & 5.47 & 53070 & 11.66 \\
\hline INQCTB-W-32744 & 137300 & 8.83 & 161766 & 5.47 & 58400 & 11.66 \\
\hline INMW16-W-32739 & 141838 & 8.83 & 173726 & 5.47 & 53475 & 11.66 \\
\hline INMW16-W-32740 & 141968 & 8.83 & 169409 & 5.47 & 57066 & 11.66 \\
\hline INMW16-W-32741 & 148545 & 8.83 & 177253 & 5.47 & 59386 & 11.66 \\
\hline INMW16-W-32742 & 138862 & 8.83 & 167279 & 5.47 & 57641 & 11.66 \\
\hline VHBLKO 1 & 137425 & 8.83 & 163447 & 5.47 & 52373 & 11.66 \\
\hline
\end{tabular}

ISI $(\mathrm{CBZ})=$ Chlorobenzene-d5

IS2 $(\mathrm{DFB})=1,4$-Difluorobenzene

IS3 $(\mathrm{DCB})=1,4-\mathrm{Di}$ chlorobenzene-d4

AREA UPPER LIMIT $=140 \%$ (Trace Volatiles) of internal standard area

AREA LOWER LIMIT $=60 \%$ (Trace Volatiles) of internal standard area

RT UPPER LIMIT $=+0.33$ (Trace Volatiles) minutes of internal standard RT

RT LOWER LIMIT $=-0.33$ (Trace Volatiles) minutes of internal standard RT

\# Column used to flag values outside contract required QC limits with an asterisk. 
$1 \mathrm{~A}$ - FORM I VOA-1

VOLATILE ORGANICS ANALYSIS DATA SHEET
EPA SAMPLE NO.

INKTW-W-32743
Lab Name: TESTAMERICA BURLINGTON

Lab Code: STLV

Contract: $8 \mathrm{E}-00302$

Matrix: (SOIL/SED/WATER) Water

Sample wt/vol: 25.0

$(\mathrm{g} / \mathrm{mL}) \quad \mathrm{mL}$

Level: (TRACE/LOW/MED) TRACE

\% Moisture: not dec.

GC Column: DB-624 ID: $0.20 \quad(\mathrm{~mm})$

Soil Extract Volume:

(uL)

(mL)
SDG No.: 200-6730

Lab Sample ID: 200-6730-5

Lab File ID: DHPU13.D

Date Received: 08/26/2011

Date Analyzed: 08/29/2011

Dilution Factor: 1.0

Soil Aliquot volume:

(uL)

CONCENTRATION UNITS: (ug/L or $u g / k g$ ) ug/L

\begin{tabular}{|c|c|c|c|}
\hline CAS NO. & COMPOUND & $\begin{array}{l}\text { CONCENTRATION UNITS: } \\
(\mathrm{ug} / \mathrm{L} \text { or } \mathrm{ug} / \mathrm{kg}) \mathrm{ug} / \mathrm{L}\end{array}$ & Q \\
\hline $75-71-8$ & Dichlorodifluoromethane & 0.50 & $\bar{U}$ \\
\hline $74-87-3$ & Chloromethane & 0.50 & $\mathrm{U}$ \\
\hline $75-01-4$ & Vinyl chloride & 0.50 & $\mathrm{U}$ \\
\hline $74-83-9$ & Bromomethane & 0.50 & $\mathrm{U}$ \\
\hline $75-00-3$ & Chloroethane & 0.50 & $\mathrm{U}$ \\
\hline $75-69-4$ & Trichlorofluoromethane & 0.50 & $\mathrm{U}$ \\
\hline $75-35-4$ & 1,1-Dichloroethene & 0.50 & $\mathrm{U}$ \\
\hline $76-13-1$ & 1,1,2-Trichloro-1,2,2-trifluoroethane & 0.50 & $\mathrm{U}$ \\
\hline $67-64-1$ & Acetone & 5.0 & $\mathrm{U}$ \\
\hline $75-15-0$ & Carbon disulfide & 0.50 & $\mathrm{U}$ \\
\hline $79-20-9$ & Methyl acetate & 0.50 & $\mathrm{U}$ \\
\hline $75-09-2$ & Methylene Chloride & 0.50 & $\mathrm{U}$ \\
\hline $156-60-5$ & trans-1,2-Dichloroethene & 0.50 & $\mathrm{U}$ \\
\hline $1634-04-4$ & Methyl tert-butyl ether & 0.50 & $\mathrm{U}$ \\
\hline $75-34-3$ & 1,1-Dichloroethane & 0.50 & $\mathrm{U}$ \\
\hline $156-59-2$ & cis-1,2-Dichloroethene & 0.50 & $\mathrm{U}$ \\
\hline $78-93-3$ & 2-Butanone & 5.0 & $\mathrm{U}$ \\
\hline $74-97-5$ & Bromochloromethane & 0.50 & $\mathrm{U}$ \\
\hline $67-66-3$ & Chloroform & 0.48 & $\mathrm{~J}$ \\
\hline $71-55-6$ & 1,1,1-Trichloroethane & 0.50 & $\mathrm{U}$ \\
\hline $110-82-7$ & Cyclohexane & 0.50 & $\mathrm{U}$ \\
\hline $56-23-5$ & Carbon tetrachloride & 12 & \\
\hline $71-43-2$ & Benzene & 0.50 & U \\
\hline $107-06-2$ & 1,2-Dichloroethane & 0.50 & $\mathrm{U}$ \\
\hline
\end{tabular}

Report 1,4-Dioxane for Low-Medium VOA analysis only 
$1 B$ - FORM I VOA-2

VOLATILE ORGANICS ANALYSIS DATA SHEET
EPA SAMPLE NO. INKTW-W-32743

Lab Name: TESTAMERICA BURLINGTON

Lab Code: STLV Case No.: INMAN Mod.

Water

Matrix: (SOIL/SED/WATER)

Sample wt/vol: 25.0 $(\mathrm{g} / \mathrm{mL}) \mathrm{mL}$

Level: (TRACE/LOW/MED)

TRACE

응 Moisture: not dec.

GC Column: DB-624 ID: 0.20 (mm)

Soil Extract Volume: (uL)

Purge volume: 25.0 (mL)
Contract: $8 \mathrm{E}-00302$

SDG No.: 200-6730

\begin{tabular}{|c|c|c|c|}
\hline CAS NO. & COMPOUND & $\begin{array}{l}\text { CONCENTRATION UNITS: } \\
(\mathrm{ug} / \mathrm{L} \text { or } \mathrm{ug} / \mathrm{kg}) \mathrm{ug} / \mathrm{I}\end{array}$ & 0 \\
\hline $79-01-6$ & Trichloroethene & 0.50 & $\overline{\mathrm{U}}$ \\
\hline $108-87-2$ & Methylcyclohexane & 0.50 & $\mathrm{U}$ \\
\hline $78-87-5$ & 1,2-Dichloropropane & 0.50 & $\mathrm{U}$ \\
\hline $75-27-4$ & Bromodichloromethane & 0.50 & $\mathrm{U}$ \\
\hline $10061-01-5$ & cis-1,3-Dichloropropene & 0.50 & $\mathrm{U}$ \\
\hline $108-10-1$ & 4-Methyl-2-pentanone & 5.0 & $\mathrm{U}$ \\
\hline $108-88-3$ & Toluene & 0.50 & $\mathrm{U}$ \\
\hline $10061-02-6$ & trans-1,3-Dichloropropene & 0.50 & $\mathrm{U}$ \\
\hline $79-00-5$ & 1,1,2-Trichloroethane & 0.50 & $\mathrm{U}$ \\
\hline $127-18-4$ & Tetrachloroethene & 0.50 & $\mathrm{U}$ \\
\hline $591-78-6$ & 2-Hexanone & 5.0 & $\mathrm{U}$ \\
\hline $124-48-1$ & Dibromochloromethane & 0.50 & $\mathrm{U}$ \\
\hline $106-93-4$ & 1,2-Dibromoethane & 0.50 & U \\
\hline $108-90-7$ & Chlorobenzene & 0.50 & $\mathrm{U}$ \\
\hline $100-41-4$ & Ethylbenzene & 0.50 & $\mathrm{U}$ \\
\hline $95-47-6$ & o-Xylene & 0.50 & $\mathrm{U}$ \\
\hline $179601-23-1$ & $\mathrm{~m}, \mathrm{p}$-Xylene & 0.50 & $\mathrm{U}$ \\
\hline $100-42-5$ & Styrene & 0.50 & U \\
\hline $75-25-2$ & Bromoform & 0.50 & $\mathrm{U}$ \\
\hline $98-82-8$ & Isopropylbenzene & 0.50 & U \\
\hline $79-34-5$ & $1,1,2,2$-Tetrachloroethane & 0.50 & $\mathrm{U}$ \\
\hline $541-73-1$ & 1,3-Dichlorobenzene & 0.50 & $\mathrm{U}$ \\
\hline $106-46-7$ & 1,4-Dichlorobenzene & 0.50 & $\mathrm{U}$ \\
\hline $95-50-1$ & 1,2-Dichlorobenzene & 0.50 & $\mathrm{U}$ \\
\hline $96-12-8$ & 1,2-Dibromo-3-Chloropropane & 0.50 & $\mathrm{U}$ \\
\hline $120-82-1$ & 1,2,4-Trichlorobenzene & 0.50 & $\mathrm{U}$ \\
\hline $87-61-6$ & 1,2,3-Trichlorobenzene & 0.50 & $\mathrm{U}$ \\
\hline
\end{tabular}


IJ - FORM I VOA-TIC

VOLATILE ORGANICS ANALYSIS DATA SHEET TENTATIVELY IDENTIFIED COMPOUNDS
EPA SAMPLE NO. INKTW-W-32743

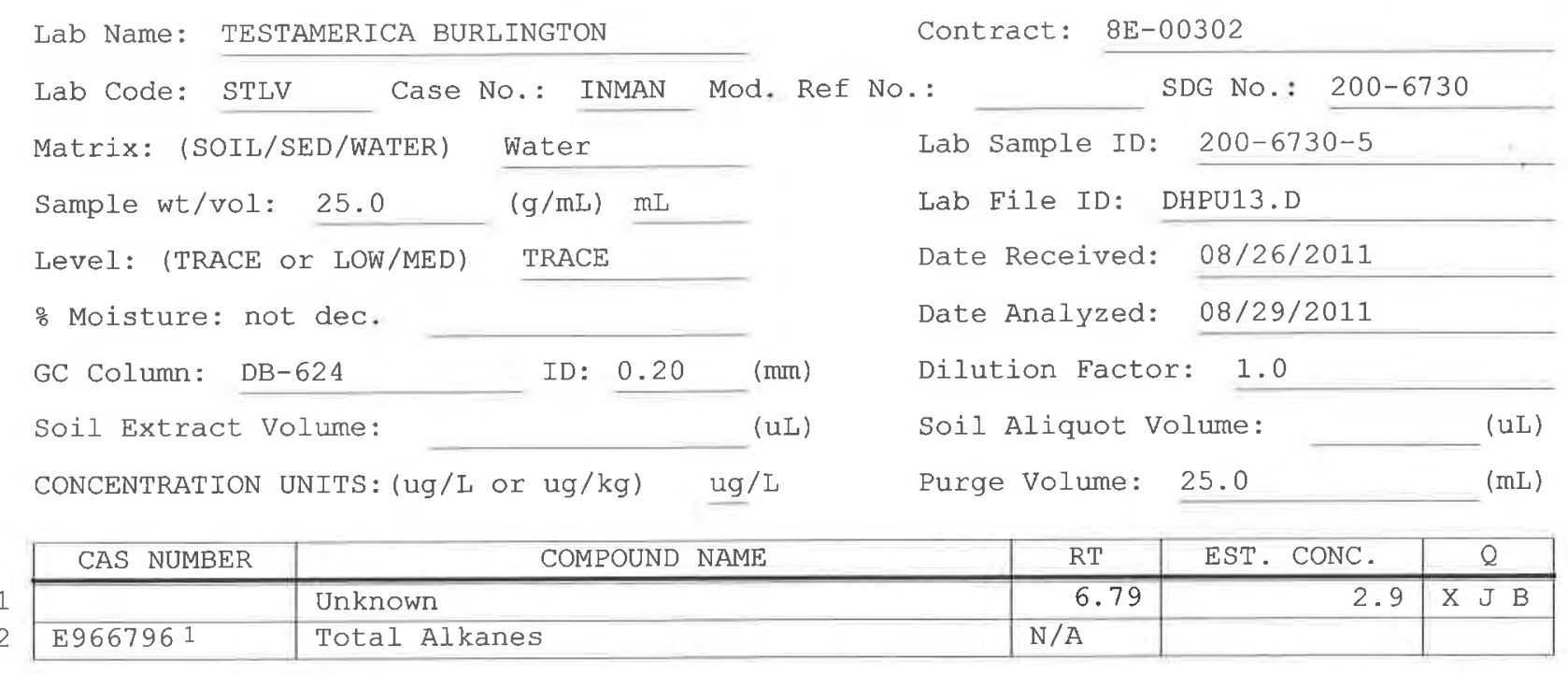

1 EPA-designated Registry Number. 
$1 \mathrm{~A}$ - FORM I VOA-1

VOLATILE ORGANICS ANALYSIS DATA SHEET
EPA SAMPLE NO.

INMW I 6-W-32739
Lab Name: TESTAMERICA BURLINGTON Case No.: INMAN

Matrix: (SOIL/SED/WATER)

Water

Sample wt/vol: 25.0

$(g / m L) \quad m L$

Level: (TRACE/LOW/MED) TRACE

\% Moisture: not dec. ID $: 0.20$

Soil Extract Volume:

Purge Volume: 25.0

(mL)
Lab Code: STLV

GC Column: DB-624

Contract: $\quad 8 \mathrm{E}-00302$

SDG No.: 200-6730

Lab Sample ID: 200-6730-1

Lab File ID: DHPU15.D

Date Received: 08/26/2011

Date Analyzed: 08/29/2011

Dilution Factor: 1.0

Soil Aliquot Volume:

(uL)

\begin{tabular}{|c|c|c|c|}
\hline CAS NO. & COMPOUND & $\begin{array}{l}\text { CONCENTRATION UNITS: } \\
(\mathrm{ug} / \mathrm{L} \text { or } \mathrm{ug} / \mathrm{kg}) \mathrm{ug} / \mathrm{L}\end{array}$ & $\mathrm{Q}$ \\
\hline $75-71-8$ & Dichlorodifluoromethane & 0.50 & $\mathrm{U}$ \\
\hline $74-87-3$ & Chloromethane & 0.50 & $\mathrm{U}$ \\
\hline $75-01-4$ & Vinyl chloride & 0.50 & $\mathrm{U}$ \\
\hline $74-83-9$ & Bromomethane & 0.50 & $\mathrm{U}$ \\
\hline $75-00-3$ & Chloroethane & 0.50 & $\mathrm{U}$ \\
\hline $75-69-4$ & 'Trichlorofluoromethane & 0.50 & $\mathrm{U}$ \\
\hline $75-35-4$ & 1,1-Dichloroethene & 0.50 & $\mathrm{U}$ \\
\hline $76-13-1$ & 1,1,2-Trichloro-1,2,2-trifluoroethane & 0.50 & $\mathrm{U}$ \\
\hline $67-64-1$ & Acetone & 5.0 & $\mathrm{U}$ \\
\hline $75-15-0$ & Carbon disulfide & 0.50 & $\mathrm{U}$ \\
\hline $79-20-9$ & Methyl acetate & 0.50 & $\mathrm{U}$ \\
\hline $75-09-2$ & Methylene Chloride & 0.50 & $\mathrm{U}$ \\
\hline $156-60-5$ & trans $-1,2-$ Dichloroethene & 0.50 & $\mathrm{U}$ \\
\hline $1634-04-4$ & Methyl tert-butyl ether & 0.50 & $\mathrm{U}$ \\
\hline $75-34-3$ & 1,1-Dichloroethane & 0.50 & $\mathrm{U}$ \\
\hline $156-59-2$ & cis-1,2-Dichloroethene & 0.50 & $\mathrm{U}$ \\
\hline $78-93-3$ & 2-Butanone & 5.0 & $\mathrm{U}$ \\
\hline $74-97-5$ & Bromochloromethane & 0.50 & $\mathrm{U}$ \\
\hline $67-66-3$ & Chloroform & 0.033 & $\mathrm{~J}$ \\
\hline $71-55-6$ & 1,1,1-Trichloroethane & 0.50 & $\mathrm{U}$ \\
\hline $110-82-7$ & Cyclohexane & 0.50 & $\mathrm{U}$ \\
\hline $56-23-5$ & Carbon tetrachloride & 0.50 & $\mathrm{U}$ \\
\hline $71-43-2$ & Benzene & 0.10 & $\mathrm{~J}$ \\
\hline $107-06-2$ & 1,2-Dichloroethane & 0.50 & $\mathrm{U}$ \\
\hline
\end{tabular}

Report 1,4-Dioxane for Low-Medium VOA analysis only 
$1 B$ - FORM I VOA-2

VOLATILE ORGANICS ANALYSIS DATA SHEET
EPA SAMPLE NO.

INMW1 6-W-32739

Lab Name: TESTAMERICA BURLINGTON

Lab Code: STLV Case

Matrix: (SOIL/SED/WATER)

Sample wt/vol: 25.0

\section{Water}

$(\mathrm{g} / \mathrm{mL}) \quad \mathrm{mL}$

Level: (TRACE/LOW/MED) TRACE

응 Moisture: not dec.

GC Column: DB-624 ID: $0.20 \quad(\mathrm{~mm})$

Soil Extract Volume:

Purge Volume: 25.0

$(\mathrm{mL})$
Contract: $8 \mathrm{E}-00302$

SDG No.: 200-6730

\begin{tabular}{|c|c|c|c|}
\hline CAS NO. & COMPOUND & $\begin{array}{l}\text { CONCENTRATION UNITS: } \\
(\mathrm{ug} / \mathrm{L} \text { or } \mathrm{ug} / \mathrm{kg}) \mathrm{ug} / \mathrm{L}\end{array}$ & $Q$ \\
\hline $79-01-6$ & Trichloroethene & 0.50 & $\bar{U}$ \\
\hline $108-87-2$ & Methylcyclohexane & 0.50 & $\mathrm{U}$ \\
\hline $78-87-5$ & 1,2-Dichloropropane & 0.50 & $\mathrm{U}$ \\
\hline $75-27-4$ & Bromodichloromethane & 0.50 & U \\
\hline $10061-01-5$ & cis-1,3-Dichloropropene & 0.50 & $\mathrm{U}$ \\
\hline $108-10-1$ & 4-Methyl-2-pentanone & 5.0 & $\mathrm{U}$ \\
\hline $108-88-3$ & Toluene & 0.053 & $\mathrm{~J}$ \\
\hline $10061-02-6$ & trans-1,3-Dichloropropene & 0.50 & $\mathrm{U}$ \\
\hline $79-00-5$ & $1,1,2$-Trichloroethane & 0.50 & $\mathrm{U}$ \\
\hline $127-18-4$ & Tetrachloroethene & 0.50 & $\mathrm{U}$ \\
\hline $591-78-6$ & 2-Hexanone & 5.0 & $\mathrm{U}$ \\
\hline $124-48-1$ & Dibromochloromethane & 0.50 & $\mathrm{U}$ \\
\hline $106-93-4$ & 1,2-Dibromoethane & 0.50 & $\mathrm{U}$ \\
\hline $108-90-7$ & Chlorobenzene & 0.50 & $\mathrm{U}$ \\
\hline $100-41-4$ & Ethylbenzene & 0.50 & $\mathrm{U}$ \\
\hline $95-47-6$ & o-Xylene & 0.50 & $\mathrm{U}$ \\
\hline $179601-23-1$ & $\mathrm{~m}, \mathrm{p}$-Xylene & 0.016 & $\mathrm{~J}$ \\
\hline $100-42-5$ & Styrene & 0.50 & $\mathrm{U}$ \\
\hline $75-25-2$ & Bromoform & 0.50 & U \\
\hline $98-82-8$ & Isopropylbenzene & 0.50 & $\mathrm{U}$ \\
\hline $79-34-5$ & $1,1,2,2$-Tetrachloroethane & 0.50 & $\mathrm{U}$ \\
\hline $541-73-1$ & 1,3-Dichlorobenzene & 0.50 & $\mathrm{U}$ \\
\hline $106-46-7$ & 1,4-Dichlorobenzene & 0.50 & $\mathrm{U}$ \\
\hline $95-50-1$ & 1,2-Dichlorobenzene & 0.50 & $\mathrm{U}$ \\
\hline $96-12-8$ & 1,2-Dibromo-3-Chloropropane & 0.50 & $\mathrm{U}$ \\
\hline $120-82-1$ & 1,2,4-Trichlorobenzene & 0.50 & $\mathrm{U}$ \\
\hline $87-61-6$ & 1,2,3-Trichlorobenzene & 0.50 & $\mathrm{U}$ \\
\hline
\end{tabular}


$1 \mathrm{~J}$ - FORM I VOA-TIC

VOLATILE ORGANICS ANALYSIS DATA SHEET TENTATIVELY IDENTIFIED COMPOUNDS
EPA SAMPLE NO.

INMW $16-W-32739$
Lab Name: TESTAMERICA BURLINGTON

Lab Code: STLV

Case No.: INMA

Matrix: (SOIL/SED/WATER)

water

Sample wt/vol: 25.0

$(\mathrm{g} / \mathrm{mL}) \mathrm{mL}$

Level: (TRACE or LOW/MED)

TRACE

음 Moisture: not dec.

GC Column: DB-624

ID: $0.20 \quad$ (mm)

Soil Extract Volume:

(uL)

CONCENTRATION UNITS: (ug/L or $\mathrm{ug} / \mathrm{kg}$ )

$\mathrm{ug} / \mathrm{L}$
Contract: $\quad 8 \mathrm{E}-00302$

SDG No.: 200-6730

Lab Sample ID: 200-6730-1

Lab File ID: DHPU15.D

Date Received: 08/26/2011

Date Analyzed: 08/29/2011

Dilution Factor: 1.0

Soil Aliquot Volume:

(uL)

Purge Volume: 25.0

$(\mathrm{mL})$

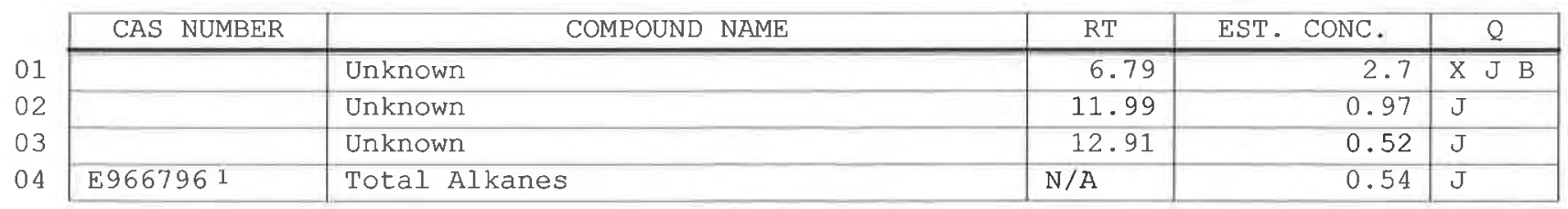

1 EPA-designated Registry Number. 
$1 A$ - FORM I VOA-1

VOLATILE ORGANICS ANALYSIS DATA SHEET
EPA SAMPLE NO.

INMW16-W-32740
Lab Name: TESTAMERICA BURLINGTON

Lab Code: STLV Case No.: INMAN Mod. Mod. Ref No.:

Contract: $8 \mathrm{E}-00302$

Matrix: (SOIL/SED/WATER)

Water

Sample wt/vol: 25.0 $(\mathrm{g} / \mathrm{mL}) \mathrm{mL}$

Leve 1: (TRACE/LOW/MED) TRACE

o Moisture: not dec.

GC Column: DB-624 ID: $0.20 \quad$ (mm)

Soil Extract Volume:

(uL) (mL)

Purge Volume: 25.0
SDG No.: 200-6730

Lab Sample ID: 200-6730-2

Lab File ID: DHPU16.D

Date Received: 08/26/2011

Date Analyzed: 08/29/2011

Dilution Factor: 1.0

Soil Aliquot Volume:

(uL)

\begin{tabular}{|c|c|c|c|}
\hline CAS NO. & COMPOUND & $\begin{array}{l}\text { CONCENTRATION UNITS: } \\
(\mathrm{ug} / \mathrm{L} \text { or } \mathrm{ug} / \mathrm{kg}) \mathrm{ug} / \mathrm{L}\end{array}$ & $Q$ \\
\hline $75-71-8$ & Dichlorodifluoromethane & 0.50 & $\overline{\mathrm{U}}$ \\
\hline $74-87-3$ & Chloromethane & 0.50 & $\mathrm{U}$ \\
\hline $75-01-4$ & Vinyl chloride & 0.50 & $\mathrm{U}$ \\
\hline $74-83-9$ & Bromomethane & 0.50 & $\mathrm{U}$ \\
\hline $75-00-3$ & Chloroethane & 0.50 & $\mathrm{U}$ \\
\hline $75-69-4$ & Trichlorofluoromethane & 0.50 & $\mathrm{U}$ \\
\hline $75-35-4$ & 1,1-Dichloroethene & 0.50 & $\mathrm{U}$ \\
\hline $76-13-1$ & 1,1,2-Trichloro-1,2,2-trifluoroethane & 0.50 & $\mathrm{U}$ \\
\hline $67-64-1$ & Acetone & 5.0 & $\mathrm{U}$ \\
\hline $75-15-0$ & Carbon disulfide & 0.50 & $\mathrm{U}$ \\
\hline $79-20-9$ & Methyl acetate & 0.50 & $\mathrm{U}$ \\
\hline $75-09-2$ & Methylene Chloride & 0.50 & $\mathrm{U}$ \\
\hline $156-60-5$ & trans-1,2-Dichloroethene & 0.50 & $\mathrm{U}$ \\
\hline $1634-04-4$ & Methyl tert-butyl ether & 0.50 & $\mathrm{U}$ \\
\hline $75-34-3$ & 1,1-Dichloroethane & 0.50 & $\mathrm{U}$ \\
\hline $156-59-2$ & cis-1,2-Dichloroethene & 0.50 & $\mathrm{U}$ \\
\hline $78-93-3$ & 2-Butanone & 5.0 & $\mathrm{U}$ \\
\hline $74-97-5$ & Bromochloromethane & 0.50 & $\mathrm{U}$ \\
\hline $67-66-3$ & Chloroform & 0.94 & \\
\hline $71-55-6$ & 1,1,1-Trichloroethane & 0.50 & U \\
\hline $110-82-7$ & Cyclohexane & 0.50 & $\mathrm{U}$ \\
\hline $56-23-5$ & Carbon tetrachloride & 13 & \\
\hline $71-43-2$ & Benzene & 0.50 & $\mathrm{U}$ \\
\hline $107-06-2$ & 1,2-Dichloroethane & 0.50 & $\mathrm{U}$ \\
\hline
\end{tabular}

Report 1,4-Dioxane for Low-Medium VOA analysis only 
$1 \mathrm{~B}$ - FORM I VOA-2

VOLATILE ORGANICS ANALYSIS DATA SHEET
EPA SAMPLE NO.

INMW16-W-32740
Lab Name: TESTAMERICA BURLINGTON

Lab Code: STLV

Matrix: (SOIL/SED/WATER)

Sample wt/vol: 25.0

Level: (TRACE/LOW/MED) TRACE

高 Moisture: not dec.

GC Column: DB-624 ID : 0.20

Soil Extract Volume:

Purge Volume: 25.0
Contract: 8E-00302

SDG No.: 200-6730
Lab Sample ID: 200-6730-2

Lab File ID: DHPU16.D

Date Received: 08/26/2011

Date Analyzed: 08/29/2011

Dilution Factor: 1.0

Soil Aliquot Volume:

(uL)

\begin{tabular}{|c|c|c|c|}
\hline CAS NO. & COMPOUND & $\begin{array}{l}\text { CONCENTRATION UNITS: } \\
(\mathrm{ug} / \mathrm{L} \text { or } \mathrm{ug} / \mathrm{kg}) \mathrm{ug} / \mathrm{L}\end{array}$ & $Q$ \\
\hline $79-01-6$ & Trichloroethene & 0.50 & $\mathrm{U}$ \\
\hline $108-87-2$ & Methylcyclohexane & 0.50 & $\mathrm{U}$ \\
\hline $78-87-5$ & 1,2-Dichloropropane & 0.50 & $\mathrm{U}$ \\
\hline $75-27-4$ & Bromodichloromethane & 0.50 & $\mathrm{U}$ \\
\hline $10061-01-5$ & cis-1,3-Dichloropropene & 0.50 & $\mathrm{U}$ \\
\hline $108-10-1$ & 4-Methyl-2-pentanone & 5.0 & $\mathrm{U}$ \\
\hline $108-88-3$ & Toluene & 0.014 & $\mathrm{~J}$ \\
\hline $10061-02-6$ & trans-1,3-Dichloropropene & 0.50 & $\mathrm{U}$ \\
\hline $79-00-5$ & 1,1,2-Trichloroethane & 0.50 & $\mathrm{U}$ \\
\hline $127-18-4$ & Tetrachloroethene & 0.50 & $\mathrm{U}$ \\
\hline $591-78-6$ & 2-Hexanone & 5.0 & $\mathrm{U}$ \\
\hline $124-48-1$ & Dibromochloromethane & 0.50 & $\mathrm{U}$ \\
\hline $106-93-4$ & 1,2-Dibromoethane & 0.50 & $\mathrm{U}$ \\
\hline $108-90-7$ & Chlorobenzene & 0.50 & $\mathrm{U}$ \\
\hline $100-41-4$ & Ethylbenzene & 0.50 & $\mathrm{U}$ \\
\hline $95-47-6$ & o-Xylene & 0.50 & $\mathrm{U}$ \\
\hline $179601-23-1$ & $\mathrm{~m}, \mathrm{p}$-Xylene & 0.50 & $\mathrm{U}$ \\
\hline $100-42-5$ & styrene & 0.50 & $\mathrm{U}$ \\
\hline $75-25-2$ & Bromoform & 0.50 & $\mathrm{U}$ \\
\hline $98-82-8$ & Isopropylbenzene & 0.50 & $\mathrm{U}$ \\
\hline $79-34-5$ & $1,1,2,2$-Tetrachloroethane & 0.50 & $\mathrm{U}$ \\
\hline $541-73-1$ & 1,3-Dichlorobenzene & 0.50 & $\mathrm{U}$ \\
\hline $106-46-7$ & 1,4-Dichlorobenzene & 0.50 & $\mathrm{U}$ \\
\hline $95-50-1$ & 1,2-Dichlorobenzene & 0.50 & $\mathrm{U}$ \\
\hline $96-12-8$ & 1,2-Dibromo-3-Chloropropane & 0.50 & $\mathrm{U}$ \\
\hline $120-82-1$ & 1,2,4-Trichlorobenzene & 0.50 & $\mathrm{U}$ \\
\hline $87-61-6$ & 1,2,3-Trichlorobenzene & 0.50 & $\mathrm{U}$ \\
\hline
\end{tabular}


$1 J$ - FORM I VOA-TIC

VOLATILE ORGANICS ANALYSIS DATA SHEET

TENTATIVELY IDENTIEIED COMPOUNDS
EPA SAMPLE NO.

INMW $16-W-32740$

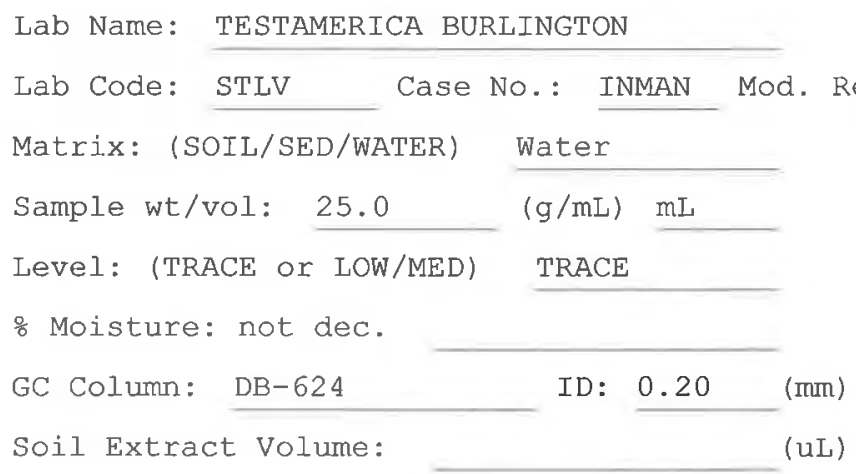

CONCENTRATION UNITS: (ug/L or $\mathrm{ug} / \mathrm{kg}$ ) ug/L
Contract: $8 \mathrm{E}-00302$

SDG No.: 200-6730

Lab Sample ID: 200-6730-2

Lab File ID: DHPU16.D

Date Received: 08/26/2011

Date Analyzed: 08/29/2011

Dilution Factor: 1.0

Soil Aliquot Volume:

(uL)

Purge Volume: 25.0

(mL)

\begin{tabular}{|l|l|l|l|r|r|}
\hline \multicolumn{1}{|c|}{ CAS NUMBER } & \multicolumn{1}{|c|}{ COMPOUND NAME } & RT & EST. CONC. & Q \\
\cline { 2 - 6 } 01 & & Unknown & 6.79 & X J B \\
\cline { 2 - 6 } & E9667961 & Total Alkanes & N/A & 3.0 & \\
\hline
\end{tabular}

1 EPA-designated Registry Number. 
$1 A$ - FORM I VOA-1

VOLATILE ORGANICS ANALYSIS DATA SHEET
EPA SAMPLE NO. INMW16-W-32741
Lab Name: TESTAMERICA BURLINGTON

Lab Code: STLV Case

Matrix: (SOIL/SED/WATER)

Water

Sample wt/vol: 25.0 $(\mathrm{g} / \mathrm{mL}) \mathrm{mL}$

Level: (TRACE/LOW/MED) TRACE

을 Moture: not dec.

GC Column: DB-624 ID: 0.20 (mm)

Soil Extract Volume: (uL) (mL)

Purge Volume: 25.0
Contract: $8 E-00302$

SDG No.: 200-6730

\begin{tabular}{|c|c|c|c|}
\hline CAS NO. & COMPOUND & $\begin{array}{l}\text { CONCENTRATION UNITS: } \\
(u g / L \text { or } u g / k g) u g / L\end{array}$ & $Q$ \\
\hline $75-71-8$ & Dichlorodifluoromethane & 0.50 & $\bar{U}$ \\
\hline $74-87-3$ & Chloromethane & 0.50 & $\mathrm{U}$ \\
\hline $75-01-4$ & Vinyl chloride & 0.50 & $\mathrm{U}$ \\
\hline $74-83-9$ & Bromomethane & 0.50 & $\mathrm{U}$ \\
\hline $75-00-3$ & Chloroethane & 0.50 & $\mathrm{U}$ \\
\hline $75-69-4$ & Trichlorofluoromethane & 0.50 & $\mathrm{U}$ \\
\hline $75-35-4$ & 1,1-Dichloroethene & 0.50 & $\mathrm{U}$ \\
\hline $76-13-1$ & 1,1,2-Trichloro-1,2,2-trifluoroethane & 0.50 & $\mathrm{U}$ \\
\hline $67-64-1$ & Acetone & 5.0 & $\mathrm{U}$ \\
\hline $75-15-0$ & Carbon disulfide & 0.50 & $\mathrm{U}$ \\
\hline $79-20-9$ & Methyl acetate & 0.50 & $\mathrm{U}$ \\
\hline $75-09-2$ & Methylene Chloride & 0.50 & U \\
\hline $156-60-5$ & trans-1,2-Dichloroethene & 0.50 & $\mathrm{U}$ \\
\hline $1634-04-4$ & Methyl tert-buty] ether & 0.50 & U \\
\hline $75-34-3$ & 1,1-Dichloroethane & 0.50 & $\mathrm{U}$ \\
\hline $156-59-2$ & cis-1,2-Dichloroethene & 0.50 & $\mathrm{U}$ \\
\hline $78-93-3$ & 2-Butanone & 5.0 & U \\
\hline $74-97-5$ & Bromochloromethane & 0.50 & U \\
\hline $67-66-3$ & Chloroform & 0.69 & \\
\hline $71-55-6$ & 1,1,1-Trichloroethane & 0.50 & $\mathrm{U}$ \\
\hline $110-82-7$ & Cyclohexane & 0.50 & $\mathrm{U}$ \\
\hline $56-23-5$ & Carbon tetrachloride & 8.9 & \\
\hline $71-43-2$ & Benzene & 0.011 & $\mathrm{~J}$ \\
\hline $107-06-2$ & 1,2-Dichloroethane & 0.50 & U \\
\hline
\end{tabular}

Report 1,4-Dioxane for Low-Medium VOA analysis only 
$1 B$ - FORM I VOA-2

VOLATILE ORGANICS ANALYSIS DATA SHEET
EPA SAMPLE NO.

INMW16-W-32741
Lab Name: TESTAMERICA BURLINGTON

Lab Code: STLV

Matrix: (SOIL/SED/WATER)

Sample wt/vol: 25.0 $(\mathrm{g} / \mathrm{mL}) \mathrm{mL}$

Level: (TRACE/LOW/MED) TRACE

응 Moisture: not dec.

GC Column: DB-624 ID $: 0.20 \quad(\mathrm{~mm})$

Soil Extract Volume: (uL)

Purge Volume: 25.0 (mL)
Contract: $\quad 8 \mathrm{E}-00302$

SDG No.: 200-6730

Lab Sample ID: 200-6730-3

Lab File ID: DHPU17.D

Date Received: 08/26/2011

Date Analyzed: 08/29/2011

Dilution Factor: 1.0

Soil Aliquot Volume:

(uL)

\begin{tabular}{|c|c|c|c|}
\hline CAS NO. & COMPOUND & $\begin{array}{l}\text { CONCENTRATION UNITS : } \\
(\mathrm{ug} / \mathrm{L} \text { or } \mathrm{ug} / \mathrm{kg}) \mathrm{ug} / \mathrm{L}\end{array}$ & $Q$ \\
\hline $79-01-6$ & Trichloroethene & 0.50 & $\overline{\mathrm{U}}$ \\
\hline $108-87-2$ & Methylcyclohexane & 0.50 & $\mathrm{U}$ \\
\hline $78-87-5$ & 1,2-Dichloropropane & 0.50 & $\mathrm{U}$ \\
\hline $75-27-4$ & Bromodichloromethane & 0.50 & $\mathrm{U}$ \\
\hline $10061-01-5$ & cis-1,3-Dichloropropene & 0.50 & $\mathrm{U}$ \\
\hline $108-10-1$ & 4-Methyl-2-pentanone & 5.0 & $\mathrm{U}$ \\
\hline $108-88-3$ & Toluene & 0.021 & $\mathrm{~J}$ \\
\hline $10061-02-6$ & trans-1,3-Dichloropropene & 0.50 & $\mathrm{U}$ \\
\hline $79-00-5$ & 1,1,2-Trichloroethane & 0.50 & $\mathrm{U}$ \\
\hline $127-18-4$ & Tetrachloroethene & 0.50 & $\mathrm{U}$ \\
\hline $591-78-6$ & 2-Hexanone & 5.0 & $\mathrm{U}$ \\
\hline $124-48-1$ & Dibromochloromethane & 0.50 & $\mathrm{U}$ \\
\hline $106-93-4$ & 1,2-Dibromoethane & 0.50 & $\mathrm{U}$ \\
\hline $108-90-7$ & Chlorobenzene & 0.50 & $\mathrm{U}$ \\
\hline $100-41-4$ & Ethylbenzene & 0.50 & $\mathrm{U}$ \\
\hline $95-47-6$ & o-Xylene & 0.50 & $\mathrm{U}$ \\
\hline $179601-23-1$ & $\mathrm{~m}, \mathrm{p}$-Xylene & 0.50 & $\mathrm{U}$ \\
\hline $100-42-5$ & Styrene & 0.50 & $\mathrm{U}$ \\
\hline $75-25-2$ & Bromoform & 0.50 & $\mathrm{U}$ \\
\hline $98-82-8$ & Isopropylbenzene & 0.50 & $\mathrm{U}$ \\
\hline $79-34-5$ & $1,1,2,2$-Tetrachloroethane & 0.50 & $\mathrm{U}$ \\
\hline $541-73-1$ & 1,3-Dichlorobenzene & 0.50 & $\mathrm{U}$ \\
\hline $106-46-7$ & 1,4-Dichlorobenzene & 0.50 & $\mathrm{U}$ \\
\hline $95-50-1$ & 1,2-Dichlorobenzene & 0.50 & $\mathrm{U}$ \\
\hline $96-12-8$ & 1,2-Dibromo-3-Chloropropane & 0.50 & $\mathrm{U}$ \\
\hline $120-82-1$ & 1,2,4-Trichlorobenzene & 0.50 & $\mathrm{U}$ \\
\hline $87-61-6$ & 1,2,3-Trichlorobenzene & 0.50 & $\mathrm{U}$ \\
\hline
\end{tabular}


$1 \mathrm{~J}-$ FORM I VOA-TIC

VOLATILE ORGANICS ANALYSIS DATA SHEET TENTATIVELY IDENTIFIED COMPOUNDS
EPA SAMPLE NO.

INMW1 6-W-32741

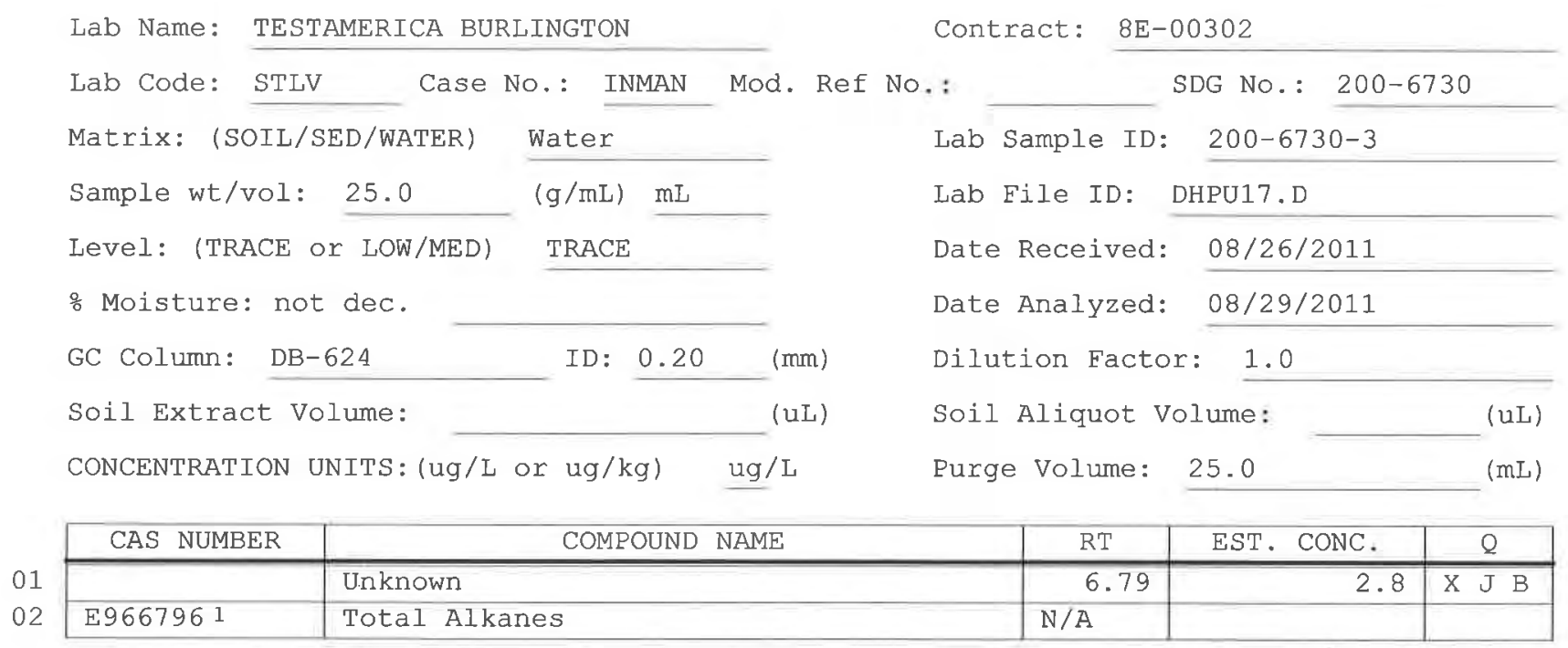

1 EPA-designated Registry Number. 
$1 A$ - FORM I VOA-1

VOLATILE ORGANICS ANALYSIS DATA SHEET
EPA SAMPLE NO.

INMW16-W-32742
Lab Name: TESTAMERICA BURLINGTON

Lab Code: STLV Case No.: INMAN

Matrix: (SOIL/SED/WATER) water

Sample wt/vol: 25.0 $(\mathrm{g} / \mathrm{mL}) \mathrm{mL}$

Level: (TRACE/LOW/MED) TRACE

o Moisture: not dec.

GC Column: DB-624 ID: 0.20

Soil Extract Volume:

Purge Volume: 25.0

Contract: $\quad 8 \mathrm{E}-00302$

Mod. Ref No.: SDG No.: $200-6730$

Lab Sample ID: 200-6730-4

Lab File ID: DHPU18.D

Date Received: 08/26/2011

Date Analyzed: 08/29/2011

Dilution Factor: 1.0

Soil Aliquot Volume:

(uL) (mL)

\begin{tabular}{|c|c|c|c|}
\hline CAS NO. & COMPOUND & $\begin{array}{l}\text { CONCENTRATION UNITS: } \\
(\mathrm{ug} / \mathrm{L} \text { or } \mathrm{ug} / \mathrm{kg}) \mathrm{ug} / \mathrm{L}\end{array}$ & $Q$ \\
\hline $75-71-8$ & Dichlorodifluoromethane & 0.50 & $\bar{U}$ \\
\hline $74-87-3$ & Chloromethane & 0.50 & U \\
\hline $75-01-4$ & Vinyl chloride & 0.50 & U \\
\hline $74-83-9$ & Bromomethane & 0.50 & $\mathrm{U}$ \\
\hline $75-00-3$ & Chloroethane & 0.50 & $\mathrm{U}$ \\
\hline $75-69-4$ & Trichlorofluoromethane & 0.50 & $\mathrm{U}$ \\
\hline $75-35-4$ & 1,1-Dichloroethene & 0.50 & $\mathrm{U}$ \\
\hline $76-13-1$ & 1,1,2-Trichloro-1,2,2-trifluoroethane & 0.50 & $\mathrm{U}$ \\
\hline $67-64-1$ & Acetone & 1.0 & $\mathrm{~J}$ \\
\hline $75-15-0$ & Carbon disulfide & 0.50 & U \\
\hline $79-20-9$ & Methyl acetate & 0.50 & $\mathrm{U}$ \\
\hline $75-09-2$ & Methylene Chloride & 0.50 & U \\
\hline $156-60-5$ & trans-1,2-Dichloroethene & 0.50 & $\mathrm{U}$ \\
\hline $1634-04-4$ & Methyl tert-butyl ether & 0.50 & $\mathrm{U}$ \\
\hline $75-34-3$ & 1,1-Dichloroethane & 0.50 & $\mathrm{U}$ \\
\hline $156-59-2$ & cis-1,2-Dichloroethene & 0.50 & $\mathrm{U}$ \\
\hline $78-93-3$ & 2-Butanone & 5.0 & $\mathrm{U}$ \\
\hline $74-97-5$ & Bromochloromethane & 0.50 & $\mathrm{U}$ \\
\hline $67-66-3$ & Chloroform & 0.50 & $\mathrm{U}$ \\
\hline $71-55-6$ & 1,1,1-Trichloroethane & 0.50 & $\mathrm{U}$ \\
\hline $110-82-7$ & Cyclohexane & 0.50 & $\mathrm{U}$ \\
\hline $56-23-5$ & Carbon tetrachloride & 0.013 & $\mathrm{~J}$ \\
\hline $71-43-2$ & Benzene & 0.033 & $\mathrm{~J}$ \\
\hline $107-06-2$ & 1,2-Dichloroethane & 0.50 & $\mathrm{U}$ \\
\hline
\end{tabular}

Report 1,4-Dioxane for Low-Medium VOA analysis only 
$1 B$ - FORM I VOA-2

VOLATILE ORGANICS ANALYSIS DATA SHEET
EPA SAMPJE NO.

INMW16-W-32742
Lab Name: TESTAMERICA BURLINGTON

Lab Code: STLV Case No.: INMAN

Matrix: (SOIL/SED/WATER)

Sample wt/vol: 25.0

water

Level: (TRACE/LOW/MED)

$(\mathrm{g} / \mathrm{mL}) \mathrm{mL}$

\% Moisture: not dec.

GC Column: DB-624

ID: 0.20 (min)

Soil Extract volume:

(uL)

Purge Volume: 25.0 (mL)
Contract: $8 \mathrm{E}-00302$

SDG No.: $200-6730$
Lab Sample ID: 200-6730-4

Lab File ID: DHPU18.D

Date Received: 08/26/2011

Date Analyzed: 08/29/2011

Dilution Factor: 1.0

Soil Aliquot Volume:

(uL)

\begin{tabular}{|c|c|c|c|}
\hline CAS NO. & COMPOUND & $\begin{array}{l}\text { CONCENTRATION UNITS: } \\
(\mathrm{ug} / \mathrm{L} \text { or } \mathrm{ug} / \mathrm{kg}) \mathrm{ug} / \mathrm{L}\end{array}$ & $Q$ \\
\hline $79-01-6$ & Trichloroethene & 0.50 & $\bar{U}$ \\
\hline $108-87-2$ & Methylcyclohexane & 0.50 & $\mathrm{U}$ \\
\hline $78-87-5$ & 1,2-Dichloropropane & 0.50 & $\mathrm{U}$ \\
\hline $75-27-4$ & Bromodichloromethane & 0.50 & $\mathrm{U}$ \\
\hline $10061-01-5$ & cis-1,3-Dichloropropene & 0.50 & $\mathrm{U}$ \\
\hline $108-10-1$ & 4-Methyl-2-pentanone & 5.0 & $\mathrm{U}$ \\
\hline $108-88-3$ & Toluene & 0.023 & $\mathrm{~J}$ \\
\hline $10061-02-6$ & trans-1,3-Dichloropropene & 0.50 & $\mathrm{U}$ \\
\hline $79-00-5$ & 1,1,2-Trichloroethane & 0.50 & $\mathrm{U}$ \\
\hline $127-18-4$ & Tetrachloroethene & 0.50 & $\mathrm{U}$ \\
\hline $591-78-6$ & 2-Hexanone & 5.0 & U \\
\hline $124-48-1$ & Dibromochloromethane & 0.50 & $\mathrm{U}$ \\
\hline $106-93-4$ & 1,2-Dibromoethane & 0.50 & $\mathrm{U}$ \\
\hline $108-90-7$ & Chlorobenzene & 0.50 & $\mathrm{U}$ \\
\hline $100-41-4$ & Ethylbenzene & 0.50 & U \\
\hline $95-47-6$ & o-Xylene & 0.50 & $\mathrm{U}$ \\
\hline $179601-23-1$ & $\mathrm{~m}, \mathrm{p}$-Xylene & 0.50 & $\mathrm{U}$ \\
\hline $100-42-5$ & Styrene & 0.50 & $\mathrm{U}$ \\
\hline $75-25-2$ & Bromoform & 0.50 & $\mathrm{U}$ \\
\hline $98-82-8$ & Isopropylbenzene & 0.50 & $\mathrm{U}$ \\
\hline $79-34-5$ & $1,1,2,2$-Tetrachloroethane & 0.50 & $\mathrm{U}$ \\
\hline $541-73-1$ & 1,3-Dichlorobenzene & 0.50 & $\mathrm{u}$ \\
\hline $106-46-7$ & 1,4-Dichlorobenzene & 0.50 & $\mathrm{U}$ \\
\hline $95-50-1$ & 1,2-Dichlorobenzene & 0.50 & $\mathrm{U}$ \\
\hline $96-12-8$ & 1,2-Dibromo-3-Chloropropane & 0.50 & $\mathrm{U}$ \\
\hline $120-82-1$ & 1,2,4-Trichlorobenzene & 0.50 & $\mathrm{U}$ \\
\hline $87-61-6$ & 1,2,3-Trichlorobenzene & 0.50 & $\mathrm{U}$ \\
\hline
\end{tabular}


$1 \mathrm{~J}$ - FORM I VOA-TIC

VOLATILE ORGANICS ANALYSIS DATA SHEET

TENTATIVELY IDENTIFIED COMPOUNDS
EPA SAMPLE NO. INMW16-W-32742
Lab Name: TESTAMERICA BURLINGTON Lab Code: STLV Cas

Matrix: (SOIL/SED/WATER)

water

Sample wt/vol: 25.0

$(\mathrm{g} / \mathrm{mL}) \mathrm{mL}$

Level: (TRACE or LOW/MED)

TRACE

o Moisture: not dec.

GC Column: DB-624

ID $: 0.20$ (mm)

Soil Extract Volume: (uL)

CONCENTRATION UNITS: (ug/L or $\mathrm{ug} / \mathrm{kg}$ ) $\mathrm{ug} / \mathrm{L}$
Contract: $\quad 8 \mathrm{E}-00302$

SDG No.: 200-6730

Lab Sample ID: 200-6730-4

Lab File ID: DHPU18.D

Date Received: 08/26/2011

Date Analyzed: 08/29/2011

Dilution Factor: 1.0

Soil Aliquot Volume:

(uL)

Purge Volume: 25.0

(mL)

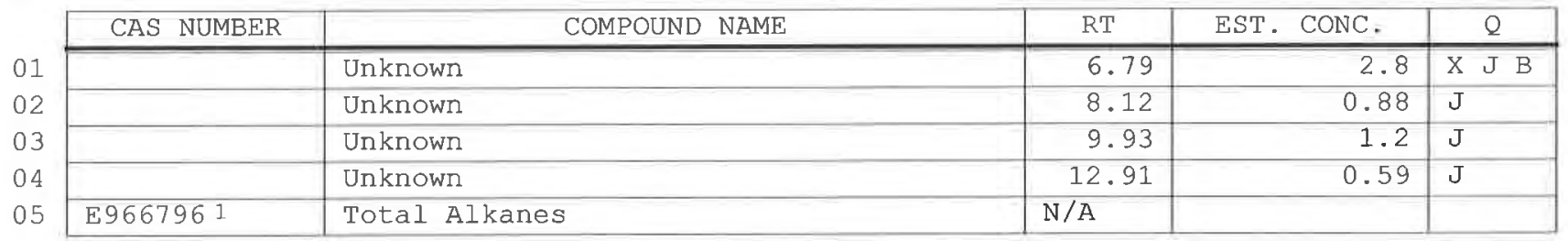

1 EPA-designated Registry Number. 
$1 A$ - FORM I VOA-1

VOLATILE ORGANICS ANALYSIS DATA SHEET
EPA SAMPLE NO.

INQCTB-W-32744
Lab Name: TESTAMERICA BURLINGTON

Lab Code: STLV Cas

Matrix: (SOIL/SED/WATER)

Sample wt/vol: 25.0

Level: (TRACE/LOW/MED) TRACE

o Moisture: not dec.

GC Column: DB-624 ID: $0.20 \quad$ (mm)

Soil Extract Volume: (uL)

Water $(\mathrm{g} / \mathrm{mL}) \mathrm{mL}$

Purge Volume: 25.0 (mL)
Contract: $8 \mathrm{E}-00302$

SDG No.: 200-6730

Lab Sample ID: 200-6730-6

Lab File ID: DHPU14.D

Date Received: 08/26/2011

Date Analyzed: 08/29/2011

Dilution Factor: 1.0

Soil Aliquot Volume:

(uL)

\begin{tabular}{|c|c|c|c|}
\hline CAS NO. & COMPOUND & $\begin{array}{l}\text { CONCENTRATION UNITS: } \\
(\mathrm{ug} / \mathrm{L} \text { or } \mathrm{ug} / \mathrm{kg}) \mathrm{ug} / \mathrm{L}\end{array}$ & $Q$ \\
\hline $75-71-8$ & Dichlorodifluoromethane & 0.50 & $\overline{\mathrm{U}}$ \\
\hline $74-87-3$ & Chloromethane & 0.50 & $\mathrm{U}$ \\
\hline $75-01-4$ & Vinyl chloride & 0.50 & $\mathrm{U}$ \\
\hline $74-83-9$ & Bromomethane & 0.50 & $\mathrm{U}$ \\
\hline $75-00-3$ & Chloroethane & 0.50 & $\mathrm{U}$ \\
\hline $75-69-4$ & Trichlorofluoromethane & 0.50 & $\mathrm{U}$ \\
\hline $75-35-4$ & 1,1-Dichloroethene & 0.50 & $\mathrm{U}$ \\
\hline $76-13-1$ & 1,1,2-Trichloro-1,2,2-trifluoroethane & 0.50 & $\mathrm{U}$ \\
\hline $67-64-1$ & Acetone & 4.5 & $\mathrm{~J}$ \\
\hline $75-15-0$ & Carbon disulfide & 0.50 & $\mathrm{U}$ \\
\hline $79-20-9$ & Methyl acetate & 0.50 & $\mathrm{U}$ \\
\hline $75-09-2$ & Methylene Chloride & 0.50 & $\mathrm{U}$ \\
\hline $156-60-5$ & trans-1,2-Dichloroethene & 0.50 & $\mathrm{U}$ \\
\hline $1634-04-4$ & Methyl tert-butyl ether & 0.50 & $\mathrm{U}$ \\
\hline $75-34-3$ & 1,1-Dichloroethane & 0.50 & $\mathrm{U}$ \\
\hline $156-59-2$ & cis-1,2-Dichloroethene & 0.50 & $\mathrm{U}$ \\
\hline $78-93-3$ & 2-Butanone & 5.0 & $\mathrm{U}$ \\
\hline $74-97-5$ & Bromochloromethane & 0.50 & $\mathrm{U}$ \\
\hline $67-66-3$ & Chloroform & 0.50 & $\mathrm{U}$ \\
\hline $71-55-6$ & 1,1,1-Trichloroethane & 0.50 & $\mathrm{U}$ \\
\hline $110-82-7$ & Cyclohexane & 0.50 & $\mathrm{U}$ \\
\hline $56-23-5$ & Carbon tetrachloride & 0.50 & $\mathrm{U}$ \\
\hline $71-43-2$ & Benzene & 0.14 & $\mathrm{~J}$ \\
\hline $107-06-2$ & 1,2-Dichloroethane & 0.50 & $\mathrm{U}$ \\
\hline
\end{tabular}

Report 1,4-Dioxane for Low-Medium VOA analysis only 
1B - FORM I VOA-2

VOLATILE ORGANICS ANALYSIS DATA SHEET
EPA SAMPLE NO.

INQCTB-W-32744
Lab Name: TESTAMERICA BURLINGTON

Lab Code: STLV

Contract: $8 \mathrm{E}-00302$

Matrix: (SOIL/SED/WATER)

Sample wt/vol: 25.0

Level: (TRACE/LOW/MED) TRACE

Water

$(\mathrm{g} / \mathrm{mL}) \quad \mathrm{mL}$

o Moisture: not dec.

GC Column: DB-624

ID $: 0.20$

(mm)

Soil Extract Volume:

(mL)

Purge Volume: 25.0
SDG No.: 200-6730

Lab Sample ID: 200-6730-6

Lab File ID: DHPU14.D

Date Received: $08 / 26 / 2011$

Date Analyzed: 08/29/2011

Dilution Factor: 1.0

Soil Aliquot Volume:

\begin{tabular}{|c|c|c|c|}
\hline CAS NO. & COMPOUND & $\begin{array}{l}\text { CONCENTRATION UNITS: } \\
(\mathrm{ug} / \mathrm{L} \text { or } \mathrm{ug} / \mathrm{kg}) \mathrm{ug} / \mathrm{L}\end{array}$ & $Q$ \\
\hline $79-01-6$ & Trichloroethene & 0.50 & $\mathrm{U}$ \\
\hline $108-87-2$ & Methylcyclohexane & 0.50 & $\mathrm{U}$ \\
\hline $78-87-5$ & 1,2-Dichloropropane & 0.50 & $\mathrm{U}$ \\
\hline $75-27-4$ & Bromodichloromethane & 0.50 & $\mathrm{U}$ \\
\hline $10061-01-5$ & cis-1,3-Dichloropropene & 0.50 & $\mathrm{U}$ \\
\hline $108-10-1$ & 4-Methyl-2-pentanone & 5.0 & $\mathrm{U}$ \\
\hline $108-88-3$ & Toluene & 0.31 & $\mathrm{~J}$ \\
\hline $10061-02-6$ & trans-1,3-Dichloropropene & 0.50 & $\mathrm{U}$ \\
\hline $79-00-5$ & 1,1,2-Trichloroethane & 0.50 & $\mathrm{U}$ \\
\hline $127-18-4$ & Tetrachloroethene & 0.50 & $\mathrm{U}$ \\
\hline $591-78-6$ & 2-Hexanone & 5.0 & $\mathrm{U}$ \\
\hline $124-48-1$ & Dibromochloromethane & 0.50 & $\mathrm{U}$ \\
\hline $106-93-4$ & 1,2-Dibromoethane & 0.50 & $\mathrm{U}$ \\
\hline $108-90-7$ & Chlorobenzene & 0.50 & $\mathrm{U}$ \\
\hline $100-41-4$ & Ethylbenzene & 0.026 & $\mathrm{~J}$ \\
\hline $95-47-6$ & o-Xylene & 0.026 & $\mathrm{~J}$ \\
\hline $179601-23-1$ & $\mathrm{~m}, \mathrm{p}$-xylene & 0.089 & $\mathrm{~J}$ \\
\hline $100-42-5$ & Styrene & 0.31 & $\mathrm{~J}$ \\
\hline $75-25-2$ & Bromoform & 0.50 & $\mathrm{U}$ \\
\hline $98-82-8$ & Isopropylbenzene & 0.50 & $\mathrm{U}$ \\
\hline $79-34-5$ & $1,1,2,2$-Tetrachloroethane & 0.50 & U \\
\hline $541-73-1$ & 1,3-Dichlorobenzene & 0.50 & $\mathrm{U}$ \\
\hline $106-46-7$ & 1,4-Dichlorobenzene & 0.50 & $\mathrm{U}$ \\
\hline $95-50-1$ & 1,2-Dichlorobenzene & 0.50 & $\mathrm{U}$ \\
\hline $96-12-8$ & 1,2-Dibromo-3-Chloropropane & 0.50 & $\mathrm{U}$ \\
\hline $120-82-1$ & 1,2,4-Trichlorobenzene & 0.50 & U \\
\hline $87-61-6$ & 1,2,3-Trichlorobenzene & 0.50 & $\mathrm{U}$ \\
\hline
\end{tabular}


$1 \mathrm{~J}$ - FORM I VOA-TIC

VOLATILE ORGANICS ANALYSIS DATA SHEET TENTATIVELY IDENTIFIED COMPOUNDS
EPA SAMPLE NO.

INQCTB-W-32744

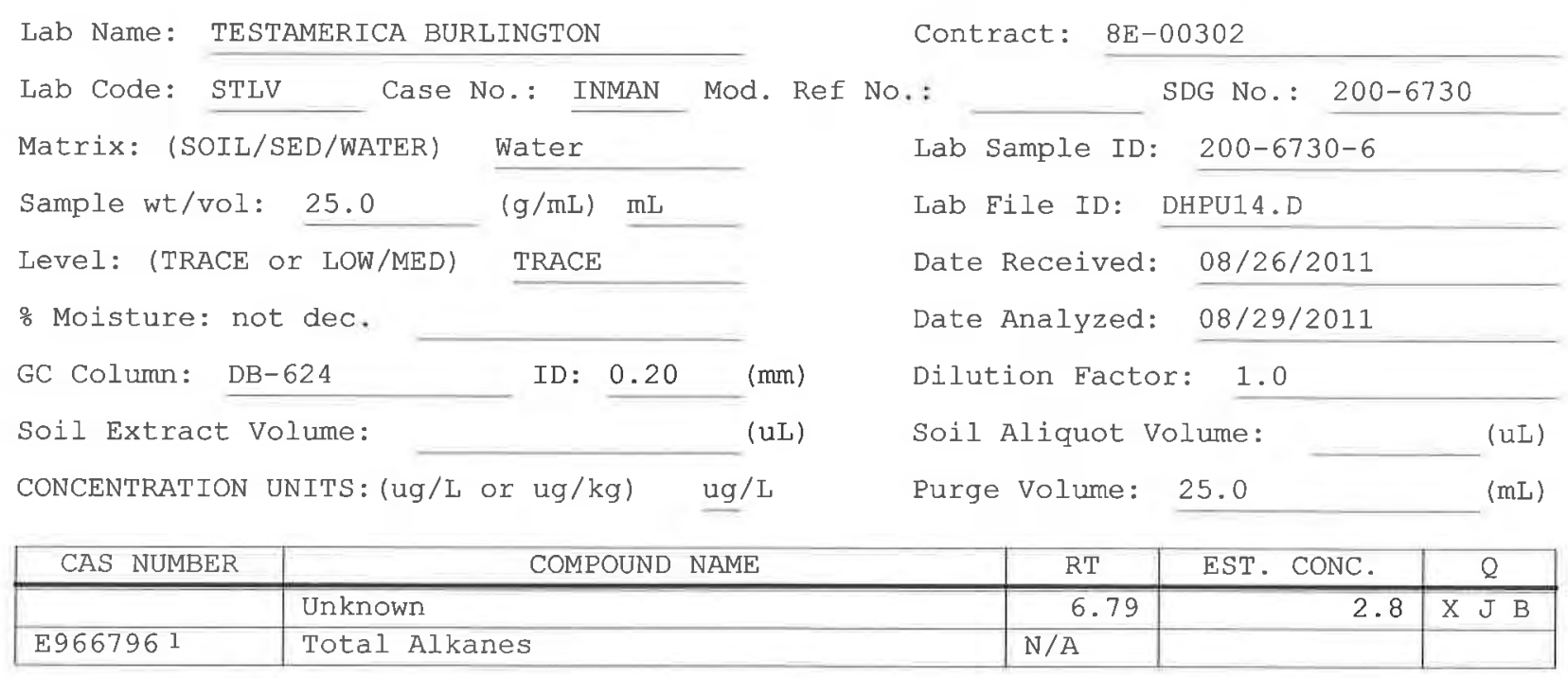

1 EPA-designated Registry Number. 
$6 A$ - FORM VI VOA-1

VOLATILE ORGANICS INITIAL CALIBRATION DATA

Lab Name: TESTAMERICA BURLINGTON

Lab Code: STLV Case No.: INMAN Mod. Ref No.:

Instrument ID: D.i

Heated Purge: (Y/N) N

Purge Volume: 25.0

GC Column: DB-624

ID: 0.20

$\mathrm{RRF} 0.5=\mathrm{DHP04} . \mathrm{D}$

$\operatorname{RRF} 10=$ DHP07.D

RRF $5.0=$ DHP06.D

(D)

\begin{tabular}{|l|r|r|r|r|}
\hline COMPOUND & RRF0.5 & RRF1.0 & RRF5.0 & RR \\
\hline Dichlorodifluoromethane & 0.418 & 0.398 & 0.390 & 0
\end{tabular}

Dichlorodifluoromethane

Chloromethane

Vinyl chloride

Bromomethane

Chloroethane

Trichlorofluoromethane

1,1-Dichloroethene

1,1,2-Trichloro-

1,2,2-trifluoroethane

Acetone

Carbon disulfide

Methyl acetate

Methylene Chloride

trans-1,2-Dichloroethene

Methyl tert-butyl ether

1,1-Dichloroethane

cis-1,2-Dichloroethene

2-Butanone

Bromochloromethane

Chloroform

1,1,1-Trichloroethane

Cyclohexane

Carbon tetrachloride

Benzene

1,2-Dichloroethane

Trichloroethene

Methylcyclohexane

\begin{tabular}{l|l}
0.418 \\
.769
\end{tabular}

0.713

0.655

$\begin{array}{ll}0.491 & 0.475\end{array}$

0.475

0.180

0.292

0.612

0.203

0.280

0.259

0.318

0.307

0.587

0.377

0.358

0.298

0.336

0.038

0.032

0.707

0.719

0.108

0.264

0.094

0.076

0.257

0.242

0.323
0.304

0.298

0.642

0.302

0.278

0.586

0.289

0.045

0.046

0.104

0.502

0.103

0.482

0.490

0.514

0.761

0.487

1. 617

0.795

0.459

1. 501

0.291

0.397

0.703

0.708

0.271

0.380

0.292

0.300

0.047

0.100

0.473

0.494

0.494

0.477

1.518

0.277

0.769

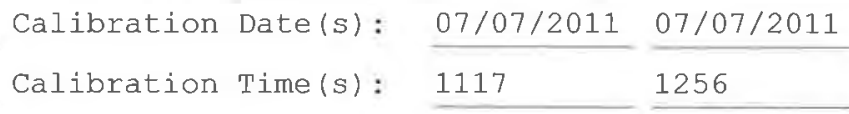

(mL)

(mm) Length: 25

(m)
RRF 1.0 = DHP05.D
RRF $20=$ DHP08.

\begin{tabular}{l|l|l|l} 
RRF 10 & RRF 20 & $\overline{\text { RRE }}$ & \%RSD \\
\hline
\end{tabular}

$\begin{array}{ll}0.409 & 0.398\end{array}$

\begin{tabular}{|l|l|}
\hline 0.403 & 2.7 \\
\hline 0.687 & 7.8
\end{tabular}

0.659

0.499

0.219

\begin{tabular}{|l|}
\hline .518 \\
\hline 0.277 \\
\hline 0.357 \\
0.769
\end{tabular}

0.355

0.757

0.608

0.303

0.358

0.032

0.760

0.079

0.248

0.312

0.319

0.312

0.049

0.099

0.493

0.490
0.909

0.474

1. 495

0.284

.757

0.398
0.639

0.403
0.687

0.491

0.239

0.496

0.209

0.272

0.611

0.297

0.347

0.606

0.305

0.355

0.030

.036

\begin{tabular}{l|l|}
0.030 & 0.036 \\
\hline 0.766 & 0.742 \\
\hline 0.074 & 0.086
\end{tabular}

0.074

0.241
0.306

0.306

0.318
0.607

0.607

0.048

0.101

0.477

0.477

0.883

0.464

1.447

0.285

0.351

0.250

0.308

0.302

0.302

0.302

0.047

0.047

0.485

0.494

0.850

0.850

1.515

0.282

\begin{tabular}{l|l|}
0.282 \\
\hline 0.368 \\
\hline 0.737 \\
\hline
\end{tabular}

\begin{tabular}{l}
768 \\
\hline
\end{tabular}

5.4

Report 1,4-Dioxane for Low-Medium VOA analysis only 
$6 \mathrm{~B}$ - FORM VI VOA-2

VOLATILE ORGANICS INITIAL CALIBRATION DATA

Lab Name: TESTAMERICA BURLINGTON

Contract: $\quad 8 \mathrm{E}-00302$

Lab Code: STLV Case No.: INMAN Mod. Ref No.:

SDG No.: $200-6730$

Instrument ID: D.i Calibration Date(s): 07/07/2011 07/07/2011

Heated Purge: (Y/N) N

Calibration Time(s): $1117 \quad 1256$

Purge Volume: 25.0

$(\mathrm{mL})$

GC Column: DB-624

ID: 0.20

(mm) Length: 25

(m)

\begin{tabular}{|c|c|c|c|c|c|c|c|}
\hline \multirow{2}{*}{$\begin{array}{l}\text { LAB FILE ID: } \\
\text { RRE } 5.0=\text { DHP06.D } \\
\text { COMPOUND }\end{array}$} & \multicolumn{3}{|c|}{$\begin{array}{l}\text { RRF 0.5 }=\text { DHP04.D } \\
\text { RRF 10 }=\text { DHP07.D }\end{array}$} & \multicolumn{4}{|c|}{$\begin{array}{l}\text { RRF 1.0 }=\text { DHPO5.D } \\
\text { RRF 20 }=\text { DHP08.D }\end{array}$} \\
\hline & $\operatorname{RRF} 0.5$ & RRF 1.0 & RRF 5.0 & RRF 10 & RRF 20 & $\overline{\mathrm{RRF}}$ & $\because \mathrm{RSD}$ \\
\hline 1,2-Dichloropropane & 0.363 & 0.373 & 0.367 & 0.371 & 0.355 & 0.366 & 2.0 \\
\hline Bromodichloromethane & 0.310 & 0.296 & 0.307 & 0.305 & 0.306 & 0.305 & 1.6 \\
\hline cis-1,3-Dichloropropene & 0.310 & 0.338 & 0.406 & 0.414 & 0.418 & 0.377 & 13.2 \\
\hline 4-Methyl-2-pentanone & 0.104 & 0.114 & 0.137 & 0.140 & 0.137 & 0.126 & 12.7 \\
\hline Toluene & 1.608 & 1.623 & 1.695 & 1.690 & 1.667 & 1.657 & 2.4 \\
\hline trans-1,3-Dichloropropene & 0.216 & 0.225 & 0.285 & 0.302 & 0.307 & 0.267 & 16.2 \\
\hline 1,1,2-Trichloroethane & 0.155 & 0.162 & 0.160 & 0.156 & 0.157 & 0.158 & 1.9 \\
\hline Tetrachloroethene & 0.311 & 0.304 & 0.306 & 0.308 & 0.299 & 0.306 & 1.5 \\
\hline 2-Hexanone & 0.076 & 0.085 & 0.096 & 0.099 & 0.096 & 0.090 & 10.6 \\
\hline Dibromochloromethane & 0.172 & 0.176 & 0.180 & 0.178 & 0.192 & 0.180 & 4.1 \\
\hline 1,2-Dibromoethane & 0.132 & 0.135 & 0.138 & 0.145 & 0.145 & 0.139 & 4.2 \\
\hline Chlorobenzene & 1.079 & 1.036 & 1.034 & 1.041 & 1.021 & 1.042 & 2.1 \\
\hline Ethylbenzene & 1.594 & 1.651 & 1.933 & 1.968 & 1.978 & 1.825 & 10.2 \\
\hline o-Xylene & 0.539 & 0.600 & 0.727 & 0.749 & 0.755 & 0.674 & 14.6 \\
\hline m,p-Xylene & 0.644 & 0.689 & 0.779 & 0.797 & 0.802 & 0.742 & 9.6 \\
\hline Styrene & 0.763 & 0.886 & 1.048 & 1.096 & 1.123 & 0.983 & 15.6 \\
\hline Bromoform & 0.107 & 0.116 & 0.126 & 0.119 & 0.137 & 0.121 & 9.2 \\
\hline Isopropylbenzene & 1.400 & 1.553 & 2.026 & 2.103 & 2.121 & 1.841 & 18.4 \\
\hline $1,1,2,2$-Tetrachloroethane & 0.168 & 0.154 & 0.168 & 0.164 & 0.164 & 0.164 & 3.6 \\
\hline 1,3-Dichlorobenzene & 1.494 & 1.468 & 1.569 & 1.629 & 1.647 & 1.562 & 5.1 \\
\hline 1,4-Dichlorobenzene & 1.827 & 1.736 & 1.615 & 1.641 & 1.610 & 1.686 & 5.5 \\
\hline 1,2-Dichlorobenzene & 1.377 & 1.388 & 1.407 & 1.392 & 1.355 & 1.384 & 1.4 \\
\hline 1,2-Dibromo-3-Chloropropane & 0.021 & 0.027 & 0.039 & 0.035 & 0.040 & 0.032 & 24.3 \\
\hline 1,2,4-Trichlorobenzene & 0.603 & 0.574 & 0.686 & 0.751 & 0.756 & 0.674 & 12.4 \\
\hline $1,2,3$-Trichlorobenzene & 0.433 & 0.469 & 0.522 & 0.573 & 0.575 & 0.514 & 12.3 \\
\hline
\end{tabular}


$6 \mathrm{C}-$ FORM VI VOA-3

VOLATILE ORGANICS INITIAL CALIBRATION DATA

Lab Name: TESTAMERICA BURLINGTON

Contract: $8 \mathrm{E}-00302$

Lab Code: STLV Case No.: INMAN Mod. Ref No.:

SDG No.: 200-6730

Instrument ID: D.i

Calibration Date(s): 07/07/2011 07/07/2011

Heated Purge: ( $\mathrm{Y} / \mathrm{N}) \mathrm{N}$

Calibration Time(s): 1117

1256

Purge Volume: 25.0

(mL)

GC Column: $\mathrm{DB}-624$

ID $: 0.20$

(mm)

Length: 25

(m)

\begin{tabular}{|c|c|c|c|c|c|c|c|}
\hline \multirow{2}{*}{$\begin{array}{l}\text { LAB FILE ID: } \\
\text { RRF } 5.0=\text { DHP06.D }\end{array}$} & \multicolumn{3}{|c|}{ RRF $0.5=$ DHPO $4 . D$} & \multicolumn{4}{|c|}{ RRF $1.0=$ DHP05.D } \\
\hline & RRF 10 & $1 \mathrm{P} 07 . \mathrm{D}$ & & RRF 20 & $=\mathrm{DHPO}$ & & \\
\hline COMPOUND & RRF 0.5 & RRF 1.0 & RRE 5.0 & RRE 10 & RRF 20 & $\overline{\mathrm{RRF}}$ & $\because \mathrm{RSD}$ \\
\hline Vinyl Chloride-d3 & 0.479 & 0.428 & 0.415 & 0.431 & 0.426 & 0.436 & 5.7 \\
\hline Chloroethane-d5 & 0.304 & 0.290 & 0.269 & 0.276 & 0.274 & 0.283 & 5.0 \\
\hline 1,1-Dichloroethene-d2 & 0.849 & 0.795 & 0.757 & 0.787 & 0.787 & 0.795 & 4.2 \\
\hline 2-Butanone-d5 & 0.041 & 0.038 & 0.040 & 0.044 & 0.043 & 0.041 & 5.5 \\
\hline Chloroform-d & 0.512 & 0.515 & 0.502 & 0.514 & 0.503 & 0.509 & 1.3 \\
\hline 1,2-Dichloroethane-d4 & 0.218 & 0.203 & 0.199 & 0.201 & 0.194 & 0.203 & 4.3 \\
\hline Benzene-d6 & 1.573 & 1.471 & 1.478 & 1.453 & 1.403 & 1.475 & 4.2 \\
\hline 1,2-Dichloropropane-d6 & 0.410 & 0.374 & 0.367 & 0.365 & 0.356 & 0.374 & 5.7 \\
\hline Toluene-d8 & 1.315 & 1.370 & 1.458 & 1.441 & 1.404 & 1.397 & 4.1 \\
\hline trans-1,3-Dichloropropene-d4 & 0.197 & 0.207 & 0.232 & 0.249 & 0.264 & 0.230 & 12.2 \\
\hline 2-Hexanone-d5 & 0.023 & 0.026 & 0.037 & 0.038 & 0.039 & 0.033 & 23.1 \\
\hline $1,1,2,2$-Tetrachloroethane-d2 & 0.151 & 0.161 & 0.167 & 0.171 & 0.169 & 0.164 & 4.8 \\
\hline 1,2-Dichlorobenzene-d4 & 0.885 & 0.843 & 0.815 & 0.807 & 0.799 & 0.830 & 4.2 \\
\hline
\end{tabular}

Report 1,4-Dioxane-d8 for Low-Medium VOA analysis only 
$7 A$ - FORM VII VOA-1

VOLATILE CONTINUING CALIBRATION DATA

Lab Name: TESTAMERICA BURLINGTON

Contract: $8 \mathrm{E}-00302$

Lab Code: STLV Case No.: INMAN

Mod. Ref No.:

SDG No.: $200-6730$

Instrument ID: D.i

Calibration Date: 08/29/2011 Time: 0939

Lab File Id: DHPU03.D Init. Calib. Date(s): 07/07/2011 07/07/2011

EPA Sample No. (VSTD\#\#\#\#): VSTD005DR

Init. Calib. Time(s): $1117 \quad 1256$

Heated Purge: $(\mathrm{Y} / \mathrm{N}) \mathrm{N}$

GC Column: DB-624

ID: $0.20(\mathrm{~mm})$ Length: 25

(m)

Purge Volume: 25.0

$(\mathrm{mL})$

\begin{tabular}{|c|c|c|c|c|c|}
\hline COMPOUND & $\overline{\mathrm{RRF}}$ & RRF5.0 & $\begin{array}{l}\text { MIN } \\
\text { RRE }\end{array}$ & $\% \mathrm{D}$ & $\mathrm{MAX}$ 음 \\
\hline Dichlorodifluoromethane & 0.403 & 0.469 & 0.010 & 16.4 & 40.0 \\
\hline Chloromethane & 0.687 & 0.717 & 0.010 & 4.4 & 40.0 \\
\hline Vinyl chloride & 0.496 & 0.514 & 0.010 & 3.6 & 30.0 \\
\hline Bromomethane & 0.209 & 0.206 & 0.010 & -1.7 & 30.0 \\
\hline Chloroethane & 0.272 & 0.264 & 0.010 & -3.0 & 40.0 \\
\hline Trichlorofluoromethane & 0.606 & 0.616 & 0.010 & 1.8 & 40.0 \\
\hline 1,1-Dichloroethene & 0.305 & 0.303 & 0.010 & -0.5 & 30.0 \\
\hline 1,1,2-Trichloro-1,2,2-trifluoroethane & 0.355 & 0.355 & 0.010 & 0.0 & 40.0 \\
\hline Acetone & 0.036 & 0.033 & 0.010 & -8.3 & 40.0 \\
\hline Carbon disulfide & 0.742 & 0.758 & 0.010 & 2.2 & 40.0 \\
\hline Methyl acetate & 0.086 & 0.079 & 0.010 & -7.8 & 40.0 \\
\hline Methylene Chloride & 0.250 & 0.253 & 0.010 & 1.0 & 40.0 \\
\hline trans-1,2-Dichloroethene & 0.308 & 0.293 & 0.010 & -4.9 & 40.0 \\
\hline Methyl tert-butyl ether & 0.302 & 0.307 & 0.010 & 1.5 & 40.0 \\
\hline 1,1-Dichloroethane & 0.609 & 0.605 & 0.010 & -0.8 & 30.0 \\
\hline cis-1,2-Dichloroethene & 0.302 & 0.299 & 0.010 & -0.8 & 40.0 \\
\hline 2-Butanone & 0.047 & 0.049 & 0.010 & 3.7 & 40.0 \\
\hline Bromochloromethane & 0.101 & 0.100 & 0.010 & -0.7 & 30.0 \\
\hline Chloroform & 0.485 & 0.482 & 0.010 & -0.8 & 30.0 \\
\hline 1,1,1-Trichloroethane & 0.494 & 0.478 & 0.010 & -3.2 & 30.0 \\
\hline Cyclohexane & 0.850 & 0.896 & 0.010 & 5.5 & 40.0 \\
\hline Carbon tetrachloride & 0.472 & 0.460 & 0.010 & -2.6 & 30.0 \\
\hline Benzene & 1.515 & 1.437 & 0.010 & -5.2 & 30.0 \\
\hline 1,2-Dichloroethane & 0.282 & 0.289 & 0.010 & 2.7 & 30.0 \\
\hline Trichloroethene & 0.368 & 0.339 & 0.010 & -7.8 & 30.0 \\
\hline Methylcyclohexane & 0.737 & 0.740 & 0.010 & 0.3 & 40.0 \\
\hline
\end{tabular}

Report 1,4-Dioxane for Low/Medium VOA analysis only 
$7 \mathrm{~B}$ - FORM VII VOA-2

VOLATILE CONTINUING CALIBRATION DATA

Lab Name: TESTAMERICA BURLINGTON

Contract: $8 \mathrm{E}-00302$

Lab Code: STLV Case No.: INMAN Mod. Ref No.:

SDG No.: 200-6730

Instrument ID: D.i

Calibration Date: 08/29/2011 Time: 0939

Lab File Id: DHPU03.D Init. Calib. Date(s): 07/07/2011 07/07/2011

EPA Sample No. (VSTD\#\#\#\#): VSTD005DR

Init. Calib. Time(s): $1117 \quad 1256$

Heated Purge: $(\mathrm{Y} / \mathrm{N}) \mathrm{N}$ GC Column: $\mathrm{DB}-624$ ID: $0.20(\mathrm{~mm})$ Length: 25

(In)

Purge Volume: 25.0 $(m L)$

\begin{tabular}{|c|c|c|c|c|c|}
\hline COMPOUND & $\overline{\mathrm{RRE}}$ & RRF5. 0 & $\begin{array}{l}\text { MIN } \\
\text { RRF }\end{array}$ & $\frac{\circ}{0} \mathrm{D}$ & $\mathrm{MAX} \div \mathrm{D}$ \\
\hline 1,2-Dichloropropane & 0.366 & 0.365 & 0.010 & -0.2 & 40.0 \\
\hline Bromodichloromethane & 0.305 & 0.290 & 0.010 & -4.8 & 30.0 \\
\hline cis-1,3-Dichloropropene & 0.377 & 0.388 & 0.010 & 2.7 & 30.0 \\
\hline 4-Methyl-2-pentanone & 0.126 & 0.132 & 0.010 & 4.2 & 40.0 \\
\hline Toluene & 1.657 & 1.622 & 0.010 & -2.1 & 30.0 \\
\hline trans-1,3-Dichloropropene & 0.267 & 0.279 & 0.010 & 4.6 & 30.0 \\
\hline 1,1,2-Trichloroethane & 0.158 & 0.156 & 0.010 & -1.5 & 30.0 \\
\hline Tetrachloroethene & 0.306 & 0.296 & 0.010 & -3.2 & 30.0 \\
\hline 2-Hexanone & 0.090 & 0.094 & 0.010 & 3.9 & 40.0 \\
\hline Dibromochloromethane & 0.180 & 0.175 & 0.010 & -2.6 & 30.0 \\
\hline 1,2-Dibromoethane & 0.139 & 0.140 & 0.010 & 0.6 & 40.0 \\
\hline Chlorobenzene & 1.042 & 1.001 & 0.010 & -4.0 & 30.0 \\
\hline Ethylbenzene & 1.825 & 1.796 & 0.010 & -1.6 & 30.0 \\
\hline o-Xylene & 0.674 & 0.688 & 0.010 & 2.0 & 30.0 \\
\hline m,p-xylene & 0.742 & 0.745 & 0.010 & 0.4 & 30.0 \\
\hline Styrene & 0.983 & 1.043 & 0.010 & 6.1 & 30.0 \\
\hline Bromoform & 0.121 & 0.127 & 0.010 & 4.9 & 30.0 \\
\hline Isopropylbenzene & 1.841 & 1.892 & 0.010 & 2.8 & 40.0 \\
\hline $1,1,2,2$-Tetrachloroethane & 0.164 & 0.156 & 0.010 & -4.9 & 30.0 \\
\hline 1,3-Dich lorobenzene & 1.562 & 1.561 & 0.010 & 0.0 & 30.0 \\
\hline 1,4-Dichlorobenzene & 1.686 & 1.662 & 0.010 & -1.4 & 30.0 \\
\hline 1,2-Dichlorobenzene & 1.384 & 1.414 & 0.010 & 2.2 & 30.0 \\
\hline 1,2-Dibromo-3-Chloropropane & 0.032 & 0.035 & 0.010 & 9.0 & 40.0 \\
\hline 1,2,4-Trichlorobenzene & 0.674 & 0.725 & 0.010 & 7.6 & 30.0 \\
\hline 1,2,3-Trichlorobenzene & 0.514 & 0.555 & 0.010 & 7.9 & 30.0 \\
\hline
\end{tabular}


$7 \mathrm{C}$ - FORM VII VOA-3

VOLATILE CONTINUING CALIBRATION DATA

Lab Name: TESTAMERICA BURLINGTON

Contract: $8 \mathrm{E}-00302$

Lab Code: STLV Case No.: INMAN Mod. Ref No.: SDG No.: 200-6730

Instrument ID: D.i

Calibration Date: 08/29/2011 Time: 0939

Lab File Id: DHPU03. D Init. Calib. Date(s): 07/07/2011 07/07/2011

EPA Sample No. (VSTD\#\#\#\#): VSTD005DR

Init. Calib. Time(s): $1117 \quad 1256$

Heated Purge: ( $\mathrm{Y} / \mathrm{N}) \quad \mathrm{N}$ GC Column: DB-624

ID: $0.20(\mathrm{~mm})$ Length: 25

(II)

Purge Volume: 25.0

$(\mathrm{mL})$

\begin{tabular}{|c|c|c|c|c|c|}
\hline COMPOUND & $\overline{\mathrm{RRF}}$ & RRF5. 0 & $\begin{array}{l}\text { MIN } \\
\text { RRE }\end{array}$ & 80 & MAX 음 \\
\hline Vinyl Chloride-d3 & 0.436 & 0.429 & 0.010 & -1.6 & 30.0 \\
\hline Chloroethane-d5 & 0.283 & 0.260 & 0.010 & -8.1 & 40.0 \\
\hline 1,1-Dichloroethene-d2 & 0.795 & 0.712 & 0.010 & -10.4 & 30.0 \\
\hline 2-Butanone-d5 & 0.041 & 0.043 & 0.010 & 4.8 & 40.0 \\
\hline Chloroform-d & 0.509 & 0.464 & 0.010 & -8.9 & 30.0 \\
\hline 1,2-Dichloroethane-d4 & 0.203 & 0.195 & 0.010 & -4.1 & 30.0 \\
\hline Benzene-d6 & 1.475 & 1.342 & 0.010 & -9.0 & 30.0 \\
\hline 1,2-Dichloropropane-d6 & 0.374 & 0.342 & 0.010 & -8.8 & 40.0 \\
\hline Toluene-d8 & 1.397 & 1.309 & 0.010 & -6.4 & 30.0 \\
\hline trans-1,3-Dichloropropene-d4 & 0.230 & 0.222 & 0.010 & -3.4 & 30.0 \\
\hline 2-Hexanone-d5 & 0.033 & 0.035 & 0.010 & 5.6 & 40.0 \\
\hline $1,1,2,2$-Tetrachloroethane-d2 & 0.164 & 0.154 & 0.010 & -6.2 & 30.0 \\
\hline 1,2-Dichlorobenzene-d4 & 0.830 & 0.790 & 0.010 & -4.7 & 30.0 \\
\hline
\end{tabular}

Report 1,4-Dioxane-d8 for Low/Medium VOA analysis only 
$7 \mathrm{~A}$ - FORM VII VOA-1

VOLATILE CONTINUING CALTBRATION DATA

Lab Name: TESTAMERICA BURLINGTON

Contract: 8E-00302

Lab Code: STLV Case No.: INMAN Mod. Ref No.:

SDG No.: $200-6730$

Instrument ID: D.i

Calibration Date: 08/29/2011 Time: 1733

Lab File Id: DHPU20.D Init. Calib. Date(s): 07/07/2011 07/07/2011

EPA Sample No. (VSTD\#\#\#\#): VSTD005RD

Init. Calib. Time(s): $1117 \quad 1256$

Heated Purge: $(\mathrm{Y} / \mathrm{N}) \mathrm{N}$ GC Column: DB-624

ID: $0.20(\mathrm{~mm})$ Length: 25

(m)

Purge Volume: 25.0

(mL)

\begin{tabular}{|c|c|c|c|c|c|}
\hline COMPOUND & $\overline{R R F}$ & RRF5.0 & $\begin{array}{l}\text { MIN } \\
\text { RRE }\end{array}$ & $\because \mathrm{D}$ & MAX $\because D$ \\
\hline Dichlorodifluoromethane & 0.403 & 0.463 & 0.010 & 14.9 & 50.0 \\
\hline Chloromethane & 0.687 & 0.727 & 0.010 & 5.9 & 50.0 \\
\hline Vinyl chloride & 0.496 & 0.503 & 0.100 & 1.5 & 50.0 \\
\hline Bromonethane & 0.209 & 0.213 & 0.100 & 1.8 & 50.0 \\
\hline Chloroethane & 0.272 & 0.270 & 0.010 & -0.9 & 50.0 \\
\hline Trichlorofluoromethane & 0.606 & 0.631 & 0.010 & 4.2 & 50.0 \\
\hline 1,1-Dichloroethene & 0.305 & 0.311 & 0.100 & 2.0 & 50.0 \\
\hline 1,1,2-Trichloro-1,2,2-trifluoroethane & 0.355 & 0.363 & 0.010 & 2.2 & 50.0 \\
\hline Acetone & 0.036 & 0.032 & 0.010 & -11.6 & 50.0 \\
\hline Carbon disulfide & 0.742 & 0.732 & 0.010 & -1.4 & 50.0 \\
\hline Methyl acetate & 0.086 & 0.081 & 0.010 & -5.4 & 50.0 \\
\hline Methylene Chloride & 0.250 & 0.256 & 0.010 & 2.4 & 50.0 \\
\hline trans-1,2-Dichloroethene & 0.308 & 0.313 & 0.010 & 1.7 & 50.0 \\
\hline Methyl tert-butyl ether & 0.302 & 0.307 & 0.010 & 1.7 & 50.0 \\
\hline 1,1-Dichloroethane & 0.609 & 0.629 & 0.200 & 3.2 & 50.0 \\
\hline cis-1,2-Dichloroethene & 0.302 & 0.305 & 0.010 & 1.2 & 50.0 \\
\hline 2-Butanone & 0.047 & 0.048 & 0.010 & 2.1 & 50.0 \\
\hline Bromochloromethane & 0.101 & 0.104 & 0.050 & 2.9 & 50.0 \\
\hline Chloroform & 0.485 & 0.499 & 0.200 & 2.8 & 50.0 \\
\hline 1,1,1-Trichloroethane & 0.494 & 0.481 & 0.100 & -2.7 & 50.0 \\
\hline Cyclohexane & 0.850 & 0.907 & 0.010 & 6.7 & 50.0 \\
\hline Carbon tetrachloride & 0.472 & 0.464 & 0.100 & -1.8 & 50.0 \\
\hline Benzene & 1.515 & 1.490 & 0.400 & -1.7 & 50.0 \\
\hline 1,2-Dichloroethane & 0.282 & 0.302 & 0.100 & 7.1 & 50.0 \\
\hline Trichloroethene & 0.368 & 0.346 & 0.300 & -6.1 & 50.0 \\
\hline Methylcyclohexane & 0.737 & 0.760 & 0.010 & 3.1 & 50.0 \\
\hline
\end{tabular}

Report 1,4-Dioxane for Low/Medium VOA analysis only 
$7 \mathrm{~B}$ - FORM VII VOA-2

VOLATILE CONTINUING CALIBRATION DATA

Lab Name: TESTAMERICA BURLINGTON

Contract: $8 \mathrm{E}-00302$

Lab Code: STLV Case No.: INMAN Mod. Ref No.:

SDG No.: 200-6730

Instrument ID: D.i

Calibration Date: 08/29/2011 Time: 1733

Lab File Id: DHPU20.D Init. Calib. Date(s): 07/07/2011 07/07/2011

EPA Sample No. (VSTD\#\#\#\#): VSTD005RD

Init. Calib. Time(s): $1117 \quad 1256$

Heated Purge: ( $\mathrm{Y} / \mathrm{N}) \mathrm{N}$ GC Column: DB-624 ID: $0.20(\mathrm{~mm})$ Length: 25 (m)

Purge Volume: 25.0 (mL)

\begin{tabular}{|c|c|c|c|c|c|}
\hline COMPOUND & $\overline{\mathrm{RRE}}$ & RRF5.0 & $\begin{array}{l}\text { MIN } \\
\text { RRF }\end{array}$ & $8 \mathrm{D}$ & MAX $\frac{\circ}{\partial} \mathrm{D}$ \\
\hline 1,2-Dichloropropane & 0.366 & 0.371 & 0.010 & 1.4 & 50.0 \\
\hline Bromodichloromethane & 0.305 & 0.300 & 0.200 & -1.5 & 50.0 \\
\hline cis-1,3-Dichloropropene & 0.377 & 0.393 & 0.200 & 4.2 & 50.0 \\
\hline 4-Methyl-2-pentanone & 0.126 & 0.135 & 0.010 & 7.1 & 50.0 \\
\hline Toluene & 1.657 & 1.674 & 0.400 & 1.0 & 50.0 \\
\hline trans-1,3-Dichloropropene & 0.267 & 0.277 & 0.100 & 3.8 & 50.0 \\
\hline 1,1,2-Trichloroethane & 0.158 & 0.160 & 0.100 & 1.5 & 50.0 \\
\hline Tetrachloroethene & 0.306 & 0.299 & 0.100 & -2.4 & 50.0 \\
\hline 2-Hexanone & 0.090 & 0.094 & 0.010 & 4.5 & 50.0 \\
\hline Dibromochloromethane & 0.180 & 0.178 & 0.100 & -0.9 & 50.0 \\
\hline 1,2-Dibromoethane & 0.139 & 0.138 & 0.010 & -0.5 & 50.0 \\
\hline Chlorobenzene & 1.042 & 1.040 & 0.500 & -0.3 & 50.0 \\
\hline Ethylbenzene & 1.825 & 1.875 & 0.100 & 2.7 & 50.0 \\
\hline o-Xylene & 0.674 & 0.724 & 0.300 & 7.3 & 50.0 \\
\hline $\mathrm{m}, \mathrm{p}$-Xylene & 0.742 & 0.767 & 0.300 & 3.4 & 50.0 \\
\hline Styrene & 0.983 & 1.077 & 0.300 & 9.6 & 50.0 \\
\hline Bromoform & 0.121 & 0.125 & 0.050 & 3.3 & 50.0 \\
\hline Isopropylbenzene & 1.841 & 1.961 & 0.010 & 6.5 & 50.0 \\
\hline $1,1,2,2$-Tetrachloroethane & 0.164 & 0.164 & 0.100 & -0.1 & 50.0 \\
\hline 1,3-Dichlorobenzene & 1.562 & 1.537 & 0.400 & -1.6 & 50.0 \\
\hline 1,4-Dichlorobenzene & 1.686 & 1.674 & 0.400 & -0.7 & 50.0 \\
\hline 1,2-Dichlorobenzene & 1.384 & 1.401 & 0.400 & 1.3 & 50.0 \\
\hline 1,2-Dibromo-3-Chloropropane & 0.032 & 0.034 & 0.010 & 3.9 & 50.0 \\
\hline 1,2,4-Trichlorobenzene & 0.674 & 0.653 & 0.200 & -3.1 & 50.0 \\
\hline $1,2,3$-Trichlorobenzene & 0.514 & 0.529 & 0.200 & 2.9 & 50.0 \\
\hline
\end{tabular}


$7 \mathrm{C}$ - FORM VII VOA-3

VOLATILE CONTINUING CALIBRATION DATA

Lab Name: TESTAMERICA BURLINGTON

Contract: $\quad 8 \mathrm{E}-00302$

Lab Code: STLV Case No.: INMAN Mod. Ref No.:

SDG No.: 200-6730

Instrument ID: D.i

Calibration Date: 08/29/2011 Time: 1733

Lab File Id: DHPU20.D Init. Calib. Date(s) : 07/07/2011 07/07/2011

EPA Sample No. (VSTD\#\#\#\#): VSTD005RD

Init. Calib. Time(s): $1117 \quad 1256$

Heated Purge: ( $\mathrm{Y} / \mathrm{N}) \quad \mathrm{N}$

GC Column: DB-624

ID: $0.20(\mathrm{~mm})$ Length: 25

(m)

Purge Volume: 25.0

(mL)

\begin{tabular}{|c|c|c|c|c|c|}
\hline COMPOUND & $\overline{\mathrm{RRF}}$ & RRE5.0 & $\begin{array}{l}\text { MIN } \\
R R F\end{array}$ & $\frac{8}{8} \mathrm{D}$ & MAX $\triangle D$ \\
\hline Vinyl Chloride-d3 & 0.436 & 0.417 & 0.010 & -4.4 & 50.0 \\
\hline Chloroethane-d5 & 0.283 & 0.271 & 0.010 & $-4 \cdot 3$ & 50.0 \\
\hline 1,1-Dichloroethene-d2 & 0.795 & 0.745 & 0.010 & -6.3 & 50.0 \\
\hline 2-Butanone-d5 & 0.041 & 0.041 & 0.010 & 0.3 & 50.0 \\
\hline Chloroform-d & 0.509 & 0.501 & 0.010 & -1.6 & 50.0 \\
\hline 1,2-Dichloroethane-d4 & 0.203 & 0.210 & 0.010 & 3.4 & 50.0 \\
\hline Benzene-d6 & 1.475 & 1.365 & 0.010 & -7.5 & 50.0 \\
\hline 1,2-Dichloropropane-d6 & 0.374 & 0.344 & 0.010 & -8.3 & 50.0 \\
\hline Toluene-d8 & 1.397 & 1.364 & 0.010 & -2.4 & 50.0 \\
\hline trans-1,3-Dichloropropene-d4 & 0.230 & 0.225 & 0.010 & -2.2 & 50.0 \\
\hline 2-Hexanone-d5 & 0.033 & 0.034 & 0.010 & 4.9 & 50.0 \\
\hline $1,1,2,2$-Tetrachloroethane-d2 & 0.164 & 0.160 & 0.010 & -2.3 & 50.0 \\
\hline 1,2-Dichlorobenzene-d4 & 0.830 & 0.813 & 0.010 & -2.0 & 50.0 \\
\hline
\end{tabular}

Report 1,4-Dioxane-d8 for Low/Medium VOA analysis only 
$1 A$ - FORM I VOA-1

VOLATILE ORGANICS ANALYSIS DATA SHEET
EPA SAMPLE NO.

VBLKDR
Lab Name: TESTAMERICA BURLINGTON

Lab Code: STLV Case No.: INMAN

water

Matrix: (SOIL/SED/WATER)

Sample wt/vol: 25.0

$(\mathrm{g} / \mathrm{mL}) \quad \mathrm{mL}$

Level: (TRACE/LOW/MED) TRACE

\% Moisture: not dec.

GC Column: DB-624

ID: 0.20

(min)

Soil Extract Volume:

(mL)

Purge Volume: 25.0
Contract: $8 \mathrm{E}-00302$

SDG No.: 200-6730

Mod. Ref No. :

Lab Sample ID: MB 200-24379/4

Lab File ID: DHPU04.D

Date Received:

Date Analyzed: 08/29/2011

Dilution Factor: 1.0

Soil Aliquot Volume:

(uL)

\begin{tabular}{|c|c|c|c|}
\hline CAS NO. & COMPOUND & $\begin{array}{l}\text { CONCENTRATION UNITS: } \\
(\mathrm{ug} / \mathrm{L} \text { or } \mathrm{ug} / \mathrm{kg}) \mathrm{ug} / \mathrm{L}\end{array}$ & 8 \\
\hline $75-71-8$ & Dichlorodifluoromethane & 0.50 & $\mathrm{U}$ \\
\hline $74-87-3$ & Chloromethane & 0.50 & $\mathrm{U}$ \\
\hline $75-01-4$ & Vinyl chloride & 0.50 & $\mathrm{U}$ \\
\hline $74-83-9$ & Bromomethane & 0.50 & $\mathrm{U}$ \\
\hline $75-00-3$ & Chloroethane & 0.50 & $\mathrm{U}$ \\
\hline $75-69-4$ & Trichlorofluoromethane & 0.50 & $\mathrm{U}$ \\
\hline $75-35-4$ & 1,1-Dichloroethene & 0.50 & $\mathrm{U}$ \\
\hline $76-13-1$ & 1,1,2-Trichloro-1,2,2-trifluoroethane & 0.50 & U \\
\hline $67-64-1$ & Acetone & 5.0 & $\mathrm{U}$ \\
\hline $75-15-0$ & Carbon disulfide & 0.50 & $\mathrm{U}$ \\
\hline $79-20-9$ & Methyl acetate & 0.50 & $\mathrm{U}$ \\
\hline $75-09-2$ & Methylene Chloride & 0.50 & $\mathrm{U}$ \\
\hline $156-60-5$ & trans-1,2-Dichloroethene & 0.50 & $\mathrm{U}$ \\
\hline $1634-04-4$ & Methyl tert-butyl ether & 0.50 & $\mathrm{U}$ \\
\hline $75-34-3$ & 1,1-Dichloroethane & 0.50 & $\mathrm{U}$ \\
\hline $156-59-2$ & cis-1,2-Dichloroethene & 0.50 & $\mathrm{U}$ \\
\hline $78-93-3$ & 2-Butanone & 5.0 & $\mathrm{U}$ \\
\hline $74-97-5$ & Bromochloromethane & 0.50 & $\mathrm{U}$ \\
\hline $67-66-3$ & Chloroform & 0.50 & $\mathrm{U}$ \\
\hline $71-55-6$ & 1,1,1-Trichloroethane & 0.50 & $\mathrm{U}$ \\
\hline $110-82-7$ & Cyclohexane & 0.50 & $\mathrm{U}$ \\
\hline $56-23-5$ & Carbon tetrachloride & 0.50 & $\mathrm{U}$ \\
\hline $71-43-2$ & Benzene & 0.50 & $\mathrm{U}$ \\
\hline $107-06-2$ & 1,2-Dichloroethane & 0.50 & $\mathrm{U}$ \\
\hline
\end{tabular}

Report 1,4-Dioxane for Low-Medium VOA analysis only 
$1 B$ - FORM I VOA-2

VOLATILE ORGANICS ANALYSIS DATA SHEET
EPA SAMPLE NO.

VBLKDR
Lab Name: TESTAMERICA BURLINGTON

Lab Code: STLV Case No.: INMAN

Matrix: (SOIL/SED/WATER)

Sample wt/vol: 25.0

Water

$(\mathrm{g} / \mathrm{mL}) \quad \mathrm{mL}$

Level: (TRACE/LOW/MED) TRACE

\% Moisture: not dec.

GC Column: DB-624

ID: 0.20

(mm)

Soil Extract Volume:

(uL)

Purge Volume: 25.0

(mL)
Contract: $8 \mathrm{E}-00302$

SDG No.: $200-6730$

Lab Sample ID: MB 200-24379/4

Lab File ID: DHPU04.D

Date Received:

Date Analyzed: 08/29/2011

Dilution Factor: 1.0

Soil Aliquot Volume:

(UL)

\begin{tabular}{|c|c|c|c|}
\hline CAS NO. & COMPOUND & $\begin{array}{l}\text { CONCENTRATION UNITS: } \\
(u g / L \text { or } u g / k g) ~ u g / L\end{array}$ & $Q$ \\
\hline $79-01-6$ & Trichloroethene & 0.50 & $\overline{\mathrm{U}}$ \\
\hline $108-87-2$ & Methylcyclohexane & 0.50 & $\mathrm{U}$ \\
\hline $78-87-5$ & 1,2-Dichloropropane & 0.50 & $\mathrm{U}$ \\
\hline $75-27-4$ & Bromodichloromethane & 0.50 & $\mathrm{U}$ \\
\hline $10061-01-5$ & cis-1,3-Dichloropropene & 0.50 & $\mathrm{U}$ \\
\hline $108-10-1$ & 4-Methyl-2-pentanone & 5.0 & $\mathrm{U}$ \\
\hline $108-88-3$ & Toluene & 0.50 & $\mathrm{U}$ \\
\hline $10061-02-6$ & trans-1,3-Dichloropropene & 0.50 & $\mathrm{U}$ \\
\hline $79-00-5$ & 1,1,2-Trichloroethane & 0.50 & $\mathrm{U}$ \\
\hline $127-18-4$ & Tetrachloroethene & 0.50 & $\mathrm{U}$ \\
\hline $591-78-6$ & 2-Hexanone & 5.0 & $\mathrm{U}$ \\
\hline $124-48-1$ & Dibromochloromethane & 0.50 & $\mathrm{U}$ \\
\hline $106-93-4$ & 1,2-Dibromoethane & 0.50 & $\mathrm{U}$ \\
\hline $108-90-7$ & Chlorobenzene & 0.50 & $\mathrm{U}$ \\
\hline $100-41-4$ & Ethylbenzene & 0.50 & $\mathrm{U}$ \\
\hline $95-47-6$ & o-Xylene & 0.50 & $\mathrm{U}$ \\
\hline $179601-23-1$ & $\mathrm{~m}, \mathrm{p}-\mathrm{xy}$ lene & 0.50 & $\mathrm{U}$ \\
\hline $100-42-5$ & Styrene & 0.50 & $\mathrm{U}$ \\
\hline $75-25-2$ & Bromoform & 0.50 & $\mathrm{U}$ \\
\hline $98-82-8$ & Isopropylbenzene & 0.50 & $\mathrm{U}$ \\
\hline $79-34-5$ & $1,1,2,2-$ Tetrachloroethane & 0.50 & $\mathrm{U}$ \\
\hline $541-73-1$ & 1,3-Dichlorobenzene & 0.50 & $\mathrm{U}$ \\
\hline $106-46-7$ & 1,4-Dichlorobenzene & 0.50 & $\mathrm{U}$ \\
\hline $95-50-1$ & 1,2-Dichlorobenzene & 0.50 & $\mathrm{U}$ \\
\hline $96-12-8$ & 1,2-Dibromo-3-Chloropropane & 0.50 & $\mathrm{U}$ \\
\hline $120-82-1$ & 1,2,4-Trichlorobenzene & 0.50 & $\mathrm{U}$ \\
\hline $87-61-6$ & 1,2,3-Trichlorobenzene & 0.50 & $\mathrm{U}$ \\
\hline
\end{tabular}


IJ - FORM I VOA-TIC

VOLATILE ORGANICS ANALYSIS DATA SHEET TENTATIVELY IDENTIFIED COMPOUNDS
EPA SAMPLE NO.

VBLKDR
Lab Name: TESTAMERICA BURLINGTON

Contract: $8 \mathrm{E}-00302$

Lab Code: STLV Case No.: INMAN

Mod. Ref No.:
Matrix: (SOIL/SED/WATER) Water

Sample wt/vol: 25.0

$(\mathrm{g} / \mathrm{mL}) \mathrm{mL}$

Level: (TRACE or LOW/MED)

TRACE

o Moisture: not dec.

GC Column: DB-624

ID: 0.20 (mm)

Soil Extract Volume: (uL)

CONCENTRATION UNITS: (ug/L or ug/ $\mathrm{kg}$ ) ug/L
SDG No.: 200-6730

Lab Sample ID: MB 200-24379/4

Lab File ID: DHPU04.D

Date Received:

Date Analyzed: 08/29/2011

Dilution Factor: 1.0

Soil Aliquot Volume:

Purge Volume: 25.0 $(\mathrm{mL})$

\begin{tabular}{|l|l|l|c|c|c|}
\hline \multicolumn{1}{|c|}{ CAS NUMBER } & \multicolumn{1}{|c|}{ COMPOUND NAME } & RT & EST. CONC. & Q \\
\cline { 2 - 6 } 01 & & Unknown & 6.79 & X J \\
\cline { 2 - 6 } 02 & E966796 I & Total Alkanes & N/A & \\
\hline
\end{tabular}

1 EPA-designated Registry Number. 
$1 A$ - FORM I VOA-1

VOLATILE ORGANICS ANALYSIS DATA SHEET
EPA SAMPLE NO.

VHBLK01
Lab Name: TESTAMERICA BURLINGTON

Lab Code: STLV Case No.: INMAN

Matrix: (SOIL/SED/WATER)

Sample wt/vol: 25.0

Water

$(\mathrm{g} / \mathrm{mL}) \mathrm{mL}$

Level: (TRACE/LOW/MED) TRACE

․․․ Moisture: not dec.

GC Column: DB-624

ID: $0.20 \quad(\mathrm{~mm})$

Soil Extract Volume:

(mL)

Purge Volume: 25.0
Contract: $8 \mathrm{E}-00302$

SDG No.: 200-6730

\begin{tabular}{|c|c|c|c|}
\hline CAS NO. & COMPOUND & $\begin{array}{l}\text { CONCENTRATION UNITS: } \\
(\mathrm{ug} / \mathrm{L} \text { or } \mathrm{ug} / \mathrm{kg}) \mathrm{ug} / \mathrm{L}\end{array}$ & $Q$ \\
\hline $75-71-8$ & Dichlorodifluoromethane & 0.50 & $\bar{U}$ \\
\hline $74-87-3$ & Chloromethane & 0.50 & $\mathrm{U}$ \\
\hline $75-01-4$ & Vinyl chloride & 0.50 & $\mathrm{U}$ \\
\hline $74-83-9$ & Bromomethane & 0.50 & $\mathrm{U}$ \\
\hline $75-00-3$ & Chloroethane & 0.50 & $\mathrm{U}$ \\
\hline $75-69-4$ & Trichlorofluoromethane & 0.50 & $\mathrm{U}$ \\
\hline $75-35-4$ & 1,1-Dichloroethene & 0.50 & $\mathrm{U}$ \\
\hline $76-13-1$ & 1,1,2-Trichloro-1,2,2-trifluoroethane & 0.50 & $\mathrm{U}$ \\
\hline $67-64-1$ & Acetone & 5.0 & $\mathrm{U}$ \\
\hline $75-15-0$ & Carbon disulfide & 0.50 & $\mathrm{U}$ \\
\hline $79-20-9$ & Methyl acetate & 0.50 & $\mathrm{U}$ \\
\hline $75-09-2$ & Methylene Chloride & 0.50 & $\mathrm{U}$ \\
\hline $156-60-5$ & trans-1,2-Dichloroethene & 0.50 & $\mathrm{U}$ \\
\hline $1634-04-4$ & Methyl tert-butyl ether & 0.50 & U \\
\hline $75-34-3$ & 1,1-Dichloroethane & 0.50 & $\mathrm{U}$ \\
\hline $156-59-2$ & cis-1,2-Dichloroethene & 0.50 & $\mathrm{U}$ \\
\hline $78-93-3$ & 2-Butanone & 5.0 & $\mathrm{U}$ \\
\hline $74-97-5$ & Bromochloromethane & 0.50 & $\mathrm{U}$ \\
\hline $67-66-3$ & Chloroform & 0.065 & $\mathrm{~J}$ \\
\hline $71-55-6$ & $1,1,1$-Trichloroethane & 0.50 & $\mathrm{U}$ \\
\hline $110-82-7$ & Cyclohexane & 0.50 & $\mathrm{U}$ \\
\hline $56-23-5$ & Carbon tetrachloride & 0.50 & $\mathrm{U}$ \\
\hline $71-43-2$ & Benzene & 0.50 & $\mathrm{U}$ \\
\hline $107-06-2$ & 1,2-Dichloroethane & 0.50 & $\mathrm{U}$ \\
\hline
\end{tabular}

Report 1,4-Dioxane for Low-Medium VOA analysis only

Lab Sample ID: $200-6730-7$

Lab File ID: DHPU19.D

Date Received:

Date Analyzed: 08/29/2011

Dilution Factor: 1.0

Soil Aliquot volume:

(uL) 
$1 B$ - FORM I VOA-2

VOLATILE ORGANICS ANALYSIS DATA SHEET
EPA SAMPLE NO.

VHBLKO1

Lab Name: TESTAMERICA BURLINGTON

Lab Code: STLV Case No.: INMAN

Matrix: (SOIL/SED/WATER)

Sample wt/vol: 25.0

Water

$(\mathrm{g} / \mathrm{mL}) \quad \mathrm{mL}$

Level: (TRACE/LOW/MED) TRACE

\% Moisture: not dec.

GC Column: DB-624

Soil Extract Volume:

ID $: 0.20$

$(\mathrm{mm})$

(mL)

Purge Volume: 25.0

Contract: $8 \mathrm{E}-00302$

SDG No.: 200-6730

Lab Sample ID: 200-6730-7

Lab File ID: DHPU19.D

Date Received:

Date Analyzed: 08/29/2011

Dilution Factor: 1.0

Soil Aliquot volume:

(uL)

\begin{tabular}{|c|c|c|c|}
\hline CAS NO. & COMPOUND & $\begin{array}{l}\text { CONCENTRATION UNITS: } \\
(\mathrm{ug} / \mathrm{L} \text { or } \mathrm{ug} / \mathrm{kg}) \mathrm{ug} / \mathrm{L}\end{array}$ & $Q$ \\
\hline $79-01-6$ & Trichloroethene & 0.50 & $\mathrm{U}$ \\
\hline $108-87-2$ & Methylcyclohexane & 0.50 & $\mathrm{U}$ \\
\hline $78-87-5$ & 1,2-Dichloropropane & 0.50 & $\mathrm{U}$ \\
\hline $75-27-4$ & Bromodichloromethane & 0.50 & $\mathrm{U}$ \\
\hline $10061-01-5$ & cis-1,3-Dichloropropene & 0.50 & $\mathrm{U}$ \\
\hline $108-10-1$ & 4-Methyl-2-pentanone & 5.0 & $\mathrm{U}$ \\
\hline $108-88-3$ & Toluene & 0.50 & $\mathrm{U}$ \\
\hline $10061-02-6$ & trans-1,3-Dichloropropene & 0.50 & $\mathrm{U}$ \\
\hline $79-00-5$ & $1,1,2-\operatorname{Trichloroethane}$ & 0.50 & $\mathrm{U}$ \\
\hline $127-18-4$ & Tetrachloroethene & 0.50 & $\mathrm{U}$ \\
\hline $591-78-6$ & 2-Hexanone & 5.0 & $\mathrm{U}$ \\
\hline $124-48-1$ & Dibromochloromethane & 0.50 & $\mathrm{U}$ \\
\hline $106-93-4$ & 1,2-Dibromoethane & 0.50 & $\mathrm{U}$ \\
\hline $108-90-7$ & Chlorobenzene & 0.50 & $\mathrm{U}$ \\
\hline $100-41-4$ & Ethylbenzene & 0.50 & $\mathrm{U}$ \\
\hline $95-47-6$ & o-Xylene & 0.50 & $\mathrm{U}$ \\
\hline $179601-23-1$ & $\mathrm{~m}, \mathrm{p}$-xylene & 0.50 & $\mathrm{U}$ \\
\hline $100-42-5$ & Styrene & 0.50 & $\mathrm{U}$ \\
\hline $75-25-2$ & Bromoform & 0.50 & $\mathrm{U}$ \\
\hline $98-82-8$ & Isopropylbenzene & 0.50 & $\mathrm{U}$ \\
\hline $79-34-5$ & $1,1,2,2$-Tetrachloroethane & 0.50 & $\mathrm{U}$ \\
\hline $541-73-1$ & 1,3-Dichlorobenzene & 0.50 & $\mathrm{U}$ \\
\hline $106-46-7$ & 1,4-Dichlorobenzene & 0.50 & $\mathrm{U}$ \\
\hline $95-50-1$ & 1,2-Dichlorobenzene & 0.50 & $\mathrm{U}$ \\
\hline $96-12-8$ & 1,2-Dibromo-3-Chloropropane & 0.50 & $\mathrm{U}$ \\
\hline $120-82-1$ & 1,2,4-Trichlorobenzene & 0.50 & $\mathrm{U}$ \\
\hline $87-61-6$ & 1,2,3-Trichlorobenzene & 0.50 & $\mathrm{U}$ \\
\hline
\end{tabular}

SOM01.2 (4/2007) 
$1 \mathrm{~J}$ - FORM I VOA-TIC

VOLATILE ORGANICS ANALYSIS DATA SHEET

TENTATIVELY IDENTIFIED COMPOUNDS
EPA SAMPLE NO.

VHBLK01

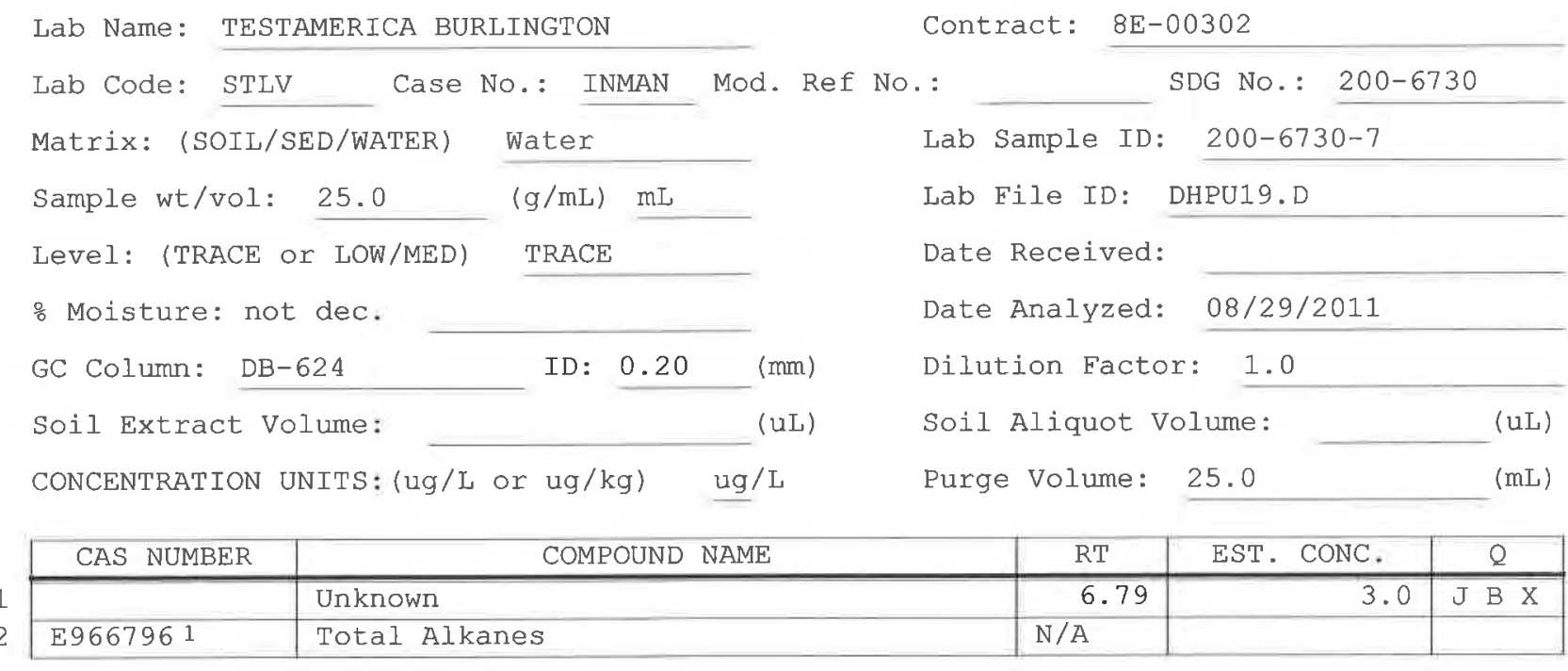

1 EPA-designated Registry Number. 


\section{TestAmerica}

THE LEADER IN ENVIRONMENTAL TESTING

\section{ANALYTICAL REPORT}

Job Number: 200-6762-1

SDG Number: 200-6762

Job Description: Inman (200-6762)

Contract Number: 1E-30401

For:

Argonne National Laboratory

9700 South Cass Avenue

Building 203

Office B-149

Argonne, IL 60439

Attention: Mr. Clyde Dennis

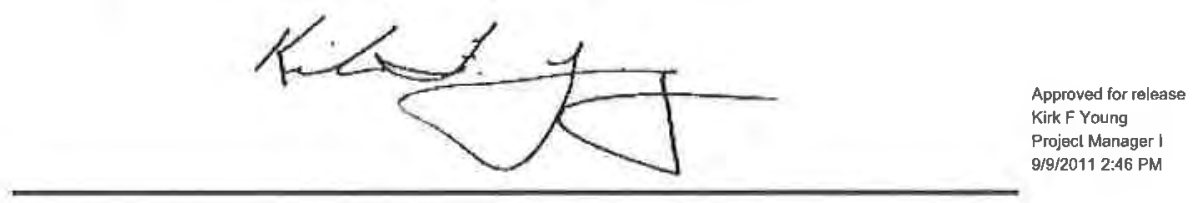

Kirk F Young

Project Manager I

kirk.young@testamericainc.com

09/09/2011

The test results in this report relate only to sample(s) as received by the laboratory. These test results were derived under a quality system that adheres to the requirements of NELAC. Pursuant to NELAC, this report may not be produced in full without written approval from the laboratory 


\section{Table of Contents}

Cover Title Page . . . . . . . . . . . . . . . . . 1

Report Narrative . . . . . . . . . . . . . . . . . . . 4

Case Narrative $\ldots \ldots \ldots \ldots \ldots \ldots \ldots \ldots \ldots \ldots \ldots \ldots \ldots \ldots$

Qualifier Definition ........................... 6

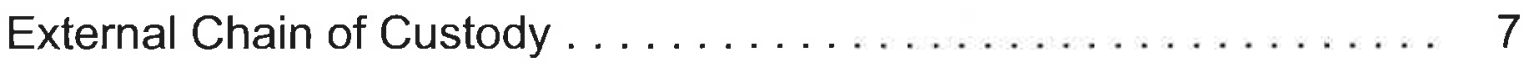

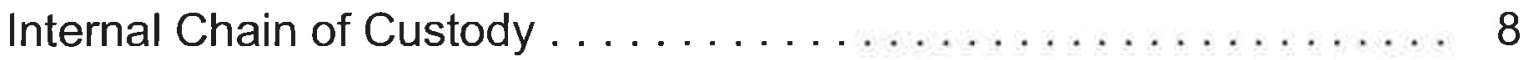

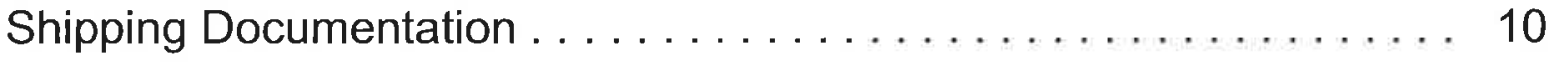

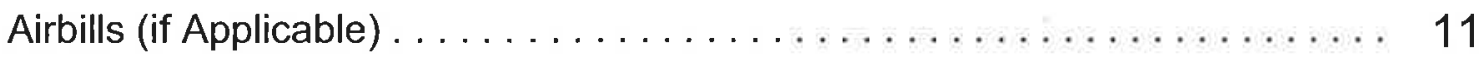

Sample Receipt and Log In Check List .................... 12

Methodology Review . . . . . . . . . . . . . . . . . . . . 14

QC Summary - SOM01.2 Volatiles-Trace . . . . . . . . . . . . . 15

QC Summary - SOM01.2 Volatiles-Trace ................... 15

Deuterated Monitoring Compound Summary $\ldots \ldots \ldots \ldots \ldots \ldots \ldots \ldots \ldots \ldots \ldots \ldots$

Method Blank $\ldots \ldots \ldots \ldots \ldots \ldots \ldots \ldots \ldots \ldots \ldots \ldots \ldots \ldots, 17$

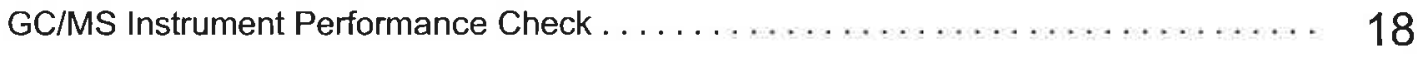

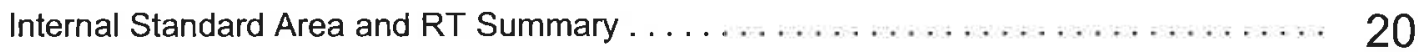

Sample Data - SOM01.2 Volatiles-Trace . . . . . . . . . . . . . 21

Sample Data - SOM01.2 Volatiles-Trace . . . . . . . . . . . . . . . 21

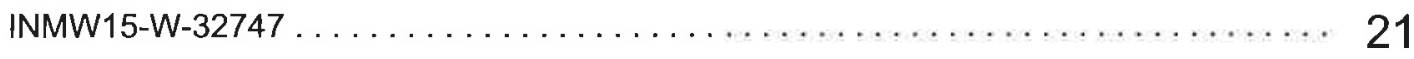

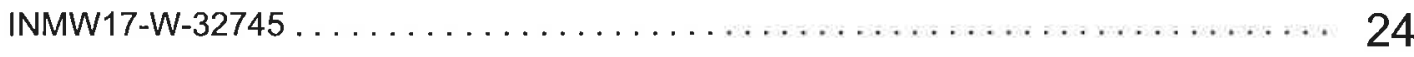

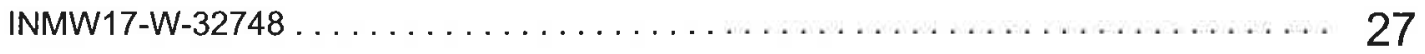

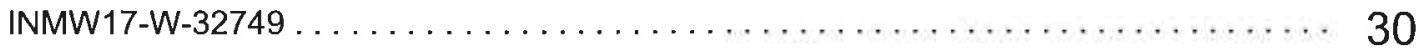

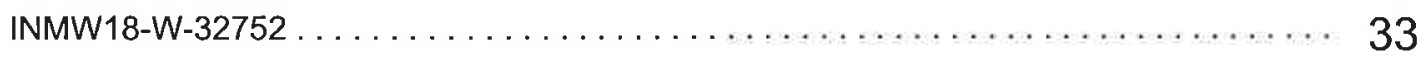

INMW18-W-32753 ......................................... 36

INMW18-W-32754 ........................................... 39

INMW18-W-32755 $\ldots \ldots \ldots \ldots \ldots \ldots \ldots \ldots \ldots \ldots \ldots \ldots \ldots \ldots \ldots \ldots \ldots \ldots \ldots \ldots \ldots, 42$ 


\section{Table of Contents}

INMW18-W-32760

INMW18-W-32761

INMW18-W-32762

INQC-W-32763

Standards - SOM01.2 Volatiles-Trace . . . . . . . . . . . . . . 57

Standards - SOM01.2 Volatiles-Trace . . . . . . . . . . . . . . . 57

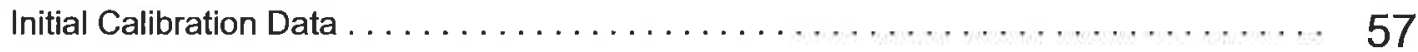

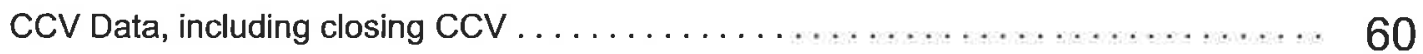

Raw Qc Data - SOM01.2 Volatiles-Trace . . . . . . . . . . . . . . . 66

Raw Qc Data - SOM01.2 Volatiles-Trace . . . . . . . . . . . . . . 66

Raw Qc Data - SOM01.2 Volatiles-Trace ........................ 66

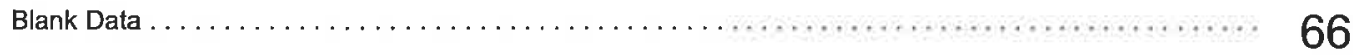




\title{
CASE NARRATIVE
}

\section{Client: Argonne National Laboratory}

\author{
Project: Inman (200-6762)
}

\author{
Report Number: 200-6762-1
}

Enclosed is the data set for the referenced project work. With the exceptions noted as flags or footnotes, standard analytical protocols were followed in performing the analytical work and the applied control limits were met.

Calculations were performed before rounding to avoid round-off errors in calculated results.

All holding times were met and proper preservation noted for the methods performed on these samples, unless otherwise detailed in the individual sections below.

\section{Receipt}

The samples were received on 08/30/2011. Documentation of the condition of the samples at the time of their receipt and any exception to the laboratory's Sample Acceptance Policy is documented in the Shipping and Receiving section of this submittal. The samples, as received, were not acid preserved. On that basis, the laboratory did provide for the analytical work to be performed within seven days of sample collection.

\section{SOM01.2 Volatile Organics (Trace Level Water)}

A storage blank was prepared for volatile organics analysis, and stored in association with the storage of the samples. That storage blank, identified as VHBLK01, was carried through the holding period with the samples, and analyzed.

Each sample in the sample set was analyzed without a dilution. Each of the analyses associated with the sample set exhibited an acceptable internal standard performance. There was an acceptable recovery of each deuterated monitoring compound (DMC) in the analysis of the method blank associated with the analytical work, and in the analysis of the storage blank associated with the sample set. The analysis of the samples in this sample set did meet the technical acceptance criteria specific to DMC recoveries, although not all DMC recoveries were within the control range in each analysis. The technical acceptance criteria does provide for the recovery of up to three DMCs to fall outside of the control range in the analysis of field samples. Matrix spike and matrix spike duplicate analyses were not performed on samples in this sample set. A trace concentration of toluene was identified in the analysis of the method blank associated with the analytical work. The concentration of toluene in that analysis was below the established reporting limit, and the analysis did meet the technical acceptance criteria for a compliant method blank analysis. The analysis of the storage blank associated with the sample set was free of analyte contamination. Present in the method blank and storage blank analyses was a non-target constituent that represents a compound that is related to the DMC formulation. The fact that the presence of this compound is not within the laboratory's control is at issue. The derived results for that compound have been qualified with an "X" qualifier to reflect the source of the contamination.

The responses for each of the target analytes met the relative standard deviation criterion in the initial calibration. The response for each target analyte met the percent difference criterion in the 
opening/continuing calibration check acquisition. The response for each target analyte met the 50.0 percent difference criterion in the closing calibration check acquisition.

The primary quantitation mass for methylcyclohexane that is specified in the Statement of Work is mass 83 . The laboratory did identify a contribution to mass 83 from 1,2-dichloropropane- $d_{6}$, one of the deuterated monitoring compounds (DMCs). The laboratory did change the primary quantitation mass assignment to mass 55 for the quantification of methylcyclohexane.

Manual integration was employed in deriving certain of the analytical results. The values that have been derived from manual integration are qualified on the quantitation reports. Extracted ion current profiles for each manual integration are included in the data package, and further documented at the end of this submittal. 


\section{DATA REPORTING QUALIFIERS}

Client: Argonne National Laboratory

Job Number: 200-6762-1

Sdg Number: 200-6762

GC/MS VOA

U

J

J

X

*

B

N
Analyzed for but not detected.

Indicates an Estimated Value for TICs

Indicates an estimated value.

See case narrative notes for explanation of the ' $X$ ' flag

Surrogate exceeds the control limit

The analyte was found in an associated blank, as well as in the sample.

This flag indicates the presumptive evidence of a compound. 


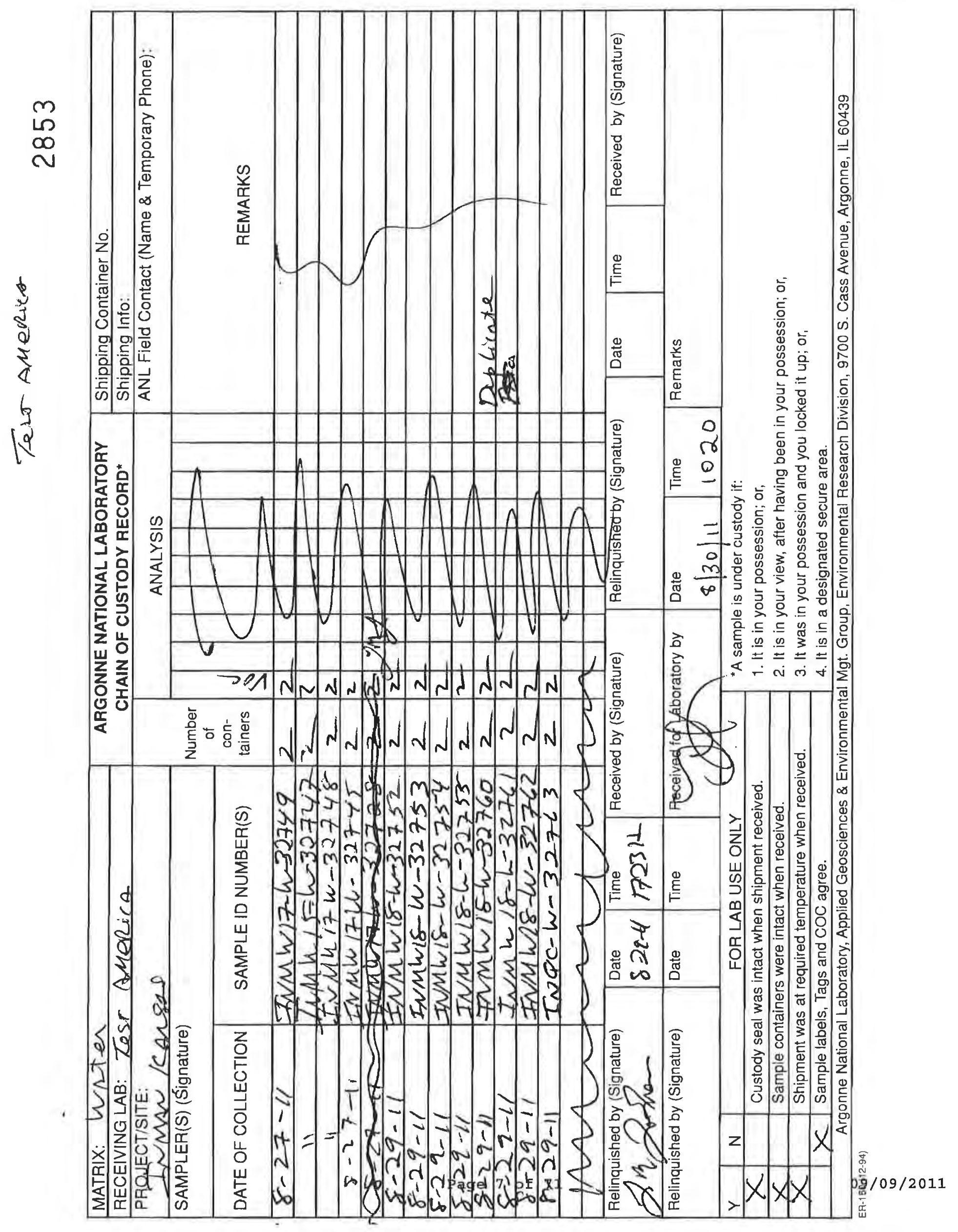




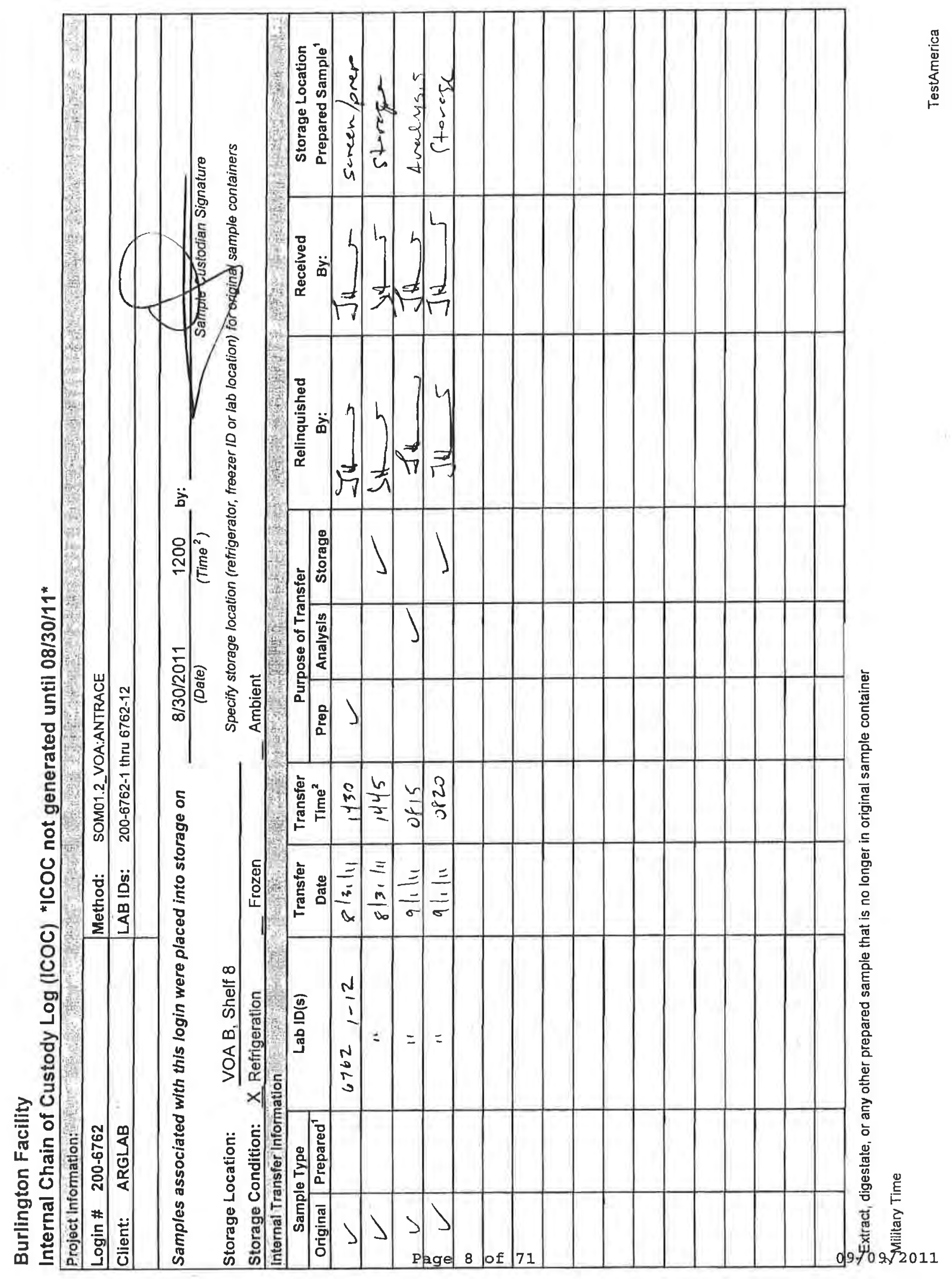




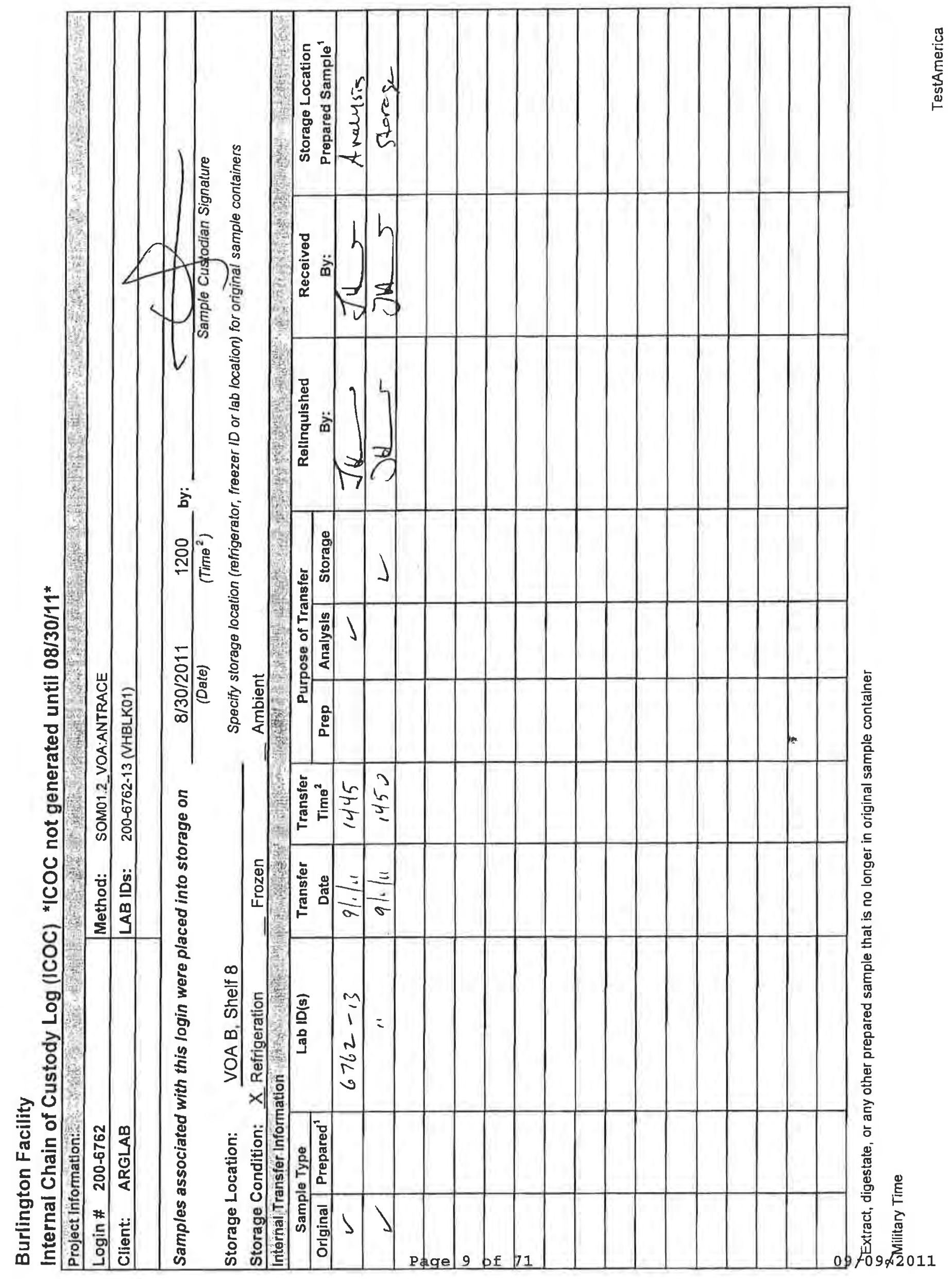




\section{Shipping and Receiving Documents}




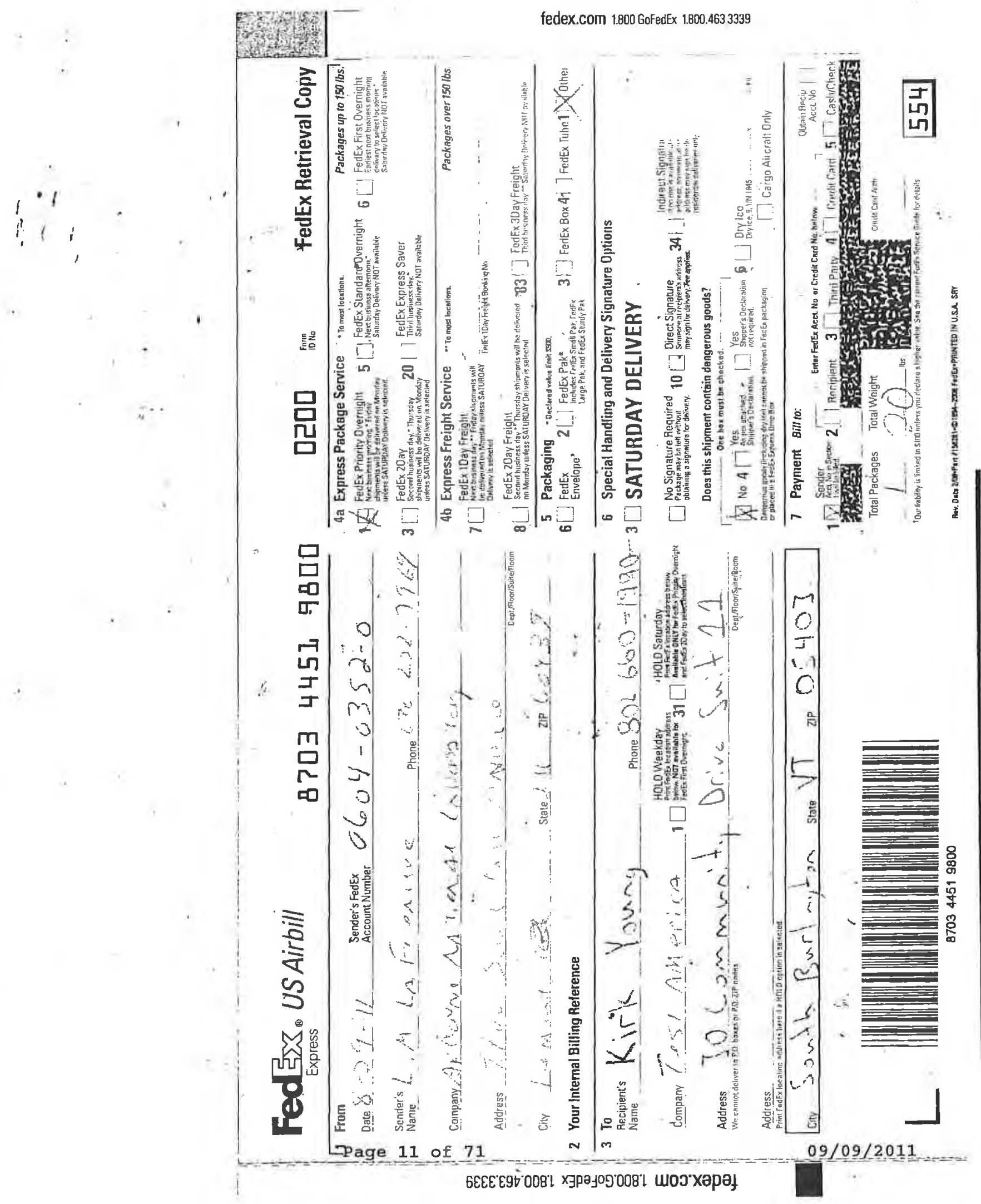




\section{Login Sample Receipt Checklist}

Client: Argonne National Laboratory

Job Number: 200-6762-1

SDG Number: 200-6762

Login Number: 6762

List Source: TestAmerica Burlington

List Number: 2

Creator: Keeton, Jamie

Question

Radioactivity either was not measured or, if measured, is at or below background

The cooler's custody seal, if present, is intact.

The cooler or samples do not appear to have been compromised or tampered with.

Samples were received on ice.

Cooler Temperature is acceptable.

Cooler Temperature is recorded.

$\mathrm{COC}$ is present.

COC is filled out in ink and legible.

$\mathrm{COC}$ is filled out with all pertinent information.

Is the Field Sampler's name present on COC?

There are no discrepancies between the sample IDs on the containers and the COC.

Samples are received within Holding Time.

Sample containers have legible labels.

Containers are not broken or leaking.

Sample collection date/times are provided.

Appropriate sample containers are used.

Sample bottles are completely filled.

Sample Preservation Verified.

There is sufficient vol. for all requested analyses, incl. any requested MS/MSDs

VOA sample vials do not have headspace or bubble is $<6 \mathrm{~mm}\left(1 / 4^{\prime \prime}\right)$ in diameter.

Multiphasic samples are not present.

Samples do not require splitting or compositing.

Residual Chlorine Checked.

\begin{tabular}{|c|c|}
\hline Answer & Comment \\
\hline N/A & Lab does not accept radioactive samples. \\
\hline True & No numbers \\
\hline \multicolumn{2}{|l|}{ True } \\
\hline \multicolumn{2}{|l|}{ True } \\
\hline \multicolumn{2}{|l|}{ True } \\
\hline True & $1.8^{\circ} \mathrm{C}, \mathrm{IR}$ gun ID $96, \mathrm{CF}=0$ \\
\hline \multicolumn{2}{|l|}{ True } \\
\hline \multicolumn{2}{|l|}{ True } \\
\hline \multicolumn{2}{|l|}{ True } \\
\hline False & Sampler's signature not recorded on $\mathrm{C}-\mathrm{O}-\mathrm{C}$. \\
\hline False & Sample collection times not listed on C-O-C. \\
\hline \multicolumn{2}{|l|}{ True } \\
\hline \multicolumn{2}{|l|}{ True } \\
\hline \multicolumn{2}{|l|}{ True } \\
\hline \multicolumn{2}{|l|}{ True } \\
\hline \multicolumn{2}{|l|}{ True } \\
\hline \multicolumn{2}{|l|}{ True } \\
\hline N/A & Sample volumes received unpreserved. \\
\hline \multicolumn{2}{|l|}{ True } \\
\hline \multicolumn{2}{|l|}{ True } \\
\hline \multicolumn{2}{|l|}{ True } \\
\hline \multicolumn{2}{|l|}{ True } \\
\hline N/A & Check done at department level if required. \\
\hline
\end{tabular}




\section{Sample Login Acknowledgement}

Job 200-6762-1

$\begin{array}{ll}\text { Client Job Description: } & \text { Inman (200-6762) } \\ \text { Purchase Order \#: } & 8 \mathrm{E}-00302 \\ \text { Work Order \#: } & 8 \mathrm{E}-00302 \\ \text { Project Manager: } & \text { Kirk F Young } \\ \text { Job Due Date: } & 9 / 13 / 2011 \\ \text { Job TAT: } & 14 \text { Days } \\ \text { Max Deliverable Level: } & \text { IV }\end{array}$

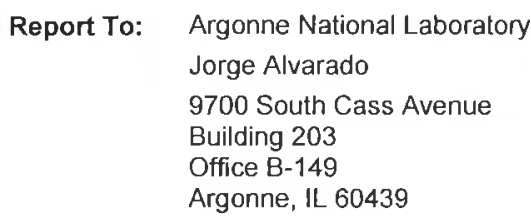

Bill To: $\quad$ Argonne National Laboratory

Accounts Payable

Chief Financial Offices

9700 S. Cass Ave.

Building 201

Argonne, IL 60439

\section{Login 200-6762}

\begin{tabular}{|c|c|c|c|c|c|}
\hline Sample Receipt: & $8 / 30 / 2011 \quad 10: 20: 00 \mathrm{AM}$ & Number of Coolers: & 1 & & \\
\hline Method of Delivery: & FedEx Priority Overnight & Cooler Temperature(s) & $\left(C^{\circ}\right): 1.8$ & & \\
\hline Lab Sample \# & Client Sample ID & Date Sampled & Matrix & & \\
\hline Method & Method Description / Work Location & & & Rpt Basis & Dry / Wet ** \\
\hline $200-6762-1$ & INMW17-W-32749 & $8 / 27 / 2011$ 12:00:00 AM & Water & & \\
\hline SOM01.2_Vol_Tr & SOM01.2 Trace Volatile Organics / In-Lab & & & Total & Wet \\
\hline 200-6762-2 & INMW15-W-32747 & 8/27/2011 12:00:00 АM & Water & & \\
\hline SOM01.2_Vol_Tr & SOM01.2 Trace Volatile Organics / In-Lab & & & Total & Wet \\
\hline $200-6762-3$ & INMW17-W-32748 & $8 / 27 / 2011$ 12:00:00 AM & Water & & \\
\hline SOM01.2_Vol_Tr & SOM01.2 Trace Volatile Organics / In-Lab & & & Total & Wet \\
\hline $200-6762-4$ & INMW17-W-32745 & $8 / 27 / 2011$ 12:00:00 AM & Water & & \\
\hline SOM01.2_Vol_Tr & SOM01.2 Trace Volatile Organics / In-Lab & & & Total & Wet \\
\hline $200-6762-5$ & INMW18-W-32752 & $8 / 29 / 2011$ 12:00:00 AM & Water & & \\
\hline SOM01.2_Vol_Tr & SOM01.2 Trace Volatile Organics / In-Lab & & & Total & Wet \\
\hline $200-6762-6$ & INMW18-W-32753 & $8 / 29 / 2011$ 12:00:00 AM & Water & & \\
\hline SOM01.2_Vol_Tr & r SOM01.2 Trace Volatile Organics / In-Lab & & & Total & Wet \\
\hline $200-6762-7$ & INMW18-W-32754 & 8/29/2011 12:00:00 AM & Water & & \\
\hline SOM01.2_Vol_Tr & SOM01.2 Trace Volatile Organics / In-Lab & & & Total & Wet \\
\hline $200-6762-8$ & INMW18-W-32755 & $8 / 29 / 2011$ 12:00:00 AM & Water & & \\
\hline SOM01.2_Vol_Tr & SOM01.2 Trace Volatile Organics / In-Lab & & & Total & Wet \\
\hline 200-6762-9 & INMW18-W-32760 & $8 / 29 / 2011$ 12:00:00 AM & Water & & \\
\hline SOM01.2_Vol_Tr & SOM01.2 Trace Volatile Organics / In-Lab & & & Total & Wet \\
\hline $200-6762-10$ & INMW18-W-32761 & $8 / 29 / 2011$ 12:00:00 AM & Water & & \\
\hline SOM01.2_Vol_Tr & SOM01.2 Trace Volatile Organics / In-Lab & & & Total & Wet \\
\hline $200-6762-11$ & INMW18-W-32762 & $8 / 29 / 2011 \quad 12: 00: 00 \mathrm{AM}$ & Water & & \\
\hline SOM01.2_Vol_Tr & SOM01.2 Trace Volatile Organics / In-Lab & & & Total & Wet \\
\hline $200-6762-12$ & INQC-W-32763 & $8 / 29 / 2011$ 12:00:00 AM & Water & & \\
\hline SOM01.2_Vol_Tr & SOM01.2 Trace Volatile Organics / In-Lab & & & Total & Wet \\
\hline $200-6762-13$ & VHBLK01 & $8 / 30 / 2011$ 11:40:00 AM & Water & & \\
\hline SOM01.2_Vol_Tr & som01.2 Trace Volatile Organics / In-Lab & & & Total & Wet \\
\hline
\end{tabular}




\section{METHODOLOGY SUMMARY}

Laboratory: TestAmerica Laboratories

Location: South Burlington, Vermont

VOA

Volatile Organics Trace - USEPA CLP SOM01.2
Project No:

SDG No: $\quad 200-6762$ 
$2 \mathrm{~A}$ - FORM II VOA-1

WATER VOLATILE DEUTERATED MONITORING COMPOUND RECOVERY

Lab Name: TESTAMERICA BURLINGTON

Lab Code: STLV Case No.: INMAN Mod. Ref No.:

Level: (TRACE or LOW) TRACE
Contract: $1 \mathrm{E}-30401$

\begin{tabular}{|c|c|c|c|c|c|c|c|}
\hline $\begin{array}{c}\text { EPA } \\
\text { SAMPLE NO. }\end{array}$ & $\begin{array}{l}\text { VDMC1 } \\
\text { (VCL) \# }\end{array}$ & $\begin{array}{l}\text { VDMC2 } \\
\text { (CLA) \# }\end{array}$ & $\begin{array}{l}\text { VDMC3 } \\
\text { (DCE) \# }\end{array}$ & $\begin{array}{l}\text { VDMC4 } \\
\text { (BUT) \# }\end{array}$ & $\begin{array}{l}\text { VDMC5 } \\
\text { (CLE) \# }\end{array}$ & $\begin{array}{l}\text { VDMC6 } \\
\text { (DCA) \# }\end{array}$ & $\begin{array}{l}\text { VDMC7 } \\
\text { (BEN) \# }\end{array}$ \\
\hline VBLKDS & 92 & 91 & 66 & 94 & 84 & 93 & 91 \\
\hline INQC-W-32763 & 98 & 96 & 70 & 93 & 88 & 98 & 96 \\
\hline INMW15-W-32747 & 92 & 92 & 66 & 182 * & 89 & 97 & 93 \\
\hline INMW17-W-32748 & 94 & 95 & 68 & 193 * & 88 & 97 & 93 \\
\hline INMW18-W-32753 & 96 & 95 & 68 & $203^{*}$ & 89 & 98 & 94 \\
\hline INMW18-W-32754 & 102 & 99 & 71 & $196 *$ & 92 & 98 & 97 \\
\hline INMW18-W-32755 & 101 & 99 & 68 & $156 *$ & 90 & 96 & 96 \\
\hline INMW18-W-32760 & 102 & 100 & 72 & 196 * & 94 & 102 & 99 \\
\hline INMW18-W-32761 & 99 & 98 & 70 & $189 *$ & 92 & 100 & 96 \\
\hline
\end{tabular}

\footnotetext{
VDMC1 $($ VCL $)=$ Vinyl Chloride-d3

VDMC2 $($ CLA $)=$ Chloroethane-d5

VDMC3 $(\mathrm{DCE})=1,1-\mathrm{Dichloroethene-d2}$

VDMC4 $($ BUT $)=2-$ Butanone-d5

VDMC5 $(\mathrm{CLF})=$ Chloroform-d

VDMC6 $(\mathrm{DCA})=1,2-\mathrm{Dich}$ loroethane-d4

VDMC7 $(B E N)=$ Benzene-d6
}

QC LIMITS

$(65-131)$

$(71-131)$

(55-104)

(49-155)

$(78-121)$

$(78-129)$

$(77-124)$

\# Column to be used to flag recovery values

* Values outside of contract required QC limits

Page 1 of 1 


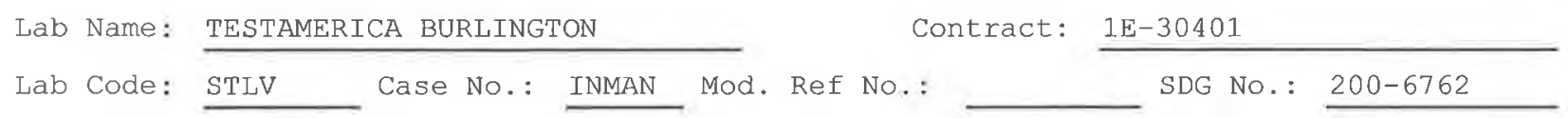

Level: (TRACE or LOW) TRACE

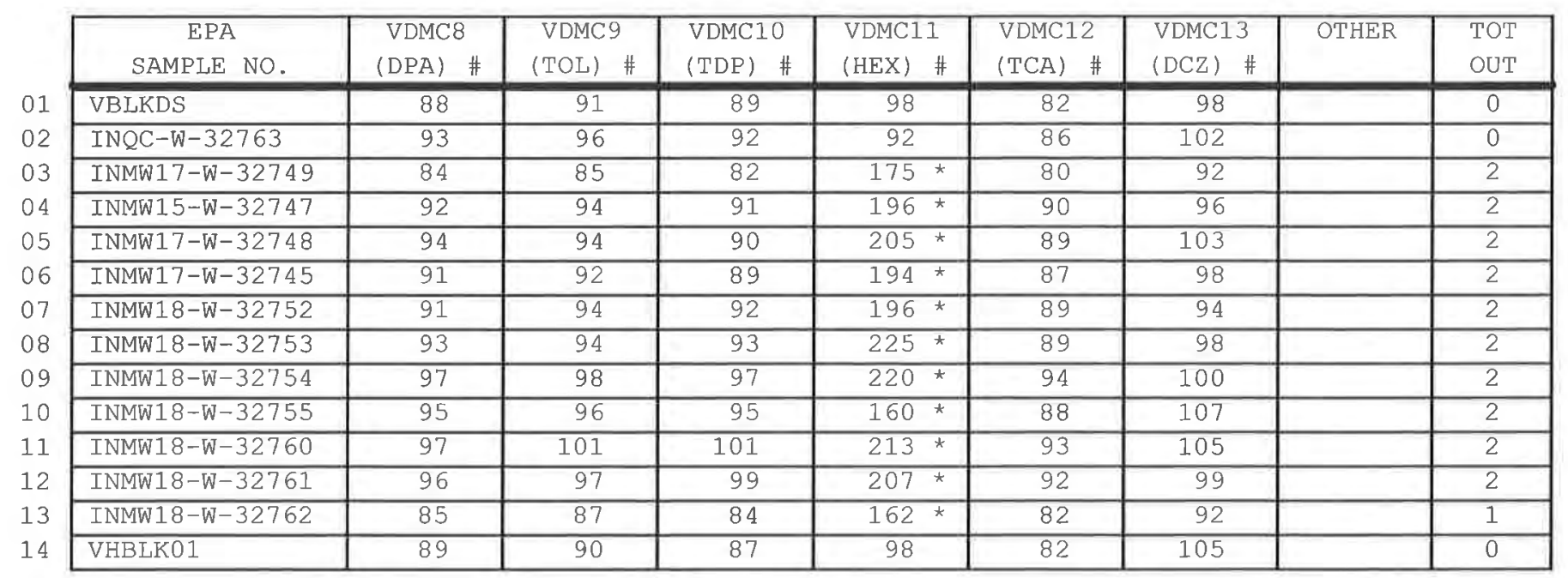

\footnotetext{
VDMC8 (DPA) $=1,2$-Dichloropropane-d6

VDMC9 (TOL) = Toluene-d8

(79-124)

VDMC10 $(\mathrm{TDP})=$ trans-1,3-Dichloropropene-d4

(77-121)

VDMC11 $(\mathrm{HEX})=2$-Hexanone-d5

(73-121)

VDMC12 $(\mathrm{TCA})=1,1,2,2$-Tetrachloroethane-d2

(28-135)

VDMC13 (DCZ) $=1,2$-Dichlorobenzene-d4

$(73-125)$

$(80-131)$
}

QC LIMITS

\# Column to be used to flag recovery values

* Values outside of contract required QC limits

Report 1,4-Dioxane-d8 for Low-Medium VOA analysis only

Page 1 of 1 
$4 A$ - FORM IV VOA

VOLATILE METHOD BLANK SUMMARY
EPA SAMPLE NO.

VBLKDS
Lab Name: TESTAMERICA BURLINGTON

Lab Code: STLV Case No.: INMAN

Lab File ID: DHPV03.D

Instrument ID: D.i

Matrix: (SOIL/SED/WATER)

Level: (TRACE or LOW/MED)

GC Column: DB-624
Water

TRACE

ID : 0.20

Contract: $1 \mathrm{E}-30401$

SDG No.: 200-6762

Lab Sample ID: MB 200-24615/3

Date Analyzed: 09/01/2011

Time Analyzed: 0812

Heated Purge: ( $\mathrm{Y} / \mathrm{N}) \mathrm{N}$

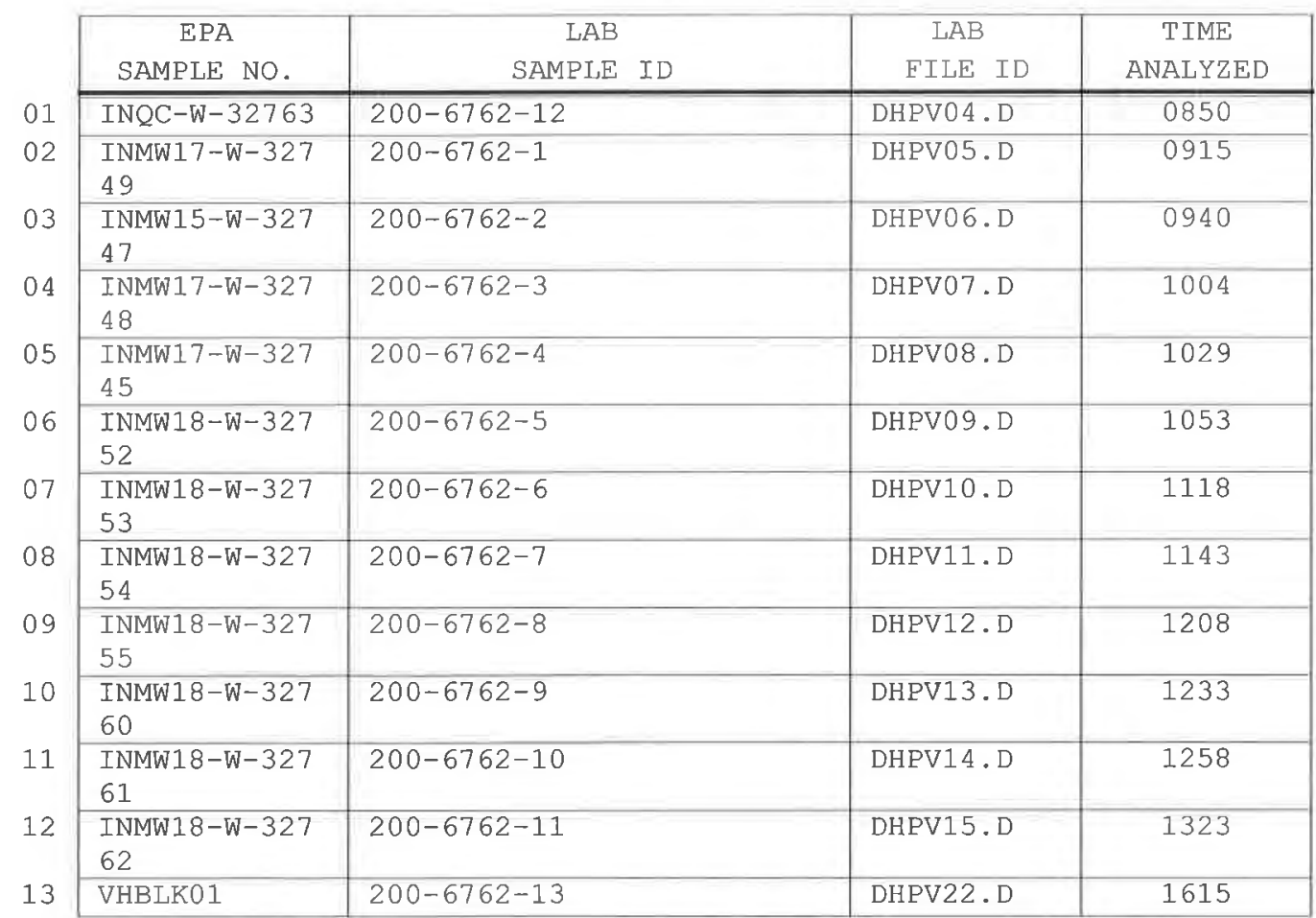

COMMENTS : 
$5 A$ - FORM V VOA

VOLATILE ORGANICS INSTRUMENT

PERFORMANCE CHECK

BROMOFLUOROBENZENE (BFB)
EPA SAMPLE NO.

BFBDV
Lab Name: TESTAMERICA BURLINGTON

Lab Code: STLV Case No.: INMAN

Lab File Id: DHPO1.D

Instrument Id: D.j

GC Column: DB-624

ID $: 0.20$
Contract: $1 \mathrm{E}-30401$

Mod. Ref No.:

SDG No.: 200-6762

BEB Injection Date: 07/07/2011

BEB Injection Time: 1005 (mm)

\begin{tabular}{|r|l|r|}
\hline m/e & ION ABUNDANCE CRITERIA & $\begin{array}{c}\text { Oo RELATIVE } \\
\text { ABUNDANCE }\end{array}$ \\
\hline 50 & $15.0-40.0 \%$ of mass 95 & 26.1 \\
\hline 75 & $30.0-80.0 \%$ of mass 95 & 46.4 \\
\hline 95 & Base peak, 100\% relative abundance & 100 \\
\hline 96 & $5.0-9.0 \%$ of mass 95 & 6.3 \\
\hline 173 & Less than 2.0\% of mass 174 & 0 \\
\hline 174 & $50.0-120 \%$ of mass 95 & 77.7 \\
\hline 175 & $5.0-9.0 \%$ of mass 174 & $6.0(1$ \\
\hline 176 & $95.0-101 \%$ of mass 174 & $78.2(101) 1$ \\
\hline 177 & $5.0-9.0 \%$ of mass 176 & $5.2(16.6) 2$ \\
\hline
\end{tabular}

1 - Value is onass 174

2 - Value is onass 176

\begin{tabular}{|c|c|c|l|c|c|}
\cline { 2 - 6 } & \multicolumn{1}{c|}{$\begin{array}{c}\text { EPA } \\
\text { SAMPLE NO. }\end{array}$} & \multicolumn{1}{c|}{ LAB } & DABE & TIME \\
SAMPLE ID & \multicolumn{1}{c|}{ FILE ID } & ANALYZED & ANALYZED \\
\cline { 2 - 7 } 01 & VSTD0.5DV & IC 200-21023/4 & DHP04.D & $07 / 07 / 2011$ & 1117 \\
\cline { 2 - 7 } 02 & VSTD001DV & IC 200-21023/5 & DHP05.D & $07 / 07 / 2011$ & 1141 \\
\cline { 2 - 7 } 03 & VSTD005DV & ICIS 200-21023/6 & DHP06.D & $07 / 07 / 2011$ & 1206 \\
\cline { 2 - 7 } 05 & VSTD010DV & IC 200-21023/7 & DHP07.D & $07 / 07 / 2011$ & 1231 \\
\cline { 2 - 7 } & VSTD020DV & IC 200-21023/8 & DHP08.D & $07 / 07 / 2011$ & 1256 \\
\hline
\end{tabular}


5A - FORM V VOA

VOLATILE ORGANICS INSTRUMENT

PERFORMANCE CHECK

BROMOFJUOROBENZENE (BEB)
EPA SAMPLE NO.

BFBDS
Lab Name: TESTAMERICA BURLINGTON Lab Code: STLV Case No.: INMAN Mod. Ref No, :

Lab File Id: DHPVO1.D

Instrument Id: D.i

GC Column: DB-624

ID: 0.20
Contract : $1 \mathrm{E}-30401$

SDG No.: 200-6762

BEB Injection Date: 09/01/2011

BFB Injection Time: 0728

\begin{tabular}{|c|c|c|}
\hline $\mathrm{m} / \mathrm{e}$ & ION ABUNDANCE CRITERIA & $\begin{array}{l}\text { \% RELATIVE } \\
\text { ABUNDANCE }\end{array}$ \\
\hline 50 & $15.0-40.0 \%$ of mass 95 & 27.5 \\
\hline 75 & $30.0-80.0 \%$ of mass 95 & 47.7 \\
\hline 95 & Base peak, $100 \%$ relative abundance & 100 \\
\hline 96 & $5.0-9.0 \%$ of mass 95 & 7.3 \\
\hline 173 & Less than $2.0 \%$ of mass 174 & 0.41 \\
\hline 174 & $50.0-120 \%$ of mass 95 & 78.8 \\
\hline 175 & $5.0-9.0 \%$ of mass 174 & 5.91 \\
\hline 176 & $95.0-101$ of of mass 174 & $75.6(95.9) 1$ \\
\hline 177 & $5.0-9.0 \%$ of mass 176 & $4.7(6.2) 2$ \\
\hline
\end{tabular}

1 - Value is omass 174

2 - Value is omass 176

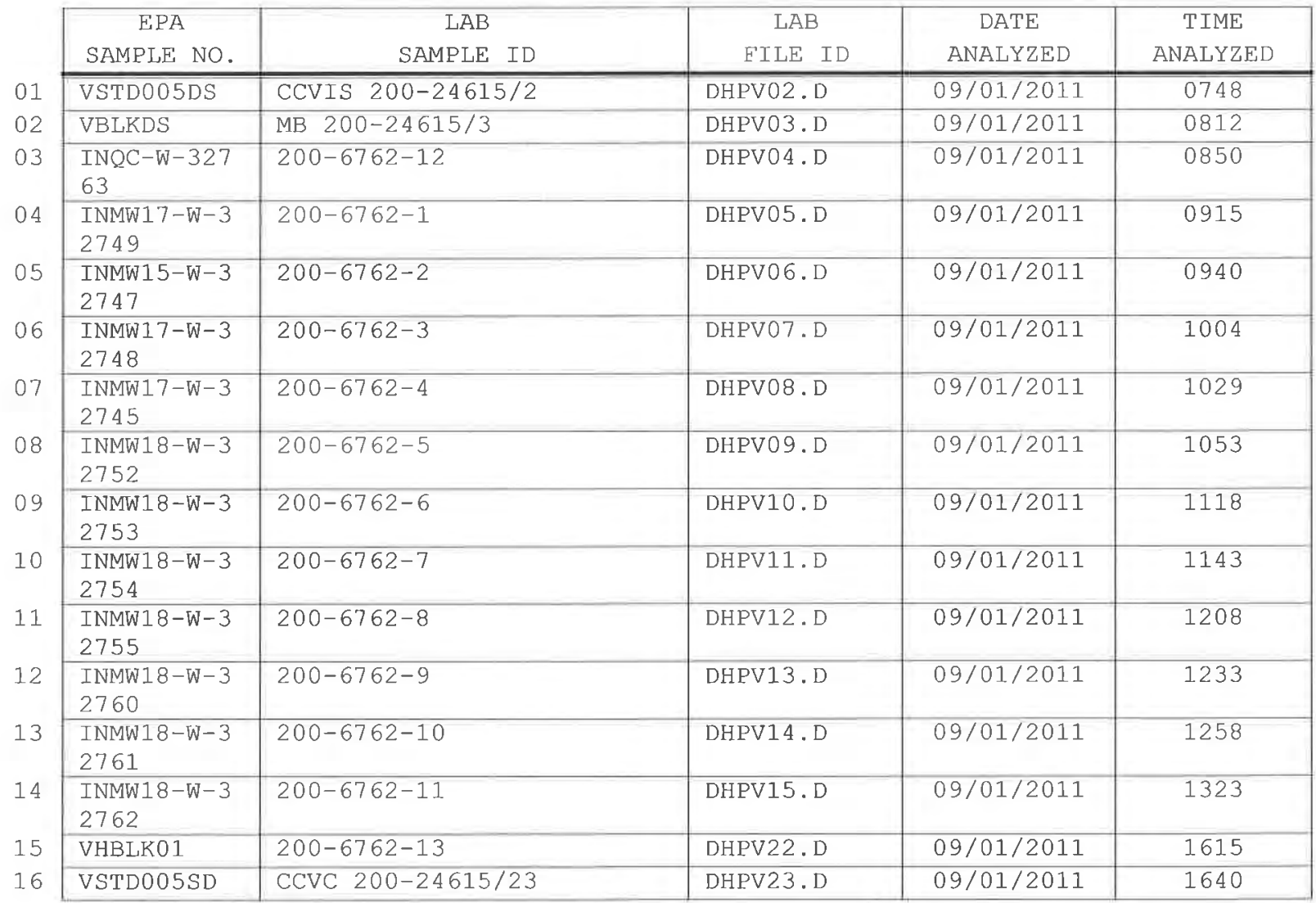


$8 A-$ FORM VIII VOA

VOLATILE INTERNAL STANDARD AREA AND RETENTION TIME SUMMARY

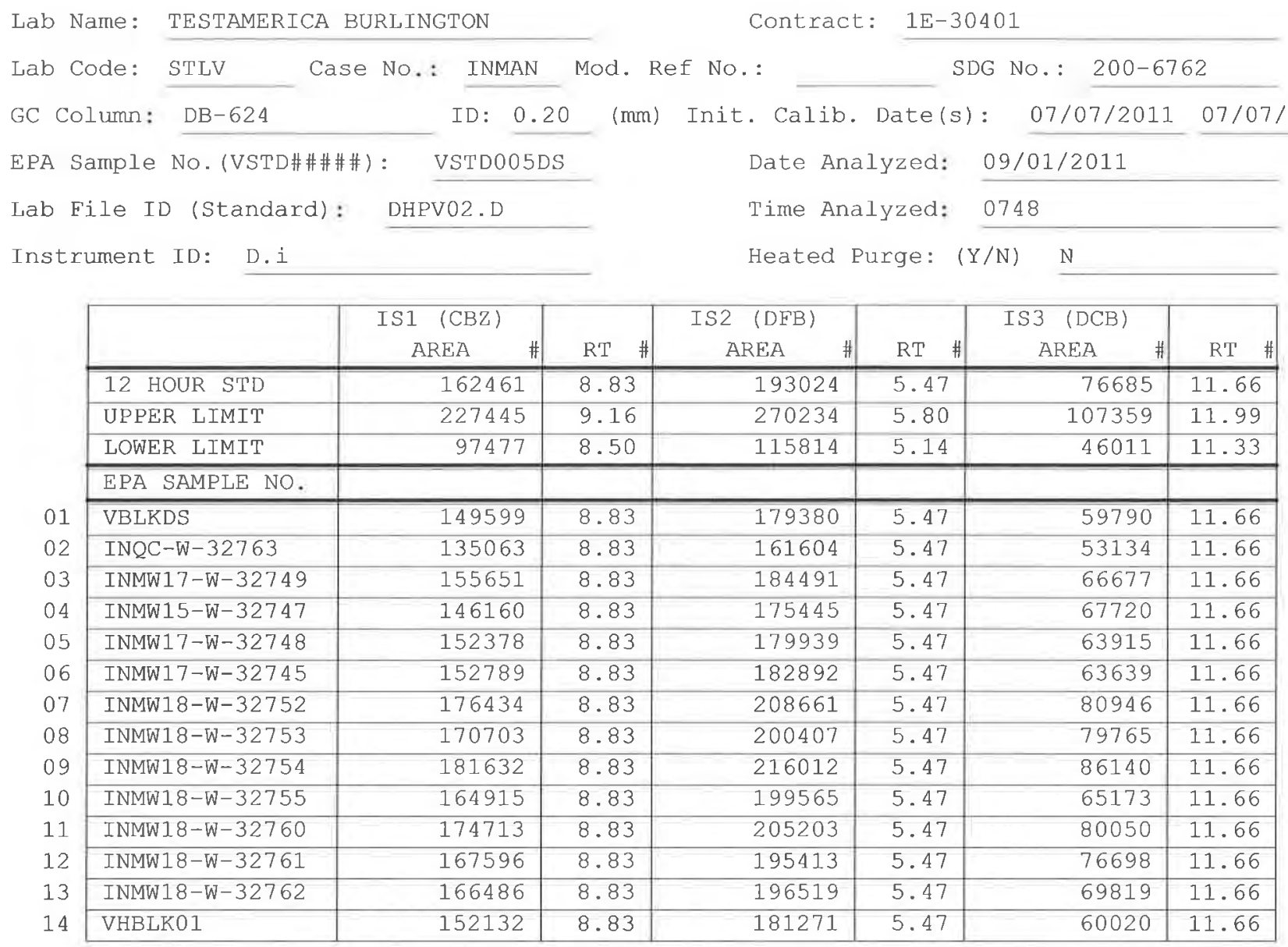

IS1 $(\mathrm{CBZ})=$ Chlorobenzene-d5

IS2 $(\mathrm{DFB})=1,4$-Difluorobenzene

IS3 $(\mathrm{DCB})=1,4-\mathrm{Dichlorobenzene-d4}$

AREA UPPER LIMIT $=140$ \% (Trace Volatiles) of internal standard area AREA LOWER LIMIT $=60 \%$ (Trace Volatiles) of internal standard area RT UPPER LIMIT $=+0.33$ (Trace Volatjles) minutes of internal standard RT RT LOWER LIMIT $=-0.33$ (Trace Volatiles) minutes of internal standard RT

\# Column used to flag values outside contract required QC limits with an asterisk. 
$1 A$ - FORM I VOA-1

VOLATIJE ORGANICS ANALYSIS DATA SHEET
EPA SAMPLE NO.

INMW $15-W-32747$
Lab Name: TESTAMERICA BURLINGTON

Lab Code: STLV Case

Matrix: (SOIL/SED/WATER)

Sample wt/vol: 25.0

water

$(\mathrm{g} / \mathrm{mL}) \mathrm{mL}$

Level: (TRACE/LOW/MED) TRACE

\% Moisture: not dec.

GC Column: DB-624 ID: $0.20 \quad$ (mm)

Soil Extract Volume: (uL) (mL)

Purge Volume: 25.0
Contract: 1E-30401

SDG No.: 200-6762

\begin{tabular}{|c|c|c|c|}
\hline CAS NO. & COMPOUND & $\begin{array}{l}\text { CONCENTRATION UNITS: } \\
(\mathrm{ug} / \mathrm{L} \text { or } \mathrm{ug} / \mathrm{kg}) \mathrm{ug} / \mathrm{L}\end{array}$ & $Q$ \\
\hline $75-71-8$ & Dichlorodifluoromethane & 0.50 & $\mathrm{U}$ \\
\hline $74-87-3$ & Chloromethane & 0.50 & $\mathrm{U}$ \\
\hline $75-01-4$ & Vinyl chloride & 0.50 & $\mathrm{U}$ \\
\hline $74-83-9$ & Bromomethane & 0.50 & U \\
\hline $75-00-3$ & Chloroethane & 0.50 & $\mathrm{U}$ \\
\hline $75-69-4$ & Trichlorofluoromethane & 0.50 & $\mathrm{U}$ \\
\hline $75-35-4$ & 1,1-Dichloroethene & 0.50 & $\mathrm{U}$ \\
\hline $76-13-1$ & 1,1,2-Trichloro-1,2,2-trifluoroethane & 0.50 & $\mathrm{U}$ \\
\hline $67-64-1$ & Acetone & 1.9 & $\mathrm{~J}$ \\
\hline $75-15-0$ & Carbon disulfide & 0.50 & $\mathrm{U}$ \\
\hline $79-20-9$ & Methyl acetate & 0.50 & $\mathrm{U}$ \\
\hline $75-09-2$ & Methylene Chloride & 0.096 & $\mathrm{~J}$ \\
\hline $156-60-5$ & trans $-1,2-$ Dichloroethene & 0.50 & $\mathrm{U}$ \\
\hline $1634-04-4$ & Methyl tert-butyl ether & 0.50 & $\mathrm{U}$ \\
\hline $75-34-3$ & 1,1-Dichloroethane & 0.50 & $\mathrm{U}$ \\
\hline $156-59-2$ & cis-1,2-Dichloroethene & 0.50 & $\mathrm{U}$ \\
\hline $78-93-3$ & 2-Butanone & 5.0 & $\mathrm{U}$ \\
\hline $74-97-5$ & Bromochloromethane & 0.11 & $\mathrm{~J}$ \\
\hline $67-66-3$ & Chloroform & 2.4 & \\
\hline $71-55-6$ & $1,1,1$-Trichloroethane & 0.50 & $\mathrm{U}$ \\
\hline $110-82-7$ & Cyclohexane & 0.50 & $\mathrm{U}$ \\
\hline $56-23-5$ & Carbon tetrachloride & 1.0 & \\
\hline $71-43-2$ & Benzene & 0.021 & $\mathrm{~J}$ \\
\hline $107-06-2$ & 1,2-Dichloroethane & 0.50 & $\mathrm{U}$ \\
\hline
\end{tabular}

Report 1,4-Dioxane for Low-Medium VOA analysis only

Lab Sample ID: 200-6762-2

Lab File ID: DHPV06.D

Date Received: 08/30/2011

Date Analyzed: 09/01/2011

Dilution Eactor: 1.0

Soil Aliquot Volume:

$(u L)$ 
$1 B$ - FORM I VOA-2

VOLATILE ORGANICS ANALYSIS DATA SHEET
EPA SAMPLE NO.

INMW15-W-32747

Lab Name: TESTAMERICA BURLINGTON

Lab Code: STLV Case No.: INMAN

Matrix: (SOIL/SED/WATER)

Sample wt/vol: 25.0

\section{Water}

$(\mathrm{g} / \mathrm{mL}) \quad \mathrm{mL}$

Level: (TRACE/LOW/MED) TRACE

\% Moisture: not dec.

GC Column: DB-624

Soil Extract Volume: ID: $0.20 \quad$ (mm)

(uL)

(mL)

Purge Volume: 25.0

Contract: 1E-30401

SDG No.: 200-6762

Lab Sample ID: 200-6762-2

Lab File ID: DHPV06.D

Date Received: 08/30/2011

Date Analyzed: 09/01/2011

Dilution Factor: 1.0

Soil Aliquot volume:

\begin{tabular}{|c|c|c|c|}
\hline CAS NO. & COMPOUND & $\begin{array}{l}\text { CONCENTRATION UNITS: } \\
(\mathrm{ug} / \mathrm{L} \text { or } \mathrm{ug} / \mathrm{kg}) \mathrm{ug} / \mathrm{L}\end{array}$ & $Q$ \\
\hline $79-01-6$ & Trichloroethene & 0.50 & $\mathrm{U}$ \\
\hline $108-87-2$ & Methylcyclohexane & 0.50 & $\mathrm{U}$ \\
\hline $78-87-5$ & 1,2-Dichloropropane & 0.50 & $\mathrm{U}$ \\
\hline $75-27-4$ & Bromodichloromethane & 1.7 & \\
\hline $10061-01-5$ & cis-1,3-Dichloropropene & 0.50 & $\mathrm{U}$ \\
\hline $108-10-1$ & 4-Methyl-2-pentanone & 5.0 & $\mathrm{U}$ \\
\hline $108-88-3$ & Toluene & 0.45 & $\mathrm{~J} B$ \\
\hline $10061-02-6$ & trans-1,3-Dichloropropene & 0.50 & $\mathrm{U}$ \\
\hline $79-00-5$ & 1,1,2-Trichloroethane & 0.50 & $\mathrm{U}$ \\
\hline $127-18-4$ & Tetrachloroethene & 0.50 & $\mathrm{U}$ \\
\hline $591-78-6$ & 2-Hexanone & 5.0 & $\mathrm{U}$ \\
\hline $124-48-1$ & Dibromochloromethane & 1.9 & \\
\hline $106-93-4$ & 1,2-Dibromoethane & 0.50 & $\mathrm{U}$ \\
\hline $108-90-7$ & Chlorobenzene & 0.50 & $\mathrm{U}$ \\
\hline $100-41-4$ & Ethylbenzene & 0.58 & \\
\hline $95-47-6$ & o-Xylene & 0.91 & \\
\hline $179601-23-1$ & $\mathrm{~m}, \mathrm{p}-\mathrm{Xy}$ lene & 2.4 & \\
\hline $100-42-5$ & Styrene & 0.50 & $\mathrm{U}$ \\
\hline $75-25-2$ & Bromoform & 0.50 & $\mathrm{U}$ \\
\hline $98-82-8$ & Isopropylbenzene & 0.50 & $\mathrm{U}$ \\
\hline $79-34-5$ & $1,1,2,2$-Tetrachloroethane & 0.50 & $\mathrm{U}$ \\
\hline $541-73-1$ & 1,3-Dichlorobenzene & 0.50 & $\mathrm{U}$ \\
\hline $106-46-7$ & 1,4-Dichlorobenzene & 0.50 & $\mathrm{U}$ \\
\hline $95-50-1$ & 1,2-Dichlorobenzene & 0.50 & $\mathrm{U}$ \\
\hline $96-12-8$ & 1,2-Dibromo-3-Chloropropane & 0.50 & $\mathrm{U}$ \\
\hline $120-82-1$ & 1,2,4-Trichlorobenzene & 0.50 & $\mathrm{U}$ \\
\hline $87-61-6$ & 1,2,3-Trichlorobenzene & 0.50 & $\mathrm{U}$ \\
\hline
\end{tabular}


$1 \mathrm{~J}$ - FORM I VOA-TIC

VOLATILE ORGANICS ANALYSIS DATA SHEET

TENTATIVELY IDENTIFIED COMPOUNDS
EPA SAMPLE NO.

INMW 15-W-32747
Lab Name: TESTAMERICA BURLINGTON

Lab Code: STLV Case No.: INMAN Mod. Ref No.:

Contract: $1 \mathrm{E}-30401$
Matrix: (SOIL/SED/WATER)

Water

Sample wt/vol: 25.0

Level: (TRACE or LOW/MED)

$(\mathrm{g} / \mathrm{mL}) \quad \mathrm{mL}$

응 Moisture: not dec.

GC Column: DB-624

Soil Extract Volume: ID: 0.20 (mm)

(uL)
TRACE

Contract: 1E-30401 SDG No.: 200-6762

Lab Sample ID: 200-6762-2

Lab File ID: DHPV06.D

Date Received: 08/30/2011

Date Analyzed: 09/01/2011

Dilution Factor: 1.0

Soil Aliquot Volume:

(uL)

CONCENTRATION UNITS: (ug/L or ug/kg) ug/L (mI)

\begin{tabular}{|c|c|c|c|c|}
\hline CAS NUMBER & COMPOUND NAME & $\mathrm{RT}$ & EST. CONC. & Q \\
\hline 01 & Unknown & 6.79 & 2.9 & $\mathrm{~B} \times \mathrm{J}$ \\
\hline $620-14-4$ & Benzene, 1-ethyl-3-methyl- & 10.70 & 0.89 & $\mathrm{~J} \mathrm{~N}$ \\
\hline $108-67-8$ & Benzene, 1,3,5-trimethyl- & 11.26 & 0.56 & $\mathrm{~J} \mathrm{~N}$ \\
\hline $622-96-8$ & Benzene, 1-ethyl-4-methyl- & 11.77 & 0.77 & $\mathrm{~J} \mathrm{~N}$ \\
\hline E9667961 & Total Alkanes & $\mathrm{N} / \mathrm{A}$ & & \\
\hline
\end{tabular}

$1 \mathrm{EPA}$-designated Registry Number. 
$1 \mathrm{~A}$ - FORM I VOA-1

VOLATILE ORGANICS ANALYSIS DATA SHEET
EPA SAMPLE NO.

INMW $17-W-32745$

Lab Name: TESTAMERICA BURLINGTON

Contract: $1 \mathrm{E}-30401$

Lab Code: STLV Case No.: INMAN Mod. Ref No.: SDG No.: 200-6762

Matrix: (SOIL/SED/WATER)

Sample wt/vol: 25.0 Water

$(\mathrm{g} / \mathrm{mL}) \quad \mathrm{mL}$

Level: (TRACE/LOW/MED) TRACE

\% Moisture: not dec.

GC Column: DB-624 ID: $0.20 \quad(\mathrm{~mm})$

Soil Extract Volume: (uL) (mL)

Purge Volume: 25.0
Lab Sample ID: 200-6762-4

Lab File ID: DHPV08.D

Date Received: 08/30/2011

Date Analyzed: 09/01/2011

Dilution Factor: 1.0

Soil Aliquot Volume:

$(u L)$

\begin{tabular}{|c|c|c|c|}
\hline CAS NO. & COMPOUND & $\begin{array}{l}\text { CONCENTRATION UNITS: } \\
(\mathrm{ug} / \mathrm{L} \text { or } \mathrm{ug} / \mathrm{kg}) \mathrm{ug} / \mathrm{L}\end{array}$ & $Q$ \\
\hline $75-71-8$ & Dichlorodifluoromethane & 0.50 & $\overline{\mathrm{U}}$ \\
\hline $74-87-3$ & Chloromethane & 0.50 & $\mathrm{U}$ \\
\hline $75-01-4$ & Vinyl chloride & 0.50 & $\mathrm{U}$ \\
\hline $74-83-9$ & Bromomethane & 0.50 & $\mathrm{U}$ \\
\hline $75-00-3$ & Chloroethane & 0.50 & $\mathrm{U}$ \\
\hline $75-69-4$ & Trichlorofluoromethane & 0.50 & $\mathrm{U}$ \\
\hline $75-35-4$ & 1,1-Dichloroethene & 0.50 & $\mathrm{U}$ \\
\hline $76-13-1$ & 1,1,2-Trichloro-1,2,2-trifluoroethane & 0.50 & $\mathrm{U}$ \\
\hline $67-64-1$ & Acetone & 1.5 & $\mathrm{~J}$ \\
\hline $75-15-0$ & Carbon disulfide & 0.50 & $\mathrm{U}$ \\
\hline $79-20-9$ & Methyl acetate & 0.50 & $\mathrm{U}$ \\
\hline $75-09-2$ & Methylene Chloride & 0.50 & $\mathrm{U}$ \\
\hline $156-60-5$ & trans $-1,2-$ Dichloroethene & 0.50 & $\mathrm{U}$ \\
\hline $1634-04-4$ & Methyl tert-butyl ether & 0.50 & $\mathrm{U}$ \\
\hline $75-34-3$ & 1,1-Dichloroethane & 0.50 & $\mathrm{U}$ \\
\hline $156-59-2$ & cis-1,2-Dichloroethene & 0.50 & $\mathrm{U}$ \\
\hline $78-93-3$ & 2-Butanone & 5.0 & $\mathrm{U}$ \\
\hline $74-97-5$ & Bromochloromethane & 0.50 & U \\
\hline $67-66-3$ & Chloroform & 0.50 & $\mathrm{U}$ \\
\hline $71-55-6$ & $1,1,1$-Trichloroethane & 0.50 & $\mathrm{U}$ \\
\hline $110-82-7$ & Cyclohexane & 0.50 & U \\
\hline $56-23-5$ & Carbon tetrachloride & 0.028 & $\mathrm{~J}$ \\
\hline $71-43-2$ & Benzene & 0.032 & $\mathrm{~J}$ \\
\hline $107-06-2$ & 1,2-Dichloroethane & 0.50 & U \\
\hline
\end{tabular}

Report 1,4-Dioxane for Low-Medium VOA analysis only 
1B - FORM I VOA-2

VOLATILE ORGANICS ANALYSIS DATA SHEET
EPA SAMPLE NO.

INMW17-W-32745

Lab Name: TESTAMERICA BURLINGTON

Lab Code: STLV

Matrix: (SOIL/SED/WATER)

Sample wt/vol: 25.0

Water

$(\mathrm{g} / \mathrm{mL}) \quad \mathrm{mL}$

Level: (TRACE/LOW/MED) TRACE

응 Moisture: not dec.

GC Column: DB-624 ID : 0.20

Soil Extract Volume:

(uL)

(mL)

Purge Volume: 25.0
Contract: $1 E-30401$

SDG No.: 200-6762

Lab Sample ID: 200-6762-4

Lab File ID: DHPV08.D

Date Received: 08/30/2011

Date Analyzed: 09/01/2011

Dilution Factor: 1.0

Soil Aliquot Volume:

(uL)

\begin{tabular}{|c|c|c|c|}
\hline CAS NO. & COMPOUND & $\begin{array}{l}\text { CONCENTRATION UNITS: } \\
(\mathrm{ug} / \mathrm{L} \text { or } \mathrm{ug} / \mathrm{kg}) \mathrm{ug} / \mathrm{L}\end{array}$ & Q \\
\hline $79-01-6$ & Trichloroethene & 0.50 & $\mathrm{U}$ \\
\hline $108-87-2$ & Methylcyclohexane & 0.50 & $\mathrm{U}$ \\
\hline $78-87-5$ & 1,2-Dichloropropane & 0.50 & $\mathrm{U}$ \\
\hline $75-27-4$ & Bromodichloromethane & 0.50 & $\mathrm{U}$ \\
\hline $10061-01-5$ & cis-1,3-Dichloropropene & 0.50 & $\mathrm{U}$ \\
\hline $108-10-1$ & 4-Methyl-2-pentanone & 5.0 & $\mathrm{U}$ \\
\hline $108-88-3$ & Toluene & 0.075 & $\mathrm{~J} \mathrm{~B}$ \\
\hline $10061-02-6$ & trans-1,3-Dichloropropene & 0.50 & $\mathrm{U}$ \\
\hline $79-00-5$ & 1,1,2-Trichloroethane & 0.50 & $\mathrm{U}$ \\
\hline $127-18-4$ & Tetrachloroethene & 0.50 & $\mathrm{U}$ \\
\hline $591-78-6$ & 2-Hexanone & 5.0 & $\mathrm{U}$ \\
\hline $124-48-1$ & Dibromochloromethane & 0.50 & $\mathrm{U}$ \\
\hline $106-93-4$ & 1,2-Dibromoethane & 0.50 & $\mathrm{U}$ \\
\hline $108-90-7$ & Chlorobenzene & 0.50 & $\mathrm{U}$ \\
\hline $100-41-4$ & Ethylbenzene & 0.50 & $\mathrm{U}$ \\
\hline $95-47-6$ & o-Xylene & 0.50 & $\mathrm{U}$ \\
\hline $179601-23-1$ & $\mathrm{~m}, \mathrm{p}$-Xylene & 0.50 & $\mathrm{U}$ \\
\hline $100-42-5$ & Styrene & 0.50 & $\mathrm{U}$ \\
\hline $75-25-2$ & Bromoform & 0.50 & $\mathrm{U}$ \\
\hline $98-82-8$ & Isopropylbenzene & 0.50 & $\mathrm{U}$ \\
\hline $79-34-5$ & $1,1,2,2$-Tetrachloroethane & 0.50 & $\mathrm{U}$ \\
\hline $541-73-1$ & 1,3-Dichlorobenzene & 0.50 & $\mathrm{U}$ \\
\hline $106-46-7$ & 1,4-Dichlorobenzene & 0.50 & $\mathrm{U}$ \\
\hline $95-50-1$ & 1,2-Dichlorobenzene & 0.50 & $\mathrm{U}$ \\
\hline $96-12-8$ & 1,2-Dibromo-3-Chloropropane & 0.50 & $\mathrm{U}$ \\
\hline $120-82-1$ & 1,2,4-Trichlorobenzene & 0.50 & $\mathrm{U}$ \\
\hline $87-61-6$ & 1,2,3-Trichlorobenzene & 0.50 & $\mathrm{U}$ \\
\hline
\end{tabular}


1J - FORM I VOA-TIC

VOLATILE ORGANICS ANALYSIS DATA SHEET TENTATIVELY IDENTIFIED COMPOUNDS
EPA SAMPLE NO.

INMW17-W-32745

Lab Name: TESTAMERICA BURLINGTON Case No.: INMAN Mod. Ref No.:

Contract: 1E-30401

Lab Code: STLV TER)

Water

$(\mathrm{g} / \mathrm{mL}) \mathrm{mL}$

Sample wt/vol: 25.0

Level: (TRACE or LOW/MED)

TRACE

응 Moisture: not dec.

GC Column: DB-624

Soil Extract Volume: ID: $0.20 \quad(\mathrm{~mm})$

(uL)
SDG No.: 200-6762.

Lab Sample ID: 200-6762-4

Lab File ID: DHPV08.D

Date Received: 08/30/2011

Date Analyzed: 09/01/2011

Dilution Factor: 1.0

Soil Aliquot Volume:

(uL)

Purge Volume: 25.0

$(\mathrm{mL})$

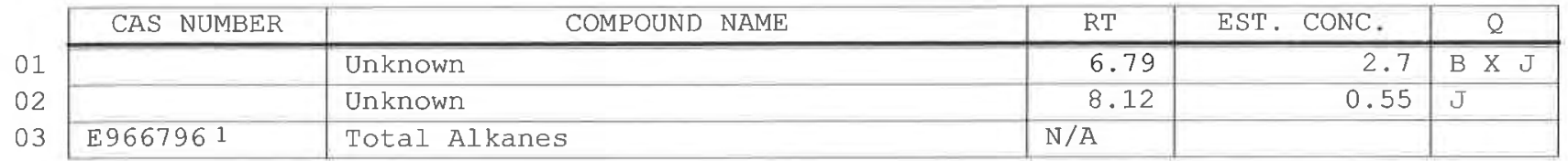

1 EPA-designated Registry Number. 
$1 A$ - EORM I VOA-1

VOLATILE ORGANICS ANALYSIS DATA SHEET
EPA SAMPLE NO.

INMW17-W-32748

Lab Name: TESTAMERICA BURLINGTON

Contract: $1 \mathrm{E}-30401$

Lab Code: STLV Case No.: INMAN Mod. Ref No.: SDG No.: 200-6762

Matrix: (SOIL/SED/WATER) Water

Sample wt/vol: 25.0

( $\mathrm{g} / \mathrm{mL}) \mathrm{mL}$

Level: (TRACE/LOW/MED) TRACE

\% Moisture: not dec.

GC Column: DB-624

ID: $0.20 \quad$ (mm)

Soil Extract Volume:

(uL)

(InL)

Purge Volume: 25.0
Lab Sample ID: 200-6762-3

Lab File ID: DHPV07.D

Date Received: $08 / 30 / 2011$

Date Analyzed: 09/01/2011

Dilution Factor: 1.0

Soil Aliquot Volume:

(uL)

\begin{tabular}{|c|c|c|c|}
\hline CAS NO. & COMPOUND & $\begin{array}{l}\text { CONCENTRATION UNITS: } \\
(u g / \mathrm{L} \text { or } \mathrm{ug} / \mathrm{kg}) \mathrm{ug} / \mathrm{L}\end{array}$ & Q \\
\hline $75-71-8$ & Dichlorodifluoromethane & 0.50 & $\overline{\mathrm{U}}$ \\
\hline $74-87-3$ & Chloromethane & 0.50 & $\mathrm{U}$ \\
\hline $75-01-4$ & Vinyl chloride & 0.50 & $\mathrm{U}$ \\
\hline $74-83-9$ & Bromomethane & 0.50 & $\mathrm{U}$ \\
\hline $75-00-3$ & Chloroethane & 0.50 & $\mathrm{U}$ \\
\hline $75-69-4$ & Trichlorofluoromethane & 0.50 & $\mathrm{U}$ \\
\hline $75-35-4$ & 1,1-Dichloroethene & 0.50 & $\mathrm{U}$ \\
\hline $76-13-1$ & 1,1,2-Trichloro-1,2,2-trifluoroethane & 0.50 & $\mathrm{U}$ \\
\hline $67-64-1$ & Acetone & 5.0 & $\mathrm{U}$ \\
\hline $75-15-0$ & Carbon disulfide & 0.50 & $\mathrm{U}$ \\
\hline $79-20-9$ & Methyl acetate & 0.50 & $\mathrm{U}$ \\
\hline $75-09-2$ & Methylene Chloride & 0.041 & $\mathrm{~J}$ \\
\hline $156-60-5$ & trans-1,2-Dichloroethene & 0.50 & $\mathrm{U}$ \\
\hline $1634-04-4$ & Methyl tert-butyl ether & 0.50 & $\mathrm{U}$ \\
\hline $75-34-3$ & 1,1-Dichloroethane & 0.50 & $\mathrm{U}$ \\
\hline $156-59-2$ & cis-1,2-Dichloroethene & 0.50 & U \\
\hline $78-93-3$ & 2-Butanone & 5.0 & $\mathrm{U}$ \\
\hline $74-97-5$ & Bromochloromethane & 0.50 & $\mathrm{U}$ \\
\hline $67-66-3$ & Chloroform & 0.26 & $\mathrm{~J}$ \\
\hline $71-55-6$ & 1,1,1-Trichloroethane & 0.50 & U \\
\hline $110-82-7$ & Cyclohexane & 0.50 & $\mathrm{U}$ \\
\hline $56-23-5$ & Carbon tetrachloride & 0.76 & \\
\hline $71-43-2$ & Benzene & 0.50 & $\mathrm{U}$ \\
\hline $107-06-2$ & 1,2-Dichloroethane & 0.50 & $\mathrm{U}$ \\
\hline
\end{tabular}

Report 1,4-Dioxane for Low-Medium VOA analysis only 
1B - FORM I VOA-2

VOLATILE ORGANICS ANALYSIS DATA SHEET
EPA SAMPLE NO.

INMW17-W-32748
Lab Name: TESTAMERICA BURLINGTON

Lab Code: STLV

Contract: 1E-30401

Matrix: (SOIL/SED/WATER)

Sample wt/vol: 25.0

Water

$(\mathrm{g} / \mathrm{mL}) \quad \mathrm{mL}$

Level: (TRACE/LOW/MED) TRACE

\% Moisture: not dec.

GC Column: DB-624

ID $: 0.20$

(mun)

Soil Extract Volume:

(uL)

(mL)

Purge Volume: 25.0

SDG No.: 200-6762

Lab Sample ID: 200-6762-3

Lab File ID: DHPV07.D

Date Received: 08/30/2011

Date Analyzed: 09/01/2011

Dilution Factor: 1.0

Soil Aliquot Volume:

\begin{tabular}{|c|c|c|c|}
\hline CAS NO. & COMPOUND & $\begin{array}{l}\text { CONCENTRATION UNITS: } \\
(\mathrm{ug} / \mathrm{L} \text { or } \mathrm{ug} / \mathrm{kg}) \mathrm{ug} / \mathrm{L}\end{array}$ & $Q$ \\
\hline $79-01-6$ & Trichloroethene & 0.50 & $\mathrm{U}$ \\
\hline $108-87-2$ & Methylcyclohexane & 0.50 & $\mathrm{U}$ \\
\hline $78-87-5$ & 1,2-Dichloropropane & 0.50 & $\mathrm{U}$ \\
\hline $75-27-4$ & Bromodichloromethane & 0.50 & $\mathrm{U}$ \\
\hline $10061-01-5$ & cis-1,3-Dichloropropene & 0.50 & $\mathrm{U}$ \\
\hline $108-10-1$ & 4-Methyl-2-pentanone & 5.0 & $\mathrm{U}$ \\
\hline $108-88-3$ & Toluene & 0.070 & $\mathrm{~J} \mathrm{~B}$ \\
\hline $10061-02-6$ & trans-1,3-Dichloropropene & 0.50 & $\mathrm{U}$ \\
\hline $79-00-5$ & 1,1,2-Trichloroethane & 0.50 & $\mathrm{U}$ \\
\hline $127-18-4$ & Tetrachloroethene & 0.50 & $\mathrm{U}$ \\
\hline $591-78-6$ & 2-Hexanone & 5.0 & $\mathrm{U}$ \\
\hline $124-48-1$ & Dibromochloromethane & 0.50 & $\mathrm{U}$ \\
\hline $106-93-4$ & 1,2-Dibromoethane & 0.50 & $\mathrm{U}$ \\
\hline $108-90-7$ & Chlorobenzene & 0.50 & $\mathrm{U}$ \\
\hline $100-41-4$ & Ethylbenzene & 0.50 & $\mathrm{U}$ \\
\hline $95-47-6$ & o-Xylene & 0.50 & $\mathrm{U}$ \\
\hline $179601-23-1$ & $\mathrm{~m}, \mathrm{p}$-Xylene & 0.50 & $\mathrm{U}$ \\
\hline $100-42-5$ & Styrene & 0.50 & $\mathrm{U}$ \\
\hline $75-25-2$ & Bromoform & 0.50 & $\mathrm{U}$ \\
\hline $98-82-8$ & Isopropylbenzene & 0.50 & $\mathrm{U}$ \\
\hline $79-34-5$ & $1,1,2,2$-Tetrachloroethane & 0.50 & $\mathrm{U}$ \\
\hline $541-73-1$ & 1,3-Dichlorobenzene & 0.50 & $\mathrm{U}$ \\
\hline $106-46-7$ & 1,4-Dichlorobenzene & 0.50 & $\mathrm{U}$ \\
\hline $95-50-1$ & 1,2-Dichlorobenzene & 0.50 & $\mathrm{U}$ \\
\hline $96-12-8$ & 1,2-Dibromo-3-Chloropropane & 0.50 & $\mathrm{U}$ \\
\hline $120-82-1$ & 1,2,4-Trichlorobenzene & 0.50 & $\mathrm{U}$ \\
\hline $87-61-6$ & 1,2,3-Trichlorobenzene & 0.50 & $\mathrm{U}$ \\
\hline
\end{tabular}


$1 \mathrm{~J}-$ FORM I VOA-TIC

VOLATILE ORGANICS ANALYSIS DATA SHEET

TENTATIVELY IDENTIFIED COMPOUNDS
EPA SAMPLE NO.

INMW17-W-32748

\begin{tabular}{|c|c|c|c|c|c|c|c|c|c|}
\hline Lab Name: TES & TAMERICA BUR & RLINGTON & & & Contr & act: $1 \mathrm{E}-$ & 30401 & & \\
\hline Lab Code: STL & Case & No.: II & JMAN & Mod. Ref & No.: & & SDG No.: & $200-6$ & 762 \\
\hline Matrix: (SOIL/ & SED/WATER) & Water & & & $\mathrm{Lab} S$ & ample ID: & $200-67$ & $52-3$ & \\
\hline Sample wt/vol: & 25.0 & $(\mathrm{~g} / \mathrm{mL})$ & $\mathrm{mL}$ & & $\mathrm{Lab} F$ & ile ID: & DHPV07.D & & \\
\hline Level: (TRACE & or LOW/MED) & TRACF & & & Date & Received: & $08 / 30 / 2$ & 2011 & \\
\hline \% Moisture: no & t dec. & & & & Date 1 & Analyzed: & $09 / 01 / 2$ & 2011 & \\
\hline GC Colunn: DB- & -624 & ID: & 0.20 & $(\mathrm{~mm})$ & Dilut & ion Facto & or: 1.0 & & \\
\hline Soil Extract Vo & olume: & & & (uL) & Soil & Aliquot V & Tolume: & & $(u L)$ \\
\hline CONCENTRATION & UNITS : (ug/L & or ug/1 & & $\mathrm{ug} / \mathrm{I}$ & Purge & Volume: & 25.0 & & $(\mathrm{~mL})$ \\
\hline CAS NUMBER & & COME & OUND & NAME & & $\mathrm{RT}$ & EST. C & ONC. & Q \\
\hline & Unknown & & & & & 6.79 & & 2.9 & $\mathrm{~B} \times \mathrm{J}$ \\
\hline E9667961 & Total Alka & anes & & & & $\mathrm{N} / \mathrm{A}$ & & & \\
\hline
\end{tabular}

1 EPA-designated Registry Number. 
Lab Name: TESTAMERICA BURLINGTON

Lab Code: STLV Case No.: INMAN Mod. Ref No.:

Matrix: (SOIL/SED/WATER)

Sample wt/vol: 25.0

Water

$(\mathrm{g} / \mathrm{mL}) \quad \mathrm{mL}$

Level: (TRACE/LOW/MED) TRACE

응 Moisture: not dec.

GC Column: DB-624

Soil Extract Volume: ID $: 0.20$ (InIn) (uL)

$(\mathrm{mL})$

Purge Volume: 25.0
Contract: 1E-30401 SDG No.: 200-6762

Lab Sample ID: 200-6762-1

Lab File ID: DHPV05.D

Date Received: 08/30/2011

Date Analyzed: 09/01/2011

Dilution Factor: 1.0

Soil Aliquot Volume: (uL)

\begin{tabular}{|c|c|c|c|}
\hline CAS NO. & COMPOUND & $\begin{array}{l}\text { CONCENTRATION UNITS: } \\
(\mathrm{ug} / \mathrm{L} \text { or } \mathrm{ug} / \mathrm{kg}) \mathrm{ug} / \mathrm{L}\end{array}$ & $Q$ \\
\hline $75-71-8$ & Dichlorodifluoromethane & 0.50 & $\overline{\mathrm{U}}$ \\
\hline $74-87-3$ & Chloromethane & 0.50 & $\mathrm{U}$ \\
\hline $75-01-4$ & Vinyl chloride & 0.50 & $\mathrm{U}$ \\
\hline $74-83-9$ & Bromomethane & 0.50 & $\mathrm{U}$ \\
\hline $75-00-3$ & Chloroethane & 0.50 & $\mathrm{U}$ \\
\hline $75-69-4$ & Trichlorofluoromethane & 0.50 & $\mathrm{U}$ \\
\hline $75-35-4$ & 1,1-Dichloroethene & 0.50 & $\mathrm{U}$ \\
\hline $76-13-1$ & 1,1,2-Trichloro-1,2,2-trifluoroethane & 0.50 & U \\
\hline $67-64-1$ & Acetone & 0.92 & $\mathrm{~J}$ \\
\hline $75-15-0$ & Carbon disulfide & 0.50 & $\mathrm{U}$ \\
\hline $79-20-9$ & Methyl acetate & 0.50 & $\mathrm{U}$ \\
\hline $75-09-2$ & Methylene Chloride & 0.037 & $\mathrm{~J}$ \\
\hline $156-60-5$ & trans-1,2-Dichloroethene & 0.50 & $\mathrm{U}$ \\
\hline $1634-04-4$ & Methyl tert-butyl ether & 0.50 & $\mathrm{U}$ \\
\hline $75-34-3$ & 1,1-Dichloroethane & 0.50 & U \\
\hline $156-59-2$ & cis-1,2-Dichloroethene & 0.50 & $\mathrm{U}$ \\
\hline $78-93-3$ & 2-Butanone & 5.0 & $\mathrm{U}$ \\
\hline $74-97-5$ & Bromochloromethane & 0.50 & $\mathrm{U}$ \\
\hline $67-66-3$ & Chloroform & 0.23 & $\mathrm{~J}$ \\
\hline $71-55-6$ & 1,1,1-Trichloroethane & 0.50 & $\mathrm{U}$ \\
\hline $110-82-7$ & Cyclohexane & 0.50 & $\mathrm{U}$ \\
\hline $56-23-5$ & Carbon tetrachloride & 0.62 & \\
\hline $71-43-2$ & Benzene & 0.0052 & $\mathrm{~J}$ \\
\hline $107-06-2$ & 1,2-Dichloroethane & 0.50 & $\mathrm{U}$ \\
\hline
\end{tabular}

Report 1,4-Dioxane for Low-Medium VOA analysis only 
1B - FORM I VOA-2

VOLATILE ORGANICS ANALYSIS DATA SHEET
EPA SAMPLE NO,

INMW17-W-32749

Lab Name: TESTAMERICA BURLINGTON

Lab Code: STLV Case No.: INMAN Mod. Ref No.:

Matrix: (SOIL/SED/WATER)

Sample wt/vol: 25.0

Water

$(\mathrm{g} / \mathrm{mL}) \quad \mathrm{mL}$

Level: (TRACE/LOW/MED) TRACE

o Moisture: not dec.

GC Column: DB-624

ID $: 0.20$

(mm)

Soil Extract Volume:

(uL)

Purge Volume: 25.0

(InL)
Contract: $1 \mathrm{E}-30401$

SDG No.: 200-6762

Lab Sample ID: 200-6762-1

Lab File ID: DHPV05.D

Date Received: 08/30/2011

Date Analyzed: 09/01/2011

Dilution Factor: 1.0

Soil Aliquot Volume:

(uL)

\begin{tabular}{|c|c|c|c|}
\hline CAS NO. & COMPOUND & $\begin{array}{l}\text { CONCENTRATION UNITS: } \\
(\mathrm{ug} / \mathrm{L} \text { or } \mathrm{ug} / \mathrm{kg}) \mathrm{ug} / \mathrm{L}\end{array}$ & $Q$ \\
\hline $79-01-6$ & Trichloroethene & 0.50 & $\bar{U}$ \\
\hline $108-87-2$ & Methylcyclohexane & 0.50 & $\mathrm{U}$ \\
\hline $78-87-5$ & 1,2-Dichloropropane & 0.50 & $\mathrm{U}$ \\
\hline $75-27-4$ & Bromodichloromethane & 0.50 & $\mathrm{U}$ \\
\hline $10061-01-5$ & cis-1,3-Dichloropropene & 0.50 & $\mathrm{U}$ \\
\hline $108-10-1$ & 4-Methyl-2-pentanone & 5.0 & $\mathrm{U}$ \\
\hline $108-88-3$ & Toluene & 0.026 & $\mathrm{~J} B$ \\
\hline $10061-02-6$ & trans $-1,3-$ Dichloropropene & 0.50 & $\mathrm{U}$ \\
\hline $79-00-5$ & 1,1,2-Trichloroethane & 0.50 & $\mathrm{U}$ \\
\hline $127-18-4$ & Tetrachloroethene & 0.50 & $\mathrm{U}$ \\
\hline $591-78-6$ & 2-Hexanone & 5.0 & $\mathrm{U}$ \\
\hline $124-48-1$ & Dibromochloromethane & 0.50 & $\mathrm{U}$ \\
\hline $106-93-4$ & 1,2-Dibromoethane & 0.50 & $\mathrm{U}$ \\
\hline $108-90-7$ & Chlorobenzene & 0.50 & $\mathrm{U}$ \\
\hline $100-41-4$ & Ethylbenzene & 0.50 & $\mathrm{U}$ \\
\hline $95-47-6$ & o-Xylene & 0.50 & $\mathrm{U}$ \\
\hline $179601-23-1$ & m,p-xylene & 0.50 & $\mathrm{U}$ \\
\hline $100-42-5$ & Styrene & 0.50 & $\mathrm{U}$ \\
\hline $75-25-2$ & Bromoform & 0.50 & $\mathrm{U}$ \\
\hline $98-82-8$ & Isopropylbenzene & 0.50 & $\mathrm{U}$ \\
\hline $79-34-5$ & $1,1,2,2$-Tetrachloroethane & 0.50 & $\mathrm{U}$ \\
\hline $541-73-1$ & 1,3-Dichlorobenzene & 0.50 & $\mathrm{U}$ \\
\hline $106-46-7$ & 1,4-Dichlorobenzene & 0.50 & $\mathrm{U}$ \\
\hline $95-50-1$ & 1,2-Dichlorobenzene & 0.50 & $\mathrm{U}$ \\
\hline $96-12-8$ & 1,2-Dibromo-3-Chloropropane & 0.50 & $\mathrm{U}$ \\
\hline $120-82-1$ & 1,2,4-Trichlorobenzene & 0.50 & $\mathrm{U}$ \\
\hline $87-61-6$ & $1,2,3$-Trichlorobenzene & 0.50 & $\mathrm{U}$ \\
\hline
\end{tabular}


1J - FORM I VOA-TIC

VOLATILE ORGANICS ANALYSIS DATA SHEET TENTATIVELY IDENTIFIED COMPOUNDS
EPA SAMPLE NO.

INMW $17-W-32749$

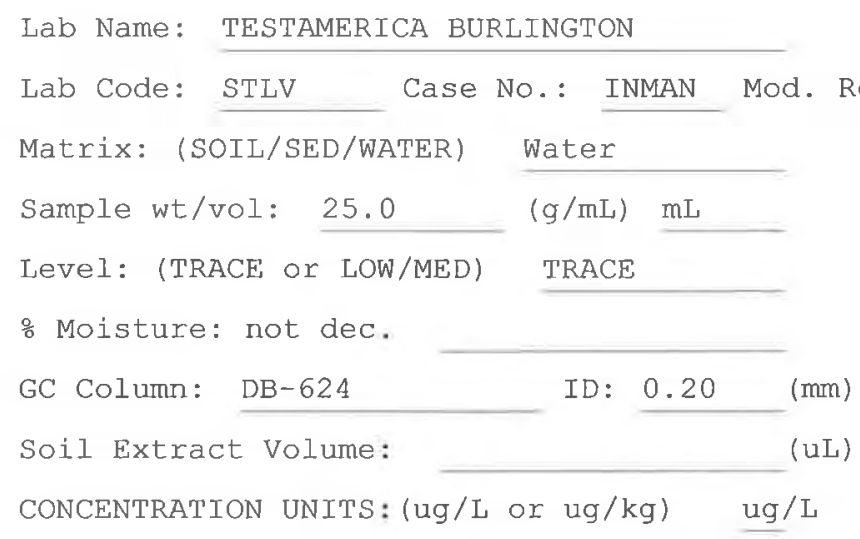

Contract: 1E-30401

Ref No.: SDG No.: 200-6762

Lab Sample ID: 200-6762-1

Lab File ID: DHPV05.D

Date Received: 08/30/2011

Date Analyzed: 09/01/2011

Dilution Factor: 1.0

Soil Aliquot volume: (uL)

Purge Volume: 25.0 (mL)

\begin{tabular}{l|l|l|r|r|r|}
\hline \multicolumn{1}{l|}{ CAS NUMBER } & \multicolumn{1}{|c|}{ COMPOUND NAME } & RT & EST. CONC. & Q \\
\cline { 2 - 7 } 01 & & Unknown & 6.79 & 2.5 & B X J \\
\hline
\end{tabular}

1 EPA-designated Registry Number. 
$1 A$ - FORM I VOA-1

VOLATILE ORGANICS ANALYSIS DATA SHEET
EPA SAMPLE NO. INMW $18-W-32752$
Lab Name: TESTAMERICA BURLINGTON

Lab Code: STLV Case No.: INMAN

Matrix: (SOIL/SED/WATER)

water

Sample wt/vol: 25.0

$(g / m L) \quad m L$

Level: (TRACE/LOW/MED) TRACE

\% Moisture: not dec.

GC Column: DB-624 ID: 0.20 (mm)

Soil Extract Volume: (uL) (mL)

Purge Volume: 25.0
Contract: $\quad 1 \mathrm{E}-30401$

SDG No.: 200-6762 Mod. Ref No.:

Lab Sample ID: 200-6762-5

Lab File ID: DHPV09.D

Date Received: 08/30/2011

Date Analyzed: 09/01/2011

Dilution Factor: 1.0

Soil Aliquot Volume:

(uL)

\begin{tabular}{|c|c|c|c|}
\hline CAS NO. & COMPOUND & $\begin{array}{l}\text { CONCENTRATION UNITS: } \\
(\mathrm{ug} / \mathrm{L} \text { or } \mathrm{ug} / \mathrm{kg}) \mathrm{ug} / \mathrm{L}\end{array}$ & Q \\
\hline $75-71-8$ & Dichlorodifluoromethane & 0.50 & $\overline{\mathrm{U}}$ \\
\hline $74-87-3$ & Chloromethane & 0.50 & $\mathrm{U}$ \\
\hline $75-01-4$ & Vinyl chloride & 0.50 & $\mathrm{U}$ \\
\hline $74-83-9$ & Bromomethane & 0.50 & $\mathrm{U}$ \\
\hline $75-00-3$ & Chloroethane & 0.50 & $\mathrm{U}$ \\
\hline $75-69-4$ & Trichlorofluoromethane & 0.50 & $\mathrm{U}$ \\
\hline $75-35-4$ & 1,1-Dichloroethene & 0.50 & $\mathrm{U}$ \\
\hline $76-13-1$ & 1,1,2-Trichloro-1,2,2-trifluoroethane & 0.50 & $\mathrm{U}$ \\
\hline $67-64-1$ & Acetone & 1.7 & $\mathrm{~J}$ \\
\hline $75-15-0$ & Carbon disulfide & 0.50 & $\mathrm{U}$ \\
\hline $79-20-9$ & Methyl acetate & 0.50 & $\mathrm{U}$ \\
\hline $75-09-2$ & Methylene Chloride & 0.50 & $\mathrm{U}$ \\
\hline $156-60-5$ & trans-1,2-Dichloroethene & 0.50 & $\mathrm{U}$ \\
\hline $1634-04-4$ & Methyl tert-butyl ether & 0.015 & $\mathrm{~J}$ \\
\hline $75-34-3$ & 1,1-Dichloroethane & 0.50 & $\mathrm{U}$ \\
\hline $156-59-2$ & cis-1,2-Dichloroethene & 0.50 & $\mathrm{U}$ \\
\hline $78-93-3$ & 2-Butanone & 5.0 & $\mathrm{U}$ \\
\hline $74-97-5$ & Bromochloromethane & 0.50 & $\mathrm{U}$ \\
\hline $67-66-3$ & Chloroform & 0.79 & \\
\hline $71-55-6$ & 1,1,1-Trichloroethane & 0.50 & $\mathrm{U}$ \\
\hline $110-82-7$ & Cyclohexane & 0.50 & $\mathrm{U}$ \\
\hline $56-23-5$ & Carbon tetrachloride & 1.1 & \\
\hline $71-43-2$ & Benzene & 0.20 & $\mathrm{~J}$ \\
\hline $107-06-2$ & 1,2-Dichloroethane & 0.50 & $\mathrm{U}$ \\
\hline
\end{tabular}

Report 1,4-Dioxane for Low-Medium VOA analysis only 
Lab Name: TESTAMERICA BURLINGTON

Lab Code: STLV

Contract: 1E-30401

Matrix: (SOIL/SED/WATER)

Sample wt/vol: 25.0

Water

$(\mathrm{g} / \mathrm{mL}) \quad \mathrm{mL}$

Level: (TRACE/LOW/MED) TRACE

응 Moisture: not dec.

GC Column: DB-624

Soil Extract Volume:

ID: 0.20 (mm)

Purge Volume: 25.0

(mL)
SDG No.: 200-6762

Lab Sample ID: 200-6762-5

Lab File ID: DHpV09.D

Date Received: 08/30/2011

Date Analyzed: 09/01/2011

Dilution Factor: 1.0

Soil Aliquot Volume:

(uL)

\begin{tabular}{|c|c|c|c|}
\hline CAS NO. & COMPOUND & $\begin{array}{l}\text { CONCENTRATION UNITS: } \\
(\mathrm{ug} / \mathrm{L} \text { or } \mathrm{ug} / \mathrm{kg}) \mathrm{ug} / \mathrm{L}\end{array}$ & $Q$ \\
\hline $79-01-6$ & Trichloroethene & 0.50 & $\bar{U}$ \\
\hline $108-87-2$ & Methylcyclohexane & 0.50 & U \\
\hline $78-87-5$ & 1,2-Dichloropropane & 0.50 & $\mathrm{U}$ \\
\hline $75-27-4$ & Bromodichloromethane & 0.50 & $\mathrm{U}$ \\
\hline $10061-01-5$ & cis-1,3-Dichloropropene & 0.50 & $\mathrm{U}$ \\
\hline $108-10-1$ & 4-Methyl-2-pentanone & 5.0 & $\mathrm{U}$ \\
\hline $108-88-3$ & Toluene & 0.12 & $\mathrm{~J} B$ \\
\hline $10061-02-6$ & trans-1,3-Dichloropropene & 0.50 & $\mathrm{U}$ \\
\hline $79-00-5$ & 1,1,2-Trichloroethane & 0.50 & $\mathrm{U}$ \\
\hline $127-18-4$ & Tetrachloroethene & 0.11 & $\mathrm{~J}$ \\
\hline $591-78-6$ & 2-Hexanone & 5.0 & $\mathrm{U}$ \\
\hline $124-48-1$ & Dibromochloromethane & 0.50 & U \\
\hline $106-93-4$ & 1,2-Dibromoethane & 0.50 & $\mathrm{U}$ \\
\hline $108-90-7$ & Chlorobenzene & 0.50 & $\mathrm{U}$ \\
\hline $100-41-4$ & Ethylbenzene & 0.020 & $\mathrm{~J}$ \\
\hline $95-47-6$ & o-Xylene & 0.50 & $\mathrm{U}$ \\
\hline $179601-23-1$ & $\mathrm{~m}, \mathrm{p}$-Xylene & 0.018 & $\mathrm{~J}$ \\
\hline $100-42-5$ & Styrene & 0.50 & $\mathrm{U}$ \\
\hline $75-25-2$ & Bromoform & 0.50 & $\mathrm{U}$ \\
\hline $98-82-8$ & Isopropylbenzene & 0.50 & $\mathrm{U}$ \\
\hline $79-34-5$ & $1,1,2,2$-Tetrachloroethane & 0.50 & $\mathrm{U}$ \\
\hline $541-73-1$ & 1,3-Dichlorobenzene & 0.50 & U \\
\hline $106-46-7$ & 1,4-Dichlorobenzene & 0.50 & $\mathrm{U}$ \\
\hline $95-50-1$ & 1,2-Dichlorobenzene & 0.50 & $\mathrm{U}$ \\
\hline $96-12-8$ & 1,2-Dibromo-3-Chloropropane & 0.50 & $\mathrm{U}$ \\
\hline $120-82-1$ & 1,2,4-Trichlorobenzene & 0.50 & $\mathrm{U}$ \\
\hline $87-61-6$ & 1,2,3-Trichlorobenzene & 0.50 & $\mathrm{U}$ \\
\hline
\end{tabular}


IJ - FORM I VOA-TIC

VOLATILE ORGANICS ANALYSIS DATA SHEET

TENTATIVELY IDENTIFIED COMPOUNDS
EPA SAMPLE NO.

INMW18-W-32752

Lab Name: TESTAMERICA BURLINGTON

Contract: 1E-30401

Lab Code: STLV Case No.: INMAN

Mod. Ref No.:
Water

$(\mathrm{g} / \mathrm{mL}) \quad \mathrm{mL}$

Sample wt/vol: 25.0

Level: (TRACE or LOW/MED)

TRACE

․ Moisture: not dec.

GC Column: DB-624

Soil Extract Volume: ID: $0.20 \quad(\mathrm{~mm})$

(uL)
: SDG No.: 200-6762

Lab Sample ID: 200-6762-5

Lab File ID: DHPVO9.D

Date Received: 08/30/2011

Date Analyzed: 09/01/2011

Dilution Factor: 1.0

Soil Aliquot Volume:

(uL)

CONCENTRATION UNITS: (ug/L or $\mathrm{ug} / \mathrm{kg}$ ) ug/L (mL)

\begin{tabular}{|c|c|c|c|c|}
\hline CAS NUMBER & COMPOUND NAME & $\mathrm{RT}$ & EST. CONC. & $Q$ \\
\hline 01 & Unknown & 6.79 & 2.9 & $\mathrm{BX} \mathrm{J}$ \\
\hline $66-25-1$ & Hexanal & 8.12 & 0.92 & $\mathrm{JN}$ \\
\hline 03 & Unknown & 9.93 & 1.2 & $\mathrm{~J}$ \\
\hline 04 & Unknown & 10.76 & 0.76 & $\mathrm{~J}$ \\
\hline 05 & Unknown & 11.99 & 2.2 & $\mathrm{~J}$ \\
\hline $124-19-6$ & Nonanal & 12.91 & 1.1 & $\mathrm{JN}$ \\
\hline E9667961 & Total Alkanes & $\mathrm{N} / \mathrm{A}$ & 0.56 & $\mathrm{~J}$ \\
\hline
\end{tabular}

1 EPA-designated Registry Number. 
$1 A$ - FORM I VOA-1

VOLATILE ORGANICS ANALYSIS DATA SHEET
EPA SAMPLE NO. INMW 18-W-32753

Lab Name: TESTAMERICA BURLINGTON

Lab Code: STLV Case No.: INMAN

Matrix: (SOIL/SED/WATER)

Sample wt/vol: 25.0

Water

$(\mathrm{g} / \mathrm{mL}) \quad \mathrm{mL}$

Level: (TRACE/LOW/MED) TRACE

$\therefore$ Moisture: not dec.

GC Column: DB-624

Soil Extract Volume: ID: $0.20 \quad$ (mm)

(uL)

(mL)

Purge Volume: 25.0

Contract: 1E-30401

SDG No.: 200-6762

Lab Sample ID: 200-6762-6

Lab File ID: DHPV10.D

Date Received: 08/30/2011

Date Analyzed: 09/01/2011

Dilution Factor: 1.0

Soil Aliquot Volume: (uL)

\begin{tabular}{|c|c|c|c|}
\hline CAS NO. & COMPOUND & $\begin{array}{l}\text { CONCENTRATION UNITS: } \\
(\mathrm{ug} / \mathrm{L} \text { or } \mathrm{ug} / \mathrm{kg}) \mathrm{ug} / \mathrm{L}\end{array}$ & $Q$ \\
\hline $75-71-8$ & Dichlorodifluoromethane & 0.50 & $\overline{\mathrm{U}}$ \\
\hline $74-87-3$ & Chloromethane & 0.50 & $\mathrm{U}$ \\
\hline $75-01-4$ & Vinyl chloride & 0.50 & $\mathrm{U}$ \\
\hline $74-83-9$ & Bromomethane & 0.50 & $\mathrm{U}$ \\
\hline $75-00-3$ & Chloroethane & 0.50 & $\mathrm{U}$ \\
\hline $75-69-4$ & Trichlorofluoromethane & 0.50 & $\mathrm{U}$ \\
\hline $75-35-4$ & 1,1-Dichloroethene & 0.50 & $\mathrm{U}$ \\
\hline $76-13-1$ & 1,1,2-Trichloro-1,2,2-trifluoroethane & 0.50 & $\mathrm{U}$ \\
\hline $67-64-1$ & Acetone & 2.3 & $\mathrm{~J}$ \\
\hline $75-15-0$ & Carbon disulfide & 0.50 & $\mathrm{U}$ \\
\hline $79-20-9$ & Methyl acetate & 0.50 & $\mathrm{U}$ \\
\hline $75-09-2$ & Methylene Chloride & 0.50 & $\mathrm{U}$ \\
\hline $156-60-5$ & trans-1,2-Dichloroethene & 0.50 & $\mathrm{U}$ \\
\hline $1634-04-4$ & Methyl tert-butyl ether & 0.50 & $\mathrm{U}$ \\
\hline $75-34-3$ & 1,1-Dichloroethane & 0.50 & $\mathrm{U}$ \\
\hline $156-59-2$ & cis-1,2-Dichloroethene & 0.50 & $\mathrm{U}$ \\
\hline $78-93-3$ & 2-Butanone & 5.0 & $\mathrm{U}$ \\
\hline $74-97-5$ & Bromochloromethane & 0.50 & $\mathrm{U}$ \\
\hline $67-66-3$ & Chloroform & 0.19 & $\mathrm{~J}$ \\
\hline $71-55-6$ & 1,1,1-Trichloroethane & 0.50 & $\mathrm{U}$ \\
\hline $110-82-7$ & Cyclohexane & 0.50 & $\mathrm{U}$ \\
\hline $56-23-5$ & Carbon tetrachloride & 0.14 & $\mathrm{~J}$ \\
\hline $71-43-2$ & Benzene & 0.073 & $\mathrm{~J}$ \\
\hline $107-06-2$ & 1,2-Dichloroethane & 0.50 & $\mathrm{U}$ \\
\hline
\end{tabular}

Report 1,4-Dioxane for Low-Medium VOA analysis only 
1B - FORM I VOA-2

VOLATILE ORGANICS ANALYSIS DATA SHEET
EPA SAMPLE NO.

INMW18-W-32753

Lab Name: TESTAMERICA BURLINGTON

Lab Code: STLV Case No.: INMA Matrix: (SOIL/SED/WATER)

Sample wt/vol: 25.0

Water

$(\mathrm{g} / \mathrm{mL}) \quad \mathrm{mL}$

Level: (TRACE/LOW/MED) TRACE

\% Moisture: not dec.

GC Column: DB-624 ID: 0.20 (mm)

Soil Extract Volume: (uL)

(mL)

Purge Volume: 25.0
Contract: $1 \mathrm{E}-30401$

SDG No.: $200-6762$
Lab Sample ID: 200-6762-6

Lab File ID: DHPV10.D

Date Received: 08/30/2011

Date Analyzed: 09/01/2011

Dilution Factor: 1.0

Soil Aliquot volume:

(uL)

\begin{tabular}{|c|c|c|c|}
\hline CAS NO. & COMPOUND & $\begin{array}{l}\text { CONCENTRATION UNITS: } \\
(\mathrm{ug} / \mathrm{L} \text { or } \mathrm{ug} / \mathrm{kg}) \mathrm{ug} / \mathrm{L}\end{array}$ & $Q$ \\
\hline $79-01-6$ & Trichloroethene & 0.50 & $\bar{U}$ \\
\hline $108-87-2$ & Methylcyclohexane & 0.50 & U \\
\hline $78-87-5$ & 1,2-Dich1oropropane & 0.50 & $\mathrm{U}$ \\
\hline $75-27-4$ & Bromodichloromethane & 0.50 & $\mathrm{U}$ \\
\hline $10061-01-5$ & cis-1,3-Dichloropropene & 0.50 & $\mathrm{U}$ \\
\hline $108-10-1$ & 4-Methyl-2-pentanone & 5.0 & $\mathrm{U}$ \\
\hline $108-88-3$ & Toluene & 0.050 & $\mathrm{~J} B$ \\
\hline $10061-02-6$ & trans-1,3-Dichloropropene & 0.50 & $\mathrm{U}$ \\
\hline $79-00-5$ & 1,1,2-Trichloroethane & 0.50 & $\mathrm{U}$ \\
\hline $127-18-4$ & Tetrachloroethene & 0.50 & $\mathrm{U}$ \\
\hline $591-78-6$ & 2-Hexanone & 5.0 & $\mathrm{U}$ \\
\hline $124-48-1$ & Dibromochloromethane & 0.50 & $\mathrm{U}$ \\
\hline $106-93-4$ & 1,2-Dibromoethane & 0.50 & $\mathrm{U}$ \\
\hline $108-90-7$ & Chlorobenzene & 0.50 & $\mathrm{U}$ \\
\hline $100-41-4$ & Ethylbenzene & 0.50 & $\mathrm{U}$ \\
\hline $95-47-6$ & o-Xylene & 0.50 & $\mathrm{U}$ \\
\hline $179601-23-1$ & $\mathrm{~m}, \mathrm{p}$-Xylene & 0.010 & $\mathrm{~J}$ \\
\hline $100-42-5$ & Styrene & 0.50 & $\mathrm{U}$ \\
\hline $75-25-2$ & Bromoform & 0.50 & $\mathrm{U}$ \\
\hline $98-82-8$ & Isopropylbenzene & 0.50 & $\mathrm{U}$ \\
\hline $79-34-5$ & $1,1,2,2$-Tetrachloroethane & 0.50 & $\mathrm{U}$ \\
\hline $541-73-1$ & 1,3-Dichlorobenzene & 0.50 & $\mathrm{U}$ \\
\hline $106-46-7$ & 1,4-Dichlorobenzene & 0.50 & $\mathrm{U}$ \\
\hline $95-50-1$ & 1,2-Dichlorobenzene & 0.50 & $\mathrm{U}$ \\
\hline $96-12-8$ & 1,2-Dibromo-3-Chloropropane & 0.50 & U \\
\hline $120-82-1$ & 1,2,4-Trichlorobenzene & 0.50 & $\mathrm{U}$ \\
\hline $87-61-6$ & 1,2,3-Trichlorobenzene & 0.50 & $\mathrm{U}$ \\
\hline
\end{tabular}


$1 \mathrm{~J}$ - FORM I VOA-TIC

VOLATILE ORGANICS ANALYSIS DATA SHEET

TENTATIVELY IDENTIFIED COMPOUNDS
EPA SAMPLE NO.

INMW $18-W-32753$

\begin{tabular}{|c|c|c|c|c|c|c|c|c|c|}
\hline Lab Name: TES & AMERICA BUF & RLINGTON & & Contr & act: & -30401 & & & \\
\hline Lab Code: STL & Case & No.: INMAN & Mod. Ref & No.: & & SDG No.: & $200-6$ & 762 & \\
\hline Matrix: (SOIL/ & SED/WATER) & Water & & Lab $S$ & Sample ID: & $200-676$ & $52-6$ & & \\
\hline Sample wt/vol: & 25.0 & $(\mathrm{~g} / \mathrm{mL}) \quad \mathrm{mL}$ & & Lab $\mathrm{E}$ & ile ID: & DHPV10.D & & & \\
\hline Level: (TRACE & L LOW/MED) & TRACE & & Date & Received: & $08 / 30 / 2$ & 011 & & \\
\hline \% Moisture: no & $=$ dec. & & & Date & Analyzed: & $09 / 01 / 2$ & 011 & & \\
\hline GC Column: DB & 624 & ID: 0.20 & $(\mathrm{~mm})$ & Dilut & ion Facto & or: 1.0 & & & \\
\hline Soil Extract $V$ & lume: & & (uL) & Soil & Aliquot V & Volume: & & & (uL) \\
\hline CONCENTRATION & JNITS: (ug/L & or $u g / k g$ ) & $\mathrm{ug} / \mathrm{L}$ & Purge & Volume: & 25.0 & & & $(\mathrm{~mL})$ \\
\hline CAS NUMBER & & COMPOUND & NAME & & $\mathrm{RT}$ & EST. CO & $\mathrm{ONC}$. & & $Q$ \\
\hline & Unknown & & & & 6.79 & & 2.9 & & $\mathrm{X} \mathrm{J}$ \\
\hline $66-25-1$ & Hexanal & & & & 8.12 & & 0.91 & & $\mathrm{~N}$ \\
\hline & Unknown & & & & 9.93 & & 1.2 & $\mathrm{~J}$ & \\
\hline & Unknown & & & & 10.76 & & 1.3 & $\mathrm{~J}$ & \\
\hline & Unknown & & & & 11.99 & & 3.2 & $\mathrm{~J}$ & \\
\hline $124-19-6$ & Nonanal & & & & 12.91 & & 1.3 & & $\mathrm{~N}$ \\
\hline & Unknown & & & & 13.70 & & 4.9 & $\mathrm{~J}$ & \\
\hline E9667961 & Total Alk & anes & & & $\mathrm{N} / \mathrm{A}$ & & & & \\
\hline
\end{tabular}

1 EPA-designated Registry Number. 
$1 \mathrm{~A}$ - FORM I VOA-1

VOLATILE ORGANICS ANALYSIS DATA SHEET
EPA SAMPLE NO. INMW18-W-32754
Iab Name: TESTAMERICA BURLINGTON

Lab Code: STLV Case

Matrix: (SOIL/SED/WATER)

Water

Sample wt/vol: 25.0

$(\mathrm{g} / \mathrm{mL}) \mathrm{mL}$

Level: (TRACE/LOW/MED) TRACE

\% Moisture: not dec.

GC Column: DB-624

ID: $0.20 \quad(\mathrm{~mm})$

Soil Extract Volume:

(uL)

(mL)

Purge Volume: 25.0
Contract: $1 \mathrm{E}-30401$

SDG No.: $200-6762$

\begin{tabular}{|c|c|c|c|}
\hline CAS NO. & COMPOUND & $\begin{array}{l}\text { CONCENTRATION UNITS: } \\
(\mathrm{ug} / \mathrm{L} \text { or } \mathrm{ug} / \mathrm{kg} \text { ) ug/L }\end{array}$ & $Q$ \\
\hline $75-71-8$ & Dichlorodifluoromethane & 0.50 & $\mathrm{U}$ \\
\hline $74-87-3$ & Chloromethane & 0.50 & $\mathrm{U}$ \\
\hline $75-01-4$ & Vinyl chloride & 0.50 & $\mathrm{U}$ \\
\hline $74-83-9$ & Bromomethane & 0.50 & $\mathrm{U}$ \\
\hline $75-00-3$ & Chloroethane & 0.50 & $\mathrm{U}$ \\
\hline $75-69-4$ & Trichlorofluoromethane & 0.50 & $\mathrm{U}$ \\
\hline $75-35-4$ & 1,1-Dichloroethene & 0.50 & $\mathrm{U}$ \\
\hline $76-13-1$ & 1,1,2-Trichloro-1,2,2-trifluoroethane & 0.50 & $\mathrm{U}$ \\
\hline $67-64-1$ & Acetone & 2.3 & $\mathrm{~J}$ \\
\hline $75-15-0$ & Carbon disulfide & 0.50 & $\mathrm{U}$ \\
\hline $79-20-9$ & Methyl acetate & 0.50 & $\mathrm{U}$ \\
\hline $75-09-2$ & Methylene Chloride & 0.50 & $\mathrm{U}$ \\
\hline $156-60-5$ & trans-1,2-Dichloroethene & 0.50 & $\mathrm{U}$ \\
\hline $1634-04-4$ & Methyl tert-butyl ether & 0.016 & $\mathrm{~J}$ \\
\hline $75-34-3$ & 1,1-Dichloroethane & 0.50 & $\mathrm{U}$ \\
\hline $156-59-2$ & cis-1,2-Dichloroethene & 0.50 & $\mathrm{U}$ \\
\hline $78-93-3$ & 2-Butanone & 5.0 & $\mathrm{U}$ \\
\hline $74-97-5$ & Bromochloromethane & 0.50 & $\mathrm{U}$ \\
\hline $67-66-3$ & Chloroform & 0.98 & \\
\hline $71-55-6$ & 1,1,1-Trichloroethane & 0.50 & $\mathrm{U}$ \\
\hline $110-82-7$ & Cyclohexane & 0.50 & $\mathrm{U}$ \\
\hline $56-23-5$ & Carbon tetrachloride & 0.34 & $\mathrm{~J}$ \\
\hline $71-43-2$ & Benzene & 0.17 & $\mathrm{~J}$ \\
\hline $107-06-2$ & 1,2-Dichloroethane & 0.50 & $\mathrm{U}$ \\
\hline
\end{tabular}

Report 1,4-Dioxane for Low-Medium VOA analysis only

Lab Sample ID: 200-6762-7

Lab Eile ID: DHPV11.D

Date Received: $08 / 30 / 2011$

Date Analyzed: 09/01/2011

Dilution Factor: 1.0

Soil Aliquot Volume:

(uL) 
IB - FORM I VOA-2

VOLATILE ORGANICS ANALYSIS DATA SHEET
EPA SAMPLE NO.

INMW18-W-32754
Lab Name: TESTAMERICA BURLINGTON

Lab Code: STLV Case No.: INMA

Matrix: (SOIL/SED/WATER)

Water

Sample wt/vol: 25.0

$(\mathrm{g} / \mathrm{mL}) \quad \mathrm{mL}$

Level: (TRACE/LOW/MED) TRACE

\% Moisture: not dec.

GC Column: DB-624

ID $: 0.20$ (mm)

Soil Extract Volume:

(uL)

$(\mathrm{mL})$

Purge Volume: 25.0

Contract: 1E-30401

SDG No.: 200-6762

Lab Sample ID: 200-6762-7

Lab File ID: DHPV11.D

Date Received: $08 / 30 / 2011$

Date Analyzed: 09/01/2011

Dilution Factor: 1.0

Soil Aliquot Volume:

(uL)

\begin{tabular}{|c|c|c|c|}
\hline CAS NO. & COMPOUND & $\begin{array}{l}\text { CONCENTRATION UNITS: } \\
(\mathrm{ug} / \mathrm{L} \text { or } \mathrm{ug} / \mathrm{kg}) \mathrm{ug} / \mathrm{L}\end{array}$ & $Q$ \\
\hline $79-01-6$ & Trichloroethene & 0.50 & $\bar{U}$ \\
\hline $108-87-2$ & Methylcyclohexane & 0.50 & $\mathrm{U}$ \\
\hline $78-87-5$ & 1,2-Dichloropropane & 0.50 & $\mathrm{U}$ \\
\hline $75-27-4$ & Bromodichloromethane & 0.50 & $\mathrm{U}$ \\
\hline $10061-01-5$ & cis-1,3-Dichloropropene & 0.50 & $\mathrm{U}$ \\
\hline $108-10-1$ & 4-Methyl-2-pentanone & 5.0 & $\mathrm{U}$ \\
\hline $108-88-3$ & Toluene & 0.094 & $\mathrm{~J} \mathrm{~B}$ \\
\hline $10061-02-6$ & trans-1,3-Dichloropropene & 0.50 & $\mathrm{U}$ \\
\hline $79-00-5$ & 1,1,2-Trichloroethane & 0.50 & $\mathrm{U}$ \\
\hline $127-18-4$ & Tetrachloroethene & 0.075 & $\mathrm{~J}$ \\
\hline $591-78-6$ & 2-Hexanone & 5.0 & $\mathrm{U}$ \\
\hline $124-48-1$ & Dibromochloromethane & 0.50 & $\mathrm{U}$ \\
\hline $106-93-4$ & 1,2-Dibromoethane & 0.50 & $\mathrm{U}$ \\
\hline $108-90-7$ & Chlorobenzene & 0.50 & $\mathrm{U}$ \\
\hline $100-41-4$ & Ethylbenzene & 0.50 & $\mathrm{U}$ \\
\hline $95-47-6$ & o-xylene & 0.50 & $\mathrm{U}$ \\
\hline $1.79601-23-1$ & m, p-Xylene & 0.014 & $\mathrm{~J}$ \\
\hline $100-42-5$ & Styrene & 0.50 & $\mathrm{U}$ \\
\hline $75-25-2$ & Bromoform & 0.50 & $\mathrm{U}$ \\
\hline $98-82-8$ & Isopropylbenzene & 0.50 & $\mathrm{U}$ \\
\hline $79-34-5$ & $1,1,2,2$-Tetrachloroethane & 0.50 & $\mathrm{U}$ \\
\hline $541-73-1$ & 1,3-Dichlorobenzene & 0.50 & $\mathrm{U}$ \\
\hline $106-46-7$ & 1,4-Dichlorobenzene & 0.50 & $\mathrm{U}$ \\
\hline $95-50-1$ & 1,2-Dichlorobenzene & 0.50 & $\mathrm{U}$ \\
\hline $96-12-8$ & 1,2-Dibromo-3-Chloropropane & 0.50 & $\mathrm{U}$ \\
\hline $120-82-1$ & 1,2,4-Trichlorobenzene & 0.50 & $\mathrm{U}$ \\
\hline $87-61-6$ & 1,2,3-Trichlorobenzene & 0.50 & $\mathrm{U}$ \\
\hline
\end{tabular}


$1 \mathrm{~J}$ - FORM I VOA-TIC

VOLATILE ORGANICS ANALYSIS DATA SHEET

TENTATIVELY IDENTIFIED COMPOUNDS
EPA SAMPLE NO.

INMW18-W-32754

Lab Name: TESTAMERICA BURLINGTON Case No.: INMAN Mod. Ref No.:

Contract: $1 \mathrm{E}-30401$

Lab Code: STLV

Water

Matrix: (SOIL/SED/WATER)

Sample wt/vol: 25.0

Level: (TRACE or LOW/MED)

$(\mathrm{g} / \mathrm{mL}) \quad \mathrm{mL}$

o Moisture: not dec.

GC Column: DB-624

Soil Extract Volume:

ID $: 0.20$

(mm)

(UL)

CONCENTRATION UNITS: (ug/L or $\mathrm{ug} / \mathrm{kg}$ ) ug/L
SDG No.: 200-6762

Lab Sample ID: 200-6762-7

Lab File ID: DHPV11.D

Date Received: 08/30/2011

Date Analyzed: 09/01/2011

Dilution Factor: 1.0

Soil Aliquot Volume: (uL)

Purge Volume: 25.0

(mL)

\begin{tabular}{|c|c|c|c|c|}
\hline CAS NUMBER & COMPOUND NAME & $\mathrm{RT}$ & EST. CONC. & Q \\
\hline 01 & Unknown & 6.79 & 3.1 & $\mathrm{BX} \mathrm{J}$ \\
\hline 02 & Unknown & 8.12 & 1.4 & $\mathrm{~J}$ \\
\hline 03 & Unknown & 9.93 & 2.0 & $\mathrm{~J}$ \\
\hline 04 & Unknown & 10.76 & 1.1 & $\mathrm{~J}$ \\
\hline 05 & Unknown & 11.54 & 0.72 & $\mathrm{~J}$ \\
\hline 06 & Unknown & 11.99 & 8.3 & J \\
\hline $124-19-6$ & Nonanal & 12.91 & 1.8 & $\mathrm{~J} \mathrm{~N}$ \\
\hline 08 & Unknown & 13.70 & 1.0 & $\mathrm{~J}$ \\
\hline E966796 I & Total Alkanes & $\mathrm{N} / \mathrm{A}$ & 0.66 & $\mathrm{~J}$ \\
\hline
\end{tabular}

1 EPA-designated Registry Number. 
$1 A$ - FORM I VOA-1

VOLATILE ORGANICS ANALYSIS DATA SHEET
EPA SAMPLE NO. INMW 18-W-32755
Lab Name: TESTAMERICA BURLINGTON

Lab Code: STLV Case No.: INMAN

Matrix: (SOIL/SED/WATER)

Water

Sample wt/vol: 25.0

$(\mathrm{g} / \mathrm{mL}) \quad \mathrm{mL}$

Level: (TRACE/LOW/MED) TRACE

응 Moisture: not dec.

GC Column: DB-624 ID: 0.20 (mm)

Soil Extract Volume: (uL)

(mL)

Purge Volume: 25.0
Contract: 1E-30401

SDG No.: 200-6762

Mod. Ref No.:

Lab Sample ID: 200-6762-8

Lab File ID: DHPV12.D

Date Received: 08/30/2011

Date Analyzed: 09/01/2011

Dilution Factor: 1.0

Soil Aliquot Volume:

(uL)

\begin{tabular}{|c|c|c|c|}
\hline CAS NO. & COMPOUND & $\begin{array}{l}\text { CONCENTRATION UNITS: } \\
(\mathrm{ug} / \mathrm{L} \text { or } u g / \mathrm{kg}) \mathrm{ug} / \mathrm{L}\end{array}$ & $\mathrm{Q}$ \\
\hline $75-71-8$ & Dichlorodifluoromethane & 0.50 & $\mathrm{U}$ \\
\hline $74-87-3$ & Chloromethane & 0.50 & $\mathrm{U}$ \\
\hline $75-01-4$ & Vinyl chloride & 0.50 & $\mathrm{U}$ \\
\hline $74-83-9$ & Bromomethane & 0.50 & $\mathrm{U}$ \\
\hline $75-00-3$ & Chloroethane & 0.50 & $\mathrm{U}$ \\
\hline $75-69-4$ & Trichlorofluoromethane & 0.50 & $\mathrm{U}$ \\
\hline $75-35-4$ & 1,1-Dichloroethene & 0.50 & $\mathrm{U}$ \\
\hline $76-13-1$ & 1,1,2-Trichloro-1,2,2-trifluoroethane & 0.50 & $\mathrm{U}$ \\
\hline $67-64-1$ & Acetone & 5.0 & $\mathrm{U}$ \\
\hline $75-15-0$ & Carbon disulfide & 0.50 & $\mathrm{U}$ \\
\hline $79-20-9$ & Methyl acetate & 0.50 & $\mathrm{U}$ \\
\hline $75-09-2$ & Methylene Chloride & 0.50 & $\mathrm{U}$ \\
\hline $156-60-5$ & trans-1,2-Dichloroethene & 0.50 & $\mathrm{U}$ \\
\hline $1634-04-4$ & Methyl tert-butyl ether & 0.50 & $\mathrm{U}$ \\
\hline $75-34-3$ & 1,1-Dichloroethane & 0.50 & $\mathrm{U}$ \\
\hline $156-59-2$ & cis-1,2-Dichloroethene & 0.50 & $\mathrm{U}$ \\
\hline $78-93-3$ & 2-Butanone & 5.0 & $\mathrm{U}$ \\
\hline $74-97-5$ & Bromochloromethane & 0.50 & $\mathrm{U}$ \\
\hline $67-66-3$ & Chloroform & 0.96 & \\
\hline $71-55-6$ & 1,1,1-Trichloroethane & 0.50 & $\mathrm{U}$ \\
\hline $110-82-7$ & Cyclohexane & 0.50 & $\mathrm{U}$ \\
\hline $56-23-5$ & Carbon tetrachloride & 3.0 & \\
\hline $71-43-2$ & Benzene & 0.50 & $\mathrm{U}$ \\
\hline $107-06-2$ & 1,2-Dichloroethane & 0.50 & $\mathrm{U}$ \\
\hline
\end{tabular}

Report 1,4-Dioxane for Low-Medium VOA analysis only 
$1 B$ - FORM I VOA-2

VOLATILE ORGANICS ANALYSIS DATA SHEET
EPA SAMPLE NO.

INMW18-W-32755

Lab Name: TESTAMERICA BURLINGTON

Lab Code: STLV Case No.: INMAN Mod. Ref No.:

Contract: 1E-30401 (mm) (uL)

Matrix: (SOIL/SED/WATER)

Sample wt/vol: 25.0

Level: (TRACE/LOW/MED) TRACF

o Moisture: not dec.

GC Column: DB-624 ID: 0.20

Soil Extract Volume:

Water

$(\mathrm{g} / \mathrm{mL}) \quad \mathrm{mL}$

Purge Volume: 25.0

(mL)

SDG NO.: 200-6762

Lab Sample ID: 200-6762-8

Lab File ID: DHPV12.D

Date Received: $08 / 30 / 2011$

Date Analyzed: 09/01/2011

Dilution Factor: 1.0

Soil Aliquot Volume:

(uI)

\begin{tabular}{|c|c|c|c|}
\hline CAS NO. & COMPOUND & $\begin{array}{l}\text { CONCENTRATION UNITS: } \\
(u g / L \text { or ug/kg) ug/L }\end{array}$ & $Q$ \\
\hline $79-01-6$ & Trichloroethene & 0.50 & $\bar{U}$ \\
\hline $108-87-2$ & Methylcyclohexane & 0.50 & $\mathrm{U}$ \\
\hline $78-87-5$ & 1,2-Dichloropropane & 0.50 & $\mathrm{U}$ \\
\hline $75-27-4$ & Bromodichloromethane & 0.50 & $\mathrm{U}$ \\
\hline $10061-01-5$ & cis-1,3-Dichloropropene & 0.50 & $\mathrm{U}$ \\
\hline $108-10-1$ & 4-Methyl-2-pentanone & 5.0 & $\mathrm{U}$ \\
\hline $108-88-3$ & Toluene & 0.016 & $J \mathrm{~B}$ \\
\hline $10061-02-6$ & trans-1,3-Dichloropropene & 0.50 & $\mathrm{U}$ \\
\hline $79-00-5$ & 1,1,2-Trichloroethane & 0.50 & $\mathrm{U}$ \\
\hline $127-18-4$ & Tetrachloroethene & 0.31 & $\mathrm{~J}$ \\
\hline $591-78-6$ & 2-Hexanone & 5.0 & $\mathrm{U}$ \\
\hline $124-48-1$ & Dibromochloromethane & 0.50 & U \\
\hline $106-93-4$ & 1,2-Dibromoethane & 0.50 & U \\
\hline $108-90-7$ & Chlorobenzene & 0.50 & $\mathrm{U}$ \\
\hline $100-41-4$ & Ethylbenzene & 0.50 & $\mathrm{U}$ \\
\hline $95-47-6$ & o-Xylene & 0.50 & $\mathrm{U}$ \\
\hline $179601-23-1$ & m,p-Xylene & 0.50 & $\mathrm{U}$ \\
\hline $100-42-5$ & Styrene & 0.50 & $\mathrm{U}$ \\
\hline $75-25-2$ & Bromoform & 0.50 & $\mathrm{U}$ \\
\hline $98-82-8$ & Isopropylbenzene & 0.50 & $\mathrm{U}$ \\
\hline $79-34-5$ & $1,1,2,2$-Tetrachloroethane & 0.50 & $\mathrm{U}$ \\
\hline $541-73-1$ & 1,3-Dichlorobenzene & 0.50 & $\mathrm{U}$ \\
\hline $106-46-7$ & 1,4-Dichlorobenzene & 0.50 & $\mathrm{U}$ \\
\hline $95-50-1$ & 1,2-Dichlorobenzene & 0.50 & $\mathrm{U}$ \\
\hline $96-12-8$ & 1,2-Dibromo-3-Chloropropane & 0.50 & $\mathrm{U}$ \\
\hline $120-82-1$ & 1,2,4-Trichlorobenzene & 0.50 & $\mathrm{U}$ \\
\hline $87-61-6$ & 1,2,3-Trichlorobenzene & 0.50 & $\mathrm{U}$ \\
\hline
\end{tabular}


1J - FORM I VOA-TIC

VOLATILE ORGANICS ANALYSIS DATA SHEET TENTATIVELY IDENTIFIED COMPOUNDS
EPA SAMPLE NO.

INMW18-W-32755

Lab Name: TESTAMERICA BURLINGTON

Contract: $1 \mathrm{E}-30401$

Lab Code: STLV Case No.: INMAN Mod. Ref No.: SDG No.: 200-6762

Matrix: (SOIL/SED/WATER)

Water

Sample wt/vol: 25.0

Level: (TRACE or LOW/MED)

$(\mathrm{g} / \mathrm{mL}) \quad \mathrm{mL}$

\% Moisture: not dec.

GC Column: DB-624

ID: 0.20

(mm)

Soil Extract Volume: (uL)

CONCENTRATION UNITS: (ug/L or ug/kg) ug/L
Lab Sample ID: 200-6762-8

Lab File ID: DHPV12.D

Date Received: 08/30/2011

Date Analyzed: 09/01/2011

Dilution Factor: 1.0

Soil Aliquot Volume: (uL)

Purge Volume: 25.0 (mL)

\begin{tabular}{|l|l|l|c|c|c|}
\hline \multicolumn{1}{|c|}{ CAS NUMBER } & \multicolumn{1}{|c|}{ COMPOUND NAME } & RT & EST. CONC. & Q \\
\cline { 2 - 6 } 01 & & Unknown & 6.79 & B X J \\
\cline { 2 - 6 } & E966796 l & Total Alkanes & N/A & & \\
\hline
\end{tabular}

IEPA-designated Registry Number. 
Lab Name: TESTAMERICA BURLINGTON

Lab Code: STLV

Contract: 1E-30401

Matrix: (SOIL/SED/WATER) Water

Sample wt/vol: 25.0 $(\mathrm{g} / \mathrm{mL}) \quad \mathrm{mL}$

Level: (TRACE/LOW/MED) TRACE

\% Moisture: not dec.

GC Column: DB-624 ID $: 0.20$

Soil Extract Volume:

(uL)

(mL)

Purge Volume: 25.0
SDG No.: 200-6762

Lab Sample ID: 200-6762-9

Lab File ID: DHPV13.D

Date Received: 08/30/2011

Date Analyzed: 09/01/2011

Dilution Factor: 1.0

Soil Aliquot Volume:

\begin{tabular}{|c|c|c|c|}
\hline CAS NO. & COMPOUND & $\begin{array}{l}\text { CONCENTRATION UNITS: } \\
(\mathrm{ug} / \mathrm{L} \text { or } \mathrm{ug} / \mathrm{kg}) \mathrm{ug} / \mathrm{L}\end{array}$ & Q \\
\hline $75-71-8$ & Dichlorodifluoromethane & 0.50 & $\bar{U}$ \\
\hline $74-87-3$ & Chloromethane & 0.50 & $\mathrm{U}$ \\
\hline $75-01-4$ & Vinyl chloride & 0.50 & $\mathrm{U}$ \\
\hline $74-83-9$ & Bromomethane & 0.50 & $\mathrm{U}$ \\
\hline $75-00-3$ & Chloroethane & 0.50 & $\mathrm{U}$ \\
\hline $75-69-4$ & Trichlorofluoromethane & 0.50 & $\mathrm{U}$ \\
\hline $75-35-4$ & 1,1-Dichloroethene & 0.50 & $\mathrm{U}$ \\
\hline $76-13-1$ & 1,1,2-Trichloro-1,2,2-trifluoroethane & 0.50 & $\mathrm{U}$ \\
\hline $67-64-1$ & Acetone & 1.6 & $\mathrm{~J}$ \\
\hline $75-15-0$ & Carbon disulfide & 0.50 & $\mathrm{U}$ \\
\hline $79-20-9$ & Methyl acetate & 0.50 & $\mathrm{U}$ \\
\hline $75-09-2$ & Methylene Chloride & 0.50 & $\mathrm{U}$ \\
\hline $156-60-5$ & trans-1,2-Dichloroethene & 0.50 & $\mathrm{U}$ \\
\hline $1634-04-4$ & Methyl tert-butyl ether & 0.50 & $\mathrm{U}$ \\
\hline $75-34-3$ & 1,1-Dichloroethane & 0.50 & $\mathrm{U}$ \\
\hline $156-59-2$ & cis-1,2-Dichloroethene & 0.50 & $\mathrm{U}$ \\
\hline $78-93-3$ & 2-Butanone & 5.0 & $\mathrm{U}$ \\
\hline $74-97-5$ & Bromochloromethane & 0.50 & $\mathrm{U}$ \\
\hline $67-66-3$ & Chloroform & 0.30 & $\mathrm{~J}$ \\
\hline $71-55-6$ & 1,1,1-Trichloroethane & 0.50 & $\mathrm{U}$ \\
\hline $110-82-7$ & Cyclohexane & 0.50 & $\mathrm{U}$ \\
\hline $56-23-5$ & Carbon tetrachloride & 1.2 & \\
\hline $71-43-2$ & Benzene & 0.18 & $\mathrm{~J}$ \\
\hline $107-06-2$ & 1,2-Dichloroethane & 0.50 & $\mathrm{U}$ \\
\hline
\end{tabular}

Report 1,4-Dioxane for Low-Medium VOA analysis only 
Lab Name: TESTAMERICA BURLINGTON

Lab Code: STLV

Matrix: (SOIL/SED/WATER)

Sample wt/vol: 25.0

Water

$(\mathrm{g} / \mathrm{mL}) \quad \mathrm{mL}$

Level: (TRACE/LOW/MED) TRACE

\% Moisture: not dec.

GC Column: DB-624 ID: 0.20 (mm)

Soil Extract Volume: (UL)

Purge Volume: 25.0

(mL)
Contract: $1 E-30401$

SDG No.: 200-6762

Lab Sample ID: 200-6762-9

Lab File ID: DHPV13.D

Date Received: 08/30/2011

Date Analyzed: 09/01/2011

Dilution Factor: 1.0

Soil Aliquot Volume:

(uL)

\begin{tabular}{|c|c|c|c|}
\hline CAS NO. & COMPOUND & $\begin{array}{l}\text { CONCENTRATION UNITS: } \\
(\mathrm{ug} / \mathrm{L} \text { or } \mathrm{ug} / \mathrm{kg}) \mathrm{ug} / \mathrm{L}\end{array}$ & Q \\
\hline $79-01-6$ & Trichloroethene & 0.50 & $\bar{U}$ \\
\hline $108-87-2$ & Methylcyclohexane & 0.50 & $\mathrm{U}$ \\
\hline $78-87-5$ & 1,2-Dichloropropane & 0.50 & $\mathrm{U}$ \\
\hline $75-27-4$ & Bromodichloromethane & 0.50 & $\mathrm{U}$ \\
\hline $10061-01-5$ & cis-1,3-Dichloropropene & 0.50 & $\mathrm{U}$ \\
\hline $108-10-1$ & 4-Methyl-2-pentanone & 5.0 & $\mathrm{U}$ \\
\hline $108-88-3$ & Toluene & 0.11 & $\mathrm{~J} B$ \\
\hline $10061-02-6$ & trans-1,3-Dichloropropene & 0.50 & $\mathrm{U}$ \\
\hline $79-00-5$ & 1,1,2-Trichloroethane & 0.50 & $\mathrm{U}$ \\
\hline $127-18-4$ & Tetrachloroethene & 0.50 & $\mathrm{U}$ \\
\hline $591-78-6$ & 2-Hexanone & 5.0 & $\mathrm{U}$ \\
\hline $124-48-1$ & Dibromochloromethane & 0.50 & $\mathrm{U}$ \\
\hline $106-93-4$ & 1,2-Dibromoethane & 0.50 & $\mathrm{U}$ \\
\hline $108-90-7$ & Chlorobenzene & 0.50 & $\mathrm{U}$ \\
\hline $100-41-4$ & Ethylbenzene & 0.50 & $\mathrm{U}$ \\
\hline $95-47-6$ & o-Xylene & 0.50 & $\mathrm{U}$ \\
\hline $179601-23-1$ & m,p-Xylene & 0.011 & $\mathrm{~J}$ \\
\hline $100-42-5$ & Styrene & 0.017 & $\mathrm{~J}$ \\
\hline $75-25-2$ & Bromoform & 0.50 & $\mathrm{U}$ \\
\hline $98-82-8$ & Isopropylbenzene & 0.50 & $\mathrm{U}$ \\
\hline $79-34-5$ & $1,1,2,2$-Tetrachloroethane & 0.50 & $\mathrm{U}$ \\
\hline $541-73-1$ & 1,3-Dichlorobenzene & 0.50 & $\mathrm{U}$ \\
\hline $106-46-7$ & 1,4-Dichlorobenzene & 0.50 & $\mathrm{U}$ \\
\hline $95-50-1$ & 1,2-Dichlorobenzene & 0.50 & $\mathrm{U}$ \\
\hline $96-12-8$ & 1,2-Dibromo-3-Chloropropane & 0.50 & $\mathrm{U}$ \\
\hline $120-82-1$ & 1,2,4-Trichlorobenzene & 0.50 & $\mathrm{U}$ \\
\hline $87-61-6$ & 1,2,3-Trichlorobenzene & 0.50 & $\mathrm{U}$ \\
\hline
\end{tabular}


$1 \mathrm{~J}$ - FORM I VOA-TIC

VOIATILE ORGANICS ANALYSTS DATA SHEET

TENTATIVELY IDENTIFIED COMPOUNDS
EPA SAMPLE NO.

INMW18-W-32760
Lab Name: TESTAMERICA BURLINGTON

Contract: 1E-30401
Case No.: INMAN

Mod. Ref No.:
SDG No.: 200-6762

Lab Sample ID: 200-6762-9

Lab File ID: DHPV13.D

Date Received: 08/30/2011

Date Analyzed: 09/01/2011

Dilution Factor: 1.0

Soil Aliquot Volume:

Purge Volume: 25.0 (mL)

CONCENTRATION UNITS: (ug/L or $\mathrm{ug} / \mathrm{kg}$ ) $\mathrm{ug} / \mathrm{L}$

(uL)
EST . CONC

01

02

03

04

05

06

07

\begin{tabular}{|c|c|c|c|c|}
\hline CAS NUMBER & COMPOUND NAME & $\mathrm{RT}$ & EST. CONC. & $Q$ \\
\hline & Unknown & 6.79 & 3.3 & $\mathrm{~B} \mathrm{X} \mathrm{J}$ \\
\hline $66-25-1$ & Hexanal & 8.12 & 1.0 & $\mathrm{JN}$ \\
\hline & Un known & 9.93 & 1.3 & $\mathrm{~J}$ \\
\hline & Unknown & 10.77 & 0.94 & $\mathrm{~J}$ \\
\hline & Unknown & 11.99 & 1.2 & $\mathrm{~J}$ \\
\hline & Unknown & 12.91 & 0.54 & $\mathrm{~J}$ \\
\hline E9667961 & Total Alkanes & $\mathrm{N} / \mathrm{A}$ & 0.51 & $\mathrm{~J}$ \\
\hline
\end{tabular}

1 EPA-designated Registry Number. 
$1 A$ - FORM I VOA-1

VOLATILE ORGANICS ANALYSIS DATA SHEET
EPA SAMPLE NO.

INMW18-W-32761

Lab Name: TESTAMERICA BURLINGTON

Contract: 1E-30401

Lab Code: STLV Case No.: INMAN Mod. Ref No.: SDG No.: 200-6762

Matrix: (SOIL/SED/WATER) Water

Sample wt/vol: 25.0

$(\mathrm{g} / \mathrm{mL}) \mathrm{mL}$

Level: (TRACE/LOW/MED) TRACE

o Moisture: not dec.

GC Column: DB-624 ID: $0.20 \quad(\mathrm{~mm})$

Soil Extract Volume: (uL)

Lab Sample ID: 200-6762-10

Purge Volume: 25.0 (mL)

Lab File ID: DHPV14.D

Date Received: 08/30/2011

Date Analyzed: 09/01/2011

Dilution Factor: 1.0

Soil Aliquot volume:

(uL)

\begin{tabular}{|c|c|c|c|}
\hline CAS NO. & COMPOUND & $\begin{array}{l}\text { CONCENTRATION UNITS: } \\
(\mathrm{ug} / \mathrm{L} \text { or } \mathrm{ug} / \mathrm{kg}) \mathrm{ug} / \mathrm{L}\end{array}$ & Q \\
\hline $75-71-8$ & Dichlorodifluoromethane & 0.50 & $\overline{\mathrm{U}}$ \\
\hline $74-87-3$ & Chloromethane & 0.50 & $\mathrm{U}$ \\
\hline $75-01-4$ & Vinyl chloride & 0.50 & $\mathrm{U}$ \\
\hline $74-83-9$ & Bromomethane & 0.50 & $\mathrm{U}$ \\
\hline $75-00-3$ & Chloroethane & 0.50 & $\mathrm{U}$ \\
\hline $75-69-4$ & Trichlorofluoromethane & 0.50 & $\mathrm{U}$ \\
\hline $75-35-4$ & 1,1-Dichloroethene & 0.50 & $\mathrm{U}$ \\
\hline $76-13-1$ & 1,1,2-Trichloro-1,2,2-trifluoroethane & 0.50 & $\mathrm{U}$ \\
\hline $67-64-1$ & Acetone & 1.6 & $\mathrm{~J}$ \\
\hline $75-15-0$ & Carbon disulfide & 0.50 & $\mathrm{U}$ \\
\hline $79-20-9$ & Methyl acetate & 0.50 & $\mathrm{U}$ \\
\hline $75-09-2$ & Methylene Chloride & 0.50 & $\mathrm{U}$ \\
\hline $156-60-5$ & trans-1,2-Dichloroethene & 0.50 & $\mathrm{U}$ \\
\hline $1634-04-4$ & Methyl tert-butyl ether & 0.50 & $\mathrm{U}$ \\
\hline $75-34-3$ & 1,1-Dichloroethane & 0.50 & $\mathrm{U}$ \\
\hline $156-59-2$ & cis-1,2-Dichloroethene & 0.50 & $\mathrm{U}$ \\
\hline $78-93-3$ & 2-Butanone & 5.0 & U \\
\hline $74-97-5$ & Bromochloromethane & 0.50 & U \\
\hline $67-66-3$ & Chloroform & 0.33 & $\mathrm{~J}$ \\
\hline $71-55-6$ & 1,1,1-Trichloroethane & 0.50 & $\mathrm{U}$ \\
\hline $110-82-7$ & Cyclohexane & 0.50 & $\mathrm{U}$ \\
\hline $56-23-5$ & Carbon tetrachloride & 1.3 & \\
\hline $71-43-2$ & Benzene & 0.16 & $\mathrm{~J}$ \\
\hline $107-06-2$ & 1,2-Dichloroethane & 0.50 & $\mathrm{U}$ \\
\hline
\end{tabular}

Report 1,4-Dioxane for Low-Medium VOA analysis only 
$1 B$ - FORM I VOA-2

VOLATILE ORGANICS ANALYSIS DATA SHEET
EPA SAMPLE NO.

INMW $18-W-32761$
Lab Name: TESTAMERICA BURLINGTON

Lab Code: STLV

Matrix: (SOIL/SED/WATER)

Sample wt/vol: 25.0

Case No.: INMAN Mod. Ref No.:

Contract: $1 \mathrm{E}-30401$

Level: (TRACE/LOW/MED) TRACE

\% Moisture: not dec.

GC Column: DB-624 ID: 0.20 (mm)

Soil Extract Volume: (uL) $(\mathrm{g} / \mathrm{mL}) \mathrm{mL}$

Purge Volume: 25.0 (mL)
Lab Sample ID: 200-6762-10

Lab File ID: DHPV14.D

SDG No.: 200-6762

Date Received: 08/30/2011

Date Analyzed: 09/01/2011

Dilution Factor: 1.0

Soil Aliquot Volume:

(uL)

\begin{tabular}{|c|c|c|c|}
\hline CAS NO. & COMPOUND & $\begin{array}{l}\text { CONCENTRATION UNITS: } \\
(\mathrm{ug} / \mathrm{L} \text { or } \mathrm{ug} / \mathrm{kg}) \mathrm{ug} / \mathrm{L}\end{array}$ & $Q$ \\
\hline $79-01-6$ & Trichloroethene & 0.50 & $\overline{\mathrm{U}}$ \\
\hline $108-87-2$ & Methylcyclohexane & 0.50 & $\mathrm{U}$ \\
\hline $78-87-5$ & 1,2-Dichloropropane & 0.50 & U \\
\hline $75-27-4$ & Bromodichloromethane & 0.50 & $\mathrm{U}$ \\
\hline $10061-01-5$ & cis-1,3-Dichloropropene & 0.50 & $\mathrm{U}$ \\
\hline $108-10-1$ & 4-Methyl-2-pentanone & 5.0 & $\mathrm{U}$ \\
\hline $108-88-3$ & Toluene & 0.10 & $\mathrm{~J} B$ \\
\hline $10061-02-6$ & trans-1,3-Dichloropropene & 0.50 & $\mathrm{U}$ \\
\hline $79-00-5$ & 1,1,2-Trichloroethane & 0.50 & $\mathrm{U}$ \\
\hline $127-18-4$ & Tetrachloroethene & 0.50 & $\mathrm{U}$ \\
\hline $591-78-6$ & 2-Hexanone & 5.0 & $\mathrm{U}$ \\
\hline $124-48-1$ & Dibromochloromethane & 0.50 & $\mathrm{U}$ \\
\hline $106-93-4$ & 1,2-Dibromoethane & 0.50 & $\mathrm{U}$ \\
\hline $108-90-7$ & Chlorobenzene & 0.50 & $\mathrm{U}$ \\
\hline $100-41-4$ & Ethylbenzene & 0.011 & $\mathrm{~J}$ \\
\hline $95-47-6$ & o-xylene & 0.50 & U \\
\hline $179601-23-1$ & $\mathrm{~m}, \mathrm{p}$-xylene & 0.015 & $\mathrm{~J}$ \\
\hline $100-42-5$ & Styrene & 0.50 & $\mathrm{U}$ \\
\hline $75-25-2$ & Bromoform & 0.50 & $\mathrm{U}$ \\
\hline $98-82-8$ & Isopropylbenzene & 0.50 & $\mathrm{U}$ \\
\hline $79-34-5$ & $1,1,2,2$-Tetrachloroethane & 0.50 & $\mathrm{U}$ \\
\hline $541-73-1$ & 1,3-Dichlorobenzene & 0.50 & $\mathrm{U}$ \\
\hline $106-46-7$ & 1,4-Dichlorobenzene & 0.50 & $\mathrm{U}$ \\
\hline $95-50-1$ & 1,2-Dichlorobenzene & 0.50 & $\mathrm{U}$ \\
\hline $96-12-8$ & 1,2-Dibromo-3-Chloropropane & 0.50 & $\mathrm{U}$ \\
\hline $120-82-1$ & $1,2,4$-Trichlorobenzene & 0.50 & $\mathrm{U}$ \\
\hline $87-61-6$ & 1,2,3-Trichlorobenzene & 0.50 & $\mathrm{U}$ \\
\hline
\end{tabular}


1J - EORM I VOA-TIC

VOLATILE ORGANICS ANALYSIS DATA SHEET TENTATIVELY IDENTIFIED COMPOUNDS
EPA SAMPLE NO,

INMW18-W-32761
Lab Name: TESTAMERICA BURLINGTON Case No.: INMAN Mod. Ref No.:

Lab Code: STLV

Matrix: (SOIL/SED/WATER)

Water

Sample wt/vol: 25.0 $(\mathrm{g} / \mathrm{mL}) \mathrm{mL}$

Level: (TRACE or LOW/MED)

TRACE

\% Moisture: not dec.

GC Column: DB-624 ID : 0.20

Soil Extract Volume: (uL)

CONCENTRATION UNITS: (ug/L or ug/kg)

$\mathrm{ug} / \mathrm{L}$
Contract: $1 \mathrm{E}-30401$

SDG No.: 200-6762

Lab Sample ID: 200-6762-10

Lab File ID: DHPV14.D

Date Received: $08 / 30 / 2011$

Date Analyzed: 09/01/2011

Dilution Factor: 1.0

Soil Aliquot Volume:

Purge Volume: 25.0 (mL)

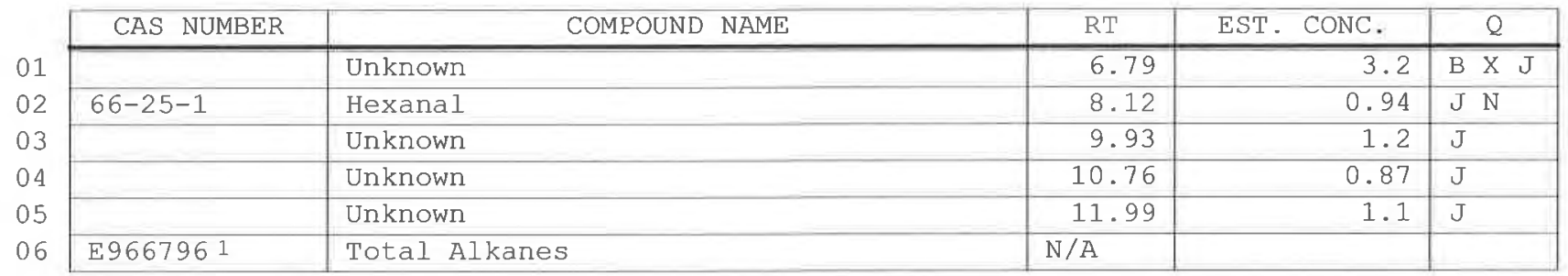

1 EPA-designated Registry Number. 
$1 A$ - FORM I VOA-1

VOLATILE ORGANICS ANALYSIS DATA SHEET
EPA SAMPLE NO.

INMW18-W-32762
Lab Name: TESTAMERICA BURLINGTON

Lab Code: STLV Case No.: INMAN

Matrix: (SOIL/SED/WATER)

Sample wt/vol: 25.0

Water

$(\mathrm{g} / \mathrm{mL}) \quad \mathrm{mL}$

Level: (TRACE/LOW/MED) TRACE

\% Moisture: not dec.

GC Column: DB-624 ID: 0.20

Soil Extract Volume:

Purge Volume: 25.0

\begin{tabular}{|c|c|c|c|}
\hline CAS NO. & COMPOUND & $\begin{array}{l}\text { CONCENTRATION UNITS: } \\
(\mathrm{ug} / \mathrm{L} \text { or } \mathrm{ug} / \mathrm{kg}) \mathrm{ug} / \mathrm{L}\end{array}$ & Q \\
\hline $75-71-8$ & Dichlorodifluoromethane & 0.50 & $\mathrm{U}$ \\
\hline $74-87-3$ & Chloromethane & 0.50 & $\mathrm{U}$ \\
\hline $75-01-4$ & Vinyl chloride & 0.50 & $\mathrm{U}$ \\
\hline $74-83-9$ & Bromomethane & 0.50 & $\mathrm{U}$ \\
\hline $75-00-3$ & Chloroethane & 0.50 & $\mathrm{U}$ \\
\hline $75-69-4$ & Trichlorofluoromethane & 0.50 & $\mathrm{U}$ \\
\hline $75-35-4$ & 1,1-Dichloroethene & 0.50 & $\mathrm{U}$ \\
\hline $76-13-1$ & 1,1,2-Trichloro-1,2,2-trifluoroethane & 0.50 & $\mathrm{U}$ \\
\hline $67-64-1$ & Acetone & 4.4 & $\mathrm{~J}$ \\
\hline $75-15-0$ & Carbon disulfide & 0.50 & $\mathrm{U}$ \\
\hline $79-20-9$ & Methyl acetate & 0.50 & $\mathrm{U}$ \\
\hline $75-09-2$ & Methylene Chloride & 0.50 & $\mathrm{U}$ \\
\hline $156-60-5$ & trans-1,2-Dichloroethene & 0.50 & $\mathrm{U}$ \\
\hline $1634-04-4$ & Methyl tert-butyl ether & 0.50 & $\mathrm{U}$ \\
\hline $75-34-3$ & 1,1-Dichloroethane & 0.50 & $\mathrm{U}$ \\
\hline $156-59-2$ & cis-1,2-Dichloroethene & 0.50 & $\mathrm{U}$ \\
\hline $78-93-3$ & 2-Butanone & 5.0 & $\mathrm{U}$ \\
\hline $74-97-5$ & Bromochloromethane & 0.50 & $\mathrm{U}$ \\
\hline $67-66-3$ & Chloroform & 0.47 & $\mathrm{~J}$ \\
\hline $71-55-6$ & 1,1,1-Trichloroethane & 0.50 & $\mathrm{U}$ \\
\hline $110-82-7$ & Cyclohexane & 0.50 & $\mathrm{U}$ \\
\hline $56-23-5$ & Carbon tetrachloride & 1.1 & \\
\hline $71-43-2$ & Benzene & 0.012 & $\mathrm{~J}$ \\
\hline $107-06-2$ & 1,2-Dichloroethane & 0.50 & $\mathrm{U}$ \\
\hline
\end{tabular}

Contract: 1E-30401

SDG No.: 200-6762

Lab Sample ID: 200-6762-11

Lab File ID: DHPV15.D

Date Received: 08/30/2011

Date Analyzed: 09/01/2011

Dilution Factor: 1.0

Soil Aliquot Volume:
(uL)

$(\mathrm{mL})$ 
$1 B$ - FORM I VOA-2

VOLATILE ORGANICS ANALYSIS DATA SHEET
EPA SAMPLE NO. INMW 18-W-32762
Lab Name: TESTAMERICA BURLINGTON

Lab Code: STLV Case No.: INMA

Matrix: (SOIL/SED/WATER) Water

Sample wt/vol: 25.0 $(\mathrm{g} / \mathrm{mL}) \quad \mathrm{mL}$

Level: (TRACE/LOW/MED) TRACE

g Moisture: not dec.

GC Column: DB-624 ID: 0.20 (mm)

Soil Extract Volume: (uL) (mL)

Purge Volume: 25.0
Contract: 1E-30401

SDG No.: 200-6762 Mod. Ref No.:

Lab Sample ID: 200-6762-11

Lab File ID: DHPV15.D

Date Received: 08/30/2011

Date Analyzed: 09/01/2011

Dilution Factor: 1.0

Soil Aliquot Volume:

(uL)

\begin{tabular}{|c|c|c|c|}
\hline CAS NO. & COMPOUND & $\begin{array}{l}\text { CONCENTRATION UNITS: } \\
(\mathrm{ug} / \mathrm{L} \text { or } \mathrm{ug} / \mathrm{kg}) \mathrm{ug} / \mathrm{L}\end{array}$ & $Q$ \\
\hline $79-01-6$ & Trichloroethene & 0.50 & $\overline{\mathrm{U} J}$ \\
\hline $108-87-2$ & Methylcyclohexane & 0.50 & U \\
\hline $78-87-5$ & 1,2-Dichloropropane & 0.50 & $\mathrm{U}$ \\
\hline $75-27-4$ & Bromodichloromethane & 0.39 & $\mathrm{~J}$ \\
\hline $10061-01-5$ & cis-1,3-Dichloropropene & 0.50 & $\mathrm{U}$ \\
\hline $108-10-1$ & 4-Methyl-2-pentanone & 5.0 & U \\
\hline $108-88-3$ & Toluene & 0.070 & $\mathrm{~J} B$ \\
\hline $10061-02-6$ & trans-1,3-Dichloropropene & 0.50 & $\mathrm{U}$ \\
\hline $79-00-5$ & 1,1,2-Trichloroethane & 0.50 & $\mathrm{U}$ \\
\hline $127-18-4$ & Tetrachloroethene & 0.50 & $\mathrm{U}$ \\
\hline $591-78-6$ & 2-Hexanone & 5.0 & $\mathrm{U}$ \\
\hline $124-48-1$ & Dibromochloromethane & 0.87 & \\
\hline $106-93-4$ & 1,2-Dibromoethane & 0.50 & $\mathrm{U}$ \\
\hline $108-90-7$ & Chlorobenzene & 0.50 & $\mathrm{U}$ \\
\hline $100-41-4$ & Ethylbenzene & 0.024 & $\mathrm{~J}$ \\
\hline $95-47-6$ & o-Xylene & 0.034 & $\mathrm{~J}$ \\
\hline $179601-23-1$ & $\mathrm{~m}, \mathrm{p}-\mathrm{Xy}$ lene & 0.054 & $\mathrm{~J}$ \\
\hline $100-42-5$ & Styrene & 0.50 & $\mathrm{U}$ \\
\hline $75-25-2$ & Bromoform & 0.50 & $\mathrm{U}$ \\
\hline $98-82-8$ & Isopropylbenzene & 0.50 & $\mathrm{U}$ \\
\hline $79-34-5$ & 1, 1,2,2-Tetrachloroethane & 0.50 & $\mathrm{U}$ \\
\hline $541-73-1$ & 1,3-Dichlorobenzene & 0.50 & $\mathrm{U}$ \\
\hline $106-46-7$ & 1,4-Dichlorobenzene & 0.50 & $\mathrm{U}$ \\
\hline $95-50-1$ & 1,2-Dichlorobenzene & 0.50 & $\mathrm{U}$ \\
\hline $96-12-8$ & 1,2-Dibromo-3-Chloropropane & 0.50 & $\mathrm{U}$ \\
\hline $120-82-1$ & 1,2,4-Trichlorobenzene & 0.50 & $\mathrm{U}$ \\
\hline $87-61-6$ & 1,2,3-Trichlorobenzene & 0.50 & $\mathrm{U}$ \\
\hline
\end{tabular}


IJ - FORM I VOA-TIC

VOLATILE ORGANICS ANALYSIS DATA SHEET TENTATIVELY IDENTIFIED COMPOUNDS
EPA SAMPLE NO.

INMW $18-W-32762$
Lab Name: TESTAMERICA BURLINGTON

Lab Code: STLV Case No.: INMAN

Matrix: (SOIL/SED/WATER)

water

Sample wt/vol: 25.0

$(\mathrm{g} / \mathrm{mL}) \mathrm{mL}$

Level: (TRACE or LOW/MED)

TRACE

\% Moisture: not dec.

GC Column: DB-624

ID : 0.20

Soil Extract Volume: (uL)

CONCENTRATION UNITS: (ug/L or $\mathrm{ug} / \mathrm{kg}$ )

$\mathrm{ug} / \mathrm{L}$
Contract: $1 \mathrm{E}-30401$

SDG No.: 200-6762

Lab Sample ID: 200-6762-11

Lab File ID: DHPV15.D

Date Received: $08 / 30 / 2011$

Date Analyzed: 09/01/2011

Dilution Factor: 1.0

Soil Aliquot volume:

Purge Volume: 25.0 $(\mathrm{mL})$

\begin{tabular}{|l|l|l|l|r|r|}
\hline \multicolumn{1}{|c|}{ CAS NUMBER } & \multicolumn{1}{|c|}{ COMPOUND NAME } & RT & EST. CONC. & Q \\
\cline { 2 - 6 } 01 & & Unknown & 6.79 & B X J \\
\cline { 2 - 6 } & E9667961 & Total Alkanes & N/A & & \\
\hline
\end{tabular}

1 EPA-designated Registry Number. 
$1 A$ - FORM I VOA-1

VOLATILE ORGANICS ANALYSIS DATA SHEET
EPA SAMPLE NO.

INQC-W-32763

Lab Name: TESTAMERICA BURLINGTON

Lab Code: STLV

Matrix: (SOIL/SED/WATER)

Sample wt/vol: 25.0

Level: (TRACE/LOW/MED)

읗 Moisture: not dec.

GC Column: DB-624

Soil Extract Volume: ID $: 0.20$

Water

$(\mathrm{g} / \mathrm{mL}) \quad \mathrm{mL}$

Purge Volume: 25.0

\begin{tabular}{|c|c|c|c|}
\hline CAS NO. & COMPOUND & $\begin{array}{l}\text { CONCENTRATION UNITS: } \\
(\mathrm{ug} / \mathrm{L} \text { or } u g / \mathrm{kg}) \mathrm{ug} / \mathrm{L}\end{array}$ & $Q$ \\
\hline $75-71-8$ & Dichlorodifluoromethane & 0.50 & $\overline{\mathrm{U}}$ \\
\hline $74-87-3$ & Chloromethane & 0.50 & $\mathrm{U}$ \\
\hline $75-01-4$ & Vinyl chloride & 0.50 & $\mathrm{U}$ \\
\hline $74-83-9$ & Bromomethane & 0.50 & $\mathrm{U}$ \\
\hline $75-00-3$ & Chloroethane & 0.50 & $\mathrm{U}$ \\
\hline $75-69-4$ & Trichlorofluoromethane & 0.50 & $\mathrm{U}$ \\
\hline $75-35-4$ & 1,1-Dichloroethene & 0.50 & $\mathrm{U}$ \\
\hline $76-13-1$ & 1,1,2-Trichloro-1,2,2-trifluoroethane & 0.50 & $\mathrm{U}$ \\
\hline $67-64-1$ & Acetone & 1.6 & $\mathrm{~J}$ \\
\hline $75-15-0$ & Carbon disulfide & 0.50 & $\mathrm{U}$ \\
\hline $79-20-9$ & Methyl acetate & 0.50 & $\mathrm{U}$ \\
\hline $75-09-2$ & Methylene Chloride & 0.50 & $\mathrm{U}$ \\
\hline $156-60-5$ & trans $-1,2-$ Dichloroethene & 0.50 & $\mathrm{U}$ \\
\hline $1634-04-4$ & Methyl tert-butyl ether & 0.50 & $\mathrm{U}$ \\
\hline $75-34-3$ & 1,1-Dichloroethane & 0.50 & $\mathrm{U}$ \\
\hline $156-59-2$ & cis-1,2-Dichloroethene & 0.50 & $\mathrm{U}$ \\
\hline $78-93-3$ & 2-Butanone & 5.0 & $\mathrm{U}$ \\
\hline $74-97-5$ & Bromochloromethane & 0.50 & $\mathrm{U}$ \\
\hline $67-66-3$ & Chloroform & 0.20 & $\mathrm{~J}$ \\
\hline $71-55-6$ & 1,1,1-Trichloroethane & 0.50 & $\mathrm{U}$ \\
\hline $110-82-7$ & Cyclohexane & 0.50 & $\mathrm{U}$ \\
\hline $56-23-5$ & Carbon tetrachloride & 0.50 & $\mathrm{U}$ \\
\hline $71-43-2$ & Benzene & 0.10 & $\mathrm{~J}$ \\
\hline $107-06-2$ & 1,2-Dichloroethane & 0.50 & $\mathrm{U}$ \\
\hline
\end{tabular}

Report 1,4-Dioxane for Low-Medium VOA analysis only 
Lab Name: TESTAMERICA BURLINGTON

Lab Code: STLV Case No.: INMAN

Water

Sample wt/vol: 25.0

$(\mathrm{g} / \mathrm{mL}) \quad \mathrm{mL}$

Level: (TRACE/LOW/MED) TRACE

․ Moisture: not dec.

GC Column: DB-624

ID: $0.20 \quad(\mathrm{~mm})$

Soil Extract Volume:

(uL)

$(\mathrm{mL})$

Purge Volume: 25.0
Matrix: (SOIL/SED/WATER)

Contract: 1E-30401

SDG No.: 200-6762

Lab Sample ID: 200-6762-12

Lab Eile ID: DHPV04.D

Date Received: 08/30/2011

Date Analyzed: 09/01/2011

Dilution Factor: 1.0

Soil Aliquot Volume:

(uL)

\begin{tabular}{|c|c|c|c|}
\hline CAS NO. & COMPOUND & $\begin{array}{l}\text { CONCENTRATION UNITS: } \\
(\mathrm{ug} / \mathrm{L} \text { or } \mathrm{ug} / \mathrm{kg}) \mathrm{ug} / \mathrm{L}\end{array}$ & $Q$ \\
\hline $79-01-6$ & Trichloroethene & 0.50 & $\mathrm{U}$ \\
\hline $108-87-2$ & Methylcyclohexane & 0.50 & $\mathrm{U}$ \\
\hline $78-87-5$ & 1,2-Dichloropropane & 0.50 & $\mathrm{U}$ \\
\hline $75-27-4$ & Bromodichloromethane & 0.50 & U \\
\hline $10061-01-5$ & cis-1,3-Dichloropropene & 0.50 & $\mathrm{U}$ \\
\hline $108-10-1$ & 4-Methyl-2-pentanone & 5.0 & $\mathrm{U}$ \\
\hline $108-88-3$ & Toluene & 1.2 & B \\
\hline $10061-02-6$ & trans-1,3-Dichloropropene & 0.50 & $\mathrm{U}$ \\
\hline $79-00-5$ & 1,1,2-Trichloroethane & 0.50 & $\mathrm{U}$ \\
\hline $127-18-4$ & Tetrachloroethene & 0.50 & $\mathrm{U}$ \\
\hline $591-78-6$ & 2-Hexanone & 5.0 & $\mathrm{U}$ \\
\hline $124-48-1$ & Dibromochloromethane & 0.50 & $\mathrm{U}$ \\
\hline $106-93-4$ & 1,2-Dibromoethane & 0.50 & $\mathrm{U}$ \\
\hline $108-90-7$ & Chlorobenzene & 0.50 & $\mathrm{U}$ \\
\hline $100-41-4$ & Ethylbenzene & 0.027 & $\mathrm{~J}$ \\
\hline $95-47-6$ & o-Xylene & 0.022 & $\mathrm{~J}$ \\
\hline $179601-23-1$ & m,p-Xylene & 0.045 & $\mathrm{~J}$ \\
\hline $100-42-5$ & Styrene & 0.072 & $\mathrm{~J}$ \\
\hline $75-25-2$ & Bromoform & 0.50 & $\mathrm{U}$ \\
\hline $98-82-8$ & Isopropylbenzene & 0.50 & $\mathrm{U}$ \\
\hline $79-34-5$ & $1,1,2,2$-Tetrachloroethane & 0.50 & $\mathrm{U}$ \\
\hline $541-73-1$ & 1,3-Dichlorobenzene & 0.50 & $\mathrm{U}$ \\
\hline $106-46-7$ & 1,4-Dichlorobenzene & 0.50 & $\mathrm{U}$ \\
\hline $95-50-1$ & 1,2-Dichlorobenzene & 0.50 & $\mathrm{U}$ \\
\hline $96-12-8$ & 1,2-Dibromo-3-Chloropropane & 0.50 & $\mathrm{U}$ \\
\hline $120-82-1$ & 1,2,4-Trichlorobenzene & 0.50 & $\mathrm{U}$ \\
\hline $87-61-6$ & 1,2,3-Trichlorobenzene & 0.50 & $\mathrm{U}$ \\
\hline
\end{tabular}


IJ - FORM I VOA-TIC

VOLATILE ORGANICS ANALYSIS DATA SHEET TENTATIVELY IDENTIEIED COMPOUNDS
EPA SAMPLE NO. INQC-W-32763
Lab Name: TESTAMERICA BURLINGTON

Lab Code: STLV Case No.: INMAN Mod.

Mod. Ref No.:

Contract: 1E-30401

Matrix: (SOIL/SED/WATER)

Water

Sample wt/vol: 25.0

Level: (TRACE or LOW/MED)

$(\mathrm{g} / \mathrm{mL}) \mathrm{mL}$

\% Moisture: not dec.

GC Column: DB-624 ID: $0.20 \quad(\mathrm{~mm})$

Soil Extract Volume: (uL)

CONCENTRATION UNITS: (ug/L or $\mathrm{ug} / \mathrm{kg}$ ) ug/L
SDG No.: 200-6762

Lab Sample ID: 200-6762-12

Lab File ID: DHPV04.D

Date Received: 08/30/2011

Date Analyzed: 09/01/2011

Dilution Factor: 1.0

Soil Aliquot Volume:

(uL)

Purge Volume: 25.0 (mL)

01

\begin{tabular}{|l|l|c|c|c|}
\hline CAS NUMBER & \multicolumn{1}{|c|}{ COMPOUND NAME } & RT & EST. CONC. & $Q$ \\
\hline & Unknown & 6.79 & 2.7 & B X J \\
\hline E9667961 & Total Alkanes & N/A & & \\
\hline
\end{tabular}

1 EPA-designated Registry Number. 
$6 A-$ FORM VI VOA-1

VOLATILE ORGANICS INITIAL CALIBRATION DATA

Lab Name: TESTAMERICA BURLINGTON

Contract: $1 \mathrm{E}-30401$

Lab Code: STLV Case No.: INMAN Mod. Ref No.:

SDG No.: 200-6762

Instrument ID: D.i Calibration Date(s): 07/07/2011 07/07/2011

Heated Purge: $(\mathrm{Y} / \mathrm{N}) \mathrm{N}$ Calibration Time(s): $1117 \quad 1256$

Purge Volume: 25.0 (mL)

GC Column: DB-624

ID: 0.20

(mm) Length: 25

(m)

\begin{tabular}{|c|c|c|c|c|c|c|c|}
\hline \multirow{3}{*}{$\begin{array}{l}\text { LAB FILE ID: } \\
\text { RRF } 5.0=\text { DHP06.D } \\
\text { COMPOUND }\end{array}$} & \multirow{2}{*}{\multicolumn{3}{|c|}{$\begin{array}{l}\operatorname{RRF} 0.5=\text { DHP04.D } \\
\operatorname{RRF10}=\text { DHP07.D }\end{array}$}} & \multicolumn{4}{|c|}{ RRF 1.0 = DHP05.D } \\
\hline & & & & RRF 20 & $=\mathrm{DHPC}$ & & \\
\hline & $\operatorname{RRF} 0.5$ & RRF 1.0 & RRF 5.0 & RRE 10 & RRF 20 & $\overline{\mathrm{RRF}}$ & $\frac{\mathrm{o}}{\mathrm{R} S \mathrm{D}}$ \\
\hline Dichlorodifluoromethane & 0.418 & 0.398 & 0.390 & 0.409 & 0.398 & 0.403 & 2.7 \\
\hline Chloromethane & 0.769 & 0.713 & 0.655 & 0.659 & 0.639 & 0.687 & 7.8 \\
\hline Vinyl chloride & 0.524 & 0.491 & 0.475 & 0.499 & 0.491 & 0.496 & 3.6 \\
\hline Bromomethane & 0.180 & 0.203 & 0.205 & 0.219 & 0.239 & 0.209 & 10.3 \\
\hline Chloroethane & 0.292 & 0.280 & 0.259 & 0.269 & 0.259 & 0.272 & 5.2 \\
\hline Trichlorofluoromethane & 0.612 & 0.609 & 0.587 & 0.608 & 0.611 & 0.606 & 1.7 \\
\hline 1,1-Dichloroethene & 0.318 & 0.307 & 0.298 & 0.303 & 0.297 & 0.305 & 2.8 \\
\hline $\begin{array}{l}\text { 1,1,2-Trichloro- } \\
1,2,2 \text {-trifluoroethane }\end{array}$ & 0.377 & 0.358 & 0.336 & 0.358 & 0.347 & 0.355 & 4.3 \\
\hline Acetone & 0.047 & 0.038 & 0.032 & 0.032 & 0.030 & 0.036 & 19.3 \\
\hline Carbon disulfide & 0.758 & 0.707 & 0.719 & 0.760 & 0.766 & 0.742 & 3.6 \\
\hline Methyl acetate & 0.108 & 0.094 & 0.076 & 0.079 & 0.074 & 0.086 & 16.7 \\
\hline Methylene Chloride & 0.264 & 0.257 & 0.242 & 0.248 & 0.241 & 0.250 & 3.9 \\
\hline trans-1,2-Dichloroethene & 0.323 & 0.298 & 0.301 & 0.312 & 0.306 & 0.308 & 3.3 \\
\hline Methyl tert-butyl ether & 0.304 & 0.278 & 0.292 & 0.319 & 0.318 & 0.302 & 5.7 \\
\hline 1,1-Dichloroethane & 0.642 & 0.586 & 0.594 & 0.617 & 0.607 & 0.609 & 3.6 \\
\hline cis-1,2-Dichloroethene & 0.302 & 0.289 & 0.300 & 0.312 & 0.306 & 0.302 & 2.7 \\
\hline 2-Butanone & 0.045 & 0.046 & 0.047 & 0.049 & 0.048 & 0.047 & 3.4 \\
\hline Bromochloromethane & 0.104 & 0.103 & 0.100 & 0.099 & 0.101 & 0.101 & 2.0 \\
\hline Chloroform & 0.502 & 0.482 & 0.473 & 0.493 & 0.477 & 0.485 & 2.5 \\
\hline $1,1,1$-Trichloroethane & 0.514 & 0.490 & 0.494 & 0.490 & 0.485 & 0.494 & 2.3 \\
\hline Cyclohexane & 0.761 & 0.795 & 0.901 & 0.909 & 0.883 & 0.850 & 7.9 \\
\hline Carbon tetrachloride & 0.487 & 0.459 & 0.477 & 0.474 & 0.464 & 0.472 & 2.4 \\
\hline Benzene & 1.617 & 1.501 & 1.518 & 1.495 & 1.447 & 1.515 & 4.1 \\
\hline 1,2-Dichloroethane & 0.291 & 0.271 & 0.277 & 0.284 & 0.285 & 0.282 & 2.7 \\
\hline Trichloroethene & 0.397 & 0.380 & 0.357 & 0.355 & 0.351 & 0.368 & 5.4 \\
\hline Methylcyclohexane & 0.703 & 0.708 & 0.769 & 0.757 & 0.751 & 0.737 & 4.1 \\
\hline
\end{tabular}

Report 1,4-Dioxane for Low-Medium VOA analysis only 
$6 B-$ EORM VI VOA-2

VOLATILE ORGANICS INITIAL CALIBRATION DATA

Lab Name: TESTAMERICA BURLINGTON

Contract: 1E-30401

Lab Code: STLV Case No.: INMAN Mod. Ref No.:

SDG No.: 200-6762

Instrument ID: D.i Calibration Date(s):

07/07/2011 07/07/2011

Heated Purge: (Y/N) N

Calibration Time(s) : 1117

1256

Purge Volume: 25.0

(mL)

GC Column: DB-624

ID: 0.20

(mm) Length: 25

(m)

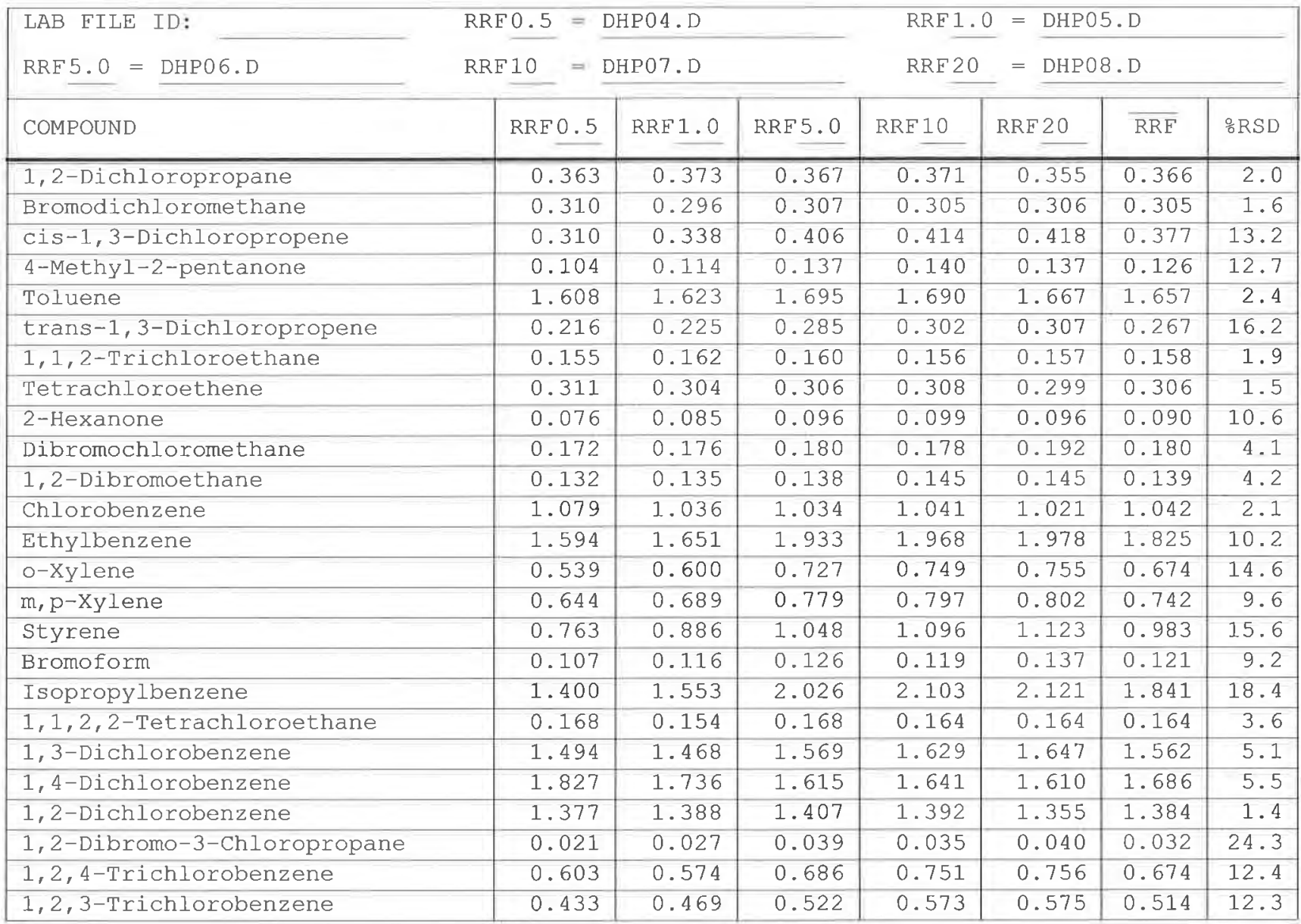


$6 \mathrm{C}-$ FORM VI VOA-3

VOLATILE ORGANICS INITIAL CALIBRATION DATA

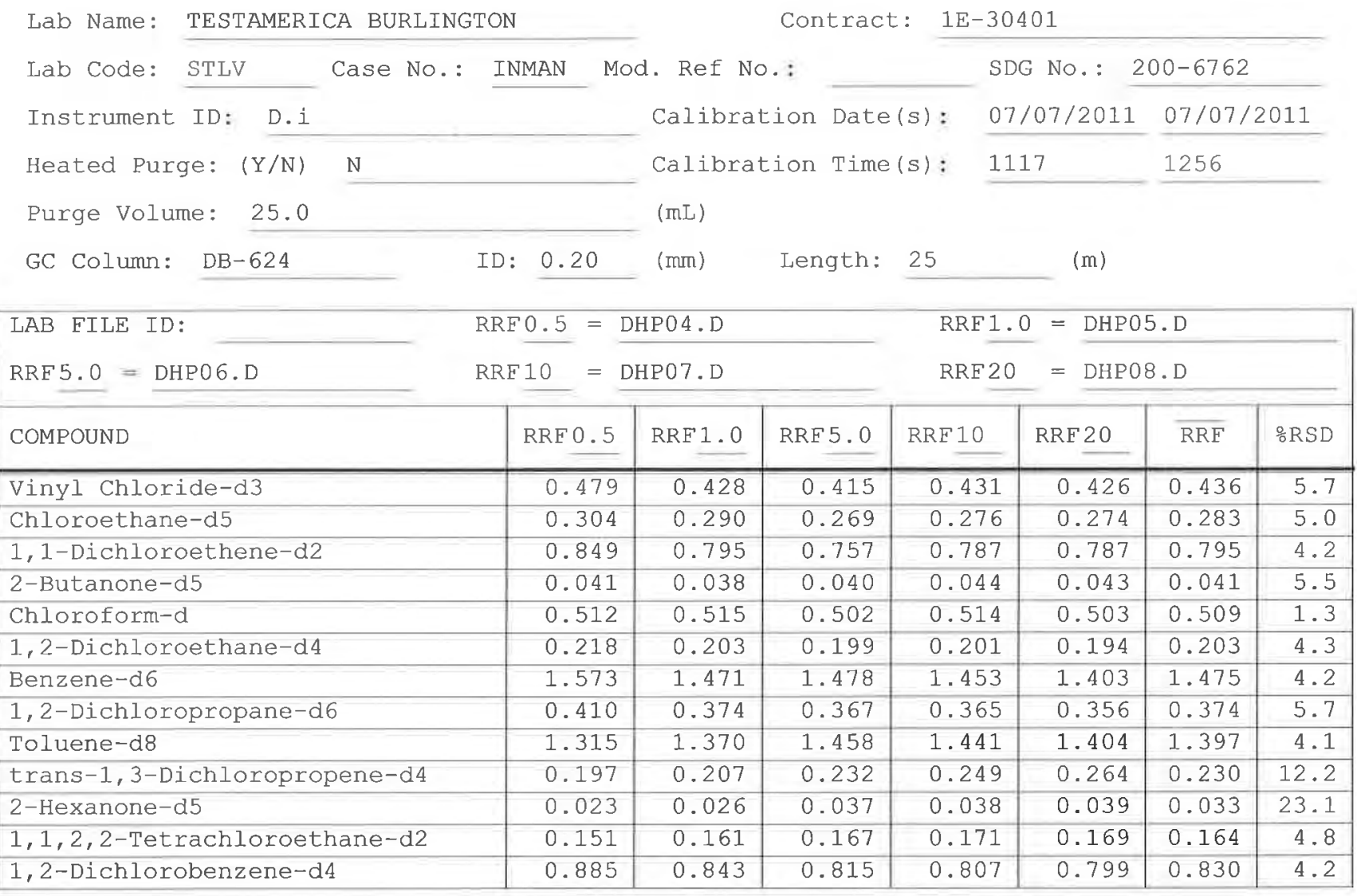

Report 1,4-Dioxane-d8 for Low-Medium VOA analysis only 
$7 \mathrm{~A}$ - FORM VII VOA-1

VOLATILE CONTINUING CALIBRATION DATA

Lab Name: TESTAMERICA BURLINGTON

Contract: 1E-30401

Lab Code: STLV Case No.: INMAN Mod. Ref No.:

SDG No.: $200-6762$

Instrument ID: D.i

Calibration Date: 09/01/2011 Time: 0748

Lab File Id: DHPV02.D Init. Calib. Date(s) : 07/07/2011 07/07/2011

EPA Sample No. (VSTD\#\#\#\#): VSTD005DS Init. Calib. Time(s): $1117 \quad 1256$

Heated Purge: ( $\mathrm{Y} / \mathrm{N}) \mathrm{N}$

GC Column: DB-624

ID: $0.20(\mathrm{~mm})$ Length: 25

(m)

Purge Volume: 25.0

(mL)

\begin{tabular}{|c|c|c|c|c|c|}
\hline COMPOUND & $\overline{\mathrm{RRE}}$ & RRE5. 0 & $\begin{array}{l}\text { MIN } \\
\text { RRF }\end{array}$ & $\div \mathrm{D}$ & MAX $\because \mathrm{D}$ \\
\hline Dichlorodifluoromethane & 0.403 & 0.428 & 0.010 & 6.3 & 40.0 \\
\hline Chloromethane & 0.687 & 0.667 & 0.010 & -3.0 & 40.0 \\
\hline Vinyl chloride & 0.496 & 0.465 & 0.010 & -6.4 & 30.0 \\
\hline Bromomethane & 0.209 & 0.188 & 0.010 & -10.1 & 30.0 \\
\hline Chloroethane & 0.272 & 0.243 & 0.010 & -10.6 & 40.0 \\
\hline Trichlorofluoromethane & 0.606 & 0.562 & 0.010 & -7.3 & 40.0 \\
\hline 1,1-Dichloroethene & 0.305 & 0.282 & 0.010 & -7.6 & 30.0 \\
\hline 1,1,2-Trichloro-1,2,2-trifluoroethane & 0.355 & 0.334 & 0.010 & -6.1 & 40.0 \\
\hline Acetone & 0.036 & 0.026 & 0.010 & -27.5 & 40.0 \\
\hline Carbon disulfide & 0.742 & 0.674 & 0.010 & -9.1 & 40.0 \\
\hline Methyl acetate & 0.086 & 0.074 & 0.010 & -13.9 & 40.0 \\
\hline Methylene Chloride & 0.250 & 0.221 & 0.010 & -11.8 & 40.0 \\
\hline trans-1,2-Dichloroethene & 0.308 & 0.274 & 0.010 & -11.0 & 40.0 \\
\hline Methyl tert-butyl ether & 0.302 & 0.242 & 0.010 & -20.0 & 40.0 \\
\hline 1,1-Dichloroethane & 0.609 & 0.557 & 0.010 & -8.6 & 30.0 \\
\hline cis-1,2-Dichloroethene & 0.302 & 0.276 & 0.010 & -8.4 & 40.0 \\
\hline 2-Butanone & 0.047 & 0.037 & 0.010 & -20.6 & 40.0 \\
\hline Bromochloromethane & 0.101 & 0.089 & 0.010 & -12.1 & 30.0 \\
\hline Chloroform & 0.485 & 0.433 & 0.010 & -10.7 & 30.0 \\
\hline 1,1,1-Trichloroethane & 0.494 & 0.453 & 0.010 & -8.4 & 30.0 \\
\hline Cyclohexane & 0.850 & 0.860 & 0.010 & 1.2 & 40.0 \\
\hline Carbon tetrachloride & 0.472 & 0.449 & 0.010 & -4.9 & 30.0 \\
\hline Benzene & 1.515 & 1.371 & 0.010 & -9.6 & 30.0 \\
\hline 1,2-Dichloroethane & 0.282 & 0.249 & 0.010 & -11.6 & 30.0 \\
\hline Trichloroethene & 0.368 & 0.331 & 0.010 & -10.0 & 30.0 \\
\hline Methylcyclohexane & 0.737 & 0.711 & 0.010 & -3.5 & 40.0 \\
\hline
\end{tabular}

Report 1,4-Dioxane for Low/Medium VOA analysis only 
$7 \mathrm{~B}$ - FORM VII VOA-2

VOLATILE CONTINUING CALIBRATION DATA

Lab Name: TESTAMERICA BURLINGTON

Contract: $1 \mathrm{E}-30401$

Lab Code: STLV Case No.: INMAN Mod. Ref No.:

SDG No.: 200-6762

Instrument ID: D.i

Calibration Date: 09/01/2011 Time: 0748

Lab File Id: DHPV02.D Init. Calib. Date(s): 07/07/2011 07/07/2011

EPA Sample No. (VSTD\#\#\#\#): VSTD005DS

Init. Calib. Time(s): $1117 \quad 1256$

Heated Purge: ( $\mathrm{Y} / \mathrm{N}) \quad \mathrm{N}$

GC Column: DB-624

ID : 0.20 (mm) Length: 25

(m)

Purge Volume: 25.0

(mL)

\begin{tabular}{|c|c|c|c|c|c|}
\hline COMPOUND & $\overline{\mathrm{RRF}}$ & RRF5.0 & $\begin{array}{l}\text { MIN } \\
\text { RRE }\end{array}$ & $\because \mathrm{D}$ & MAX $\div D$ \\
\hline 1,2-Dichloropropane & 0.366 & 0.336 & 0.010 & -8.1 & 40.0 \\
\hline Bromodichloromethane & 0.305 & 0.269 & 0.010 & -11.7 & 30.0 \\
\hline cis-1,3-Dichloropropene & 0.377 & 0.355 & 0.010 & -6.1 & 30.0 \\
\hline 4-Methyl-2-pentanone & 0.126 & 0.108 & 0.010 & -14.5 & 40.0 \\
\hline Toluene & 1.657 & 1.560 & 0.010 & -5.8 & 30.0 \\
\hline trans-1,3-Dichloropropene & 0.267 & 0.248 & 0.010 & -7.1 & 30.0 \\
\hline $1,1,2$-Trichloroethane & 0.158 & 0.138 & 0.010 & -12.5 & 30.0 \\
\hline Tetrachloroethene & 0.306 & 0.276 & 0.010 & -9.8 & 30.0 \\
\hline 2-Hexanone & 0.090 & 0.076 & 0.010 & -15.6 & 40.0 \\
\hline Dibromochloromethane & 0.180 & 0.156 & 0.010 & -13.3 & 30.0 \\
\hline 1.2-Dibromoethane & 0.139 & 0.121 & 0.010 & -13.1 & 40.0 \\
\hline Chlorobenzene & 1.042 & 0.949 & 0.010 & -9.0 & 30.0 \\
\hline Ethylbenzene & 1.825 & 1.731 & 0.010 & -5.1 & 30.0 \\
\hline o-Xylene & 0.674 & 0.656 & 0.010 & -2.7 & 30.0 \\
\hline $\mathrm{m}, \mathrm{p}$-Xylene & 0.742 & 0.711 & 0.010 & -4.1 & 30.0 \\
\hline Styrene & 0.983 & 0.946 & 0.010 & -3.8 & 30.0 \\
\hline Bromoform & 0.121 & 0.100 & 0.010 & -17.1 & 30.0 \\
\hline Isopropylbenzene & 1.841 & 1.827 & 0.010 & -0.7 & 40.0 \\
\hline $1,1,2,2$-Tetrachloroethane & 0.164 & 0.132 & 0.010 & -19.7 & 30.0 \\
\hline 1,3-Dichlorobenzene & 1.562 & 1.428 & 0.010 & -8.6 & 30.0 \\
\hline 1,4-Dichlorobenzene & 1.686 & 1.501 & 0.010 & -11.0 & 30.0 \\
\hline 1,2-Dichlorobenzene & 1.384 & 1.268 & 0.010 & -8.4 & 30.0 \\
\hline 1,2-Dibromo-3-Chloropropane & 0.032 & 0.032 & 0.010 & -0.9 & 40.0 \\
\hline 1,2,4-Trichlorobenzene & 0.674 & 0.663 & 0.010 & -1.6 & 30.0 \\
\hline 1,2,3-Trichlorobenzene & 0.514 & 0.496 & 0.010 & -3.5 & 30.0 \\
\hline
\end{tabular}


7C - FORM VII VOA-3

VOLATILE CONTINUING CALIBRATION DATA

Lab Name: TESTAMERICA BURLINGTON

Contract: 1E-30401

Lab Code: STLV Case No.: INMAN Mod. Ref No.:

SDG No.: 200-6762

Instrument ID: D.i

Calibration Date: 09/01/2011 Time: 0748

Lab File Id: DHPV02.D

Init. Calib. Date(s) : 07/07/2011 07/07/2011

EPA Sample No. (VSTD\#\#\#\#): VSTD005DS

Init. Calib. Time(s): $1117 \quad 1256$

Heated Purge: $(\mathrm{Y} / \mathrm{N}) \mathrm{N}$

GC Column: DB-624

ID: $0.20(\mathrm{~mm})$ Length: 25

(m)

Purge Volume: 25.0

$(\mathrm{mL})$

\begin{tabular}{|c|c|c|c|c|c|}
\hline COMPOUND & RRF & RRF5. 0 & $\begin{array}{l}\text { MIN } \\
\text { RRF }\end{array}$ & $\because \mathrm{D}$ & MAX $\because D$ \\
\hline Vinyl Chloride-d3 & 0.436 & 0.395 & 0.010 & -9.4 & 30.0 \\
\hline Chloroethane-d5 & 0.283 & 0.249 & 0.010 & -12.1 & 40.0 \\
\hline 1,1-Dichloroethene-d2 & 0.795 & 0.657 & 0.010 & -17.4 & 30.0 \\
\hline 2-Butanone-d5 & 0.041 & 0.033 & 0.010 & $-19 \cdot 3$ & 40.0 \\
\hline Chloroform-d & 0.509 & 0.429 & 0.010 & -15.7 & 30.0 \\
\hline 1,2-Dichloroethane-d4 & 0.203 & 0.174 & 0.010 & -14.5 & 30.0 \\
\hline Benzene-d6 & 1.475 & 1.277 & 0.010 & $-13 \cdot 4$ & 30.0 \\
\hline 1,2-Dichloropropane-d6 & 0.374 & 0.311 & 0.010 & -17.1 & 40.0 \\
\hline Toluene-d8 & 1.397 & 1.272 & 0.010 & -9.0 & 30.0 \\
\hline trans-1,3-Dichloropropene-d4 & 0.230 & 0.193 & 0.010 & -16.1 & 30.0 \\
\hline 2-Hexanone-d5 & 0.033 & 0.028 & 0.010 & -15.2 & 40.0 \\
\hline $1,1,2,2$-Tetrachloroethane-d2 & 0.164 & 0.131 & 0.010 & -20.2 & 30.0 \\
\hline 1,2-Dichlorobenzene-d4 & 0.830 & 0.712 & 0.010 & -14.2 & 30.0 \\
\hline
\end{tabular}

Report 1,4-Dioxane-d8 for Low/Medium VOA analysis only 
$7 A$ - FORM VII VOA-1

VOLATILE CONTINUING CALIBRATION DATA

Lab Name: TESTAMERICA BURLINGTON

Contract: $1 \mathrm{E}-30401$

Lab Code: STLV Case No.: INMAN Mod. Ref No.:

SDG No.: $200-6762$

Instrument ID: D.i

Calibration Date: 09/01/2011 Time: 1640

Lab File Id: DHPV23.D Init. Calib. Date(s): 07/07/2011 07/07/2011

EPA Sample No. (VSTD\#\#\#\#): VSTD005SD

Init. Calib. Time(s): $1117 \quad 1256$

Heated Purge: ( $\mathrm{Y} / \mathrm{N})$

GC Column: DB-624

ID: $0.20(\mathrm{~mm})$ Length: 25

(m)

Purge Volume: 25.0

$(\mathrm{mL})$

\begin{tabular}{|c|c|c|c|c|c|}
\hline COMPOUND & $\mathrm{RRF}$ & RRF5.0 & $\begin{array}{l}\text { MIN } \\
\text { RRE }\end{array}$ & $\frac{\circ}{\partial}$ & $\mathrm{MAX} \because \mathrm{D}$ \\
\hline Dichlorodifluoromethane & 0.403 & 0.451 & 0.010 & 11.9 & 50.0 \\
\hline Chloromethane & 0.687 & 0.699 & 0.010 & 1.7 & 50.0 \\
\hline Vinyl chloride & 0.496 & 0.489 & 0.100 & -1.5 & 50.0 \\
\hline Bromomethane & 0.209 & 0.206 & 0.100 & -1.6 & 50.0 \\
\hline Chloroethane & 0.272 & 0.263 & 0.010 & -3.4 & 50.0 \\
\hline Trichlorofluoromethane & 0.606 & 0.605 & 0.010 & -0.1 & 50.0 \\
\hline 1,1-Dichloroethene & 0.305 & 0.302 & 0.100 & -0.8 & 50.0 \\
\hline 1,1,2-Trichloro-1,2,2-trifluoroethane & 0.355 & 0.349 & 0.010 & -1.8 & 50.0 \\
\hline Acetone & 0.036 & 0.030 & 0.010 & -15.8 & 50.0 \\
\hline Carbon disulfide & 0.742 & 0.692 & 0.010 & -6.7 & 50.0 \\
\hline Methyl acetate & 0.086 & 0.084 & 0.010 & -2.2 & 50.0 \\
\hline Methylene Chloride & 0.250 & 0.239 & 0.010 & -4.6 & 50.0 \\
\hline trans-1,2-Dichloroethene & 0.308 & 0.298 & 0.010 & -3.2 & 50.0 \\
\hline Methyl tert-butyl ether & 0.302 & 0.302 & 0.010 & -0.1 & 50.0 \\
\hline 1,1-Dichloroethane & 0.609 & 0.609 & 0.200 & -0.1 & 50.0 \\
\hline cis-1,2-Dichloroethene & 0.302 & 0.298 & 0.010 & -1.2 & 50.0 \\
\hline 2-Butanone & 0.047 & 0.048 & 0.010 & 1.5 & 50.0 \\
\hline Bromochloromethane & 0.101 & 0.100 & 0.050 & -1.0 & 50.0 \\
\hline Chloroform & 0.485 & 0.486 & 0.200 & 0.1 & 50.0 \\
\hline 1,1,1-Trichloroethane & 0.494 & 0.484 & 0.100 & -2.1 & 50.0 \\
\hline Cyclohexane & 0.850 & 0.900 & 0.010 & 5.9 & 50.0 \\
\hline Carbon tetrachloride & 0.472 & 0.452 & 0.100 & -4.2 & 50.0 \\
\hline Benzene & 1.515 & 1.458 & 0.400 & -3.8 & 50.0 \\
\hline 1,2-Dichloroethane & 0.282 & 0.289 & 0.100 & 2.6 & 50.0 \\
\hline Trichloroethene & 0.368 & 0.339 & 0.300 & -7.8 & 50.0 \\
\hline Methylcyclohexane & 0.737 & 0.749 & 0.010 & 1.6 & 50.0 \\
\hline
\end{tabular}

Report 1,4-Dioxane for Low/Medium VOA analysis only 
$7 B$ - FORM VII VOA-2

VOLATILE CONTINUING CALIBRATION DATA

Lab Name: TESTAMERICA BURIJINGTON

Contract: 1E-30401

Lab Code: STLV Case No.: INMAN Mod. Ref No.:

SDG No.: 200-6762

Instrument ID: D.i

Calibration Date: 09/01/2011 Time: 1640

Lab File Id: DHPV23.D Init. Calib. Date(s): 07/07/2011 07/07/2011

EPA Sample No. (VSTD\#\#\#\#): VSTD005SD

Init. Calib. Time(s): $1117 \quad 1256$

Heated Purge: (Y/N) N

GC Column: DB-624

ID: $0.20(\mathrm{~mm})$ Length: 25

(m)

Purge Volume: 25.0

(mL)

\begin{tabular}{|c|c|c|c|c|c|}
\hline COMPOUND & $\overline{\mathrm{RRF}}$ & RRF5.0 & $\begin{array}{l}\text { MIN } \\
\text { RRF }\end{array}$ & $\circ \mathrm{D}$ & MAX $\div \mathrm{D}$ \\
\hline 1,2-Dichloropropane & 0.366 & 0.362 & 0.010 & -1.0 & 50.0 \\
\hline Bromodichloromethane & 0.305 & 0.294 & 0.200 & -3.6 & 50.0 \\
\hline cis-1,3-Dichloropropene & 0.377 & 0.384 & 0.200 & 1.8 & 50.0 \\
\hline 4-Methyl-2-pentanone & 0.126 & 0.132 & 0.010 & 4.5 & 50.0 \\
\hline Toluene & 1.657 & 1.655 & 0.400 & -0.1 & 50.0 \\
\hline trans-1,3-Dichloropropene & 0.267 & 0.273 & 0.100 & 2.3 & 50.0 \\
\hline 1,1,2-Trichloroethane & 0.158 & 0.158 & 0.100 & 0.0 & 50.0 \\
\hline Tetrachloroethene & 0.306 & 0.295 & 0.100 & -3.6 & 50.0 \\
\hline 2-Hexanone & 0.090 & 0.093 & 0.010 & 2.7 & 50.0 \\
\hline Dibromochloromethane & 0.180 & 0.173 & 0.100 & -3.6 & 50.0 \\
\hline 1,2-Dibromoethane & 0.139 & 0.139 & 0.010 & 0.2 & 50.0 \\
\hline Chlorobenzene & 1.042 & 1.020 & 0.500 & -2.1 & 50.0 \\
\hline Ethylbenzene & 1.825 & 1.834 & 0.100 & 0.5 & 50.0 \\
\hline o-Xylene & 0.674 & 0.708 & 0.300 & 5.0 & 50.0 \\
\hline $\mathrm{m}, \mathrm{p}$-xylene & 0.742 & 0.766 & 0.300 & 3.2 & 50.0 \\
\hline Styrene & 0.983 & 1.075 & 0.300 & 9.4 & 50.0 \\
\hline Bromoform & 0.121 & 0.115 & 0.050 & -5.0 & 50.0 \\
\hline Isopropylbenzene & 1.841 & 1.945 & 0.010 & 5.7 & 50.0 \\
\hline $1,1,2,2$-Tetrachloroethane & 0.164 & 0.159 & 0.100 & -2.8 & 50.0 \\
\hline 1,3-Dichlorobenzene & 1.562 & 1.479 & 0.400 & -5.3 & 50.0 \\
\hline 1,4-Dichlorobenzene & 1.686 & 1.582 & 0.400 & -6.1 & 50.0 \\
\hline 1,2-Dichlorobenzene & 1.384 & 1.371 & 0.400 & -0.9 & 50.0 \\
\hline 1,2-Dibromo-3-Chloropropane & 0.032 & 0.030 & 0.010 & -7.9 & 50.0 \\
\hline 1,2,4-Trichlorobenzene & 0.674 & 0.643 & 0.200 & -4.6 & 50.0 \\
\hline 1,2,3-Trichlorobenzene & 0.514 & 0.523 & 0.200 & 1.6 & 50.0 \\
\hline
\end{tabular}


Lab Name: TESTAMERICA BURLINGTON

Contract: $1 \mathrm{E}-30401$

Lab Code: STLV Case No.: INMAN

Mod. Ref No.:

SDG No.: 200-6762

Instrument ID: D.i

Calibration Date: 09/01/2011 Time: 1640

Lab File Id: DHPV23.D Init. Calib. Date(s): 07/07/2011 07/07/2011

EPA Sample No. (VSTD\#\#\#\#): VSTD005SD

Init. Calib. Time(s): $1117 \quad 1256$

Heated Purge: ( $\mathrm{Y} / \mathrm{N}) \quad \mathrm{N}$

GC Column: DB-624

ID: $0.20(\mathrm{~mm})$ Length: 25

(m)

Purge Volume: 25.0

$(m L)$

\begin{tabular}{|c|c|c|c|c|c|}
\hline COMPOUND & $\overline{\mathrm{RRF}}$ & RRF5.0 & $\begin{array}{l}\text { MIN } \\
\text { RRF }\end{array}$ & $\frac{\circ}{0}$ & MAX $\div D$ \\
\hline Vinyl Chloride-d3 & 0.436 & 0.406 & 0.010 & -6.8 & 50.0 \\
\hline Chloroethane-d5 & 0.283 & 0.267 & 0.010 & -5.7 & 50.0 \\
\hline 1,1-Dichloroethene-d2 & 0.795 & 0.711 & 0.010 & -10.5 & 50.0 \\
\hline 2-Butanone-d5 & 0.041 & 0.040 & 0.010 & -3.0 & 50.0 \\
\hline Chloroform-d & 0.509 & 0.476 & 0.010 & -6.5 & 50.0 \\
\hline 1,2-Dichloroethane-d4 & 0.203 & 0.201 & 0.010 & -1.1 & 50.0 \\
\hline Benzene-d6 & 1.475 & 1.371 & 0.010 & -7.1 & 50.0 \\
\hline 1,2-Dichloropropane-d6 & 0.374 & 0.343 & 0.010 & -8.3 & 50.0 \\
\hline Toluene-d8 & 1.397 & 1.344 & 0.010 & -3.8 & 50.0 \\
\hline trans-1,3-Dichloropropene-d4 & 0.230 & 0.222 & 0.010 & -3.4 & 50.0 \\
\hline 2-Hexanone-d5 & 0.033 & 0.034 & 0.010 & 3.4 & 50.0 \\
\hline $1,1,2,2$-Tetrachloroethane-d2 & 0.164 & 0.153 & 0.010 & -6.6 & 50.0 \\
\hline 1,2-Dichlorobenzene-d4 & 0.830 & 0.762 & 0.010 & -8.1 & 50.0 \\
\hline
\end{tabular}

Report 1,4-Dioxane-d8 for Low/Medium VOA analysis only 
$1 A$ - FORM I VOA-1

VOLATILE ORGANICS ANALYSIS DATA SHEET
EPA SAMPLE NO. VBLKDS
Lab Name: TESTAMERICA BURLINGTON

Lab Code: STLV Case No.: INMA
Matrix: (SOIL/SED/WATER)

Sample wt/vol: 25.0

Water

$(\mathrm{g} / \mathrm{mL}) \quad \mathrm{mL}$

Level: (TRACE/LOW/MED) TRACE

\% Moisture: not dec.

GC Column: DB-624

Soil Extract Volume: ID: $0.20 \quad(\mathrm{~mm})$

Purge Volume: 25.0
Contract: 1E-30401

SDG No.: 200-6762
Lab Sample ID: MB 200-24615/3

Lab File ID: DHPV03.D

Date Received:

Date Analyzed: 09/01/2011

Dilution Factor: 1.0

(uL)
Soil Aliquot Volume:

(mL)

\begin{tabular}{|c|c|c|c|}
\hline CAS NO. & COMPOUND & $\begin{array}{l}\text { CONCENTRATION UNITS: } \\
(\mathrm{ug} / \mathrm{L} \text { or } \mathrm{ug} / \mathrm{kg}) \mathrm{ug} / \mathrm{L}\end{array}$ & $Q$ \\
\hline $75-71-8$ & Dichlorodifluoromethane & 0.50 & U \\
\hline $74-87-3$ & Chloromethane & 0.50 & $\mathrm{U}$ \\
\hline $75-01-4$ & Vinyl chloride & 0.50 & $\mathrm{U}$ \\
\hline $74-83-9$ & Bromomethane & 0.50 & $\mathrm{U}$ \\
\hline $75-00-3$ & Chloroethane & 0.50 & $\mathrm{U}$ \\
\hline $75-69-4$ & Trichlorofluoromethane & 0.50 & $\mathrm{U}$ \\
\hline $75-35-4$ & 1,1-Dichloroethene & 0.50 & $\mathrm{U}$ \\
\hline $76-13-1$ & 1,1,2-Trichloro-1,2,2-trifluoroethane & 0.50 & $\mathrm{U}$ \\
\hline $67-64-1$ & Acetone & 5.0 & $\mathrm{U}$ \\
\hline $75-15-0$ & Carbon disulfide & 0.50 & $\mathrm{U}$ \\
\hline $79-20-9$ & Methyl acetate & 0.50 & $\mathrm{U}$ \\
\hline $75-09-2$ & Methylene Chloride & 0.50 & $\mathrm{U}$ \\
\hline $156-60-5$ & trans-1,2-Dichloroethene & 0.50 & $\mathrm{U}$ \\
\hline $1634-04-4$ & Methyl tert-butyl ether & 0.50 & $\mathrm{U}$ \\
\hline $75-34-3$ & 1,1-Dichloroethane & 0.50 & $\mathrm{U}$ \\
\hline $156-59-2$ & cis-1,2-Dichloroethene & 0.50 & $\mathrm{U}$ \\
\hline $78-93-3$ & 2-Butanone & 5.0 & $\mathrm{U}$ \\
\hline $74-97-5$ & Bromochloromethane & 0.50 & $\mathrm{U}$ \\
\hline $67-66-3$ & Chloroform & 0.50 & $\mathrm{U}$ \\
\hline $71-55-6$ & 1,1,1-Trichloroethane & 0.50 & $\mathrm{U}$ \\
\hline $110-82-7$ & Cyclohexane & 0.50 & $\mathrm{U}$ \\
\hline $56-23-5$ & Carbon tetrachloride & 0.50 & $\mathrm{U}$ \\
\hline $71-43-2$ & Benzene & 0.50 & $\mathrm{U}$ \\
\hline $107-06-2$ & 1,2-Dichloroethane & 0.50 & $\mathrm{U}$ \\
\hline
\end{tabular}

Report 1,4-Dioxane for Low-Medium VOA analysis only 
EPA SAMPLE NO.

VOLATILE ORGANICS ANALYSIS DATA SHEET

VBLKDS

Lab Name: TESTAMERICA BURLINGTON

Lab Code: STLV Case No.: INMA

Matrix: (SOIL/SED/WATER)

Water

Sample wt/vol: 25.0

$(\mathrm{g} / \mathrm{mL}) \quad \mathrm{mL}$

Level: (TRACE/LOW/MED) TRACE

응 Moisture: not dec.

GC Column: DB-624 ID $: 0.20$ (mm)

Soil Extract Volume: (uL) $(\mathrm{mL})$

Purge Volume: 25.0
Contract: 1E-30401

SDG No.: 200-6762 Mod. Ref No.:

Lab Sample ID: MB 200-24615/3

Lab File ID: DHPV03.D

Date Received:

Date Analyzed: 09/01/2011

Dilution Factor: 1.0

Soil Aliquot Volume:

(uL)

\begin{tabular}{|c|c|c|c|}
\hline CAS NO. & COMPOUND & $\begin{array}{l}\text { CONCENTRATION UNITS: } \\
(\mathrm{ug} / \mathrm{L} \text { or } \mathrm{ug} / \mathrm{kg}) \mathrm{ug} / \mathrm{L}\end{array}$ & $Q$ \\
\hline $79-01-6$ & Trichloroethene & 0.50 & $\mathrm{U}$ \\
\hline $108-87-2$ & Methylcyclohexane & 0.50 & $\mathrm{U}$ \\
\hline $78-87-5$ & 1,2-Dichloropropane & 0.50 & $\mathrm{U}$ \\
\hline $75-27-4$ & Bromodichloromethane & 0.50 & $\mathrm{U}$ \\
\hline $10061-01-5$ & cis-1,3-Dichloropropene & 0.50 & $\mathrm{U}$ \\
\hline $108-10-1$ & 4-Methyl-2-pentanone & 5.0 & $\mathrm{U}$ \\
\hline $1.08-88-3$ & Toluene & 0.0094 & $\mathrm{~J}$ \\
\hline $10061-02-6$ & trans-1,3-Dichloropropene & 0.50 & $\mathrm{U}$ \\
\hline $79-00-5$ & 1,1,2-Trichloroethane & 0.50 & $\mathrm{U}$ \\
\hline $127-18-4$ & Tetrachloroethene & 0.50 & $\mathrm{U}$ \\
\hline $591-78-6$ & 2-Hexanone & 5.0 & $\mathrm{U}$ \\
\hline $124-48-1$ & Dibromochloromethane & 0.50 & $\mathrm{U}$ \\
\hline $106-93-4$ & 1,2-Dibromoethane & 0.50 & $\mathrm{U}$ \\
\hline $108-90-7$ & Chlorobenzene & 0.50 & $\mathrm{U}$ \\
\hline $100-41-4$ & Ethylbenzene & 0.50 & $\mathrm{U}$ \\
\hline $95-47-6$ & o-Xylene & 0.50 & $\mathrm{U}$ \\
\hline $179601-23-1$ & $\mathrm{~m}, \mathrm{p}$-xylene & 0.50 & $\mathrm{U}$ \\
\hline $100-42-5$ & Styrene & 0.50 & $\mathrm{U}$ \\
\hline $75-25-2$ & Bromoform & 0.50 & $\mathrm{U}$ \\
\hline $98-82-8$ & Isopropylbenzene & 0.50 & $\mathrm{U}$ \\
\hline $79-34-5$ & $1,1,2,2$-Tetrachloroethane & 0.50 & $\mathrm{U}$ \\
\hline $541-73-1$ & 1,3-Dichlorobenzene & 0.50 & $\mathrm{U}$ \\
\hline $106-46-7$ & 1,4-Dichlorobenzene & 0.50 & $\mathrm{U}$ \\
\hline $95-50-1$ & 1,2-Dichlorobenzene & 0.50 & $\mathrm{U}$ \\
\hline $96-12-8$ & 1,2-Dibromo-3-Chloropropane & 0.50 & $\mathrm{U}$ \\
\hline $120-82-1$ & 1,2,4-Trichlorobenzene & 0.50 & $\mathrm{U}$ \\
\hline $87-61-6$ & 1,2,3-Trichlorobenzene & 0.50 & $\mathrm{U}$ \\
\hline
\end{tabular}


$1 \mathrm{~J}$ - FORM I VOA-TIC

VOLATILE ORGANICS ANALYSIS DATA SHEET

TENTATIVELY IDENTIEIED COMPOUNDS
EPA SAMPLE NO.

VBLKDS
Lab Name: TESTAMERICA BURLINGTON Case No.: INMAN

Lab Code: STLV Matrix: (SOIL/SED/WATER)

Water

Sample wt/vol: 25.0 $(\mathrm{g} / \mathrm{mL}) \mathrm{mL}$

Level: (TRACE or LOW/MED)

TRACE

응 Moisture: not dec.

GC Column: DB-624 ID: $0.20 \quad$ (mm)

Soil Extract Volume: (uL)

CONCENTRATION UNITS: (ug/L or $\mathrm{ug} / \mathrm{kg}$ ) ug/L
Contract: 1E-30401 SDG No.: 200-6762
Lab Sample ID: MB 200-24615/3

Lab File ID: DHPV03.D

Date Received:

Date Analyzed: 09/01/2011

Dilution Factor: 1.0

Soil Aliquot Volume:

Purge Volume: 25.0 (mL)

\begin{tabular}{|l|l|c|c|c|}
\hline CAS NUMBER & \multicolumn{1}{|c|}{ COMPOUND NAME } & RT & EST. CONC. & Q \\
\hline & Unknown & 6.79 & N & X J \\
\hline E9667961 & Total Alkanes & N/A & & \\
\hline
\end{tabular}

1 EPA-designated Registry Number. 
Lab Name: TESTAMERICA BURLINGTON

Lab Code: STLV

Matrix: (SOIL/SED/WATER)

Sample wt/vol: 25.0

Water

$(\mathrm{g} / \mathrm{mL}) \quad \mathrm{mL}$

Level: (TRACE/LOW/MED) TRACE

Moisture: not dec.

GC Column: DB-624 ID: $0.20 \quad(\mathrm{~mm})$

Soil Extract Volume:

(mL)

Purge Volume: 25.0

Contract: 1E-30401

: : SDG No.: 200-6762

\begin{tabular}{|c|c|c|c|}
\hline CAS NO. & COMPOUND & $\begin{array}{l}\text { CONCENTRATION UNITS: } \\
(\mathrm{ug} / \mathrm{L} \text { or } \mathrm{ug} / \mathrm{kg}) \mathrm{ug} / \mathrm{L}\end{array}$ & $Q$ \\
\hline $75-71-8$ & Dichlorodifluoromethane & 0.50 & $\overline{\mathrm{U}}$ \\
\hline $74-87-3$ & Chloromethane & 0.50 & $\mathrm{U}$ \\
\hline $75-01-4$ & Vinyl chloride & 0.50 & $\mathrm{U}$ \\
\hline $74-83-9$ & Bromomethane & 0.50 & $\mathrm{U}$ \\
\hline $75-00-3$ & Chloroethane & 0.50 & $\mathrm{U}$ \\
\hline $75-69-4$ & Trichlorofluoromethane & 0.50 & $\mathrm{U}$ \\
\hline $75-35-4$ & 1,1-Dichloroethene & 0.50 & $\mathrm{U}$ \\
\hline $76-13-1$ & 1,1,2-Trichloro-1,2,2-trifluoroethane & 0.50 & $\mathrm{U}$ \\
\hline $67-64-1$ & Acetone & 5.0 & $\mathrm{U}$ \\
\hline $75-15-0$ & Carbon disulfide & 0.50 & $\mathrm{U}$ \\
\hline $79-20-9$ & Methyl acetate & 0.50 & $\mathrm{U}$ \\
\hline $75-09-2$ & Methylene Chloride & 0.50 & $\mathrm{U}$ \\
\hline $156-60-5$ & trans-1,2-Dichloroethene & 0.50 & $\mathrm{U}$ \\
\hline $1634-04-4$ & Methyl tert-butyl ether & 0.50 & U \\
\hline $75-34-3$ & 1,1-Dichloroethane & 0.50 & $\mathrm{U}$ \\
\hline $156-59-2$ & cis-1,2-Dichloroethene & 0.50 & $\mathrm{U}$ \\
\hline $78-93-3$ & 2-Butanone & 5.0 & $\mathrm{U}$ \\
\hline $74-97-5$ & Bromochloromethane & 0.50 & $\mathrm{U}$ \\
\hline $67-66-3$ & Chloroform & 0.50 & $\mathrm{U}$ \\
\hline $71-55-6$ & 1,1,1-Trichloroethane & 0.50 & $\mathrm{U}$ \\
\hline $110-82-7$ & Cyclohexane & 0.50 & $\mathrm{U}$ \\
\hline $56-23-5$ & Carbon tetrachloride & 0.50 & U \\
\hline $71-43-2$ & Benzene & 0.50 & $\mathrm{U}$ \\
\hline $107-06-2$ & 1,2-Dichloroethane & 0.50 & U \\
\hline
\end{tabular}

Report 1,4-Dioxane for Low-Medium VOA analysis only

Lab Sample ID: 200-6762-13

Lab File ID: DHPV22.D

Date Received:

Date Analyzed: 09/01/2011

Dilution Factor: 1.0

Soil Aliquot Volume: 
$1 \mathrm{~B}$ - FORM I VOA-2

VOLATILE ORGANICS ANALYSIS DATA SHEET
EPA SAMPLE NO.

VHBLK01

Lab Name: TESTAMERICA BURLINGTON

Lab Code: STLV Case No.: INMAN Mod

Water

$(\mathrm{g} / \mathrm{mL}) \mathrm{mL}$

Sample wt/vol: 25.0

TRACE

Level: (TRACE/LOW/MED)

$\%$ Moisture: not dec.

GC Column: DB-624

ID: 0.20

$(\mathrm{mm})$

Soil Extract Volume:

(uL)

Purge Volume: 25.0

(mL)
Contract: 1E-30401

SDG No.: 200-6762

Lab Sample ID: 200-6762-13

Lab File ID: DHPV22.D

Date Received:

Date Analyzed: 09/01/2011

Dilution Factor: 1.0

Soil Aliquot Volume:

(uL)

\begin{tabular}{|c|c|c|c|}
\hline CAS NO. & COMPOUND & $\begin{array}{l}\text { CONCENTRATION UNITS: } \\
(\mathrm{ug} / \mathrm{L} \text { or } \mathrm{ug} / \mathrm{kg}) \mathrm{ug} / \mathrm{L}\end{array}$ & $Q$ \\
\hline $79-01-6$ & Trichloroethene & 0.50 & $\overline{\mathrm{U}}$ \\
\hline $108-87-2$ & Methylcyclohexane & 0.50 & $\mathrm{U}$ \\
\hline $78-87-5$ & 1,2-Dichloropropane & 0.50 & $\mathrm{U}$ \\
\hline $75-27-4$ & Bromodichloromethane & 0.50 & $\mathrm{U}$ \\
\hline $10061-01-5$ & cis-1,3-Dichloropropene & 0.50 & $\mathrm{U}$ \\
\hline $108-10-1$ & 4-Methyl-2-pentanone & 5.0 & $\mathrm{U}$ \\
\hline $108-88-3$ & Toluene & 0.50 & $\mathrm{U}$ \\
\hline $10061-02-6$ & trans-1,3-Dichloropropene & 0.50 & $\mathrm{U}$ \\
\hline $79-00-5$ & 1,1,2-Trichloroethane & 0.50 & $\mathrm{U}$ \\
\hline $127-18-4$ & Tetrachloroethene & 0.50 & $\mathrm{U}$ \\
\hline $591-78-6$ & 2-Hexanone & 5.0 & $\mathrm{U}$ \\
\hline $124-48-1$ & Dibromochloromethane & 0.50 & $\mathrm{U}$ \\
\hline $106-93-4$ & 1,2-Dibromoethane & 0.50 & $\mathrm{U}$ \\
\hline $108-90-7$ & Chlorobenzene & 0.50 & $\mathrm{U}$ \\
\hline $100-4.1-4$ & Ethylbenzene & 0.50 & $\mathrm{U}$ \\
\hline $95-47-6$ & o-Xylene & 0.50 & $\mathrm{U}$ \\
\hline $179601-23-1$ & $\mathrm{~m}, \mathrm{p}$-Xylene & 0.50 & $\mathrm{U}$ \\
\hline $100-42-5$ & Styrene & 0.50 & $\mathrm{U}$ \\
\hline $75-25-2$ & Bromoform & 0.50 & $\mathrm{U}$ \\
\hline $98-82-8$ & Isopropylbenzene & 0.50 & $\mathrm{U}$ \\
\hline $79-34-5$ & $1,1,2,2$-Tetrachloroethane & 0.50 & $\mathrm{U}$ \\
\hline $541-73-1$ & 1,3-Dichlorobenzene & 0.50 & $\mathrm{U}$ \\
\hline $106-46-7$ & 1,4-Dichlorobenzene & 0.50 & $\mathrm{U}$ \\
\hline $95-50-1$ & 1,2-Dichlorobenzene & 0.50 & $\mathrm{U}$ \\
\hline $96-12-8$ & 1,2-Dibromo-3-Chloropropane & 0.50 & $\mathrm{U}$ \\
\hline $120-82-1$ & 1,2,4-Trichlorobenzene & 0.50 & $\mathrm{U}$ \\
\hline $87-61-6$ & 1,2,3-Trichlorobenzene & 0.50 & $\mathrm{U}$ \\
\hline
\end{tabular}


$1 \mathrm{~J}$ - FORM I VOA-TIC

VOLATILE ORGANICS ANALYSIS DATA SHEET TENTATIVELY IDENTIFIED COMPOUNDS
EPA SAMPLE NO.

VHBLKO1
Lab Name: TESTAMERICA BURLINGTON Case No.: INMAN Mod. Ref No.:

Contract: 1E-30401
Water

Matrix: (SOIL/SED/WATER)

Sample wt/vol: 25.0

Level: (TRACE or LOW/MED)

$(\mathrm{g} / \mathrm{mL}) \quad \mathrm{mL}$

TRACE

음 Moisture: not dec.

GC Column: DB-624

ID: 0.20 (mm)

Soil Extract Volume: (UL)

CONCENTRATION UNITS: (ug/L or $\mathrm{ug} / \mathrm{kg}$ )

$\mathrm{ug} / \mathrm{L}$
SDG No.: 200-6762

Lab Sample ID: 200-6762-13

Lab File ID: DHPV22.D

Date Received:

Date Analyzed: 09/01/2011

Dilution Factor: 1.0

Soil Aliquot Volume:

(UL)

Purge Volume: 25.0

(mL)

\begin{tabular}{|l|l|l|r|r|r|}
\hline \multicolumn{1}{c|}{ CAS NUMBER } & \multicolumn{1}{|c|}{ COMPOUND NAME } & RT & EST. CONC. & Q \\
\cline { 2 - 6 } 01 & & Unknown & 6.79 & 2.7 & B X J \\
\cline { 2 - 6 } & E9667961 & Total Alkanes & N/A & & \\
\hline
\end{tabular}

1 EPA-designated Registry Number. 


\section{TestAmerica}

THE LEADER IN ENYIRONMENTAL TESTING

\section{ANALYTICAL REPORT}

Job Number: 200-6783-1

SDG Number: 200-6783

Job Description: Inman (200-6783)

Contract Number: 1E-30401

For:

Argonne National Laboratory

9700 South Cass Avenue

Building 203

Office B-149

Argonne, IL 60439

Attention: Mr. Clyde Dennis

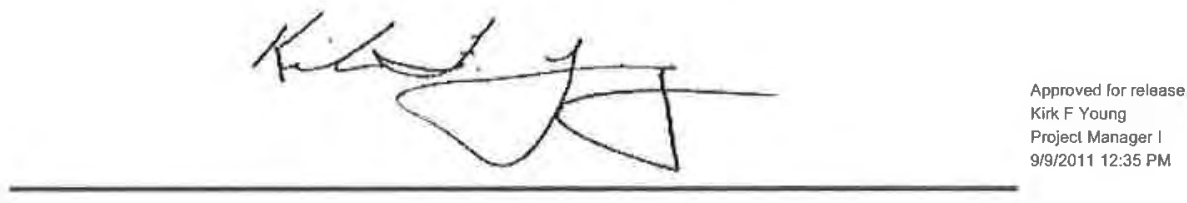

Kirk F Young

Project Manager I

kirk.young@testamericainc.com

09/09/2011

The test results in this report relate only to sample(s) as received by the laboratory. These test results were derived under a quality system that adheres to the requirements of NELAC. Pursuant to NELAC, this report may not be produced in full without written approval from the laboratory 


\section{Table of Contents}

Cover Title Page ........................ 1

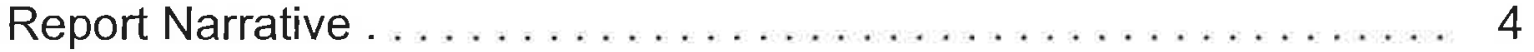

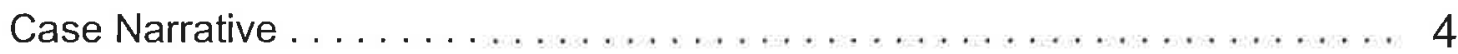

Qualifier Definition ............................. 6

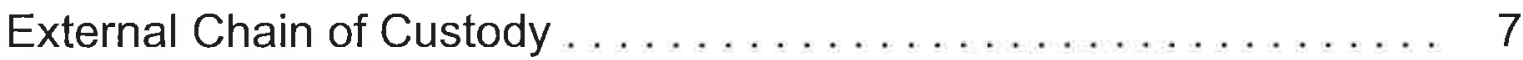

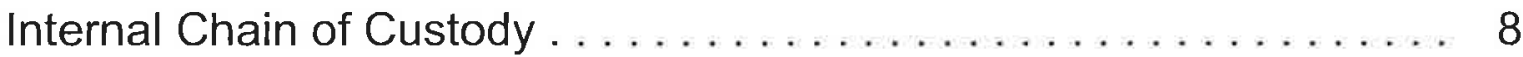

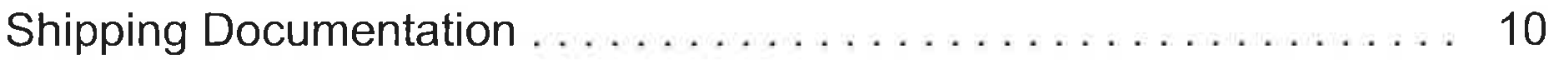

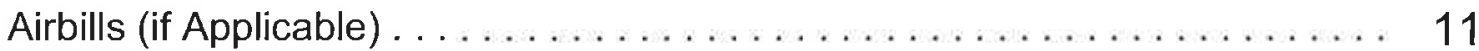

Sample Receipt and Log In Check List . .................... 12

Methodology Review . . . . . . . . . . . . . . . . . . . 14

QC Summary - SOM01.2 Volatiles-Trace . . . . . . . . . . . . . . . 15

QC Summary - SOM01.2 Volatiles-Trace . . . . . . . . . . . . . . . . 15

Deuterated Monitoring Compound Summary $\ldots \ldots \ldots \ldots \ldots \ldots \ldots \ldots \ldots \ldots \ldots, 15$

Method Blank $\ldots \ldots \ldots \ldots \ldots \ldots \ldots \ldots \ldots \ldots \ldots \ldots \ldots \ldots \ldots \ldots \ldots \ldots \ldots, 17$

GC/MS Instrument Performance Check . . . . . . . . . . . . . . . . 19

Internal Standard Area and RT Summary . . . . . . . . . . . . . . . . . . . . . . 22

Sample Data - SOM01.2 Volatiles-Trace . . . . . . . . . . . . . . 24

Sample Data - SOM01.2 Volatiles-Trace .................... 24

INMW16-W-32764 .......................................... 24

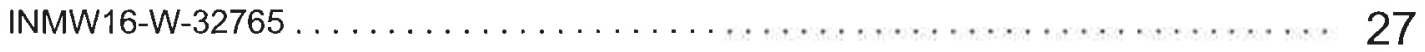

INMW16-W-32767 ....................................... 30

INMW19-W-32768 ...................................... 33

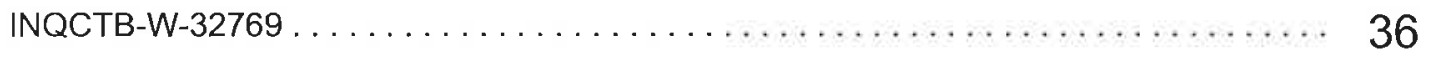

Standards - SOM01.2 Volatiles-Trace . . . . . . . . . . . . . . . 39

Standards - SOM01.2 Volatiles-Trace . . . . . . . . . . . . . . . . 39

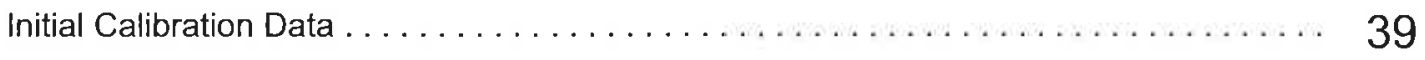

$\begin{array}{ll}\text { Page } 2 \text { of } 62 & 09 / 09 / 2011\end{array}$ 


\section{Table of Contents}

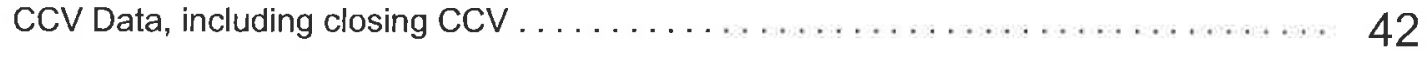

Raw Qc Data - SOM01.2 Volatiles-Trace . . . . . . . . . . . . . . . . . . . . 54

Raw Qc Data - SOM01.2 Volatiles-Trace . . . . . . . . . . . . . . . . . . . . . 54

Raw Qc Data - SOM01.2 Volatiles-Trace ...................... 54

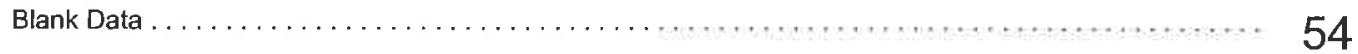




\title{
CASE NARRATIVE
}

\section{Client: Argonne National Laboratory}

\author{
Project: Inman (200-6783)
}

\author{
Report Number: 200-6783-1
}

Enclosed is the data set for the referenced project work. With the exceptions noted as flags or footnotes, standard analytical protocols were followed in performing the analytical work and the applied control limits were met.

Calculations were performed before rounding to avoid round-off errors in calculated results.

All holding times were met and proper preservation noted for the methods performed on these samples, unless otherwise detailed in the individual sections below.

\section{Receipt}

The samples were received on 08/31/2011. Documentation of the condition of the samples at the time of their receipt and any exception to the laboratory's Sample Acceptance Policy is documented in the Shipping and Receiving section of this submittal. The samples, as received, were not acid preserved. On that basis, the laboratory did provide for the analytical work to be performed within seven days of sample collection.

\section{SOM01.2 Volatile Organics (Trace Level Water)}

A storage blank was prepared for volatile organics analysis, and stored in association with the storage of the samples. That storage blank, identified as VHBLK01, was carried through the holding period with the samples, and analyzed.

Each sample in the sample set was analyzed without a dilution. Each of the analyses associated with the sample set exhibited an acceptable internal standard performance. There was an acceptable recovery of each deuterated monitoring compound (DMC) in the analysis of the method blanks associated with the analytical work, and in the analysis of the storage blank associated with the sample set. The analysis of the samples in this sample set did meet the technical acceptance criteria specific to DMC recoveries, although not all DMC recoveries were within the control range in each analysis. The technical acceptance criteria does provide for the recovery of up to three DMCs to fall outside of the control range in the analysis of field samples. Matrix spike and matrix spike duplicate analyses were not performed on samples in this sample set. A trace concentration of toluene was identified in the analysis of one of the method blanks associated with the analytical work (VBLKDS). The concentration of toluene in that analysis was below the established reporting limit, and the analysis did meet the technical acceptance criteria for a compliant method blank analysis. The analysis of the storage blank associated with the sample set was free of analyte contamination. Present in the method blank and storage blank analyses was a non-target constituent that represents a compound that is related to the DMC formulation. The fact that the presence of this compound is not within the laboratory's control is at issue. The derived results for that compound have been qualified with an " $X$ " qualifier to reflect the source of the contamination.

The responses for each of the target analytes met the relative standard deviation criterion in the initial calibration. The response for each target analyte met the percent difference criterion in 
each opening/continuing calibration check acquisition. The response for each target analyte met the 50.0 percent difference criterion in each closing calibration check acquisition.

The primary quantitation mass for methylcyclohexane that is specified in the Statement of Work is mass 83 . The laboratory did identify a contribution to mass 83 from 1,2-dichloropropane- $d_{6}$, one of the deuterated monitoring compounds (DMCs). The laboratory did change the primary quantitation mass assignment to mass 55 for the quantification of methylcyclohexane.

Manual integration was employed in deriving certain of the analytical results. The values that have been derived from manual integration are qualified on the quantitation reports. Extracted ion current profiles for each manual integration are included in the data package, and further documented at the end of this submittal. 


\section{DATA REPORTING QUALIFIERS}

Client: Argonne National Laboratory

Job Number: $200-6783-1$

Sdg Number: $200-6783$

GC/MS VOA

U

$J$

$J$

X

B

N
Analyzed for but not detected.

Indicates an Estimated Value for TICs

Indicates an estimated value.

See case narrative notes for explanation of the ' $X$ ' flag

Surrogate exceeds the control limit

The analyte was found in an associated blank, as well as in the sample.

This flag indicates the presumptive evidence of a compound. 


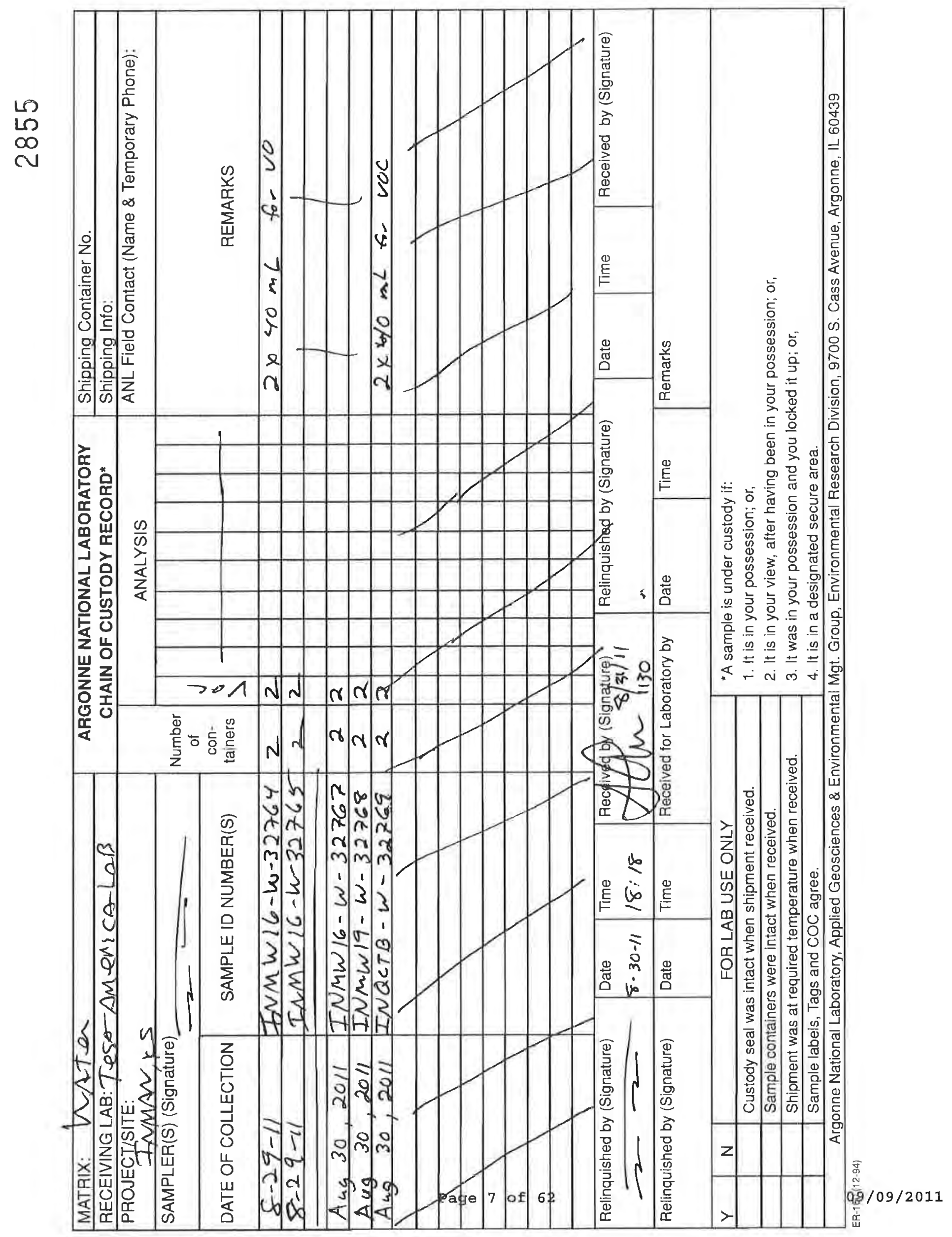




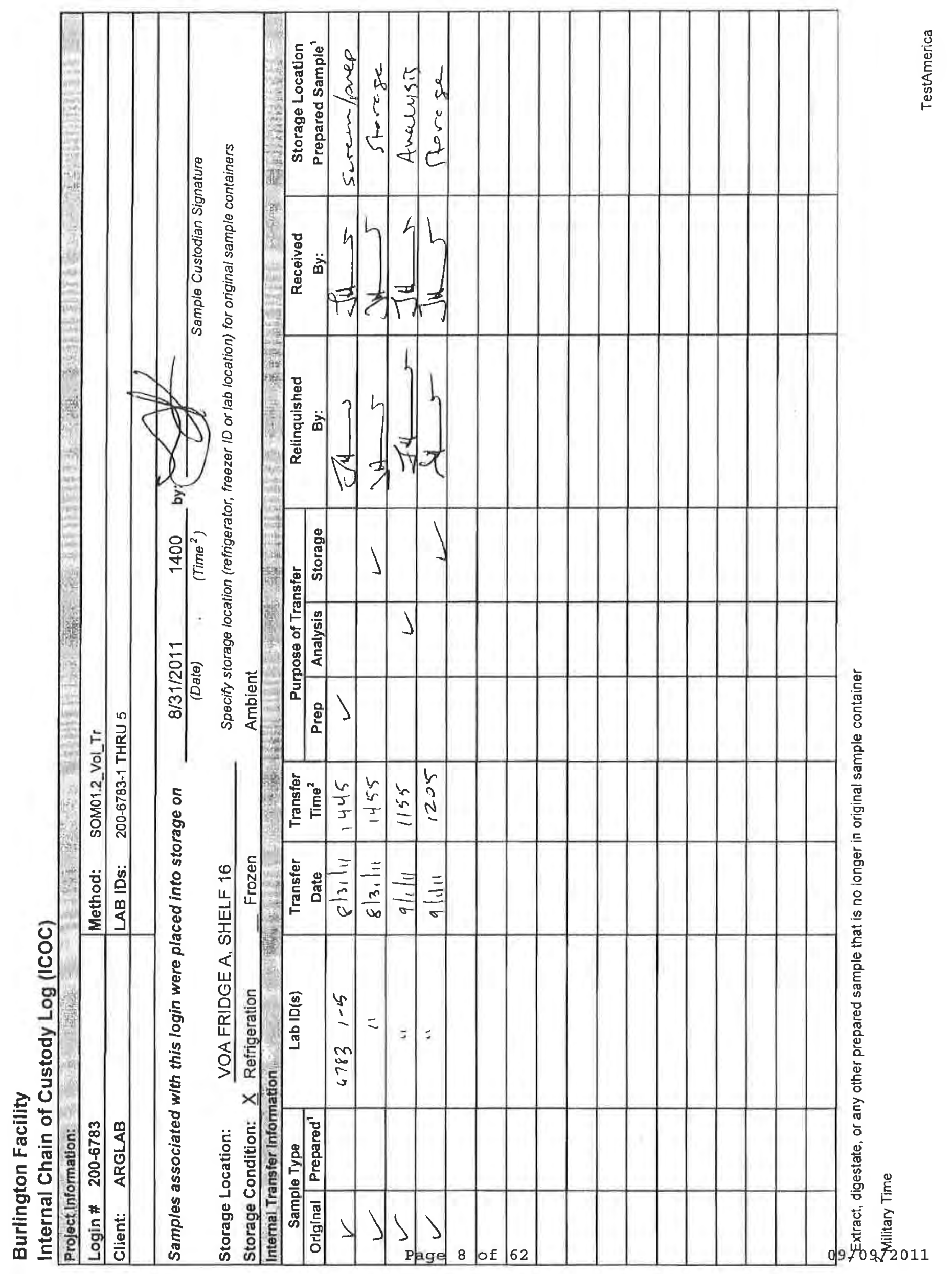




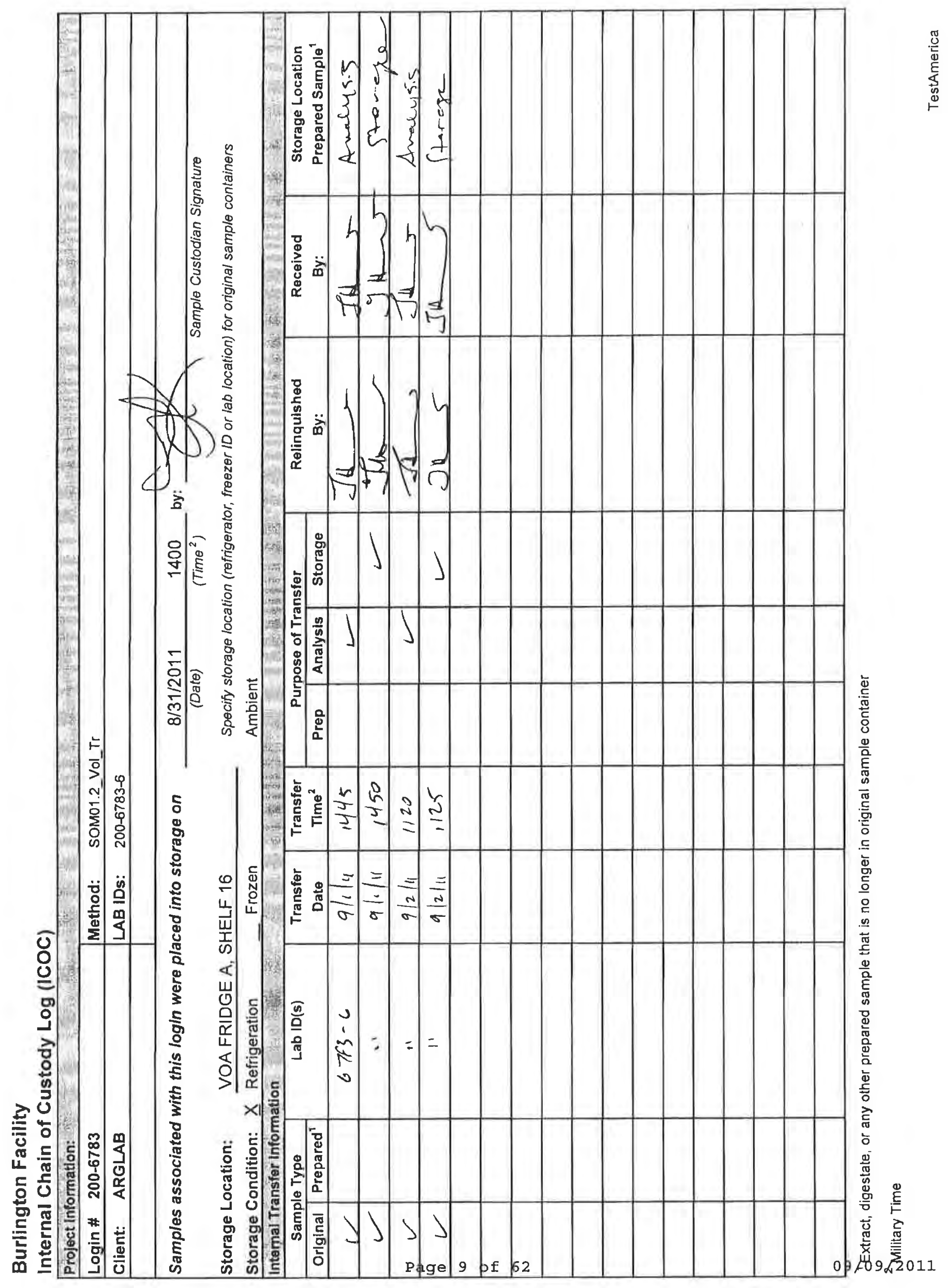




\section{Shipping and Receiving Documents}


Fed $\mathrm{IZ}_{\mathrm{B}}$ NEW Package Express US Airbill

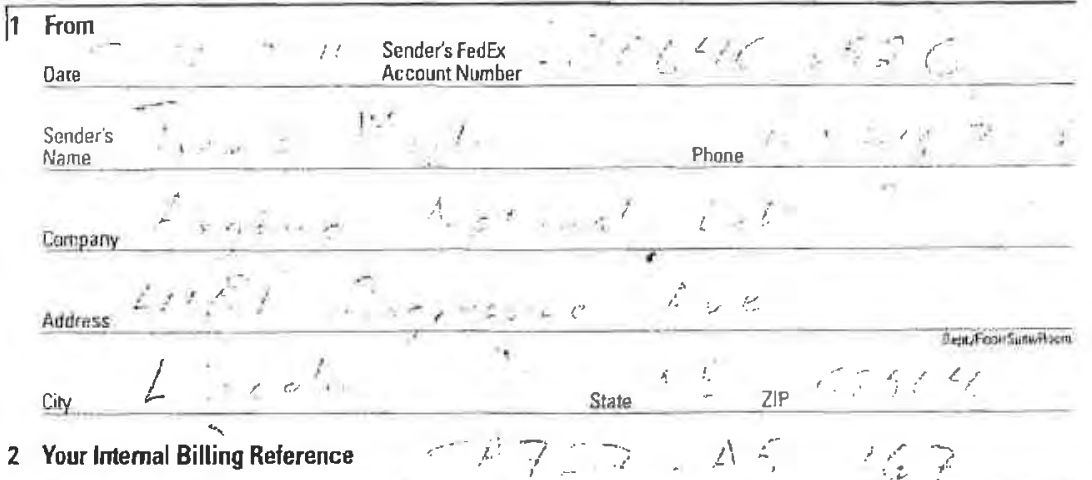

To
Recipient's $K, k$, $K$ Name

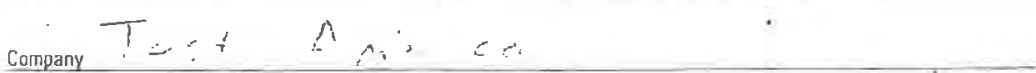
Zi

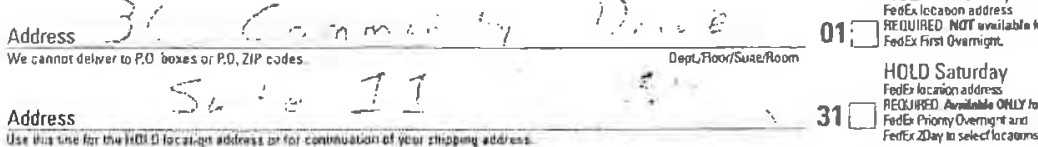

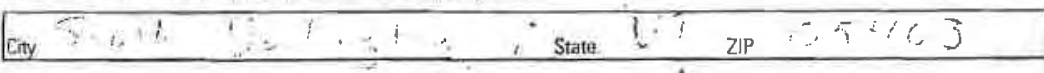
in

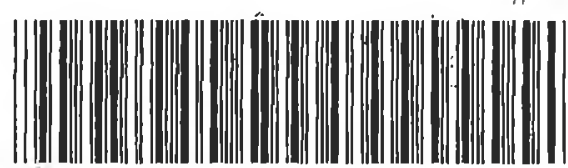

प्रिए

FedEx Patiovel Copi

4 Express Package Servic

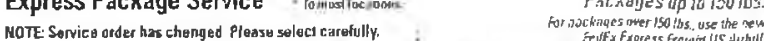

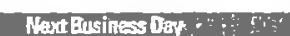

06, Fed Ex First Overnigin

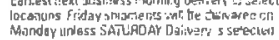

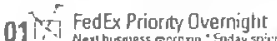

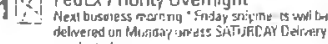

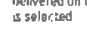

$05:-7$ FedEx Stándard Dverright

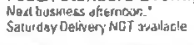

5 Packaging : Ond wod yedua limi Ison

06. JedEx Envelope ${ }^{*} 02$.] FedEx Pak ${ }^{*}$

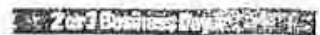

49!: NEN FedE\& 20ay A.A.

03 FEsEx 2Day

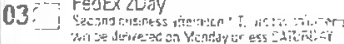

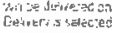

20 C FeiEx Express Saver

\section{Special Handling and Delivery Signature Options} 03!_ SATURDAY DEUVERY

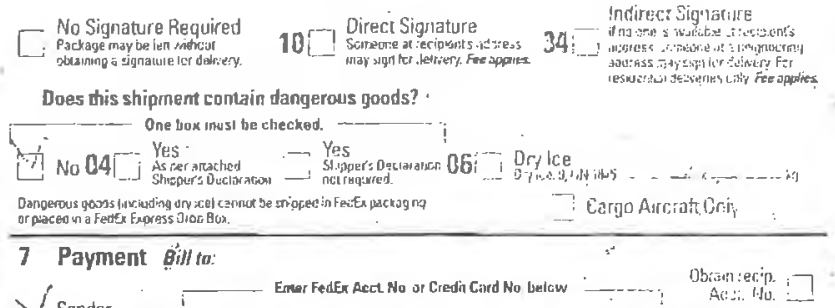
Sender
1

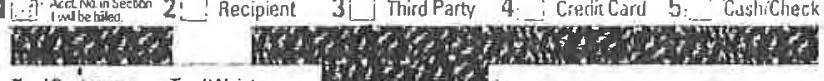
Total Packages Total Weąight

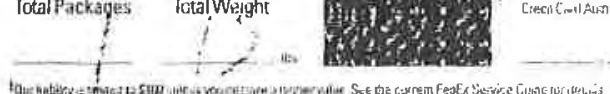

61 그 


\section{Login Sample Receipt Checklist}

Client: Argonne National Laboratory

Job Number: 200-6783-1

SDG Number: 200-6783

Login Number: 6783

List Source: TestAmerica Burlington

List Number: 1

Creator: Marion, Greg T

Question

Answer

Comment

Radioactivity either was not measured or, if measured, is at or below

N/A

Lab does not accept radioactive samples.

background

The cooler's custody seal, if present, is intact.

NO CUSTODY SEAL NUMBERS

The cooler or samples do not appear to have been compromised or

True

tampered with

Samples were received on ice.

True

True

Cooler Temperature is acceptable.

Cooler Temperature is recorded.

True

COC is present.

True

$5.2^{\circ} \mathrm{C}$, IR GUN ID 96, CF 0

COC is filled out in ink and legible.

True

$\mathrm{COC}$ is filled out with all pertinent information.

True

Is the Field Sampler's name present on COC?

False

Sample times not listed on COC

True

There are no discrepancies between the sample IDs on the containers and

True

he COC

Samples are received within Holding Time.

True

Sample containers have legible labels.

True

Containers are not broken or leaking.

True

Sample collection date/times are provided.

True

Appropriate sample containers are used.

True

Sample bottles are completely filled.

True

Sample Preservation Verified.

N/A

Sample volumes were received unpreserved.

There is sufficient vol. for all requested analyses, incl. any requested

MS/MSDs

VOA sample vials do not have headspace or bubble is $<6 \mathrm{~mm}\left(1 / 4^{\prime \prime}\right)$ in

True

diameter.

Multiphasic samples are not present.

True

Samples do not require splitting or compositing.

Residual Chlorine Checked.

True

N/A

Check done at department level if required. 


\section{Sample Login Acknowledgement}

Job 200-6783-1

$\begin{array}{ll}\text { Client Job Description: } & \text { Inman (200-6783) } \\ \text { Purchase Order \#: } & 1 \mathrm{E}-30401 \\ \text { Work Order \#: } & 1 \mathrm{E}-30401 \\ \text { Project Manager: } & \text { Kirk F Young } \\ \text { Job Due Date: } & 9 / 14 / 2011 \\ \text { Job TAT: } & 14 \text { Days } \\ \text { Max Deliverable Level: } & \text { IV } \\ & \\ \text { Earliest Deliverable Due: } & 9 / 14 / 2011\end{array}$

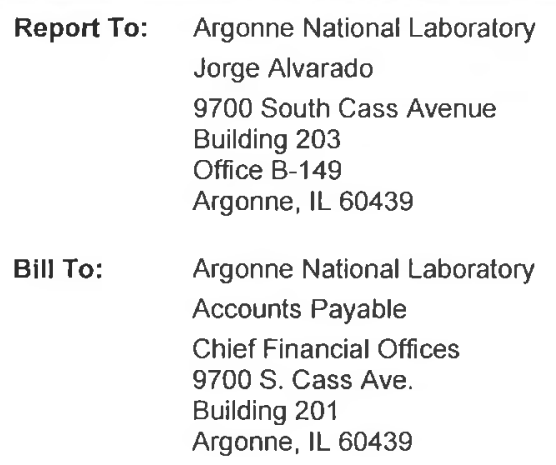

Login 200-6783

\begin{tabular}{|c|c|c|c|c|c|c|}
\hline Sample Receipt: & 8/31/2011 11:30:00 AM & \multirow{2}{*}{\multicolumn{3}{|c|}{$\begin{array}{lc}\text { Number of Coolers: } & 1 \\
\text { Cooler Temperature }(\mathrm{s})\left(\mathrm{C}^{\circ}\right): & 5.2\end{array}$}} & & \\
\hline Method of Delivery: & FedEx Priority Overnight & & & & & \\
\hline Lab Sample \# & Client Sample ID & Date Samp & oled & Matrix & & \\
\hline Method & Method Description / Work Location & & & & Rpt Basis & Dry / Wet ** \\
\hline $200-6783-1$ & INMW16-W-32764 & $8 / 29 / 2011$ & 5:50:00 PM & Water & & \\
\hline SOM01.2_Vol_Tr & SOM01.2 Trace Volatile Organics / In-Lab & & & & Total & Wet \\
\hline $200-6783-2$ & INMW16-W-32765 & $8 / 29 / 2011$ & 5:55:00 PM & Water & & \\
\hline SOM01.2_Vol_Tr & SOM01.2 Trace Volatile Organics / In-Lab & & & & Total & Wet \\
\hline $200-6783-3$ & INMW16-W-32767 & $8 / 30 / 2011$ & 1:30:00 PM & Water & & \\
\hline SOM01.2_Vol_Tr & SOM01.2 Trace Volatile Organics / In-Lab & & & & Total & Wet \\
\hline $200-6783-4$ & INMW19-W-32768 & $8 / 30 / 2011$ & 2:35:00 PM & Water & & \\
\hline SOM01.2_Vol_Tr & SOM01.2 Trace Volatile Organics / In-Lab & & & & Total & Wet \\
\hline $200-6783-5$ & INQCTB-W-32769 & $8 / 30 / 2011$ & 5:45:00 PM & Water & & \\
\hline SOM01.2_Vol_Tr & SOM01.2 Trace Volatile Organics / In-Lab & & & & Total & Wet \\
\hline $200-6783-6$ & VHBLK01 & $8 / 31 / 2011$ & 1:50:00 PM & Water & & \\
\hline SOM01.2_Vol_Tr & SOM01.2 Trace Volatile Organics / In-Lab & & & & Total & Wet \\
\hline
\end{tabular}




\section{METHODOLOGY SUMMARY}

$\begin{array}{llll}\text { Laboratory: } & \text { TestAmerica Laboratories } & \text { Project No: } \\ \text { Location: } & \text { South Burlington, Vermont } & \text { SDG No: } & \text { 200-6783 }\end{array}$

VOA

Volatile Organics Trace - USEPA CLP SOM01.2 
$2 A$ - FORM II VOA-1

WATER VOLATILE DEUTERATED MONITORING COMPOUND RECOVERY

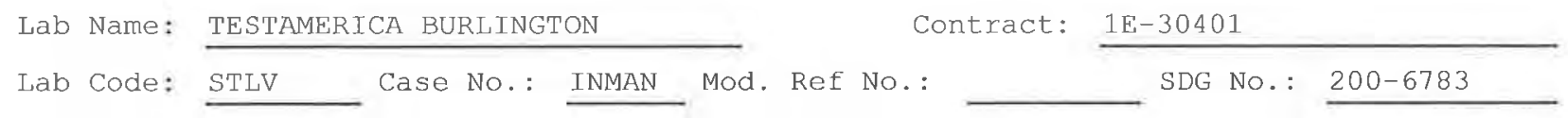

Level: (TRACE or LOW) TRACE

\begin{tabular}{|c|c|c|c|c|c|c|c|}
\hline $\begin{array}{c}\text { EPA } \\
\text { SAMPLE NO. }\end{array}$ & $\begin{array}{l}\text { VDMC1 } \\
\text { (VCL) \# }\end{array}$ & $\begin{array}{l}\text { VDMC2 } \\
\text { (CLA) \# }\end{array}$ & $\begin{array}{l}\text { VDMC3 } \\
\text { (DCE) \# }\end{array}$ & $\begin{array}{l}\text { VDMC4 } \\
\text { (BUT) \# }\end{array}$ & $\begin{array}{l}\text { VDMC5 } \\
\text { (CLE) \# }\end{array}$ & $\begin{array}{l}\text { VDMC6 } \\
\text { (DCA) \# }\end{array}$ & $\begin{array}{l}\text { VDMC7 } \\
\text { (BEN) \# }\end{array}$ \\
\hline VBLKDS & 92 & 91 & 66 & 94 & 84 & 93 & 91 \\
\hline INMW16-W-32764 & 93 & 92 & 66 & $165 *$ & 86 & 93 & 91 \\
\hline INMW16-W-32767 & 86 & 87 & 62 & 139 & 79 & 88 & 86 \\
\hline INMW19-W-32768 & 94 & 92 & 66 & 148 & 86 & 95 & 91 \\
\hline VHBLK01 & 88 & 88 & 66 & 95 & 85 & 95 & $\overline{88}$ \\
\hline
\end{tabular}

\footnotetext{
VDMC1 $($ VCL $)=$ Vinyl Chloride-d3

VDMC2 (CLA) = Chloroethane-d5

VDMC3 $(\mathrm{DCE})=1,1-$ Dichloroethene-d2

VDMC4 $(B U T)=2$-Butanone-d5

VDMC5 $(\mathrm{CLF})=$ Chloroform-d

VDMC6 $(D C A)=1,2-$ Dichloroethane-d4

VDMC7 (BEN) = Benzene-d6
}

$$
\begin{gathered}
\text { QC LIMITS } \\
\hline(65-131) \\
(71-131) \\
(55-104) \\
(49-155) \\
(78-121) \\
(78-129) \\
(77-124)
\end{gathered}
$$

\# Column to be used to flag recovery values

* Values outside of contract required QC limits

Page 1 of 1 
$2 \mathrm{~B}$ - FORM II VOA-2

WATER VOLATILE DEUTERATED MONITORING COMPOUND RECOVERY

Lab Name: TESTAMERICA BURLINGTON

Contract: $1 \mathrm{E}-30401$

Lab Code: STLV Case No.: INMAN Mod. Ref No.:

SDG No.: 200-6783

Level: (TRACE or LOW) TRACE

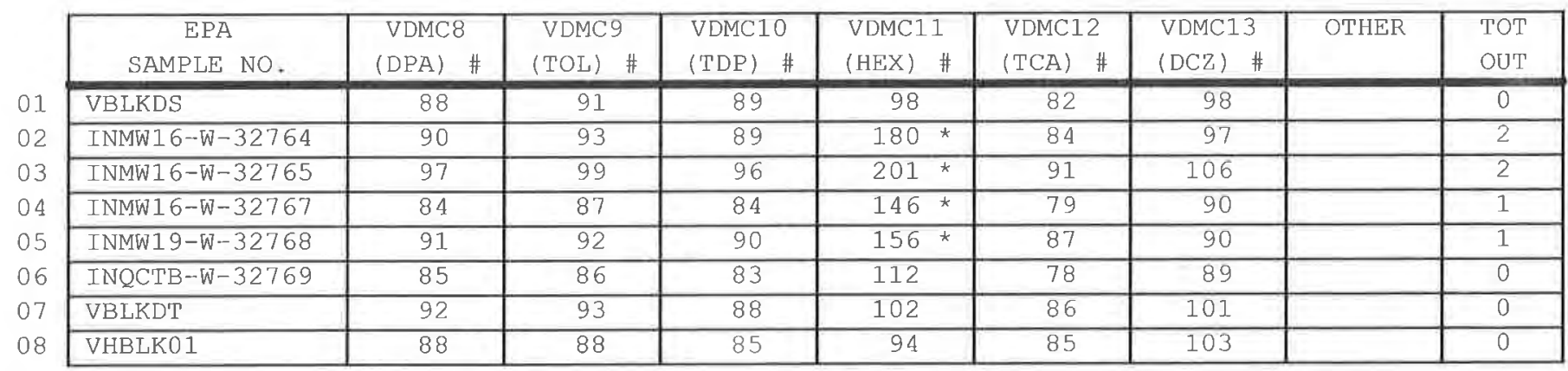

VDMC $(D P A)=1,2-$ Dichloropropane-d6

QC LIMITS

VDMC9 $($ TOL $)=$ Toluene-d8

$(79-124)$

VDMC10 $(\mathrm{TDP})=$ trans $-1,3-\mathrm{Dich}$ loropropene-d4

$(77-121)$

VDMC1 $1(\mathrm{HEX})=2-$ Hexanone-d5

$(73-121)$

VDMC12 $(\mathrm{TCA})=1,1,2,2$-Tetrachloroethane-d2

$(28-135)$

VDMC13 $($ DCZ $)=1,2$-Dichlorobenzene-d4

$(73-125)$

$(80-131)$

\# Column to be used to flag recovery values

* Values outside of contract required QC limits

Report 1,4-Dioxane-d8 for Low-Medium VOA analysis only

Page 1 of 1 
$4 A$ - FORM IV VOA

VOLATILE METHOD BLANK SUMMARY
EPA SAMPLE NO.

VBLKDS
Lab Name: TESTAMERICA BURLINGTON

Lab Code: STLV Case No.

Lab File ID: DHPV03.D

Instrument ID: D.i

Matrix: (SOIL/SED/WATER)

Water

Level: (TRACE or LOW/MED)

TRACE

GC Column: DB-624

ID: 0.20 (mm)
Contract: $1 \mathrm{E}-30401$

SDG No.: 200-6783

Lab Sample ID: MB 200-24615/3

Date Analyzed: 09/01/2011

Time Analyzed: 0812

Heated Purge: ( $\mathrm{Y} / \mathrm{N}$ ) N

\begin{tabular}{|l|l|l|l|c|}
\hline \multicolumn{1}{c|}{$\begin{array}{c}\text { EPA } \\
\text { SAMPLE NO. }\end{array}$} & \multicolumn{1}{|c|}{ LAB } & \multicolumn{1}{c|}{ LAB } \\
SAMPLE ID & $\begin{array}{c}\text { TIME } \\
\text { FILE ID }\end{array}$ & ANALYZED \\
\cline { 2 - 5 } 01 & $\begin{array}{l}\text { INMW16-W-327 } \\
64\end{array}$ & $200-6783-1$ & DHPV16.D & 1347 \\
\cline { 2 - 5 } 02 & $\begin{array}{l}\text { INMW16-W-327 } \\
65\end{array}$ & $200-6783-2$ & DHPV17.D & 1412 \\
\cline { 2 - 5 } 03 & $\begin{array}{l}\text { INMW16-W-327 } \\
67\end{array}$ & $200-6783-3$ & DHPV18.D & 1436 \\
\cline { 2 - 5 } 04 & $\begin{array}{l}\text { INMW19-W-327 } \\
68\end{array}$ & $200-6783-4$ & DHPV19.D & 1501 \\
\hline \multirow{2}{*}{05} & $\begin{array}{l}\text { INQCTB-W-327 } \\
69\end{array}$ & $200-6783-5$ & DHPV20.D & 1526 \\
\hline
\end{tabular}

COMMENTS:

Page 1 of 1 
$4 A$ - FORM IV VOA

VOLATILE METHOD BLANK SUMMARY
EPA SAMPLE NO.

VBLKDT
Lab Name: TESTAMERICA BURLINGTON

Lab Code: STLV Case No.: INMA

Lab File ID: DHPW04.D

Instrument ID: D.i

Matrix: (SOIL/SED/WATER)

Level: (TRACE or LOW/MED)

GC Column: DB-624

ater

TRACE

ID : 0.20
Contract: $1 E-30401$

SDG No.: 200-6783

Lab Sample ID: MB 200-24648/4

Date Analyzed: 09/02/2011

Time Analyzed: 0907

Heated Purge: $(\mathrm{Y} / \mathrm{N}) \mathrm{N}$
01

\begin{tabular}{c} 
EPA \\
SAMPLE NO. \\
\hline VHBIKO1
\end{tabular}

$\mathrm{LAB}$ SAMPLE ID

\begin{tabular}{|c|c|}
\hline $\begin{array}{c}\text { LAB } \\
\text { FILE ID }\end{array}$ & $\begin{array}{c}\text { TIME } \\
\text { ANALYZED }\end{array}$ \\
\hline DHPW13.D & 1303 \\
\hline
\end{tabular}

COMMENTS :

Page 1 of 1 
$5 A$ - FORM V VOA

VOLATILE ORGANICS INSTRUMENT

PERFORMANCE CHECK

BROMOFLUOROBENZENE (BEB)
EPA SAMPLE NO.

BE'BDV
Lab Name: TESTAMERICA BURLINGTON

Contract: $1 \mathrm{E}-30401$

Lab Code: STLV Case No.: INMAN Mod. Ref No.:

SDG No.: $200-6783$

Lab File Id: DHP01.D

Instrument Id: D. i

GC Column: DB-624

ID: 0.20
BEB Injection Date: 07/07/2011

BEB Injection Time: 1005

\begin{tabular}{|r|l|r|}
\hline m/e & ION ABUNDANCE CRITERIA & $\begin{array}{c}\text { RELATIVE } \\
\text { ABUNDANCE }\end{array}$ \\
\hline 50 & $15.0-40.0 \%$ of mass 95 & 26.1 \\
\hline 75 & $30.0-80.0 \%$ of mass 95 & 46.4 \\
\hline 95 & Base peak, 100\% relative abundance & 100 \\
\hline 96 & $5.0-9.0 \%$ of mass 95 & 6.3 \\
\hline 173 & Less than $2.0 \%$ of mass 174 & 0 \\
\hline 174 & $50.0-120 \%$ of mass 95 & $0) 1$ \\
\hline 175 & $5.0-9.0 \%$ of mass 174 & 77.7 \\
\hline 176 & $95.0-101 \%$ of mass 174 & 7.0 \\
\hline 177 & $5.0-9.0 \%$ of mass 176 & $7.8) 1$ \\
\hline
\end{tabular}

1 - Value is omass $174 \quad 2$ - Value is omass 176

\begin{tabular}{|c|c|c|c|c|}
\hline $\begin{array}{c}\text { EPA } \\
\text { SAMPLE NO. }\end{array}$ & $\begin{array}{r}\text { IAB } \\
\text { SAMPLE ID }\end{array}$ & $\begin{array}{c}\text { LAB } \\
\text { FILE ID }\end{array}$ & $\begin{array}{c}\text { DATE } \\
\text { ANALYZED }\end{array}$ & $\begin{array}{c}\text { TIME } \\
\text { ANALYZED }\end{array}$ \\
\hline VSTDO.5DV & IC $200-21023 / 4$ & DHPO 4.D & $07 / 07 / 2011$ & 1117 \\
\hline VSTD001DV & IC $200-21023 / 5$ & DHPO5.D & $07 / 07 / 2011$ & 1141 \\
\hline VSTD005DV & ICIS $200-21023 / 6$ & DHPO 6.D & $07 / 07 / 2011$ & 1206 \\
\hline VSTD010DV & IC $200-21023 / 7$ & DHPO7.D & $07 / 07 / 2011$ & 1231 \\
\hline VSTD020DV & IC $200-21023 / 8$ & DHP08.D & $07 / 07 / 2011$ & 1256 \\
\hline
\end{tabular}


$5 A$ - FORM V VOA

VOLATILE ORGANICS INSTRUMENT

PERFORMANCE CHECK

BROMOFLUOROBENZENE (BEB)
EPA SAMPLE NO. BFBDS

Lab Name: TESTAMERICA BURLINGTON

Contract: 1E-30401

Lab Code: STLV Case No.: INMAN Mod. Ref No.: SDG NO.: 200-6783

Lab File Id: DHPV01.D

BFB Injection Date: 09/01/2011

Instrument Id: D.i

BFB Injection Time: 0728

GC Column: DB-624

ID: 0.20

(mm)

\begin{tabular}{|c|c|c|}
\hline $\mathrm{m} / \mathrm{e}$ & ION ABUNDANCE CRITERIA & $\begin{array}{l}\text { O RELATIVE } \\
\text { ABUNDANCE }\end{array}$ \\
\hline 50 & $15.0-40.0 \%$ of mass 95 & 27.5 \\
\hline 75 & $30.0-80.0 \%$ of mass 95 & 47.7 \\
\hline 95 & Base peak, $100 \%$ relative abundance & 100 \\
\hline 96 & $5.0-9.0 \%$ of mass 95 & 7.3 \\
\hline 173 & Less than $2.0 \%$ of mass 174 & $0.4 \imath$ \\
\hline 174 & $50.0-120 \%$ of mass 95 & 78.8 \\
\hline 175 & $5.0-9.0 \%$ of mass 174 & 5.91 \\
\hline 176 & $95.0-101 \%$ of mass 174 & $75.6(95.9) 1$ \\
\hline 177 & $5.0-9.0 \%$ of mass 176 & $4.7(6.2) 2$ \\
\hline
\end{tabular}

1 - Value is omass 174

2 - Value is omass 176

\begin{tabular}{|c|c|c|c|c|}
\hline $\begin{array}{c}\text { EPA } \\
\text { SAMPLE NO. }\end{array}$ & $\begin{array}{r}\text { LAB } \\
\text { SAMPLE ID }\end{array}$ & $\begin{array}{c}\text { LAB } \\
\text { FILE ID }\end{array}$ & $\begin{array}{c}\text { DATE } \\
\text { ANALYZED }\end{array}$ & $\begin{array}{c}\text { TIME } \\
\text { ANALYQED }\end{array}$ \\
\hline VSTD005DS & CCVIS $200-24615 / 2$ & DHPV02.D & $09 / 01 / 2011$ & 0748 \\
\hline VBLKDS & MB 200-24615/3 & DHPV03.D & $09 / 01 / 2011$ & 0812 \\
\hline $\begin{array}{l}\text { INMW16-W-3 } \\
2764\end{array}$ & $200-6783-1$ & DHPV16.D & $09 / 01 / 2011$ & 1347 \\
\hline $\begin{array}{l}\text { INMW16-W-3 } \\
2765\end{array}$ & $200-6783-2$ & DHPV17.D & $09 / 01 / 2011$ & 1412 \\
\hline $\begin{array}{l}\text { INMW16-W-3 } \\
2767\end{array}$ & $200-6783-3$ & DHPV18.D & $09 / 01 / 2011$ & 1436 \\
\hline $\begin{array}{l}\text { INMW19-W-3 } \\
2768\end{array}$ & $200-6783-4$ & DHPV19.D & $09 / 01 / 2011$ & 1501 \\
\hline $\begin{array}{l}\text { INQCTB-W-3 } \\
2769\end{array}$ & $200-6783-5$ & DHPV20.D & $09 / 01 / 2011$ & 1526 \\
\hline VSTD005SD & CCVC $200-24615 / 23$ & DHPV23.D & $09 / 01 / 2011$ & 1640 \\
\hline
\end{tabular}

Page 1 of 1 
$5 A$ - FORM V VOA

VOLATILE ORGANICS INSTRUMENT

PEREORMANCE CHECK

BROMOFLUOROBENZENE (BEB)
EPA SAMPLE NO.

BFBDT
Lab Name: TESTAMERICA BURIINGTON

Lab Code: STLV

Case No.: INMAN

Contract: $1 \mathrm{E}-30401$

Lab File Id: DHPW01.D

Instrument Id: D. i

GC Column: DB-624

ID: 0.20

(mm)

\begin{tabular}{|c|c|c|}
\hline $\mathrm{m} / \mathrm{e}$ & ION ABUNDANCE CRITERIA & $\begin{array}{l}\text { 음 RELATIVE } \\
\text { ABUNDANCE }\end{array}$ \\
\hline 50 & $15.0-40.0 \%$ of mass 95 & 26.8 \\
\hline 75 & $30.0-80.0 \%$ of mass 95 & 48.2 \\
\hline 95 & Base peak, 100응 relative abundance & 100 \\
\hline 96 & $5.0-9.0 \%$ of mass 95 & 7.1 \\
\hline 173 & Less than $2.0 \%$ of mass 174 & 1.01 \\
\hline 174 & $50.0-120 \%$ of mass 95 & 79.0 \\
\hline 175 & $5.0-9.0 \%$ of mass 174 & $5.7(7.3) 1$ \\
\hline 176 & $95.0-101 \%$ of mass 174 & $78.7(99.7) 1$ \\
\hline 177 & $5.0-9.0 \%$ of mass 176 & $4.4(5.6) 2$ \\
\hline
\end{tabular}

1 - Value is onass $174 \quad 2$ - Value is omass 176

\begin{tabular}{|c|c|c|c|c|}
\hline $\begin{array}{c}\text { EPA } \\
\text { SAMPLE NO. }\end{array}$ & $\begin{array}{r}\text { LAB } \\
\text { SAMPLE ID }\end{array}$ & $\begin{array}{c}\text { LAB } \\
\text { FILE ID }\end{array}$ & $\begin{array}{c}\text { DATE } \\
\text { ANALYZED }\end{array}$ & $\begin{array}{c}\text { TIME } \\
\text { ANALYZED }\end{array}$ \\
\hline VSTD005DT & CCVIS $200-24648 / 3$ & DHPW03.D & $09 / 02 / 2011$ & 0842 \\
\hline VBLKDT & MB $200-24648 / 4$ & DHPW04.D & $09 / 02 / 2011$ & 0907 \\
\hline VHBLKO1 & $200-6783-6$ & DHPW13.D & $09 / 02 / 2011$ & 1303 \\
\hline VSTD005TD & CCVC $200-24648 / 14$ & DHPW1 4.D & $09 / 02 / 2011$ & 1327 \\
\hline
\end{tabular}

Page 1 of 1 
Lab Code: STLV Case No.: INMAN Mod. Ref No.: SDG No.: 200-6783

GC Column: DB-624 ID $: 0.20$ (min) Init

EPA Sample No.(VSTD\#\#\#\#\#): VSTD005DS

Date Analyzed: 09/01/2011

Lab File ID (Standard): DHPV02.D

Time Analyzed: 0748

Instrument ID: D.i Heated Purge: (Y/N) N

\begin{tabular}{|c|c|c|c|c|c|c|}
\hline & $\begin{array}{c}\text { IS1 (CBZ) } \\
\text { AREA }\end{array}$ & $\mathrm{RT}$ ㅇ & $\begin{array}{c}\text { IS2 (DFB) } \\
\text { AREA }\end{array}$ & RT \# & $\begin{array}{c}\text { IS3 (DCB) } \\
\text { AREA } \\
\end{array}$ & $\mathrm{RT}$ \\
\hline 12 HOUR STD & 162461 & 8.83 & 193024 & 5.47 & 76685 & 11.66 \\
\hline UPPER LIMIT & 227445 & 9.16 & 270234 & 5.80 & 107359 & 11.99 \\
\hline LOWER LIMIT & 97477 & 8.50 & 115814 & 5.14 & 46011 & 11.33 \\
\hline EPA SAMPLE NO. & & & & & & \\
\hline$\overline{\text { VBLKDS }}$ & 149599 & 8.83 & 179380 & 5.47 & 59790 & 11.66 \\
\hline INMW16-W-32764 & 155658 & 8.83 & 184446 & 5.47 & 65883 & 11.66 \\
\hline INMW16-W-32765 & 160877 & 8.83 & 188508 & 5.47 & 70699 & 11.66 \\
\hline INMW16-W-32767 & 169393 & 8.83 & 202064 & 5.47 & 73428 & 11.66 \\
\hline INMW19-W-32768 & 181489 & 8.83 & 213589 & 5.47 & 87378 & 11.66 \\
\hline INQCTB-W-32769 & 168661 & 8.83 & 198543 & 5.47 & 73304 & 11.66 \\
\hline
\end{tabular}

IS1 $(\mathrm{CBZ})=$ Chlorobenzene-d5

IS2 $(\mathrm{DFB})=1,4-\mathrm{Difluorobenzene}$

IS3 $(\mathrm{DCB})=1,4-\mathrm{Dich}$ lorobenzene-d4

AREA UPPER LIMIT $=140 \%$ (Trace Volatiles) of internal standard area AREA LOWER LIMTT $=60 \%$ (Trace Volatiles) of internal standard area RT UPPER LIMIT $=+0.33$ (Trace Volatiles) minutes of internal standard RT RT LOWER LIMIT $=-0.33$ (Trace Volatiles) minutes of internal standard RT

\# Column used to flag values outside contract required QC limits with an asterisk. 
Lab Code: STLV Case No.: INMAN Mod. Ref No.: SDG No.: 200-6783

GC Column: DB-624 ID: 0.20 (mm) Init. Calib. Date(s): 07/07/2011 07/07/2011

EPA Sample No. (VSTD\#\#\#\#\#): VSTD005DT Date Analyzed: 09/02/2011

Lab File ID (Standard): DHPw03.D Time Analyzed: 0842

Instrument ID: D.i Heated Purge: $(\mathrm{Y} / \mathrm{N}) \mathrm{N}$

\begin{tabular}{|c|c|c|c|c|c|c|}
\hline & $\begin{array}{c}\text { IS1 (CBZ) } \\
\text { AREA }\end{array}$ & RT \# & $\begin{array}{c}\text { IS2 (DFB) } \\
\text { AREA }\end{array}$ & RT \# & $\begin{array}{c}\text { IS3 (DCB) } \\
\text { AREA }\end{array}$ & RT \# \\
\hline 12 HOUR STD & 172970 & 8.83 & 199477 & 5.47 & 85243 & 11.66 \\
\hline UPPER LIMIT & 242158 & 9.16 & 279268 & 5.80 & 119340 & 11.99 \\
\hline LOWER LIMIT & 103782 & 8.50 & 119686 & 5.14 & 51146 & 11.33 \\
\hline EPA SAMPLE NO. & & & & & & \\
\hline$\overline{\text { VBLKDT }}$ & 165625 & 8.83 & 195396 & 5.47 & 66988 & 11.66 \\
\hline VHBLKOI & 138515 & 8.83 & 162133 & 5.47 & 53603 & 11.66 \\
\hline
\end{tabular}

IS1 (CBZ) = Chlorobenzene-d5

Is2 $(\mathrm{DFB})=1,4$-Difluorobenzene

IS3 $(\mathrm{DCB})=1,4-\mathrm{Dich}$ lorobenzene-d4

AREA UPPER LIMIT $=140 \%$ (Trace Volatiles) of internal standard area AREA LOWER LIMIT $=60 \%$ (Trace Volatiles) of internal standard area RT UPPER LIMIT $=+0.33$ (Trace Volatiles) minutes of internal standard RT RT LOWER LIMIT $=-0.33$ (Trace Volatiles) minutes of internal standard RT

\# Column used to flag values outside contract required QC limits with an asterisk. 
Lab Name: TESTAMERICA BURLINGTON

Lab Code: STLV Case No.: INMA

\section{Water}

Matrix: (SOIL/SED/WATER)

$(\mathrm{g} / \mathrm{mL}) \mathrm{mL}$

Sample wt/vol: 25.0

TRACE

Level: (TRACE/LOW/MED)

\% Moisture: not dec.

GC Column: DB-624

ID $: 0.20 \quad(\mathrm{~mm})$

Soil Extract Volume:

(uL)

(mL)

Purge Volune: 25.0
Contract: 1E-30401

SDG No.: 200-6783

\begin{tabular}{|c|c|c|c|}
\hline CAS NO. & COMPOUND & $\begin{array}{l}\text { CONCENTRATION UNITS: } \\
(\mathrm{ug} / \mathrm{L} \text { or } \mathrm{ug} / \mathrm{kg}) \mathrm{ug} / \mathrm{L}\end{array}$ & Q \\
\hline $75-71-8$ & Dichlorodifluoromethane & 0.50 & $\overline{\mathrm{U}}$ \\
\hline $74-87-3$ & Chloromethane & 0.50 & $\mathrm{U}$ \\
\hline $75-01-4$ & Vinyl chloride & 0.50 & $\mathrm{U}$ \\
\hline $74-83-9$ & Bromomethane & 0.50 & $\mathrm{U}$ \\
\hline $75-00-3$ & Chloroethane & 0.50 & $\mathrm{U}$ \\
\hline $75-69-4$ & Trichlorofluoromethane & 0.50 & $\mathrm{U}$ \\
\hline $75-35-4$ & 1,1-Dichloroethene & 0.50 & $\mathrm{U}$ \\
\hline $76-13-1$ & 1,1,2-Trichloro-1,2,2-trifluoroethane & 0.50 & $\mathrm{U}$ \\
\hline $67-64-1$ & Acetone & 5.0 & $\mathrm{U}$ \\
\hline $75-15-0$ & Carbon disulfide & 0.50 & $\mathrm{U}$ \\
\hline $79-20-9$ & Methyl acetate & 0.50 & $\mathrm{U}$ \\
\hline $75-09-2$ & Methylene Chloride & 0.50 & $\mathrm{U}$ \\
\hline $156-60-5$ & trans-1,2-Dichloroethene & 0.50 & $\mathrm{U}$ \\
\hline $1634-04-4$ & Methyl tert-butyl ether & 0.50 & $\mathrm{U}$ \\
\hline $75-34-3$ & 1,1-Dichloroethane & 0.50 & $\mathrm{U}$ \\
\hline $156-59-2$ & cis-1,2-Dichloroethene & 0.50 & $\mathrm{U}$ \\
\hline $78-93-3$ & 2-Butanone & 5.0 & $\mathrm{U}$ \\
\hline $74-97-5$ & Bromochloromethane & 0.50 & $\mathrm{U}$ \\
\hline $67-66-3$ & Chloroform & 0.44 & $\mathrm{~J}$ \\
\hline $71-55-6$ & 1,1,1-Trichloroethane & 0.50 & $\mathrm{U}$ \\
\hline $110-82-7$ & Cyclohexane & 0.50 & $\mathrm{U}$ \\
\hline $56-23-5$ & Carbon tetrachloride & 0.014 & $\mathrm{~J}$ \\
\hline $71-43-2$ & Benzene & 0.50 & $\mathrm{U}$ \\
\hline $107-06-2$ & 1,2-Dichloroethane & 0.50 & $\mathrm{U}$ \\
\hline
\end{tabular}

Report 1,4-Dioxane for Low-Medium VOA analysis only

Lab Sample ID: 200-6783-1

Lab File ID: DHPV16.D

Date Received: 08/31/2011

Date Analyzed: 09/01/2011

Dilution Factor: 1.0

Soil Aliquot Volume:

(uL) 
$1 B$ - EORM I VOA-2

VOLATILE ORGANICS ANALYSIS DATA SHEET
EPA SAMPLE NO.

INMW16-W-32764

Lab Name: TESTAMERICA BURLINGTON

Contract: 1E-30401

Lab Code: STLV Case No.: INMAN Mod. Ref No.:

Matrix: (SOIL/SED/WATER)

Sample wt/vol: 25.0

Water

$(\mathrm{g} / \mathrm{mL}) \mathrm{mL}$

Level: (TRACE/LOW/MED) TRACE

\% Moisture: not dec.

GC Column: DB-624

Soil Extract Volume: ID: $0.20 \quad(\mathrm{~mm})$

(uL)

(mL)

Purge Volume: 25.0
SDG No.: 200-6783

Lab Sample ID: 200-6783-1

Lab File ID: DHPV16.D

Date Received: 08/31/2011

Date Analyzed: 09/01/2011

Dilution Factor: 1.0

Soil Aliquot Volume:

(uL)

\begin{tabular}{|c|c|c|c|}
\hline CAS NO. & COMPOUND & $\begin{array}{l}\text { CONCENTRATION UNITS: } \\
(\mathrm{ug} / \mathrm{L} \text { or } \mathrm{ug} / \mathrm{kg}) \mathrm{ug} / \mathrm{L}\end{array}$ & $Q$ \\
\hline $79-01-6$ & Trichloroethene & 0.50 & $\mathrm{U}$ \\
\hline $108-87-2$ & Methylcyclohexane & 0.50 & $\mathrm{U}$ \\
\hline $78-87-5$ & 1,2-Dichloropropane & 0.50 & $\mathrm{U}$ \\
\hline $75-27-4$ & Bromodichloromethane & 0.53 & \\
\hline $10061-01-5$ & cis-1,3-Dichloropropene & 0.50 & $\mathrm{U}$ \\
\hline $108-10-1$ & 4-Methyl-2-pentanone & 5.0 & $\mathrm{U}$ \\
\hline $108-88-3$ & Toluene & 0.055 & $\mathrm{~J} \mathrm{~B}$ \\
\hline $10061-02-6$ & trans-1,3-Dichloropropene & 0.50 & $\mathrm{U}$ \\
\hline $79-00-5$ & 1,1,2-Trichloroethane & 0.50 & $\mathrm{U}$ \\
\hline $127-18-4$ & Tetrachloroethene & 0.50 & $\mathrm{U}$ \\
\hline $591-78-6$ & 2-Hexanone & 5.0 & $\mathrm{U}$ \\
\hline $124-48-1$ & Dibromochloromethane & 0.56 & \\
\hline $106-93-4$ & 1,2-Dibromoethane & 0.50 & $\mathrm{U}$ \\
\hline $108-90-7$ & Chlorobenzene & 0.50 & $\mathrm{U}$ \\
\hline $100-41-4$ & Ethylbenzene & 0.064 & $\mathrm{~J}$ \\
\hline $95-47-6$ & o-xylene & 0.077 & $\mathrm{~J}$ \\
\hline $179601-23-1$ & $\mathrm{~m}, \mathrm{p}$-Xylene & 0.17 & $\mathrm{~J}$ \\
\hline $100-42-5$ & Styrene & 0.50 & $\mathrm{U}$ \\
\hline $75-25-2$ & Bromoform & 0.50 & $\mathrm{U}$ \\
\hline $98-82-8$ & Isopropylbenzene & 0.50 & $\mathrm{U}$ \\
\hline $79-34-5$ & $1,1,2,2$-Tetrachloroethane & 0.50 & $\mathrm{U}$ \\
\hline $541-73-1$ & 1,3-Dichlorobenzene & 0.50 & $\mathrm{U}$ \\
\hline $106-46-7$ & 1,4-Dichlorobenzene & 0.50 & $\mathrm{U}$ \\
\hline $95-50-1$ & 1,2-Dichlorobenzene & 0.50 & $\mathrm{U}$ \\
\hline $96-12-8$ & 1,2-Dibromo-3-Chloropropane & 0.50 & $\mathrm{U}$ \\
\hline $120-82-1$ & 1,2,4-Trichlorobenzene & 0.50 & $\mathrm{U}$ \\
\hline $87-61-6$ & 1,2,3-Trichlorobenzene & 0.50 & $\mathrm{U}$ \\
\hline
\end{tabular}


$1 \mathrm{~J}$ - FORM I VOA-TIC

VOLATILE ORGANICS ANALYSIS DATA SHEET TENTATIVELY IDENTIFIED COMPOUNDS
EPA SAMPLE NO.

INMW1 6-W-32764

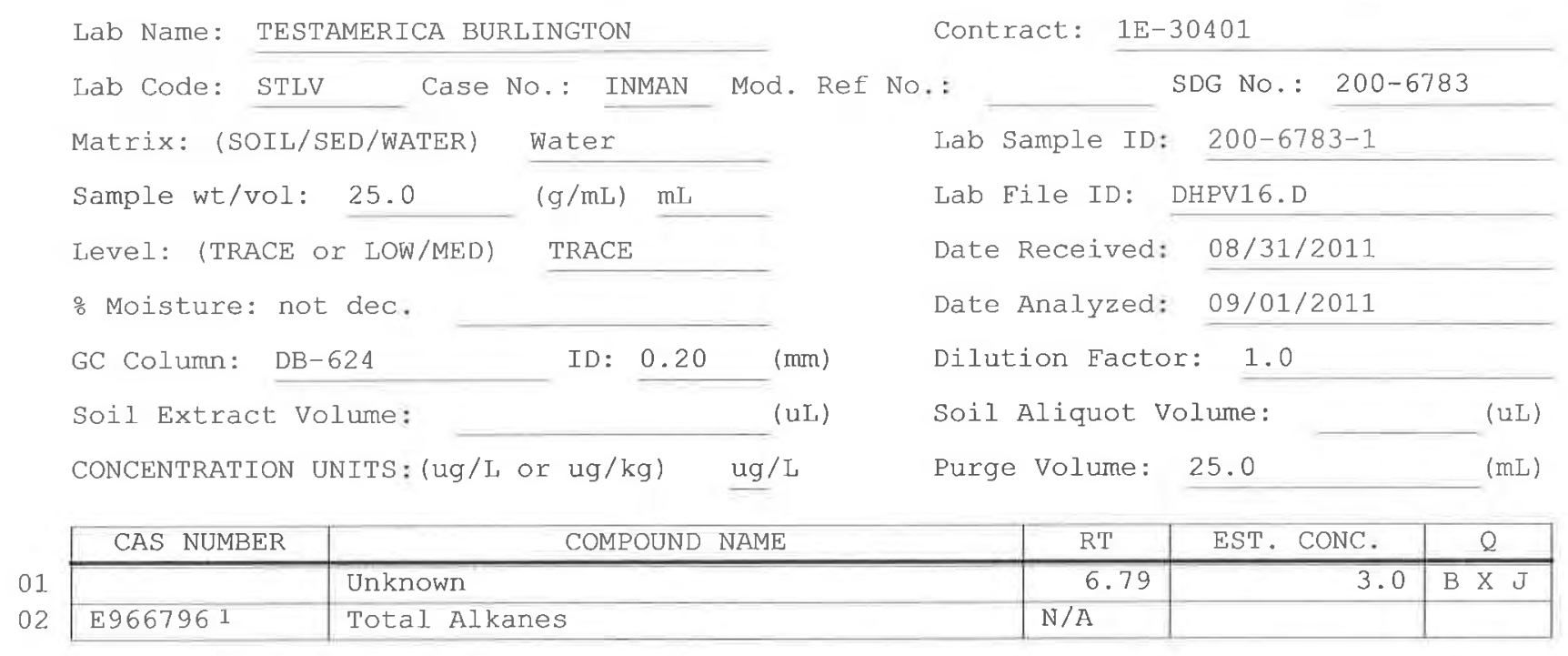

1 EPA-designated Registry Number. 
Lab Name: TESTAMERICA BURLINGTON

Lab Code: STLV Case No.: INMAN

Matrix: (SOIL/SED/WATER)

Water

Sample wt/vol: 25.0

$(\mathrm{g} / \mathrm{mL}) \quad \mathrm{mL}$

Level: (TRACE/IOW/MED) TRACE

음 Moisture: not dec.

GC Column: DB-624

ID : 0.20

$(\mathrm{mm})$

Soil Extract Volume:

(uL)

Contract: 1E-30401

Purge Volume: 25.0

$(\mathrm{mL})$
SDG No : 200-6783

Lab Sample ID: 200-6783-2

Lab File ID: DHPV17.D

Date Received: 08/31/2011

Date Analyzed: 09/01/2011

Dilution Factor: 1.0

Soil Aliquot Volume:

(uL)

\begin{tabular}{|c|c|c|c|}
\hline CAS NO. & COMPOUND & $\begin{array}{l}\text { CONCENTRATION UNITS: } \\
(\mathrm{ug} / \mathrm{L} \text { or } \mathrm{ug} / \mathrm{kg}) \mathrm{ug} / \mathrm{L}\end{array}$ & $Q$ \\
\hline $75-71-8$ & Dichlorodifluoromethane & 0.50 & $\mathrm{U}$ \\
\hline $74-87-3$ & Chloromethane & 0.50 & $\mathrm{U}$ \\
\hline $75-01-4$ & Vinyl chloride & 0.50 & $\mathrm{U}$ \\
\hline $74-83-9$ & Bromomethane & 0.50 & $\mathrm{U}$ \\
\hline $75-00-3$ & Chloroethane & 0.50 & $\mathrm{U}$ \\
\hline $75-69-4$ & Trichlorofluoromethane & 0.50 & $\mathrm{U}$ \\
\hline $75-35-4$ & 1,1-Dichloroethene & 0.50 & $\mathrm{U}$ \\
\hline $76-13-1$ & 1,1,2-Trichloro-1,2,2-trifluoroethane & 0.50 & $\mathrm{U}$ \\
\hline $67-64-1$ & Acetone & 2.2 & $\mathrm{~J}$ \\
\hline $75-15-0$ & Carbon disulfide & 0.50 & $\mathrm{U}$ \\
\hline $79-20-9$ & Methyl acetate & 0.50 & $\mathrm{U}$ \\
\hline $75-09-2$ & Methylene Chloride & 0.50 & $\mathrm{U}$ \\
\hline $156-60-5$ & trans-1,2-Dichloroethene & 0.50 & $\mathrm{U}$ \\
\hline $1634-04-4$ & Methyl tert-butyl ether & 0.50 & $\mathrm{U}$ \\
\hline $75-34-3$ & 1,1-Dichloroethane & 0.50 & $\mathrm{U}$ \\
\hline $156-59-2$ & cis-1,2-Dichloroethene & 0.50 & $\mathrm{U}$ \\
\hline $78-93-3$ & 2-Butanone & 1.9 & $\mathrm{~J}$ \\
\hline $74-97-5$ & Bromochloromethane & 0.50 & $\mathrm{U}$ \\
\hline $67-66-3$ & Chloroform & 0.42 & $\mathrm{~J}$ \\
\hline $71-55-6$ & 1,1,1-Trichloroethane & 0.50 & $\mathrm{U}$ \\
\hline $110-82-7$ & Cyclohexane & 0.50 & $\mathrm{U}$ \\
\hline $56-23-5$ & Carbon tetrachloride & 2.0 & \\
\hline $71-43-2$ & Benzene & 0.011 & $\mathrm{~J}$ \\
\hline $107-06-2$ & 1,2-Dichloroethane & 0.50 & $\mathrm{U}$ \\
\hline
\end{tabular}

Report 1,4-Dioxane for Low-Medium VOA analysis only 
$1 B$ - FORM I VOA-2

VOLATILE ORGANICS ANALYSIS DATA SHEET
EPA SAMPLE NO.

INMW16-W-32765
Lab Name: TESTAMERICA BURLINGTON

Lab Code: STLV

Case No.: INMAN

Matrix: (SOIL/SED/WATER)

Water

Sample wt/vol: 25.0

$(\mathrm{g} / \mathrm{mL}) \mathrm{mL}$

Level: (TRACE/LOW/MED) TRACE

\% Moisture: not dec.

GC Column: DB-624

ID: 0.20

( $\mathrm{mmn})$

Soil Extract Volume:

(uL)

(InL)

Purge Volume: 25.0
Contract: $1 \mathrm{E}-30401$

SDG No.: 200-6783

Lab Sample ID: 200-6783-2

Lab File ID: DHPV17.D

Date Received: 08/31/2011

Date Analyzed: 09/01/2011

Dilution Factor: 1.0

Soil Aliquot Volume:

(uL)

\begin{tabular}{|c|c|c|c|}
\hline CAS NO. & COMPOUND & $\begin{array}{l}\text { CONCENTRATION UNITS: } \\
(\mathrm{ug} / \mathrm{L} \text { or } \mathrm{ug} / \mathrm{kg}) \mathrm{ug} / \mathrm{L}\end{array}$ & $Q$ \\
\hline $79-01-6$ & Trichloroethene & 0.50 & $\overline{\mathrm{U}}$ \\
\hline $108-87-2$ & Methylcyclohexane & 0.50 & $\mathrm{U}$ \\
\hline $78-87-5$ & 1,2-Dichloropropane & 0.50 & $\mathrm{U}$ \\
\hline $75-27-4$ & Bromodichloromethane & 0.50 & $\mathrm{U}$ \\
\hline $10061-01-5$ & cis-1,3-Dichloropropene & 0.50 & $\mathrm{U}$ \\
\hline $108-10-1$ & 4-Methyl-2-pentanone & 5.0 & $\mathrm{U}$ \\
\hline $108-88-3$ & Toluene & 0.040 & $\mathrm{~J} B$ \\
\hline $10061-02-6$ & trans-1,3-Dichloropropene & 0.50 & $\mathrm{U}$ \\
\hline $79-00-5$ & 1,1,2-Trichloroethane & 0.50 & $\mathrm{U}$ \\
\hline $127-18-4$ & Tetrachloroethene & 0.50 & $\mathrm{U}$ \\
\hline $591-78-6$ & 2-Hexanone & 5.0 & $\mathrm{U}$ \\
\hline $124-48-1$ & Dibromochloromethane & 0.20 & $\mathrm{~J}$ \\
\hline $106-93-4$ & 1,2-Dibromoethane & 0.50 & $\mathrm{U}$ \\
\hline $108-90-7$ & Chlorobenzene & 0.50 & $\mathrm{U}$ \\
\hline $100-41-4$ & Ethylbenzene & 0.50 & $\mathrm{U}$ \\
\hline $95-47-6$ & o-Xylene & 0.50 & $\mathrm{U}$ \\
\hline $179601-23-1$ & $\mathrm{~m}, \mathrm{p}$-Xylene & 0.0051 & $\mathrm{~J}$ \\
\hline $100-42-5$ & Styrene & 0.50 & $\mathrm{U}$ \\
\hline $75-25-2$ & Bromoform & 0.50 & $\mathrm{U}$ \\
\hline $98-82-8$ & Isopropylbenzene & 0.50 & $\mathrm{U}$ \\
\hline $79-34-5$ & $1,1,2,2$-Tetrachloroethane & 0.50 & $\mathrm{U}$ \\
\hline $541-73-1$ & 1,3-Dichlorobenzene & 0.50 & $\mathrm{U}$ \\
\hline $106-46-7$ & 1,4-Dichlorobenzene & 0.50 & $\mathrm{U}$ \\
\hline $95-50-1$ & 1,2-Dichlorobenzene & 0.50 & $\mathrm{U}$ \\
\hline $96-12-8$ & 1,2-Dibromo-3-Ch1oropropane & 0.50 & $U$ \\
\hline $120-82-1$ & 1,2,4-Trichlorobenzene & 0.50 & $\mathrm{U}$ \\
\hline $87-61-6$ & 1,2,3-Trichlorobenzene & 0.50 & $\mathrm{U}$ \\
\hline
\end{tabular}


IJ - FORM I VOA-TIC

VOLATILE ORGANICS ANALYSIS DATA SHEET TENTATIVELY IDENTIFIED COMPOUNDS
EPA SAMPLE NO.

INMW 16-W-32765
Lab Name: TESTAMERICA BURLINGTON

Lab Code: STLV Case No.: INMAN

Matrix: (SOIL/SED/WATER)

Sample wt/vol: 25.0

Water

$(\mathrm{g} / \mathrm{mL}) \mathrm{mL}$

Level: (TRACE or LOW/MED)

TRACE

응 Moisture: not dec.

GC Column: DB-624

ID: 0.20

Soil Extract Volume:

(uL)

CONCEN'TRATION UNITS: (ug/I or $\mathrm{ug} / \mathrm{kg}$ ) ug/L
Contract: 1E-30401

SDG No.: $200-6783$

Lab Sample ID: 200-6783-2

Lab File ID: DHPV17.D

Date Received: 08/31/2011

Date Analyzed: 09/01/2011

Dilution Factor: 1.0

Soil Aliquot Volume:

Purge Volume: 25.0 $(\mathrm{mL})$

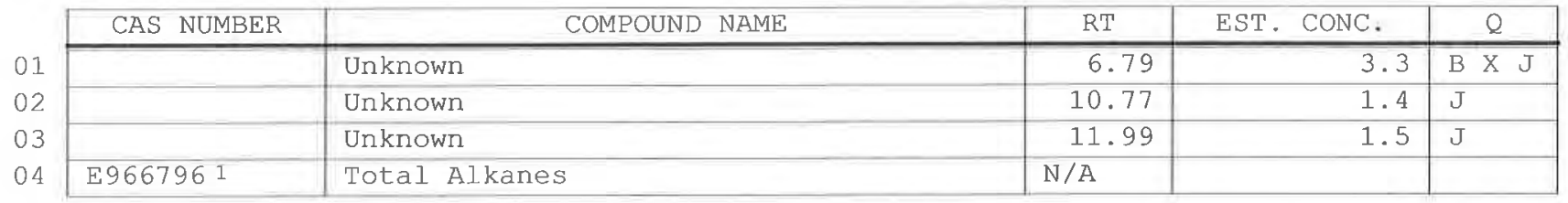

1 EPA-designated Registry Number. 
$1 A$ - FORM I VOA-1

VOLATILE ORGANICS ANALYSIS DATA SHEET
EPA SAMPLE NO.

INMW1 6-W-32767
Lab Name: TESTAMERICA BURLINGTON

Lab Code: STLV Case No.: INMAN Mod. Ref No.:
Water

$(\mathrm{g} / \mathrm{mL}) \quad \mathrm{mL}$

Sample wt/vol: 25.0

Leve l: (TRACE/LOW/MED)

TRACE

\% Moisture: not dec.

GC Column: DB-624

Soil Extract Volume: ID $: 0.20$
Contract: 1E-30401

SDG No.: 200-6783

Lab Sample ID: 200-6783-3

Lab File ID: DHPV18.D

Date Received: 08/31/2011

Date Analyzed: 09/01/2011

Dilution Factor: 1.0

Soil Aliquot volume:

(uL)

Purge Volume: 25.0

(mL)

\begin{tabular}{|c|c|c|c|}
\hline CAS NO. & COMPOUND & $\begin{array}{l}\text { CONCENTRATION UNITS: } \\
(\mathrm{ug} / \mathrm{L} \text { or } \mathrm{ug} / \mathrm{kg}) \mathrm{ug} / \mathrm{L}\end{array}$ & $Q$ \\
\hline $75-71-8$ & Dichlorodifluoromethane & 0.50 & $\overline{\mathrm{U}}$ \\
\hline $74-87-3$ & Chloromethane & 0.50 & $\mathrm{U}$ \\
\hline $75-01-4$ & Vinyl chloride & 0.50 & $\mathrm{U}$ \\
\hline $74-83-9$ & Bromomethane & 0.50 & $\mathrm{U}$ \\
\hline $75-00-3$ & Chloroethane & 0.50 & $\mathrm{U}$ \\
\hline $75-69-4$ & Trichlorofluoromethane & 0.50 & $\mathrm{U}$ \\
\hline $75-35-4$ & 1,1-Dichloroethene & 0.50 & $\mathrm{U}$ \\
\hline $76-13-1$ & 1,1,2-Trichloro-1,2,2-trifluoroethane & 0.50 & $\mathrm{U}$ \\
\hline $67-64-1$ & Acetone & 5.0 & $\mathrm{U}$ \\
\hline $75-15-0$ & Carbon disulfide & 0.50 & $\mathrm{U}$ \\
\hline $79-20-9$ & Methyl acetate & 0.50 & $\mathrm{U}$ \\
\hline $75-09-2$ & Methylene Chloride & 0.50 & $\mathrm{U}$ \\
\hline $156-60-5$ & trans 1,2 -Dichloroethene & 0.50 & $\mathrm{U}$ \\
\hline $1634-04-4$ & Methyl tert-butyl ether & 0.50 & $\mathrm{U}$ \\
\hline $75-34-3$ & 1,1-Dichloroethane & 0.50 & $\mathrm{U}$ \\
\hline $156-59-2$ & cis-1,2-Dichloroethene & 0.50 & $\mathrm{U}$ \\
\hline $78-93-3$ & 2-Butanone & 5.0 & $\mathrm{U}$ \\
\hline $74-97-5$ & Bromochloromethane & 0.50 & $\mathrm{U}$ \\
\hline $67-66-3$ & Chloroform & 0.41 & $\mathrm{~J}$ \\
\hline $71-55-6$ & 1,1,1-Trichloroethane & 0.50 & $\mathrm{U}$ \\
\hline $110-82-7$ & Cyclohexane & 0.50 & $\mathrm{U}$ \\
\hline $56-23-5$ & Carbon tetrachloride & 0.012 & $\mathrm{~J}$ \\
\hline $71-43-2$ & Benzene & 0.0084 & $\mathrm{~J}$ \\
\hline $107-06-2$ & 1,2-Dichloroethane & 0.50 & $\mathrm{U}$ \\
\hline
\end{tabular}

Report 1,4-Dioxane for Low-Medium VOA analysis only 
$1 B$ - FORM I VOA-2

VOLATILE ORGANICS ANALYSIS DATA SHEET
EPA SAMPLE NO.

INMW $16-W-32767$
Lab Name: TESTAMERICA BURLINGTON

Lab Code: STLV Case

Matrix: (SOIL/SED/WATER)

Sample wt/vol: 25.0

$(\mathrm{g} / \mathrm{mL}) \mathrm{mL}$

Level: (TRACE/LOW/MED) TRACE

응 Moisture: not dec.

GC Column: DB-624

ID: 0.20 $(\mathrm{mm})$

Soil Extract Volume:

(uL)

$(\mathrm{mL})$

Purge Volume: 25.0
Contract: 1E-30401

SDG No.: 200-6783

\section{:}

Lab Sample ID: 200-6783-3

Lab File ID: DHPV18.D

Date Received: 08/31/2011

Date Analyzed: 09/01/2011

Dilution Factor: 1.0

Soil Aliquot Volume:

(uL)

Purge volume: 25.0 (mT)

\begin{tabular}{|c|c|c|c|}
\hline CAS NO. & COMPOUND & $\begin{array}{l}\text { CONCENTRATION UNITS: } \\
(\mathrm{ug} / \mathrm{L} \text { or } \mathrm{ug} / \mathrm{kg}) \mathrm{ug} / \mathrm{L}\end{array}$ & $Q$ \\
\hline $79-01-6$ & Trichloroethene & 0.50 & $\overline{\mathrm{U}}$ \\
\hline $108-87-2$ & Methylcyclohexane & 0.50 & $\mathrm{U}$ \\
\hline $78-87-5$ & 1,2-Dichloropropane & 0.50 & $\mathrm{U}$ \\
\hline $75-27-4$ & Bromodichloromethane & 0.50 & $\mathrm{U}$ \\
\hline $10061-01-5$ & cis-1,3-Dichloropropene & 0.50 & $\mathrm{U}$ \\
\hline $108-10-1$ & 4-Methyl-2-pentanone & 5.0 & $\mathrm{U}$ \\
\hline $108-88-3$ & Toluene & 0.20 & $\mathrm{~J} \mathrm{~B}$ \\
\hline $10061-02-6$ & trans-1,3-Dichloropropene & 0.50 & $\mathrm{U}$ \\
\hline $79-00-5$ & 1,1,2-Trichloroethane & 0.50 & $\mathrm{U}$ \\
\hline $127-18-4$ & Tetrachloroethene & 0.50 & $\mathrm{U}$ \\
\hline $591-78-6$ & 2-Hexanone & 5.0 & $\mathrm{U}$ \\
\hline $124-48-1$ & Dibromochloromethane & 0.40 & $\mathrm{~J}$ \\
\hline $106-93-4$ & 1,2-Dibromoethane & 0.50 & $\mathrm{U}$ \\
\hline $108-90-7$ & Chlorobenzene & 0.50 & U \\
\hline $100-41-4$ & Ethylbenzene & 0.13 & $\mathrm{~J}$ \\
\hline $95-47-6$ & o-Xylene & 0.18 & $\mathrm{~J}$ \\
\hline $179601-23-1$ & $\mathrm{~m}, \mathrm{p}$-Xylene & 0.37 & $\mathrm{~J}$ \\
\hline $100-42-5$ & styrene & 0.50 & $\mathrm{U}$ \\
\hline $75-25-2$ & Bromoform & 0.50 & $\mathrm{U}$ \\
\hline $98-82-8$ & Isopropylbenzene & 0.50 & $\mathrm{U}$ \\
\hline $79-34-5$ & $1,1,2,2$-Tetrachloroethane & 0.50 & $\mathrm{U}$ \\
\hline $541-73-1$ & 1,3-Dichlorobenzene & 0.50 & $\mathrm{U}$ \\
\hline $106-46-7$ & 1,4-Dichlorobenzene & 0.50 & $\mathrm{U}$ \\
\hline $95-50-1$ & 1,2-Dichlorobenzene & 0.50 & $\mathrm{U}$ \\
\hline $96-12-8$ & 1,2-Dibromo-3-Chloropropane & 0.50 & $\mathrm{U}$ \\
\hline $120-82-1$ & 1,2,4-Trichlorobenzene & 0.50 & $\mathrm{U}$ \\
\hline $87-61-6$ & 1,2,3-Trichlorobenzene & 0.50 & $\mathrm{U}$ \\
\hline
\end{tabular}


$1 \mathrm{~J}$ - FORM I VOA-TIC

VOLATILE ORGANICS ANALYSIS DATA SHEET TENTATIVELY IDENTIFIED COMPOUNDS
EPA SAMPLE NO.

INMW16-W-32767
Lab Name: TESTAMERICA BURLINGION

Lab Code: STLV Case No.: INMAN Mod. Ref No.:

Matrix: (SOIL/SED/WATER) Wat

Sample wt/vol: 25.0

$(\mathrm{g} / \mathrm{mL}) \quad \mathrm{mL}$

Level: (TRACE or LOW/MED)

TRACE

음 Moisture: not dec.

GC Column: DB-624

ID: 0.20 (mm)

Soil Extract Volume: (uL)

CONCENTRATION UNITS: (ug/I or ug/kg) ug/L
Contract: $1 \mathrm{E}-30401$

SDG No.: 200-6783

Lab Sample ID: $200-6783-3$

Lab Eile ID: DHPV18.D

Date Received: 08/31/2011

Date Analyzed: 09/01/2011

Dilution Factor: 1.0

Soil Aliquot Volume: (uL)

Purge Volume: 25.0 (mL)

\begin{tabular}{|c|c|c|c|c|}
\hline CAS NUMBER & COMPOUND NAME & RT & EST. CONC. & $Q$ \\
\hline & Unknown & 6.79 & 2.7 & $\mathrm{~B} X \mathrm{~J}$ \\
\hline E9667961 & Total Alkanes & $N / A$ & & \\
\hline
\end{tabular}

1 EPA-designated Registry Number. 
1A - FORM I VOA-1

VOLATILE ORGANICS ANALYSIS DATA SHEET
EPA SAMPLE NO. INMW19-W-32768
Lab Name: TESTAMERICA BURLINGTON

Lab Code: STLV Case No.: INMAN

Matrix: (SOIL/SED/WATER)

Sample wt/vol: 25.0

Level: (TRACE/LOW/MED) TRACE

응 Moisture: not dec.

GC Column: DB-62.4 ID: $0.20 \quad$ (mm)

Soil Extract Volume:

Purge Volume: 25.0

(mL)
Contract: 1E-30401

SDG No.: 200-6783

Lab Sample ID: 200-6783-4

Lab File ID: DHPV19.D

Date Received: $08 / 31 / 2011$

Date Analyzed: 09/01/2011

Dilution Factor: 1.0

Soil Aliquot Volume:

(uL)

\begin{tabular}{|c|c|c|c|}
\hline CAS NO. & COMPOUND & $\begin{array}{l}\text { CONCENTRATION UNITS: } \\
(\mathrm{ug} / \mathrm{L} \text { or } \mathrm{ug} / \mathrm{kg}) \mathrm{ug} / \mathrm{L}\end{array}$ & Q \\
\hline $75-71-8$ & Dichlorodifluoromethane & 0.50 & $\overline{\mathrm{U}}$ \\
\hline $74-87-3$ & Chloromethane & 0.50 & $\mathrm{U}$ \\
\hline $75-01-4$ & Vinyl chloride & 0.50 & $\mathrm{U}$ \\
\hline $74-83-9$ & Bromomethane & 0.50 & $\mathrm{U}$ \\
\hline $75-00-3$ & Chloroethane & 0.50 & $\mathrm{U}$ \\
\hline $75-69-4$ & Trichlorofluoromethane & 0.50 & $\mathrm{U}$ \\
\hline $75-35-4$ & 1,1-Dichloroethene & 0.50 & $\mathrm{U}$ \\
\hline $76-13-1$ & $1,1,2$-Trichloro-1,2,2-trifluoroethane & 0.50 & U \\
\hline $67-64-1$ & Acetone & 1.5 & $\mathrm{~J}$ \\
\hline $75-15-0$ & Carbon disulfide & 0.50 & $\mathrm{U}$ \\
\hline $79-20-9$ & Methyl acetate & 0.50 & $\mathrm{U}$ \\
\hline $75-09-2$ & Methylene Chloride & 0.50 & $\mathrm{U}$ \\
\hline $156-60-5$ & trans $-1,2-$ Dichloroethene & 0.50 & $\mathrm{U}$ \\
\hline $1634-04-4$ & Methyl tert-butyl ether & 0.50 & $\mathrm{U}$ \\
\hline $75-34-3$ & 1,1-Dichloroethane & 0.50 & $\mathrm{U}$ \\
\hline $156-59-2$ & cis-1,2-Dichloroethene & 0.50 & U \\
\hline $78-93-3$ & 2-Butanone & 5.0 & $\mathrm{U}$ \\
\hline $74-97-5$ & Bromochloromethane & 0.50 & $\mathrm{U}$ \\
\hline $67-66-3$ & Chloroform & 0.50 & $\mathrm{U}$ \\
\hline $71-55-6$ & 1,1,1-Trichloroethane & 0.50 & $\mathrm{U}$ \\
\hline $110-82-7$ & Cyclohexane & 0.50 & $\mathrm{U}$ \\
\hline $56-23-5$ & Carbon tetrachloride & 0.12 & $\mathrm{~J}$ \\
\hline $71-43-2$ & Benzene & 0.046 & $\mathrm{~J}$ \\
\hline $107-06-2$ & 1,2-Dichloroethane & 0.50 & U \\
\hline
\end{tabular}

Report 1,4-Dioxane for Low-Medium VOA analysis only 
$1 B$ - FORM I VOA-2

VOLATILE ORGANICS ANALYSIS DATA SHEET
EPA SAMPLE NO. INMW 19-W-32768
Lab Name: TESTAMERICA BURLINGTON

Lab Code: STLV Case No.: INMA

Matrix: (SOIL/SED/WATER)

Water

Sample wt/vol: 25.0

$(\mathrm{g} / \mathrm{mL}) \mathrm{mL}$

Level: (TRACE/LOW/MED) TRACE

\% Moisture: not dec.

GC Colunn: DB-624

ID: 0.20 (mm)

Soil Extract Volume: (uL) $(\mathrm{mL})$

Purge Volume: 25.0
Contract: 1E-30401

SDG No.: 200-6783

Mod. Ref No.:

Lab Sample ID: 200-6783-4

Lab File ID: DHPV19.D

Date Received: 08/31/2011

Date Analyzed: 09/01/2011

Dilution Factor: 1.0

Soil Aliquot Volume:

(uL)

\begin{tabular}{|c|c|c|c|}
\hline CAS NO. & COMPOUND & $\begin{array}{l}\text { CONCENTRATION UNITS: } \\
(\mathrm{ug} / \mathrm{L} \text { or } \mathrm{ug} / \mathrm{kg}) \mathrm{ug} / \mathrm{L}\end{array}$ & $Q$ \\
\hline $79-01-6$ & Trichloroethene & 0.50 & $\mathrm{U}$ \\
\hline $108-87-2$ & Methylcyclohexane & 0.50 & $\mathrm{U}$ \\
\hline $78-87-5$ & 1,2-Dichloropropane & 0.50 & $\mathrm{U}$ \\
\hline $75-27-4$ & Bromodichloromethane & 0.50 & $\mathrm{U}$ \\
\hline $10061-01-5$ & cis-1,3-Dichloropropene & 0.50 & $\mathrm{U}$ \\
\hline $108-10-1$ & 4-Methyl-2-pentanone & 5.0 & $\mathrm{U}$ \\
\hline $108-88-3$ & Toluene & 0.054 & $\mathrm{~J} B$ \\
\hline $10061-02-6$ & trans-1,3-Dichloropropene & 0.50 & $\mathrm{U}$ \\
\hline $79-00-5$ & 1,1,2-Trichloroethane & 0.50 & $\mathrm{U}$ \\
\hline $127-18-4$ & Tetrachloroethene & 0.54 & \\
\hline $591-78-6$ & 2-Hexanone & 5.0 & $\mathrm{u}$ \\
\hline $124-48-1$ & Dibromochloromethane & 0.50 & $\mathrm{U}$ \\
\hline $106-93-4$ & 1,2-Dibromoethane & 0.50 & $\mathrm{U}$ \\
\hline $108-90-7$ & Chlorobenzene & 0.50 & $\mathrm{U}$ \\
\hline $100-41-4$ & Ethylbenzene & 0.50 & $\mathrm{U}$ \\
\hline $95-47-6$ & o-Xylene & 0.50 & $\mathrm{U}$ \\
\hline $179601-23-1$ & $\mathrm{~m}, \mathrm{p}$-xylene & 0.50 & $\mathrm{U}$ \\
\hline $100-42-5$ & Styrene & 0.50 & $\mathrm{U}$ \\
\hline $75-25-2$ & Bromoform & 0.50 & $\mathrm{U}$ \\
\hline $98-82-8$ & Isopropylbenzene & 0.50 & $\mathrm{U}$ \\
\hline $79-34-5$ & $1,1,2,2$-Tetrachloroethane & 0.50 & $\mathrm{U}$ \\
\hline $541-73-1$ & 1,3-Dichlorobenzene & 0.50 & $\mathrm{U}$ \\
\hline $106-46-7$ & 1,4-Dichlorobenzene & 0.50 & $\mathrm{U}$ \\
\hline $95-50-1$ & 1,2-Dichlorobenzene & 0.50 & $\mathrm{U}$ \\
\hline $96-12-8$ & 1,2-Dibromo-3-Chloropropane & 0.50 & $\mathrm{U}$ \\
\hline $120-82-1$ & 1,2,4-Trichlorobenzene & 0.50 & $\mathrm{U}$ \\
\hline $87-61-6$ & 1,2,3-Trichlorobenzene & 0.50 & $\mathrm{U}$ \\
\hline
\end{tabular}


1J - FORM I VOA-TIC

VOLATILE ORGANICS ANALYSIS DATA SHEET

TENTATIVELY IDENTIFIED COMPOUNDS
EPA SAMPLE NO.

INMW 19-W-32768

Lab Name: TESTAMERICA BURLINGTON

Lab Code: STLV Case No.: INMAN

Water

$(g / m L) \quad m L$

Sample wt/vol: 25.0

TRACE

Level: (TRACE or LOW/MED)

음 Moisture: not dec.

GC Column: DB-624

ID: 0.20

(mm)

Soil Extract Volume:

(uL)

CONCENTRATION UNITS: (ug/L or $\mathrm{ug} / \mathrm{kg}$ ) ug/L
Contract: 1E-30401

SDG No.: 200-6783

Lab Sample ID: 200-6783-4

Lab File ID: DHPV19.D

Date Received: 08/31/2011

Date Analyzed: 09/01/2011

Dilution Factor: 1.0

Soil Aliquot volume:

Purge Volume: 25.0 (mL)

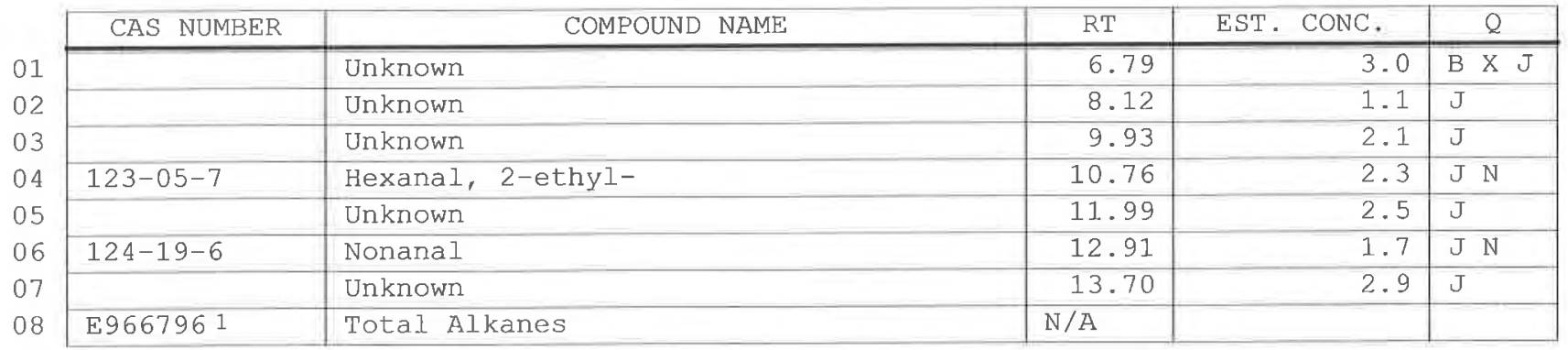

1 EPA-designated Registry Number. 
$1 A$ - EORM I VOA-1

VOLATILE ORGANICS ANALYSIS DATA SHEET
FPA SAMPLE NO.

INQCTB-W-32769
Lab Name: TESTAMERICA BURLINGTON

Lab Code: STLV Case No.: INMAN

Matrix: (SOIL/SED/WATER) Water

Sample wt/vol: 25.0

$(\mathrm{g} / \mathrm{mL}) \quad \mathrm{mL}$

Level: (TRACE/LOW/MED) TRACE

\% Moisture: not dec.

GC Column: DB-624 ID: $0.20 \quad$ (mm)

Soil Extract Volume:

Purge Volume: 25.0

(mL)
Contract: 1E-30401

SDG No.: 200-6783

\begin{tabular}{|c|c|c|c|}
\hline CAS NO. & COMPOUND & $\begin{array}{l}\text { CONCENTRATION UNITS: } \\
(\mathrm{ug} / \mathrm{L} \text { or } \mathrm{ug} / \mathrm{kg}) \mathrm{ug} / \mathrm{L}\end{array}$ & $Q$ \\
\hline $75-71-8$ & Dichlorodifluoromethane & 0.50 & $\bar{U}$ \\
\hline $74-87-3$ & Chloromethane & 0.50 & $\mathrm{U}$ \\
\hline $75-01-4$ & Vinyl chloride & 0.50 & $\mathrm{U}$ \\
\hline $74-83-9$ & Bromomethane & 0.50 & $\mathrm{U}$ \\
\hline $75-00-3$ & Chloroethane & 0.50 & $\mathrm{U}$ \\
\hline $75-69-4$ & Trichlorofluoromethane & 0.50 & $\mathrm{U}$ \\
\hline $75-35-4$ & 1,1-Dichloroethene & 0.50 & $\mathrm{U}$ \\
\hline $76-13-1$ & 1,1,2-Trichloro-1,2,2-trifluoroethane & 0.50 & $\mathrm{U}$ \\
\hline $67-64-1$ & Acetone & 1.4 & $\mathrm{~J}$ \\
\hline $75-15-0$ & Carbon disulfide & 0.50 & $\mathrm{U}$ \\
\hline $79-20-9$ & Methyl acetate & 0.50 & $\mathrm{U}$ \\
\hline $75-09-2$ & Methylene Chloride & 0.50 & $\mathrm{U}$ \\
\hline $156-60-5$ & trans-1,2-Dichloroethene & 0.50 & $\mathrm{U}$ \\
\hline $1634-04-4$ & Methyl tert-butyl ether & 0.50 & $\mathrm{U}$ \\
\hline $75-34-3$ & 1,1-Dichloroethane & 0.50 & $\mathrm{U}$ \\
\hline $156-59-2$ & cis-1,2-Dichloroethene & 0.50 & $\mathrm{U}$ \\
\hline $78-93-3$ & 2-Butanone & 5.0 & $\mathrm{U}$ \\
\hline $74-97-5$ & Bromochloromethane & 0.50 & $\mathrm{U}$ \\
\hline $67-66-3$ & Chloroform & 0.13 & $\mathrm{~J}$ \\
\hline $71-55-6$ & 1,1,1-Trichloroethane & 0.50 & $\mathrm{U}$ \\
\hline $110-82-7$ & Cyclohexane & 0.50 & $\mathrm{U}$ \\
\hline $56-23-5$ & Carbon tetrachloride & 0.50 & $\mathrm{U}$ \\
\hline $71-43-2$ & Benzene & 0.038 & $\mathrm{~J}$ \\
\hline $107-06-2$ & 1,2-Dichloroethane & 0.50 & $\mathrm{U}$ \\
\hline
\end{tabular}

Report 1,4-Dioxane for Low-Medium VOA analysis only

Lab Sample ID: 200-6783-5

Lab File ID: DHPV20.D

Date Received: 08/31/2011

Date Analyzed: 09/01/2011

Dilution Factor: 1.0

Soil Aliquot Volune:

(uL) 
$1 B$ - FORM I VOA-2

VOIATILE ORGANICS ANALYSIS DATA SHEET
EPA SAMPLE NO.

INQCTB-W-32769
Lab Name: TESTAMERICA BURLINGTON

Lab Code: STLV

Matrix: (SOIL/SED/WATER)

Sample wt/vol: 25.0

Level: (TRACE/LOW/MED) TRACE

\% Moisture: not dec.

GC Column: DB-624 ID: 0.20

Soil Extract volume:

Purge Volume: 25.0
Contract: 1E-30401

SDG No.: 200-6783
Lab Sample ID: 200-6783-5

Lab File ID: DHPV20.D

Date Received: 08/31/2011.

Date Analyzed: 09/01/2011

Dilution Factor: 1.0

Soil Aliquot Volume:

(uL)

\begin{tabular}{|c|c|c|c|}
\hline CAS NO. & COMPOUND & $\begin{array}{l}\text { CONCENTRATION UNITS: } \\
(\mathrm{ug} / \mathrm{L} \text { or } \mathrm{ug} / \mathrm{kg}) \mathrm{ug} / \mathrm{L}\end{array}$ & $\mathrm{Q}$ \\
\hline $79-01-6$ & Trichloroethene & 0.50 & $\mathrm{U}$ \\
\hline $108-87-2$ & Methylcyclohexane & 0.50 & $\mathrm{U}$ \\
\hline $78-87-5$ & 1,2-Dichloropropane & 0.50 & $\mathrm{U}$ \\
\hline $75-27-4$ & Bromodichloromethane & 0.50 & $\mathrm{U}$ \\
\hline $10061-01-5$ & cis-1,3-Dichloropropene & 0.50 & $\mathrm{U}$ \\
\hline $108-10-1$ & 4-Methyl-2-pentanone & 5.0 & $\mathrm{U}$ \\
\hline $108-88-3$ & Toluene & 0.35 & $\mathrm{~J} B$ \\
\hline $10061-02-6$ & trans-1,3-Dichloropropene & 0.50 & $\mathrm{U}$ \\
\hline $79-00-5$ & 1,1,2-Trichloroethane & 0.50 & $\mathrm{U}$ \\
\hline $127-18-4$ & Tetrachloroethene & 0.50 & $\mathrm{U}$ \\
\hline $591-78-6$ & 2-Hexanone & 5.0 & $\mathrm{U}$ \\
\hline $124-48-1$ & Dibromochloromethane & 0.50 & $\mathrm{U}$ \\
\hline $106-93-4$ & 1,2-Dibromoethane & 0.50 & $\mathrm{U}$ \\
\hline $108-90-7$ & Chlorobenzene & 0.50 & $\mathrm{U}$ \\
\hline $100-41-4$ & Ethylbenzene & 0.028 & $\mathrm{~J}$ \\
\hline $95-47-6$ & o-xylene & 0.50 & $\mathrm{U}$ \\
\hline $179601-23-1$ & $\mathrm{~m}, \mathrm{p}$-Xylene & 0.081 & $\mathrm{~J}$ \\
\hline $100-42-5$ & Styrene & 0.34 & $\mathrm{~J}$ \\
\hline $75-25-2$ & Bromoform & 0.50 & $\mathrm{U}$ \\
\hline $98-82-8$ & Isopropylbenzene & 0.50 & $\mathrm{U}$ \\
\hline $79-34-5$ & $1,1,2,2$-Tetrachloroethane & 0.50 & $\mathrm{U}$ \\
\hline $541-73-1$ & 1,3-Dichlorobenzene & 0.50 & $\mathrm{U}$ \\
\hline $106-46-7$ & 1,4-Dichlorobenzene & 0.50 & $\mathrm{U}$ \\
\hline $95-50-1$ & 1,2-Dichlorobenzene & 0.50 & $\mathrm{U}$ \\
\hline $96-12-8$ & 1,2-Dibromo-3-Chloropropane & 0.50 & $\mathrm{U}$ \\
\hline $120-82-1$ & 1,2,4-Trichlorobenzene & 0.50 & $\mathrm{U}$ \\
\hline $87-61-6$ & 1,2,3-Trichlorobenzene & 0.50 & $\mathrm{U}$ \\
\hline
\end{tabular}


$1 \mathrm{~J}$ - FORM I VOA-TIC

VOLATILE ORGANICS ANALYSIS DATA SHEET TENTATIVELY IDENTIFIED COMPOUNDS
EPA SAMPLE NO.

INQCTB-W-32769

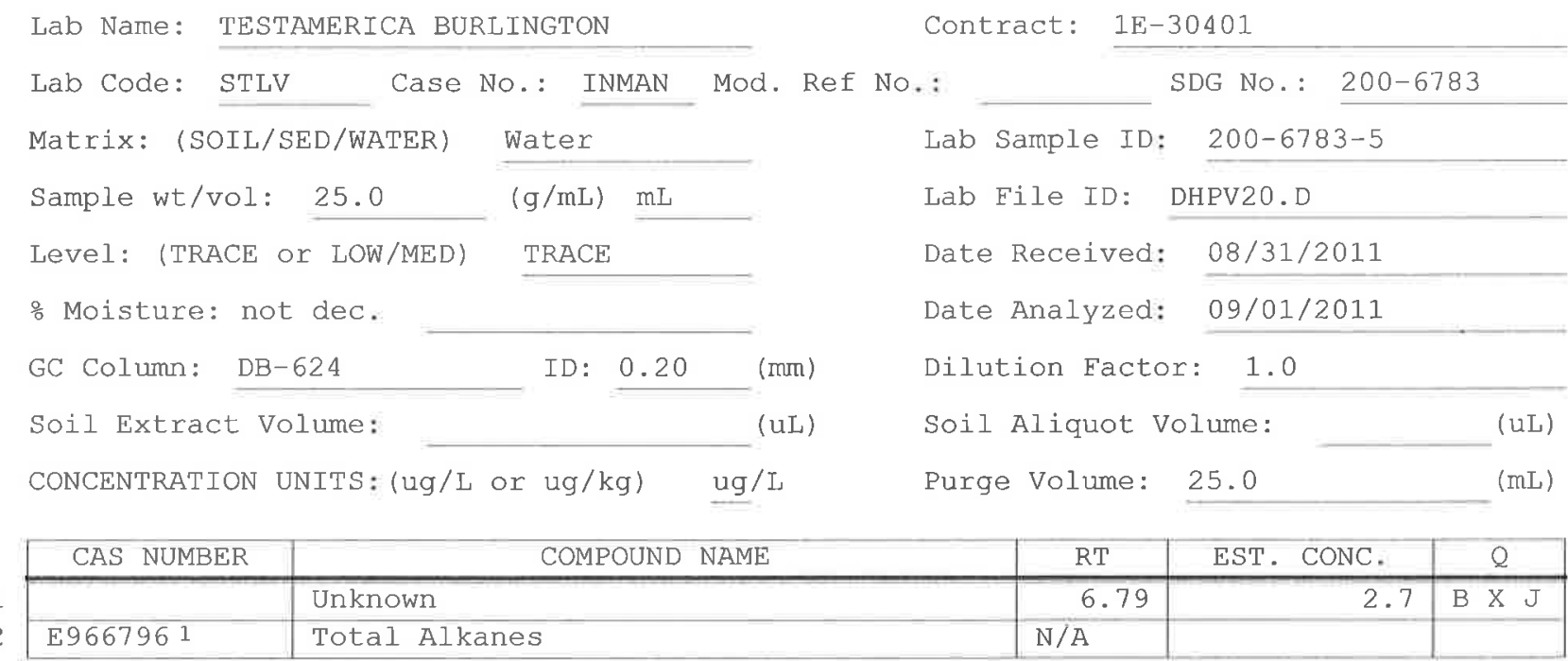

1 EPA-designated Registry Number. 
$6 \mathrm{~A}$ - FORM VI VOA-1

VOLATILE ORGANTCS INITIAL CALIBRATION DATA

Lab Name: TESTAMERICA BURLINGTON

Contract: 1E-30401

Lab Code: STLV Case No.: INMAN Mod. Ref No.:

Instrument ID: D.i Calibration Date(s): 07/07/2011 07/07/2011

Heated Purge: (Y/N) N

Calibration Time(s): $1117 \quad 1256$

Purge Volume: 25.0

(mL)

GC Column: DB-624

ID: 0.20

(mm) Length: 25

(m)

\begin{tabular}{|c|c|c|c|c|c|c|c|}
\hline \multirow{2}{*}{$\begin{array}{l}\text { LAB FILE ID: } \\
\text { RRE } 5.0=\text { DHP06.D }\end{array}$} & \multicolumn{3}{|c|}{ RRF0.5 = DHP04.D } & \multicolumn{4}{|c|}{ RRE $1.0=$ DHP05.D } \\
\hline & RRF 10 & IP07.D & & RRF 20 & $=\mathrm{DHPC}$ & . D & \\
\hline COMPOUND & RRF 0.5 & RRE 1.0 & RRF 5.0 & RRF'10 & RRE20 & $\overline{\mathrm{RRE}}$ & RSD \\
\hline Dichlorodifluoromethane & 0.418 & 0.398 & 0.390 & 0.409 & 0.398 & 0.403 & 2.7 \\
\hline Chloromethane & 0.769 & 0.713 & 0.655 & 0.659 & 0.639 & 0.687 & 7.8 \\
\hline Vinyl chloride & 0.524 & 0.491 & 0.475 & 0.499 & 0.491 & 0.496 & 3.6 \\
\hline Bromomethane & 0.180 & 0.203 & 0.205 & 0.219 & 0.239 & 0.209 & 10.3 \\
\hline Chloroethane & 0.292 & 0.280 & 0.259 & 0.269 & 0.259 & 0.272 & 5.2 \\
\hline Trichlorofluoromethane & 0.612 & 0.609 & 0.587 & 0.608 & 0.611 & 0.606 & 1.7 \\
\hline 1,1-Dichloroethene & 0.318 & 0.307 & 0.298 & 0.303 & 0.297 & 0.305 & 2.8 \\
\hline $\begin{array}{l}1,1,2 \text {-Trichloro- } \\
1,2,2 \text {-trifluoroethane }\end{array}$ & 0.377 & 0.358 & 0.336 & 0.358 & 0.347 & 0.355 & 4.3 \\
\hline Acetone & 0.047 & 0.038 & 0.032 & 0.032 & 0.030 & 0.036 & 19.3 \\
\hline Carbon disulfide & 0.758 & 0.707 & 0.719 & 0.760 & 0.766 & 0.742 & 3.6 \\
\hline Methyl acetate & 0.108 & 0.094 & 0.076 & 0.079 & 0.074 & 0.086 & 16.7 \\
\hline Methylene Chloride & 0.264 & 0.257 & 0.242 & 0.248 & 0.241 & 0.250 & 3.9 \\
\hline trans-1,2-Dichloroethene & 0.323 & 0.298 & 0.301 & 0.312 & 0.306 & 0.308 & 3.3 \\
\hline Methyl tert-butyl ether & 0.304 & 0.278 & 0.292 & 0.319 & 0.318 & 0.302 & 5.7 \\
\hline 1,1-Dichloroethane & 0.642 & 0.586 & 0.594 & 0.617 & 0.607 & 0.609 & 3.6 \\
\hline cis-1,2-Dichloroethene & 0.302 & 0.289 & 0.300 & 0.312 & 0.306 & 0.302 & 2.7 \\
\hline 2-Butanone & 0.045 & 0.046 & 0.047 & 0.049 & 0.048 & 0.047 & 3.4 \\
\hline Bromochloromethane & 0.104 & 0.103 & 0.100 & 0.099 & 0.101 & 0.101 & 2.0 \\
\hline Chloroform & 0.502 & 0.482 & 0.473 & 0.493 & 0.477 & 0.485 & 2.5 \\
\hline 1,1,1-Trichloroethane & 0.514 & 0.490 & 0.494 & 0.490 & 0.485 & 0.494 & 2.3 \\
\hline Cyclohexane & 0.761 & 0.795 & 0.901 & 0.909 & 0.883 & 0.850 & 7.9 \\
\hline Carbon tetrachloride & 0.487 & 0.459 & 0.477 & 0.474 & 0.464 & 0.472 & 2.4 \\
\hline Benzene & 1.617 & 1.501 & 1.518 & 1.495 & 1.447 & 1.515 & 4.1 \\
\hline 1,2-Dichloroethane & 0.291 & 0.271 & 0.277 & 0.284 & 0.285 & 0.282 & 2.7 \\
\hline Trichloroethene & 0.397 & 0.380 & 0.357 & 0.355 & 0.351 & 0.368 & 5.4 \\
\hline Methylcyclohexane & 0.703 & 0.708 & 0.769 & 0.757 & 0.751 & 0.737 & 4.1 \\
\hline
\end{tabular}

Report 1,4-Dioxane for Low-Medium VOA analysis only 
$6 \mathrm{~B}-$ FORM VI VOA-2

VOLATILE ORGANICS INITIAL CALIBRATION DATA

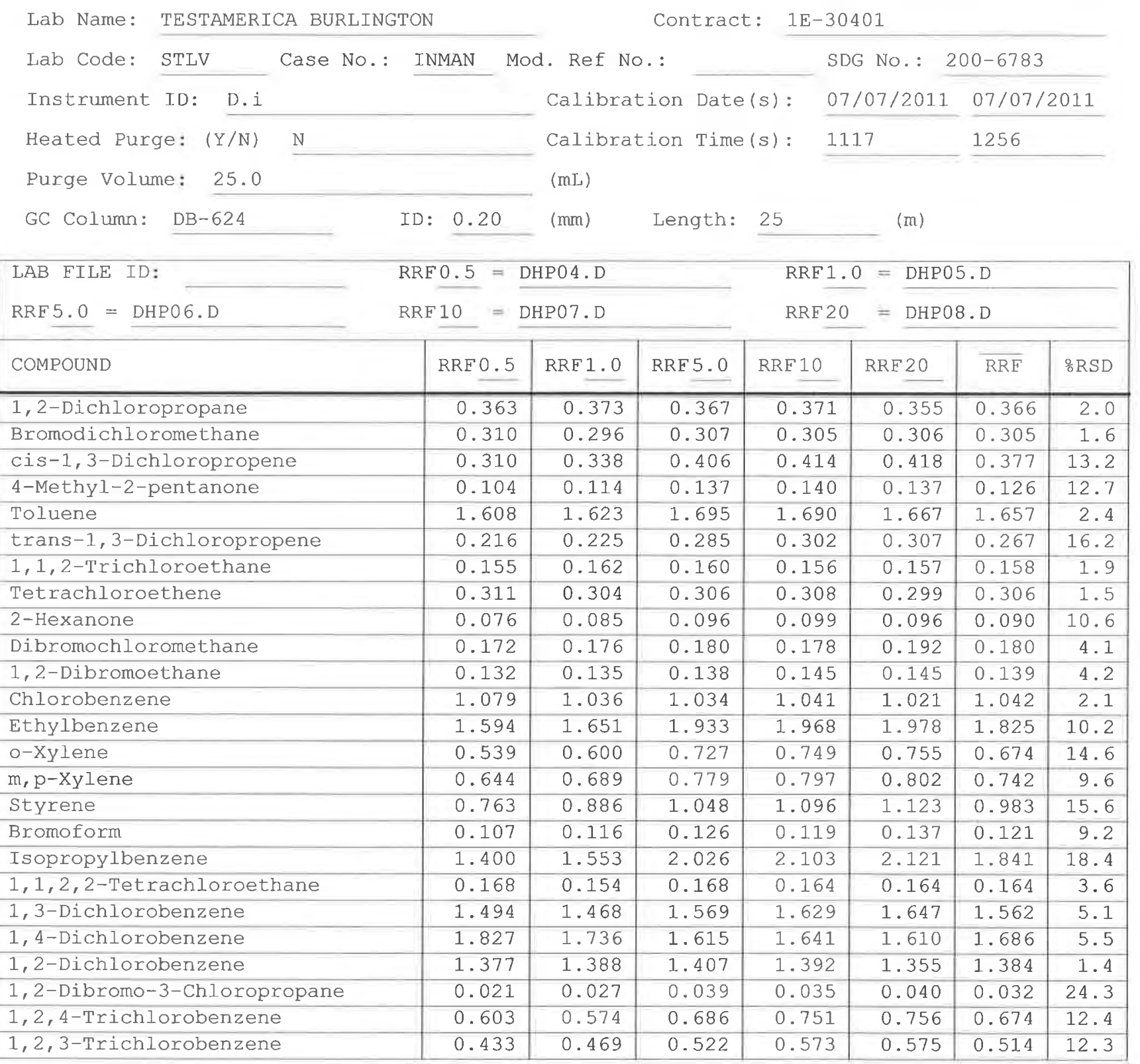


$6 \mathrm{C}-$ FORM VI VOA-3

VOLATILE ORGANICS INITIAL CALIBRATION DATA

Lab Name: TESTAMERICA BURLINGTON

Contract: 1E-30401

Lab Code: STLV Case No.: INMAN Mod. Ref No.:

SDG No.: 200-6783

Instrument ID: D.i Calibration Date(s): 07/07/2011 07/07/2011

Heated Purge: (Y/N) N

Calibration Time(s): 1117

1256

Purge Volume: 25.0

$(\mathrm{mL})$

GC Column: DB-624

ID: 0.20

(mm) Length: 25

(m)

\begin{tabular}{|c|c|c|c|c|c|c|c|}
\hline \multirow{2}{*}{$\begin{array}{l}\text { LAB FILE ID: } \\
\text { RRF } 5.0=\text { DHP06.D }\end{array}$} & \multicolumn{3}{|c|}{ RRF $0.5=$ DHPO4.D } & \multicolumn{4}{|c|}{ RRF1.0 = DHP05.D } \\
\hline & RRE 10 & PP07.D & & RRF 20 & $=\mathrm{DHPC}$ & D & \\
\hline COMPOUND & RRE 0.5 & RRE 1.0 & RRF 5.0 & RRF 10 & RRF 20 & $\overline{\mathrm{RRF}}$ & $\because \mathrm{RSD}$ \\
\hline Vinyl Chloride-d3 & 0.479 & 0.428 & 0.415 & 0.431 & 0.426 & 0.436 & 5.7 \\
\hline Chloroethane-d5 & 0.304 & 0.290 & 0.269 & 0.276 & 0.274 & 0.283 & 5.0 \\
\hline 1,1-Dichloroethene-d2 & 0.849 & 0.795 & 0.757 & 0.787 & 0.787 & 0.795 & 4.2 \\
\hline 2-Butanone-d5 & 0.041 & 0.038 & 0.040 & 0.044 & 0.043 & 0.041 & 5.5 \\
\hline Chloroform-d & 0.512 & 0.515 & 0.502 & 0.514 & 0.503 & 0.509 & 1.3 \\
\hline 1,2-Dichloroethane-d4 & 0.218 & 0.203 & 0.199 & 0.201 & 0.194 & 0.203 & 4.3 \\
\hline Benzene-d6 & 1.573 & 1.471 & 1.478 & 1.453 & 1.403 & 1.475 & 4.2 \\
\hline 1,2-Dichloropropane-d6 & 0.410 & 0.374 & 0.367 & 0.365 & 0.356 & 0.374 & 5.7 \\
\hline Toluene-d8 & 1.315 & 1.370 & 1.458 & 1.441 & 1.404 & 1.397 & 4.1 \\
\hline trans-1,3-Dichloropropene-d 4 & 0.197 & 0.207 & 0.232 & 0.249 & 0.264 & 0.230 & 12.2 \\
\hline 2-Hexanone-d5 & 0.023 & 0.026 & 0.037 & 0.038 & 0.039 & 0.033 & 23.1 \\
\hline $1,1,2,2$-Tetrachloroethane-d2 & 0.151 & 0.161 & 0.167 & 0.171 & 0.169 & 0.164 & 4.8 \\
\hline 1,2-Dichlorobenzene-d4 & 0.885 & 0.843 & 0.815 & 0.807 & 0.799 & 0.830 & 4.2 \\
\hline
\end{tabular}

Report 1,4-Dioxane-d8 for Low-Medium VOA analysis only 
$7 A-$ FORM VII VOA-1

VOLATILE CONTINUING CALIBRATION DATA

Lab Name: TESTAMERICA BURLINGTON Contract: 1E-30401

Lab Code: STLV Case No.: INMAN Mod. Ref No.: SDG No.: 200-6783

Instrument ID: D.i Calibration Date: 09/01/2011 Time: 0748

Lab File Id: DHPV02.D Init. Calib. Date(s): 07/07/2011 07/07/2011

EPA Sample No.(VSTD\#\#\#): VSTD005DS Init. Calib. Time(s): $1117 \quad 1256$

Heated Purge: (Y/N) N GC Column: DB-624 ID: 0.20 (mm) Length: 25 (m)

Purge Volume: $25.0 \quad$ (mL)

\begin{tabular}{|c|c|c|c|c|c|}
\hline COMPOUND & $\overline{\mathrm{RRF}}$ & RRF5.0 & $\begin{array}{l}\text { MIN } \\
\text { RRE }\end{array}$ & $\because \mathrm{D}$ & $\mathrm{MAX} \div \mathrm{D}$ \\
\hline Dichlorodifluoromethane & 0.403 & 0.428 & 0.010 & 6.3 & 40.0 \\
\hline Chloromethane & 0.687 & 0.667 & 0.010 & -3.0 & 40.0 \\
\hline Vinyl chloride & 0.496 & 0.465 & 0.010 & -6.4 & 30.0 \\
\hline Bromomethane & 0.209 & 0.188 & 0.010 & -10.1 & 30.0 \\
\hline Chloroethane & 0.272 & 0.243 & 0.010 & -10.6 & 40.0 \\
\hline Trichlorofluoromethane & 0.606 & 0.562 & 0.010 & -7.3 & 40.0 \\
\hline 1,1-Dichloroethene & 0.305 & 0.282 & 0.010 & -7.6 & 30.0 \\
\hline 1, 1,2-Trichloro-1,2,2-trifluoroethane & 0.355 & 0.334 & 0.010 & -6.1 & 40.0 \\
\hline Acetone & 0.036 & 0.026 & 0.010 & -27.5 & 40.0 \\
\hline Carbon disulfide & 0.742 & 0.674 & 0.010 & -9.1 & 40.0 \\
\hline Methyl acetate & 0.086 & 0.074 & 0.010 & -13.9 & 40.0 \\
\hline Methylene Chloride & 0.250 & 0.221 & 0.010 & -11.8 & 40.0 \\
\hline trans-1,2--Dichloroethene & 0.308 & 0.274 & 0.010 & -11.0 & 40.0 \\
\hline Methyl tert-butyl ether & 0.302 & 0.242 & 0.010 & -20.0 & 40.0 \\
\hline 1,1-Dichloroethane & 0.609 & 0.557 & 0.010 & -8.6 & 30.0 \\
\hline cis-1,2-Dichloroethene & 0.302 & 0.276 & 0.010 & -8.4 & 40.0 \\
\hline 2-Butanone & 0.047 & 0.037 & 0.010 & -20.6 & 40.0 \\
\hline Bromochloromethane & 0.101 & 0.089 & 0.010 & -12.1 & 30.0 \\
\hline Chloroform & 0.485 & 0.433 & 0.010 & -10.7 & 30.0 \\
\hline $1,1,1$-Trichloroethane & 0.494 & 0.453 & 0.010 & -8.4 & 30.0 \\
\hline Cyclohexane & 0.850 & 0.860 & 0.010 & 1.2 & 40.0 \\
\hline Carbon tetrachloride & 0.472 & 0.449 & 0.010 & -4.9 & 30.0 \\
\hline Benzene & 1.515 & 1.371 & 0.010 & -9.6 & 30.0 \\
\hline 1,2-Dichloroethane & 0.282 & 0.249 & 0.010 & -11.6 & 30.0 \\
\hline Trichloroethene & 0.368 & 0.331 & 0.010 & -10.0 & 30.0 \\
\hline Methylcyclohexane & 0.737 & 0.711 & 0.010 & -3.5 & 40.0 \\
\hline
\end{tabular}

Report 1,4-Dioxane for Low/Medium VOA analysis only 
$7 \mathrm{~B}$ - FORM VII VOA-2

VOLATILE CONTINUING CALIBRATION DATA

Lab Name: TESTAMERICA BURJINGTON

Contract: $1 \mathrm{E}-30401$

Lab Code: STLV

Case No.: INMAN Mod. Ref No.:

SDG No.: 200-6783

Instrument ID: D.i

Calibration Date: 09/01/2011 Time: 0748

Lab File Id: DHPV02.D Init. Calib. Date(s): 07/07/2011 07/07/2011

EPA Sample No. (VSTD\#\#\#\#): VSTD005DS

Init. Calib. Time(s): $1117 \quad 1256$

Heated Purge: ( $\mathrm{Y} / \mathrm{N}) \mathrm{N}$

GC Column: DB-624

ID: $0.20(\mathrm{~mm})$ Length: 25

(m)

Purge Volume: 25.0

(mL)

\begin{tabular}{|c|c|c|c|c|c|}
\hline COMPOUND & $\overline{\mathrm{RRF}}$ & RRF5. 0 & $\begin{array}{l}\text { MIN } \\
\text { RRE }\end{array}$ & $\because \mathrm{D}$ & MAX $\cong D$ \\
\hline 1,2-Dichloropropane & 0.366 & 0.336 & 0.010 & -8.1 & 40.0 \\
\hline Bromodichloromethane & 0.305 & 0.269 & 0.010 & -11.7 & 30.0 \\
\hline cis-1,3-Dichloropropene & 0.377 & 0.355 & 0.010 & -6.1 & 30.0 \\
\hline 4-Methyl-2-pentanone & 0.126 & 0.108 & 0.010 & -14.5 & 40.0 \\
\hline Toluene & 1.657 & 1.560 & 0.010 & -5.8 & 30.0 \\
\hline trans-1,3-Dichloropropene & 0.267 & 0.248 & 0.010 & -7.1 & 30.0 \\
\hline 1,1,2-Trichloroethane & 0.158 & 0.138 & 0.010 & -12.5 & 30.0 \\
\hline Tetrachloroethene & 0.306 & 0.276 & 0.010 & -9.8 & 30.0 \\
\hline 2-Hexanone & 0.090 & 0.076 & 0.010 & -15.6 & 40.0 \\
\hline Dibromochloromethane & 0.180 & 0.156 & 0.010 & -13.3 & 30.0 \\
\hline 1,2-Dibromoethane & 0.139 & 0.121 & 0.010 & -13.1 & 40.0 \\
\hline Chlorobenzene & 1.042 & 0.949 & 0.010 & -9.0 & 30.0 \\
\hline Ethylbenzene & 1.825 & 1.731 & 0.010 & -5.1 & 30.0 \\
\hline o-Xylene & 0.674 & 0.656 & 0.010 & -2.7 & 30.0 \\
\hline $\mathrm{m}, \mathrm{p}$-XYlene & 0.742 & 0.711 & 0.010 & -4.1 & 30.0 \\
\hline Styrene & 0.983 & 0.946 & 0.010 & -3.8 & 30.0 \\
\hline Bromoform & 0.121 & 0.100 & 0.010 & -17.1 & 30.0 \\
\hline Isopropylbenzene & 1.841 & 1.827 & 0.010 & -0.7 & 40.0 \\
\hline $1,1,2,2$-Tetrachloroethane & 0.164 & 0.132 & 0.010 & -19.7 & 30.0 \\
\hline 1,3-Dichlorobenzene & 1.562 & 1.428 & 0.010 & -8.6 & 30.0 \\
\hline 1,4-Dichlorobenzene & 1.686 & 1.501 & 0.010 & -11.0 & 30.0 \\
\hline 1,2-Dichlorobenzene & 1.384 & 1.268 & 0.010 & -8.4 & 30.0 \\
\hline 1,2-Dibromo-3-Chloropropane & 0.032 & 0.032 & 0.010 & -0.9 & 40.0 \\
\hline 1,2,4-Trichlorobenzene & 0.674 & 0.663 & 0.010 & -1.6 & 30.0 \\
\hline 1,2,3-Trichlorobenzene & 0.514 & 0.496 & 0.010 & -3.5 & 30.0 \\
\hline
\end{tabular}


$7 \mathrm{C}$ - FORM VII VOA-3

VOLATILE CONTINUING CALIBRATION DATA

Lab Name: TESTAMERICA BURLINGTON

Contract: 1E-30401

Lab Code: STLV Case No.: INMAN Mod. Ref No.:

SDG No.: 200-6783

Instrument ID: D.i

Calibration Date: 09/01/2011 Time: 0748

Lab File Id: DHPV02.D Init. Calib. Date(s): 07/07/2011 07/07/2011

EPA Sample No. (VSTD\#\#\#\#): VSTD005DS

Init. Calib. Time(s): $1117 \quad 1256$

Heated Purge: ( $\mathrm{Y} / \mathrm{N}) \mathrm{N}$

GC Column: DB-624

ID: $0.20(\mathrm{~mm})$ Length: 25

(In)

Purge Volume: 25.0

(mL)

\begin{tabular}{|c|c|c|c|c|c|}
\hline COMPOUND & $\overline{\mathrm{RRF}}$ & RRF5. 0 & $\begin{array}{l}\text { MIN } \\
\text { RRF }\end{array}$ & $\frac{\circ}{8} \mathrm{D}$ & $\mathrm{MAX} \div \mathrm{D}$ \\
\hline Vinyl Chloride-d3 & 0.436 & 0.395 & 0.010 & -9.4 & 30.0 \\
\hline Chloroethane-d5 & 0.283 & 0.249 & 0.010 & $-12 \cdot 1$ & 40.0 \\
\hline 1,1-Dichloroethene-d2 & 0.795 & 0.657 & 0.010 & -17.4 & 30.0 \\
\hline 2-Butanone-d5 & 0.041 & 0.033 & 0.010 & -19.3 & 40.0 \\
\hline Chloroform-d & 0.509 & 0.429 & 0.010 & -15.7 & 30.0 \\
\hline 1,2-Dichloroethane-d4 & 0.203 & 0.174 & 0.010 & -14.5 & 30.0 \\
\hline Benzene-d6 & 1.475 & 1.277 & 0.010 & -13.4 & 30.0 \\
\hline 1,2-Dichloropropane-d6 & 0.374 & 0.311 & 0.010 & -17.1 & 40.0 \\
\hline Toluene-d8 & 1.397 & 1.272 & 0.010 & -9.0 & 30.0 \\
\hline trans $-1,3-$ Dichloropropene-d4 & 0.230 & 0.193 & 0.010 & -16.1 & 30.0 \\
\hline 2-Hexanone-d5 & 0.033 & 0.028 & 0.010 & -15.2 & 40.0 \\
\hline $1,1,2,2$-Tetrachloroethane-d2 & 0.164 & 0.131 & 0.010 & -20.2 & 30.0 \\
\hline 1,2-Dichlorobenzene-d4 & 0.830 & 0.712 & 0.010 & -14.2 & 30.0 \\
\hline
\end{tabular}

Report 1,4-Dioxane-d8 for Low/Medium VOA analysis only 
$7 A-$ FORM VII VOA-1

VOLATILE CONTINUING CALIBRATION DATA

Lab Name: TESTAMERICA BURLINGTON

Contract: 1E-30401

Lab Code: STLV Case No.: INMAN Mod. Ref No.:

SDG No.: 200-6783

Instrument ID: D.i

Calibration Date: 09/01/2011 Time: 1640

Lab File Id: DHPV23.D

Init. Calib. Date(s): 07/07/2011 07/07/2011

EPA Sample No. (VSTD\#\#\#): VSTD005SD

Init. Calib. Time(s): $1117 \quad 1256$

Heated Purge: $(\mathrm{Y} / \mathrm{N}) \quad \mathrm{N}$

GC Column: DB-624

ID: $0.20(\mathrm{~mm})$ Length: 25

(m)

Purge Volume: 25.0

$(\mathrm{mL})$

\begin{tabular}{|c|c|c|c|c|c|}
\hline COMPOUND & $\overline{R R F}$ & RRF5. 0 & $\begin{array}{l}\text { MIN } \\
\text { RRF }\end{array}$ & $\because \mathrm{D}$ & MAX $\because D$ \\
\hline Dichlorodifluoromethane & 0.403 & 0.451 & 0.010 & 11.9 & 50.0 \\
\hline Chloromethane & 0.687 & 0.699 & 0.010 & 1.7 & 50.0 \\
\hline Vinyl chloride & 0.496 & 0.489 & 0.100 & -1.5 & 50.0 \\
\hline Bromomethane & 0.209 & 0.206 & 0.100 & -1.6 & 50.0 \\
\hline Chloroethane & 0.272 & 0.263 & 0.010 & -3.4 & 50.0 \\
\hline Trichlorofluoromethane & 0.606 & 0.605 & 0.010 & -0.1 & 50.0 \\
\hline 1,1-Dichloroethene & 0.305 & 0.302 & 0.100 & -0.8 & 50.0 \\
\hline 1,1,2-Trichloro-1,2,2-trifluoroethane & 0.355 & 0.349 & 0.010 & -1.8 & 50.0 \\
\hline Acetone & 0.036 & 0.030 & 0.010 & -15.8 & 50.0 \\
\hline Carbon disulfide & 0.742 & 0.692 & 0.010 & -6.7 & 50.0 \\
\hline Methyl acetate & 0.086 & 0.084 & 0.010 & -2.2 & 50.0 \\
\hline Methylene Chloride & 0.250 & 0.239 & 0.010 & -4.6 & 50.0 \\
\hline trans-1,2-Dichloroethene & 0.308 & 0.298 & 0.010 & -3.2 & 50.0 \\
\hline Methyl tert-butyl ether & 0.302 & 0.302 & 0.010 & -0.1 & 50.0 \\
\hline 1,1-Dichloroethane & 0.609 & 0.609 & 0.200 & -0.1 & 50.0 \\
\hline cis-1,2-Dichloroethene & 0.302 & 0.298 & 0.010 & -1.2 & 50.0 \\
\hline 2-Butanone & 0.047 & 0.048 & 0.010 & 1.5 & 50.0 \\
\hline Bromoch loromethane & 0.101 & 0.100 & 0.050 & -1.0 & 50.0 \\
\hline Chloroform & 0.485 & 0.486 & 0.200 & 0.1 & 50.0 \\
\hline 1,1,1-Trichloroethane & 0.494 & 0.484 & 0.100 & -2.1 & 50.0 \\
\hline Cyclohexane & 0.850 & 0.900 & 0.010 & 5.9 & 50.0 \\
\hline Carbon tetrachloride & 0.472 & 0.452 & 0.100 & -4.2 & 50.0 \\
\hline Benzene & 1.515 & 1.458 & 0.400 & -3.8 & 50.0 \\
\hline 1,2-Dichloroethane & 0.282 & 0.289 & 0.100 & 2.6 & 50.0 \\
\hline Trichloroethene & 0.368 & 0.339 & 0.300 & -7.8 & 50.0 \\
\hline Methyl.cyclohexane & 0.737 & 0.749 & 0.010 & 1.6 & 50.0 \\
\hline
\end{tabular}

Report 1,4-Dioxane for Low/Medium VOA analysis only 
Lab Name: TESTAMERICA BURLINGTON

Lab Code: STLV Case No.: INMAN

Instrument ID: D.i

Lab File Id: DHPV23.D

EPA Sample No. (VSTD\#\#\#\#):

Heated Purge: $(\mathrm{Y} / \mathrm{N}) \mathrm{N}$

Purge Volume: 25.0
Contract: 1E-30401

SDG No.: 200-6783

Calibration Date: 09/01/2011 Time: 1640 Init. Calib. Date(s): 07/07/2011 07/07/2011

Init. Calib. 'Time(s): $1117 \quad 1256$

ID: $0.20(\mathrm{~mm})$ Length: 25 (m)

(mL)

\begin{tabular}{|c|c|c|c|c|c|}
\hline COMPOUND & $\overline{\mathrm{RRF}}$ & $\operatorname{RRF} 5.0$ & $\begin{array}{l}\text { MIN } \\
\text { RRF }\end{array}$ & $\frac{\circ}{\partial} \mathrm{D}$ & MAX $\because D$ \\
\hline 1,2-Dichloropropane & 0.366 & 0.362 & 0.010 & -1.0 & 50.0 \\
\hline Bromodichloromethane & 0.305 & 0.294 & 0.200 & -3.6 & 50.0 \\
\hline cis-1,3-Dichloropropene & 0.377 & 0.384 & 0.200 & 1.8 & 50.0 \\
\hline 4-Methyl-2-pentanone & 0.126 & 0.132 & 0.010 & 4.5 & 50.0 \\
\hline Toluene & 1.657 & 1.655 & 0.400 & -0.1 & 50.0 \\
\hline trans-1,3-Dichloropropene & 0.267 & 0.273 & 0.100 & 2.3 & 50.0 \\
\hline 1,1,2-Trichloroethane & 0.158 & 0.158 & 0.100 & 0.0 & 50.0 \\
\hline Tetrachloroethene & 0.306 & 0.295 & 0.100 & -3.6 & 50.0 \\
\hline 2-Hexanone & 0.090 & 0.093 & 0.010 & 2.7 & 50.0 \\
\hline Dibromochloromethane & 0.180 & 0.173 & 0.100 & -3.6 & 50.0 \\
\hline 1,2-Dibromoethane & 0.139 & 0.139 & 0.010 & 0.2 & 50.0 \\
\hline Chlorobenzene & 1.042 & 1.020 & 0.500 & -2.1 & 50.0 \\
\hline Ethylbenzene & 1.825 & 1.834 & 0.100 & 0.5 & 50.0 \\
\hline o-Xylene & 0.674 & 0.708 & 0.300 & 5.0 & 50.0 \\
\hline $\mathrm{m}, \mathrm{p}$-Xylene & 0.742 & 0.766 & 0.300 & 3.2 & 50.0 \\
\hline Styrene & 0.983 & 1.075 & 0.300 & 9.4 & 50.0 \\
\hline Bromoform & 0.121 & 0.115 & 0.050 & -5.0 & 50.0 \\
\hline Isopropylbenzene & 1.841 & 1.945 & 0.010 & 5.7 & 50.0 \\
\hline $1,1,2,2$-Tetrachloroethane & 0.164 & 0.159 & 0.100 & -2.8 & 50.0 \\
\hline 1,3-Dichlorobenzene & 1.562 & 1.479 & 0.400 & -5.3 & 50.0 \\
\hline 1,4-Dichlorobenzene & 1.686 & 1.582 & 0.400 & -6.1 & 50.0 \\
\hline 1,2-Dichlorobenzene & 1.384 & 1.371 & 0.400 & -0.9 & 50.0 \\
\hline 1,2-Dibromo-3-Chloropropane & 0.032 & 0.030 & 0.010 & -7.9 & 50.0 \\
\hline 1,2,4-Trichlorobenzene & 0.674 & 0.643 & 0.200 & -4.6 & 50.0 \\
\hline 1,2,3-Trichlorobenzene & 0.514 & 0.523 & 0.200 & 1.6 & 50.0 \\
\hline
\end{tabular}


7C - FORM VII VOA-3

VOLATILE CONTINUING CALIBRATION DATA

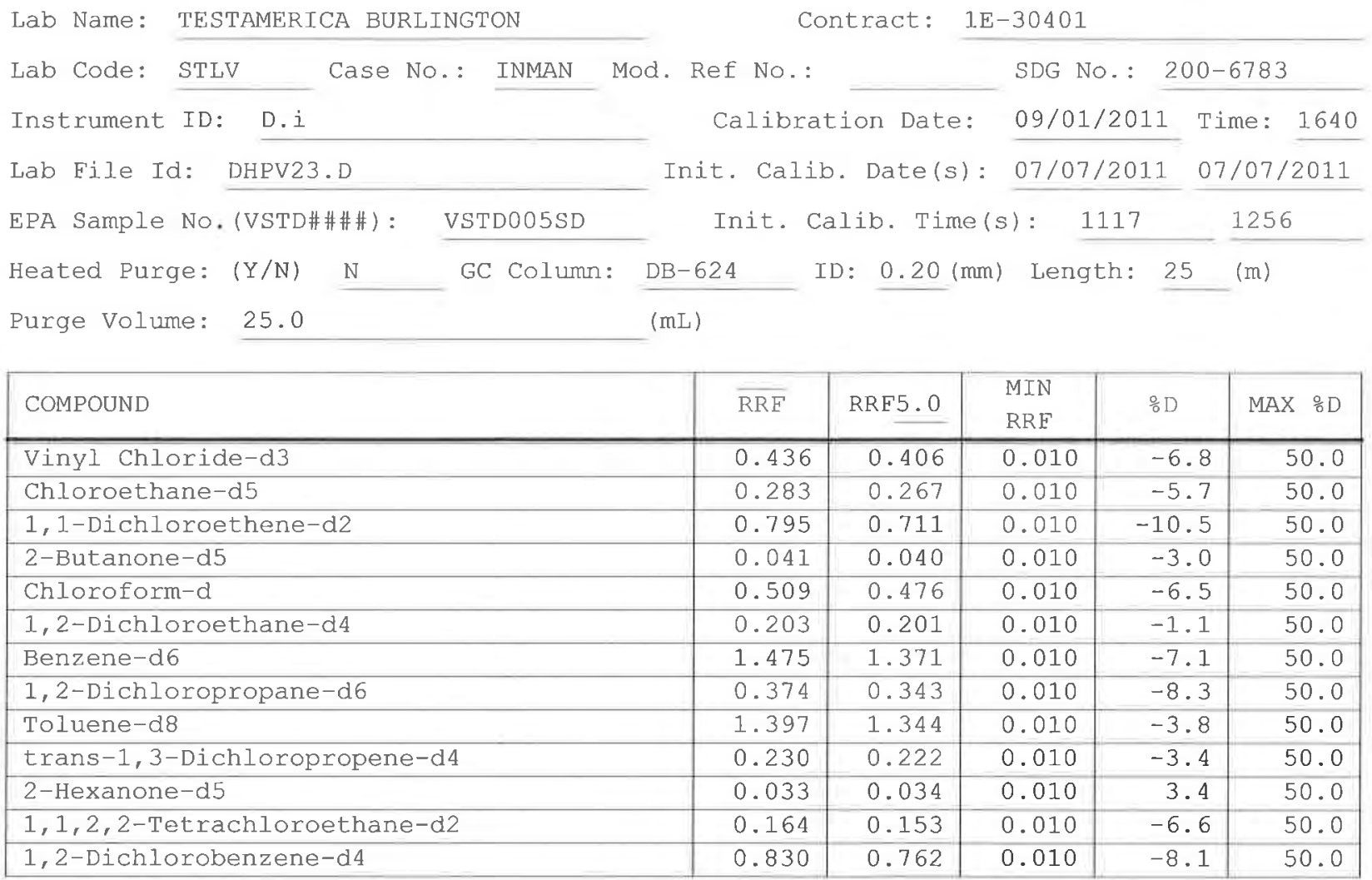


$7 A$ - FORM VII VOA-1

VOLATILE CONTINUING CALIBRATION DATA

Lab Name: TESTAMERICA BURLINGTON

Contract: 1E-30401

Lab Code: STLV Case No.: INMAN Mod. Ref No.: SDG No.: 200-6783

Instrument ID: D.i

Calibration Date: 09/02/2011 Time: 0842

Lab File Id: DHPW03.D Init. Calib. Date(s): 07/07/2011 07/07/2011

EPA Sample No. (VSTD\#\#\#\#): VSTD005DT

Init. Calib. Time(s): $1117 \quad 1256$

Heated Purge: ( $Y / N) \quad N$ GC Column: DB-624 ID: 0.20 (mm) Length: 25

(III)

Purge Volume: 25.0 (mL)

\begin{tabular}{|c|c|c|c|c|c|}
\hline COMPOUND & $\overline{\mathrm{RRF}}$ & RRF5.0 & $\begin{array}{l}\text { MIN } \\
\text { RRE }\end{array}$ & $\frac{\circ}{6} \mathrm{D}$ & $\mathrm{MAX} \div \mathrm{D}$ \\
\hline Dichlorodifluoromethane & 0.403 & 0.426 & 0.010 & 5.9 & 40.0 \\
\hline Chloromethane & 0.687 & 0.653 & 0.010 & -5.0 & 40.0 \\
\hline Vinyl chloride & 0.496 & 0.455 & 0.010 & -8.2 & 30.0 \\
\hline Bromomethane & 0.209 & 0.193 & 0.010 & -7.5 & 30.0 \\
\hline Chloroethane & 0.272 & 0.239 & 0.010 & -12.2 & 40.0 \\
\hline Trichlorofluoromethane & 0.606 & 0.576 & 0.010 & -4.8 & 40.0 \\
\hline 1,1-Dichloroethene & 0.305 & 0.276 & 0.010 & -9.3 & 30.0 \\
\hline 1,1,2-Trichloro-1,2,2-trifluoroethane & 0.355 & 0.328 & 0.010 & -7.6 & 40.0 \\
\hline Acetone & 0.036 & 0.029 & 0.010 & -17.7 & 40.0 \\
\hline Carbon disulfide & 0.742 & 0.686 & 0.010 & -7.5 & 40.0 \\
\hline Methyl acetate & 0.086 & 0.077 & 0.010 & -10.3 & 40.0 \\
\hline Methylene Chloride & 0.250 & 0.223 & 0.010 & -10.9 & 40.0 \\
\hline trans $-1,2-$ Dichloroethene & 0.308 & 0.274 & 0.010 & -11.1 & 40.0 \\
\hline Methyl tert-butyl ether & 0.302 & 0.285 & 0.010 & -5.7 & 40.0 \\
\hline 1,1-Dichloroethane & 0.609 & 0.573 & 0.010 & -6.0 & 30.0 \\
\hline cis-1,2-Dichloroethene & 0.302 & 0.281 & 0.010 & -6.8 & 40.0 \\
\hline 2-Butanone & 0.047 & 0.043 & 0.010 & -7.6 & 40.0 \\
\hline Bromochloromethane & 0.101 & 0.095 & 0.010 & -6.0 & 30.0 \\
\hline Chloroform & 0.485 & 0.457 & 0.010 & -5.8 & 30.0 \\
\hline 1,1,1-Trichloroethane & 0.494 & 0.456 & 0.010 & -7.8 & 30.0 \\
\hline Cyclohexane & 0.850 & 0.852 & 0.010 & 0.3 & 40.0 \\
\hline Carbon tetrachloride & 0.472 & 0.436 & 0.010 & -7.6 & 30.0 \\
\hline Benzene & 1.515 & 1.375 & 0.010 & -9.3 & 30.0 \\
\hline 1,2-Dichloroethane & 0.282 & 0.272 & 0.010 & -3.3 & 30.0 \\
\hline Trichloroethene & 0.368 & 0.327 & 0.010 & -11.2 & 30.0 \\
\hline Methylcyclohexane & 0.737 & 0.709 & 0.010 & -3.9 & 40.0 \\
\hline
\end{tabular}

Report 1,4-Dioxane for Low/Medium VoA analysis only 
$7 \mathrm{~B}$ - FORM VII VOA-2

VOLATILE CONTINUING CALIBRATION DATA

Lab Name: TESTAMERICA BURLINGTON

Contract: 1E-30401

Lab Code: STLV

Mod. Ref No.:

SDG No.: 200-6783

Instrument ID: D.i

Calibration Date: 09/02/2011 Time: 0842

Lab File Id: DHPW03.D

Init. Calib. Date(s): 07/07/2011 07/07/2011

EPA Sample No. (VSTD\#\#\#\#): VSTD005DT

Init. Calib. Time(s): $1117 \quad 1256$

Heated Purge: ( $\mathrm{Y} / \mathrm{N}) \mathrm{N}$

GC Column: DB-624

ID: $0.20(\mathrm{~mm})$ Length: 25

(m)

Purge Volume: 25.0

(mL)

\begin{tabular}{|c|c|c|c|c|c|}
\hline COMPOUND & $\overline{\mathrm{RRE}}$ & RRF5.0 & $\begin{array}{l}\text { MIN } \\
\text { RRE }\end{array}$ & $\because \mathrm{D}$ & MAX $\because D$ \\
\hline 1,2-Dichloropropane & 0.366 & 0.347 & 0.010 & -5.0 & 40.0 \\
\hline Bromodichloromethane & 0.305 & 0.281 & 0.010 & -7.9 & 30.0 \\
\hline cis-1,3-Dichloropropene & 0.377 & 0.375 & 0.010 & -0.7 & 30.0 \\
\hline 4-Methyl-2-pentanone & 0.126 & 0.125 & 0.010 & -1.1 & 40.0 \\
\hline Toluene & 1.657 & 1.553 & 0.010 & -6.3 & 30.0 \\
\hline trans-1,3-Dichloropropene & 0.267 & 0.260 & 0.010 & -2.7 & 30.0 \\
\hline $1,1,2$-Trichloroethane & 0.158 & 0.146 & 0.010 & -7.3 & 30.0 \\
\hline Tetrachloroethene & 0.306 & 0.276 & 0.010 & -9.7 & 30.0 \\
\hline 2-Hexanone & 0.090 & 0.087 & 0.010 & -3.9 & 40.0 \\
\hline Dibromochloromethane & 0.180 & 0.171 & 0.010 & -4.9 & 30.0 \\
\hline 1,2-Dibromoethane & 0.139 & 0.133 & 0.010 & -4.3 & 40.0 \\
\hline Chlorobenzene & 1.042 & 0.964 & 0.010 & -7.5 & 30.0 \\
\hline Ethylbenzene & 1.825 & 1.765 & 0.010 & -3.3 & 30.0 \\
\hline o-Xylene & 0.674 & 0.674 & 0.010 & -0.1 & 30.0 \\
\hline $\mathrm{m}, \mathrm{p}$-Xylene & 0.742 & 0.733 & 0.010 & -1.1 & 30.0 \\
\hline styrene & 0.983 & 1.000 & 0.010 & 1.7 & 30.0 \\
\hline Bromoform & 0.121 & 0.110 & 0.010 & -9.0 & 30.0 \\
\hline Isopropylbenzene & 1.841 & 1.831 & 0.010 & -0.5 & 40.0 \\
\hline $1,1,2,2$-Tetrachloroethane & 0.164 & 0.151 & 0.010 & -7.9 & 30.0 \\
\hline 1,3-Dichlorobenzene & 1.562 & 1.412 & 0.010 & -9.6 & 30.0 \\
\hline 1,4-Dichlorobenzene & 1.686 & 1.513 & 0.010 & -10.2 & 30.0 \\
\hline 1,2-Dichlorobenzene & 1.384 & 1.281 & 0.010 & -7.5 & 30.0 \\
\hline 1,2-Dibromo-3-Chloropropane & 0.032 & 0.035 & 0.010 & 8.0 & 40.0 \\
\hline 1,2,4-Trichlorobenzene & 0.674 & 0.636 & 0.010 & -5.6 & 30.0 \\
\hline 1,2,3-Trichlorobenzene & 0.514 & 0.506 & 0.010 & -1.7 & 30.0 \\
\hline
\end{tabular}


7C - FORM VII VOA-3

VOLATILE CONTINUING CALIBRATION DATA

Lab Name: TESTAMERICA BURLINGTON

Contract: $1 \mathrm{E}-30401$

Lab Code: STLV Case No.: INMAN Mod. Ref No.:

SDG No.: 200-6783

Instrument ID: D.i

Calibration Date: 09/02/2011 Time: 0842

Lab File Id: DHPW03.D Init. Calib. Date(s) : 07/07/2011 07/07/2011

EPA Sample No.(VSTD\#\#\#\#): VSTD005DT

Init. Calib. Time(s): $1117 \quad 1256$

Heated Purge: ( $\mathrm{Y} / \mathrm{N}) \mathrm{N}$

GC Column: DB-624

ID: $0.20(\mathrm{~mm})$ Length: 25

$(\mathrm{m})$

Purge Volume: 25.0

$(\mathrm{mL})$

\begin{tabular}{|c|c|c|c|c|c|}
\hline COMPOUND & $\overline{\mathrm{RRE}}$ & RRF5. 0 & $\begin{array}{l}\text { MIN } \\
\text { RRF }\end{array}$ & 음 & MAX $\because D$ \\
\hline Vinyl Chloride-d3 & 0.436 & 0.384 & 0.010 & -11.9 & 30.0 \\
\hline Chloroethane-d5 & 0.283 & 0.249 & 0.010 & $-11 \cdot 9$ & 40.0 \\
\hline 1,1-Dichloroethene-d2 & 0.795 & 0.663 & 0.010 & -16.6 & 30.0 \\
\hline 2-Butanone-d5 & 0.041 & 0.038 & 0.010 & -7.0 & 40.0 \\
\hline Chloroform-d & 0.509 & 0.455 & 0.010 & -10.7 & 30.0 \\
\hline 1,2-Dichloroethane-d4 & 0.203 & 0.192 & 0.010 & -5.6 & 30.0 \\
\hline Benzene-d6 & 1.475 & 1.295 & 0.010 & -12.2 & 30.0 \\
\hline 1,2-Dichloropropane-d6 & 0.374 & 0.321 & 0.010 & -14.3 & 40.0 \\
\hline Toluene-d8 & 1.397 & 1.274 & 0.010 & -8.8 & 30.0 \\
\hline trans-1,3-Dichloropropene-d4 & 0.230 & 0.210 & 0.010 & -8.5 & 30.0 \\
\hline 2-Hexanone-d5 & 0.033 & 0.034 & 0.010 & 3.1 & 40.0 \\
\hline $1,1,2,2$-Tetrachloroethane-d2 & 0.164 & 0.145 & 0.010 & $-11 \cdot 4$ & 30.0 \\
\hline 1,2-Dichlorobenzene-d4 & 0.830 & 0.727 & 0.010 & -12.4 & 30.0 \\
\hline
\end{tabular}

Report 1,4-Dioxane-d8 for Low/Medium VOA analysis only 
$7 \mathrm{~A}$ - FORM VII VOA-1

VOLATILE CONTINUING CALIBRATION DATA

Lab Name: TESTAMERICA BURLINGTON

Contract: 1E-30401

Lab Code: STLV Case No.: INMAN Mod. Ref No.:

SDG No.: 200-6783

Instrument ID: D.i

Calibration Date: 09/02/2011 Time: 1327

Lab File Id: DHPW14.D Init. Calib. Date(s): 07/07/2011 07/07/2011

EPA Sample No. (VSTD\#\#\#\#): VSTD005TD

Init. Calib. Time(s): $1117 \quad 1256$

Heated Purge: $(\mathrm{Y} / \mathrm{N}) \mathrm{N}$

GC Column: DB-624

ID: $0.20(\mathrm{~mm})$ Length: 25

(m)

Purge Volume: 25.0

(mL)

\begin{tabular}{|c|c|c|c|c|c|}
\hline COMPOUND & $\overline{\mathrm{RRF}}$ & RRE5. 0 & $\begin{array}{l}\text { MIN } \\
\text { RRF }\end{array}$ & $\because \mathrm{D}$ & $\mathrm{MAX} \div \mathrm{D}$ \\
\hline Dichlorodifluoromethane & 0.403 & 0.467 & 0.010 & 16.0 & 50.0 \\
\hline Chloromethane & 0.687 & 0.701 & 0.010 & 2.1 & 50.0 \\
\hline Vinyl chloride & 0.496 & 0.494 & 0.100 & -0.5 & 50.0 \\
\hline Bromomethane & 0.209 & 0.202 & 0.100 & -3.3 & 50.0 \\
\hline Chloroethane & 0.272 & 0.256 & 0.010 & -5.8 & 50.0 \\
\hline Trichlorofluoromethane & 0.606 & 0.612 & 0.010 & 1.0 & 50.0 \\
\hline 1,1-Dichloroethene & 0.305 & 0.296 & 0.100 & -3.0 & 50.0 \\
\hline 1,1,2-Trichloro-1,2,2-trifluoroethane & 0.355 & 0.362 & 0.010 & 1.8 & 50.0 \\
\hline Acetone & 0.036 & 0.032 & 0.010 & -10.9 & 50.0 \\
\hline Carbon disulfide & 0.742 & 0.687 & 0.010 & -7.4 & 50.0 \\
\hline Methyl acetate & 0.086 & 0.088 & 0.010 & 1.9 & 50.0 \\
\hline Methylene Chloride & 0.250 & 0.246 & 0.010 & -1.6 & 50.0 \\
\hline trans-1,2-Dichloroethene & 0.308 & 0.305 & 0.010 & -0.8 & 50.0 \\
\hline Methyl tert-butyl ether & 0.302 & 0.300 & 0.010 & -0.7 & 50.0 \\
\hline 1,1-Dichloroethane & 0.609 & 0.609 & 0.200 & 0.0 & 50.0 \\
\hline cis-1,2-Dichloroethene & 0.302 & 0.302 & 0.010 & 0.2 & 50.0 \\
\hline 2-Butanone & 0.047 & 0.048 & 0.010 & 1.4 & 50.0 \\
\hline Bromochloromethane & 0.101 & 0.104 & 0.050 & 3.2 & 50.0 \\
\hline Chloroform & 0.485 & 0.497 & 0.200 & 2.4 & 50.0 \\
\hline 1,1,1-Trichloroethane & 0.494 & 0.475 & 0.100 & -3.9 & 50.0 \\
\hline Cyclohexane & 0.850 & 0.891 & 0.010 & 4.9 & 50.0 \\
\hline Carbon tetrachloride & 0.472 & 0.466 & 0.100 & -1.4 & 50.0 \\
\hline Benzene & 1.515 & 1.442 & 0.400 & -4.8 & 50.0 \\
\hline 1,2-Dichloroethane & 0.282 & 0.300 & 0.100 & 6.5 & 50.0 \\
\hline Trichloroethene & 0.368 & 0.341 & 0.300 & -7.3 & 50.0 \\
\hline Methylcyclohexane & 0.737 & 0.758 & 0.010 & 2.7 & 50.0 \\
\hline
\end{tabular}

Report 1,4-Dioxane for Low/Medium VOA analysis only 
$7 \mathrm{~B}$ - FORM VII VOA-2

VOLATILE CONTINUING CALIBRATION DATA

Lab Name: TESTAMERICA BURLINGTON

Contract: 1E-30401

Lab Code: STLV Case No.: INMAN

Mod. Ref No.:

SDG No.: 200-6783

Instrument ID: D.i

Calibration Date: 09/02/2011 Time: 1327

Lab File Id: DHPW14.D

Init. Calib. Date(s): 07/07/2011 07/07/2011

EPA Sample No. (VSTD\#\#\#\#): VSTD005TD

Init. Calib. Time(s): $1117 \quad 1256$

Heated Purge: ( $\mathrm{Y} / \mathrm{N}) \mathrm{N}$

GC Column: DB-624

ID: $0.20(\mathrm{~mm})$ Length: 25

(m)

Purge Volume: 25.0

(mL)

\begin{tabular}{|c|c|c|c|c|c|}
\hline COMPOUND & $\overline{\mathrm{RRF}}$ & RRF5. 0 & $\begin{array}{l}\text { MIN } \\
\text { RRE }\end{array}$ & $\because \mathrm{D}$ & MAX $\div D$ \\
\hline 1,2-Dichloropropane & 0.366 & 0.369 & 0.010 & 0.9 & 50.0 \\
\hline Bromodichloromethane & 0.305 & 0.296 & 0.200 & -2.9 & 50.0 \\
\hline cis-1,3-Dichloropropene & 0.377 & 0.386 & 0.200 & 2.2 & 50.0 \\
\hline 4-Methyl-2-pentanone & 0.126 & 0.133 & 0.010 & 5.6 & 50.0 \\
\hline Toluene & 1.657 & 1.620 & 0.400 & -2.2 & 50.0 \\
\hline trans-1,3-Dichloropropene & 0.267 & 0.273 & 0.100 & 2.2 & 50.0 \\
\hline 1,1,2-Trichloroethane & 0.158 & 0.156 & 0.100 & -1.5 & 50.0 \\
\hline Tetrachloroethene & 0.306 & 0.294 & 0.100 & -4.0 & 50.0 \\
\hline 2-Hexanone & 0.090 & 0.095 & 0.010 & 5.5 & 50.0 \\
\hline Dibromochloromethane & 0.180 & 0.169 & 0.100 & -5.9 & 50.0 \\
\hline 1,2-Dibromoethane & 0.139 & 0.140 & 0.010 & 0.6 & 50.0 \\
\hline Chlorobenzene & 1.042 & 1.024 & 0.500 & -1.7 & 50.0 \\
\hline Ethylbenzene & 1.825 & 1.833 & 0.100 & 0.5 & 50.0 \\
\hline o-Xylene & 0.674 & 0.702 & 0.300 & 4.1 & 50.0 \\
\hline $\mathrm{m}, \mathrm{p}$-Xylene & 0.742 & 0.767 & 0.300 & 3.4 & 50.0 \\
\hline Styrene & 0.983 & 1.073 & 0.300 & 9.1 & 50.0 \\
\hline Bromoform & 0.121 & 0.110 & 0.050 & -9.0 & 50.0 \\
\hline Isopropylbenzene & 1.841 & 1.940 & 0.010 & 5.4 & 50.0 \\
\hline $1,1,2,2$-Tetrachloroethane & 0.164 & 0.161 & 0.100 & -1.6 & 50.0 \\
\hline 1,3-Dichlorobenzene & 1.562 & 1.460 & 0.400 & -6.5 & 50.0 \\
\hline 1,4-Dichlorobenzene & 1.686 & 1.597 & 0.400 & -5.3 & 50.0 \\
\hline 1,2-Dichlorobenzene & 1.384 & 1.378 & 0.400 & -0.4 & 50.0 \\
\hline 1,2-Dibromo-3-Chloropropane & 0.032 & 0.036 & 0.010 & 12.5 & 50.0 \\
\hline 1,2,4-Trichlorobenzene & 0.674 & 0.626 & 0.200 & -7.1 & 50.0 \\
\hline 1,2,3-Trichlorobenzene & 0.514 & 0.500 & 0.200 & -2.8 & 50.0 \\
\hline
\end{tabular}


$7 \mathrm{C}-$ FORM VII VOA-3

VOLATILE CONTINUING CALIBRATION DATA

Lab Name: TESTAMERICA BURLINGTON

Contract: 1E-30401

Lab Code: STLV Case No.: INMAN Mod. Ref No.:

SDG No.: 200-6783

Instrument ID: D.i

Calibration Date: 09/02/2011 Time: 1327

Lab File Id: DHPW14.D Init. Calib. Date(s): 07/07/2011 07/07/2011

EPA Sample No.(VSTD\#\#\#): VSTD005'TD

Init. Calib. Time(s): $1117 \quad 1256$

Heated Purge: ( $\mathrm{Y} / \mathrm{N}) \mathrm{N}$

GC Colurnn: DB-624

ID $: 0.20(\mathrm{~mm})$ Length: 25

$(\mathrm{m})$

Purge Volume: 25.0

$(\mathrm{mL})$

\begin{tabular}{|c|c|c|c|c|c|}
\hline COMPOUND & $\overline{\mathrm{RRF}}$ & RRF5.0 & $\begin{array}{l}\text { MIN } \\
\text { RRE }\end{array}$ & $\frac{\circ}{0} \mathrm{D}$ & MAX $\% D$ \\
\hline Vinyl Chloride-d3 & 0.436 & 0.404 & 0.010 & -7.2 & 50.0 \\
\hline Chloroethane-d5 & 0.283 & 0.263 & 0.010 & -6.9 & 50.0 \\
\hline 1,1-Dichloroethene-d2 & 0.795 & 0.738 & 0.010 & -7.2 & 50.0 \\
\hline 2-Butanone-d5 & 0.041 & 0.042 & 0.010 & 0.9 & 50.0 \\
\hline Chloroform-d & 0.509 & 0.482 & 0.010 & -5.4 & 50.0 \\
\hline 1,2-Dichloroethane-d4 & 0.203 & 0.206 & 0.010 & 1.6 & 50.0 \\
\hline Benzene-d6 & 1.475 & 1.348 & 0.010 & -8.7 & 50.0 \\
\hline 1,2-Dichloropropane-d6 & 0.374 & 0.346 & 0.010 & -7.6 & 50.0 \\
\hline Toluene-d8 & 1.397 & 1.348 & 0.010 & -3.6 & 50.0 \\
\hline trans-1,3-Dichloropropene-d4 & 0.230 & 0.217 & 0.010 & -5.6 & 50.0 \\
\hline 2-Hexanone-d5 & 0.033 & 0.035 & 0.010 & 6.5 & 50.0 \\
\hline $1,1,2,2$-Tetrachloroethane-d2 & 0.164 & 0.154 & 0.010 & -6.1 & 50.0 \\
\hline 1,2-Dichlorobenzene-d4 & 0.830 & 0.727 & 0.010 & -12.3 & 50.0 \\
\hline
\end{tabular}

Report 1,4-Dioxane-d8 for Low/Medium VOA analysis only 
$1 A$ - FORM I VOA-1

VOLATILE ORGANICS ANALYSIS DATA SHEET
EPA SAMPLE NO.

VBLKDS
Lab Name: TESTAMERICA BURLINGTON

Lab Code: STLV Case No.: INMAN

Matrix: (SOIL/SED/WATER)

Water

Sample wt/vol: 25.0 $(\mathrm{g} / \mathrm{mL}) \mathrm{mL}$

Level: (TRACE/LOW/MED) TRACE

응 Moisture: not dec.

GC Column: DB-624

ID: 0.20

$(\mathrm{mm})$

Soil Extract Volume:

(uL)

(mL)

Purge Volume: 25.0
Contract: 1E-30401

SDG No.: 200-6783

. :

Lab Sample ID: MB 200-24615/3

Lab File ID: DHPV03.D

Date Received:

Date Analyzed: 09/01/2011

Dilution Factor: 1.0

Soil Aliquot Volume:

(uL)

\begin{tabular}{|c|c|c|c|}
\hline CAS NO. & COMPOUND & $\begin{array}{l}\text { CONCENTRATION UNITS: } \\
(\mathrm{ug} / \mathrm{L} \text { or } \mathrm{ug} / \mathrm{kg}) \mathrm{ug} / \mathrm{L}\end{array}$ & Q \\
\hline $75-71-8$ & Dichlorodifluoromethane & 0.50 & $\mathrm{U}$ \\
\hline $74-87-3$ & Chloromethane & 0.50 & $\mathrm{U}$ \\
\hline $75-01-4$ & Vinyl chloride & 0.50 & $\mathrm{U}$ \\
\hline $74-83-9$ & Bromomethane & 0.50 & $\mathrm{U}$ \\
\hline $75-00-3$ & Chloroethane & 0.50 & $\mathrm{U}$ \\
\hline $75-69-4$ & Trichlorofluoromethane & 0.50 & $\mathrm{U}$ \\
\hline $75-35-4$ & 1,1-Dichloroethene & 0.50 & $\mathrm{U}$ \\
\hline $76-13-1$ & 1,1,2-Trichloro-1,2,2-trifluoroethane & 0.50 & $\mathrm{U}$ \\
\hline $67-64-1$ & Acetone & 5.0 & $\mathrm{U}$ \\
\hline $75-15-0$ & Carbon disulfide & 0.50 & $\mathrm{U}$ \\
\hline $79-20-9$ & Methyl acetate & 0.50 & $\mathrm{U}$ \\
\hline $75-09-2$ & Methylene Chloride & 0.50 & $\mathrm{U}$ \\
\hline $156-60-5$ & trans-1,2-Dichloroethene & 0.50 & $\mathrm{U}$ \\
\hline $1634-04-4$ & Methyl tert-butyl ether & 0.50 & $\mathrm{U}$ \\
\hline $75-34-3$ & 1,1-Dichloroethane & 0.50 & $\mathrm{U}$ \\
\hline $156-59-2$ & cis-1,2-Dichloroethene & 0.50 & $\mathrm{U}$ \\
\hline $78-93-3$ & 2-Butanone & 5.0 & $\mathrm{U}$ \\
\hline $74-97-5$ & Bromochloromethane & 0.50 & U \\
\hline $67-66-3$ & Chloroform & 0.50 & $\mathrm{U}$ \\
\hline $71-55-6$ & 1,1,1-Trichloroethane & 0.50 & $\mathrm{U}$ \\
\hline $110-82-7$ & Cyclohexane & 0.50 & $\mathrm{U}$ \\
\hline $56-23-5$ & Carbon tetrachloride & 0.50 & $\mathrm{U}$ \\
\hline $71-43-2$ & Benzene & 0.50 & $\mathrm{U}$ \\
\hline $107-06-2$ & 1,2-Dichloroethane & 0.50 & $\mathrm{U}$ \\
\hline
\end{tabular}

Report 1,4-Dioxane for Low-Medium VOA analysis only 
Lab Name: TESTAMERICA BURLINGTON

Lab Code: STLV Case No.: INMA Mod. Ref No.:

Water

$(\mathrm{g} / \mathrm{mL}) \quad \mathrm{mL}$

Sample wt/vol: 25.0

TRACE

Level: (TRACE/LOW/MED)

\% Moisture: not dec.

GC Column: DB-624

ID: 0.20 (mm)

Soil Extract Volume:

Purge Volume: 25.0 $(\mathrm{mL})$
Contract: 1E-30401

SDG No.: 200-6783

Lab Sample ID: MB 200-24615/3

Lab File ID: DHPV03.D

Date Received:

Date Analyzed: 09/01/2011

Dilution Factor: 1.0

Soil Aliquot Volume:

\begin{tabular}{|c|c|c|c|}
\hline CAS NO. & COMPOUND & $\begin{array}{l}\text { CONCENTRATION UNITS: } \\
(\mathrm{ug} / \mathrm{L} \text { or } \mathrm{ug} / \mathrm{kg}) \mathrm{ug} / \mathrm{L}\end{array}$ & $Q$ \\
\hline $79-01-6$ & Trichloroethene & 0.50 & $\bar{U}$ \\
\hline $108-87-2$ & Methylcyclohexane & 0.50 & $\mathrm{U}$ \\
\hline $78-87-5$ & 1,2-Dichloropropane & 0.50 & $\mathrm{U}$ \\
\hline $75-27-4$ & Bromodichloromethane & 0.50 & $\mathrm{U}$ \\
\hline $10061-01-5$ & cis-1,3-Dichloropropene & 0.50 & $\mathrm{U}$ \\
\hline $108-10-1$ & 4-Methyl-2-pentanone & 5.0 & $\mathrm{U}$ \\
\hline $108-88-3$ & Toluene & 0.0094 & $\mathrm{~J}$ \\
\hline $10061-02-6$ & trans-1,3-Dichloropropene & 0.50 & $\mathrm{U}$ \\
\hline $79-00-5$ & 1, 1,2-Trichloroethane & 0.50 & $\mathrm{U}$ \\
\hline $127-18-4$ & Tetrachloroethene & 0.50 & $\mathrm{U}$ \\
\hline $591-78-6$ & 2-Hexanone & 5.0 & $\mathrm{U}$ \\
\hline $124-48-1$ & Dibromochloromethane & 0.50 & $\mathrm{U}$ \\
\hline $106-93-4$ & 1,2-Dibromoethane & 0.50 & $\mathrm{U}$ \\
\hline $108-90-7$ & Chlorobenzene & 0.50 & $\mathrm{U}$ \\
\hline $100-41-4$ & Ethylbenzene & 0.50 & $\mathrm{U}$ \\
\hline $95-47-6$ & o-xylene & 0.50 & $\mathrm{U}$ \\
\hline $179601-23-1$ & m,p-Xylene & 0.50 & $\mathrm{U}$ \\
\hline $100-42-5$ & Styrene & 0.50 & $\mathrm{U}$ \\
\hline $75-25-2$ & Bromoform & 0.50 & $\mathrm{U}$ \\
\hline $98-82-8$ & Isopropylbenzene & 0.50 & $\mathrm{U}$ \\
\hline $79-34-5$ & $1,1,2,2$-Tetrachloroethane & 0.50 & $\mathrm{U}$ \\
\hline $541-73-1$ & 1,3-Dichlorobenzene & 0.50 & $\mathrm{U}$ \\
\hline $106-46-7$ & 1,4-Dichlorobenzene & 0.50 & $\mathrm{U}$ \\
\hline $95-50-1$ & 1,2-Dichlorobenzene & 0.50 & $\mathrm{U}$ \\
\hline $96-12-8$ & 1,2-Dibromo-3-Chloropropane & 0.50 & $\mathrm{U}$ \\
\hline $120-82-1$ & 1,2,4-Trichlorobenzene & 0.50 & $\mathrm{U}$ \\
\hline $87-61-6$ & 1,2,3-Trichlorobenzene & 0.50 & $\mathrm{U}$ \\
\hline
\end{tabular}


IJ - FORM I VOA-TIC

VOLATILE ORGANICS ANALYSIS DATA SHEET TENTATIVELY IDENTTEIED COMPOUNDS
EPA SAMPLE NO.

VBLKDS
Lab Name: TESTAMERICA BURLINGTON

Lab Code: STLV Case No.: INMAN

Matrix: (SOIL/SED/WATER)

Water

Sample wt/vol: 25.0 $(\mathrm{g} / \mathrm{mL}) \quad \mathrm{mL}$

Level: (TRACE or LOW/MED)

TRACE

\% Moisture: not dec.

GC Column: DB-624

Soil Extract Volume: ID: 0.20 (mn)

(uL)

CONCENTRATION UNITS: (ug/L or ug/kg) ug/L
Contract: $1 \mathrm{E}-30401$ SDG No.: 200-6783

Lab Sample ID: MB 200-24615/3

Lab File ID: DHPV03.D

Date Received:

Date Analyzed: 09/01/2011

Dilution Factor: 1.0

Soil Aliquot Volume:

Purge Volume: 25.0 $(\mathrm{mL})$

\begin{tabular}{|l|l|l|l|r|r|}
\hline \multicolumn{1}{|c|}{ CAS NUMBER } & \multicolumn{1}{|c|}{ COMPOUND NAME } & RT & EST. CONC. & Q \\
\cline { 2 - 6 } 01 & & Unknown & 6.79 & J \\
\hline
\end{tabular}

1 EPA-designated Registry Number. 
Lab Name: TESTAMERICA BURLINGTON

Lab Code: STLV

Case No.: INMAN

Matrix: (SOIL/SED/WATER)

Sample wt/vol: 25.0

Water

$(\mathrm{g} / \mathrm{mL}) \mathrm{mL}$

Level: (TRACE/LOW/MED) TRACE

\% Moisture: not dec.

GC Column: DB-624

ID: $0.20 \quad(\mathrm{~mm})$

Soil Extract Volume:

(uL)

(mL)

Purge Volume: 25.0
Contract: 1E-30401

SDG No.: 200-6783

: :

Lab Sample ID: MB 200-24648/4

Lab File ID: DHPW04.D

Date Received:

Date Analyzed: 09/02/2011

Dilution Factor: 1.0

Soil Aliquot Volume:

(uL)

\begin{tabular}{|c|c|c|c|}
\hline CAS NO. & COMPOUND & $\begin{array}{l}\text { CONCENTRATION UNITS: } \\
(\mathrm{ug} / \mathrm{L} \text { or } \mathrm{ug} / \mathrm{kg}) \mathrm{ug} / \mathrm{L}\end{array}$ & $Q$ \\
\hline $75-71-8$ & Dichlorodifluoromethane & 0.50 & $\mathrm{U}$ \\
\hline $74-87-3$ & Chloromethane & 0.50 & $\mathrm{U}$ \\
\hline $75-01-4$ & Vinyl chloride & 0.50 & $\mathrm{U}$ \\
\hline $74-83-9$ & Bromomethane & 0.50 & $\mathrm{U}$ \\
\hline $75-00-3$ & Chloroethane & 0.50 & $\mathrm{U}$ \\
\hline $75-69-4$ & Trichlorofluoromethane & 0.50 & $\mathrm{U}$ \\
\hline $75-35-4$ & 1,1-Dichloroethene & 0.50 & $\mathrm{U}$ \\
\hline $76-13-1$ & 1,1,2-Trichloro-1,2,2-trifluoroethane & 0.50 & $\mathrm{U}$ \\
\hline $67-64-1$ & Acetone & 5.0 & $\mathrm{U}$ \\
\hline $75-15-0$ & Carbon disulfide & 0.50 & $\mathrm{U}$ \\
\hline $79-20-9$ & Methyl acetate & 0.50 & $\mathrm{U}$ \\
\hline $75-09-2$ & Methylene Chloride & 0.50 & $\mathrm{U}$ \\
\hline $156-60-5$ & trans $-1,2-$ Dichloroethene & 0.50 & $\mathrm{U}$ \\
\hline $1634-04-4$ & Methyl tert-butyl ether & 0.50 & $\mathrm{U}$ \\
\hline $75-34-3$ & 1,1-Dichloroethane & 0.50 & $\mathrm{U}$ \\
\hline $156-59-2$ & cis-1,2-Dichloroethene & 0.50 & $\mathrm{U}$ \\
\hline $78-93-3$ & 2-Butanone & 5.0 & $\mathrm{U}$ \\
\hline $74-97-5$ & Bromochloromethane & 0.50 & $\mathrm{U}$ \\
\hline $67-66-3$ & Chloroform & 0.50 & U \\
\hline $71-55-6$ & 1,1,1-Trichloroethane & 0.50 & $\mathrm{U}$ \\
\hline $110-82-7$ & Cyclohexane & 0.50 & $\mathrm{U}$ \\
\hline $56-23-5$ & Carbon tetrachloride & 0.50 & $\mathrm{U}$ \\
\hline $71-43-2$ & Benzene & 0.50 & $\mathrm{U}$ \\
\hline $107-06-2$ & 1,2-Dichloroethane & 0.50 & $\mathrm{U}$ \\
\hline
\end{tabular}

Report 1,4-Dioxane for Low-Medium VOA analysis only 
$1 B$ - FORM I VOA-2

VOLATILE ORGANICS ANALYSIS DATA SHEET
EPA SAMPLE NO.

VBLKDT
Lab Name: TESTAMERICA BURLINGTON

Contract: 1E-30401

Lab Code: STLV Case No.: INMAN Mod. Ref No.:

Matrix: (SOIL/SED/WATER) Water

Sample wt/vol: 25.0

$(\mathrm{g} / \mathrm{mL}) \mathrm{mL}$

Level: (TRACE/LOW/MED) TRACE

\% Moisture: not dec.

GC Column: DB-624

ID: 0.20 (mm)

Soil Extract Volume:

(uL)

(mL)

Date Received:

Purge Volume: 25.0
SDG No.: 200-6783

Lab Sample ID: MB 200-24648/4

Lab Eile ID: DHPW04.D

Date Analyzed: 09/02/2011

Dilution Factor: 1.0

Soil Aliquot volume:

(uL)

\begin{tabular}{|c|c|c|c|}
\hline CAS NO. & COMPOUND & $\begin{array}{l}\text { CONCENTRATION UNITS: } \\
(\mathrm{ug} / \mathrm{L} \text { or } \mathrm{ug} / \mathrm{kg}) \mathrm{ug} / \mathrm{L}\end{array}$ & $Q$ \\
\hline $79-01-6$ & Trichloroethene & 0.50 & $\bar{U}$ \\
\hline $108-87-2$ & Methylcyclohexane & 0.50 & $\mathrm{U}$ \\
\hline $78-87-5$ & 1,2-Dichloropropane & 0.50 & $\mathrm{U}$ \\
\hline $75-27-4$ & Bromodichloromethane & 0.50 & $\mathrm{U}$ \\
\hline $10061-01-5$ & cis-1,3-Dichloropropene & 0.50 & $\mathrm{U}$ \\
\hline $108-10-1$ & 4-Methyl-2-pentanone & 5.0 & $\mathrm{U}$ \\
\hline $108-88-3$ & Toluene & 0.50 & $\mathrm{U}$ \\
\hline $10061-02-6$ & trans-1,3-Dichloropropene & 0.50 & $\mathrm{U}$ \\
\hline $79-00-5$ & 1,1,2-Trichloroethane & 0.50 & $\mathrm{U}$ \\
\hline $127-18-4$ & Tetrachloroethene & 0.50 & U \\
\hline $591-78-6$ & 2-Hexanone & 5.0 & $\mathrm{U}$ \\
\hline $124-48-1$ & Dibromochloromethane & 0.50 & $\mathrm{U}$ \\
\hline $106-93-4$ & 1,2-Dibromoethane & 0.50 & $\mathrm{U}$ \\
\hline $108-90-7$ & Chlorobenzene & 0.50 & $\mathrm{U}$ \\
\hline $100-41-4$ & Ethylbenzene & 0.50 & $\mathrm{U}$ \\
\hline $95-47-6$ & o-Xylene & 0.50 & U \\
\hline $179601-23-1$ & $\mathrm{~m}, \mathrm{p}-\mathrm{X}_{Y}$ lene & 0.50 & $\mathrm{U}$ \\
\hline $100-42-5$ & Styrene & 0.50 & $\mathrm{U}$ \\
\hline $75-25-2$ & Bromoform & 0.50 & $\mathrm{U}$ \\
\hline $98-82-8$ & Isopropylbenzene & 0.50 & $\mathrm{U}$ \\
\hline $79-34-5$ & $1,1,2,2$-Tetrachloroethane & 0.50 & $\mathrm{U}$ \\
\hline $541-73-1$ & 1,3-Dichlorobenzene & 0.50 & $\mathrm{U}$ \\
\hline $106-46-7$ & 1,4-Dichlorobenzene & 0.50 & $\mathrm{U}$ \\
\hline $95-50-1$ & 1,2-Dichlorobenzene & 0.50 & $\mathrm{U}$ \\
\hline $96-12-8$ & 1,2-Dibromo-3-Chloropropane & 0.50 & $\mathrm{U}$ \\
\hline $120-82-1$ & 1,2,4-Trichlorobenzene & 0.50 & $\mathrm{U}$ \\
\hline $87-61-6$ & 1,2,3-Trichlorobenzene & 0.50 & $\mathrm{U}$ \\
\hline
\end{tabular}


$1 \mathrm{~J}$ - FORM I VOA-TIC

VOLATILE ORGANICS ANALYSIS DATA SHEET TENTATIVELY IDENTIFIED COMPOUNDS
EPA SAMPLE NO.

VBLKDT
Lab Name: TESTAMERICA BURLINGTON

Lab Code: STLV Case No.: INMAN

Matrix: (SOIL/SED/WATER)

Water

Sample wt/vol: 25.0 $(\mathrm{g} / \mathrm{mL}) \mathrm{mL}$

Level: (TRACE or LOW/MED)

TRACE

\% Moisture: not dec.

GC Column: DB-624

ID: 0.20 (mm)

Soil Extract Volume:
Contract: $1 \mathrm{E}-30401$

SDG No.: 200-6783

CONCENTRATION UNITS: (ug/L or $\mathrm{ug} / \mathrm{kg}$ ) ug/L

Lab Sample ID: MB 200-24648/4

Lab File ID: DHPWO4.D

Date Received:

Date Analyzed: 09/02/2011

Dilution Factor: 1.0

Soil Aliquot Volume:

(uL)

Purge Volume: 25.0

$(\mathrm{mL})$

\begin{tabular}{|l|l|l|l|r|r|}
\hline \multicolumn{1}{|c|}{ CAS NUMBER } & \multicolumn{1}{|c|}{ COMPOUND NAME } & RT & EST. CONC. & Q \\
\cline { 2 - 6 } 01 & & Unknown & 6.79 & X J \\
\cline { 2 - 6 } & E9667961 & Total Alkanes & N/A & \\
\hline
\end{tabular}

1EPA-designated Registry Number. 
Lab Name: TESTAMERICA BURLINGTON

Lab Code: STLV Case No.:- INMA

Matrix: (SOIL/SED/WATER)

Water

Sample wt/vol: 25.0

$(\mathrm{g} / \mathrm{mL}) \quad \mathrm{mL}$

Level: (TRACE/LOW/MED) TRACE

응 Moisture: not dec.

GC Column: DB-624 ID: $0.20 \quad(\mathrm{~mm})$

Soil Extract Volume: (mL)

Purge Volume: 25.0
Contract: 1E-30401

SDG No.: 200-6783

\begin{tabular}{|c|c|c|c|}
\hline CAS NO. & COMPOUND & $\begin{array}{l}\text { CONCENTRATION UNITS: } \\
(\mathrm{ug} / \mathrm{L} \text { or } \mathrm{ug} / \mathrm{kg}) \mathrm{ug} / \mathrm{L}\end{array}$ & $Q$ \\
\hline $75-71-8$ & Dichlorodifluoromethane & 0.50 & $\mathrm{U}$ \\
\hline $74-87-3$ & Chloromethane & 0.50 & $\mathrm{U}$ \\
\hline $75-01-4$ & Vinyl chloride & 0.50 & $\mathrm{U}$ \\
\hline $74-83-9$ & Bromomethane & 0.50 & U \\
\hline $75-00-3$ & Chloroethane & 0.50 & $\mathrm{U}$ \\
\hline $75-69-4$ & Trichlorofluoromethane & 0.50 & $\mathrm{U}$ \\
\hline $75-35-4$ & 1,1-Dichloroethene & 0.50 & $\mathrm{U}$ \\
\hline $76-13-1$ & 1,1,2-Trichloro-1,2,2-trifluoroethane & 0.50 & $\mathrm{U}$ \\
\hline $67-64-1$ & Acetone & 5.0 & $\mathrm{U}$ \\
\hline $75-15-0$ & Carbon disulfide & 0.50 & $\mathrm{U}$ \\
\hline $79-20-9$ & Methyl acetate & 0.50 & $\mathrm{U}$ \\
\hline $75-09-2$ & Methylene Chloride & 0.50 & $\mathrm{U}$ \\
\hline $156-60-5$ & trans-1,2-Dichloroethene & 0.50 & $\mathrm{U}$ \\
\hline $1634-04-4$ & Methyl tert-butyl ether & 0.50 & $\mathrm{U}$ \\
\hline $75-34-3$ & 1,1-Dichloroethane & 0.50 & $\mathrm{U}$ \\
\hline $156-59-2$ & cis-1,2-Dichloroethene & 0.50 & $\mathrm{U}$ \\
\hline $78-93-3$ & 2-Butanone & 5.0 & $\mathrm{U}$ \\
\hline $74-97-5$ & Bromoch loromethane & 0.50 & $\mathrm{U}$ \\
\hline $67-66-3$ & Chloroform & 0.50 & $\mathrm{U}$ \\
\hline $71-55-6$ & 1,1,1-Trichloroethane & 0.50 & $\mathrm{U}$ \\
\hline $110-82-7$ & Cyclohexane & 0.50 & $\mathrm{U}$ \\
\hline $56-23-5$ & Carbon tetrachloride & 0.50 & $\mathrm{U}$ \\
\hline $71-43-2$ & Benzene & 0.50 & $\mathrm{U}$ \\
\hline $107-06-2$ & 1,2-Dichloroethane & 0.50 & $\mathrm{U}$ \\
\hline
\end{tabular}

Report 1,4-Dioxane for Low-Medium VOA analysis only 
$1 B$ - FORM I VOA-2

VOLATILE ORGANICS ANALYSIS DATA SHEET
EPA SAMPLE NO.

VHBLK01
Lab Name: TESTAMERICA BURLINGTON

Lab Code: STLV Case No.: INMAN Mod. Ref No, :

Matrix: (SOIL/SED/WATER)

Sample wt/vol: 25.0

Water

$(\mathrm{g} / \mathrm{mL}) \mathrm{mL}$

Level: (TRACE/LOW/MED) TRACE

\% Moisture: not dec.

GC Column: DB-624

ID $: 0.20$ (mm)

Soil Extract Volume:

(uL)

$(\mathrm{mL})$

Purge Volume: 25.0
Contract: 1E-30401

SDG No.: 200-6783

Lab Sample ID: 200-6783-6

Lab File ID: DHPW13.D

Date Received:

Date Analyzed: 09/02/2011

Dilution Factor: 1.0

Soil Aliquot Volume:

(uL)

\begin{tabular}{|c|c|c|c|}
\hline CAS NO. & COMPOUND & $\begin{array}{l}\text { CONCENTRATION UNITS: } \\
(\mathrm{ug} / \mathrm{L} \text { or } \mathrm{ug} / \mathrm{kg}) \mathrm{ug} / \mathrm{L}\end{array}$ & $Q$ \\
\hline $79-01-6$ & Trichloroethene & 0.50 & $\overline{\mathrm{U}}$ \\
\hline $108-87-2$ & Methylcyclohexane & 0.50 & U \\
\hline $78-87-5$ & 1,2-Dichloropropane & 0.50 & $\mathrm{U}$ \\
\hline $75-27-4$ & Bromodichloromethane & 0.50 & $\mathrm{U}$ \\
\hline $10061-01-5$ & cis-1,3-Dichloropropene & 0.50 & $\mathrm{U}$ \\
\hline $108-10-1$ & 4-Methyl-2-pentanone & 5.0 & U \\
\hline $108-88-3$ & Toluene & 0.50 & $\mathrm{U}$ \\
\hline $10061-02-6$ & trans-1,3-Dichloropropene & 0.50 & $\mathrm{U}$ \\
\hline $79-00-5$ & 1,1,2-Trichloroethane & 0.50 & $\mathrm{U}$ \\
\hline $127-18-4$ & Tetrachloroethene & 0.50 & $\mathrm{U}$ \\
\hline $591-78-6$ & 2-Hexanone & 5.0 & $\mathrm{U}$ \\
\hline $124-48-1$ & Dibromochloromethane & 0.50 & $\mathrm{U}$ \\
\hline $106-93-4$ & 1,2-Dibromoethane & 0.50 & U \\
\hline $108-90-7$ & Chlorobenzene & 0.50 & U \\
\hline $100-41-4$ & Ethylbenzene & 0.50 & $\mathrm{U}$ \\
\hline $95-47-6$ & o-Xylene & 0.50 & $\mathrm{U}$ \\
\hline $179601-23-1$ & $\mathrm{~m}, \mathrm{p}$-xylene & 0.50 & $\mathrm{U}$ \\
\hline $100-42-5$ & Styrene & 0.50 & $\mathrm{U}$ \\
\hline $75-25-2$ & Bromoform & 0.50 & $\mathrm{U}$ \\
\hline $98-82-8$ & Isopropylbenzene & 0.50 & U \\
\hline $79-34-5$ & $1,1,2,2-$ Tetrachloroethane & 0.50 & $\mathrm{U}$ \\
\hline $541-73-1$ & 1,3-Dichlorobenzene & 0.50 & $\mathrm{U}$ \\
\hline $106-46-7$ & 1,4-Dichlorobenzene & 0.50 & $\mathrm{U}$ \\
\hline $95-50-1$ & 1,2-Dichlorobenzene & 0.50 & $\mathrm{U}$ \\
\hline $96-12-8$ & 1,2-Dibromo-3-Chloropropane & 0.50 & U \\
\hline $120-82-1$ & 1,2,4-Trichlorobenzene & 0.50 & $\mathrm{U}$ \\
\hline $87-61-6$ & 1,2,3-Trichlorobenzene & 0.50 & $\mathrm{U}$ \\
\hline
\end{tabular}


IJ - FORM I VOA-TIC

VOLATILE ORGANICS ANALYSIS DATA SHEET TENTATIVELY IDENTIFIED COMPOUNDS
EPA SAMPLE NO.

VHBLK01

Lab Name: TESTAMERICA BURLINGTON Case No.: INMAN Mod. Ref No.:

Contract: 1E-30401

Lab Code: STLV

Water

$(\mathrm{g} / \mathrm{mL}) \mathrm{mL}$

Sample wt/vol: 25.0

TRACE

Level: (TRACE or IJOW/MED)

\% Moisture: not dec.

GC Column: DB-624

ID $: 0.20 \quad(\mathrm{~mm})$

Soil Extract Volume:

(uL)
Lab Sample ID: 200-6783-6

Lab File ID: DHPW13.D

Date Received:

Date Analyzed: 09/02/2011

Dilution Factor: 1.0

Soil Aliquot volume:

(uL)

Purge Volume: 25.0

$(\mathrm{mL})$

$$
0
$$

CONCENTRATION UNITS: (ug/L or $\mathrm{ug} / \mathrm{kg}$ ) ug/L

\begin{tabular}{|l|l|l|r|r|}
\hline \multicolumn{1}{|c|}{ CAS NUMBER } & \multicolumn{1}{|c|}{ COMPOUND NAME } & RT & EST. CONC. & Q \\
\hline & Unknown & 6.79 & 2.6 & B X J \\
\hline
\end{tabular}

1 EPA-designated Registry Number. 


\section{TestAmerica}

THE LEADER IN ENVIRONMENTAL TESTING

ANALYTICAL REPORT

Job Number: 200-6846-1

SDG Number: 200-6846

Job Description: Inman (200-6846)

Contract Number: 1E-30401

For:

Argonne National Laboratory

9700 South Cass Avenue

Building 203

Office B-149

Argonne, IL 60439

Attention: Mr. Clyde Dennis

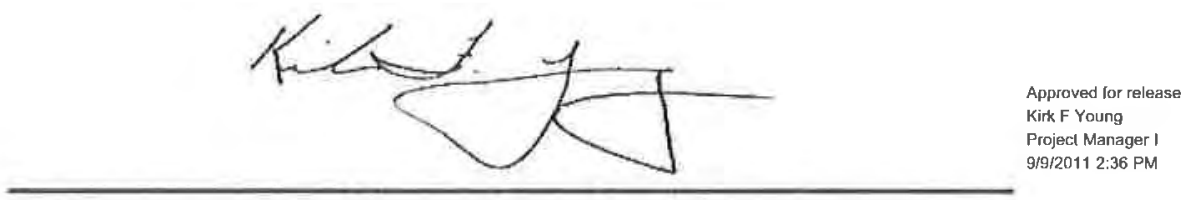

Kirk F Young

Project Manager I

kirk.young@testamericainc.com

09/09/2011

The test results in this report relate only to sample(s) as received by the laboratory. These test results were derived under a quality system that adheres to the requirements of NELAC. Pursuant to NELAC, this report may not be produced in full without written approval from the laboratory 


\section{Table of Contents}

Cover Title Page ............................ 1

Report Narrative .............................. 4

Case Narrative ................................. 4

Qualifier Definition ............................... 6

External Chain of Custody ...................... 7

Internal Chain of Custody . . . . . . . . . . . . . . . . . 8

Shipping Documentation ........................ 10

Airbills (if Applicable) ............................. 11

Sample Receipt and Log In Check List ...................... 12

Methodology Review............................. 14

QC Summary - SOM01.2 Volatiles-Trace .................. 15

QC Summary - SOM01.2 Volatiles-Trace ...................... 15

Deuterated Monitoring Compound Summary . . . . . . . . . . . . . . 15

Method Blank . . . . . . . . . . . . . . . . 17

GC/MS Instrument Performance Check ......................... 18

Internal Standard Area and RT Summary .......................... 20

Sample Data - SOM01.2 Volatiles-Trace ................. 21

Sample Data - SOM01.2 Volatiles-Trace ...................... 21

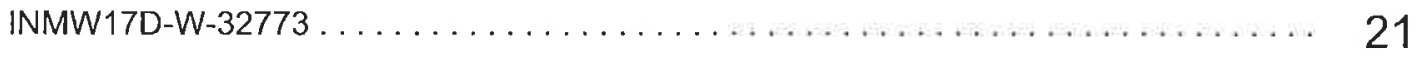

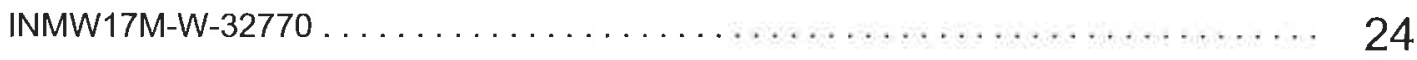

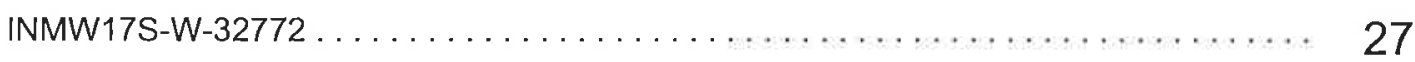

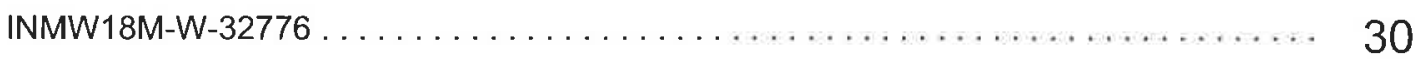

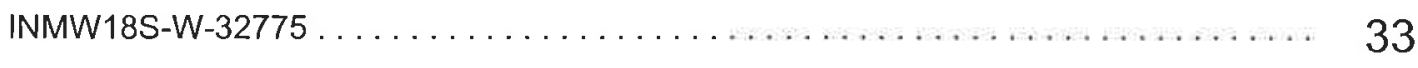

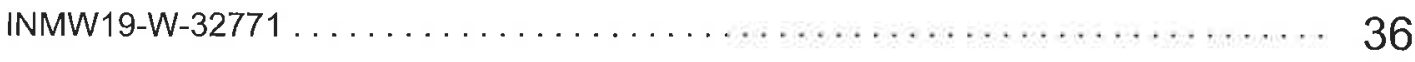

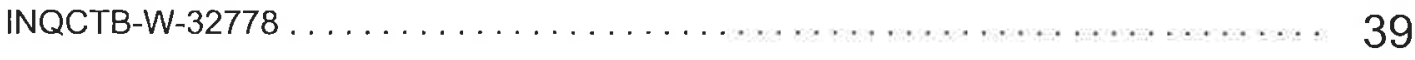

Standards - SOM01.2 Volatiles-Trace .................. 42 


\section{Table of Contents}

Standards - SOM01.2 Volatiles-Trace . . . . . . . . . . . . . . . . 42

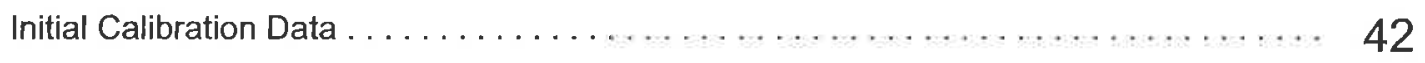

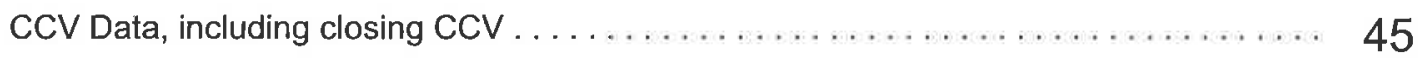

Raw Qc Data - SOM01.2 Volatiles-Trace . . . . . . . . . . . . . . . . 51

Raw Qc Data - SOM01.2 Volatiles-Trace . . . . . . . . . . . . . . . . . . 51

Raw Qc Data - SOM01.2 Volatiles-Trace . . . . . . . . . . . . . . . . . 51

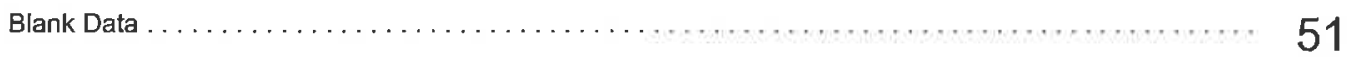




\title{
CASE NARRATIVE
}

\section{Client: Argonne National Laboratory}

\author{
Project: Inman (200-6846)
}

\author{
Report Number: 200-6846-1
}

\begin{abstract}
Enclosed is the data set for the referenced project work. With the exceptions noted as flags or footnotes, standard analytical protocols were followed in performing the analytical work and the applied control limits were met.
\end{abstract}

Calculations were performed before rounding to avoid round-off errors in calculated results.

All holding times were met and proper preservation noted for the methods performed on these samples, unless otherwise detailed in the individual sections below.

\section{Receipt}

The samples were received on 09/02/2011. Documentation of the condition of the samples at the time of their receipt and any exception to the laboratory's Sample Acceptance Policy is documented in the Shipping and Receiving section of this submittal. The samples, as received, were not acid preserved. On that basis, the laboratory did provide for the analytical work to be performed within seven days of sample collection.

\section{SOM01.2 Volatile Organics (Trace Level Water)}

A storage blank was prepared for volatile organics analysis, and stored in association with the storage of the samples. That storage blank, identified as VHBLK01, was carried through the holding period with the samples, and analyzed.

Each sample in the sample set was analyzed without a dilution. Each of the analyses associated with the sample set exhibited an acceptable internal standard performance. There was an acceptable recovery of each deuterated monitoring compound (DMC) in the analysis of the method blank associated with the analytical work, and in the analysis of the storage blank associated with the sample set. The analysis of the samples in this sample set did meet the technical acceptance criteria specific to DMC recoveries, although not all DMC recoveries were within the control range in each analysis. The technical acceptance criteria does provide for the recovery of up to three DMCs to fall outside of the control range in the analysis of field samples. Matrix spike and matrix spike duplicate analyses were not performed on samples in this sample set. A trace concentration of toluene was identified in the analysis of the method blank associated with the analytical work. The concentration of toluene in that analysis was below the established reporting limit, and the analysis did meet the technical acceptance criteria for a compliant method blank analysis. A trace concentration of toluene was also identified in the analysis of the storage blank associated with the sample set. The concentration of toluene in that analysis was below the established reporting limit, and the analysis did meet the technical acceptance criteria for a compliant storage blank analysis. Present in the method blank and storage blank analyses was a non-target constituent that represents a compound that is related to the DMC formulation. The fact that the presence of this compound is not within the laboratory's control is at issue. The derived results for that compound have been qualified with an " $X$ " qualifier to reflect the source of the contamination. 
The responses for each of the target analytes met the relative standard deviation criterion in the initial calibration. The response for each target analyte met the percent difference criterion in the opening/continuing calibration check acquisition. The response for each target analyte met the 50.0 percent difference criterion in the closing calibration check acquisition.

The primary quantitation mass for methylcyclohexane that is specified in the Statement of Work is mass 83 . The laboratory did identify a contribution to mass 83 from 1,2-dichloropropane- $d_{6}$, one of the deuterated monitoring compounds (DMCs). The laboratory did change the primary quantitation mass assignment to mass 55 for the quantification of methylcyclohexane.

Manual integration was employed in deriving certain of the analytical results. The values that have been derived from manual integration are qualified on the quantitation reports. Extracted ion current profiles for each manual integration are included in the data package, and further documented at the end of this submittal. 
DATA REPORTING QUALIFIERS

Client: Argonne National Laboratory

Job Number: 200-6846-1

Sdg Number: $200-6846$

Lab Section

Qualifier

Description

GC/MS VOA

U

J

$J$

X

*

B

N
Analyzed for but not detected.

Indicates an Estimated Value for TICs

Indicates an estimated value.

See case narrative notes for explanation of the ' $X$ ' flag

Surrogate exceeds the control limit

The analyte was found in an associated blank, as well as in the sample.

This flag indicates the presumptive evidence of a compound. 


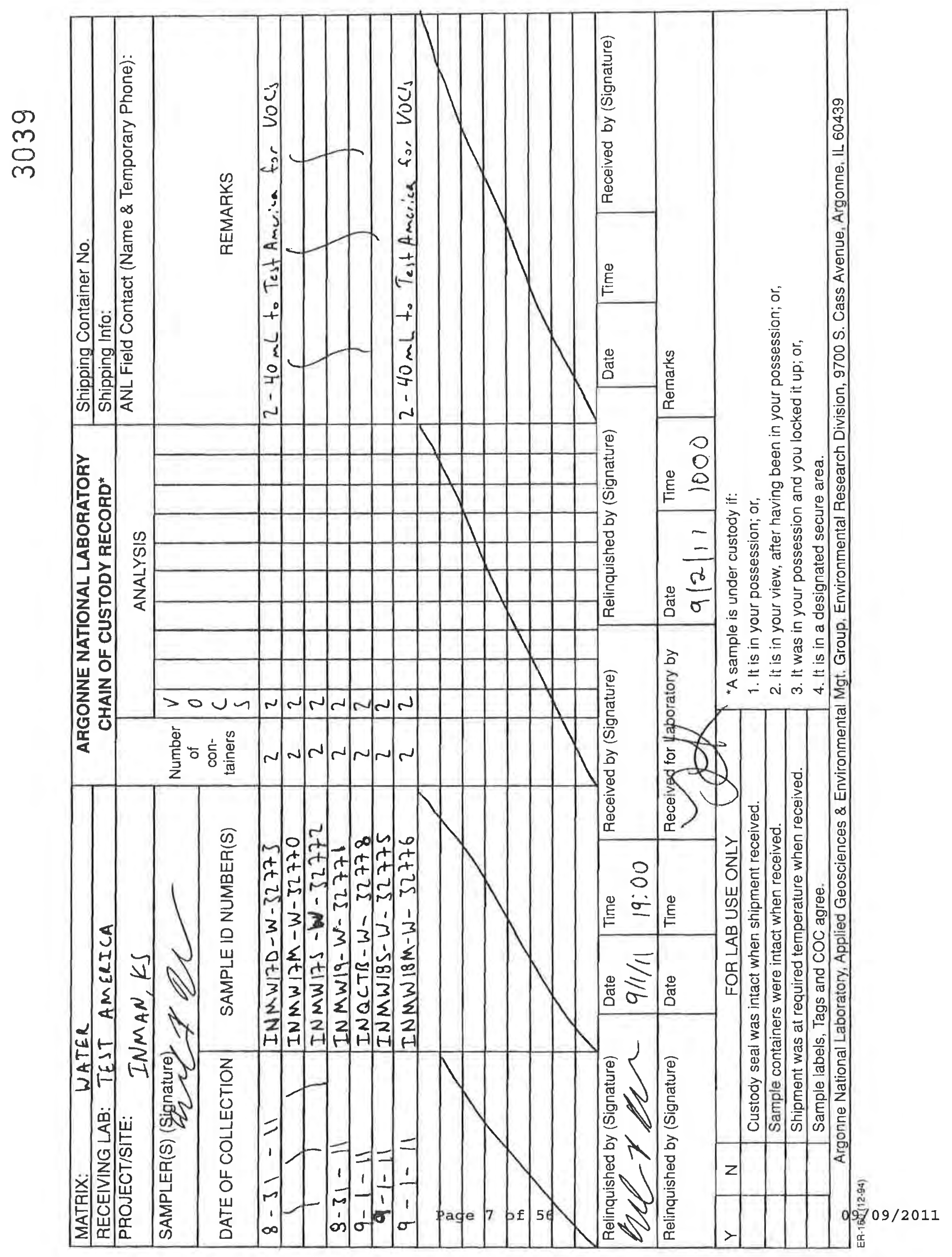




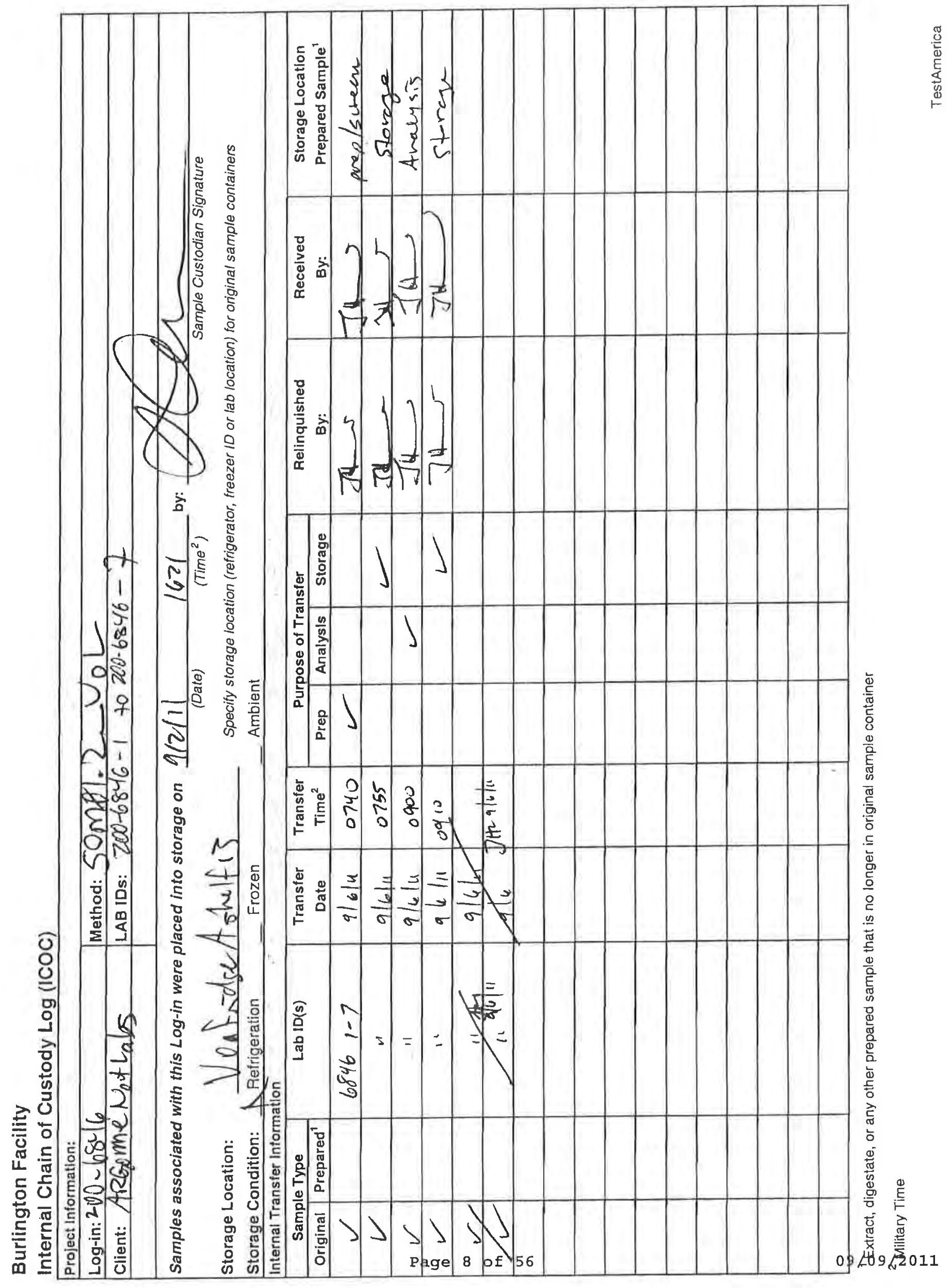




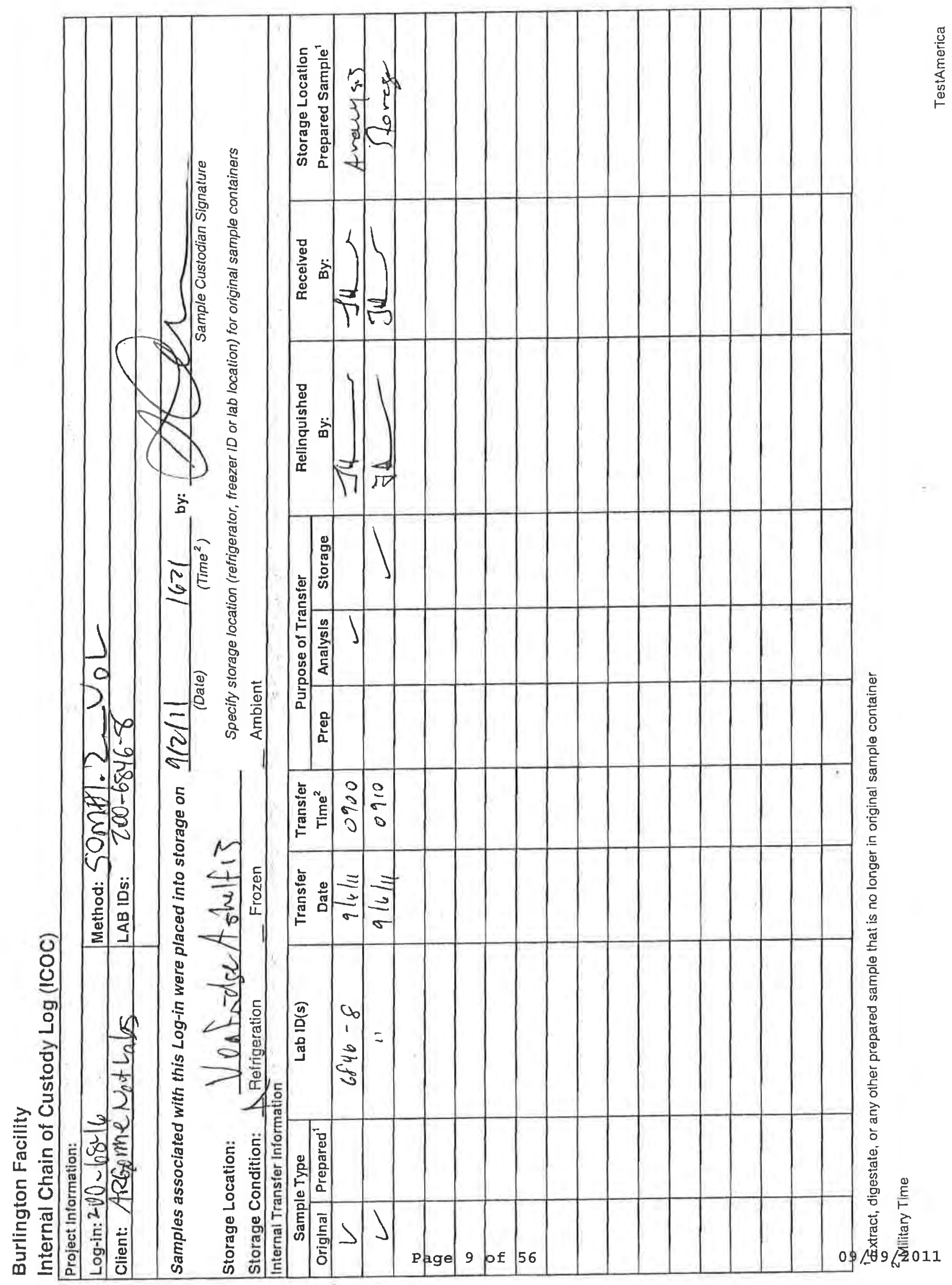


Shipping and

Receiving

Documents 


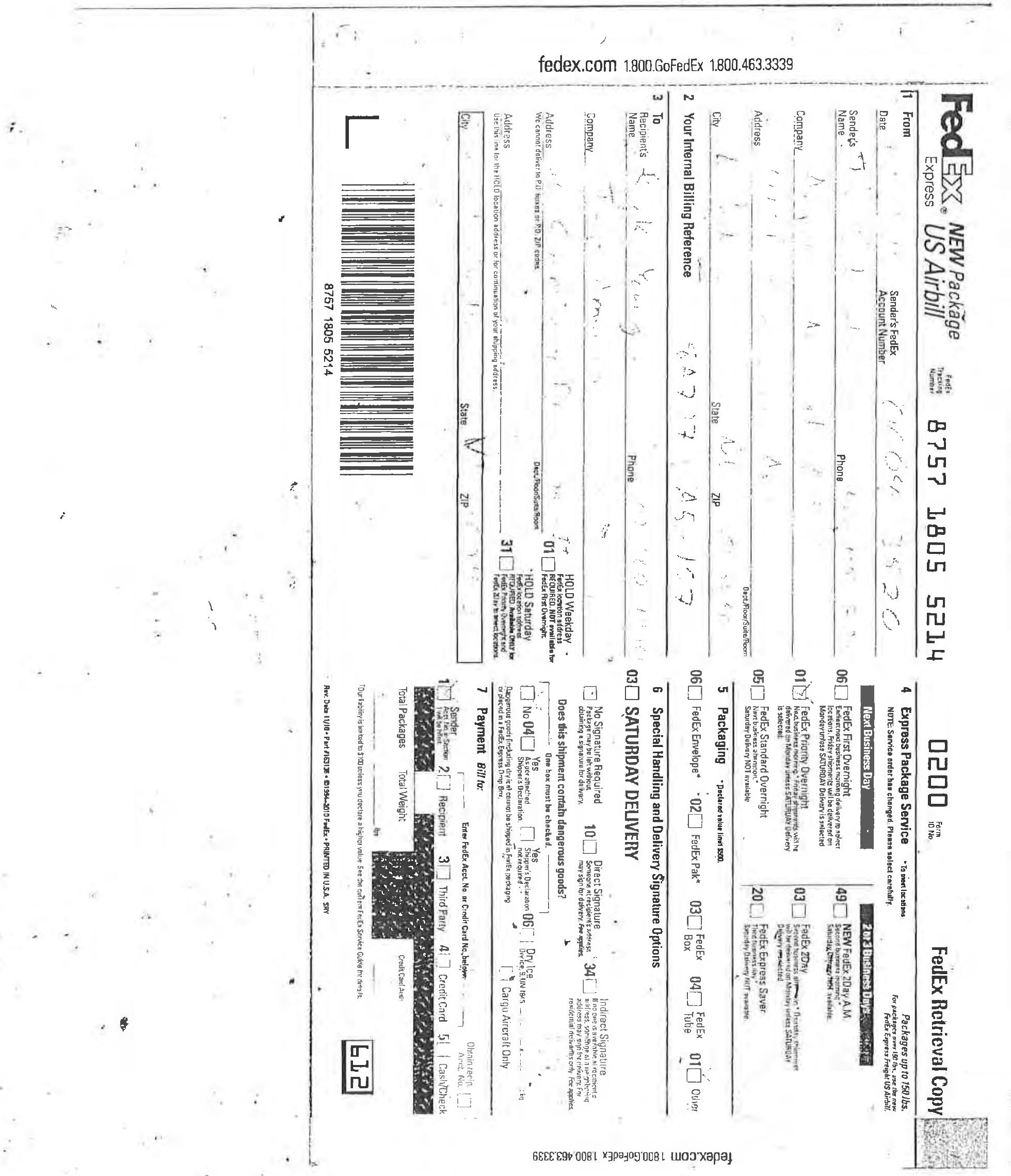




\section{Login Sample Receipt Checklist}

Creator: Marion, Greg T

Question

Radioactivity either was not measured or, if measured, is at or below background

The cooler's custody seal, if present, is intact.

The cooler or samples do not appear to have been compromised or tampered with.

Samples were received on ice.

Cooler Temperature is acceptable.

Cooler Temperature is recorded.

$\mathrm{COC}$ is present.

COC is filled out in ink and legible.

$\mathrm{COC}$ is filled out with all pertinent information.

Is the Field Sampler's name present on COC?

There are no discrepancies between the sample IDs on the containers and the COC.

Samples are received within Holding Time.

Sample containers have legible labels

Containers are not broken or leaking.

Sample collection date/times are provided.

Appropriate sample containers are used.

Sample bottles are completely filled.

Sample Preservation Verified.

There is sufficient vol. for all requested analyses, incl. any requested MS/MSDs

VOA sample vials do not have headspace or bubble is $<6 \mathrm{~mm}(1 / 4 ")$ in diameter.

Multiphasic samples are not present.

$\begin{array}{ll}\text { Answer } & \text { Comment } \\ \text { N/A } & \text { Lab does not accept radioactive samples. } \\ \text { True } & \text { NO SEAL NUMBERS } \\ \text { True } & \\ \text { True } & \\ \text { True } & \\ \text { True } & 3.5^{\circ} \text { C IR GUN ID 96/CF=0 } \\ \text { True } & \\ \text { True } & \\ \text { True } & \\ \text { True } & \\ \text { True } & \\ \text { True } & \\ \text { True } & \\ \text { True } & \\ \text { True } & \text { Sample collection times not listed on COC, but } \\ \text { True } & \text { referenced on sample labels. } \\ \text { True } & \\ \text { N/A } & \\ \text { True } & \\ \text { True } & \\ \text { N/A } & \\ \text { N/A } & \\ \text { N/A } & \\ & \\ & \\ & \\ & \\ & \end{array}$




\section{Sample Login Acknowledgement}

Job 200-6846-1

$\begin{array}{ll}\text { Client Job Description: } & \text { Inman (200-6846) } \\ \text { Purchase Order \#: } & \text { 1E-30401 } \\ \text { Work Order \#: } & \text { 1E-30401 } \\ \text { Project Manager: } & \text { Kirk F Young } \\ \text { Job Due Date: } & 9 / 16 / 2011 \\ \text { Job TAT: } & \text { 14 Days } \\ \text { Max Deliverable Level: } & \text { IV } \\ & \\ \text { Earliest Deliverable Due: } & 9 / 16 / 2011\end{array}$

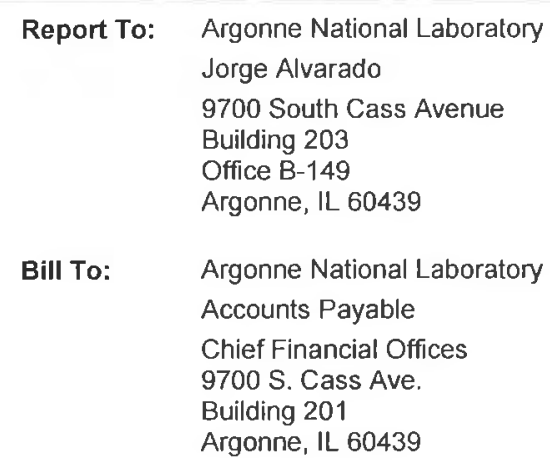

Login 200-6846

\begin{tabular}{|c|c|}
\hline $\begin{array}{l}\text { Sample Receipt: } \\
\text { Method of Delivery: }\end{array}$ & $\begin{array}{l}\text { 9/2/2011 10:00:00 AM } \\
\text { FedEx Priority Overnight }\end{array}$ \\
\hline $\begin{array}{l}\text { Lab Sample \# } \\
\text { Method }\end{array}$ & $\begin{array}{l}\text { Client Sample ID } \\
\text { Method Description / Work Location }\end{array}$ \\
\hline 200-6846-1 & INMW17D-W-32773 \\
\hline SOM01.2_Vol_Tr & SOM01.2 Trace Volatile Organics / In-Lab \\
\hline $200-6846-2$ & INMW17M-W-32770 \\
\hline SOM01.2_Vol_Tr & SOM01.2 Trace Volatile Organics / In-Lab \\
\hline $200-6846-3$ & INMW17S-W-32772 \\
\hline SOM01.2_Vol_Tr & SOM01.2 Trace Volatile Organics / In-Lab \\
\hline $200-6846-4$ & INMW19-W-32771 \\
\hline SOM01.2_Vol_Tr & SOM01.2 Trace Vol \\
\hline 200-6846-5 & INQCTB-W-32778 \\
\hline SOM01.2_Vol_Tr & SOM01.2 Trace Volatile Organics / In-Lab \\
\hline $200-6846-6$ & INMW18S-W-32775 \\
\hline SOM01.2_Vol_Tr & SOM01.2 Trace Volatile Organics / In-Lab \\
\hline 200-6846-7 & INMW18M-W-32776 \\
\hline SOM01.2_Vol_Tr & SOM01.2 Trace Volatile Organics / In-Lab \\
\hline 200-6846-8 & VHBLK01 \\
\hline SOM01.2 & о ои \\
\hline
\end{tabular}

$\begin{array}{ll}\text { Number of Coolers: } & 1 \\ \text { Cooler Temperature(s) }\left(\mathbf{C}^{\circ}\right): & 3.5 ;\end{array}$

\begin{tabular}{|c|c|c|c|}
\hline \multirow[t]{2}{*}{ Date Sampled } & \multirow[t]{2}{*}{ Matrix } & & \\
\hline & & Rpt Basis & Dry / Wet *k \\
\hline \multirow{2}{*}{ 8/31/2011 12:00:00 AM } & Water & & \\
\hline & & Total & Wet \\
\hline \multirow[t]{2}{*}{$8 / 31 / 2011 \quad 12: 00: 00$ AM } & Water & & \\
\hline & & Total & Wet \\
\hline \multirow[t]{2}{*}{ 8/31/2011 12:00:00 AM } & Water & & \\
\hline & & Total & Wet \\
\hline \multirow[t]{2}{*}{$8 / 31 / 2011$ 12:00:00 AM } & Water & & \\
\hline & & Total & Wet \\
\hline \multirow[t]{2}{*}{ 9/1/2011 12:00:00 AM } & Water & & \\
\hline & & Total & Wet \\
\hline \multirow[t]{2}{*}{ 9/1/2011 12:00:00 AM } & Water & & \\
\hline & & Total & Wet \\
\hline \multirow[t]{2}{*}{ 9/1/2011 12:00:00 АM } & Water & & \\
\hline & & Total & Wet \\
\hline \multirow[t]{2}{*}{ 9/2/2011 4:10:00 PM } & Water & & \\
\hline & & Total & Wet \\
\hline
\end{tabular}




\section{METHODOLOGY SUMMARY}

$\begin{array}{llll}\text { Laboratory: } & \text { TestAmerica Laboratories } & \text { Project No: } & \\ \text { Location: } & \text { South Burlington, Vermont } & \text { SDG No: } & \end{array}$

VOA

Volatile Organics Trace - USEPA CLP SOM01.2 
Lab Name: TESTAMERICA BURLINGTON

Lab Code: STLV Case No.: INMAN Mod. Ref No.:

Level: (TRACE or LOW) TRACE
Contract: $\quad 8 \mathrm{E}-00302$

SDG No.: 200-6846

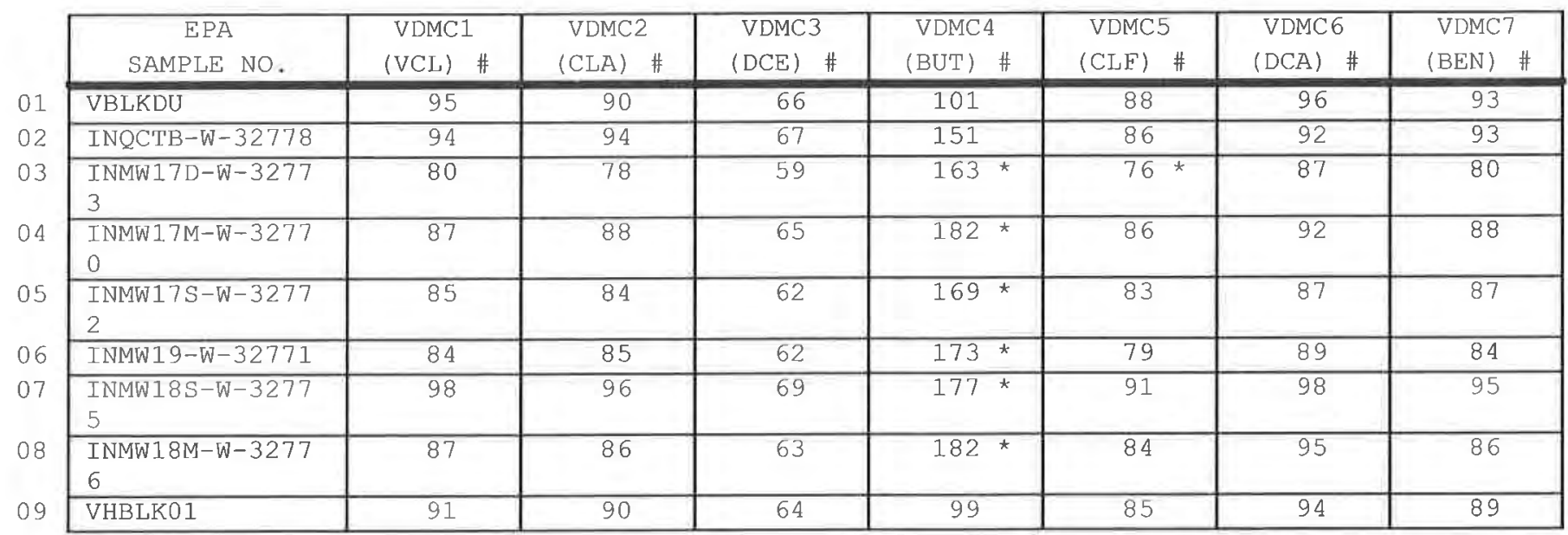

VDMCl (VCL) = Vinyl Chloride-d3

VDMC2 $(\mathrm{CLA})=$ Chloroethane $-\mathrm{d} 5$

VDMC3 $(\mathrm{DCE})=1,1-\mathrm{Dich}$ loroethene-d2

VDMC4 (BUT) = 2-Butanone-d5

VDMC5 $(\mathrm{CLF})=$ Chloroform-d

VDMC6 $($ DCA $)=1,2-$ Dichloroethane-d4

VDMC7 (BEN) = Benzene-d6

$$
\begin{gathered}
\text { QC LIMITS } \\
\hline(65-131) \\
(71-131) \\
(55-104) \\
(49-155) \\
(78-121) \\
(78-129) \\
(77-124)
\end{gathered}
$$

* Column to be used to flag recovery values

* Values outside of contract required QC limits 
Lab Name: TESTAMERICA BURLINGTON

Lab Code: STLV Case No.: INMAN Mod. Ref No.:

Level: (TRACE or LOW) TRACE
Contract: $8 \mathrm{E}-00302$

SDG No.: 200-6846

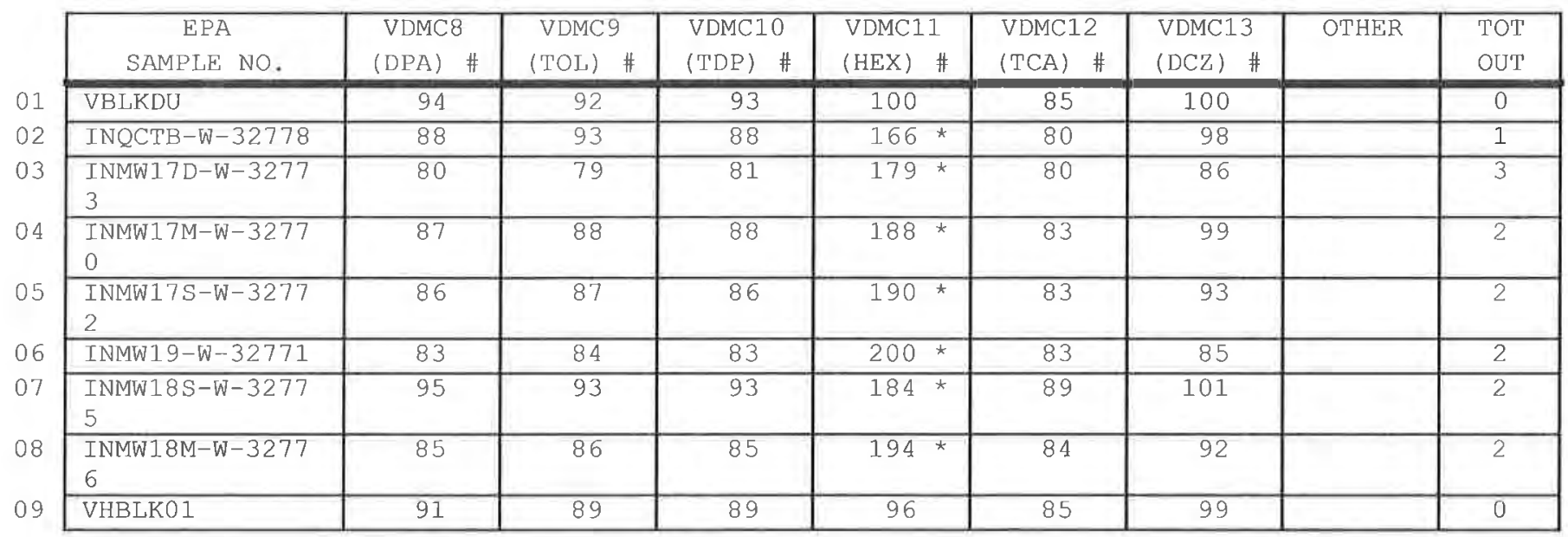

VDMC8 $(D P A)=1,2-$-Dichloropropane-d6

VDMC9 $\quad$ (TOL) $=$ Toluene-d8

VDMC10 $(T D P)=$ trans-1,3-Dichloropropene-d4

VDMC11 $(\mathrm{HEX})=2-$ Hexanone-d5

VDMC12 $(\mathrm{TCA})=1,1,2,2$-Tetrachloroethane-d2

VDMC13 $($ DCZ $)=1,2-$ Dichlorobenzene-d4
QC LIMITS

(79-124)

$(77-121)$

(73-121)

(28-135)

$(73-125)$

$(80-131)$

\# Column to be used to flag recovery values

* Values outside of contract required QC limits

Report 1,4-Dioxane-d8 for Low-Medium VOA analysis only

Page 1 of 1 
$4 A$ - FORM IV VOA

VOLATILE METHOD BLANK SUMMARY
EPA SAMPLE NO.

VBLKDU
Lab Name: TESTAMERICA BURLINGTON

Lab Code: STLV Case No.: INMAN

Lab File ID: DHPX03.D

Instrument ID: D.i

Matrix: (SOIL/SED/WATER)

Level: (TRACE or LOW/MED)

GC Column: DB-624

\begin{tabular}{|c|c|c|c|}
\hline $\begin{array}{c}\text { EPA } \\
\text { SAMPLE NO. }\end{array}$ & $\begin{array}{c}\text { LAB } \\
\text { SAMPLE ID }\end{array}$ & $\begin{array}{l}\text { LAB } \\
\text { FILE ID }\end{array}$ & $\begin{array}{c}\text { TIME } \\
\text { ANALYZED }\end{array}$ \\
\hline $\begin{array}{l}\text { INQCTB-W-327 } \\
78\end{array}$ & $200-6846-5$ & DHPX04.D & 0929 \\
\hline $\begin{array}{l}\text { INMW17D-W-32 } \\
773\end{array}$ & $200-6846-1$ & DHPX05.D & 0953 \\
\hline $\begin{array}{l}\text { INMW1 } 17 \mathrm{M}-\mathrm{W}-32 \\
770\end{array}$ & $200-6846-2$ & DHPX06.D & 1018 \\
\hline $\begin{array}{l}\text { INMW17S-W-32 } \\
772\end{array}$ & $200-6846-3$ & DHPX07.D & 1043 \\
\hline $\begin{array}{l}\text { INMW19-W-327 } \\
71\end{array}$ & $200-6846-4$ & DHPX08.D & 1107 \\
\hline $\begin{array}{l}\text { INMW18S-W-32 } \\
775\end{array}$ & $200-6846-6$ & DHPX09.D & 1132 \\
\hline $\begin{array}{l}\text { INMW18M-W-32 } \\
776\end{array}$ & $200-6846-7$ & DHPX10.D & 1157 \\
\hline VHBLK01 & $200-6846-8$ & DHPX11.D & 1222 \\
\hline
\end{tabular}

Contract: $\quad 8 \mathrm{E}-00302$

SDG No.: 200-6846

Lab Sample ID: MB 200-24749/3

Date Analyzed: 09/06/2011

Time Analyzed: 0851

Heated Purge: ( $Y / N) \quad N$
TRACE

ID: 0.20 (mm)

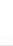

$+$


$5 A$ - EORM V VOA

VOIATILE ORGANICS INSTRUMENT

PEREORMANCE CHECK

BROMOFLUOROBENZENE (BFB)
EPA SAMPLE NO.

BEBDV

Lab Name: TESTAMERICA BURLINGTON

Contract: $\quad 8 \mathrm{E}-00302$

Lab Code: STLV Case No.: INMAN Mod. Ref No. SDG No.: $200-6846$

Lab File Id: DHP01.D

Instrument Id: D.i

GC Column: DB-624

\begin{tabular}{|l|l}
\hline $\mathrm{m} / \mathrm{e}$ & ION ABUNDANCE CRITERI
\end{tabular}

$50-15.0-40.0 \%$ of mass 95

$75 \quad 30.0-80.0 \%$ of mass 95

95 Base peak, 100\% relative abundance

$965.0-9.0 \%$ of mass 95

173 Less than 2.0 ? of mass 174

$17450.0-120 \%$ of mass 95

$175 \quad 5.0-9.0 \%$ of mass 174

$17695.0-101 \%$ of mass 174

$1775.0-9.0 \%$ of mass 176

\begin{abstract}
BFB Injection Date: 07/07/2011
BFB Injection Time: 1005
\end{abstract}

1 - Value is omass 174

2 - Value is onass 176

\begin{tabular}{|c|c|c|c|c|c|}
\hline & $\begin{array}{c}\text { EPA } \\
\text { SAMPLE NO. }\end{array}$ & $\begin{array}{r}\text { LAB } \\
\text { SAMPLE ID }\end{array}$ & $\begin{array}{c}\text { LAB } \\
\text { FILE ID }\end{array}$ & $\begin{array}{c}\text { DATE } \\
\text { ANALYZED }\end{array}$ & $\begin{array}{c}\text { TIME } \\
\text { ANALYZED }\end{array}$ \\
\hline 01 & VSTD0.5DV & IC $200-21023 / 4$ & $\mathrm{DHPO} 4 . \mathrm{D}$ & $07 / 07 / 2011$ & 1117 \\
\hline 02 & VSTD001DV & IC $200-21023 / 5$ & DHPO5.D & $07 / 07 / 2011$ & 1141 \\
\hline 03 & VSTD005DV & ICIS $200-21023 / 6$ & DHPO6.D & $07 / 07 / 2011$ & 1206 \\
\hline 04 & VSTD010DV & IC 200-21023/7 & DHPO7.D & $07 / 07 / 2011$ & 1231 \\
\hline 05 & VSTD020DV & IC $200-21023 / 8$ & DHP08.D & $07 / 07 / 2011$ & 1256 \\
\hline
\end{tabular}


$5 A$ - FORM V VOA

VOLATILE ORGANICS INSTRUMENT

PEREORMANCE CHECK

BROMOFLUOROBENZENE (BEB)
EPA SAMPLE NO.

BFBDU
Lab Name: TESTAMERICA BURLINGTON

Lab Code: STLV

Case No.: INMAN

Lab File Id: DHPX01. D

Instrument Id: D.i

GC Column: DB-624
ID : 0.20 (mm)
Contract : $8 \mathrm{E}-00302$

SDG No.: 200-6846

BFB Injection Date: 09/06/2011

BF'B Injection Time: 0803

\begin{tabular}{|c|c|c|}
\hline $\mathrm{m} / \mathrm{e}$ & ION ABUNDANCE CRITERIA & $\begin{array}{r}\text { RELATIVE } \\
\text { ABUNDANCE }\end{array}$ \\
\hline 50 & $15.0-40.0 \%$ of mass 95 & 25.5 \\
\hline 75 & $30.0-80.0 \%$ of mass 95 & 43.8 \\
\hline 95 & Base peak, $100 \%$ relative abundance & 100 \\
\hline 96 & $5.0-9.0 \%$ of mass 95 & 6.1 \\
\hline 173 & Less than $2.0 \%$ of mass 174 & 01 \\
\hline 174 & $50.0-120 \%$ of Inass 95 & 78.6 \\
\hline 175 & $5.0-9.0 \%$ of mass 174 & 5.91 \\
\hline 176 & $95.0-101 \%$ of mass 174 & $76.8(97.8) 1$ \\
\hline 177 & $5.0-9.0 \%$ of mass 176 & $4.9(6.4) 2$ \\
\hline
\end{tabular}

1 - Value is omass 174

2 - Value is omass 176

\begin{tabular}{|c|c|c|c|c|}
\hline $\begin{array}{c}\text { EPA } \\
\text { SAMPLE NO. }\end{array}$ & $\begin{array}{r}\text { IAB } \\
\text { SAMPLE ID }\end{array}$ & $\begin{array}{c}\text { LAB } \\
\text { EILE ID }\end{array}$ & $\begin{array}{c}\text { DATE } \\
\text { ANALYZED }\end{array}$ & $\begin{array}{c}\text { TIME } \\
\text { ANALYZED }\end{array}$ \\
\hline VSTD005DU & CCVIS $200-24749 / 2$ & $\mathrm{DHPXO2.D}$ & $09 / 06 / 2011$ & 0826 \\
\hline VBLKDU & MB $200-24749 / 3$ & DHPX03.D & $09 / 06 / 2011$ & 0851 \\
\hline $\begin{array}{l}\text { INQCTB-W-3 } \\
2778\end{array}$ & $200-6846-5$ & DHPX04.D & $09 / 06 / 2011$ & 0929 \\
\hline $\begin{array}{l}\text { INMW17D-W- } \\
32773\end{array}$ & $200-6846-1$ & DHPX05.D & $09 / 06 / 2011$ & 0953 \\
\hline $\begin{array}{l}\text { INMW } 17 \mathrm{M}-\mathrm{W}- \\
32770\end{array}$ & $200-6846-2$ & DHPX06.D & $09 / 06 / 2011$ & 1018 \\
\hline $\begin{array}{l}\text { INMW } 17 \mathrm{~S}-\mathrm{W}- \\
32772\end{array}$ & $200-6846-3$ & DHPX07.D & $09 / 06 / 2011$ & 1043 \\
\hline $\begin{array}{l}\text { INMW19-W-3 } \\
2771\end{array}$ & $200-6846-4$ & DHPX08.D & $09 / 06 / 2011$ & 1107 \\
\hline $\begin{array}{l}\text { INMW } 18 \mathrm{~S}-W- \\
32775\end{array}$ & $200-6846-6$ & DHPX09.D & $09 / 06 / 2011$ & 1132 \\
\hline $\begin{array}{l}\text { INMW18M-W- } \\
32776\end{array}$ & $200-6846-7$ & DHPX10.D & $09 / 06 / 2011$ & 1157 \\
\hline VHBLKO1 & $200-6846-8$ & DHPX11.D & $09 / 06 / 2011$ & 1222 \\
\hline VSTD005UD & CCVC $200-24749 / 18$ & DHPX18.D & $09 / 06 / 2011$ & 1514 \\
\hline
\end{tabular}


Lab Name: TESTAMERICA BURLINGTON

Contract: $8 \mathrm{E}-00302$

Lab Code: STLV Case No.: INMAN Mod. Ref No.: SDG No.: 200-6846

GC Column: DB-624 ID: 0.20 (mm) Init. Calib. Date(s): 07/07/2011 07/07/2011

EPA Sample No.(VSTD\#\#\#\#): VSTD005DU

Date Analyzed: 09/06/2011

Lab File ID (Standard): DHPX02.D

Time Analyzed: 0826

Instrument ID: D.i

Heated Purge: ( $\mathrm{Y} / \mathrm{N}) \mathrm{N}$

\begin{tabular}{|c|c|c|c|c|c|c|}
\hline & $\begin{array}{c}\text { IS1 (CBZ) } \\
\text { AREA }\end{array}$ & RT \# & $\begin{array}{c}\text { IS2 (DFB) } \\
\text { AREA }\end{array}$ & RT \# & $\begin{array}{c}\text { IS3 (DCB) } \\
\text { AREA }\end{array}$ & RT \# \\
\hline 12 HOUR STD & 149346 & 8.83 & 179388 & 5.47 & 71.938 & 11.66 \\
\hline UPPER IIMIT & 209084 & 9.16 & 251143 & 5.80 & 100713 & 11.99 \\
\hline LOWER LIMIT & 89608 & 8.50 & 107633 & 5.14 & 43163 & 11.33 \\
\hline EPA SAMPLE NO. & & & & & & \\
\hline$\overline{\text { VBLKDU }}$ & 149345 & 8.83 & 178136 & 5.47 & 61438 & 11.66 \\
\hline INQCTB-W-32778 & 160215 & 8.83 & 192414 & 5.47 & 66371 & 11.66 \\
\hline $\begin{array}{l}\text { INMW17D-W-3277 } \\
3\end{array}$ & 163951 & 8.83 & 192189 & 5.47 & 68196 & 11.66 \\
\hline $\begin{array}{l}\text { INMW17M-W-3277 } \\
0\end{array}$ & 154979 & 8.83 & 179956 & 5.47 & 62353 & 11.66 \\
\hline $\begin{array}{l}\text { INMW17S-W-3277 } \\
2\end{array}$ & 173091 & 8.83 & 208380 & 5.47 & 74389 & 11.66 \\
\hline INMW19-W-32771 & 174638 & 8.83 & 205584 & 5.47 & 83043 & 11.66 \\
\hline $\begin{array}{l}\text { INMW18S-W-3277 } \\
5\end{array}$ & 147405 & 8.83 & 173628 & 5.47 & 60363 & 11.66 \\
\hline $\begin{array}{l}\text { INMW18M-W-3277 } \\
6\end{array}$ & 156835 & 8.83 & 182423 & 5.47 & 67129 & 11.66 \\
\hline VHBLK01 & 140531 & 8.84 & 164814 & 5.47 & 54082 & 11.66 \\
\hline
\end{tabular}

IS1 (CBZ) $=$ Chlorobenzene-d5

IS2 $(\mathrm{DFB})=1,4-$ Difluorobenzene

IS3 $(\mathrm{DCB})=1,4$-Dichlorobenzene-d4

AREA UPPER LIMIT $=140 \%$ (Trace Volatiles) of internal standard area AREA LOWER LIMIT $=60 \%$ (Trace Volatiles) of internal standard area RT UPPER LIMIT $=+0.33$ (Trace Volatiles) minutes of internal standard RT RT LOWER LIMIT $=-0.33$ (Trace Volatiles) minutes of internal standard RT

\# Column used to flag values outside contract required QC limits with an asterisk. 
$1 \mathrm{~A}-$ FORM I VOA-1

VOLATILE ORGANICS ANALYSIS DATA SHEET
EPA SAMPLE NO.

INMW17D-W-32773
Lab Name: TESTAMERICA BURLINGTON

Lab Code: STLV Case

Matrix: (SOIL/SED/WATER)

Water

Sample wt/vol: 25.0

$(\mathrm{g} / \mathrm{mL}) \mathrm{mL}$

Level: (TRACE/LOW/MED) TRACE

음 Moisture: not dec.

GC Column: DB-624

ID : 0.20

(min)

Soil Extract Volume:

(uL)

Purge Volume: 25.0

(mL)
Contract: $8 \mathrm{E}-00302$

SDG No.: 200-6846

\begin{tabular}{|c|c|c|c|}
\hline CAS NO. & COMPOUND & $\begin{array}{l}\text { CONCENTRATION UNITS: } \\
(\mathrm{ug} / \mathrm{L} \text { or } \mathrm{ug} / \mathrm{kg}) \mathrm{ug} / \mathrm{L}\end{array}$ & $Q$ \\
\hline $75-71-8$ & Dichlorodifluoromethane & 0.50 & $\mathrm{U}$ \\
\hline $74-87-3$ & Chloromethane & 0.50 & $\mathrm{U}$ \\
\hline $75-01-4$ & Vinyl chloride & 0.50 & $\mathrm{U}$ \\
\hline $74-83-9$ & Bromomethane & 0.50 & $\mathrm{U}$ \\
\hline $75-00-3$ & Chloroethane & 0.50 & $\mathrm{U}$ \\
\hline $75-69-4$ & Trichlorofluoromethane & 0.50 & $\mathrm{U}$ \\
\hline $75-35-4$ & 1,1-Dichloroethene & 0.50 & $\mathrm{U}$ \\
\hline $76-13-1$ & 1,1,2-Trichloro-1,2,2-trifluoroethane & 0.50 & $\mathrm{U}$ \\
\hline $67-64-1$ & Acetone & 5.0 & $\mathrm{U}$ \\
\hline $75-15-0$ & Carbon disulfide & 0.50 & $\mathrm{U}$ \\
\hline $79-20-9$ & Methyl acetate & 0.50 & $\mathrm{U}$ \\
\hline $75-09-2$ & Methylene Chloride & 0.50 & $\mathrm{U}$ \\
\hline $156-60-5$ & trans-1,2-Dichloroethene & 0.50 & $\mathrm{U}$ \\
\hline $1634-04-4$ & Methyl tert-butyl ether & 0.50 & $\mathrm{U}$ \\
\hline $75-34-3$ & 1,1-Dichloroethane & 0.50 & $\mathrm{U}$ \\
\hline $156-59-2$ & cis-1,2-Dichloroethene & 0.50 & $\mathrm{U}$ \\
\hline $78-93-3$ & 2-Butanone & 5.0 & $\mathrm{U}$ \\
\hline $74-97-5$ & Bromochloromethane & 0.50 & $\mathrm{U}$ \\
\hline $67-66-3$ & Chloroform & 0.13 & $\mathrm{~J}$ \\
\hline $71-55-6$ & 1,1,1-Trichloroethane & 0.50 & $\mathrm{U}$ \\
\hline $110-82-7$ & Cyclohexane & 0.50 & $\mathrm{U}$ \\
\hline $56-23-5$ & Carbon tetrachloride & 0.50 & $\mathrm{U}$ \\
\hline $71-43-2$ & Benzene & 0.50 & $\mathrm{U}$ \\
\hline $107-06-2$ & 1,2-Dichloroethane & 0.50 & $\mathrm{U}$ \\
\hline
\end{tabular}

Report 1,4-Dioxane for Low-Medium VOA analysis only

Lab Sample ID: 200-6846-1

Lab File ID: DHPX05.D

Date Received: 09/02/2011

Date Analyzed: 09/06/2011

Dilution Eactor: 1.0

Soil Aliquot Volume:

(uL) 
$1 \mathrm{~B}$ - FORM I VOA-2

VOLATILE ORGANICS ANALYSIS DATA SHEET
EPA SAMPI_E NO.

INMW17D-W-32773

Lab Name: TESTAMERICA BURLINGTON

Lab Code: STLV Case No.: INMAN Mod.

Water

$(\mathrm{g} / \mathrm{mL}) \quad \mathrm{mL}$

Sample wt/vol: 25.0

TRACE

Level: (TRACE/LOW/MED)

o Moisture: not dec.

GC Column: DB-624

ID $: 0.20$ (mm)

Soil Extract Volume: (uL)

(mL)

Purge Volume: 25.0
Contract: $8 \mathrm{E}-00302$

SDG No.: 200-6846 o.:

Lab Sample ID: 200-6846-1

Lab File ID: DHPX05.D

Date Received: 09/02/2011

Date Analyzed: 09/06/2011

Dilution Factor: 1.0

Soil Aliquot Volume:

(uL)

\begin{tabular}{|c|c|c|c|}
\hline CAS NO. & COMPOUND & $\begin{array}{l}\text { CONCENTRATION UNITS: } \\
(\mathrm{ug} / \mathrm{L} \text { or } \mathrm{ug} / \mathrm{kg}) \mathrm{ug} / \mathrm{L}\end{array}$ & $Q$ \\
\hline $79-01-6$ & Trichloroethene & 0.50 & $\bar{U}$ \\
\hline $108-87-2$ & Methylcyclohexane & 0.50 & $\mathrm{U}$ \\
\hline $78-87-5$ & 1,2-Dichloropropane & 0.50 & $\mathrm{U}$ \\
\hline $75-27-4$ & Bromodichloromethane & 0.50 & $\mathrm{U}$ \\
\hline $10061-01-5$ & cis-1,3-Dichloropropene & 0.50 & $\mathrm{U}$ \\
\hline $108-10-1$ & 4-Methyl-2-pentanone & 5.0 & $\mathrm{U}$ \\
\hline $108-88-3$ & Toluene & 0.070 & $\mathrm{~J} B$ \\
\hline $10061-02-6$ & trans $-1,3-$ Dichloropropene & 0.50 & $\mathrm{U}$ \\
\hline $79-00-5$ & 1,1,2-Trichloroethane & 0.50 & $\mathrm{U}$ \\
\hline $127-18-4$ & Tetrachloroethene & 0.50 & $\mathrm{U}$ \\
\hline $591-78-6$ & 2-Hexanone & 5.0 & $\mathrm{U}$ \\
\hline $124-48-1$ & Dibromochloromethane & 0.50 & $\mathrm{U}$ \\
\hline $106-93-4$ & 1,2-Dibromoethane & 0.50 & $\mathrm{U}$ \\
\hline $108-90-7$ & Chlorobenzene & 0.50 & $\mathrm{U}$ \\
\hline $100-41-4$ & Ethylbenzene & 0.068 & $\mathrm{~J}$ \\
\hline $95-47-6$ & o-Xylene & 0.073 & $\mathrm{~J}$ \\
\hline $179601-23-1$ & $\mathrm{~m}, \mathrm{p}$-Xylene & 0.080 & $\mathrm{~J}$ \\
\hline $100-42-5$ & Styrene & 0.50 & $\mathrm{U}$ \\
\hline $75-25-2$ & Bromoform & 0.50 & $\mathrm{U}$ \\
\hline $98-82-8$ & Isopropylbenzene & 0.50 & $\mathrm{U}$ \\
\hline $79-34-5$ & $1,1,2,2$-Tetrachloroethane & 0.50 & $\mathrm{U}$ \\
\hline $541-73-1$ & 1,3-Dichlorobenzene & 0.50 & $\mathrm{U}$ \\
\hline $106-46-7$ & 1,4-Dichlorobenzene & 0.50 & $\mathrm{U}$ \\
\hline $95-50-1$ & 1,2-Dichlorobenzene & 0.50 & $\mathrm{U}$ \\
\hline $96-12-8$ & 1,2-Dibromo-3-Chloropropane & 0.50 & $\mathrm{U}$ \\
\hline $120-82-1$ & 1,2,4-Trichlorobenzene & 0.50 & $\mathrm{U}$ \\
\hline $87-61-6$ & 1,2,3-Trichlorobenzene & 0.50 & $\mathrm{U}$ \\
\hline
\end{tabular}


$1 \mathrm{~J}-$ FORM I VOA-TIC

VOLATILE ORGANICS ANALYSIS DATA SHEET TENTATIVELY IDENTIFIED COMPOUNDS
EPA SAMPLE NO.

INMW17D-W-32773
Lab Name: TESTAMERICA BURLINGTON Lab Code: STLV Case No.: INMAN

Matrix: (SOIL/SED/WATER)

Sample wt/vol: 25.0

water

$(\mathrm{g} / \mathrm{mL}) \mathrm{mL}$

Level: (TRACE or LOW/MED)

TRACE

음 Moisture: not dec.

GC Column: DB-624 ID: $0.20 \quad(\mathrm{~mm})$

Soil Extract Volume: (uL)
Contract: $\quad 8 \mathrm{E}-00302$

SDG No.: $200-6846$

Lab Sample ID: 200-6846-1

Lab File ID: DHPX05.D

Date Received: 09/02/2011

Date Analyzed: 09/06/2011

Dilution Factor: 1.0

Soil Aliquot Volume: (uL)

CONCENTRATION UNITS: (ug/L or ug/kg) ug/L

Purge Volume: 25.0 (mL)

\begin{tabular}{|c|c|c|c|c|}
\hline CAS NUMBER & COMPOUND NAME & $\mathrm{RT}$ & EST. CONC. & 0 \\
\hline & Unknown & 6.79 & 2.5 & $\mathrm{~B} \times \mathrm{J}$ \\
\hline E9667961 & Total Alkanes & $N / A$ & & \\
\hline
\end{tabular}

1 EPA-designated Registry Number. 
$1 A$ - FORM I VOA-1

VOLATILE ORGANICS ANALYSIS DATA SHEET
EPA SAMPLE NO.

INMW 17M-W-32770
Lab Name: TESTAMERICA BURLINGTON

Lab Code: STLV Case No.: INMAN

Matrix: (SOIL/SED/WATER)

Water

Sample wt/vol: 25.0

$(\mathrm{g} / \mathrm{mL}) \mathrm{mL}$

Level: (TRACE/LOW/MED) TRACE

믕 Moisture: not dec.

GC Column: DB-624

ID $: 0.20$

Soil Extract Volume:

Purge Volume: 25.0

\begin{tabular}{|c|c|c|c|}
\hline CAS NO. & COMPOUND & $\begin{array}{l}\text { CONCENTRATION UNITS: } \\
(\mathrm{ug} / \mathrm{L} \text { or } \mathrm{ug} / \mathrm{kg}) \mathrm{ug} / \mathrm{L}\end{array}$ & $Q$ \\
\hline $75-71-8$ & Dichlorodifluoromethane & 0.50 & $\bar{U}$ \\
\hline $74-87-3$ & Chloromethane & 0.50 & $\mathrm{U}$ \\
\hline $75-01-4$ & Vinyl chloride & 0.50 & $\mathrm{U}$ \\
\hline $74-83-9$ & Bromomethane & 0.50 & $\mathrm{U}$ \\
\hline $75-00-3$ & Chloroethane & 0.50 & $\mathrm{U}$ \\
\hline $75-69-4$ & Trichlorofluoromethane & 0.50 & $\mathrm{U}$ \\
\hline $75-35-4$ & 1,1-Dichloroethene & 0.50 & $\mathrm{U}$ \\
\hline $76-13-1$ & 1,1,2-Trichloro-1,2,2-trifluoroethane & 0.50 & U \\
\hline $67-64-1$ & Acetone & 5.0 & U \\
\hline $75-15-0$ & Carbon disulfide & 0.50 & $\mathrm{U}$ \\
\hline $79-20-9$ & Methyl acetate & 0.50 & $\mathrm{U}$ \\
\hline $75-09-2$ & Methylene Chloride & 0.049 & $\mathrm{~J}$ \\
\hline $156-60-5$ & trans-1,2-Dichloroethene & 0.50 & $\mathrm{U}$ \\
\hline $1634-04-4$ & Methyl tert-butyl ether & 0.50 & $\mathrm{U}$ \\
\hline $75-34-3$ & 1,1-Dichloroethane & 0.50 & $\mathrm{U}$ \\
\hline $156-59-2$ & cis-1,2-Dichloroethene & 0.50 & $\mathrm{U}$ \\
\hline $78-93-3$ & 2-Butanone & 5.0 & $\mathrm{U}$ \\
\hline $74-97-5$ & Bromochloromethane & 0.50 & $\mathrm{U}$ \\
\hline $67-66-3$ & Chloroform & 0.40 & $\mathrm{~J}$ \\
\hline $71-55-6$ & 1,1,1-Trichloroethane & 0.50 & $\mathrm{U}$ \\
\hline $110-82-7$ & Cyclohexane & 0.50 & $\mathrm{U}$ \\
\hline $56-23-5$ & Carbon tetrachloride & 0.72 & \\
\hline $71-43-2$ & Benzene & 0.0039 & $\mathrm{~J}$ \\
\hline $107-06-2$ & 1,2-Dichloroethane & 0.50 & $\mathrm{U}$ \\
\hline
\end{tabular}

Contract: $8 \mathrm{E}-00302$

SDG No.: 200-6846

Lab Sample ID: 200-6846-2

Lab File ID: DHPX06.D

Date Received: 09/02/2011

Date Analyzed: 09/06/2011

Dilution Factor: 1.0

Soil Aliquot Volume:

(uL)

(uL)

$(\mathrm{mL})$ 
$1 B$ - FORM I VOA-2

VOLATILE ORGANICS ANALYSIS DATA SHEET
EPA SAMPLE NO.

INMW17M-W-32770
Lab Name: TESTAMERICA BURLINGTON

Lab Code: STLV Case No.: INMA

Matrix: (SOIL/SED/WATER)

Water

Sample wt/vol: 25.0

$(\mathrm{g} / \mathrm{mL}) \quad \mathrm{mI}$

Level: (TRACE/LOW/MED) TRACE

음 Moisture: not dec.

GC Column: DB-624

ID: $0.20 \quad(\mathrm{~mm})$

Soil Extract Volume:

(uL)

\section{(mL)}

Contract: $\quad 8 \mathrm{E}-00302$

SDG No.: 200-6846

Lab Sample ID: 200-6846-2

Lab File ID: DHPX06.D

Date Received: 09/02/2011

Date Analyzed: 09/06/2011

Dilution Factor: 1.0

Soil Aliquot Volume:

(uL)

\begin{tabular}{|c|c|c|c|}
\hline CAS NO. & COMPOUND & $\begin{array}{l}\text { CONCENTRATION UNITS: } \\
(\mathrm{ug} / \mathrm{L} \text { or } \mathrm{ug} / \mathrm{kg}) \mathrm{ug} / \mathrm{L}\end{array}$ & 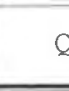 \\
\hline $79-01-6$ & Trichloroethene & 0.50 & $\mathrm{U}$ \\
\hline $108-87-2$ & Methylcyclohexane & 0.50 & $\mathrm{U}$ \\
\hline $78-87-5$ & 1,2-Dichloropropane & 0.50 & $\mathrm{U}$ \\
\hline $75-27-4$ & Bromodichloromethane & 0.50 & $\mathrm{U}$ \\
\hline $10061-01-5$ & cis-1,3-Dichloropropene & 0.50 & $\mathrm{U}$ \\
\hline $108-10-1$ & 4-Methyl-2-pentanone & 5.0 & $\mathrm{U}$ \\
\hline $108-88-3$ & Toluene & 0.067 & $\mathrm{~J} \mathrm{~B}$ \\
\hline $10061-02-6$ & trans-1,3-Dichloropropene & 0.50 & $\mathrm{U}$ \\
\hline $79-00-5$ & 1,1,2-Trichloroethane & 0.50 & $\mathrm{U}$ \\
\hline $127-18-4$ & Tetrachloroethene & 0.50 & $\mathrm{U}$ \\
\hline $591-78-6$ & 2-Hexanone & 5.0 & $\mathrm{U}$ \\
\hline $124-48-1$ & Dibromochloromethane & 0.15 & $\mathrm{~J}$ \\
\hline $106-93-4$ & 1,2-Dibromoethane & 0.50 & $\mathrm{U}$ \\
\hline $108-90-7$ & Chlorobenzene & 0.50 & $\mathrm{U}$ \\
\hline $100-41-4$ & Ethylbenzene & 0.049 & $\mathrm{~J}$ \\
\hline $95-47-6$ & o-xylene & 0.059 & $\mathrm{~J}$ \\
\hline $179601-23-1$ & $\mathrm{~m}, \mathrm{p}$-Xylene & 0.044 & $\mathrm{~J}$ \\
\hline $100-42-5$ & Styrene & 0.50 & $\mathrm{U}$ \\
\hline $75-25-2$ & Bromoform & 0.50 & $\mathrm{U}$ \\
\hline $98-82-8$ & Isopropylbenzene & 0.50 & $\mathrm{U}$ \\
\hline $79-34-5$ & $1,1,2,2$-Tetrachloroethane & 0.50 & $\mathrm{U}$ \\
\hline $541-73-1$ & 1,3-Dichlorobenzene & 0.50 & $\mathrm{U}$ \\
\hline $106-46-7$ & 1,4-Dichlorobenzene & 0.50 & $\mathrm{U}$ \\
\hline $95-50-1$ & 1,2-Dichlorobenzene & 0.50 & $\mathrm{U}$ \\
\hline $96-12-8$ & 1,2-Dibromo-3-Chloropropane & 0.50 & $\mathrm{U}$ \\
\hline $120-82-1$ & $1,2,4$-Trichlorobenzene & 0.50 & $\mathrm{U}$ \\
\hline $87-61-6$ & 1,2,3-Trichlorobenzene & 0.50 & $\mathrm{U}$ \\
\hline
\end{tabular}


1J - EORM I VOA-TIC

VOLATILE ORGANICS ANALYSIS DATA SHEET

TENTATIVELY IDENTIFIED COMPOUNDS
EPA SAMPLE NO.

INMW I7M-W-32770
Lab Name: TESTAMERICA BURLINGTON

Lab Code: STLV Case No.: INMAN Mod. Ref No.:

Matrix: (SOIL/SED/WATER) Water

Sample wt/vol: 25.0

$(\mathrm{g} / \mathrm{mL}) \quad \mathrm{mL}$

Level: (TRACE or LOW/MED)

TRACE

o Moisture: not dec.

GC Column: DB-624 ID: 0.20 (mm)

Soil Extract Volume: (uL)

CONCENTRATION UNITS: (ug/L or ug/kg)
Contract: $8 \mathrm{E}-00302$

SDG No.: 200-6846

Lab Sample ID: 200-6846-2

Lab File ID: DHPX06.D

Date Received: 09/02/2011

Date Analyzed: 09/06/2011

Dilution Factor: 1.0

Soil Aliquot Volume:

(uL)

Purge Volume: 25.0 (mL)

01

\begin{tabular}{|c|l|c|c|c|}
\hline CAS NUMBER & \multicolumn{1}{|c|}{ COMPOUND NAME } & RT & EST. CONC. & Q \\
\hline & Unknown & 6.79 & 2.8 & B X J \\
\hline E9667961 & Total Alkanes & N/A & & \\
\hline
\end{tabular}

1 EPA-designated Registry Number. 
$1 A$ - EORM I VOA-1

VOLATILE ORGANICS ANALYSIS DATA SHEET
EPA SAMPLE NO.

INMW17S-W-32772
Lab Name: TESTAMERICA BURLINGTON

Lab Code: STLV Case No.: INMA

Matrix: (SOIL/SED/WATER)

water

Sample wt/vol: 25.0

$(g / \mathrm{mL}) \quad \mathrm{mL}$

Level: (TRACE/LOW/MED) TRACE

\% Moisture: not dec.

GC Column: DB-624 ID: 0.20 (rim)

Soil Extract Volume: (uL) (mL)

Purge Volume: 25.0
Contract: $\quad 8 \mathrm{E}-00302$

SDG No.: $200-6846$

Mod. Ref No.:

Lab Sample ID: 200-6846-3

Lab File ID: DHPX07.D

Date Received: 09/02/2011

Date Analyzed: 09/06/2011

Dilution Factor: 1.0

Soil Aliquot Volume:

(uL)

\begin{tabular}{|c|c|c|c|}
\hline CAS NO. & COMPOUND & $\begin{array}{l}\text { CONCENTRATION UNITS: } \\
(\mathrm{ug} / \mathrm{L} \text { or } \mathrm{ug} / \mathrm{kg}) \mathrm{ug} / \mathrm{L}\end{array}$ & Q \\
\hline $75-71-8$ & Dichlorodifluoromethane & 0.50 & $\mathrm{U}$ \\
\hline $74-87-3$ & Chloromethane & 0.50 & $\mathrm{U}$ \\
\hline $75-01-4$ & Vinyl chloride & 0.50 & $\mathrm{U}$ \\
\hline $74-83-9$ & Bromomethane & 0.50 & $\mathrm{U}$ \\
\hline $75-00-3$ & Chloroethane & 0.50 & $\mathrm{U}$ \\
\hline $75-69-4$ & Trichlorofluoromethane & 0.50 & $\mathrm{U}$ \\
\hline $75-35-4$ & 1,1-Dichloroethene & 0.50 & $\mathrm{U}$ \\
\hline $76-13-1$ & 1,1,2-Trichloro-1,2,2-trifluoroethane & 0.50 & $\mathrm{U}$ \\
\hline $67-64-1$ & Acetone & 5.5 & \\
\hline $75-15-0$ & Carbon disulfide & 0.50 & $\mathrm{U}$ \\
\hline $79-20-9$ & Methyl acetate & 0.50 & $\mathrm{U}$ \\
\hline $75-09-2$ & Methylene Chloride & 0.50 & $\mathrm{U}$ \\
\hline $156-60-5$ & trans-1,2-Dichloroethene & 0.50 & $\mathrm{U}$ \\
\hline $1634-04-4$ & Methyl tert-butyl ether & 0.50 & $\mathrm{U}$ \\
\hline $75-34-3$ & 1,1-Dichloroethane & 0.50 & $\mathrm{U}$ \\
\hline $156-59-2$ & cis-1,2-Dichloroethene & 0.50 & $\mathrm{U}$ \\
\hline $78-93-3$ & 2-Butanone & 5.0 & $\mathrm{U}$ \\
\hline $74-97-5$ & Bromochloromethane & 0.50 & $\mathrm{U}$ \\
\hline $67-66-3$ & Chloroform & 0.63 & \\
\hline $71-55-6$ & 1,1,1-Trichloroethane & 0.50 & $\mathrm{U}$ \\
\hline $110-82-7$ & Cyclohexane & 0.50 & $\mathrm{U}$ \\
\hline $56-23-5$ & Carbon tetrachloride & 0.027 & $\mathrm{~J}$ \\
\hline $71-43-2$ & Benzene & 0.023 & $\mathrm{~J}$ \\
\hline $107-06-2$ & 1,2-Dichloroethane & 0.50 & $\mathrm{U}$ \\
\hline
\end{tabular}

Report 1,4-Dioxane for Low-Medium VOA analysis only 
Lab Name: TESTAMERICA BURLINGTON

Contract: $\quad 8 \mathrm{E}-00302$

Lab Code: STLV Case No.: INMAN Mod. Ref No.:
Matrix: (SOIL/SED/WATER) Water

Sample wt/vol: 25.0

$(\mathrm{g} / \mathrm{mL}) \mathrm{mL}$

Level: (TRACE/LOW/MED) TRACE

응 Moisture: not dec.

GC Column: DB-624 ID: $0.20 \quad$ (mm)

Soil Extract Volume:

(uL) (mL)

Purge Volume: 25.0
SDG No.: 200-6846

Lab Sample ID: 200-6846-3

Lab File ID: DHPX07.D

Date Received: 09/02/2011

Date Analyzed: 09/06/2011

Dilution Factor: 1.0

Soil Aliquot Volume:

\begin{tabular}{|c|c|c|c|}
\hline CAS NO. & COMPOUND & $\begin{array}{l}\text { CONCENTRATION UNITS: } \\
(\mathrm{ug} / \mathrm{L} \text { or } \mathrm{ug} / \mathrm{kg}) \mathrm{ug} / \mathrm{L}\end{array}$ & $Q$ \\
\hline $79-0.1-6$ & Trichloroethene & 0.50 & $\mathrm{U}$ \\
\hline $108-87-2$ & Methylcyclohexane & 0.50 & $\mathrm{U}$ \\
\hline $78-87-5$ & 1,2-Dichloropropane & 0.50 & U \\
\hline $75-27-4$ & Bromodichloromethane & 0.48 & $\mathrm{~J}$ \\
\hline $10061-01-5$ & cis-1,3-Dichloropropene & 0.50 & $\mathrm{U}$ \\
\hline $108-10-1$ & 4-Methyl-2-pentanone & 5.0 & $\mathrm{U}$ \\
\hline $108-88-3$ & Toluene & 0.33 & $\mathrm{JB}$ \\
\hline $10061-02-6$ & trans-1,3-Dichloropropene & 0.50 & $\mathrm{U}$ \\
\hline $79-00-5$ & 1,1,2-Trichloroethane & 0.50 & $\mathrm{U}$ \\
\hline $127-18-4$ & Tetrachloroethene & 0.50 & $\mathrm{U}$ \\
\hline $591-78-6$ & 2-Hexanone & 5.0 & $\mathrm{U}$ \\
\hline $124-48-1$ & Dibromochloromethane & 0.58 & \\
\hline $106-93-4$ & 1,2-Dibromoethane & 0.50 & $\mathrm{U}$ \\
\hline $108-90-7$ & Chlorobenzene & 0.50 & $\mathrm{U}$ \\
\hline $100-41-4$ & Ethylbenzene & 0.16 & $\mathrm{~J}$ \\
\hline $95-47-6$ & o-Xylene & 0.44 & $\mathrm{~J}$ \\
\hline $179601-23-1$ & $\mathrm{~m}, \mathrm{p}-\mathrm{XY}_{\mathrm{Y}} \mathrm{ene}$ & 0.50 & $\mathrm{U}$ \\
\hline $100-42-5$ & Styrene & 0.50 & $\mathrm{U}$ \\
\hline $75-25-2$ & Bromoform & 0.50 & $\mathrm{U}$ \\
\hline $98-82-8$ & Isopropylbenzene & 0.50 & $\mathrm{U}$ \\
\hline $79-34-5$ & $1,1,2,2$-Tetrachloroethane & 0.50 & $\mathrm{U}$ \\
\hline $541-73-1$ & 1,3-Dichlorobenzene & 0.50 & $\mathrm{U}$ \\
\hline $106-46-7$ & 1,4-Dichlorobenzene & 0.50 & $\mathrm{U}$ \\
\hline $95-50-1$ & 1,2-Dichlorobenzene & 0.50 & $\mathrm{U}$ \\
\hline $96-12-8$ & 1,2-Dibromo-3-Chloropropane & 0.50 & $\mathrm{U}$ \\
\hline $120-82-1$ & 1,2,4-Trichlorobenzene & 0.50 & $\mathrm{U}$ \\
\hline $87-61-6$ & 1,2,3-Trichlorobenzene & 0.50 & $\mathrm{U}$ \\
\hline
\end{tabular}


$1 \mathrm{~J}$ - EORM I VOA-TIC

VOLATILE ORGANICS ANALYSIS DATA SHEET

TENTATIVELY IDENTIFIED COMPOUNDS
EPA SAMPLE NO.

INMW17S-W-32772

Lab Name: TESTAMERICA BURLINGTON

Contract: $8 E-00302$

Lab Code: STLV Case No.: INMAN Mod. Ref No.: SDG No.: 200-6846

Matrix: (SOIL/SED/WATER)

water

Sample wt/vol: 25.0 $(\mathrm{g} / \mathrm{mL}) \quad \mathrm{mL}$

Level: (TRACE or LOW/MED)

TRACE

응 Moisture: not dec.

GC Column: DB-624 ID: $0.20 \quad(\mathrm{~mm})$

Soil Extract Volume: (uL)

CONCENTRATION UNITS: (ug/L or $\mathrm{ug} / \mathrm{kg}$ ) ug/L
Lab Sample ID: 200-6846-3

Lab File ID: DHPX07.D

Date Received: 09/02/2011

Date Analyzed: 09/06/2011

Dilution Factor: 1.0

Soil Aliquot Volume: (uL)

Purge Volume: 25.0 $(\mathrm{mL})$

\begin{tabular}{|l|l|l|l|r|r|}
\hline \multicolumn{1}{|c|}{ CAS NUMBER } & \multicolumn{1}{|c|}{ COMPOUND NAME } & RT & EST. CONC. & Q \\
\cline { 2 - 6 } 01 & & Unknown & 6.79 & B X J \\
\cline { 2 - 6 } & E966796I & Total Alkanes & N/A & \\
\hline
\end{tabular}

1 EPA-designated Registry Number. 
$1 \mathrm{~A}$ - FORM I VOA-1

VOLATILE ORGANICS ANALYSIS DATA SHEET
EPA SAMPLE NO.

INMW18M-W-32776
Lab Name: TESTAMERICA BURLINGTON

Lab Code: STLV Case No.: INMAN

Matrix: (SOIL/SED/WATER)

Sample wt/vol: 25.0

Water

$(\mathrm{g} / \mathrm{mL}) \quad \mathrm{mL}$

Level: (TRACE/LOW/MED) TRACE

\% Moisture: not dec.

GC Column: DB-624

ID $: 0.20$

$(\mathrm{mm})$

Soil Extract volume:

(uL)

(mL)

Purge Volume: 25.0
Contract: $8 \mathrm{E}-00302$

SDG No.: 200-6846

\begin{tabular}{|c|c|c|c|}
\hline CAS NO. & COMPOUND & $\begin{array}{l}\text { CONCENTRATION UNITS: } \\
(\mathrm{ug} / \mathrm{L} \text { or } \mathrm{ug} / \mathrm{kg}) \mathrm{ug} / \mathrm{L}\end{array}$ & $Q$ \\
\hline $75-71-8$ & Dichlorodifluoromethane & 0.50 & $\mathrm{U}$ \\
\hline $74-87-3$ & Chloromethane & 0.50 & $\mathrm{U}$ \\
\hline $75-01-4$ & Vinyl chloride & 0.50 & $\mathrm{U}$ \\
\hline $74-83-9$ & Bromomethane & 0.50 & $\mathrm{U}$ \\
\hline $75-00-3$ & Chloroethane & 0.50 & $\mathrm{U}$ \\
\hline $75-69-4$ & Trichlorofluoromethane & 0.50 & $\mathrm{U}$ \\
\hline $75-35-4$ & 1,1-Dichloroethene & 0.50 & $\mathrm{U}$ \\
\hline $76-13-1$ & 1,1,2-Trichloro-1,2,2-trifluoroethane & 0.50 & $\mathrm{U}$ \\
\hline $67-64-1$ & Acetone & 1.6 & $\mathrm{~J}$ \\
\hline $75-15-0$ & Carbon disulfide & 0.50 & $\mathrm{U}$ \\
\hline $79-20-9$ & Methyl acetate & 0.50 & $\mathrm{U}$ \\
\hline $75-09-2$ & Methylene Chloride & 0.50 & $\mathrm{U}$ \\
\hline $156-60-5$ & trans-1,2-Dichloroethene & 0.50 & $\mathrm{U}$ \\
\hline $1634-04-4$ & Methyl tert-butyl ether & 0.50 & $\mathrm{U}$ \\
\hline $75-34-3$ & 1,1-Dichloroethane & 0.50 & $\mathrm{U}$ \\
\hline $156-59-2$ & cis-1,2-Dichloroethene & 0.50 & $\mathrm{U}$ \\
\hline $78-93-3$ & 2-Butanone & 5.0 & $\mathrm{U}$ \\
\hline $74-97-5$ & Bromochloromethane & 0.50 & $\mathrm{U}$ \\
\hline $67-66-3$ & Chloroform & 0.15 & $\mathrm{~J}$ \\
\hline $71-55-6$ & 1,1,1-Trichloroethane & 0.50 & $\mathrm{U}$ \\
\hline $110-82-7$ & Cyclohexane & 0.50 & $\mathrm{U}$ \\
\hline $56-23-5$ & Carbon tetrachloride & 0.50 & $\mathrm{U}$ \\
\hline $71-43-2$ & Benzene & 0.038 & $\mathrm{~J}$ \\
\hline $107-06-2$ & 1,2-Dichloroethane & 0.50 & $\mathrm{U}$ \\
\hline
\end{tabular}

Report 1,4-Dioxane for Low-Medium VOA analysis only

Lab Sample ID: 200-6846-7

Lab File ID: DHPX10.D

Date Received: 09/02/2011

Date Analyzed: 09/06/2011

Dilution Factor: 1.0

Soil Aliquot Volume:

(uL) 
$1 B$ - EORM I VOA-2

VOLATILE ORGANICS ANALYSIS DATA SHEET
EPA SAMPLE NO.

INMW18M-W-32776
Lab Name: TESTAMERICA BURLINGTON

Lab Code: STLV Cas

Matrix: (SOIL/SED/WATER)

Water

Sample wt/vol: 25.0

$(\mathrm{g} / \mathrm{mL}) \mathrm{mL}$

Level: (TRACE/LOW/MED) TRACE

o Moisture: not dec.

GC Column: DB-624 ID $: 0.20 \quad(\mathrm{~mm})$

Soil Extract Volume:

Purge Volume: $\quad 25.0$

(mL)
Contract: $\quad 8 \mathrm{E}-00302$

SDG No.: 200-6846

Lab Sample ID: $\quad 200-6846-7$

Lab File ID: DHPX10.D

Date Received: 09/02/2011

Date Analyzed: 09/06/2011

Dilution Factor: 1.0

Soil Aliquot Volume:

(uL)

\begin{tabular}{|c|c|c|c|}
\hline CAS NO. & COMPOUND & $\begin{array}{l}\text { CONCENTRATION UNITS: } \\
(\mathrm{ug} / \mathrm{L} \text { or } \mathrm{ug} / \mathrm{kg}) \mathrm{ug} / \mathrm{L}\end{array}$ & $Q$ \\
\hline $79-01-6$ & Trichloroethene & 0.50 & $\mathrm{U}$ \\
\hline $108-87-2$ & Methylcyclohexane & 0.50 & $\mathrm{U}$ \\
\hline $78-87-5$ & 1,2-Dichloropropane & 0.50 & $\mathrm{U}$ \\
\hline $75-27-4$ & Bromodichloromethane & 0.50 & $\mathrm{U}$ \\
\hline $10061-01-5$ & cis-1,3-Dichloropropene & 0.50 & $\mathrm{U}$ \\
\hline $108-10-1$ & 4-Methyl-2-pentanone & 5.0 & U \\
\hline $108-88-3$ & Toluene & 0.32 & J $\mathrm{B}$ \\
\hline $10061-02-6$ & trans-1,3-Dichloropropene & 0.50 & U \\
\hline $79-00-5$ & $1,1,2$-Trichloroethane & 0.50 & U \\
\hline $127-18-4$ & Tetrachloroethene & 0.50 & $\mathrm{U}$ \\
\hline $591-78-6$ & 2-Hexanone & 5.0 & $\mathrm{U}$ \\
\hline $124-48-1$ & Dibromochloromethane & 0.50 & U \\
\hline $106-93-4$ & 1,2-Dibromoethane & 0.50 & $\mathrm{U}$ \\
\hline $108-90-7$ & Chlorobenzene & 0.50 & $\mathrm{U}$ \\
\hline $100-41-4$ & Ethylbenzene & 0.022 & $\mathrm{~J}$ \\
\hline $95-47-6$ & o-Xylene & 0.028 & $\mathrm{~J}$ \\
\hline $179601-23-1$ & $\mathrm{~m}, \mathrm{p}-\mathrm{xy}$ lene & 0.096 & $\mathrm{~J}$ \\
\hline $100-42-5$ & Styrene & 0.32 & $\mathrm{~J}$ \\
\hline $75-25-2$ & Bromoform & 0.50 & $\mathrm{U}$ \\
\hline $98-82-8$ & Isopropylbenzene & 0.50 & $\mathrm{U}$ \\
\hline $79-34-5$ & $1,1,2,2$-Tetrachloroethane & 0.50 & $\mathrm{U}$ \\
\hline $541-73-1$ & 1,3-Dichlorobenzene & 0.50 & $\mathrm{U}$ \\
\hline $106-46-7$ & 1,4-Dichlorobenzene & 0.50 & $\mathrm{U}$ \\
\hline $95-50-1$ & 1,2-Dichlorobenzene & 0.50 & $\mathrm{U}$ \\
\hline $96-12-8$ & 1,2-Dibromo-3-Chloropropane & 0.50 & $\mathrm{U}$ \\
\hline $120-82-1$ & $1,2,4$-Trichlorobenzene & 0.50 & $\mathrm{U}$ \\
\hline $87-61-6$ & $1,2,3$-Trichlorobenzene & 0.50 & $\mathrm{U}$ \\
\hline
\end{tabular}


$1 \mathrm{~J}$ - FORM I VOA-TIC

VOLATILE ORGANICS ANALYSIS DATA SHEET TENTATIVELY IDENTIFIED COMPOUNDS
EPA SAMPLE NO.

INMW18M-W-32776
Lab Name: TESTAMERICA BURLINGTON

Lab code: STLV Case No.: INMAN

Matrix: (SOIL/SED/WATER)

Sample wt/vol: 25.0

Water

$(\mathrm{g} / \mathrm{mL}) \quad \mathrm{mL}$

Level: (TRACE or LOW/MED)

TRACE

\% Moisture: not dec.

GC Column: DB-624 ID $: 0.20 \quad$ (mm)

Soil Extract Volume: (uL)

CONCENTRATION UNITS: (ug/L Or $\mathrm{ug} / \mathrm{kg}$ ) ug/L
Contract: $\quad 8 \mathrm{E}-00302$

SDG No.: 200-6846

Lab Sample ID: 200-6846-7

Lab File ID: DHPX10.D

Date Received: 09/02/2011

Date Analyzed: 09/06/2011

Dilution Factor: 1.0

Soil Aliquot Volume:

(uL)

Purge Volume: 25.0 (mL)

01

02

\begin{tabular}{|l|l|c|c|c|}
\hline \multicolumn{1}{|c|}{ CAS NUMBER } & \multicolumn{1}{|c|}{ COMPOUND NAME } & RT & EST. CONC. & Q \\
\hline & Unknown & 6.79 & 2.7 & $\mathrm{~B} \mathrm{X} \mathrm{J}$ \\
\hline E9667961 & Total Alkanes & N/A & & \\
\hline
\end{tabular}

1 EPA-designated Registry Number. 
Lab Name: TESTAMERICA BURLINGTON

Lab Code: STLV Case No.: INMA

Matrix: (SOIL/SED/WATER)

Sample wt/vol: 25.0

Water

$(\mathrm{g} / \mathrm{mL}) \mathrm{mL}$

Level: (TRACE/LOW/MED) TRACE

응 Moisture: not dec.

GC Column: DB-624

ID : 0.20

Soil Extract Volume:

Purge Volume: 25.0
Contract: $\quad 8 \mathrm{E}-00302$

SDG No. : 200-6846

\begin{tabular}{|c|c|c|c|}
\hline CAS NO. & COMPOUND & $\begin{array}{l}\text { CONCENTRATION UNITS: } \\
(\mathrm{ug} / \mathrm{L} \text { or } \mathrm{ug} / \mathrm{kg}) \mathrm{ug} / \mathrm{L}\end{array}$ & $Q$ \\
\hline $75-71-8$ & Dichlorodifluoromethane & 0.50 & $\bar{U}$ \\
\hline $74-87-3$ & Chloromethane & 0.50 & $\mathrm{U}$ \\
\hline $75-01-4$ & Vinyl chloride & 0.50 & $\mathrm{U}$ \\
\hline $74-83-9$ & Bromomethane & 0.50 & $\mathrm{U}$ \\
\hline $75-00-3$ & Chloroethane & 0.50 & $\mathrm{U}$ \\
\hline $75-69-4$ & Trichlorofluoromethane & 0.50 & $\mathrm{U}$ \\
\hline $75-35-4$ & 1,1-Dichloroethene & 0.50 & $\mathrm{U}$ \\
\hline $76-13-1$ & 1,1,2-Trichloro-1,2,2-trifluoroethane & 0.50 & $\mathrm{U}$ \\
\hline $67-64-1$ & Acetone & 5.0 & $\mathrm{U}$ \\
\hline $75-15-0$ & Carbon disulfide & 0.50 & $\mathrm{U}$ \\
\hline $79-20-9$ & Methyl acetate & 0.50 & $\mathrm{U}$ \\
\hline $75-09-2$ & Methylene Chloride & 0.50 & $\mathrm{U}$ \\
\hline $156-60-5$ & trans-1,2-Dichloroethene & 0.50 & U \\
\hline $1634-04-4$ & Methyl tert-butyl ether & 0.50 & $\mathrm{U}$ \\
\hline $75-34-3$ & 1,1-Dichloroethane & 0.50 & $\mathrm{U}$ \\
\hline $156-59-2$ & cis-1,2-Dichloroethene & 0.50 & $\mathrm{U}$ \\
\hline $78-93-3$ & 2-Butanone & 5.0 & $\mathrm{U}$ \\
\hline $74-97-5$ & Bromochloromethane & 0.50 & $\mathrm{U}$ \\
\hline $67-66-3$ & Chloroform & 1.0 & \\
\hline $71-55-6$ & 1,1,1-Trichloroethane & 0.50 & $\mathrm{U}$ \\
\hline $110-82-7$ & Cyclohexane & 0.50 & $\mathrm{U}$ \\
\hline $56-23-5$ & Carbon tetrachloride & 3.0 & \\
\hline $71-43-2$ & Benzene & 0.0084 & $\mathrm{~J}$ \\
\hline $107-06-2$ & 1,2-Dichloroethane & 0.50 & $\mathrm{U}$ \\
\hline
\end{tabular}

Lab Sample ID: 200-6846-6

Lab File ID: DHPX09.D

Date Received: 09/02/2011

Date Analyzed: 09/06/2011

Dilution Factor: 1.0

Soil Aliquot Volume:

(uL)

(uL) (mL) 
Lab Name: TESTAMERICA BURLINGTON

Lab Code: STLV Case No.: INMAN

Matrix: (SOIJ/SED/WATER)

Water

Sample wt/vol: 25.0

$(\mathrm{g} / \mathrm{mL}) \quad \mathrm{mL}$

Level: (TRACE/LOW/MED) TRACE

o Moisture: not dec.

GC Column: DB-624

ID: 0.20 (mm)

Soil Extract Volume:

(uL) (mL)

Purge Volume: 25.0
Contract: $8 E-00302$

SDG No.: $200-6846$

Lab Sample ID: 200-6846-6

Lab File ID: DHPX09.D

Date Received: 09/02/2011

Date Analyzed: 09/06/2011

Dilution Factor: 1.0

Soil Aliquot Volume:

(uL)

\begin{tabular}{|c|c|c|c|}
\hline CAS NO. & COMPOUND & $\begin{array}{l}\text { CONCENTRATION UNITS: } \\
(\mathrm{ug} / \mathrm{L} \text { or } \mathrm{ug} / \mathrm{kg} \text { ) } \mathrm{ug} / \mathrm{L}\end{array}$ & $\mathrm{Q}$ \\
\hline $79-01-6$ & Trichloroethene & 0.50 & $\mathrm{U}$ \\
\hline $108-87-2$ & Methylcyclohexane & 0.50 & $\mathrm{U}$ \\
\hline $78-87-5$ & 1,2-Dichloropropane & 0.50 & $\mathrm{U}$ \\
\hline $75-27-4$ & Bromodichloromethane & 0.50 & $\mathrm{U}$ \\
\hline $10061-01-5$ & cis-1,3-Dichloropropene & 0.50 & $\mathrm{U}$ \\
\hline $108-10-1$ & 4-Methyl-2-pentanone & 5.0 & $\mathrm{U}$ \\
\hline $108-88-3$ & Toluene & 0.076 & $J B$ \\
\hline $10061-02-6$ & trans-1,3-Dichloropropene & 0.50 & $\mathrm{U}$ \\
\hline $79-00-5$ & 1,1,2-Trichloroethane & 0.50 & $\mathrm{U}$ \\
\hline $127-18-4$ & Tetrachloroethene & 0.29 & $\mathrm{~J}$ \\
\hline $591-78-6$ & 2-Hexanone & 5.0 & $\mathrm{U}$ \\
\hline $124-48-1$ & Dibromochloromethane & 0.13 & $\mathrm{~J}$ \\
\hline $106-93-4$ & 1,2-Dibromoethane & 0.50 & $\mathrm{U}$ \\
\hline $108-90-7$ & Chlorobenzene & 0.50 & $\mathrm{U}$ \\
\hline $100-41-4$ & Ethylbenzene & 0.032 & J \\
\hline $95-47-6$ & o-Xylene & 0.037 & $\mathrm{~J}$ \\
\hline $179601-23-1$ & $\mathrm{~m}, \mathrm{p}$-Xylene & 0.11 & $\mathrm{~J}$ \\
\hline $100-42-5$ & Styrene & 0.50 & $\mathrm{U}$ \\
\hline $75-25-2$ & Bromoform & 0.50 & $\mathrm{U}$ \\
\hline $98-82-8$ & Isopropylbenzene & 0.50 & $\mathrm{U}$ \\
\hline $79-34-5$ & $1,1,2,2$-Tetrachloroethane & 0.50 & $\mathrm{U}$ \\
\hline $541-73-1$ & 1,3-Dichlorobenzene & 0.50 & $\mathrm{U}$ \\
\hline $106-46-7$ & 1,4-Dichlorobenzene & 0.50 & $\mathrm{U}$ \\
\hline $95-50-1$ & 1,2-Dichlorobenzene & 0.50 & $\mathrm{U}$ \\
\hline $96-12-8$ & 1,2-Dibromo-3-Chloropropane & 0.50 & $\mathrm{U}$ \\
\hline $120-82-1$ & 1,2,4-Trichlorobenzene & 0.50 & $\mathrm{U}$ \\
\hline $87-61-6$ & 1,2,3-Trichlorobenzene & 0.50 & $\mathrm{U}$ \\
\hline
\end{tabular}


$1 \mathrm{~J}$ - FORM I VOA-TIC

VOLATILE ORGANICS ANALYSIS DATA SHEET TENTATIVELY IDENTIFIED COMPOUNDS
EPA SAMPLE NO.

INMW18S-W-32775

Lab Name: TESTAMERICA BURLINGTON Case No.: INMAN Mod. Ref No.:

Contract: $8 \mathrm{E}-00302$

Lab Code: STLV

Water

$(\mathrm{g} / \mathrm{mL}) \quad \mathrm{mL}$

Sample wt/vol: 25.0 TRACE

Level: (TRACE or LOW/MED)

\% Moisture: not dec.

GC Column: DB-624

ID: 0.20

(mm)

Soil Extract Volume:

CONCENTRATION UNITS: (ug/L or $\mathrm{ug} / \mathrm{kg}$ ) ug/L
SDG No.: 200-6846

Lab Sample ID: $200-6846-6$

Lab File ID: DHPX09.D

Date Received: 09/02/2011

Date Analyzed: 09/06/2011

Dilution Factor: 1.0

Soil Aliquot volume:

(uL)

Purge Volume: 25.0

(mL)

\begin{tabular}{|l|l|l|r|r|r|}
\hline \multicolumn{1}{|c|}{ CAS NUMBER } & \multicolumn{1}{|c|}{ COMPOUND NAME } & RT & EST. CONC. & Q \\
\cline { 2 - 6 } 01 & & 6.79 & 2.9 & B X J \\
\hline
\end{tabular}

1 EPA-designated Registry Number. 
$1 \mathrm{~A}$ - FORM I VOA-1

VOLATILE ORGANICS ANALYSIS DATA SHEET
EPA SAMPLE NO.

INMW19-W-32771
Lab Name: TESTAMERICA BURLINGTON

Lab Code: STLV Case

Matrix: (SOIL/SED/WATER)

Water

Sample wt/vol: 25.0

$(\mathrm{g} / \mathrm{mL}) \mathrm{mL}$

Level: (TRACE/LOW/MED) TRACE

음 Moisture: not dec.

GC Column: DB-624 ID: $0.20 \quad(\mathrm{~mm})$

Soil Extract Volume:

(mL)

Purge Volune: 25.0
Contract: $8 \mathrm{E}-00302$

SDG No.: 200-6846

\begin{tabular}{|c|c|c|c|}
\hline CAS NO. & COMPOUND & $\begin{array}{l}\text { CONCENTRATION UNITS: } \\
(\mathrm{ug} / \mathrm{L} \text { or } \mathrm{ug} / \mathrm{kg}) \mathrm{ug} / \mathrm{L}\end{array}$ & $Q$ \\
\hline $75-71-8$ & Dichlorodifluoromethane & 0.50 & $\mathrm{U}$ \\
\hline $74-87-3$ & Chloromethane & 0.50 & $\mathrm{U}$ \\
\hline $75-01-4$ & Vinyl chloride & 0.50 & $\mathrm{U}$ \\
\hline $74-83-9$ & Bromomethane & 0.50 & $\mathrm{U}$ \\
\hline $75-00-3$ & Chloroethane & 0.50 & $\mathrm{U}$ \\
\hline $75-69-4$ & Trichlorofluoromethane & 0.50 & U \\
\hline $75-35-4$ & 1,1-Dichloroethene & 0.50 & $\mathrm{U}$ \\
\hline $76-13-1$ & 1,1,2-Trichloro-1,2,2-trifluoroethane & 0.50 & $\mathrm{U}$ \\
\hline $67-64-1$ & Acetone & 1.6 & $\mathrm{~J}$ \\
\hline $75-15-0$ & Carbon disulfide & 0.50 & $\mathrm{U}$ \\
\hline $79-20-9$ & Methyl acetate & 0.50 & $\mathrm{U}$ \\
\hline $75-09-2$ & Methylene Chloride & 0.50 & $\mathrm{U}$ \\
\hline $156-60-5$ & trans-1,2-Dichloroethene & 0.50 & $\mathrm{U}$ \\
\hline $1634-04-4$ & Methyl tert-butyl ether & 0.50 & $\mathrm{U}$ \\
\hline $75-34-3$ & 1,1-Dichloroethane & 0.50 & $\mathrm{U}$ \\
\hline $156-59-2$ & cis-1,2-Dichloroethene & 0.50 & $\mathrm{U}$ \\
\hline $78-93-3$ & 2-Butanone & 5.0 & $\mathrm{U}$ \\
\hline $74-97-5$ & Bromochloromethane & 0.50 & $\mathrm{U}$ \\
\hline $67-66-3$ & Chloroform & 0.19 & $\mathrm{~J}$ \\
\hline $71-55-6$ & 1,1,1-Trichloroethane & 0.50 & $\mathrm{U}$ \\
\hline $110-82-7$ & Cyclohexane & 0.50 & $\mathrm{U}$ \\
\hline $56-23-5$ & Carbon tetrachloride & 0.19 & $\mathrm{~J}$ \\
\hline $71-43-2$ & Benzene & 0.025 & $\mathrm{~J}$ \\
\hline $107-06-2$ & 1,2-Dichloroethane & 0.50 & $\mathrm{U}$ \\
\hline
\end{tabular}

Report 1,4-Dioxane for Low-Medium VOA analysis only

Lab Sample ID: 200-6846-4

Lab File ID: DHPX08.D

Date Received: 09/02/2011

Date Analyzed: 09/06/2011

Dilution F'actor: 1.0

Soil Aliquot Volume:

(uL) 
$1 \mathrm{~B}$ - FORM I VOA-2

VOLATILE ORGANICS ANALYSIS DATA SHEET
EPA SAMPLE NO.

INMW19-W-32771
Lab Name: TESTAMERICA BURLINGTON

Lab Code: STLV Case No.: INMAN

Matrix: (SOIL/SED/WATER)

Water

Sample wt/vol: 25.0

$(\mathrm{g} / \mathrm{mL}) \mathrm{mL}$

Level: (TRACE/LOW/MED) TRACE

응 Moisture: not dec.

GC Column: DB-624

ID: 0.20

$(\mathrm{mm})$

Soil Extract Volume:

(mL)

Purge Volume: 25.0
Contract: $8 \mathrm{E}-00302$
SDG No.: 200-6846

. :

Lab Sample ID: 200-6846-4

Lab File ID: DHPX08.D

Date Received: 09/02/2011

Date Analyzed: 09/06/2011

Dilution Factor: 1.0

Soil Aliquot Volume:

(uL)

\begin{tabular}{|c|c|c|c|}
\hline CAS NO. & COMPOUND & $\begin{array}{l}\text { CONCENTRATION UNITS: } \\
(\mathrm{ug} / \mathrm{L} \text { or } \mathrm{ug} / \mathrm{kg}) \mathrm{ug} / \mathrm{L}\end{array}$ & $Q$ \\
\hline $79-01-6$ & Trichloroethene & 0.50 & $\mathrm{U}$ \\
\hline $108-87-2$ & Methylcyclohexane & 0.50 & U \\
\hline $78-87-5$ & 1,2-Dichloropropane & 0.50 & U \\
\hline $75-27-4$ & Bromodichloromethane & 0.50 & U \\
\hline $10061-01-5$ & cis-1,3-Dichloropropene & 0.50 & $\mathrm{U}$ \\
\hline $108-10-1$ & 4-Methyl-2-pentanone & 5.0 & $\mathrm{U}$ \\
\hline $108-88-3$ & Toluene & 0.031 & $\mathrm{~J} B$ \\
\hline $10061-02-6$ & trans-1,3-Dichloropropene & 0.50 & $\mathrm{U}$ \\
\hline $79-00-5$ & 1,1,2-Trichloroethane & 0.50 & $\mathrm{U}$ \\
\hline $127-18-4$ & Tetrachloroethene & 0.50 & $\mathrm{U}$ \\
\hline $591-78-6$ & 2-Hexanone & 5.0 & $\mathrm{U}$ \\
\hline $124-48-1$ & Dibromochloromethane & 0.50 & $\mathrm{U}$ \\
\hline $106-93-4$ & 1,2-Dibromoethane & 0.50 & $\mathrm{U}$ \\
\hline $108-90-7$ & Chlorobenzene & 0.50 & $\mathrm{U}$ \\
\hline $100-41-4$ & Ethylbenzene & 0.50 & $\mathrm{U}$ \\
\hline $95-47-6$ & o-xylene & 0.50 & $\mathrm{U}$ \\
\hline $179601-23-1$ & $\mathrm{~m}, \mathrm{p}$-Xylene & 0.50 & $\mathrm{U}$ \\
\hline $100-42-5$ & Styrene & 0.50 & $\mathrm{U}$ \\
\hline $75-25-2$ & Bromoform & 0.50 & $\mathrm{U}$ \\
\hline $98-82-8$ & Isopropylbenzene & 0.50 & $\mathrm{U}$ \\
\hline $79-34-5$ & $1,1,2,2$-Tetrachloroethane & 0.50 & U \\
\hline $541-73-1$ & 1,3-Dichlorobenzene & 0.50 & $\mathrm{U}$ \\
\hline $106-46-7$ & 1,4-Dichlorobenzene & 0.50 & $\mathrm{~J}$ \\
\hline $95-50-1$ & 1,2-Dichlorobenzene & 0.50 & $\mathrm{U}$ \\
\hline $96-12-8$ & 1,2-Dibromo-3-Chloropropane & 0.50 & $\mathrm{U}$ \\
\hline $120-82-1$ & $1,2,4$-Trichlorobenzene & 0.50 & U \\
\hline $87-61-6$ & $1,2,3$-Trichlorobenzene & 0.50 & $\mathrm{U}$ \\
\hline
\end{tabular}


$1 \mathrm{~J}-$ FORM I VOA-TIC

VOLATIIE, ORGANICS ANALYSIS DATA SHEET TENTATIVELY IDENTIEIED COMPOUNDS
EPA SAMPLE NO.

INMW19-W-32771
Lab Name: TESTAMERICA BURLINGTON

Lab Code: STLV Case No.: INMAN

Matrix: (SOIL/SED/WATER)

Water

Sample wt/vol: 25.0

$(\mathrm{g} / \mathrm{mL}) \mathrm{mL}$

Level: (TRACE or LOW/MED)

TRACE

음 Moisture: not dec.

GC Column: DB-624

ID : 0.20

(mm)

Soil Extract Volume:

(uL)

CONCENTRATION UNITS: (ug/L or $\mathrm{ug} / \mathrm{kg}$ ) ug/L
Contract: $8 \mathrm{E}-00302$

SDG No.: 200-6846

Lab Sample ID: 200-6846-4

Lab File ID: DHPX08.D

Date Received: 09/02/2011

Date Analyzed: 09/06/2011

Dilution Factor: 1.0

Soil Aliquot Volume:

(uL)

Purge Volume: 25.0

(mL)

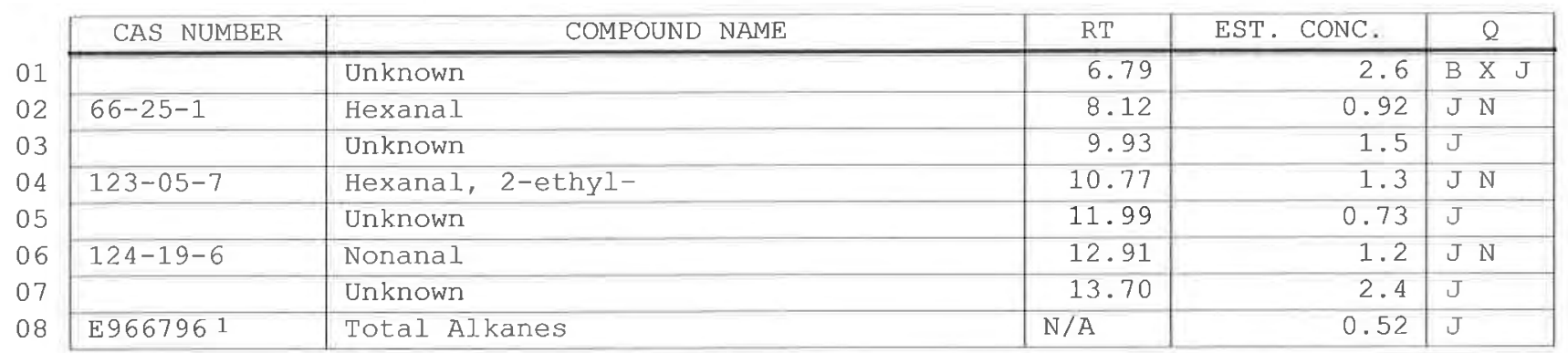

1 EPA-designated Registry Number. 
$1 A$ - FORM I VOA-1

VOLATILE ORGANICS ANALYSIS DATA SHEET
EPA SAMPLE NO. INQCTB-W-32778
Lab Name: TESTAMERICA BURLINGTON

Lab Code: STLV Case No.: INMAN

Matrix: (SOIL/SED/WATER)

Sample wt/vol: 25.0

Water

$(\mathrm{g} / \mathrm{mL}) \mathrm{mL}$

Level: (TRACE/LOW/MED) TRACE

Moisture: not dec.

GC Column: DB-624 ID: $0.20 \quad(\mathrm{~mm})$

Soil Extract Volume:

(mL)

Purge Volume: 25.0
Contract: $8 \mathrm{E}-00302$

SDG No.: 200-6846

Lab Sample ID: 200-6846-5

Lab File ID: DHPX04.D

Date Received: 09/02/2011

Date Analyzed: 09/06/2011

Dilution Factor: 1.0

Soil Aliquot Volume:

(uL)

\begin{tabular}{|c|c|c|c|}
\hline CAS NO. & COMPOUND & $\begin{array}{l}\text { CONCENTRATION UNITS: } \\
(\mathrm{ug} / \mathrm{L} \text { or } \mathrm{ug} / \mathrm{kg}) \mathrm{ug} / \mathrm{L}\end{array}$ & $Q$ \\
\hline $75-71-8$ & Dichlorodifluoromethane & 0.50 & $\mathrm{U}$ \\
\hline $74-87-3$ & Chloromethane & 0.50 & $\mathrm{U}$ \\
\hline $75-01-4$ & Vinyl chloride & 0.50 & $\mathrm{U}$ \\
\hline $74-83-9$ & Bromomethane & 0.50 & $\mathrm{U}$ \\
\hline $75-00-3$ & Chloroethane & 0.50 & $\mathrm{U}$ \\
\hline $75-69-4$ & Trichlorofluoromethane & 0.50 & $\mathrm{U}$ \\
\hline $75-35-4$ & 1,1-Dichloroethene & 0.50 & $\mathrm{U}$ \\
\hline $76-13-1$ & $1,1,2$-Trichloro-1,2,2-trifluoroethane & 0.50 & $\mathrm{U}$ \\
\hline $67-64-1$ & Acetone & 5.0 & $\mathrm{U}$ \\
\hline $75-15-0$ & Carbon disulfide & 0.50 & $\mathrm{U}$ \\
\hline $79-20-9$ & Methyl acetate & 0.50 & $\mathrm{U}$ \\
\hline $75-09-2$ & Methylene Chloride & 0.50 & $\mathrm{U}$ \\
\hline $156-60-5$ & trans-1,2-Dichloroethene & 0.50 & $\mathrm{U}$ \\
\hline $1634-04-4$ & Methyl tert-butyl ether & 0.50 & $\mathrm{U}$ \\
\hline $75-34-3$ & 1,1-Dichloroethane & 0.50 & $\mathrm{U}$ \\
\hline $156-59-2$ & cis-1,2-Dichloroethene & 0.50 & $\mathrm{U}$ \\
\hline $78-93-3$ & 2-Butanone & 5.0 & $\mathrm{U}$ \\
\hline $74-97-5$ & Bromochloromethane & 0.50 & $\mathrm{U}$ \\
\hline $67-66-3$ & Chloroform & 0.99 & \\
\hline $71-55-6$ & 1,1,1-Trichloroethane & 0.50 & U \\
\hline $110-82-7$ & Cyclohexane & 0.50 & $\mathrm{U}$ \\
\hline $56-23-5$ & Carbon tetrachloride & 4.1 & \\
\hline $71-43-2$ & Benzene & 0.50 & $\mathrm{U}$ \\
\hline $107-06-2$ & 1,2-Dichloroethane & 0.50 & $\mathrm{U}$ \\
\hline
\end{tabular}

Report 1,4-Dioxane for Low-Medium VOA analysis only 
$1 B$ - FORM I VOA-2

VOLATILE ORGANICS ANALYSIS DATA SHEET
EPA SAMPLE NO. INQCTB-W-32778
Lab Name: TESTAMERICA BURLINGTON

Lab Code: STLV Case No.: INMAN

Water

Matrix: (SOIL/SED/WATER)

$(\mathrm{g} / \mathrm{mL}) \mathrm{mL}$

Sample wt/vol: 25.0

Level: (TRACE/LOW/MED) TRACE

\% Moisture: not dec.

GC Column: DB-624 ID: $0.20 \quad(\mathrm{~mm})$

Soil Extract Volume:

(uL)

(mL)

Purge Volume: 25.0
Contract: $8 \mathrm{E}-00302$

SDG No.: 200-6846

o. :

Lab Sample ID: 200-6846-5

Lab File ID: DHPX04.D

Date Received: 09/02/2011

Date Analyzed: 09/06/2011

Dilution Factor: 1.0

Soil Aliquot Volume:

(uL)

\begin{tabular}{|c|c|c|c|}
\hline CAS NO. & COMPOUND & $\begin{array}{l}\text { CONCENTRATION UNITS: } \\
(\mathrm{ug} / \mathrm{L} \text { or } u g / \mathrm{kg}) \mathrm{ug} / \mathrm{L}\end{array}$ & Q \\
\hline $79-01-6$ & Trichloroethene & 0.50 & $\mathrm{U}$ \\
\hline $108-87-2$ & Methylcyclohexane & 0.50 & $\mathrm{U}$ \\
\hline $78-87-5$ & 1,2-Dichloropropane & 0.50 & $\mathrm{U}$ \\
\hline $75-27-4$ & Bromodichloromethane & 0.50 & $\mathrm{U}$ \\
\hline $10061-01-5$ & cis-1,3-Dichloropropene & 0.50 & $\mathrm{U}$ \\
\hline $108-10-1$ & 4-Methyl-2-pentanone & 5.0 & $\mathrm{U}$ \\
\hline $108-88-3$ & Toluene & 0.13 & $\mathrm{~J} \mathrm{~B}$ \\
\hline $10061-02-6$ & trans-1,3-Dichloropropene & 0.50 & $\mathrm{U}$ \\
\hline $79-00-5$ & 1,1,2-Trichloroethane & 0.50 & $\mathrm{U}$ \\
\hline $127-18-4$ & Tetrachloroethene & 0.077 & $\mathrm{~J}$ \\
\hline $591-78-6$ & 2-Hexanone & 5.0 & $\mathrm{U}$ \\
\hline $124-48-1$ & Dibromochloromethane & 0.31 & $\mathrm{~J}$ \\
\hline $106-93-4$ & 1,2-Dibromoethane & 0.50 & $\mathrm{U}$ \\
\hline $108-90-7$ & Chlorobenzene & 0.50 & $\mathrm{U}$ \\
\hline $100-41-4$ & Ethylbenzene & 0.085 & $\mathrm{~J}$ \\
\hline $95-47-6$ & o-Xylene & 0.090 & $\mathrm{~J}$ \\
\hline $179601-23-1$ & $\mathrm{~m}, \mathrm{p}$-Xylene & 0.14 & $\mathrm{~J}$ \\
\hline $100-42-5$ & Styrene & 0.016 & $\mathrm{~J}$ \\
\hline $75-25-2$ & Bromoform & 0.50 & $\mathrm{U}$ \\
\hline $98-82-8$ & Isopropylbenzene & 0.50 & $\mathrm{U}$ \\
\hline $79-34-5$ & $1,1,2,2$-Tetrachloroethane & 0.50 & $\mathrm{U}$ \\
\hline $541-73-1$ & 1,3-Dichlorobenzene & 0.50 & $\mathrm{U}$ \\
\hline $106-46-7$ & 1,4-Dichlorobenzene & 0.50 & $\mathrm{U}$ \\
\hline $95-50-1$ & 1,2-Dichlorobenzene & 0.50 & $\mathrm{U}$ \\
\hline $96-12-8$ & 1,2-Dibromo-3-Chloropropane & 0.50 & $\mathrm{U}$ \\
\hline $120-82-1$ & 1,2,4-Trichlorobenzene & 0.50 & $\mathrm{U}$ \\
\hline $87-61-6$ & 1,2,3-Trichlorobenzene & 0.50 & $\mathrm{U}$ \\
\hline
\end{tabular}


IJ - FORM I VOA-TIC

VOLATILE ORGANICS ANALYSIS DATA SHEET TENTATIVELY IDENTIEIED COMPOUNDS
EPA SAMPLE NO.

INQCTB-W-32778
Lab Name: TESTAMERICA BURLINGTON Case No.: INMAN Mod. Ref No.:

Contract: $8 \mathrm{E}-00302$

Lab Code: STLV

Water

Matrix: (SOIL/SED/WATER)

$(\mathrm{g} / \mathrm{mL}) \mathrm{mL}$

Sample wt/vol: 25.0

TRACE

Level: (TRACE or LOW/MED)

음 Moisture: not dec.

GC Column: DB-624

ID $: 0.20 \quad$ (mm)

Soil Extract Volume:

(uL)

CONCENTRATION UNITS: $(\mathrm{ug} / \mathrm{L}$ or $\mathrm{ug} / \mathrm{kg}) \mathrm{ug} / \mathrm{L}$
SDG No.: 200-6846

Lab Sample ID: 200-6846-5

Lab File ID: DHPX04.D

Date Received: 09/02/2011

Date Analyzed: 09/06/2011

Dilution Factor: 1.0

Soil Aliquot Volume:

(uL)

Purge Volume: 25.0

$(\mathrm{mL})$

\begin{tabular}{|l|l|l|c|c|c|}
\hline \multicolumn{1}{|c|}{ CAS NUMBER } & \multicolumn{1}{|c|}{ COMPOUND NAME } & RT & EST. CONC. & Q \\
\cline { 2 - 6 } 01 & & Unknown & 6.79 & B X J \\
\cline { 2 - 6 } 02 & E9667961 & Total Alkanes & N/A & \\
\hline
\end{tabular}

1.EPA-designated Registry Number. 
$6 A$ - FORM VI VOA-1

VOLATILE ORGANICS INITIAL CALIBRATION DATA

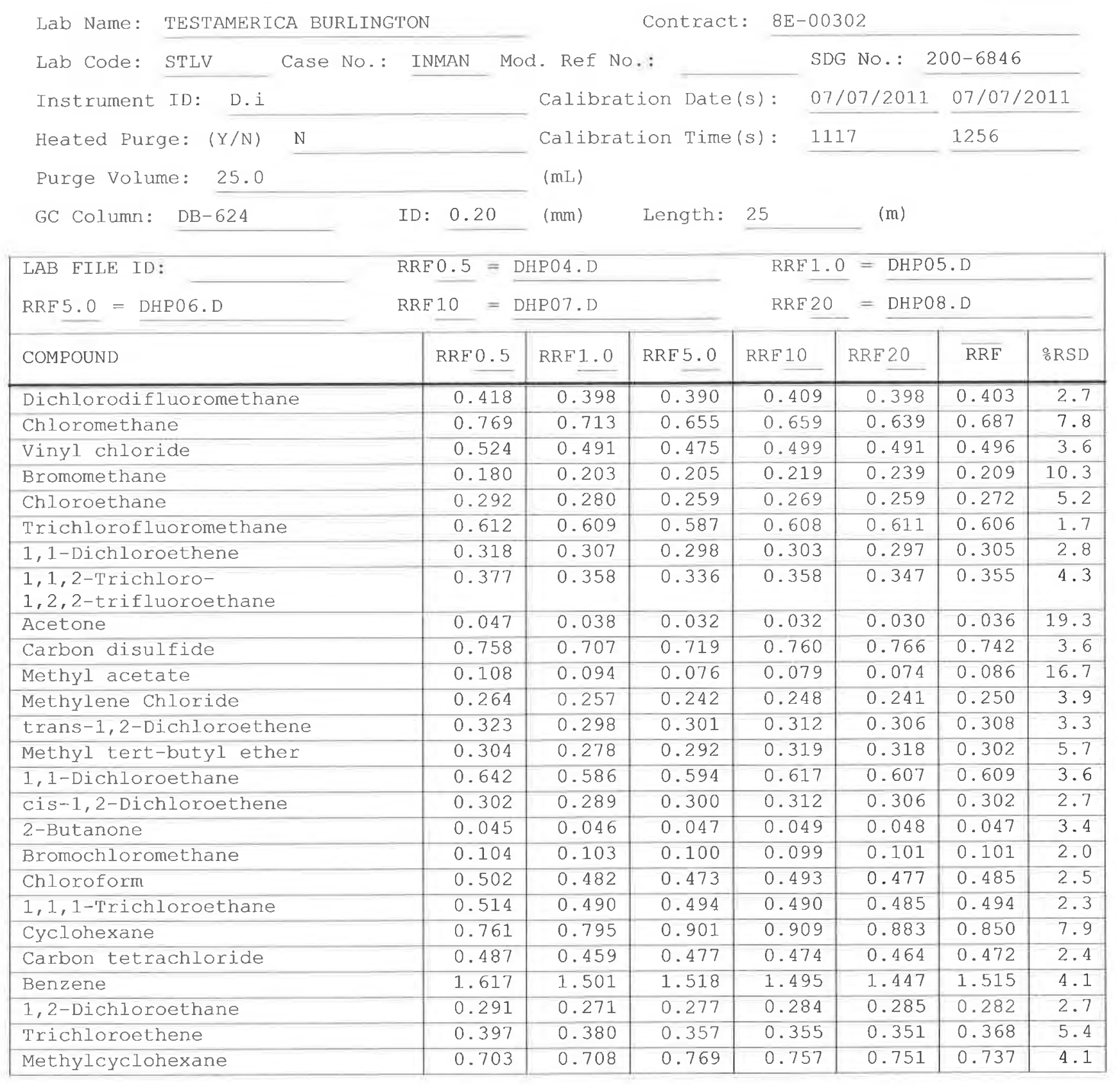

Report 1,4-Dioxane for Low-Medium VOA analysis only 
$6 \mathrm{~B}-$ FORM VI VOA-2

VOLATILE ORGANICS INITIAL CALIBRATION DATA

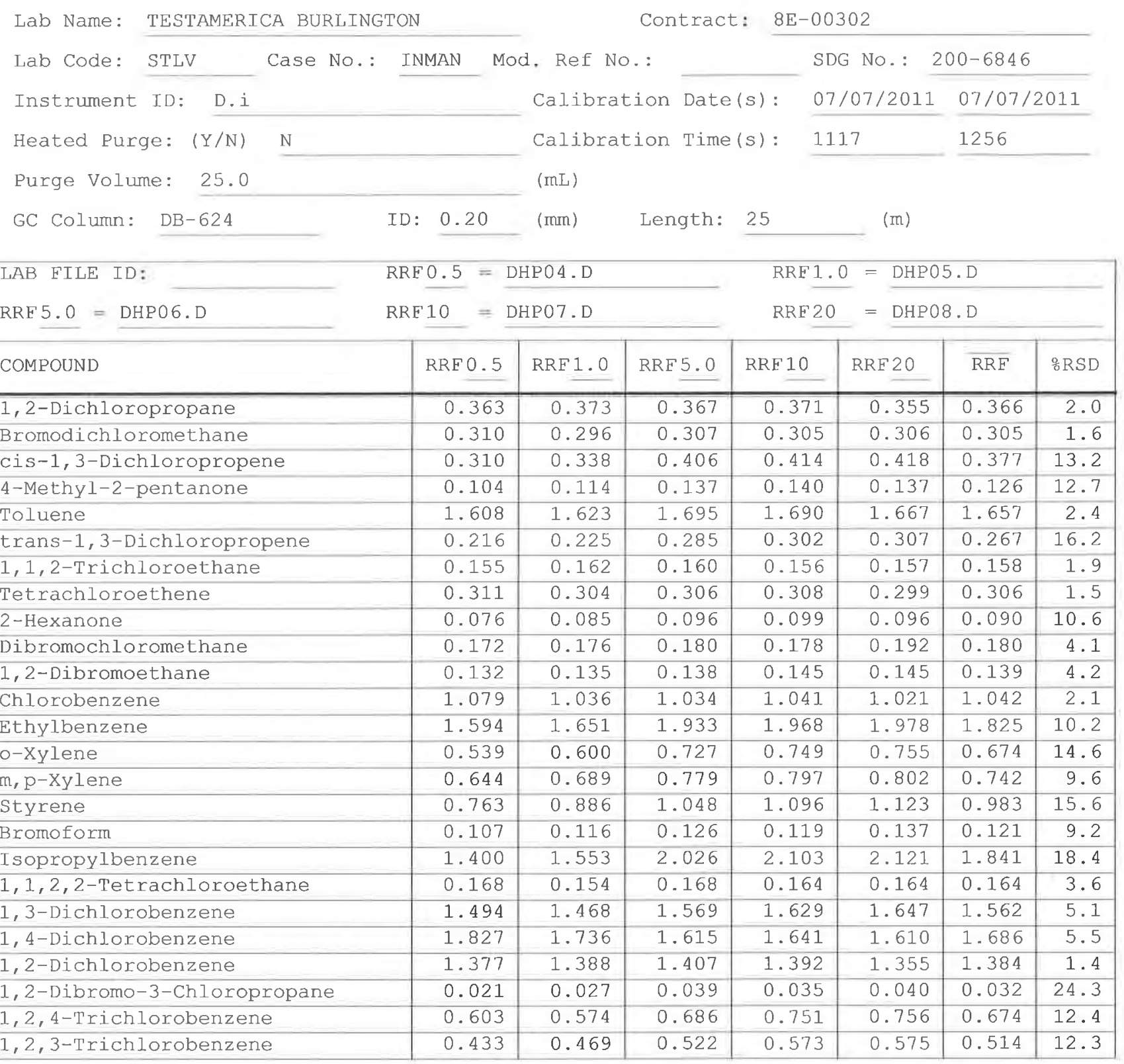


$6 \mathrm{C}-$ FORM VI VOA-3

VOLATILE ORGANICS INITIAL CALIBRATION DATA

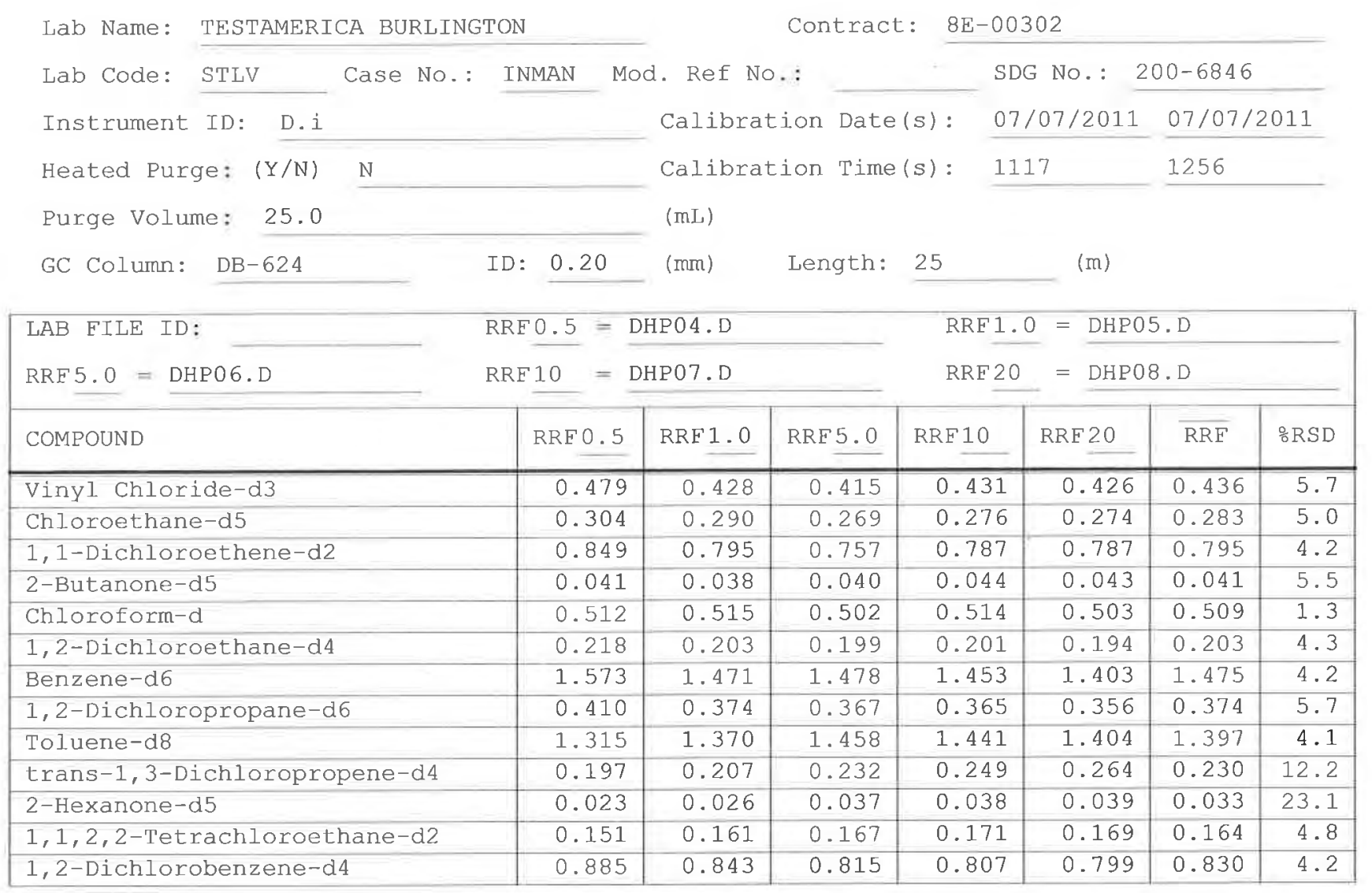

Report 1,4-Dioxane-d8 for Low-Medium VOA analysis only 
$7 \mathrm{~A}$ - FORM VII VOA-1

VOLATILE CONTINUING CALIBRATION DATA

Lab Name: TESTAMERICA BURLINGTON

Contract: $8 \mathrm{E}-00302$

Lab Code: STLV Case No.: INMAN Mod. Ref No.:

SDG No.: $200-6846$

Instrument ID: D.i

Calibration Date: 09/06/2011 Time: 0826

Lab File Id: DHPX02.D Init. Calib. Date(s) : 07/07/2011 07/07/2011

EPA Sample No. (VSTD\#\#\#): VSTD005DU

Init. Calib. Time(s): $1117 \quad 1256$

Heated Purge: $(\mathrm{Y} / \mathrm{N}) \mathrm{N}$

GC Column: DB-624

ID: $0.20(\mathrm{~mm})$ Length: 25

(m)

Purge Volume: 25.0

(mL)

\begin{tabular}{|c|c|c|c|c|c|}
\hline COMPOUND & $\overline{\mathrm{RRF}}$ & RRF5.0 & $\begin{array}{l}\text { MIN } \\
\text { RRF }\end{array}$ & 음 & $\operatorname{MAX} \cong \mathrm{D}$ \\
\hline Dichlorodifluoromethane & 0.403 & 0.517 & 0.010 & 28.3 & 40.0 \\
\hline Chloromethane & 0.687 & 0.765 & 0.010 & 11.3 & 40.0 \\
\hline Vinyl chloride & 0.496 & 0.552 & 0.010 & 11.3 & 30.0 \\
\hline Bromomethane & 0.209 & 0.232 & 0.010 & 10.8 & 30.0 \\
\hline Chloroethane & 0.272 & 0.281 & 0.010 & 3.2 & 40.0 \\
\hline Trichlorofluoromethane & 0.606 & 0.658 & 0.010 & 8.7 & 40.0 \\
\hline 1,1-Dichloroethene & 0.305 & 0.317 & 0.010 & 4.0 & 30.0 \\
\hline 1,1,2-Trichloro-1,2,2-trifluoroethane & 0.355 & 0.377 & 0.010 & 6.1 & 40.0 \\
\hline Acetone & 0.036 & 0.031 & 0.010 & -13.8 & 40.0 \\
\hline Carbon disulfide & 0.742 & 0.767 & 0.010 & 3.4 & 40.0 \\
\hline Methyl acetate & 0.086 & 0.086 & 0.010 & 0.2 & 40.0 \\
\hline Methylene Chloride & 0.250 & 0.246 & 0.010 & -1.6 & 40.0 \\
\hline trans-1,2-Dichloroethene & 0.308 & 0.313 & 0.010 & 1.7 & 40.0 \\
\hline Methyl tert-butyl ether & 0.302 & 0.275 & 0.010 & -9.1 & 40.0 \\
\hline 1,1-Dichloroethane & 0.609 & 0.619 & 0.010 & 1.6 & 30.0 \\
\hline cis-1,2-Dichloroethene & 0.302 & 0.305 & 0.010 & 1.0 & 40.0 \\
\hline 2-Butanone & 0.047 & 0.045 & 0.010 & -4.9 & 40.0 \\
\hline Bromochloromethane & 0.101 & 0.102 & 0.010 & 0.5 & 30.0 \\
\hline Chloroform & 0.485 & 0.487 & 0.010 & 0.3 & 30.0 \\
\hline 1,1,1-Trichloroethane & 0.494 & 0.524 & 0.010 & 5.9 & 30.0 \\
\hline Cyclohexane & 0.850 & 0.982 & 0.010 & 15.6 & 40.0 \\
\hline Carbon tetrachloride & 0.472 & 0.511 & 0.010 & 8.3 & 30.0 \\
\hline Benzene & 1.515 & 1.544 & 0.010 & 1.9 & 30.0 \\
\hline 1,2-Dichloroethane & 0.282 & 0.286 & 0.010 & 1.5 & 30.0 \\
\hline Trichloroethene & 0.368 & 0.370 & 0.010 & 0.7 & 30.0 \\
\hline Methylcyclohexane & 0.737 & 0.814 & 0.010 & 10.3 & 40.0 \\
\hline
\end{tabular}

Report 1,4-Dioxane for Low/Medium VOA analysis only 
$7 B$ - FORM VII VOA-2

VOLATILE CONTINUING CALIBRATION DATA

Lab Name: TESTAMERICA BURLINGTON

Contract: $8 \mathrm{E}-00302$

Lab Code: STLV Case No.: INMAN Mod. Ref No.:

SDG No.: 200-6846

Instrument ID: D.i

Calibration Date: 09/06/2011 Time: 0826

Lab File Id: DHPX02.D

EPA Sample No. (VSTD\#\#\#\#): VSTD005DU Init. Calib. Date(s): 07/07/2011 07/07/2011

Heated Purge: ( $\mathrm{Y} / \mathrm{N})$ GC Column: DB-624

Init. Calib. Time(s): $1117 \quad 1256$

Purge Volume: 25.0 (mL)

\begin{tabular}{|c|c|c|c|c|c|}
\hline COMPOUND & $\mathrm{RRF}$ & RRE5.0 & $\begin{array}{l}\text { MIN } \\
\text { RRF }\end{array}$ & $\frac{\mathrm{g}}{\mathrm{D}} \mathrm{D}$ & MAX $\div D$ \\
\hline 1,2-Dichloropropane & 0.366 & 0.384 & 0.010 & 4.9 & 40.0 \\
\hline Bromodichloromethane & 0.305 & 0.303 & 0.010 & -0.6 & 30.0 \\
\hline cis-1,3-Dichloropropene & 0.377 & 0.401 & 0.010 & 6.3 & 30.0 \\
\hline 4-Methyl-2-pentanone & 0.126 & 0.122 & 0.010 & -3.5 & 40.0 \\
\hline Toluene & 1.657 & 1.729 & 0.010 & 4.3 & 30.0 \\
\hline trans-1,3-Dichloropropene & 0.267 & 0.276 & 0.010 & 3.5 & 30.0 \\
\hline 1,1,2-Trichloroethane & 0.158 & 0.158 & 0.010 & -0.2 & 30.0 \\
\hline Tetrachloroethene & 0.306 & 0.317 & 0.010 & 3.6 & 30.0 \\
\hline 2-Hexanone & 0.090 & 0.086 & 0.010 & -4.9 & 40.0 \\
\hline Dibromochloromethane & 0.180 & 0.174 & 0.010 & -3.2 & 30.0 \\
\hline 1,2-Dibromoethane & 0.139 & 0.136 & 0.010 & -2.0 & 40.0 \\
\hline Chlorobenzene & 1.042 & 1.070 & 0.010 & 2.6 & 30.0 \\
\hline Ethylbenzene & 1.825 & 1.929 & 0.010 & 5.7 & 30.0 \\
\hline o-Xylene & 0.674 & 0.736 & 0.010 & 9.1 & 30.0 \\
\hline m,p-xylene & 0.742 & 0.793 & 0.010 & 6.9 & 30.0 \\
\hline Styrene & 0.983 & 1.080 & 0.010 & 9.8 & 30.0 \\
\hline Bromoform & 0.121 & 0.122 & 0.010 & 0.6 & 30.0 \\
\hline Isopropylbenzene & 1.841 & 2.064 & 0.010 & 12.1 & 40.0 \\
\hline $1,1,2,2$-Tetrachloroethane & 0.164 & 0.152 & 0.010 & -7.0 & 30.0 \\
\hline 1,3-Dichlorobenzene & 1.562 & 1.575 & 0.010 & 0.8 & 30.0 \\
\hline 1,4-Dichlorobenzene & 1.686 & 1.666 & 0.010 & -1.2 & 30.0 \\
\hline 1,2-Dichlorobenzene & 1.384 & 1.386 & 0.010 & 0.2 & 30.0 \\
\hline 1,2-Dibromo-3-Chloropropane & 0.032 & 0.032 & 0.010 & 0.3 & 40.0 \\
\hline 1,2,4-Trichlorobenzene & 0.674 & 0.712 & 0.010 & 5.6 & 30.0 \\
\hline 1,2,3-Trichlorobenzene & 0.514 & 0.540 & 0.010 & 5.1 & 30.0 \\
\hline
\end{tabular}


$7 \mathrm{C}-$ FORM VII VOA-3

VOLATILE CONTINUING CALIBRATION DATA

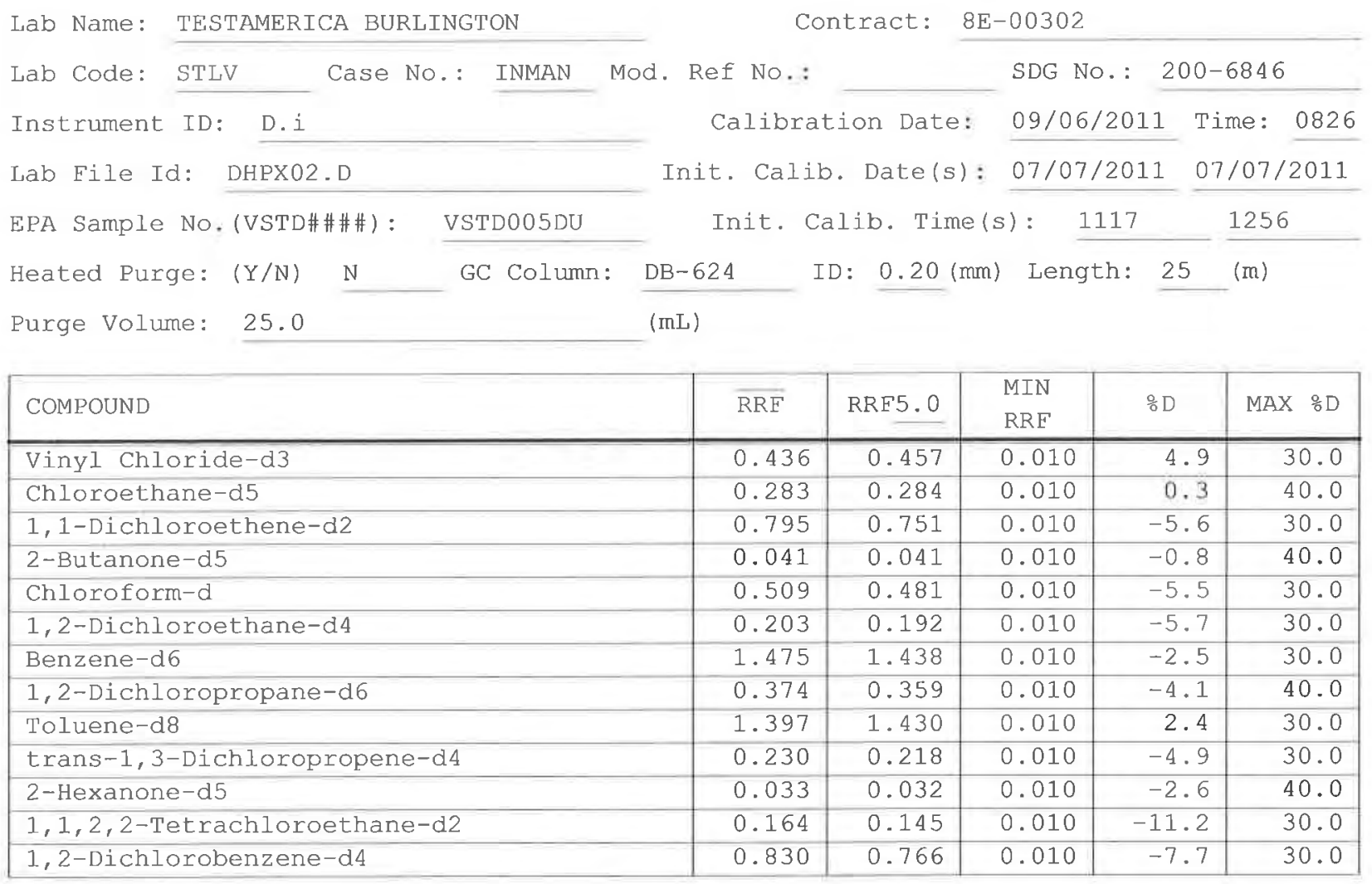

Report 1,4-Dioxane-d8 for Low/Medium VOA analysis only 
$7 A$ - FORM VII VOA-1

VOLATILE CONTINUING CALIBRATION DATA

Lab Name: TESTAMERICA BURLINGTON

Contract: $8 \mathrm{E}-00302$

Lab Code: STLV Case No.: INMAN Mod. Ref No.:

SDG No.: 200-6846

Instrument ID: D.i

Calibration Date: 09/06/2011 Time: 1514

Lab File Id: DHPX18.D

EPA Sample No. (VSTD\#\#\#\#): VSTD005Ud Init. Calib. Date(s): 07/07/2011 07/07/2011

STDO05UD

Init. Calib. Time(s): 1117 1256

Heated Purge: ( $Y / N)$

GC Column: DB-624

ID: $0.20(\mathrm{~mm})$ Length: 25

(m)

Purge Volume: 25.0 (mL)

\begin{tabular}{|c|c|c|c|c|c|}
\hline COMPOUND & $\overline{R R F}$ & RRF5.0 & $\begin{array}{l}\text { MIN } \\
\text { RRE }\end{array}$ & $\frac{\circ}{\partial} \mathrm{D}$ & $\operatorname{MAX} \div \mathrm{D}$ \\
\hline Dichlorodifluoromethane & 0.403 & 0.479 & 0.010 & 19.0 & 50.0 \\
\hline Chloromethane & 0.687 & 0.747 & 0.010 & 8.8 & 50.0 \\
\hline Vinyl chloride & 0.496 & 0.510 & 0.100 & 2.8 & 50.0 \\
\hline Bromomethane & 0.209 & 0.212 & 0.100 & 1.6 & 50.0 \\
\hline Chloroethane & 0.272 & 0.270 & 0.010 & -0.6 & 50.0 \\
\hline Trichlorofluoromethane & 0.606 & 0.649 & 0.010 & 7.2 & 50.0 \\
\hline 1,1-Dichloroethene & 0.305 & 0.308 & 0.100 & 1.0 & 50.0 \\
\hline 1,1,2-Trichloro-1,2,2-trifluoroethane & 0.355 & 0.380 & 0.010 & 7.1 & 50.0 \\
\hline Acetone & 0.036 & 0.033 & 0.010 & -7.2 & 50.0 \\
\hline Carbon disulfide & 0.742 & 0.736 & 0.010 & -0.7 & 50.0 \\
\hline Methyl acetate & 0.086 & 0.093 & 0.010 & 7.9 & 50.0 \\
\hline Methylene Chloride & 0.250 & 0.260 & 0.010 & 3.9 & 50.0 \\
\hline trans-1,2-Dichloroethene & 0.308 & 0.321 & 0.010 & 4.3 & 50.0 \\
\hline Methyl tert-butyl ether & 0.302 & 0.318 & 0.010 & 5.1 & 50.0 \\
\hline 1,1-Dichloroethane & 0.609 & 0.644 & 0.200 & 5.7 & 50.0 \\
\hline cis-1,2-Dichloroethene & 0.302 & 0.314 & 0.010 & 4.0 & 50.0 \\
\hline 2-Butanone & 0.047 & 0.051 & 0.010 & 7.3 & 50.0 \\
\hline Bromochloromethane & 0.101 & 0.107 & 0.050 & 5.7 & 50.0 \\
\hline Chloroform & 0.485 & 0.517 & 0.200 & 6.5 & 50.0 \\
\hline 1,1,1-Trichloroethane & 0.494 & 0.497 & 0.100 & 0.6 & 50.0 \\
\hline Cyclohexane & 0.850 & 0.939 & 0.010 & 10.5 & 50.0 \\
\hline Carbon tetrachloride & 0.472 & 0.495 & 0.100 & 4.8 & 50.0 \\
\hline Benzene & 1.515 & 1.560 & 0.400 & 2.9 & 50.0 \\
\hline 1,2-Dichloroethane & 0.282 & 0.299 & 0.100 & 6.2 & 50.0 \\
\hline Trichloroethene & 0.368 & 0.358 & 0.300 & -2.8 & 50.0 \\
\hline Methylcyclohexane & 0.737 & 0.802 & 0.010 & 8.7 & 50.0 \\
\hline
\end{tabular}

Report 1,4-Dioxane for Low/Medium VOA analysis only 
$7 \mathrm{~B}$ - EORM VII VOA-2

VOLATILE CONTINUING CALIBRATION DATA

Lab Name: TESTAMERICA BURLINGTON

Contract: $\quad 8 \mathrm{E}-00302$

Lab Code: STLV Case No.: INMAN Mod. Ref No.:

SDG No.: 200-6846

Instrument ID: D.i

Calibration Date: 09/06/2011 Time: 1514

Lab File Id: DHPX18.D

Init. Calib. Date(s): 07/07/2011 07/07/2011

EPA Sample No. (VSTD\#\#\#\#): VSTD005UD

Init. Calib. Time(s): $1117 \quad \underline{1256}$

Heated Purge: ( $\mathrm{Y} / \mathrm{N}) \mathrm{N}$

GC Column: DB-624

ID: $0.20(\mathrm{~mm})$ Length: 25

(m)

Purge Volume: 25.0

(m工)

\begin{tabular}{|c|c|c|c|c|c|}
\hline COMPOUND & $\overline{\mathrm{RRF}}$ & RRF5.0 & $\begin{array}{l}\text { MIN } \\
\text { RRE }\end{array}$ & $\because \mathrm{D}$ & $\operatorname{MAX} \div \mathrm{D}$ \\
\hline 1,2-Dichloropropane & 0.366 & 0.384 & 0.010 & 5.1 & 50.0 \\
\hline Bromodichloromethane & 0.305 & 0.311 & 0.200 & 2.0 & 50.0 \\
\hline cis-1,3-Dichloropropene & 0.377 & 0.407 & 0.200 & 7.8 & 50.0 \\
\hline 4-Methyl-2-pentanone & 0.126 & 0.141 & 0.010 & 11.5 & 50.0 \\
\hline Toluene & 1.657 & 1.733 & 0.400 & 4.6 & 50.0 \\
\hline trans-1,3-Dichloropropene & 0.267 & 0.292 & 0.100 & 9.3 & 50.0 \\
\hline 1,1,2-Trichloroethane & 0.158 & 0.168 & 0.100 & 6.5 & 50.0 \\
\hline Tetrachloroethene & 0.306 & 0.319 & 0.100 & 4.3 & 50.0 \\
\hline 2-Hexanone & 0.090 & 0.100 & 0.010 & 10.2 & 50.0 \\
\hline Dibromochloromethane & 0.180 & 0.188 & 0.100 & 4.8 & 50.0 \\
\hline 1,2-Dibromoethane & 0.139 & 0.145 & 0.010 & 4.6 & 50.0 \\
\hline Chlorobenzene & 1.042 & 1.086 & 0.500 & 4.2 & 50.0 \\
\hline Ethylbenzene & 1.825 & 1.968 & 0.100 & 7.9 & 50.0 \\
\hline o-Xylene & 0.674 & 0.754 & 0.300 & 11.8 & 50.0 \\
\hline $\mathrm{m}, \mathrm{p}$-Xylene & 0.742 & 0.809 & 0.300 & 9.0 & 50.0 \\
\hline Styrene & 0.983 & 1.123 & 0.300 & 14.3 & 50.0 \\
\hline Bromoform & 0.121 & 0.116 & 0.050 & -4.2 & 50.0 \\
\hline Isopropylbenzene & 1.841 & 2.055 & 0.010 & 11.6 & 50.0 \\
\hline $1,1,2,2$-Tetrachloroethane & 0.164 & 0.165 & 0.100 & 1.0 & 50.0 \\
\hline 1,3-Dichlorobenzene & 1.562 & 1.527 & 0.400 & -2.2 & 50.0 \\
\hline 1,4-Dichlorobenzene & 1.686 & 1.671 & 0.400 & -0.9 & 50.0 \\
\hline 1,2-Dichlorobenzene & 1.384 & 1.441 & 0.400 & 4.1 & 50.0 \\
\hline 1,2-Dibromo-3-Chloropropane & 0.032 & 0.035 & 0.010 & 8.4 & 50.0 \\
\hline 1,2,4-Trichlorobenzene & 0.674 & 0.679 & 0.200 & 0.7 & 50.0 \\
\hline 1,2,3-Trichlorobenzene & 0.514 & 0.545 & 0.200 & 5.9 & 50.0 \\
\hline
\end{tabular}


$7 \mathrm{C}$ - FORM VII VOA-3

VOLATILE CONTINUING CALIBRATION DATA

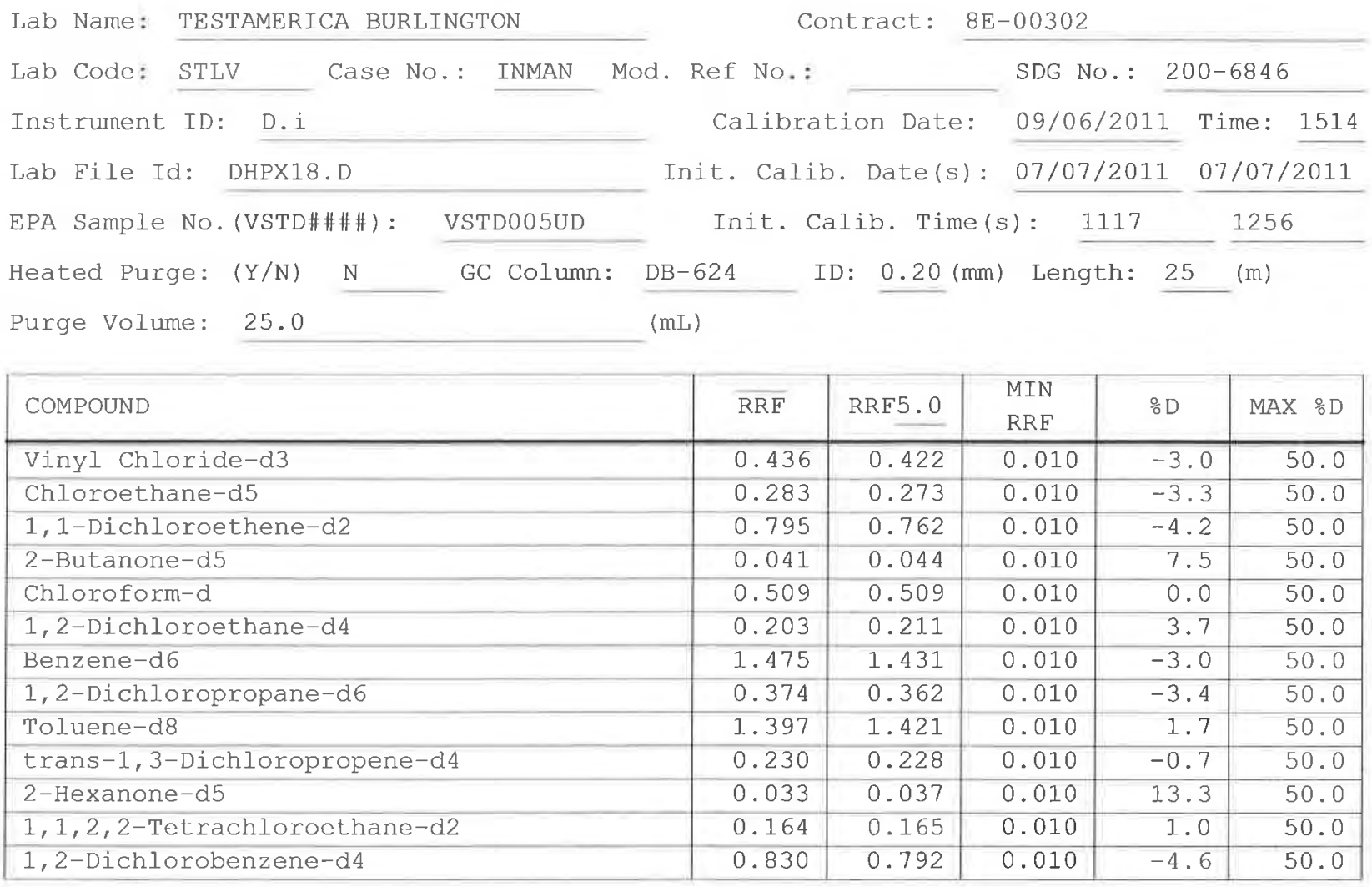


$1 A$ - FORM I VOA-1

VOLATILE ORGANICS ANALYSIS DATA SHEET
EPA SAMPLE NO.

VBLKDU
Lab Name: TESTAMERICA BURLINGTON

Lab Code: STLV

Case No.: INMAN

Matrix: (SOIL/SED/WATER)

Water

Sample wt/vol: 25.0

$(\mathrm{g} / \mathrm{mL}) \quad \mathrm{mL}$

Level: (TRACE/LOW/MED) TRACE

응 Moisture: not dec.

GC Column: DB-624 ID : $0.20 \quad$ (mm)

Soil Extract Volume:

(uL)

(mL)

Purge Volume: 25.0

Contract: 8E-00302

Mod. Ref No.:

SDG No.: 200-6846

Lab Sample ID: MB 200-24749/3

Lab File ID: DHPX03.D

Date Received:

Date Analyzed: 09/06/2011

Dilution Factor: 1.0

Soil Aliquot Volume:

(uL)

\begin{tabular}{|c|c|c|c|}
\hline CAS NO. & COMPOUND & $\begin{array}{l}\text { CONCENTRATION UNITS: } \\
(\mathrm{ug} / \mathrm{L} \text { or } \mathrm{ug} / \mathrm{kg}) \mathrm{ug} / \mathrm{L}\end{array}$ & $Q$ \\
\hline $75-71-8$ & Dichlorodifluoromethane & 0.50 & $\mathrm{U}$ \\
\hline $74-87-3$ & Chloromethane & 0.036 & $\mathrm{~J}$ \\
\hline $75-01-4$ & Vinyl chloride & 0.50 & $\mathrm{U}$ \\
\hline $74-83-9$ & Bromomethane & 0.50 & $\mathrm{U}$ \\
\hline $75-00-3$ & Chloroethane & 0.50 & $\mathrm{U}$ \\
\hline $75-69-4$ & Trichlorofluoromethane & 0.50 & $\mathrm{U}$ \\
\hline $75-35-4$ & 1,1-Dichloroethene & 0.50 & $\mathrm{U}$ \\
\hline $76-13-1$ & 1, 1,2-Trichloro-1,2,2-trifluoroethane & 0.50 & $\mathrm{U}$ \\
\hline $67-64-1$ & Acetone & 5.0 & $\mathrm{U}$ \\
\hline $75-15-0$ & Carbon disulfide & 0.50 & $\mathrm{U}$ \\
\hline $79-20-9$ & Methyl acetate & 0.50 & $\mathrm{U}$ \\
\hline $75-09-2$ & Methylene Chloride & 0.50 & $\mathrm{U}$ \\
\hline $156-60-5$ & trans-1,2-Dichloroethene & 0.50 & $\mathrm{U}$ \\
\hline $1634-04-4$ & Methyl tert-butyl ether & 0.50 & $\mathrm{U}$ \\
\hline $75-34-3$ & 1,1-Dichloroethane & 0.50 & $\mathrm{U}$ \\
\hline $156-59-2$ & cis-1,2-Dichloroethene & 0.50 & $\mathrm{U}$ \\
\hline $78-93-3$ & 2-Butanone & 5.0 & $\mathrm{U}$ \\
\hline $74-97-5$ & Bromochloromethane & 0.50 & U \\
\hline $67-66-3$ & Chloroform & 0.50 & $\mathrm{U}$ \\
\hline $71-55-6$ & 1,1,1-Trichloroethane & 0.50 & $\mathrm{U}$ \\
\hline $110-82-7$ & Cyclohexane & 0.50 & $\mathrm{U}$ \\
\hline $56-23-5$ & Carbon tetrachloride & 0.50 & $\mathrm{U}$ \\
\hline $71-43-2$ & Benzene & 0.50 & $\mathrm{U}$ \\
\hline $107-06-2$ & 1,2-Dichloroethane & 0.50 & $\mathrm{U}$ \\
\hline
\end{tabular}

Report 1,4-Dioxane for Low-Medium VOA analysis only 
Lab Name: TESTAMERICA BURLINGTON

Lab Code: STLV Case

Matrix: (SOIL/SED/WATER)

Water

Sample wt/vol: 25.0 $(\mathrm{g} / \mathrm{mL}) \quad \mathrm{mL}$

Level: (TRACE/LOW/MED) TRACE

응 Moisture: not dec.

GC Column: DB-624

ID: 0.20 (mm)

Soil Extract Volume:

(uL) (mL)

Purge Volume: 25.0
Contract: $8 \mathrm{E}-00302$

SDG No.: 200-6846

\begin{tabular}{|c|c|c|c|}
\hline CAS NO. & COMPOUND & $\begin{array}{l}\text { CONCENTRATION UNITS: } \\
(\mathrm{ug} / \mathrm{L} \text { or } \mathrm{ug} / \mathrm{kg}) \mathrm{ug} / \mathrm{L}\end{array}$ & $Q$ \\
\hline $79-01-6$ & Trichloroethene & 0.50 & $\mathrm{U}$ \\
\hline $108-87-2$ & Methylcyclohexane & 0.50 & $\mathrm{U}$ \\
\hline $78-87-5$ & 1,2-Dichloropropane & 0.50 & $\mathrm{U}$ \\
\hline $75-27-4$ & Bromodichloromethane & 0.50 & $\mathrm{U}$ \\
\hline $10061-01-5$ & cis-1,3-Dichloropropene & 0.50 & $\mathrm{U}$ \\
\hline $108-10-1$ & 4-Methyl-2-pentanone & 5.0 & $\mathrm{U}$ \\
\hline $108-88-3$ & Toluene & 0.017 & $\mathrm{~J}$ \\
\hline $10061-02-6$ & trans $-1,3-$ Dichloropropene & 0.50 & $\mathrm{U}$ \\
\hline $79-00-5$ & 1,1,2-Trichloroethane & 0.50 & $\mathrm{U}$ \\
\hline $127-18-4$ & Tetrachloroethene & 0.50 & $\mathrm{U}$ \\
\hline $591-78-6$ & 2-Hexanone & 5.0 & $\mathrm{U}$ \\
\hline $124-48-1$ & Dibromochloromethane & 0.50 & $\mathrm{U}$ \\
\hline $106-93-4$ & 1,2-Dibromoethane & 0.50 & $\mathrm{U}$ \\
\hline $108-90-7$ & Chlorobenzene & 0.50 & $\mathrm{U}$ \\
\hline $100-41-4$ & Ethylbenzene & 0.50 & $\mathrm{U}$ \\
\hline $95-47-6$ & o-Xylene & 0.50 & $\mathrm{U}$ \\
\hline $179601-23-1$ & $\mathrm{~m}, \mathrm{p}$-Xylene & 0.50 & $\mathrm{U}$ \\
\hline $100-42-5$ & Styrene & 0.50 & $\mathrm{U}$ \\
\hline $75-25-2$ & Bromoform & 0.50 & $\mathrm{U}$ \\
\hline $98-82-8$ & Isopropylbenzene & 0.50 & $\mathrm{U}$ \\
\hline $79-34-5$ & $1,1,2,2$-Tetrachloroethane & 0.50 & $\mathrm{U}$ \\
\hline $541-73-1$ & 1,3-Dichlorobenzene & 0.50 & $\mathrm{U}$ \\
\hline $106-46-7$ & 1,4-Dichlorobenzene & 0.50 & $\mathrm{U}$ \\
\hline $95-50-1$ & 1,2-Dichlorobenzene & 0.50 & $\mathrm{U}$ \\
\hline $96-12-8$ & 1,2-Dibromo-3-Chloropropane & 0.50 & $\mathrm{U}$ \\
\hline $120-82-1$ & 1,2,4-Trichlorobenzene & 0.50 & $\mathrm{U}$ \\
\hline $87-61-6$ & 1,2,3-Trichlorobenzene & 0.50 & $\mathrm{U}$ \\
\hline
\end{tabular}


1J - FORM I VOA-TIC

VOLATILE ORGANICS ANALYSIS DATA SHEET TENTATIVELY IDENTIFIED COMPOUNDS
EPA SAMPLE NO.

VBLKDU
Lab Name: TESTAMERICA BURLINGTON

Lab Code: STLV Case No.: INMAN

Matrix: (SOIL/SED/WATER)

Sample wt/vol: 25.0

water

Level: (TRACE or LOW/MED)

$(\mathrm{g} / \mathrm{mL}) \mathrm{mL}$

\% Moisture: not dec.

GC Column: DB-624

ID: 0.20

(IIIIn)

Soil Extract Volume:

(uL)

CONCENTRATION UNITS: (ug/L or $\mathrm{ug} / \mathrm{kg}$ ) $\mathrm{ug} / \mathrm{L}$
Contract: $8 \mathrm{E}-00302$

SDG No.: 200-6846

Lab Sample ID: MB 200-24749/3

Lab File ID: DHPX03.D

Date Received:

Date Analyzed: 09/06/2011

Dilution Factor: 1.0

Soil Aliquot Volume: (uL)

Purge Volume: 25.0 (mL)

\begin{tabular}{l|l|l|l|c|c|}
\hline \multicolumn{1}{|c|}{ CAS NUMBER } & \multicolumn{1}{|c|}{ COMPOUND NAME } & RT & EST. CONC. & Q \\
\cline { 2 - 6 } 01 & & Unknown & 6.79 & $\mathrm{~J}$ \\
\hline & E9667961 & Total Alkanes & N/A & 2.8 & \\
\hline
\end{tabular}

\footnotetext{
1 EPA-designated Registry Number.
} 
$1 \mathrm{~A}$ - FORM I VOA-1

VOLATILE ORGANICS ANALYSIS DATA SHEET
EPA SAMPLE NO.

VHBLK01
Lab Name: TESTAMERICA BURLINGTON

Lab Code: STLV Case No.: INMAN

Matrix: (SOIL/SED/WATER)

Sample wt/vol: 25.0

water

$(\mathrm{g} / \mathrm{mL}) \quad \mathrm{mL}$

Level: (TRACE/LOW/MED) TRACE

\% Moisture: not dec.

GC Column: DB-624

ID: 0.20

Soil Extract Volume:

(uL)

(mL)

Purge Volume: 25.0
Contract: $\quad 8 \mathrm{E}-00302$

SDG No.: 200-6846

Lab Sample' ID: $\quad 200-6846-8$

Lab File ID: DHPX11.D

Date Received:

Date Analyzed: 09/06/2011

Dilution Eactor: 1.0

Soil Aliquot Volume:

(uL)

\begin{tabular}{|c|c|c|c|}
\hline CAS NO. & COMPOUND & $\begin{array}{l}\text { CONCENTRATION UNITS: } \\
(\mathrm{ug} / \mathrm{L} \text { or } \mathrm{ug} / \mathrm{kg}) \mathrm{ug} / \mathrm{L}\end{array}$ & $Q$ \\
\hline $75-71-8$ & Dichlorodifluoromethane & 0.50 & $\overline{\mathrm{U}}$ \\
\hline $74-87-3$ & Chloromethane & 0.50 & $\mathrm{U}$ \\
\hline $75-01-4$ & Vinyl chloride & 0.50 & $\mathrm{U}$ \\
\hline $74-83-9$ & Bromomethane & 0.50 & $\mathrm{U}$ \\
\hline $75-00-3$ & Chloroethane & 0.50 & $\mathrm{U}$ \\
\hline $75-69-4$ & Trichlorofluoromethane & 0.50 & $\mathrm{U}$ \\
\hline $75-35-4$ & 1,1-Dichloroethene & 0.50 & $\mathrm{U}$ \\
\hline $76-13-1$ & 1,1,2-Trichloro-1,2,2-trifluoroethane & 0.50 & $\mathrm{U}$ \\
\hline $67-64-1$ & Acetone & 5.0 & $\mathrm{U}$ \\
\hline $75-15-0$ & Carbon disulfide & 0.50 & $\mathrm{U}$ \\
\hline $79-20-9$ & Methyl acetate & 0.50 & $\mathrm{U}$ \\
\hline $75-09-2$ & Methylene Chloride & 0.50 & $\mathrm{U}$ \\
\hline $156-60-5$ & trans-1,2-Dichloroethene & 0.50 & $\mathrm{U}$ \\
\hline $1634-04-4$ & Methyl tert-butyl ether & 0.50 & $\mathrm{U}$ \\
\hline $75-34-3$ & 1,1-Dichloroethane & 0.50 & $\mathrm{U}$ \\
\hline $156-59-2$ & cis-1,2-Dichloroethene & 0.50 & $\mathrm{U}$ \\
\hline $78-93-3$ & 2-Butanone & 5.0 & $\mathrm{U}$ \\
\hline $74-97-5$ & Bromochloromethane & 0.50 & $\mathrm{U}$ \\
\hline $67-66-3$ & Chloroform & 0.50 & $\mathrm{U}$ \\
\hline $71-55-6$ & 1,1,1-Trichloroethane & 0.50 & $\mathrm{U}$ \\
\hline $110-82-7$ & Cyclohexane & 0.50 & $\mathrm{U}$ \\
\hline $56-23-5$ & Carbon tetrachloride & 0.50 & $\mathrm{U}$ \\
\hline $71-43-2$ & Benzene & 0.50 & $\mathrm{U}$ \\
\hline $107-06-2$ & 1,2-Dichloroethane & 0.50 & $\mathrm{U}$ \\
\hline
\end{tabular}

Report 1,4-Dioxane for Low-Medium VOA analysis only 
$1 B$ - FORM I VOA-2

VOLATILE ORGANICS ANALYSIS DATA SHEET
EPA SAMPLE NO.

VHBLKO1
Lab Name: TESTAMERICA BURLINGTON

Lab Code: STLV

Case No.: INMAN

Matrix: (SOIL/SED/WATER)

Water

Sample wt/vol: 25.0

$(\mathrm{g} / \mathrm{mL}) \mathrm{mL}$

Level: (TRACE/LOW/MED) TRACE

o Moisture: not dec.

GC Column: DB-624

ID : 0.20 (nm)

Soil Extract Volume:

Purge Volume: 25.0

(uL)

(mL)
Contract: $8 \mathrm{E}-00302$

SDG No.: 200-6846

Lab Sample ID: 200-6846-8

Lab File ID: DHPX11.D

Date Received:

Date Analyzed: 09/06/2011

Dilution Factor: 1.0

Soil Aliquot Volume:

(uL)

\begin{tabular}{|c|c|c|c|}
\hline CAS NO. & COMPOUND & $\begin{array}{l}\text { CONCENTRATION UNITS: } \\
\text { (ug/L or } \mathrm{ug} / \mathrm{kg} \text { ) ug/L }\end{array}$ & $Q$ \\
\hline $79-01-6$ & Trichloroethene & 0.50 & $\bar{U}$ \\
\hline $108-87-2$ & Methylcyclohexane & 0.50 & $\mathrm{U}$ \\
\hline $78-87-5$ & 1,2-Dichloropropane & 0.50 & $\mathrm{U}$ \\
\hline $75-27-4$ & Bromodichloromethane & 0.50 & $\mathrm{U}$ \\
\hline $10061-01-5$ & cis-1,3-Dichloropropene & 0.50 & $\mathrm{U}$ \\
\hline $108-10-1$ & 4-Methyl-2-pentanone & 5.0 & $\mathrm{U}$ \\
\hline $108-88-3$ & Toluene & 0.020 & $\mathrm{~J} \mathrm{~B}$ \\
\hline $10061-02-6$ & trans-1,3-Dichloropropene & 0.50 & $\mathrm{U}$ \\
\hline $79-00-5$ & 1,1,2-Trichloroethane & 0.50 & $\mathrm{U}$ \\
\hline $127-18-4$ & Tetrachloroethene & 0.50 & $\mathrm{U}$ \\
\hline $591-78-6$ & 2-Hexanone & 5.0 & $\mathrm{U}$ \\
\hline $124-48-1$ & Dibromochloromethane & 0.50 & $\mathrm{U}$ \\
\hline $106-93-4$ & 1,2-Dibromoethane & 0.50 & $\mathrm{U}$ \\
\hline $108-90-7$ & Chlorobenzene & 0.50 & $\mathrm{U}$ \\
\hline $100-41-4$ & Ethylbenzene & 0.50 & $\mathrm{U}$ \\
\hline $95-47-6$ & o-xylene & 0.50 & $\mathrm{U}$ \\
\hline $179601-23-1$ & $\mathrm{~m}, \mathrm{p}$-Xylene & 0.50 & U \\
\hline $100-42-5$ & Styrene & 0.50 & $\mathrm{U}$ \\
\hline $75-25-2$ & Bromoform & 0.50 & U \\
\hline $98-82-8$ & Isopropylbenzene & 0.50 & $\mathrm{U}$ \\
\hline $79-34-5$ & $1,1,2,2$-Tetrachloroethane & 0.50 & $\mathrm{U}$ \\
\hline $541-73-1$ & 1,3-Dichlorobenzene & 0.50 & U \\
\hline $106-46-7$ & 1,4-Dichlorobenzene & 0.50 & $\mathrm{U}$ \\
\hline $95-50-1$ & 1,2-Dichlorobenzene & 0.50 & $\mathrm{U}$ \\
\hline $96-12-8$ & 1,2-Dibromo-3-Chloropropane & 0.50 & $\mathrm{U}$ \\
\hline $120-82-1$ & 1,2,4-Trichlorobenzene & 0.50 & $\mathrm{U}$ \\
\hline $87-61-6$ & 1,2,3-Trichlorobenzene & 0.50 & $\mathrm{U}$ \\
\hline
\end{tabular}


$1 \mathrm{~J}$ - FORM I VOA-TIC

VOLATILE ORGANICS ANALYSIS DATA SHEET TENTATIVELY IDENTIFIED COMPOUNDS
EPA SAMPLE NO.

VHBLKO1
Lab Name: TESTAMERICA BURLINGTON

Lab Code: STLV Case No.: INMAN Mod. Ref No.:

Contract: $\quad 8 \mathrm{E}-00302$ SDG No.: 200-6846

Matrix: (SOIL/SED/WATER)

Sample wt/vol: 25.0

ter

$(\mathrm{g} / \mathrm{mL}) \mathrm{mL}$

Level: (TRACE or LOW/MED)

TRACE

\% Moisture: not dec.

GC Column: DB-624

Soil Extract Volume: ID: $0.20 \quad(\mathrm{~mm})$

(uL)

CONCENTRATION UNITS: (ug/L or ug/kg) ug/L
Lab Sample ID: $\quad 200-6846-8$

Lab File ID: DHPX11.D

Date Received:

Date Analyzed: 09/06/2011

Dilution Factor: 1.0

Soil Aliquot Volume:

(uL)

Purge Volume: 25.0

\begin{tabular}{|l|l|l|r|r|r|r|}
\hline \multicolumn{1}{|c|}{ CAS NUMBER } & COMPOUND NAME & RT & EST. CONC. & Q \\
\cline { 2 - 6 } 01 & & Unknown & 6.79 & 2.7 & B X J \\
\hline & E9667961 & Total Alkanes & N/A & \\
\hline
\end{tabular}

1 EPA-designated Registry Number. 


\section{TestAmerica}

THE LEADER IN ENVIRONMENTAL TESTING

\section{ANALYTICAL REPORT}

Job Number: 200-7006-1

SDG Number: 200-7006

Job Description: Inman (200-7006)

Contract Number: 1E-30401

For:

Argonne National Laboratory

9700 South Cass Avenue

Building 203

Office B-149

Argonne, IL 60439

Attention: Mr. Clyde Dennis

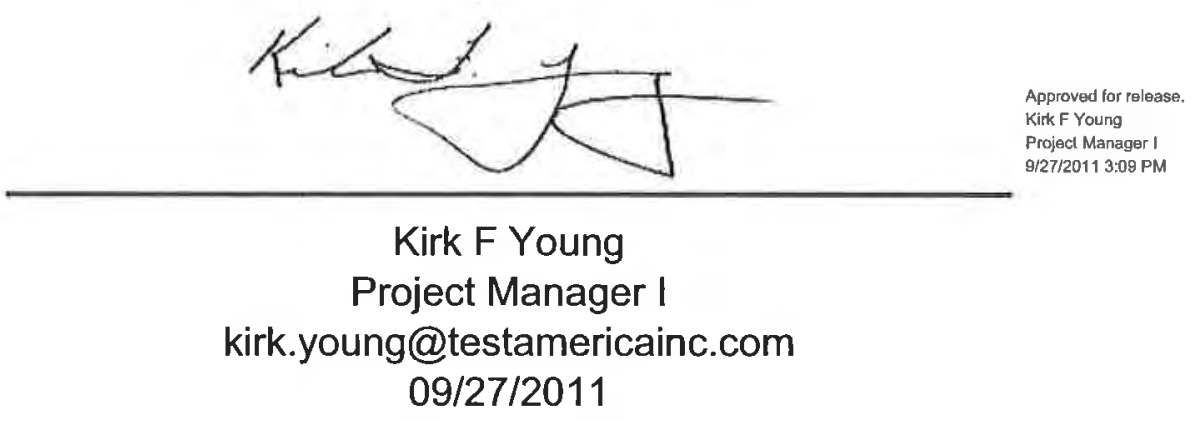

The test results in this report relate only to sample(s) as received by the laboratory. These test results were derived under a quality system that adheres to the requirements of NELAC. Pursuant to NELAC, this report may not be produced in full without written approval from the laboratory 


\section{Table of Contents}

Cover Title Page ........................ 1

Report Narrative . . . . . . . . . . . . . . . . . . . . . . 4

Case Narrative ............................. 4

Qualifier Definition ........................... 6

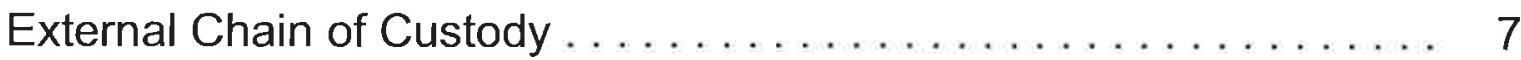

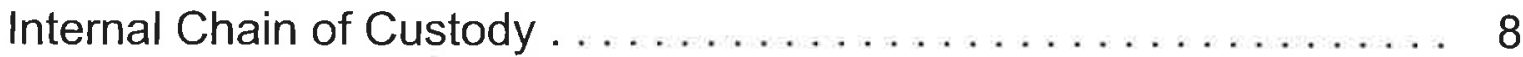

Shipping Documentation . . . . . . . . . . . . . . . . . 10

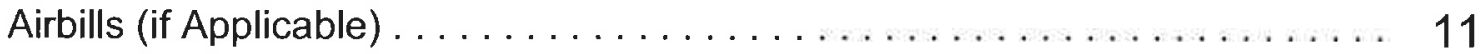

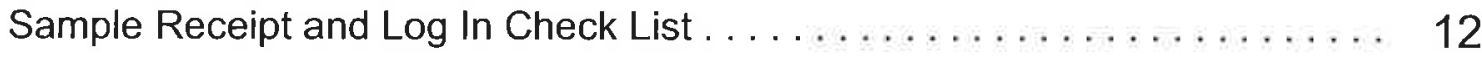

Methodology Review . . . . . . . . . . . . . . . . . . . . . . . . . 14

QC Summary - SOM01.2 Volatiles-Trace . . . . . . . . . . . . . 15

QC Summary - SOM01.2 Volatiles-Trace . . . . . . . . . . . . . . . 15

Deuterated Monitoring Compound Summary $\ldots \ldots \ldots \ldots \ldots \ldots \ldots \ldots \ldots \ldots \ldots \ldots \ldots \ldots \ldots \ldots$

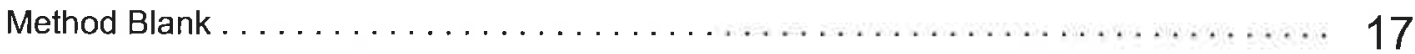

GC/MS Instrument Performance Check $\ldots \ldots \ldots \ldots \ldots \ldots \ldots \ldots \ldots \ldots \ldots, 18$

Internal Standard Area and RT Summary $\ldots \ldots \ldots \ldots \ldots \ldots \ldots \ldots \ldots \ldots \ldots \ldots \ldots \ldots \ldots, 20$

Sample Data - SOM01.2 Volatiles-Trace . . . . . . . . . . . . . 21

Sample Data - SOM01.2 Volatiles-Trace . . . . . . . . . . . . . . . 21

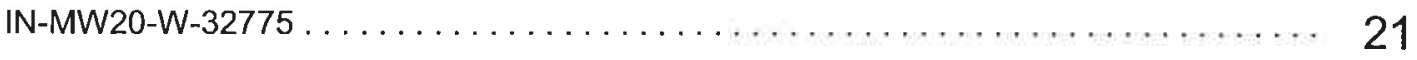

IN-MW20-W-32776 $\ldots \ldots \ldots \ldots \ldots \ldots \ldots \ldots \ldots \ldots \ldots \ldots \ldots \ldots \ldots \ldots \ldots \ldots \ldots \ldots \ldots, 24$

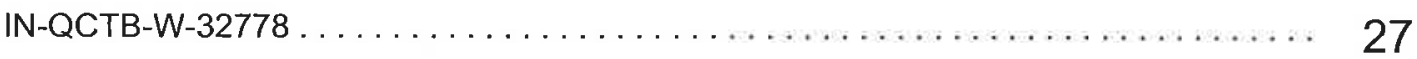

Standards - SOM01.2 Volatiles-Trace . . . . . . . . . . . . . . . 30

Standards - SOM01.2 Volatiles-Trace $\ldots \ldots \ldots \ldots \ldots \ldots \ldots \ldots \ldots \ldots, 30$

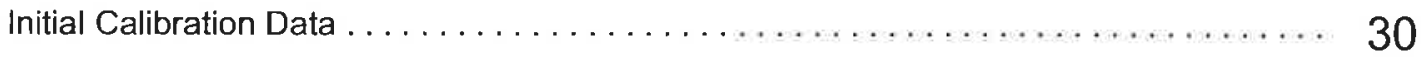

CCV Data, including closing CCV $\ldots \ldots \ldots \ldots \ldots \ldots \ldots \ldots \ldots \ldots \ldots \ldots \ldots \ldots \ldots \ldots$

Raw Qc Data - SOM01.2 Volatiles-Trace . . . . . . . . . . . . . . . . 39 


\section{Table of Contents}

Raw Qc Data - SOM01.2 Volatiles-Trace . . . . . . . . . . . . . . . . 39

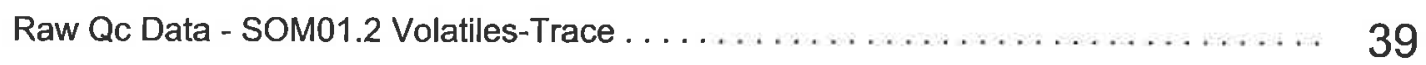

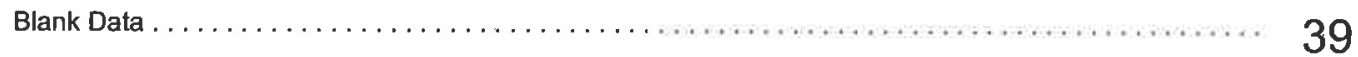




\title{
CASE NARRATIVE
}

\section{Client: Argonne National Laboratory}

\author{
Project: Inman (200-7006)
}

\author{
Report Number: 200-7006-1
}

\begin{abstract}
Enclosed is the data set for the referenced project work. With the exceptions noted as flags or footnotes, standard analytical protocols were followed in performing the analytical work and the applied control limits were met.
\end{abstract}

Calculations were performed before rounding to avoid round-off errors in calculated results.

All holding times were met and proper preservation noted for the methods performed on these samples, unless otherwise detailed in the individual sections below.

\section{Receipt}

The samples were received on 09/15/2011. Documentation of the condition of the samples at the time of their receipt and any exception to the laboratory's Sample Acceptance Policy is documented in the Shipping and Receiving section of this submittal. The samples, as received, were not acid preserved. On that basis, the laboratory did provide for the analytical work to be performed within seven days of sample collection.

\section{SOM01.2 Volatile Organics (Trace Level Water)}

A storage blank was prepared for volatile organics analysis, and stored in association with the storage of the samples. That storage blank, identified as VHBLK01, was carried through the holding period with the samples, and analyzed.

Each sample in the sample set was analyzed without a dilution. Each of the analyses associated with the sample set exhibited an acceptable internal standard performance. There was an acceptable recovery of each deuterated monitoring compound (DMC) in the analysis of the method blank associated with the analytical work, and in the analysis of the storage blank associated with the sample set. The analysis of the samples in this sample set did meet the technical acceptance criteria specific to DMC recoveries, although not all DMC recoveries were within the control range in each analysis. The technical acceptance criteria does provide for the recovery of up to three DMCs to fall outside of the control range in the analysis of field samples. Matrix spike and matrix spike duplicate analyses were not performed on samples in this sample set. A trace concentration of toluene was identified in the analysis of the method blank associated with the analytical work. The concentration of toluene in that analysis was below the established reporting limit, and the analysis did meet the technical acceptance criteria for a compliant method blank analysis. Trace concentrations of chloroform and toluene were identified in the analysis of the storage blank associated with the sample set. The concentration of each analyte in that analysis was below the established reporting limit, and the analysis did meet the technical acceptance criteria for a compliant storage blank analysis. Present in the method blank and storage blank analyses was a non-target constituent that represents a compound that is related to the DMC formulation. The fact that the presence of this compound is not within the laboratory's control is at issue. The derived results for that compound have been qualified with an " $X$ " qualifier to reflect the source of the contamination. 
The responses for each of the target analytes met the relative standard deviation criterion in the initial calibration. The response for each target analyte met the percent difference criterion in the opening/continuing calibration check acquisition. The response for each target analyte met the 50.0 percent difference criterion in the closing calibration check acquisition.

The primary quantitation mass for methylcyclohexane that is specified in the Statement of Work is mass 83 . The laboratory did identify a contribution to mass 83 from 1,2-dichloropropane- $d_{6}$, one of the deuterated monitoring compounds (DMCs). The laboratory did change the primary quantitation mass assignment to mass 55 for the quantification of methylcyclohexane.

Manual integration was employed in deriving certain of the analytical results. The values that have been derived from manual integration are qualified on the quantitation reports. Extracted ion current profiles for each manual integration are included in the data package, and further documented at the end of this submittal. 


\section{DATA REPORTING QUALIFIERS}

Client: Argonne National Laboratory

Job Number: $200-7006-1$

Sdg Number: 200-7006

Lab Section

Description

GC/MS VOA

$\mathrm{U}$

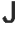

$J$

$X$

B

N
Analyzed for but not detected.

Indicates an Estimated Value for TICs

Indicates an estimated value.

See case narrative notes for explanation of the ' $X$ ' flag

Surrogate exceeds the control limit

The analyte was found in an associated blank, as well as in the sample.

This flag indicates the presumptive evidence of a compound. 


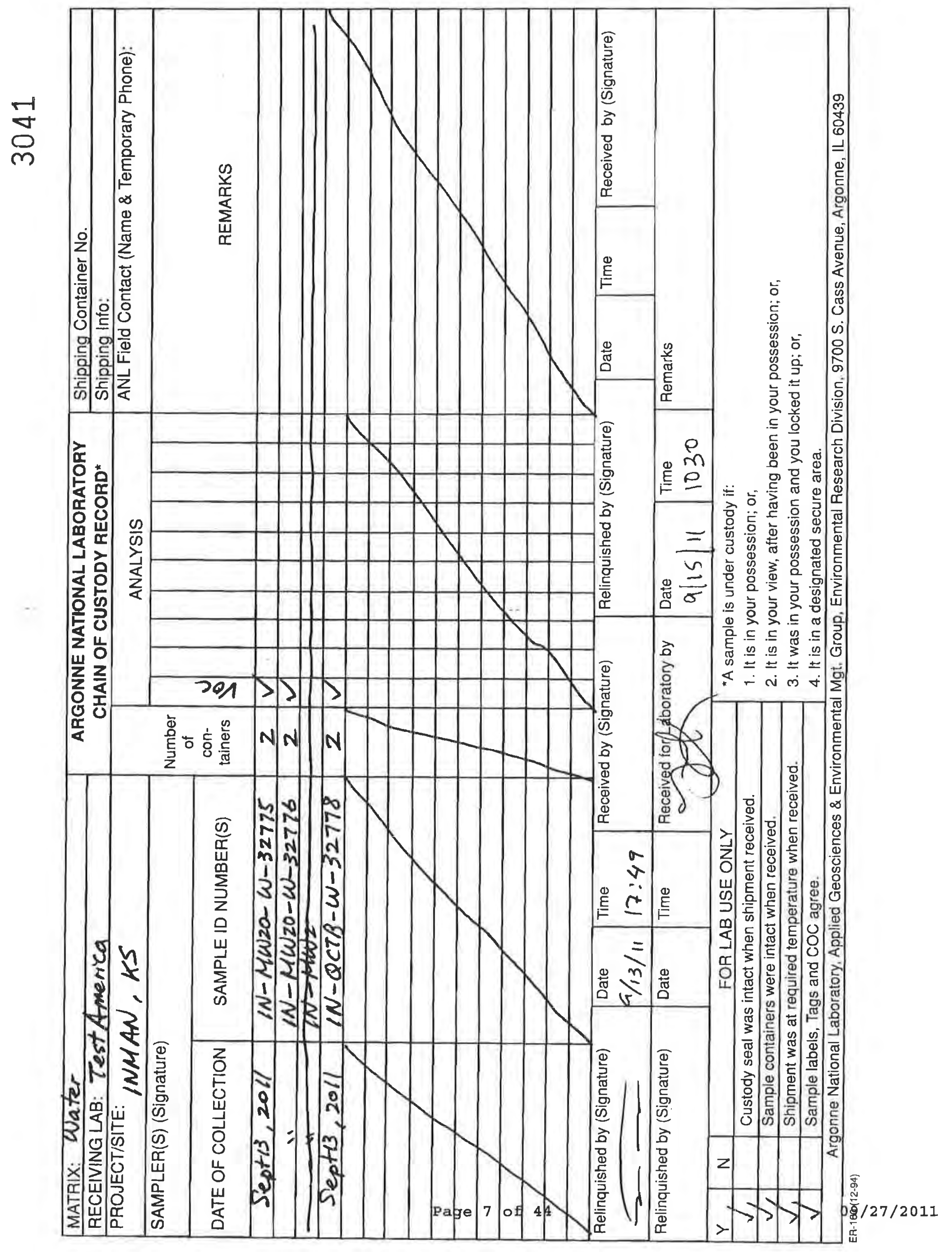




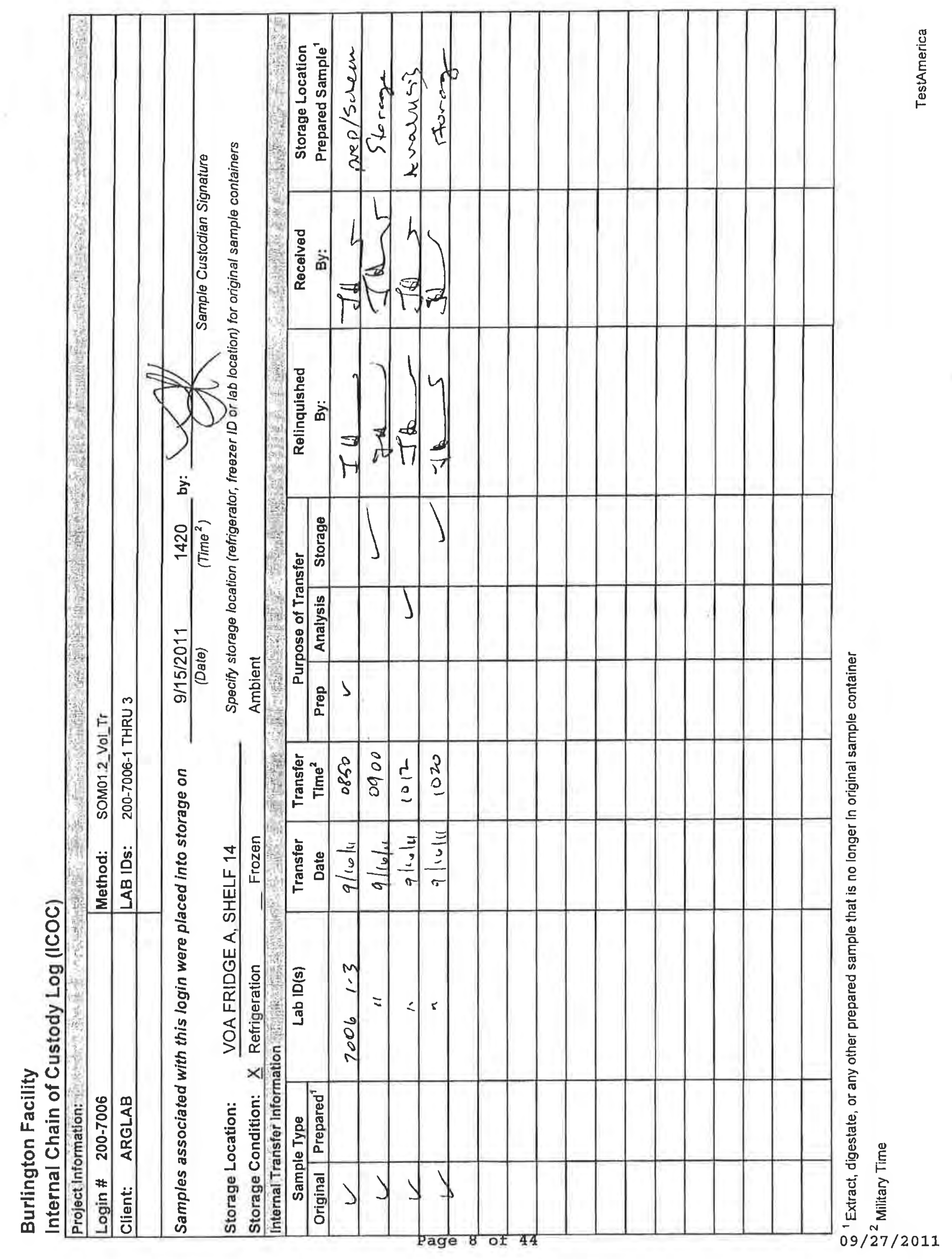




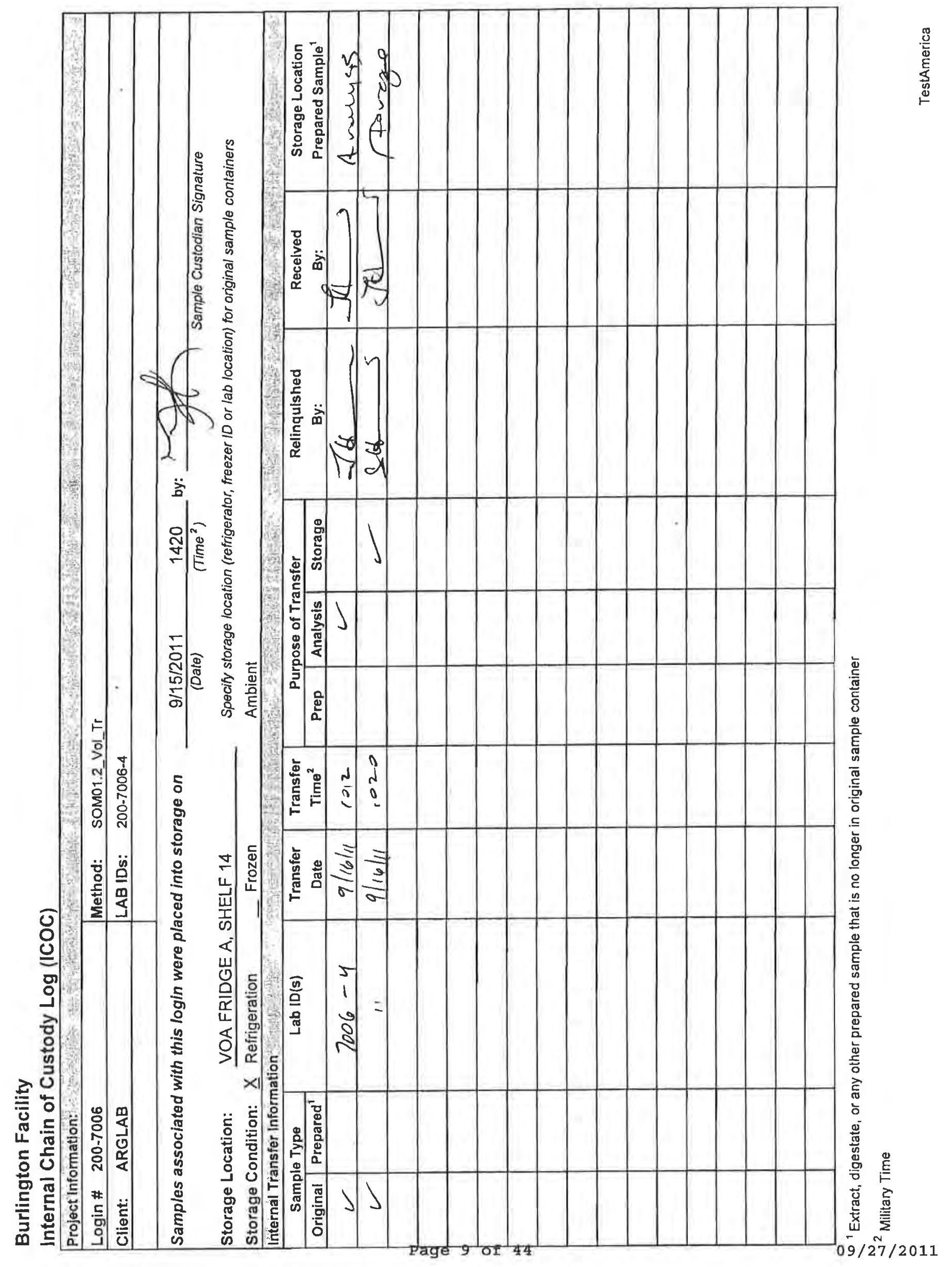


Shipping and

Receiving

Documents 


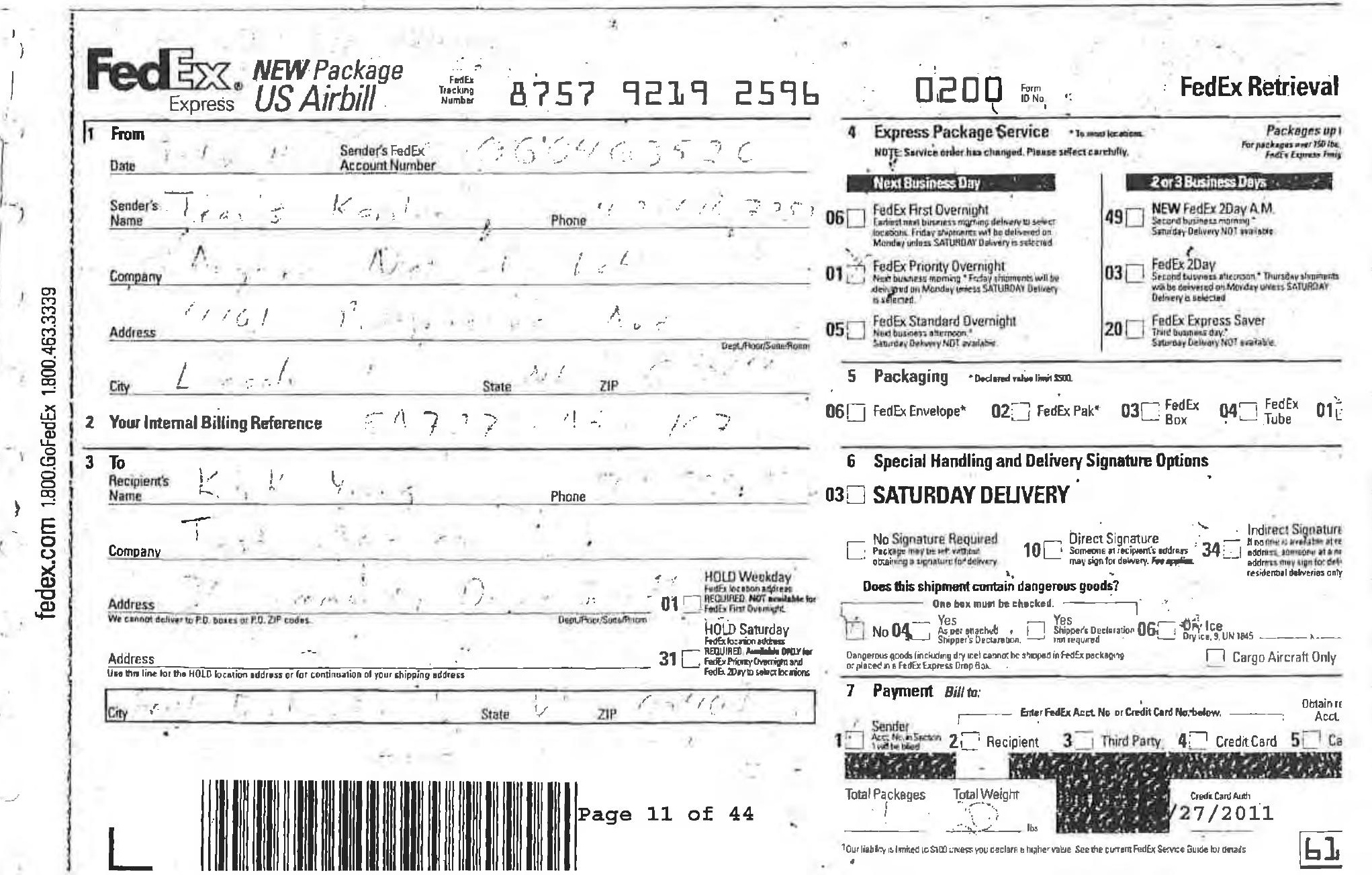




\section{Login Sample Receipt Checklist}

\section{Question}

Radioactivity either was not measured or, if measured, is at or below background

The cooler's custody seal, if present, is intact.

The cooler or samples do not appear to have been compromised or tampered with.

Samples were received on ice.

Cooler Temperature is acceptable.

Cooler Temperature is recorded.

$\mathrm{COC}$ is present.

$\mathrm{COC}$ is filled out in ink and legible.

$\mathrm{COC}$ is filled out with all pertinent information.

Is the Field Sampler's name present on COC?

There are no discrepancies between the sample IDs on the containers and the COC.

Samples are received within Holding Time.

Sample containers have legible labels.

Containers are not broken or leaking.

Sample collection date/times are provided.

Appropriate sample containers are used.

Sample bottles are completely filled.

Sample Preservation Verified.

There is sufficient vol. for all requested analyses, incl. any requested MS/MSDs

VOA sample vials do not have headspace or bubble is $<6 \mathrm{~mm}\left(1 / 4^{\prime \prime}\right)$ in diameter.

Multiphasic samples are not present.

Samples do not require splitting or compositing.

Residual Chlorine Checked.

Answer

N/A

True

True

True

True

True

True

True

False

N/A

True

True

True

True

True

True

True

N/A

True

True

True

True

N/A

\section{Comment}

Lab does not accept radioactive samples

NO CUSTODY SEAL NUMBERS

$3.0^{\circ} \mathrm{C}$, IR GUN ID $96, \mathrm{CF} 0$

No sample time on $\mathrm{COC}$

Not requested on $\mathrm{COC}$

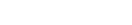

Sample volumes were unpreserved. 


\section{Sample Login Acknowledgement}

Job 200-7006-1

$\begin{array}{ll}\text { Client Job Description: } & \text { Inman (200-7006) } \\ \text { Purchase Order \#: } & 1 \mathrm{E}-30401 \\ \text { Work Order \#: } & 1 \mathrm{E}-30401 \\ \text { Project Manager: } & \text { Kirk F Young } \\ \text { Job Due Date: } & 9 / 29 / 2011 \\ \text { Job TAT: } & \text { 14 Days } \\ \text { Max Deliverable Level: } & \text { IV }\end{array}$

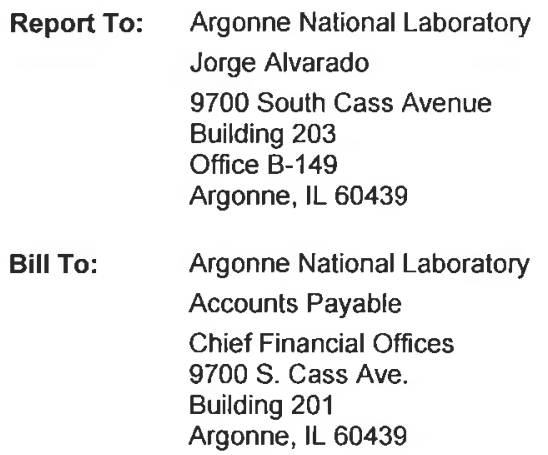

Login 200-7006

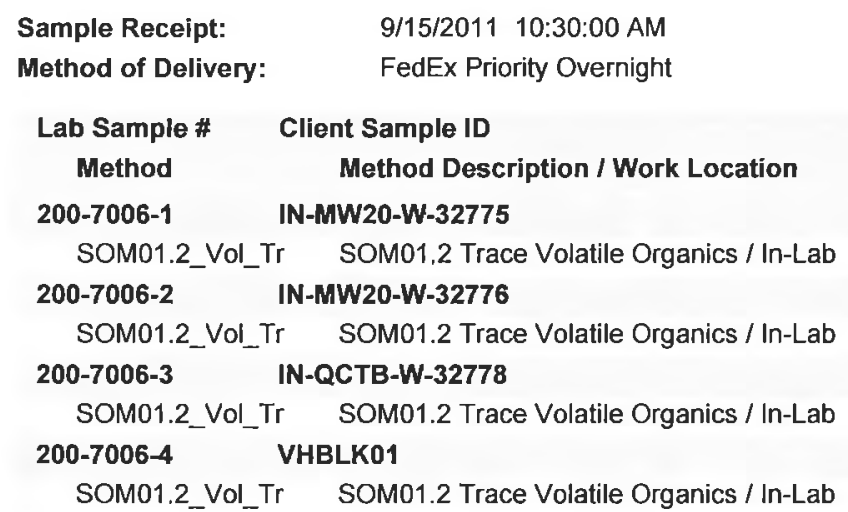

Number of Coolers: $\quad 1$

Cooler Temperature(s) $\left(\mathbf{C}^{\circ}\right): 3.0$;

\begin{tabular}{|c|c|c|c|}
\hline \multirow[t]{2}{*}{ Date Sampled } & \multirow[t]{2}{*}{ Matrix } & & \\
\hline & & Rpt Basis & Dry / Wet $\star \star$ \\
\hline 9/13/2011 12:00:00 AM & Water & & \\
\hline & & Total & Wet \\
\hline 9/13/2011 12:00:00 AM & Water & & \\
\hline & & Total & Wet \\
\hline 9/13/2011 12:00:00 AM & Water & & \\
\hline & & Total & Wet \\
\hline 9/15/2011 2:00:00 PM & Water & & \\
\hline & & Total & Wet \\
\hline
\end{tabular}




\section{METHODOLOGY SUMMARY}

\begin{tabular}{|c|c|c|}
\hline Laboratory: & TestAmerica Laboratories & Project No: \\
\hline Location: & South Burlington, Vermont & SDG No: \\
\hline
\end{tabular}

VOA

Volatile Organics Trace - USEPA CLP SOM01.2 
$2 A$ - FORM II VOA-1

WATER VOLATILE DEUTERATED MONITORING COMPOUND RECOVERY

Lab Name: TESTAMERICA BURLINGTON

Contract: $8 \mathrm{E}-00302$

Lab Code: STLV Case No.: INMAN Mod. Ref No.: SDG No.: 200-7006

Level: (TRACE or LOW) TRACE

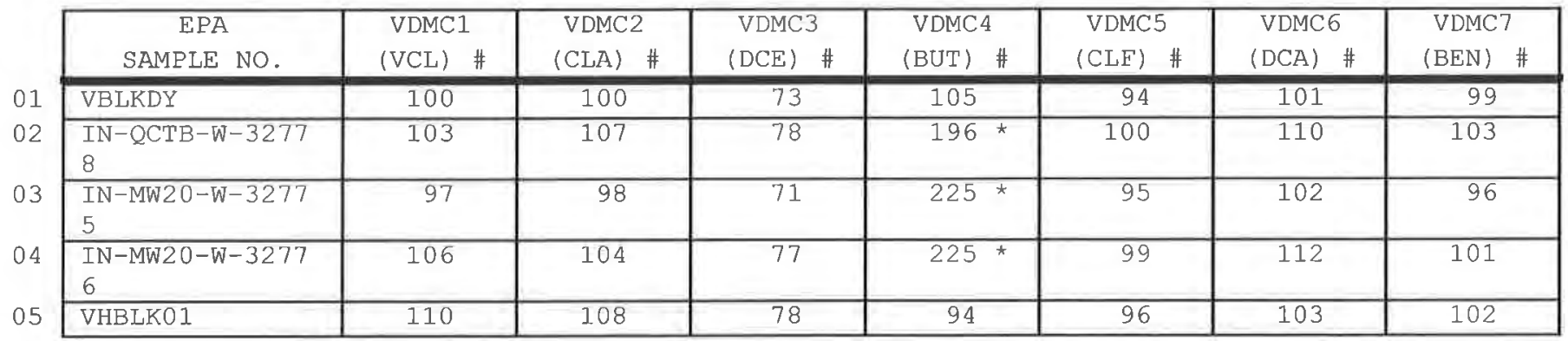

\footnotetext{
VDMC1 (VCL) = Vinyl Chloride-d3

VDMC2 $\quad(\mathrm{CLA})=$ Chloroethane $-\mathrm{d} 5$

VDMC3 $($ DCE $)=1,1$-Dichloroethene-d2

VDMC4 $\quad(B U T)=2$-Butanone-d5

VDMC5 $(\mathrm{CLF})=$ Chloroform-d

VDMC6 $($ DCA $)=1,2-$ Dichloroethane-d4

VDMC7 $(\mathrm{BEN})=$ Benzene-d6
}

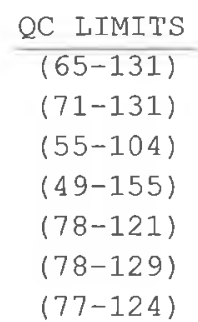

\# Column to be used to flag recovery values

* Values outside of contract required QC limits

Page 1 of 1
SOM01.2 (4/2007) 
2B - FORM II VOA-2

WATER VOLATILE DEUTERATED MONITORING COMPOUND RECOVERY

Lab Name: TESTAMERICA BURLINGTON

Contract: $8 \mathrm{E}-00302$

Lab Code: STLV Case No.: INMAN Mod. Ref No.:

SDG No.: 200-7006

Level: (TRACE or LOW) TRACE

\begin{tabular}{|c|c|c|c|c|c|c|c|c|}
\hline $\begin{array}{c}\text { EPA } \\
\text { SAMPLE NO. }\end{array}$ & $\begin{array}{l}\text { VDMC8 } \\
\text { (DPA) \# }\end{array}$ & $\begin{array}{l}\text { VDMC9 } \\
\text { (TOL) \# }\end{array}$ & $\begin{array}{l}\text { VDMC10 } \\
\text { (TDP) \# }\end{array}$ & $\begin{array}{l}\text { VDMCI1 } \\
(\operatorname{HEX}) \quad \#\end{array}$ & $\begin{array}{l}\text { VDMC12 } \\
\text { (TCA) \# }\end{array}$ & $\begin{array}{l}\text { VDMC13 } \\
\text { (DCZ) \# }\end{array}$ & OTHER & $\begin{array}{l}\text { TOT } \\
\text { OUT }\end{array}$ \\
\hline VBLKDY & 97 & 97 & 96 & 106 & 91 & 108 & & 0 \\
\hline $\begin{array}{l}\text { IN-QCTB-W-3277 } \\
8\end{array}$ & 103 & 102 & 98 & 204 * & 97 & 113 & & 2 \\
\hline $\begin{array}{l}\text { IN-MW20-W-3277 } \\
5\end{array}$ & 92 & 96 & 96 & 250 * & 97 & 99 & & 2 \\
\hline $\begin{array}{l}\text { IN-MW2 } 2-W-3277 \\
6\end{array}$ & 98 & 101 & 102 & 230 * & 103 & 101 & & 2 \\
\hline
\end{tabular}

VDMC8 $(D P A)=1,2-D i c h l o r o p r o p a n e-d 6$

VDMC9 (TOL) = Toluene-d8

VDMC10 $(\mathrm{TDP})=$ trans-1,3-Dichloropropene-d4

VDMC11 $(\mathrm{HEX})=2-$ Hexanone-d5

VDMC12 $(\mathrm{TCA})=1,1,2,2-\mathrm{Tet}$ rachloroethane-d2

VDMC13 $(\mathrm{DCZ})=1,2-$ Dichlorobenzene-d4
QC LIMITS

$(79-124)$

$(77-121)$

(73-121)

$(28-135)$

$(73-125)$

$(80-131)$

\# Column to be used to flag recovery values

* Values outside of contract required QC limits

Report 1,4-Dioxane-d8 for Low-Medium VOA analysis only

Page 1 of 1 
$4 A$ - FORM IV VOA

VOLATIIE METHOD BLANK SUMMARY
EPA SAMPLE NO.

VBLKDY
Lab Name: TESTAMERICA BURLINGTON

Lab Code: STLV Case No.: INMAN Mod. Ref No.:

Lab File ID: DHPAA03.D

Instrument ID: D.i

Matrix: (SOIL/SED/WATER)

Level: (TRACE or LOW/MED)

GC Column: DB-624

\begin{tabular}{c|l|l|l|c|}
\hline \multicolumn{1}{c|}{$\begin{array}{c}\text { EPA } \\
\text { SAMPLE NO. }\end{array}$} & \multicolumn{1}{c|}{$\begin{array}{c}\text { LAB } \\
\text { SAMPLE ID }\end{array}$} & $\begin{array}{c}\text { LAB } \\
\text { FILE ID }\end{array}$ & $\begin{array}{c}\text { TIME } \\
\text { ANALYZED }\end{array}$ \\
\cline { 2 - 5 } 01 & $\begin{array}{l}\text { IN-QCTB-W-32 } \\
778\end{array}$ & $200-7006-3$ & DHPAA06.D & 1146 \\
\cline { 2 - 5 } 02 & $\begin{array}{l}\text { IN-MW20-W-32 } \\
775\end{array}$ & $200-7006-1$ & DHPAA07.D & 1211 \\
\cline { 2 - 5 } 03 & $\begin{array}{l}\text { IN-MW20-W-32 } \\
776\end{array}$ & $200-7006-2$ & DHPAA08.D & 1235 \\
\cline { 2 - 5 } 04 & VHBLK01 & $200-7006-4$ & DHPAA09.D & 1333 \\
\hline
\end{tabular}

Contract: $8 \mathrm{E}-00302$

SDG No.: 200-7006

Lab Sample ID: MB 200-25361/3

Date Analyzed: 09/16/2011

Time Analyzed: 0956

Heated Purge: ( $\mathrm{Y} / \mathrm{N}) \mathrm{N}$ 
$5 A$ - FORM V VOA

VOLATILE ORGANICS INSTRUMENT

PEREORMANCE CHECK BROMOFLUOROBENZENE (BEB)
EPA SAMPLE NO.

BFBDV

Lab Name: TESTAMERICA BURLINGTON

Contract: $\quad 8 E-00302$

Lab Code: STLV Case No.: INMAN Mod. Ref No.: SDG No.: 200-7006

Lab File Id: DHP01.D

BFB Injection Date: $07 / 07 / 2011$

Instrument Id: D.i

BFB Injection Time: 1005

GC Column: DB-624

ID : 0.20 (mm)

\begin{tabular}{|r|l|r|}
\hline $\mathrm{m} / \mathrm{e}$ & ION ABUNDANCE CRITERIA & $\begin{array}{c}\text { RELATIVE } \\
\text { ABUNDANCE }\end{array}$ \\
\hline 50 & $15.0-40.0 \%$ of mass 95 & 26.1 \\
\hline 75 & $30.0-80.0 \%$ of mass 95 & 46.4 \\
\hline 95 & Base peak, 100\% relative abundance & 100 \\
\hline 96 & $5.0-9.0 \%$ of mass 95 & 6.3 \\
\hline 173 & Less than $2.0 \%$ of mass 174 & 0 \\
\hline 174 & $50.0-120 \%$ of mass 95 & $0) 1$ \\
\hline 175 & $5.0-9.0 \%$ of mass 174 & 77.7 \\
\hline 176 & $95.0-101 \%$ of mass 174 & 6.0 \\
\hline 177 & $5.0-9.0 \%$ of mass 176 & $7.8) 1$ \\
\hline
\end{tabular}

1 - Value is omass 174

2 - Value is omass 176

\begin{tabular}{|c|c|c|c|c|}
\hline $\begin{array}{c}\text { EPA } \\
\text { SAMPLE NO. }\end{array}$ & $\begin{array}{r}\text { LAB } \\
\text { SAMPLE ID }\end{array}$ & $\begin{array}{c}\text { LAB } \\
\text { FIIE ID }\end{array}$ & $\begin{array}{c}\text { DATE } \\
\text { ANALYZED }\end{array}$ & $\begin{array}{c}\text { TIME } \\
\text { ANALYZED }\end{array}$ \\
\hline VSTDO.5DV & IC $200-21023 / 4$ & $\mathrm{DHPO} 4 . \mathrm{D}$ & $07 / 07 / 2011$ & 1117 \\
\hline VSTD001DV & IC $200-21023 / 5$ & DHP05.D & $07 / 07 / 2011$ & 1141 \\
\hline VSTD005DV & ICIS $200-21023 / 6$ & DHP06.D & $07 / 07 / 2011$ & 1206 \\
\hline VSTD010DV & IC $200-21023 / 7$ & DHP07.D & $07 / 07 / 2011$ & 1231 \\
\hline VSTD020DV & IC $200-21023 / 8$ & DHP08.D & $07 / 07 / 2011$ & 1256 \\
\hline
\end{tabular}


$5 A$ - FORM V VOA

VOLATILE ORGANICS INSTRUMENT

PERFORMANCE CHECK

BROMOFLUOROBENZENE (BFB)
EPA SAMPLE NO.

BEBDY
Lab Name: TESTAMERICA BURLINGTON

Lab Code: STLV

Case No.: INMAN

Lab File Id: DHPAA01.D

Instrument Id: D.i

GC Column: DB-624

\begin{tabular}{|l|l}
\hline $\mathrm{m} / \mathrm{e}$ & ION ABUNDANCE CRITERIA
\end{tabular}

\begin{tabular}{|r|l|r|}
\hline \hline 50 & $15.0-40.0 \%$ of mass 95 & 26.2 \\
\hline 75 & $30.0-80.0 \%$ of mass 95 & 45.6 \\
\hline 95 & Base peak, 100\% relative abundance & 100 \\
\hline 96 & $5.0-9.0 \%$ of mass 95 & 6.7 \\
\hline 173 & Less than 2.0\% of mass 174 & 0 \\
\hline 174 & $50.0-120 \%$ of mass 95 & $0) 1$ \\
\hline 175 & $5.0-9.0 \%$ of mass 174 & 8.5 \\
\hline 176 & $95.0-101 \%$ of mass 174 & $7.8) 1$ \\
\hline 177 & $5.0-9.0 \%$ of mass 176 & $96.3) 1$ \\
\hline
\end{tabular}

1 - Value is omass 174

2 - Value is omass 176

\begin{tabular}{|c|c|c|c|c|}
\hline $\begin{array}{c}\text { EPA } \\
\text { SAMPLE NO. }\end{array}$ & $\begin{array}{r}\text { LAB } \\
\text { SAMPLE } \text { ID }\end{array}$ & $\begin{array}{c}\text { LAB } \\
\text { FILE ID }\end{array}$ & $\begin{array}{c}\text { DATE } \\
\text { ANALYZED }\end{array}$ & $\begin{array}{c}\text { TIME } \\
\text { ANALYZED }\end{array}$ \\
\hline VSTD005DY & CCVIS $200-25361 / 2$ & DHPAAO2.D & $09 / 16 / 2011$ & 0932 \\
\hline VBLKDY & MB $200-25361 / 3$ & DHPAAO $3 . \mathrm{D}$ & $09 / 16 / 2011$ & 0956 \\
\hline $\begin{array}{l}\text { IN-QCTB-W- } \\
32778\end{array}$ & $200-7006-3$ & DHPAAO $6 . \mathrm{D}$ & $09 / 16 / 2011$ & 1146 \\
\hline $\begin{array}{l}\text { IN-MW20-W- } \\
32775\end{array}$ & $200-7006-1$ & DHPAAO7.D & $09 / 16 / 2011$ & 1211 \\
\hline $\begin{array}{l}\text { IN-MW20-W- } \\
32776\end{array}$ & $200-7006-2$ & DHPAA08.D & $09 / 16 / 2011$ & 1235 \\
\hline VHBLKOI & $200-7006-4$ & DHPAAO9.D & $09 / 16 / 2011$ & 1333 \\
\hline VSTD005YD & CCVC $200-25361 / 10$ & DHPAA10.D & $09 / 16 / 2011$ & 1357 \\
\hline
\end{tabular}

BEB Injection Date: 09/16/2011

BFB Injection Time: 0916 (mm) 
Lab Name: TESTAMERICA BURLINGTON

Lab Code: STLV Case No.: INMAN Mod. Ref No.:
GC Column: DB-624

EPA Sample No.(VSTD\#\#\#\#):

Lab File ID (Standard)

Instrument ID: D.i
Contract: $8 \mathrm{E}-00302$

SDG No.: 200-7006

ID: 0.20 (mm) Init, Calib. Date(s): 07/07/2011 07/07/2011

Date Analyzed: 09/16/2011

Time Analyzed: 0932

Heated Purge: ( $\mathrm{Y} / \mathrm{N}) \mathrm{N}$

\begin{tabular}{|c|c|c|c|c|c|c|}
\hline & $\begin{array}{c}\text { IS1 (CBZ) } \\
\text { AREA }\end{array}$ & RT \# & $\begin{array}{c}\text { IS2 (DFB) } \\
\text { AREA }\end{array}$ & RT \# & $\begin{array}{c}\text { IS3 (DCB) } \\
\text { AREA }\end{array}$ & RT \\
\hline 12 HOUR STD & 143170 & 8.83 & 170167 & 5.47 & 69004 & 11.66 \\
\hline UPPER LIMIT & 200438 & 9.16 & 238234 & 5.80 & 96606 & 11.99 \\
\hline LOWER LIMIT & 85902 & 8.50 & 102100 & 5.14 & 41402 & 11.33 \\
\hline EPA SAMPLE NO. & & & & & & \\
\hline$\overline{\text { VBLKDY }}$ & 142021 & 8.83 & 168894 & 5.47 & $\overline{56972}$ & 11.66 \\
\hline $\begin{array}{l}\text { IN-QCTB-W-3277 } \\
8\end{array}$ & 121416 & 8.83 & 141313 & 5.47 & 45805 & 11.66 \\
\hline $\begin{array}{l}\text { IN-MW20-W-3277 } \\
5\end{array}$ & 148833 & 8.83 & 171271 & 5.47 & 69832 & 11.66 \\
\hline $\begin{array}{l}\text { IN-MW20-W-3277 } \\
6\end{array}$ & 152131 & 8.83 & 172916 & 5.47 & 73053 & 11.66 \\
\hline VHBLK01 & 121937 & 8.83 & 146186 & 5.47 & 46138 & 11.66 \\
\hline
\end{tabular}

IS1 $(\mathrm{CBZ})=$ Chlorobenzene-d5

IS2 $(\mathrm{DFB})=1,4$-Difluorobenzene

IS $3(\mathrm{DCB})=1,4$-Dichlorobenzene-d4

AREA UPPER LIMIT $=140 \%$ (Trace Volatiles) of internal standard area

AREA LOWER LIMIT $=60 \%$ (Trace Volatiles) of internal standard area

RT UPPER LIMIT $=+0.33$ (Trace Volatiles) minutes of internal standard RT

RT LOWER LIMIT $=-0.33$ (Trace Volatiles) minutes of internal standard RT

\# Colurnn used to flag values outside contract required QC limits with an asterisk. 
Lab Name: TESTAMERICA BURLINGTON

Lab Code: S'TLV Case No.: INMAN

Matrix: (SOIL/SED/WATER)

Water

Sample wt/vol: 25.0

$(\mathrm{g} / \mathrm{mL}) \mathrm{mL}$

Level: (TRACE/LOW/MED) TRACE

o Moisture: not dec.

GC Column: DB-624 ID $: 0.20 \quad(\mathrm{~mm})$

Soil Extract Volume: (uL) (mL)

Purge Volume: 25.0
Contract : $\quad 8 \mathrm{E}-00302$

SDG No.: 200-7006

Mod. Ref No.:

Lab Sample ID: 200-7006-1

Lab File ID: DHPAA07.D

Date Received: 09/15/2011

Date Analyzed: 09/16/2011

Dilution Eactor: 1.0

Soil Aliquot Volume:

(uL)

\begin{tabular}{|c|c|c|c|}
\hline CAS NO. & COMPOUND & $\begin{array}{l}\text { CONCENTRATION UNITS: } \\
(\mathrm{ug} / \mathrm{L} \text { or } \mathrm{ug} / \mathrm{kg}) \mathrm{ug} / \mathrm{L}\end{array}$ & $Q$ \\
\hline $75-71-8$ & Dichlorodifluoromethane & 0.50 & $\mathrm{U}$ \\
\hline $74-87-3$ & Chloromethane & 0.50 & $\mathrm{U}$ \\
\hline $75-01-4$ & Vinyl chloride & 0.50 & $\mathrm{U}$ \\
\hline $74-83-9$ & Bromomethane & 0.50 & $\mathrm{U}$ \\
\hline $75-00-3$ & Chloroethane & 0.50 & $\mathrm{U}$ \\
\hline $75-69-4$ & Trichlorofluoromethane & 0.50 & $\mathrm{U}$ \\
\hline $75-35-4$ & 1,1-Dichloroethene & 0.50 & $\mathrm{U}$ \\
\hline $76-13-1$ & 1,1,2-Trichloro-1,2,2-trifluoroethane & 0.50 & $\mathrm{U}$ \\
\hline $67-64-1$ & Acetone & 4.6 & $\mathrm{~J}$ \\
\hline $75-15-0$ & Carbon disulfide & 0.50 & $\mathrm{U}$ \\
\hline $79-20-9$ & Methyl acetate & 0.50 & $\mathrm{U}$ \\
\hline $75-09-2$ & Methylene Chloride & 0.50 & $\mathrm{U}$ \\
\hline $156-60-5$ & trans-1,2-Dichloroethene & 0.50 & $\mathrm{U}$ \\
\hline $1634-04-4$ & Methyl tert-butyl ether & 0.50 & $\mathrm{U}$ \\
\hline $75-34-3$ & 1,1-Dichloroethane & 0.50 & $\mathrm{U}$ \\
\hline $156-59-2$ & cis-1,2-Dichloroethene & 0.50 & $\mathrm{U}$ \\
\hline $78-93-3$ & 2-Butanone & 5.0 & $\mathrm{U}$ \\
\hline $74-97-5$ & Bromochloromethane & 0.50 & $\mathrm{U}$ \\
\hline $67-66-3$ & Chloroform & 0.040 & $\mathrm{~J}$ \\
\hline $71-55-6$ & $1,1,1$-Trichloroethane & 0.50 & $\mathrm{U}$ \\
\hline $110-82-7$ & Cyclohexane & 0.50 & $\mathrm{U}$ \\
\hline $56-23-5$ & Carbon tetrachloride & 0.50 & $\mathrm{U}$ \\
\hline $71-43-2$ & Benzene & 0.024 & $\mathrm{~J}$ \\
\hline $107-06-2$ & 1,2-Dichloroethane & 0.50 & $\mathrm{U}$ \\
\hline
\end{tabular}

Report 1,4-Dioxane for Low-Mediun VOA analysis only 
$1 B$ - FORM I VOA-2

VOLATILE ORGANICS ANALYSIS DATA SHEET
EPA SAMPLE NO.

IN-MW20-W-32775
Lab Name: TESTAMERICA BURLINGTON

Lab Code: STLV Case No.: INMA

Matrix: (SOIL/SED/WATER)

Sample wt/vol: 25.0

Water

$(\mathrm{g} / \mathrm{mL}) \quad \mathrm{mL}$

Level: (TRACE/LOW/MED) TRACE

응 Moisture: not dec.

GC Column: DB-624

ID: 0.20 (mm)

Soil Extract Volume:

(uL)

(mL)

Purge Volume: 25.0
Contract: $8 \mathrm{E}-00302$

SDG No.: 200-7006

:

Lab Sample ID: 200-7006-1

Lab File ID: DHPAA07.D

Date Received: 09/15/2011

Date Analyzed: 09/16/2011

Dilution Factor: 1.0

Soil Aliquot Volume:

( $L L$ L)

\begin{tabular}{|c|c|c|c|}
\hline CAS NO. & COMPOUND & $\begin{array}{l}\text { CONCENTRATION UNITS: } \\
(\mathrm{ug} / \mathrm{L} \text { or } \mathrm{ug} / \mathrm{kg}) \mathrm{ug} / \mathrm{L}\end{array}$ & $Q$ \\
\hline $79-01-6$ & Trichloroethene & 0.50 & $\mathrm{U}$ \\
\hline $108-87-2$ & Methylcyclohexane & 0.50 & $\mathrm{U}$ \\
\hline $78-87-5$ & 1,2-Dichloropropane & 0.50 & $\mathrm{U}$ \\
\hline $75-27-4$ & Bromodichloromethane & 0.50 & $\mathrm{U}$ \\
\hline $10061-01-5$ & cis-1,3-Dichloropropene & 0.50 & $\mathrm{U}$ \\
\hline $108-10-1$ & 4-Methyl-2-pentanone & 5.0 & $\mathrm{U}$ \\
\hline $108-88-3$ & Toluene & 0.18 & $\mathrm{~J} \mathrm{~B}$ \\
\hline $10061-02-6$ & trans $-1,3$-Dichloropropene & 0.50 & $\mathrm{U}$ \\
\hline $79-00-5$ & 1,1,2-Trichloroethane & 0.50 & $\mathrm{U}$ \\
\hline $127-18-4$ & Tetrachloroethene & 0.50 & $\mathrm{U}$ \\
\hline $591-78-6$ & 2-Hexanone & 5.0 & $\mathrm{U}$ \\
\hline $124-48-1$ & Dibromochloromethane & 0.50 & $\mathrm{U}$ \\
\hline $106-93-4$ & 1,2-Dibromoethane & 0.50 & $\mathrm{U}$ \\
\hline $108-90-7$ & Chlorobenzene & 0.50 & $\mathrm{U}$ \\
\hline $100-41-4$ & Ethylbenzene & 0.50 & $\mathrm{U}$ \\
\hline $95-47-6$ & o-Xylene & 0.50 & $\mathrm{U}$ \\
\hline $179601-23-1$ & $\mathrm{~m}, \mathrm{p}$-Xylene & 0.50 & $\mathrm{U}$ \\
\hline $100-42-5$ & styrene & 0.50 & $\mathrm{U}$ \\
\hline $75-25-2$ & Bromoform & 0.50 & $\mathrm{U}$ \\
\hline $98-82-8$ & Isopropylbenzene & 0.50 & $\mathrm{U}$ \\
\hline $79-34-5$ & $1,1,2,2$-Tetrachloroethane & 0.50 & $\mathrm{U}$ \\
\hline $541-73-1$ & 1,3-Dichlorobenzene & 0.50 & $\mathrm{U}$ \\
\hline $106-46-7$ & 1,4-Dichlorobenzene & 0.50 & $\mathrm{U}$ \\
\hline $95-50-1$ & 1,2-Dichlorobenzene & 0.50 & $\mathrm{U}$ \\
\hline $96-12-8$ & 1,2-Dibromo-3-Chloropropane & 0.50 & $\mathrm{U}$ \\
\hline $120-82-1$ & 1,2,4-Trichlorobenzene & 0.50 & $\mathrm{U}$ \\
\hline $87-61-6$ & $1,2,3$-Trichlorobenzene & 0.50 & $\mathrm{U}$ \\
\hline
\end{tabular}


$1 \mathrm{~J}$ - FORM I VOA-TIC

VOLATILE ORGANICS ANALYSIS DATA SHEET TENTATIVELY IDENTIFIED COMPOUNDS
EPA SAMPLE NO.

IN-MW20-W-32775

Lab Name: TESTAMERICA BURLINGTON Case No.: INMAN Mod. Ref No.: Case No.:

Matrix: (SOIL/SED/WATER)

Sample wt/vol: 25.0

Level: (TRACE or LOW/MED)

$(\mathrm{g} / \mathrm{mL}) \quad \mathrm{mL}$

TRACE

o Moisture: not dec.

GC Column: DB-624

Soil Extract Volume: ID: 0.20 (mun)

(uL)

CONCENTRATION UNITS: (ug/L or $\mathrm{ug} / \mathrm{kg}$ ) ug/L
Contract: $8 E-00302$

SDG No.: 200-7006

Lab Sample ID: 200-7006-1

Lab File ID: DHPAA07.D

Date Received: 09/15/2011

Date Analyzed: 09/16/2011

Dilution Factor: 1.0

Soil Aliquot Volume:

(uL)

Purge Volume: 25.0

(mL)

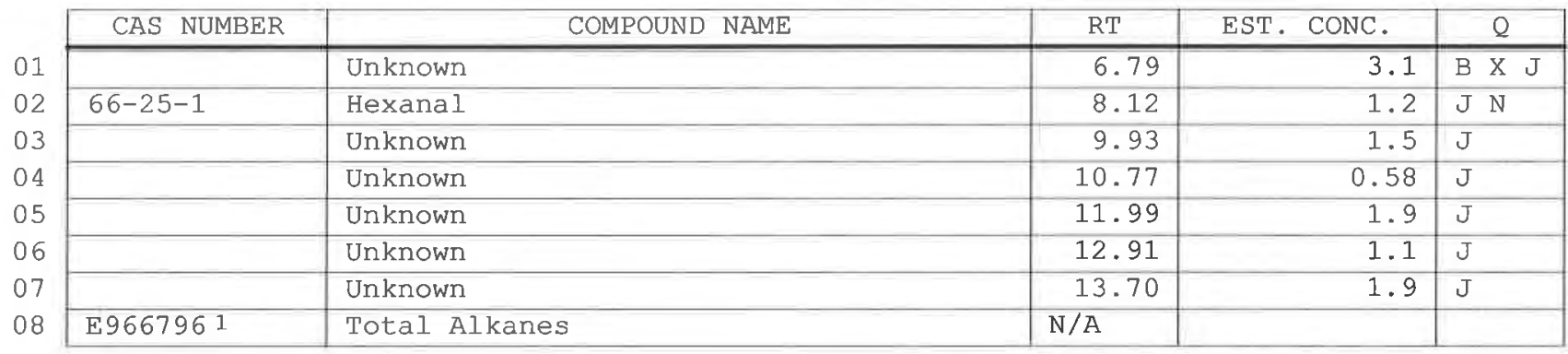

1 EPA-designated Registry Number. 
$1 A$ - FORM I VOA-1

VOLATILE ORGANICS ANALYSIS DATA SHEET
EPA SAMPLE NO.

IN-MW20-W-32776
Lab Name: TESTAMERICA BURLINGTON

Lab Code: STLV Case

Matrix: (SOIL/SED/WATER)

Sample wt/vol: 25.0

water

$(\mathrm{g} / \mathrm{mL}) \mathrm{mL}$

Level: (TRACE/LOW/MED) TRACE

\% Moisture: not dec.

GC Column: DB-624 ID: $0.20 \quad$ (mm)

Soil Extract Volume:

(uL)

(mL)

Purge Volume: 25.0
Contract: $8 \mathrm{E}-00302$

SDG No.: 200-7006

\begin{tabular}{|c|c|c|c|}
\hline CAS NO. & COMPOUND & $\begin{array}{l}\text { CONCENTRATION UNITS: } \\
(\mathrm{ug} / \mathrm{L} \text { or } \mathrm{ug} / \mathrm{kg}) \mathrm{ug} / \mathrm{L}\end{array}$ & $Q$ \\
\hline $75-71-8$ & Dichlorodifluoromethane & 0.50 & $\overline{\mathrm{U}}$ \\
\hline $74-87-3$ & Chloromethane & 0.50 & $\mathrm{U}$ \\
\hline $75-01-4$ & Vinyl chloride & 0.50 & $\mathrm{U}$ \\
\hline $74-83-9$ & Bromomethane & 0.50 & $\mathrm{U}$ \\
\hline $75-00-3$ & Chloroethane & 0.50 & $\mathrm{U}$ \\
\hline $75-69-4$ & Trichlorofluoromethane & 0.50 & $\mathrm{U}$ \\
\hline $75-35-4$ & 1,1-Dichloroethene & 0.50 & $\mathrm{U}$ \\
\hline $76-13-1$ & 1,1,2-Trichloro-1,2,2-trifluoroethane & 0.50 & $\mathrm{U}$ \\
\hline $67-64-1$ & Acetone & 4.7 & $\mathrm{~J}$ \\
\hline $75-15-0$ & Carbon disulfide & 0.50 & $\mathrm{U}$ \\
\hline $79-20-9$ & Methyl acetate & 0.50 & $\mathrm{U}$ \\
\hline $75-09-2$ & Methylene Chloride & 0.50 & $\mathrm{U}$ \\
\hline $156-60-5$ & trans-1,2-Dichloroethene & 0.50 & $\mathrm{U}$ \\
\hline $1634-04-4$ & Methyl tert-butyl ether & 0.50 & $\mathrm{U}$ \\
\hline $75-34-3$ & 1,1-Dichloroethane & 0.50 & $\mathrm{U}$ \\
\hline $156-59-2$ & cis-1,2-Dichloroethene & 0.50 & $\mathrm{U}$ \\
\hline $78-93-3$ & 2-Butanone & 2.1 & $\mathrm{~J}$ \\
\hline $74-97-5$ & Bromochloromethane & 0.50 & $\mathrm{U}$ \\
\hline $67-66-3$ & Chloroform & 0.032 & $\mathrm{~J}$ \\
\hline $71-55-6$ & 1,1,1-Trichloroethane & 0.50 & $\mathrm{U}$ \\
\hline $110-82-7$ & Cyclohexane & 0.50 & $\mathrm{U}$ \\
\hline $56-23-5$ & Carbon tetrachloride & 0.50 & $\mathrm{U}$ \\
\hline $71-43-2$ & Benzene & 0.16 & $\mathrm{~J}$ \\
\hline $107-06-2$ & 1,2-Dichloroethane & 0.50 & $\mathrm{U}$ \\
\hline
\end{tabular}

Report 1,4-Dioxane for Low-Medium VOA analysis only

Lab Sample ID: 200-7006-2

Lab File ID: DHPAA08.D

Date Received: 09/15/2011

Date Analyzed: 09/16/2011

Dilution Eactor: 1.0

Soil Aliquot Volume: 
$1 B$ - FORM I VOA-2

VOLATILE ORGANICS ANALYSIS DATA SHEET
EPA SAMPLE NO.

IN-MW20-W-32776
Lab Name: TESTAMERICA BURLINGTON

Lab Code: STLV Case No.: INMAN Mod Mod. Ref No.:

Contract: $8 \mathrm{E}-00302$

Matrix: (SOIL/SED/WATER)

Water

Sample wt/vol: 25.0

$(\mathrm{g} / \mathrm{mL}) \quad \mathrm{mL}$

Level: (TRACE/LOW/MED) TRACE

\% Moisture: not dec.

GC Column: DB-624 ID: 0.20 (mm)

Soil Extract Volume:

(uL)

$(\mathrm{mL})$

Purge Volume: 25.0
SDG NO.: 200-7006

\begin{tabular}{|c|c|c|c|}
\hline CAS NO. & COMPOUND & $\begin{array}{l}\text { CONCENTRATION UNITS: } \\
(\mathrm{ug} / \mathrm{L} \text { or } \mathrm{ug} / \mathrm{kg} \text { ) ug/L }\end{array}$ & $Q$ \\
\hline $79-01-6$ & Trichloroethene & 0.50 & $\overline{\mathrm{U}}$ \\
\hline $108-87-2$ & Methylcyclohexane & 0.50 & $\mathrm{U}$ \\
\hline $78-87-5$ & 1,2-Dichloropropane & 0.50 & $\mathrm{U}$ \\
\hline $75-27-4$ & Bromodichloromethane & 0.50 & $\mathrm{U}$ \\
\hline $10061-01-5$ & cis-1,3-Dichloropropene & 0.50 & $\mathrm{U}$ \\
\hline $108-10-1$ & 4-Methyl-2-pentanone & 5.0 & $\mathrm{U}$ \\
\hline $108-88-3$ & Toluene & 0.14 & $\mathrm{JB}$ \\
\hline $10061-02-6$ & trans-1,3-Dichloropropene & 0.50 & $\mathrm{U}$ \\
\hline $79-00-5$ & 1,1,2-Trichloroethane & 0.50 & $\mathrm{U}$ \\
\hline $127-18-4$ & Tetrachloroethene & 0.50 & $\mathrm{U}$ \\
\hline $591-78-6$ & 2-Hexanone & 5.0 & $\mathrm{U}$ \\
\hline $124-48-1$ & Dibromochloromethane & 0.50 & $\mathrm{U}$ \\
\hline $106-93-4$ & 1,2-Dibromoethane & 0.50 & U \\
\hline $108-90-7$ & Chlorobenzene & 0.50 & U \\
\hline $100-41-4$ & Ethylbenzene & 0.50 & $\mathrm{U}$ \\
\hline $95-47-6$ & o-Xylene & 0.50 & $\mathrm{U}$ \\
\hline $179601-23-1$ & m,p-xylene & 0.017 & $\mathrm{~J}$ \\
\hline $100-42-5$ & Styrene & 0.50 & $\mathrm{U}$ \\
\hline $75-25-2$ & Bromoform & 0.50 & $\mathrm{U}$ \\
\hline $98-82-8$ & Isopropylbenzene & 0.50 & $\mathrm{U}$ \\
\hline $79-34-5$ & $1,1,2,2$-Tetrachloroethane & 0.50 & $\mathrm{~J}$ \\
\hline $541-73-1$ & 1,3-Dichlorobenzene & 0.50 & $\mathrm{U}$ \\
\hline $106-46-7$ & 1,4-Dichlorobenzene & 0.50 & $\mathrm{U}$ \\
\hline $95-50-1$ & 1,2-Dichlorobenzene & 0.50 & $\mathrm{U}$ \\
\hline $96-12-8$ & 1,2-Dibromo-3-Chloropropane & 0.50 & $\mathrm{U}$ \\
\hline $120-82-1$ & 1,2,4-Trichlorobenzene & 0.50 & $\mathrm{U}$ \\
\hline $87-61-6$ & 1,2,3-Trichlorobenzene & 0.50 & $\mathrm{U}$ \\
\hline
\end{tabular}


IJ - FORM I VOA-TIC

VOLATILE ORGANICS ANALYSIS DATA SHEET TENTATIVELY IDENTIEIED COMPOUNDS
EPA SAMPLE NO.

IN-MW20-W-32776
Lab Name: TESTAMERICA BURLINGTON

Contract: $8 \mathrm{E}-00302$

Lab Code: STLV Case No.: INMAN Mod. Ref No.: SDG No.: 200-7006

Matrix: (SOIL/SED/WATER) Water

Sample wt/vol: 25.0

$(\mathrm{g} / \mathrm{mL}) \quad \mathrm{mL}$

Level: (TRACE or LOW/MED)

TRACE

\% Moisture: not dec.

GC Column: DB-624 ID: $0.20 \quad$ (mm)

Soil Extract Volume:

(uL)

CONCENTRATION UNITS: (ug/L or $\mathrm{ug} / \mathrm{kg}$ ) ug/L
Lab Sample ID: 200-7006-2

Lab File ID: DHPAA08.D

Date Received: 09/15/2011

Date Analyzed: 09/16/2011

Dilution Factor: 1.0

Soil Aliquot Volume:

(UL)

Purge Volume: 25.0

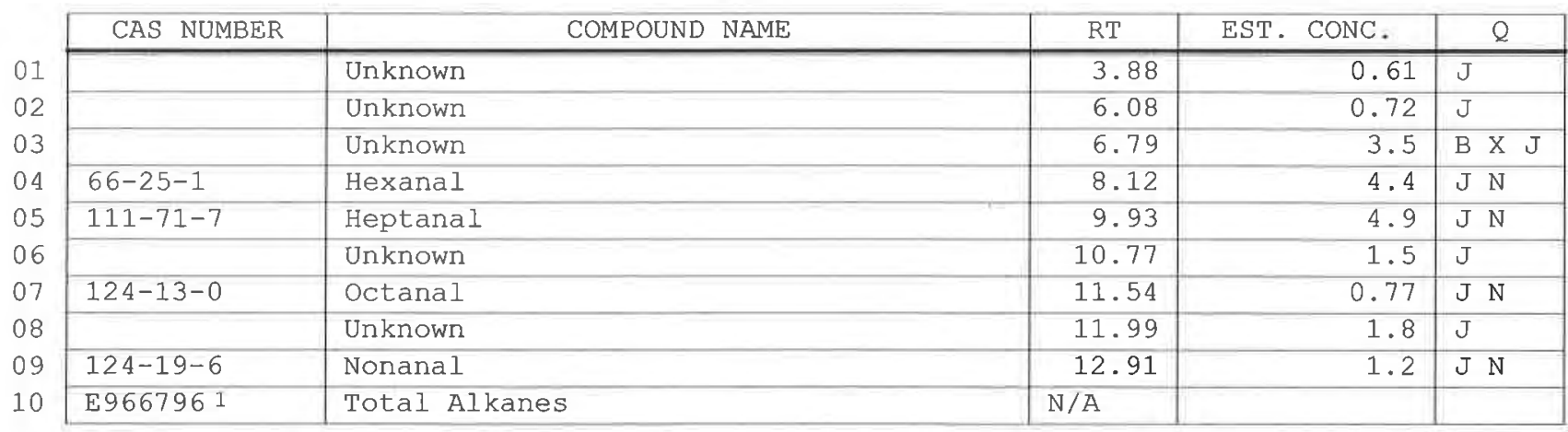

1 EPA-designated Registry Number. 
Lab Name: TESTAMERICA BURLINGTON

Lab Code: STLV Case No.: INMA

Matrix: (SOIL/SED/WATER)

Water

Sample wt/vol: 25.0

$(\mathrm{g} / \mathrm{mL}) \quad \mathrm{mL}$

Level: (TRACE/LOW/MED) TRACE

응 Moisture: not dec.

GC Column: DB-624

ID: 0.20 (mm)

Soil Extract Volume:

(uL) (mL)

Purge Volume: 25.0
Contract: 8E-00302

SDG No.: 200-7006 Mod, Ref No.:

Lab Sample ID: 200-7006-3

Lab File ID: DHPAA06.D

Date Received: 09/15/2011

Date Analyzed: 09/16/2011

Dilution Factor: 1.0

Soil Aliquot Volume:

(uL)

\begin{tabular}{|c|c|c|c|}
\hline CAS NO. & COMPOUND & $\begin{array}{l}\text { CONCENTRATION UNITS: } \\
(\mathrm{ug} / \mathrm{L} \text { or } \mathrm{ug} / \mathrm{kg}) \mathrm{ug} / \mathrm{L}\end{array}$ & $Q$ \\
\hline $75-71-8$ & Dichlorodifluoromethane & 0.50 & $\bar{U}$ \\
\hline $74-87-3$ & Chloromethane & 0.50 & $\mathrm{U}$ \\
\hline $75-01-4$ & Vinyl chloride & 0.50 & $\mathrm{U}$ \\
\hline $74-83-9$ & Bromomethane & 0.50 & $\mathrm{U}$ \\
\hline $75-00-3$ & Chloroethane & 0.50 & $\mathrm{U}$ \\
\hline $75-69-4$ & Trichlorofluoromethane & 0.50 & $\mathrm{U}$ \\
\hline $75-35-4$ & 1,1-Dichloroethene & 0.50 & $\mathrm{U}$ \\
\hline $76-13-1$ & 1,1,2-Trichloro-1,2,2-trifluoroethane & 0.50 & $\mathrm{U}$ \\
\hline $67-64-1$ & Acetone & 5.0 & $\mathrm{U}$ \\
\hline $75-15-0$ & Carbon disulfide & 0.50 & $\mathrm{U}$ \\
\hline $79-20-9$ & Methyl acetate & 0.50 & $\mathrm{U}$ \\
\hline $75-09-2$ & Methylene Chloride & 0.50 & $\mathrm{U}$ \\
\hline $156-60-5$ & trans-1,2-Dichloroethene & 0.50 & $\mathrm{U}$ \\
\hline $1634-04-4$ & Methyl tert-butyl ether & 0.50 & $\mathrm{U}$ \\
\hline $75-34-3$ & 1,1-Dichloroethane & 0.50 & $\mathrm{U}$ \\
\hline $156-59-2$ & cis-1,2-Dichloroethene & 0.50 & $\mathrm{U}$ \\
\hline $78-93-3$ & 2-Butanone & 5.0 & $\mathrm{U}$ \\
\hline $74-97-5$ & Bromochloromethane & 0.50 & $\mathrm{U}$ \\
\hline $67-66-3$ & Chloroform & 0.17 & $\mathrm{~J}$ \\
\hline $71-55-6$ & 1,1,1-Trichloroethane & 0.50 & $\mathrm{U}$ \\
\hline $110-82-7$ & Cyclohexane & 0.50 & $\mathrm{U}$ \\
\hline $56-23-5$ & Carbon tetrachloride & 0.50 & $\mathrm{U}$ \\
\hline $71-43-2$ & Benzene & 0.070 & $\mathrm{~J}$ \\
\hline $107-06-2$ & 1,2-Dichloroethane & 0.50 & $\mathrm{U}$ \\
\hline
\end{tabular}

Report 1,4-Dioxane for Low-Medium VOA analysis only 
Lab Name: TESTAMERICA BURLINGTON

Lab Code: STLV Case No.: INMAN

Matrix: (SOIL/SED/WATER)

Water

sample wt/vol: 25.0

$(g / m L) \quad m L$

Level: (TRACE/LOW/MED)

TRACE

\% Moisture: not dec.

GC Column: DB-624

ID $: 0.20$

$(\mathrm{mm})$

Soil Extract Volume:

(uL)

Purge Volume: 25.0

(mL)
Contract: $\quad 8 \mathrm{E}-00302$

SDG No.: 200-7006

\begin{tabular}{|c|c|c|c|}
\hline CAS NO. & COMPOUND & $\begin{array}{l}\text { CONCENTRATION UNITS: } \\
(\mathrm{ug} / \mathrm{L} \text { or } \mathrm{ug} / \mathrm{kg} \text { ) ug } / \mathrm{L}\end{array}$ & Q \\
\hline $79-01-6$ & Trichloroethene & 0.50 & $\bar{U}$ \\
\hline $108-87-2$ & Methylcyclohexane & 0.50 & $\mathrm{U}$ \\
\hline $78-87-5$ & 1,2-Dichloropropane & 0.50 & $\mathrm{U}$ \\
\hline $75-27-4$ & Bromodichloromethane & 0.50 & $\mathrm{u}$ \\
\hline $10061-01-5$ & cis-1,3-Dichloropropene & 0.50 & U \\
\hline $108-10-1$ & 4-Methyl-2-pentanone & 5.0 & $\mathrm{U}$ \\
\hline $108-88-3$ & Toluene & 0.58 & B \\
\hline $10061-02-6$ & trans-1,3-Dichloropropene & 0.50 & $\mathrm{U}$ \\
\hline $79-00-5$ & 1,1,2-Trichloroethane & 0.50 & $\mathrm{U}$ \\
\hline $127-18-4$ & Tetrachloroethene & 0.50 & $\mathrm{U}$ \\
\hline $591-78-6$ & 2-Hexanone & 5.0 & $\mathrm{U}$ \\
\hline $124-48-1$ & Dibromochloromethane & 0.50 & $\mathrm{U}$ \\
\hline $106-93-4$ & 1,2-Dibromoethane & 0.50 & $\mathrm{U}$ \\
\hline $108-90-7$ & Chlorobenzene & 0.50 & $\mathrm{U}$ \\
\hline $100-41-4$ & Ethylbenzene & 0.018 & $\mathrm{~J}$ \\
\hline $95-47-6$ & o-Xylene & 0.0070 & $\mathrm{~J}$ \\
\hline $179601-23-1$ & $\mathrm{~m}, \mathrm{p}$-Xylene & 0.021 & $\mathrm{~J}$ \\
\hline $100-42-5$ & Styrene & 0.035 & $\mathrm{~J}$ \\
\hline $75-25-2$ & Bromoform & 0.50 & $\mathrm{U}$ \\
\hline $98-82-8$ & Isopropylbenzene & 0.50 & $\mathrm{U}$ \\
\hline $79-34-5$ & $1,1,2,2$-Tetrachloroethane & 0.50 & $\mathrm{U}$ \\
\hline $541-73-1$ & 1,3-Dichlorobenzene & 0.50 & U \\
\hline $106-46-7$ & 1,4-Dichlorobenzene & 0.50 & $\mathrm{U}$ \\
\hline $95-50-1$ & 1,2-Dichlorobenzene & 0.50 & $\mathrm{U}$ \\
\hline $96-12-8$ & 1,2-Dibromo-3-Chloropropane & 0.50 & U \\
\hline $120-82-1$ & 1,2,4-Trichlorobenzene & 0.50 & $\mathrm{U}$ \\
\hline $87-61-6$ & 1,2,3-Trichlorobenzene & 0.50 & $\mathrm{U}$ \\
\hline
\end{tabular}


$1 \mathrm{~J}$ - FORM I VOA-TIC

VOLATILE ORGANICS ANALYSIS DATA SHEET TENTATIVELY IDENTIFIED COMPOUNDS
EPA SAMPLE NO.

IN-QCTB-W-32778

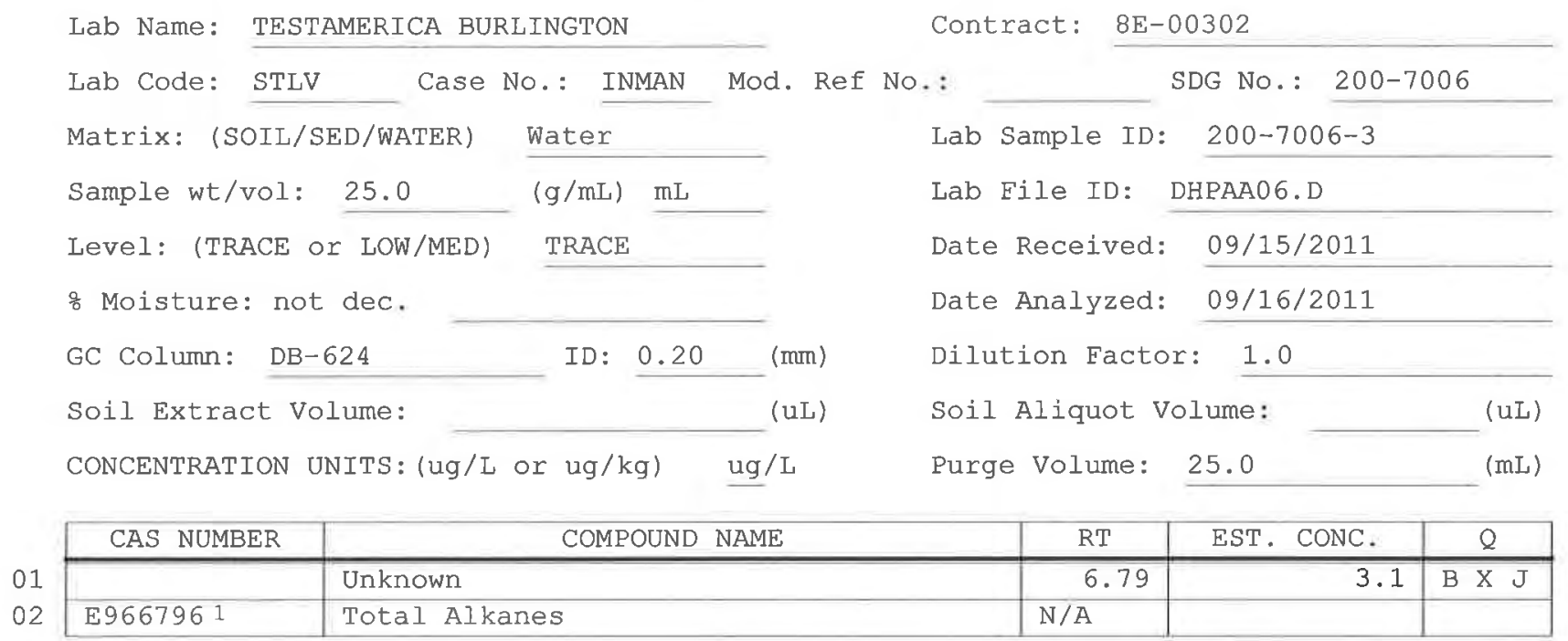

1 EPA-designated Registry Number. 
$6 A$ - FORM VI VOA-1

VOLATILE ORGANICS INITIAL CALIBRATION DATA

Lab Name: TESTAMERICA BURLINGTON

Contract: $\quad 8 \mathrm{E}-00302$

Lab Code: STLV Case No.: INMAN Mod. Ref No.:

SDG No.: 200-7006

Instrument ID: D.i Calibration Date(s): 07/07/2011 07/07/2011

Heated Purge: (Y/N) N

Calibration Time(s) : 1117

1256

Purge Volume: 25.0 ( $\mathrm{nL}$ )

GC Column: DB-624

ID: 0.20

(min) Length: 25

(m)

\begin{tabular}{|c|c|c|c|c|c|c|c|}
\hline \multirow{3}{*}{$\begin{array}{l}\text { LAB FILE ID: } \\
\text { RRF } 5.0=\text { DHP06.D } \\
\text { COMPOUND }\end{array}$} & \multirow{2}{*}{\multicolumn{3}{|c|}{$\begin{array}{l}\text { RRF0.5 }=\text { DHP04.D } \\
\text { RRF10 }=\text { DHP07.D }\end{array}$}} & \multirow{2}{*}{\multicolumn{4}{|c|}{$\begin{array}{l}\text { RRF1.0 }=\text { DHP05.D } \\
\text { RRE20 }=\text { DHP08.D }\end{array}$}} \\
\hline & & & & & & & \\
\hline & RRE 0.5 & RRF 1.0 & RRF 5.0 & RRE 10 & RRE 20 & $\overline{\mathrm{RRF}}$ & $\because R S D$ \\
\hline Dichlorodifluoromethane & 0.418 & 0.398 & 0.390 & 0.409 & 0.398 & 0.403 & 2.7 \\
\hline Chloromethane & 0.769 & 0.713 & 0.655 & 0.659 & 0.639 & 0.687 & 7.8 \\
\hline Vinyl chloride & 0.524 & 0.491 & 0.475 & 0.499 & 0.491 & 0.496 & 3.6 \\
\hline Bromomethane & 0.180 & 0.203 & 0.205 & 0.219 & 0.239 & 0.209 & 10.3 \\
\hline Chloroethane & 0.292 & 0.280 & 0.259 & 0.269 & 0.259 & 0.272 & 5.2 \\
\hline Trichlorofluoromethane & 0.612 & 0.609 & 0.587 & 0.608 & 0.611 & 0.606 & 1.7 \\
\hline 1,1-Dichloroethene & 0.318 & 0.307 & 0.298 & 0.303 & 0.297 & 0.305 & 2.8 \\
\hline $\begin{array}{l}1,1,2 \text {-Trichloro- } \\
1,2,2 \text {-trifluoroethane }\end{array}$ & 0.377 & 0.358 & 0.336 & 0.358 & 0.347 & 0.355 & 4.3 \\
\hline Acetone & 0.047 & 0.038 & 0.032 & 0.032 & 0.030 & 0.036 & 19.3 \\
\hline Carbon disulfide & 0.758 & 0.707 & 0.719 & 0.760 & 0.766 & 0.742 & 3.6 \\
\hline Methyl acetate & 0.108 & 0.094 & 0.076 & 0.079 & 0.074 & 0.086 & 16.7 \\
\hline Methylene Chloride & 0.264 & 0.257 & 0.242 & 0.248 & 0.241 & 0.250 & 3.9 \\
\hline trans-1,2-Dichloroethene & 0.323 & 0.298 & 0.301 & 0.312 & 0.306 & 0.308 & 3.3 \\
\hline Methyl tert-butyl ether & 0.304 & 0.278 & 0.292 & 0.319 & 0.318 & 0.302 & 5.7 \\
\hline 1,1-Dichloroethane & 0.642 & 0.586 & 0.594 & 0.617 & 0.607 & 0.609 & 3.6 \\
\hline cis-1,2-Dichloroethene & 0.302 & 0.289 & 0.300 & 0.312 & 0.306 & 0.302 & 2.7 \\
\hline 2-Butanone & 0.045 & 0.046 & 0.047 & 0.049 & 0.048 & 0.047 & 3.4 \\
\hline Bromochloromethane & 0.104 & 0.103 & 0.100 & 0.099 & 0.101 & 0.101 & 2.0 \\
\hline Chloroform & 0.502 & 0.482 & 0.473 & 0.493 & 0.477 & 0.485 & 2.5 \\
\hline 1,1,1-Trichloroethane & 0.514 & 0.490 & 0.494 & 0.490 & 0.485 & 0.494 & 2.3 \\
\hline Cyclohexane & 0.761 & 0.795 & 0.901 & 0.909 & 0.883 & 0.850 & 7.9 \\
\hline Carbon tetrachloride & 0.487 & 0.459 & 0.477 & 0.474 & 0.464 & 0.472 & 2.4 \\
\hline Benzene & 1.617 & 1.501 & 1.518 & 1.495 & 1.447 & 1.515 & 4.1 \\
\hline 1,2-Dichloroethane & 0.291 & 0.271 & 0.277 & 0.284 & 0.285 & 0.282 & 2.7 \\
\hline Trichloroethene & 0.397 & 0.380 & 0.357 & 0.355 & 0.351 & 0.368 & 5.4 \\
\hline Methylcyclohexane & 0.703 & 0.708 & 0.769 & 0.757 & 0.751 & 0.737 & 4.1 \\
\hline
\end{tabular}

Report 1,4-Dioxane for Low-Medium VOA analysis only 
$6 \mathrm{~B}-$ FORM VI VOA-2

VOLATILE ORGANICS INITIAL CALIBRATION DATA

Lab Name: TESTAMERICA BURLINGTON

Contract: $\quad 8 \mathrm{E}-00302$

Lab Code: STLV Case No.: INMAN Mod. Ref No.:

SDG NO.: 200-7006

Instrument ID: D.i

Calibration Date(s): 07/07/2011 07/07/2011

Heated Purge: $(\mathrm{Y} / \mathrm{N}) \mathrm{N}$

Calibration Time(s): 1117 1256

Purge Volume: 25.0 (mL)

GC Column: DB-624

ID: 0.20

(mm) Length: 25

(m)

\begin{tabular}{|c|c|c|c|c|c|c|c|}
\hline \multirow{2}{*}{$\begin{array}{l}\text { LAB FILE ID: } \\
\text { RRF } 5.0=\text { DHPO6.D }\end{array}$} & \multicolumn{3}{|c|}{$\mathrm{RRF} 0.5=\mathrm{DHPO} 4 . \mathrm{D}$} & \multicolumn{4}{|c|}{ RRF $1.0=$ DHP05.D } \\
\hline & $\operatorname{RRF} 10=\square$ & IP07.D & & RRE 20 & $=\mathrm{DHPO}$ &. $\mathrm{D}$ & \\
\hline COMPOUND & RRF 0.5 & $\mathrm{RRF} 1.0$ & RRE 5.0 & RRF 10 & RRF 20 & $\overline{\mathrm{RRF}}$ & $\stackrel{\circ}{R} S D$ \\
\hline 1,2-Dichloropropane & 0.363 & 0.373 & 0.367 & 0.371 & 0.355 & 0.366 & 2.0 \\
\hline Bromodichloromethane & 0.310 & 0.296 & 0.307 & 0.305 & 0.306 & 0.305 & 1.6 \\
\hline cis-1,3-Dichloropropene & 0.310 & 0.338 & 0.406 & 0.414 & 0.418 & 0.377 & 13.2 \\
\hline 4-Methyl-2-pentanone & 0.104 & 0.114 & 0.137 & 0.140 & 0.137 & 0.126 & 12.7 \\
\hline Toluene & 1.608 & 1.623 & 1.695 & 1.690 & 1.667 & 1.657 & 2.4 \\
\hline trans-1,3-Dichloropropene & 0.216 & 0.225 & 0.285 & 0.302 & 0.307 & 0.267 & 16.2 \\
\hline 1,1,2-Trichloroethane & 0.155 & 0.162 & 0.160 & 0.156 & 0.157 & 0.158 & 1.9 \\
\hline Tetrachloroethene & 0.311 & 0.304 & 0.306 & 0.308 & 0.299 & 0.306 & 1.5 \\
\hline 2-Hexanone & 0.076 & 0.085 & 0.096 & 0.099 & 0.096 & 0.090 & 10.6 \\
\hline Dibromochloromethane & 0.172 & 0.176 & 0.180 & 0.178 & 0.192 & 0.180 & 4.1 \\
\hline 1,2-Dibromoethane & 0.132 & 0.135 & 0.138 & 0.145 & 0.145 & 0.139 & 4.2 \\
\hline Chlorobenzene & 1.079 & 1.036 & 1.034 & 1.041 & 1.021 & 1.042 & 2.1 \\
\hline Ethylbenzene & 1.594 & 1.651 & 1.933 & 1.968 & 1.978 & 1.825 & 10.2 \\
\hline o-Xylene & 0.539 & 0.600 & 0.727 & 0.749 & 0.755 & 0.674 & 14.6 \\
\hline $\mathrm{m}, \mathrm{p}-\mathrm{xy}$ lene & 0.644 & 0.689 & 0.779 & 0.797 & 0.802 & 0.742 & 9.6 \\
\hline Styrene & 0.763 & 0.886 & 1.048 & 1.096 & 1.123 & 0.983 & 15.6 \\
\hline Bromoform & 0.107 & 0.116 & 0.126 & 0.119 & 0.137 & 0.121 & 9.2 \\
\hline Isopropylbenzene & 1.400 & 1.553 & 2.026 & 2.103 & 2.121 & 1.841 & 18.4 \\
\hline $1,1,2,2$-Tetrachloroethane & 0.168 & 0.154 & 0.168 & 0.164 & 0.164 & 0.164 & 3.6 \\
\hline 1,3-Dichlorobenzene & 1.494 & 1.468 & 1.569 & 1.629 & 1.647 & 1.562 & 5.1 \\
\hline 1,4-Dichlorobenzene & 1.827 & 1.736 & 1.615 & 1.641 & 1.610 & 1.686 & 5.5 \\
\hline 1,2-Dichlorobenzene & 1.377 & 1.388 & 1.407 & 1.392 & 1.355 & 1.384 & 1.4 \\
\hline 1,2-Dibromo-3-Chloropropane & 0.021 & 0.027 & 0.039 & 0.035 & 0.040 & 0.032 & 24.3 \\
\hline 1,2,4-Trichlorobenzene & 0.603 & 0.574 & 0.686 & 0.751 & 0.756 & 0.674 & 12.4 \\
\hline 1,2,3-Trichlorobenzene & 0.433 & 0.469 & 0.522 & 0.573 & 0.575 & 0.514 & 12.3 \\
\hline
\end{tabular}


$6 C$ - FORM VI VOA-3

VOLATILE ORGANICS INITIAL CALIBRATION DATA

Lab Name: TESTAMERICA BURLINGTON

Contract: $8 \mathrm{E}-00302$

Lab Code: STLV Case No.: INMAN Mod. Ref No.:

SDG No.: 200-7006

Instrument ID: D.i

Calibration Date(s): 07/07/2011 07/07/2011

Heated Purge: $(\mathrm{Y} / \mathrm{N}) \mathrm{N}$

Calibration Time(s): 1117 1256

Purge Volume: 25.0 $(\mathrm{mL})$

GC Column: DB-624

ID: 0.20

(mm) Length: 25

(m)

\begin{tabular}{|c|c|c|c|c|c|c|c|}
\hline \multirow{2}{*}{$\begin{array}{l}\text { LAB FILE ID: } \\
\text { RRF } 5.0=\text { DHP06.D }\end{array}$} & \multicolumn{3}{|c|}{ RRF $0.5=$ DHP0 $4 . D$} & \multicolumn{4}{|c|}{ RRF1.0 $=$ DHP05.D } \\
\hline & 10 & IP07.D & & RRF 20 & $=\mathrm{DHP}$ &. $\mathrm{D}$ & \\
\hline COMPOUND & RRF 0.5 & RRF 1.0 & $\operatorname{RRE} 5.0$ & RRF 10 & RRF 20 & $\overline{\mathrm{RRF}}$ & $\%$ RSD \\
\hline Vinyl Chloride-d3 & 0.479 & 0.428 & 0.415 & 0.431 & 0.426 & 0.436 & 5.7 \\
\hline Chloroethane-d5 & 0.304 & 0.290 & 0.269 & 0.276 & 0.274 & 0.283 & 5.0 \\
\hline 1,1-Dichloroethene-d2 & 0.849 & 0.795 & 0.757 & 0.787 & 0.787 & 0.795 & 4.2 \\
\hline 2-Butanone-d5 & 0.041 & 0.038 & 0.040 & 0.044 & 0.043 & 0.041 & 5.5 \\
\hline Chloroform-d & 0.512 & 0.515 & 0.502 & 0.514 & 0.503 & 0.509 & 1.3 \\
\hline 1,2-Dichloroethane-d4 & 0.218 & 0.203 & 0.199 & 0.201 & 0.194 & 0.203 & 4.3 \\
\hline Benzene-d6 & 1.573 & 1.471 & 1.478 & 1.453 & 1.403 & 1.475 & 4.2 \\
\hline 1,2-Dichloropropane-d6 & 0.410 & 0.374 & 0.367 & 0.365 & 0.356 & 0.374 & 5.7 \\
\hline Toluene-d8 & 1.315 & 1.370 & 1.458 & 1.441 & 1.404 & 1.397 & 4.1 \\
\hline trans-1,3-Dichloropropene-d4 & 0.197 & 0.207 & 0.232 & 0.249 & 0.264 & 0.230 & 12.2 \\
\hline 2-Hexanone-d5 & 0.023 & 0.026 & 0.037 & 0.038 & 0.039 & 0.033 & 23.1 \\
\hline $1,1,2,2$-Tetrachloroethane-d2 & 0.151 & 0.161 & 0.167 & 0.171 & 0.169 & 0.164 & 4.8 \\
\hline 1,2-Dichlorobenzene-d4 & 0.885 & 0.843 & 0.815 & 0.807 & 0.799 & 0.830 & 4.2 \\
\hline
\end{tabular}

Report 1,4-Dioxane-d8 for Low-Medium VOA analysis only 
$7 A$ - FORM VII VOA-1

VOLATILE CONTINUING CALIBRATION DATA

Lab Name: TESTAMERICA BURLINGTON

Contract: $8 \mathrm{E}-00302$

Lab Code: STLV Case No.: INMAN Mod. Ref No.:

SDG No.: 200-7006

Instrument ID: D.i

Calibration Date: 09/16/2011 Time: 0932

Lab File Id: DHPAA02.D

EPA Sample No. (VSTD\#\#\#\#): VSTD005DY Init. Calib. Date(s): 07/07/2011 07/07/2011

Heated Purge: $(\mathrm{Y} / \mathrm{N}) \quad \mathrm{N}$ GC Column: DB-624

Inịt. Calib. Time(s): $1117 \quad 1256$

Purge Volume: 25.0 (mL)

\begin{tabular}{|c|c|c|c|c|c|}
\hline COMPOUND & $\overline{\mathrm{RRF}}$ & RRF5.0 & $\begin{array}{l}\text { MIN } \\
\text { RRF }\end{array}$ & $\frac{9}{8} \mathrm{D}$ & $\mathrm{MAX} \div \mathrm{D}$ \\
\hline Dichlorodifluoromethane & 0.403 & 0.468 & 0.010 & 16.2 & 40.0 \\
\hline Chloromethane & 0.687 & 0.738 & 0.010 & 7.5 & 40.0 \\
\hline Vinyl chloride & 0.496 & 0.524 & 0.010 & 5.6 & 30.0 \\
\hline Bromomethane & 0.209 & 0.225 & 0.010 & 7.4 & 30.0 \\
\hline Chloroethane & 0.272 & 0.274 & 0.010 & 0.7 & 40.0 \\
\hline Trichlorofluoromethane & 0.606 & 0.641 & 0.010 & 5.9 & 40.0 \\
\hline 1,1-Dichloroethene & 0.305 & 0.310 & 0.010 & 1.9 & 30.0 \\
\hline 1,1,2-Trichloro-1,2,2-trifluoroethane & 0.355 & 0.365 & 0.010 & 2.9 & 40.0 \\
\hline Acetone & 0.036 & 0.035 & 0.010 & -1.5 & 40.0 \\
\hline Carbon disulfide & 0.742 & 0.799 & 0.010 & 7.7 & 40.0 \\
\hline Methyl acetate & 0.086 & 0.083 & 0.010 & -3.4 & 40.0 \\
\hline Methylene Chloride & 0.250 & 0.248 & 0.010 & -1.1 & 40.0 \\
\hline trans-1,2-Dichloroethene & 0.308 & 0.299 & 0.010 & -2.8 & 40.0 \\
\hline Methyl tert-butyl ether & 0.302 & 0.251 & 0.010 & -17.0 & 40.0 \\
\hline 1,1-Dichloroethane & 0.609 & 0.606 & 0.010 & -0.5 & 30.0 \\
\hline cis-1,2-Dichloroethene & 0.302 & 0.295 & 0.010 & -2.3 & 40.0 \\
\hline 2-Butanone & 0.047 & 0.042 & 0.010 & -10.5 & 40.0 \\
\hline Bromochloromethane & 0.101 & 0.095 & 0.010 & -6.3 & 30.0 \\
\hline Chloroform & 0.485 & 0.467 & 0.010 & -3.7 & 30.0 \\
\hline 1,1,1-Trichloroethane & 0.494 & 0.500 & 0.010 & 1.1 & 30.0 \\
\hline Cyclohexane & 0.850 & 0.928 & 0.010 & 9.3 & 40.0 \\
\hline Carbon tetrachloride & 0.472 & 0.484 & 0.010 & 2.5 & 30.0 \\
\hline Benzene & 1.515 & 1.513 & 0.010 & -0.2 & 30.0 \\
\hline 1,2-Dichloroethane & 0.282 & 0.269 & 0.010 & -4.3 & 30.0 \\
\hline Trichloroethene & 0.368 & 0.348 & 0.010 & -5.3 & 30.0 \\
\hline Methylcyclohexane & 0.737 & 0.768 & 0.010 & 4.1 & 40.0 \\
\hline
\end{tabular}

Report 1,4-Dioxane for Low/Medium VOA analysis only 
$7 B$ - FORM VII VOA-2

VOLATILE CONTINUING CALIBRATION DATA

Lab Name: TESTAMERICA BURLINGTON

Contract: $8 \mathrm{E}-00302$

Lab Code: STlu Case No.: INMAN Mod. Ref No.:

SDG No.: 200-7006

Instrument ID: D.i

Calibration Date: 09/16/2011 Time: 0932

Lab File Id: DHPAAO2.D

EPA Sample No. (VSTD\#\#\#\#): VSTDO05DY Init. Calib. Date(s): 07/07/2011 07/07/2011

Heated Purge: (Y/N)

$\mathrm{N} \quad$ GC Column: DB-624

Init. Calib. Time(s): $1117 \quad 1256$

Purge Volume: 25.0

(mI)

\begin{tabular}{|c|c|c|c|c|c|}
\hline COMPOUND & $\overline{\mathrm{RRE}}$ & RRF5.0 & $\begin{array}{l}\text { MIN } \\
\text { RRE }\end{array}$ & $\because \mathrm{D}$ & $\operatorname{MAX} \div \mathrm{D}$ \\
\hline 1,2-Dichloropropane & 0.366 & 0.352 & 0.010 & -3.6 & 40.0 \\
\hline Bromodichloromethane & 0.305 & 0.295 & 0.010 & -3.2 & 30.0 \\
\hline cis-1,3-Dichloropropene & 0.377 & 0.378 & 0.010 & 0.2 & 30.0 \\
\hline 4-Methyl-2-pentanone & 0.126 & 0.114 & 0.010 & -9.9 & 40.0 \\
\hline Toluene & 1.657 & 1.666 & 0.010 & 0.5 & 30.0 \\
\hline trans-1,3-Dichloropropene & 0.267 & 0.263 & 0.010 & -1.3 & 30.0 \\
\hline 1,1,2-Trichloroethane & 0.158 & 0.149 & 0.010 & -5.7 & 30.0 \\
\hline Tetrachloroethene & 0.306 & 0.301 & 0.010 & -1.6 & 30.0 \\
\hline 2-Hexanone & 0.090 & 0.081 & 0.010 & -10.9 & 40.0 \\
\hline Dibromochloromethane & 0.180 & 0.170 & 0.010 & -5.6 & 30.0 \\
\hline 1,2-Dibromoethane & 0.139 & 0.131 & 0.010 & -6.0 & 40.0 \\
\hline Chlorobenzene & 1.042 & 1.003 & 0.010 & -3.8 & 30.0 \\
\hline Ethylbenzene & 1.825 & 1.855 & 0.010 & 1.6 & 30.0 \\
\hline o-Xylene & 0.674 & 0.701 & 0.010 & 3.9 & 30.0 \\
\hline $\mathrm{m}, \mathrm{p}$-Xylene & 0.742 & 0.760 & 0.010 & 2.4 & 30.0 \\
\hline Styrene & 0.983 & 1.031 & 0.010 & 4.9 & 30.0 \\
\hline Bromoform & 0.121 & 0.117 & 0.010 & -3.3 & 30.0 \\
\hline Isopropylbenzene & 1.841 & 1.951 & 0.010 & 6.0 & 40.0 \\
\hline $1,1,2,2$-Tetrachloroethane & 0.164 & 0.143 & 0.010 & -12.4 & 30.0 \\
\hline 1,3-Dichlorobenzene & 1.562 & 1.499 & 0.010 & -4.0 & 30.0 \\
\hline 1,4-Dichlorobenzene & 1.686 & 1.570 & 0.010 & -6.9 & 30.0 \\
\hline 1,2-Dichlorobenzene & 1.384 & 1.308 & 0.010 & -5.5 & 30.0 \\
\hline 1,2-Dibromo-3-Chloropropane & 0.032 & 0.032 & 0.010 & -0.1 & 40.0 \\
\hline 1,2,4-Trichlorobenzene & 0.674 & 0.655 & 0.010 & -2.8 & 30.0 \\
\hline 1,2,3-Trichlorobenzene & 0.514 & 0.505 & 0.010 & -1.9 & 30.0 \\
\hline
\end{tabular}


7C - FORM VII VOA-3

VOLATILE CONTINUING CALIBRATION DATA

Lab Name: TESTAMERICA BURLINGTON

Contract: $8 \mathrm{E}-00302$

Lab Code: STLV Case No.: INMAN Mod. Ref No.:

SDG No.: 200-7006

Instrument ID: D.i

Calibration Date: 09/16/2011 Time: 0932

Lab File Id: DHPAA02.D Init. Calib. Date(s): 07/07/2011 07/07/2011

EPA Sample No.(VSTD\#\#\#\#): VSTD005DY

Init. Calib. Time(s): $1117 \quad 1256$

Heated Purge: ( $\mathrm{Y} / \mathrm{N}) \mathrm{N}$

GC Column: DB-624

ID: $0.20(\mathrm{~mm})$ Length: 25

(m)

Purge Volume: 25.0

$(\mathrm{mL})$

\begin{tabular}{|c|c|c|c|c|c|}
\hline COMPOUND & $\overline{\mathrm{RRF}}$ & RRE5. 0 & $\begin{array}{l}\text { MIN } \\
\text { RRE }\end{array}$ & $\frac{2}{0} \mathrm{D}$ & MAX $\because D$ \\
\hline Vinyl Chloride-d3 & 0.436 & 0.438 & 0.010 & 0.6 & 30.0 \\
\hline Chloroethane-d5 & 0.283 & 0.269 & 0.010 & -4.8 & 40.0 \\
\hline 1,1-Dichloroethene-d2 & 0.795 & 0.747 & 0.010 & -6.1 & 30.0 \\
\hline 2-Butanone-d5 & 0.041 & 0.037 & 0.010 & -11.3 & 40.0 \\
\hline Chloroform-d & 0.509 & 0.470 & 0.010 & -7.8 & 30.0 \\
\hline 1,2-Dichloroethane-d4 & 0.203 & 0.182 & 0.010 & -10.3 & 30.0 \\
\hline Benzene-d6 & 1.475 & 1.390 & 0.010 & -5.8 & 30.0 \\
\hline 1,2-Dich1oropropane-d6 & 0.374 & 0.340 & 0.010 & -9.2 & 40.0 \\
\hline Toluene-d8 & 1.397 & 1.365 & 0.010 & -2.3 & 30.0 \\
\hline trans-1,3-Dichloropropene-d4 & 0.230 & 0.208 & 0.010 & -9.4 & 30.0 \\
\hline 2-Hexanone-d5 & 0.033 & 0.029 & 0.010 & -11.9 & 40.0 \\
\hline $1,1,2,2$-Tetrachloroethane-d2 & 0.164 & 0.141 & 0.010 & -14.0 & 30.0 \\
\hline 1,2-Dichlorobenzene-d4 & 0.830 & 0.730 & 0.010 & -12.0 & 30.0 \\
\hline
\end{tabular}

Report 1,4-Dioxane-d8 for Low/Medium VOA analysis only 
$7 A$ - FORM VII VOA-1

VOLATILE CONTINUING CALIBRATION DATA

Lab Name: TESTAMERICA BURLINGTON

Contract: 8E-00302

Lab Code: STLV Case No.: INMAN Mod. Ref No.:

SDG No.: 200-7006

Instrument ID: D.i

Calibration Date: 09/16/2011 Time: 1357

Lab File Id: DHPAAIO.D

EPA Sample No. (VSTD\#\#\#\#): VSTD005YD Init. Calib. Date(s): 07/07/2011 07/07/2011

Heated Purge: $(Y / N)$ GC Column: DB-624

Init. Calib. Time(s): $1117 \quad 1256$

Purge Volume: 25.0 ID: $0.20(\mathrm{~mm})$ Length: 25

(m) (mL)

\begin{tabular}{|c|c|c|c|c|c|}
\hline COMPOUND & $\overline{\mathrm{RRF}}$ & RRF5. 0 & $\begin{array}{l}\text { MIN } \\
\text { RRF }\end{array}$ & $\frac{\circ}{\partial} \mathrm{D}$ & MAX $\div D$ \\
\hline Dichlorodifluoxomethane & 0.403 & 0.460 & 0.010 & 14.2 & 50.0 \\
\hline Chloromethane & 0.687 & 0.704 & 0.010 & 2.5 & 50.0 \\
\hline Vinyl chloride & 0.496 & 0.509 & 0.100 & 2.5 & 50.0 \\
\hline Bromomethane & 0.209 & 0.206 & 0.100 & -1.3 & 50.0 \\
\hline Chloroethane & 0.272 & 0.266 & 0.010 & -2.4 & 50.0 \\
\hline Trichlorofluoromethane & 0.606 & 0.639 & 0.010 & 5.6 & 50.0 \\
\hline 1,1-Dichloroethene & 0.305 & 0.299 & 0.100 & -2.0 & 50.0 \\
\hline 1,1,2-Trichloro-1,2,2-trifluoroethane & 0.355 & 0.370 & 0.010 & 4.1 & 50.0 \\
\hline Acetone & 0.036 & 0.033 & 0.010 & -7.0 & 50.0 \\
\hline Carbon disulfide & 0.742 & 0.763 & 0.010 & 2.9 & 50.0 \\
\hline Methyl acetate & 0.086 & 0.092 & 0.010 & 6.7 & 50.0 \\
\hline Methylene Chloride & 0.250 & 0.262 & 0.010 & 4.6 & 50.0 \\
\hline trans $-1,2-$ Dichloroethene & 0.308 & 0.307 & 0.010 & -0.3 & 50.0 \\
\hline Methyl tert-butyl ether & 0.302 & 0.317 & 0.010 & 4.8 & 50.0 \\
\hline 1,1-Dichloroethane & 0.609 & 0.632 & 0.200 & 3.7 & 50.0 \\
\hline cis-1,2-Dichloroethene & 0.302 & 0.320 & 0.010 & 6.2 & 50.0 \\
\hline 2-Butanone & 0.047 & 0.052 & 0.010 & 11.3 & 50.0 \\
\hline Bromochloromethane & 0.101 & 0.110 & 0.050 & 8.7 & 50.0 \\
\hline Chloroform & 0.485 & 0.510 & 0.200 & 5.0 & 50.0 \\
\hline 1,1,1-Trichloroethane & 0.494 & 0.505 & 0.100 & 2.1 & 50.0 \\
\hline Cyclohexane & 0.850 & 0.927 & 0.010 & 9.1 & 50.0 \\
\hline Carbon tetrachloride & 0.472 & 0.485 & 0.100 & 2.7 & 50.0 \\
\hline Benzene & 1.515 & 1.509 & 0.400 & -0.4 & 50.0 \\
\hline 1,2-Dichloroethane & 0.282 & 0.309 & 0.100 & 9.6 & 50.0 \\
\hline Trichloroethene & 0.368 & 0.350 & 0.300 & -5.0 & 50.0 \\
\hline Methylcyclohexane & 0.737 & 0.774 & 0.010 & 5.0 & 50.0 \\
\hline
\end{tabular}

Report 1,4-Dioxane for Low/Medium VOA analysis only 
$7 B$ - EORM VII VOA-2

VOLATILE CONTINUING CALIBRATION DATA

Lab Name: TESTAMERICA BURLINGTON

Contract: $8 \mathrm{E}-00302$

Lab Code: STLV Case No.: INMAN Mod. Ref No.:

SDG No.: 200-7006

Instrument ID: D.i

Calibration Date: 09/16/2011 Time: 1357

Lab File Id: DHPAA10.D Init. Calib. Date(s): 07/07/2011 07/07/2011

EPA Sample No. (VSTD\#\#\#\#): VSTD005YD

Init. Calib. Time(s): $1117 \quad 1256$

Heated Purge: $(\mathrm{Y} / \mathrm{N})$ N GC Column: DB-624

ID: $0.20(\mathrm{~mm})$ Length: 25

(m)

Purge Volume: 25.0 $(\mathrm{mL})$

\begin{tabular}{|c|c|c|c|c|c|}
\hline COMPOUND & $\overline{\mathrm{RRF}}$ & RRF5. 0 & $\begin{array}{l}\text { MIN } \\
\text { RRF }\end{array}$ & $\% \mathrm{D}$ & $\operatorname{MAX} \because \mathrm{D}$ \\
\hline 1,2-Dichloropropane & 0.366 & 0.383 & 0.010 & 4.7 & 50.0 \\
\hline Bromodichloromethane & 0.305 & 0.308 & 0.200 & 1.2 & 50.0 \\
\hline cis-1,3-Dichloropropene & 0.377 & 0.411 & 0.200 & 8.8 & 50.0 \\
\hline 4-Methyl-2-pentanone & 0.126 & 0.141 & 0.010 & 11.9 & 50.0 \\
\hline Toluene & 1.657 & 1.684 & 0.400 & 1.6 & 50.0 \\
\hline trans-1,3-Dichloropropene & 0.267 & 0.298 & 0.100 & 11.6 & 50.0 \\
\hline 1,1,2-Trichloroethane & 0.158 & 0.170 & 0.100 & 7.8 & 50.0 \\
\hline Tetrachloroethene & 0.306 & 0.302 & 0.100 & -1.4 & 50.0 \\
\hline 2-Hexanone & 0.090 & 0.101 & 0.010 & 12.0 & 50.0 \\
\hline Dibromochloromethane & 0.180 & 0.190 & 0.100 & 6.0 & 50.0 \\
\hline 1,2-Dibromoethane & 0.139 & 0.146 & 0.010 & 5.3 & 50.0 \\
\hline Chlorobenzene & 1.042 & 1.066 & 0.500 & 2.3 & 50.0 \\
\hline Ethylbenzene & 1.825 & 1.906 & 0.100 & 4.5 & 50.0 \\
\hline o-xylene & 0.674 & 0.731 & 0.300 & 8.4 & 50.0 \\
\hline $\mathrm{m}, \mathrm{p}$-xylene & 0.742 & 0.791 & 0.300 & 6.6 & 50.0 \\
\hline Styrene & 0.983 & 1.100 & 0.300 & 11.9 & 50.0 \\
\hline Bromoform & 0.121 & 0.135 & 0.050 & 11.5 & 50.0 \\
\hline Isopropylbenzene & 1.841 & 1.989 & 0.010 & 8.1 & 50.0 \\
\hline $1,1,2,2$-Tetrachloroethane & 0.164 & 0.170 & 0.100 & 3.8 & 50.0 \\
\hline 1,3-Dichlorobenzene & 1.562 & 1.532 & 0.400 & -1.9 & 50.0 \\
\hline 1,4-Dichlorobenzene & 1.686 & 1.673 & 0.400 & -0.8 & 50.0 \\
\hline 1,2-Dichlorobenzene & 1.384 & 1.415 & 0.400 & 2.3 & 50.0 \\
\hline 1,2-Dibromo-3-Chloropropane & 0.032 & 0.036 & 0.010 & 12.3 & 50.0 \\
\hline 1,2,4-Trichlorobenzene & 0.674 & 0.667 & 0.200 & -1.1 & 50.0 \\
\hline 1,2,3-Trichlorobenzene & 0.514 & 0.531 & 0.200 & 3.2 & 50.0 \\
\hline
\end{tabular}


7C - FORM VII VOA-3

VOLATILE CONTINUING CALIBRATION DATA

Lab Name: TESTAMERICA BURLINGTON

Contract: $8 \mathrm{E}-00302$

Lab Code: STLV Case No.: INMAN Mod. Ref No.: SDG No.: 200-7006

Instrument ID: D.i

Calibration Date: 09/16/2011 Time: 1357

Lab File Id: DHPAA10. D Init. Calib. Date(s): 07/07/2011 07/07/2011

EPA Sample No. (VSTD\#\#\#\#): VSTD005YD

Init. Calib. Time(s): $1117 \quad 1256$

Heated Purge: $(\mathrm{Y} / \mathrm{N}) \mathrm{N}$ GC Column: DB-624 ID: $0.20(\mathrm{~mm})$ Length: 25

(m)

Purge Volume: 25.0 $(\mathrm{mL})$

\begin{tabular}{|c|c|c|c|c|c|}
\hline COMPOUND & $\overline{\mathrm{RRF}}$ & RRF5.0 & $\begin{array}{l}\text { MIN } \\
\text { RRE }\end{array}$ & $\frac{\circ}{8}$ & MAX $\because \mathrm{D}$ \\
\hline Vinyl Chloride-d3 & 0.436 & 0.412 & 0.010 & -5.4 & 50.0 \\
\hline Chloroethane-d5 & 0.283 & 0.264 & 0.010 & -6.5 & 50.0 \\
\hline 1,1-Dichloroethene-d2 & 0.795 & 0.742 & 0.010 & -6.7 & 50.0 \\
\hline 2-Butanone-d5 & 0.041 & 0.047 & 0.010 & 13.7 & 50.0 \\
\hline Chloroform-d & 0.509 & 0.511 & 0.010 & 0.2 & 50.0 \\
\hline 1,2-Dichloroethane-d4 & 0.203 & 0.212 & 0.010 & 4.4 & 50.0 \\
\hline Benzene-d6 & 1.475 & 1.426 & 0.010 & -3.4 & 50.0 \\
\hline 1,2-Dichloropropane-d6 & 0.374 & 0.356 & 0.010 & -4.9 & 50.0 \\
\hline Toluene-d8 & 1.397 & 1.385 & 0.010 & -0.9 & 50.0 \\
\hline trans-1,3-Dichloropropene-d4 & 0.230 & 0.228 & 0.010 & -0.8 & 50.0 \\
\hline 2-Hexanone-d5 & 0.033 & 0.037 & 0.010 & 12.0 & 50.0 \\
\hline $1,1,2,2$-Tetrachloroethane-d2 & 0.164 & 0.170 & 0.010 & 3.7 & 50.0 \\
\hline 1,2-Dichlorobenzene-d4 & 0.830 & 0.771 & 0.010 & -7.0 & 50.0 \\
\hline
\end{tabular}


$1 A$ - FORM I VOA-1

VOLATILE ORGANICS ANALYSIS DATA SHEET
EPA SAMPLE NO.

VBLKDY
Lab Name: TESTAMERICA BURLINGTON

Lab Code: STLV Cas

Matrix: (SOIL/SED/WATER)

Sample wt/vol: 25.0

$(\mathrm{g} / \mathrm{mL}) \mathrm{mL}$

Level: (TRACE/LOW/MED) TRACE

o Moisture: not dec.

GC Column: DB-624

ID: 0.20 (mm)

Soil Extract Volume:

(uL)

(mL)

Purge Volume: 25.0
Contract: $8 \mathrm{E}-00302$

SDG No.: 200-7006

: $\quad$ SDG No.: 200-7006

Lab Sample ID: MB 200-25361/3

Lab File ID: DHPAA03.D

Date Received:

Date Analyzed: 09/16/2011

Dilution Factor: 1.0

Soil Aliquot Volume:

(uL)

\begin{tabular}{|c|c|c|c|}
\hline CAS NO. & COMPOUND & $\begin{array}{l}\text { CONCENTRATION UNITS: } \\
(u g / L \text { or } u g / k g) ~ u g / L\end{array}$ & Q \\
\hline $75-71-8$ & Dichlorodifluoromethane & 0.50 & $\overline{\mathrm{U}}$ \\
\hline $74-87-3$ & Chloromethane & 0.50 & $\mathrm{U}$ \\
\hline $75-01-4$ & Vinyl chloride & 0.50 & $\mathrm{U}$ \\
\hline $74-83-9$ & Bromomethane & 0.50 & $\mathrm{U}$ \\
\hline $75-00-3$ & Chloroethane & 0.50 & $\mathrm{U}$ \\
\hline $75-69-4$ & Trichlorofluoromethane & 0.50 & $\mathrm{U}$ \\
\hline $75-35-4$ & 1,1-Dichloroethene & 0.50 & $\mathrm{U}$ \\
\hline $76-13-1$ & 1,1,2-Trichloro-1,2,2-trifluoroethane & 0.50 & $\mathrm{U}$ \\
\hline $67-64-1$ & Acetone & 5.0 & $\mathrm{U}$ \\
\hline $75-15-0$ & Carbon disulfide & 0.50 & $\mathrm{U}$ \\
\hline $79-20-9$ & Methyl acetate & 0.50 & $\mathrm{U}$ \\
\hline $75-09-2$ & Methylene Chloride & 0.50 & $\mathrm{U}$ \\
\hline $156-60-5$ & trans-1,2-Dichloroethene & 0.50 & $\mathrm{U}$ \\
\hline $1634-04-4$ & Methyl tert-butyl ether & 0.50 & $\mathrm{U}$ \\
\hline $75-34-3$ & 1,1-Dichloroethane & 0.50 & $\mathrm{U}$ \\
\hline $156-59-2$ & cis-1,2-Dichloroethene & 0.50 & $\mathrm{U}$ \\
\hline $78-93-3$ & 2-Butanone & 5.0 & $\mathrm{U}$ \\
\hline $74-97-5$ & Bromochloromethane & 0.50 & $\mathrm{U}$ \\
\hline $67-66-3$ & Chloroform & 0.50 & $\mathrm{U}$ \\
\hline $71-55-6$ & 1,1,1-Trichloroethane & 0.50 & $\mathrm{U}$ \\
\hline $110-82-7$ & Cyclohexane & 0.50 & $\mathrm{U}$ \\
\hline $56-23-5$ & Carbon tetrachloride & 0.50 & $\mathrm{U}$ \\
\hline $71-43-2$ & Benzene & 0.50 & $\mathrm{U}$ \\
\hline $107-06-2$ & 1,2-Dichloroethane & 0.50 & $\mathrm{U}$ \\
\hline
\end{tabular}

Report 1,4-Dioxane for Low-Medium VOA analysis only 
$1 \mathrm{~B}-$ FORM I VOA-2

VOLATILE ORGANICS ANALYSIS DATA SHEET
EPA SAMPLE NO.

VBLKDY
Lab Name: TESTAMERICA BURLINGTON

Lab Code: STLV

Contract: $\quad 8 \mathrm{E}-00302$

Matrix: (SOIL/SED/WATER)

Sample wt/vol: 25.0

Water

$(\mathrm{g} / \mathrm{mL}) \quad \mathrm{mL}$

Level: (TRACE/LOW/MED) TRACE

今 Moisture: not dec.

GC Column: DB-624 ID : $0.20 \quad$ (mm)

Soil Extract Volume:

$(m L)$
SDG No.: 200-7006

Lab Sample ID: MB 200-25361/3

Lab File ID: DHPAA03.D

Date Received:

Date Analyzed: 09/16/2011

Dilution Factor: 1.0

Soil Aliquot Volume:
CONCENTRATION UNITS: (ug/L or $u g / k g) ~ u g / L$

\begin{tabular}{|c|c|c|c|}
\hline CAS NO. & COMPOUND & $\begin{array}{l}\text { CONCENTRATION UNITS: } \\
(\mathrm{ug} / \mathrm{L} \text { or } u g / \mathrm{kg}) \mathrm{ug} / \mathrm{L}\end{array}$ & $Q$ \\
\hline $79-01-6$ & Trichloroethene & 0.50 & $\mathrm{U}$ \\
\hline $108-87-2$ & Methylcyclohexane & 0.50 & $\mathrm{U}$ \\
\hline $78-87-5$ & 1,2-Dichloropropane & 0.50 & $\mathrm{U}$ \\
\hline $75-27-4$ & Bromodichloromethane & 0.50 & $\mathrm{U}$ \\
\hline $10061-01-5$ & cis-1,3-Dichloropropene & 0.50 & $\mathrm{U}$ \\
\hline $108-10-1$ & 4-Methy1-2-pentanone & 5.0 & $\mathrm{U}$ \\
\hline $108-88-3$ & Toluene & 0.0089 & $\mathrm{~J}$ \\
\hline $10061-02-6$ & trans-1,3-Dichloropropene & 0.50 & $\mathrm{U}$ \\
\hline $79-00-5$ & 1,1,2-Trichloroethane & 0.50 & $\mathrm{U}$ \\
\hline $127-18-4$ & Tetrachloroethene & 0.50 & $\mathrm{U}$ \\
\hline $591-78-6$ & 2-Hexanone & 5.0 & $\mathrm{U}$ \\
\hline $124-48-1$ & Dibromoch loromethane & 0.50 & $\mathrm{U}$ \\
\hline $106-93-4$ & 1,2-Dibromoethane & 0.50 & $\mathrm{U}$ \\
\hline $108-90-7$ & Chlorobenzene & 0.50 & $\mathrm{U}$ \\
\hline $100-41-4$ & Ethylbenzene & 0.50 & $\mathrm{U}$ \\
\hline $95-47-6$ & o-xylene & 0.50 & $\mathrm{U}$ \\
\hline $179601-23-1$ & m, p-Xylene & 0.50 & $\mathrm{U}$ \\
\hline $100-42-5$ & Styrene & 0.50 & $\mathrm{U}$ \\
\hline $75-25-2$ & Bromoform & 0.50 & $\mathrm{U}$ \\
\hline $98-82-8$ & Isopropylbenzene & 0.50 & $\mathrm{U}$ \\
\hline $79-34-5$ & $1,1,2,2$-Tetrachloroethane & 0.50 & $\mathrm{U}$ \\
\hline $541-73-1$ & 1,3-Dichlorobenzene & 0.50 & $\mathrm{U}$ \\
\hline $106-46-7$ & 1,4-Dichlorobenzene & 0.50 & $\mathrm{U}$ \\
\hline $95-50-1$ & 1,2-Dichlorobenzene & 0.50 & $\mathrm{U}$ \\
\hline $96-12-8$ & 1,2-Dibromo-3-Chloropropane & 0.50 & $\mathrm{U}$ \\
\hline $120-82-1$ & 1,2,4-Trichlorobenzene & 0.50 & $\mathrm{U}$ \\
\hline $87-61-6$ & 1,2,3-Trichlorobenzene & 0.50 & $\mathrm{U}$ \\
\hline
\end{tabular}


IJ - FORM I VOA-TIC

VOLATILE ORGANICS ANALYSIS DATA SHEET TENTATIVELY IDENTIEIED COMPOUNDS
EPA SAMPLE NO.

VBLKDY

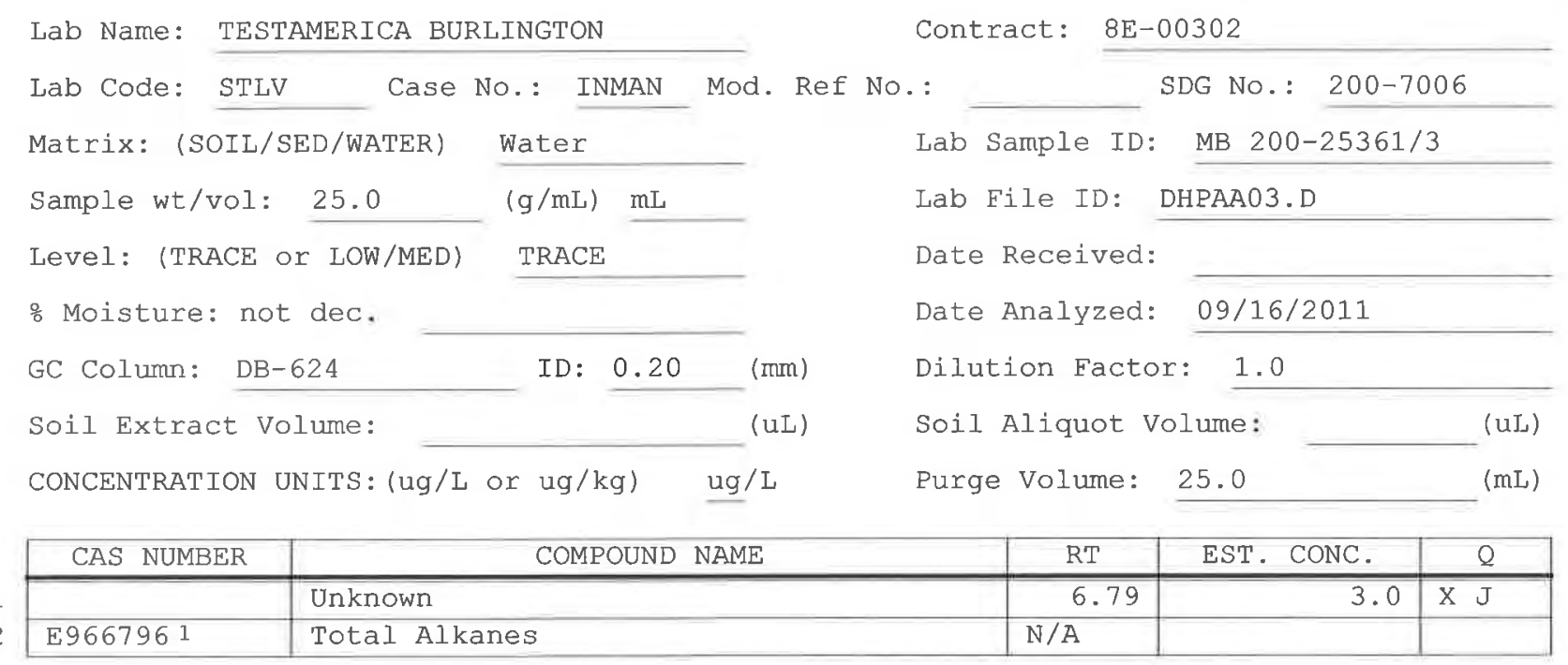

1 EPA-designated Registry Number. 
Lab Name: TESTAMERICA BURLINGTON

Contract: $8 \mathrm{E}-00302$

Lab Code: STLV Cas

Matrix: (SOIL/SED/WATER)

Water

Sample wt/vol: 25.0

$(\mathrm{g} / \mathrm{mL}) \quad \mathrm{mL}$

Level: (TRACE/LOW/MED)

TRACE

음 Moisture: not dec.

GC Column: DB-624 ID: $0.20 \quad(\mathrm{~mm})$

Soil Extract Volume:

Purge Volume: 25.0 $(m L)$

Lab File ID: DHPAA09.D

Date Received:

Dilution Factor: 1.0

Soil Aliquot Volume:

SDG NO.: 200-7006

Lab Sample ID: 200-7006-4

Date Analyzed: 09/16/2011

\begin{tabular}{|c|c|c|c|}
\hline CAS NO. & COMPOUND & $\begin{array}{l}\text { CONCENTRATION UNITS: } \\
(\mathrm{ug} / \mathrm{L} \text { or } \mathrm{ug} / \mathrm{kg}) \mathrm{ug} / \mathrm{L}\end{array}$ & Q \\
\hline $75-71-8$ & Dichlorodifluoromethane & 0.50 & $\mathrm{U}$ \\
\hline $74-87-3$ & Chloromethane & 0.50 & $\mathrm{U}$ \\
\hline $75-01-4$ & Vinyl chloride & 0.50 & $\mathrm{U}$ \\
\hline $74-83-9$ & Bromomethane & 0.50 & $\mathrm{U}$ \\
\hline $75-00-3$ & Chloroethane & 0.50 & $\mathrm{U}$ \\
\hline $75-69-4$ & Trichlorofluoromethane & 0.50 & $\mathrm{U}$ \\
\hline $75-35-4$ & 1,1-Dichloroethene & 0.50 & $\mathrm{U}$ \\
\hline $76-13-1$ & 1,1,2-Trichloro-1,2,2-trifluoroethane & 0.50 & U \\
\hline $67-64-1$ & Acetone & 5.0 & $\mathrm{U}$ \\
\hline $75-15-0$ & Carbon disulfide & 0.50 & $\mathrm{U}$ \\
\hline $79-20-9$ & Methyl acetate & 0.50 & $\mathrm{U}$ \\
\hline $75-09-2$ & Methylene Chloride & 0.50 & $\mathrm{U}$ \\
\hline $156-60-5$ & trans-1,2-Dichloroethene & 0.50 & $\mathrm{U}$ \\
\hline $1634-04-4$ & Methyl tert-butyl ether & 0.50 & $\mathrm{U}$ \\
\hline $75-34-3$ & 1,1-Dichloroethane & 0.50 & $\mathrm{U}$ \\
\hline $156-59-2$ & cis-1,2-Dichloroethene & 0.50 & $\mathrm{U}$ \\
\hline $78-93-3$ & 2-Butanone & 5.0 & $\mathrm{U}$ \\
\hline $74-97-5$ & Bromochloromethane & 0.50 & $\mathrm{U}$ \\
\hline $67-66-3$ & Chloroform & 0.061 & $\mathrm{~J}$ \\
\hline $71-55-6$ & 1,1,1-Trichloroethane & 0.50 & $\mathrm{U}$ \\
\hline $110-82-7$ & Cyclohexane & 0.50 & $\mathrm{U}$ \\
\hline $56-23-5$ & Carbon tetrachloride & 0.50 & U \\
\hline $71-43-2$ & Benzene & 0.50 & $\mathrm{U}$ \\
\hline $107-06-2$ & 1,2-Dichloroethane & 0.50 & $\mathrm{U}$ \\
\hline
\end{tabular}

Report 1,4-Dioxane for Low-Medium VOA analysis only 
EPA SAMPJE NO.

VOLATILE ORGANICS ANALYSIS DATA SHEET

VHBLKO 1

Lab Name: TESTAMERICA BURLINGTON

Contract: $\quad 8 \mathrm{E}-00302$

Lab Code: STLV Case No.: INMAN Mod. Ref No.:

SDG No.: 200-7006

Matrix: (SOIL/SED/WATER)

Sample wt/vol: 25.0

Water

$(\mathrm{g} / \mathrm{mL}) \mathrm{mL}$

Level: ('TRACE/LOW/MED) TRACE

음 Moisture: not dec.

GC Column: DB-624 ID: $0.20 \quad$ ( $\mathrm{mm})$

Soil Extract Volume: (uL)

(mL)

Purge Volume: 25.0
Lab Sample ID: 200-7006-4

Lab File ID: DHPAA09.D

Date Received:

Date Analyzed: 09/16/2011

Dilution Factor: 1.0

Soil Aliquot Volume:

(uL)

\begin{tabular}{|c|c|c|c|}
\hline CAS NO. & COMPOUND & $\begin{array}{l}\text { CONCENTRATION UNITS: } \\
(\mathrm{ug} / \mathrm{L} \text { or } \mathrm{ug} / \mathrm{kg} \text { ) ug/L }\end{array}$ & $Q$ \\
\hline $79-01-6$ & Trichloroethene & 0.50 & $\bar{U}$ \\
\hline $108-87-2$ & Methylcyclohexane & 0.50 & $\mathrm{U}$ \\
\hline $78-87-5$ & 1,2-Dichloropropane & 0.50 & $\mathrm{U}$ \\
\hline $75-27-4$ & Bromodichloromethane & 0.50 & $\mathrm{U}$ \\
\hline $10061-01-5$ & cis-1,3-Dichloropropene & 0.50 & $\mathrm{U}$ \\
\hline $108-10-1$ & 4-Methyl-2-pentanone & 5.0 & $\mathrm{U}$ \\
\hline $108-88-3$ & Toluene & 0.012 & $\mathrm{~J} B$ \\
\hline $10061-02-6$ & trans-1,3-Dichloropropene & 0.50 & $\mathrm{U}$ \\
\hline $79-00-5$ & 1,1,2-Trichloroethane & 0.50 & $\mathrm{U}$ \\
\hline $127-18-4$ & Tetrachloroethene & 0.50 & $\mathrm{U}$ \\
\hline $591-78-6$ & 2-Hexanone & 5.0 & $\mathrm{U}$ \\
\hline $124-48-1$ & Dibromochloromethane & 0.50 & $\mathrm{u}$ \\
\hline $106-93-4$ & 1,2-Dibromoethane & 0.50 & $\mathrm{U}$ \\
\hline $108-90-7$ & Chlorobenzene & 0.50 & $\mathrm{U}$ \\
\hline $100-41-4$ & Ethylbenzene & 0.50 & $\mathrm{U}$ \\
\hline $95-47-6$ & o-xylene & 0.50 & $\mathrm{U}$ \\
\hline $179601-23-1$ & m,p-Xylene & 0.50 & $\mathrm{U}$ \\
\hline $100-42-5$ & Styrene & 0.50 & $\mathrm{U}$ \\
\hline $75-25-2$ & Bromoform & 0.50 & $\mathrm{U}$ \\
\hline $98-82-8$ & Isopropylbenzene & 0.50 & $\mathrm{U}$ \\
\hline $79-34-5$ & $1,1,2,2$-Tetrachloroethane & 0.50 & $\mathrm{U}$ \\
\hline $541-73-1$ & 1,3-Dichlorobenzene & 0.50 & $\mathrm{U}$ \\
\hline $106-46-7$ & 1,4-Dichlorobenzene & 0.50 & $\mathrm{U}$ \\
\hline $95-50-1$ & 1,2-Dichlorobenzene & 0.50 & $\mathrm{U}$ \\
\hline $96-12-8$ & 1,2-Dibromo-3-Chloropropane & 0.50 & $\mathrm{U}$ \\
\hline $120-82-1$ & 1,2,4-Trichlorobenzene & 0.50 & $\mathrm{U}$ \\
\hline $87-61-6$ & 1,2,3-Trichlorobenzene & 0.50 & $\mathrm{U}$ \\
\hline
\end{tabular}


1J - FORM I VOA-TIC

VOLATILE ORGANICS ANALYSIS DATA SHEET TENTATIVELY IDENTIFIED COMPOUNDS
EPA SAMPLE NO.

VHBLK01

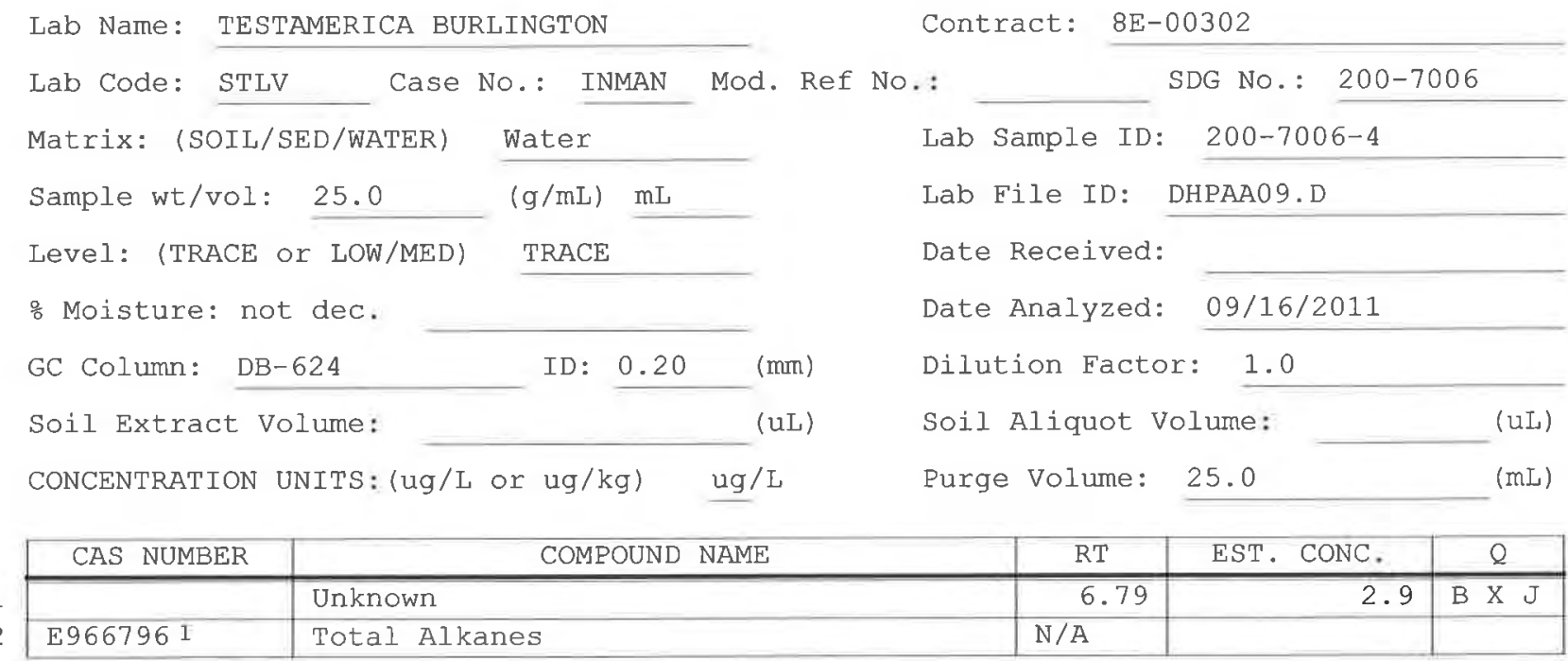

1 EPA-designated Registry Number. 


\section{TestAmerica}

THE LEADER IN ENVIRONMENTAL TESTING

\section{ANALYTICAL REPORT}

Job Number: 200-7045-1

SDG Number: 200-7045

Job Description: Inman (200-7045)

Contract Number: 1E-30401

For:

Argonne National Laboratory

9700 South Cass Avenue

Building 203

Office B-149

Argonne, IL 60439

Attention: Mr. Clyde Dennis

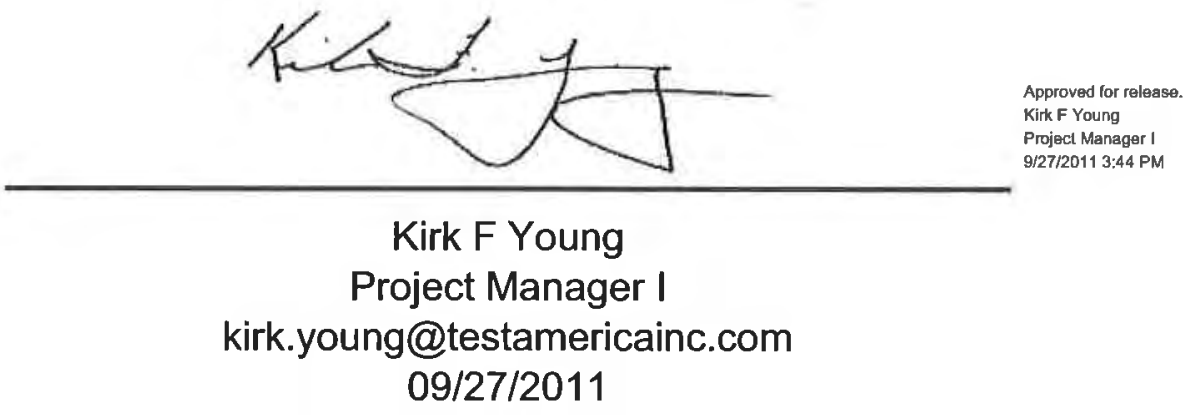

The test results in this report relate only to sample(s) as received by the laboratory. These test results were derived under a quality system that adheres to the requirements of NELAC. Pursuant to NELAC, this report may not be produced in full without written approval from the laboratory 


\section{Table of Contents}

Cover Title Page . . . . . . . . . . . . . . . . . 1

Report Narrative . . . . . . . . . . . . . . . . . . . 4

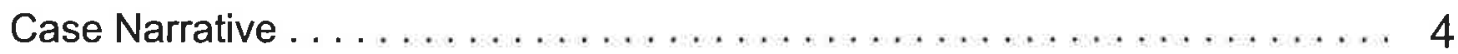

Qualifier Definition ............................. 6

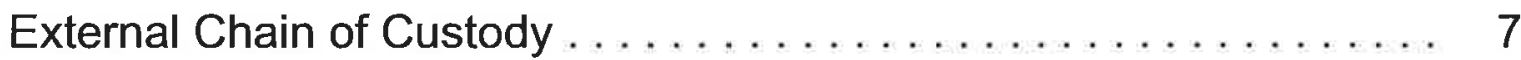

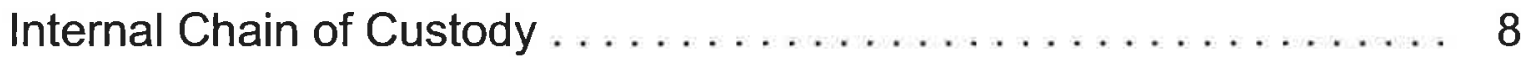

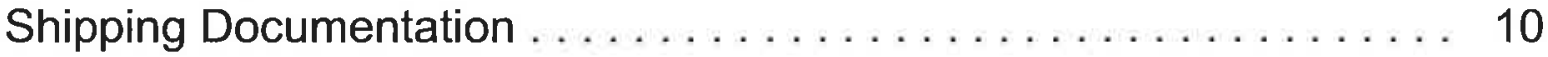

Airbills (if Applicable) . . . . . . . . . . . . . . . . . . . . . 11

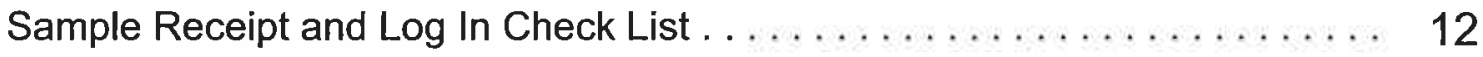

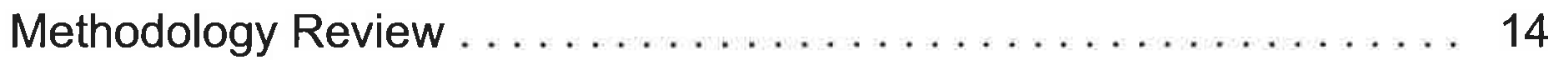

QC Summary - SOM01.2 Volatiles-Trace . . . . . . . . . . . . . . 15

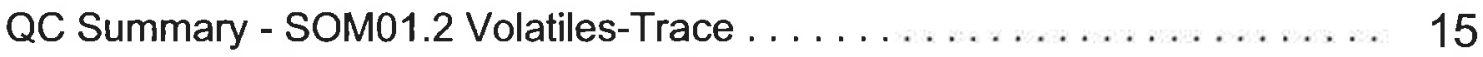

Deuterated Monitoring Compound Summary $\ldots \ldots \ldots \ldots \ldots \ldots \ldots \ldots \ldots \ldots \ldots \ldots \ldots$

Method Blank $\ldots \ldots \ldots \ldots \ldots \ldots \ldots \ldots \ldots \ldots \ldots \ldots \ldots \ldots \ldots \ldots, 17$

GC/MS Instrument Performance Check .......................... 18

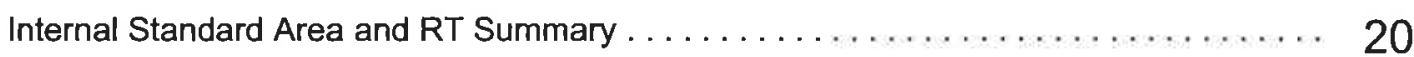

Sample Data - SOM01.2 Volatiles-Trace . . . . . . . . . . . . . 21

Sample Data - SOM01.2 Volatiles-Trace . . . . . . . . . . . . . . . 21

INMW14D-W-32782 ....................................... 21

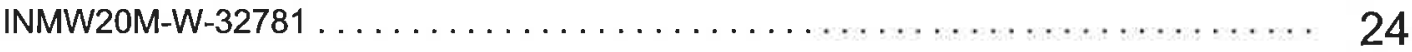

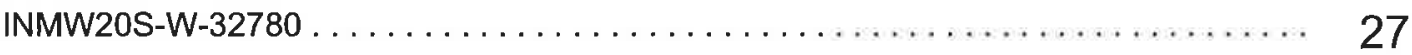

INQCTB-W-32783 ...................................... 30

Standards - SOM01.2 Volatiles-Trace . . . . . . . . . . . . . . 33

Standards - SOM01.2 Volatiles-Trace . ................... 33

Initial Calibration Data $\ldots \ldots \ldots \ldots \ldots \ldots \ldots \ldots \ldots \ldots \ldots \ldots \ldots \ldots \ldots \ldots \ldots \ldots \ldots \ldots \ldots, \quad 33$

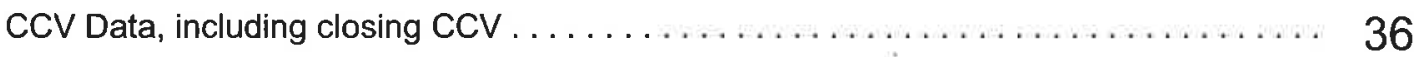

$\begin{array}{ll}\text { Page } 2 \text { of } 47 & 09 / 27 / 2011\end{array}$ 


\section{Table of Contents}

Raw Qc Data - SOM01.2 Volatiles-Trace . . . . . . . . . . . . . . . . 42

Raw Qc Data - SOM01.2 Volatiles-Trace . . . . . . . . . . . . . . . . 42

Raw Qc Data - SOM01.2 Volatiles-Trace $\ldots \ldots \ldots \ldots \ldots \ldots \ldots \ldots \ldots \ldots \ldots, 42$

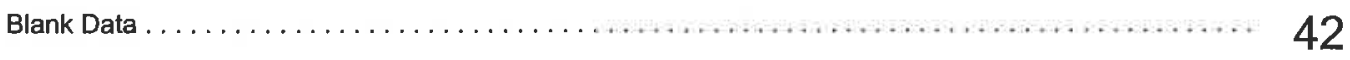




\title{
CASE NARRATIVE
}

\section{Client: Argonne National Laboratory}

\author{
Project: Inman (200-7045)
}

Report Number: 200-7045-1

Enclosed is the data set for the referenced project work. With the exceptions noted as flags or footnotes, standard analytical protocols were followed in performing the analytical work and the applied control limits were met.

Calculations were performed before rounding to avoid round-off errors in calculated results.

All holding times were met and proper preșervation noted for the methods performed on these samples, unless otherwise detailed in the individual sections below.

\section{Receipt}

The samples were received on 09/16/2011. Documentation of the condition of the samples at the time of their receipt and any exception to the laboratory's Sample Acceptance Policy is documented in the Shipping and Receiving section of this submittal. The samples, as received, were not acid preserved. On that basis, the laboratory did provide for the analytical work to be performed within seven days of sample collection.

\section{SOM01.2 Volatile Organics (Trace Level Water)}

A storage blank was prepared for volatile organics analysis, and stored in association with the storage of the samples. That storage blank, identified as VHBLK01, was carried through the holding period with the samples, and analyzed.

Each sample in the sample set was analyzed without a dilution. Each of the analyses associated with the sample set exhibited an acceptable internal standard performance. There was an acceptable recovery of each deuterated monitoring compound (DMC) in the analysis of the method blank associated with the analytical work, and in the analysis of the storage blank associated with the sample set. The analysis of the samples in this sample set did meet the technical acceptance criteria specific to DMC recoveries, although not all DMC recoveries were within the control range in each analysis. The technical acceptance criteria does provide for the recovery of up to three DMCs to fall outside of the control range in the analysis of field samples. Matrix spike and matrix spike duplicate analyses were not performed on samples in this sample set. Trace concentrations of acetone, chloroform, and toluene were identified in the analysis of the method blank associated with the analytical work. The concentration of each analyte in that analysis was below the established reporting limit, and the analysis did meet the technical acceptance criteria for a compliant method blank analysis. A trace concentration of chioroform was identified in the analysis of the storage blank associated with the sample set. The concentration of chloroform in that analysis was below the established reporting limit, and the analysis did meet the technical acceptance criteria for a compliant storage blank analysis. Present in the method blank and storage blank analyses was a non-target constituent that represents a compound that is related to the DMC formulation. The fact that the presence of this compound is not within the laboratory's control is at issue. The derived results for that compound have been qualified with an "X" qualifier to reflect the source of the contamination. 
The responses for each of the target analytes met the relative standard deviation criterion in the initial calibration. The response for each target analyte met the percent difference criterion in the opening/continuing calibration check acquisition. The response for each target analyte met the 50.0 percent difference criterion in the closing calibration check acquisition.

The primary quantitation mass for methylcyclohexane that is specified in the Statement of Work is mass 83 . The laboratory did identify a contribution to mass 83 from 1,2-dichloropropane- $d_{6}$, one of the deuterated monitoring compounds (DMCs). The laboratory did change the primary quantitation mass assignment to mass 55 for the quantification of methylcyclohexane.

Manual integration was employed in deriving certain of the analytical results. The values that have been derived from manual integration are qualified on the quantitation reports. Extracted ion current profiles for each manual integration are included in the data package, and further documented at the end of this submittal. 
DATA REPORTING QUALIFIERS

Client: Argonne National Laboratory

Job Number: $200-7045-1$

Sdg Number: $200-7045$

GC/MS VOA

U

$\mathrm{J}$

$J$

$\mathrm{X}$

*

B
Analyzed for but not detected.

Indicates an Estimated Value for TICs

Indicates an estimated value.

See case narrative notes for explanation of the ' $X$ ' flag

Surrogate exceeds the control limit

The analyte was found in an associated blank, as well as in the sample. 


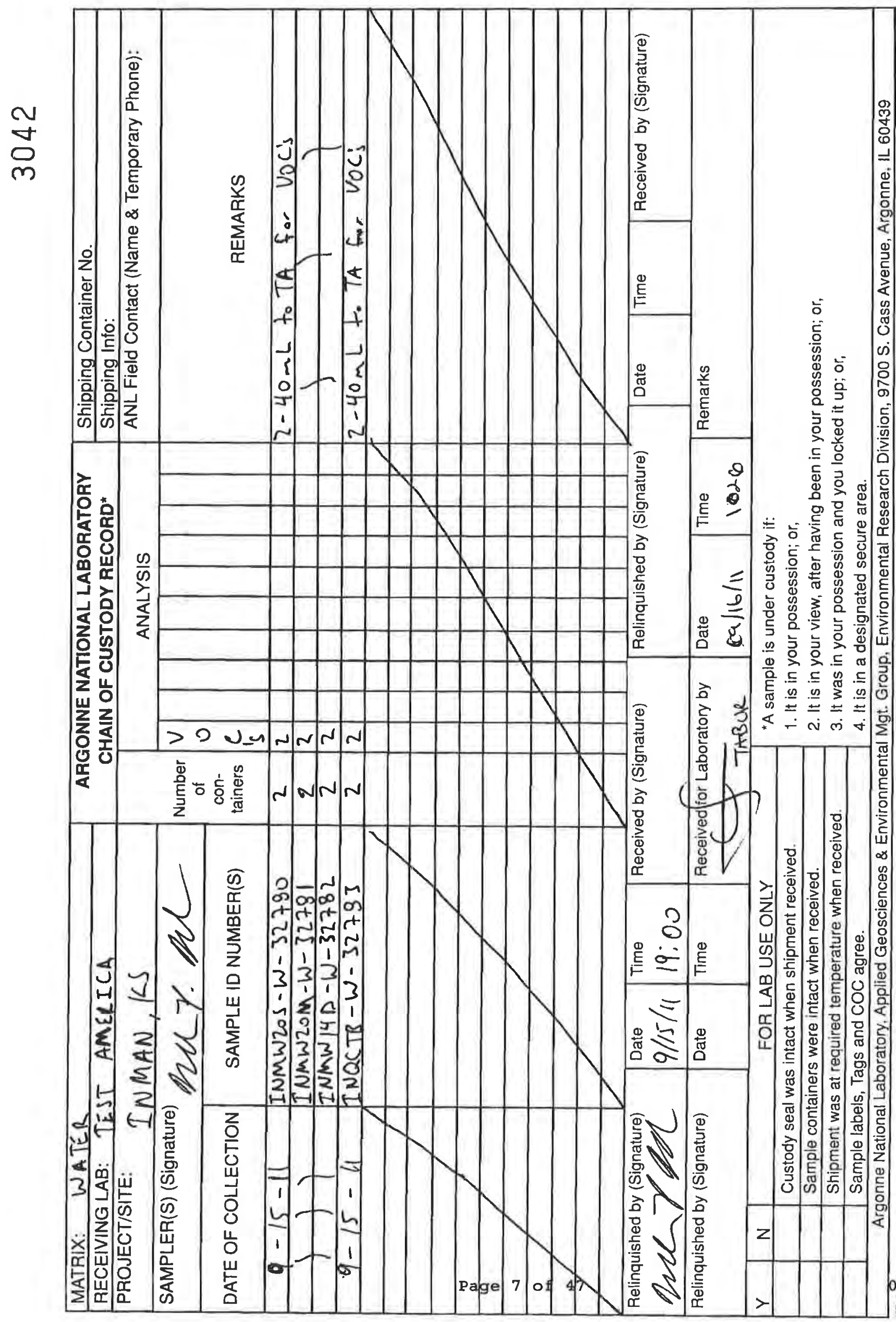




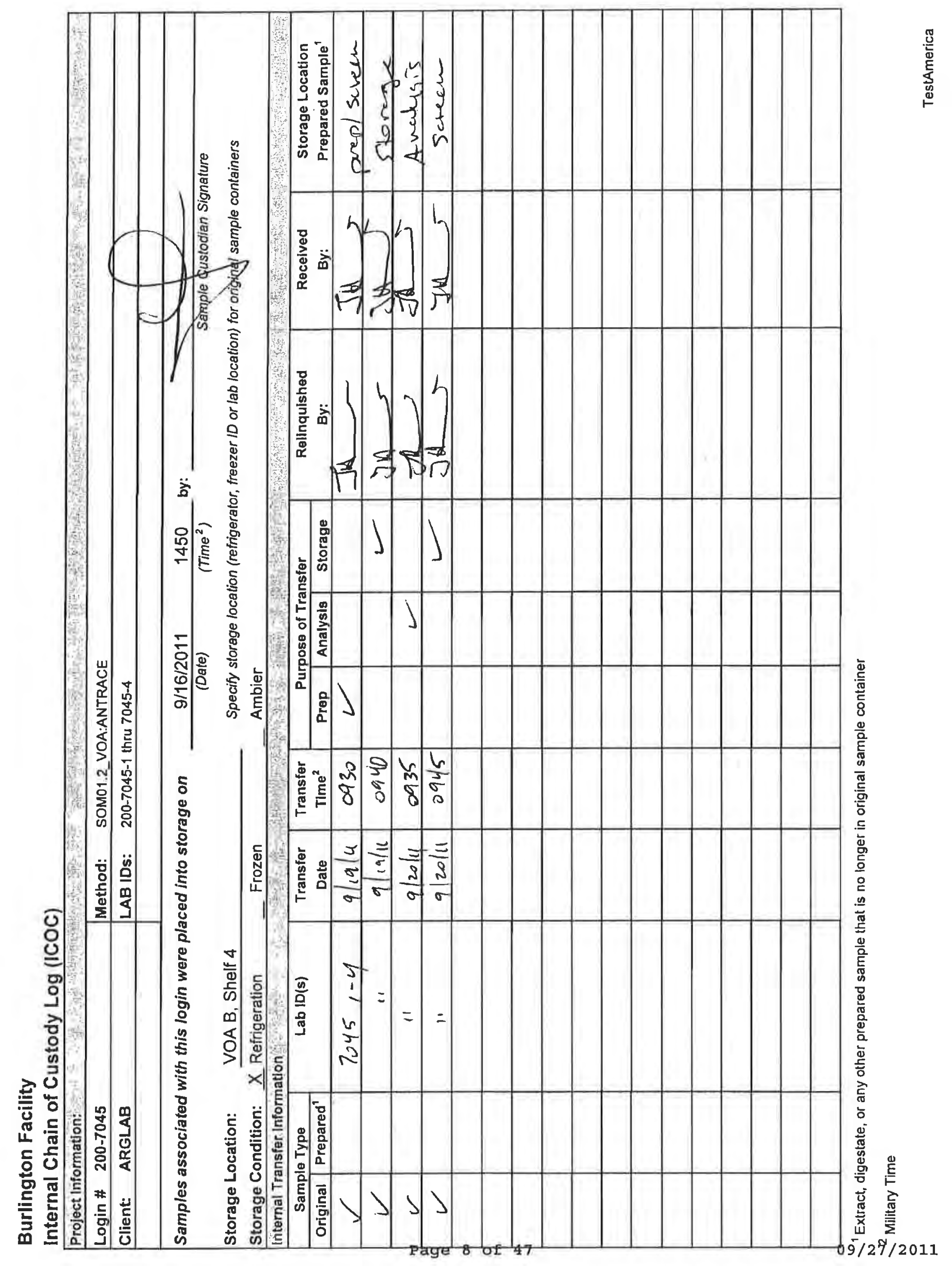




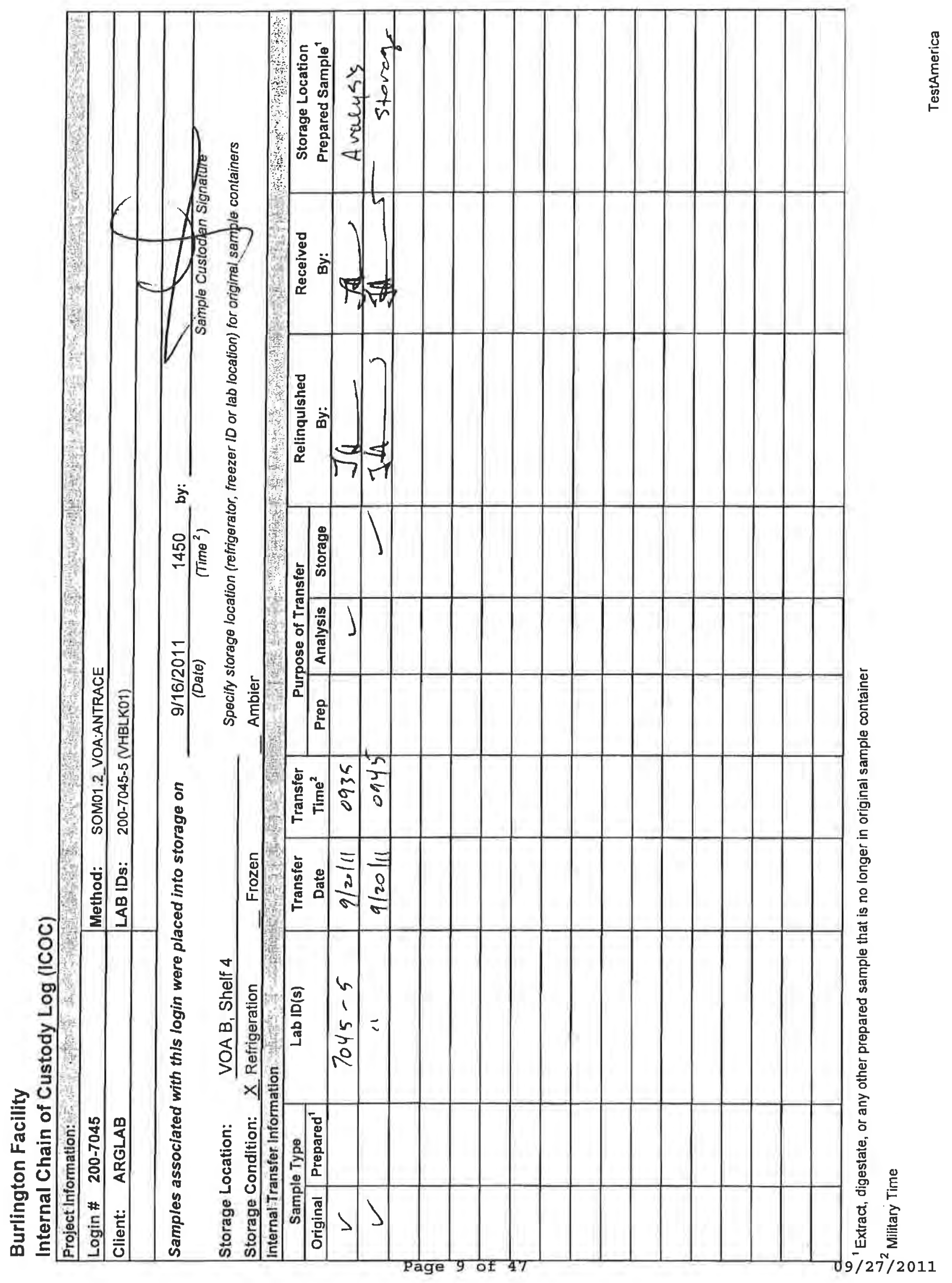




\section{Shipping and Receiving Documents}




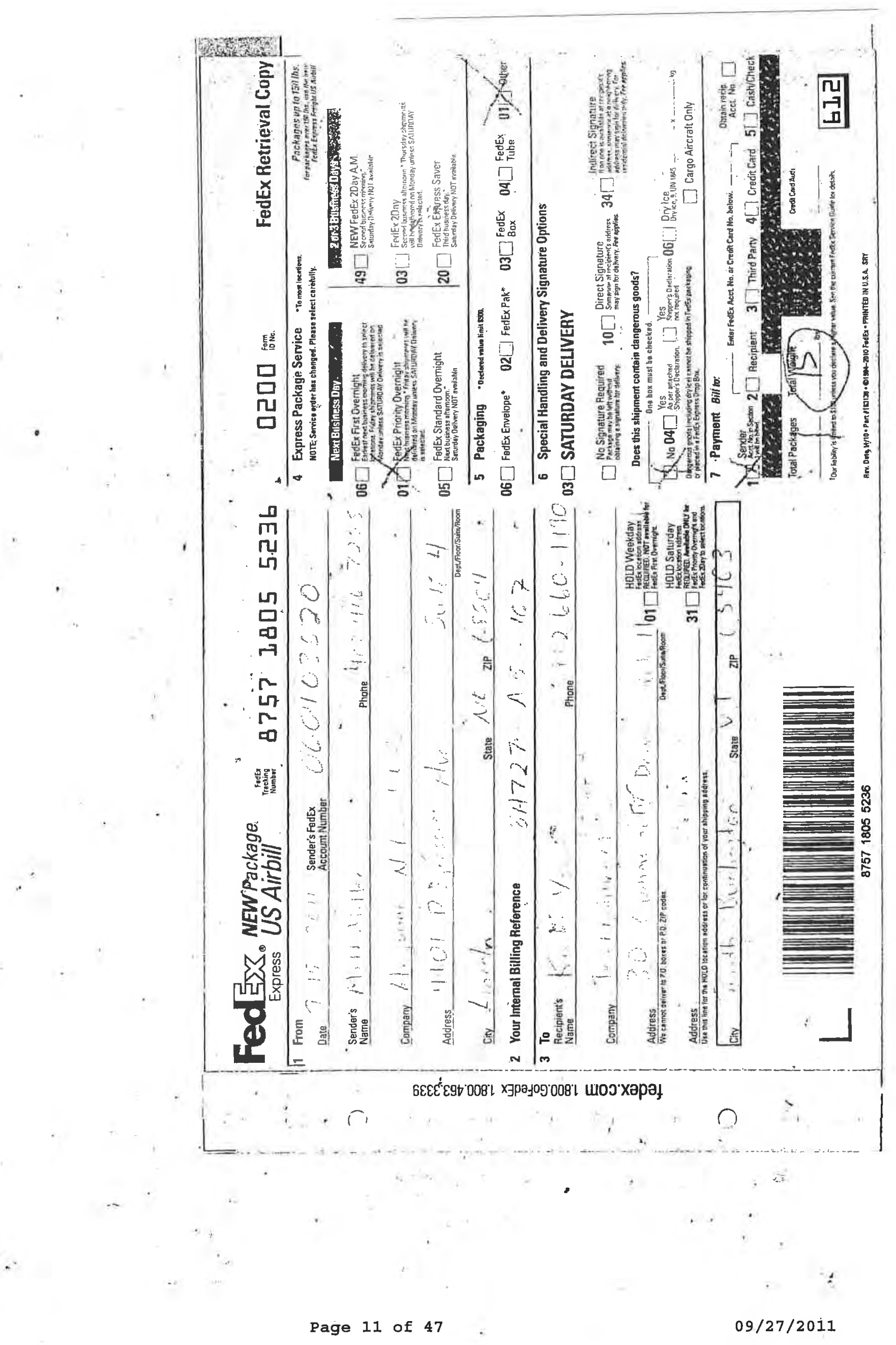




\section{Login Sample Receipt Checklist}

Client: Argonne National Laboratory

Job Number: 200-7045-1

SDG Number: 200-7045

Login Number: 7045

List Source: TestAmerica Burlington

List Number: 2

Creator: Matot, Wade $\mathbf{M}$

Question

Answer

Radioactivity either was not measured or, if measured, is at or below background

The cooler's custody seal, if present, is intact.

The cooler or samples do not appear to have been compromised or tampered with.

Samples were received on ice.

Cooler Temperature is acceptable.

Cooler Temperature is recorded.

$\mathrm{COC}$ is present.

COC is filled out in ink and legible.

COC is filled out with all pertinent information.

Is the Field Sampler's name present on COC?

There are no discrepancies between the sample IDs on the containers and the COC.

Samples are received within Holding Time.

Sample containers have legible labels.

Containers are not broken or leaking.

Sample collection date/times are provided

Appropriate sample containers are used.

Sample bottles are completely filled.

Sample Preservation Verified

There is sufficient vol. for all requested analyses, incl. any requested

MS/MSDs

VOA sample vials do not have headspace or bubble is $<6 \mathrm{~mm}\left(1 / 4^{\prime \prime}\right)$ in

diameter.

Multiphasic samples are not present.

Samples do not require splitting or compositing

Residual Chlorine Checked.

N/A
True $\quad$ Lab does not accept radioactive samples.
True
True
True
True
True
True
True
True
False
True ${ }^{\circ}$ IR gun ID $96, \mathrm{CF}=0$
True
True
True
True
True
N/A
True
True
True
True
N/A




\section{Sample Login Acknowledgement}

Job 200-7045-1

$\begin{array}{ll}\text { Client Job Description: } & \text { Inman (200-7045) } \\ \text { Purchase Order \#: } & \text { 1E-30401 } \\ \text { Work Order \#: } & \text { 1E-30401 } \\ \text { Project Manager: } & \text { Kirk F Young } \\ \text { Job Due Date: } & 9 / 30 / 2011 \\ \text { Job TAT: } & 14 \text { Days } \\ \text { Max Deliverable Level: } & \text { IV }\end{array}$

Earliest Deliverable Due: 9/30/2011

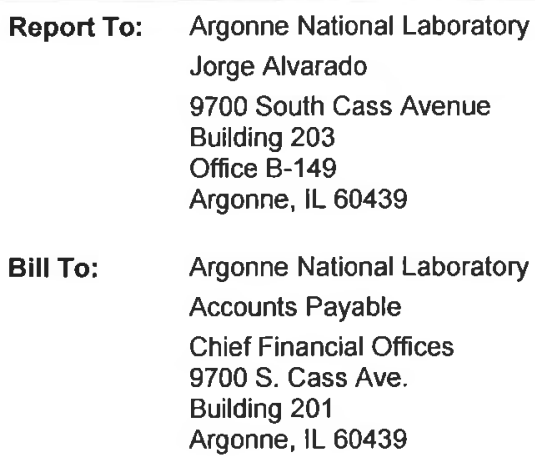

Login 200-7045

\begin{tabular}{|c|c|}
\hline Sample Receipt: & 9/16/2011 10:20:00 AM \\
\hline Method of Delivery: & FedEx Priority Overnight \\
\hline \multicolumn{2}{|c|}{ Lab Sample \# Client Sample ID } \\
\hline Method & $\begin{array}{l}\text { ient Sample ID } \\
\text { Method Description / Work Location }\end{array}$ \\
\hline 200-7045-1 & INMW20S-W-32780 \\
\hline \multicolumn{2}{|l|}{ SOM01.2_Vol_Tr } \\
\hline 200-7045-2 & INMW20M-W-32781 \\
\hline SOM01.2_Vol_Tr & SOM01.2 Trace Volatile Organics / In-Lab \\
\hline 200-7045-3 & INMW14D-W-32782 \\
\hline SOM01.2_Vol_Tr & SOM01.2 Trace Volatile Organics / In-Lab \\
\hline 200-7045-4 & INQCTB-W-32783 \\
\hline SOM01.2_Vol_Tr & SOM01.2 Trace Volatile Organics / In-Lab \\
\hline 200-7045-5 & VHBLK01 \\
\hline SOM01.2_Vol_Tr & SOM01.2 Tr \\
\hline
\end{tabular}

Number of Coolers: $\quad 1$

Cooler Temperature(s) $\left(C^{\circ}\right): 0.1$;

\begin{tabular}{|c|c|c|c|}
\hline \multirow[t]{2}{*}{ Date Sampled } & \multicolumn{3}{|l|}{ Matrix } \\
\hline & & Rpt Basis & Dry / Wet ** \\
\hline 9/15/2011 12:00:00 AM & Water & & \\
\hline & & Total & Wet \\
\hline 9/15/2011 12:00:00 AM & Water & & \\
\hline & & Total & Wet \\
\hline 9/15/2011 12:00:00 AM & Water & & \\
\hline & & Total & Wet \\
\hline 9/15/2011 12:00:00 AM & Water & & \\
\hline & & Total & Wet \\
\hline 9/16/2011 2:25:00 PM & Water & & \\
\hline & & Total & Wet \\
\hline
\end{tabular}




\section{METHODOLOGY SUMMARY}

\begin{tabular}{lll} 
Laboratory: & TestAmerica Laboratories & Project No: \\
Location: $\quad$ South Burlington, Vermont & SDG No: & $200-7045$ \\
\hline VOA & \\
Volatile Organics Trace - USEPA CLP SOM01.2 &
\end{tabular}


Lab Name: TESTAMERICA BURLINGTON

Lab Code: STIV Case No.: INMAN Mod. Ref No.:

Level: (TRACE or LOW) TRACE
Contract: 8E-00302

SDG No.: 200-7045

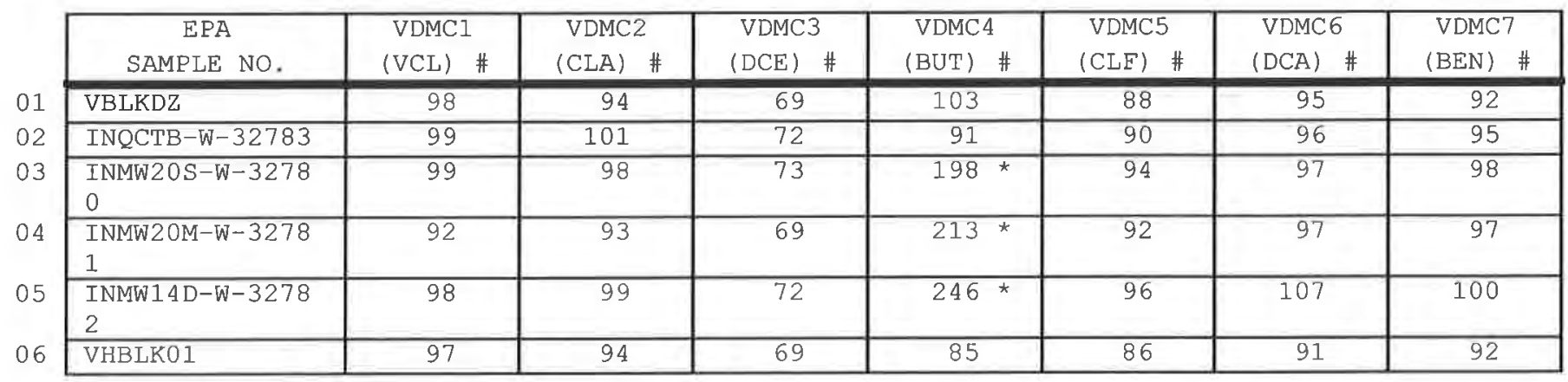

\footnotetext{
VDMC1 (VCL) = Vinyl Chloride-d3

VDMC2 $($ CLA $)=$ Chloroethane $-\mathrm{d} 5$

VDMC3 $(\mathrm{DCE})=1,1-\mathrm{Dich}$ loroethene-d2

VDMC4 $\quad(B U T)=2-B u t a n o n e-d 5$

VDMC5 $(\mathrm{CLF})=$ Chloroform-d

VDMC6 $(\mathrm{DCA})=1,2-\mathrm{Dich}$ loroethane-d4

VDMC7 $(\mathrm{BEN})=$ Benzene-d6
}

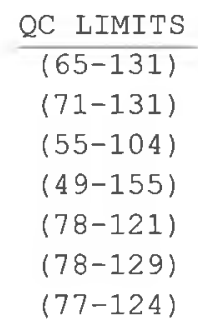

\# Column to be used to flag recovery values

* Values outside of contract required QC limits
Page 1 of 1
SOM01.2 (4/2007) 
$2 \mathrm{~B}-$ FORM II VOA-2

WATER VOLATILE DEUTERATED MONITORING COMPOUND RECOVERY

Lab Name: TESTAMERICA BURLINGTON

Contract: $8 \mathrm{E}-00302$

Lab Code: STLV Case No.: INMAN Mod. Ref No.:

SDG No.: 200-7045

Level: (TRACE or LOW) TRACE

\begin{tabular}{|c|c|c|c|c|c|c|c|c|}
\hline $\begin{array}{c}\text { EPA } \\
\text { SAMPLE NO. }\end{array}$ & $\begin{array}{l}\text { VDMC8 } \\
\text { (DPA) \# }\end{array}$ & $\begin{array}{l}\text { VDMC9 } \\
\text { (TOL) \# }\end{array}$ & $\begin{array}{l}\text { VDMC10 } \\
\text { (TDP) \# }\end{array}$ & $\begin{array}{l}\text { VDMC11 } \\
(\mathrm{HEX}) \#\end{array}$ & $\begin{array}{l}\text { VDMC12 } \\
\text { (TCA) \# }\end{array}$ & $\begin{array}{l}\text { VDMC13 } \\
\text { (DCZ) \# }\end{array}$ & OTHER & $\begin{array}{l}\text { TOT } \\
\text { OUT }\end{array}$ \\
\hline VBLKDZ & 91 & 91 & 92 & 97 & 82 & 100 & & 0 \\
\hline INQCTB-W-32783 & 92 & 94 & 90 & 90 & 81 & 96 & & 0 \\
\hline $\begin{array}{l}\text { INMW20M-W-3278 } \\
1\end{array}$ & 94 & 95 & 95 & $253 *$ & 88 & 103 & & 2 \\
\hline VHBLKO1 & 89 & 92 & 83 & 82 & 76 & 104 & & 0 \\
\hline
\end{tabular}

VDMC8 $(D P A)=1,2-$ Dichloropropane-d6

QC LIMITS

VDMC9 $($ TOL) = Toluene-d8

$(79-124)$

VDMC10 $($ TDP $)=$ trans-1,3-Dichloropropene-d4

$(77-121)$

VDMC11 $($ HEX) $=2$-Hexanone-d5

$(73-121)$

VDMC12 $(\mathrm{TCA})=1,1,2,2$-Tetrachloroethane-d2

(28-135)

VDMC13 $(\mathrm{DCZ})=1,2-\mathrm{Dich}$ lorobenzene-d4

$(73-125)$

$(80-131)$

\# Column to be used to flag recovery values

* Values outside of contract required QC limits

Report 1,4-Dioxane-d8 for Low-Medium VOA analysis only

Page 1 of 1 
$4 A$ - FORM IV VOA

VOLATILE METHOD BLANK SUMMARY
EPA SAMPLE NO.

VBLKDZ
Lab Name: TESTAMERICA BURLINGTON

Lab Code: STLV Case No.

INMAN

Lab File ID: DHPABO3.D

Instrument ID: D.i

Matrix: (SOII/SED/WATER)

Level: (TRACE or LOW/MED)

Water

TRACE

GC Column: DB-624

ID $: 0.20$

(mm)
Contract: $\quad 8 \mathrm{E}-00302$

SDG No.: 200-7045

Lab Sample ID: MB 200-25508/3

Date Analyzed: 09/20/2011

Time Analyzed: 0926

Heated Purge: $(\mathrm{Y} / \mathrm{N}) \mathrm{N}$

\begin{tabular}{|l|l|l|l|c|}
\cline { 2 - 5 } \multicolumn{1}{c|}{ EPA } & \multicolumn{1}{|c|}{ LAB } & LAB & TIME \\
SAMPLE NO. & \multicolumn{1}{|c|}{ SAMPLE ID } & FILE ID & ANALYZED \\
\cline { 2 - 5 } 01 & $\begin{array}{l}\text { INQCTB-W-327 } \\
83\end{array}$ & $200-7045-4$ & DHPAB04.D & 1002 \\
\cline { 2 - 5 } 02 & $\begin{array}{l}\text { INMW20S-W-32 } \\
780\end{array}$ & $200-7045-1$ & DHPAB05.D & 1027 \\
\cline { 2 - 5 } 03 & $\begin{array}{l}\text { INMW20M-W-32 } \\
781\end{array}$ & $200-7045-2$ & DHPAB06.D & 1051 \\
\cline { 2 - 5 } 04 & $\begin{array}{l}\text { INMW14D-W-32 } \\
782\end{array}$ & $200-7045-3$ & DHPAB07.D & 1116 \\
\cline { 2 - 5 } 05 & VHBLK01 & $200-7045-5$ & DHPAB08.D & 1154 \\
\hline
\end{tabular}

COMMENTS:

Page 1 of 1 
$5 A$ - FORM V VOA

VOLATILE ORGANICS INSTRUMENT

PERFORMANCE CHECK

BROMOFLUOROBENZENE (BEB)
EPA SAMPLE NO.

BEBDV
Lab Name: TESTAMERICA BURLINGTON

Lab Code: STLV Case No.: INMA

Lab File Id: DHP01.D

Instrument Id: D.i

GC Column: DB-624

ID: 0.20 (mm)

Contract: $\quad 8 \mathrm{E}-00302$

\begin{tabular}{|r|l|r|}
\hline $\mathrm{m} / \mathrm{e}$ & ION ABUNDANCE CRITERIA & $\begin{array}{c}\text { RELATIVE } \\
\text { ABUNDANCE }\end{array}$ \\
\hline 50 & $15.0-40.0 \%$ of mass 95 & 26.1 \\
\hline 75 & $30.0-80.0 \%$ of mass 95 & 46.4 \\
\hline 95 & Base peak, 100\% relative abundance & 100 \\
\hline 96 & $5.0-9.0 \%$ of mass 95 & 6.3 \\
\hline 173 & Less than $2.0 \%$ of mass 174 & 0 \\
\hline 174 & $50.0-120 \%$ of mass 95 & $0) 1$ \\
\hline 175 & $5.0-9.0 \%$ of mass 174 & 77.7 \\
\hline 176 & $95.0-101 \%$ of mass 174 & 6.0 \\
\hline 177 & $5.0-9.0 \%$ of mass 176 & $7.8) 1$ \\
\hline
\end{tabular}

1 - Value is ormass $174 \quad 2$ - Value is ormass 176

\begin{tabular}{|c|c|c|c|c|}
\hline $\begin{array}{c}\text { EPA } \\
\text { SAMPLE NO. }\end{array}$ & $\begin{array}{r}\text { LAB } \\
\text { SAMPLE ID }\end{array}$ & $\begin{array}{c}\text { LAB } \\
\text { FILE ID }\end{array}$ & $\begin{array}{c}\text { DATE } \\
\text { ANALYZED }\end{array}$ & $\begin{array}{c}\text { TIME } \\
\text { ANALYZED }\end{array}$ \\
\hline VSTD0.5DV & IC $200-21023 / 4$ & DHPO4.D & $07 / 07 / 2011$ & 1117 \\
\hline VSTD001DV & IC $200-21023 / 5$ & DHPO5.D & $07 / 07 / 2011$ & 1141 \\
\hline VSTD005DV & ICIS $200-21023 / 6$ & DHPO 6. D & $07 / 07 / 2011$ & 1206 \\
\hline VSTD010DV & IC $200-21023 / 7$ & DHP07.D & $07 / 07 / 2011$ & 1231 \\
\hline VSTD020DV & IC $200-21023 / 8$ & DHPO8.D & $07 / 07 / 2011$ & 1256 \\
\hline
\end{tabular}

Page 1 of 1 
$5 A$ - FORM V VOA

VOLATILE ORGANICS INSTRUMENT

PERFORMANCE CHECK

BROMOFLUOROBENZENE (BEB)
EPA SAMPLE NO.

BFBDZ

Lab Name: TESTAMERICA BURLINGTON

Contract: $\quad 8 \mathrm{E}-00302$

Lab Code: STLV Case No.: INMAN Mod. Ref No.: SDG No.: 200-7045

Lab File Id: DHPABO1.D

Instrument Id: D.i

GC Column: DB-624

\begin{tabular}{|l|l}
\hline $\mathrm{m} / \mathrm{e}$ & ION ABUNDANCE CRITERIA \\
\hline
\end{tabular}

$50 \quad 15.0-40.0 \%$ of mass 95

$75 \quad 30.0-80.0 \%$ of mass 95

95 Base peak, 100\% relative abundance

$965.0-9.0 \%$ of mass 95

173 Less than $2.0 \%$ of mass 174

$17450.0-120 \%$ of mass 95

$175 \quad 5.0-9.0 \%$ of mass 174

$17695.0-101 \%$ of mass 174

$1775.0-9.0$ o of mass 176
BFB Injection Date: 09/20/2011

BFB Injection Time: 0845

1 - Value is omass $174 \quad 2$ - Value is omass 176

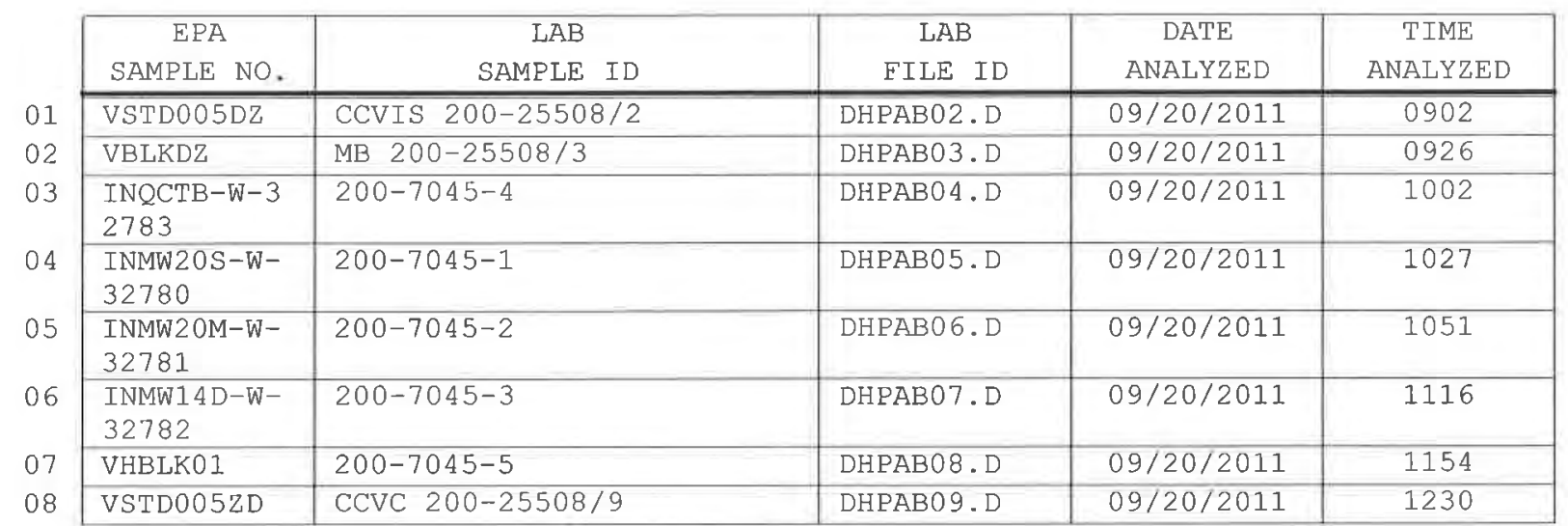


Lab Name: TESTAMERICA BURLINGTON

Lab Code: STLV Case No. :

GC Column: DB-624

INMAN Mod. Ref No. :

Contract: $\quad 8 \mathrm{E}-00302$

EPA Sample No. (VSTD\#\#\#\#) :

Lab File ID (Standard):

ID: 0.20

(mm) Init.

VSTD005DZ

Instrument ID: D.i
DHPAB02.D
SDG No.: 200-7045

Calib. Date(s) : 07/07/2011 07/07/2011

Date Analyzed: 09/20/2011

Time Analyzed: 0902

Heated Purge: $(\mathrm{Y} / \mathrm{N}) \mathrm{N}$

\begin{tabular}{|c|c|c|c|c|c|c|}
\hline & $\begin{array}{c}\text { IS1 (CBZ) } \\
\text { AREA }\end{array}$ & $\mathrm{RT} \#$ & $\begin{array}{c}\text { IS2 (DFB) } \\
\text { AREA }\end{array}$ & RT \# & $\begin{array}{c}\text { IS3 (DCB) } \\
\text { AREA }\end{array}$ & $\mathrm{RT}$ \\
\hline 12 HOUR STD & 163488 & 8.83 & 190870 & 5.47 & 79865 & 11.66 \\
\hline UPPER LIMIT & 228883 & 9.16 & 267218 & 5.80 & 111811 & 11.99 \\
\hline LOWER LIMIT & 98093 & 8.50 & 114522 & 5.14 & 47919 & 11.33 \\
\hline EPA SAMPLE NO. & & & & & & \\
\hline$\overline{\text { VBLKDZ }}$ & 156136 & 8.83 & 183963 & 5.47 & 62588 & 11.66 \\
\hline INQCTB-W-32783 & 145068 & 8.83 & 171033 & 5.47 & 61737 & 11.66 \\
\hline $\begin{array}{l}\text { INMW20S-W-3278 } \\
0\end{array}$ & 146335 & 8.83 & 173131 & 5.47 & 64724 & 11.66 \\
\hline $\begin{array}{l}\text { INMW2 } 0 M-W-3278 \\
1\end{array}$ & 164578 & 8.83 & 198076 & 5.47 & 69148 & 11.66 \\
\hline $\begin{array}{l}\text { INMW1 4D-W-3278 } \\
2\end{array}$ & 152557 & 8.83 & 179499 & 5.47 & 61619 & 11.66 \\
\hline VHBLKO 1 & 143973 & 8.83 & 171001 & 5.47 & 54072 & 11.66 \\
\hline
\end{tabular}

IS1 $(\mathrm{CBZ})=$ Chlorobenzene- $d 5$

IS2 $(\mathrm{DFB})=1,4$-Difluorobenzene

IS3 $(\mathrm{DCB})=1,4-\mathrm{Dichlorobenzene-d4}$

AREA UPPER LIMIT $=140 \%$ (Trace Volatiles) of internal standard area AREA LOWER LIMIT $=60 \%$ (Trace Volatiles) of internal standard area RT UPPER LIMIT $=+0.33$ (Trace Volatiles) minutes of internal standard RT RT LOWER LIMIT $=-0.33$ (Trace Volatiles) minutes of internal standard RT

\# Column used to flag values outside contract required QC limits with an asterisk. 
$1 A$ - FORM I VOA-1

VOLATILE ORGANICS ANALYSIS DATA SHEET
EPA SAMPLE NO.

INMW14D-W-32782
Lab Name: TESTAMERICA BURLINGTON

Lab Code: STLV

Case No.: INMAN

Matrix: (SOIL/SED/WATER)

Water

Sample wt/vol: 25.0

$(\mathrm{g} / \mathrm{mL}) \mathrm{mL}$

Level: (TRACE/LOW/MED) TRACE

o Moisture: not dec.

GC Column: DB-624

ID $: 0.20$ (mm)

Soil Extract Volume:

Purge Volume: 25.0
Contract: $8 \mathrm{E}-00302$

SDG No.: 200-7045

Lab Sample ID: 200-7045-3

Lab File ID: DHPAB07.D

Date Received: 09/16/2011

Date Analyzed: 09/20/2011

Dilution Factor: 1.0

Soil Aliquot Volume:

(uL)

\begin{tabular}{|c|c|c|c|}
\hline CAS NO. & COMPOUND & $\begin{array}{l}\text { CONCENTRATION UNITS: } \\
(\mathrm{ug} / \mathrm{L} \text { or } \mathrm{ug} / \mathrm{kg}) \mathrm{ug} / \mathrm{L}\end{array}$ & $Q$ \\
\hline $75-71-8$ & Dichlorodifluoromethane & 0.50 & $\bar{U}$ \\
\hline $74-87-3$ & Chloromethane & 0.054 & $\mathrm{~J}$ \\
\hline $75-01-4$ & Vinyl chloride & 0.50 & $\mathrm{U}$ \\
\hline $74-83-9$ & Bromomethane & 0.50 & $\mathrm{U}$ \\
\hline $75-00-3$ & Chloroethane & 0.50 & $\mathrm{U}$ \\
\hline $75-69-4$ & Trichlorofluoromethane & 0.50 & $\mathrm{U}$ \\
\hline $75-35-4$ & 1,1-Dichloroethene & 0.50 & $\mathrm{U}$ \\
\hline $76-13-1$ & 1,1,2-Trichloro-1,2,2-trifluoroethane & 0.50 & $\mathrm{U}$ \\
\hline $67-64-1$ & Acetone & 32 & $\mathrm{~B}$ \\
\hline $75-15-0$ & Carbon disulfide & 0.50 & $\mathrm{U}$ \\
\hline $79-20-9$ & Methyl acetate & 0.50 & $\mathrm{U}$ \\
\hline $75-09-2$ & Methylene Chloride & 0.15 & $\mathrm{~J}$ \\
\hline $156-60-5$ & trans-1,2-Dichloroethene & 0.50 & $\mathrm{U}$ \\
\hline $1634-04-4$ & Methyl tert-butyl ether & 0.50 & $\mathrm{U}$ \\
\hline $75-34-3$ & 1,1-Dichloroethane & 0.50 & U \\
\hline $156-59-2$ & cis-1,2-Dichloroethene & 0.50 & $\mathrm{U}$ \\
\hline $78-93-3$ & 2-Butanone & 29 & \\
\hline $74-97-5$ & Bromochloromethane & 0.088 & $\mathrm{~J}$ \\
\hline $67-66-3$ & Chloroform & 0.56 & $\mathrm{~B}$ \\
\hline $71-55-6$ & 1,1,1-Trichloroethane & 0.50 & $\mathrm{U}$ \\
\hline $110-82-7$ & Cyclohexane & 0.50 & $\mathrm{U}$ \\
\hline $56-23-5$ & Carbon tetrachloride & 0.50 & $\mathrm{U}$ \\
\hline $71-43-2$ & Benzene & 0.021 & $\mathrm{~J}$ \\
\hline $107-06-2$ & 1,2-Dichloroethane & 0.50 & U \\
\hline
\end{tabular}

Report 1,4-Dioxane for Low-Medium VOA analysis only 
$1 \mathrm{~B}$ - FORM I VOA-2

VOLATILE ORGANICS ANATYSIS DATA SHEET
EPA SAMPLE NO.

INMW14D-W-32782
Lab Name: TESTAMERICA BURLINGTON

Lab Code: STLV Case No.: INMA Mod
Water

$(\mathrm{g} / \mathrm{mL}) \quad \mathrm{mL}$

Sample wt/vol: 25.0

Level: (TRACE/LOW/MED) TRACE

o Moisture: not dec.

GC Column: DB-624

ID: 0.20 (mm)

Soil Extract Volume:

Purge Volume: 25.0 $(\mathrm{mL})$
Contract: $8 \mathrm{E}-00302$

SDG No.: 200-7045
Lab Sample ID: 200-7045-3

Lab File ID: DHPAB07.D

Date Received: 09/16/2011

Date Analyzed: 09/20/2011

Dilution Factor: 1.0

Soil Aliquot Volume:

(UL)

\begin{tabular}{|c|c|c|c|}
\hline CAS NO. & COMPOUND & $\begin{array}{l}\text { CONCENTRATION UNITS: } \\
(\mathrm{ug} / \mathrm{L} \text { or } \mathrm{ug} / \mathrm{kg}) \mathrm{ug} / \mathrm{L}\end{array}$ & $Q$ \\
\hline $79-01-6$ & Trichloroethene & 0.50 & $\mathrm{U}$ \\
\hline $108-87-2$ & Methylcyclohexane & 0.50 & $\mathrm{U}$ \\
\hline $78-87-5$ & 1,2-Dichloropropane & 0.50 & $\mathrm{U}$ \\
\hline $75-27-4$ & Bromodichloromethane & 0.50 & $\mathrm{U}$ \\
\hline $10061-01-5$ & cis-1,3-Dichloropropene & 0.50 & $\mathrm{U}$ \\
\hline $108-10-1$ & 4-Methyl-2-pentanone & 5.0 & $\mathrm{U}$ \\
\hline $108-88-3$ & Toluene & 0.021 & $\mathrm{~J} \mathrm{~B}$ \\
\hline $10061-02-6$ & trans-1,3-Dichloropropene & 0.50 & $\mathrm{U}$ \\
\hline $79-00-5$ & 1,1,2-Trichloroethane & 0.50 & $\mathrm{U}$ \\
\hline $127-18-4$ & Tetrachloroethene & 0.50 & $\mathrm{U}$ \\
\hline $591-78-6$ & 2-Hexanone & 5.0 & $\mathrm{U}$ \\
\hline $124-48-1$ & Dibromochloromethane & 0.50 & $\mathrm{U}$ \\
\hline $106-93-4$ & 1,2-Dibromoethane & 0.50 & $\mathrm{U}$ \\
\hline $108-90-7$ & Chlorobenzene & 0.50 & $\mathrm{U}$ \\
\hline $100-41-4$ & Ethylbenzene & 0.011 & $\mathrm{~J}$ \\
\hline $95-47-6$ & o-xylene & 0.0062 & $\mathrm{~J}$ \\
\hline $179601-23-1$ & $\mathrm{~m}, \mathrm{p}-\mathrm{XYl}_{\mathrm{l}} \mathrm{ene}$ & 0.014 & $\mathrm{~J}$ \\
\hline $100-42-5$ & Styrene & 0.50 & $\mathrm{U}$ \\
\hline $75-25-2$ & Bromoform & 0.50 & $\mathrm{U}$ \\
\hline $98-82-8$ & Isopropylbenzene & 0.50 & $\mathrm{U}$ \\
\hline $79-34-5$ & $1,1,2,2$-Tetrachloroethane & 0.50 & $\mathrm{U}$ \\
\hline $541-73-1$ & 1,3-Dichlorobenzene & 0.50 & $\mathrm{U}$ \\
\hline $106-46-7$ & 1,4-Dichlorobenzene & 0.50 & $\mathrm{U}$ \\
\hline $95-50-1$ & 1,2-Dichlorobenzene & 0.50 & $\mathrm{U}$ \\
\hline $96-12-8$ & 1,2-Dibromo-3-Chloropropane & 0.50 & $\mathrm{U}$ \\
\hline $120-82-1$ & 1,2,4-Trichlorobenzene & 0.50 & $\mathrm{U}$ \\
\hline $87-61-6$ & 1,2,3-Trichlorobenzene & 0.50 & $\mathrm{U}$ \\
\hline
\end{tabular}


1J - FORM I VOA-TIC

VOLATILE ORGANICS ANALYSIS DATA SHEET

TENTATIVELY IDENTIFIED COMPOUNDS
EPA SAMPLE NO.

INMW14D-W-32782

Lab Name: TESTAMERICA BURLINGTON

Contract: $8 \mathrm{E}-00302$

Lab Code: STLV Case No.: INMAN Mod. Ref No.: SDG No.: $200-7045$

Matrix: (SOIL/SED/WATER)

Water

Sample wt/vol: 25.0 $(\mathrm{g} / \mathrm{mL}) \quad \mathrm{mL}$

Level: (TRACE or LOW/MED) TRACE

ㅇ Moisture: not dec.

GC Column: DB-624 ID: 0.20 (mm)

Soil Extract Volume: (uL)

CONCENTRATION UNITS: (ug/L or $\mathrm{ug} / \mathrm{kg}$ )
Lab Sample ID: 200-7045-3

Lab File ID: DHPAB07.D

Date Received: 09/16/2011

Date Analyzed: 09/20/2011

Dilution Factor: 1.0

Soil Aliquot Volume: (uL)

Purge Volume: 25.0 (mL)

\begin{tabular}{|l|l|l|l|r|r|}
\hline \multicolumn{1}{|c|}{ CAS NUMBER } & \multicolumn{1}{|c|}{ COMPOUND NAME } & RT & EST. CONC. & Q \\
\cline { 2 - 6 } 01 & & Unknown & 6.79 & B X J \\
\cline { 2 - 6 } 02 & E9667961 & Total Alkanes & N/A & & \\
\hline
\end{tabular}

1 EPA-designated Registry Number. 
Lab Name: TESTAMERICA BURLINGTON

Contract: $8 \mathrm{E}-00302$

Lab Code: STLV Case No.: INMAN Mod. Ref No.:

SDG No.: 200-7045

Matrix: (SOIL/SED/WATER)

Sample wt/vol: 25.0

Water

$(\mathrm{g} / \mathrm{mL}) \quad \mathrm{mL}$

Level: (TRACE/LOW/MED) TRACE

o Moisture: not dec.

GC Column: DB-624 ID: 0.20 (mm)

Soil Extract Volume: (uL) (mL)

Purge Volume: 25.0
Lab Sample ID: 200-7045-2

Lab File ID: DHPABO6.D

Date Received: 09/16/2011

Date Analyzed: 09/20/2011

Dilution Factor: 1.0

Soil Aliquot Volume:

(uL)

\begin{tabular}{|c|c|c|c|}
\hline CAS NO. & COMPOUND & $\begin{array}{l}\text { CONCENTRATION UNITS: } \\
(\mathrm{ug} / \mathrm{L} \text { or } \mathrm{ug} / \mathrm{kg}) \mathrm{ug} / \mathrm{L}\end{array}$ & Q \\
\hline $75-71-8$ & Dichlorodifluoromethane & 0.50 & $\mathrm{U}$ \\
\hline $74-87-3$ & Chloromethane & 0.50 & $\mathrm{U}$ \\
\hline $75-01-4$ & Vinyl chloride & 0.50 & $\mathrm{U}$ \\
\hline $74-83-9$ & Bromomethane & 0.50 & $\mathrm{U}$ \\
\hline $75-00-3$ & Chloroethane & 0.50 & $\mathrm{U}$ \\
\hline $75-69-4$ & Trichlorofluoromethane & 0.50 & $\mathrm{U}$ \\
\hline $75-35-4$ & 1,1-Dichloroethene & 0.50 & $\mathrm{U}$ \\
\hline $76-13-1$ & 1,1,2-Trichloro-1,2,2-trifluoroethane & 0.50 & $\mathrm{U}$ \\
\hline $67-64-1$ & Acetone & 55 & B \\
\hline $75-15-0$ & Carbon disulfide & 0.50 & $\mathrm{U}$ \\
\hline $79-20-9$ & Methyl acetate & 0.50 & $\mathrm{U}$ \\
\hline $75-09-2$ & Methylene Chloride & 0.50 & $\mathrm{U}$ \\
\hline $156-60-5$ & trans-1,2-Dichloroethene & 0.50 & $\mathrm{U}$ \\
\hline $1634-04-4$ & Methyl tert-butyl ether & 0.50 & $\mathrm{U}$ \\
\hline $75-34-3$ & 1,1-Dichloroethane & 0.50 & $\mathrm{U}$ \\
\hline $156-59-2$ & cis-1,2-Dichloroethene & 0.50 & $\mathrm{U}$ \\
\hline $78-93-3$ & 2-Butanone & 180 & \\
\hline $74-97-5$ & Bromochloromethane & 0.50 & $\mathrm{U}$ \\
\hline $67-66-3$ & Chloroform & 0.32 & $\mathrm{~J} \mathrm{~B}$ \\
\hline $71-55-6$ & 1,1,1-Trichloroethane & 0.50 & $\mathrm{U}$ \\
\hline $110-82-7$ & Cyclohexane & 0.50 & $\mathrm{U}$ \\
\hline $56-23-5$ & Carbon tetrachloride & 0.50 & $\mathrm{U}$ \\
\hline $71-43-2$ & Benzene & 0.012 & $\mathrm{~J}$ \\
\hline $107-06-2$ & 1,2-Dichloroethane & 0.50 & $\mathrm{U}$ \\
\hline
\end{tabular}

Report 1,4-Dioxane for Low-Medium VOA analysis only 
$1 B$ - FORM I VOA-2

VOLATILE ORGANICS ANALYSIS DATA SHEET
EPA SAMPLE NO.

INMW20M-W-32781
Lab Name: TESTAMERICA BURLINGTON

Lab Code: STLV Cas

Matrix: (SOIL/SED/WATER)

Sample wt/vol: 25.0

Water

Sample wt/VOl: 25.0

$(\mathrm{g} / \mathrm{mL}) \mathrm{mL}$

Level: (TRACE/LOW/MED) TRACE

응 Moisture: not dec.

GC Column: DB-624 ID: 0.20 (mm)

Soil Extract Volume:

(uL)

(mL)

Purge Volume: 25.0
Contract: $\quad 8 \mathrm{E}-00302$

SDG No.: 200-7045

\begin{tabular}{|c|c|c|c|}
\hline CAS NO. & COMPOUND & $\begin{array}{l}\text { CONCENTRATION UNITS: } \\
(\mathrm{ug} / \mathrm{L} \text { or } \mathrm{ug} / \mathrm{kg}) \mathrm{ug} / \mathrm{L}\end{array}$ & $Q$ \\
\hline $79-01-6$ & Trichloroethene & 0.50 & $\bar{U}$ \\
\hline $108-87-2$ & Methylcyclohexane & 0.50 & $\mathrm{U}$ \\
\hline $78-87-5$ & 1,2-Dichloropropane & 0.50 & $\mathrm{U}$ \\
\hline $75-27-4$ & Bromodichloromethane & 0.50 & \\
\hline $10061-01-5$ & cis-1,3-Dichloropropene & 0.50 & $\mathrm{U}$ \\
\hline $108-10-1$ & 4-Methyl-2-pentanone & 5.0 & $\mathrm{U}$ \\
\hline $108-88-3$ & Toluene & 0.054 & $\mathrm{~J} B$ \\
\hline $10061-02-6$ & trans-1,3-Dichloropropene & 0.50 & $\mathrm{U}$ \\
\hline $79-00-5$ & 1,1,2-Trichloroethane & 0.50 & $\mathrm{U}$ \\
\hline $127-18-4$ & Tetrachloroethene & 0.50 & $\mathrm{U}$ \\
\hline $591-78-6$ & 2-Hexanone & 5.0 & $\mathrm{U}$ \\
\hline $124-48-1$ & Dibromochloromethane & 0.95 & \\
\hline $106-93-4$ & 1,2-Dibromoethane & 0.50 & $\mathrm{U}$ \\
\hline $108-90-7$ & Chlorobenzene & 0.50 & $\mathrm{U}$ \\
\hline $100-41-4$ & Ethylbenzene & 0.053 & $\mathrm{~J}$ \\
\hline $95-47-6$ & o-Xylene & 0.054 & $\mathrm{~J}$ \\
\hline $179601-23-1$ & $\mathrm{~m}, \mathrm{p}$-Xylene & 0.16 & $\mathrm{~J}$ \\
\hline $100-42-5$ & Styrene & 0.50 & $\mathrm{U}$ \\
\hline $75-25-2$ & Bromoform & 0.50 & $\mathrm{U}$ \\
\hline $98-82-8$ & Isopropylbenzene & 0.50 & $\mathrm{U}$ \\
\hline $79-34-5$ & $1,1,2,2$-Tetrachloroethane & 0.50 & $\mathrm{U}$ \\
\hline $541-73-1$ & 1,3-Dichlorobenzene & 0.50 & $\mathrm{U}$ \\
\hline $106-46-7$ & 1,4-Dichlorobenzene & 0.50 & $\mathrm{U}$ \\
\hline $95-50-1$ & 1,2-Dichlorobenzene & 0.50 & $\mathrm{U}$ \\
\hline $96-12-8$ & 1,2-Dibromo-3-Chloropropane & 0.50 & $\mathrm{U}$ \\
\hline $120-82-1$ & 1,2,4-Trichlorobenzene & 0.50 & $\mathrm{U}$ \\
\hline $87-61-6$ & 1,2,3-Trichlorobenzene & 0.50 & $\mathrm{U}$ \\
\hline
\end{tabular}

Lab Sample ID: 200-7045-2

Lab File ID: DHPAB06.D

Date Received: 09/16/2011

Date Analyzed: 09/20/2011

Dilution Factor: 1.0

Soil Aliquot Volume:

(uL) 
$1 \mathrm{~J}$ - FORM I VOA-TIC

VOLATILE ORGANICS ANALYSIS DATA SHEET

TENTATIVELY IDENTIFIED COMPOUNDS
EPA SAMPLE NO.

INMW20M-W-32781

Lab Name: TESTAMERICA BURLINGTON

Contract: $8 \mathrm{E}-00302$

Lab Code: STLV Case No.: INMAN Mod. Ref No.: SDG No.: 200-7045

Matrix: (SOIL/SED/WATER)

Water

Sample wt/vol: 25.0 $(\mathrm{g} / \mathrm{mL}) \mathrm{mL}$

Level: (TRACE or LOW/MED) TRACE

o Moisture: not dec.

GC Column: DB-624 ID: 0.20 (mm)

Soil Extract Volume: (uL)

CONCENTRATION UNITS: (ug/I or $\mathrm{ug} / \mathrm{kg}) \quad \underline{\mathrm{ug}} / \mathrm{L}$
Lab Sample ID: 200-7045-2

Lab File ID: DHPAB06.D

Date Received: 09/16/2011

Date Analyzed: 09/20/2011

Dilution Factor: 1.0

Soil Aliquot Volume: (uL)

Purge Volume: 25.0 $(\mathrm{mL})$

01

\begin{tabular}{|c|l|c|r|r|}
\hline \multicolumn{1}{|c|}{ CAS NUMBER } & \multicolumn{1}{|c|}{ COMPOUND NAME } & RT & EST. CONC. & Q \\
\hline & Unknown & 6.79 & 2.9 & B X J \\
\hline E966796 I & Total Alkanes & N/A & & \\
\hline
\end{tabular}

1 EPA-designated Registry Number. 
$1 A$ - FORM I VOA-1

. VOLATILE ORGANICS ANALYSIS DATA SHEET
EPA SAMPLE NO. INMW20S-W-32780
Lab Name: TESTAMERICA BURLINGTON

Lab Code: STLV Case No.: INMAN

Mod. Ref No.:

Contract: $8 \mathrm{E}-00302$

Matrix: (SOIL/SED/WATER)

Water

Sample wt/vol: 25.0

$(\mathrm{g} / \mathrm{mL}) \quad \mathrm{mL}$

Level: (TRACE/LOW/MED) TRACE

\% Moisture: not dec.

GC Column: DB-624

ID $: 0.20$ (mm)

Soil Extract Volume:

(uL) (mL)

Purge Volume: 25.0

Lab File ID: DHPAB05.D

Dilution Factor: 1.0

Soil Aliquot Volume:

SDG No.: 200-7045

Lab Sample ID: 200-7045-1

Date Received: 09/16/2011

Date Analyzed: 09/20/2011

(uL)

\begin{tabular}{|c|c|c|c|}
\hline CAS NO. & COMPOUND & $\begin{array}{l}\text { CONCENTRATION UNITS: } \\
(\mathrm{ug} / \mathrm{L} \text { or } \mathrm{ug} / \mathrm{kg}) \mathrm{ug} / \mathrm{L}\end{array}$ & $Q$ \\
\hline $75-71-8$ & Dichlorodifluoromethane & 0.50 & $\mathrm{U}$ \\
\hline $74-87-3$ & Chloromethane & 0.50 & $\mathrm{U}$ \\
\hline $75-01-4$ & Vinyl chloride & 0.50 & $\mathrm{U}$ \\
\hline $74-83-9$ & Bromomethane & 0.50 & $\mathrm{U}$ \\
\hline $75-00-3$ & Chloroethane & 0.50 & $\mathrm{U}$ \\
\hline $75-69-4$ & Trichlorofluoromethane & 0.50 & $\mathrm{U}$ \\
\hline $75-35-4$ & 1,1-Dichloroethene & 0.50 & $\mathrm{U}$ \\
\hline $76-13-1$ & 1,1,2-Trichloro-1,2,2-trifluoroethane & 0.50 & $\mathrm{U}$ \\
\hline $67-64-1$ & Acetone & 35 & $\mathrm{~B}$ \\
\hline $75-15-0$ & Carbon disulfide & 0.50 & $\mathrm{U}$ \\
\hline $79-20-9$ & Methyl acetate & 0.50 & $\mathrm{U}$ \\
\hline $75-09-2$ & Methylene Chloride & 0.50 & $\mathrm{U}$ \\
\hline $156-60-5$ & trans-1,2-Dichloroethene & 0.50 & $\mathrm{U}$ \\
\hline $1634-04-4$ & Methyl tert-butyl ether & 0.50 & $\mathrm{U}$ \\
\hline $75-34-3$ & 1,1-Dichloroethane & 0.50 & $\mathrm{U}$ \\
\hline $156-59-2$ & cis-1,2-Dichloroethene & 0.50 & $\mathrm{U}$ \\
\hline $78-93-3$ & 2-Butanone & 98 & \\
\hline $74-97-5$ & Bromochloromethane & 0.50 & $\mathrm{U}$ \\
\hline $67-66-3$ & Chloroform & 0.73 & B \\
\hline $71-55-6$ & 1,1,1-Trichloroethane & 0.50 & $\mathrm{U}$ \\
\hline $110-82-7$ & Cyclohexane & 0.50 & $\mathrm{U}$ \\
\hline $56-23-5$ & Carbon tetrachloride & 0.50 & $\mathrm{U}$ \\
\hline $71-43-2$ & Benzene & 0.50 & $\mathrm{U}$ \\
\hline $107-06-2$ & 1,2-Dichloroethane & 0.50 & $\mathrm{U}$ \\
\hline
\end{tabular}

Report 1,4-Dioxane for Low-Medium $V O A$ analysis only 
Lab Name: TESTAMERICA BURLINGTON

Lab Code: STLV Cas

Matrix: (SOIL/SED/WATER)

Water

Sample wt/vol: 25.0

$(\mathrm{g} / \mathrm{mL}) \mathrm{mL}$

Level: (TRACE/LOW/MED) TRACE

음 Moisture: not dec.

GC Column: DB-624

ID: 0.20 $(\mathrm{mm})$

Soil Extract Volume:

(uL)

(mL)

Purge volume: 25.0
Contract: $8 \mathrm{E}-00302$

SDG No.: 200-7045

\begin{tabular}{|c|c|c|c|}
\hline CAS NO. & COMPOUND & $\begin{array}{l}\text { CONCENTRATION UNITS: } \\
(\mathrm{ug} / \mathrm{L} \text { or } \mathrm{ug} / \mathrm{kg}) \mathrm{ug} / \mathrm{L}\end{array}$ & $\varnothing$ \\
\hline $79-01-6$ & Trichloroethene & 0.50 & $\bar{U}$ \\
\hline $108-87-2$ & Methylcyclohexane & 0.50 & $\mathrm{U}$ \\
\hline $78-87-5$ & 1,2-Dichloropropane & 0.50 & $\mathrm{U}$ \\
\hline $75-27-4$ & Bromodichloromethane & 1.3 & \\
\hline $10061-01-5$ & cis-1,3-Dichloropropene & 0.50 & $\mathrm{U}$ \\
\hline $108-10-1$ & 4-Methyl-2-pentanone & 5.0 & $\mathrm{U}$ \\
\hline $108-88-3$ & Toluene & 0.31 & $\mathrm{~J} B$ \\
\hline $10061-02-6$ & trans-1,3-Dichloropropene & 0.50 & $\mathrm{U}$ \\
\hline $79-00-5$ & 1,1,2-Trichloroethane & 0.50 & $\mathrm{U}$ \\
\hline $127-18-4$ & Tetrachloroethene & 0.50 & $\mathrm{U}$ \\
\hline $591-78-6$ & 2-Hexanone & 5.0 & $\mathrm{U}$ \\
\hline $124-48-1$ & Dibromochloromethane & 2.5 & \\
\hline $106-93-4$ & 1,2-Dibromoethane & 0.50 & $\mathrm{U}$ \\
\hline $108-90-7$ & Chlorobenzene & 0.50 & U \\
\hline $100-41-4$ & Ethylbenzene & 0.12 & $\mathrm{~J}$ \\
\hline $95-47-6$ & o-xylene & 0.14 & $\mathrm{~J}$ \\
\hline $179601-23-1$ & $\mathrm{~m}, \mathrm{p}$-Xylene & 0.39 & $\mathrm{~J}$ \\
\hline $100-42-5$ & Styrene & 0.50 & $\mathrm{U}$ \\
\hline $75-25-2$ & Bromoform & 2.5 & \\
\hline $98-82-8$ & Isopropylbenzene & 0.50 & $\mathrm{U}$ \\
\hline $79-34-5$ & $1,1,2,2$-Tetrachloroethane & 0.50 & $\mathrm{U}$ \\
\hline $541-73-1$ & 1,3-Dichlorobenzene & 0.50 & $\mathrm{U}$ \\
\hline $106-46-7$ & 1,4-Dichlorobenzene & 0.50 & $\mathrm{U}$ \\
\hline $95-50-1$ & 1,2-Dichlorobenzene & 0.50 & $\mathrm{U}$ \\
\hline $96-12-8$ & 1,2-Dibromo-3-Chloropropane & 0.50 & $\mathrm{U}$ \\
\hline $120-82-1$ & 1,2,4-Trichlorobenzene & 0.50 & $\mathrm{U}$ \\
\hline $87-61-6$ & 1,2,3-Trichlorobenzene & 0.50 & $\mathrm{U}$ \\
\hline
\end{tabular}

Lab Sample ID: 200-7045-1

Lab File ID: DHPAB05.D

Date Received: 09/16/2011

Date Analyzed: 09/20/2011

Dilution Factor: 1.0

Soil Aliquot Volume:

(uL)

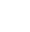


$1 \mathrm{~J}$ - FORM I VOA-TIC

VOLATILE ORGANICS ANALYSIS DATA SHEET

TENTATIVELY IDENTIEIED COMPOUNDS
EPA SAMPLE NO.

INMW $20 S-W-32780$

Lab Name: TESTAMERICA BURLINGTON

Contract: $\quad 8 \mathrm{E}-00302$

Lab Code: STLV Case No.: INMAN Mod. Ref No.:

SDG NO.: 200-7045

Matrix: (SOIL/SED/WATER)

Water

Sample wt/vol: 25.0

$(\mathrm{g} / \mathrm{mL}) \mathrm{mL}$

Level: (TRACE or LOW/MED)

TRACE

웅 Moisture: not dec.

GC Column: DB-624

ID: 0.20 (mm)

Soil Extract Volume: (uL)

CONCENTRATION UNITS: (ug/L or ug/kg) ug/L
Lab Sample ID: 200-7045-1

Lab File ID: DHPAB05.D

Date Received: 09/16/2011

Date Analyzed: 09/20/2011

Dilution Factor: 1.0

Soil Aliquot Volume: (uL)

Purge Volume: 25.0 (mL)

\begin{tabular}{|l|l|l|r|r|r|}
\hline \multicolumn{1}{|c|}{ CAS NUMBER } & \multicolumn{1}{|c|}{ COMPOUND NAME } & RT & EST. CONC. & Q \\
\cline { 2 - 6 } 01 & & Unknown & 6.79 & 3.0 & B X J \\
\cline { 2 - 6 } & E966796 I & Total Alkanes & N/A & \\
\hline
\end{tabular}

1 EPA-designated Registry Number. 
IA - FORM I VOA-1

VOLATILE ORGANICS ANALYSIS DATA SHEET
EPA SAMPLE NO.

INQCTB-W-32783
Lab Name: TESTAMERICA BURLINGTON

Lab Code: STLV Case No.: INMAN

Matrix: (SOIL/SED/WATER)

Water

Sample wt/vol: 25.0

$(\mathrm{g} / \mathrm{mL}) \quad \mathrm{mL}$

Level: (TRACE/LOW/MED) TRACE

o Moisture: not dec.

GC Column: $\mathrm{DB}-624$

ID: 0.20 $(\mathrm{mm})$

Soil Extract Volume:

(uL) $(\mathrm{mL})$

Purge Volume: 25.0
Contract: $8 E-00302$

SDG No.: 200-7045

Mod. Ref No.:

Lab Sample ID: 200-7045-4

Lab File ID: DHPABO4.D

Date Received: 09/16/2011

Date Analyzed: 09/20/2011

Dilution Factor: 1.0

Soil Aliquot Volume:

(uL)

\begin{tabular}{|c|c|c|c|}
\hline CAS NO. & COMPOUND & $\begin{array}{l}\text { CONCENTRATION UNITS: } \\
(\mathrm{ug} / \mathrm{L} \text { or } \mathrm{ug} / \mathrm{kg}) \mathrm{ug} / \mathrm{L}\end{array}$ & Q \\
\hline $75-71-8$ & Dichlorodifluoromethane & 0.50 & $\mathrm{U}$ \\
\hline $74-87-3$ & Chloromethane & 0.50 & $\mathrm{U}$ \\
\hline $75-01-4$ & Vinyl chloride & 0.50 & $\mathrm{U}$ \\
\hline $74-83-9$ & Bromomethane & 0.50 & $\mathrm{U}$ \\
\hline $75-00-3$ & Chloroethane & 0.50 & $\mathrm{U}$ \\
\hline $75-69-4$ & Trichlorofluoromethane & 0.50 & $\mathrm{U}$ \\
\hline $75-35-4$ & 1,1-Dichloroethene & 0.50 & $\mathrm{U}$ \\
\hline $76-13-1$ & 1,1,2-Trichloro-1,2,2-trifluoroethane & 0.50 & U \\
\hline $67-64-1$ & Acetone & 1.9 & $\mathrm{~J} \mathrm{~B}$ \\
\hline $75-15-0$ & Carbon disulfide & 0.50 & U \\
\hline $79-20-9$ & Methyl acetate & 0.50 & $\mathrm{U}$ \\
\hline $75-09-2$ & Methylene Chloride & 0.50 & $\mathrm{U}$ \\
\hline $156-60-5$ & trans-1,2-Dichloroethene & 0.50 & $\mathrm{U}$ \\
\hline $1634-04-4$ & Methyl tert-butyl ether & 0.50 & $\mathrm{U}$ \\
\hline $75-34-3$ & 1,1-Dichloroethane & 0.50 & $\mathrm{U}$ \\
\hline $156-59-2$ & cis-1,2-Dichloroethene & 0.50 & $\mathrm{U}$ \\
\hline $78-93-3$ & 2-Butanone & 5.0 & $\mathrm{U}$ \\
\hline $74-97-5$ & Bromochloromethane & 0.50 & $\mathrm{U}$ \\
\hline $67-66-3$ & Chloroform & 0.12 & $J \mathrm{~B}$ \\
\hline $71-55-6$ & 1,1,1-Trichloroethane & 0.50 & $\mathrm{U}$ \\
\hline $110-82-7$ & Cyclohexane & 0.50 & $\mathrm{U}$ \\
\hline $56-23-5$ & Carbon tetrachloride & 0.50 & $\mathrm{U}$ \\
\hline $71-43-2$ & Benzene & 0.074 & $\mathrm{~J}$ \\
\hline $107-06-2$ & 1,2-Dichloroethane & 0.50 & $\mathrm{U}$ \\
\hline
\end{tabular}

Report 1,4-Dioxane for Low-Medium VOA analysis only 
$1 \mathrm{~B}$ - FORM I VOA-2

VOLATILE ORGANICS ANALYSIS DATA SHEET
EPA SAMPLE NO.

INQCT'B-W-32783
Lab Name: TESTAMERICA BURLINGTON

Lab Code: STLV Case No.: INMAN

Matrix: (SOIL/SED/WATER)

Sample wt/vol: 25.0

Water

$(\mathrm{g} / \mathrm{mL}) \quad \mathrm{mL}$

Level: (TRACE/LOW/MED) TRACE

․ Moisture: not dec.

GC Column: DB-624 ID $: 0.20$ (mm)

Soil Extract Volume:

(uL)

(mL)

Purge Volume: 25.0
Contract: $\quad 8 \mathrm{E}-00302$

SDG No.: 200-7045

\begin{tabular}{|c|c|c|c|}
\hline CAS NO. & COMPOUND & $\begin{array}{l}\text { CONCENTRATION UNITS: } \\
(\mathrm{ug} / \mathrm{L} \text { or } \mathrm{ug} / \mathrm{kg}) \mathrm{ug} / \mathrm{L}\end{array}$ & $Q$ \\
\hline $79-01-6$ & Trichloroethene & 0.50 & $\mathrm{U}$ \\
\hline $108-87-2$ & Methylcyclohexane & 0.50 & $\mathrm{U}$ \\
\hline $78-87-5$ & 1,2-Dichloropropane & 0.50 & $\mathrm{U}$ \\
\hline $75-27-4$ & Bromodichloromethane & 0.50 & $\mathrm{U}$ \\
\hline $10061-01-5$ & cis-1,3-Dichloropropene & 0.50 & $\mathrm{U}$ \\
\hline $108-10-1$ & 4-Methyl-2-pentanone & 5.0 & $\mathrm{U}$ \\
\hline $108-88-3$ & Toluene & 0.19 & $\mathrm{~J} B$ \\
\hline $10061-02-6$ & trans-1,3-Dichloropropene & 0.50 & $\mathrm{U}$ \\
\hline $79-00-5$ & $1,1,2$-Trichloroethane & 0.50 & $\mathrm{U}$ \\
\hline $127-18-4$ & Tetrachloroethene & 0.50 & $\mathrm{U}$ \\
\hline $591-78-6$ & 2-Hexanone & 5.0 & $\mathrm{U}$ \\
\hline $124-48-1$ & Dibromochloromethane & 0.50 & $\mathrm{U}$ \\
\hline $106-93-4$ & 1,2-Dibromoethane & 0.50 & $\mathrm{U}$ \\
\hline $108-90-7$ & Chlorobenzene & 0.50 & $\mathrm{U}$ \\
\hline $100-41-4$ & Ethylbenzene & 0.50 & $\mathrm{U}$ \\
\hline $95-47-6$ & o-Xylene & 0.012 & $\mathrm{~J}$ \\
\hline $179601-23-1$ & $\mathrm{~m}, \mathrm{p}$-Xylene & 0.045 & $\mathrm{~J}$ \\
\hline $100-42-5$ & styrene & 0.13 & $\mathrm{~J}$ \\
\hline $75-25-2$ & Bromoform & 0.50 & $\mathrm{U}$ \\
\hline $98-82-8$ & Isopropylbenzene & 0.50 & $\mathrm{U}$ \\
\hline $79-34-5$ & $1,1,2,2$-Tetrachloroethane & 0.50 & $\mathrm{U}$ \\
\hline $541-73-1$ & 1,3-Dichlorobenzene & 0.50 & $\mathrm{U}$ \\
\hline $106-46-7$ & 1,4-Dichlorobenzene & 0.50 & $\mathrm{U}$ \\
\hline $95-50-1$ & 1,2-Dichlorobenzene & 0.50 & $\mathrm{U}$ \\
\hline $96-12-8$ & 1,2-Dibromo-3-Chloropropane & 0.50 & $\mathrm{U}$ \\
\hline $120-82-1$ & 1,2,4-Trichlorobenzene & 0.50 & $\mathrm{U}$ \\
\hline $87-61-6$ & 1,2,3-Trichlorobenzene & 0.50 & $\mathrm{U}$ \\
\hline
\end{tabular}

Lab Sample ID: 200-7045-4

Lab File ID: DHPAB04.D

Date Received: 09/16/2011

Date Analyzed: 09/20/2011

Dilution Factor: 1.0

Soil Aliquot Volume:

(uL) 
$1 \mathrm{~J}$ - FORM I VOA-TIC

VOLATILE ORGANICS ANALYSIS DATA SHEET

TENTATIVELY IDENTIFIED COMPOUNDS
EPA SAMPLE NO.

INQCTB-W-32783

Lab Name: TESTAMERICA BURLINGTON

Contract: $8 \mathrm{E}-00302$

Lab Code: STLV Case No.: INMAN Mod. Ref No.: SDG No.: 200-7045

Matrix: (SOIL/SED/WATER) Water

Sample wt/vol: 25.0 $(\mathrm{g} / \mathrm{mL}) \mathrm{mL}$

Level: (TRACE or LOW/MED) TRACE

음 Moisture: not dec.

GC Column: DB-624 ID: 0.20 (mm)

Soil Extract Volume: (uL)
Lab Sample ID: 200-7045-4

Lab File ID: DHPAB04.D

Date Received: 09/16/2011

Date Analyzed: 09/20/2011

Dilution Factor: 1.0

Soil Aliquot Volume: (uL)

Purge Volume: 25.0 (mL)

\begin{tabular}{|l|l|l|r|r|r|}
\hline \multicolumn{1}{|c|}{ CAS NUMBER } & \multicolumn{1}{|c|}{ COMPOUND NAME } & RT & EST. CONC. & Q \\
\cline { 2 - 6 } 01 & & Unknown & 6.79 & 2.8 & B X J \\
\cline { 2 - 6 } & E9667961 & Total Alkanes & N/A & & \\
\hline
\end{tabular}

1 EPA-designated Registry Number. 
$6 \mathrm{~A}$ - FORM VI VOA-1

VOLATILE ORGANICS INITIAL CALIBRATION DATA

Lab Name: TESTAMERICA BURLINGTON

Lab Code: STLV Case No.: INMAN Mod. Ref No.:

Instrument ID: D.i

Heated Purge: $(\mathrm{Y} / \mathrm{N}) \mathrm{N}$

Purge Volume: 25.0

GC Column: DB-624
Calibration Date(s):

Calibration Time (s)

$(\mathrm{mL})$

ID: 0.20

(mm) Length: 25
Contract: $8 \mathrm{E}-00302$

SDG NO.: 200-7045

$07 / 07 / 2011 \quad 07 / 07 / 2011$

1117 1256 (m)

\begin{tabular}{|c|c|c|c|c|c|c|c|}
\hline \multirow{2}{*}{$\begin{array}{l}\text { LAB FILE ID: } \\
\text { RRF } 5.0=\text { DHP06.D }\end{array}$} & \multicolumn{3}{|c|}{ RRF $0.5=$ DHP0 4.D } & \multicolumn{4}{|c|}{ RRE1.0 $=$ DHP05.D } \\
\hline & RRF 10 & IP07.D & & RRF 20 & $=\mathrm{DHP}$ & . D & \\
\hline COMPOUND & RRF 0.5 & RRE 1.0 & RRE 5.0 & RRE 10 & RRF 20 & $\overline{R R F}$ & 을 \\
\hline Dichlorodifluoromethane & 0.418 & 0.398 & 0.390 & 0.409 & 0.398 & 0.403 & 2.7 \\
\hline Chloromethane & 0.769 & 0.713 & 0.655 & 0.659 & 0.639 & 0.687 & 7.8 \\
\hline Vinyl chloride & 0.524 & 0.491 & 0.475 & 0.499 & 0.491 & 0.496 & 3.6 \\
\hline Bromomethane & 0.180 & 0.203 & 0.205 & 0.219 & 0.239 & 0.209 & 10.3 \\
\hline Chloroethane & 0.292 & 0.280 & 0.259 & 0.269 & 0.259 & 0.272 & 5.2 \\
\hline Trichlorofluoromethane & 0.612 & 0.609 & 0.587 & 0.608 & 0.611 & 0.606 & 1.7 \\
\hline 1,1-Dichloroethene & 0.318 & 0.307 & 0.298 & 0.303 & 0.297 & 0.305 & 2.8 \\
\hline $\begin{array}{l}1,1,2 \text {-Trichloro- } \\
1,2,2 \text {-trifluoroethane }\end{array}$ & 0.377 & 0.358 & 0.336 & 0.358 & 0.347 & 0.355 & 4.3 \\
\hline Acetone & 0.047 & 0.038 & 0.032 & 0.032 & 0.030 & 0.036 & 19.3 \\
\hline Carbon disulfide & 0.758 & 0.707 & 0.719 & 0.760 & 0.766 & 0.742 & 3.6 \\
\hline Methyl acetate & 0.108 & 0.094 & 0.076 & 0.079 & 0.074 & 0.086 & 16.7 \\
\hline Methylene Chloride & 0.264 & 0.257 & 0.242 & 0.248 & 0.241 & 0.250 & 3.9 \\
\hline trans-1,2-Dichloroethene & 0.323 & 0.298 & 0.301 & 0.312 & 0.306 & 0.308 & 3.3 \\
\hline Methyl tert-butyl ether & 0.304 & 0.278 & 0.292 & 0.319 & 0.318 & 0.302 & 5.7 \\
\hline 1,1-Dichloroethane & 0.642 & 0.586 & 0.594 & 0.617 & 0.607 & 0.609 & 3.6 \\
\hline cis-1,2-Dichloroethene & 0.302 & 0.289 & 0.300 & 0.312 & 0.306 & 0.302 & 2.7 \\
\hline 2-Butanone & 0.045 & 0.046 & 0.047 & 0.049 & 0.048 & 0.047 & 3.4 \\
\hline Bromochloromethane & 0.104 & 0.103 & 0.100 & 0.099 & 0.101 & 0.101 & 2.0 \\
\hline Chloroform & 0.502 & 0.482 & 0.473 & 0.493 & 0.477 & 0.485 & 2.5 \\
\hline 1,1,1-Trichloroethane & 0.514 & 0.490 & 0.494 & 0.490 & 0.485 & 0.494 & 2.3 \\
\hline Cyclohexane & 0.761 & 0.795 & 0.901 & 0.909 & 0.883 & 0.850 & 7.9 \\
\hline Carbon tetrachloride & 0.487 & 0.459 & 0.477 & 0.474 & 0.464 & 0.472 & 2.4 \\
\hline Benzene & 1.617 & 1.501 & 1.518 & 1.495 & 1.447 & 1.515 & 4.1 \\
\hline 1,2-Dichloroethane & 0.291 & 0.271 & 0.277 & 0.284 & 0.285 & 0.282 & 2.7 \\
\hline Trichloroethene & 0.397 & 0.380 & 0.357 & 0.355 & 0.351 & 0.368 & 5.4 \\
\hline Methylcyclohexane & 0.703 & 0.708 & 0.769 & 0.757 & 0.751 & 0.737 & 4.1 \\
\hline
\end{tabular}

Report 1,4-Dioxane for Low-Medium VOA analysis only 
$6 \mathrm{~B}$ - FORM VI VOA-2

VOLATILE ORGANICS INITIAL CALIBRATION DATA

Lab Name: TESTAMERICA BURLINGTON

Lab Code: STLV Case No.: INMAN Mod. Ref No.:

Instrument ID: D.i

Heated Purge: $(\mathrm{Y} / \mathrm{N}) \mathrm{N}$

Purge Volume: 25.0

GC Column: DB-624

$\mathrm{N}$

LAB FILE ID:
RRF $5.0=$ DHPO6.D

COMPOUND

1,2-Dichloropropane

Bromodichloromethane

cis-1,3-Dichloropropene

4-Methyl-2-pentanone

Toluene

trans-1,3-Dichloropropene

1,1,2-Trichloroethane

Tetrachloroethene

2-Hexanone

Dibromochloromethane

1,2-Dibromoethane

Chlorobenzene

Ethylbenzene

o-xylene

m, p-xylene

styrene

Bromoform

Isopropylbenzene

1, 1,2,2-Tetrachloroethane

1,3-Dichlorobenzene

1,4-Dichlorobenzene

1,2-Dichlorobenzene

1,2-Dibromo-3-Chloropropane

1,2,4-Trichlorobenzene

1,2,3-Trichlorobenzene
ID: 0.20

$$
\begin{aligned}
& \text { RRF 0.5 }=\underline{\text { DHP04.D }} \\
& \text { RRE10 }=\text { DHP07.D }
\end{aligned}
$$

Calibration Date(s):

Calibration Time(s): 1117

(mL)

(mm) Length: 25

(m)
SDG No.: 200-7045

$07 / 07 / 2011 \quad 07 / 07 / 2011$

\begin{tabular}{|c|c|c|c|c|c|c|}
\hline RRF 0.5 & RRF 1.0 & RRF 5.0 & RRF 10 & RRE 20 & $\overline{\mathrm{RRF}}$ & $\frac{\circ}{0} \mathrm{RSD}$ \\
\hline 0.363 & 0.373 & 0.367 & 0.371 & 0.355 & 0.366 & 2.0 \\
\hline 0.310 & 0.296 & 0.307 & 0.305 & 0.306 & 0.305 & 1.6 \\
\hline 0.310 & 0.338 & 0.406 & 0.414 & 0.418 & 0.377 & 13.2 \\
\hline 0.104 & 0.114 & 0.137 & 0.140 & 0.137 & 0.126 & 12.7 \\
\hline 1.608 & 1.623 & 1.695 & 1.690 & 1.667 & 1.657 & 2.4 \\
\hline 0.216 & 0.225 & 0.285 & 0.302 & 0.307 & 0.267 & 16.2 \\
\hline 0.155 & 0.162 & 0.160 & 0.156 & 0.157 & 0.158 & 1.9 \\
\hline 0.311 & 0.304 & 0.306 & 0.308 & 0.299 & 0.306 & 1.5 \\
\hline 0.076 & 0.085 & 0.096 & 0.099 & 0.096 & 0.090 & 10.6 \\
\hline 0.172 & 0.176 & 0.180 & 0.178 & 0.192 & 0.180 & 4.1 \\
\hline 0.132 & 0.135 & 0.138 & 0.145 & 0.145 & 0.139 & 4.2 \\
\hline 1.079 & 1.036 & 1.034 & 1.041 & 1.021 & 1.042 & 2.1 \\
\hline 1.594 & 1.651 & 1.933 & 1.968 & 1.978 & 1.825 & 10.2 \\
\hline 0.539 & 0.600 & 0.727 & 0.749 & 0.755 & 0.674 & 14.6 \\
\hline 0.644 & 0.689 & 0.779 & 0.797 & 0.802 & 0.742 & 9.6 \\
\hline 0.763 & 0.886 & 1.048 & 1.096 & 1.123 & 0.983 & 15.6 \\
\hline 0.107 & 0.116 & 0.126 & 0.119 & 0.137 & 0.121 & 9.2 \\
\hline 1.400 & 1.553 & 2.026 & 2.103 & 2.121 & 1.841 & 18.4 \\
\hline 0.168 & 0.154 & 0.168 & 0.164 & 0.164 & 0.164 & 3.6 \\
\hline 1.494 & 1.468 & 1.569 & 1.629 & 1.647 & 1.562 & 5.1 \\
\hline 1.827 & 1.736 & 1.615 & 1.641 & 1.610 & 1.686 & 5.5 \\
\hline 1.377 & 1.388 & 1.407 & 1.392 & 1.355 & 1.384 & 1.4 \\
\hline 0.021 & 0.027 & 0.039 & 0.035 & 0.040 & 0.032 & 24.3 \\
\hline 0.603 & 0.574 & 0.686 & 0.751 & 0.756 & 0.674 & 12.4 \\
\hline 0.433 & 0.469 & 0.522 & 0.573 & 0.575 & 0.514 & 12.3 \\
\hline
\end{tabular}

1256
RRF 1.0 = DHP05.D

$\mathrm{RRF} 20=\mathrm{DHP08} \cdot \mathrm{D}$ 
$6 \mathrm{C}$ - FORM VI VOA-3

VOLATILE ORGANICS INITIAL CALIBRATION DATA

Lab Name: TESTAMERICA BURLINGTON

Contract: $8 \mathrm{E}-00302$

Lab Code: STLV Case No.: INMAN Mod. Ref No,

SDG No.: 200-7045

Instrument ID: D.i

Calibration Date(s):

$07 / 07 / 2011 \quad 07 / 07 / 2011$

Heated Purge: $(\mathrm{Y} / \mathrm{N}) \mathrm{N}$

Calibration Time(s): 1117

1256

Purge Volume: 25.0 $(\mathrm{mL})$

GC Column: DB-624

ID : 0.20

(mm) Length: 25

(m)

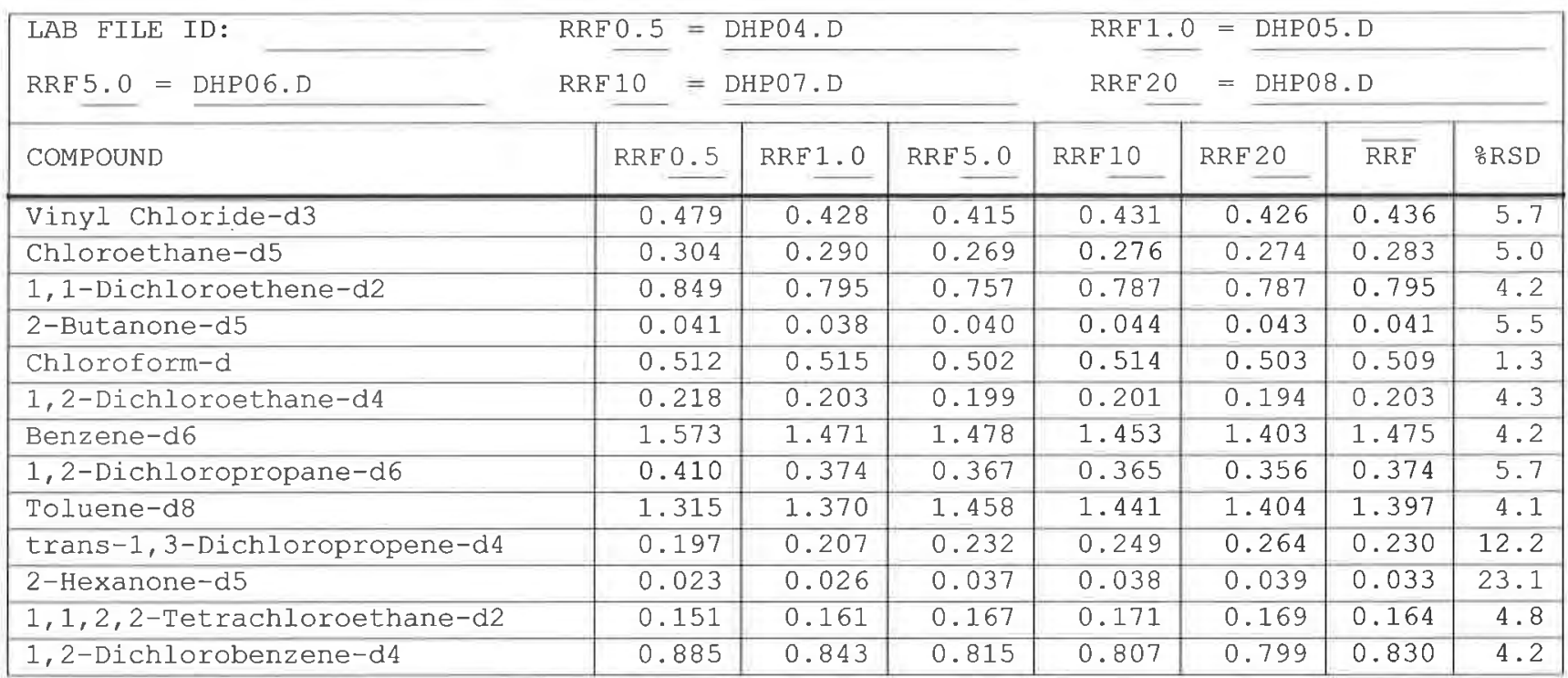

Report 1,4-Dioxane-d8 for Low-Medium VOA analysis only 
Lab Name: TESTAMERICA BURLINGTON

Lab Code: STLV Case No.: INMAN Mod. Ref No.:

Contract: $8 \mathrm{E}-00302$

Instrument ID: D.i

Lab File Id: DHPABO2.D

EPA Sample No. (VSTD\#\#\#\#): VSTD005DZ

Heated Purge: ( $\mathrm{Y} / \mathrm{N}) \mathrm{N}$

N GC Column: DB-624

Purge Volume: 25.0 (mL)

SDG No.: 200-7045

Calibration Date: 09/20/2011 Time: 0902 Init. Calib. Date(s): 07/07/2011 07/07/2011

Init. Calib. Time(s): $1117 \quad 1256$

ID: $0.20(\mathrm{~mm})$ Length: 25 (m)

\begin{tabular}{|c|c|c|c|c|c|}
\hline COMPOUND & $\overline{\mathrm{RRF}}$ & RRF5.0 & $\begin{array}{l}\text { MIN } \\
\text { RRE }\end{array}$ & $\because \mathrm{D}$ & $\operatorname{MAX} \div \mathrm{D}$ \\
\hline Dichlorodifluoromethane & 0.403 & 0.450 & 0.010 & 11.8 & 40.0 \\
\hline Chloromethane & 0.687 & 0.744 & 0.010 & 8.3 & 40.0 \\
\hline Vinyl chloride & 0.496 & 0.527 & 0.010 & 6.2 & 30.0 \\
\hline Bromomethane & 0.209 & 0.231 & 0.010 & 10.4 & 30.0 \\
\hline Chloroethane & 0.272 & 0.269 & 0.010 & -1.0 & 40.0 \\
\hline Trichlorofluoromethane & 0.606 & 0.642 & 0.010 & 6.0 & 40.0 \\
\hline 1,1-Dichloroethene & 0.305 & 0.313 & 0.010 & 2.8 & 30.0 \\
\hline 1,1,2-Trichloro-1,2,2-trifluoroethane & 0.355 & 0.377 & 0.010 & 6.1 & 40.0 \\
\hline Acetone & 0.036 & 0.040 & 0.010 & 11.5 & 40.0 \\
\hline Carbon disulfide & 0.742 & 0.847 & 0.010 & 14.2 & 40.0 \\
\hline Methyl acetate & 0.086 & 0.079 & 0.010 & -8.7 & 40.0 \\
\hline Methylene Chloride & 0.250 & 0.242 & 0.010 & -3.1 & 40.0 \\
\hline trans-1,2-Dichloroethene & 0.308 & 0.316 & 0.010 & 2.5 & 40.0 \\
\hline Methyl tert-butyl ether & 0.302 & 0.262 & 0.010 & -13.2 & 40.0 \\
\hline 1,1-Dichloroethane & 0.609 & 0.619 & 0.010 & 1.6 & 30.0 \\
\hline cis-1,2-Dichloroethene & 0.302 & 0.304 & 0.010 & 0.8 & 40.0 \\
\hline 2-Butanone & 0.047 & 0.042 & 0.010 & -10.3 & 40.0 \\
\hline Bromochloromethane & 0.101 & 0.096 & 0.010 & -5.4 & 30.0 \\
\hline Chloroform & 0.485 & 0.487 & 0.010 & 0.4 & 30.0 \\
\hline $1,1,1-$ Trichloroethane & 0.494 & 0.501 & 0.010 & 1.3 & 30.0 \\
\hline Cyclohexane & 0.850 & 0.924 & 0.010 & 8.7 & 40.0 \\
\hline Carbon tetrachloride & 0.472 & 0.489 & 0.010 & 3.5 & 30.0 \\
\hline Benzene & 1.515 & 1.525 & 0.010 & 0.6 & 30.0 \\
\hline 1,2-Dichloroethane & 0.282 & 0.274 & 0.010 & -2.6 & 30.0 \\
\hline Trichloroethene & 0.368 & 0.354 & 0.010 & -3.8 & 30.0 \\
\hline Methylcyclohexane & 0.737 & 0.771 & 0.010 & 4.5 & 40.0 \\
\hline
\end{tabular}

Report 1,4-Dioxane for Low/Medium VOA analysis only 
7B - FORM VII VOA-2

VOLATILE CONTINUING CALIBRATION DATA

Lab Name: TESTAMERICA BURLINGTON

Contract: $\quad 8 \mathrm{E}-00302$

Lab Code: STLV Case No.: INMAN Mod. Ref No.:

SDG No.: 200-7045

Instrument ID: D.i

Calibration Date: 09/20/2011 Time: 0902

Lab File Id: DHPAB02.D

EPA Sample No. (VSTD\#\#\#\#): VSTD005DZ Init. Calib. Date(s): 07/07/2011 07/07/2011

Heated Purge: ( $\mathrm{Y} / \mathrm{N})$ GC Colunn: DB-624

Init. Calib. Time(s): 1117 1256

Purge Volume: 25.0

(mL)

\begin{tabular}{|c|c|c|c|c|c|}
\hline COMPOUND & $\overline{\mathrm{RRE}}$ & RRE5.0 & $\begin{array}{l}\text { MIN } \\
\text { RRF }\end{array}$ & 음 & MAX $\div D$ \\
\hline 1,2-Dichloropropane & 0.366 & 0.364 & 0.010 & -0.4 & 40.0 \\
\hline Bromodichloromethane & 0.305 & 0.294 & 0.010 & -3.5 & 30.0 \\
\hline cis-1,3-Dichloropropene & 0.377 & 0.375 & 0.010 & -0.6 & 30.0 \\
\hline 4-Methyl-2-pentanone & 0.126 & 0.111 & 0.010 & -12.1 & 40.0 \\
\hline Toluene & 1.657 & 1.690 & 0.010 & 2.0 & 30.0 \\
\hline trans-1,3-Dichloropropene & 0.267 & 0.264 & 0.010 & -1.1 & 30.0 \\
\hline 1,1,2-Trichloroethane & 0.158 & 0.146 & 0.010 & -7.7 & 30.0 \\
\hline Tetrachloroethene & 0.306 & 0.305 & 0.010 & -0.3 & 30.0 \\
\hline 2-Hexanone & 0.090 & 0.077 & 0.010 & -14.7 & 40.0 \\
\hline Dibromochloromethane & 0.180 & 0.168 & 0.010 & -6.6 & 30.0 \\
\hline 1,2-Dibromoethane & 0.139 & 0.126 & 0.010 & -9.3 & 40.0 \\
\hline Chlorobenzene & 1.042 & 1.010 & 0.010 & -3.1 & 30.0 \\
\hline Ethylbenzene & 1.825 & 1.858 & 0.010 & 1.8 & 30.0 \\
\hline o-Xylene & 0.674 & 0.703 & 0.010 & 4.3 & 30.0 \\
\hline $\mathrm{m}, \mathrm{p}$-Xylene & 0.742 & 0.775 & 0.010 & 4.5 & 30.0 \\
\hline Styrene & 0.983 & 1.043 & 0.010 & 6.1 & 30.0 \\
\hline Bromoform & 0.121 & 0.122 & 0.010 & 0.7 & 30.0 \\
\hline Isopropylbenzene & 1.841 & 1.971 & 0.010 & 7.1 & 40.0 \\
\hline $1,1,2,2$-Tetrachloroethane & 0.164 & 0.136 & 0.010 & -17.0 & 30.0 \\
\hline 1,3-Dichlorobenzene & 1.562 & 1.533 & 0.010 & -1.8 & 30.0 \\
\hline 1,4-Dichlorobenzene & 1.686 & 1.556 & 0.010 & -7.7 & 30.0 \\
\hline 1,2-Dichlorobenzene & 1.384 & 1.270 & 0.010 & -8.2 & 30.0 \\
\hline 1,2-Dibromo-3-Chloropropane & 0.032 & 0.034 & 0.010 & 5.3 & 40.0 \\
\hline 1,2,4-Trichlorobenzene & 0.674 & 0.715 & 0.010 & 6.1 & 30.0 \\
\hline 1,2,3-Trichlorobenzene & 0.514 & 0.533 & 0.010 & 3.5 & 30.0 \\
\hline
\end{tabular}


7C - FORM VII VOA-3

VOLATILE CONTINUING CALIBRATION DATA

Lab Name: TESTAMERICA BURLINGTON

Contract: $8 \mathrm{E}-00302$

Lab Code: STLV Case No.: INMAN Mod. Ref No.:

SDG No.: 200-7045

Instrument ID: D.i

Calibration Date: 09/20/2011 Time: 0902

Lab File Id: DHPAB02.D Init. Calib. Date(s): 07/07/2011 07/07/2011

EPA Sample No. (VSTD\#\#\#\#): VSTD005DZ

Init. Calib. Time(s): $1117 \quad 1256$

Heated Purge: ( $\mathrm{Y} / \mathrm{N})$

$\mathrm{N} \quad \mathrm{GC}$ Column: $\mathrm{DB}-624$

ID: $0.20(\mathrm{~mm})$ Length: 25

(m)

Purge Volume: 25.0

$(\mathrm{mL})$

\begin{tabular}{|c|c|c|c|c|c|}
\hline COMPOUND & $\overline{\mathrm{RRF}}$ & RRF5. 0 & $\begin{array}{l}\text { MIN } \\
\text { RRF }\end{array}$ & $\stackrel{\circ}{D}$ & MAX $\% D$ \\
\hline Vinyl Chloride-d3 & 0.436 & 0.435 & 0.010 & -0.2 & 30.0 \\
\hline Chloroethane-d5 & 0.283 & 0.277 & 0.010 & -1.9 & 40.0 \\
\hline 1,1-Dichloroethene-d2 & 0.795 & 0.736 & 0.010 & -7.4 & 30.0 \\
\hline 2-Butanone-d5 & 0.041 & 0.037 & 0.010 & -9.7 & 40.0 \\
\hline Chloroform-d & 0.509 & 0.477 & 0.010 & -6.3 & 30.0 \\
\hline 1,2-Dichloroethane-d4 & 0.203 & 0.184 & 0.010 & -9.5 & 30.0 \\
\hline Benzene-d6 & 1.475 & 1.389 & 0.010 & -5.9 & 30.0 \\
\hline 1,2-Dichloropropane-d6 & 0.374 & 0.339 & 0.010 & -9.5 & 40.0 \\
\hline Toluene-d8 & 1.397 & 1.351 & 0.010 & -3.3 & 30.0 \\
\hline trans-1,3-Dichloropropene-d4 & 0.230 & 0.213 & 0.010 & -7.5 & 30.0 \\
\hline 2-Hexanone-d5 & 0.033 & 0.029 & 0.010 & -10.4 & 40.0 \\
\hline $1,1,2,2$-Tetrachloroethane-d2 & 0.164 & 0.138 & 0.010 & -15.9 & 30.0 \\
\hline 1,2-Dichlorobenzene-d4 & 0.830 & 0.735 & 0.010 & -11.4 & 30.0 \\
\hline
\end{tabular}

Report 1,4-Dioxane-d8 for Low/Medium VOA analysis only 
$7 A$ - EORM VII VOA-1

VOLATILE CONTINUING CALIBRATION DATA

Lab Name: TESTAMERICA BURLINGTON

Contract: $8 \mathrm{E}-00302$

Lab Code: STLV Case No.: INMAN Mod. Ref No.:

SDG NO.: 200-7045

Instrument ID: D.i

Calibration Date: 09/20/2011 Time: 1230

Lab File Id: DHPAB09.D

EPA Sample No. (VSTD\#\#\#\#): VSTD005ZD Init. Calib. Date(s): 07/07/2011 07/07/2011

Heated Purge: (Y/N)

GC Column: DB-624

Init. Calib. Time(s): 1117 1256

Purge Volume: 25.0 (mL)

\begin{tabular}{|c|c|c|c|c|c|}
\hline COMPOUND & $\overline{\mathrm{RRF}}$ & RRF5. 0 & $\begin{array}{l}\text { MIN } \\
\text { RRF }\end{array}$ & $\because \mathrm{D}$ & MAX $\frac{\circ}{\partial}$ \\
\hline Dichlorodifluoromethane & 0.403 & 0.424 & 0.010 & 5.2 & 50.0 \\
\hline Chloromethane & 0.687 & 0.654 & 0.010 & -4.8 & 50.0 \\
\hline Vinyl chloride & 0.496 & 0.465 & 0.100 & -6.3 & 50.0 \\
\hline Bromomethane & 0.209 & 0.190 & 0.100 & -9.2 & 50.0 \\
\hline Chloroethane & 0.272 & 0.248 & 0.010 & -9.0 & 50.0 \\
\hline Trichlorofluoromethane & 0.606 & 0.584 & 0.010 & -3.6 & 50.0 \\
\hline 1,1-Dichloroethene & 0.305 & 0.287 & 0.100 & -5.8 & 50.0 \\
\hline 1,1,2-Trichloro-1,2,2-trifluoroethane & 0.355 & 0.341 & 0.010 & -4.0 & 50.0 \\
\hline Acetone & 0.036 & 0.027 & 0.010 & -24.3 & 50.0 \\
\hline Carbon disulfide & 0.742 & 0.699 & 0.010 & -5.8 & 50.0 \\
\hline Methyl acetate & 0.086 & 0.073 & 0.010 & -14.9 & 50.0 \\
\hline Methylene Chloride & 0.250 & 0.224 & 0.010 & -10.3 & 50.0 \\
\hline trans-1,2-Dichloroethene & 0.308 & 0.289 & 0.010 & -6.2 & 50.0 \\
\hline Methyl tert-butyl ether & 0.302 & 0.259 & 0.010 & -14.5 & 50.0 \\
\hline 1,1-Dichloroethane & 0.609 & 0.578 & 0.200 & -5.2 & 50.0 \\
\hline cis-1,2-Dichloroethene & 0.302 & 0.278 & 0.010 & -7.8 & 50.0 \\
\hline 2-Butanone & 0.047 & 0.042 & 0.010 & -11.4 & 50.0 \\
\hline Bromochloromethane & 0.101 & 0.092 & 0.050 & -9.1 & 50.0 \\
\hline Chloroform & 0.485 & 0.460 & 0.200 & -5.1 & 50.0 \\
\hline 1,1,1-Trichloroethane & 0.494 & 0.451 & 0.100 & -8.8 & 50.0 \\
\hline Cyclohexane & 0.850 & 0.843 & 0.010 & -0.7 & 50.0 \\
\hline Carbon tetrachloride & 0.472 & 0.442 & 0.100 & -6.4 & 50.0 \\
\hline Benzene & 1.515 & 1.387 & 0.400 & -8.4 & 50.0 \\
\hline 1,2-Dichloroethane & 0.282 & 0.270 & 0.100 & -4.0 & 50.0 \\
\hline Trichloroethene & 0.368 & 0.330 & 0.300 & -10.5 & 50.0 \\
\hline Methylcyclohexane & 0.737 & 0.709 & 0.010 & -3.9 & 50.0 \\
\hline
\end{tabular}

Report 1,4-Dioxane for Low/Medium VOA analysis only 
$7 B$ - FORM VII VOA-2

VOLATILE CONTINUING CALIBRATION DATA

Lab Name: TESTAMERICA BURLINGTON

Contract: $\quad 8 \mathrm{E}-00302$

Lab Code: STLV Case No.: INMAN Mod. Ref No.:

SDG No.: 200-7045

Instrument ID: D.i

Calibration Date: 09/20/2011 Time: 1230

Lab File Id: DHPAB09.D Init. Calib. Date(s): 07/07/2011 07/07/2011

EPA Sample No. (VSTD\#\#\#\#): VSTD005zD

Init. Calib. Time(s): 1117 1256

Heated Purge: (Y/N) N GC Column: DB-624 ID: $0.20(\mathrm{~mm})$ Length: 25 (m)

Purge Volume: $25.0 \quad$ (mL)

\begin{tabular}{|c|c|c|c|c|c|}
\hline COMPOUND & $\overline{\mathrm{RRF}}$ & RRF5. 0 & $\begin{array}{l}\text { MIN } \\
\text { RRF }\end{array}$ & $8 \mathrm{D}$ & MAX $\div D$ \\
\hline 1,2-Dichloropropane & 0.366 & 0.335 & 0.010 & -8.5 & 50.0 \\
\hline Bromodichloromethane & 0.305 & 0.274 & 0.200 & -10.0 & 50.0 \\
\hline cis-1,3-Dichloropropene & 0.377 & 0.361 & 0.200 & -4.3 & 50.0 \\
\hline 4-Methy1-2-pentanone & 0.126 & 0.111 & 0.010 & -12.4 & 50.0 \\
\hline Toluene & 1.657 & 1.559 & 0.400 & -5.9 & 50.0 \\
\hline trans-1,3-Dichloropropene & 0.267 & 0.241 & 0.100 & -9.7 & 50.0 \\
\hline 1,1,2-Trichloroethane & 0.158 & 0.142 & 0.100 & -10.3 & 50.0 \\
\hline Tetrachloroethene & 0.306 & 0.280 & 0.100 & -8.6 & 50.0 \\
\hline 2-Hexanone & 0.090 & 0.078 & 0.010 & -14.1 & 50.0 \\
\hline Dibromochloromethane & 0.180 & 0.160 & 0.100 & -10.8 & 50.0 \\
\hline 1,2-Dibromoethane & 0.139 & 0.126 & 0.010 & -9.1 & 50.0 \\
\hline Chlorobenzene & 1.042 & 0.972 & 0.500 & -6.8 & 50.0 \\
\hline Ethylbenzene & 1.825 & 1.757 & 0.100 & -3.7 & 50.0 \\
\hline o-Xylene & 0.674 & 0.667 & 0.300 & -1.0 & 50.0 \\
\hline $\mathrm{m}, \mathrm{p}$-Xylene & 0.742 & 0.722 & 0.300 & -2.7 & 50.0 \\
\hline Styrene & 0.983 & 0.994 & 0.300 & 1.1 & 50.0 \\
\hline Bromoform & 0.121 & 0.108 & 0.050 & -11.0 & 50.0 \\
\hline Isopropylbenzene & 1.841 & 1.868 & 0.010 & 1.5 & 50.0 \\
\hline $1,1,2,2$-Tetrachloroethane & 0.164 & 0.134 & 0.100 & $-18 \cdot 3$ & 50.0 \\
\hline 1,3-Dichlorobenzene & 1.562 & 1.400 & 0.400 & -10.4 & 50.0 \\
\hline 1,4-Dichlorobenzene & 1.686 & 1.510 & 0.400 & -10.4 & 50.0 \\
\hline 1,2-Dichlorobenzene & 1.384 & 1.273 & 0.400 & -8.0 & 50.0 \\
\hline 1,2-Dibromo-3-Chloropropane & 0.032 & 0.030 & 0.010 & -8.1 & 50.0 \\
\hline 1,2,4-Trichlorobenzene & 0.674 & 0.594 & 0.200 & -11.9 & 50.0 \\
\hline 1,2,3-Trichlorobenzene & 0.514 & 0.453 & 0.200 & -11.9 & 50.0 \\
\hline
\end{tabular}


7C - FORM VII VOA-3

VOLATILE CONTINUING CALIBRATION DATA

Lab Name: TESTAMERICA BURLINGTON

Contract: $8 \mathrm{E}-00302$

Lab Code: STLV Case No.: INMAN Mod. Ref No.:

SDG No.: 200-7045

Instrument ID: D.i

Calibration Date: 09/20/2011 Time: 1230

Lab File Id: DHPAB09.D Init. Calib. Date(s): 07/07/2011 07/07/2011

EPA Sample No. (VSTD\#\#\#\#): VSTD005zD

Init. Calib. Time(s): $1117 \quad 1256$

Heated Purge: $(\mathrm{Y} / \mathrm{N}) \quad \mathrm{N}$ GC Column: $\mathrm{DB}-624$

ID: $0.20(\mathrm{~mm})$ Length:

25

(II)

Purge Volume: 25.0

(mL)

\begin{tabular}{|c|c|c|c|c|c|}
\hline COMPOUND & $\overline{\mathrm{RRF}}$ & RRF5.0 & $\begin{array}{l}\text { MIN } \\
\text { RRF }\end{array}$ & $\because D$ & MAX $\because D$ \\
\hline Vinyl Chloride-d3 & 0.436 & 0.385 & 0.010 & -11.7 & 50.0 \\
\hline Chloroethane-d5 & 0.283 & 0.249 & 0.010 & -11.9 & 50.0 \\
\hline 1,1-Dichloroethene-d2 & 0.795 & 0.684 & 0.010 & -13.9 & 50.0 \\
\hline 2-Butanone-d5 & 0.041 & 0.037 & 0.010 & -10.8 & 50.0 \\
\hline Chloroform-d & 0.509 & 0.456 & 0.010 & -10.4 & 50.0 \\
\hline 1,2-Dichloroethane-d4 & 0.203 & 0.184 & 0.010 & -9.5 & 50.0 \\
\hline Benzene-d6 & 1.475 & 1.288 & 0.010 & -12.7 & 50.0 \\
\hline 1,2-Dichloropropane-d6 & 0.374 & 0.321 & 0.010 & -14.4 & 50.0 \\
\hline Toluene-d8 & 1.397 & 1.267 & 0.010 & -9.4 & 50.0 \\
\hline trans-1,3-Dichloropropene-d4 & 0.230 & 0.200 & 0.010 & -12.7 & 50.0 \\
\hline 2-Hexanone-d5 & 0.033 & 0.030 & 0.010 & -9.6 & 50.0 \\
\hline $1,1,2,2$-Tetrachloroethane-d2 & 0.164 & 0.137 & 0.010 & -16.4 & 50.0 \\
\hline 1,2-Dichlorobenzene-d4 & 0.830 & 0.703 & 0.010 & -15.3 & 50.0 \\
\hline
\end{tabular}

Report 1,4-Dioxane-d8 for Low/Medium VOA analysis only 
Lab Name: TESTAMERICA BURLINGTON

Lab Code: STLV Case No.: INMAN

Matrix: (SOIL/SED/WATER)

water

Sample wt/vol: 25.0

$(\mathrm{g} / \mathrm{mL}) \mathrm{mL}$

Level: (TRACE/LOW/MED)

TRACE

o Moisture: not dec.

GC Column: DB-624

ID: 0.20

(mm)

Soil Extract Volume:

Purge Volume: 25.0

(uL)

(mL)
Contract: $8 \mathrm{E}-00302$

SDG No.: 200-7045

Lab Sample ID: MB 200-25508/3

Lab File ID: DHPAB03.D

Date Received:

Date Analyzed: 09/20/2011

Dilution Factor: 1.0

Soil Aliquot Volume:

(uL)

\begin{tabular}{|c|c|c|c|}
\hline CAS NO. & COMPOUND & $\begin{array}{l}\text { CONCENTRATION UNITS: } \\
(\mathrm{ug} / \mathrm{L} \text { or } \mathrm{ug} / \mathrm{kg}) \mathrm{ug} / \mathrm{I}\end{array}$ & Q \\
\hline $75-71-8$ & Dichlorodifluoromethane & 0.50 & $\overline{\mathrm{U}}$ \\
\hline $74-87-3$ & Chloromethane & 0.50 & $\mathrm{U}$ \\
\hline $75-01-4$ & Vinyl chloride & 0.50 & $\mathrm{U}$ \\
\hline $74-83-9$ & Bromomethane & 0.50 & $\mathrm{U}$ \\
\hline $75-00-3$ & Chloroethane & 0.50 & $\mathrm{U}$ \\
\hline $75-69-4$ & Trichlorofluoromethane & 0.50 & $\mathrm{U}$ \\
\hline $75-35-4$ & 1,1-Dichloroethene & 0.50 & $\mathrm{U}$ \\
\hline $76-13-1$ & 1,1,2-Trichloro-1,2,2-trifluoroethane & 0.50 & $\mathrm{U}$ \\
\hline $67-64-1$ & Acetone & 1.3 & $\mathrm{~J}$ \\
\hline $75-15-0$ & Carbon disulfide & 0.50 & $\mathrm{U}$ \\
\hline $79-20-9$ & Methyl acetate & 0.50 & $\mathrm{U}$ \\
\hline $75-09-2$ & Methylene Chloride & 0.50 & $\mathrm{~J}$ \\
\hline $156-60-5$ & trans-1,2-Dichloroethene & 0.50 & $\mathrm{~J}$ \\
\hline $1634-04-4$ & Methyl tert-butyl ether & 0.50 & $\mathrm{U}$ \\
\hline $75-34-3$ & 1,1-Dichloroethane & 0.50 & $\mathrm{U}$ \\
\hline $156-59-2$ & cis-1,2-Dichloroethene & 0.50 & $\mathrm{U}$ \\
\hline $78-93-3$ & 2-Butanone & 5.0 & $\mathrm{U}$ \\
\hline $74-97-5$ & Bromochloromethane & 0.50 & $\mathrm{U}$ \\
\hline $67-66-3$ & Chloroform & 0.044 & $\mathrm{~J}$ \\
\hline $71-55-6$ & 1,1,1-Trichloroethane & 0.50 & $\mathrm{U}$ \\
\hline $110-82-7$ & Cyclohexane & 0.50 & $\mathrm{U}$ \\
\hline $56-23-5$ & Carbon tetrachloride & 0.50 & $\mathrm{U}$ \\
\hline $71-43-2$ & Benzene & 0.50 & $\mathrm{U}$ \\
\hline $107-06-2$ & 1,2-Dichloroethane & 0.50 & $\mathrm{U}$ \\
\hline
\end{tabular}

Report 1,4-Dioxane for Low-Medium VOA analysis only 
$1 \mathrm{~B}$ - FORM I VOA-2

VOLATILE ORGANICS ANAI,YSIS DATA SHEET
EPA SAMPLE NO.

VBLKDZ
Lab Name: TESTAMERICA BURLINGTON

Iuab Code: STLV

Case No.: INMA

Matrix: (SOIL/SED/WATER)

Sample wt/vol: 25.0

Water

Level: (TRACE/LOW/MED) TRACE

\% Moisture: not dec.

GC Column: DB-624 (mm)

Soil Extract Volume: (mL)

Purge Volume: 25.0 ID: 0.20

(uL)

$(\mathrm{g} / \mathrm{mL}) \mathrm{mL}$
Contract: $\quad 8 \mathrm{E}-00302$

SDG No.: 200-7045

\begin{tabular}{|c|c|c|c|}
\hline CAS NO. & COMPOUND & $\begin{array}{l}\text { CONCENTRATION UNITS: } \\
(\mathrm{ug} / \mathrm{L} \text { or } \mathrm{ug} / \mathrm{kg}) \mathrm{ug} / \mathrm{L}\end{array}$ & $Q$ \\
\hline $79-01-6$ & Trichloroethene & 0.50 & $\mathrm{U}$ \\
\hline $108-87-2$ & Methylcyclohexane & 0.50 & $\mathrm{U}$ \\
\hline $78-87-5$ & 1,2-Dichloropropane & 0.50 & $\mathrm{U}$ \\
\hline $75-27-4$ & Bromodichloromethane & 0.50 & $\mathrm{U}$ \\
\hline $10061-01-5$ & cis-1,3-Dichloropropene & 0.50 & $\mathrm{U}$ \\
\hline $108-10-1$ & 4-Methyl-2-pentanone & 5.0 & $\mathrm{U}$ \\
\hline $108-88-3$ & Toluene & 0.018 & $\mathrm{~J}$ \\
\hline $10061-02-6$ & trans-1,3-Dichloropropene & 0.50 & U \\
\hline $79-00-5$ & 1,1,2-Trichloroethane & 0.50 & $\mathrm{U}$ \\
\hline $127-18-4$ & Tetrachloroethene & 0.50 & $\mathrm{U}$ \\
\hline $591-78-6$ & 2-Hexanone & 5.0 & $\mathrm{U}$ \\
\hline $124-48-1$ & Dibromochloromethane & 0.50 & $\mathrm{U}$ \\
\hline $106-93-4$ & 1,2-Dibromoethane & 0.50 & $\mathrm{U}$ \\
\hline $108-90-7$ & Chlorobenzene & 0.50 & $\mathrm{U}$ \\
\hline $100-41-4$ & Ethylbenzene & 0.50 & $\mathrm{U}$ \\
\hline $95-47-6$ & o-Xylene & 0.50 & $\mathrm{U}$ \\
\hline $179601-23-1$ & $\mathrm{~m}, \mathrm{p}$-Xylene & 0.50 & $\mathrm{U}$ \\
\hline $100-42-5$ & Styrene & 0.50 & $\mathrm{U}$ \\
\hline $75-25-2$ & Bromoform & 0.50 & $\mathrm{U}$ \\
\hline $98-82-8$ & Isopropylbenzene & 0.50 & $\mathrm{U}$ \\
\hline $79-34-5$ & $1,1,2,2$-Tetrachloroethane & 0.50 & $\mathrm{U}$ \\
\hline $541-73-1$ & 1,3-Dichlorobenzene & 0.50 & $\mathrm{U}$ \\
\hline $106-46-7$ & 1,4-Dichlorobenzene & 0.50 & $\mathrm{U}$ \\
\hline $95-50-1$ & 1,2-Dichlorobenzene & 0.50 & $\mathrm{U}$ \\
\hline $96-12-8$ & 1,2-Dibromo-3-Chloropropane & 0.50 & $\mathrm{U}$ \\
\hline $120-82-1$ & 1,2,4-Trichlorobenzene & 0.50 & $\mathrm{U}$ \\
\hline $87-61-6$ & 1,2,3-Trichlorobenzene & 0.50 & $\mathrm{U}$ \\
\hline
\end{tabular}

Lab Sample ID: MB 200-25508/3

Lab File ID: DHPABO3.D

Date Received:

Date Analyzed: 09/20/2011

Dilution Factor: 1.0

Soil Aliquot Volume:

(uL) 
$1 \mathrm{~J}-$ FORM I VOA-TIC

VOLATILE ORGANICS ANALYSIS DATA SHEET TENTATIVELY IDENTIEIED COMPOUNDS
EPA SAMPLE NO.

VBLKDZ
Lab Name: TESTAMERICA BURLINGTON

Lab Code: STLV Case No.: INMAN

Matrix: (SOIL/SED/WATER)

Water

Sample wt/vol: 25.0 $(\mathrm{g} / \mathrm{mL}) \mathrm{mL}$

Level: (TRACE or LOW/MED) TRACE

읗 Moisture: not dec.

GC Column: DB-624 ID: 0.20 (mm)

Soil Extract Volume: (uL) CONCENTRATION UNITS: (ug/L or ug/kg) ug/L
Contract: $\quad 8 \mathrm{E}-00302$

SDG No.: $200-7045$

Lab Sample ID: MB 200-25508/3

Lab File ID: DHPAB03.D

Date Received:

Date Analyzed: 09/20/2011

Dilution Eactor: 1.0

Soil Aliquot Volume: (uL)

Purge Volume: 25.0 (mL)

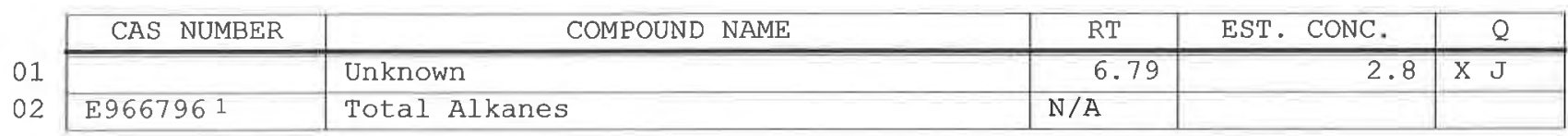

1 EPA-designated Registry Number. 
$1 A$ - FORM I VOA-1

VOLATILE ORGANICS ANALYSIS DATA SHEET
EPA SAMPLE NO.

VHBLK01
Lab Name: TESTAMERICA BURLINGTON

Lab Code: STLV

Case No.: INMAN

Water

Matrix: (SOIL/SED/WATER)

Sample wt/vol: 25.0 $(\mathrm{g} / \mathrm{mL}) \quad \mathrm{mL}$

Level: (TRACE/LOW/MED) TRACE

o Moisture: not dec.

GC Column: DB-624 ID : 0.20 $(\mathrm{mm})$

Soil Extract Volume: (uL) (mL)

Purge Volume: 25.0
Contract: $\quad 8 \mathrm{E}-00302$

SDG No.: 200-7045

Mod. Ref No.:

Lab Sample ID: 200-7045-5

Lab File ID: DHPAB08.D

Date Received:

Date Analyzed: 09/20/2011

Dilution Factor: 1.0

Soil Aliquot Volume:

(UL)

\begin{tabular}{|c|c|c|c|}
\hline CAS NO. & COMPOUND & $\begin{array}{l}\text { CONCENTRATION UNITS: } \\
(\mathrm{ug} / \mathrm{L} \text { or } \mathrm{ug} / \mathrm{kg}) \mathrm{ug} / \mathrm{L}\end{array}$ & $Q$ \\
\hline $75-71-8$ & Dichlorodifluoromethane & 0.50 & $\mathrm{U}$ \\
\hline $74-87-3$ & Chloromethane & 0.50 & $\mathrm{U}$ \\
\hline $75-01-4$ & Vinyl chloride & 0.50 & $\mathrm{U}$ \\
\hline $74-83-9$ & Bromomethane & 0.50 & $\mathrm{U}$ \\
\hline $75-00-3$ & Chloroethane & 0.50 & $\mathrm{U}$ \\
\hline $75-69-4$ & Trichlorofluoromethane & 0.50 & $\mathrm{U}$ \\
\hline $75-35-4$ & 1,1-Dichloroethene & 0.50 & $\mathrm{U}$ \\
\hline $76-13-1$ & 1,1,2-Trichloro-1,2,2-trifluoroethane & 0.50 & $\mathrm{U}$ \\
\hline $67-64-1$ & Acetone & 5.0 & $\mathrm{U}$ \\
\hline $75-15-0$ & Carbon disulfide & 0.50 & $\mathrm{U}$ \\
\hline $79-20-9$ & Methyl acetate & 0.50 & $\mathrm{U}$ \\
\hline $75-09-2$ & Methylene Chloride & 0.50 & $\mathrm{U}$ \\
\hline $156-60-5$ & trans-1,2-Dichloroethene & 0.50 & $\mathrm{U}$ \\
\hline $1634-04-4$ & Methyl tert-butyl ether & 0.50 & $\mathrm{U}$ \\
\hline $75-34-3$ & 1,1-Dichloroethane & 0.50 & $\mathrm{U}$ \\
\hline $156-59-2$ & cis-1,2-Dichloroethene & 0.50 & $\mathrm{U}$ \\
\hline $78-93-3$ & 2-Butanone & 5.0 & $\mathrm{U}$ \\
\hline $74-97-5$ & Bromochloromethane & 0.50 & $\mathrm{U}$ \\
\hline $67-66-3$ & Chloroform & 0.057 & $\mathrm{~J} B$ \\
\hline $71-55-6$ & $1,1,1$-Trichloroethane & 0.50 & $\mathrm{U}$ \\
\hline $110-82-7$ & Cyclohexane & 0.50 & $\mathrm{U}$ \\
\hline $56-23-5$ & Carbon tetrachloride & 0.50 & $\mathrm{U}$ \\
\hline $71-43-2$ & Benzene & 0.50 & $\mathrm{UJ}$ \\
\hline $107-06-2$ & 1,2-Dichloroethane & 0.50 & $\mathrm{U}$ \\
\hline
\end{tabular}

Report 1,4-Dioxane for Low-Medium VOA analysis only 
EPA SAMPLE NO. VHBLK01
Lab Name: TESTAMERICA BURLINGTON

Lab Code: STLV Case

Matrix: (SOIL/SED/WATER)

Water

Sample wt/vol: 25.0

$(\mathrm{g} / \mathrm{mL}) \mathrm{mL}$

Level: (TRACE/LOW/MED) TRACE

o Moisture: not dec.

GC Column: DB-624

ID: 0.20 (mm)

Soil Extract Volume:

(uL) (mL)

Purge Volume: 25.0
Contract: $8 \mathrm{E}-00302$

SDG No.: 200-7045

\begin{tabular}{|c|c|c|c|}
\hline CAS NO. & COMPOUND & $\begin{array}{l}\text { CONCENTRATION UNITS: } \\
(\mathrm{ug} / \mathrm{L} \text { or } \mathrm{ug} / \mathrm{kg}) \mathrm{ug} / \mathrm{L}\end{array}$ & $Q$ \\
\hline $79-01-6$ & Trichloroethene & 0.50 & $\mathrm{U}$ \\
\hline $108-87-2$ & Methylcyclohexane & 0.50 & $\mathrm{U}$ \\
\hline $78-87-5$ & 1,2-Dichloropropane & 0.50 & $\mathrm{U}$ \\
\hline $75-27-4$ & Bromodichloromethane & 0.50 & $\mathrm{U}$ \\
\hline $10061-01-5$ & cis-1,3-Dichloropropene & 0.50 & $\mathrm{U}$ \\
\hline $108-10-1$ & 4-Methyl-2-pentanone & 5.0 & $\mathrm{U}$ \\
\hline $108-88-3$ & Toluene & 0.50 & $\mathrm{U}$ \\
\hline $10061-02-6$ & trans-1,3-Dichloropropene & 0.50 & $\mathrm{U}$ \\
\hline $79-00-5$ & 1,1,2-Trichloroethane & 0.50 & $\mathrm{U}$ \\
\hline $127-18-4$ & Tetrachloroethene & 0.50 & $\mathrm{U}$ \\
\hline $591-78-6$ & 2-Hexanone & 5.0 & $\mathrm{U}$ \\
\hline $124-48-1$ & Dibromochloromethane & 0.50 & $\mathrm{U}$ \\
\hline $106-93-4$ & 1,2-Dibromoethane & 0.50 & $\mathrm{U}$ \\
\hline $108-90-7$ & Chlorobenzene & 0.50 & $\mathrm{U}$ \\
\hline $100-41-4$ & Ethylbenzene & 0.50 & $\mathrm{U}$ \\
\hline $95-47-6$ & o-Xylene & 0.50 & $\mathrm{U}$ \\
\hline $179601-23-1$ & $\mathrm{~m}, \mathrm{p}$-Xylene & 0.50 & $\mathrm{U}$ \\
\hline $100-42-5$ & Styrene & 0.50 & $\mathrm{U}$ \\
\hline $75-25-2$ & Bromoform & 0.50 & $\mathrm{U}$ \\
\hline $98-82-8$ & Isopropylbenzene & 0.50 & $\mathrm{U}$ \\
\hline $79-34-5$ & $1,1,2,2$-Tetrachloroethane & 0.50 & $\mathrm{U}$ \\
\hline $541-73-1$ & 1,3-Dichlorobenzene & 0.50 & $\mathrm{U}$ \\
\hline $106-46-7$ & 1,4-Dichlorobenzene & 0.50 & $\mathrm{U}$ \\
\hline $95-50-1$ & 1,2-Dichlorobenzene & 0.50 & $\mathrm{U}$ \\
\hline $96-12-8$ & 1,2-Dibromo-3-Chloropropane & 0.50 & U \\
\hline $120-82-1$ & 1,2,4-Trichlorobenzene & 0.50 & U \\
\hline $87-61-6$ & $1,2,3$-Trichlorobenzene & 0.50 & $\mathrm{U}$ \\
\hline
\end{tabular}

Lab Sample ID: 200-7045-5

Lab File ID: DHPAB08.D

Date Received:

Date Analyzed: 09/20/2011

Dilution Factor: 1.0

Soil Aliquot Volume:

(uL) 
$1 \mathrm{~J}$ - FORM I VOA-TIC

VOLATILE ORGANICS ANALYSIS DATA SHEET TENTATIVELY IDENTIFIED COMPOUNDS
EPA SAMPLE NO.

VHBLK01
Lab Name: TESTAMERICA BURLINGTON Case No.: INMAN Mod. Ref No.:

Contract: $8 \mathrm{E}-00302$

\begin{abstract}
Lab Code: STLV
\end{abstract}
Water

Matrix: (SOIL/SED/WATER) $(\mathrm{g} / \mathrm{mL}) \mathrm{mL}$

Sample wt/vol: 25.0

TRACE

Level: (TRACE or LOW/MED)

응 Moisture: not dec.

GC Column: DB-624

ID: 0.20 (mm)

Soil Extract Volume: (uL)

CONCENTRATION UNITS: (ug/L or $u g / k g) \quad u g / L$
SDG No.: 200-7045

Lab Sample ID: 200-7045-5

Lab File ID: DHPAB08.D

Date Received:

Date Analyzed: 09/20/2011

Dilution Factor: 1.0

Soil Aliquot Volume: (uL)

Purge Volume: 25.0 (mL)

\begin{tabular}{l|l|l|r|r|r|}
\hline \multicolumn{1}{|c|}{ CAS NUMBER } & \multicolumn{1}{|c|}{ COMPOUND NAME } & \multicolumn{1}{|c|}{ RT } & EST. CONC. & Q \\
\cline { 2 - 6 } 01 & & Unknown & 6.79 & 2.6 & B X J \\
\cline { 2 - 6 } 02 & E9667961 & Total Alkanes & N/A & & \\
\hline
\end{tabular}

1 EPA-designated Registry Number. 


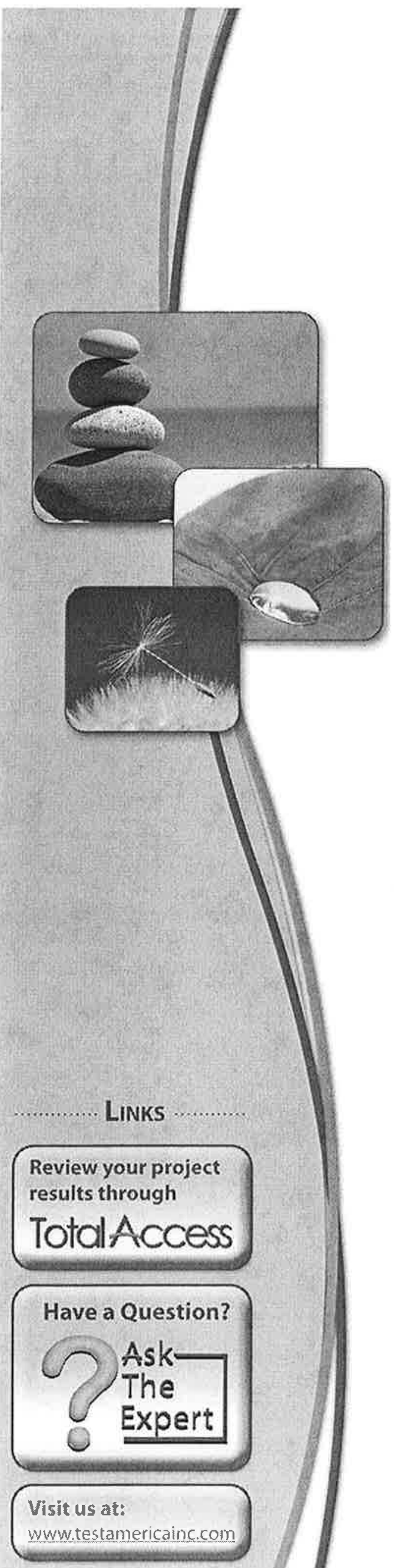

\section{TestAmerica}

THE LEADER IN ENVIRONMENTAL. TESTING

\section{ANALYTICAL REPORT}

TestAmerica Laboratories, Inc.

TestAmerica Chicago

2417 Bond Street

University Park, IL 60484

Tel: (708)534-5200

TestAmerica Job ID: 500-34569-1

Client Project/Site: Murdock Creek

For:

Argonne National Laboratory

9700 South Cass Avenue

Building 203

Office B-149

Argonne, Illinois 60439

Attn: Mr. Clyde Dennis

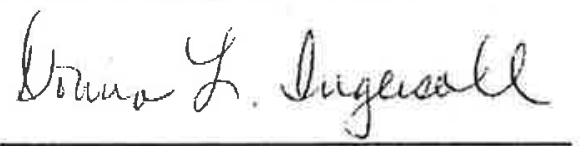

Authorized for release by:

06/09/2011 04:59:00 PM

Donna Ingersoll

Project Manager II

donna.ingersoll@testamericainc.com

Designee for

Jim Knapp

Customer Service Manager

jim.knapp@testamericainc.com

Results relate only to the items tested and the sample(s) as received by the laboratory. The test results in this report meet all 2003 NELAC requirements for accredited parameters, exceptions are noted in this report. Pursuant to NELAC, this report may not be reproduced except in full, and with written approval from the laboratory. For questions please contact the Project Manager at the e-mail address or telephone number listed on this page. 


\section{Table of Contents}

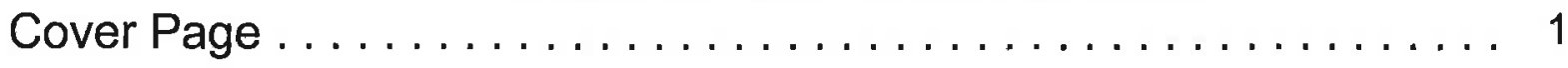

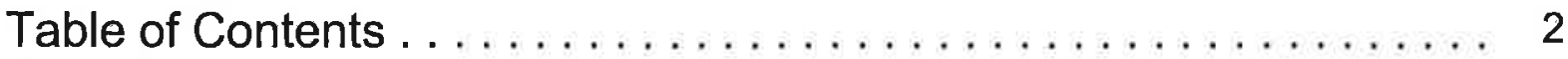

Case Narrative ......................... 3

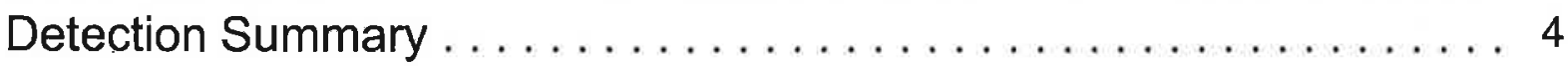

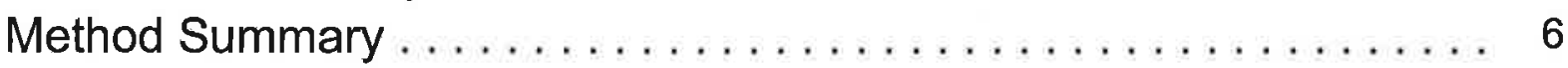

Sample Summary . . . . . . . . . . . . . . . . . . . . . . 7

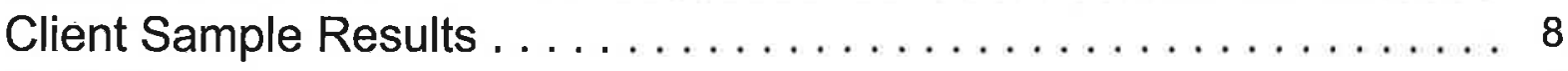

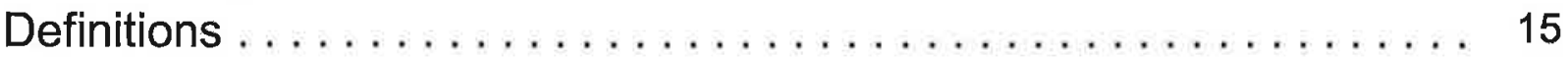

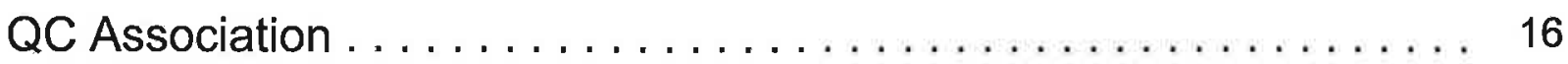

QC Sample Results . . . . . . . . . . . . . . . . . . . . . 19

Certification Summary . . . . . . . . . . . . . . . . . 23

Sample Receipt Checklist . . . . . . . . . . . . . . . . . 24 


\section{Case Narrative}

Client: Argonne National Laboratory

TestAmerica Job ID: 500-34569-1

Project/Site: Murdock Creek

\section{Job ID: 500-34569-1}

\section{Laboratory: TestAmerica Chicago}

Narrative

\section{Job Narrative}

500-34569-1

\section{Comments}

No additional comments.

Receipt

All samples were received in good condition within temperature requirements.

Metals

No analytical or quality issues were noted.

\section{General Chemistry}

Method(s) 300.0: The orthophosphorus matrix spike / matrix spike duplicate (MS/MSD) recoveries for sample INTHIESSEN-W-32685 (500-34569-1) in batch 114678 were outside control limits. The associated laboratory control sample (LCS) recovery met acceptance criteria.

Method(s) 300.0, 9056: The following IC samples in batch 114678 were diluted due to the abundance of non-target analytes: INGOERTZEN-W-32688 (500-34569-4), INHULL-W-32691 (500-34569-7), INISAAC-W-32689 (500-34569-5), INKLASSEN-W-32867 (500-34569-3), INPANKRATZ-W-32690 (500-34569-6), INTHIESSEN-W-32685 (500-34569-1), Elevated reporting limits (RLs) for nitrite are provided.

Method(s) 300.0, 9056: The continuing calibration blank (CCB) for lon Chromatography batch 115146 contained chloride, nitrate, and sulfate above the reporting limit (RL). The associated samples containing detects for chloride and sulfate analytes were at concentrations greater than $10 \mathrm{X}$ the value found in the $\mathrm{CCB}$; therefore, re-analysis of the samples was not performed. The associated samples containing a detect for nitrate were either nondetects for the compound, or the detects were reported due to holding time constraints. INBROWN-W-32686 (500-34569-2)

Method(s) 300.0: The following IC sample in batch 115146 was diluted due to the abundance of non-target analytes: INBROWN-W-32686 (500-34569-2). Elevated reporting limits (RLs) for nitrate are provided.

Also, the continuing calibration verifications ( $C \mathrm{CVs}$ ) throughout the batch were consistently just below the acceptance control limits for nitrite but due to hold time constraints, the results have been reported.

Method(s) 300.0: The following IC samples in batch 115833 was diluted due to the abundance of non-target analytes: INGOERTZEN-W-32688 (500-34569-4), INPANKRATZ-W-32690 (500-34569-6), INTHIESSEN-W-32685 (500-34569-1). Elevated reporting limits (RLs) for bromide are provided.

No other analytical or quality issues were noted. 
Client Sample ID: INTHIESSEN-W-32685

Lab Sample ID: 500-34569-1

\begin{tabular}{llrl} 
Analyte & Result & Qualifier \\
\hline Calcium & 170 & & \\
Magnesium & 50 & \\
Potassium & 1.9 & $\mathrm{~J}$ \\
Sodium & 78 & $\mathrm{~B}$ \\
Zinc & 0.032 & $\mathrm{~B}$ \\
Silicon - RA & 13 & $\mathrm{~B}$ \\
Chloride & 260 & \\
Nitrate as N & 38 & \\
Sulfate & 21 & 180
\end{tabular}

\begin{tabular}{|c|c|c|}
\hline RL & MDL & Unit \\
\hline 5.0 & 0.12 & $\mathrm{mg} / \mathrm{L}$ \\
\hline 5.0 & 0.064 & $\mathrm{mg} / \mathrm{l}$ \\
\hline 5,0 & 0.18 & $\mathrm{mg} / \mathrm{L}$ \\
\hline 5.0 & 0,051 & $\mathrm{mg} / \mathrm{L}$ \\
\hline 0.020 & 0.00075 & $\mathrm{ng} / \mathrm{L}$ \\
\hline 0.10 & 0.0047 & $\mathrm{mg} / \mathrm{L}$ \\
\hline 10 & 4.2 & $\mathrm{mg} / \mathrm{L}$ \\
\hline 1.0 & 0.23 & $\mathrm{mg} / \mathrm{L}$ \\
\hline 2,0 & 0,90 & $\mathrm{mg} / \mathrm{L}$ \\
\hline 5.0 & 1,3 & $\mathrm{mg}$ \\
\hline
\end{tabular}

\begin{tabular}{rll} 
Dil Fac & D & Method \\
\hline 1 & & $6010 \mathrm{~B}$ \\
1 & $6010 \mathrm{~B}$ \\
1 & $6010 \mathrm{~B}$ \\
1 & $6010 \mathrm{~B}$ \\
1 & $6010 \mathrm{~B}$ \\
1 & $6010 \mathrm{~B}$ \\
50 & 300,0 \\
10 & 300,0 \\
10 & 300.0 \\
1 & SM $2320 \mathrm{~B}$
\end{tabular}

Prep Type Dissolved Dissolved Dissolved Dissolved Dissolved Dissolved Total/NA Total/NA Total/NA Total/NA

\section{Client Sample ID: INBROWN-W-32686}

Lab Sample ID: 500-34569-2

\begin{tabular}{lrl} 
Analyte & Result & Qualifier \\
\cline { 3 - 3 } Calcium & 440 & 70 \\
Magnesium & $0.0098 \mathrm{~J}$ \\
Panganese & $3.4 \mathrm{~J}$ \\
Zinc & $0.0036 \mathrm{~J} \mathrm{~B}$ \\
Sodium - DL & 130 & \\
Silicon - RA & $12 \mathrm{~B}$ \\
Chloride & $44 \wedge$ \\
Sulfate & $86 \wedge$ \\
Bicarbonate Alkalinity as CaCO3 & 240
\end{tabular}

$\mathbf{R L}$
5.0
5.0
0.015
5.0
0.020
50
0.10
10
10
5.0

$\begin{array}{rl}\text { MDL } & \text { Unit } \\ 0.12 & \mathrm{mg} / \mathrm{L} \\ 0.064 & \mathrm{mg} / \mathrm{L} \\ 0.0019 & \mathrm{mg} / \mathrm{L} \\ 0.18 & \mathrm{mg} / \mathrm{L} \\ 0.00075 & \mathrm{mg} / \mathrm{L} \\ 0.51 & \mathrm{mg} / \mathrm{L} \\ 0.0047 & \mathrm{mg} / \mathrm{L} \\ 4.2 & \mathrm{mg} / \mathrm{L} \\ 4.5 & \mathrm{mg} / \mathrm{L} \\ 1.3 & \mathrm{mg} / \mathrm{L}\end{array}$

\begin{tabular}{rll} 
Dil Fac & D & Method \\
\hline 1 & & $6010 \mathrm{~B}$ \\
1 & $6010 \mathrm{~B}$ \\
1 & $6010 \mathrm{~B}$ \\
1 & $6010 \mathrm{~B}$ \\
1 & $6010 \mathrm{~B}$ \\
10 & $6010 \mathrm{~B}$ \\
1 & $6010 \mathrm{~B}$ \\
50 & 300.0 \\
50 & 300.0 \\
1 & SM $2320 \mathrm{~B}$
\end{tabular}

Prep Type
Dissolved
Dissolved
Dissolved
Dissolved
Dissolved
Dissolved
Dissolved
Total/NA
Total/NA
Total/NA

Client Sample ID: INKLASSEN-W-32867

Lab. Sample ID: 500-34569-3

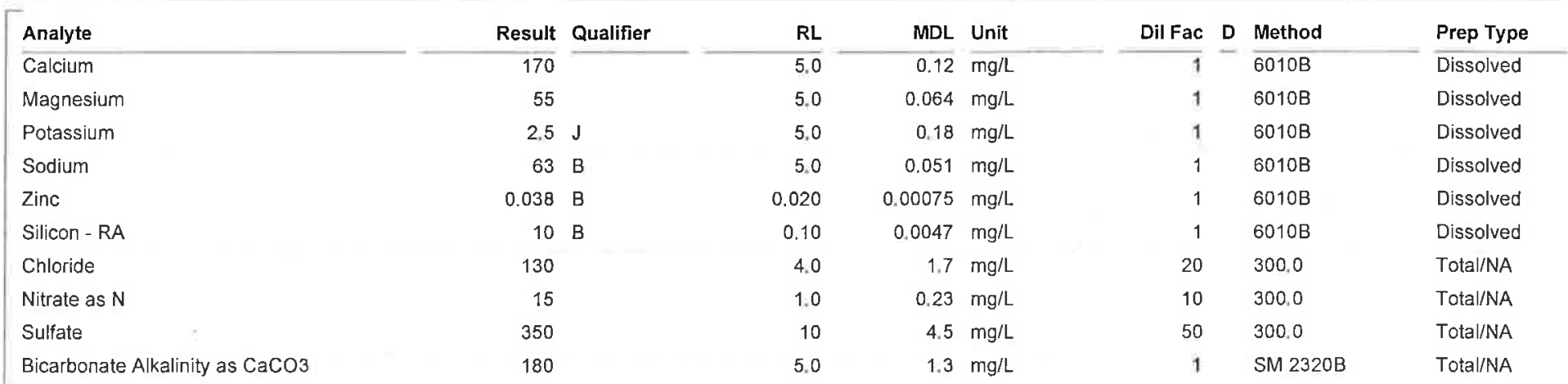

Client Sample ID: INGOERTZEN-W-32688

Lab Sample ID: 500-34569-4

\begin{tabular}{|c|c|c|c|c|c|c|c|c|}
\hline Analyte & Result & Qualifier & RL & MDL & Unit & Dil Fac & D Method & Prep Type \\
\hline Calcium & 150 & & 5.0 & 0.12 & $\mathrm{mg} / \mathrm{L}$ & 1 & $6010 \mathrm{~B}$ & Dissolved \\
\hline Magnesium & 38 & & 5.0 & 0.064 & $\mathrm{mg} / \mathrm{L}$ & 1 & $6010 \mathrm{~B}$ & Dissolved \\
\hline Phosphorus & 5.6 & $\mathrm{~J}$ & 250 & 5.2 & $\mathrm{ug} / \mathrm{L}$ & 1 & $6010 \mathrm{~B}$ & Dissolved \\
\hline Potassium & 1.3 & $J$ & 5.0 & 0.18 & $\mathrm{mg} / \mathrm{L}$ & 1 & $6010 \mathrm{~B}$ & Dissolved \\
\hline Sodium & 70 & $B$ & 5.0 & 0,051 & $\mathrm{mg} / \mathrm{L}$ & 1 & $6010 \mathrm{~B}$ & Dissolved \\
\hline Zinc & 0.063 & $B$ & 0.020 & 0.00075 & $\mathrm{mg} / \mathrm{L}$ & 1 & $6010 \mathrm{~B}$ & Dissolved \\
\hline Silicon - RA & 13 & $\mathrm{~B}$ & 0.10 & 0,0047 & $\mathrm{mg} / \mathrm{L}$ & 1 & $6010 \mathrm{~B}$ & Dissolved \\
\hline Chloride & 300 & & 10 & 4,2 & $\mathrm{mg} / \mathrm{t}$ & 50 & 300.0 & Total/NA \\
\hline Nitrate as $\mathrm{N}$ & 5.9 & & 1.0 & 0.23 & $\mathrm{mg} / \mathrm{L}$ & 10 & 300.0 & Total/NA \\
\hline Sulfate & 23 & & 2.0 & 0,90 & $\mathrm{mg} / \mathrm{L}$ & 10 & 300,0 & Total/NA \\
\hline Bicarbonate Alkalinity as $\mathrm{CaCO} 3$ & 190 & & 5.0 & 1.3 & $\mathrm{mg} / \mathrm{L}$ & 1 & SM 2320B & Total/NA \\
\hline
\end{tabular}




\section{Detection Summary}

Client: Argonne National Laboratory

Client Sample ID: INISAAC-W-32689

Lab Sample ID: 500-34569-5

\begin{tabular}{|c|c|c|c|c|c|c|c|c|}
\hline Analyte & Result & Qualifier & $R \mathbf{R}$ & MDL & Unit & Dil Fac $D$ & Method & Prep Type \\
\hline Calcium & 220 & & 5.0 & 0.12 & $\mathrm{mg} / \mathrm{L}$ & 1 & $6010 \mathrm{~B}$ & Dissolved \\
\hline Magnesium & 50 & & 5.0 & 0.064 & $\mathrm{mg} / \mathrm{L}$ & 1 & $6010 \mathrm{~B}$ & Dissolved \\
\hline Phosphorus & 6.4 & J & 250 & 5.2 & $\mathrm{ug} / \mathrm{h}$ & 1 & $6010 \mathrm{~B}$ & Dissolved \\
\hline Potassium & 2.1 & J & 5.0 & 0.18 & $\mathrm{mg} / \mathrm{L}$ & 1 & $6010 \mathrm{~B}$ & Dissolved \\
\hline Sodium & 62 & B & 5.0 & 0.051 & $\mathrm{mg} / \mathrm{L}$ & 1 & $6010 \mathrm{~B}$ & Dissolved \\
\hline Zinc & 0.022 & 8 & 0.020 & 0.00075 & $\mathrm{mg} / \mathrm{L}$ & 1 & $6010 \mathrm{~B}$ & Dissolved \\
\hline Silicon - RA & 12 & B & 0.10 & 0.0047 & $\mathrm{mg} / \mathrm{L}$ & 1 & $6010 \mathrm{~B}$ & Dissolved \\
\hline Chloride & 130 & & 4.0 & 1.7 & $\mathrm{mg} / \mathrm{L}$ & 20 & 300.0 & Total/NA \\
\hline Nitrate as $\mathrm{N}$ & 7.5 & & 1.0 & 0.23 & $\mathrm{mg} / \mathrm{L}$ & 10 & 300.0 & Total/NA \\
\hline Sulfate & 340 & & 10 & 4.5 & $\mathrm{mg} / \mathrm{L}$ & 50 & 300.0 & Total/NA \\
\hline Bicarbonate Alkalinity as $\mathrm{CaCO} 3$ & 230 & & 5.0 & 1.3 & $\mathrm{mg} / \mathrm{L}$ & 1 & SM 2320B & Total/NA \\
\hline
\end{tabular}

Client Sample ID: INPANKRATZ-W-32690

Lab Sample ID: 500-34569-6

\begin{tabular}{llll} 
Analyte & Result & Qualifier \\
\cline { 1 - 2 } Calcium & 210 & \\
Magnesium & 59 & \\
Potassium & 3.0 & $\mathrm{~J}$ \\
Sodium & 58 & $\mathrm{~B}$ \\
Zinc & 0.022 & $\mathrm{~B}$ \\
Silicon - RA & 15 & $\mathrm{~B}$ \\
Chloride & 230 & \\
Nitrate as N & 14 & \\
Sulfate & 420 \\
Bicarbonate Alkalinity as $\mathrm{CaCO}$ & 160
\end{tabular}

\begin{tabular}{rrrr} 
RL & MDL & Unit \\
\hline 5.0 & 0.12 & $\mathrm{mg} / \mathrm{L}$ \\
5.0 & 0.064 & $\mathrm{mg} / \mathrm{L}$ \\
5.0 & 0.18 & $\mathrm{mg} / \mathrm{L}$ \\
5.0 & 0.051 & $\mathrm{mg} / \mathrm{L}$ \\
0.020 & 0.00075 & $\mathrm{mg} / \mathrm{L}$ \\
0.10 & 0.0047 & $\mathrm{mg} / \mathrm{L}$ \\
10 & 4.2 & $\mathrm{mg} / \mathrm{L}$ \\
1.0 & 0.23 & $\mathrm{mg} / \mathrm{L}$ \\
10 & 4.5 & $\mathrm{mg} / \mathrm{L}$ \\
5.0 & 1.3 & $\mathrm{mg} / \mathrm{L}$
\end{tabular}

\begin{tabular}{rll} 
Dil Fac & D & Method \\
\hline 1 & & $6010 \mathrm{~B}$ \\
1 & $6010 \mathrm{~B}$ \\
1 & $6010 \mathrm{~B}$ \\
1 & $6010 \mathrm{~B}$ \\
1 & $6010 \mathrm{~B}$ \\
1 & $6010 \mathrm{~B}$ \\
50 & 300.0 \\
10 & 300.0 \\
50 & 300.0 \\
1 & $\mathrm{SM} 2320 \mathrm{~B}$
\end{tabular}

Prep Type Dissolved Dissolved Dissolved Dissolved Dissolved Dissolved Total/NA Total/NA Total/NA Total/NA

Client Sample ID: INHULL-W-32691

Lab Sample ID: 500-34569-7

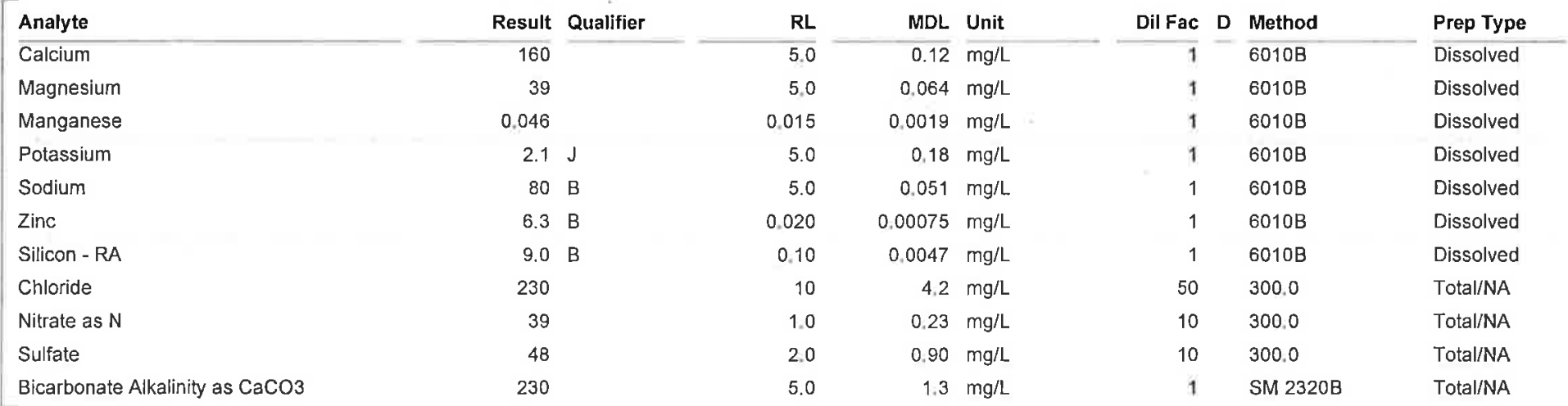




\section{Method Summary}

Client: Argonne National Laboratory Project/Site: Murdock Creek

\begin{tabular}{|c|c|c|c|}
\hline Method & Method Description & Protocol & Laboratory \\
\hline $6010 B$ & Metals (ICP) & SW846 & TAL BUR \\
\hline 300.0 & Anions, Ion Chromatography & MCAWW & TAL CHI \\
\hline SM 2320B & Alkalinity & SM & TAL CHI \\
\hline
\end{tabular}

Protocol References:

MCAWW = "Methods For Chemical Analysis Of Water And Wastes", EPA-600/4-79-020, March 1983 And Subsequent Revisions.

$\mathrm{SM}=$ "Standard Methods For The Examination Of Water And Wastewater",

SW846 = "Test Methods For Evaluating Solid Waste, Physical/Chemical Methods", Third Edition, November 1986 And Its Updates.

Laboratory References:

TAL BUR = TestAmerica Burlington, 30 Community Drive, Suite 11, South Burlington, VT 05403, TEL (802)660-1990

TAL CHI = TestAmerica Chicago, 2417 Bond Street, University Park, IL 60484, TEL (708)534-5200 


\section{Sample Summary}

Client: Argonne National Laboratory

TestAmerica Job ID: 500-34569-1

\section{Project/Site: Murdock Creek}

\begin{tabular}{l} 
Lab Sample ID \\
\hline $500-34569-1$ \\
$500-34569-2$ \\
$500-34569-3$ \\
$500-34569-4$ \\
$500-34569-5$ \\
$500-34569-6$ \\
$500-34569-7$
\end{tabular}

Client Sample ID
INTHIESSEN-W-32685
INBROWN-W-32686
INKLASSEN-W-32867
INGOERTZEN-W-32688
INISAAC-W-32689
INPANKRATZ-W-32690
INHULL-W-32691

Matrix

Collected

Received

05/24/1116:30 05/25/1110:00

05/24/1116:05 05/25/1110:00

05/24/11 13:10 05/25/1110:00

05/24/11 13:22 05/25/11 10:00

$05 / 24 / 1113: 40 \quad 05 / 25 / 1110: 00$

05/24/11 13:50 05/25/11 10:00

INHULL-W-32691

$05 / 24 / 1114: 06$

$05 / 25 / 1110: 00$ 
Method: 6010B - Metals (ICP) - Dissolved

\section{Analyte}

Aluminum

Calcium

Iron

Magnesium

Manganese

Phosphorus

Potassium

Sodium

Zinc
Result Qualifier

$<0.20$

170

$<0.20$

50

$<0.015$

$<250$

$1.9 \mathrm{~J}$

$78 \mathrm{~B}$

$0.032 \mathrm{~B}$
RL

0.20
5.0

5.0
0.20

5.0

0.015

250

5.0

5.0

0.020

$\begin{array}{rll}\text { MDL } & \text { Unit } \\ & \mathrm{mg} / \mathrm{L} \\ 0.12 & \mathrm{mg} / \mathrm{L} \\ 0.039 & \mathrm{mg} / \mathrm{L} \\ 0.064 & \mathrm{mg} / \mathrm{L} \\ 0.0019 & \mathrm{mg} / \mathrm{L} \\ 5.2 & \mathrm{ug} / \mathrm{L} \\ 0.18 & \mathrm{mg} / \mathrm{L} \\ 0.051 & \mathrm{mg} / \mathrm{L} \\ 0.00075 & \mathrm{mg} / \mathrm{L}\end{array}$

D

\begin{tabular}{c} 
Prepared \\
\hline 06/02/11 08:20 \\
06/02/11 08:20 \\
06/02/11 08:20 \\
06/02/11 08:20 \\
06/02/11 08:20 \\
06/02/11 08:20 \\
06/02/11 08:20 \\
06/02/11 08:20 \\
06/02/11 08:20
\end{tabular}

\begin{tabular}{|c|c|}
\hline Analyzed & \\
\hline $06 / 05 / 1120: 42$ & \\
\hline $06 / 05 / 1120: 42$ & \\
\hline 06/05/11 20:42 & \\
\hline $06 / 05 / 1120: 42$ & \\
\hline $06 / 05 / 1120: 42$ & \\
\hline 06/05/11 20:42 & \\
\hline $06 / 05 / 1120: 42$ & \\
\hline $06 / 05 / 1120: 42$ & \\
\hline $08 / 05 / 1120: 42$ & \\
\hline
\end{tabular}

Method: 6010B - Metals (ICP) - Dissolved - RA

Analyte

Result Qualifier

$13 \bar{B}$

$\frac{{ }^{R L}}{0.10}$

MDL Unit $0.0047 \mathrm{mg} / \mathrm{L}$

$\frac{\text { Prepared }}{06 / 02 / 1108: 20}$

Analyzed

Dil Fac 06/06/11 12:02

\begin{tabular}{|c|c|}
\hline Analyzed & \\
\hline 8/07/11 18:36 & \\
\hline $66 / 07 / 1118: 51$ & \\
\hline $05 / 25 / 1120: 33$ & \\
\hline $5 / 25 / 4120: 33$ & \\
\hline $55 / 25 / 1120: 33$ & \\
\hline $5 / 25 / 1120: 18$ & \\
\hline $55 / 27 / 1115: 48$ & \\
\hline$/ 27 / 1115: 48$ & \\
\hline
\end{tabular}

\section{General Chemistry}

Analyte

Bromide

Chloride

Nitrate as $\mathrm{N}$

Nitrite as $\mathrm{N}$

Sulfate

Orthophosphate as $\mathrm{P}$

Bicarbonate Alkalinity as $\mathrm{CaCO} 3$

Carbonate Alkallnity as $\mathrm{CaCO}$

\begin{tabular}{rr} 
Result & Qualifier \\
\hline$<0.40$ & \\
260 & \\
38 & $<1.0$ \\
21 \\
$<0.20$ \\
180 \\
$<5.0$
\end{tabular}

\begin{tabular}{rrrr} 
RL & MDL & Unit & D Prepared \\
\cline { 2 - 2 } 0.40 & 0.11 & $\mathrm{mg} / \mathrm{L}$ \\
10 & $4.2 \mathrm{mg} / \mathrm{L}$ & \\
1.0 & $0.23 \mathrm{mg} / \mathrm{L}$ \\
1.0 & $0.23 \mathrm{mg} / \mathrm{L}$ \\
2.0 & $0.90 \mathrm{mg} / \mathrm{L}$ \\
0.20 & $0.13 \mathrm{mg} / \mathrm{L}$ \\
5.0 & $1.3 \mathrm{mg} / \mathrm{L}$ \\
5.0 & $1.3 \mathrm{mg} / \mathrm{L}$
\end{tabular}




\section{Client Sample Results}

Lab Sample ID: 500-34569-2

Client Sample ID: INBROWN-W-32686

Matrix: Water

Date Collected: 05/24/11 16:05

Date Received: 05/25/11 10:00

Method: 6010B - Metals (ICP) - Dissolved

\section{Analyte}

Aluminum

Calcium

Iron

Magnesium

Manganese

Phosphorus

Potassium

Zinc
Result Qualifier

$<0.20$

440

$<0.20$

70

$0.0098 \mathrm{~J}$

$<250$

$3.4 \mathrm{~J}$

$0.0036 \mathrm{~J} \mathrm{~B}$

$\mathbf{R L}$
0.20
5.0
0.20
5.0
0.015
250
5.0
0.020

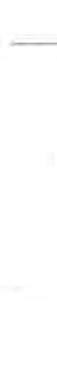

MDL Unit

$0.039 \mathrm{mg} / \mathrm{L}$

$0.12 \mathrm{mg} / \mathrm{L}$

$0.039 \mathrm{mg} / \mathrm{L}$

$0.064 \mathrm{mg} / \mathrm{L}$

$0.0019 \mathrm{mg} / \mathrm{L}$

$5.2 \mathrm{ug} / \mathrm{L}$

$0.18 \mathrm{mg} / \mathrm{L}$

$0.00075 \mathrm{mg} / \mathrm{L}$
D

\begin{tabular}{|c|c|}
\hline Prepared & Analyzed \\
\hline 06/02/11 08:20 & $06 / 05 / 1120: 47$ \\
\hline 06/02/11 08:20 & $06 / 05 / 1120: 47$ \\
\hline $06 / 02 / 1108: 20$ & $06 / 05 / 1120: 47$ \\
\hline $06 / 02 / 1108: 20$ & $06 / 05 / 1120: 47$ \\
\hline $06 / 02 / 1108: 20$ & $06 / 05 / 1120: 47$ \\
\hline 06/02/11 08:20 & $06 / 05 / 1120: 47$ \\
\hline 06/02/11 08:20 & $06 / 05 / 1120: 47$ \\
\hline 06/02/11 08:20 & $06 / 05 / 1120: 47$ \\
\hline
\end{tabular}

Method: 6010B - Metals (ICP) - Dissolved - DL

Analyte

Result Qualifier

Sodium

130

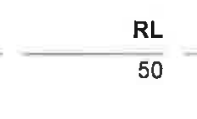

MDL Unit

$\frac{\text { Prepared }}{06 / 02 / 1108: 20} \quad \frac{\text { Analyzed }}{06 / 06 / 1143: 13} \stackrel{\text { Dil Fac }}{10}$

Method: 6010B - Metals (ICP) - Dissolved - RA

Analyte

Result Qualifier

$12 \mathrm{~B}$

RL

MDL Unit

$0.0047 \overline{\mathrm{mg} / \mathrm{L}}$

D

$\frac{\text { Prepared }}{06 / 02 / 1108: 20} \frac{\text { Analyzed }}{06 / 06 / 1112: 07} \frac{\text { Dil Fac }}{1}$

General Chemistry

\begin{tabular}{|c|c|c|c|c|c|}
\hline Analyte & Result & Qualifier & RL & MDL & Unit \\
\hline Bromide & $<0.20$ & & 0.20 & 0.057 & $\mathrm{mg} / \mathrm{L}$ \\
\hline Chloride & 44 & ᄉ & 10 & 4.2 & $\mathrm{mg} / \mathrm{L}$ \\
\hline Nitrate as $\mathbf{N}$ & $<0.10$ & & 0.10 & 0.023 & $\mathrm{mg} / \mathrm{L}$ \\
\hline Nitrite as $\mathrm{N}$ & $<5.0$ & $H^{\wedge} \star$ & 5.0 & 1.2 & $\mathrm{mg} / \mathrm{L}$ \\
\hline Sulfate & 86 & $\wedge$ & 10 & 4.5 & $\mathrm{mg} / \mathrm{L}$ \\
\hline Orthophosphate as $P$ & $<0.20$ & & 0.20 & 0.13 & $\mathrm{mg} / \mathrm{L}$ \\
\hline Bicarbonate Alkalinity as $\mathrm{CaCO} 3$ & 240 & & 5.0 & 1.3 & $\mathrm{mg} / \mathrm{L}$ \\
\hline Carbonate Alkalinity as $\mathrm{CaCO} 3$ & $<5.0$ & & 5.0 & 1.3 & $\mathrm{mg} / \mathrm{L}$ \\
\hline
\end{tabular}

Carbonate Alkalinity as $\mathrm{CaCO} 3$

$<5.0$

$1.3 \mathrm{mg} / \mathrm{L}$ 


\section{Client Sample Results}

Project/Site: Murdock Creek

Client Sample ID: INKLASSEN-W-32867

Lab Sample ID: 500-34569-3

Date Collected: 05/24/11 13:10

Matrix: Water

Date Received: 05/25/11 10:00

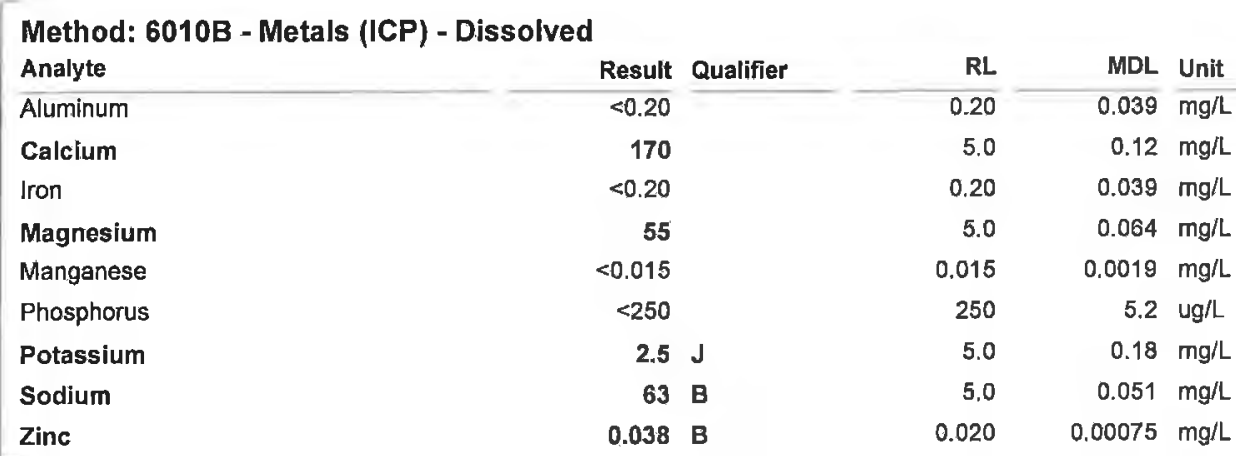

Method: 6010B - Metals (ICP) - Dissolved - RA

Analyte

Silicon

Result Qualifier

$10 \mathrm{~B}$

Result Qualifier

$<0.20$

Bromide

130

Chloride

Nitrate as $\mathbf{N}$

Nitrite as $\mathrm{N}$

15

$<1.0$

Sulfate

Orthophosphate as $\mathrm{P}$

350

$<0.20$

180

Bicarbonate Alkalinity as $\mathrm{CaCO} 3$

$<5.0$
MDL Unit

\begin{tabular}{r} 
RL \\
\hline 0.10 \\
\\
$\mathbf{R L}$ \\
\hline 0.20 \\
4.0 \\
1.0 \\
1.0 \\
10 \\
0.20 \\
5.0 \\
5.0
\end{tabular}

MDL Unit $0.0047 \mathrm{mg} / \mathrm{L}$ $0.057 \mathrm{mg} / \mathrm{L}$

$1.7 \mathrm{mg} / \mathrm{L}$

$0.23 \mathrm{mg} / \mathrm{L}$

$0.23 \mathrm{mg} / \mathrm{L}$

$4.5 \mathrm{mg} / \mathrm{L}$

$0.13 \mathrm{mg} / \mathrm{L}$

$1.3 \mathrm{mg} / \mathrm{L}$

$1.3 \mathrm{mg} / \mathrm{L}$
D

\begin{tabular}{c} 
Prepared \\
\hline 06/02/11 08:20 \\
06/02/11 08:20 \\
06/02/11 08:20 \\
06/02/11 08:20 \\
06/02/11 08:20 \\
06/02/11 08:20 \\
06/02/11 08:20 \\
06/02/11 08:20 \\
06/02/11 08:20
\end{tabular}

\begin{tabular}{c} 
Prepared \\
\hline 06/02/11 08:20 \\
06/02/11 08:20 \\
06/02/11 08:20 \\
06/02/11 08:20 \\
06/02/11 08:20 \\
06/02/11 08:20 \\
06/02/11 08:20 \\
06/02/11 08:20 \\
06/02/11 08:20
\end{tabular}

\begin{tabular}{|c|c|}
\hline Analyzed & \\
\hline $06 / 05 / 1120: 52$ & \\
\hline $06 / 05 / 1120: 52$ & \\
\hline $06 / 05 / 1120: 52$ & \\
\hline $06 / 05 / 1120.52$ & \\
\hline $06 / 05 / 1120: 52$ & \\
\hline $06 / 05 / 1120: 52$ & \\
\hline $06 / 05 / 1120: 52$ & \\
\hline $06 / 05 / 1120: 52$ & \\
\hline $06 / 05 / 1120: 52$ & \\
\hline
\end{tabular}

D

$\frac{\text { Prepared }}{\text { 06/02/11 08:20 }}$

Analyzed 06/06/11 12:12

Dil Fac
D

Prepared

\begin{tabular}{|c|c|}
\hline Analyzed & Dil Fac \\
\hline $06 / 07 / 1119: 22$ & \\
\hline $06 / 07 / 1119: 38$ & 2 \\
\hline 05/25/11 23:01 & 10 \\
\hline $05 / 25 / 1123: 01$ & \\
\hline $06 / 07 / 1119: 53$ & 5 \\
\hline $05 / 25 / 1122: 47$ & \\
\hline $05 / 27 / 1116: 03$ & \\
\hline $05 / 27 / 1116: 03$ & \\
\hline
\end{tabular}




\section{Client Sample Results}

Client: Argonne National Laboratory

Project/Site: Murdock Creek

Client Sample ID: INGOERTZEN-W-32688

Lab Sample ID: 500-34569-4

Date Collected: 05/24/11 13:22

Matrix: Water

Date Received: 05/25/11 10:00

Method: 6010B - Metals (ICP) - Dissolved

Analyte

Aluminum

Calcium

Iron

Magnesium

Manganese

Potassium

Sodium

Zinc
Phosphorus
Result Qualifier

$<0.20$

150

$<0.20$

38

$<0.015$

$5.6 \mathrm{~J}$

$1.3 \mathrm{~J}$

$70 \mathrm{~B}$

$0.063 \mathrm{~B}$

\begin{abstract}
RL
\end{abstract}

0.20

5.0

0.20

5.0

0.015

015
250

5.0

5.0

0,020

$\begin{array}{rll}\text { MDL } & \text { Unit } \\ 0.039 & \mathrm{mg} / \mathrm{L} \\ 0.12 & \mathrm{mg} / \mathrm{L} \\ 0.039 & \mathrm{mg} / \mathrm{L} \\ 0.064 & \mathrm{mg} / \mathrm{L} \\ 0.0019 & \mathrm{mg} / \mathrm{L} \\ 5.2 & \mathrm{ug} / \mathrm{L} \\ 0.18 & \mathrm{mg} / \mathrm{L} \\ 0.051 & \mathrm{mg} / \mathrm{L} \\ 0.00075 & \mathrm{mg} / \mathrm{L}\end{array}$

D

Prepared
06/02/11 08:20
06/02/11 08:20
06/02/11 08:20
06/02/11 08:20
06/02/11 08:20
06/02/11 08:20
06/02/11 08:20
06/02/11 08:20
06/02/11 08:20

\begin{tabular}{crr} 
Analyzed & & Dil Fac \\
\cline { 1 - 1 } 06/05/11 20:57 & 1 \\
06/05/11 20:57 & 1 \\
06/05/11 20:57 & 1 \\
06/05/11 20:57 & 1 \\
06/05/11 20:57 & 1 \\
06/05/11 20:57 & 1 \\
06/05/11 20:57 & 1 \\
$06 / 05 / 1120: 57$ & 1 \\
$06 / 05 / 1120: 57$ & 1
\end{tabular}

Method: 6010B - Metals (ICP) - Dissolved - RA
MDL Unit

$0.0047 \overline{\mathrm{mg} / \mathrm{L}}$
Analyzed 06/06/11 12:17
Analyte

Silicon

Result Qualifier

$13 \mathrm{~B}$
D

$\frac{\text { Prepared }}{06 / 02 / 1108: 20}$
Dil Fac

\section{General Chemistry}

Analyte

Bromide

Chloride

Nitrate as $\mathbf{N}$

Nitrite as $\mathbf{N}$

Sulfate

Orthophosphate as $P$

Bicarbonate Alkalinity as $\mathrm{CaCO}$

Carbonate Alkalinity as $\mathrm{CaCO} 3$

Result Qualifier
$<0.40$
300
5.9
$<1.0$
23
$<0.20$
190
$<5.0$

\begin{tabular}{|c|c|c|c|c|}
\hline $\mathbf{R L}$ & MDL & Unit & D & Prepared \\
\hline 0.40 & 0.11 & $\mathrm{mg} / \mathrm{L}$ & & \\
\hline 10 & 4,2 & $\mathrm{mg} / \mathrm{L}$ & & \\
\hline 1.0 & 0.23 & $\mathrm{mg} / \mathrm{L}$ & & \\
\hline 1.0 & 0.23 & $\mathrm{mg} / \mathrm{L}$ & & \\
\hline 2.0 & 0.90 & $\mathrm{mg} / \mathrm{L}$ & & \\
\hline 0.20 & 0.13 & $\mathrm{mg} / \mathrm{L}$ & & \\
\hline 5.0 & 1.3 & $\mathrm{mg} / \mathrm{L}$ & & \\
\hline 5.0 & 1.3 & $\mathrm{mg} / \mathrm{L}$ & - & \\
\hline
\end{tabular}

\begin{tabular}{|c|c|}
\hline Analyzed & Dil Fac \\
\hline $06 / 07 / 1120: 08$ & 2 \\
\hline $06 / 08 / 1101: 15$ & 50 \\
\hline 05/25/11 23:31 & 10 \\
\hline $05 / 25 / 1123: 31$ & 10 \\
\hline 05/25/11 23:31 & 10 \\
\hline 05/25/11 23:16 & 1 \\
\hline $05 / 27 / 1116: 09$ & 1 \\
\hline $05 / 27 / 1116: 09$ & 1 \\
\hline
\end{tabular}




\section{Client Sample Results}

Project/Site: Murdock Creek

Client Sample ID: INISAAC-W-32689

Lab Sample ID: 500-34569-5

Date Collected: 05/24/11 13:40

Matrix: Water

Date Received: 05/25/11 10:00

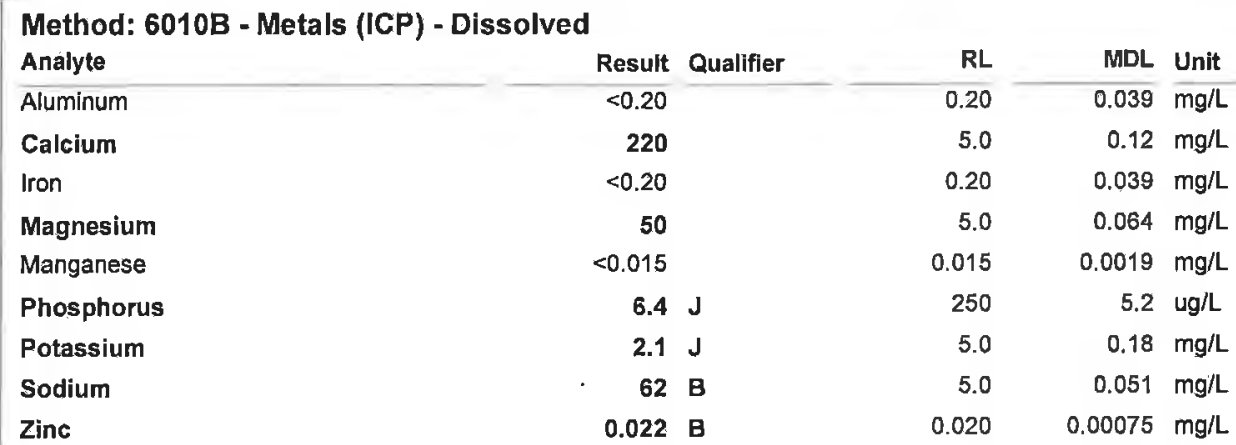

Method: 6010B - Metals (ICP) - Dissolved - RA

Analyte

Silicon

Resut

12

Qualifier

General Chemistry

Analyte

Result Qualifier

Bromide

$<0.20$

Nitrate as $\mathbf{N}$

7.5

Nitrite as $\mathrm{N}$

$<1.0$

Sulfate

Orthophosphate as $\mathbf{P}$

340

$<0,20$

230

Bicarbonate Alkalinity as $\mathrm{CaCO} 3$

$<5.0$
MDL Unit

$0.057 \mathrm{mg} / \mathrm{L}$

$1.7 \mathrm{mg} / \mathrm{L}$

$0.23 \mathrm{mg} / \mathrm{L}$

$\mathbf{R L}$

MDL Unit

$0.23 \mathrm{mg} / \mathrm{L}$

$4.5 \mathrm{mg} / \mathrm{L}$

$0.13 \mathrm{mg} / \mathrm{L}$

$1.3 \mathrm{mg} / \mathrm{L}$

$1.3 \mathrm{mg} / \mathrm{L}$
Carbonate Alkalinity as $\mathrm{CaCO} 3$
5.0

5.0
D

\begin{tabular}{cccrr} 
Prepared & & Analyzed & & Dil Fac \\
\cline { 5 - 5 } $06 / 02 / 11$ 08:20 & & $06 / 05 / 1 \uparrow 21: 02$ & & 1 \\
06/02/11 08:20 & & $06 / 05 / 1121: 02$ & & 1 \\
06/02/11 08:20 & & $06 / 05 / 1121: 02$ & & 1 \\
06/02/11 08:20 & $06 / 05 / 1121: 02$ & & 1 \\
06/02/11 08:20 & $06 / 05 / 1121: 02$ & & 1 \\
06/02/11 08:20 & $06 / 05 / 1121: 02$ & & 1 \\
06/02/11 08:20 & $06 / 05 / 1121: 02$ & & 1 \\
06/02/11 08:20 & $06 / 05 / 1121: 02$ & & 1 \\
06/02/11 08:20 & $06 / 05 / 1121: 02$ & & 1
\end{tabular}

$\frac{\text { Prepared }}{06 / 02 / 1108: 20} \frac{\text { Analyzed }}{06 / 06 / 1112: 22} \frac{\text { Dil Fac }}{1}$

D

Prepared

Analyzed 06/07/11 21:10

Dịl Fac

06/07/11 21:25 20

05/26/11 00:01 10

05/26/11 00:01 10

06/07/11 21:40 50

05/25/11 23:46

05/27/11 16:35 1

05/27/11 16:35 1 


\section{Client Sample Results}

Client: Argonne National Laboratory

Project/Site: Murdock Creek

Client Sample ID: INPANKRATZ-W-32690

Lab Sample ID: 500-34569-6

Date Collected: 05/24/11 13:50

Matrix: Water

Date Received: 05/25/11 10:00

\begin{tabular}{|c|c|c|c|c|c|c|c|c|c|}
\hline Analyte & Result & Qualifier & $\mathbf{R L}$ & MDL & Unit & $\mathbf{D}$ & Prepared & Analyzed & Dil Fac \\
\hline Aluminum & $<0.20$ & & 0.20 & 0.039 & $\mathrm{mg} / \mathrm{L}$ & & $06 / 02 / 1108: 20$ & $06 / 05 / 1121: 08$ & 1 \\
\hline Calcium & 210 & & 5.0 & 0,12 & $\mathrm{mg} / \mathrm{L}$ & & $06 / 02 / 1108: 20$ & $06 / 05 / 1121: 08$ & 1 \\
\hline Iron & $<0,20$ & & 0.20 & 0.039 & $\mathrm{mg} / \mathrm{L}$ & & $06 / 02 / 1108: 20$ & $06 / 05 / 1121: 08$ & 1 \\
\hline Magnesium & 59 & & 5.0 & 0.064 & $\mathrm{mg} / \mathrm{L}$ & & $06 / 02 / 1108: 20$ & $06 / 05 / 1121: 08$ & 1 \\
\hline Manganese & $<0.015$ & & 0.015 & 0.0019 & $\mathrm{mg} / \mathrm{L}$ & & $06 / 02 / 1108: 20$ & $06 / 05 / 1121: 08$ & 1 \\
\hline Phosphorus & $<250$ & & 250 & 5.2 & $u g / L$ & & $06 / 02 / 1108: 20$ & $06 / 05 / 1121: 08$ & 1 \\
\hline Potassium & 3.0 & $J$ & 5.0 & 0.18 & $\mathrm{mg} / \mathrm{L}$ & & $06 / 02 / 1108: 20$ & $06 / 05 / 1121: 08$ & 1 \\
\hline Sodium & 58 & $\mathrm{~B}$ & 5.0 & 0.051 & $\mathrm{mg} / \mathrm{L}$ & & $06 / 02 / 1108: 20$ & $06 / 05 / 1121: 08$ & 1 \\
\hline Zinc & 0.022 & B & 0.020 & 0.00075 & $\mathrm{mg} / \mathrm{L}$ & & $06 / 02 / 1108: 20$ & 06/05/11 21:08 & . \\
\hline
\end{tabular}

Method: 6010B - Metals (ICP) - Dissolved - RA

\begin{tabular}{|c|c|c|c|c|c|c|c|c|c|}
\hline Analyte & Result & Qualifier & $\mathbf{R L}$ & MDL & Unit & D & Prepared & Analyzed & Dil Fac \\
\hline Silicon & 15 & $B$ & 0.10 & 0.0047 & $\mathrm{mg} / \mathrm{L}$ & & 06/02/11 08:20 & $06 / 06 / 1112: 27$ & 1 \\
\hline
\end{tabular}

General Chemistry

\begin{tabular}{|c|c|c|c|c|c|c|c|c|c|}
\hline Analyte & Result & Qualifier & RL & MDL & Unit & D & Prepared & Analyzed & Dil Fac \\
\hline Bromide & $<0.40$ & & 0.40 & 0.11 & $\mathrm{mg} / \mathrm{L}$ & & & $06 / 07 / 1121: 56$ & 2 \\
\hline Chloride & 230 & & 10 & 4.2 & $\mathrm{mg} / \mathrm{L}$ & & & $06 / 07 / 1122: 11$ & 50 \\
\hline Nitrate as $\mathrm{N}$ & 14 & & 1.0 & 0.23 & $\mathrm{mg} / \mathrm{L}$ & & & 05/26/11 01:00 & 10 \\
\hline Nitrite as $\mathrm{N}$ & $<1.0$ & & 1.0 & 0.23 & $\mathrm{mg} / \mathrm{L}$ & & & $05 / 26 / 1101: 00$ & 10 \\
\hline Sulfate & 420 & & 10 & 4.5 & $\mathrm{mg} / \mathrm{L}$ & & & $06 / 07 / 1122: 11$ & 50 \\
\hline Orthophosphate as P & $<0.20$ & & 0.20 & 0.13 & $\mathrm{mg} / \mathrm{L}$ & & & $05 / 26 / 1100: 16$ & 1 \\
\hline Bicarbonate Alkalinity as $\mathrm{CaCO}$ & 160 & & 5.0 & 1.3 & $\mathrm{mg} / \mathrm{L}$ & & & $05 / 27 / 1116: 41$ & 1 \\
\hline Carbonate Alkalinity as $\mathrm{CaCO} 3$ & $<5.0$ & & 5.0 & 1.3 & $\mathrm{mg} / \mathrm{L}$ & & & $05 / 27 / 1116: 41$ & 1 \\
\hline
\end{tabular}




\section{Client Sample Results}

Method: 6010B - Metals (ICP) - Dissolved

\section{Analyte}

Aluminum

Calcium

Iron

Magnesium

Manganese

Phosphorus

Potassium

Sodium

Zinc
Result Qualifier $<0.20$

160

$<0.20$

39

0.046

$<250$

$2.1 \mathrm{~J}$

$80 \mathrm{~B}$

$6.3 \mathrm{~B}$

\begin{tabular}{|c|c|}
\hline MDL & \\
\hline 0.039 & \\
\hline 0.12 & \\
\hline 0.039 & \\
\hline 0.064 & \\
\hline 0.0019 & \\
\hline 5.2 & \\
\hline 0.18 & \\
\hline 0.051 & \\
\hline 0.00075 & \\
\hline
\end{tabular}

D

Prepared
06/02/11 08:20
06/02/11 08:20
06/02/11 08:20
06/02/11 08:20
06/02/11 08:20
06/02/11 08:20
06/02/11 08:20
06/02/11 08:20
06/02/11 08:20

\begin{tabular}{crr}
\multicolumn{1}{c}{ Analyzed } & Dil Fac \\
\cline { 1 - 1 } $06 / 05 / 1121: 13$ & & 1 \\
$06 / 05 / 1121: 13$ & & 1 \\
$06 / 05 / 1121: 13$ & & 1 \\
$06 / 05 / 1121: 13$ & & 1 \\
$06 / 05 / 1121: 13$ & & 1 \\
$06 / 05 / 1121: 13$ & & 1 \\
$06 / 05 / 1121: 13$ & & 1 \\
$06 / 05 / 1121: 13$ & & 1 \\
$06 / 05 / 1121: 13$ & & 1
\end{tabular}

Method: 6010B - Metals (ICP) - Dissolved - RA

Analyte

Silicon
Result Qualifier

9.0 B
MDL Unit $0.0047 \mathrm{mg} / \mathrm{L}$
Analyzed 06/06/11 12:33

Dil Fac

\section{General Chemistry}

Analyte

Bromide

Chloride

Nitrate as $\mathbf{N}$

Nitrite as $\mathrm{N}$

Sulfate

Orthophosphate as $\mathrm{P}$

Bicarbonate Alkalinity as $\mathrm{CaCO} 3$

Carbonate Alkalinity as $\mathrm{CaCO} 3$

Result Qualifier
$<0.40$
230
39
$<1.0$
48
$<0.20$
230
$<5.0$

\begin{tabular}{|c|c|c|}
\hline MDL & Unit & \multirow[t]{8}{*}{ D } \\
\hline 0.11 & $\mathrm{mg} / \mathrm{L}$ & \\
\hline 4.2 & $\mathrm{mg} / \mathrm{L}$ & \\
\hline 0.23 & $\mathrm{mg} / \mathrm{L}$ & \\
\hline 0.23 & $\mathrm{mg} / \mathrm{L}$ & \\
\hline 0.90 & $\mathrm{mg} / \mathrm{L}$ & \\
\hline 0.13 & $\mathrm{mg} / \mathrm{L}$ & \\
\hline .3 & $\mathrm{mg} / \mathrm{L}$ & \\
\hline .3 & $\mathrm{mg} / \mathrm{L}$ & \\
\hline
\end{tabular}

\begin{tabular}{|c|c|}
\hline Analyzed & Dil Fac \\
\hline $06 / 07 / 1123: 28$ & 2 \\
\hline $06 / 08 / 1100: 14$ & 50 \\
\hline 05/26/11 01:30 & 10 \\
\hline $05 / 26 / 1101: 30$ & 10 \\
\hline $05 / 26 / 1101: 30$ & 10 \\
\hline $05 / 26 / 1101: 15$ & \\
\hline $06 / 06 / 1118: 20$ & \\
\hline $06 / 06 / 1118: 20$ & \\
\hline
\end{tabular}




\section{Definitions/Glossary}

Client: Argonne National Laboratory

Project/Site: Murdock Creek

\section{Qualifiers}

Metals

Qualifier

Qualifier Description

B Compound was found in the blank and sample.

$\mathrm{J}$ Result is less than the RL but greater than or equal to the MDL and the cancentration is an approximate value.

\section{General Chemistry}

Qualifier Qualifier Description

- $\quad$ LCS or LCSD exceeds the control limits

A ICV,CCV,ICB,CCB, ISA, ISB, CRI, CRA, DLCK or MRL standard: Instrument related QC exceeds the control limits.

$F \quad$ MS or MSD exceeds the control limits

$\mathrm{H} \quad$ Sample was prepped or analyzed beyond the specified holding time

\section{Glossary}

Abbreviation

察

EPA

ND

MDL

$\mathrm{RL}$

RE, RE1 (etc.)

$\% R$

RPD
These commonly used abbreviations may or may not be present in this report.

Listed under the " $\mathrm{D}$ " column to designate that the result is reported on a dry weight basis.

United States Environmental Protection Agency

Not Detected above the reporting level.

Method Detection Limit

Reporting Limit

Indicates a Re-extraction or Reanalysis of the sample.

Percent Recovery

Relative Percent Difference, a measure of the relative difference between two points. 


\section{Metals}

\section{Prep Batch: 18903}

Lab Sample ID
MB 200-18903/1-A
MB 200-18903/1-A - RA
LCS 200-18903/2-A
LCS 200-18903/2-A - RA
PB 200-18832/1-B PB
PB 200-18832/1-B PB - RA
$500-34569-1$
$500-34569-1$ - RA
$500-34569-2$
$500-34569-2$ - RA
$500-34569-2$ - DL
$500-34569-3$
$500-34569-3-$ RA
$500-34569-4$
$500-34569-4-$ RA
$500-34569-5$
$500-34569-5$ - RA
$500-34569-6$
$500-34569-6$ - RA
$500-34569-7$
$500-34569-7$ - RA

Client Sample ID
Method Blank
Method Blank
Lab Control Sample
Lab Control Sample
Method Blank
Method Blank
INTHIESSEN-W-32685
INTHIESSEN-W-32685
INBROWN-W-32686
INBROWN-W-32686
INBROWN-W-32686
INKLASSEN-W-32867
INKLASSEN-W-32867
INGOERTZEN-W-32688
INGOERTZEN-W-32688
INISAAC-W-32689
INISAAC-W-32689
INPANKRATZ-W-32690
INPANKRATZ-W-32690
INHULL-W-32691
INHULL-W-32691

\begin{tabular}{lll} 
Prep Type & Matrix & Method \\
\cline { 1 - 2 } Total/NA & Water & $3010 \mathrm{~A}$ \\
Total/NA & Water & $3010 \mathrm{~A}$ \\
Total/NA & Water & $3010 \mathrm{~A}$ \\
Total/NA & Water & $3010 \mathrm{~A}$ \\
Dissolved & Water & $3010 \mathrm{~A}$ \\
Dissolved & Water & $3010 \mathrm{~A}$ \\
Dissolved & Water & $3010 \mathrm{~A}$ \\
Dissolved & Water & $3010 \mathrm{~A}$ \\
Dissolved & Water & $3010 \mathrm{~A}$ \\
Dissolved & Water & $3010 \mathrm{~A}$ \\
Dissolved & Water & $3010 \mathrm{~A}$ \\
Dissolved & Water & $3010 \mathrm{~A}$ \\
Dissolved & Water & $3010 \mathrm{~A}$ \\
Dissolved & Water & $3010 \mathrm{~A}$ \\
Dissolved & Water & $3010 \mathrm{~A}$ \\
Dissolved & Water & $3010 \mathrm{~A}$ \\
Dissolved & Water & $3010 \mathrm{~A}$ \\
Dissolved & Water & $3010 \mathrm{~A}$ \\
Dissolved & Water & $3010 \mathrm{~A}$ \\
Dissolved & Water & $3010 \mathrm{~A}$ \\
Dissolved & Water & $3010 \mathrm{~A}$
\end{tabular}

Analysis Batch: 19110

Lab Sample ID
MB 200-18903/1-A
LCS 200-18903/2-A
PB 200-18832/1-B PB
$500-34569-1$
$500-34569-2$
$500-34569-3$
$500-34569-4$
$500-34569-5$
$500-34569-6$
$500-34569-7$

Analysis Batch: 19148

Lab Sample ID
MB 200-18903/1-A - RA
LCS 200-18903/2-A - RA
PB 200-18832/1-B PB - RA
$500-34569-1$ - RA
$500-34569-2$ - RA
$500-34569-3$ - RA
$500-34569-4$ - RA
$500-34569-5$ - RA
$500-34569-6$ - RA
$500-34569-7$ - RA
$500-34569-2$ - DL

Client Sample ID

Method Blank

Lab Control Sample

Method Blank

INTHIESSEN-W-32685

INBROWN-W-32686

INKLASSEN-W-32867

INGOERTZEN-W-32688

INISAAC-W-32689

INPANKRATZ-W-32690

INHULL-W-32691

Client Sample ID
Method Blank
Lab Control Sample
Method Blank
INTHIESSEN-W-32685
INBROWN-W-32686
INKLASSEN-W-32867
INGOERTZEN-W-32688
INISAAC-W-32689
INPANKRATZ-W-32690
INHULL-W-32691
INBROWN-W-32686

\begin{tabular}{lllr} 
Prep Type & Matrix & Method & Prep Batch \\
\cline { 1 - 2 } Total/NA & Water & $6010 \mathrm{~B}$ & 18903 \\
Total/NA & Water & $6010 \mathrm{~B}$ & 18903 \\
Dissolved & Water & $6010 \mathrm{~B}$ & 18903 \\
Dissolved & Water & $6010 \mathrm{~B}$ & 18903 \\
Dissolved & Water & $6010 \mathrm{~B}$ & 18903 \\
Dissolved & Water & $6010 \mathrm{~B}$ & 18903 \\
Dissolved & Water & $6010 \mathrm{~B}$ & 18903 \\
Dissolved & Water & $6010 \mathrm{~B}$ & 18903 \\
Dissolved & Water & $6010 \mathrm{~B}$ & 18903 \\
Dissolved & Water & $6010 \mathrm{~B}$ & 18903
\end{tabular}

\begin{tabular}{lllr} 
Prep Type & Matrix & Method & Prep Batch \\
\cline { 1 - 2 } Total/NA & Water & $6010 \mathrm{~B}$ & 18903 \\
Total/NA & Water & $6010 \mathrm{~B}$ & 18903 \\
Dissolved & Water & $6010 \mathrm{~B}$ & 18903 \\
Dissolved & Water & $6010 \mathrm{~B}$ & 18903 \\
Dissolved & Water & $6010 \mathrm{~B}$ & 18903 \\
Dissolved & Water & $6010 \mathrm{~B}$ & 18903 \\
Dissolved & Water & $6010 \mathrm{~B}$ & 18903 \\
Dissolved & Water & $6010 \mathrm{~B}$ & 18903 \\
Dissolved & Water & $6010 \mathrm{~B}$ & 18903 \\
Dissolved & Water & $6010 \mathrm{~B}$ & 18903 \\
Dissolved & Water & $6010 \mathrm{~B}$ & 18903
\end{tabular}




\section{General Chemistry}

\section{Analysis Batch: 114678}

\begin{tabular}{l} 
Lab Sample ID \\
\hline MB 500-114678/33 \\
LCS 500-114678/34 \\
$500-34569-1$ \\
$500-34569-1$ \\
$500-34569-1$ MS \\
$500-34569-1$ MS \\
$500-34569-1$ MSD \\
$500-34569-1$ MSD \\
$500-34569-2$ \\
$500-34569-3$ \\
$500-34569-3$ \\
$500-34569-4$ \\
$500-34569-4$ \\
$500-34569-5$ \\
$500-34569-5$ \\
$500-34569-6$ \\
$500-34569-6$ \\
$500-34569-7$ \\
$500-34569-7$
\end{tabular}

\begin{tabular}{|c|c|c|c|c|}
\hline Client Sample ID & Prep Type & Matrix & Method & Prep Batch \\
\hline Method Blank & Total/NA & Water & 300.0 & \\
\hline Lab Control Sample & Total/NA & Water & 300.0 & \\
\hline INTHIESSEN-W-32685 & Total/NA & Water & 300.0 & \\
\hline INTHIESSEN-W-32685 & Total/NA & Water & 300.0 & \\
\hline INTHIESSEN-W-32685 & Total/NA & Water & 300.0 & \\
\hline INTHIESSEN-W-32685 & Total/NA & Water & 300.0 & \\
\hline INTHIESSEN-W-32685 & Total/NA & Water & 300.0 & \\
\hline INTHIESSEN-W-32685 & Total/NA & Water & 300.0 & \\
\hline INBROWN-W-32686 & Total/NA & Water & 300.0 & \\
\hline INKLASSEN-W-32867 & Total/NA & Water & 300.0 & \\
\hline INKLASSEN-W-32867 & Total/NA & Water & 300.0 & \\
\hline INGOERTZEN-W-32688 & Total/NA & Water & 300.0 & \\
\hline INGOERTZEN-W-32688 & Total/NA & Water & 300.0 & \\
\hline INISAAC-W-32689 & Total/NA & Water & 300.0 & \\
\hline INISAAC-W-32689 & Total/NA & Water & 300.0 & \\
\hline INPANKRATZ-W-32690 & Total/NA & Water & 300.0 & \\
\hline INPANKRATZ-W-32690 & Total/NA & Water & 300.0 & \\
\hline INHULL-W-32691 & Total/NA & Water & 300.0 & \\
\hline INHULL-W-32691 & Total/NA & Water & 300.0 & \\
\hline
\end{tabular}

\section{Analysis Batch: 114772}

\begin{tabular}{l} 
Lab Sample ID \\
\hline MB 500-114772/2 \\
$500-34569-1$ \\
$500-34569-2$ \\
$500-34569-3$ \\
$500-34569-4$ \\
$500-34569-5$ \\
$500-34569-6$ \\
LCS 500-114772/26
\end{tabular}

Analysis Batch: 115146

Lab Sample ID MB 500-115146/3

LCS 500-115146/4

500-34569-2

Analysis Batch: 115721

Lab Sample ID MB 500-115721/2

LCS 500-115721/3

500-34569-7

\section{Analysis Batch: 115833}

Lab Sample ID

MB 500-115833/3

LCS 500-115833/4

500-34569-1

500-34569-1

500-34569-2

500-34569-3

500-34569-3

\section{Client Sample ID \\ Method Blank \\ INTHIESSEN-W-32685 \\ INBROWN-W-32686 \\ INKLASSEN-W-32867 \\ INGOERTZEN-W-32688 \\ INISAAC-W-32689 \\ INPANKRATZ-W-32690}

Lab Control Sample

Client Sample ID

Method Blank

Lab Control Sample

INBROWN-W-32686
Prep Type

\section{Total/NA}

Total/NA

Total/NA

Total/NA

Total/NA

Total/NA

Total/NA

Total/NA
Matrix

Water

Water

Water

Water

Water

Water

Water

Water
Method

SM 2320B

SM $2320 B$

SM $2320 B$

SM $2320 B$

SM $2320 B$

SM $2320 B$

SM 2320B

SM 2320B
Prep Type

Total/NA

Total/NA

Total/NA
Matrix

Water

Water

Water

Prep Type

Total/NA

Total/NA

Total/NA
Matrix

Water

Water

Water
Method

300.0

300.0

300.0
Prep Type

Total/NA

Total/NA

Total/NA

Total/NA

Total/NA

Total/NA

Total/NA

$\begin{array}{lll}\text { Matrix } & \text { Method } & \text { Prep Batch } \\ \text { Water } & 300.0 & 300.0 \\ \text { Water } & 300.0 \\ \text { Water } & 300.0 \\ \text { Water } & 300.0 \\ \text { Water } & 300.0 \\ \text { Water } & 3000\end{array}$




\section{QC Association Summary}

Client: Argonne National Laboratory

Project/Site: Murdock Creek

\section{General Chemistry (Continued)}

\section{Analysis Batch: 115833 (Continued)}

\begin{tabular}{l} 
Lab Sample ID \\
\hline $500-34569-3$ \\
$500-34569-4$ \\
$500-34569-5$ \\
$500-34569-5$ \\
$500-34569-5$ \\
$500-34569-6$ \\
$500-34569-6$ \\
$500-34569-7$ \\
$500-34569-7$ \\
$500-34569-4$
\end{tabular}

Client Sample ID

INKLASSEN-W-32867

Prep Type

INGOERTZEN-W-32688

Total/NA

Method

300.0

Total/NA

INISAAC-W-32689

INISAAC-W-32689

INISAAC-W-32689

Total/NA

Total/NA

Total/NA

Total/NA

INPANKRATZ-W-32690

INPANKRATZ-W-32690

INHULL-W-32691

Total/NA

Matrix

Water

$\begin{array}{ll}\text { Water } & 300.0 \\ \text { Water } & 300.0\end{array}$

$\begin{array}{ll}\text { Water } & 3000\end{array}$

Water $\quad 300.0$

Water $\quad 300.0$

INHULL-W-32691

Total/NA

Water

300.0

Total/NA

Water

300.0

INGOERTZEN-W-32688

Total/NA

Water

300.0

300.0 


\section{QC Sample Results}

Method: 6010B - Metals (ICP)

Lab Sample ID: MB 200-18903/1-A

Matrix: Water

Analysis Batch: 19110

\begin{tabular}{|c|c|c|c|c|c|}
\hline Analyte & $\begin{array}{r}\text { MB } \\
\text { Result }\end{array}$ & $\begin{array}{l}\text { MB } \\
\text { Qualifier }\end{array}$ & $\mathbf{R L}$ & MDL & Unit \\
\hline Aluminum & 0.0396 & $\mathrm{~J}$ & 0.20 & 0.039 & $\mathrm{mg} / \mathrm{L}$ \\
\hline Calcium & $<5.0$ & & 5.0 & 0.12 & $\mathrm{mg} / \mathrm{L}$ \\
\hline Iron & $<0.20$ & & 0.20 & 0.039 & $\mathrm{mg} / \mathrm{L}$ \\
\hline Magnesium & $<5.0$ & & 5.0 & 0.064 & $\mathrm{mg} / \mathrm{L}$ \\
\hline Manganese & $<0.015$ & & 0.015 & 0.0019 & $\mathrm{mg} / \mathrm{L}$ \\
\hline Phosphorus & $<250$ & & 250 & 5.2 & $\mathrm{ug} / \mathrm{L}$ \\
\hline Potassium & $<5.0$ & & 5.0 & 0.18 & $\mathrm{mg} / \mathrm{L}$ \\
\hline Sodium & $<5.0$ & & 5.0 & 0.051 & $\mathrm{mg} / \mathrm{L}$ \\
\hline Zinc & $<0.020$ & & 0.020 & 0.00075 & $\mathrm{mg} / \mathrm{L}$ \\
\hline
\end{tabular}

Client Sample ID: Method Blank Prep Type: Total/NA Prep Batch: 18903

Lab Sample ID: MB 200-18903/1-A

Matrix: Water

Analysis Batch: 19148

\begin{tabular}{|c|c|c|c|c|}
\hline \multirow{2}{*}{ Analyte } & \multicolumn{4}{|c|}{ MB MB } \\
\hline & Result & Qualifier & RL & MDL \\
\hline Bilicon - RA & 0.0209 & J & 0.10 & 0.0047 \\
\hline
\end{tabular}

Lab Sample ID: LCS 200-18903/2-A

$0047 \mathrm{mg} / \mathrm{L}$
Prepared 06/02/11 08:20 06/02/11 08:20 06/02/11 08:20 $06 / 02 / 1108: 20$ 06/02/11 08:20 06/02/11 08:20 06/02/11 08:20 06/02/11 08:20 06/02/11 08:20

\begin{tabular}{|c|c|}
\hline Analyzed & \\
\hline $06 / 05 / 1120: 27$ & \\
\hline $06 / 05 / 1120 ; 27$ & \\
\hline $06 / 05 / 1120: 27$ & \\
\hline $06 / 05 / 1120: 27$ & \\
\hline $06 / 05 / 1120: 27$ & \\
\hline $06 / 05 / 1120: 27$ & \\
\hline $06 / 05 / 1120: 27$ & \\
\hline $06 / 05 / 1120: 27$ & \\
\hline $06 / 05 / 1120: 27$ & \\
\hline
\end{tabular}

Client Sample ID: Method Blank Prep Type: Total/NA Prep Batch: 18903 Matrix: Water Analysis Batch: 19110

\begin{tabular}{|c|c|c|c|}
\hline Analyte & $\begin{array}{r}\text { Spike } \\
\text { Added }\end{array}$ & $\begin{array}{r}\text { LCS } \\
\text { Result }\end{array}$ & $\begin{array}{l}\text { LCS } \\
\text { Qualifier }\end{array}$ \\
\hline Aluminum & 51.0 & 45.6 & \\
\hline Calcium & 50.0 & 44.5 & \\
\hline Iron & 50.5 & 46.1 & \\
\hline Magnesium & 50.0 & 44.8 & \\
\hline Manganese & 0.500 & 0.452 & \\
\hline Phosphorus & 1000 & 900 & \\
\hline Potassium & 50.0 & 44.5 & \\
\hline Sodium & 50.0 & 45.0 & \\
\hline Zinc & 0.500 & 0.473 & \\
\hline
\end{tabular}

Client Sample ID: Lab Control Sample Prep Type: Total/NA Prep Batch: 18903 $\%$ Rec.

nit $\quad$ D

\begin{tabular}{rr} 
\% Rec & Limits \\
\cline { 2 - 2 } 89 & $80-120$ \\
89 & $80-120$ \\
91 & $80-120$ \\
90 & $80-120$ \\
90 & $80-120$ \\
90 & $80-120$ \\
89 & $80-120$ \\
90 & $80-120$ \\
95 & $80-120$
\end{tabular}

Client Sample ID: Lab Control Sample Prep Type: Total/NA Prep Batch: 18903 Matrix: Water

Analysis Batch: 19148

Analyte

Spike

LCS LCS

Silicon - RA

Added Result Qualifier

Lab Sample ID: PB 200-18832/1-B PB

Matrix: Water

Analysis Batch: 19110

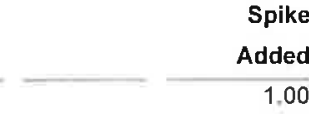

0.962

Unit

D $\frac{\% \text { Rec }}{96} \frac{\text { Limits }}{80-120}$

Client Sample ID: Method Blank Prep Type: Dissolved

\begin{tabular}{lrrrrr} 
Analyte & Result & Qualifier & RL & MDL Unit \\
\cline { 2 - 2 } & $<0.20$ & $<5.0$ & 0.20 & 0.039 & $\frac{\mathrm{mg} / \mathrm{L}}{0.0}$ \\
Calcium & $<0.20$ & 5.0 & $0.12 \mathrm{mg} / \mathrm{L}$ \\
Iron & $<5.0$ & 0.20 & $0.039 \mathrm{mg} / \mathrm{L}$ \\
Magnesium & $<0.015$ & 5.0 & $0.064 \mathrm{mg} / \mathrm{L}$ \\
Manganese & $<250$ & 0.015 & $0.0019 \mathrm{mg} / \mathrm{L}$ \\
Phosphorus & 250 & $5.2 \mathrm{ug} / \mathrm{L}$
\end{tabular}

Prep Batch: 18903

$\begin{array}{cr}\text { Analyzed } & \text { Dil Fac } \\ 20: 37 & 1 \\ 06 / 05 / 1120: 37 & 1 \\ 06 / 05 / 1120: 37 & 1 \\ 06 / 05 / 1120: 37 & 1 \\ 06 / 05 / 1120: 37 & 1 \\ 06 / 05 / 1120: 37 & 1\end{array}$




\section{Method: 6010B - Metals (ICP) (Continued)}

Lab Sample ID: PB 200-18832/1-B PB

Matrix: Water

Analysis Batch: 19110

\begin{tabular}{|c|c|c|c|c|}
\hline Analyte & Result & Qualifier & RL & MDL \\
\hline Potassium & $<5.0$ & & 5.0 & 0.18 \\
\hline Sodium & 0.0718 & $\downarrow$ & 5.0 & 0.051 \\
\hline Zinc & 0.00154 & $\mathrm{~J}$ & 0.020 & 0.00075 \\
\hline
\end{tabular}

Client Sample ID: Method Blank Prep Type: Dissolved Prep Batch: 18903
Lab Sample ID: PB 200-18832/1-B PB

Matrix: Water

Analysis Batch: 19148

Silicon - RA
PB PB Result Qualifier $0.0259 \mathrm{~J}$
Client Sample ID: Method Blank Prep Type: Total/NA
D 06/02/11 08:20
06/02/11 08:20
06/02/11 08:20

Client Sample ID: Method Blank Prep Type: Dissolved Prep Batch: 18903

\section{Method: 300.0 - Anions, Ion Chromatography}

Lab Sample ID: MB 500-114678/33

Matrix: Water

Analysis Batch: 114678

\section{Analyte}

Nitrate as $\mathbf{N}$

Nitrite as $N$

Sulfate

Orthophosphate as $\mathrm{P}$

Lab Sample ID: LCS 500-114678/34

Matrix: Water

Analysis Batch: 114678

\section{Analyte}

Nitrate as $\mathrm{N}$

Nitrite as $\mathrm{N}$

Sulfate

Orthophosphate as $\mathrm{P}$

Lab Sample ID: 500-34569-1 MS

Matrix: Water

Analysis Batch: 114678

\begin{tabular}{ll} 
Analyte & $\begin{array}{r}\text { Sample Sample } \\
\text { Result Qualifier }\end{array}$ \\
\hline Orthophosphate as P & $<0.20$
\end{tabular}

Lab Sample ID: 500-34569-1 MS

Matrix: Water

Analysis Batch: 114678

$\begin{array}{lrl}\text { Analyte } & \begin{array}{r}\text { Sample } \\ \text { Sesult }\end{array} \\ \text { Nitrate as N } & 38 \\ \text { Nitrite as N } & <1.0 \\ \text { Sulfate } & 2 \uparrow\end{array}$

MB MB
Result Qualifier
$<0.10$
$<0.10$
$<0.20$
$<0.20$

$\mathbf{R L}$
0.10
0.10
0.20
0.20

\begin{tabular}{rl} 
MDL & Unit \\
\cline { 2 - 2 } 0.023 & $\mathrm{mg} / \mathrm{L}$ \\
0.023 & $\mathrm{mg} / \mathrm{L}$ \\
0.090 & $\mathrm{mg} / \mathrm{L}$ \\
0.13 & $\mathrm{mg} / \mathrm{L}$
\end{tabular}

D Prepared

\begin{tabular}{cr} 
Analyzed & Dil Fac \\
\hline $05 / 25 / 1117: 50$ & 1 \\
$05 / 25 / 1117: 50$ & 1 \\
$05 / 25 / 1117: 50$ & 1 \\
$05 / 25 / 1117: 50$ & 1
\end{tabular}

Client Sample ID: Lab Control Sample Prep Type: Total/NA

\begin{tabular}{|c|c|c|c|c|c|c|}
\hline $\begin{array}{l}\text { Spike } \\
\text { Added }\end{array}$ & $\begin{array}{r}\text { LCS } \\
\text { Result }\end{array}$ & $\begin{array}{l}\text { LCS } \\
\text { Qualifier }\end{array}$ & Unit & D & $\%$ Rec & $\begin{array}{l}\% \text { Rec. } \\
\text { Limits }\end{array}$ \\
\hline 2.00 & 2.03 & & $\mathrm{mg} / \mathrm{L}$ & & 101 & $90-110$ \\
\hline 2.00 & 1.85 & & $\mathrm{mg} / \mathrm{L}$ & & 92 & $90-110$ \\
\hline 5,00 & 4.89 & & $\mathrm{mg} / \mathrm{L}$ & & 98 & $90-110$ \\
\hline 2.00 & 2.06 & & $\mathrm{mg} / \mathrm{L}$ & & 103 & $90-110$ \\
\hline
\end{tabular}

Client Sample ID: INTHIESSEN-W-32685

Prep Type: Total/NA

$\%$ Rec.

MS MS

Result Qualifier

Added

$1.07 \overline{\mathrm{F}} \frac{\mathrm{m} / \mathrm{L}}{\mathrm{mg}}$

$\underline{\mathbf{D}}$

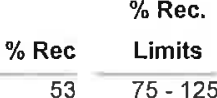

Client Sample ID: INTHIESSEN-W-32685

Prep Type: Total/NA

\begin{tabular}{|c|c|c|c|c|c|c|}
\hline Spike & MS & MS & & & & $\%$ Rec. \\
\hline Added & Result & Qualifier & Unit & D & $\%$ Rec & Limits \\
\hline 20.0 & 61.1 & & $\mathrm{mg} / \mathrm{L}$ & & 114 & $75 \cdot 125$ \\
\hline 20.0 & 17.7 & & $\mathrm{mg} / \mathrm{L}$ & & 89 & $75-125$ \\
\hline 50.0 & 69.6 & & $\mathrm{mg} / \mathrm{L}$ & & 97 & $75-125$ \\
\hline
\end{tabular}




\section{Method: 300.0 - Anions, Ion Chromatography (Continued)}

Lab Sample ID: 500-34569-1 MSD

Matrix: Water

Analysis Batch: 114678

Analyte

Lab Sample ID: 500-34569-1 MSD

Matrix: Water

Analysis Batch: 114678

\section{Analyte}

Nitrate as $\mathrm{N}$

Nitrite as $\mathrm{N}$

Sulfate

Lab Sample ID: MB 500-115146/3

Matrix: Water

Analysis Batch: 115146

Analyte

Chloride

Nitrite as $\mathrm{N}$

Sulfate

Lab Sample ID: LCS 500-115146/4

Matrix: Water

Analysis Batch: 115146

Lab Sample ID: MB 500-115833/3

Matrix: Water

Sample Sample

Result Qualifier

$<0.20$

MB MB

Result Qualifier

$<0.20$

$<0.10$

\begin{tabular}{lr} 
Analyte & $\begin{array}{r}\text { Spike } \\
\text { Added }\end{array}$ \\
\hline Chloride & 3.00 \\
Nitrite as $N$ & 2.00 \\
Sulfate & 5.00
\end{tabular}

Client Sample ID: INTHIESSEN-W-32685

Prep Type: Total/NA

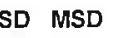

Result Qualifier

\begin{tabular}{|c|c|c|c|c|c|}
\hline & & Rec & $\begin{array}{l}\% \text { Rec. } \\
\text { Limits }\end{array}$ & & \\
\hline $\mathrm{mol}$ & & 61 & $75-125$ & 13 & 20 \\
\hline
\end{tabular}

Client Sample ID: INTHIESSEN-W-32685

Prep Type: Total/NA

\begin{tabular}{|c|c|c|c|c|c|c|c|c|c|c|}
\hline Resule & $\begin{array}{l}\text { Sample } \\
\text { Qualifier }\end{array}$ & $\begin{array}{l}\text { Spike } \\
\text { Added }\end{array}$ & $\begin{array}{r}\text { MSD } \\
\text { Result }\end{array}$ & $\begin{array}{l}\text { MSD } \\
\text { Oualifier }\end{array}$ & Unit & D & $\% \mathrm{Rer}$ & $\begin{array}{l}\% \text { Rec. } \\
\text { Limits }\end{array}$ & RPD & $\begin{array}{r}\text { RPD } \\
\text { Limit }\end{array}$ \\
\hline 38 & & 20.0 & 61.0 & & $\mathrm{mg} / \mathrm{L}$ & & 114 & $75-125$ & 0 & 20 \\
\hline$<1.0$ & & 20.0 & 17.8 & & $\mathrm{mg} / \mathrm{L}$ & & 89 & $75-125$ & 0 & 20 \\
\hline 21 & & 50.0 & 69.7 & & $\mathrm{mg} / \mathrm{L}$ & & 97 & $75-125$ & 0 & 20 \\
\hline
\end{tabular}

Client Sample ID: Method Blank Prep Type: Total/NA

Analysis Batch: 115833

\begin{tabular}{|c|c|c|c|c|c|}
\hline Analyte & Result & Qualifier & RL & MDL & Unit \\
\hline Bromide & $<0.20$ & & 0.20 & 0.057 & $\mathrm{mg} / \mathrm{L}$ \\
\hline Chloride & $<0.20$ & & 0.20 & 0.083 & $\mathrm{mg} / \mathrm{L}$ \\
\hline Sulfate & $<0.20$ & & 0.20 & 0.090 & $\mathrm{mg} / \mathrm{L}$ \\
\hline
\end{tabular}

Lab Sample ID: LCS 500-115833/4

$\frac{\mathrm{RL}}{0.20}$

MDL Unit

D Prepared

\begin{tabular}{crr}
\multicolumn{1}{c}{ Analyzed } & & Dil Fac \\
\cline { 1 - 1 } 05/26/11 18:26 & & 1 \\
$05 / 26 / 1118: 26$ & & 1 \\
$05 / 26 / 1118: 26$ & & 1
\end{tabular}

0.10

$0.023 \mathrm{mg} / \mathrm{L}$

$0.090 \mathrm{mg} / \mathrm{L}$

05/26/1118:26

Client Sample ID: Lab Control Sample Prep Type: Total/NA

Matrix: Water

Analysis Batch: 115833

\begin{tabular}{|c|c|c|c|}
\hline Analyte & $\begin{array}{l}\text { Spike } \\
\text { Added }\end{array}$ & $\begin{array}{r}\text { LCS } \\
\text { Result }\end{array}$ & $\begin{array}{l}\text { LCS } \\
\text { Qualifier }\end{array}$ \\
\hline Bromide & 2.00 & 1.95 & \\
\hline Chloride & 3.00 & 2.75 & \\
\hline Sulfate & 5.00 & 5.13 & \\
\hline
\end{tabular}

\begin{aligned} & LCS LCS \\ & Result Qualifier \\ & \hline 2.70 \\ & 1.77 \\ & 4.77\end{aligned}

\begin{tabular}{l} 
Unit \\
\hline $\mathrm{mg} / \mathrm{L}$ \\
$\mathrm{mg} / \mathrm{L}$ \\
$\mathrm{mg} / \mathrm{L}$
\end{tabular}

Client Sample ID: Method Blank Prep Type: Total/NA 
Project/Site: Murdock Creek

\section{Method: SM 2320B - Alkalinity}

Lab Sample ID: MB 500-114772/2

Matrix: Water

Client Sample ID: Method Blank

Analysis Batch: 114772

Analyte

Bicarbonate Alkalinity as $\mathrm{CaCO} 3$

Carbonate Alkalinity as $\mathrm{CaCO} 3$

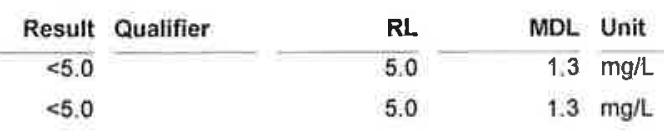

Lab Sample ID: MB 500-115721/2

Matrix: Water

Prep Type: Total/NA

Analysis Batch: 115721

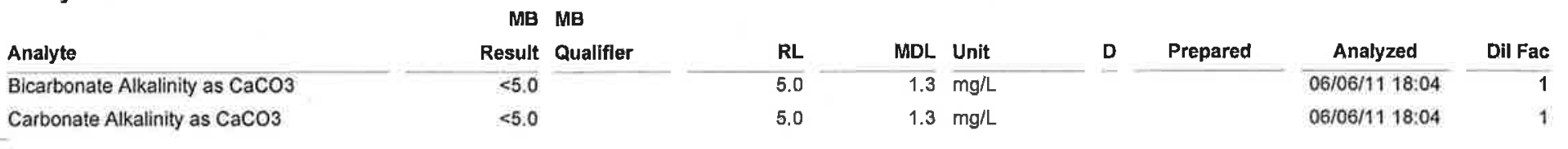




\section{Certification Summary}

\section{Project/Site: Murdock Creek}

\begin{tabular}{|c|c|c|c|c|}
\hline Laboratory & Authority & Program & EPA Region & Certification ID \\
\hline TestAmerica Chicago & ACLASS & DoD ELAP & 0 & ADE-1429 \\
\hline TestAmerica Chicago & ACLASS & ISO/IEC 17025 & 0 & AT-1428 \\
\hline TestAmerica Chicago & Alabama & State Program & 4 & 40461 \\
\hline TestAmerica Chicago & California & NELAC & 9 & $01132 \mathrm{CA}$ \\
\hline TestAmerica Chicago & Florida & NELAC & 4 & $\mathrm{E} 871072$ \\
\hline TestAmerica Chicago & Georgia & Georgia EPD & 4 & N/A \\
\hline TestAmerica Chicago & Georgia & State Program & 4 & 939 \\
\hline TestAmerica Chicago & Hawaii & State Program & 9 & $N / A$ \\
\hline TestAmerica Chicago & Illinois & NELAC & 5 & 100201 \\
\hline TestAmerica Chicago & Indiana & State Program & 5 & C-IL-02 \\
\hline TestAmerica Chicago & lowa & State Program & 7 & 82 \\
\hline TestAmerica Chicago & Kansas & NELAC & 7 & E-10161 \\
\hline TestAmerica Chicago & Kentucky & Kentucky UST & 4 & 66 \\
\hline TestAmerica Chicago & Kentucky & State Program & 4 & 90023 \\
\hline TestAmerica Chicago & Louisiana & NELAC & 6 & 30720 \\
\hline TestAmerica Chicago & Massachusetts & State Program & 1 & M-IL035 \\
\hline TestAmerica Chicago & Mississippi & State Program & 4 & $\mathrm{~N} / \mathrm{A}$ \\
\hline TestAmerica Chicago & North Carolina & North Carolina DENR & 4 & 291 \\
\hline TestAmerica Chicago & Oklahoma & State Program & 6 & 8908 \\
\hline TestAmerica Chicago & South Carolina & State Program & 4 & 77001 \\
\hline TestAmerica Chicago & Texas & NELAC & 6 & T104704252-09-TX \\
\hline TestAmerica Chicago & USDA & USDA & 0 & P330-09-00027 \\
\hline TestAmerica Chicago & Wisconsin & State Program & 5 & 999580010 \\
\hline TestAmerica Chicago & Wyoming & State Program & 8 & 8TMS-Q \\
\hline TestAmerica Burlington & ACLASS & DoD ELAP & 0 & ADE-1492 \\
\hline TestAmerica Burlington & Connecticut & State Program & 1 & $\mathrm{PH}-0751$ \\
\hline TestAmerica Burlington & Delaware & Delaware DNREC & 3 & NA \\
\hline TestAmerica Burlington & Maine & State Program & 1 & VT00008 \\
\hline TestAmerica Burlington & Minnesota & State Program & 5 & $050-999-436$ \\
\hline TestAmerica Burlington & New Hampshire & NELAC & 1 & 200610 \\
\hline TestAmerica Burlington & New Jersey & NELAC & 2 & VT972 \\
\hline TestAmerica Burlington & New York & NELAC & 2 & 10391 \\
\hline TestAmerica Burlington & Pennsylvania & NELAC & 3 & $68-00489$ \\
\hline TestAmerica Burlington & Rhode Island & State Program & 1 & LAO00298 \\
\hline TestAmerica Burlington & USDA & USDA & 0 & P330-11-00093 \\
\hline TestAmerica Burlington & Vermont & State Program & 1 & VT-4000 \\
\hline
\end{tabular}

Accreditation may not be offered or required for all methods and analytes reported in this package. Please contact your project manager for the laboratory's current list of certified methods and analytes. 


\section{Login Sample Receipt Checklist}

List Number: 1

Creator: Lunt, Jeff $\mathrm{T}$

Question

Radioactivity either was not measured or, if measured, is at or below background

The cooler's custody seal, if present, is intact.

The cooler or samples do not appear to have been compromised or tampered with.

Samples were received on ice.

Cooler Temperature is acceptable.

Cooler Temperature is recorded.

COC is present.

$\mathrm{COC}$ is filled out in ink and legible.

COC is filled out with all pertinent information.

Is the Field Sampler's name present on COC?

There are no discrepancies between the sample IDs on the containers and the COC.

Samples are received within Holding Time.

Sample containers have legible labels.

Containers are not broken or leaking.

Sample collection date/times are provided.

Appropriate sample containers are used.

Sample bottles are completely filled.

Sample Preservation Verified.

There is sufficient vol. for all requested analyses, incl. any requested MS/MSDs

VOA sample vials do not have headspace or bubble is $<6 \mathrm{~mm}\left(1 / 4^{\prime \prime}\right)$ in diameter.

Multiphasic samples are not present.

Samples do not require splitting or compositing.

Residual Chlorine Checked.
Answer Comment

True

True

True

True

True

True

True

True

True

True

True

True

True

True

True

True

True

True

True

True

True

True

True 


\section{Login Sample Receipt Checklist}

Client: Argonne National Laboratory

Job Number: $500-34569-1$

Login Number: 34569

List Source: TestAmerica Burlington

List Number: 1

List Creation: 05/26/11 12:28 PM

Creator: Matot, Wade M

Question

Radioactivity either was not measured or, if measured, is at or below background

The cooler's custody seal, if present, is intact.

The cooler or samples do not appear to have been compromised or tampered with.

Samples were received on ice.

Cooler Temperature is acceptable.

Cooler Temperature is recorded.

COC is present.

$\mathrm{COC}$ is filled out in ink and legible.

$\mathrm{COC}$ is filled out with all pertinent information.

Is the Field Sampler's name present on COC?

There are no discrepancies between the sample IDs on the containers and the COC.

Samples are received within Holding Time.

Sample containers have legible labels.

Containers are not broken or leaking.

Sample collection date/times are provided.

Appropriate sample containers are used.

Sample bottles are completely filled.

Sample Preservation Verifjed.

There is sufficient vol. for all requested analyses, incl. any requested MS/MSDs

VOA sample vials do not have headspace or bubble is $<6 \mathrm{~mm}\left(1 / 4^{\prime \prime}\right)$ in diameter.

Multiphasic samples are not present.

Samples do not require splitting or compositing.

Residual Chlorine Checked.

$\begin{array}{ll}\text { Answer } & \text { Comment } \\ \text { N/A } & \text { Lab does not accept radioactive samples. } \\ \text { True } & \\ \text { True } & \\ \text { True } & \\ \text { True } & \\ \text { True } & \text { o1.0C IR gun ID 96, CF= 0 } \\ \text { True } & \\ \text { True } & \\ \text { True } & \\ \text { N/A } & \\ \text { True } & \text { Received project as a subcontract. } \\ \text { True } & \\ \text { True } & \\ \text { True } \\ \text { True } \\ \text { True } \\ \text { True } \\ \text { N/A } \\ \text { True } \\ \text { N/A }\end{array}$




\section{TestAmerica South Burlington, VT Sample Data Summary Package}

134662 


\section{TestAmerica}

THE LEADER IN ENVIRONMENTAL TESTING

TestAmerica Laboratories, Inc.

December 15, 2009

Mr. Clyde Dennis

Argonne National Laboratory

9700 S. Cass Avenue,

Bldg. 203, Office B149

Argonne, IL 60439

Re: Laboratory Project No. 21005

Case: INMAN; SDG: 134662

Dear Mr. Dennis:

Enclosed are analytical results for samples that were received by TestAmerica Burlington on November $13^{\text {th }}, 2009$. Laboratory identification numbers were assigned, and designated as follows:

\begin{tabular}{llll} 
Lab ID & $\begin{array}{l}\text { Client } \\
\text { Sample ID }\end{array}$ & $\begin{array}{l}\text { Sample } \\
\text { Date }\end{array}$ & $\begin{array}{l}\text { Sample } \\
\text { Matrix }\end{array}$ \\
\cline { 2 - 4 } & Received: & $\begin{array}{l}\text { 11/13/09 ETR No: } \\
134662\end{array}$ & \\
813472 & IN-S-30672 & $11 / 12 / 09$ & LIQUID \\
813473 & IN-S-30695 & $11 / 12 / 09$ & LIQUID \\
813474 & IN-S-30676 & $11 / 12 / 09$ & LIQUID \\
813475 & IN-S-30696 & $11 / 12 / 09$ & LIQUID \\
813476 & IN-S-30667 & $11 / 12 / 09$ & LIQUID \\
813477 & IN-S-30734 & $11 / 12 / 09$ & LIQUID \\
813478 & IN-S-30628 & $11 / 12 / 09$ & LIQUID \\
813479 & IN-S-30633 & $11 / 12 / 09$ & LIQUID \\
813480 & IN-S-30662 & $11 / 12 / 09$ & LIQUID \\
813481 & IN-S-30655 & $11 / 12 / 09$ & LIQUID \\
813482 & IN-S-30670 & $11 / 12 / 09$ & LIQUID \\
813483 & IN-S-MEOH-BLANK & $11 / 12 / 09$ & LIQUID
\end{tabular}

Documentation of the condition of the samples at the time of their receipt and any exception to the laboratory's Sample Acceptance Policy is documented in the Sample Handling section of this submittal.

The samples were analyzed by Method $8260 \mathrm{~B}$, using a low-level calibration. In performing the analytical work, 500 microliters of the methanol extract were added to the 5 milliliter purge volume. The analysis of samples IN-S-30667, IN-S-30734, IN-S-30628, IN-S-30633, IN-S-30662, IN-S-30655, and IN-S-30670 did exhibit low internal standard performance. The 


\section{TestAmerica}

THE LEADER IN ENVIRONMENTAL TESTING

derived recoveries of the surrogate controls were high in the analysis of samples IN-S-30667 and IN-S-30670, and low in the analysis of samples IN-S-30734, IN-S-30628, IN-S-30633, IN-S-30662, and IN-S-30655. The laboratory's method of preparing the test volumes for the analysis consumes 4.4 milliliters of methanol extract. There was not a sufficient amount of extract remaining to pursue a further analysis of the referenced samples. Two types of laboratory control sample analyses were performed in the analytical sequence. One was performed to evaluate method performance, and one was performed with 500 microliters of methanol added to the purge volume in order to characterize the affect on the analytical process. With the exception of that for chloroprene, there was an acceptable recovery of each target analyte in the laboratory control sample analysis that defined method performance. The recovery of chloroprene in that analysis was 79 percent. The laboratory uses a lower control limit of 80 percent in evaluating the recovery performance of chloroprene in a laboratory control sample analysis. In the laboratory control sample analysis with methanol, several of the earlier eluting compounds did exhibit a lower recovery performance. Most significantly affected was the recovery performance of acrolein, acetone, methyl iodide, carbon disulfide, allyl chloride, and propionitrile, for which the recovery was below 50 percent. There was an acceptable recovery of chloroform and carbon tetrachloride in each of the laboratory control sample analyses. Matrix spike and matrix spike duplicate analyses were not performed on samples in this sample set. Trace concentrations of 1,1,1-trichloroethane and chlorobenzene were identified in the analysis sample IN-S-MEOH-BLANK. The laboratory did associate the analysis of IN-S-MEOH-BLANK with the analysis of each of the field samples in order to reference the blank association, and accordingly qualify the reported results. The analysis of the instrument blank associated with the analytical work was free of analyte contamination. The laboratory did provide for a methanol blank in the analytical sequence in order to properly characterize the results of the laboratory control sample analysis that was performed with 500 microliters of methanol added to the purge volume. The analysis of that method blank was free of analyte contamination.

Any reference within this report to Severn Trent Laboratories, Inc. or STL, should be understood to refer to TestAmerica Laboratories, Inc. (formerly known as Severn Trent Laboratories, Inc.) The analytical results associated with the samples presented in this test report were generated under a quality system that adheres to requirements specified in the NELAC standard. Release of the data in this test report and any associated electronic deliverables is authorized by the Laboratory Director's designee as verified by the following signature.

If there are any questions regarding this submittal, please contact me at 802 660-1990.

Sincerely,

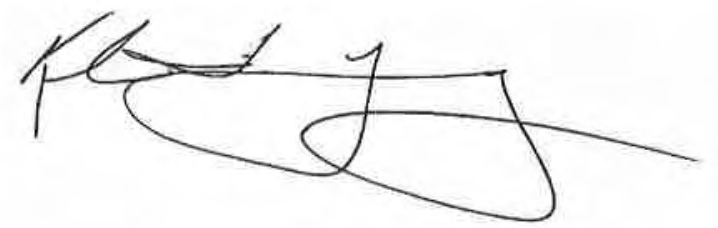

Kirk F. Young

Project Manager

$\mathrm{KFY/hsf}$

Enclosure 


\section{TestAmerica Burlington Data Qualifier Definitions}

\section{Organic}

U: Compound analyzed but not detected at a concentration above the reporting limit.

J: $\quad$ Estimated value.

$\mathrm{N}$ : Indicates presumptive evidence of a compound. This flag is used only for tentatively identified compounds (TICs) where the identification of a compound is based on a mass spectral library search.

P: $\quad$ SW-846: The relative percent difference for detected concentrations between two GC columns is greater than $40 \%$. Unless otherwise specified the higher of the two values is reported on the Form I.

CLP SOW: Greater than 25\% difference for detected concentrations between two $\mathrm{GC}$ columns. Unless otherwise specified the lower of the two values is reported on the Form I.

C: Pesticide result whose identification has been confirmed by GC/MS.

B: $\quad$ Analyte is found in the sample and the associated method blank. The flag is used for tentatively identified compounds as well as positively identified compounds.

E: Compounds whose concentrations exceed the upper limit of the calibration range of the instrument for that specific analysis.

D: Concentrations identified from analysis of the sample at a secondary dilution.

A: Tentatively identified compound is a suspected aldol condensation product.

$X, Y, Z$ : Laboratory defined flags that may be used alone or combined, as needed. If used, the description of the flag is defined in the project narrative.

\section{Inorganic/Metals}

E: $\quad$ Reported value is estimated due to the presence of interference.

$\mathrm{N}$ : Matrix spike sample recovery is not within control limits.

* Duplicate sample analysis is not within control limits.

B: The result reported is less than the reporting limit but greater than the instrument detection limit.

U: $\quad$ Analyte was analyzed for but not detected above the reporting limit.

Method Codes:

P ICP-AES

MS ICP-MS

CV Cold Vapor AA

AS Semi-Automated Spectrophotometric 


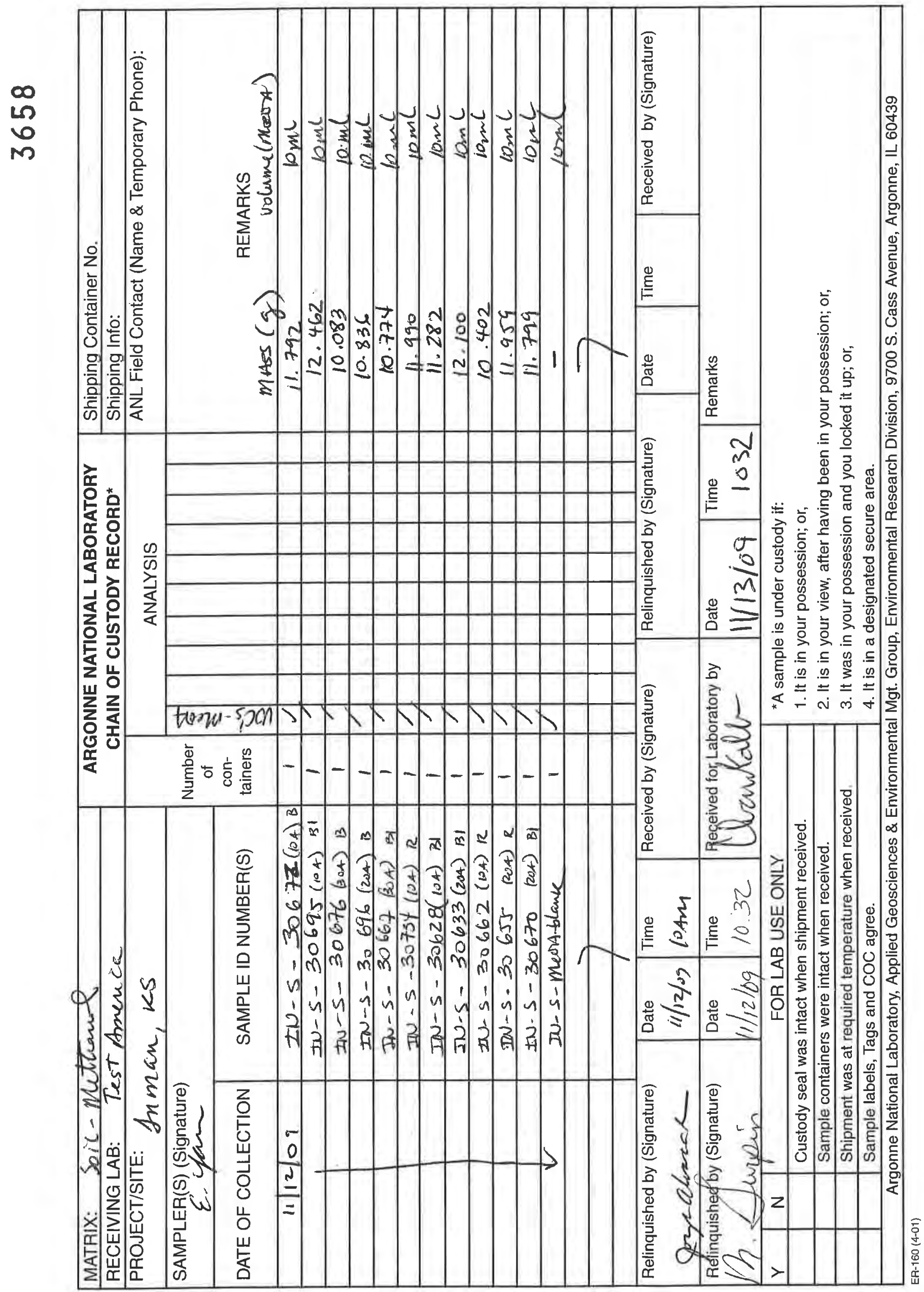




\section{TestAmerica}

THE LEADER IN ENYIRONMENTAL TESTING

\section{Sample Data Summary - 8260B Low Waters}


FORM 1

VOLATILE ORGANICS ANALYSIS DATA SHEET
ARGLAB SAMPLE NO.

IN-S-30628
Lab Name: TESTAMERICA BURLINGTON

Lab Code: STLV

Case No.: INMAN

Matrix: (soil/water) SOIL

sample wt/vol:

$11.3(\mathrm{~g} / \mathrm{mL}) \mathrm{G}$

Level: (low/med) MED

$\div$ Moisture: not dec.

GC Column: DB-624 ID: 0.53 (mm)

Soil Extract Volume: 10000 (uL)
Contract: $8 \mathrm{E}-00302$

SAS NO. :

SDG No.: 134662

Lab Sample ID: 813478

Lab File ID: $\quad 813478$

Date Received: 11/13/09

Date Analyzed: 11/19/09

Dilution Factor: 1.0

Soil Aliquot Volume:

500 (uL)
CAS NO.
COMPOUND
$(\mathrm{ug} / \mathrm{L}$ or $\mathrm{ug} / \mathrm{Kg})$ UG/KG

CONCENTRATION UNITS :

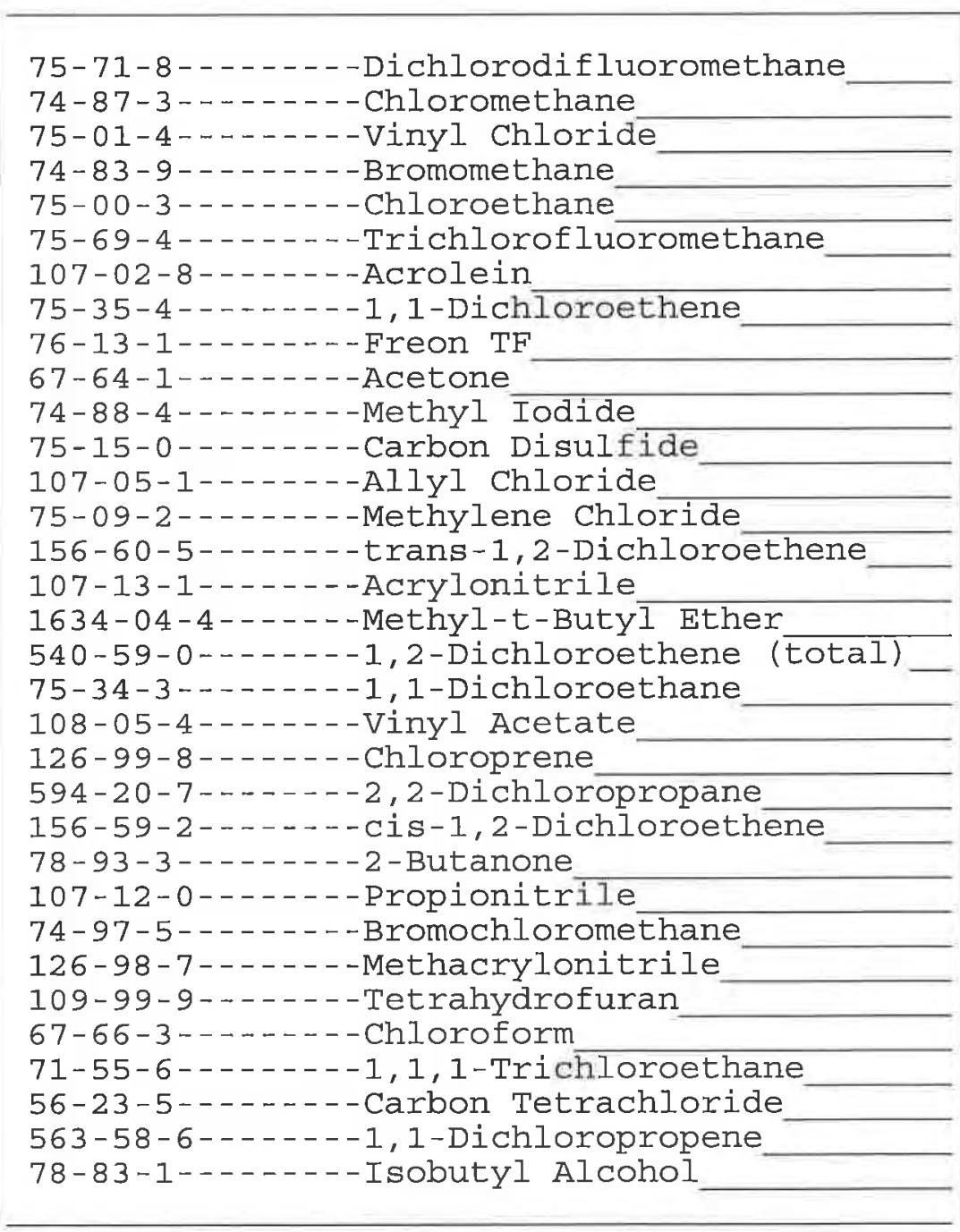

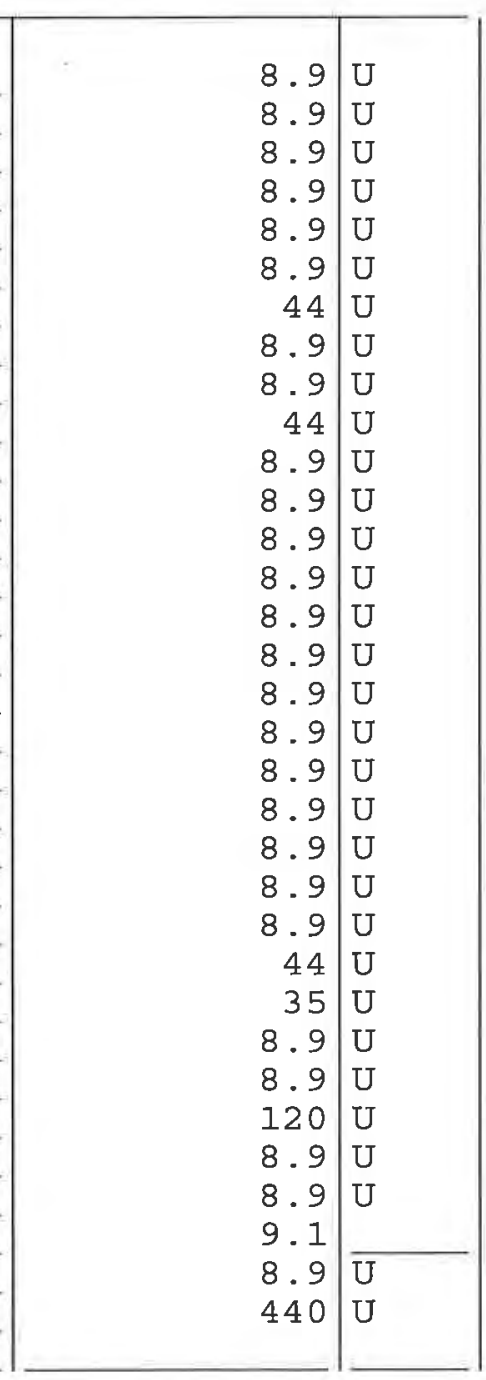


FORM 1

VOLATILE ORGANICS ANALYSIS DATA SHEET
Lab Name: TESTAMERICA BURLINGTON

Lab Code: STLV

Case No.: INMAN

Matrix: (soil/water) SOIL

Sample wt/vol:

$11.3(\mathrm{~g} / \mathrm{mL}) \mathrm{G}$

Level: (low/med) MED

\% Moisture: not dec.

GC Column: DB-624 ID: 0.53 (mm)

Soil Extract Volume: 10000 (uL)
Contract: $8 \mathrm{E}-00302$

SAS NO.:
ARGLAB SAMPLE NO.

IN-S-30628

SDG No.: 134662

Lab Sample ID: 813478

Lab File ID: $\quad 813478$

Date Received: 11/13/09

Date Analyzed: 11/19/09

Dilution Factor: 1.0

Soil Aliquot Volume:

500 (uL)

CONCENTRATION UNITS :

(ug/L or $\mathrm{ug} / \mathrm{Kg}$ ) UG/KG

CAS NO.

COMPOUND

Q

\begin{tabular}{l|l}
8.9 & $\mathrm{U}$ \\
8.9 & $\mathrm{U}$ \\
8.9 & $\mathrm{U}$ \\
8.9 & $\mathrm{U}$ \\
8.9 & $\mathrm{U}$ \\
8.9 & $\mathrm{U}$ \\
440 & $\mathrm{U}$ \\
8.9 & $\mathrm{U}$ \\
8.9 & $\mathrm{U}$ \\
8.9 & $\mathrm{U}$ \\
44 & $\mathrm{U}$ \\
8.9 & $\mathrm{U}$ \\
8.9 & $\mathrm{U}$ \\
8.9 & $\mathrm{U}$ \\
8.9 & $\mathrm{U}$ \\
8.9 & $\mathrm{U}$ \\
8.9 & $\mathrm{U}$ \\
44 & $\mathrm{U}$ \\
8.9 & $\mathrm{U}$ \\
8.9 & $\mathrm{U}$ \\
5.1 & $\mathrm{JB}$ \\
8.9 & $\mathrm{U}$ \\
8.9 & $\mathrm{U}$ \\
8.9 & $\mathrm{U}$ \\
8.9 & $\mathrm{U}$ \\
8.9 & $\mathrm{U}$ \\
8.9 & $\mathrm{U}$ \\
8.9 & $\mathrm{U}$ \\
8.9 & $\mathrm{U}$ \\
8.9 & $\mathrm{U}$ \\
8.9 & $\mathrm{U}$ \\
8.9 & $\mathrm{U}$ \\
8.9 & $\mathrm{U}$ \\
& \\
\hline
\end{tabular}


FORM 1

VOLATILE ORGANICS ANALYSIS DATA SHEET
Lab Name: TESTAMERICA BURLINGTON

Lab Code: STLV

Case No.: INMAN

Matrix: (soil/water) SoIL

Sample wt/vol:

$11.3(\mathrm{~g} / \mathrm{mL}) \quad \mathrm{G}$

Level: (low/med) MED

\% Moisture: not dec.

GC Column: DB-624 ID: 0.53 (mm)

Soil Extract Volume: 10000 (uL)
Contract: $8 \mathrm{E}-00302$

SAS NO. :
ARGLAB SAMPLE NO.

IN-S-30628

SDG No. : 134662

Lab Sample ID: 813478

Lab File ID: $\quad 813478$

Date Received: 11/13/09

Date Analyzed: 11/19/09

Dilution Factor: 1.0

Soil Aliquot Volume:

500 (uL)

CAS NO.

COMPOUND

CONCENTRATION UNITS :

(ug/L or ug/Kg) UG/KG

$Q$

$8.9 \mathrm{U}$

8. $9 \mathrm{U}$

$8.9 \mathrm{U}$

$8.9 \mathrm{U}$

$8.9 \mathrm{U}$

$8.9 \mathrm{U}$

$8.9 \mathrm{U}$

$8.9 \mathrm{U}$

$8.9 \mathrm{U}$

$8.9 \mathrm{U}$

$8.9 \mathrm{U}$

8.9 U

8.9 U

$8.9 \mathrm{U}$

$8.9 \mathrm{U}$

$8.9 \mathrm{U}$

$8.9 \mathrm{U}$ 
FORM 1

VOLATILE ORGANICS ANALYSIS DATA SHEET
Lab Name: TESTAMERICA BURLINGTON

Lab Code: STLV

Case No.: INMAN

Matrix: (soil/water) SOIL

Sample wt/vol:

$12.1(\mathrm{~g} / \mathrm{mL}) \quad \mathrm{G}$

Level : (low $/$ med) MED

\% Moisture: not dec.

GC Column: DB-624 ID: 0.53 (mm)

Soil Extract Volume: 10000 (uL)
Contract : $8 \mathrm{E}-00302$

SAS NO. :

SDG No.: 134662
IN $-S-30633$

Lab Sample ID: 813479

Lab File ID: $\quad 813479$

Date Received: 11/13/09

Date Analyzed: 11/19/09

Dilution Factor: 1.0

Soil Aliquot Volume:

500 (uL)

CAS NO.

COMPOUND

(ug/L or $\mathrm{ug} / \mathrm{Kg}$ ) UG/KG

Q

$8.3 U$

$8.3 \mathrm{U}$

$8.3 \mathrm{U}$

$8.3 \mathrm{U}$

$8.3 \mathrm{U}$

$8.3 \mathrm{U}$

$41 \mathrm{U}$

$8.3 \mathrm{U}$

$8.3 \mathrm{U}$

$41 \mathrm{U}$

$8.3 \mathrm{U}$

$8.3 \mathrm{U}$

$8.3 \mathrm{U}$

$8.3 \mathrm{U}$

$8.3 \mathrm{U}$

$8.3 \mathrm{U}$

$8.3 \mathrm{U}$

$8.3 \mathrm{U}$

$8.3 \mathrm{U}$

$8.3 \mathrm{U}$

$8.3 \mathrm{U}$

$8.3 \mathrm{U}$

$8.3 \mathrm{U}$

$41 \mathrm{U}$

$33 \mathrm{U}$

$8.3 \mathrm{U}$

$8.3 \mathrm{U}$

$120 \mathrm{U}$

$8.3 \mathrm{U}$

$8.3 \mathrm{U}$

$8.3 \mathrm{U}$

$8.3 \mathrm{U}$

$410 \mathrm{U}$ 
FORM 1

VOLATILE ORGANICS ANALYSIS DATA SHEET
Lab Name: TESTAMERICA BURLINGTON

Lab Code: STLV

Case No.: INMAN

Matrix: (soil/water) soIL

Sample wt/vol:

$12.1(\mathrm{~g} / \mathrm{mL}) \mathrm{G}$

Level: (low/med) MED

$\div$ Moisture: not dec.

GC Column: DB-624 ID: 0.53 (mm)

Soil Extract Volume: 10000 (uL)
Contract: $8 \mathrm{E}-00302$

SAS NO. :
ARGLAB SAMPLE NO.

IN-S-30633

SDG No.: 134662

Lab Sample ID: 813479

Lab File ID: $\quad 813479$

Date Received: 11/13/09

Date Analyzed: 11/19/09

Dilution Factor: 1.0

Soil Aliquot Volume:

500 (uL)

CONCENTRATION UNITS:

(ug/L or $\mathrm{ug} / \mathrm{Kg}$ ) UG/KG

Q

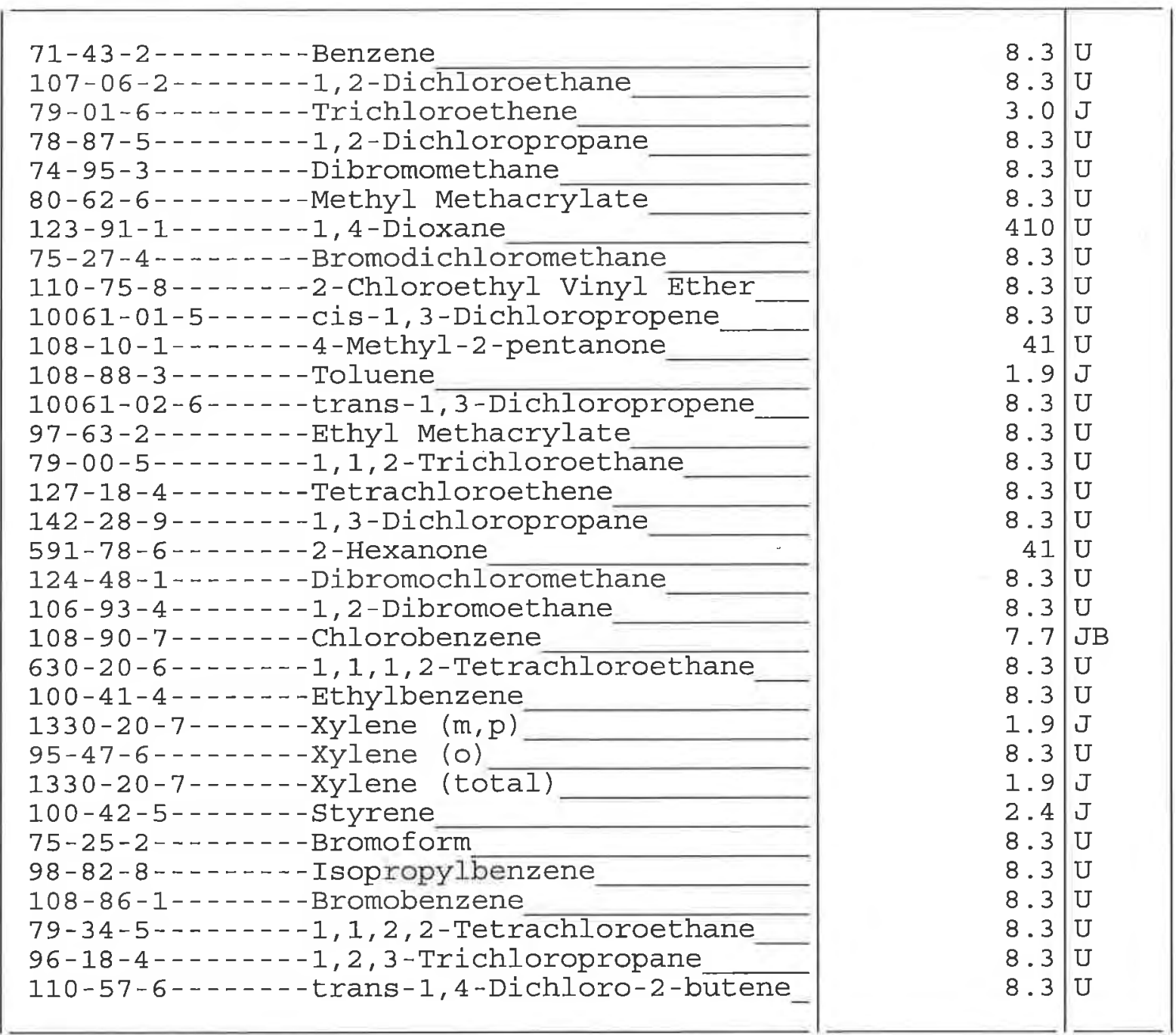


FORM 1

VOLATILE ORGANICS ANALYSIS DATA SHEET

Lab Name: TESTAMERICA BURLINGTON

Lab Code: STLV

Case No.: INMAN

Matrix: (soil/water) soIL

Sample wt/vol:

$12.1(\mathrm{~g} / \mathrm{mL}) \mathrm{G}$

Level : (low/med) MED

․․․ Moisture: not dec.

GC Column: DB-624 ID: 0.53 (mm)

Soil Extract Volume: 10000 (uL)

CAS NO.
Contract: 8E-00302

SAS NO. :

SDG No.: 134662
IN-S-30633

Lab Sample ID: 813479

Lab File ID: $\quad 813479$

Date Received: 11/13/09

Date Analyzed: 11/19/09

Dilution Factor: 1.0

Soil Aliquot Volume:

500 (uL)

CONCENTRATION UNITS :

(ug/L or ug/Kg) UG/KG Q

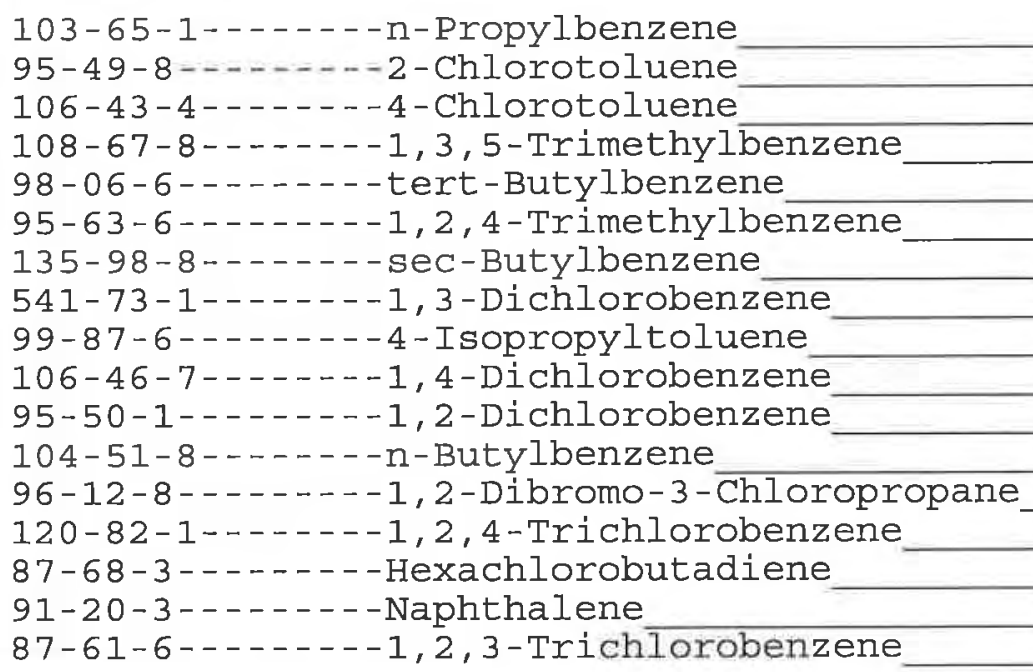

$8.3 \mathrm{U}$

$8.3 \mathrm{U}$

$8.3 \mathrm{U}$

$8.3 \mathrm{U}$

$8.3 \mathrm{U}$

$8.3 \mathrm{U}$

$8.3 \mathrm{U}$

$8.3 \mathrm{U}$

$8.3 \mathrm{U}$

$8.3 \mathrm{U}$

$8.3 \mathrm{U}$

$8.3 \mathrm{U}$

$8.3 \mathrm{U}$

$8.3 \mathrm{U}$

$8.3 \mathrm{U}$

$8.3 \mathrm{U}$

$8.3 \mathrm{U}$ 
FORM 1

VOLATILE ORGANICS ANALYSIS DATA SHEET
Lab Name: TESTAMERICA BURLINGTON

Lab Code: STLV

Case No.: INMAN

Matrix: (soil/water) SOIL

Sample wt/vol:

$12.0(\mathrm{~g} / \mathrm{mL}) \quad \mathrm{G}$

Level: (low/med) MED

\% Moisture: not dec.

GC Column: DB-624 ID: 0.53 (mm)

Soil Extract Volume: 10000 (uL)
Contract: $8 \mathrm{E}-00302$

SAS NO.:
ARGLAB SAMPLE NO.

IN-S-30655

SDG NO.: 134662

Lab Sample ID: 813481

Lab File ID: 813481

Date Received: 11/13/09

Date Analyzed: 11/19/09

Dilution Factor: 1.0

Soil Aliquot Volume: $\quad 500$ (uL)

CONCENTRATION UNTTS:

CAS NO. COMPOUND (ug/L or ug/Kg) UG/KG

$Q$

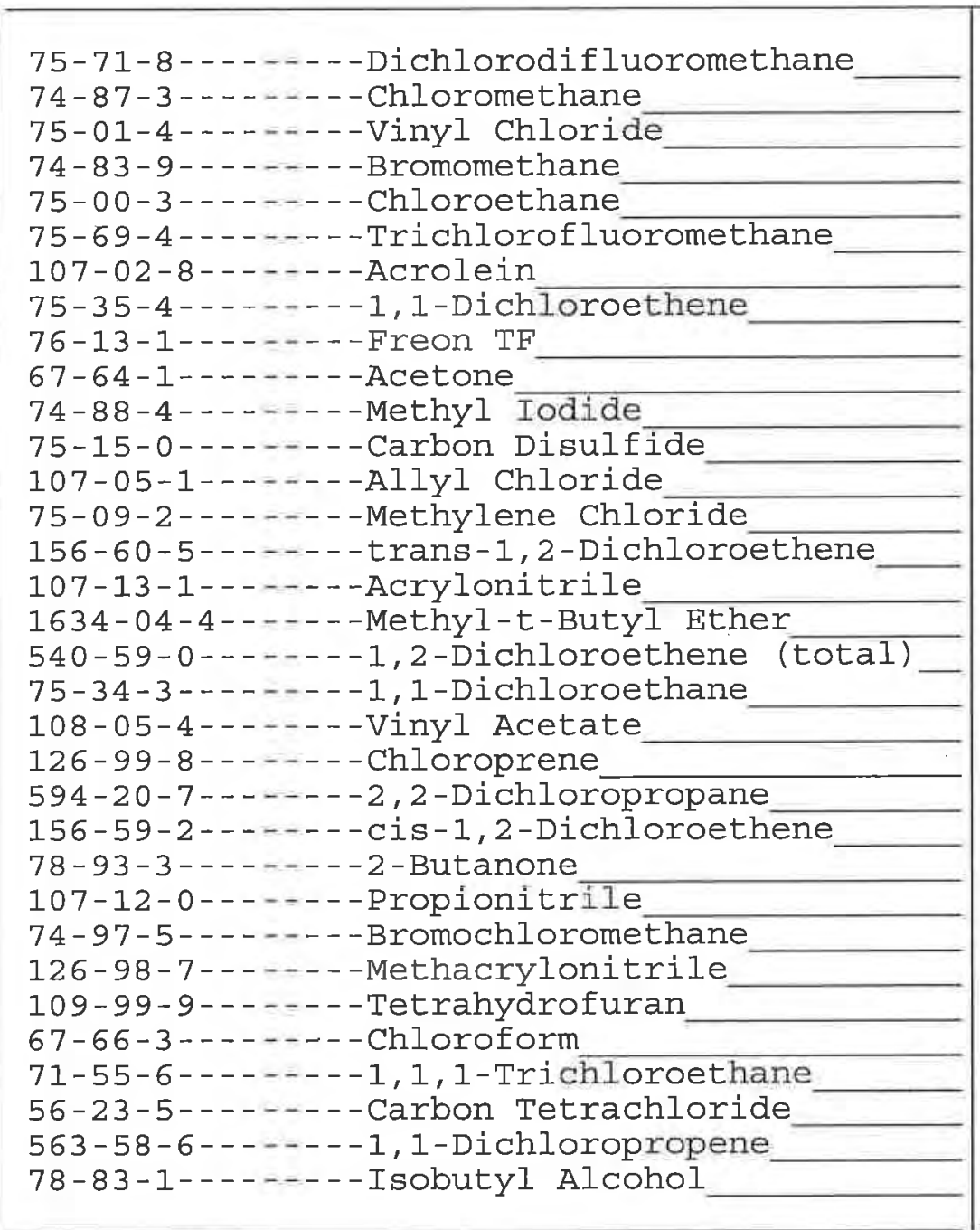

\begin{tabular}{|r|r|}
8.4 & $\mathrm{U}$ \\
8.4 & $\mathrm{U}$ \\
8.4 & $\mathrm{U}$ \\
8.4 & $\mathrm{U}$ \\
8.4 & $\mathrm{U}$ \\
8.4 & $\mathrm{U}$ \\
42 & $\mathrm{U}$ \\
8.4 & $\mathrm{U}$ \\
8.4 & $\mathrm{U}$ \\
42 & $\mathrm{U}$ \\
8.4 & $\mathrm{U}$ \\
8.4 & $\mathrm{U}$ \\
8.4 & $\mathrm{U}$ \\
8.4 & $\mathrm{U}$ \\
8.4 & $\mathrm{U}$ \\
8.4 & $\mathrm{U}$ \\
8.4 & $\mathrm{U}$ \\
8.4 & $\mathrm{U}$ \\
8.4 & $\mathrm{U}$ \\
8.4 & $\mathrm{U}$ \\
8.4 & $\mathrm{U}$ \\
8.4 & $\mathrm{U}$ \\
8.4 & $\mathrm{U}$ \\
42 & $\mathrm{U}$ \\
33 & $\mathrm{U}$ \\
8.4 & $\mathrm{U}$ \\
8.4 & $\mathrm{U}$ \\
120 & $\mathrm{U}$ \\
8.4 & $\mathrm{U}$ \\
8.4 & $\mathrm{U}$ \\
8.4 & $\mathrm{U}$ \\
8.4 & $\mathrm{U}$ \\
420 & $\mathrm{U}$ \\
& \\
\hline
\end{tabular}


FORM 1

VOLATILE ORGANICS ANALYSIS DATA SHEET
Lab Name: TESTAMERICA BURLINGTON

Lab Code: STLV

Case No.: INMAN

Matrix: (soil/water) solL

Sample wt/vol:

$12.0(\mathrm{~g} / \mathrm{mL}) \mathrm{G}$

Level: (low/med) MED

\% Moisture: not dec.

GC Column: DB-624 ID: $0.53 \quad(\mathrm{~mm})$

Soil Extract Volume: 10000 (uL)
Contract: $8 \mathrm{E}-00302$

SAS NO.:
ARGLAB SAMPLE NO.

IN-S-30655

SDG No.: 134662

Lab Sample ID: 813481

Lab File ID: 813481

Date Received: 11/13/09

Date Analyzed: 11/19/09

Dilution Factor: 1.0

Soil Aliquot Volume:

500 (UL)

CONCENTRATION UNITS:

( $\mathrm{ug} / \mathrm{L}$ or $\mathrm{ug} / \mathrm{Kg}$ ) UG/KG

Q

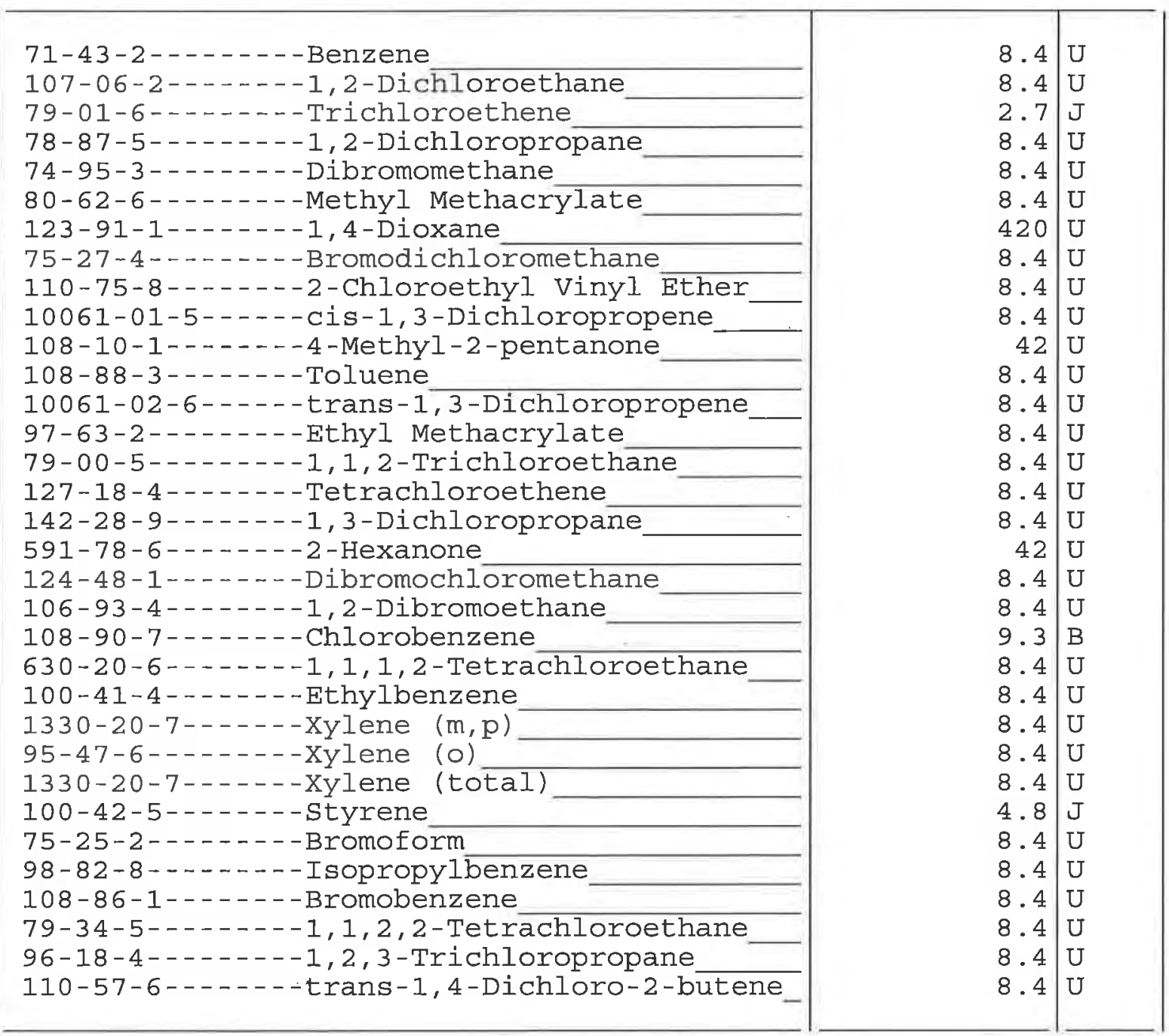


FORM 1

VOLATILE ORGANICS ANALYSIS DATA SHEET
Lab Name: TESTAMERICA BURLINGTON

Lab Code: STLV

Case No.: INMAN

Matrix: (soil/water) soIL

Sample wt/vol:

$12.0(\mathrm{~g} / \mathrm{mL}) \mathrm{G}$

Level : (low/med) MED

\% Moisture: not dec.

GC Column: DB-624 ID: 0.53 (mm)

Soil Extract Volume: 10000 (uL)
Contract : $8 \mathrm{E}-00302$

SAS No. :

SDG No.: 134662

Lab Sample ID: 813481

Lab File ID: $\quad 813481$

Date Received: 11/13/09

Date Analyzed: 11/19/09

Dilution Factor: 1.0

Soil Aliquot Volume:

$500(u L)$

(ug/L or ug/Kg) UG/KG

Q

103-65-1-------n-Propylbenzene

95-49-8-...---2-Chlorotoluene

106-43-4--.-.--4-Chlorotoluene

108-67-8------1, 3,5-Trimethylbenzene

98-06-6-------tert-Butylbenzene

95-63-6--..--1, 2, 4-Trimethylbenzene

135-98-8------sec-Butylbenzene

541-73-1_-..--1,3-Dichlorobenzene

99-87-6--- - - - 4-Isopropyltoluene

106-46-7--..--1,4-Dichlorobenzene

95-50-1-...---1,2-Dichlorobenzene

104-51-8------n-Butylbenzene

$96-12-8-------1,2-$ Dibromo-3-Chloropropane

120-82-1------1, 2,4-Trichlorobenzene

87-68-3------- Hexachlorobutadiene

91-20-3-- - - - Naphthalene

87-61-6-------1,2,3-Trichlorobenzene

\begin{tabular}{|l|l|l|}
8.4 & $\mathrm{U}$ \\
8.4 & $\mathrm{U}$ \\
8.4 & $\mathrm{U}$ \\
8.4 & $\mathrm{U}$ \\
8.4 & $\mathrm{U}$ \\
8.4 & $\mathrm{U}$ \\
8.4 & $\mathrm{U}$ \\
8.4 & $\mathrm{U}$ \\
8.4 & $\mathrm{U}$ \\
1.8 & $\mathrm{~J}$ \\
8.4 & $\mathrm{U}$ \\
8.4 & $\mathrm{U}$ \\
8.4 & $\mathrm{U}$ \\
8.4 & $\mathrm{U}$ \\
8.4 & $\mathrm{U}$ \\
8.4 & $\mathrm{U}$ \\
8.4 & $\mathrm{U}$ \\
\hline
\end{tabular}


FORM 1

VOLATILE ORGANICS ANALYSIS DATA SHEET
Lab Name: TESTAMERICA BURLINGTON

Lab Code: STLV

Case No.: INMAN

Matrix: (soil/water) SoIL

Sample wt/vol:

$10.4(\mathrm{~g} / \mathrm{mL}) \mathrm{G}$

Level: (low/med) MED

$\%$ Moisture: not dec.

GC Column: DB-624 ID: 0.53 (mm)

Soil Extract Volume: 10000 (uL)
Contract: $8 \mathrm{E}-00302$

ARGLAB SAMPLE NO.

IN-S-30662

SDG No. : 134662

Lab Sample ID: 813480

Lab File ID: 813480

Date Received: 11/13/09

Date Analyzed: 11/19/09

Dilution Factor: 1.0

Soil Aliquot Volume:

500 (uL)

CONCENTRATION UNITS:

(ug/L or ug/Kg) UG/KG

$Q$

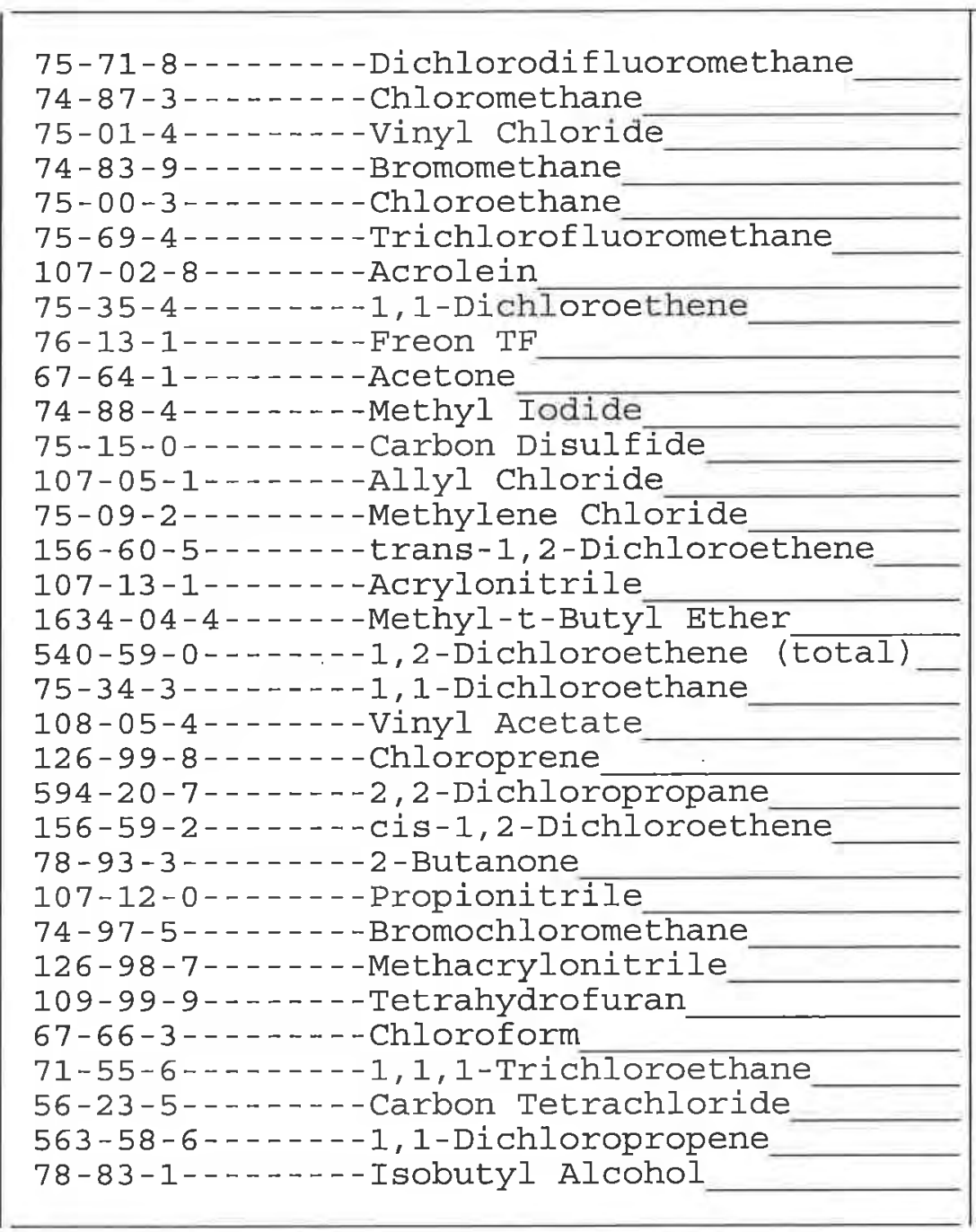


FORM 1

VOLATILE ORGANICS ANALYSIS DATA SHEET
ARGLAB SAMPLE NO.

IN $-S-30662$
Lab Name: TESTAMERICA BURLINGTON

Lab Code: STLV

Case No.: INMAN

Matrix: (soil/water) soII

Sample wt/vol:

$10.4(\mathrm{~g} / \mathrm{mL}) \mathrm{G}$

Level: (low/med) MED

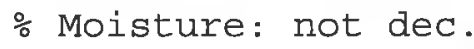

GC Column: DB-624 ID: 0.53 (mm)

Soil Extract Volume: 10000 (uL)
Contract: $8 \mathrm{E}-00302$

SAS NO. :

SDG No. : 134662
Lab Sample ID: 813480

Lab File ID: 813480

Date Received: 11/13/09

Date Analyzed: 11/19/09

Dilution Factor: 1.0

Soil Aliquot Volume:

500 (uL)

CAS NO.

COMPOUND

CONCENTRATION UNITS :

( $\mathrm{ug} / \mathrm{L}$ or $\mathrm{ug} / \mathrm{Kg}$ ) UG/KG

$Q$

$9.6 \mathrm{U}$

$9.6 \mathrm{U}$

$9.6 \mathrm{U}$

$9.6 \mathrm{U}$

$9.6 \mathrm{U}$

$9.6 \mathrm{U}$

$480 \mathrm{U}$

$9.6 \mathrm{U}$

$9.6 \mathrm{U}$

$9.6 \mathrm{U}$

$48 \mathrm{U}$

$9.6 \mathrm{U}$

$9.6 \mathrm{U}$

$9.6 \mathrm{U}$

$9.6 \mathrm{U}$

$9.6 \mathrm{U}$

$9.6 \mathrm{U}$

$48 \mathrm{U}$

$9.6 \mathrm{U}$

$9.6 \mathrm{U}$

$5.8 \mathrm{JB}$

$9.6 \mathrm{U}$

$9.6 \mathrm{U}$

$9.6 \mathrm{U}$

$9.6 \mathrm{U}$

$9.6 \mathrm{U}$

$9.6 \mathrm{U}$

$9.6 \mathrm{U}$

$9.6 \mathrm{U}$

$9.6 \mathrm{U}$

$9.6 \mathrm{U}$

$9.6 \mathrm{U}$

$9.6 \mathrm{U}$ 
FORM 1

VOLATILE ORGANICS ANALYSIS DATA SHEET
ARGLAB SAMPLE NO.

IN-S-30662
Lab Name: TESTAMERICA BURLINGTON

Lab Code: STLV
Case No.: INMAN

Matrix: (soil/water) SOIL

Sample wt/vol:

$10.4(\mathrm{~g} / \mathrm{mL}) \mathrm{G}$

Level: (low/med) MED

$\div$ Moisture: not dec.

GC Column: DB-624 ID: 0.53 (mm)

Soil Extract Volume: 10000 (uL)
Contract: $8 \mathrm{E}-00302$

SAS NO.:

SDG NO.: 134662

Lab Sample ID: 813480

Lab File ID: $\quad 813480$

Date Received: 11/13/09

Date Analyzed: 11/19/09

Dilution Factor: 1.0

Soil Aliquot Volume:

500 (UL)

CAS NO.

COMPOUND

CONCENTRATION UNITS:

(ug/L or $\mathrm{ug} / \mathrm{Kg}$ ) UG/KG

Q

$9.6 \mathrm{U}$

$9.6 \mathrm{U}$

$9.6 \mathrm{U}$

$9.6 \mathrm{U}$

$9.6 \mathrm{U}$

$9.6 \mathrm{U}$

$9.6 \mathrm{U}$

$9.6 \mathrm{U}$

$9.6 \mathrm{U}$

$9.6 \mathrm{U}$

$9.6 \mathrm{U}$

$9.6 \mathrm{U}$

$9.6 \mathrm{U}$

$9.6 \mathrm{U}$

$9.6 \mathrm{U}$

$9.6 \mathrm{U}$

$9.6 \mathrm{U}$ 
FORM 1

VOLATILE ORGANICS ANALYSIS DATA SHEET

Lab Name: TESTAMERICA BURLINGTON

Lab Code: STLV

Case No.: INMAN

Matrix: (soil/water) SOIL

Sample wt/vol: $\quad 10.8(\mathrm{~g} / \mathrm{mL}) \mathrm{G}$

Level : (low/med) MED

\% Moisture: not dec.

GC Column: DB-624 ID: 0.53 (mm)

Soil Extract Volume: 10000 (uL)
Contract: $8 \mathrm{E}-00302$

SAS NO. :
ARGLAB SAMPLE NO.

IN-S-30667

SDG No.: 134662

Lab Sample ID: 813476

Lab File ID: $\quad 813476$

Date Received: 11/13/09

Date Analyzed: 11/19/09

Dilution Factor: 1.0

Soil Aliquot Volume:

500 (uL)

CONCENTRATION UNITS :

(ug/L or ug/Kg) UG/KG

$Q$

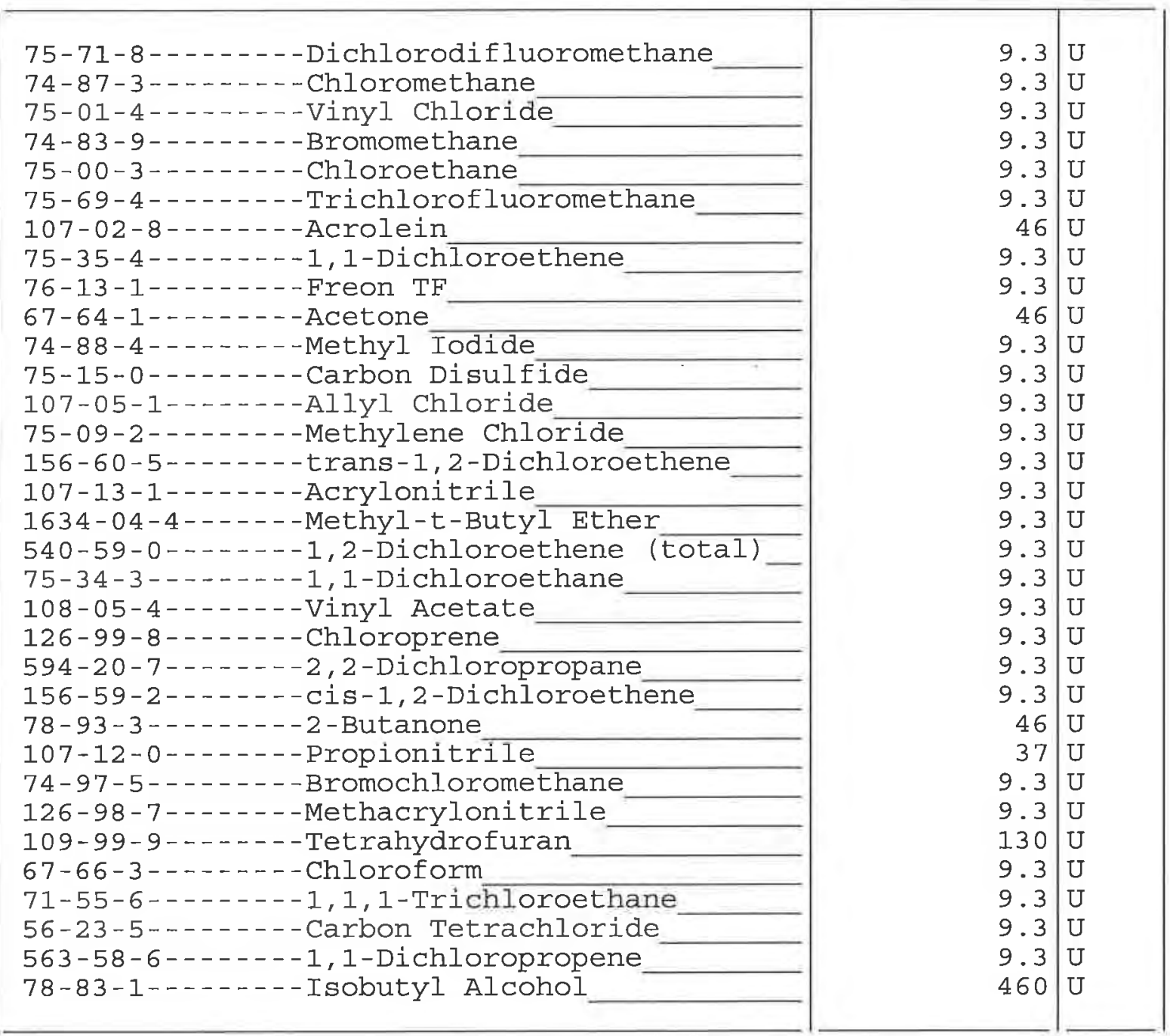


FORM 1

VOLATILE ORGANICS ANALYSIS DATA SHEET
ARGLAB SAMPLE NO.

IN-S-30667
Lab Name: TESTAMERICA BURLINGTON

Lab Code: STLV

Case No.: INMAN

Matrix: (soil/water) soIL

Sample wt/vol:

$10.8(\mathrm{~g} / \mathrm{mL}) \mathrm{G}$

Level: (low/med) MED

$\%$ Moisture: not dec.

GC Column: DB-624 ID: 0.53 (mm)

Soil Extract Volume: 10000 (uL)
Contract: $8 E-00302$

SAS NO.:

SDG No.: 134662

Lab Sample ID: 813476

Lab File ID: $\quad 813476$

Date Received: 11/13/09

Date Analyzed: 11/19/09

Dilution Factor: 1.0

Soil Aliquot Volume:

500 (UL)

CAS NO.

COMPOUND

CONCENTRATION UNITS :

(ug/L or $\mathrm{ug} / \mathrm{Kg}$ ) UG/KG

Q

71-43-2-....---Benzene

107-06-2-----1,2-Dichloroethane

79-01-6-------Trichloroethene

78-87-5--.---1, 2-Dichloropropane

74-95-3---n-Dibromomethane

80-62-6-...---Methyl Methacrylate

123-91-1--.--1,4-Dioxane

75-27-4------Bromodichloromethane

110-75-8-...-2-Chloroethyl Vinyl Ether

10061-01-5-----cis-1,3-Dichloropropene

108-10-1--- --4-Methyl-2-pentanone

108-88-3--.----Toluene

10061-02-6-----trans-1, 3-Dichloropropene

97-63-2--..--Ethyl Methacrylate

79-00-5-...-1, 1,2-Trichloroethane

127-18-4--1---Tetrachloroethene

142-28-9------1, 3-Dichloropropane

591-78-6------2-Hexanone.

124-48-1-------Dibromochloromethane

106-93-4-..--1,2-Dibromoethane

108-90-7-...--Chlorobenzene

630-20-6--.---1, 1, 1,2-Tetrachloroethane

100-41-4------Ethylbenzene

1330-20-7-...-Xylene (m, p)

95-47-6.......Xylene (o)

1330-20-7--.--xylene (total)

100-42-5--.---styrene

75-25-2-------Bromoform

98-82-8-------Isopropyl benzene

108-86-1-...-.-Bromobenzene

79-34-5------1, 1,2,2-Tetrachloroethane

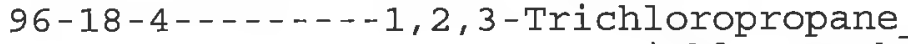

110-57-6------trans-1,4-Dichloro-2-butene

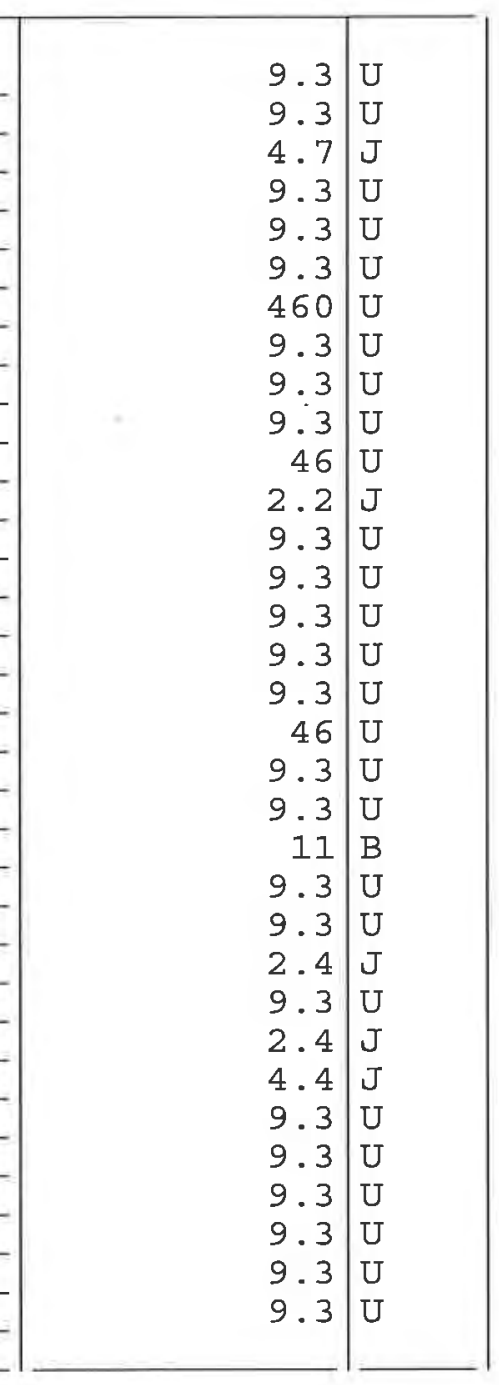


FORM 1

VOLATILE ORGANICS ANALYSIS DATA SHEET
Lab Name: TESTAMERICA BURLINGTON

Lab Code: STLV

Case No.: INMAN

Matrix: (soil/water) SoIL

Sample wt/vol:

$10.8(\mathrm{~g} / \mathrm{mL}) \quad \mathrm{G}$

Level : (low/med) MED

$\div$ Moisture: not dec.

GC Column: DB-624 ID: 0.53 (mm)

Soil Extract Volume: 10000 (uL)
Contract: $8 \mathrm{E}-00302$

SAS NO.:

SDG No.: 134662

Lab Sample ID: 813476

Lab File ID: $\quad 813476$

Date Received: 11/13/09

Date Analyzed: 11/19/09

Dilution Factor: 1.0

Soil Aliquot Volume:

500 (uL)

CAS NO.

COMPOUND

CONCENTRATION UNITS :

(ug/L or $\mathrm{ug} / \mathrm{Kg}$ ) UG/KG

Q

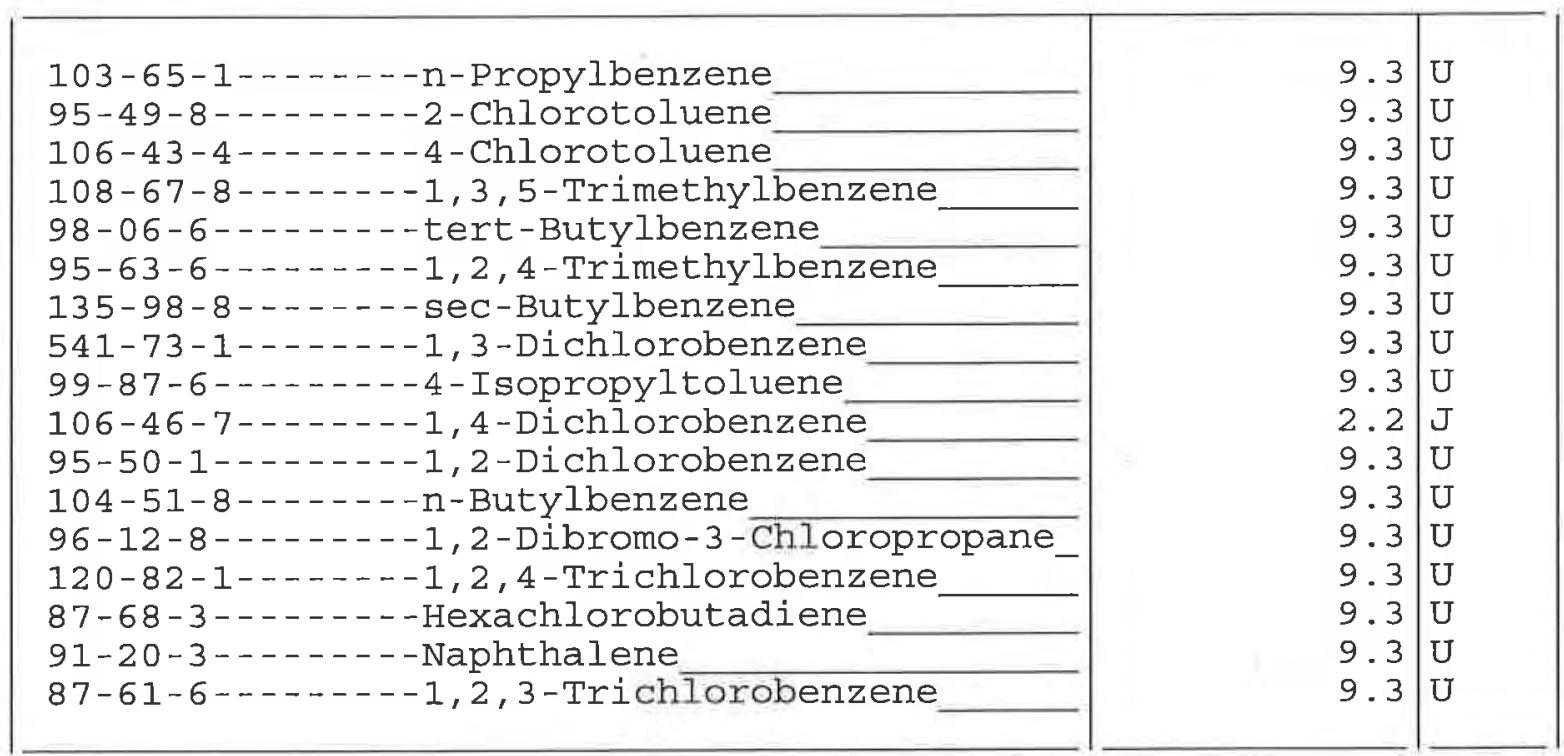


FORM 1

VOLATILE ORGANICS ANALYSIS DATA SHEET
ARGLAB SAMPLE NO.

IN-S-30670
Lab Name: TESTAMERICA BURLINGTON

Lab Code: STLV

Case No.: INMAN

Matrix: (soil/water) SOIL

Sample wt/vol:

$11.8(\mathrm{~g} / \mathrm{mL}) \quad \mathrm{G}$

Level: (low/med) MED

\% Moisture: not dec.

GC Column: DB-624 ID: 0.53 (mm)

Soil Extract Volume: 10000 (uL)
Contract: 8E-00302

SAS NO.:

SDG No.: 134662
Lab Sample ID: 813482

Lab File ID: $\quad 813482$

Date Received: 11/13/09

Date Analyzed: 11/19/09

Dilution Factor: 1.0

Soil Aliquot Volume:

$500(u L)$

CAS NO.

COMPOUND

CONCENTRATION UNITS:

(ug/L or ug/Kg) UG/KG

Q

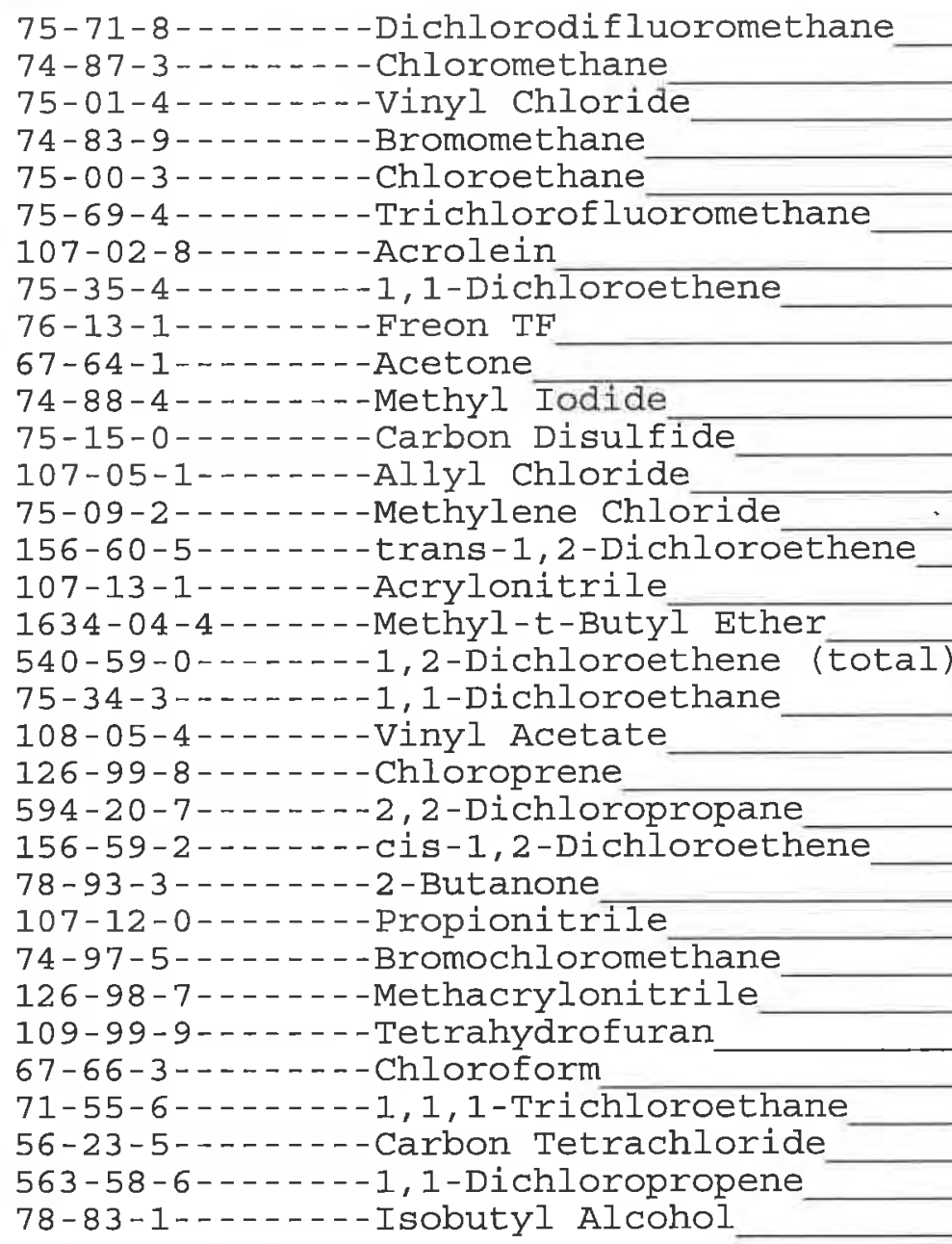

\begin{tabular}{r|l}
8.5 & $\mathrm{U}$ \\
8.5 & $\mathrm{U}$ \\
8.5 & $\mathrm{U}$ \\
8.5 & $\mathrm{U}$ \\
8.5 & $\mathrm{U}$ \\
8.5 & $\mathrm{U}$ \\
42 & $\mathrm{U}$ \\
8.5 & $\mathrm{U}$ \\
8.5 & $\mathrm{U}$ \\
42 & $\mathrm{U}$ \\
8.5 & $\mathrm{U}$ \\
8.5 & $\mathrm{U}$ \\
8.5 & $\mathrm{U}$ \\
8.5 & $\mathrm{U}$ \\
8.5 & $\mathrm{U}$ \\
8.5 & $\mathrm{U}$ \\
8.5 & $\mathrm{U}$ \\
8.5 & $\mathrm{U}$ \\
8.5 & $\mathrm{U}$ \\
8.5 & $\mathrm{U}$ \\
8.5 & $\mathrm{U}$ \\
8.5 & $\mathrm{U}$ \\
8.5 & $\mathrm{U}$ \\
42 & $\mathrm{U}$ \\
34 & $\mathrm{U}$ \\
8.5 & $\mathrm{U}$ \\
8.5 & $\mathrm{U}$ \\
120 & $\mathrm{U}$ \\
8.5 & $\mathrm{U}$ \\
8.5 & $\mathrm{U}$ \\
8.5 & $\mathrm{U}$ \\
8.5 & $\mathrm{U}$ \\
420 & $\mathrm{U}$ \\
&
\end{tabular}


FORM 1

VOLATILE ORGANICS ANALYSIS DATA SHEET
ARGLAB SAMPLE NO.

IN-S-30670
Lab Name: TESTAMERICA BURLINGTON

Lab Code: STLV
Case No.: INMAN

Matrix: (soil/water) SOIL

sample wt/vol:

Level: (low/med) MED

$\div$ Moisture: not dec.

GC Column: DB-624 ID: 0.53 (mm)

Soil Extract Volume: 10000 (uL)
Contract: $8 \mathrm{E}-00302$

SAS No. :

SDG No.: 134662
Lab Sample ID: 813482

Lab File ID: $\quad 813482$

Date Received: 11/13/09

Date Analyzed: 11/19/09

Dilution Factor: 1.0

Soil Aliquot Volume:

$500(u L)$

CAS NO.

COMPOUND

CONCENTRATION UNITS :

( $\mathrm{ug} / \mathrm{L}$ or $\mathrm{ug} / \mathrm{Kg}$ ) UG/KG

Q

\begin{tabular}{r|l}
8.5 & $\mathrm{U}$ \\
8.5 & $\mathrm{U}$ \\
6.0 & $\mathrm{~J}$ \\
8.5 & $\mathrm{U}$ \\
8.5 & $\mathrm{U}$ \\
8.5 & $\mathrm{U}$ \\
420 & $\mathrm{U}$ \\
8.5 & $\mathrm{U}$ \\
8.5 & $\mathrm{U}$ \\
8.5 & $\mathrm{U}$ \\
42 & $\mathrm{U}$ \\
3.0 & $\mathrm{~J}$ \\
8.5 & $\mathrm{U}$ \\
8.5 & $\mathrm{U}$ \\
8.5 & $\mathrm{U}$ \\
8.5 & $\mathrm{U}$ \\
8.5 & $\mathrm{U}$ \\
42 & $\mathrm{U}$ \\
8.5 & $\mathrm{U}$ \\
8.5 & $\mathrm{U}$ \\
13 & $\mathrm{~B}$ \\
8.5 & $\mathrm{U}$ \\
8.5 & $\mathrm{U}$ \\
8.5 & $\mathrm{U}$ \\
8.5 & $\mathrm{U}$ \\
8.5 & $\mathrm{U}$ \\
6.6 & $\mathrm{~J}$ \\
8.5 & $\mathrm{U}$ \\
8.5 & $\mathrm{U}$ \\
8.5 & $\mathrm{U}$ \\
8.5 & $\mathrm{U}$ \\
8.5 & $\mathrm{U}$ \\
8.5 & $\mathrm{U}$ \\
& \\
8
\end{tabular}


FORM 1

VOLATILE ORGANICS ANALYSIS DATA SHEET
ARGIAB SAMPLE NO.

IN-S-30670
Lab Name: TESTAMERICA BURLINGTON

Lab Code: STLV

Case No.: INMAN

Matrix: (soil/water) soIL

Sample wt/vol:

$11.8(\mathrm{~g} / \mathrm{mL}) \quad \mathrm{G}$

Level: (low/med) MED

$\div$ Moisture: not dec.

GC Column: DB-624 ID: 0.53 (mm)

Soil Extract Volume: 10000 (uL)
Contract: $8 \mathrm{E}-00302$

SAS NO.:

SDG No.: 134662

Lab Sample ID: 813482

Lab File ID: $\quad 813482$

Date Received: 11/13/09

Date Analyzed: 11/19/09

Dilution Factor: 1.0

Soil Aliquot Volume:

$500(u L)$

CAS NO.

COMPOUND

CONCENTRATION UNITS:

(ug/L or ug/Kg) UG/KG

Q

$8.5 \mathrm{U}$

$8.5 \mathrm{U}$

$8.5 \mathrm{U}$

$8.5 \mathrm{U}$

$8.5 \mathrm{U}$

$8.5 \mathrm{U}$

$8.5 \mathrm{U}$

$8.5 \mathrm{U}$

$8.5 \mathrm{U}$

$2.7 \mathrm{~J}$

$8.5 \mathrm{U}$

$8.5 \mathrm{U}$

$8.5 \mathrm{U}$

$8.5 \mathrm{U}$

$8.5 \mathrm{U}$

$8.5 \mathrm{U}$

$8.5 \mathrm{U}$ 
FORM 1

VOLATILE ORGANICS ANALYSIS DATA SHEET

Lab Name: TESTAMERICA BURLINGTON

Lab Code: STLV

Case No.: INMAN

Matrix: (soil/water) SOIL

Sample wt/vol:

$11.8(\mathrm{~g} / \mathrm{mL}) \mathrm{G}$

Level: (low/med) MED

$\%$ Moisture: not dec.

GC Column: DB-624 ID: 0.53 (mm)

Soil Extract Volume: 10000 (uL)
Contract: 8E-00302

SAS NO.:
ARGLAB SAMPLE NO.

IN-S-30672

SDG No.: 134662

Lab Sample ID: 813472

Lab File ID: $\quad 813472$

Date Received: 11/13/09

Date Analyzed: 11/19/09

Dilution Factor: 1.0

Soil Aliquot Volume:

500 (UL)

CONCENTRATION UNITS :

CAS NO.

COMPOUND

Q

$8.5 \mathrm{U}$

$8.5 \mathrm{U}$

$8.5 \mathrm{U}$

$8.5 \mathrm{U}$

$8.5 \mathrm{U}$

$8.5 \mathrm{U}$

$42 \mathrm{U}$

$8.5 \mathrm{U}$

$8.5 \mathrm{U}$

$42 \mathrm{U}$

$8.5 \mathrm{U}$

$8.5 \mathrm{U}$

$8.5 \mathrm{U}$

$8.5 \mathrm{U}$

$8.5 \mathrm{U}$

$8.5 \mathrm{U}$

$8.5 \mathrm{U}$

$8.5 \mathrm{U}$

$8.5 \mathrm{U}$

$8.5 \mathrm{U}$

$8.5 \mathrm{U}$

$8.5 \mathrm{U}$

$8.5 \mathrm{U}$

$42 \mathrm{U}$

$34 \mathrm{U}$

$8.5 \mathrm{U}$

$8.5 \mathrm{U}$

$120 \mathrm{U}$

$8.5 \mathrm{U}$

$8.5 \mathrm{U}$

$8.5 \mathrm{U}$

$8.5 \mathrm{U}$

$420 \mathrm{U}$ 
FORM 1

VOLATILE ORGANICS ANALYSIS DATA SHEET
ARGLAB SAMPLE NO.

IN-S-30672
Lab Name: TESTAMERICA BURLINGTON

Lab Code: STLV
Case No.: INMAN

Matrix: (soil/water) SOIL

Sample wt/vol:

$11.8(\mathrm{~g} / \mathrm{mL}) \mathrm{G}$

Level : (low/med) MED

\% Moisture: not dec.

GC Column: DB-624 ID: 0.53 (mm)

Soil Extract Volume: 10000 (uL)
Contract : 8E-00302

SAS No.:

SDG No.: 134662
Lab Sample ID: 813472

Lab File ID: $\quad 813472$

Date Received: 11/13/09

Date Analyzed: 11/19/09

Dilution Factor: 1.0

500 (uL)

CAS NO. COMPOUND (ug/L or ug/Kg) UG/KG

Q

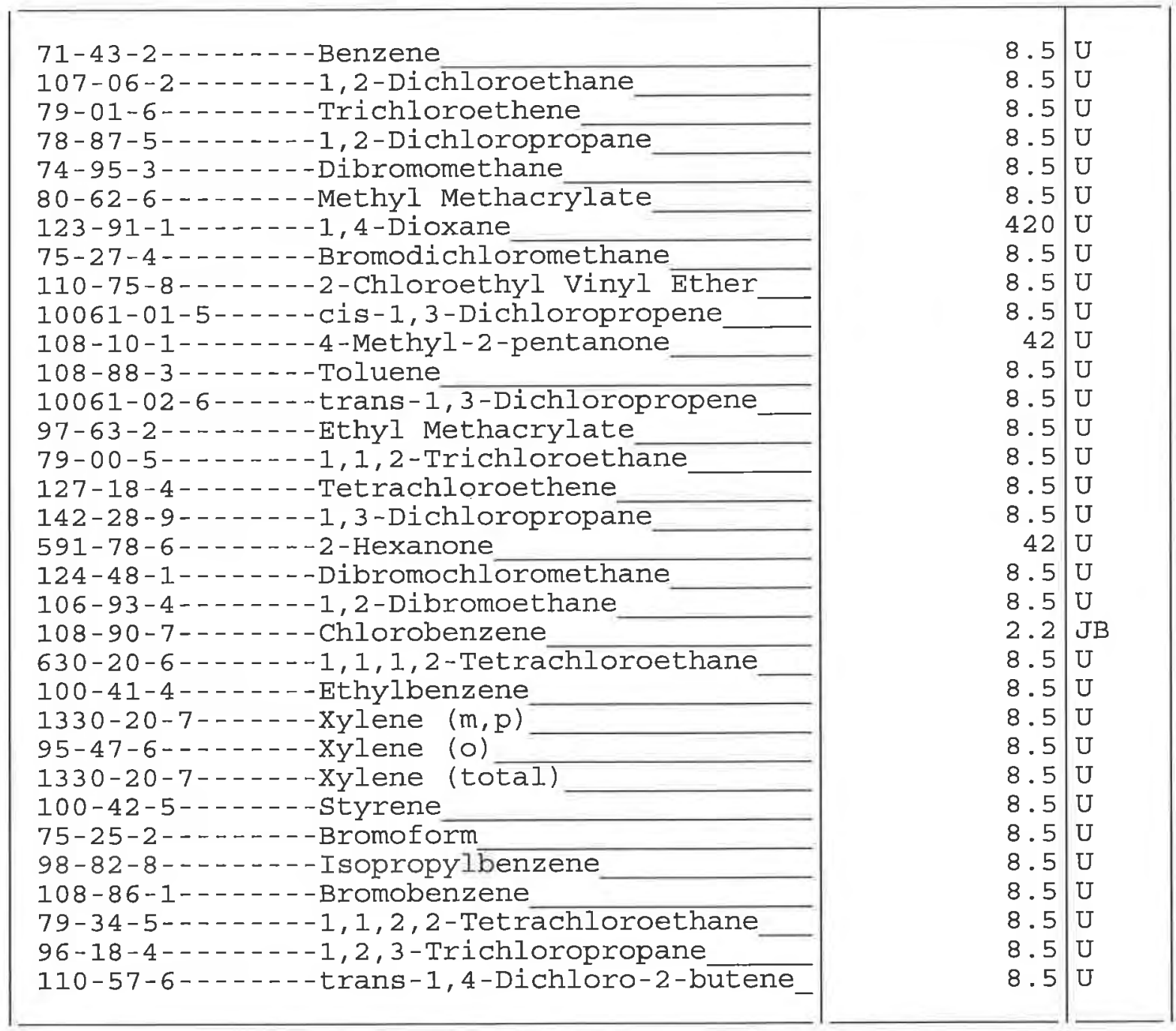


FORM 1

VOLATILE ORGANICS ANALYSIS DATA SHEET
ARGLAB SAMPLE NO.

IN-S-30672
Lab Name: TESTAMERICA BURLINGTON

Lab Code: STLV

Case No.: INMAN

Matrix: (soil/water) SOIL

Sample wt/vol:

$11.8(\mathrm{~g} / \mathrm{mL}) \quad \mathrm{G}$

Level : (low/med) MED

$\%$ Moisture: not dec.

GC Column: DB-624 ID : 0.53 (mm)

Soil Extract Volume: 10000 (uL)
Contract : 8E-00302

SAS No. :

SDG No.: 134662
Lab Sample ID: 813472

Lab File ID: $\quad 813472$

Date Received: 11/13/09

Date Analyzed: 11/19/09

Dilution Factor: 1.0

Soil Aliquot Volume:

500 (uL)

CAS NO.

COMPOUND

CONCENTRATION UNITS:

(ug/L or ug/Kg) UG/KG

Q

$8.5 \mathrm{U}$

$8.5 \mathrm{U}$

$8.5 \mathrm{U}$

$8.5 \mathrm{U}$

$8.5 \mathrm{U}$

$8.5 \mathrm{U}$

$8.5 \mathrm{U}$

$8.5 \mathrm{U}$

$8.5 \mathrm{U}$

$8.5 \mathrm{U}$

$8.5 \mathrm{U}$

$8.5 \mathrm{U}$

$8.5 \mathrm{U}$

$8.5 \mathrm{U}$

$8.5 \mathrm{U}$

$8.5 \mathrm{U}$

$8.5 \mathrm{U}$ 
FORM 1

VOLATILE ORGANICS ANALYSIS DATA SHEET
Lab Name: TESTAMERICA BURLINGTON

Lab Code: STLV

Case No.: INMAN

Matrix: (soil/water) SOIL

Sample wt/vol: $\quad 10.1(\mathrm{~g} / \mathrm{mL}) \mathrm{G}$

Level: (low/med) MED

$\%$ Moisture: not dec.

GC Column: DB-624 ID: 0.53 (mm)

Soil Extract Volume: 10000 (uL)
Contract: $8 \mathrm{E}-00302$

SAS NO.:
ARGLAB SAMPLE NO.

IN-S-30676

SDG No.: 134662

Lab Sample ID: 813474

Lab File ID: $\quad 813474$

Date Received: 11/13/09

Date Analyzed: 11/19/09

Dilution Factor: 1.0

Soil Aliquot Volume:

$500(\mathrm{uL})$

CONCENTRATION UNITS:

CAS NO.

COMPOUND

Q

$9.9 \mathrm{U}$

$9.9 \mathrm{U}$

$9.9 \mathrm{U}$

$9.9 \mathrm{U}$

$9.9 \mathrm{U}$

$9.9 \mathrm{U}$

$50 \mathrm{U}$

$9.9 \mathrm{U}$

$9.9 \mathrm{U}$

$50 \mathrm{U}$

$9.9 \mathrm{U}$

$9.9 \mathrm{U}$

$9.9 \mathrm{U}$

$9.9 \mathrm{U}$

$9.9 \mathrm{U}$

$9.9 \mathrm{U}$

$9.9 \mathrm{U}$

$9.9 \mathrm{U}$

$9.9 \mathrm{U}$

$9.9 \mathrm{U}$

$9.9 \mathrm{U}$

$9.9 \mathrm{U}$

$9.9 \mathrm{U}$

$50 \mathrm{U}$

$40 \mathrm{U}$

$9.9 \mathrm{U}$

$9.9 \mathrm{U}$

$140 \mathrm{U}$

$9.9 \mathrm{U}$

$9.9 \mathrm{U}$

$9.9 \mathrm{U}$

$9.9 \mathrm{U}$

$500 \mathrm{U}$ 
FORM 1

VOLATILE ORGANICS ANALYSIS DATA SHEET
ARGLAB SAMPLE NO.

IN-S-30676
Lab Name: TESTAMERICA BURLINGTON

Lab Code: STLV
Case No.: INMAN

Matrix: (soil/water) sol工

Sample wt/vol:

$10.1(\mathrm{~g} / \mathrm{mL}) \mathrm{G}$

Level: (low/med) MED

\% Moisture: not dec.

GC Column: DB-624 ID: 0.53 (mm)

Soil Extract Volume: 10000 (uL)
Contract: $8 \mathrm{E}-00302$

SAS NO.:

SDG NO.: 134662
Lab Sample ID: 813474

Lab File ID: $\quad 813474$

Date Received: 11/13/09

Date Analyzed: 11/19/09

Dilution Factor: 1.0

Soil Aliquot Volume:

$500(\mathrm{uL})$

CAS NO.

COMPOUND

CONCENTRATION UNITS:

(ug/L or $\mathrm{ug} / \mathrm{Kg}$ ) UG/KG

Q

\begin{tabular}{r|l}
9.9 & $\mathrm{U}$ \\
9.9 & $\mathrm{U}$ \\
9.9 & $\mathrm{U}$ \\
9.9 & $\mathrm{U}$ \\
9.9 & $\mathrm{U}$ \\
9.9 & $\mathrm{U}$ \\
500 & $\mathrm{U}$ \\
9.9 & $\mathrm{U}$ \\
9.9 & $\mathrm{U}$ \\
9.9 & $\mathrm{U}$ \\
50 & $\mathrm{U}$ \\
9.9 & $\mathrm{U}$ \\
9.9 & $\mathrm{U}$ \\
9.9 & $\mathrm{U}$ \\
9.9 & $\mathrm{U}$ \\
9.9 & $\mathrm{U}$ \\
9.9 & $\mathrm{U}$ \\
50 & $\mathrm{U}$ \\
9.9 & $\mathrm{U}$ \\
9.9 & $\mathrm{U}$ \\
9.9 & $\mathrm{U}$ \\
9.9 & $\mathrm{U}$ \\
9.9 & $\mathrm{U}$ \\
9.9 & $\mathrm{U}$ \\
9.9 & $\mathrm{U}$ \\
9.9 & $\mathrm{U}$ \\
9.9 & $\mathrm{U}$ \\
9.9 & $\mathrm{U}$ \\
9.9 & $\mathrm{U}$ \\
9.9 & $\mathrm{U}$ \\
9.9 & $\mathrm{U}$ \\
9.9 & $\mathrm{U}$ \\
9.9 & $\mathrm{U}$ \\
& \\
\hline
\end{tabular}


FORM 1

VOLATILE ORGANICS ANALYSIS DATA SHEET
ARGLAB SAMPLE NO.

IN-S-30676
Lab Name: TESTAMERICA BURLINGTON

Lab Code: STLV

Case No.: INMAN

Matrix: (soil/water) soIL

Sample wt/vol:

$10.1(\mathrm{~g} / \mathrm{mL}) \mathrm{G}$

Level: (low/med) MED

$\%$ Moisture: not dec.

GC Column: DB-624

ID : $0.53(\mathrm{~mm})$

Soil Extract Volume: 10000 (uL)
Contract: $8 \mathrm{E}-00302$

SAS NO.:

SDG No.: 134662

Lab Sample ID: 813474

Lab File ID: $\quad 813474$

Date Received: 11/13/09

Date Analyzed: 11/19/09

Dilution Factor: 1.0

Soil Aliquot Volume:

500 (uL)

CAS NO.

COMPOUND

CONCENTRATION UNITS:

( $\mathrm{ug} / \mathrm{L}$ or $\mathrm{ug} / \mathrm{Kg}$ ) UG/KG

Q

$9.9 \mathrm{U}$

$9.9 \mathrm{U}$

$9.9 \mathrm{U}$

$9.9 \mathrm{U}$

$.9 .9 \mathrm{U}$

$9.9 \mathrm{U}$

$9.9 \mathrm{U}$

$9.9 \mathrm{U}$

$9.9 \mathrm{U}$

9. $9 \mathrm{U}$

$9.9 \mathrm{U}$

9. $9 \mathrm{U}$

$9.9 \mathrm{U}$

$9.9 \mathrm{U}$

$9.9 \mathrm{U}$

9. $9 \mathrm{U}$

$9.9 \mathrm{U}$ 
FORM 1

VOLATILE ORGANICS ANALYSIS DATA SHEET
ARGLAB SAMPLE NO.

IN-S-30695
Lab Name: TESTAMERICA BURLINGTON

Lab Code: STIV

Matrix: (soil/water) soIL

Sample wt/vol:

$12.5(\mathrm{~g} / \mathrm{mL}) \mathrm{G}$

Level: (low/med) MED

\% Moisture: not dec.

GC Column: DB-624 ID: 0.53 (mm)

Soil Extract Volume: 10000 (uL)
Contract: $8 E-00302$

SAS NO.:

SDG No. : 134662

Lab Sample ID: 813473

Lab File ID: 813473

Date Received: 11/13/09

Date Analyzed: 11/19/09

Dilution Factor: 1.0

Soil Aliquot Volume:

500 (uL)

CAS NO.

COMPOUND

(ug/L or ug/Kg) UG/KG

$Q$

$8.0 \mathrm{U}$

$8.0 \mathrm{U}$

8. $0 \mathrm{U}$

$8.0 \mathrm{U}$

$8.0 \mathrm{U}$

$8.0 \mathrm{U}$

$40 \mathrm{U}$

$8.0 \mathrm{U}$

$8.0 \mathrm{U}$

$40 \mathrm{U}$

$8.0 \mathrm{U}$

8. $0 \mathrm{U}$

$8.0 \mathrm{U}$

$8.0 \mathrm{U}$

$8.0 \mathrm{U}$

$8.0 \mathrm{U}$

$8.0 \mathrm{U}$

$8.0 \mathrm{U}$

$8.0 \mathrm{U}$

$8.0 \mathrm{U}$

$8.0 \mathrm{U}$

$8.0 \mathrm{U}$

$8.0 \mathrm{U}$

$40 \mathrm{U}$

$32 \mathrm{U}$

8. $0 \mathrm{U}$

$8.0 \mathrm{U}$

$110 \mathrm{U}$

$8.0 \mathrm{U}$

$8.0 \mathrm{U}$

$2.0 \mathrm{~J}$

$8.0 \mathrm{U}$

$400 \mathrm{U}$

78-83-1-..-.-.-Isobutyl Alcohol 
FORM 1

VOLATILE ORGANICS ANALYSIS DATA SHEET
ARGLAB SAMPLE NO.

IN-S-30695
Lab Name: TESTAMERICA BURLINGTON
Lab Code: STLV

Case No.: INMAN

Matrix: (soil/water) SOIL

Sample wt/vol:

Level: (low/med) MED

\% Moisture: not dec.

GC Column: DB-624 ID: 0.53 (mm)

Soil Extract Volume: 10000 (uL)
Contract: $8 \mathrm{E}-00302$

SAS No.:

SDG No.: 134662
Lab Sample ID: 813473

Lab File ID: 813473

Date Received: 11/13/09

Date Analyzed: 11/19/09

Dilution Factor: 1.0

Soil Aliquot Volume:

500 (uL)

CAS NO.

COMPOUND

CONCENTRATION UNITS :

(ug/L or ug/Kg) UG/KG

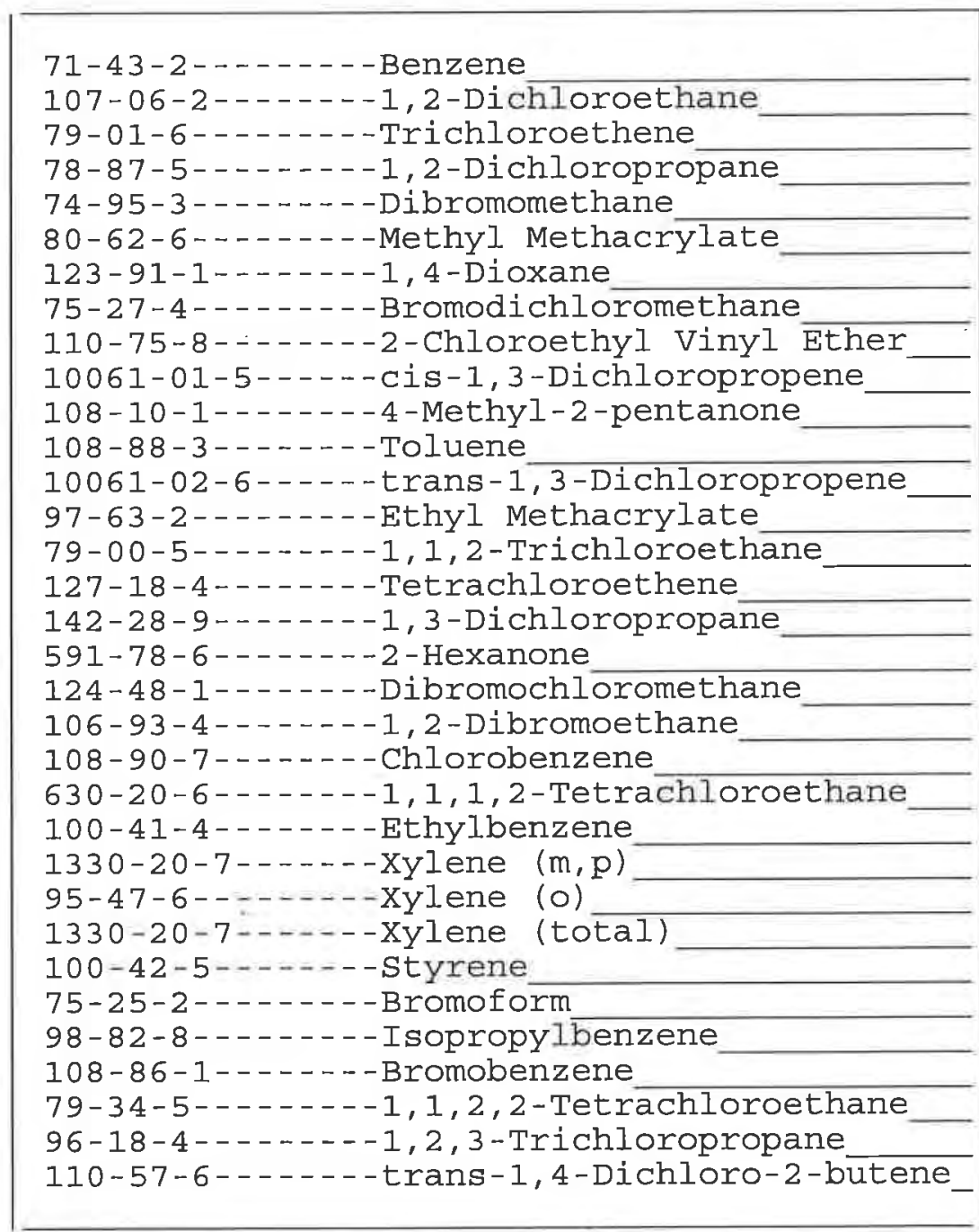

\begin{tabular}{|l|l|l|}
8.0 & $\mathrm{U}$ \\
8.0 & $\mathrm{U}$ \\
8.0 & $\mathrm{U}$ \\
8.0 & $\mathrm{U}$ \\
8.0 & $\mathrm{U}$ \\
8.0 & $\mathrm{U}$ \\
400 & $\mathrm{U}$ \\
8.0 & $\mathrm{U}$ \\
8.0 & $\mathrm{U}$ \\
8.0 & $\mathrm{U}$ \\
40 & $\mathrm{U}$ \\
8.0 & $\mathrm{U}$ \\
8.0 & $\mathrm{U}$ \\
8.0 & $\mathrm{U}$ \\
8.0 & $\mathrm{U}$ \\
8.0 & $\mathrm{U}$ \\
8.0 & $\mathrm{U}$ \\
40 & $\mathrm{U}$ \\
8.0 & $\mathrm{U}$ \\
8.0 & $\mathrm{U}$ \\
8.0 & $\mathrm{U}$ \\
8.0 & $\mathrm{U}$ \\
8.0 & $\mathrm{U}$ \\
8.0 & $\mathrm{U}$ \\
8.0 & $\mathrm{U}$ \\
8.0 & $\mathrm{U}$ \\
8.0 & $\mathrm{U}$ \\
8.0 & $\mathrm{U}$ \\
8.0 & $\mathrm{U}$ \\
8.0 & $\mathrm{U}$ \\
8.0 & $\mathrm{U}$ \\
8.0 & $\mathrm{U}$ \\
8.0 & $\mathrm{U}$ \\
& & \\
\hline & \\
8
\end{tabular}


FORM 1

VOLATILE ORGANICS ANALYSIS DATA SHEET
ARGLAB SAMPLE NO.

IN-S-30695
Lab Name: TESTAMERICA BURLINGTON

Lab Code: STLV

Case No.: INMAN

Matrix: (soil/water) soIL

Sample wt/vol:

$12.5(\mathrm{~g} / \mathrm{mL}) \mathrm{G}$

Level: (low/med) MED

\% Moisture: not dec.

GC Column: DB-624 ID: 0.53 (mm)

Soil Extract Volume: 10000 (uL)
Contract: 8E-00302

SAS NO.:

SDG No.: 134662

Lab Sample ID: 813473

Lab File ID: $\quad 813473$

Date Received: 11/13/09

Date Analyzed: 11/19/09

Dilution Factor: 1.0

Soil Aliquot Volume:

500 (UL)

CAS NO.

COMPOUND

CONCENTRATION UNITS :

(ug/L or ug/Kg) UG/KG

Q

$8.0 \mathrm{U}$

$8.0 \mathrm{U}$

$8.0 \mathrm{U}$

$8.0 \mathrm{U}$

$8.0 \mathrm{U}$

$8.0 \mathrm{U}$

$8.0 \mathrm{U}$

$8.0 \mathrm{U}$

$8.0 \mathrm{U}$

$8.0 \mathrm{U}$

$8.0 \mathrm{U}$

$8.0 \mathrm{U}$

$8.0 \mathrm{U}$

$8.0 \mathrm{U}$

$8.0 \mathrm{U}$

$8.0 \mathrm{U}$

$8.0 \mathrm{U}$ 
FORM 1

VOLATILE ORGANICS ANALYSIS DATA SHEET
Lab Name: TESTAMERICA BURLINGTON

Lab Code: STLV

Case No.: INMAN

Matrix: (soil/water) SOIL

Sample wt/vol:

$10.8(\mathrm{~g} / \mathrm{mL}) \quad \mathrm{G}$

Level: (low/med) MED

\% Moisture: not dec.

GC Column: DB-624 ID: 0.53 (mm)

Soil Extract Volume: 10000 (uL)
Contract: 8E-00302

IN-S-30696
ARGLAB SAMPLE NO.

SAS No. :

SDG No. : 134662
Lab Sample ID: 813475

Lab File ID: 813475

Date Received: 11/13/09

Date Analyzed: 11/19/09

Dilution Factor: 1.0

Soil Aliquot Volume:

500 (uL)

CAS NO.

COMPOUND

CONCENTRATION UNITS:

(ug/L or $u g / \mathrm{Kg}$ ) UG/KG

$Q$

$9.2 \mathrm{U}$

$9.2 \mathrm{U}$

$9.2 \mathrm{U}$

$9.2 \mathrm{U}$

$9.2 \mathrm{U}$

$9.2 \mathrm{U}$

$46 \mathrm{U}$

$9.2 \mathrm{U}$

$9.2 \mathrm{U}$

$46 \mathrm{U}$

$9.2 \mathrm{U}$

$9.2 \mathrm{U}$

$9.2 \mathrm{U}$

$9.2 \mathrm{U}$

$9.2 \mathrm{U}$

$9.2 \mathrm{U}$

$9.2 \mathrm{U}$

$9.2 \mathrm{U}$

$9.2 \mathrm{U}$

$9.2 \mathrm{U}$

$9.2 \mathrm{U}$

$9.2 \mathrm{U}$

$9.2 \mathrm{U}$

$46 \mathrm{U}$

$37 \mathrm{U}$

$9.2 \mathrm{U}$

$9.2 \mathrm{U}$

$130 \mathrm{U}$

$9.2 \mathrm{U}$

$9.2 \mathrm{U}$

$9.2 \mathrm{U}$

$9.2 \mathrm{U}$

$460 \mathrm{U}$ 
FORM 1

VOLATILE ORGANICS ANALYSIS DATA SHEET
ARGLAB SAMPLE NO.

IN-S-30696
Lab Name: TESTAMERICA BURLINGTON

Lab Code: STLV
Case No.: INMAN

Matrix: (soil/water) SOIL

Sample wt/vol:

$10.8(\mathrm{~g} / \mathrm{mL}) \quad \mathrm{G}$

Level: (low/med) MED

\% Moisture: not dec.

GC Column: DB-624 ID: 0.53 (mm)

Soil Extract Volume: 10000 (uL)
Contract: 8E-00302

SAS NO. :

SDG No.: 134662
Lab Sample ID: 813475

Lab File ID: 813475

Date Received: 11/13/09

Date Analyzed: 11/19/09

Dilution Factor: 1.0

Soil Aliquot Volume:

500 (uL)

CONCENTRATION UNITS:
(ug/L or $u g / \mathrm{Kg}$ ) UG/KG

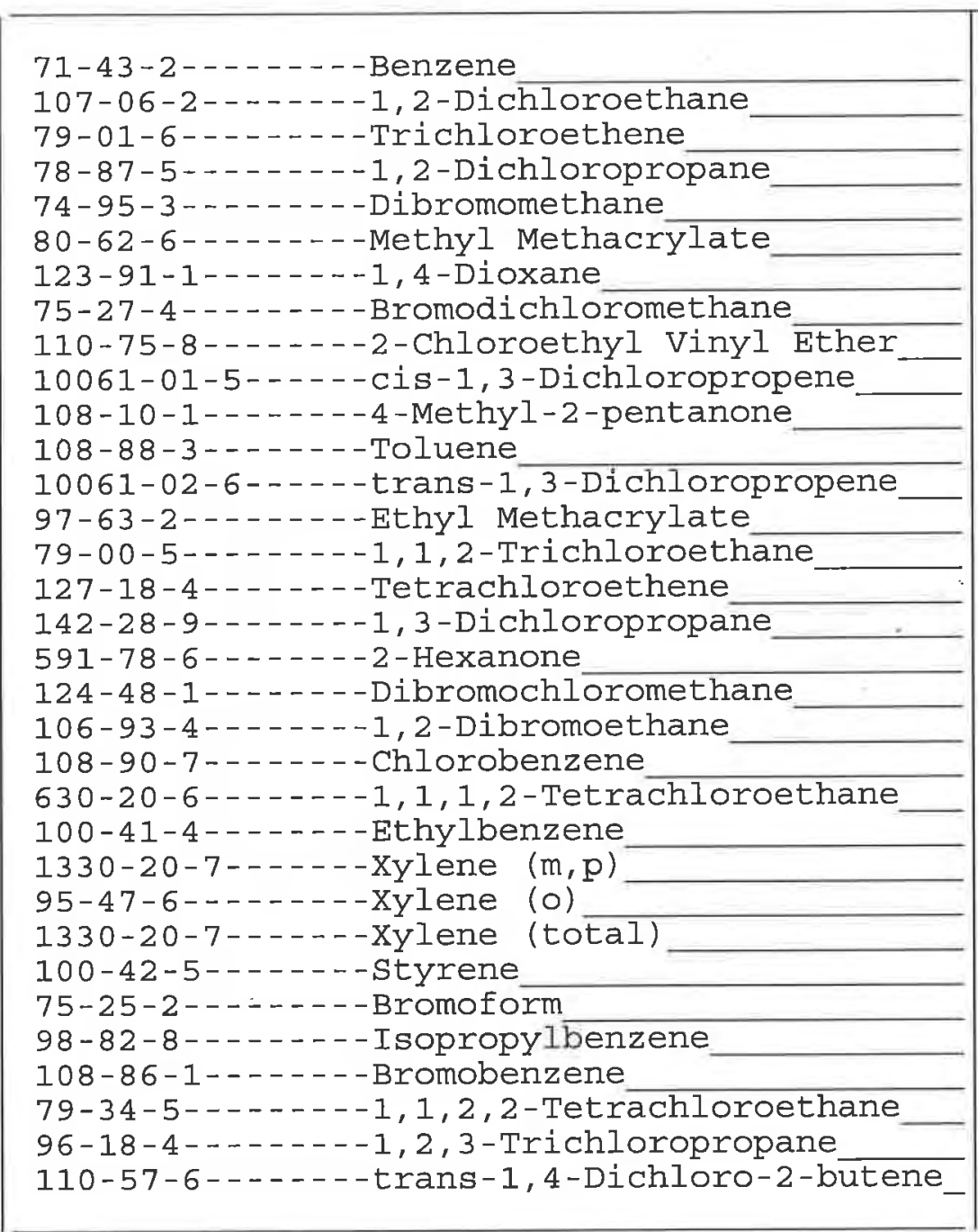

\begin{tabular}{l|l}
9.2 & $\mathrm{U}$ \\
9.2 & $\mathrm{U}$ \\
9.2 & $\mathrm{U}$ \\
9.2 & $\mathrm{U}$ \\
9.2 & $\mathrm{U}$ \\
9.2 & $\mathrm{U}$ \\
460 & $\mathrm{U}$ \\
9.2 & $\mathrm{U}$ \\
9.2 & $\mathrm{U}$ \\
9.2 & $\mathrm{U}$ \\
46 & $\mathrm{U}$ \\
9.2 & $\mathrm{U}$ \\
9.2 & $\mathrm{U}$ \\
9.2 & $\mathrm{U}$ \\
9.2 & $\mathrm{U}$ \\
9.2 & $\mathrm{U}$ \\
9.2 & $\mathrm{U}$ \\
46 & $\mathrm{U}$ \\
9.2 & $\mathrm{U}$ \\
9.2 & $\mathrm{U}$ \\
9.2 & $\mathrm{U}$ \\
9.2 & $\mathrm{U}$ \\
9.2 & $\mathrm{U}$ \\
9.2 & $\mathrm{U}$ \\
9.2 & $\mathrm{U}$ \\
9.2 & $\mathrm{U}$ \\
9.2 & $\mathrm{U}$ \\
9.2 & $\mathrm{U}$ \\
9.2 & $\mathrm{U}$ \\
9.2 & $\mathrm{U}$ \\
9.2 & $\mathrm{U}$ \\
9.2 & $\mathrm{U}$ \\
9.2 & $\mathrm{U}$ \\
& \\
\hline &
\end{tabular}


FORM 1 YQLATILE ORGANICS ANALYSIS DATA SHEET

Lab Name: TESTAMERICA BURLINGTON Case No.: INMAN

Lab Code: STLV

Matrix: (soil/water) soIL

Sample wt/vol: $\quad 10.8(\mathrm{~g} / \mathrm{mL}) \mathrm{G}$

Level: (low/med) MED

$\div$ Moisture: not dec.

GC Column: DB-624 ID: 0.53 (mm)

Soil Extract Volume: 10000 (uL)
Contract: $8 \mathrm{E}-00302$

SAS NO.:
ARGLAB SAMPLE NO.

IN-S-30696

SDG No.: 134662

Lab Sample ID: 813475

Lab File ID: $\quad 813475$

Date Received: 11/13/09

Date Analyzed: 11/19/09

Dilution Factor: 1.0

Soil Aliquot Volume:

500 (uL)

CONCENTRATION UNITS:

CAS NO.

COMPOUND

103-65-1-..--n--nropylbenzene 95-49-8-1-n--2-Chlorotoluene 106-43-4-----4-Chlorotoluene 108-67-8--.--1, 3,5-Trimethyl benzene 98-06-6---.--tert-Butylbenzene 95-63-6------1, 2,4-Trimethylbenzene 135-98-8-..---sec-Butylbenzene 541-73-1------1,3-Dichlorobenzene 99-87-6-- - - - 4-Isopropyltoluene 106-46-7-_-n-1,4-Dichlorobenzene 95-50-1------1, 2-Dichlorobenzene 104-51-8------n-Butylbenzene 96-12-8--- - - -1,2-Dibromo-3-Chloropropane 120-82-1-...-1,2,4-Trichlorobenzene 87-68-3-1..--Hexachlorobutadiene 91-20-3--.---Naphthalene 87-61-6-..---1, 2,3-Trichlorobenzene
$9.2 \mathrm{U}$

$9.2 \mathrm{U}$

$9.2 \mathrm{U}$

$9.2 \mathrm{U}$

$9.2 \mathrm{U}$

$9.2 \mathrm{U}$

$9.2 \mathrm{U}$

$9.2 \mathrm{U}$

$9.2 \mathrm{U}$

$9.2 \mathrm{U}$

$9.2 \mathrm{U}$

$9.2 \mathrm{U}$

$9.2 \mathrm{U}$

$9.2 \mathrm{U}$

$9.2 \mathrm{U}$

$9.2 \mathrm{U}$

$9.2 \mathrm{U}$ 
FORM 1

VOLATILE ORGANICS ANALYSIS DATA SHEET
ARGLAB SAMPLE NO.

IN-S-30734
Lab Name: TESTAMERICA BURLINGTON

Lab Code: STLV

Case NO.: INMAN

Matrix: (soil/water) SOIL

Sample wt/vol:

$12.0(\mathrm{~g} / \mathrm{mL}) \mathrm{G}$

Level: (low/med) MED

\% Moisture: not dec.

GC Column: DB-624 ID: 0.53 (mm)

Soil Extract Volume: 10000 (uL)
Contract: $8 \mathrm{E}-00302$

SAS NO. :

SDG No.: 134662
Lab Sample ID: 813477

Lab File ID: $\quad 813477$

Date Received: 11/13/09

Date Analyzed: 11/19/09

Dilution Factor: 1.0

Soil Aliquot Volume:

500 (uL)

CAS NO.

COMPOUND

CONCENTRATION UNITS:

(ug/L or ug/Kg) UG/KG

Q

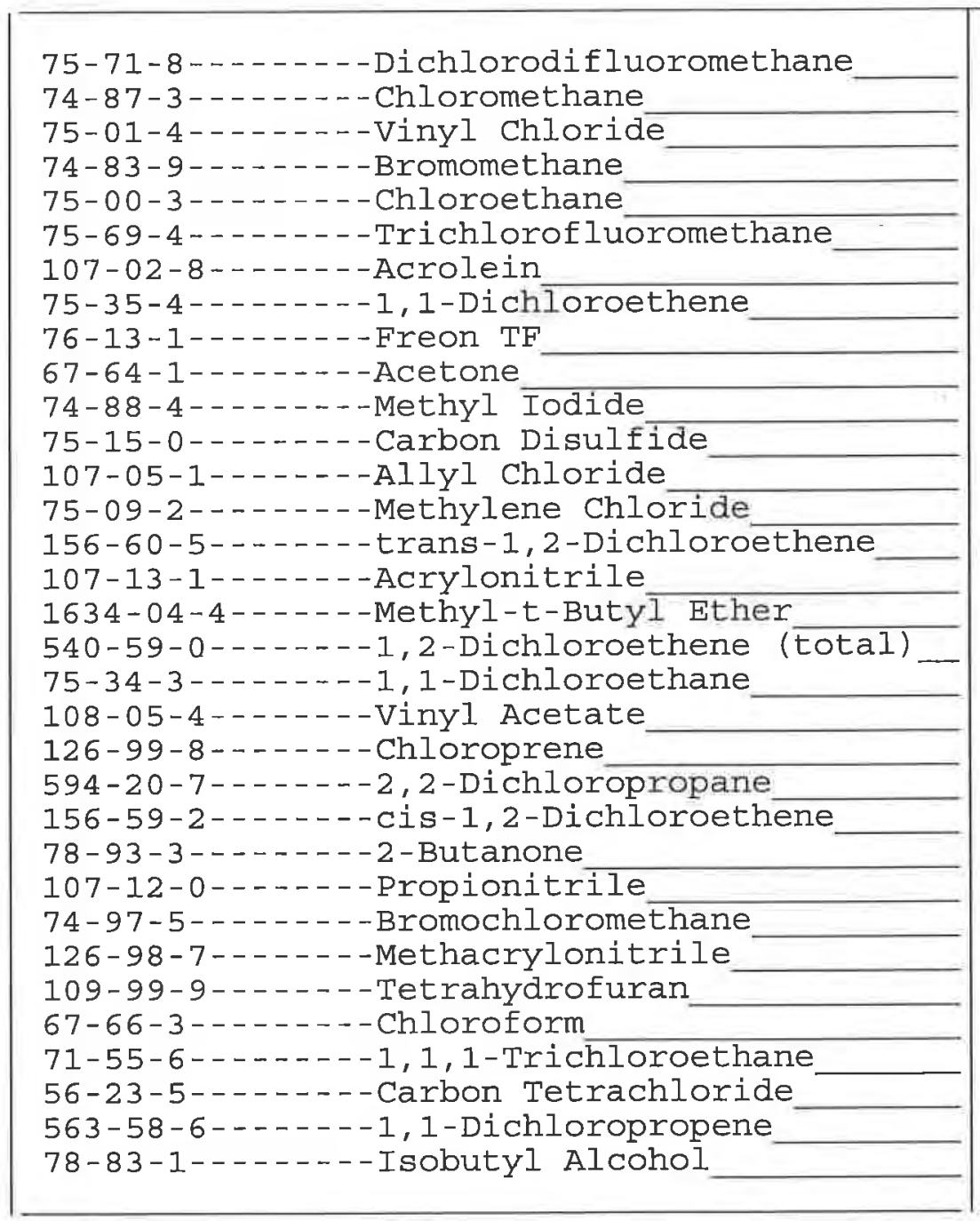

\begin{tabular}{|c|c|}
\hline 8.3 & $\tau$ \\
\hline 8.3 & $\tau$ \\
\hline 8.3 & [ \\
\hline 8.3 & $\tau$ \\
\hline 8.3 & $\tau$ \\
\hline 8.3 & $\tau$ \\
\hline 42 & $T$ \\
\hline 8.3 & $T$ \\
\hline 8.3 & $\tau$ \\
\hline 42 & $\tau$ \\
\hline 8.3 & T \\
\hline 8.3 & $\tau$ \\
\hline 8.3 & $\tau$ \\
\hline 8.3 & $\tau$ \\
\hline 8.3 & I \\
\hline 8.3 & $\tau$ \\
\hline 8.3 & 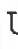 \\
\hline 8.3 & $T$ \\
\hline 8.3 & $\tau$ \\
\hline 8.3 & $\tau$ \\
\hline 8.3 & 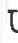 \\
\hline 8.3 & I \\
\hline 8.3 & $\tau$ \\
\hline 42 & I \\
\hline 33 & $\tau$ \\
\hline 8.3 & $\tau$ \\
\hline 8.3 & 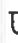 \\
\hline 120 & \\
\hline 17 & \\
\hline 42 & \\
\hline 8.3 & I \\
\hline 8.3 & \\
\hline 420 & \\
\hline
\end{tabular}


FORM 1

VOLATILE ORGANICS ANALYSIS DATA SHEET
ARGLAB SAMPLE NO.

IN-S-30734
Lab Name: TESTAMERICA BURLINGTON

Lab Code: STLV
Case No.: INMAN

Matrix: (soil/water) SOIL

Sample wt/vol:

Level: (low/med) MED

$\div$ Moisture: not dec.

GC Column: DB-624 ID: 0.53 (mm)

Soil Extract Volume: 10000 (uL)
Contract: $8 \mathrm{E}-00302$

SAS NO. :

SDG No.: 134662
Lab Sample ID: 813477

Lab File ID: $\quad 813477$

Date Received: 11/13/09

Date Analyzed: 11/19/09

Dilution Factor: 1.0

Soil Aliquot Volume:

500 (UL)

CAS NO.

COMPOUND

CONCENTRATION UNITS :

(ug/L or ug/Kg) UG/KG

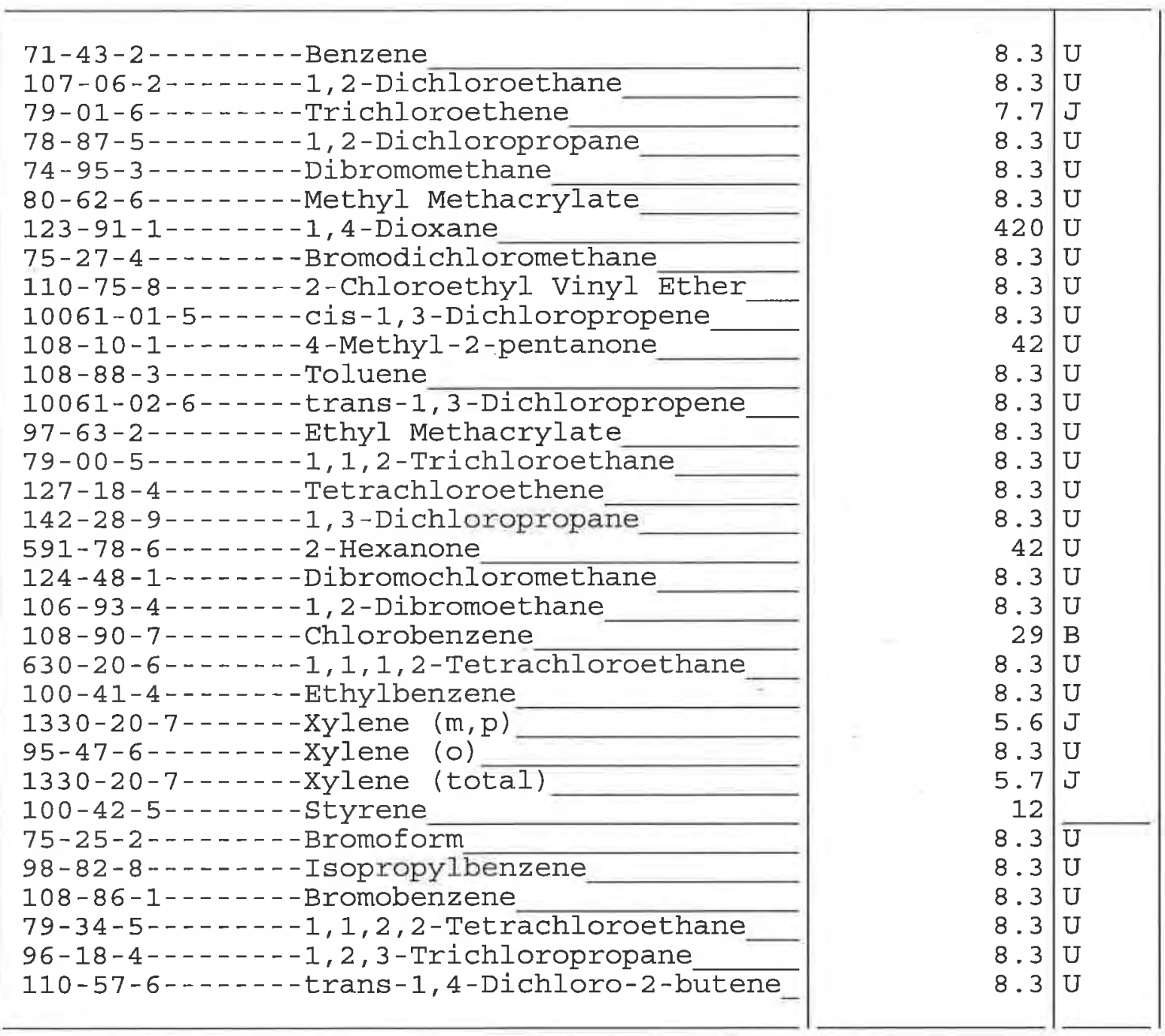


FORM 1

VOLATILE ORGANICS ANALYSIS DATA SHEET
Lab Name: TESTAMERICA BURLINGTON

Lab Code: STLV

Case No.: INMAN

Matrix: (soil/water) soIL

Sample wt/vol:

$$
12.0(\mathrm{~g} / \mathrm{mL}) \mathrm{G}
$$

Level: (low/med) MED

: Moisture: not dec.

GC Column: DB-624 ID: 0.53 (mm)

Soil Extract Volume: $10000(u L)$
Contract: $8 \mathrm{E}-00302$

IN-S-30734
SAS NO. :

SDG No.: 134662
Lab Sample ID: 813477

Lab File ID: $\quad 813477$

Date Received: 11/13/09

Date Analyzed: 11/19/09

Dilution Factor: 1.0

Soil Aliquot Volume:

500 (uL)

CONCENTRATION UNITS :

CAS NO.

COMPOUND

103-65-1-..--n-Propylbenzene

95-49-8-....-2-Chlorotoluene 106-43-4-1-.-4-Chlorotoluene 108-67-8-.---1,3,5-Trimethylbenzene 98-06-6--.---tert-Butylbenzene 95-63-6--.---1, 2, 4-Trimethylbenzene 135-98-8-----sec-Butylbenzene 541-73-1--.--1, 3-Dichlorobenzene 99-87-6-------4-Isopropyltoluene 106-46-7-...-1,4-Dichlorobenzene 95-50-1-...-1,2-Dichlorobenzene 104-51-8-.---n-Butylbenzene 96-12-8-- - - - 1,2-Dibromo-3-Chloropropane 120-82-1--.--1,2,4-Trichlorobenzene 87-68-3-...---Hexachlorobutadiene 91-20-3--.---Naphthalene 87-61-6------1, 2, 3-Trichlorobenzene
$8.3 \mathrm{U}$

$8.3 \mathrm{U}$

$8.3 \mathrm{U}$

$8.3 \mathrm{U}$

$8.3 \mathrm{U}$

$8.3 \mathrm{U}$

$8.3 \mathrm{U}$

$2.2 \mathrm{~J}$

$8.3 \mathrm{U}$

$5.9 \mathrm{~J}$

$8.3 \mathrm{U}$

$8.3 \mathrm{U}$

$8.3 \mathrm{U}$

$3.3 \mathrm{~J}$

$8.3 \mathrm{U}$

$4.3 \mathrm{~J}$

$8.3 \mathrm{U}$ 
FORM 1

VOLATILE ORGANICS ANALYSIS DATA SHEET
ARGLAB SAMPLE NO.

IN-S-MEOHBLK
Lab Name: TESTAMERICA BURLINGTON

Lab Code: STLV
Case No.: INMAN

Matrix: (soil/water) soIL

Sample wt/vol:

Level: (low/med) MED

\% Moisture: not dec.

GC Column: DB-624 ID: 0.53 (mm)

Soil Extract Volume: 10000 (uL)
Contract: 8E-00302

SAS No. :

SDG NO.: 134662
Lab Sample ID: 813483

Lab File ID: 813483

Date Received: 11/13/09

Date Analyzed: 11/19/09

Dilution Factor: 1.0

Soil Aliquot Volume:

$500(\mathrm{uL})$

CONCENTRATION UNITS:

COMPOUND (ug/I or $\mathrm{ug} / \mathrm{Kg}$ ) UG/KG

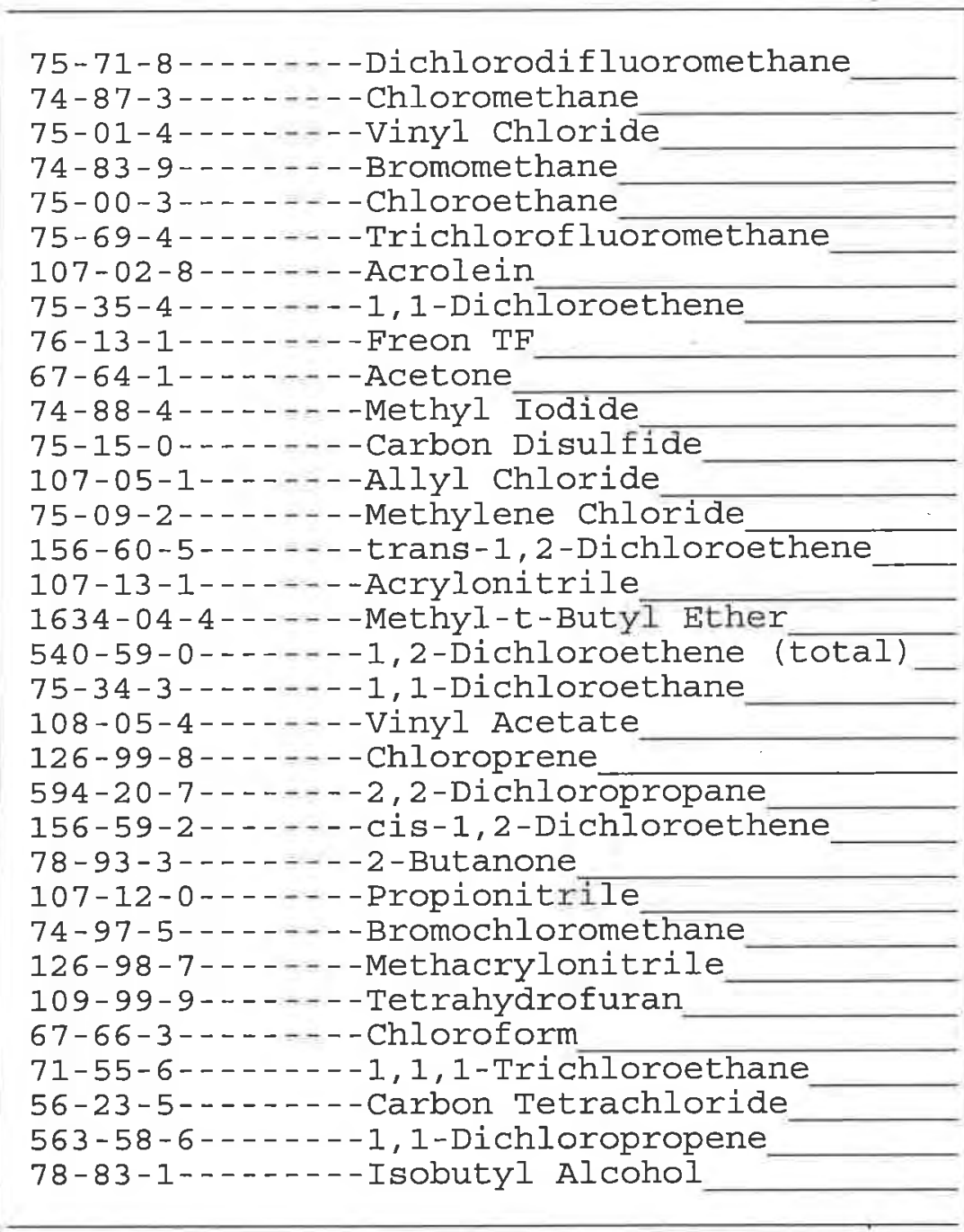

\begin{tabular}{|r|r|l|}
\hline 10 & $\mathrm{U}$ \\
10 & $\mathrm{U}$ \\
10 & $\mathrm{U}$ \\
10 & $\mathrm{U}$ \\
10 & $\mathrm{U}$ \\
10 & $\mathrm{U}$ \\
50 & $\mathrm{U}$ \\
10 & $\mathrm{U}$ \\
10 & $\mathrm{U}$ \\
50 & $\mathrm{U}$ \\
10 & $\mathrm{U}$ \\
10 & $\mathrm{U}$ \\
10 & $\mathrm{U}$ \\
10 & $\mathrm{U}$ \\
10 & $\mathrm{U}$ \\
10 & $\mathrm{U}$ \\
10 & $\mathrm{U}$ \\
10 & $\mathrm{U}$ \\
10 & $\mathrm{U}$ \\
10 & $\mathrm{U}$ \\
10 & $\mathrm{U}$ \\
10 & $\mathrm{U}$ \\
10 & $\mathrm{U}$ \\
50 & $\mathrm{U}$ \\
40 & $\mathrm{U}$ \\
10 & $\mathrm{U}$ \\
10 & $\mathrm{U}$ \\
140 & $\mathrm{U}$ \\
10 & $\mathrm{U}$ \\
2.7 & $\mathrm{~J}$ \\
10 & $\mathrm{U}$ \\
10 & $\mathrm{U}$ \\
500 & $\mathrm{U}$ \\
& \\
\hline
\end{tabular}


FORM 1

VOLATILE ORGANICS ANALYSIS DATA SHEET
ARGLAB SAMPLE NO.

IN-S-MEOHBLK
Lab Name: TESTAMERICA BURLINGTON

Lab Code: STLV
Case No.: INMAN

Matrix: (soil/water) soIL

Sample wt/vol:

$10.0(\mathrm{~g} / \mathrm{mL}) \quad \mathrm{G}$

Level: (low/med) MED

\% Moisture: not dec.

GC Column: DB-624 ID: 0.53 (mm)

Soil Extract Volume: 10000 (uL)
Contract: $8 \mathrm{E}-00302$

SAS NO.:

SDG NO.: 134662
Lab Sample ID: 813483

Lab File ID: $\quad 813483$

Date Received: 11/13/09

Date Analyzed: 11/19/09

Dilution Factor: 1.0

Soil Aliquot Volume:

$500(u L)$

CONCENTRATION UNITS:

COMPOUND NO. (ug/L or ug/ $\mathrm{Kg}$ ) UG/KG

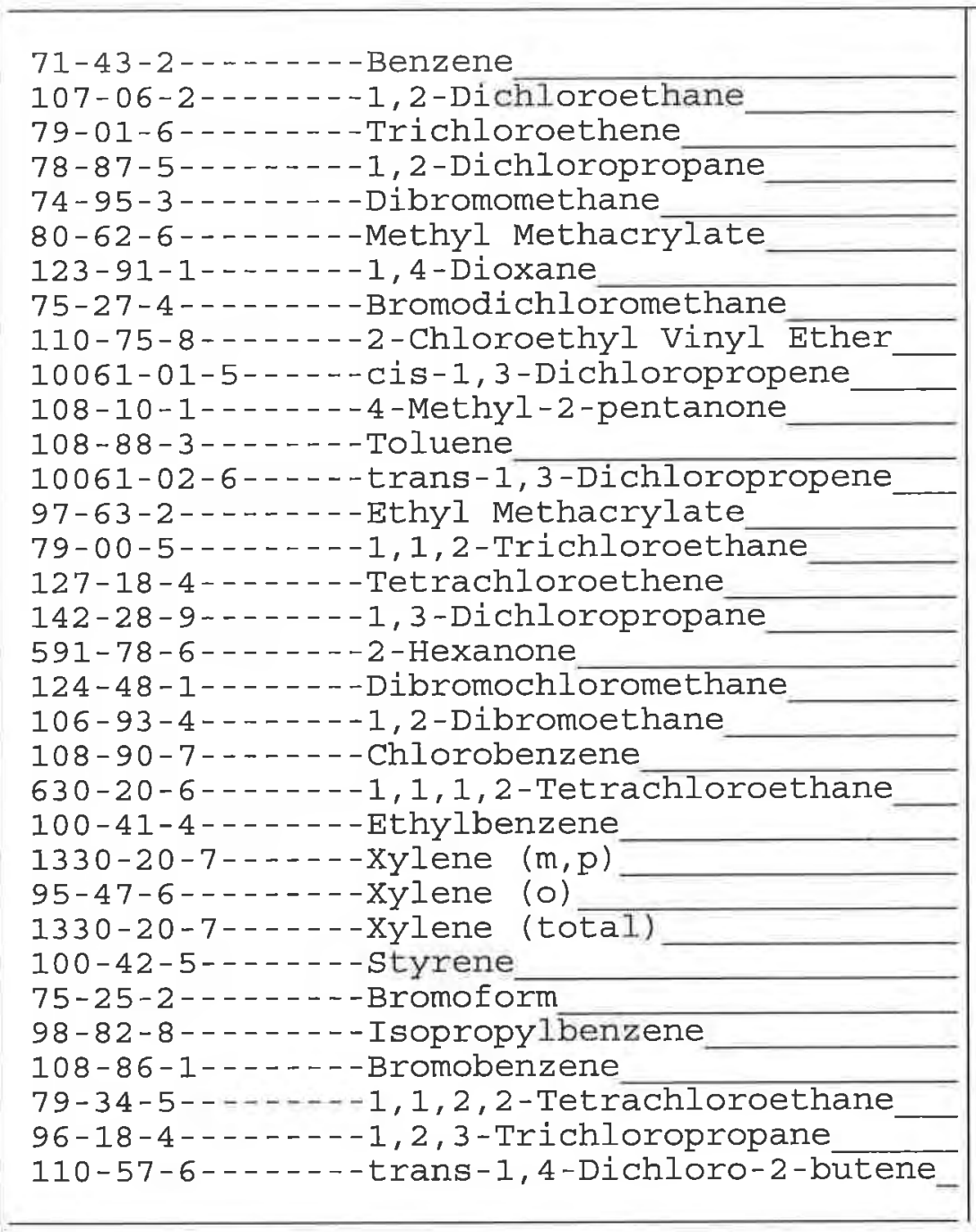

\begin{tabular}{r|l|l|}
10 & $\mathrm{U}$ \\
10 & $\mathrm{U}$ \\
10 & $\mathrm{U}$ \\
10 & $\mathrm{U}$ \\
10 & $\mathrm{U}$ \\
10 & $\mathrm{U}$ \\
500 & $\mathrm{U}$ \\
10 & $\mathrm{U}$ \\
10 & $\mathrm{U}$ \\
10 & $\mathrm{U}$ \\
50 & $\mathrm{U}$ \\
10 & $\mathrm{U}$ \\
10 & $\mathrm{U}$ \\
10 & $\mathrm{U}$ \\
10 & $\mathrm{U}$ \\
10 & $\mathrm{U}$ \\
10 & $\mathrm{U}$ \\
50 & $\mathrm{U}$ \\
10 & $\mathrm{U}$ \\
10 & $\mathrm{U}$ \\
2.6 & $\mathrm{~J}$ \\
10 & $\mathrm{U}$ \\
10 & $\mathrm{U}$ \\
10 & $\mathrm{U}$ \\
10 & $\mathrm{U}$ \\
10 & $\mathrm{U}$ \\
10 & $\mathrm{U}$ \\
10 & $\mathrm{U}$ \\
10 & $\mathrm{U}$ \\
10 & $\mathrm{U}$ \\
10 & $\mathrm{U}$ \\
10 & $\mathrm{U}$ \\
10 & $\mathrm{U}$ \\
& \\
\hline & \\
10 & \\
10 &
\end{tabular}


FORM 1

VOLATILE ORGANICS ANALYSIS DATA SHEET
ARGLAB SAMPLE NO.

IN-S-MEOHBLK
Lab Name: TESTAMERICA BURLINGTON

Lab Code: STLV . Case No.: INMAN

Matrix: (soil/water) soIL

Sample wt/vol:

$10.0(\mathrm{~g} / \mathrm{mL}) \mathrm{G}$

Level: (low/med) MED

\% Moisture: not dec.

GC Column: DB-624 ID: 0.53 (mm)

Soil Extract Volume: 10000 (uL)
Contract: 8E-00302

SAS NO. :

SDG No.: 134662

Lab Sample ID: 813483

Lab File ID: 813483

Date Received: 11/13/09

Date Analyzed: 11/19/09

Dilution Factor: 1.0

Soil Aliquot Volume:

500 (uL)

CAS NO.

COMPOUND

CONCENTRATION UNITS :

( ug/L or ug/Kg) UG/KG Q

\begin{tabular}{|c|}
\hline 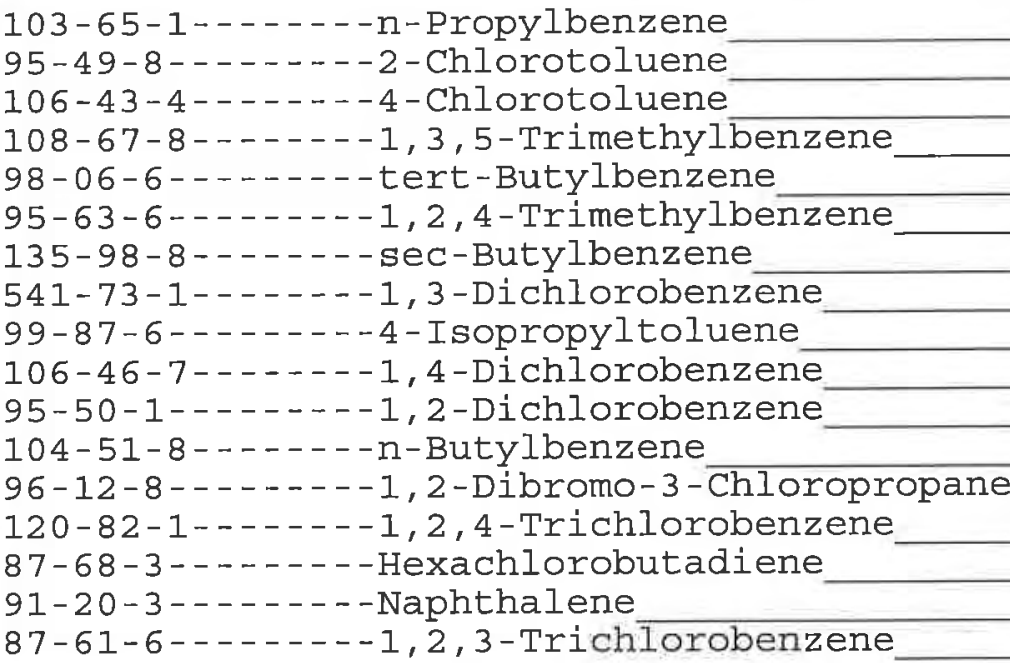 \\
\hline
\end{tabular}

\begin{tabular}{|l|l|l|}
\hline 10 & $\mathrm{U}$ \\
10 & $\mathrm{U}$ \\
10 & $\mathrm{U}$ \\
10 & $\mathrm{U}$ \\
10 & $\mathrm{U}$ \\
10 & $\mathrm{U}$ \\
10 & $\mathrm{U}$ \\
10 & $\mathrm{U}$ \\
10 & $\mathrm{U}$ \\
10 & $\mathrm{U}$ \\
10 & $\mathrm{U}$ \\
10 & $\mathrm{U}$ \\
10 & $\mathrm{U}$ \\
10 & $\mathrm{U}$ \\
10 & $\mathrm{U}$ \\
10 & $\mathrm{U}$ \\
10 & $\mathrm{U}$ \\
\hline
\end{tabular}


FORM 1

VOLATILE ORGANICS ANALYSIS DATA SHEET
CLIENT SAMPLE NO.

MBLK111909NA
Lab Name: TESTAMERICA BURLINGTON

Lab Code: STLV
Case No.: INMAN

Matrix: (soil/water) WATER

Sample wt/vol:

$5.000(\mathrm{~g} / \mathrm{mL}) \mathrm{ML}$

Level: (low/med) LOW

\% Moisture: not dec.

GC Column: DB-624 ID: 0.53 (mm)

Soil Extract Volume:
Contract: 8E-00302

SAS NO.:

SDG No.: 134662
Lab Sample ID: MBLK111909NA

Lab File ID: NXOB03D

Date Received:

Date Analyzed: 11/19/09

Dilution Factor: 1.0

Soil Aliquot Volume: (uL)

CONCENTRATION UNITS :
(ug/L or $u g / \mathrm{Kg})$ UG/I

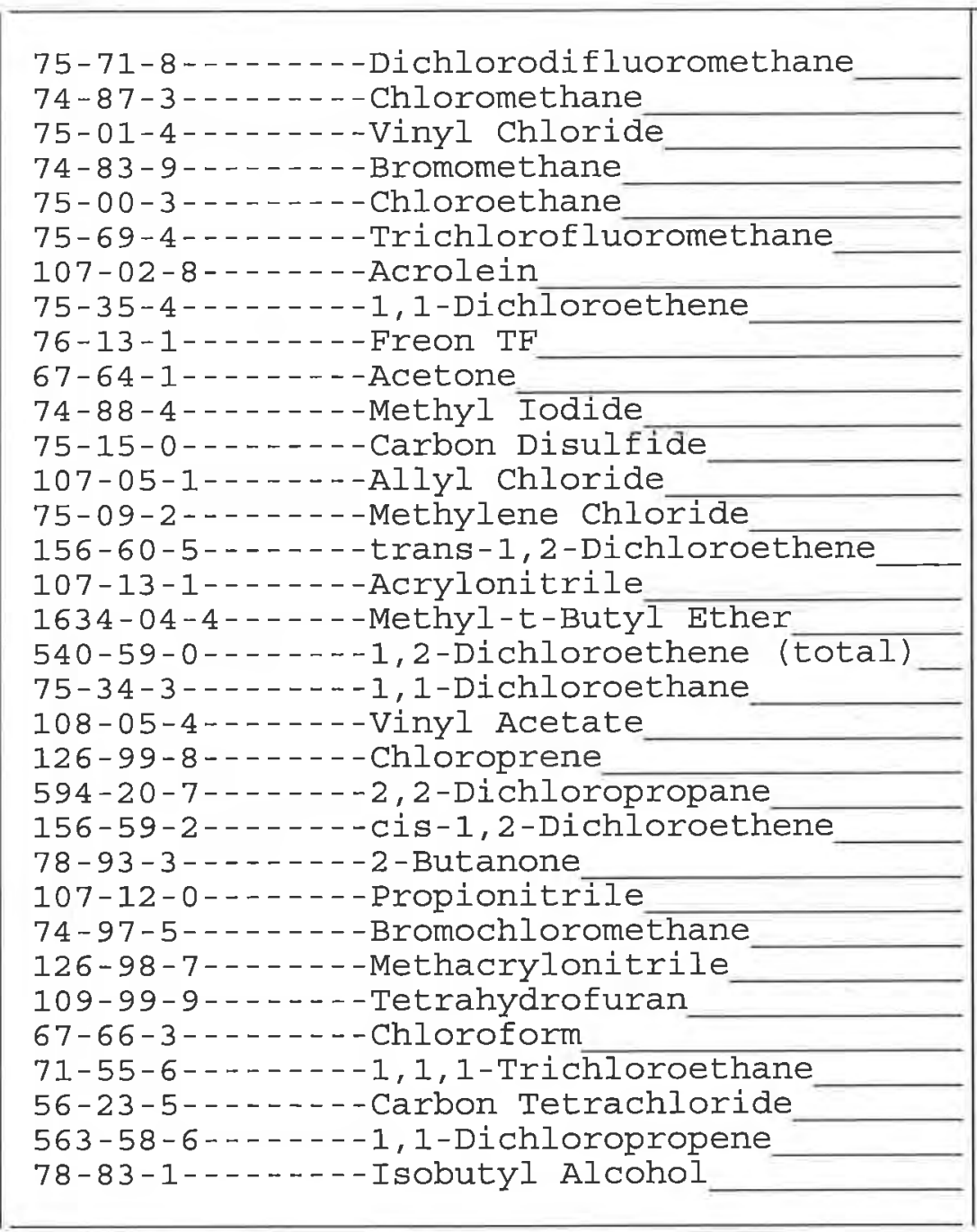

\begin{tabular}{|r|r|l|}
\hline 1.0 & $\mathrm{U}$ \\
1.0 & $\mathrm{U}$ \\
1.0 & $\mathrm{U}$ \\
1.0 & $\mathrm{U}$ \\
1.0 & $\mathrm{U}$ \\
1.0 & $\mathrm{U}$ \\
5.0 & $\mathrm{U}$ \\
1.0 & $\mathrm{U}$ \\
1.0 & $\mathrm{U}$ \\
5.0 & $\mathrm{U}$ \\
1.0 & $\mathrm{U}$ \\
1.0 & $\mathrm{U}$ \\
1.0 & $\mathrm{U}$ \\
1.0 & $\mathrm{U}$ \\
1.0 & $\mathrm{U}$ \\
1.0 & $\mathrm{U}$ \\
1.0 & $\mathrm{U}$ \\
1.0 & $\mathrm{U}$ \\
1.0 & $\mathrm{U}$ \\
1.0 & $\mathrm{U}$ \\
1.0 & $\mathrm{U}$ \\
1.0 & $\mathrm{U}$ \\
1.0 & $\mathrm{U}$ \\
5.0 & $\mathrm{U}$ \\
4.0 & $\mathrm{U}$ \\
1.0 & $\mathrm{U}$ \\
1.0 & $\mathrm{U}$ \\
14 & $\mathrm{U}$ \\
1.0 & $\mathrm{U}$ \\
1.0 & $\mathrm{U}$ \\
1.0 & $\mathrm{U}$ \\
1.0 & $\mathrm{U}$ \\
50 & $\mathrm{U}$ \\
& & \\
\hline & \\
\hline
\end{tabular}


FORM 1

VOLATILE ORGANICS ANALYSIS DATA SHEET
CLIENT SAMPLE NO.

MBLK111909NA
Lab Name: TESTAMERICA BURLINGTON

Lab Code: STLV

Case No.: INMAN

Matrix: (soil/water) WATER

Sample wt/vol:

$5.000(\mathrm{~g} / \mathrm{mL}) \mathrm{ML}$

Level : (low/med) LOW

\% Moisture: not dec.

GC Column: DB-624 ID: 0.53 (mm)

Soil Extract Volume:

(uL)
Contract: 8E-00302

SAS No. :

SDG No.: 134662

Lab Sample ID: MBLK111909NA

Lab File ID: NXOB03D

Date Received:

Date Analyzed: 11/19/09

Dilution Factor: 1.0

Soil Aliquot Volume:

(uL)

(ug/L or ug/Kg) UG/L

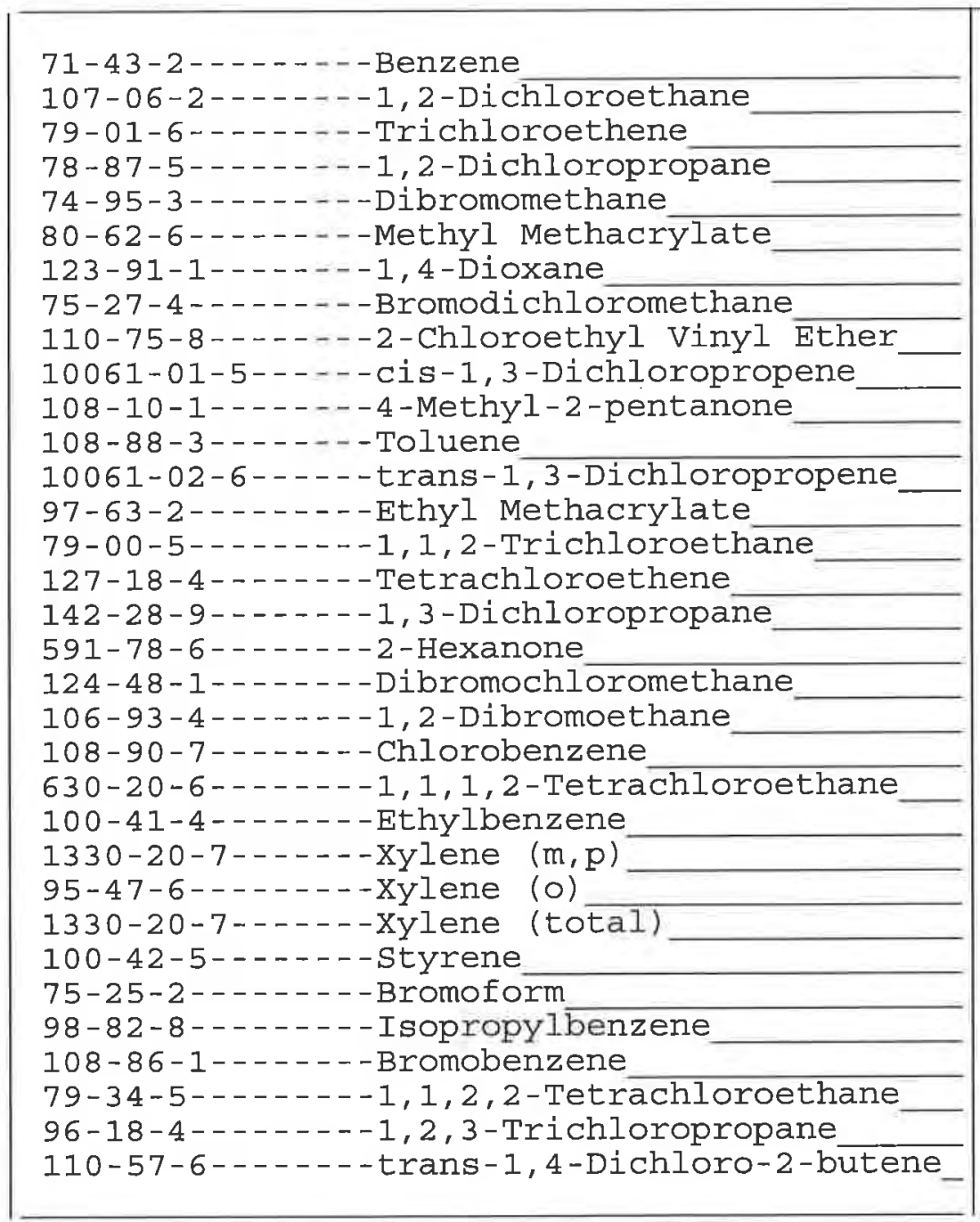

\begin{tabular}{ll|l}
1.1 .0 & $\mathrm{U}$ \\
1.0 & $\mathrm{U}$ \\
1.0 & $\mathrm{U}$ \\
1.0 & $\mathrm{U}$ \\
1.0 & $\mathrm{U}$ \\
1.0 & $\mathrm{U}$ \\
50 & $\mathrm{U}$ \\
1.0 & $\mathrm{U}$ \\
1.0 & $\mathrm{U}$ \\
1.0 & $\mathrm{U}$ \\
5.0 & $\mathrm{U}$ \\
1.0 & $\mathrm{U}$ \\
1.0 & $\mathrm{U}$ \\
1.0 & $\mathrm{U}$ \\
1.0 & $\mathrm{U}$ \\
1.0 & $\mathrm{U}$ \\
1.0 & $\mathrm{U}$ \\
5.0 & $\mathrm{U}$ \\
1.0 & $\mathrm{U}$ \\
1.0 & $\mathrm{U}$ \\
1.0 & $\mathrm{U}$ \\
1.0 & $\mathrm{U}$ \\
1.0 & $\mathrm{U}$ \\
1.0 & $\mathrm{U}$ \\
1.0 & $\mathrm{U}$ \\
1.0 & $\mathrm{U}$ \\
1.0 & $\mathrm{U}$ \\
1.0 & $\mathrm{U}$ \\
1.0 & $\mathrm{U}$ \\
1.0 & $\mathrm{U}$ \\
1.0 & $\mathrm{U}$ \\
1.0 & $\mathrm{U}$ \\
1.0 & $\mathrm{U}$ \\
& \\
1 & \\
1.01
\end{tabular}


FORM 1

VOLATILE ORGANICS ANALYSIS DATA SHEET

Lab Name: TESTAMERICA BURLINGTON

Contract : 8E-00302

Case No.: INMAN SAS No.:

CLIENT SAMPLE NO.

Lab Code: STLV

Matrix: (soil/water) WATER

Sample wt/vol:

$5.000(\mathrm{~g} / \mathrm{mL}) \quad$ ML

Level: (low/med) LOW

\% Moisture: not dec.

GC Column: DB- 624

ID : $0.53 \quad(\mathrm{~mm})$

Soil Extract Volume:

(uL)
MBLK111909NA

SDG NO.: 134662

Lab Sample ID: MBLK111909NA

Lab File ID: NXOB03D

Date Received:

Date Analyzed: 11/19/09

Dilution Factor: 1.0

Soil Aliquot Volume: (UL)

CAS NO. COMPOUND (ug/L or ug/Kg) UG/L

CONCENTRATION UNITS:
$(\mathrm{ug} / \mathrm{L}$ or $\mathrm{ug} / \mathrm{Kg}$ ) UG/L

Q

1. $0 \mathrm{U}$

$1.0 \mathrm{U}$

$1.0 \mathrm{U}$

1. $0 \mathrm{U}$

$1.0 \mathrm{U}$

$1.0 \mathrm{U}$

I. $0 \mathrm{U}$

$1.0 \mathrm{U}$

$1.0 \mathrm{U}$

1. $0 \mathrm{U}$

$1.0 \mathrm{U}$

$1.0 \mathrm{U}$

$1.0 \mathrm{U}$

1. $0 \mathrm{U}$

1. $0 \mathrm{U}$

$1.0 \mathrm{U}$

1. $0 \mathrm{U}$ 
FORM 1

VOLATILE ORGANICS ANALYSIS DATA SHEET
CLIENT SAMPLE NO.

MBLK111909ME
Lab Name: TESTAMERICA BURLINGTON

Lab Code: STLV
Case No.: INMAN

Matrix: (soil/water) soIL

Sample wt/vol:

$10.0(\mathrm{~g} / \mathrm{mL}) \mathrm{G}$

Level: (low/med) MED

\% Moisture: not dec.

GC Column: DB-624 ID: $0.53 \quad(\mathrm{~mm})$

Soil Extract Volume: 10000 (uL)
Contract: 8E-00302

SAS NO. :

SDG No.: 134662

Lab Sample ID: MBIıK111909ME

Lab File ID: NXOB04D

Date Received:

Date Analyzed: 11/19/09

Dilution Factor: 1.0

Soil Aliquot Volume:

500 (uL)
CAS NO.
COMPOUND
( $\mathrm{ug} / \mathrm{L}$ or $\mathrm{ug} / \mathrm{Kg}$ ) UG/KG
Q

CONCENTRATION UNITS:

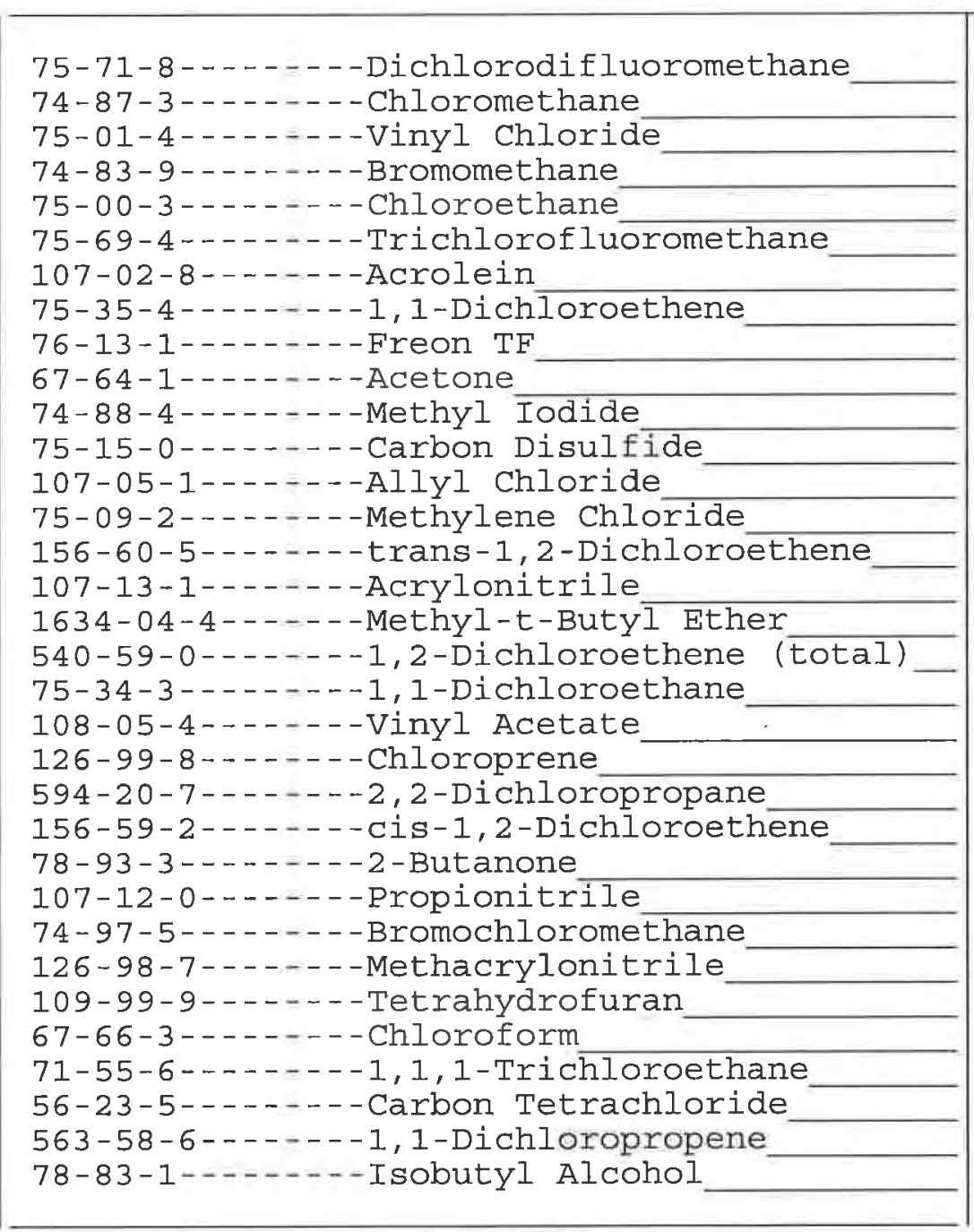

\begin{tabular}{|r|r|}
\hline 10 & $\mathrm{U}$ \\
10 & $\mathrm{U}$ \\
10 & $\mathrm{U}$ \\
10 & $\mathrm{U}$ \\
10 & $\mathrm{U}$ \\
10 & $\mathrm{U}$ \\
50 & $\mathrm{U}$ \\
10 & $\mathrm{U}$ \\
10 & $\mathrm{U}$ \\
50 & $\mathrm{U}$ \\
10 & $\mathrm{U}$ \\
10 & $\mathrm{U}$ \\
10 & $\mathrm{U}$ \\
10 & $\mathrm{U}$ \\
10 & $\mathrm{U}$ \\
10 & $\mathrm{U}$ \\
10 & $\mathrm{U}$ \\
10 & $\mathrm{U}$ \\
10 & $\mathrm{U}$ \\
10 & $\mathrm{U}$ \\
10 & $\mathrm{U}$ \\
10 & $\mathrm{U}$ \\
10 & $\mathrm{U}$ \\
50 & $\mathrm{U}$ \\
40 & $\mathrm{U}$ \\
10 & $\mathrm{U}$ \\
10 & $\mathrm{U}$ \\
140 & $\mathrm{U}$ \\
10 & $\mathrm{U}$ \\
10 & $\mathrm{U}$ \\
10 & $\mathrm{U}$ \\
10 & $\mathrm{U}$ \\
500 & $\mathrm{U}$ \\
& \\
\hline &
\end{tabular}


FORM 1

VOLATILE ORGANICS ANALYSIS DATA SHEET
CLIENT SAMPLE NO.

MBLK111909ME
Lab Name: TESTAMERICA BURLINGTON

Lab Code: STLV
Case No.: INMAN

Matrix: (soil/water) solL

Sample wt/vol:

Level: (low/med) MED

\% Moisture: not dec.

GC Column: DB-624 ID: 0.53 (mm)

Soil Extract Volume: 10000 (uL)
Contract: $8 \mathrm{E}-00302$

SAS NO.:

SDG No.: 134662

Lab Sample ID: MBLK111909ME

Lab File ID: NXOB04D

Date Received:

Date Analyzed: 11/19/09

Dilution Factor: 1.0

Soil Aliquot Volume:

500 (uL)

CAS NO.

COMPOUND

CONCENTRATION UNITS :

(ug/L or ug/Kg) UG/KG

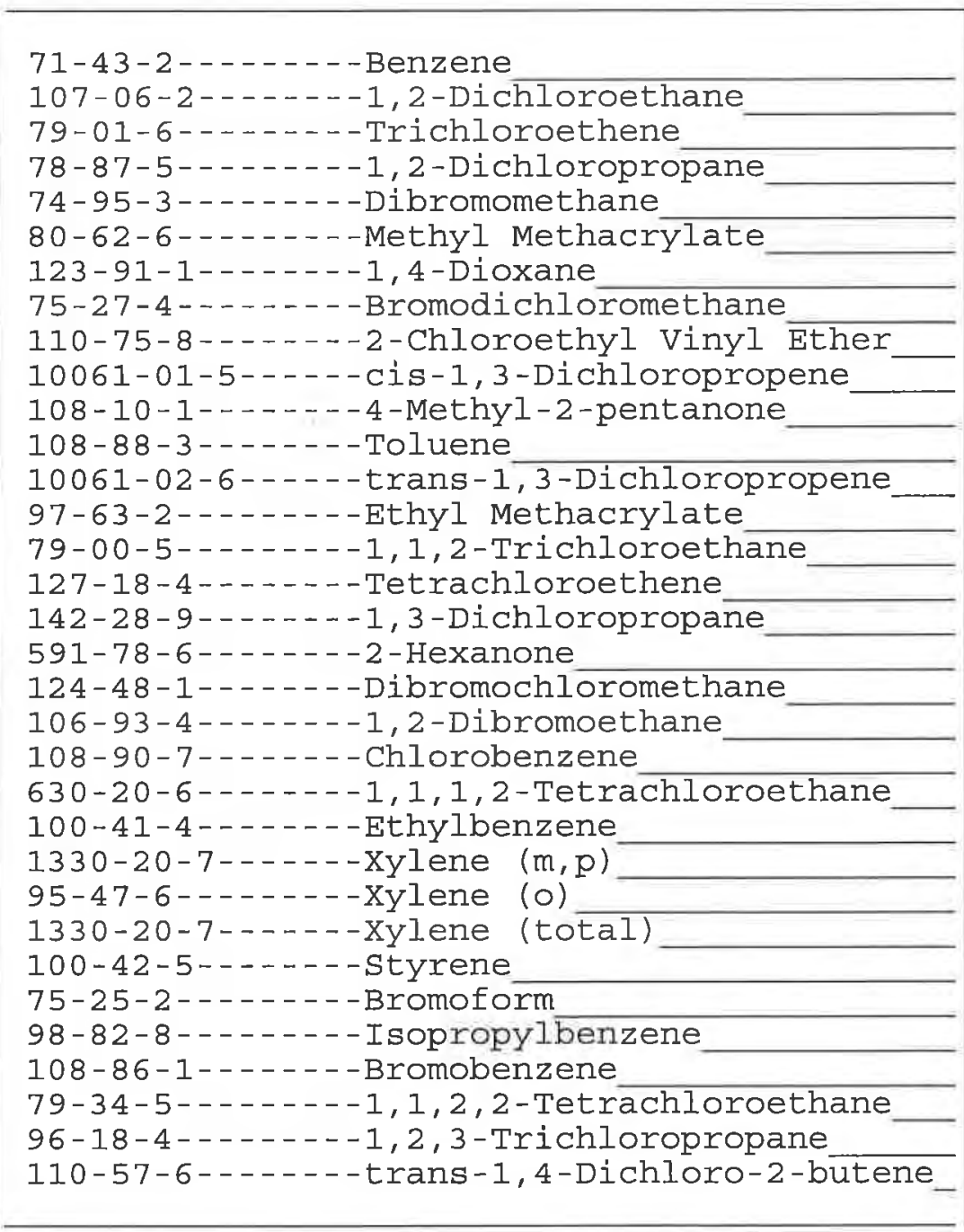

\begin{tabular}{|r|r|l|}
10 & $\mathrm{U}$ \\
10 & $\mathrm{U}$ \\
10 & $\mathrm{U}$ \\
10 & $\mathrm{U}$ \\
10 & $\mathrm{U}$ \\
10 & $\mathrm{U}$ \\
500 & $\mathrm{U}$ \\
10 & $\mathrm{U}$ \\
10 & $\mathrm{U}$ \\
10 & $\mathrm{U}$ \\
50 & $\mathrm{U}$ \\
10 & $\mathrm{U}$ \\
10 & $\mathrm{U}$ \\
10 & $\mathrm{U}$ \\
10 & $\mathrm{U}$ \\
10 & $\mathrm{U}$ \\
10 & $\mathrm{U}$ \\
50 & $\mathrm{U}$ \\
10 & $\mathrm{U}$ \\
10 & $\mathrm{U}$ \\
10 & $\mathrm{U}$ \\
10 & $\mathrm{U}$ \\
10 & $\mathrm{U}$ \\
10 & $\mathrm{U}$ \\
10 & $\mathrm{U}$ \\
10 & $\mathrm{U}$ \\
10 & $\mathrm{U}$ \\
10 & $\mathrm{U}$ \\
10 & $\mathrm{U}$ \\
10 & $\mathrm{U}$ \\
10 & $\mathrm{U}$ \\
10 & $\mathrm{U}$ \\
10 & $\mathrm{U}$ \\
& & \\
\hline &
\end{tabular}


FORM 1

VOLATILE ORGANICS ANALYSIS DATA SHEET
CLIENT SAMPLE NO.

MBLKI11909ME
Lab Name: TESTAMERICA BURLINGTON

Lab Code: STLV

Case No.: INMAN

Matrix: (soil/water) soIL

Sample wt/vol:

$10.0(\mathrm{~g} / \mathrm{mL}) \quad \mathrm{G}$

Level : (low/med) MED

\% Moisture: not dec.

GC Column: DB-624 ID: 0.53 (mm)

Soil Extract Volume: 10000 (uL)
Contract: 8E-00302

SAS NO.:

SDG No.: 134662

Lab Sample ID: MBLK111909ME

Lab File ID: NXOB04D

Date Received:

Date Analyzed: 11/19/09

Dilution Factor: 1.0

Soil Aliquot Volume:

500 (uL)

CAS NO.

COMPOUND

CONCENTRATION UNITS :

( $\mathrm{ug} / \mathrm{L}$ or $\mathrm{ug} / \mathrm{Kg}$ ) UG/KG

Q

103-65-1--.---n-Propylbenzene

95-49-8--1---2-Chlorotoluene

106-43-4-----4-Chlorotoluene

108-67-8------1,3,5-Trimethyl benzene

98-06-6-------tert-Butylbenzene

95-63-6-- - - - 1,2,4-Trimethylbenzene

135-98-8------sec-Butylbenzene

541-73-1----1, 3-Dichlorobenzene

99-87-6--...-4-4sopropyltoluene

106-46-7-----1, 4-Dichlorobenzene

95-50-1-----1,2-Dichlorobenzene

104-51-8------n-Butylbenzene

96-12-8--- - - - 1, 2-Dibromo-3-Chloropropane_

120-82-1------1,2,4-Trichlorobenzene

87-68-3--..--Hexachlorobutadiene

91-20-3.....--Naphthalene

87-61-6-...-.1,2,3-Trichlorobenzene

\begin{tabular}{|l|l|l|} 
& \\
10 & $\mathrm{U}$ \\
10 & $\mathrm{U}$ \\
10 & $\mathrm{U}$ \\
10 & $\mathrm{U}$ \\
10 & $\mathrm{U}$ \\
10 & $\mathrm{U}$ \\
10 & $\mathrm{U}$ \\
10 & $\mathrm{U}$ \\
10 & $\mathrm{U}$ \\
10 & $\mathrm{U}$ \\
10 & $\mathrm{U}$ \\
10 & $\mathrm{U}$ \\
10 & $\mathrm{U}$ \\
10 & $\mathrm{U}$ \\
10 & $\mathrm{U}$ \\
10 & $\mathrm{U}$ \\
10 & $\mathrm{U}$ \\
\hline
\end{tabular}


FORM 1

VOLATILE ORGANICS ANALYSIS DATA SHEET
CLIENT SAMPLE NO.

NA111909LCS
Lab Name: TESTAMERICA BURLINGTON

Lab Code: STLV
Case No.: INMAN

Matrix: (soil/water) WATER

Sample wt/vol:

Level: (low/med) Low

$\%$ Moisture: not dec.

GC Column: DB-624 ID: 0.53 (mm)

Soil Extract Volume:
Contract: $8 \mathrm{E}-00302$

SAS NO. :

SDG No.: 134662

Lab Sample ID: NA111909LCS

Lab File ID: NXO10CQ

Date Received:

Date Analyzed: 11/19/09

Dilution Factor: 1.0

Soil Aliquot Volume: (uL)
CAS NO.
COMPOUND
(ug/L or $\mathrm{ug} / \mathrm{Kg}) \mathrm{UG} / \mathrm{L}$
Q

CONCENTRATION UNITS:

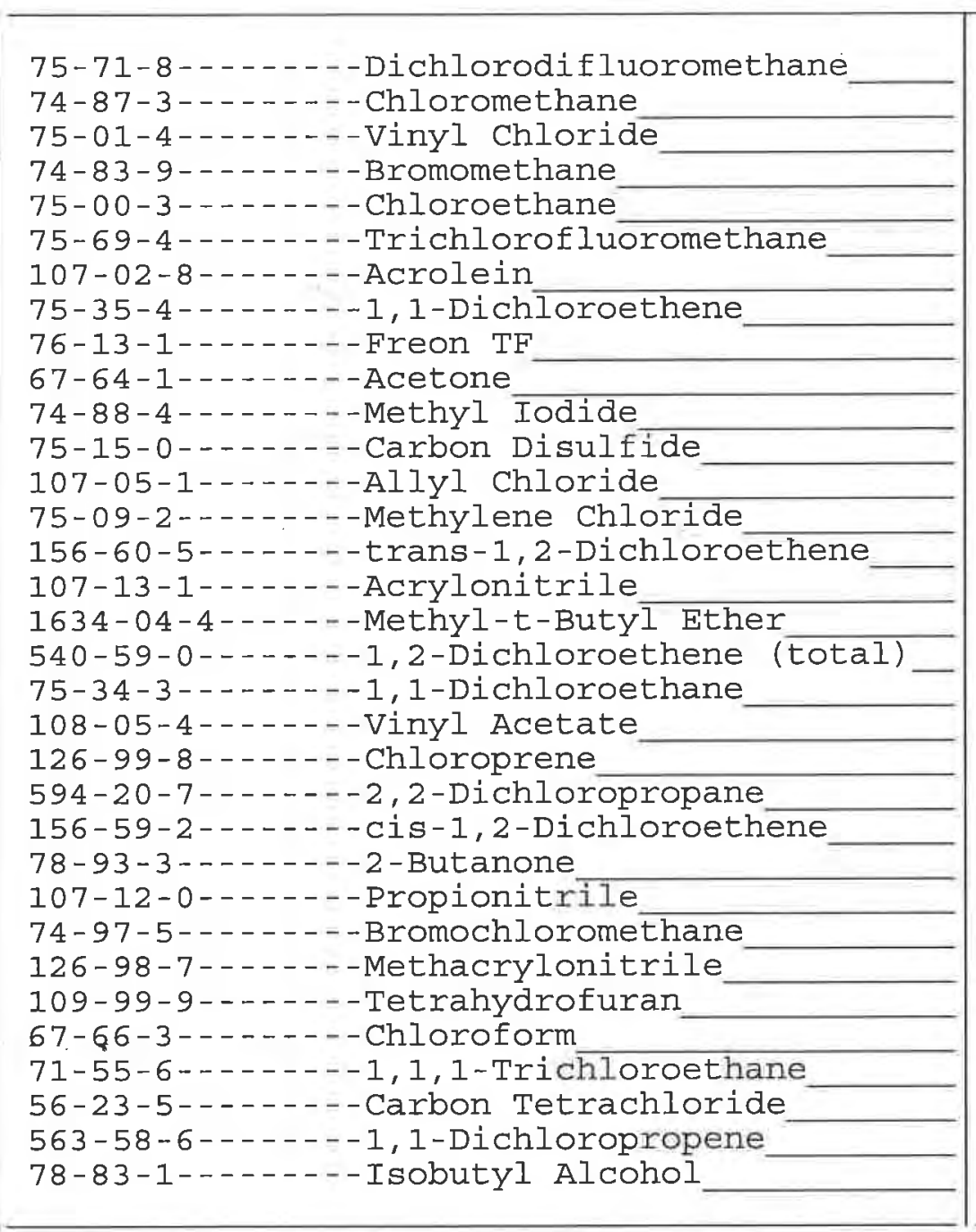

\begin{tabular}{r|l}
9.4 \\
8.9 \\
9.6 \\
9.7 \\
9.9 \\
9.4 \\
42 \\
9.9 \\
9.3 \\
40 \\
11 \\
9.3 \\
8.9 \\
9.8 \\
9.8 \\
9.8 \\
9.3 \\
19 \\
9.5 \\
\hline .8 \\
7.9 \\
9.4 \\
9.4 \\
47 \\
40 \\
\hline \\
\hline \\
\hline \\
130 \\
9.6 \\
9.4 \\
9.4 \\
9.6 \\
460 \\
\end{tabular}


FORM 1

VOLATILE ORGANICS ANALYSIS DATA SHEET
CLIENT SAMPLE NO.

NA111909LCS
Lab Name: TESTAMERICA BURLINGTON

Lab Code: STLV

Case No.: INMAN

Matrix: (soil/water) WATER

Sample wt/vol:

$5.000(\mathrm{~g} / \mathrm{mL}) \mathrm{ML}$

Level: (low/med) LOW

\% Moisture: not dec.

GC Column: DB-624 ID: 0.53 (mm)

Soil Extract Volume:
(uL)
Contract: 8E-00302

SAS NO. :

SDG No.: 134662
Lab Sample ID: NA111909LCS

Lab File ID: NXO10CQ

Date Received:

Date Analyzed: 11/19/09

Dilution Factor: 1.0
CAS NO.
COMPOUND
Soil Aliquot Volume: CONCENTRATION UNITS : (ug/L or $\mathrm{ug} / \mathrm{Kg}) \mathrm{UG} / \mathrm{L}$ $71-43-2-\ldots-\cdots-$ Benzene

107-06-2--.---1,2-Dichloroethane

79-01-6-...-. - Trichloroethene

78-87-5-- - - - 1, 2-Dichloropropane

74-95-3-------Dibromomethane

80-62-6-...---Methyl Methacrylate

123-91-1--.---1, 4-Dioxane

75-27-4-.-----Bromodichloromethane

110-75-8------2-Chloroethyl Vinyl Ether

10061-01-5-----cis-1,3-Dichloropropene

108-10-1-------4-Methyl-2-pentanone

108-88-3--.---Toluene

10061-02-6-----trans-1,3-Dichloropropene

97-63-2-------Ethyl Methacrylate

79-00-5-...--1, 1, 2-Trichloroethane

127-18-4--.--- Tetrachloroethene

142-28-9--.---1,3-Dichloropropane

591-78-6------ - 2-Hexanone

124-48-1----n-Dibromochloromethane

106-93-4-...--1, 2-Dibromoethane

108-90-7-...-- Chlorobenzene

630-20-6---.-1, 1, 1, 2-Tetrachloroethane

100-41-4------- Ethylbenzene

$1330-20-7-\ldots-\ldots y l$ ene $(m, p)$

95-47-6--.-.--xylene (o)

1330-20-7-----xylene (total)

100-42-5--.----styrene

75-25-2-- - - - - Bromoform

98-82-8--------Isopropyl benzene

108-86-1--.----Bromobenzene

79-34-5-- - - - 1, 1,2,2-Tetrachloroethane

96-18-4--- - - - 1, 2, 3-Trichloropropane

110-57-6------trans-1,4-Dichloro-2-butene

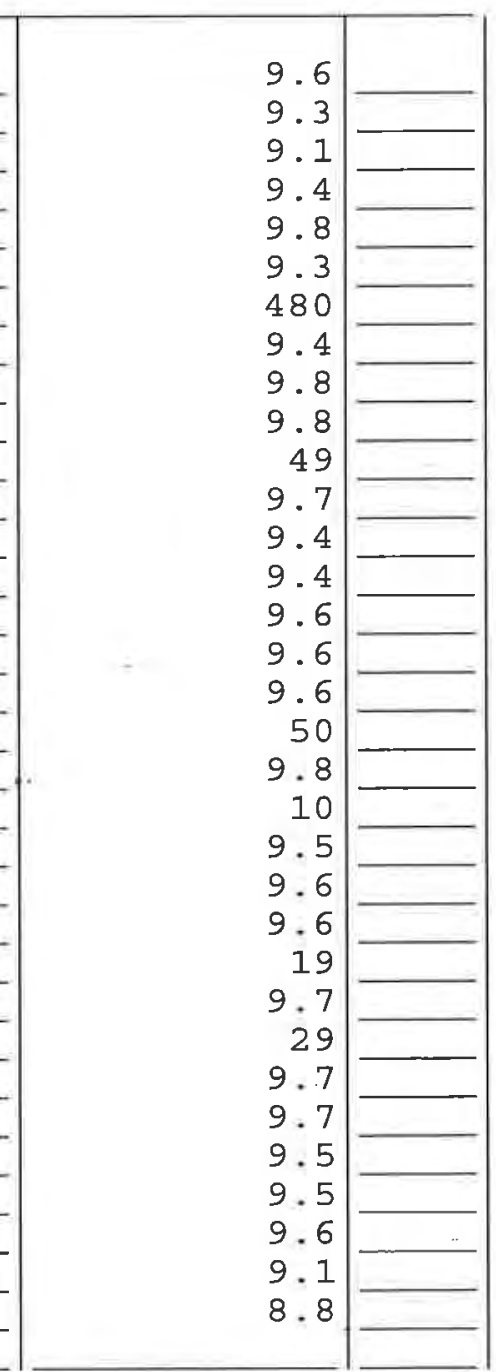


FORM 1

VOLATILE ORGANICS ANALYSIS DATA SHEET
CLIENT SAMPLE NO.

NA111909LCS
Lab Name: TESTAMERICA BURLINGTON

Lab Code: STLV

Case No.: INMAN

Matrix: (soil/water) WATER

sample wt/vol:

$5.000(\mathrm{~g} / \mathrm{mL}) \mathrm{ML}$

Level: (low/med) LOW

\% Moisture: not dec.

GC Column: DB-624 ID: 0.53 (mm)

Soil Extract Volume: (uL)
Contract: 8E-00302

SAS NO.:

SDG No.: 134662

Lab Sample ID: NA111909LCS

Lab File ID: NXO10CQ

Date Received:

Date Analyzed: 11/19/09

Dilution Factor: 1.0

Soil Aliquot Volume: (uL)

$(\mathrm{ug} / \mathrm{L}$ or $\mathrm{ug} / \mathrm{Kg}) \mathrm{UG} / \mathrm{L}$

Q

103-65-1--..--n-Propylbenzene

95-49-8-...--2-Chlorotoluene

106-43-4--.---4-Chlorotoluene

108-67-8------1, 3,5-Trimethylbenzene

98-06-6-------tert-Butylbenzene

95-63-6-- - ---1,2,4-Trimethylbenzene

135-98-8------sec-Butylbenzene

541-73-1--.--1, 3-Dichlorobenzene

99-87-6--.---4-Isopropyltoluene

106-46-7------1,4-Dichlorobenzene

95-50-1-...--1,2-Dichlorobenzene

104-51-8--.---n-Butylbenzene

96-12-8-------1,2-Dibromo-3-Chloropropane

120-82-1------1,2,4-Trichlorobenzene

87-68-3-...-.-Hexachlorobutadiene

91-20-3-...---Naphthalene

87-61-6--.---1,2,3-Trichlorobenzene

\begin{tabular}{|r|}
9.6 \\
9.9 \\
9.7 \\
9.3 \\
9.5 \\
9.6 \\
9.5 \\
$\overline{ }$ \\
$-\square$ \\
9.3 \\
9.4 \\
9.6 \\
9.3 \\
9.4 \\
9.3 \\
9.5 \\
9.8 \\
9.5 \\
\end{tabular}


FORM 1

VOLATILE ORGANICS ANALYSIS DATA SHEET
CLIENT SAMPLE NO.

ME111909LCS

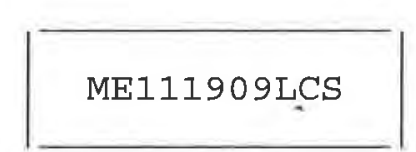

SDG No.: 134662

Lab Code: STLV Case No.: INMAN SAS No.:

Lab Sample ID: ME111909LCS

Matrix: (soil/water) SOIL

Lab File ID: NXO10DQ3

Sample wt/vol:

$10.0(\mathrm{~g} / \mathrm{mL}) \mathrm{G}$

Level: (low/med) MED

Date Received:

\% Moisture: not dec.

GC Column: DB-624 ID: 0.53 (mm)

Date Analyzed: 11/19/09

Soil Extract Volume: 10000 (uL)

Dilution Factor: 1.0

Soil Aliquot Volume:

$500(u L)$

CONCENTRATION UNITS :

CAS NO. COMPOUND (ug/L or $\mathrm{ug} / \mathrm{Kg}) \quad \mathrm{UG} / \mathrm{KG}$

G Q

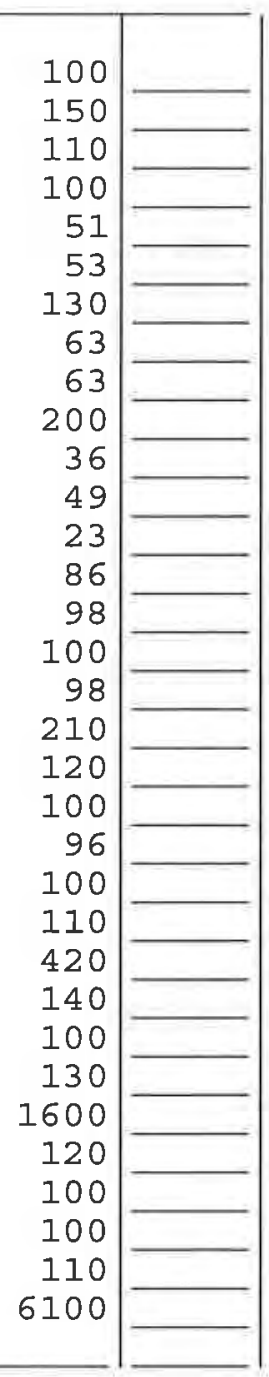


FORM 1

VOLATILE ORGANICS ANALYSIS DATA SHEET
CLIENT SAMPLE NO.

ME111909LCS
Lab Name: TESTAMERICA BURLINGTON

Lab Code: STLV
Case No.: INMAN

Matrix: (soil/water) SOIL

Sample wt/vol:

$10.0(\mathrm{~g} / \mathrm{mL}) \mathrm{G}$

Level: (low/med) MED

$\div$ Moisture: not dec.

GC Column: DB-624 ID: 0.53 (mm)

Soil Extract Volume: 10000 (uL)
Contract: 8E-00302

SAS No.:

SDG NO.: 134662

Lab Sample ID: ME111909LCS

Lab File ID: NXO10DQ3

Date Received:

Date Analyzed: 11/19/09

Dilution Factor: 1.0

Soil Aliquot Volume:

500 (uL)
CAS NO.
COMPOUND
(ug/L or $\mathrm{ug} / \mathrm{Kg}) \mathrm{UG} / \mathrm{KG}$

CONCENTRATION UNITS:

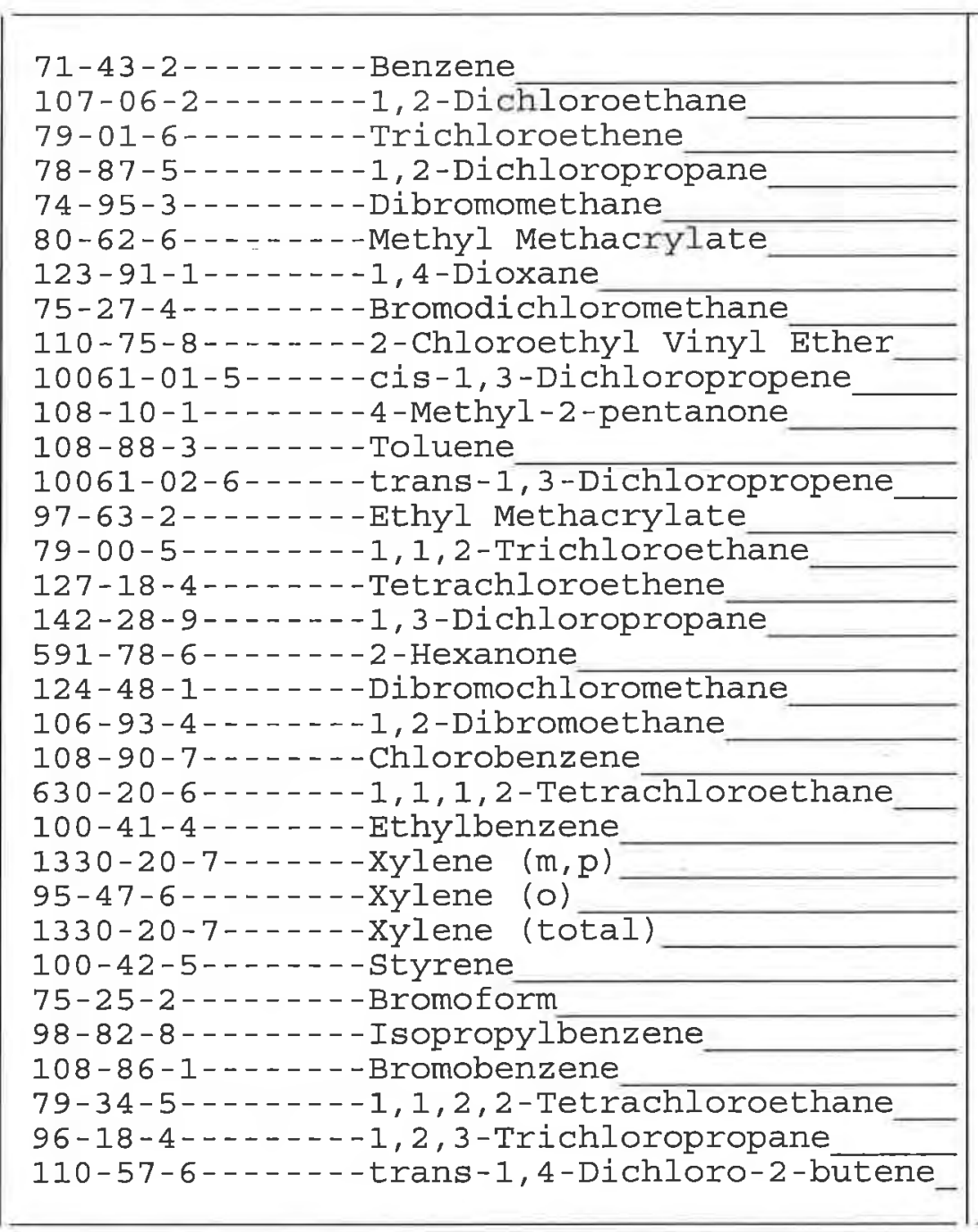

\begin{tabular}{rr|l|}
110 \\
110 \\
100 \\
110 \\
110 \\
88 & $\square$ \\
6500 \\
110 \\
110 \\
110 \\
480 \\
100 \\
94 \\
97 \\
94 \\
97 \\
96 \\
390 \\
95 \\
95 \\
100 \\
98 \\
100 \\
210 \\
100 \\
310 \\
100 \\
84 \\
100 \\
100 \\
84 \\
79 \\
73 \\
\\
\end{tabular}


FORM 1

VOLATILE ORGANICS ANALYSIS DATA SHEET
CLIENT SAMPLE NO.

ME111909LCS
Lab Name: TESTAMERICA BURLINGTON

Lab Code: STLV

Case No.: INMAN

Matrix: (soil/water) SOIL

Sample wt/vol:

$10.0(\mathrm{~g} / \mathrm{mL}) \mathrm{G}$

Level: (low/med) MED

$\%$ Moisture: not dec.

GC Column: DB-624

ID : $0.53 \quad(\mathrm{~mm})$

Soil Extract Volume: 10000 (uL)
Contract: 8E-00302

SAS NO.:

SDG No.: 134662

Lab Sample ID: ME111909LCS

Lab File ID: NXO10DQ3

Date Received:

Date Analyzed: 11/19/09

Dilution Factor: 1.0

Soil Aliquot Volume:

$500(u L)$

CAS NO.

COMPOUND

CONCENTRATION UNITS :

( $\mathrm{ug} / \mathrm{L}$ or $\mathrm{ug} / \mathrm{Kg}$ ) UG/KG

Q

103-65-1--.---n-Propylbenzene

95-49-8-------2-Chlorotoluene

106-43-4------4-Chlorotoluene

108-67-8------1,3,5-Trimethylbenzene

98-06-6-------tert-Butylbenzene

95-63-6-...--1,2,4-Trimethylbenzene

135-98-8-...--sec-Butylbenzene

541-73-1--.--1, 3-Dichlorobenzene

99-87-6------4-Isopropyltoluene

106-46-7-----1,4-Dichlorobenzene

95-50-1------1,2-Dichlorobenzene

104-51-8------n-Butylbenzene

96-12-8-- - - - 1, 2-Dibromo-3-Chloropropane

120-82-1-...-1, 2,4-Trichlorobenzene

87-68-3--1----Hexachlorobutadiene

91-20-3-..---Naphthalene

87-61-6-..---1, 2,3-Trichlorobenzene

\begin{tabular}{rr|l}
100 & \\
100 & $\square$ \\
100 & $\square$ \\
100 & $\square$ \\
100 & $\square$ \\
110 & $\square$ \\
100 \\
100 \\
100 \\
96 \\
110 \\
78 \\
99 \\
110 \\
75 \\
95 \\
\end{tabular}


FORM 2

SOIL VOLATILE SYSTEM MONITORING COMPOUND RECOVERY

Lab Name: TESTAMERICA BURLINGTON

Lab Code: STLV

Case No.: INMAN
Contract: $8 \mathrm{E}-00302$

SAS NO.:
SDG No.: 134662

Level: (low/med) MED

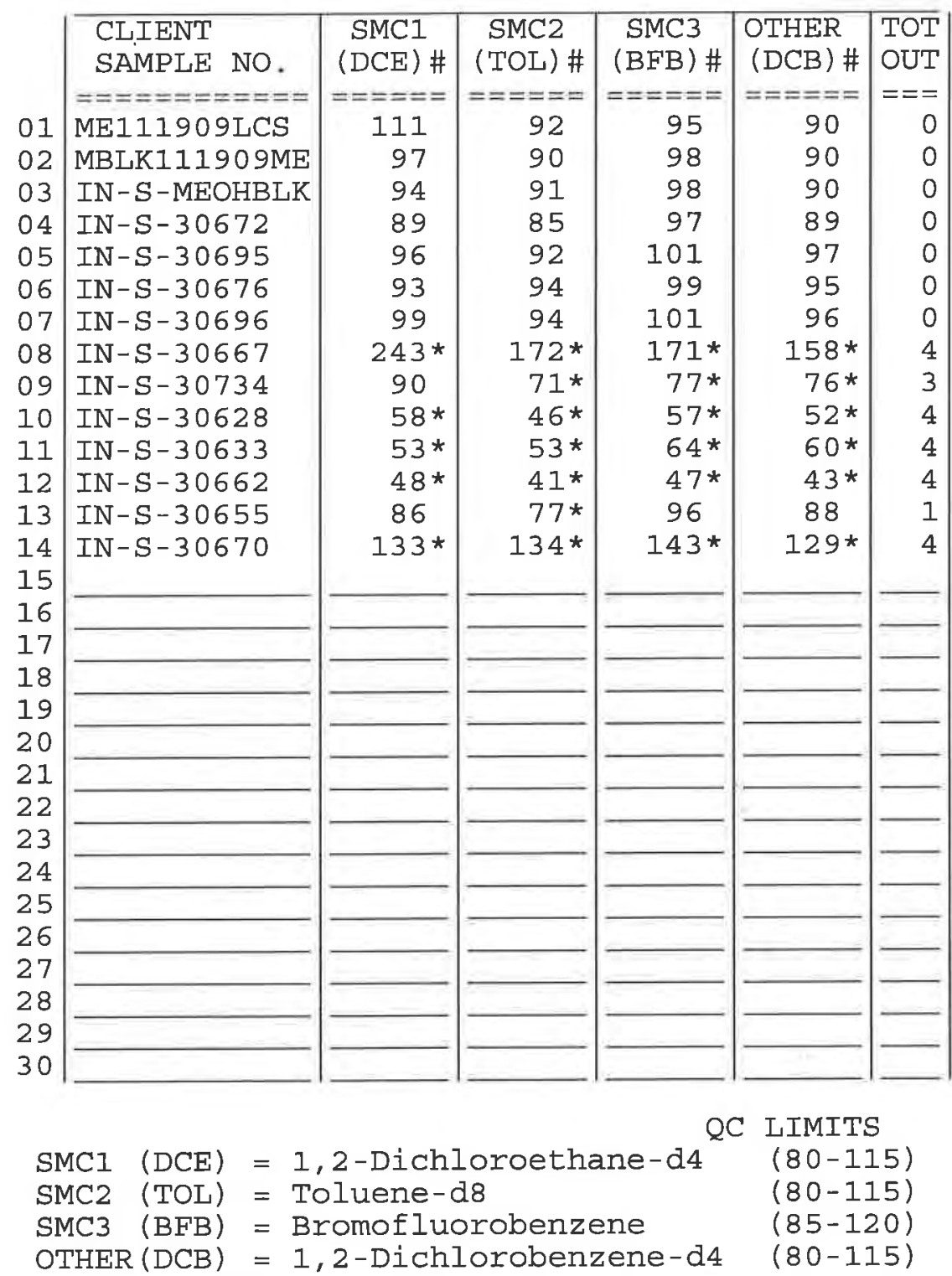

\# Column to be used to flag recovery values

* Values outside of contract required QC limits

D System Monitoring Compound diluted out 
FORM 2

WATER VOLATILE SYSTEM MONITORING COMPOUND RECOVERY

Lab Name: TESTAMERICA BURLINGTON

Lab Code: STLV
Case No.: INMAN

Contract: $8 \mathrm{E}-00302$

SAS No. :

SDG No.: 134662

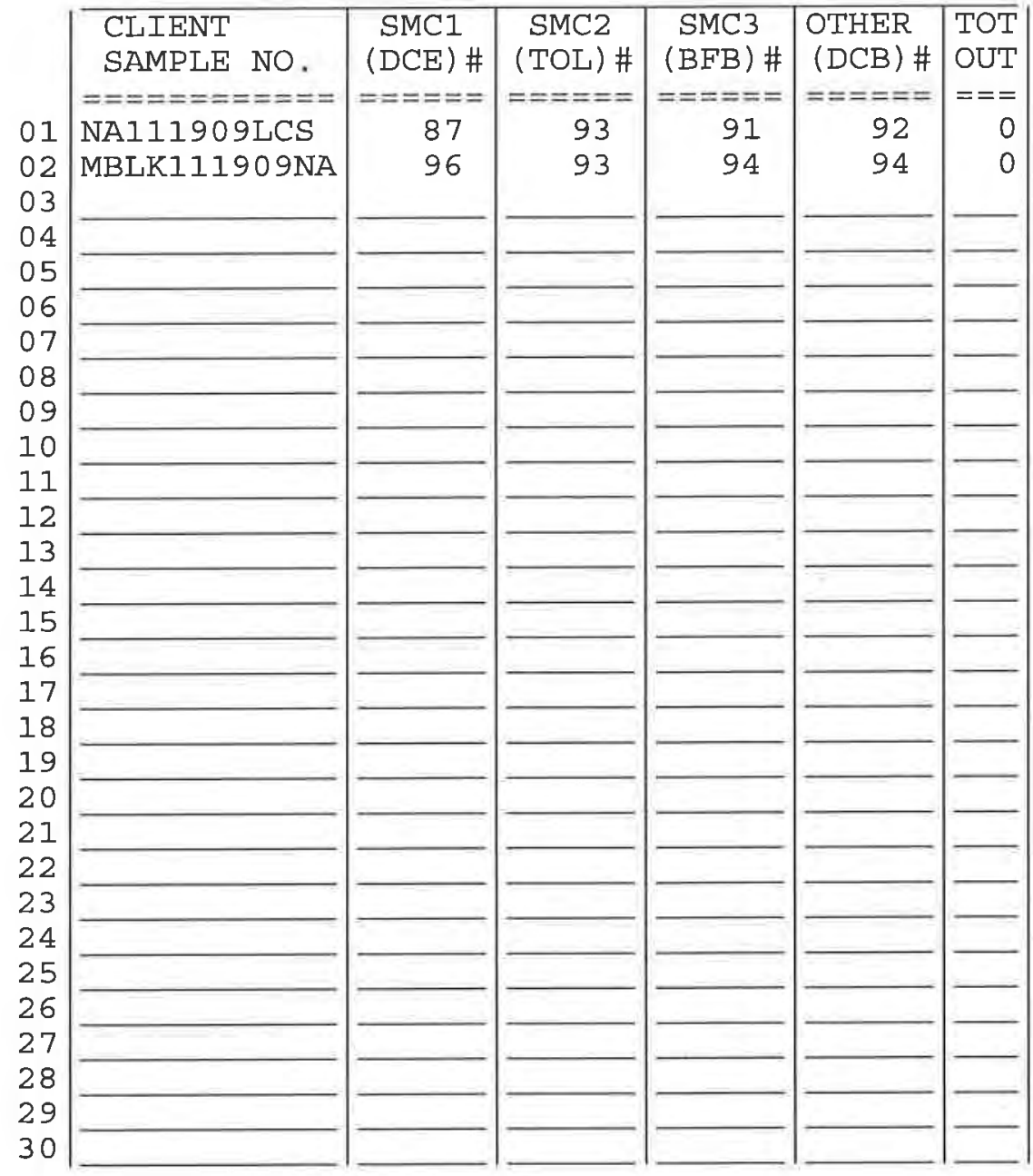

QC LIMITS

SMC1 $(D C E)=1,2-D i c h l o r o e t h a n e-d 4 \quad(80-115)$

SMC2 $($ TOL $)=$ Toluene-d8 $(80-115)$

SMC3 $(\mathrm{BFB})=$ Bromofluorobenzene $(85-120)$

OTHER $(\mathrm{DCB})=1,2-\mathrm{Dichlorobenzene-d4}(80-115)$

\# Column to be used to flag recovery values

* Values outside of contract required QC limits

D System Monitoring Compound diluted out 
FORM 3

SOIL VOLATILE LAB CONTROL SAMPLE

Lab Name: TESTAMERICA BURLINGTON Contract: 8E-00302

Lab Code: STLV Case No.: INMAN SAS No.: $\quad$ SDG No.: 134662

Matrix Spike - Sample No.: ME111909LCS Level:(low/med) MED

\begin{tabular}{|c|c|c|c|c|c|}
\hline COMPOUND & $\begin{array}{l}\text { SPIKE } \\
\text { ADDED } \\
\text { (ug/Kg) }\end{array}$ & $\begin{array}{c}\text { SAMPLE } \\
\text { CONCENTRATION } \\
\text { (ug/Kg) }\end{array}$ & $\begin{array}{c}\text { LCS } \\
\text { CONCENTRATION } \\
(\mathrm{ug} / \mathrm{Kg})\end{array}$ & $\begin{array}{l}\text { LCS } \\
\text { \% } \\
\text { REC \# }\end{array}$ & $\begin{array}{l}\text { QC. } \\
\text { LIMITS } \\
\text { REC. }\end{array}$ \\
\hline 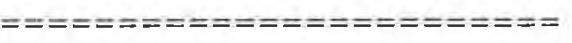 & $=====e==0$ & 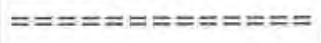 & 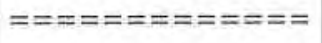 & $======$ & $======$ \\
\hline Dichlorodifluoromethane & 100 & & 100 & 100 & $50-160$ \\
\hline Chloromethane & 100 & & 150 & $150 *$ & $55-140$ \\
\hline Vinyl Chloride & 100 & & 110 & 110 & $75-135$ \\
\hline Bromomethane & 100 & & 100 & 100 & $70-130$ \\
\hline Chloroethane & 100 & & 51 & $51 *$ & $70-135$ \\
\hline Trichlorofluoromethane & 100 & & 53 & 53 * & $80-115$ \\
\hline Acrolein & 500 & & 130 & 26 * & $35-170$ \\
\hline 1,1-Dichloroethene & 100 & & 63 & 63 * & $80-120$ \\
\hline Freon $\mathrm{TF}$ & 100 & & 63 & 63 * & $80-120$ \\
\hline Acetone & 500 & & 200 & 40 & $40-175$ \\
\hline Methyl Iodide & 100 & & 36 & 36 * & $60-140$ \\
\hline Carbon Disulfide & 100 & & 49 & 49 * & $80-125$ \\
\hline Allyl Chloride & 100 & & 23 & 23 * & $75-120$ \\
\hline Methylene Chloride & 100 & & 86 & 86 & $85-120$ \\
\hline trans-1,2-Dichloroethen & 100 & & 98 & 98 & $80-115$ \\
\hline Acrylonitrile & 100 & & 100 & 100 & $70-130$ \\
\hline Methyl-t-Butyl Ether & 100 & & 98 & 98 & $80-120$ \\
\hline 1,2-Dichloroethene (tot & 200 & & 210 & 105 & $80-115$ \\
\hline 1,1-Dichloroethane & 100 & & 120 & 120 & $85-120$ \\
\hline Vinyl Acetate & 100 & & 100 & 100 & $70-145$ \\
\hline Chloroprene & 100 & & 96 & 96 & $80-125$ \\
\hline 2,2-Dichloropropane & 100 & & 100 & 100 & $80-120$ \\
\hline cis-1,2-Dichloroethene & 100 & & 110 & 110 & $80-115$ \\
\hline 2-Butanone & 500 & & 420 & 84 & $70-140$ \\
\hline Propionitrile & 400 & & 140 & $35 *$ & $75-130$ \\
\hline Bromochloromethane & 100 & & 100 & 100 & $80-115$ \\
\hline Methacrylonitrile & 100 & & 130 & $130 *$ & $75-120$ \\
\hline Tetrahydrofuran & 1400 & & 1600 & 114 & $75-125$ \\
\hline
\end{tabular}

\# Column to be used to flag recovery and RPD values with an asterisk

* Values outside of QC limits

COMMENTS : 
FORM 3

SOIL VOLATILE LAAB CONTROL SAMPLE

Lab Name: TESTAMERICA BURLINGTON Contract: 8E-00302

Lab Code: STLV Case No.: INMAN SAS No.: $\quad$ SDG No.: 134662

Matrix Spike - Sample No.: ME111909LCS Level:(low/med) MED

\begin{tabular}{|c|c|c|c|c|c|}
\hline COMPOUND & $\begin{array}{l}\text { SPIKE } \\
\text { ADDED } \\
(\mathrm{ug} / \mathrm{Kg})\end{array}$ & $\begin{array}{c}\text { SAMPLE } \\
\text { CONCENTRATION } \\
\text { (ug/Kg) }\end{array}$ & $\begin{array}{c}\text { LCS } \\
\text { CONCENTRATION } \\
(\mathrm{ug} / \mathrm{Kg})\end{array}$ & $\begin{array}{l}\text { LCS } \\
\frac{\circ}{0} \\
\text { REC \# }\end{array}$ & $\begin{array}{c}\text { QC. } \\
\text { LIMITS } \\
\text { REC. }\end{array}$ \\
\hline 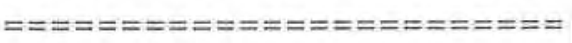 & $========$ & 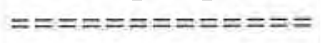 & 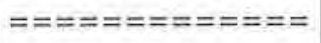 & $======$ & $======$ \\
\hline Chloroform & 100 & & 120 & 120 & $85-120$ \\
\hline 1,1,1-Trichloroethane & 100 & & 100 & 100 & $80-115$ \\
\hline Carbon Tetrachloride & 100 & & 100 & 100 & $80-115$ \\
\hline 1,1-Dichloropropene & 100 & & 110 & 110 & $80-115$ \\
\hline Isobutyl Alcohol & 5000 & & 6100 & 122 & $65-145$ \\
\hline Benzene & 100 & & 110 & 110 & $80-125$ \\
\hline 1,2-Dichloroethane & 100 & & 110 & 110 & $80-120$ \\
\hline Trichloroethene & 100 & & 100 & 100 & $80-120$ \\
\hline 1,2-Dichloropropane & 100 & & 110 & 110 & $80-120$ \\
\hline Dibromomethane & 100 & & 110 & 110 & $85-120$ \\
\hline Methyl Methacrylate & 100 & & 88 & 88 & $75-120$ \\
\hline 1,4-Dioxane & 5000 & & 6500 & 130 & $45-170$ \\
\hline Bromodichloromethane & 100 & & 110 & 110 & $85-120$ \\
\hline 2-Chloroethyl Vinyl Eth & 100 & & 110 & 110 & $80-115$ \\
\hline cis-1,3-Dichloropropene & 100 & & 110 & 110 & $85-120$ \\
\hline 4-Methyl-2-pentanone & 500 & & 480 & 96 & $75-130$ \\
\hline Toluene & 100 & & 100 & 100 & $85-120$ \\
\hline trans-1,3-Dichloroprope & 100 & & 94 & 94 & $85-120$ \\
\hline Ethyl Methacrylate & 100 & & 97 & 97 & $80-120$ \\
\hline 1,1,2-Trichloroethane & 100 & & 94 & 94 & $80-125$ \\
\hline Tetrachloroethene & 100 & & 97 & 97 & $80-115$ \\
\hline 1,3-Dichloropropane & 100 & & 96 & 96 & $85-120$ \\
\hline 2-Hexanone & 500 & & 390 & 78 & $60-165$ \\
\hline Dibromochloromethane & 100 & & 95 & 95 & $85-120$ \\
\hline 1,2-Dibromoethane & 100 & & 95 & 95 & $85-120$ \\
\hline Chlorobenzene & 100 & & 100 & 100 & $80-120$ \\
\hline $1,1,1,2$-Tetrachloroetha & 100 & & 98 & 98 & $80-115$ \\
\hline Ethylbenzene & 100 & & 100 & 100 & $85-120$ \\
\hline
\end{tabular}

\# Column to be used to flag recovery and RPD values with an asterisk

* Values outside of QC limits

COMMENTS : 
FORM 3

SOIL VOLATILE LAB CONTROL SAMPLE

Lab Name: TESTAMERICA BURLINGTON

Lab Code: STLV

Case No.: INMAN

Contract: $8 E-00302$

Matrix Spike - Sample No.: ME111909LCS Level:(low/med) MED

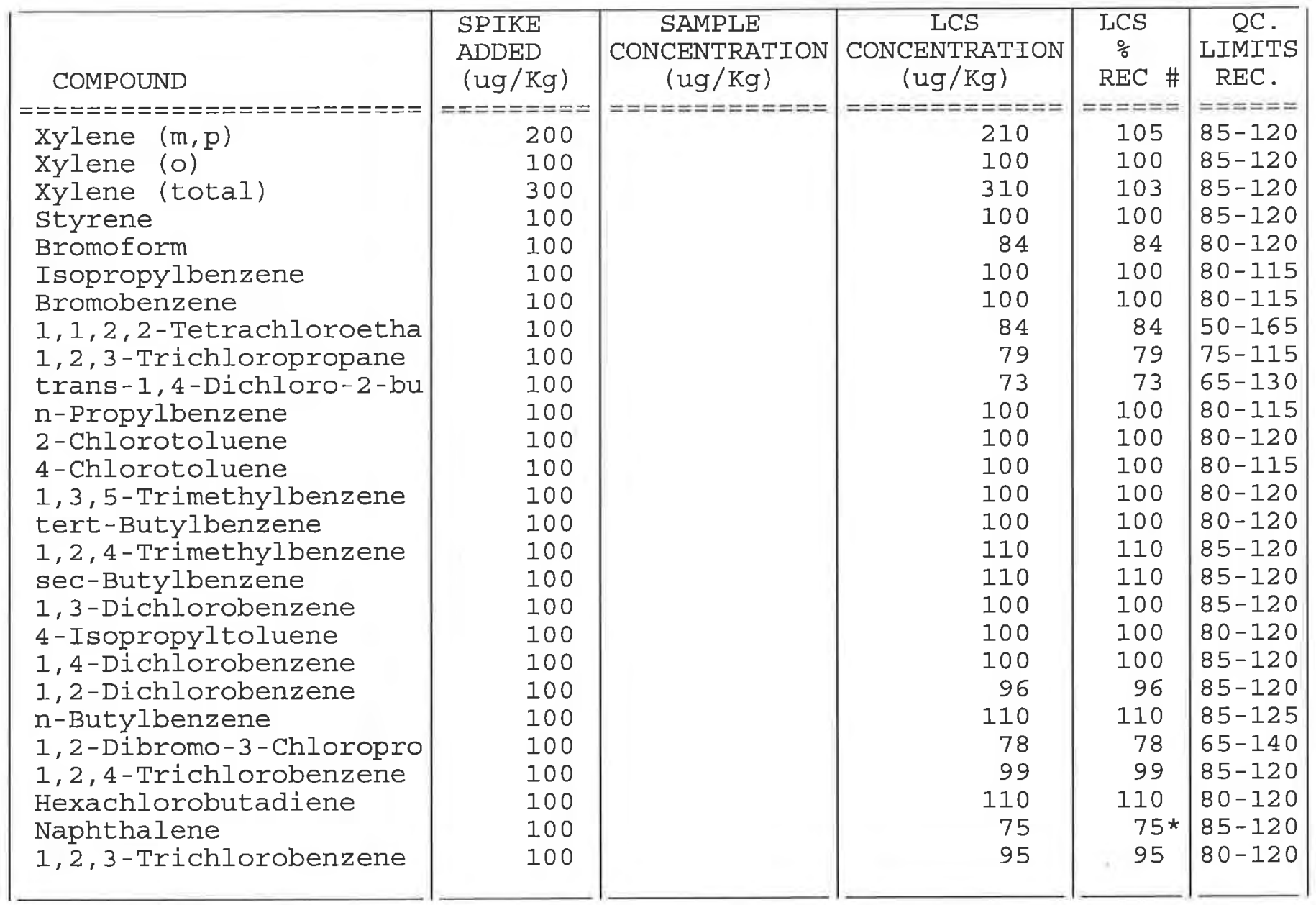

\# Column to be used to flag recovery and RPD values with an asterisk

* Values outside of QC limits

RPD: 0 out of 0 outside limits

Spike Recovery: 12 out of 83 outside limits

COMMENTS : 
FORM 3

WATER VOLATILE LAB CONTROL SAMPLE

Lab Name: TESTAMERICA BURLINGTON Contract: 8E-00302

Lab Code: STLV Case No.: INMAN SAS No.: $\quad$ SDG No.: 134662

Matrix Spike - Sample No.: NA111909LCS

\begin{tabular}{|c|c|c|c|c|c|}
\hline COMPOUND & $\begin{array}{l}\text { SPIKE } \\
\text { ADDED } \\
\text { (ug/L) }\end{array}$ & $\begin{array}{c}\text { SAMPLE } \\
\text { CONCENTRATION } \\
(\mathrm{ug} / \mathrm{L})\end{array}$ & $\begin{array}{c}\text { LCS } \\
\text { CONCENTRATION } \\
(u g / L)\end{array}$ & $\begin{array}{l}\text { LCS } \\
\vdots \\
\text { REC \# }\end{array}$ & $\begin{array}{l}\text { QC. } \\
\text { LIMITS } \\
\text { REC. }\end{array}$ \\
\hline 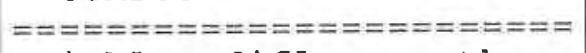 & $========$ & $======= \pm=== \pm=$ & $=============$ & $======$ & $======$ \\
\hline Dichlorodifluoromethane & 10 & & 9.4 & 94 & $50-160$ \\
\hline Chloromethane & 10 & & 8.9 & 89 & $55-140$ \\
\hline Vinyl Chloride & 10 & & 9.6 & 96 & $75-135$ \\
\hline Bromomethane & 10 & & 9.7 & 97 & $70-130$ \\
\hline Chloroethane & 10 & & 9.9 & 99 & $70-135$ \\
\hline Trichlorofluoromethane & 10 & & 9.4 & 94 & $80-115$ \\
\hline Acrolein & 50 & & 42 & 84 & $35-170$ \\
\hline 1,1-Dichloroethene & 10 & & 9.9 & 99 & $80-120$ \\
\hline Freon TF & 10 & & 9.3 & 93 & $80-120$ \\
\hline Acetone & 50 & & 40 & 80 & $40-175$ \\
\hline Methyl Iodide & 10 & & 11 & 110 & $60-140$ \\
\hline Carbon Disulfide & 10 & & 9.3 & 93 & $80-125$ \\
\hline Allyl Chloride & 10 & & 8.9 & 89 & $75-120$ \\
\hline Methylene Chloride & 10 & & 9.8 & 98 & $85-120$ \\
\hline trans-1,2-Dichloroethen & 10 & & 9.8 & 98 & $80-115$ \\
\hline Acrylonitrile & 10 & & 9.8 & 98 & $70-130$ \\
\hline Methyl-t-Butyl Ether & 10 & & 9.3 & 93 & $80-120$ \\
\hline 1,2-Dichloroethene (tot & 20 & & 19 & 95 & $80-115$ \\
\hline 1,1-Dichloroethane & 10 & & 9.5 & 95 & $85-120$ \\
\hline Vinyl Acetate & 10 & & 8.8 & 88 & $70-145$ \\
\hline Chloroprene & 10 & & 7.9 & $79 *$ & $80-125$ \\
\hline 2,2-Dichloropropane & 10 & & 9.4 & 94 & $80-120$ \\
\hline cis-1,2-Dichloroethene & 10 & & 9.4 & 94 & $80-115$ \\
\hline 2 -Butanone & 50 & & 47 & 94 & $70-140$ \\
\hline Propionitrile & 40 & & 40 & 100 & $75-130$ \\
\hline Bromochloromethane & 10 & & 9.6 & 96 & $80-115$ \\
\hline Methacrylonitrile & 10 & & 9.1 & 91 & $75-120$ \\
\hline Tetrahydrofuran & 140 & & 130 & 93 & $75-125$ \\
\hline
\end{tabular}

\# Column to be used to flag recovery and RPD values with an asterisk

* Values outside of QC limits

COMMENTS : 
FORM 3

WATER VOLATILE LAB CONTROL SAMPLE

Lab Name: TESTAMERICA BURLINGTON Contract: 8E-00302

Lab Code: STLV Case No.: INMAN SAS No.: $\quad$ SDG No.: 134662

Matrix Spike - Sample No.: NA111909LCS

\begin{tabular}{|c|c|c|c|c|c|}
\hline COMPOUND & $\begin{array}{l}\text { SPIKE } \\
\text { ADDED } \\
\text { (ug/L) }\end{array}$ & $\begin{array}{c}\text { SAMPLE } \\
\text { CONCENTRATION } \\
(\mathrm{ug} / \mathrm{L})\end{array}$ & $\begin{array}{c}\text { LCS } \\
\text { CONCENTRATION } \\
(\mathrm{ug} / \mathrm{L})\end{array}$ & $\begin{array}{l}\text { LCS } \\
\% \\
\text { REC \# }\end{array}$ & $\begin{array}{l}\text { QC. } \\
\text { LIMITS } \\
\text { REC. }\end{array}$ \\
\hline 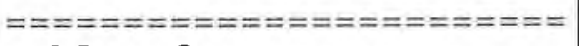 & $==== \pm==x=$ & & 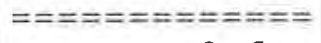 & & \\
\hline Chloroform & 10 & & 9.6 & 96 & $85-120$ \\
\hline 1,1,1-Trichloroethane & 10 & & 9.4 & 94 & $80-115$ \\
\hline Carbon Tetrachloride & 10 & & 9.4 & 94 & $80-115$ \\
\hline 1,1-Dichloropropene & 10 & & 9.6 & 96 & $80-115$ \\
\hline Isobutyl Alcohol & 500 & & 460 & 92 & $65-145$ \\
\hline Benzene & 10 & & 9.6 & 96 & $80-125$ \\
\hline 1,2-Dichloroethane & 10 & & 9.3 & 93 & $80-120$ \\
\hline Trichloroethene & 10 & & 9.1 & 91 & $80-120$ \\
\hline 1,2-Dichloropropane & 10 & & 9.4 & 94 & $80-120$ \\
\hline Dibromomethane & 10 & & 9.8 & 98 & $85-120$ \\
\hline Methyl Methacrylate & 10 & & 9.3 & 93 & $75-120$ \\
\hline 1,4-Dioxane & 500 & & 480 & 96 & $45-170$ \\
\hline Bromodichloromethane & 10 & & 9.4 & 94 & $85-120$ \\
\hline 2-Chloroethyl Vinyl Eth & 10 & & 9.8 & 98 & $80-115$ \\
\hline cis-1,3-Dichloropropene & 10 & & 9.8 & 98 & $85-120$ \\
\hline 4-Methyl-2-pentanone & 50 & & 49 & 98 & $75-130$ \\
\hline Toluene & 10 & & 9.7 & 97 & $85-120$ \\
\hline trans-1,3-Dichloroprope & 10 & & 9.4 & 94 & $85-120$ \\
\hline Ethyl Methacrylate & 10 & & 9.4 & 94 & $80-120$ \\
\hline 1,1,2-Trichloroethane & 10 & & 9.6 & 96 & $80-125$ \\
\hline Tetrachloroethene & 10 & & 9.6 & 96 & $80-115$ \\
\hline 1,3-Dichloropropane & 10 & & 9.6 & 96 & $85-120$ \\
\hline 2-Hexanone & 50 & & 50 & 100 & $60-165$ \\
\hline Dibromochloromethane & 10 & & 9.8 & 98 & $85-120$ \\
\hline 1,2-Dibromoethane & 10 & & 10 & 100 & $85-120$ \\
\hline Chlorobenzene & 10 & & 9.5 & 95 & $80-120$ \\
\hline $1,1,1,2$-Tetrachloroetha & 10 & & 9.6 & 96 & $80-115$ \\
\hline Ethylbenzene & 10 & & 9.6 & 96 & $85-120$ \\
\hline
\end{tabular}

\# Column to be used to flag recovery and RPD values with an asterisk

* Values outside of QC limits

COMMENTS : 
FORM 3

WATER VOLATILE LAB CONTROL SAMPLE

Lab Name: TESTAMERICA BURLINGTON Contract: 8E-00302

Lab Code: STLV Case No.: INMAN SAS No.: $\quad$ SDG No.: 134662

Matrix Spike - Sample No.: NA111909LCS

\begin{tabular}{|c|c|c|c|c|c|}
\hline COMPOUND & $\begin{array}{l}\text { SPIKE } \\
\text { ADDED } \\
(\mathrm{ug} / \mathrm{L})\end{array}$ & $\begin{array}{c}\text { SAMPLE } \\
\text { CONCENTRATION } \\
(u g / L)\end{array}$ & $\begin{array}{c}\text { LCS } \\
\text { CONCENTRATION } \\
(\mathrm{ug} / \mathrm{L})\end{array}$ & $\begin{array}{l}\text { LCS } \\
\frac{\circ}{\circ} \\
\text { REC \# }\end{array}$ & $\begin{array}{c}\text { QC. } \\
\text { LIMITS } \\
\text { REC. }\end{array}$ \\
\hline 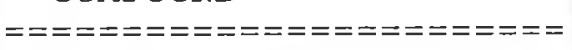 & $========$ & $============$ & $=============$ & $=====$ & $=====$ \\
\hline Xylene $(m, p)$ & 20 & & 19 & 95 & $85-120$ \\
\hline Xylene (o) & 10 & & 9.7 & 97 & $85-120$ \\
\hline xylene (total) & 30 & & 29 & 97 & $85-120$ \\
\hline styrene & 10 & & 9.7 & 97 & $85-120$ \\
\hline Bromoform & 10 & & 9.7 & 97 & $80-120$ \\
\hline Isopropylbenzene & 10 & & 9.5 & 95 & $80-115$ \\
\hline Bromobenzene & 10 & & 9.5 & 95 & $80-115$ \\
\hline $1,1,2,2$-Tetrachloroetha & 10 & & 9.6 & 96 & $50-165$ \\
\hline 1,2,3-Trichloropropane & 10 & & 9.1 & 91 & $75-115$ \\
\hline trans-1,4-Dichloro-2-bu & 10 & & 8.8 & 88 & $65-130$ \\
\hline n-Propylbenzene & 10 & & 9.6 & 96 & $80-115$ \\
\hline 2-Chlorotoluene & 10 & & 9.9 & 99 & $80-120$ \\
\hline 4-Chlorotoluene & 10 & & 9.7 & 97 & $80-115$ \\
\hline 1,3,5-Trimethylbenzene & 10 & & 9.3 & 93 & $80-120$ \\
\hline tert-Butylbenzene & 10 & & 9.5 & 95 & $80-120$ \\
\hline $1,2,4$-Trimethylbenzene & 10 & & 9.6 & 96 & $85-120$ \\
\hline sec-Butylbenzene & 10 & & 9.5 & 95 & $85-120$ \\
\hline 1,3-Dichlorobenzene & 10 & & 9.6 & 96 & $85-120$ \\
\hline 4-Isopropyltoluene & 10 & & 9.3 & 93 & $80-120$ \\
\hline 1,4-Dichlorobenzene & 10 & & 9.4 & 94 & $85-120$ \\
\hline 1,2-Dichlorobenzene & 10 & & 9.6 & 96 & $85-120$ \\
\hline n-Butylbenzene & 10 & & 9.3 & 93 & $85-125$ \\
\hline 1,2-Dibromo-3-Chloropro & 10 & & 9.4 & 94 & $65-140$ \\
\hline 1,2,4-Trichlorobenzene & 10 & & 9.3 & 93 & $85-120$ \\
\hline Hexachlorobutadiene & 10 & & 9.5 & 95 & $80-120$ \\
\hline Naphthalene & 10 & & 9.8 & 98 & $85-120$ \\
\hline $1,2,3$-Trichlorobenzene & 10 & & 9.5 & 95 & $80-120$ \\
\hline
\end{tabular}

\# Column to be used to flag recovery and RPD values with an asterisk

* Values outside of QC limits

RPD: 0 out of 0 outside limits

Spike Recovery: 1 out of 83 outside limits

COMMENTS : 
FORM 4

VOLATILE METHOD BLANK SUMMARY

Lab Name: TESTAMERICA BURLINGTON

Contract: $8 E-00302$

Case No.: INMAN

SAS NO.:

SDG No.: 134662

Lab Code: STLV

Lab File ID: 813483

Date Analyzed: 11/19/09

GC Column: DB-624 ID: 0.53 (mm)

Instrument ID: $\mathrm{N}$

Lab Sample ID: 813483

Time Analyzed: 1245

Heated Purge: (Y/N) N
ARGLAB SAMPLE NO.

IN-S-MEOHBLK

THIS METHOD BLANK APPLIES TO THE FOLLOWING SAMPLES, MS and MSD:

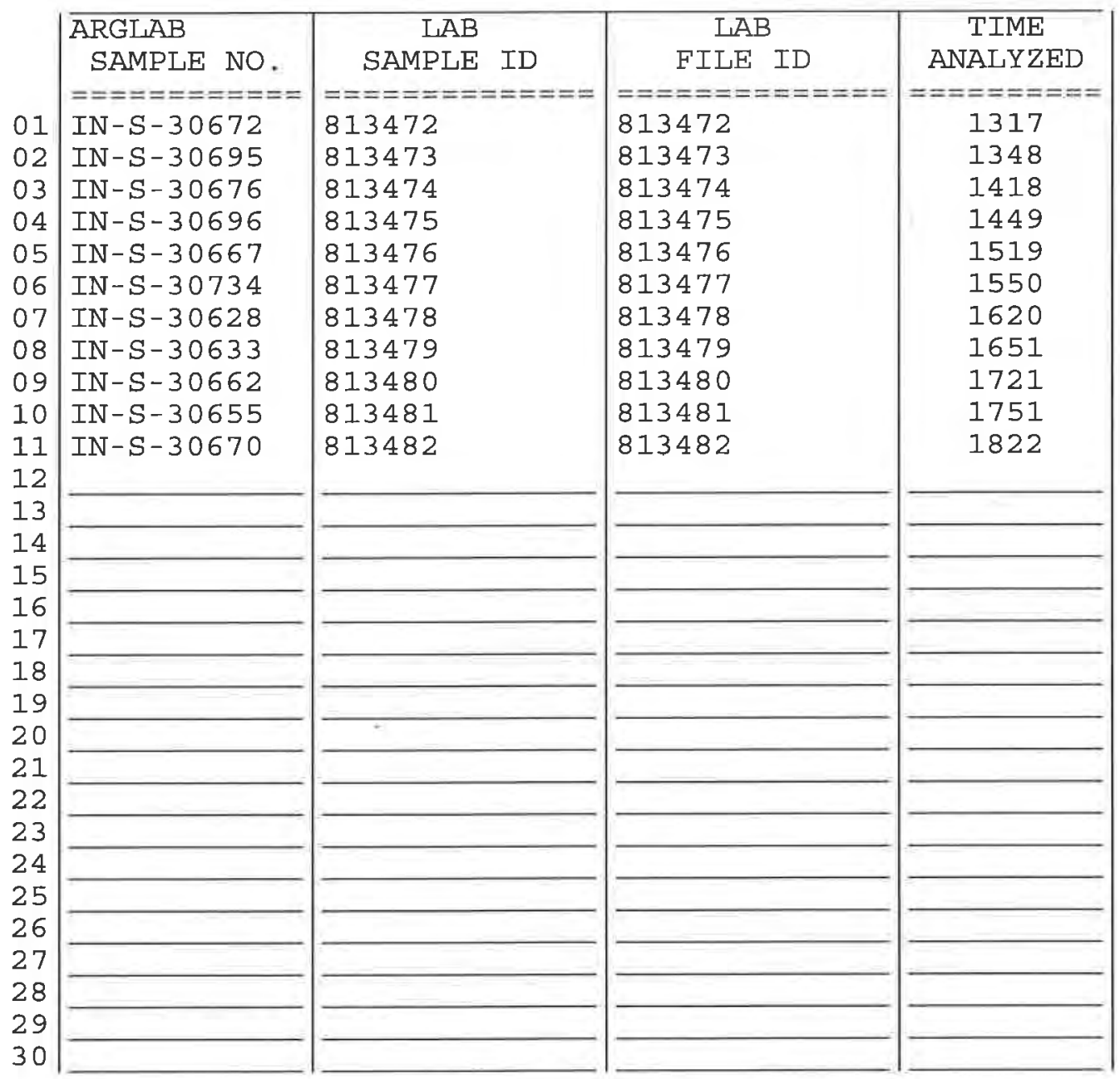

COMMENTS :

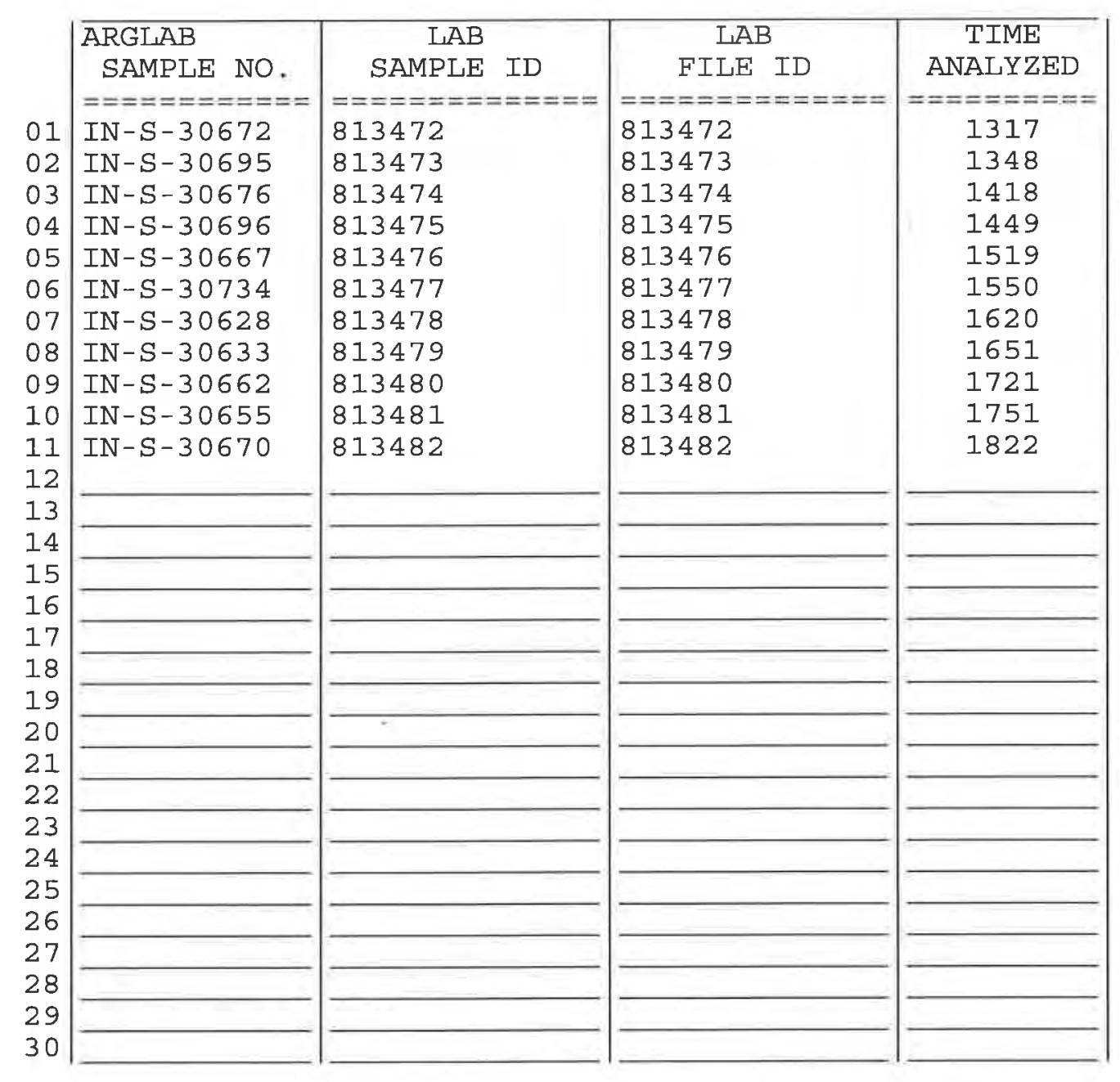

page 1 of 1 
FORM 4

VOLATILE METHOD BLANK SUMMARY

Lab Name: TESTAMERICA BURLINGTON

Contract: $8 \mathrm{E}-00302$

Case No.: INMAN

SAS NO.:

Lab Sample ID: MBLK111909ME

Date Analyzed: 11/19/09

GC Column: DB-624 ID: 0.53 (mm)

Time Analyzed: 1215

Heated Purge: $(\mathrm{Y} / \mathrm{N}) \mathrm{N}$

Instrument ID: $\mathrm{N}$

THIS METHOD BLANK APPLIES TO THE FOLLOWING SAMPLES, MS and MSD:

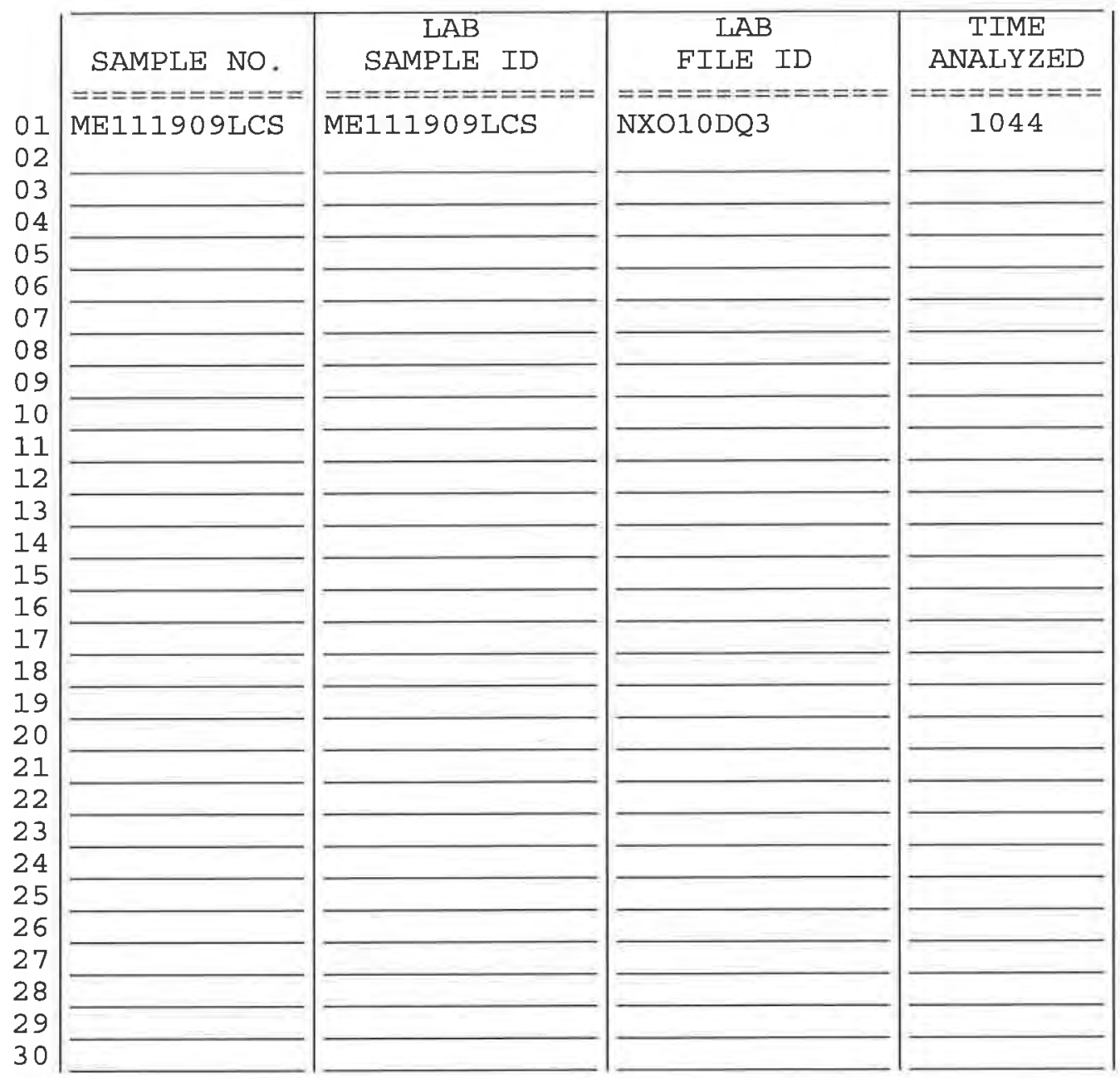

COMMENTS :

page 1 of 1 
FORM 4

VOLATILE METHOD BLANK SUMMARY
CLIENT SAMPLE NO.

MBLK111909NA
Lab Name: TESTAMERICA BURLINGTON

Lab Code: STLV Case No.: INMAN

Lab File ID: NXOB03D

Date Analyzed: 11/19/09

GC Column: DB-624 ID: $0.53 \quad$ (mm)

Instrument ID: $\mathrm{N}$
Contract: $8 \mathrm{E}-00302$

SAS NO.:

SDG No.: 134662

Lab Sample ID: MBLK111909NA

Time Analyzed: 1145

Heated Purge: (Y/N) N

THIS METHOD BLANK APPLIES TO THE FOLLOWING SAMPLES, MS and MSD:

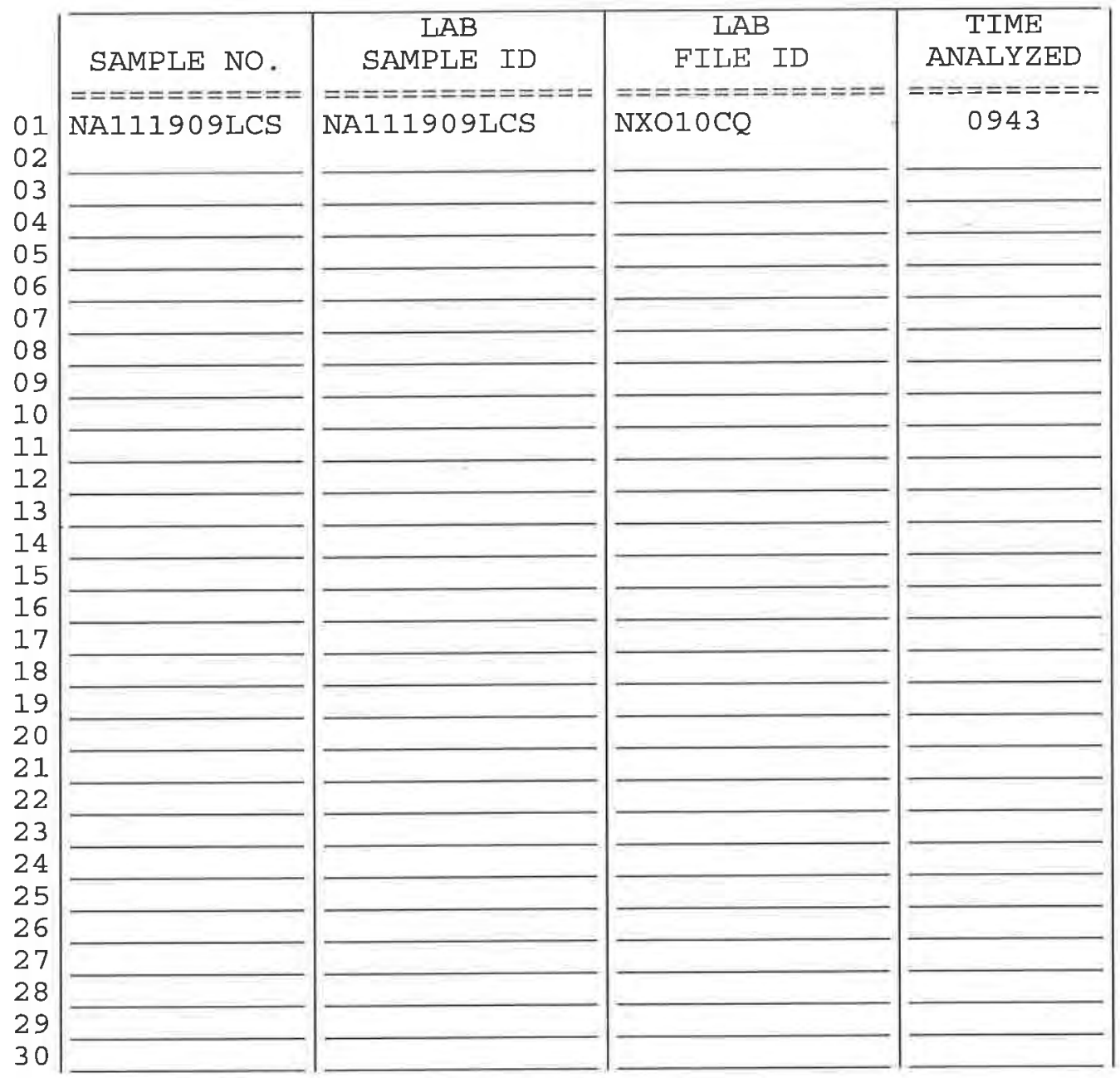

COMMENTS :

page 1 of 1 
FORM 5

VOLATILE ORGANIC INSTRUMENT PERFORMANCE CHECK BROMOFLUOROBENZENE (BFB)

Lab Name: TESTAMERICA BURLINGTON

Lab Code: STLV Case No.: INMAN

Lab File ID: NX001PV

Instrument ID: $\mathrm{N}$

GC Column: DB-624
Contract: $8 \mathrm{E}-00302$

SAS NO.:
SDG No.: 134662

BFB Injection Date: 11/16/09

BFB Injection Time: 1411

Heated Purge: $(\mathrm{Y} / \mathrm{N}) \mathrm{N}$

\begin{tabular}{|c|c|c|}
\hline $\begin{array}{c}\mathrm{m} / \mathrm{e} \\
====\end{array}$ & 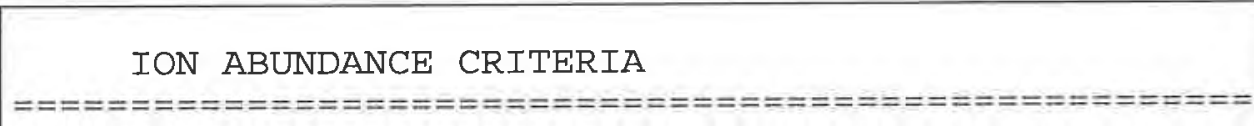 & $\begin{array}{c}\% \text { RELATIVE } \\
\text { ABUNDANCE } \\
=============\end{array}$ \\
\hline 50 & $15.0-40.0 \%$ of mass 95 & 22.5 \\
\hline 75 & $30.0-60.0 \%$ of mass 95 & 51.9 \\
\hline 95 & Base Peak, $100 \%$ relative abundance & 100.0 \\
\hline 96 & $5.0-9.0 \%$ of mass 95 & 6.6 \\
\hline 173 & Less than $2.0 \%$ of mass 174 & $0 . 0 \longdiv { ( 0 . 0 ) 1 }$ \\
\hline 174 & $50.0-120.0 \%$ of mass 95 & 60.3 \\
\hline 175 & $5.0-9.0 \%$ of mass 174 & $4.6 \bar{T}$ \\
\hline 176 & $95.0-101.0 \%$ of mass 174 & $58.7(97.4) 1$ \\
\hline 177 & $5.0-9.0 \%$ of mass 176 & $4.2(7.2) 2$ \\
\hline
\end{tabular}

THIS CHECK APPLIES TO THE FOLLOWING SAMPLES, MS, MSD, BIANKS, AND STANDARDS:

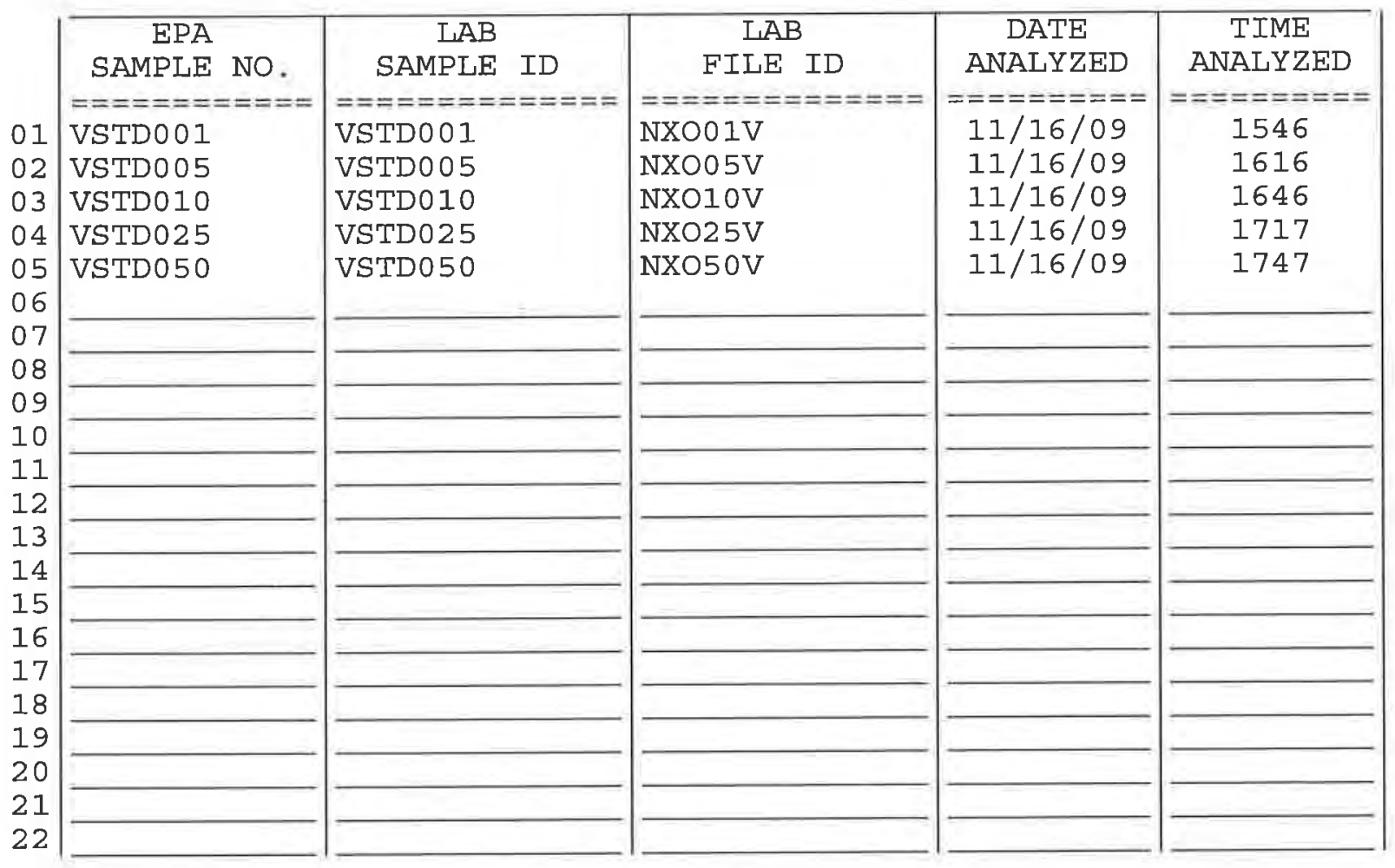


FORM 5

VOLATILE ORGANIC INSTRUMENT PERFORMANCE CHECK

BROMOFLUOROBENZENE (BFB)

Lab Name: TESTAMERICA BURLINGTON

Lab Code: STLV

Case No.: INMAN

Lab File ID: NX007PV

Instrument ID: $\mathrm{N}$

GC Column: DB-624

ID : $0.53 \quad(\mathrm{~mm})$
Contract: $8 \mathrm{E}-00302$

SAS No.:

SDG No.: 134662

BFB Injection Date: 11/19/09

BFB Injection Time: 0812

Heated Purge: ( $\mathrm{Y} / \mathrm{N}) \mathrm{N}$

\begin{tabular}{|c|c|c|}
\hline $\mathrm{m} / \mathrm{e}$ & ION ABUNDANCE CRITERIA & $\begin{array}{l}\text { RELATIVE } \\
\text { ABUNDANCE }\end{array}$ \\
\hline$=====$ & 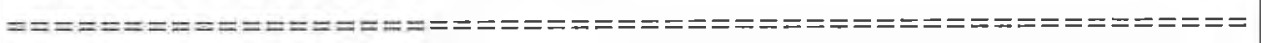 & 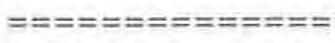 \\
\hline 50 & $15.0-40.0 \%$ of mass 95 & 21.5 \\
\hline 75 & $30.0-60.0 \%$ of mass 95 & 48.1 \\
\hline 95 & Base Peak, $100 \%$ relative abundance & 100.0 \\
\hline 96 & $5.0-9.0 \%$ of mass 95 & 6.9 \\
\hline 173 & Less than $2.0 \%$ of mass 174 & $0.0 \overline{(}$ \\
\hline 174 & $50.0-120.0 \%$ of mass 95 & 64.2 \\
\hline 175 & $5.0-9.0 \%$ of mass 174 & 4.57 \\
\hline 176 & $95.0-101.0 \%$ of mass $\overline{174}$ & $62.8(97.8) 1$ \\
\hline 177 & $5.0-9.0 \%$ of mass 176 & $4.2(6.8) 2$ \\
\hline
\end{tabular}

THIS CHECK APPLIES TO THE FOLLOWING SAMPLES, MS, MSD, BLANKS, AND STANDARDS:

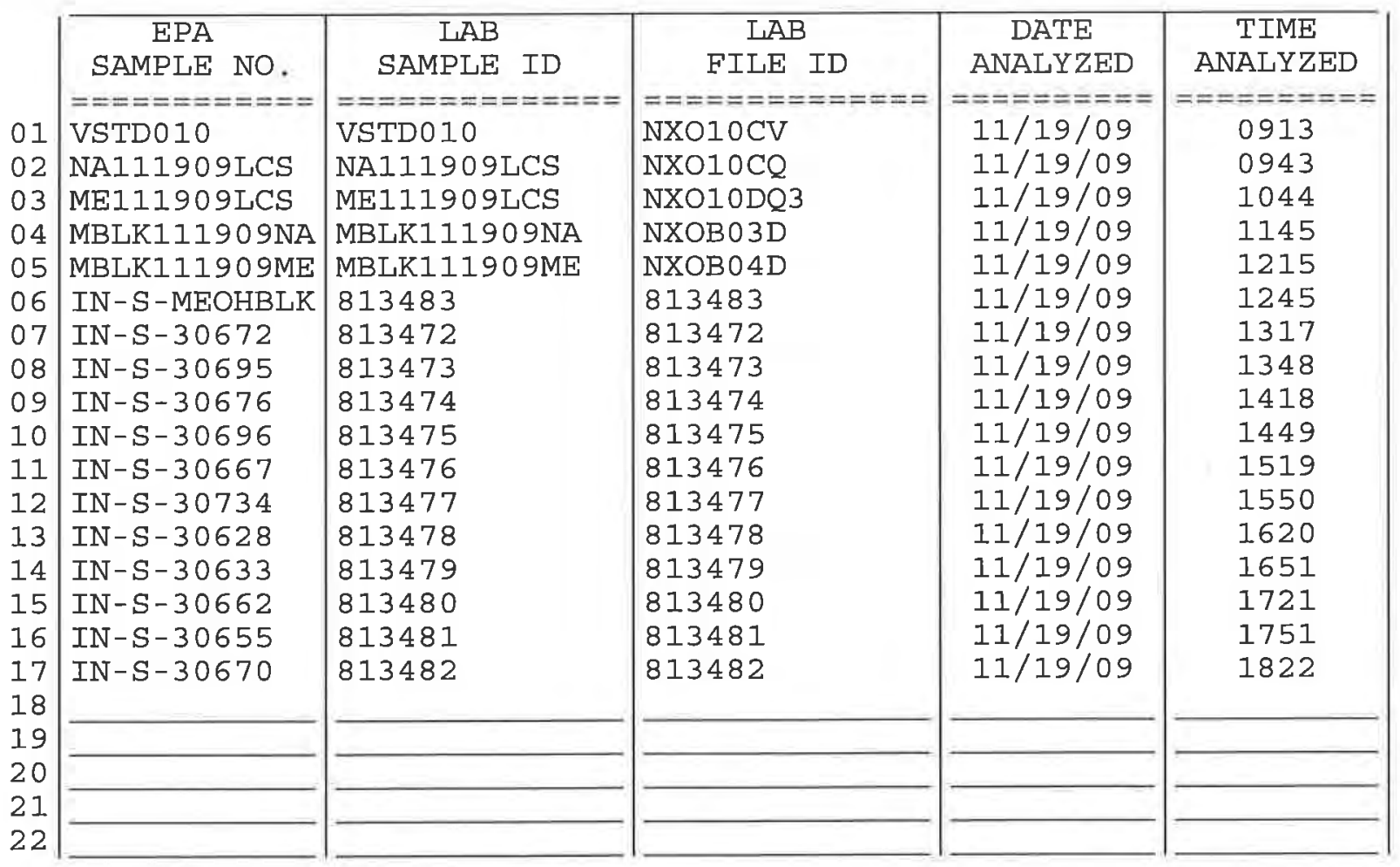

page 1 of 1 


\section{FORM 6 \\ VOLATILE ORGANICS INITIAL CALIBRATION DATA}

Lab Name: TESTAMERICA BURLINGTON

Contract: $8 \mathrm{E}-00302$

Lab Code: STLV

Case No.: INMAN SAS No.:

SDG NO.: 134662

Instrument ID: N

Calibration Date(s): 11/16/09

$11 / 16 / 09$

Heated Purge: ( $\mathrm{Y} / \mathrm{N}) \mathrm{N}$

Calibration Time(s) : 1546

1747

GC Column: DB-624 ID: $0.53 \quad(\mathrm{~mm})$

\begin{tabular}{|c|c|c|c|c|c|c|c|}
\hline $\begin{array}{l}\text { LAB FILE ID: } \\
\text { RRF10 =NXO10V }\end{array}$ & $\begin{array}{l}=\mathrm{NXO} 01 \\
=\mathrm{NXO} 25\end{array}$ & & $\begin{array}{l}\text { RRF5 } \\
\text { RRF5 }\end{array}$ & $\begin{array}{l}=\mathrm{NXO0} \\
0=\mathrm{NXO5}\end{array}$ & & & \\
\hline & & & & & & & תא \\
\hline 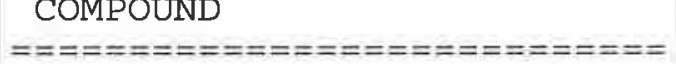 & $\begin{array}{l}\mathrm{RRF} 1 \\
======\end{array}$ & $\begin{array}{l}\text { RRF'5 } \\
======\end{array}$ & $\begin{array}{l}\operatorname{RRF} 10 \\
======\end{array}$ & $\begin{array}{l}\operatorname{RRF} 25 \\
======\end{array}$ & $\begin{array}{l}\mathrm{RRF} b 0 \\
=====\end{array}$ & $\begin{array}{c}\mathrm{RRF} \\
======\end{array}$ & $\begin{array}{l}\text { RSD } \\
=====\end{array}$ \\
\hline Dichlorodifluoromethane & * 0.791 & 0.684 & 0.706 & 0.714 & 0.667 & 0.712 & $6.7 *$ \\
\hline Chloromethane & * 0.577 & 0.455 & 0.465 & 0.451 & 0.401 & 0.470 & $13.8 *$ \\
\hline Vinyl Chloride & * 0.431 & 0.384 & 0.394 & 0.404 & 0.383 & 0.399 & $4.9 *$ \\
\hline Bromomethane & * 0.312 & 0.272 & 0.284 & 0.315 & 0.344 & 0.305 & $9.3 *$ \\
\hline Chloroethane & * 0.280 & 0.243 & 0.250 & 0.265 & 0.239 & 0.255 & $6.7 *$ \\
\hline Trichlorofluoromethane & * 0.972 & 0.848 & 0.881 & 0.894 & 0.851 & 0.889 & $5.6 *$ \\
\hline Acrolein & $\star 0.033$ & 0.031 & 0.031 & 0.033 & 0.033 & 0.032 & $2.8 *$ \\
\hline 1,1-Dichloroethene & $\star 0.376$ & 0.361 & 0.365 & 0.377 & 0.364 & 0.369 & $2.0 *$ \\
\hline Freon $\mathrm{TF}$ & $\star 0.822$ & 0.813 & 0.816 & 0.826 & 0.800 & 0.815 & $1.2 *$ \\
\hline Acetone & $\star 0.079$ & 0.073 & 0.074 & 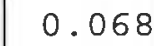 & 0 . & 0.072 & $6.4 *$ \\
\hline Methyl Iodide & $\star 0.326$ & 0.460 & 0.542 & 0.714 & 0.783 & 0.565 & $32.9 *$ \\
\hline Carbon Disulfide & $\star 1.211$ & 1.141 & 1.144 & 1.180 & 1.142 & 1.164 & $2.7 *$ \\
\hline Allyl Chloride & * 0.599 & 0.572 & 0.578 & 0.607 & 0.590 & 0.589 & $2 \cdot 5^{\star}$ \\
\hline Methylene Chloride & * 0.412 & 0.386 & 0.394 & 0.407 & 0.401 & 0.400 & $2.6 *$ \\
\hline trans-1,2-Dichloroethene & $* 0.409$ & 0.405 & 0.389 & 0.411 & 0.401 & 0.403 & $2.2 *$ \\
\hline Acrylonitrile & $\star 0.053$ & 0.070 & 0.076 & 0.078 & 0.079 & 0.071 & $15.0 *$ \\
\hline Methyl-t-Butyl Ether & $\star 0.784$ & 0.817 & 0.786 & 0.827 & 0.803 & 0.803 & 2.4 * \\
\hline 1,2-Dichloroethene (total) & $\star 0.428$ & 0.411 & 0.398 & 0.417 & 0.412 & 0.413 & $2.7 *$ \\
\hline 1,1-Dichloroethane & * 0.768 & 0.789 & 0.779 & 0.810 & 0.801 & 0.789 & $2.1 *$ \\
\hline Vinyl Acetate & $\star 0.823$ & 0.847 & 0.846 & 0.878 & 0.879 & 0.855 & $2.8 *$ \\
\hline Chloroprene & * 0.577 & 0.575 & 0.576 & 0.614 & 0.602 & 0.589 & $3.1 *$ \\
\hline 2,2-Dichloropropane & * 0.676 & 0.638 & 0.617 & 0.618 & 0.605 & 0.631 & $4.4^{*}$ \\
\hline cis-1,2-Dichloroethene & * 0.448 & 0.417 & 0.407 & 0.424 & 0.422 & 0.424 & $3.5 *$ \\
\hline 2-Butanone & $* 0.031$ & 0 . & 0.026 & 0.026 & 27 & 0.027 & $8.2 *$ \\
\hline Propionitrile & $\star 0.022$ & 0.026 & 0.025 & 0.026 & 0.026 & 0.025 & $7.3 *$ \\
\hline Bromochloromethane & $* 0.265$ & 0.275 & 0.262 & 0.279 & 0.268 & 0.270 & $2.6 *$ \\
\hline Methacrylonitrile & * 0.095 & 0.081 & 0.092 & 0.088 & 0.088 & 0.089 & $6.2 *$ \\
\hline Tetrahydrofuran & * 0.078 & 0.078 & 0.078 & 0. & & 78 & $1.3 *$ \\
\hline Chloroform & * 0.812 & 0.829 & 0.837 & 0.862 & 0.833 & 0.835 & $2.2^{\star}$ \\
\hline $1,1,1$-Trichloroethane & $\star 0.700$ & 0.690 & 0.666 & 0.684 & 0.664 & 0.681 & $2.3 *$ \\
\hline Carbon Tetrachloride & * 0.632 & 0.629 & 0.627 & 0.643 & 0.626 & 0.631 & $1.1 *$ \\
\hline 1,1-Dichloropropene & $\star 0.621$ & 0.568 & 0.565 & 0.585 & 0.570 & 0.582 & $4.0 *$ \\
\hline Isobutyl Alcohol & $\star 0.012$ & 0.010 & 0.010 & 0.010 & 0.010 & 0.010 & $9.8 *$ \\
\hline Benzene & * 0.966 & 0.972 & 0.948 & 0.985 & 0.977 & 0.970 & 1.4 * \\
\hline 1,2-Dichloroethane & $\star 0.498$ & 0.482 & 0.452 & 0.474 & 0.466 & 0.474 & $3.5 *$ \\
\hline Trichloroethene & * 0.722 & 0.578 & 0.561 & 0.563 & 0.541 & 0.593 & 12.4 * \\
\hline 1,2-Dichloropropane & * 0.555 & 0.539 & 0.529 & 0.540 & 0.520 & 0.537 & $2.4^{\star}$ \\
\hline
\end{tabular}

Compounds with required minimum $R R F$ and maximim $\% R S D$ values.

All other compounds must meet a minimim RRF of 0.010 .

page 1 of 3 


\section{FORM 6 \\ VOLATILE ORGANICS INITIAL CALIBRATION DATA}

Lab Name: TESTAMERICA BURLINGTON Contract: 8E-00302

Lab Code: STLV Case No.: INMAN SAS No.: $\quad$ SDG No.: 134662

Instrument ID: N Calibration Date(s): 11/16/09 11/16/09

Heated Purge: (Y/N) N $\quad$ Calibration Time(s): $1546 \quad 1747$

GC Column: DB-624 ID: $0.53 \quad$ (mm)

\begin{tabular}{|c|c|c|c|c|c|c|c|}
\hline $\begin{array}{l}\text { LAB FILE ID: } \\
\text { RRF10 =NXOIOV }\end{array}$ & $\begin{array}{l}=\mathrm{NXO} 01 \\
=\mathrm{NXO} 25\end{array}$ & & $\begin{array}{l}\text { RRF5 } \\
\text { RRF5 }\end{array}$ & $\begin{array}{l}=\text { NXO0 } \\
0=\text { NXO5 }\end{array}$ & & & \\
\hline COMPOUND & RRF1 & & F1 & RRF2 & RRF : & $\overline{R R F}$ & RSD \\
\hline 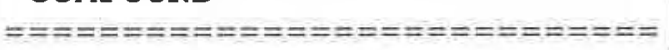 & $======$ & $==$ & $==== \pm=$ & $=$ & & $======$ & $===$ \\
\hline Dibromomethane & $\star 0.516$ & 0.528 & 0.532 & 0.548 & 0.537 & 0.532 & 2.2 \\
\hline Methyl Methacrylate & $\star 0.293$ & 0.263 & 0.265 & 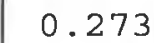 & .275 & & \\
\hline 1,4-Dioxane & $\star 0.003$ & 0.003 & 0.003 & 0.003 & 0.003 & 0.003 & $5.2 *$ \\
\hline Bromodichloromethane & * 0.980 & 0.987 & 0.983 & 1.025 & 0.994 & 0.994 & 1.8 \\
\hline 2-Chloroethyl Vinyl Ether & * 0.295 & 0.260 & 0.271 & 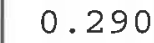 & 84 & & $\perp$ \\
\hline cis-1,3-Dichlo & * 0.715 & & 0.734 & & & & \\
\hline 4-Methyl-2-pent & * 0.382 & 0. & 0.383 & 0.388 & 78 & 0. & 1.6 \\
\hline Toluene & $\star 0.734$ & & 0.732 & & 0.740 & & 1.7 \\
\hline trans $-1,3-$ Dic & $\star 0.660$ & & 0.658 & & & & 2.6 \\
\hline Ethyl Methac & & & 0 . & & & & .9 \\
\hline $1,1,2$-Trichlo & * 0 . & & 0.418 & & & & 3.1 \\
\hline Tetrachloroet. & $\star 0.526$ & 0 . & 0.531 & 53 & & 2 & 2.9 \\
\hline 1,3-Dichloropropane & $\star 0.733$ & & 0.756 & & & & 2.2 \\
\hline 2-Hexanone & & & 54 & & & & 1.9 \\
\hline ibromochloro & & & 18 & & & & 3.1 \\
\hline ibromoet & * 0.729 & & 0.751 & 33 & & & 2.8 \\
\hline Chlorobenzene & $\star 1.028$ & 1.036 & 0.998 & 1.040 & 21 & 1.025 & 1.6 \\
\hline $1,1,1,2$-Tetrachloroethane & $\star 0.547$ & & 0.561 & & & & 4. \\
\hline Ethylbenzene & $\star 1.464$ & & 1.526 & & & & 2 . \\
\hline ylene $(m, p)$ & $\star 0.536$ & 0 . & 0.532 & & & & 3.3 \\
\hline Xylene (o) & * 0.519 & 0.542 & 0.537 & 0.5 & $=0$ & 0.541 & 3.1 \\
\hline xylene (total) & * 0.519 & 0.542 & 0.537 & & 0 & 0.541 & 3. \\
\hline styrene & * 0. & & 40 & & & & \\
\hline comoform & * 0. & & 72 & 0 & & 3 & 5. \\
\hline Isopropylbenzene & $\star 2.501$ & 2.5 & 2.466 & 2.5 & 54 & 2.525 & 1.6 \\
\hline Bromobenzene & $\star 0.910$ & 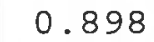 & 0.903 & 0.915 & & 0.910 & 1.2 \\
\hline $1,1,2,2$-Tetra & $\star 1.442$ & & & & & & \\
\hline $2,3-\operatorname{Trich} 10$ & $\star 0.303$ & & 11 & 3 & & 3 & 2 \\
\hline ans-1,4-Dichloro-2 & $\star 0.277$ & 0. & 0.258 & 0.264 & & 0.266 & 2.5 \\
\hline n-Propylbenzene & $\star 0.617$ & 0.618 & 0.620 & 0.639 & 0.6 & 0.624 & 1.4 \\
\hline 2-Chlorotoluene & $\star 0.615$ & 0.642 & 0.637 & & & & \\
\hline 4-Chlorotoluene & $\star 0.678$ & & 0.648 & & & & \\
\hline $1,3,5$-Trimethyl be & $\star 2.004$ & 1.5 & 1.897 & 1. & 1 . & 1 . & $\cdot-$ \\
\hline tert-Butylben & $\star 1.926$ & 1. & 1.966 & 1.991 & 1.978 & 1.966 & 1.2 \\
\hline $1,2,4$-Trimethylbenzene & $\star 1.878$ & 1.920 & 1.930 & 1.934 & 1.941 & 1.921 & 1.3 \\
\hline sec-Butylber & $\star 2.670$ & 2.6 & 2.682 & 2.6 & & & $0 . \varepsilon$ \\
\hline 1,3-Dichlorobenze & * 1.387 & 1.399 & 1.407 & 1.385 & 1.406 & 1.397 & $0 . \varepsilon$ \\
\hline
\end{tabular}

Compounds with required minimum RRF and maximim $\%$ RSD values.

All other compounds must meet a minimim RRF of 0.010 .

page 2 of 3

FORM VI VOA 


\section{FORM 6 \\ VOLATILE ORGANICS INITIAL CALIBRATION DATA}

Lab Name: TESTAMERICA BURLINGTON

Contract: $8 \mathrm{E}-00302$

Lab Code: STLV

Case No.: INMAN SAS No.:

SDG No.: 134662

Instrument ID: N

Calibration Date(s): 11/16/09 11/16/09

Heated Purge: ( $Y / N)$ N

Calibration Time(s): 1546

1747

GC Column: DB-624 ID: 0.53 (mm)

\begin{tabular}{|c|c|c|c|c|c|c|c|}
\hline $\begin{array}{ll}\text { LAB FILE ID : } & \text { RRF1 } \\
\text { RRF } 10=\text { NXO10V } & \text { RRF25 }\end{array}$ & $\begin{array}{l}=\mathrm{NXO01 \textrm {T }} \\
=\mathrm{NXO} 25 \mathrm{~V}\end{array}$ & & $\begin{array}{l}\text { RRF5 } \\
\text { RRF5 }\end{array}$ & $\begin{array}{l}=\mathrm{NXOO5} \\
=\mathrm{NXO5}\end{array}$ & & & \\
\hline COMPOUND & RRF1 & RRF5 & RRF10 & RRF25 & RRF50 & $\mathrm{RRF}$ & RSD \\
\hline 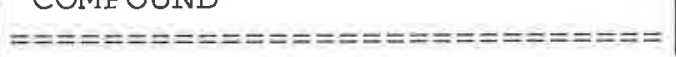 & $=====$ & $=====$ & $======$ & $===-==$ & $=====$ & $==-===$ & $=====$ \\
\hline 4-Isopropyltoluene & * 2.059 & 2.070 & 2.064 & 2.110 & 2.127 & 2.086 & $1.5^{\prime}$ \\
\hline 1,4-Dichlorobenzene & $\star 1.604$ & 1.595 & 1.521 & 1.605 & 1.556 & 1.576 & $2.3 \times$ \\
\hline 1,2-Dichlorobenzene & $\times 1.365$ & 1.357 & 1.392 & 1.380 & 1.373 & 1.373 & 1.0 \\
\hline n-Butylbenzene & * 1.976 & 2.058 & 2.058 & 2.091 & 2.113 & 2.059 & $2.5^{\prime}$ \\
\hline 1,2-Dibromo-3-Chloropropane & $* 0.271$ & 0.303 & 0.294 & 0.292 & 0.299 & 0.292 & 4.2 \\
\hline $1,2,4$-Trichlorobenzene & $\star 0.937$ & 0.978 & 0.978 & 0.997 & 1.042 & 0.986 & $3.8 *$ \\
\hline Hexachlorobutadiene & $\star 0.443$ & 0.436 & 0.433 & 0.438 & 0.434 & 0.437 & $0.9 *$ \\
\hline Naphthalene & * 1.557 & 1.728 & 1.575 & 1.655 & 1.765 & 1.656 & $5.5 *$ \\
\hline $1,2,3$-Trichlorobenzene & $\star 0.818$ & 0.860 & 0.877 & 0.872 & 0.905 & 0.866 & $3.7 *$ \\
\hline 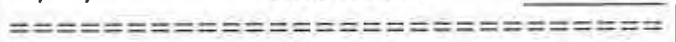 & |====== & $======$ & $===x=\approx$ & $======$ & $==ニ==ニ$ & $======$ & $=====\mid$ \\
\hline 1,2 -Dichloroethane-d4 & 0.419 & 0.391 & 0.392 & 0.398 & 0.380 & 0.396 & $3.6 *$ \\
\hline Toluene-d8 & $\star 1.081$ & 1.153 & 1.108 & 1.190 & 1.136 & 1.134 & $3.7 *$ \\
\hline Bromofluorobenzene & $\star 1.519$ & 1.442 & 1.430 & 1.448 & 1.472 & 1.462 & $2.4 *$ \\
\hline 1,2 -Dichlorobenzene-d4 & * 0.964 & 0.890 & 0.895 & 0.906 & 0.900 & 0.911 & $3.3 *$ \\
\hline & & & & & & & \\
\hline & & & & & & & \\
\hline & & & & & & & \\
\hline & & & & & & & \\
\hline & & & & & & & \\
\hline & & & & & & & \\
\hline & & & & & & & \\
\hline & & & & & & & \\
\hline & & & & & & & \\
\hline & & & & & & & \\
\hline & & & & & & & \\
\hline & & & & & & & \\
\hline & & & & & & & \\
\hline & & & & & & & \\
\hline & & & & & & & \\
\hline & & & & & & & \\
\hline & & & & & & & \\
\hline
\end{tabular}

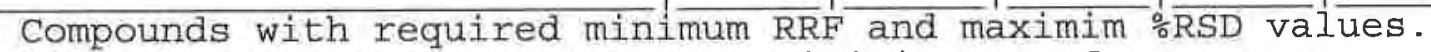

All other compounds must meet a minimim RRF of 0.010 .

page 3 of 3 
FORM 7

VOLATILE CONTINUING CALIBRATION CHECK

Lab Name: TESTAMERICA BURLINGTON

Contract: $8 \mathrm{E}-00302$

Lab Code: STLV Case No.: INMAN SAS No.:

SDG NO.: 134662

Instrument ID: $\mathrm{N}$

Calibration Date: 11/19/09 Time: 0913

Lab File ID: NXO10CV

Init. Calib. Date(s): 11/16/09 11/16/09

Heated Purge: ( $\mathrm{Y} / \mathrm{N}) \mathrm{N}$

Init. Calib. Times: 1546

1747

GC Column: DB-624 ID: 0.53 (mm)

\begin{tabular}{|c|c|c|c|c|c|}
\hline & & & MIN & & MAX \\
\hline COMPOUND & $\overline{R R F}$ & RRF10 & $\mathrm{RRF}$ & $\begin{array}{c}\because \mathrm{D} \\
======\end{array}$ & $\div \mathrm{D}$ \\
\hline 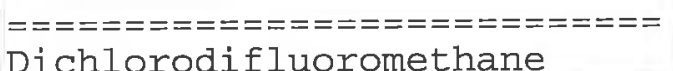 & $=======1$ & $\begin{aligned}==== & ==== \\
& 0.697\end{aligned}$ & $========$ & $\begin{array}{r}===== \\
2.1\end{array}$ & $\begin{array}{l}==== \\
20.0\end{array}$ \\
\hline Chloromethane & 0.470 & 0.424 & 0.1 & 9.8 & 20.0 \\
\hline Vinyl Chloride & 0.399 & 0.381 & & 4.5 & 20.0 \\
\hline Bromomethane & 0.305 & 0.308 & & 1.0 & 20.0 \\
\hline Chloroethane & 0.247 & 0.249 & & 0.8 & 20.0 \\
\hline Trichlorofluoromethane. & 0.889 & 0.872 & & 1.9 & 20.0 \\
\hline Acrolein & 0.032 & 0.029 & & 9.4 & 20.0 \\
\hline 1,1-Dichloroethene & 0.369 & 0.327 & & 11.4 & 20.0 \\
\hline Freon $\mathrm{TF}$ & 0.815 & 0.757 & & 7.1 & 20.0 \\
\hline Acetone & 0.072 & 0.059 & & 18.0 & 20.0 \\
\hline Methyl Iodide & 0.565 & 0.615 & & 8.8 & 20.0 \\
\hline Carbon Disulfide & 1.164 & 0.994 & & 14.6 & 20.0 \\
\hline Allyl Chloride & 0.589 & 0.526 & & 10.7 & 20.0 \\
\hline Methylene Chloride & 0.400 & 0.363 & & 9.2 & 20.0 \\
\hline trans-1,2-Dichloroethene & 0.403 & 0.362 & & 10.2 & 20.0 \\
\hline Acrylonitrile & 0.071 & 0.073 & & 2.8 & 20.0 \\
\hline Methyl-t-Butyl Ether & 0.803 & 0.732 & & 8.8 & 20.0 \\
\hline 1,2-Dichloroethene (total) & 0.413 & 0.375 & & 9.2 & 20.0 \\
\hline 1,1-Dichloroethane & 0.789 & 0.712 & 0.1 & 9.8 & 20.0 \\
\hline Vinyl Acetate & 0.855 & 0.806 & & 5.7 & 20.0 \\
\hline Chloroprene & 0.589 & 0.538 & & 8.6 & 20.0 \\
\hline 2,2-Dichloropropane & 0.631 & 0.588 & & 6.8 & 20.0 \\
\hline cis-1,2-Dichloroethene & 0.424 & 0.388 & & 8.5 & 20.0 \\
\hline 2-Butanone & 0.027 & 0.024 & & 11.1 & 20.0 \\
\hline Propionitrile & 0.025 & 0.024 & & 4.0 & 20.0 \\
\hline Bromochloromethane & 0.270 & 0.248 & & 8.1 & 20.0 \\
\hline Methacrylonitrile & 0.089 & 0.078 & & 12.4 & 20.0 \\
\hline Tetrahydrofuran & 0.078 & 0.071 & & 9.0 & 20.0 \\
\hline Chloroform & 0.835 & 0.794 & & 4.9 & 20.0 \\
\hline $1,1,1$-Trichloroethane & 0.681 & 0.612 & & 10.1 & 20.0 \\
\hline Carbon Tetrachloride & 0.631 & 0.591 & & 6.3 & 20.0 \\
\hline 1,1-Dichloropropene & 0.582 & 0.515 & & 11.5 & 20.0 \\
\hline Isobutyl Alcohol & 0.010 & 0.010 & & 0.0 & 20.0 \\
\hline Benzene & 0.970 & 0.919 & & 5.2 & 20.0 \\
\hline 1,2-Dichloroethane & 0.474 & 0.427 & & 9.9 & 20.0 \\
\hline Trichloroethene & 0.593 & 0.527 & & 11.1 & 20.0 \\
\hline 1,2-Dichloropropane & 0.537 & 0.518 & & 3.5 & 20.0 \\
\hline
\end{tabular}


FORM 7

VOLATILE CONTINUING CALIBRATION CHECK

Lab Name: TESTAMERICA BURLINGTON Contract: 8E-00302

Lab Code: STLV Case No.: INMAN SAS No.:

SDG No.: 134662

Instrument ID: $\mathrm{N}$

Calibration Date: 11/19/09 Time: 0913

Lab File ID: NXO10CV

Init. Calib. Date(s): 11/16/09 11/16/09

Heated Purge: ( $\mathrm{Y} / \mathrm{N}) \mathrm{N}$

Init. Calib. Times: 1546

1747

GC Column: DB-624 ID: $0.53 \quad(\mathrm{~mm})$

\begin{tabular}{|c|c|c|c|c|c|}
\hline COMPOUND & $\overline{\mathrm{RRF}}$ & RRF10 & $\begin{array}{l}\text { MIN } \\
\text { RRF }\end{array}$ & $\because \mathrm{D}$ & $\begin{array}{l}\text { MAX } \\
\div D\end{array}$ \\
\hline 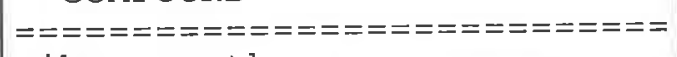 & $=======-=$ & $====== \pm==$ & $========$ & $======$ & $====$ \\
\hline Dibromomethane & 0.532 & 0.515 & & 3.2 & 20.0 \\
\hline Methyl Methacrylate. & 0.274 & 0.262 & & 4.4 & 20.0 \\
\hline 1,4 -Dioxane & 0.003 & 0.003 & & 0.0 & 20.0 \\
\hline Bromodichloromethane & 0.994 & 0.979 & & 1.5 & 20.0 \\
\hline 2-Chloroethyl Vinyl Ether & 0.280 & 0.256 & & 8.6 & 20.0 \\
\hline Cis-1,3-Dichloropropene & 0.739 & 0.728 & & 1.5 & 20.0 \\
\hline 4-Methyl-2-pentanone & 0.380 & 0.357 & & 6.0 & 20.0 \\
\hline Toluene & 0.743 & 0.706 & & 5.0 & 20.0 \\
\hline trans-1,3-Dichloropropene & 0.669 & 0.633 & & 5.4 & 20.0 \\
\hline Ethyl Methacrylate & 0.568 & 0.558 & & 1.8 & 20.0 \\
\hline 1,1,2-Trichloroethane & 0.434 & 0.406 & & 6.4 & 20.0 \\
\hline Tetrachloroethene & 0.542 & 0.525 & & 3.1 & 20.0 \\
\hline 1,3-Dichloropropane & 0.756 & 0.716 & & 5.3 & 20.0 \\
\hline 2 -Hexanone & 0.255 & 0.230 & & 9.8 & 20.0 \\
\hline Dibromochloromethane & 0.827 & 0.794 & & 4.0 & 20.0 \\
\hline 1,2-Dibromoethane & 0.758 & 0.727 & & 4.1 & 20.0 \\
\hline Chlorobenzene & 1.025 & 0.997 & 0.3 & 2.7 & 20.0 \\
\hline $1,1,1,2$-Tetrachloroethane & 0.566 & 0.544 & & 3.9 & 20.0 \\
\hline Ethylbenzene & 1.529 & 1.484 & & 2.9 & 20.0 \\
\hline Xylene $(\mathrm{m}, \mathrm{p})$ & 0.550 & 0.530 & & 3.6 & 20.0 \\
\hline Xylene (o) & 0.541 & 0.528 & & 2.4 & 20.0 \\
\hline xylene (total) & 0.541 & 0.528 & & 2.4 & 20.0 \\
\hline styrene & 0.938 & 0.918 & & 2.1 & 20.0 \\
\hline Bromoform & 0.583 & 0.568 & 0.1 & 2.6 & 20.0 \\
\hline Isopropylbenzene & 2.525 & 2.483 & & 1.7 & 20.0 \\
\hline Bromobenzene & 0.910 & 0.899 & & 1.2 & 20.0 \\
\hline $1,1,2,2$-Tetrachloroethane & 1.432 & 1.429 & 0.3 & 0.2 & 20.0 \\
\hline $1,2,3$-Trichloropropane & 0.313 & 0.306 & & 2.2 & 20.0 \\
\hline trans-1,4-Dichloro-2-butene & 0.266 & 0.254 & & 4.5 & 20.0 \\
\hline n-Propylbenzene & 0.624 & 0.623 & & 0.2 & 20.0 \\
\hline 2-Chlorotoluene & 0.638 & 0.641 & & 0.5 & 20.0 \\
\hline 4-Chlorotoluene & 0.672 & 0.663 & & 1.3 & 20.0 \\
\hline $1,3,5$-Trimethyl benzene. & 1.949 & 1.906 & & 2.2 & 20.0 \\
\hline tert-Butylbenzene & 1.966 & 1.938 & & 1.4 & 20.0 \\
\hline 1,2,4-Trimethylbenzene & 1.921 & 1.899 & & 1.1 & 20.0 \\
\hline sec-Butylbenzene & 2.684 & 2.676 & & 0.3 & 20.0 \\
\hline 1,3 -Dichlorobenzene & 1.397 & 1.426 & & 2.1 & 20.0 \\
\hline
\end{tabular}


FORM 7

VOLATILE CONTINUING CALIBRATION CHECK

Lab Name: TESTAMERICA BURLINGTON Contract: 8E-00302

Lab Code: STLV Case No.: INMAN SAS No.:

SDG No.: 134662

Instrument ID: $\mathrm{N}$

Calibration Date: 11/19/09 Time: 0913

Lab File ID: NXO10CV

Init. Calib. Date(s): 11/16/09 11/16/09

Heated Purge: $(\mathrm{Y} / \mathrm{N}) \mathrm{N}$

Init. Calib. Times: 1546

1747

GC Column: DB-624 ID: 0.53 (mm)

\begin{tabular}{|c|c|c|c|c|c|}
\hline COMPOUND & $\mathrm{RRF}$ & RRF10 & $\begin{array}{l}\text { MIN } \\
\text { RRF }\end{array}$ & $\because \mathrm{D}$ & $\begin{array}{l}\text { MAX } \\
\because D\end{array}$ \\
\hline 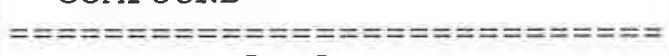 & $========$ & $=========$ & $=======$ & $=====$ & $====$ \\
\hline 4-Isopropyltoluene & 2.086 & 2.091 & & 0.2 & 20.0 \\
\hline 1,4-Dichlorobenzene & 1.576 & 1.551 & & 1.6 & 20.0 \\
\hline 1,2-Dichlorobenzene & 1.373 & 1.346 & & 2.0 & 20.0 \\
\hline n-Butylbenzene & 2.059 & 2.043 & & 0.8 & 20.0 \\
\hline $1,2-D$ & 0.292 & 0.282 & & 3.4 & 20.0 \\
\hline $1,2,4$ & 0.986 & 0.980 & & 0.6 & 20.0 \\
\hline Hexac & 0.437 & 0.433 & & 0.9 & 20.0 \\
\hline Napht & 1.656 & 1.696 & & 2.4 & 20.0 \\
\hline chlorobenzene & 0.866 & 0.859 & & 0.8 & 20.0 \\
\hline$====$ & $==\mathbf{=}=\mathbf{=}=\mathbf{=}=$ & $=========$ & $==x===x=$ & $=====$ & $== \pm=$ \\
\hline oroethane-d4 & 0.396 & 0.362 & & 8.6 & 20.0 \\
\hline Tolue & 1.134 & 1.079 & & 4.8 & 20.0 \\
\hline Bromo & 1.462 & 1.426 & & 2.5 & 20.0 \\
\hline 1,2-Dichlorobenzene-d4 & 0.911 & 0.883 & & 3.1 & 20.0 \\
\hline
\end{tabular}

page 3 of 3 
FORM 8

VOLATILE INTERNAL STANDARD AREA AND RT SUMMARY

Lab Name: TESTAMERICA BURLINGTON

Lab Code: STLV Case No.: INMAN

Lab File ID (Standard): NXO10CV

Instrument ID: $\mathrm{N}$

GC Column: DB-624 ID: 0.53 (mm)
Contract: $8 \mathrm{E}-00302$

SAS No.: $\quad$ SDG No.: 134662

Date Analyzed: 11/19/09

Time Analyzed: 0913

Heated Purge: (Y/N) N

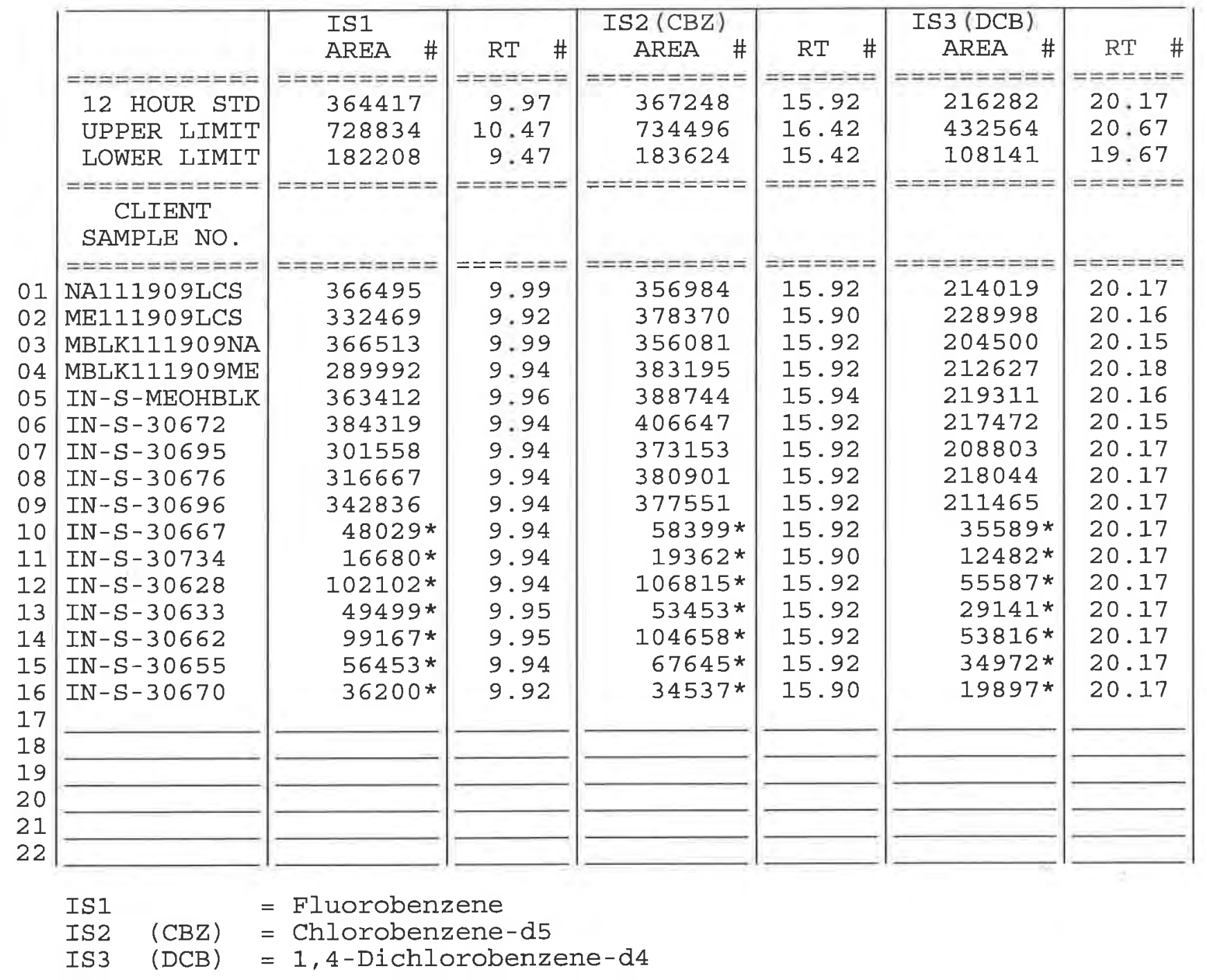

AREA UPPER IIMIT $=+100 \%$ of internal standard area AREA LOWER LIMIT $=-50 \%$ of internal standard area RT UPPER LIMIT $=+0.50$ minutes of internal standard RT RT LOWER LIMIT $=-0.50$ minutes of internal standard RT

\# Column used to flag values outside QC limits with an asterisk. * Values outside of QC limits.

page 1 of 1 
Argonne

Environmental Science Division

Argonne National Laboratory

9700 South Cass Avenue, BIdg. 203

Argonne, IL 60439-4843

www.anl.gov 